Supplement of Hydrol. Earth Syst. Sci., 20, 4503-4524, 2016

http://www.hydrol-earth-syst-sci.net/20/4503/2016/

doi:10.5194/hess-20-4503-2016-supplement

(C) Author(s) 2016. CC Attribution 3.0 License.

(c) (1)

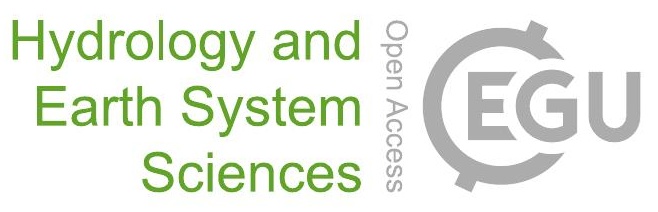

Supplement of

\title{
Climate elasticity of streamflow revisited - an elasticity index based on long-term hydrometeorological records
}

\author{
V. Andréassian et al. \\ Correspondence to: Vazken Andréassian
}

The copyright of individual parts of the supplement might differ from the CC-BY 3.0 licence. 

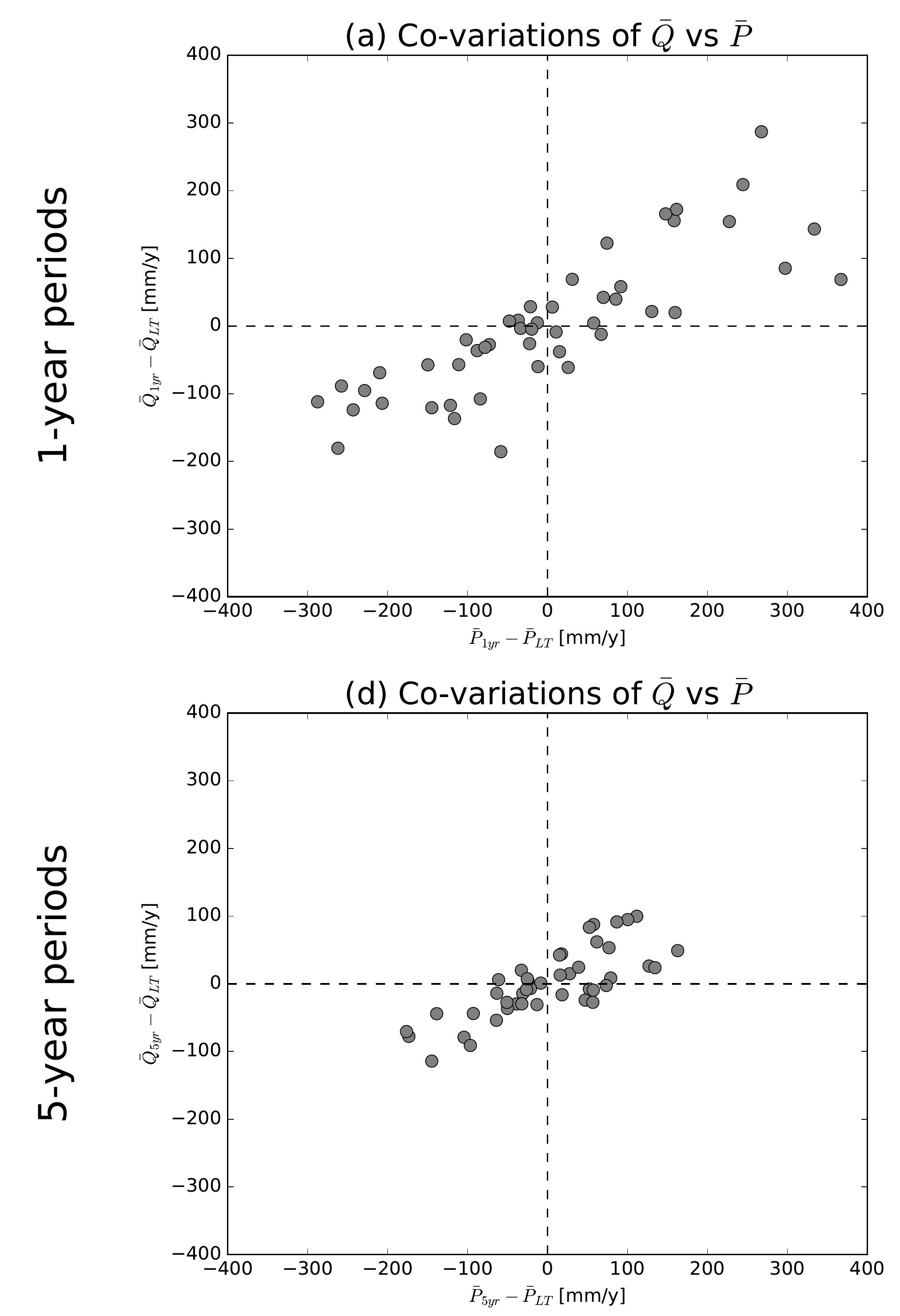

(g) Co-variations of $\bar{Q}$ vs $\bar{P}$

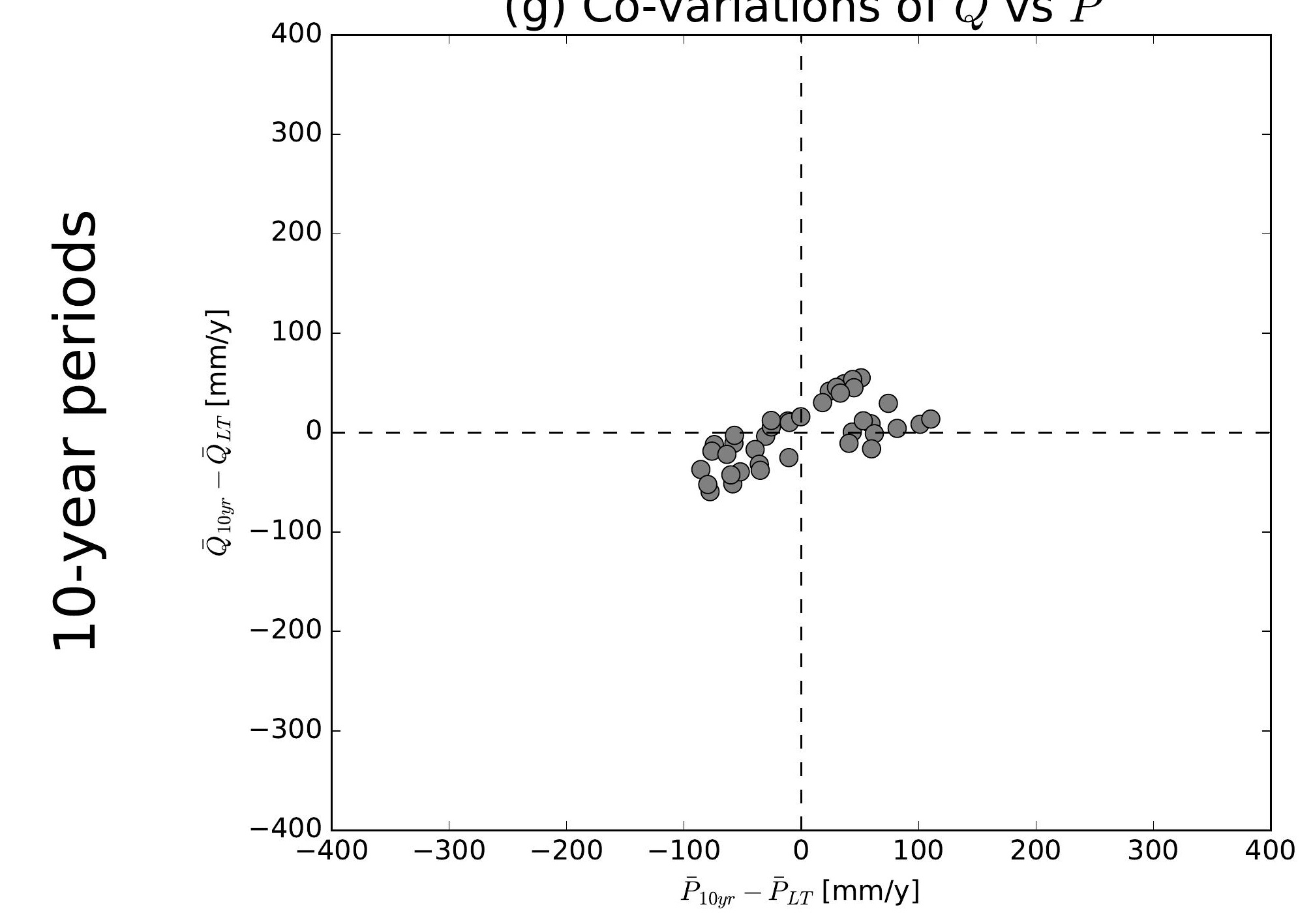

(j) Co-variations of $\bar{Q}$ vs $\bar{P}$

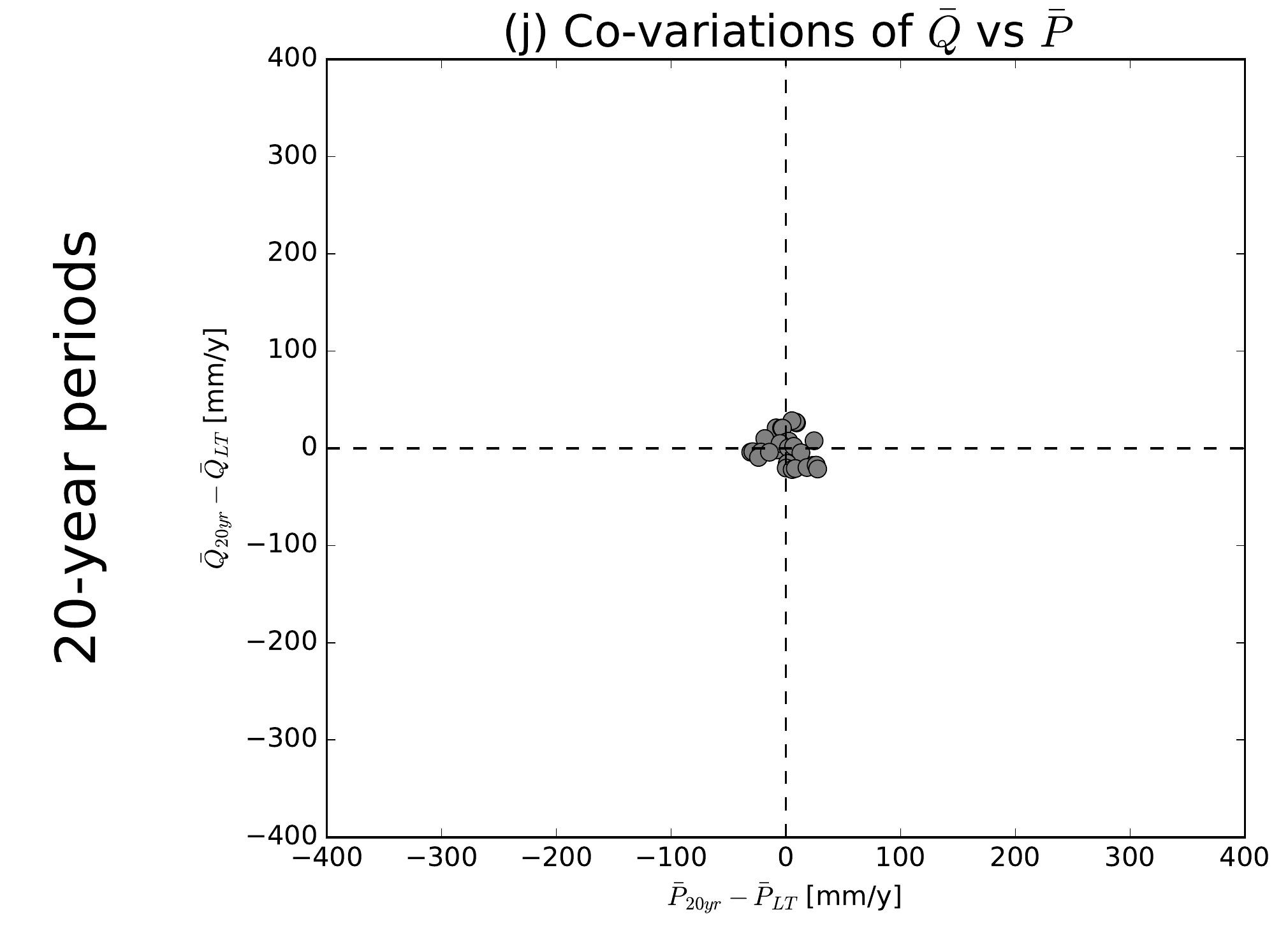

(b) Co-variations of $\bar{Q}$ vs $\overline{E 0}$

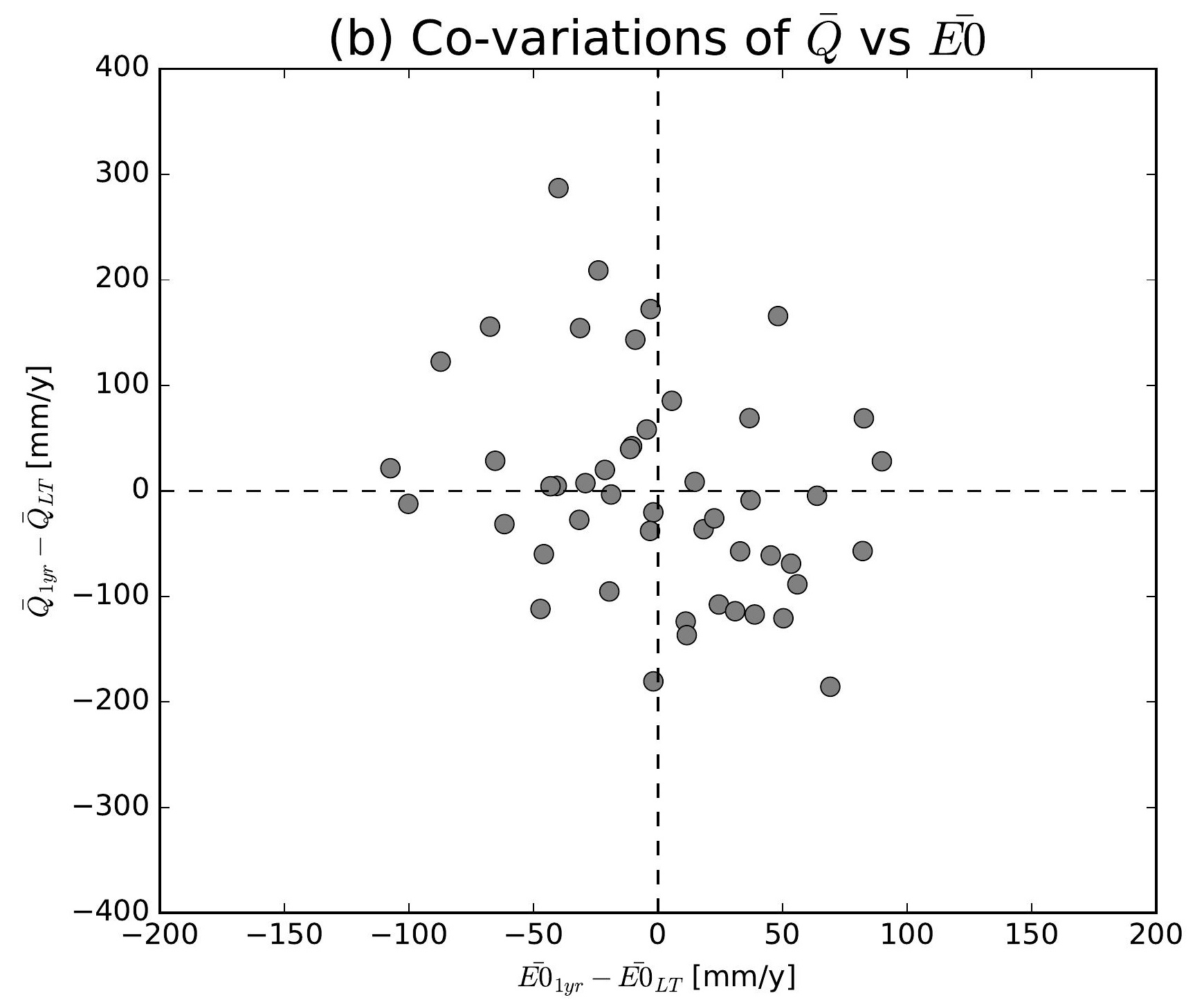

(e) Co-variations of $\bar{Q}$ vs $\overline{E 0}$

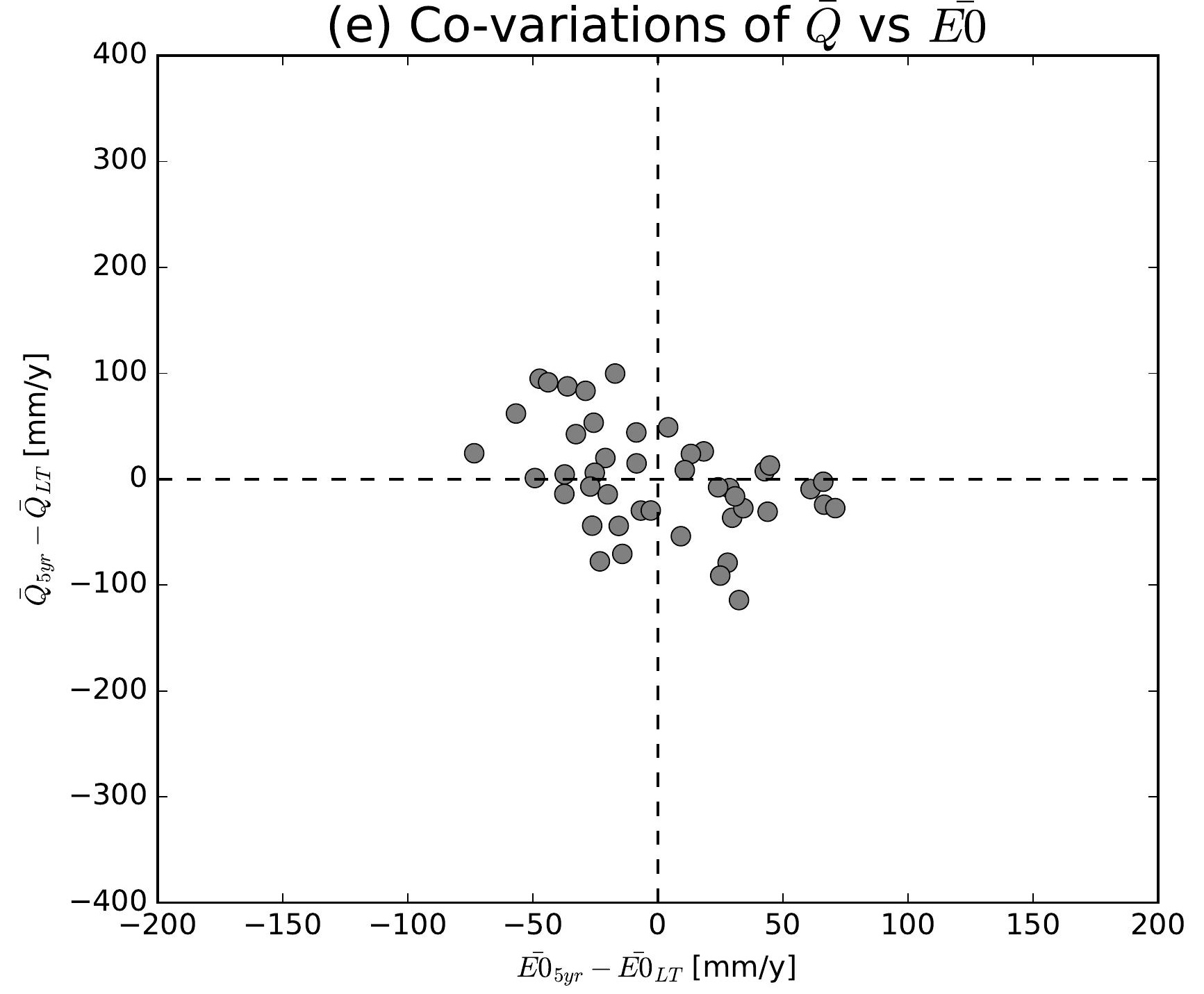

(h) Co-variations of $\bar{Q}$ vs $\overline{E 0}$

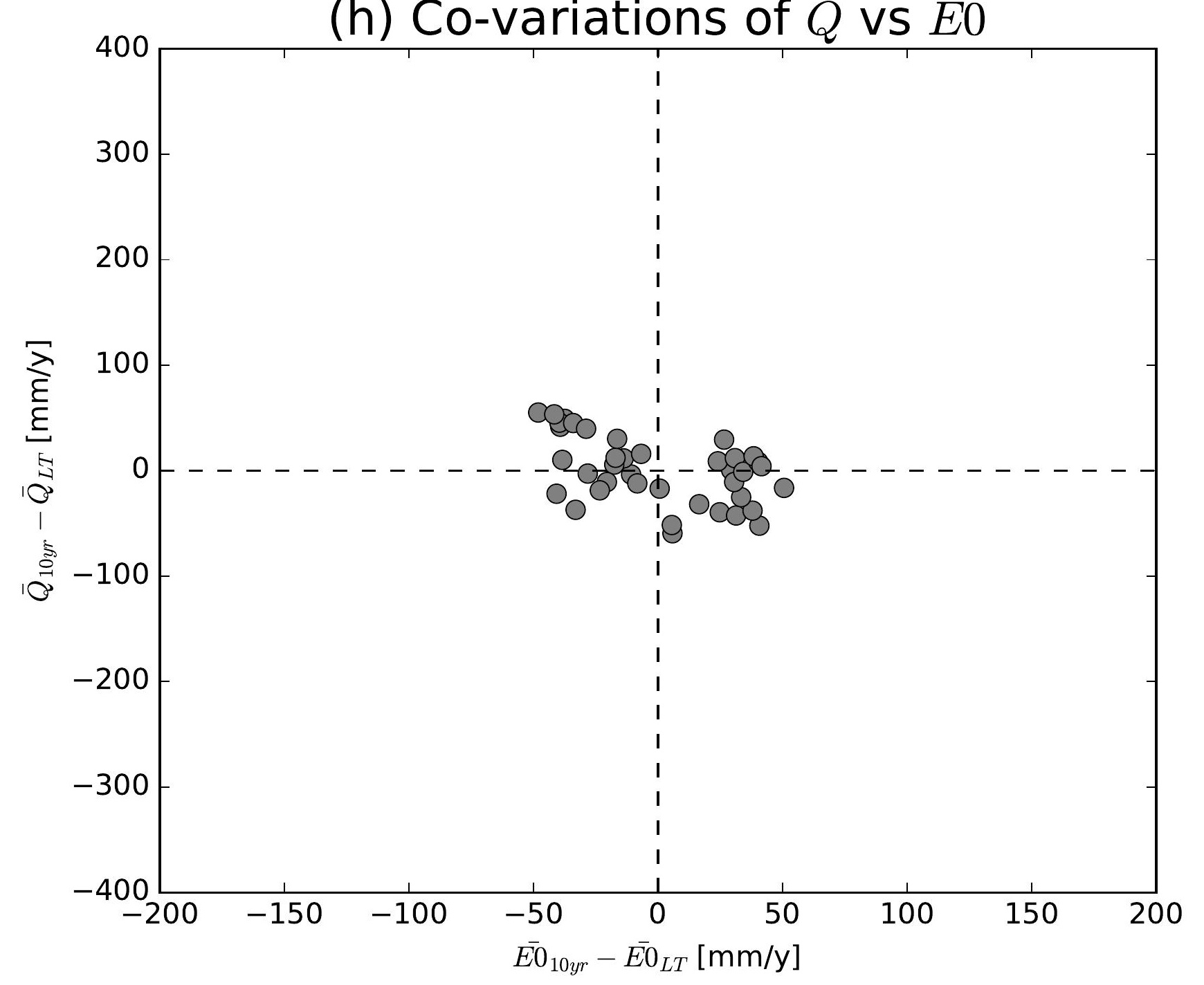

(k) Co-variations of $\bar{Q}$ vs $\overline{E 0}$

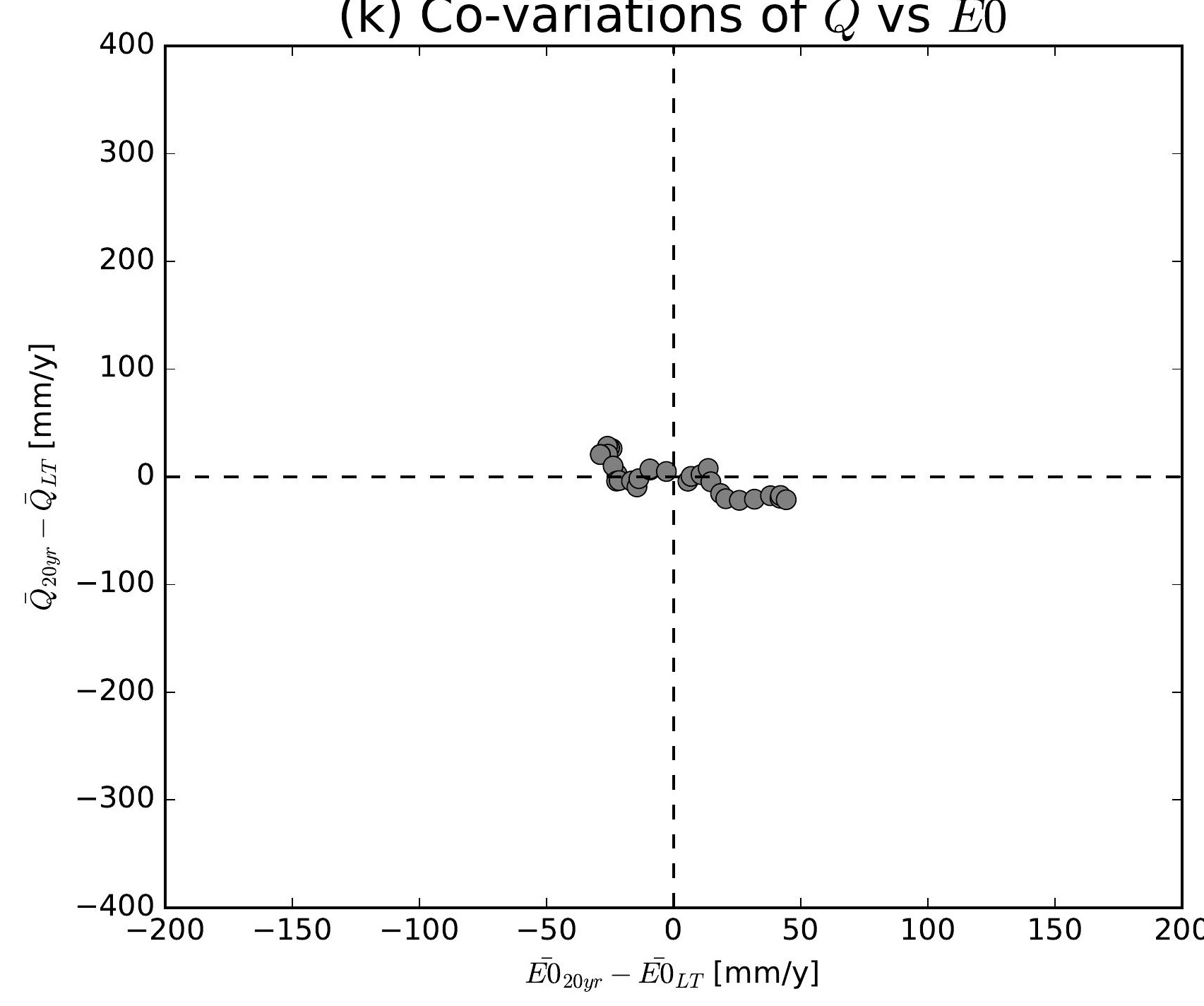

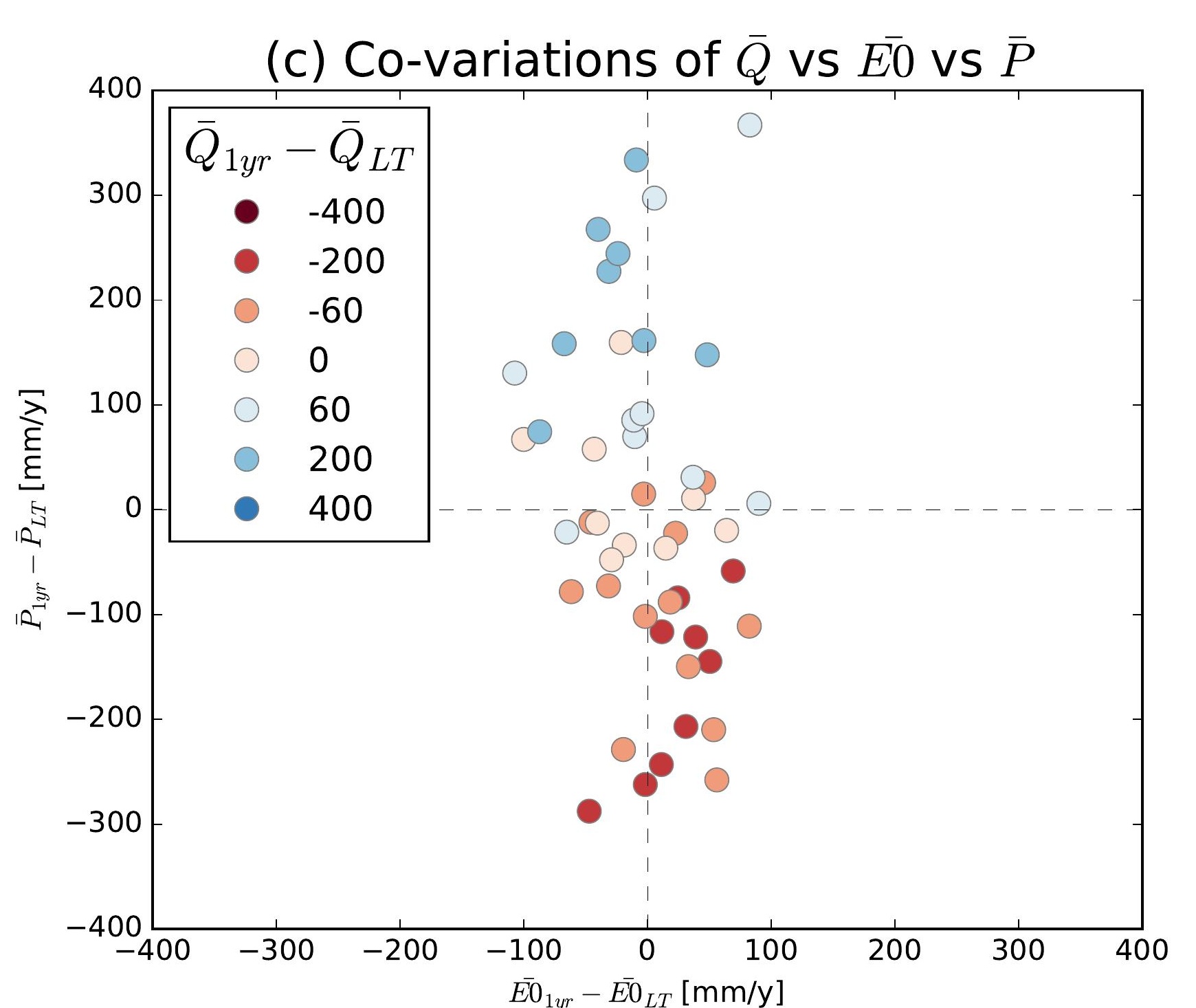
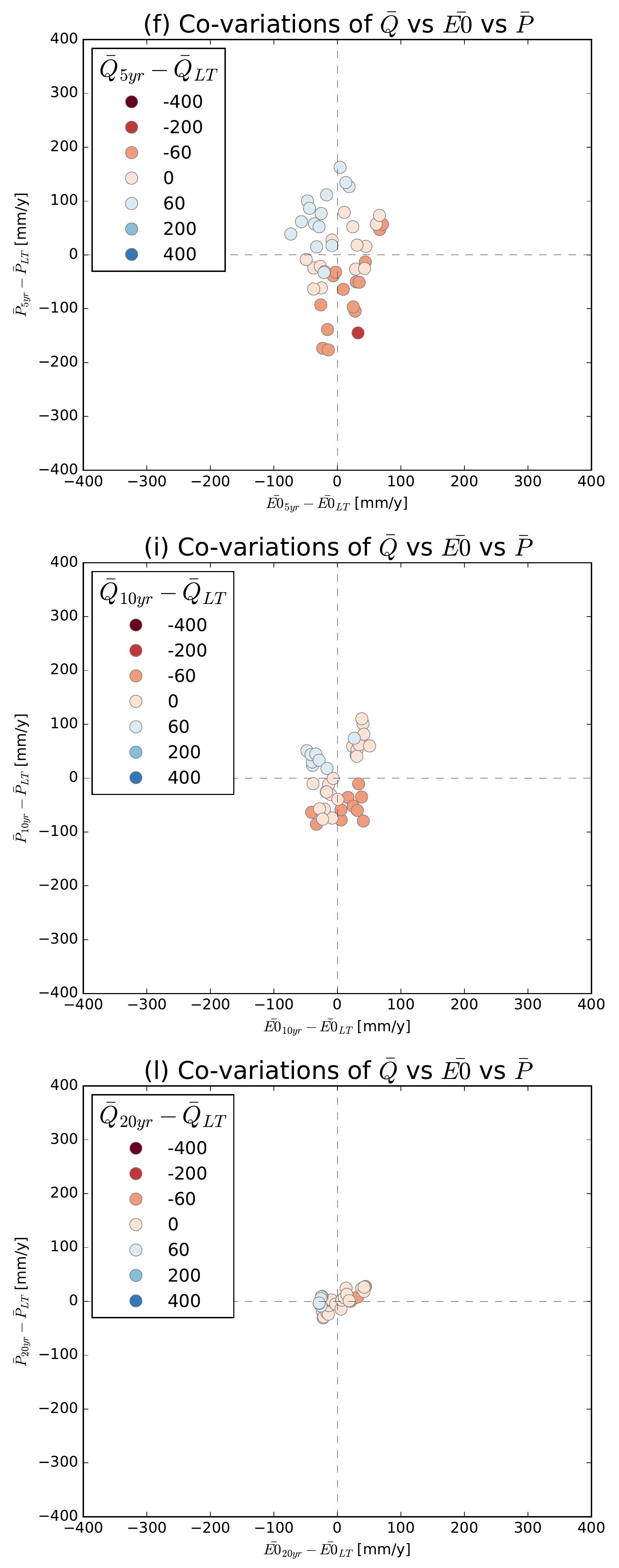

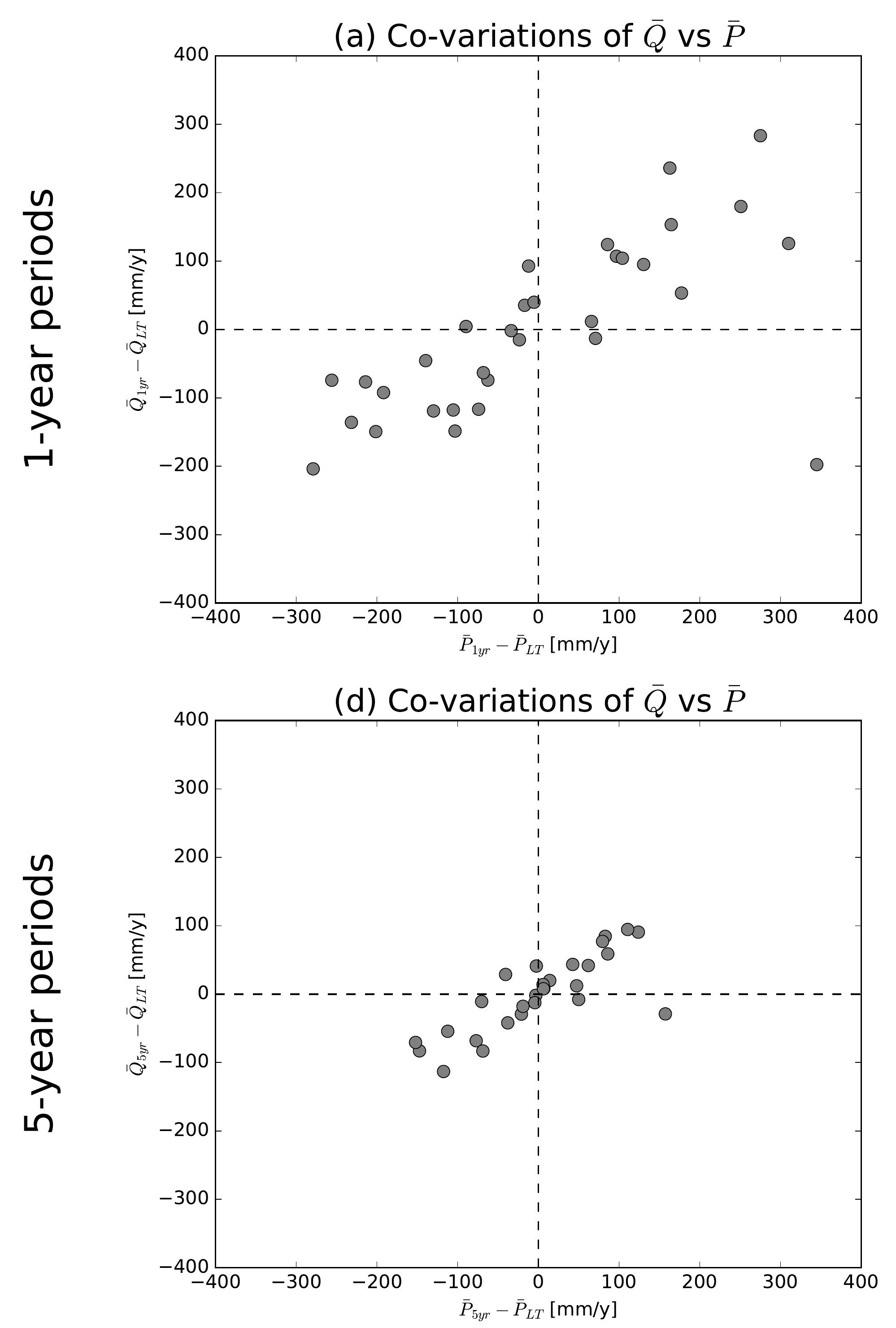

(g) Co-variations of $\bar{Q}$ vs $\bar{P}$

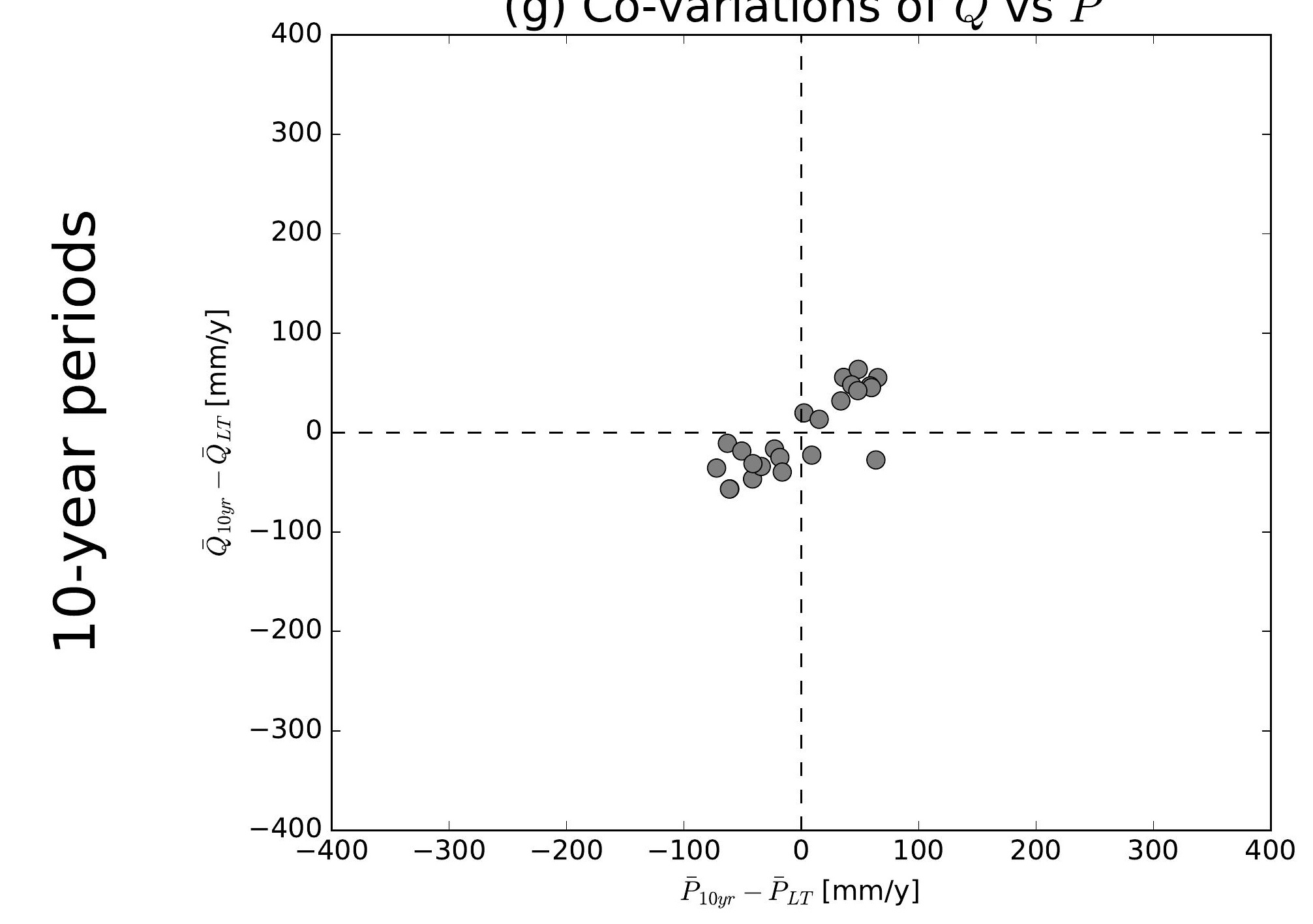

(j) Co-variations of $\bar{Q}$ vs $\bar{P}$

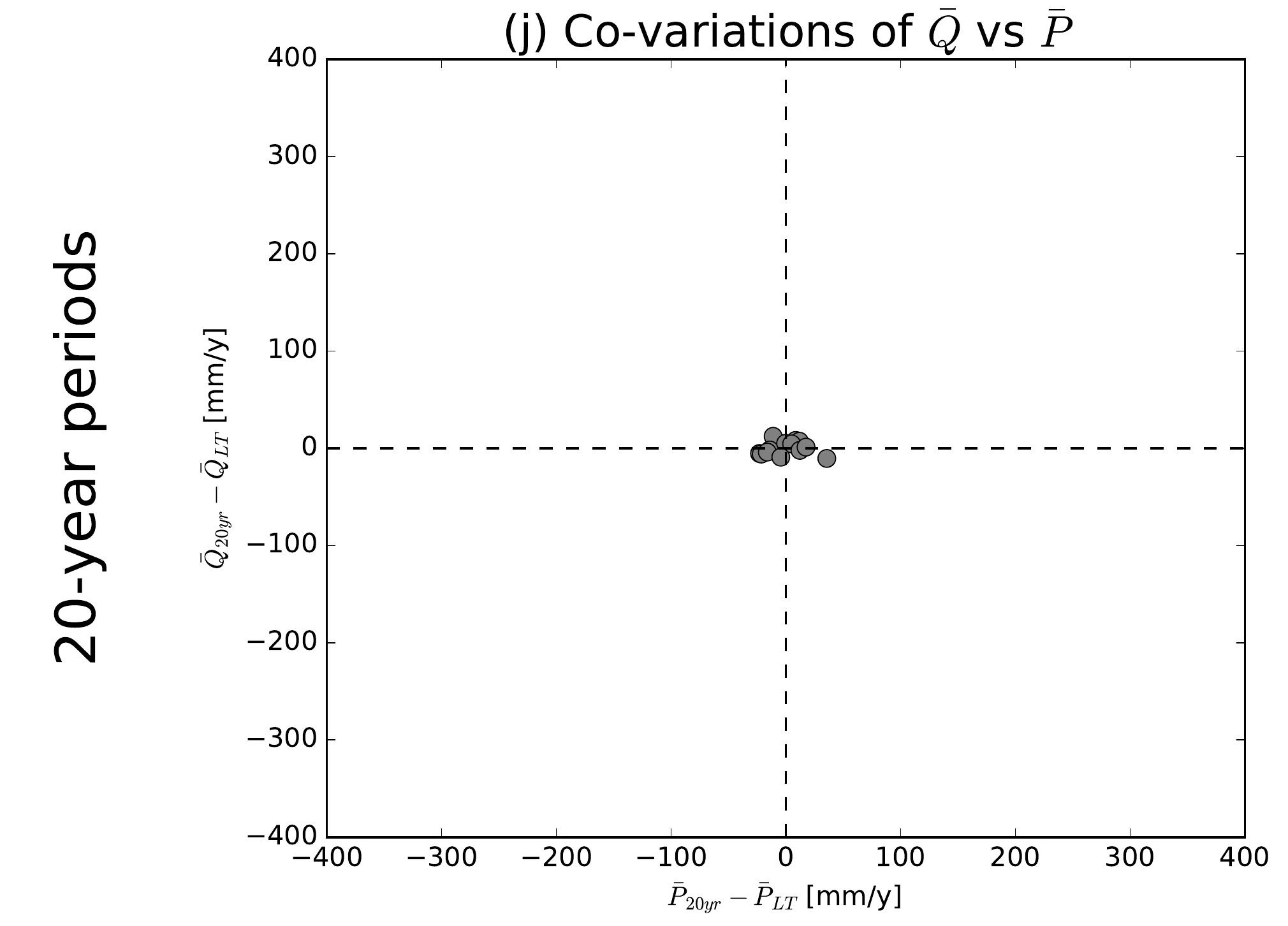

(b) Co-variations of $\bar{Q}$ vs $\overline{E 0}$

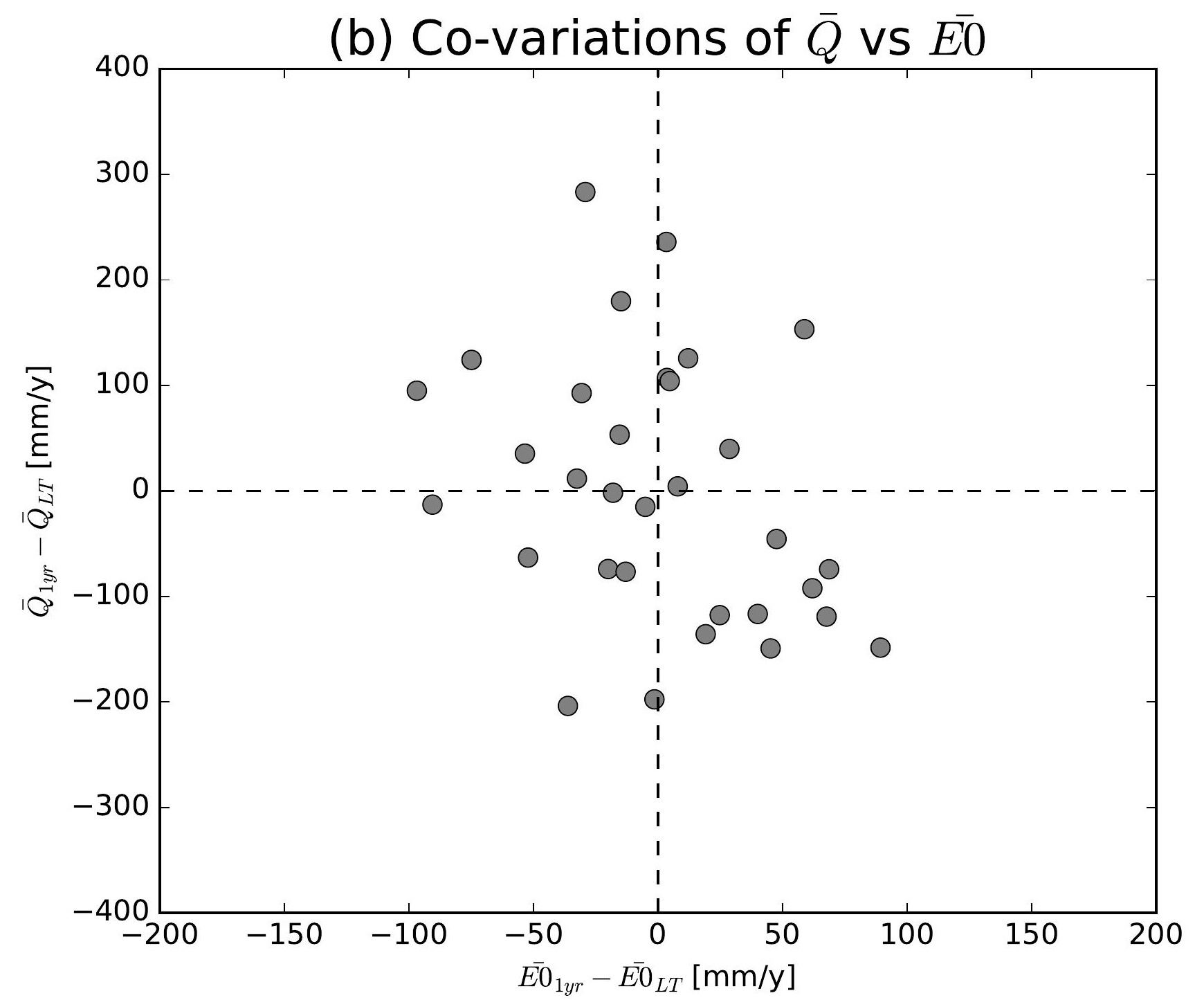

(e) Co-variations of $\bar{Q}$ vs $\overline{E 0}$

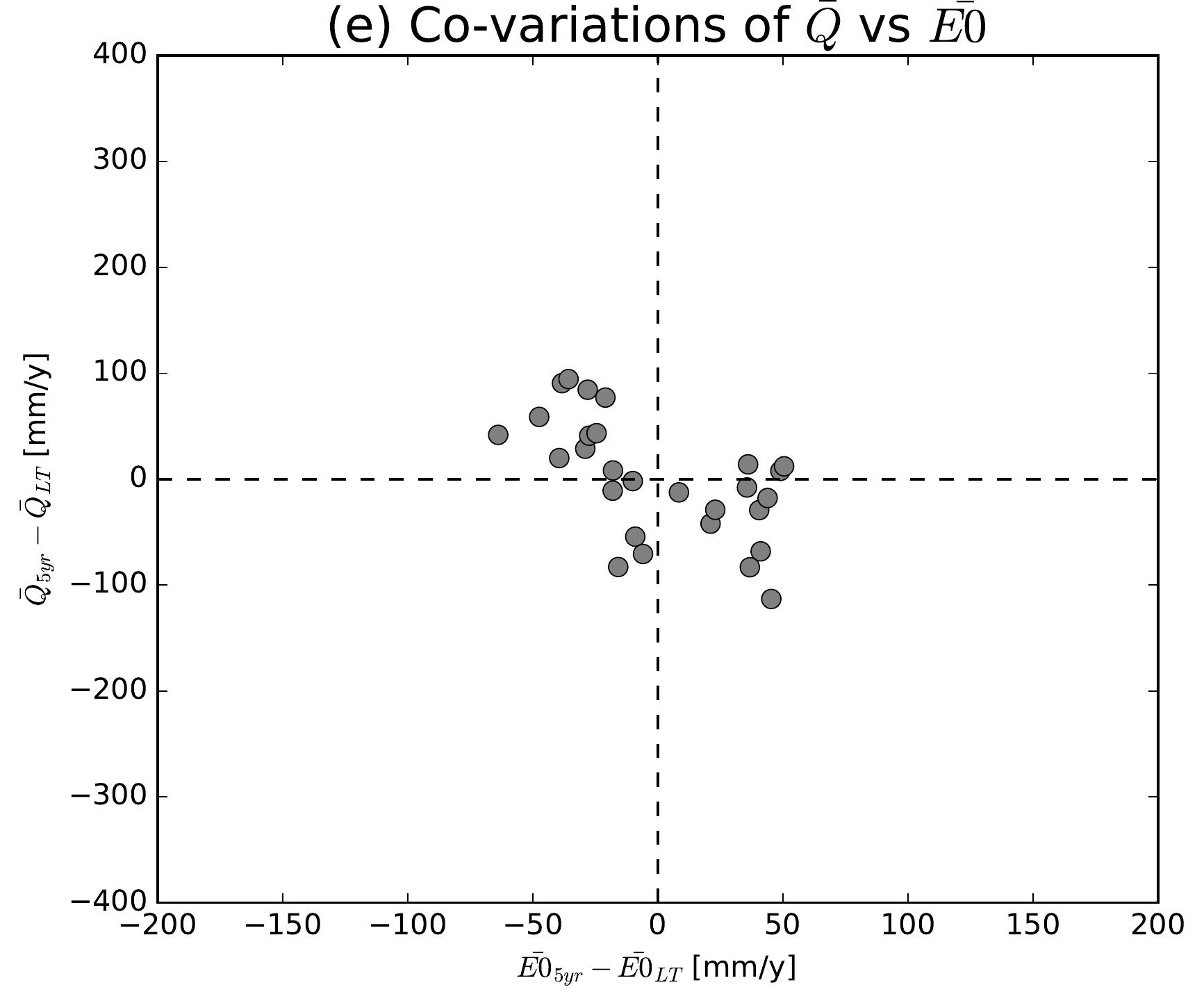

(h) Co-variations of $\bar{Q}$ vs $\overline{E 0}$

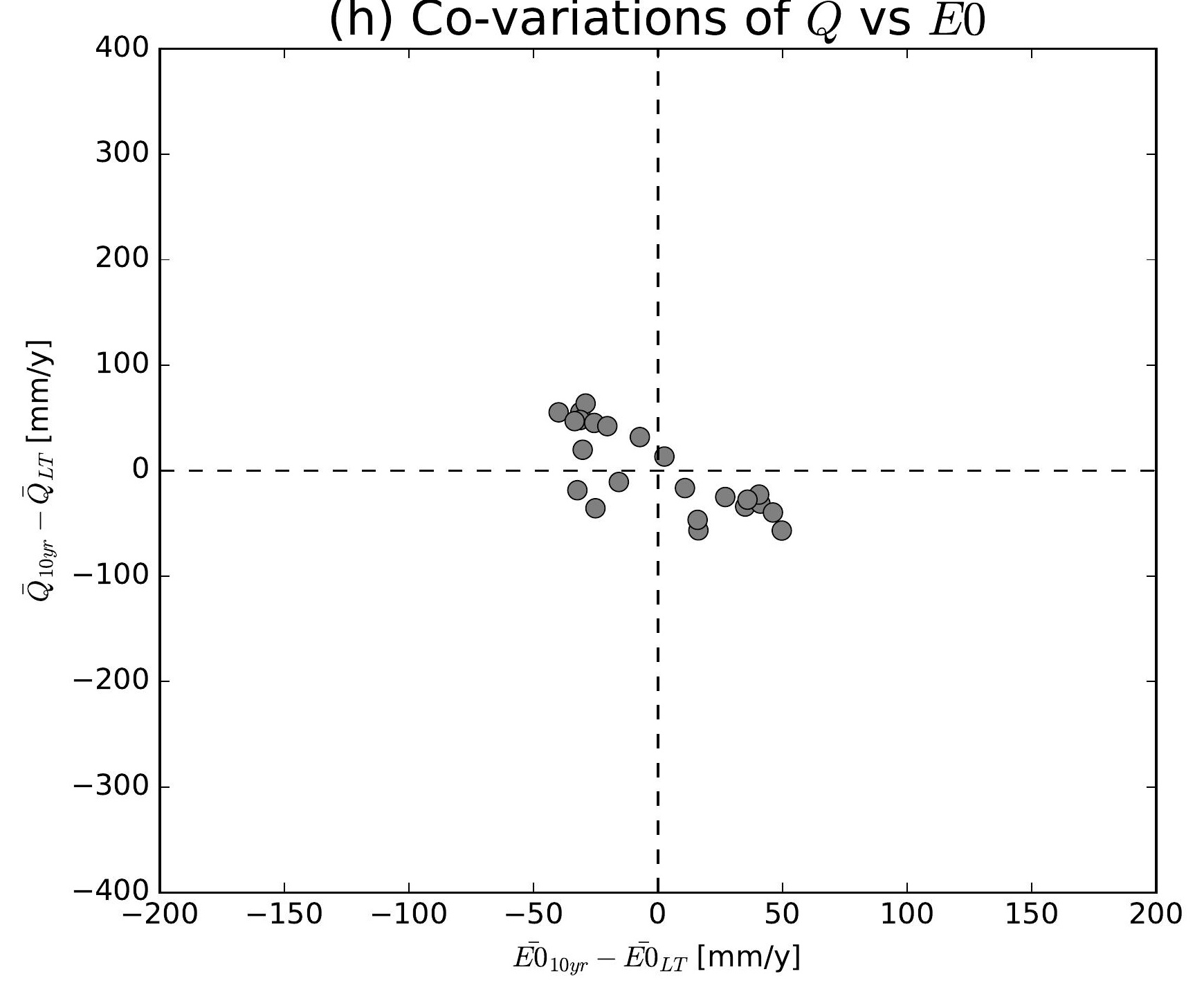

(k) Co-variations of $\bar{Q}$ vs $\overline{E 0}$

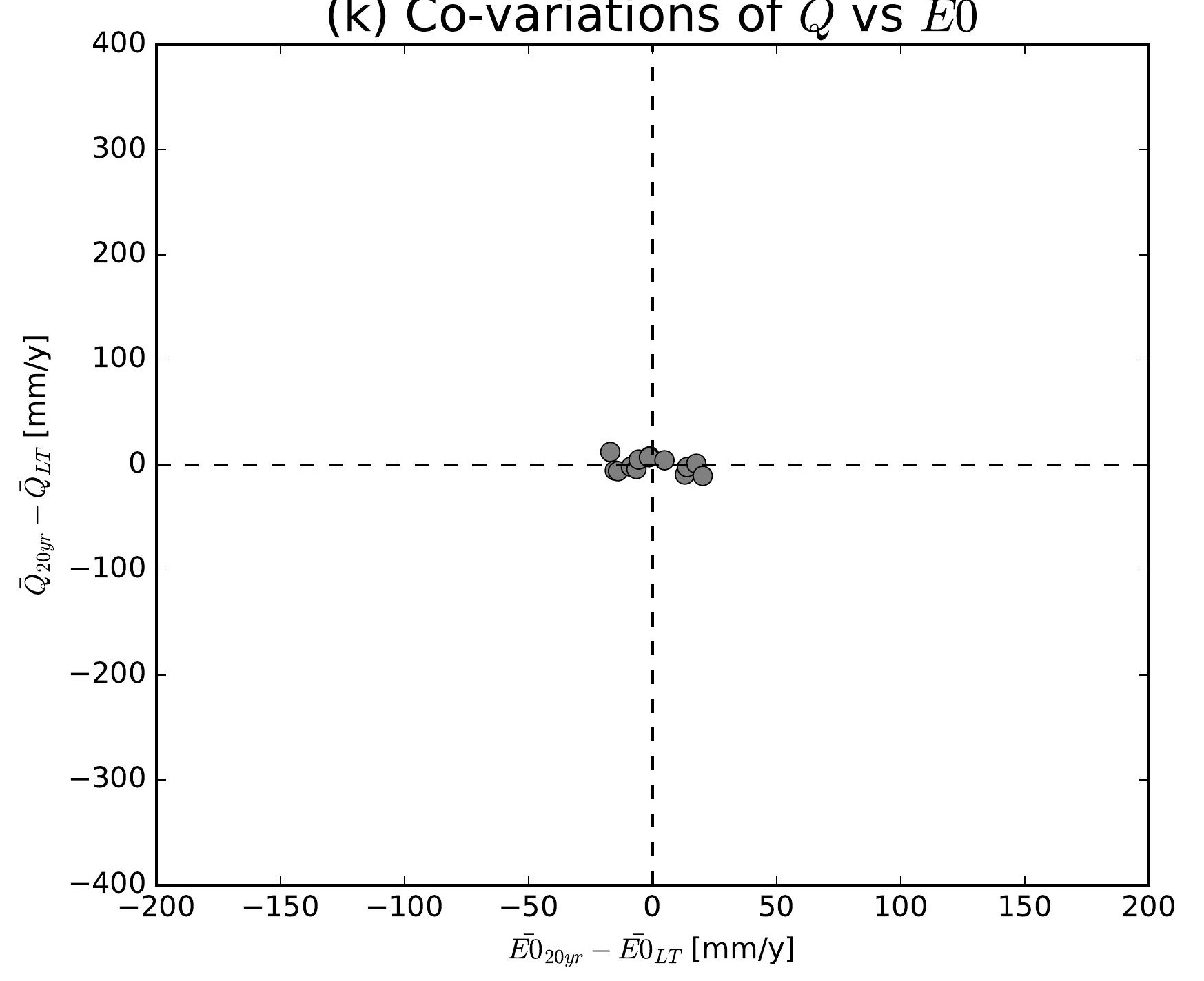

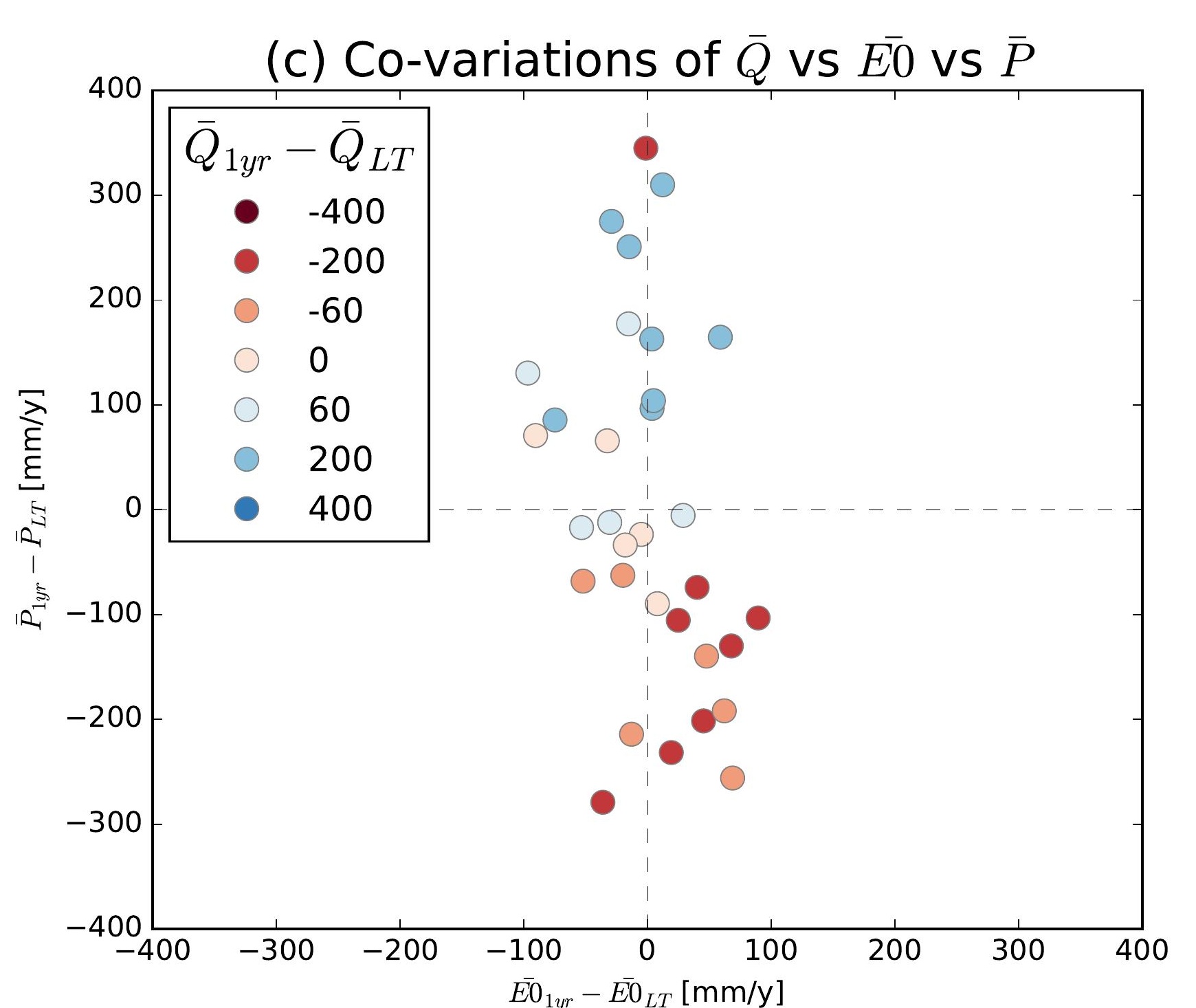
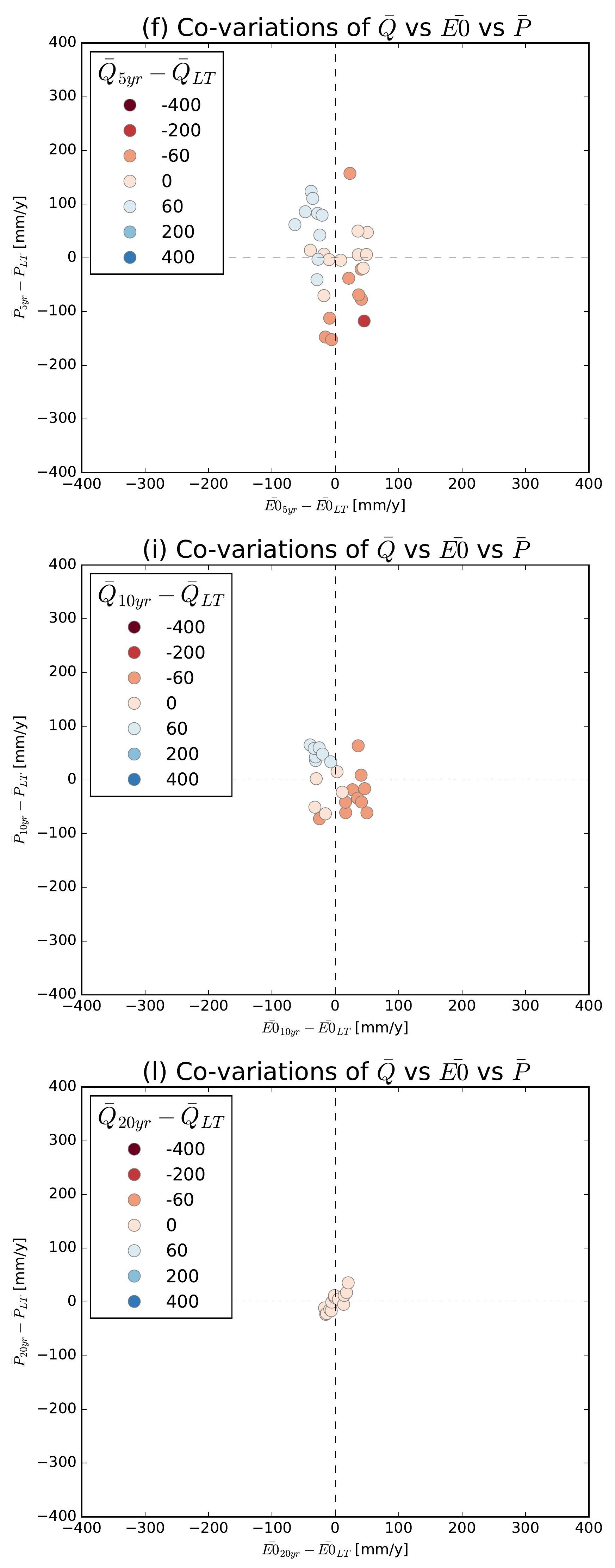

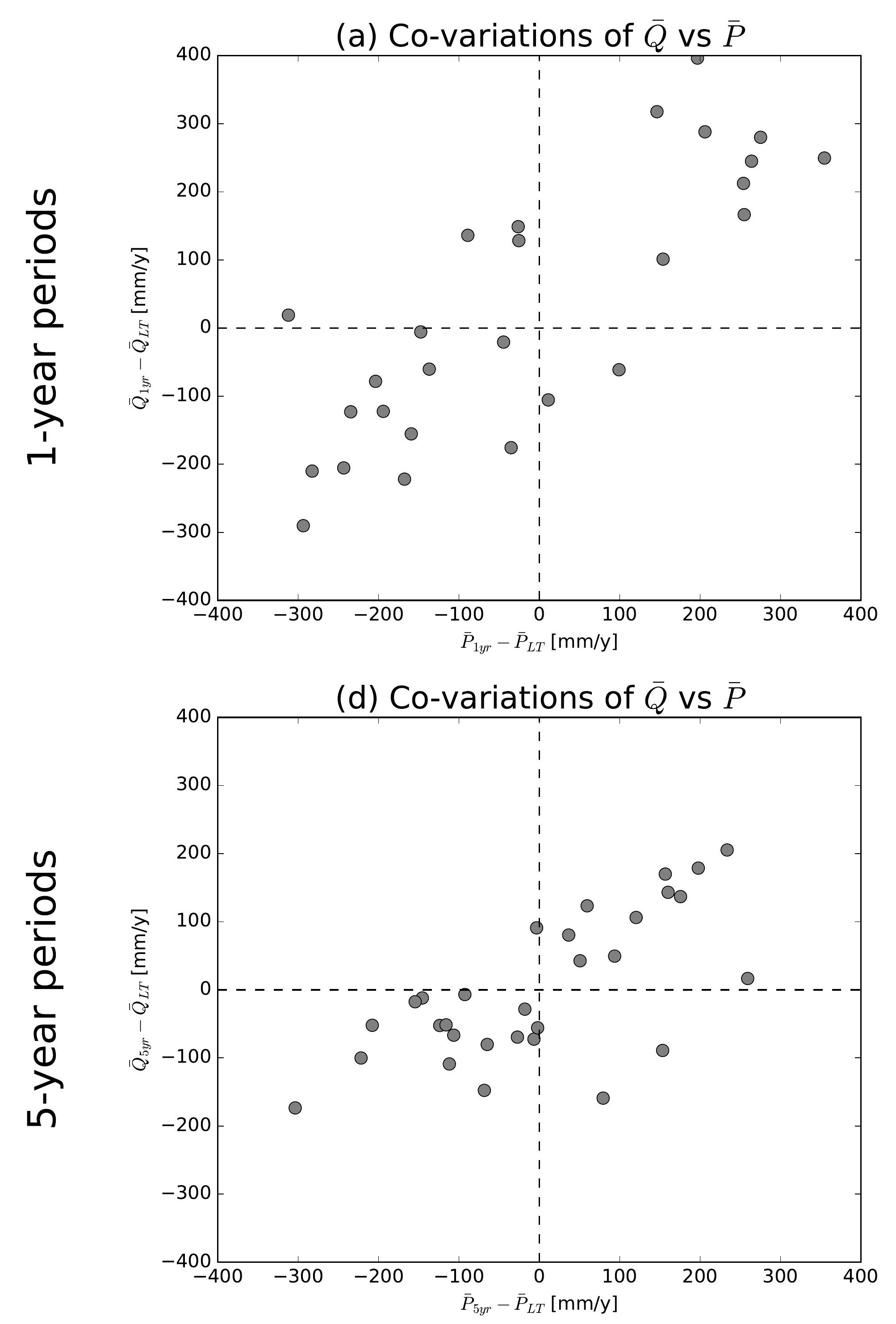

(g) Co-variations of $\bar{Q}$ vs $\bar{P}$

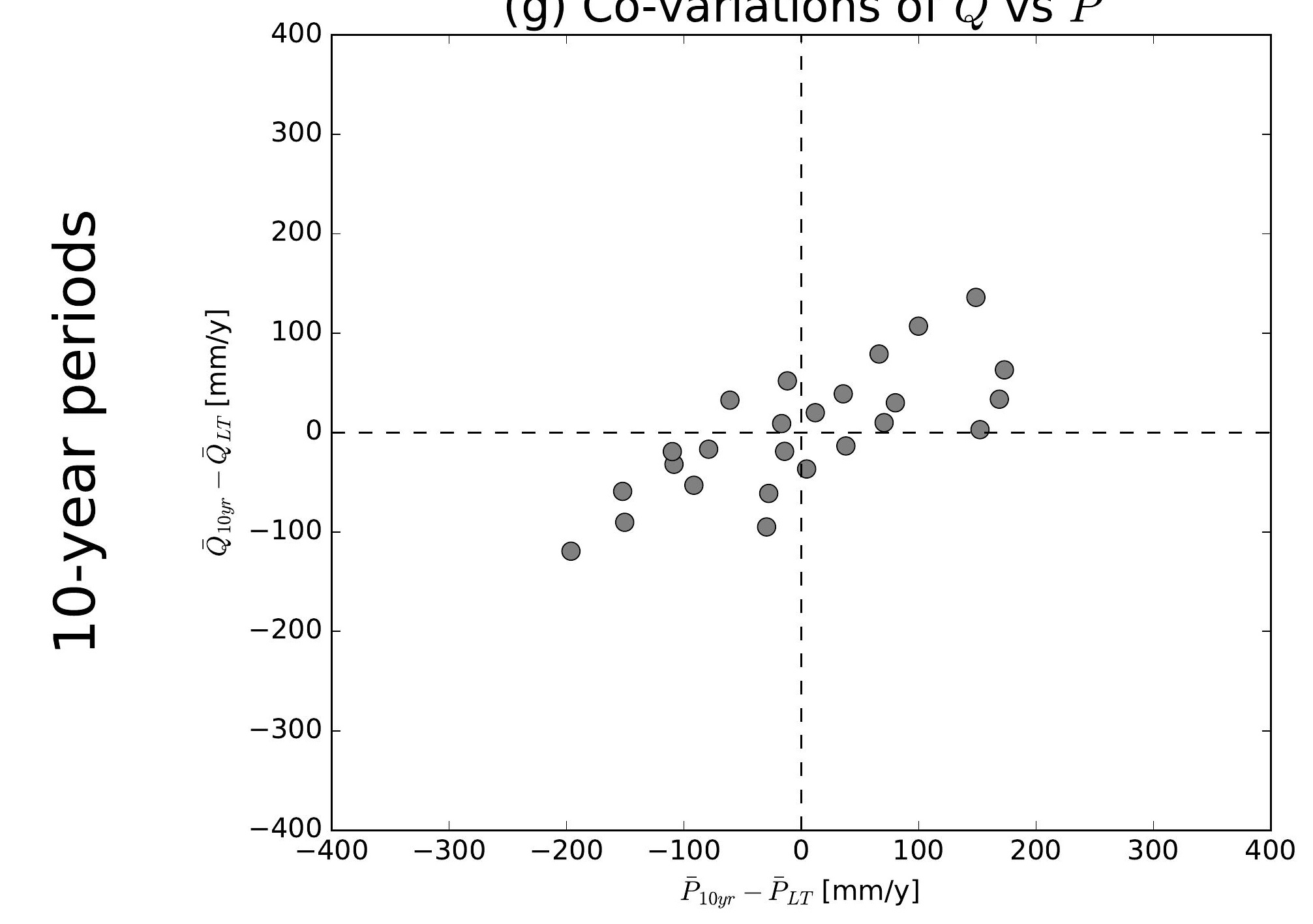

(j) Co-variations of $\bar{Q}$ vs $\bar{P}$

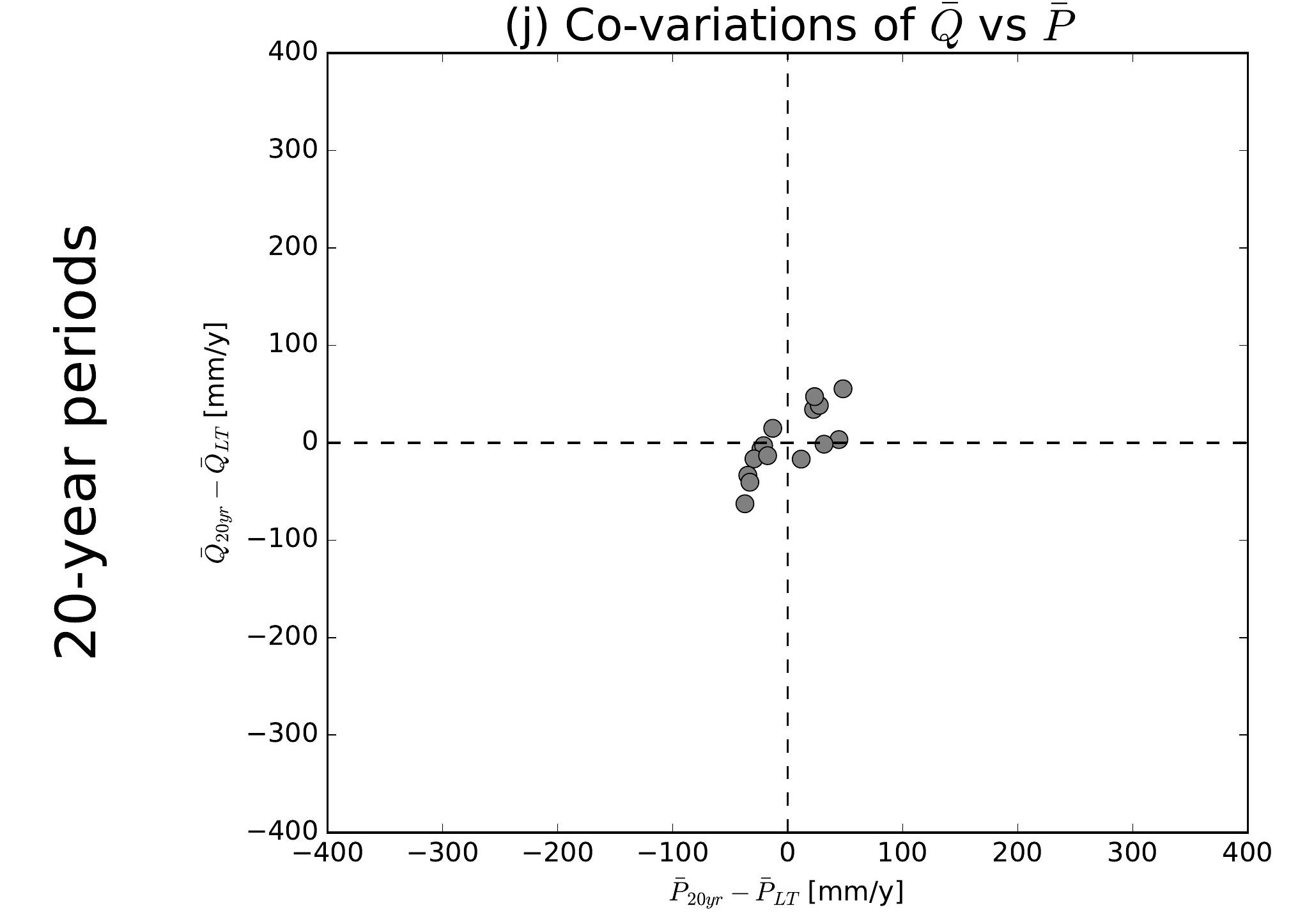

(b) Co-variations of $\bar{Q}$ vs $\overline{E 0}$

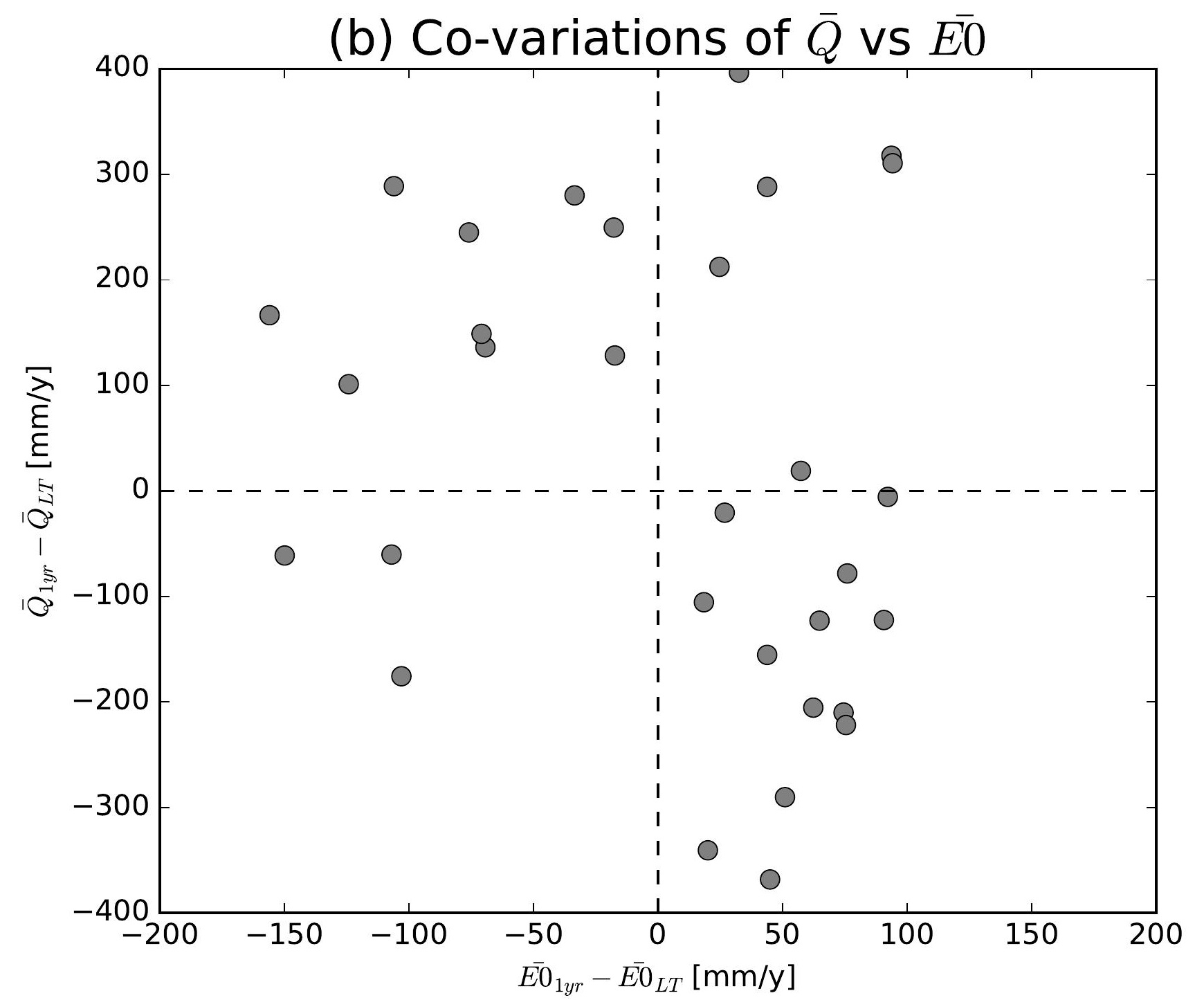

(e) Co-variations of $\bar{Q}$ vs $\overline{E 0}$

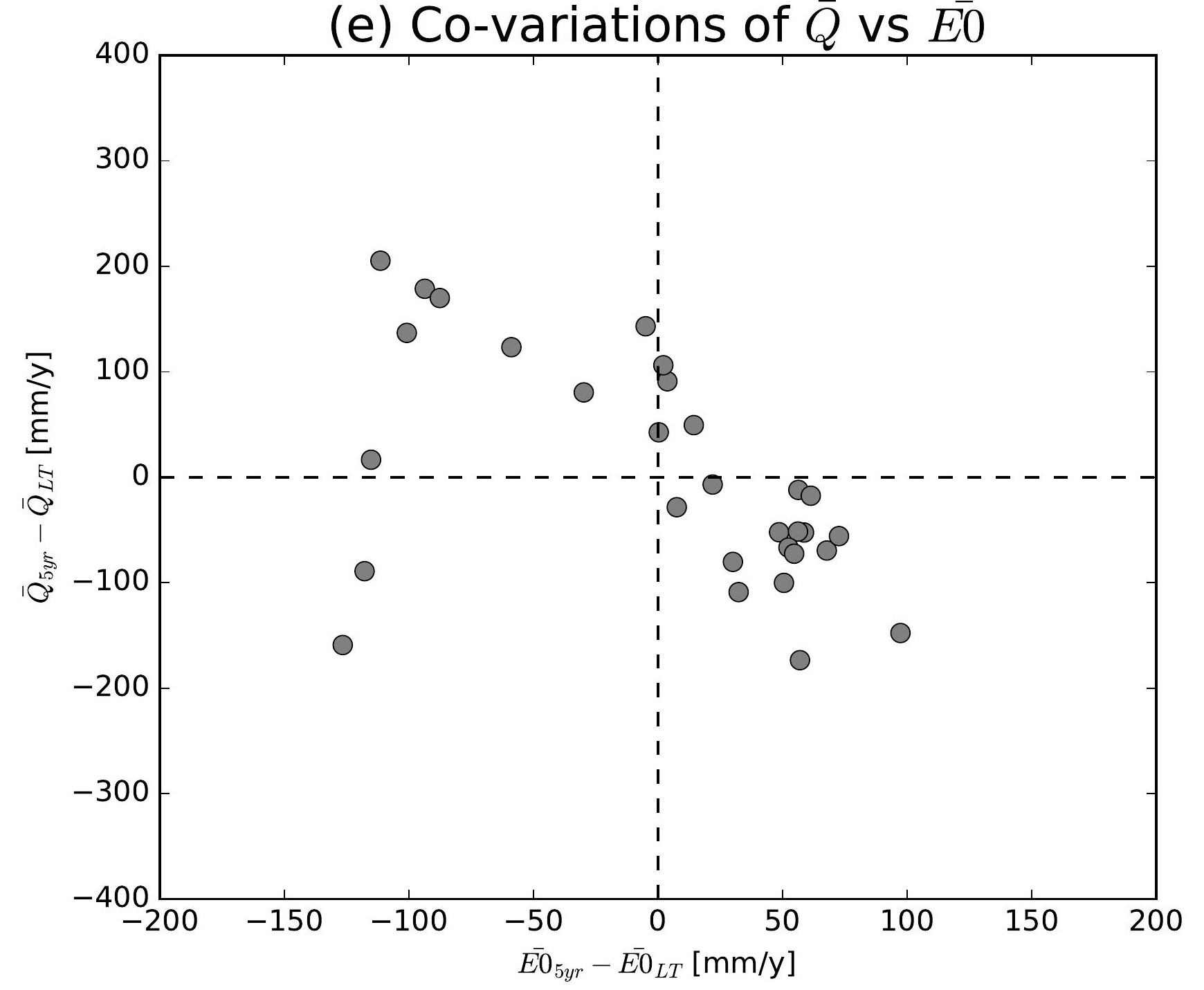

(h) Co-variations of $\bar{Q}$ vs $\overline{E 0}$

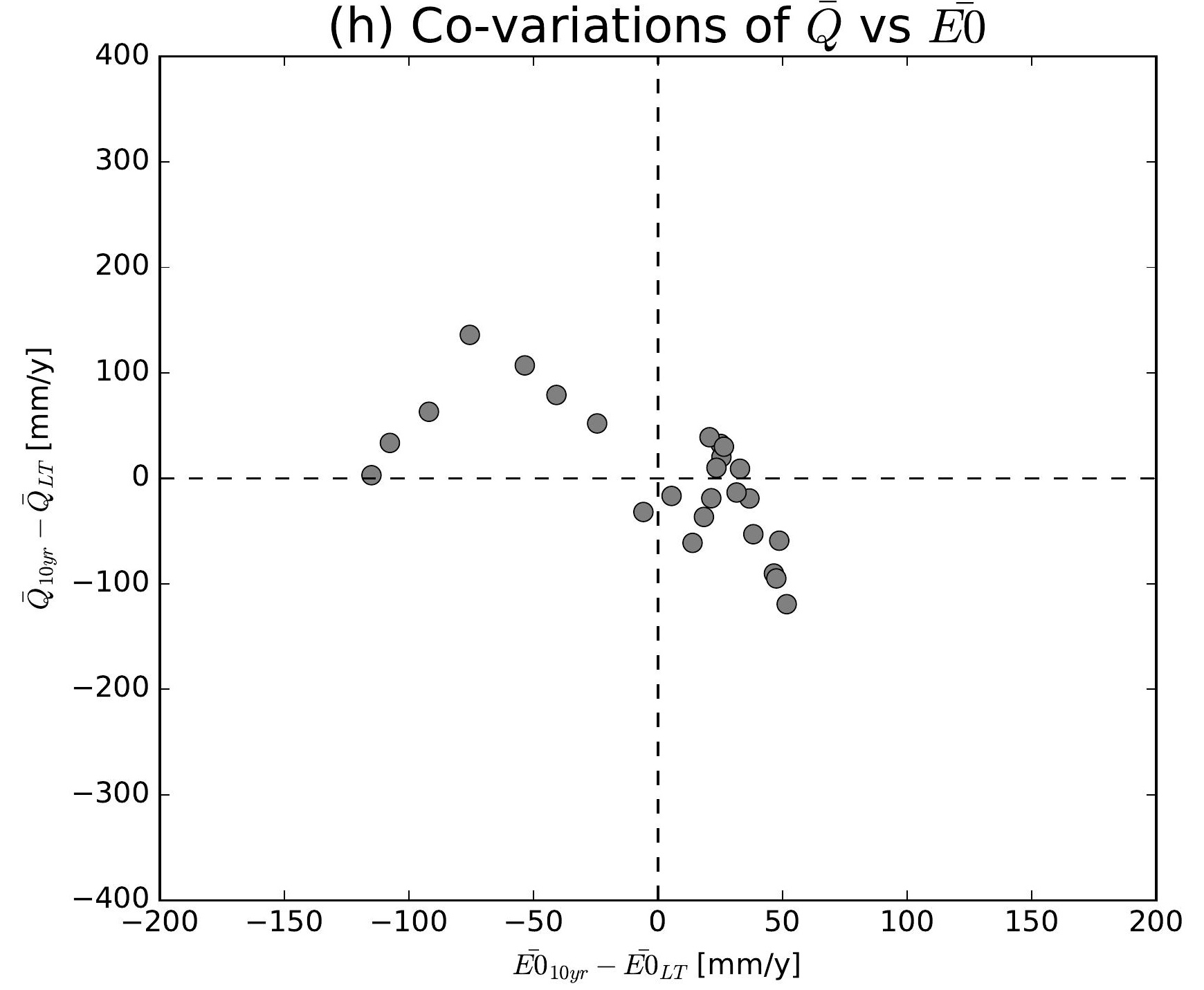

(k) Co-variations of $\bar{Q}$ vs $\overline{E 0}$

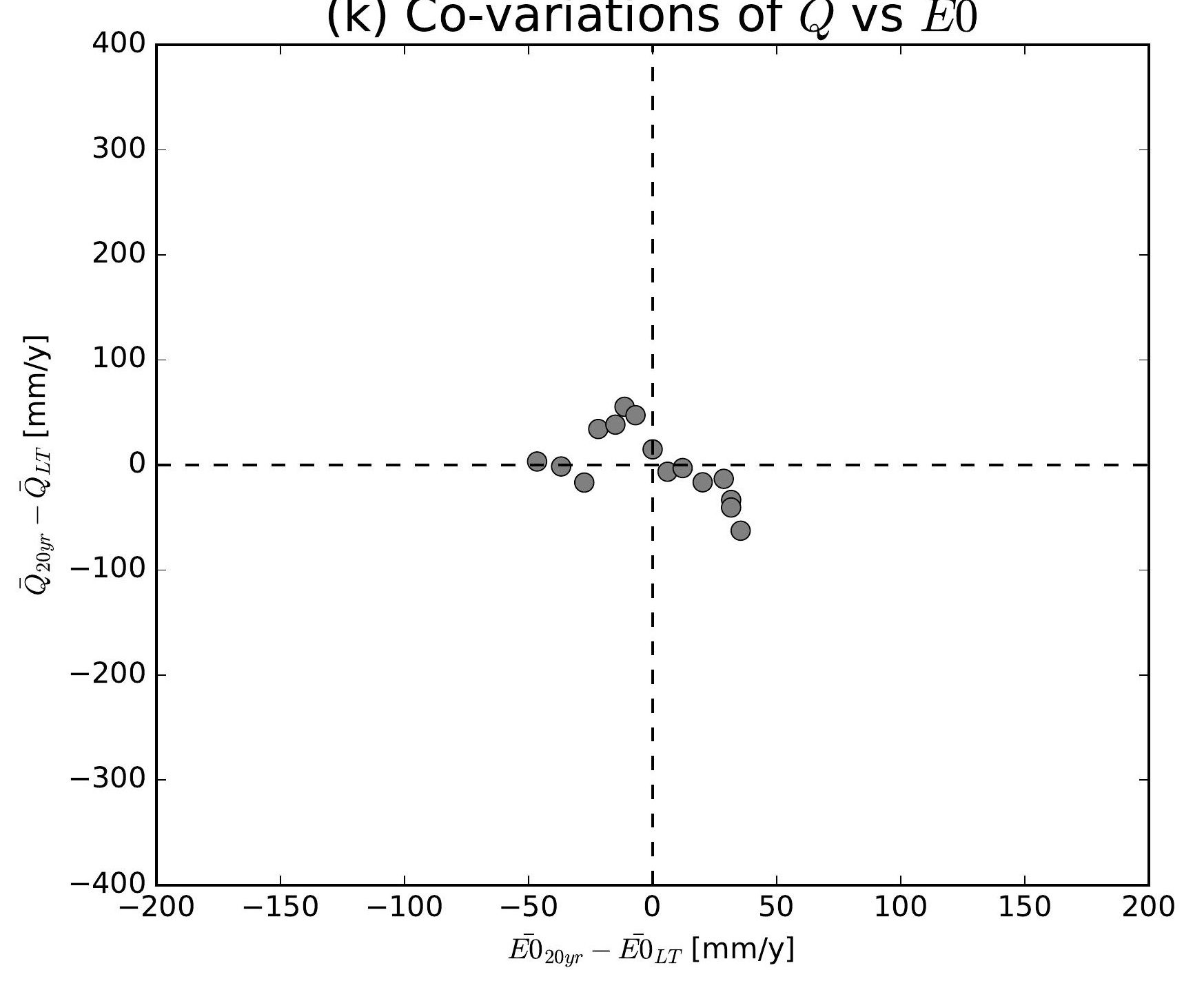

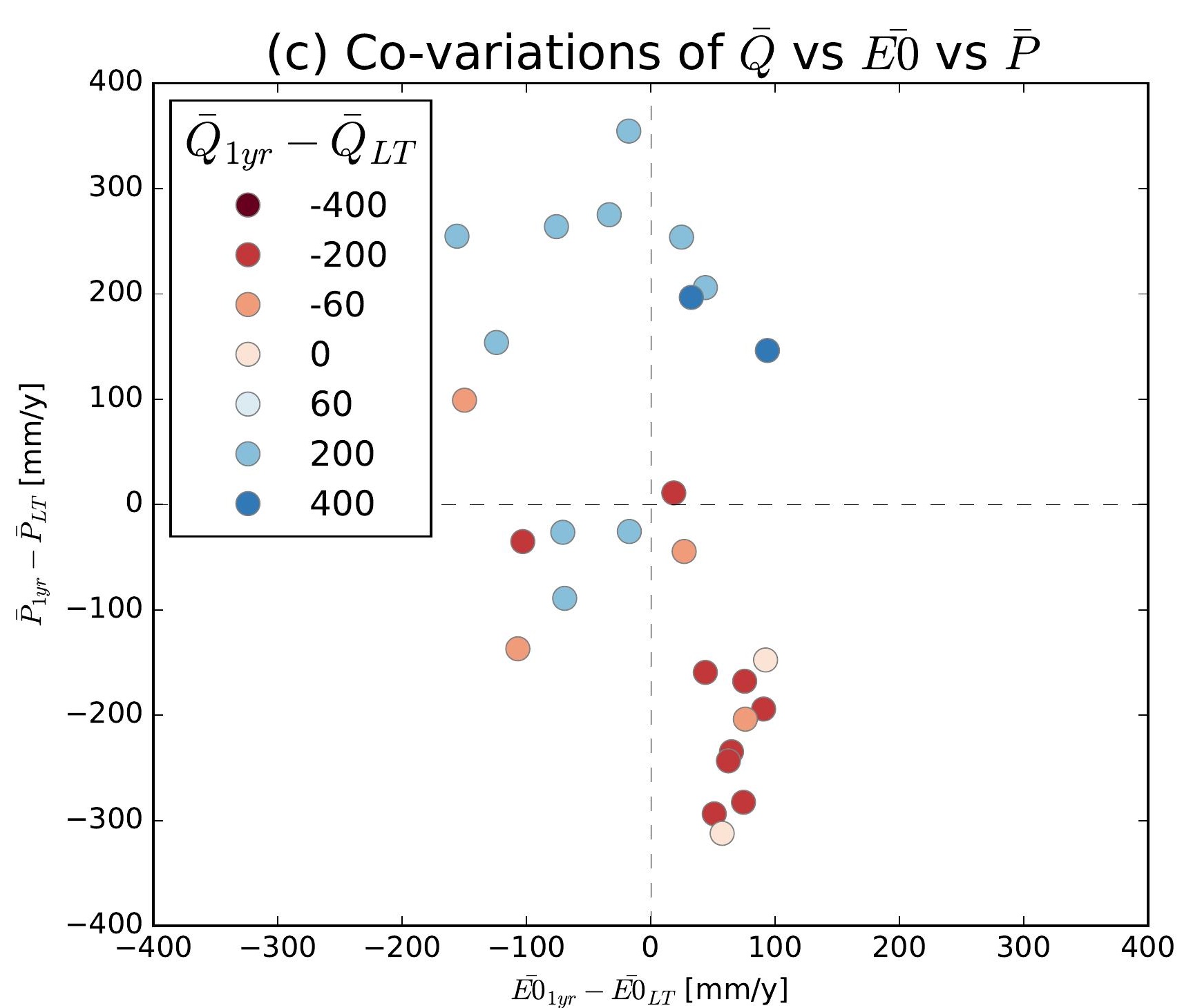
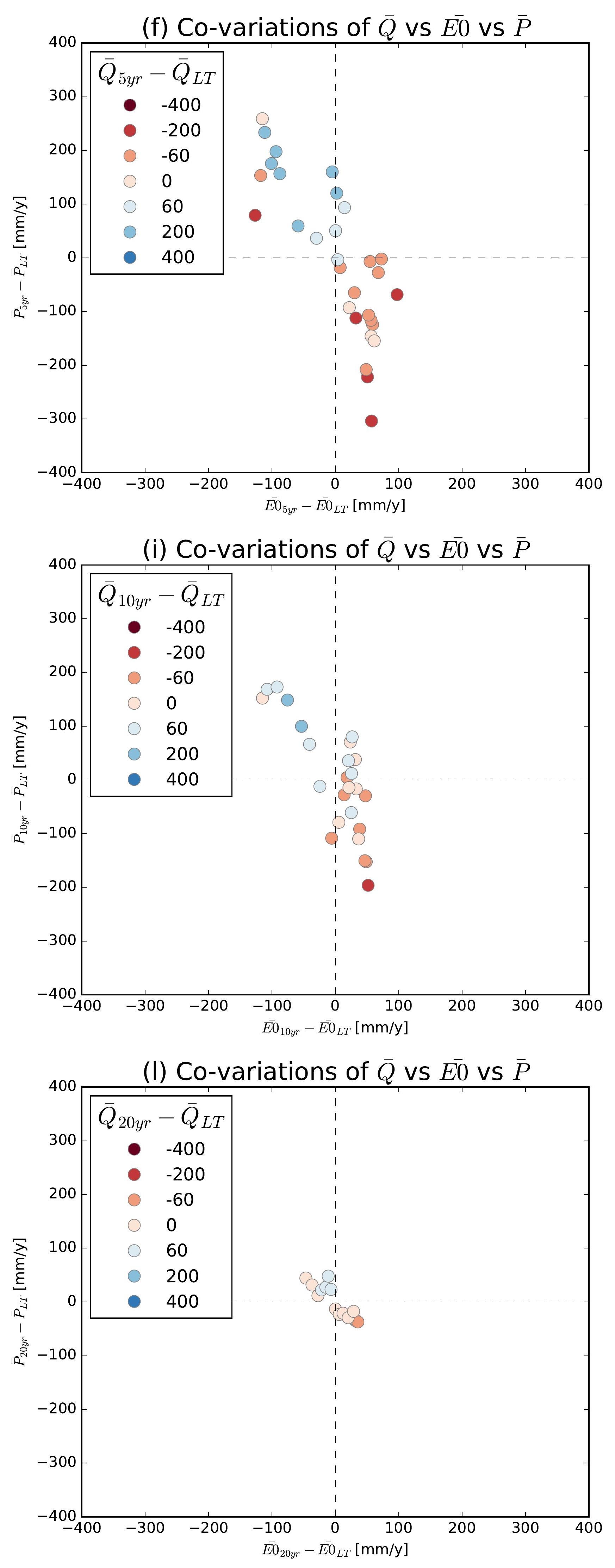

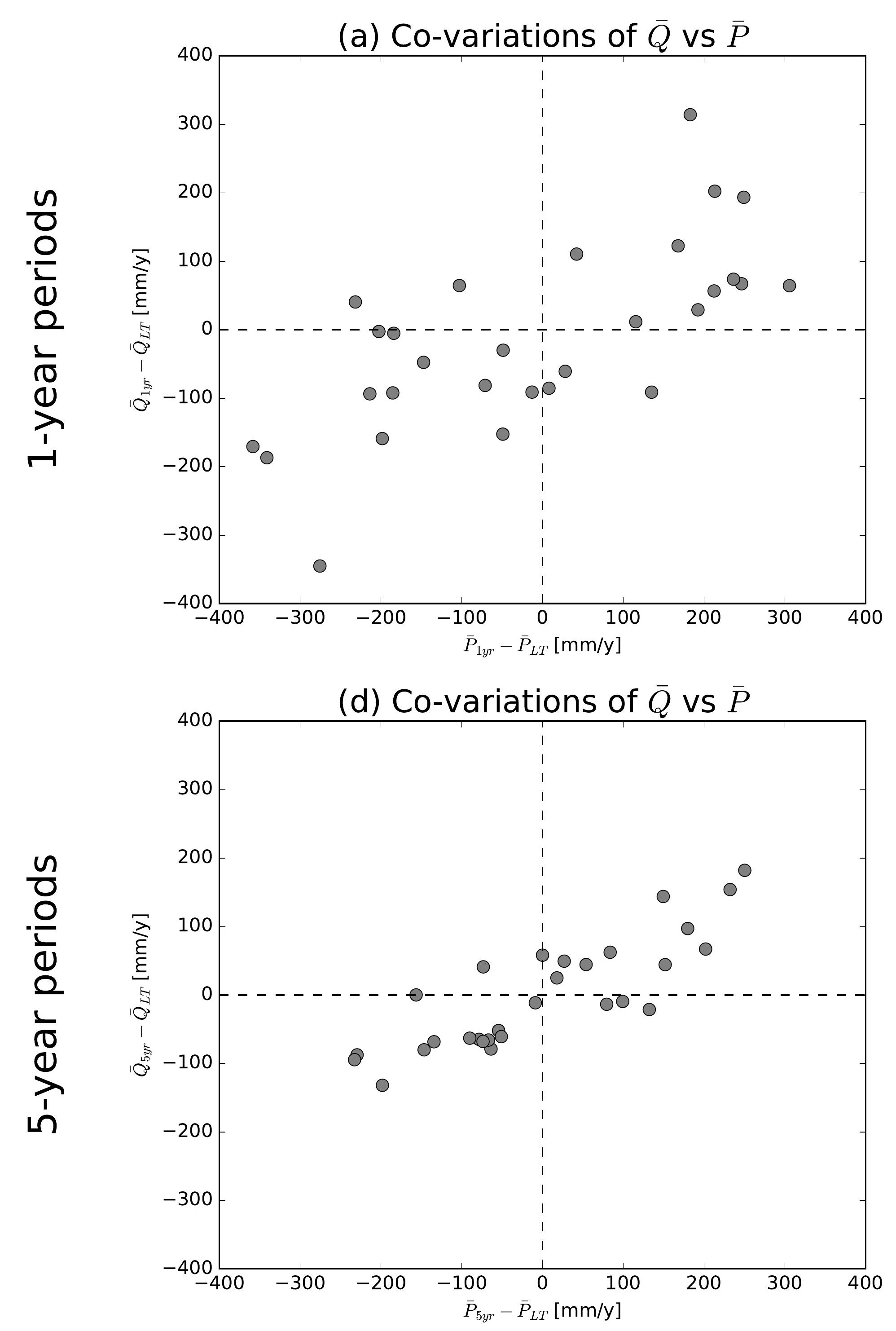

(g) Co-variations of $\bar{Q}$ vs $\bar{P}$

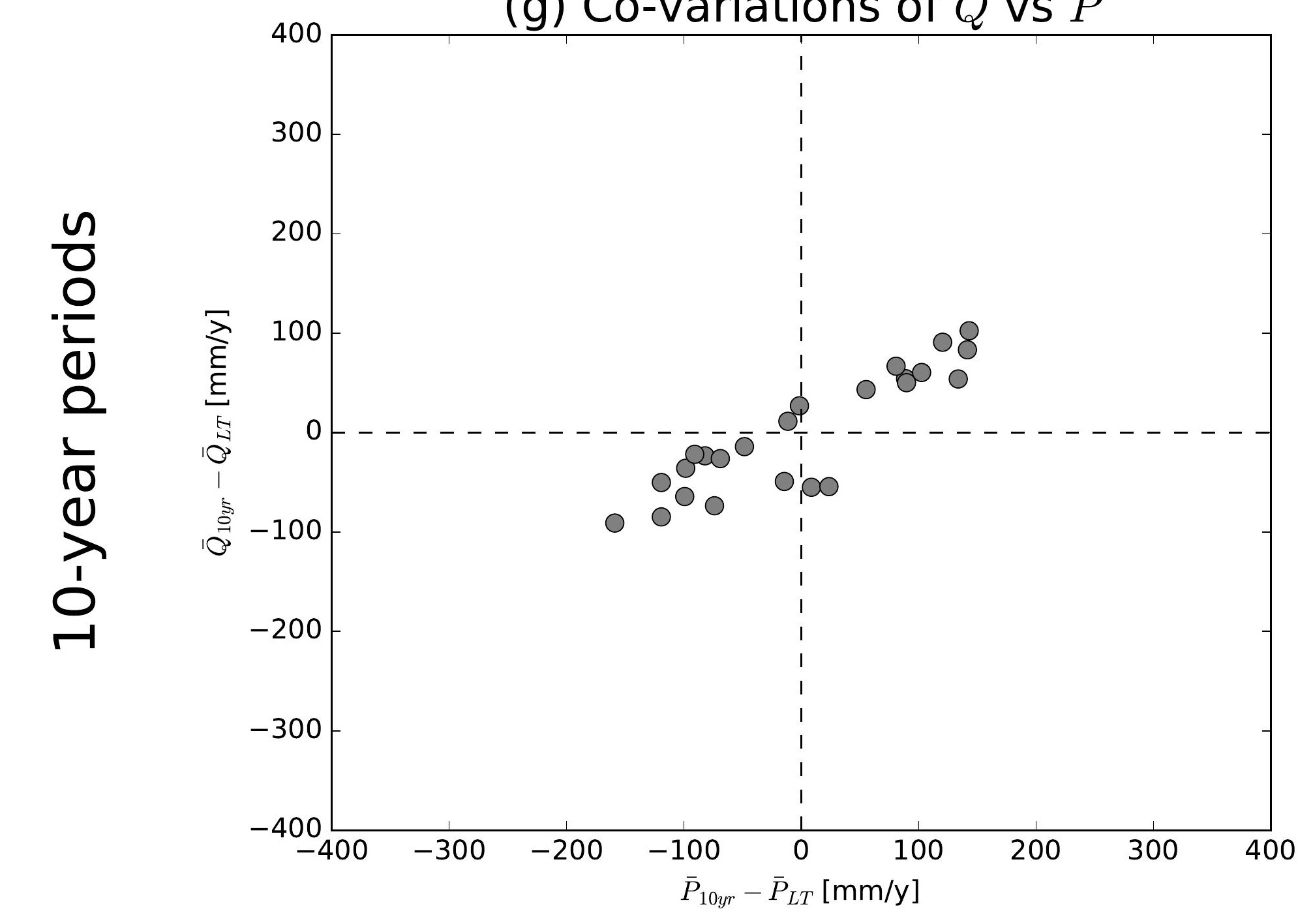

(j) Co-variations of $\bar{Q}$ vs $\bar{P}$

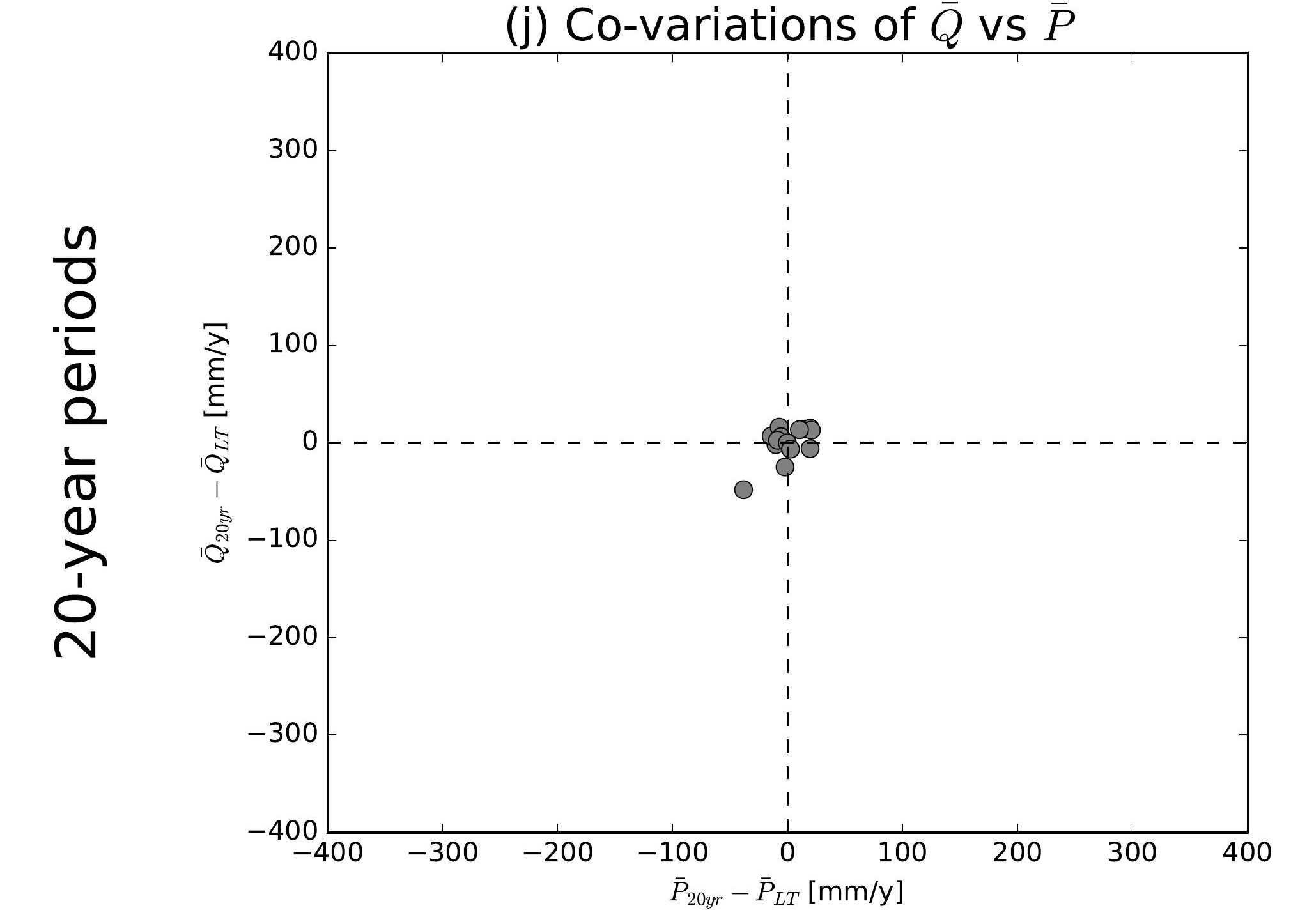

(b) Co-variations of $\bar{Q}$ vs $\overline{E 0}$

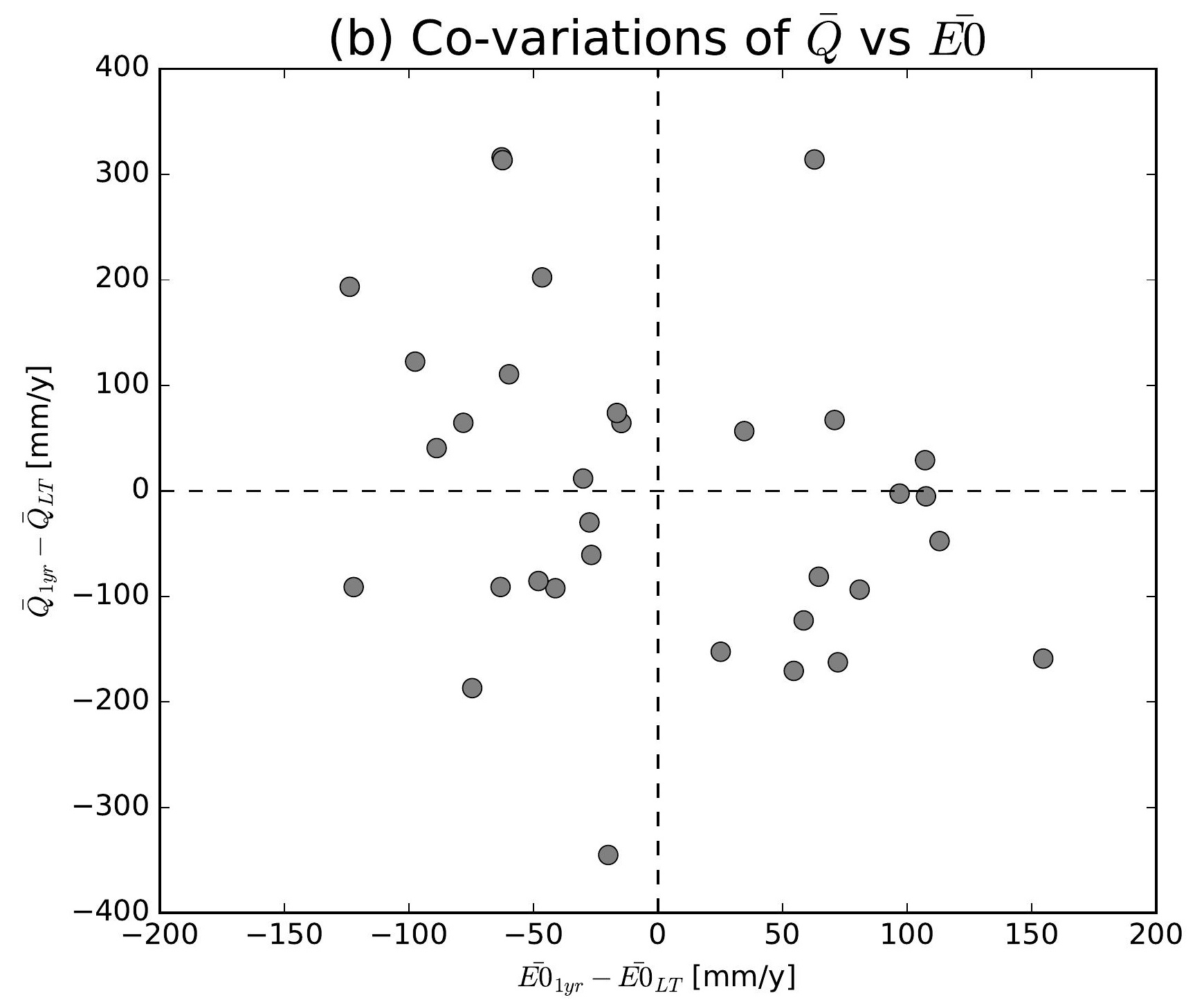

(e) Co-variations of $\bar{Q}$ vs $\overline{E 0}$

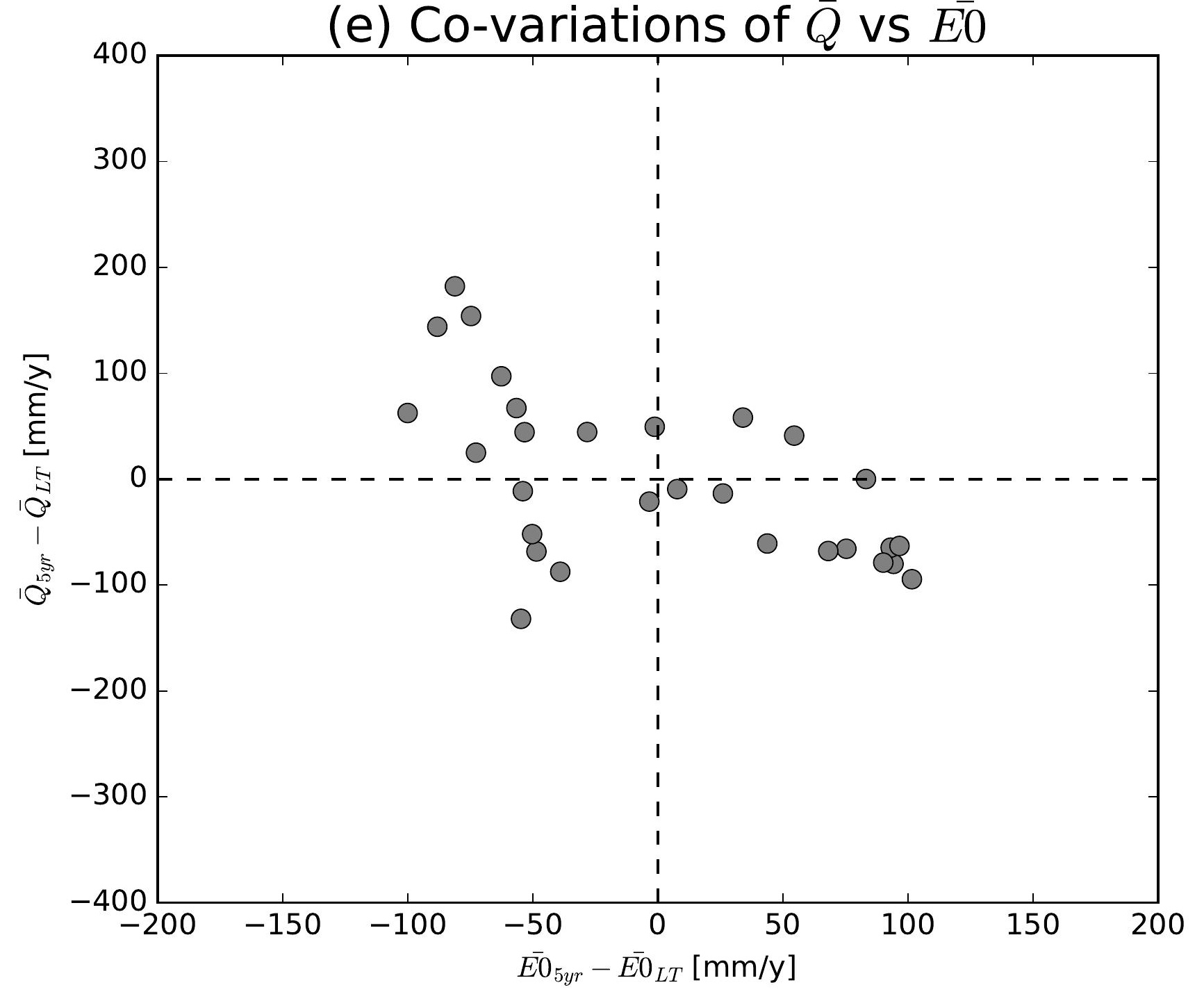

(h) Co-variations of $\bar{Q}$ vs $\overline{E 0}$

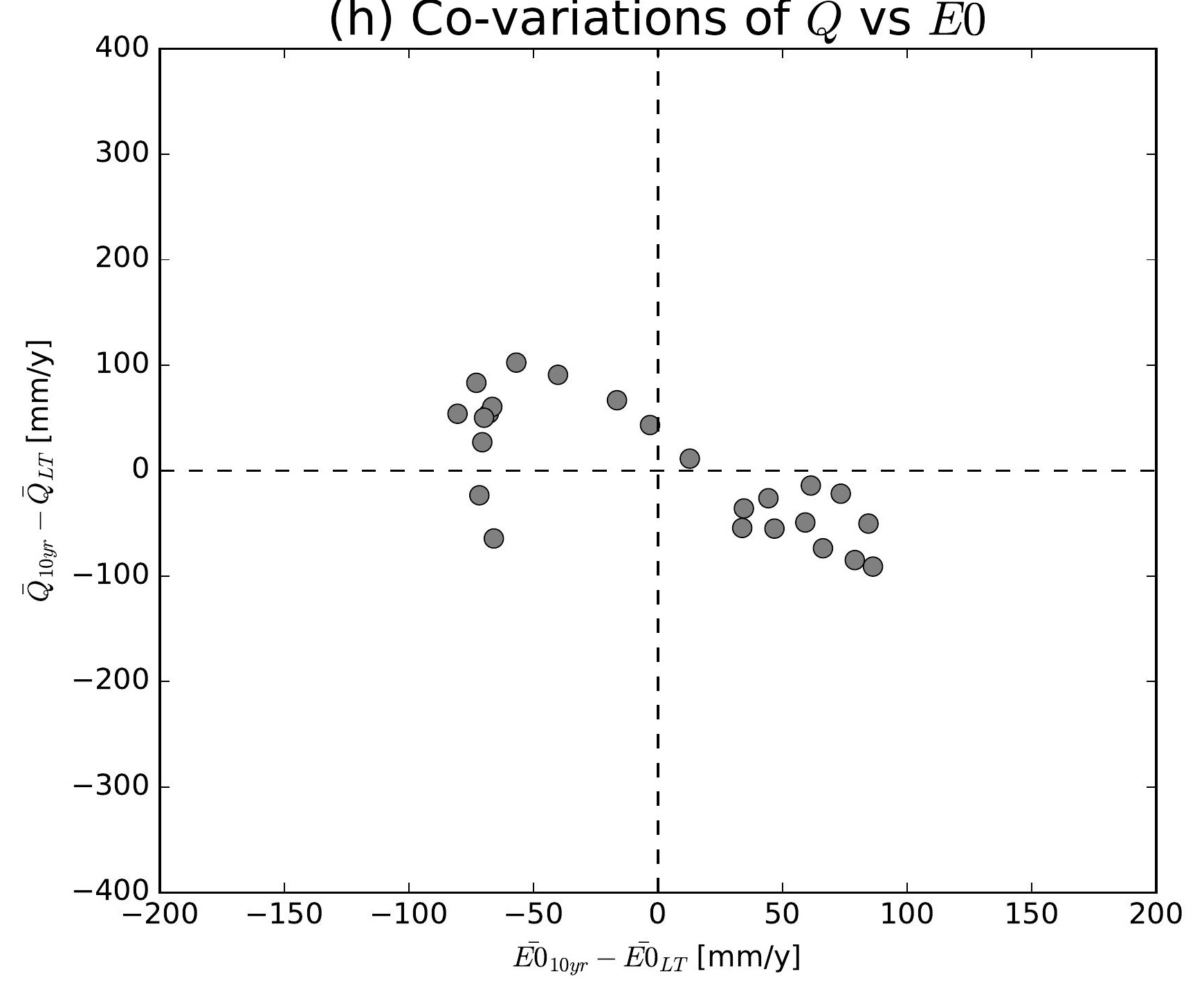

(k) Co-variations of $\bar{Q}$ vs $\overline{E 0}$

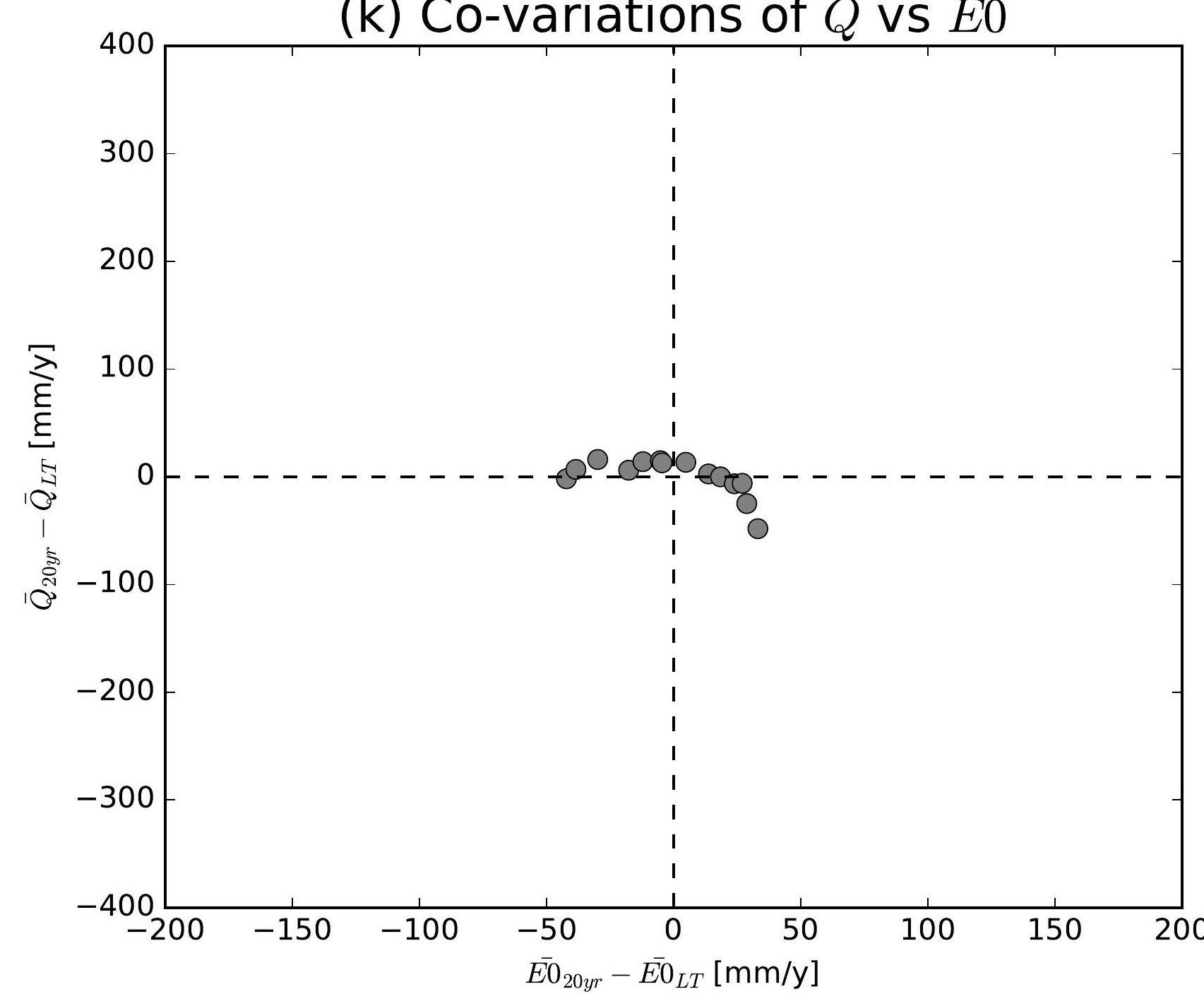

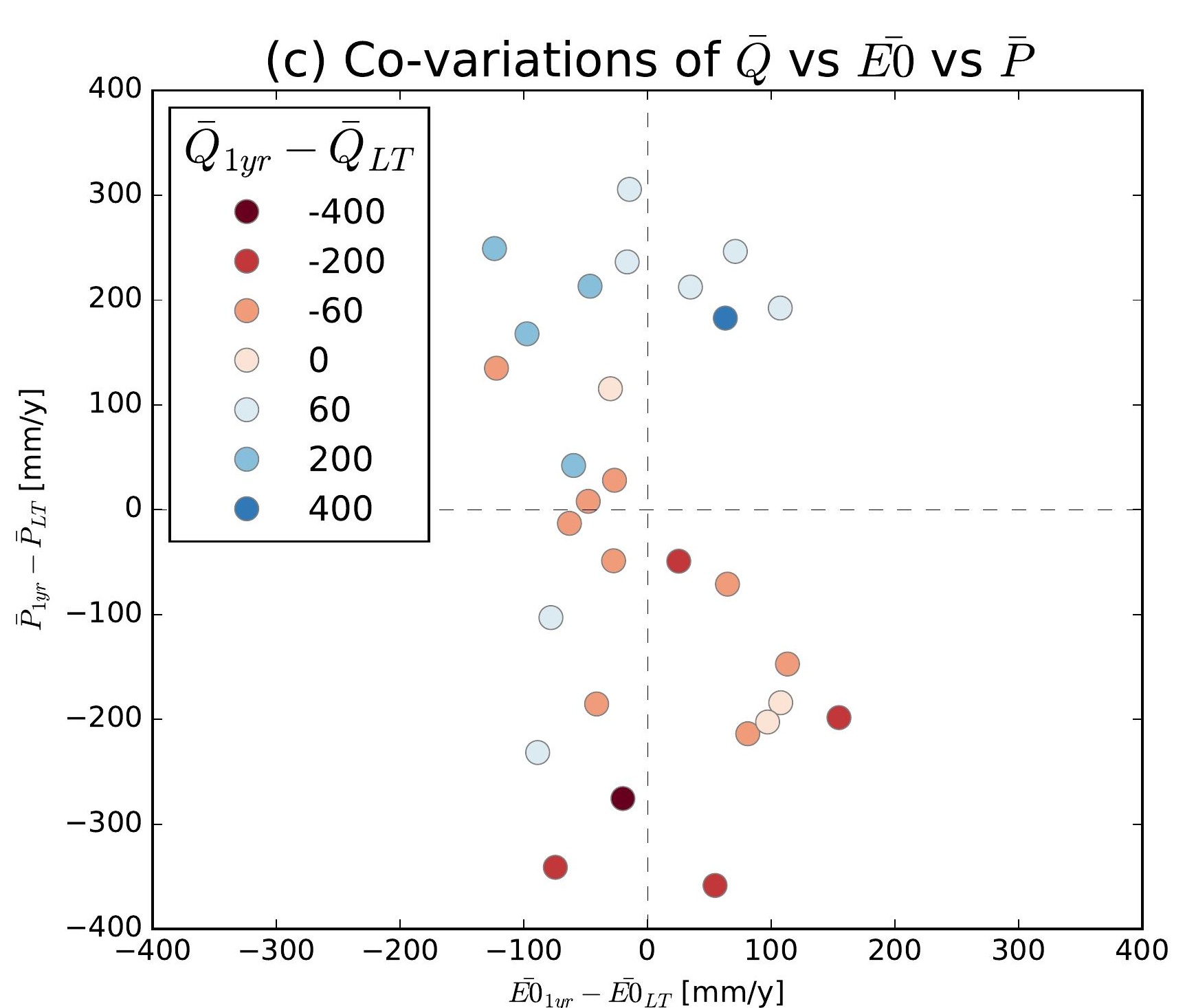
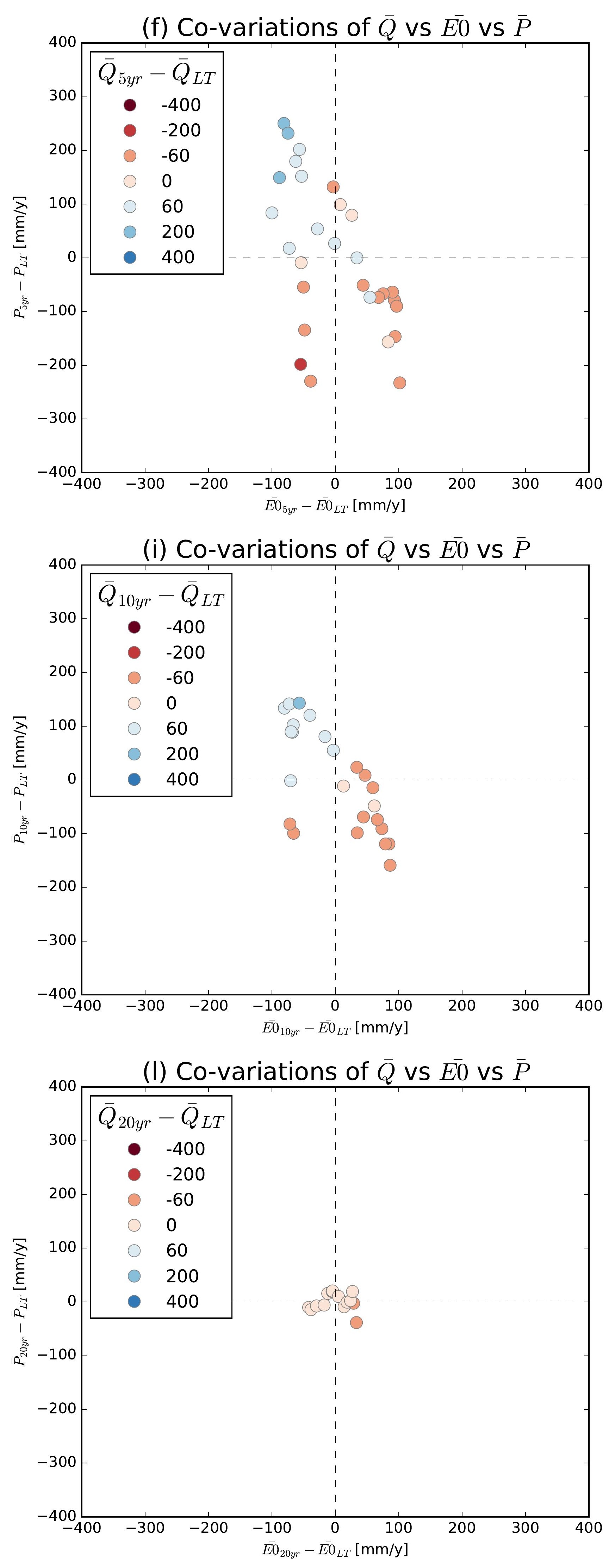

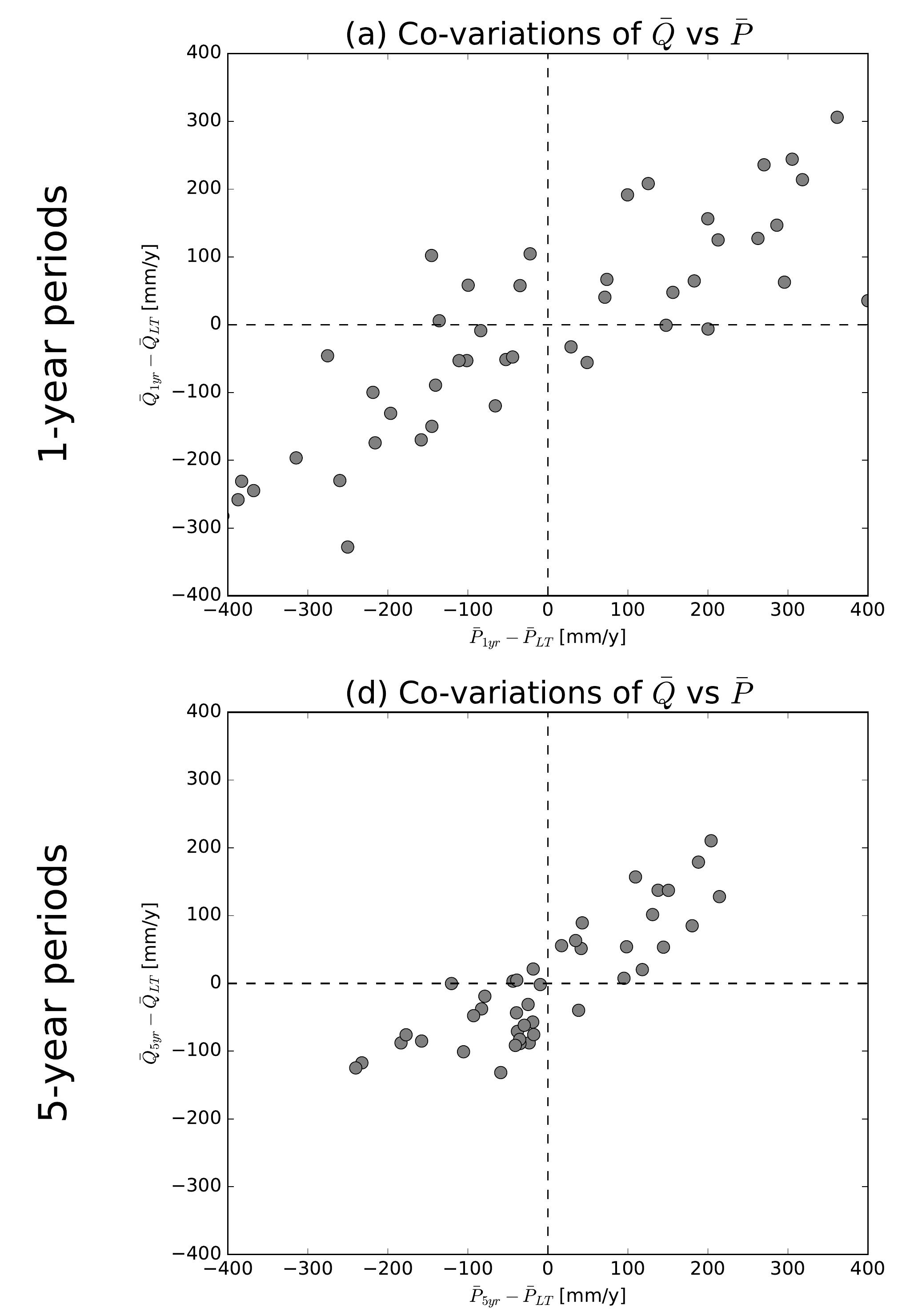

(g) Co-variations of $\bar{Q}$ vs $\bar{P}$
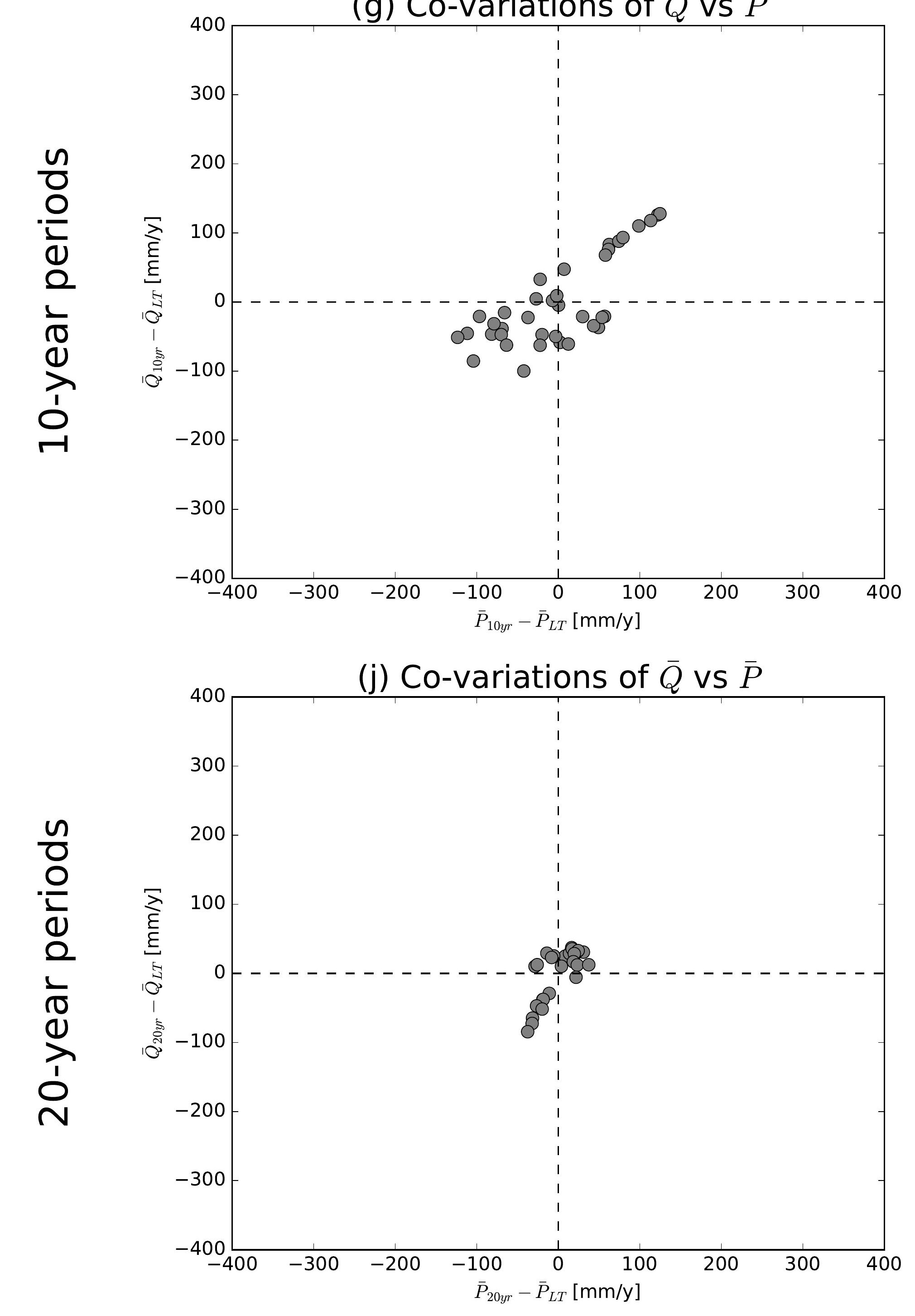

(b) Co-variations of $\bar{Q}$ vs $\overline{E 0}$

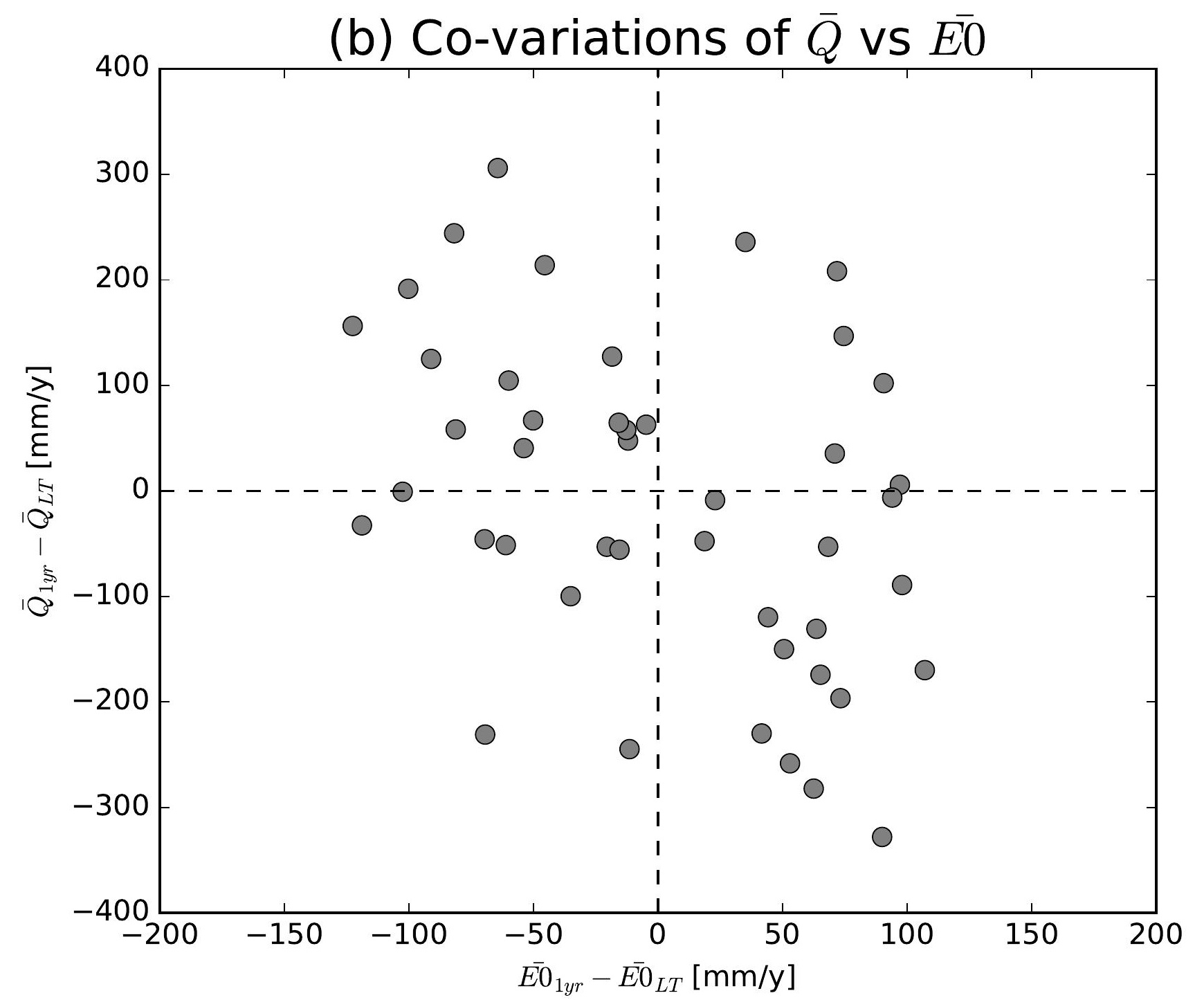

(e) Co-variations of $\bar{Q}$ vs $\overline{E 0}$

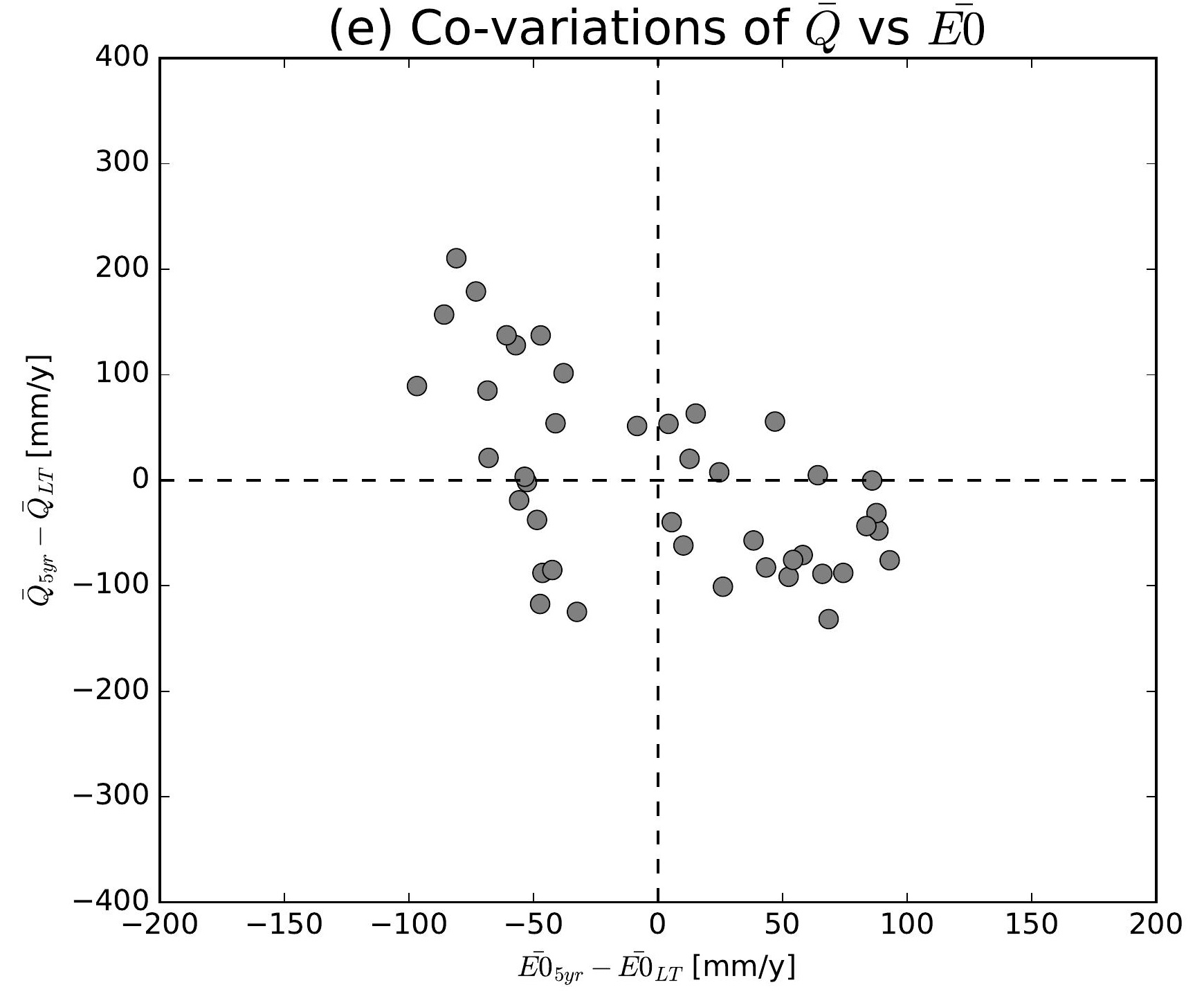

(h) Co-variations of $\bar{Q}$ vs $\overline{E 0}$

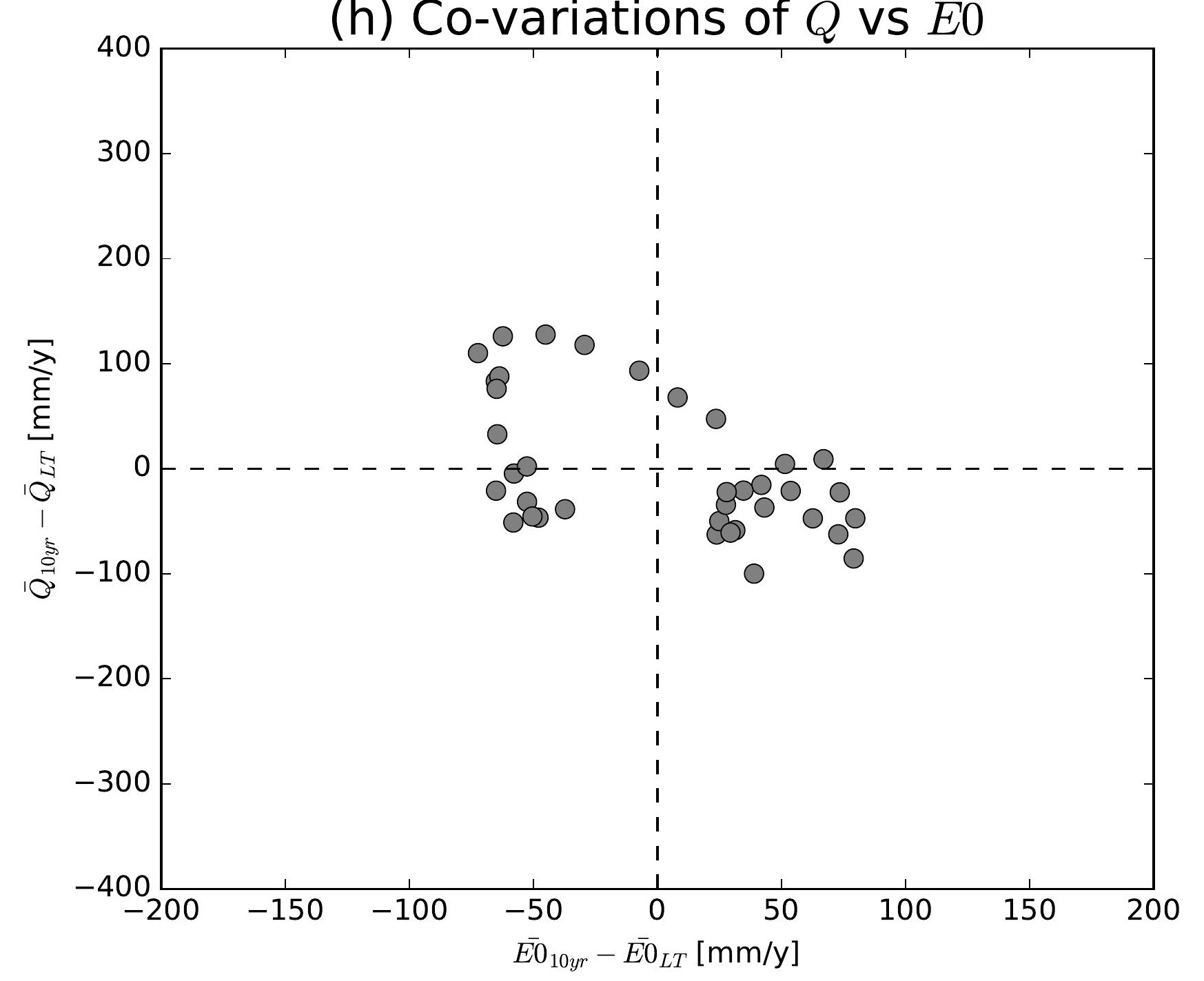

(k) Co-variations of $\bar{Q}$ vs $\overline{E 0}$

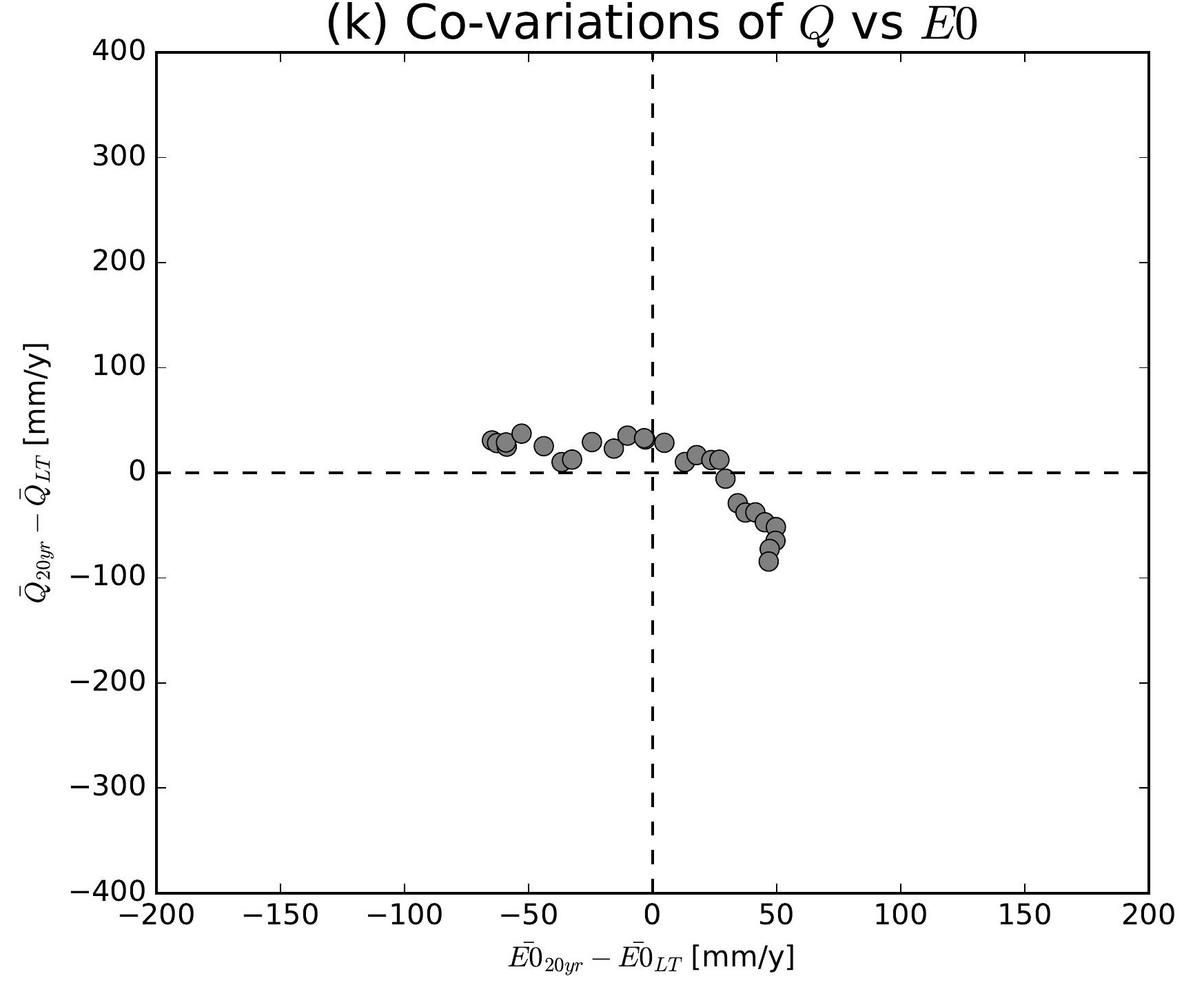

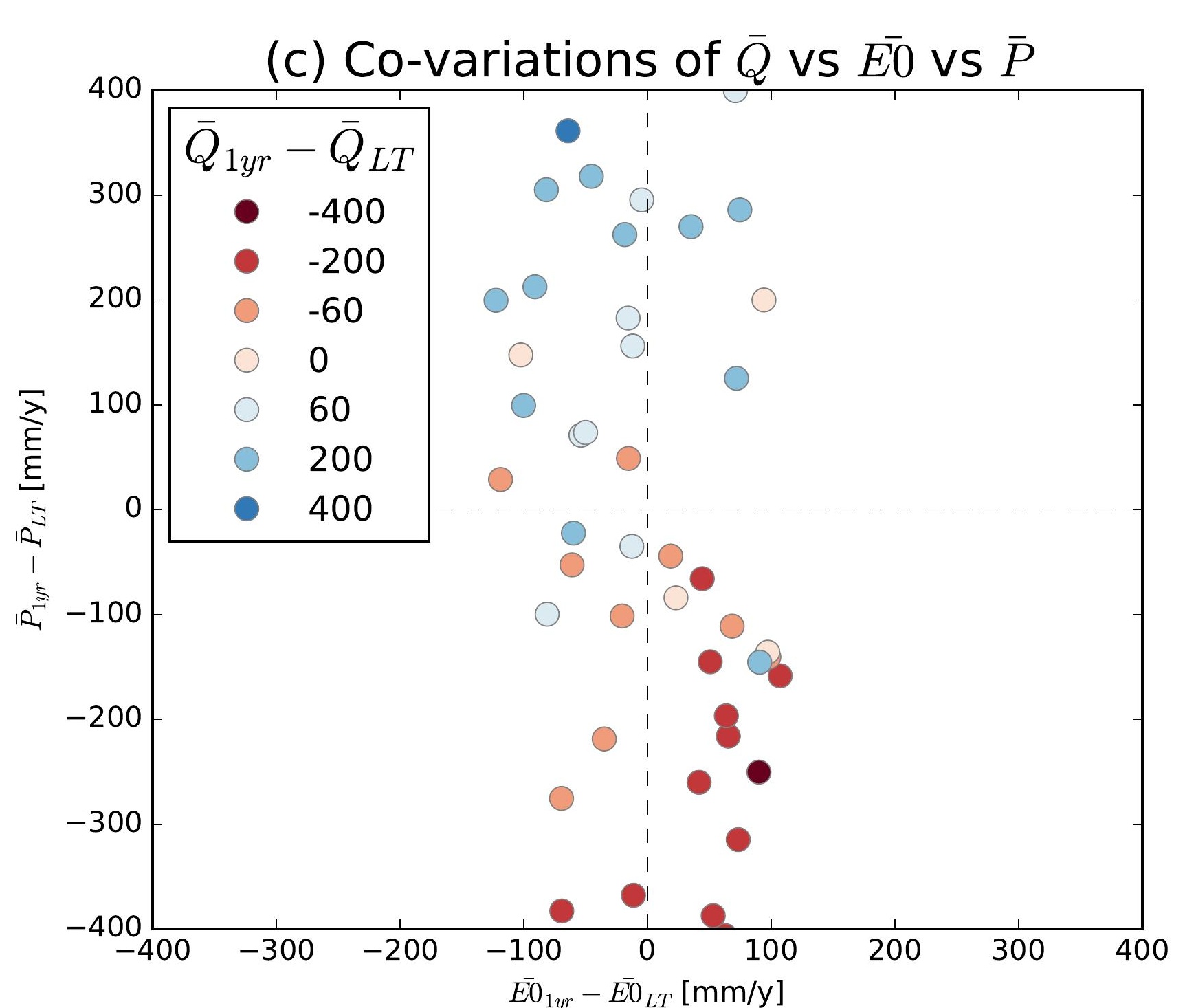
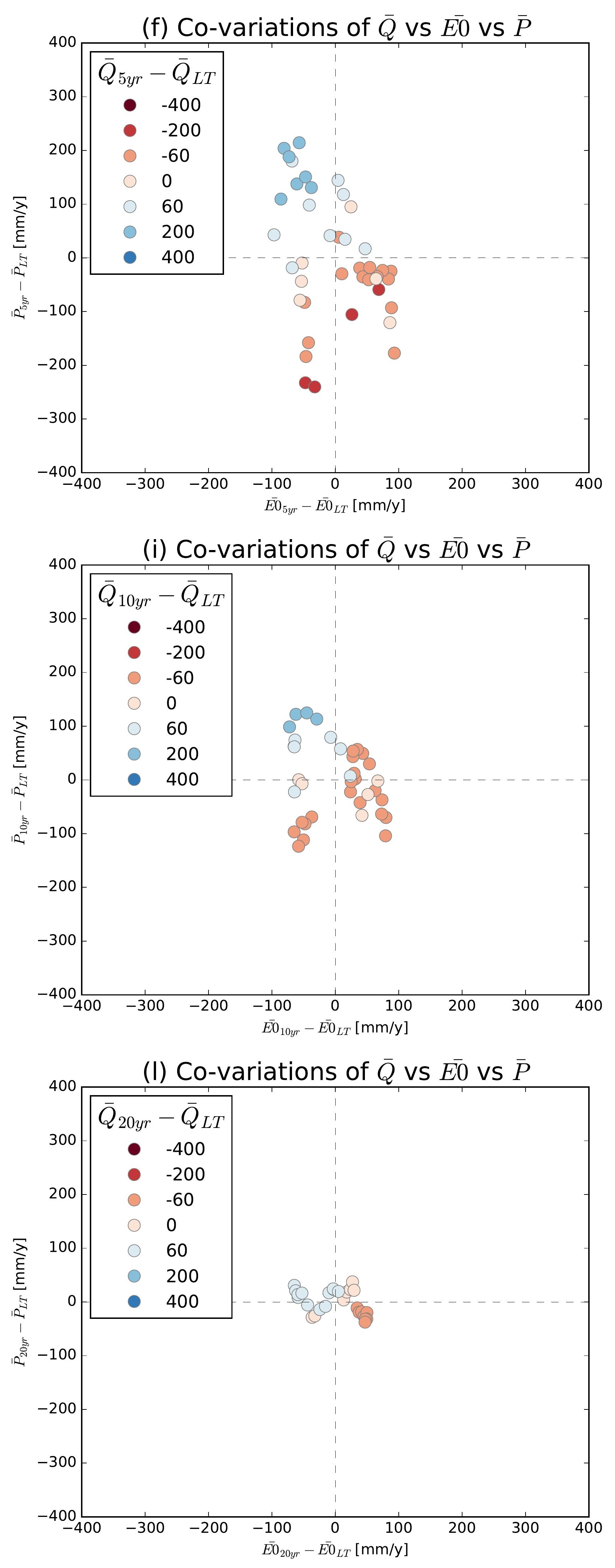

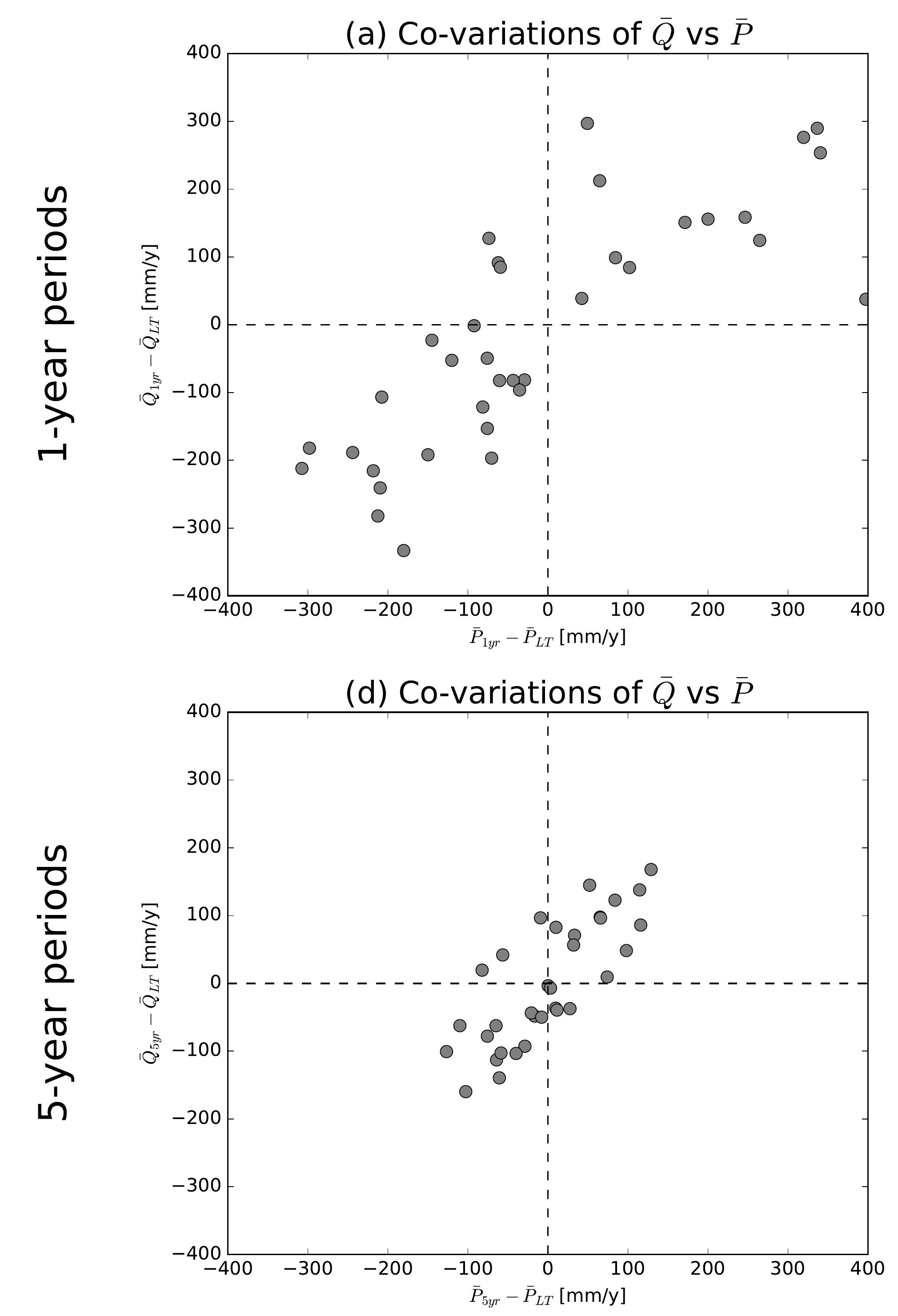

(g) Co-variations of $\bar{Q}$ vs $\bar{P}$
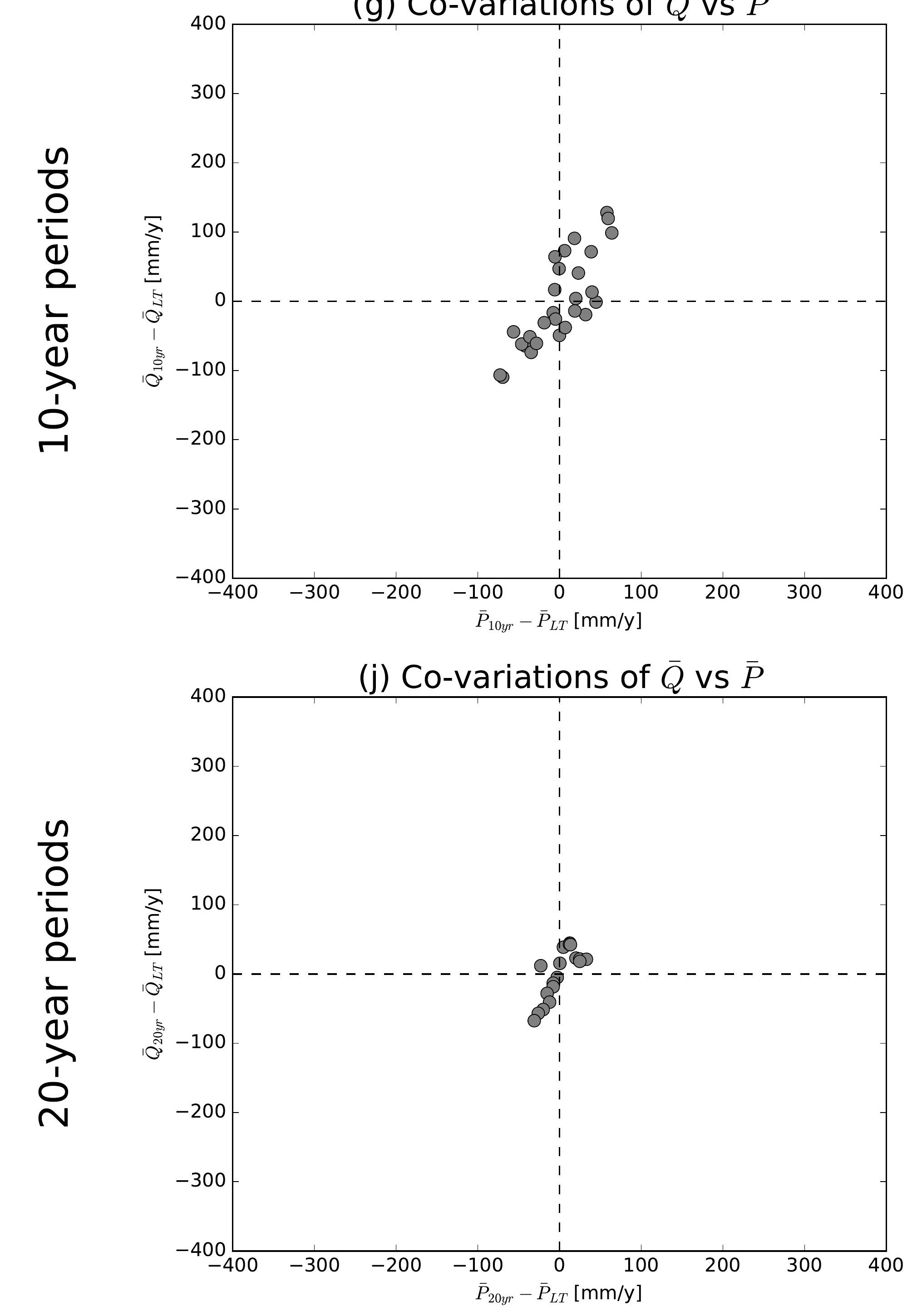

(b) Co-variations of $\bar{Q}$ vs $\overline{E 0}$

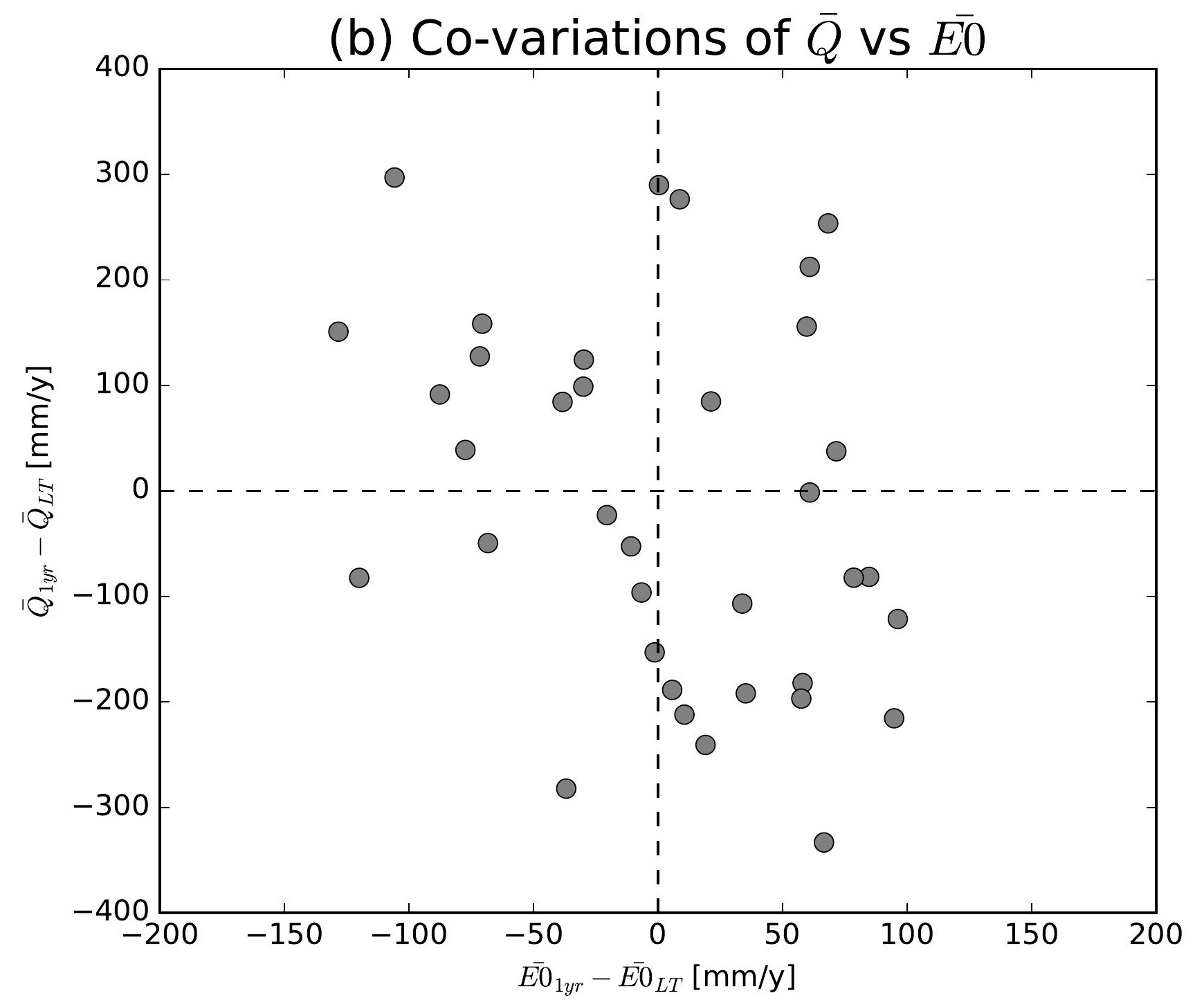

(e) Co-variations of $\bar{Q}$ vs $\overline{E 0}$

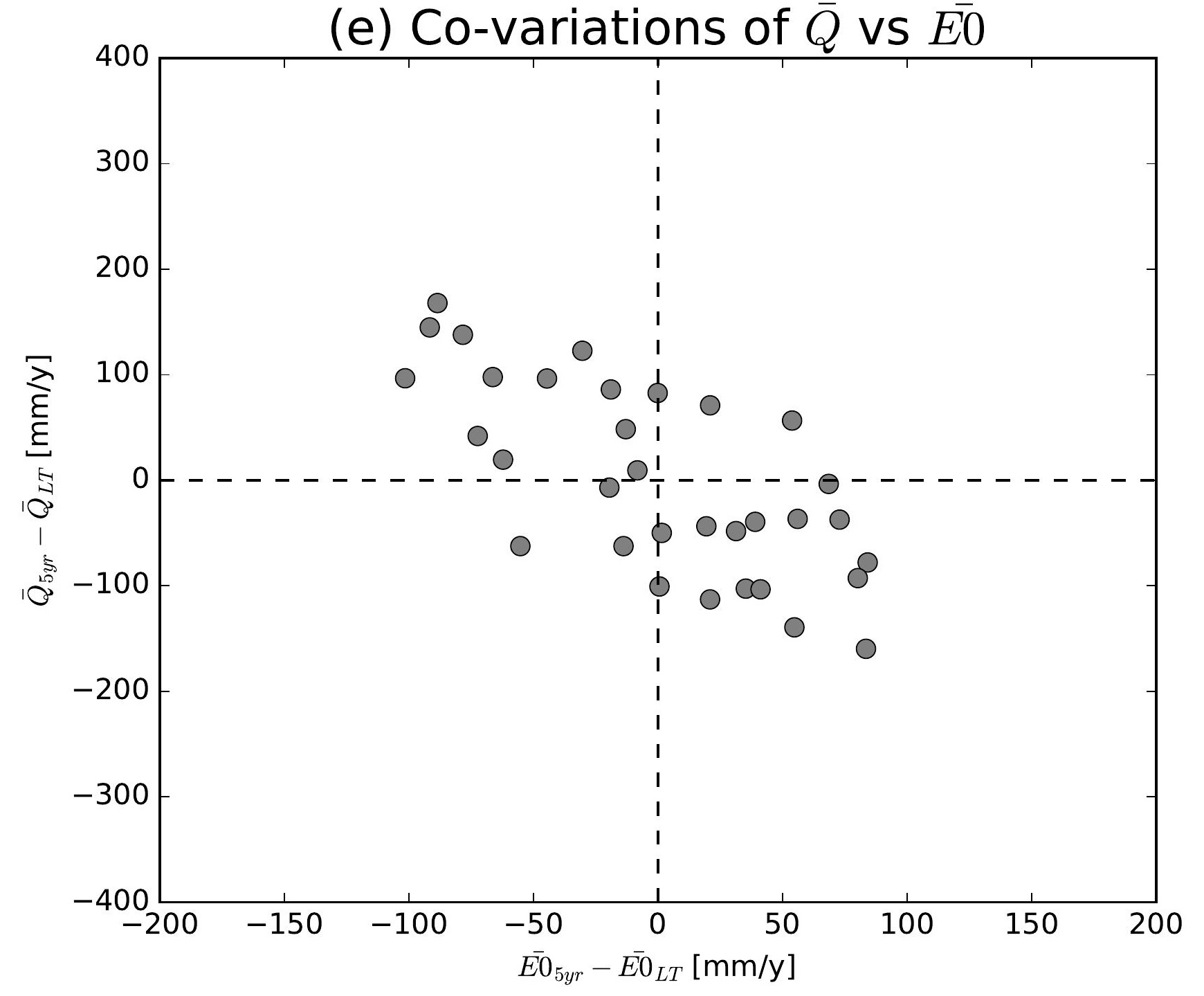

(h) Co-variations of $\bar{Q}$ vs $\overline{E 0}$

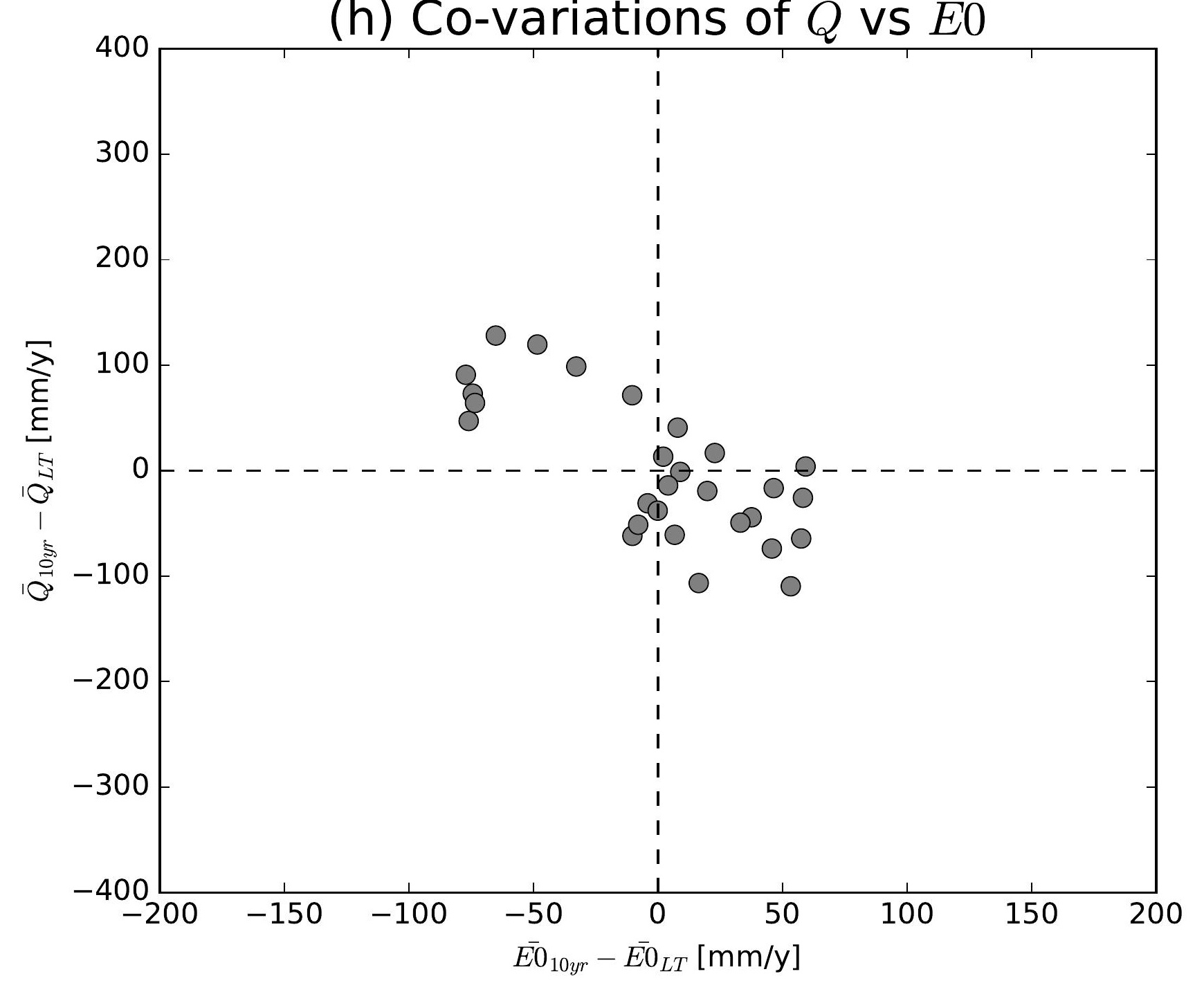

(k) Co-variations of $\bar{Q}$ vs $\overline{E 0}$

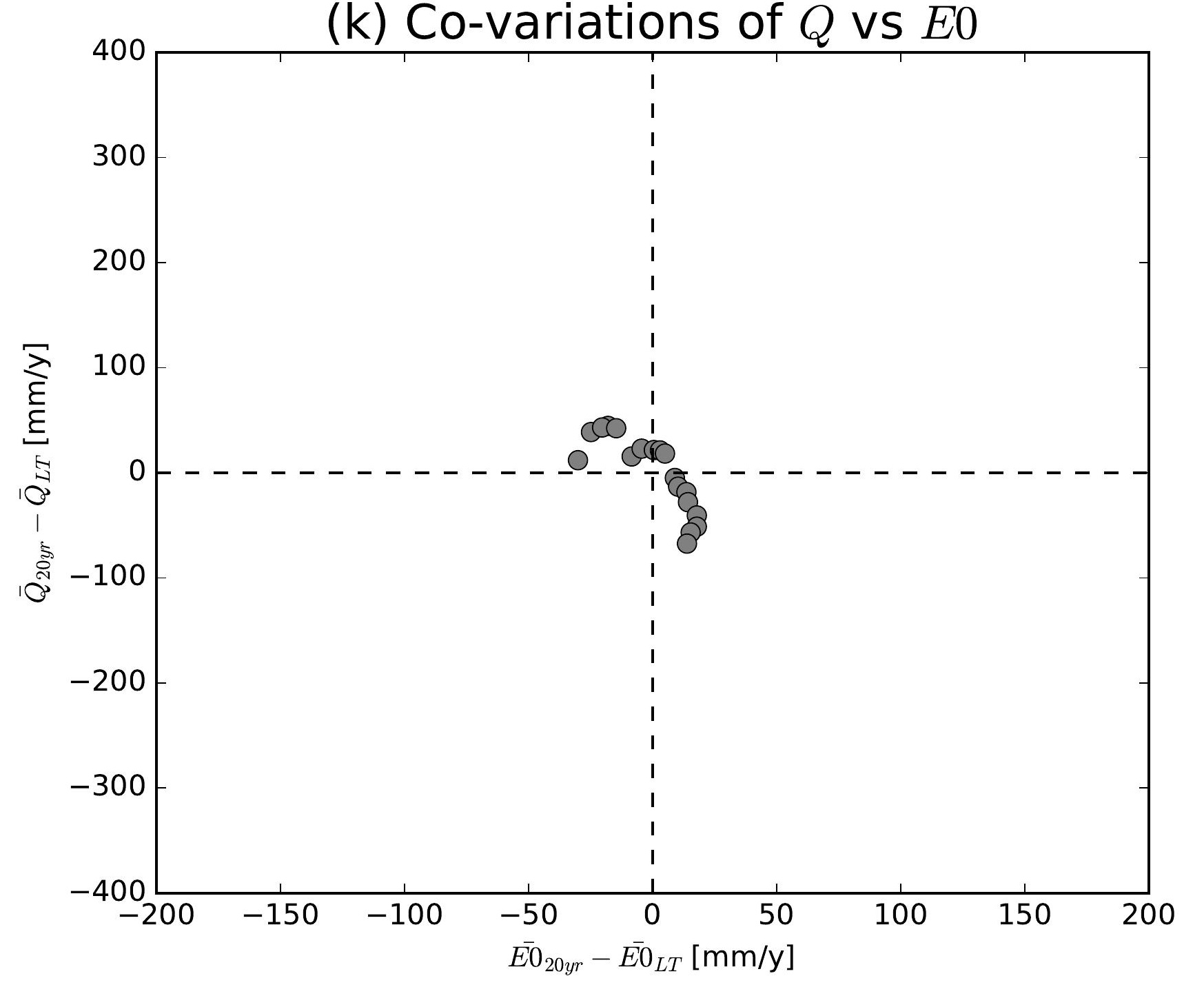

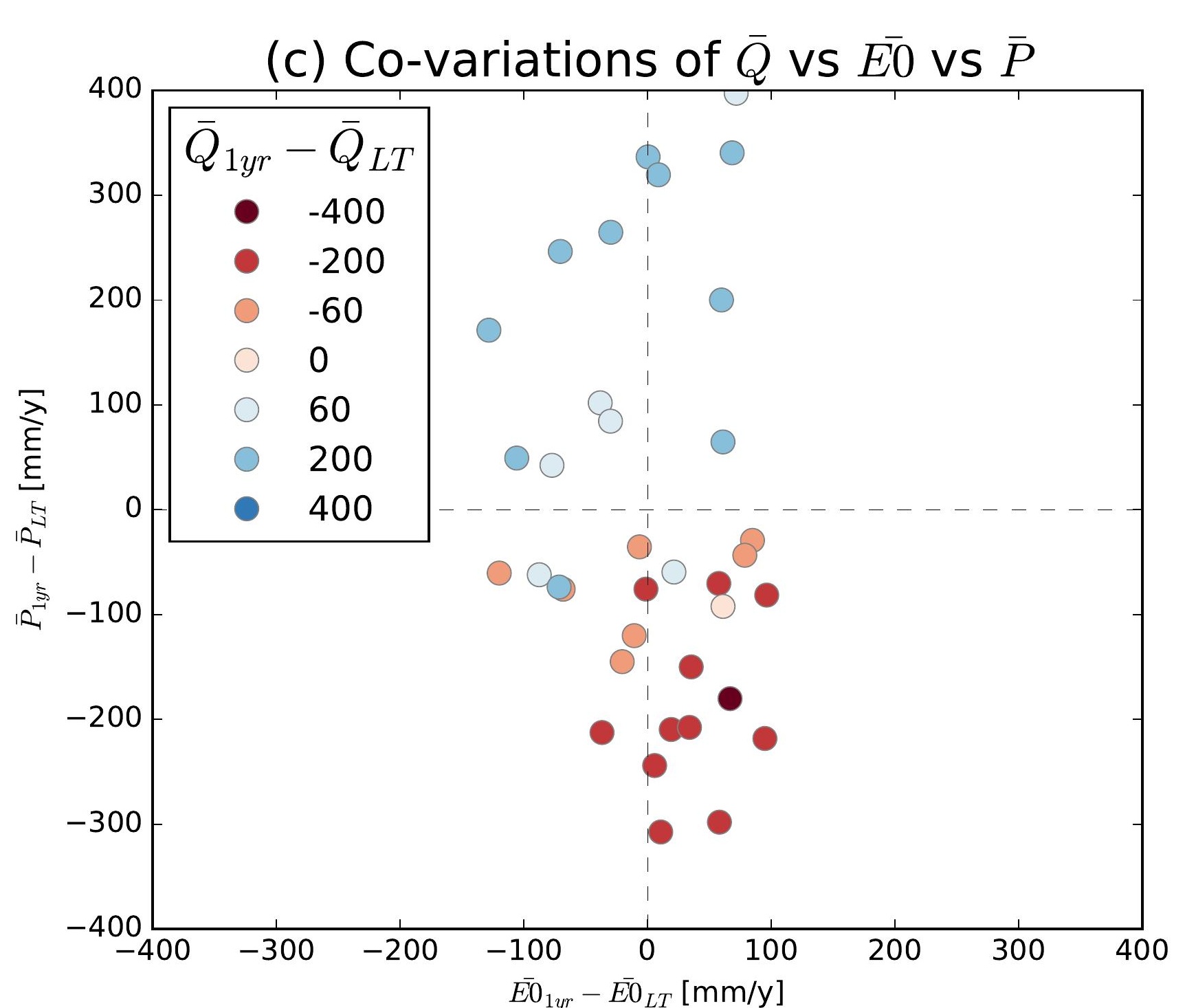
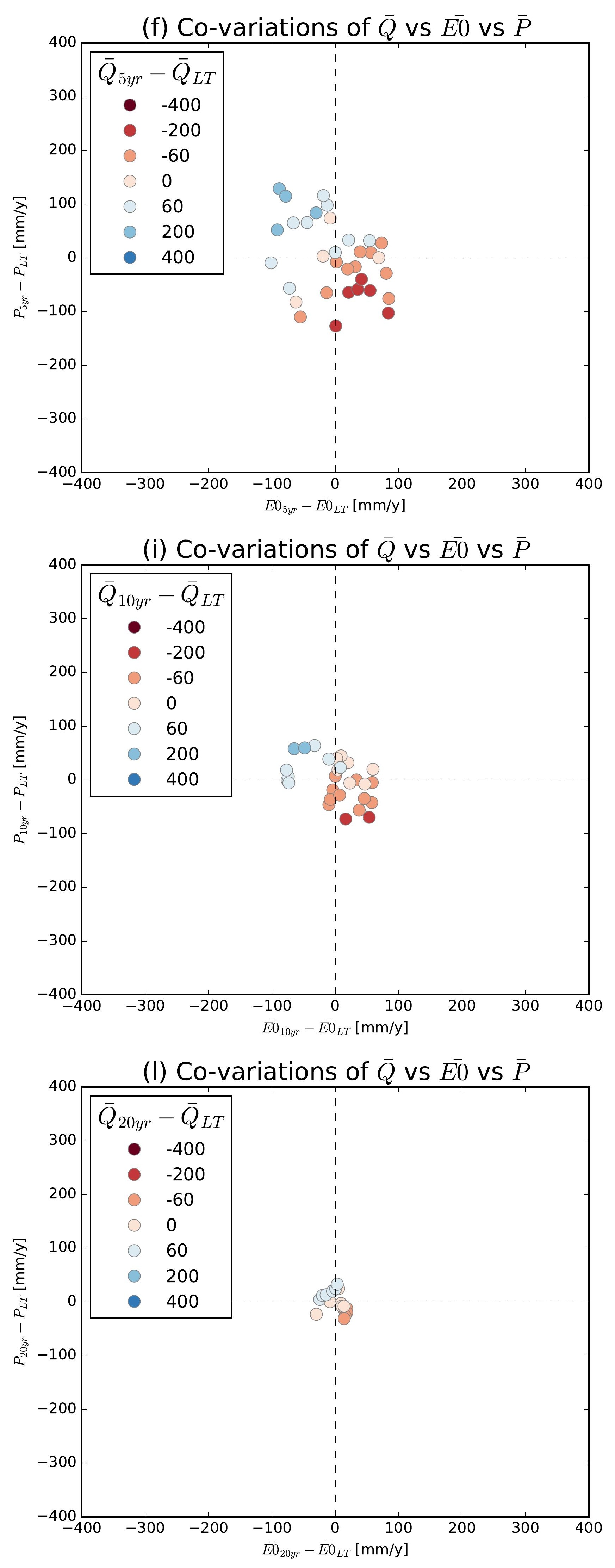

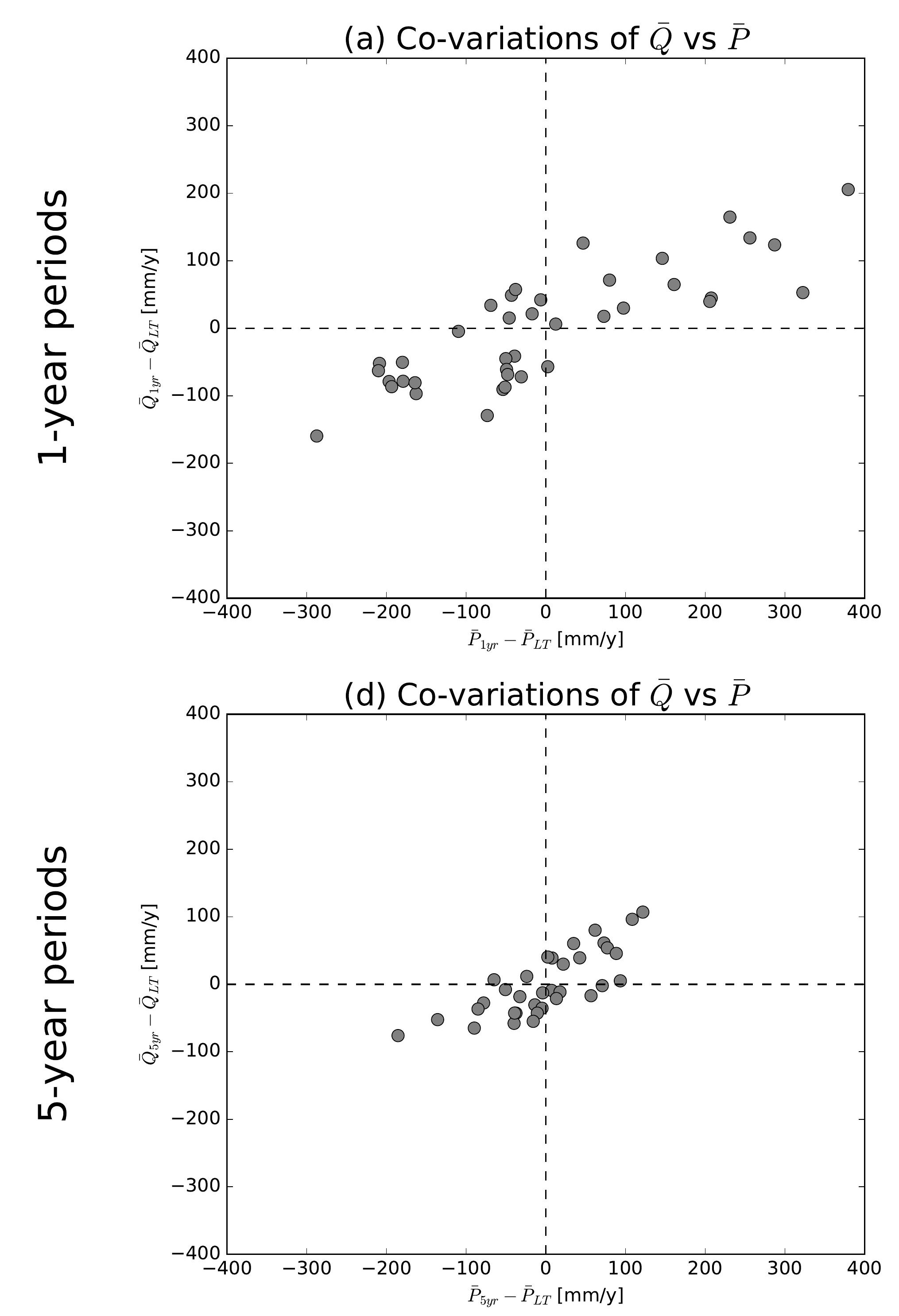

(g) Co-variations of $\bar{Q}$ vs $\bar{P}$

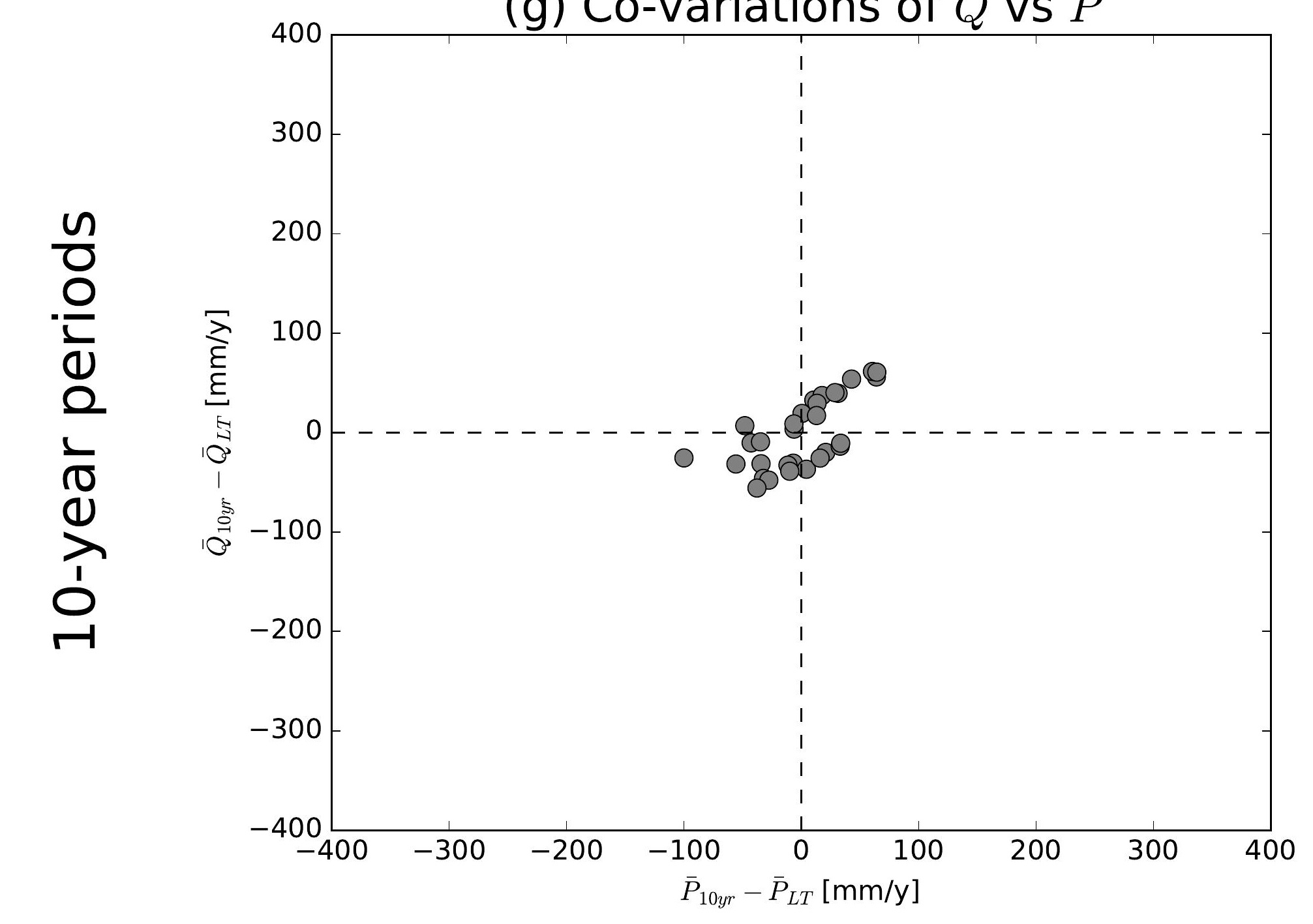

(j) Co-variations of $\bar{Q}$ vs $\bar{P}$

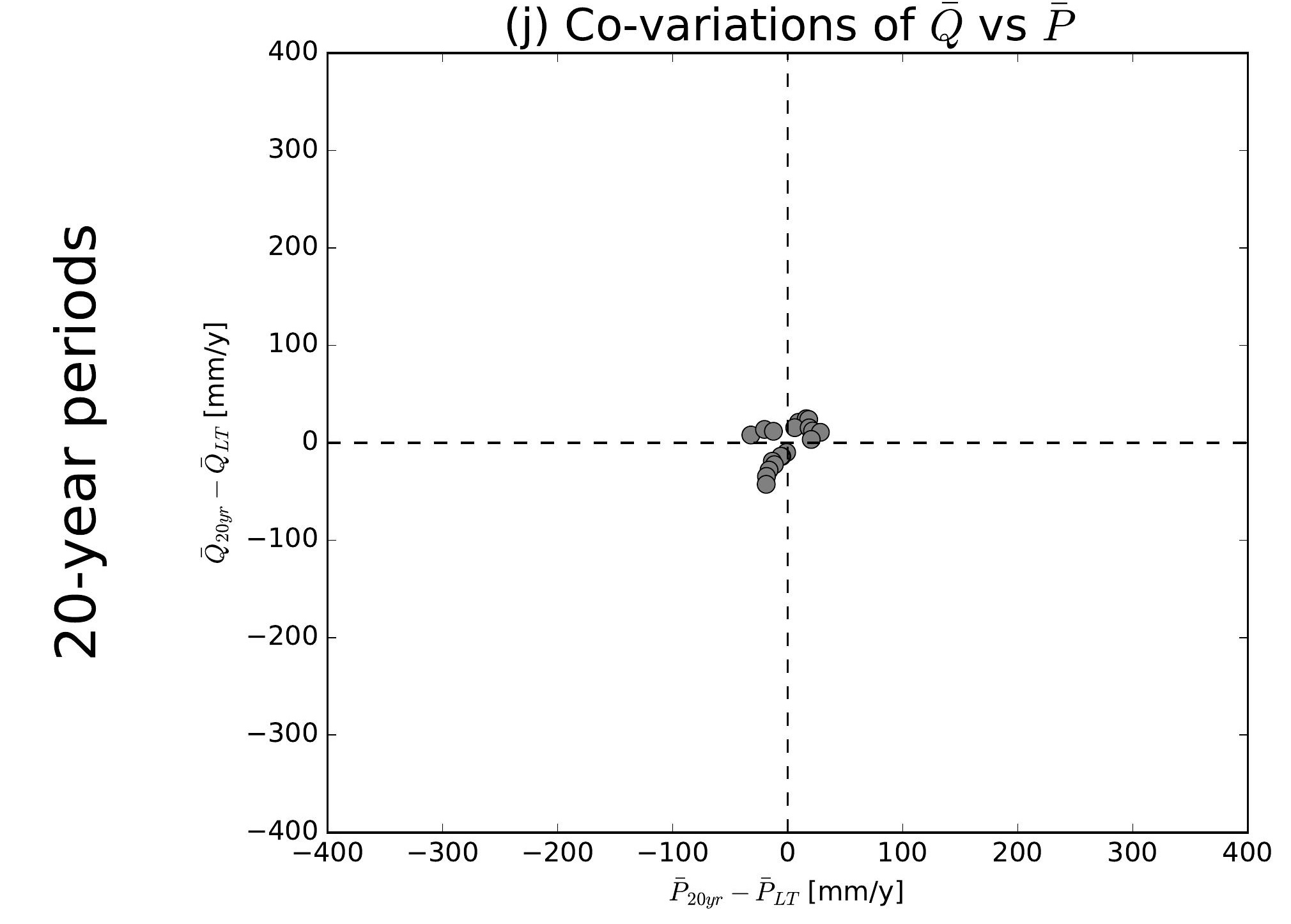

(b) Co-variations of $\bar{Q}$ vs $\overline{E 0}$

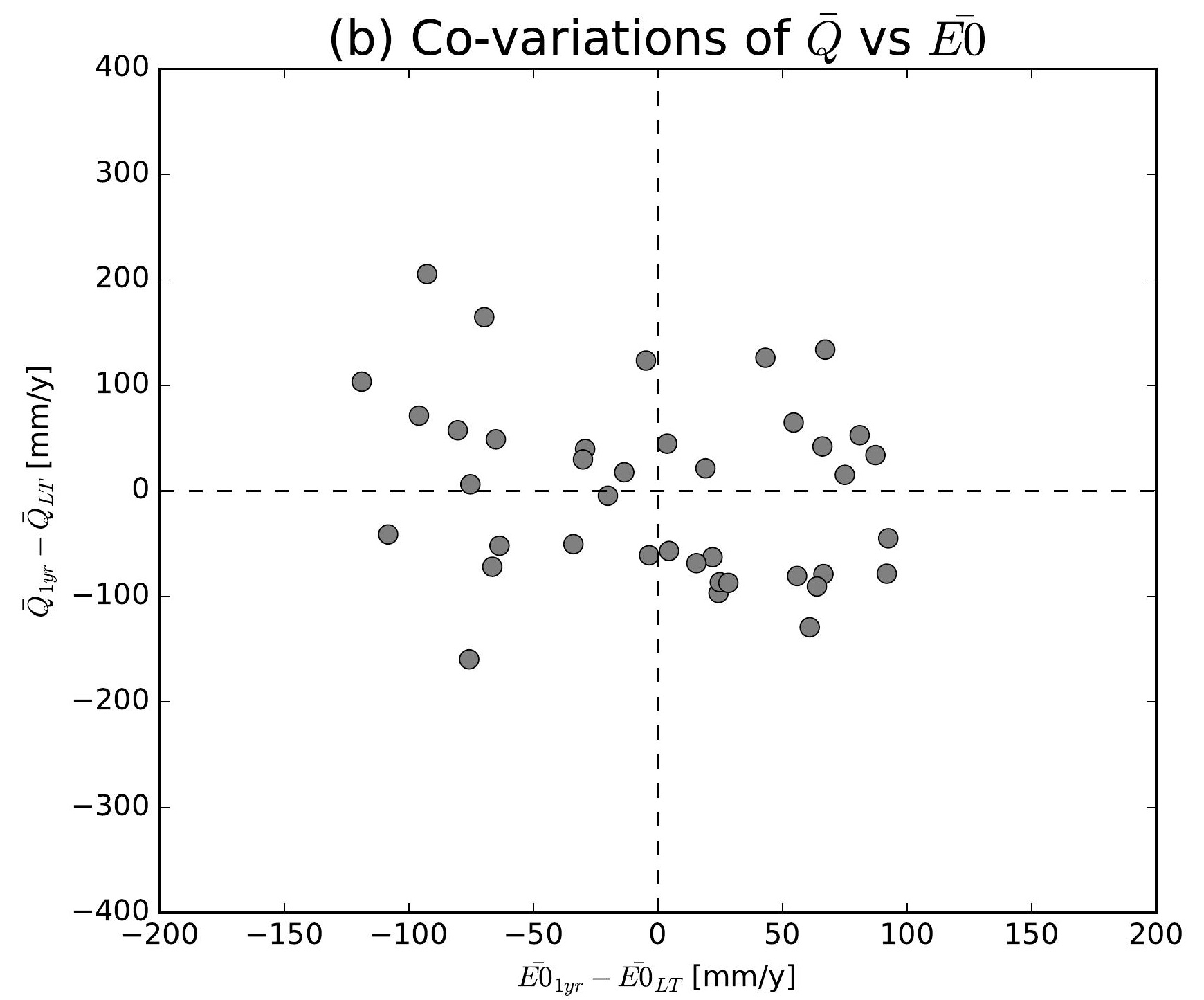

(e) Co-variations of $\bar{Q}$ vs $\overline{E 0}$

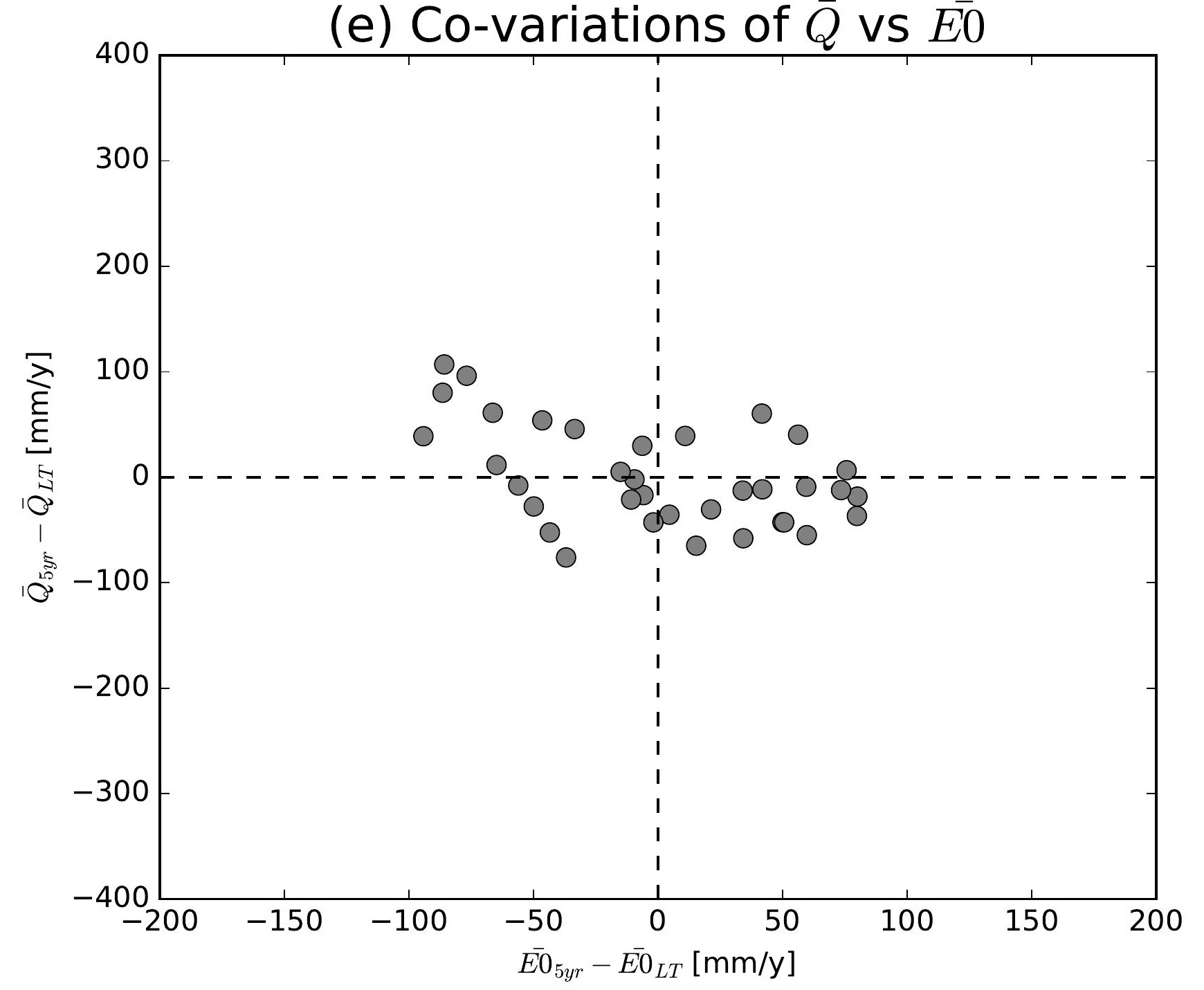

(h) Co-variations of $\bar{Q}$ vs $\overline{E 0}$

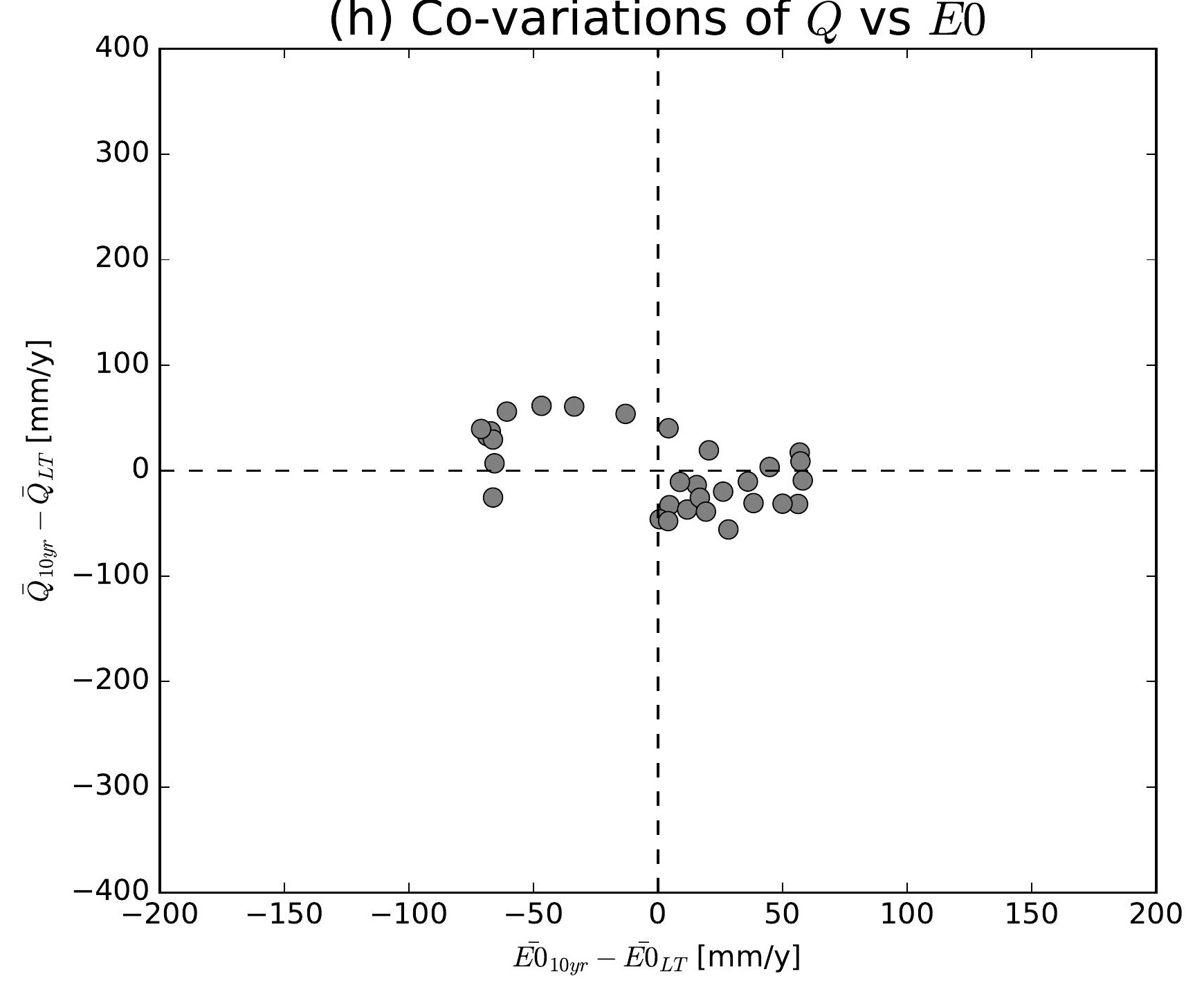

(k) Co-variations of $\bar{Q}$ vs $\overline{E 0}$

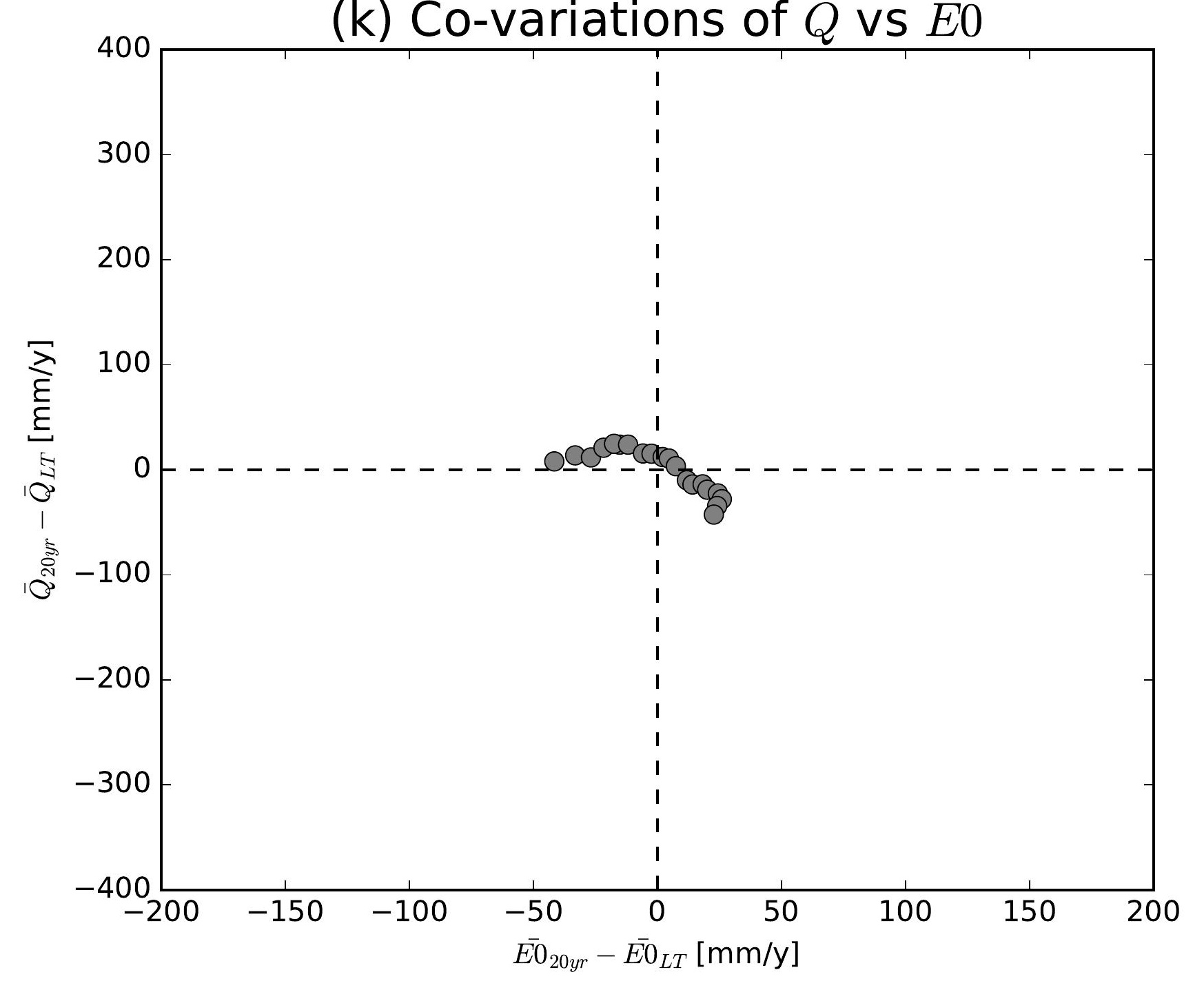

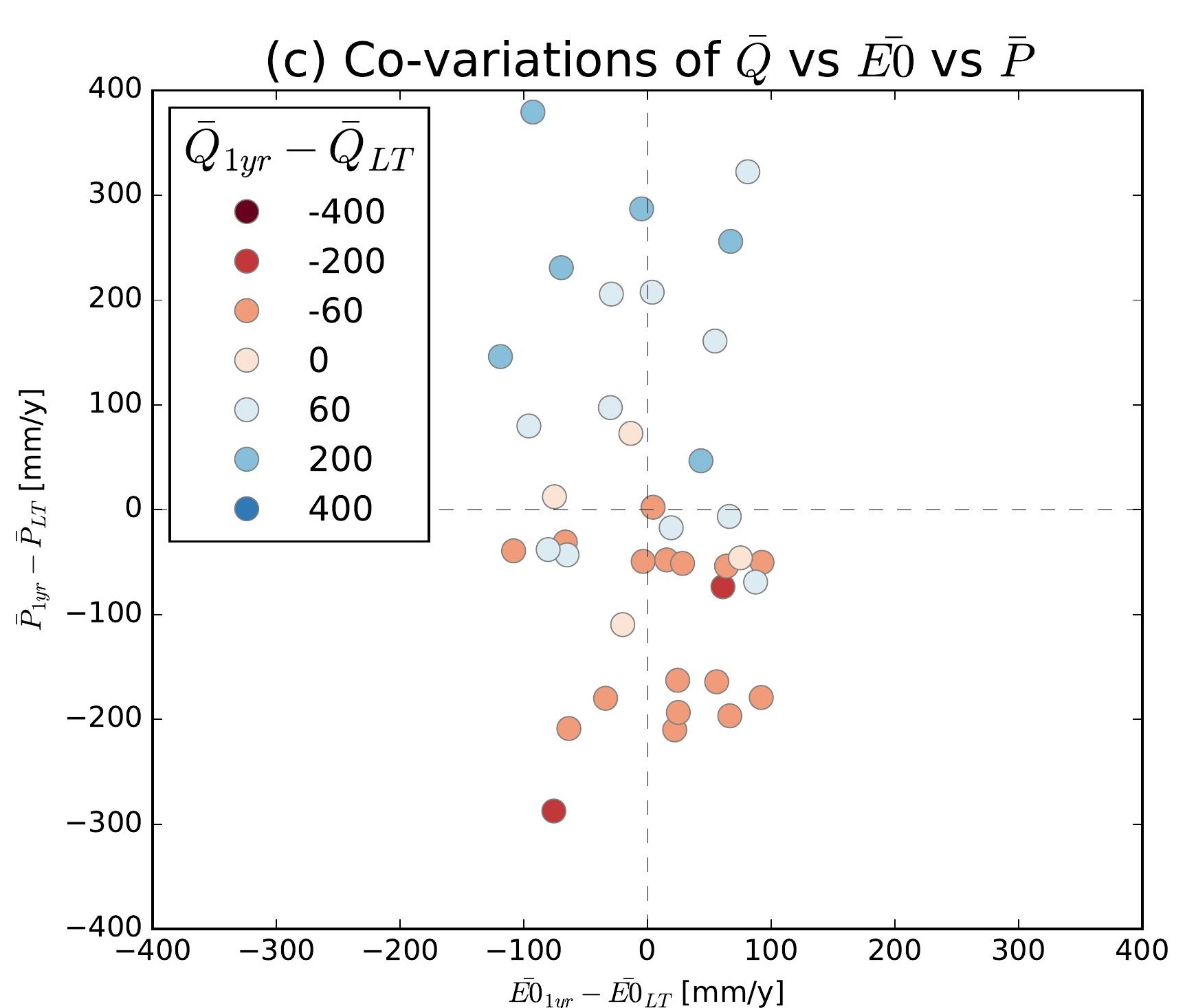
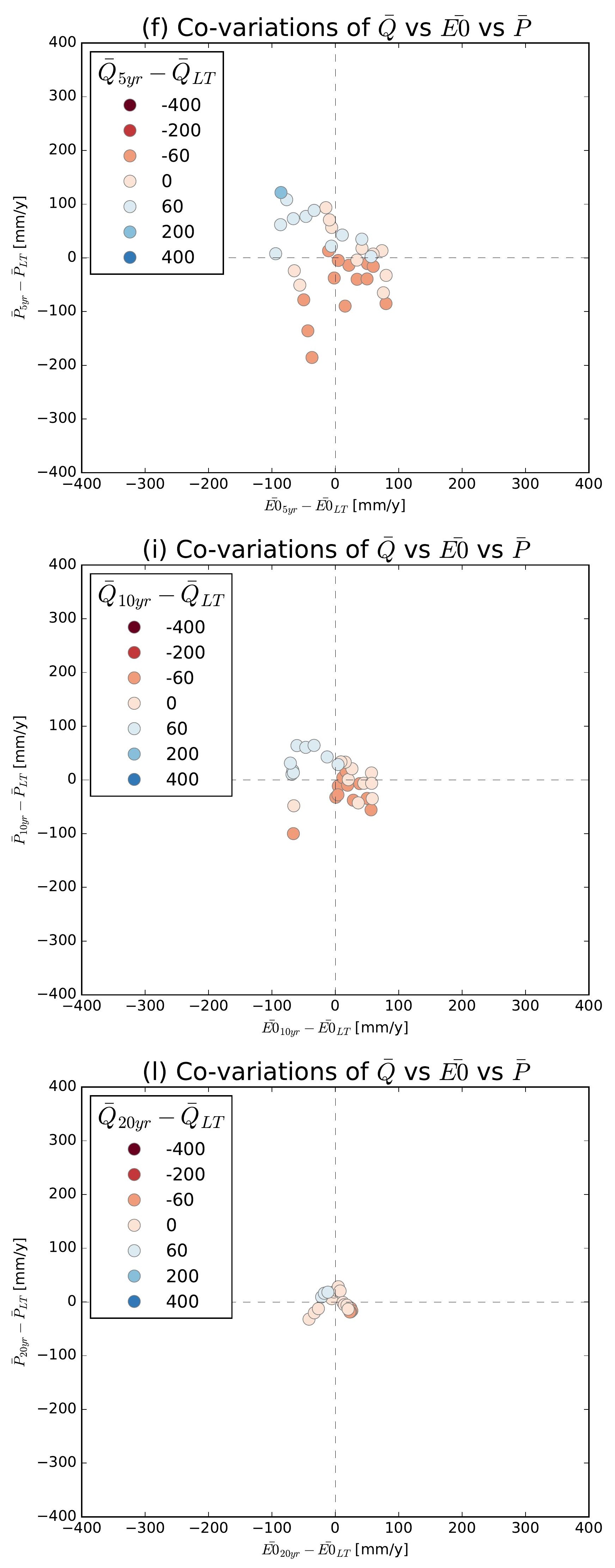

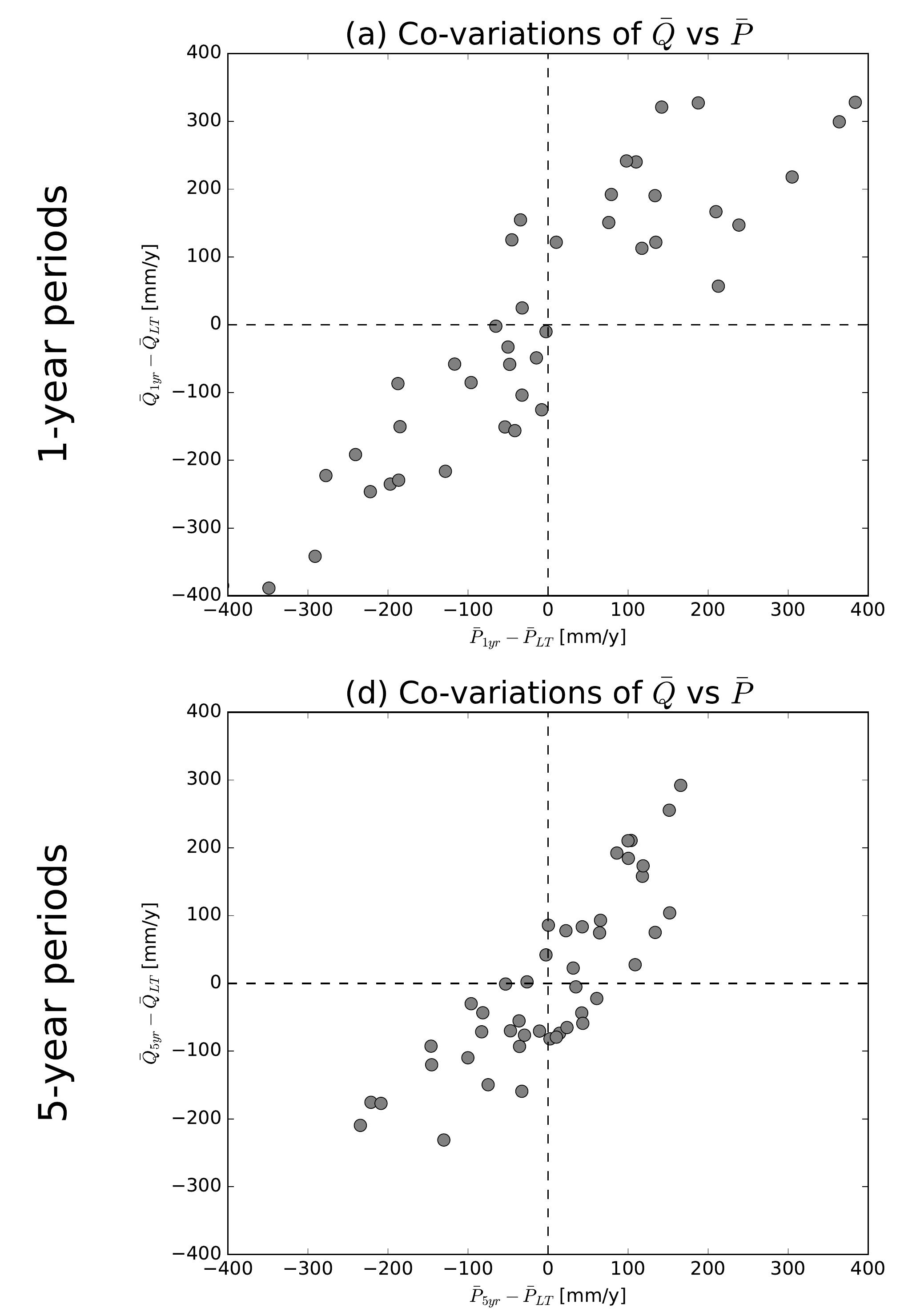

(g) Co-variations of $\bar{Q}$ vs $\bar{P}$
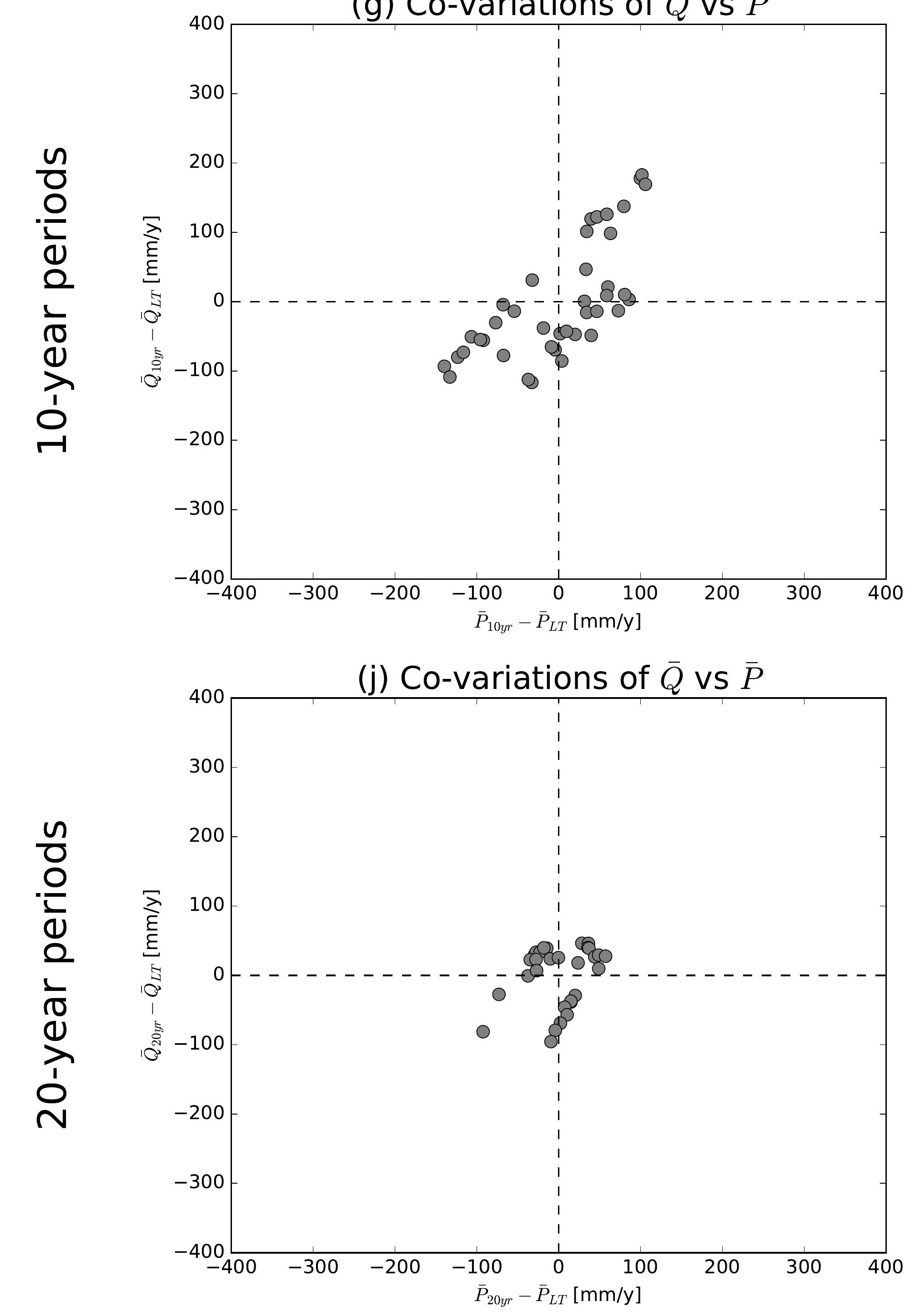

(b) Co-variations of $\bar{Q}$ vs $\overline{E 0}$

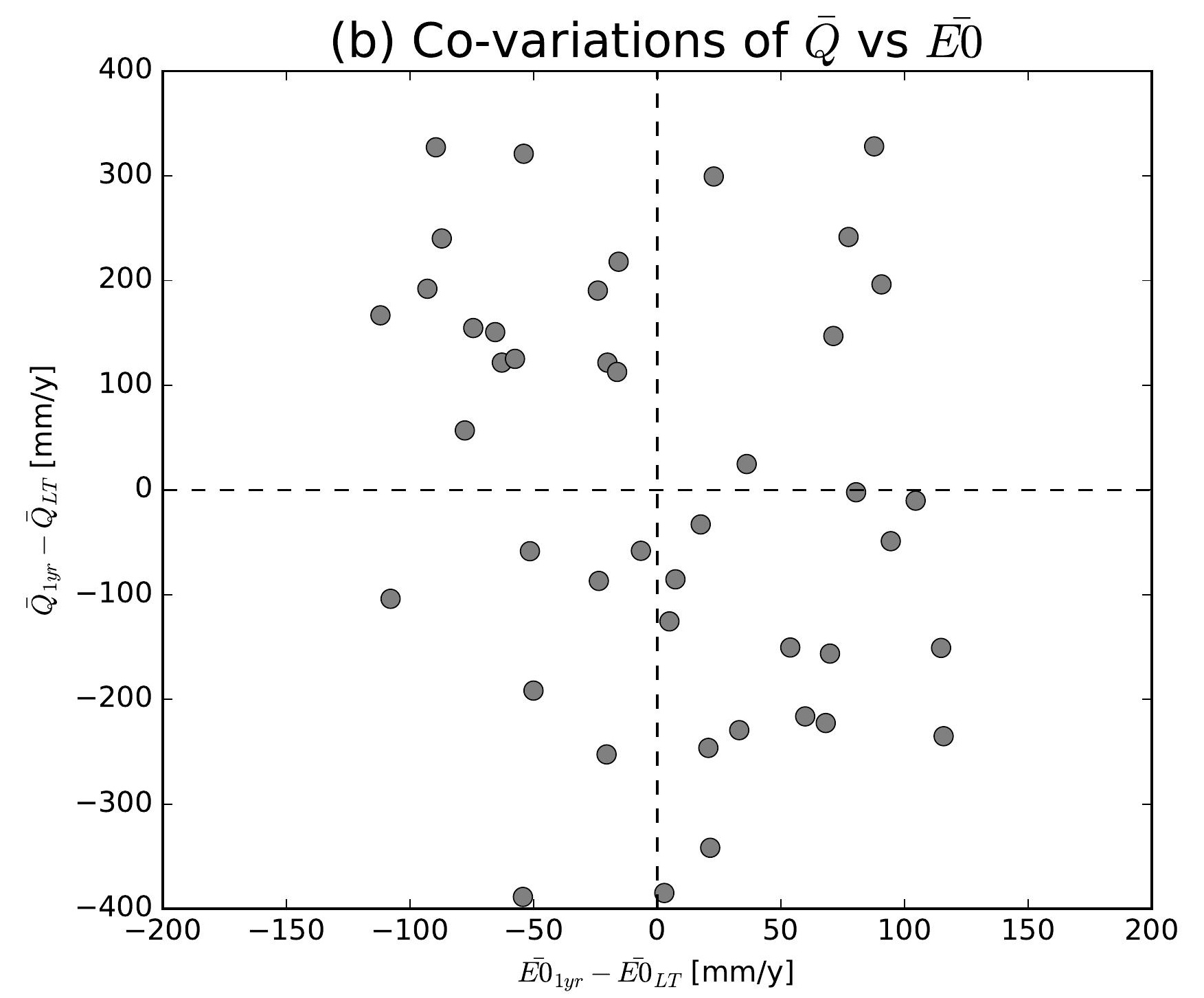

(e) Co-variations of $\bar{Q}$ vs $\overline{E 0}$

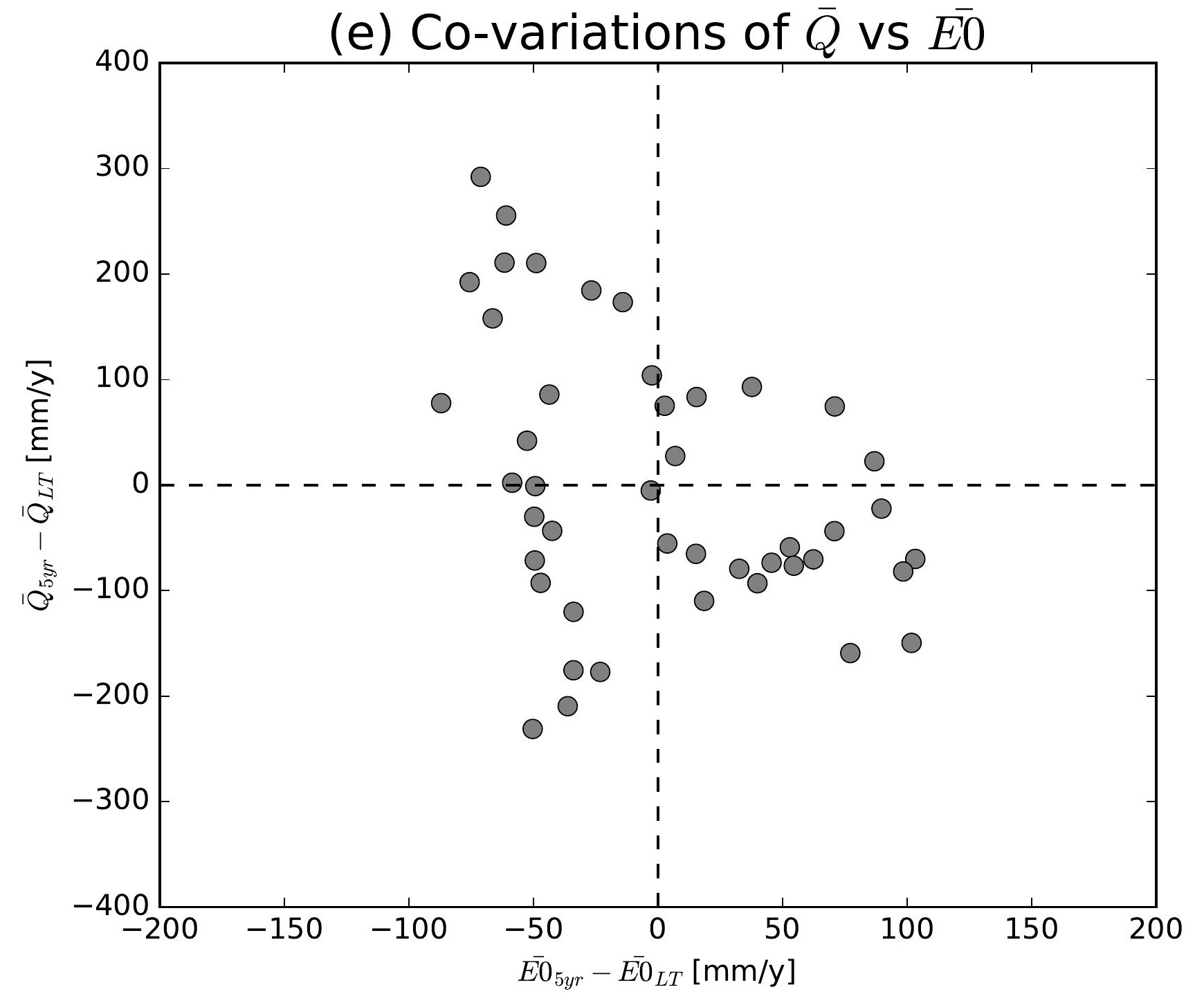

(h) Co-variations of $\bar{Q}$ vs $\overline{E 0}$

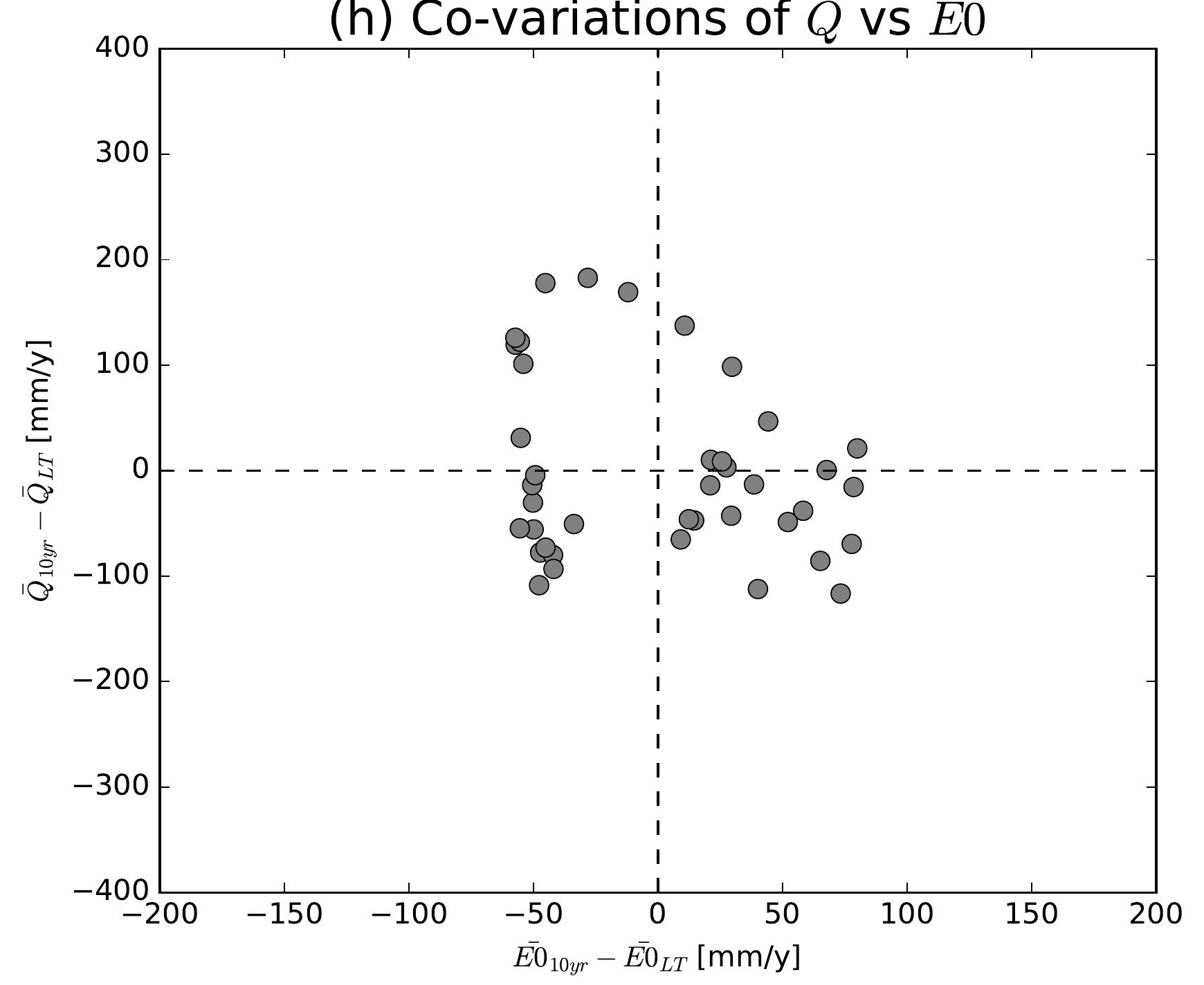

(k) Co-variations of $\bar{Q}$ vs $\overline{E 0}$

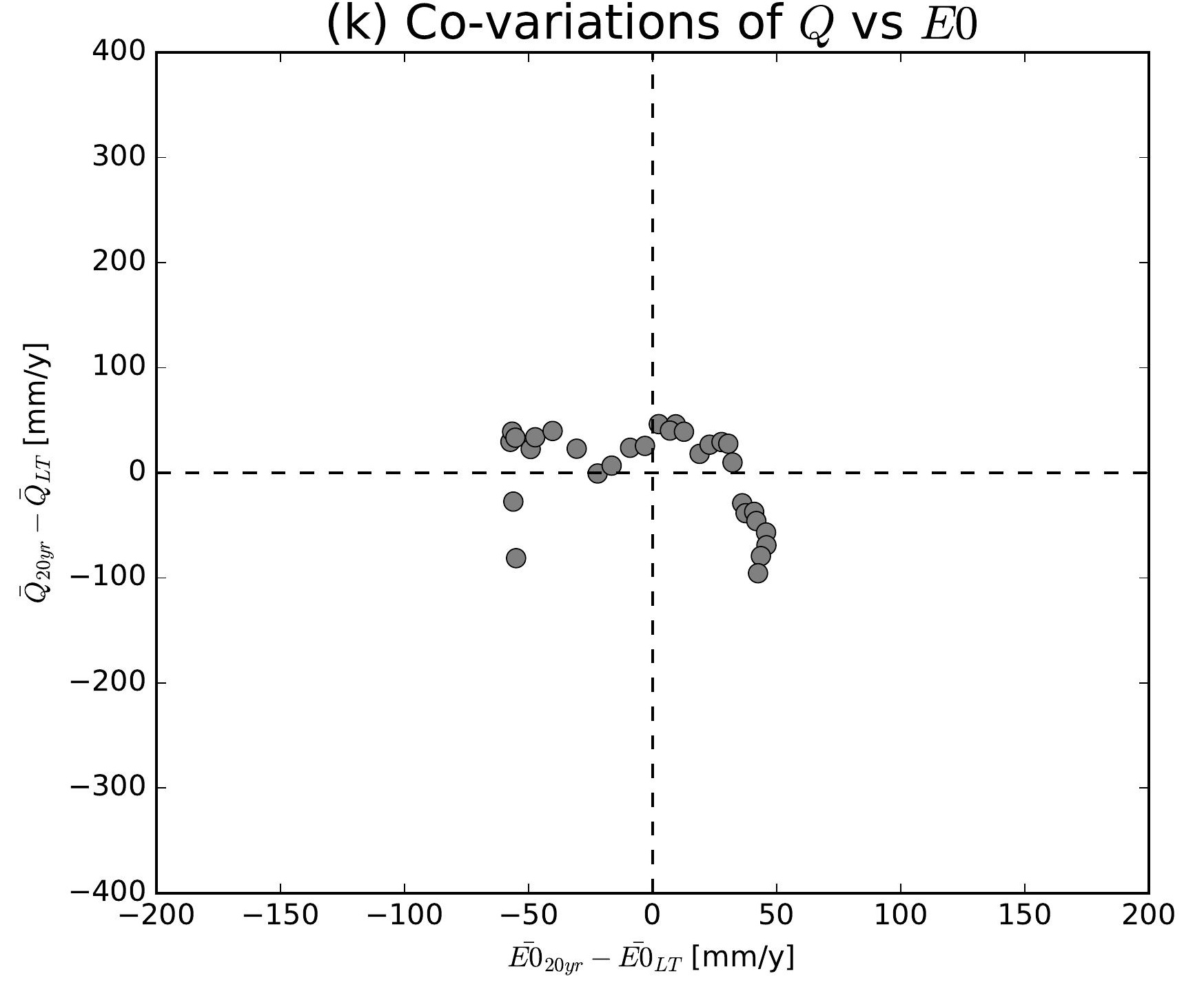

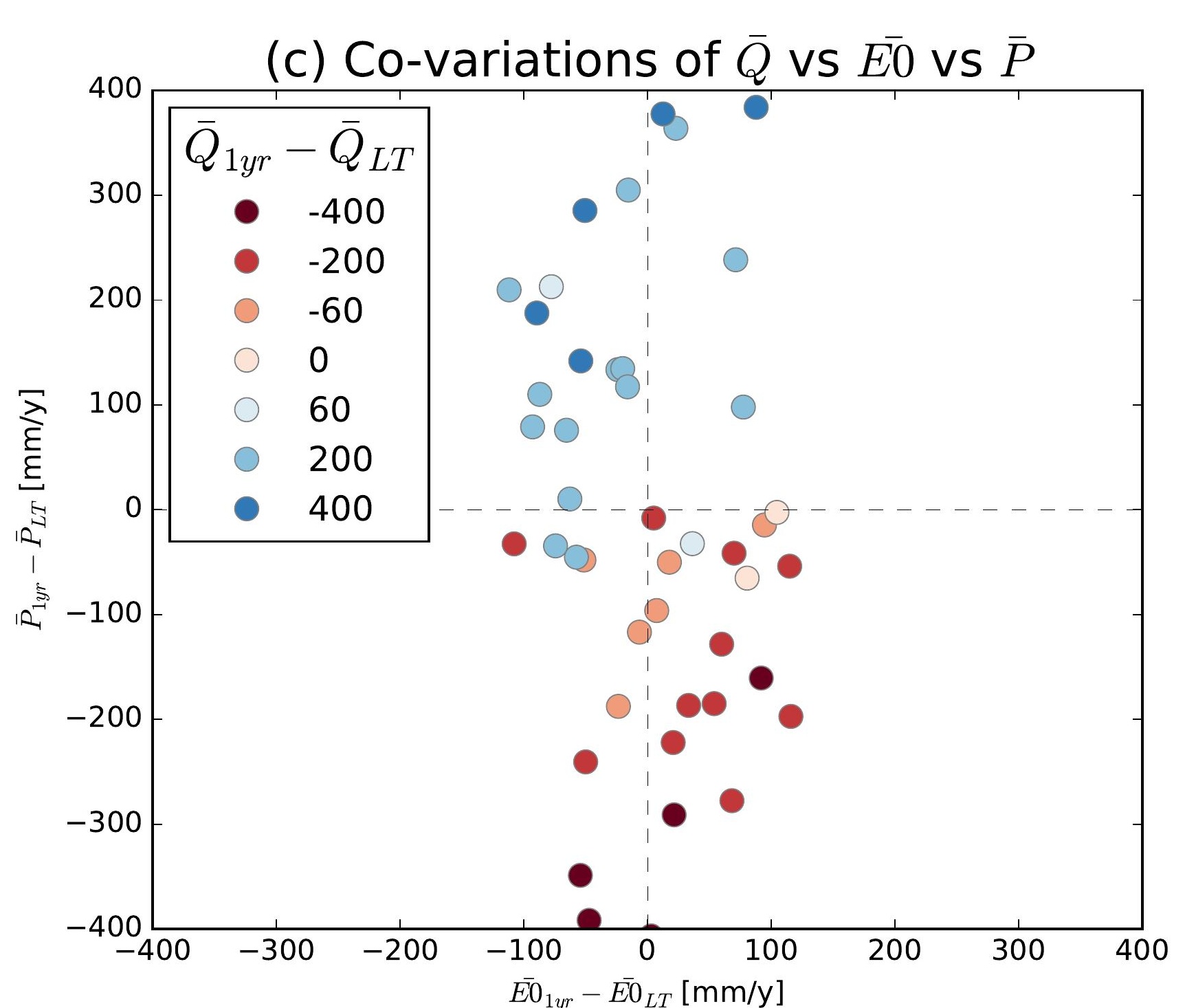
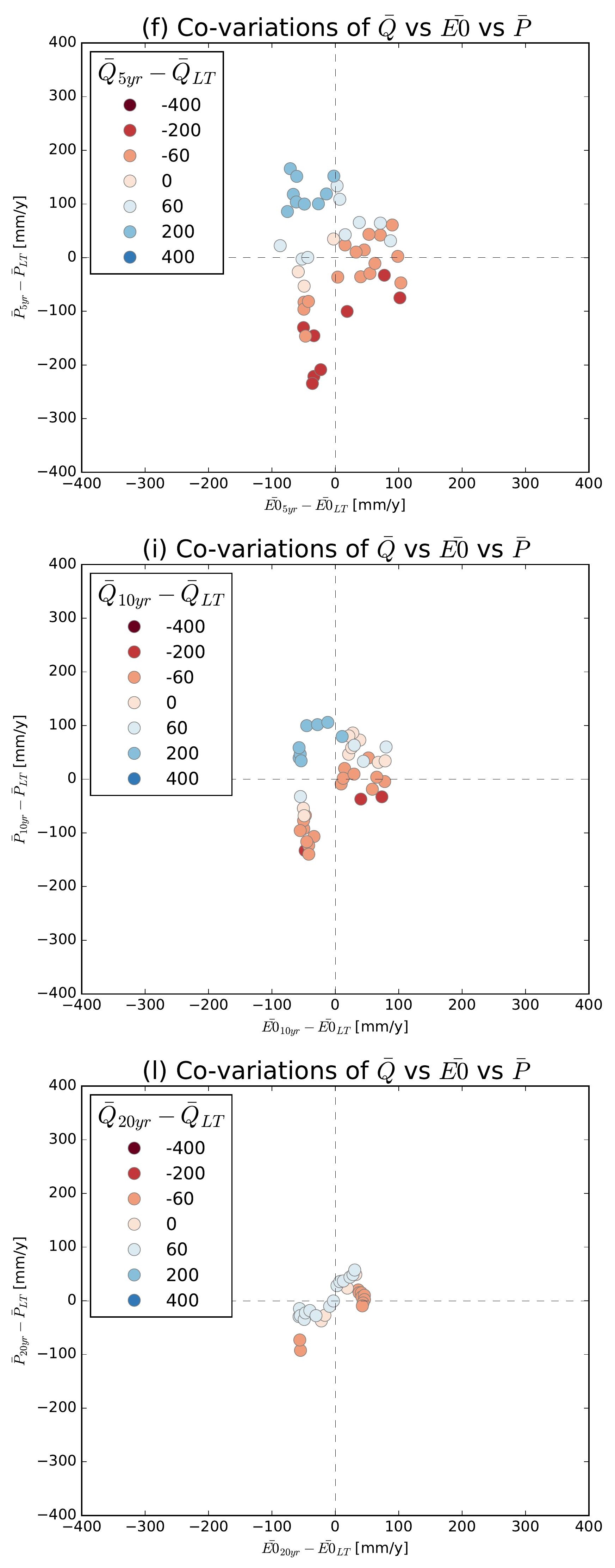

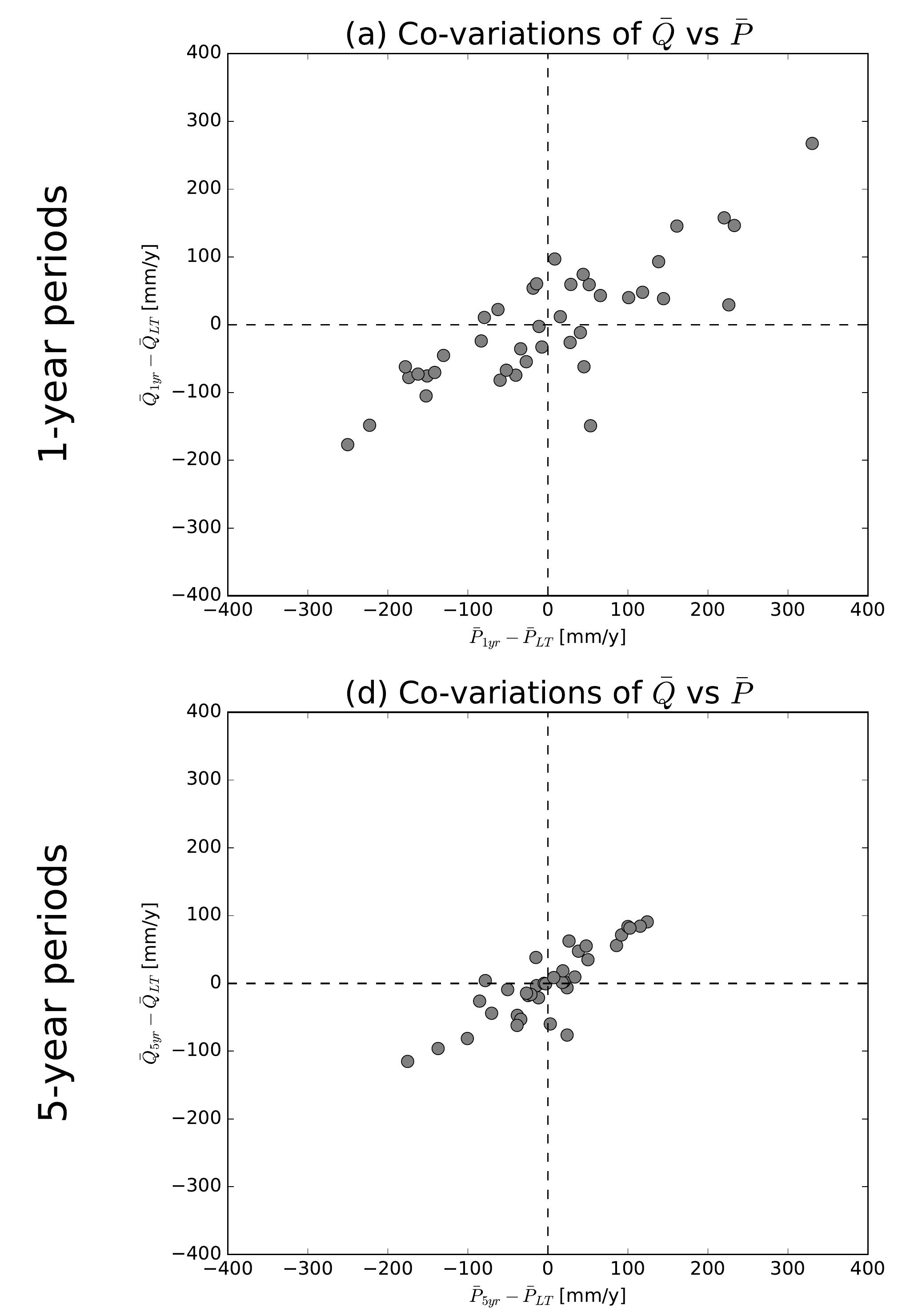

(g) Co-variations of $\bar{Q}$ vs $\bar{P}$
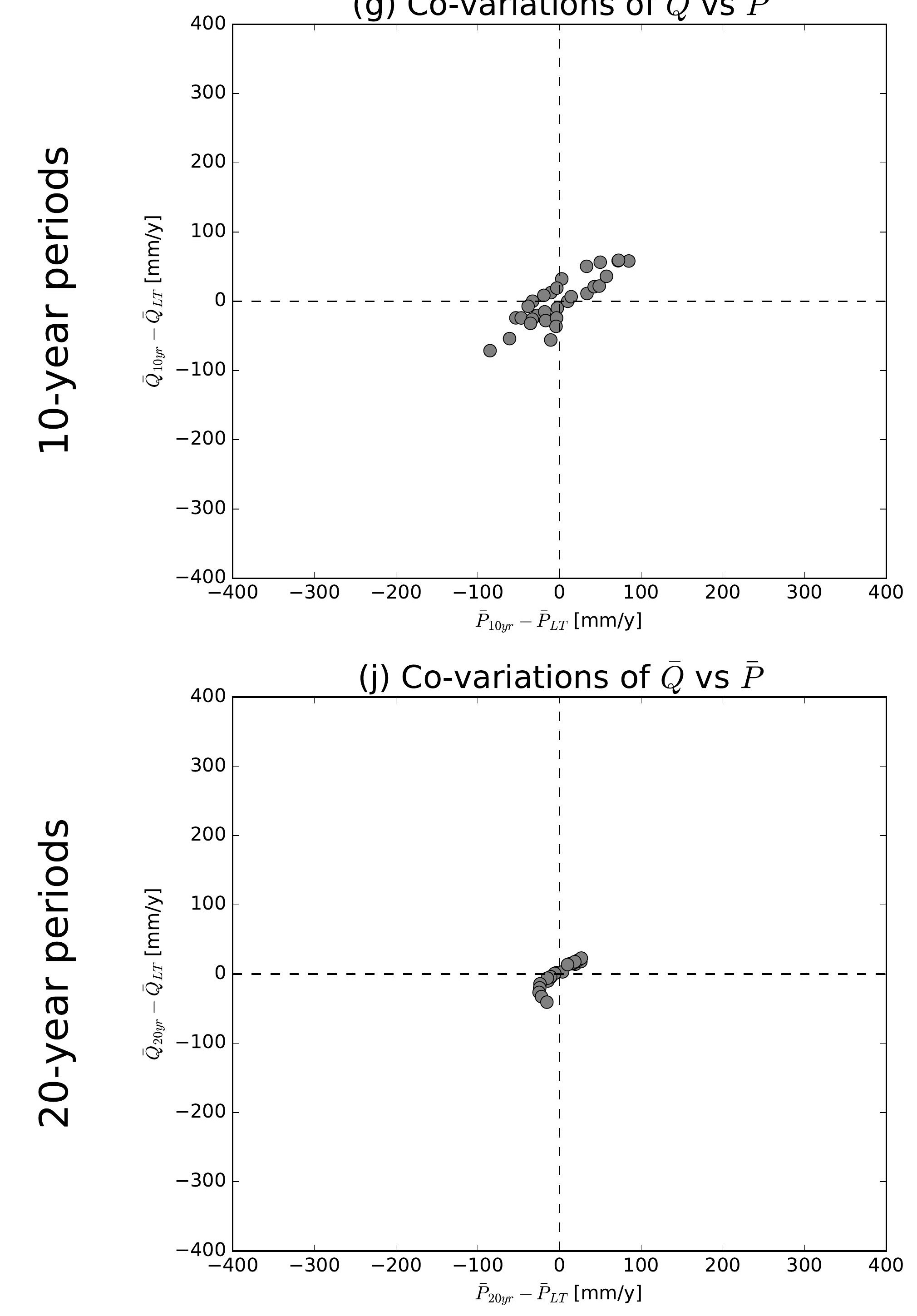

(b) Co-variations of $\bar{Q}$ vs $\overline{E 0}$

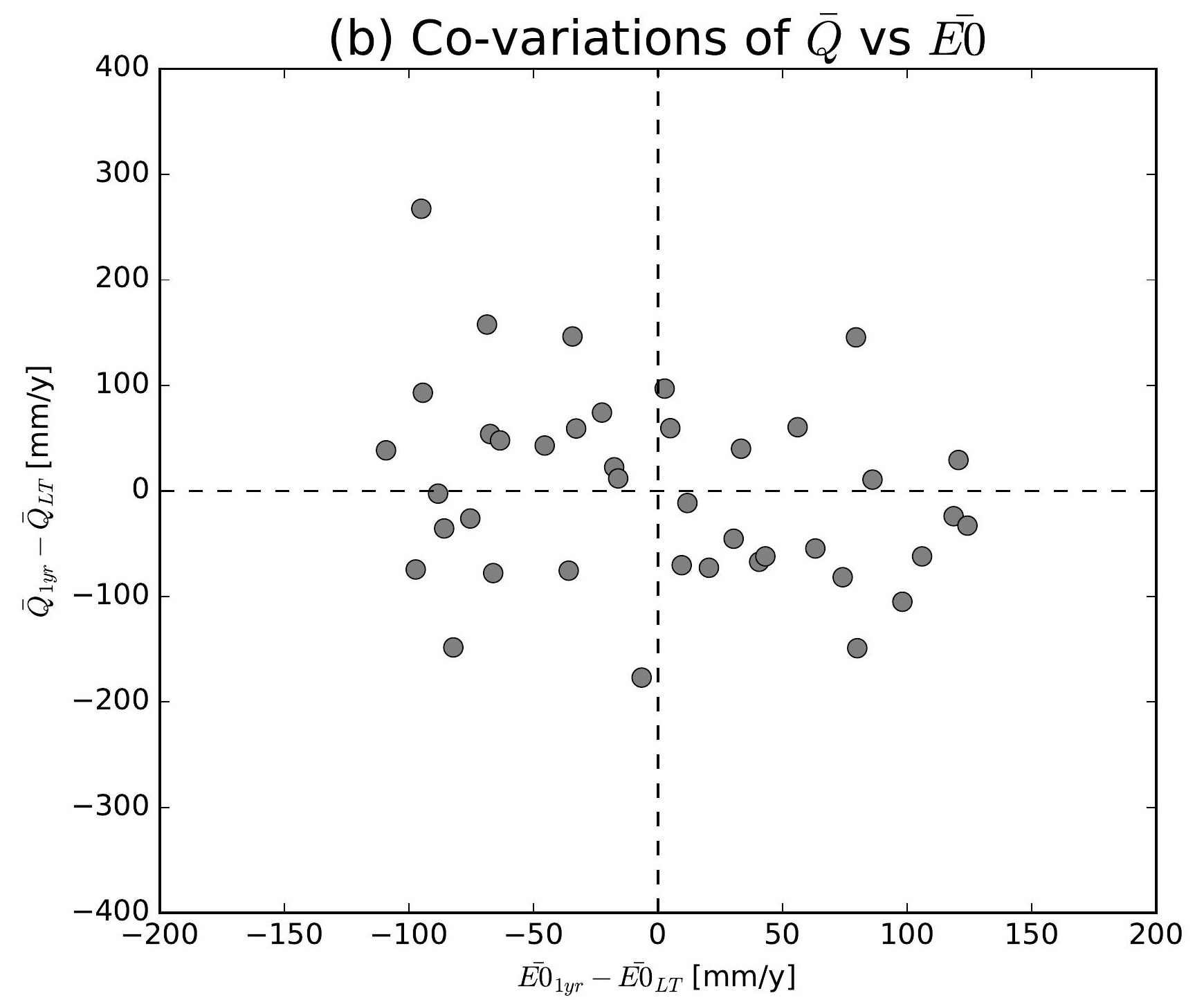

(e) Co-variations of $\bar{Q}$ vs $\overline{E 0}$

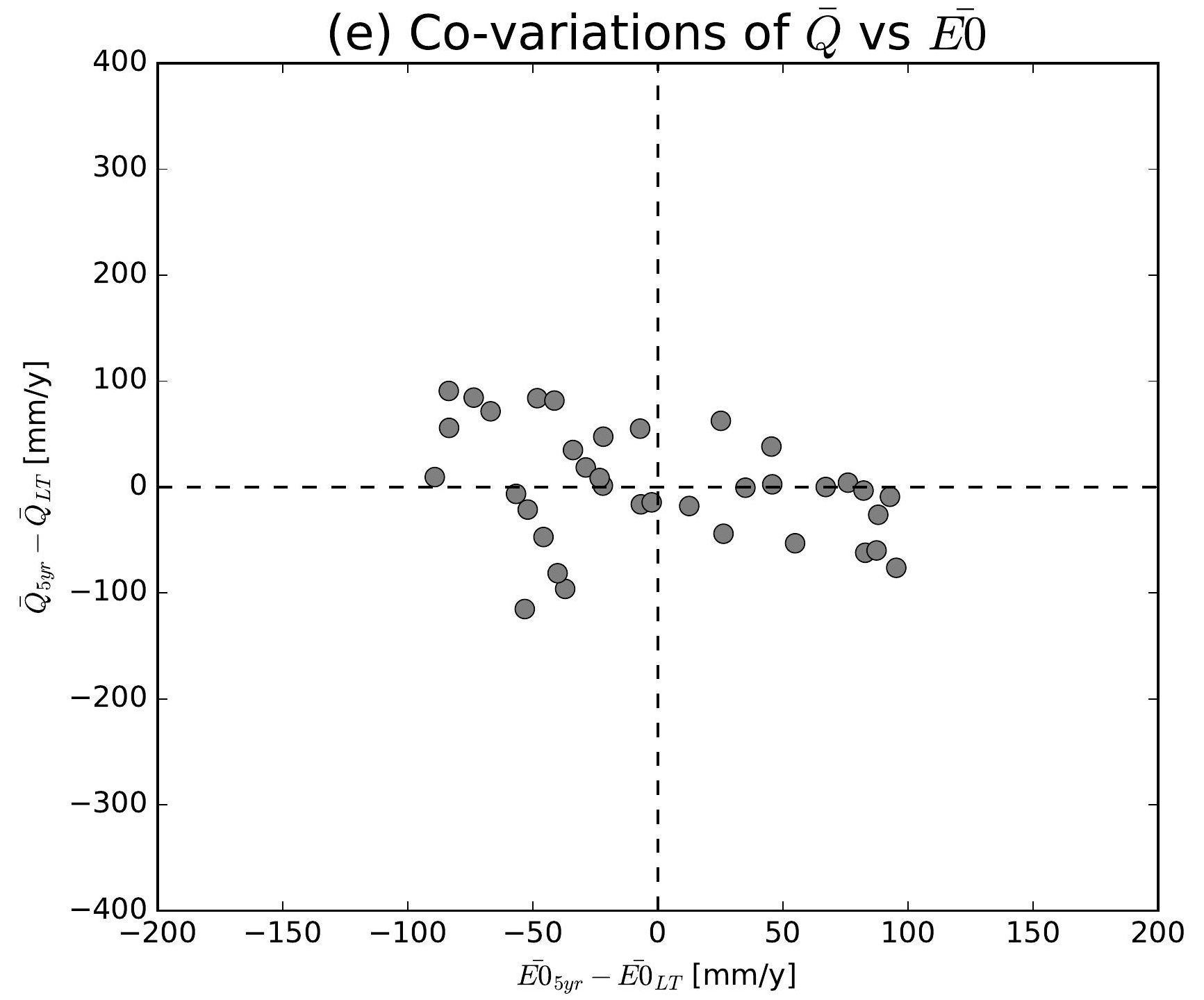

(h) Co-variations of $\bar{Q}$ vs $\overline{E 0}$

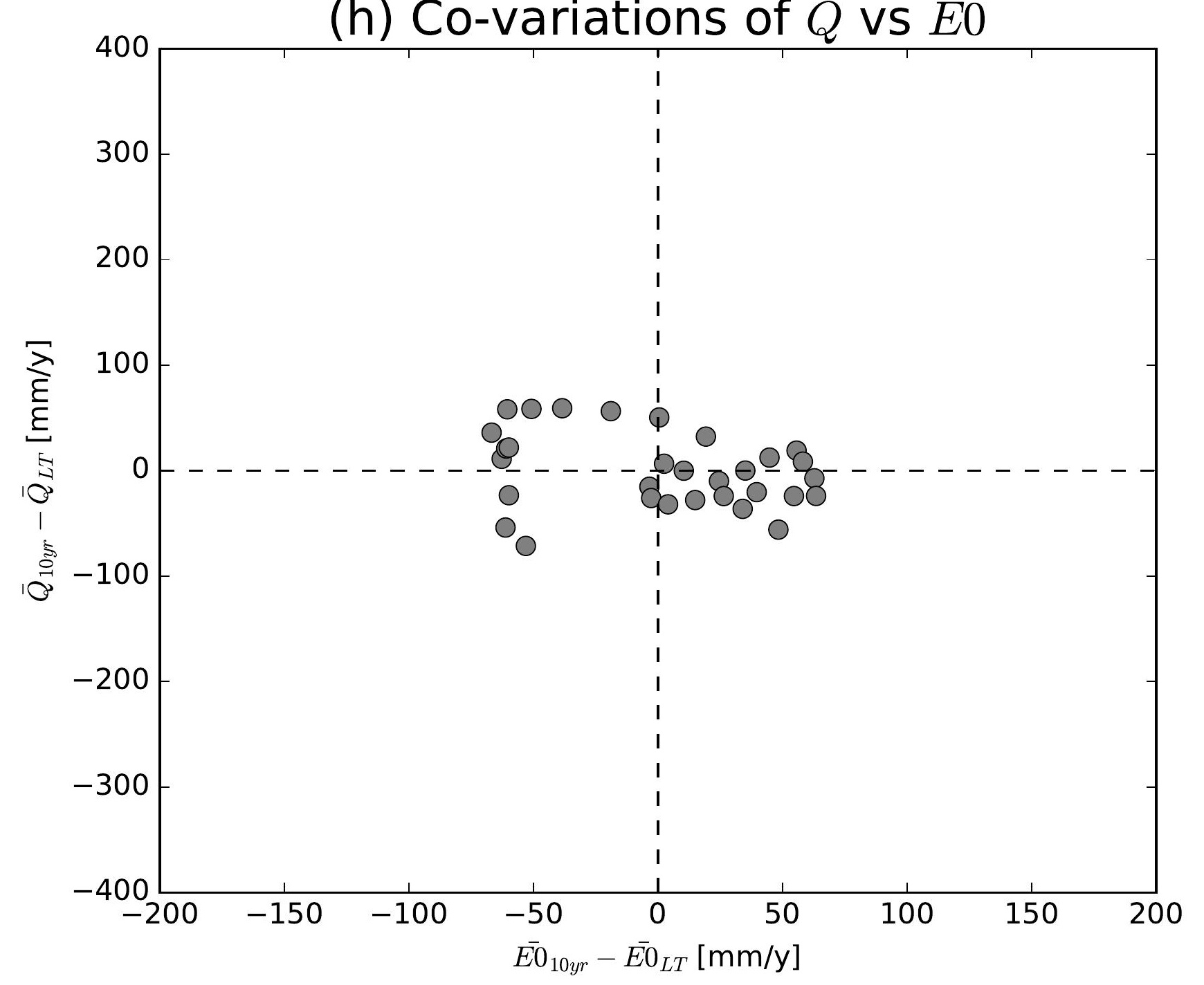

(k) Co-variations of $\bar{Q}$ vs $\overline{E 0}$

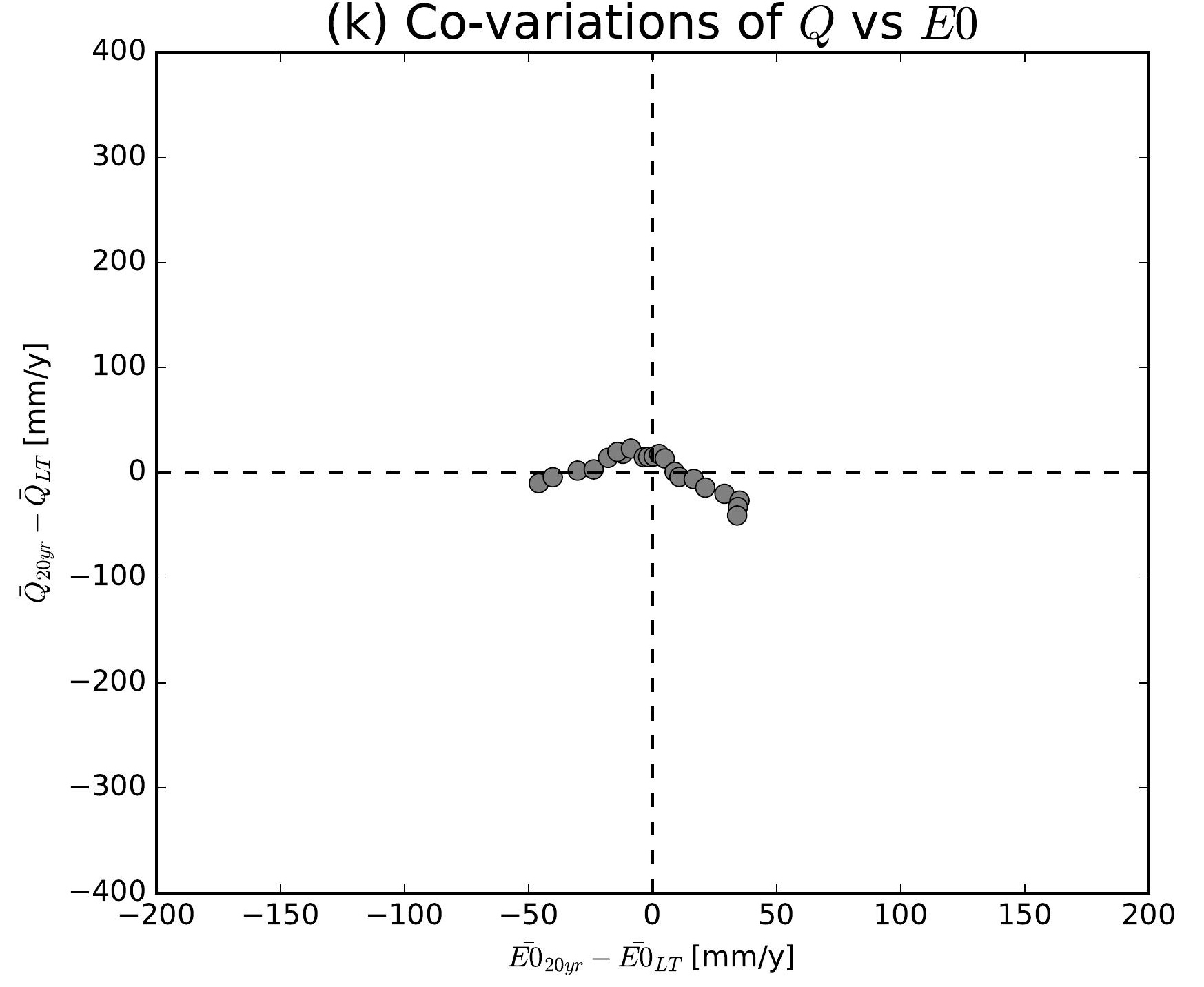

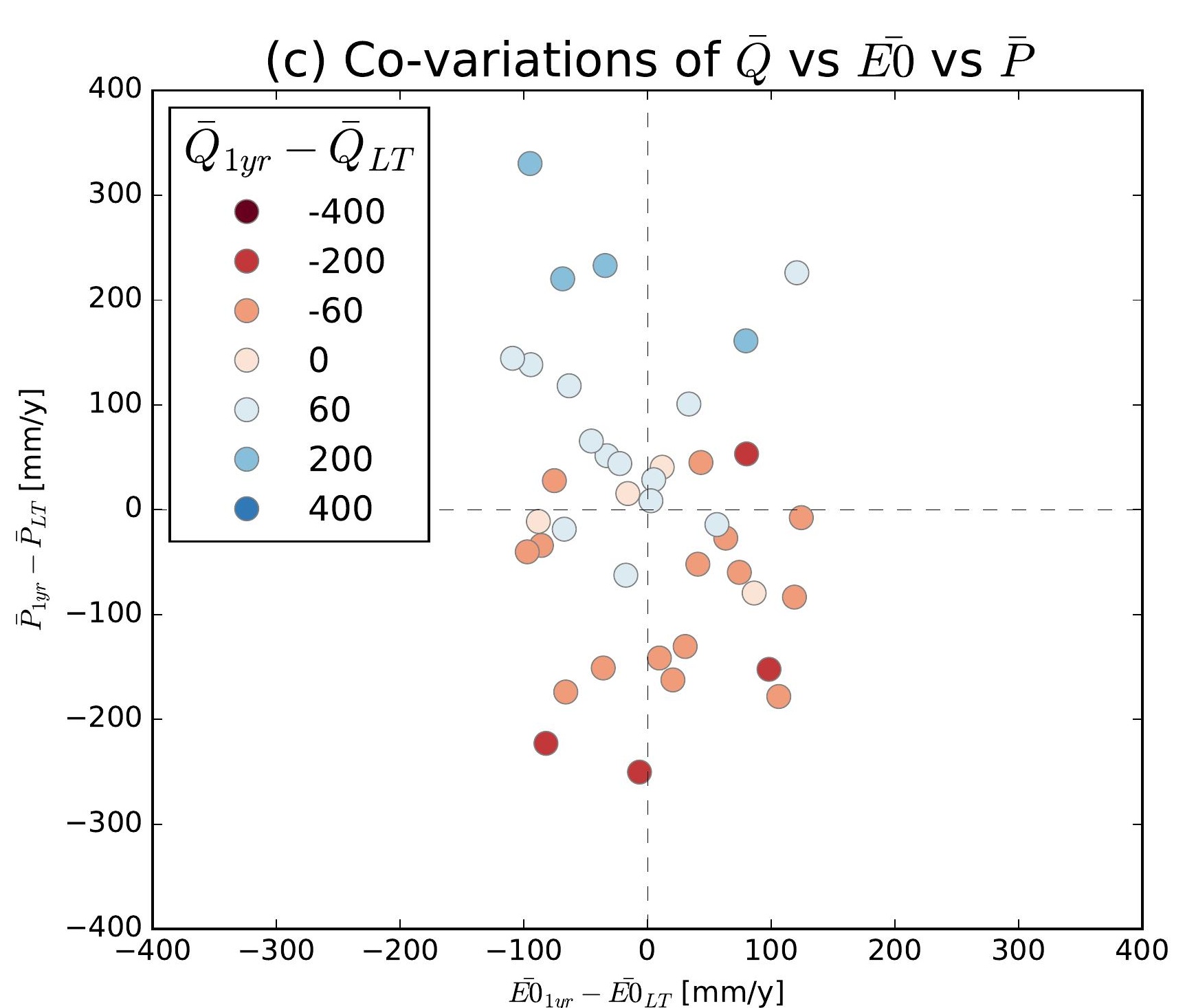
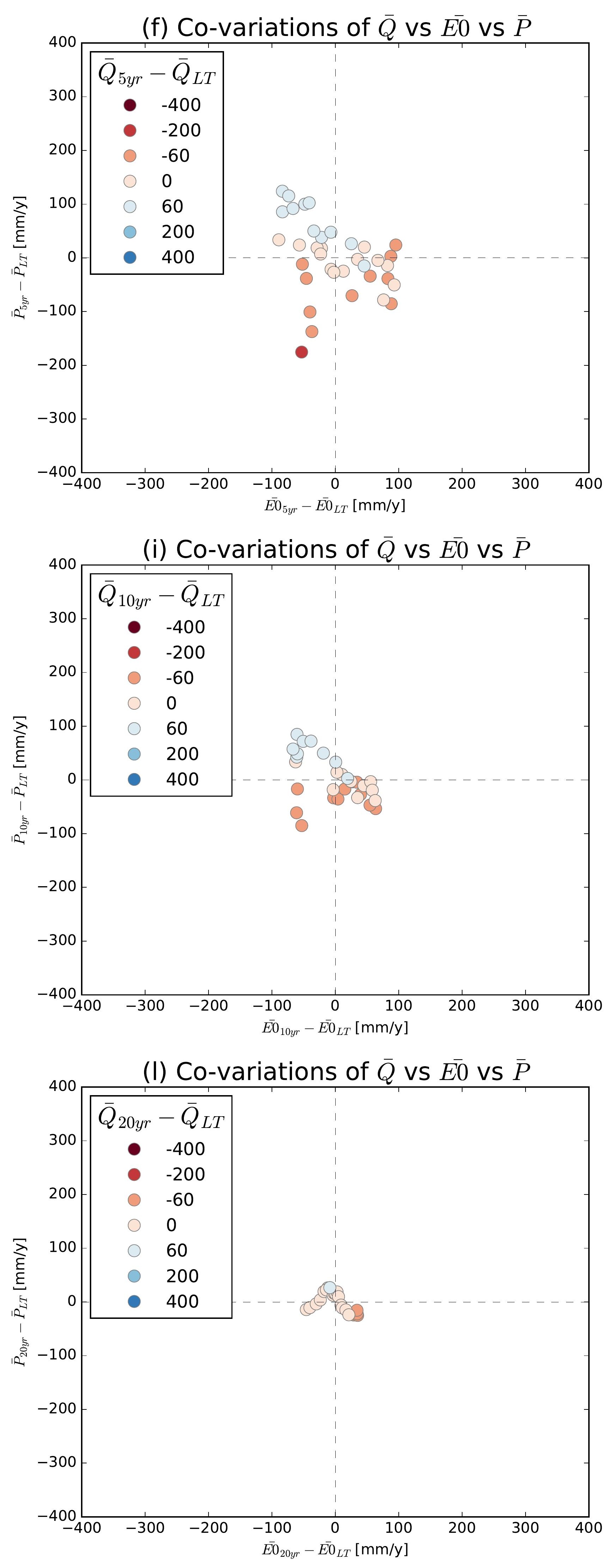

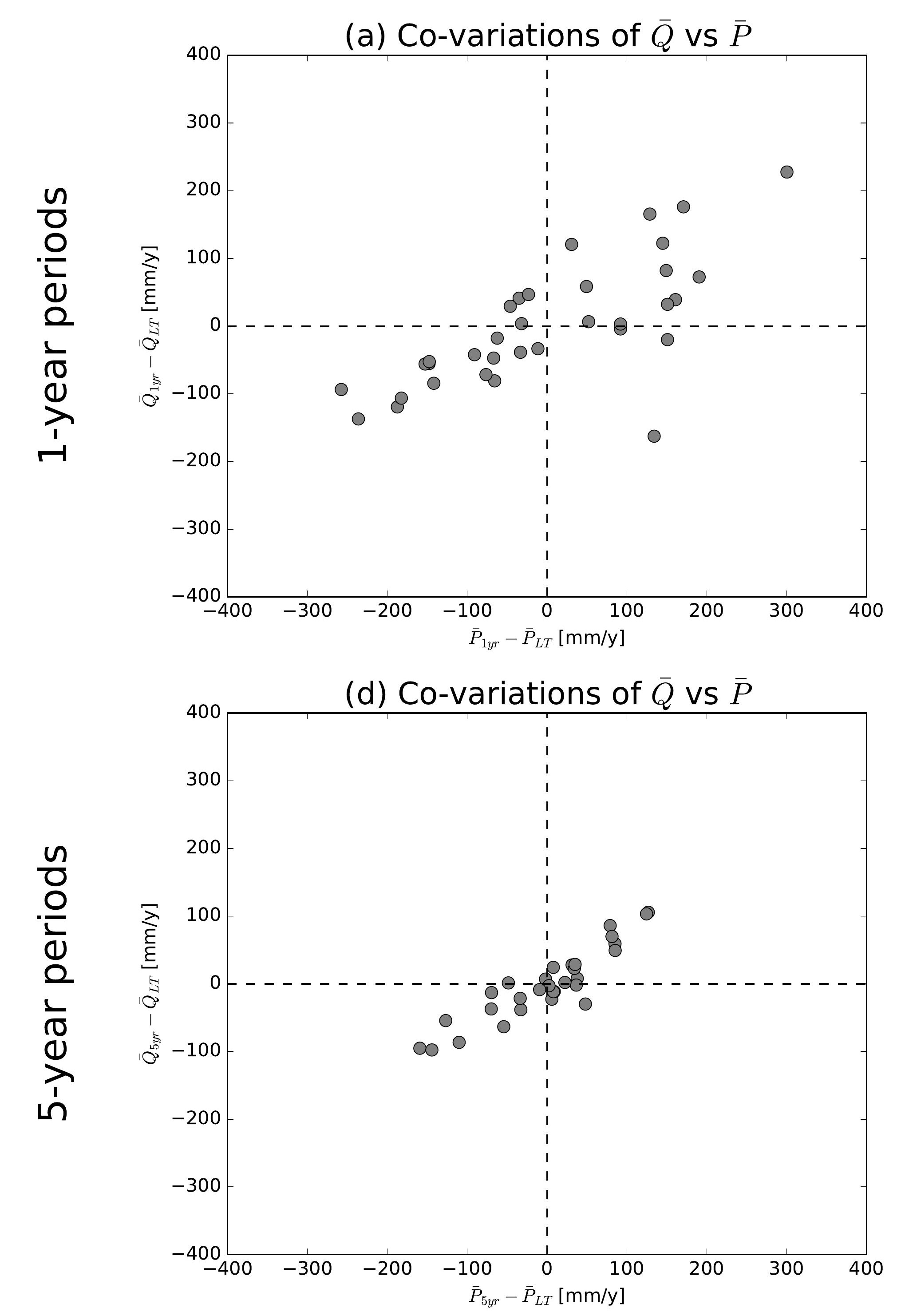

(g) Co-variations of $\bar{Q}$ vs $\bar{P}$

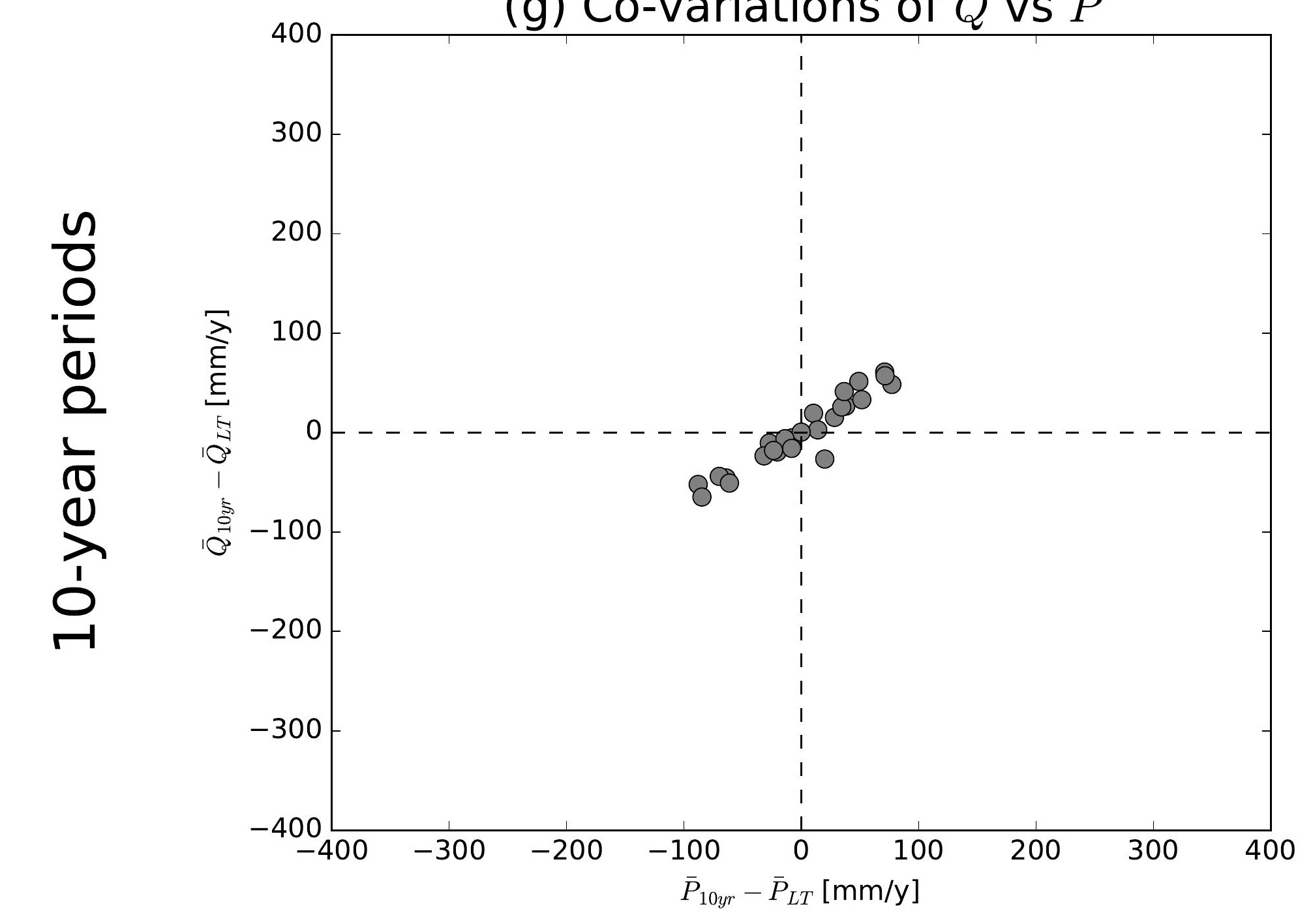

(j) Co-variations of $\bar{Q}$ vs $\bar{P}$

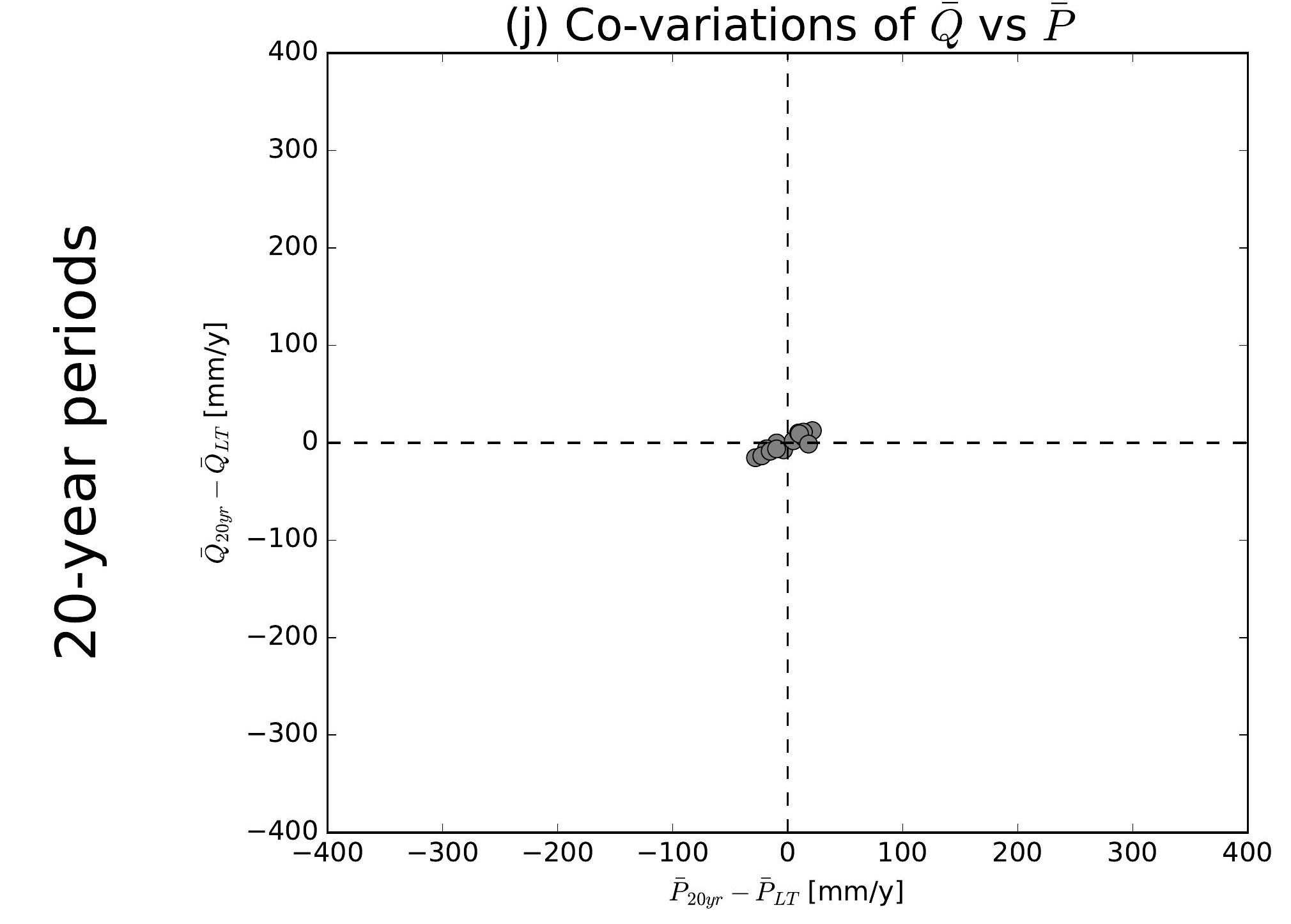

(b) Co-variations of $\bar{Q}$ vs $\overline{E 0}$

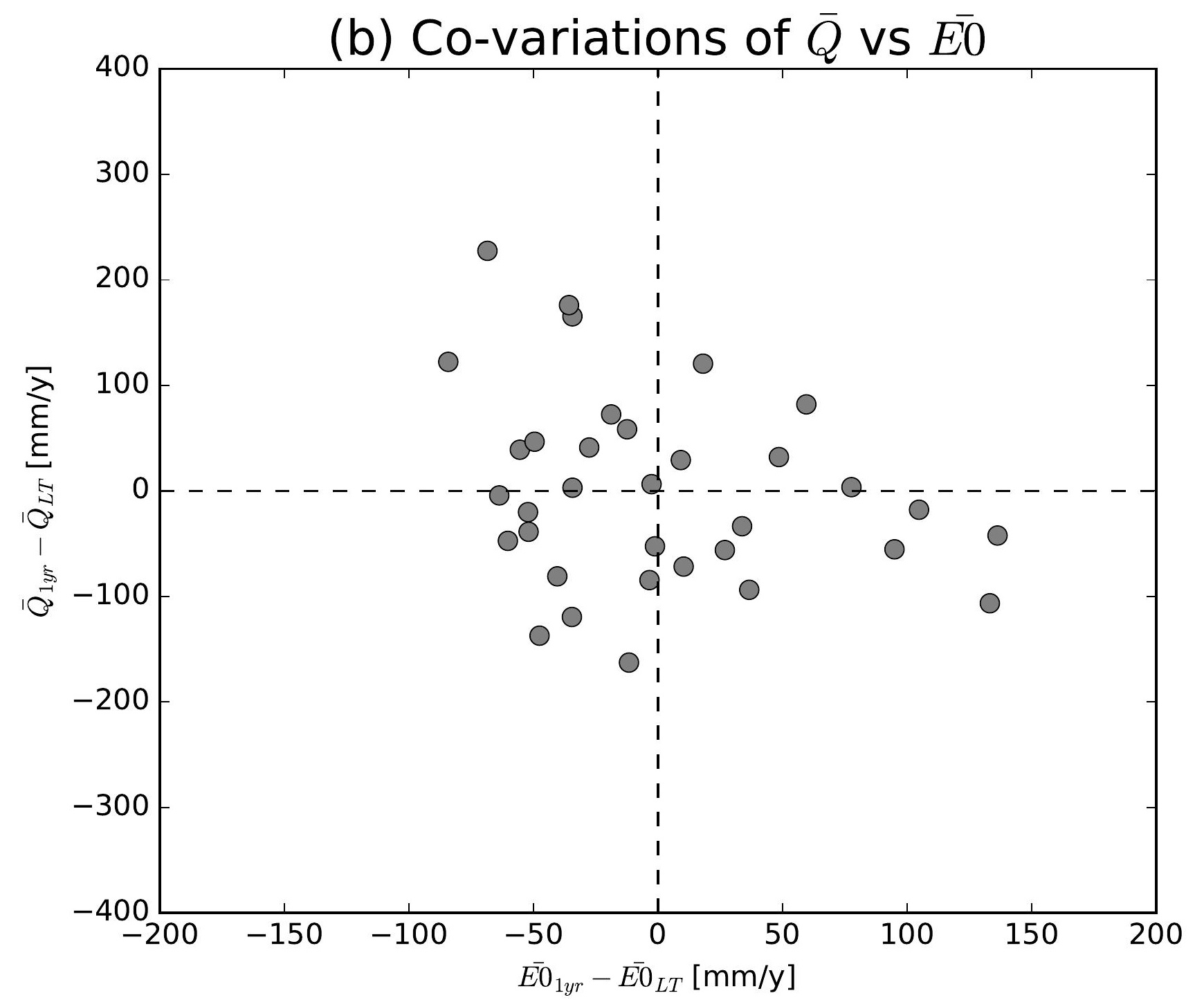

(e) Co-variations of $\bar{Q}$ vs $\overline{E 0}$

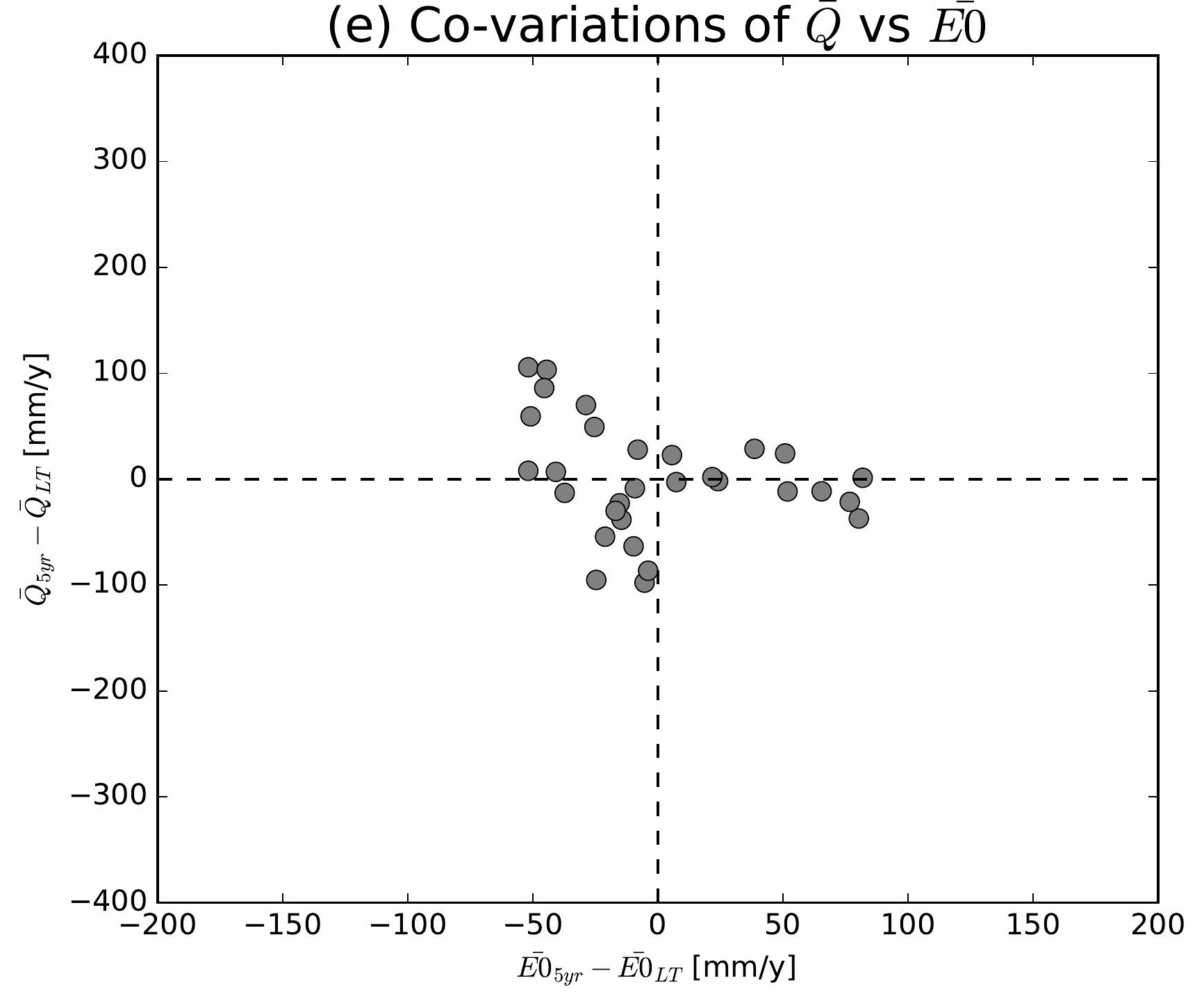

(h) Co-variations of $\bar{Q}$ vs $\overline{E 0}$

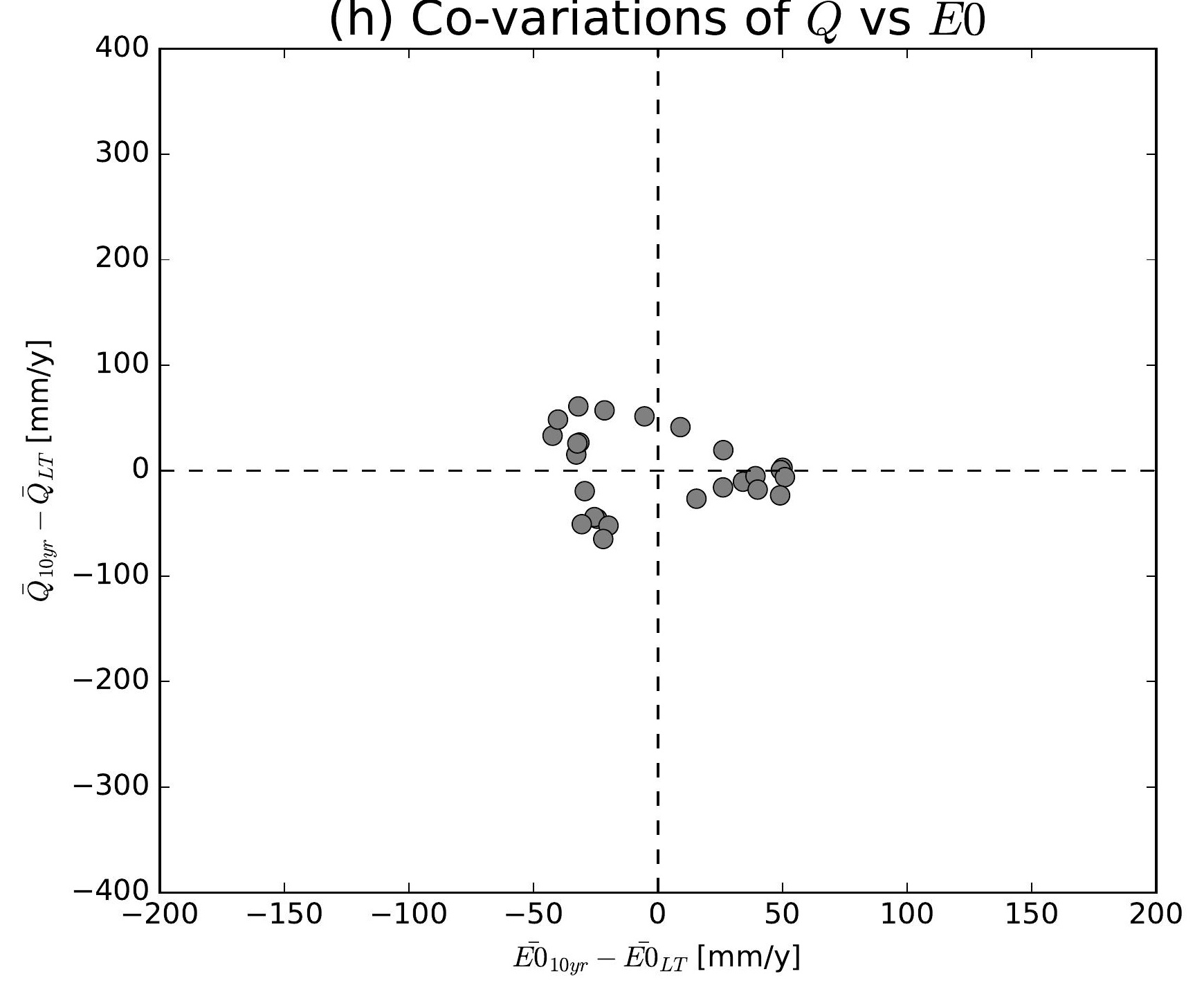

(k) Co-variations of $\bar{Q}$ vs $\overline{E 0}$

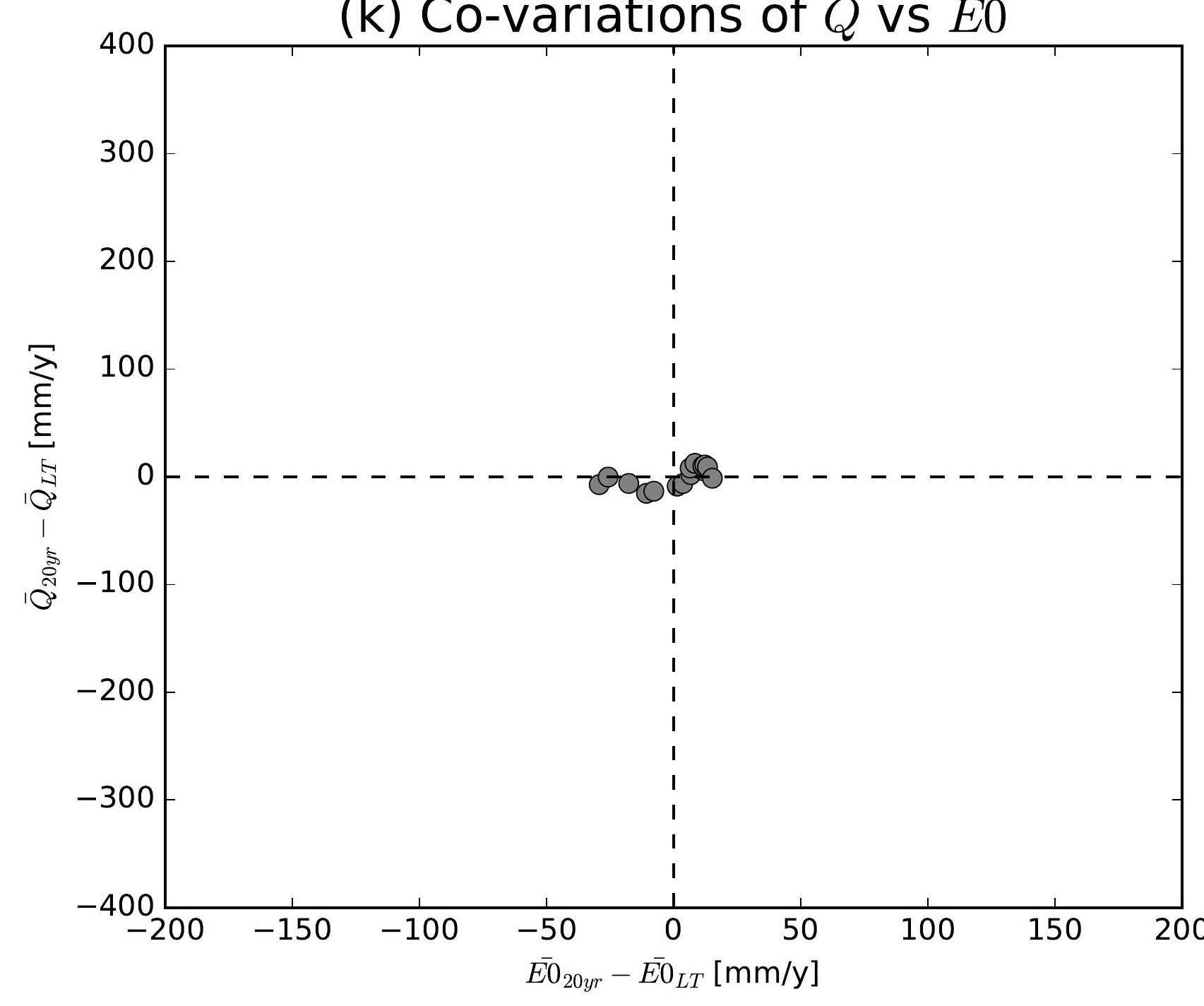

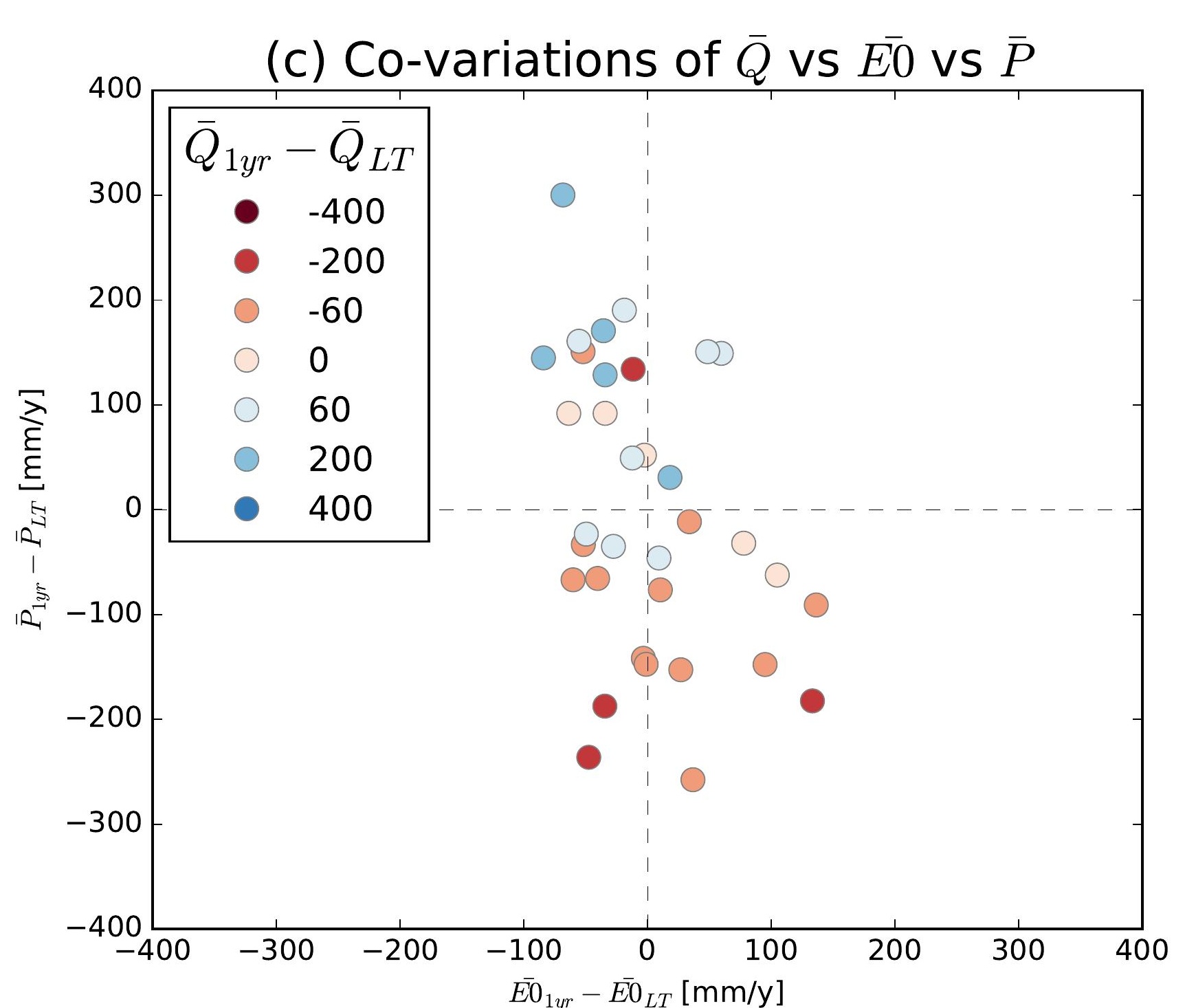
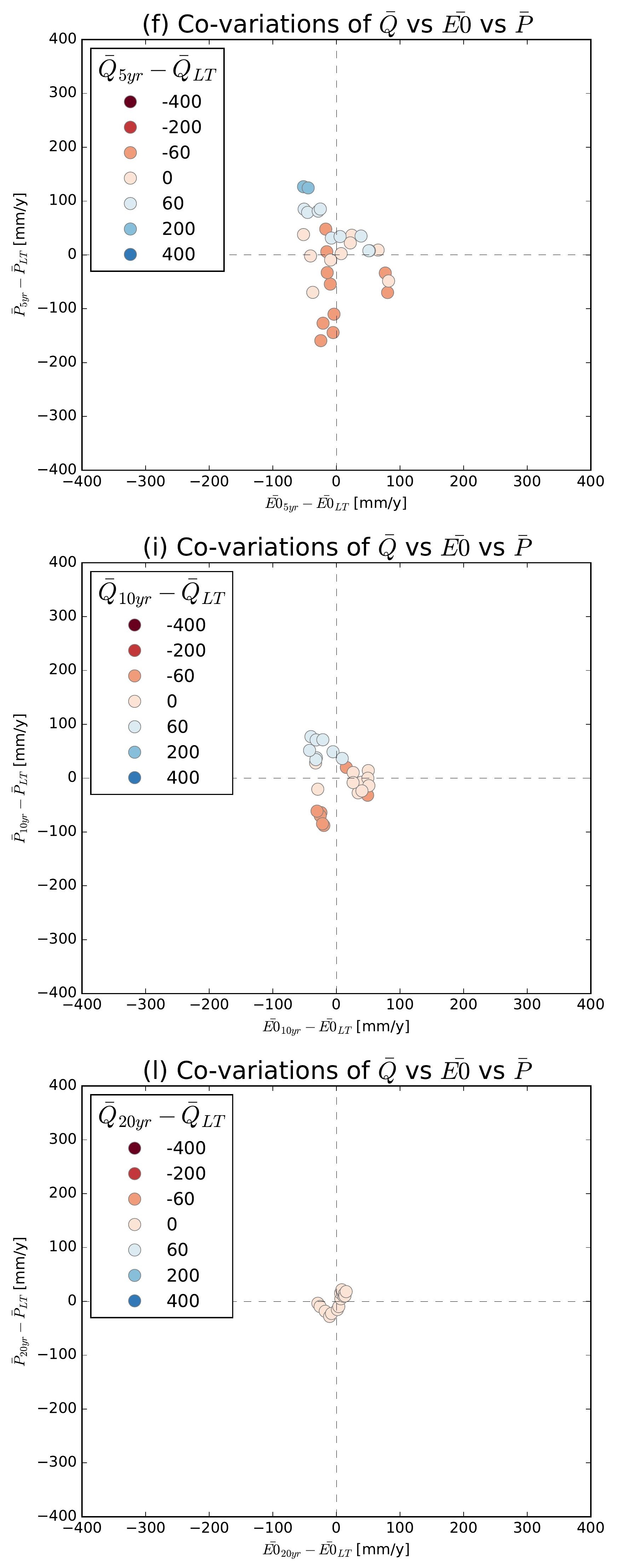

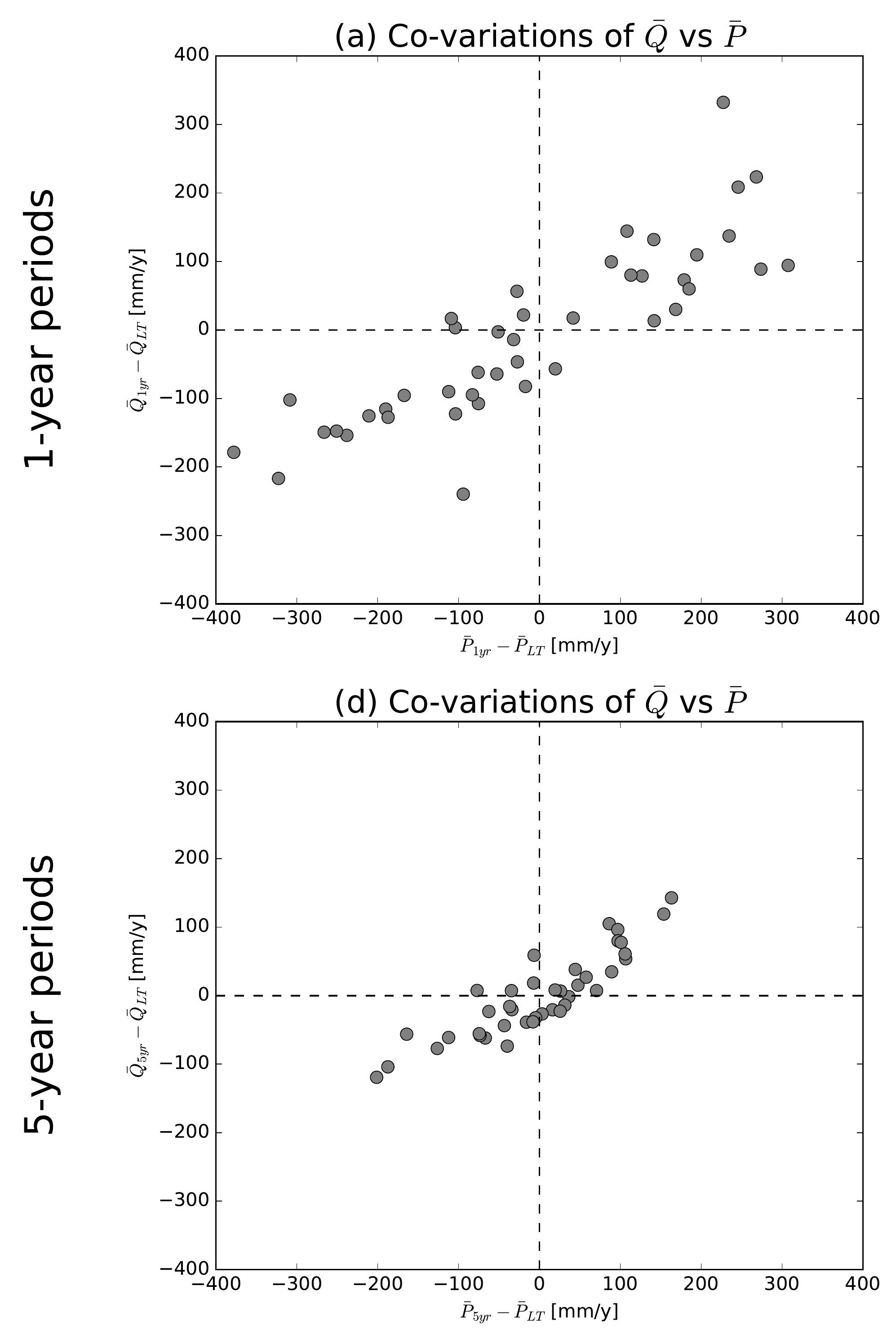

(g) Co-variations of $\bar{Q}$ vs $\bar{P}$

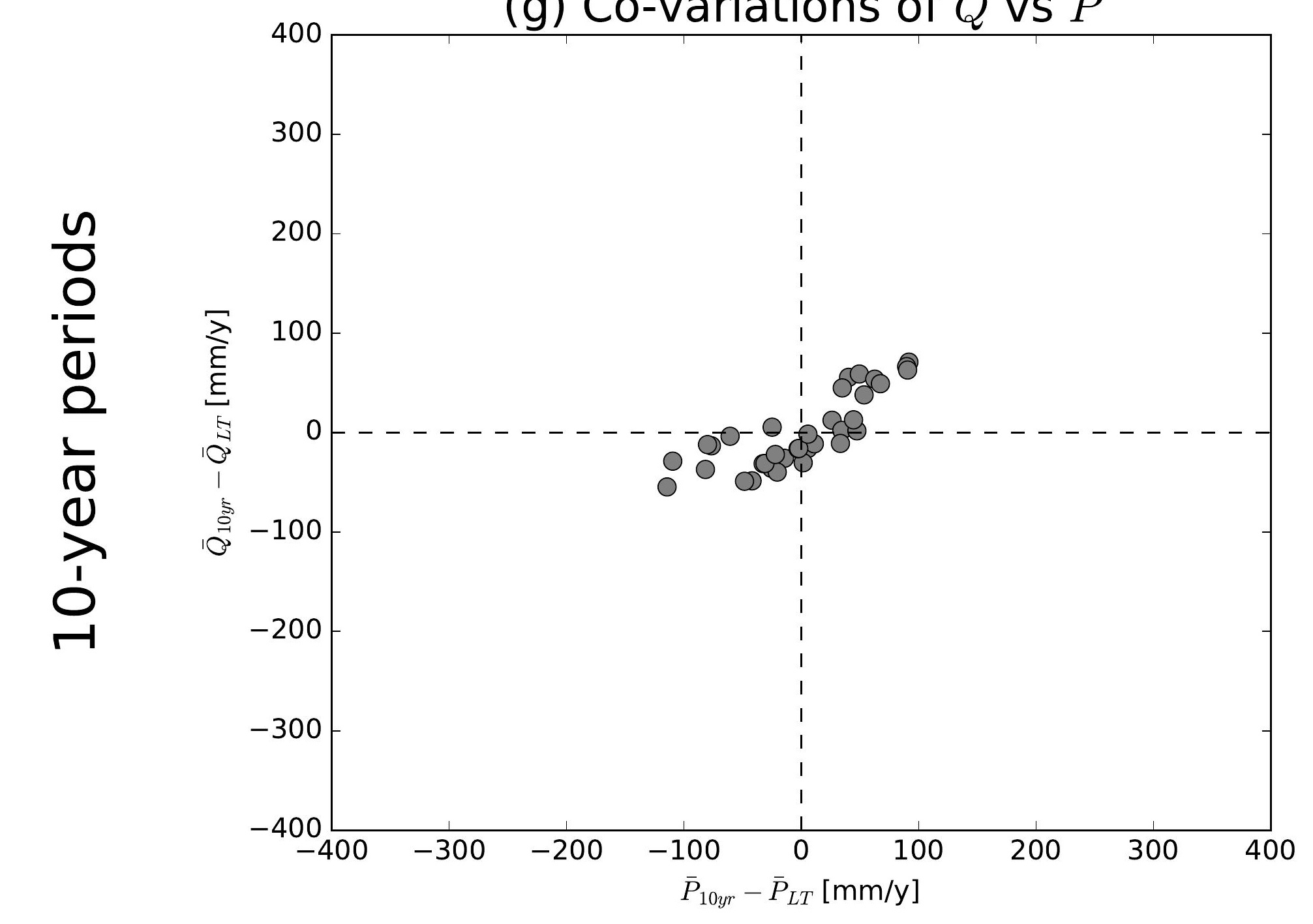

(j) Co-variations of $\bar{Q}$ vs $\bar{P}$

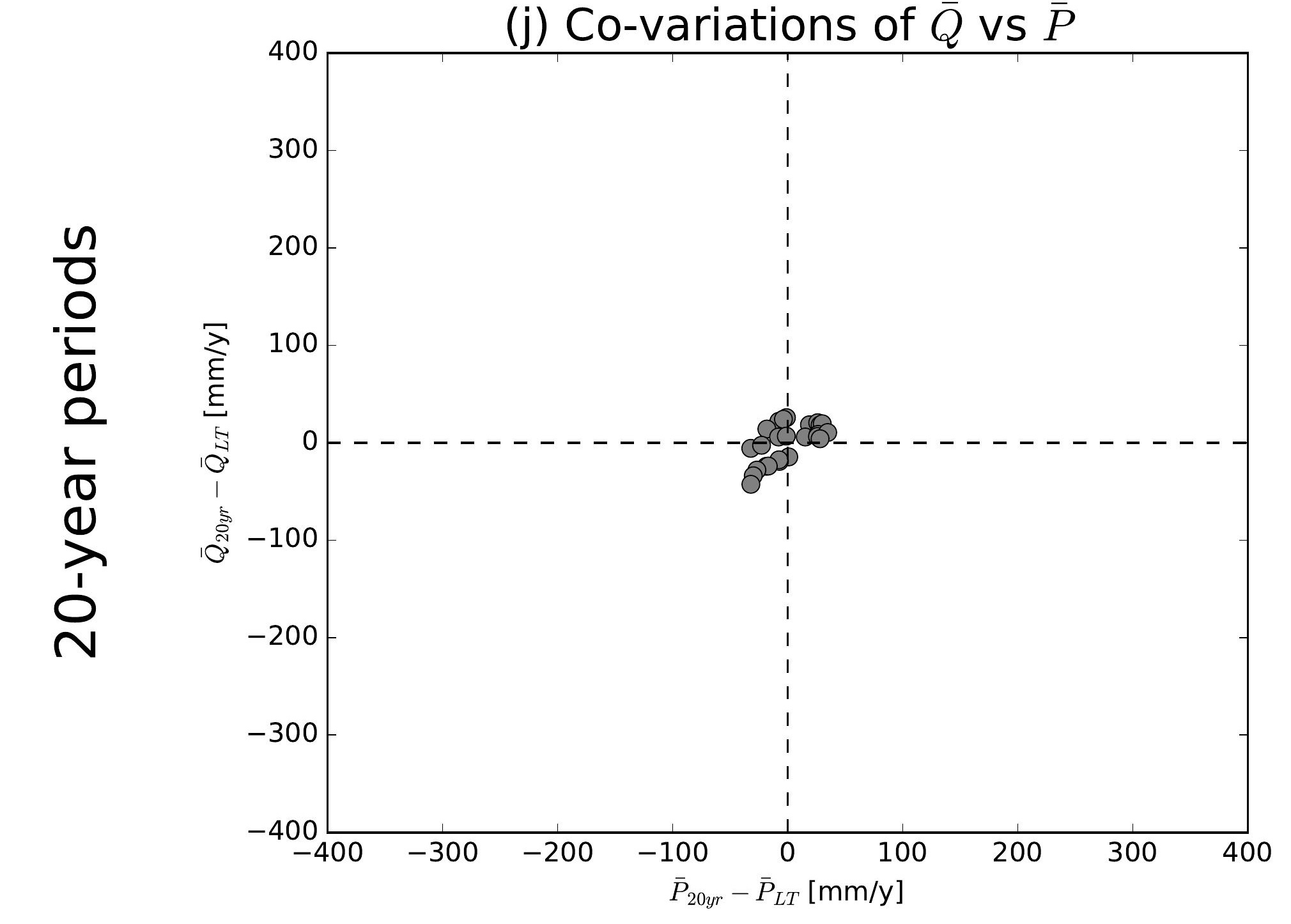

(b) Co-variations of $\bar{Q}$ vs $\overline{E 0}$

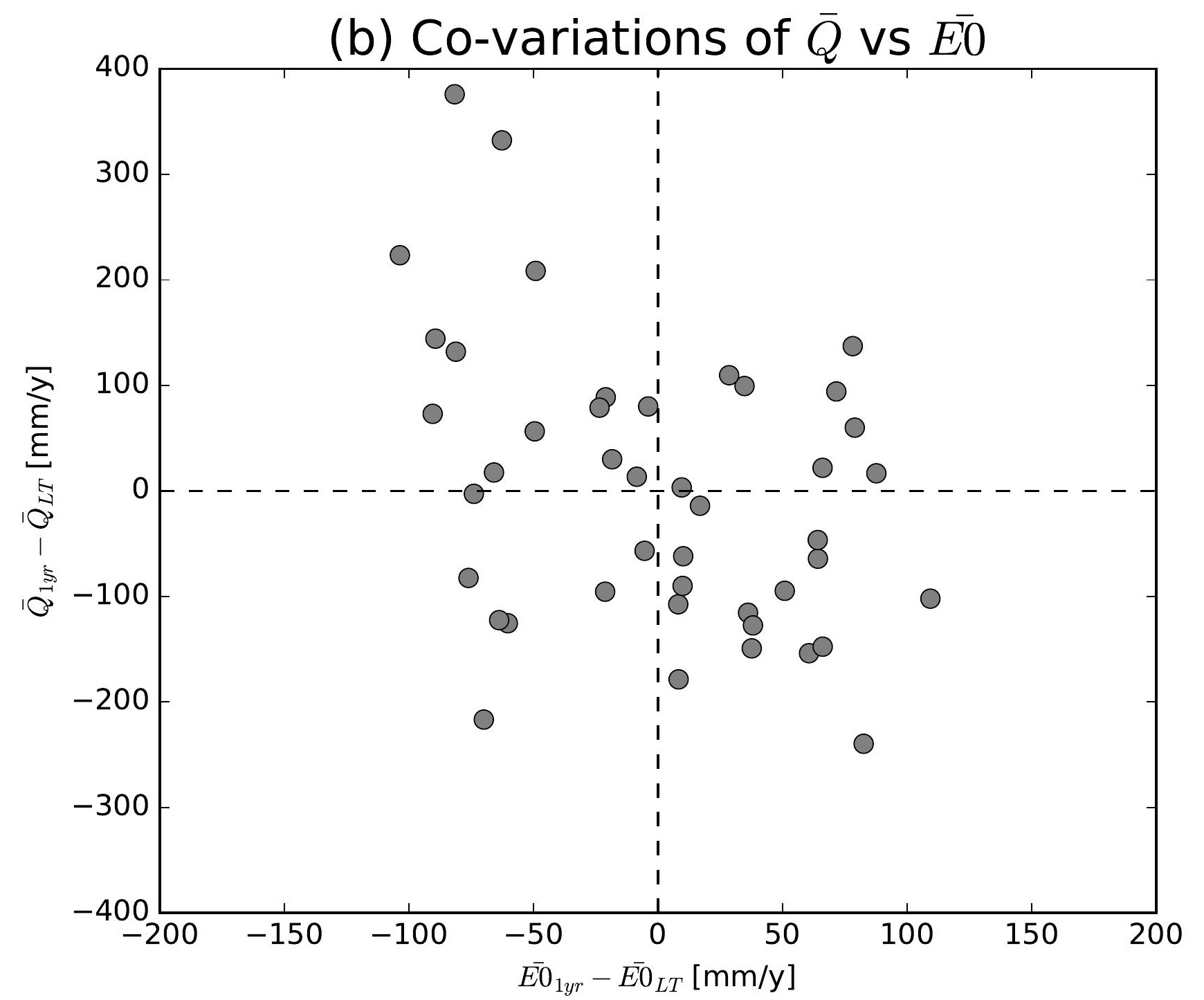

(e) Co-variations of $\bar{Q}$ vs $\overline{E 0}$

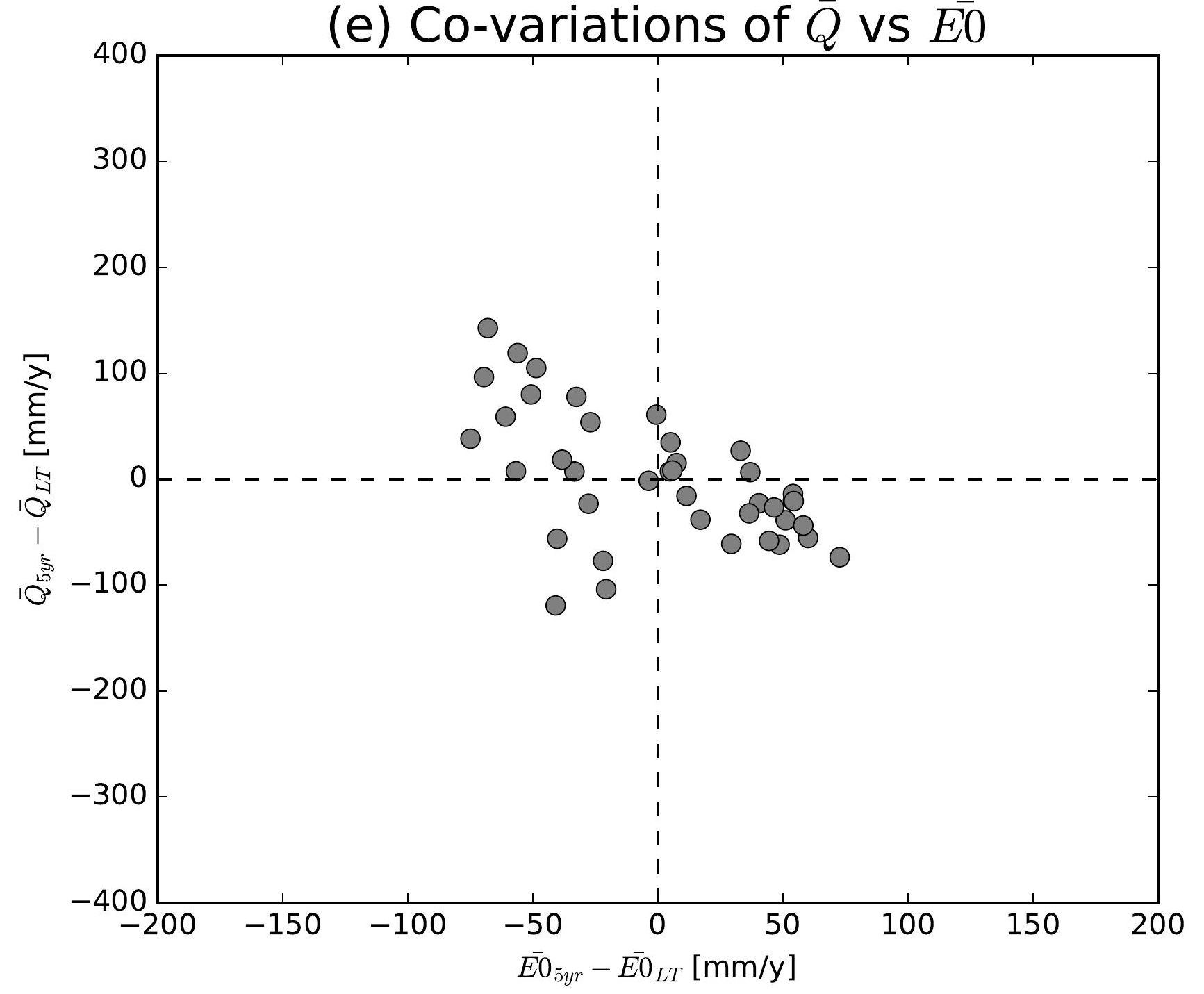

(h) Co-variations of $\bar{Q}$ vs $\overline{E 0}$

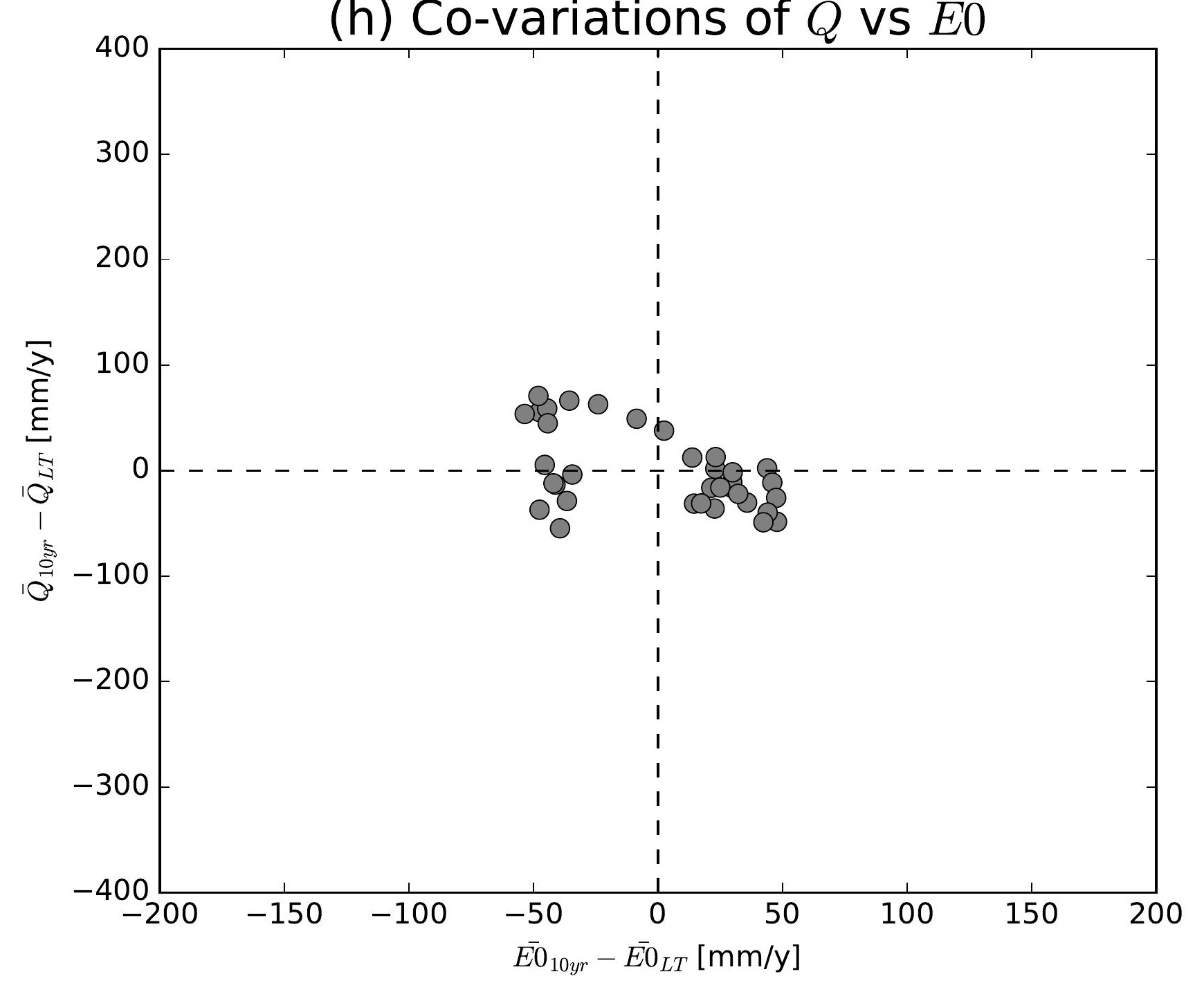

(k) Co-variations of $\bar{Q}$ vs $\overline{E 0}$

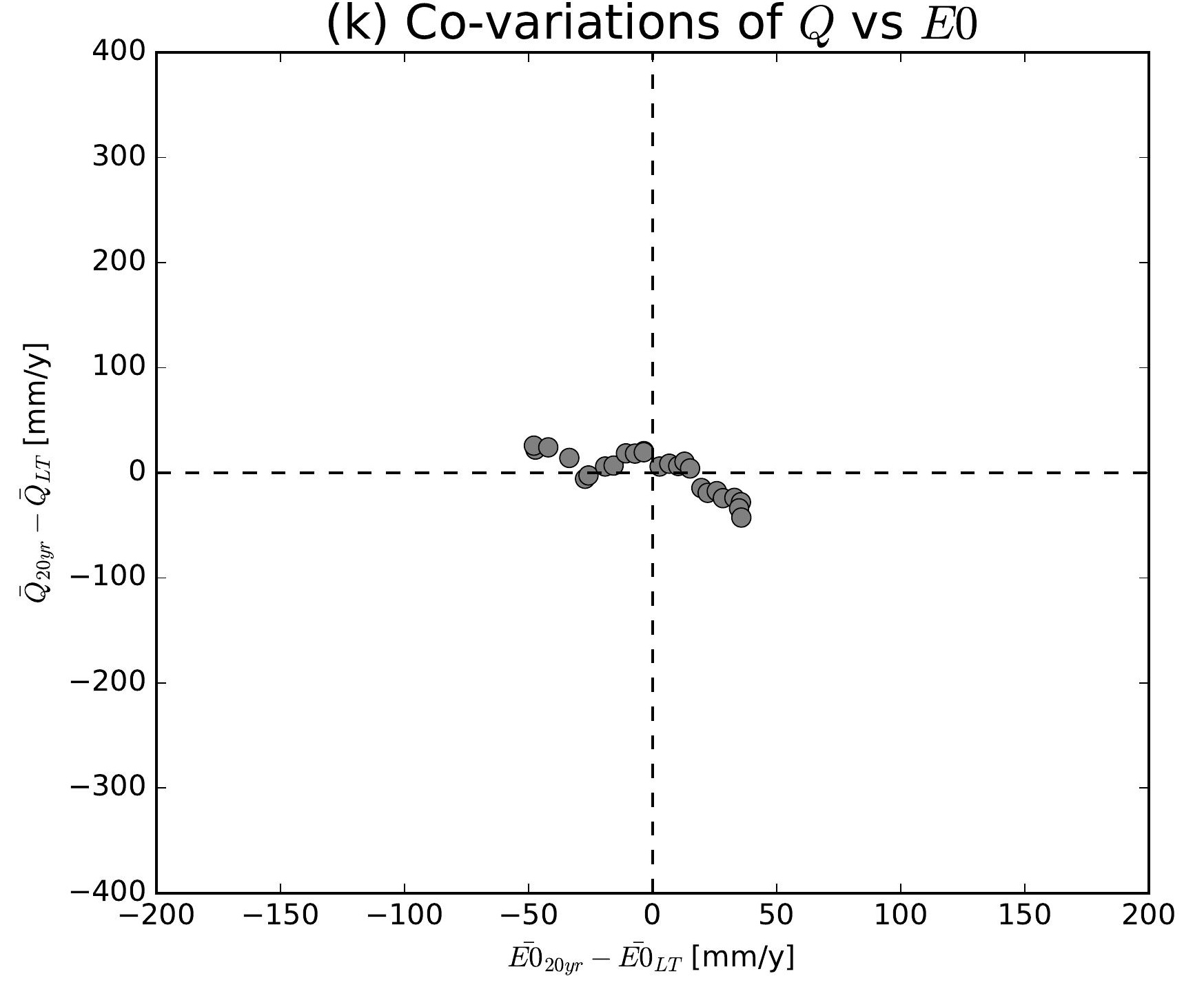

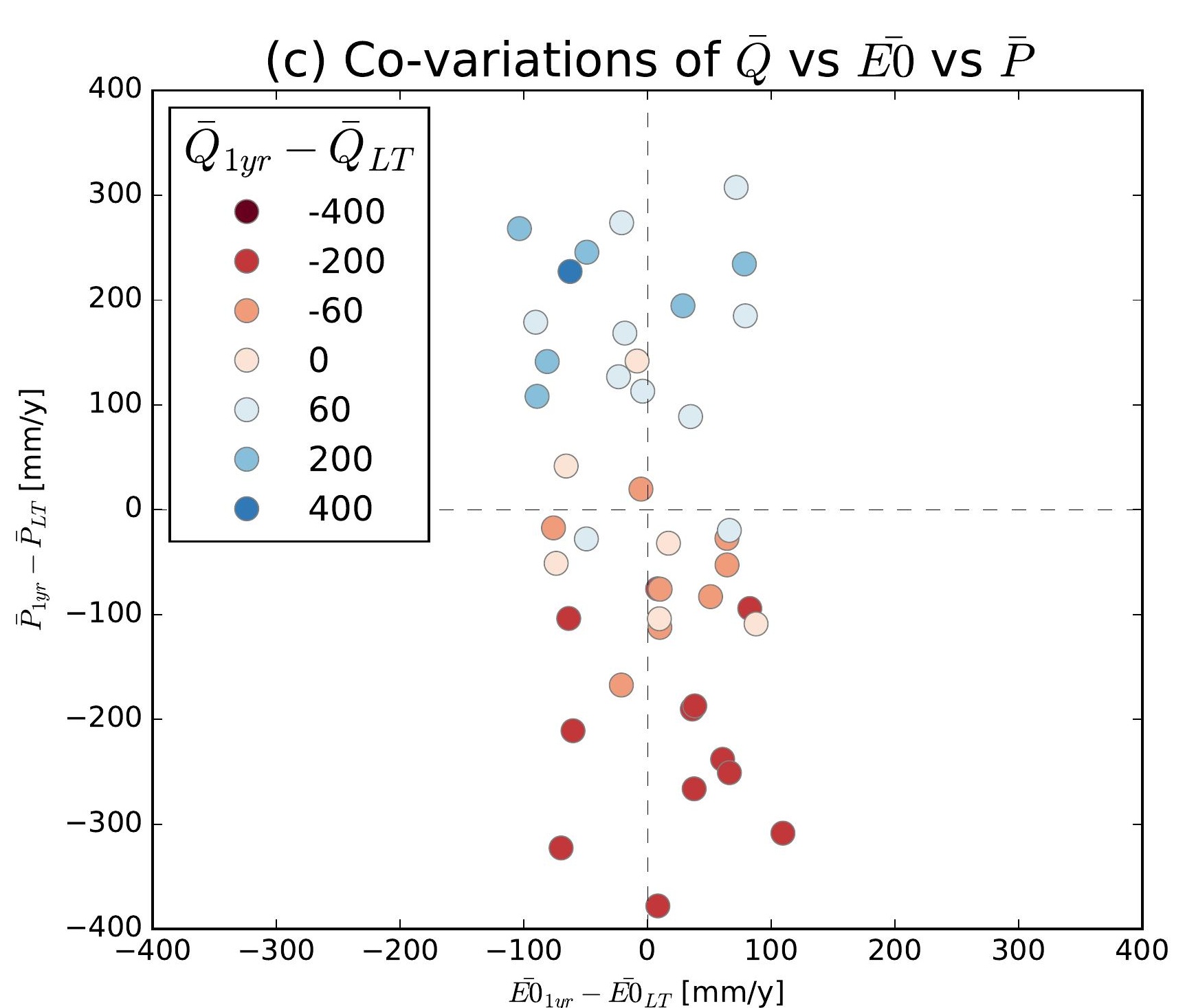
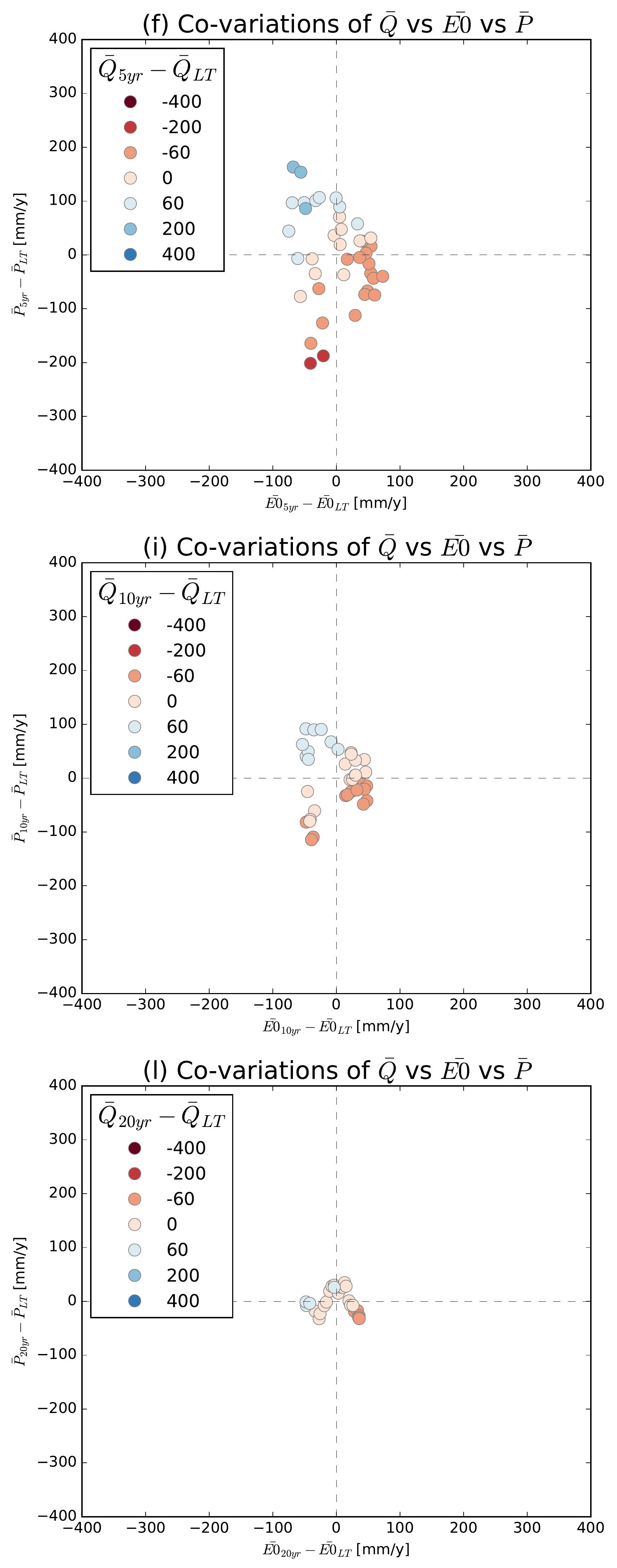

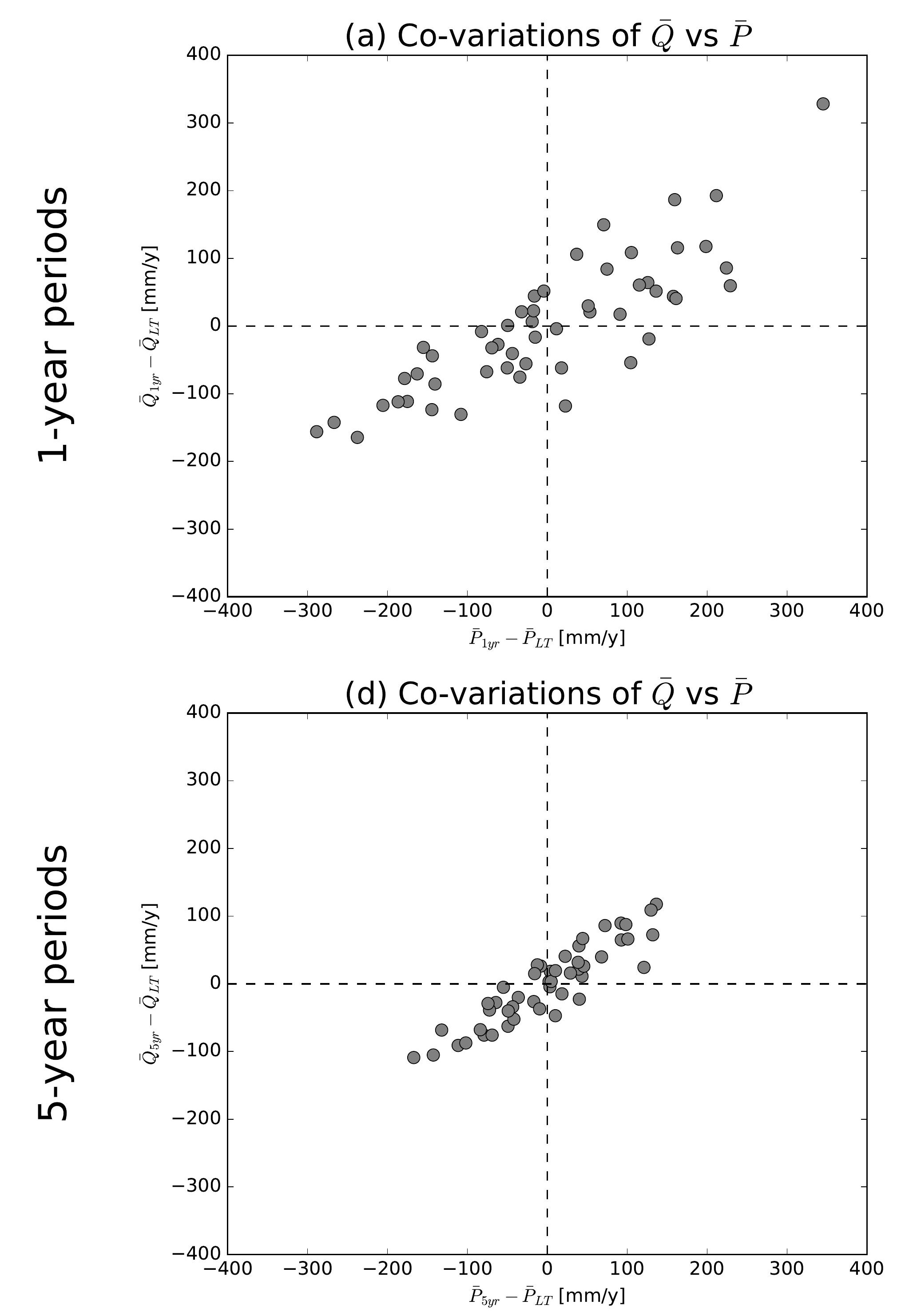

(g) Co-variations of $\bar{Q}$ vs $\bar{P}$

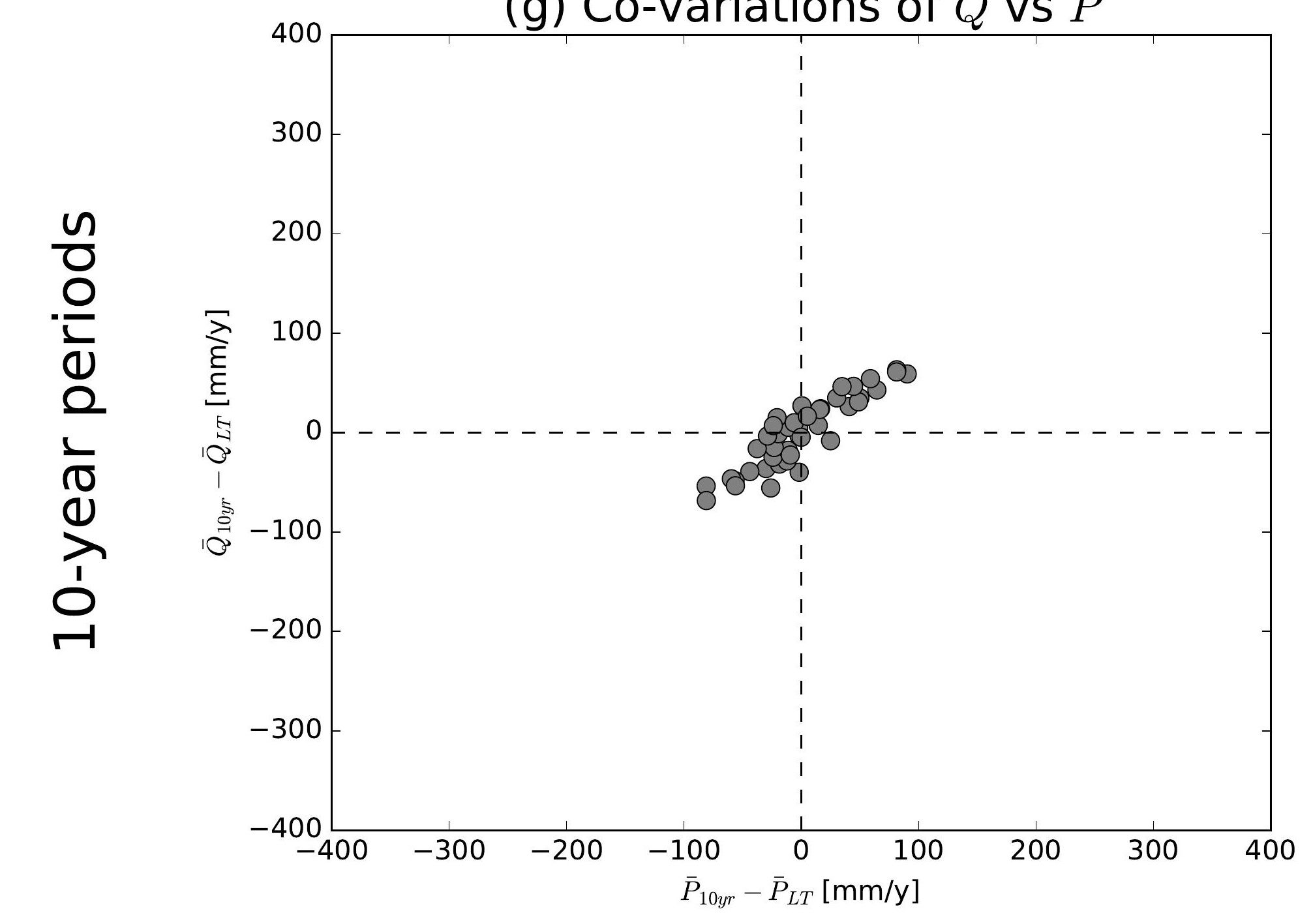

(j) Co-variations of $\bar{Q}$ vs $\bar{P}$

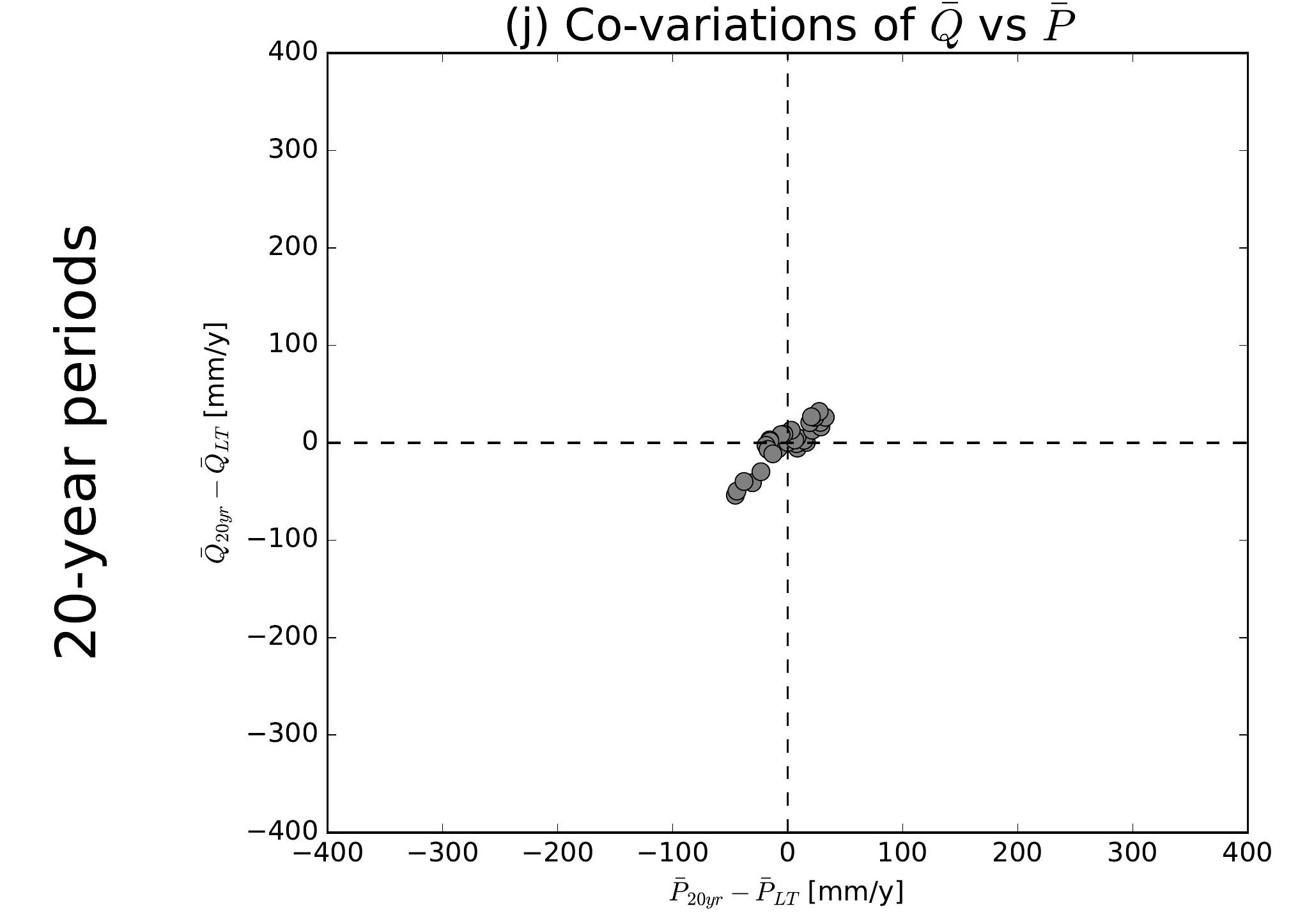

(b) Co-variations of $\bar{Q}$ vs $\overline{E 0}$

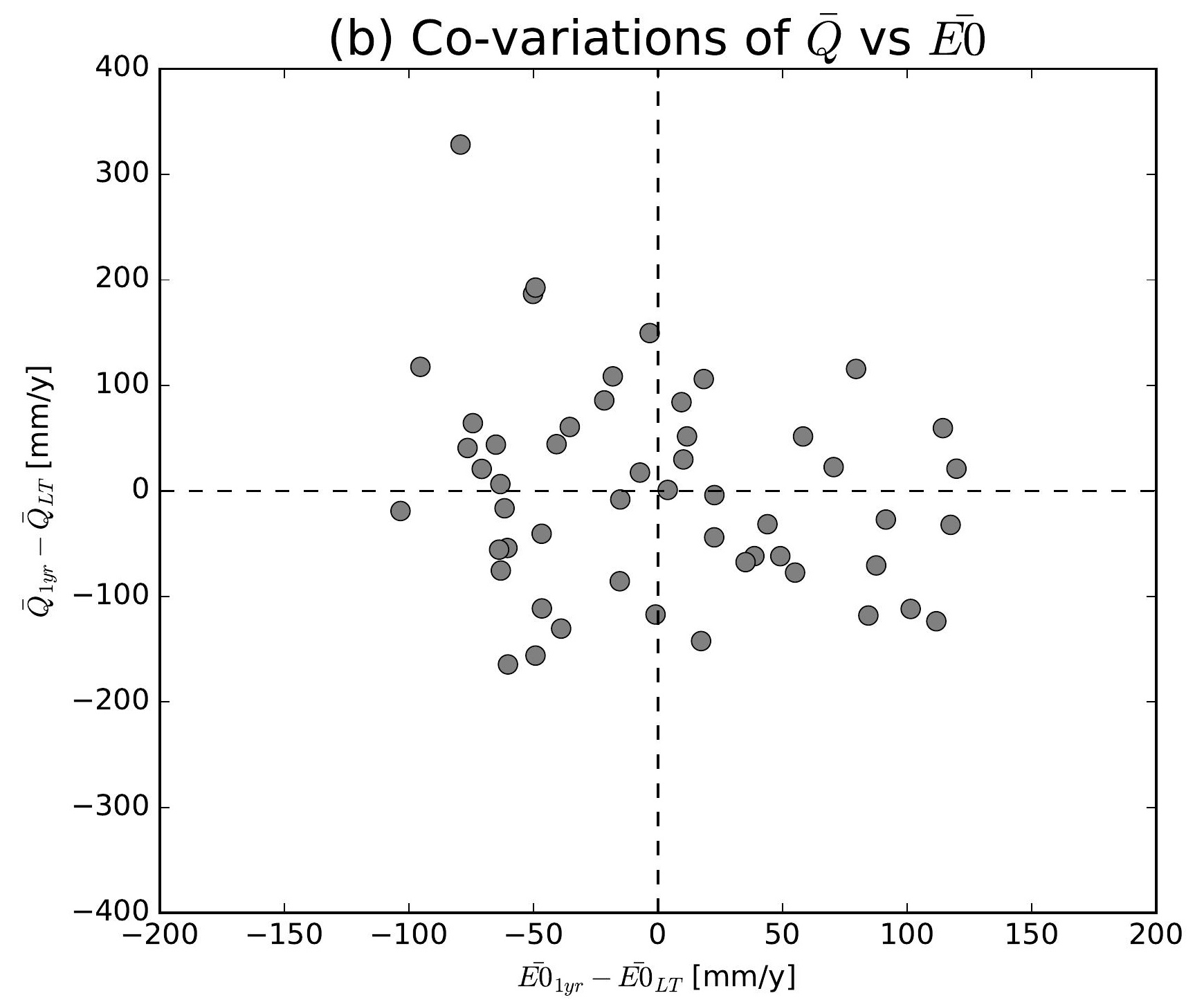

(e) Co-variations of $\bar{Q}$ vs $\overline{E 0}$

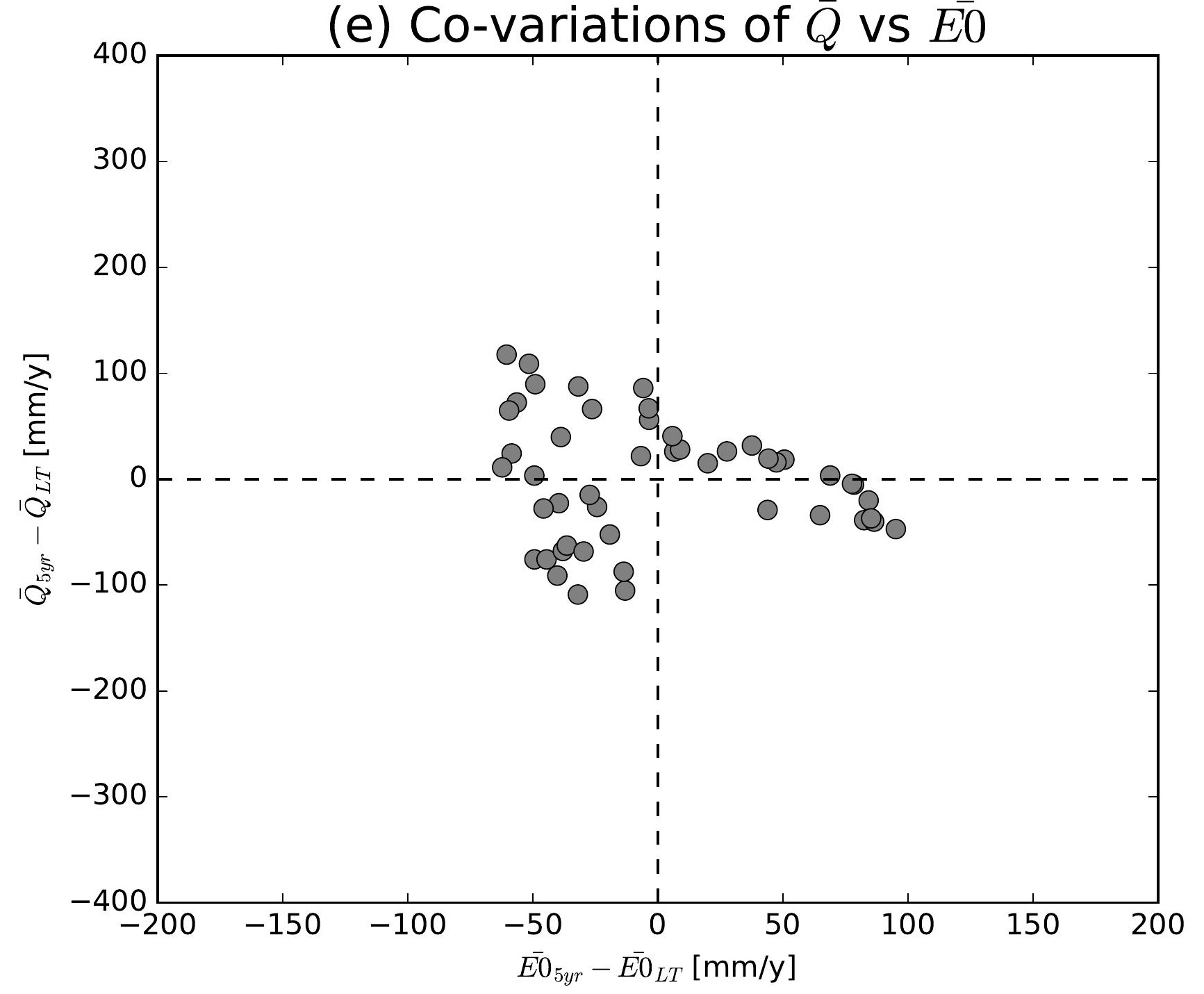

(h) Co-variations of $\bar{Q}$ vs $\overline{E 0}$

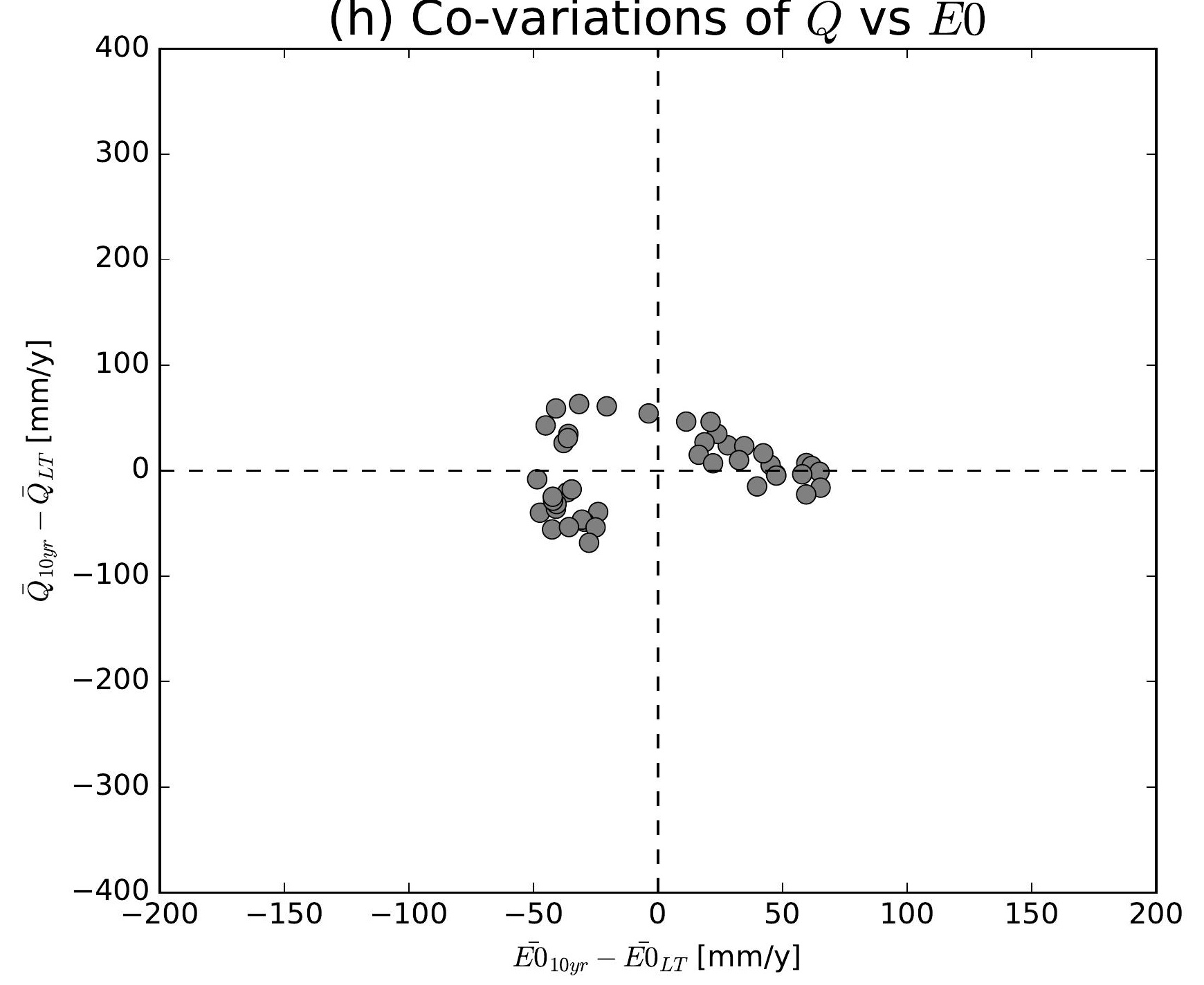

(k) Co-variations of $\bar{Q}$ vs $\overline{E 0}$

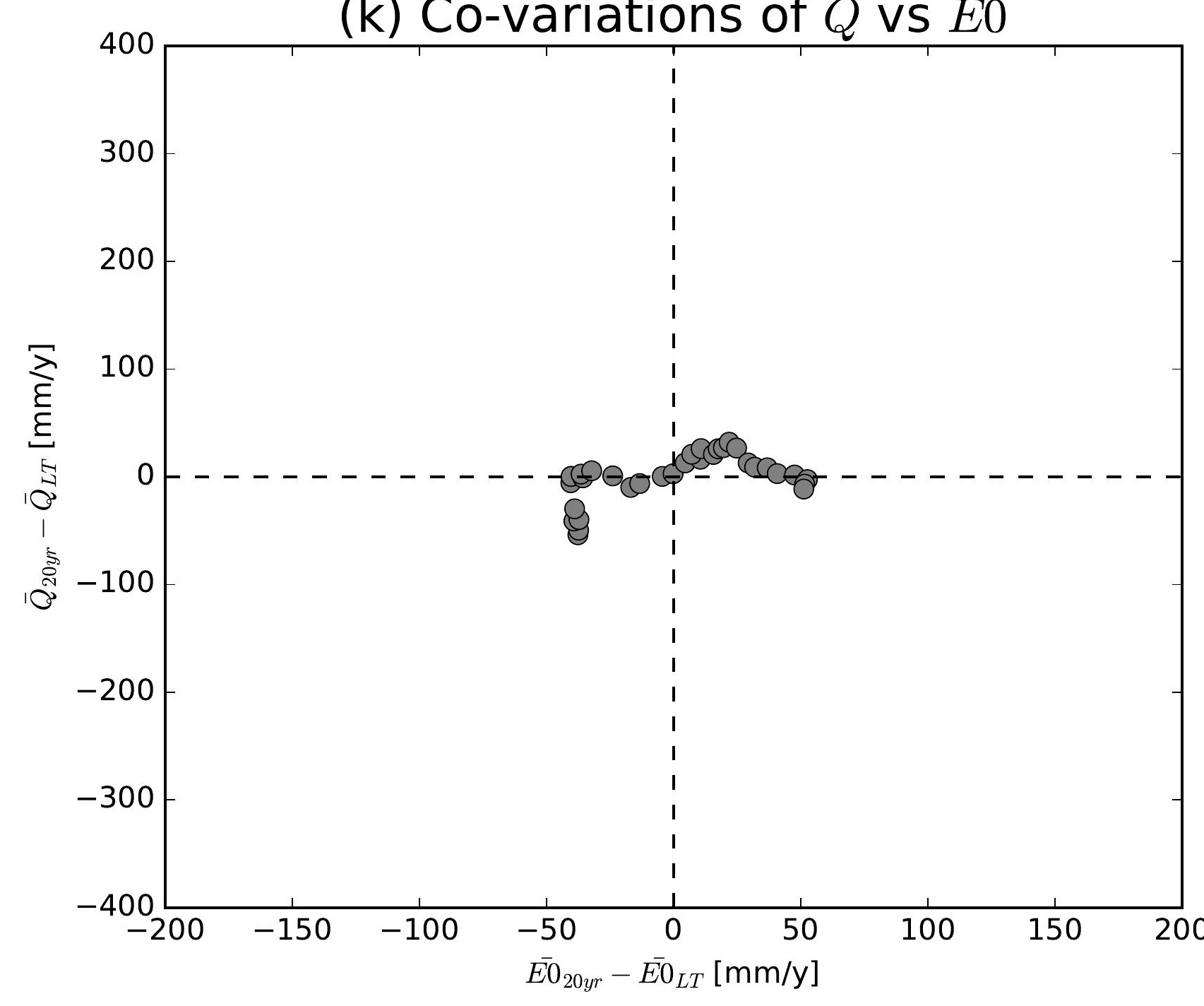

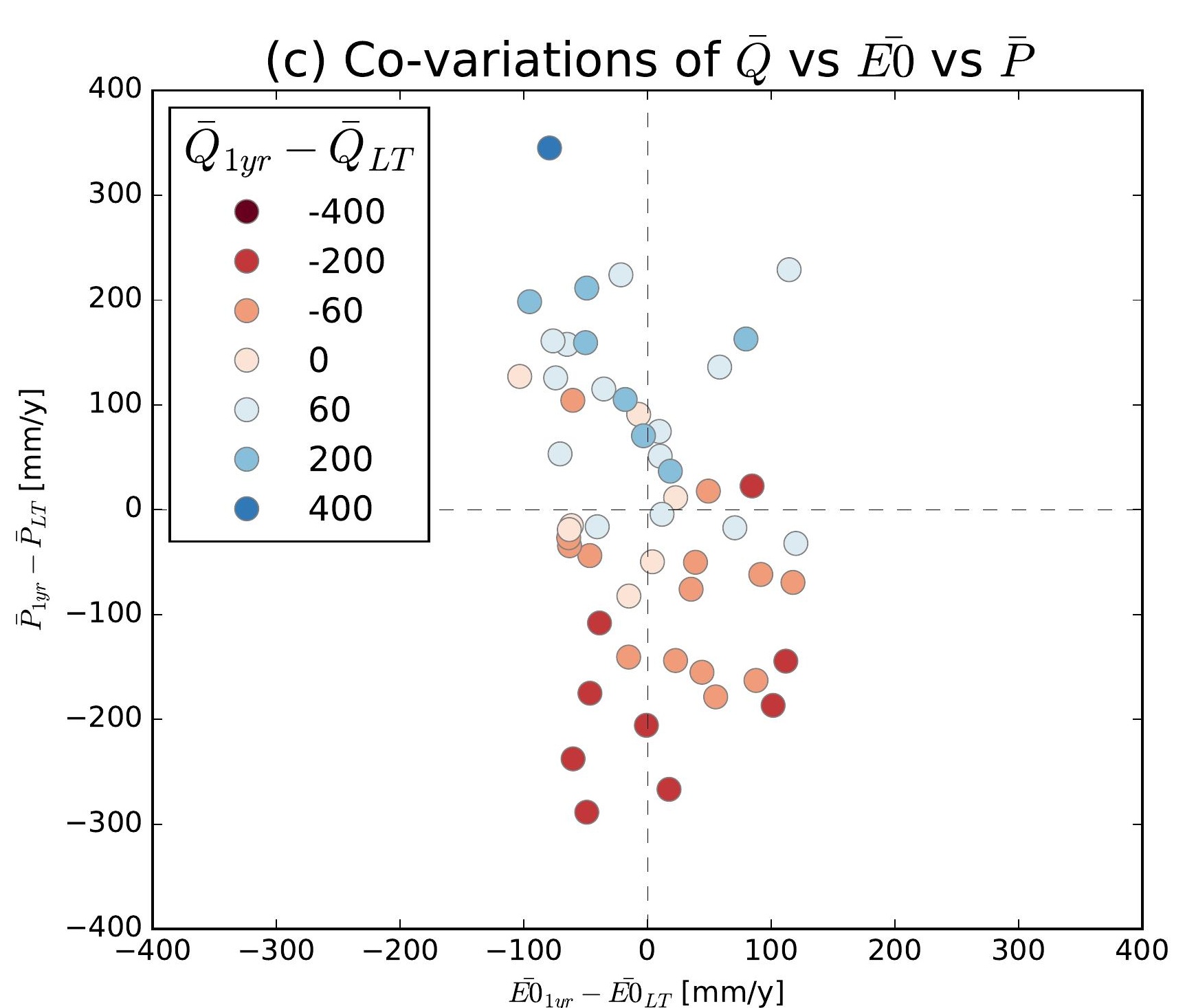
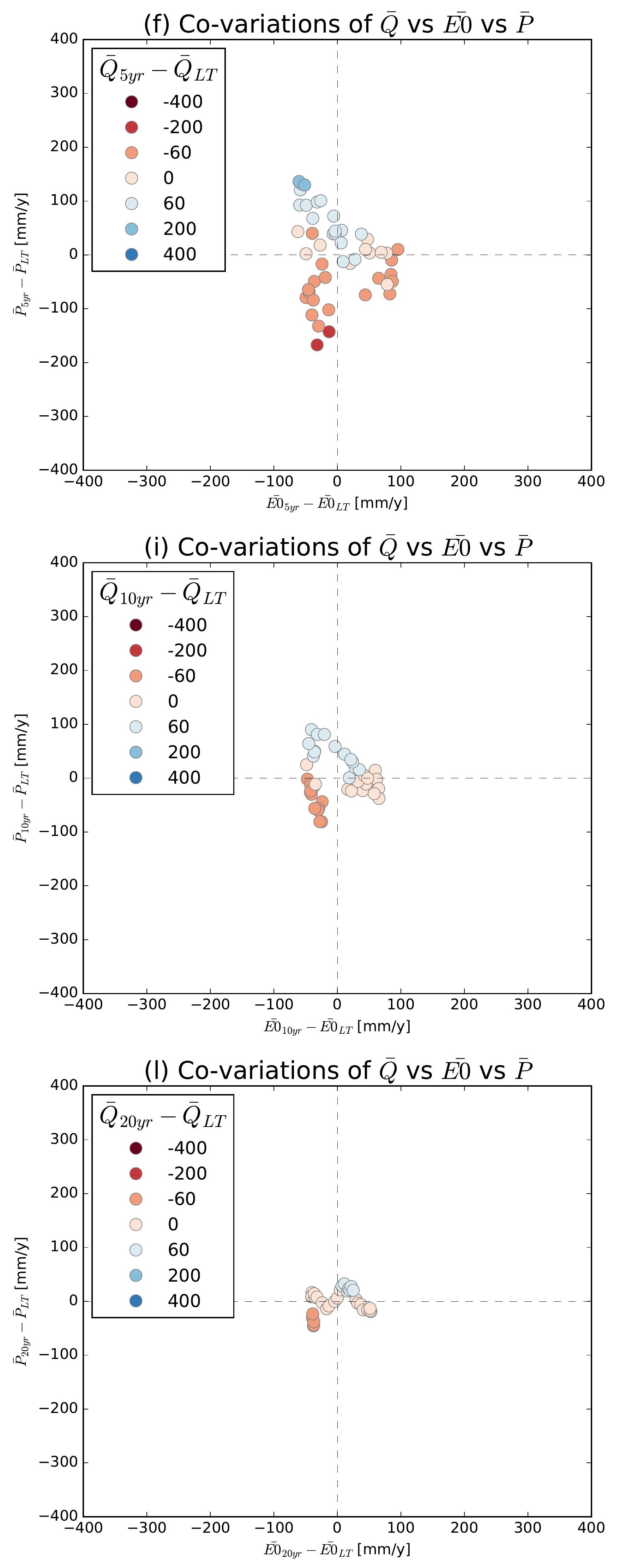

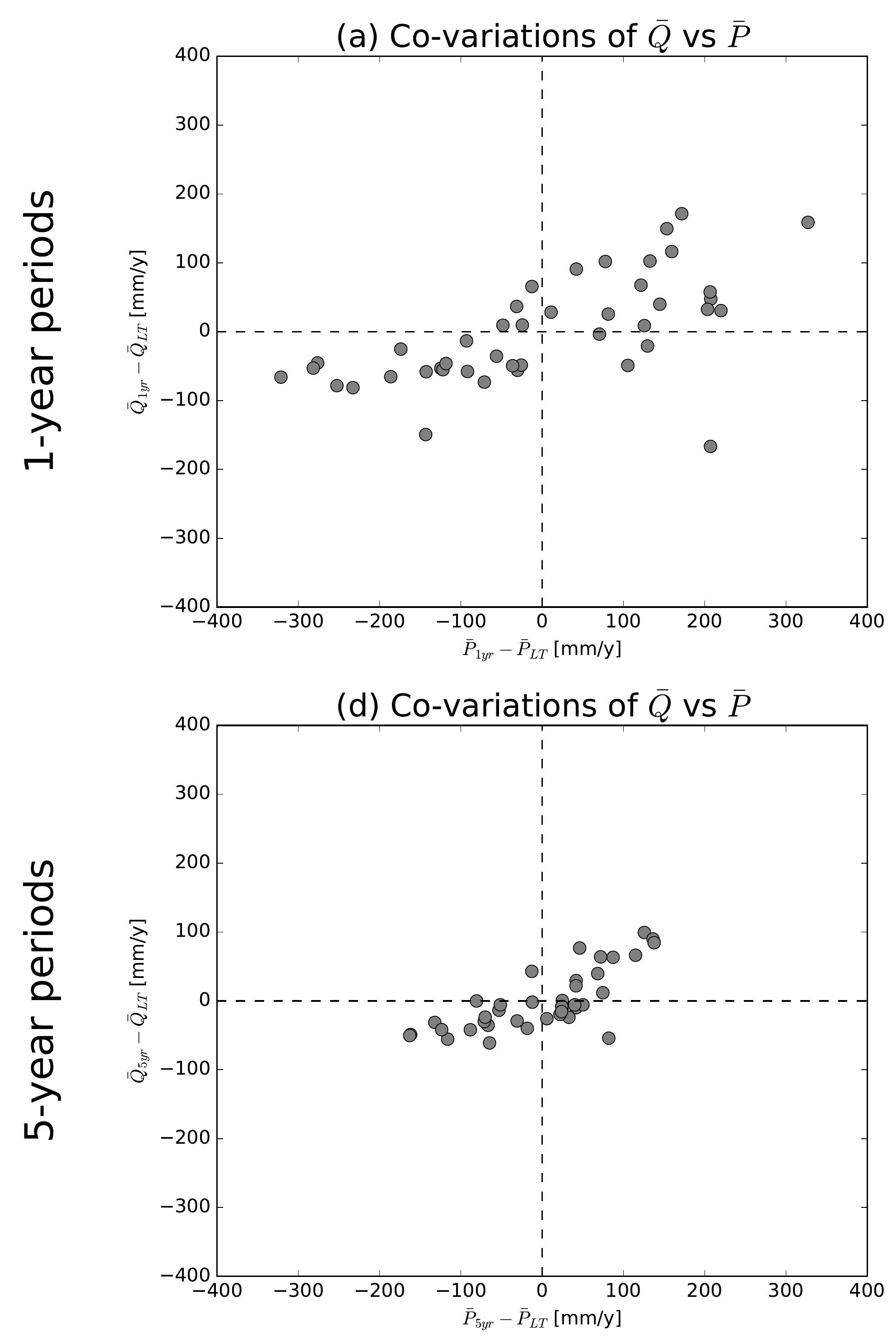

(g) Co-variations of $\bar{Q}$ vs $\bar{P}$

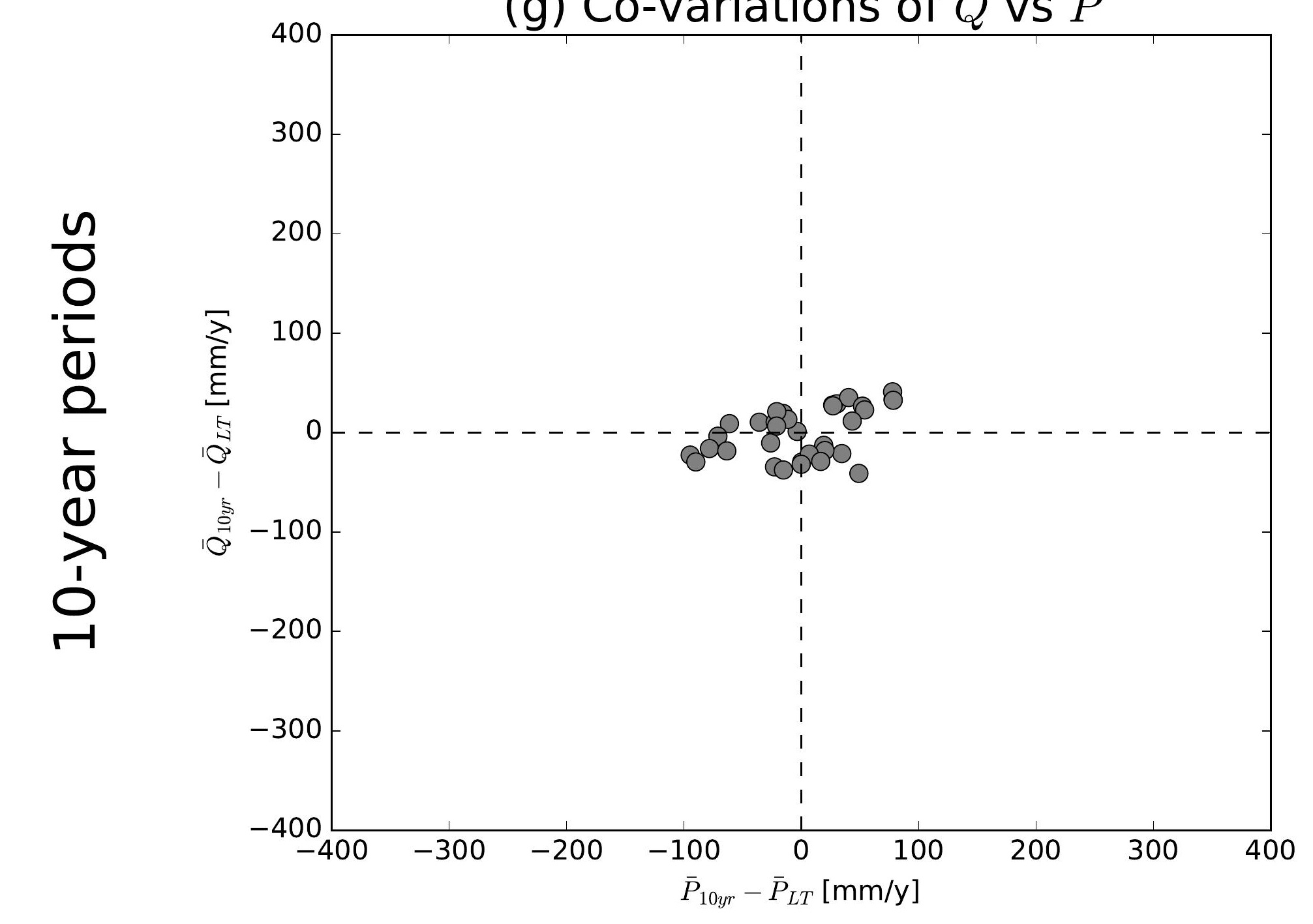

(j) Co-variations of $\bar{Q}$ vs $\bar{P}$

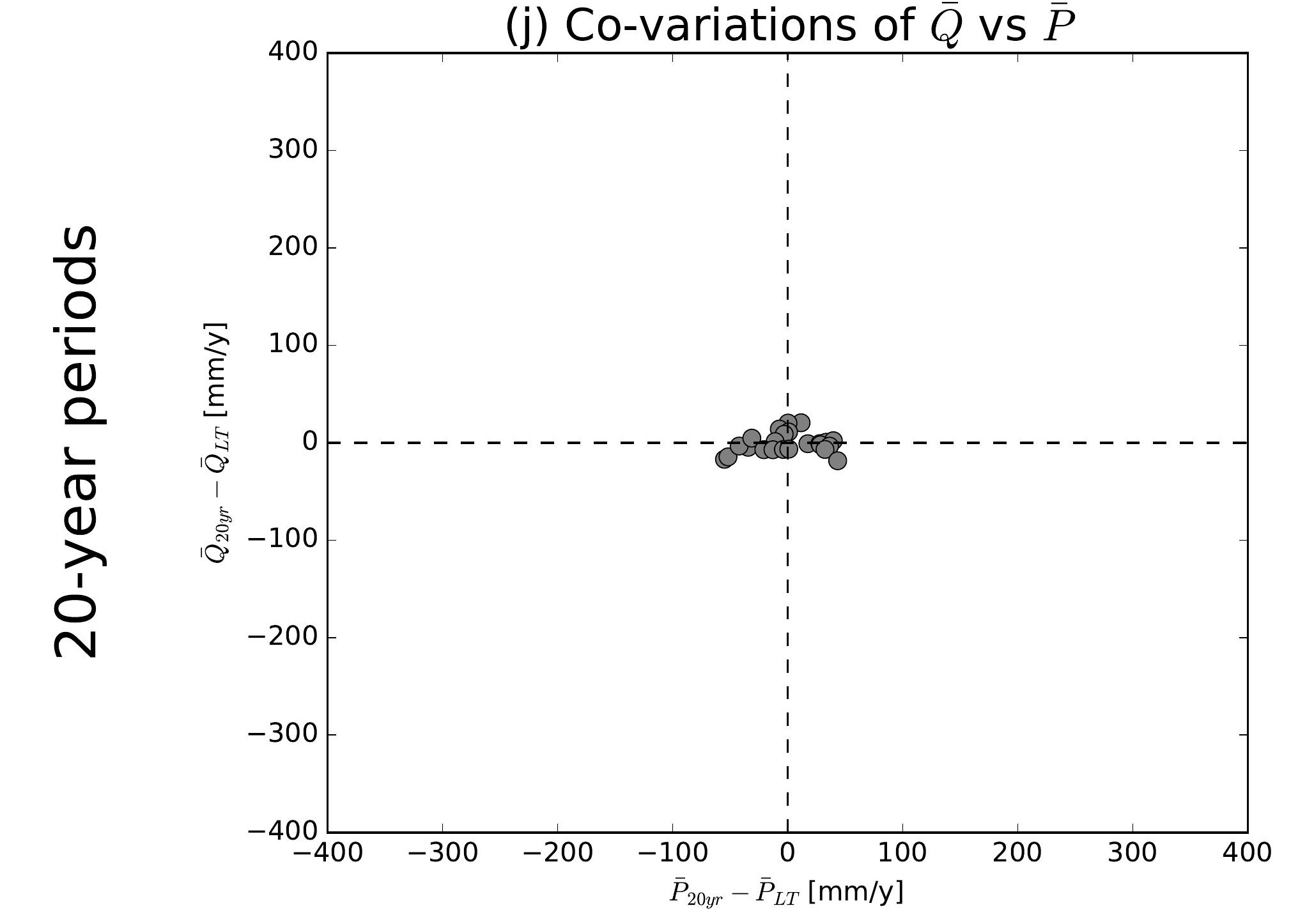

(b) Co-variations of $\bar{Q}$ vs $\overline{E 0}$

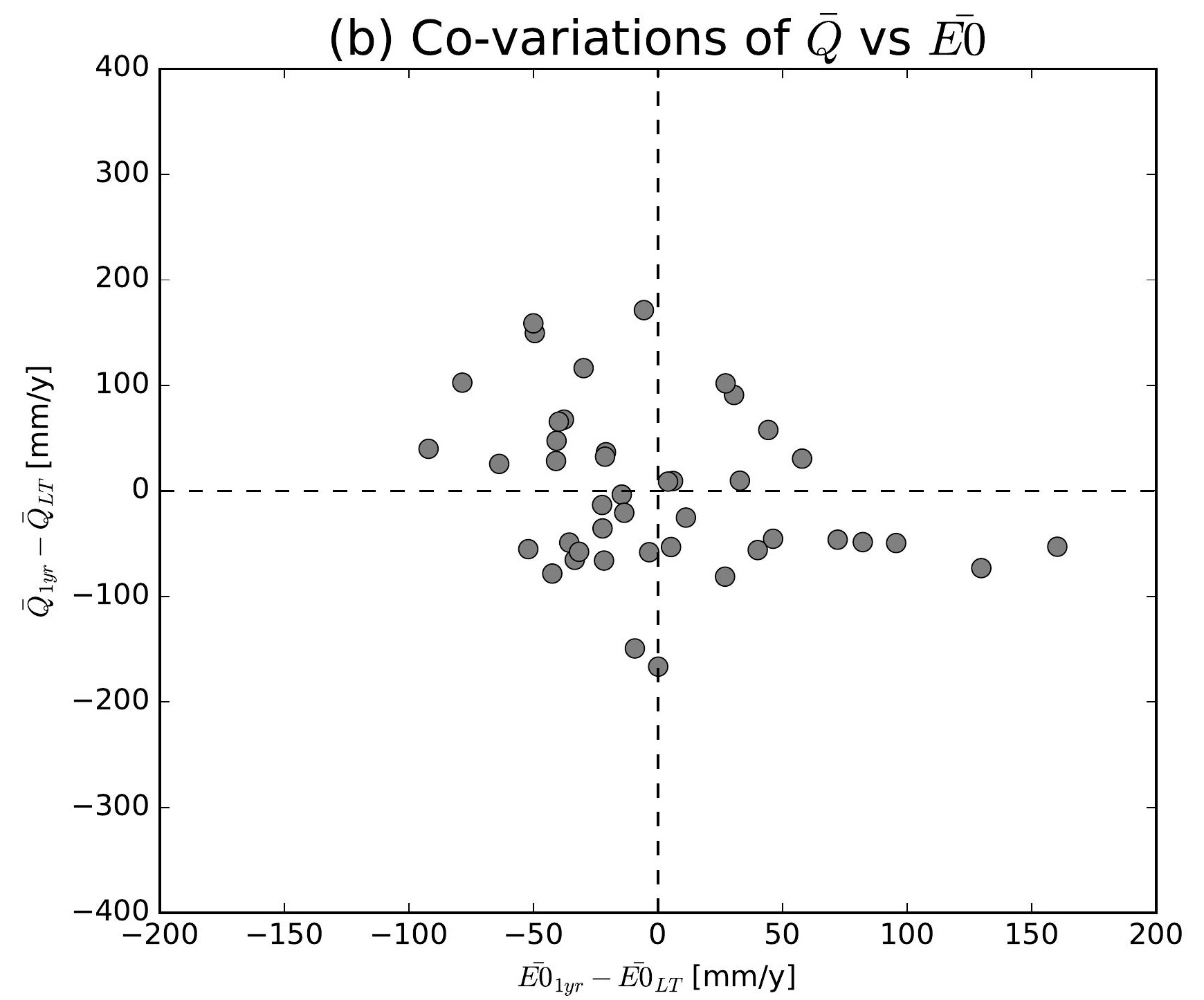

(e) Co-variations of $\bar{Q}$ vs $\overline{E 0}$

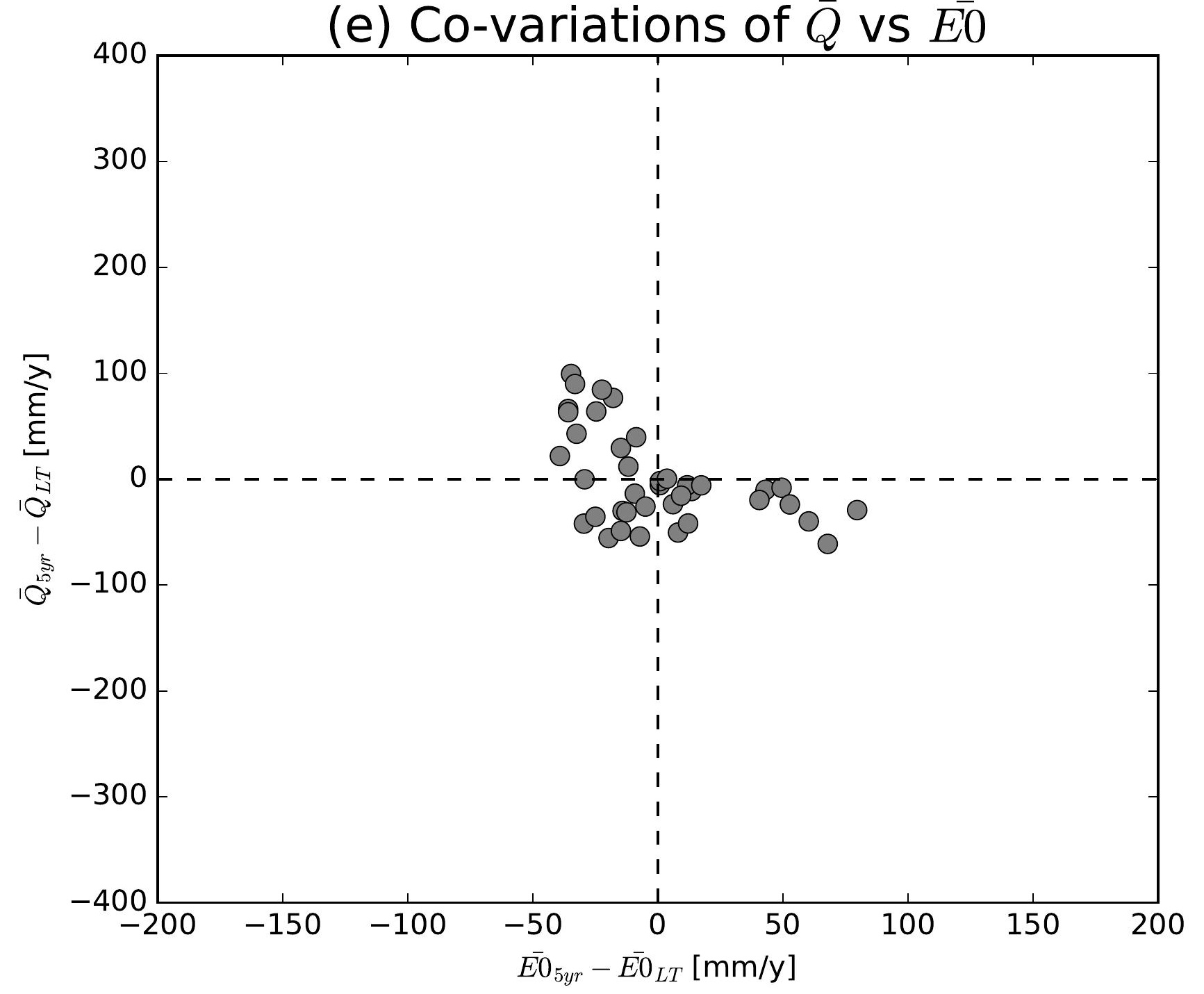

(h) Co-variations of $\bar{Q}$ vs $\overline{E 0}$

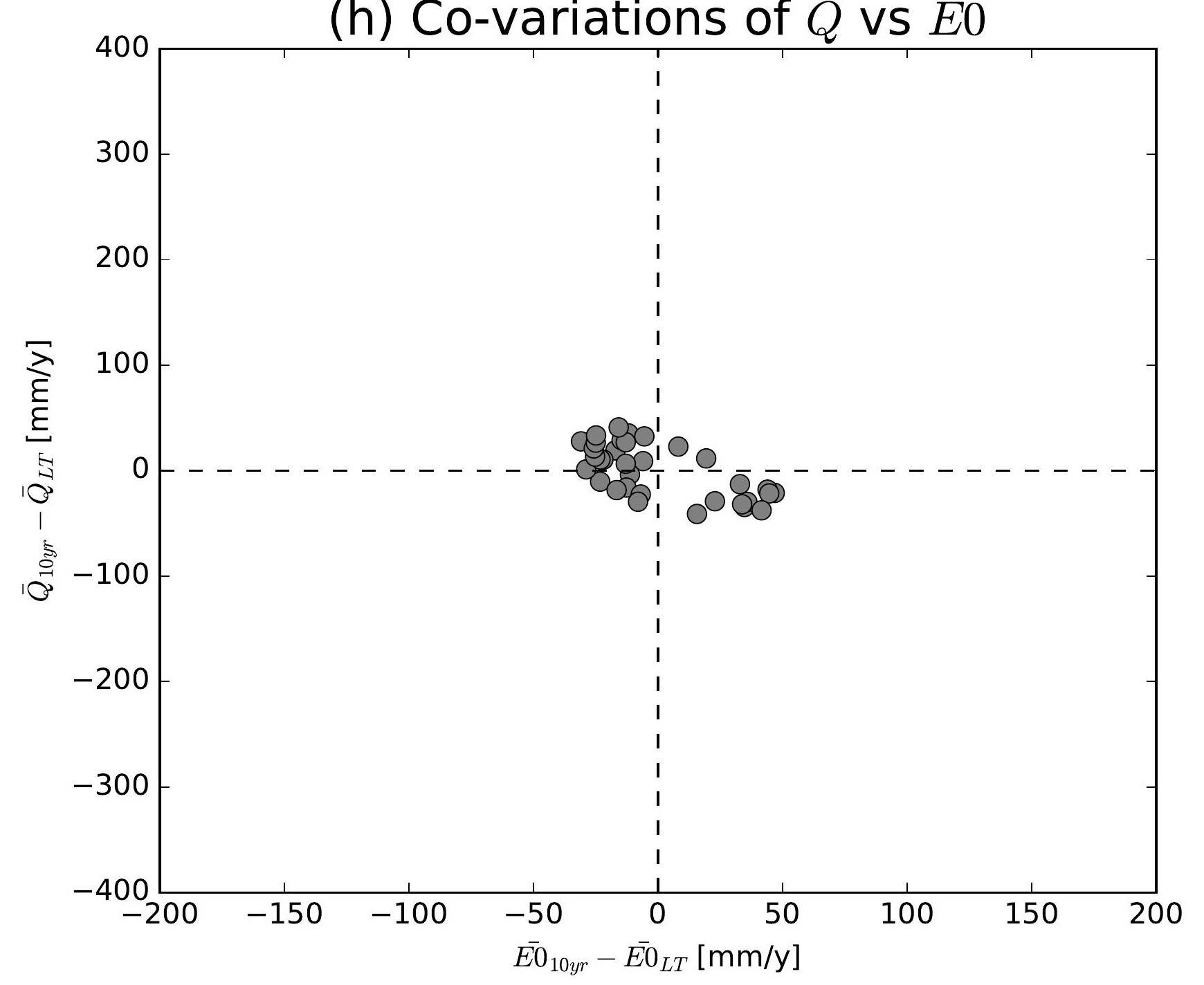

(k) Co-variations of $\bar{Q}$ vs $\overline{E 0}$

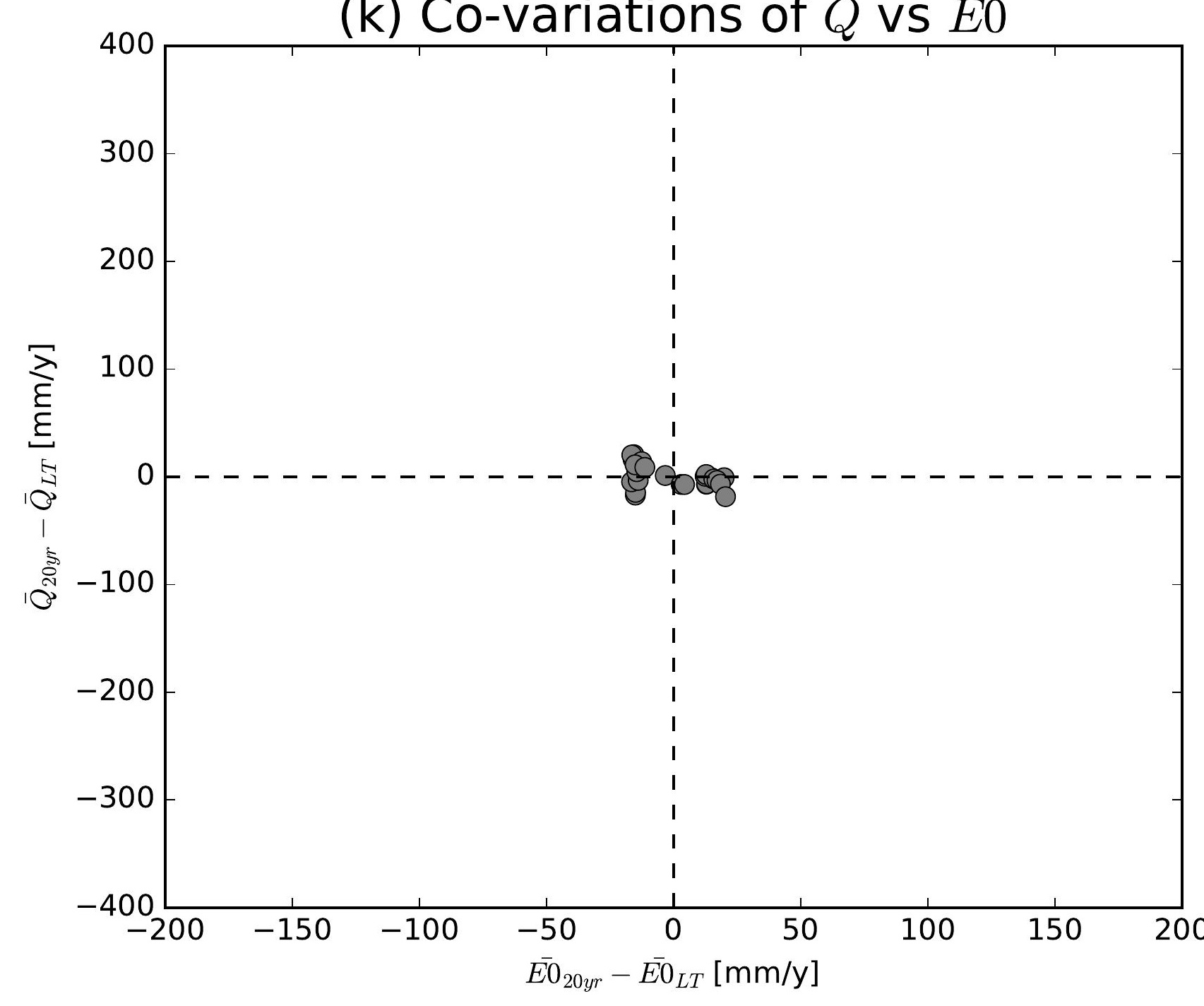

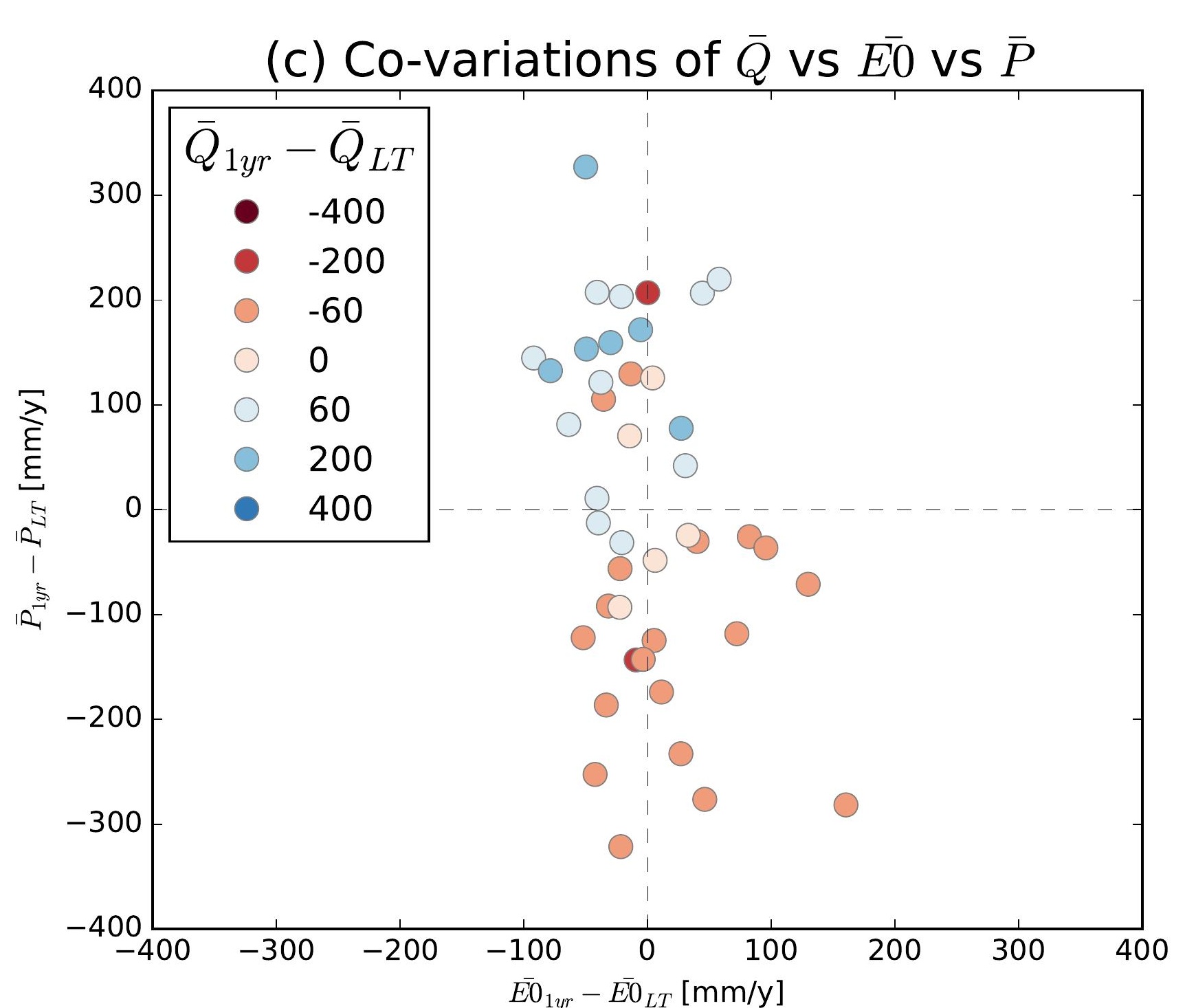
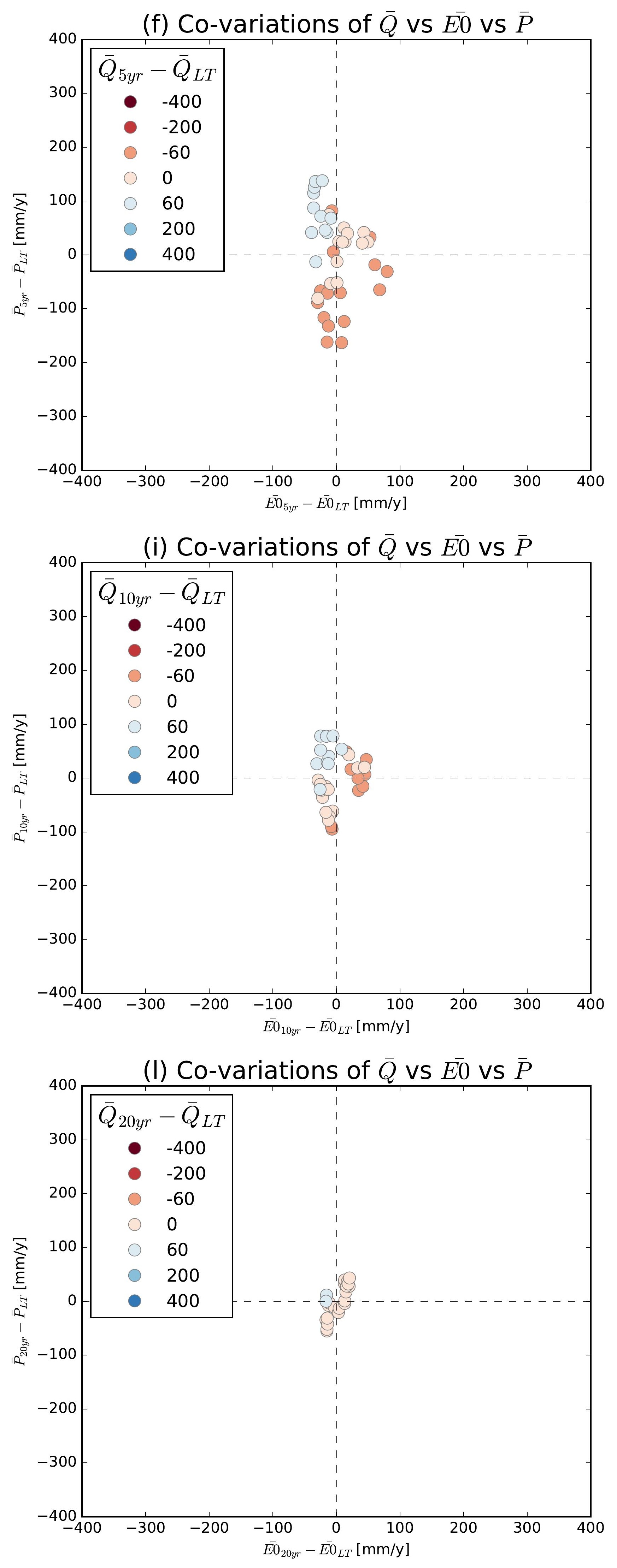

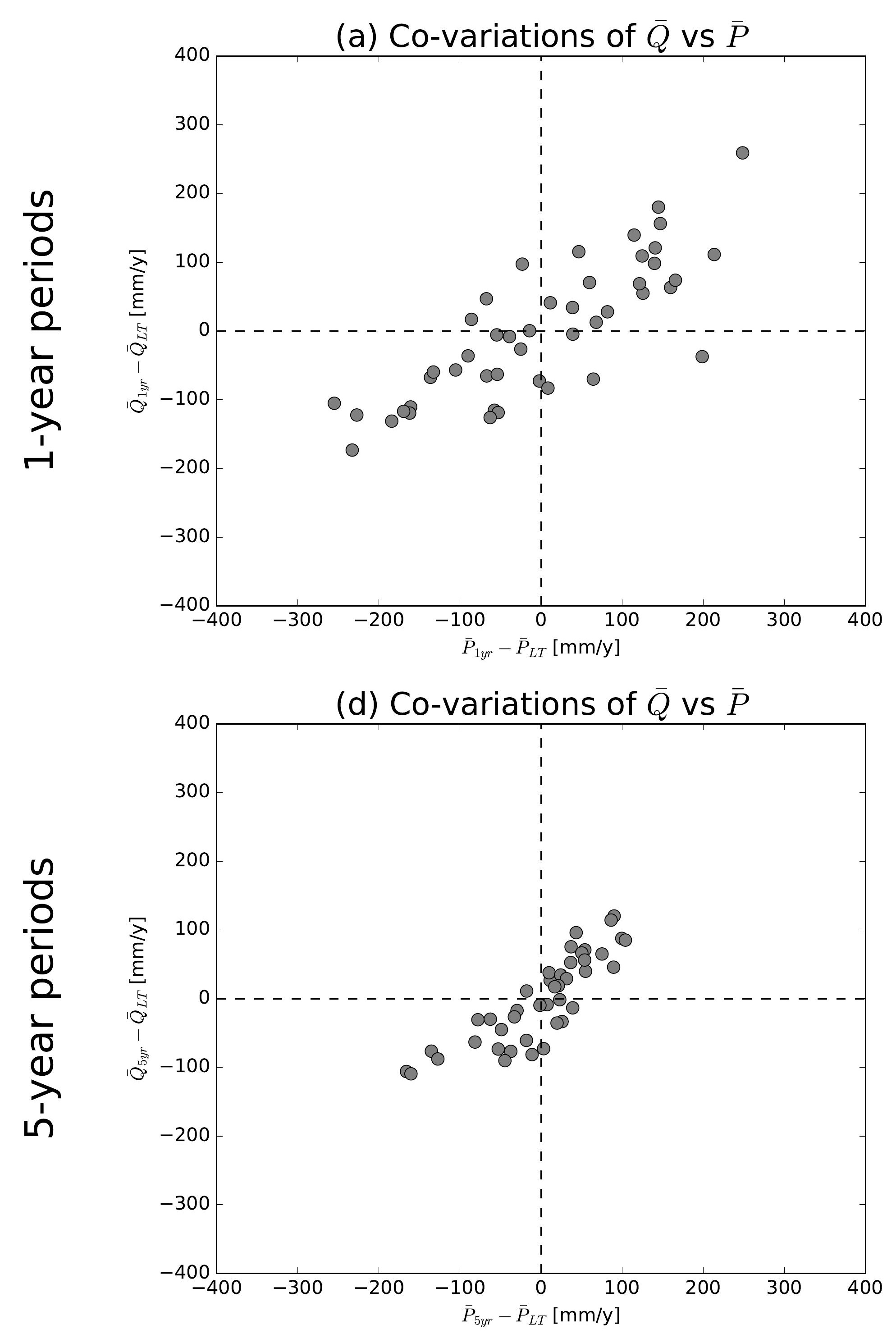

(g) Co-variations of $\bar{Q}$ vs $\bar{P}$

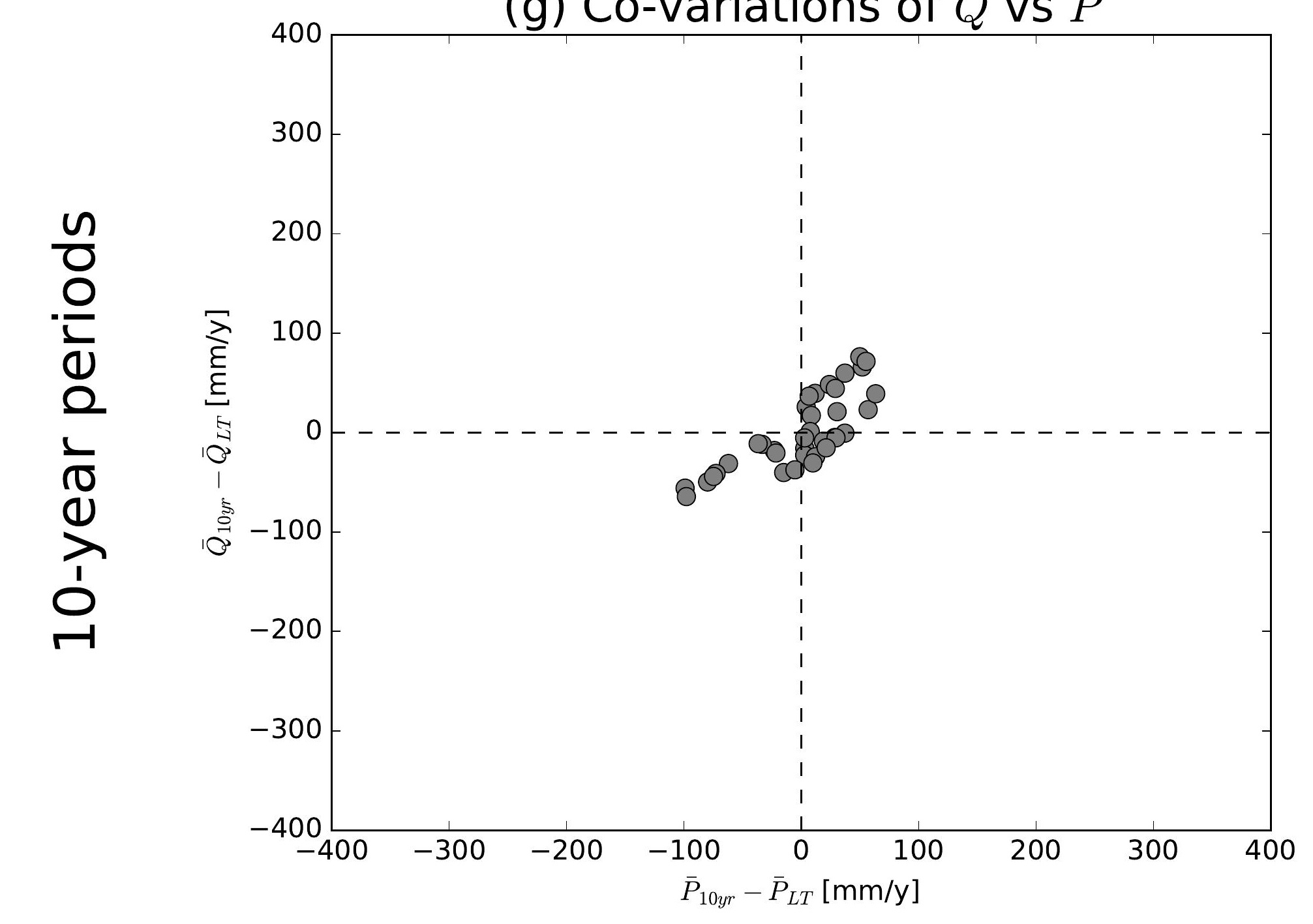

(j) Co-variations of $\bar{Q}$ vs $\bar{P}$

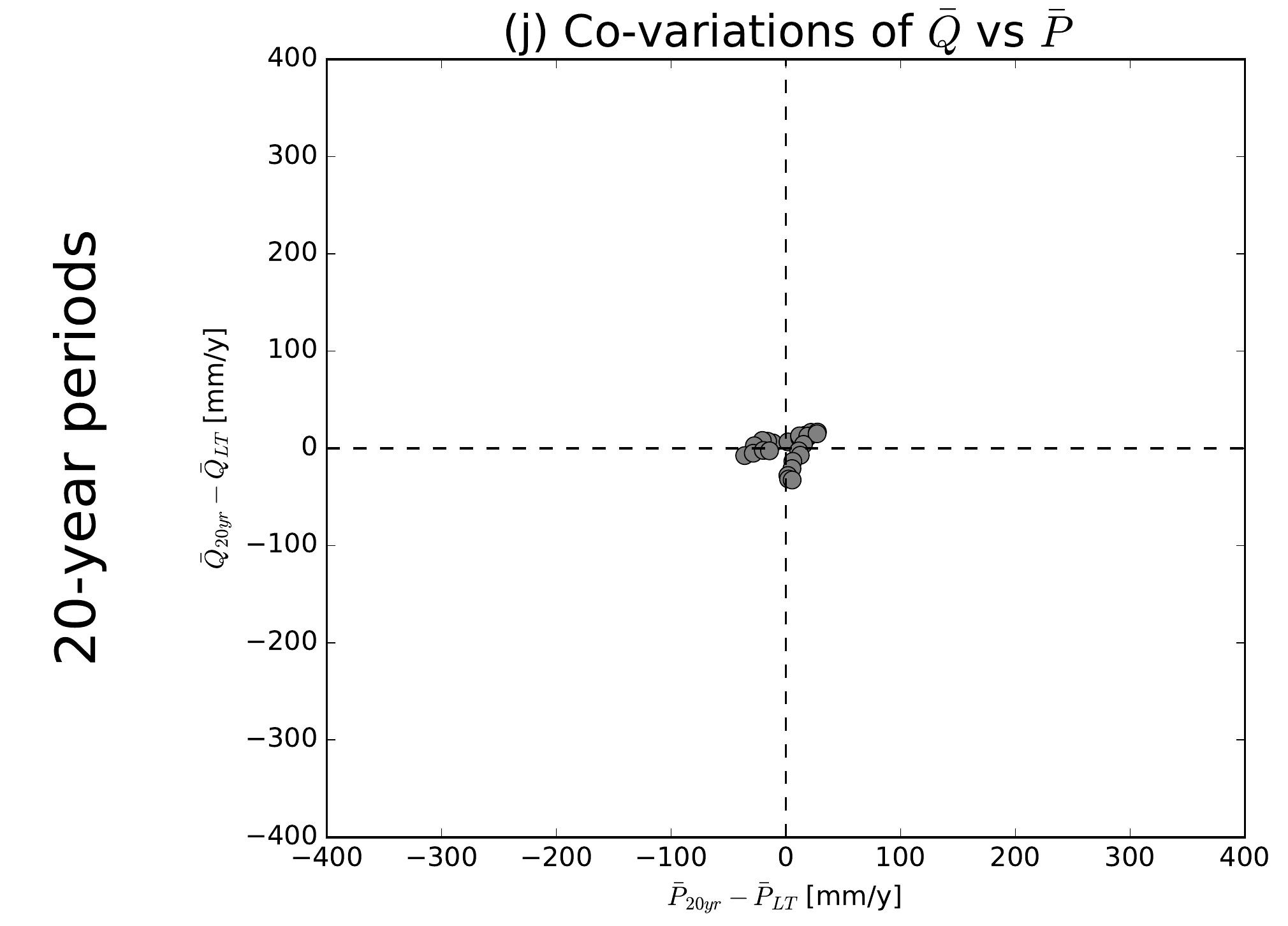

(b) Co-variations of $\bar{Q}$ vs $\overline{E 0}$

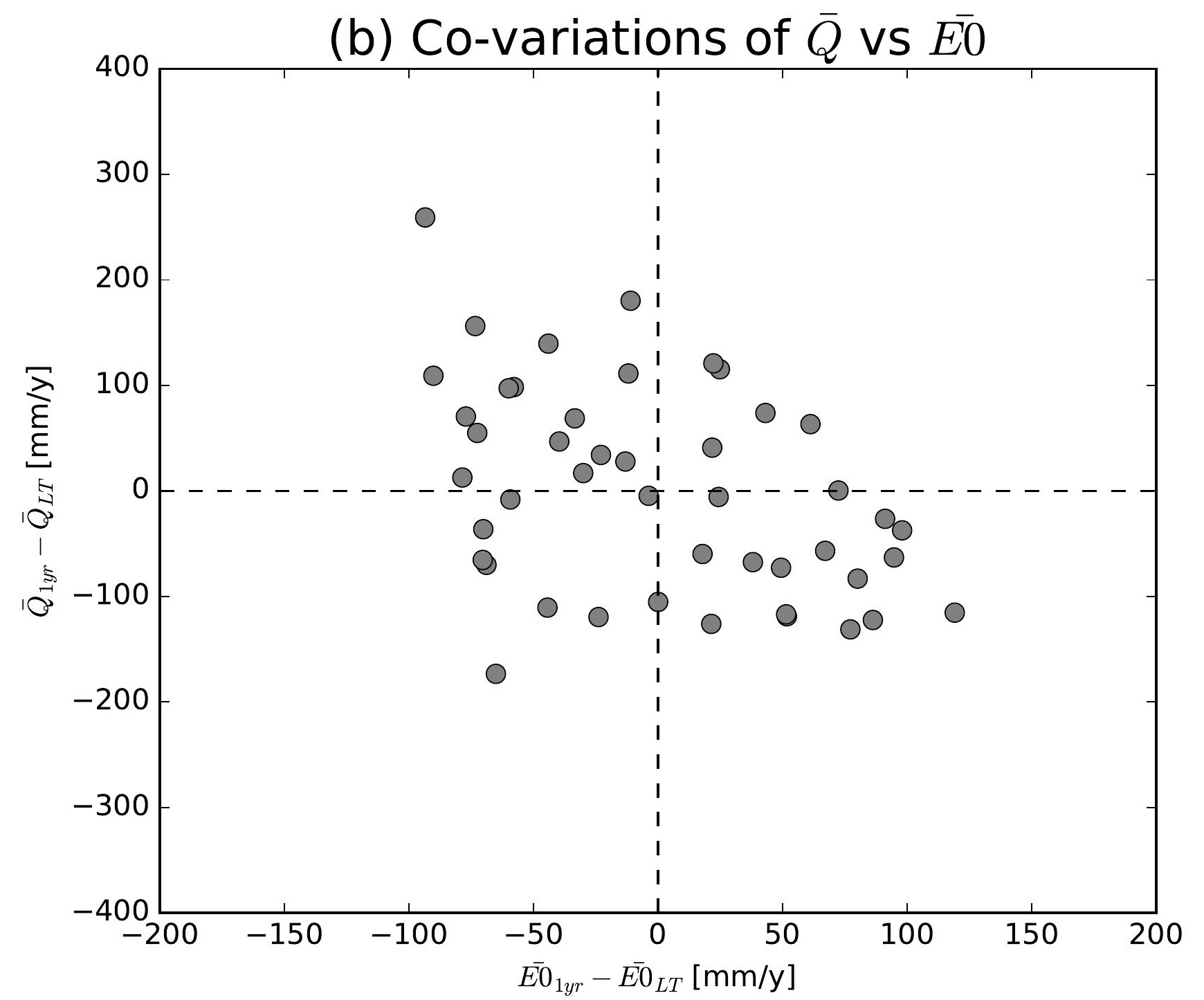

(e) Co-variations of $\bar{Q}$ vs $\overline{E 0}$

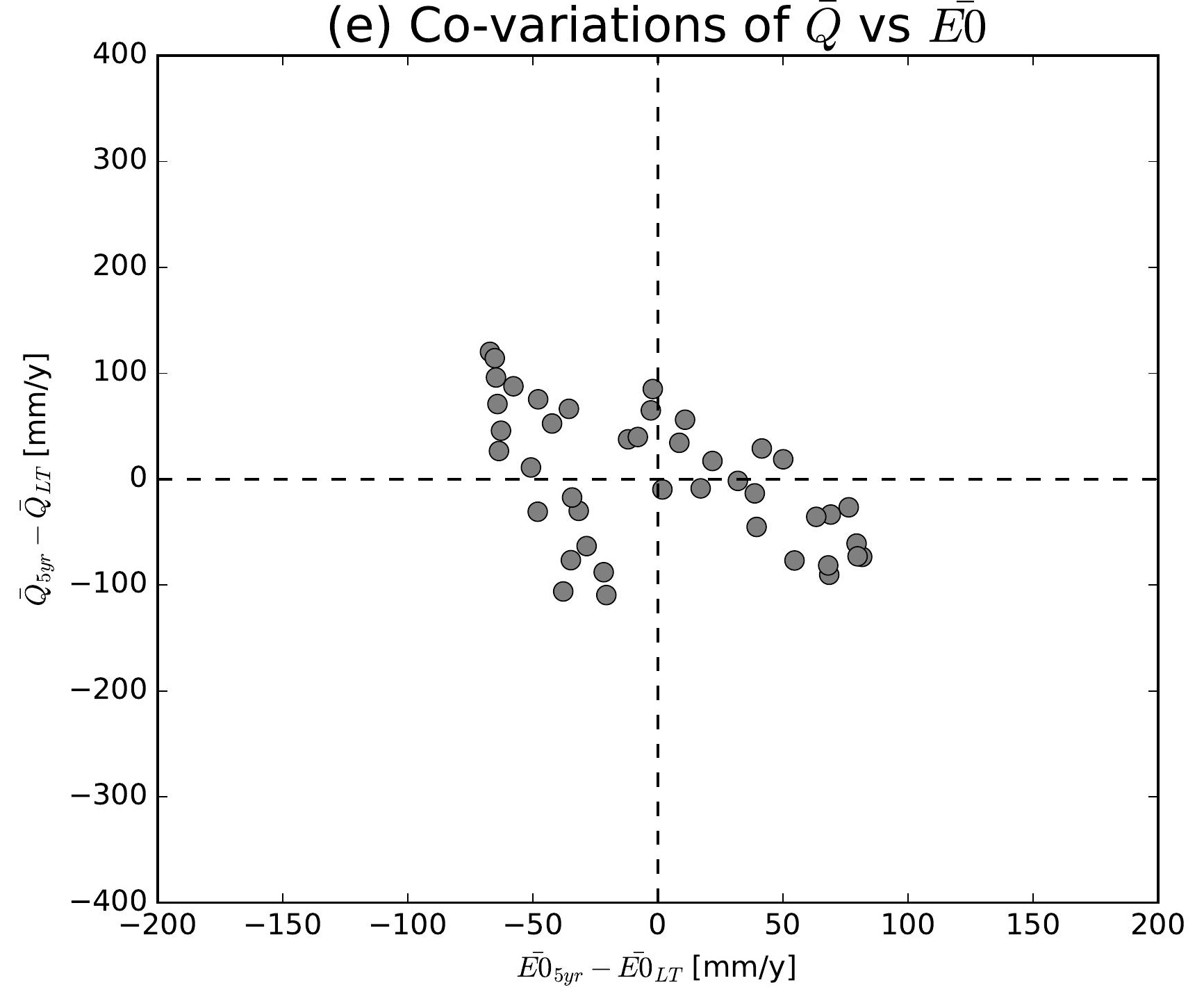

(h) Co-variations of $\bar{Q}$ vs $\overline{E 0}$

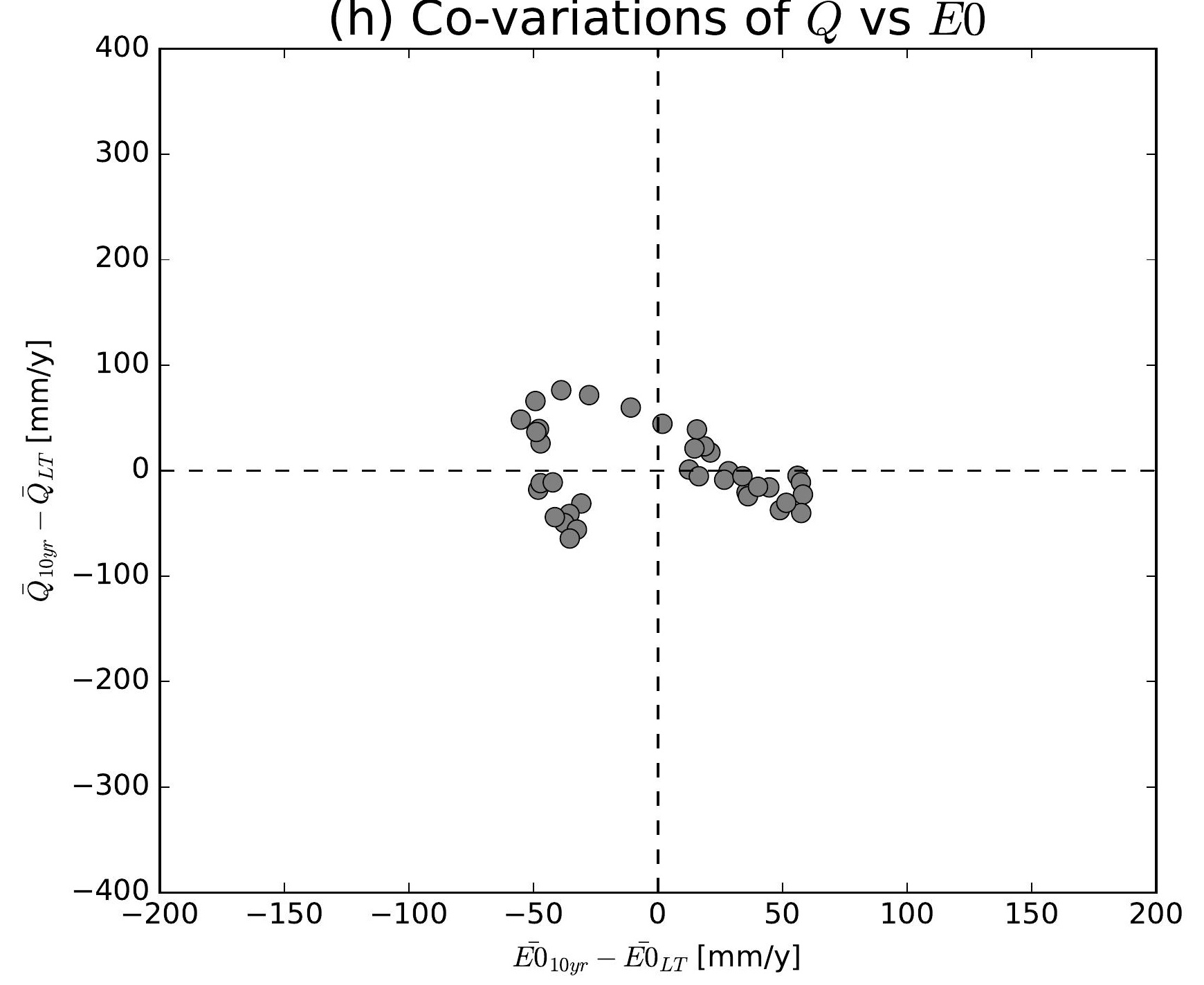

(k) Co-variations of $\bar{Q}$ vs $\overline{E 0}$

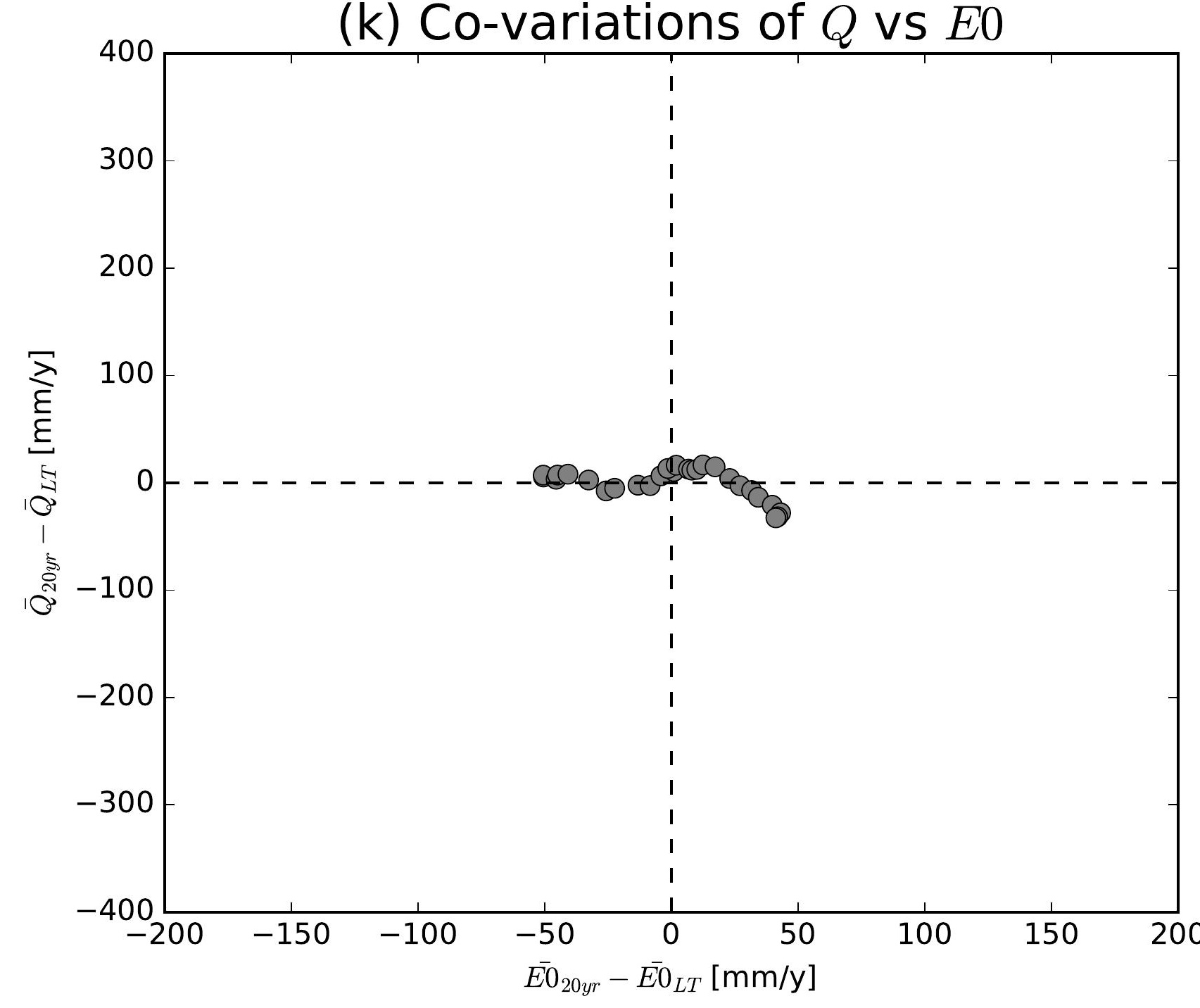

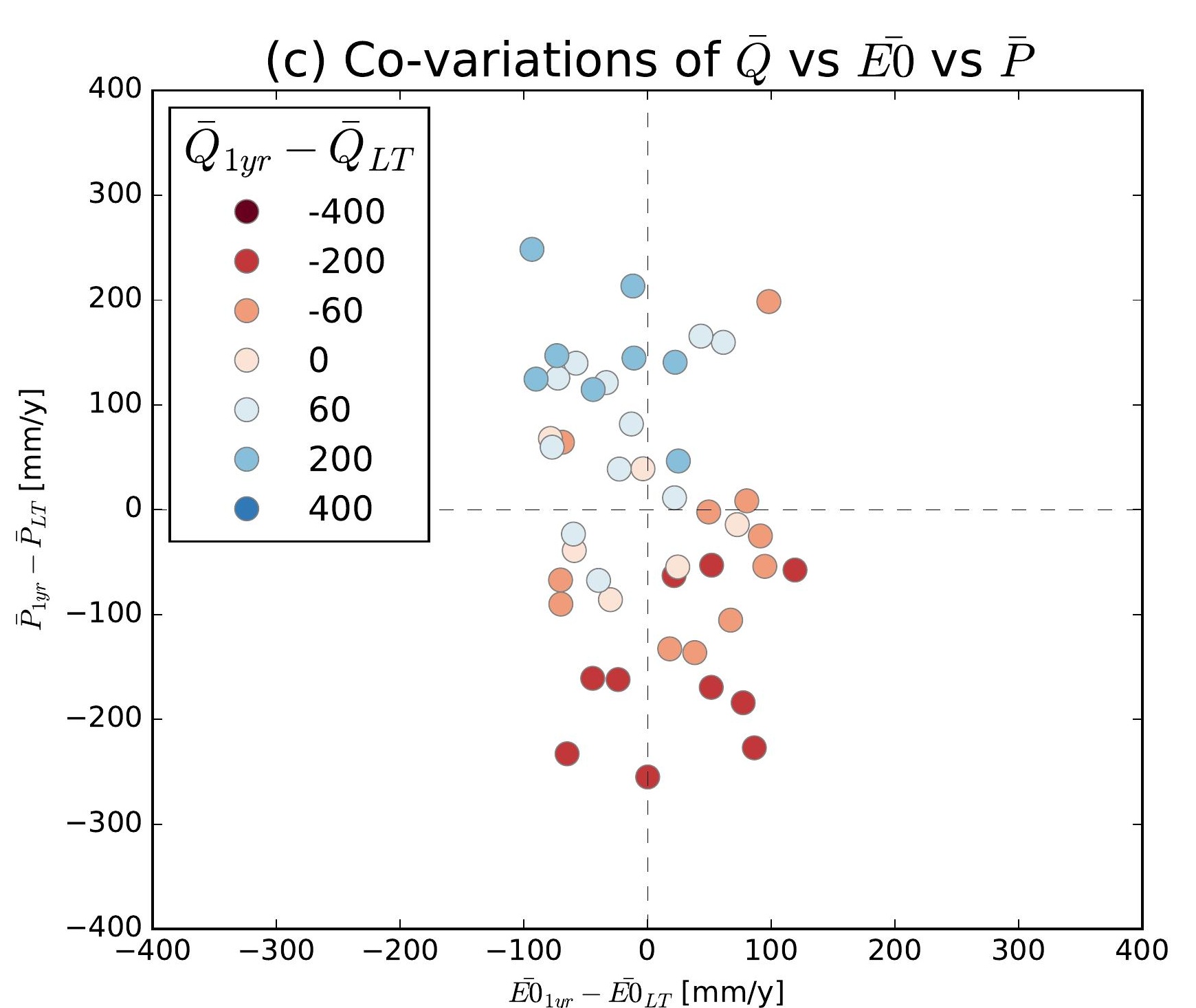
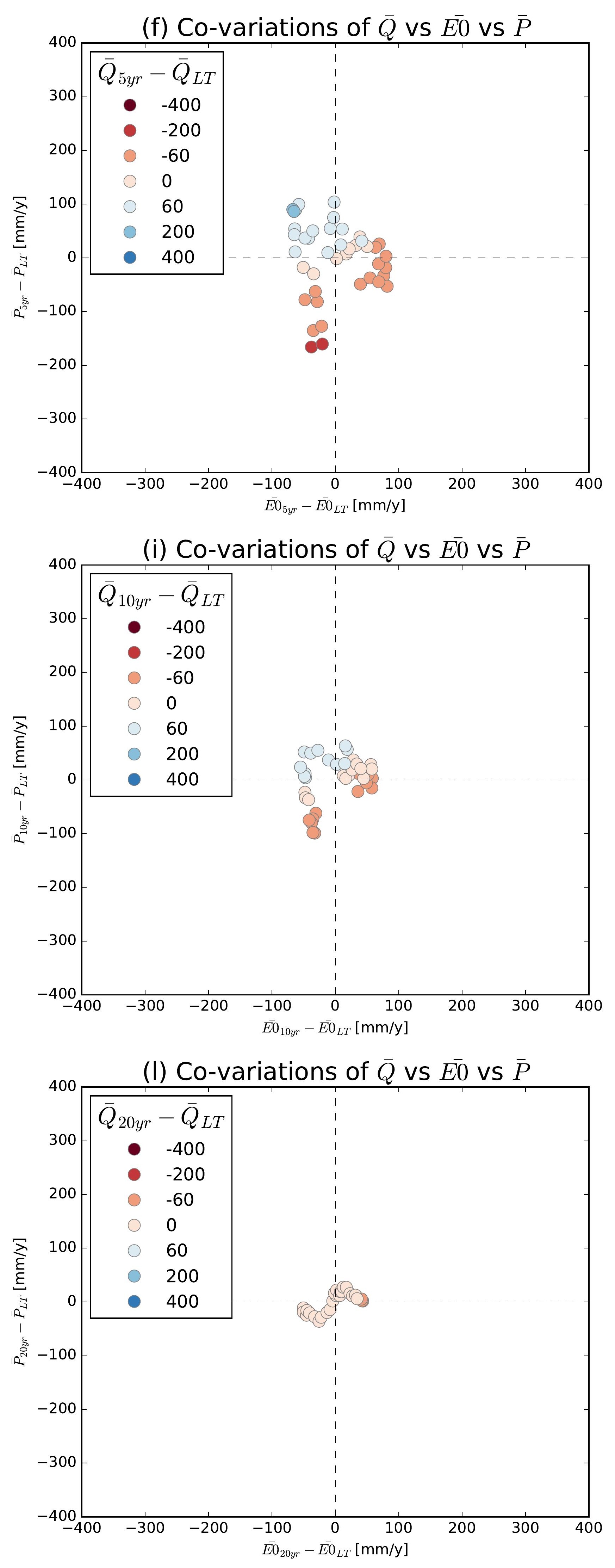

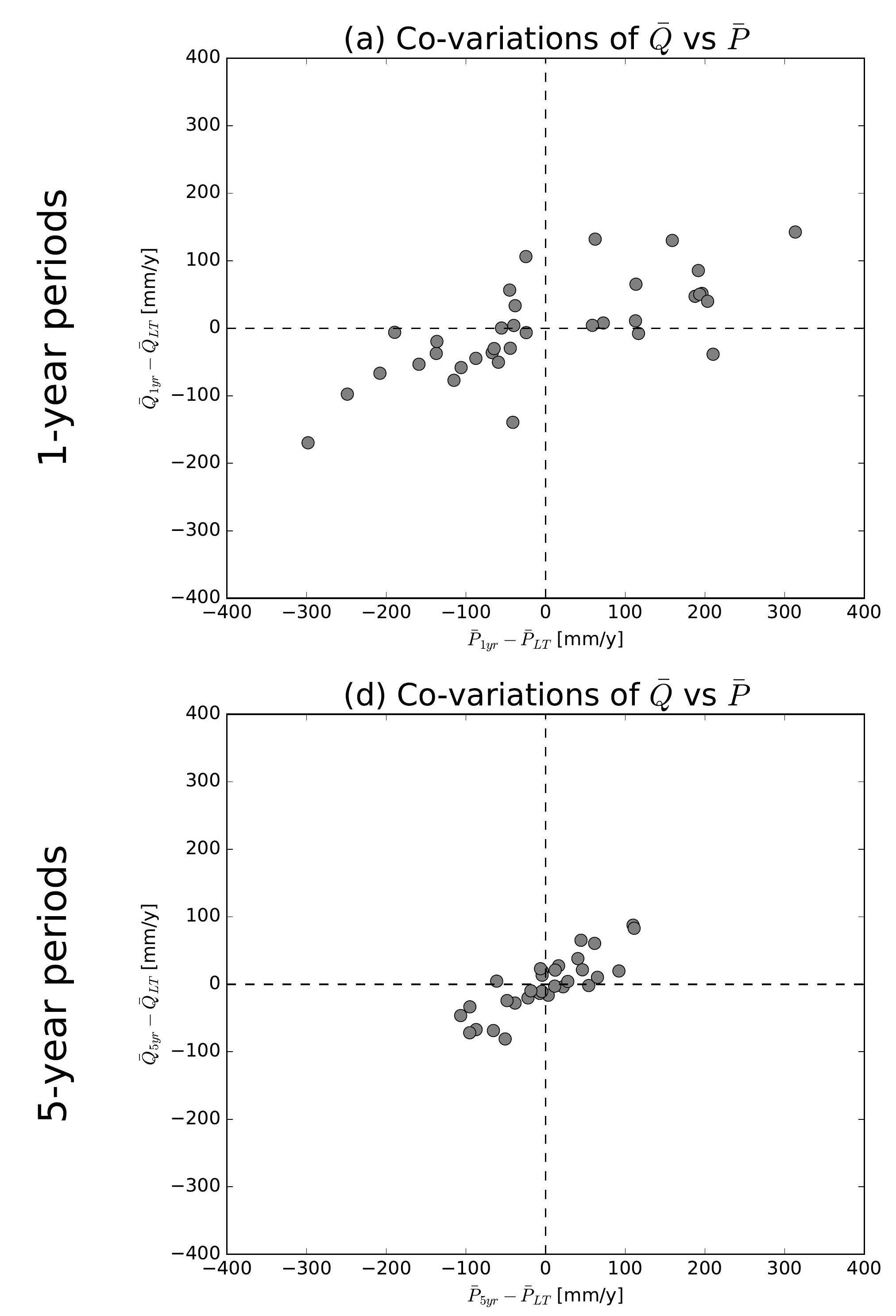

(g) Co-variations of $\bar{Q}$ vs $\bar{P}$
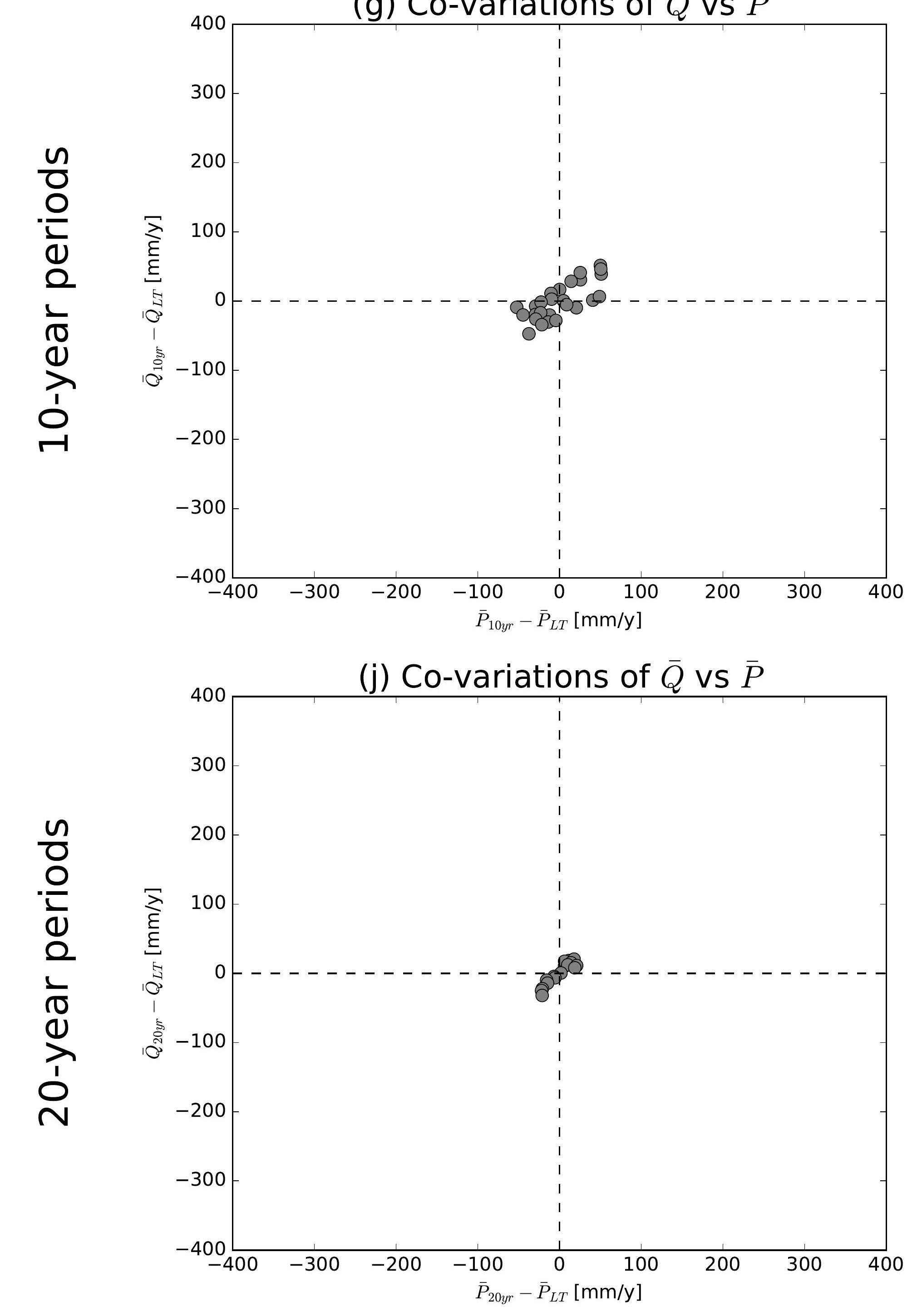

(b) Co-variations of $\bar{Q}$ vs $\overline{E 0}$

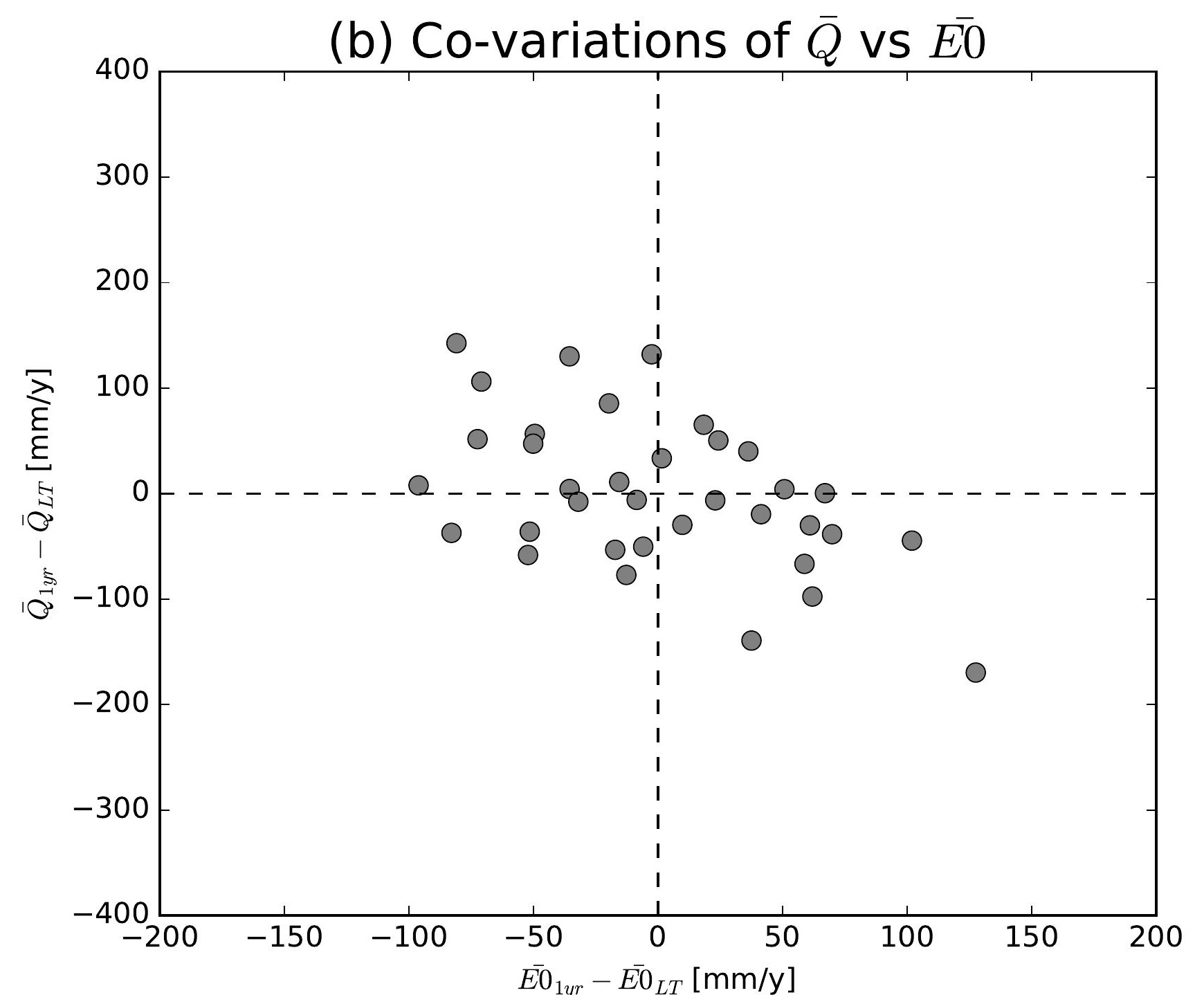

(e) Co-variations of $\bar{Q}$ vs $\overline{E 0}$

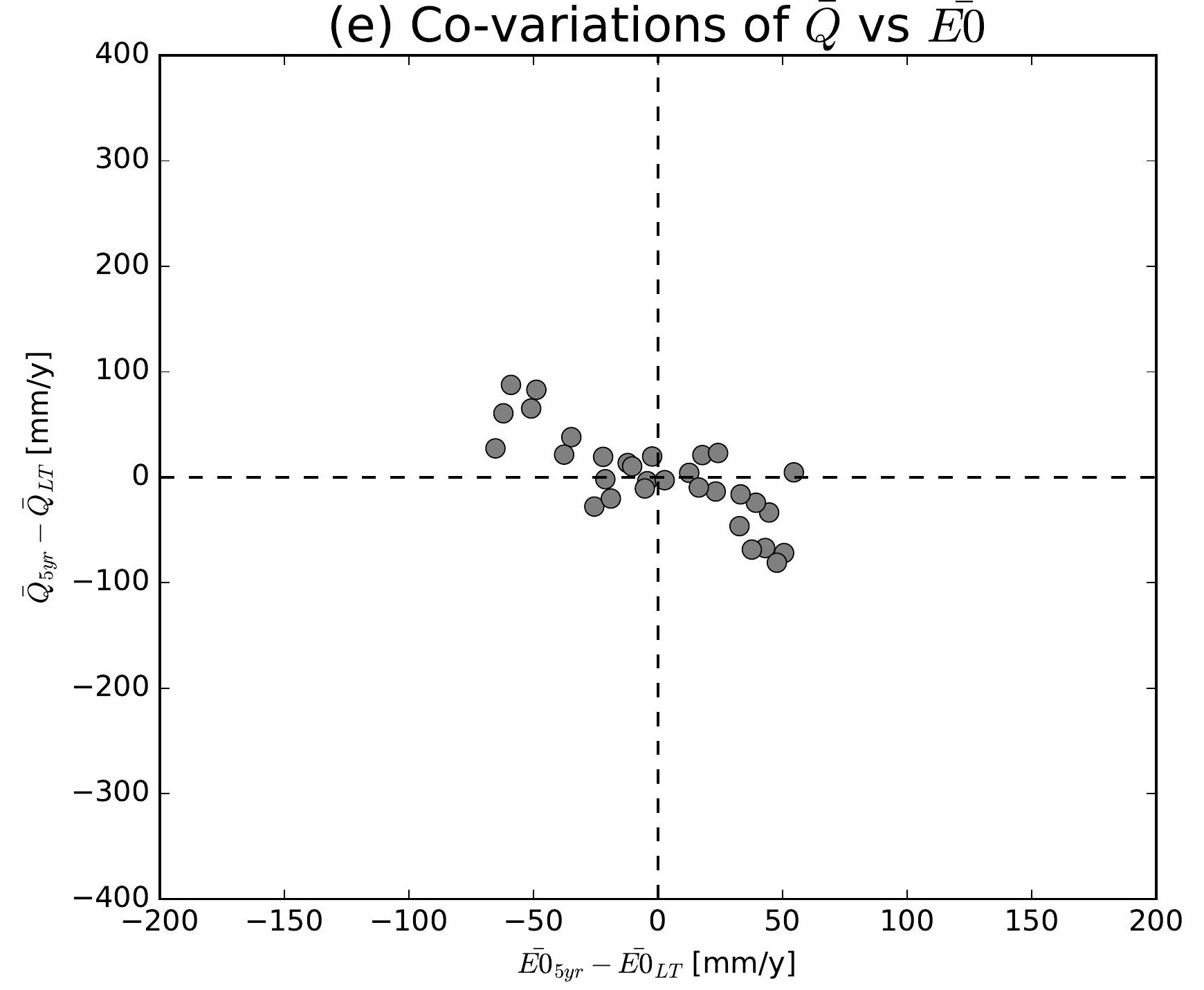

(h) Co-variations of $\bar{Q}$ vs $\overline{E 0}$

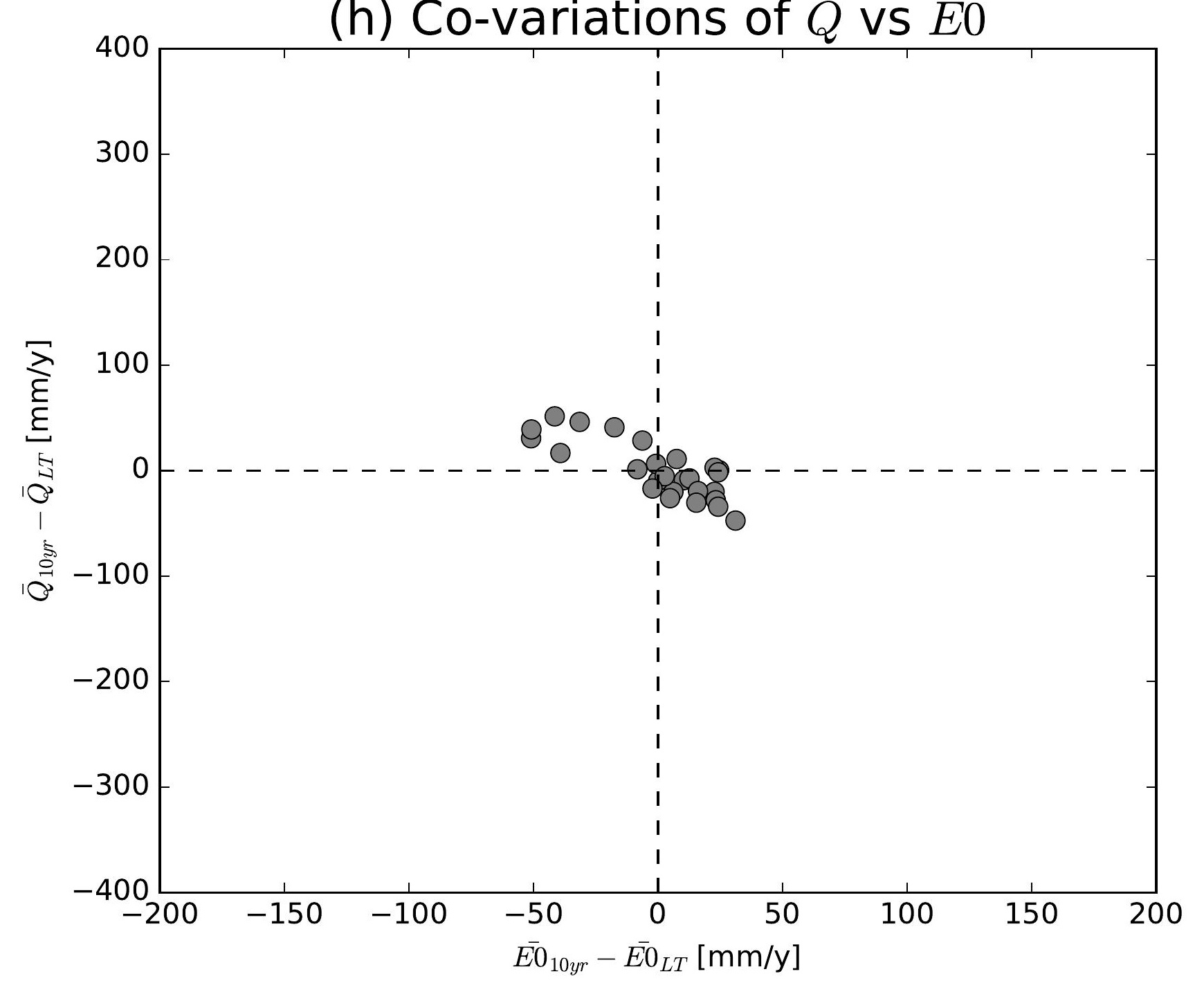

(k) Co-variations of $\bar{Q}$ vs $\overline{E 0}$

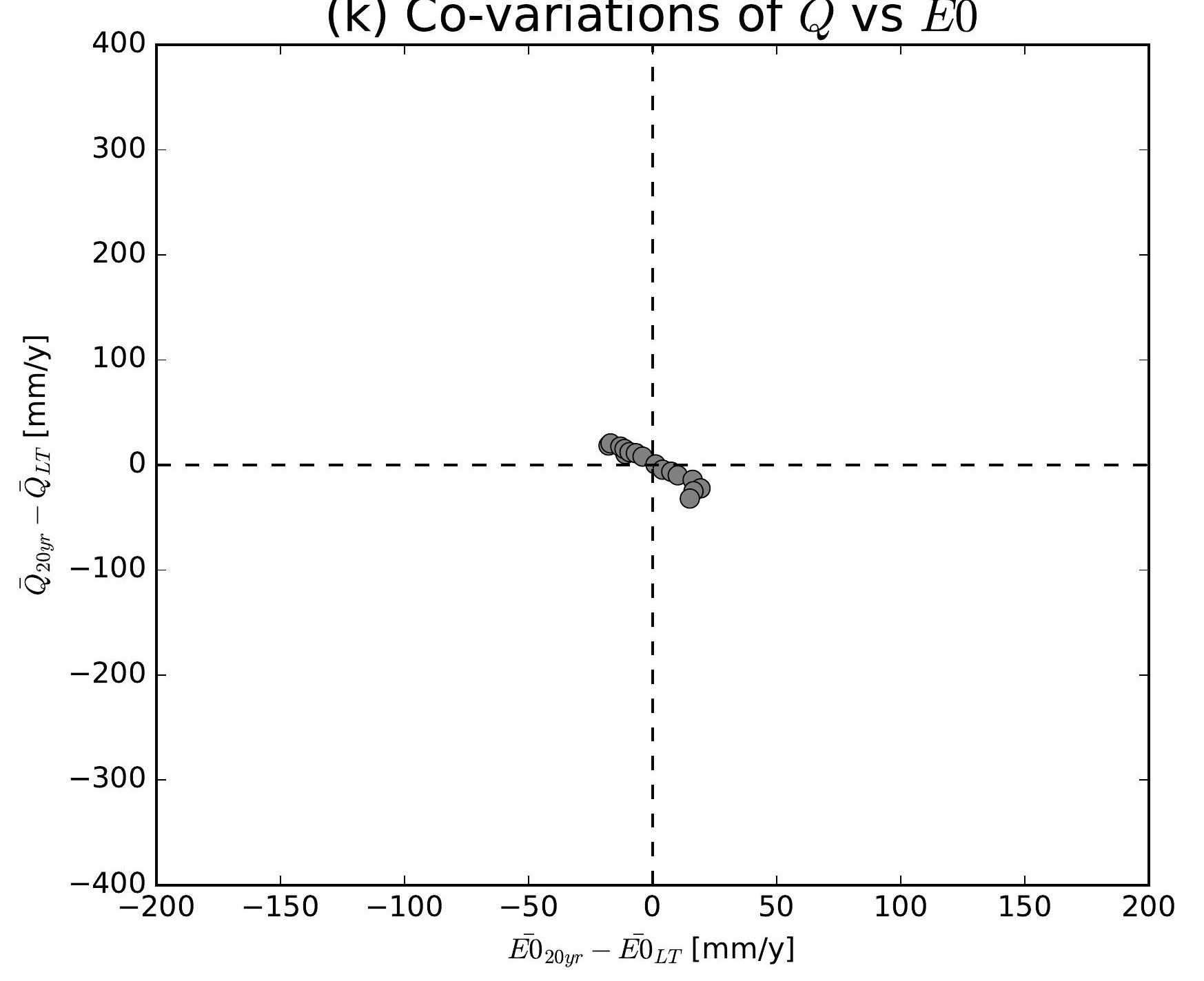

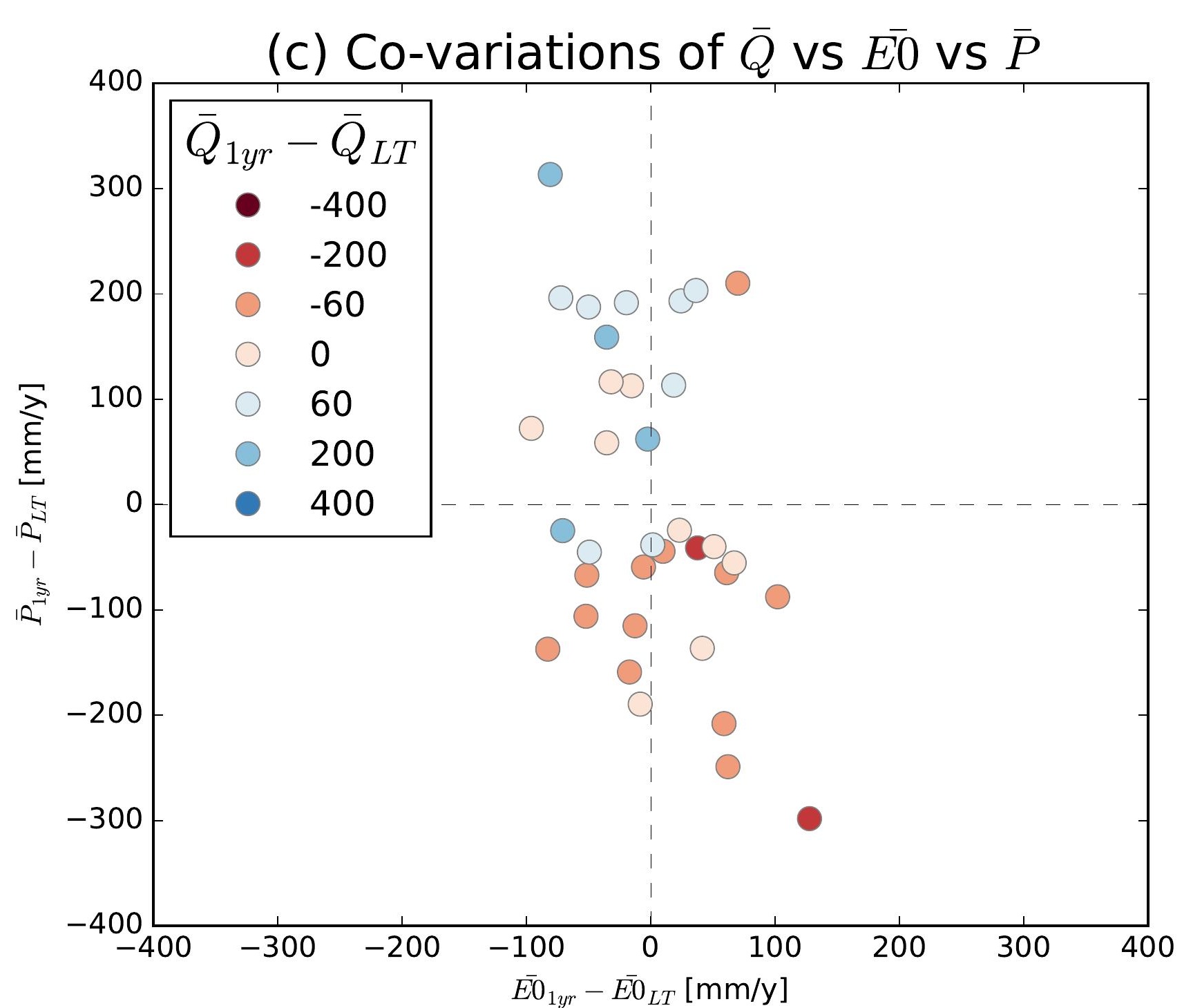
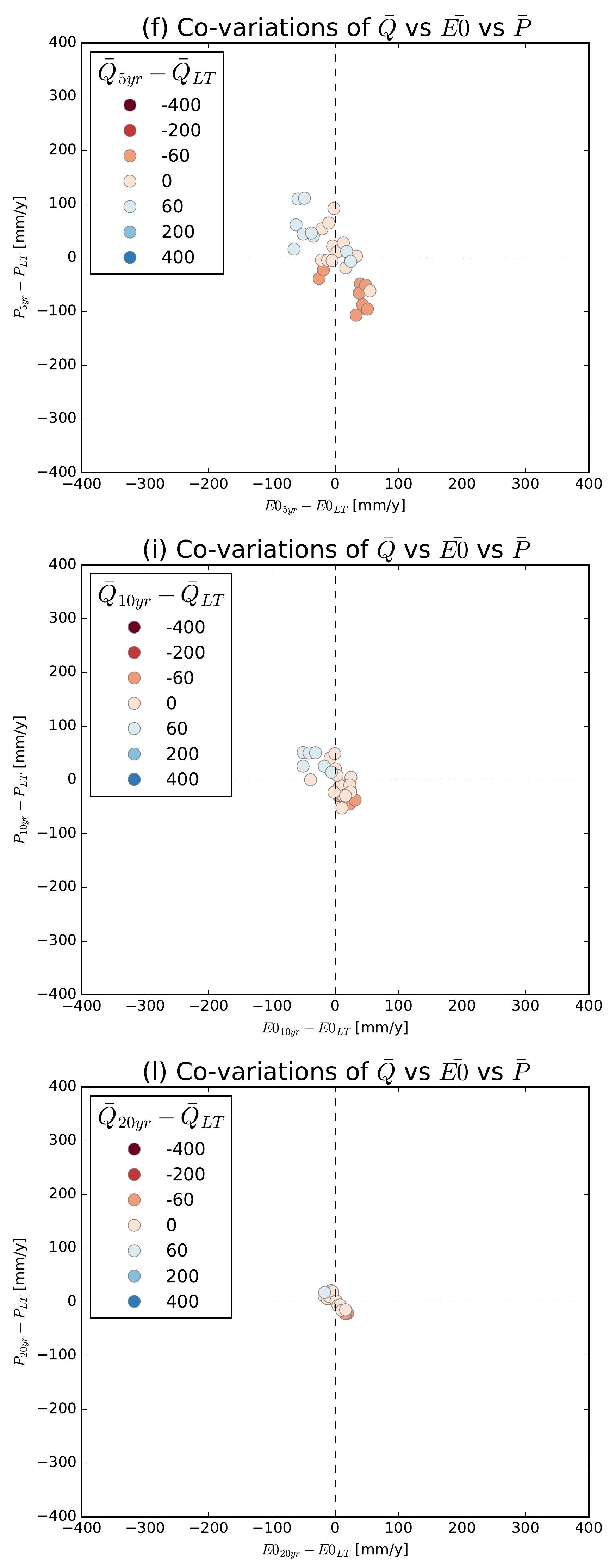

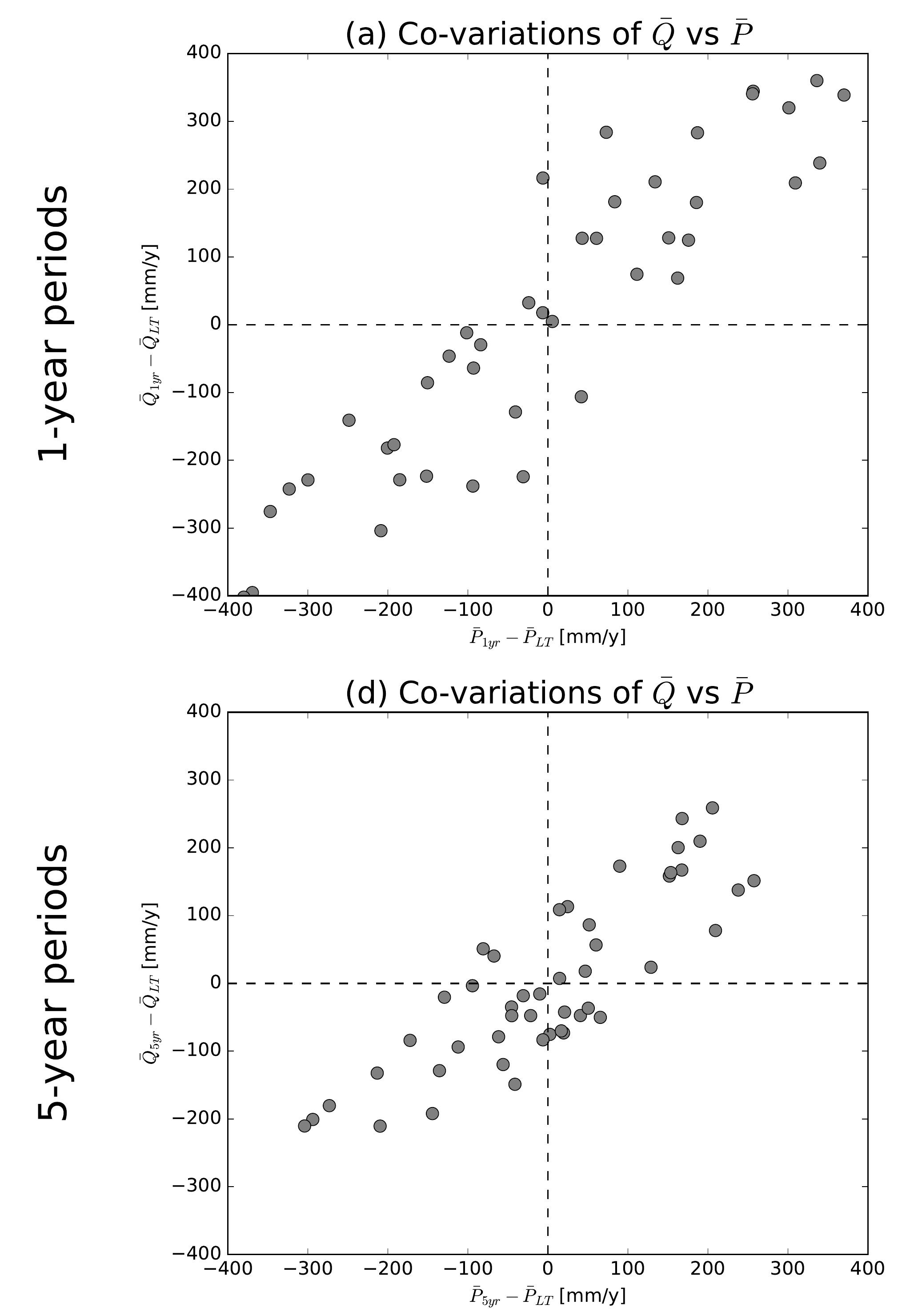

(g) Co-variations of $\bar{Q}$ vs $\bar{P}$
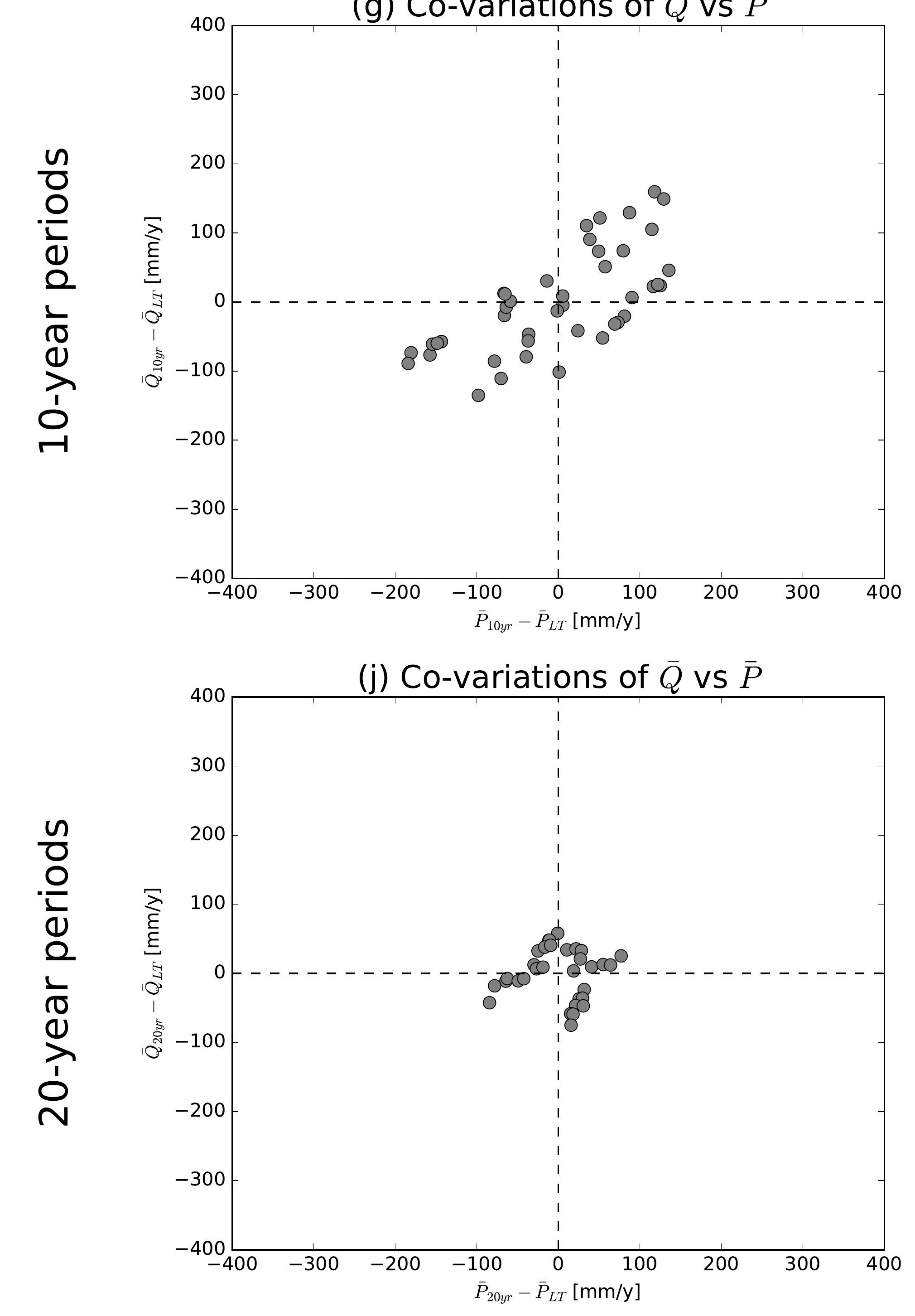

(b) Co-variations of $\bar{Q}$ vs $\overline{E 0}$

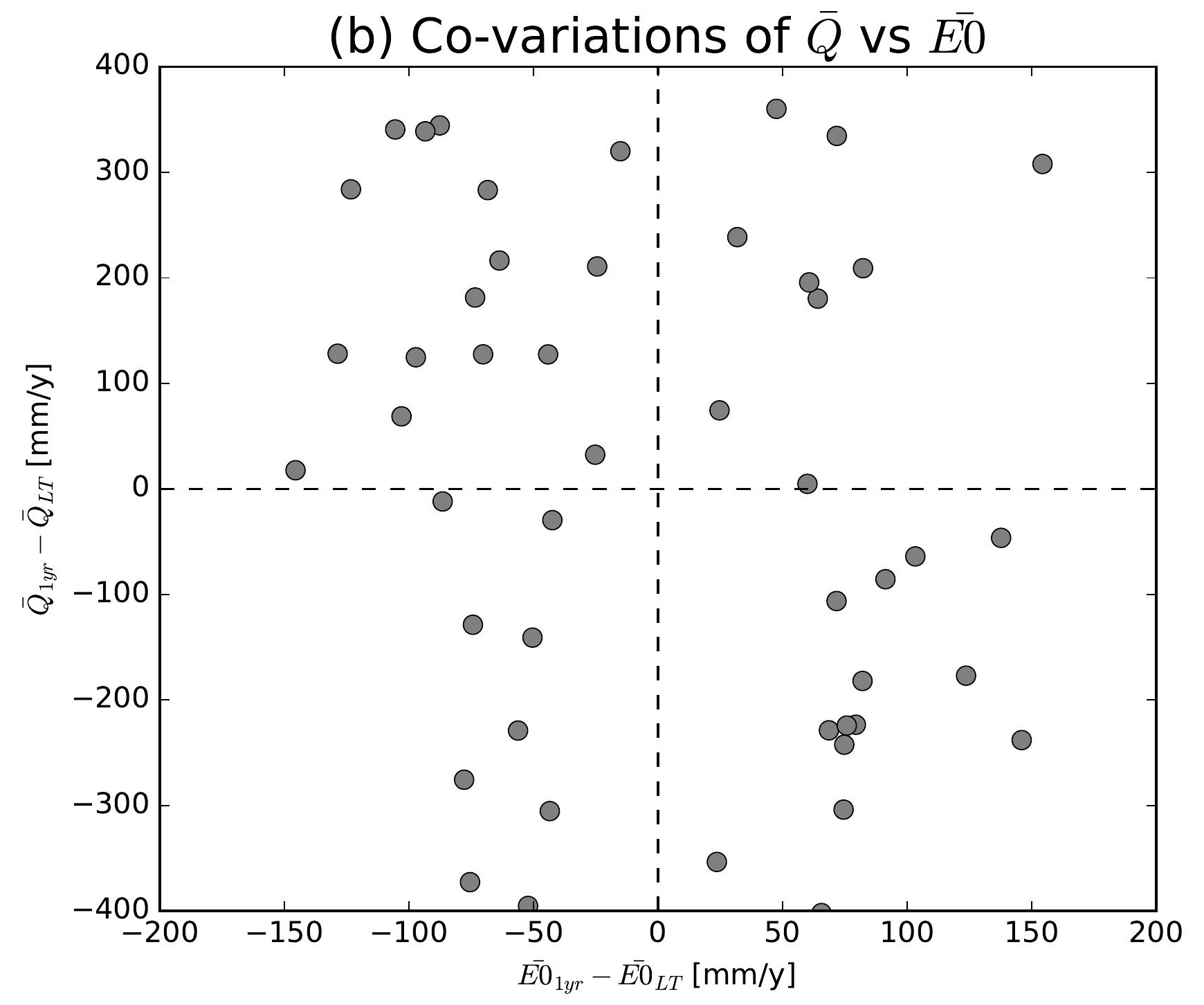

(e) Co-variations of $\bar{Q}$ vs $\overline{E 0}$

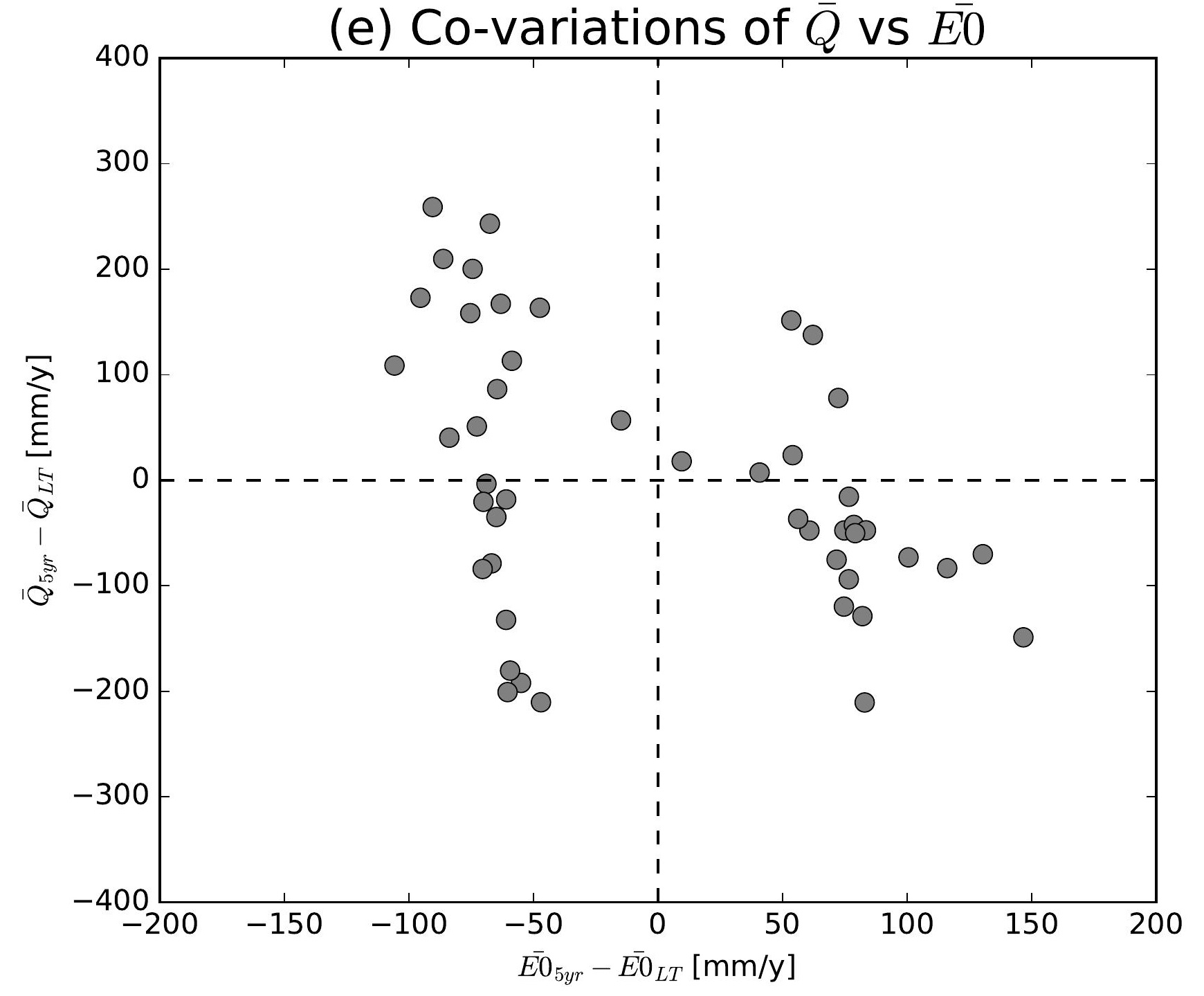

(h) Co-variations of $\bar{Q}$ vs $\overline{E 0}$

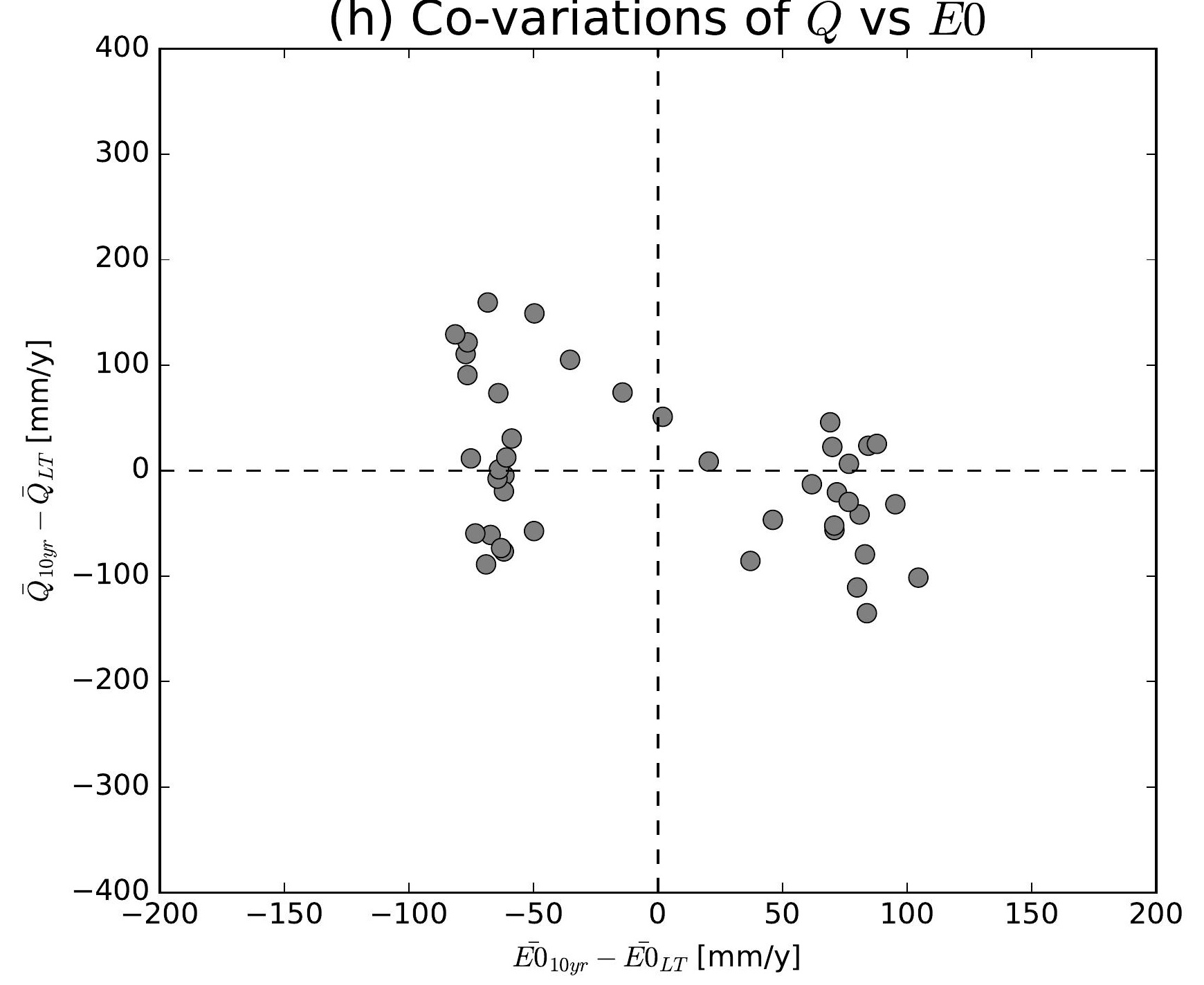

(k) Co-variations of $\bar{Q}$ vs $\overline{E 0}$

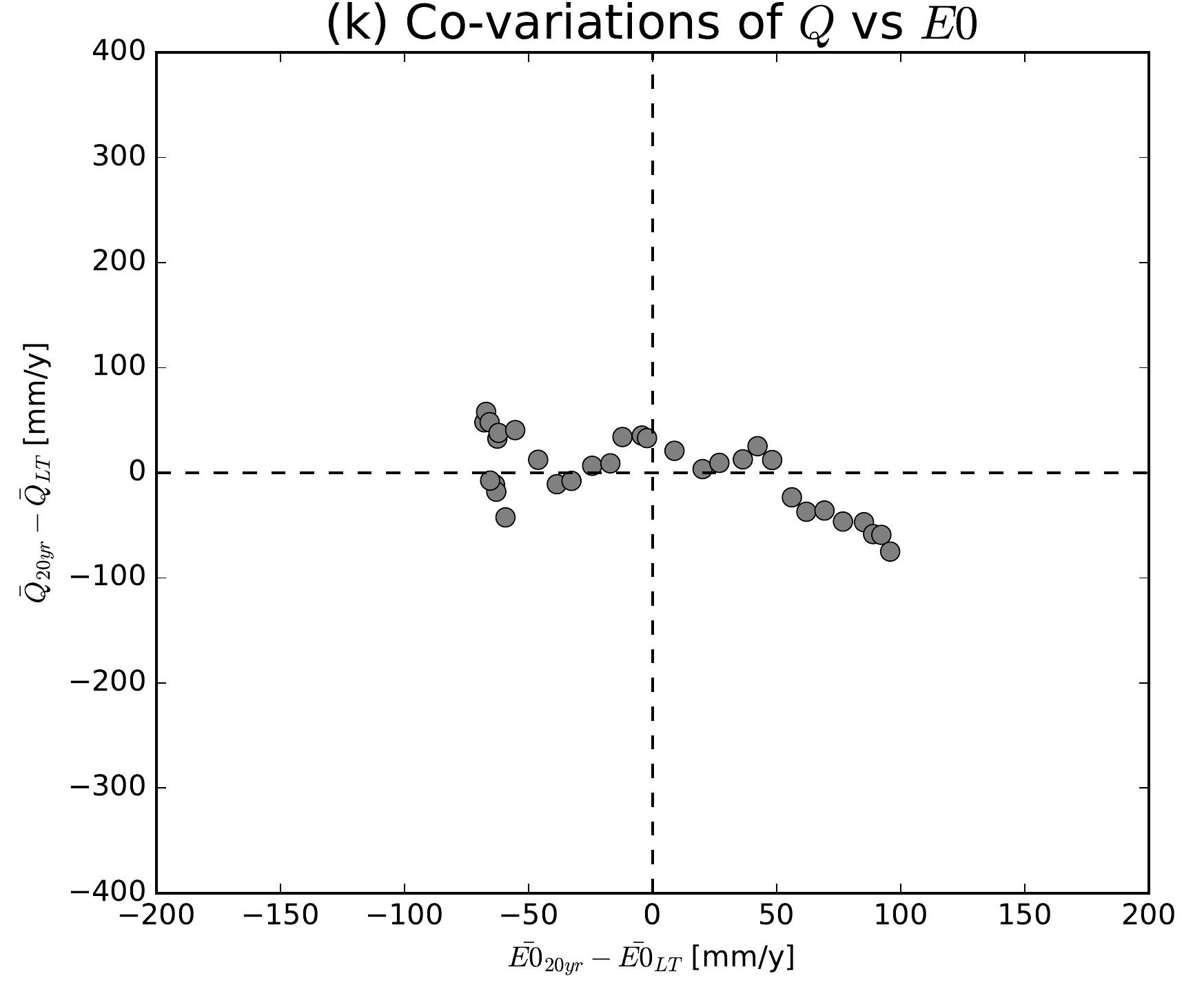

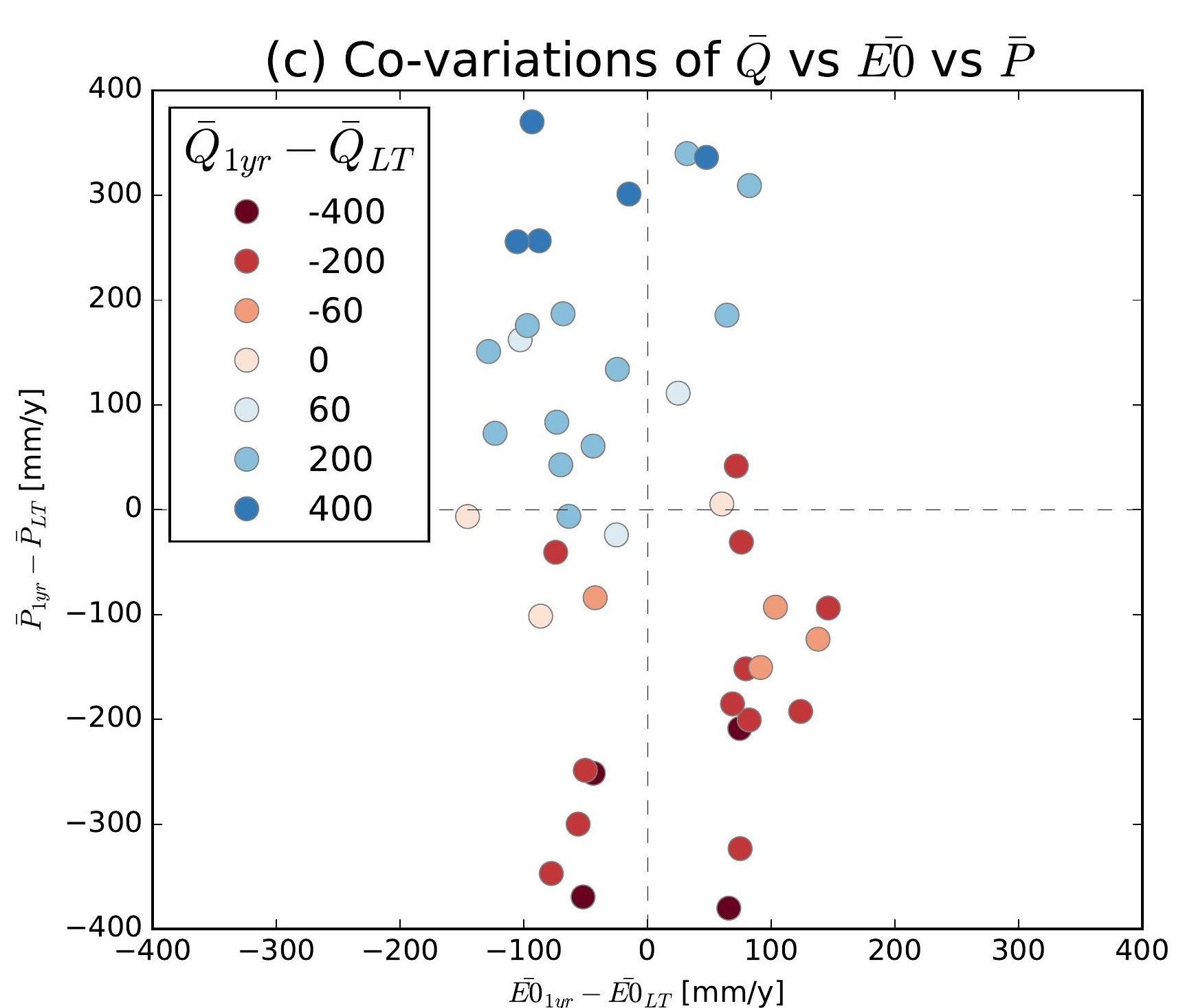
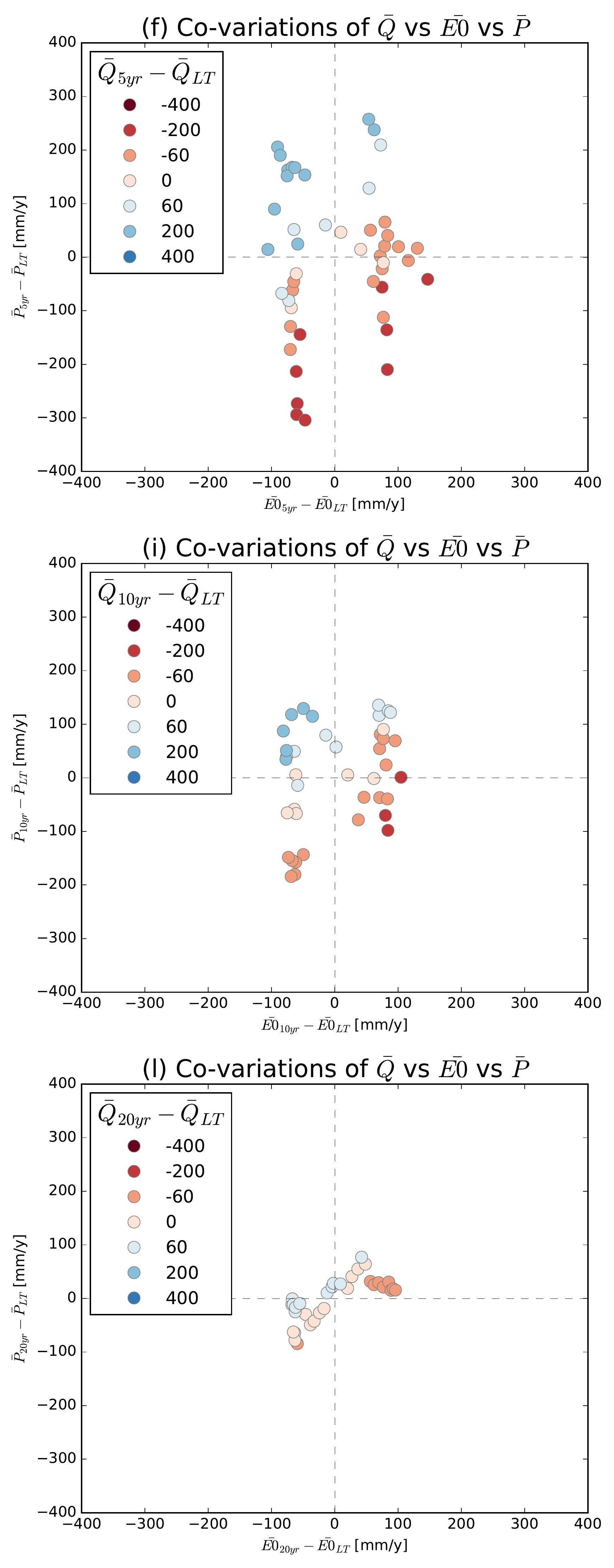

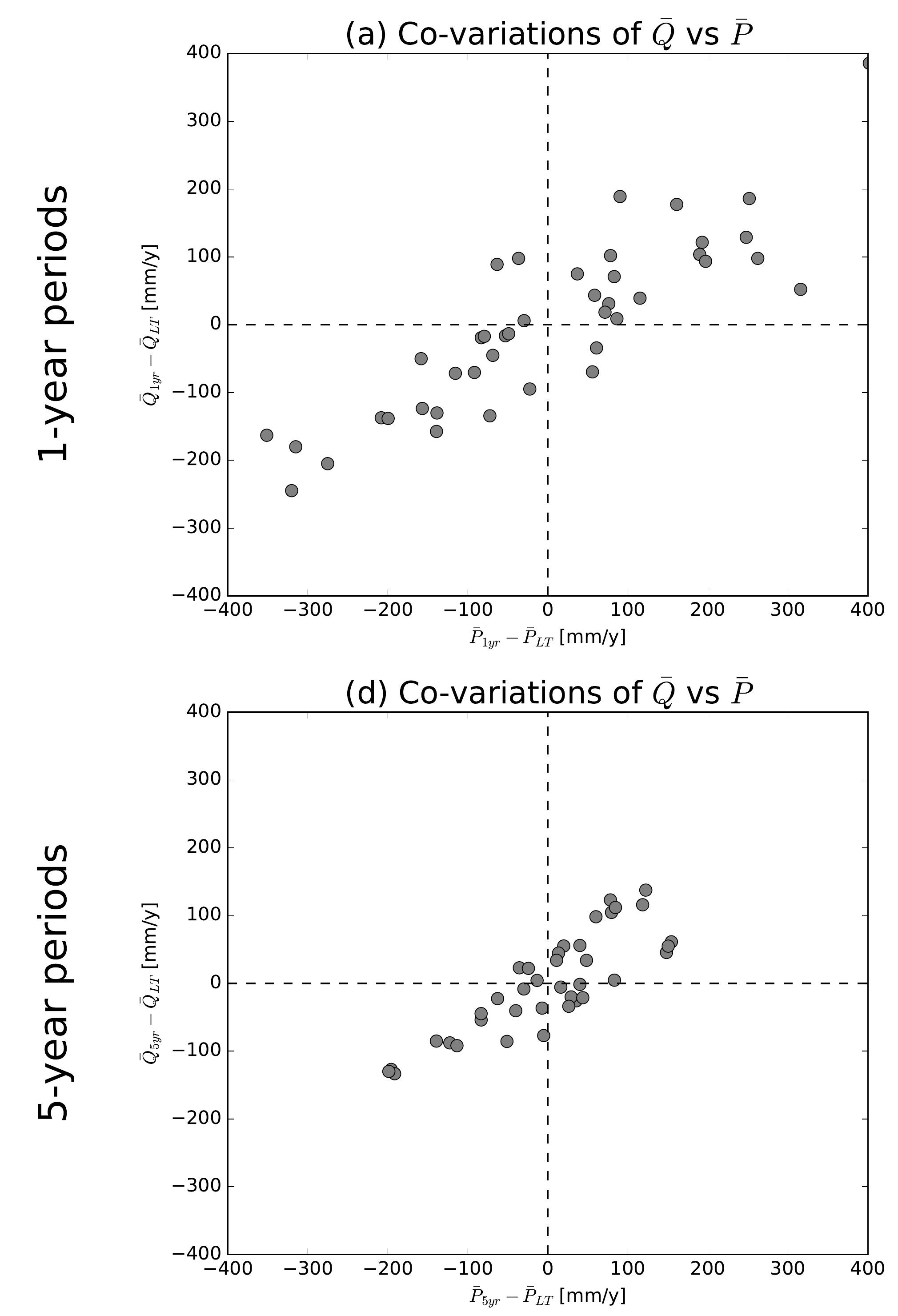

(g) Co-variations of $\bar{Q}$ vs $\bar{P}$
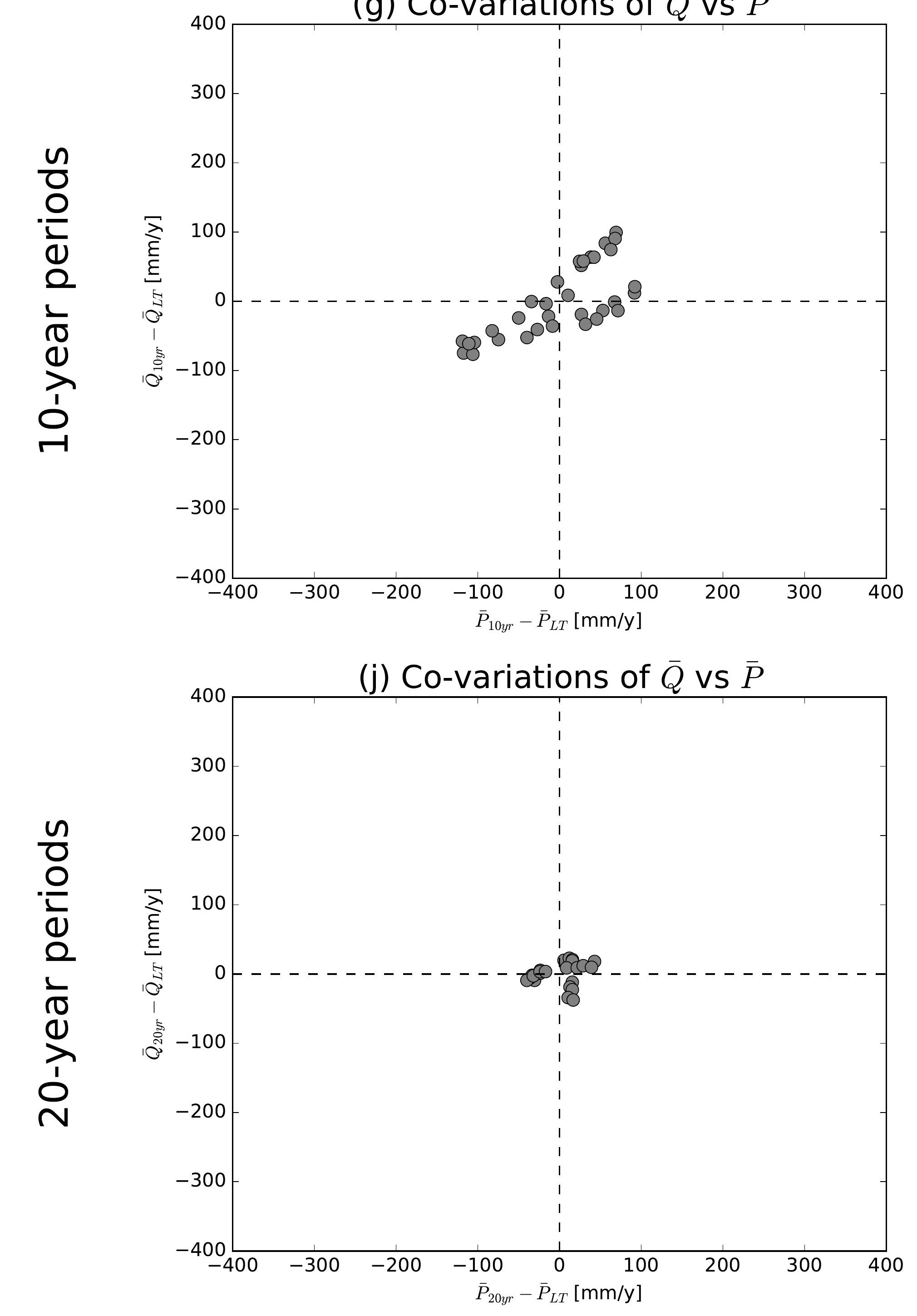

(b) Co-variations of $\bar{Q}$ vs $\overline{E 0}$

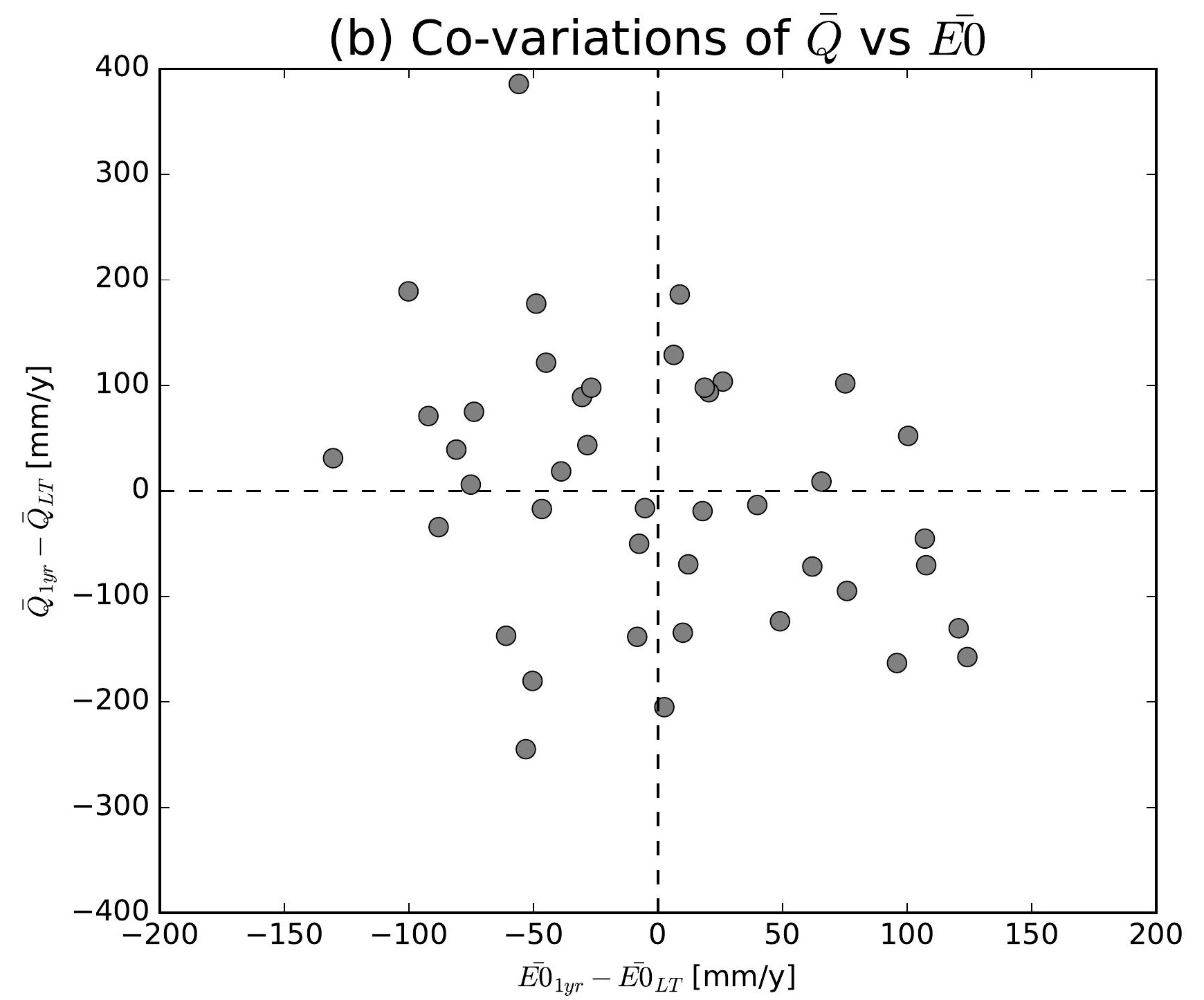

(e) Co-variations of $\bar{Q}$ vs $\overline{E 0}$

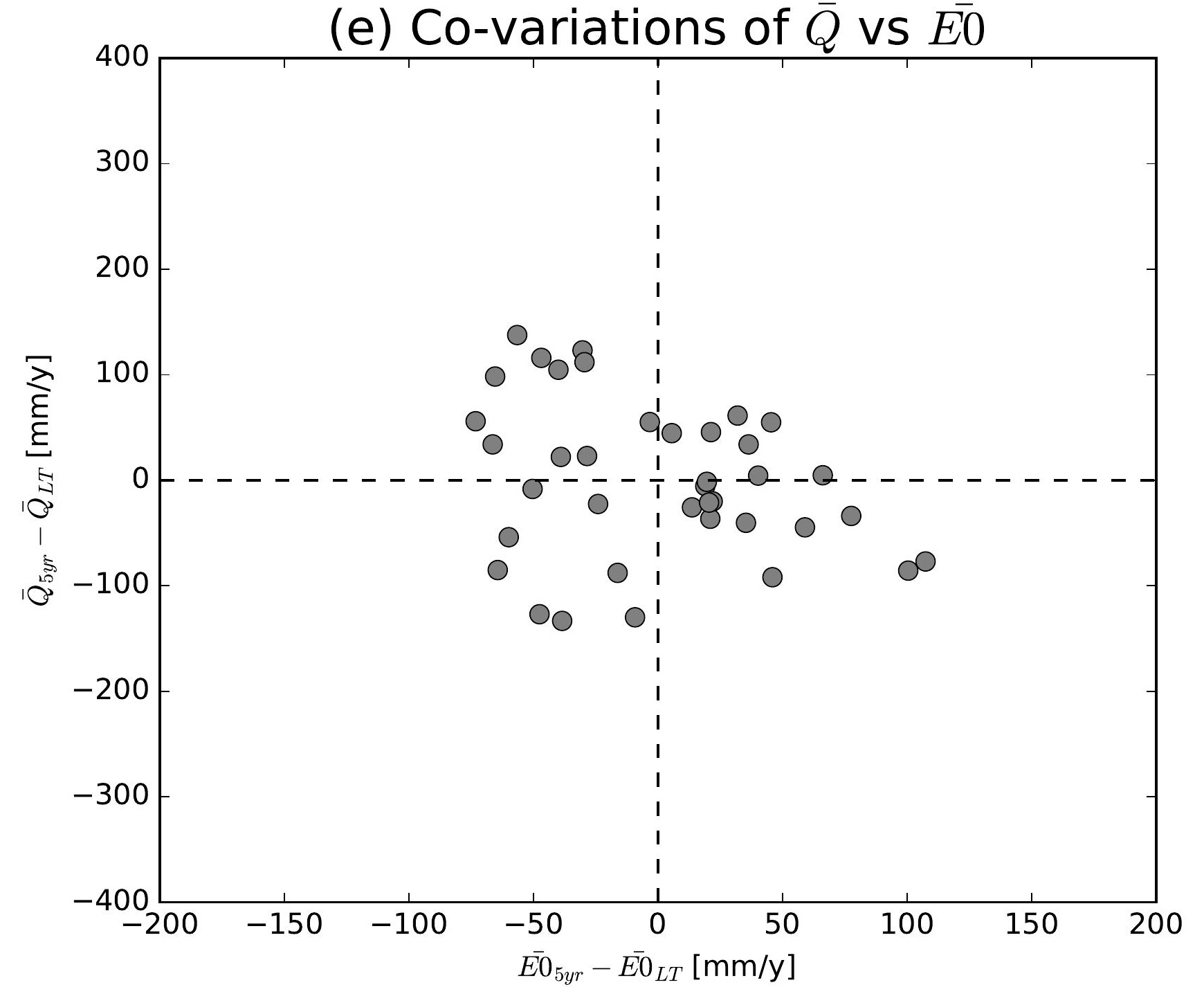

(h) Co-variations of $\bar{Q}$ vs $\overline{E 0}$

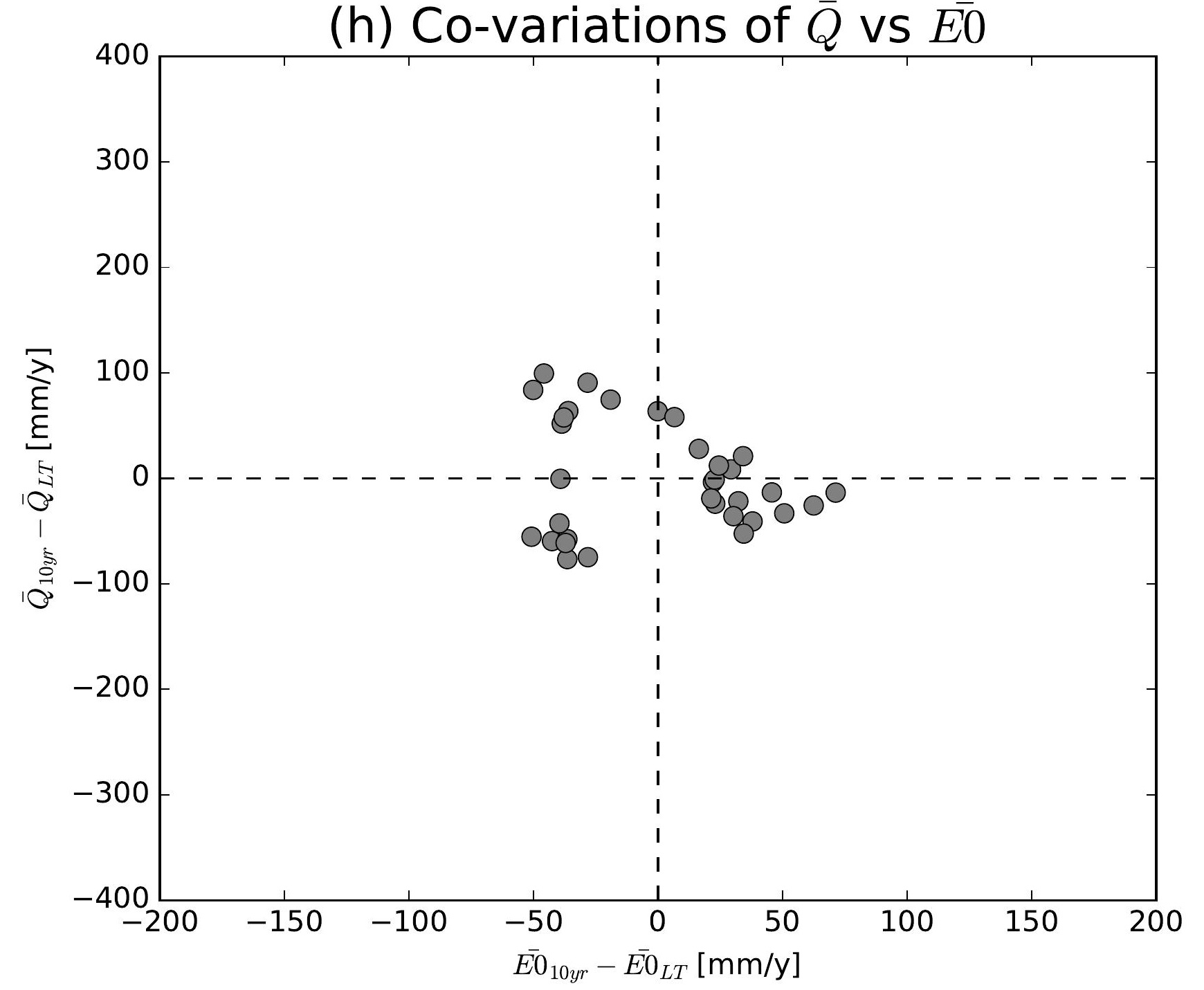

(k) Co-variations of $\bar{Q}$ vs $\overline{E 0}$

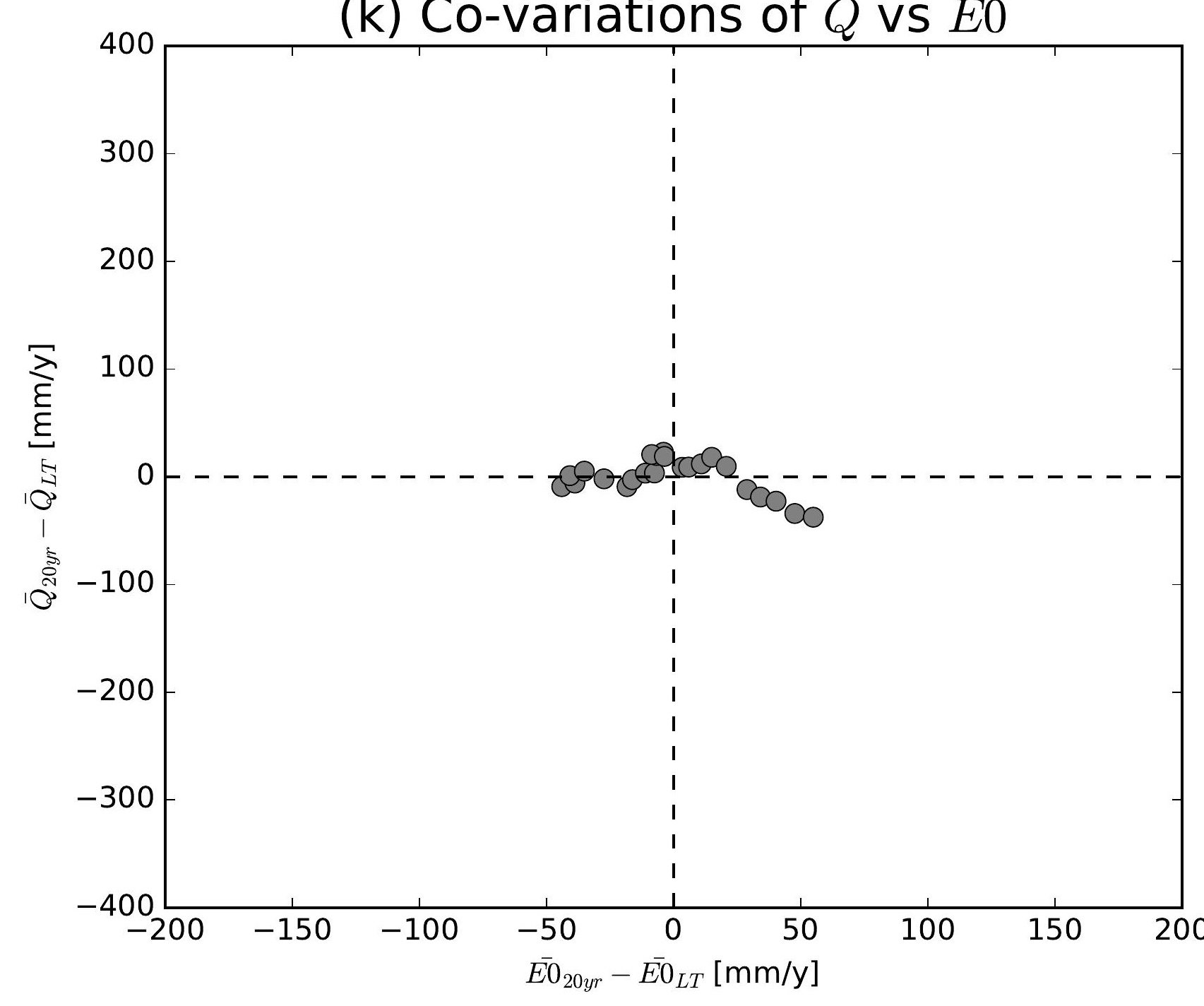

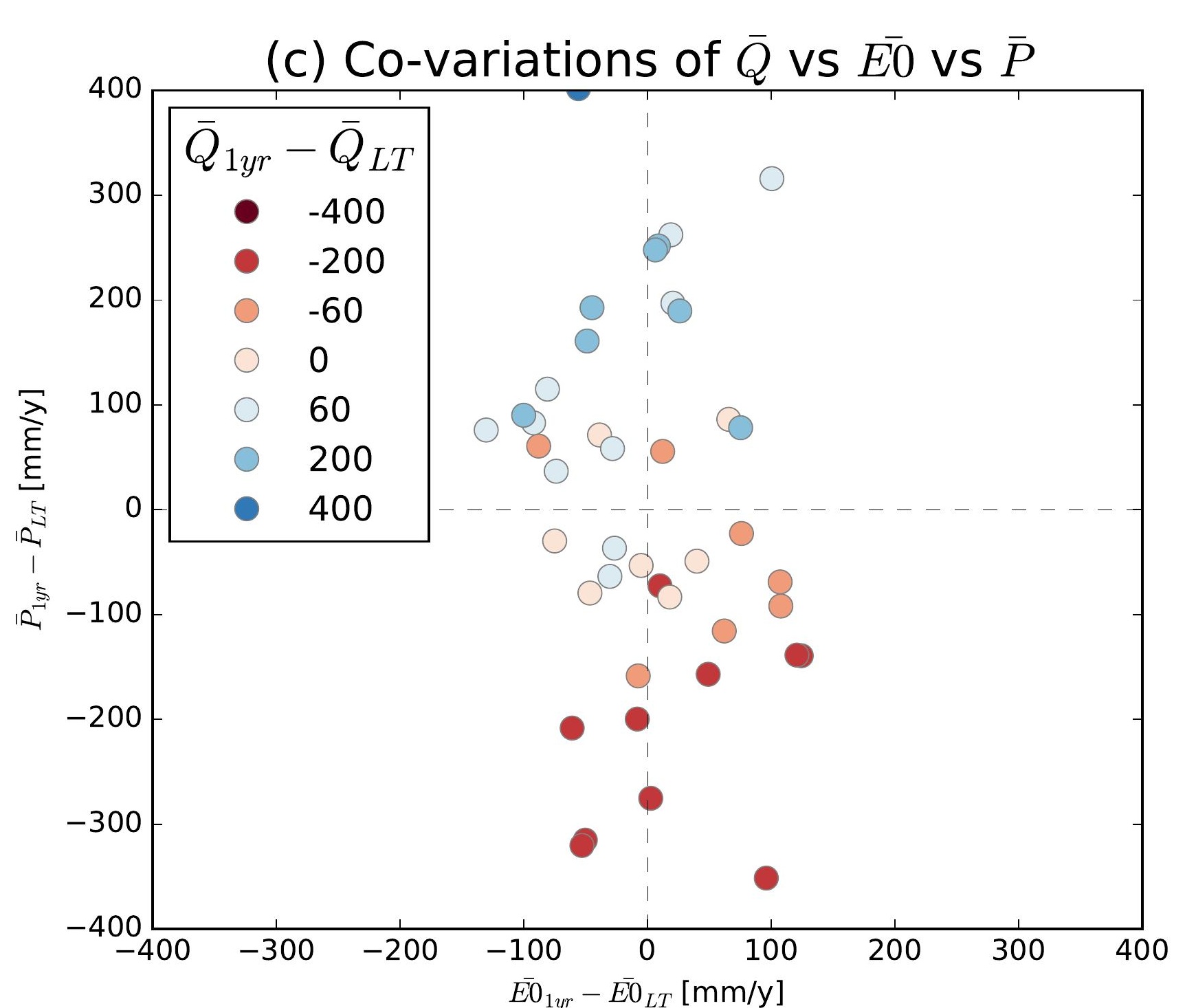
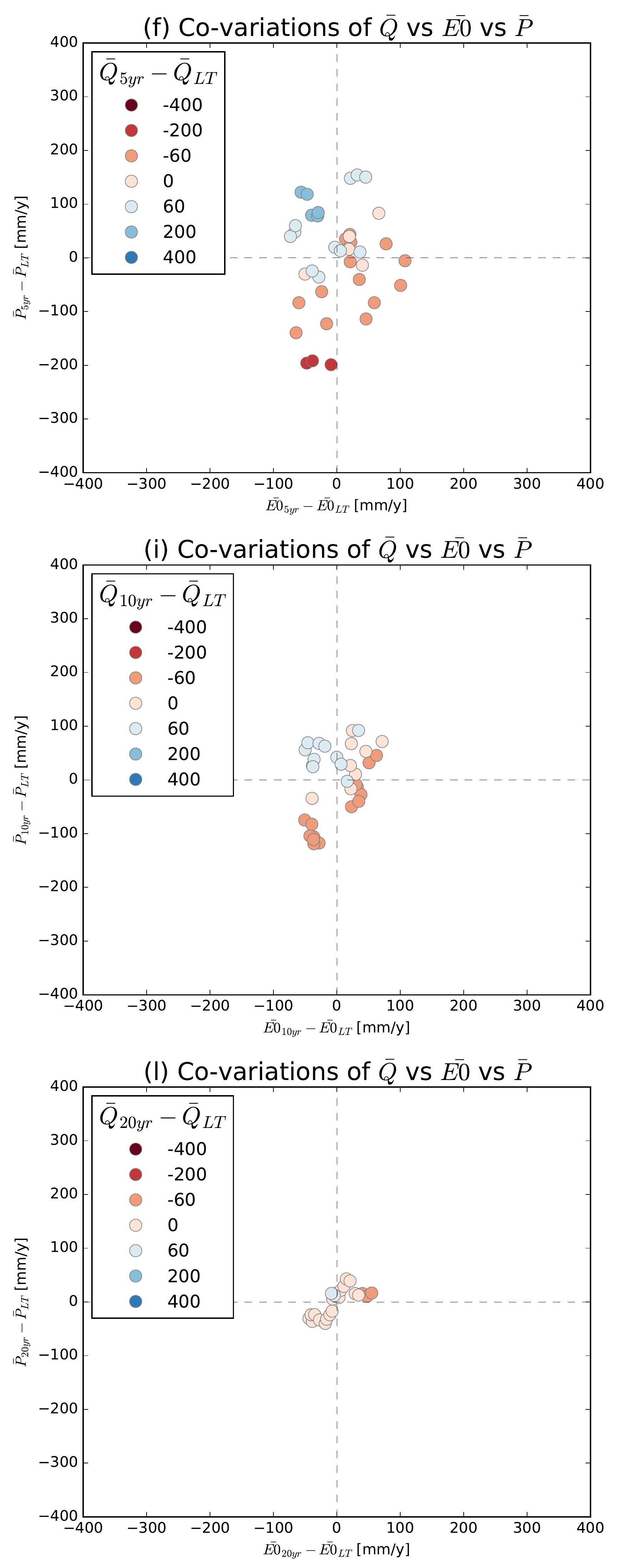

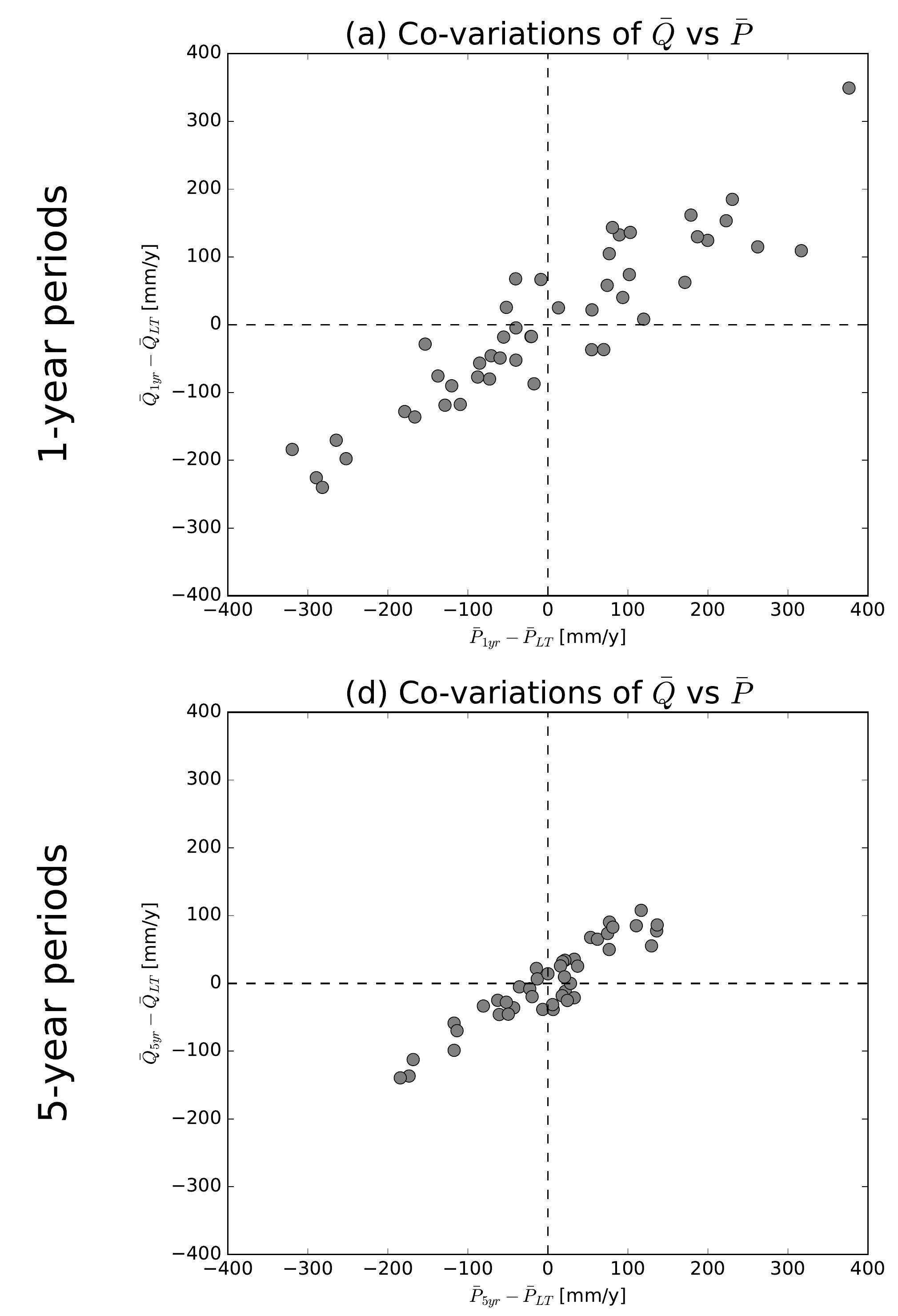

(g) Co-variations of $\bar{Q}$ vs $\bar{P}$
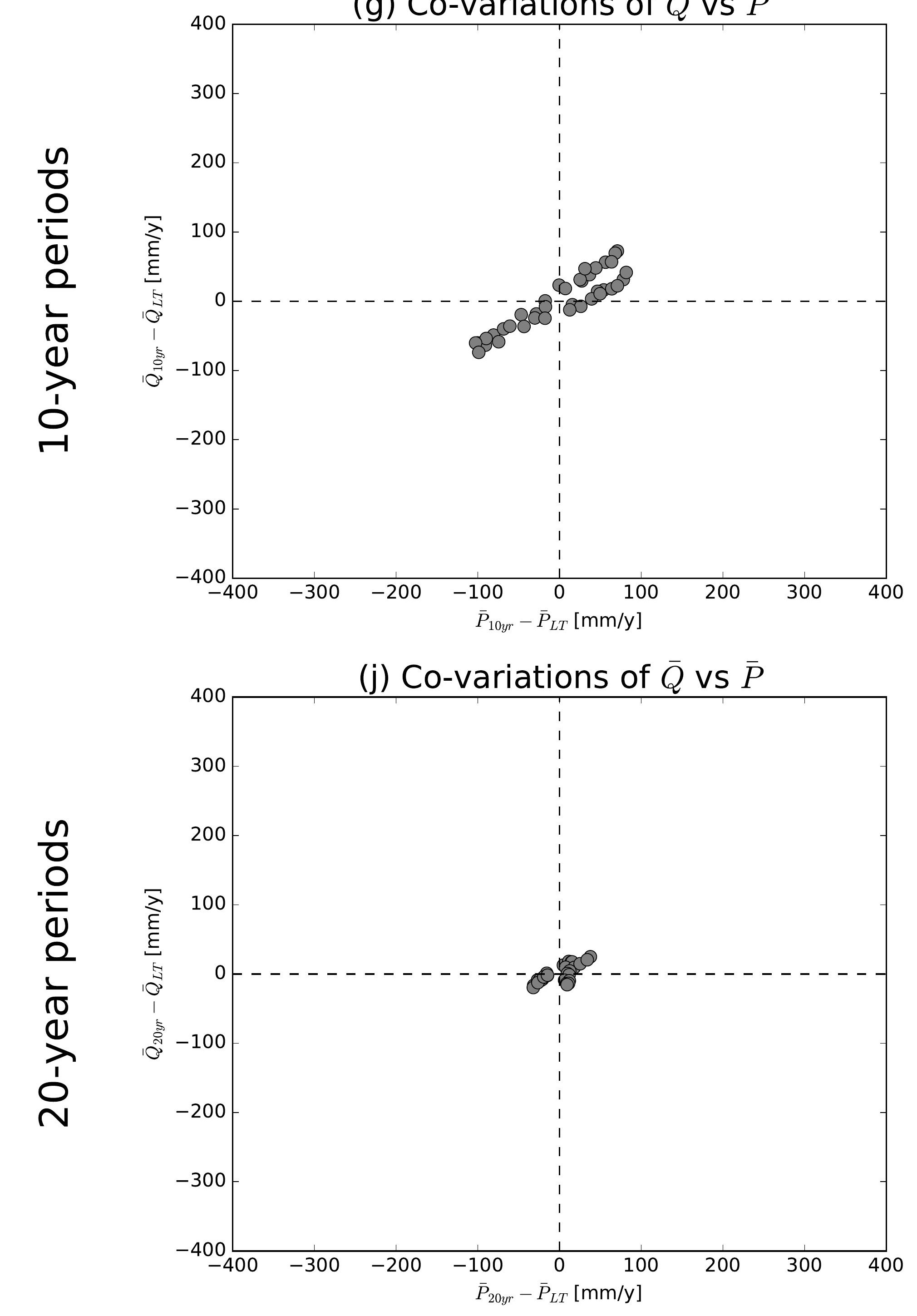

(b) Co-variations of $\bar{Q}$ vs $\overline{E 0}$

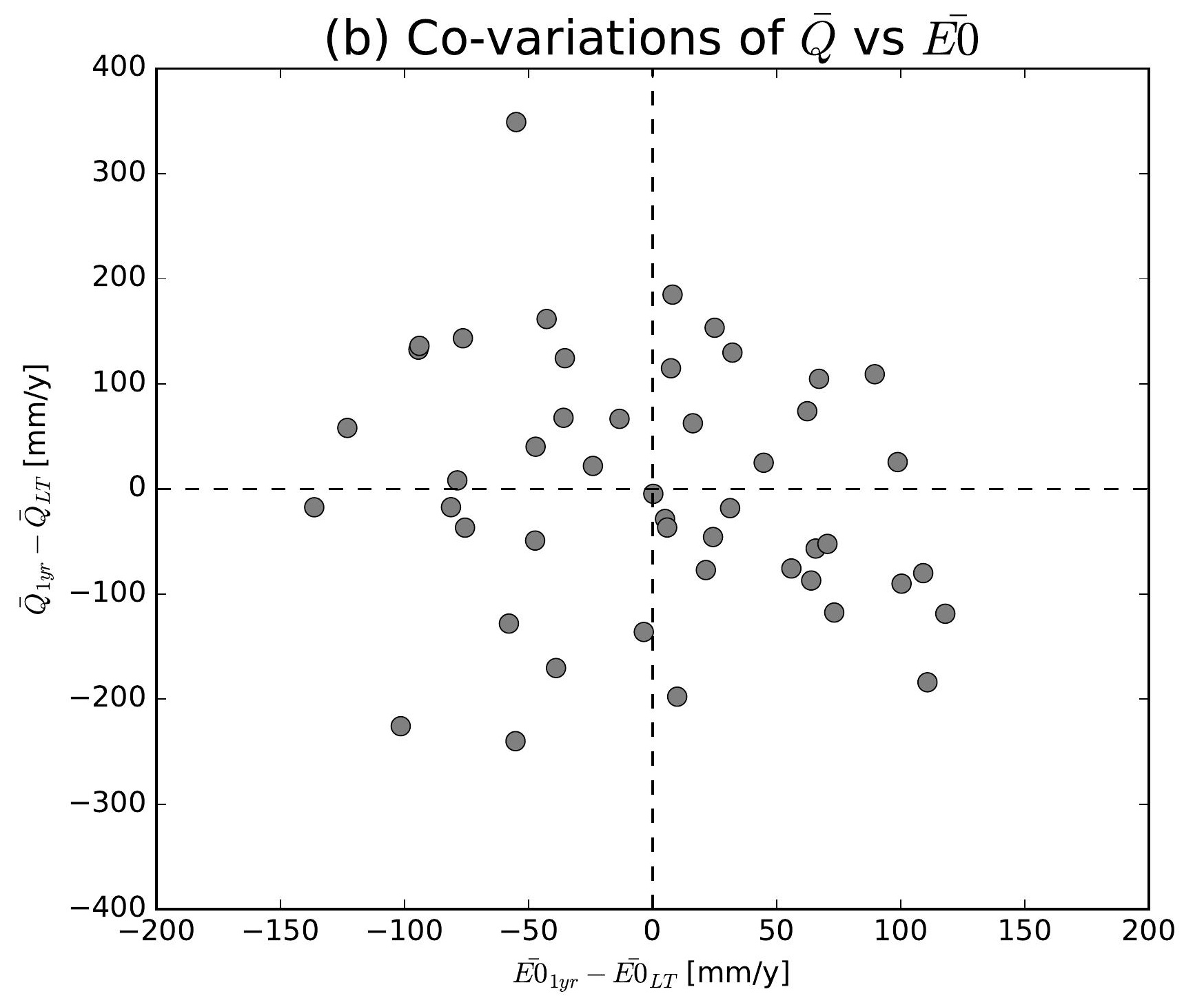

(e) Co-variations of $\bar{Q}$ vs $\overline{E 0}$

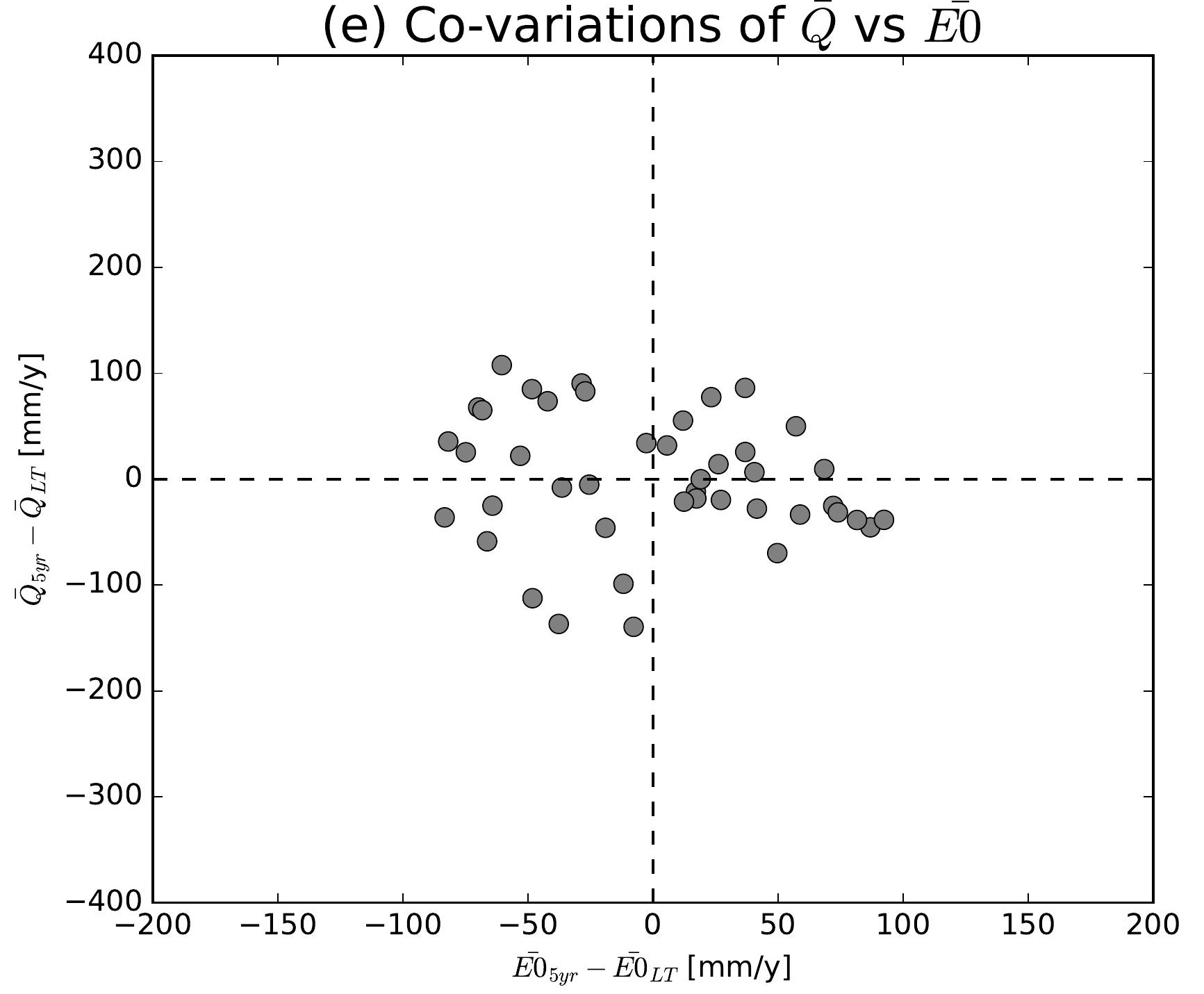

(h) Co-variations of $\bar{Q}$ vs $\overline{E 0}$

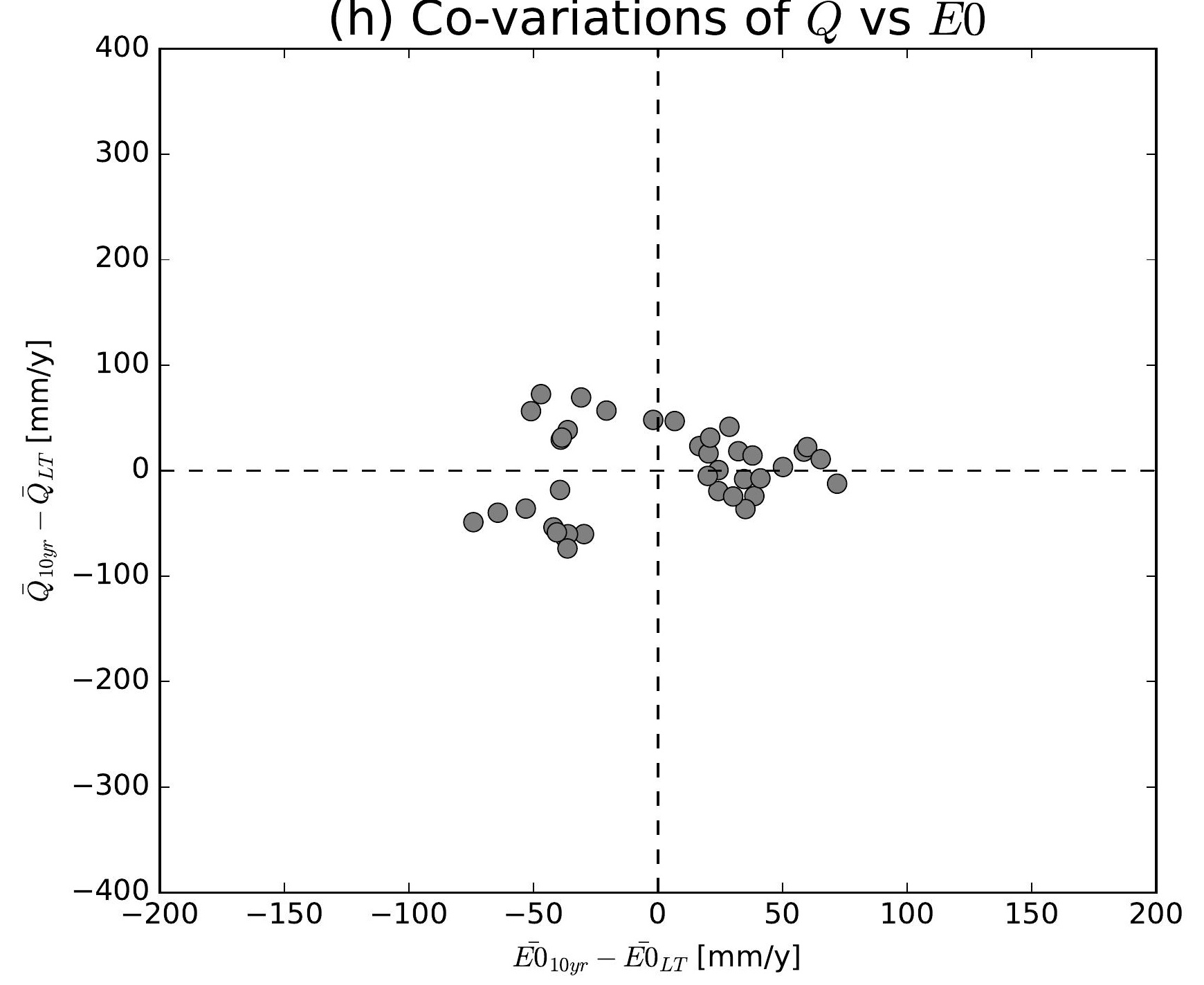

(k) Co-variations of $\bar{Q}$ vs $\overline{E 0}$

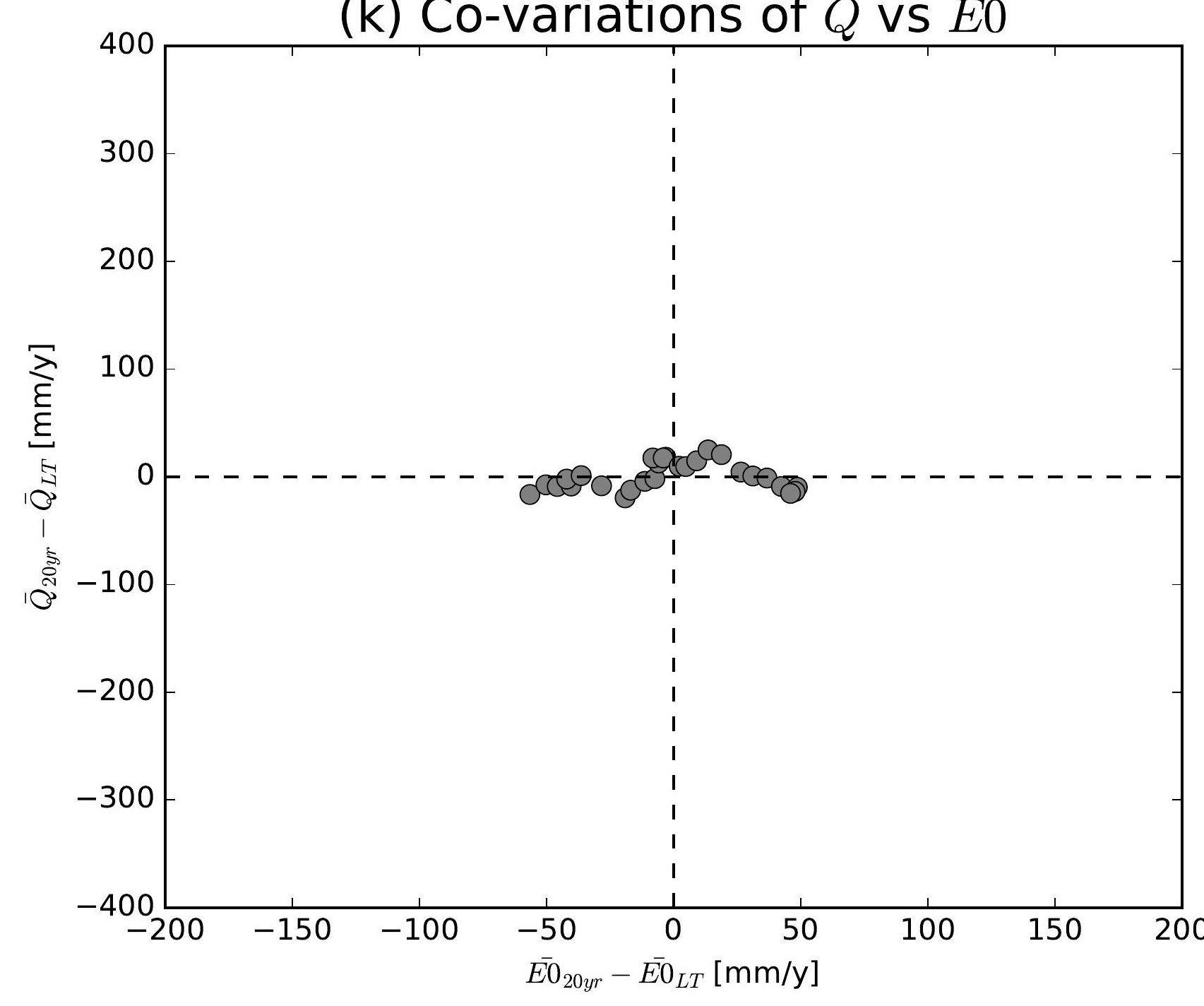

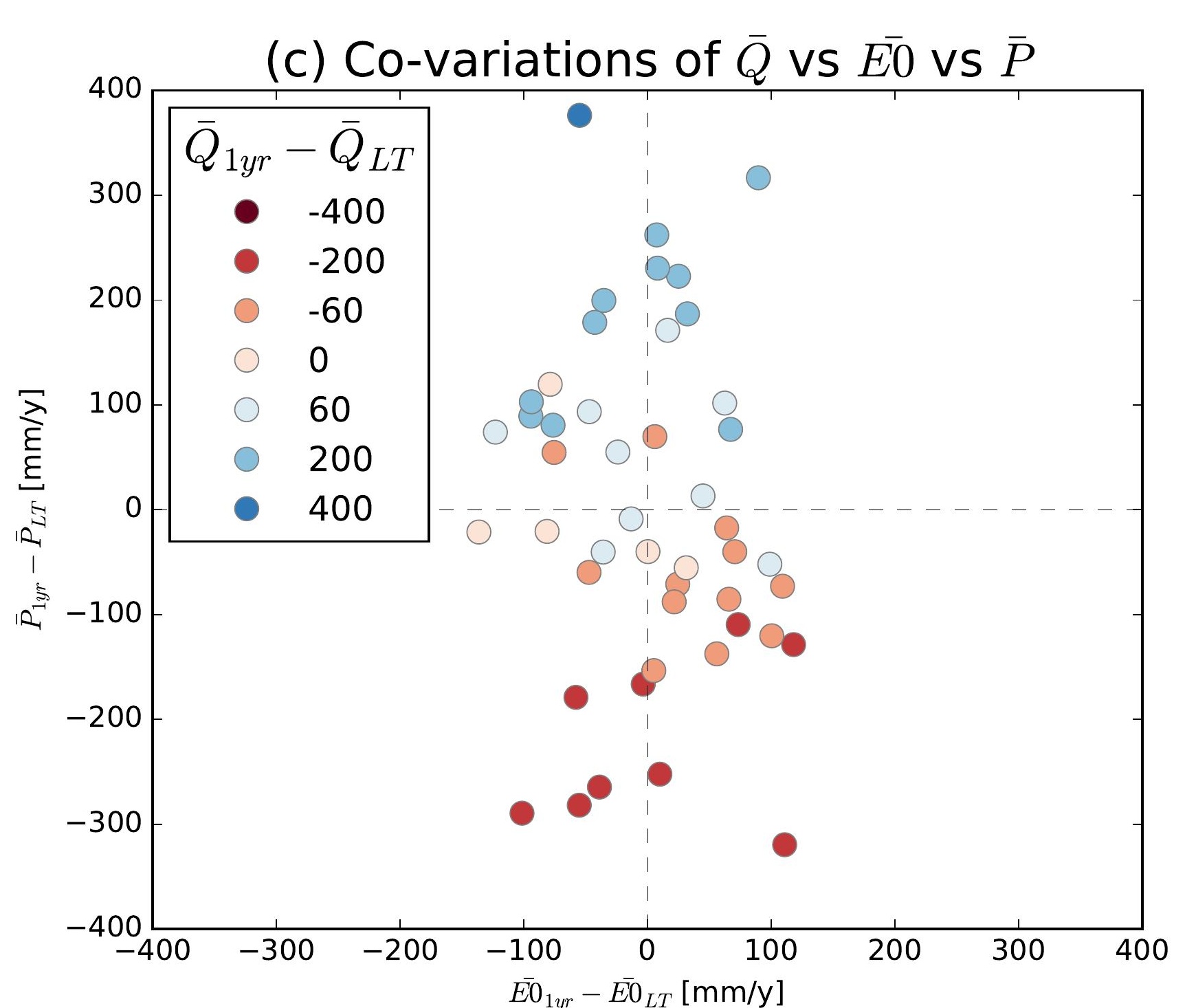
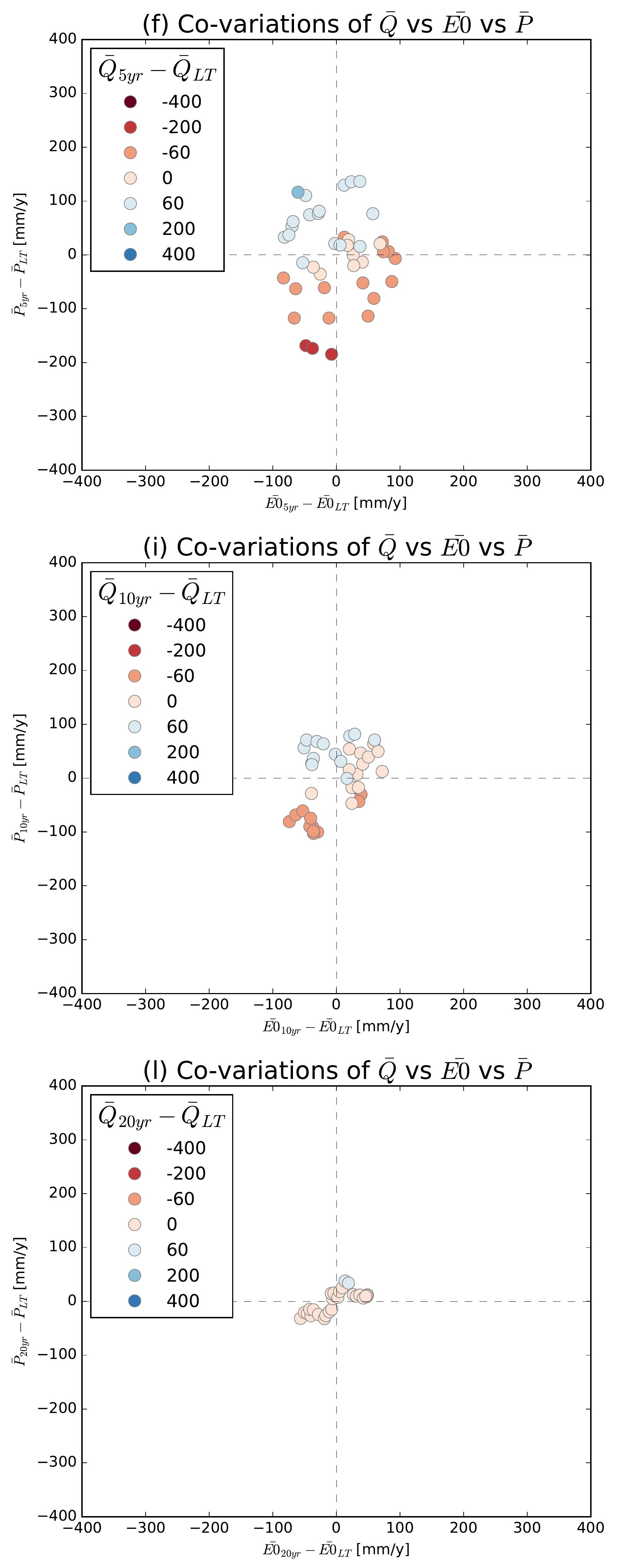

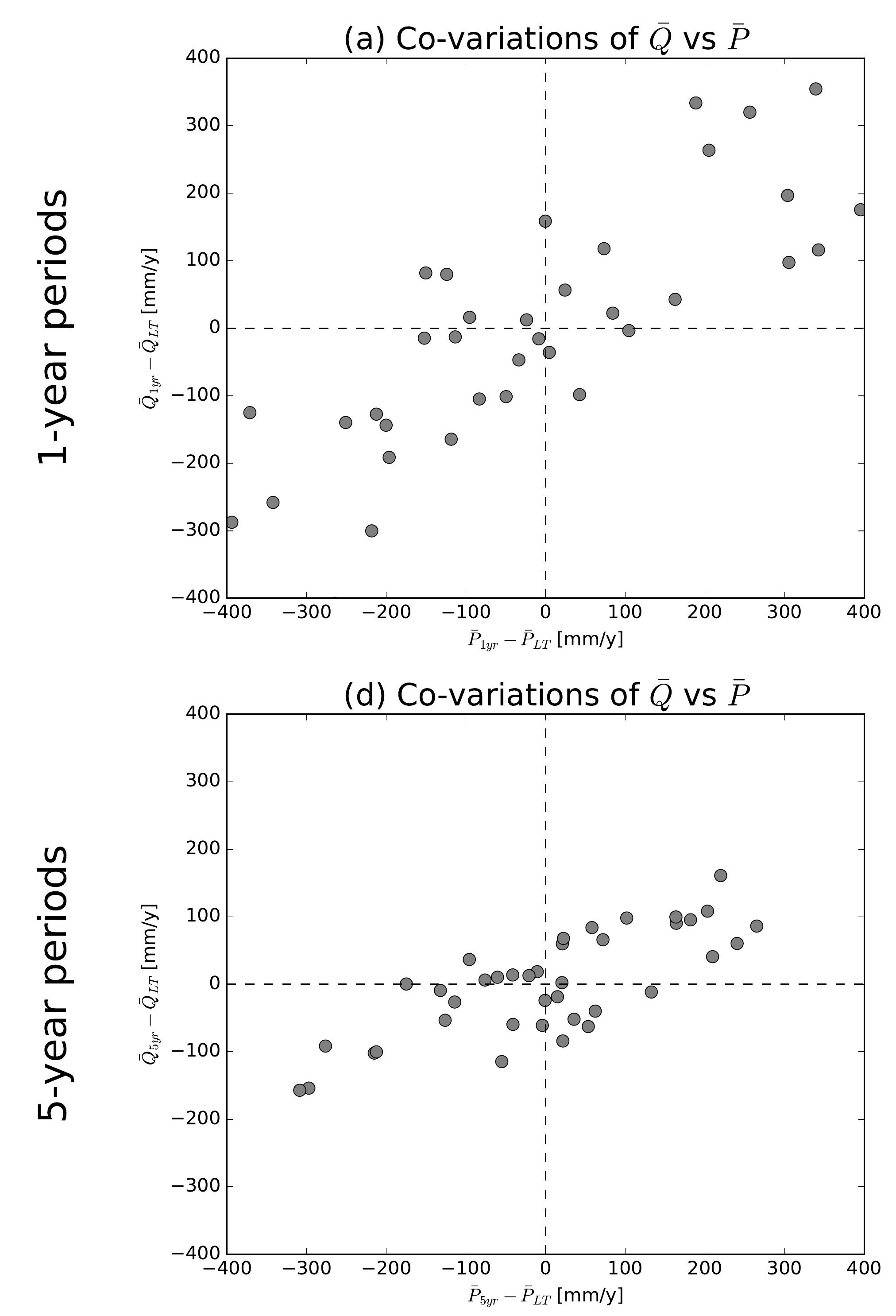

(g) Co-variations of $\bar{Q}$ vs $\bar{P}$

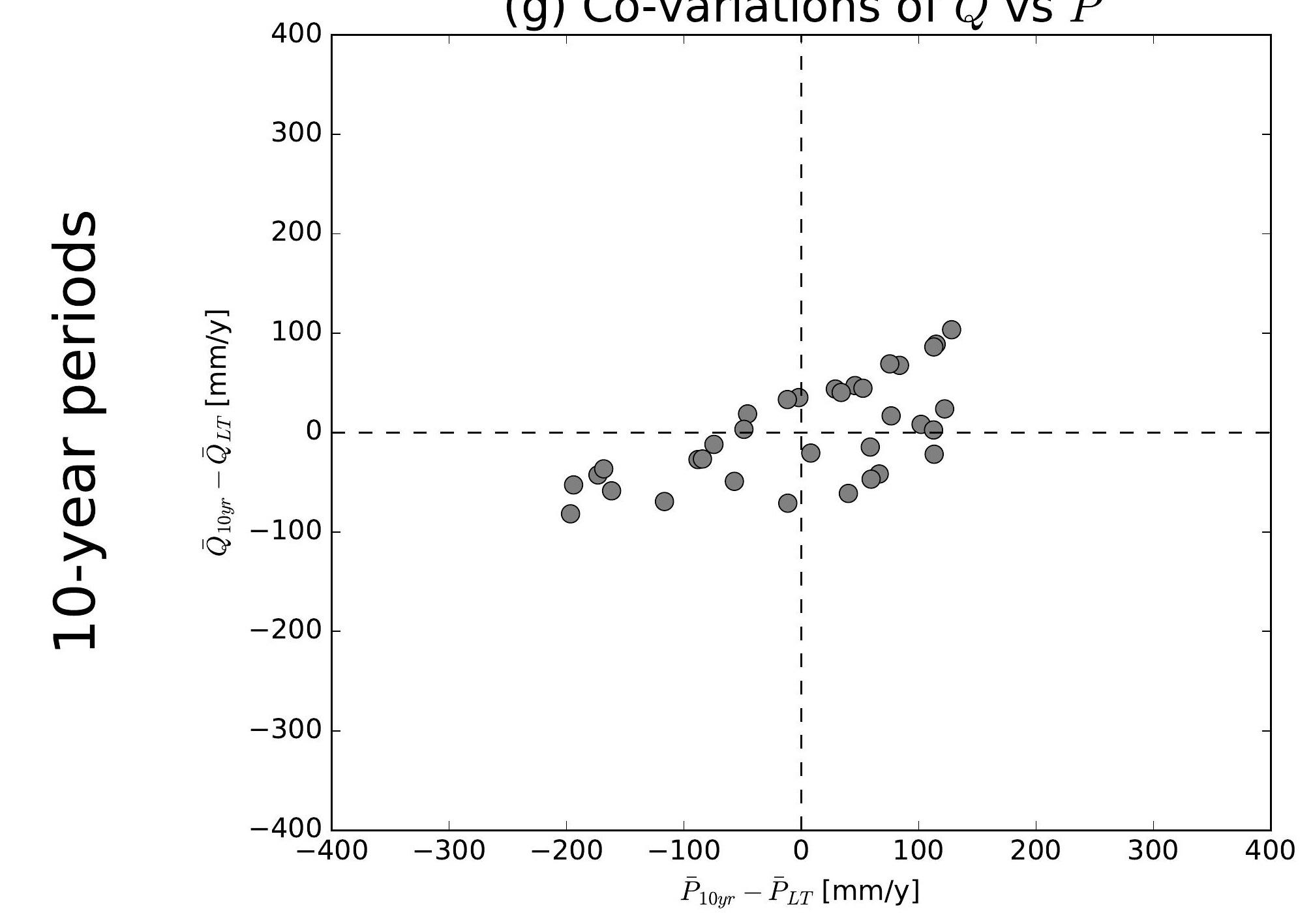

(j) Co-variations of $\bar{Q}$ vs $\bar{P}$

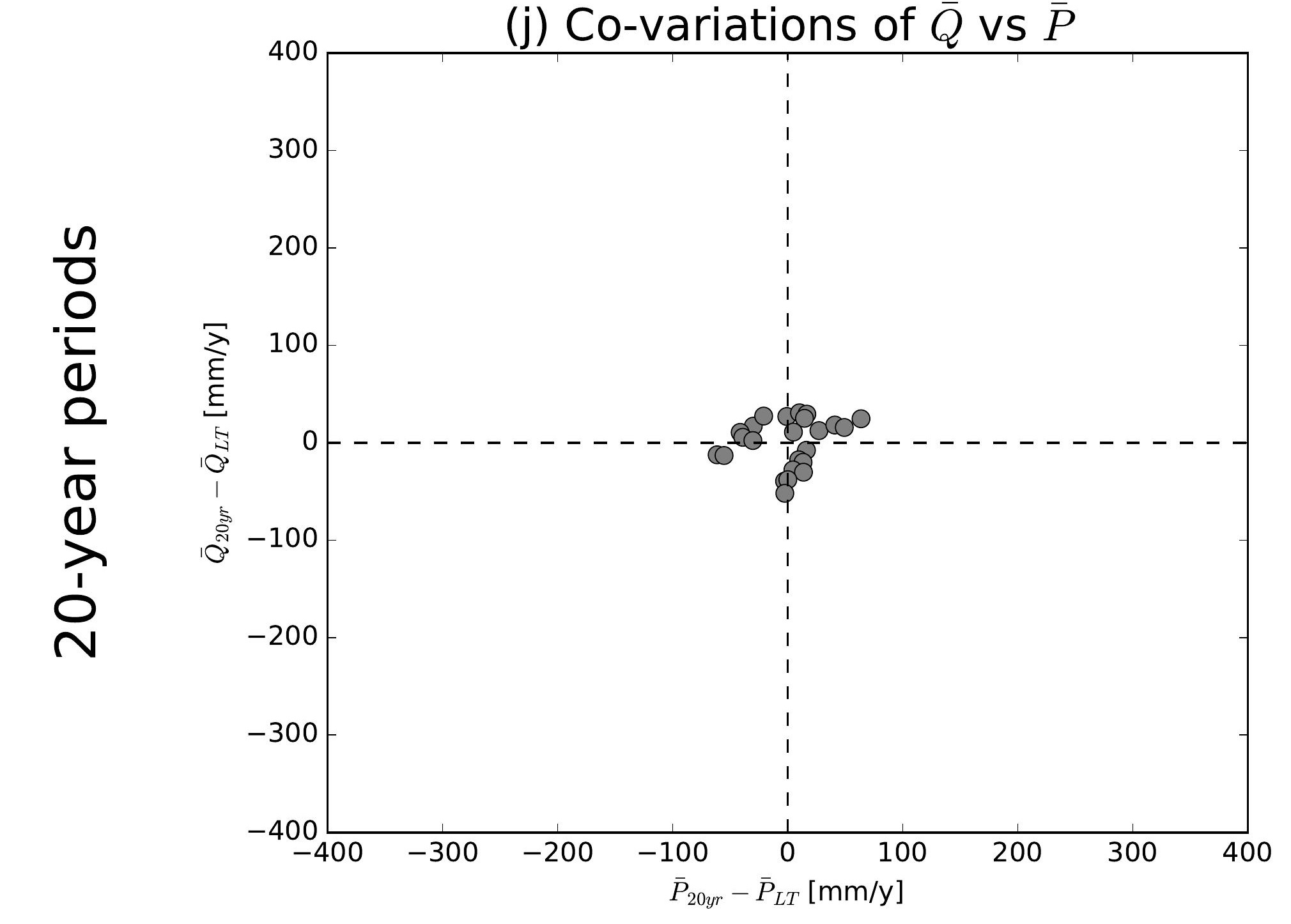

(b) Co-variations of $\bar{Q}$ vs $\overline{E 0}$

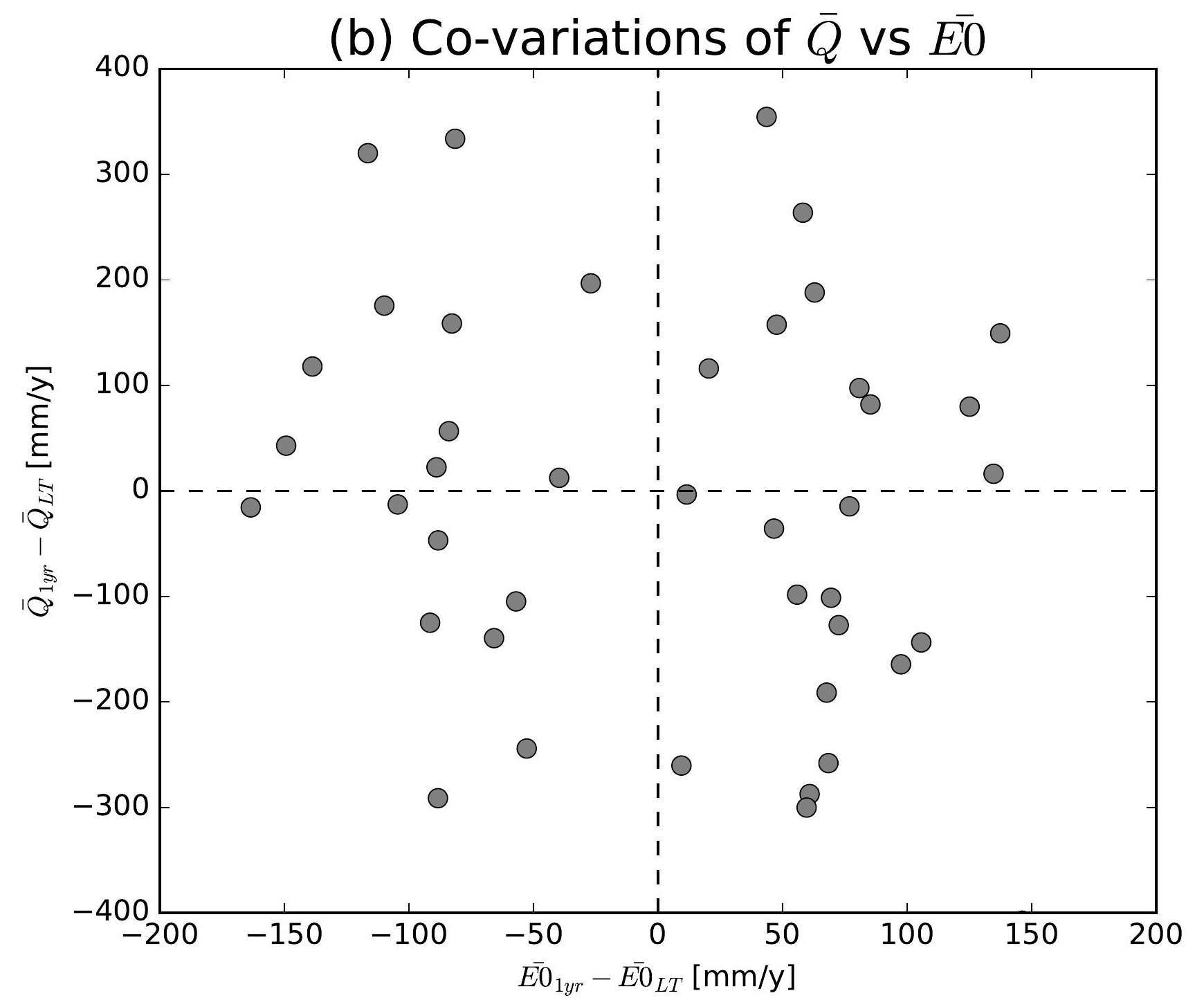

(e) Co-variations of $\bar{Q}$ vs $\overline{E 0}$

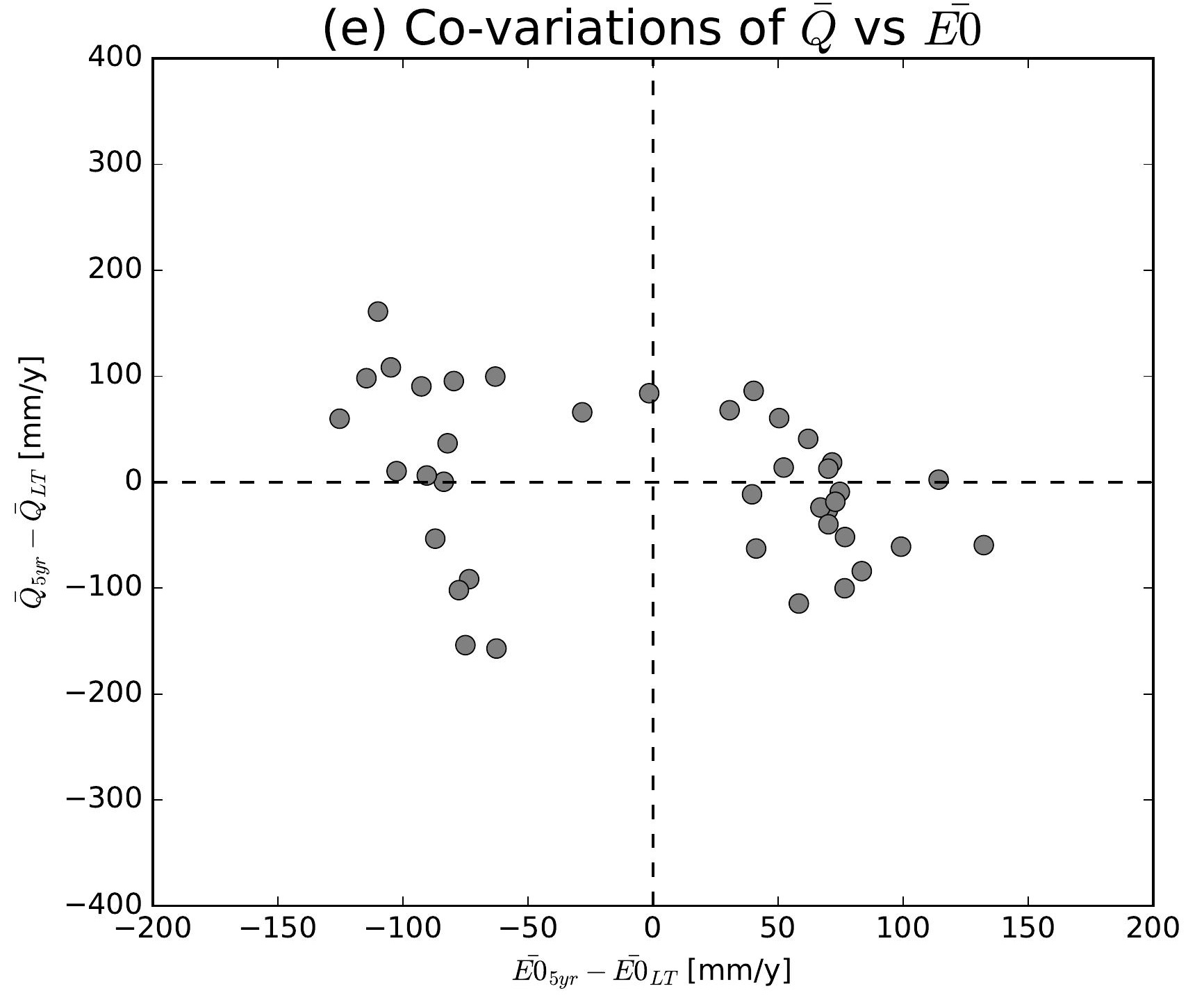

(h) Co-variations of $\bar{Q}$ vs $\overline{E 0}$

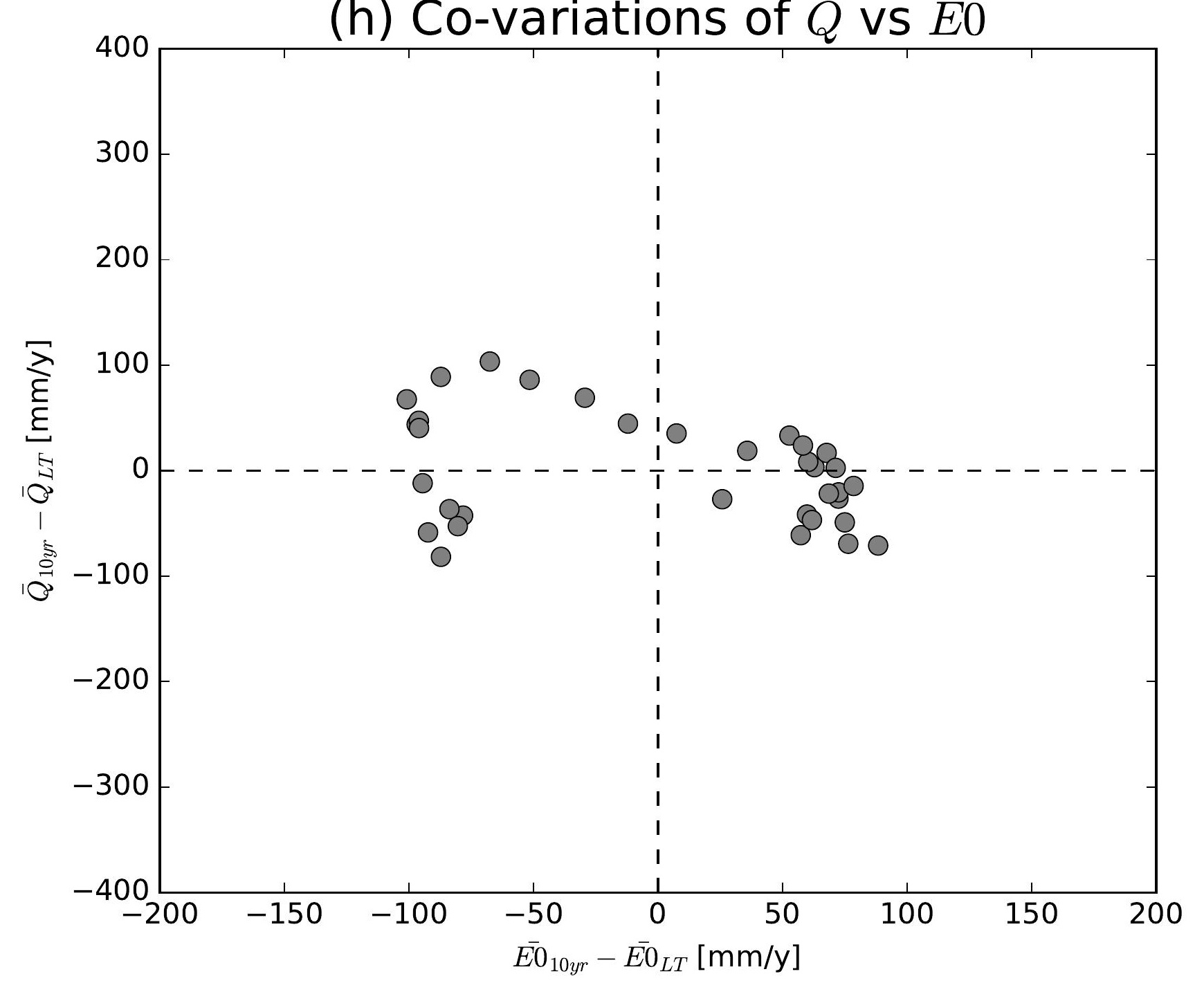

(k) Co-variations of $\bar{Q}$ vs $\overline{E 0}$

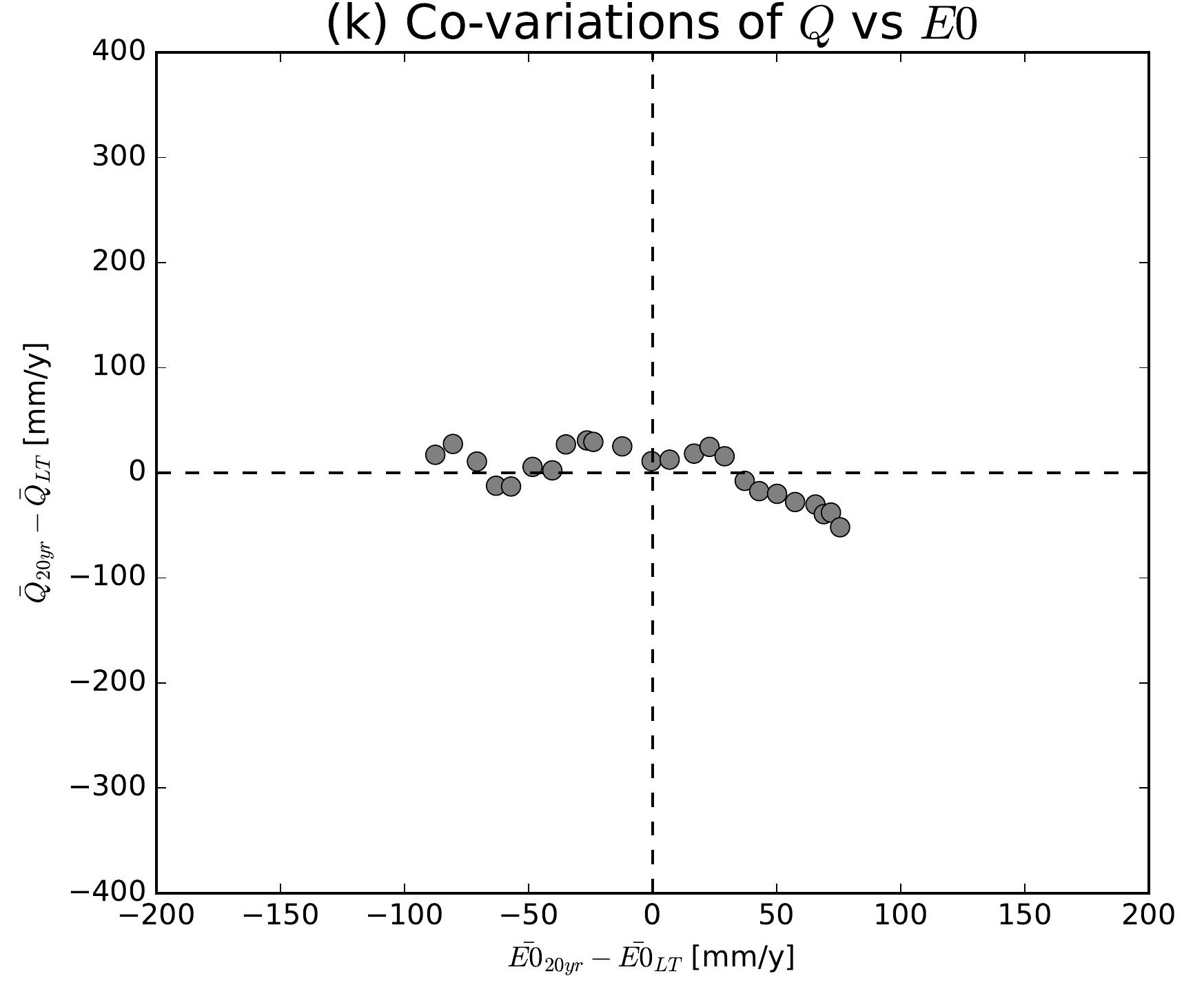

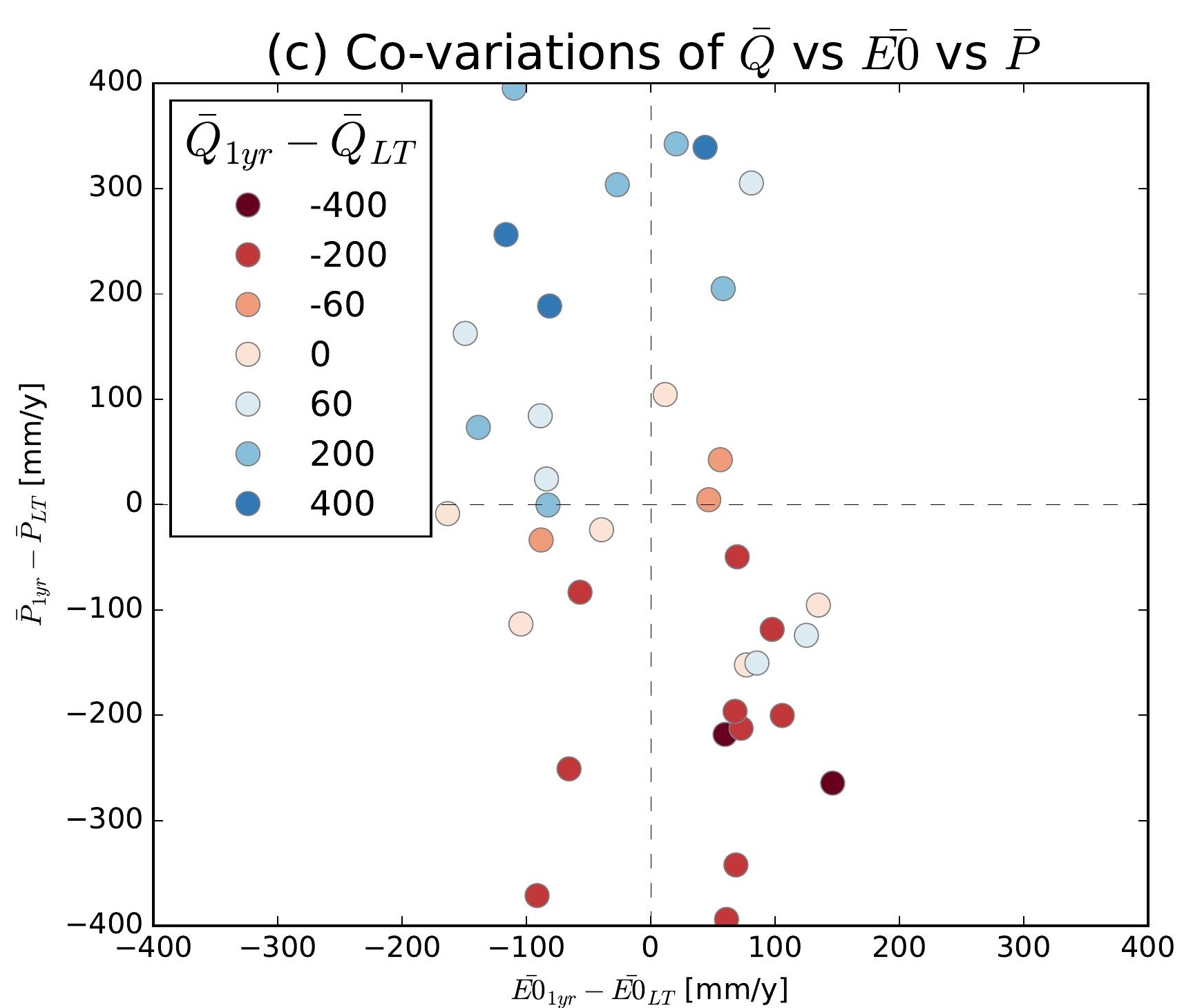
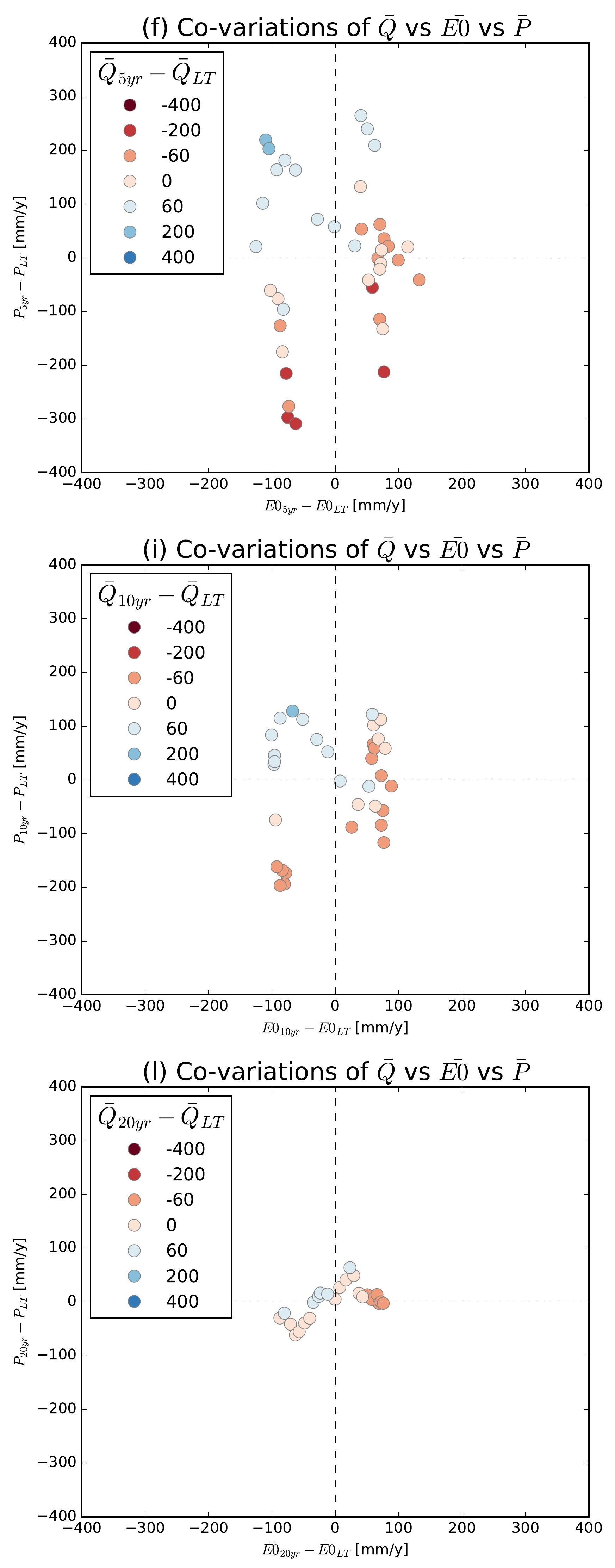

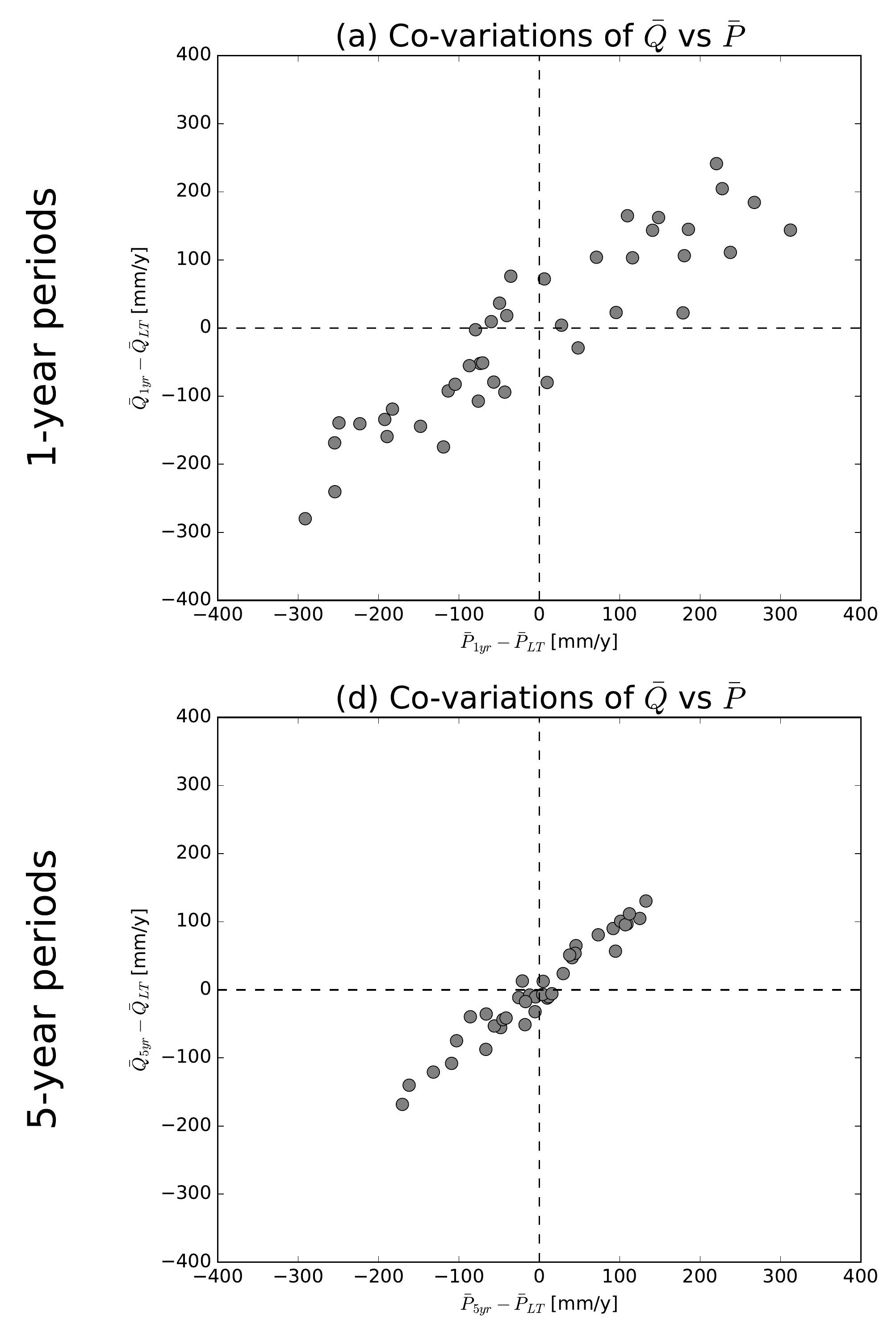

(g) Co-variations of $\bar{Q}$ vs $\bar{P}$

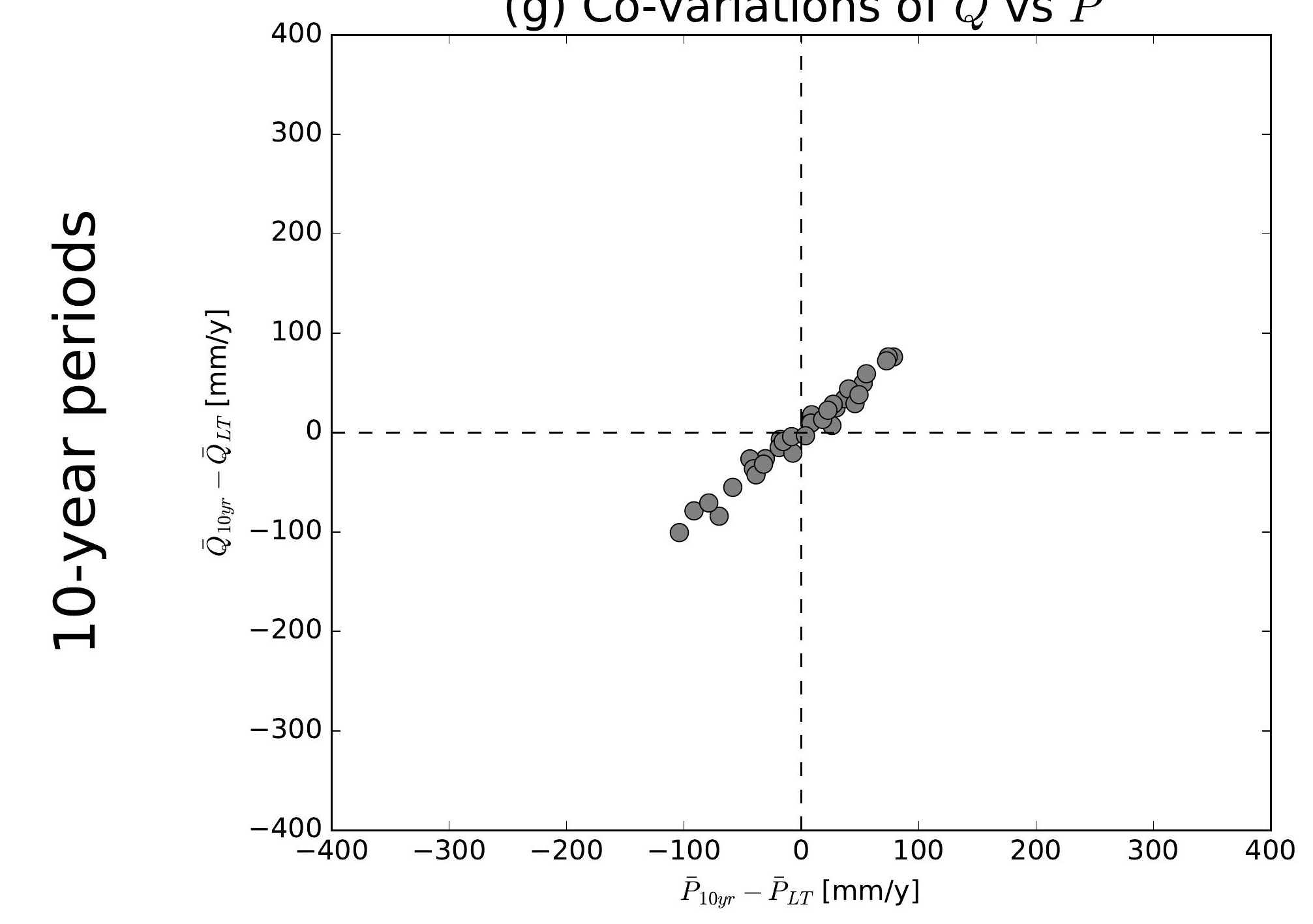

(j) Co-variations of $\bar{Q}$ vs $\bar{P}$

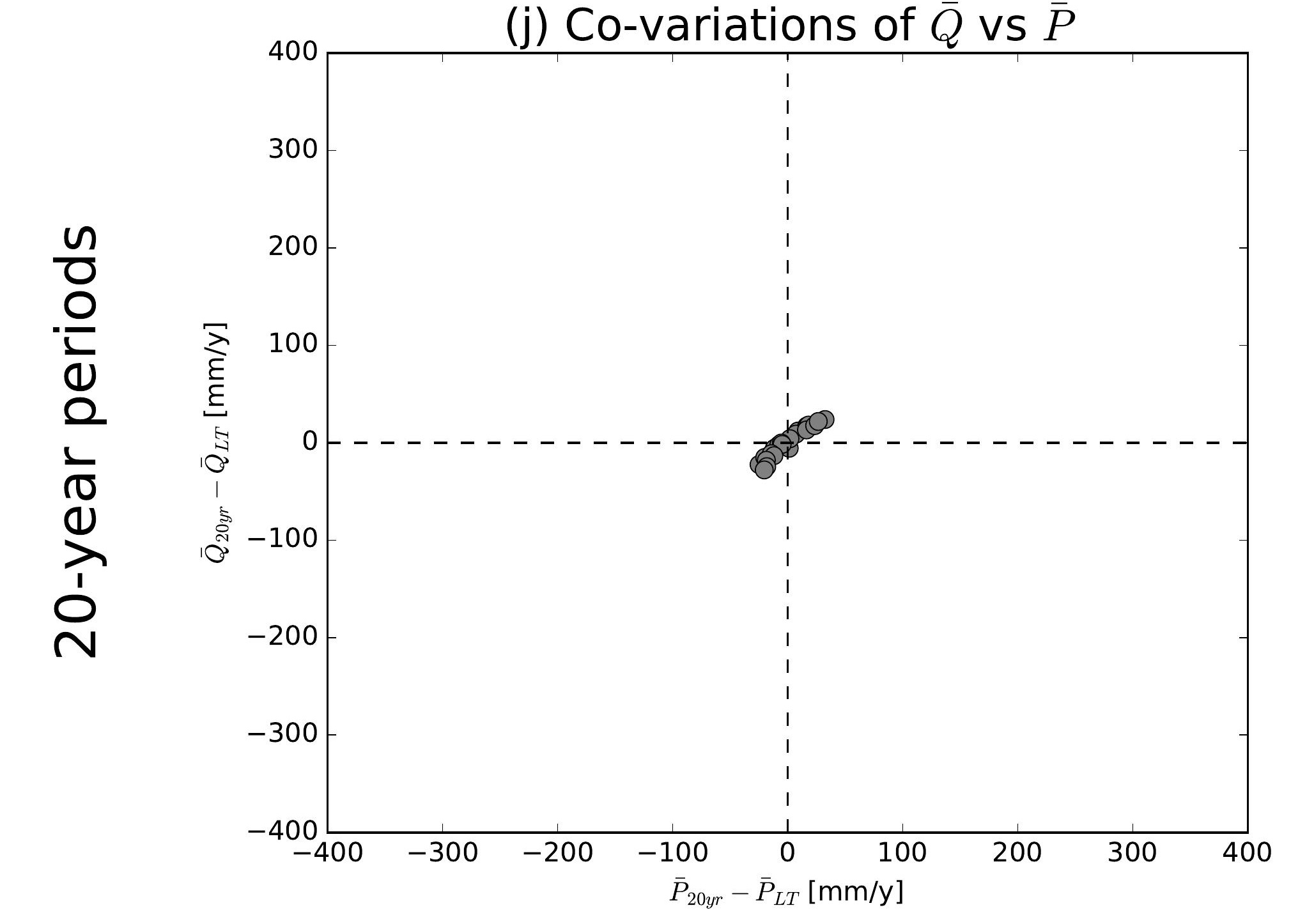

(b) Co-variations of $\bar{Q}$ vs $\overline{E 0}$

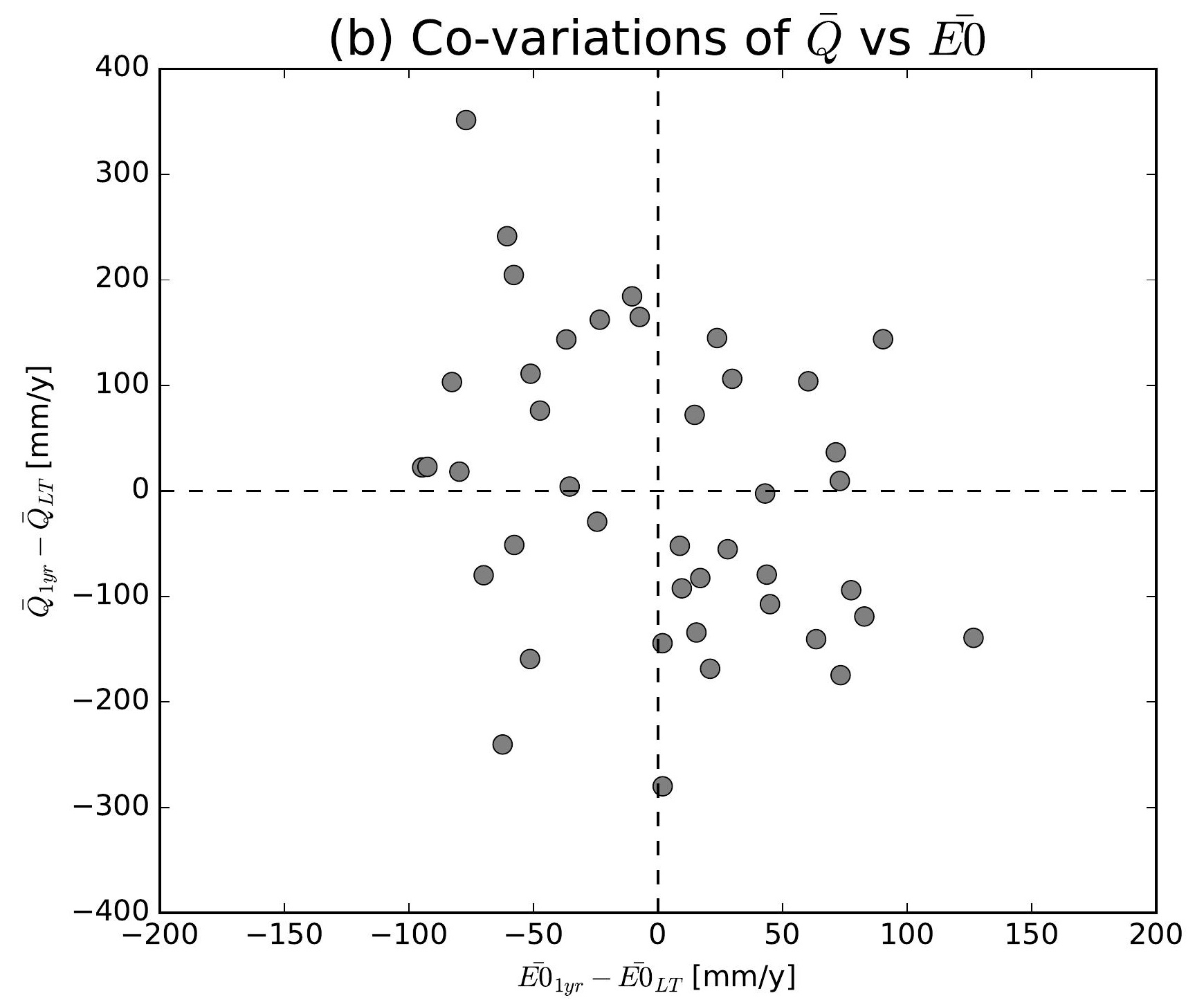

(e) Co-variations of $\bar{Q}$ vs $\overline{E 0}$

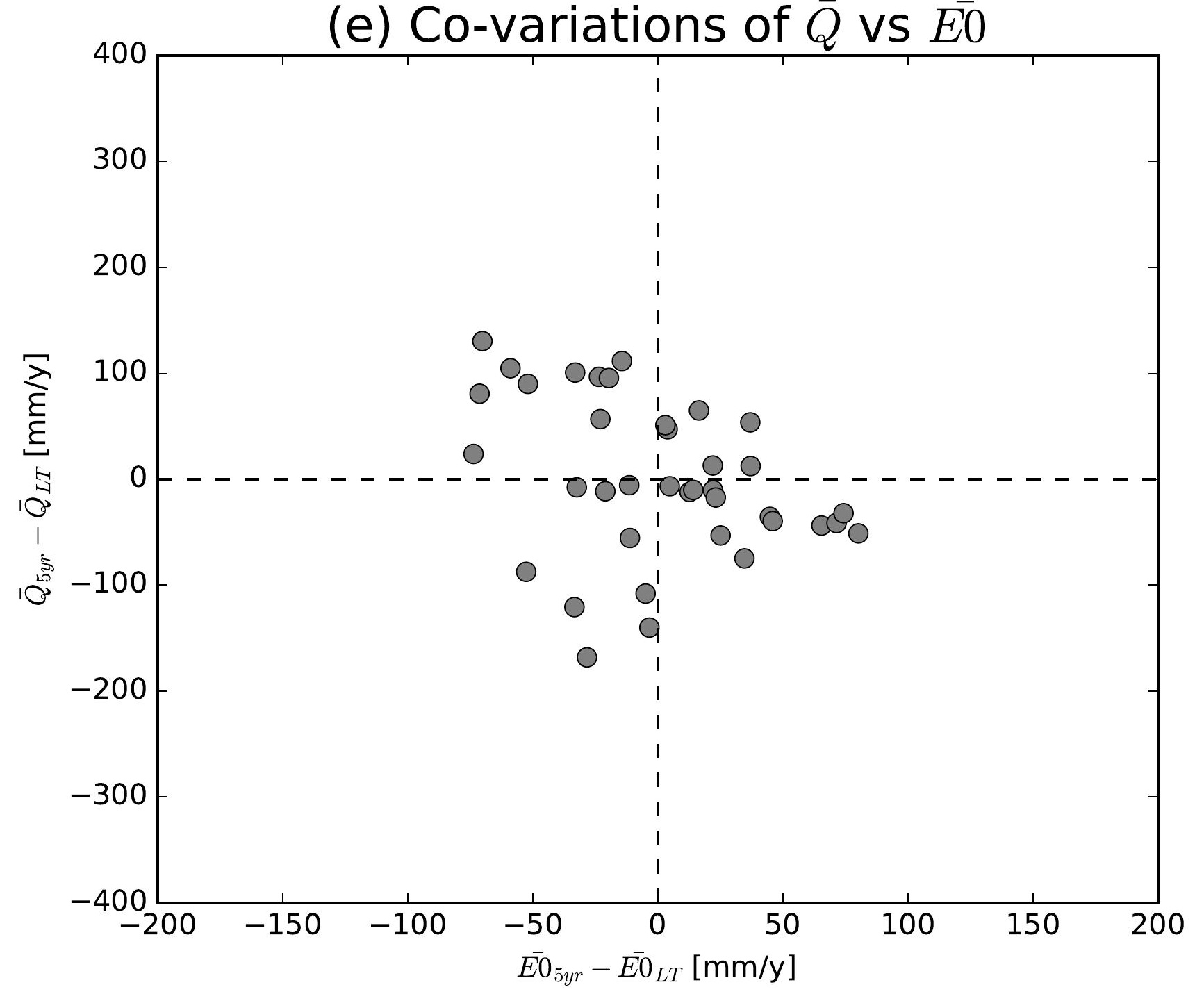

(h) Co-variations of $\bar{Q}$ vs $\overline{E 0}$

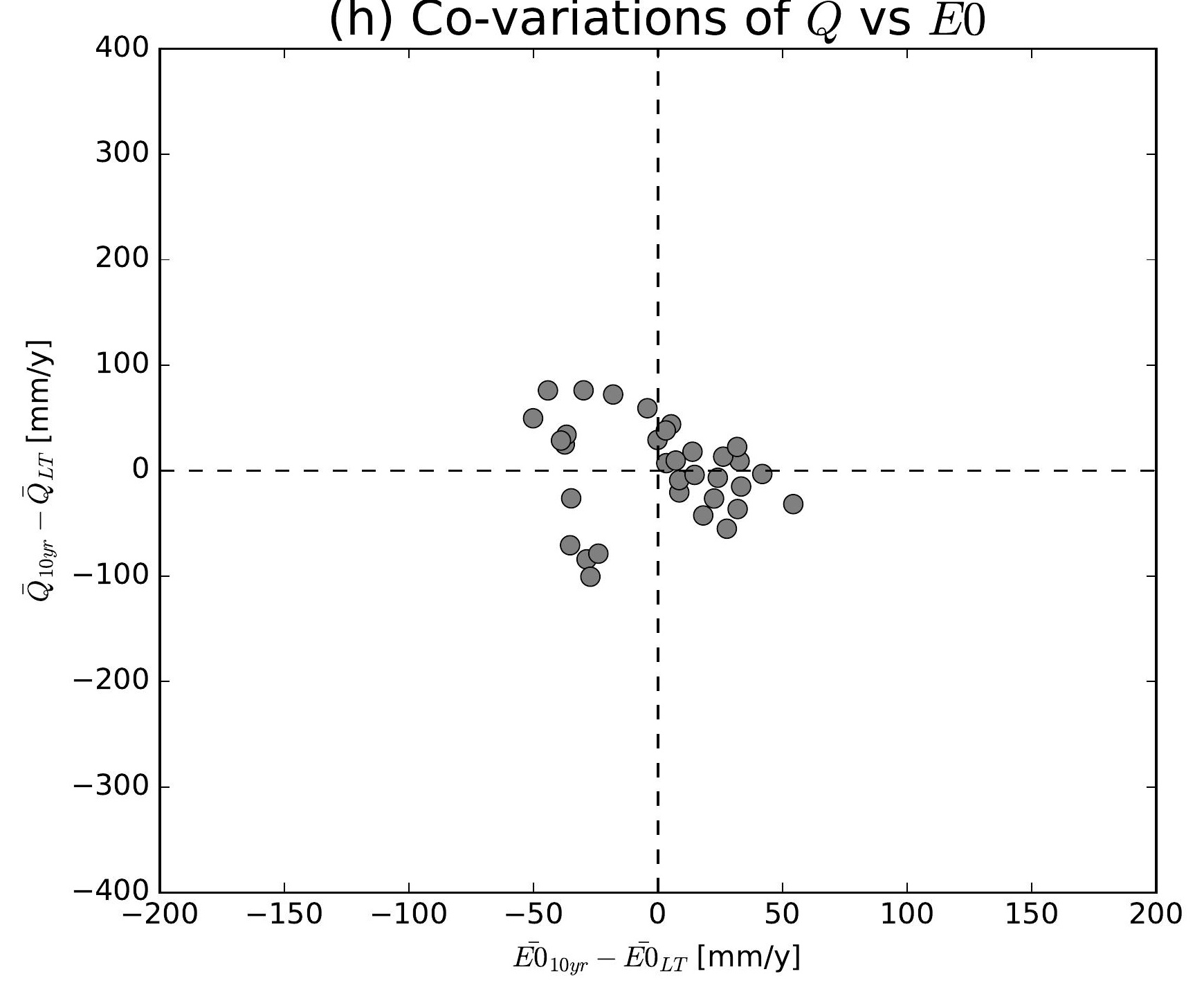

(k) Co-variations of $\bar{Q}$ vs $\overline{E 0}$

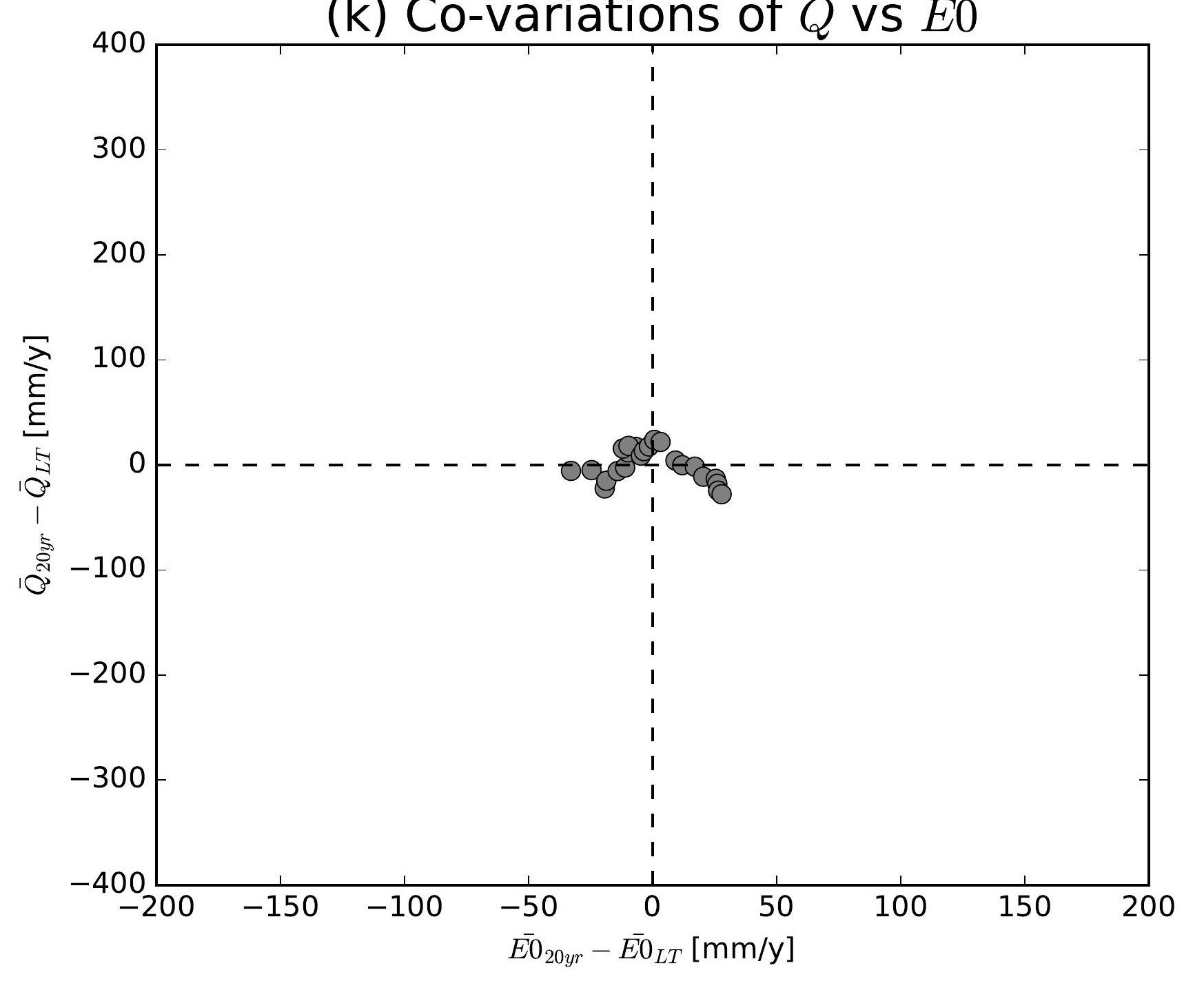

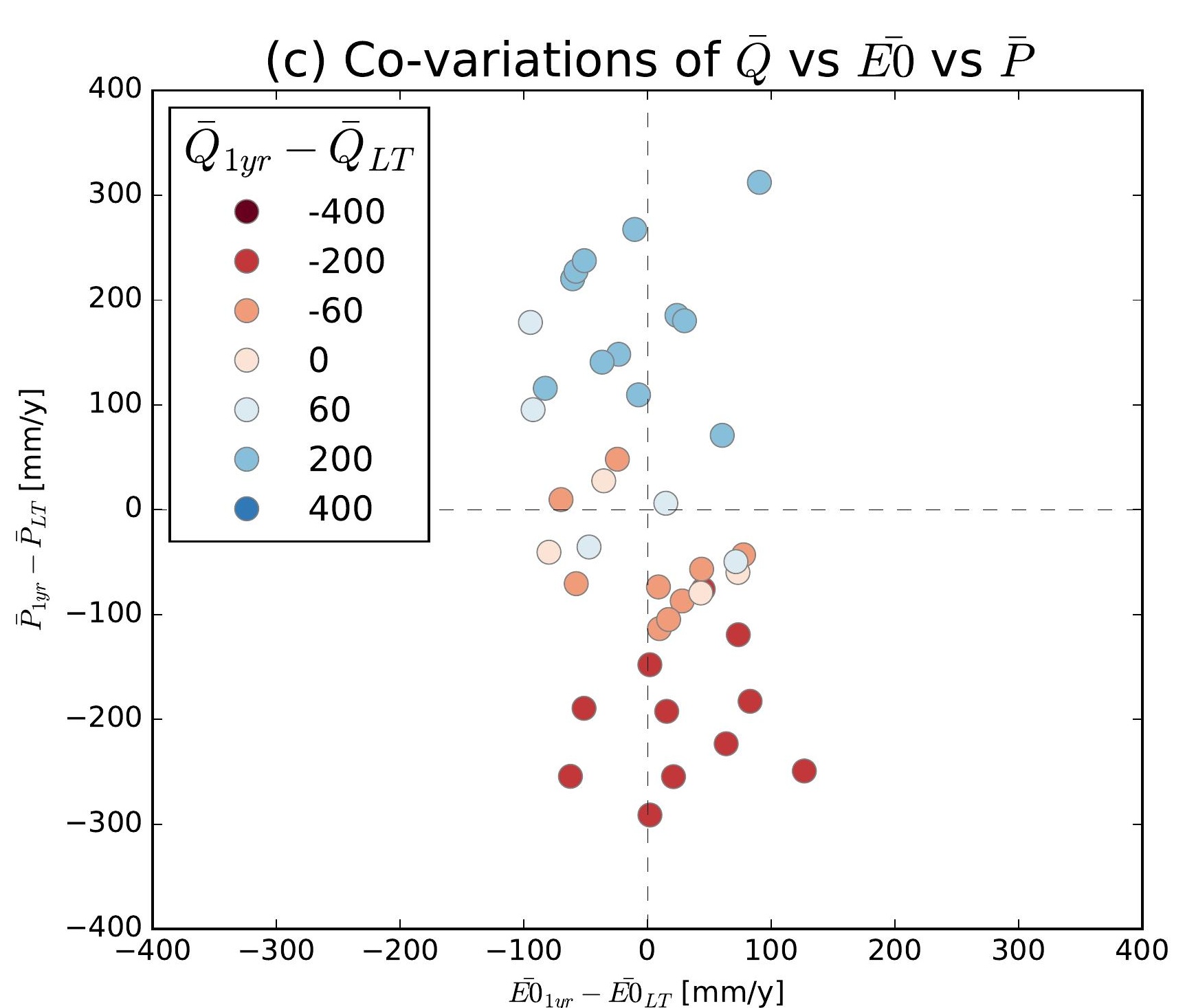
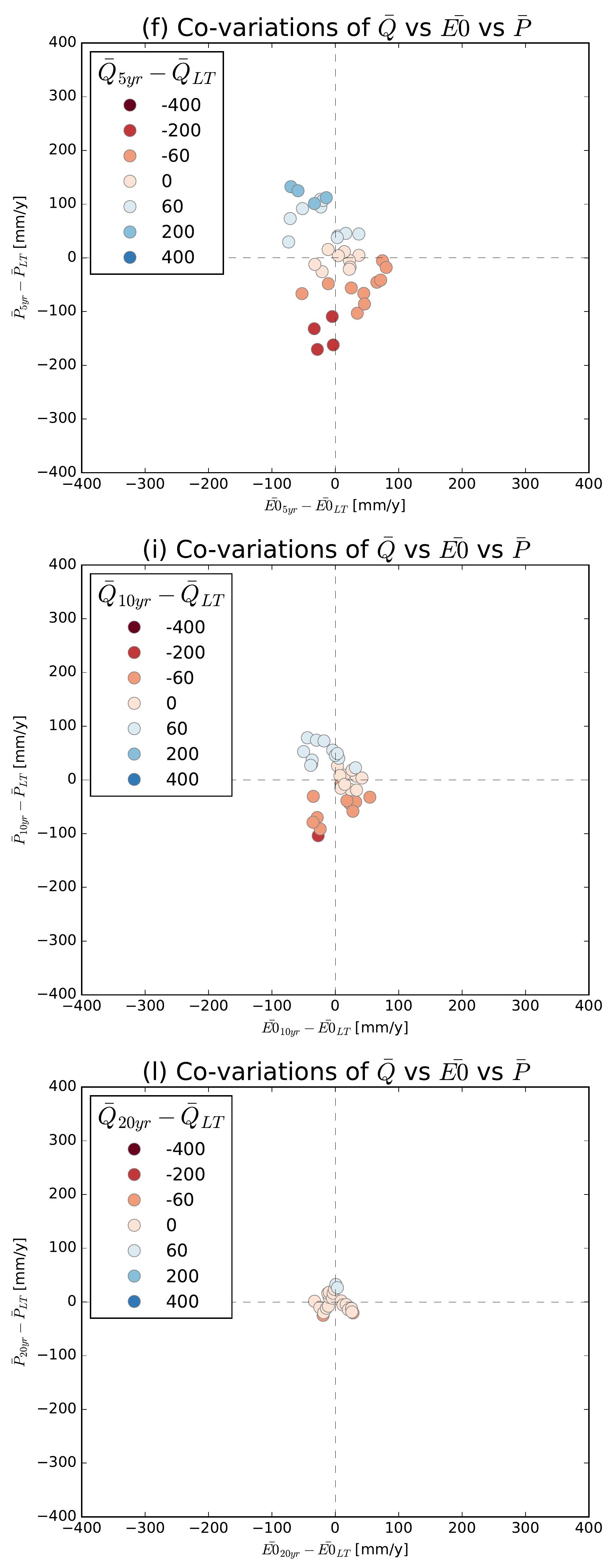

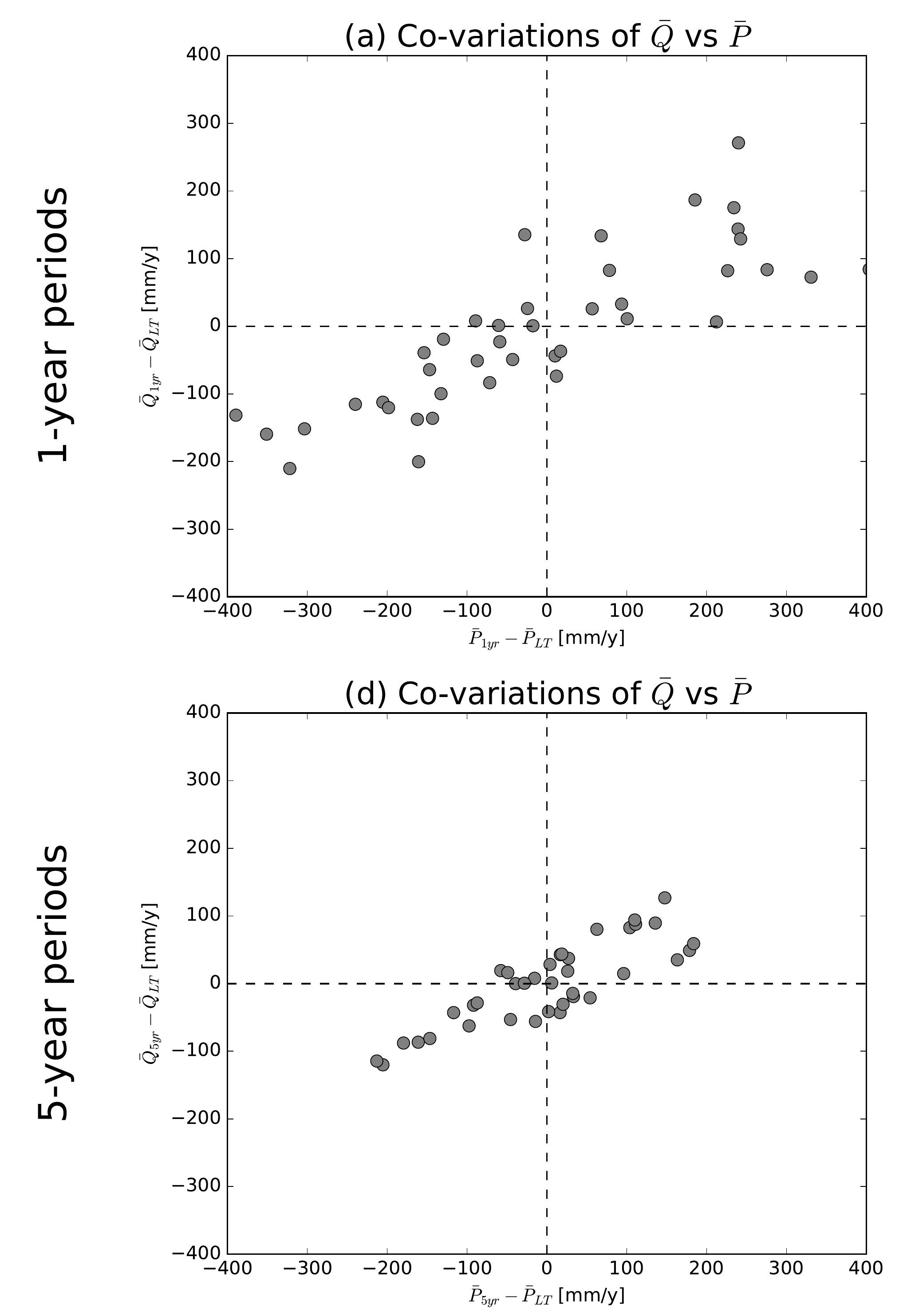

(g) Co-variations of $\bar{Q}$ vs $\bar{P}$

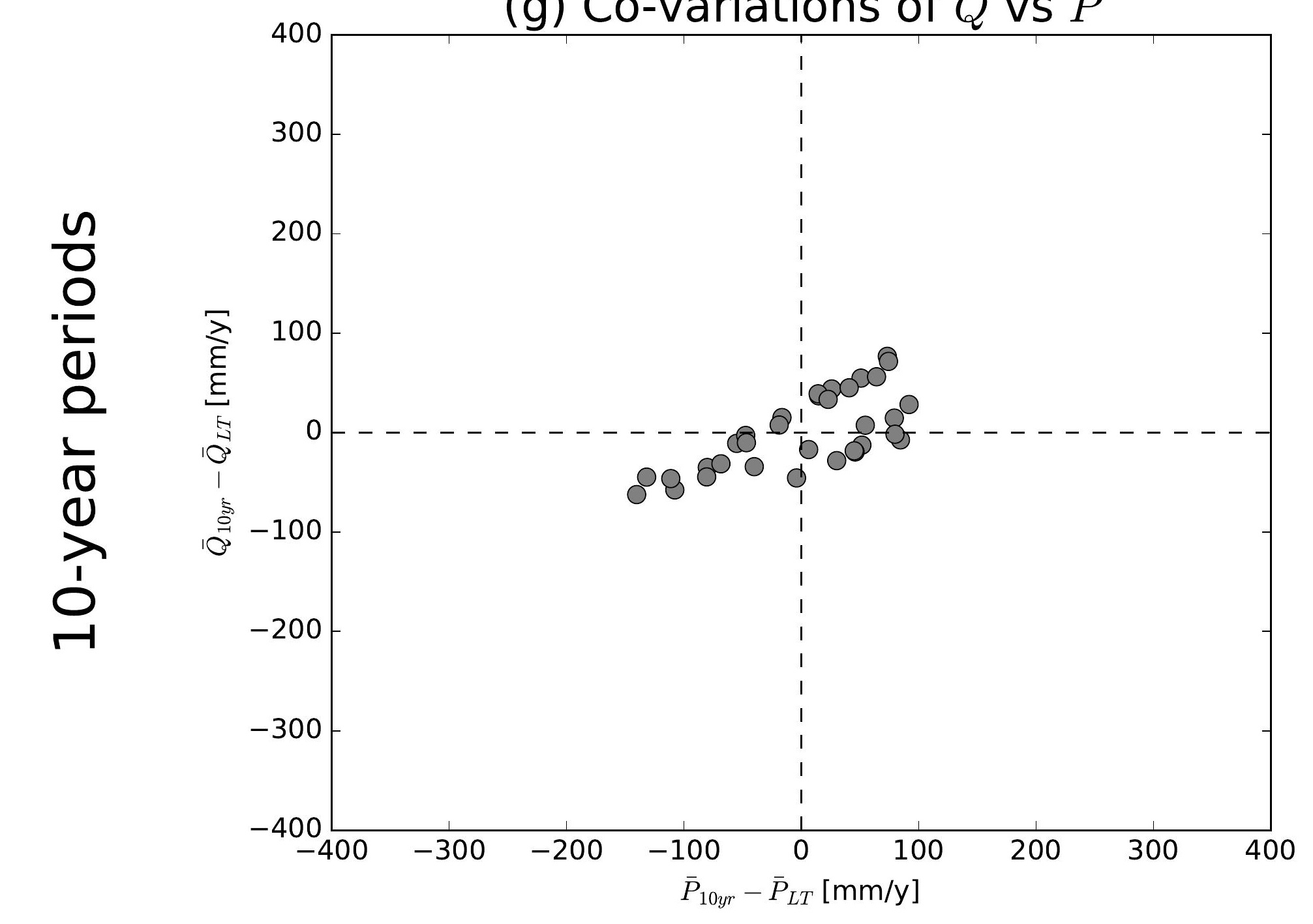

(j) Co-variations of $\bar{Q}$ vs $\bar{P}$

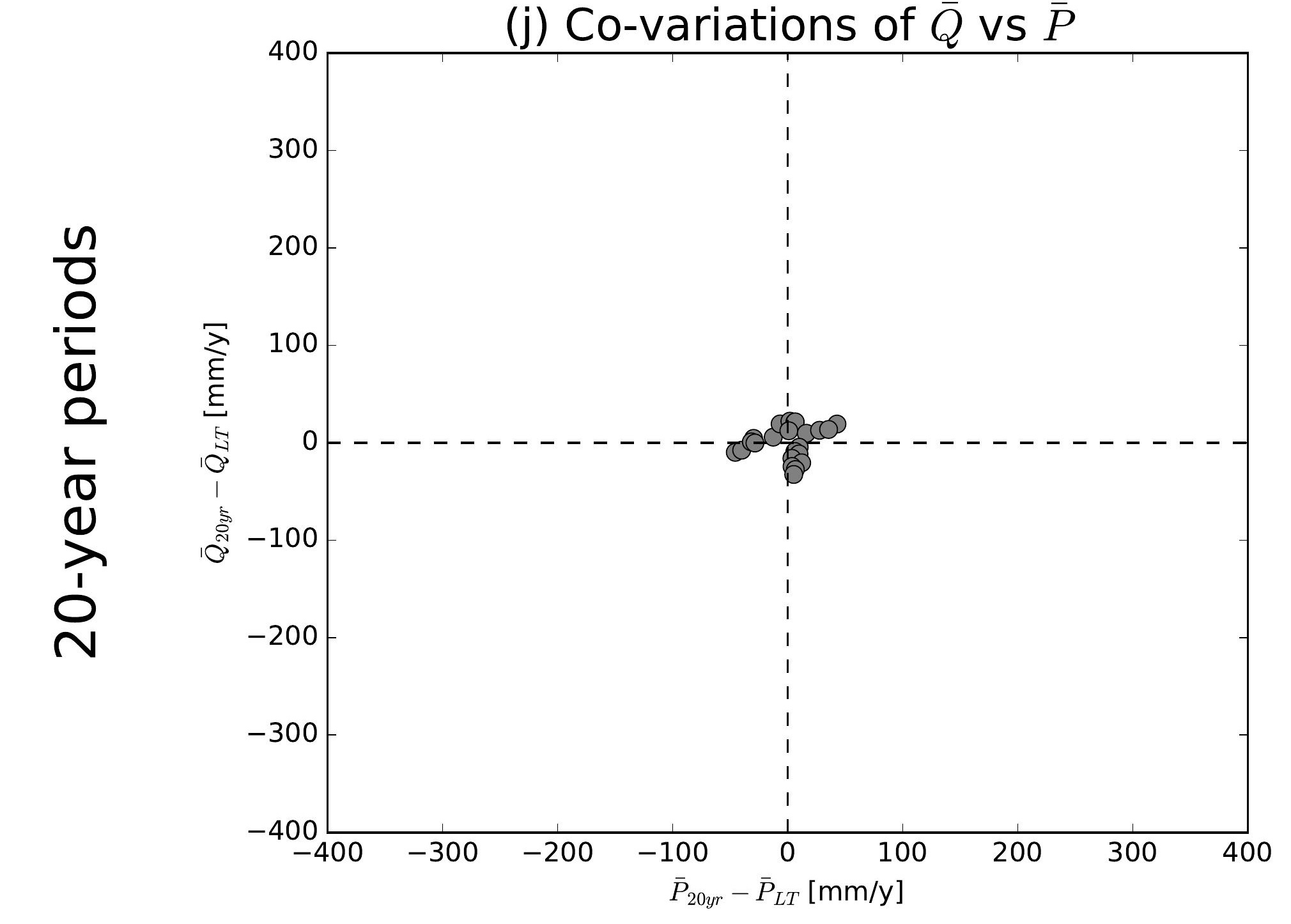

(b) Co-variations of $\bar{Q}$ vs $\overline{E 0}$

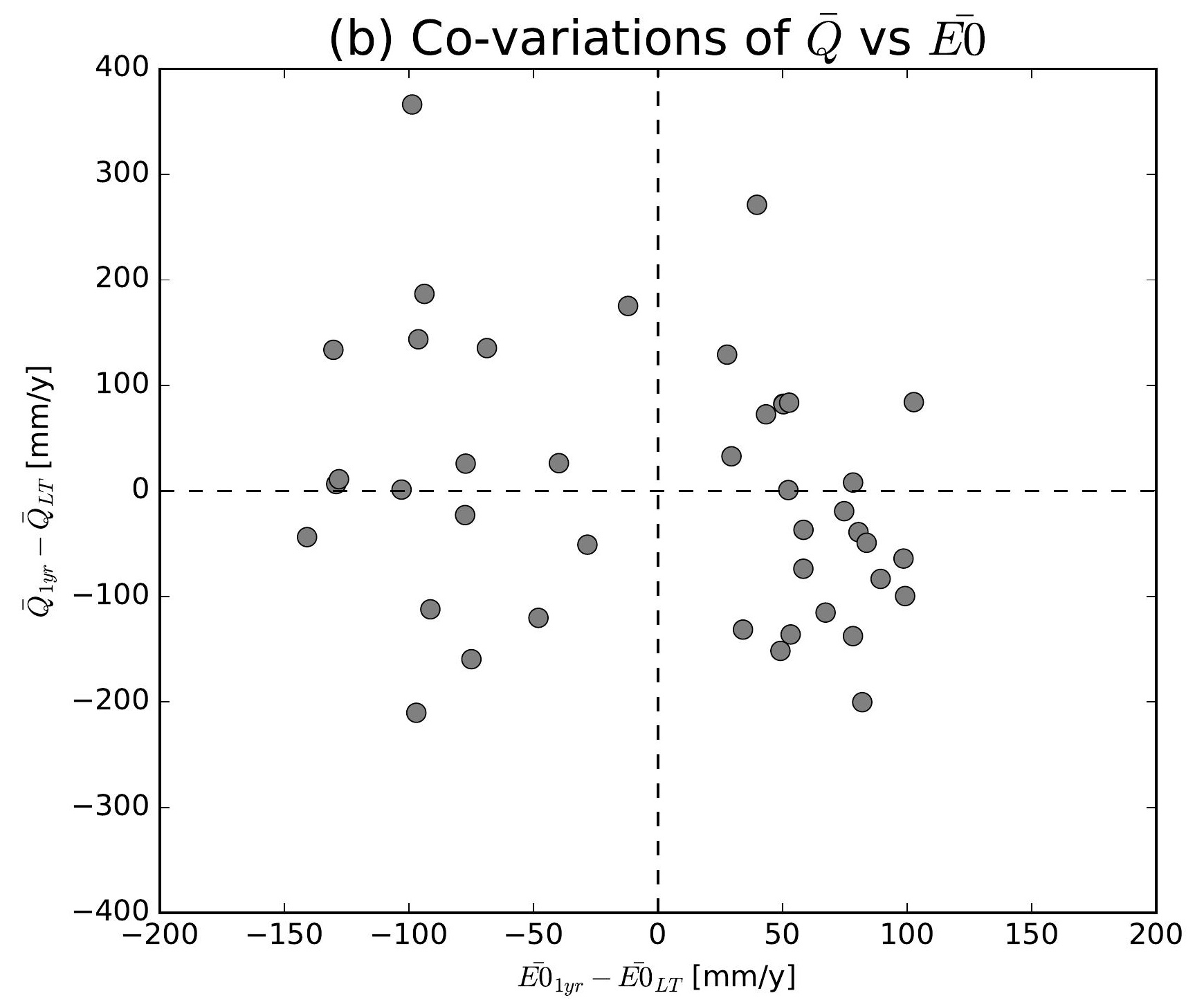

(e) Co-variations of $\bar{Q}$ vs $\overline{E 0}$

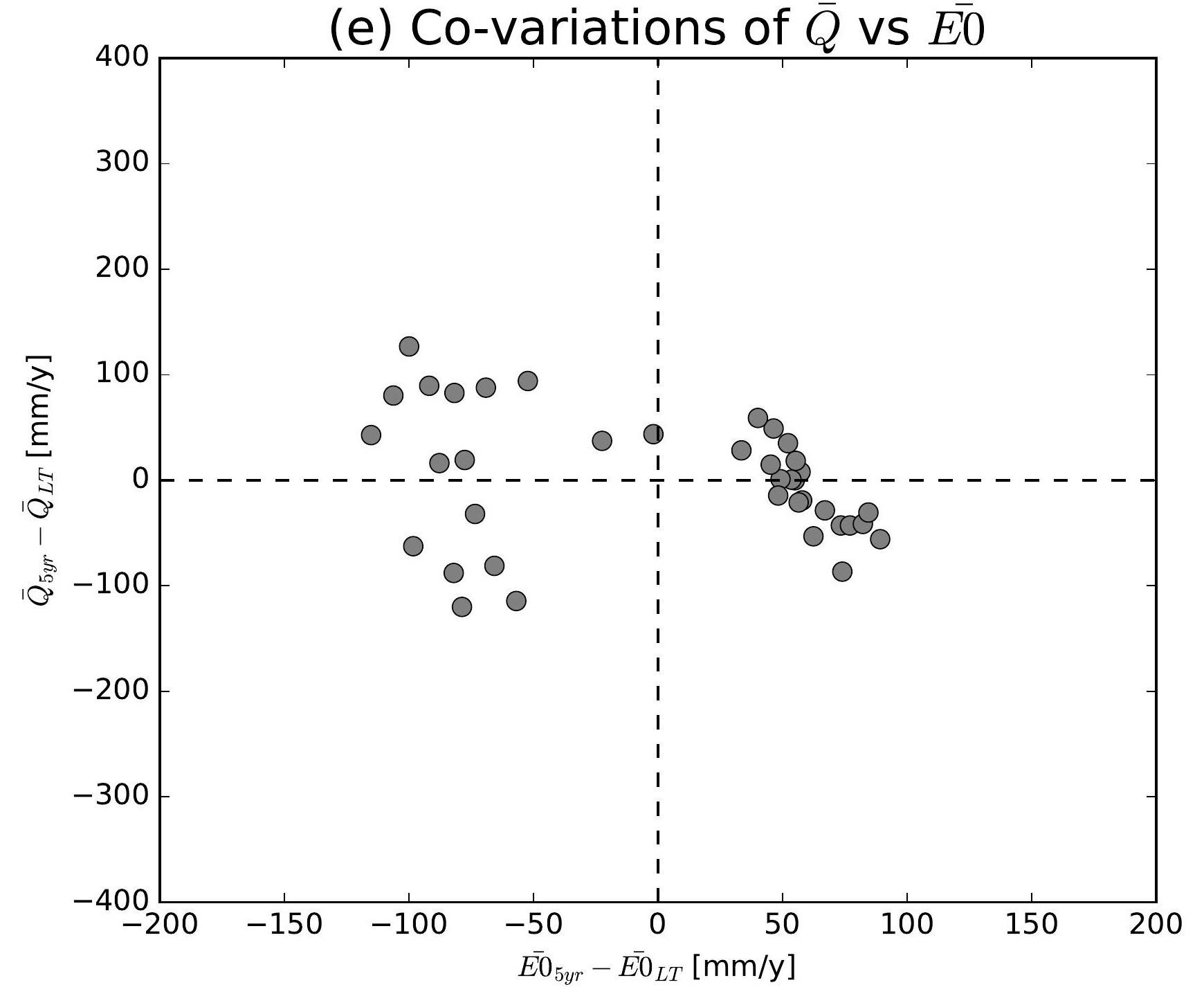

(h) Co-variations of $\bar{Q}$ vs $\overline{E 0}$

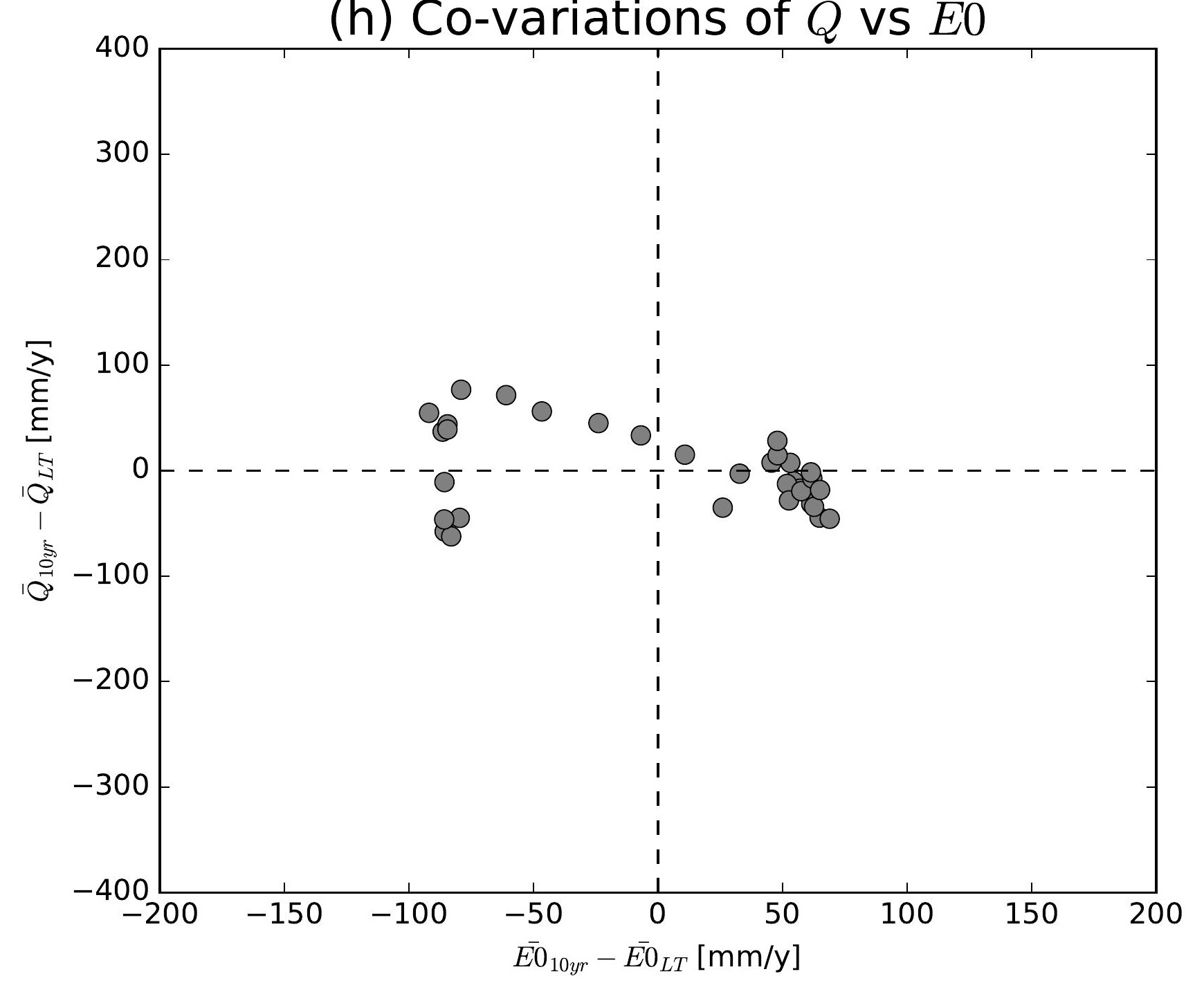

(k) Co-variations of $\bar{Q}$ vs $\overline{E 0}$

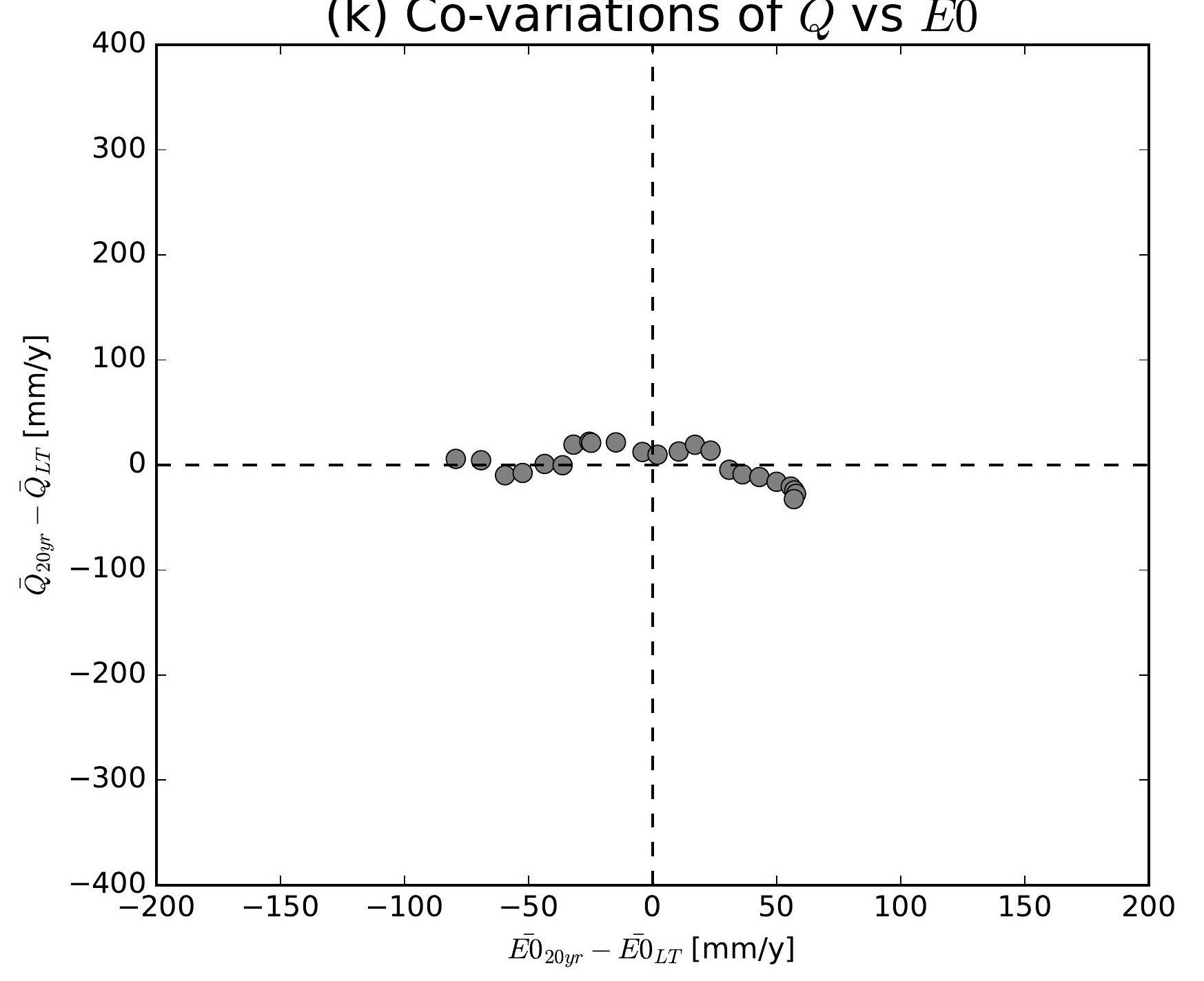

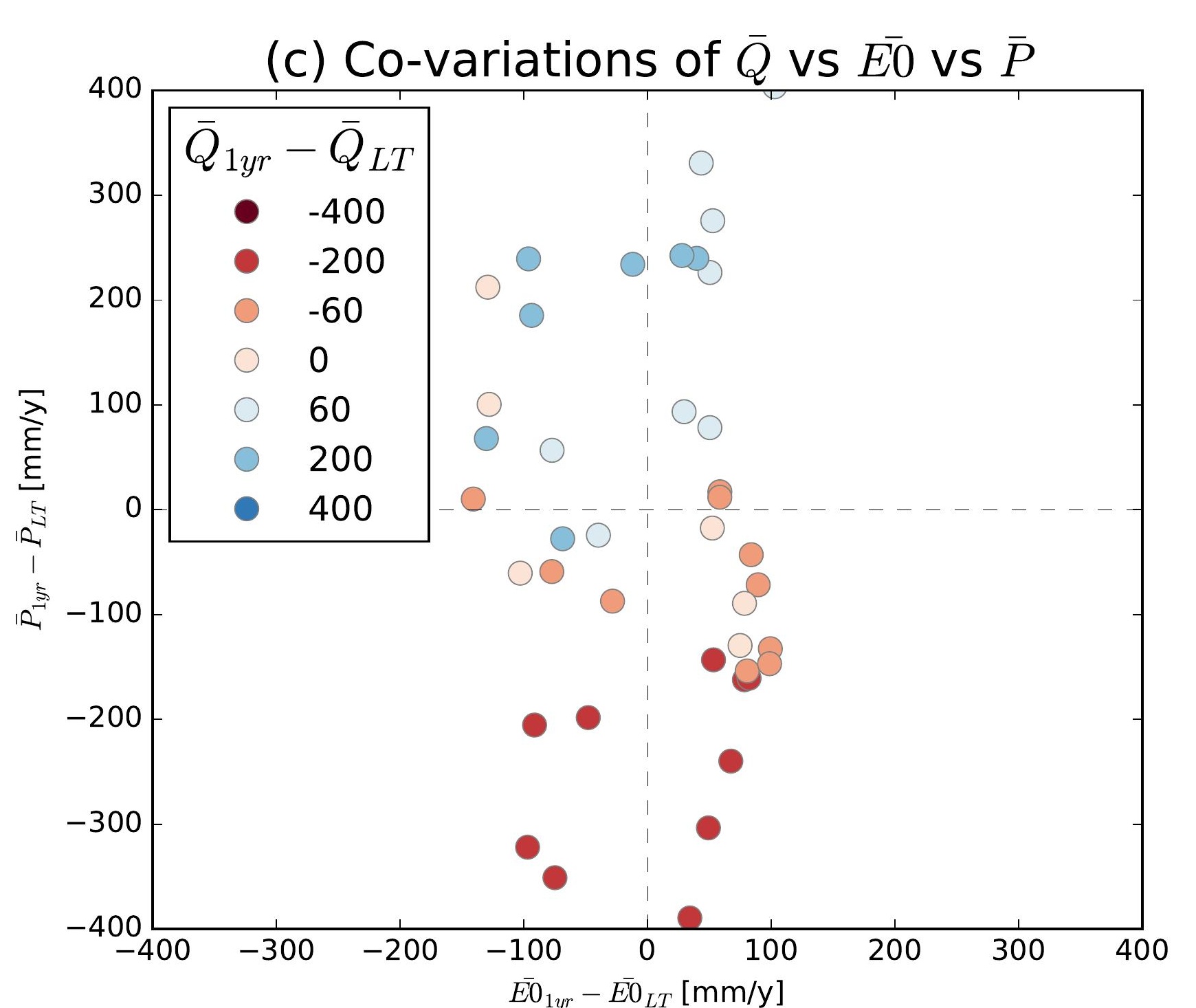
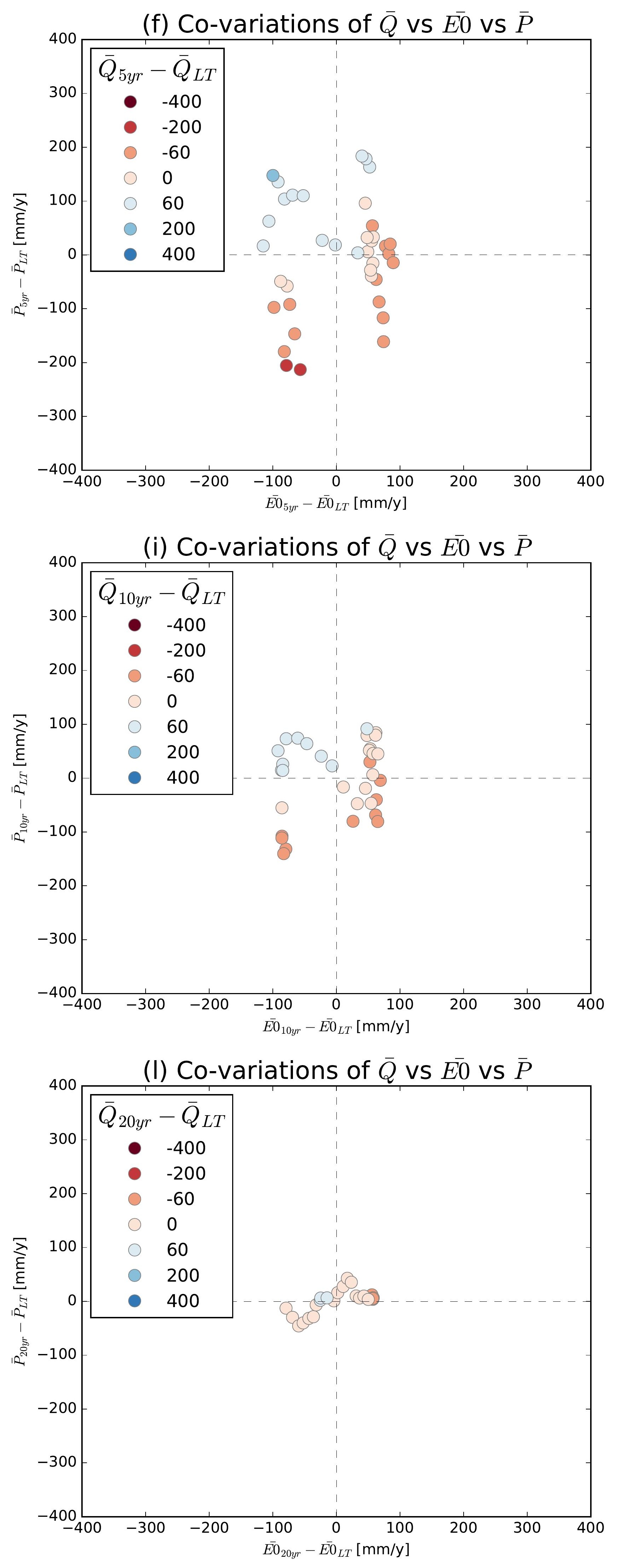

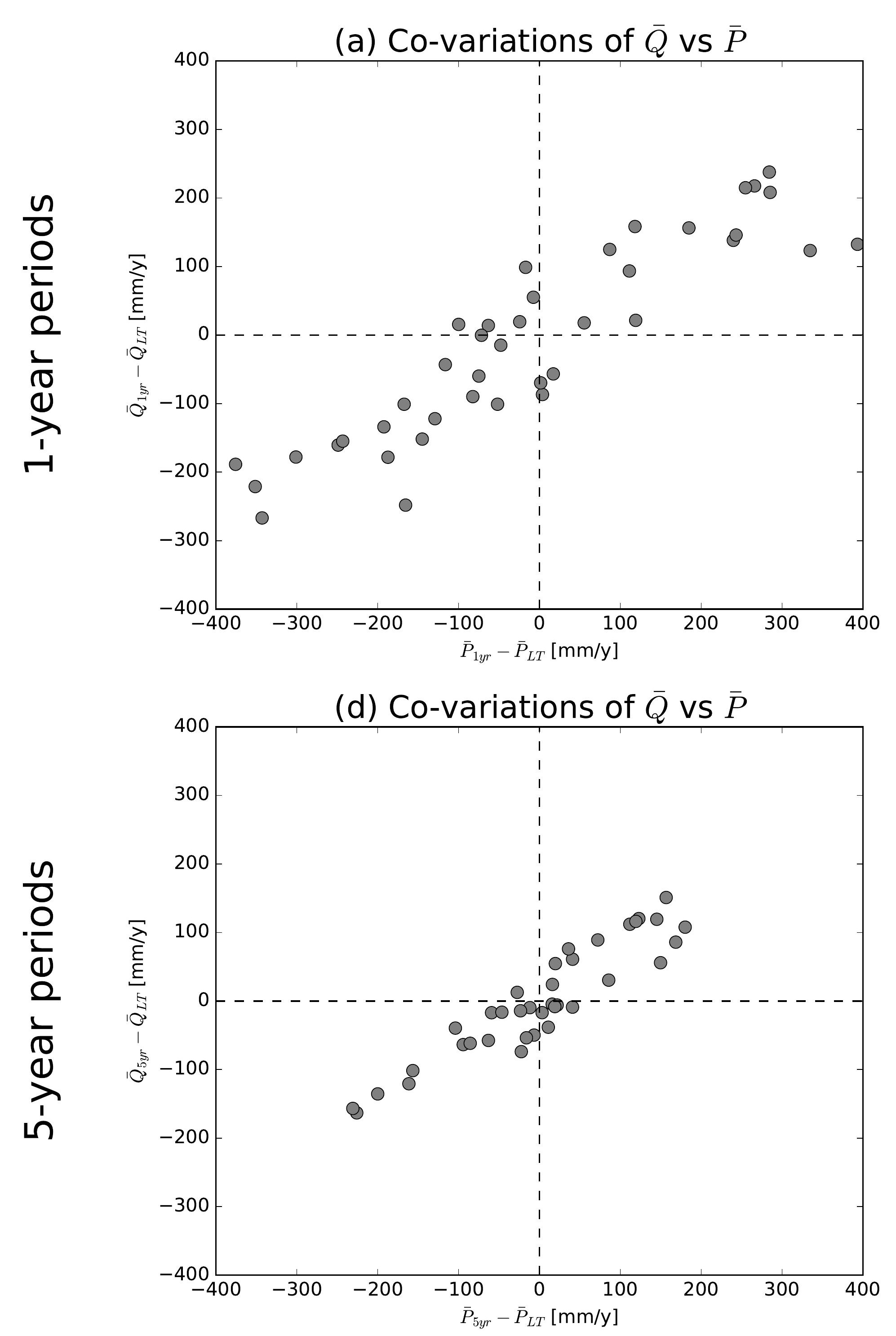

(g) Co-variations of $\bar{Q}$ vs $\bar{P}$
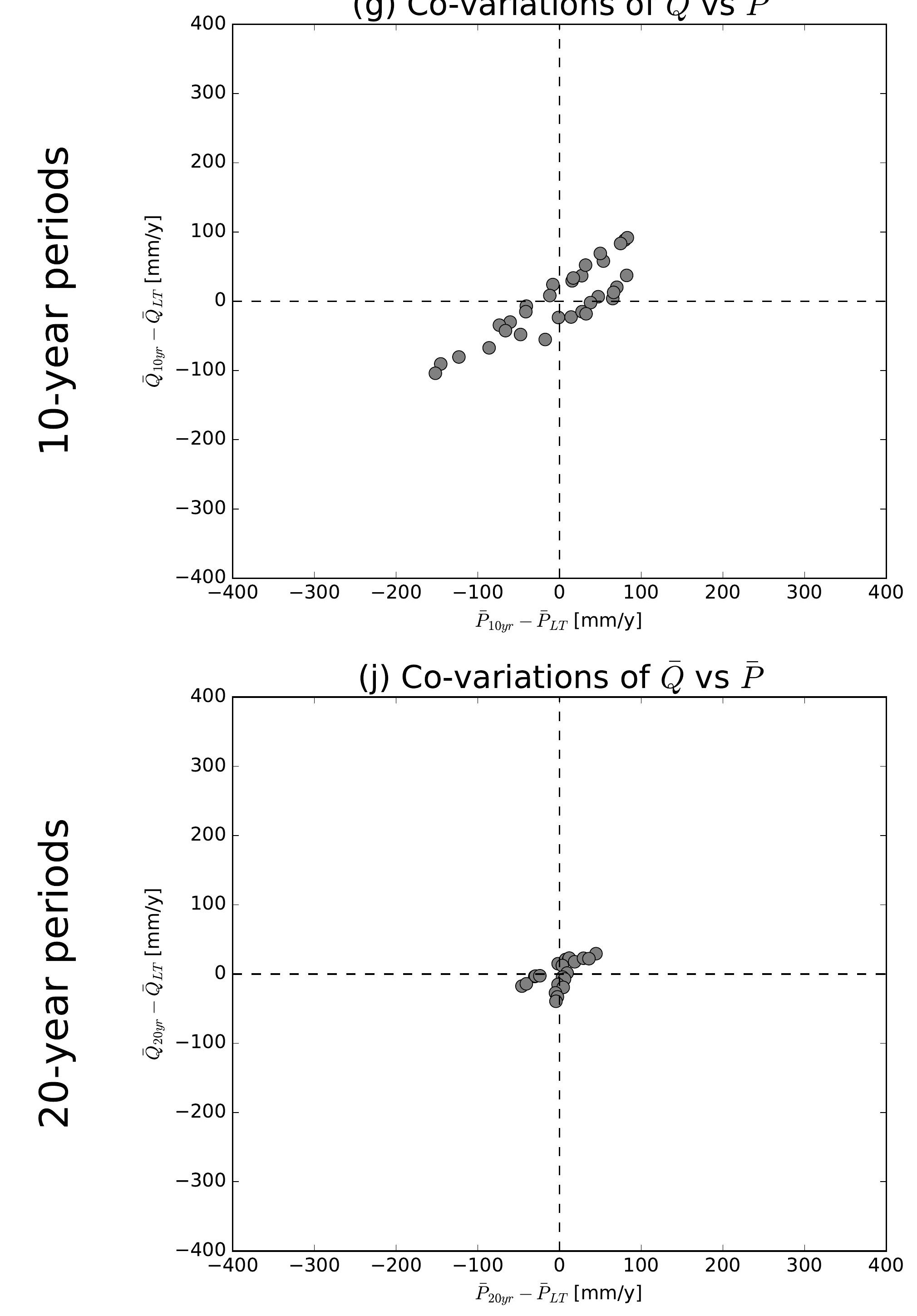

(b) Co-variations of $\bar{Q}$ vs $\overline{E 0}$

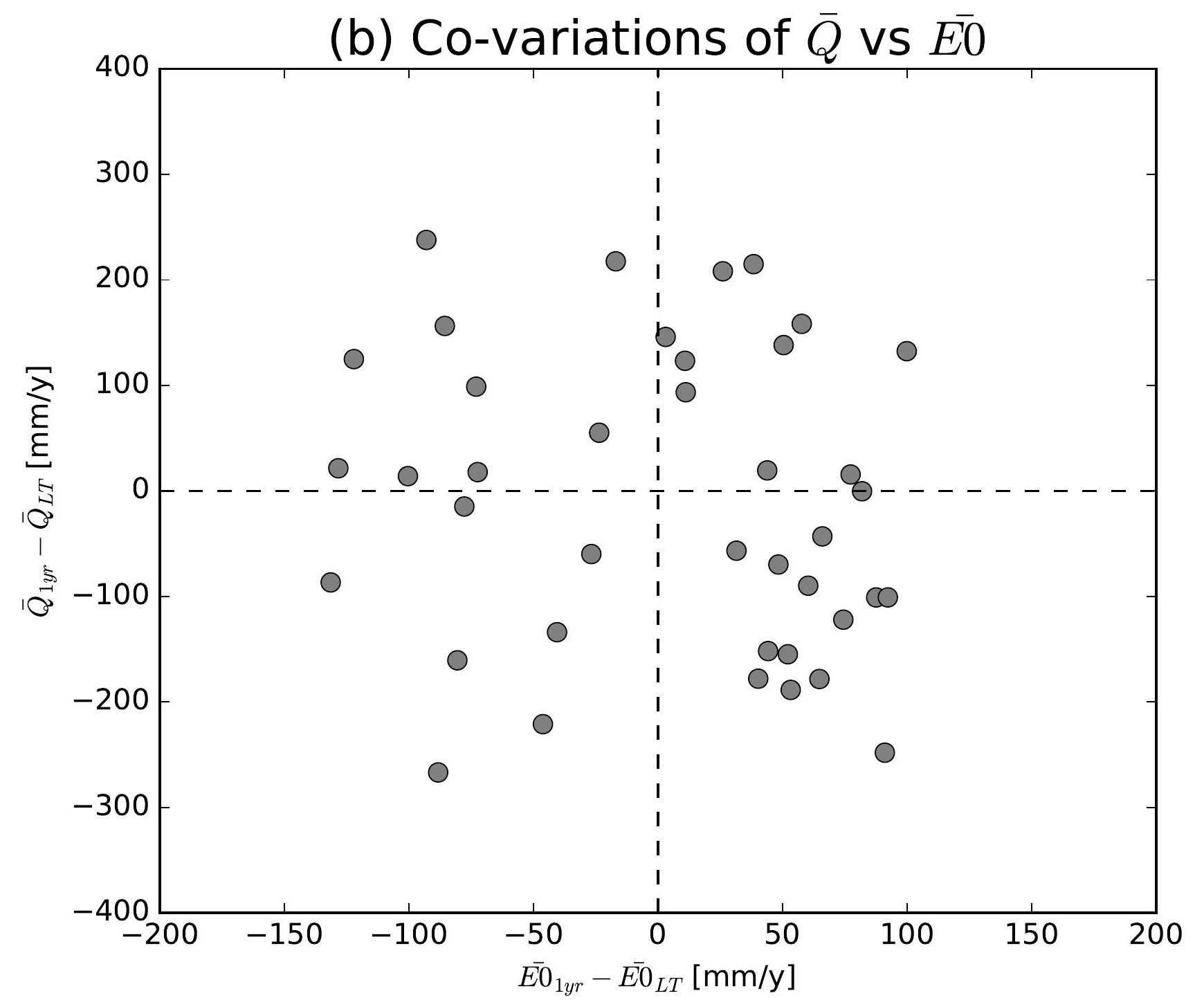

(e) Co-variations of $\bar{Q}$ vs $\overline{E 0}$

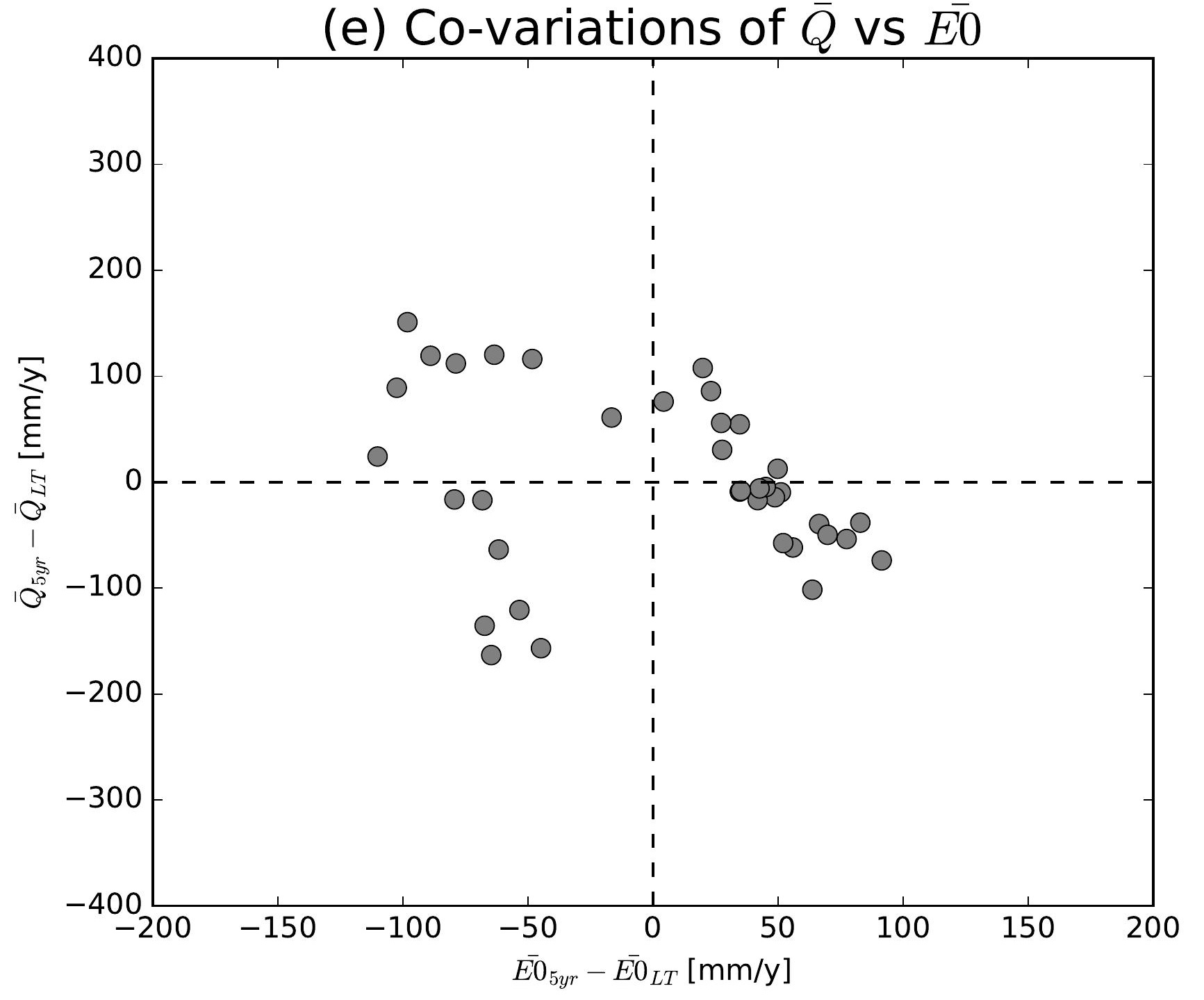

(h) Co-variations of $\bar{Q}$ vs $\overline{E 0}$

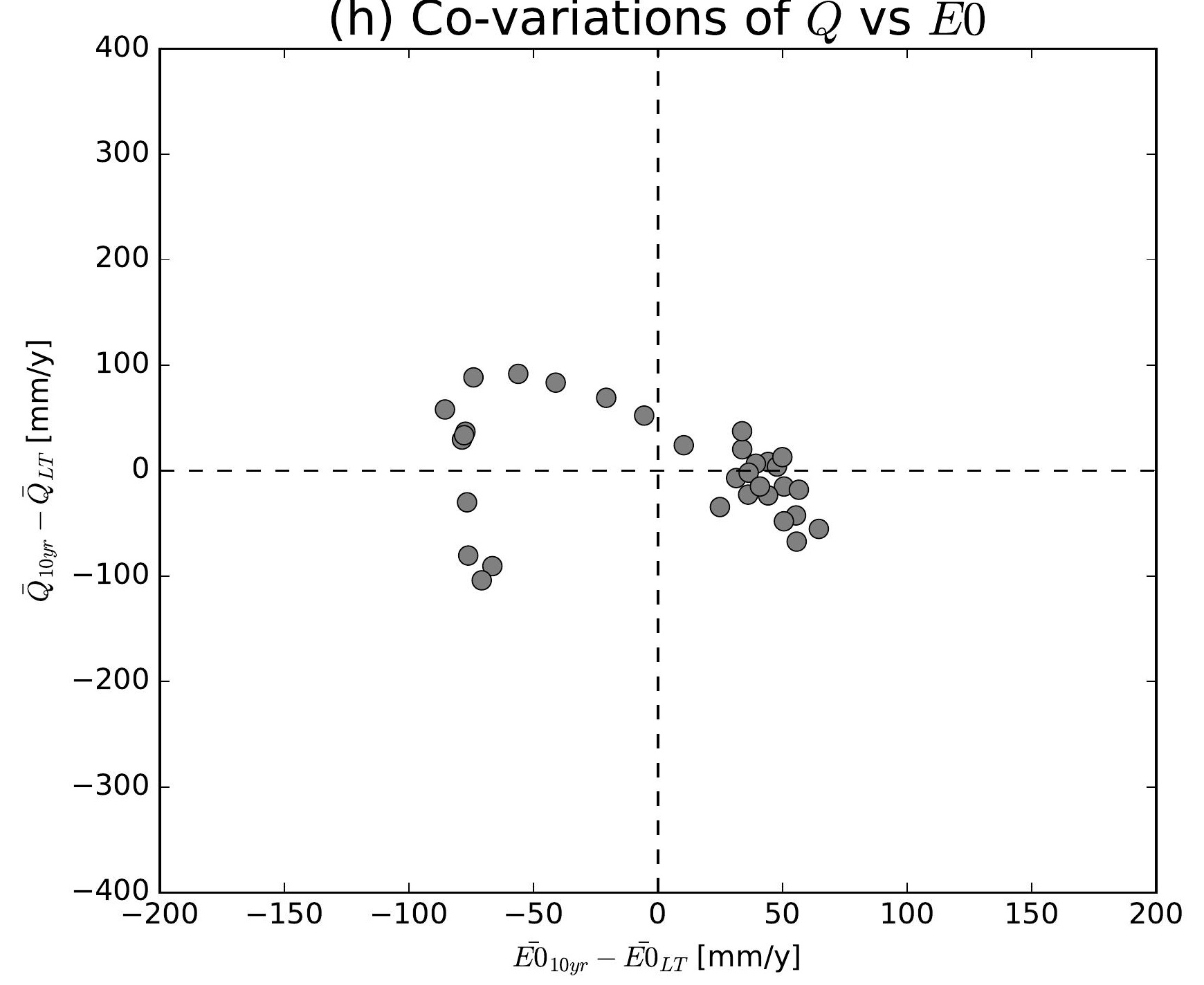

(k) Co-variations of $\bar{Q}$ vs $\overline{E 0}$

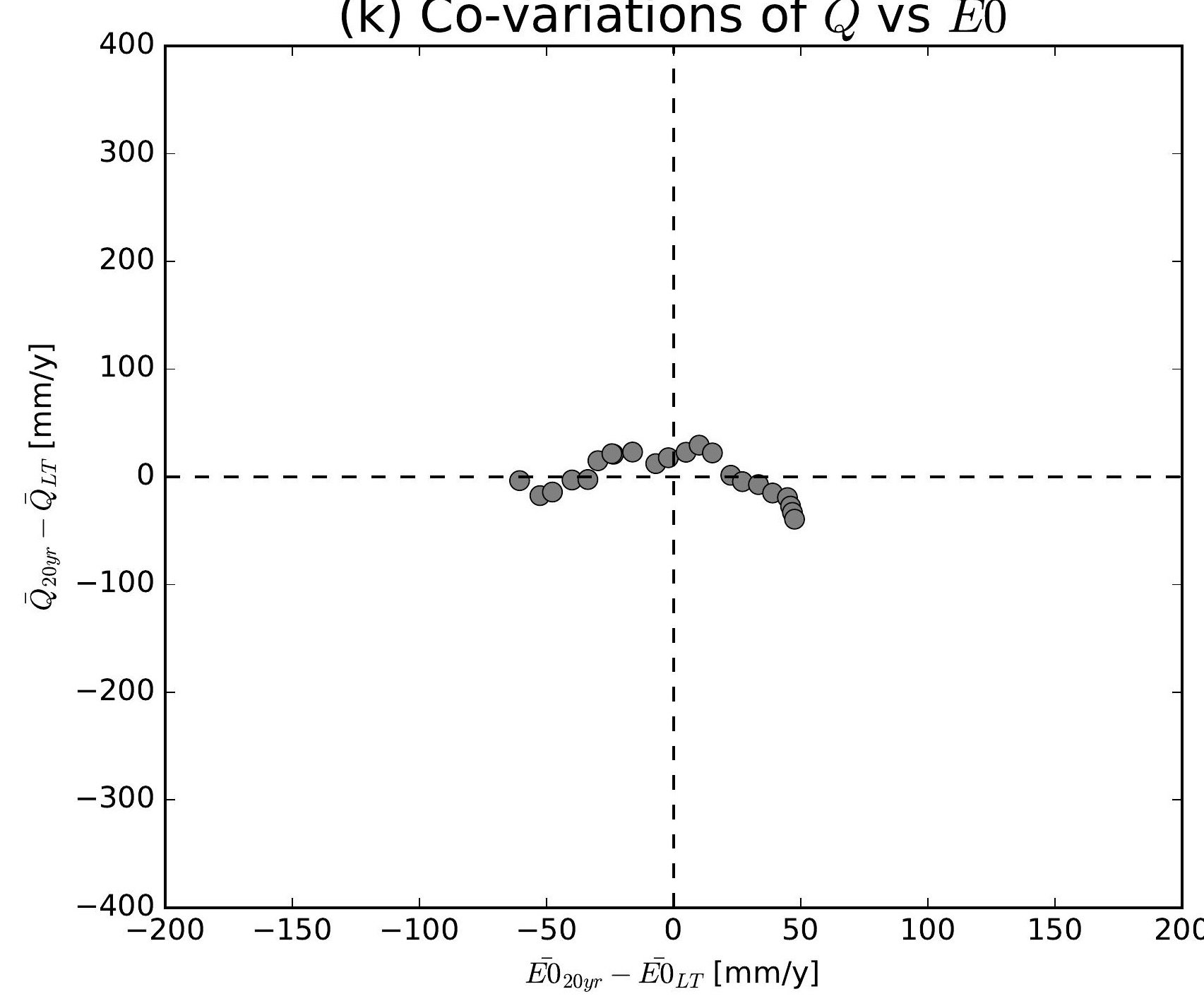

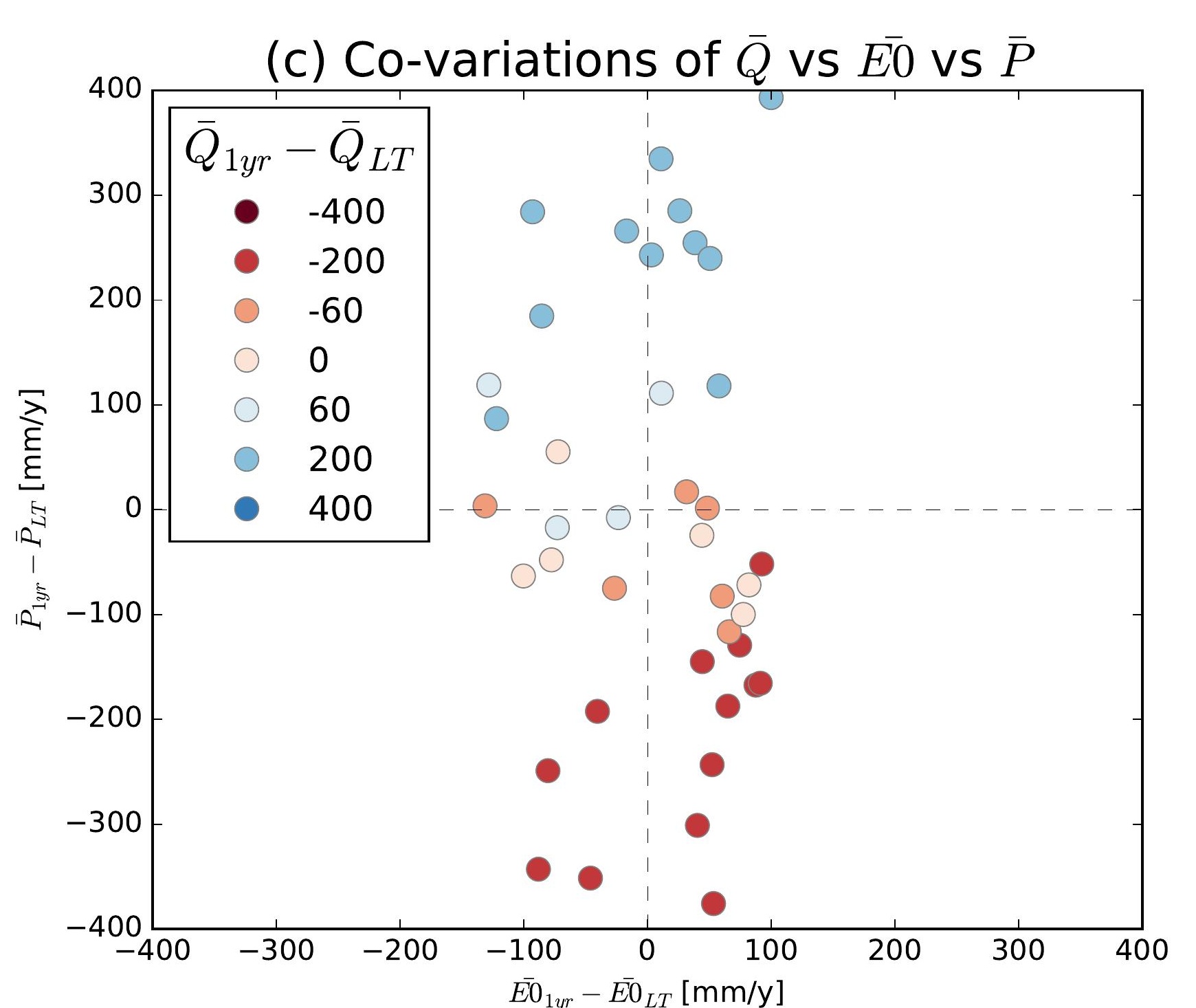
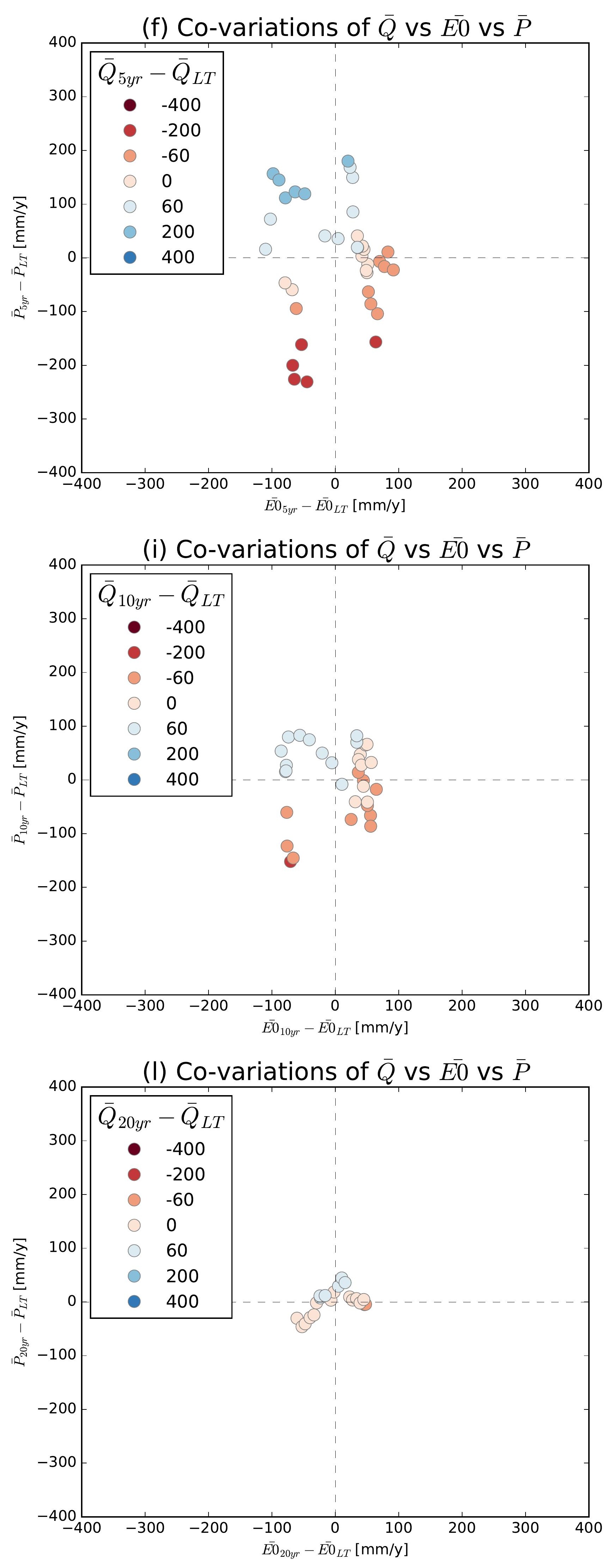

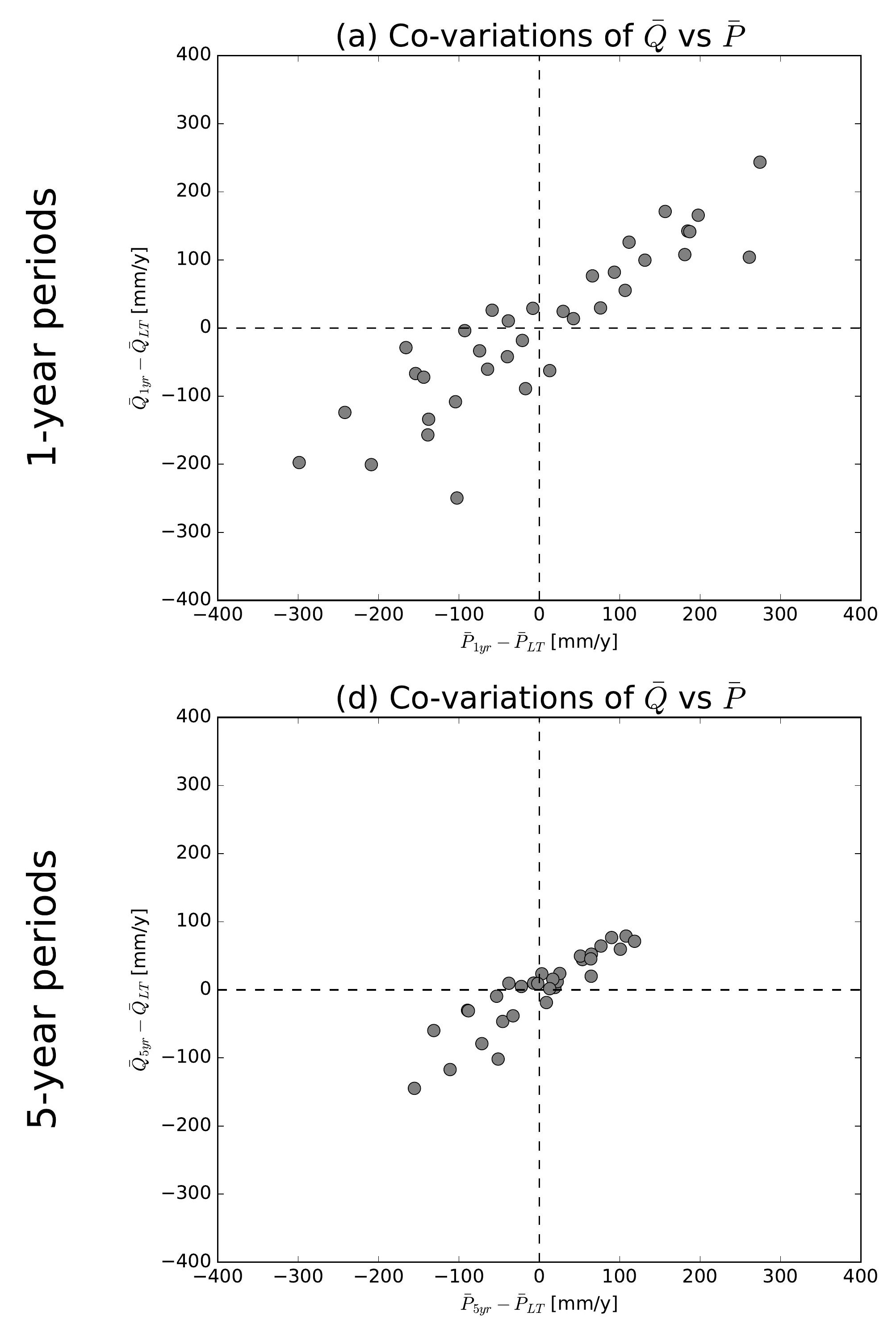

(g) Co-variations of $\bar{Q}$ vs $\bar{P}$

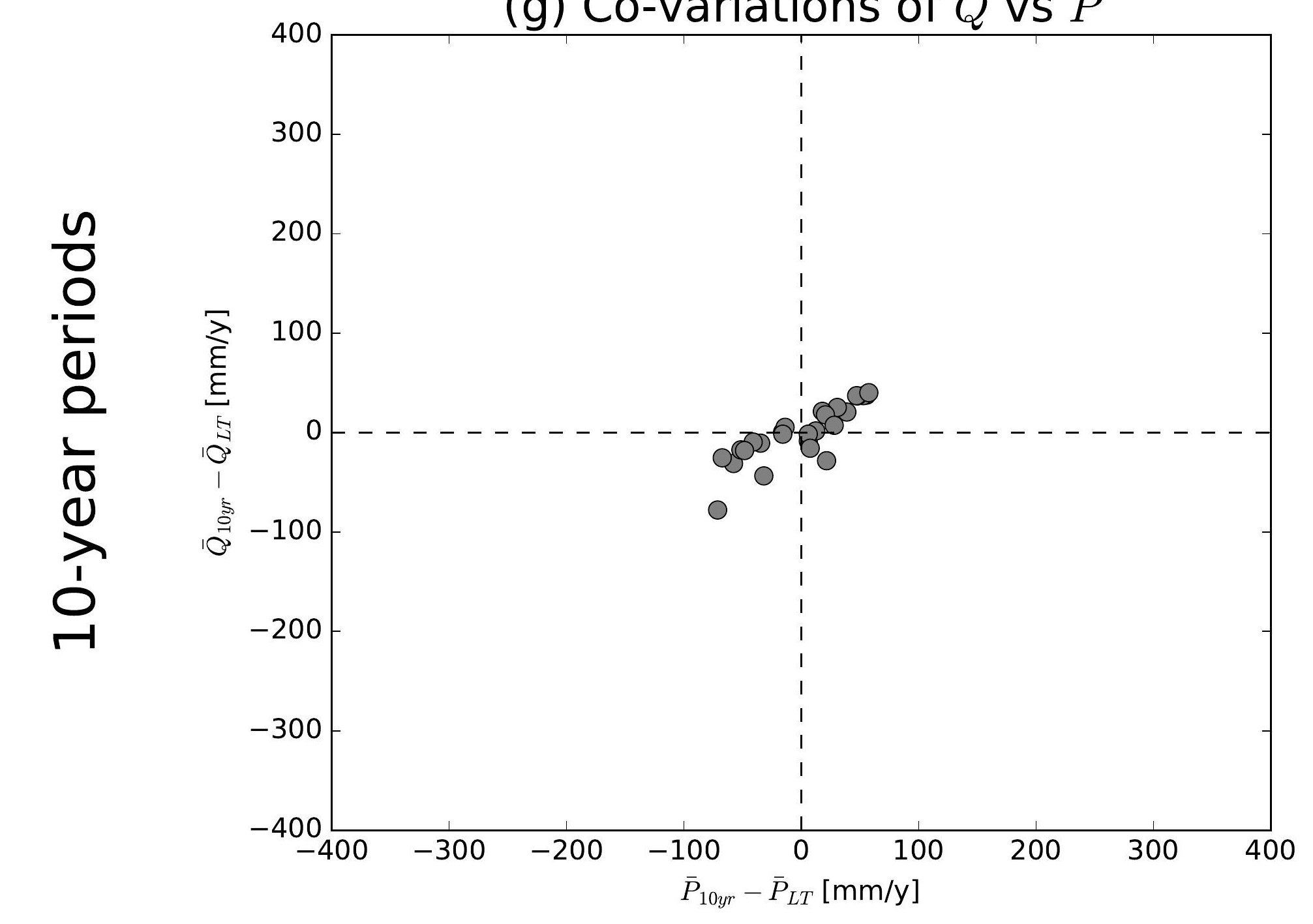

(j) Co-variations of $\bar{Q}$ vs $\bar{P}$

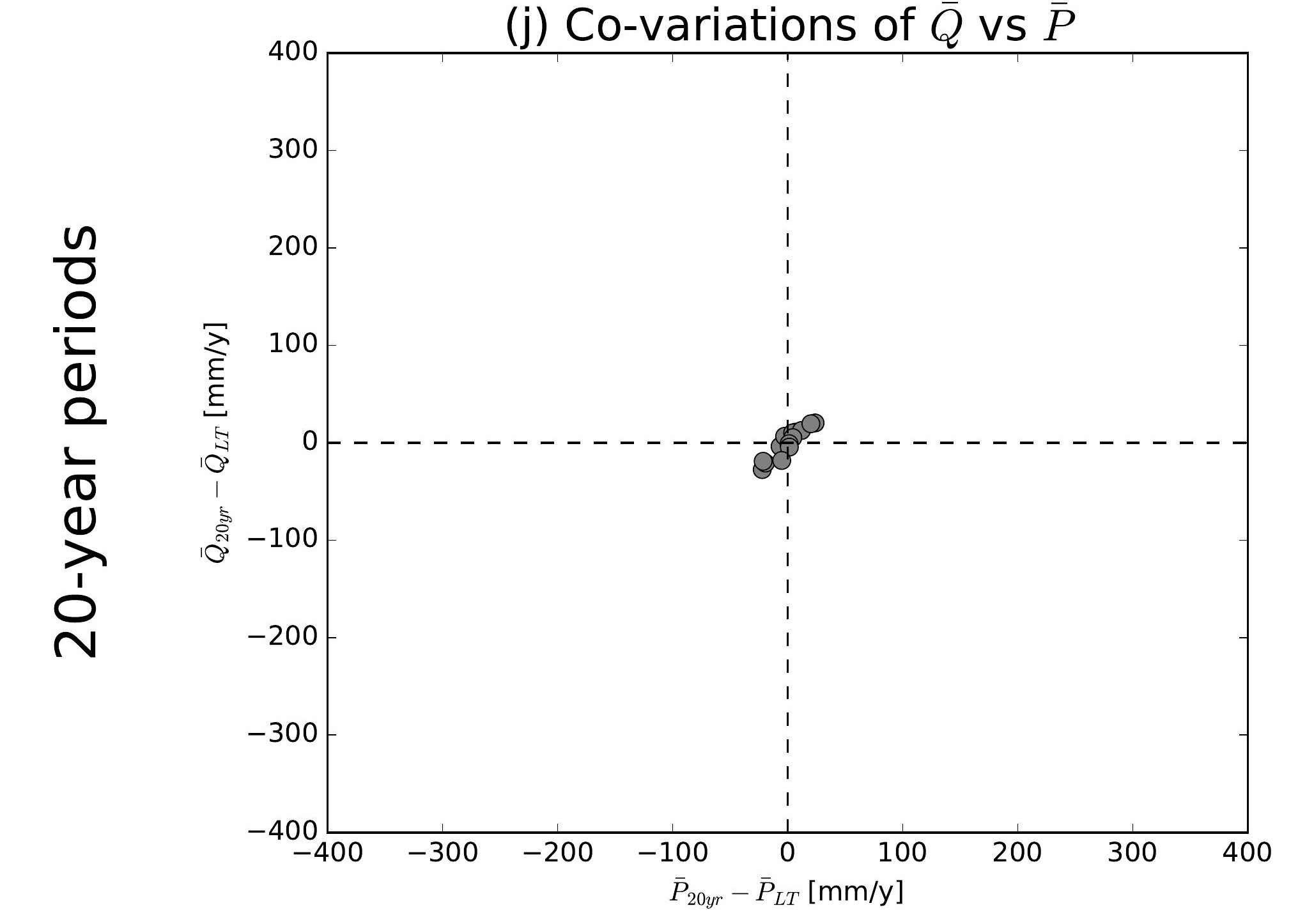

(b) Co-variations of $\bar{Q}$ vs $\overline{E 0}$

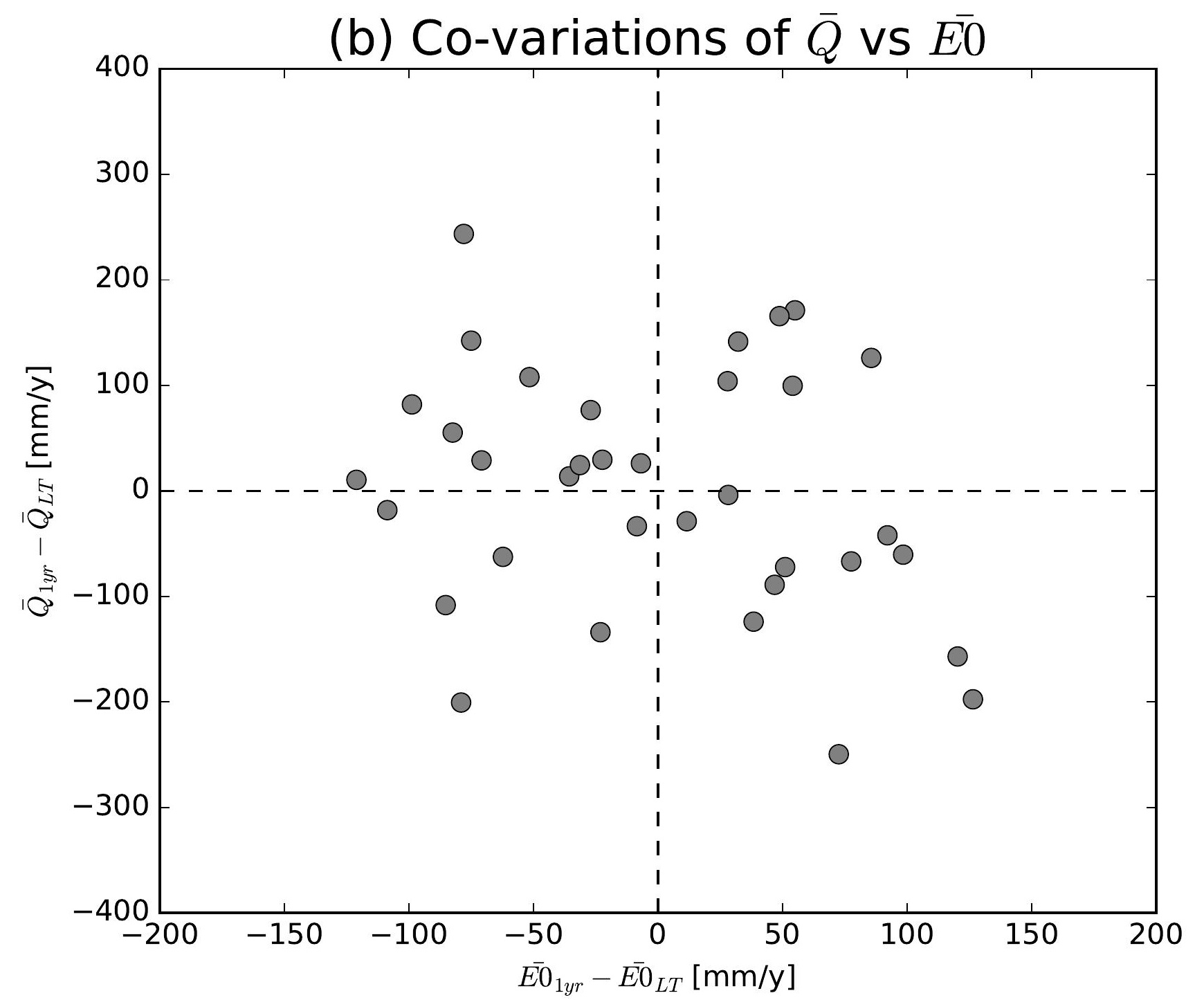

(e) Co-variations of $\bar{Q}$ vs $\overline{E 0}$

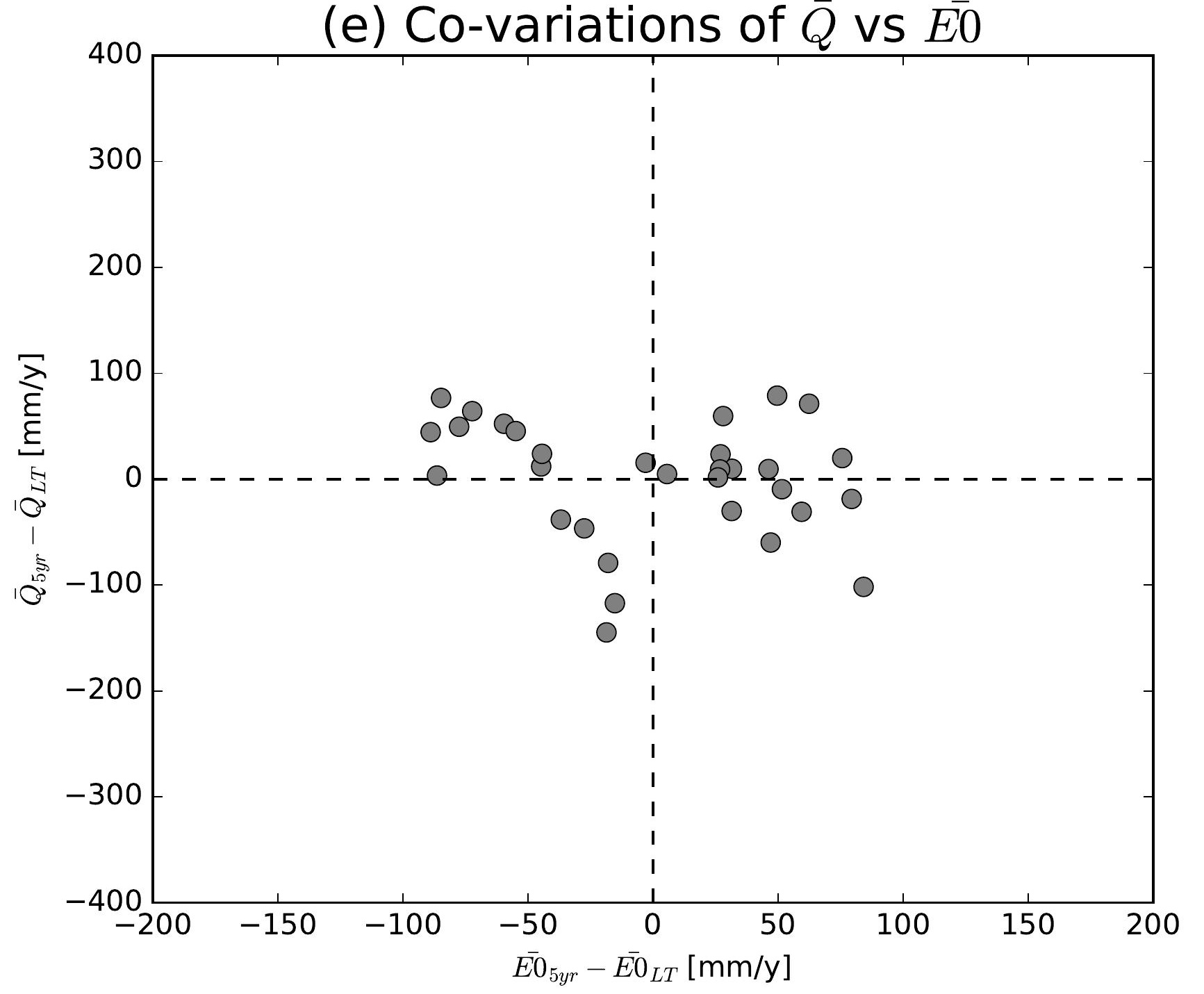

(h) Co-variations of $\bar{Q}$ vs $\overline{E 0}$

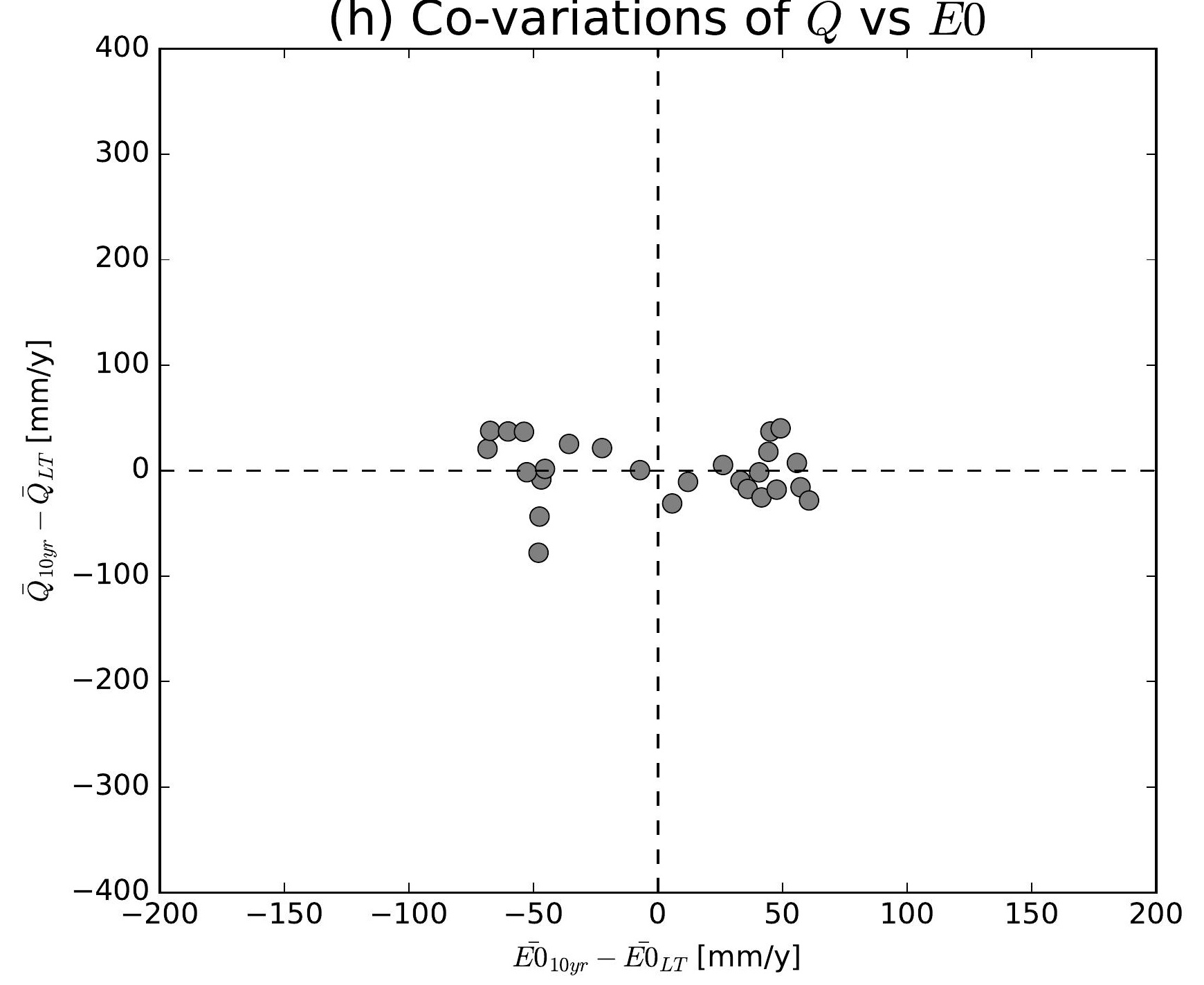

(k) Co-variations of $\bar{Q}$ vs $\overline{E 0}$

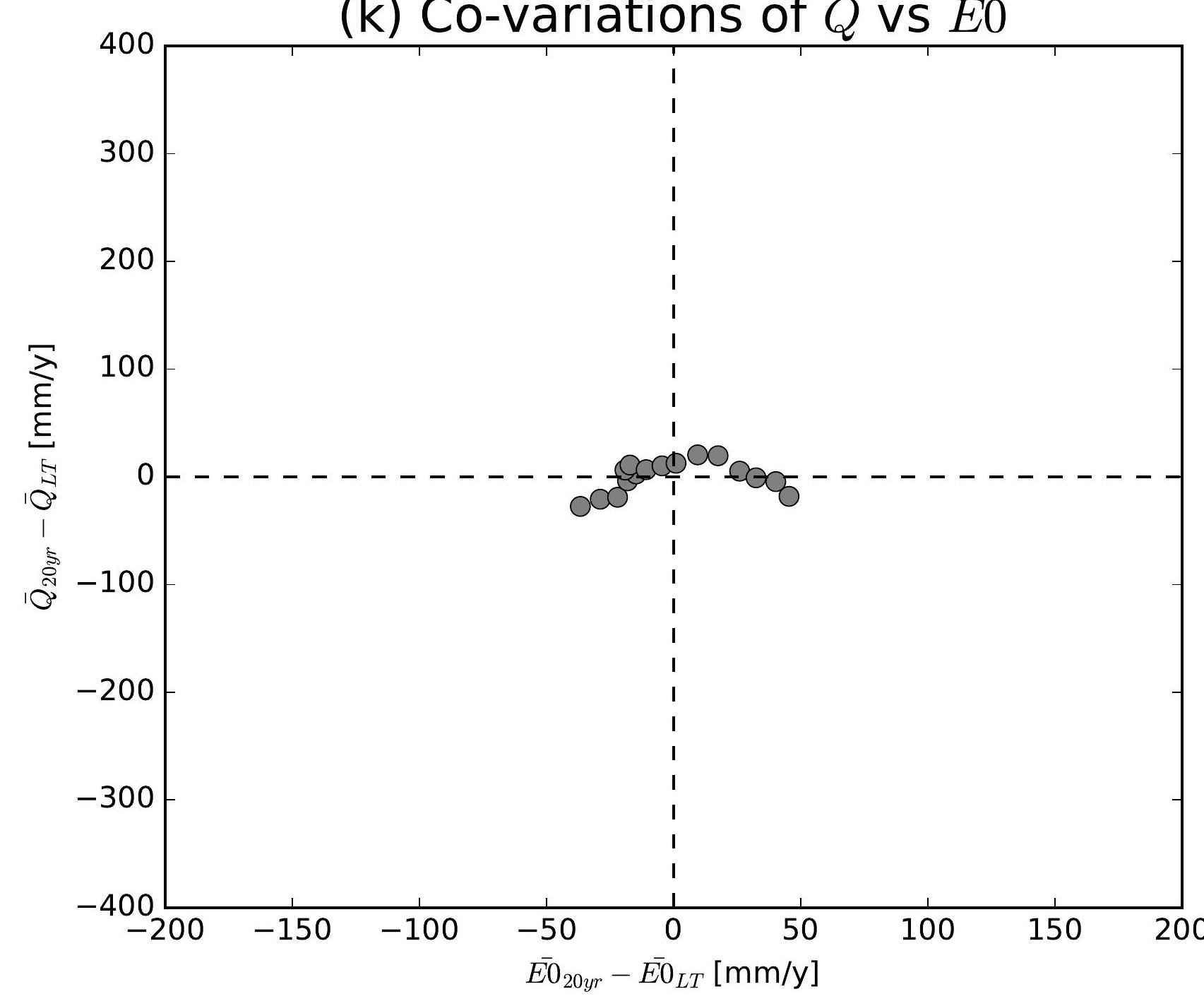

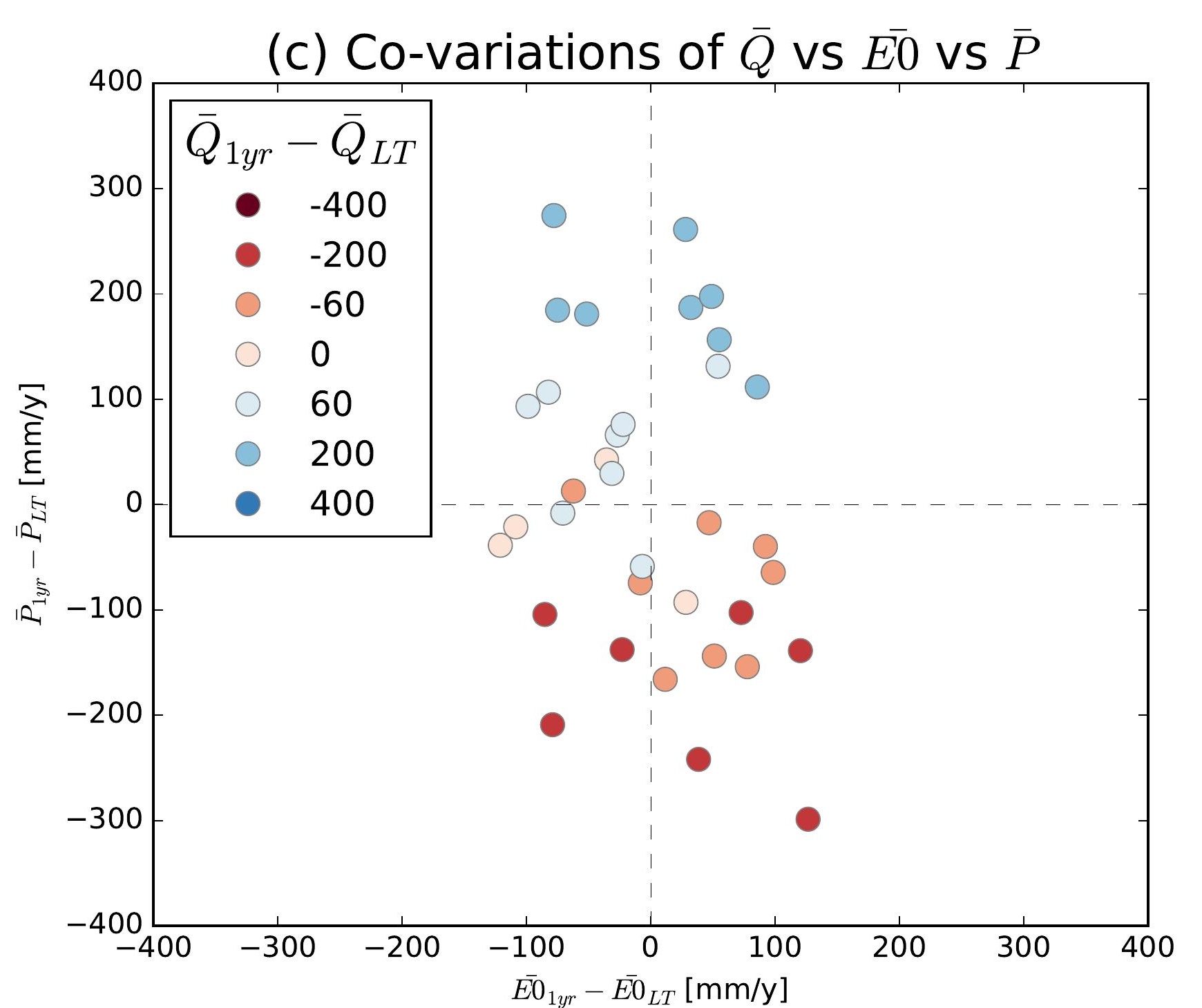
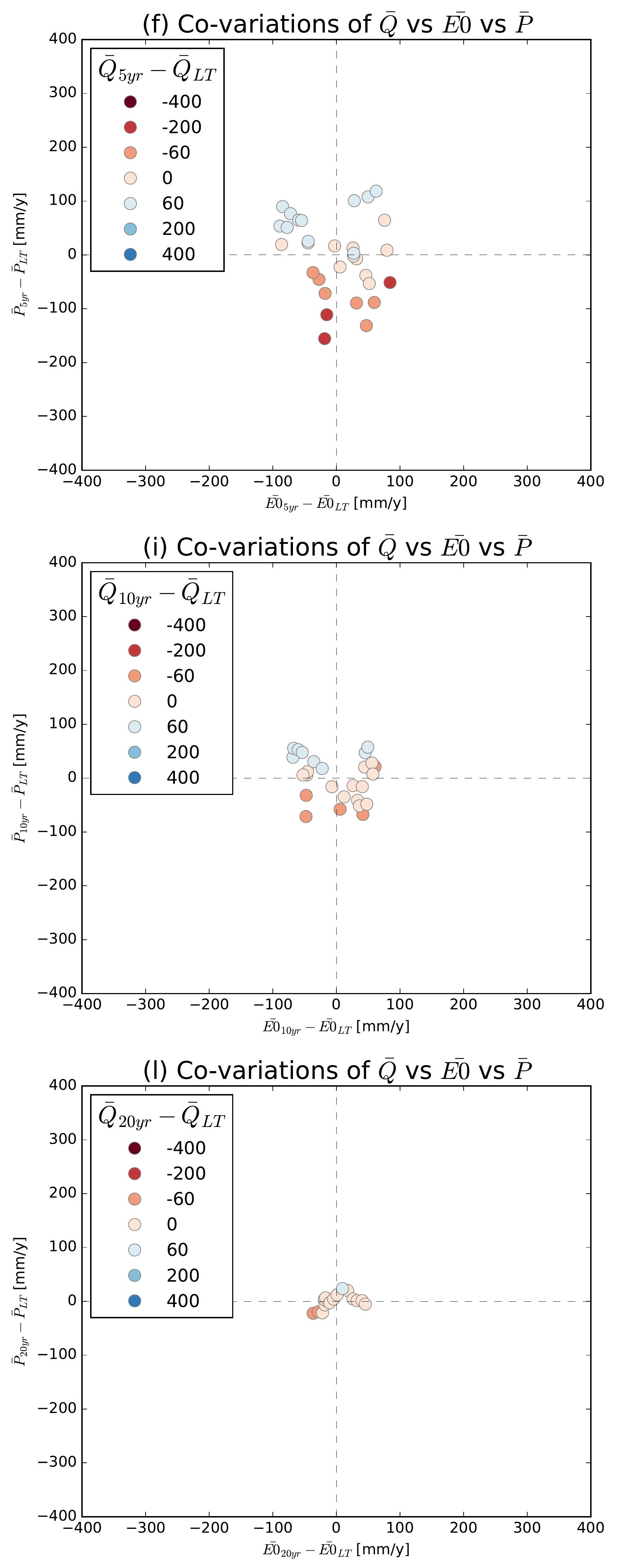

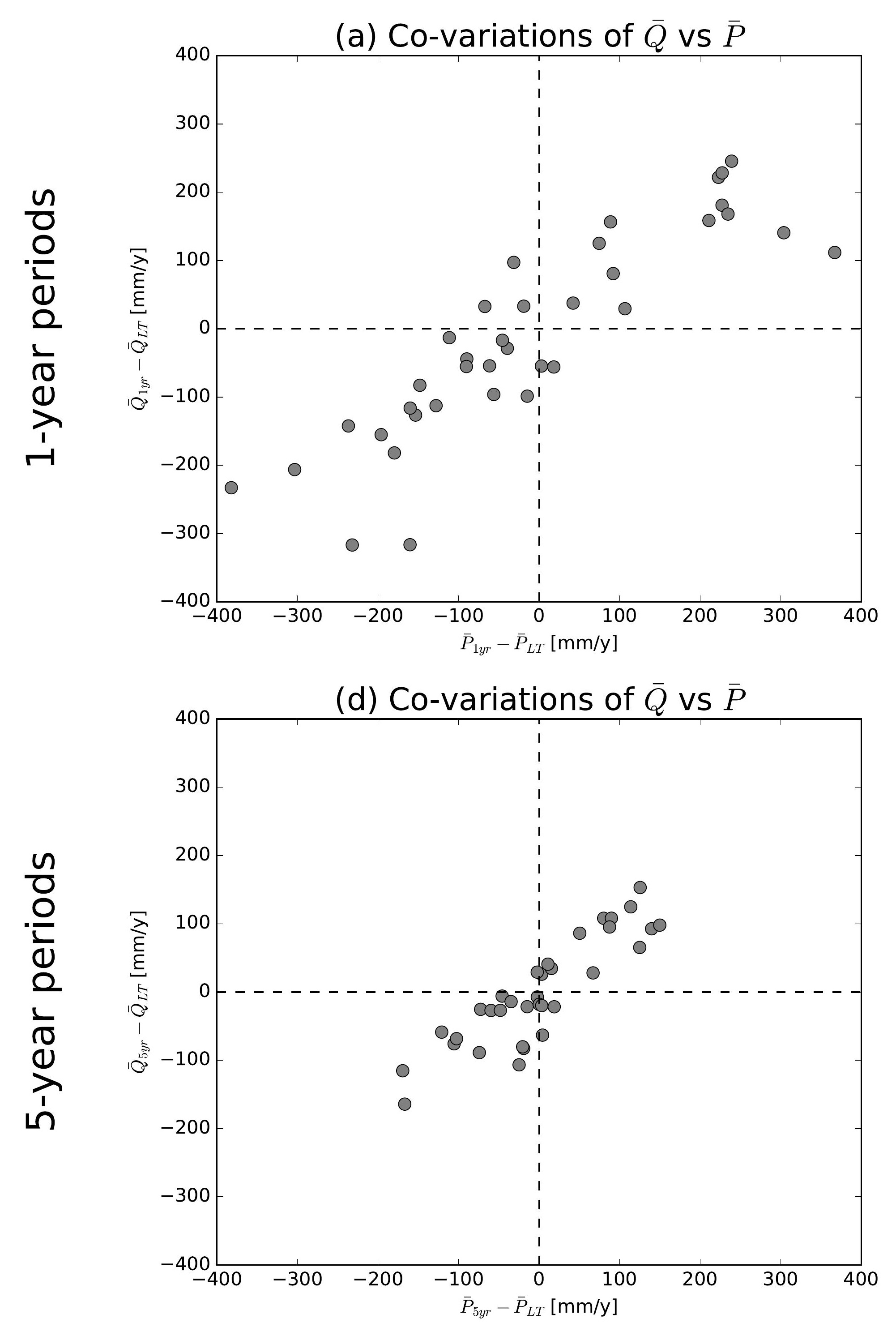

(g) Co-variations of $\bar{Q}$ vs $\bar{P}$
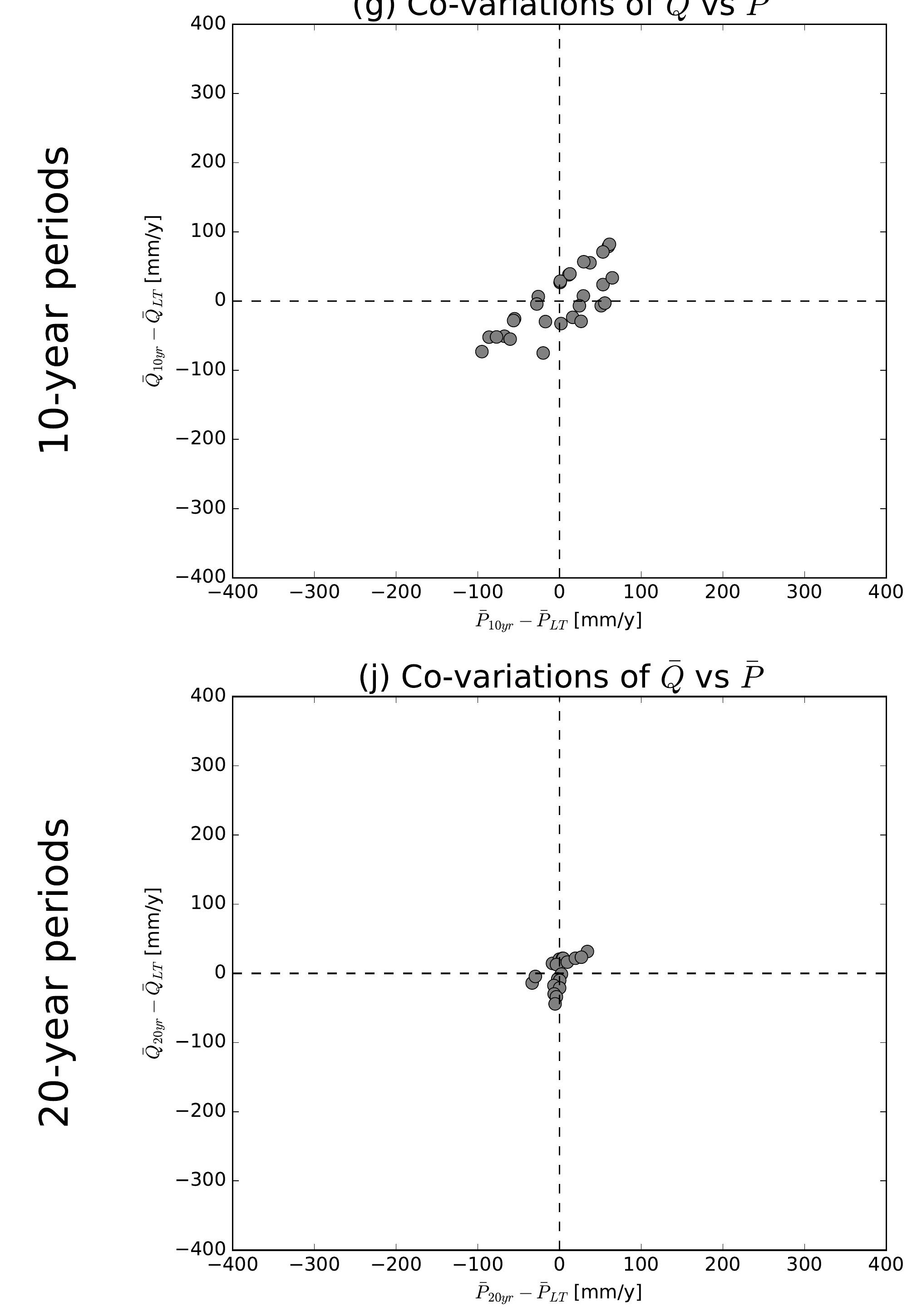

(b) Co-variations of $\bar{Q}$ vs $\overline{E 0}$

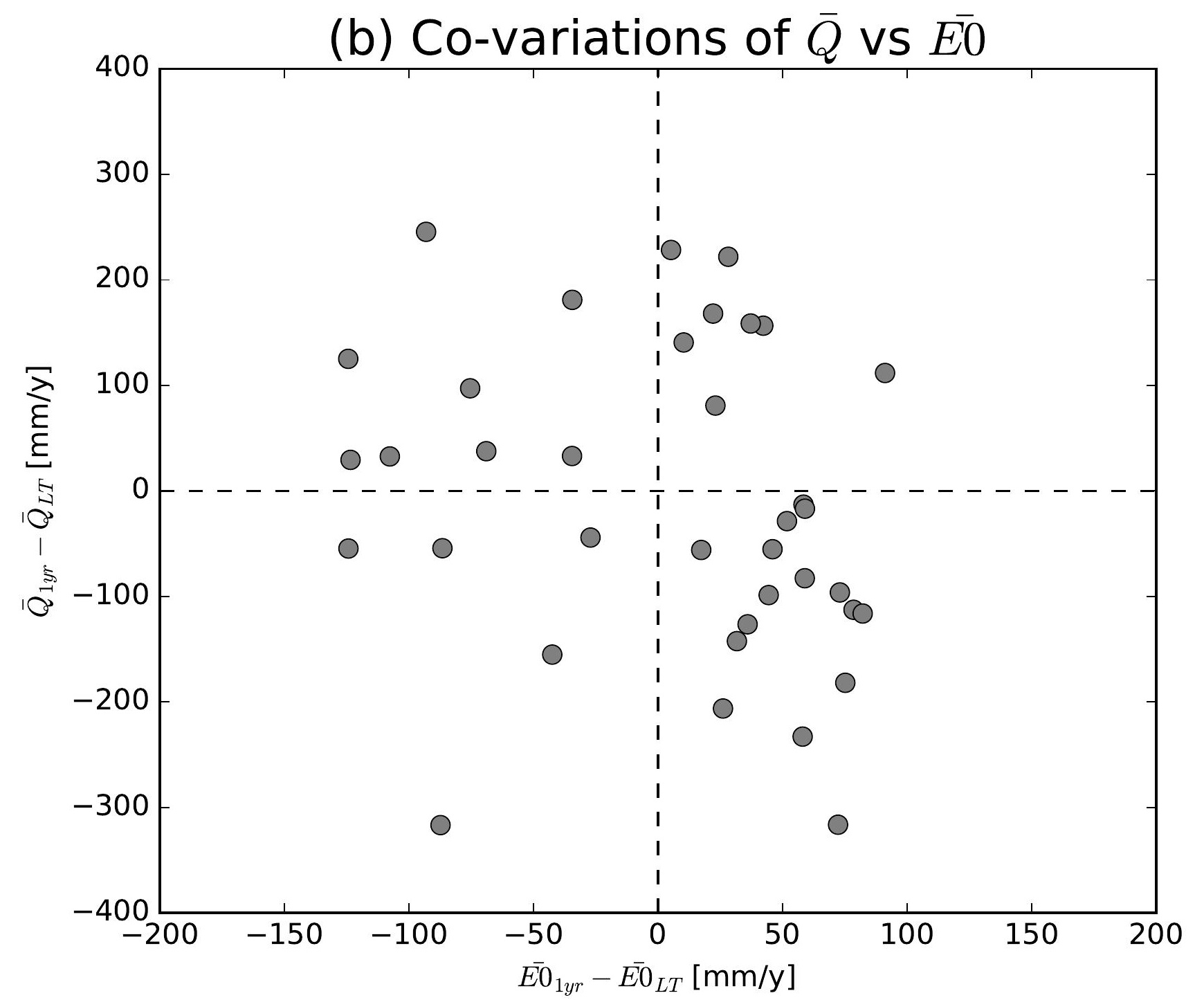

(e) Co-variations of $\bar{Q}$ vs $\overline{E 0}$

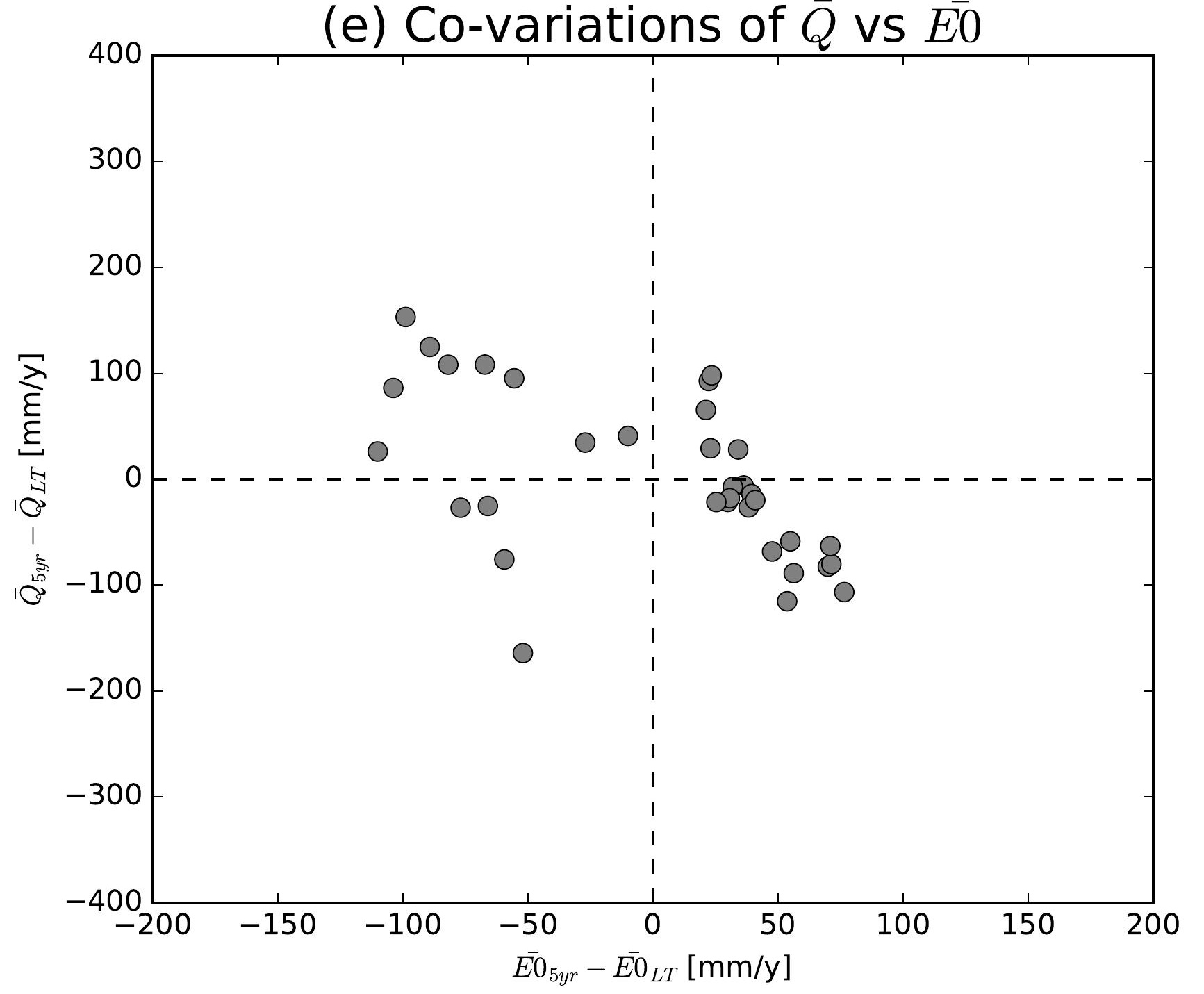

(h) Co-variations of $\bar{Q}$ vs $\overline{E 0}$

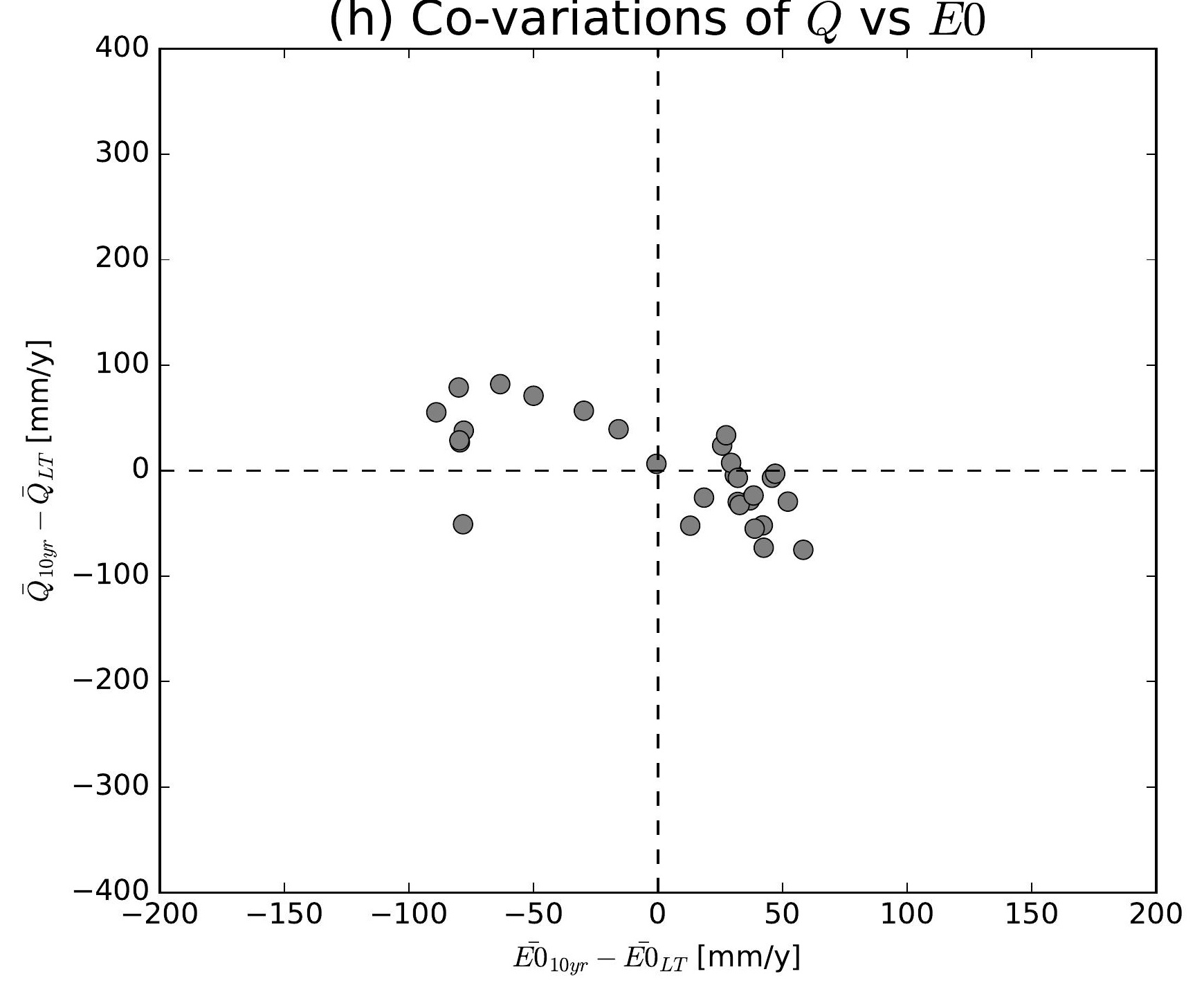

(k) Co-variations of $\bar{Q}$ vs $\overline{E 0}$

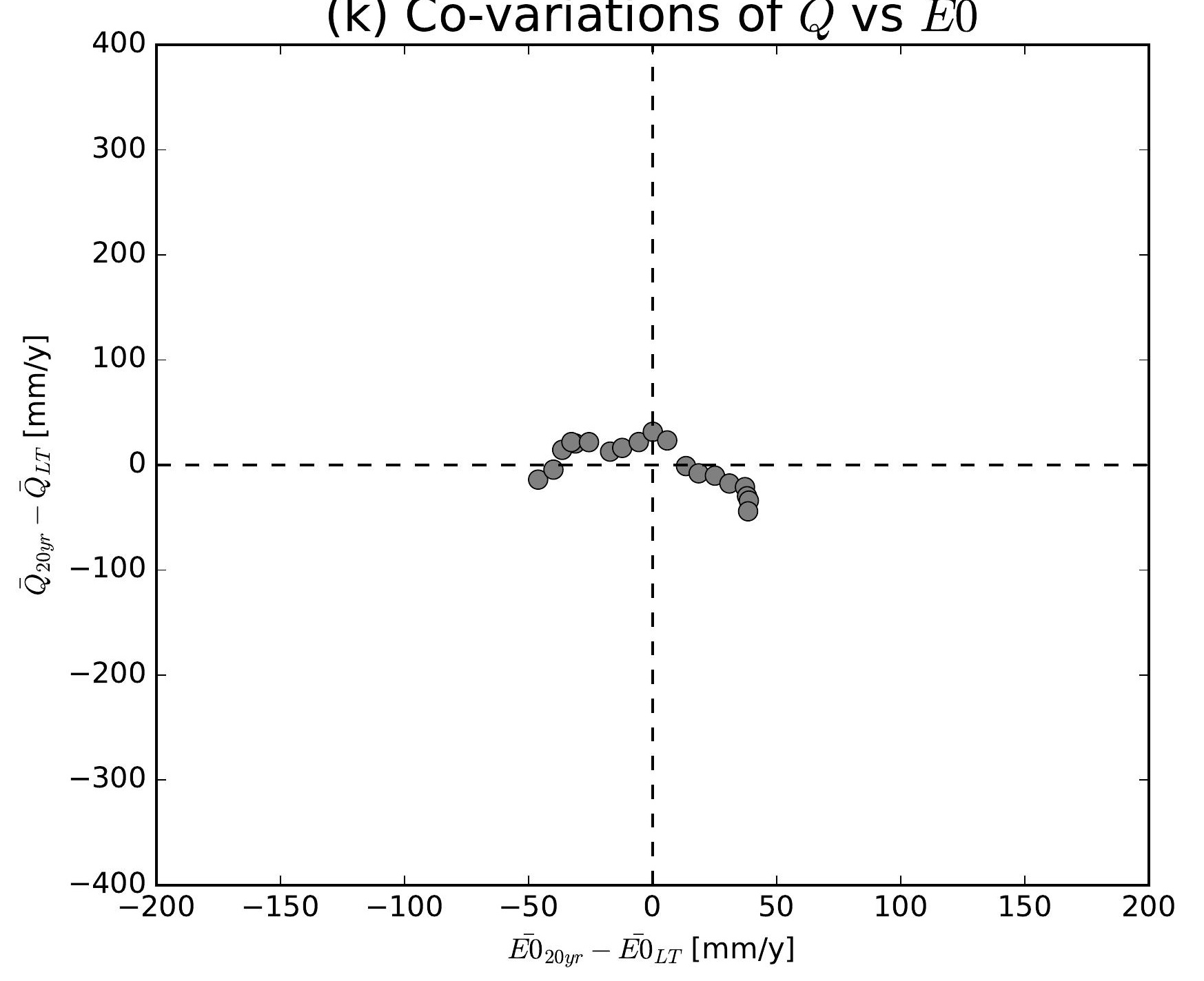

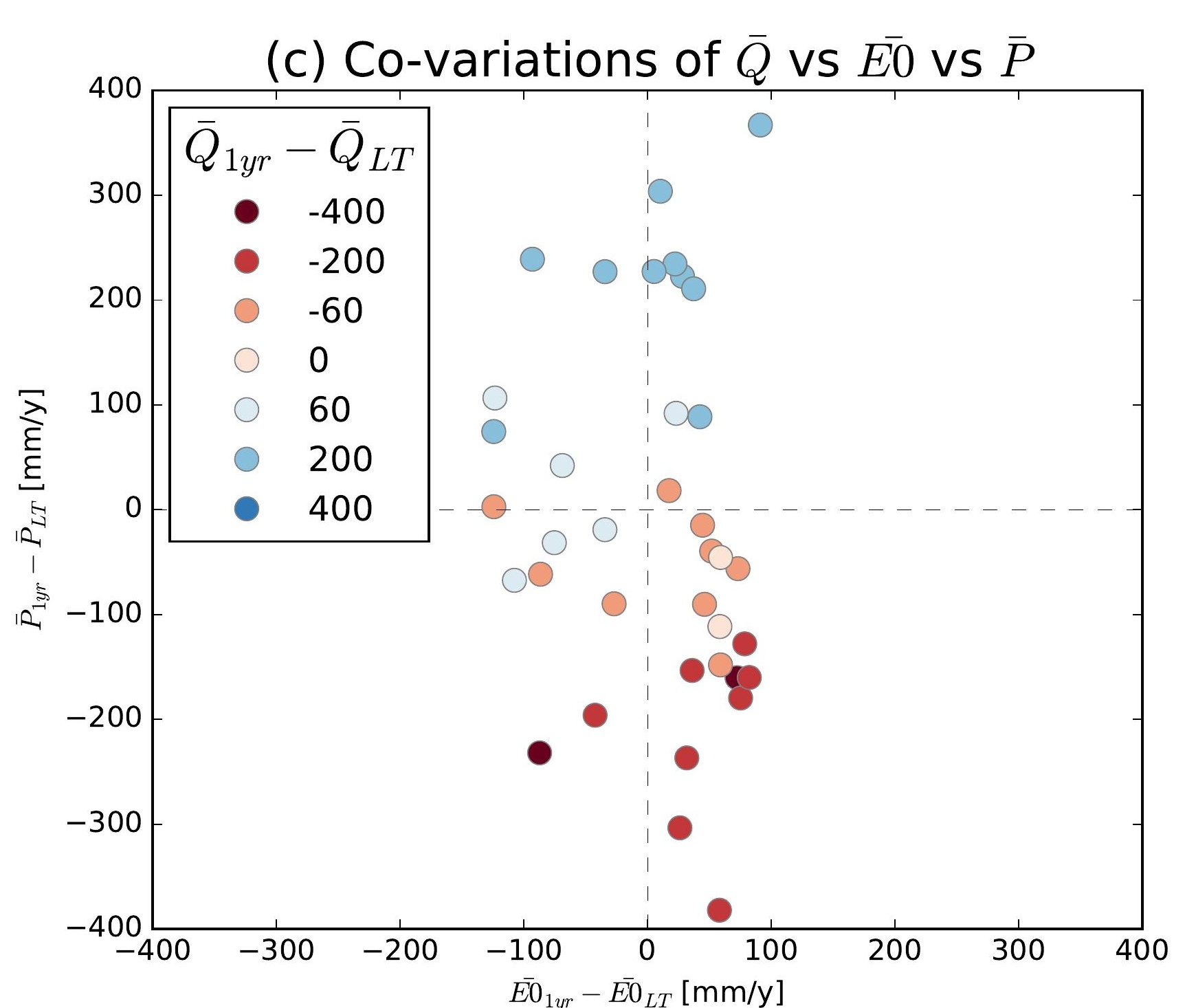
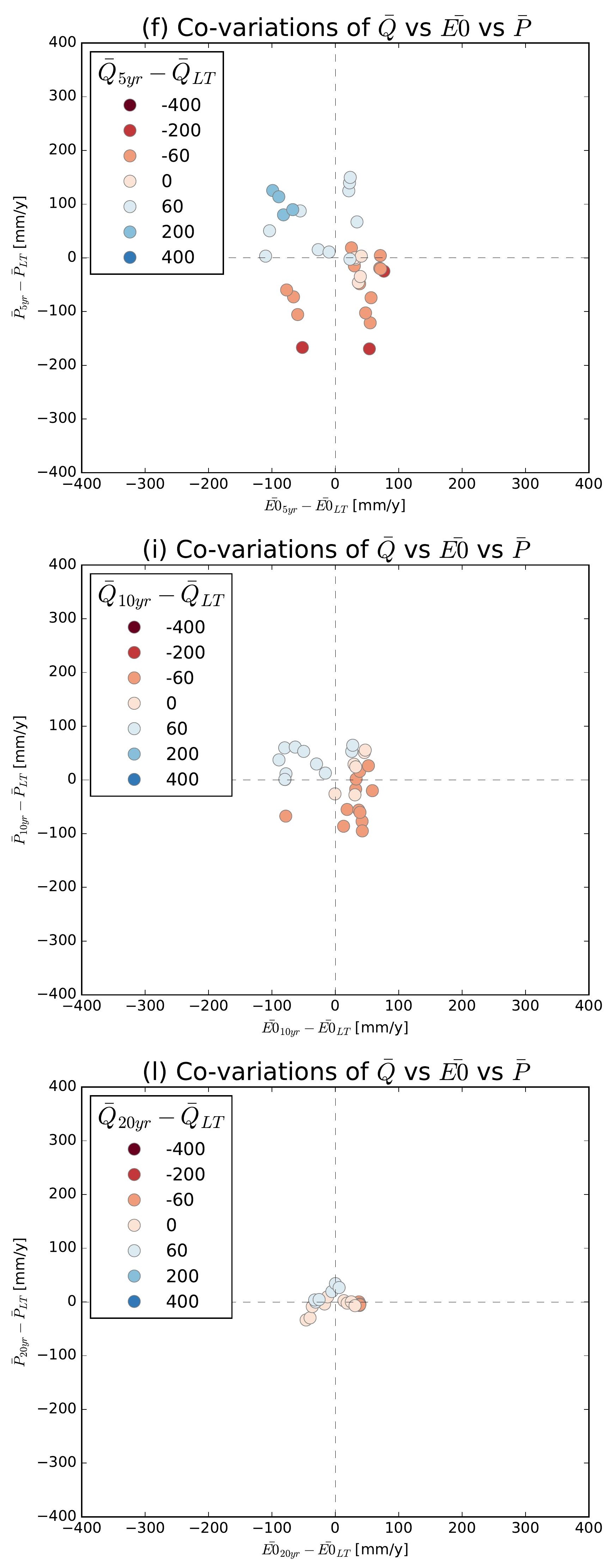

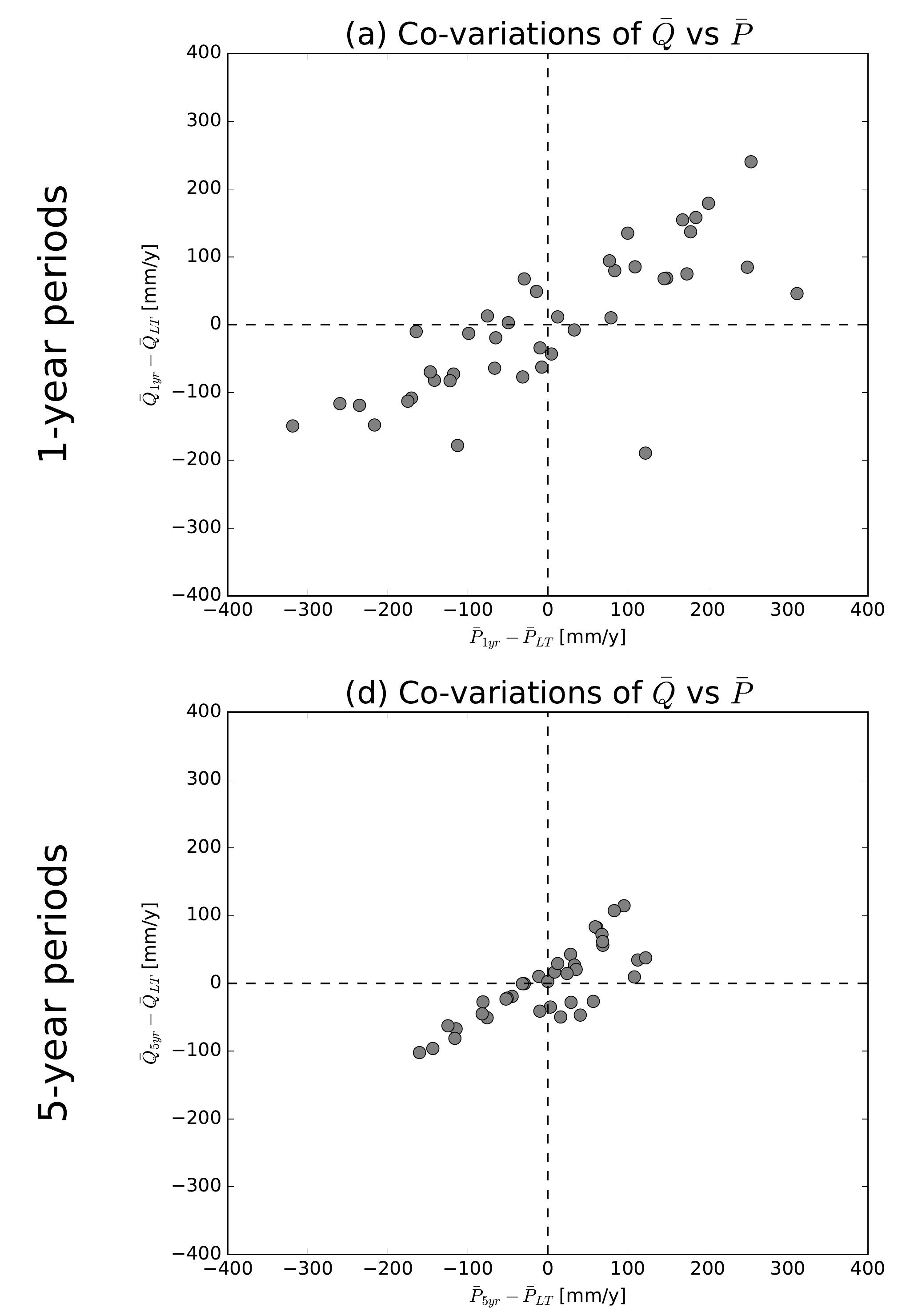

(g) Co-variations of $\bar{Q}$ vs $\bar{P}$
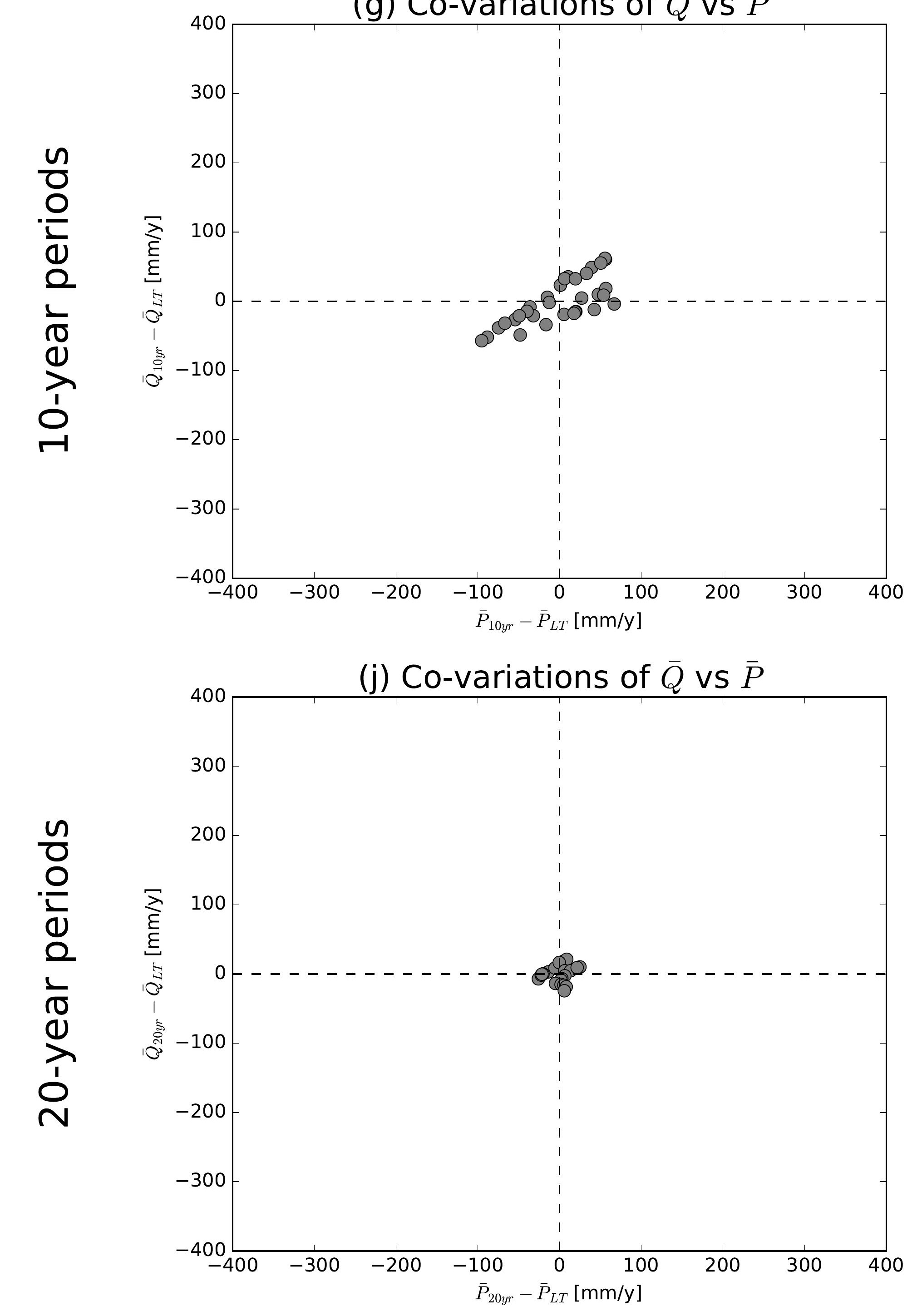

(b) Co-variations of $\bar{Q}$ vs $\overline{E 0}$

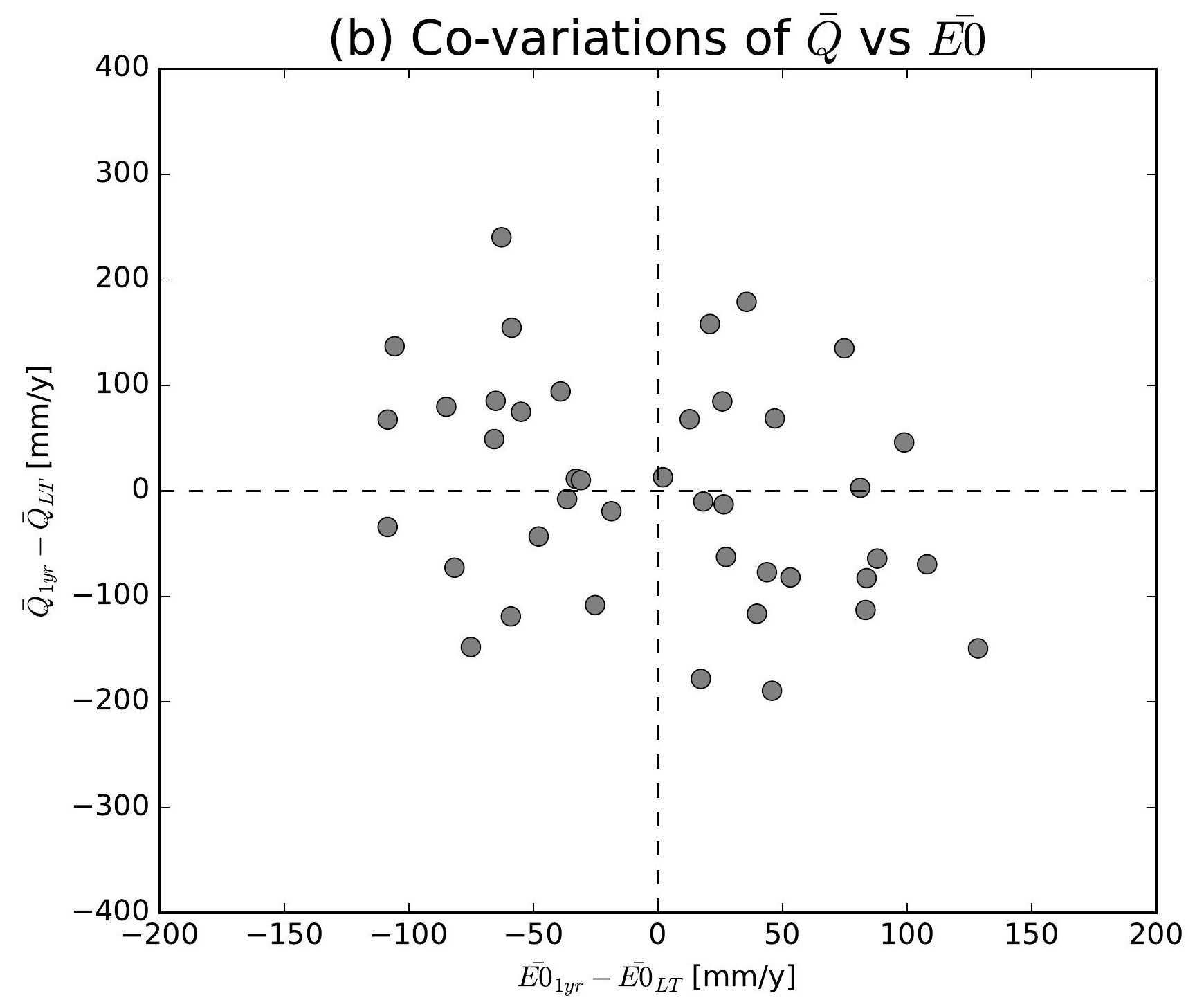

(e) Co-variations of $\bar{Q}$ vs $\overline{E 0}$

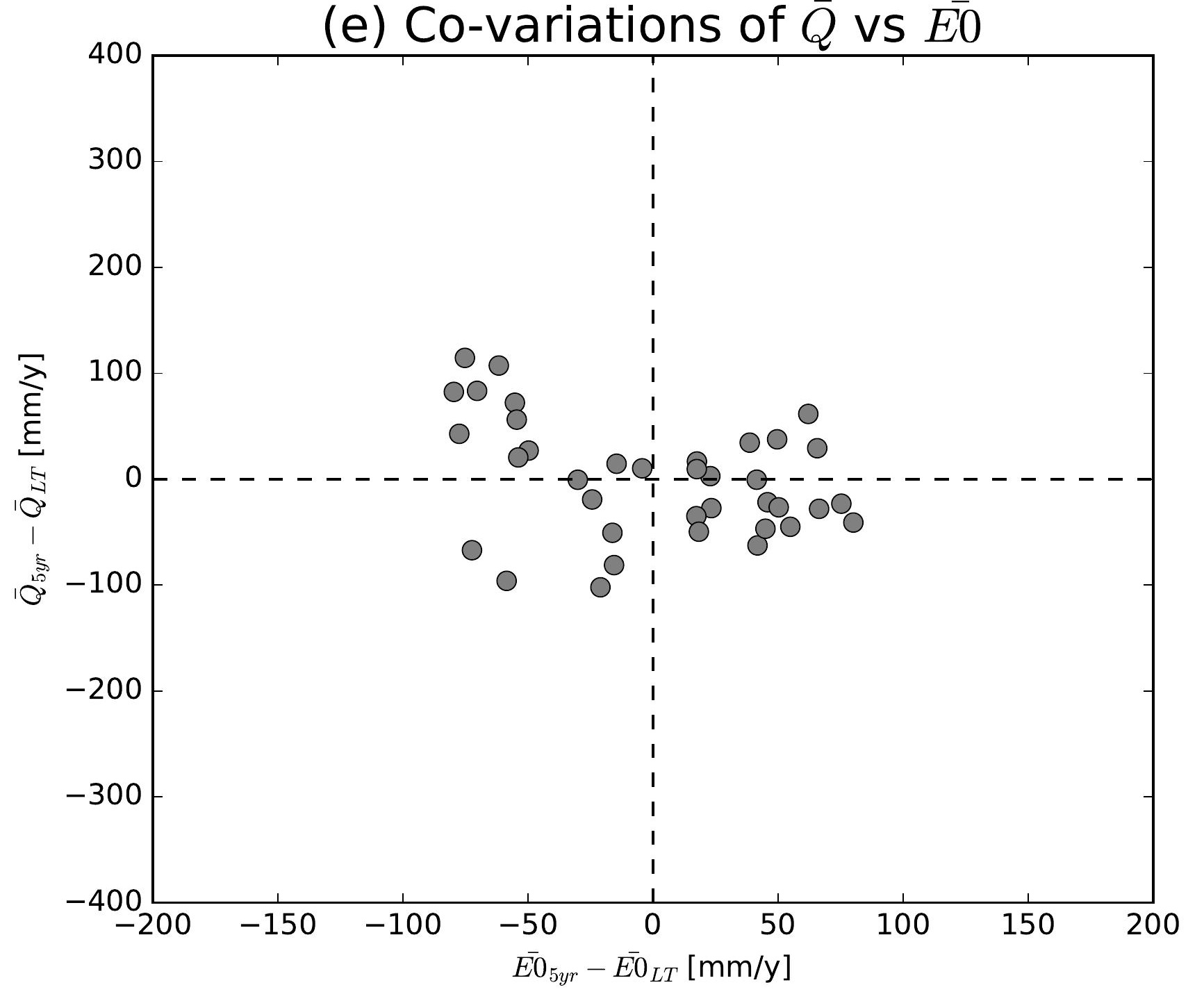

(h) Co-variations of $\bar{Q}$ vs $\overline{E 0}$

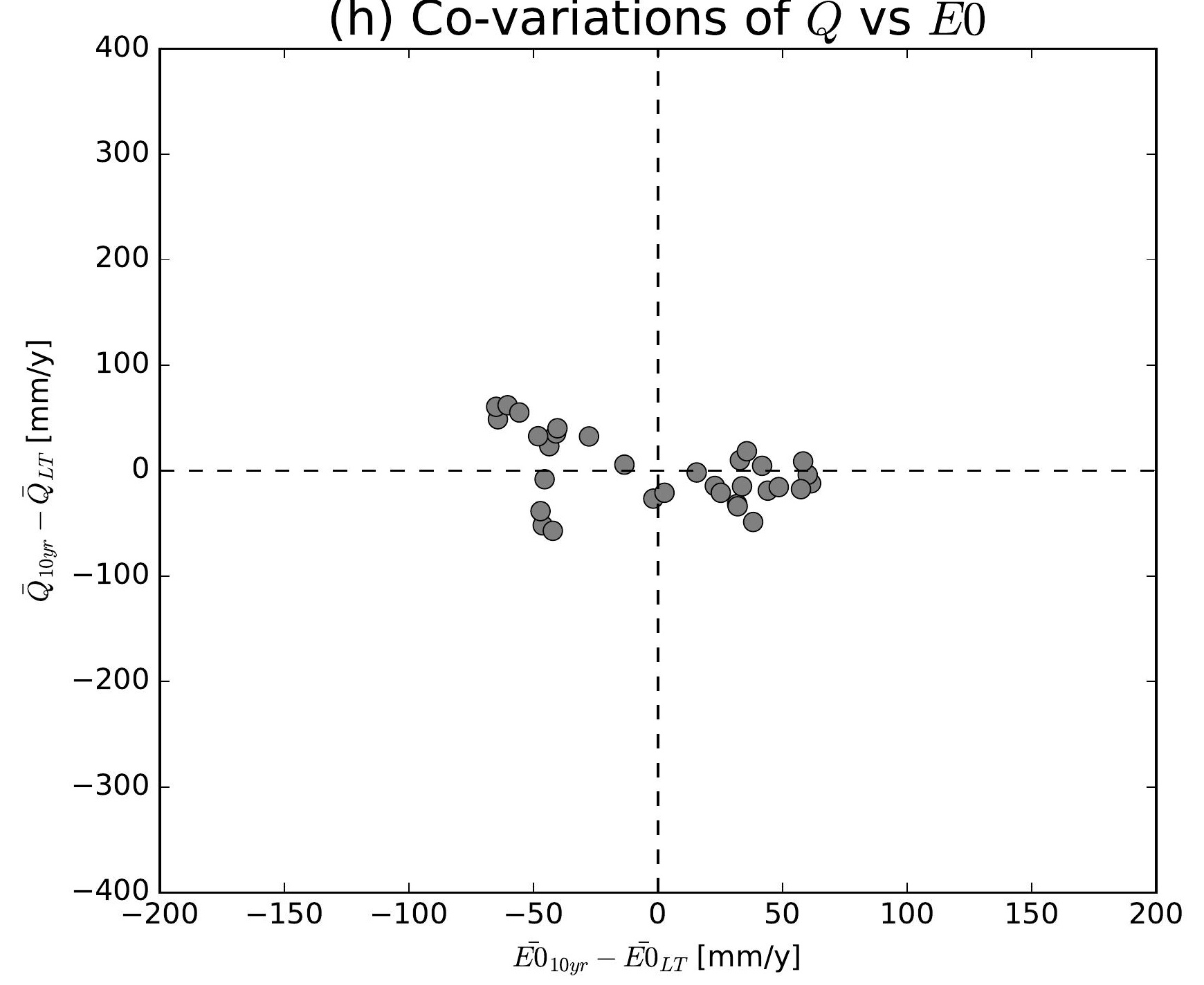

(k) Co-variations of $\bar{Q}$ vs $\overline{E 0}$

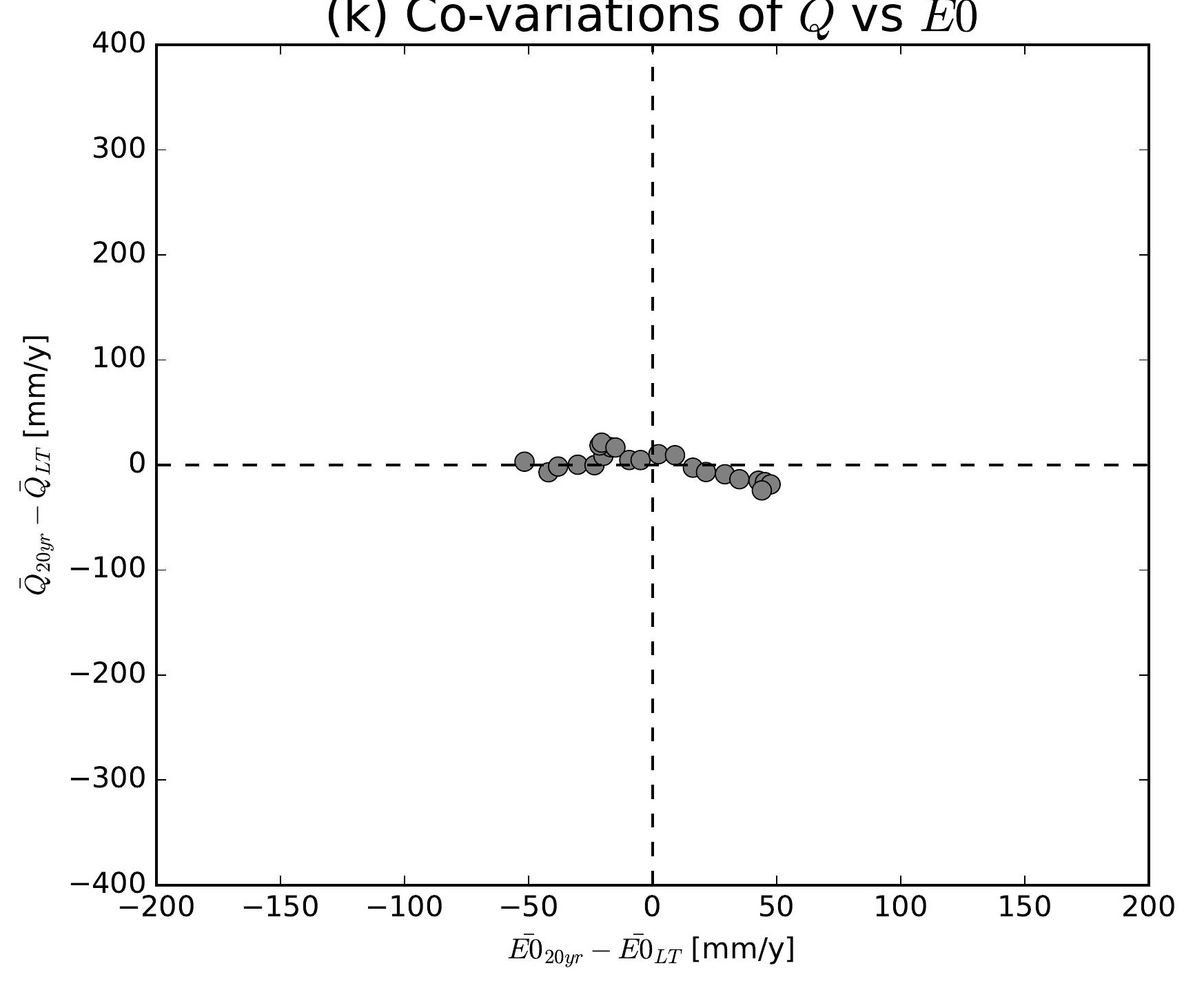

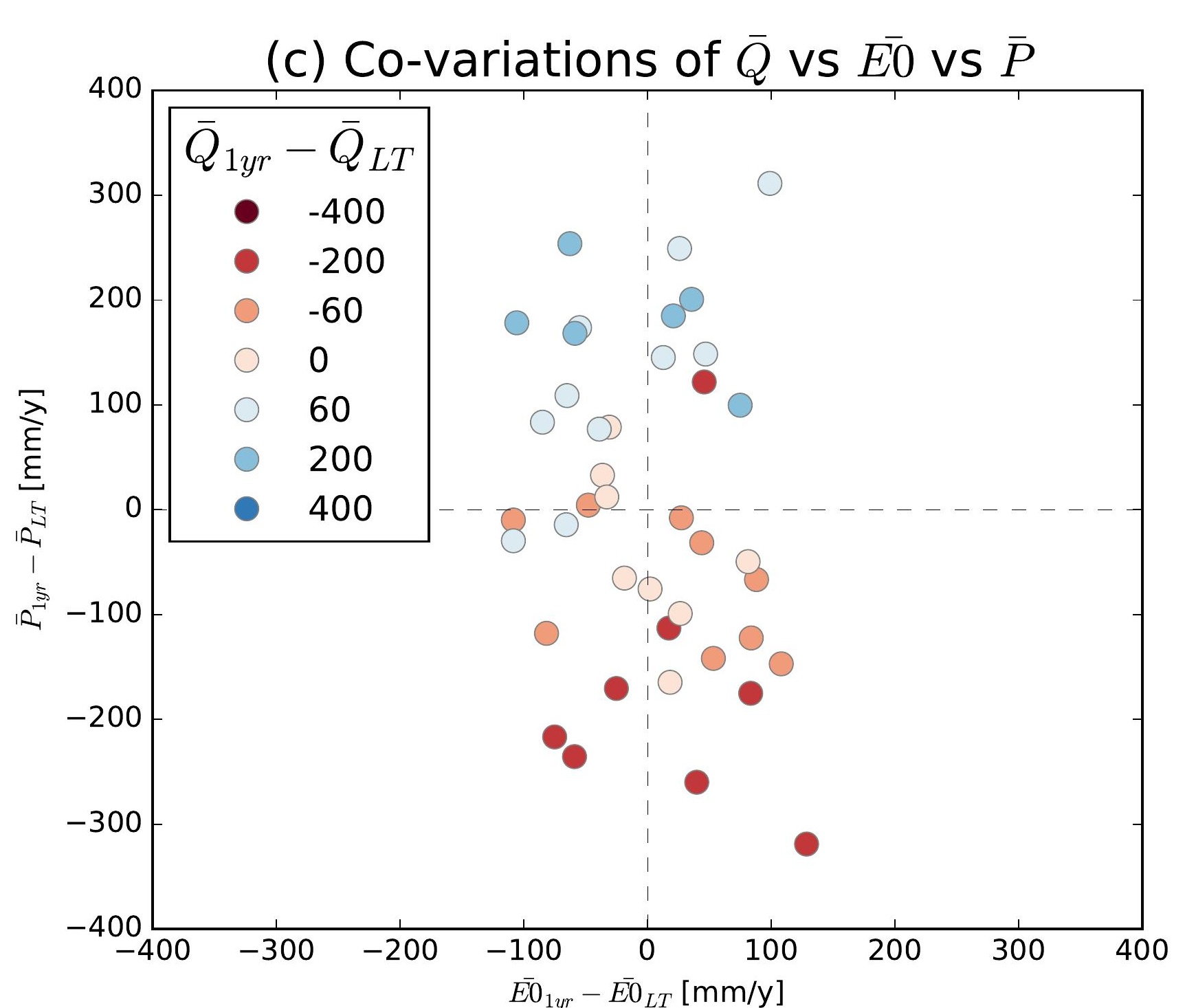
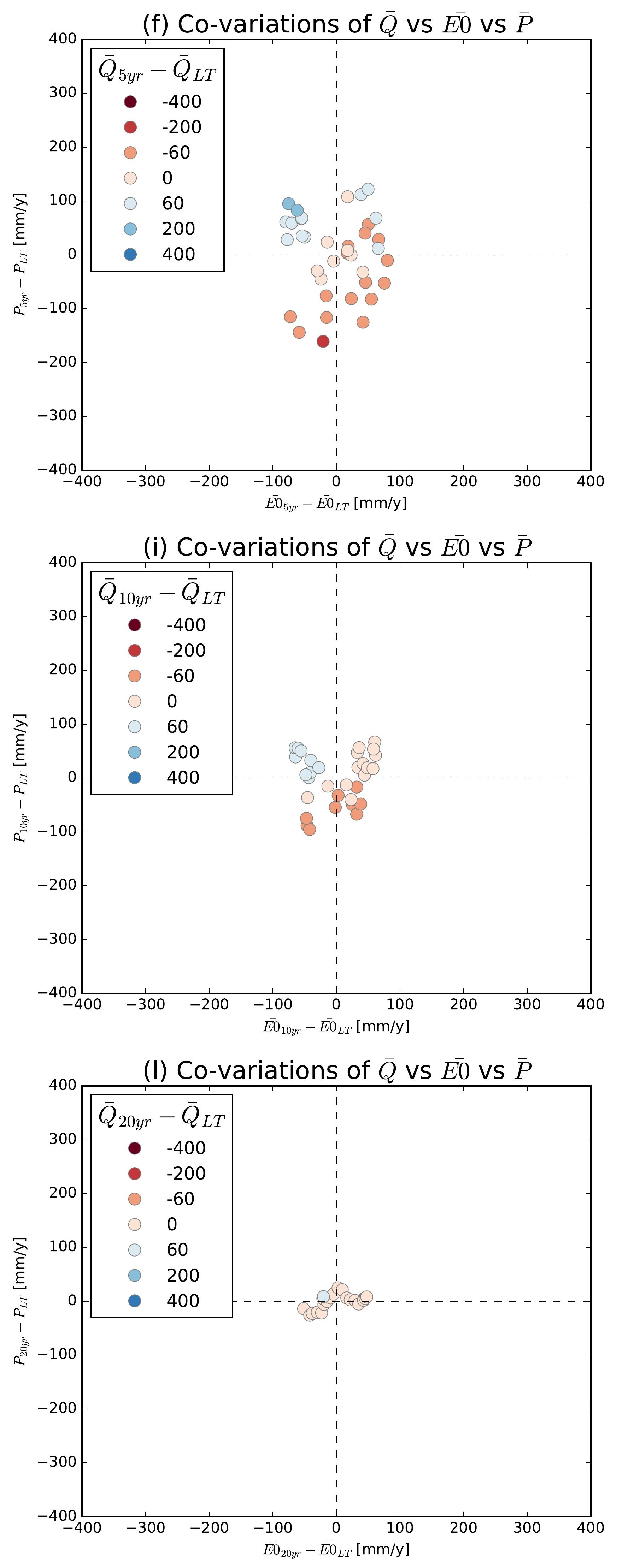

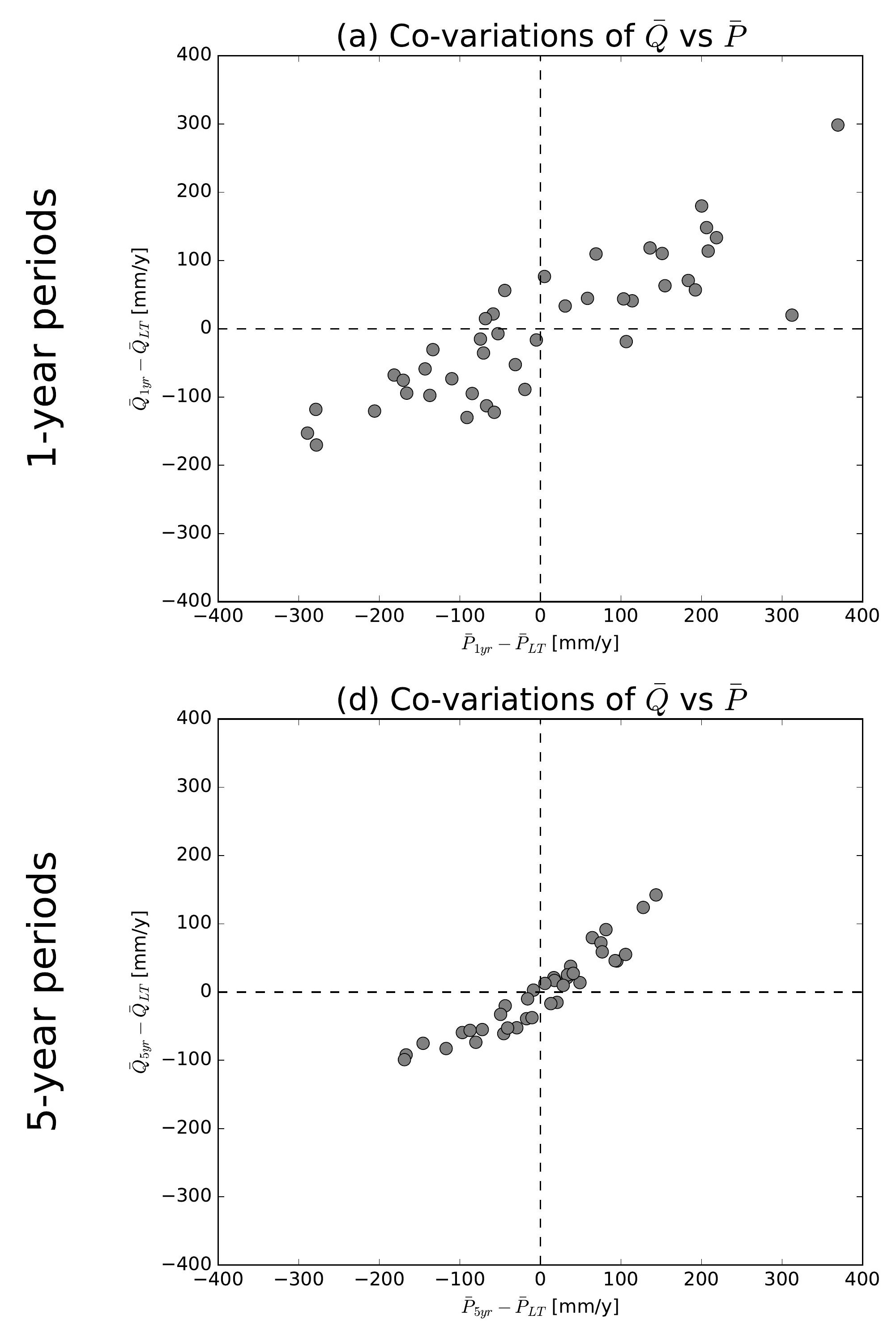

(g) Co-variations of $\bar{Q}$ vs $\bar{P}$

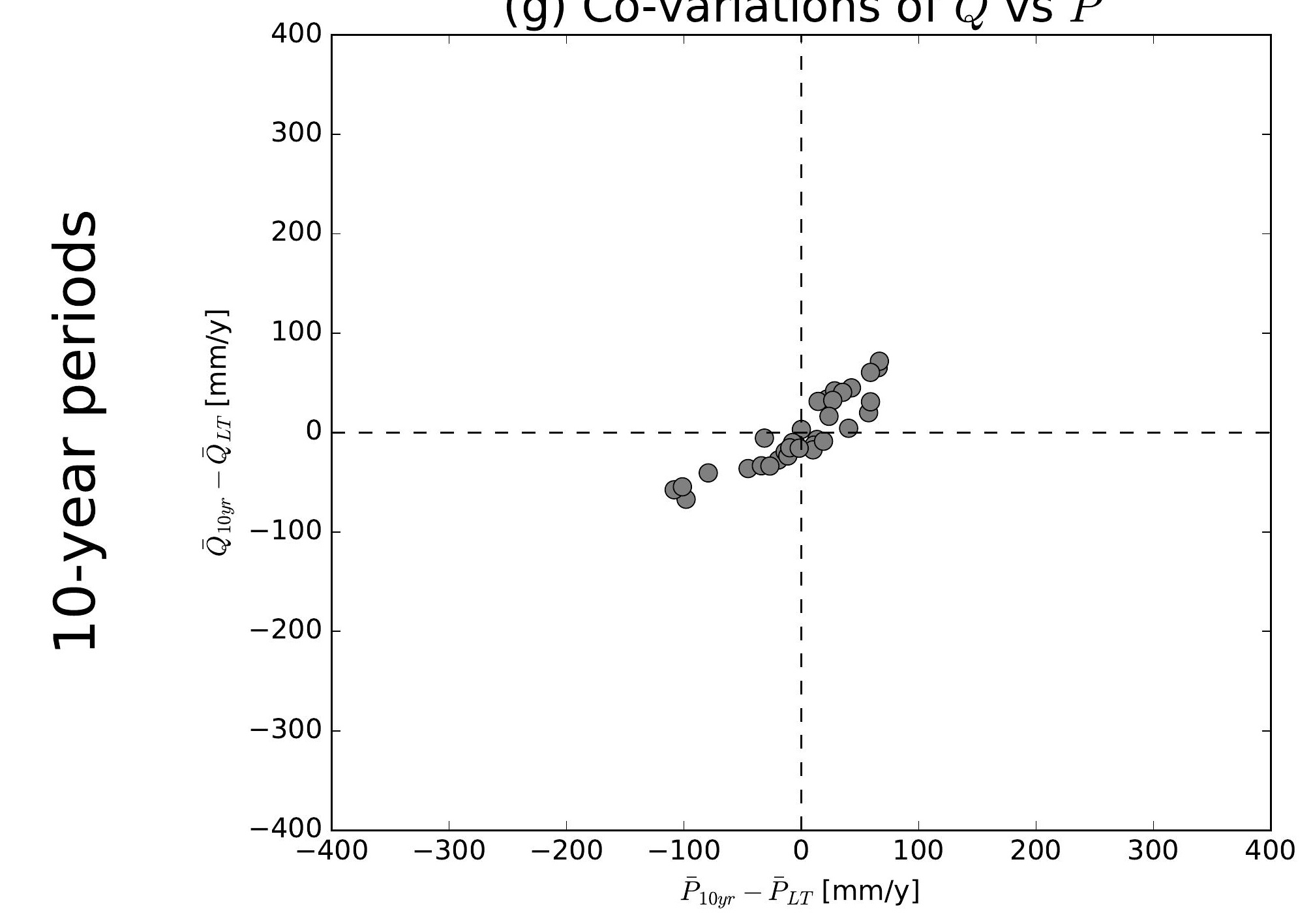

(j) Co-variations of $\bar{Q}$ vs $\bar{P}$

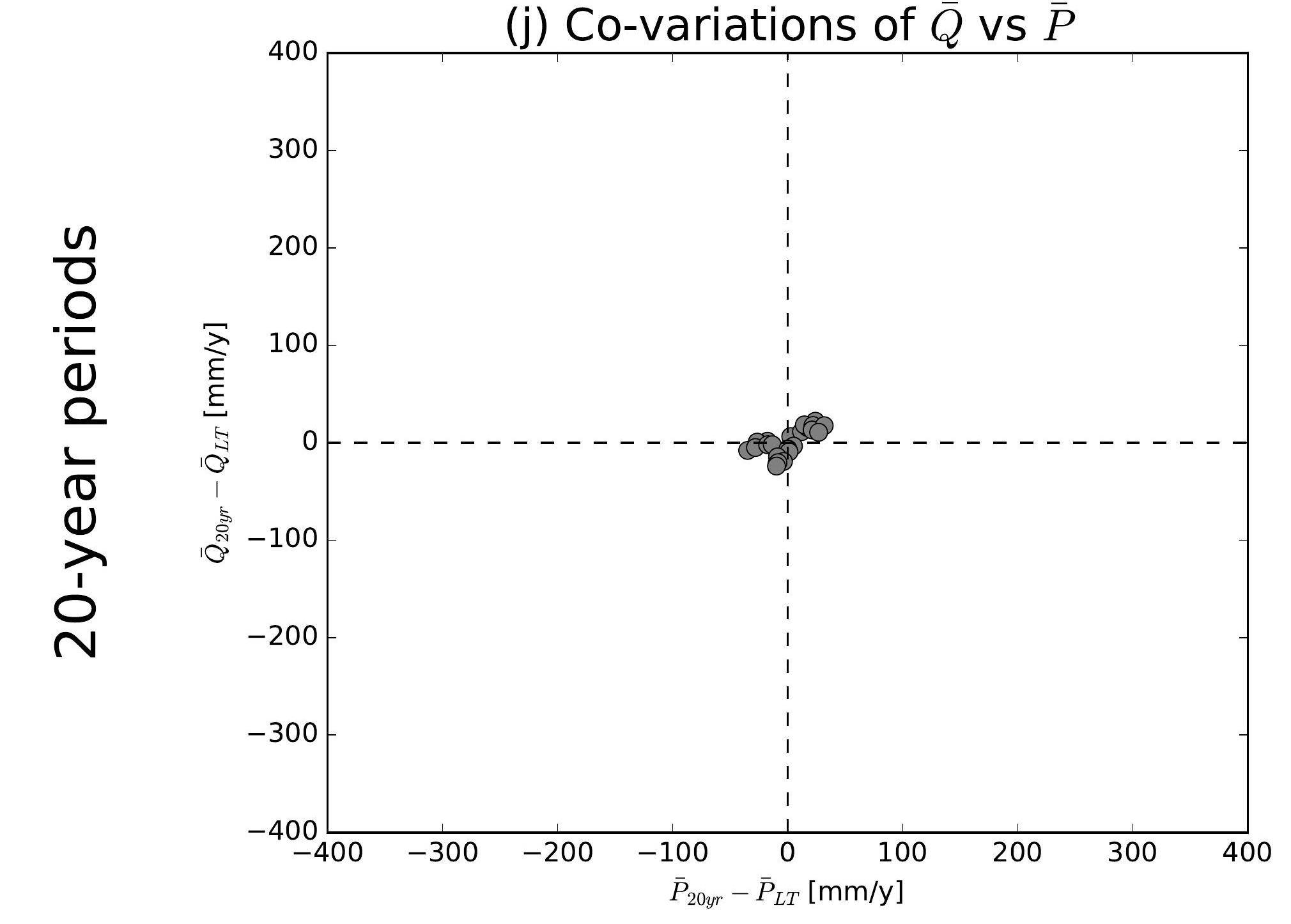

(b) Co-variations of $\bar{Q}$ vs $\overline{E 0}$

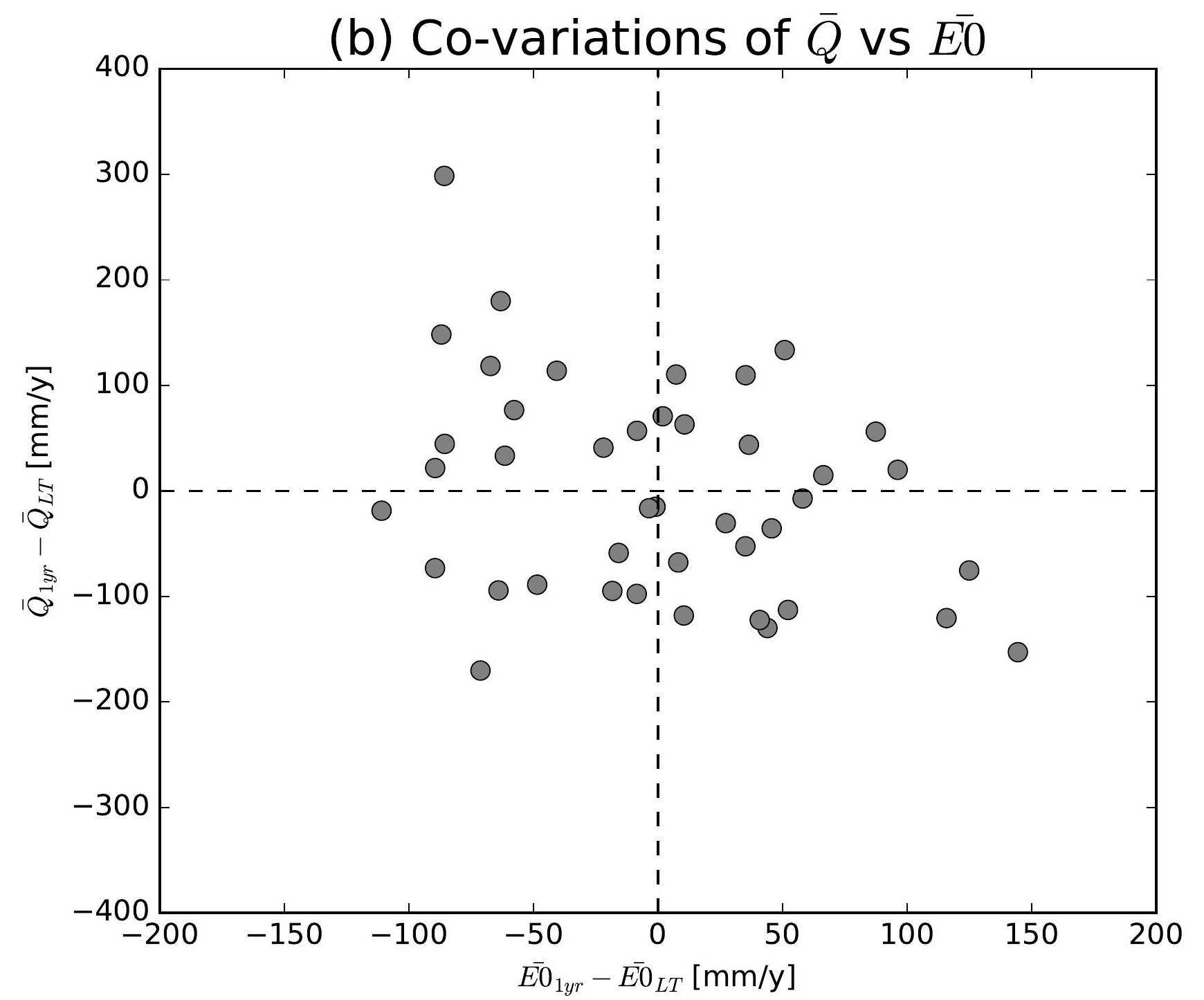

(e) Co-variations of $\bar{Q}$ vs $\overline{E 0}$

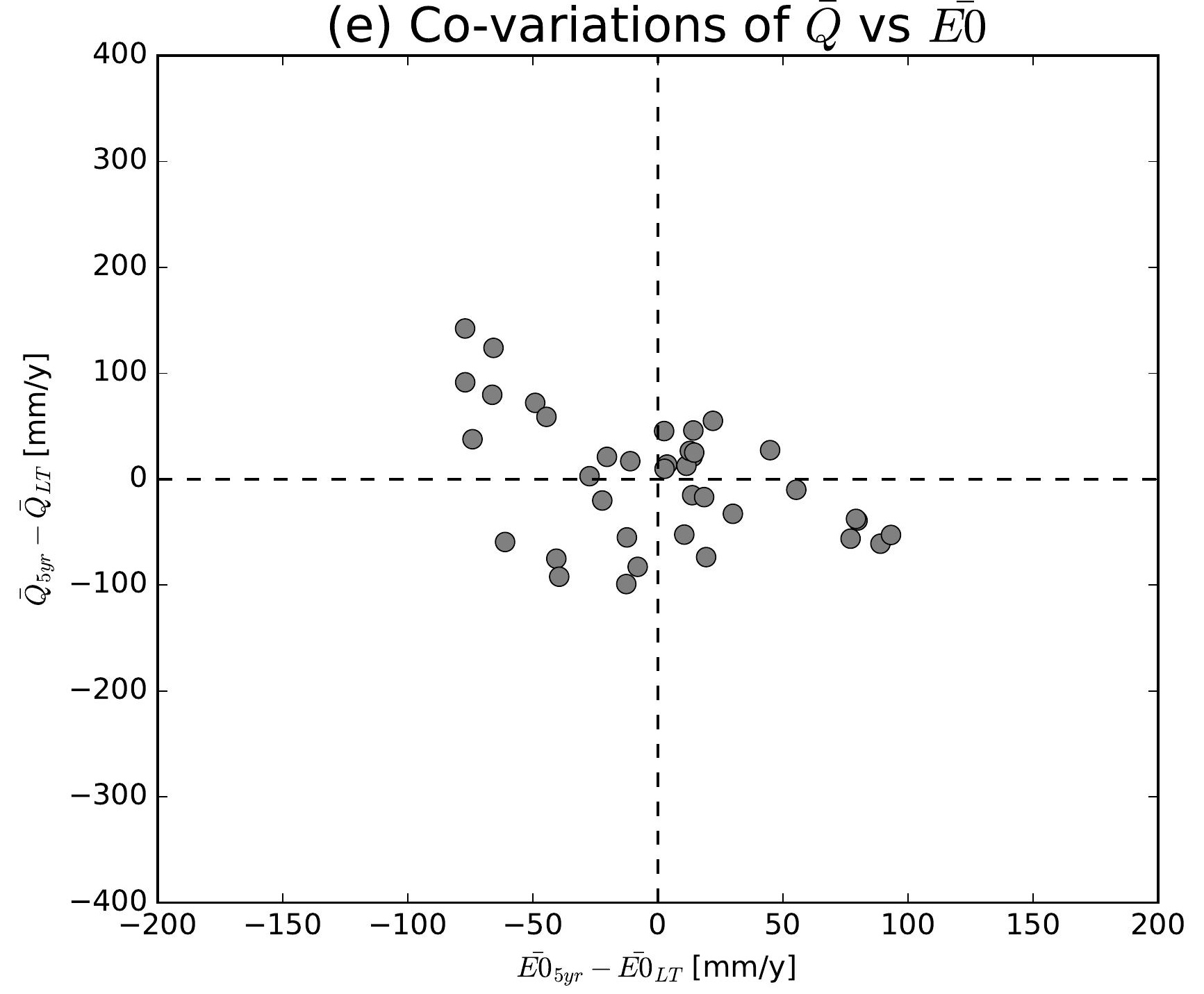

(h) Co-variations of $\bar{Q}$ vs $\overline{E 0}$

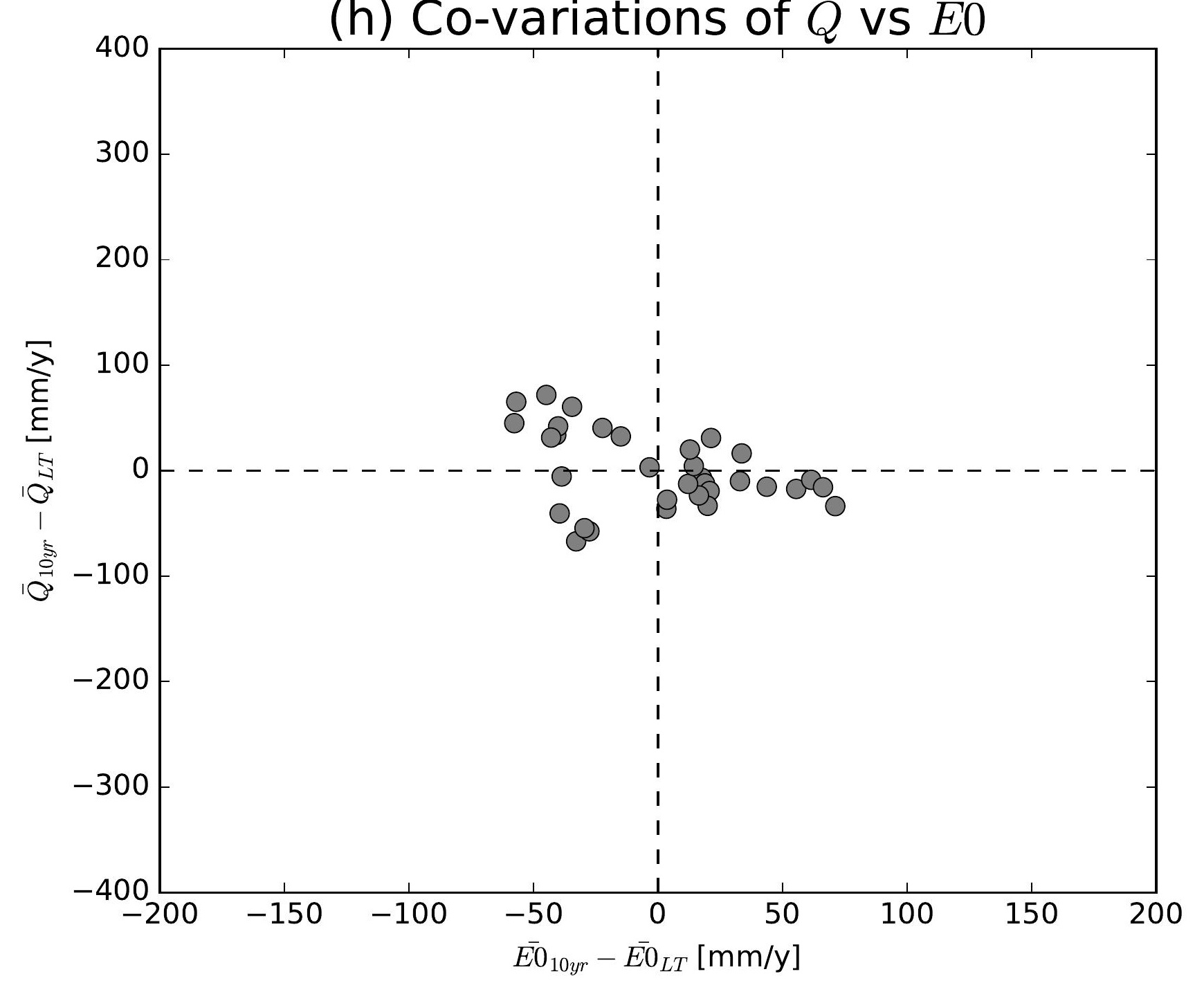

(k) Co-variations of $\bar{Q}$ vs $\overline{E 0}$

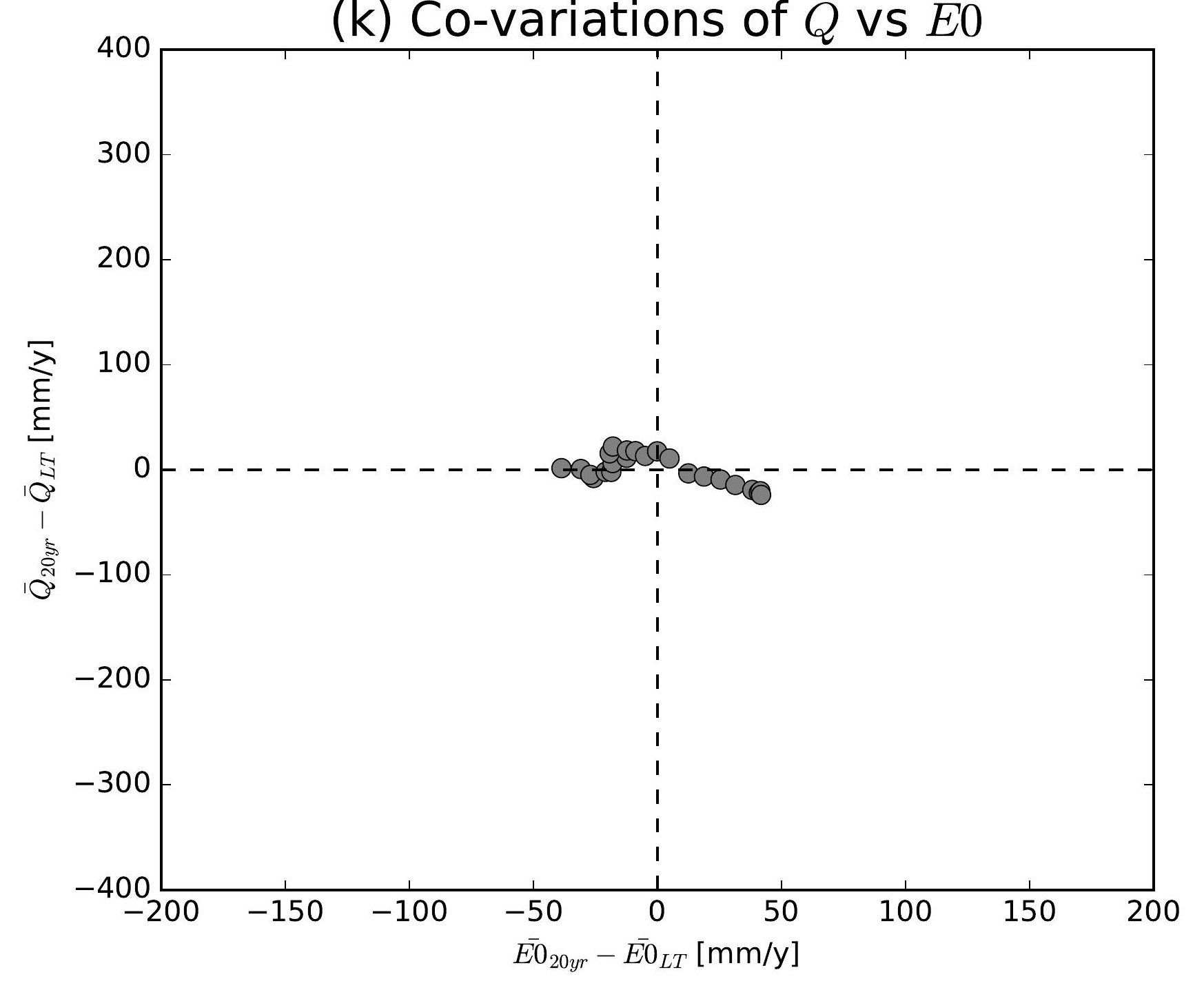

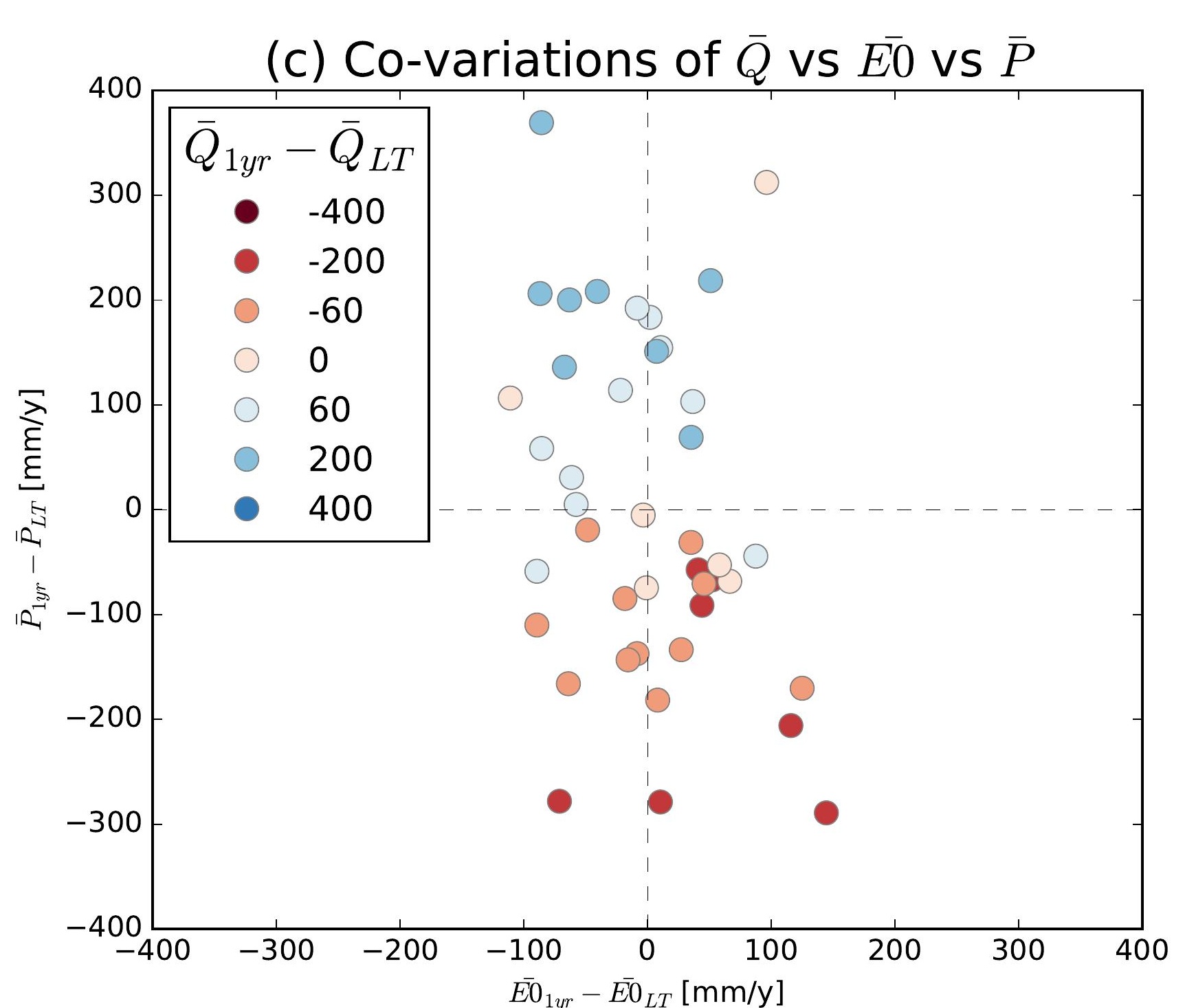
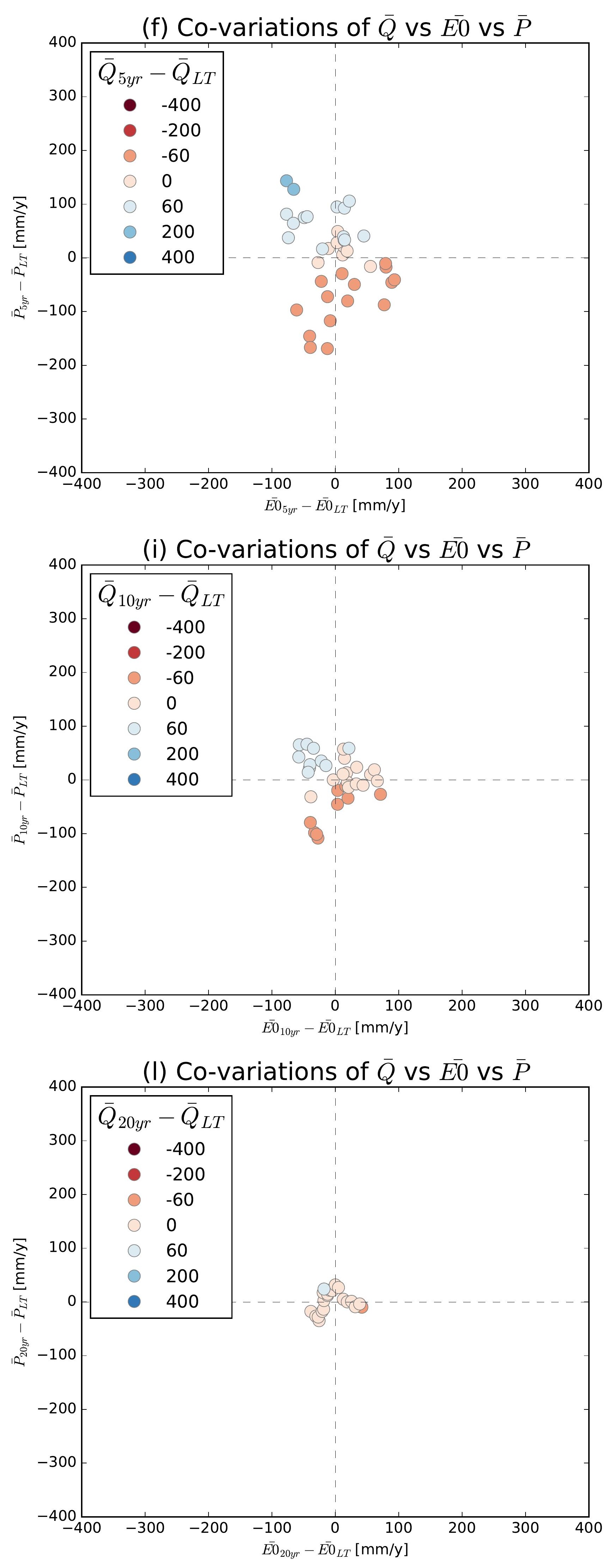

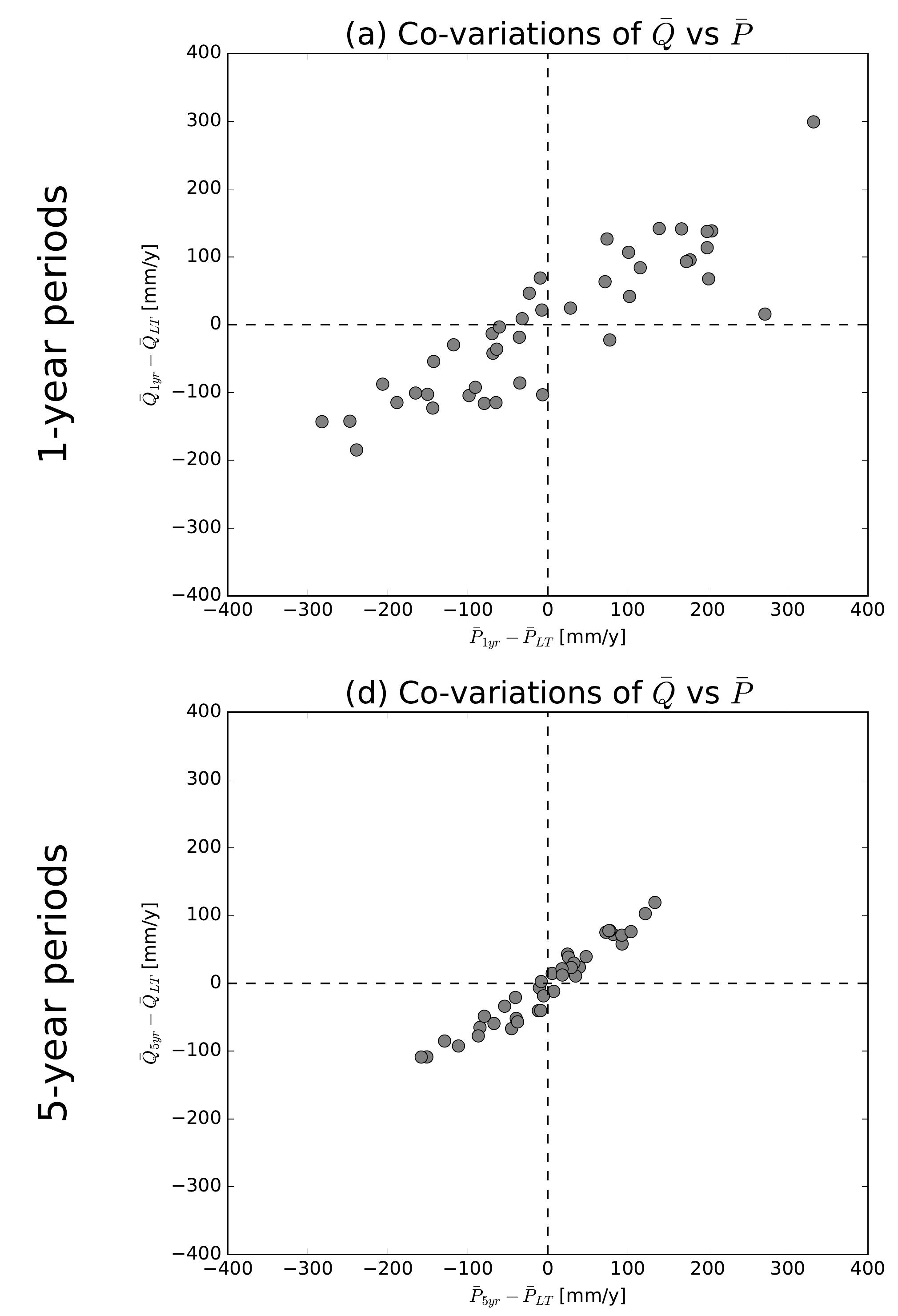

(g) Co-variations of $\bar{Q}$ vs $\bar{P}$
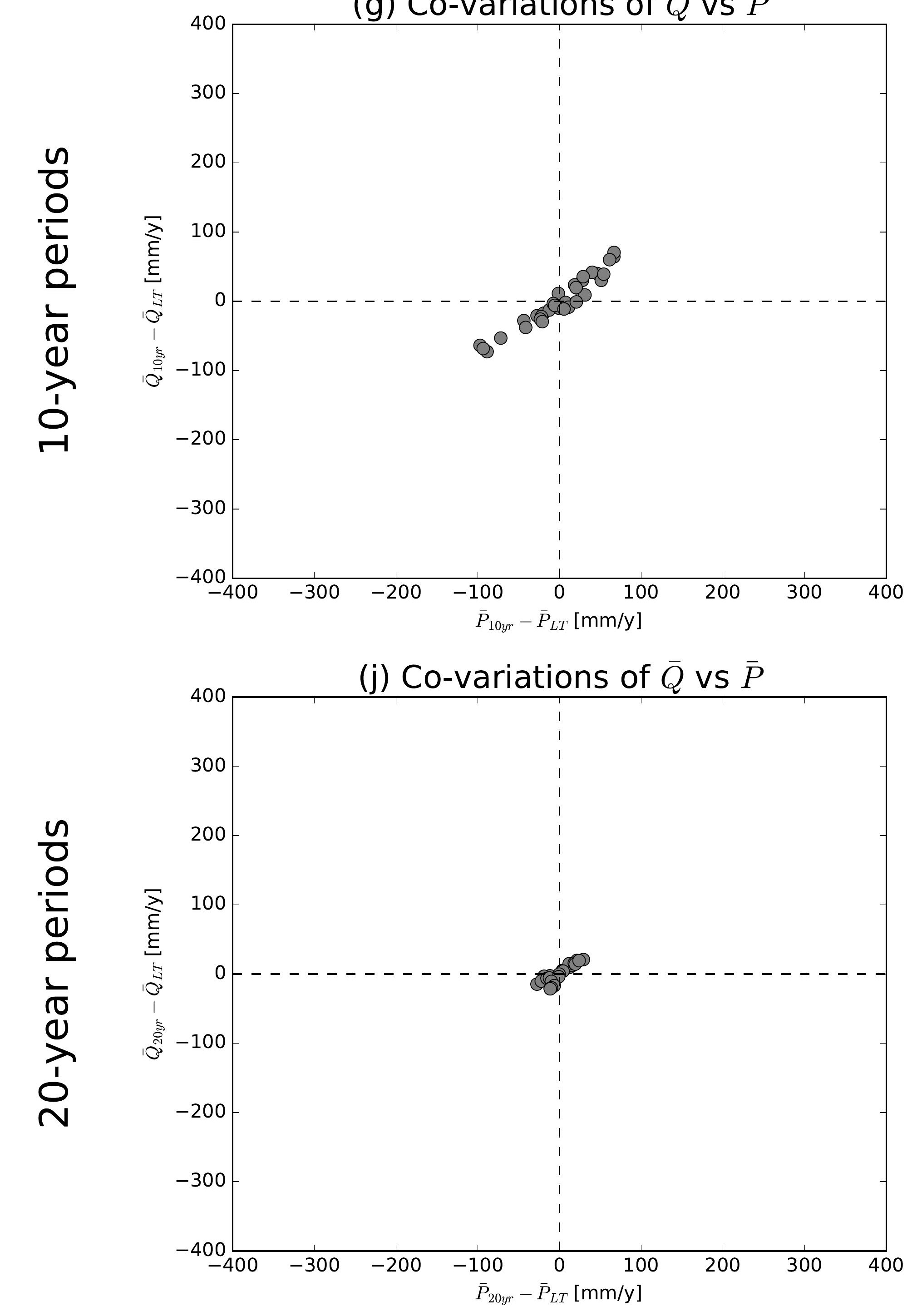

(b) Co-variations of $\bar{Q}$ vs $\overline{E 0}$

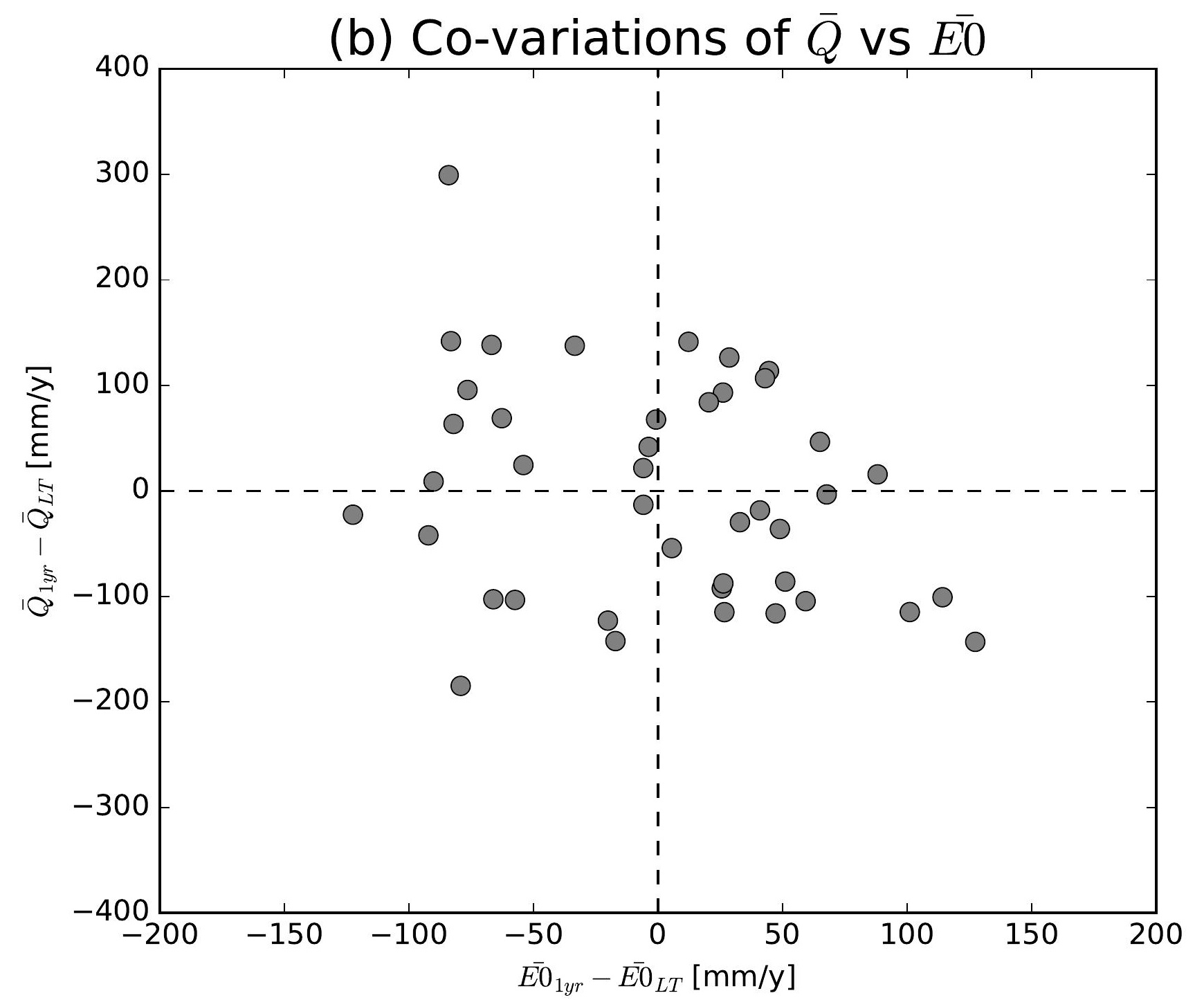

(e) Co-variations of $\bar{Q}$ vs $\overline{E 0}$

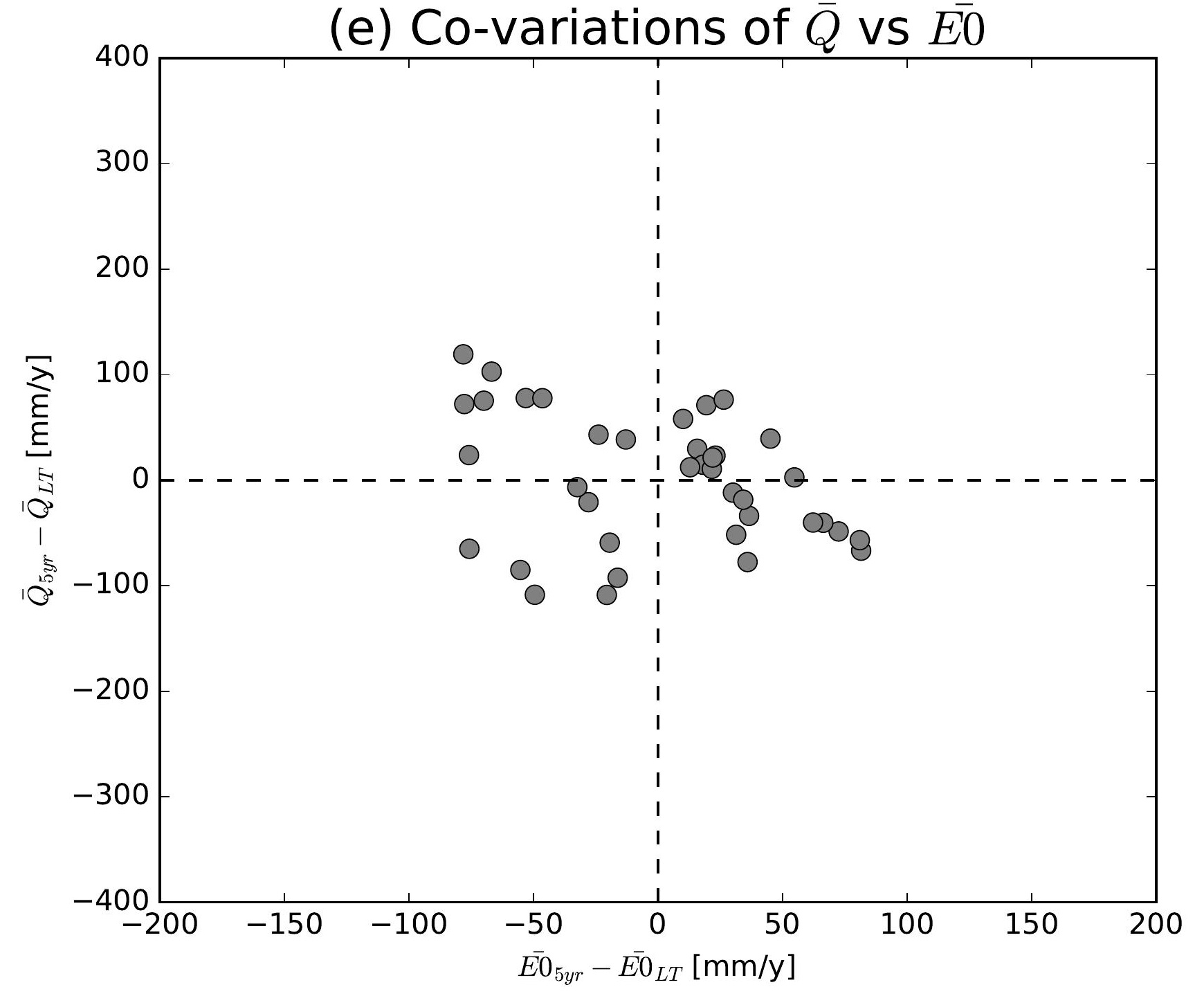

(h) Co-variations of $\bar{Q}$ vs $\overline{E 0}$

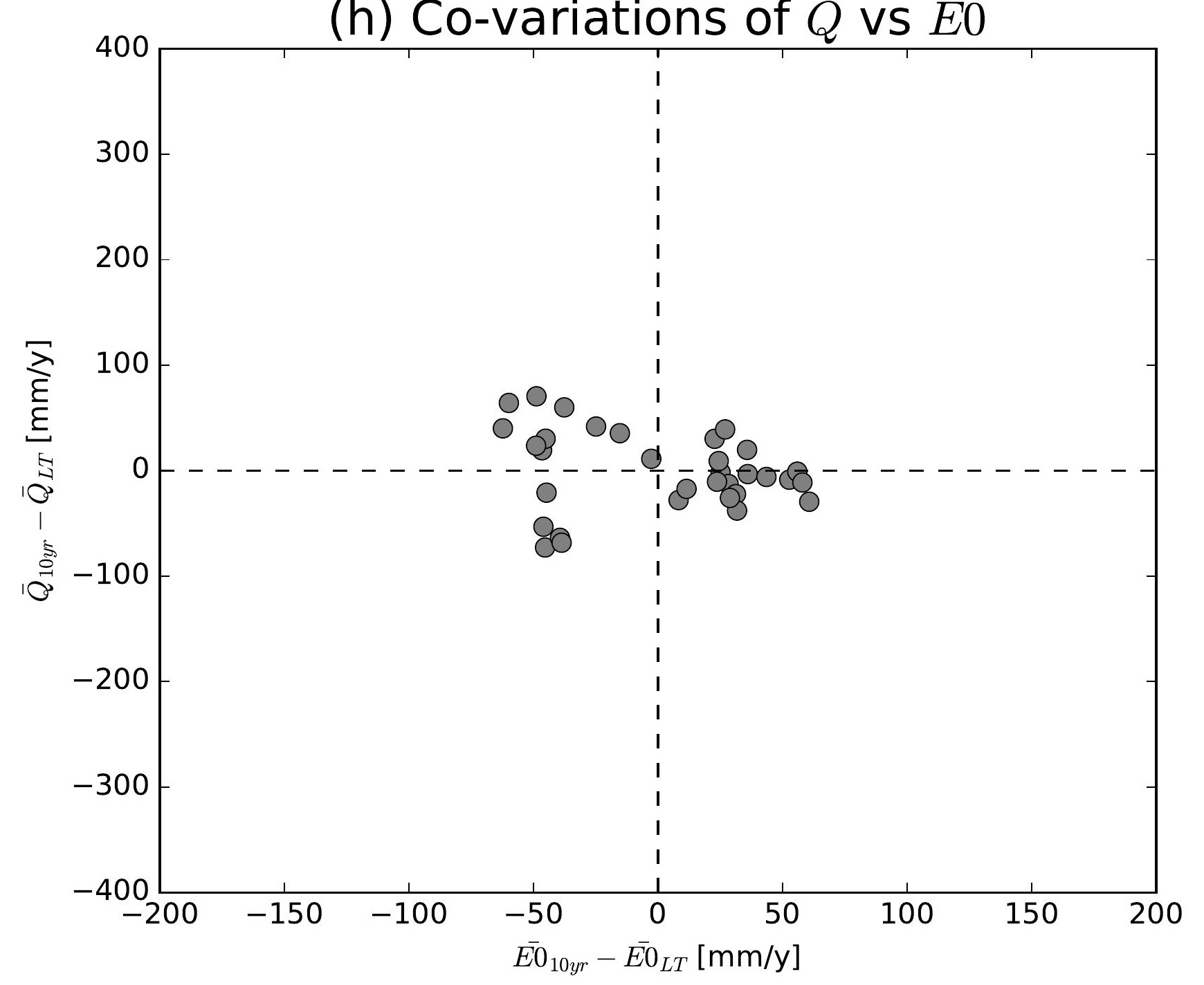

(k) Co-variations of $\bar{Q}$ vs $\overline{E 0}$

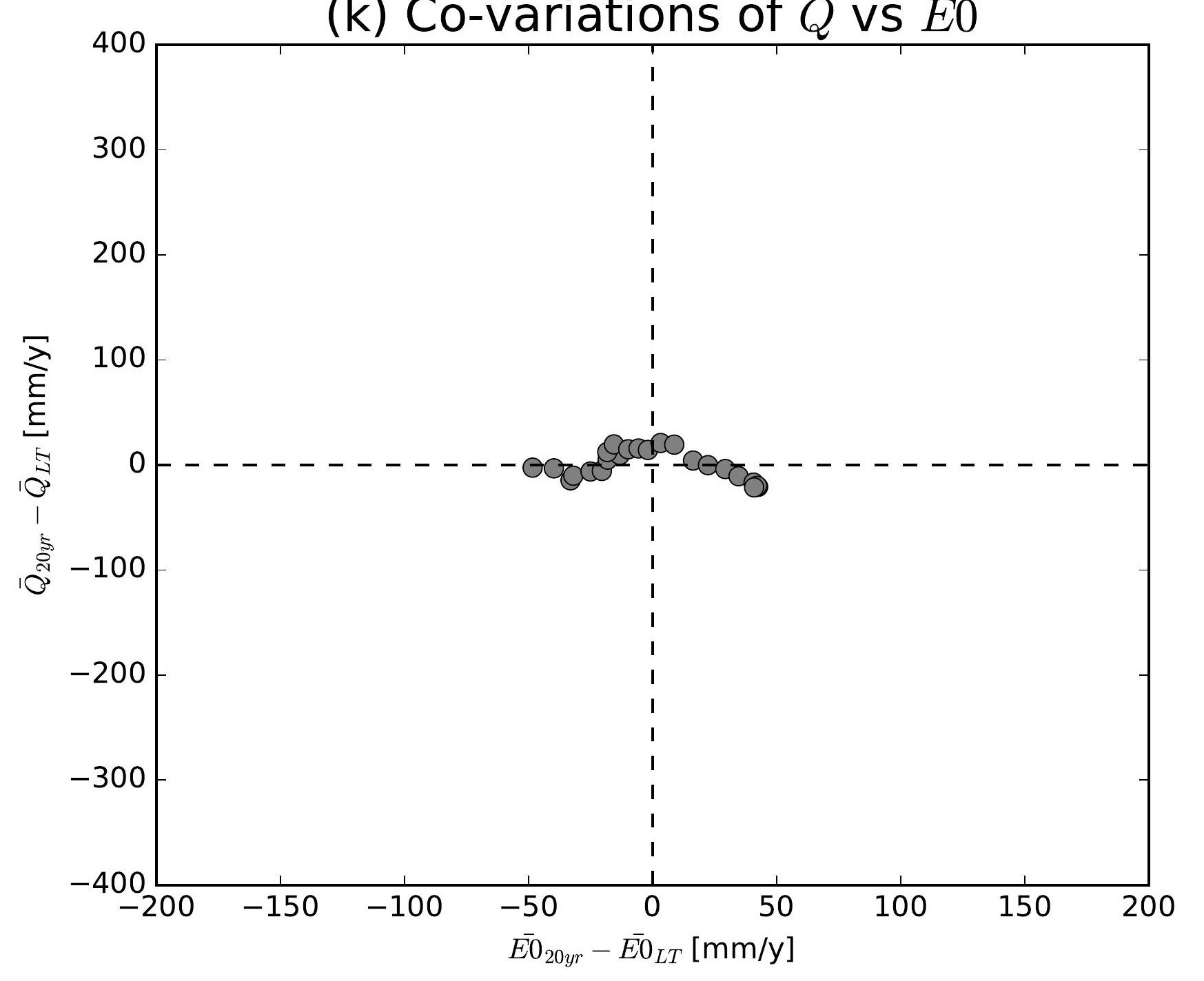

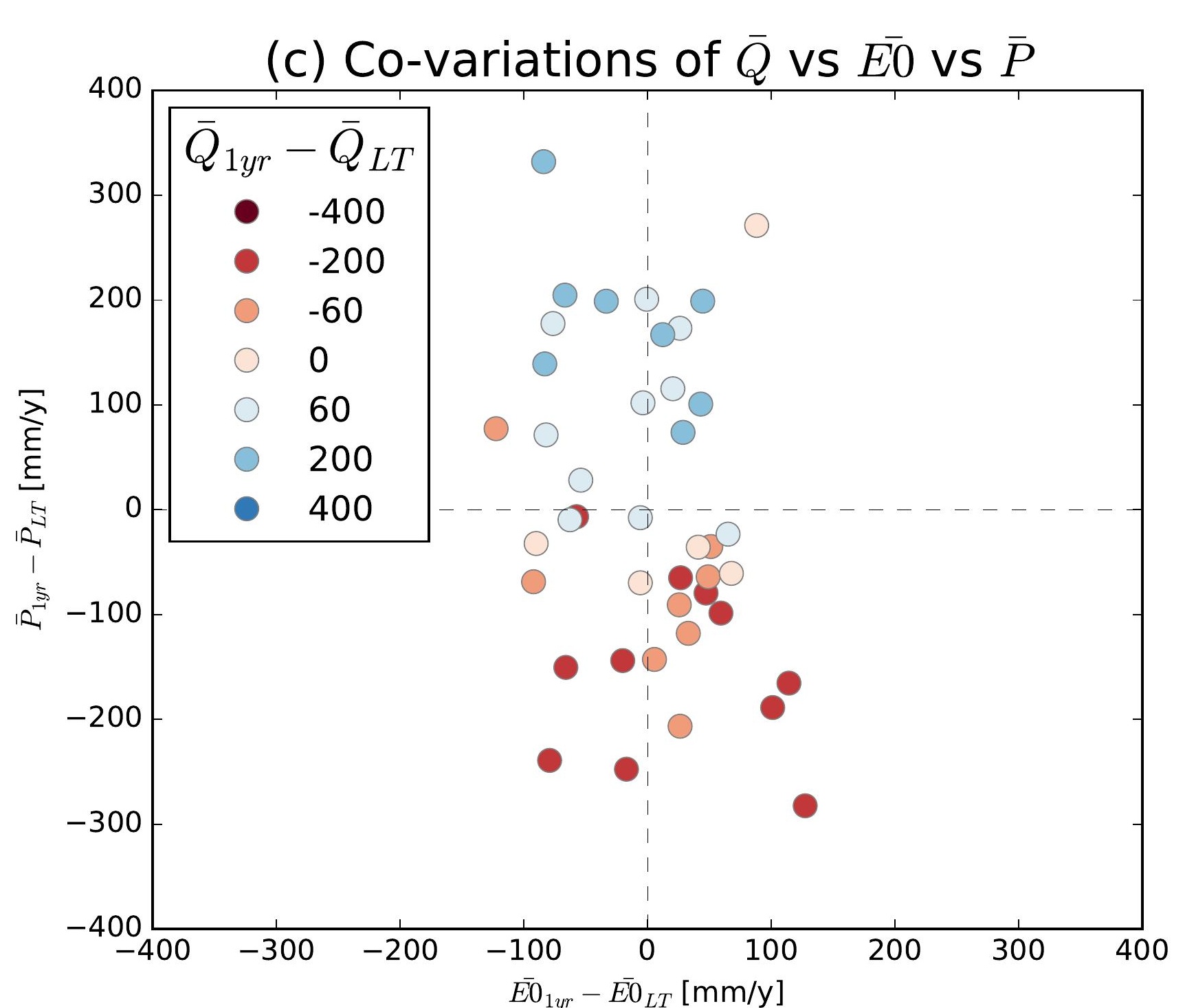
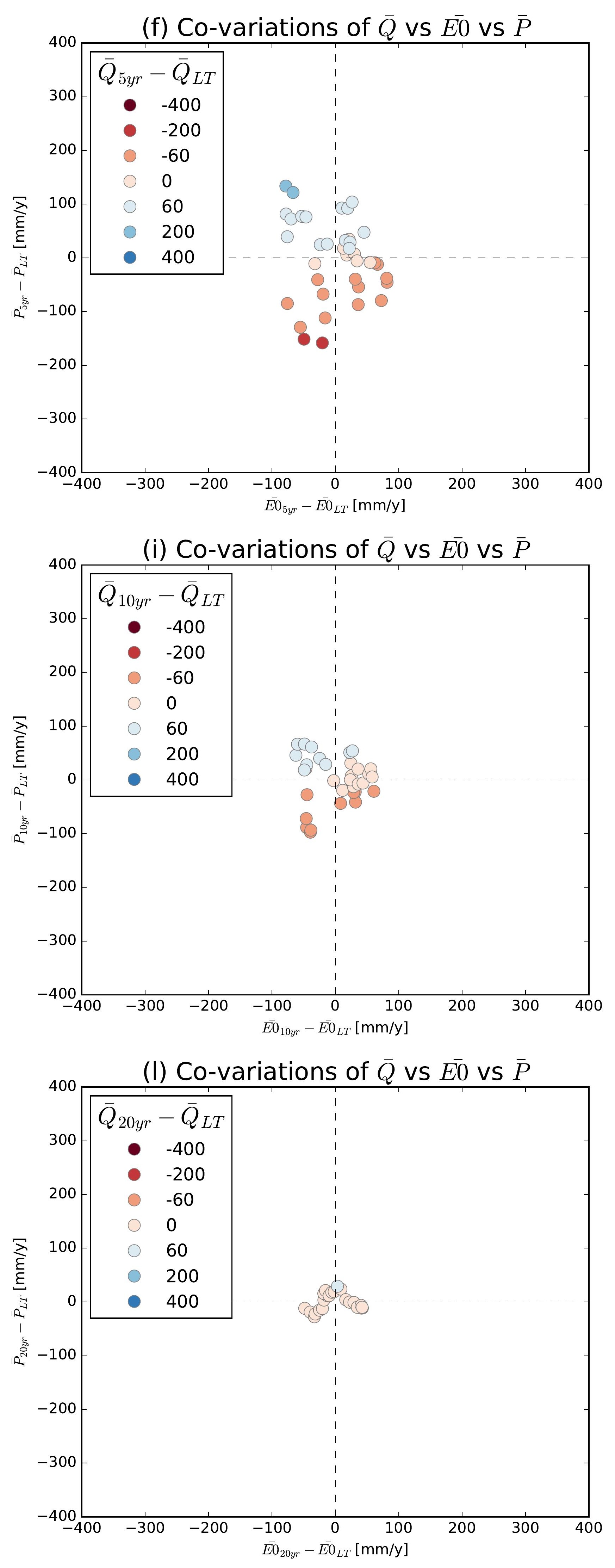

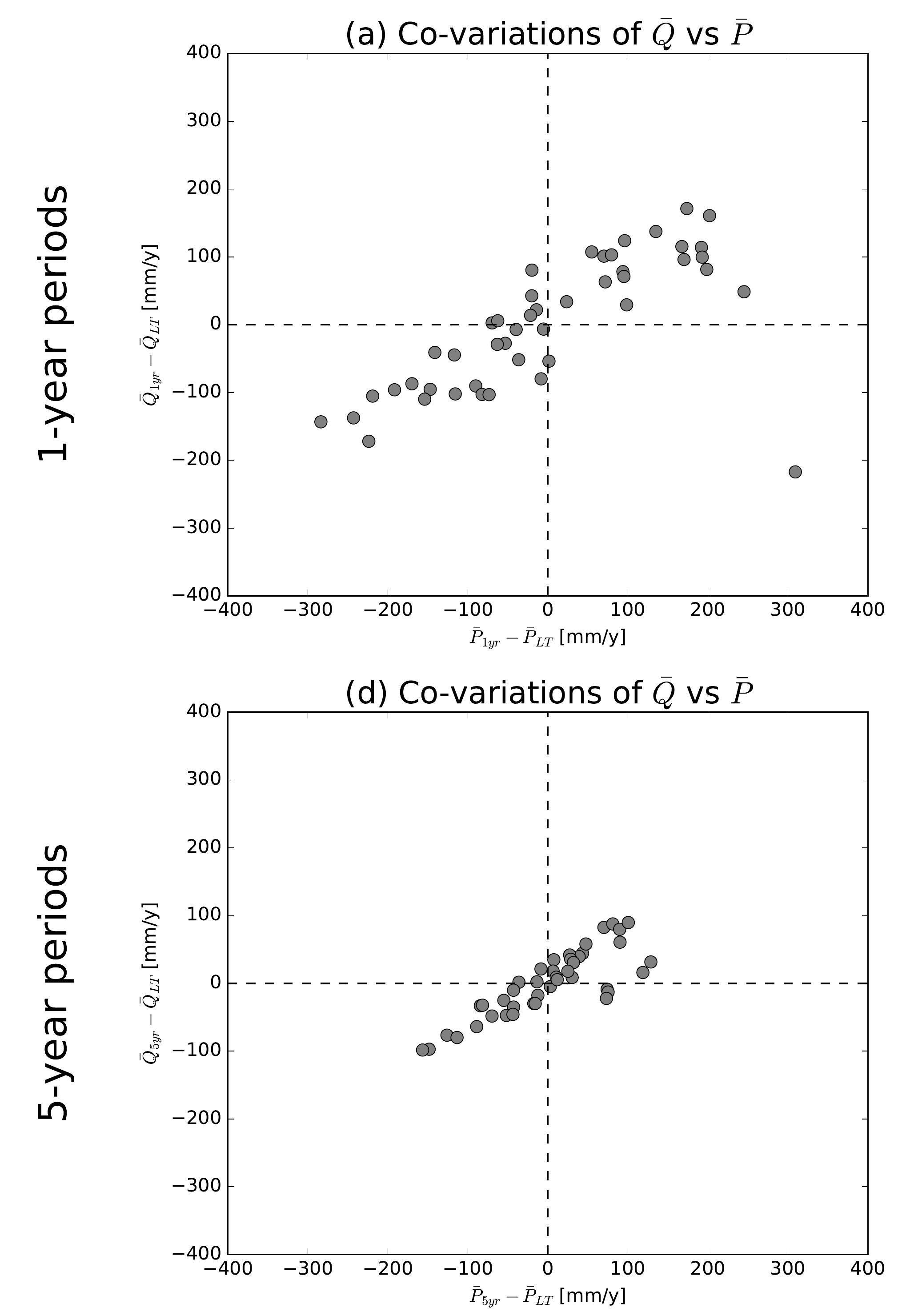

(g) Co-variations of $\bar{Q}$ vs $\bar{P}$
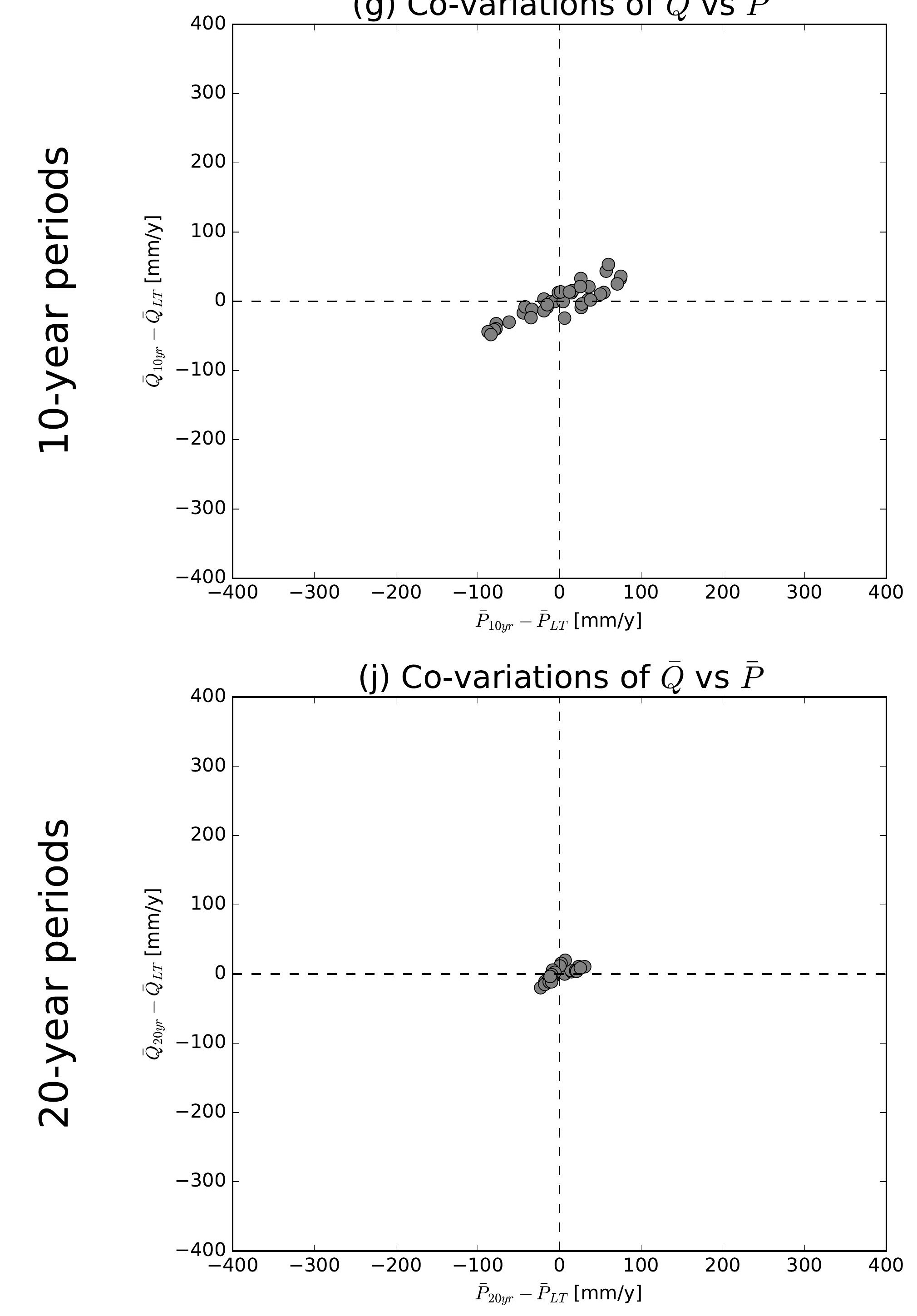

(b) Co-variations of $\bar{Q}$ vs $\overline{E 0}$

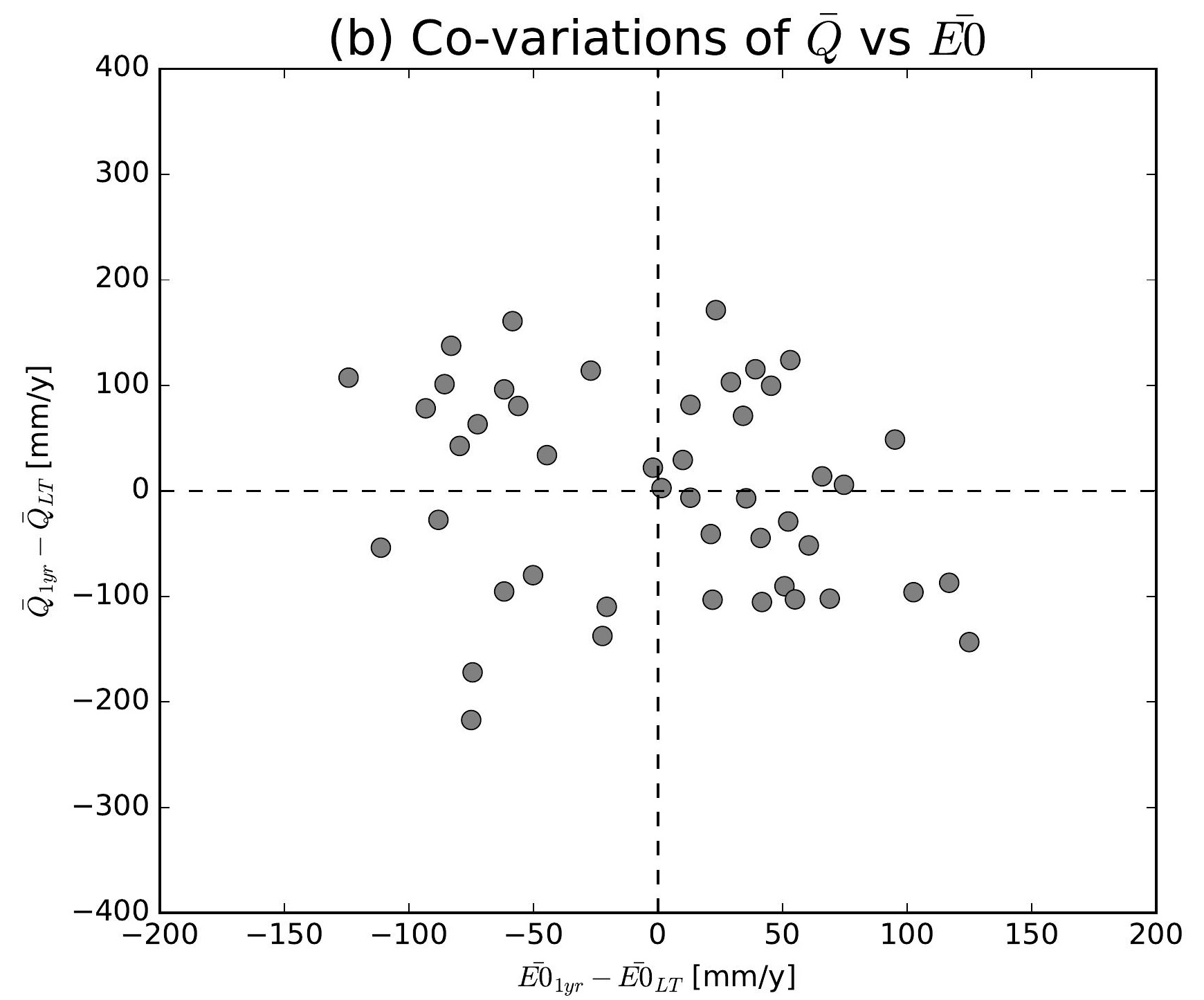

(e) Co-variations of $\bar{Q}$ vs $\overline{E 0}$

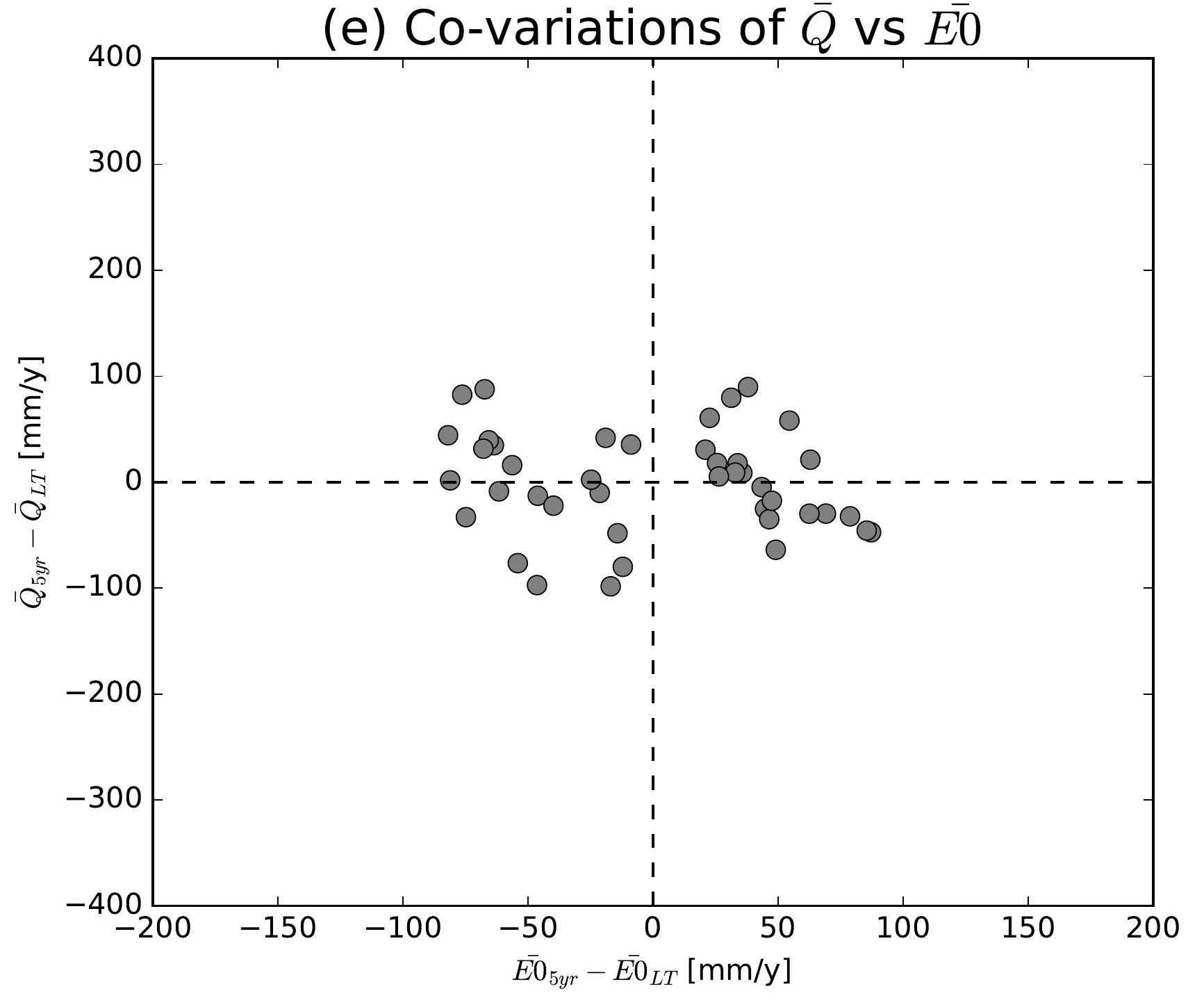

(h) Co-variations of $\bar{Q}$ vs $\overline{E 0}$

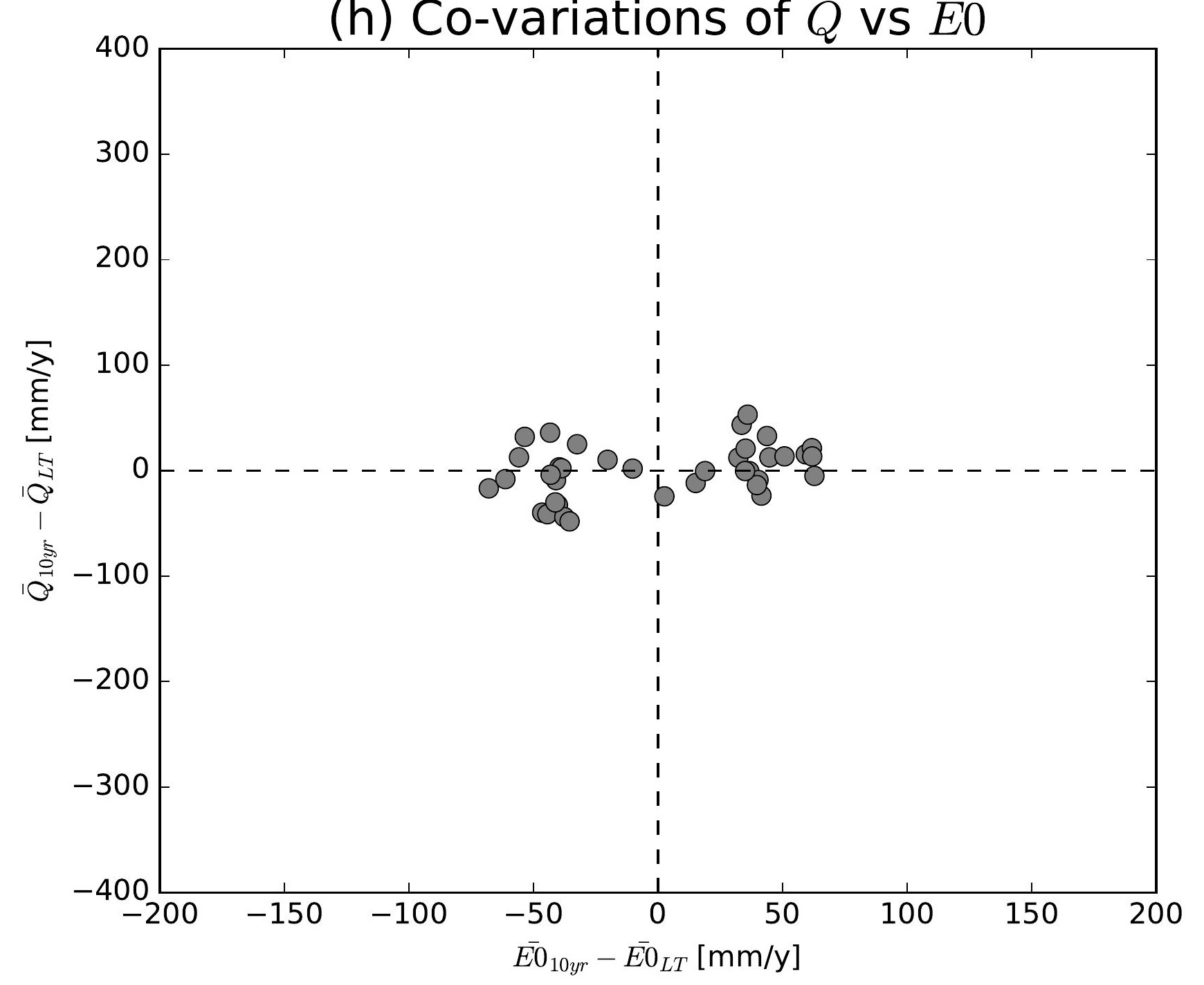

(k) Co-variations of $\bar{Q}$ vs $\overline{E 0}$

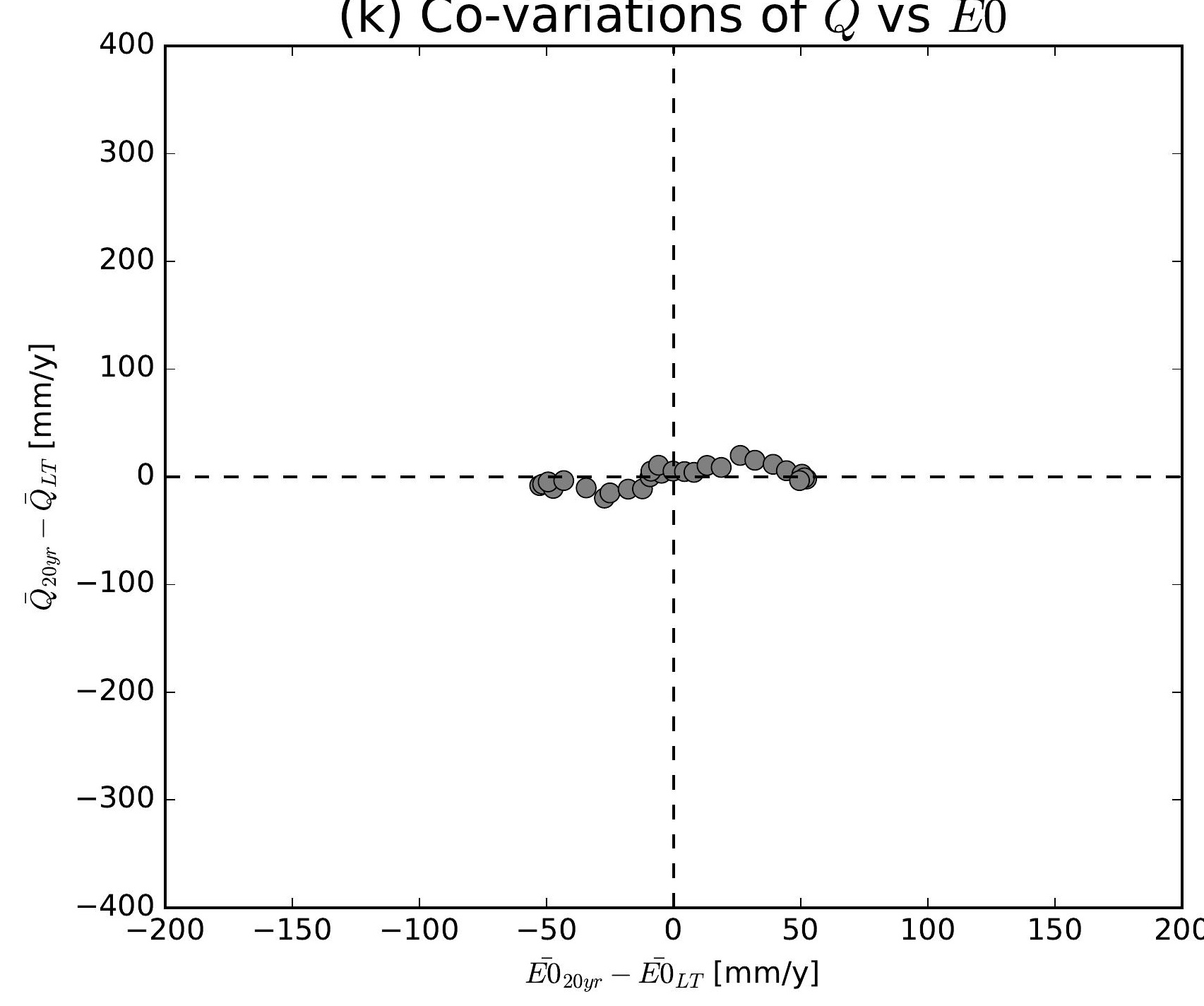

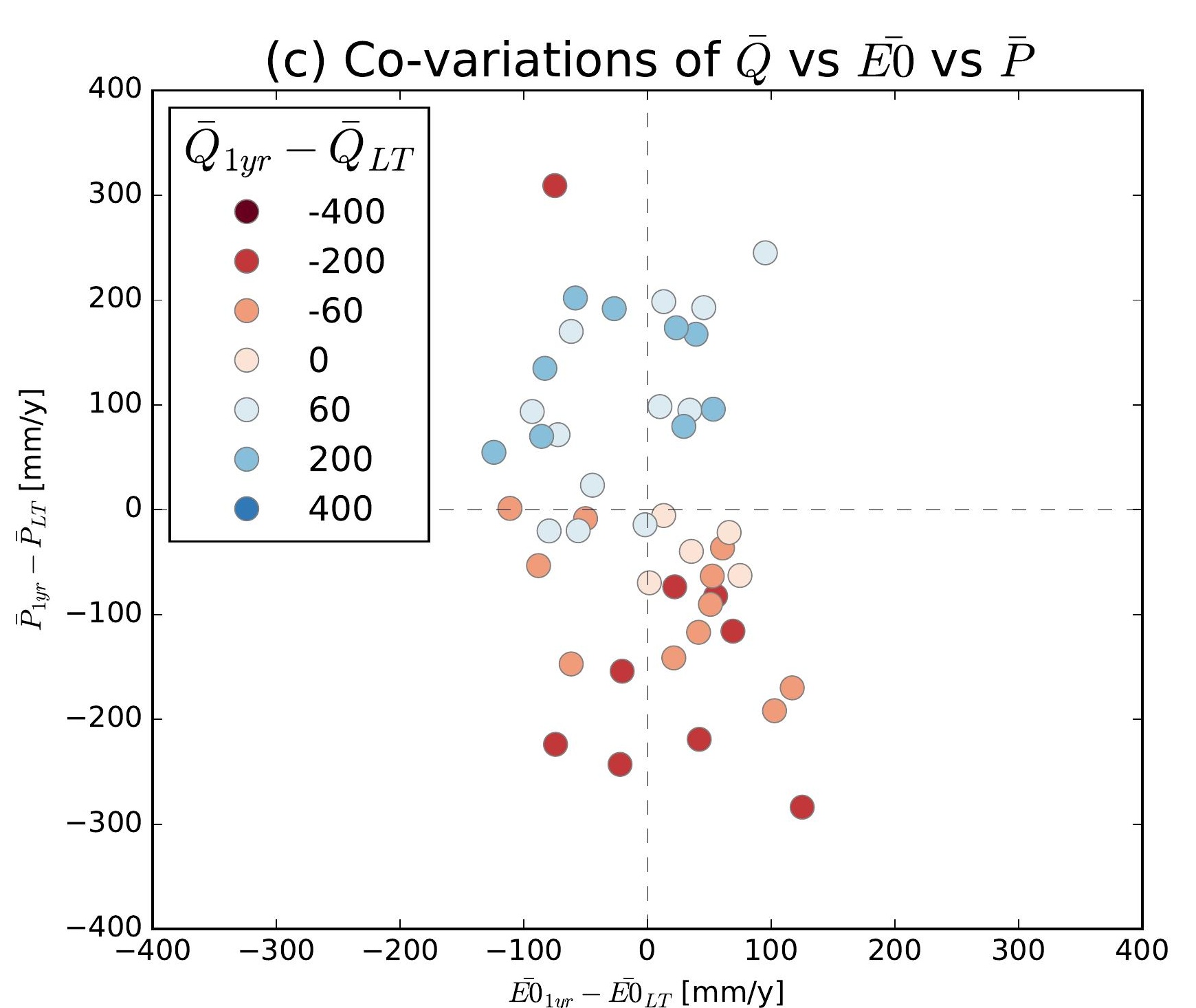
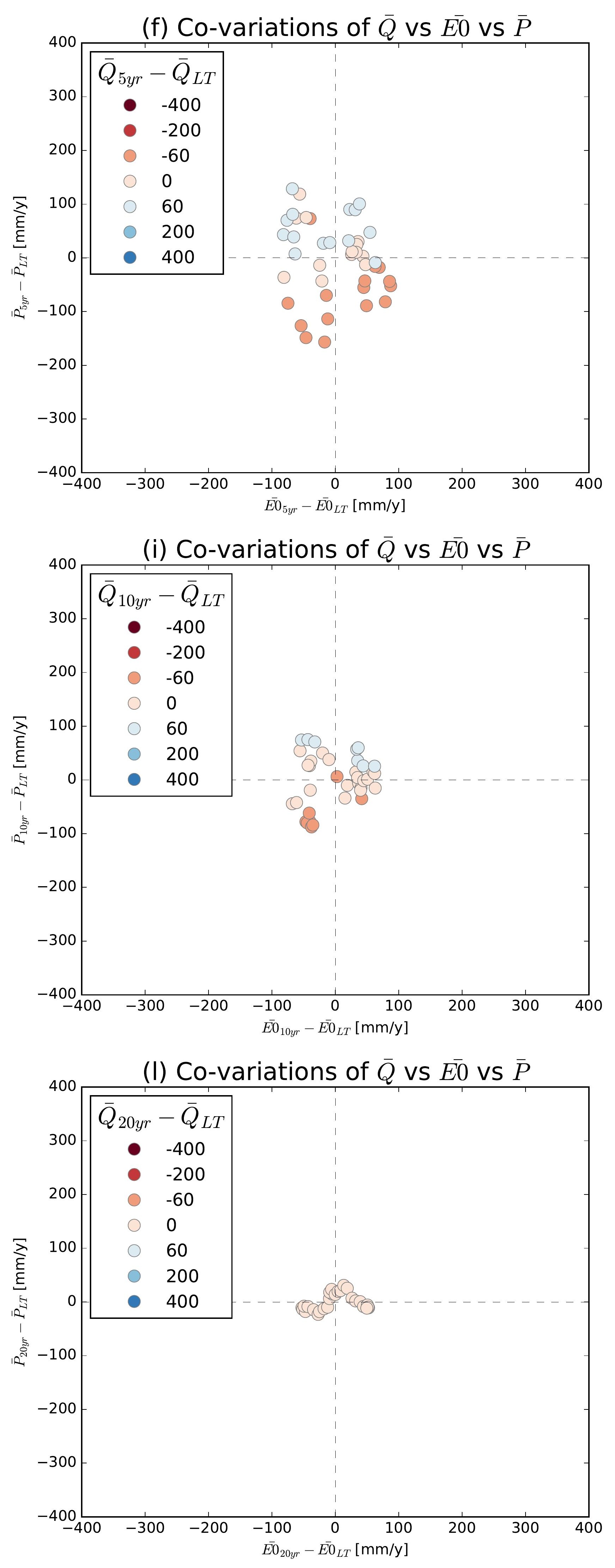

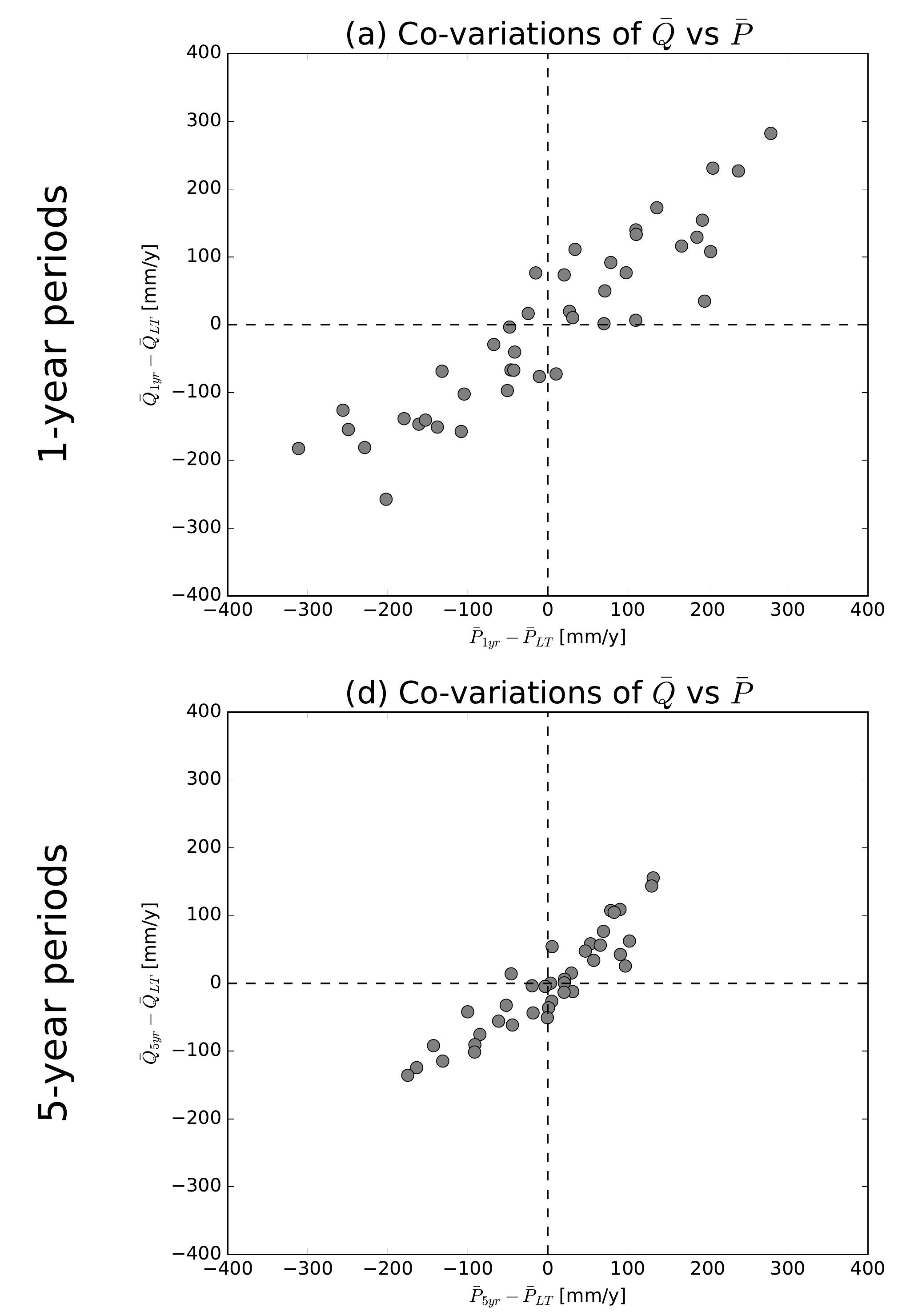

(g) Co-variations of $\bar{Q}$ vs $\bar{P}$
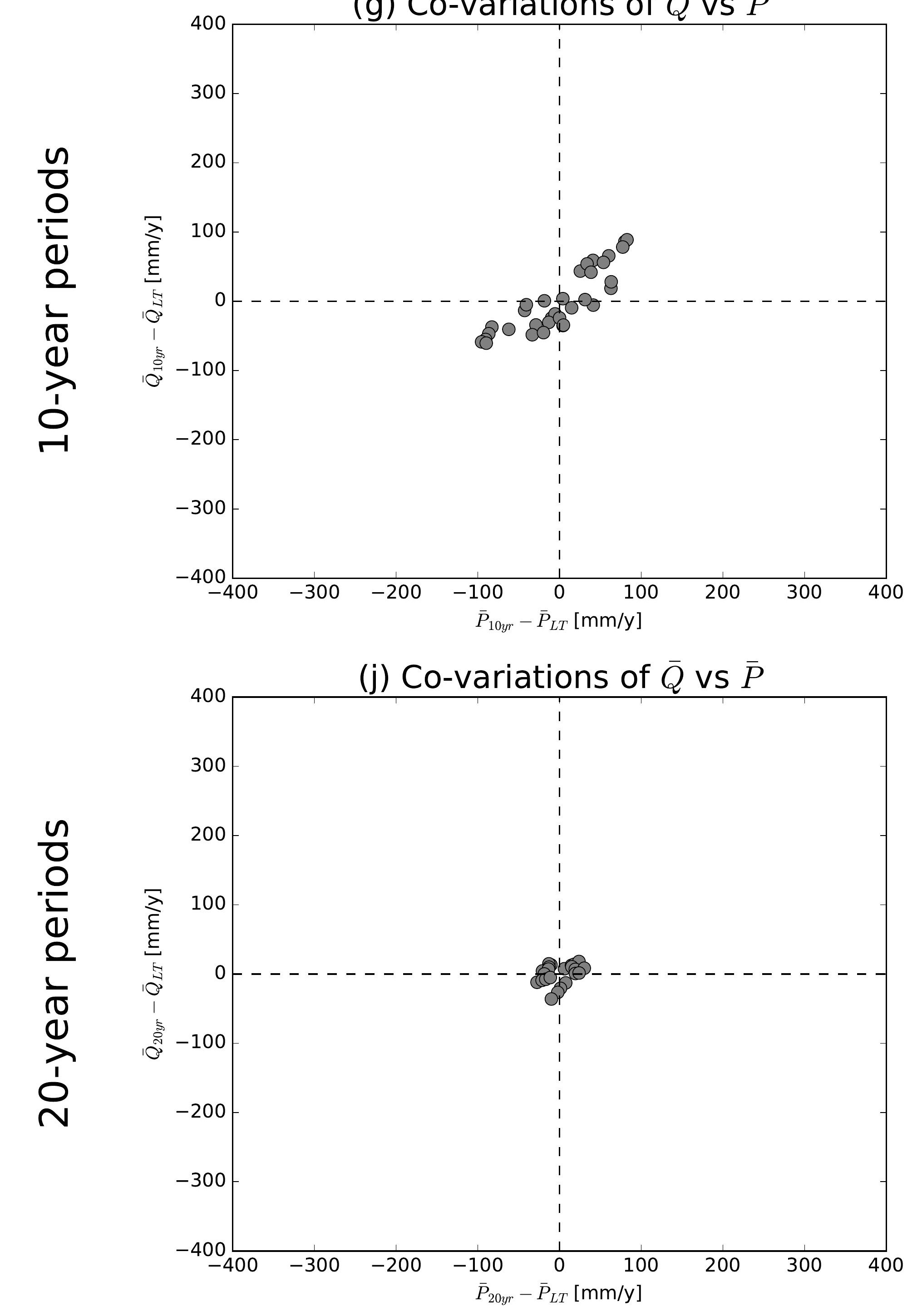

(b) Co-variations of $\bar{Q}$ vs $\overline{E 0}$

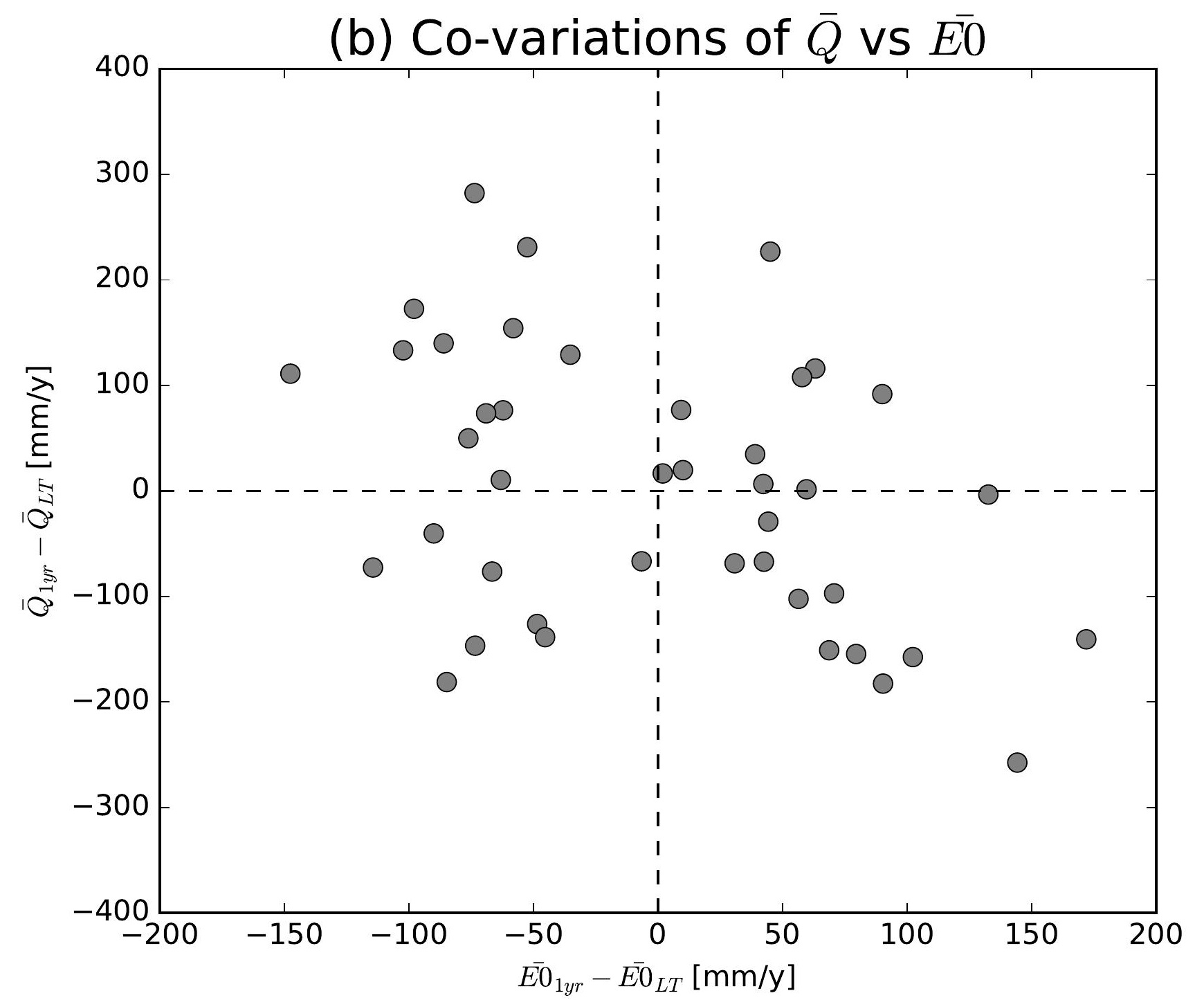

(e) Co-variations of $\bar{Q}$ vs $\overline{E 0}$

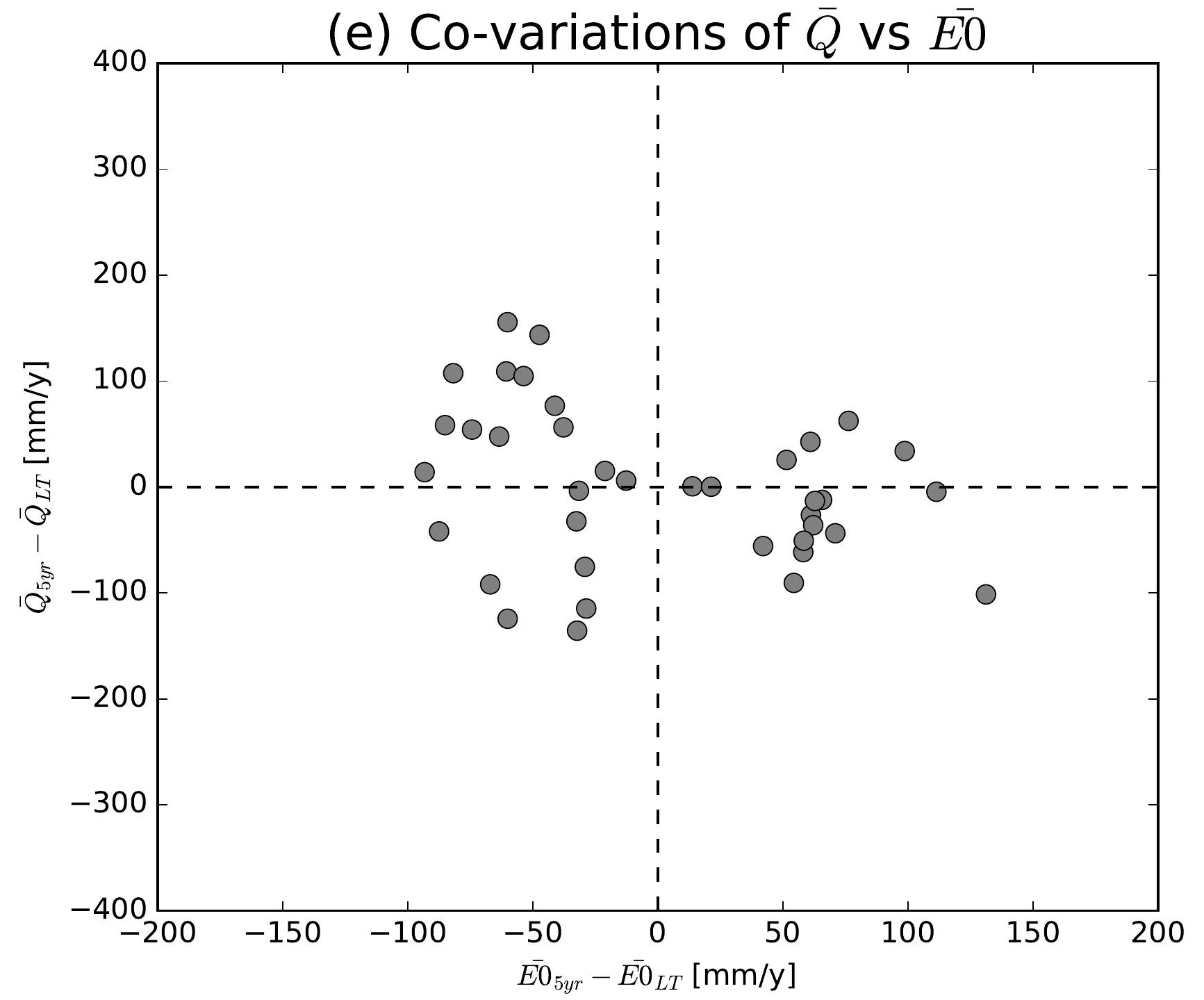

(h) Co-variations of $\bar{Q}$ vs $\overline{E 0}$

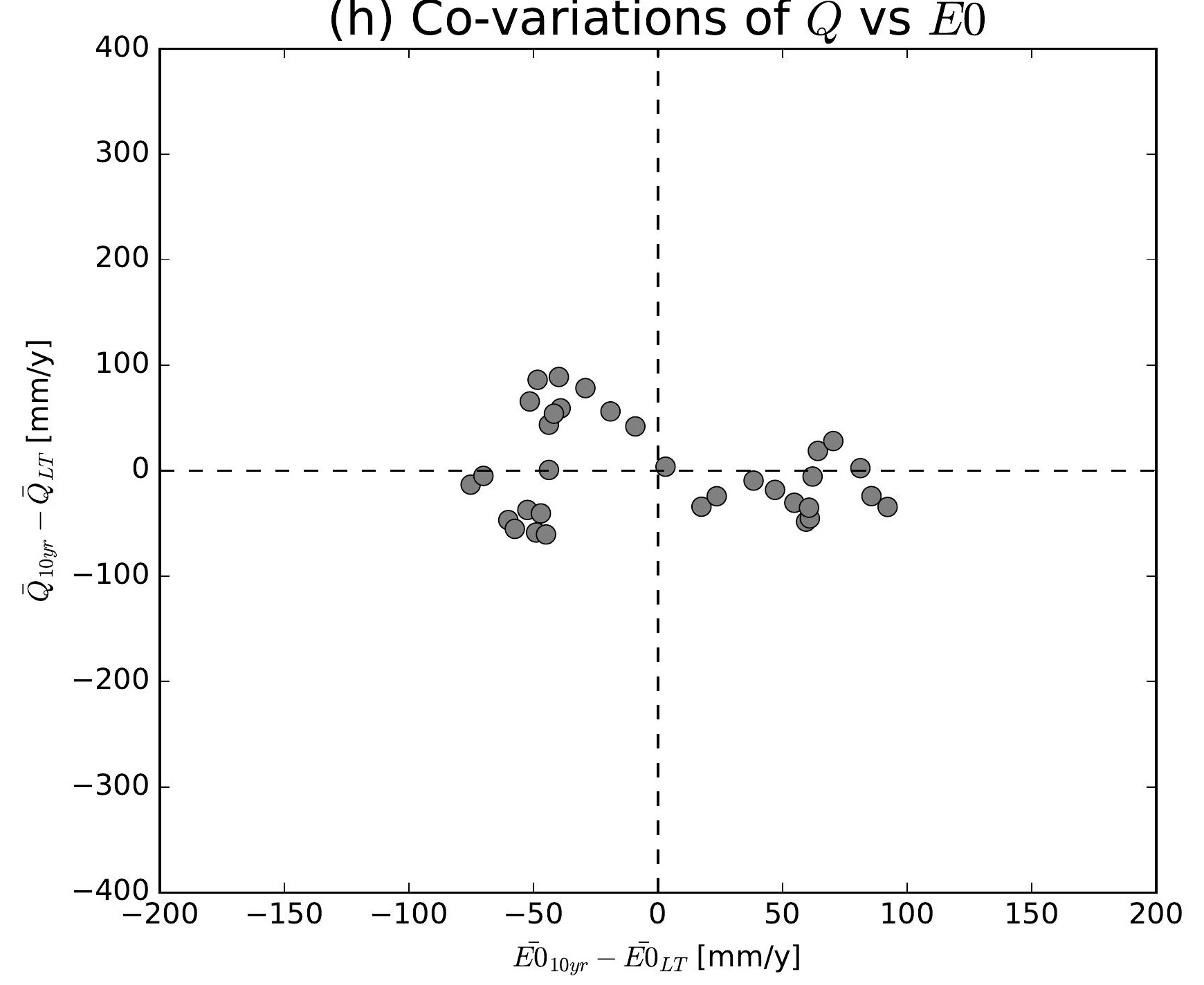

(k) Co-variations of $\bar{Q}$ vs $\overline{E 0}$

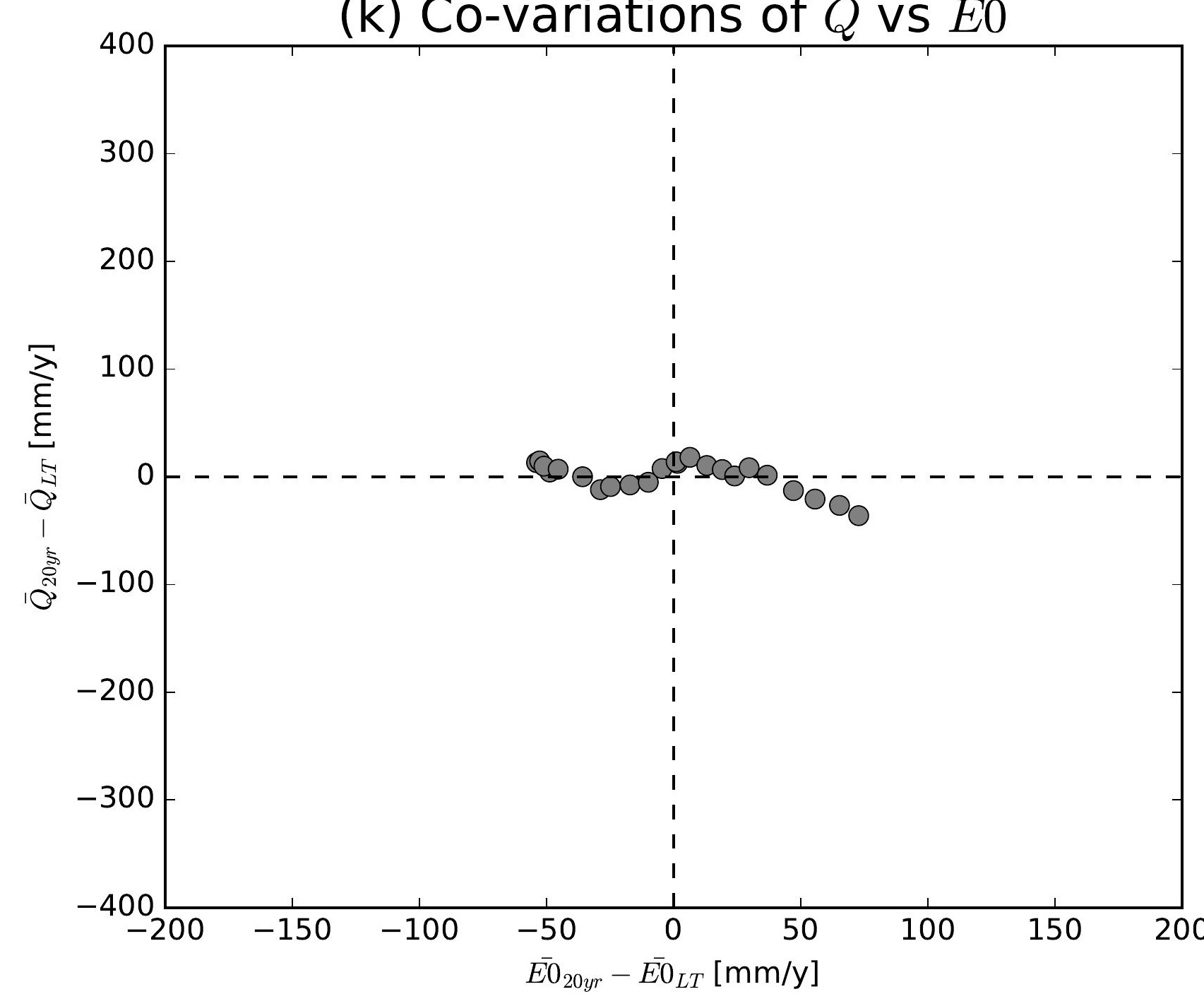

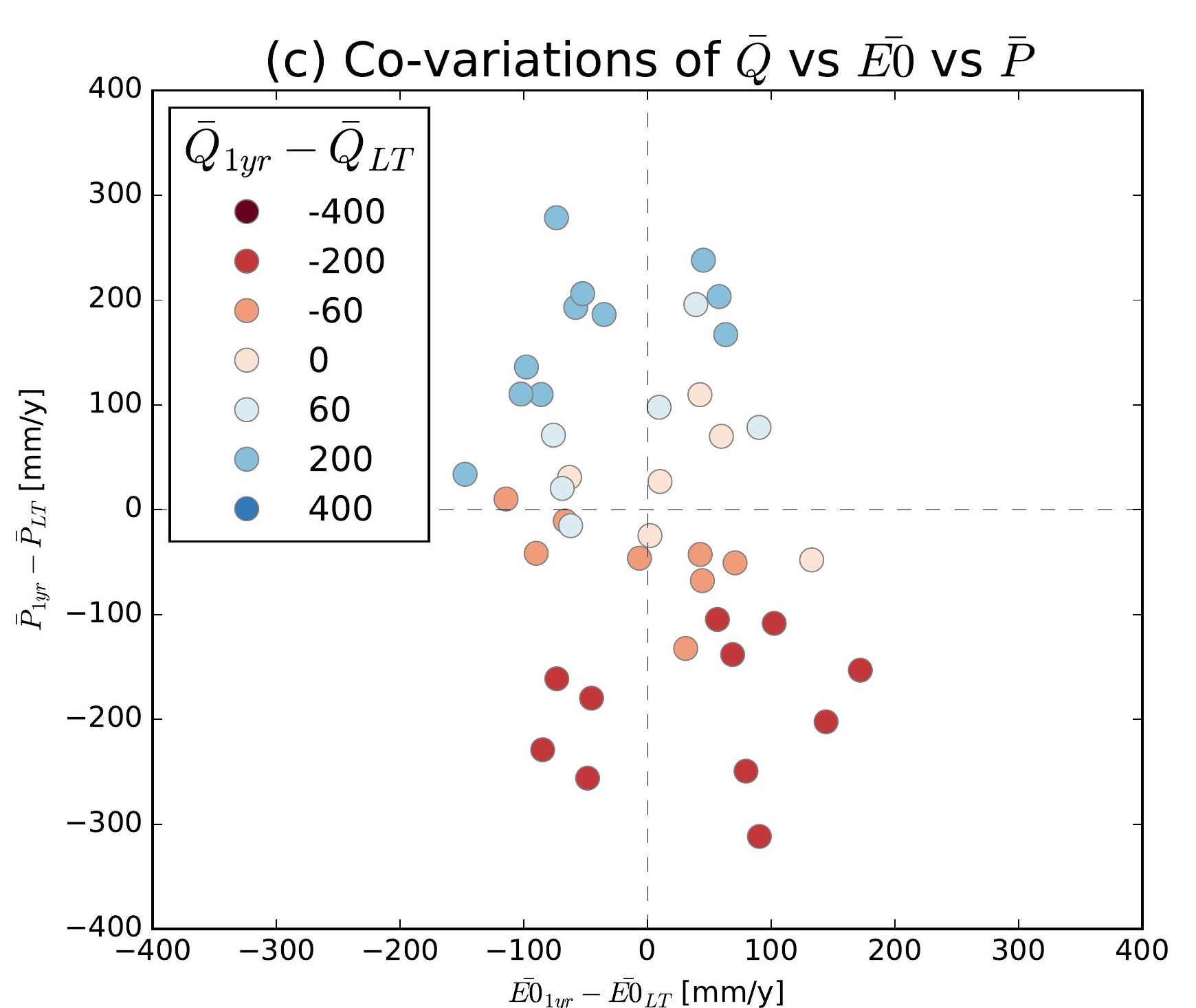
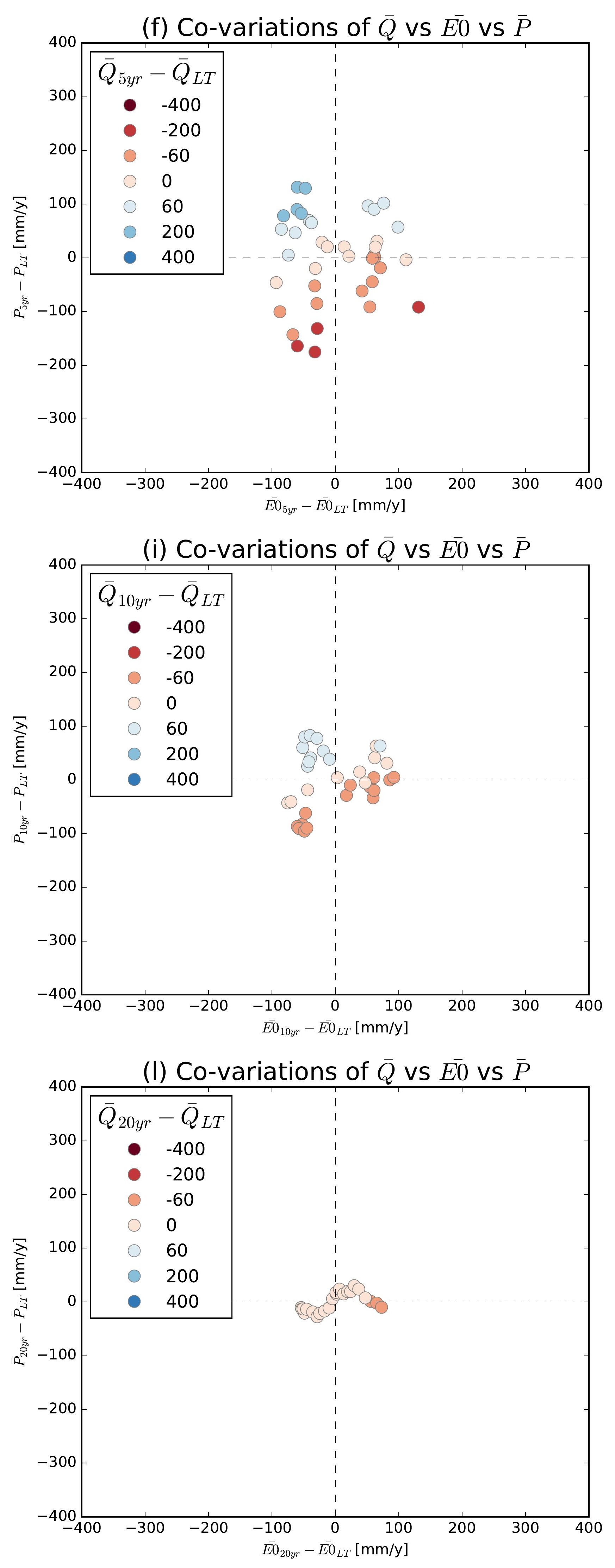

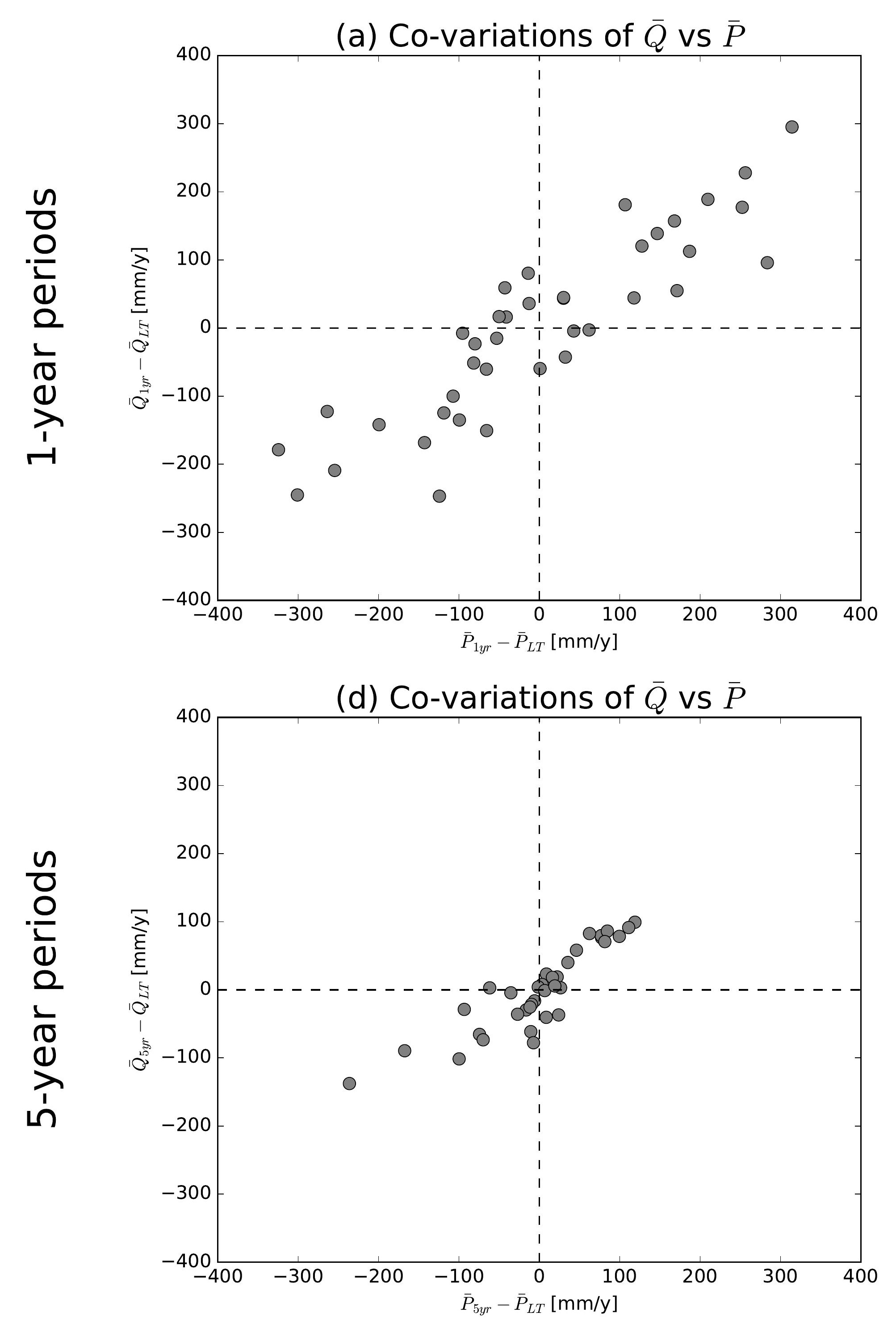

(g) Co-variations of $\bar{Q}$ vs $\bar{P}$

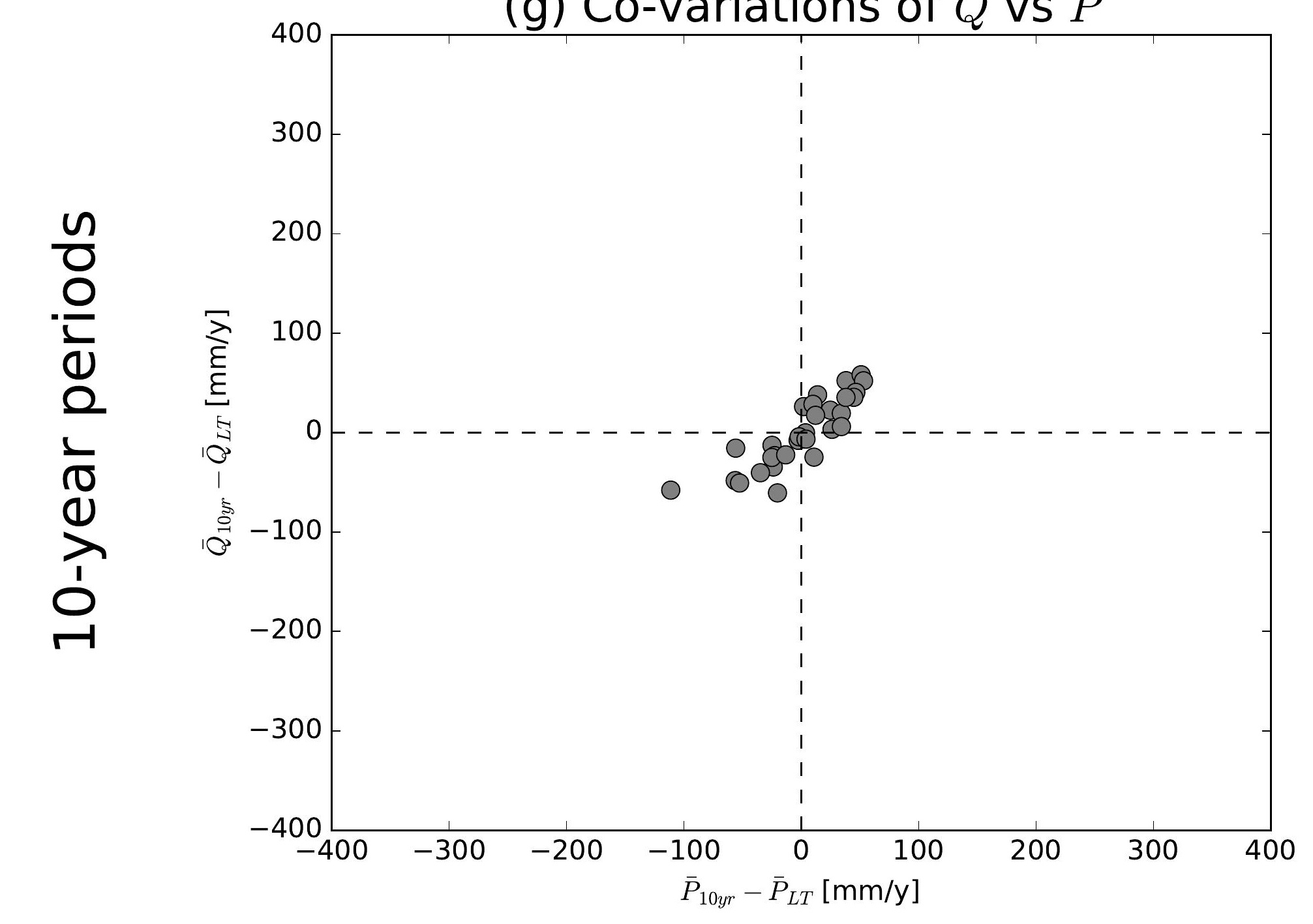

(j) Co-variations of $\bar{Q}$ vs $\bar{P}$

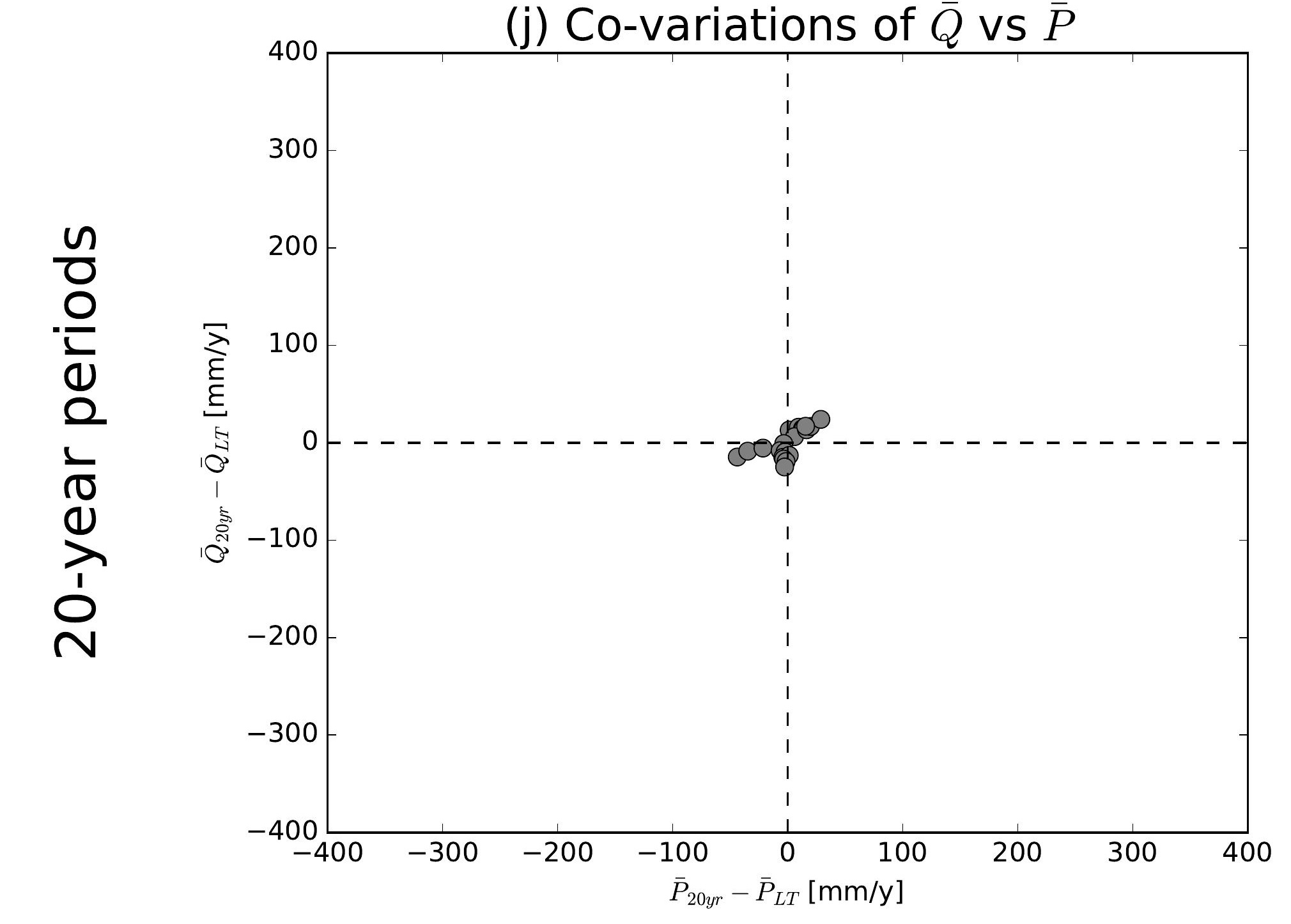

(b) Co-variations of $\bar{Q}$ vs $\overline{E 0}$

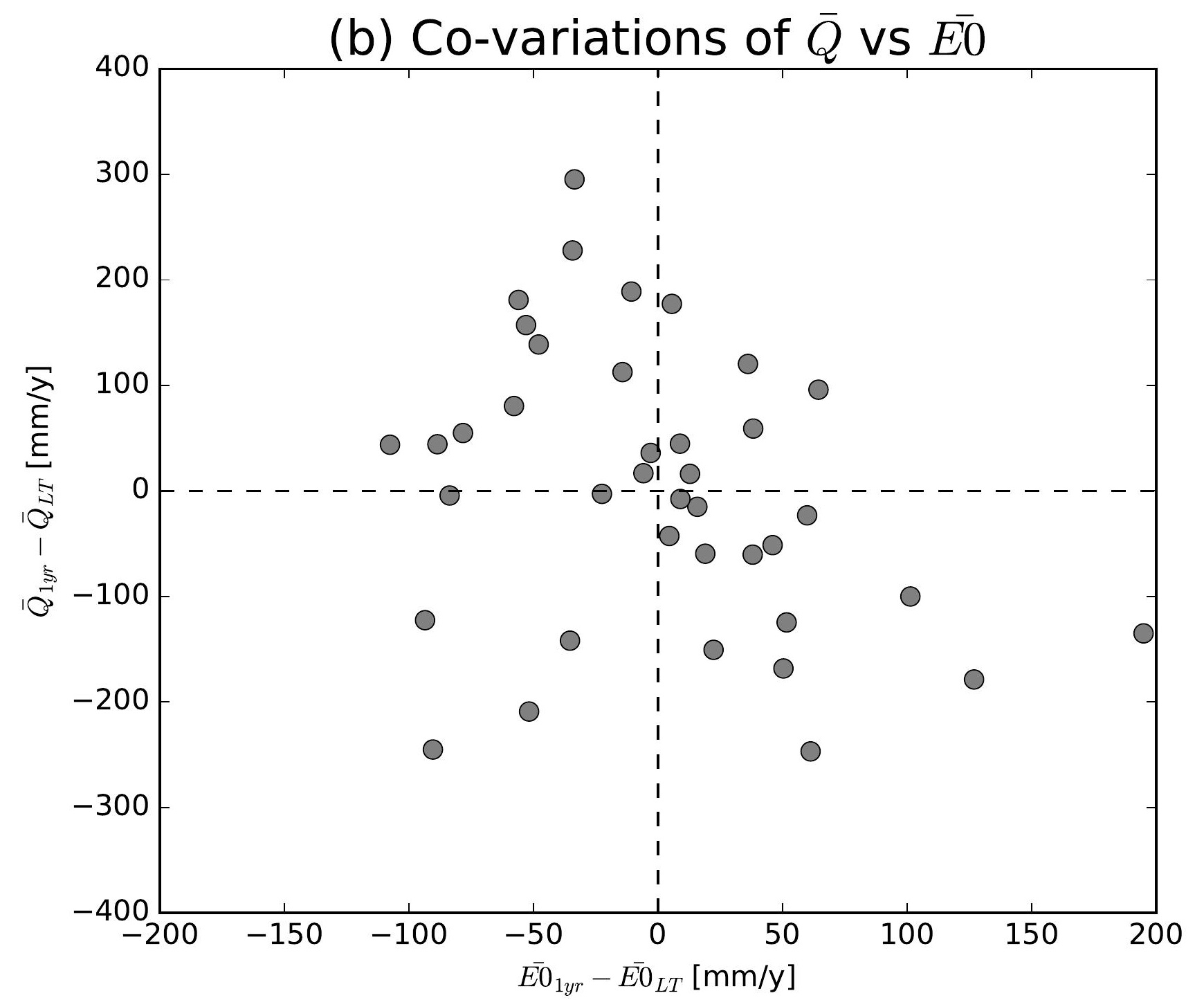

(e) Co-variations of $\bar{Q}$ vs $\overline{E 0}$

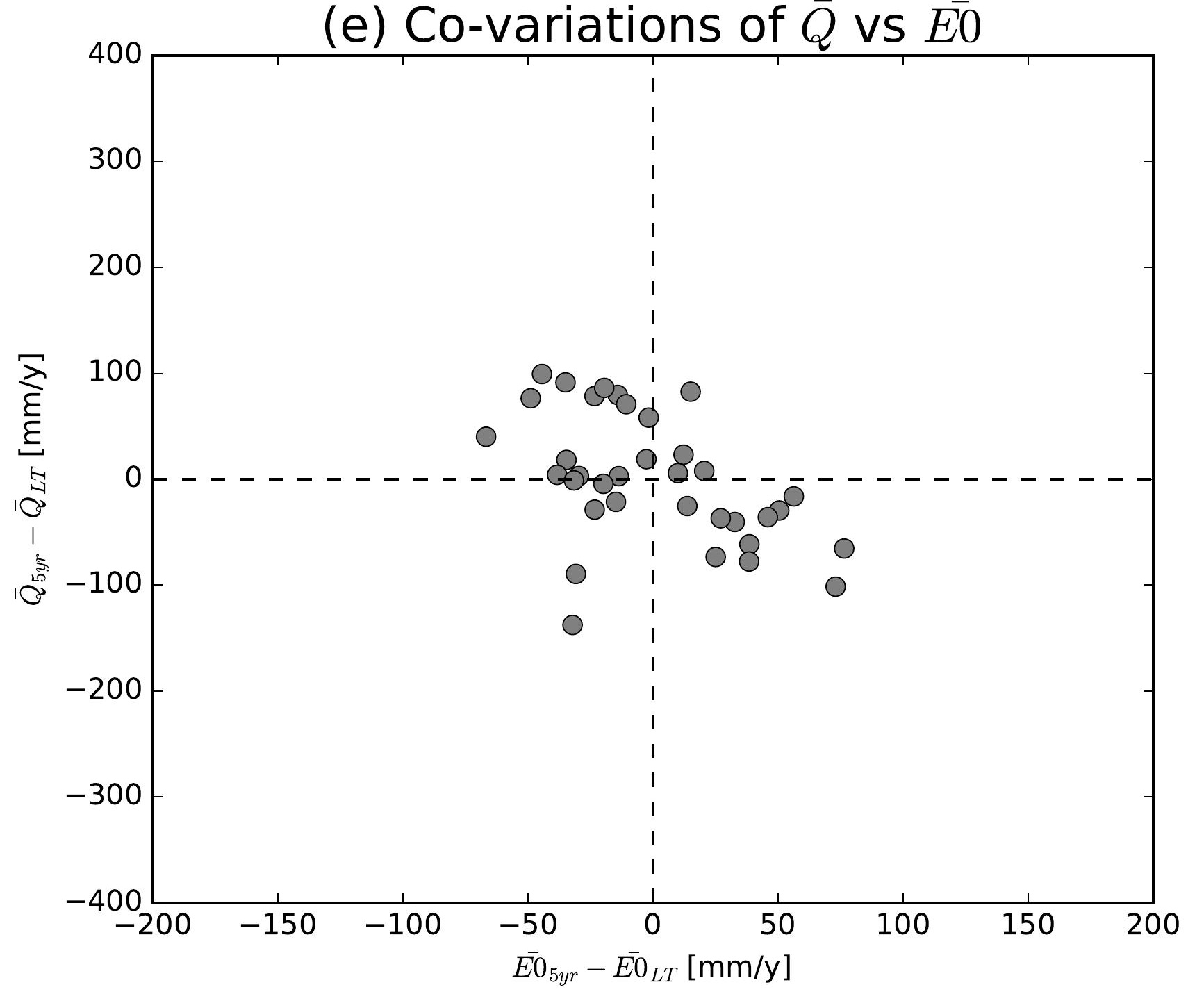

(h) Co-variations of $\bar{Q}$ vs $\overline{E 0}$

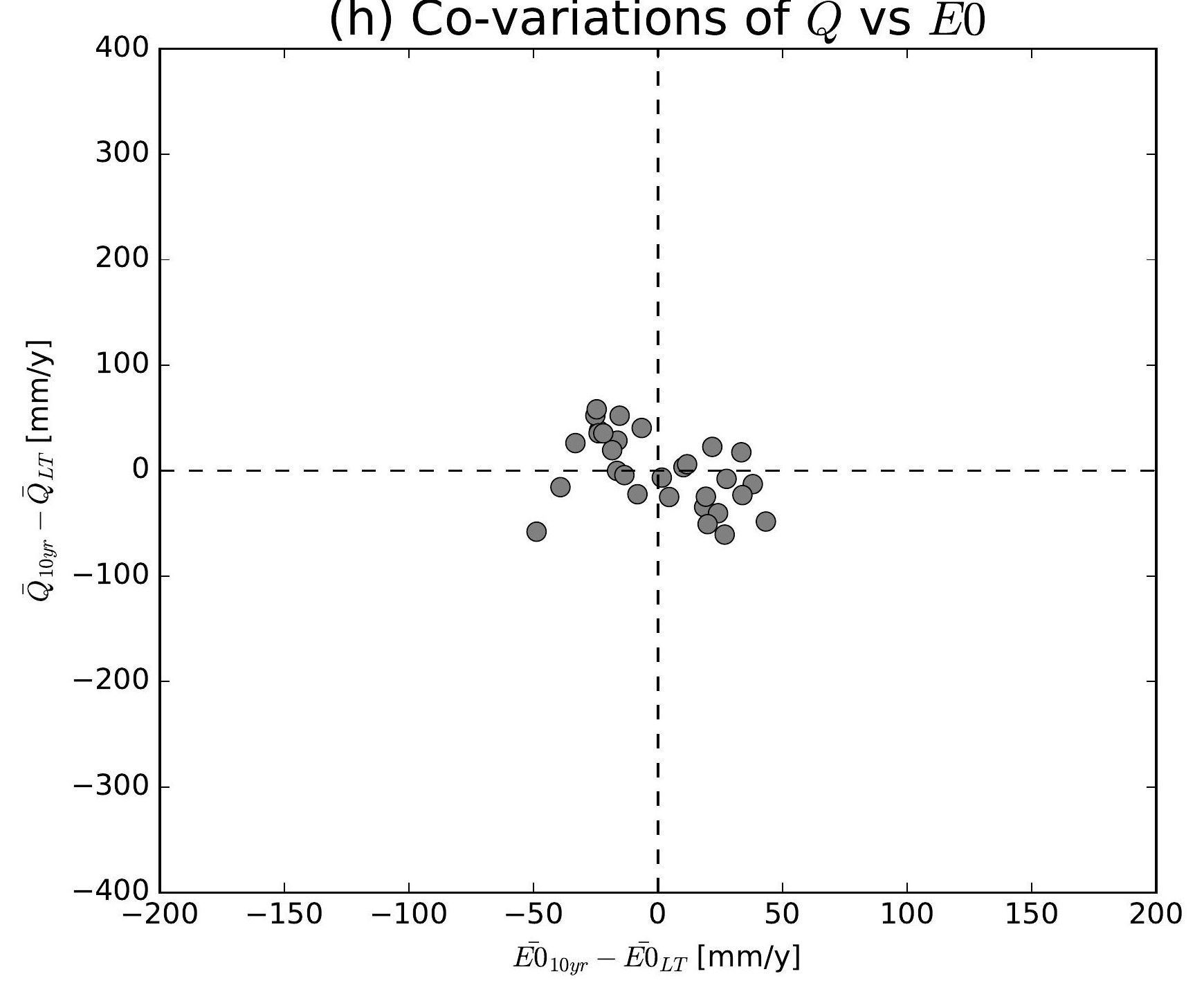

(k) Co-variations of $\bar{Q}$ vs $\overline{E 0}$

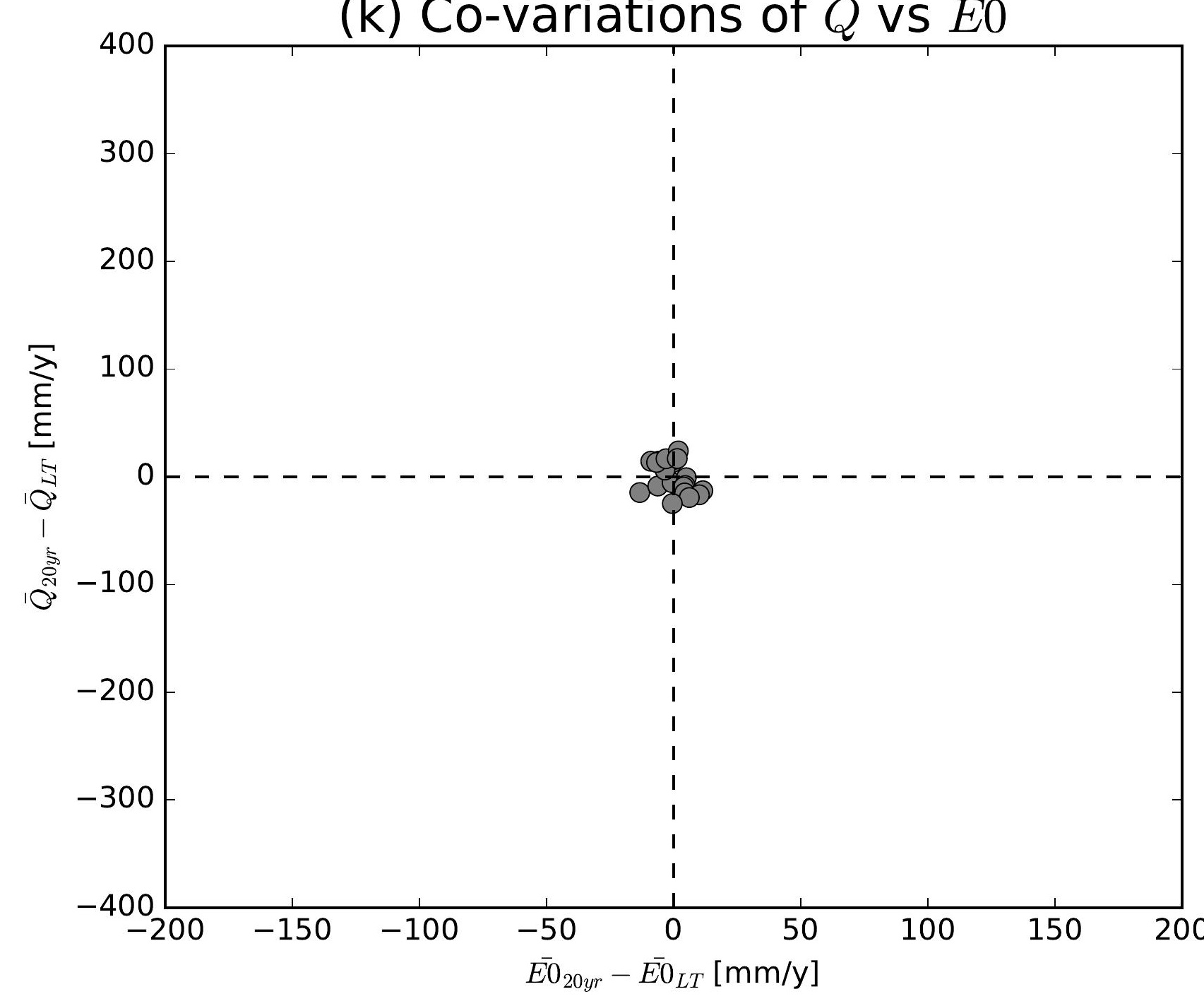

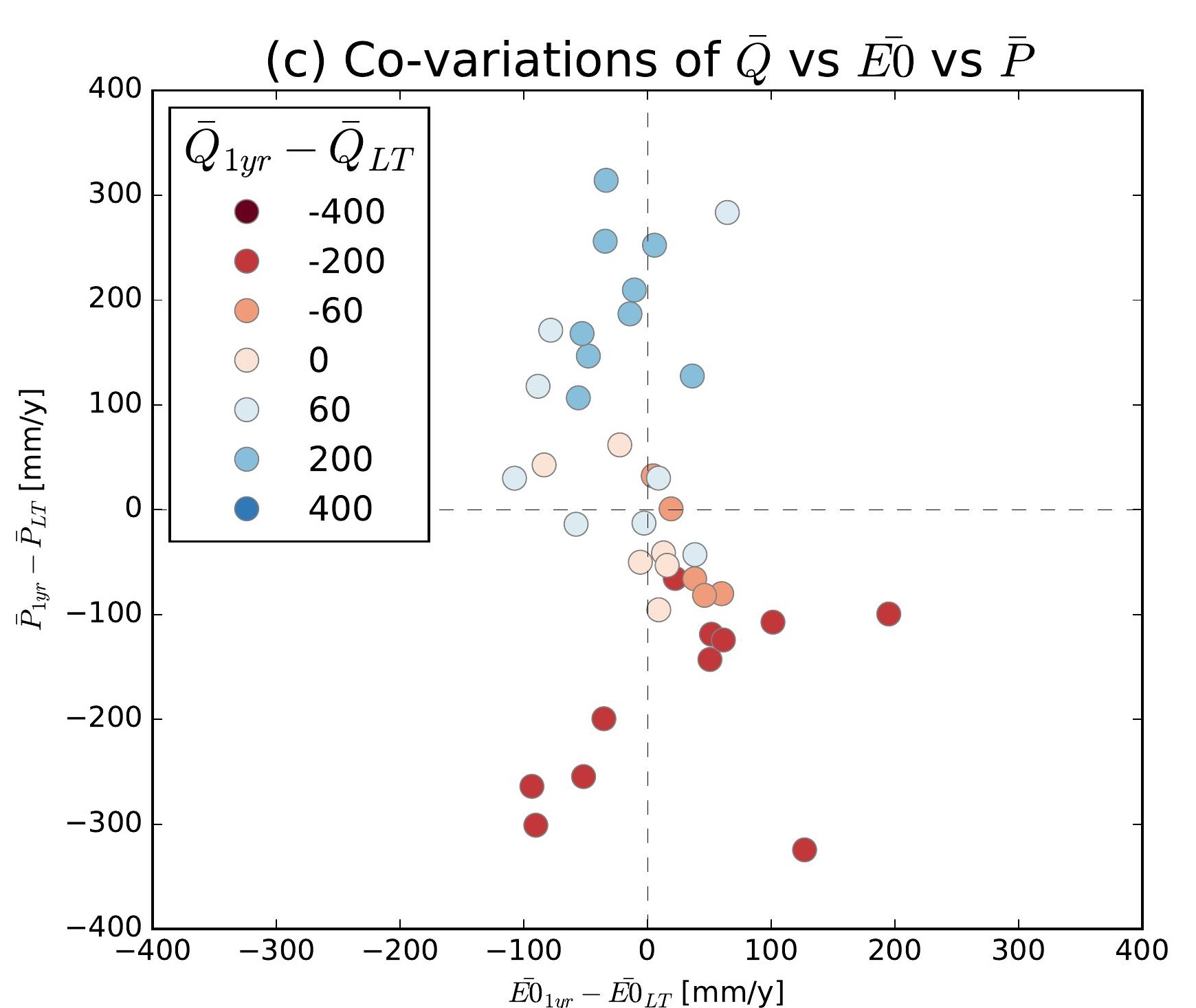
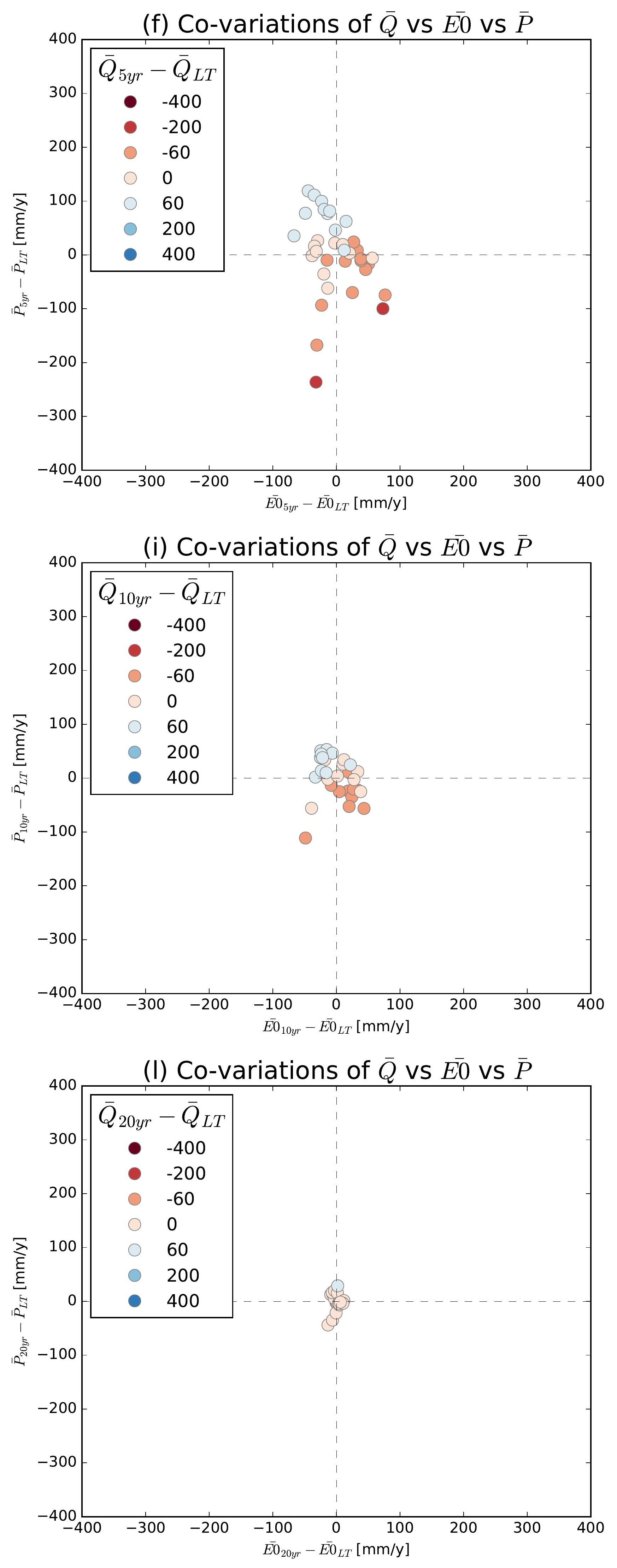

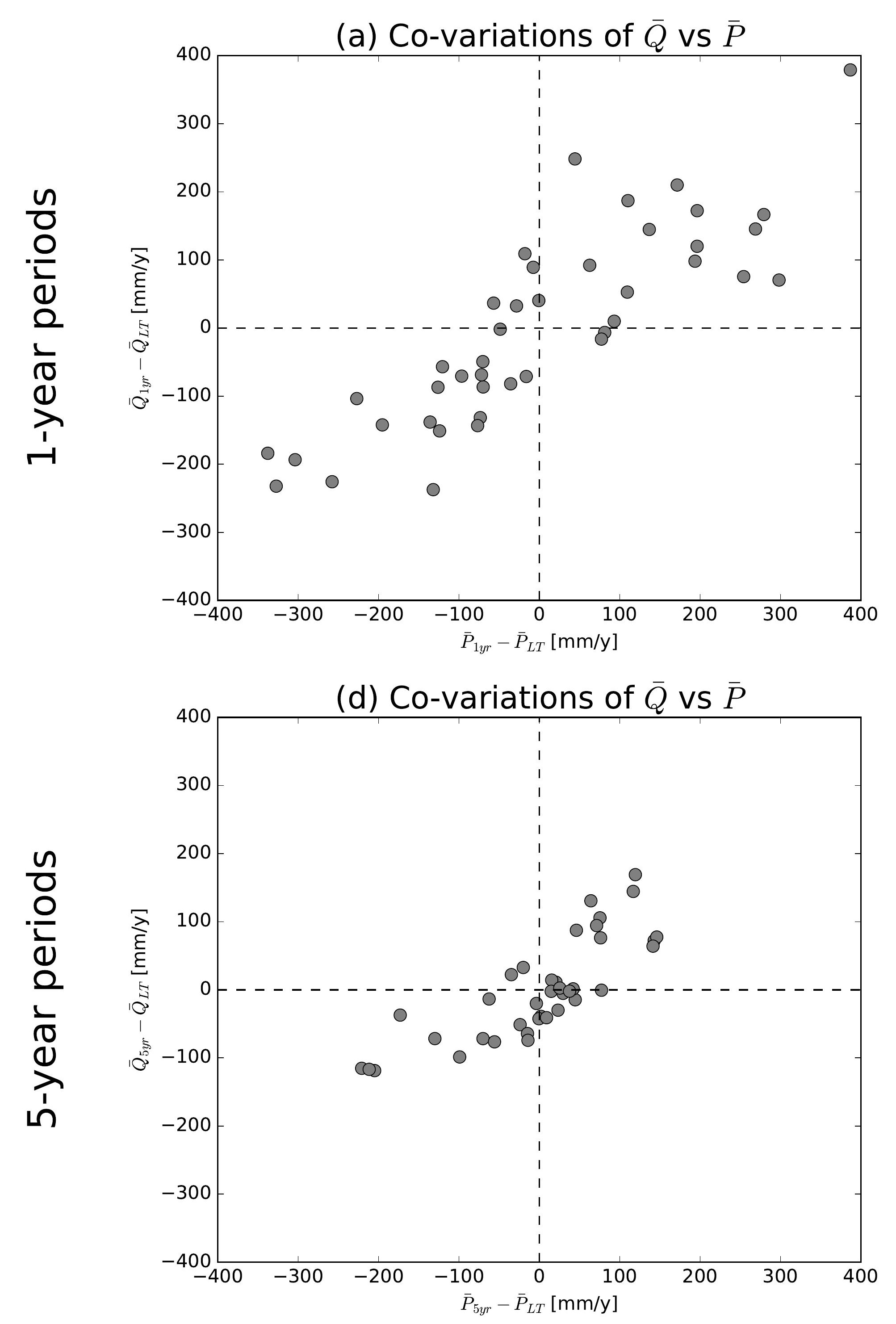

(g) Co-variations of $\bar{Q}$ vs $\bar{P}$

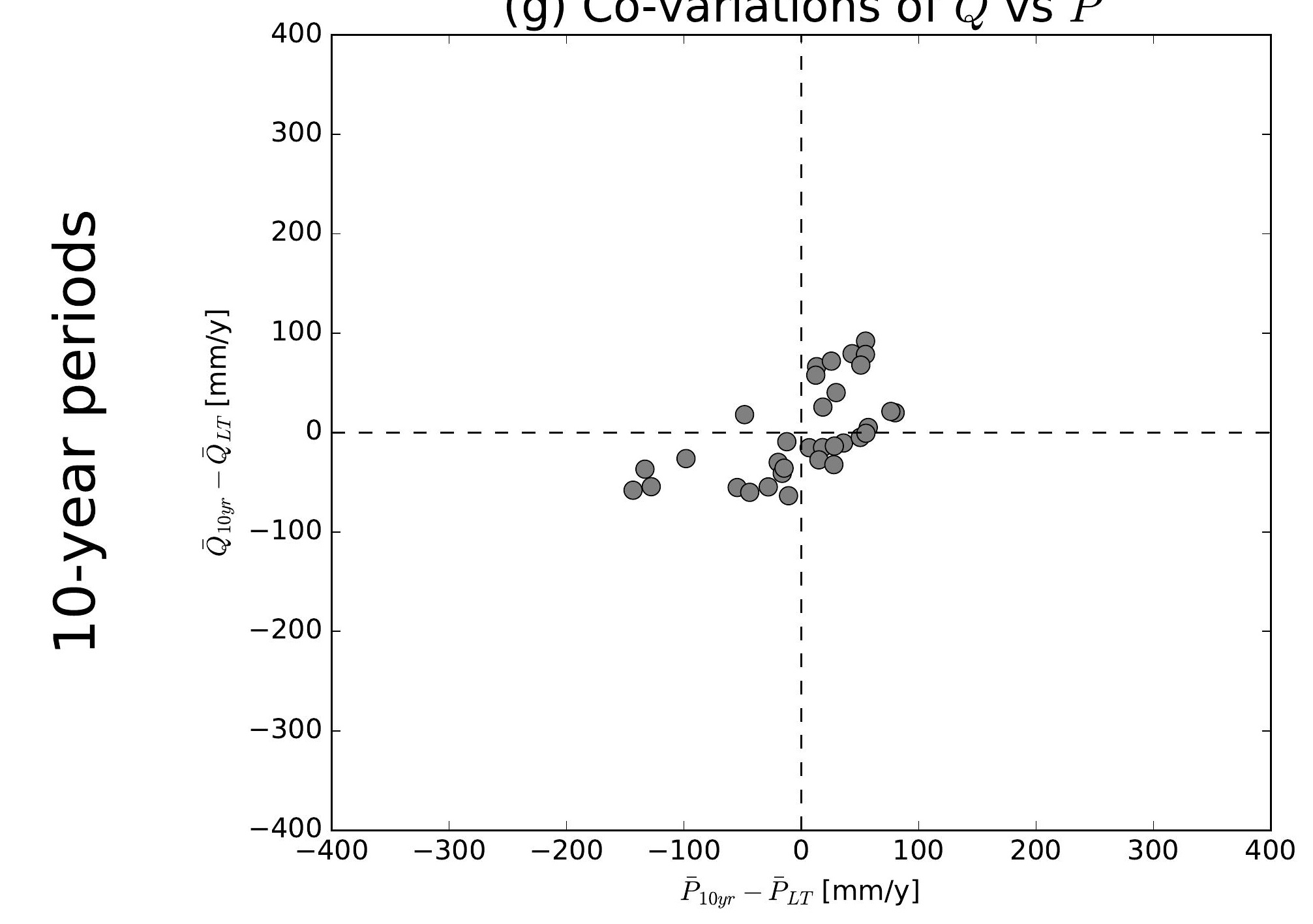

(j) Co-variations of $\bar{Q}$ vs $\bar{P}$

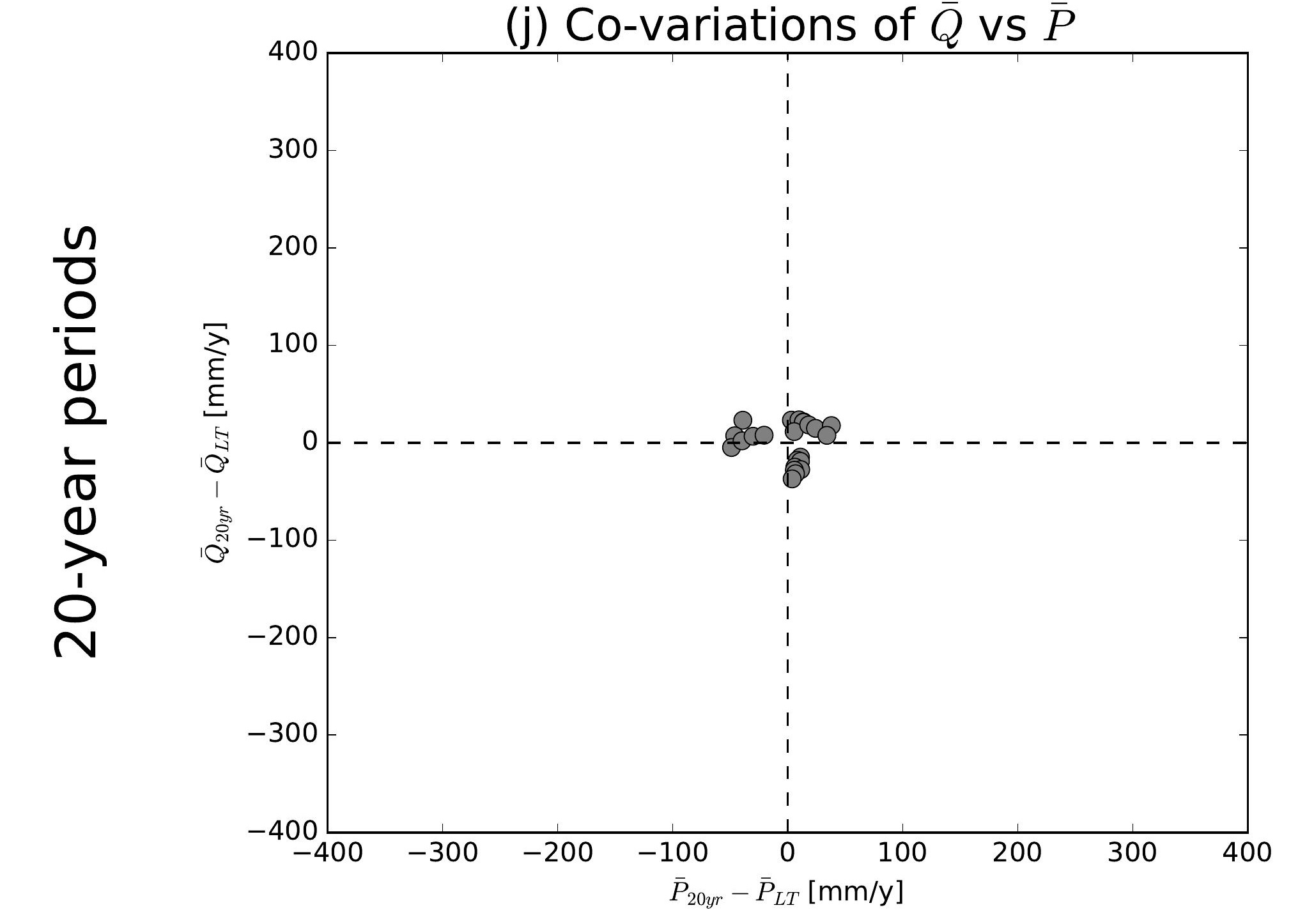

(b) Co-variations of $\bar{Q}$ vs $\overline{E 0}$

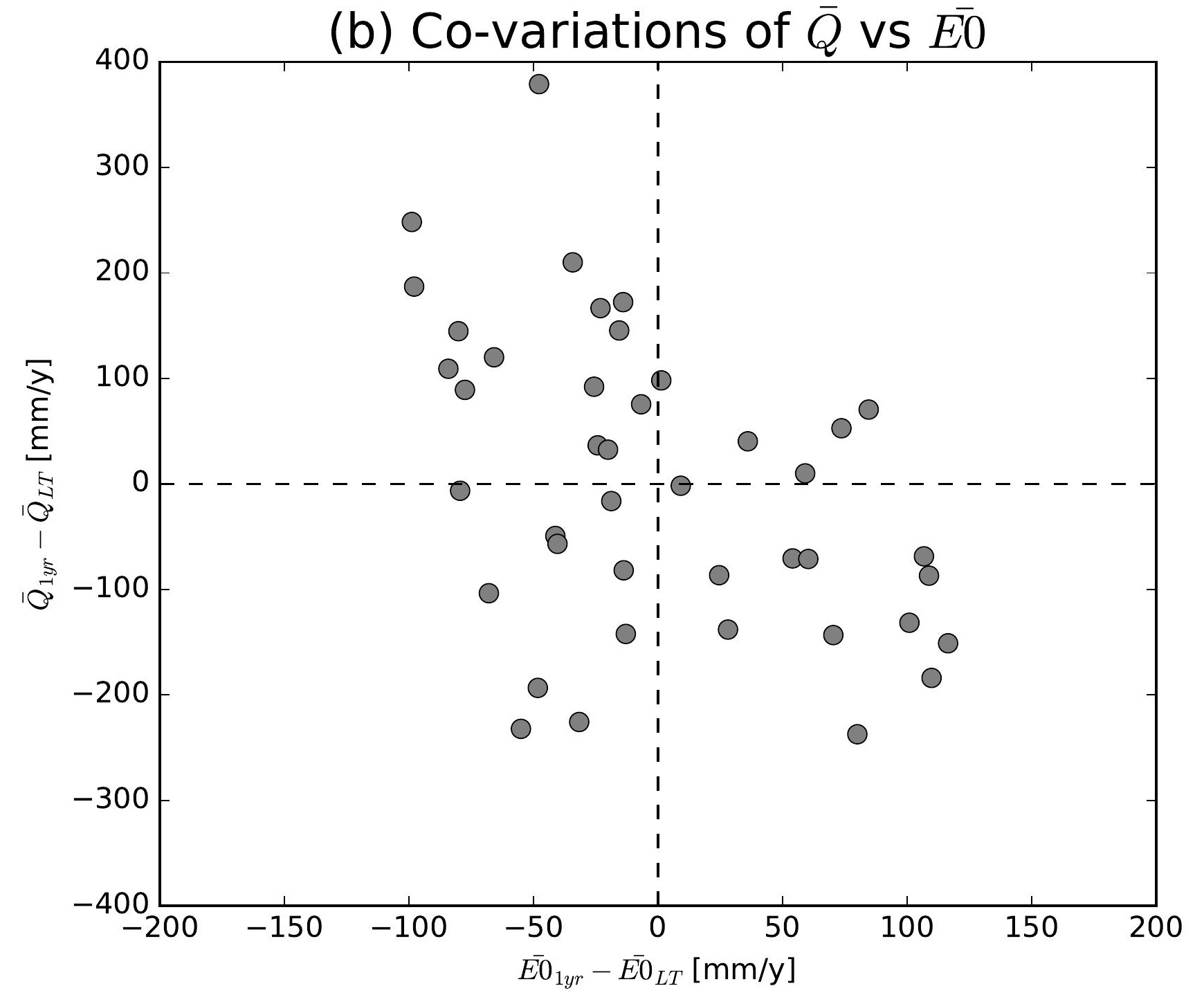

(e) Co-variations of $\bar{Q}$ vs $\overline{E 0}$

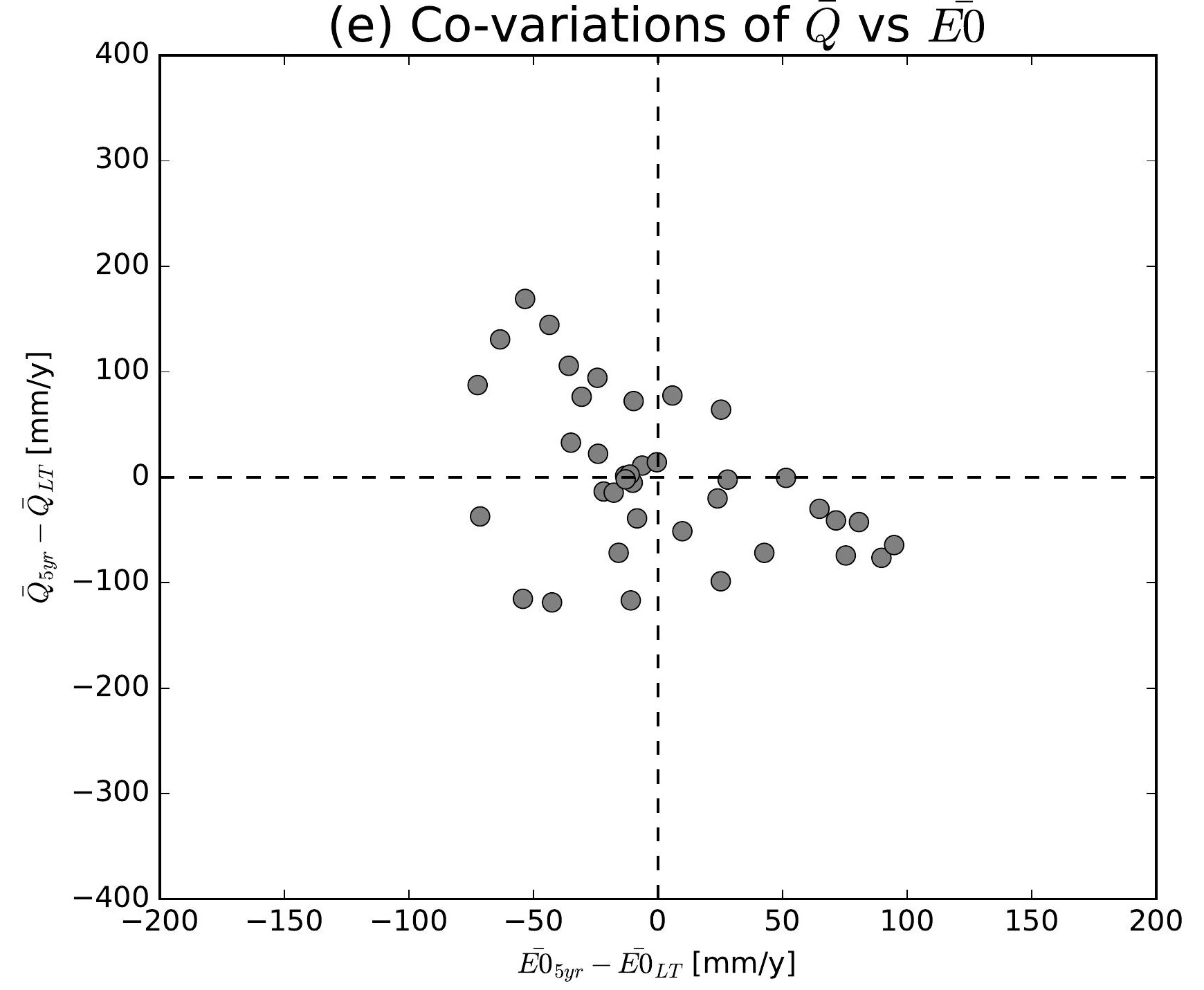

(h) Co-variations of $\bar{Q}$ vs $\overline{E 0}$

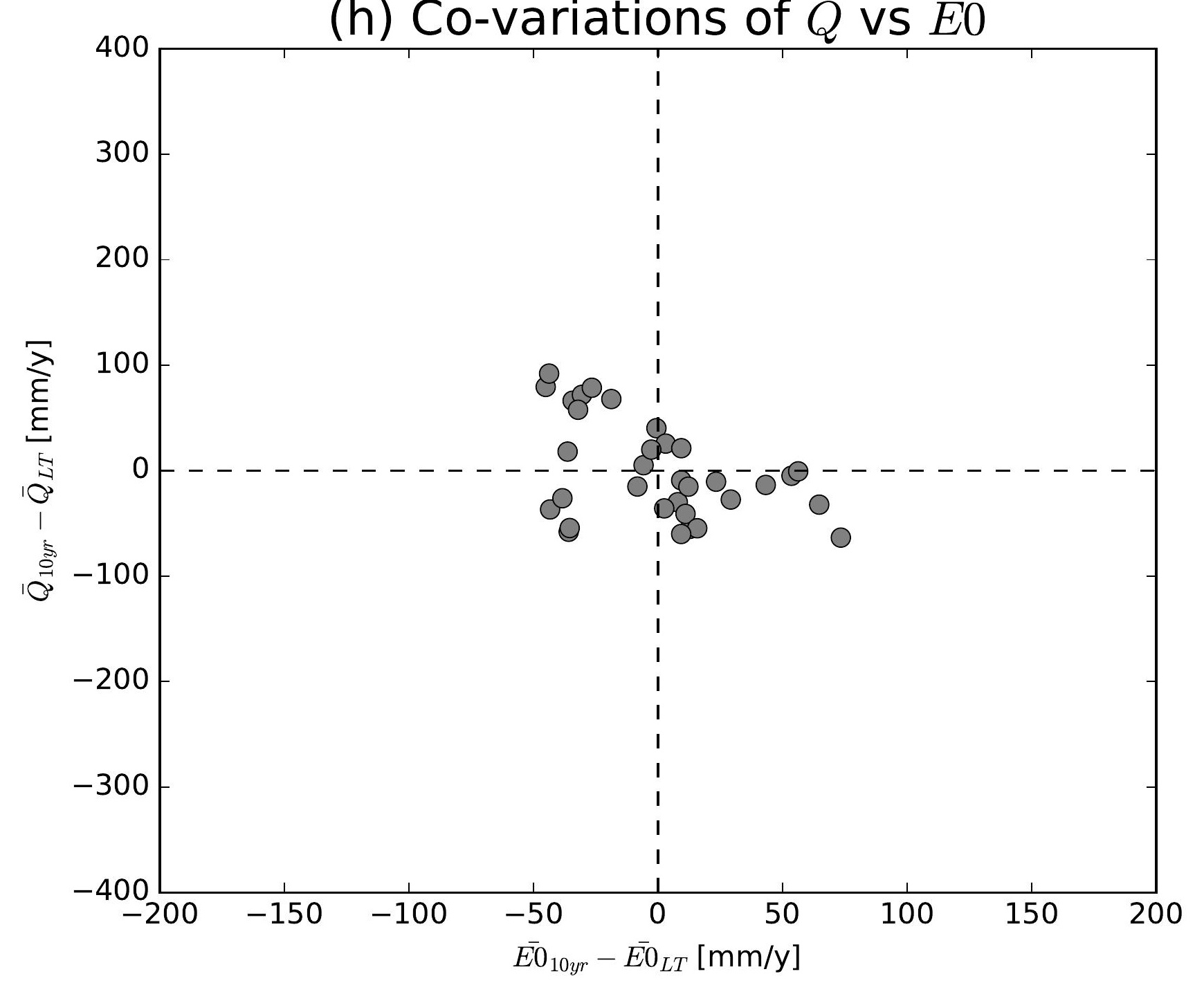

(k) Co-variations of $\bar{Q}$ vs $\overline{E 0}$

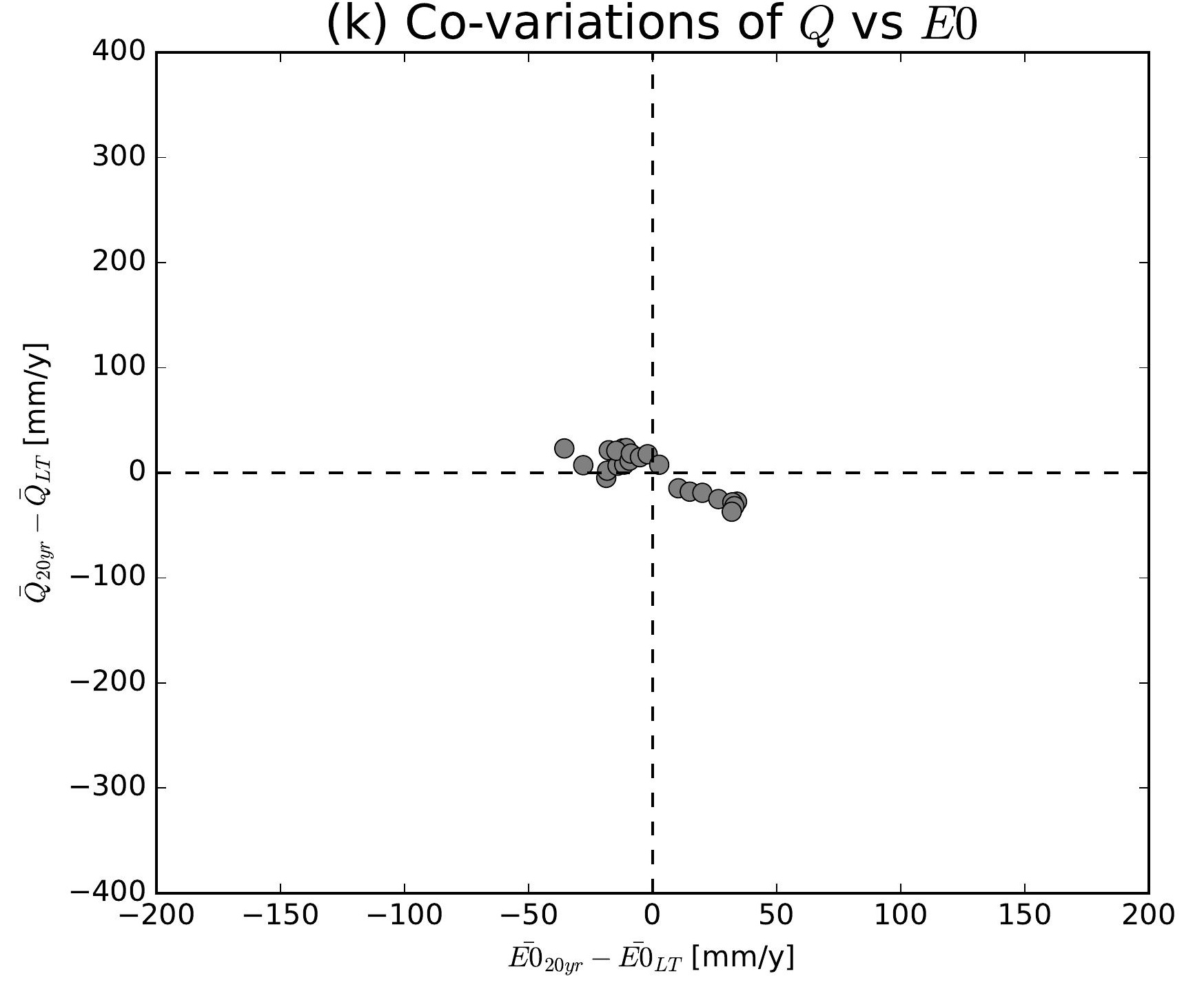

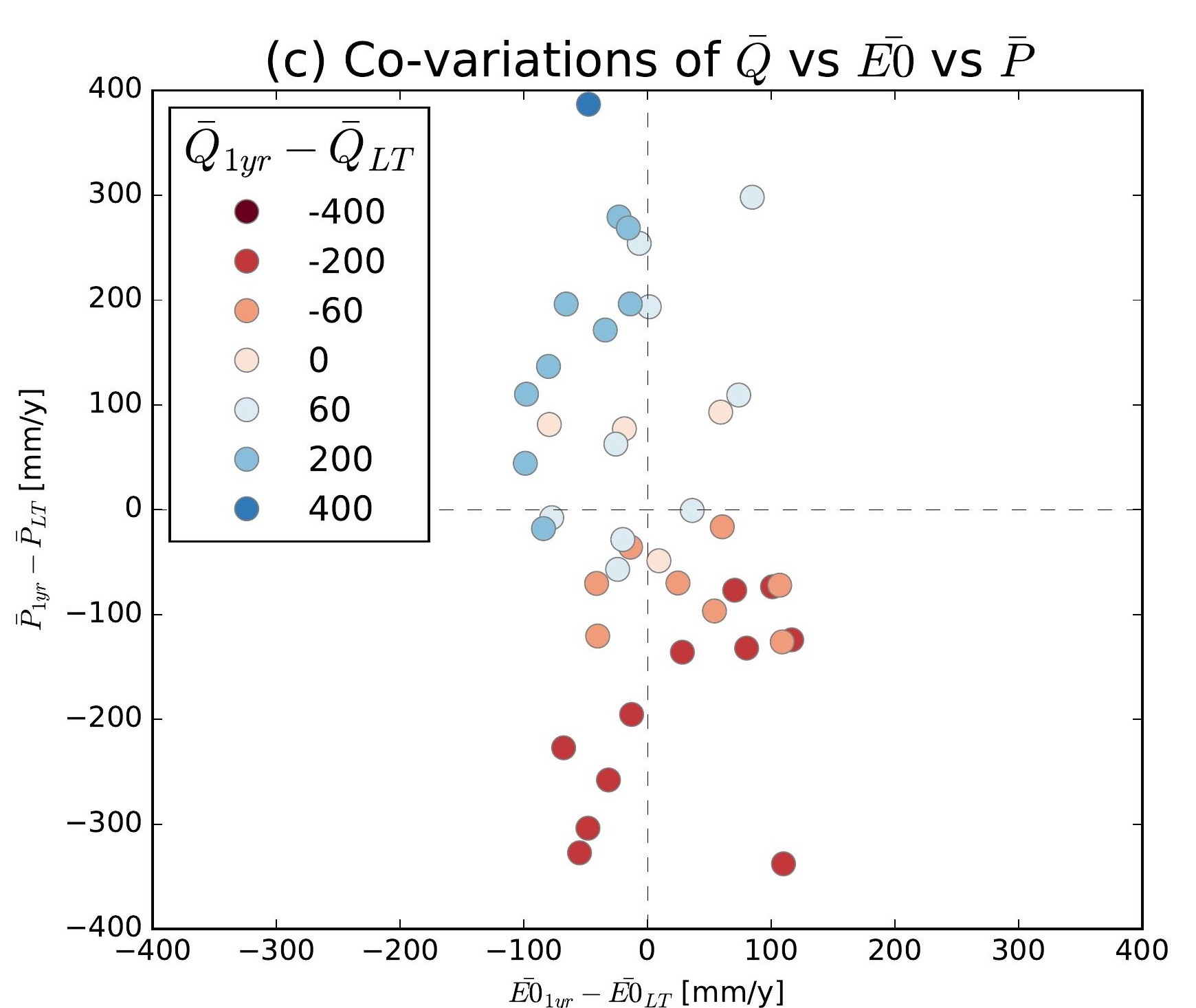
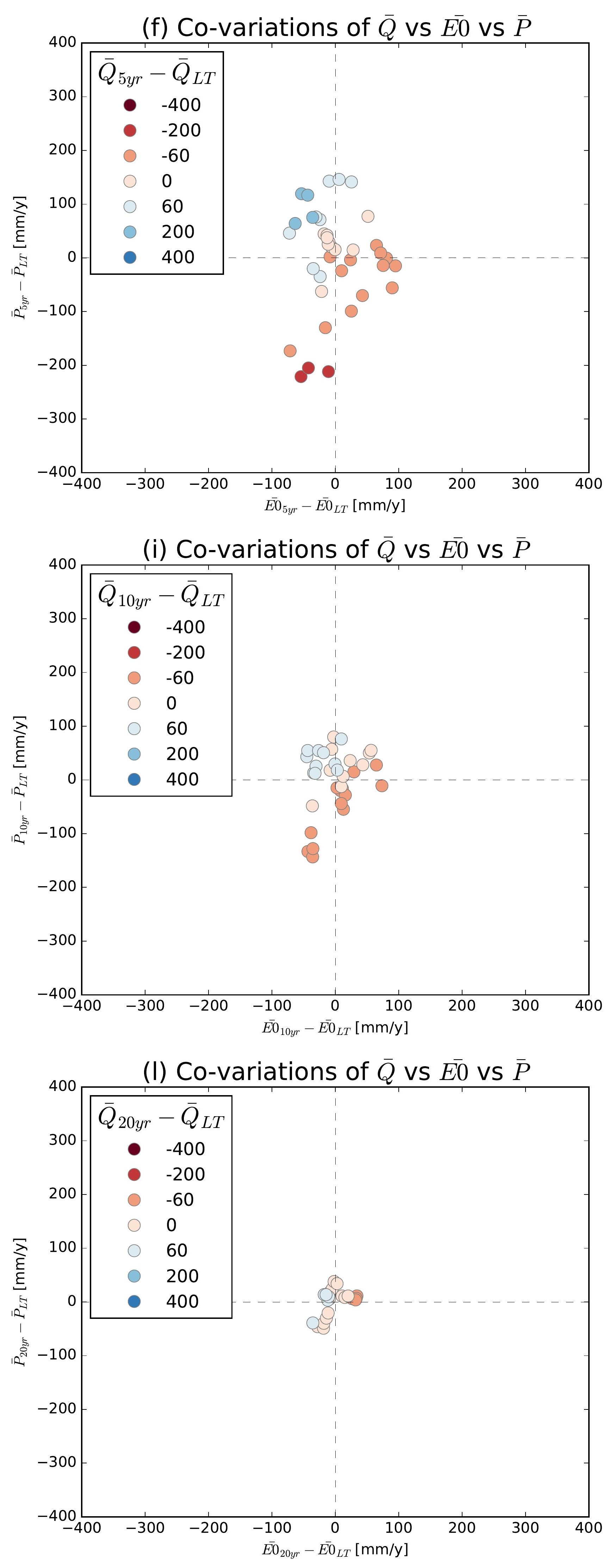

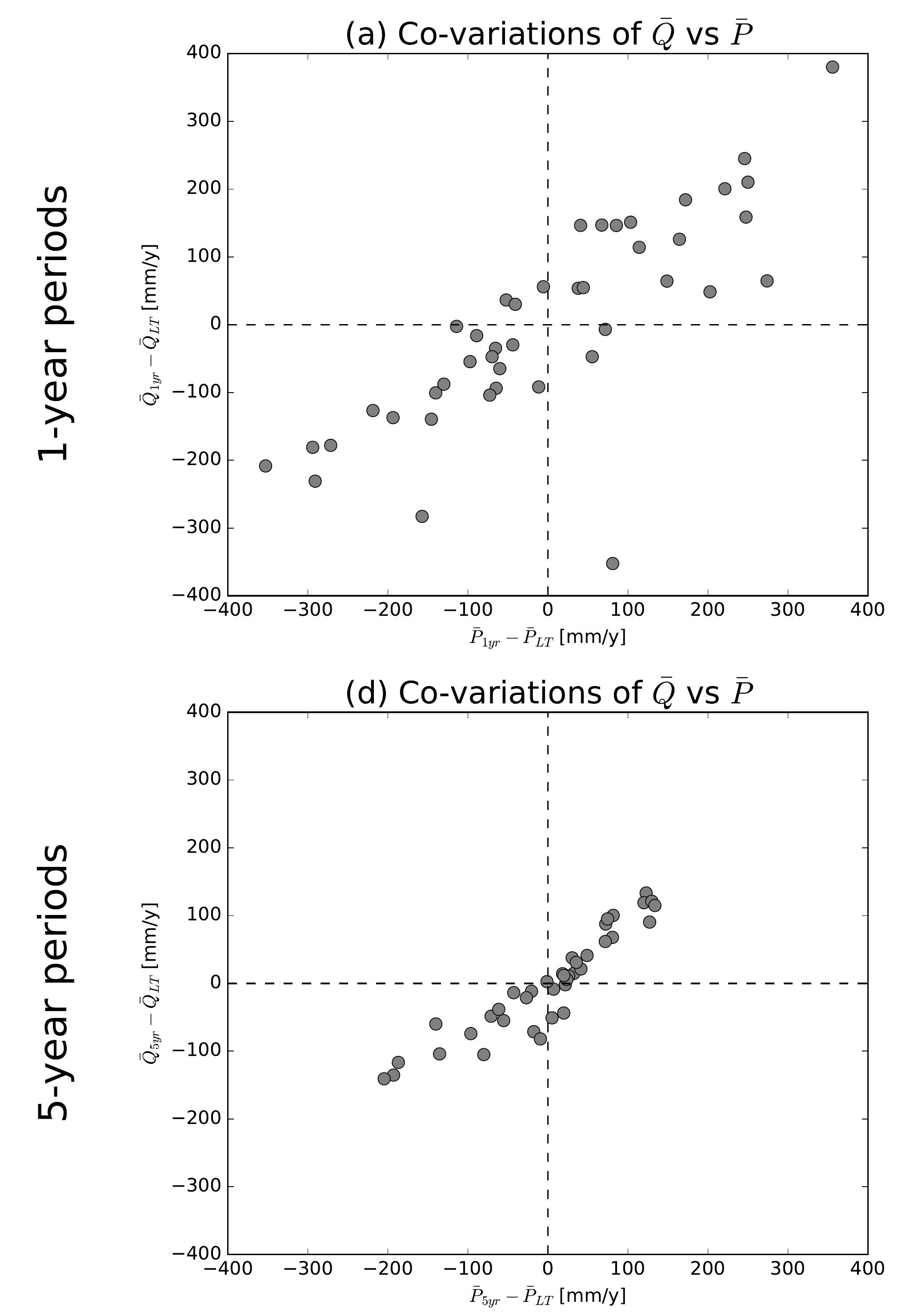

(g) Co-variations of $\bar{Q}$ vs $\bar{P}$
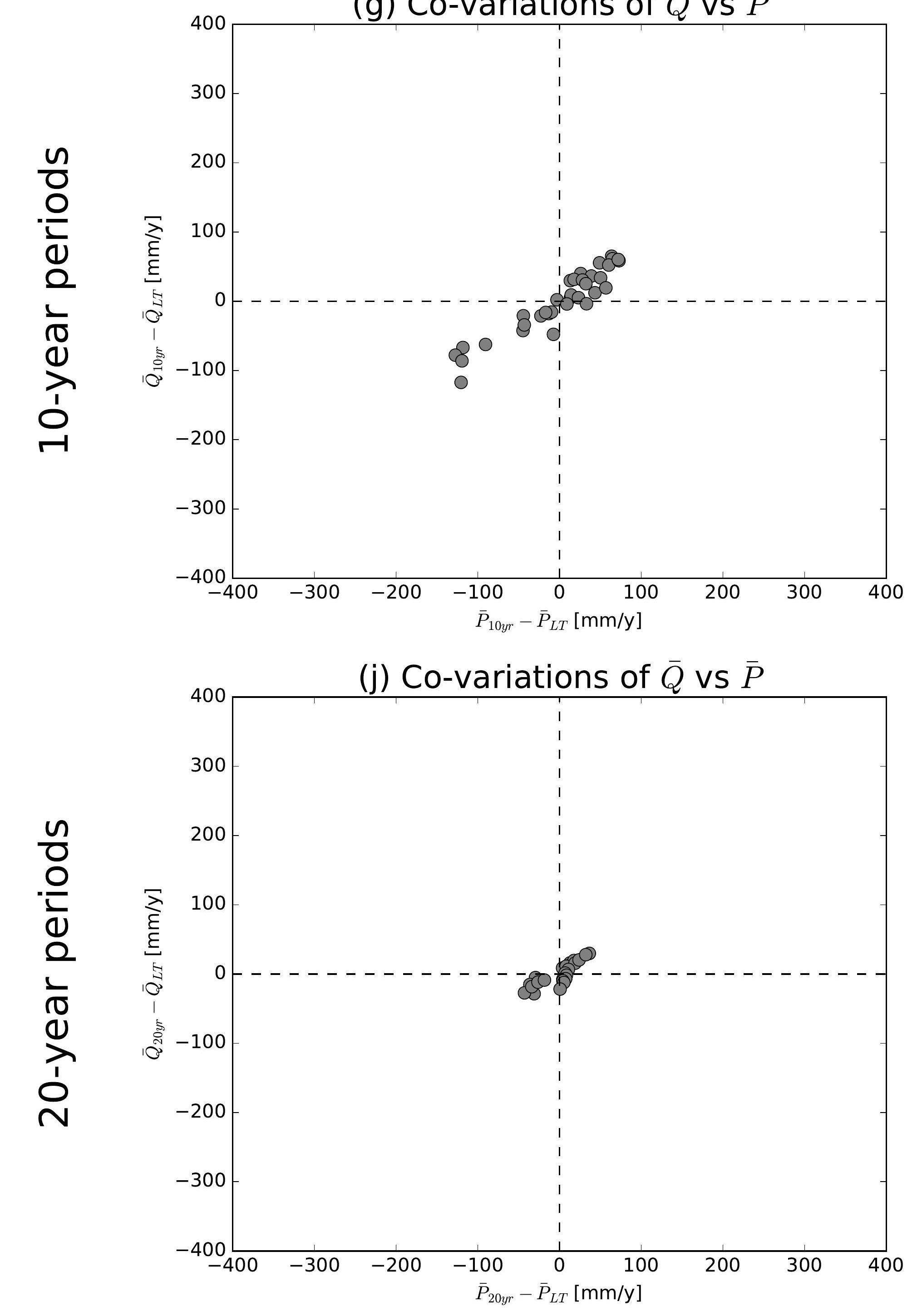

(b) Co-variations of $\bar{Q}$ vs $\overline{E 0}$

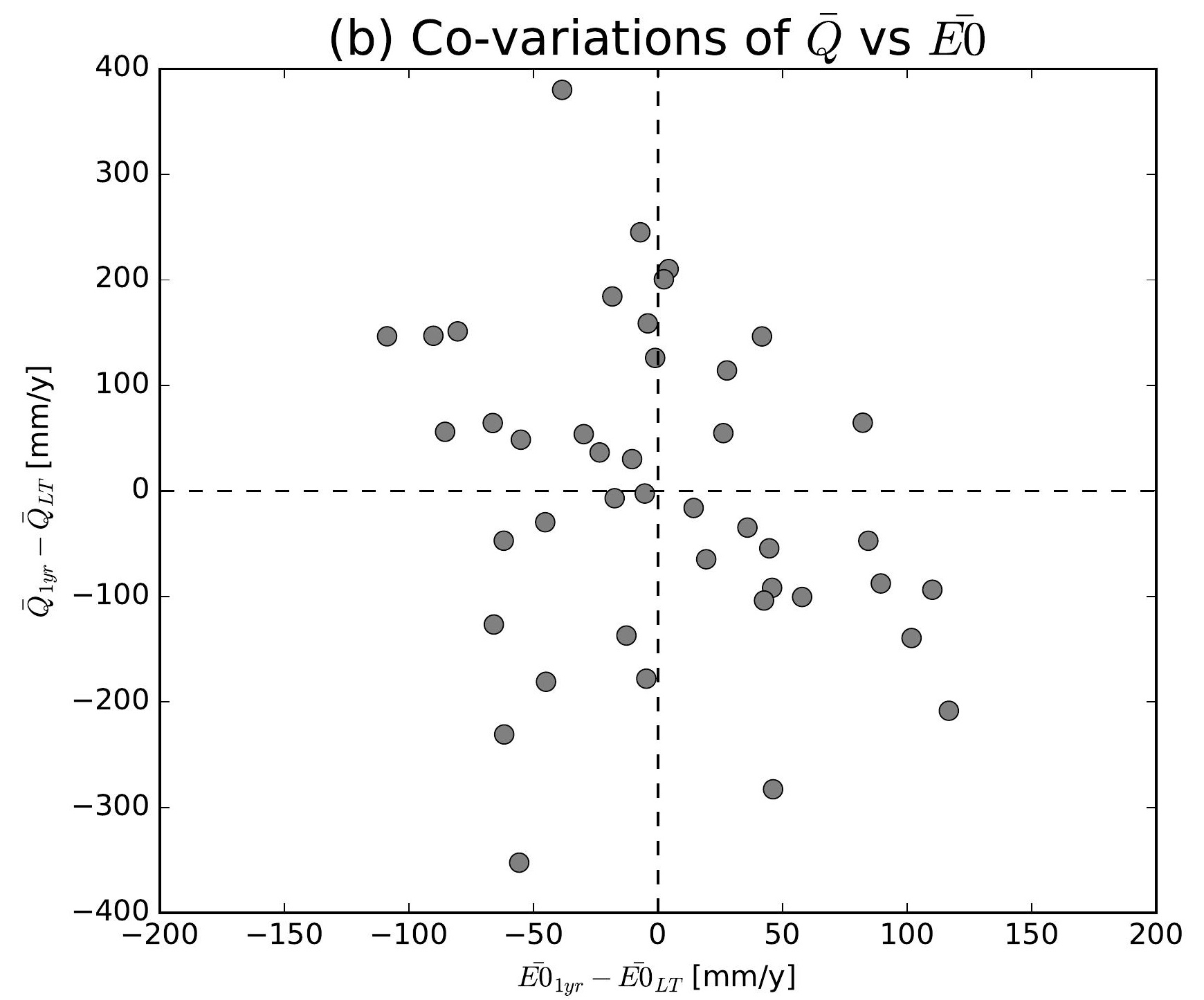

(e) Co-variations of $\bar{Q}$ vs $\overline{E 0}$

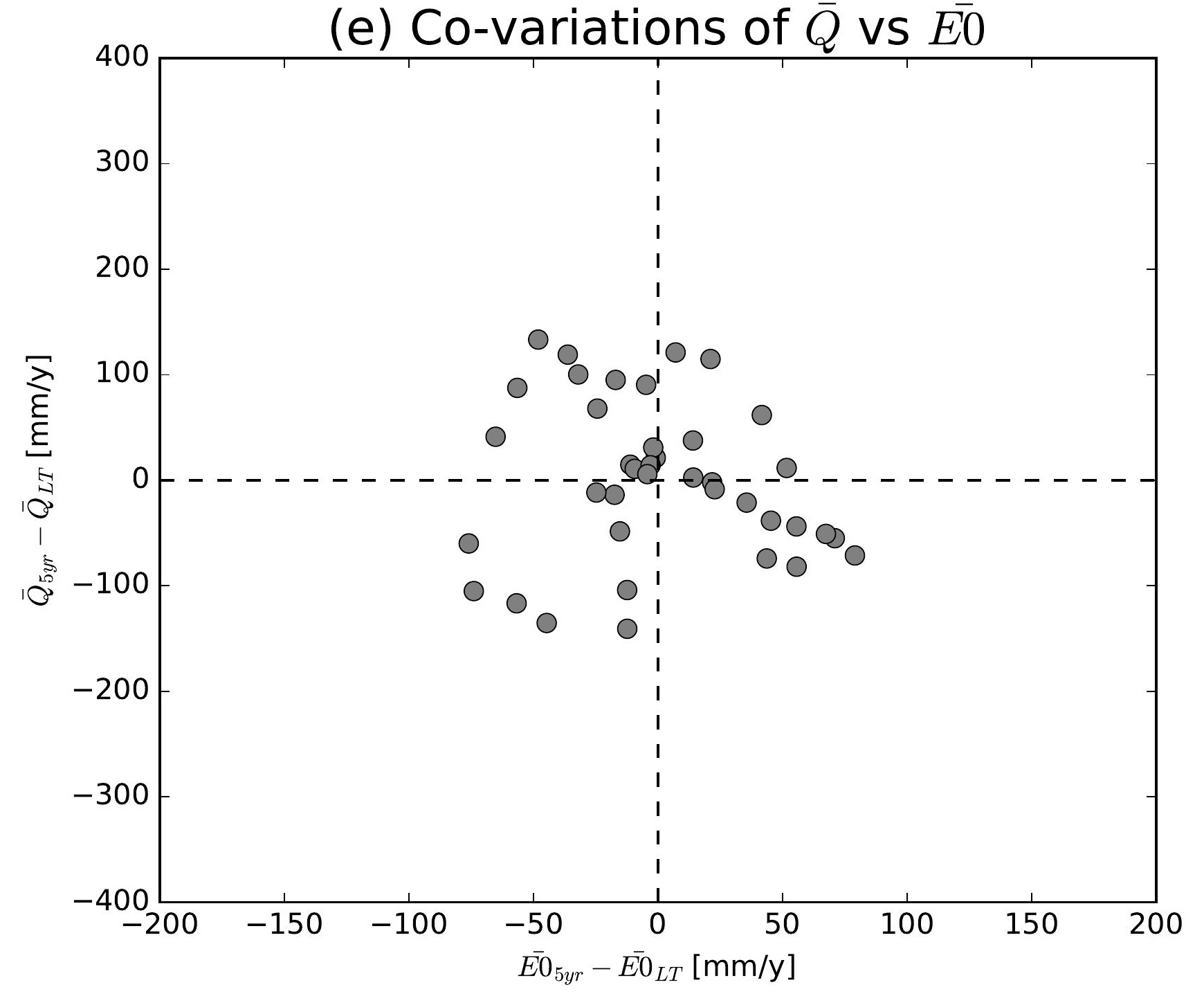

(h) Co-variations of $\bar{Q}$ vs $\overline{E 0}$

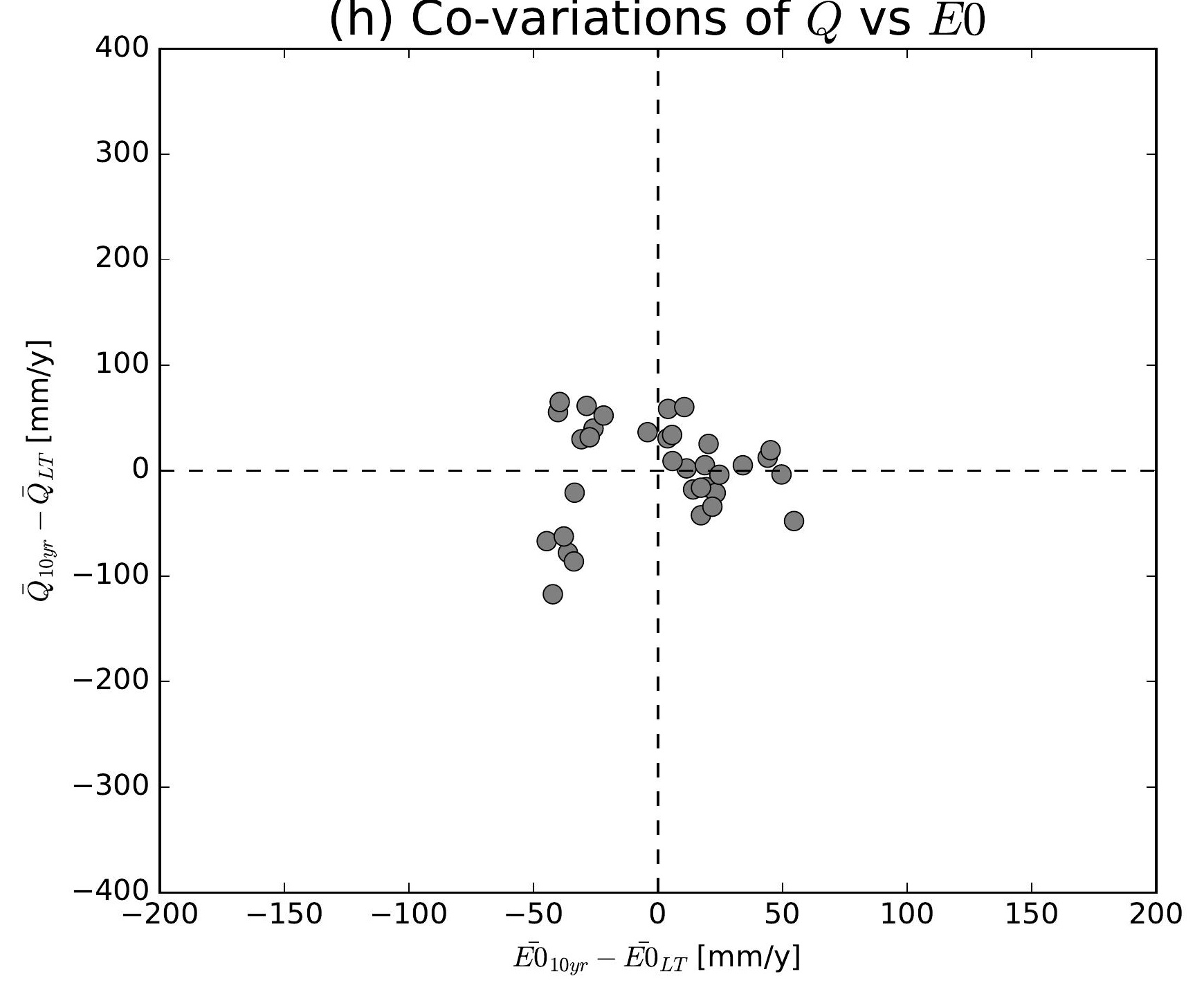

(k) Co-variations of $\bar{Q}$ vs $\overline{E 0}$

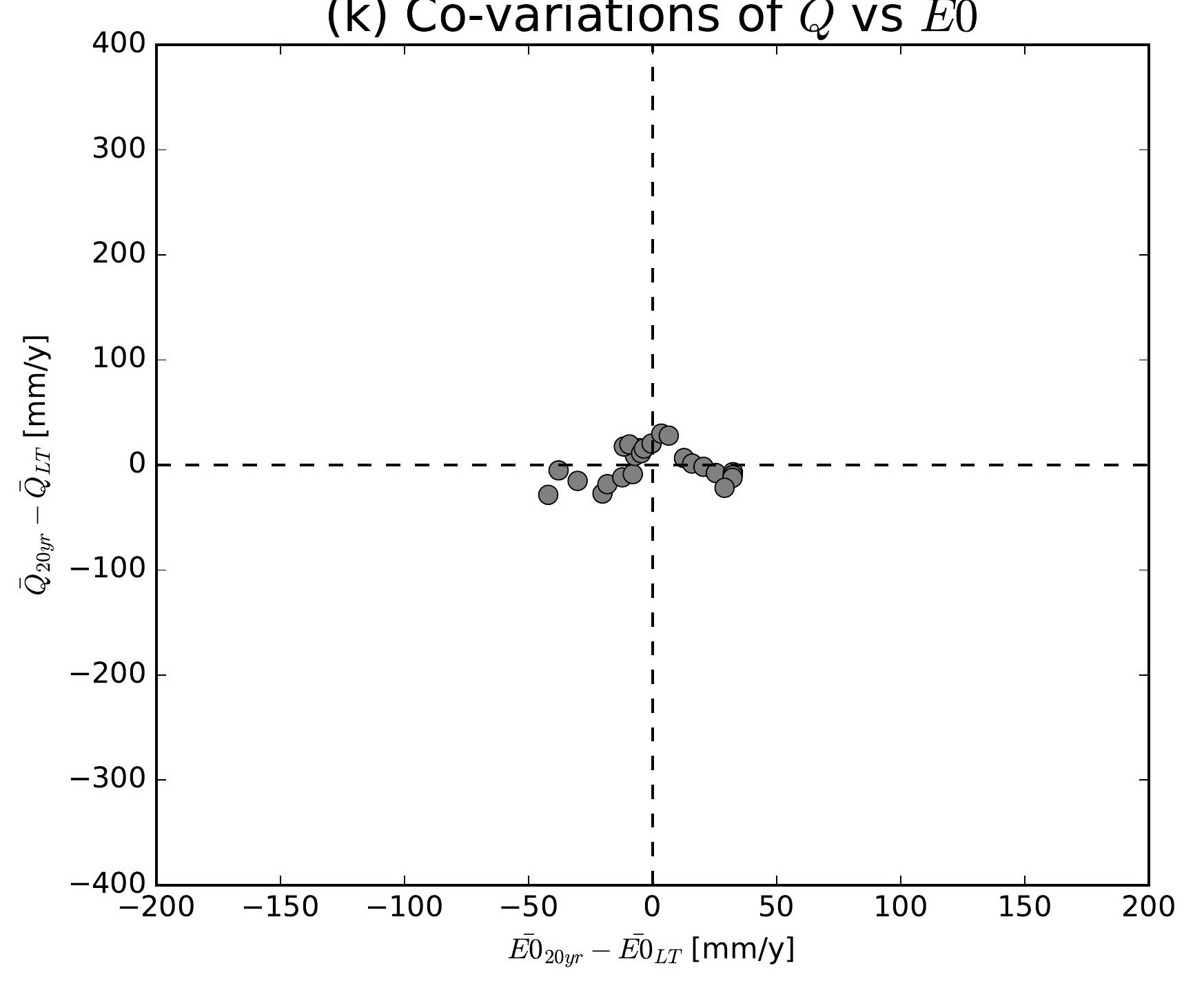

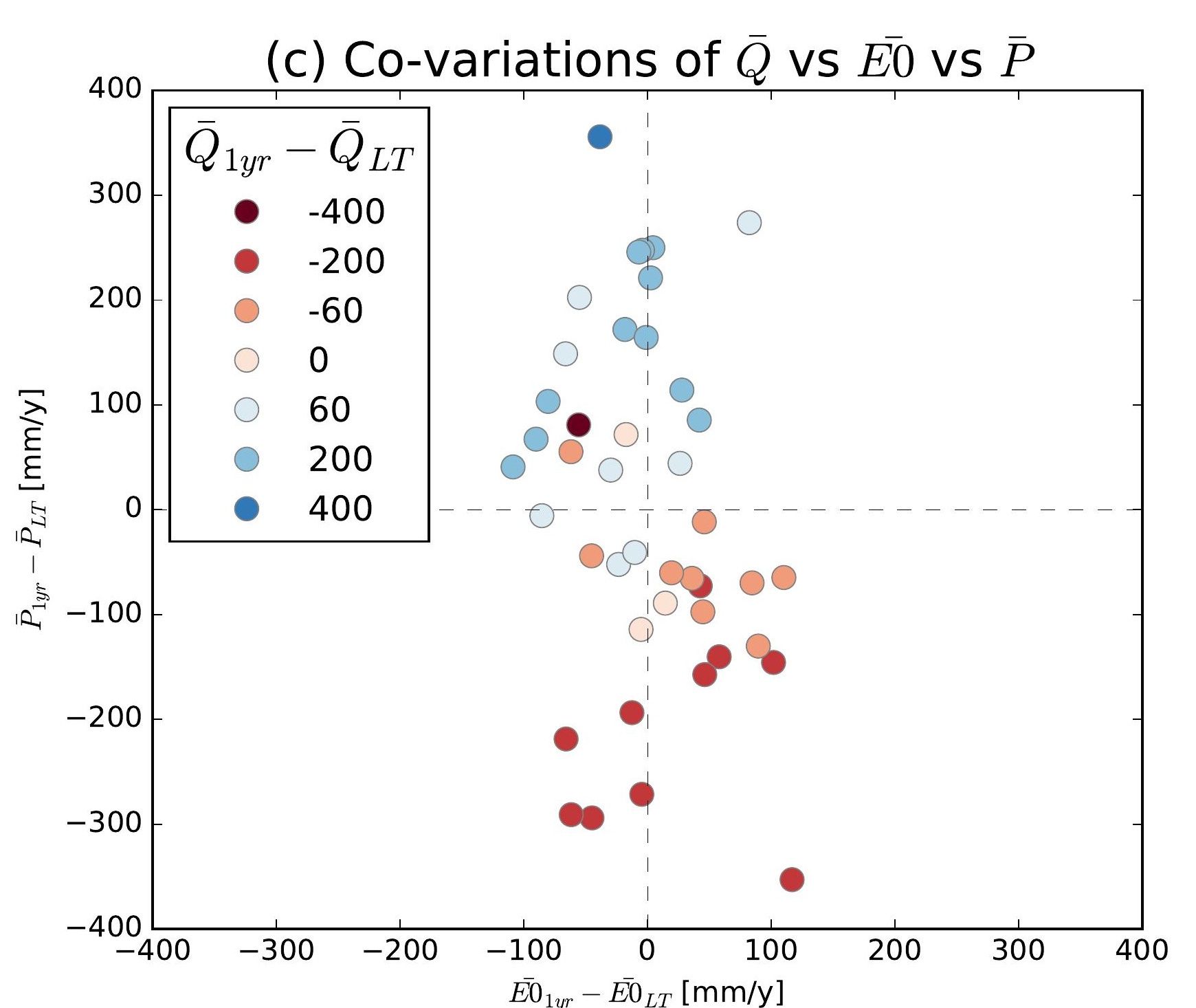
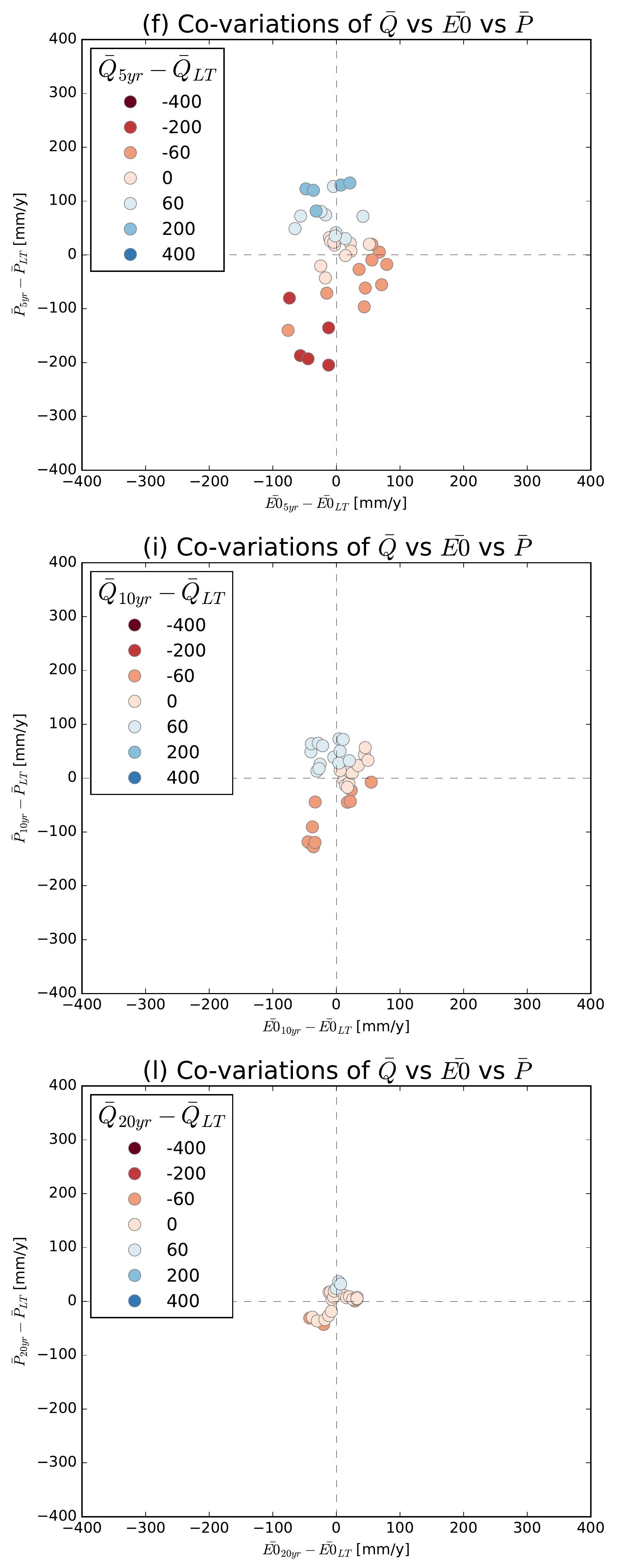

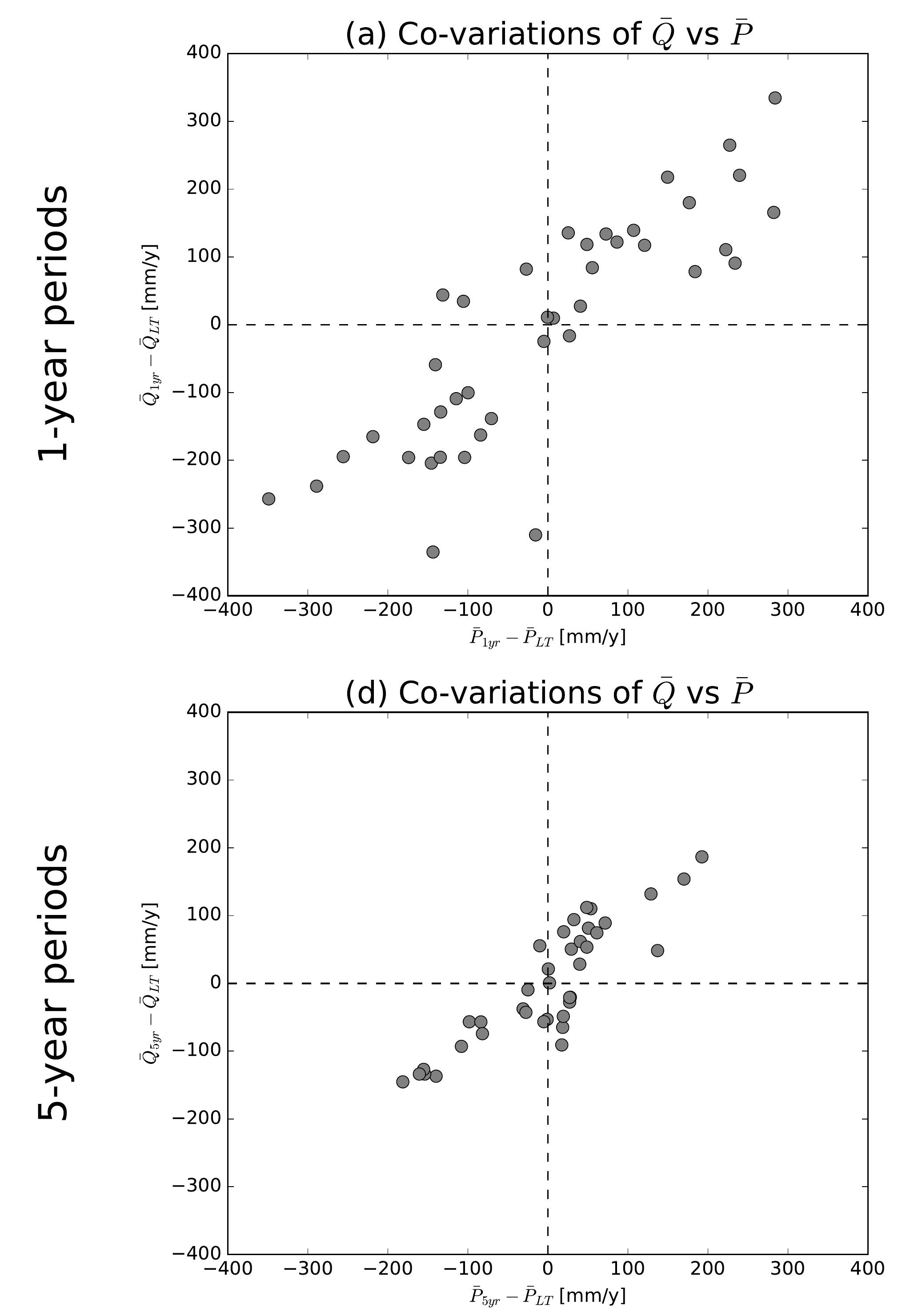

(g) Co-variations of $\bar{Q}$ vs $\bar{P}$

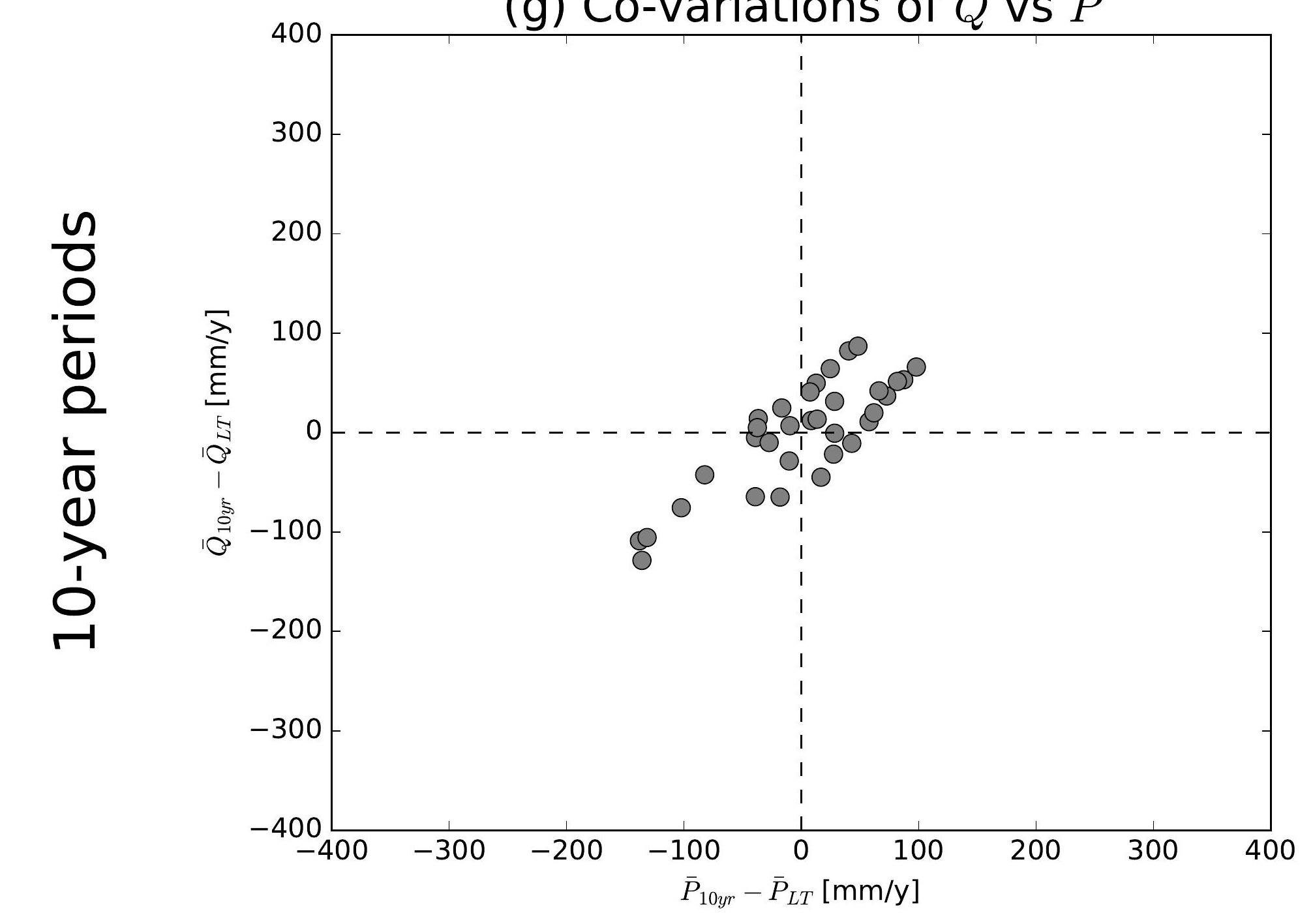

(j) Co-variations of $\bar{Q}$ vs $\bar{P}$

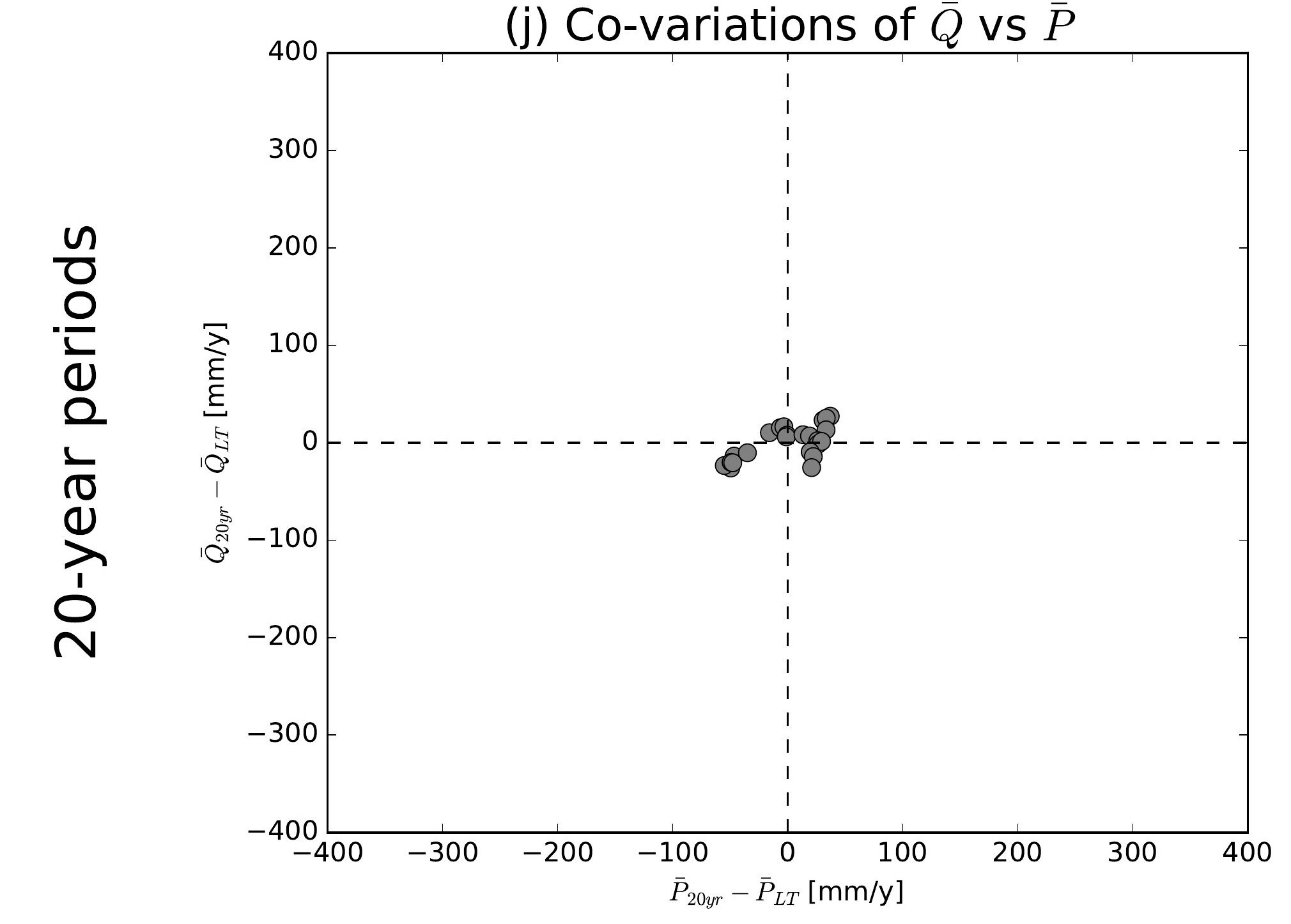

(b) Co-variations of $\bar{Q}$ vs $\overline{E 0}$

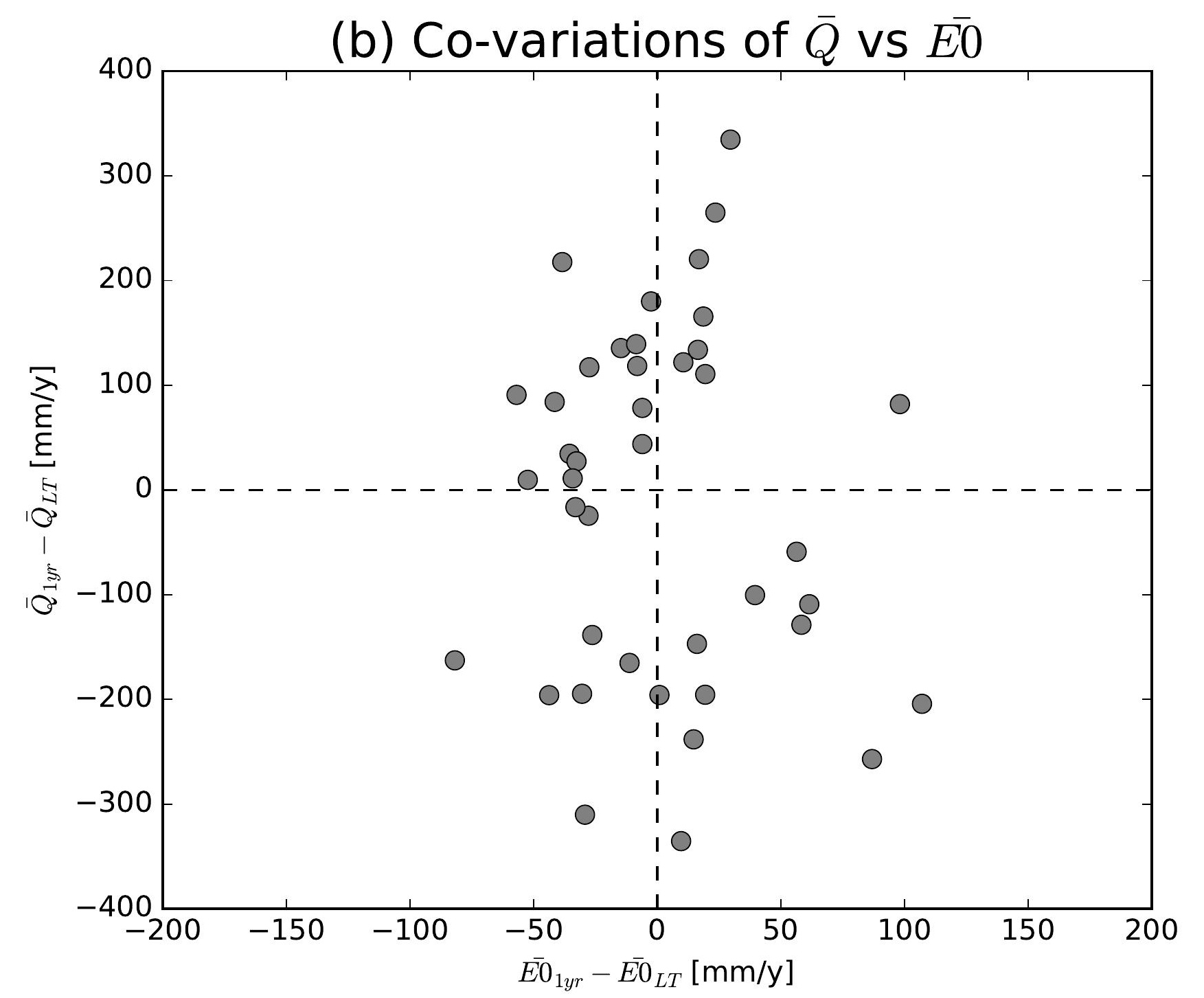

(e) Co-variations of $\bar{Q}$ vs $\overline{E 0}$

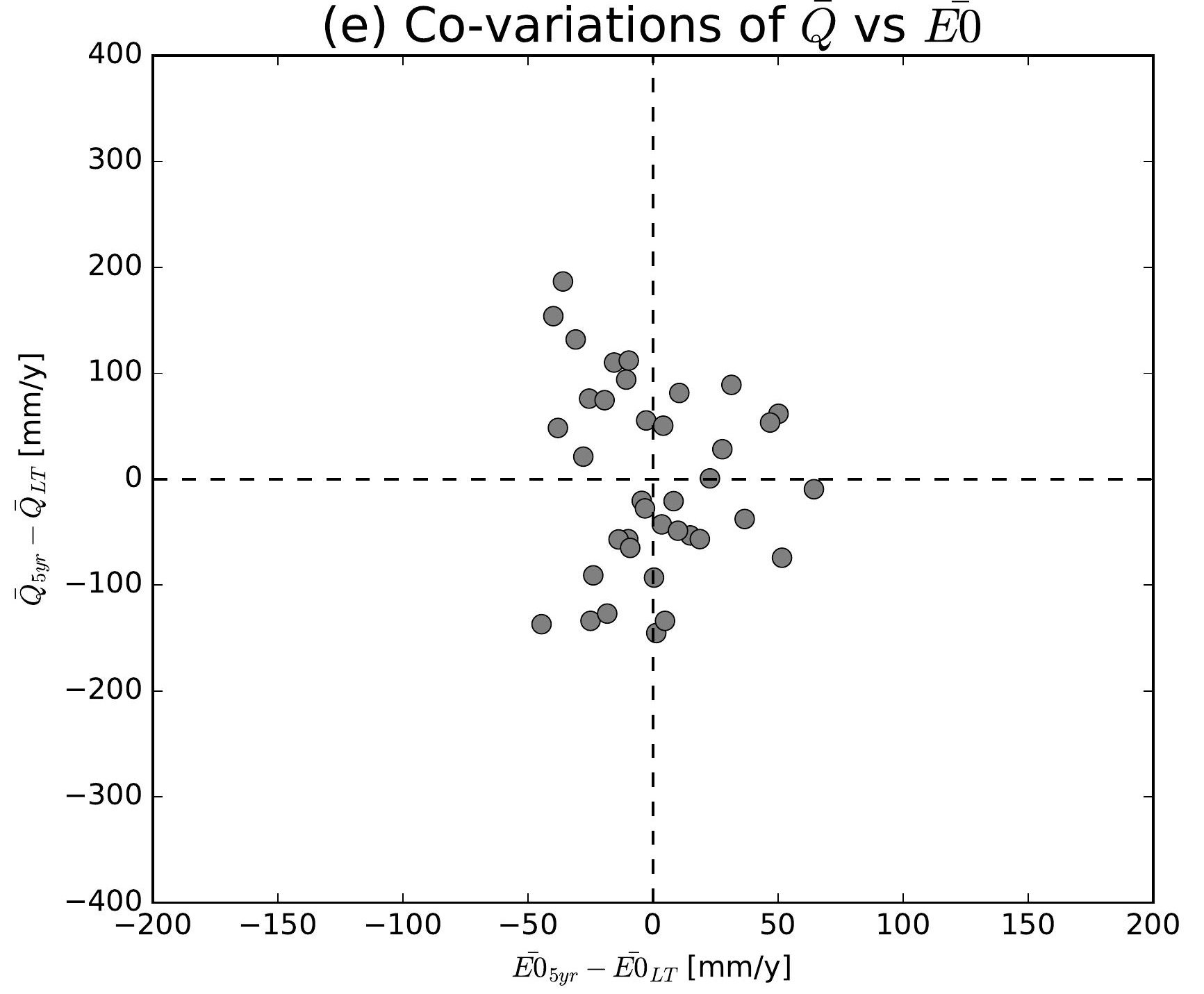

(h) Co-variations of $\bar{Q}$ vs $\overline{E 0}$

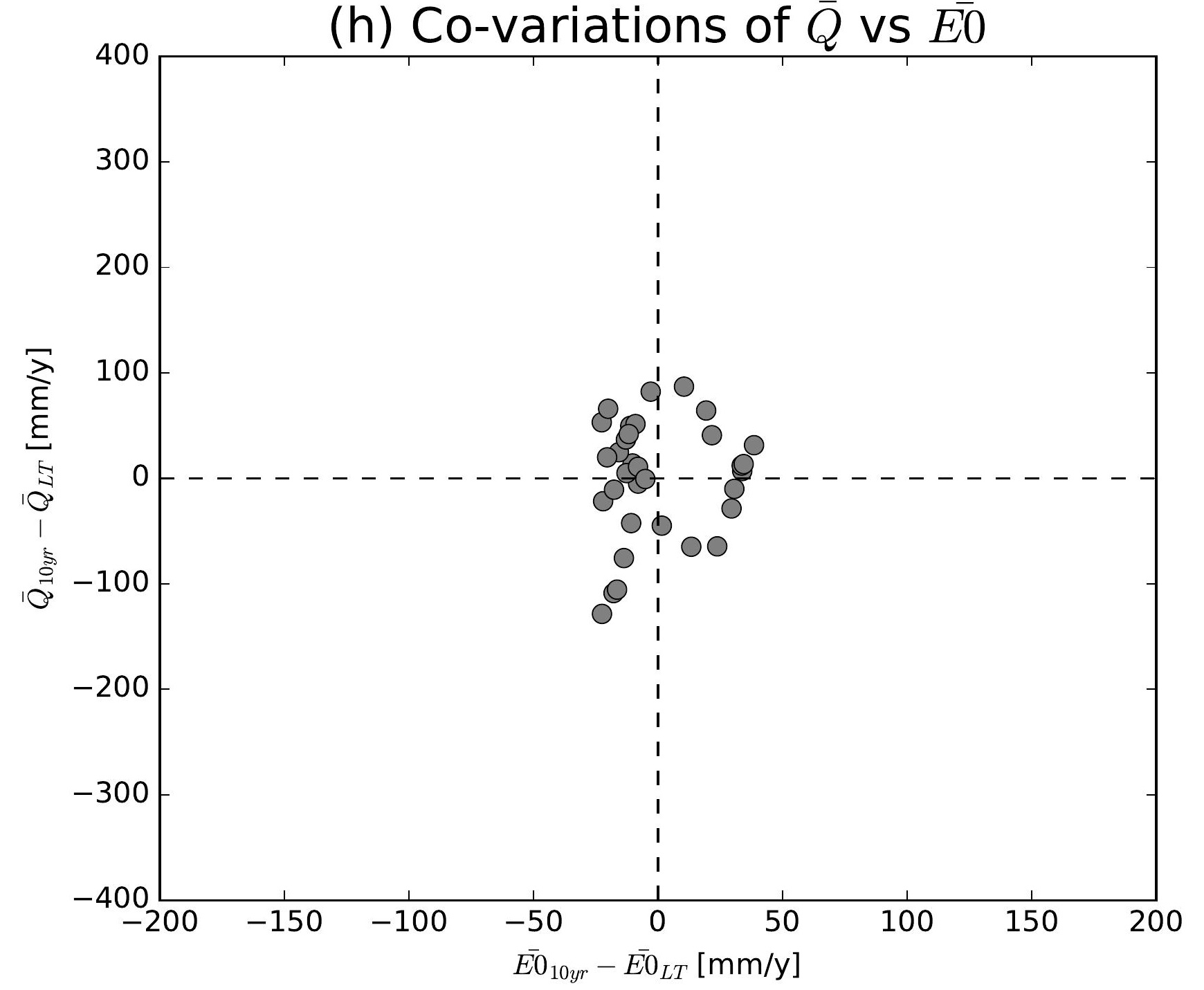

(k) Co-variations of $\bar{Q}$ vs $\overline{E 0}$

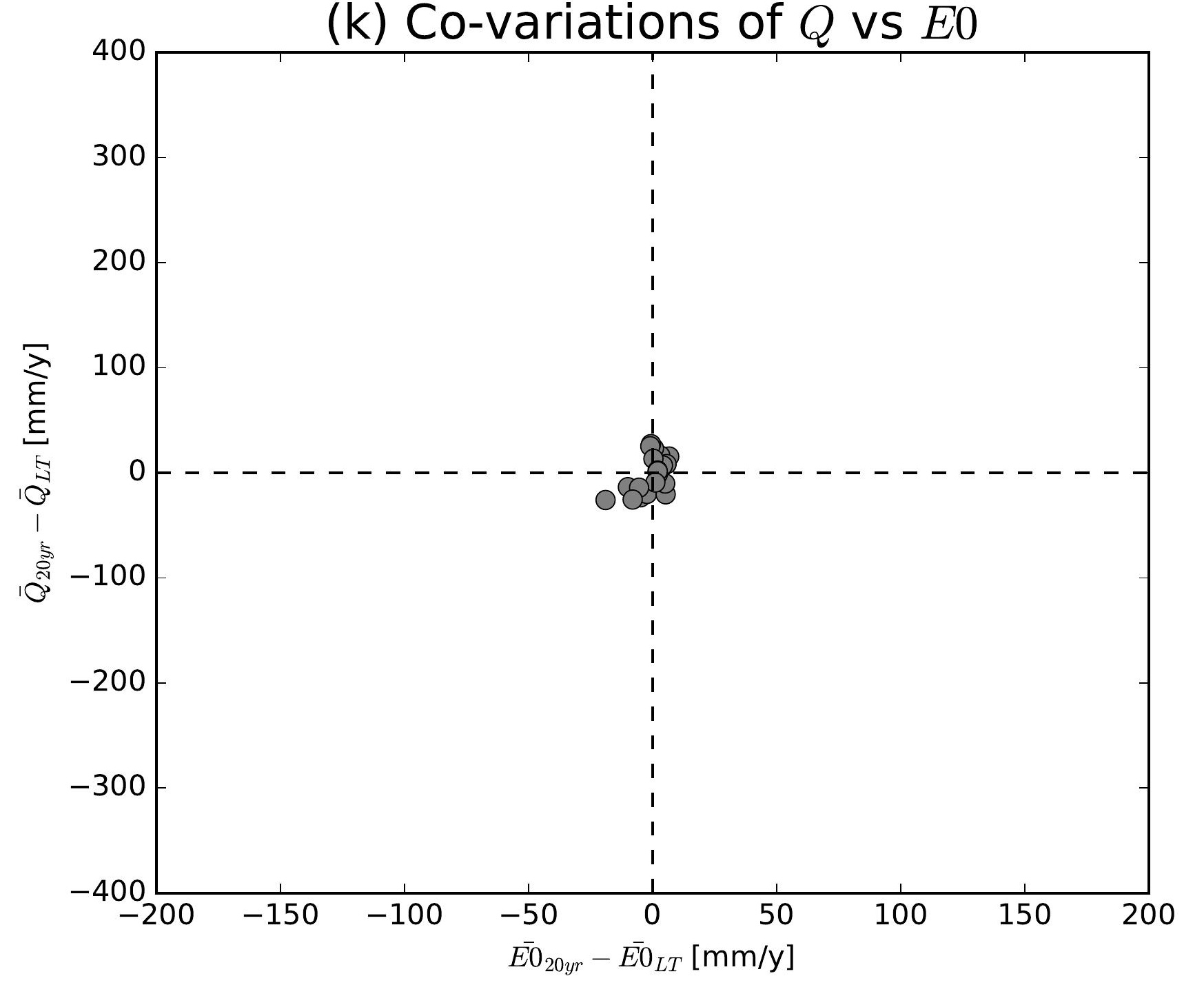

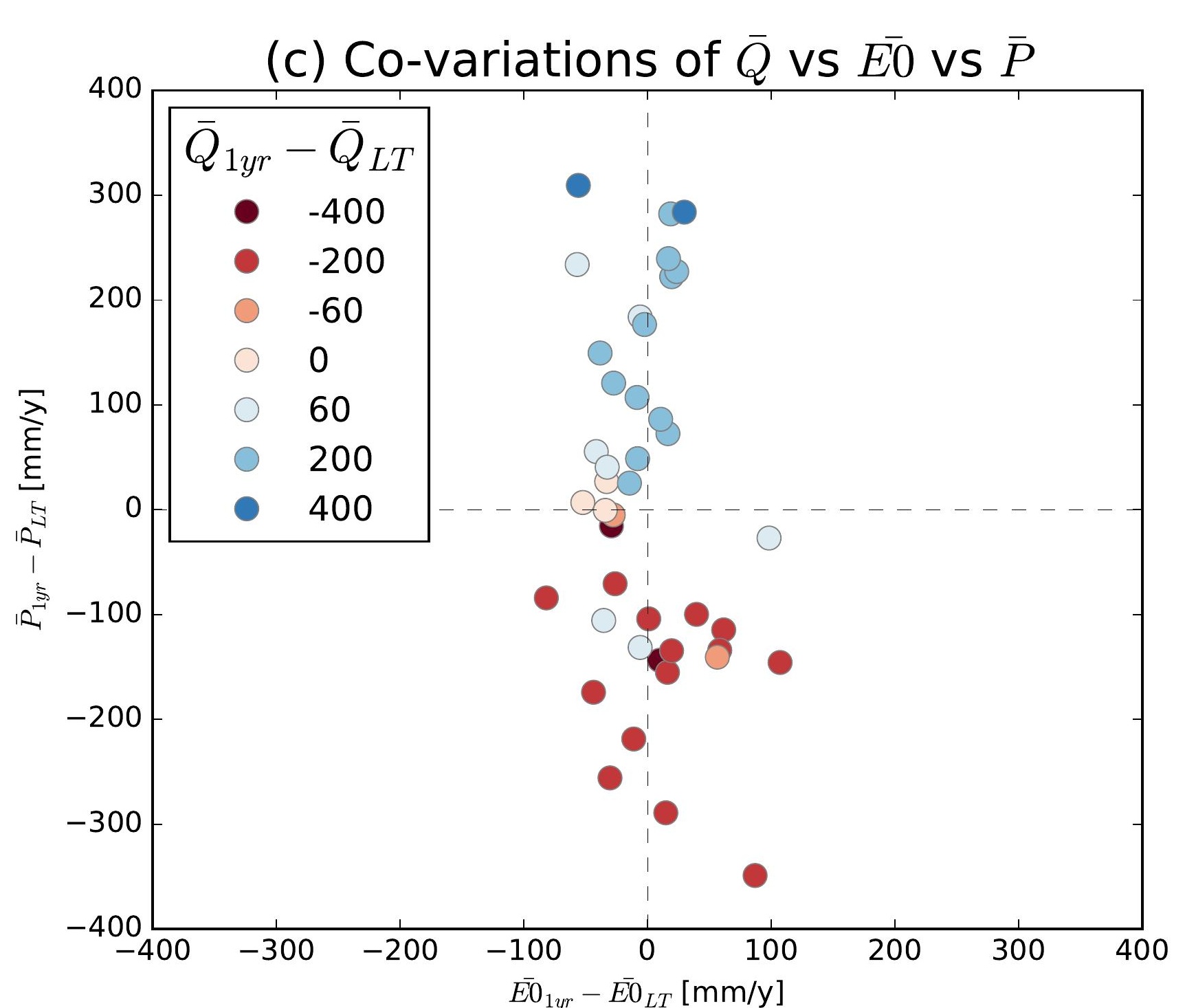
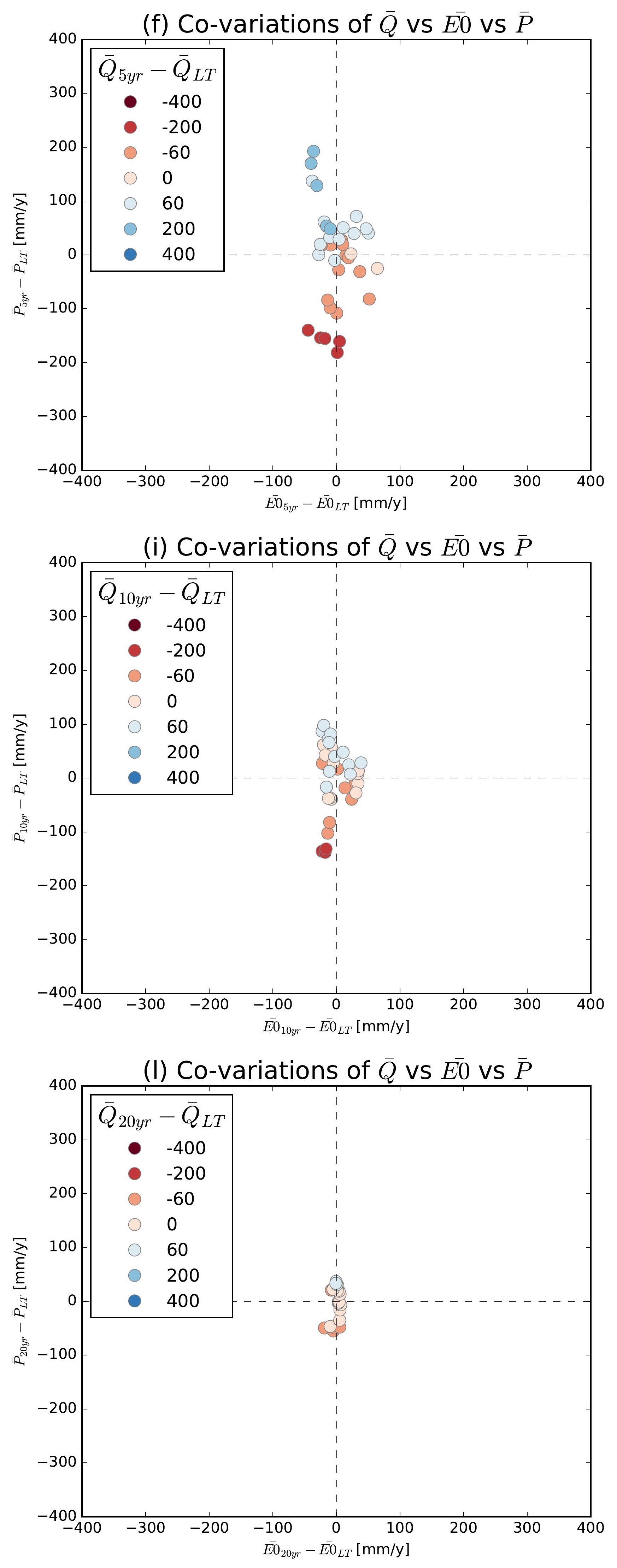

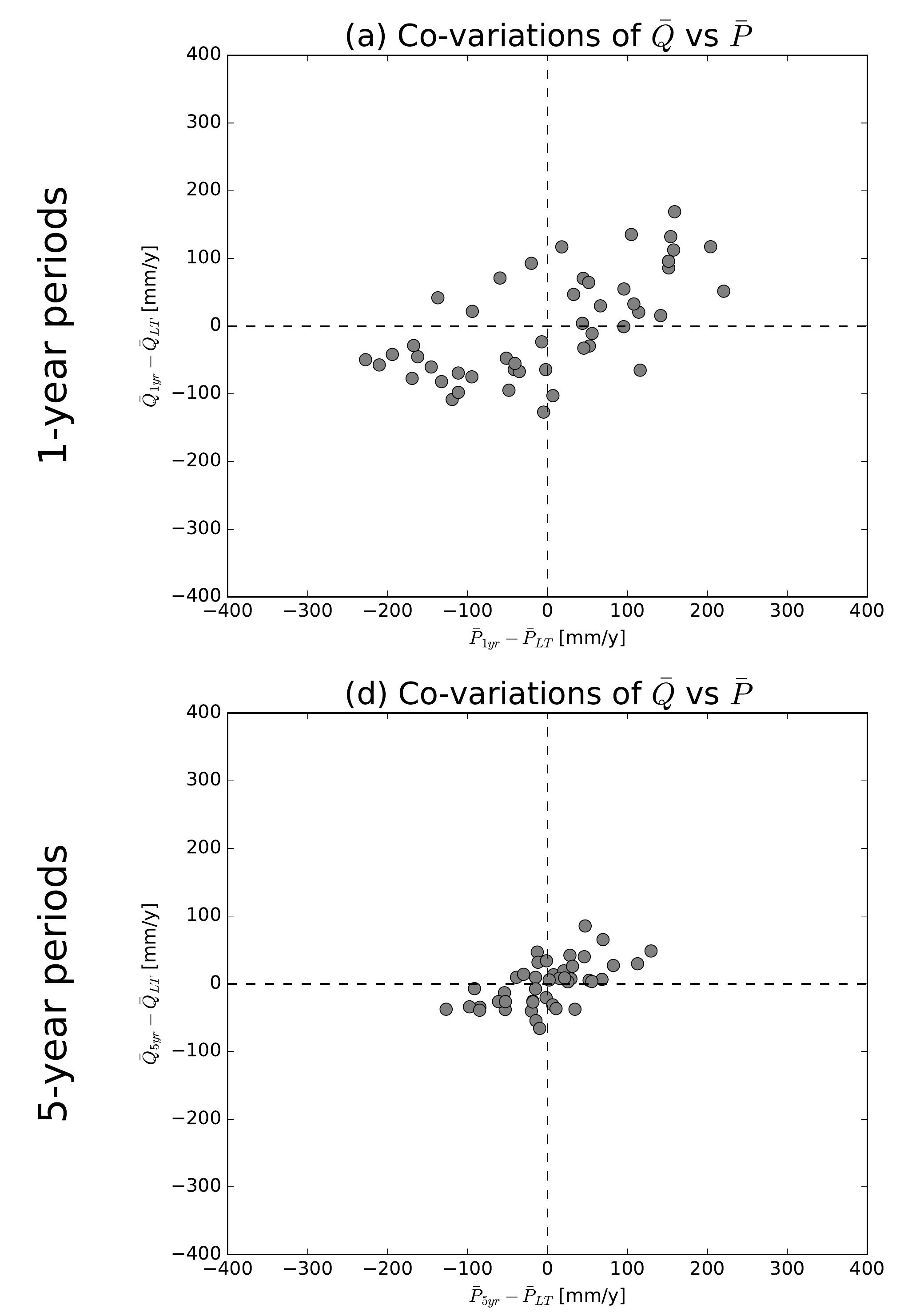

(g) Co-variations of $\bar{Q}$ vs $\bar{P}$
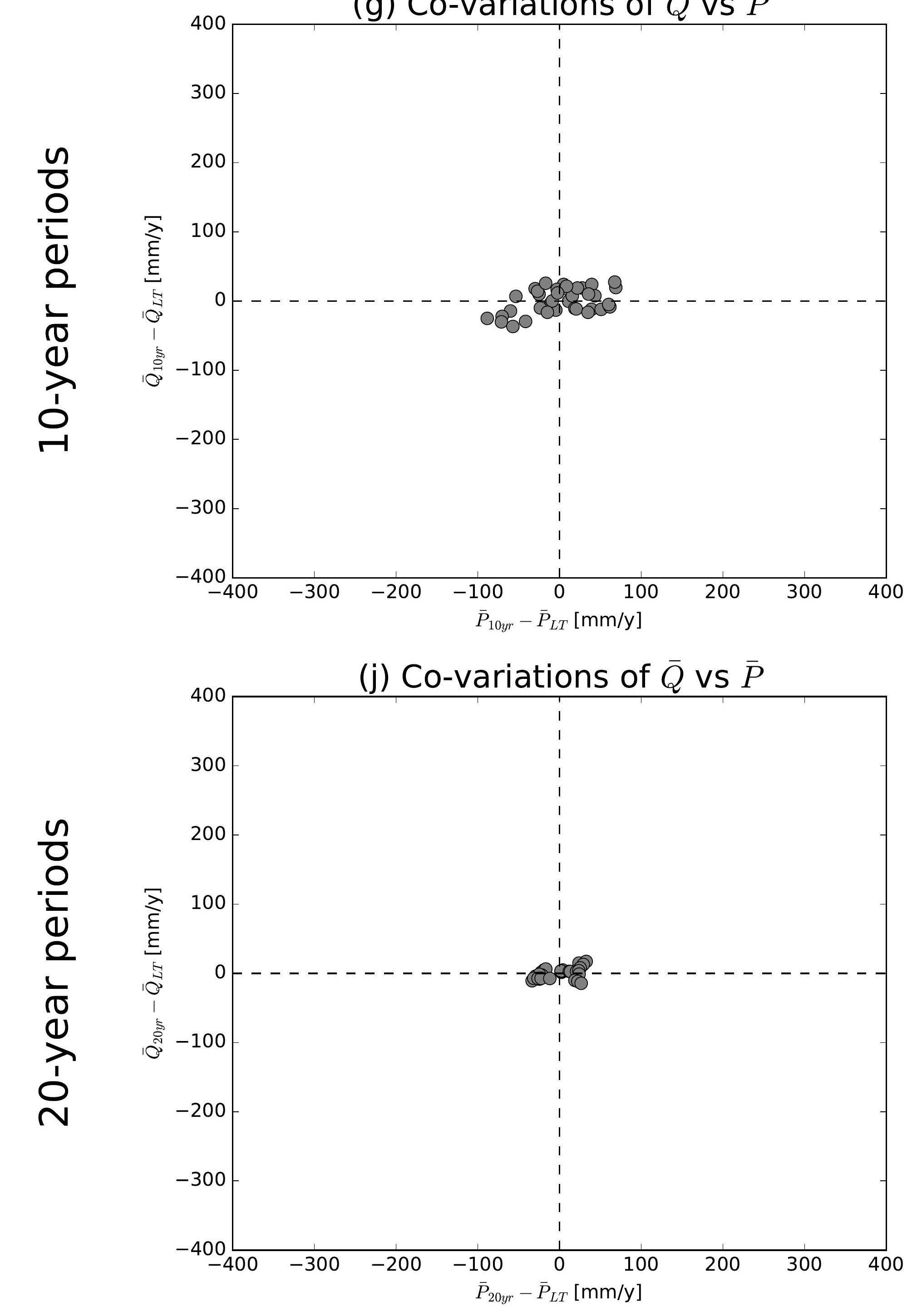

(b) Co-variations of $\bar{Q}$ vs $\overline{E 0}$

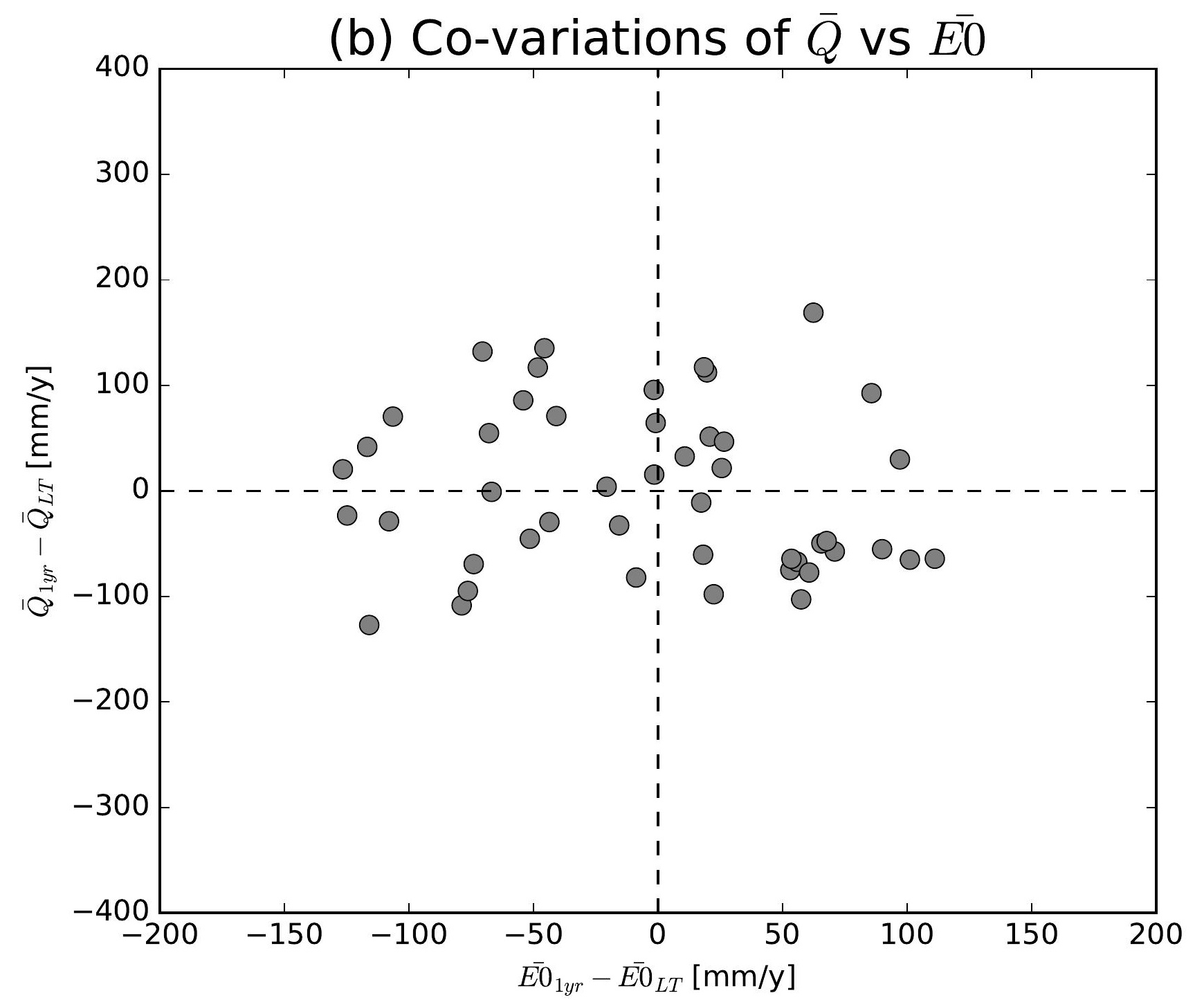

(e) Co-variations of $\bar{Q}$ vs $\overline{E 0}$

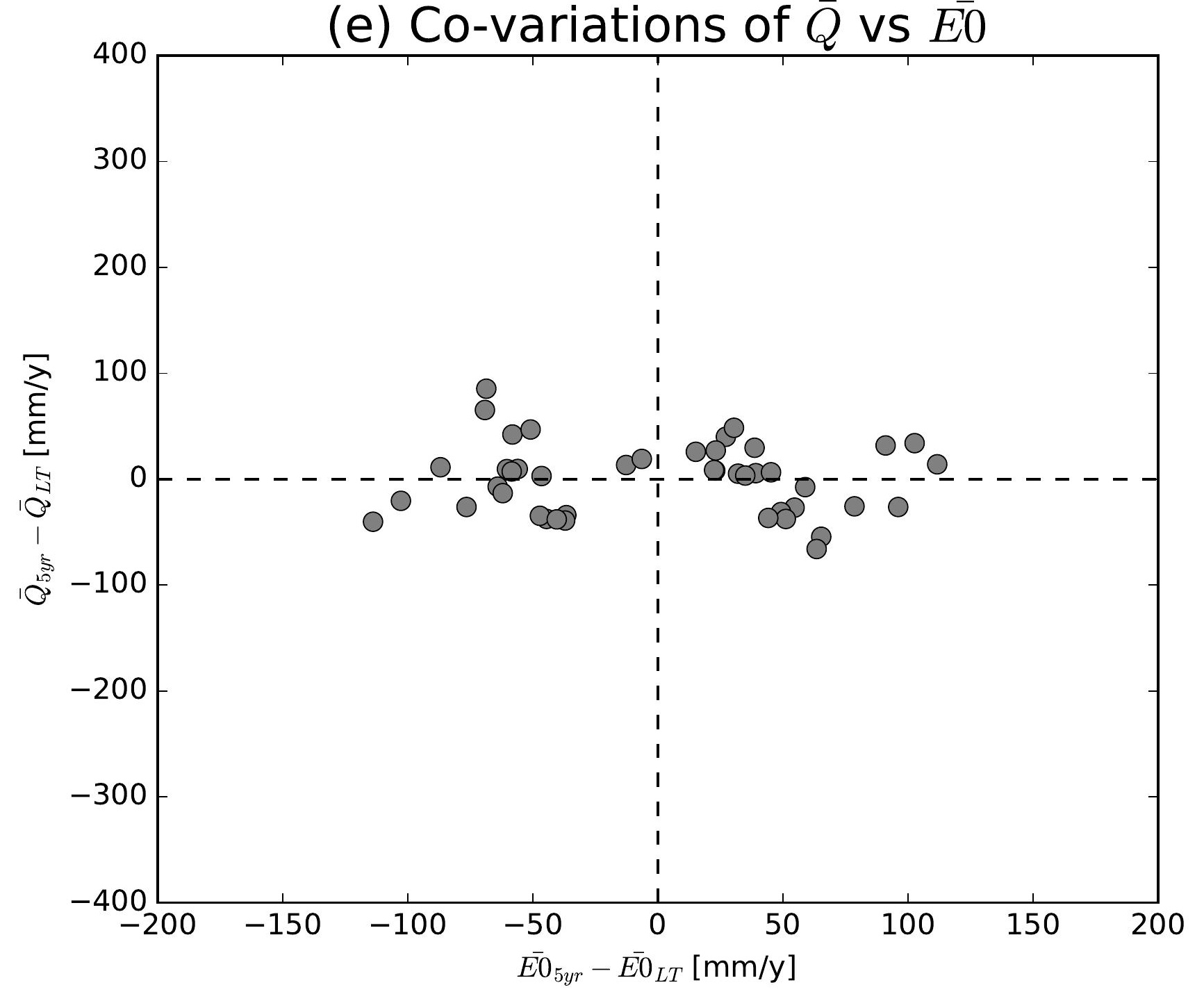

(h) Co-variations of $\bar{Q}$ vs $\overline{E 0}$

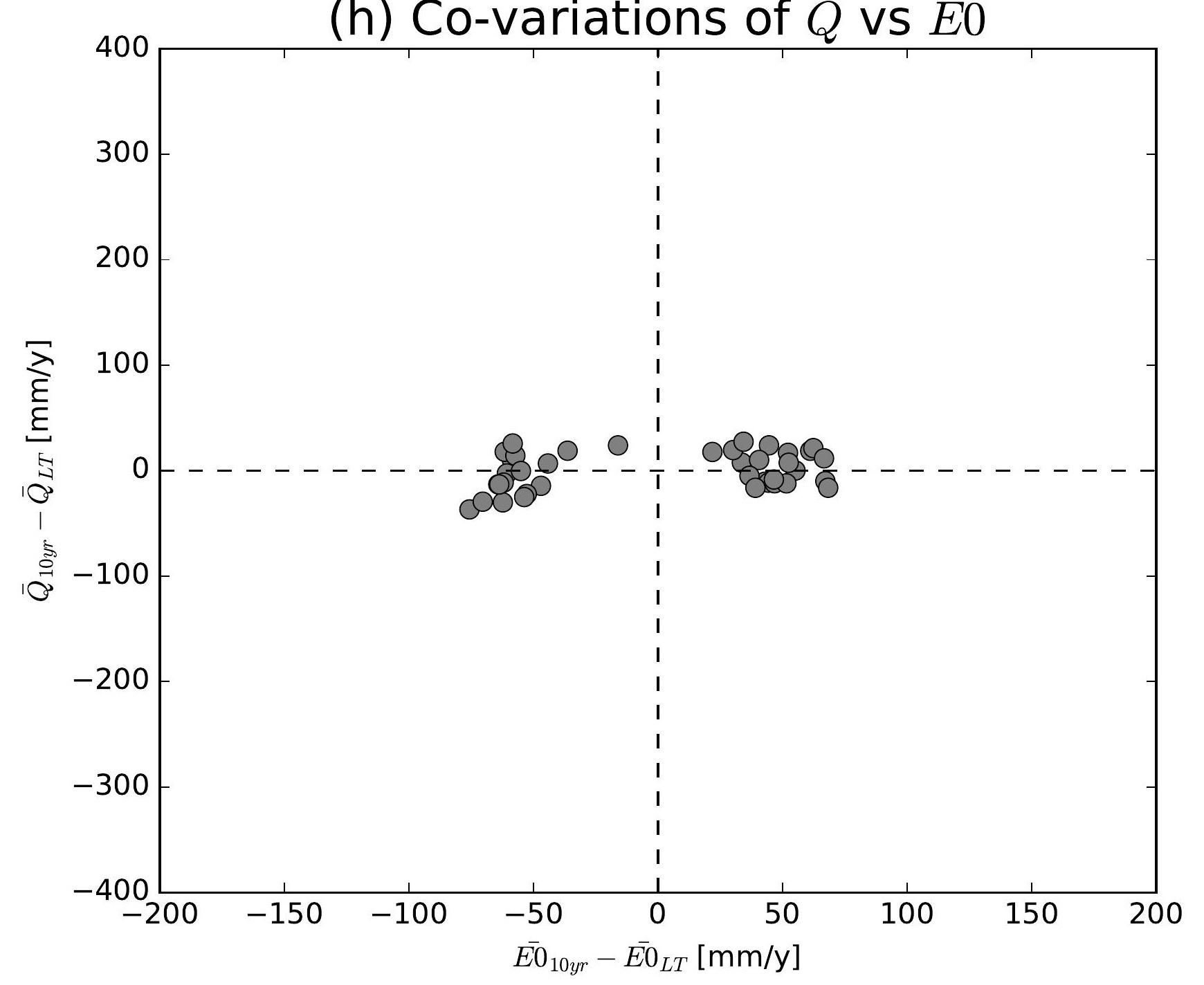

(k) Co-variations of $\bar{Q}$ vs $\overline{E 0}$

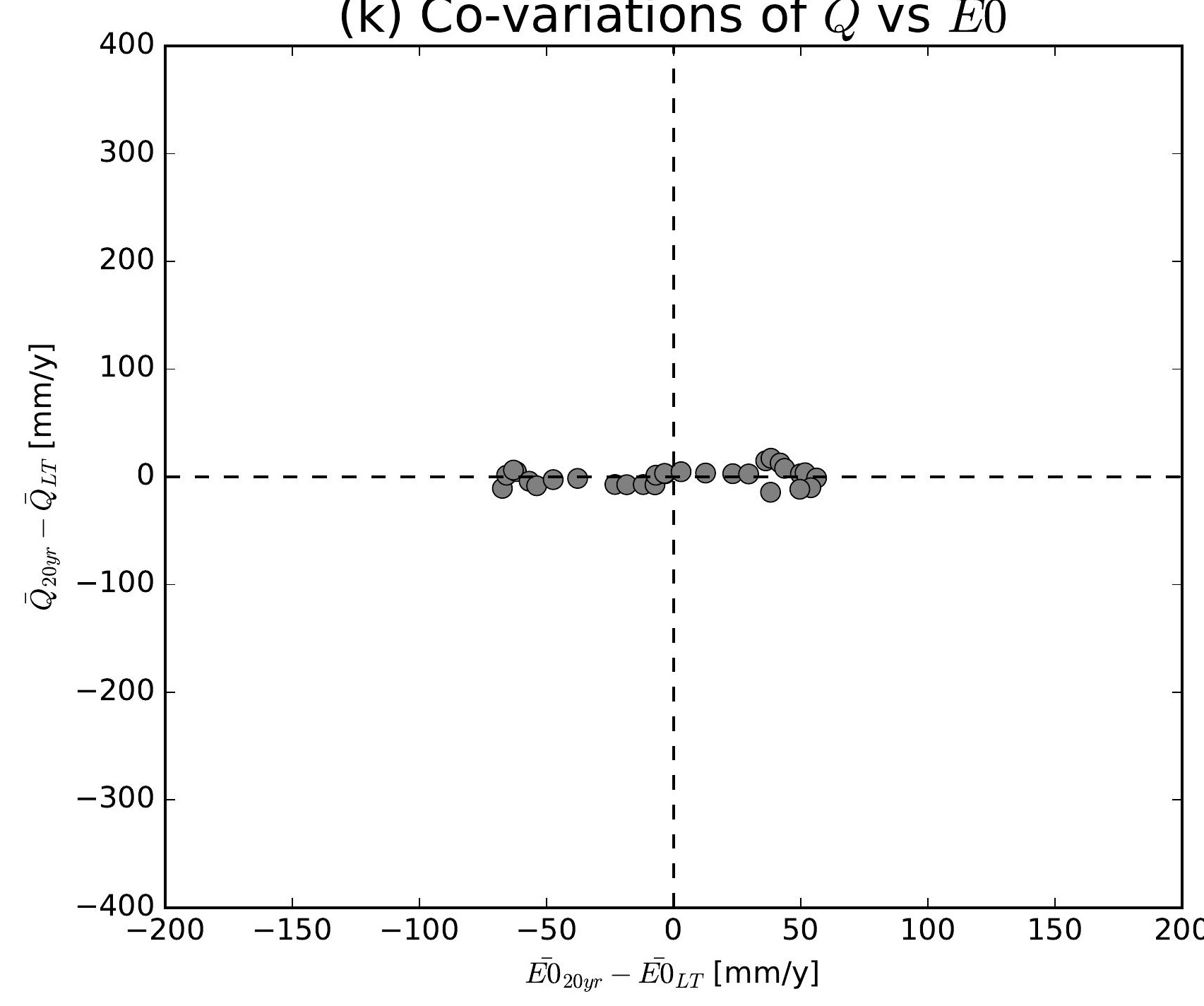

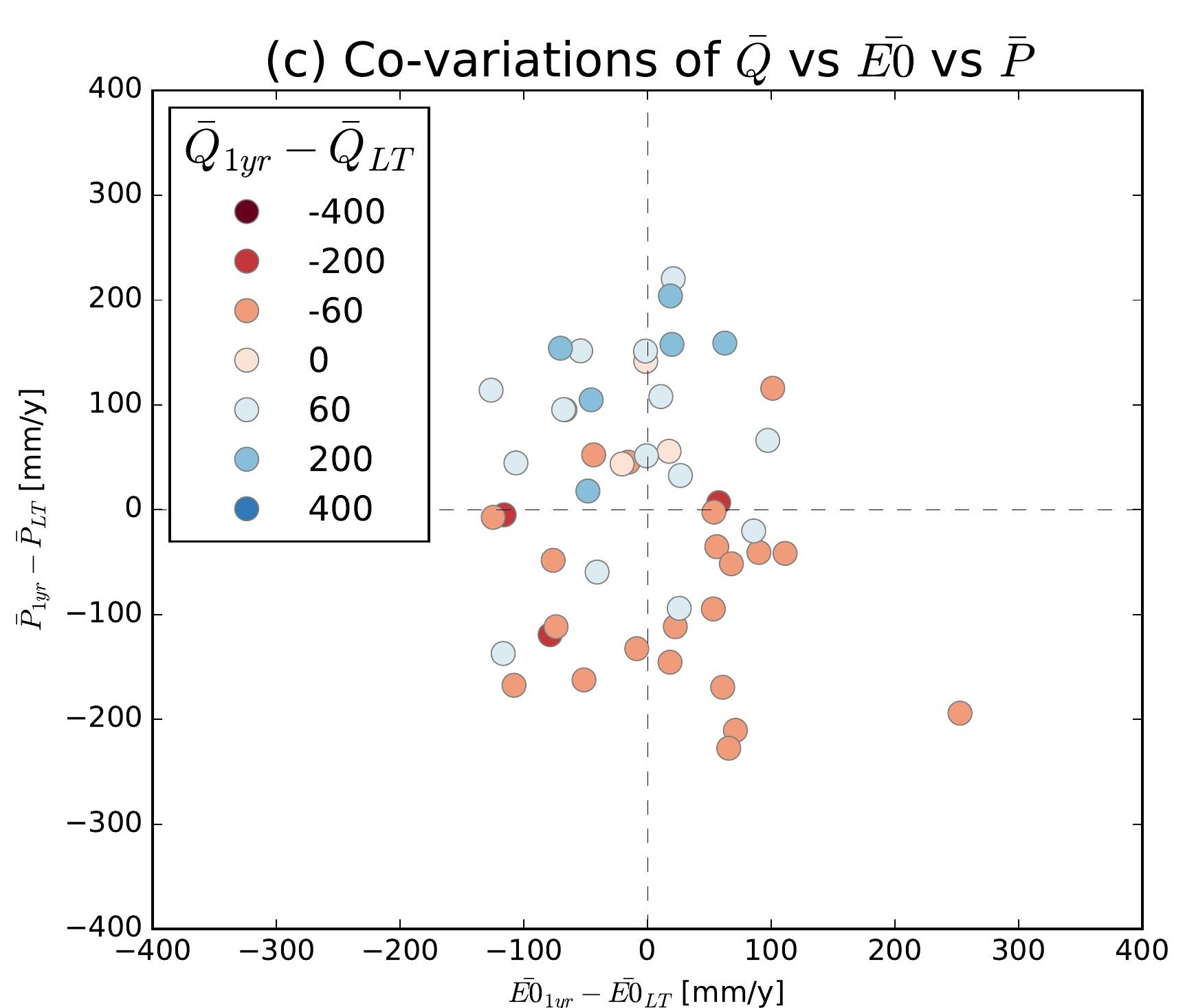
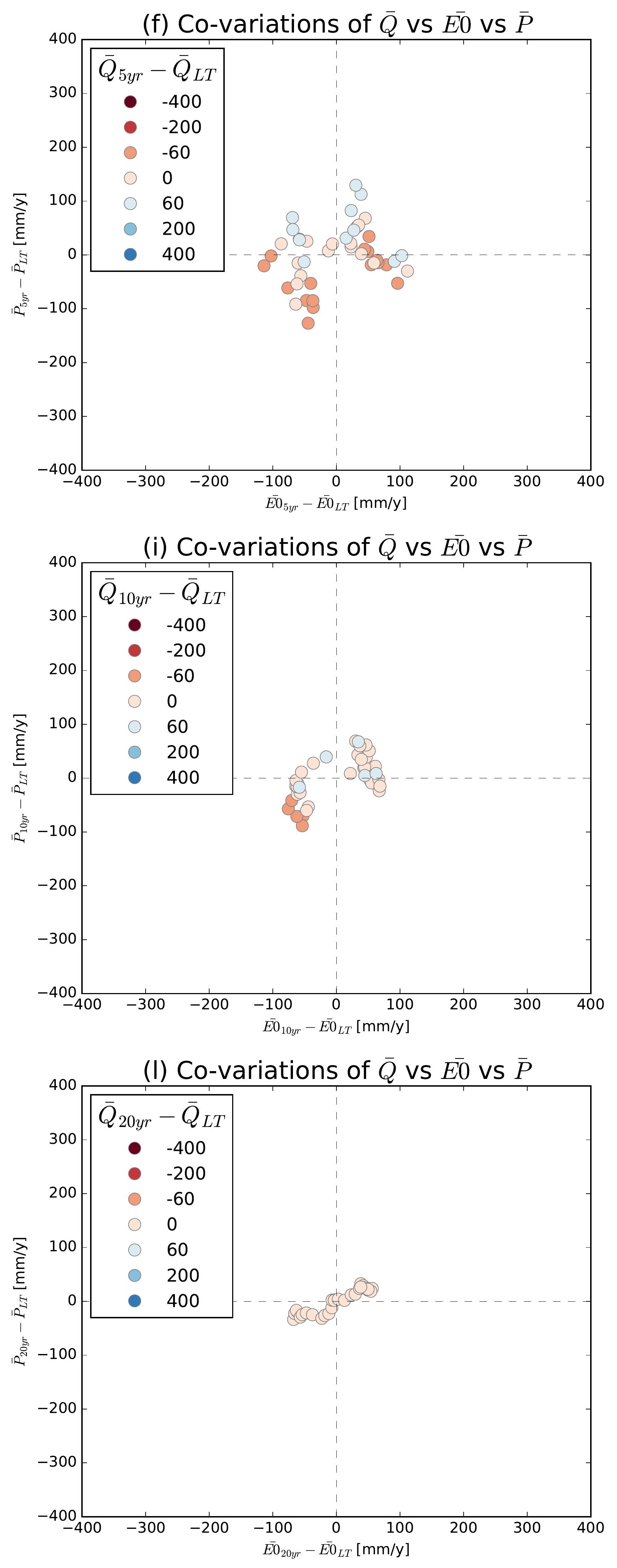

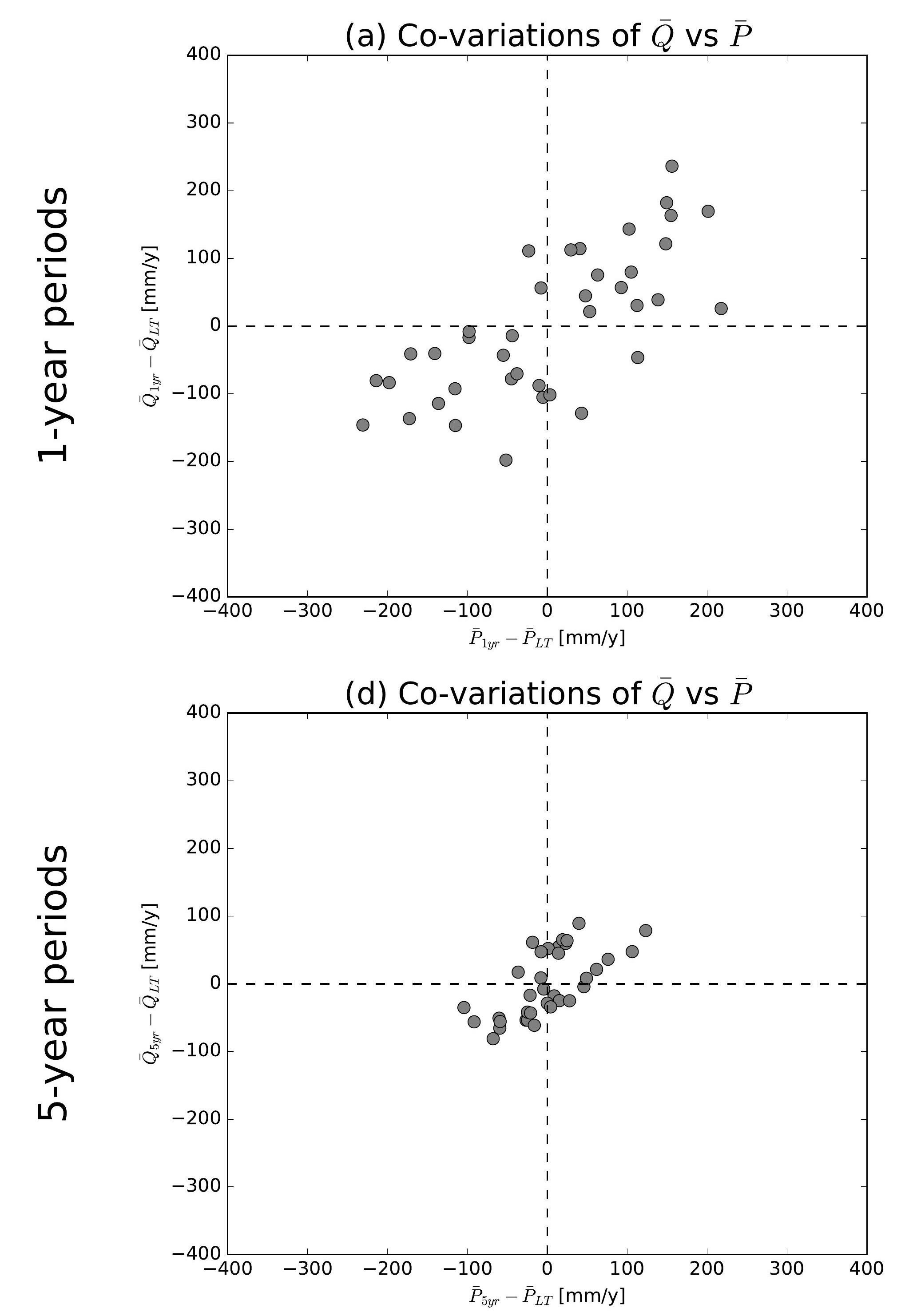

(g) Co-variations of $\bar{Q}$ vs $\bar{P}$
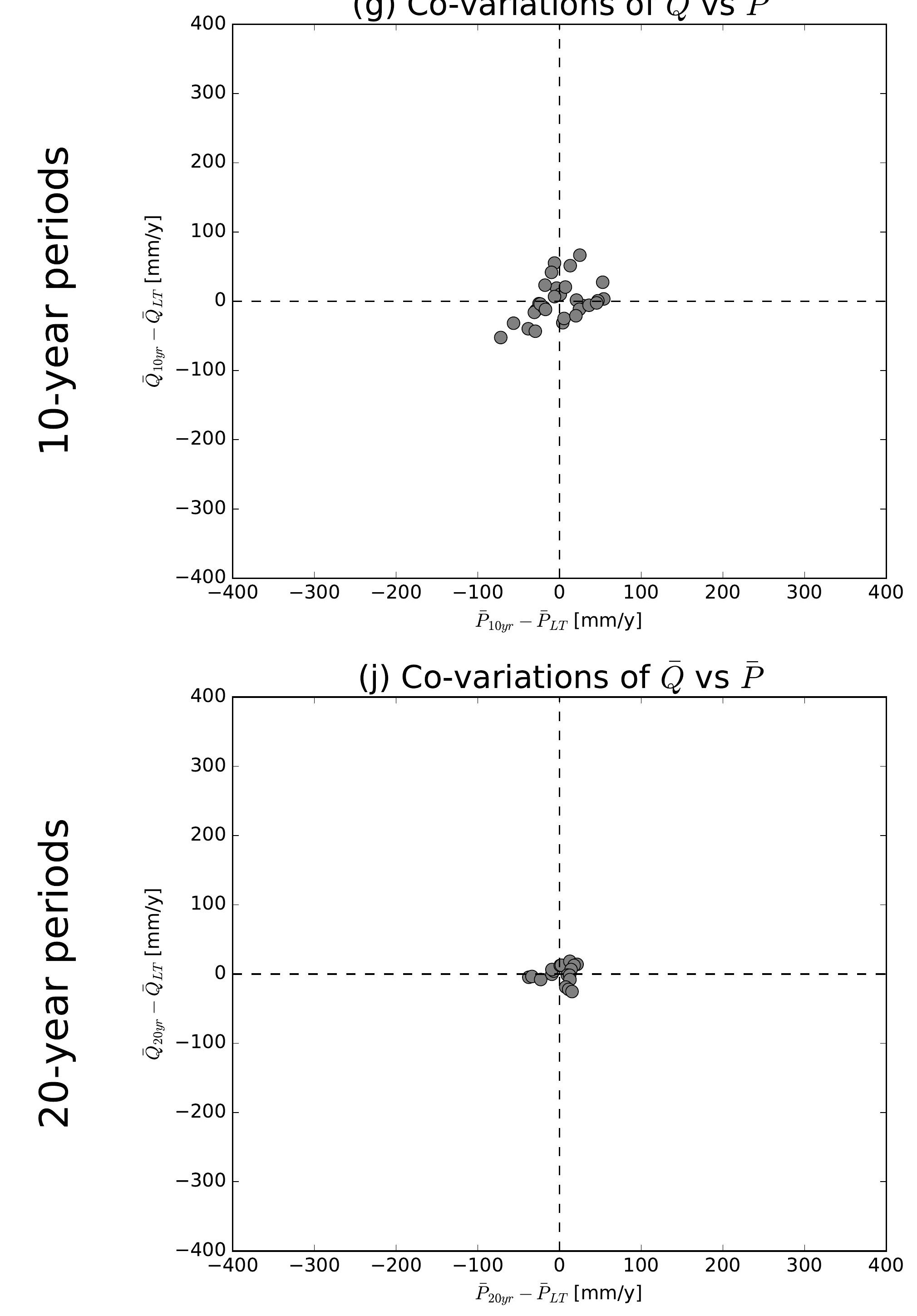

(b) Co-variations of $\bar{Q}$ vs $\overline{E 0}$

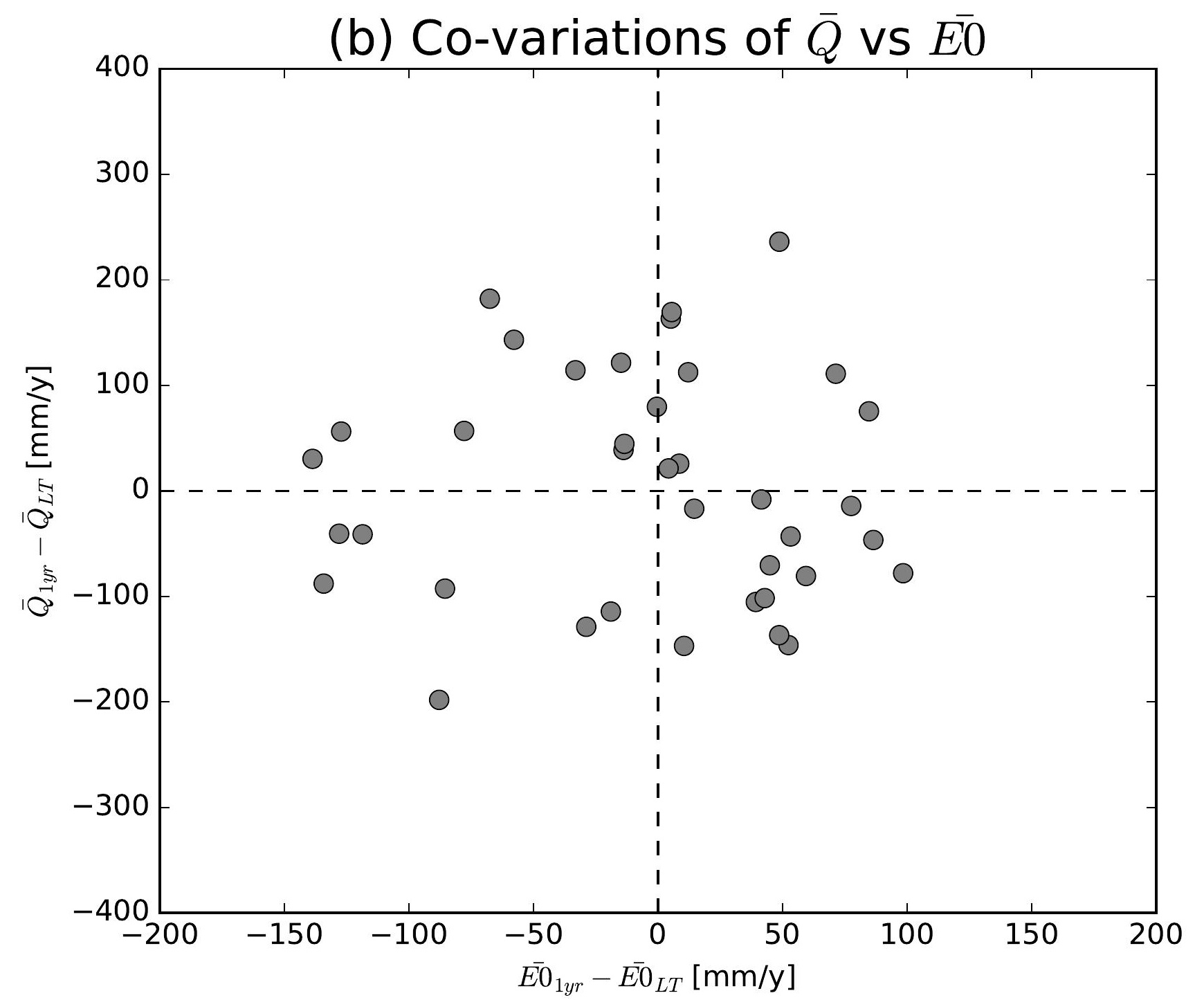

(e) Co-variations of $\bar{Q}$ vs $\overline{E 0}$

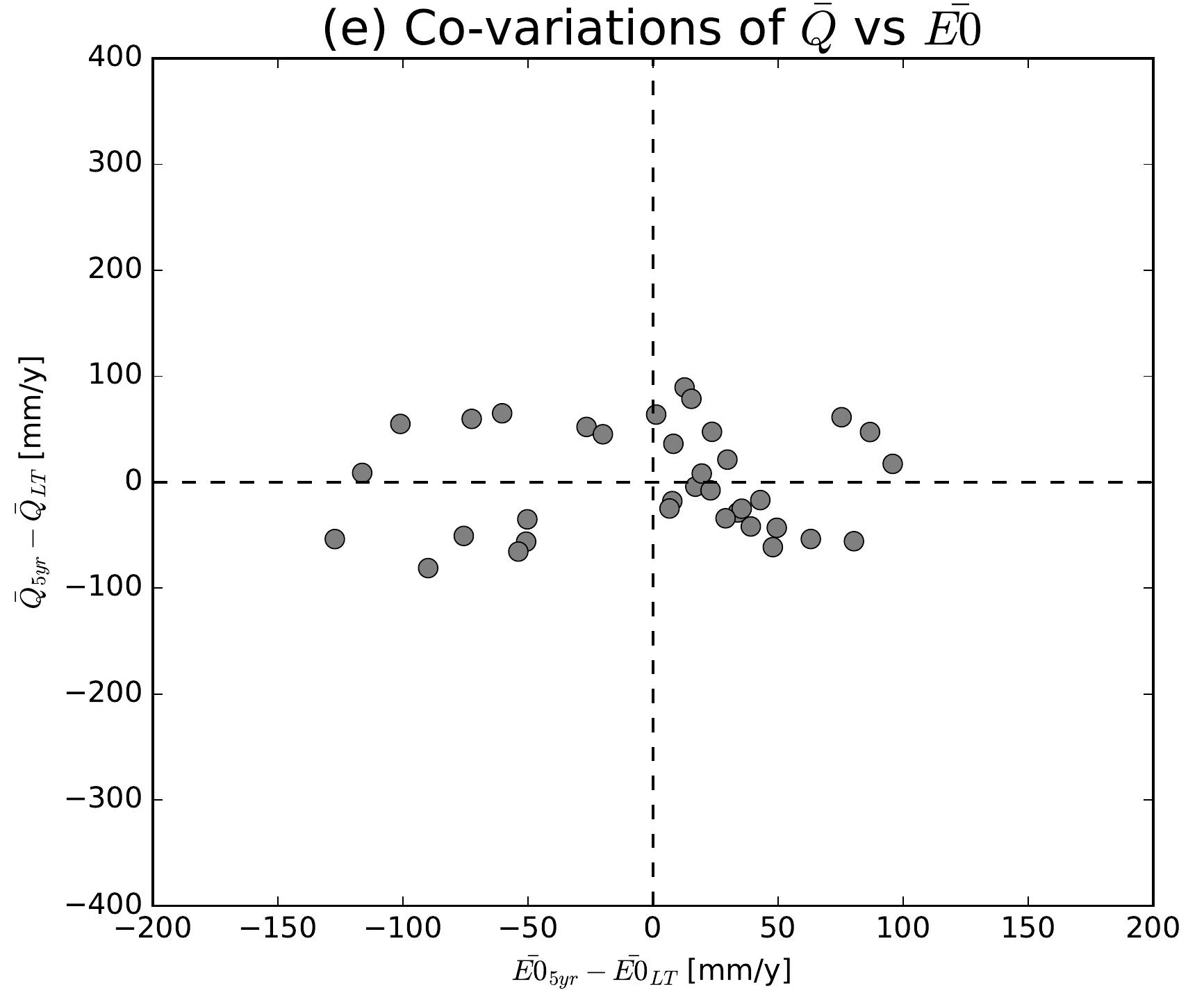

(h) Co-variations of $\bar{Q}$ vs $\overline{E 0}$

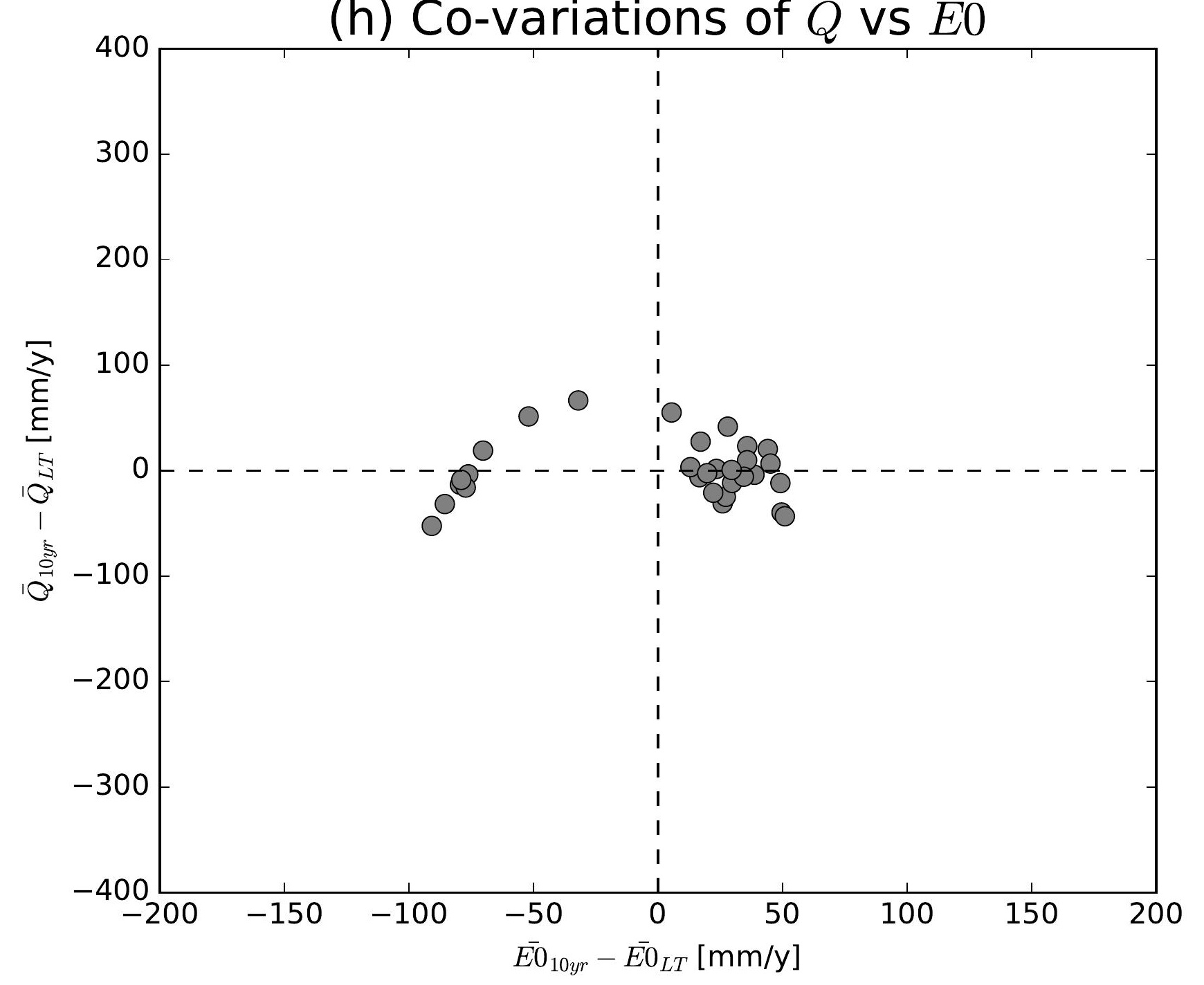

(k) Co-variations of $\bar{Q}$ vs $\overline{E 0}$

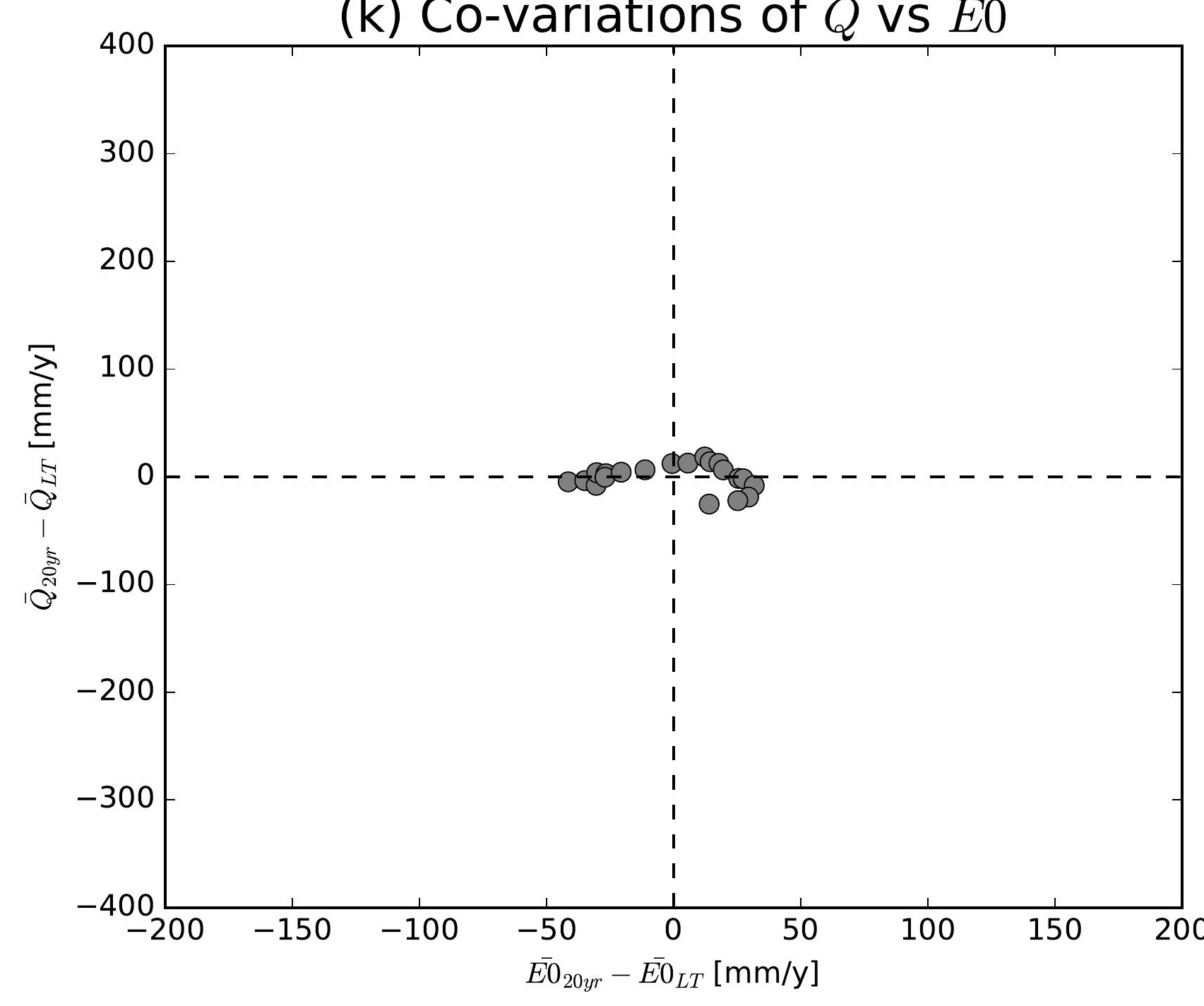

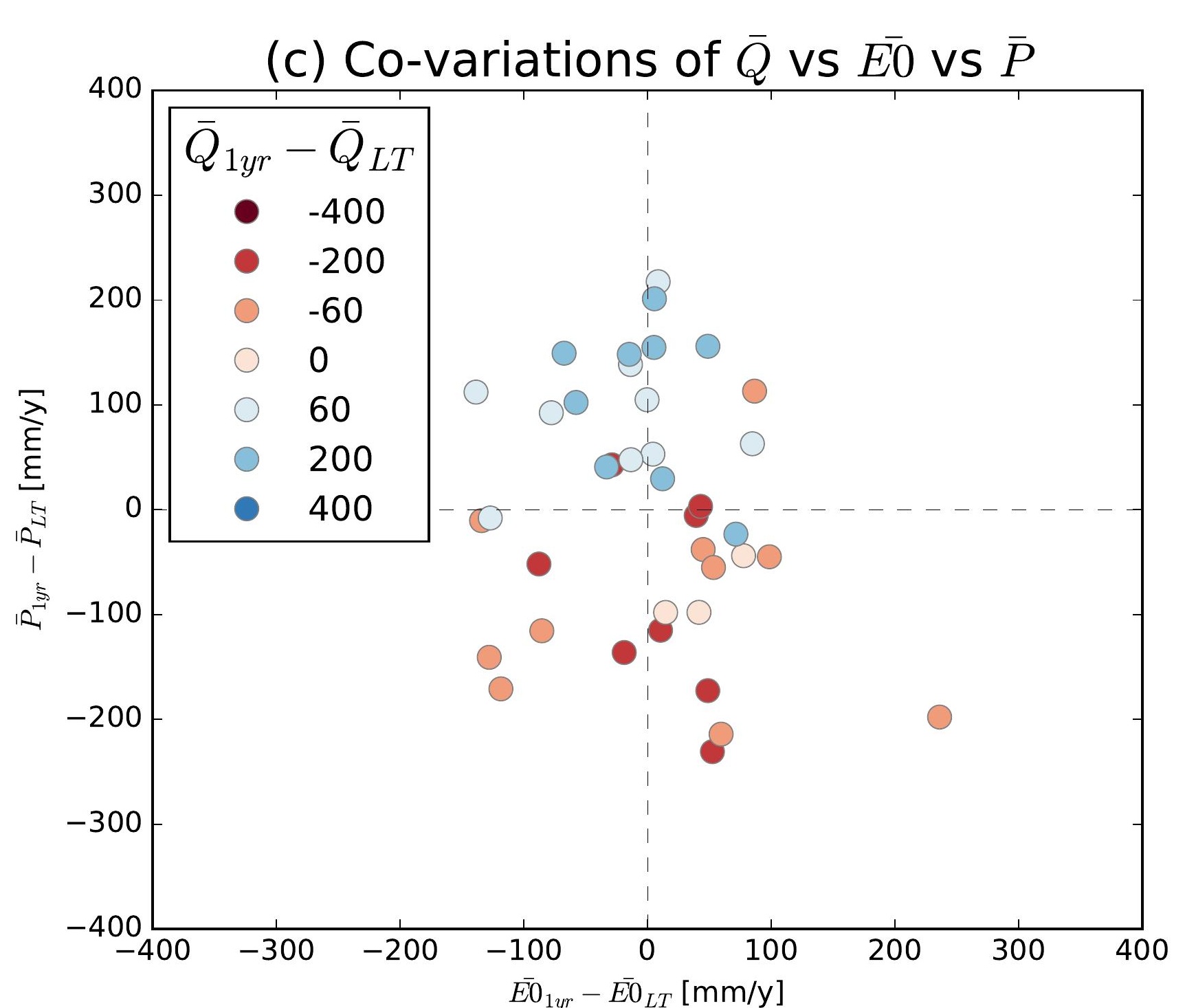
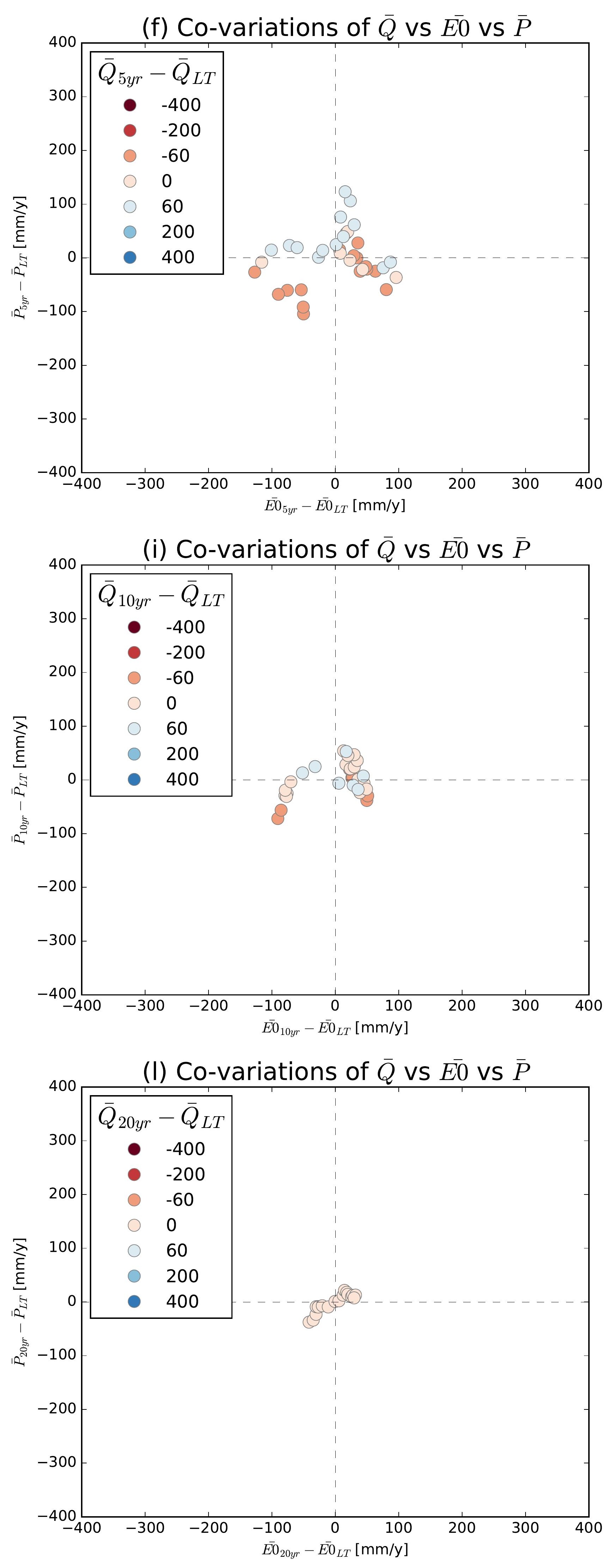

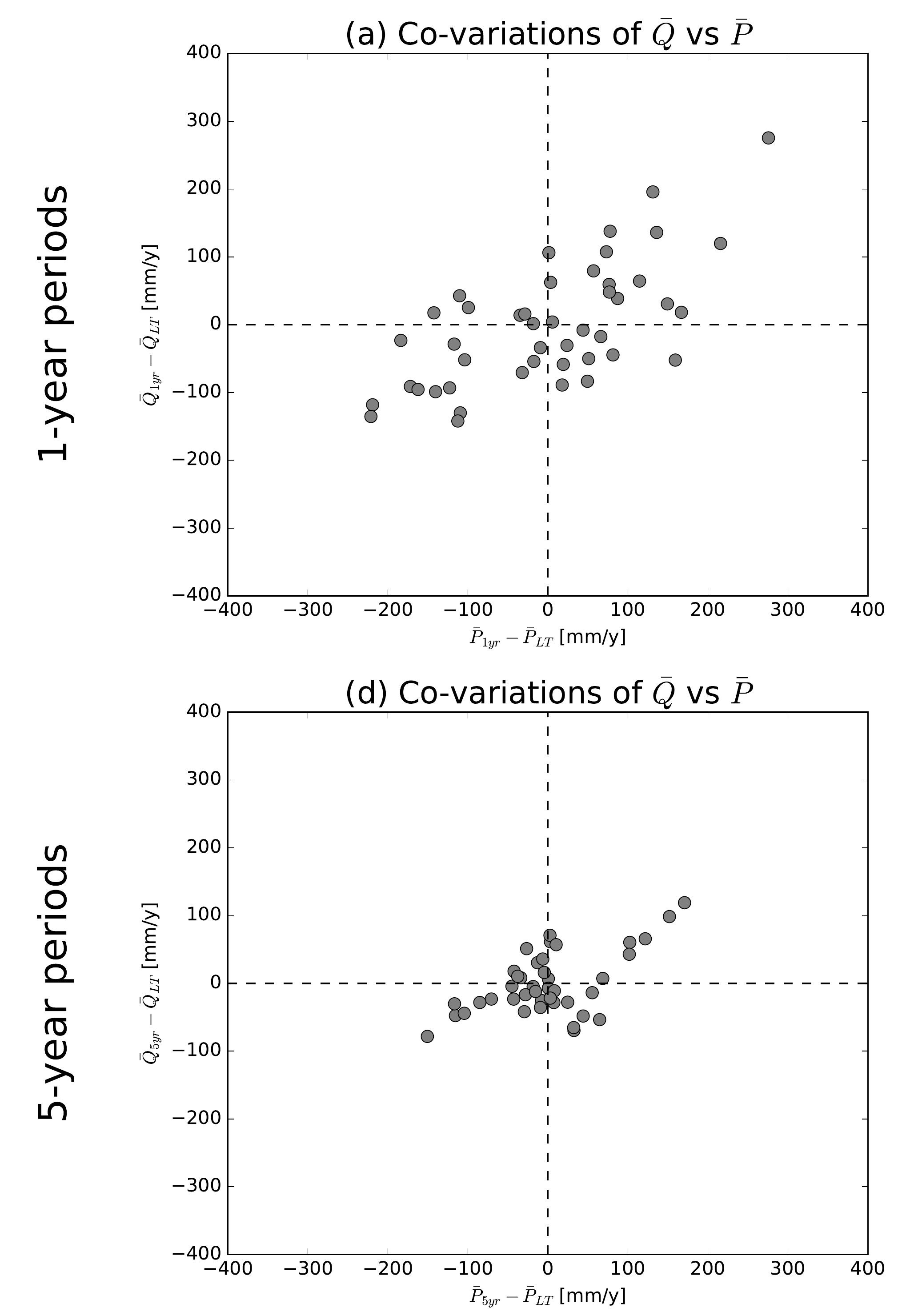

(g) Co-variations of $\bar{Q}$ vs $\bar{P}$
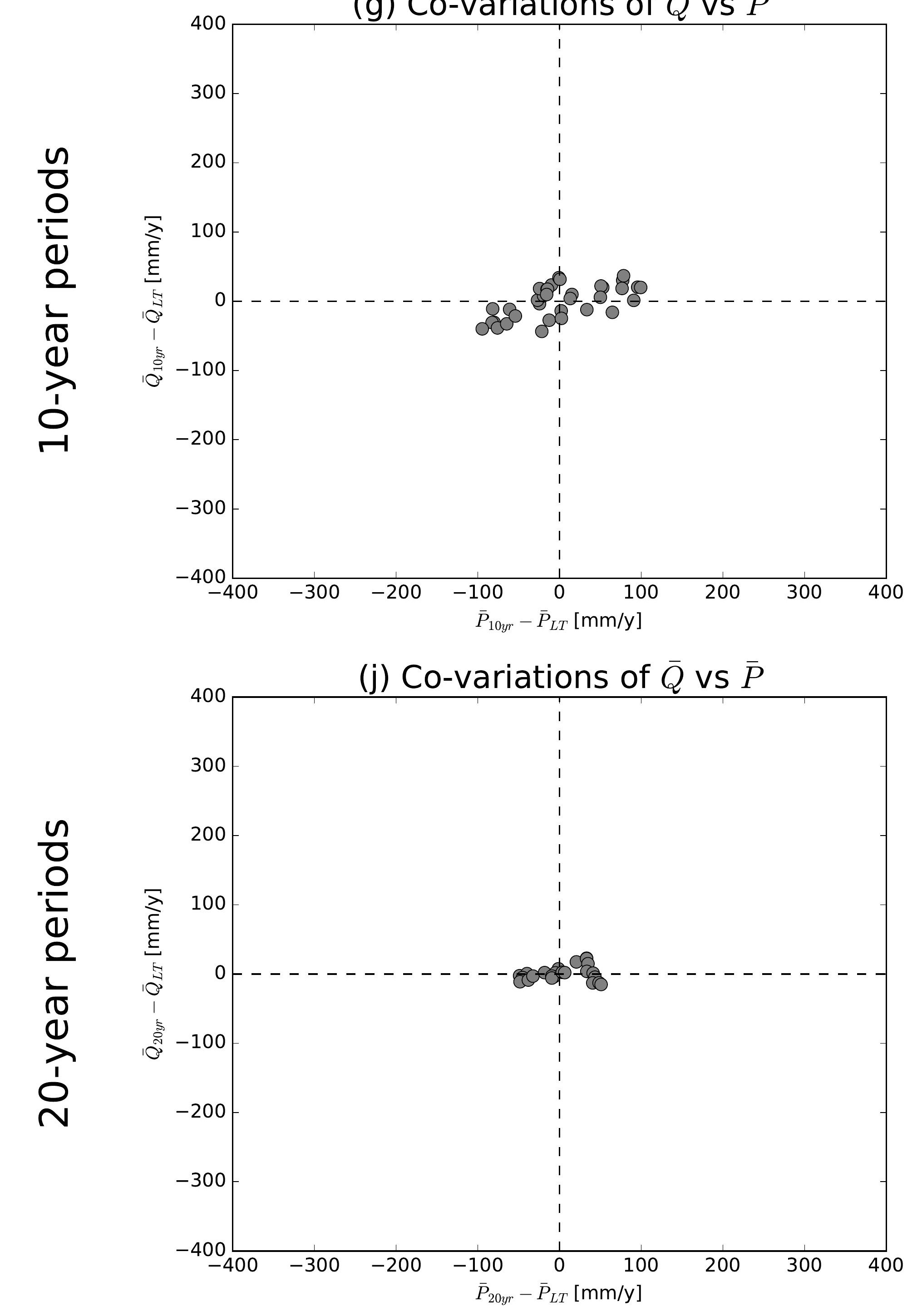

(b) Co-variations of $\bar{Q}$ vs $\overline{E 0}$

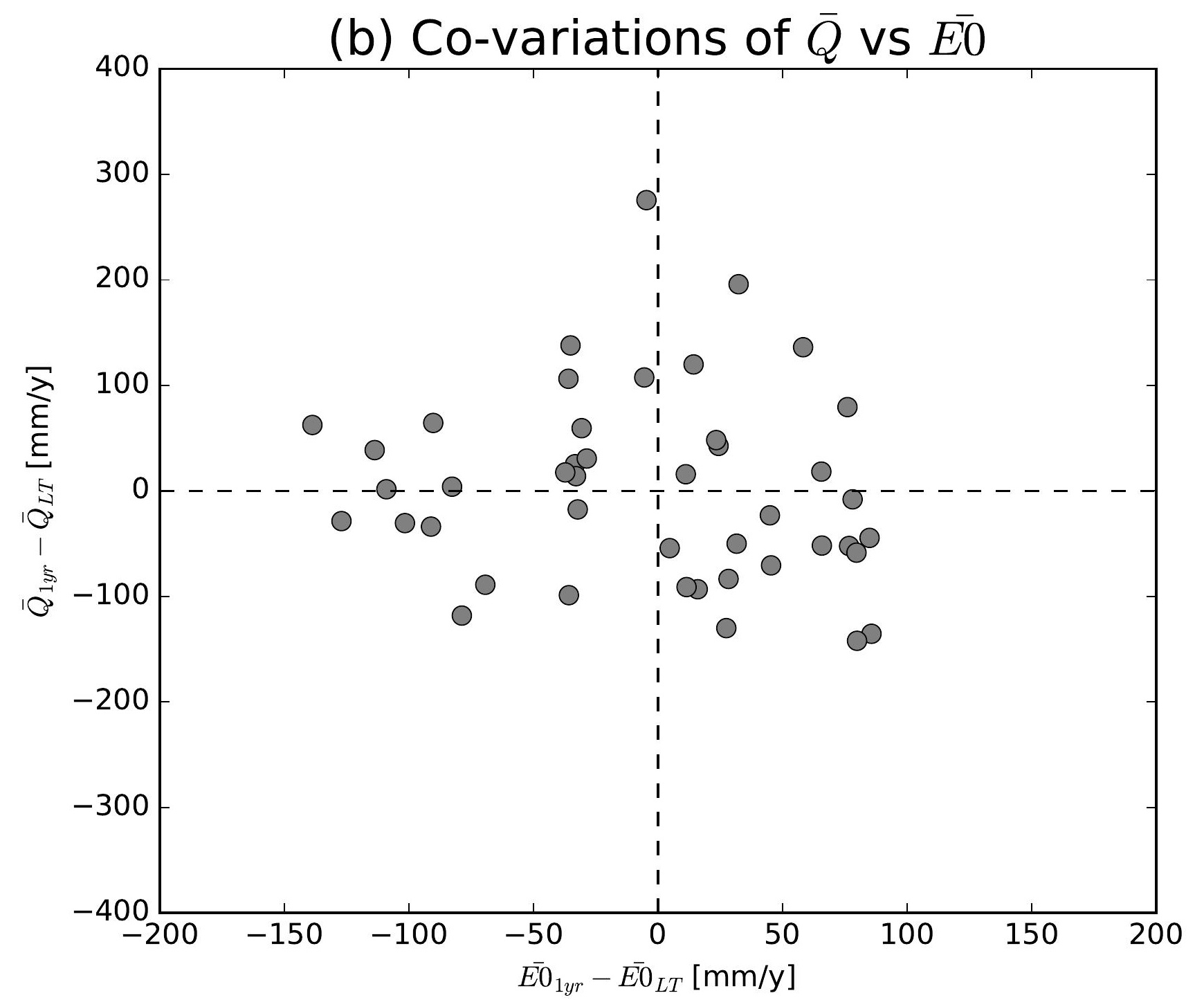

(e) Co-variations of $\bar{Q}$ vs $\overline{E 0}$

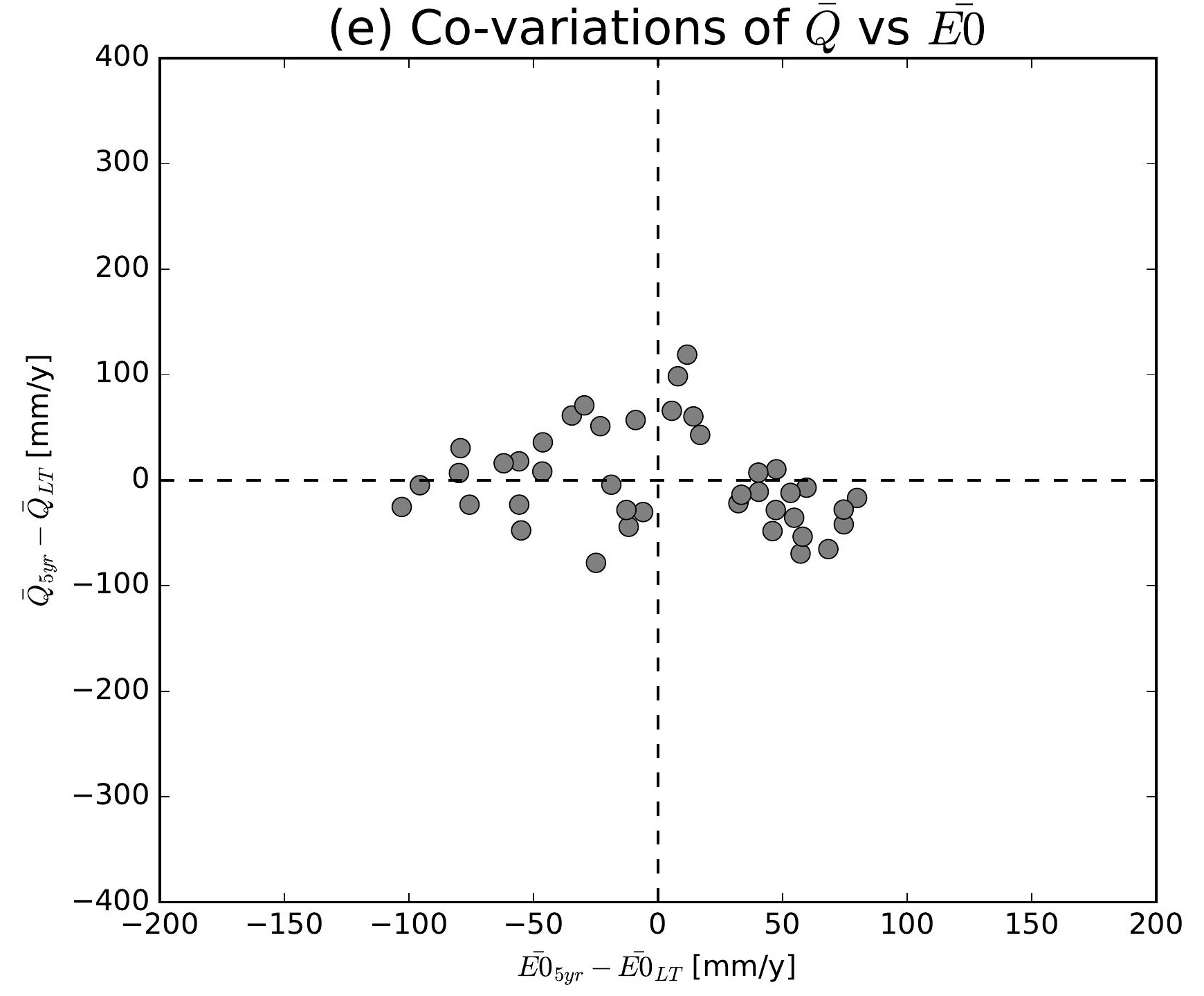

(h) Co-variations of $\bar{Q}$ vs $\overline{E 0}$

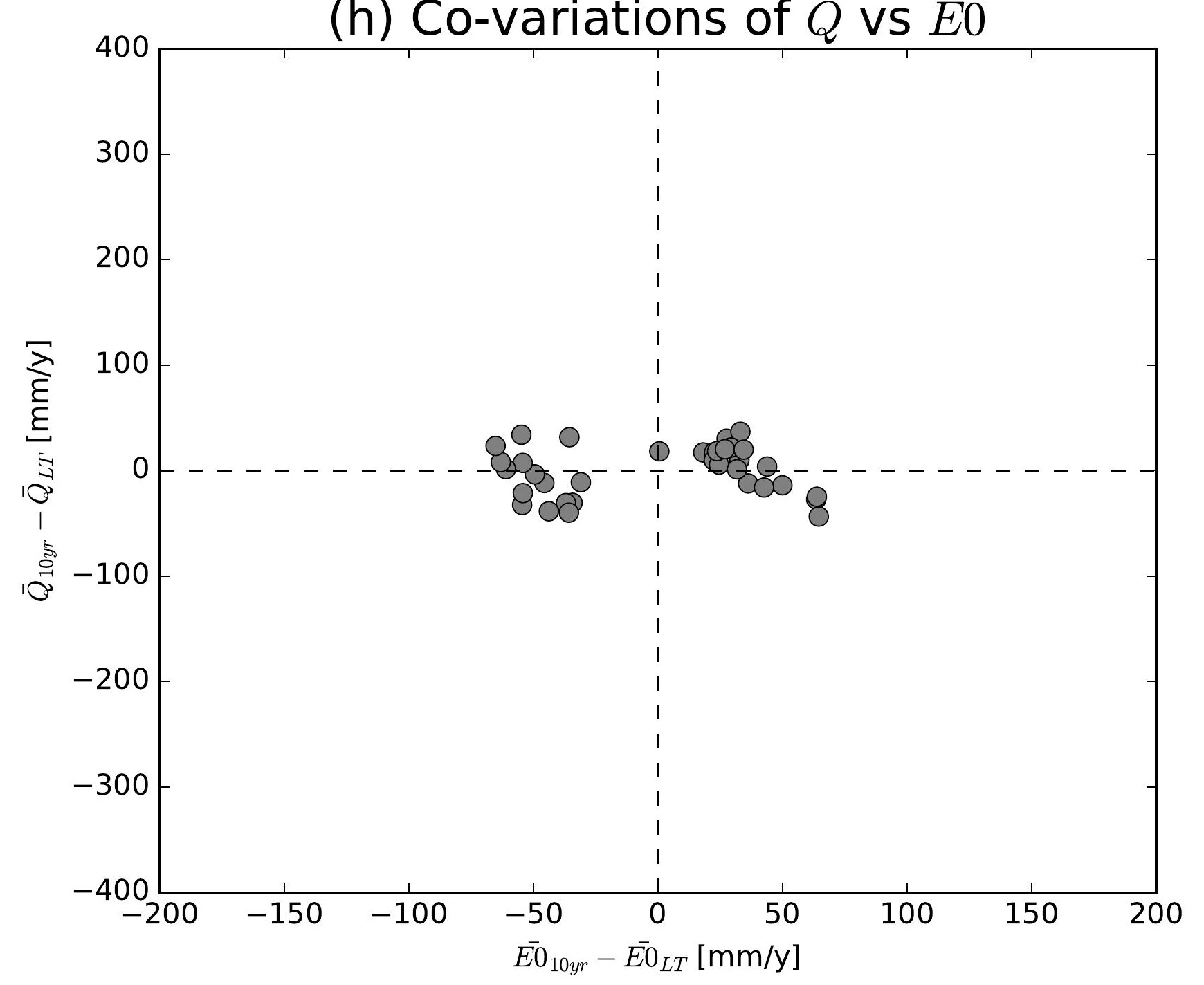

(k) Co-variations of $\bar{Q}$ vs $\overline{E 0}$

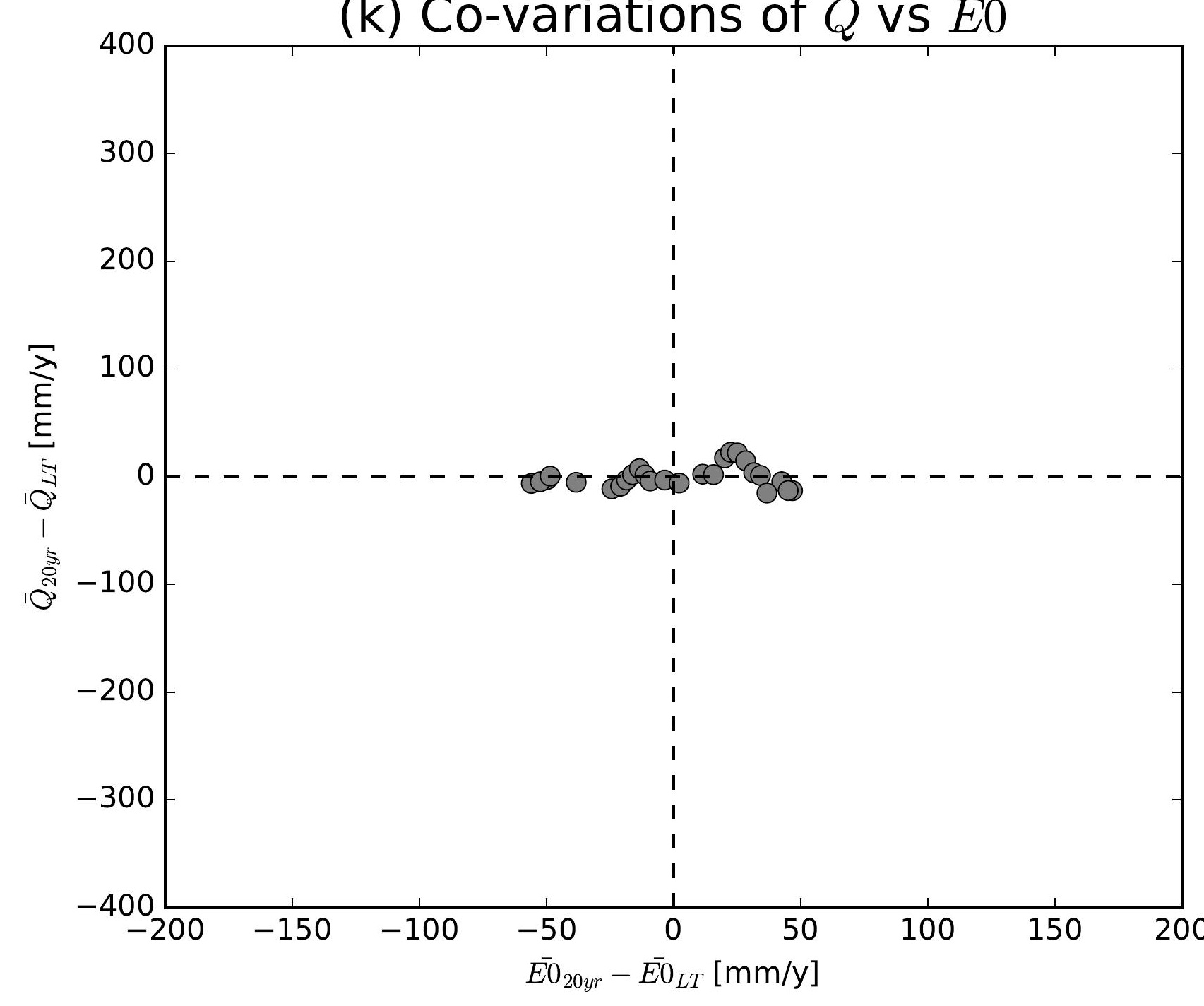

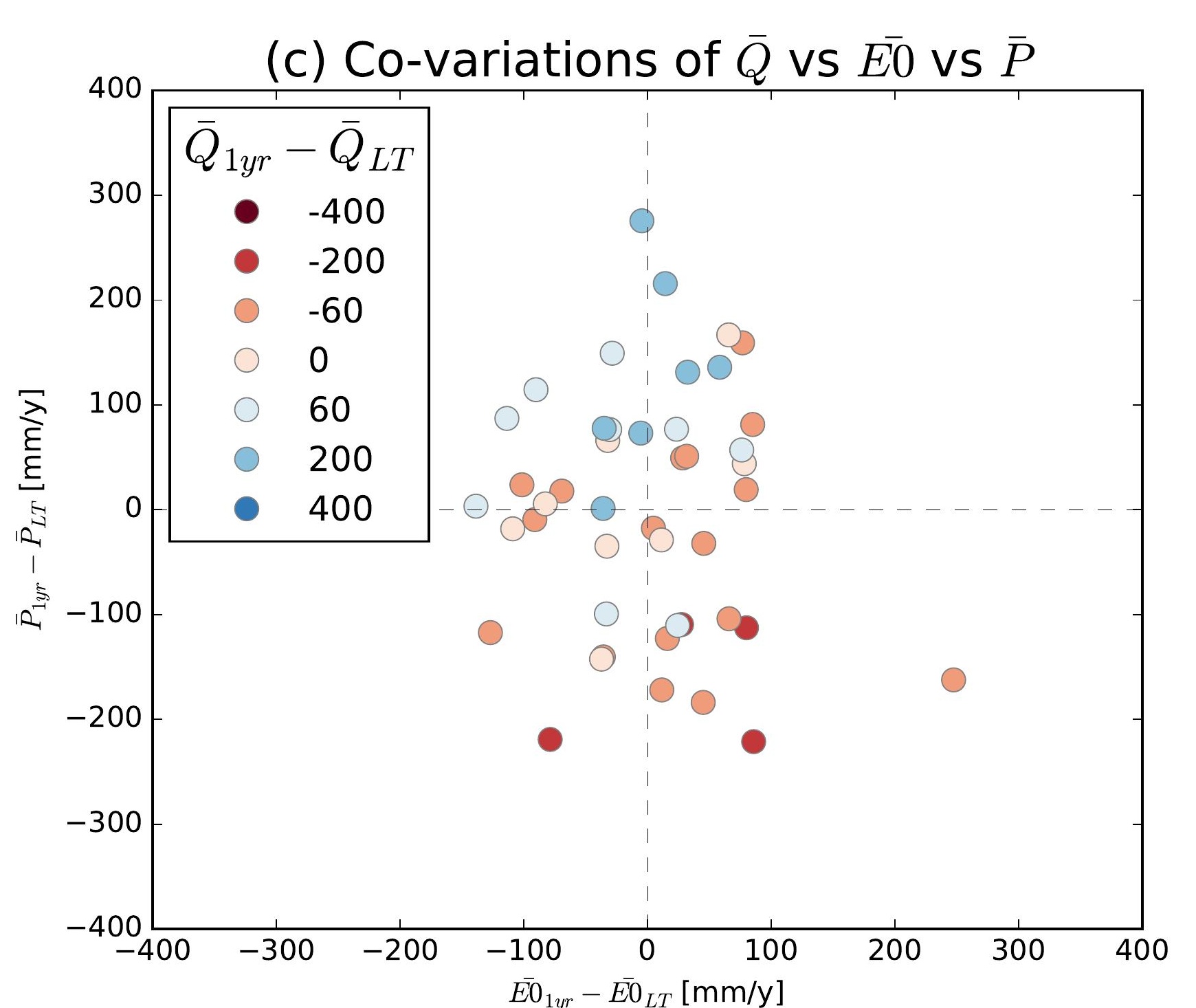
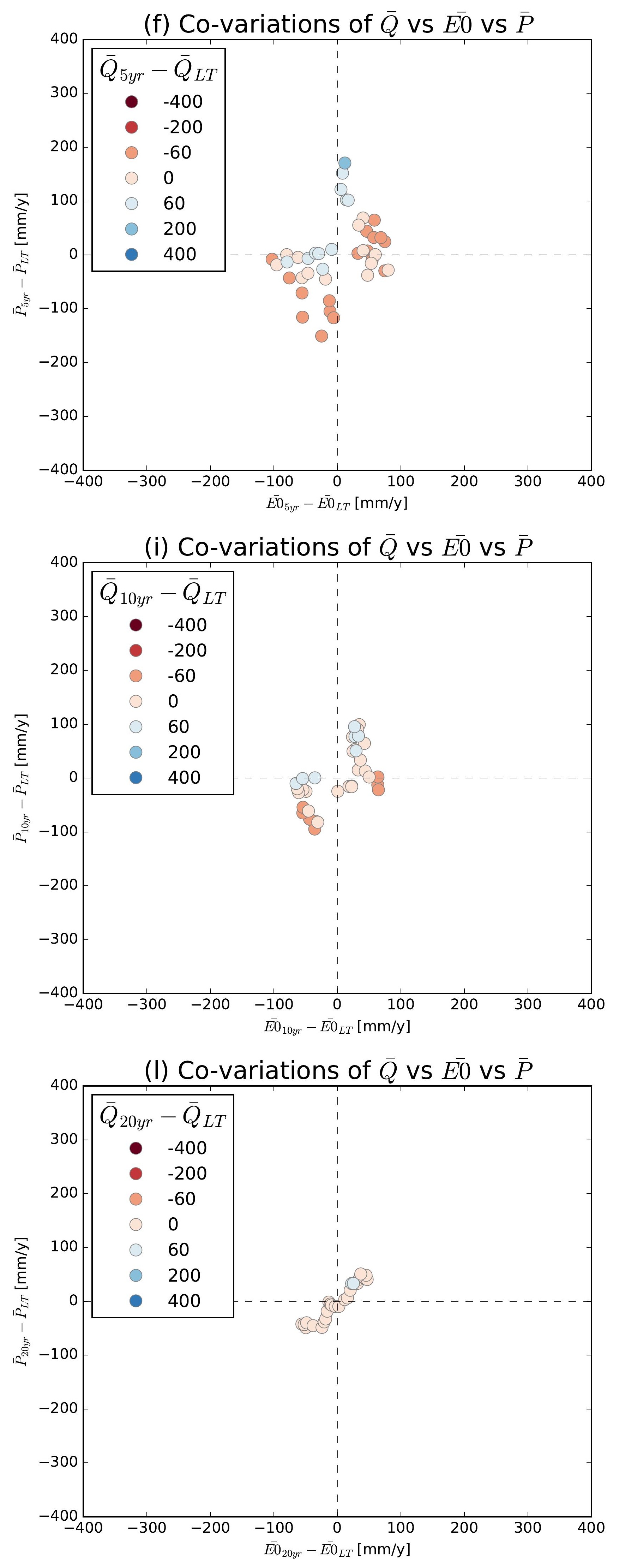

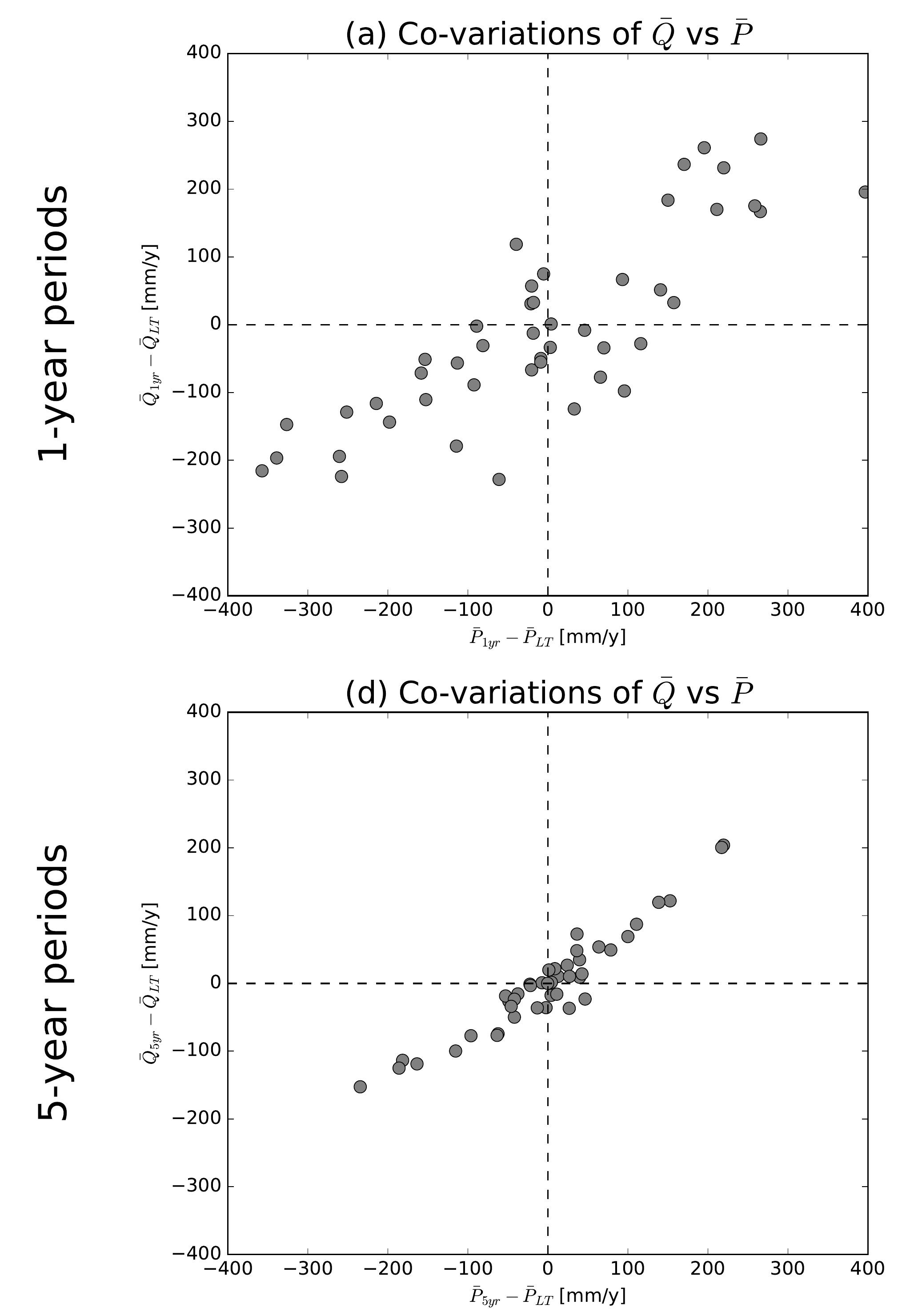

(g) Co-variations of $\bar{Q}$ vs $\bar{P}$

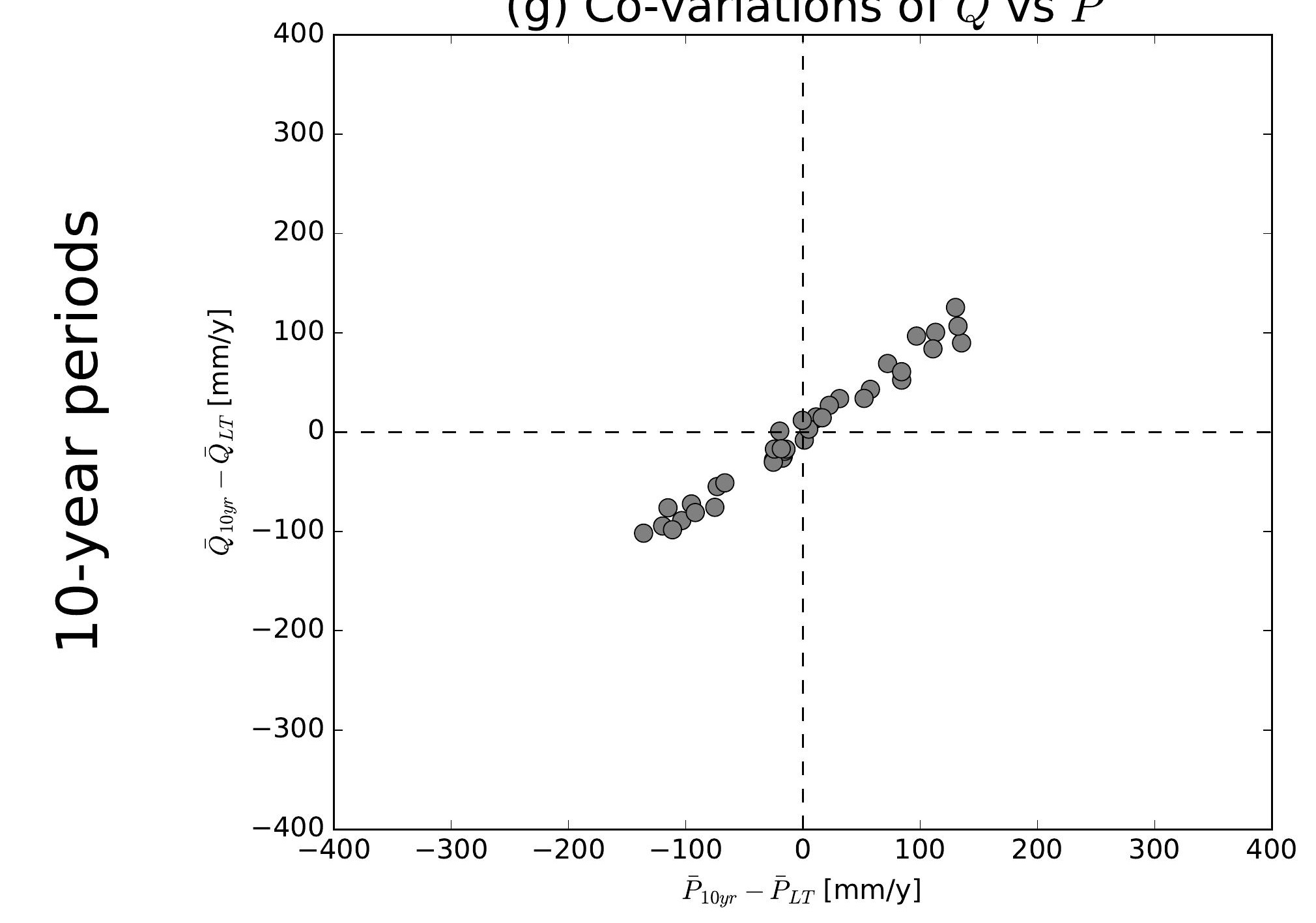

(j) Co-variations of $\bar{Q}$ vs $\bar{P}$

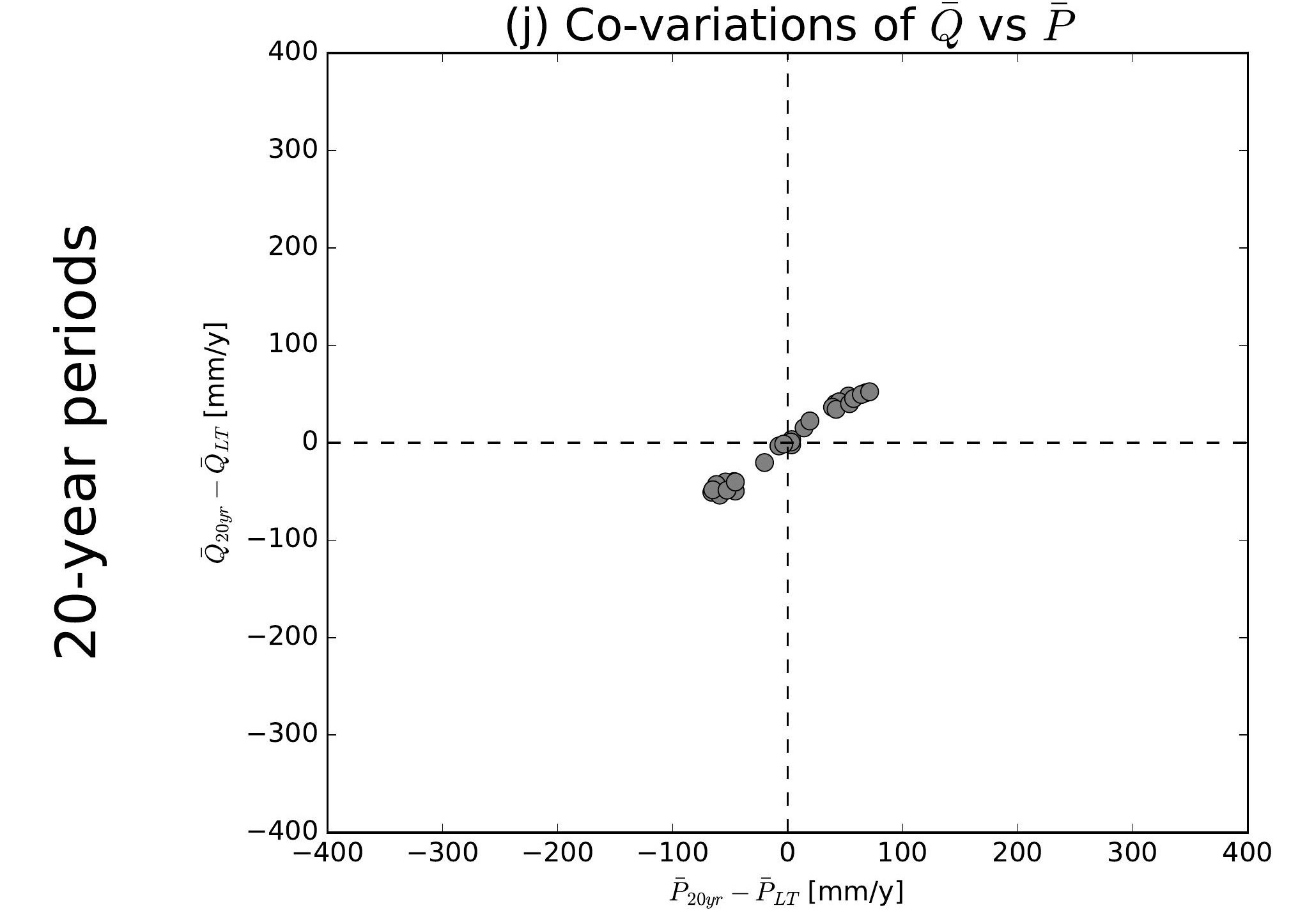

(b) Co-variations of $\bar{Q}$ vs $\overline{E 0}$

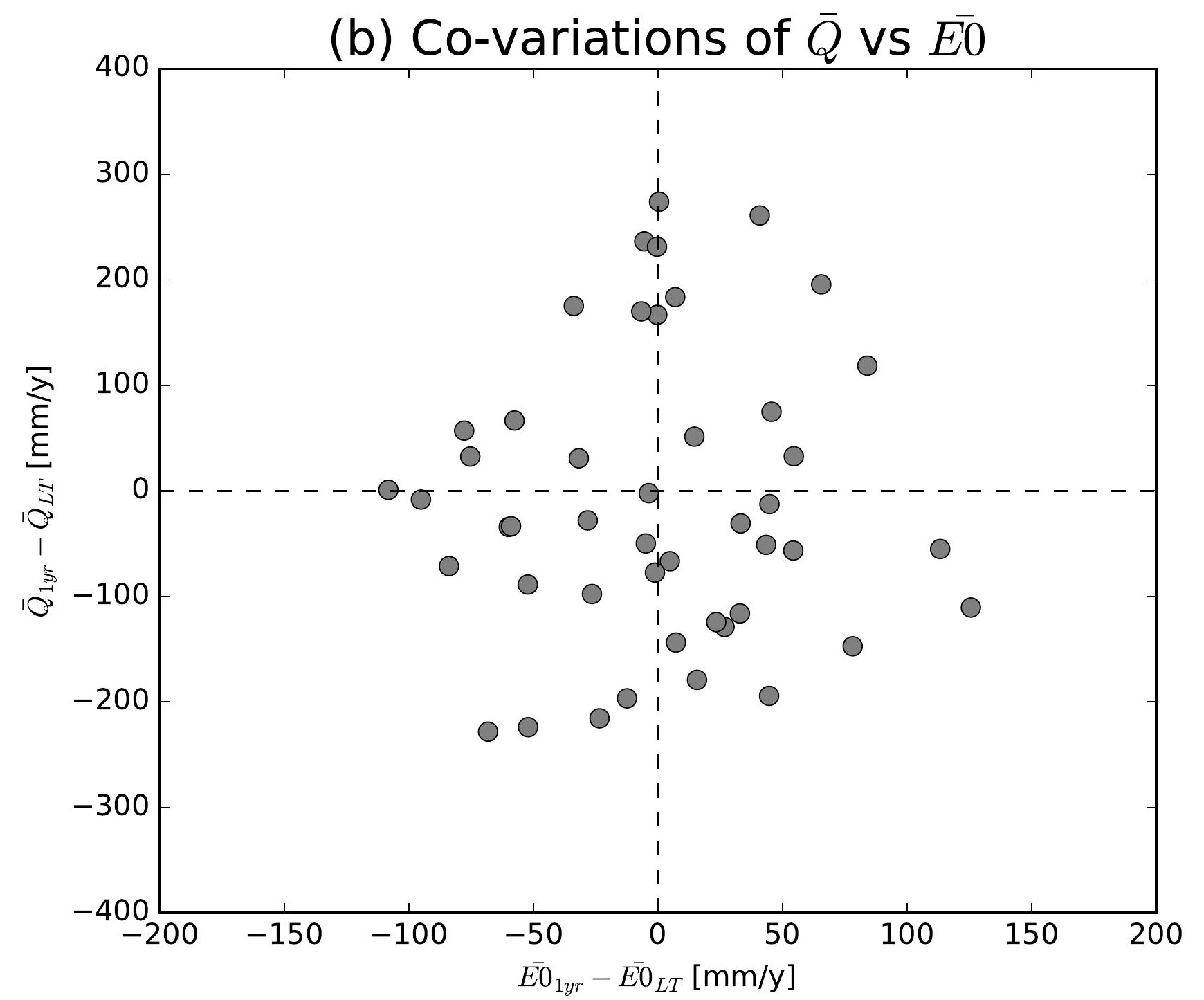

(e) Co-variations of $\bar{Q}$ vs $\overline{E 0}$

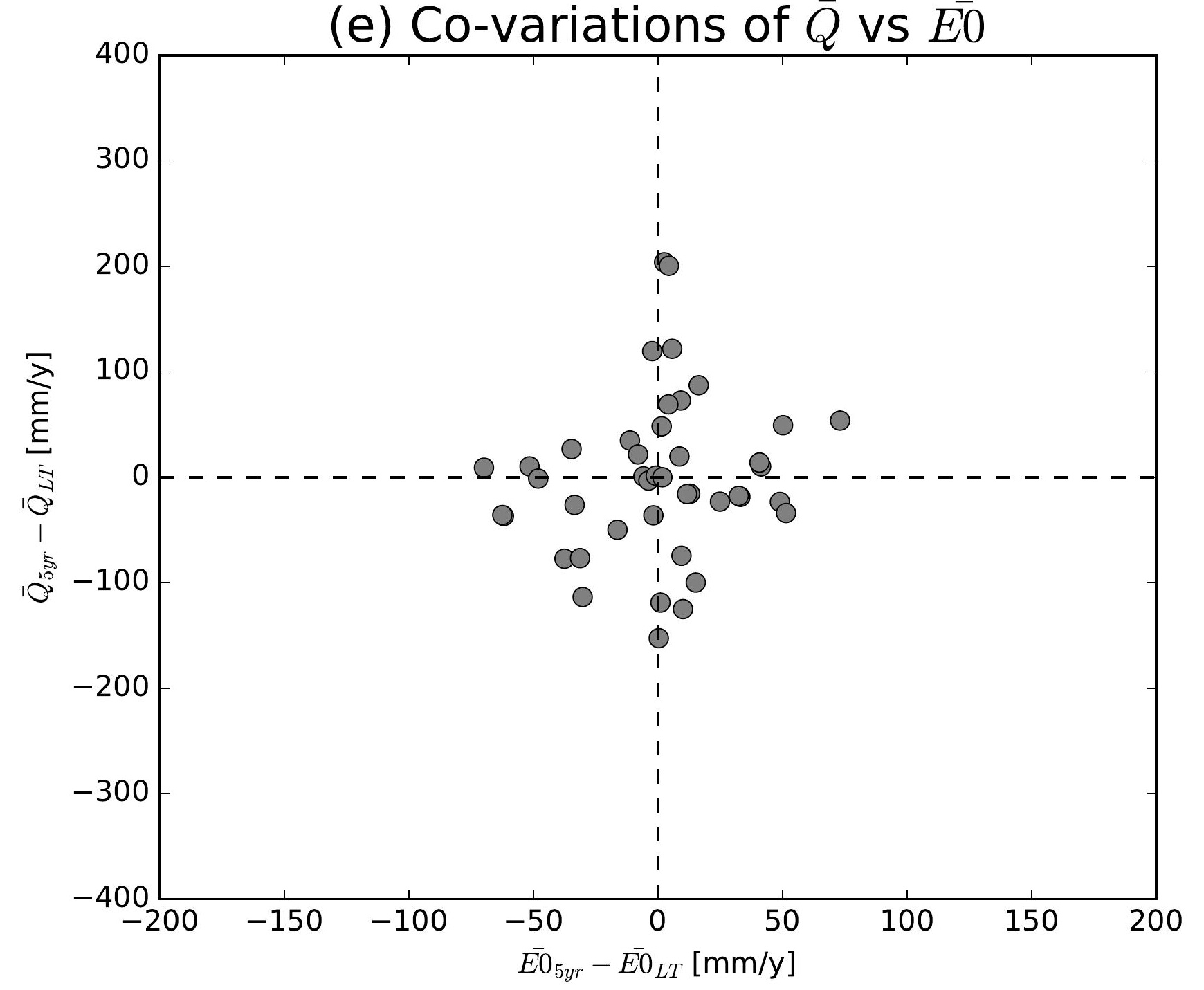

(h) Co-variations of $\bar{Q}$ vs $\overline{E 0}$

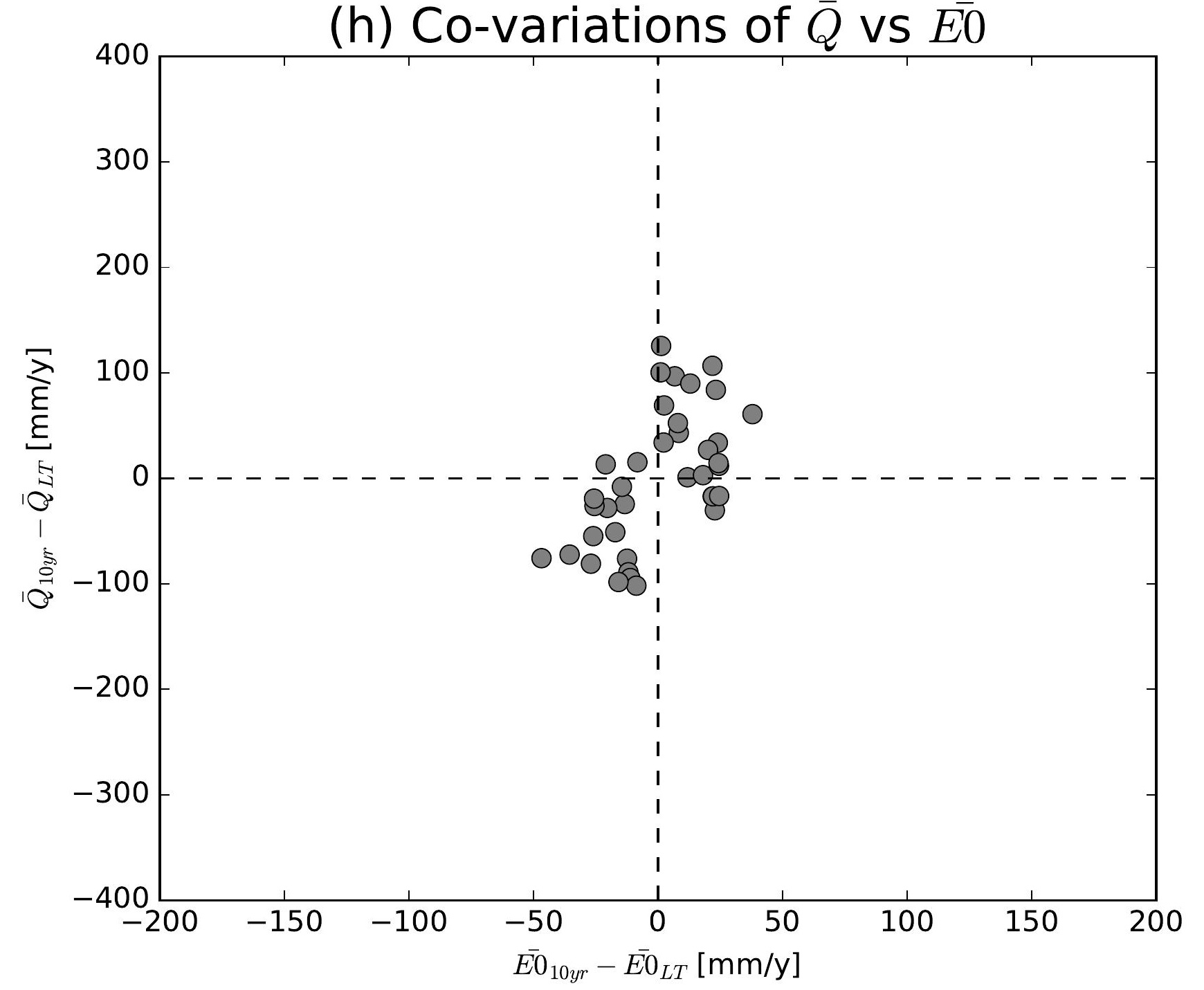

(k) Co-variations of $\bar{Q}$ vs $\overline{E 0}$

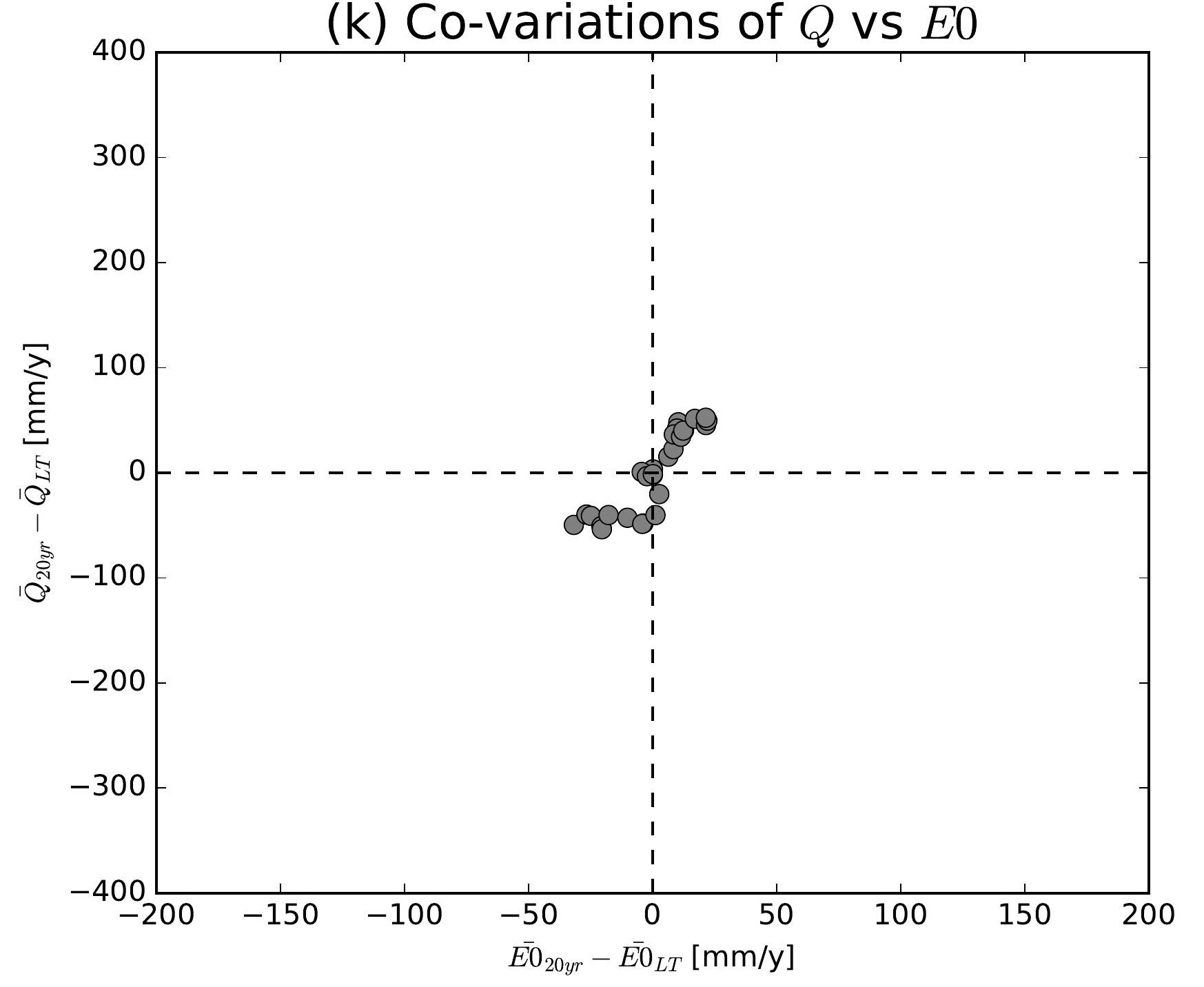

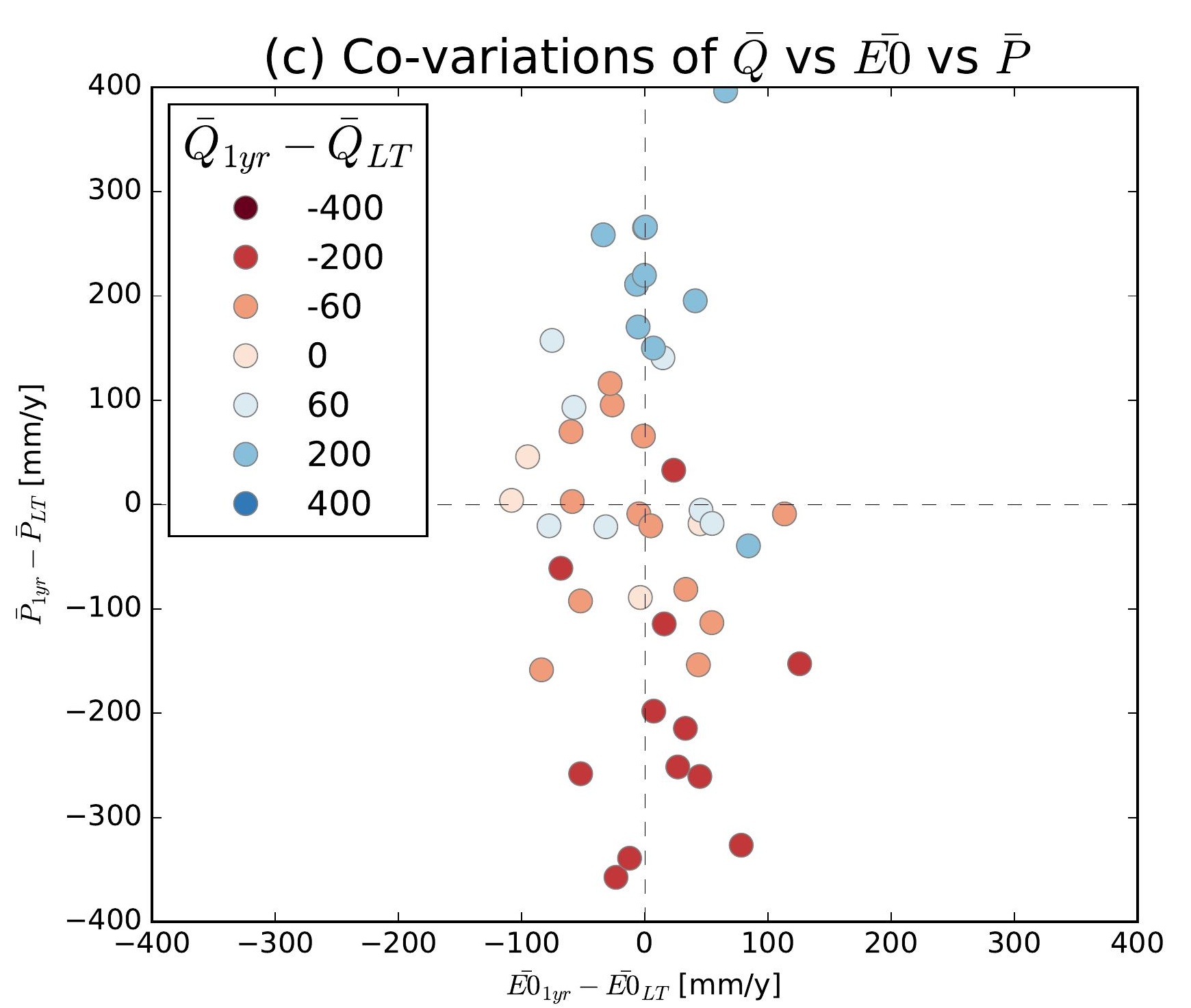
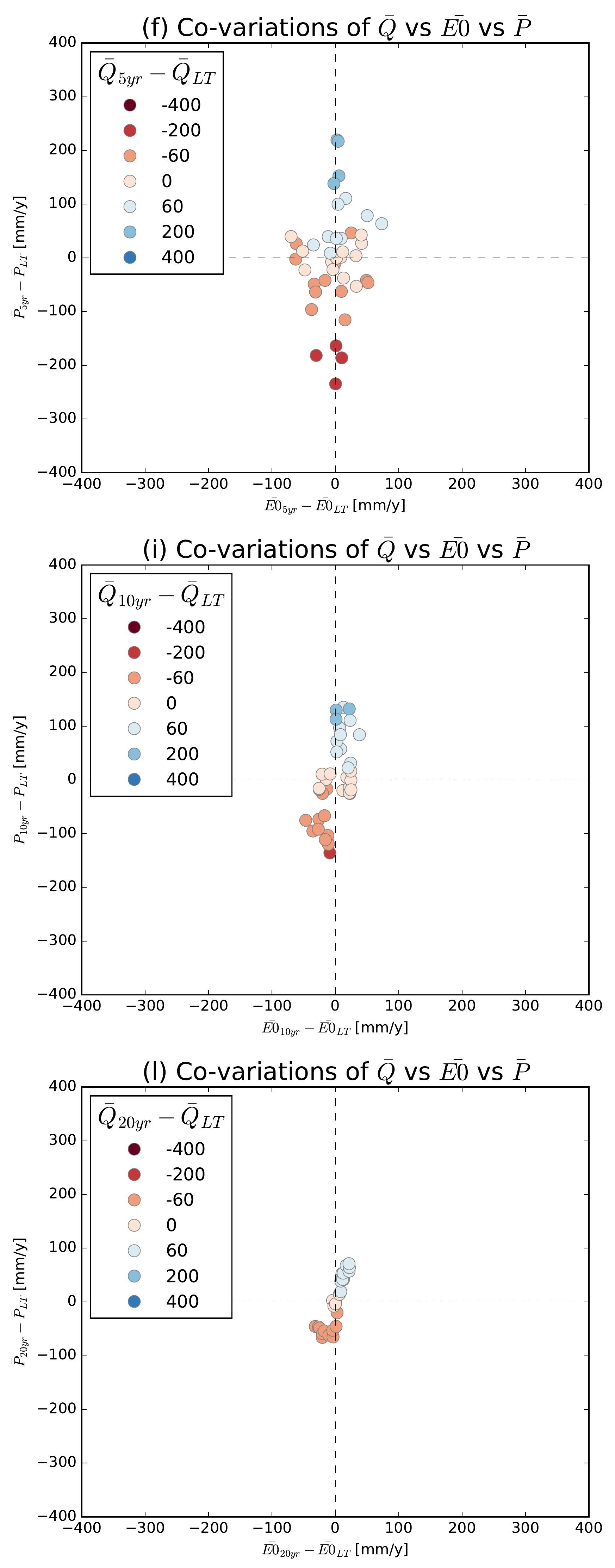

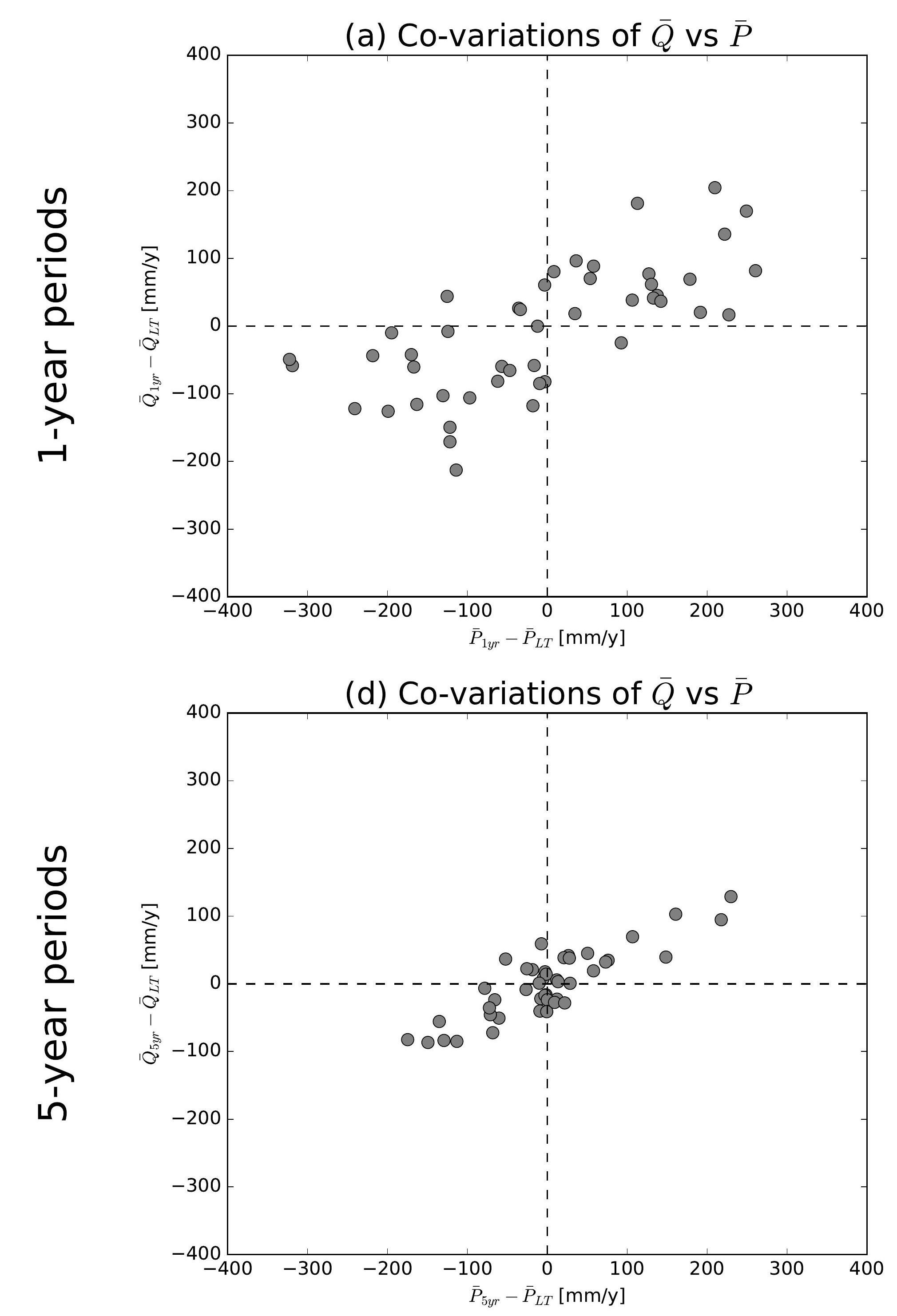

(g) Co-variations of $\bar{Q}$ vs $\bar{P}$
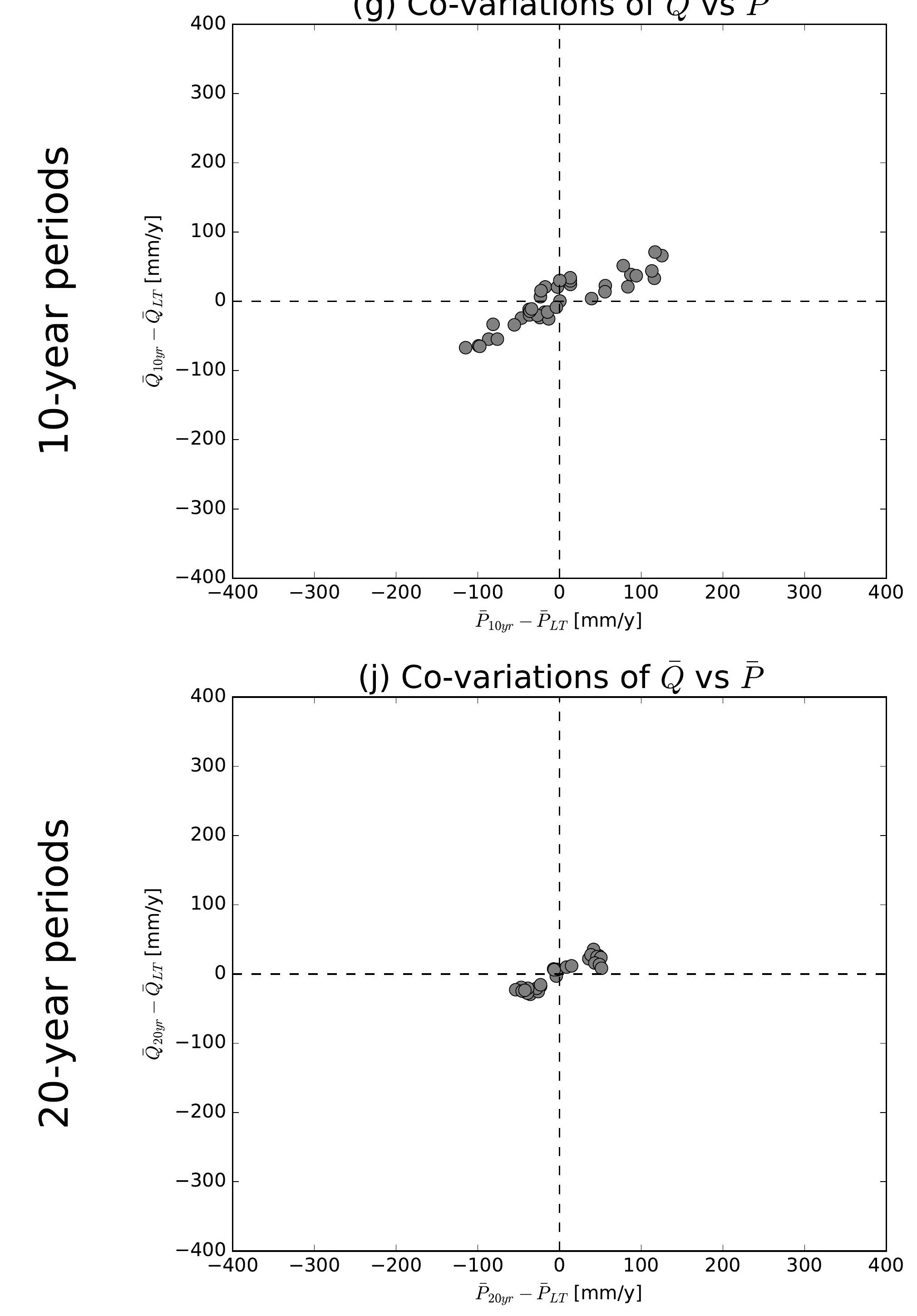

(b) Co-variations of $\bar{Q}$ vs $\overline{E 0}$

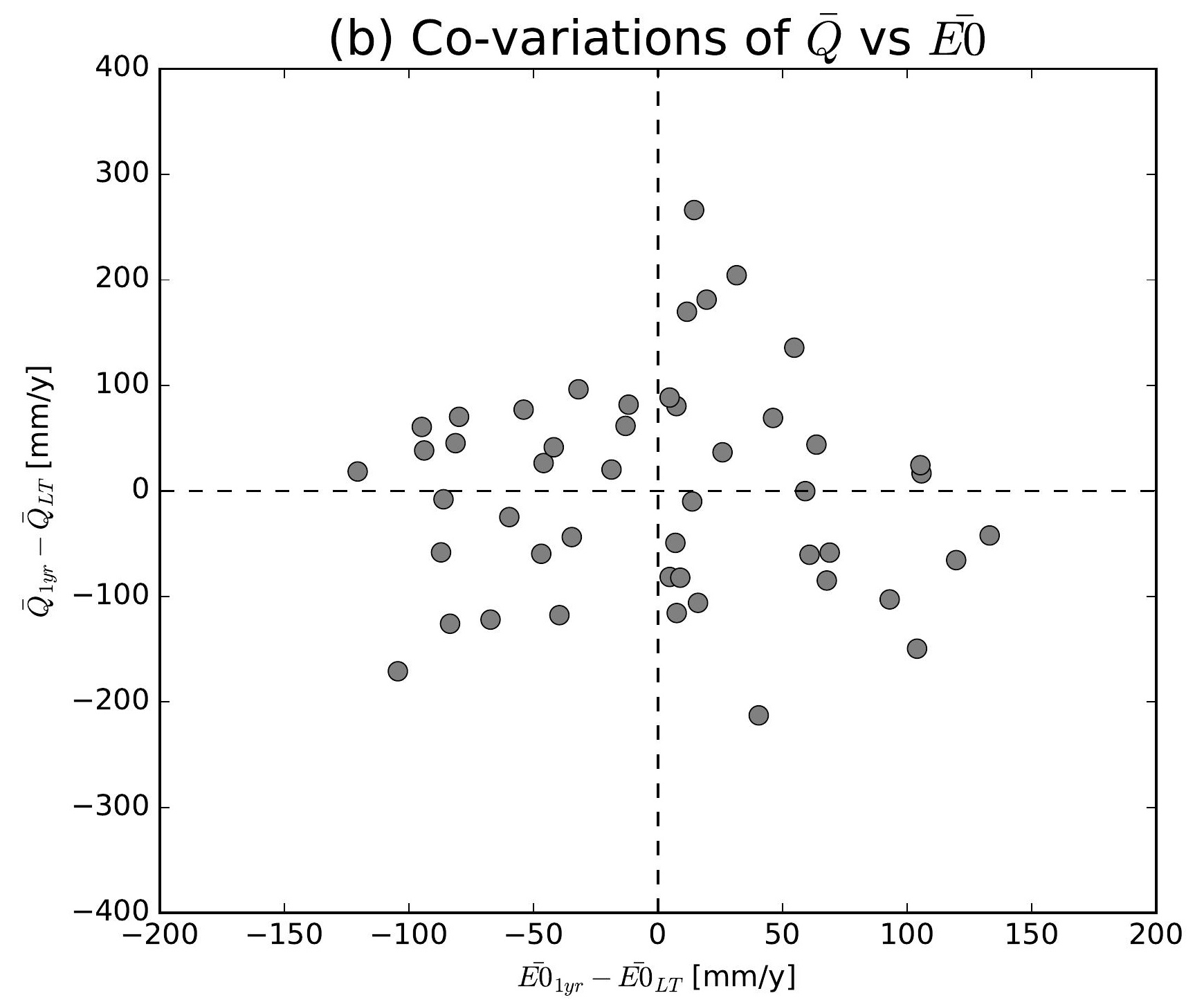

(e) Co-variations of $\bar{Q}$ vs $\overline{E 0}$

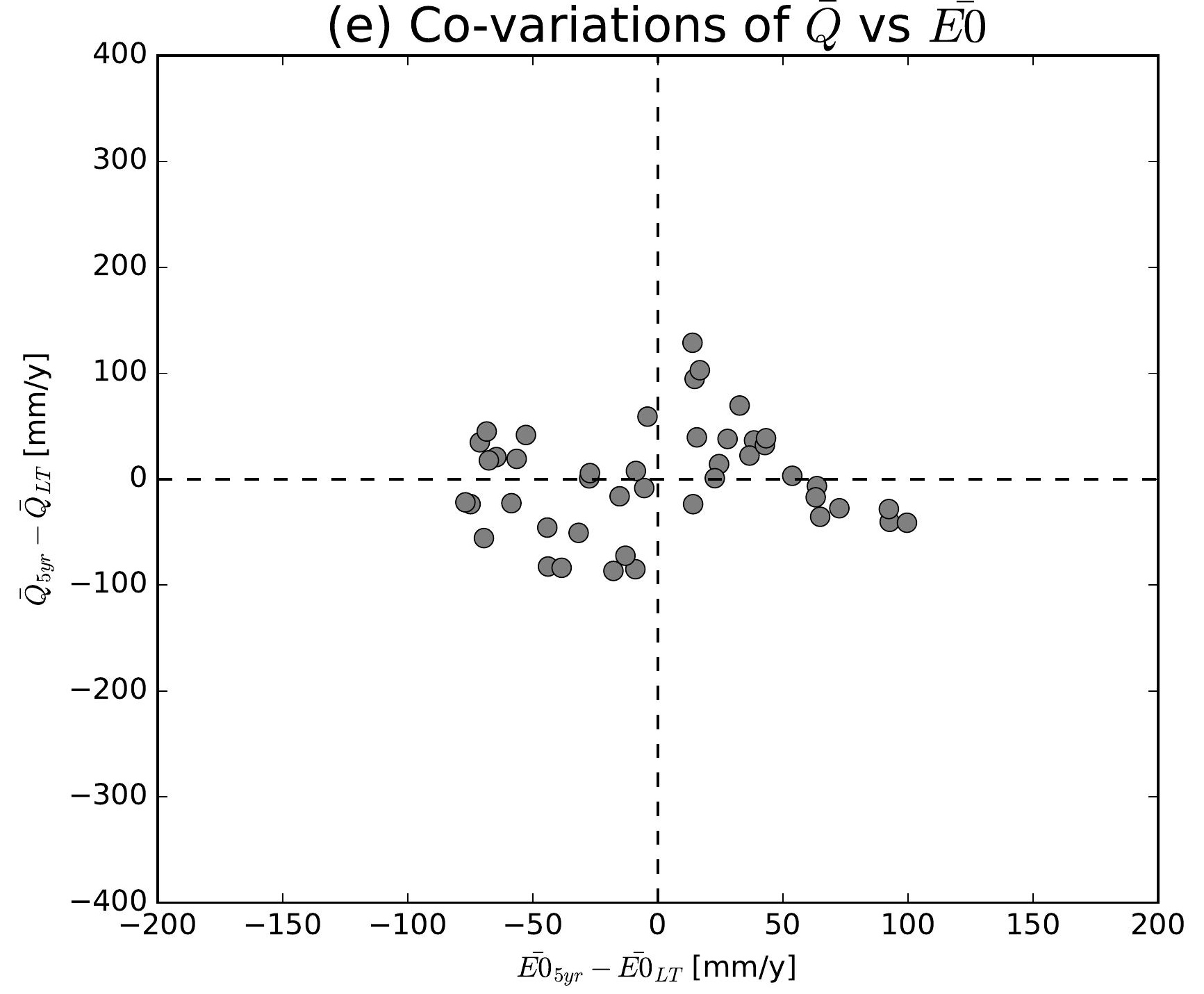

(h) Co-variations of $\bar{Q}$ vs $\overline{E 0}$

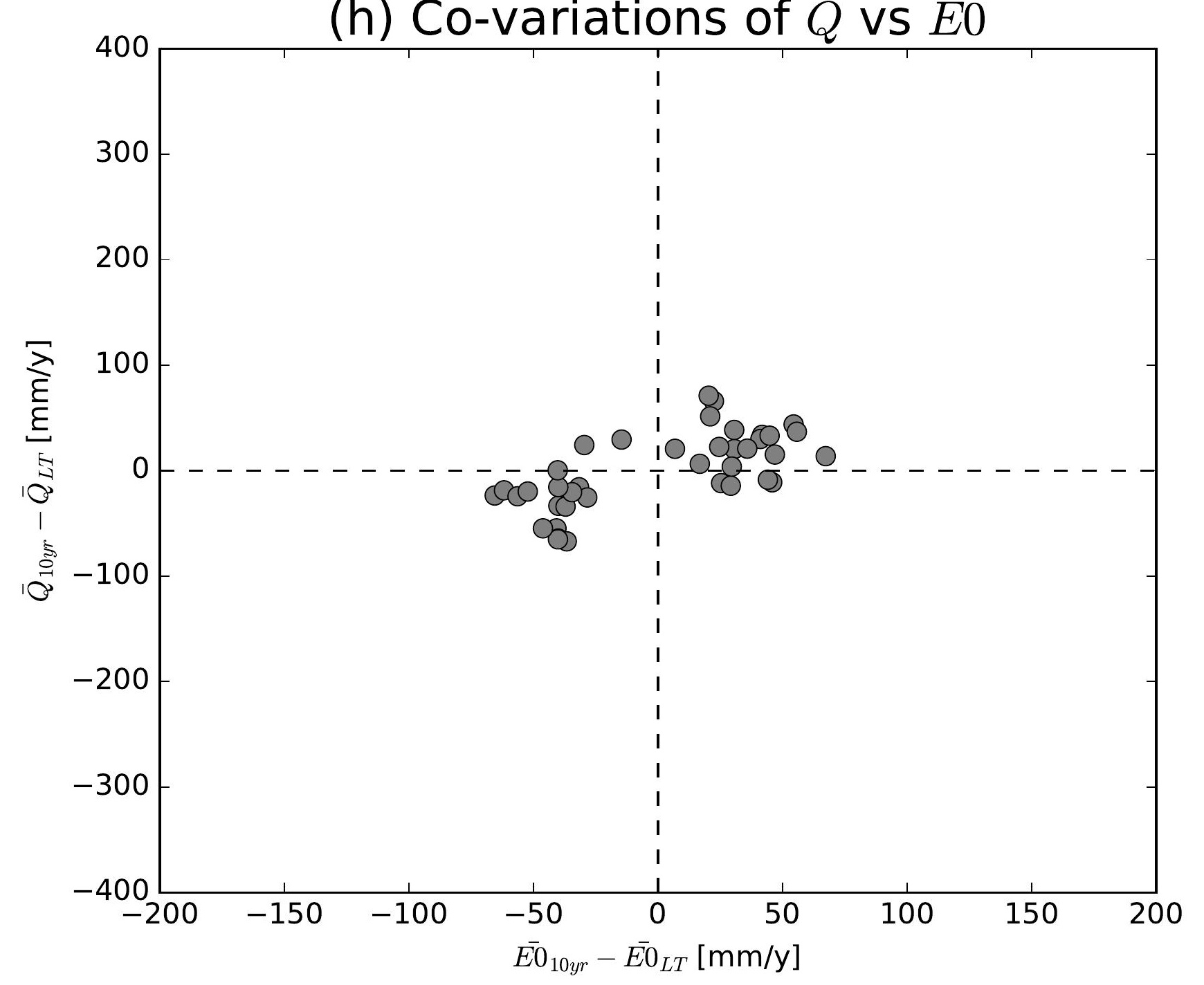

(k) Co-variations of $\bar{Q}$ vs $\overline{E 0}$

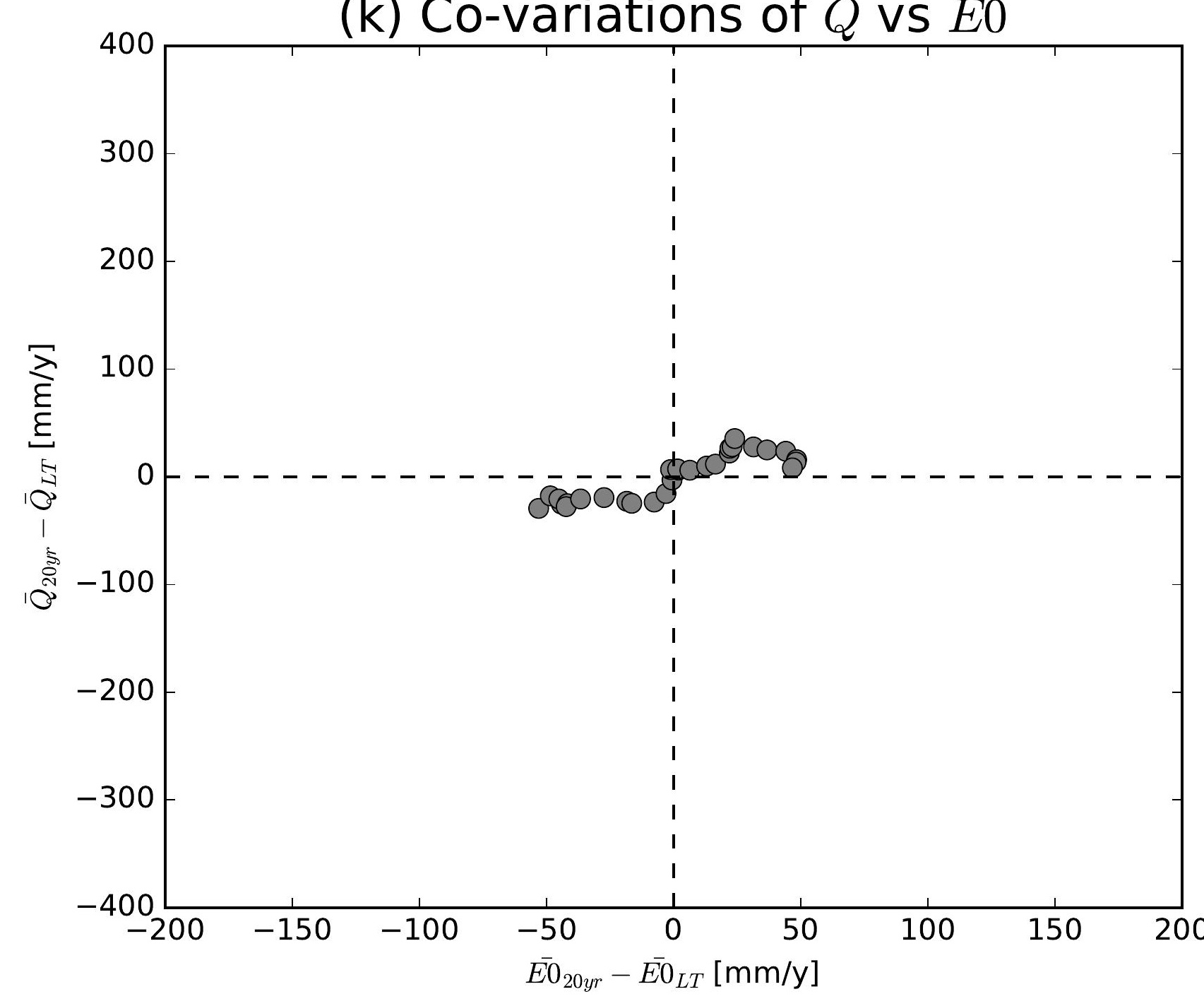

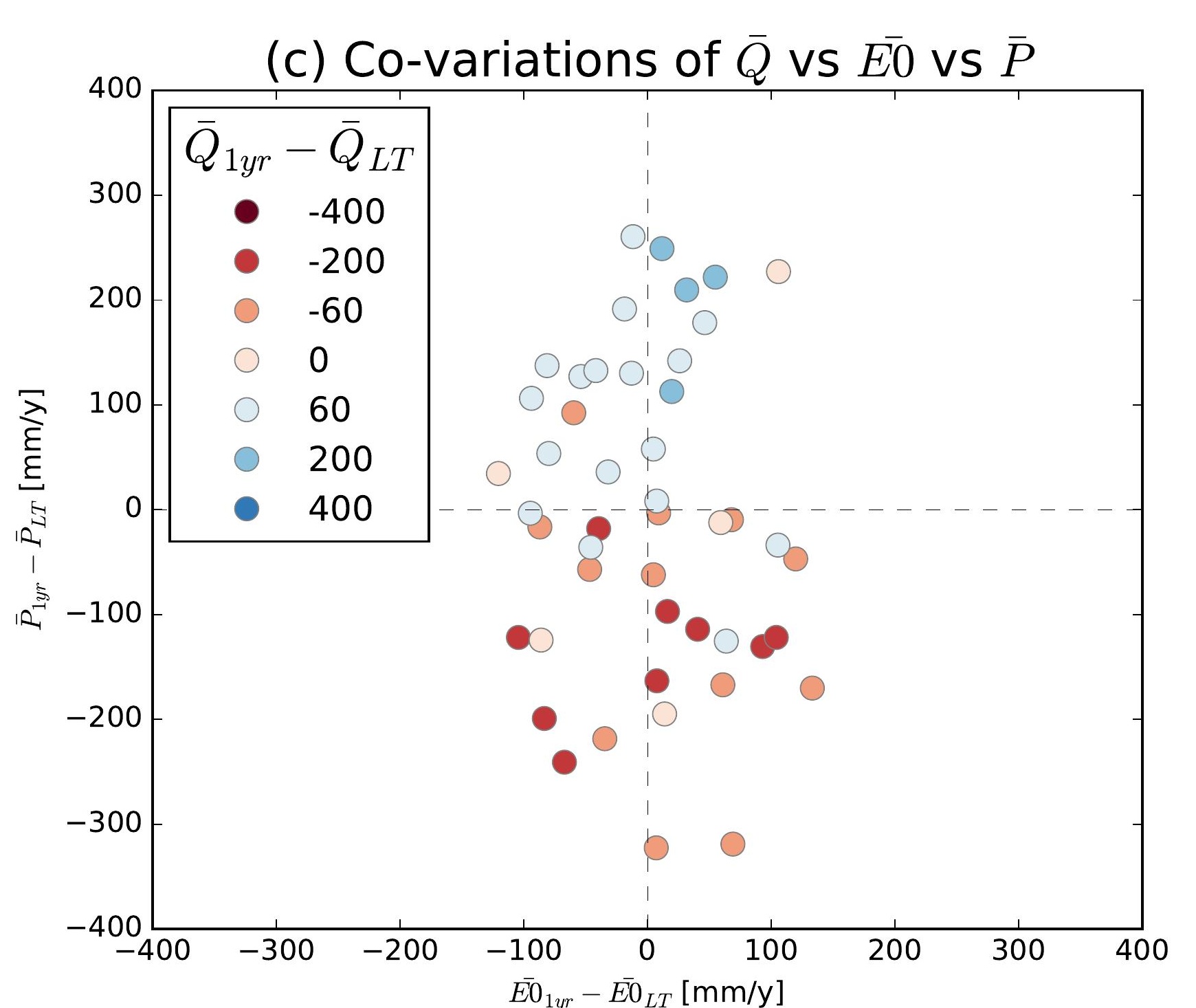
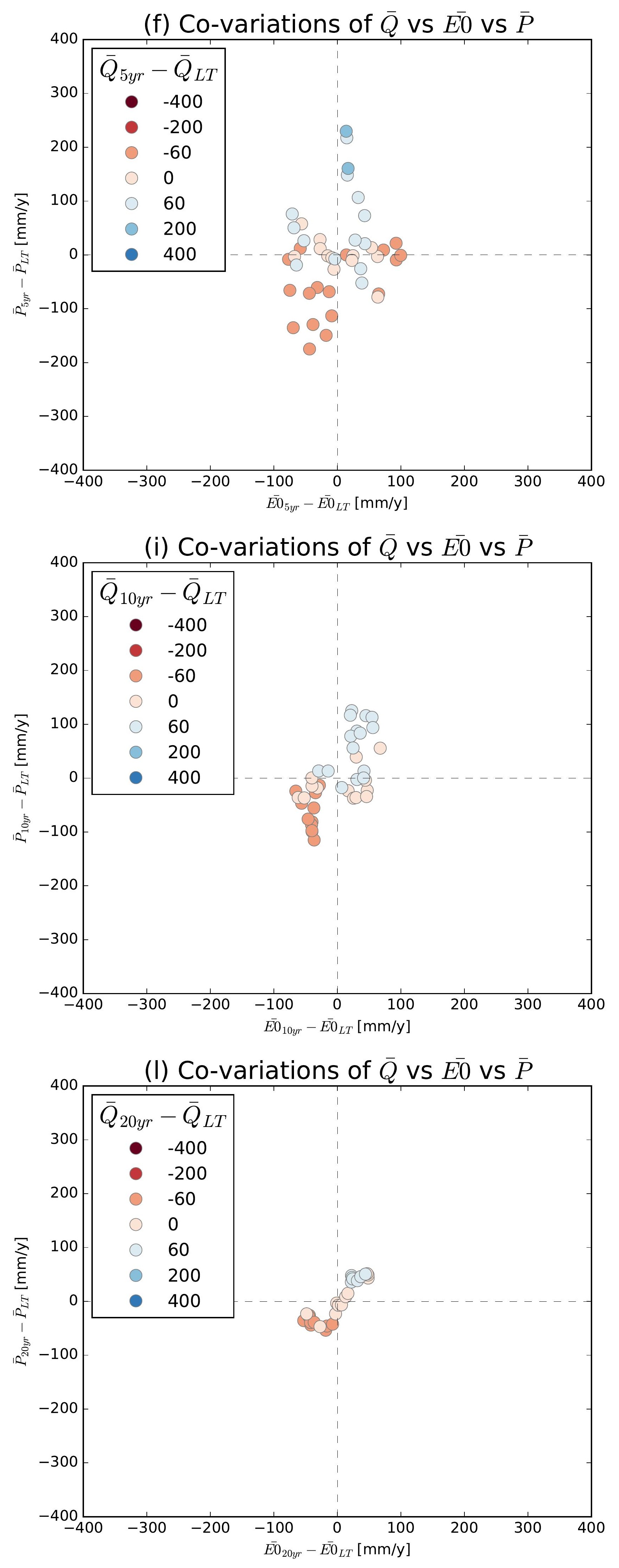

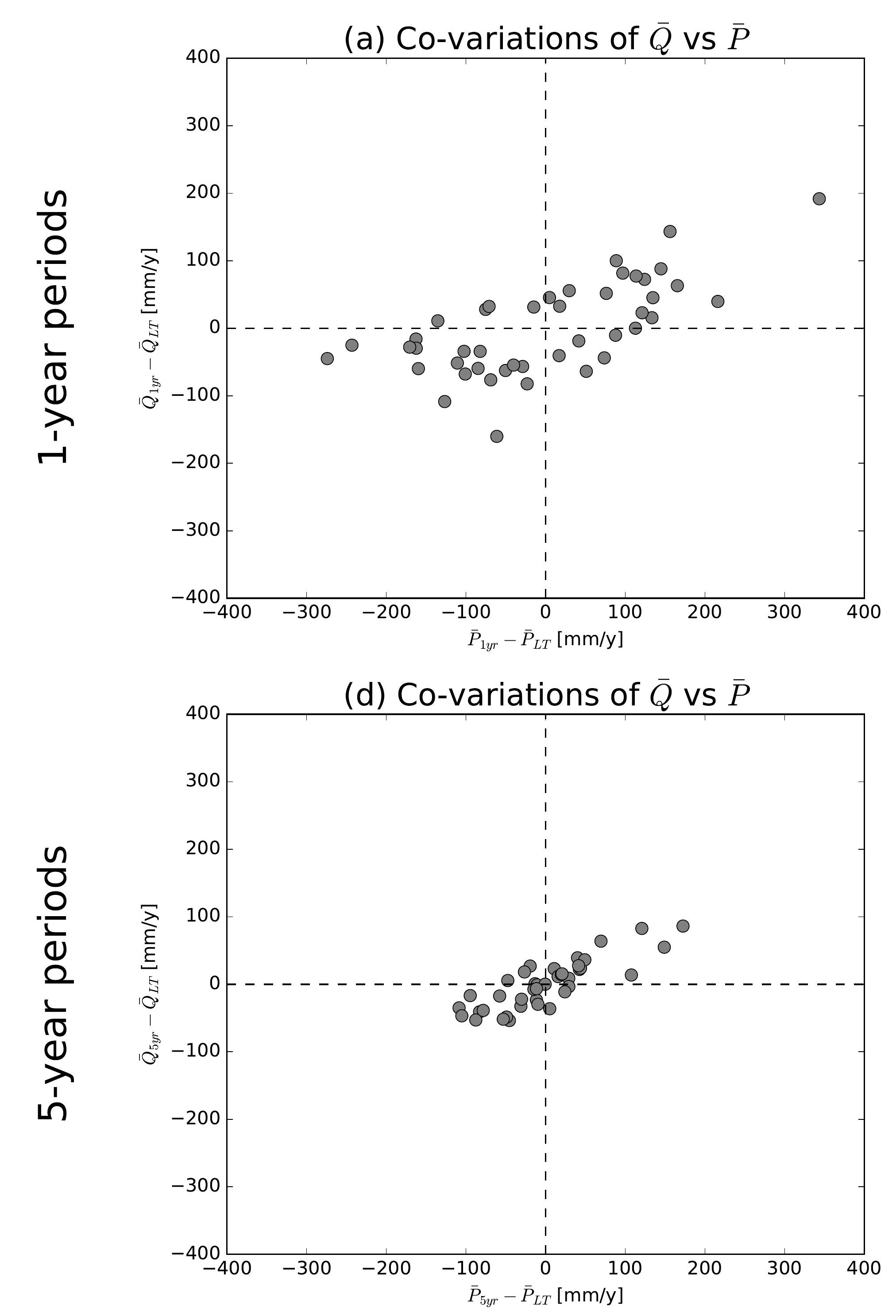

(g) Co-variations of $\bar{Q}$ vs $\bar{P}$

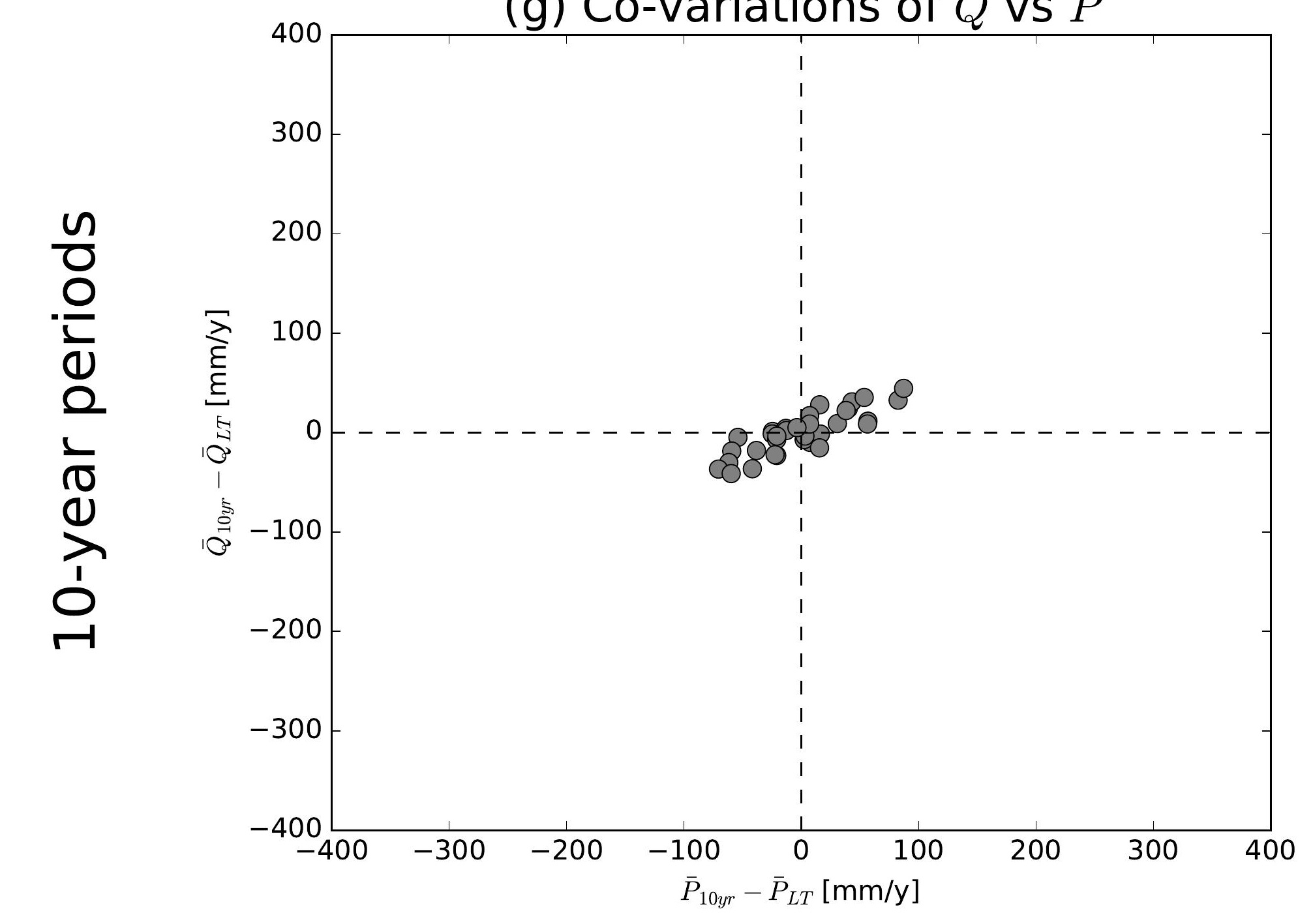

(j) Co-variations of $\bar{Q}$ vs $\bar{P}$

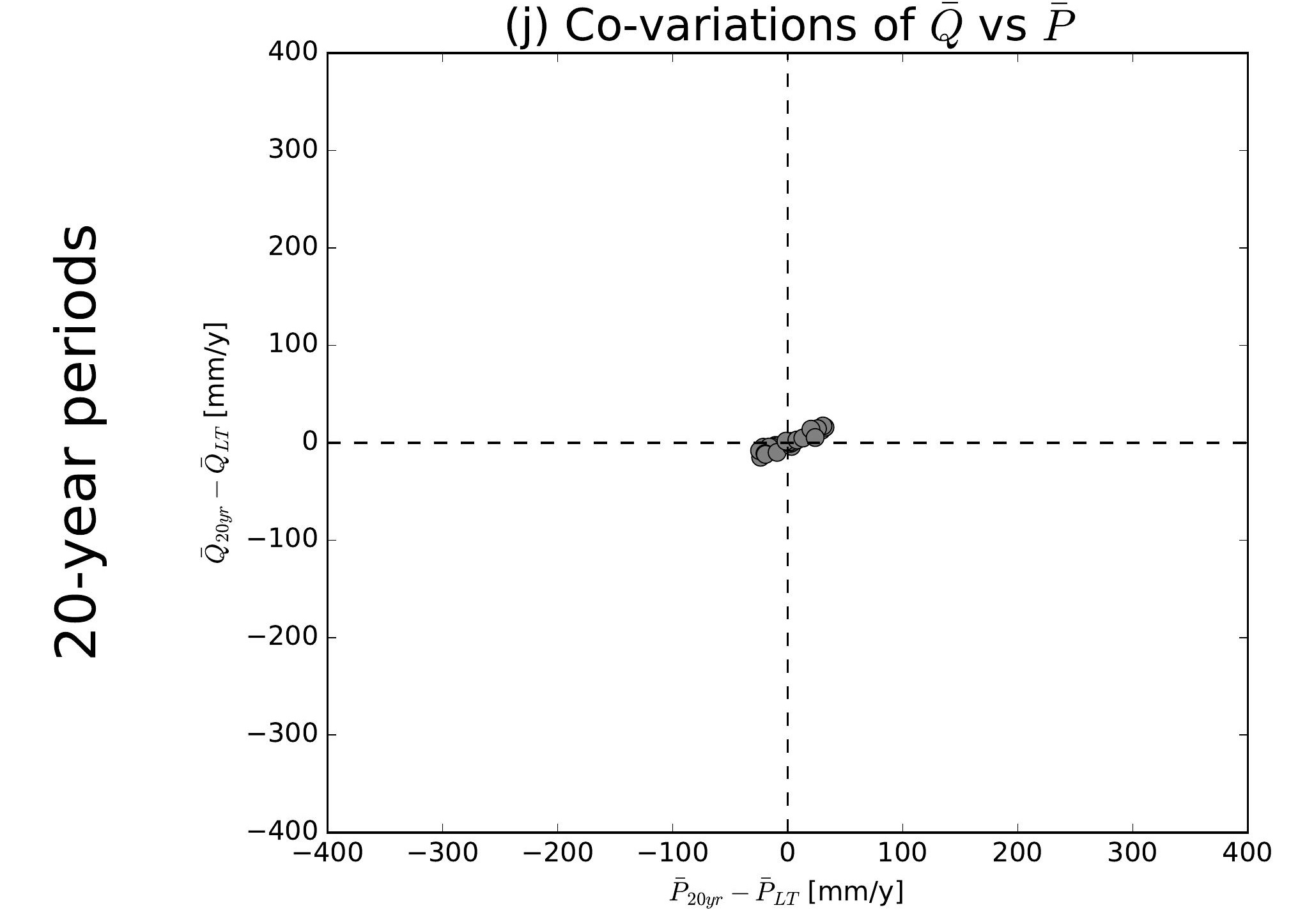

(b) Co-variations of $\bar{Q}$ vs $\overline{E 0}$

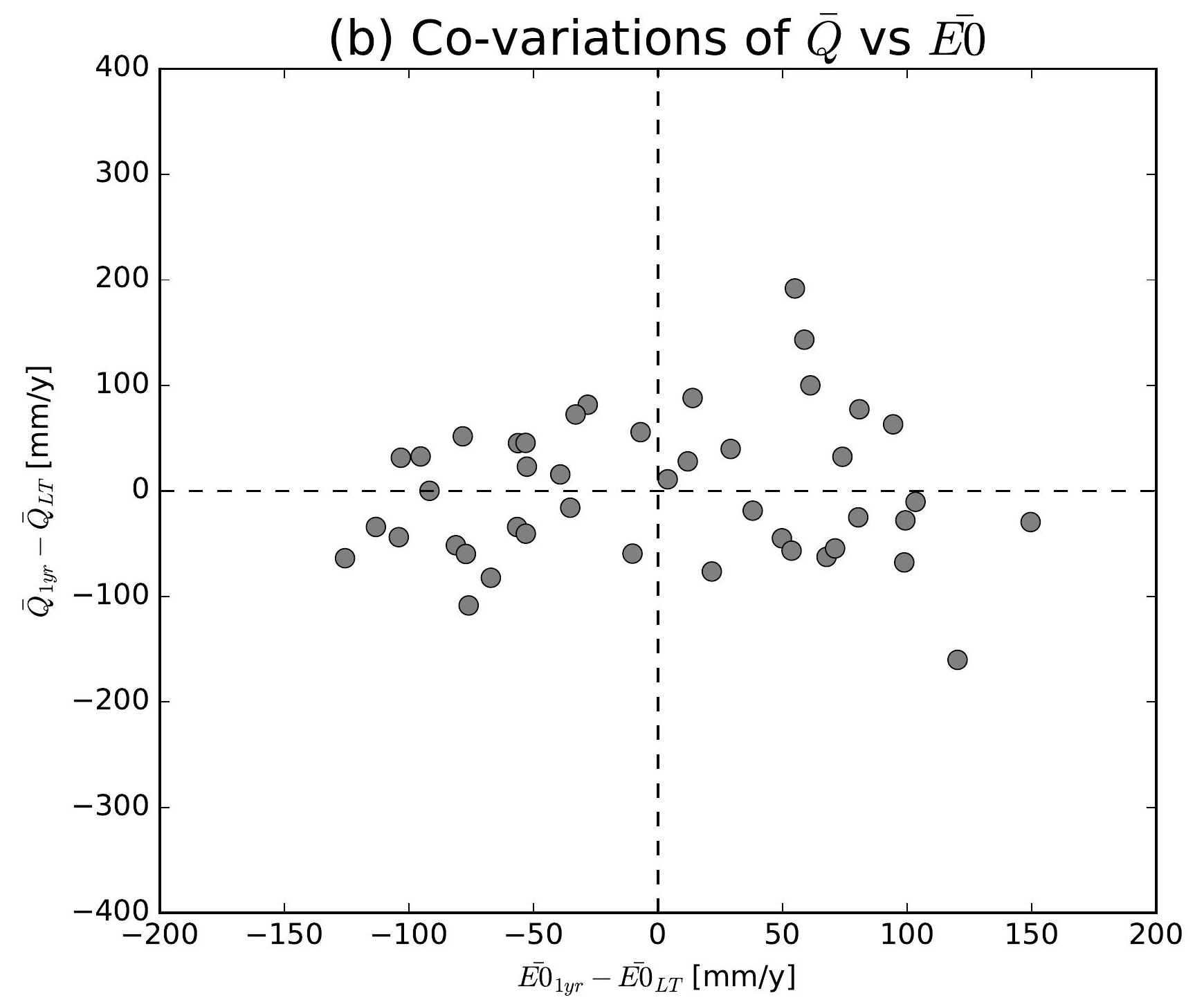

(e) Co-variations of $\bar{Q}$ vs $\overline{E 0}$

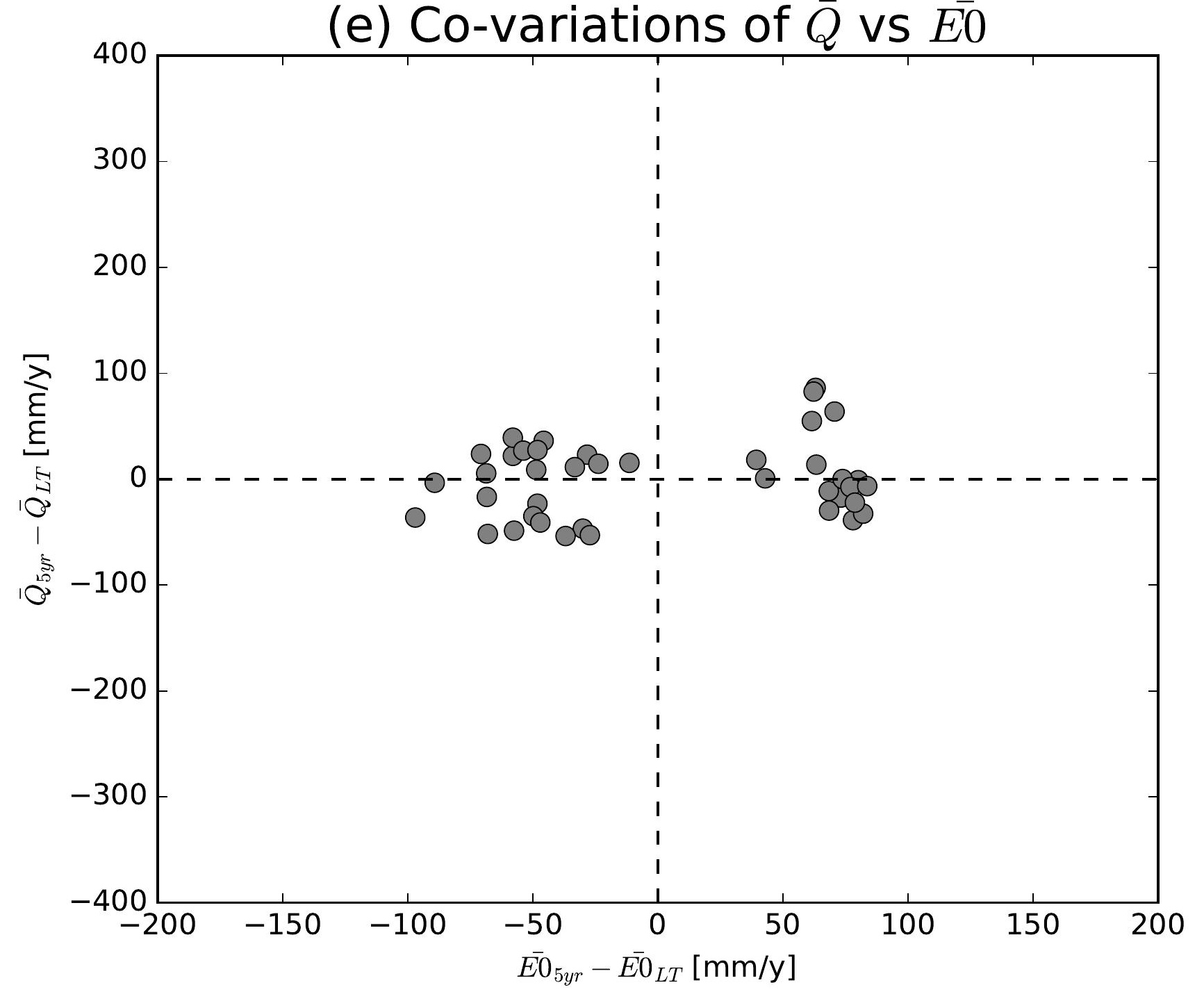

(h) Co-variations of $\bar{Q}$ vs $\overline{E 0}$

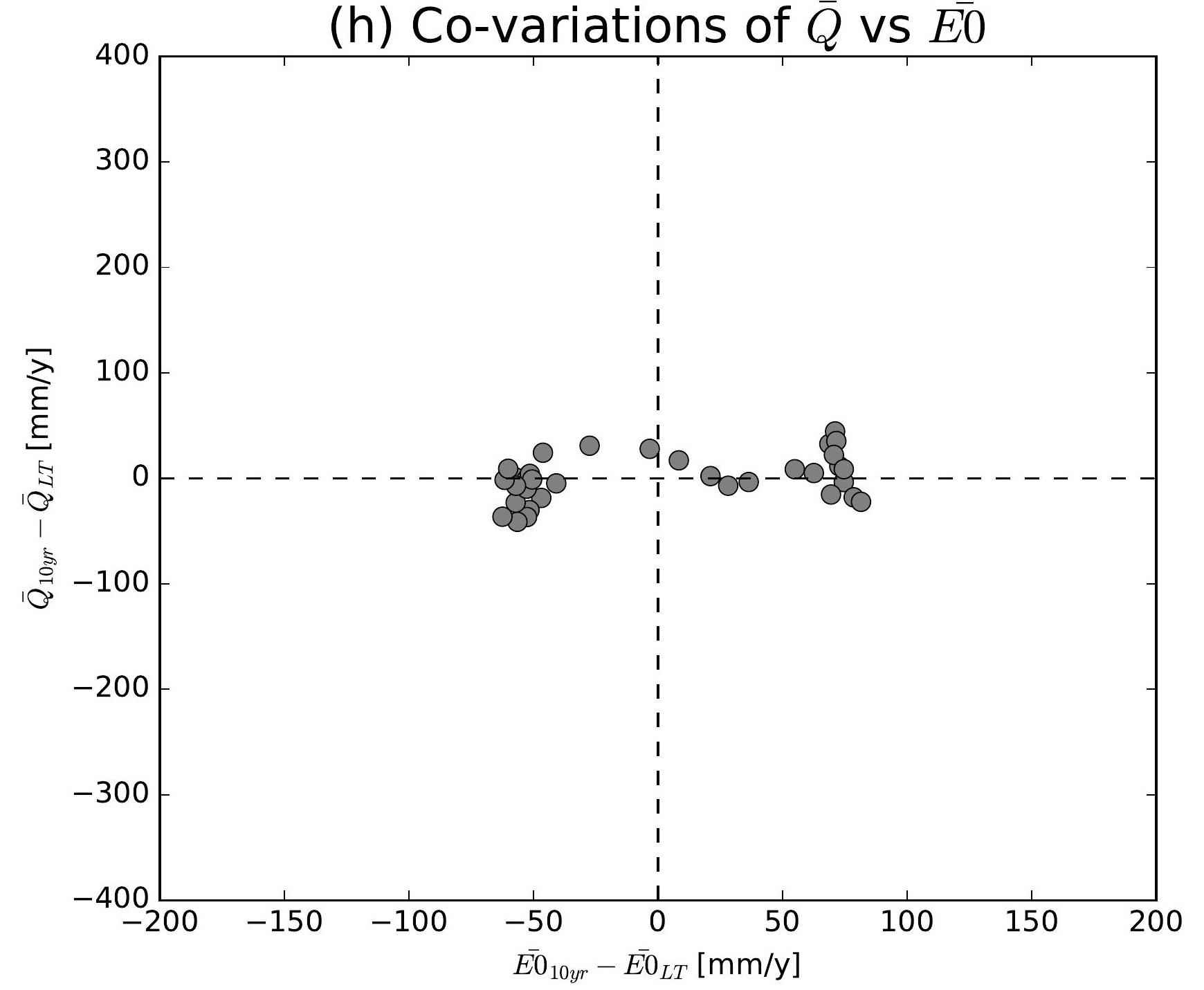

(k) Co-variations of $\bar{Q}$ vs $\overline{E 0}$

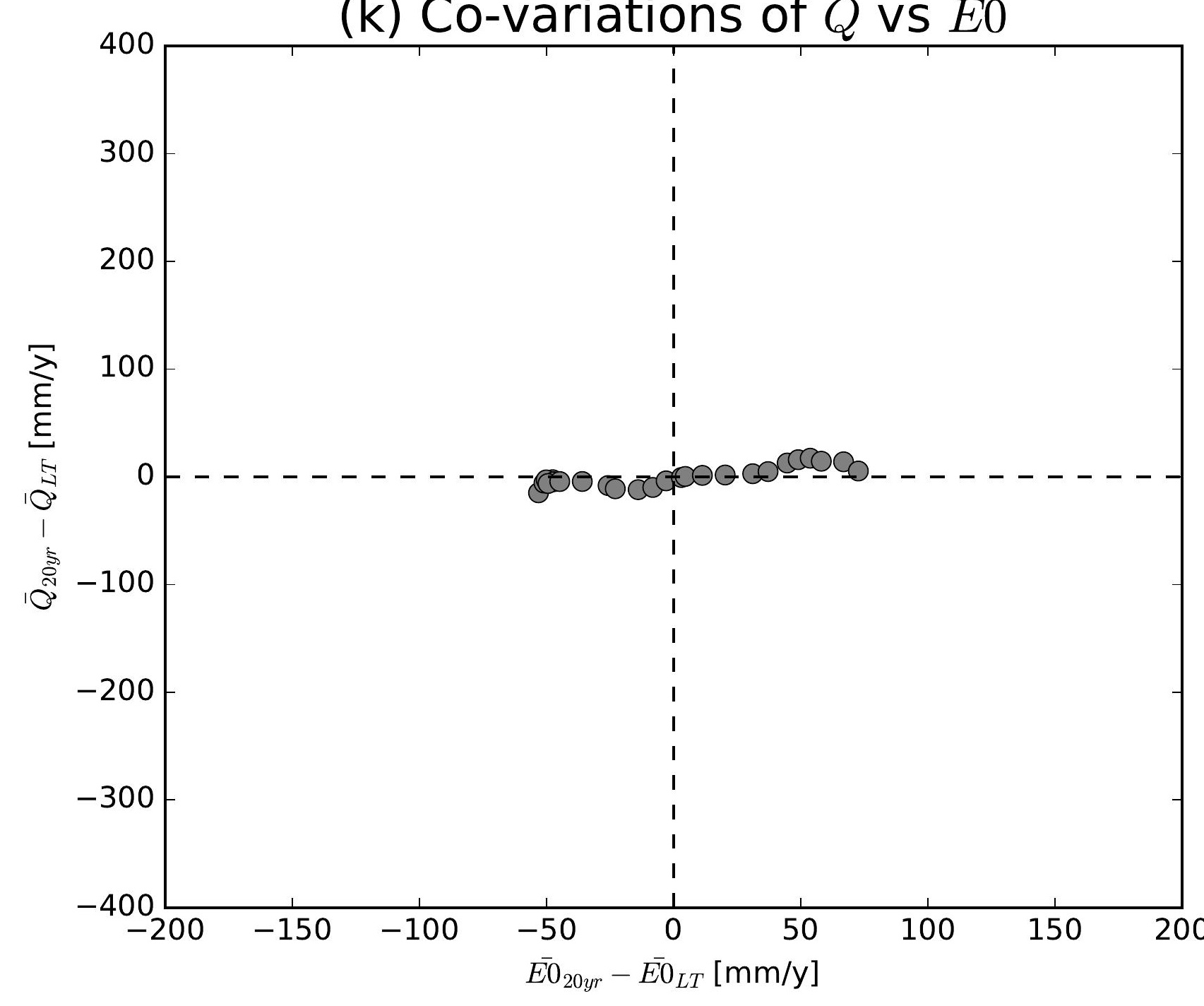

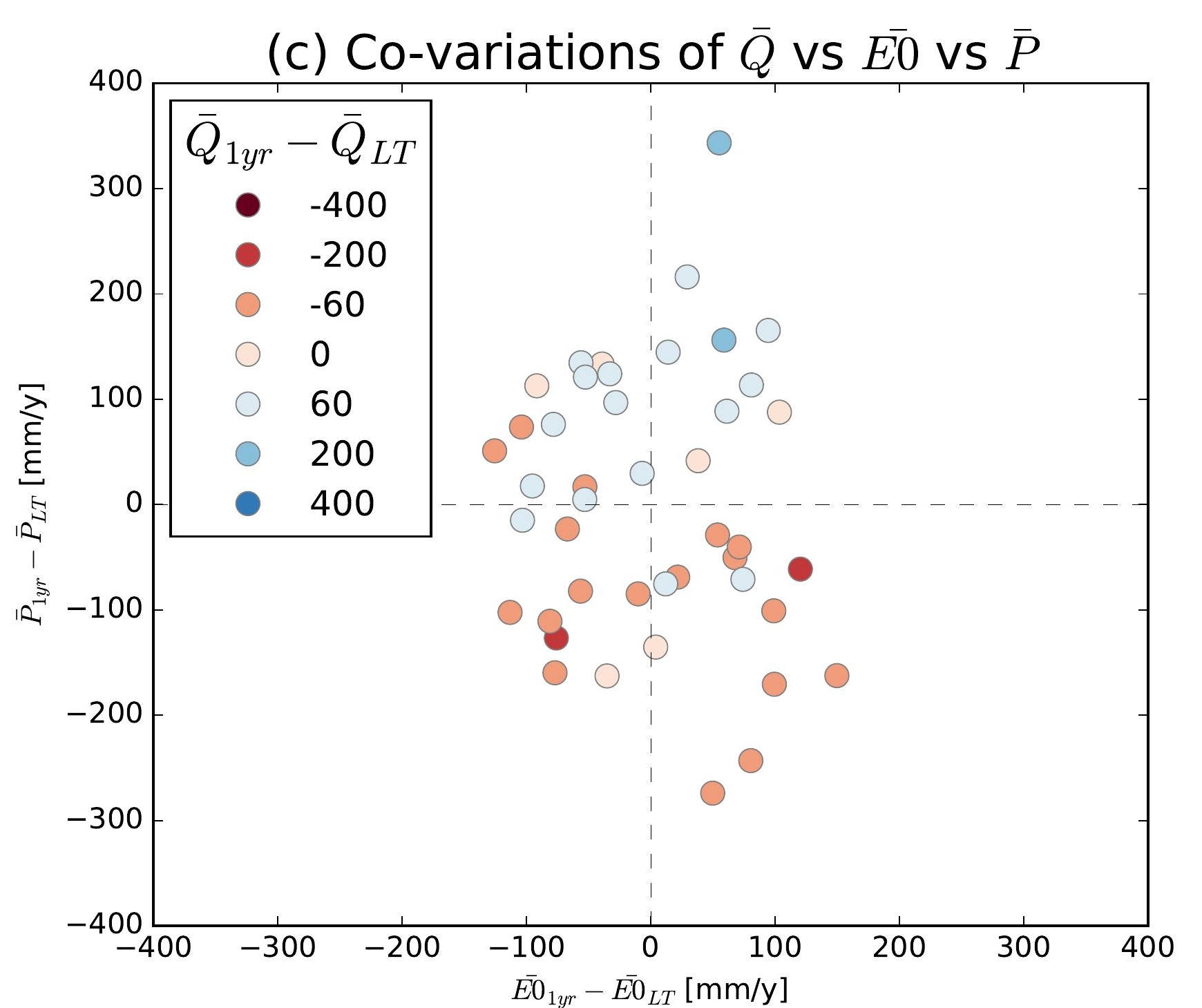
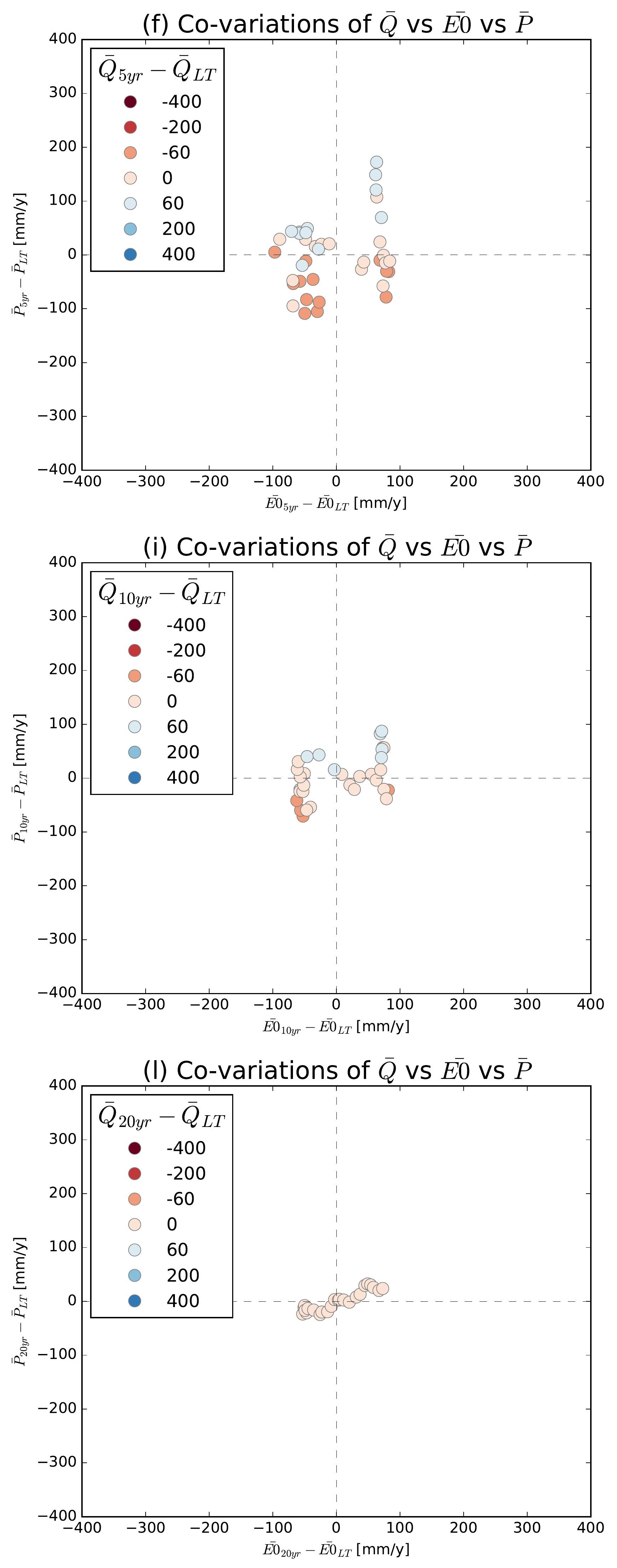

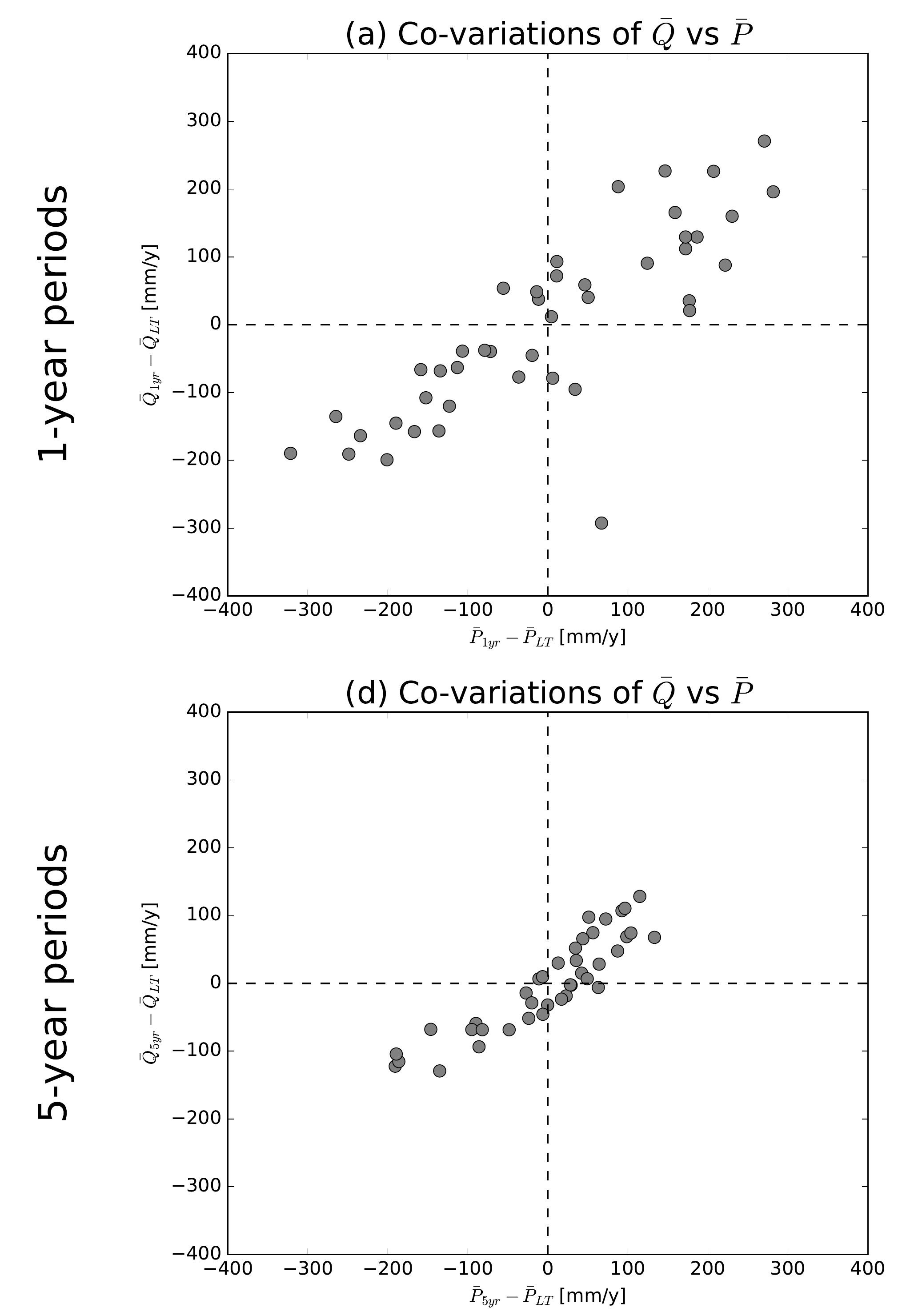

(g) Co-variations of $\bar{Q}$ vs $\bar{P}$
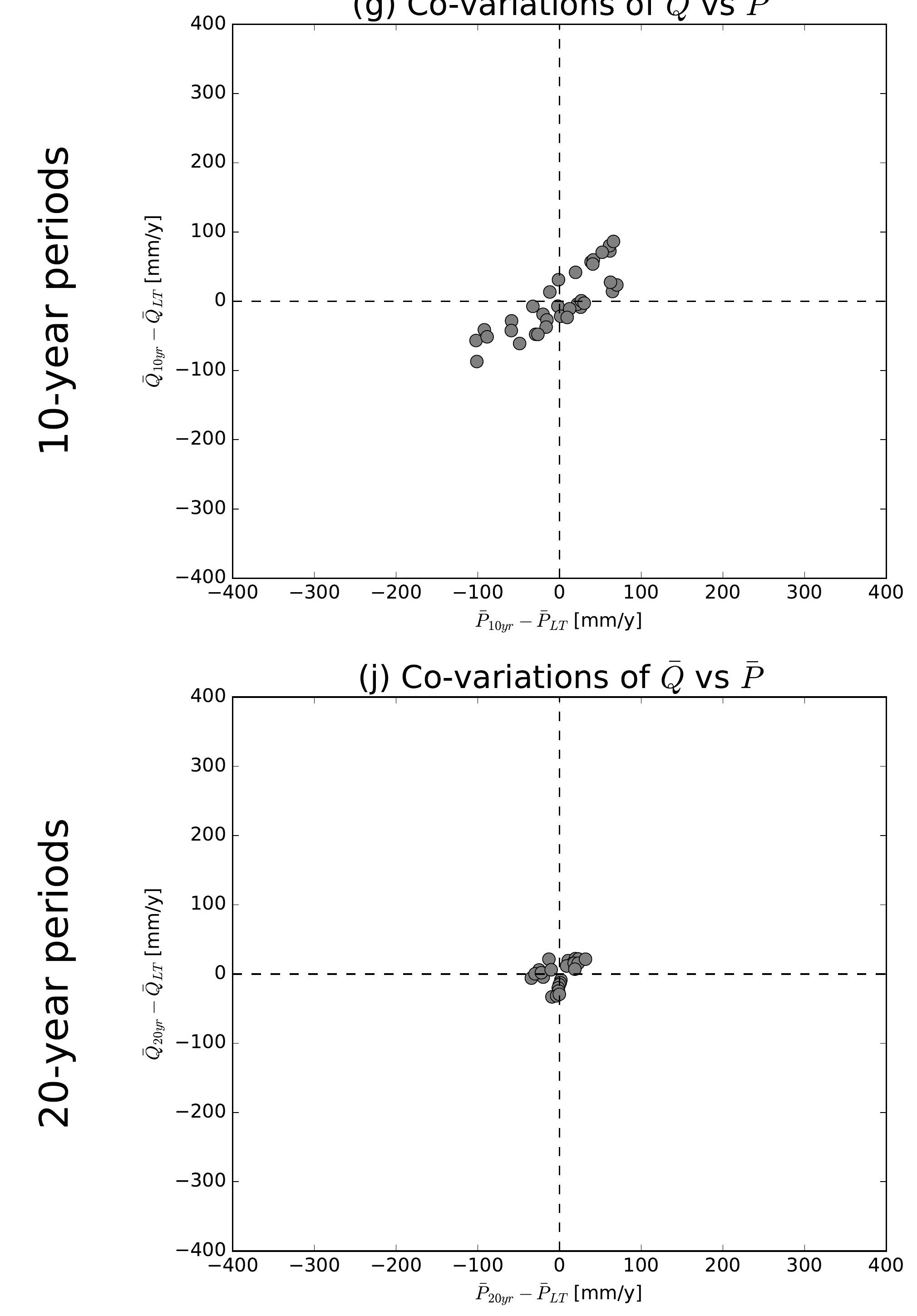

(b) Co-variations of $\bar{Q}$ vs $\overline{E 0}$

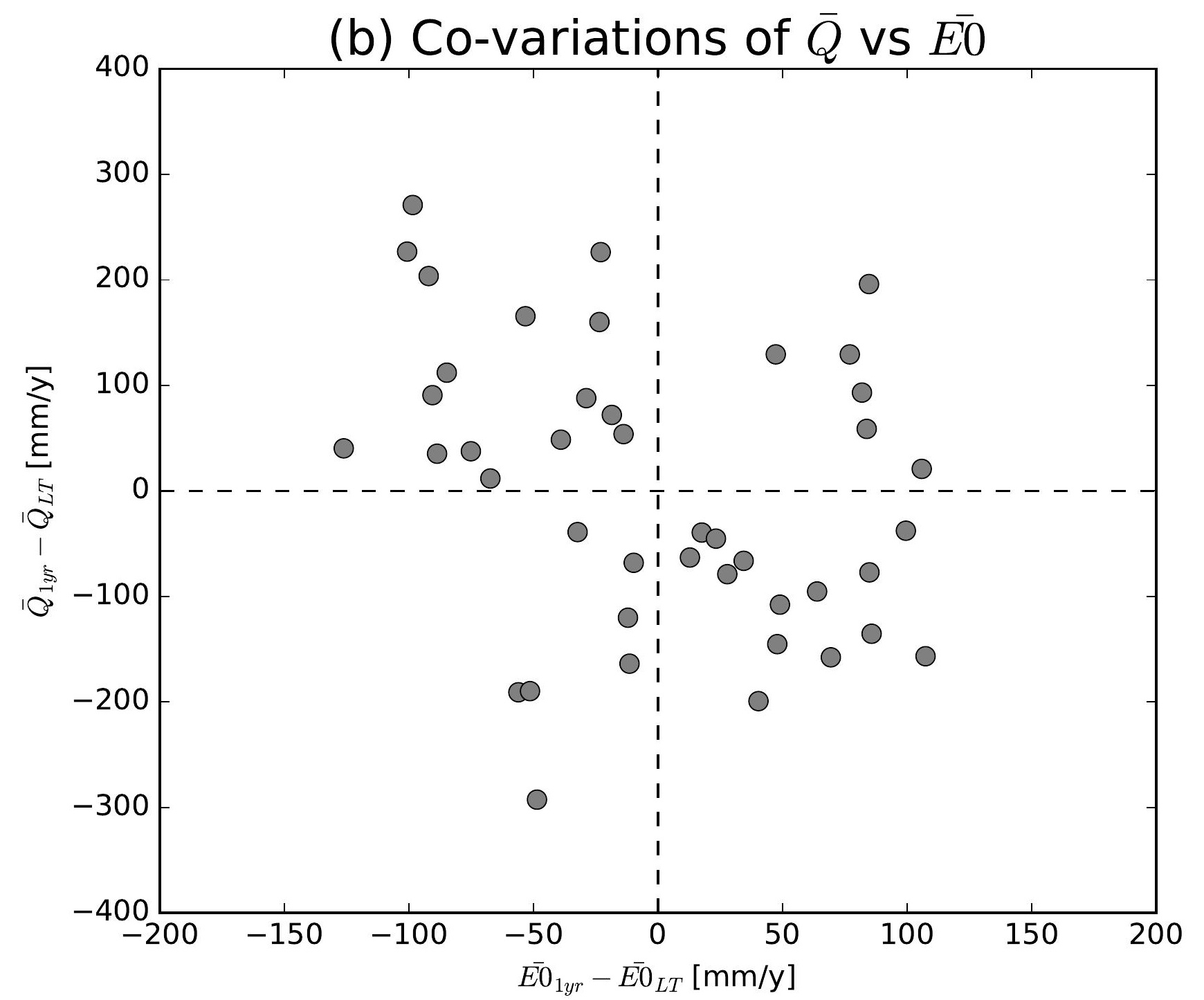

(e) Co-variations of $\bar{Q}$ vs $\overline{E 0}$

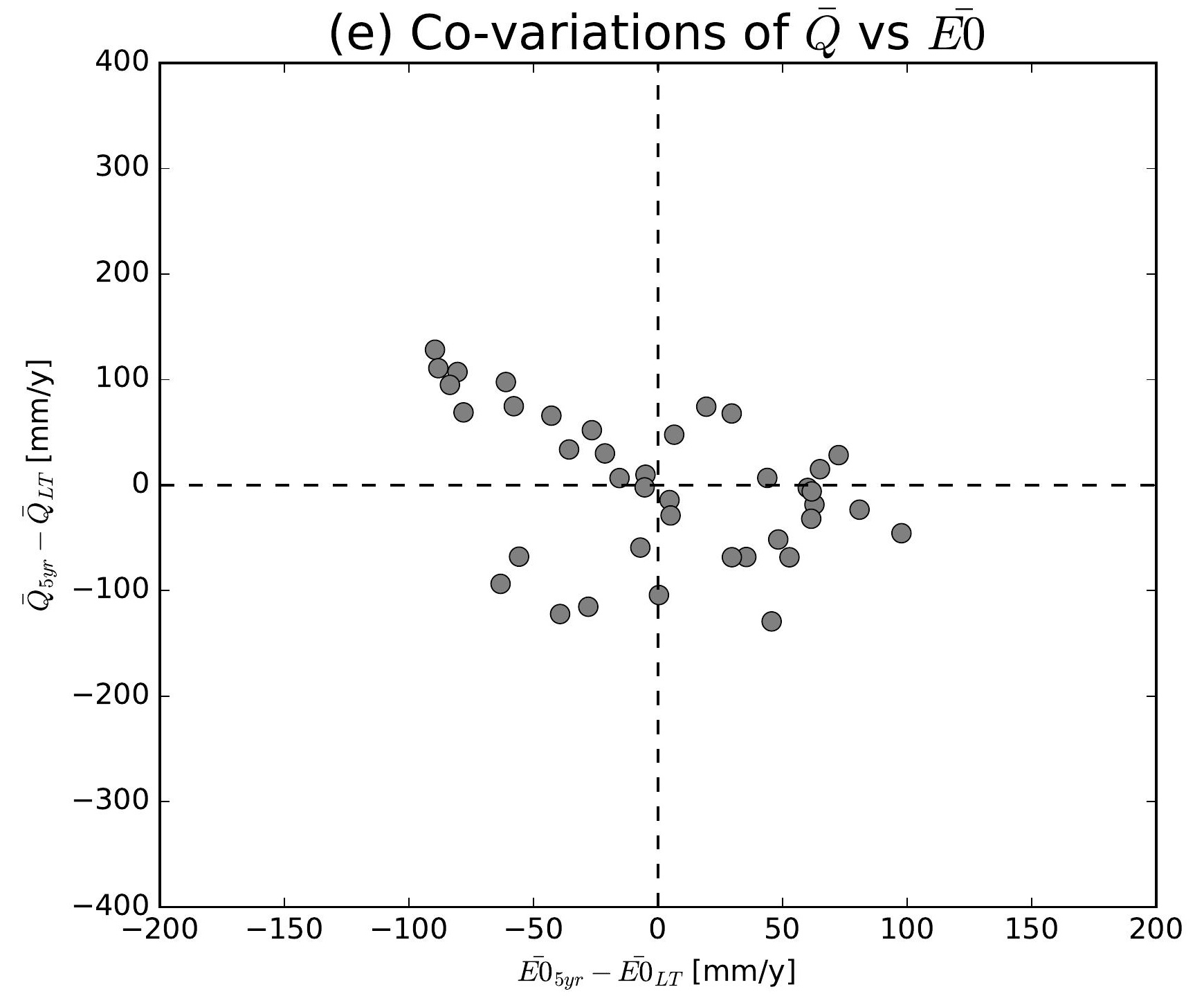

(h) Co-variations of $\bar{Q}$ vs $\overline{E 0}$

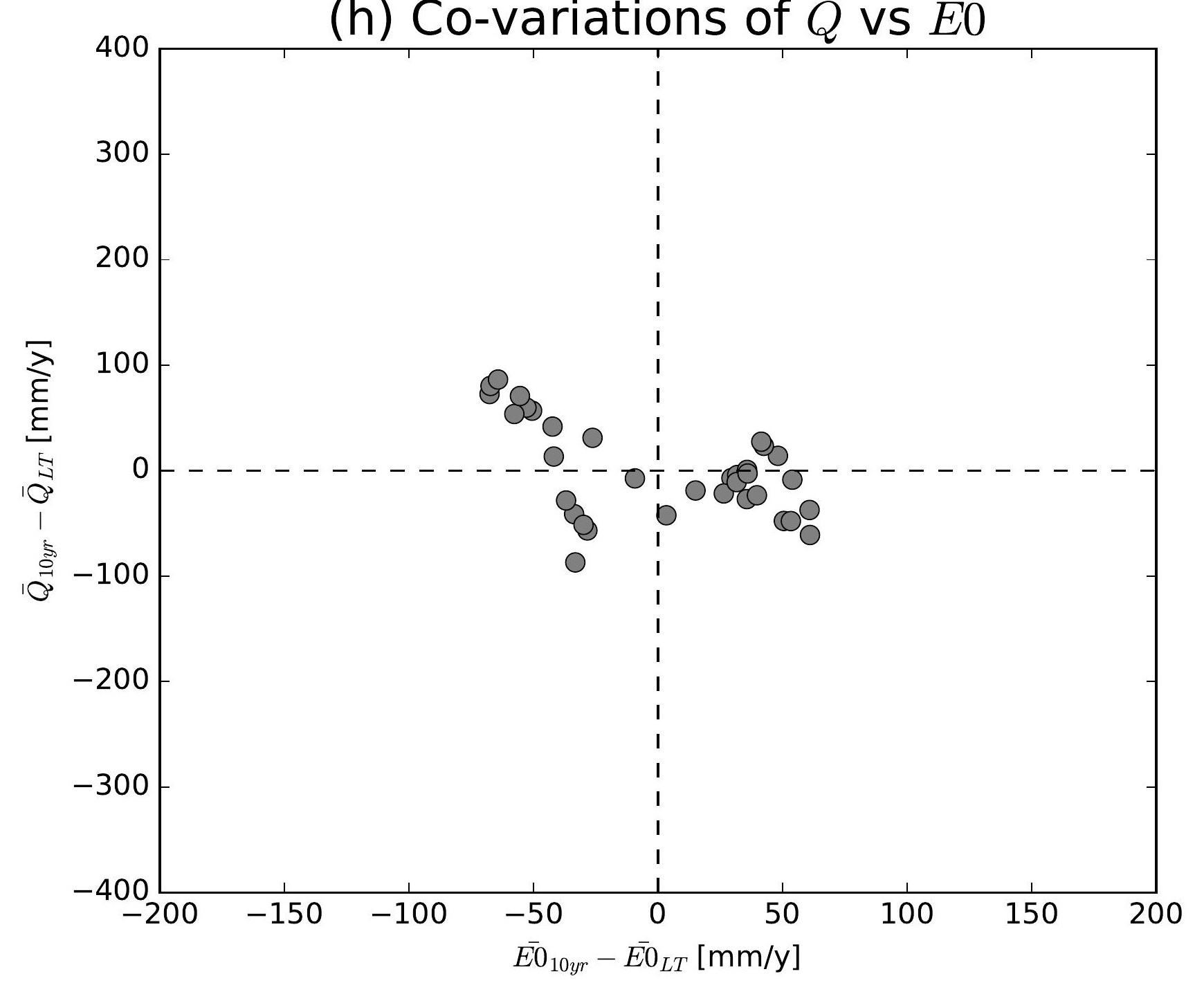

(k) Co-variations of $\bar{Q}$ vs $\overline{E 0}$

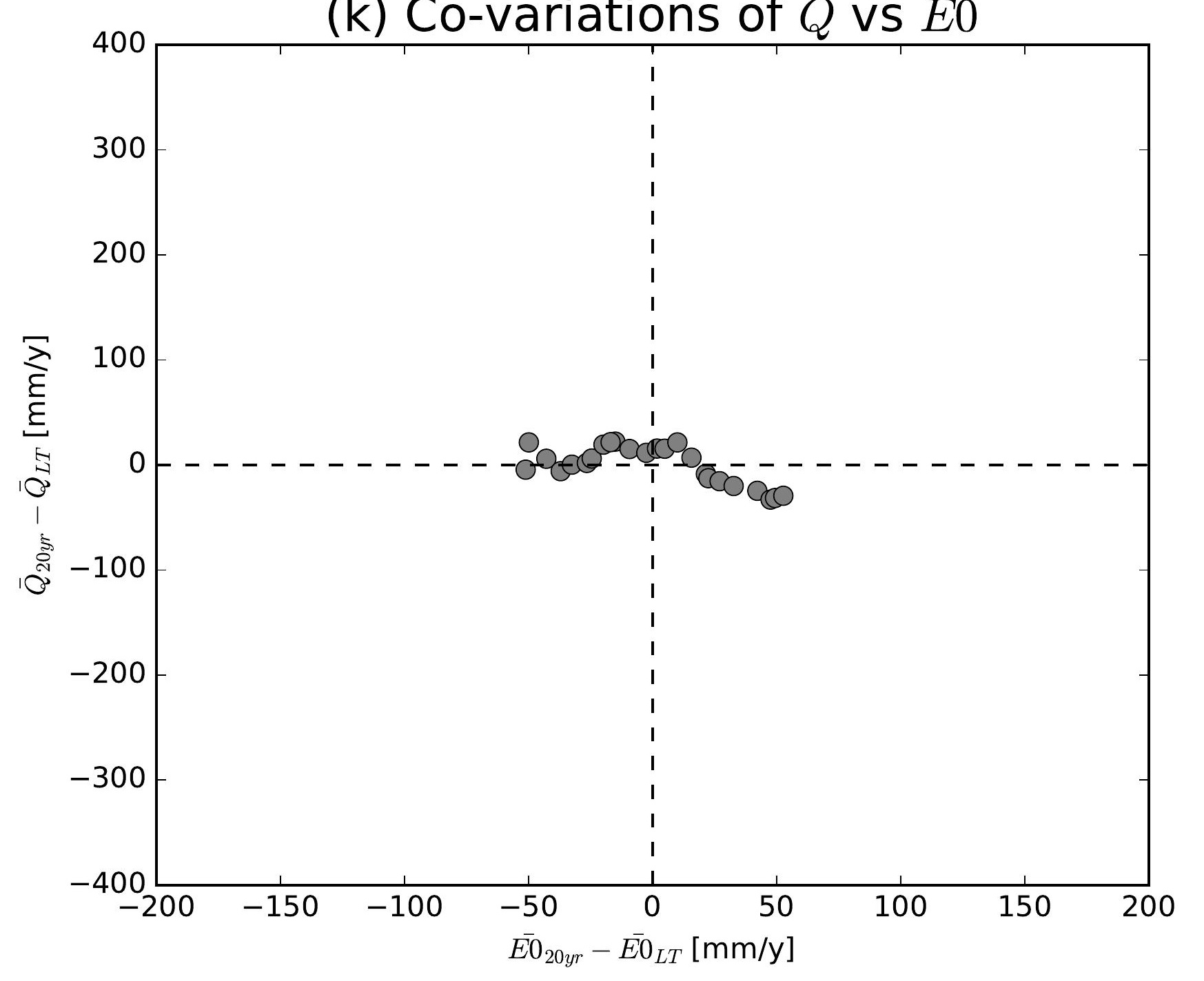

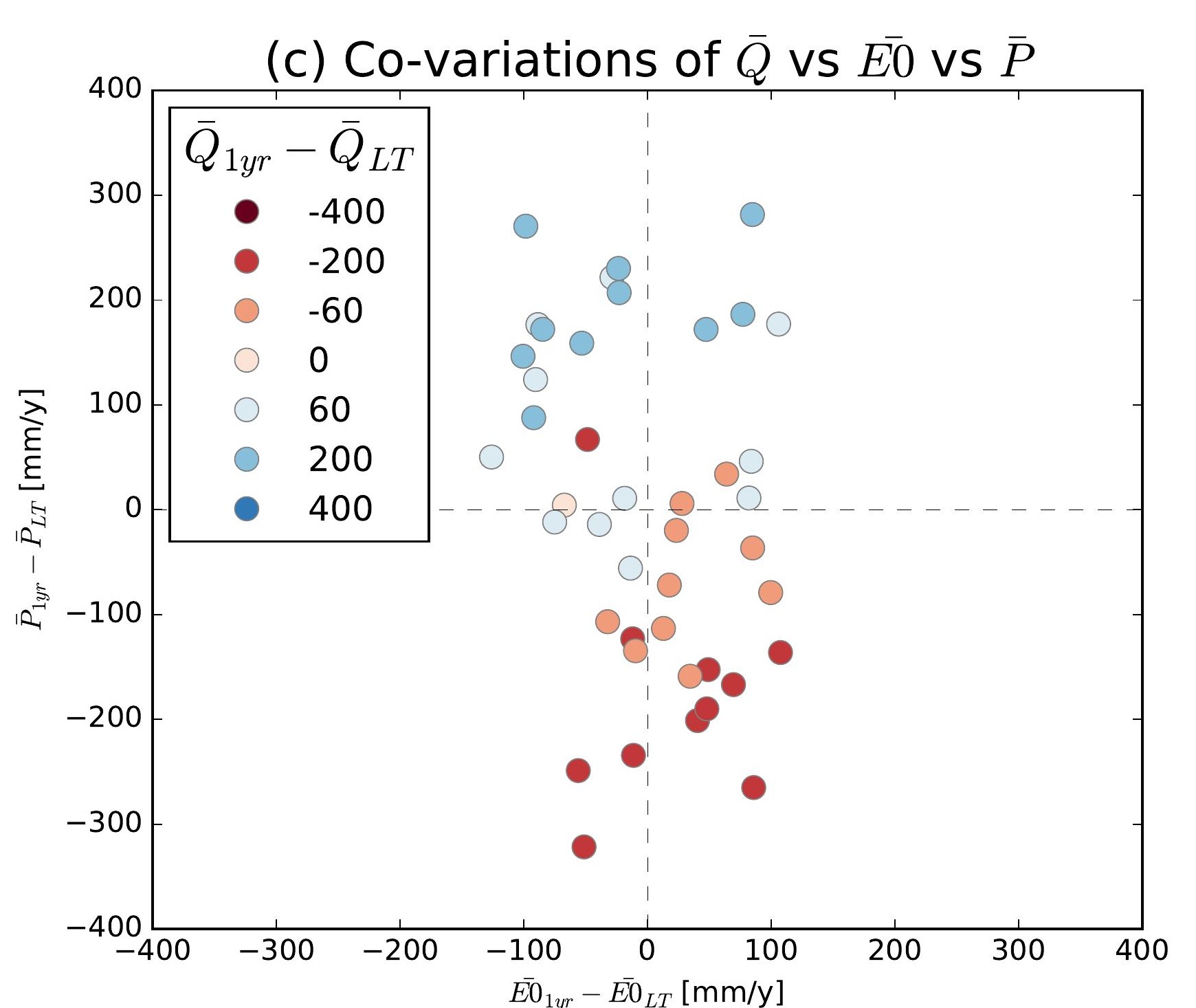
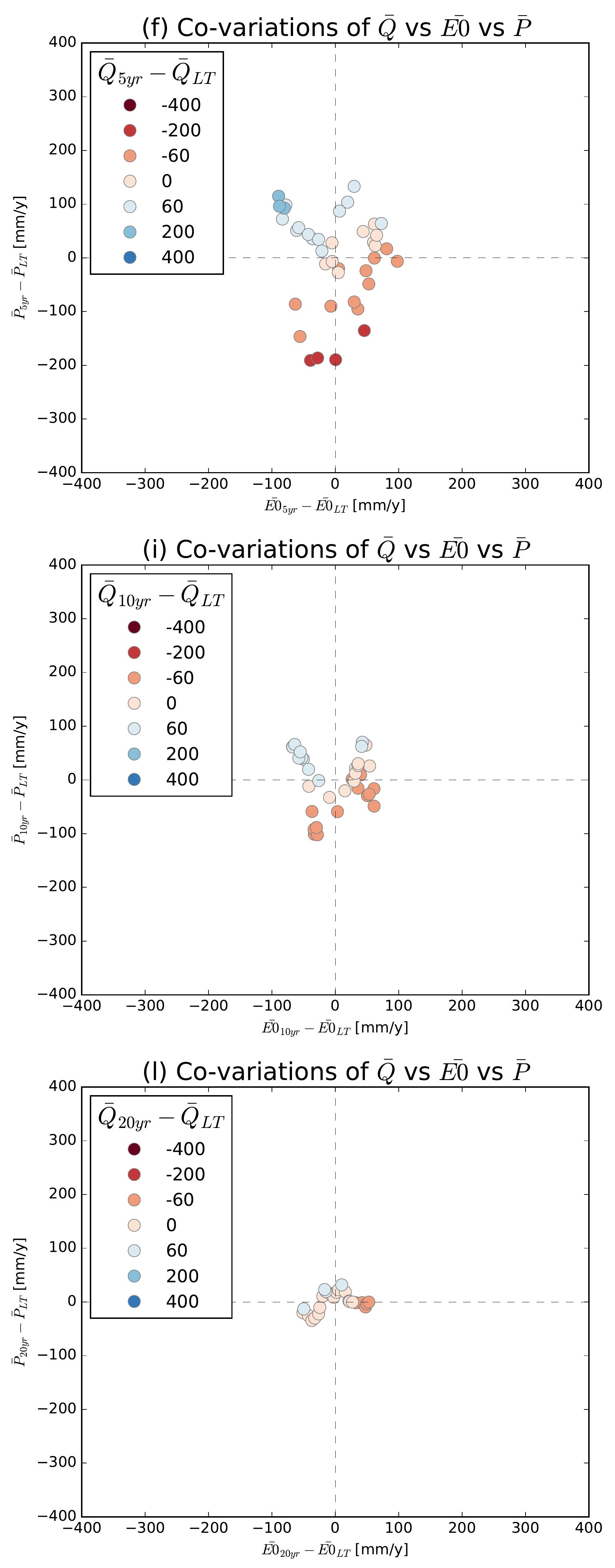

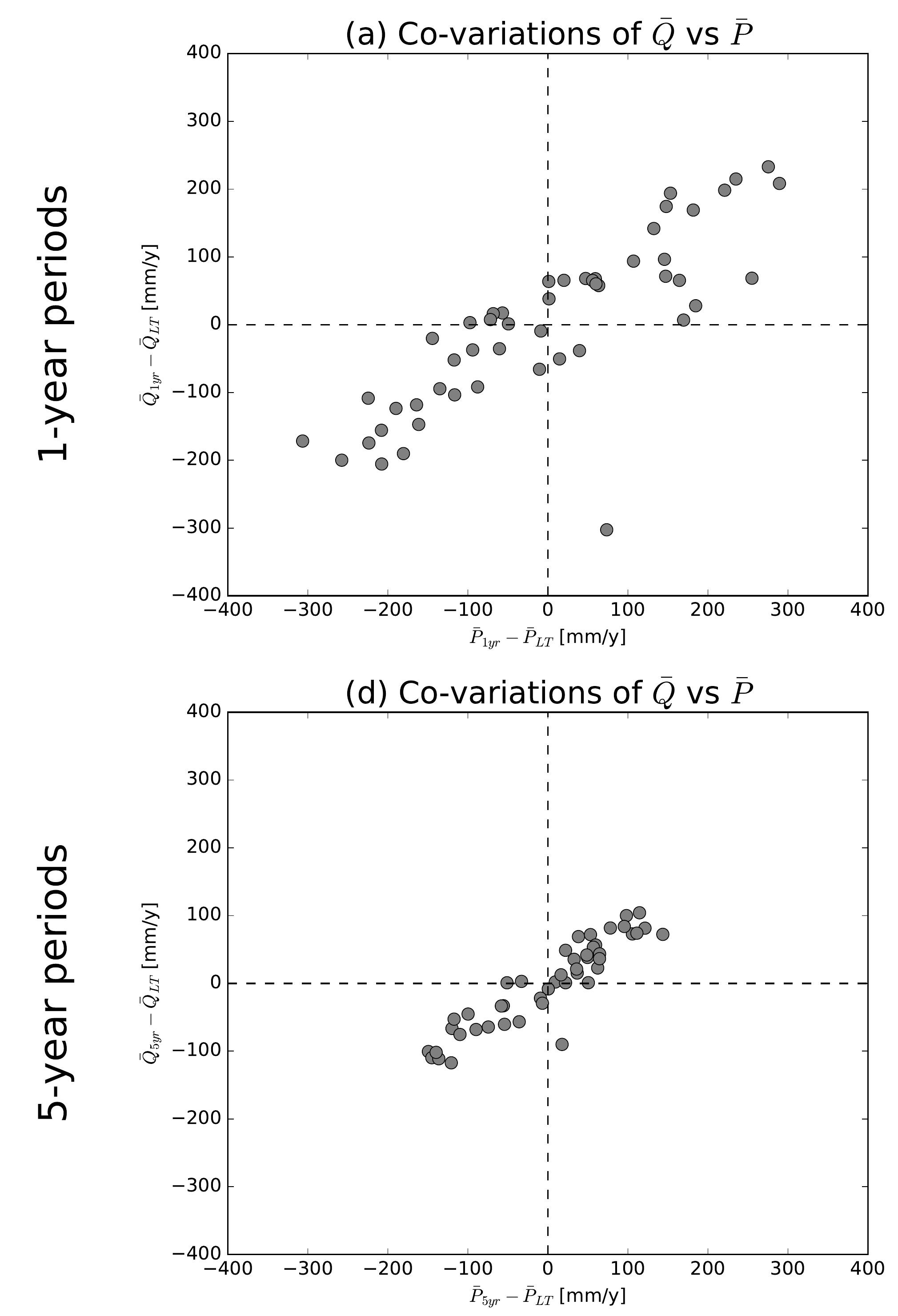

(g) Co-variations of $\bar{Q}$ vs $\bar{P}$
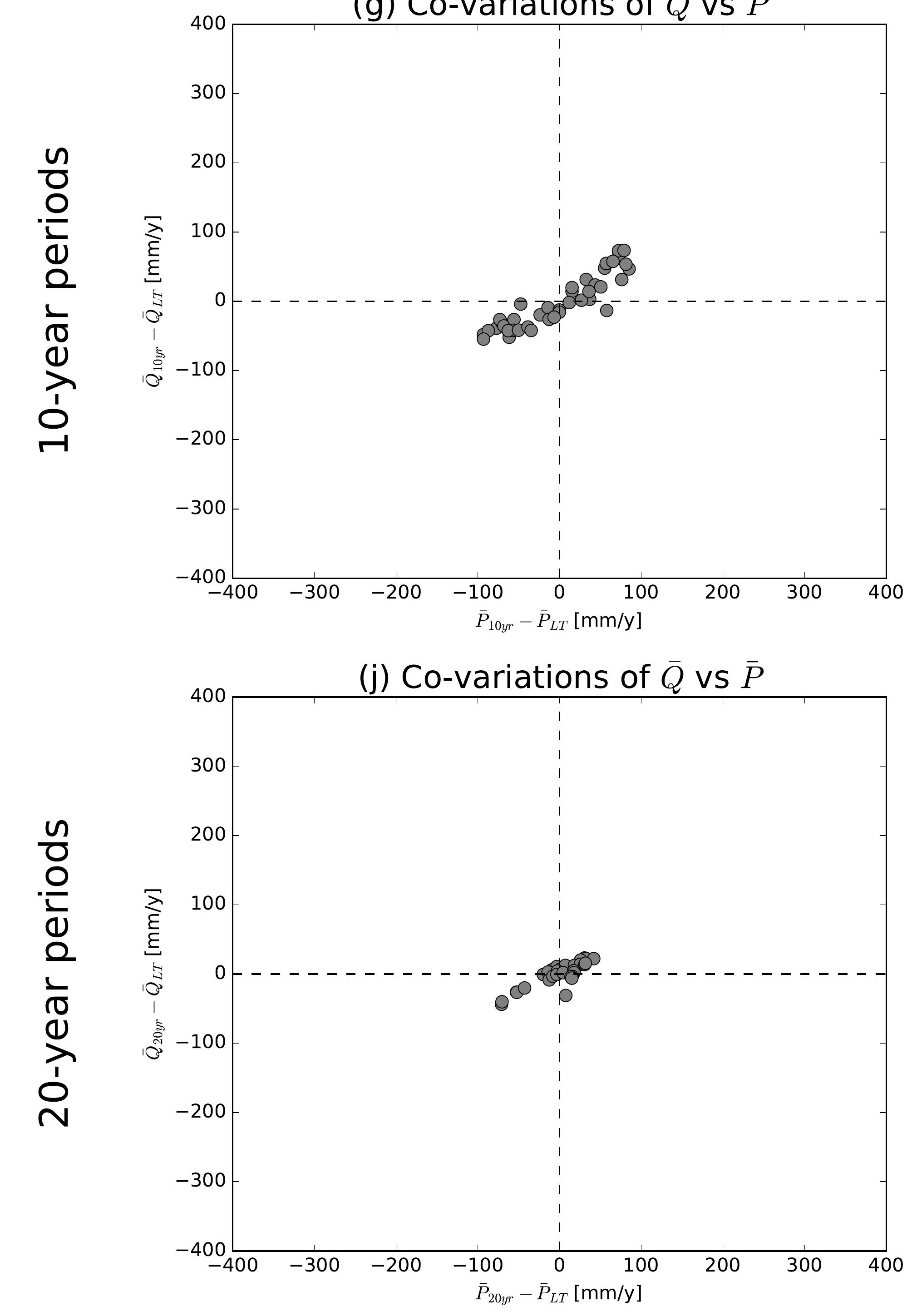

(b) Co-variations of $\bar{Q}$ vs $\overline{E 0}$

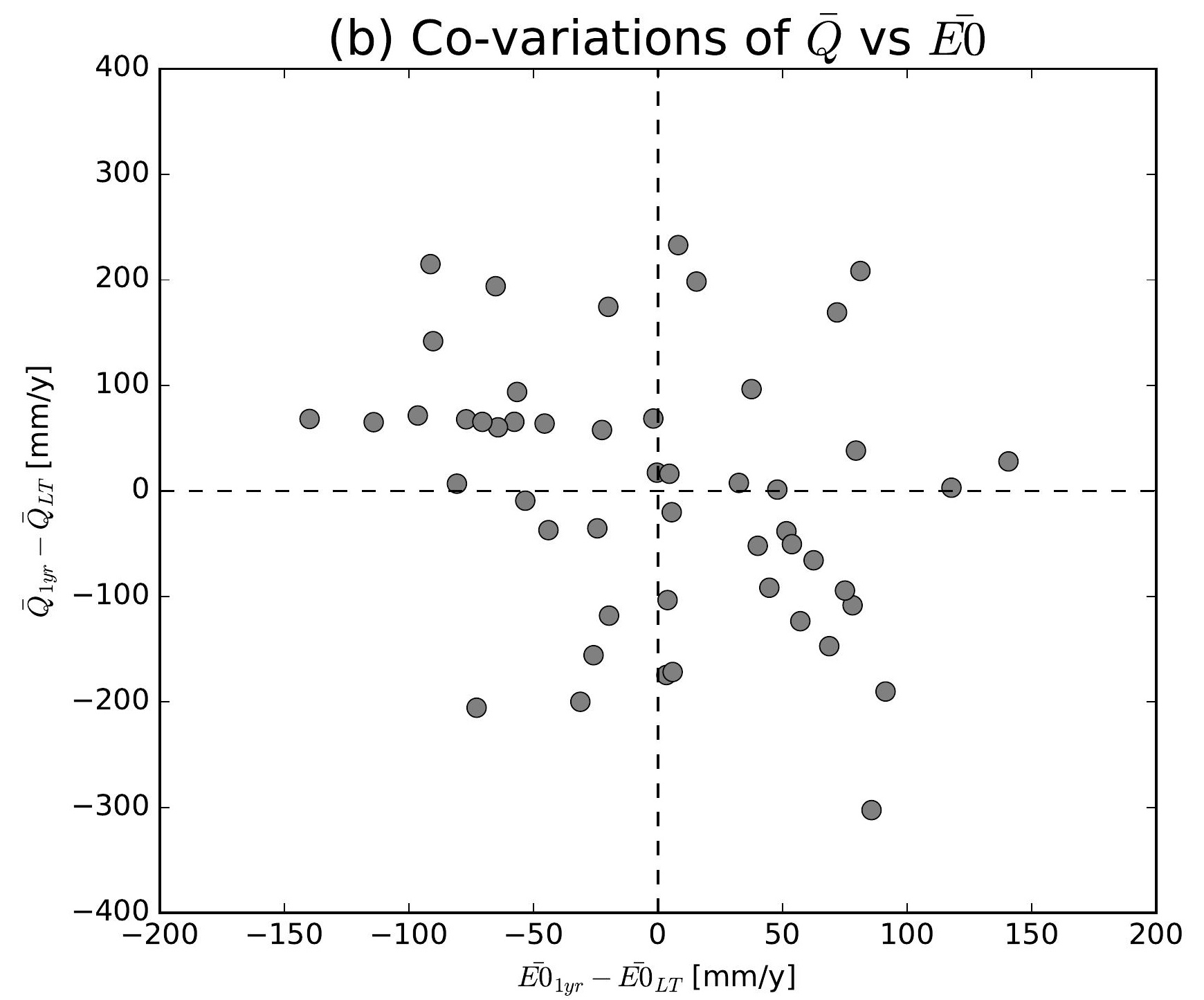

(e) Co-variations of $\bar{Q}$ vs $\overline{E 0}$

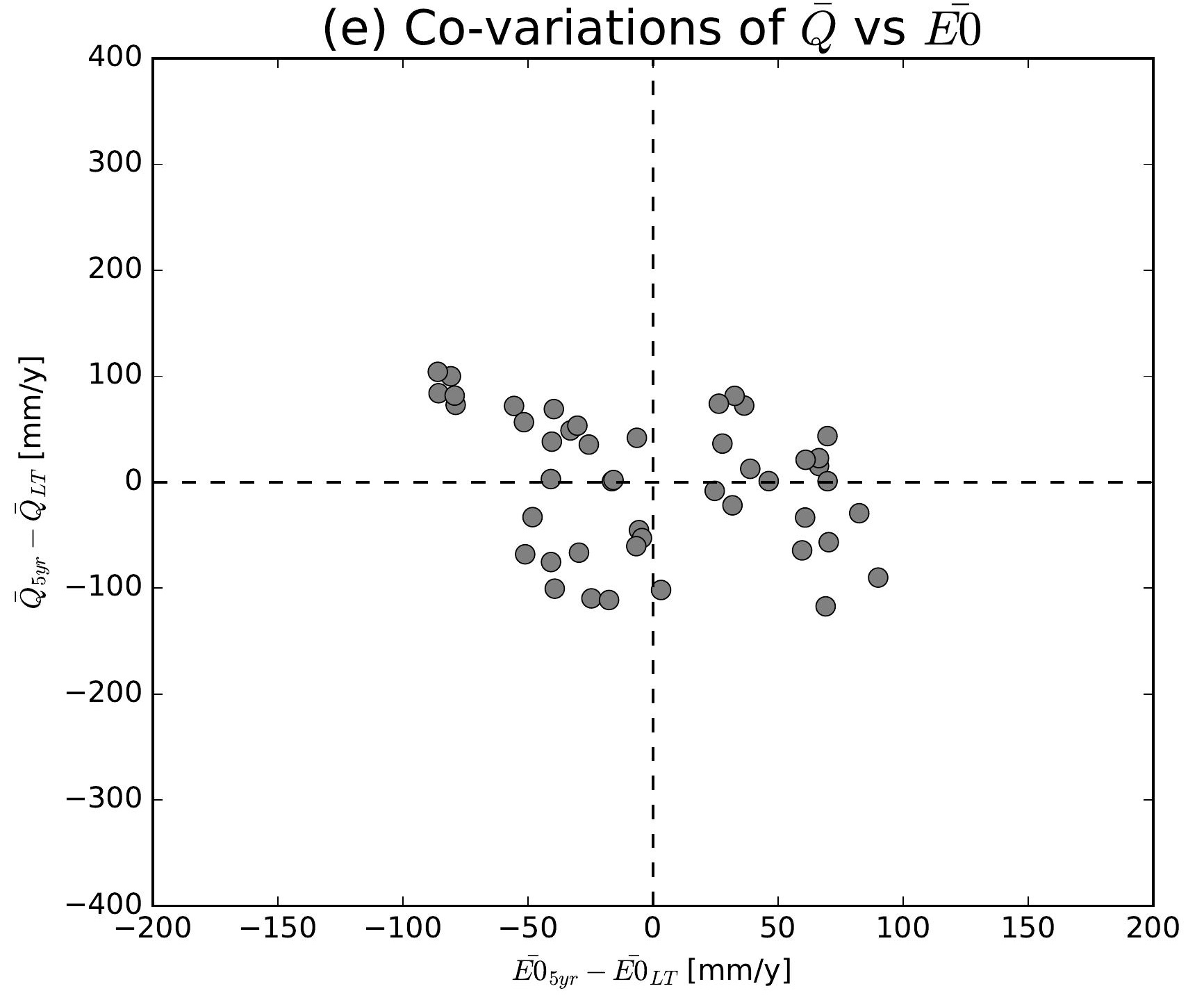

(h) Co-variations of $\bar{Q}$ vs $\overline{E 0}$

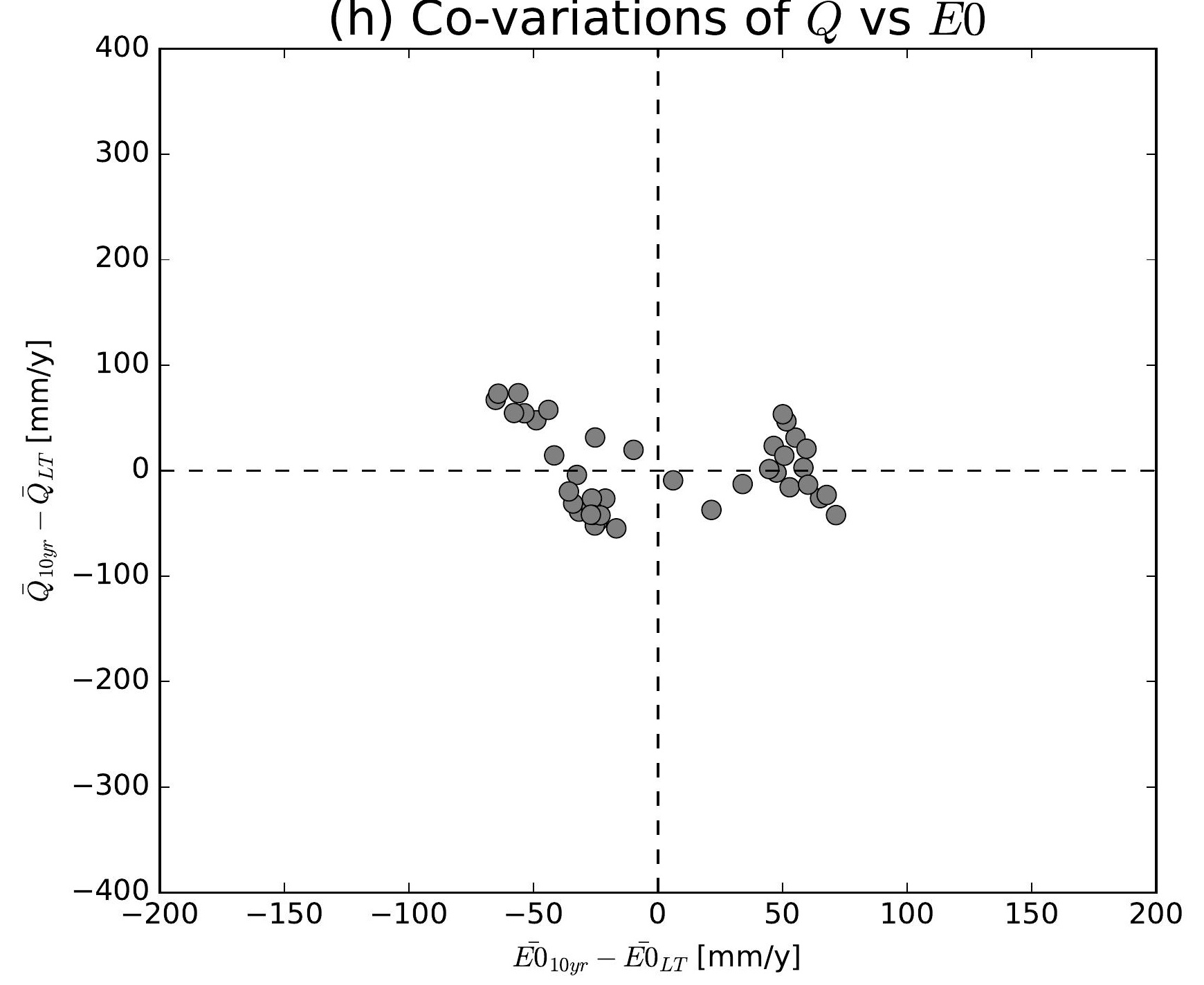

(k) Co-variations of $\bar{Q}$ vs $\overline{E 0}$

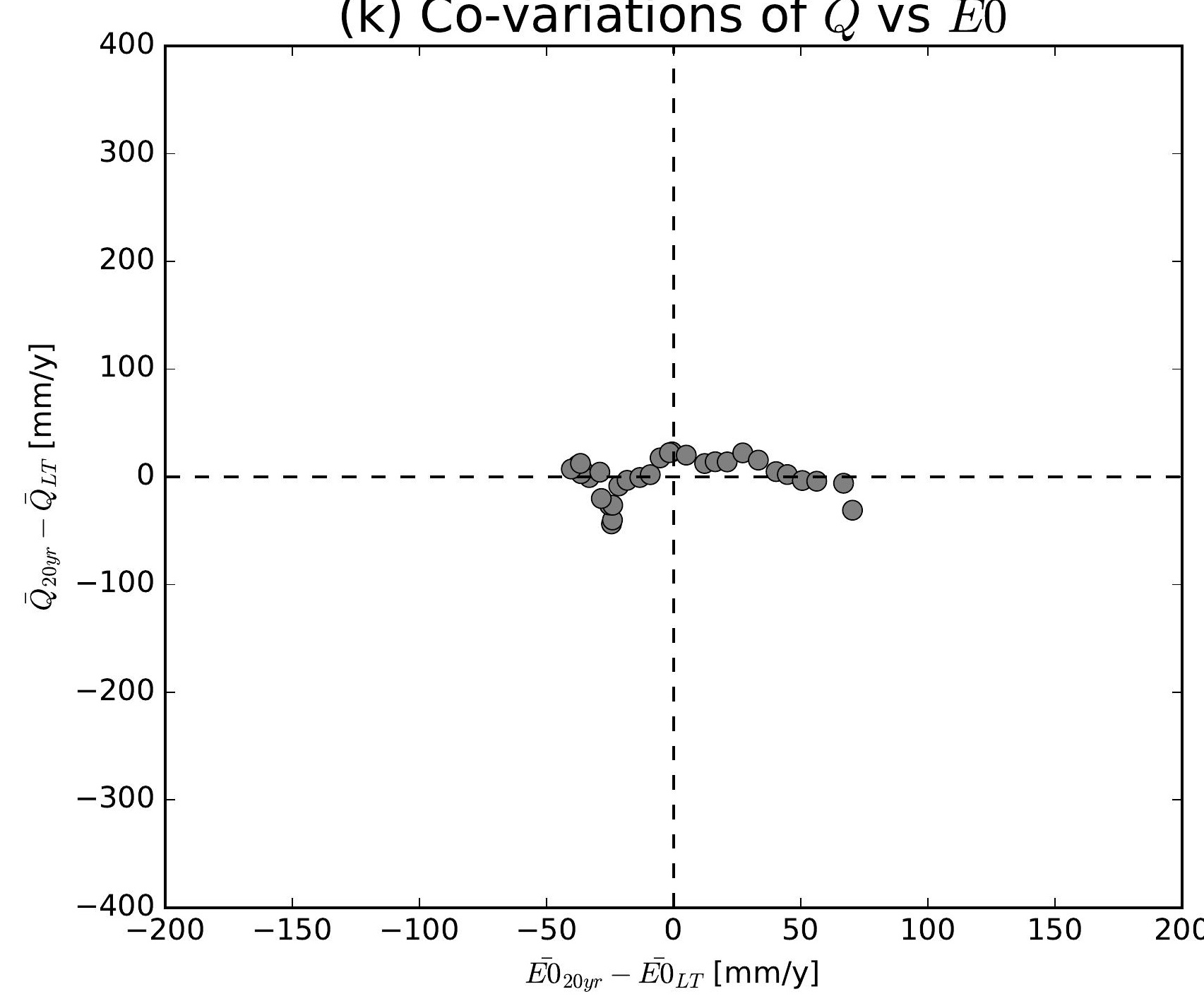

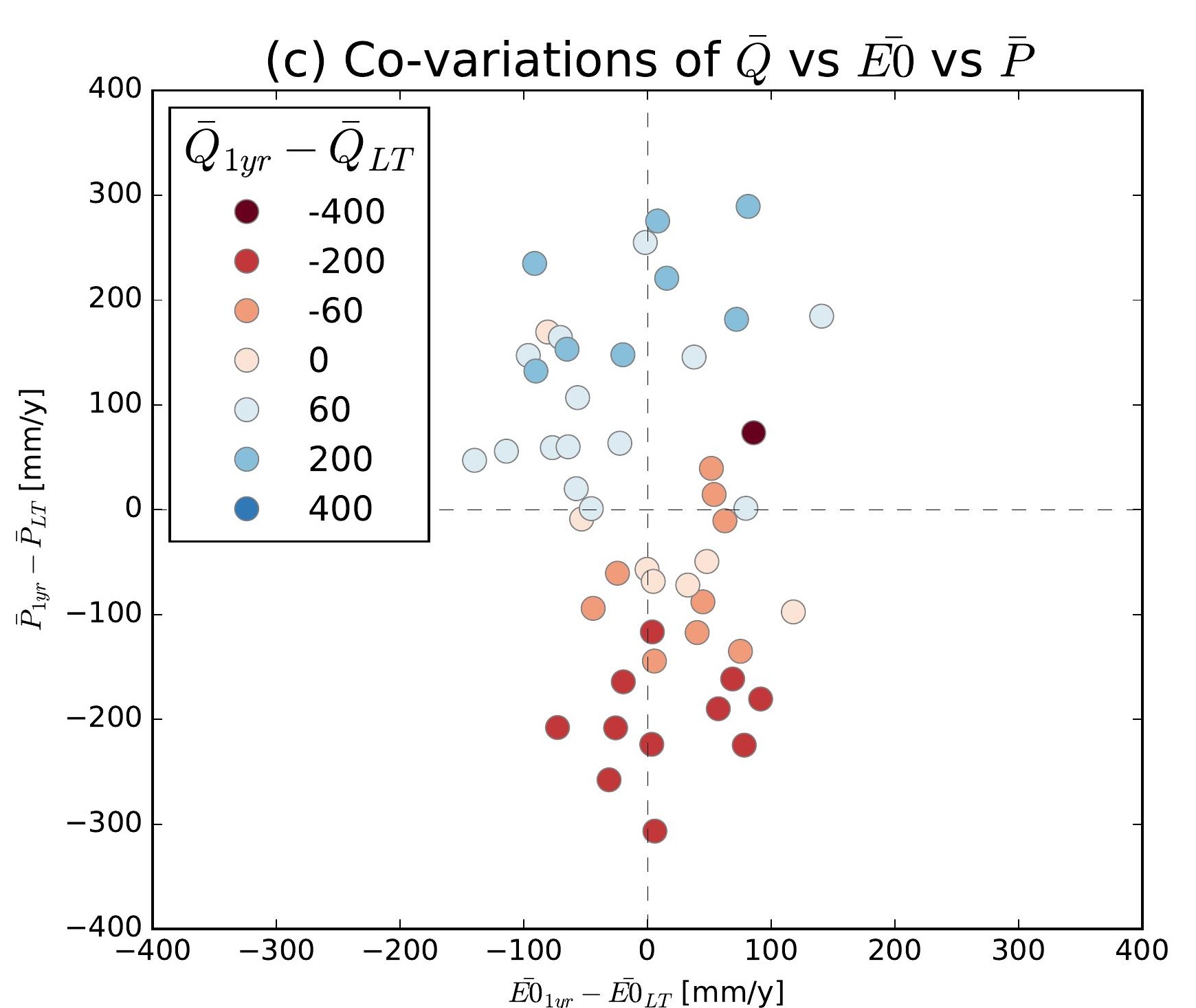
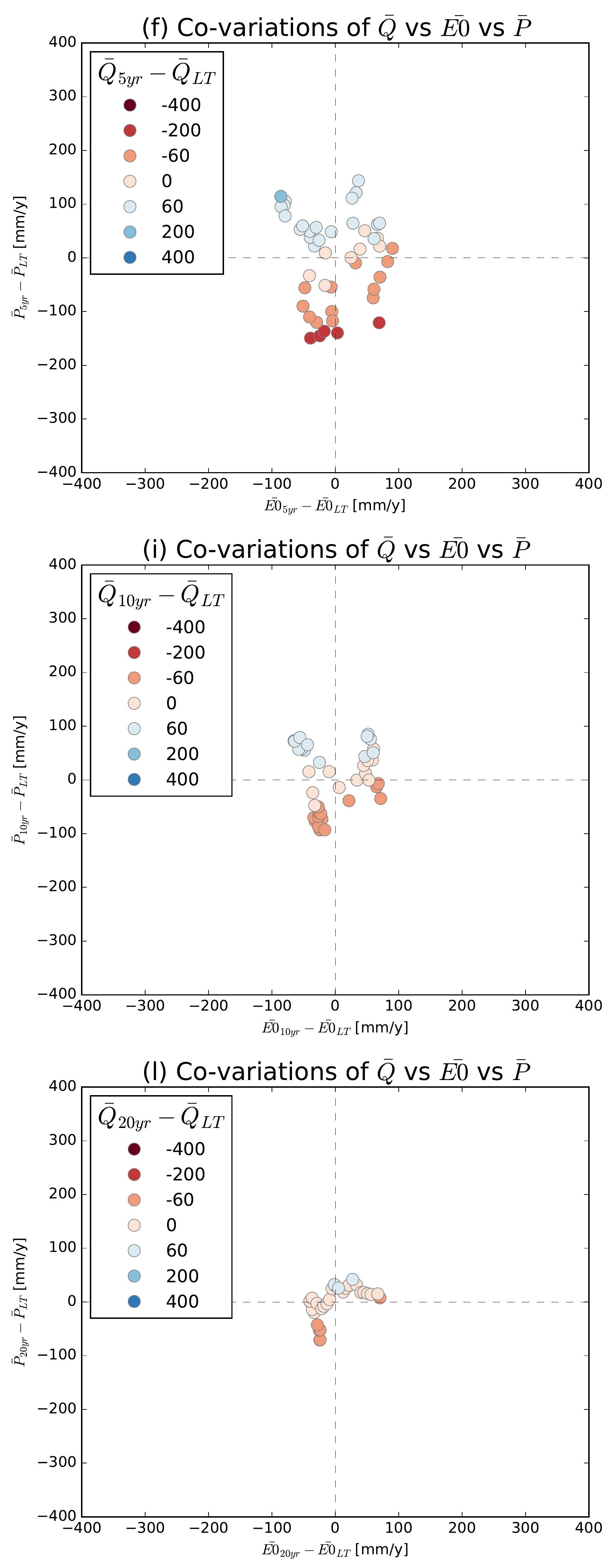

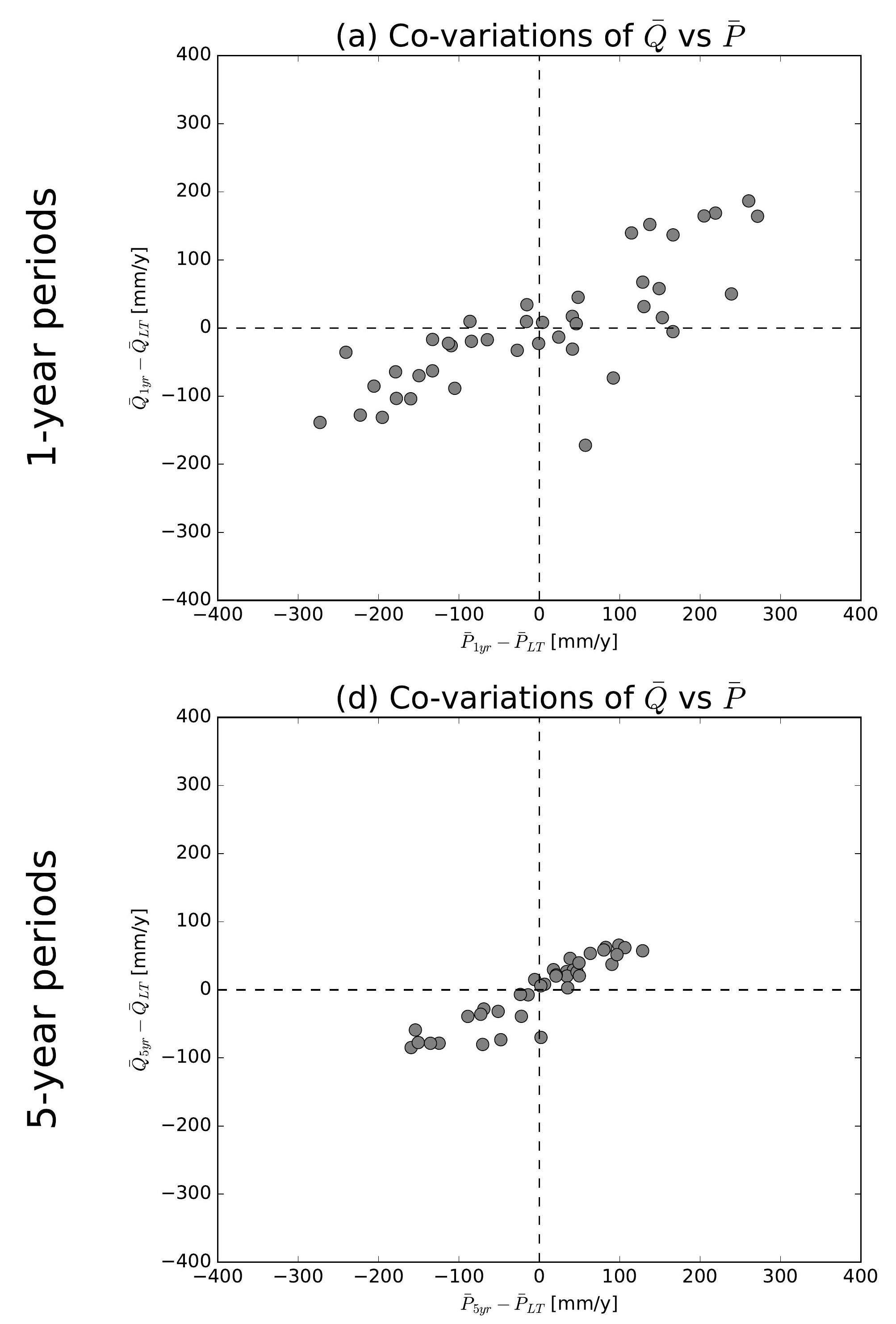

(g) Co-variations of $\bar{Q}$ vs $\bar{P}$

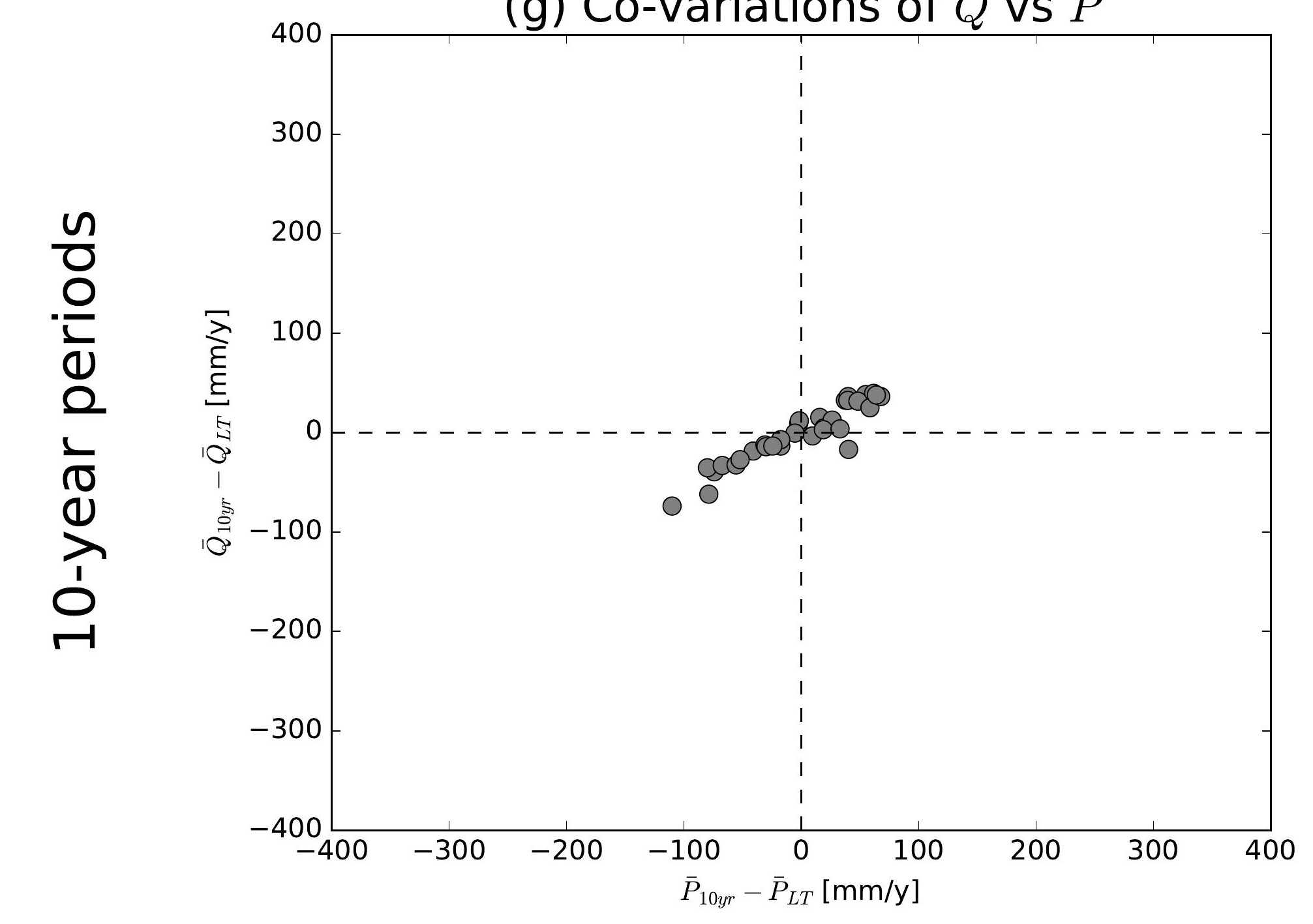

(j) Co-variations of $\bar{Q}$ vs $\bar{P}$

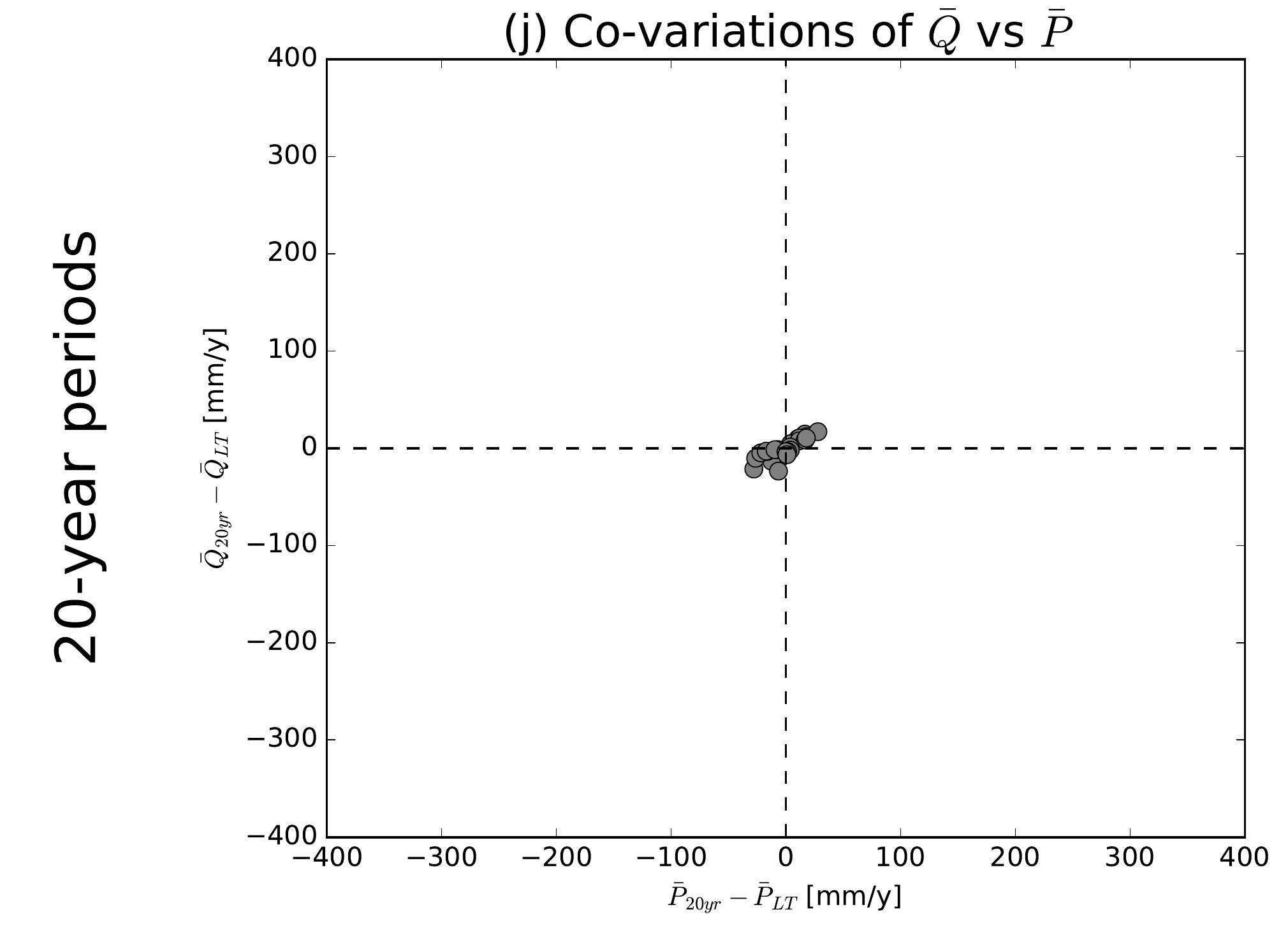

(b) Co-variations of $\bar{Q}$ vs $\overline{E 0}$

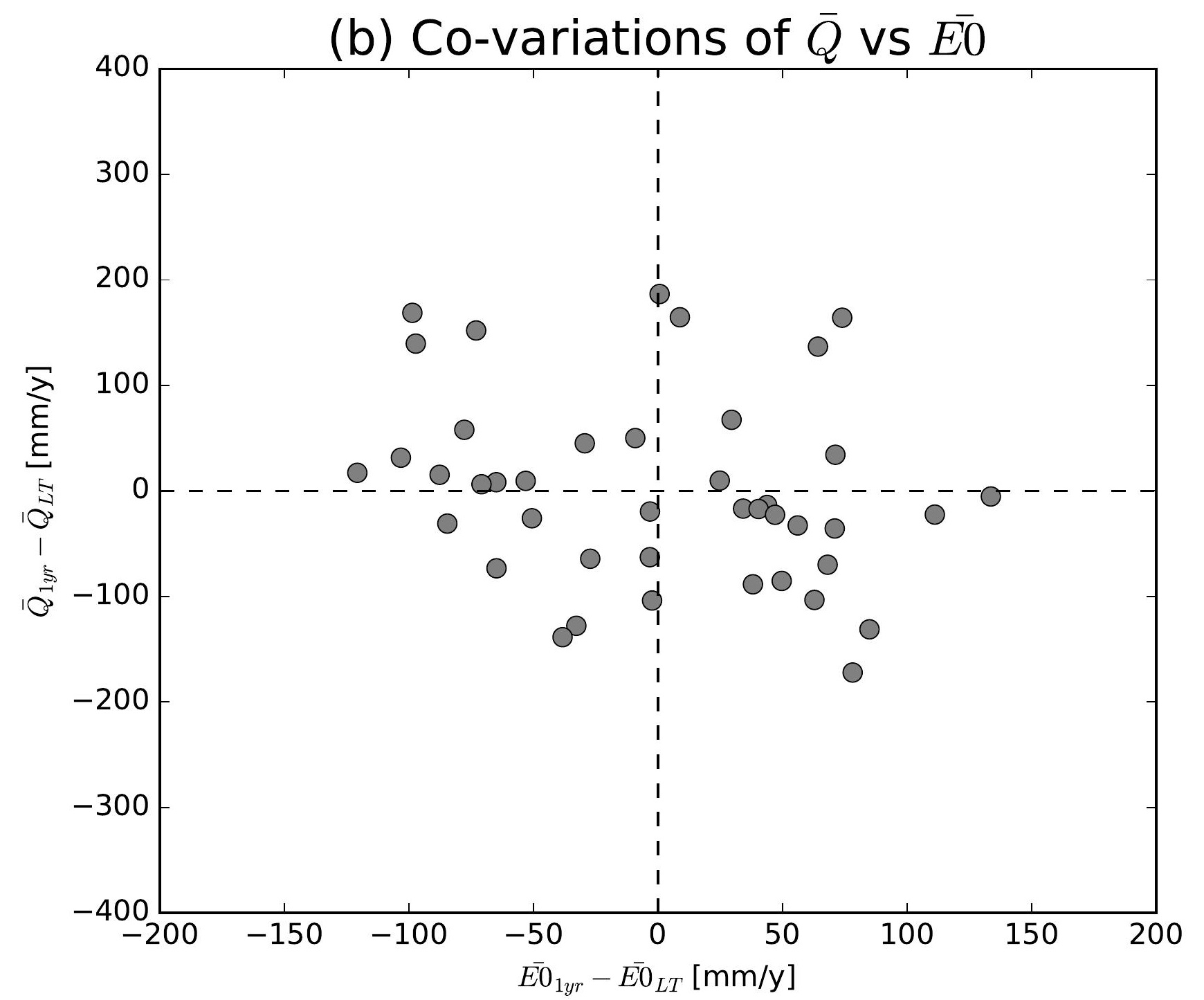

(e) Co-variations of $\bar{Q}$ vs $\overline{E 0}$

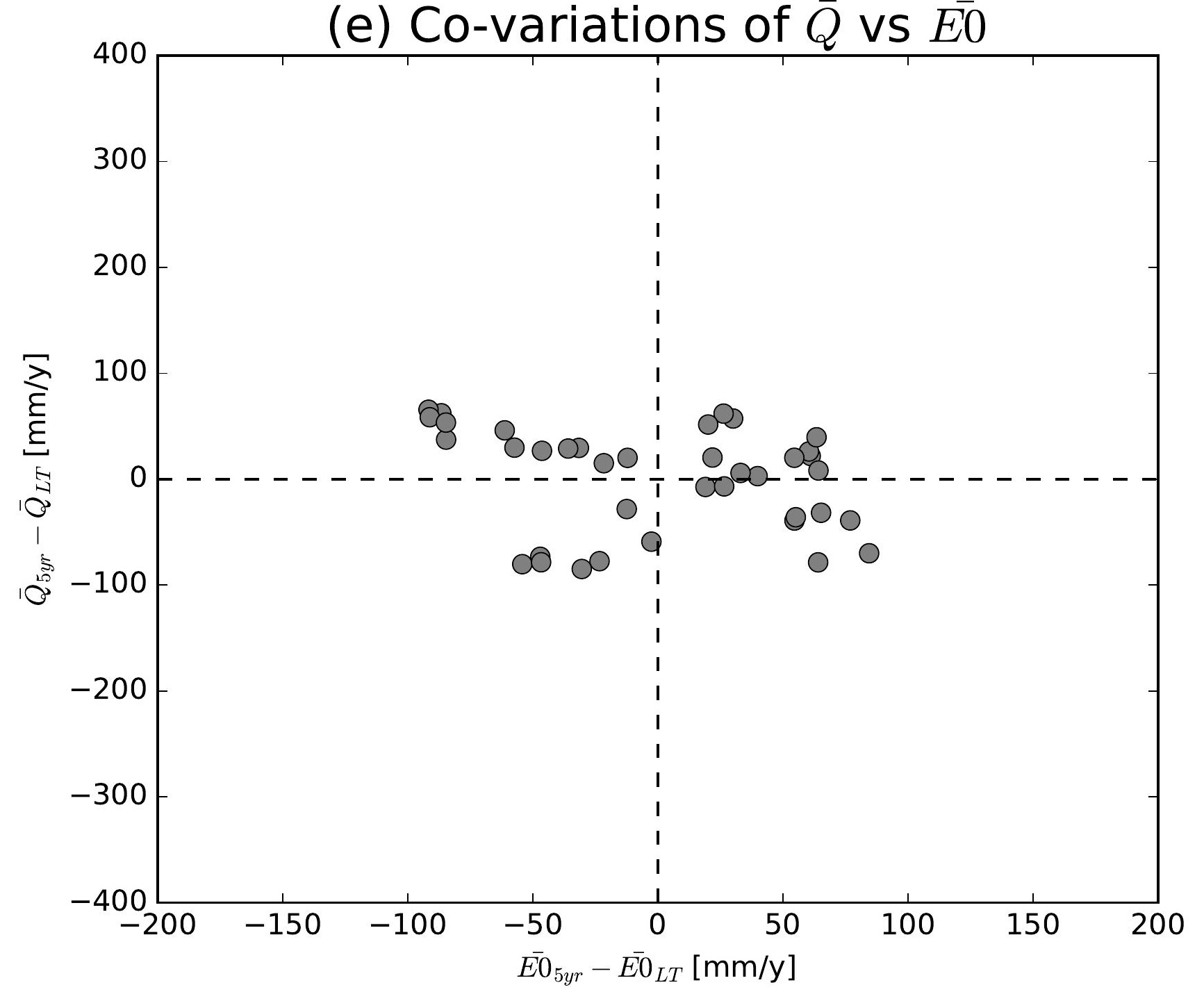

(h) Co-variations of $\bar{Q}$ vs $\overline{E 0}$

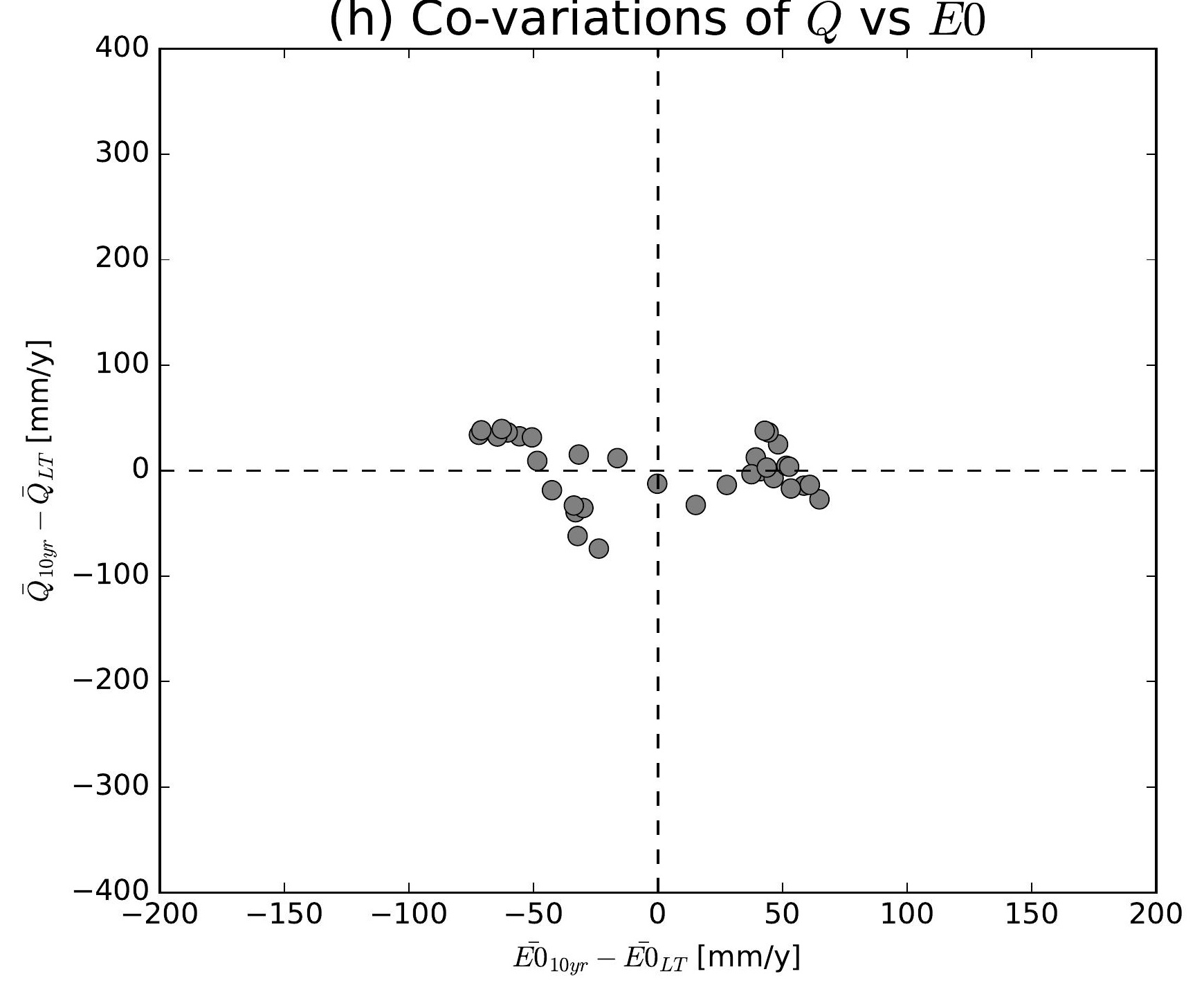

(k) Co-variations of $\bar{Q}$ vs $\overline{E 0}$

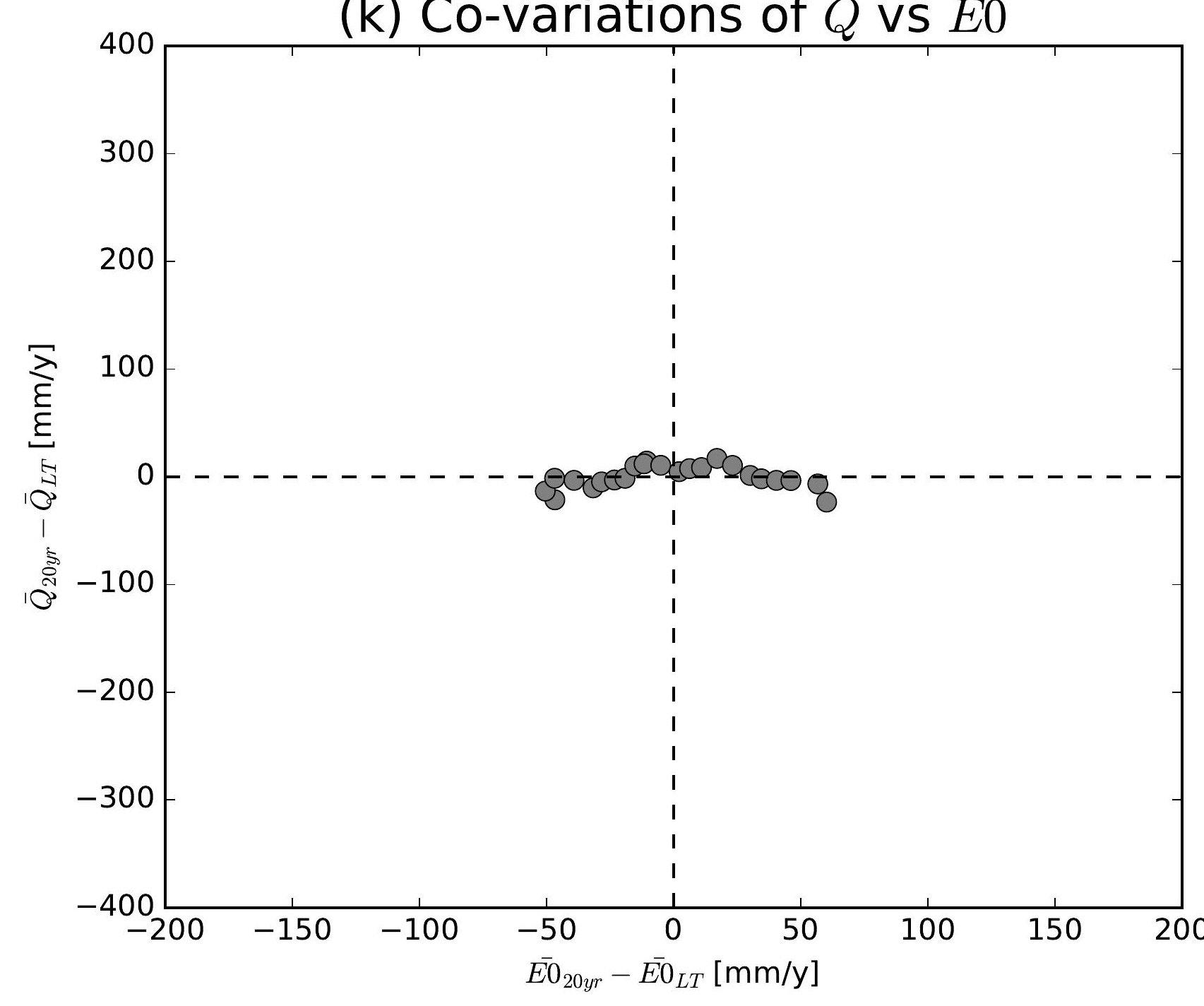

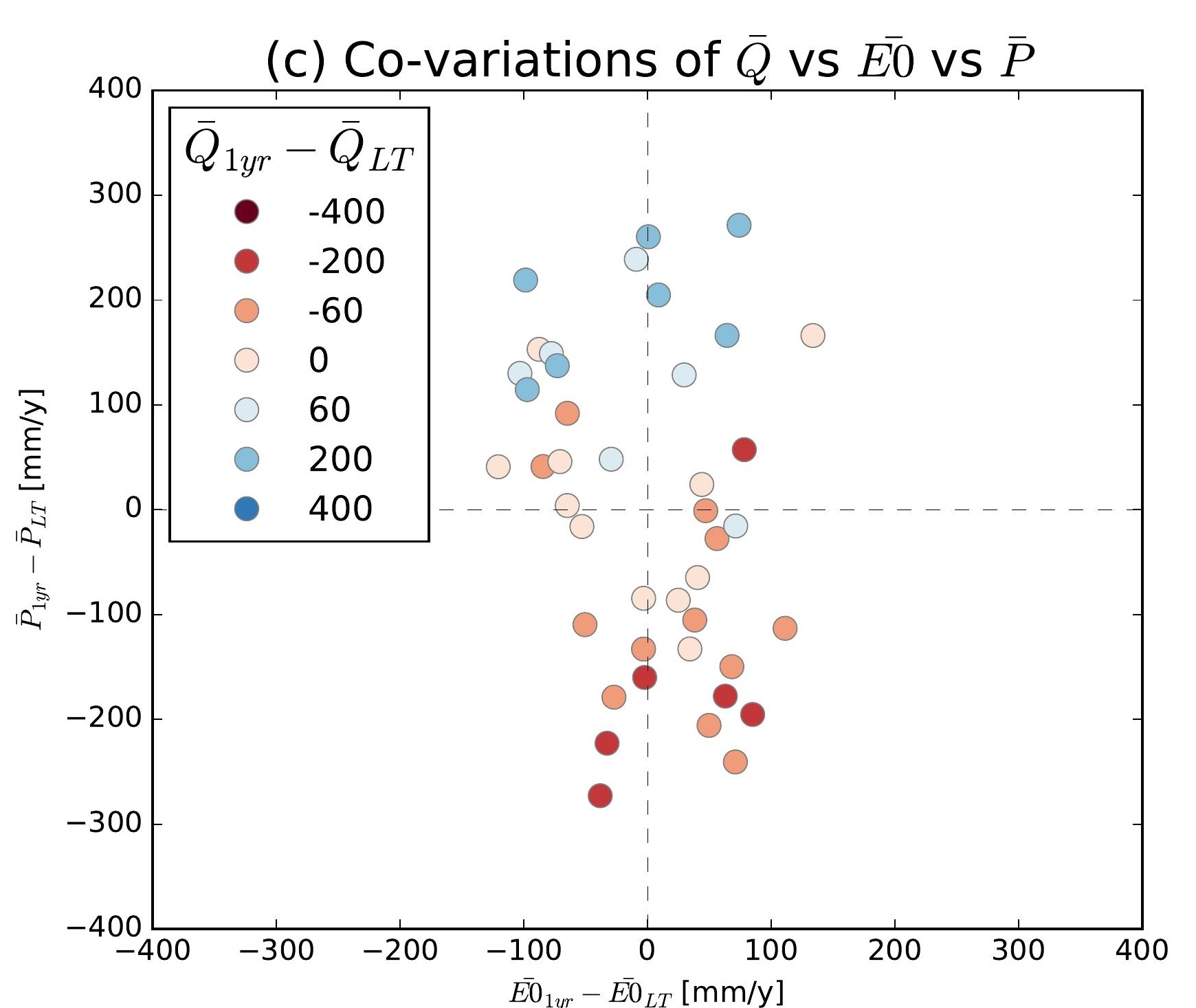
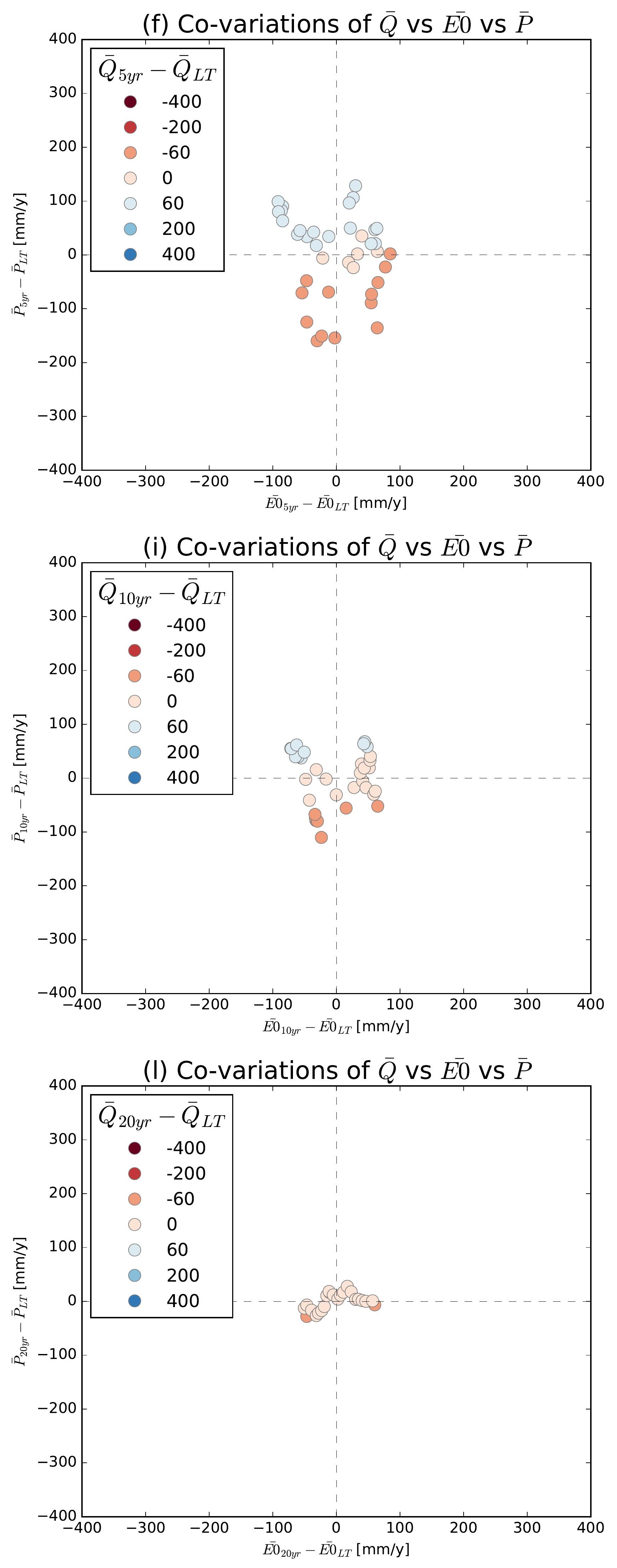

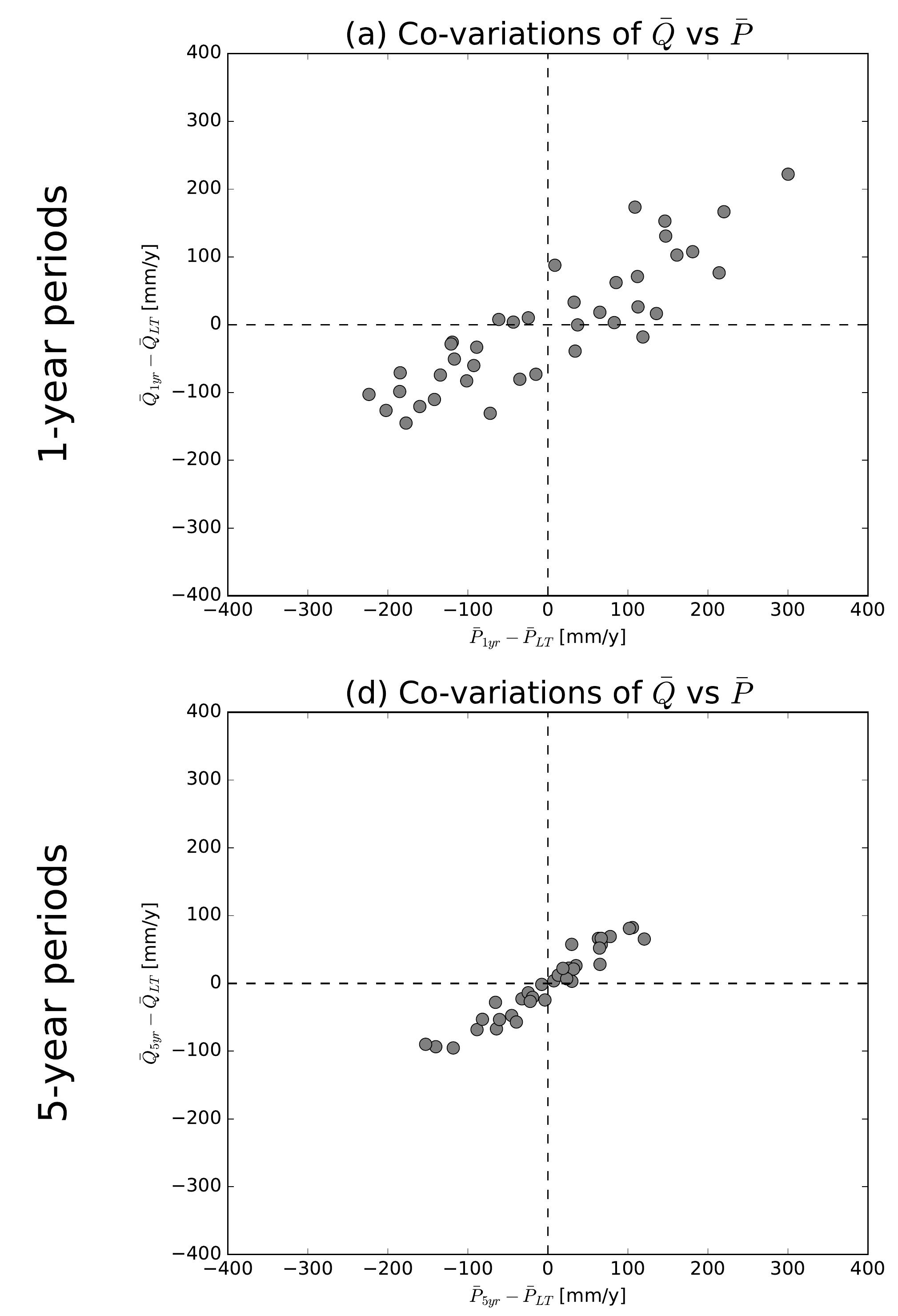

(g) Co-variations of $\bar{Q}$ vs $\bar{P}$
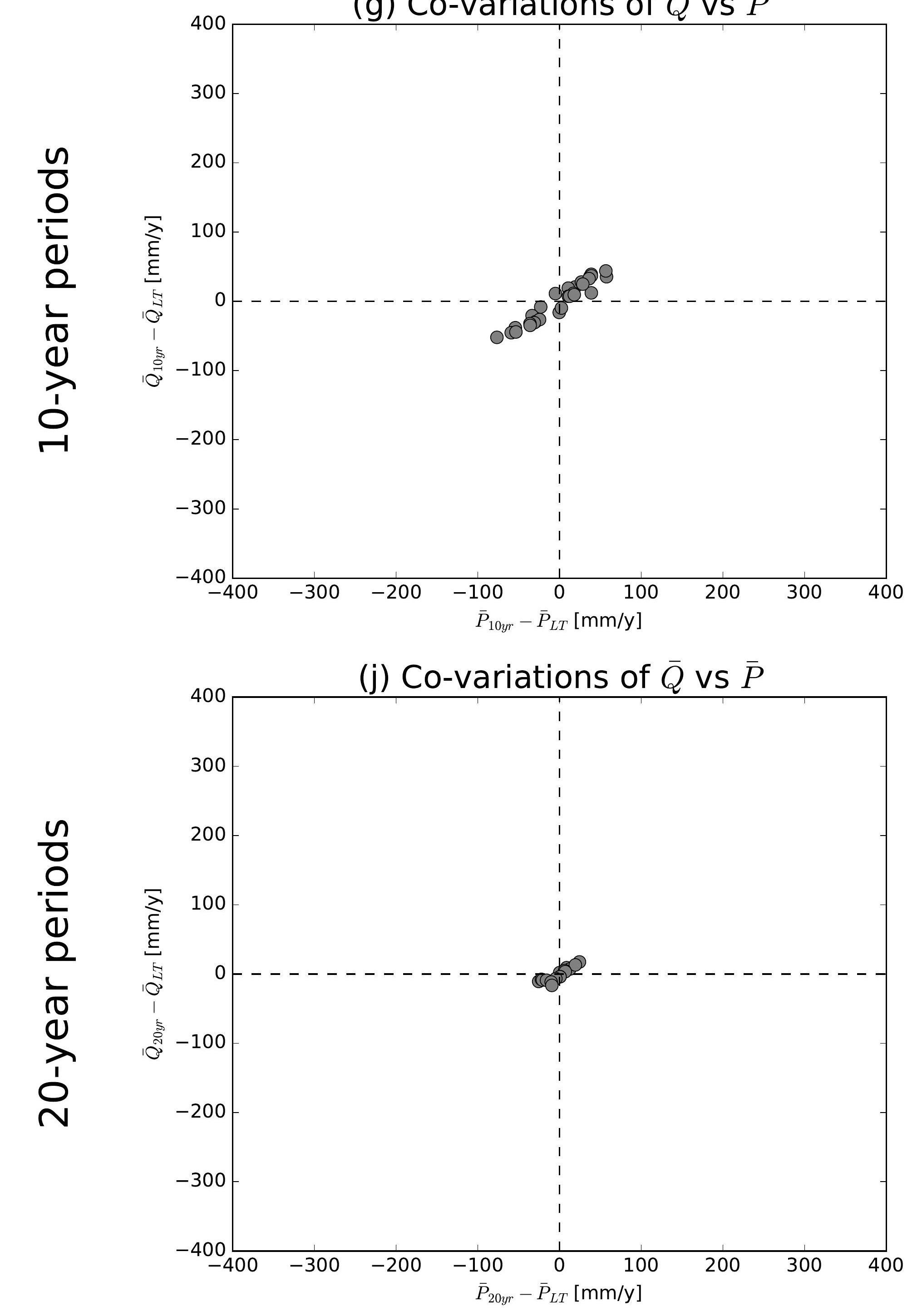

(b) Co-variations of $\bar{Q}$ vs $\overline{E 0}$

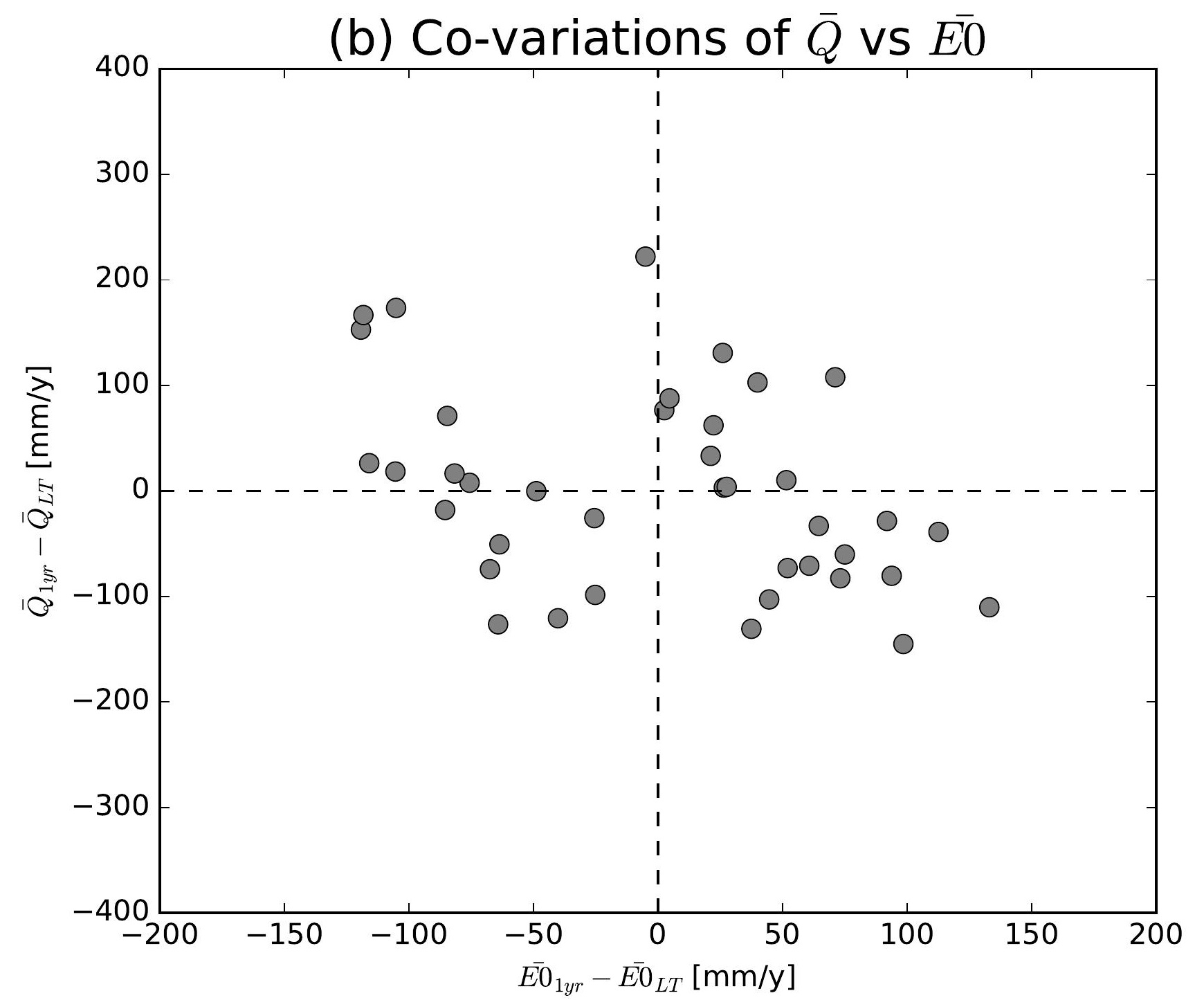

(e) Co-variations of $\bar{Q}$ vs $\overline{E 0}$

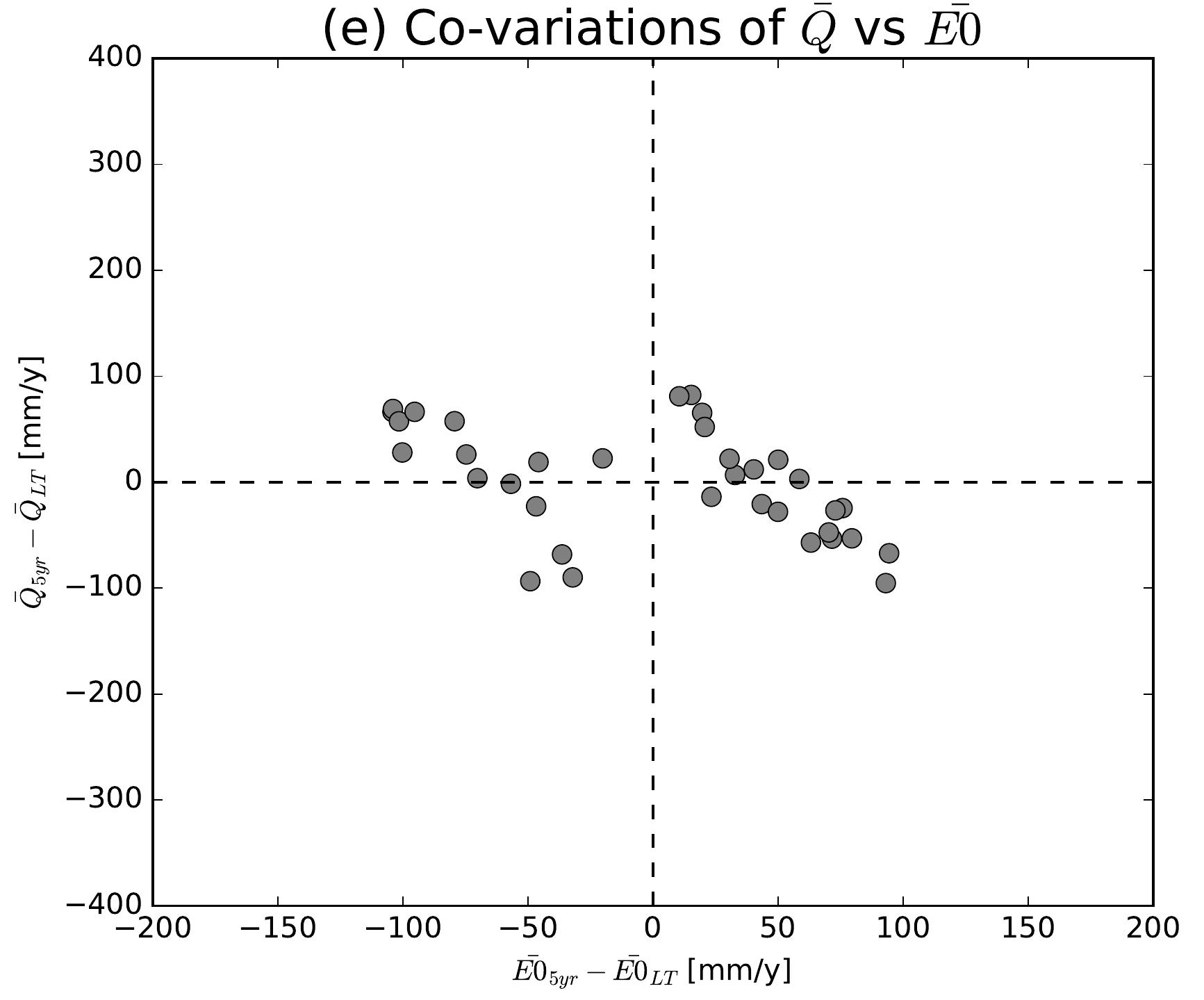

(h) Co-variations of $\bar{Q}$ vs $\overline{E 0}$

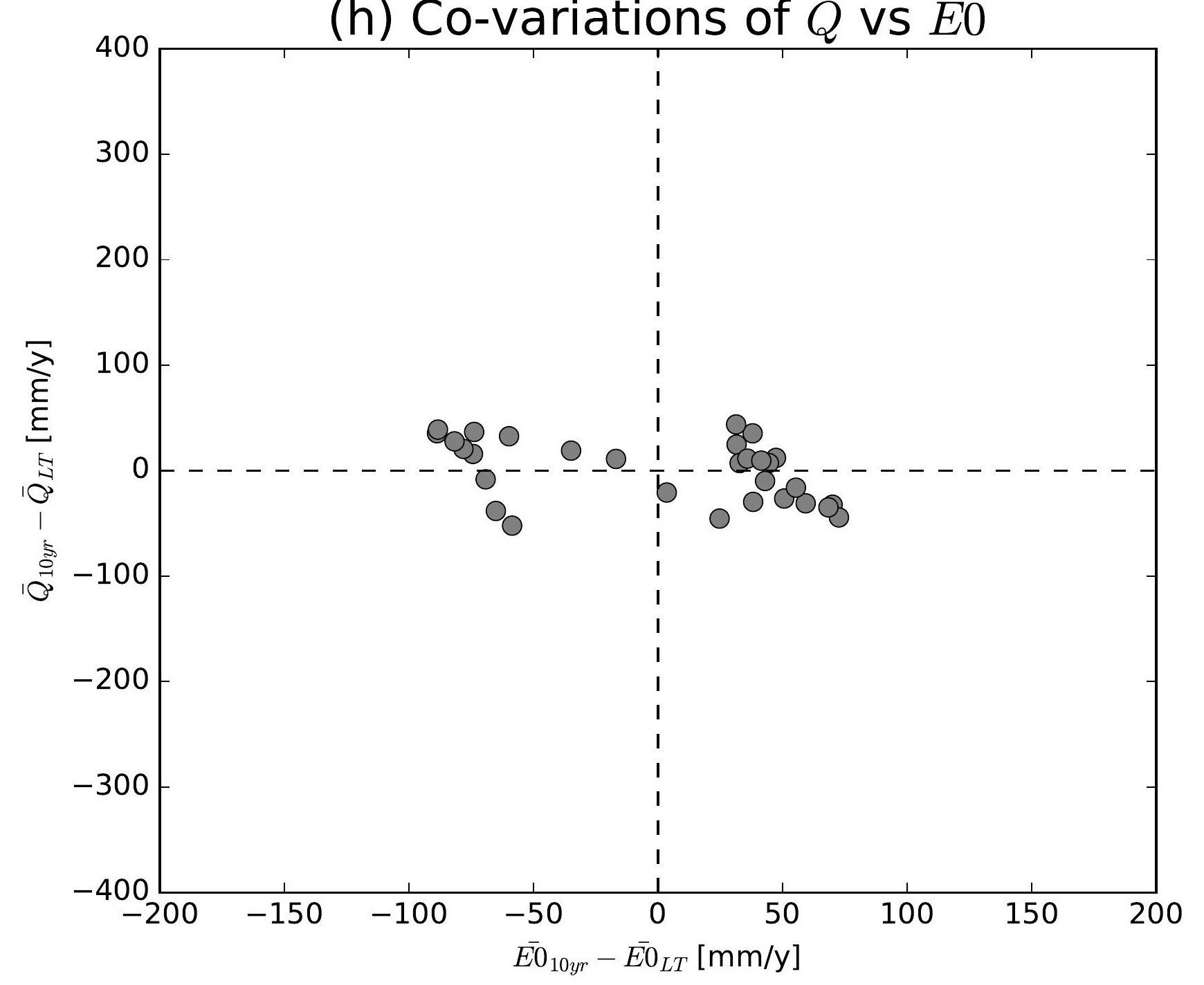

(k) Co-variations of $\bar{Q}$ vs $\overline{E 0}$

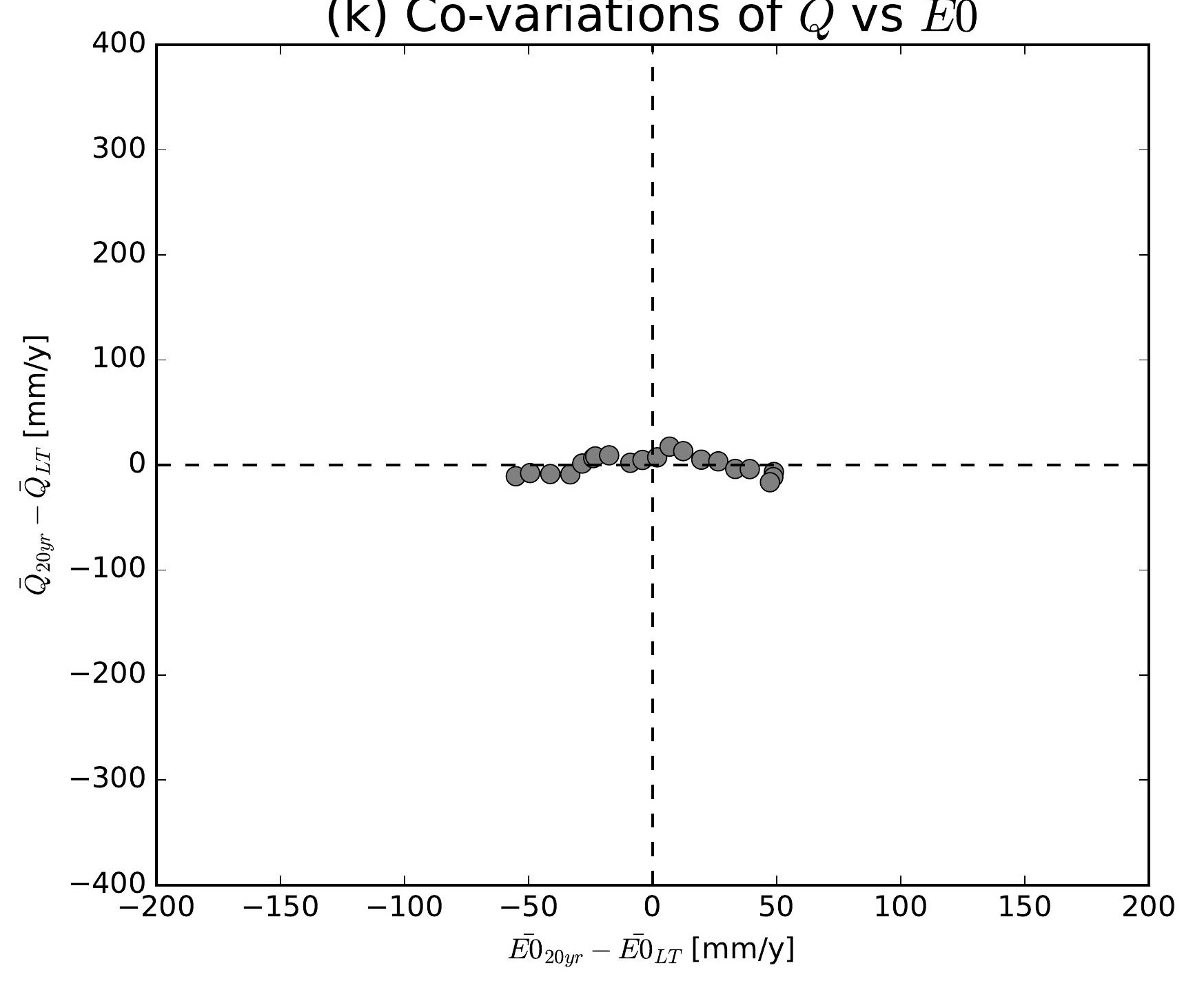

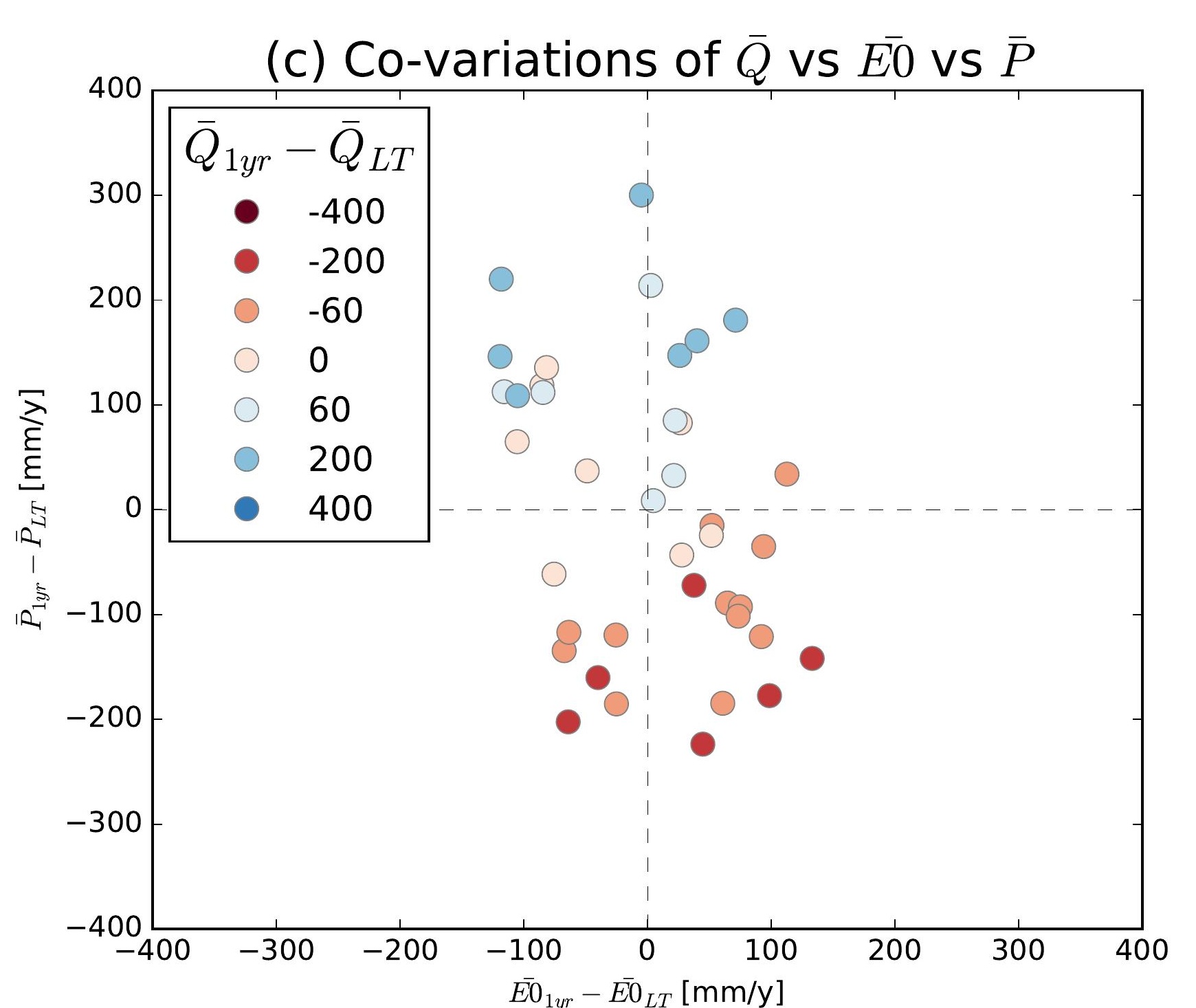
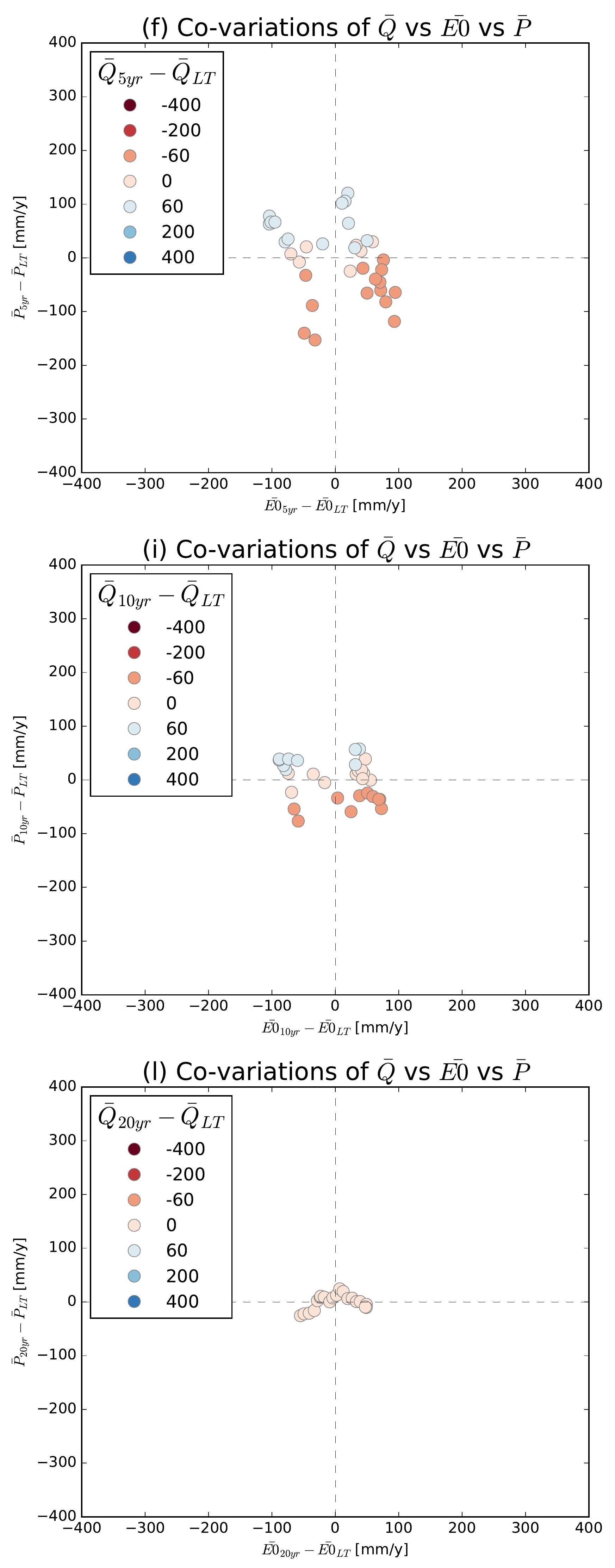

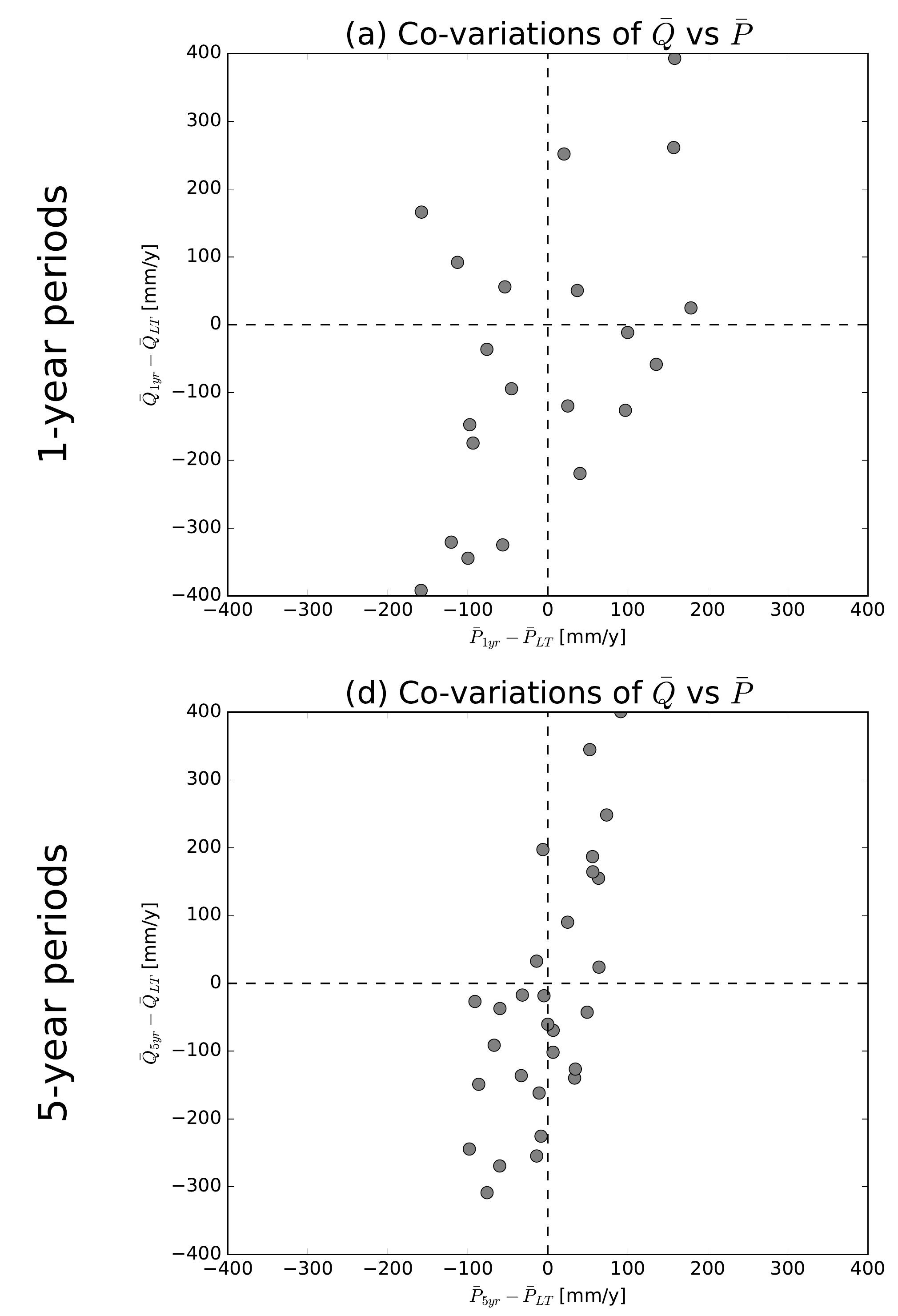

(g) Co-variations of $\bar{Q}$ vs $\bar{P}$
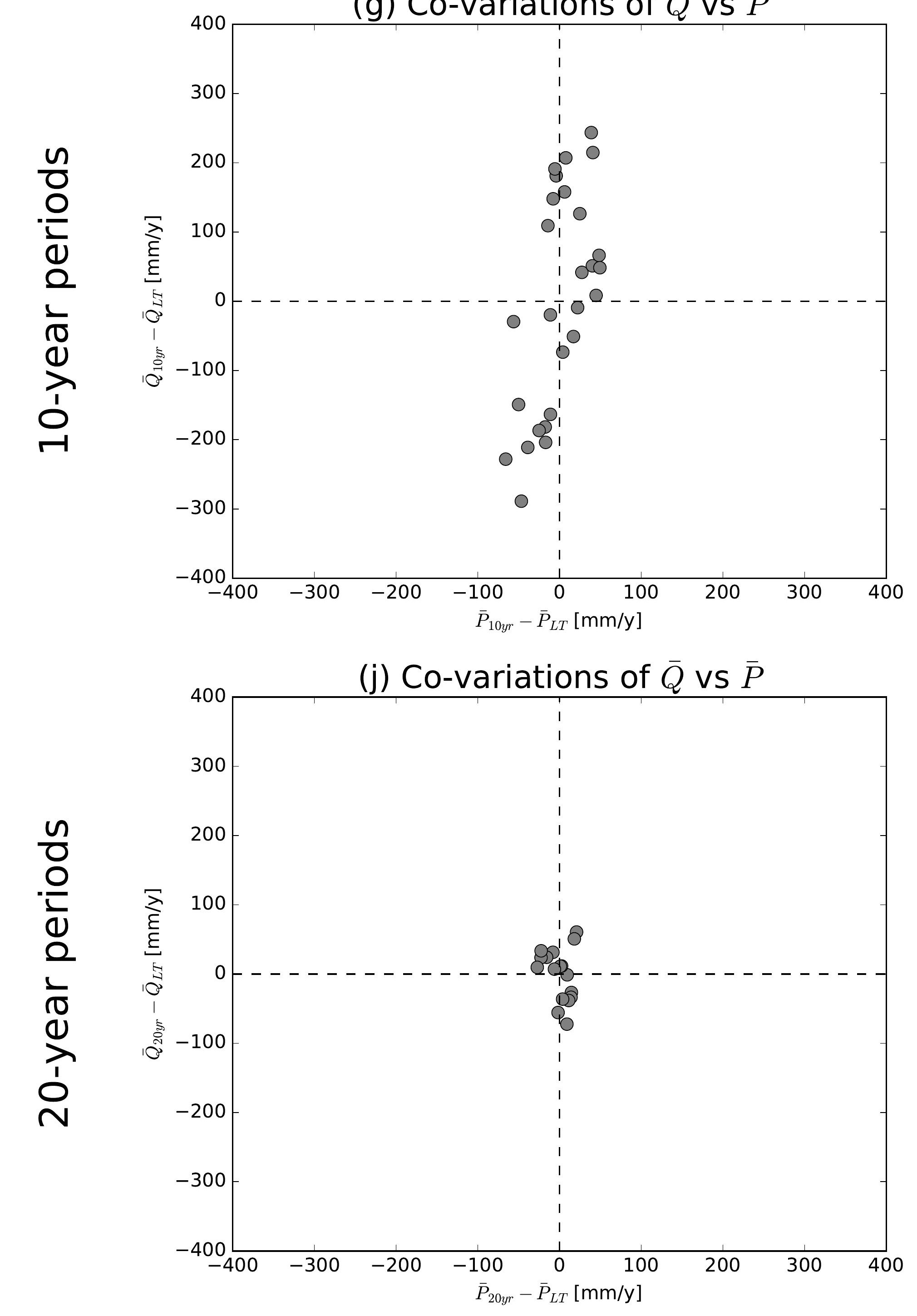

(b) Co-variations of $\bar{Q}$ vs $\overline{E 0}$

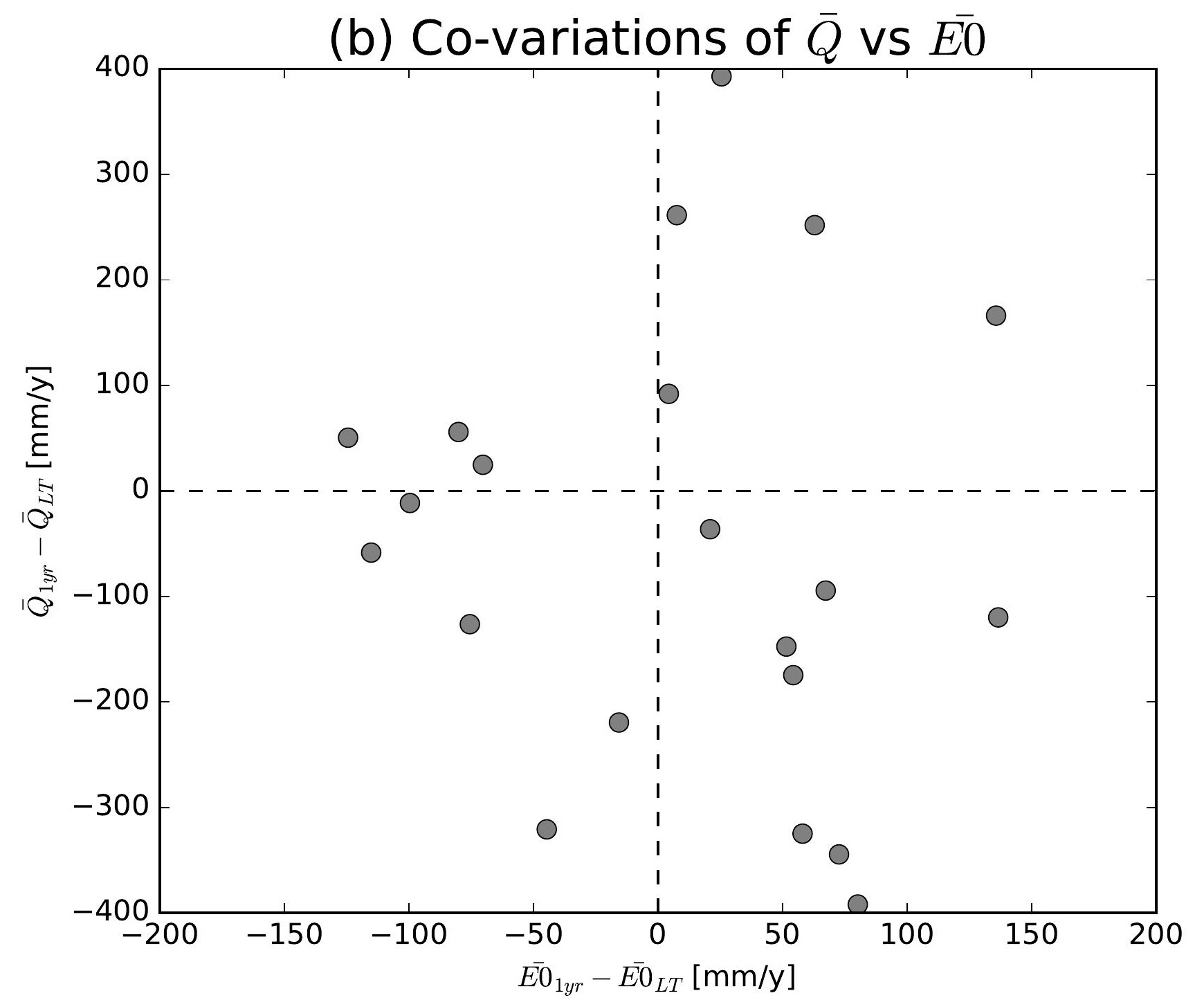

(e) Co-variations of $\bar{Q}$ vs $\overline{E 0}$

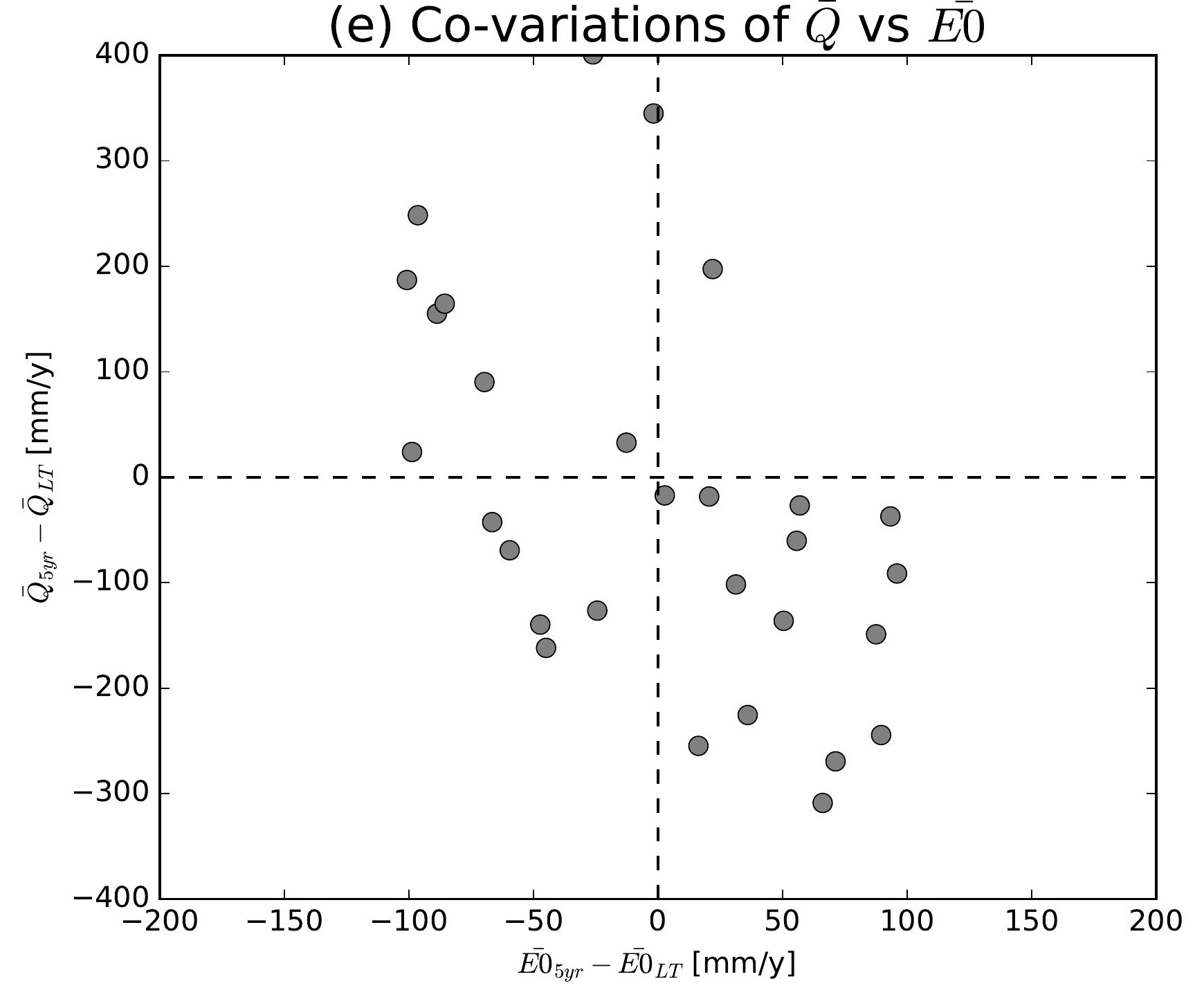

(h) Co-variations of $\bar{Q}$ vs $\overline{E 0}$

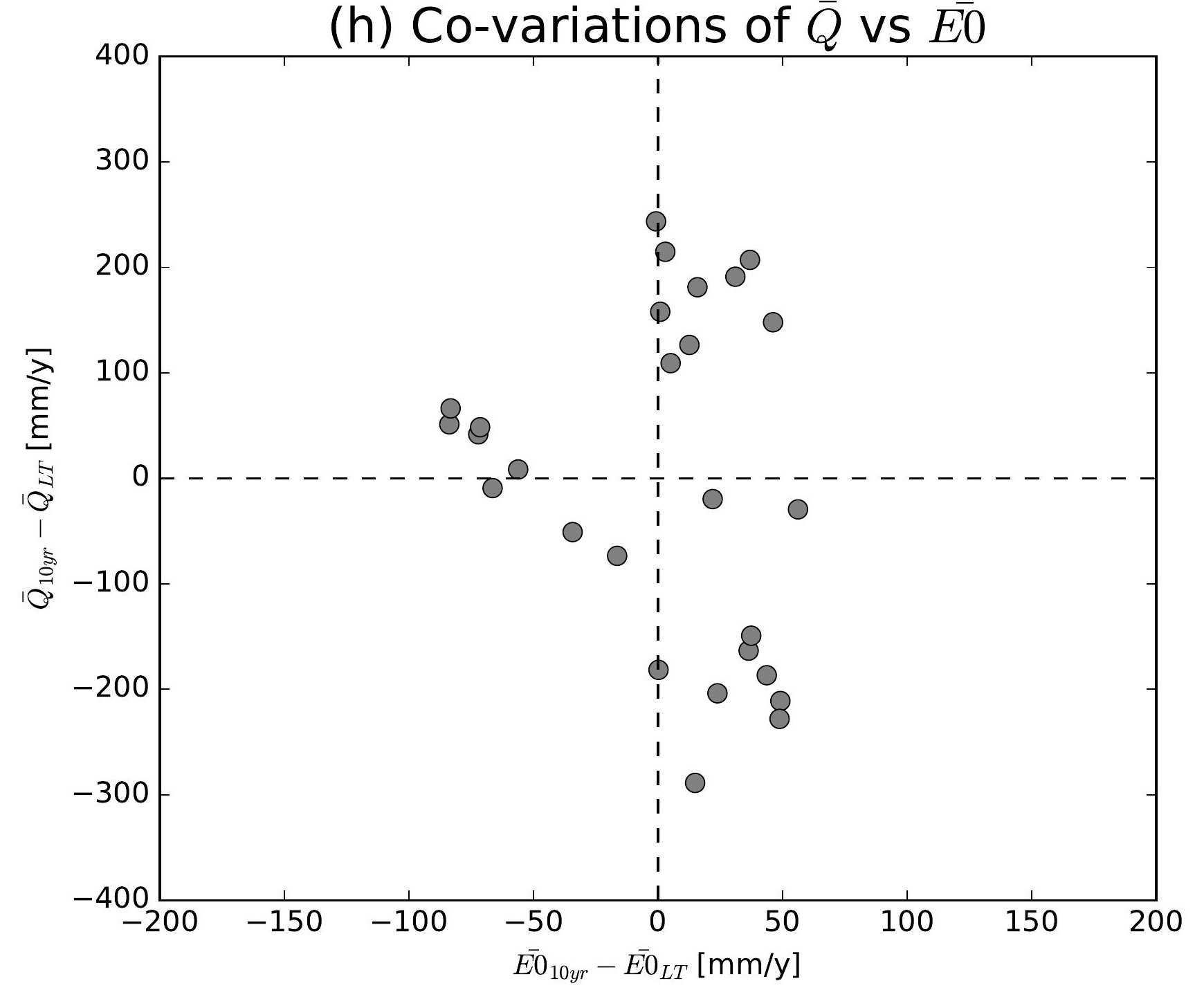

(k) Co-variations of $\bar{Q}$ vs $\overline{E 0}$

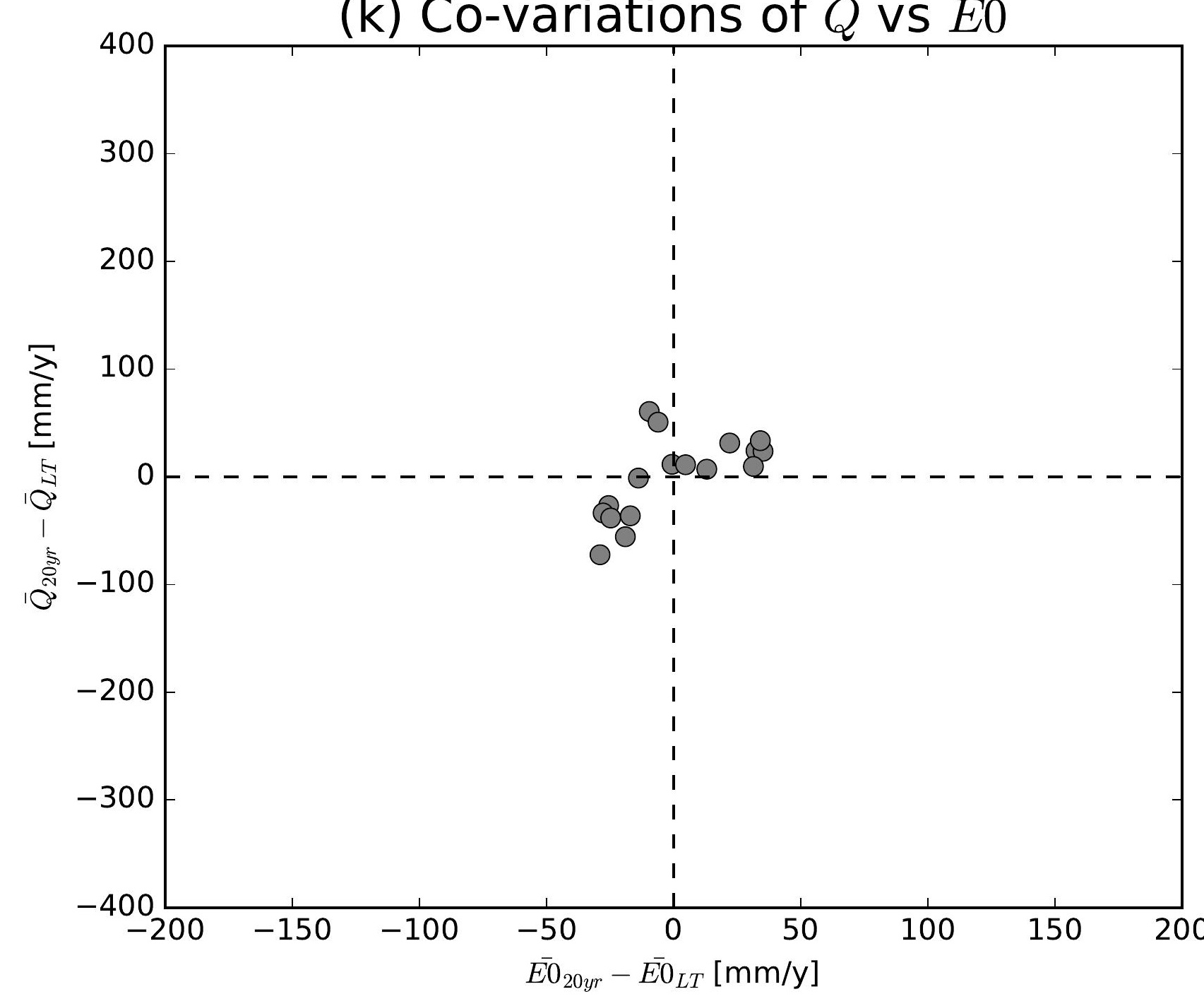

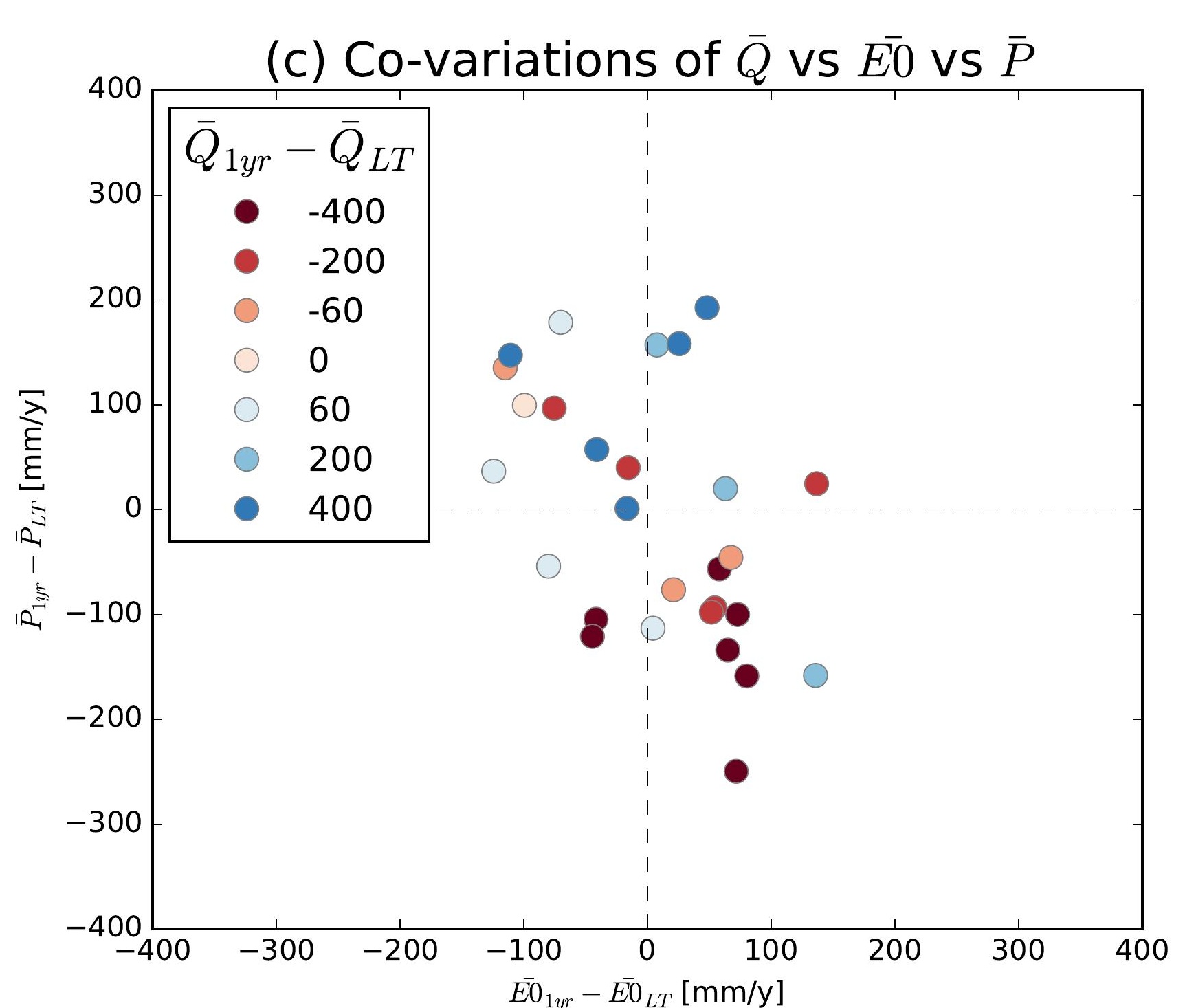
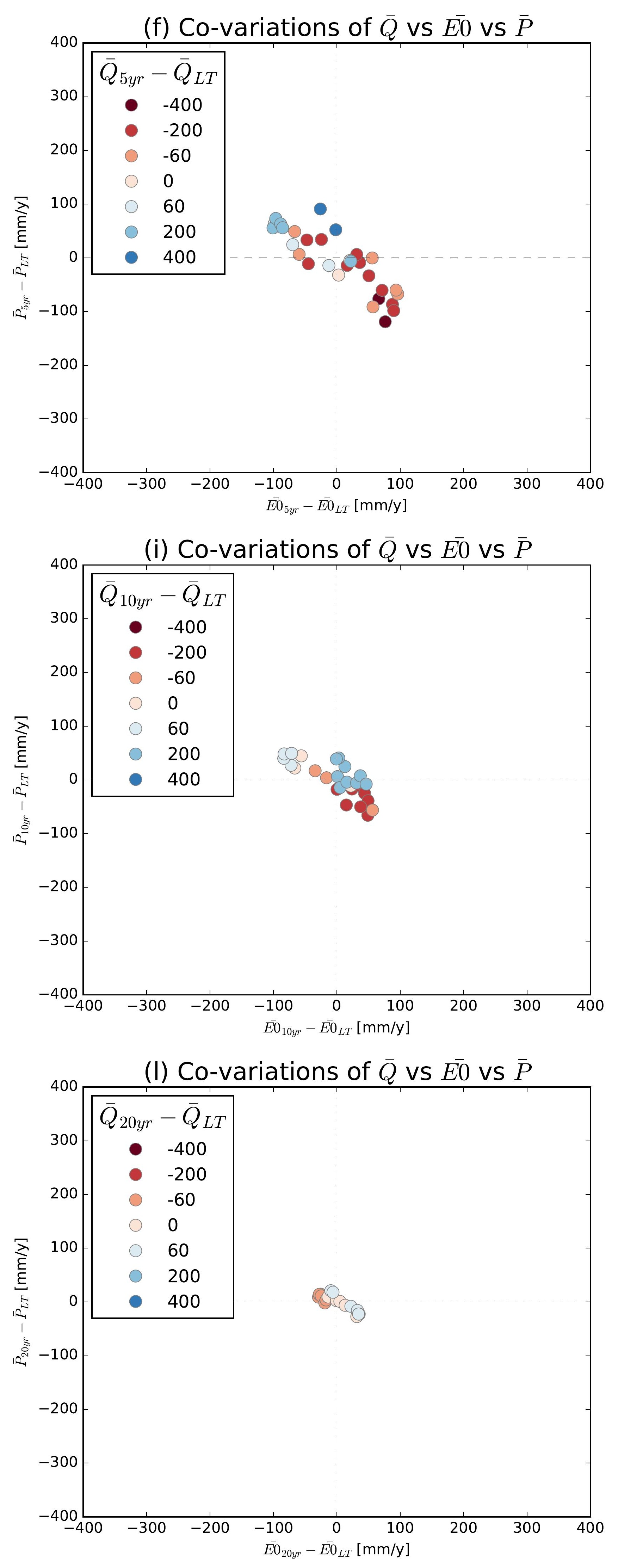

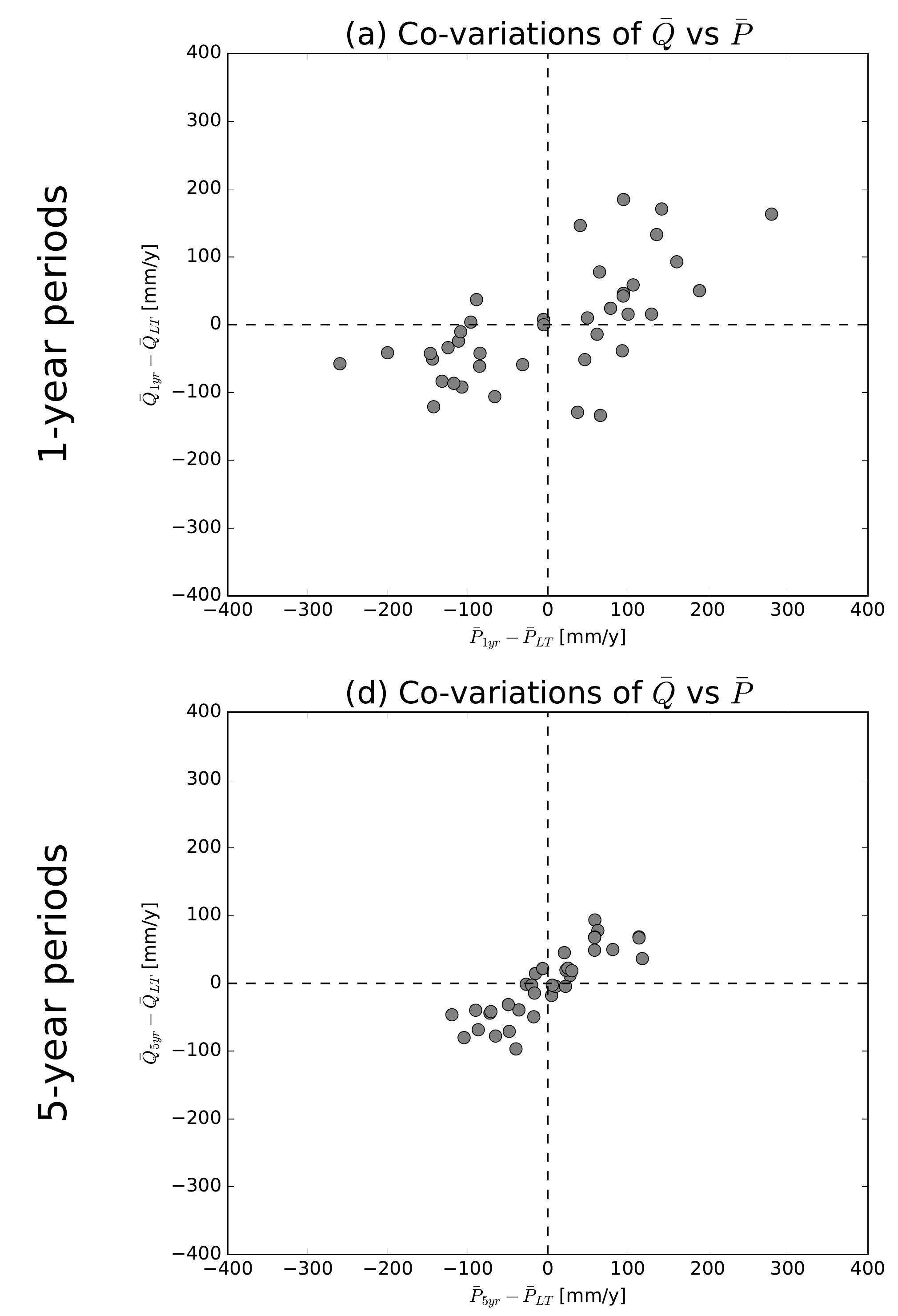

(g) Co-variations of $\bar{Q}$ vs $\bar{P}$
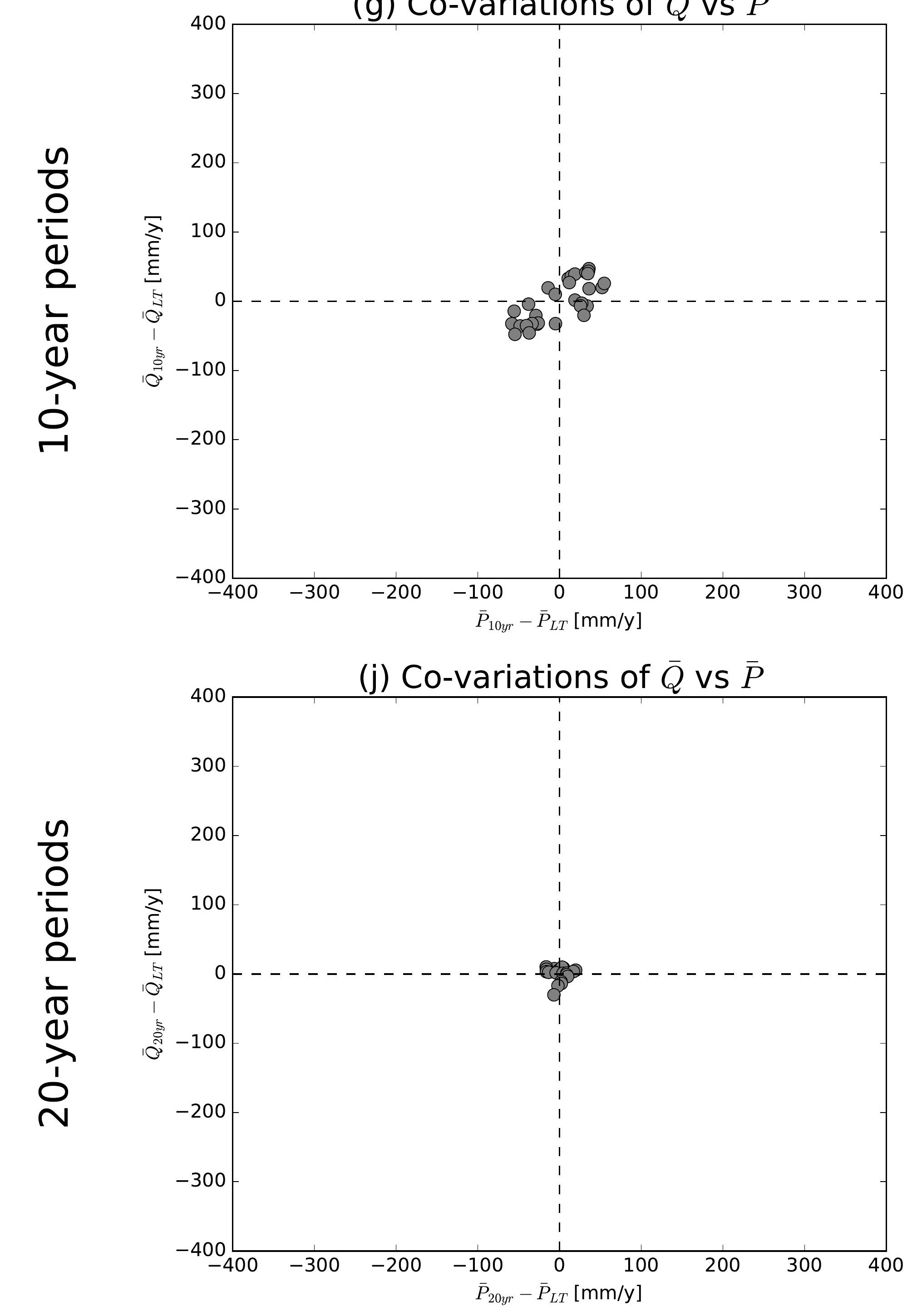

(b) Co-variations of $\bar{Q}$ vs $\overline{E 0}$

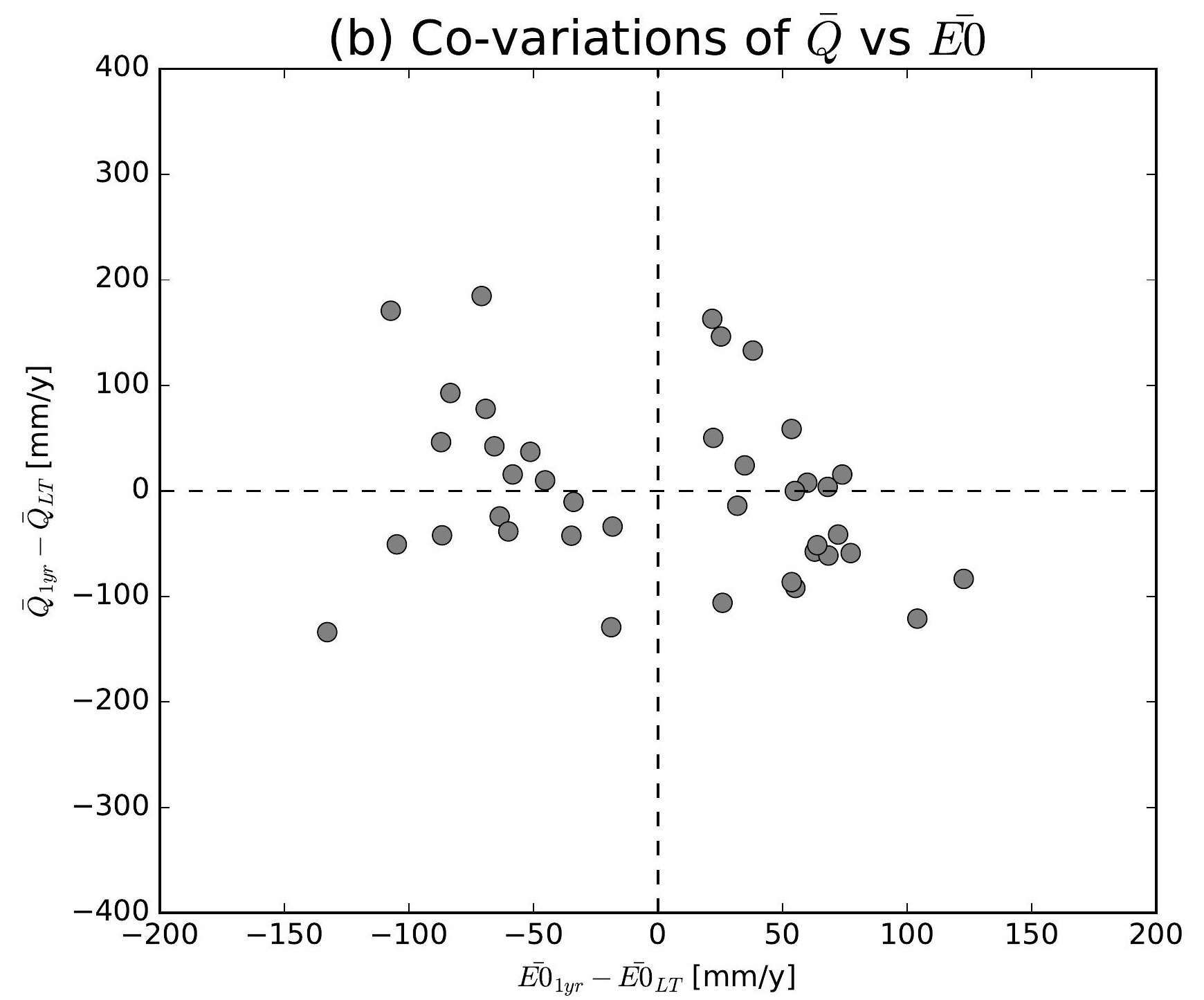

(e) Co-variations of $\bar{Q}$ vs $\overline{E 0}$

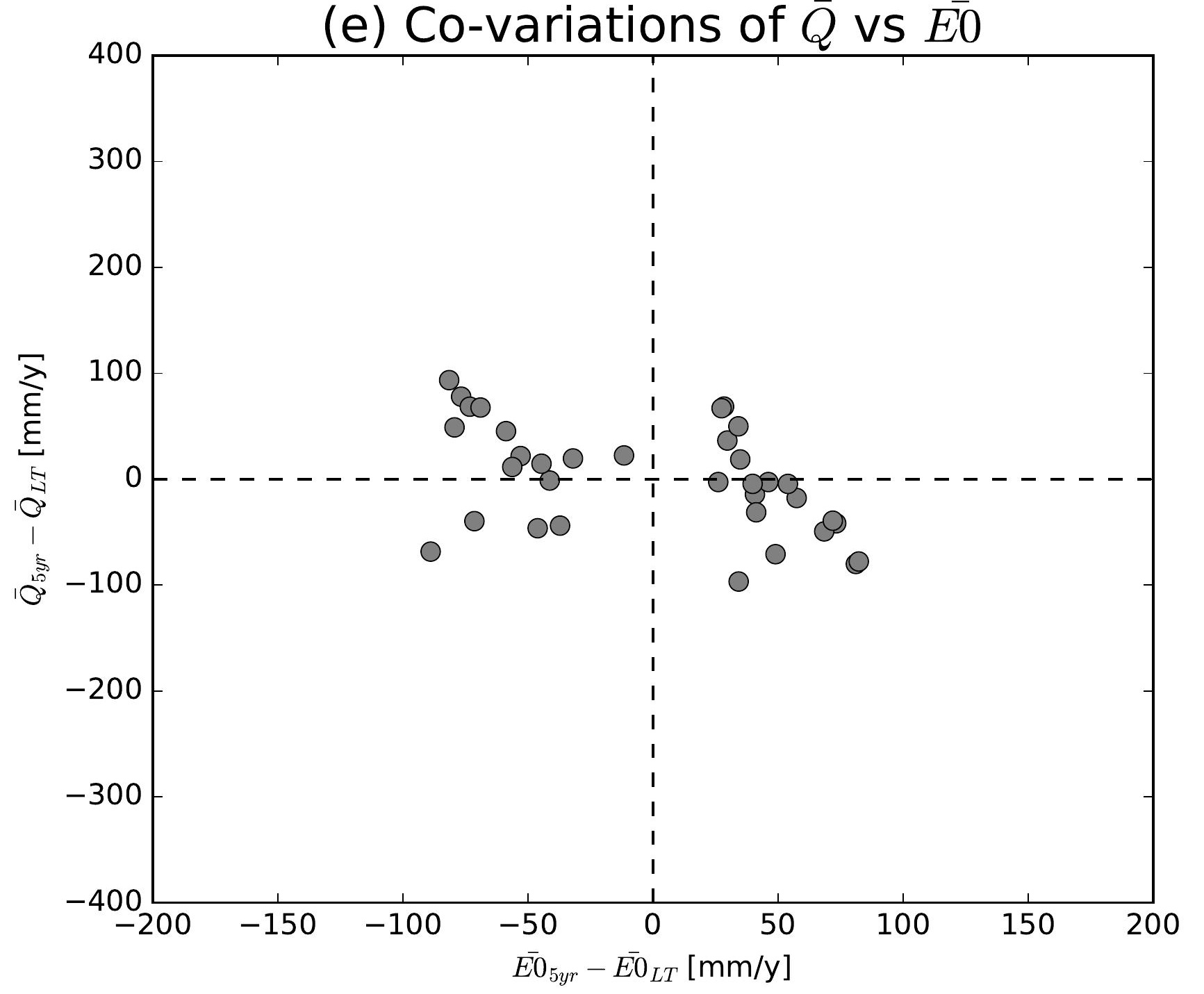

(h) Co-variations of $\bar{Q}$ vs $\overline{E 0}$

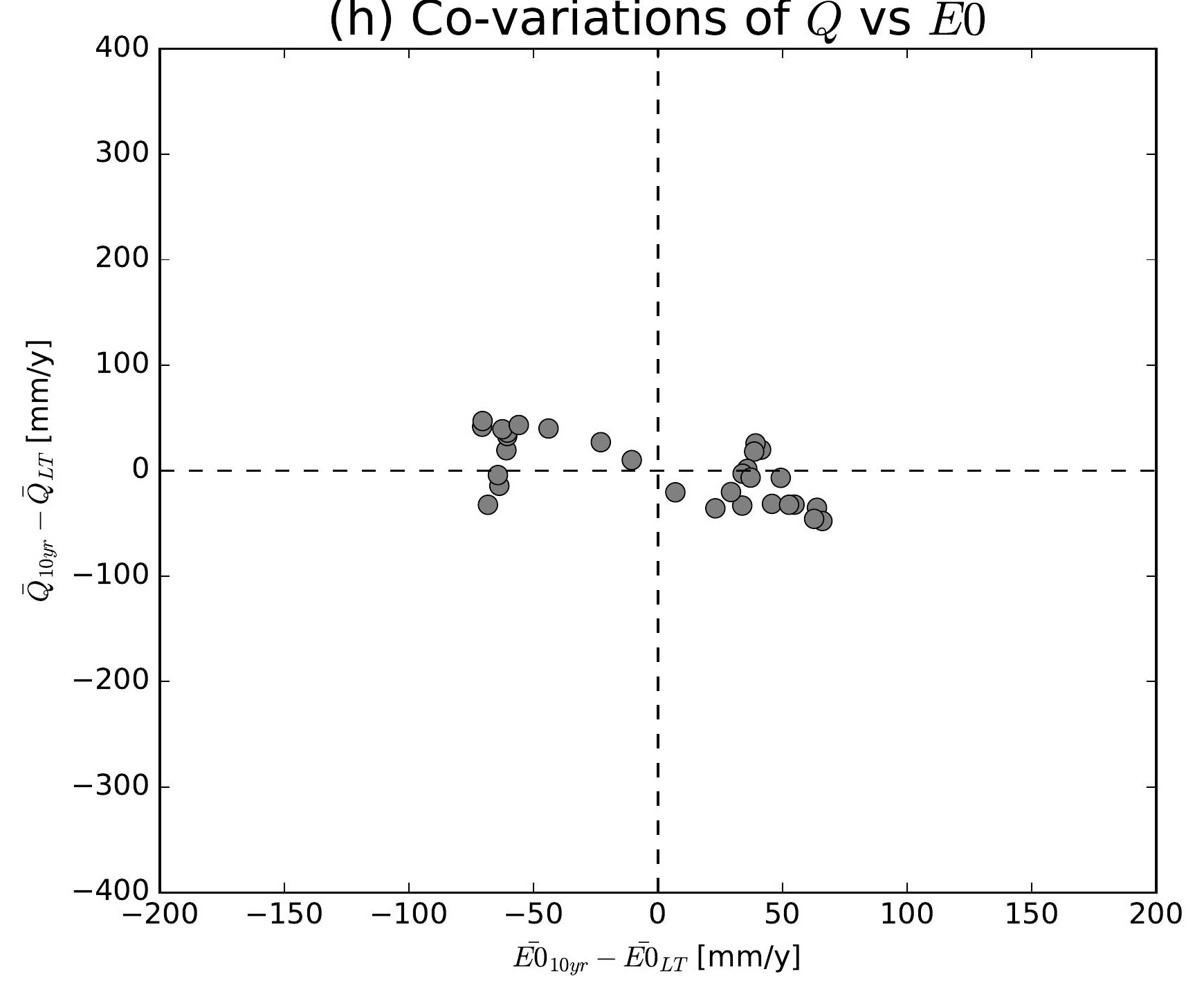

(k) Co-variations of $\bar{Q}$ vs $\overline{E 0}$

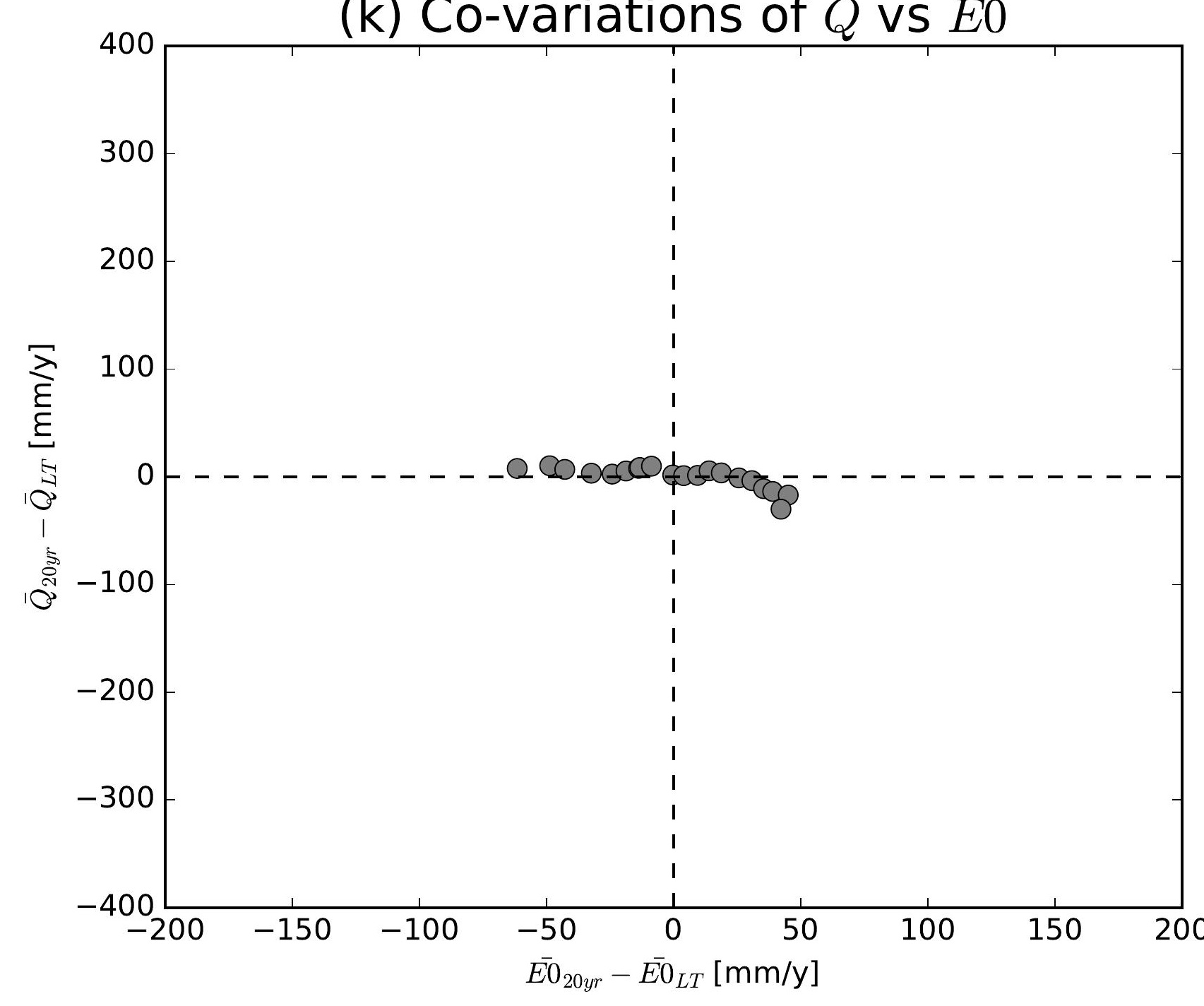

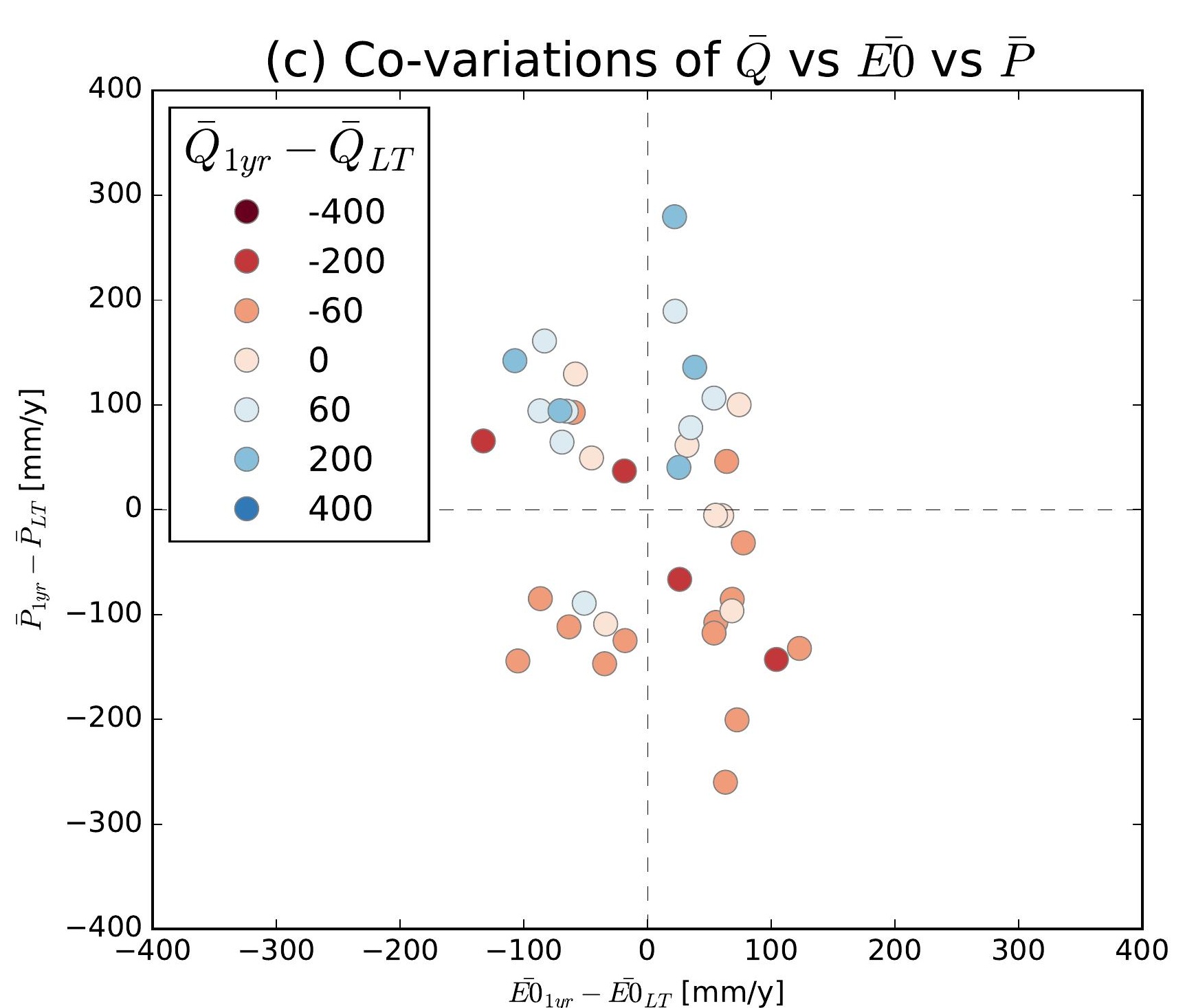
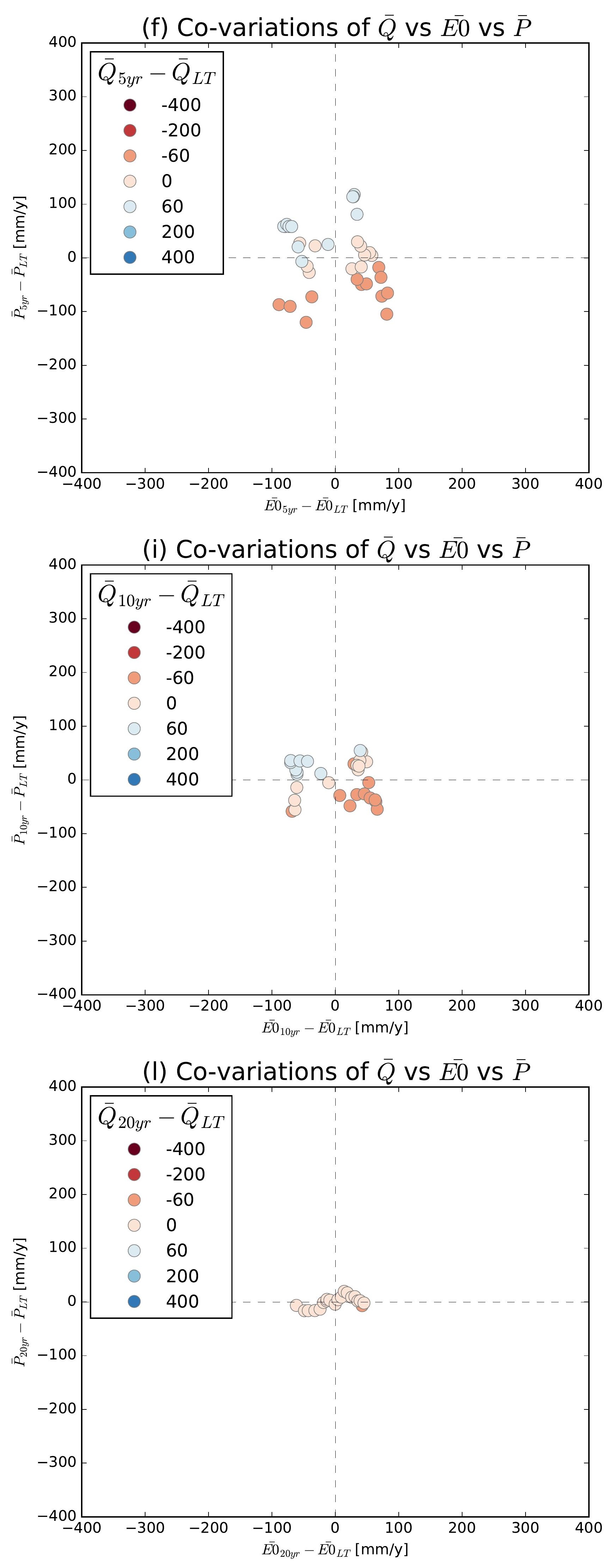

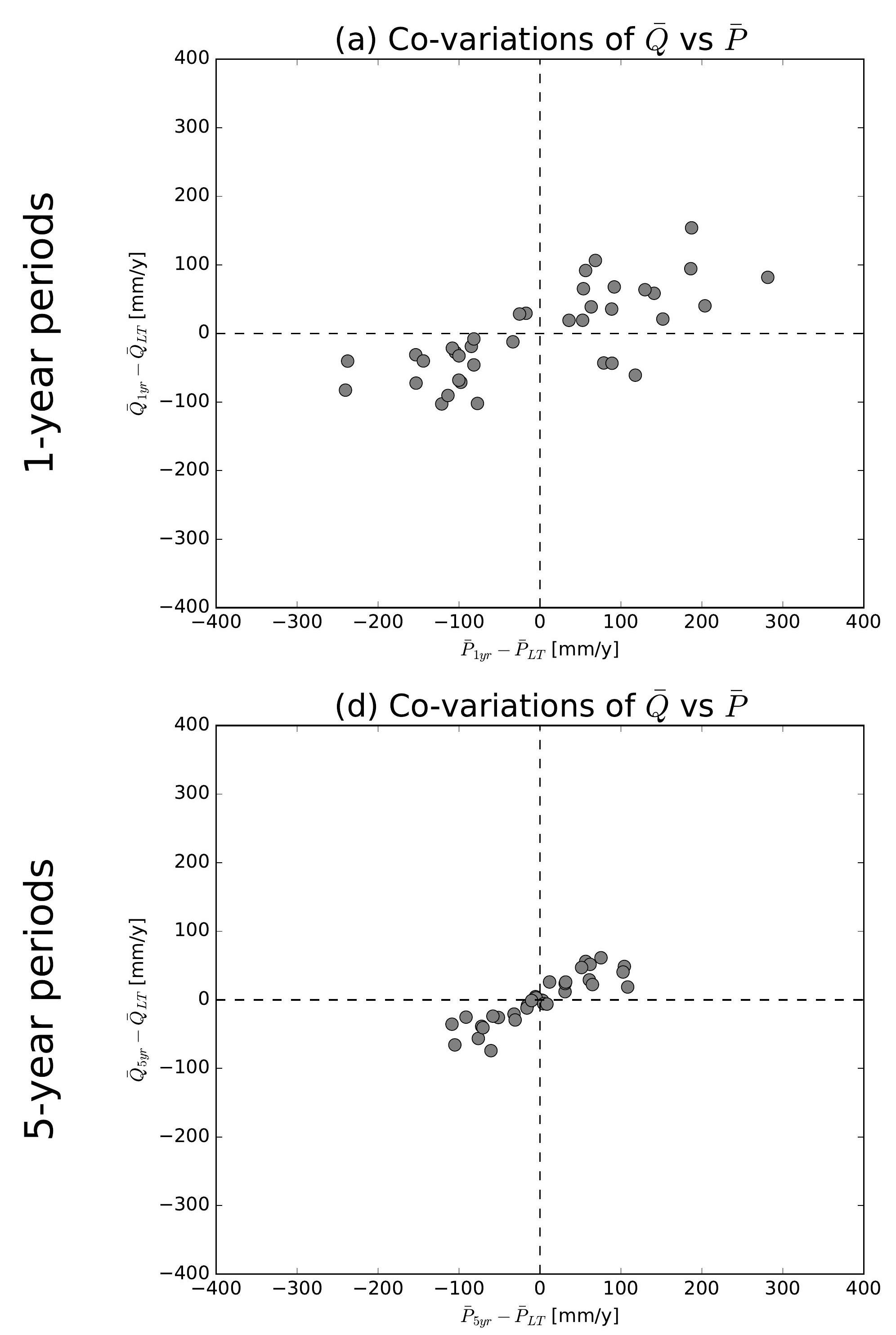

(g) Co-variations of $\bar{Q}$ vs $\bar{P}$
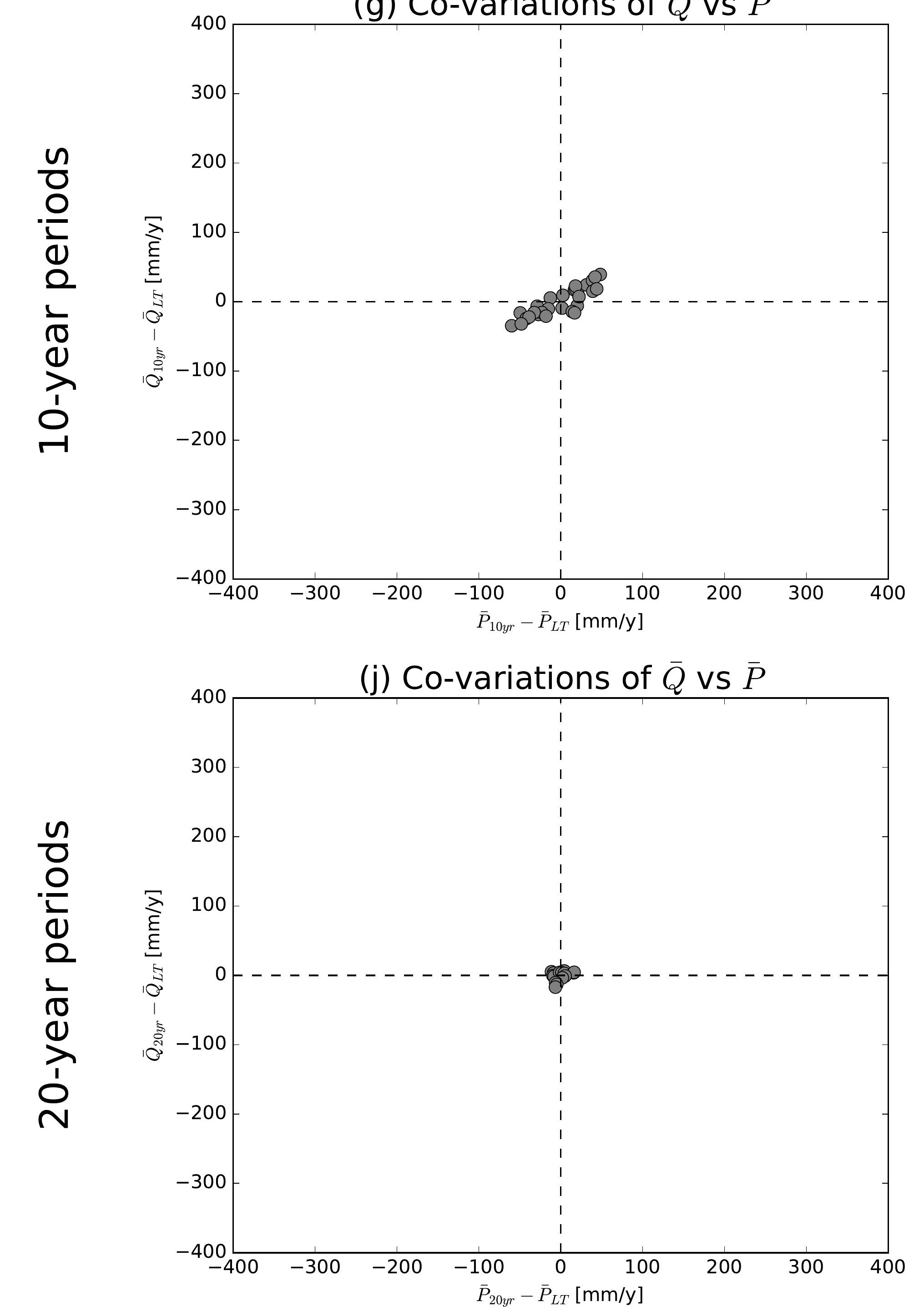

(b) Co-variations of $\bar{Q}$ vs $\overline{E 0}$

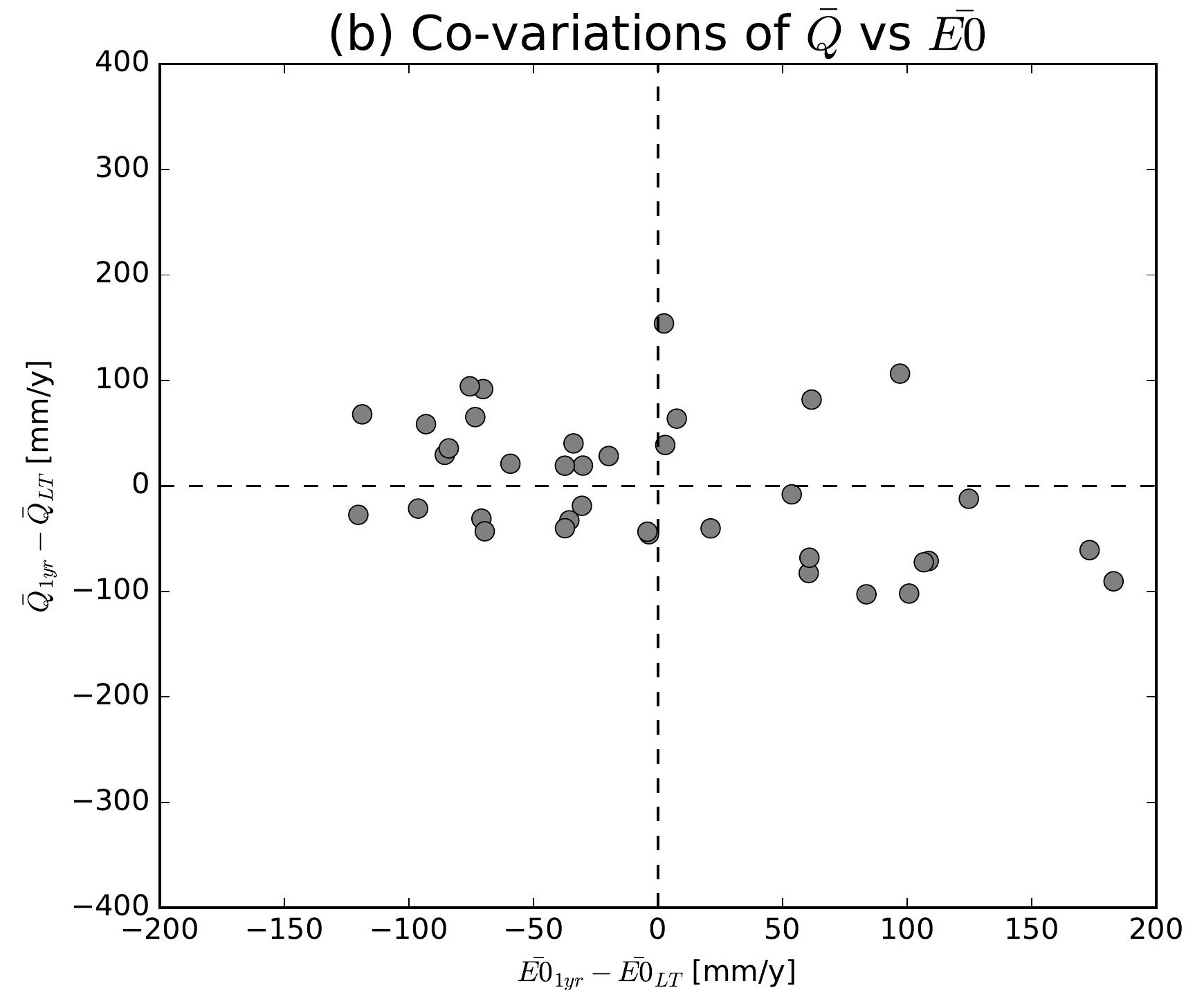

(e) Co-variations of $\bar{Q}$ vs $\overline{E 0}$

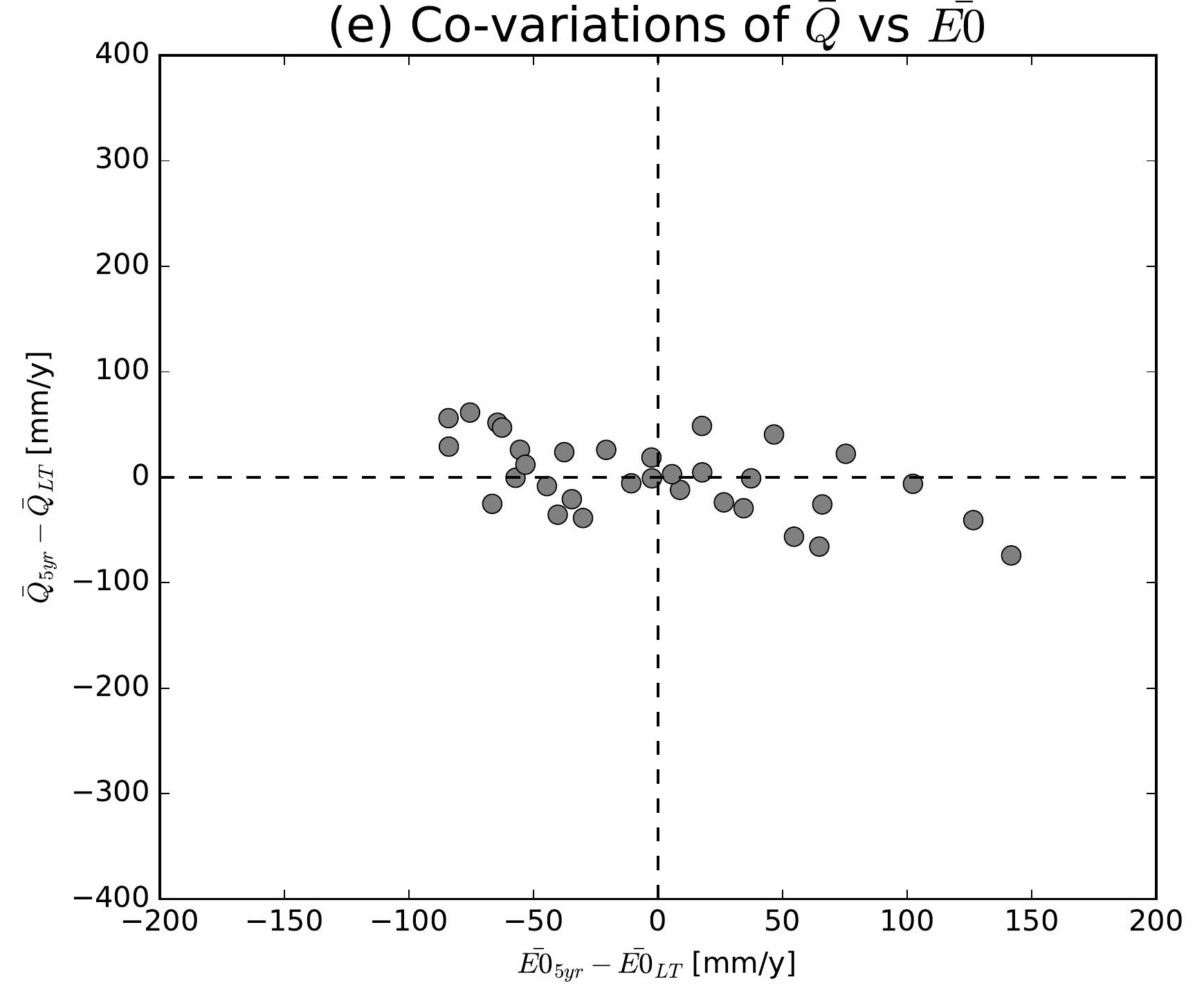

(h) Co-variations of $\bar{Q}$ vs $\overline{E 0}$

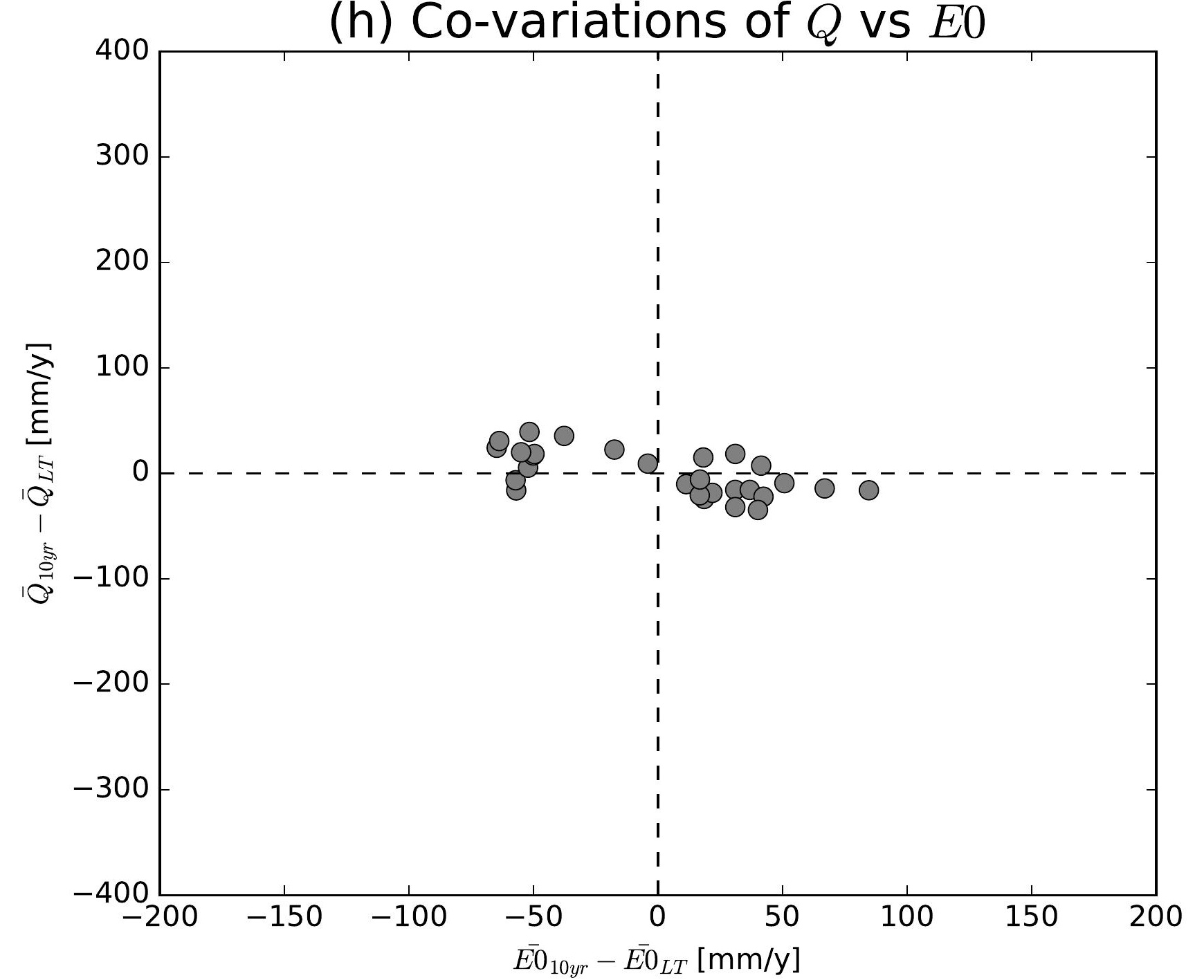

(k) Co-variations of $\bar{Q}$ vs $\overline{E 0}$

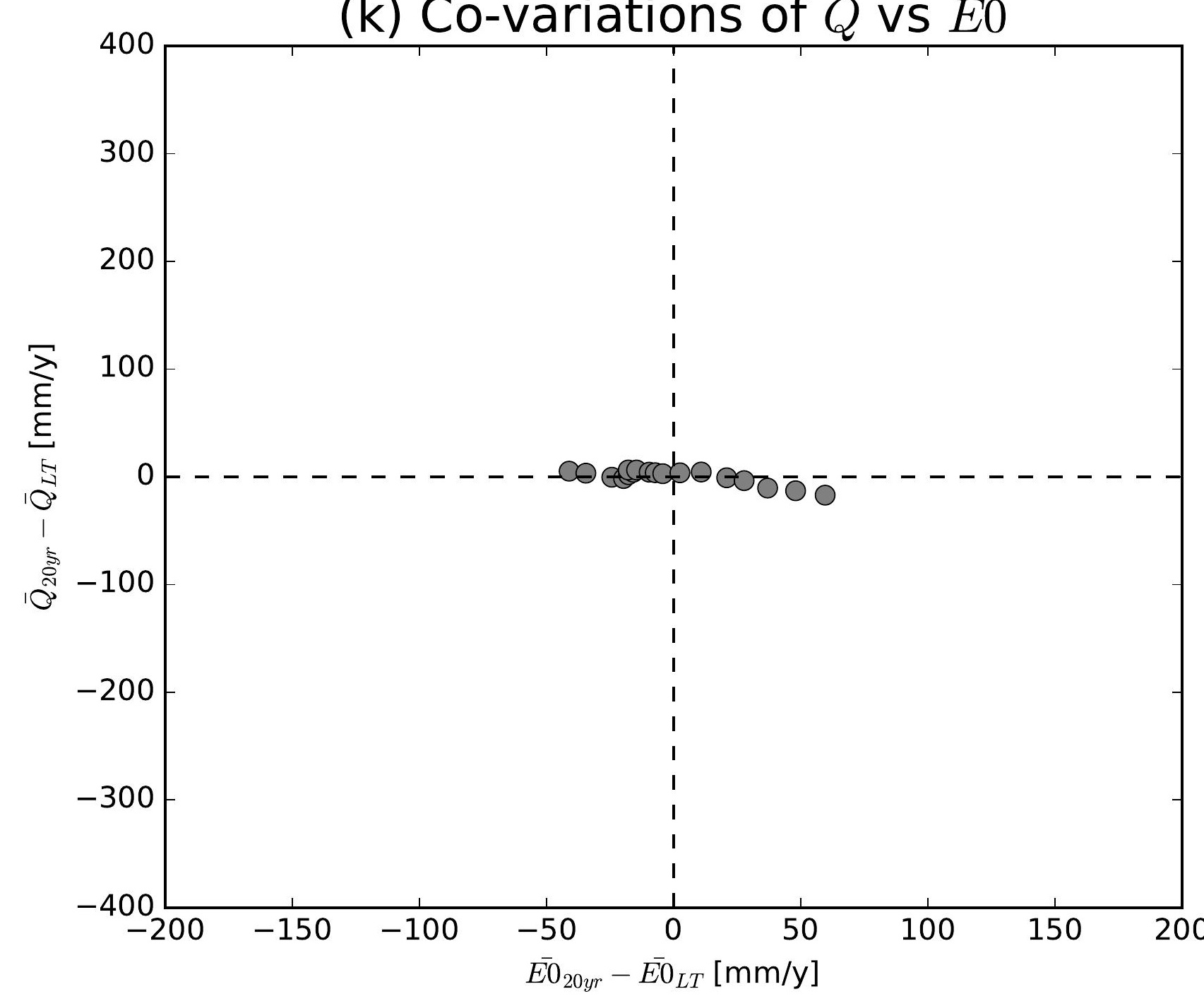

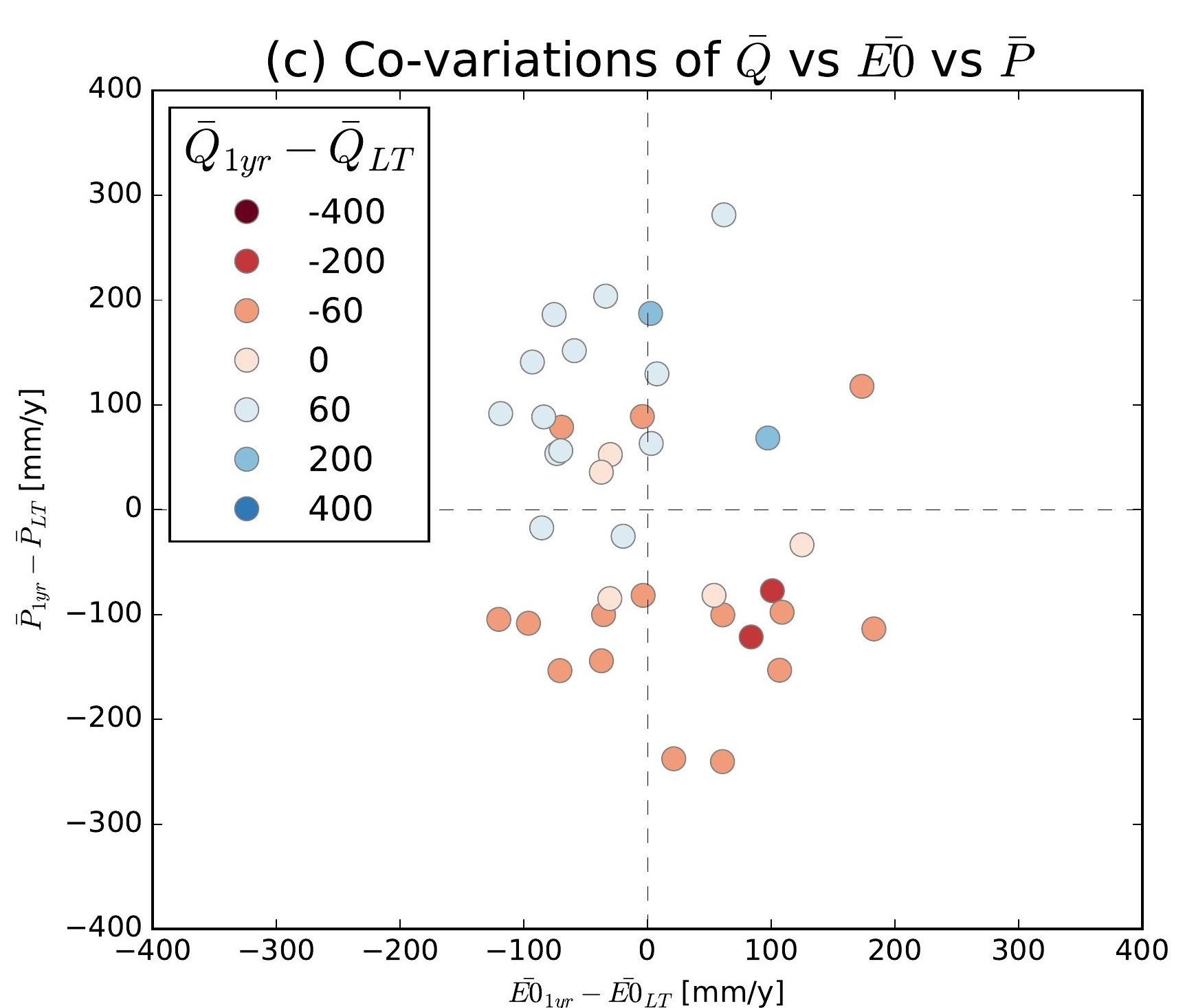
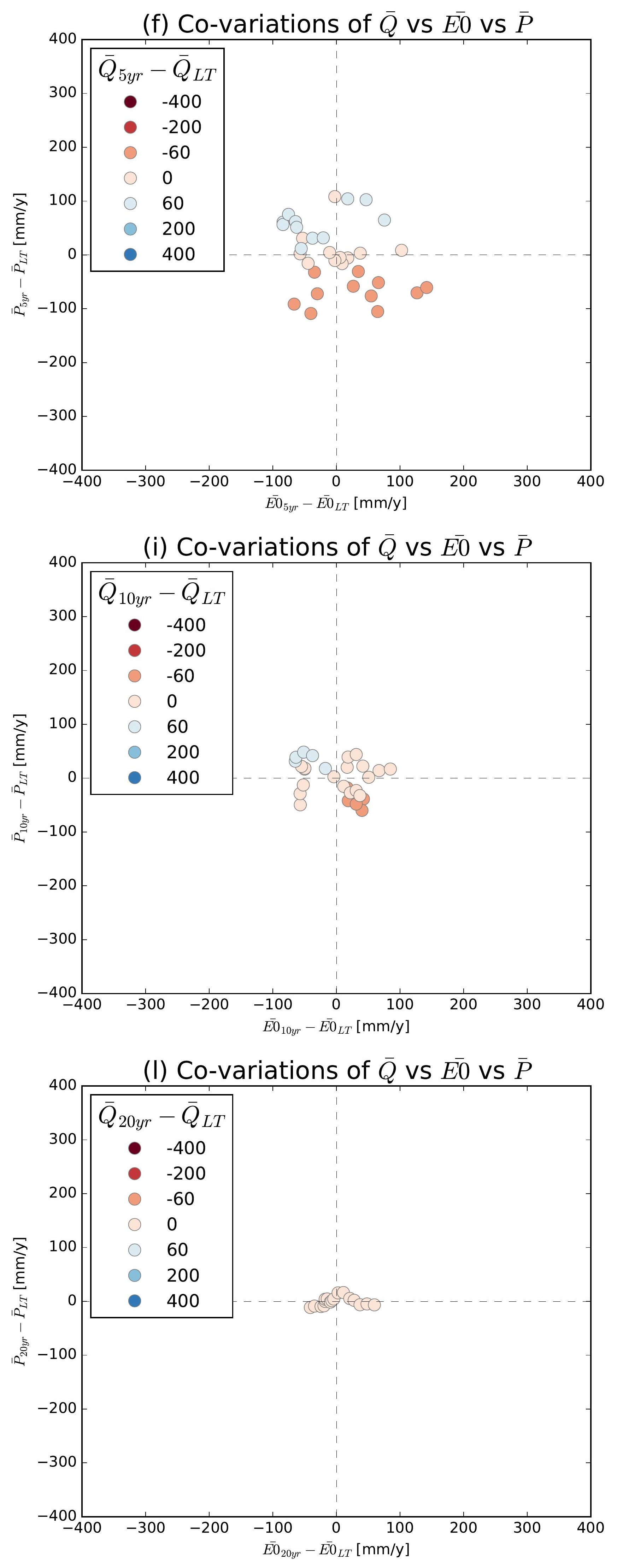

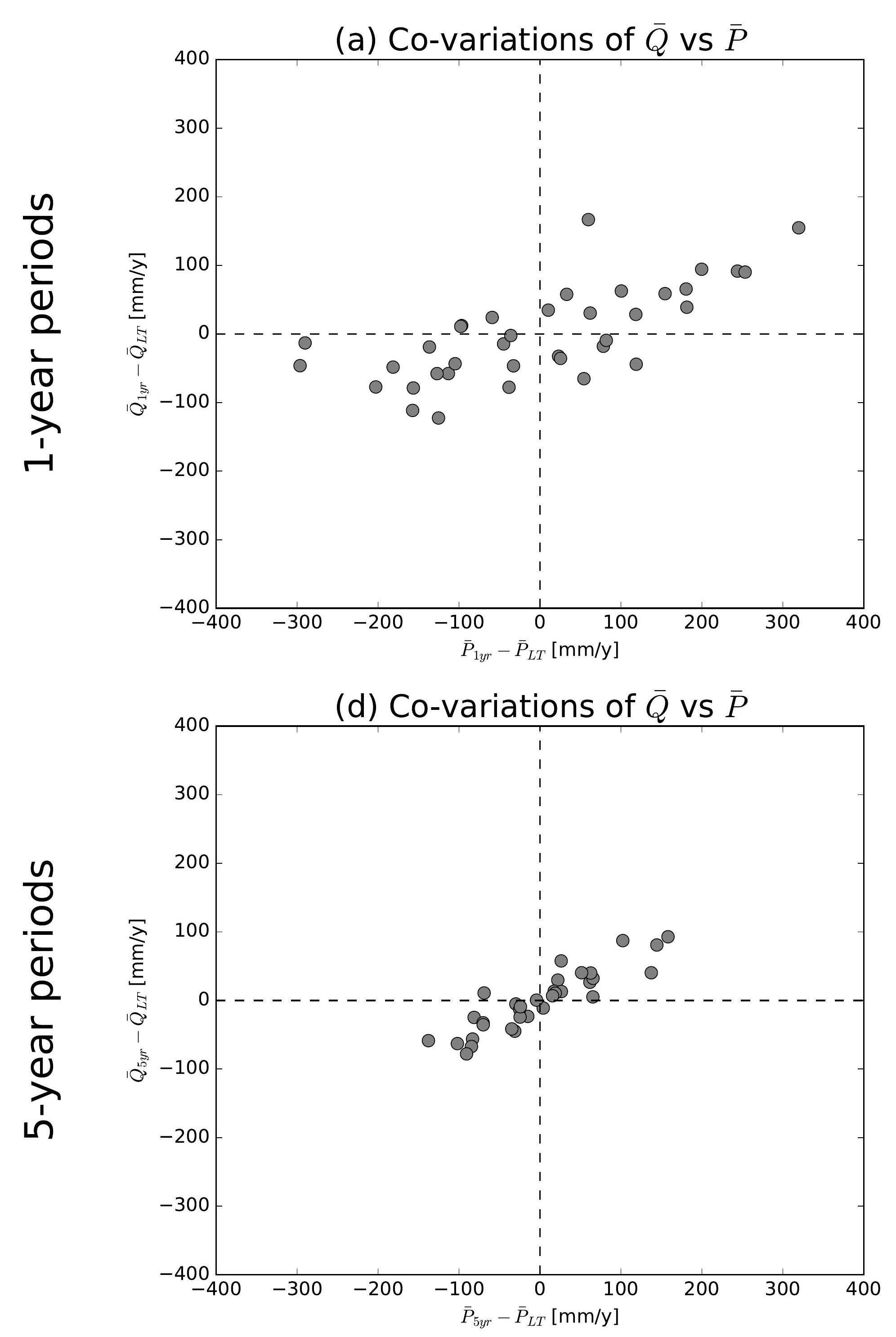

(g) Co-variations of $\bar{Q}$ vs $\bar{P}$
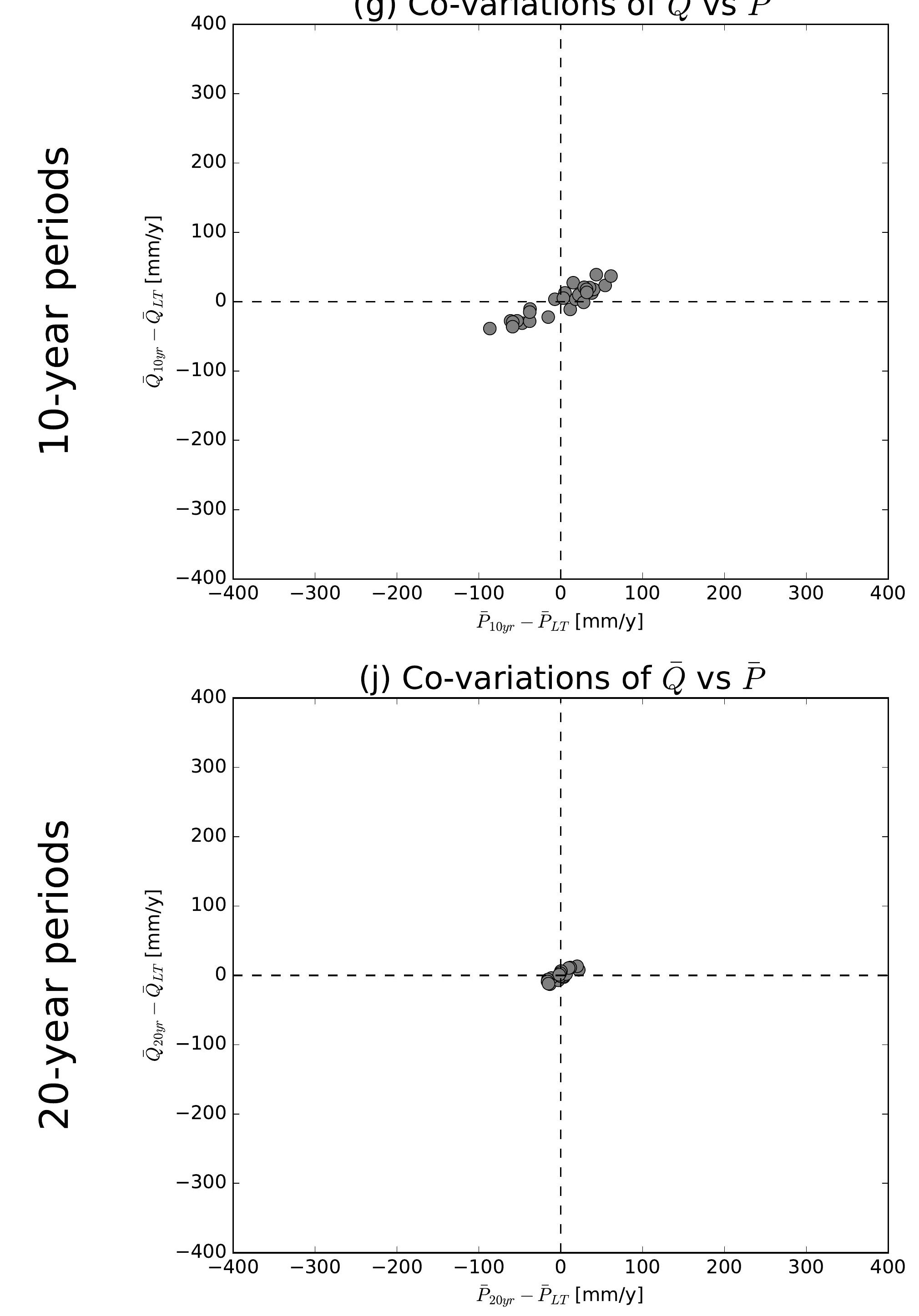

(b) Co-variations of $\bar{Q}$ vs $\overline{E 0}$

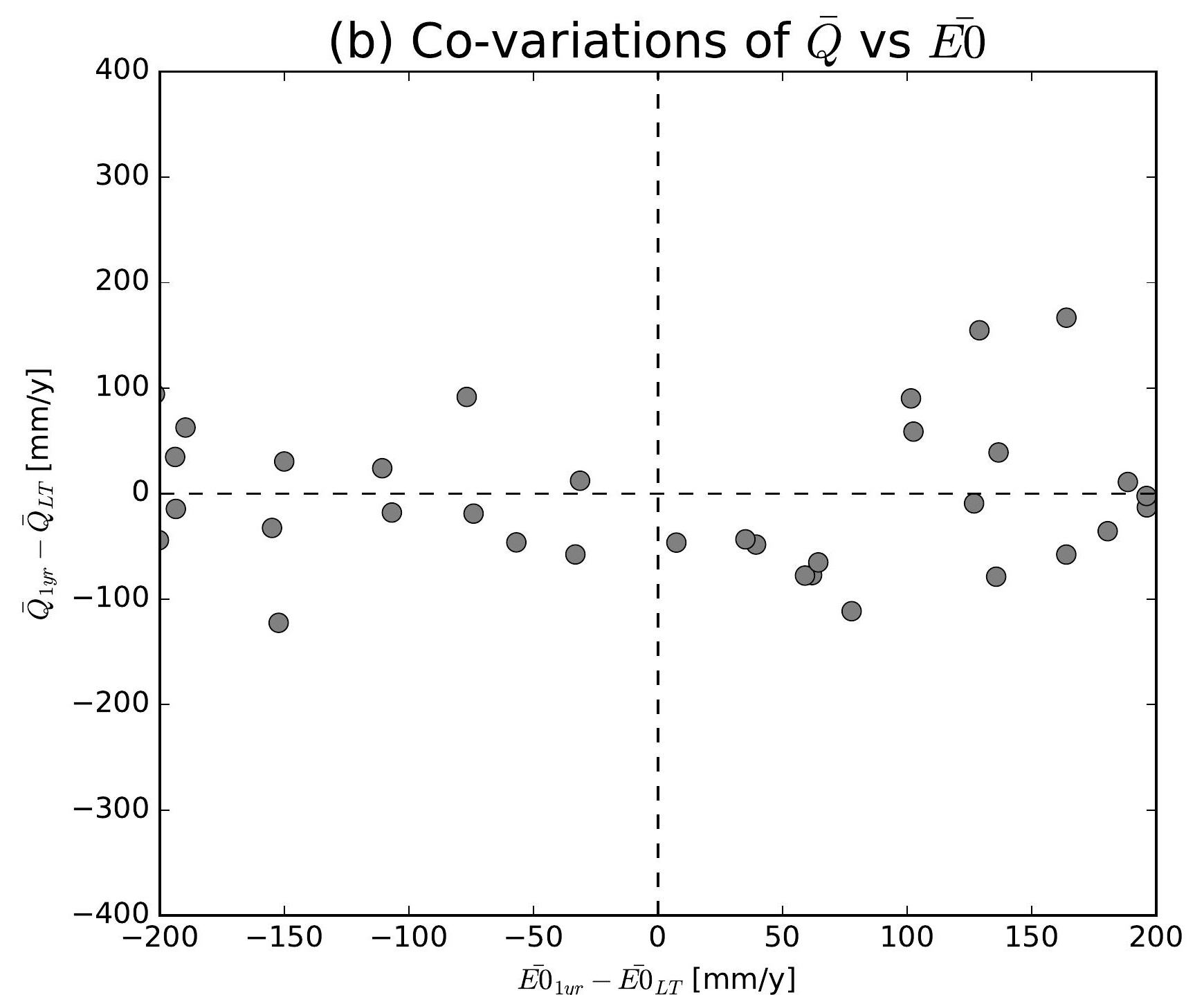

(e) Co-variations of $\bar{Q}$ vs $\overline{E 0}$

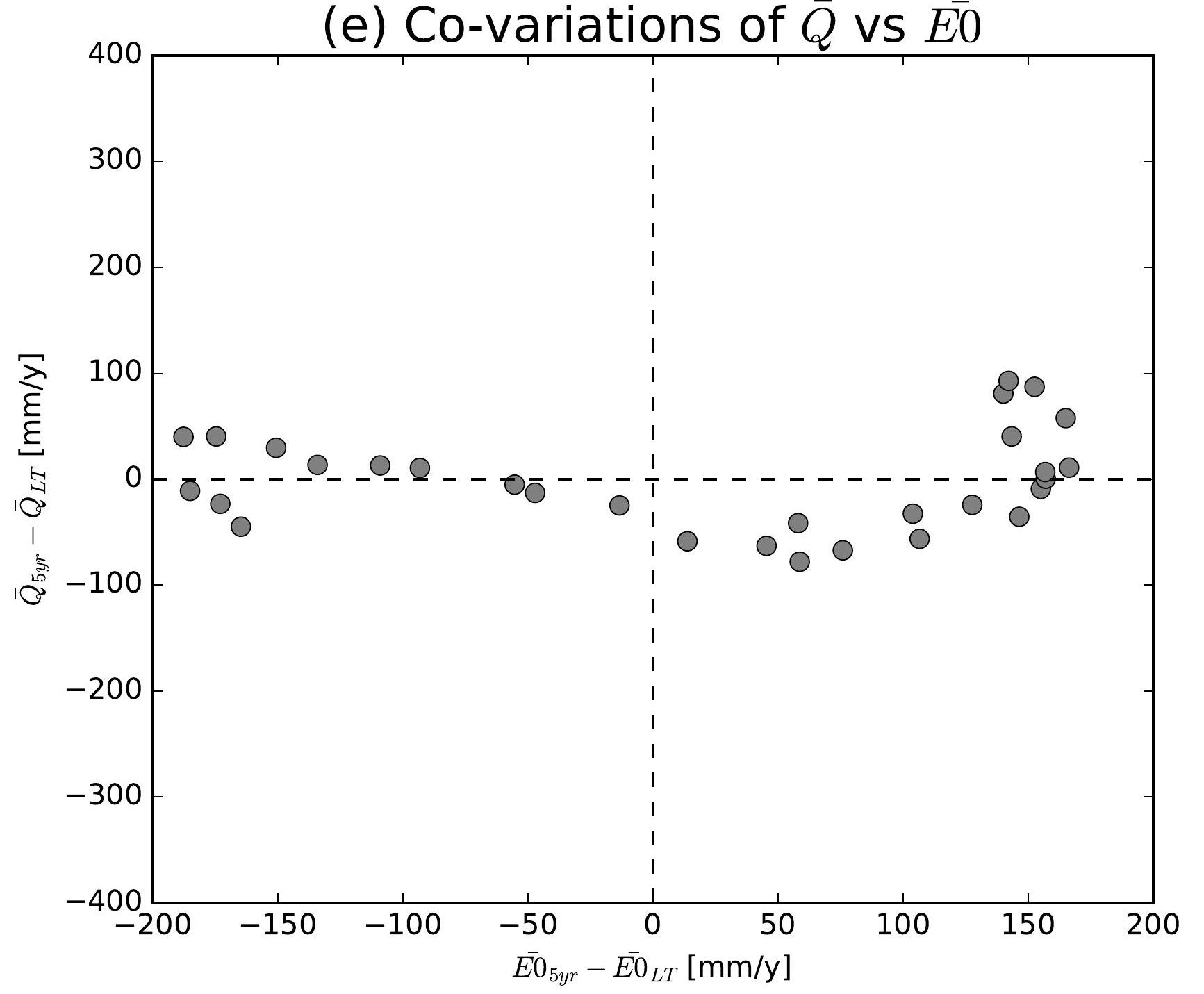

(h) Co-variations of $\bar{Q}$ vs $\overline{E 0}$

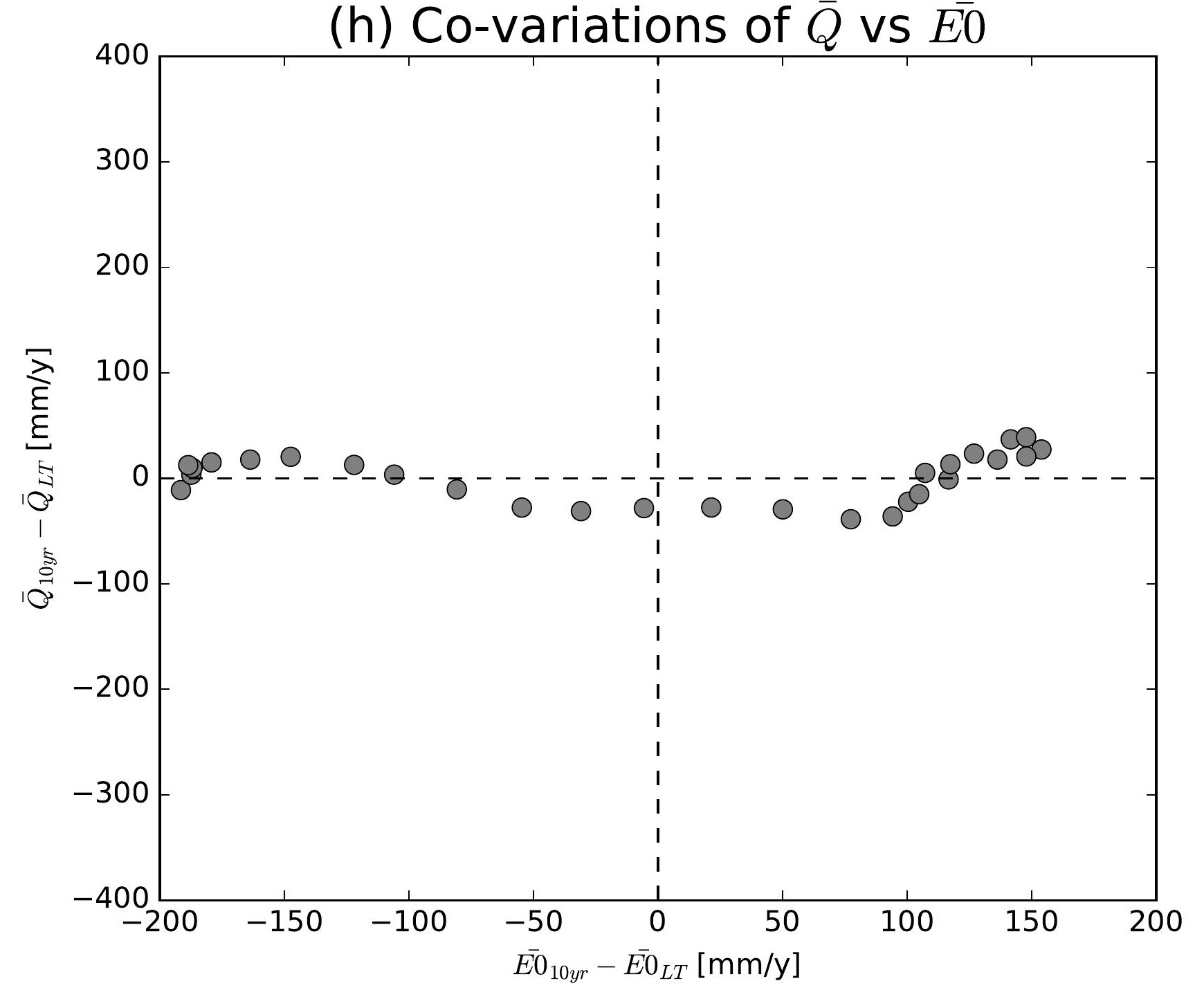

(k) Co-variations of $\bar{Q}$ vs $\overline{E 0}$

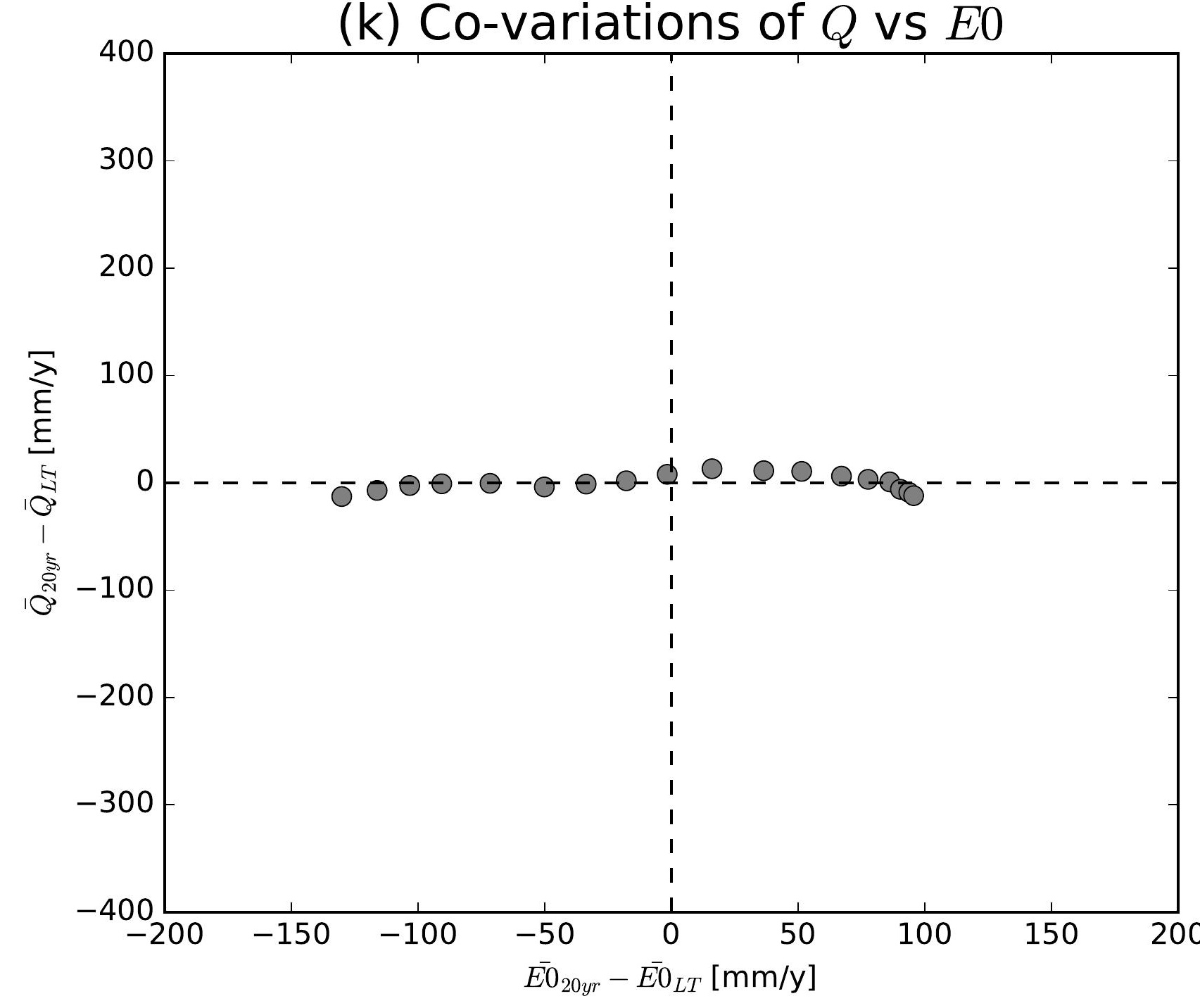

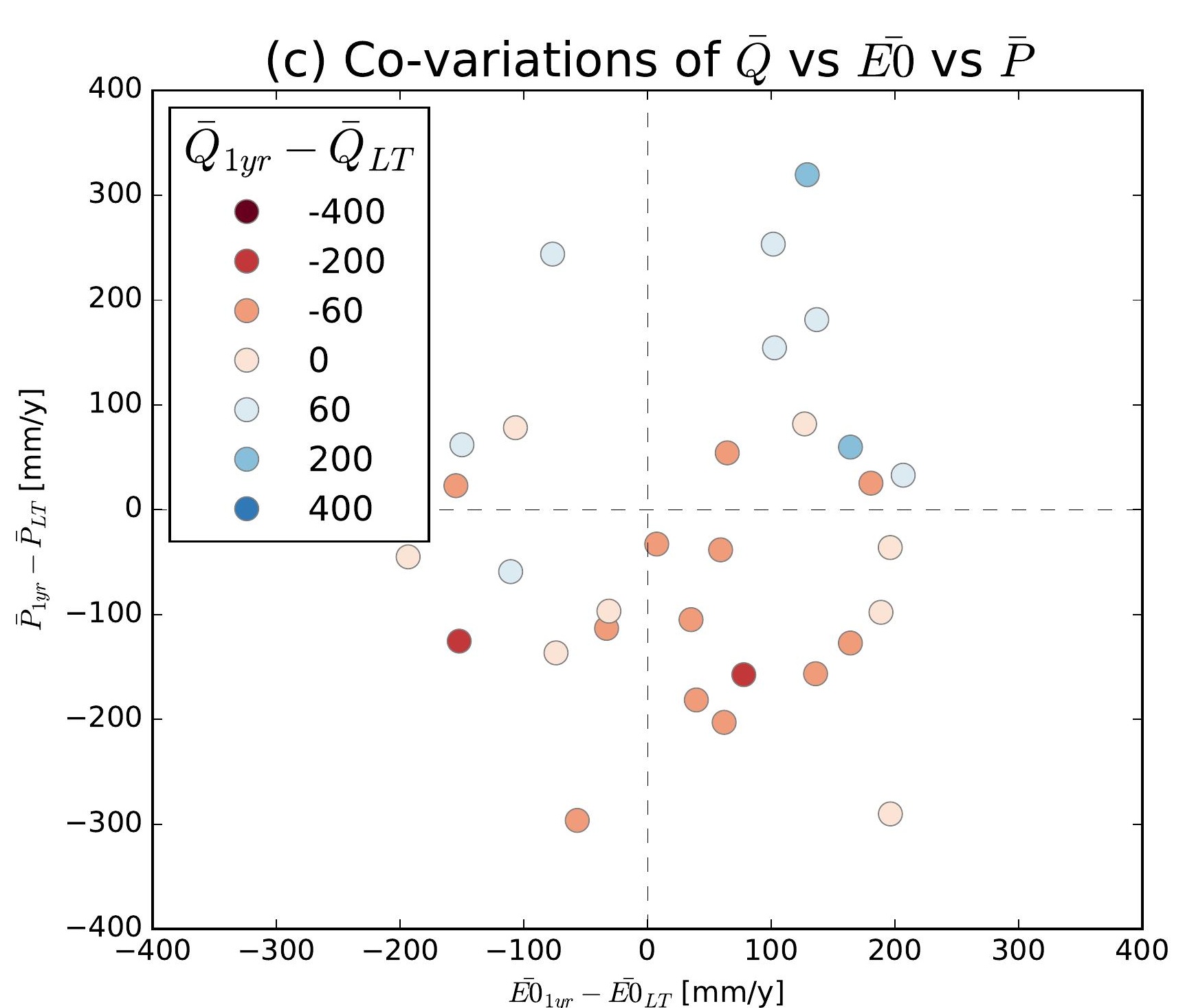
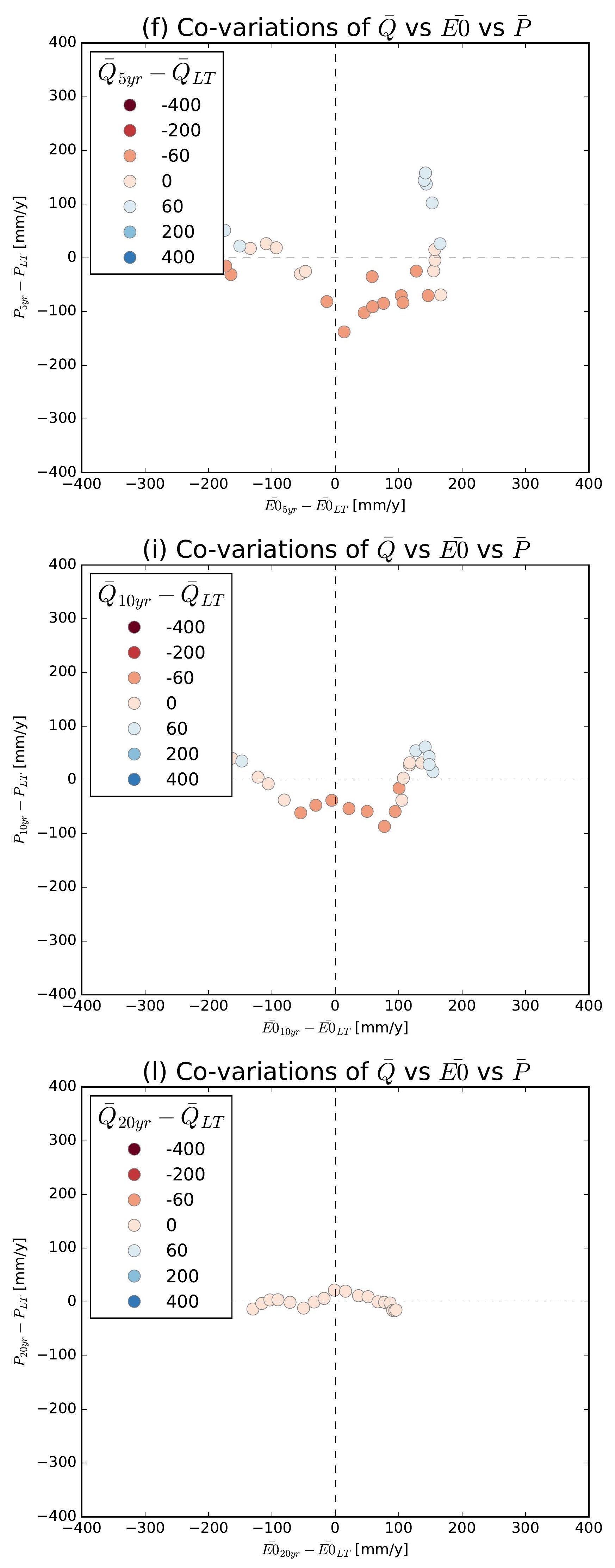

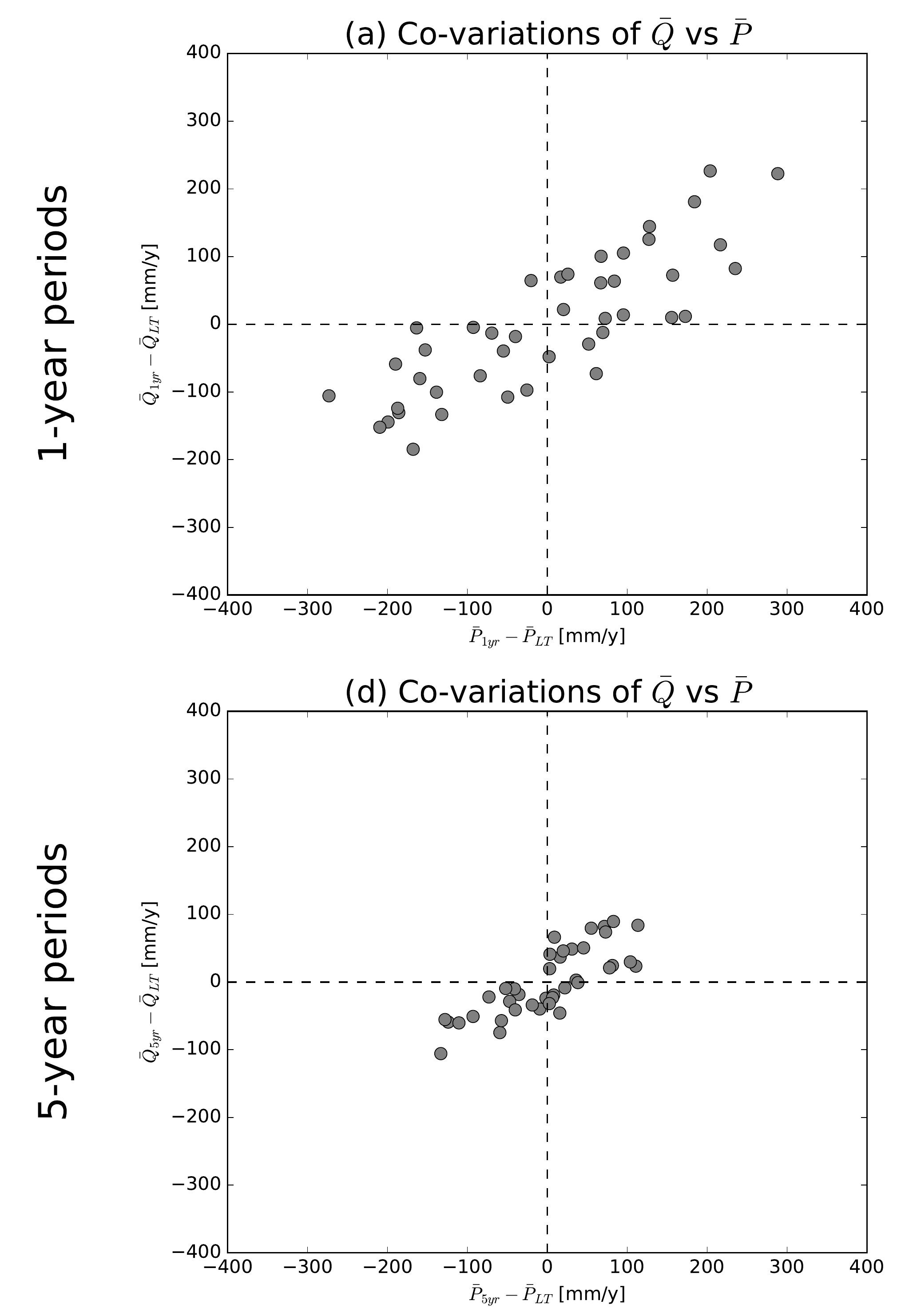

(g) Co-variations of $\bar{Q}$ vs $\bar{P}$
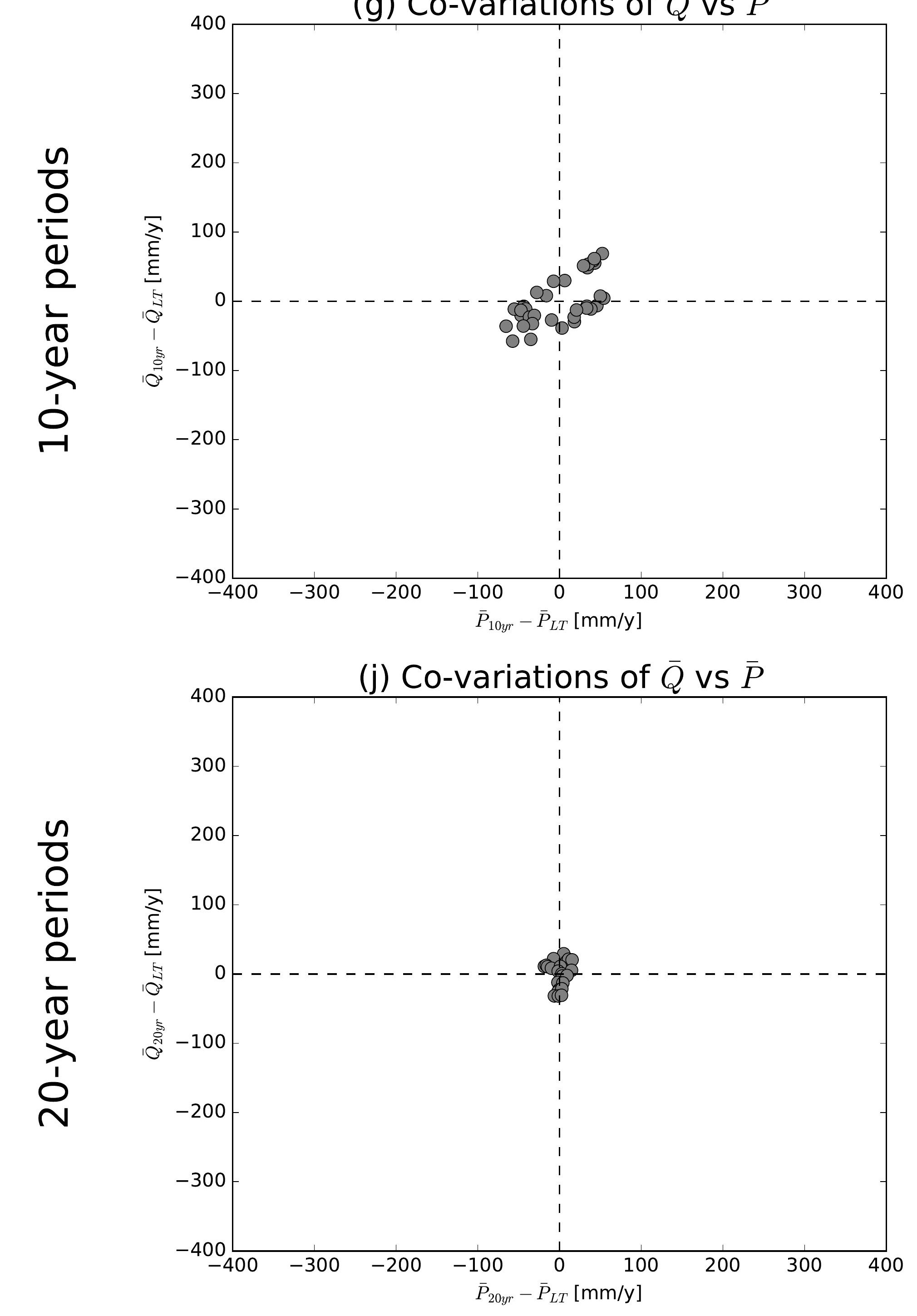

(b) Co-variations of $\bar{Q}$ vs $\overline{E 0}$

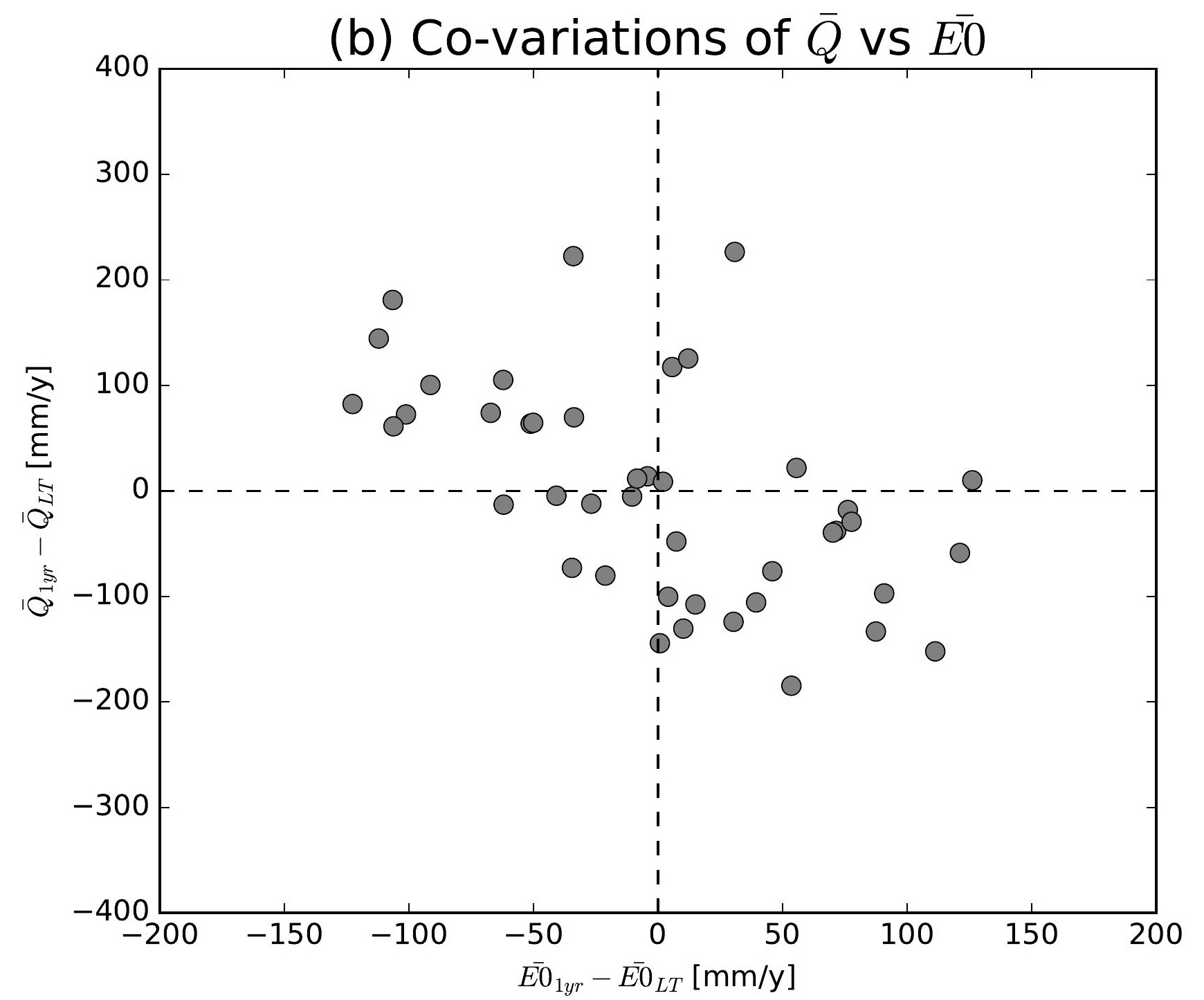

(e) Co-variations of $\bar{Q}$ vs $\overline{E 0}$

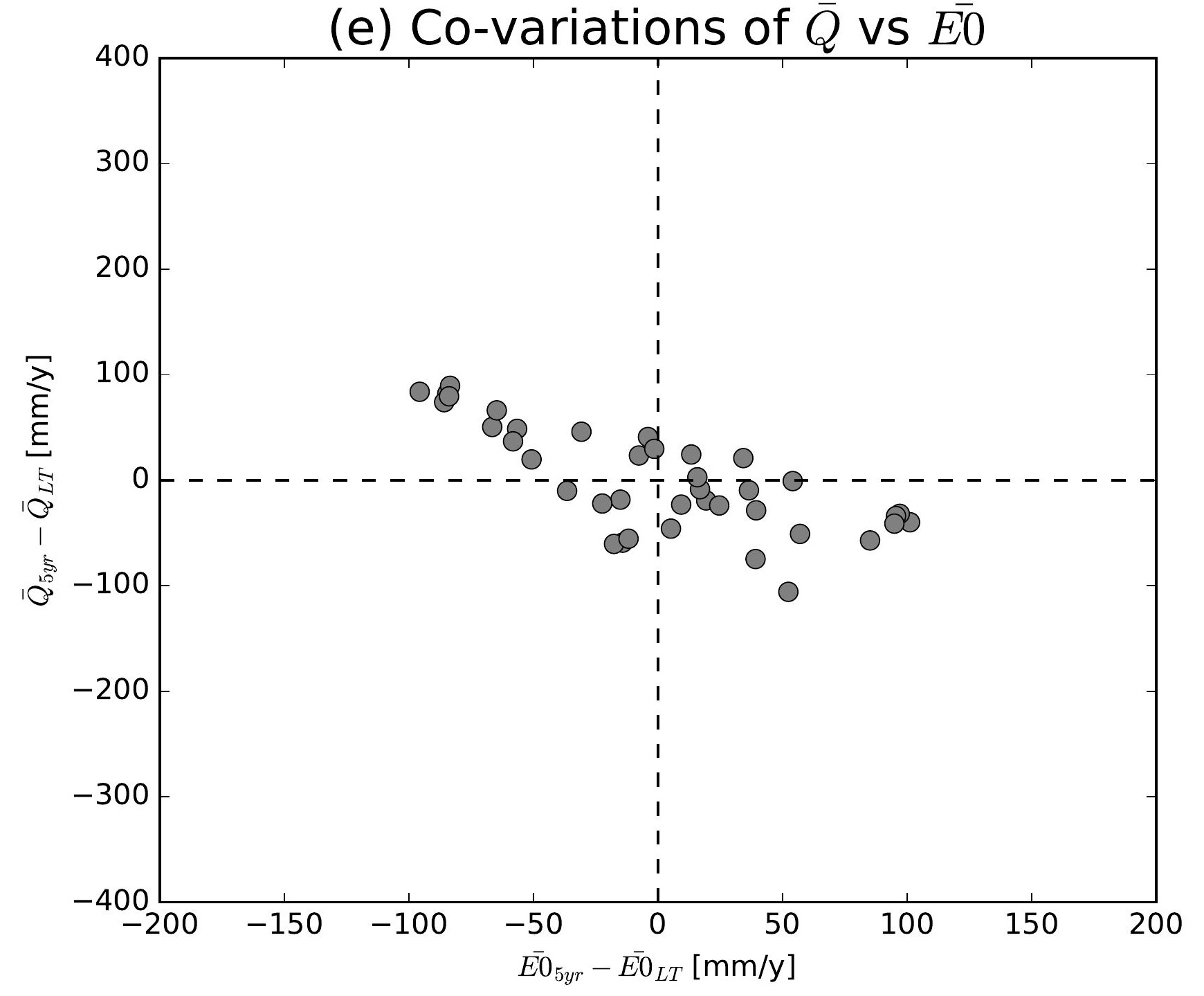

(h) Co-variations of $\bar{Q}$ vs $\overline{E 0}$

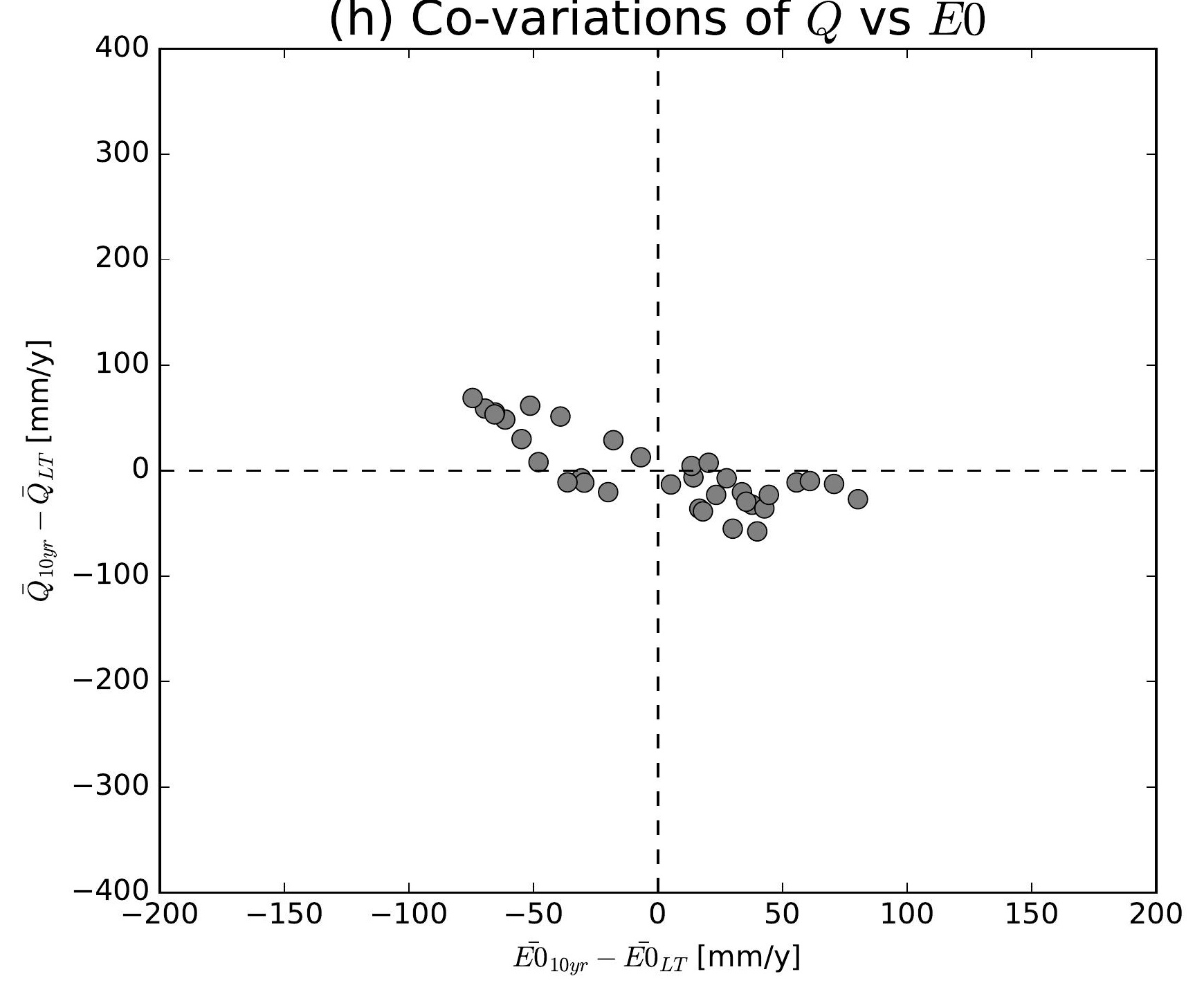

(k) Co-variations of $\bar{Q}$ vs $\overline{E 0}$

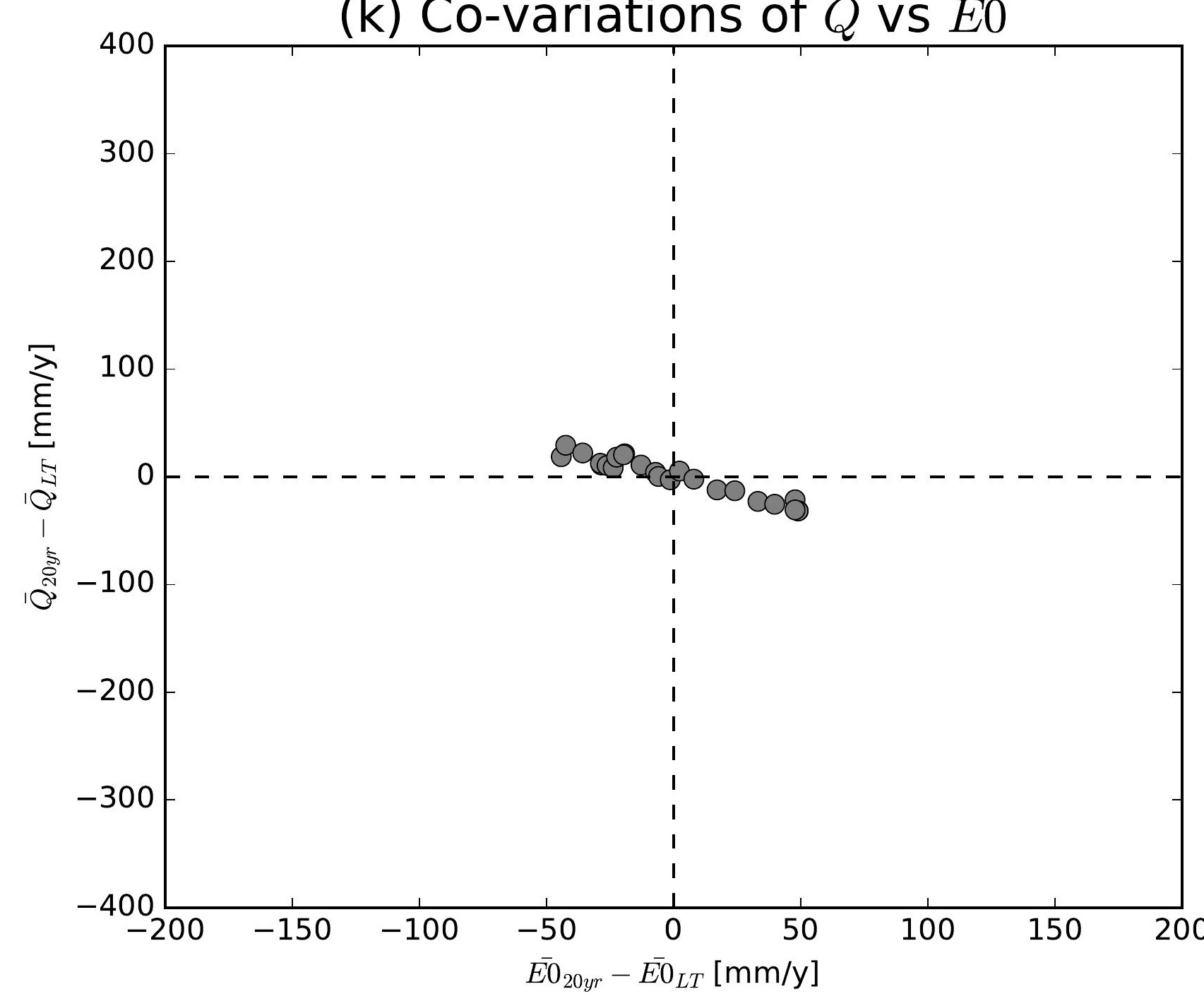

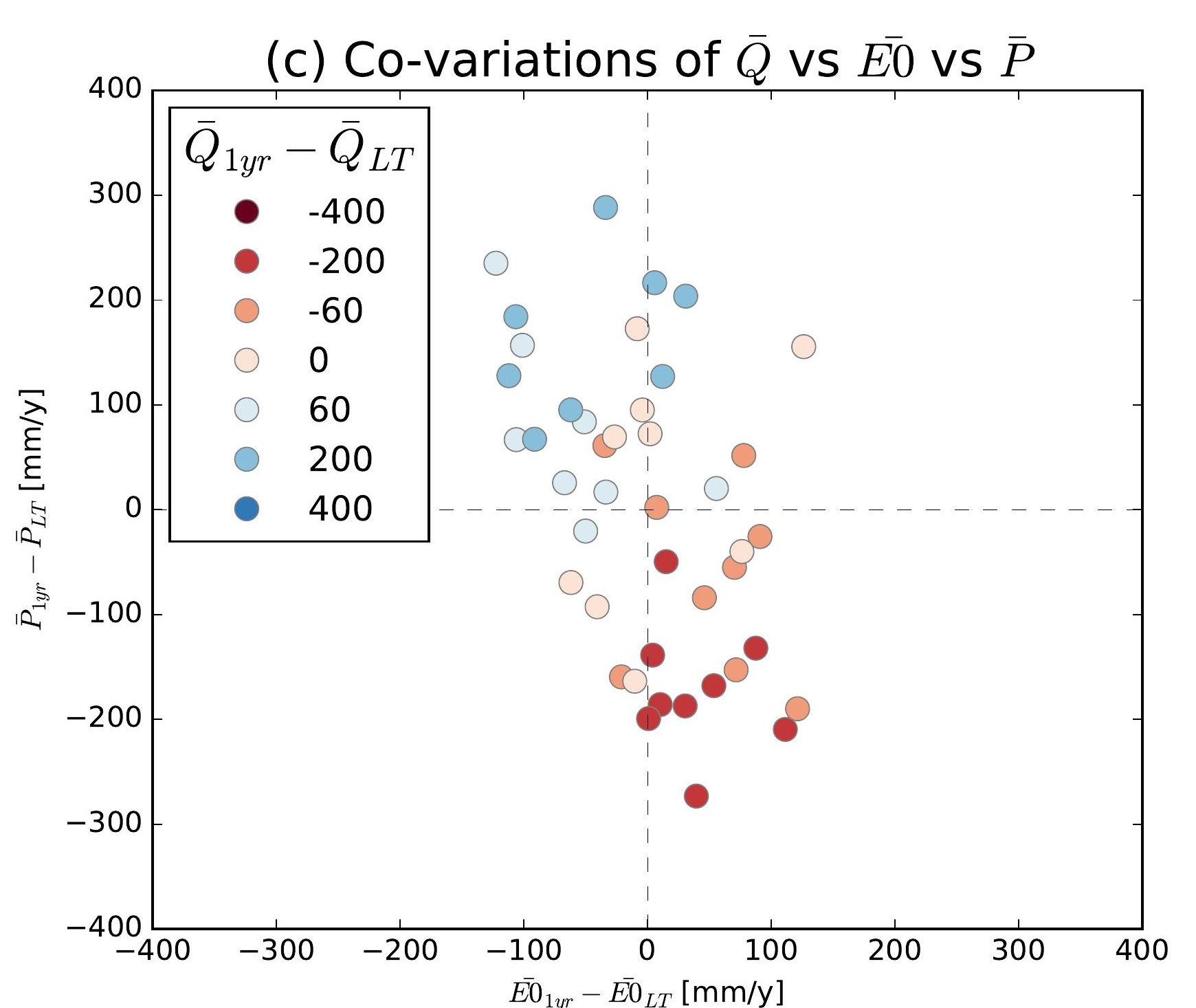
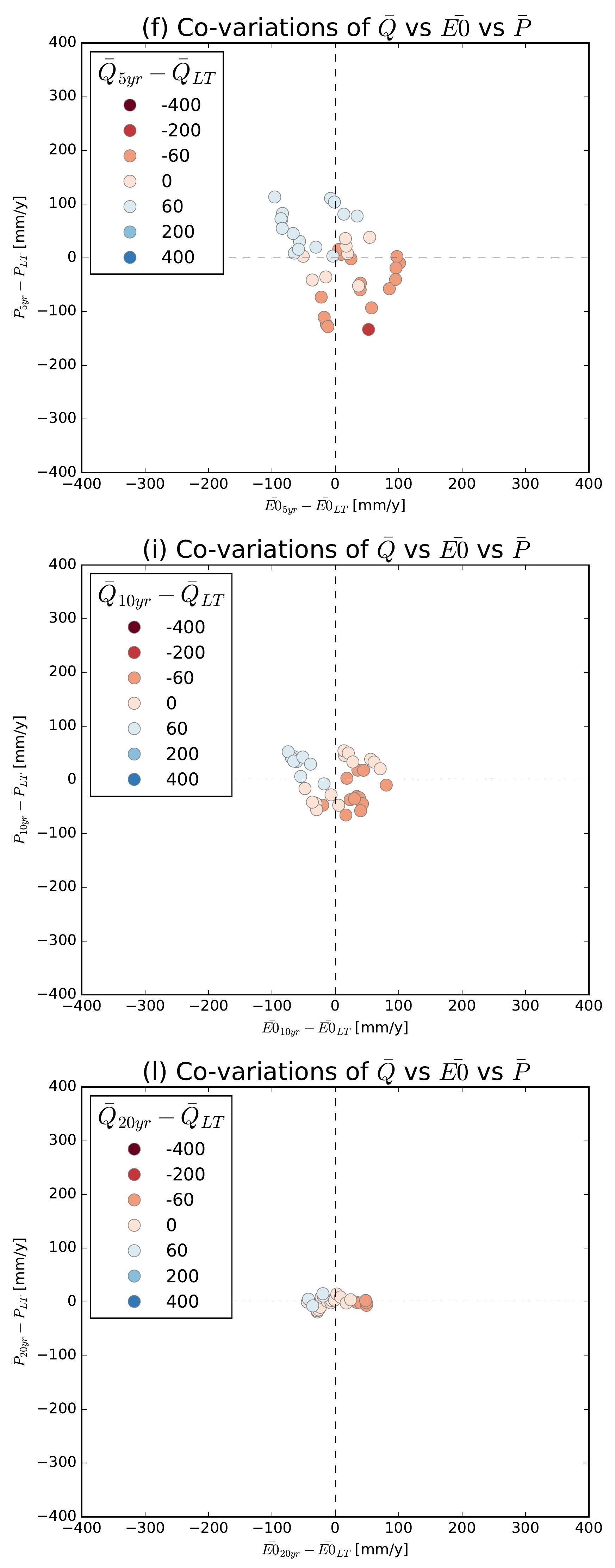

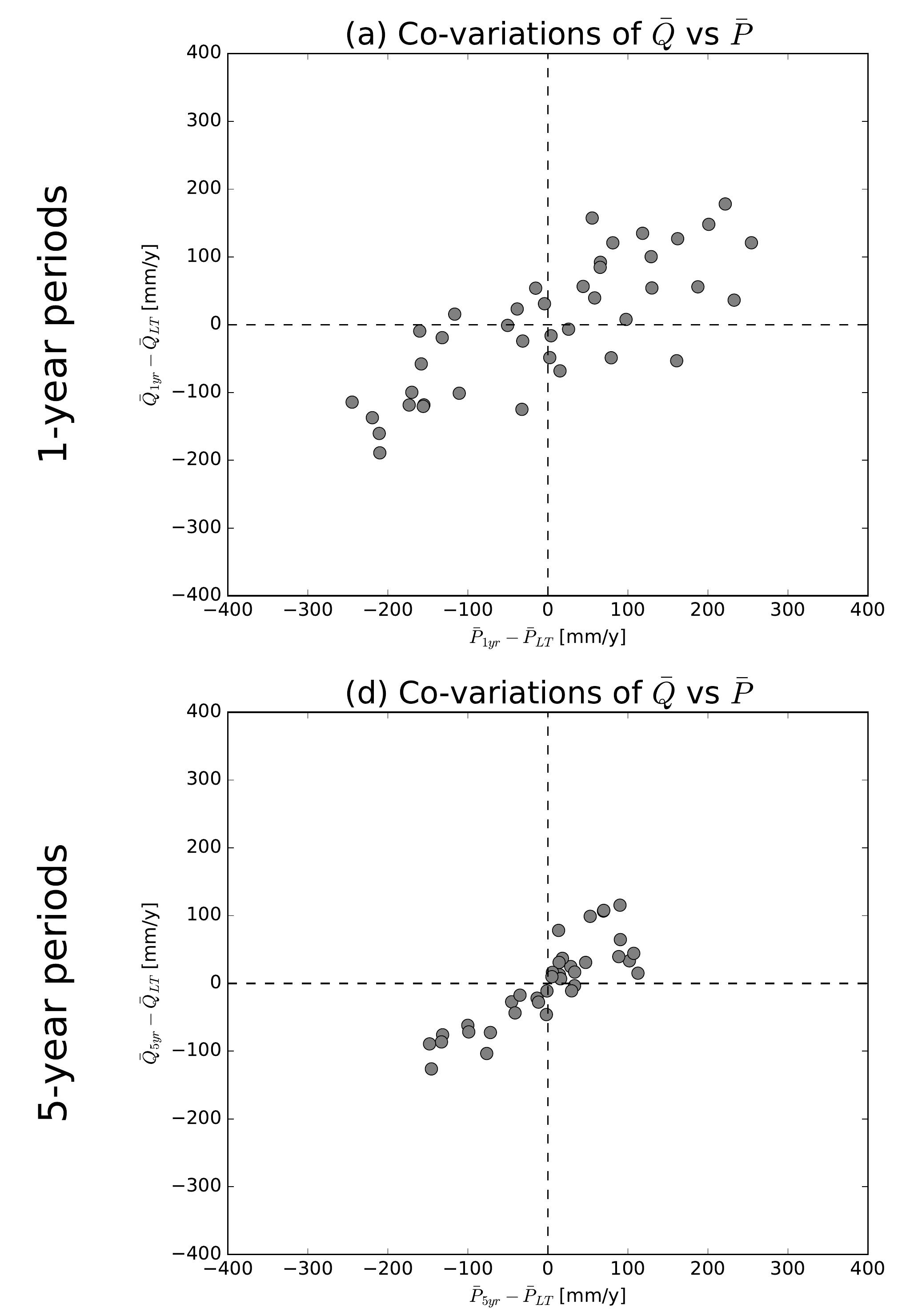

(g) Co-variations of $\bar{Q}$ vs $\bar{P}$
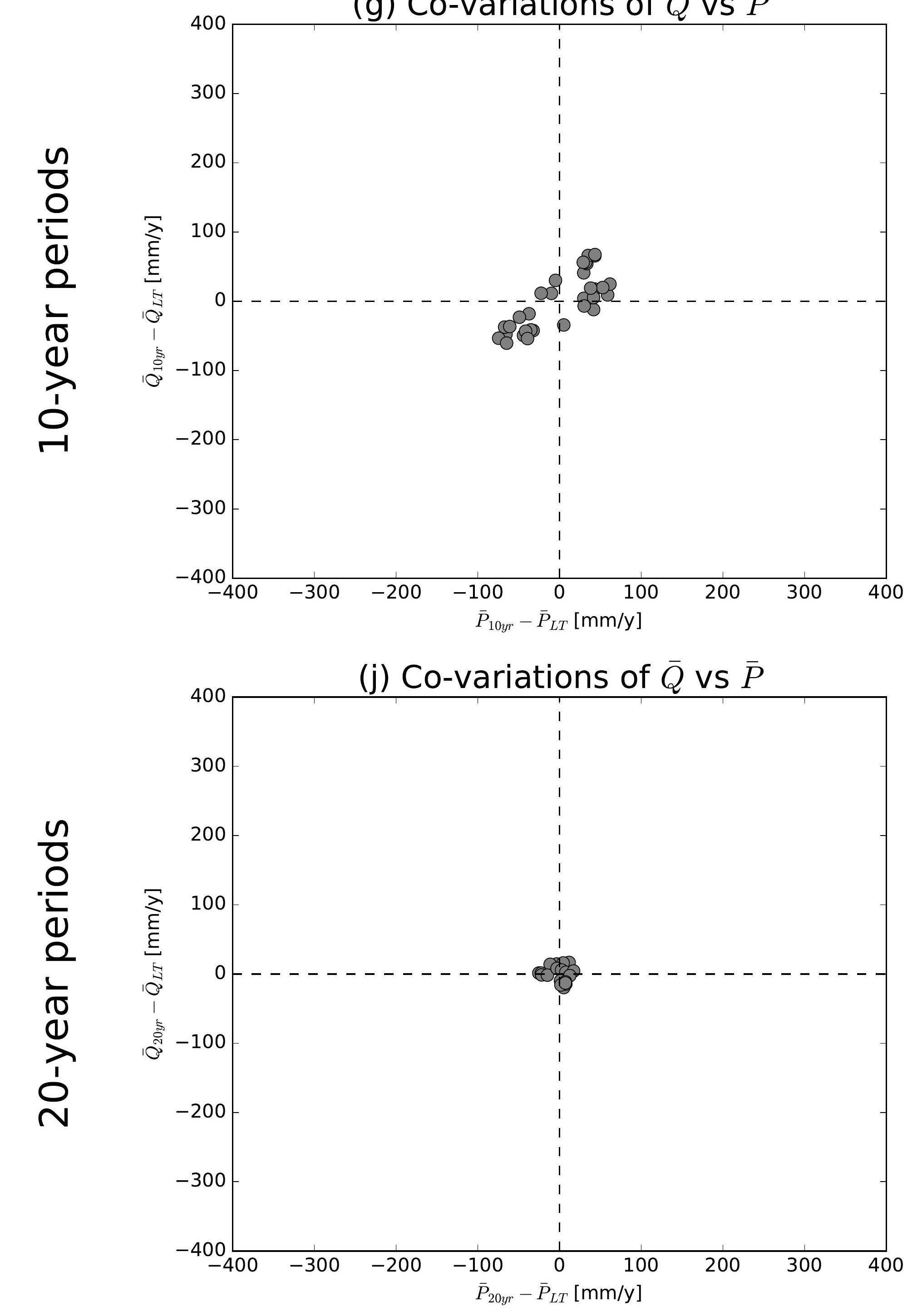

(b) Co-variations of $\bar{Q}$ vs $\overline{E 0}$

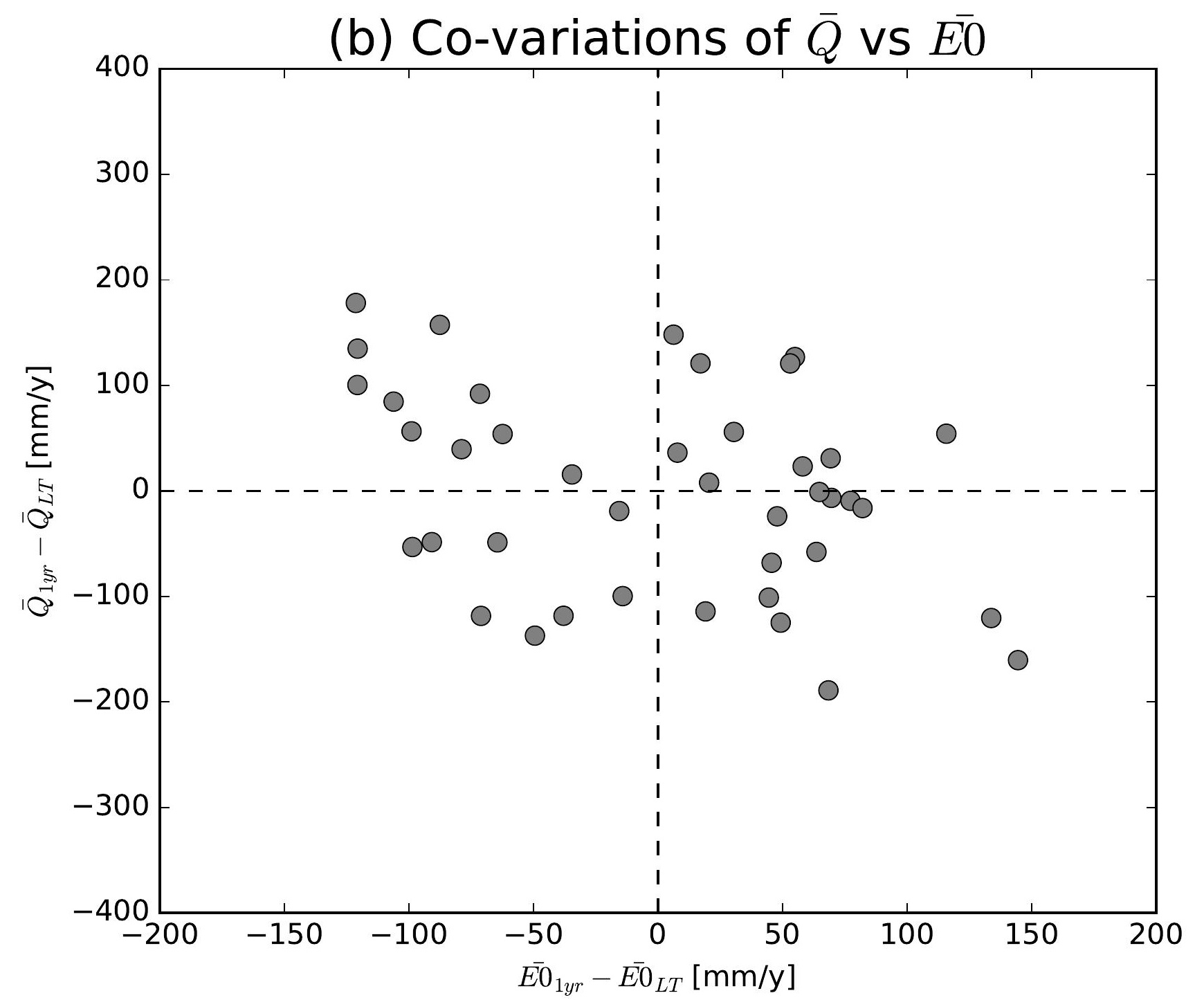

(e) Co-variations of $\bar{Q}$ vs $\overline{E 0}$

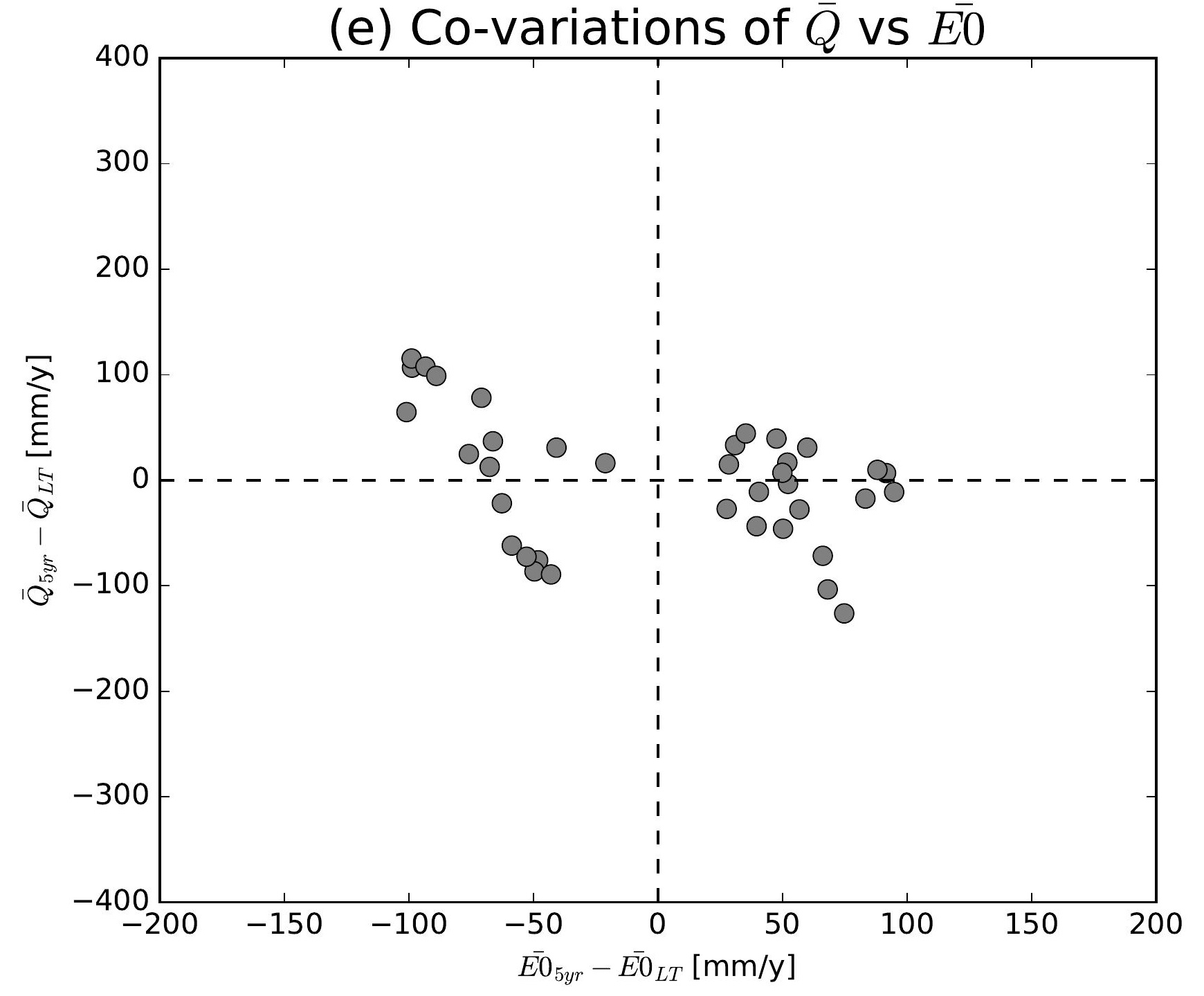

(h) Co-variations of $\bar{Q}$ vs $\overline{E 0}$

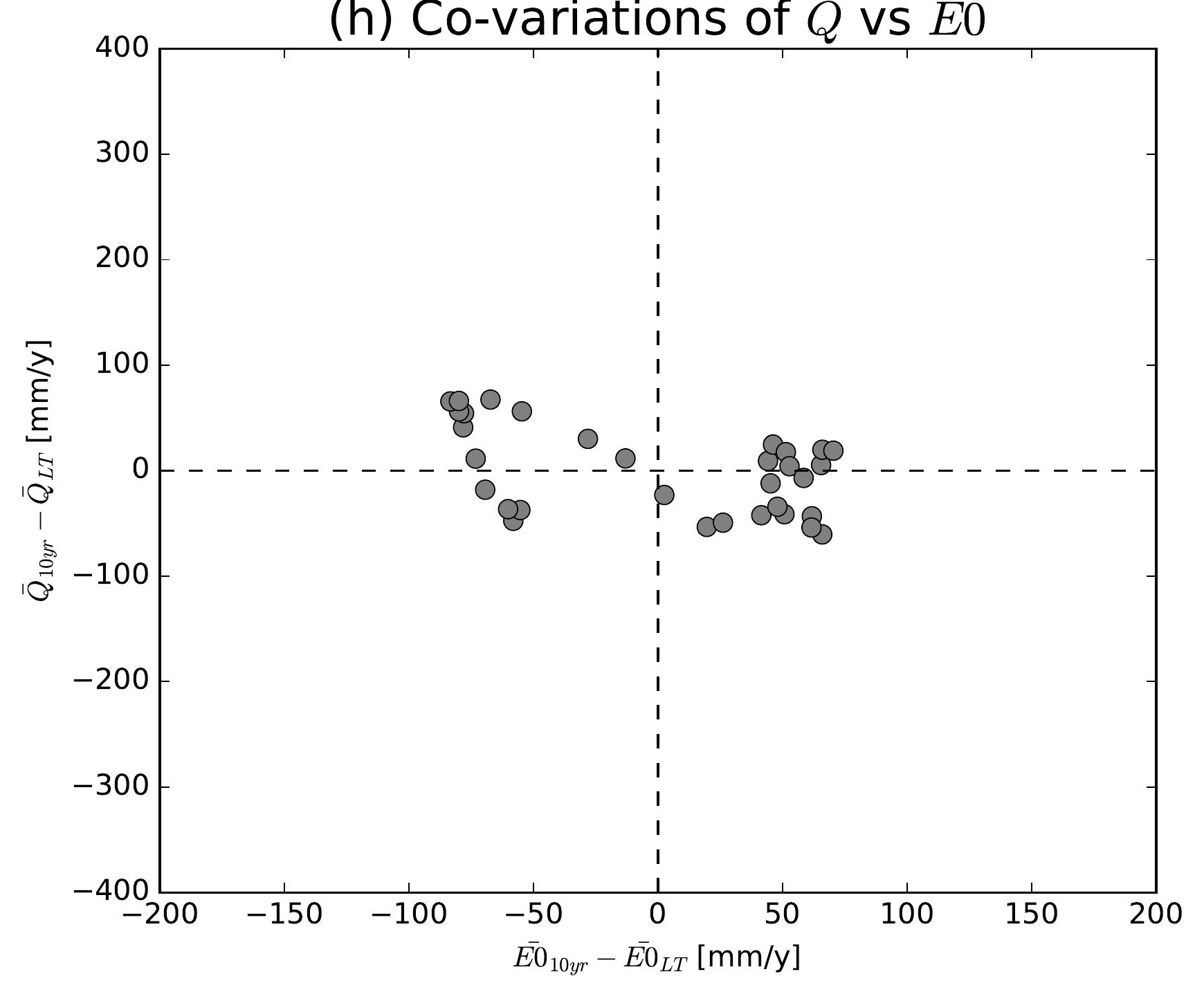

(k) Co-variations of $\bar{Q}$ vs $\overline{E 0}$

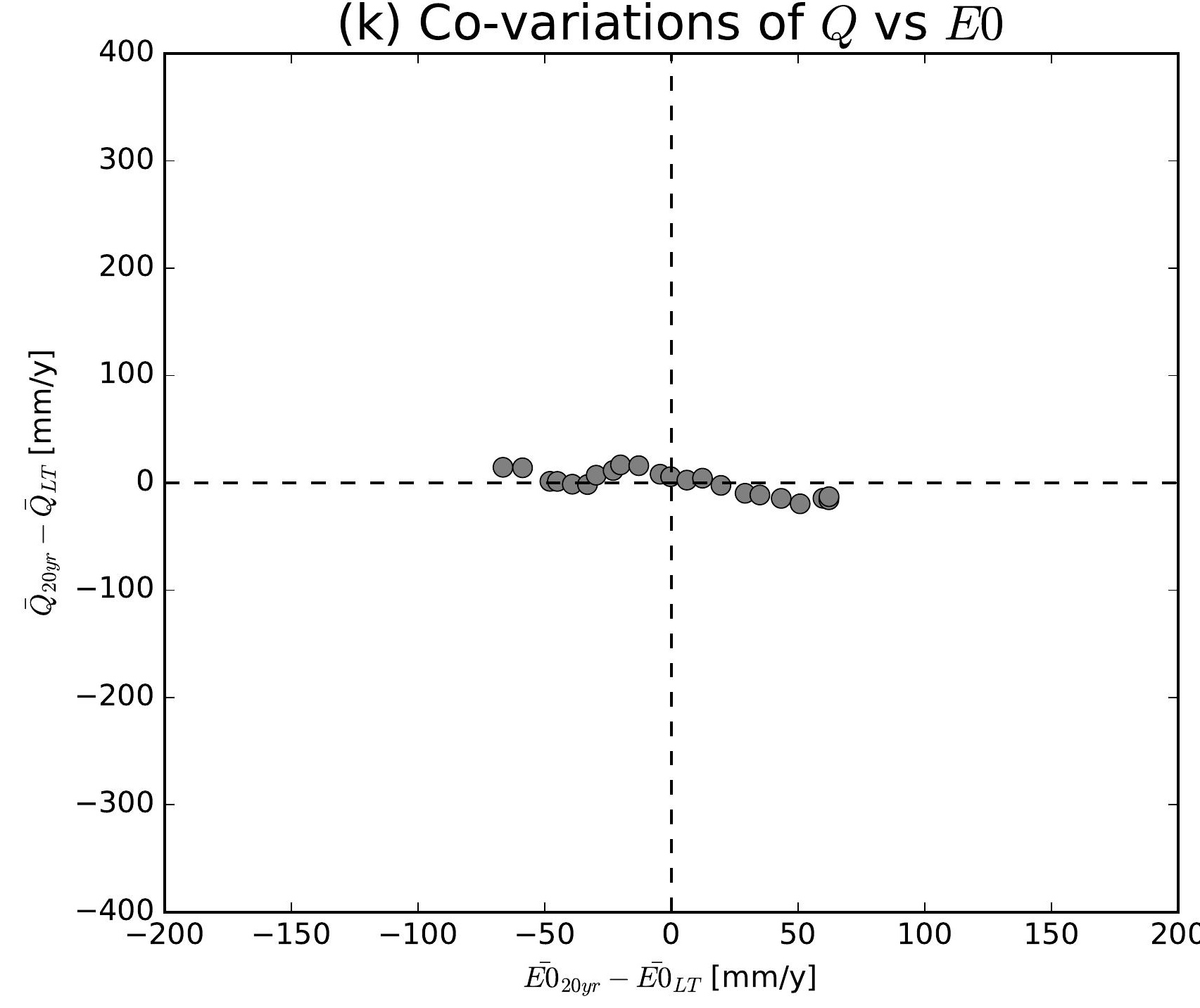

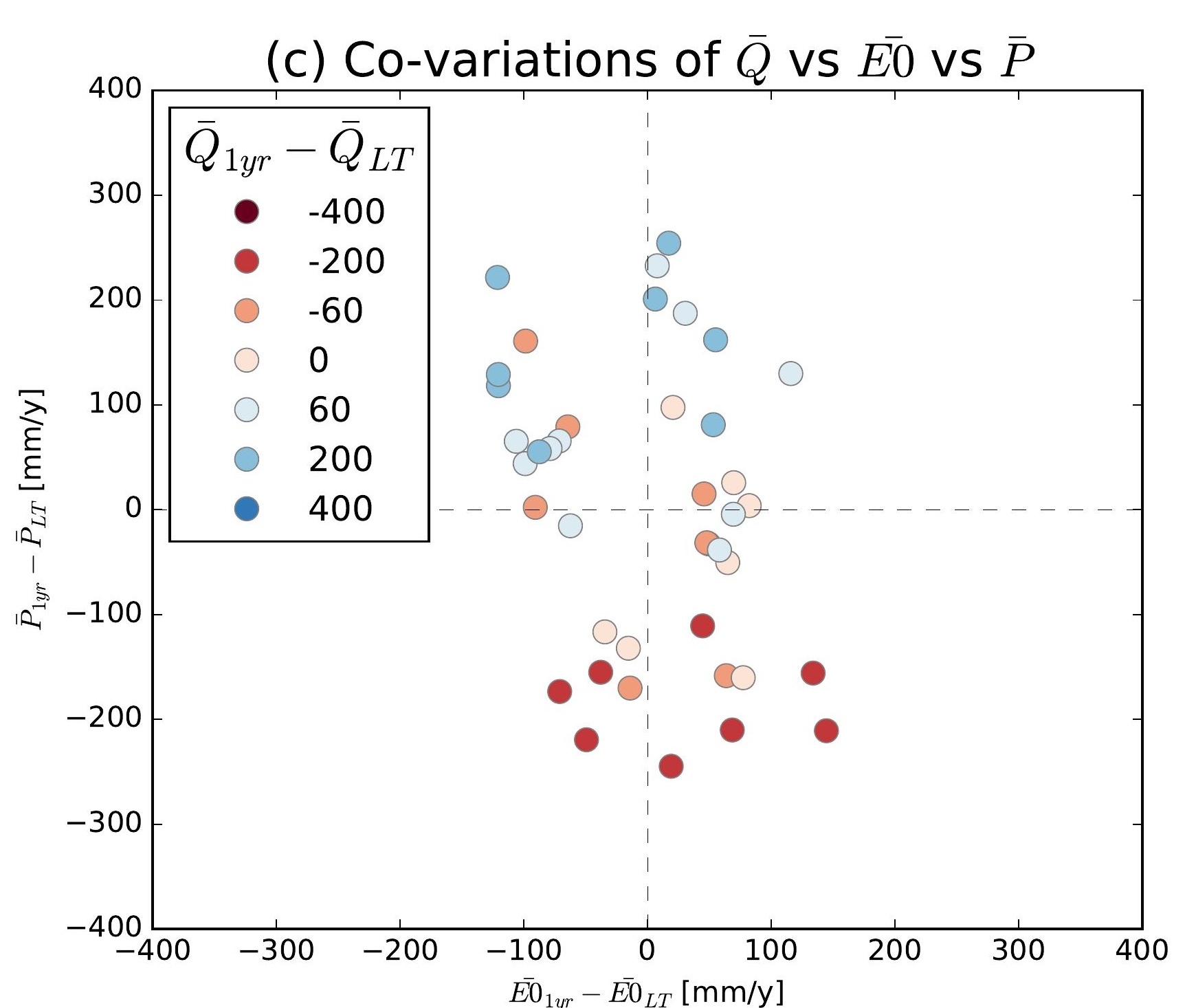
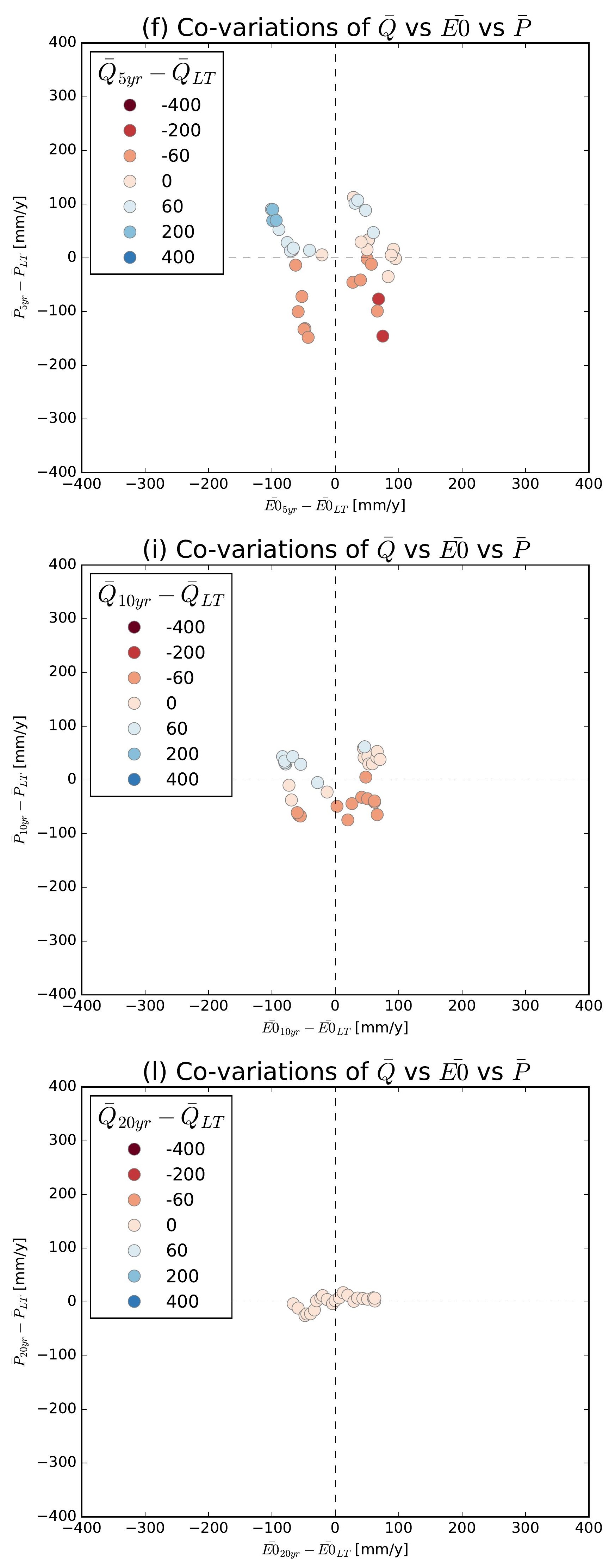

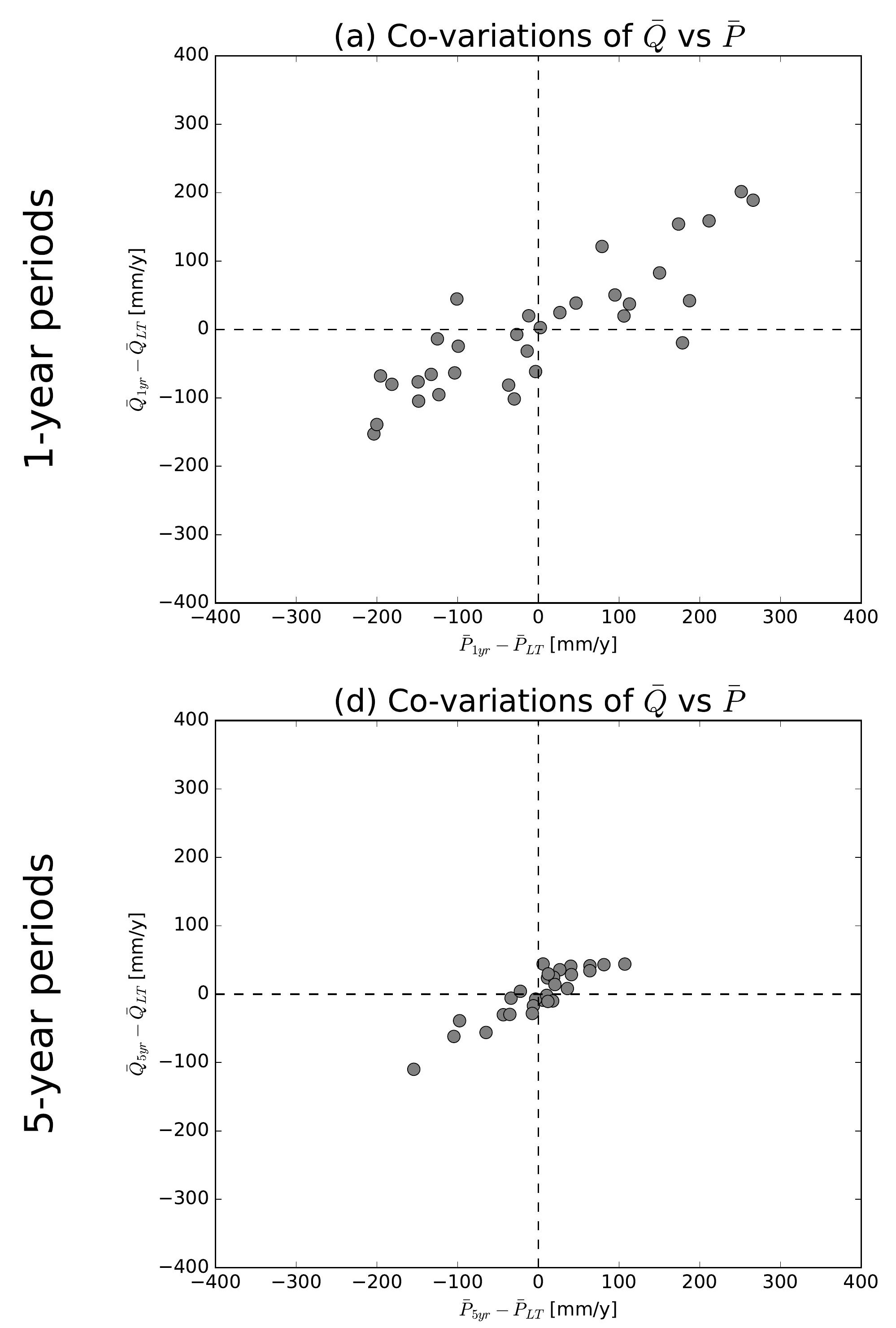

(g) Co-variations of $\bar{Q}$ vs $\bar{P}$
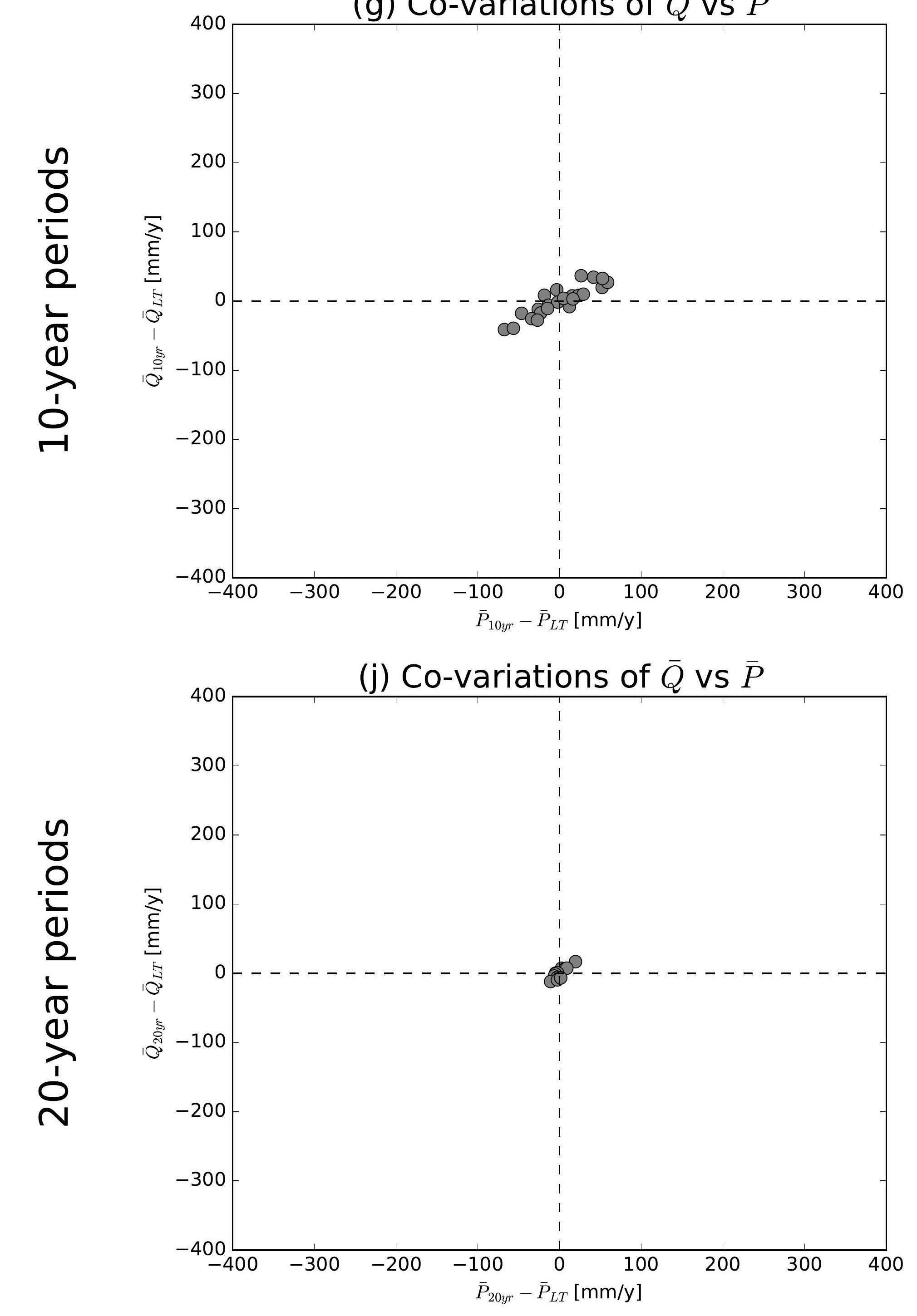

(b) Co-variations of $\bar{Q}$ vs $\overline{E 0}$

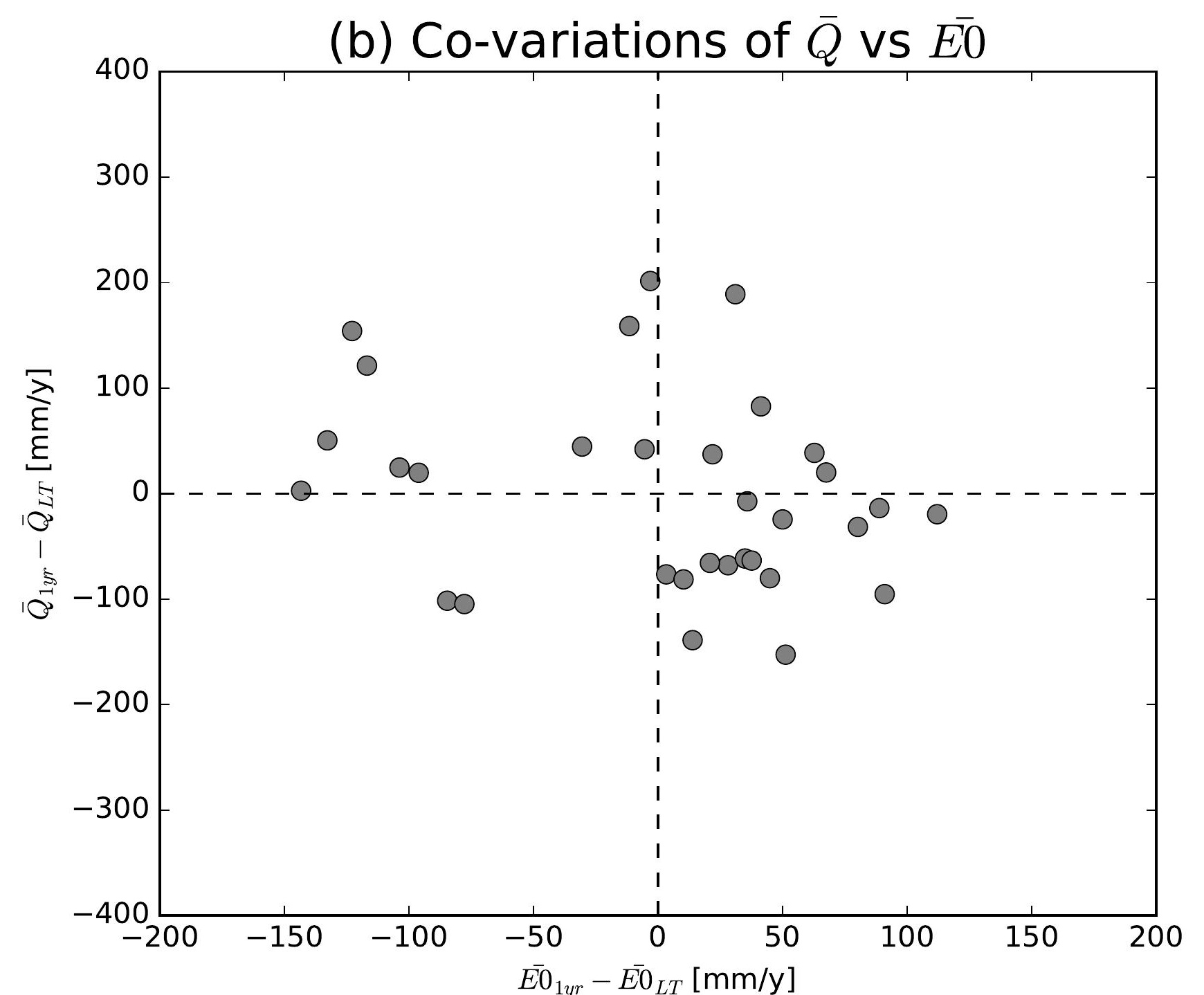

(e) Co-variations of $\bar{Q}$ vs $\overline{E 0}$

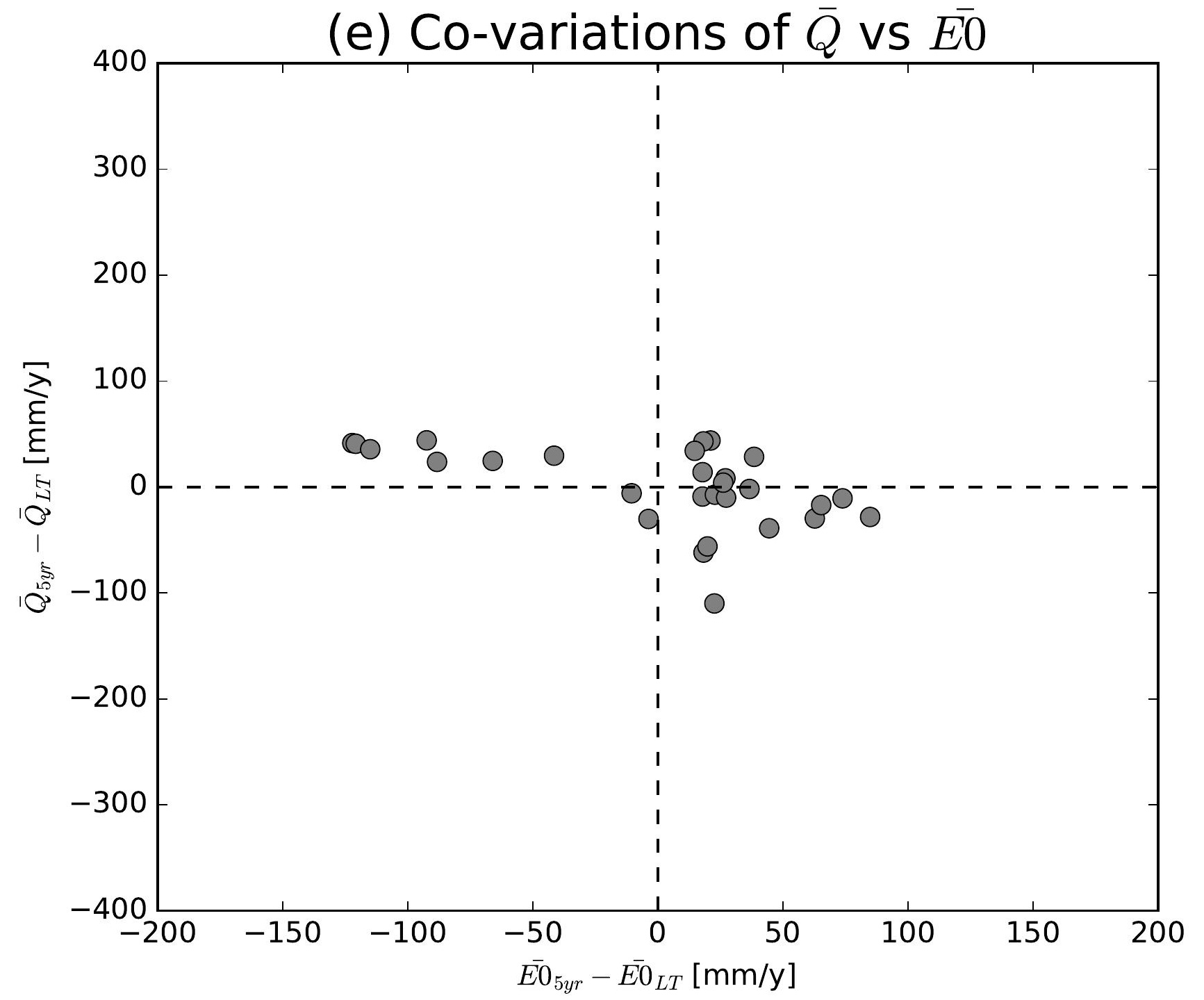

(h) Co-variations of $\bar{Q}$ vs $\overline{E 0}$

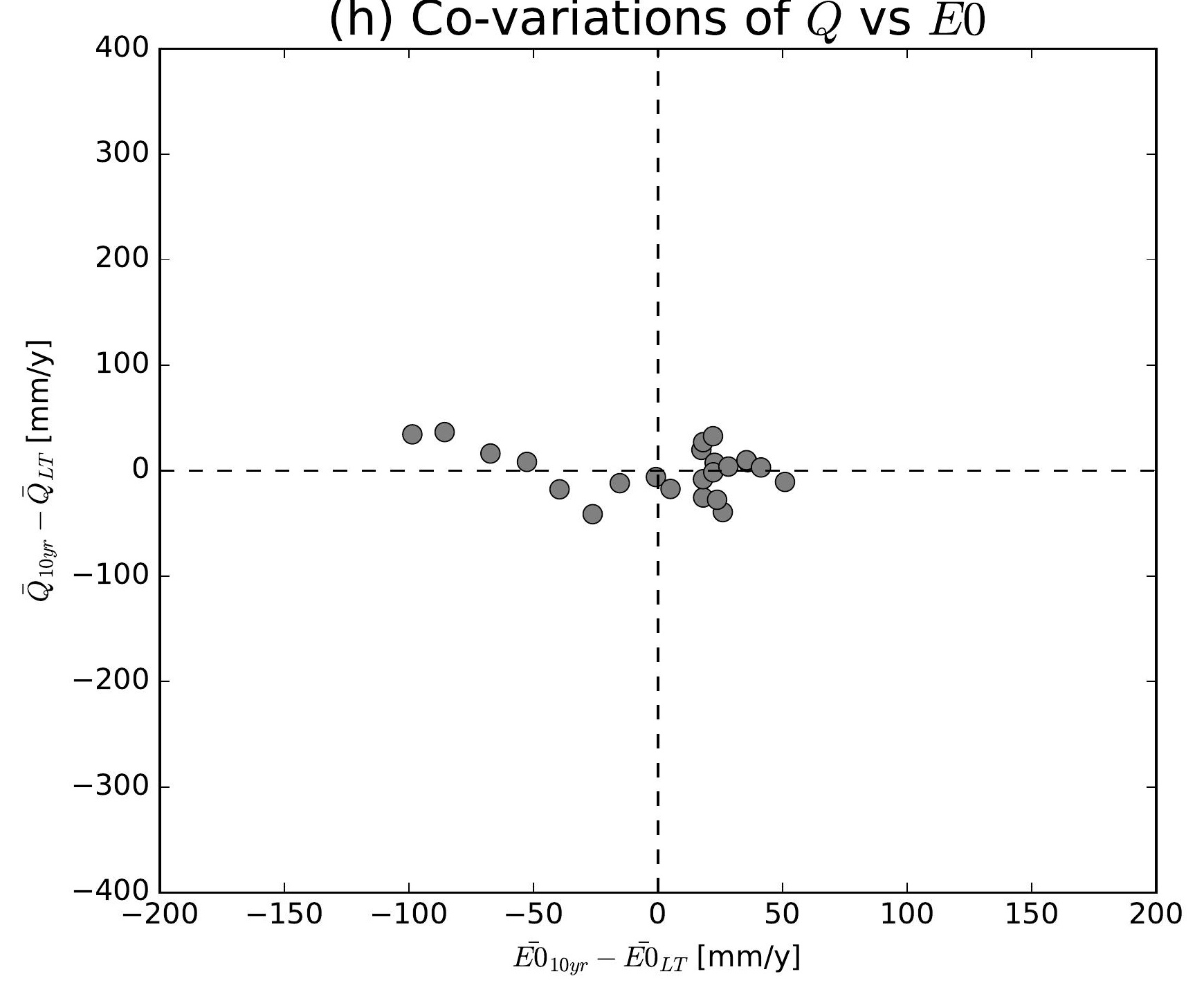

(k) Co-variations of $\bar{Q}$ vs $\overline{E 0}$

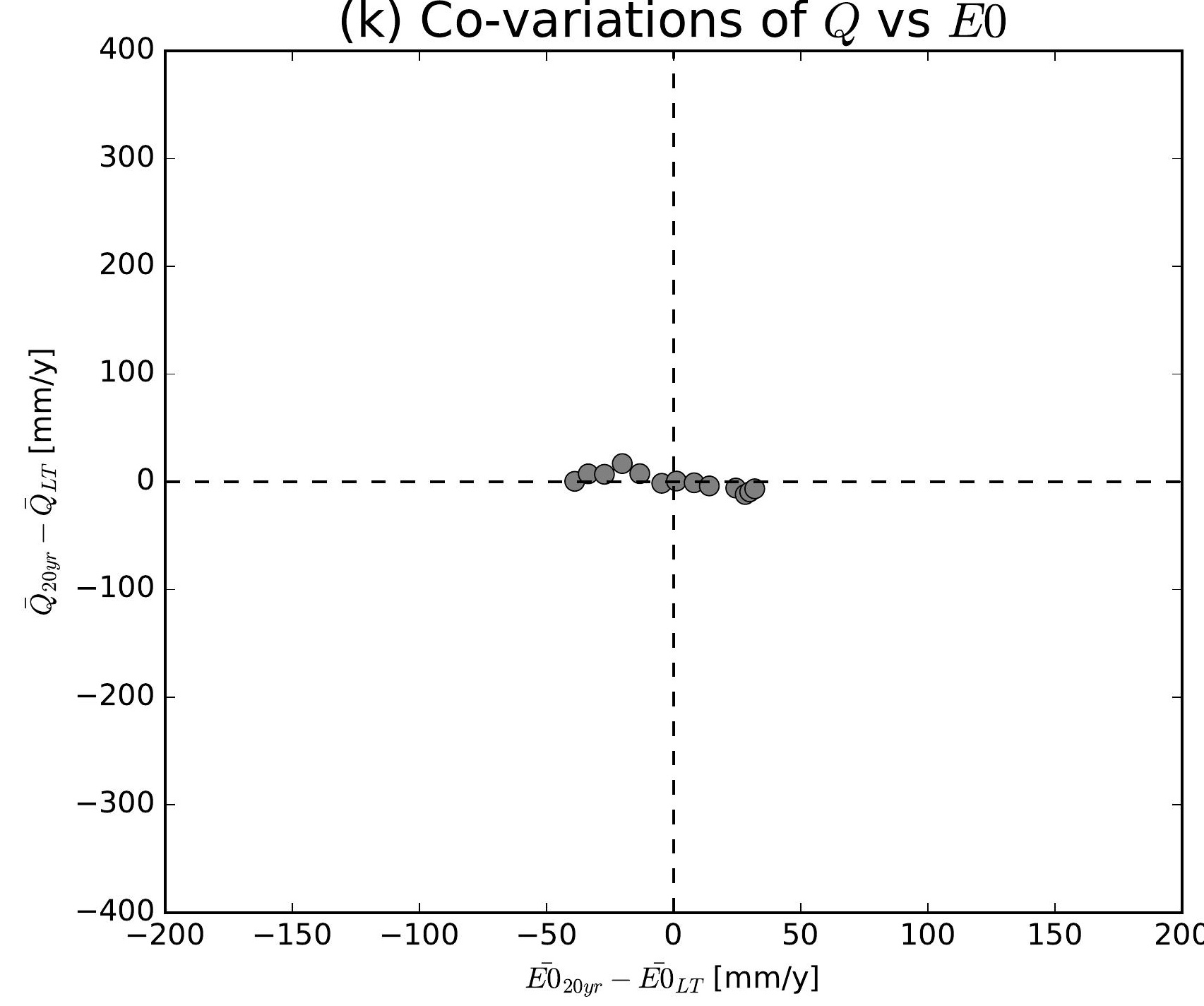

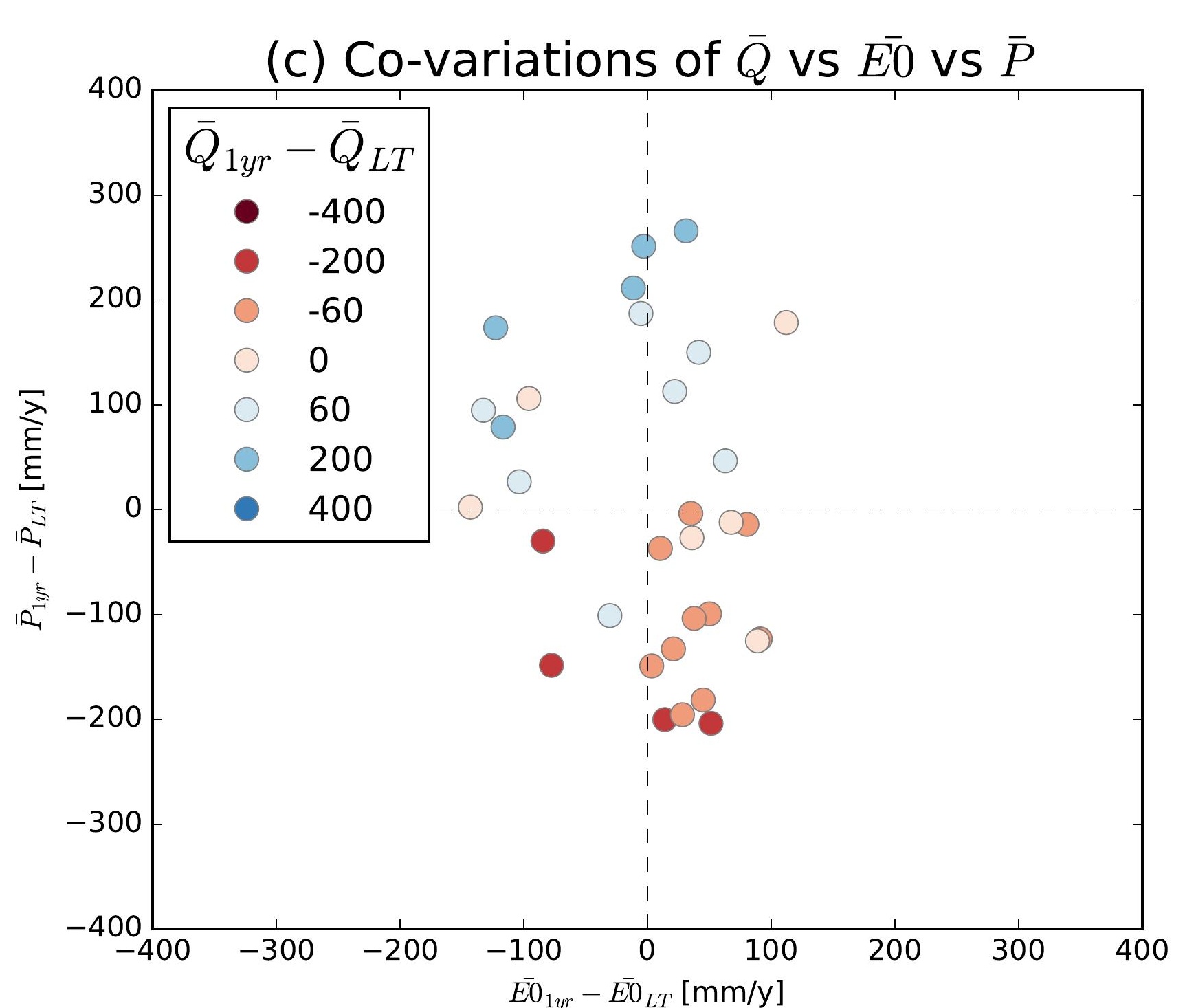
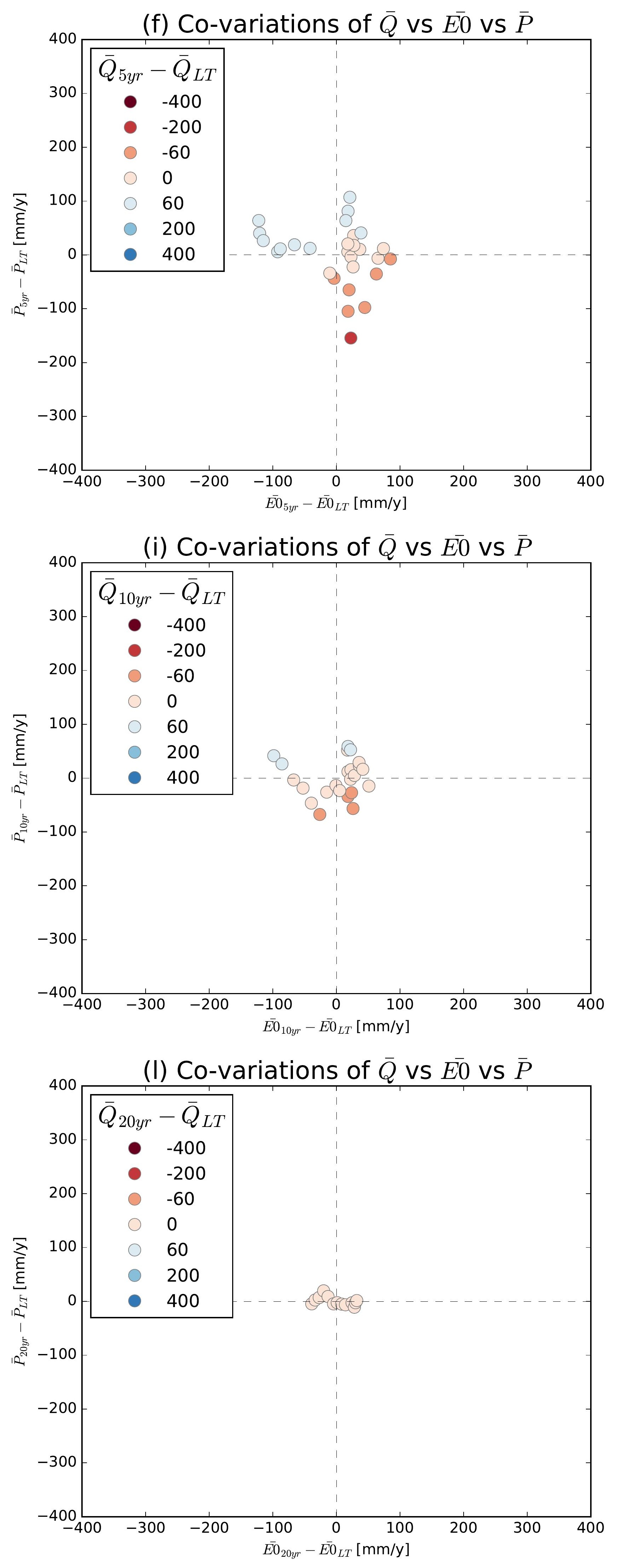

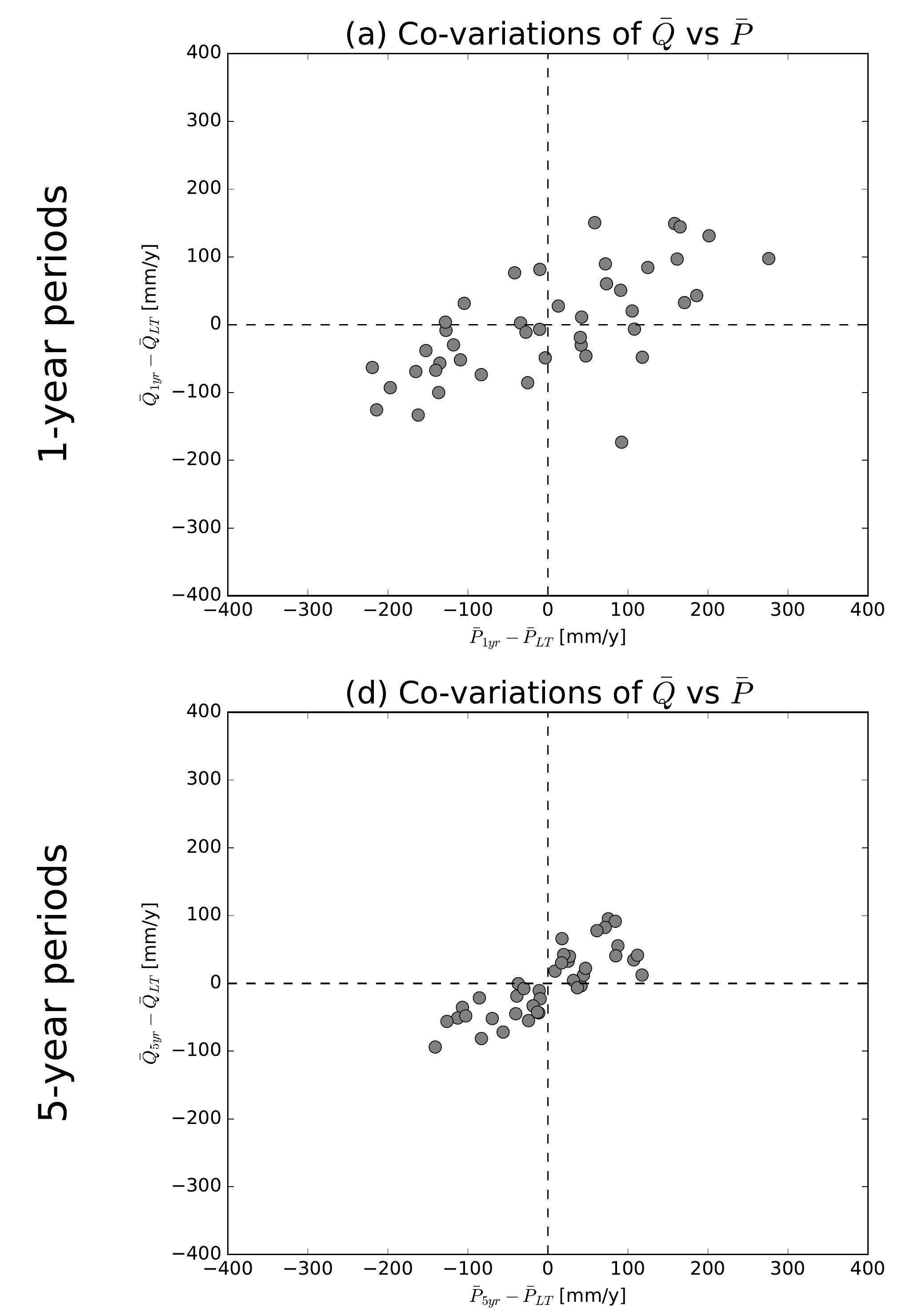

(g) Co-variations of $\bar{Q}$ vs $\bar{P}$
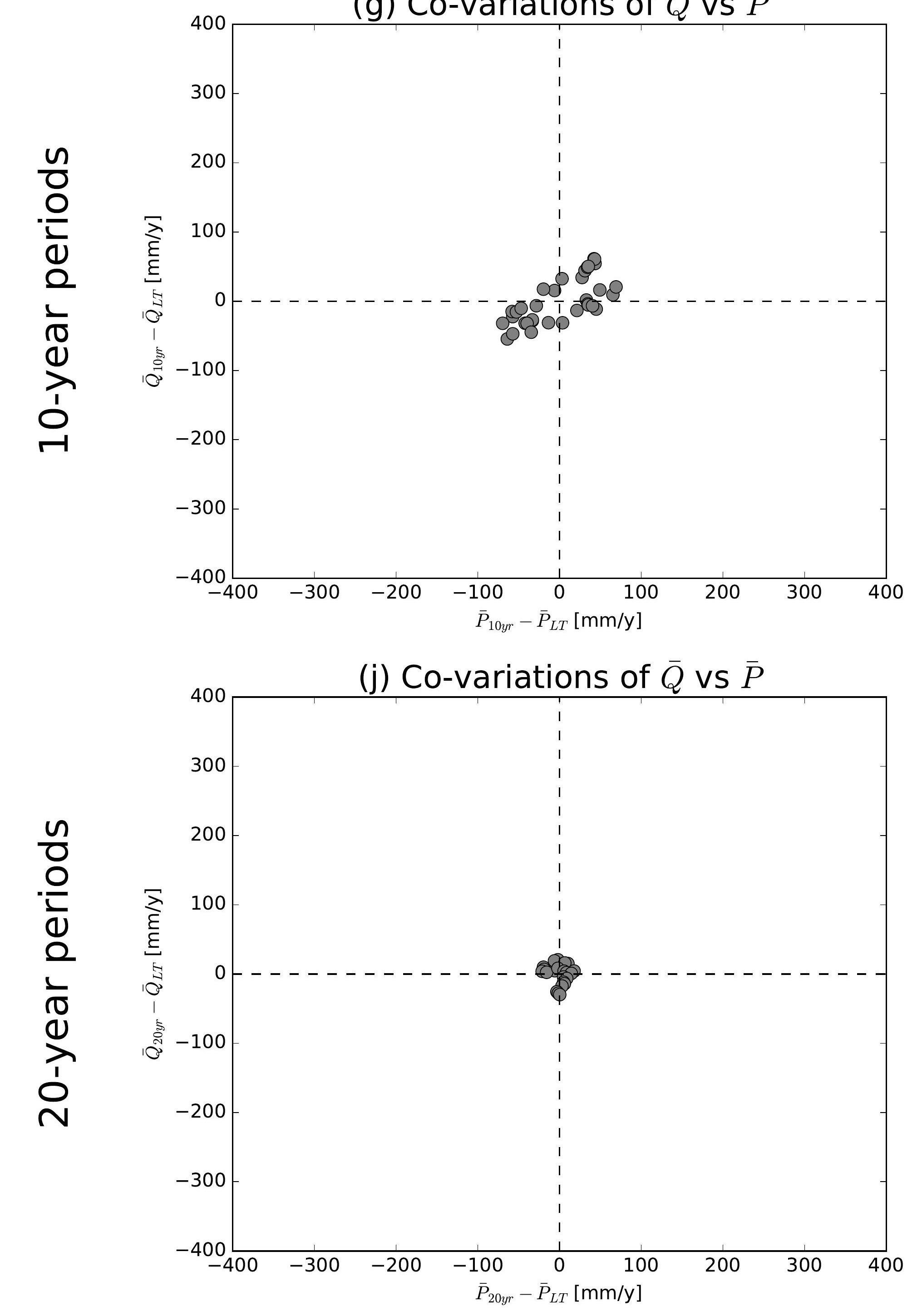

(b) Co-variations of $\bar{Q}$ vs $\overline{E 0}$

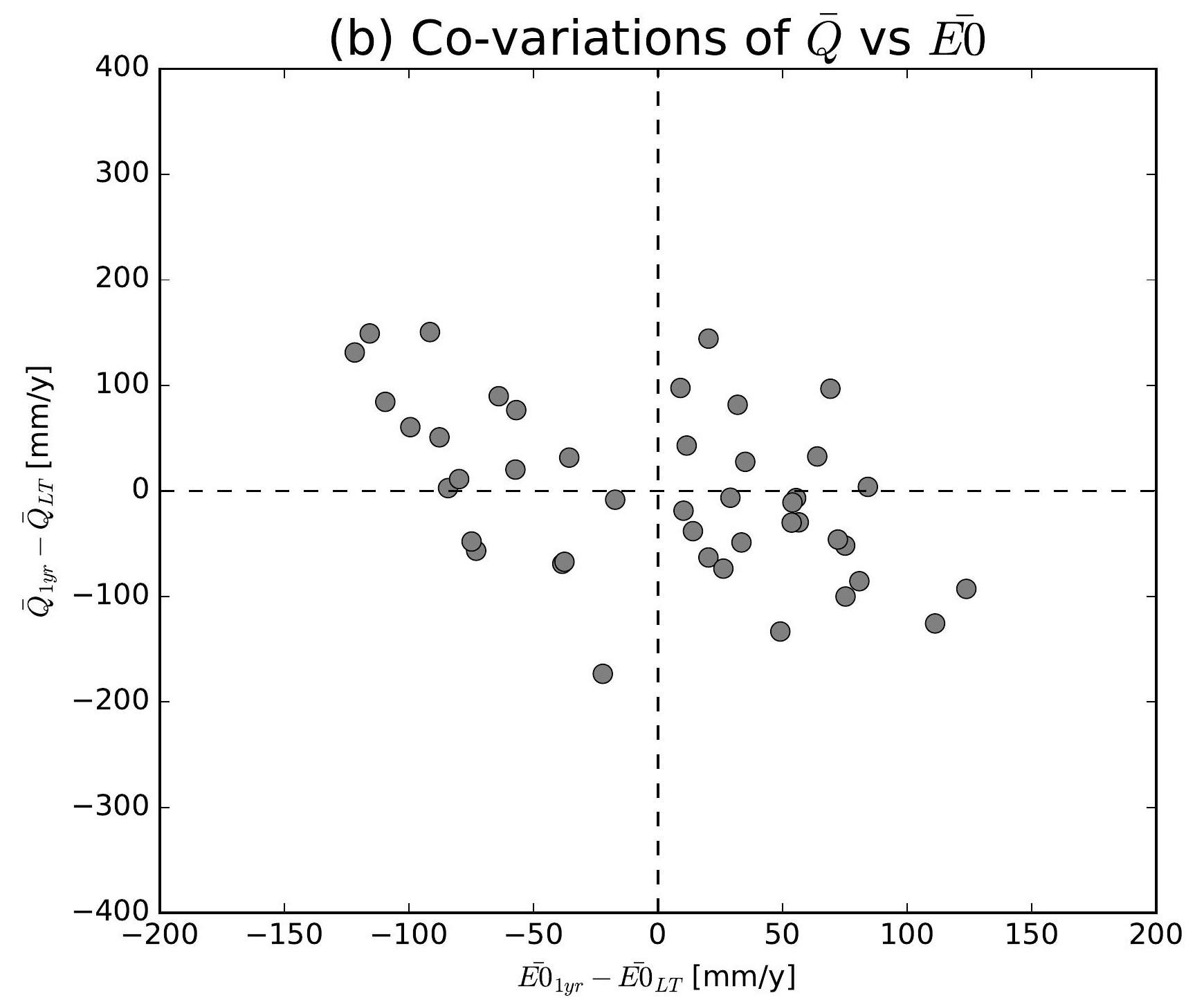

(e) Co-variations of $\bar{Q}$ vs $\overline{E 0}$

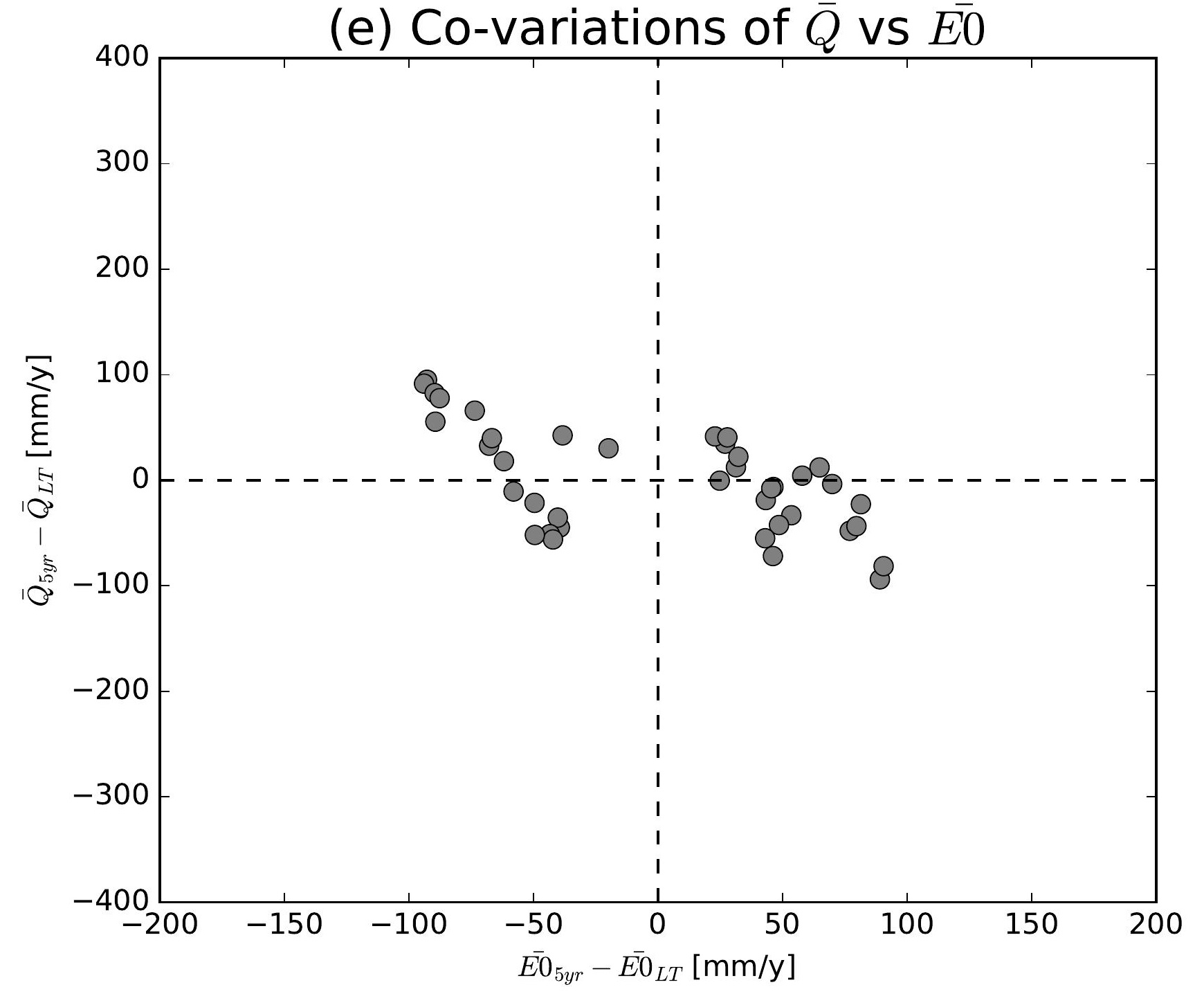

(h) Co-variations of $\bar{Q}$ vs $\overline{E 0}$

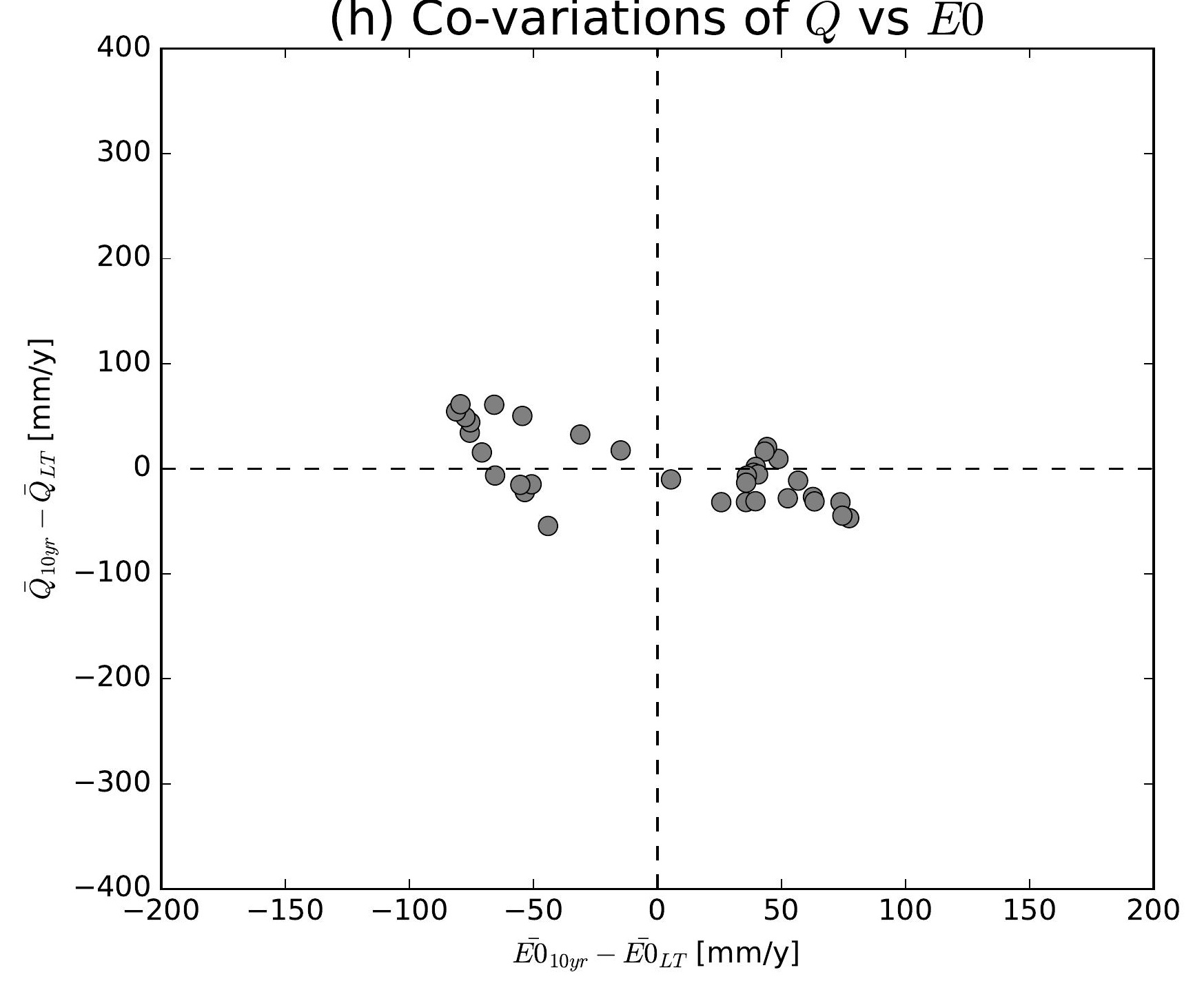

(k) Co-variations of $\bar{Q}$ vs $\overline{E 0}$

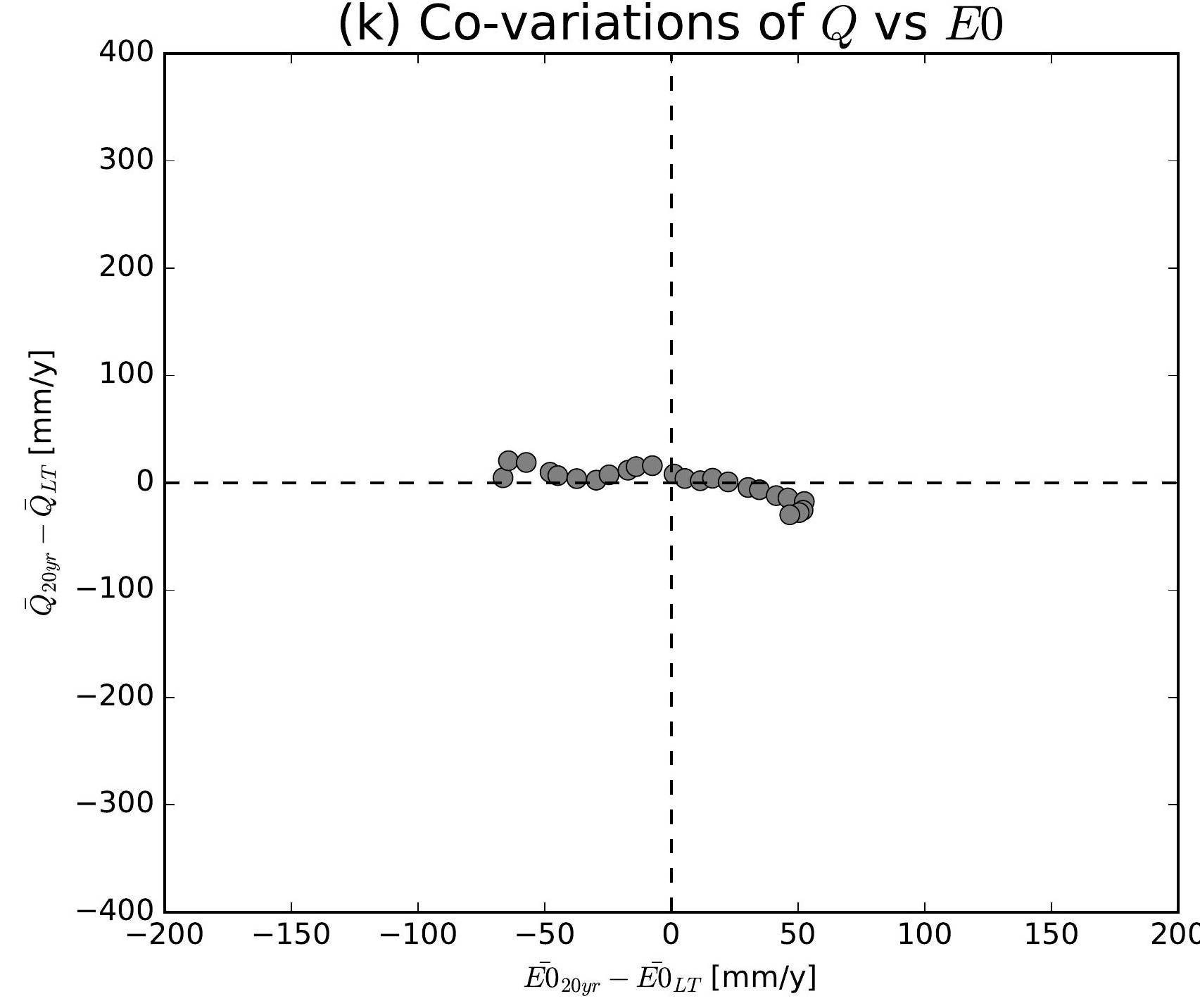

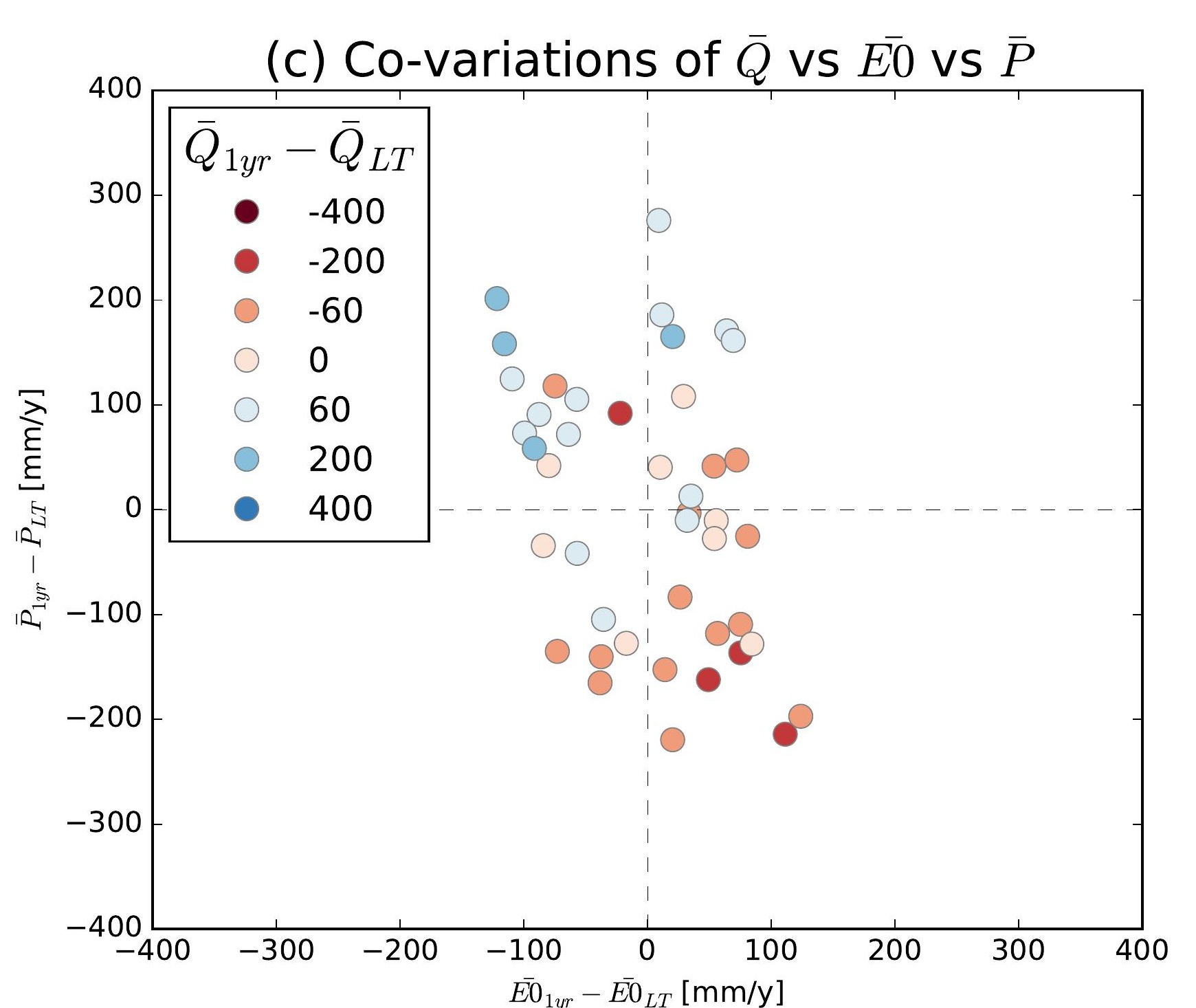
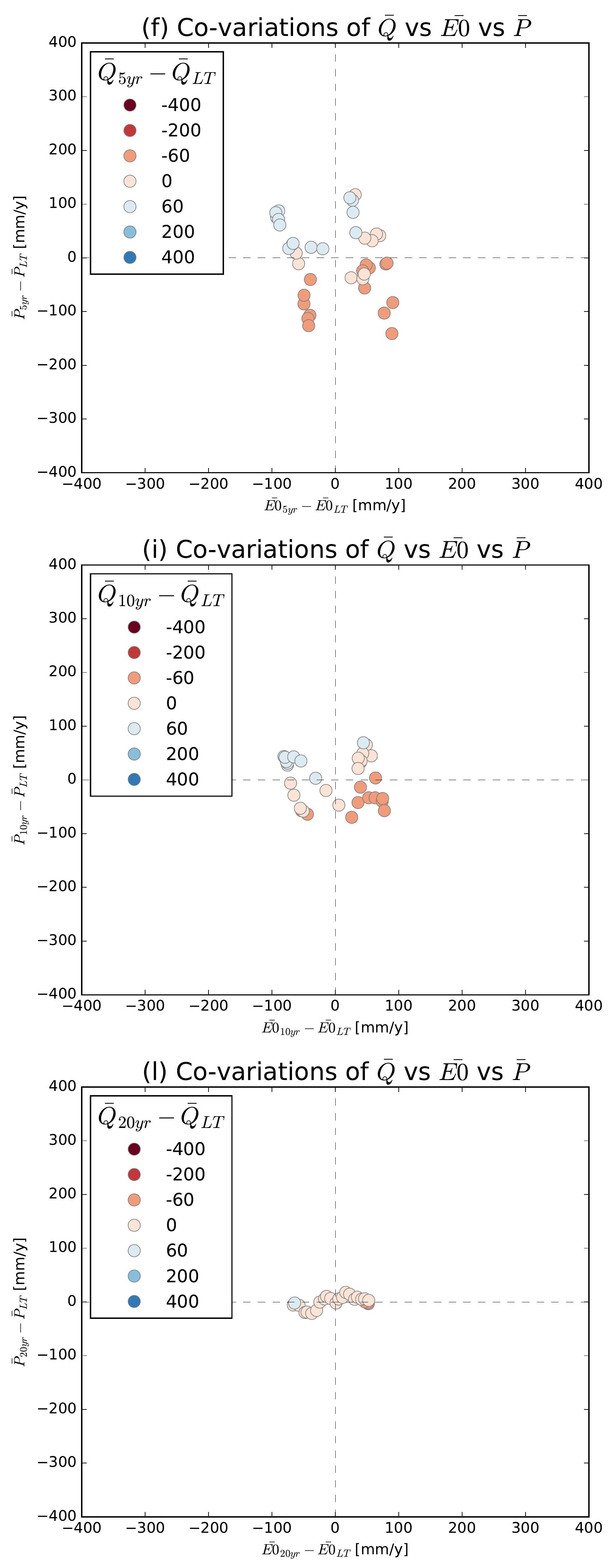

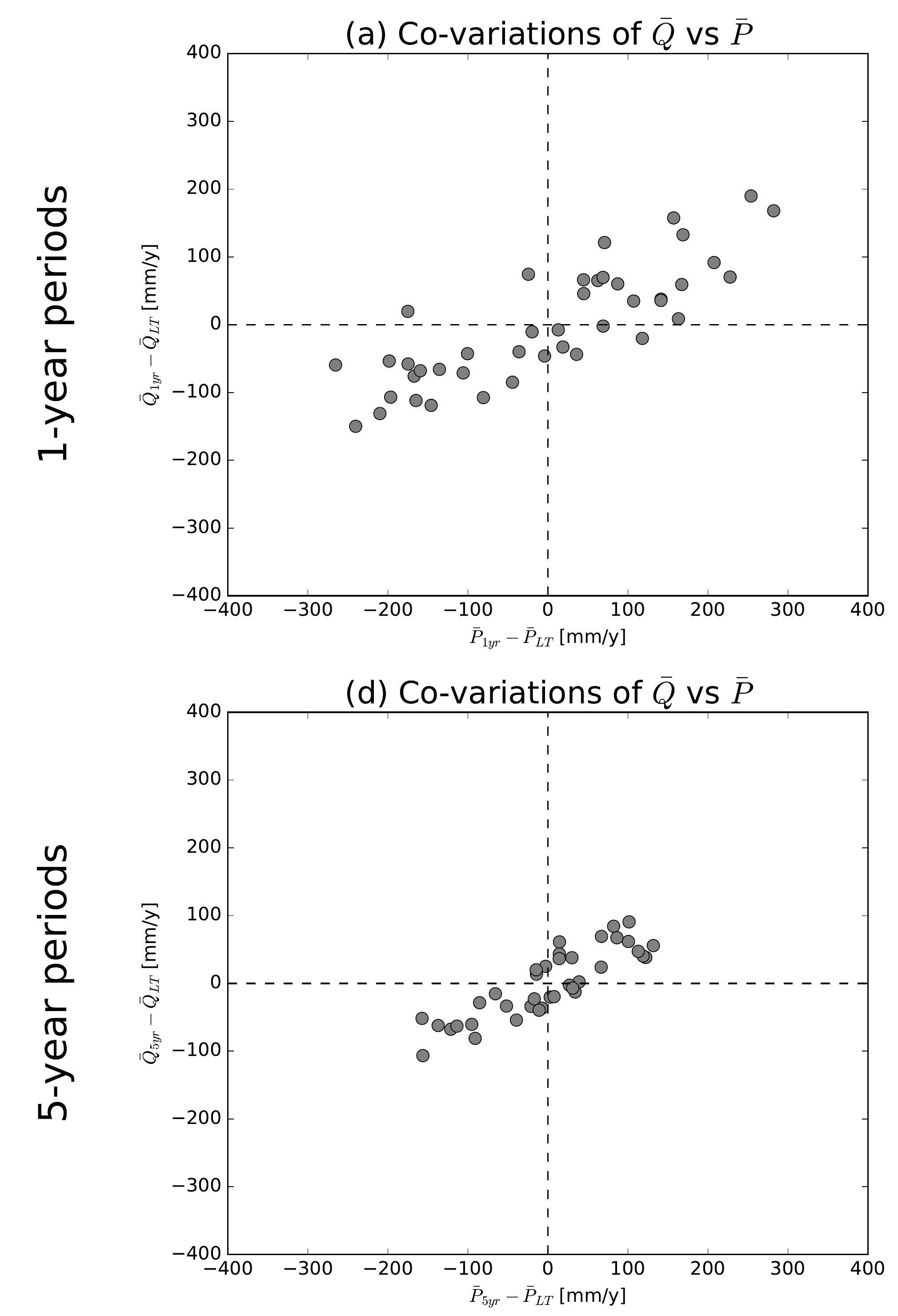

(g) Co-variations of $\bar{Q}$ vs $\bar{P}$
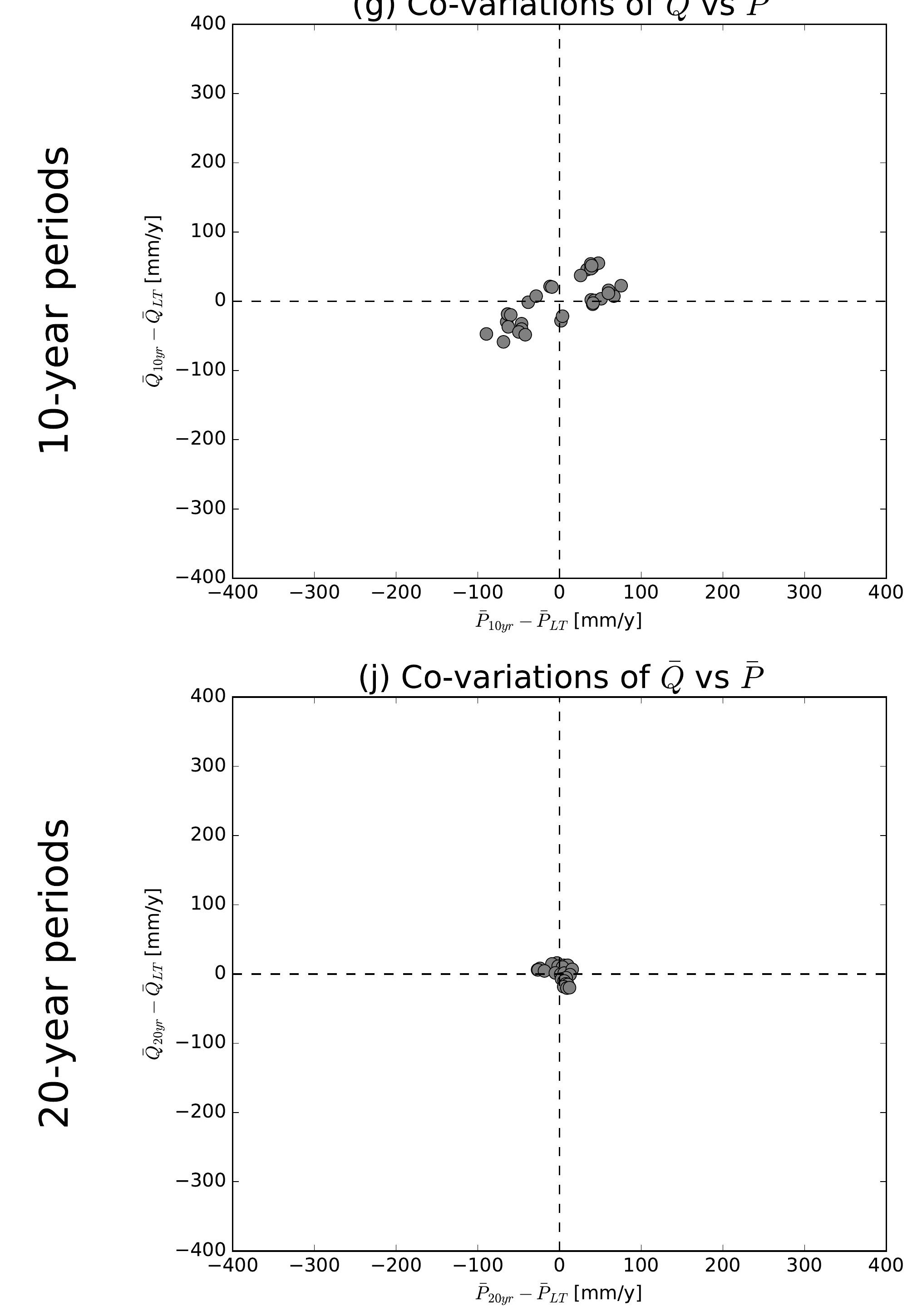

(b) Co-variations of $\bar{Q}$ vs $\overline{E 0}$

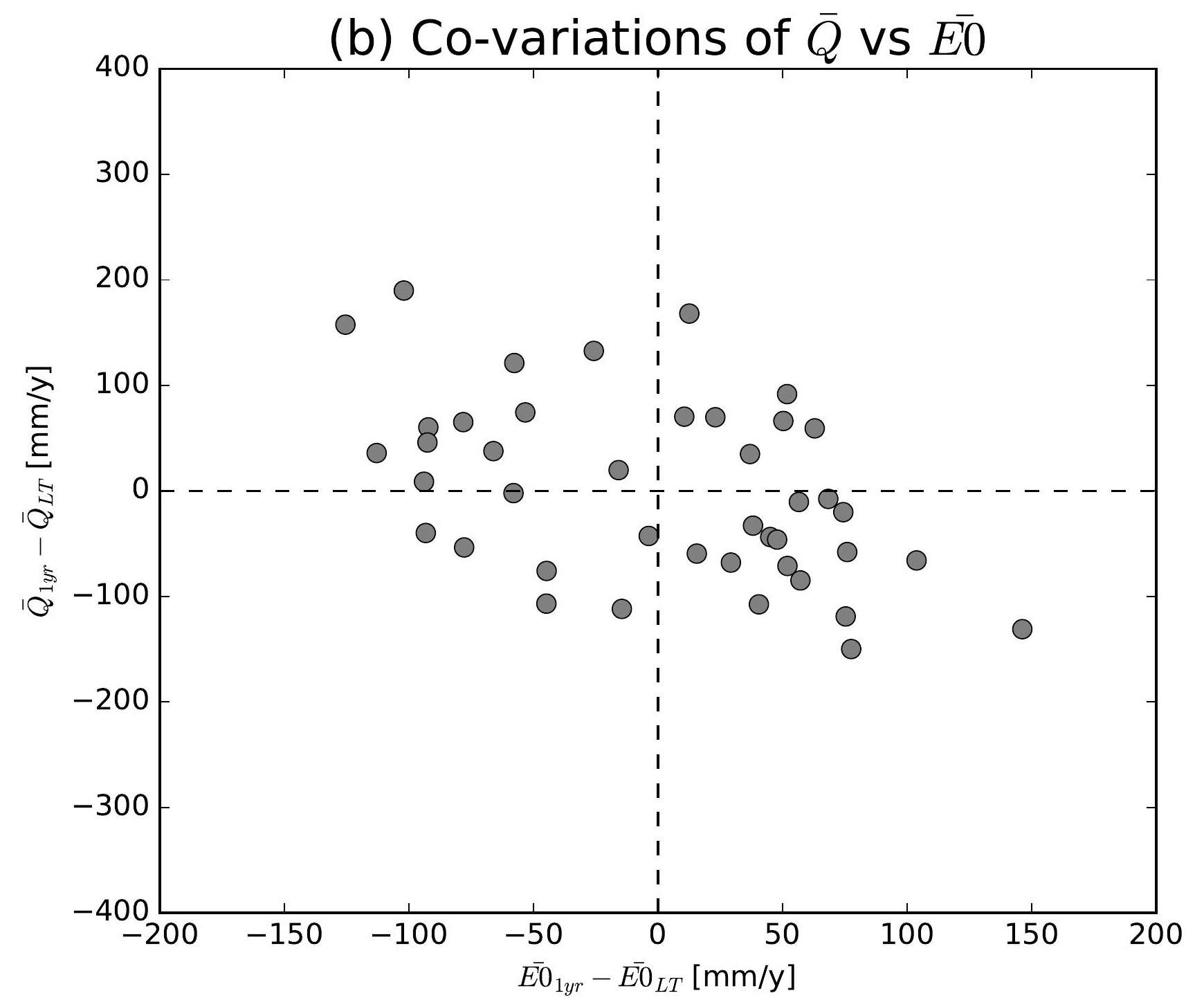

(e) Co-variations of $\bar{Q}$ vs $\overline{E 0}$

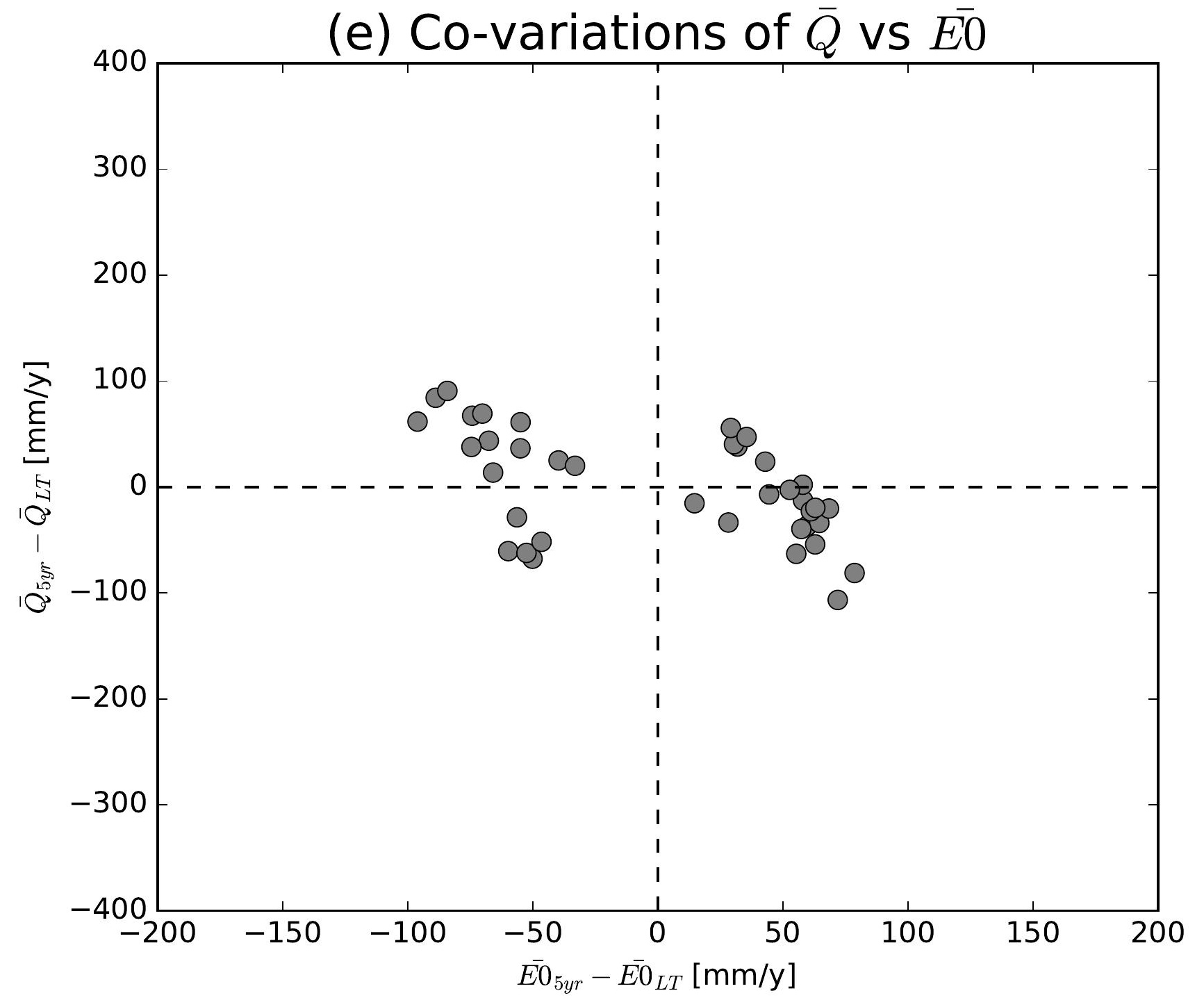

(h) Co-variations of $\bar{Q}$ vs $\overline{E 0}$

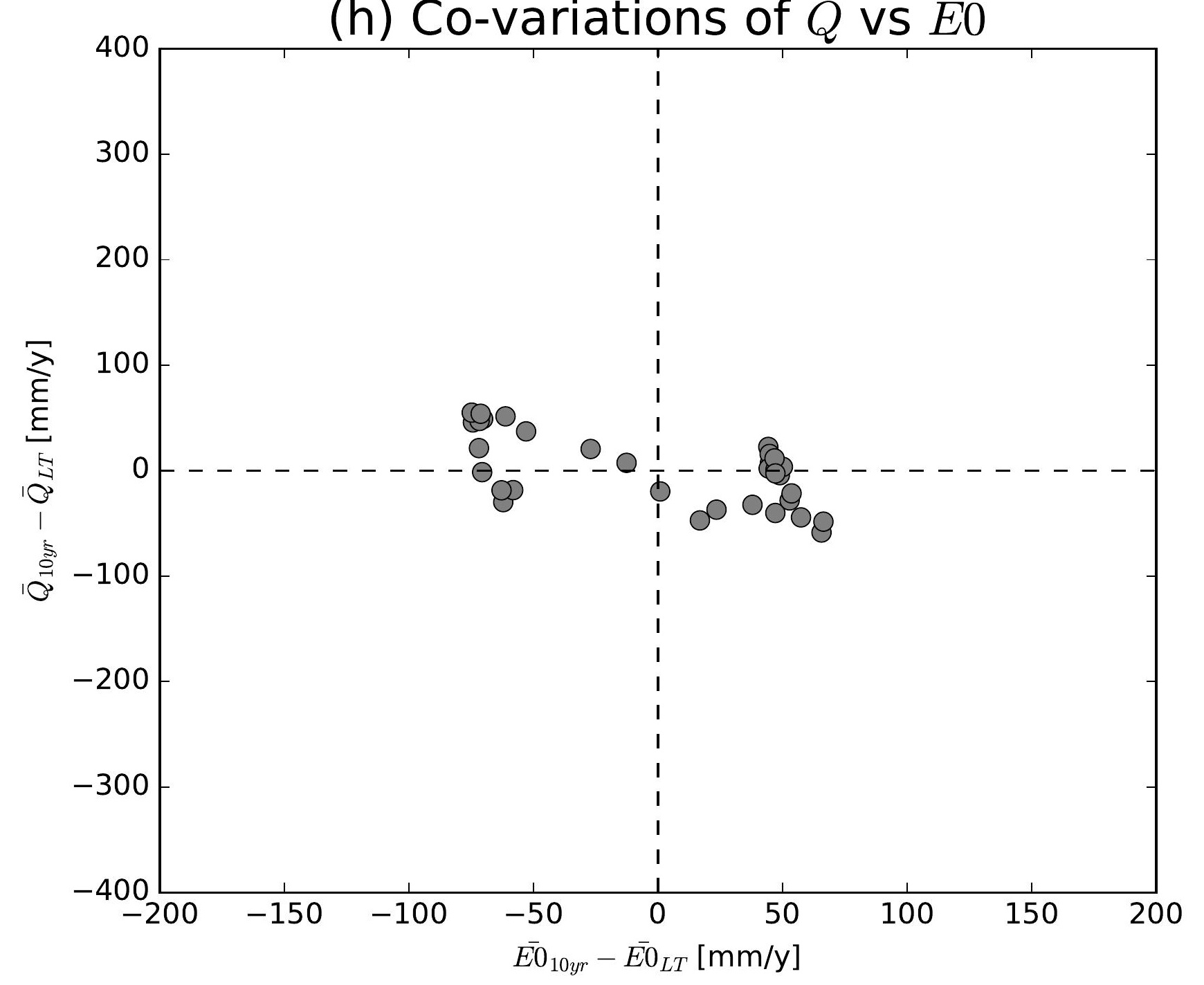

(k) Co-variations of $\bar{Q}$ vs $\overline{E 0}$

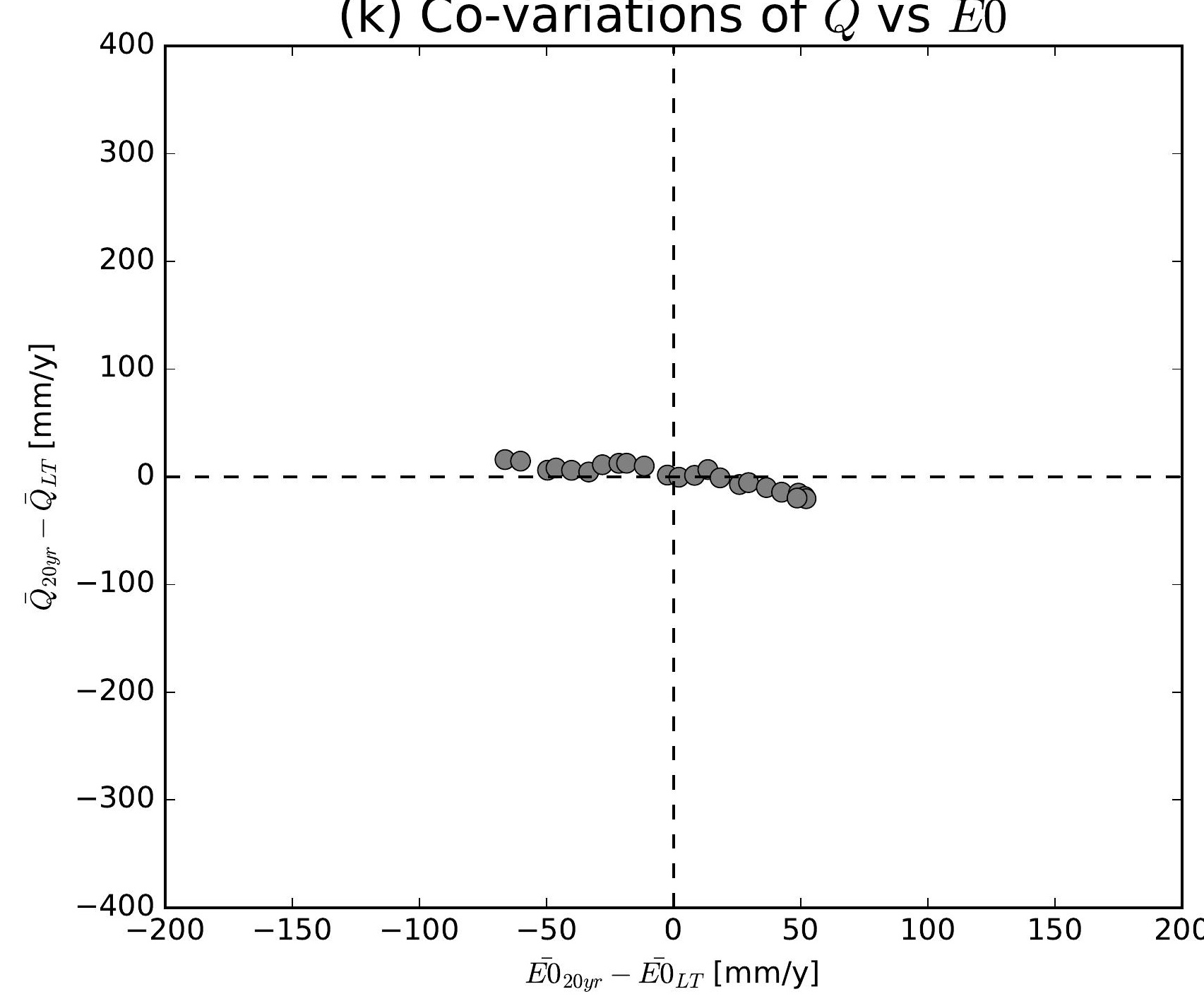

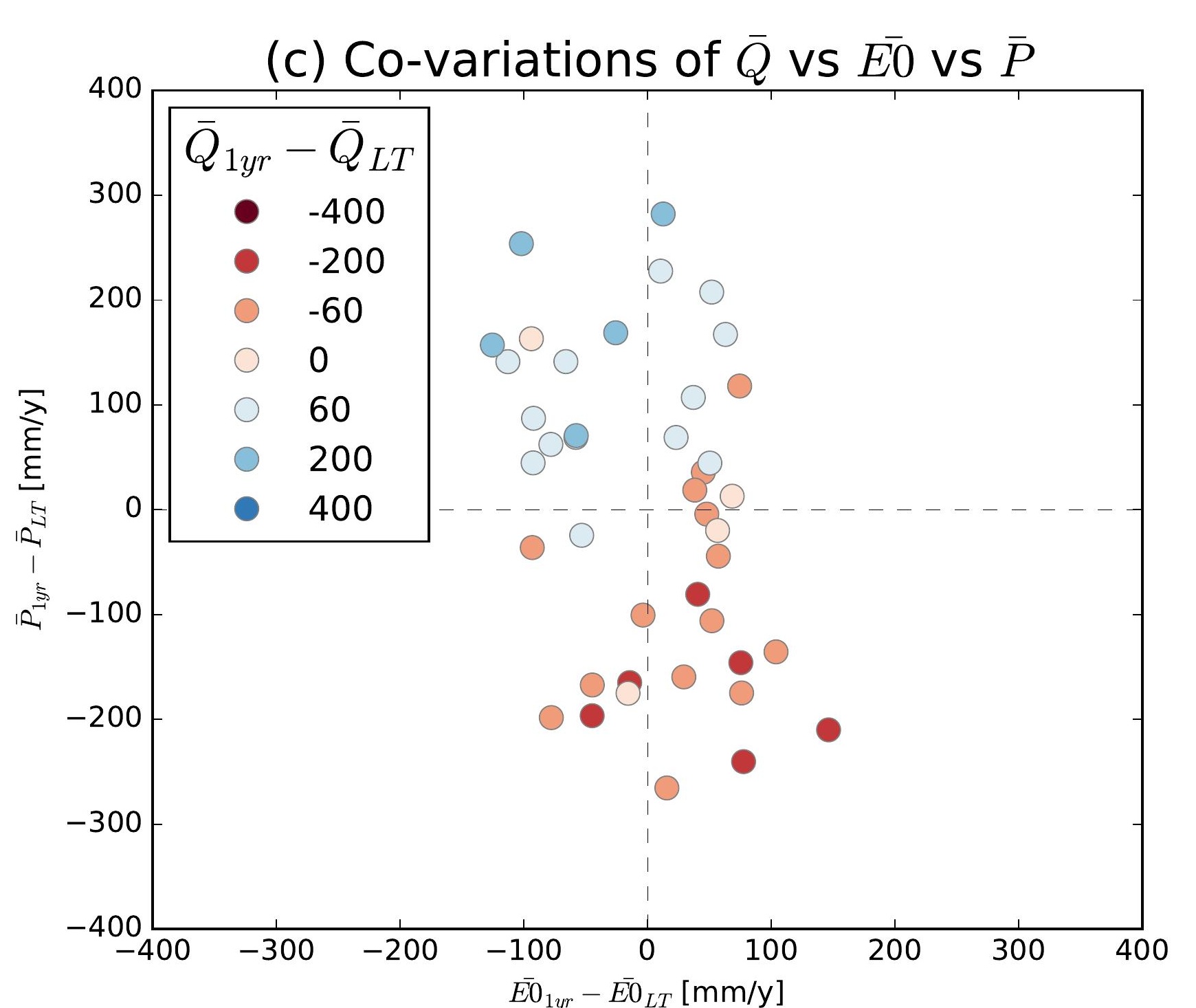
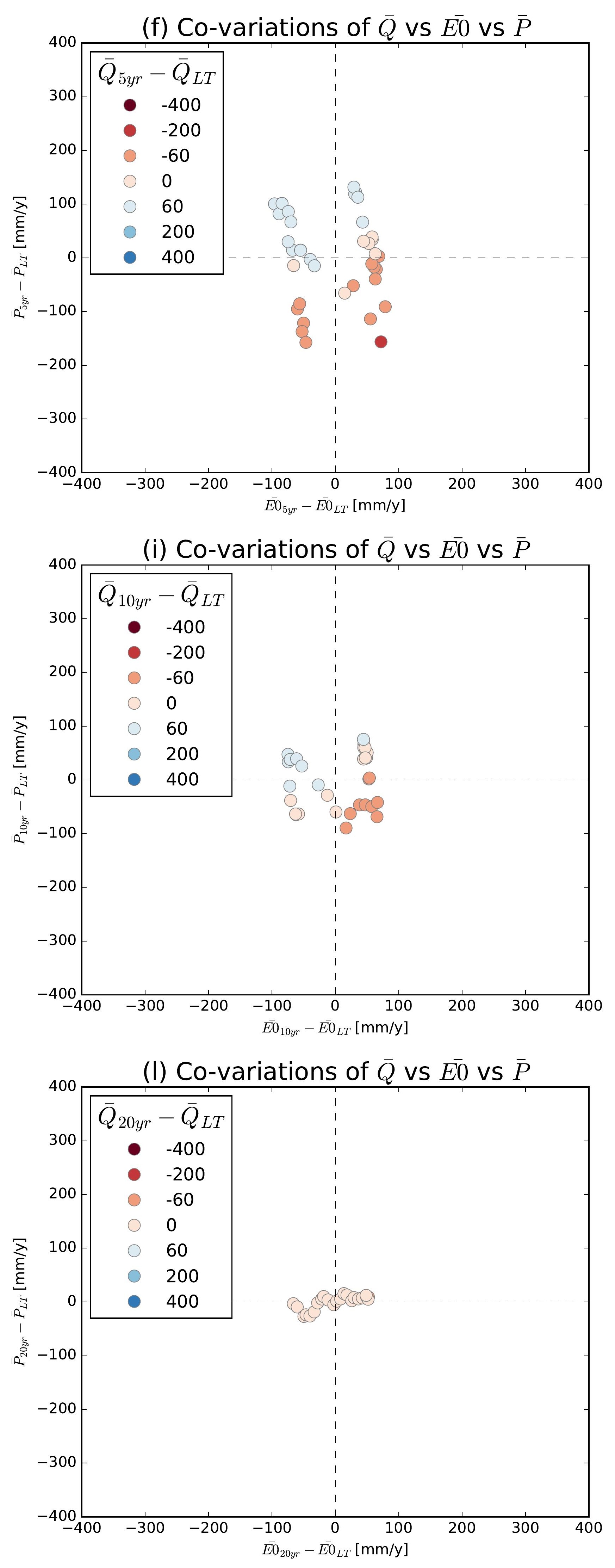

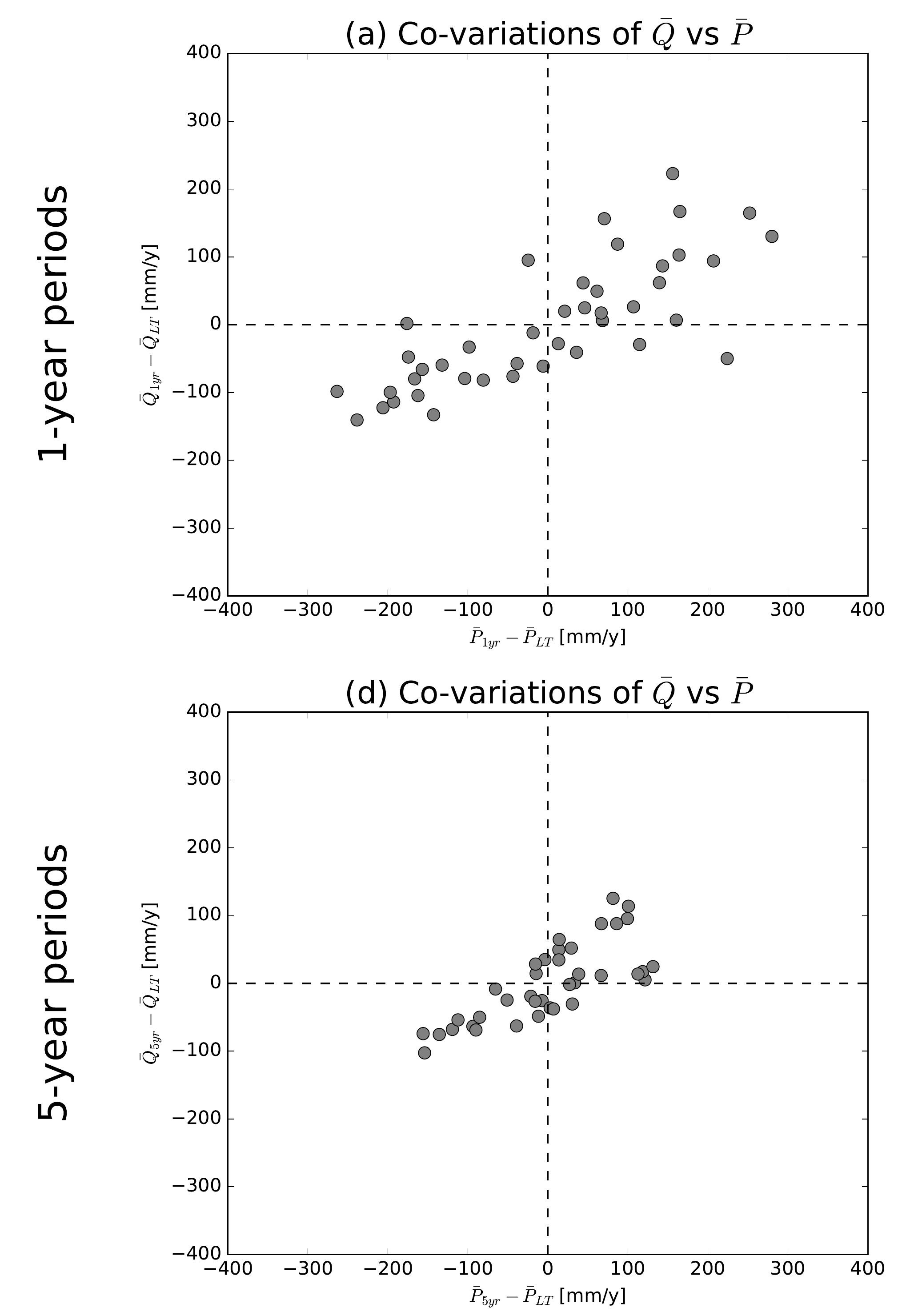

(g) Co-variations of $\bar{Q}$ vs $\bar{P}$
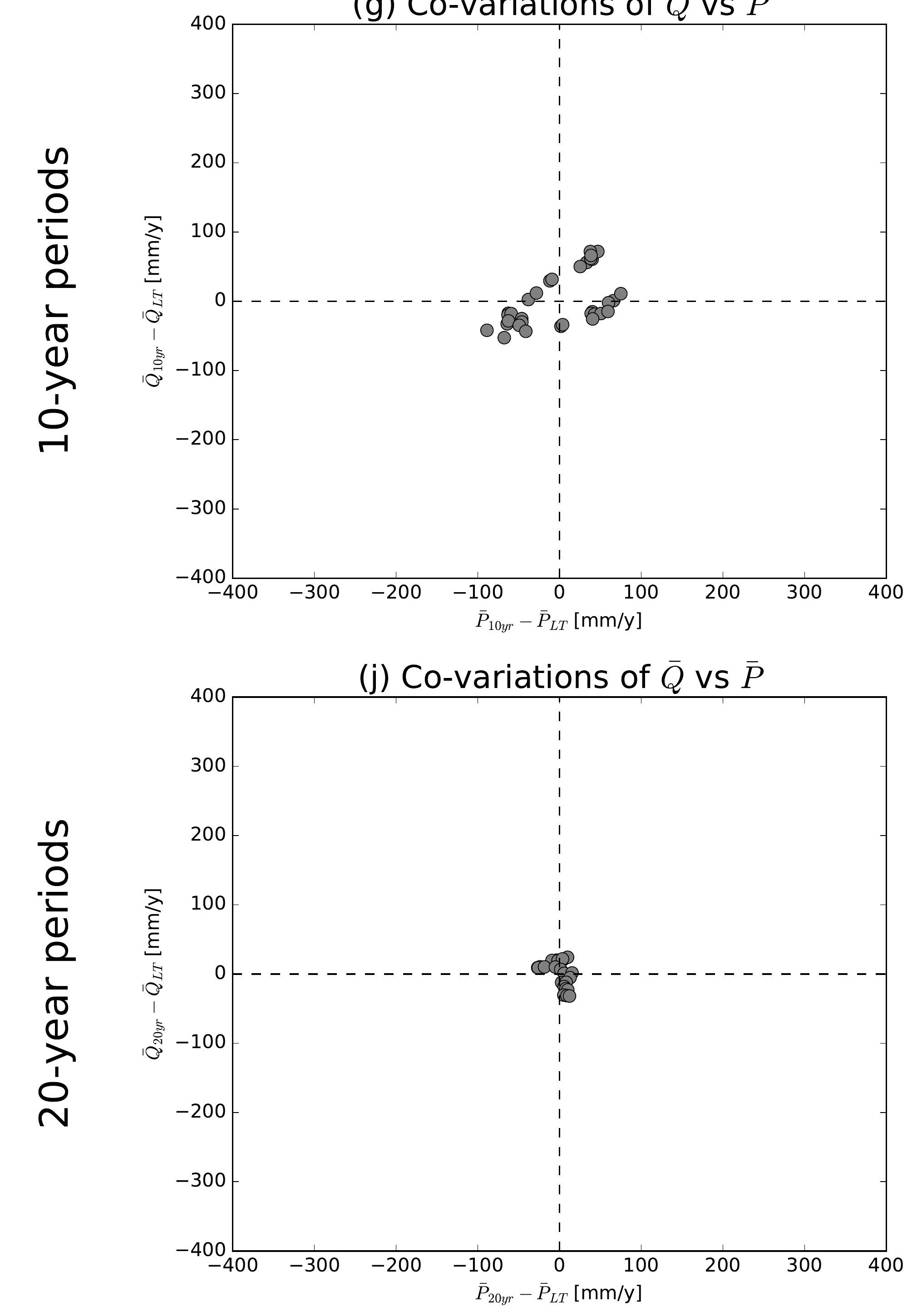

(b) Co-variations of $\bar{Q}$ vs $\overline{E 0}$

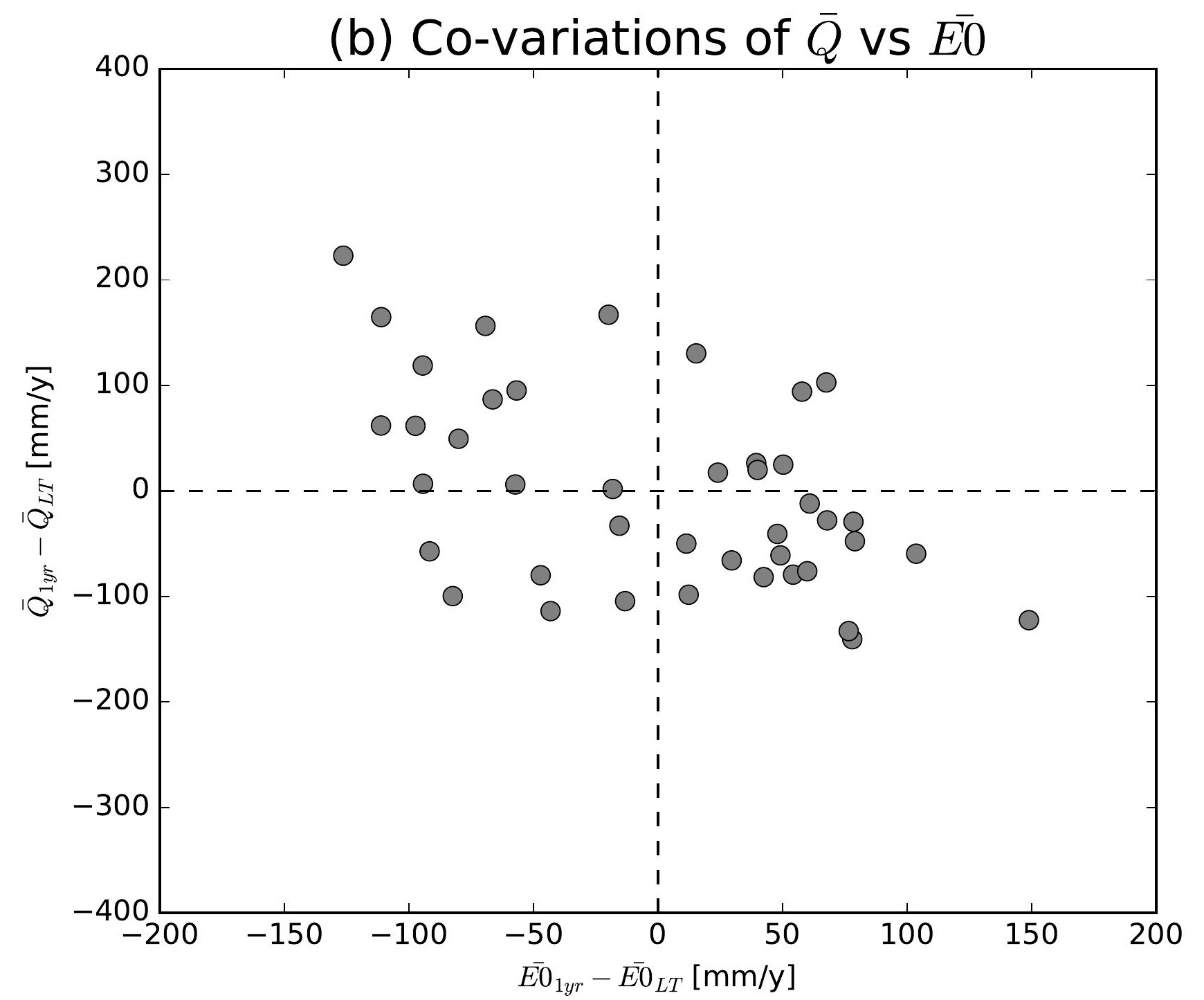

(e) Co-variations of $\bar{Q}$ vs $\overline{E 0}$

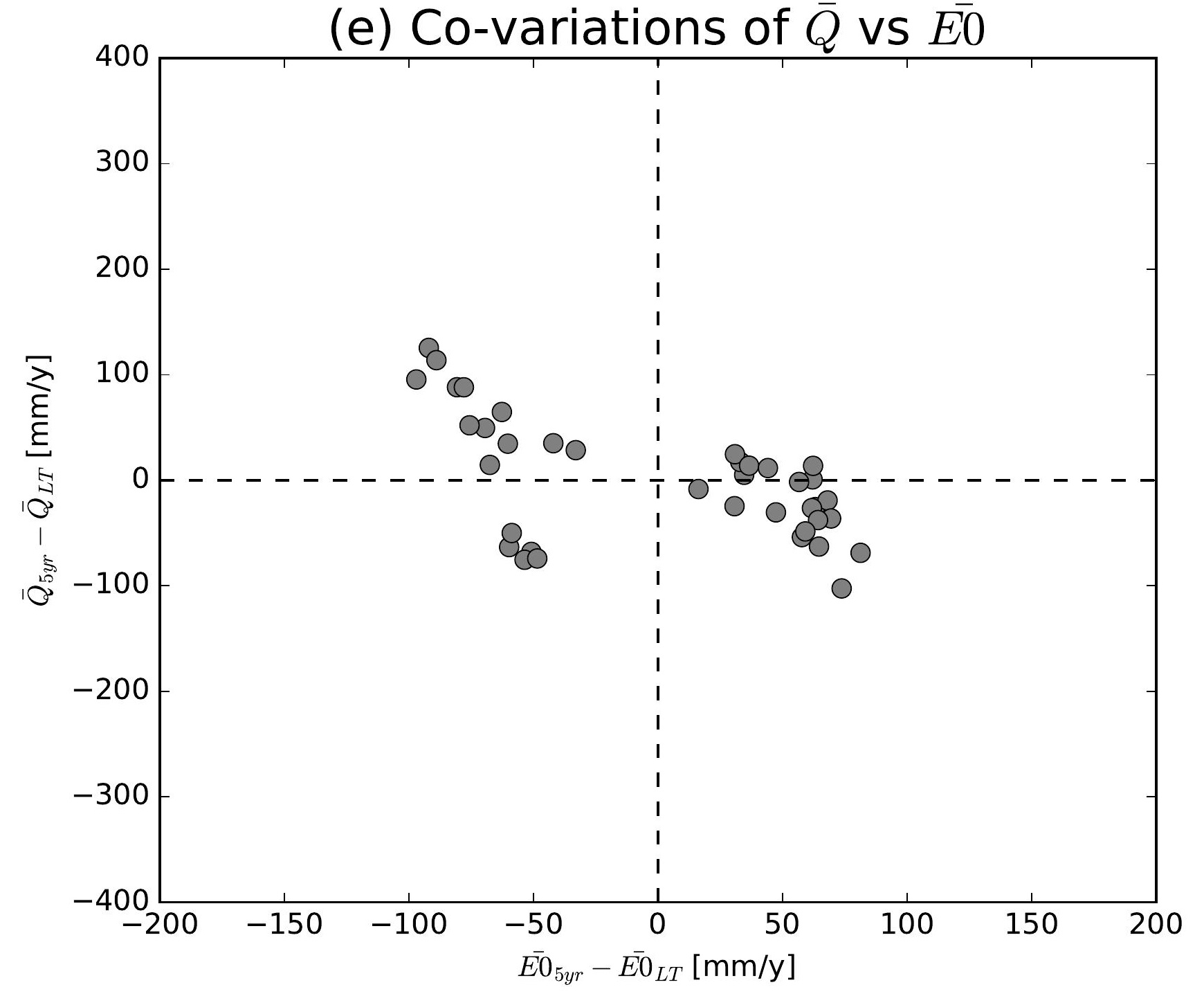

(h) Co-variations of $\bar{Q}$ vs $\overline{E 0}$

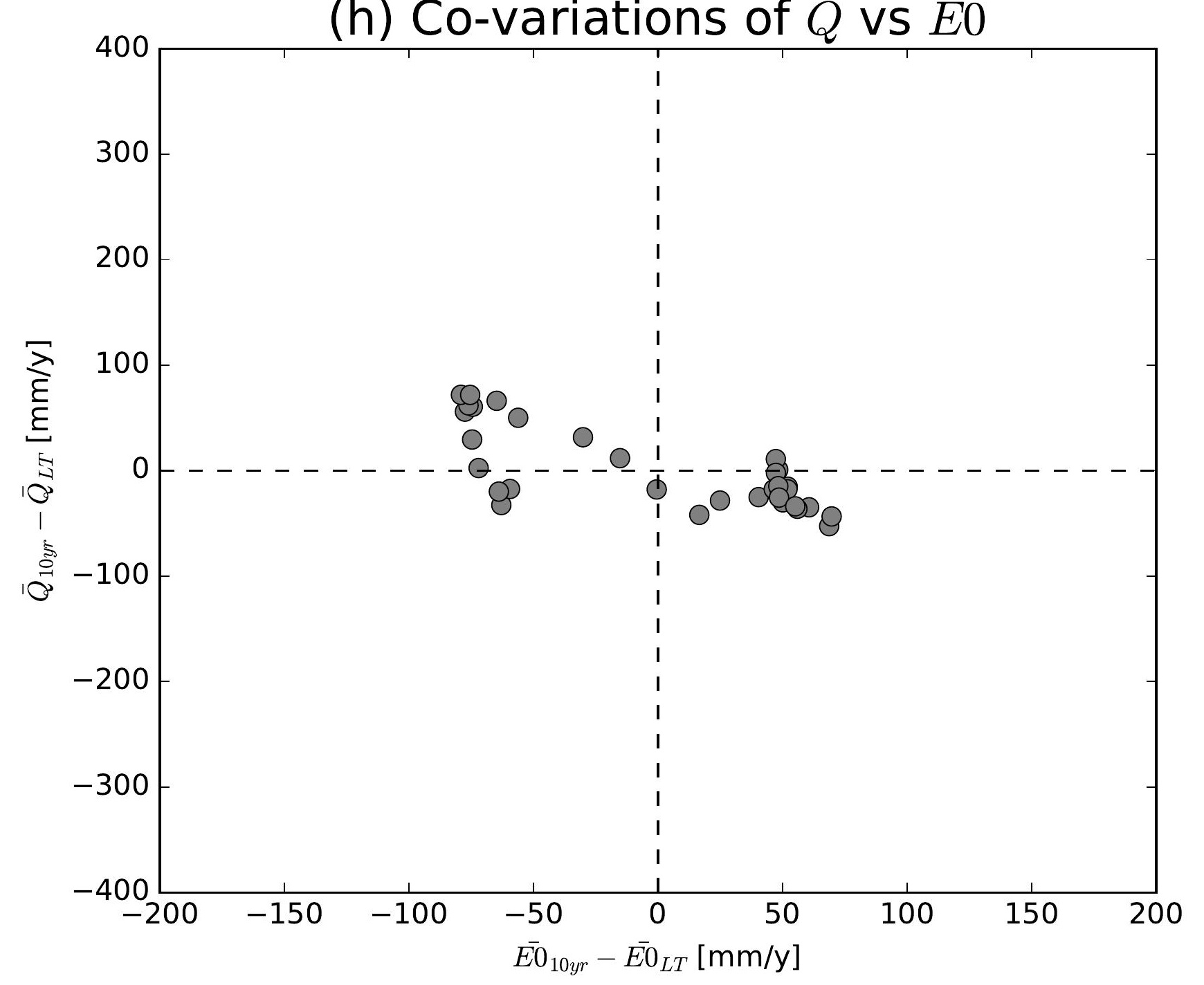

(k) Co-variations of $\bar{Q}$ vs $\overline{E 0}$

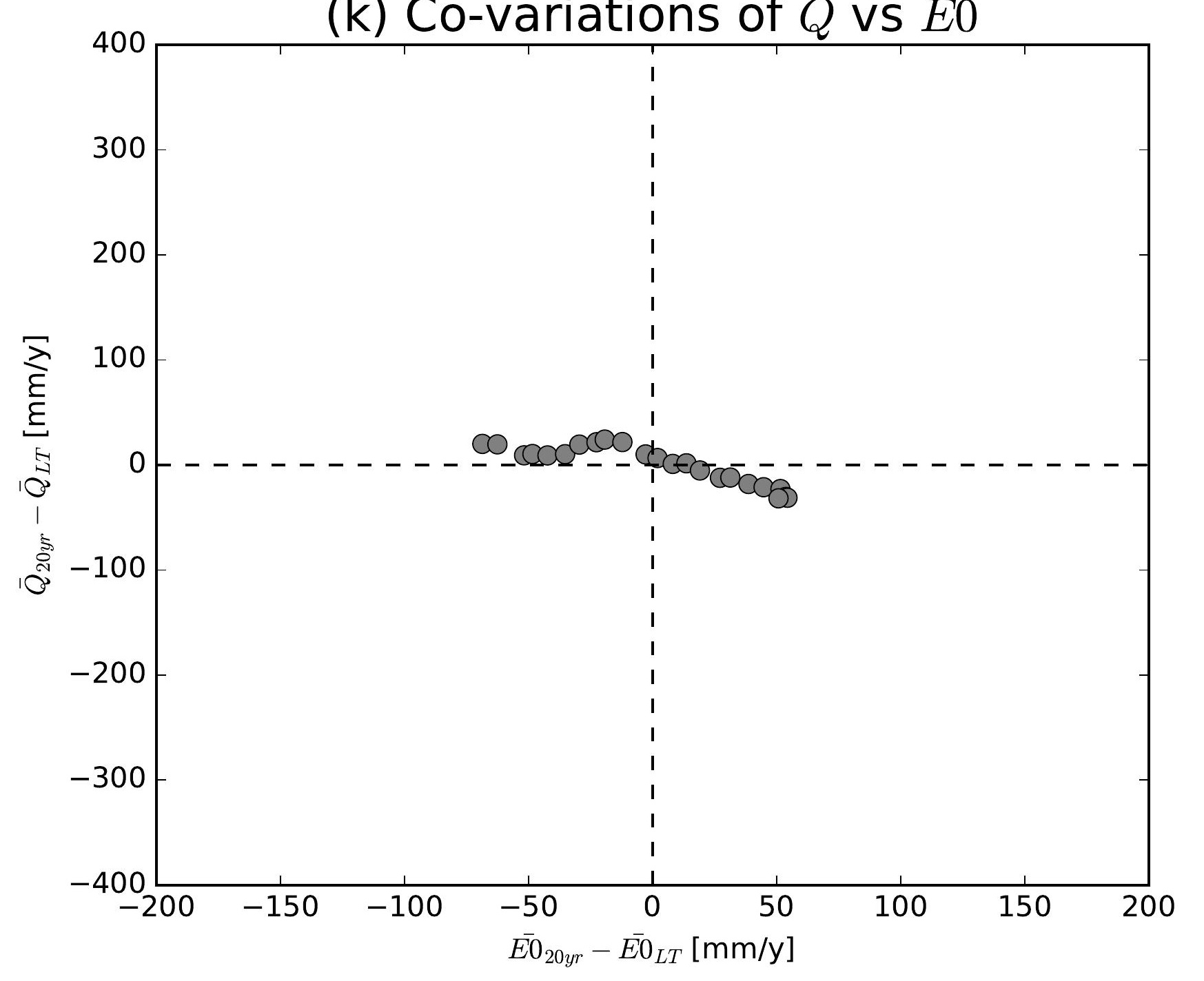

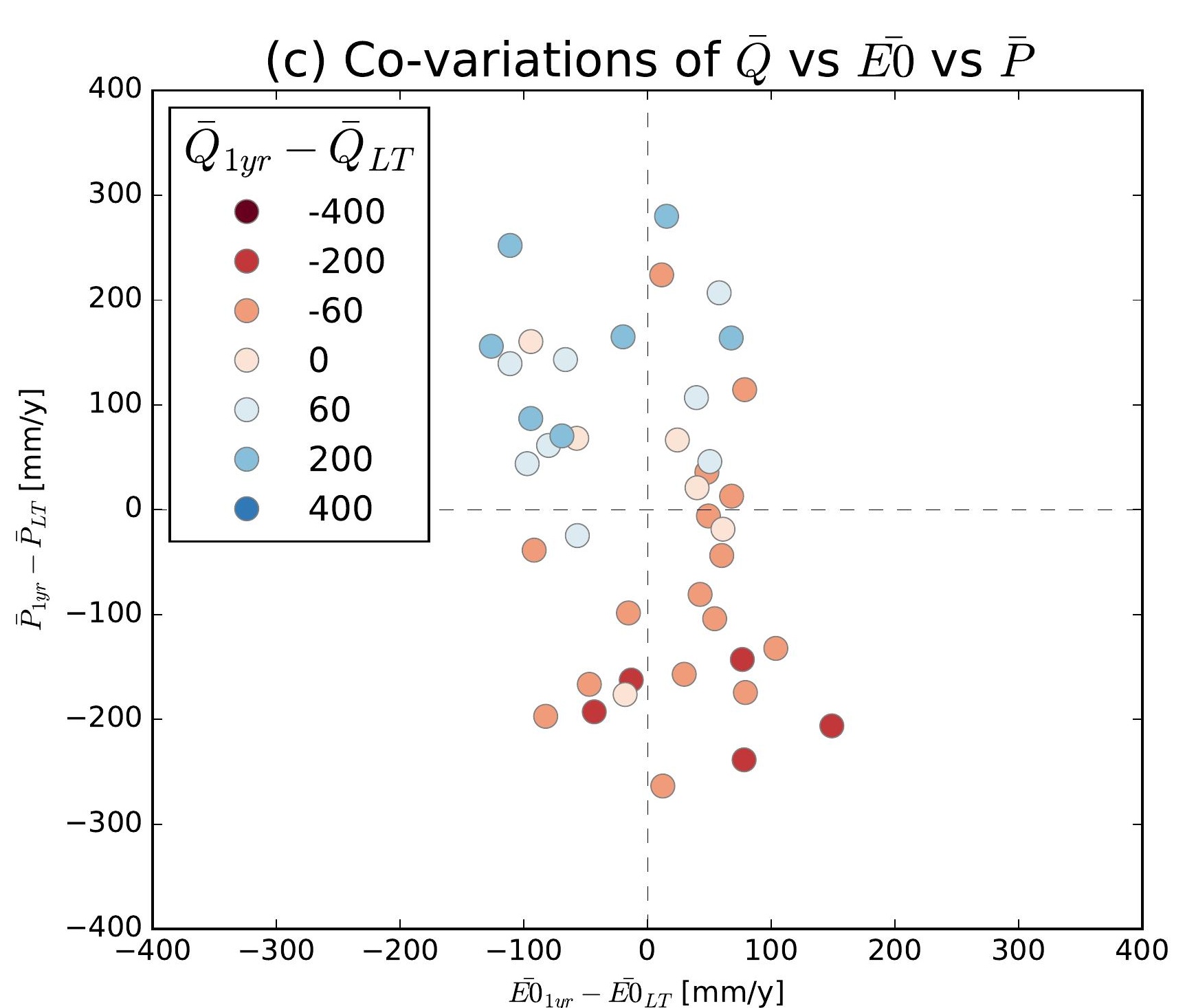
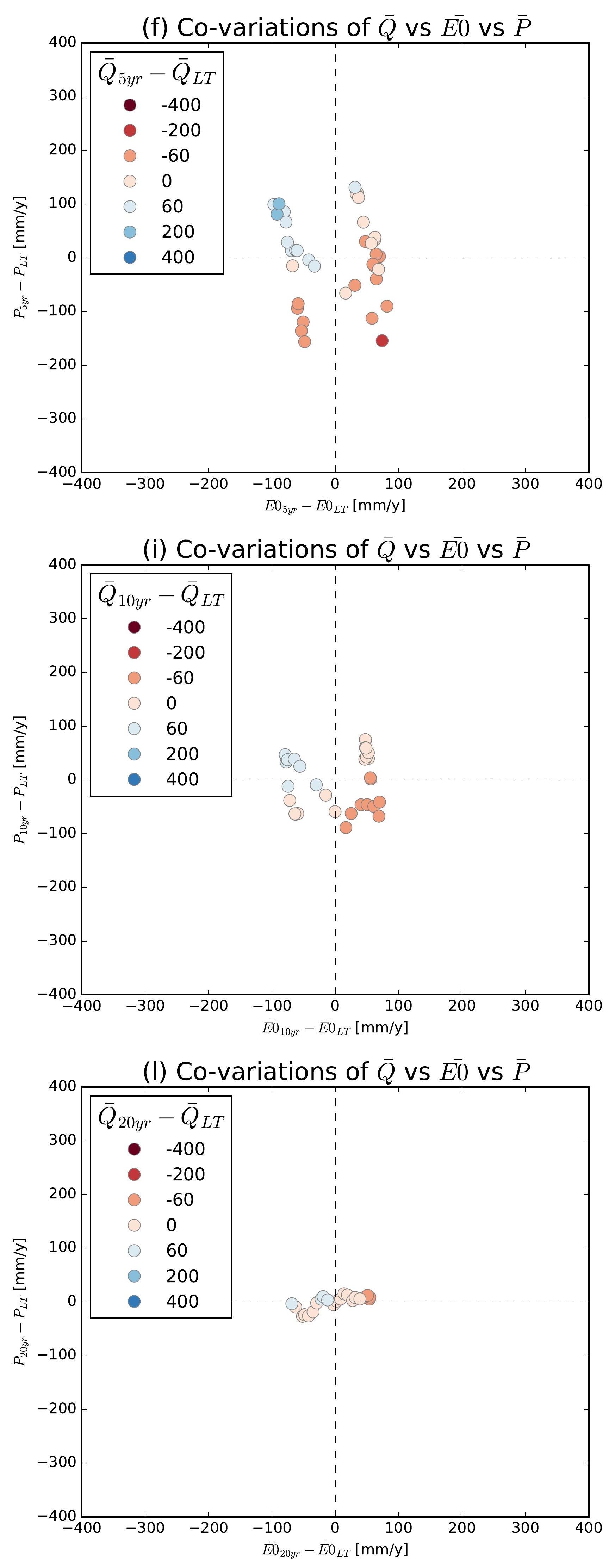

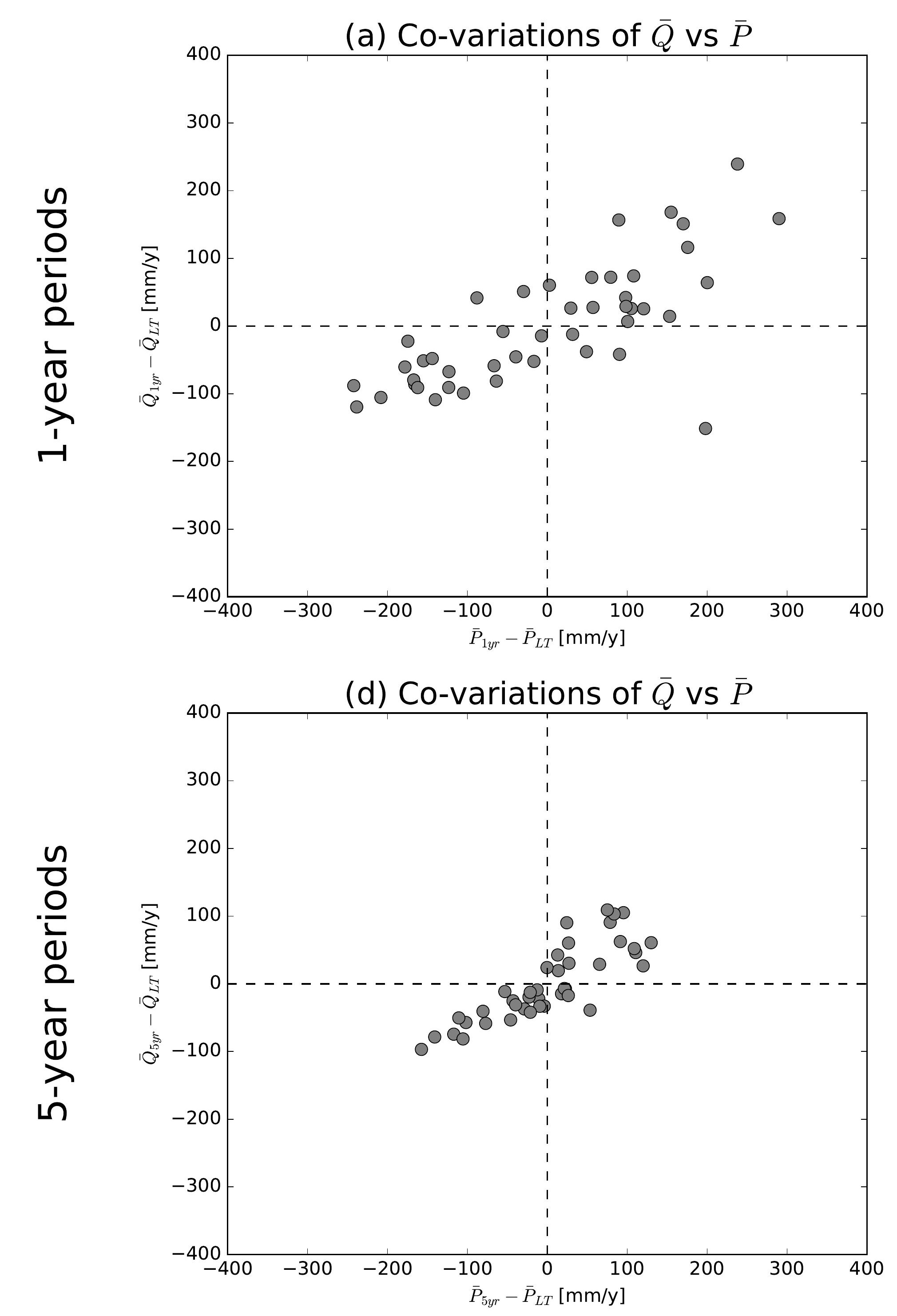

(g) Co-variations of $\bar{Q}$ vs $\bar{P}$
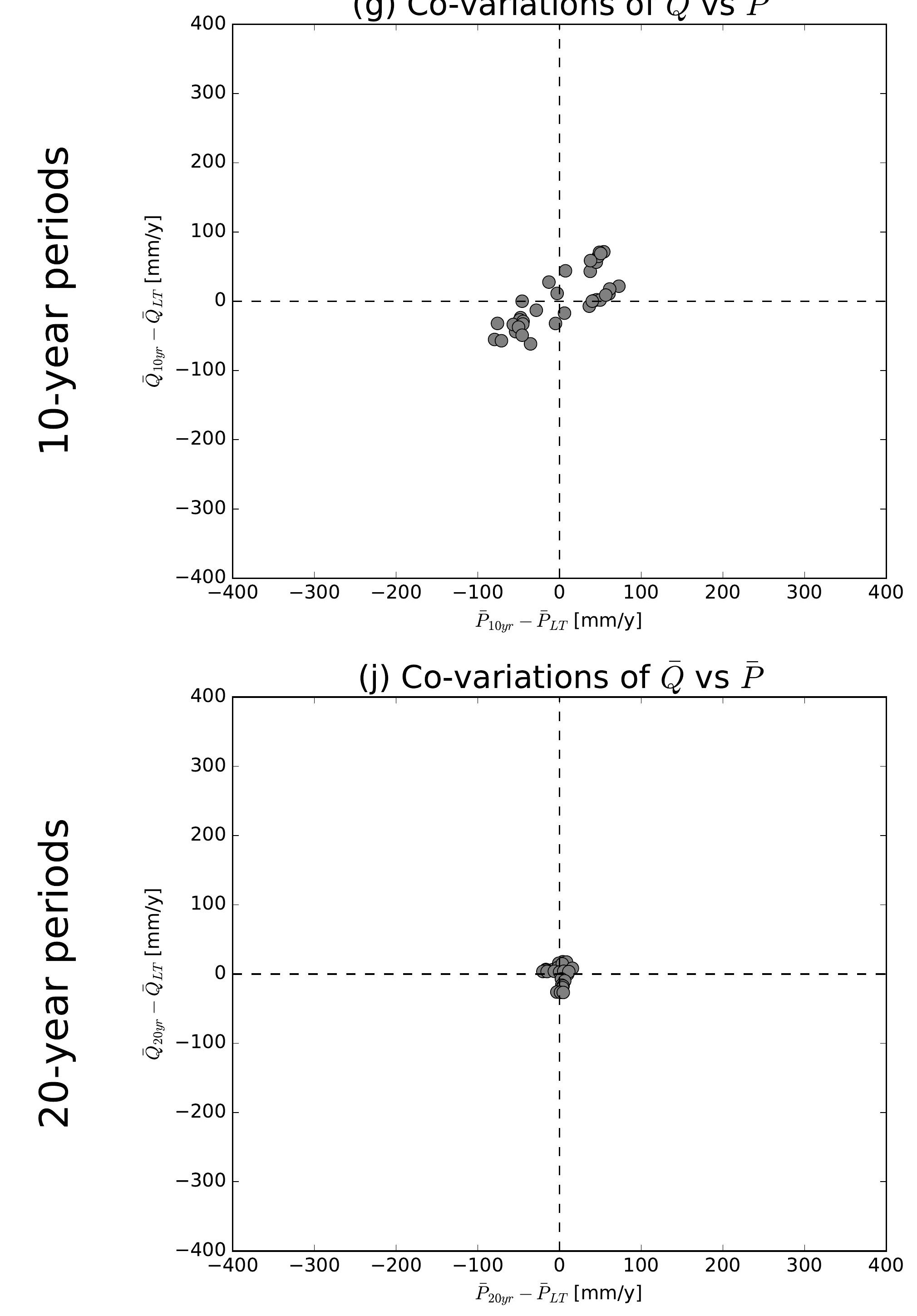

(b) Co-variations of $\bar{Q}$ vs $\overline{E 0}$

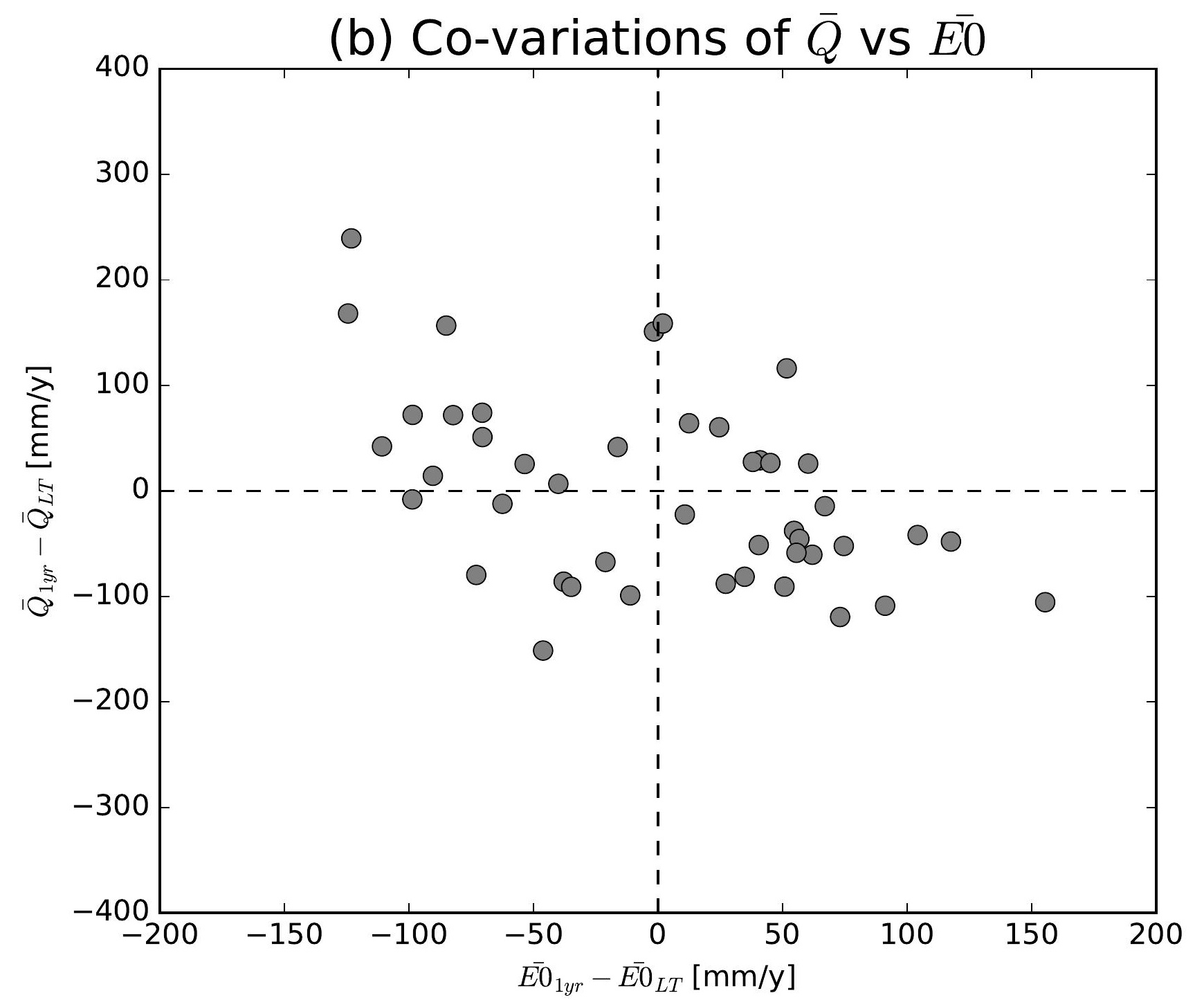

(e) Co-variations of $\bar{Q}$ vs $\overline{E 0}$

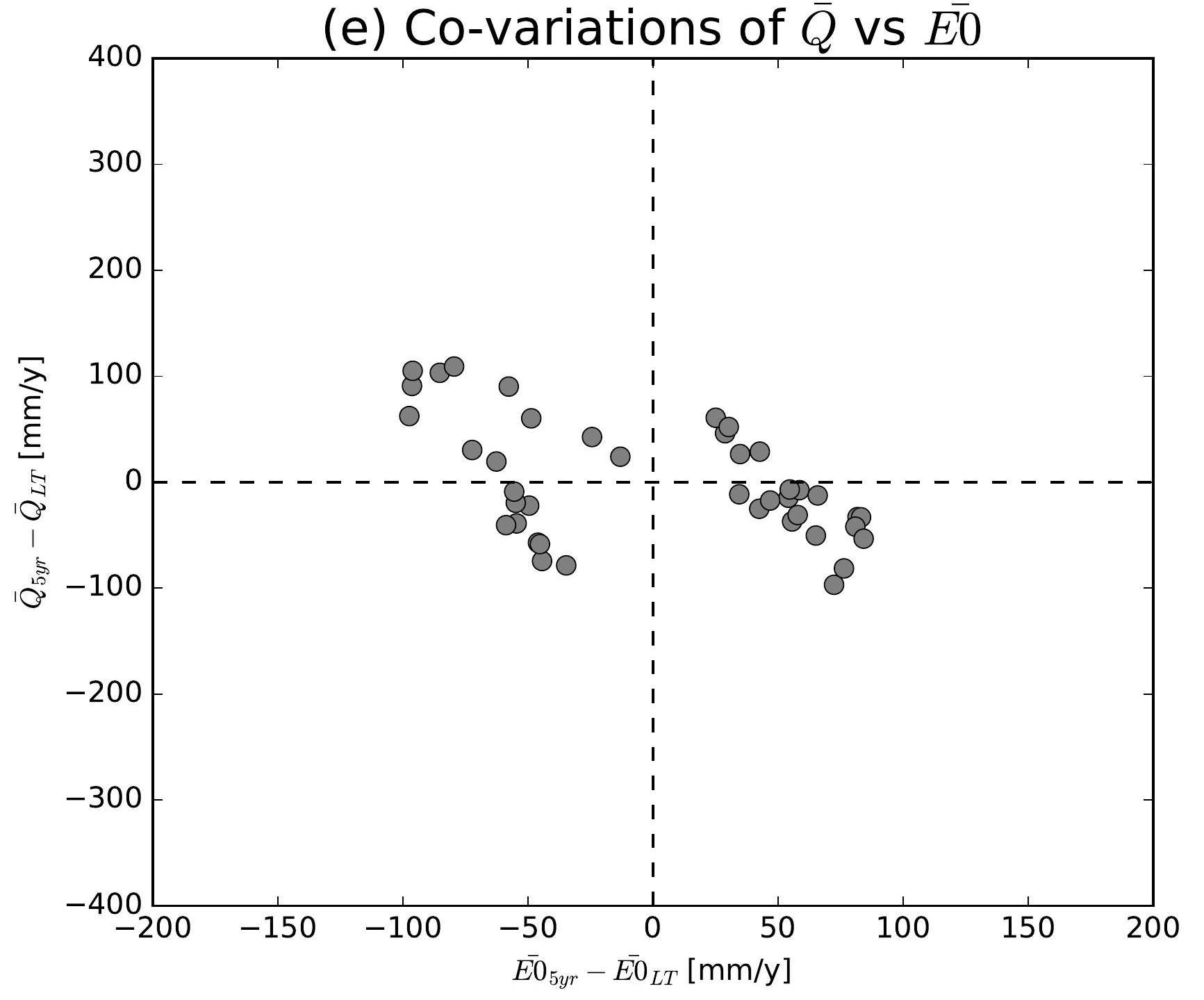

(h) Co-variations of $\bar{Q}$ vs $\overline{E 0}$

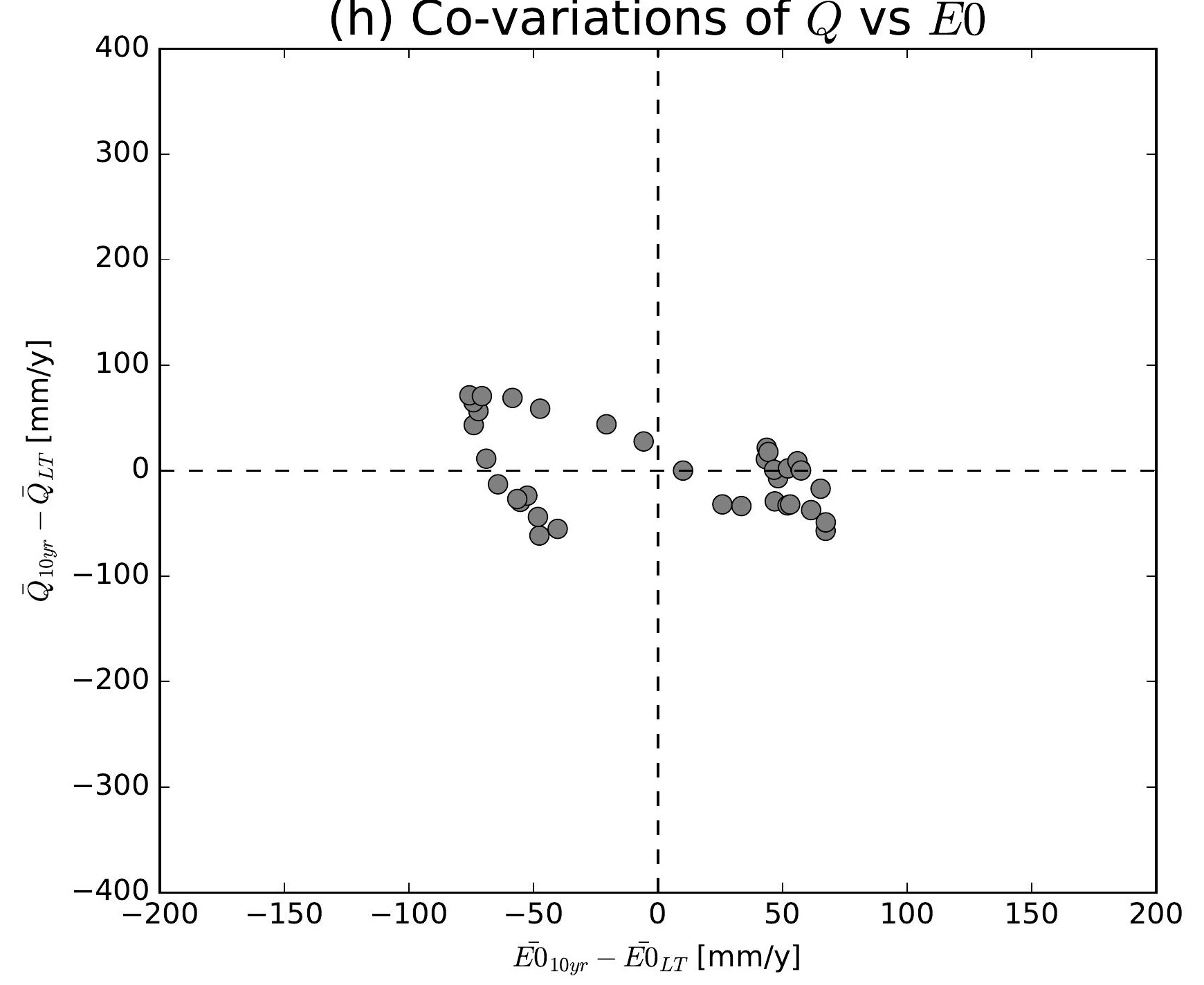

(k) Co-variations of $\bar{Q}$ vs $\overline{E 0}$

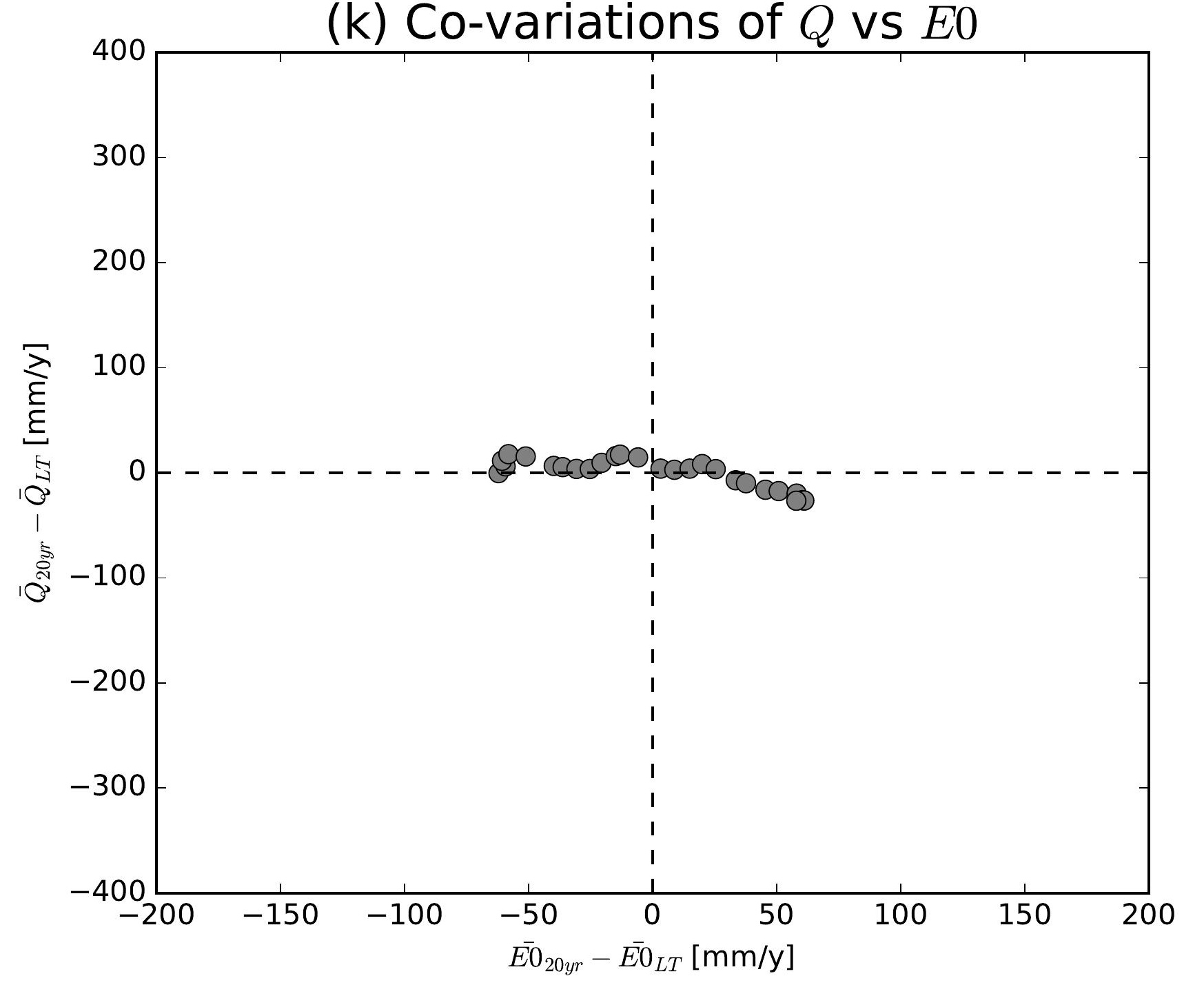

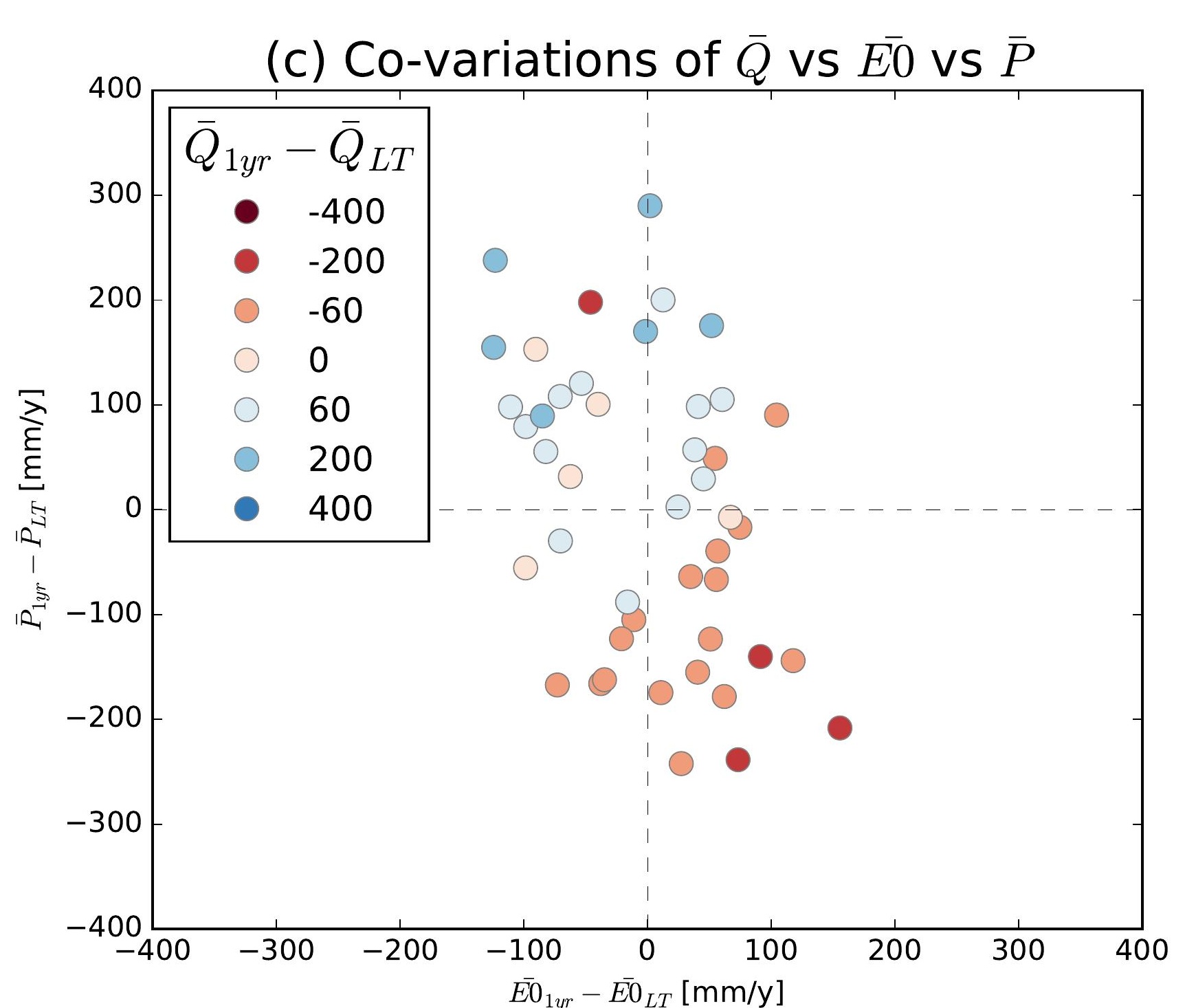
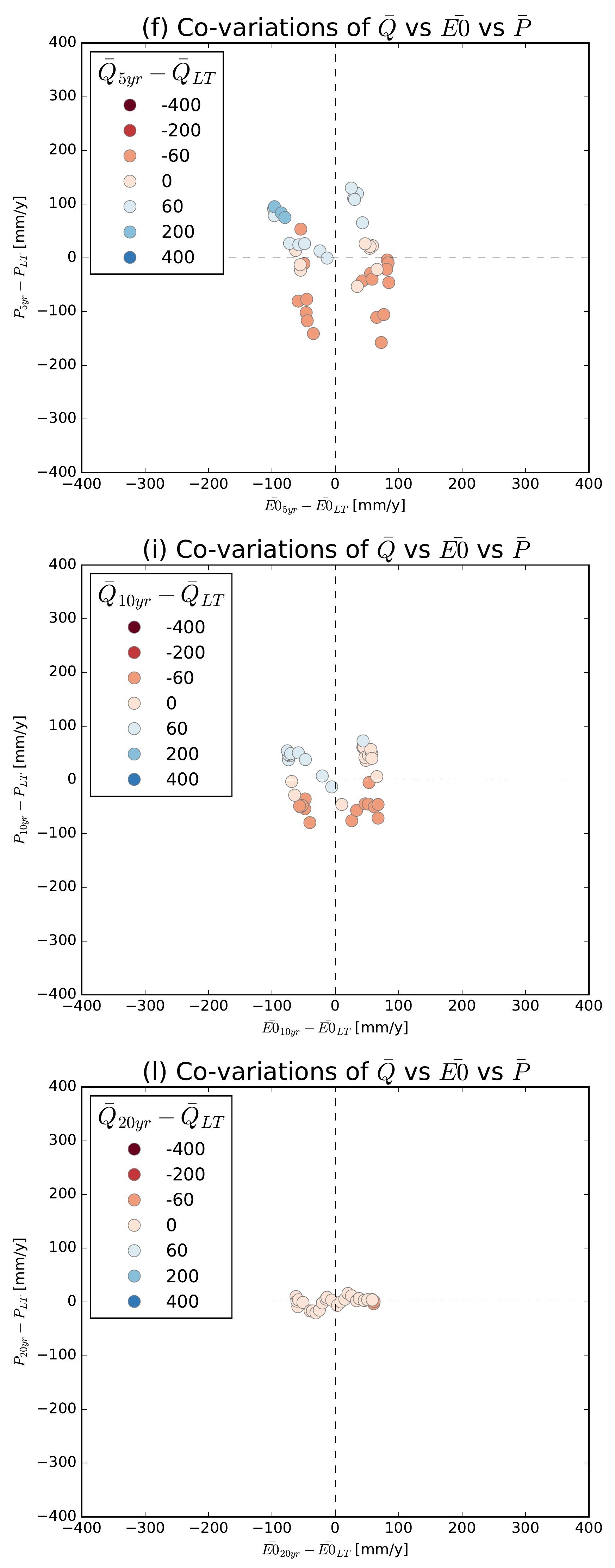

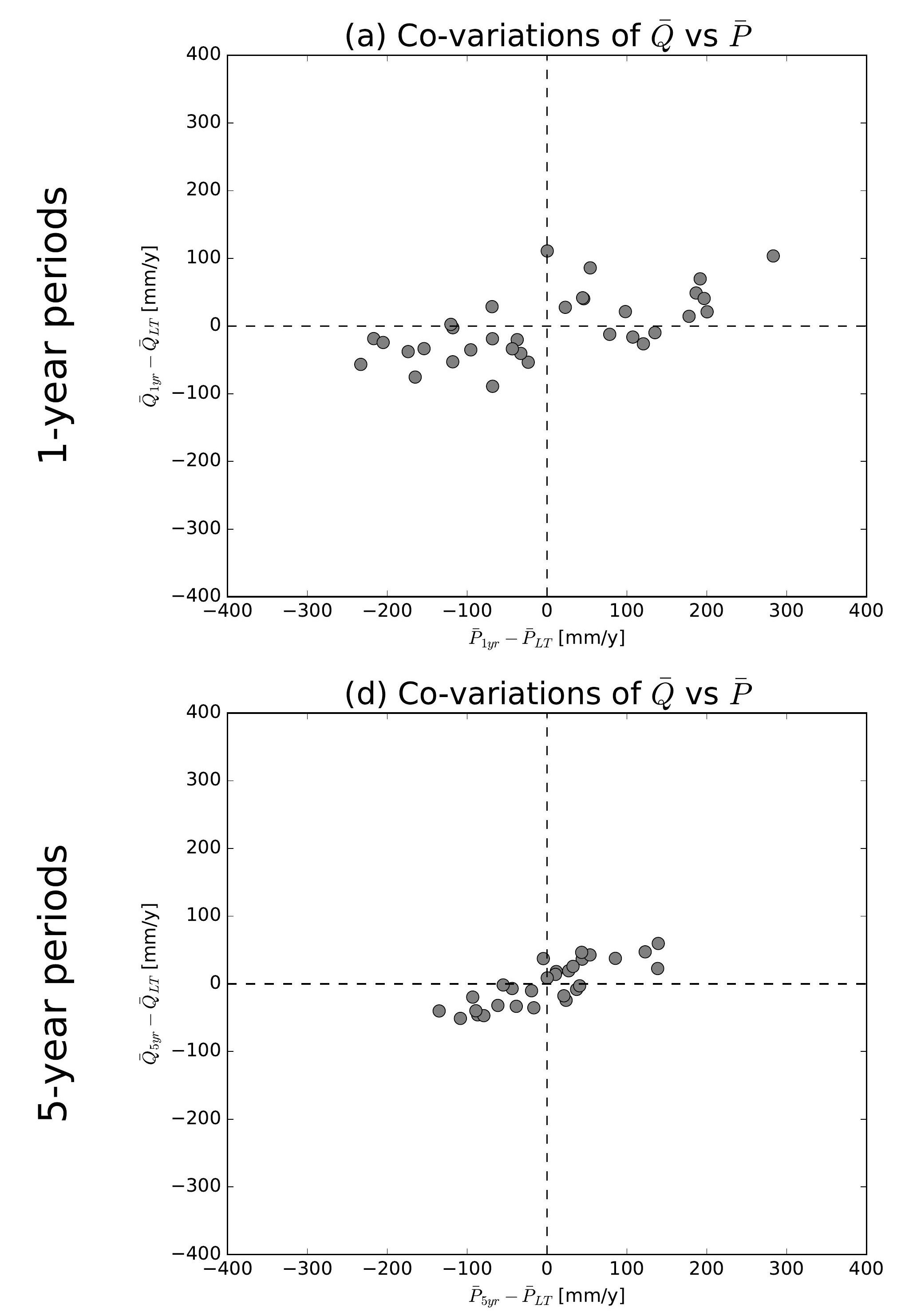

(g) Co-variations of $\bar{Q}$ vs $\bar{P}$
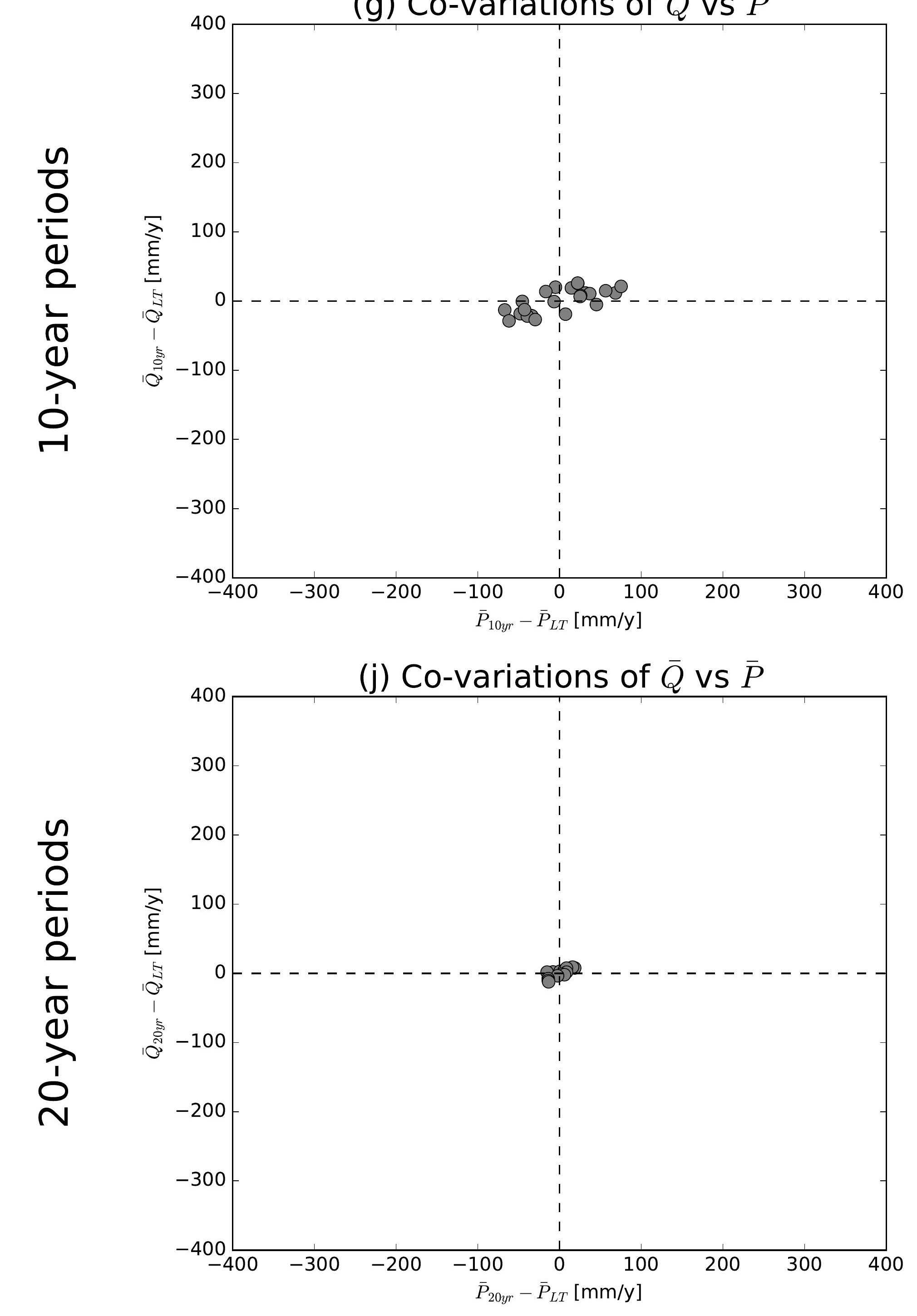

(b) Co-variations of $\bar{Q}$ vs $\overline{E 0}$

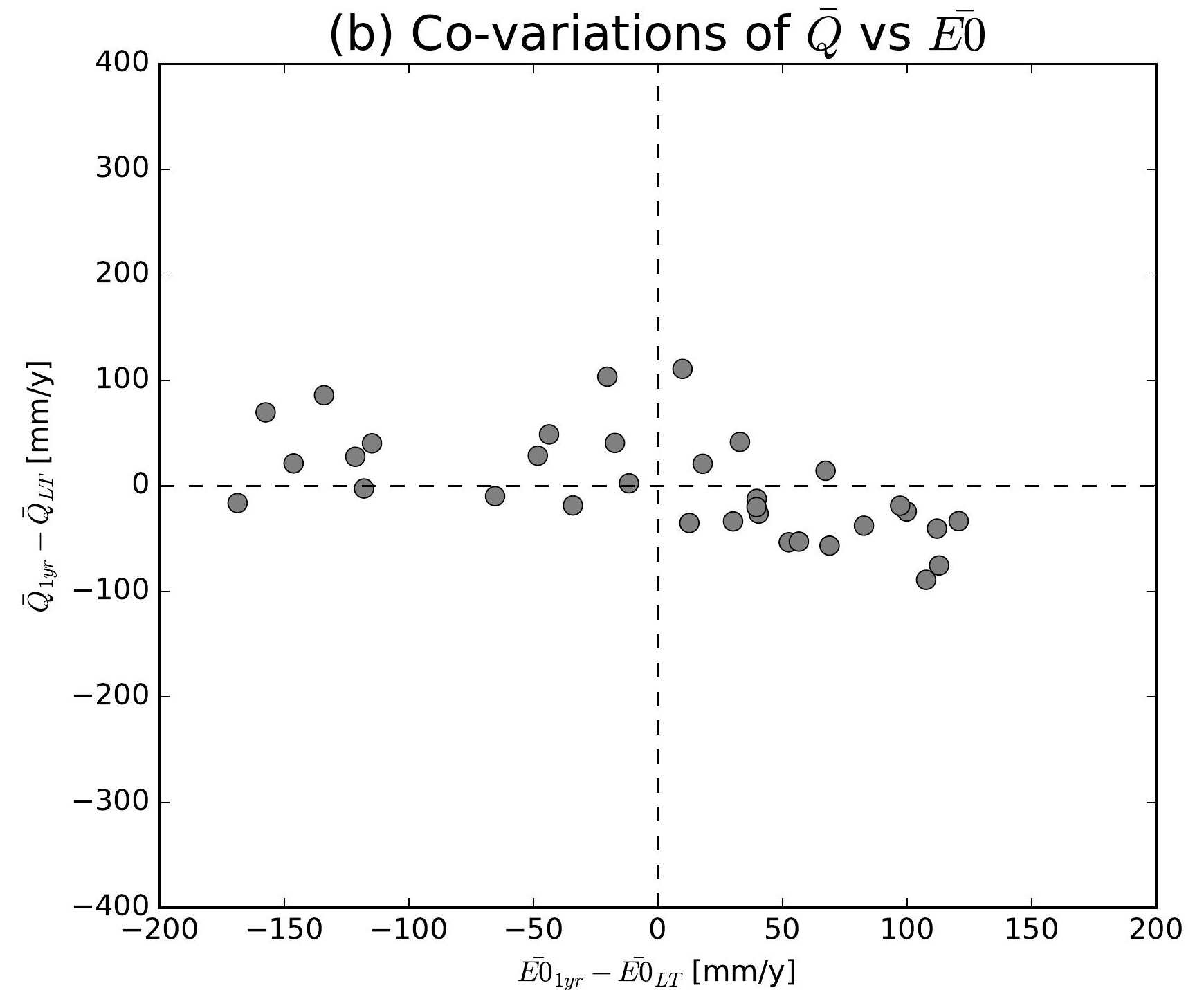

(e) Co-variations of $\bar{Q}$ vs $\overline{E 0}$

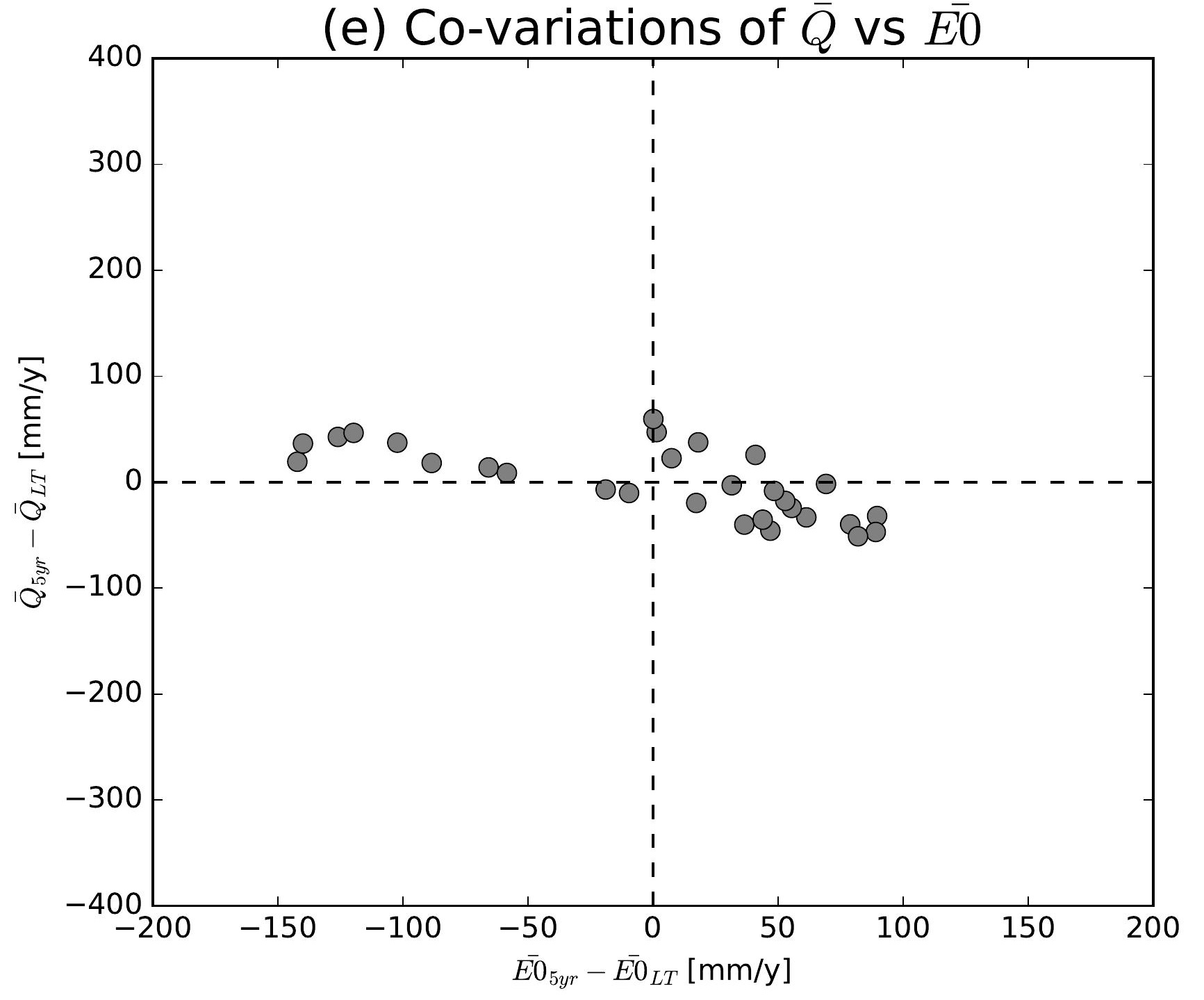

(h) Co-variations of $\bar{Q}$ vs $\overline{E 0}$

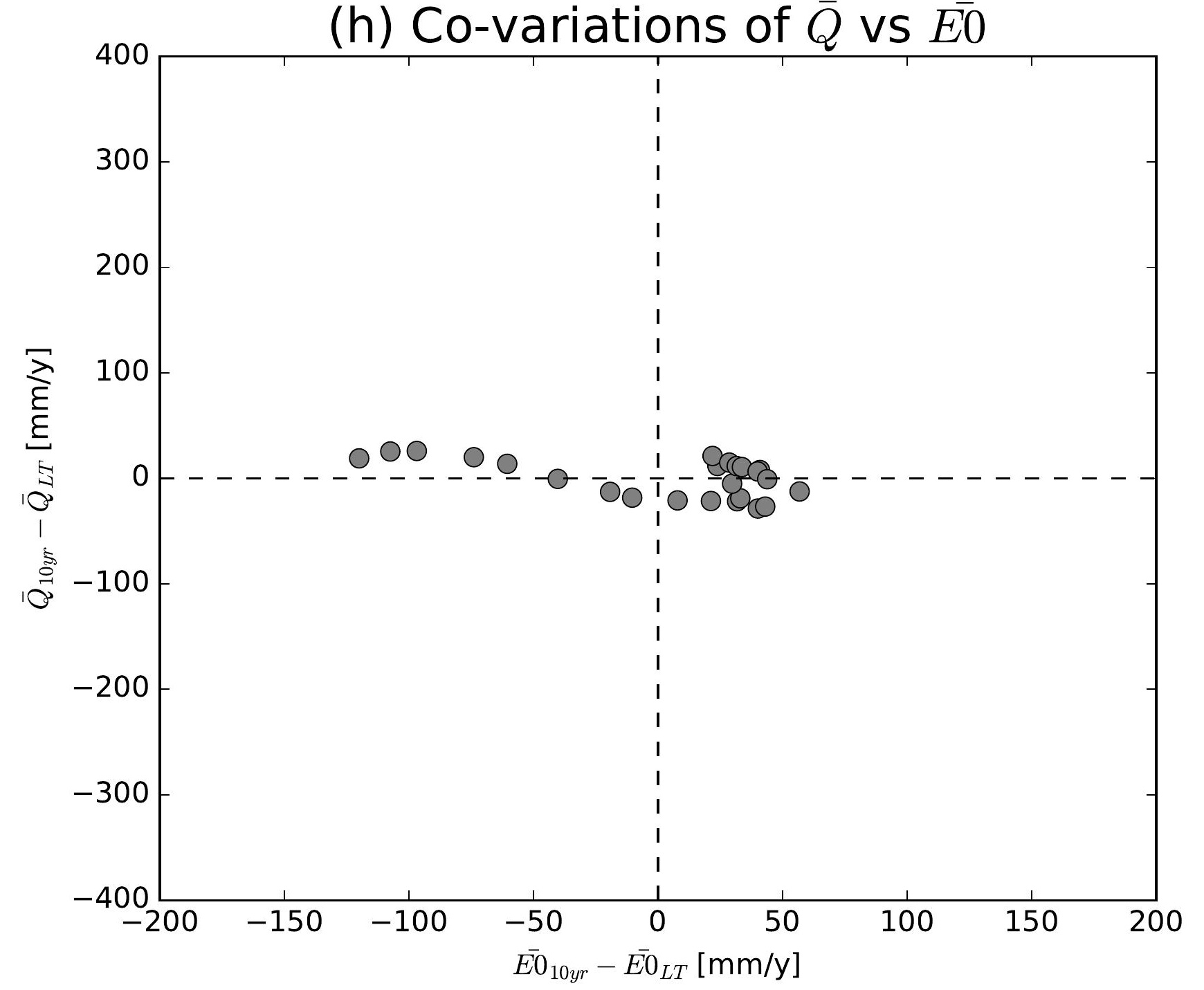

(k) Co-variations of $\bar{Q}$ vs $\overline{E 0}$

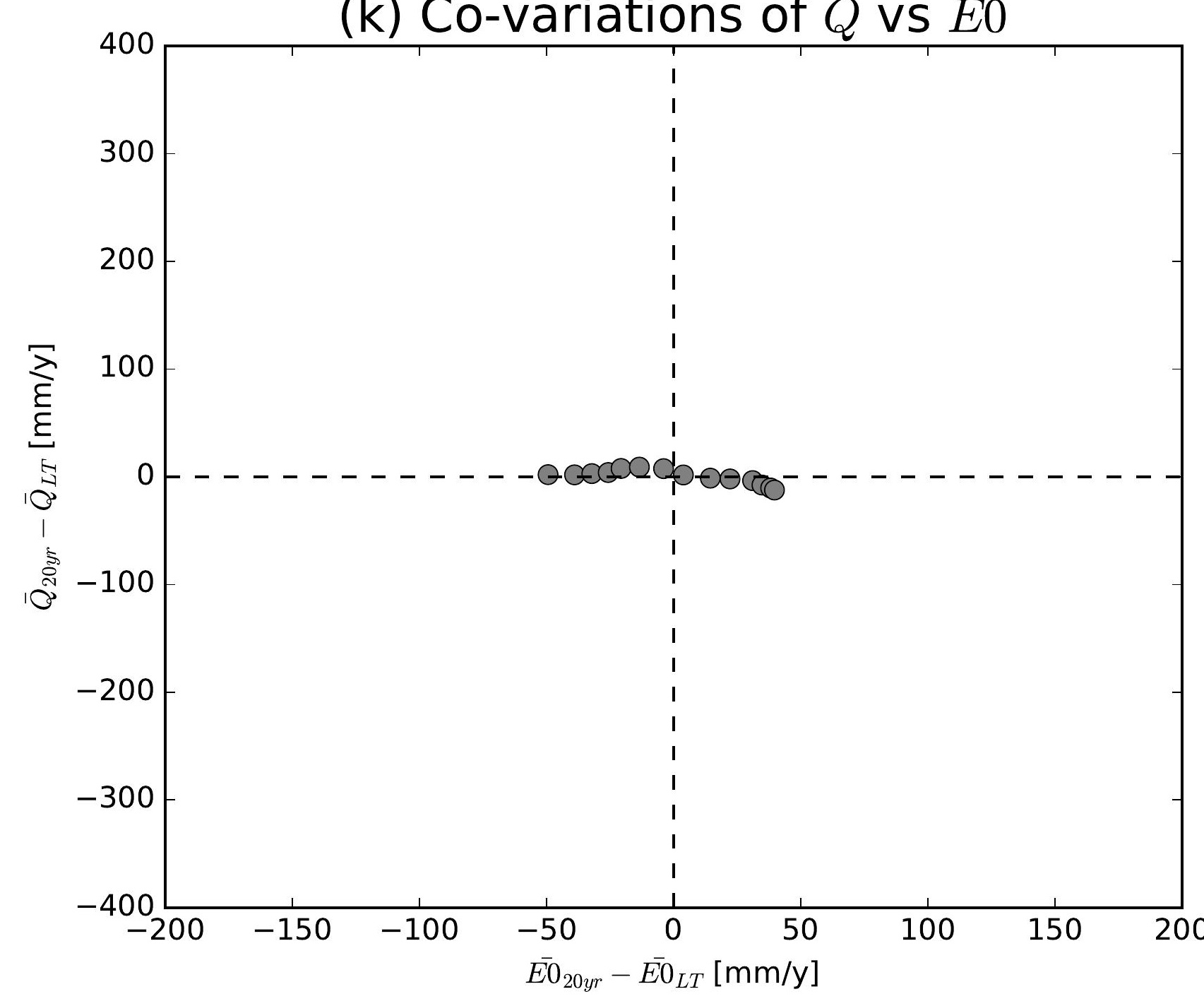

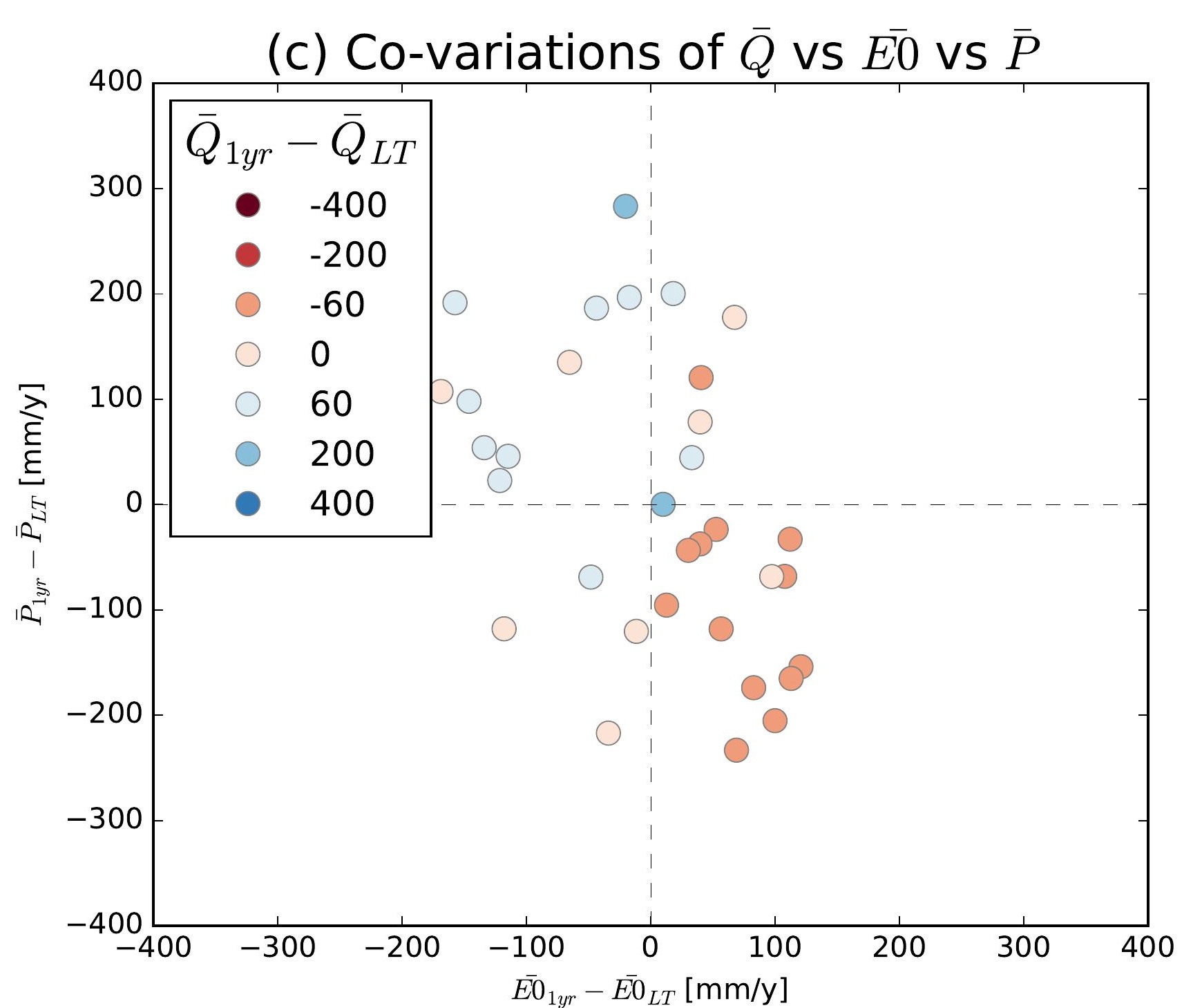
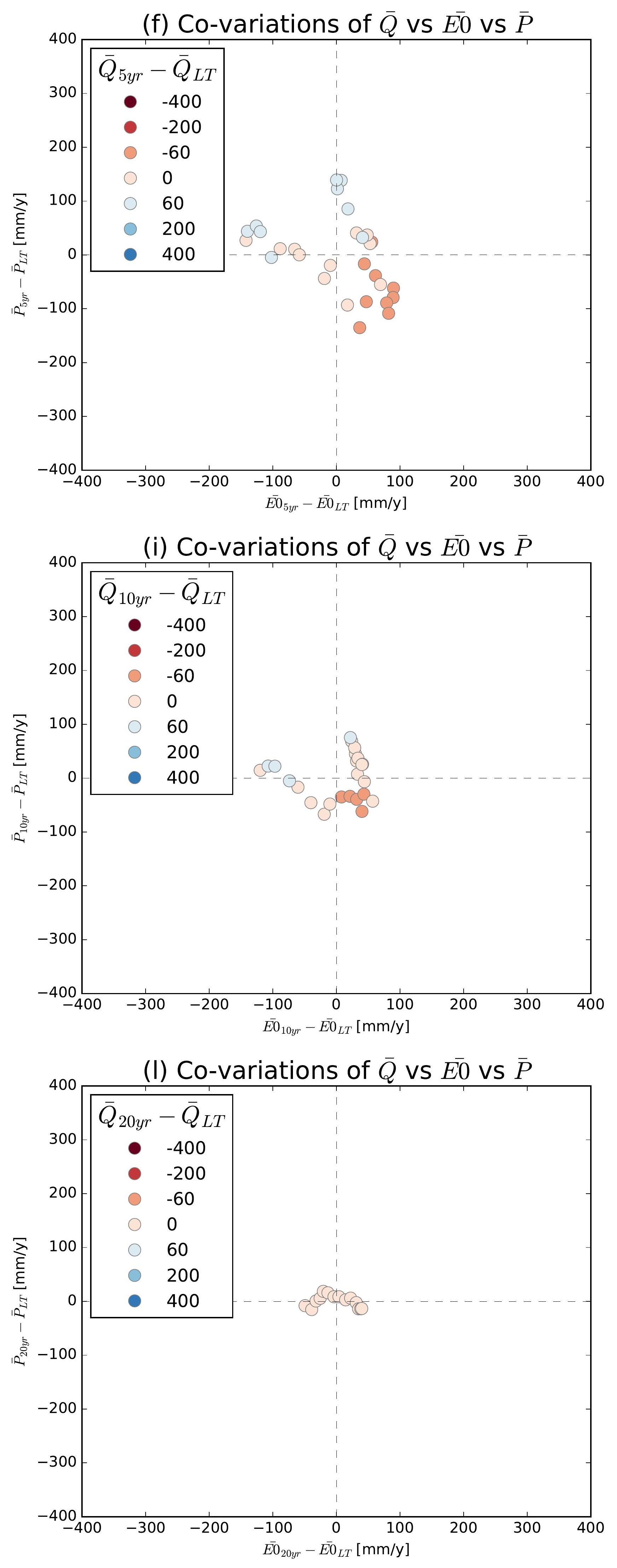

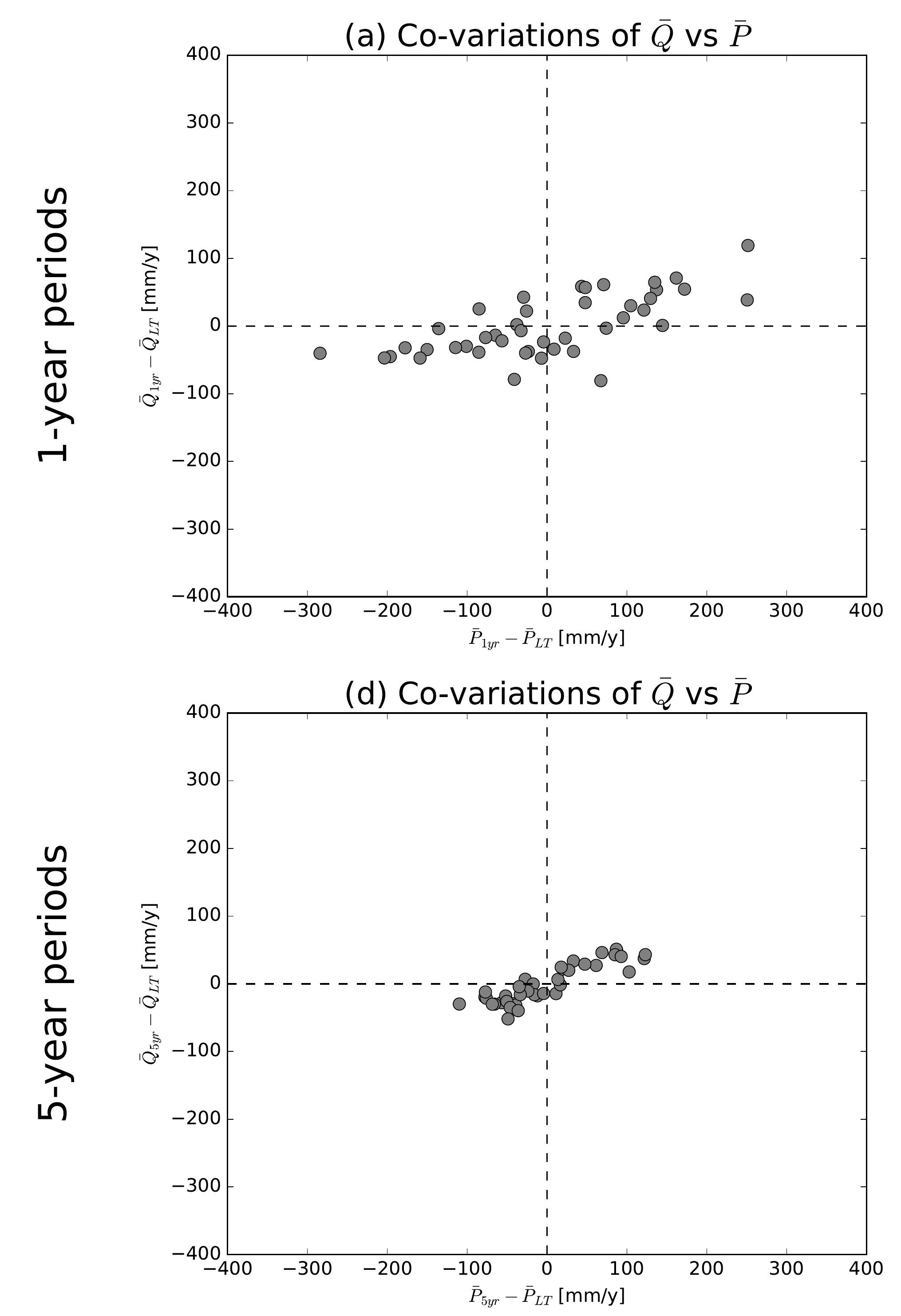

(g) Co-variations of $\bar{Q}$ vs $\bar{P}$
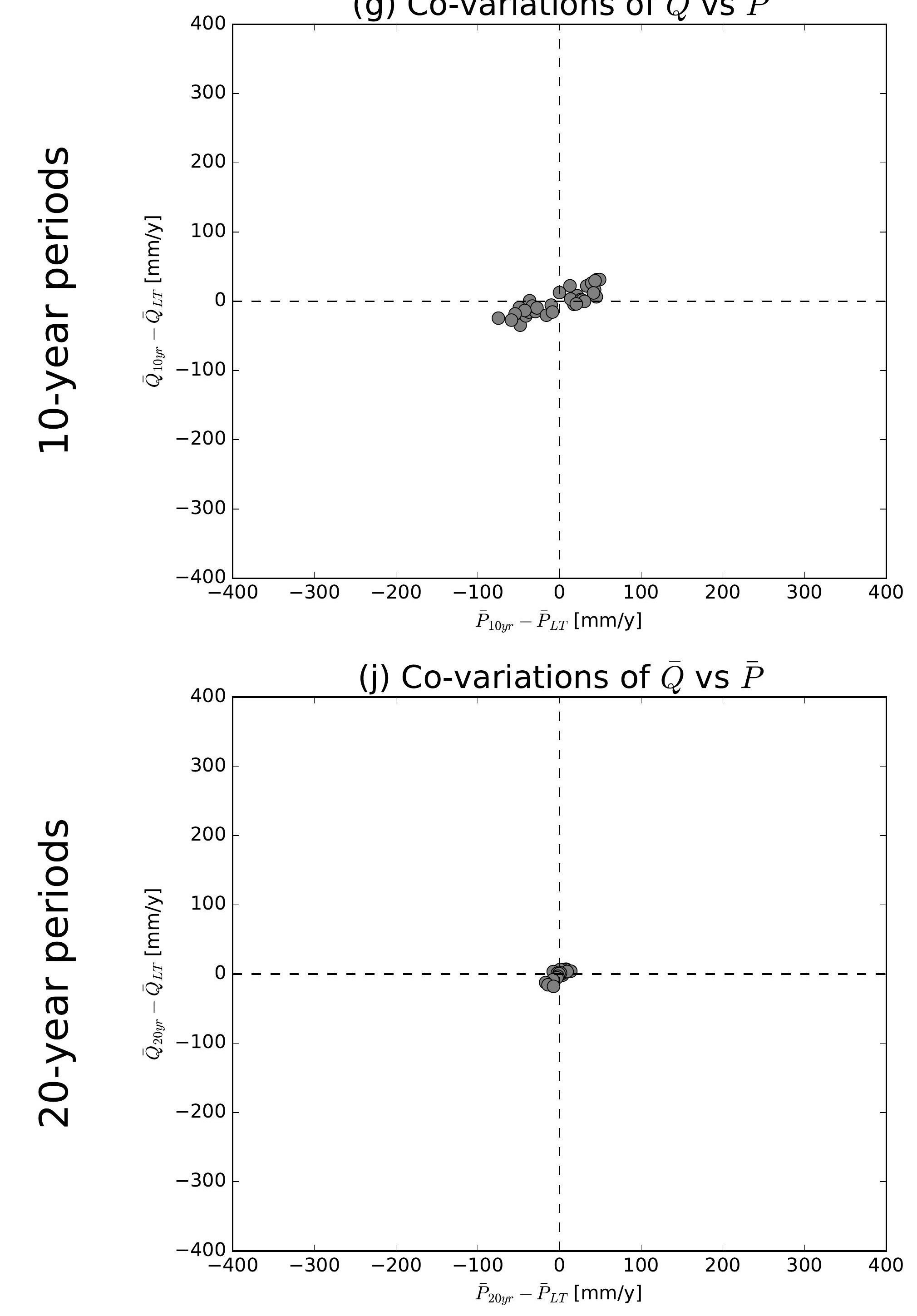

(b) Co-variations of $\bar{Q}$ vs $\overline{E 0}$

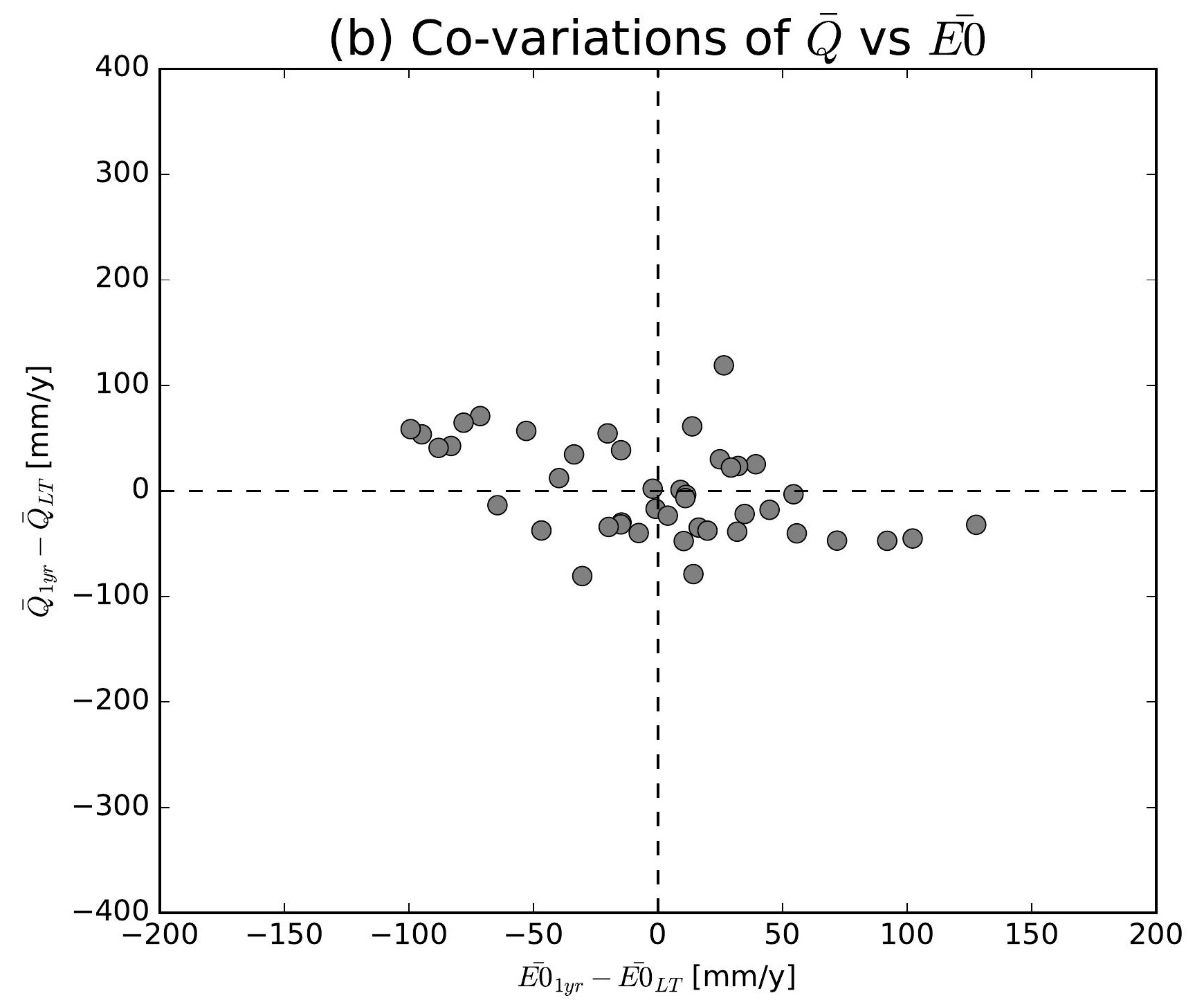

(e) Co-variations of $\bar{Q}$ vs $\overline{E 0}$

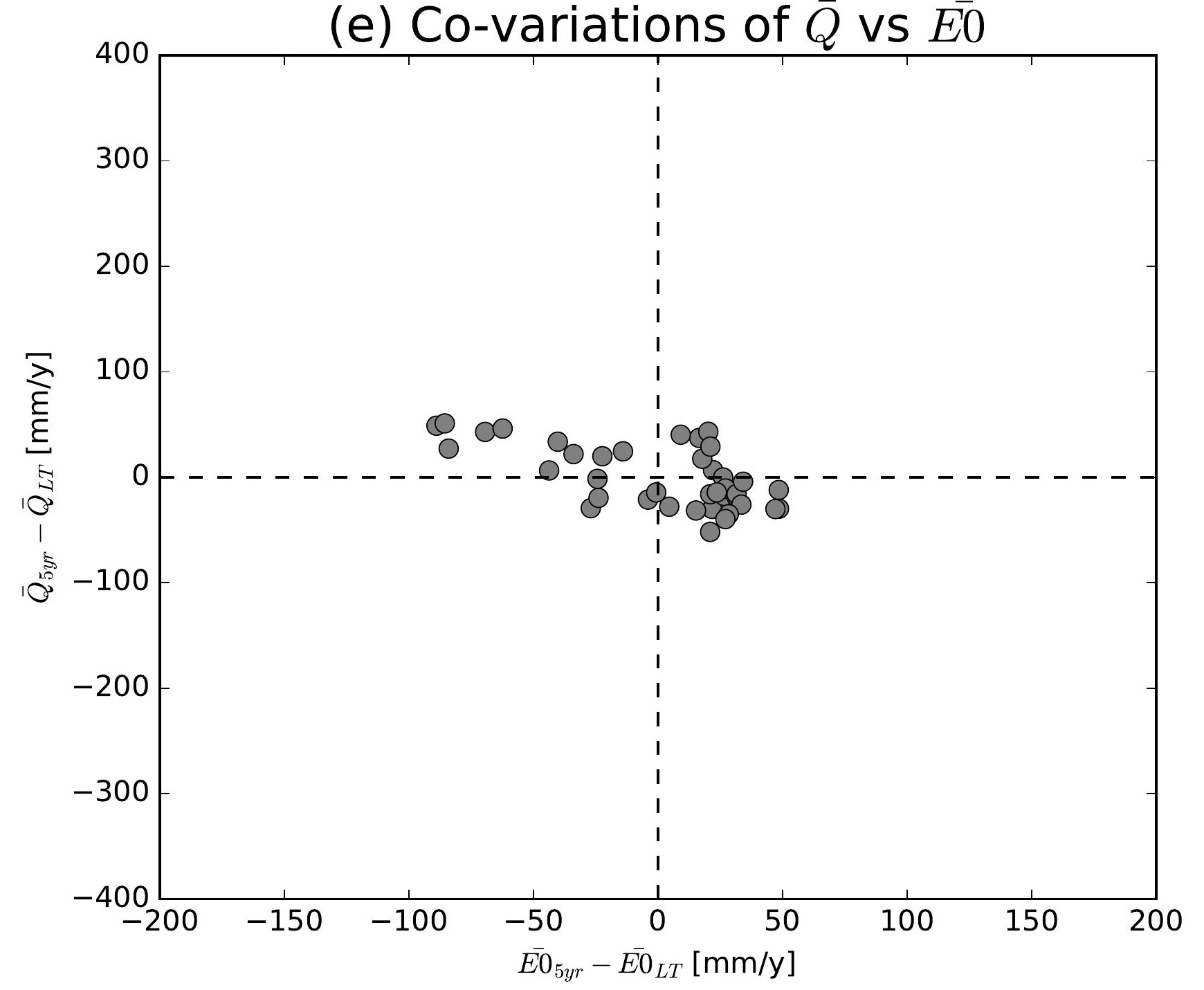

(h) Co-variations of $\bar{Q}$ vs $\overline{E 0}$

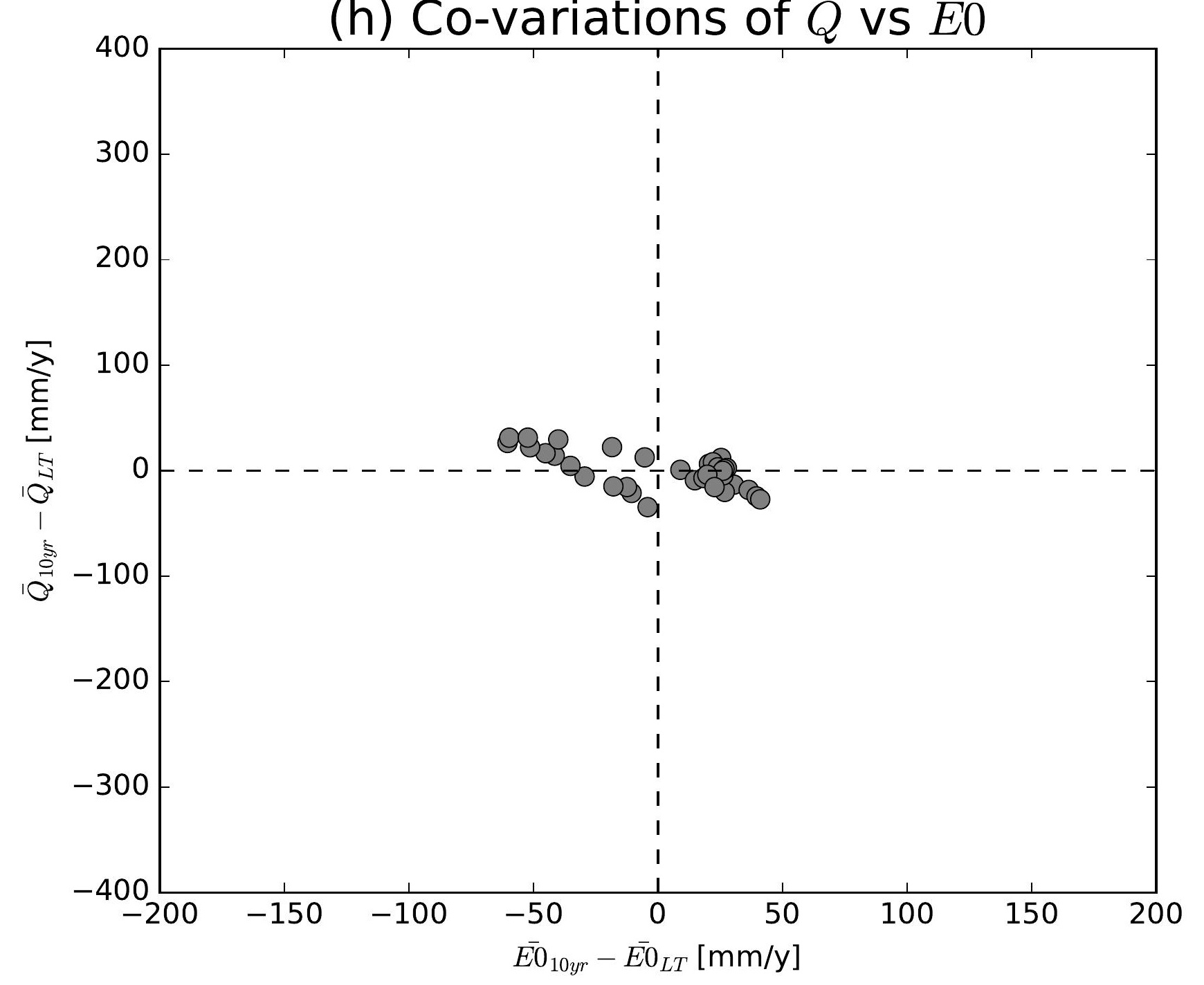

(k) Co-variations of $\bar{Q}$ vs $\overline{E 0}$

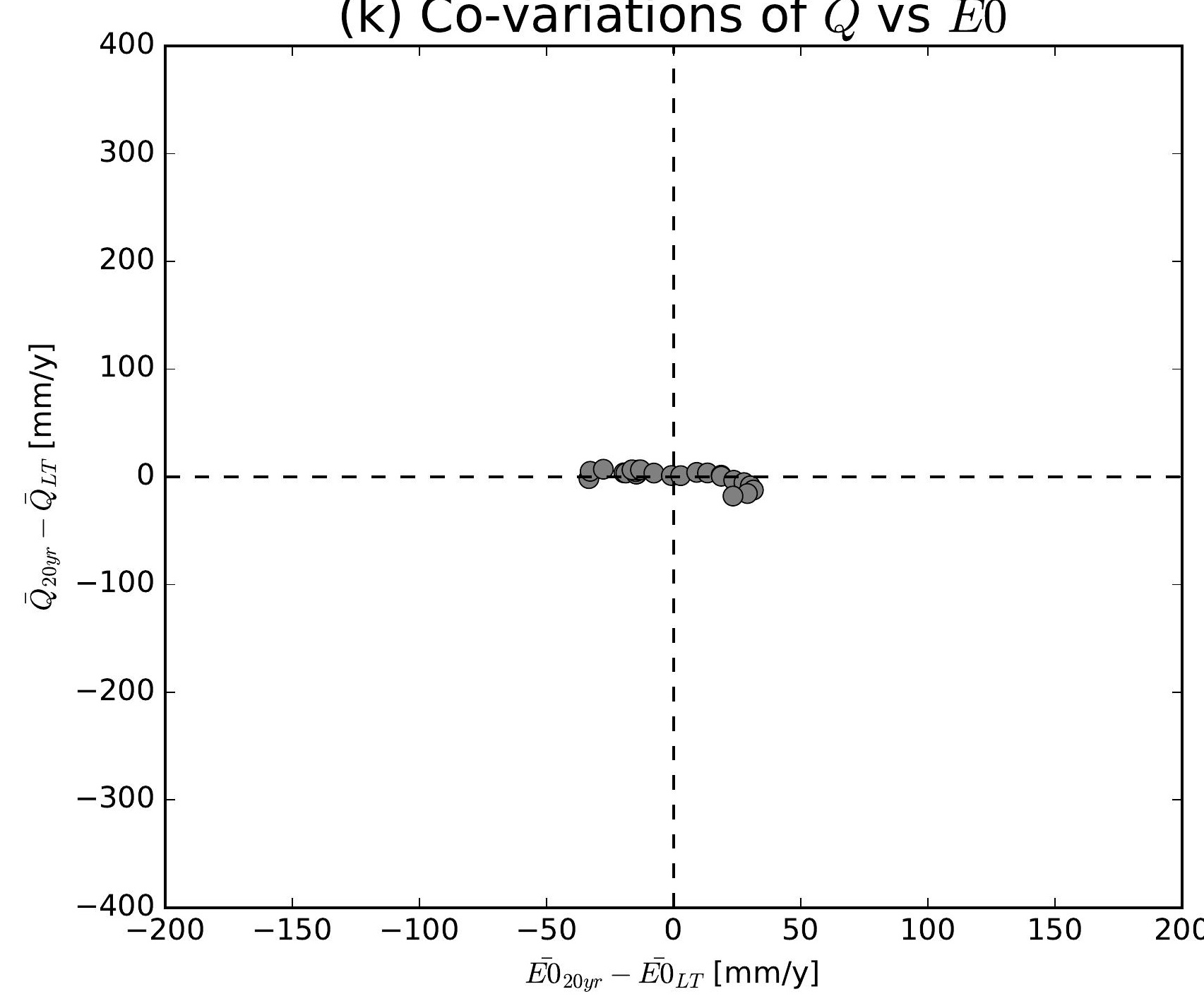

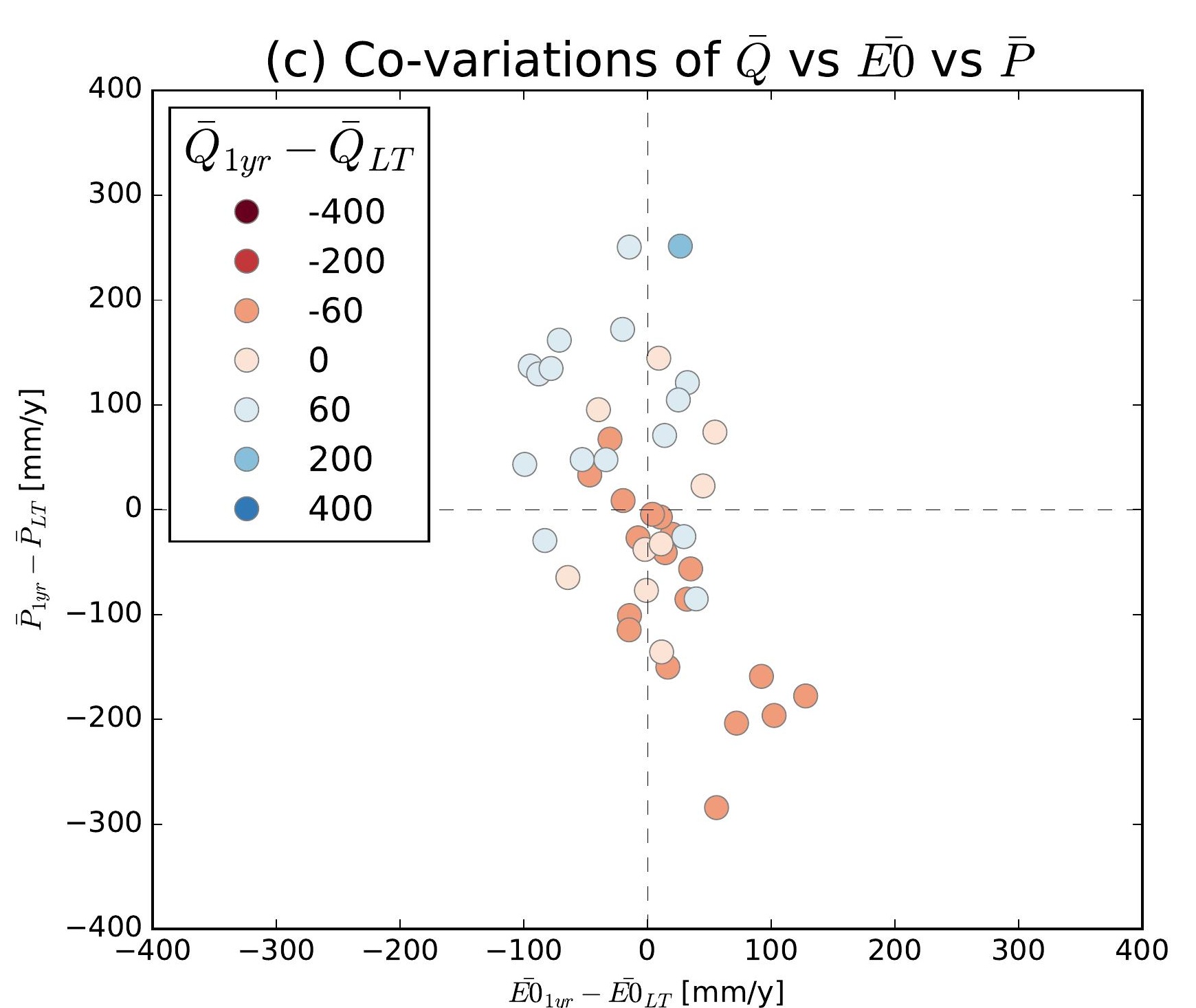
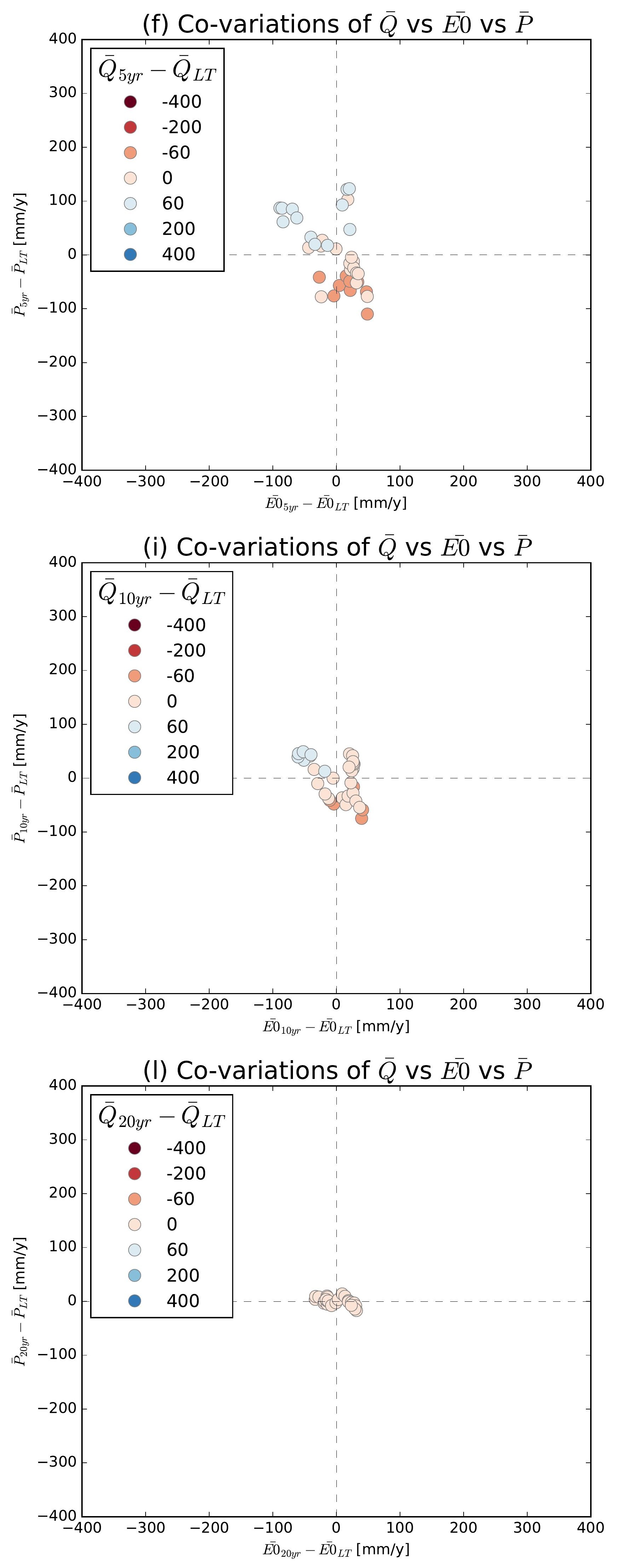

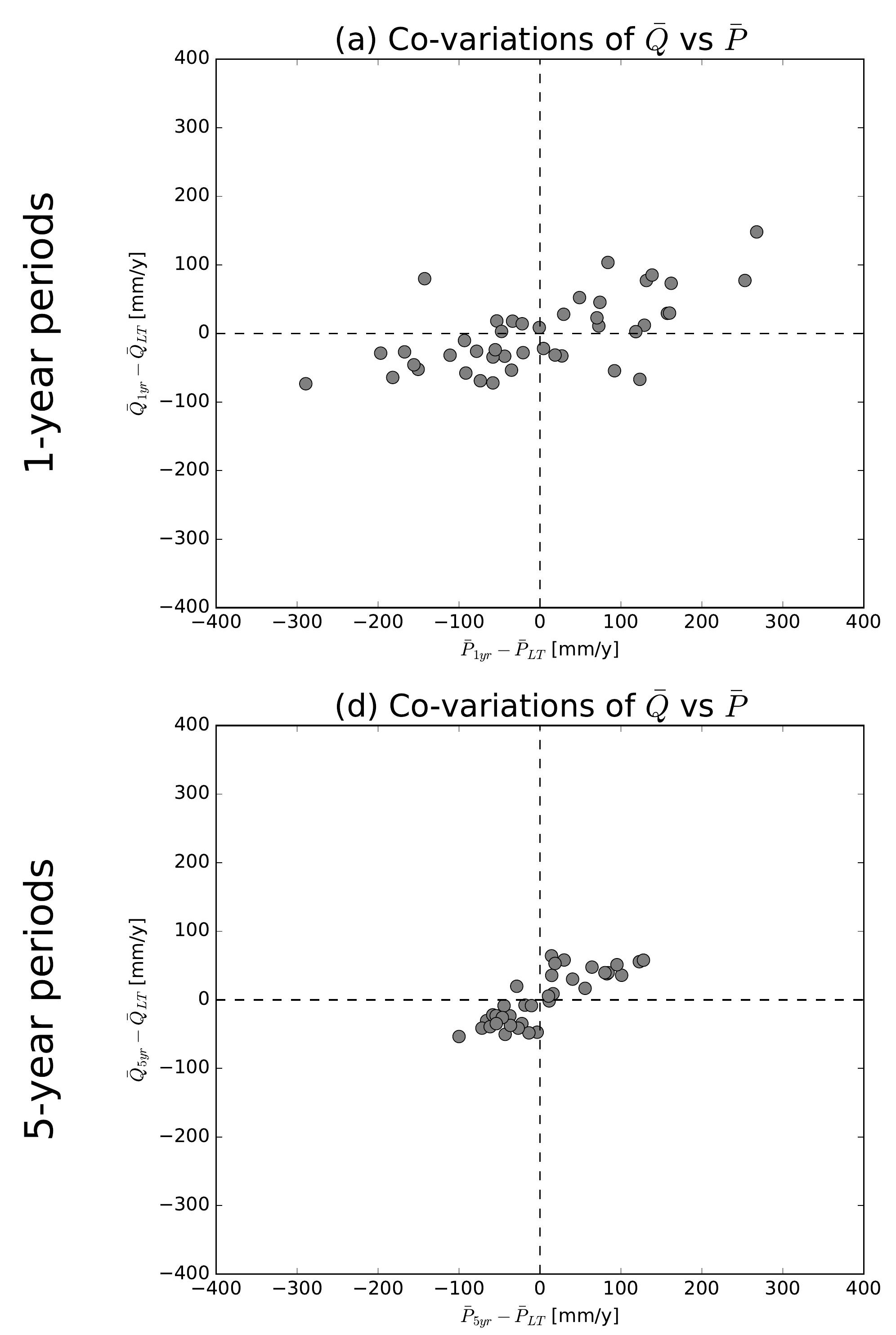

(g) Co-variations of $\bar{Q}$ vs $\bar{P}$
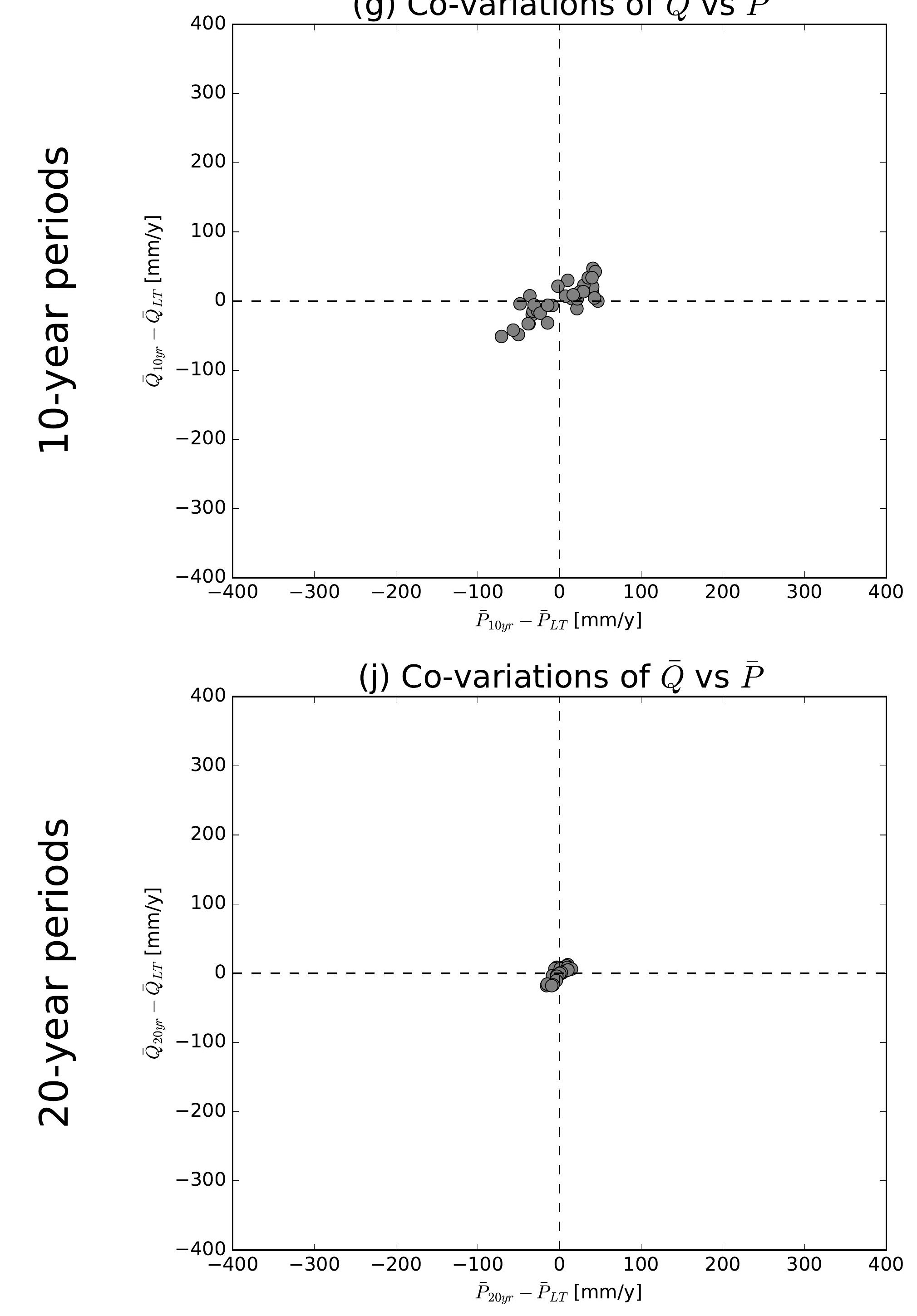

(b) Co-variations of $\bar{Q}$ vs $\overline{E 0}$

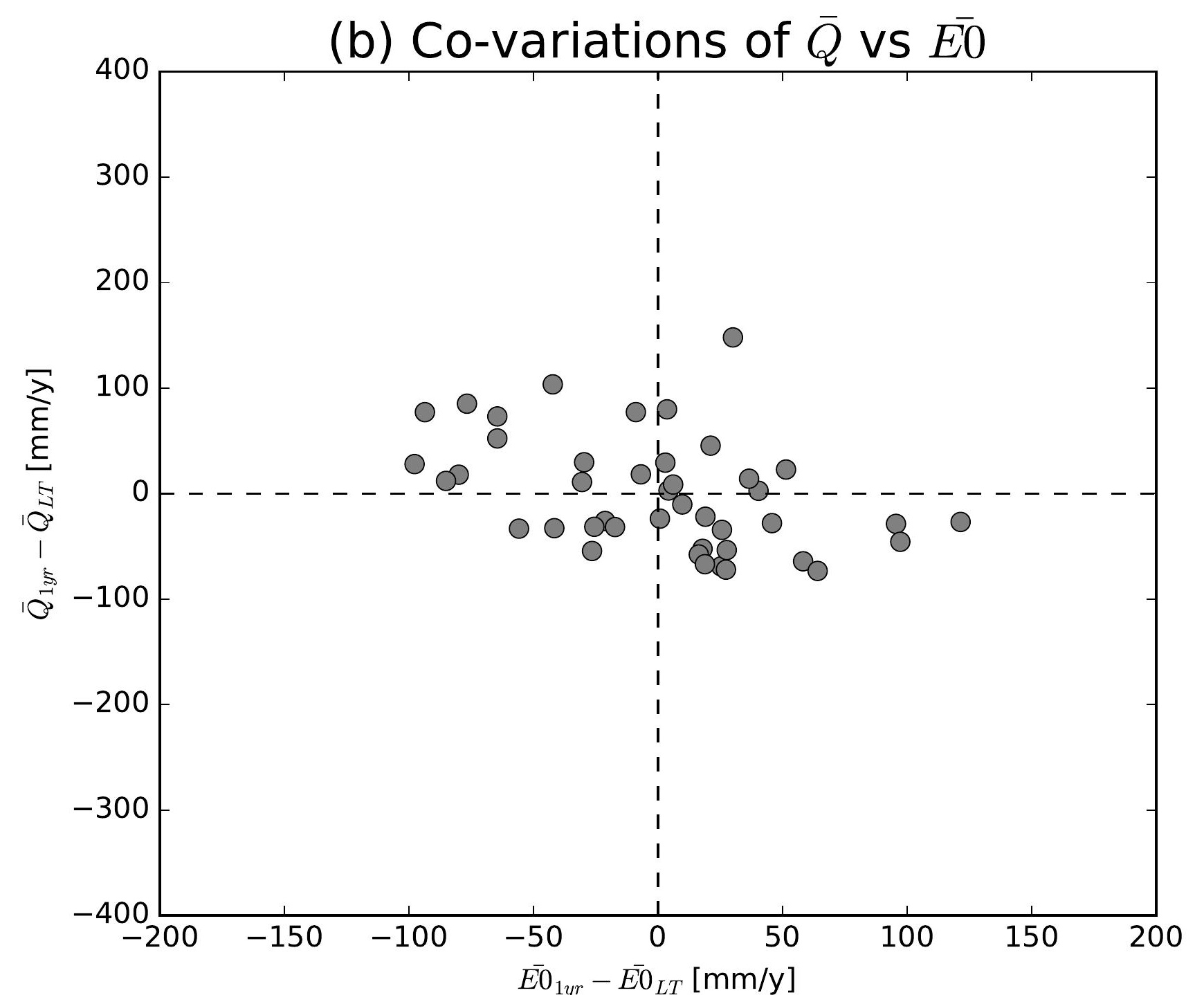

(e) Co-variations of $\bar{Q}$ vs $\overline{E 0}$

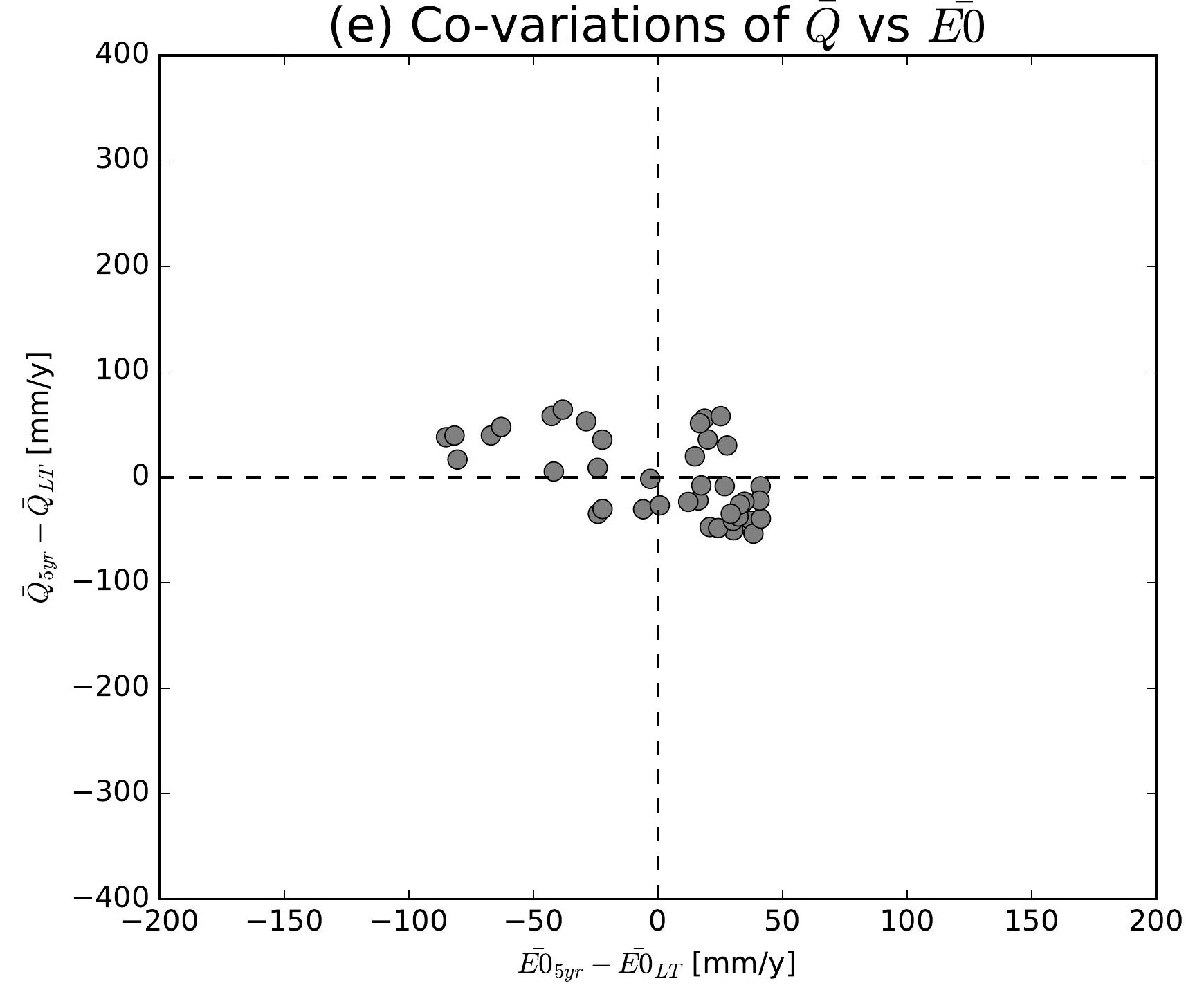

(h) Co-variations of $\bar{Q}$ vs $\overline{E 0}$

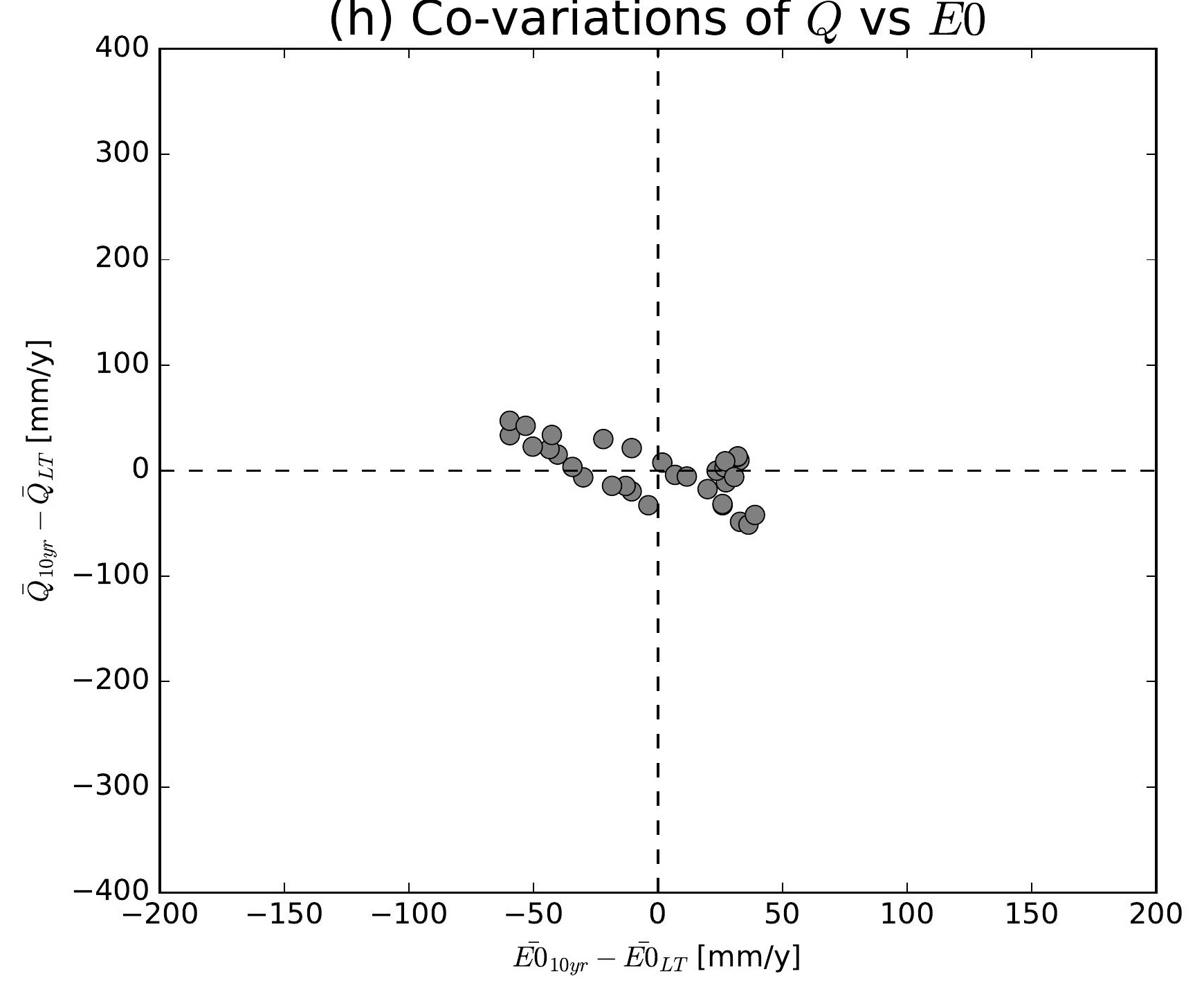

(k) Co-variations of $\bar{Q}$ vs $\overline{E 0}$

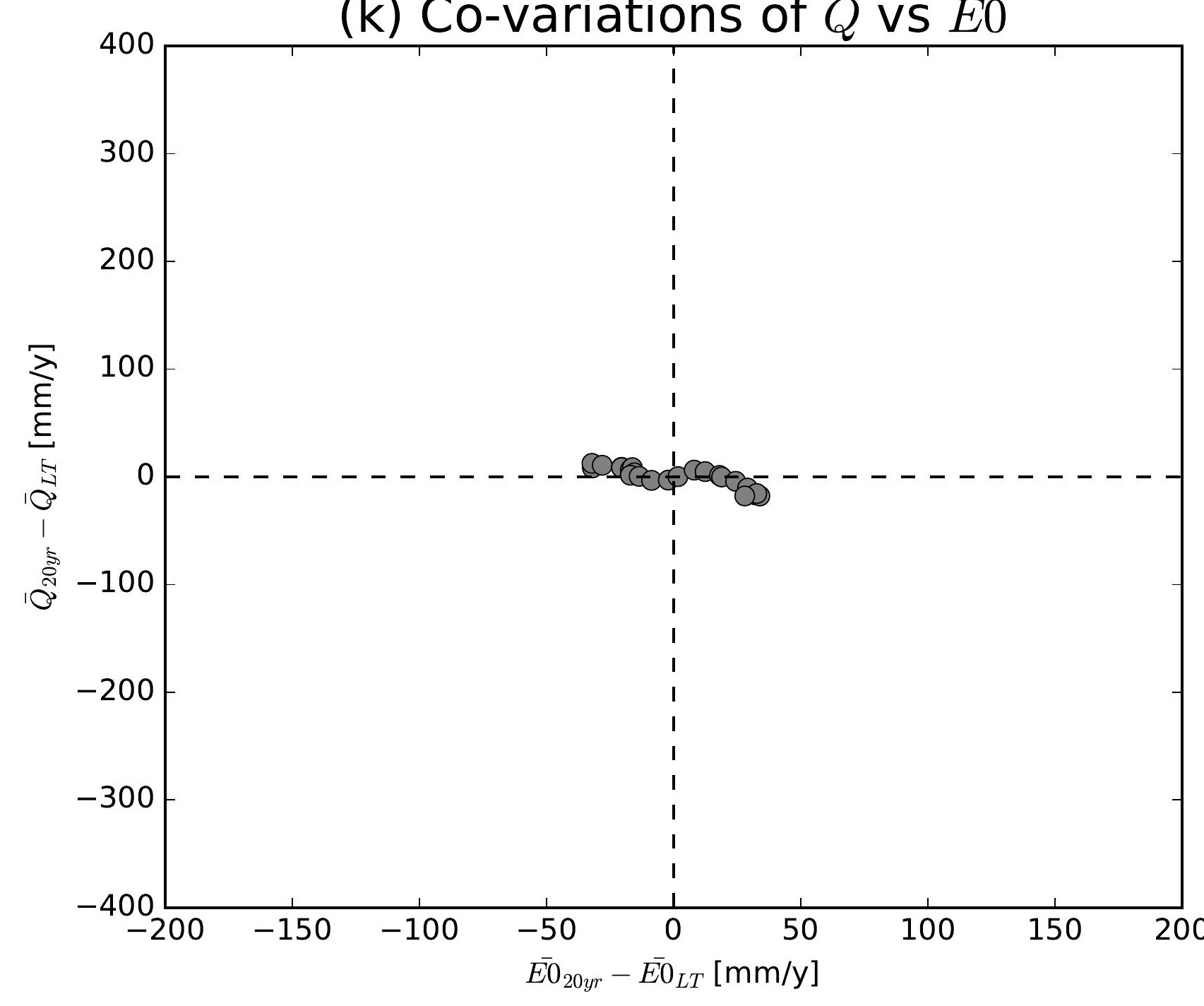

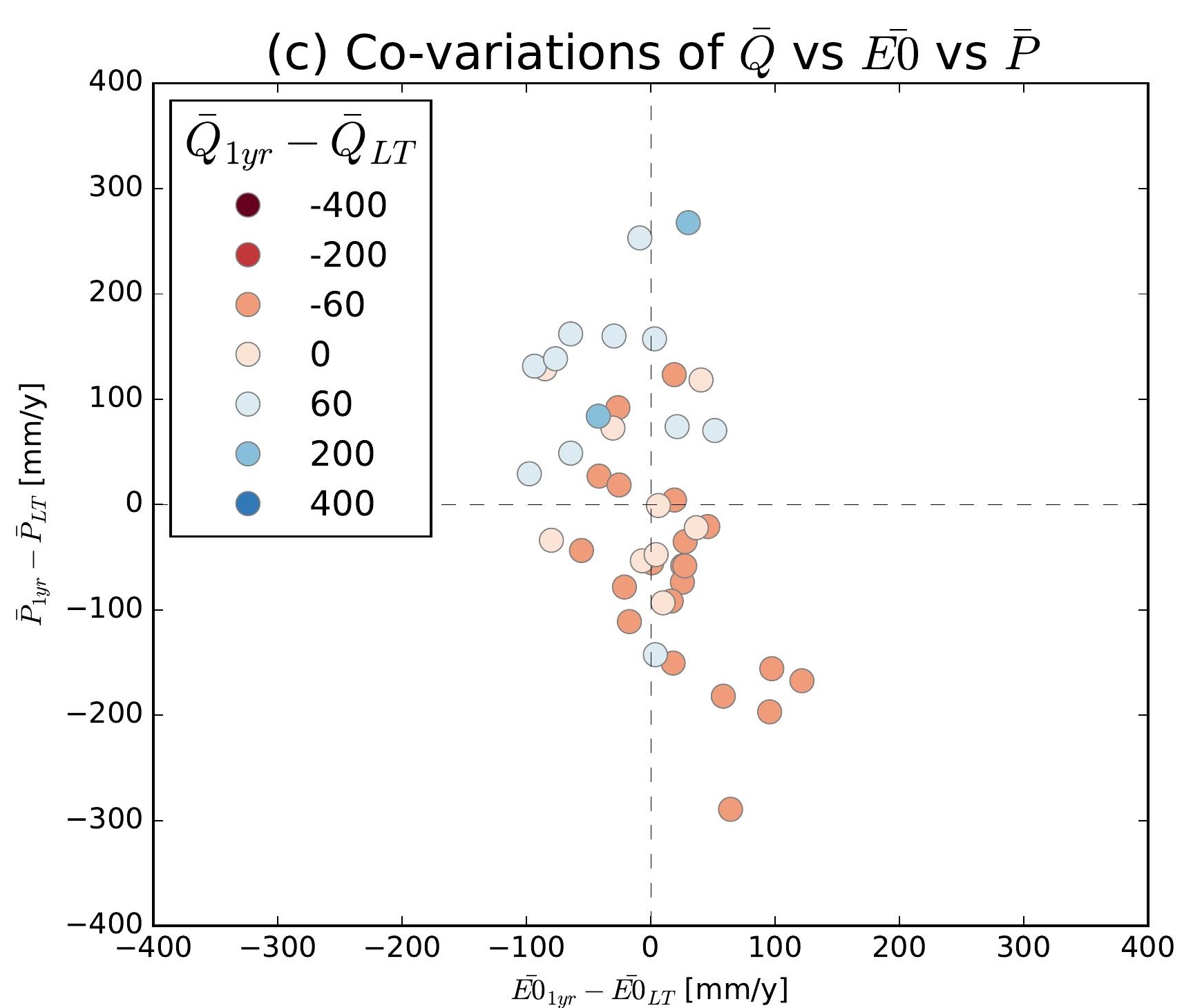
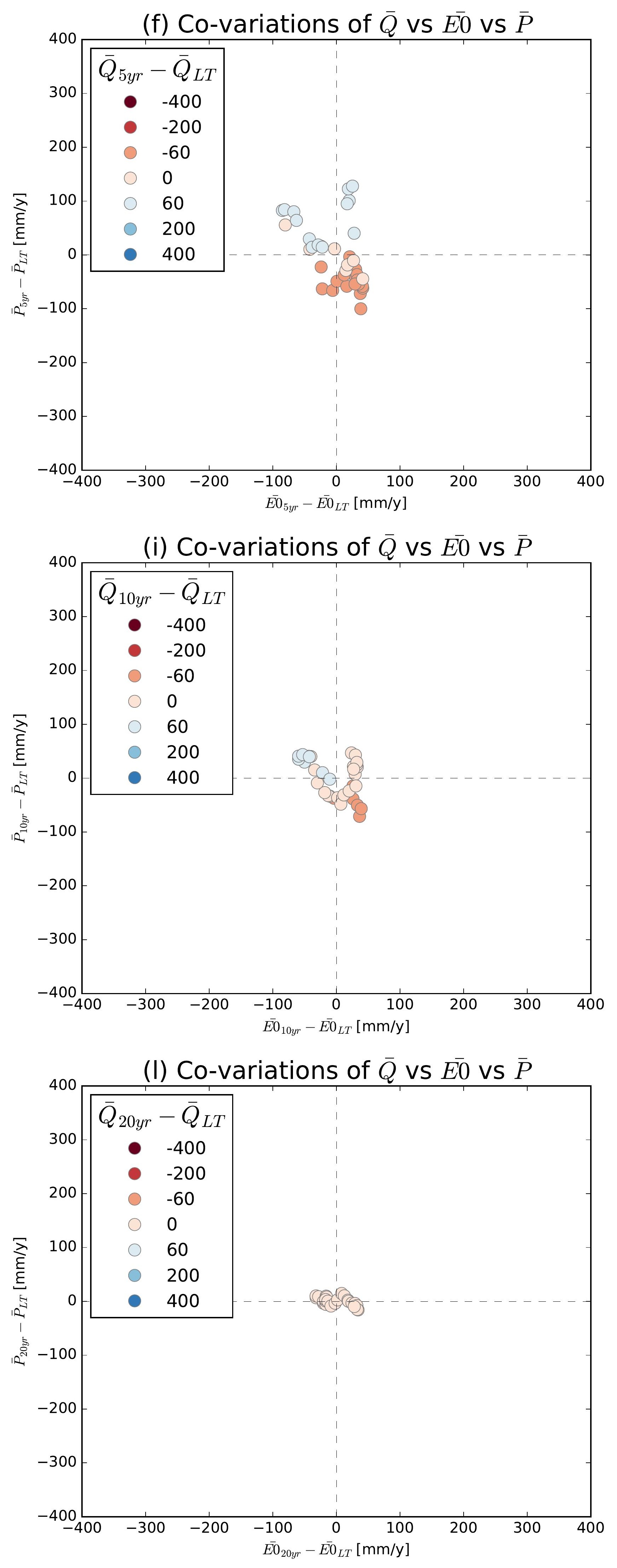

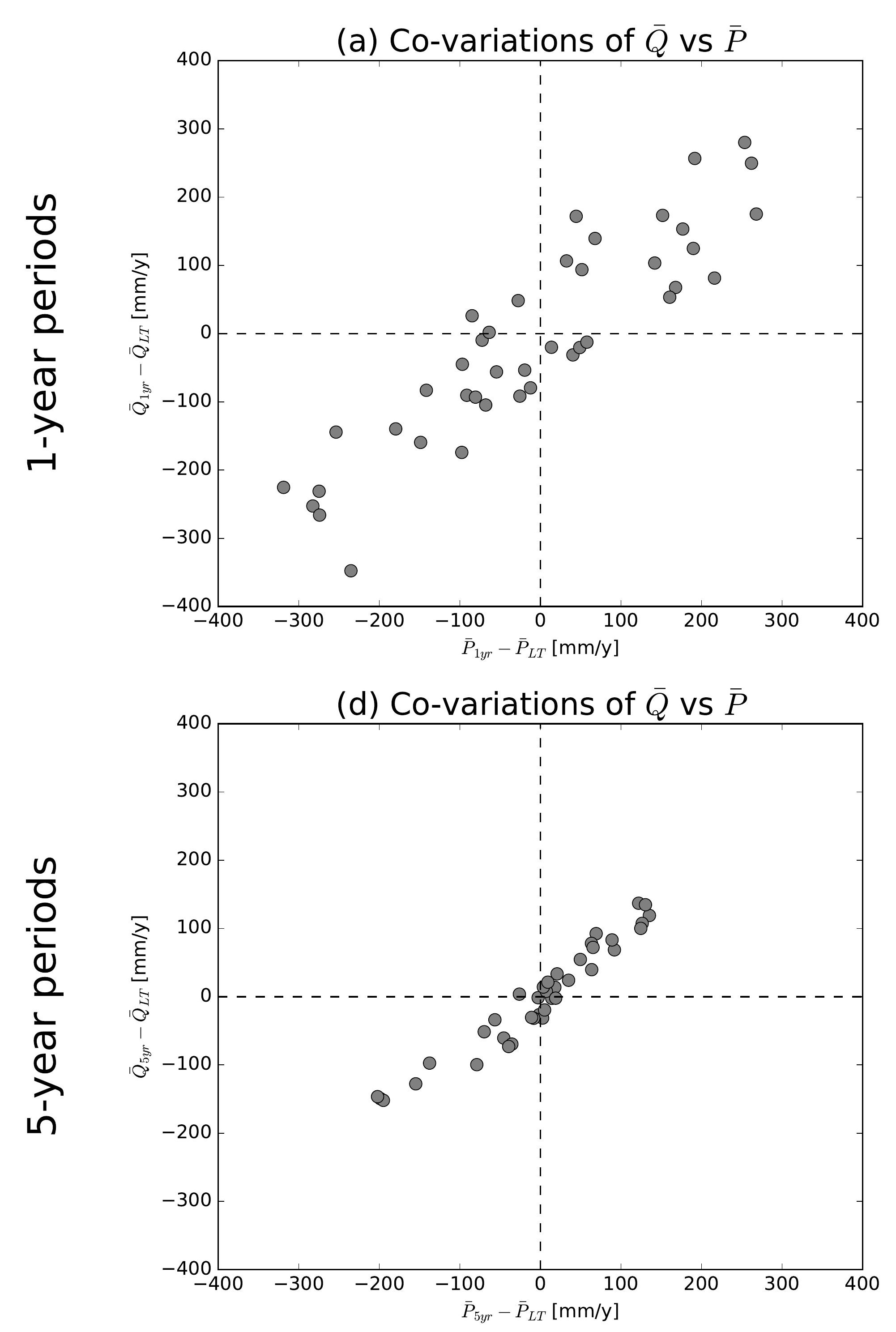

(g) Co-variations of $\bar{Q}$ vs $\bar{P}$
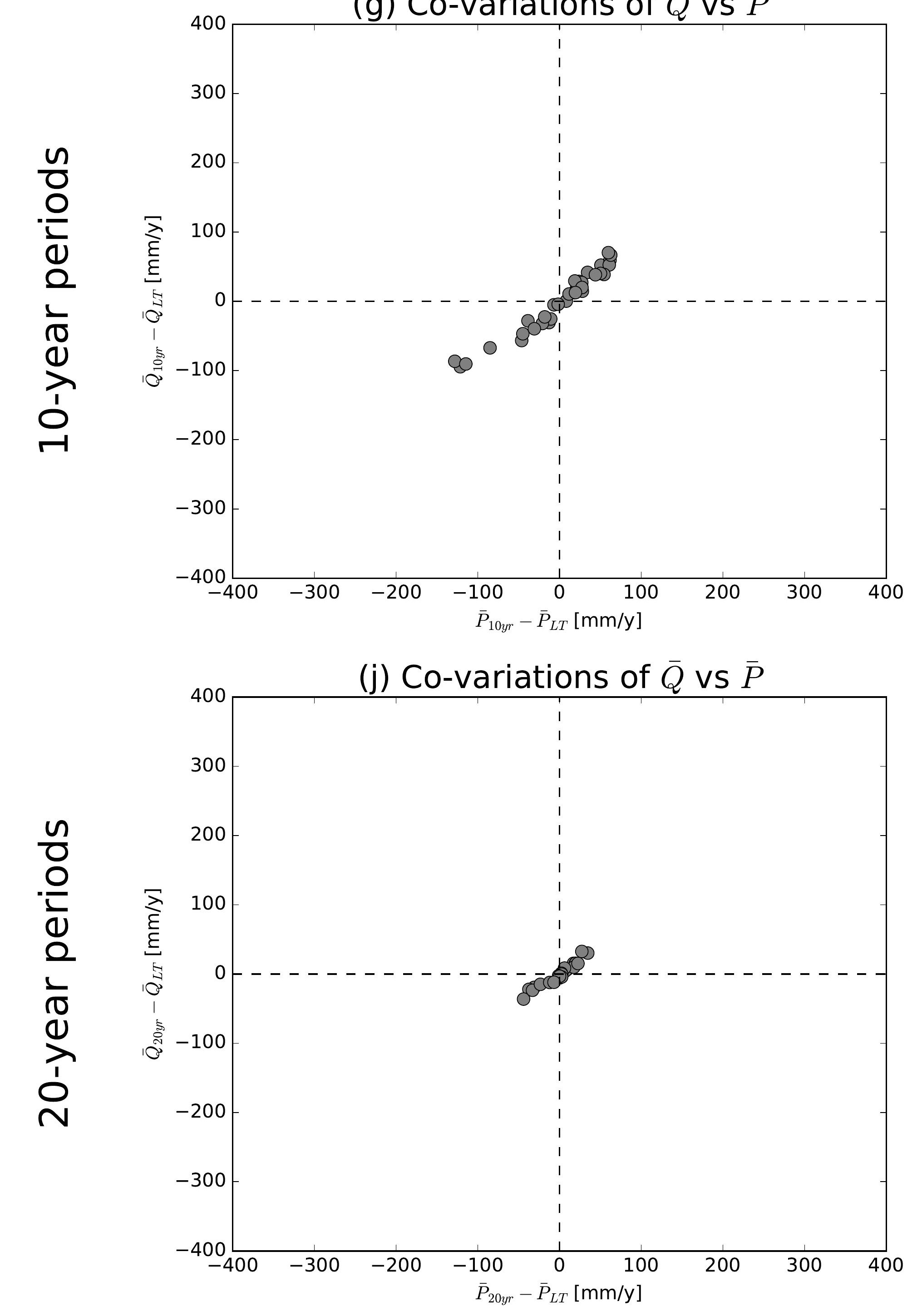

(b) Co-variations of $\bar{Q}$ vs $\overline{E 0}$

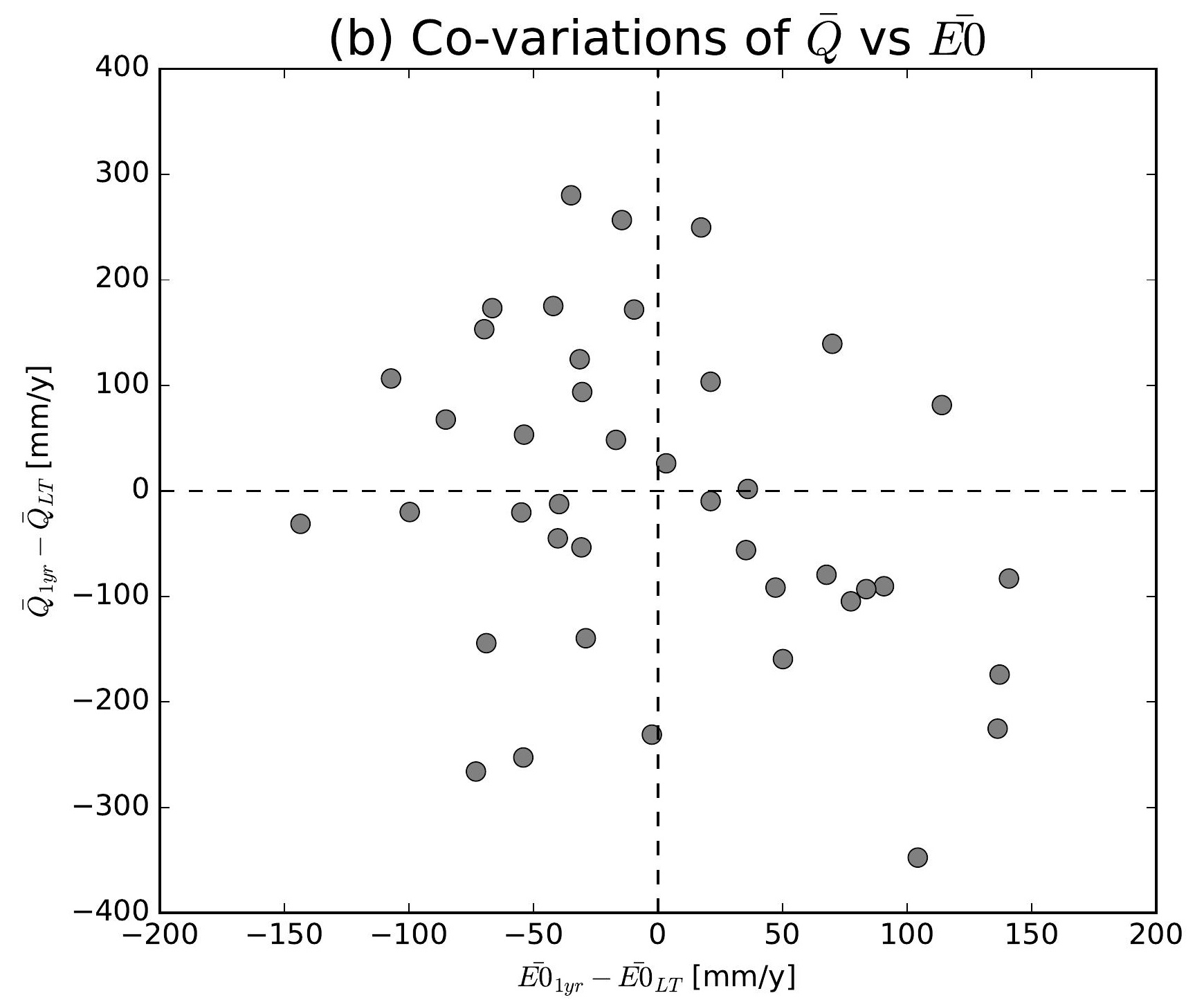

(e) Co-variations of $\bar{Q}$ vs $\overline{E 0}$

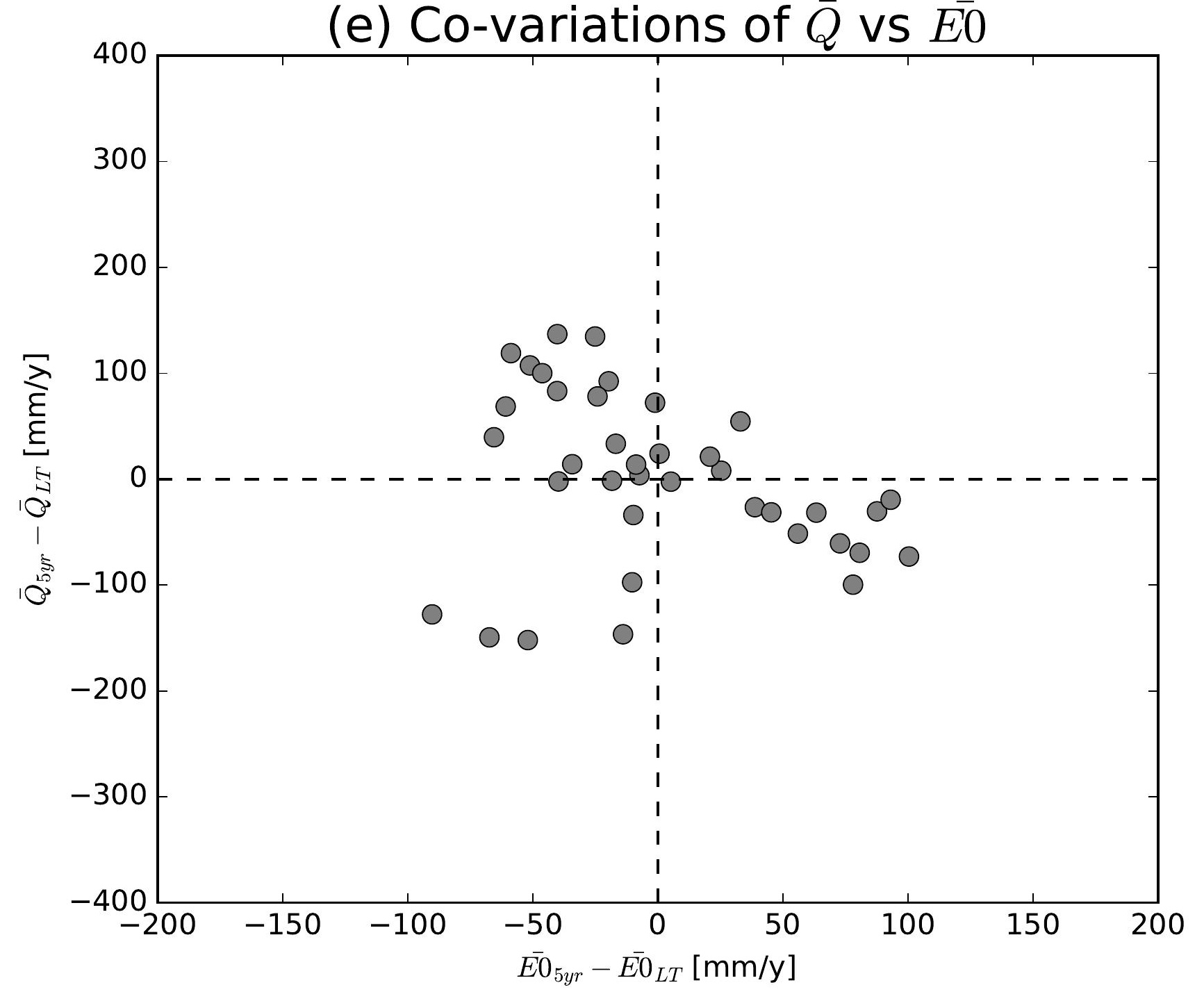

(h) Co-variations of $\bar{Q}$ vs $\overline{E 0}$

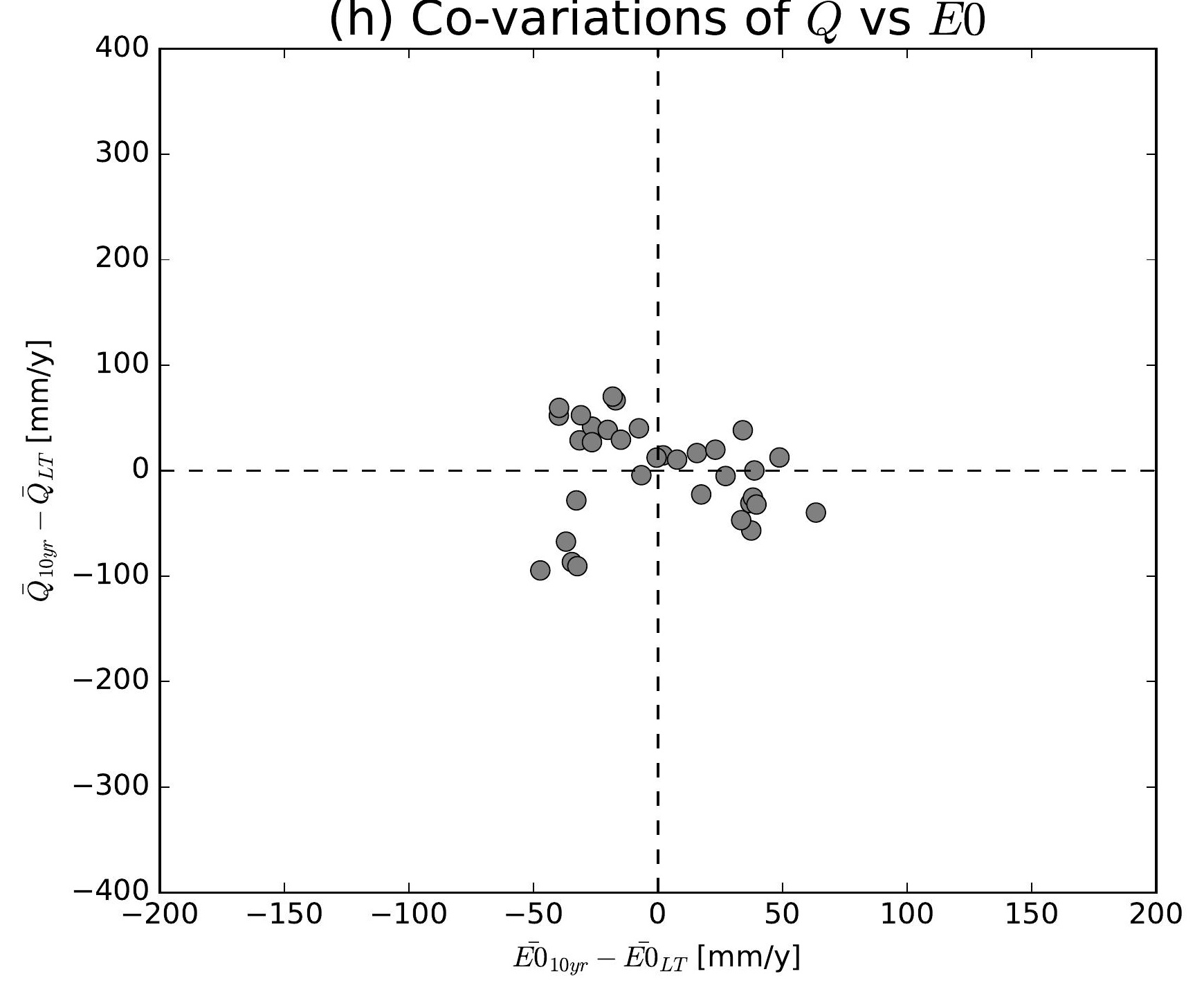

(k) Co-variations of $\bar{Q}$ vs $\overline{E 0}$

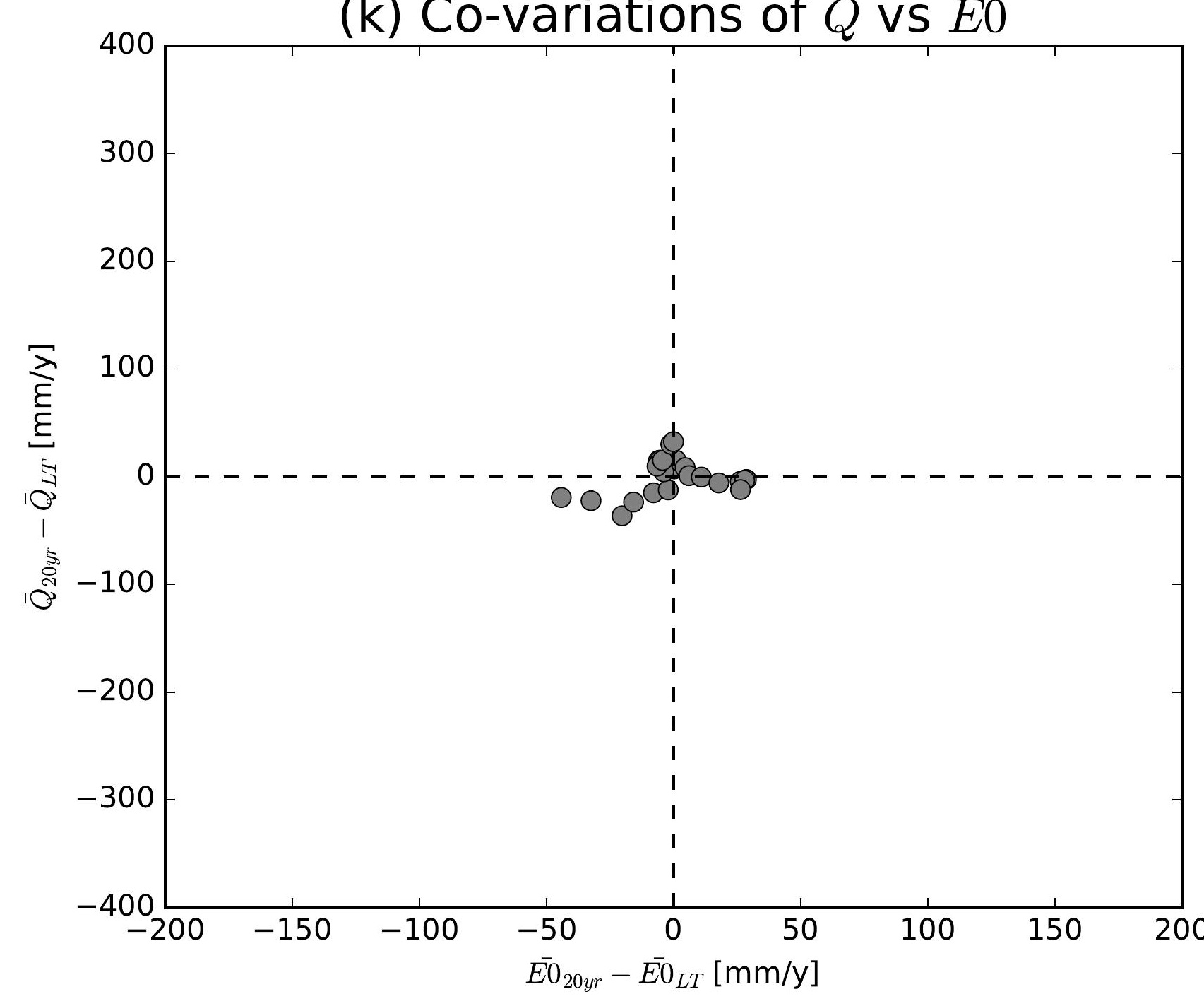

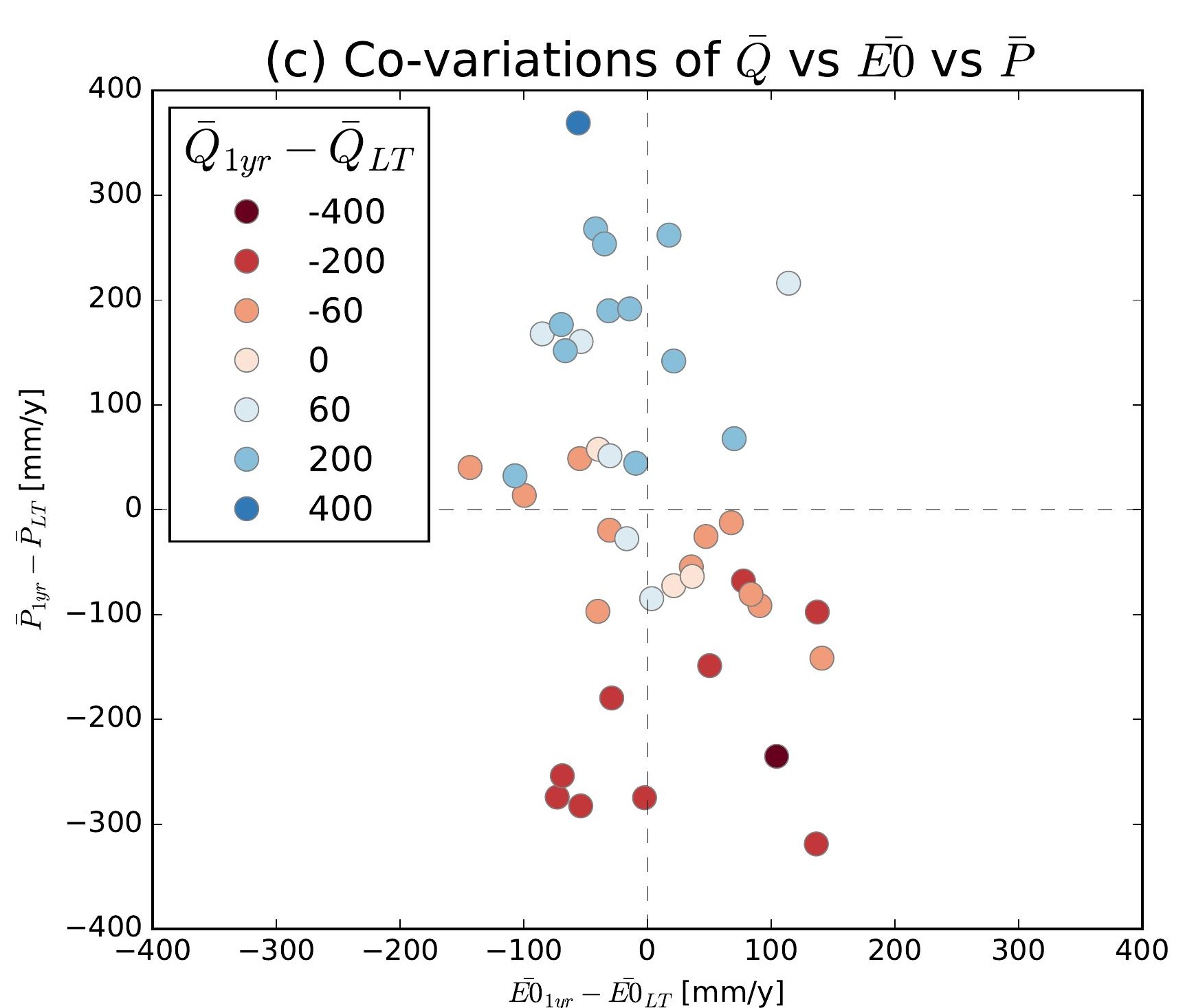
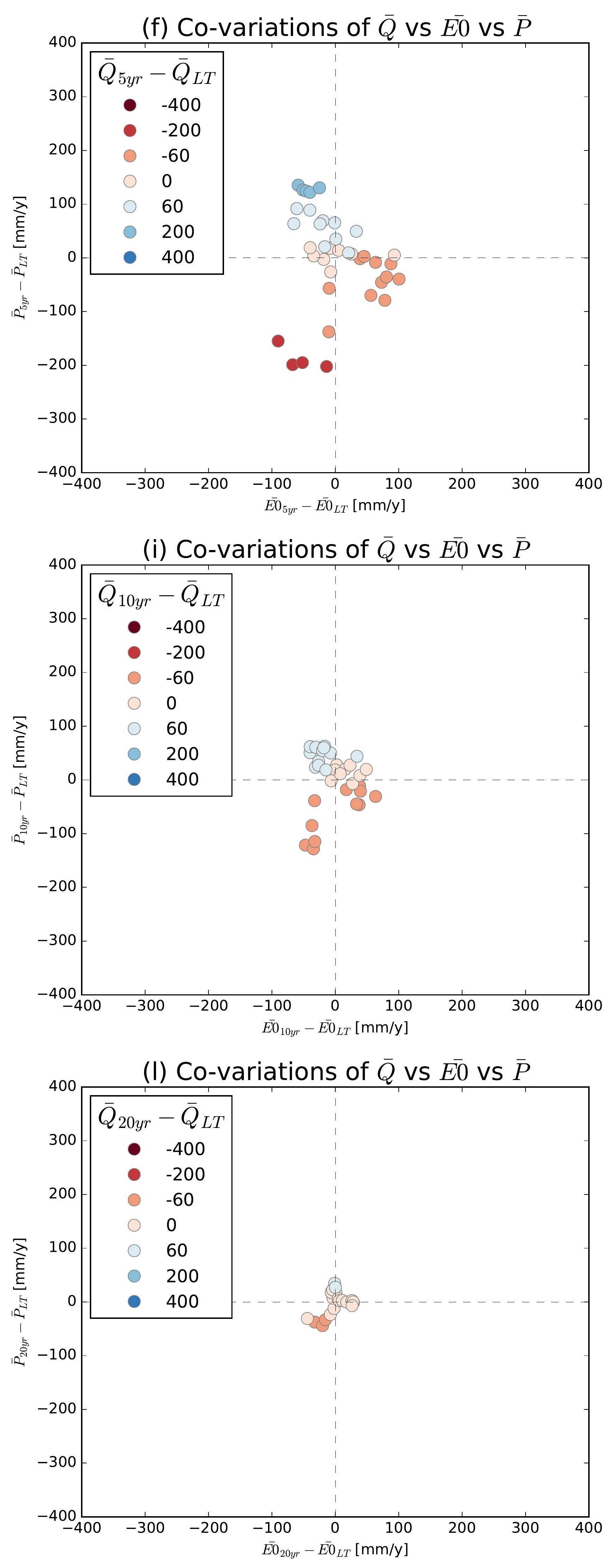

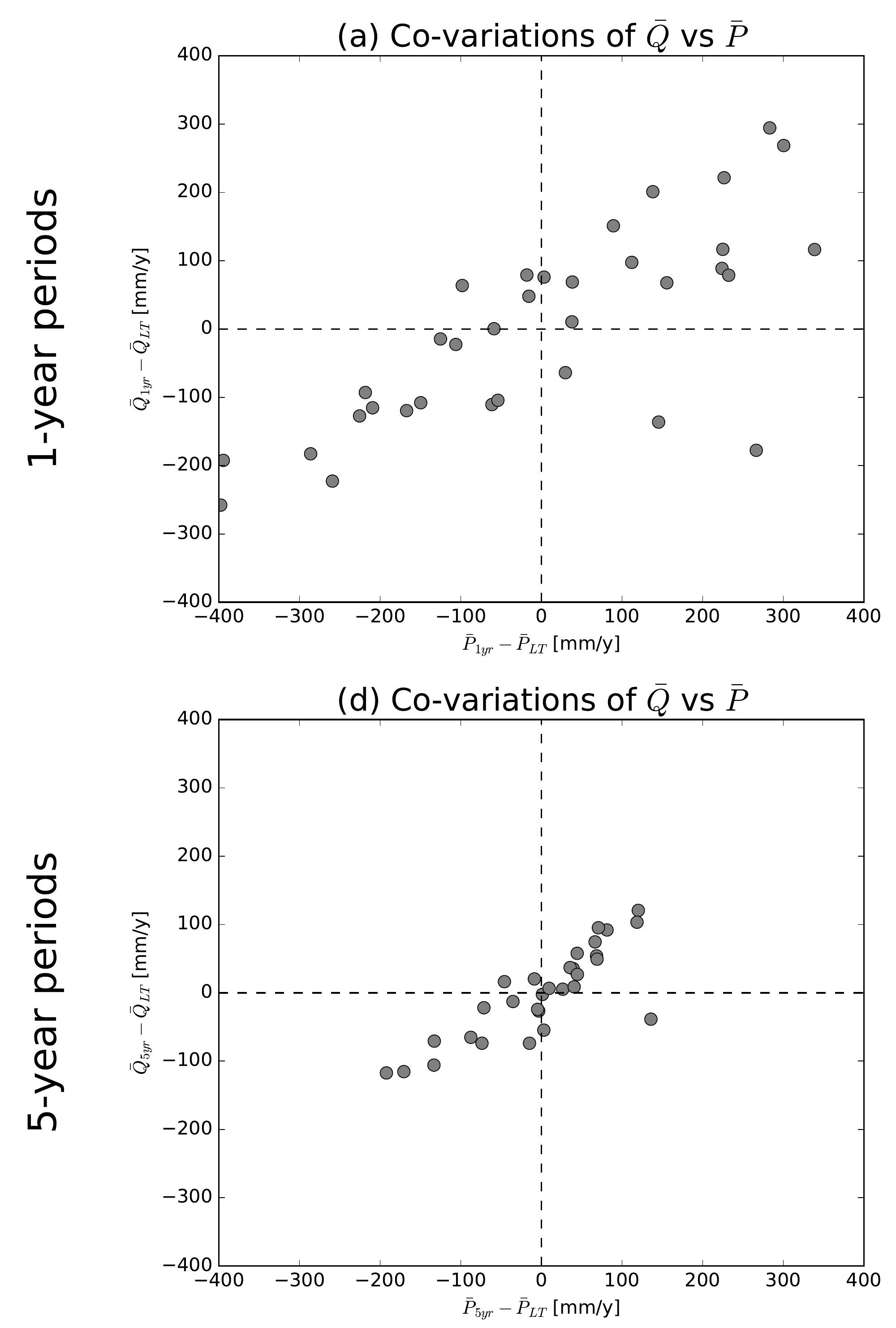

(g) Co-variations of $\bar{Q}$ vs $\bar{P}$

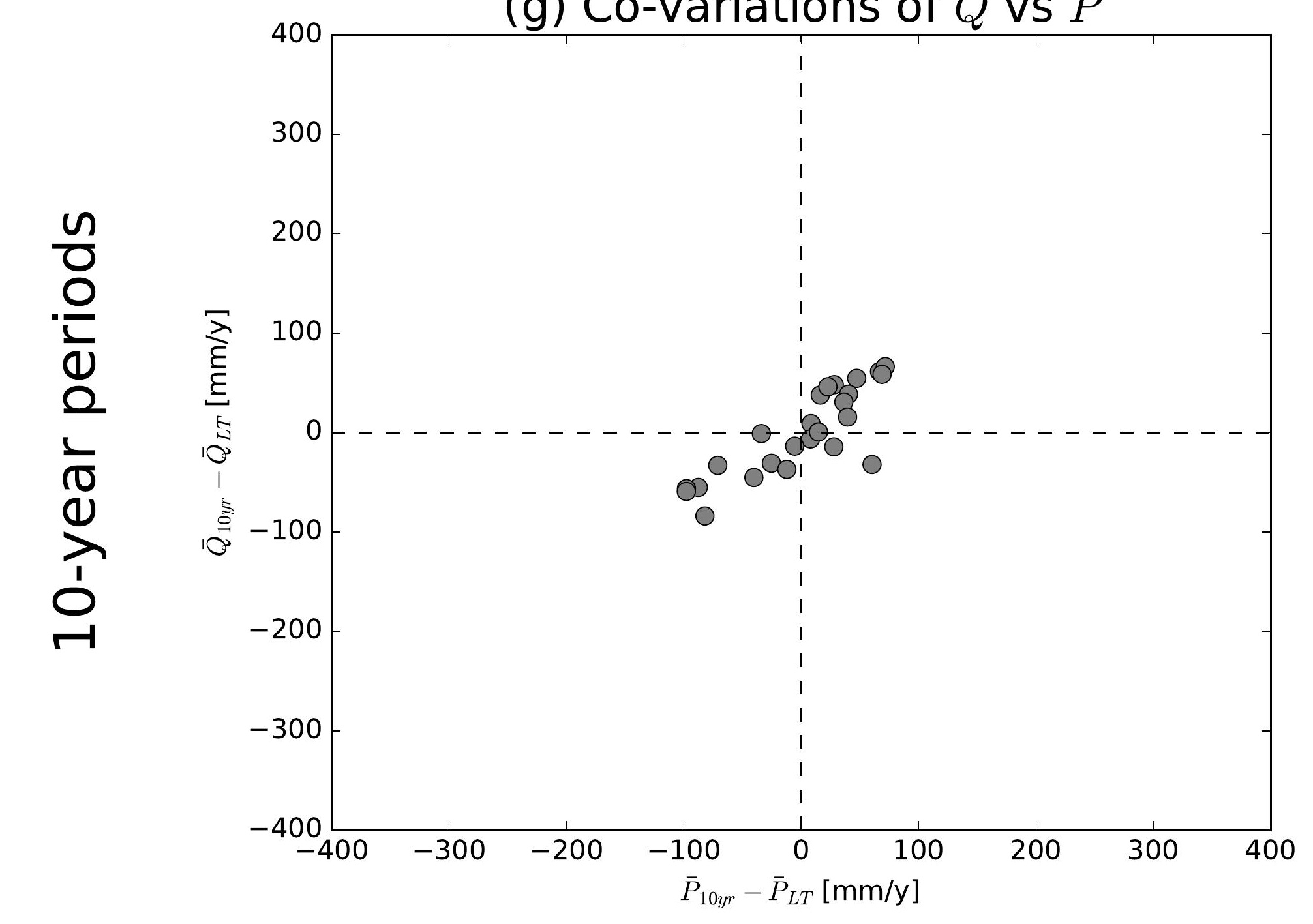

(j) Co-variations of $\bar{Q}$ vs $\bar{P}$

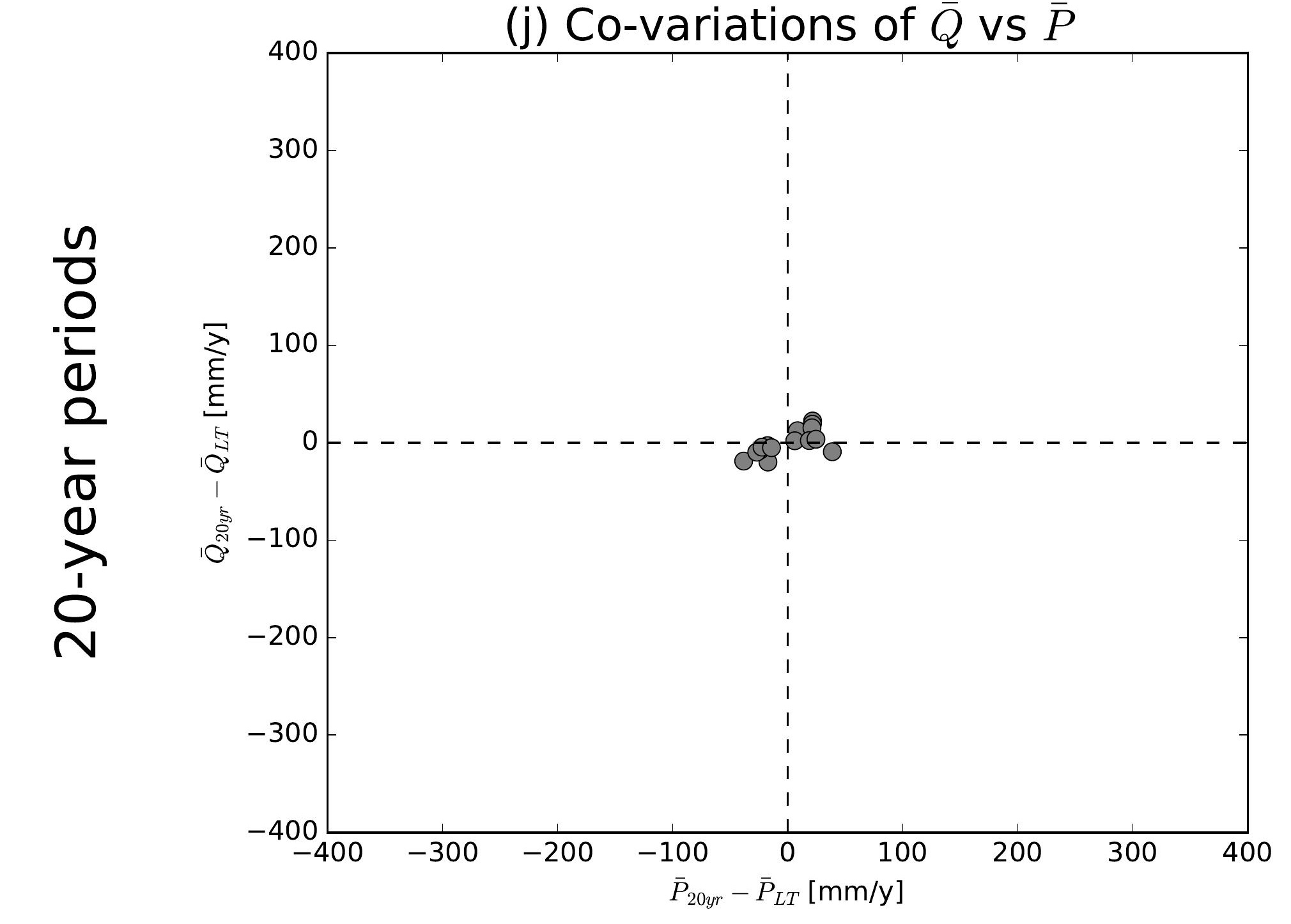

(b) Co-variations of $\bar{Q}$ vs $\overline{E 0}$

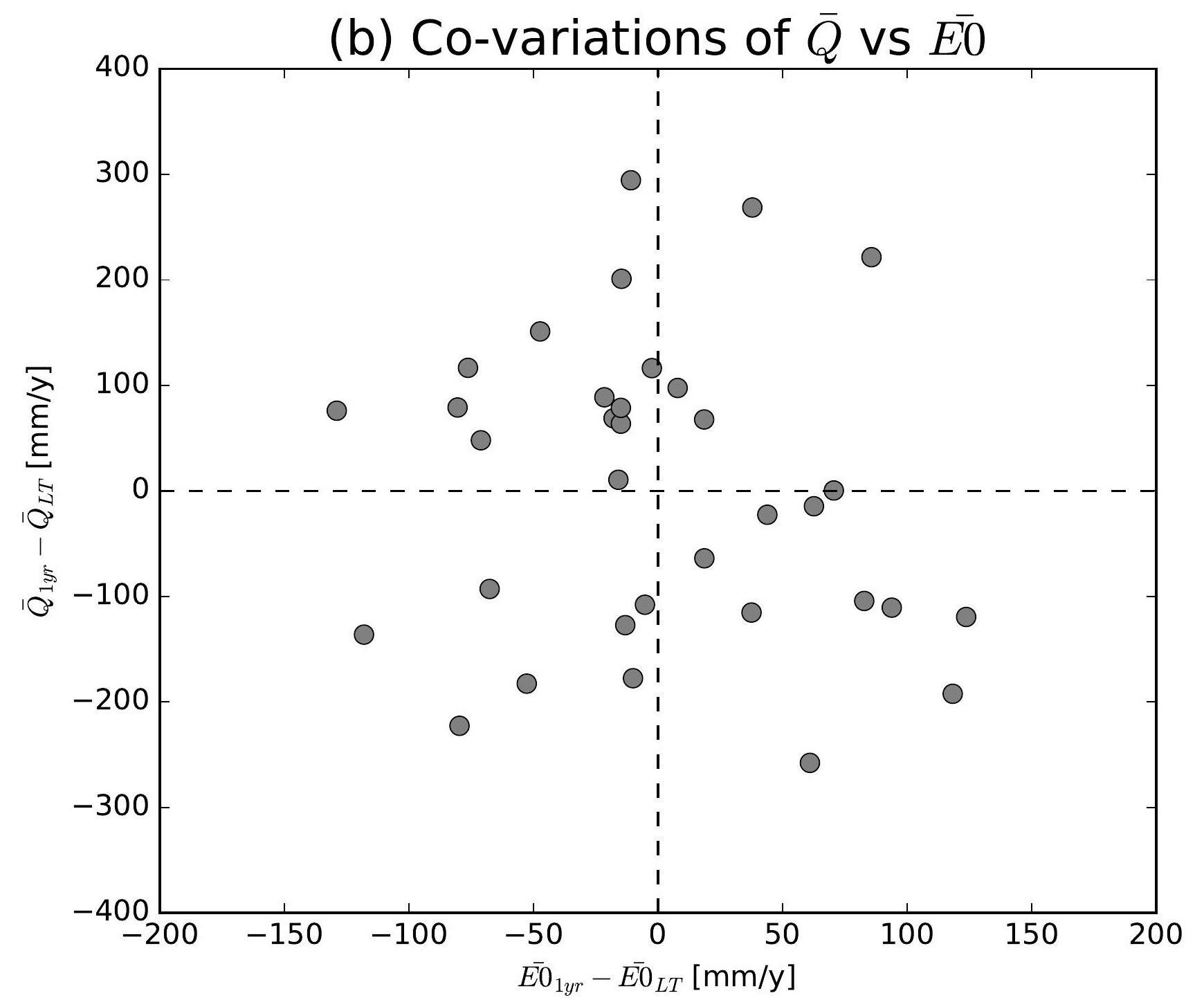

(e) Co-variations of $\bar{Q}$ vs $\overline{E 0}$

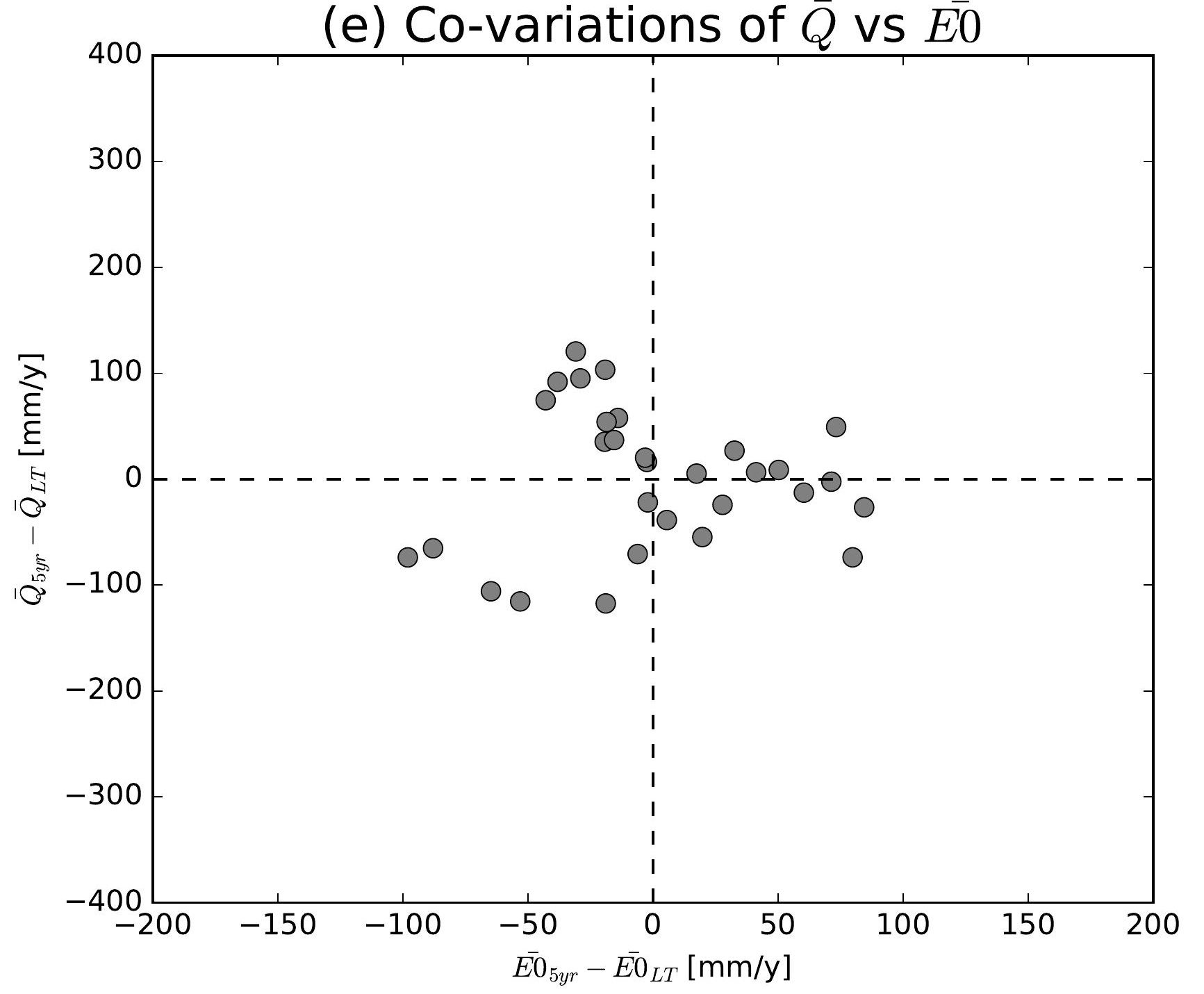

(h) Co-variations of $\bar{Q}$ vs $\overline{E 0}$

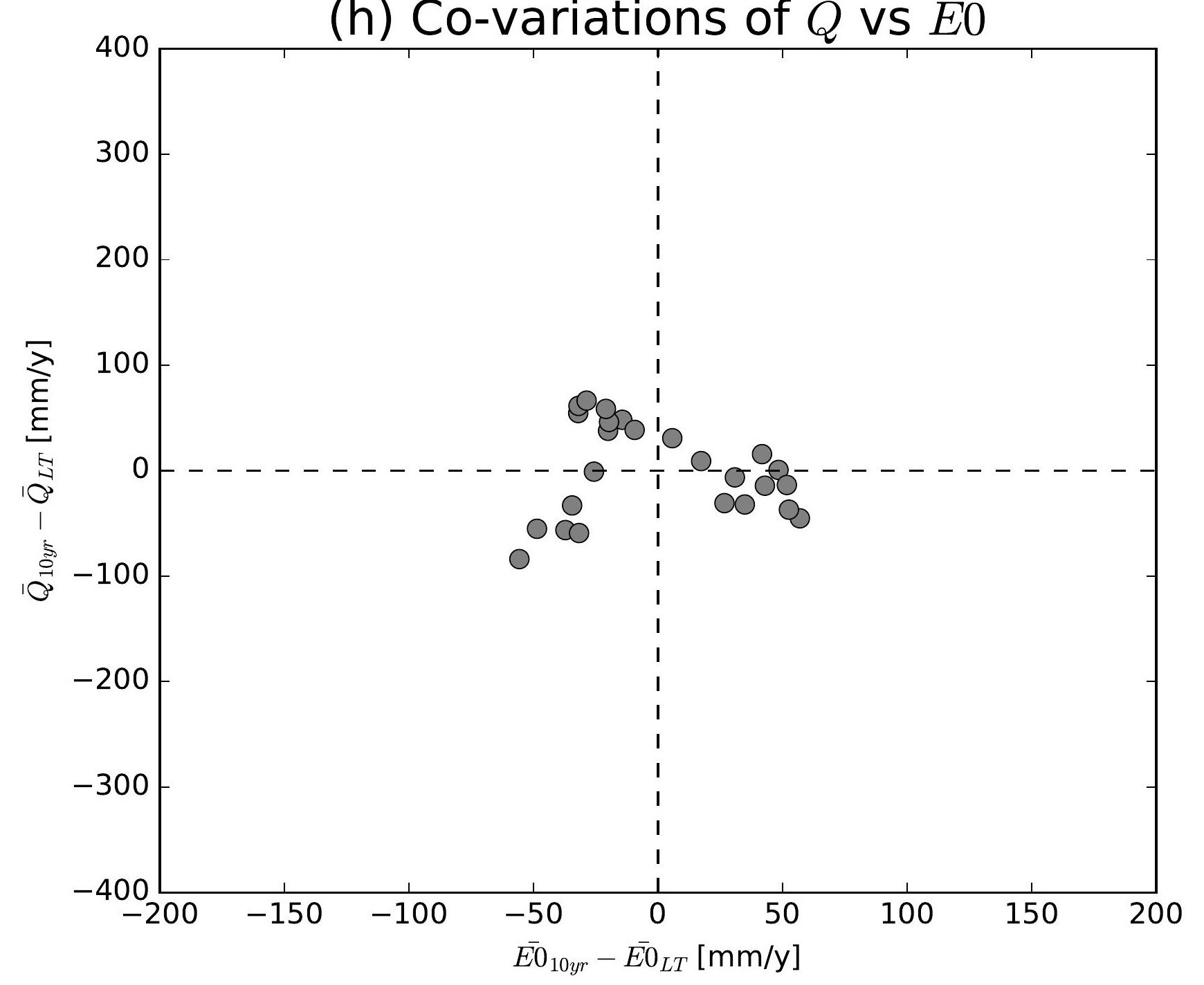

(k) Co-variations of $\bar{Q}$ vs $\overline{E 0}$

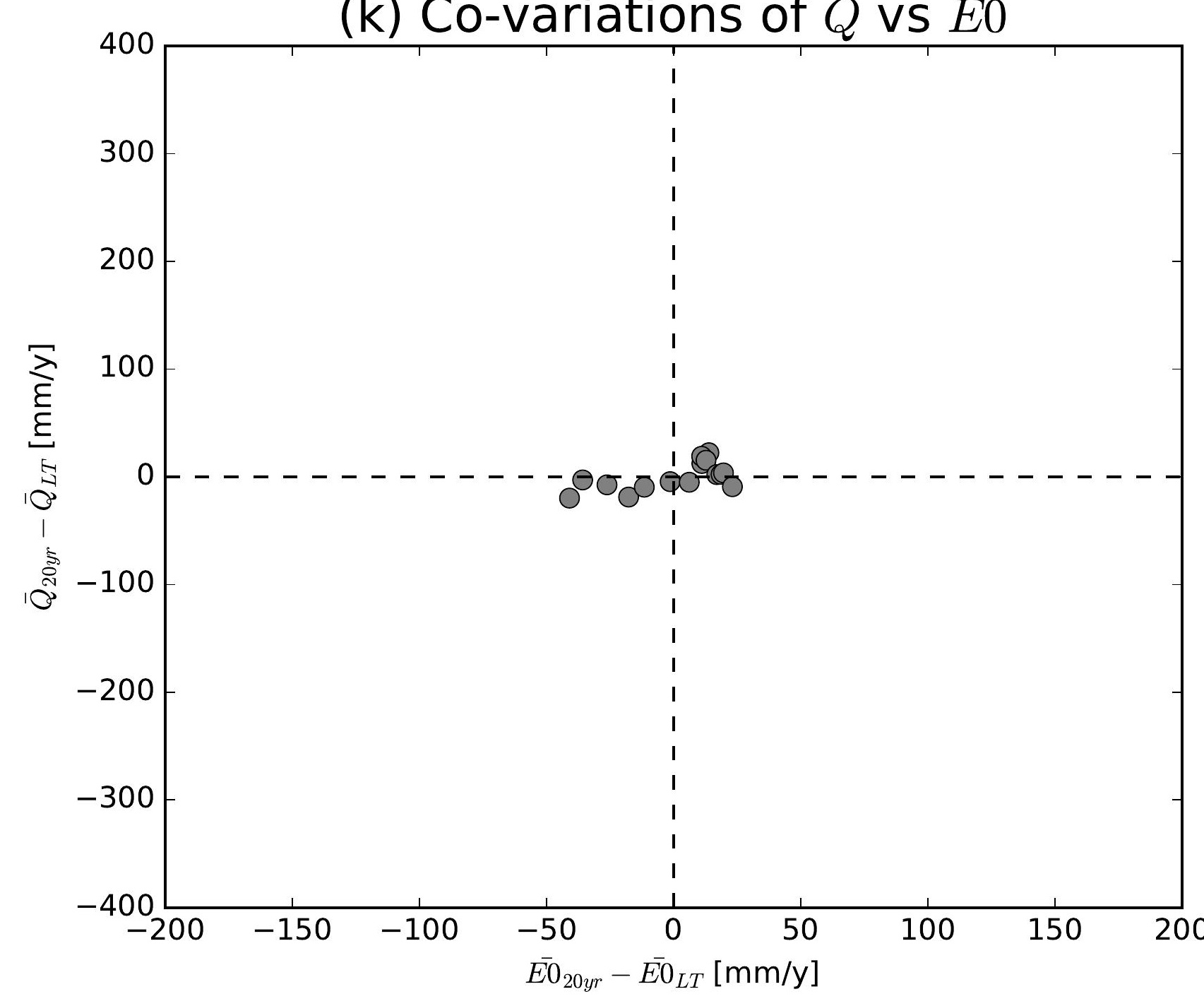

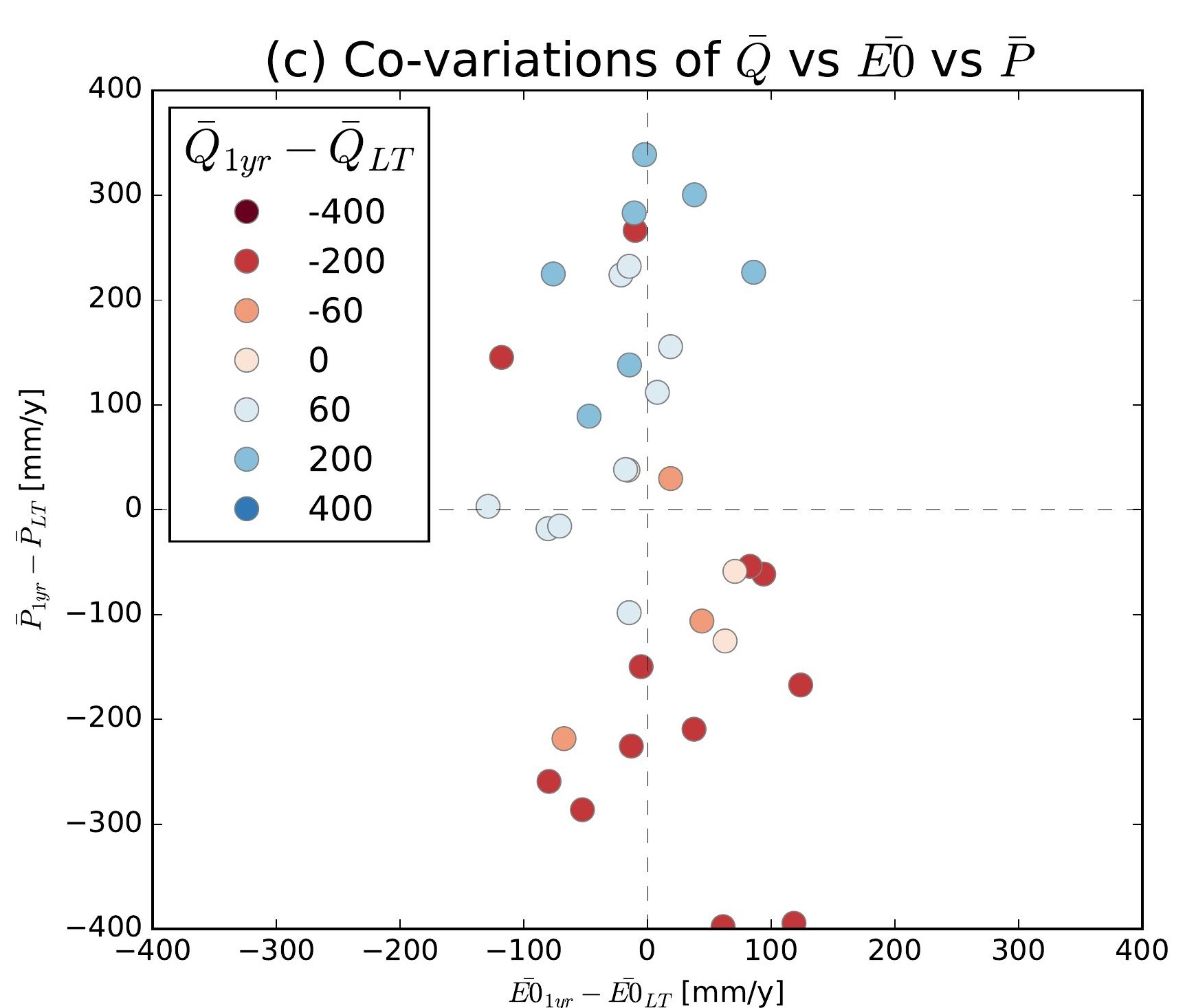
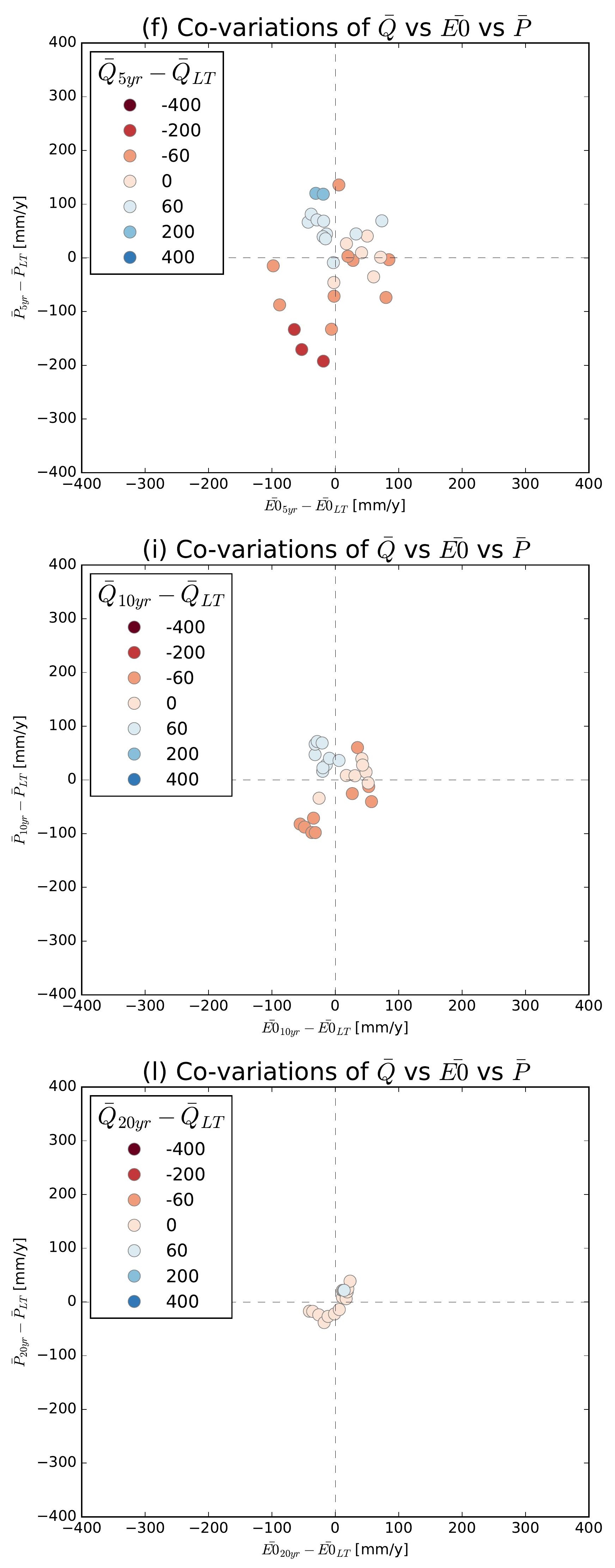

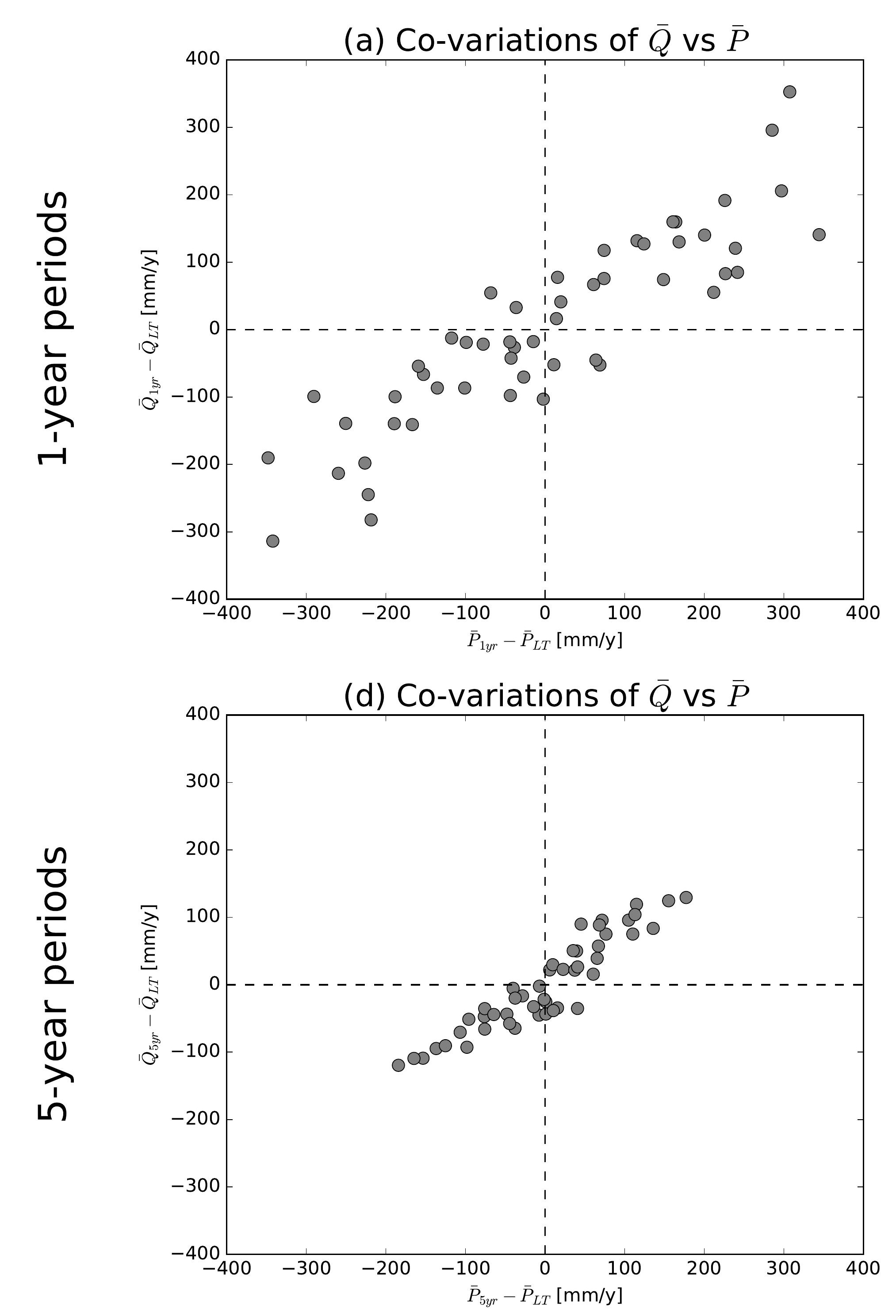

(g) Co-variations of $\bar{Q}$ vs $\bar{P}$
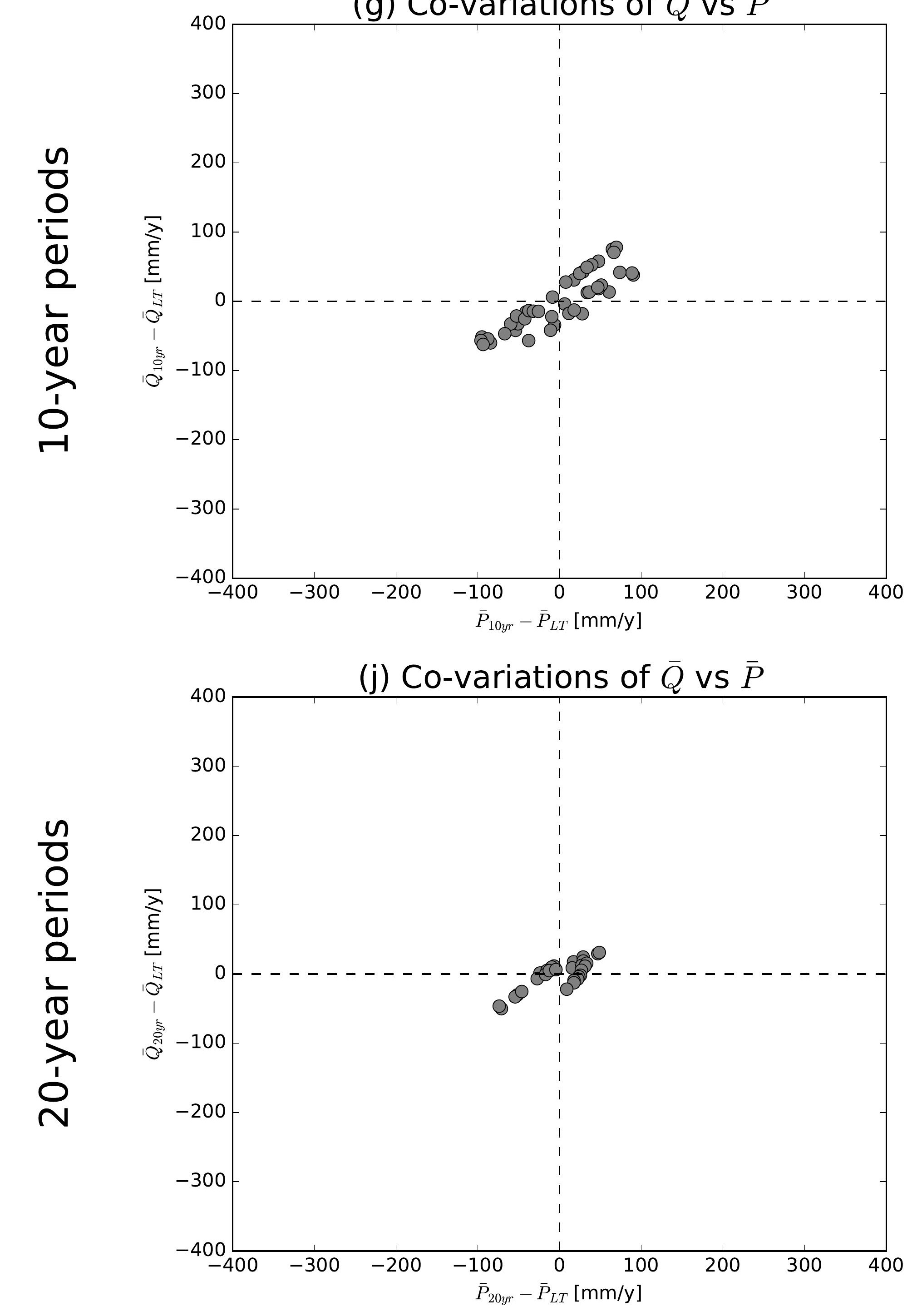

(b) Co-variations of $\bar{Q}$ vs $\overline{E 0}$

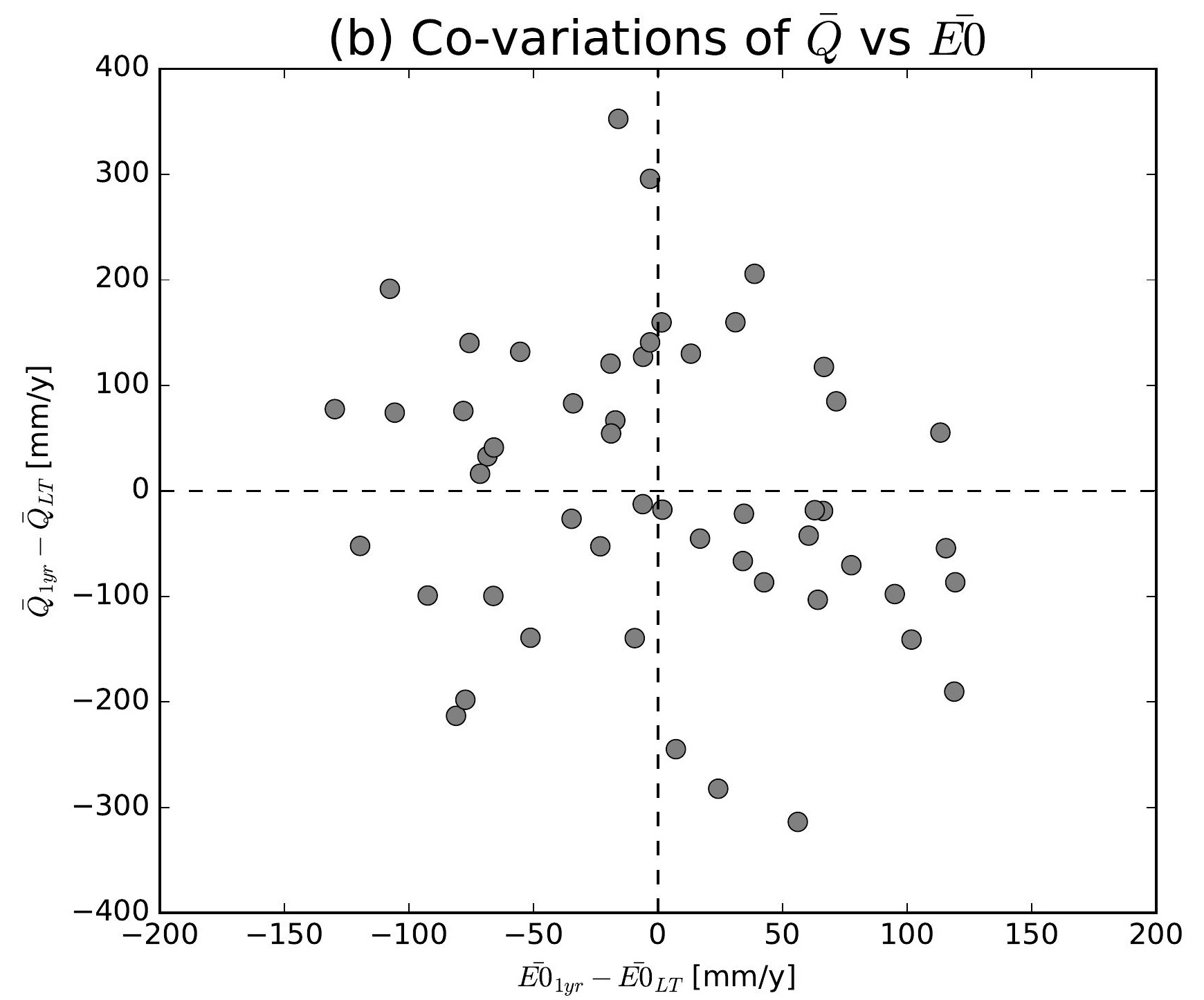

(e) Co-variations of $\bar{Q}$ vs $\overline{E 0}$

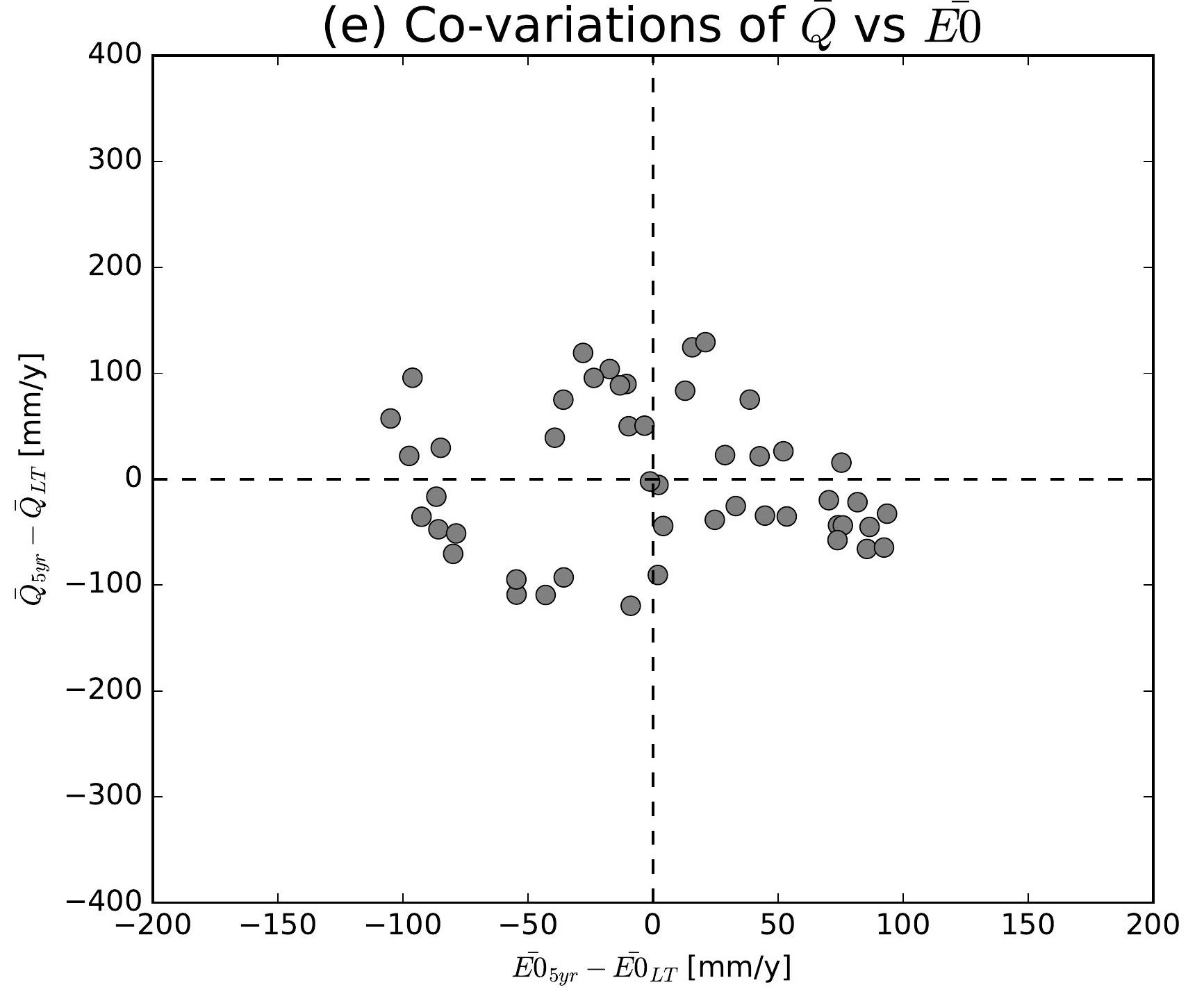

(h) Co-variations of $\bar{Q}$ vs $\overline{E 0}$

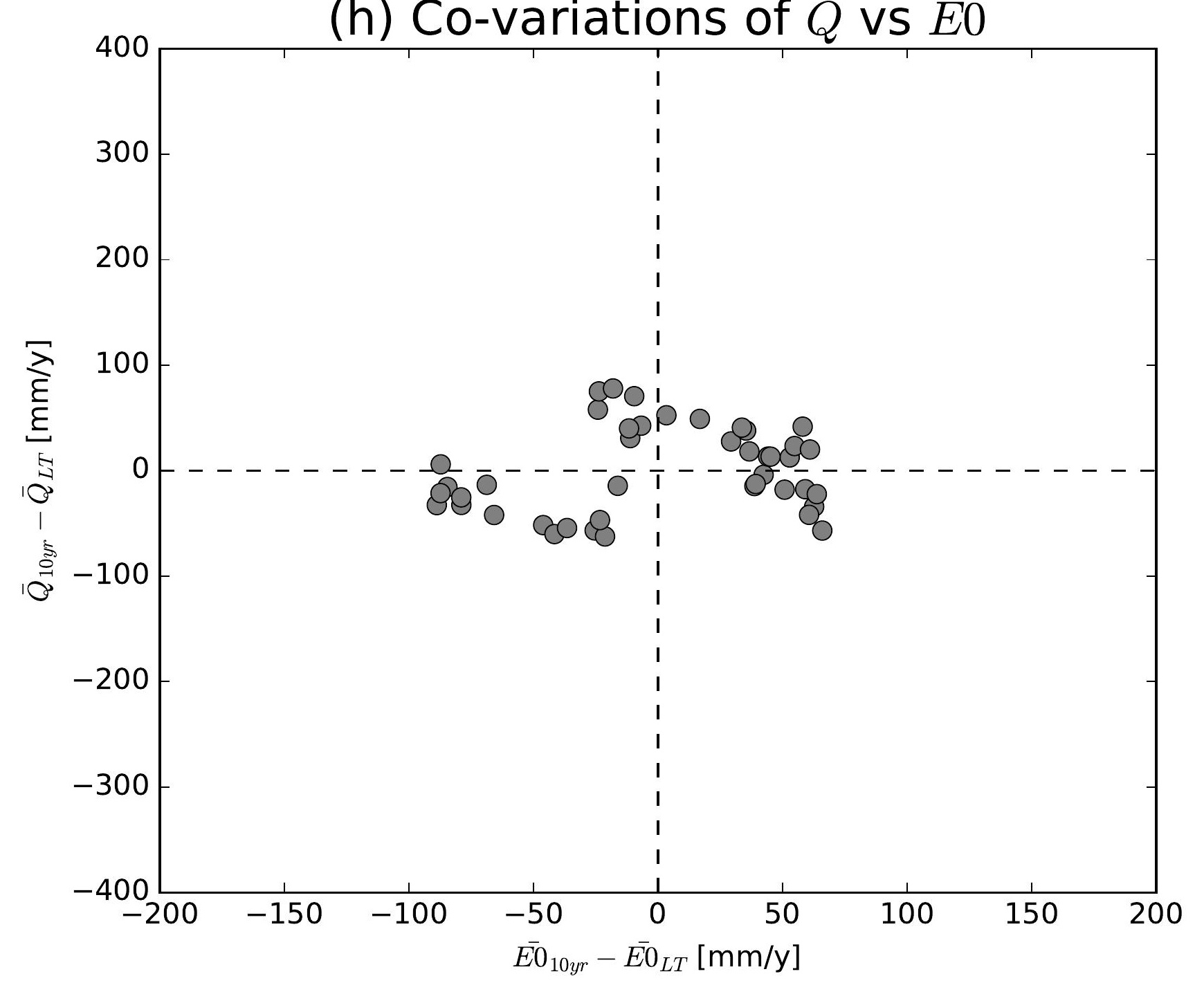

(k) Co-variations of $\bar{Q}$ vs $\overline{E 0}$

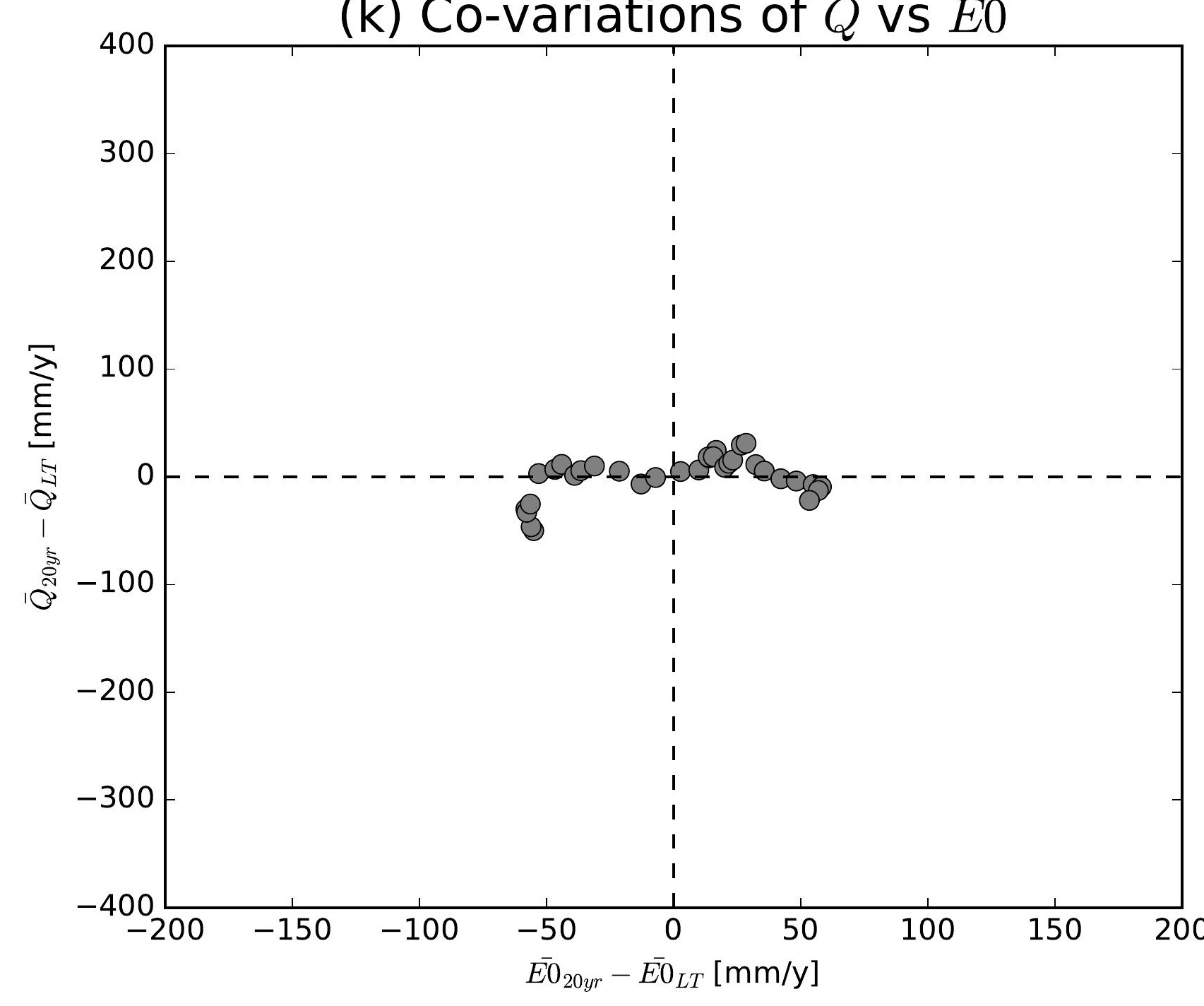

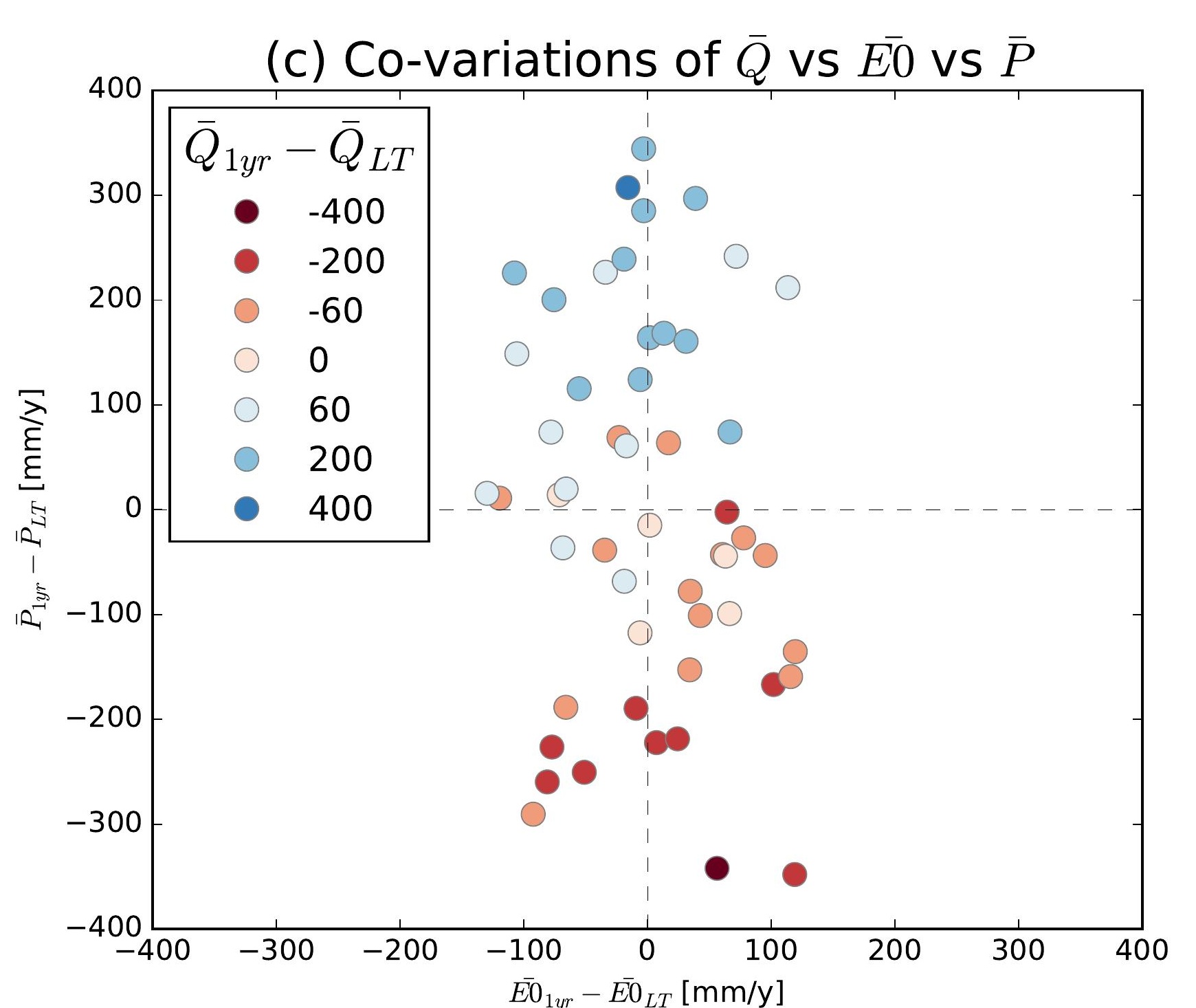
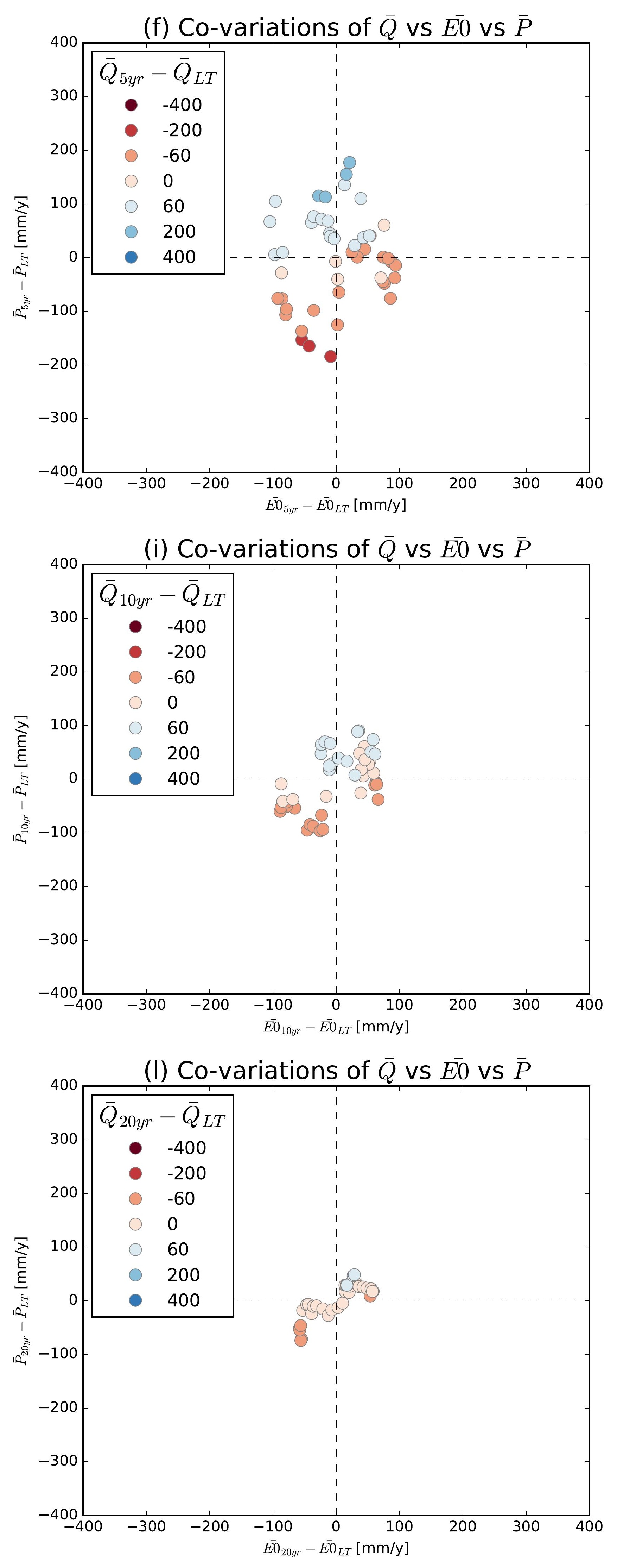

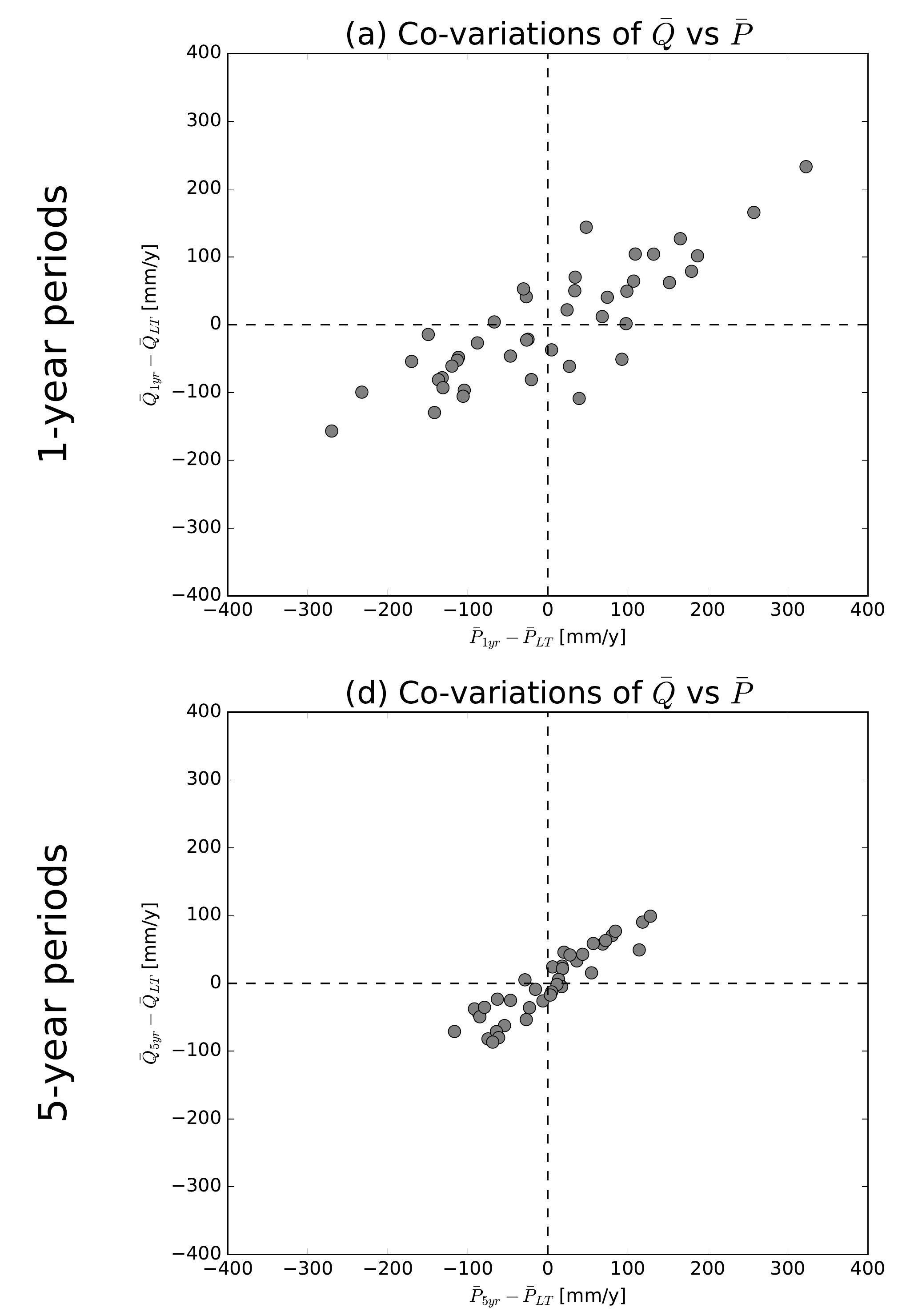

(g) Co-variations of $\bar{Q}$ vs $\bar{P}$
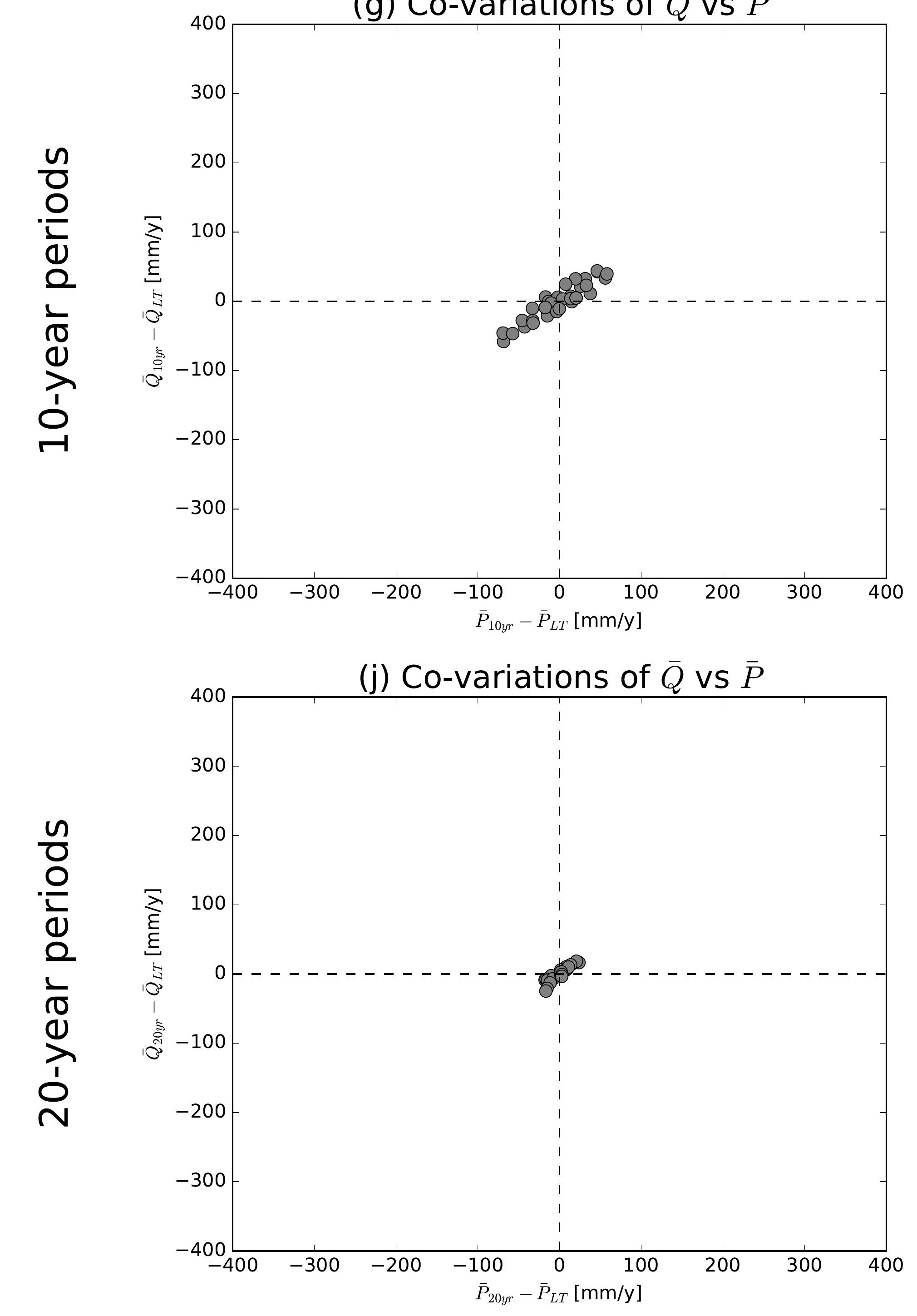

(b) Co-variations of $\bar{Q}$ vs $\overline{E 0}$

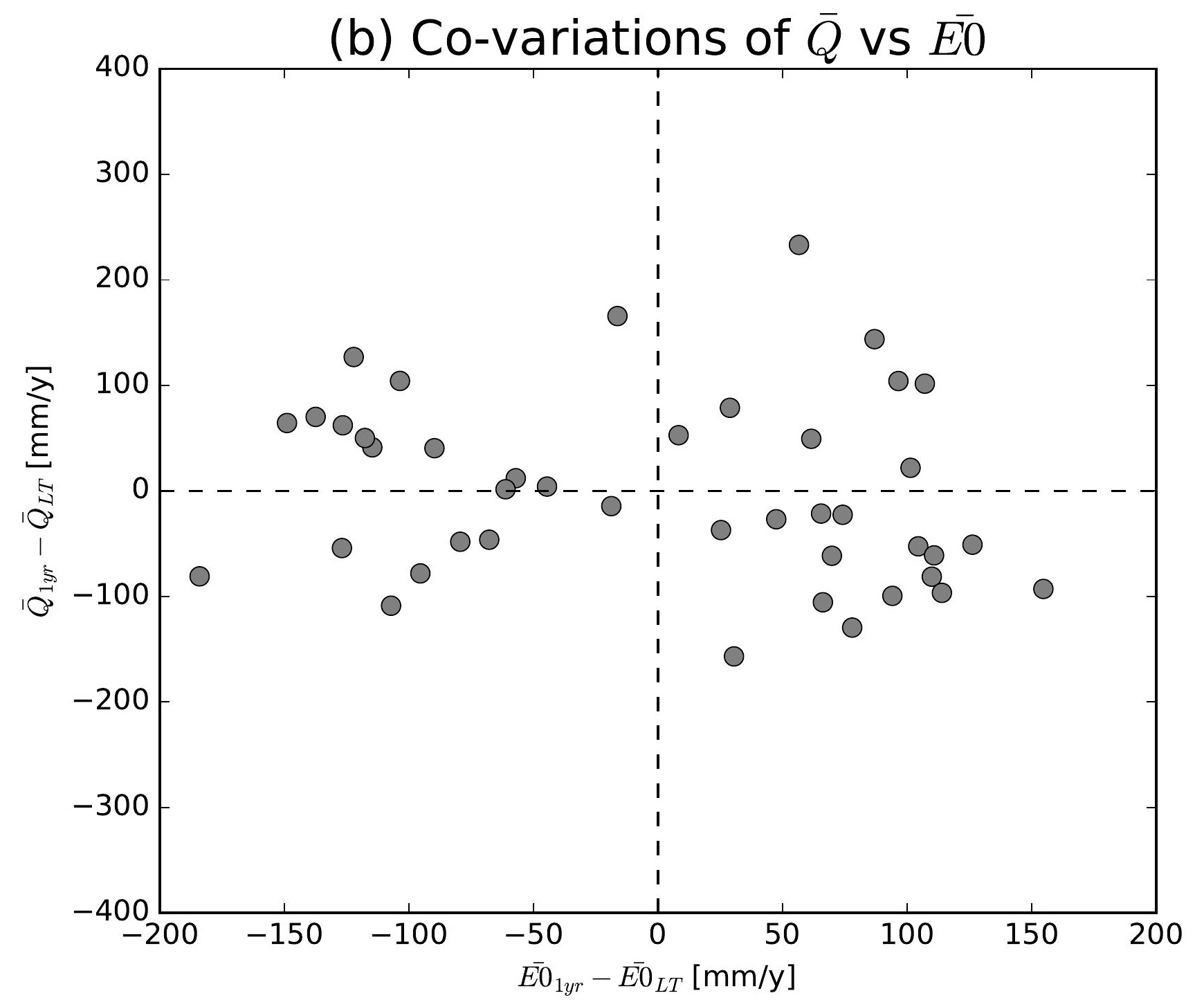

(e) Co-variations of $\bar{Q}$ vs $\overline{E 0}$

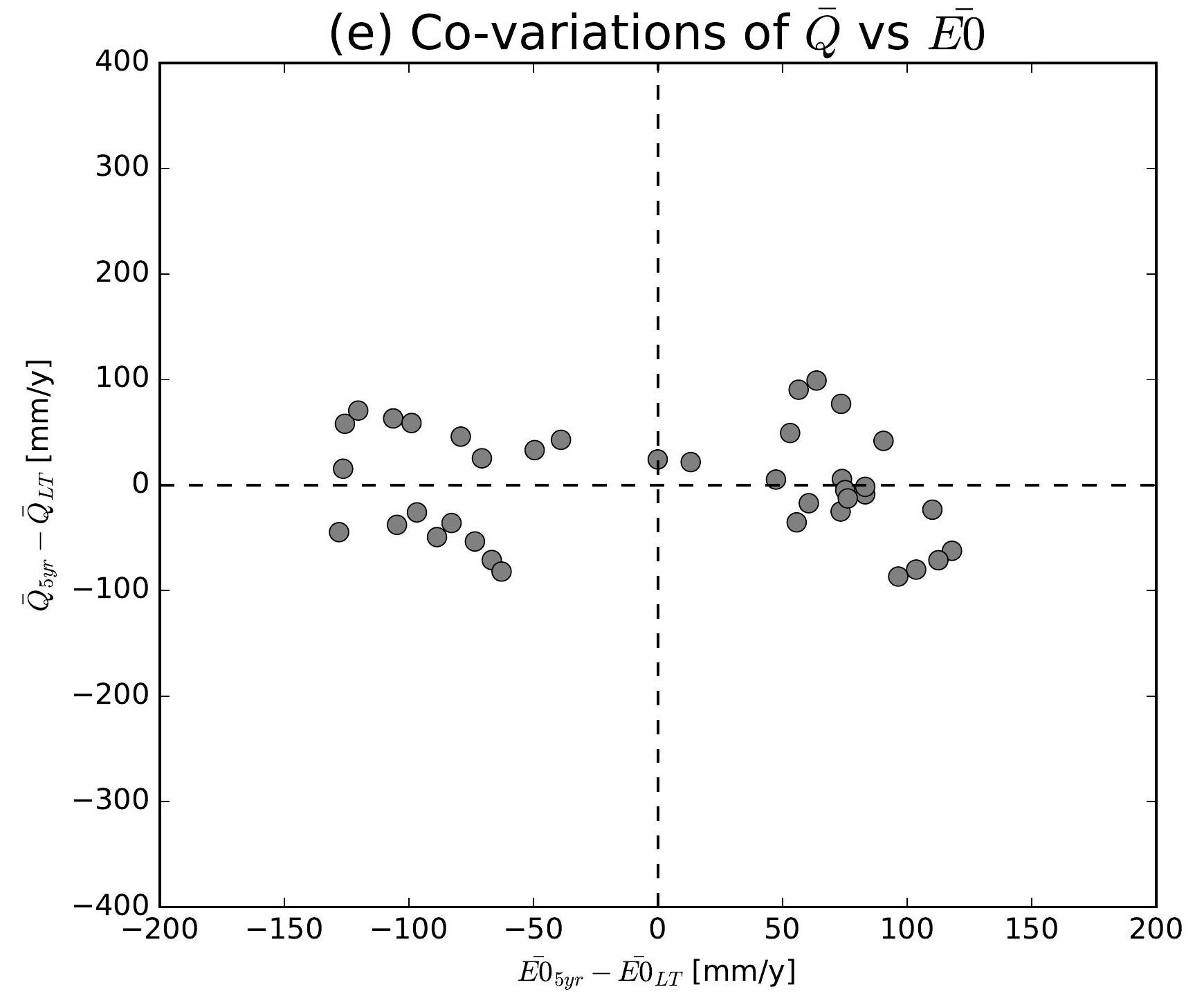

(h) Co-variations of $\bar{Q}$ vs $\overline{E 0}$

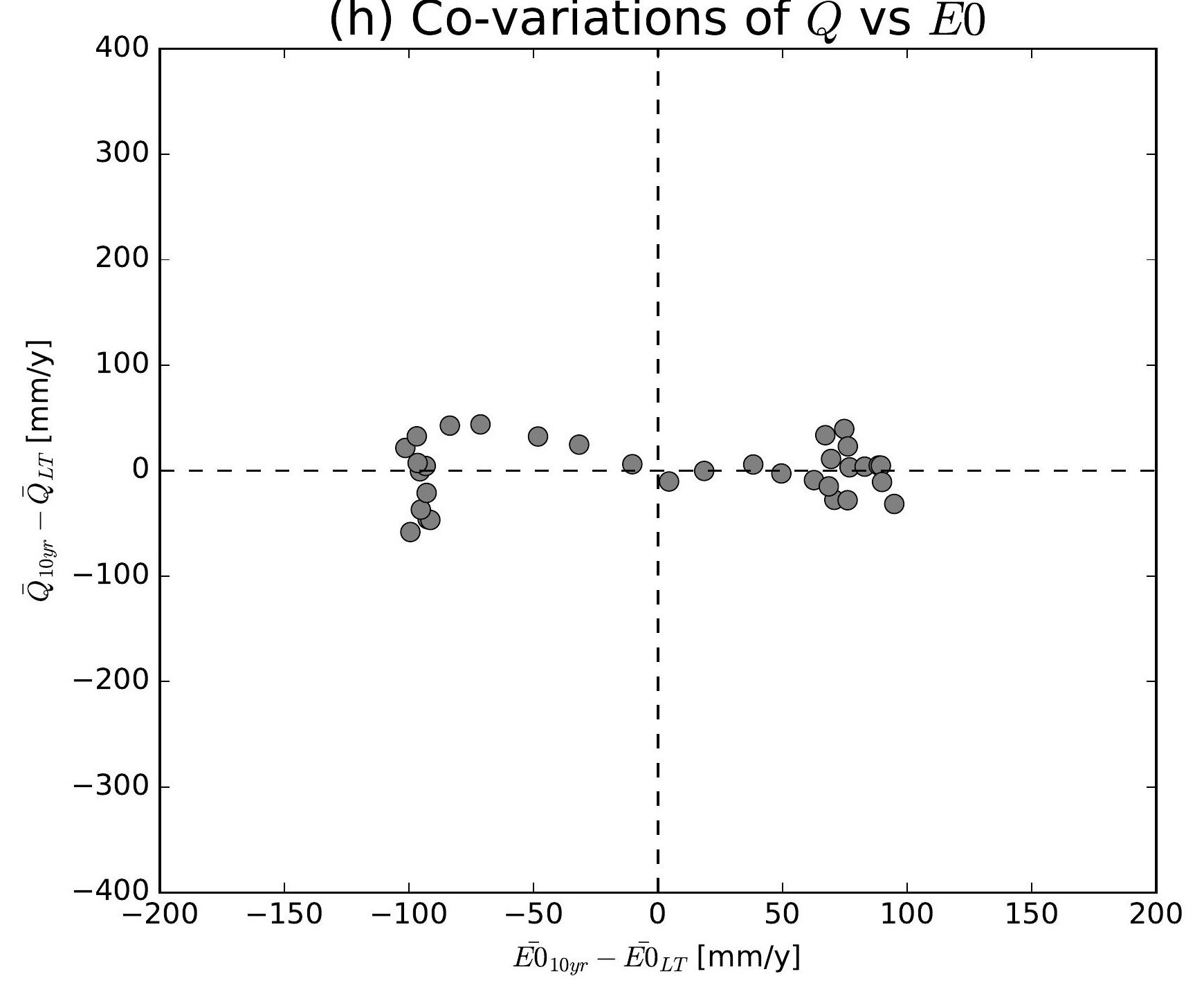

(k) Co-variations of $\bar{Q}$ vs $\overline{E 0}$

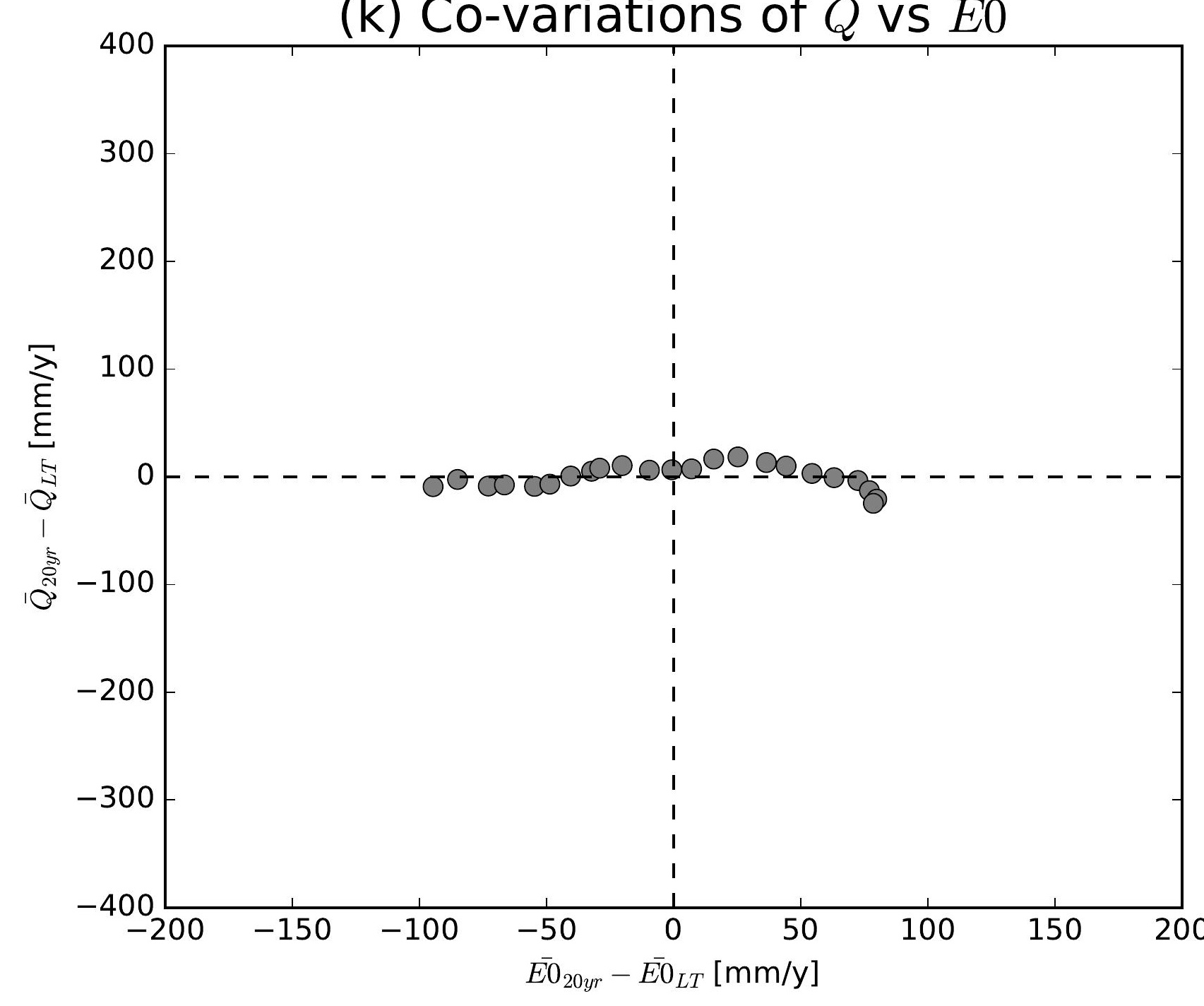

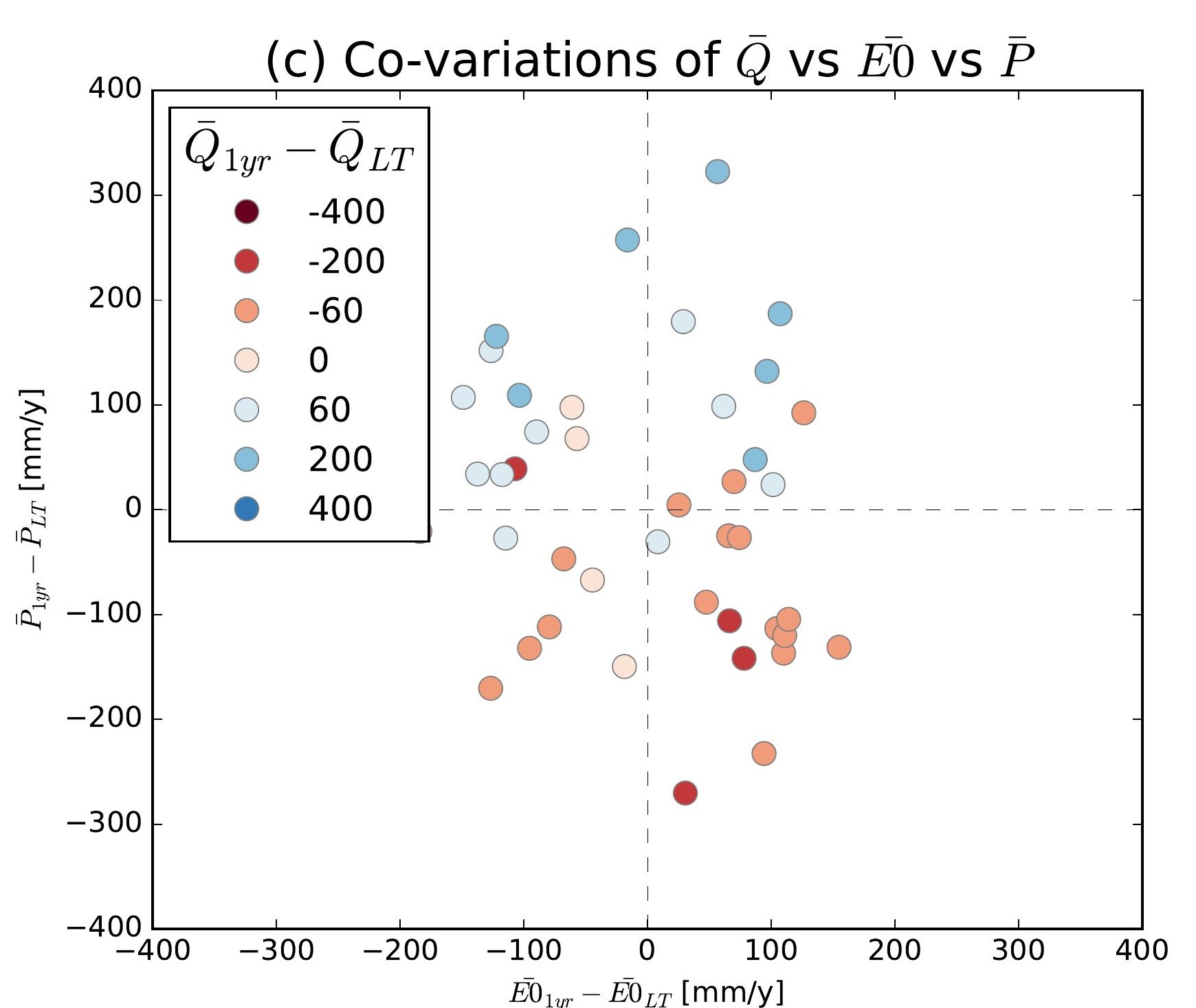
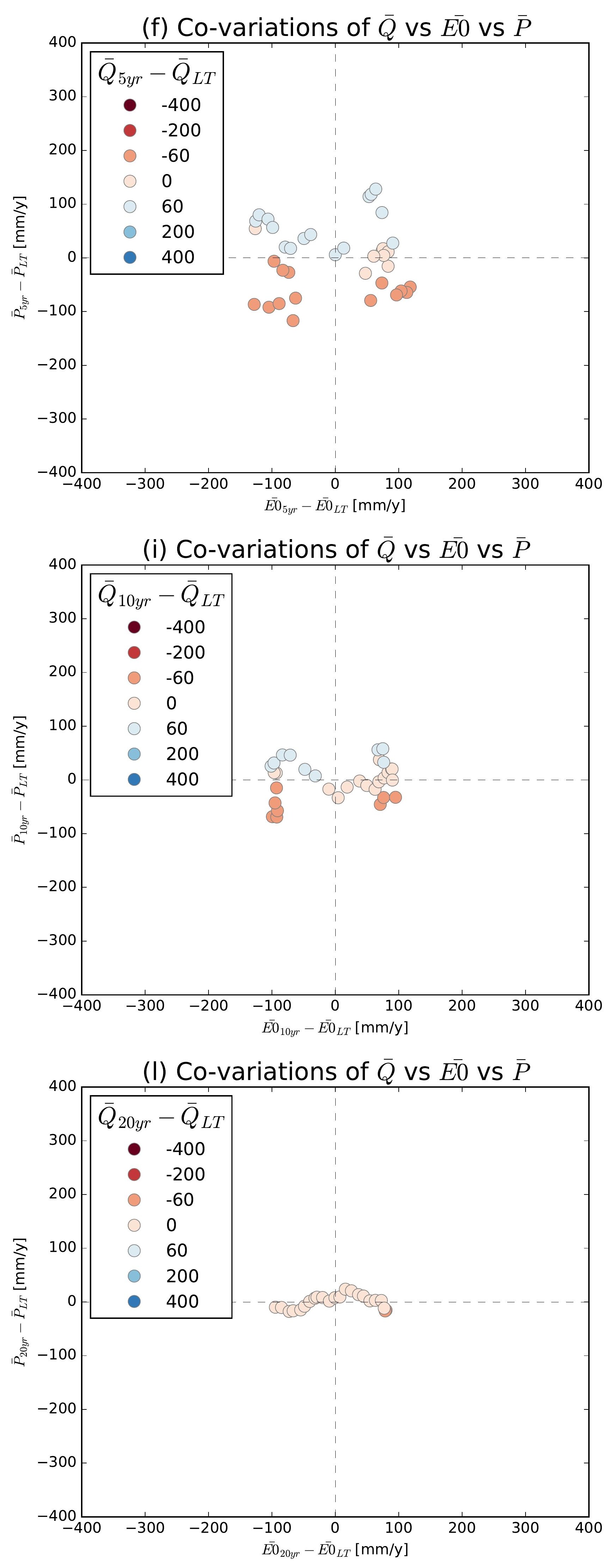

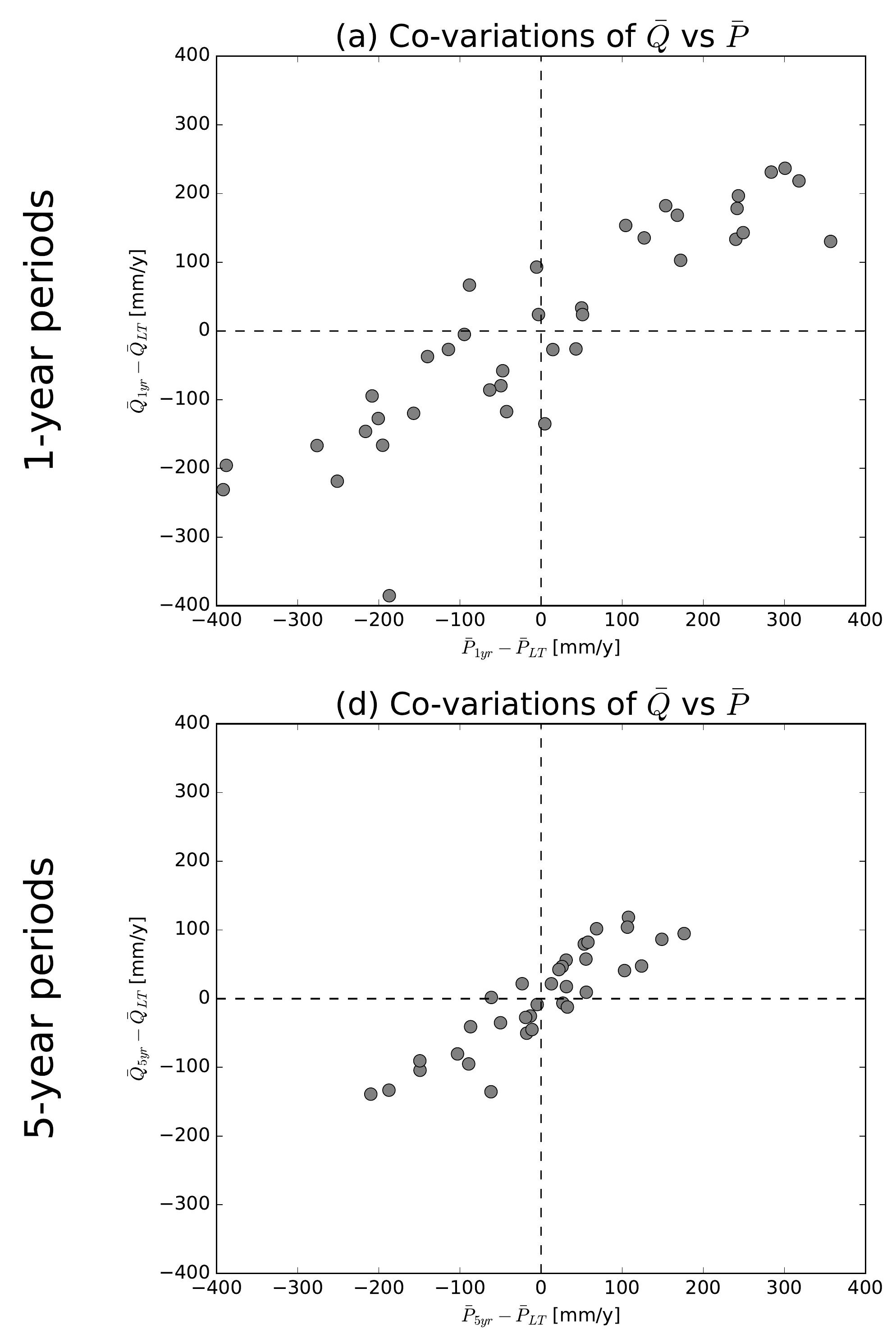

(g) Co-variations of $\bar{Q}$ vs $\bar{P}$
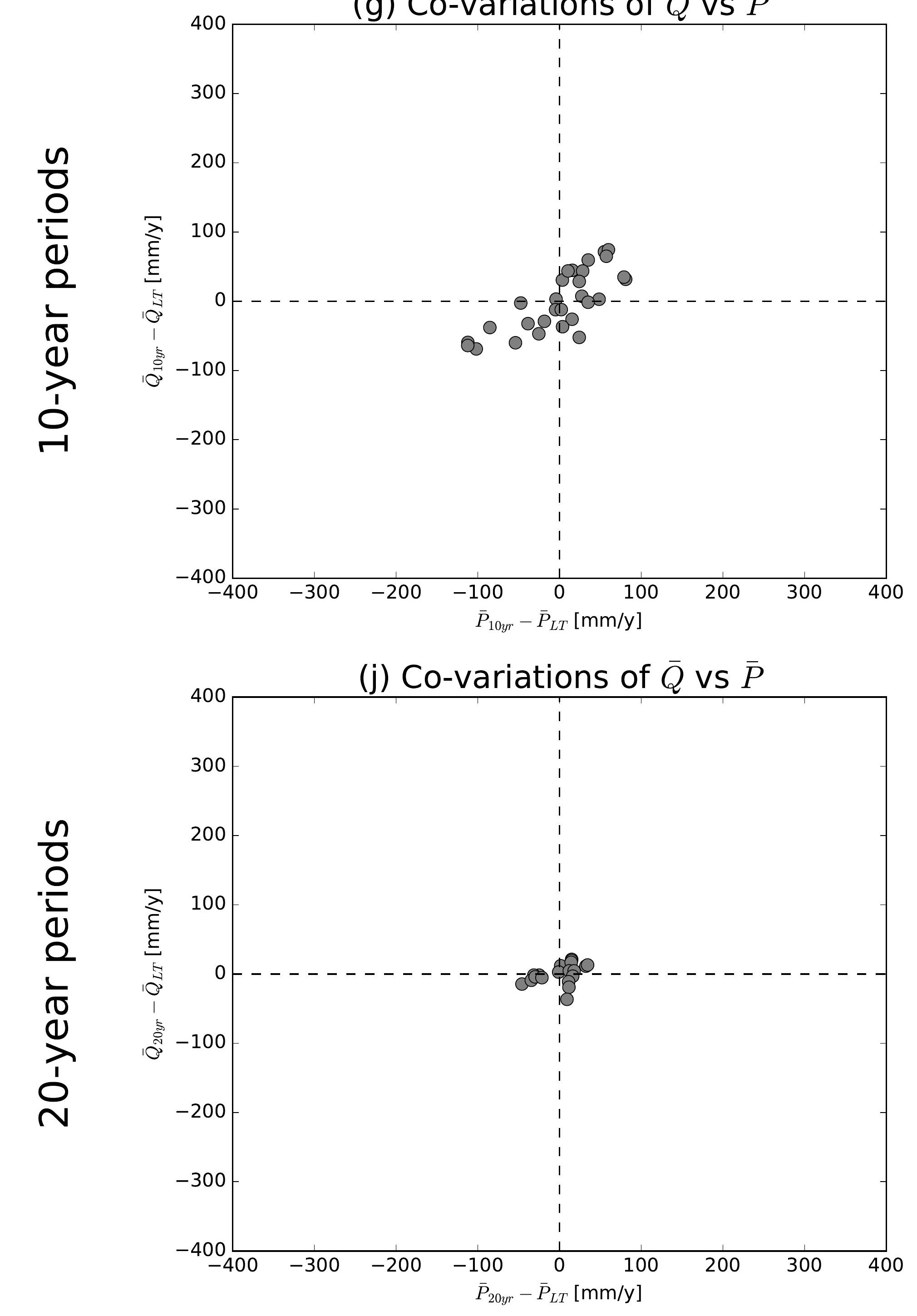

(b) Co-variations of $\bar{Q}$ vs $\overline{E 0}$

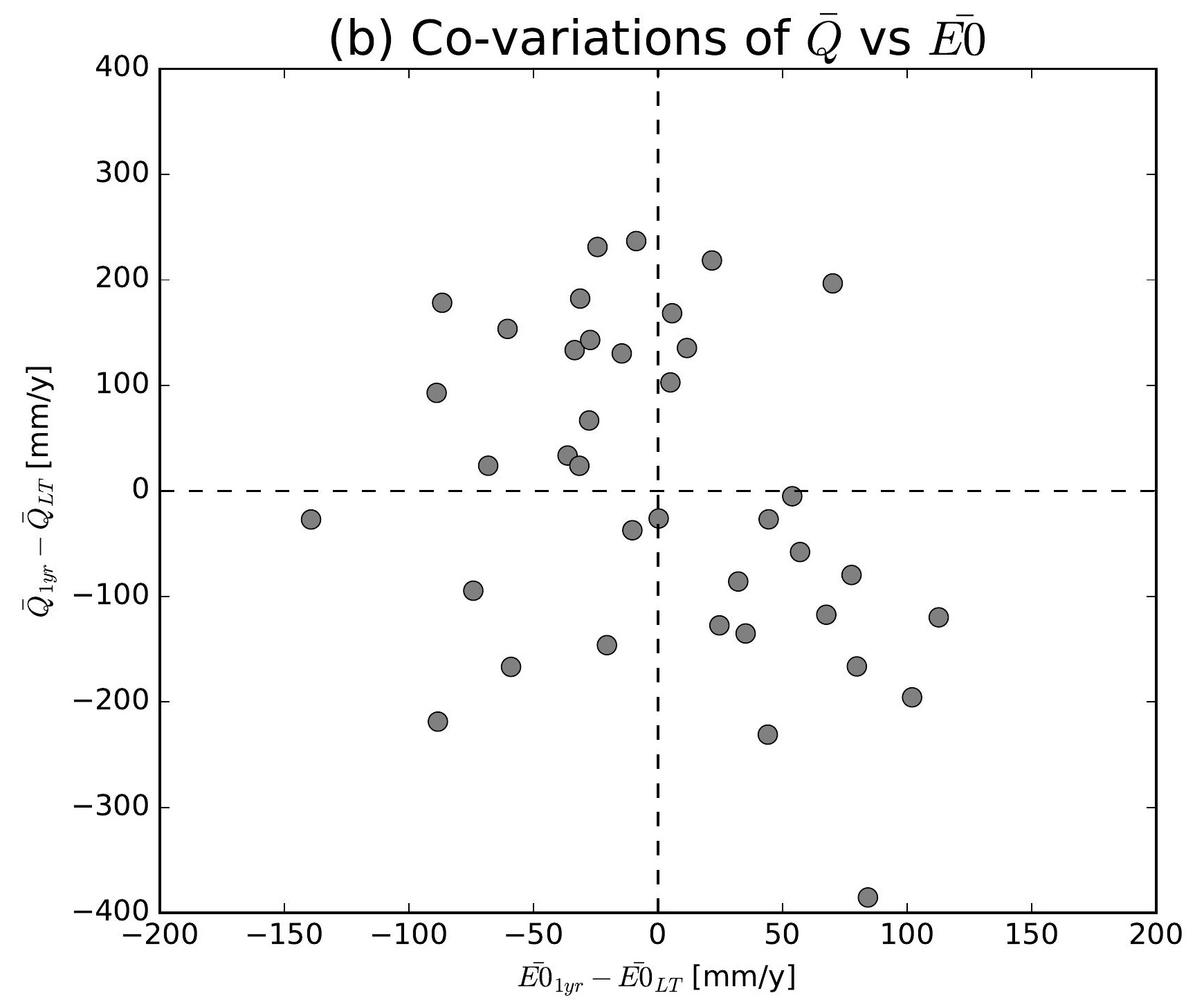

(e) Co-variations of $\bar{Q}$ vs $\overline{E 0}$

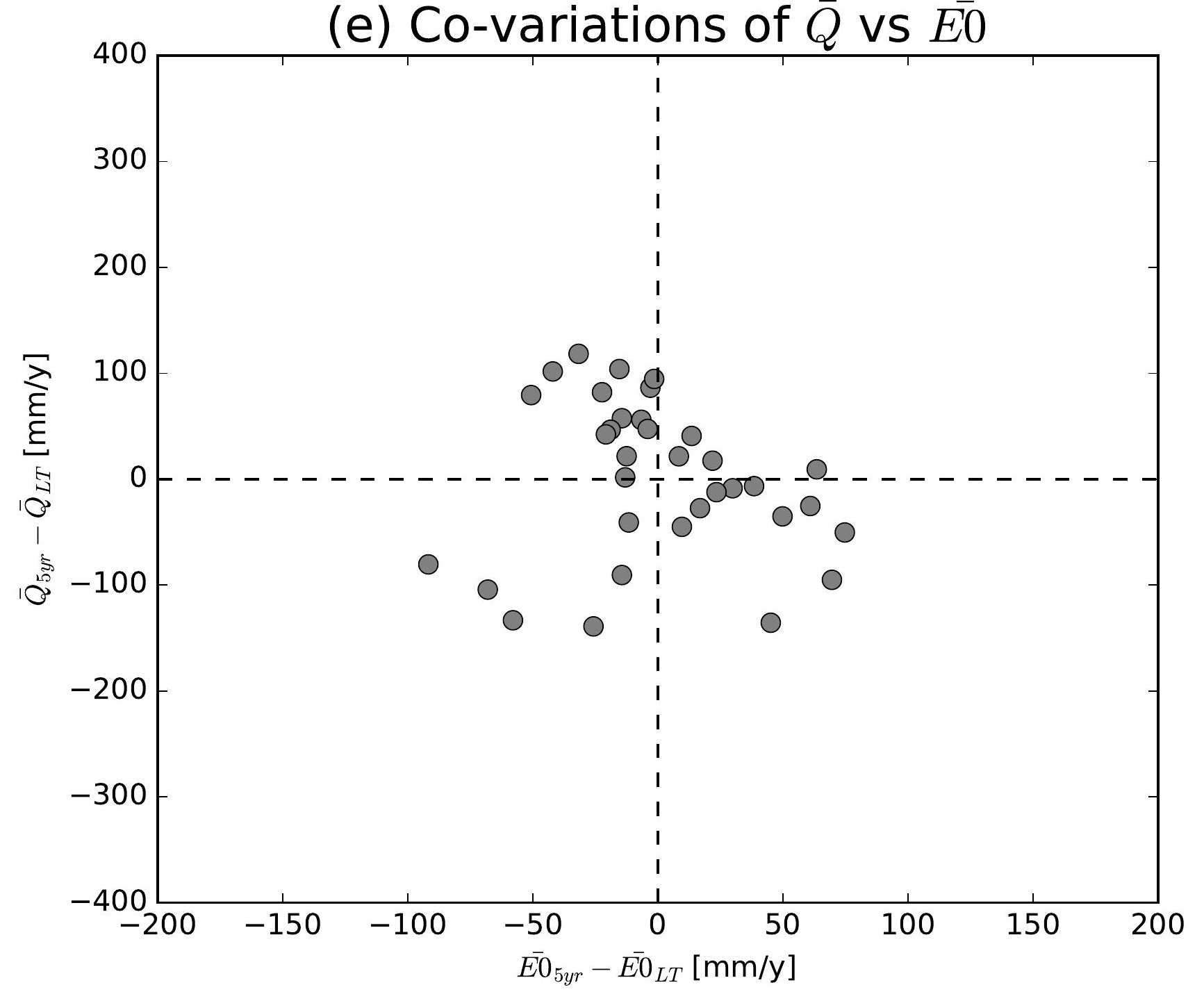

(h) Co-variations of $\bar{Q}$ vs $\overline{E 0}$

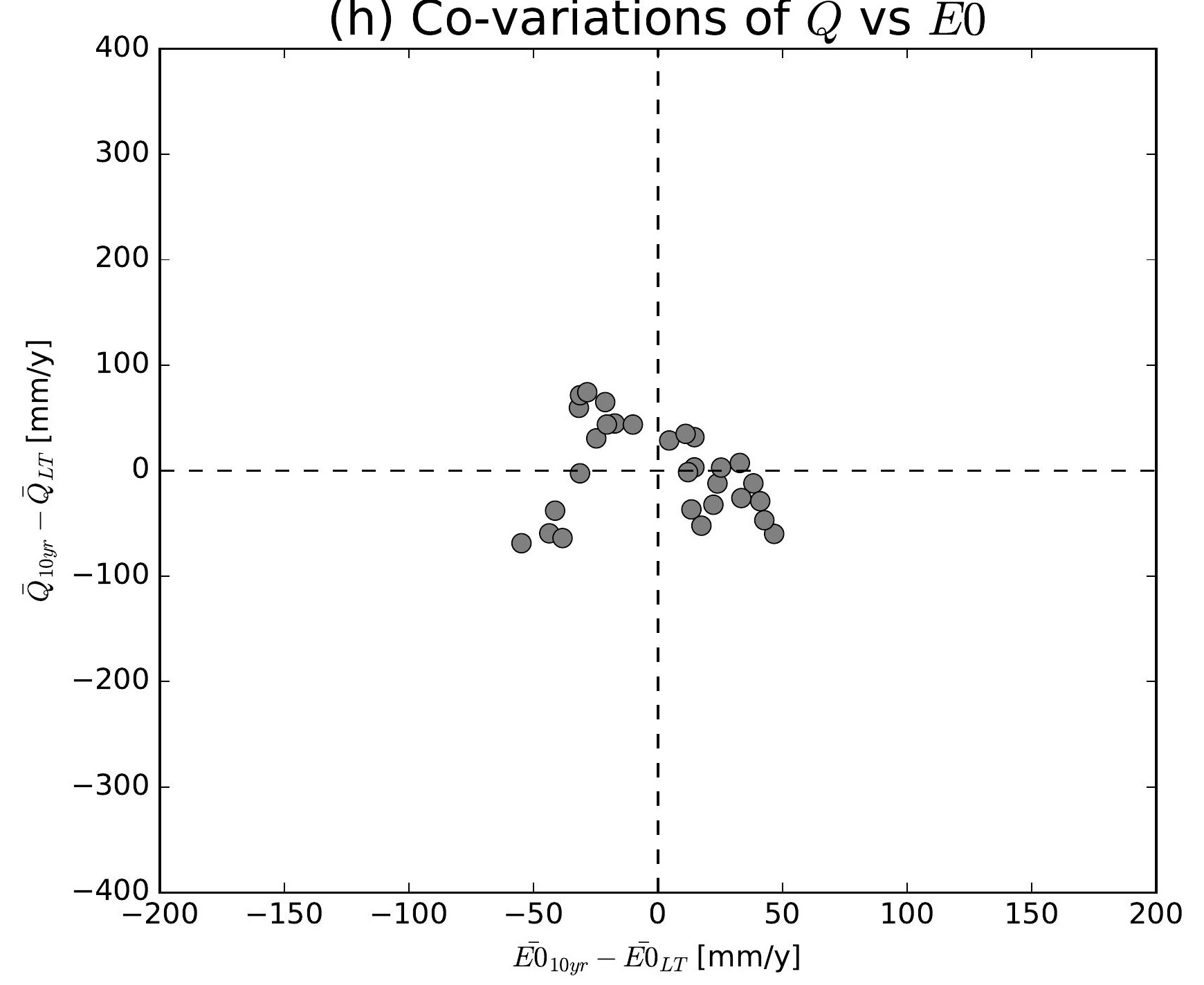

(k) Co-variations of $\bar{Q}$ vs $\overline{E 0}$

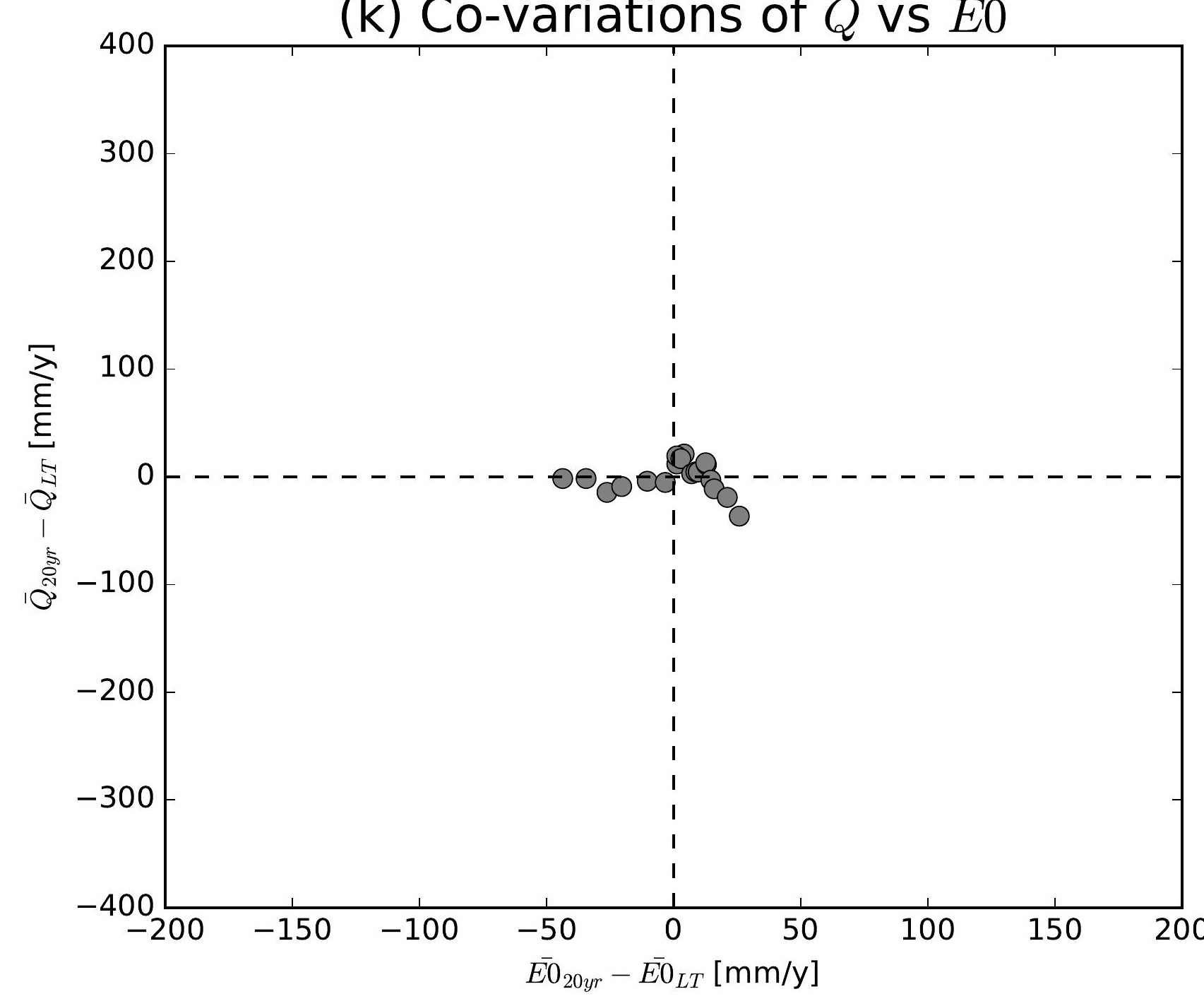

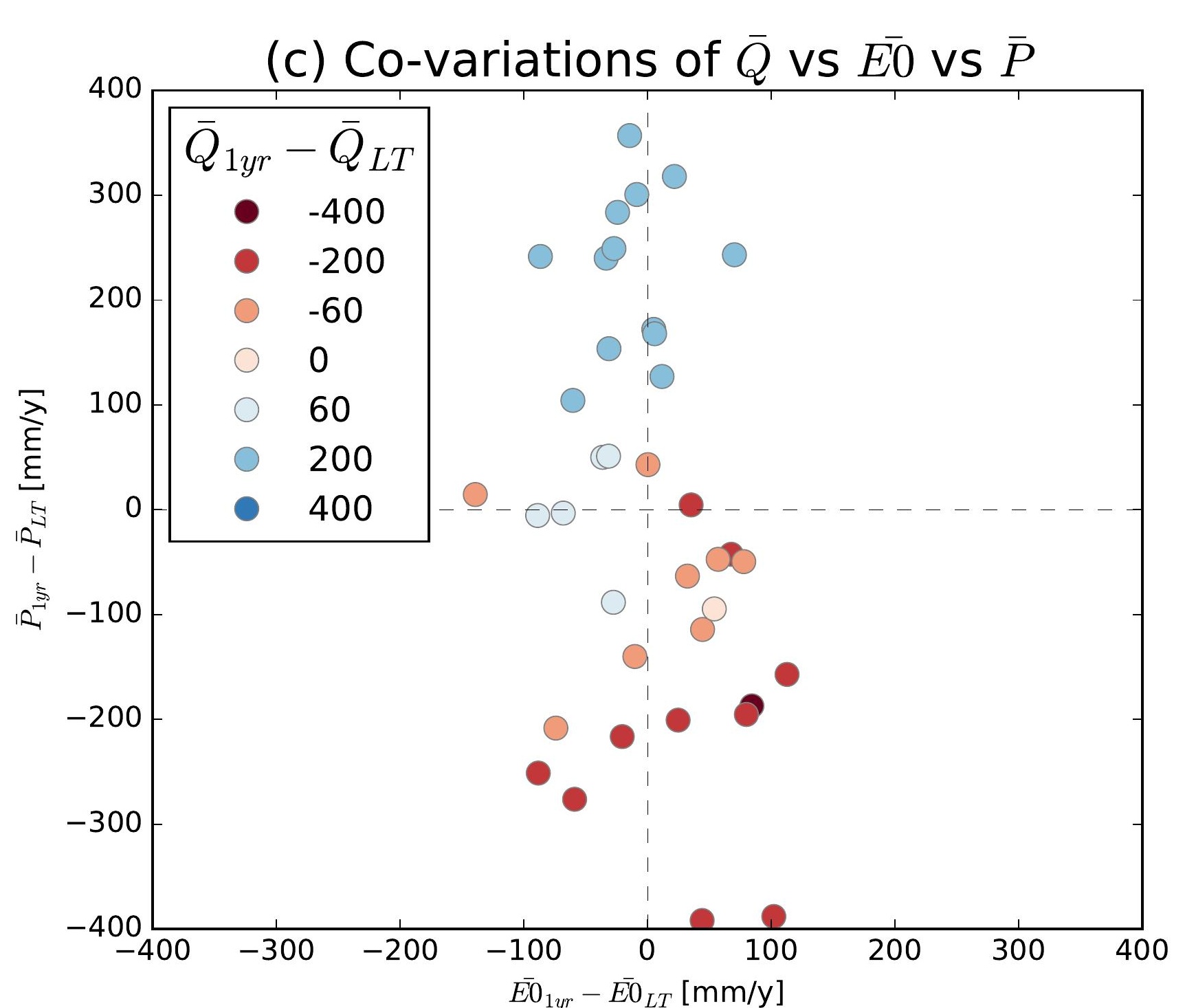
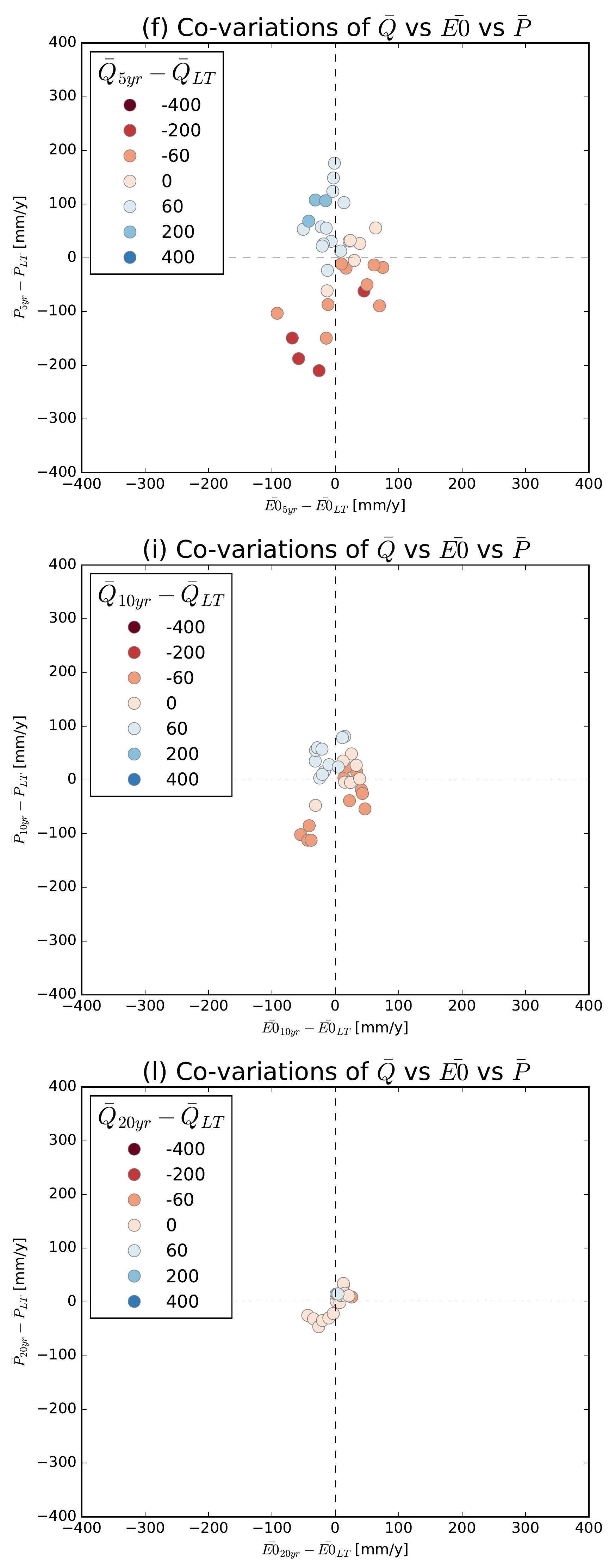

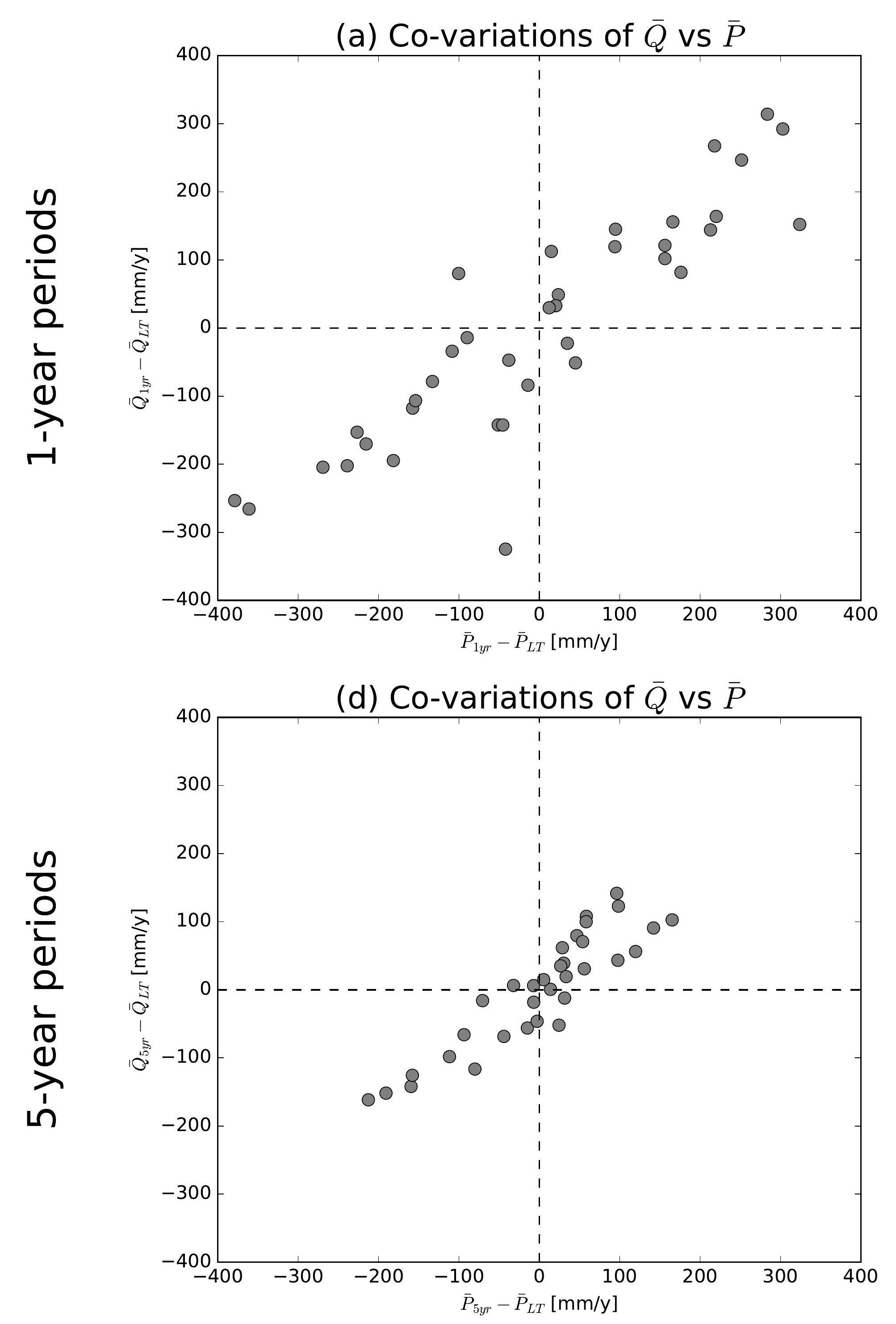

(g) Co-variations of $\bar{Q}$ vs $\bar{P}$

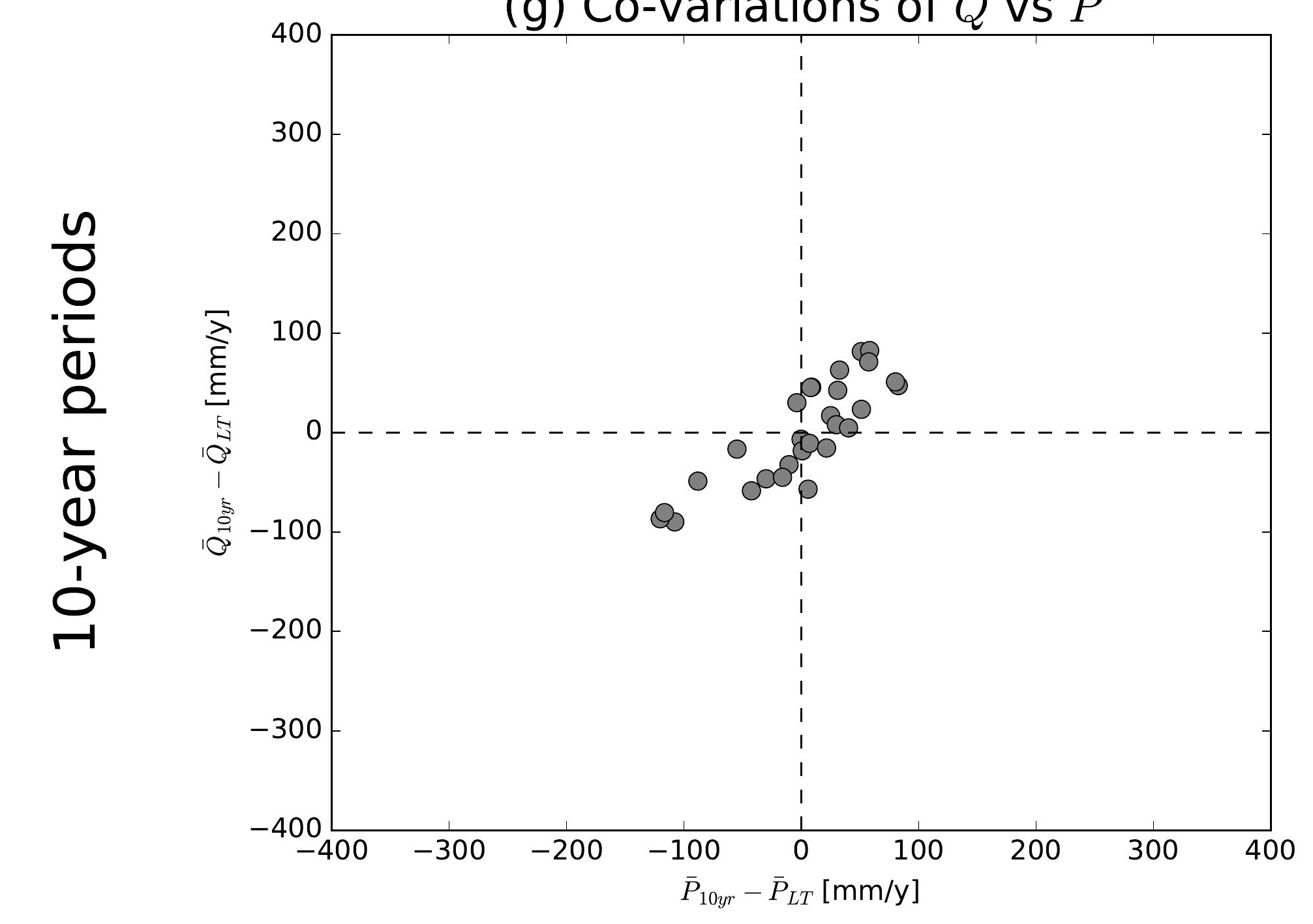

(j) Co-variations of $\bar{Q}$ vs $\bar{P}$

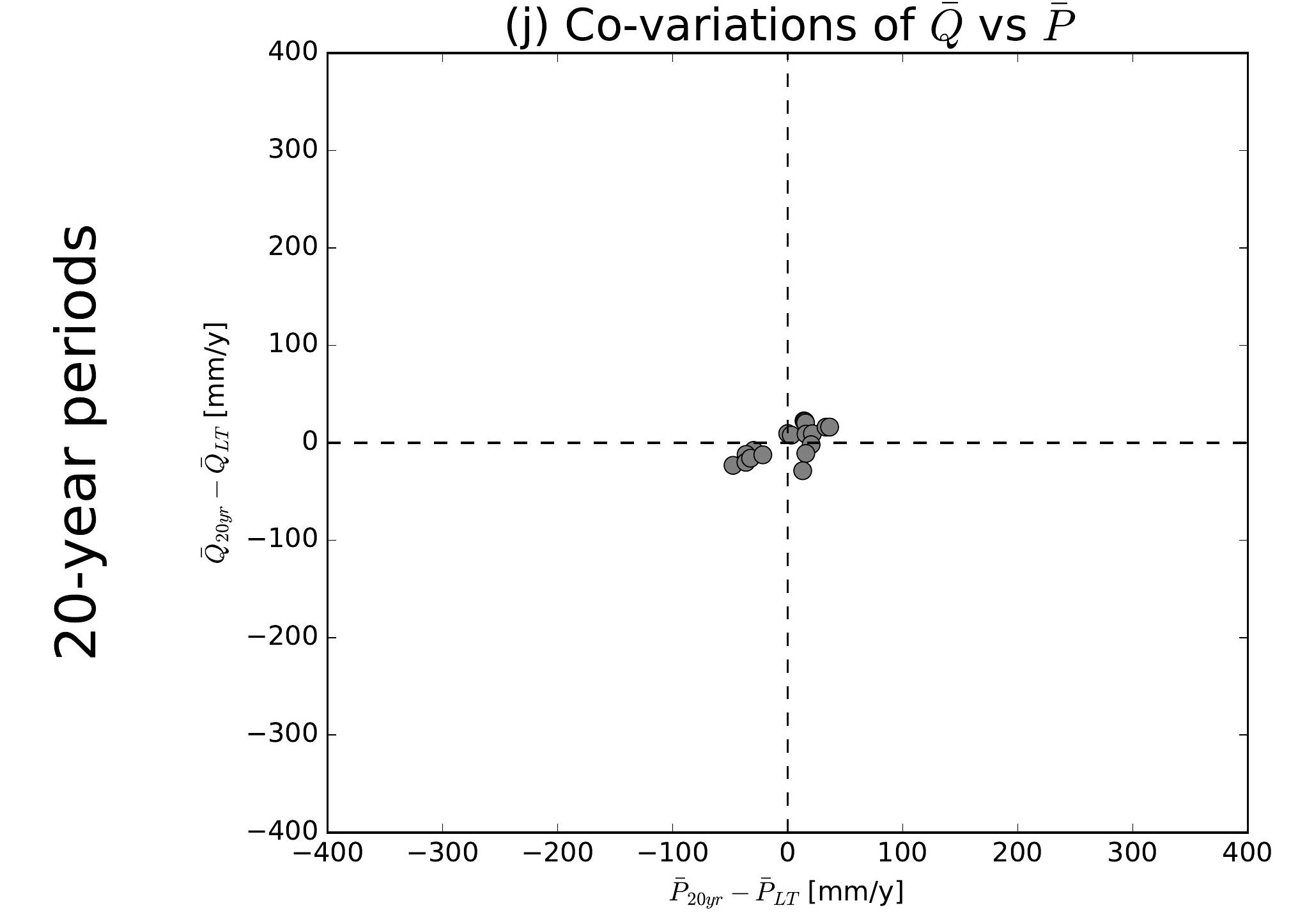

(b) Co-variations of $\bar{Q}$ vs $\overline{E 0}$

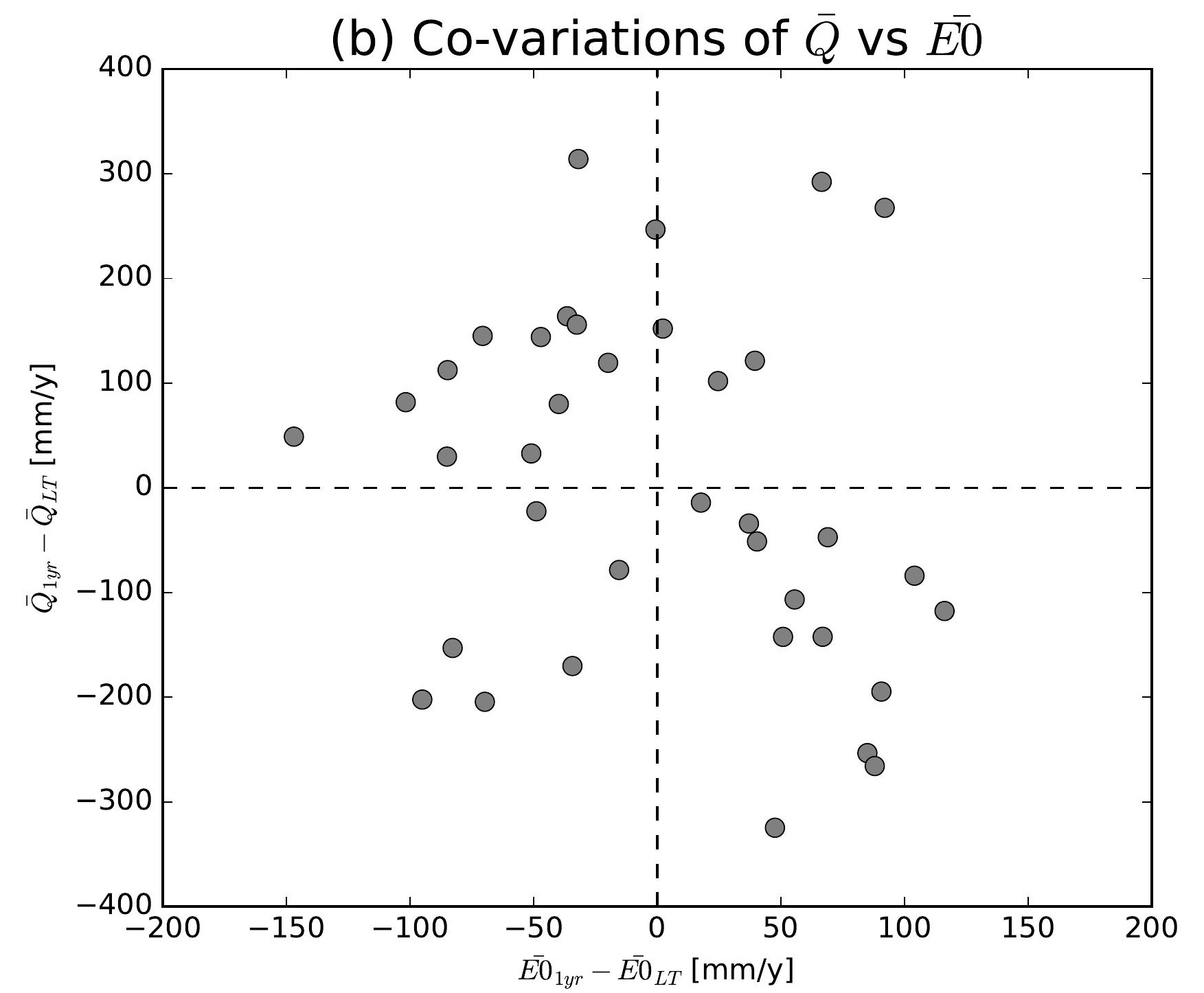

(e) Co-variations of $\bar{Q}$ vs $\overline{E 0}$

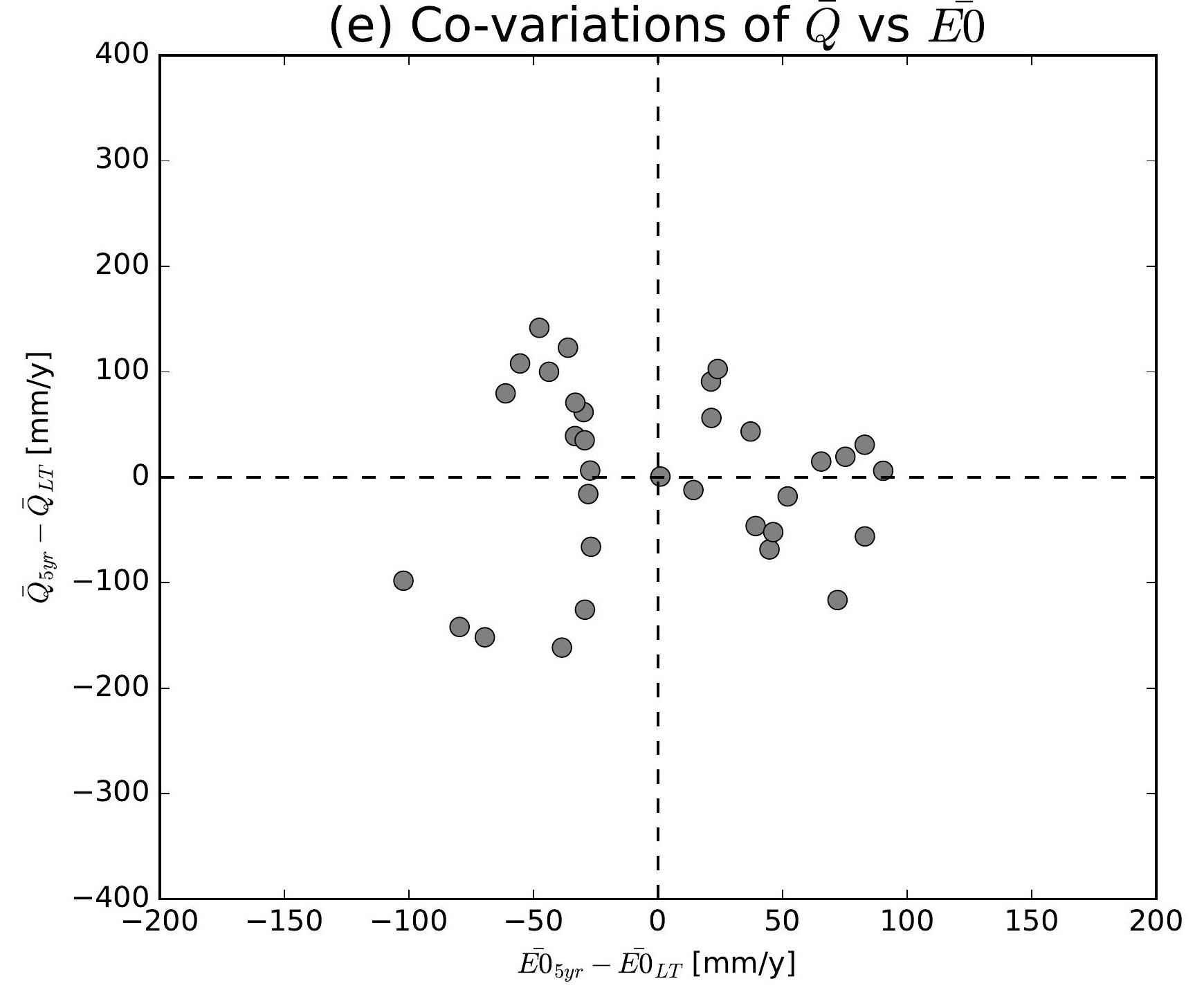

(h) Co-variations of $\bar{Q}$ vs $\overline{E 0}$

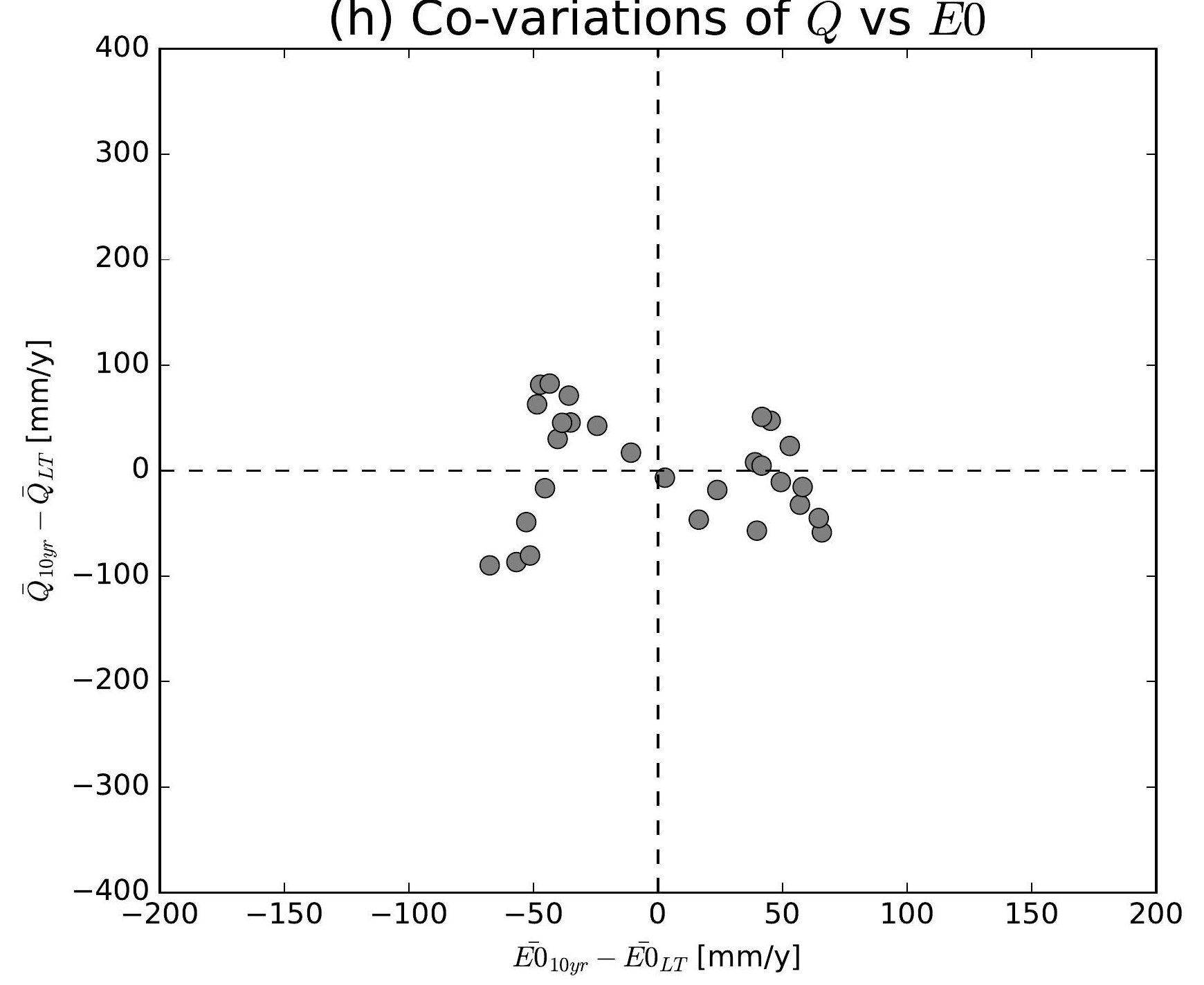

(k) Co-variations of $\bar{Q}$ vs $\overline{E 0}$

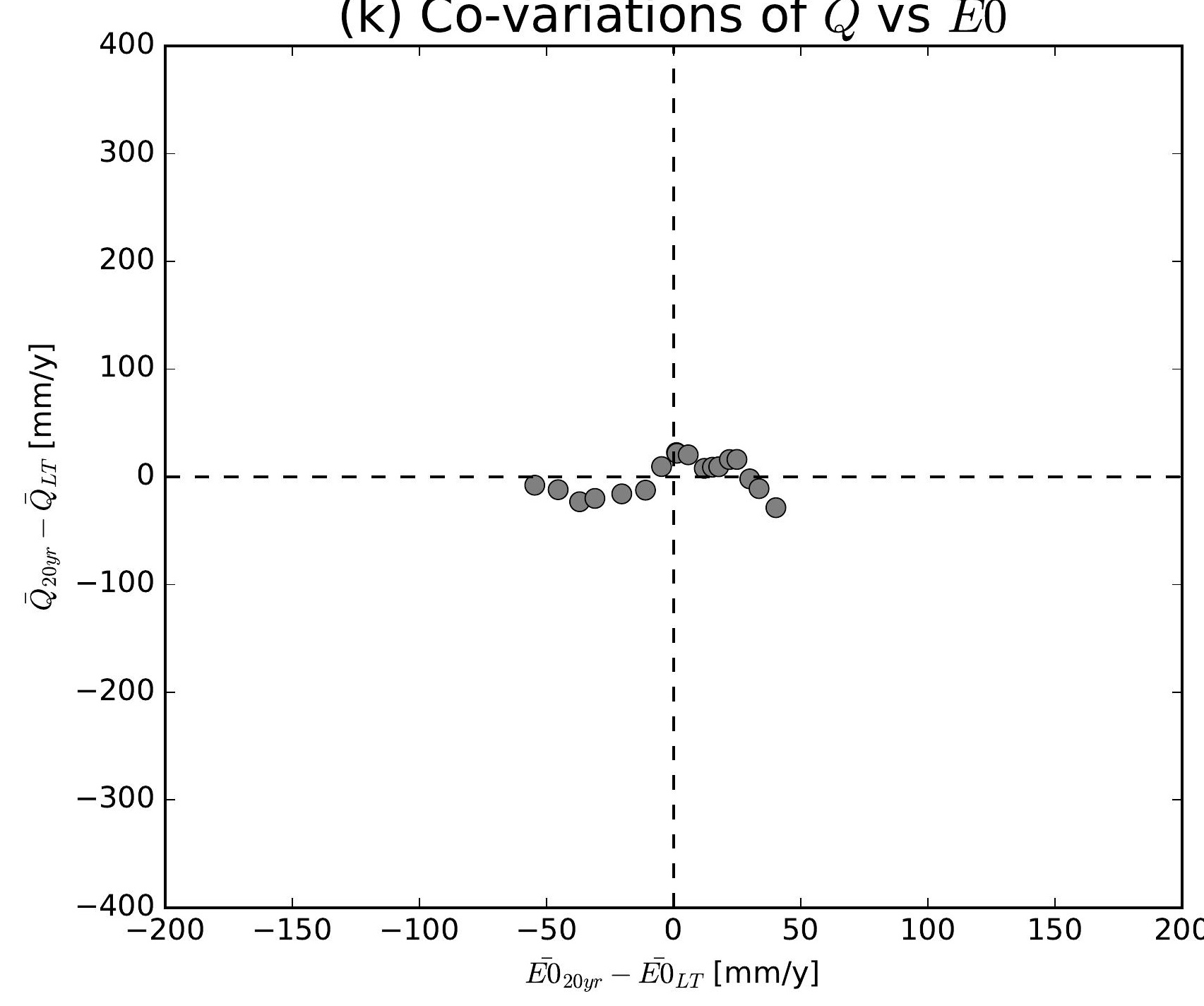

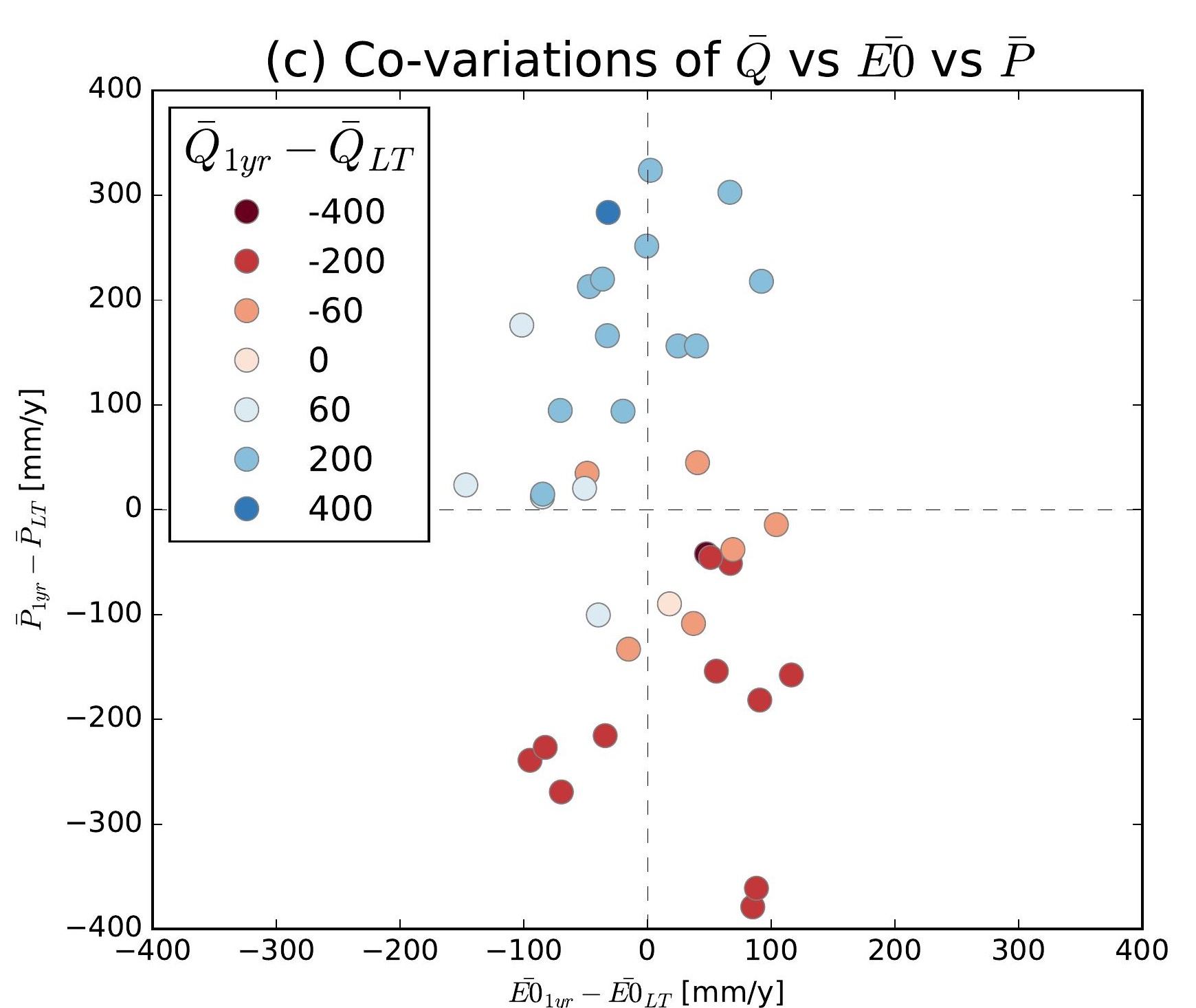
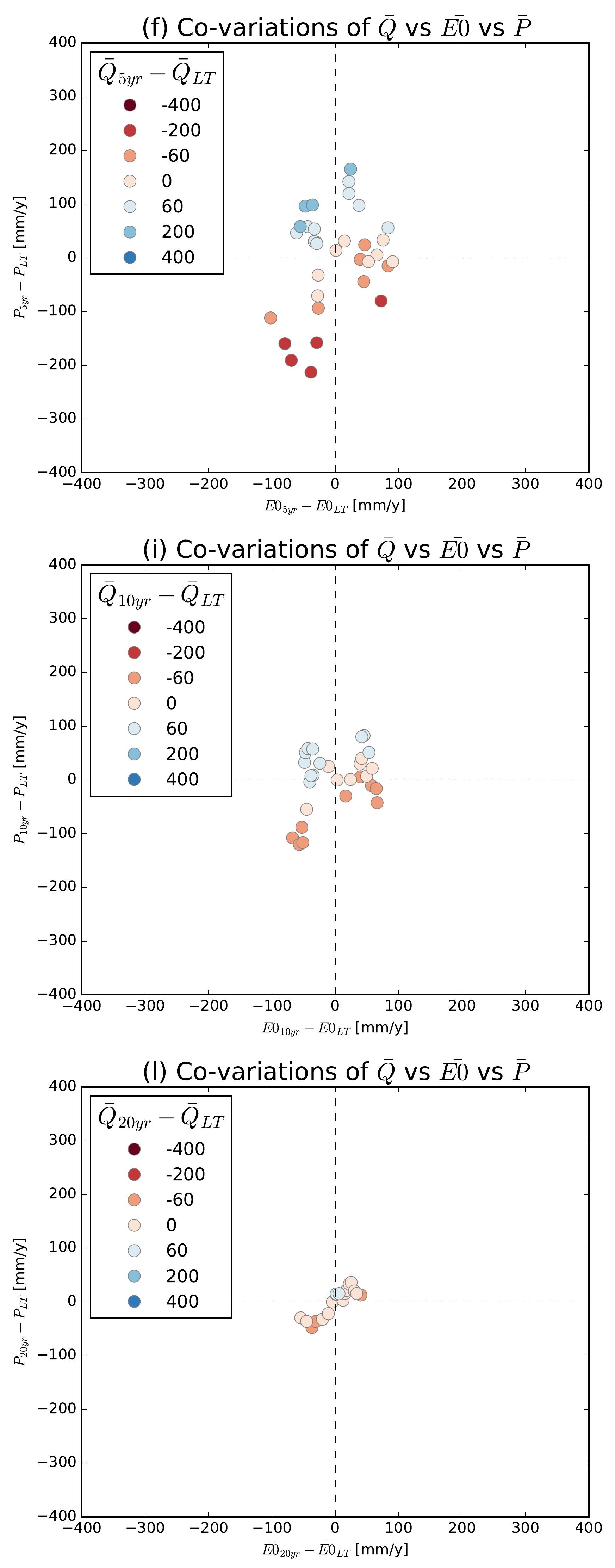

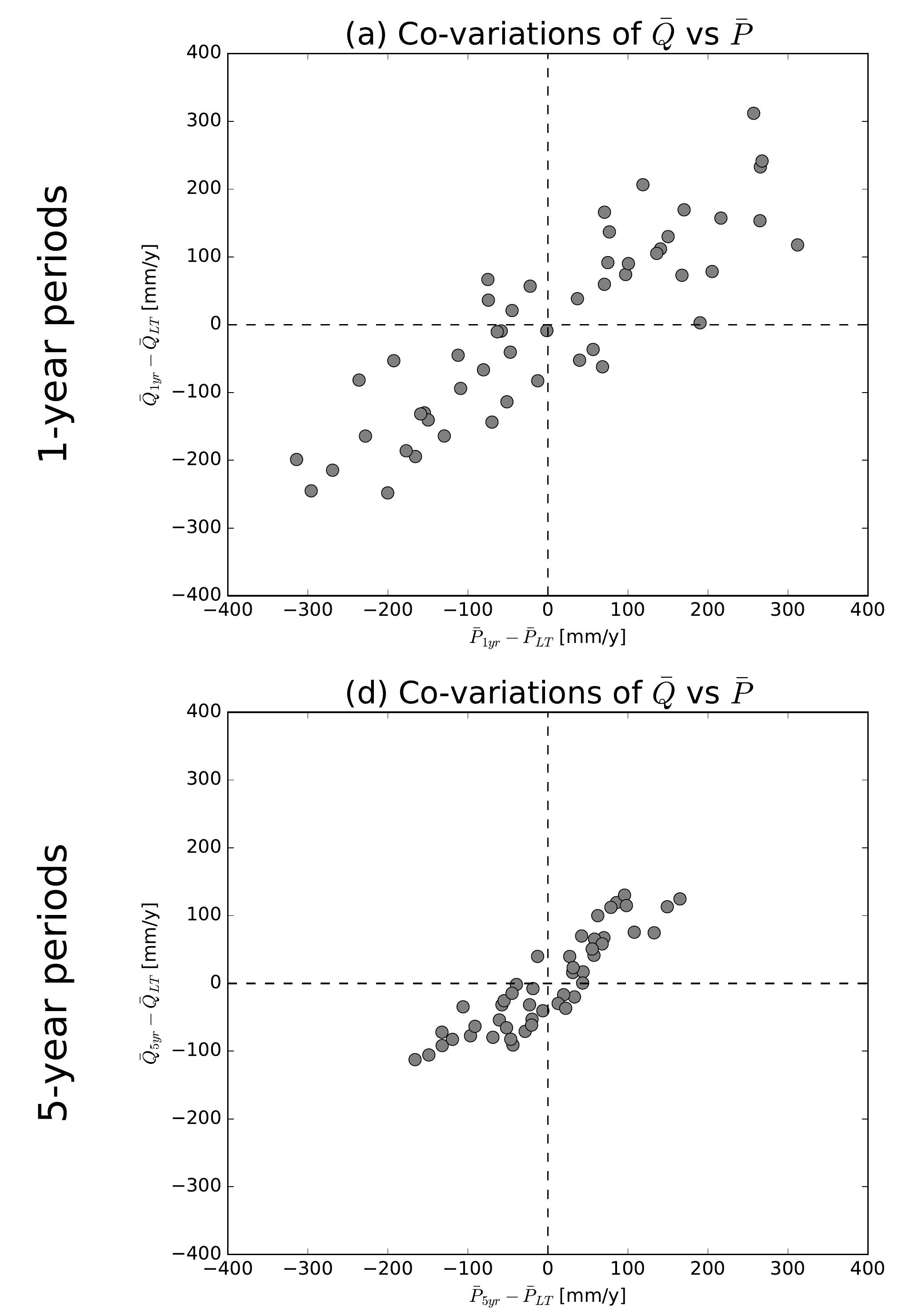

(g) Co-variations of $\bar{Q}$ vs $\bar{P}$
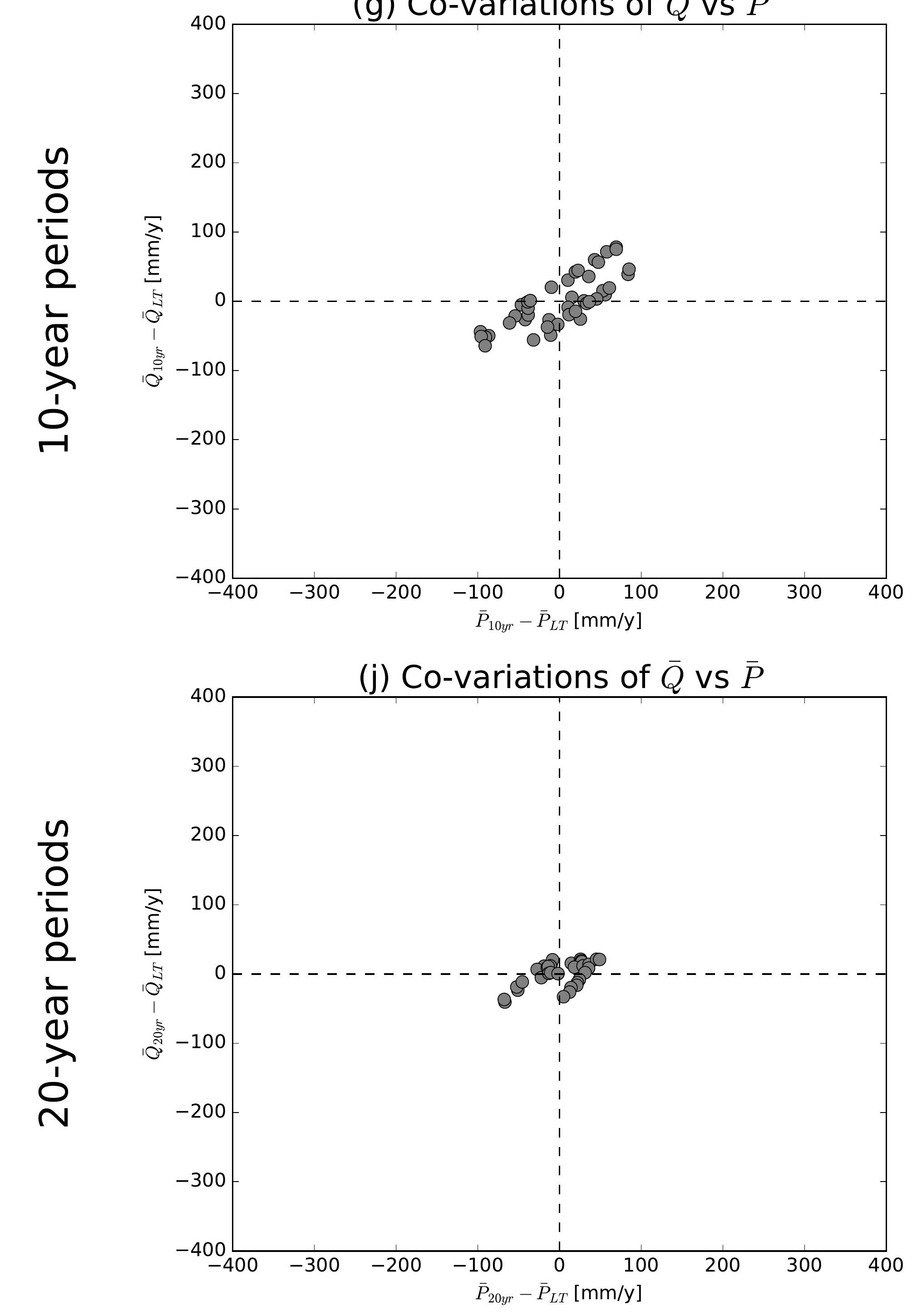

(b) Co-variations of $\bar{Q}$ vs $\overline{E 0}$

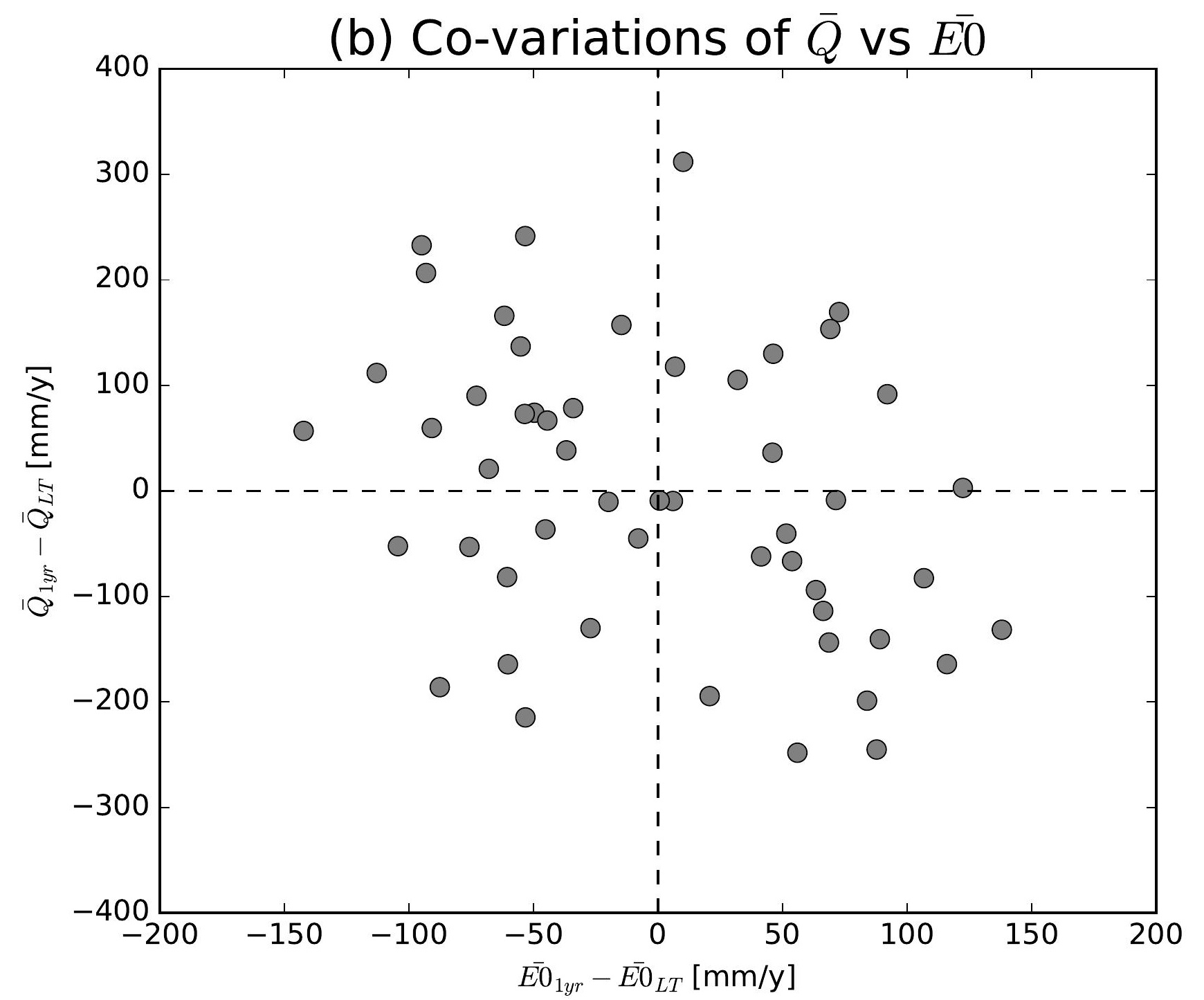

(e) Co-variations of $\bar{Q}$ vs $\overline{E 0}$

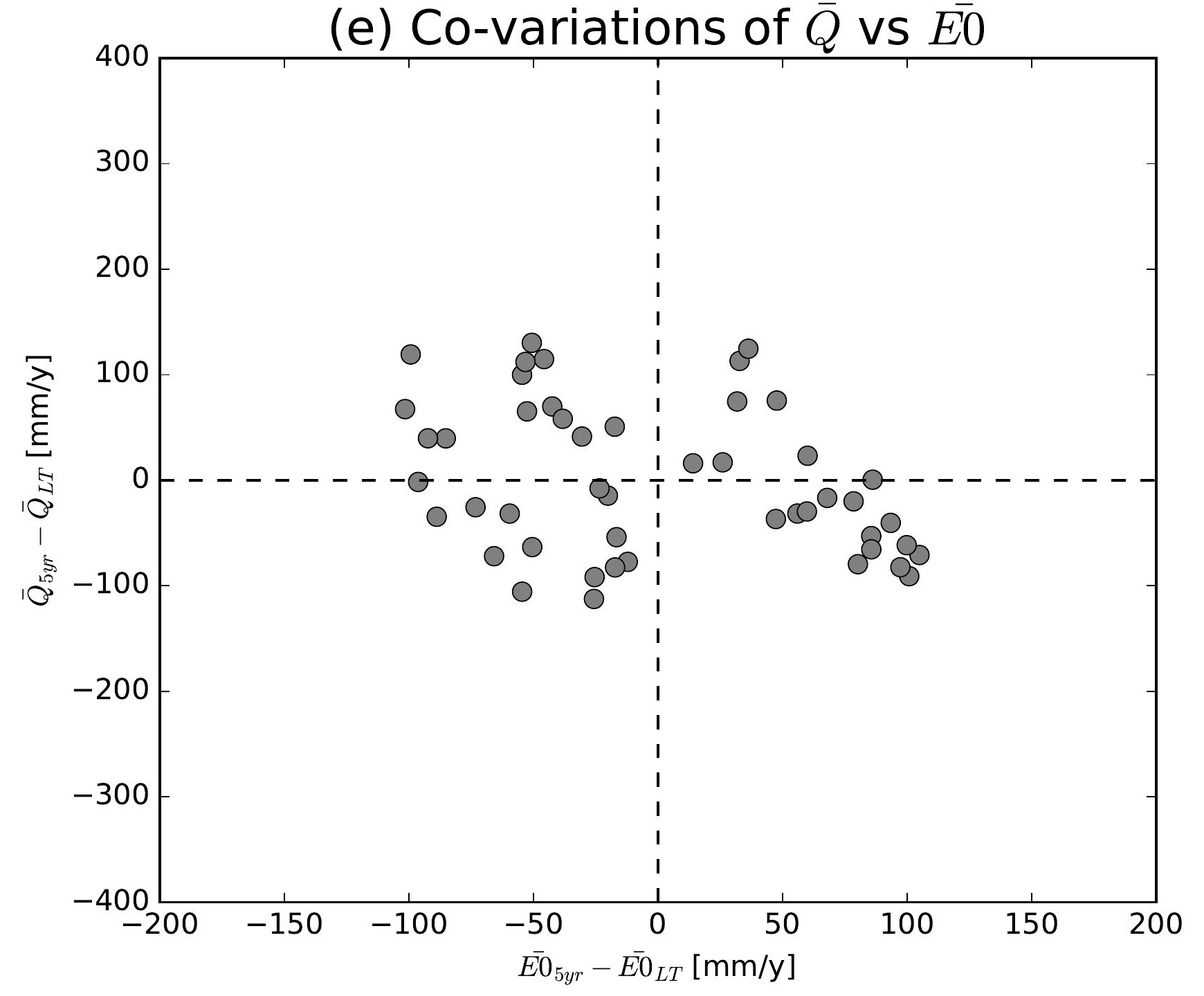

(h) Co-variations of $\bar{Q}$ vs $\overline{E 0}$

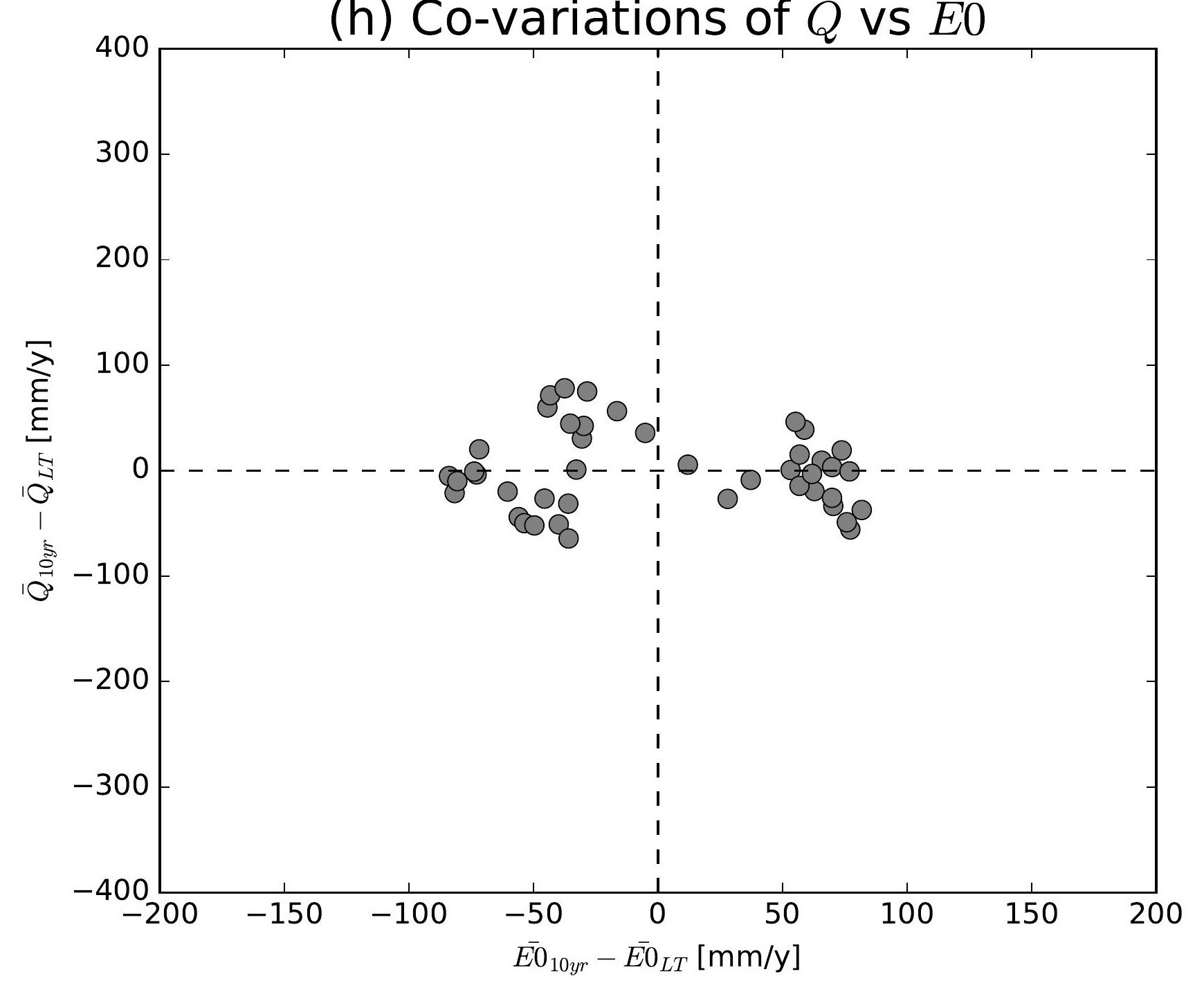

(k) Co-variations of $\bar{Q}$ vs $\overline{E 0}$

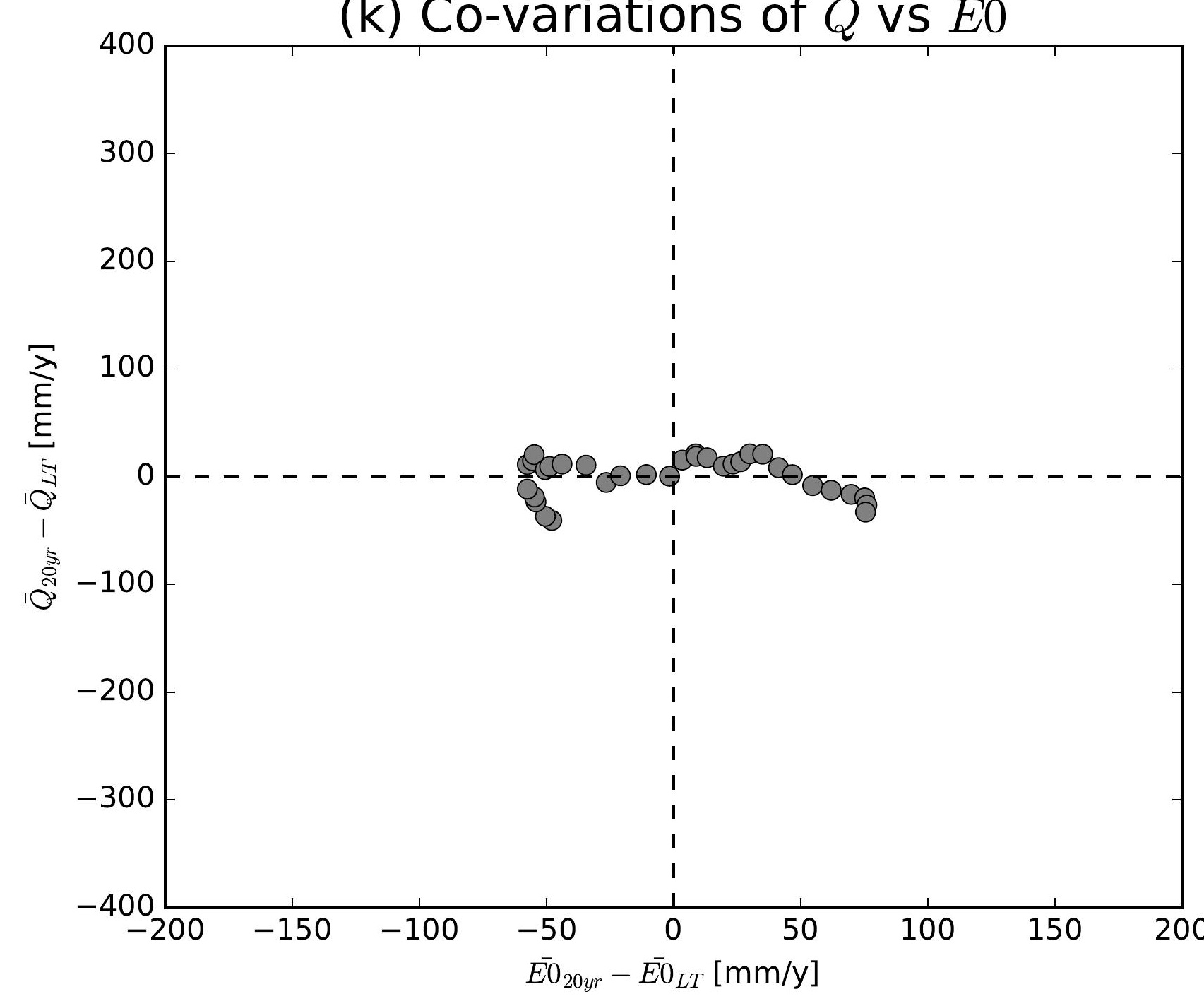

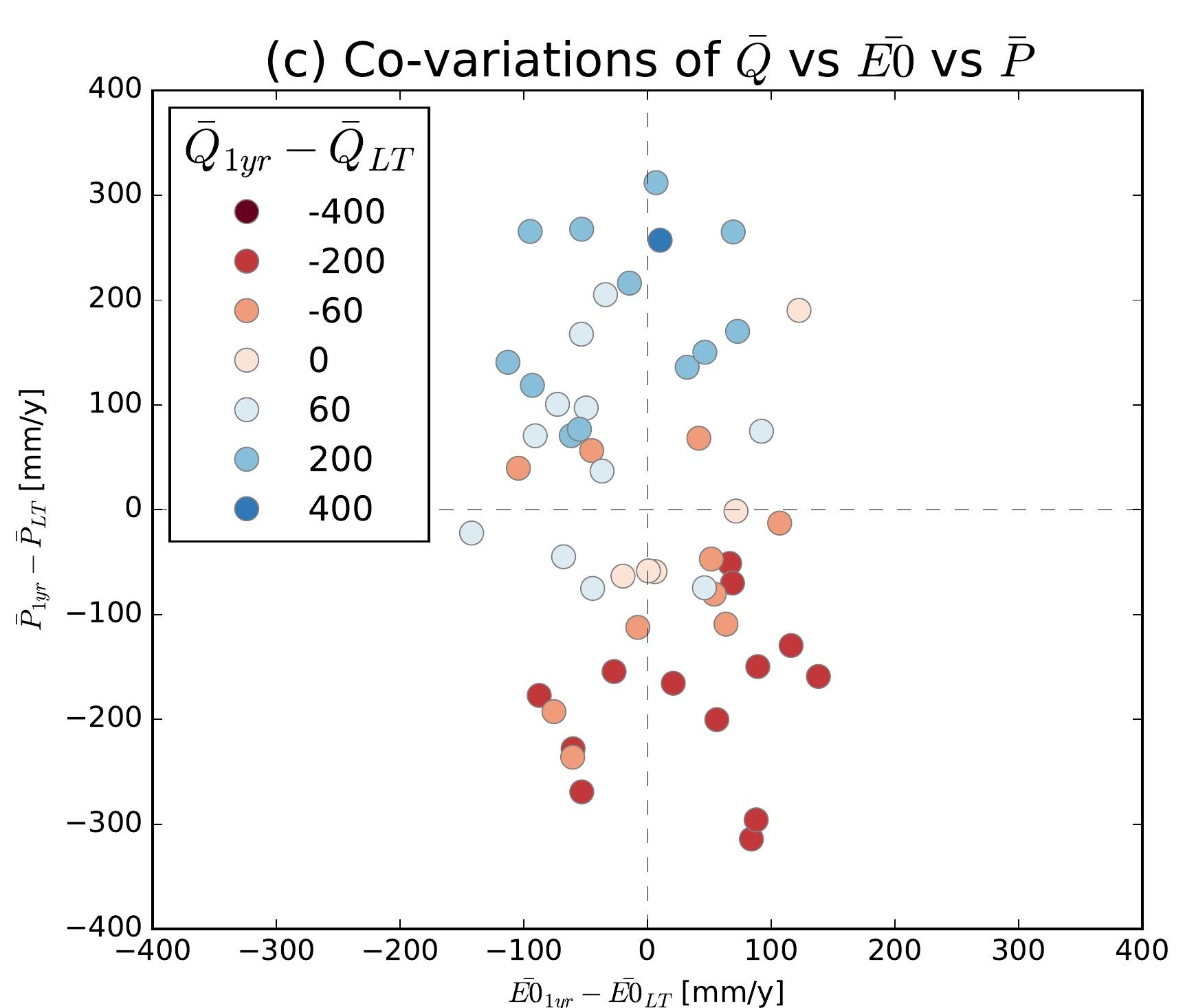
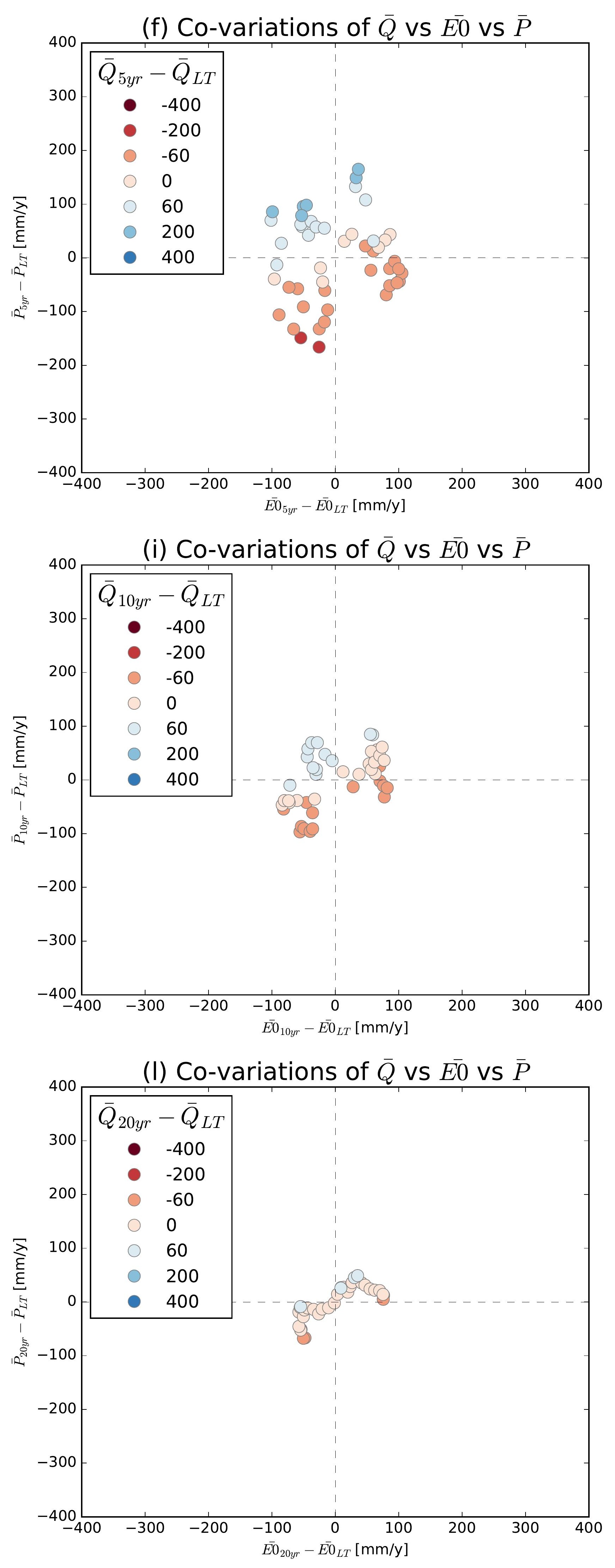

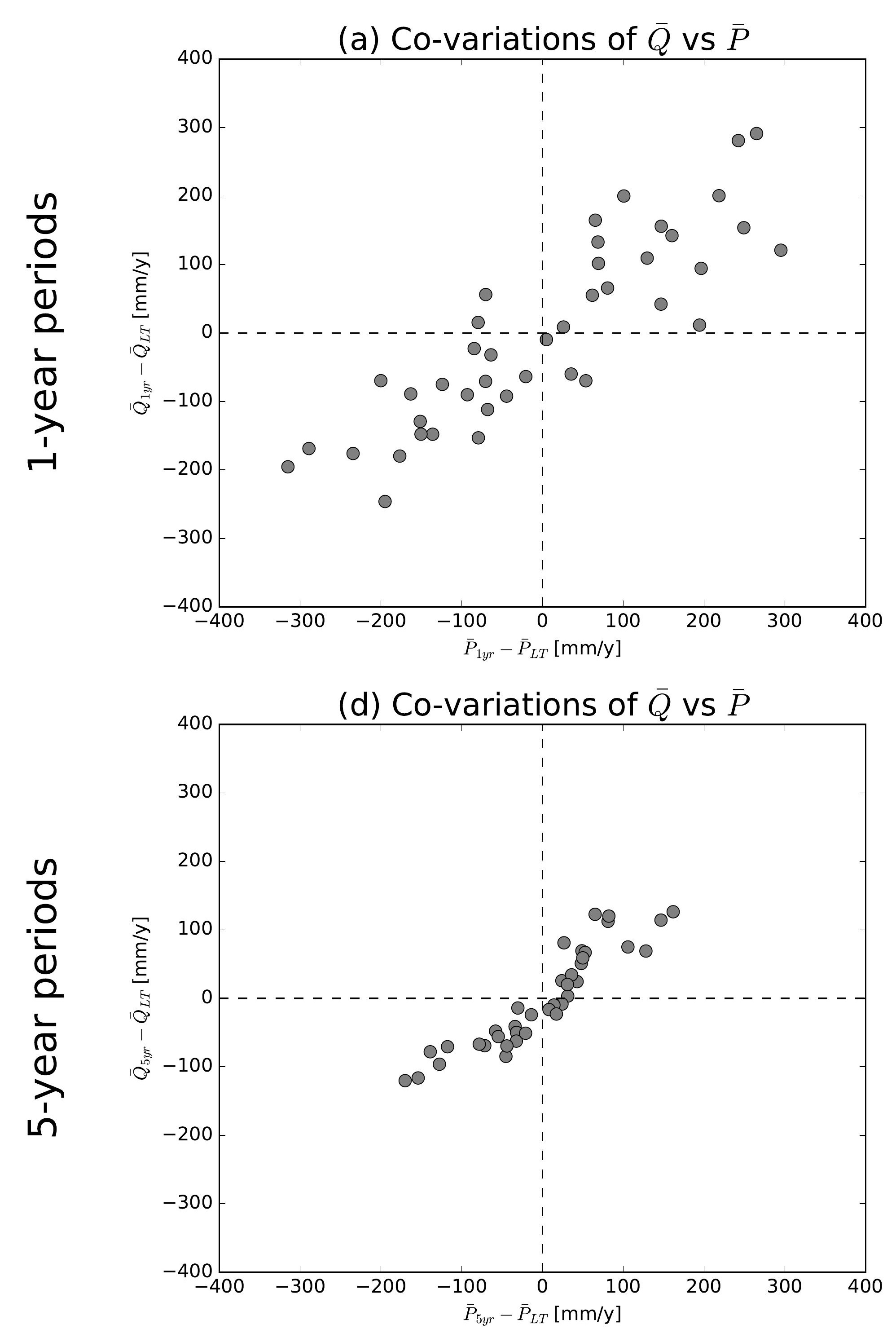

(g) Co-variations of $\bar{Q}$ vs $\bar{P}$

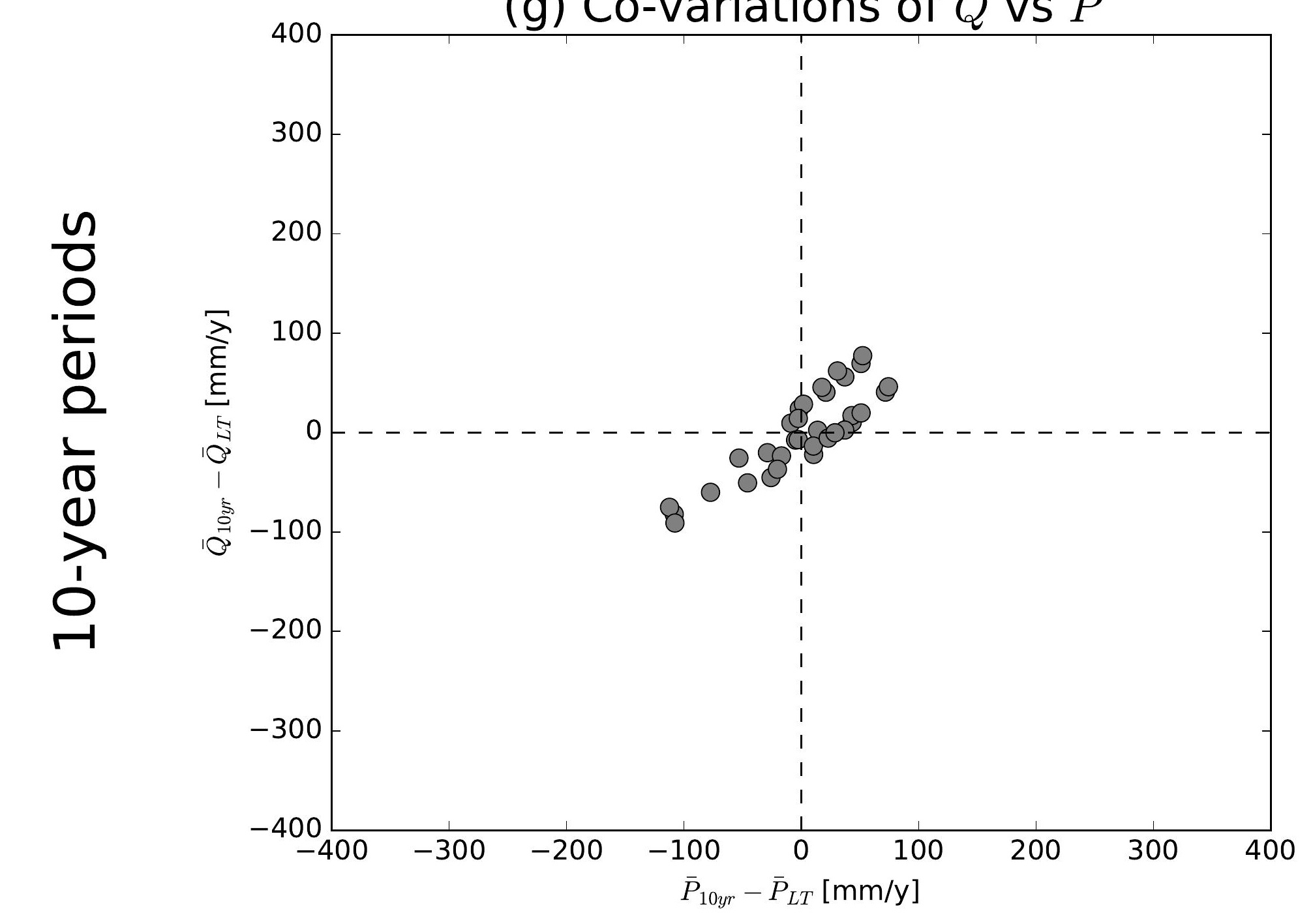

(j) Co-variations of $\bar{Q}$ vs $\bar{P}$

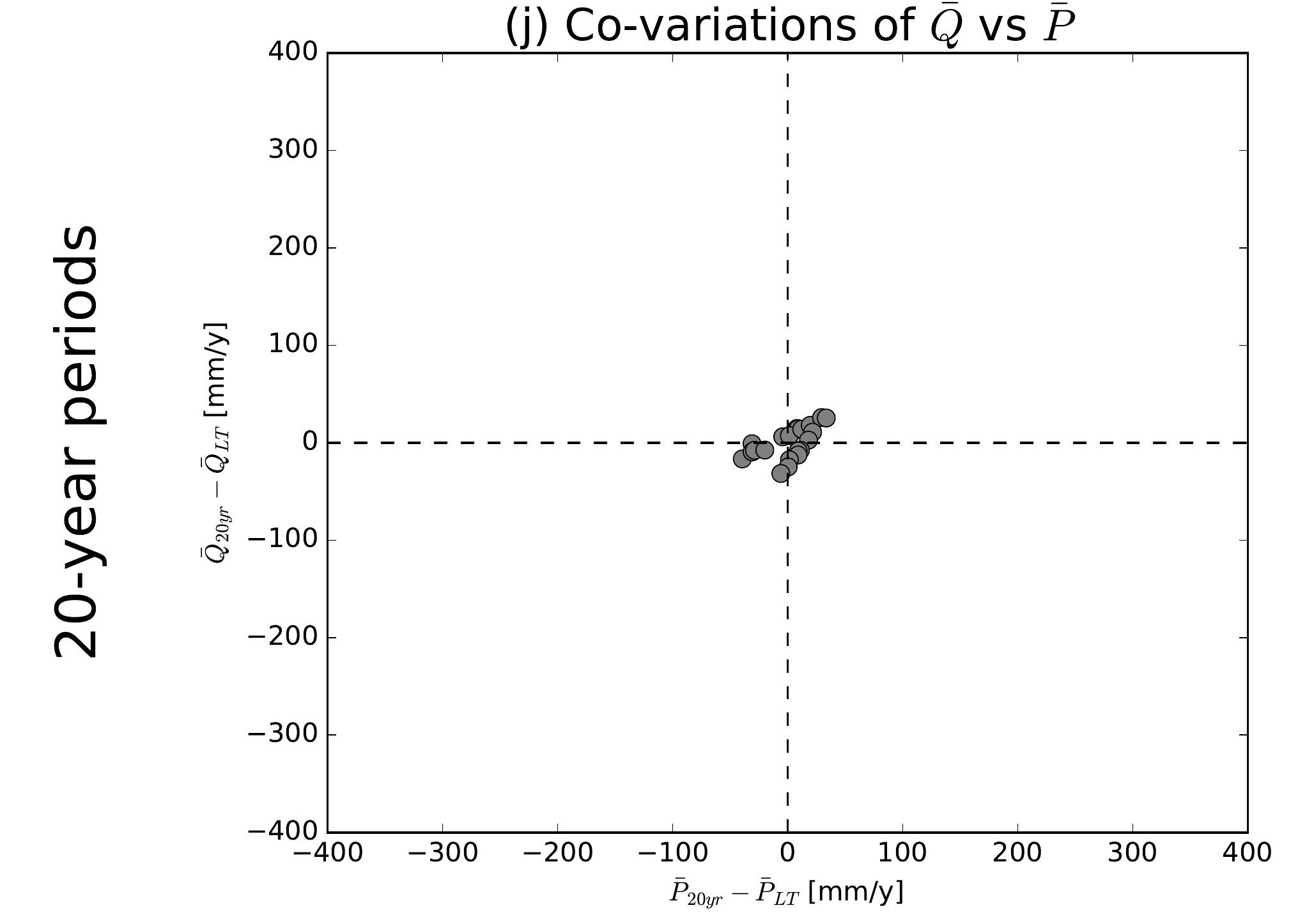

(b) Co-variations of $\bar{Q}$ vs $\overline{E 0}$

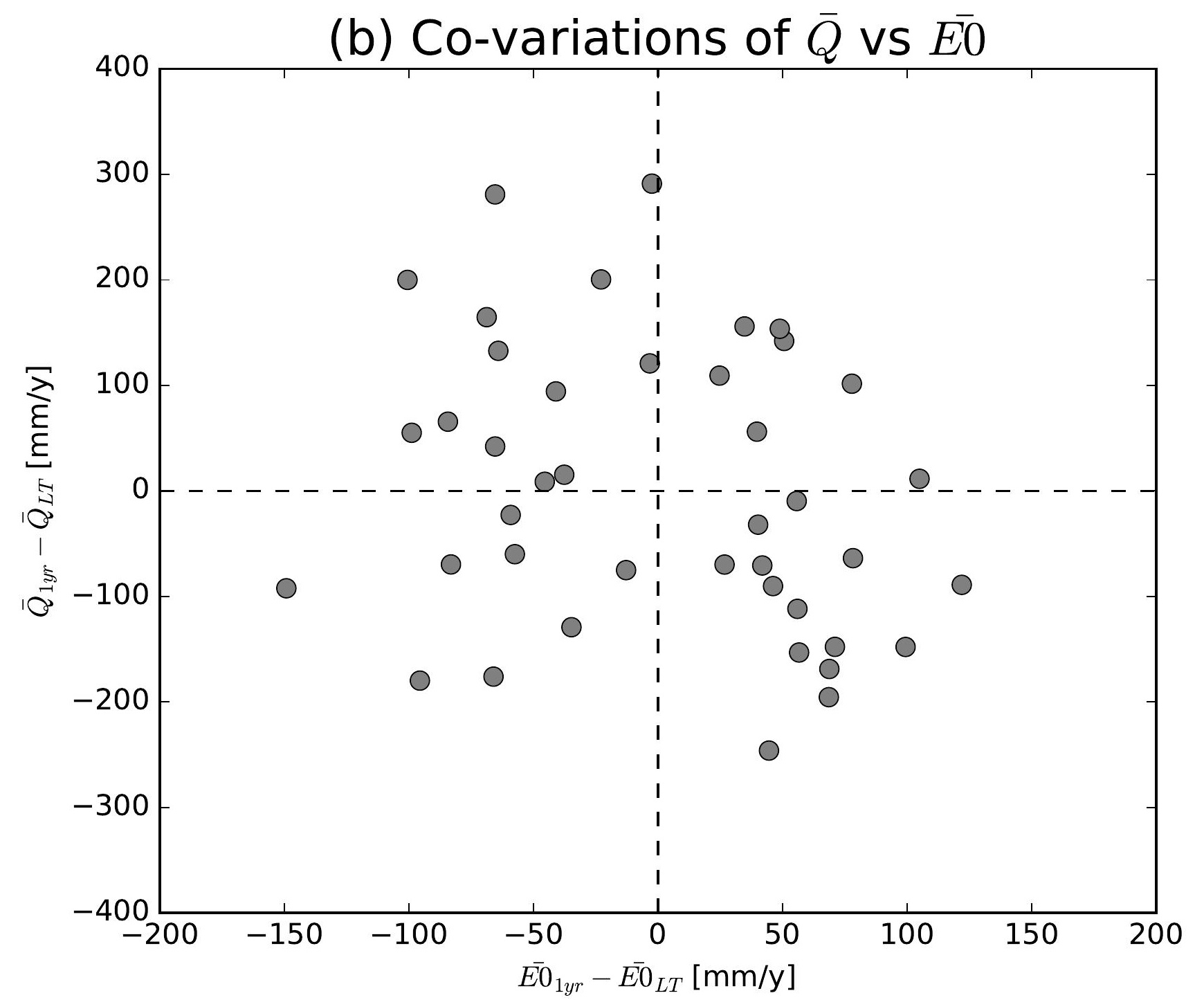

(e) Co-variations of $\bar{Q}$ vs $\overline{E 0}$

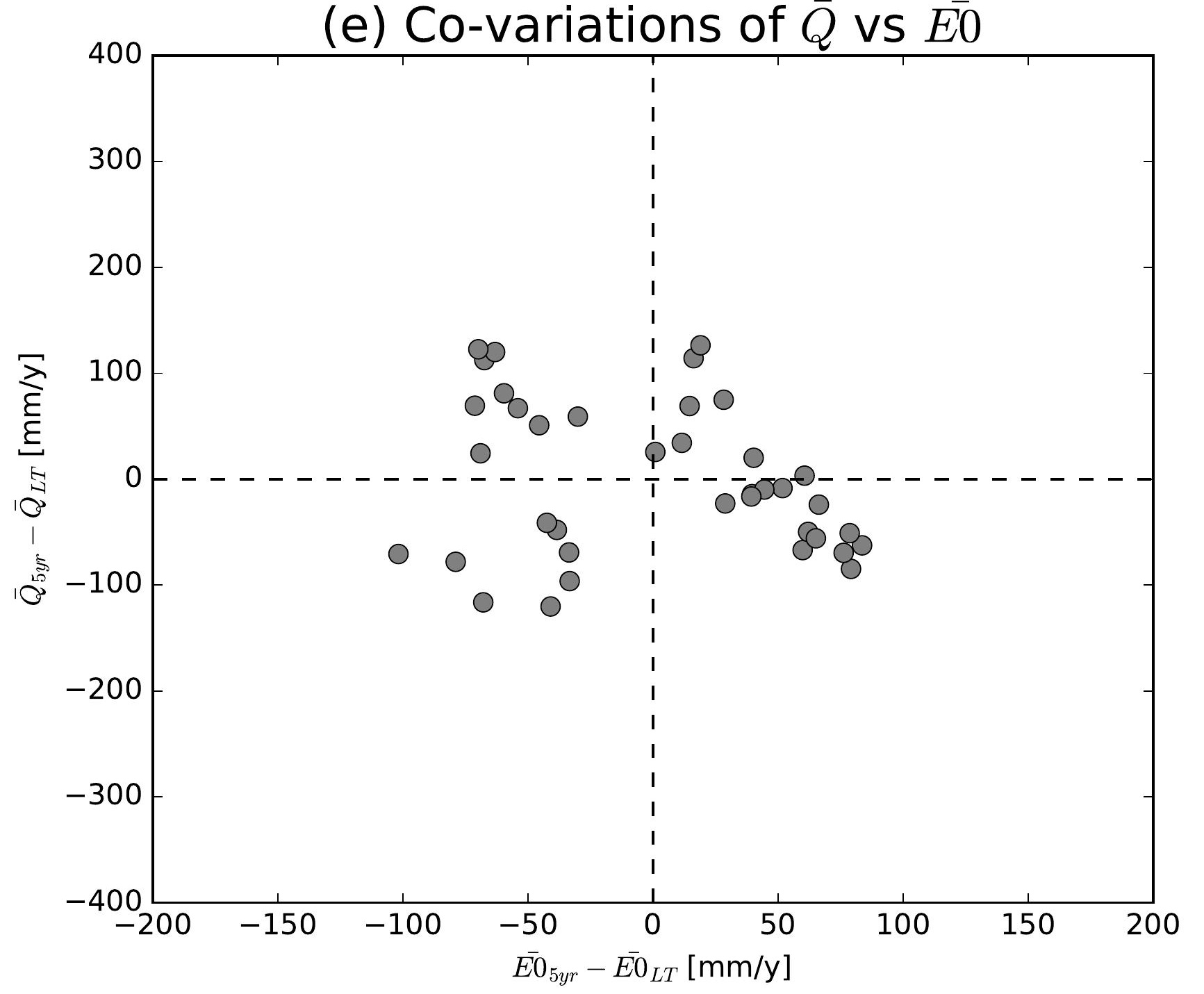

(h) Co-variations of $\bar{Q}$ vs $\overline{E 0}$

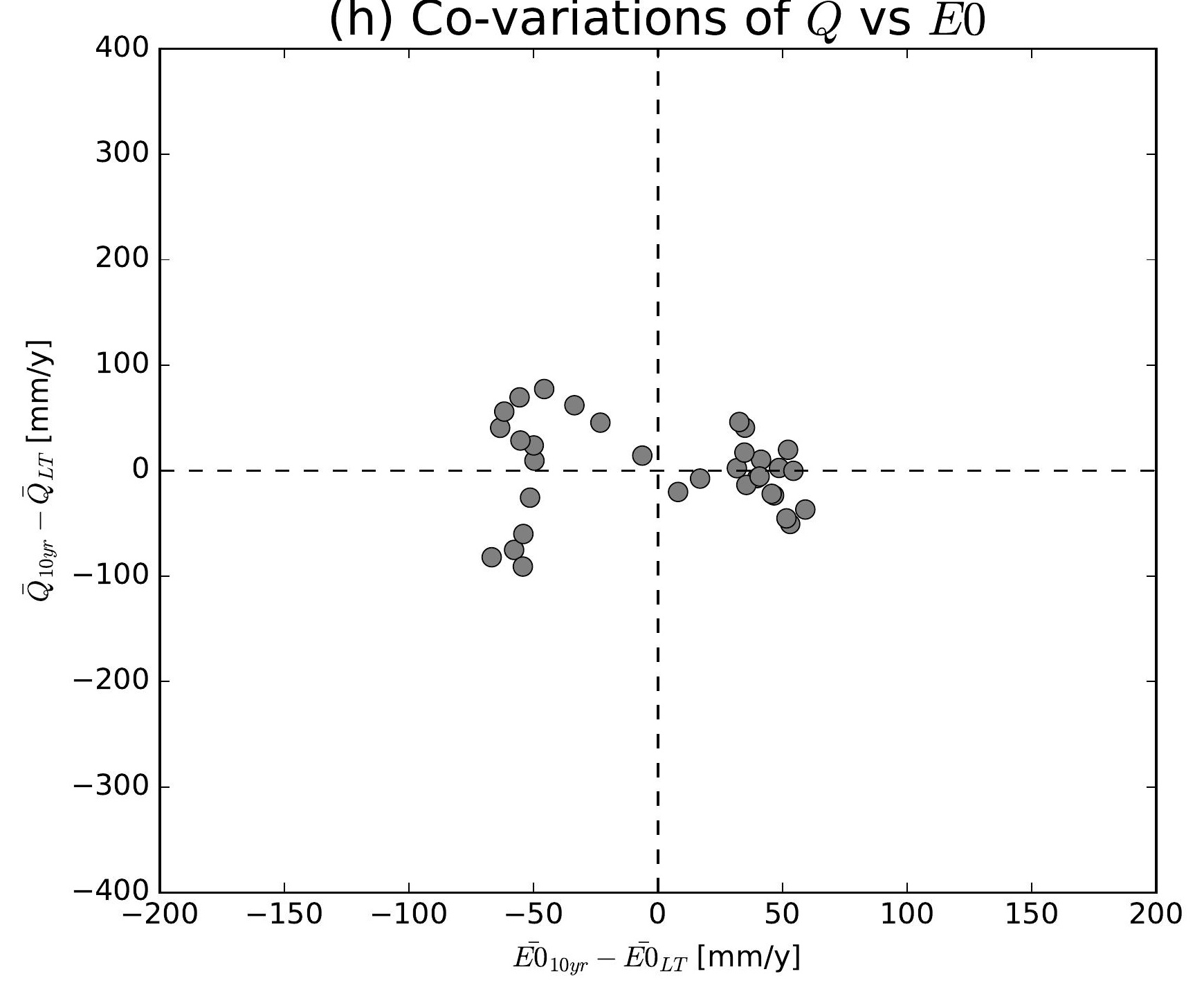

(k) Co-variations of $\bar{Q}$ vs $\overline{E 0}$

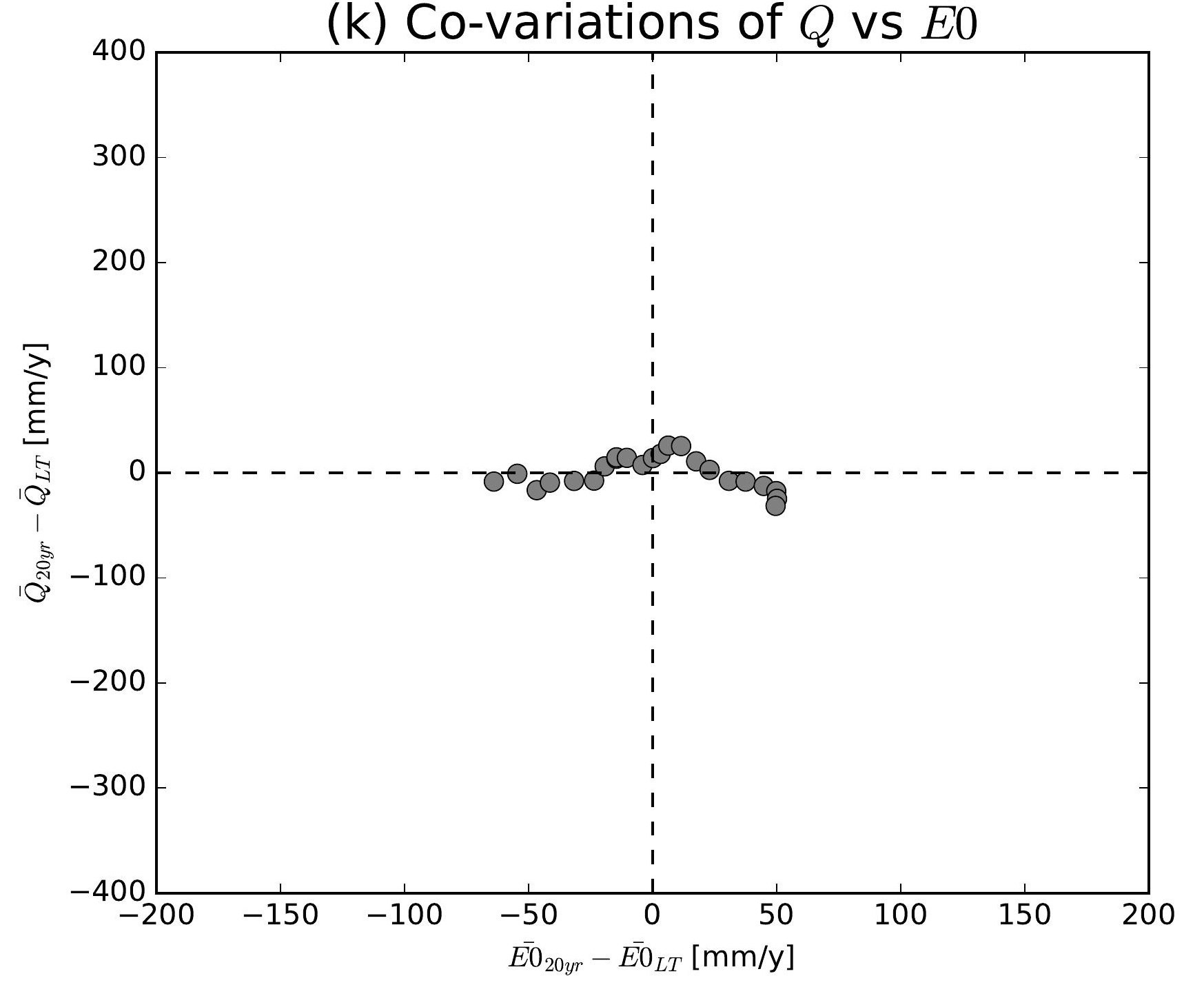

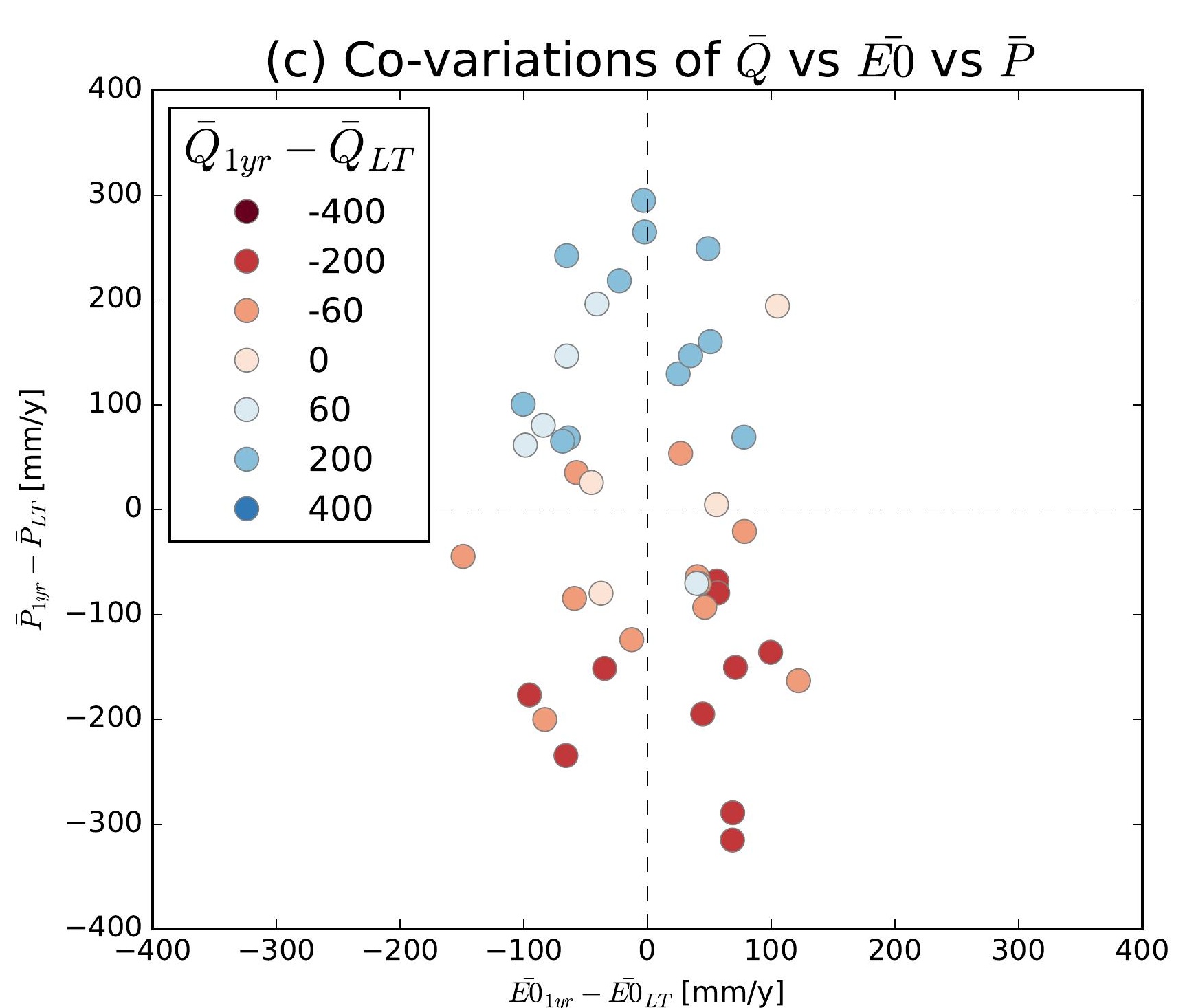
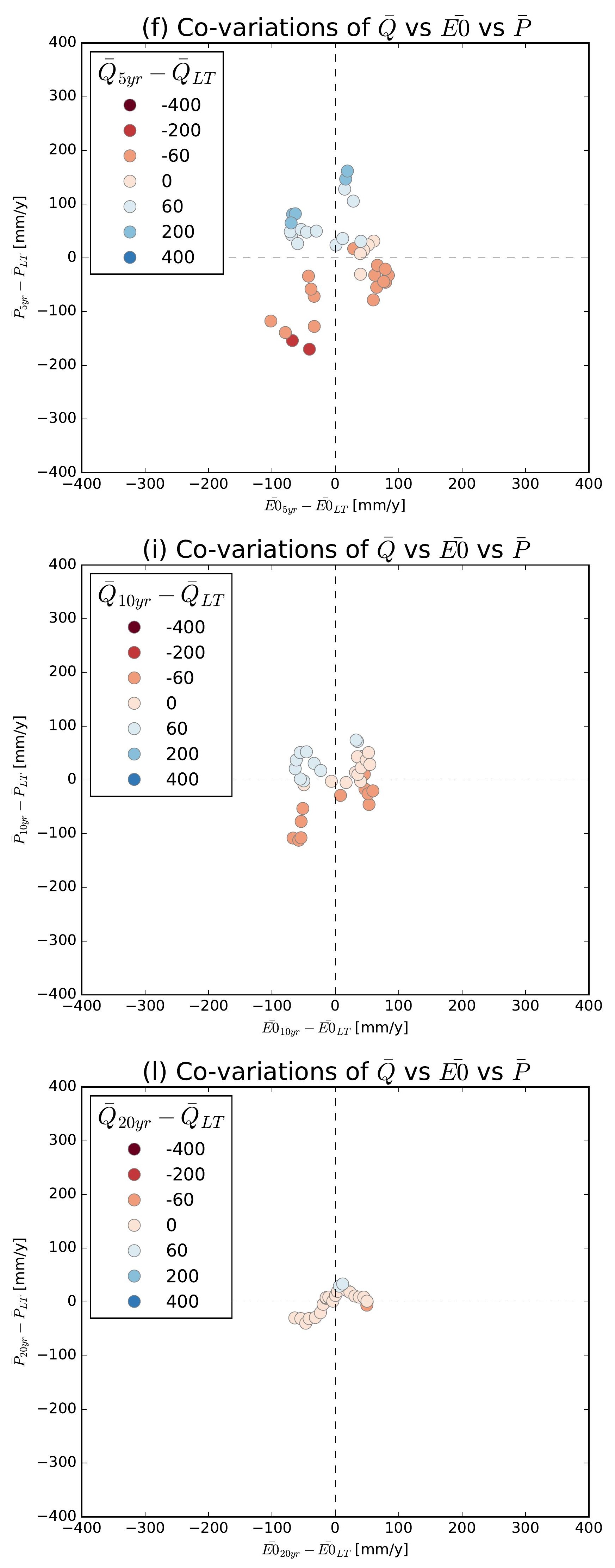

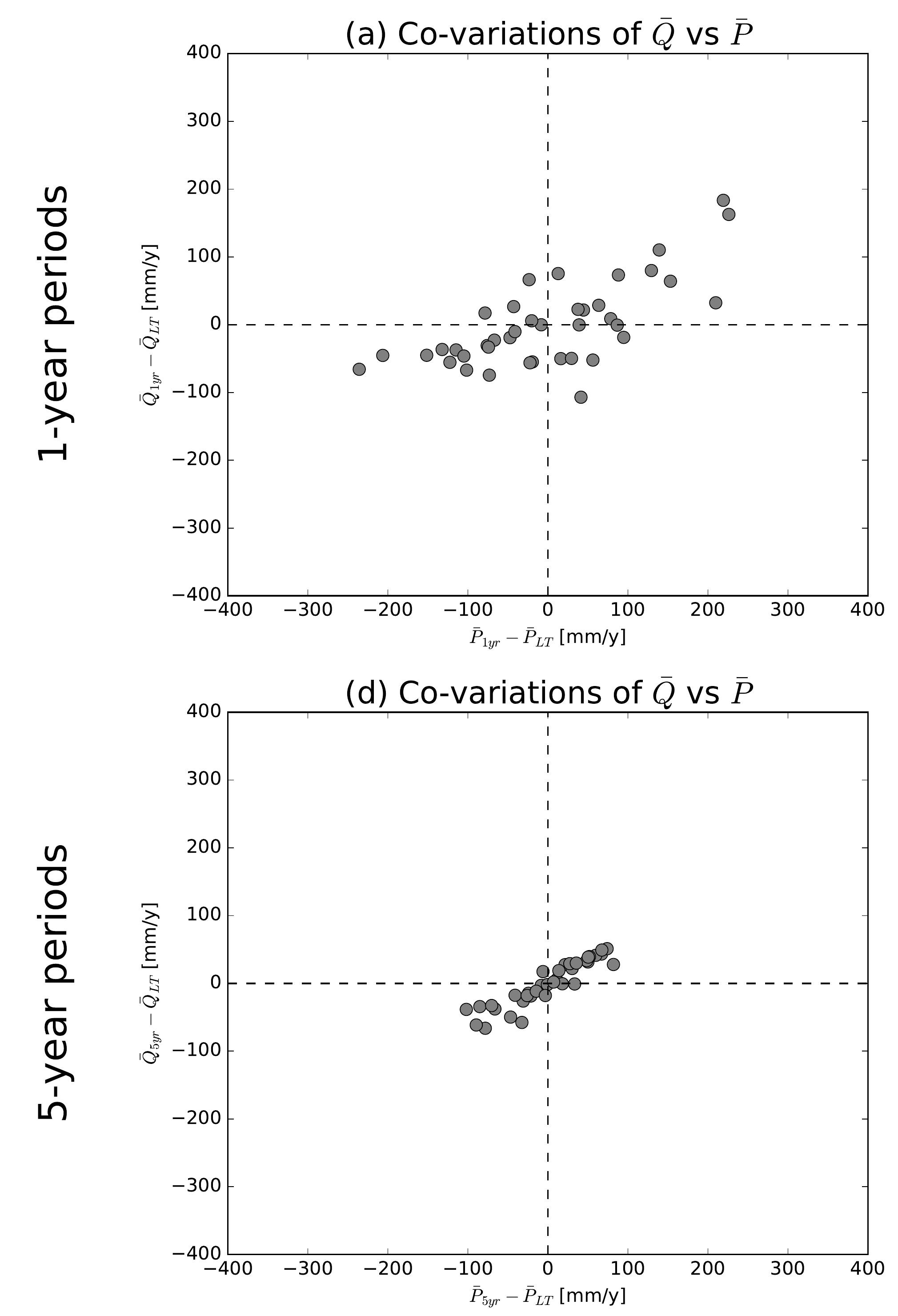

(g) Co-variations of $\bar{Q}$ vs $\bar{P}$
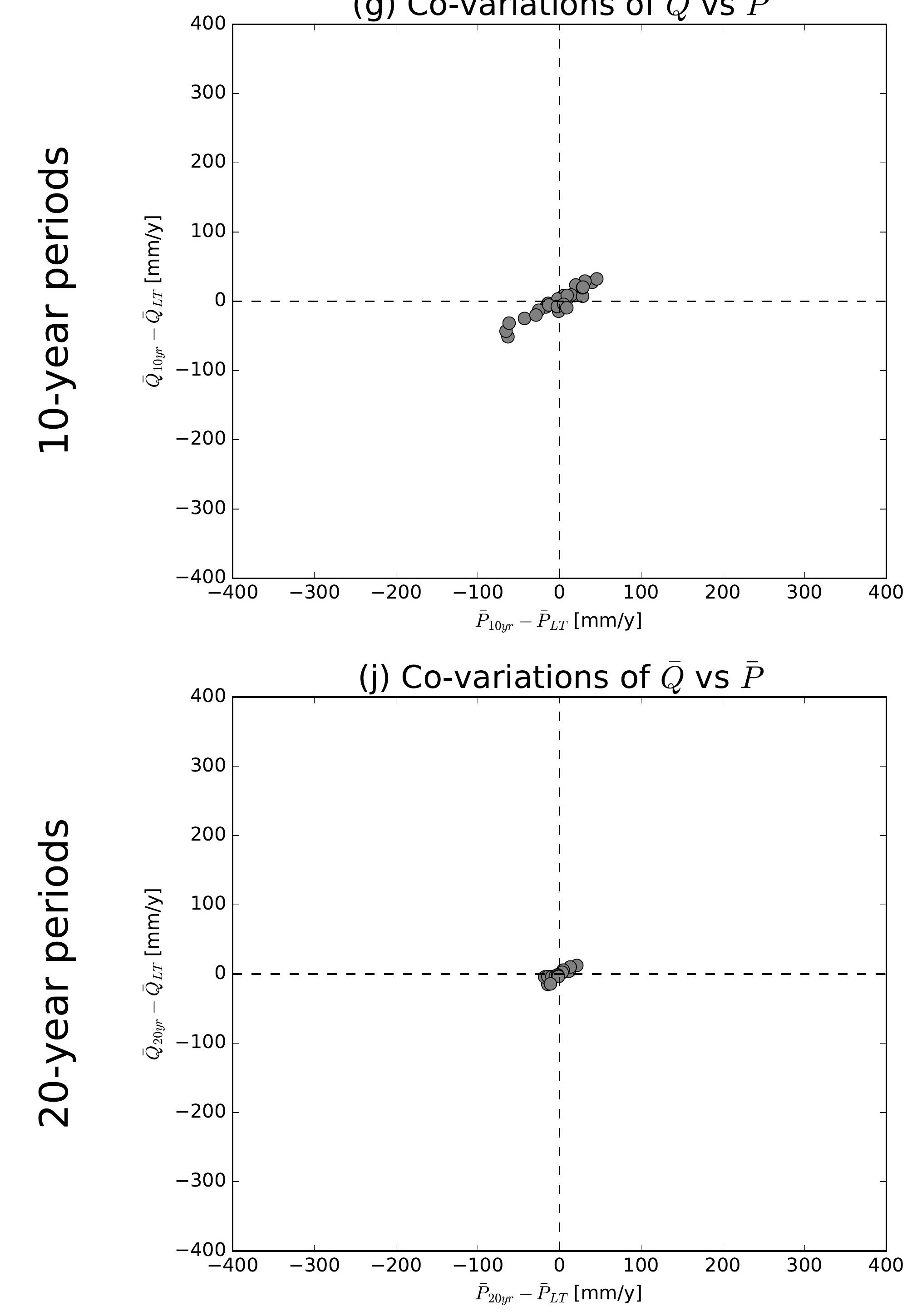

(b) Co-variations of $\bar{Q}$ vs $\overline{E 0}$

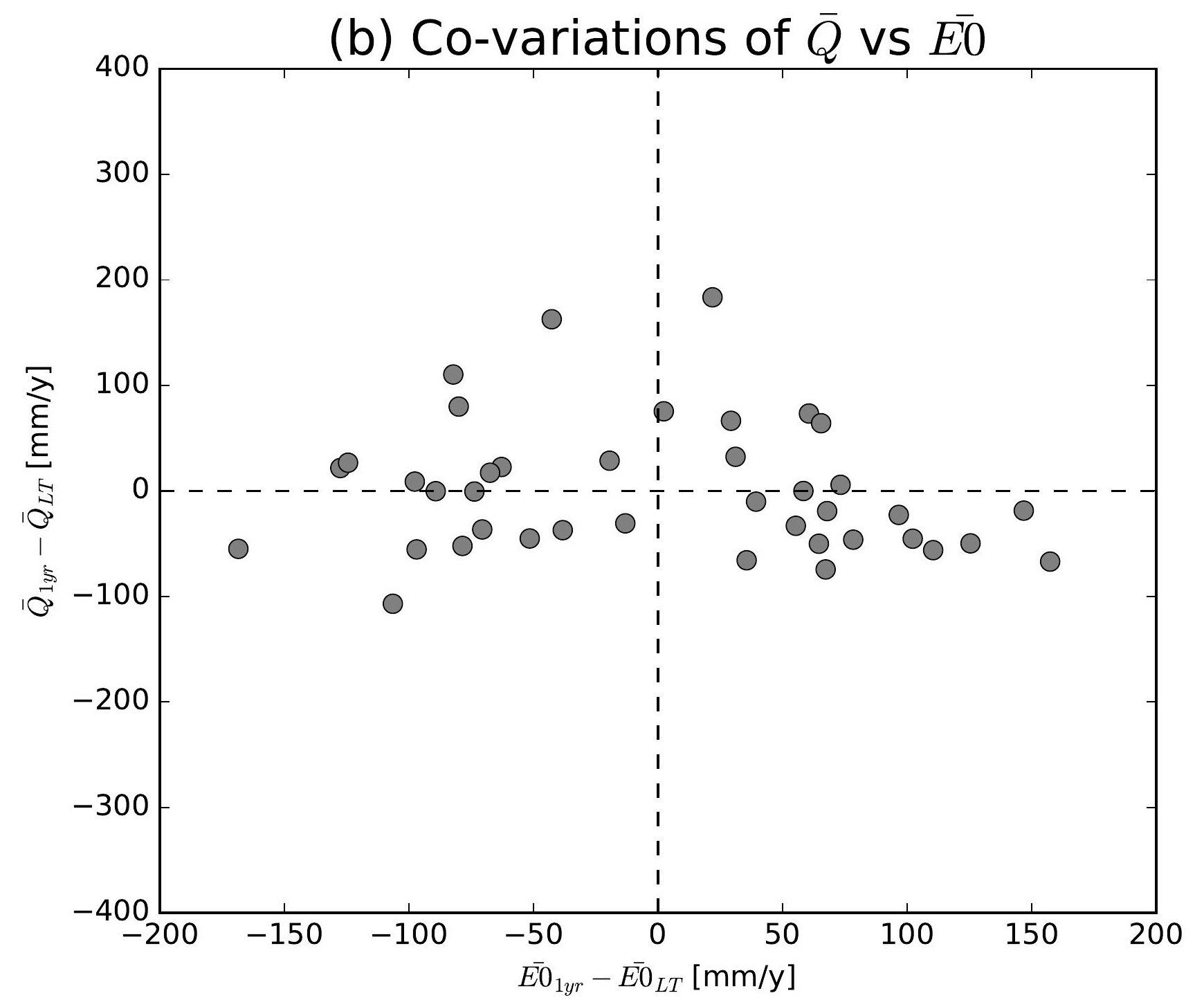

(e) Co-variations of $\bar{Q}$ vs $\overline{E 0}$

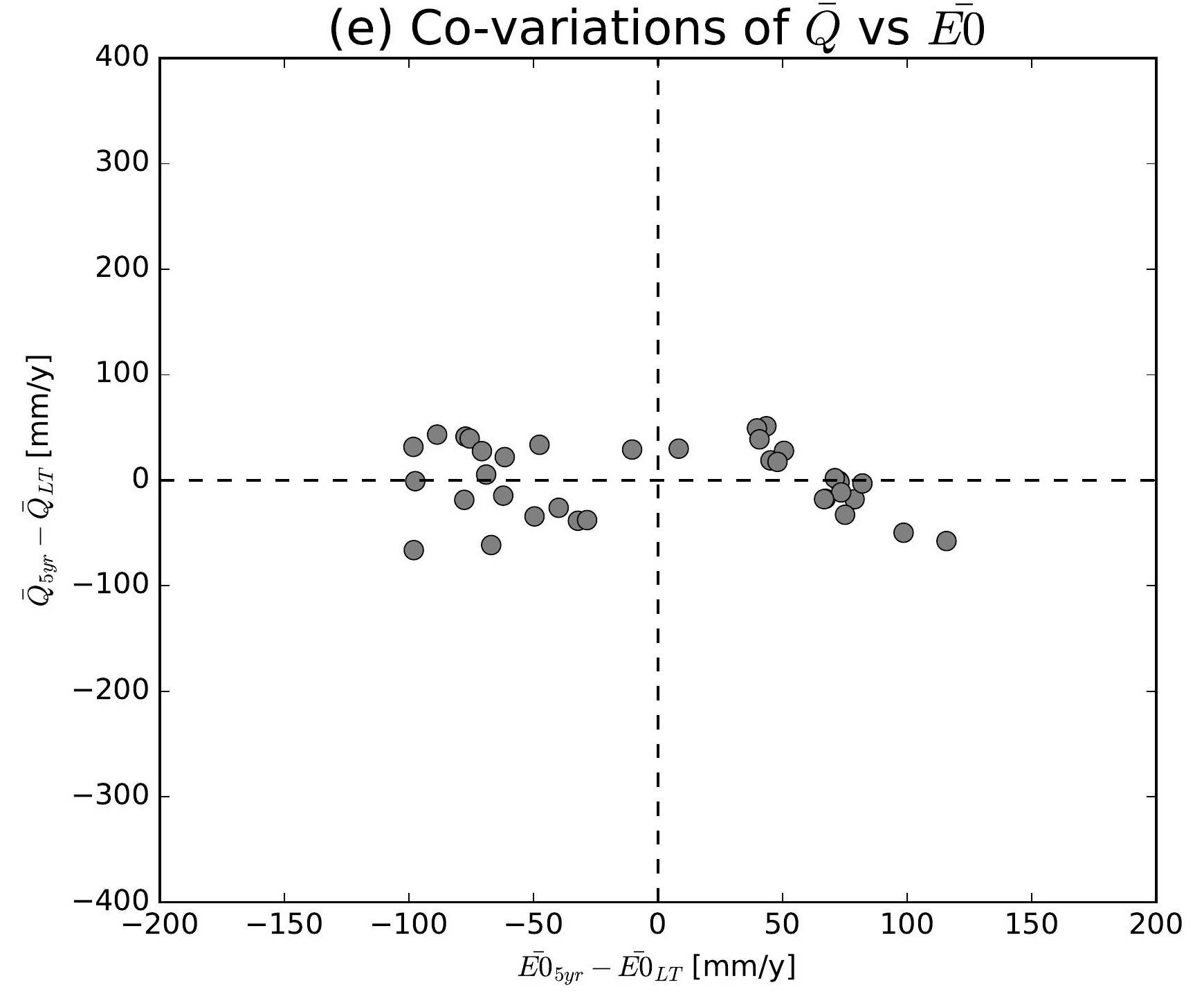

(h) Co-variations of $\bar{Q}$ vs $\overline{E 0}$

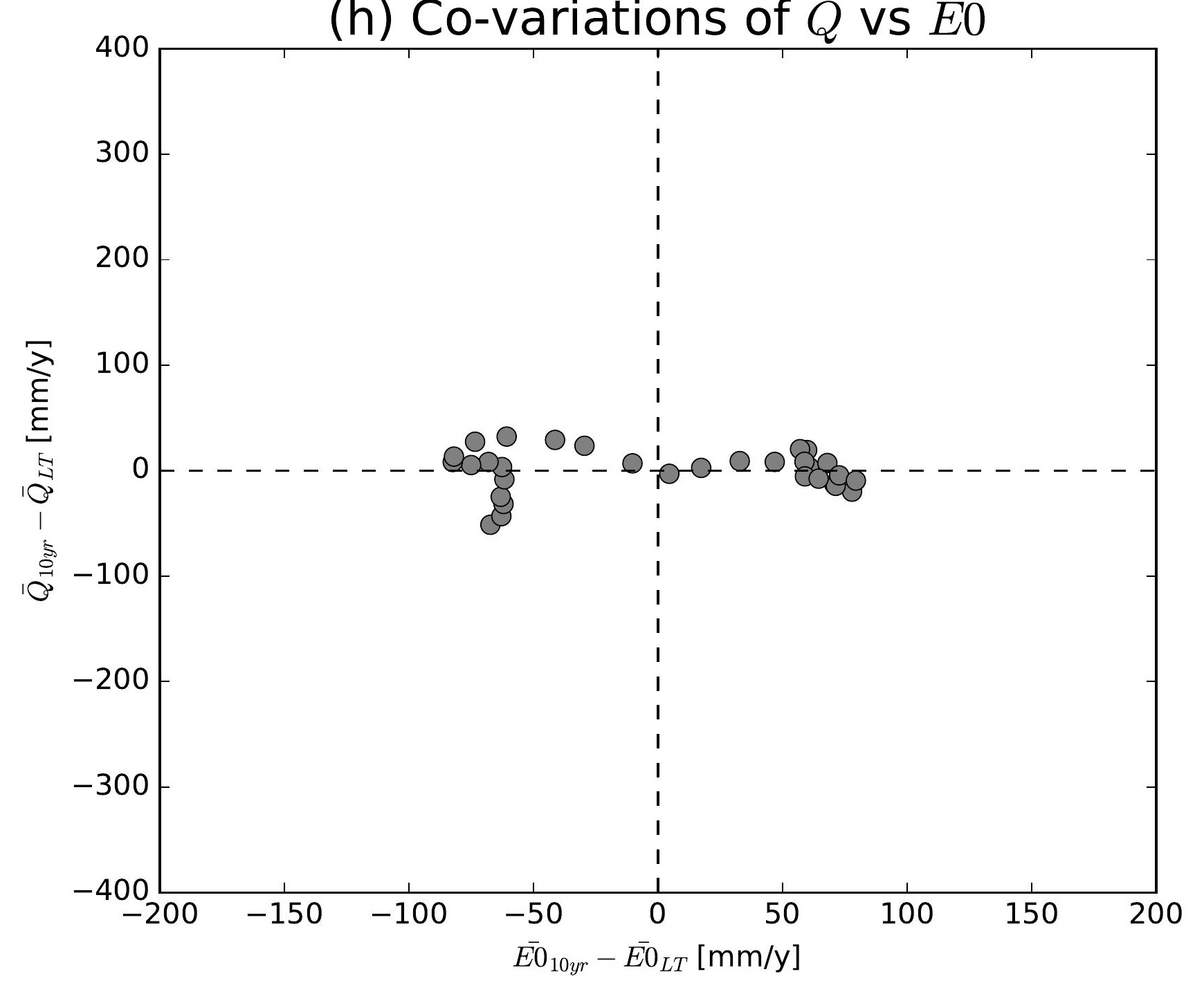

(k) Co-variations of $\bar{Q}$ vs $\overline{E 0}$

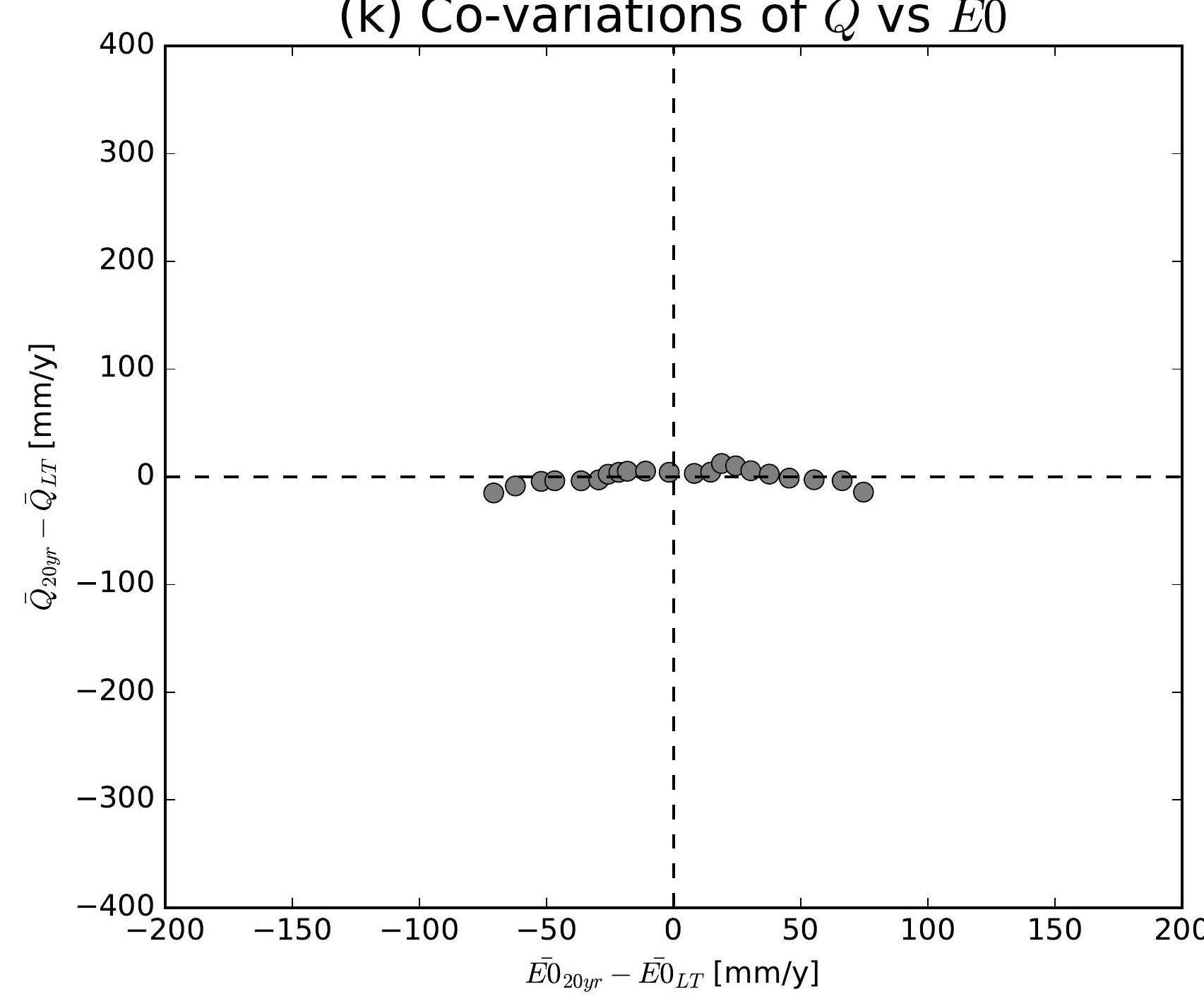

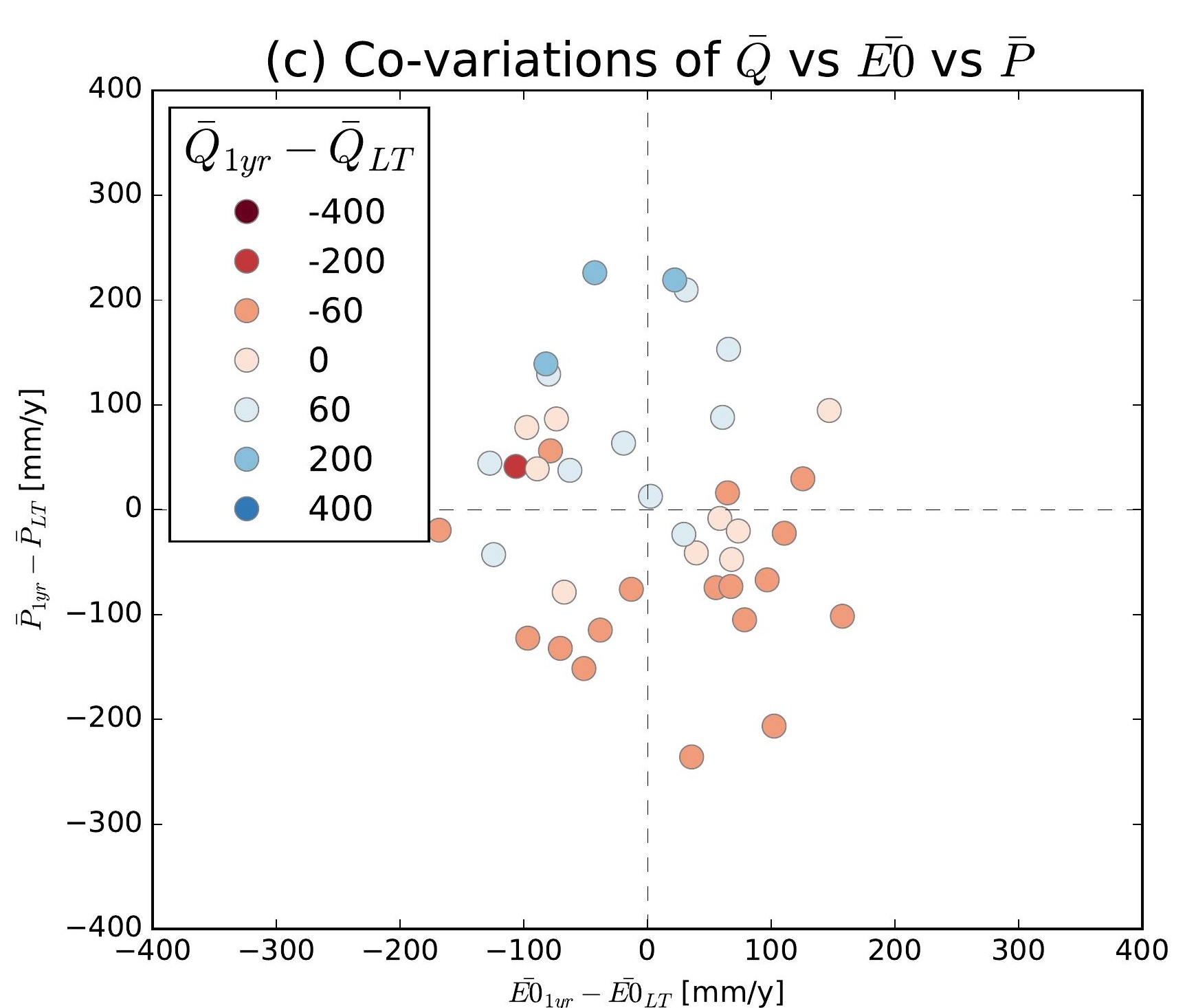
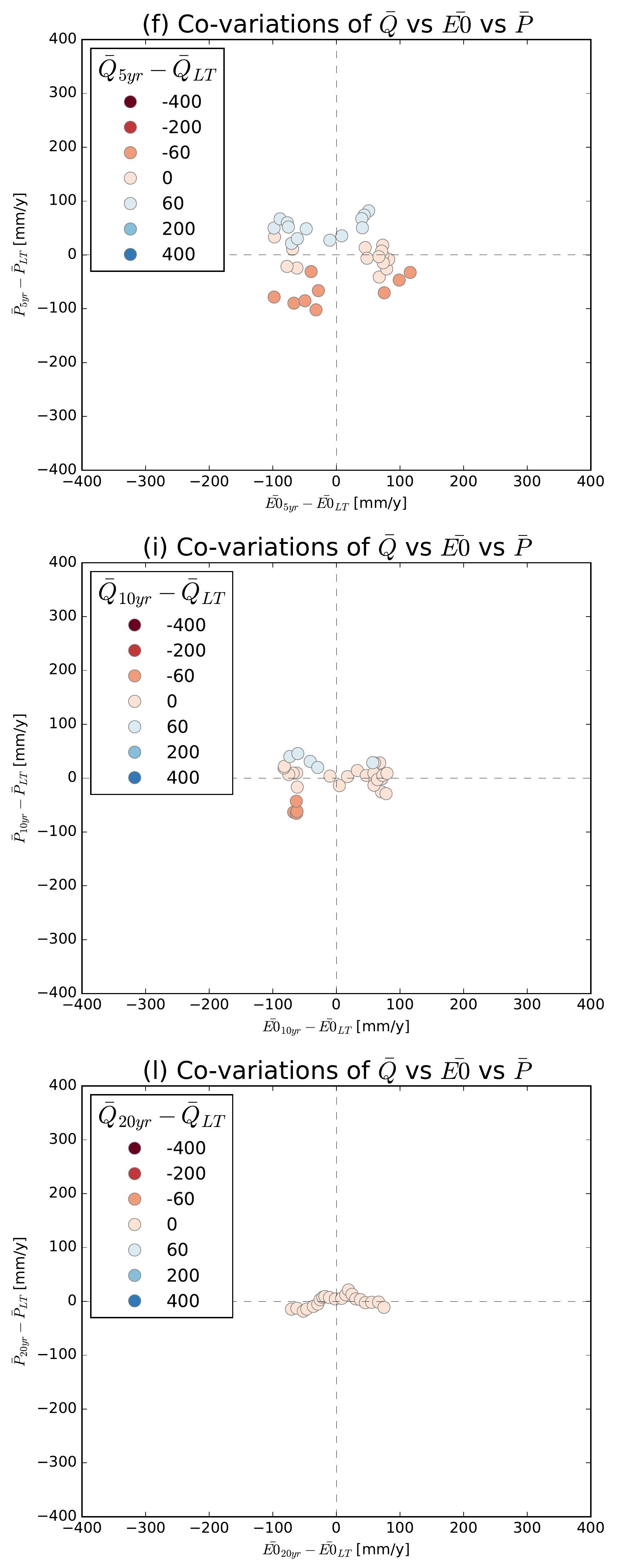

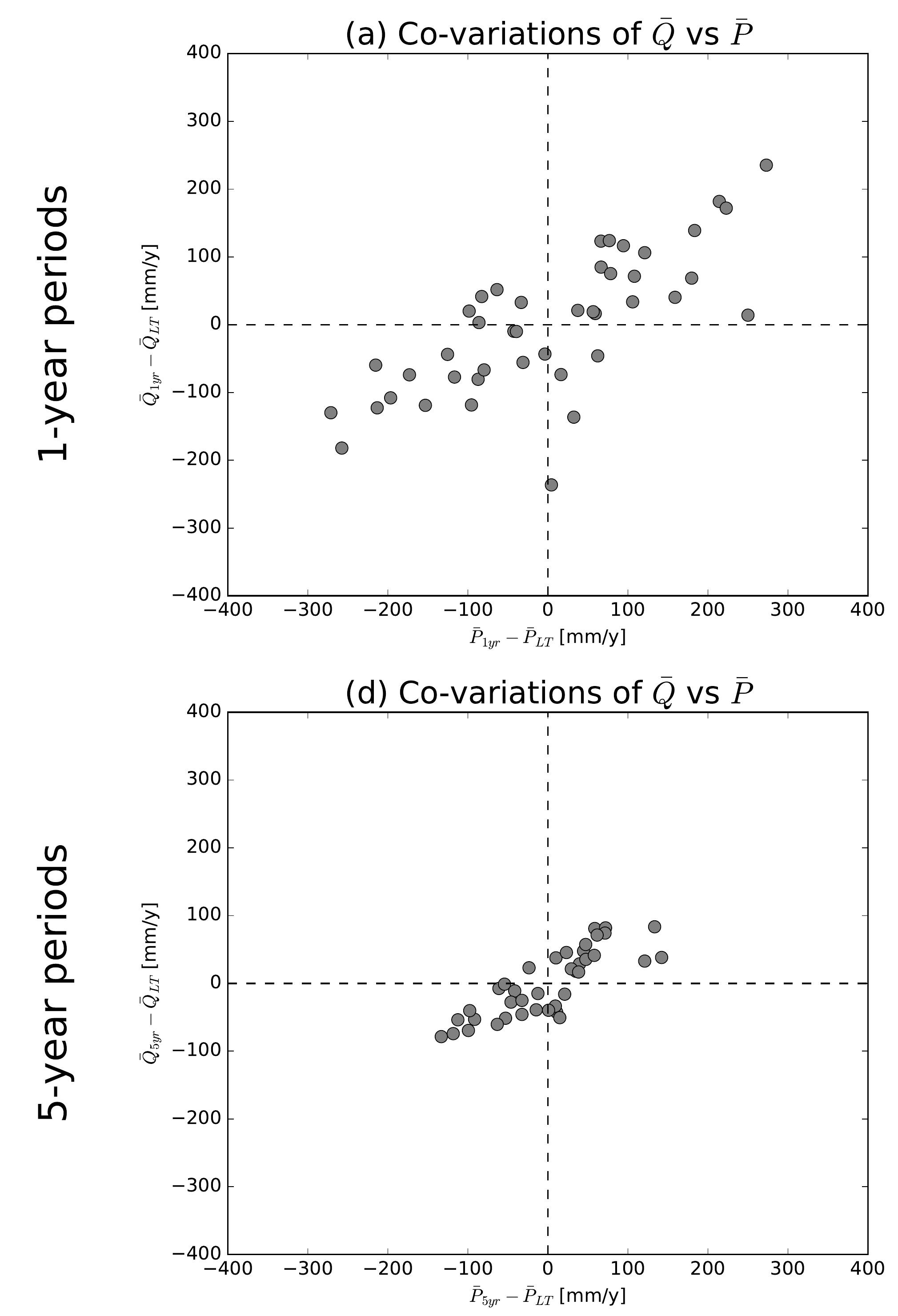

(g) Co-variations of $\bar{Q}$ vs $\bar{P}$
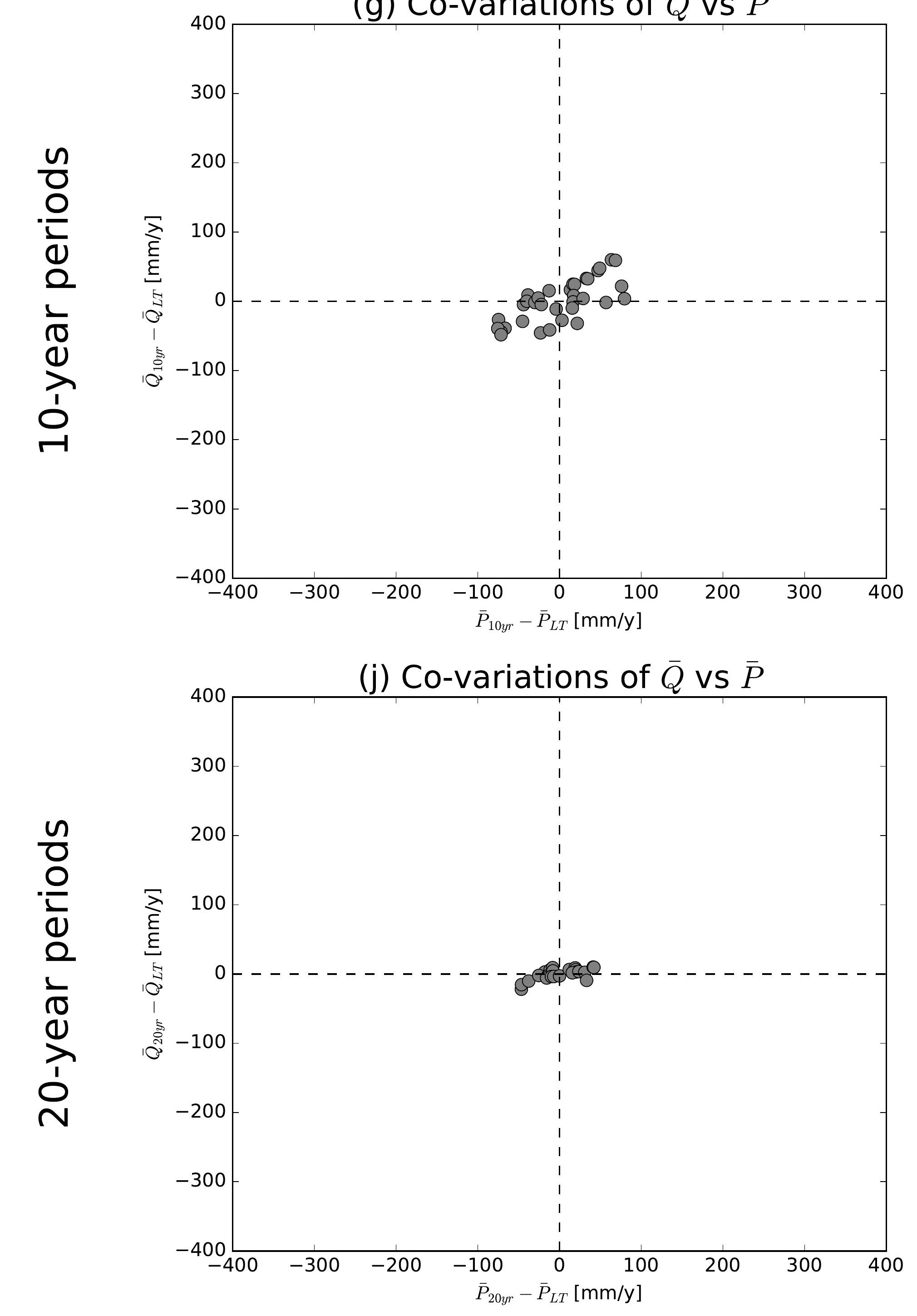

(b) Co-variations of $\bar{Q}$ vs $\overline{E 0}$

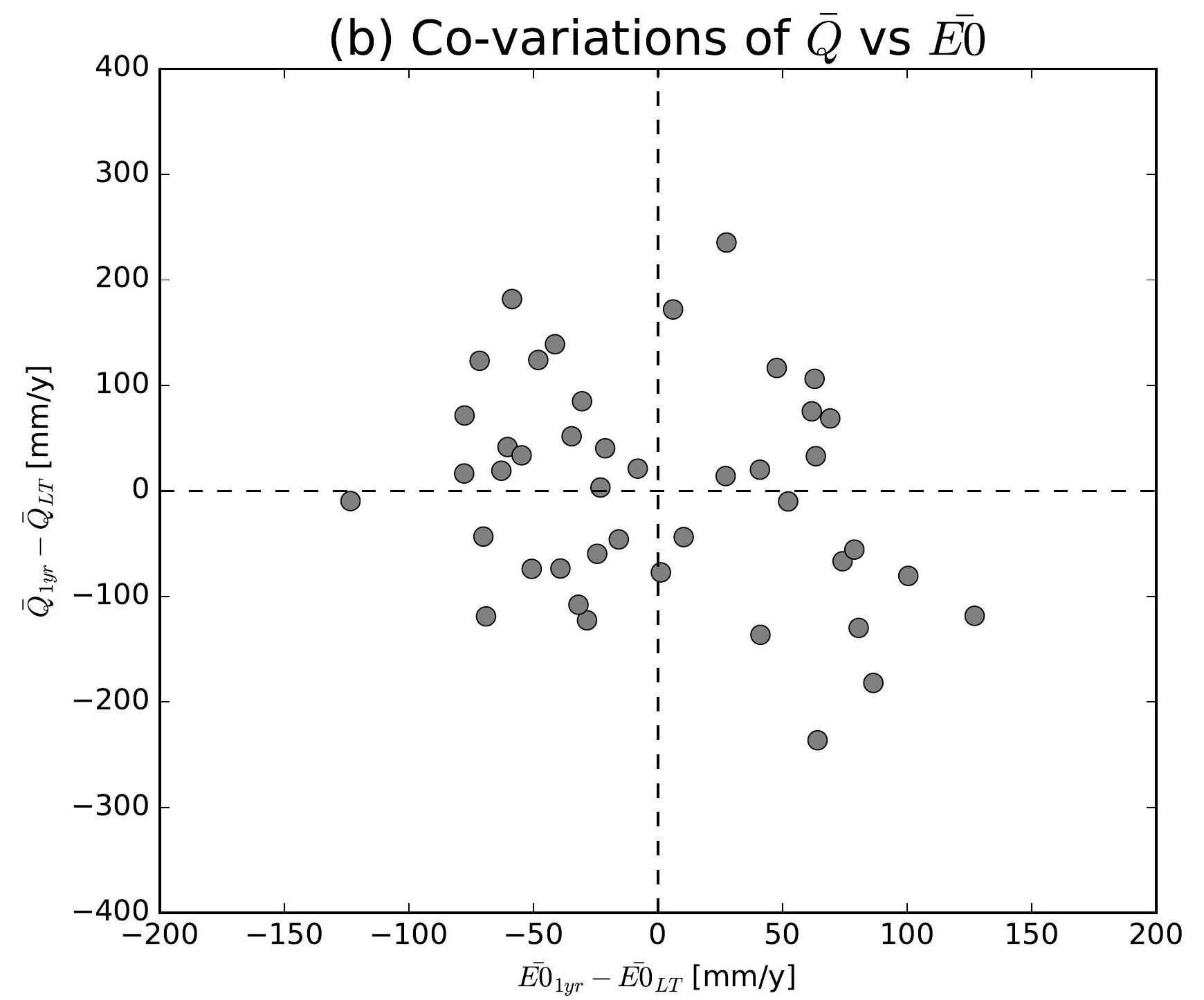

(e) Co-variations of $\bar{Q}$ vs $\overline{E 0}$

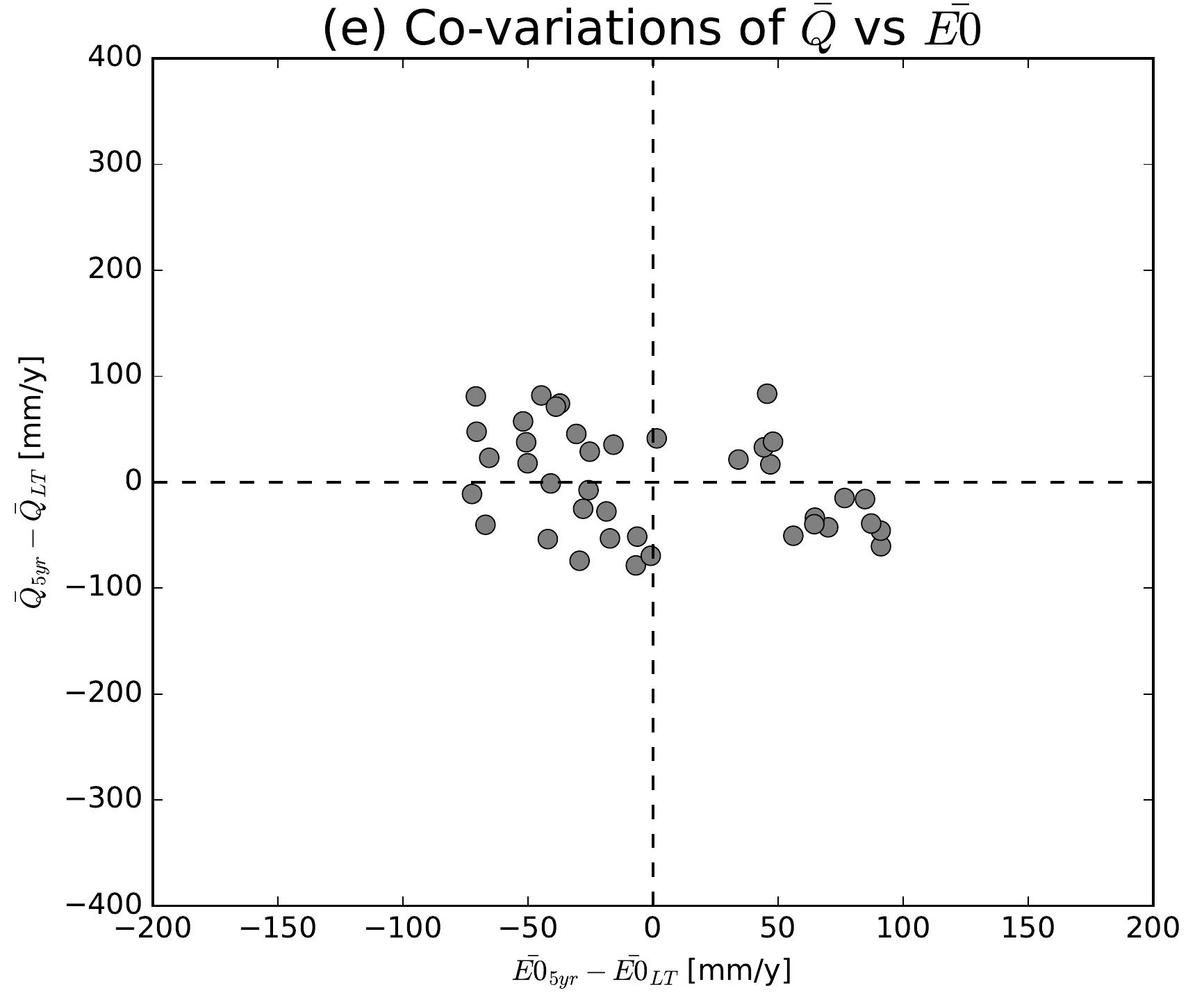

(h) Co-variations of $\bar{Q}$ vs $\overline{E 0}$

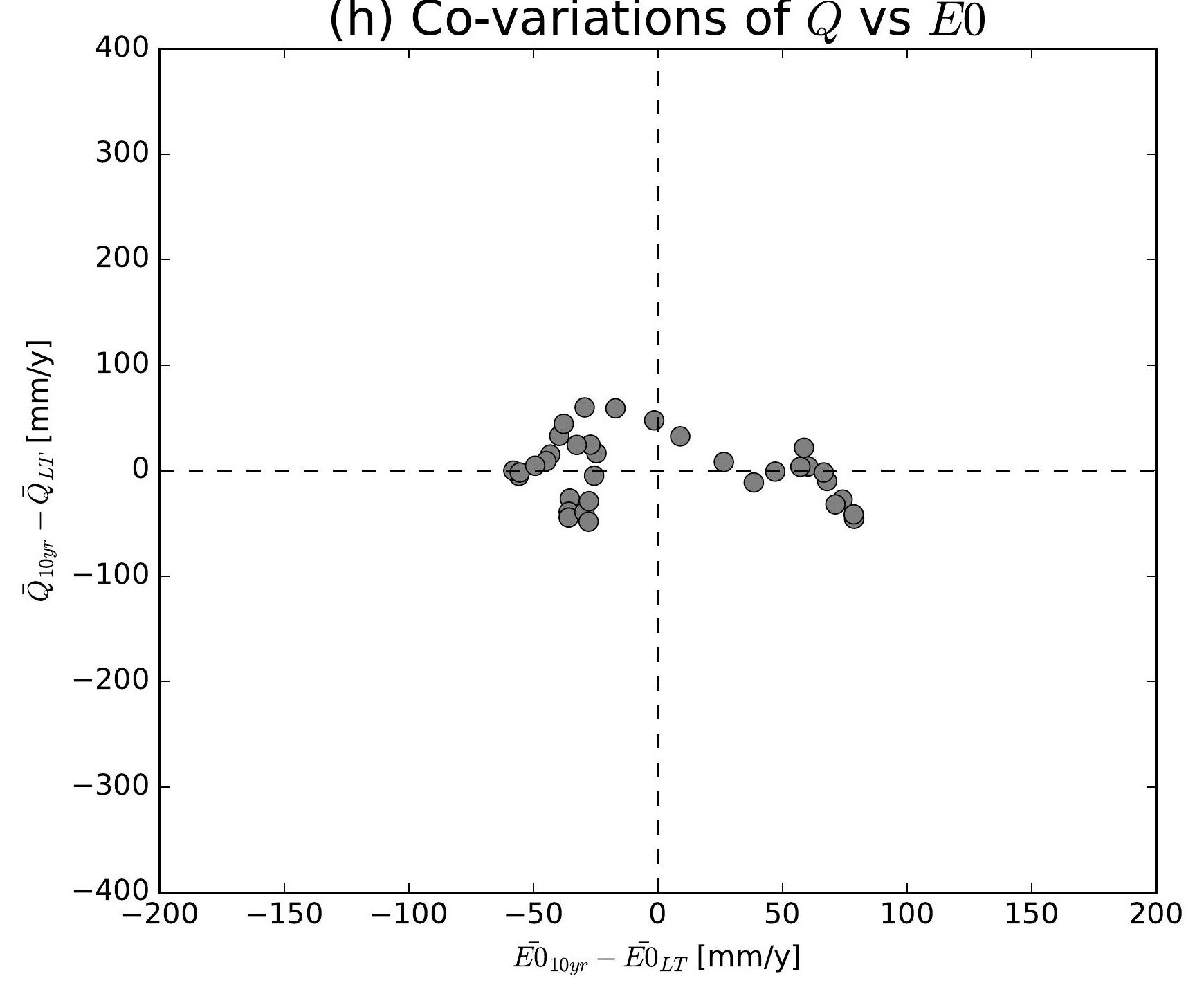

(k) Co-variations of $\bar{Q}$ vs $\overline{E 0}$

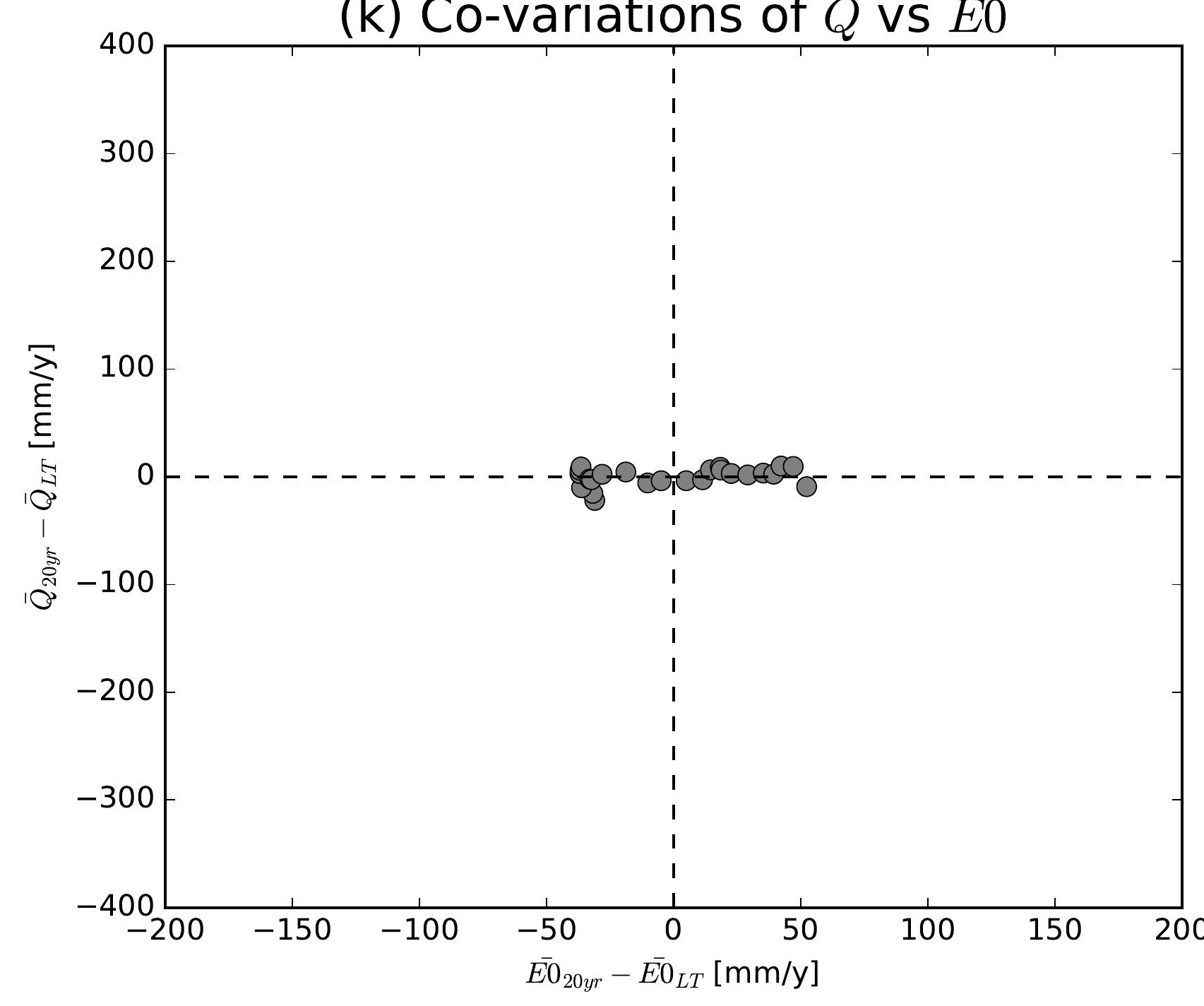

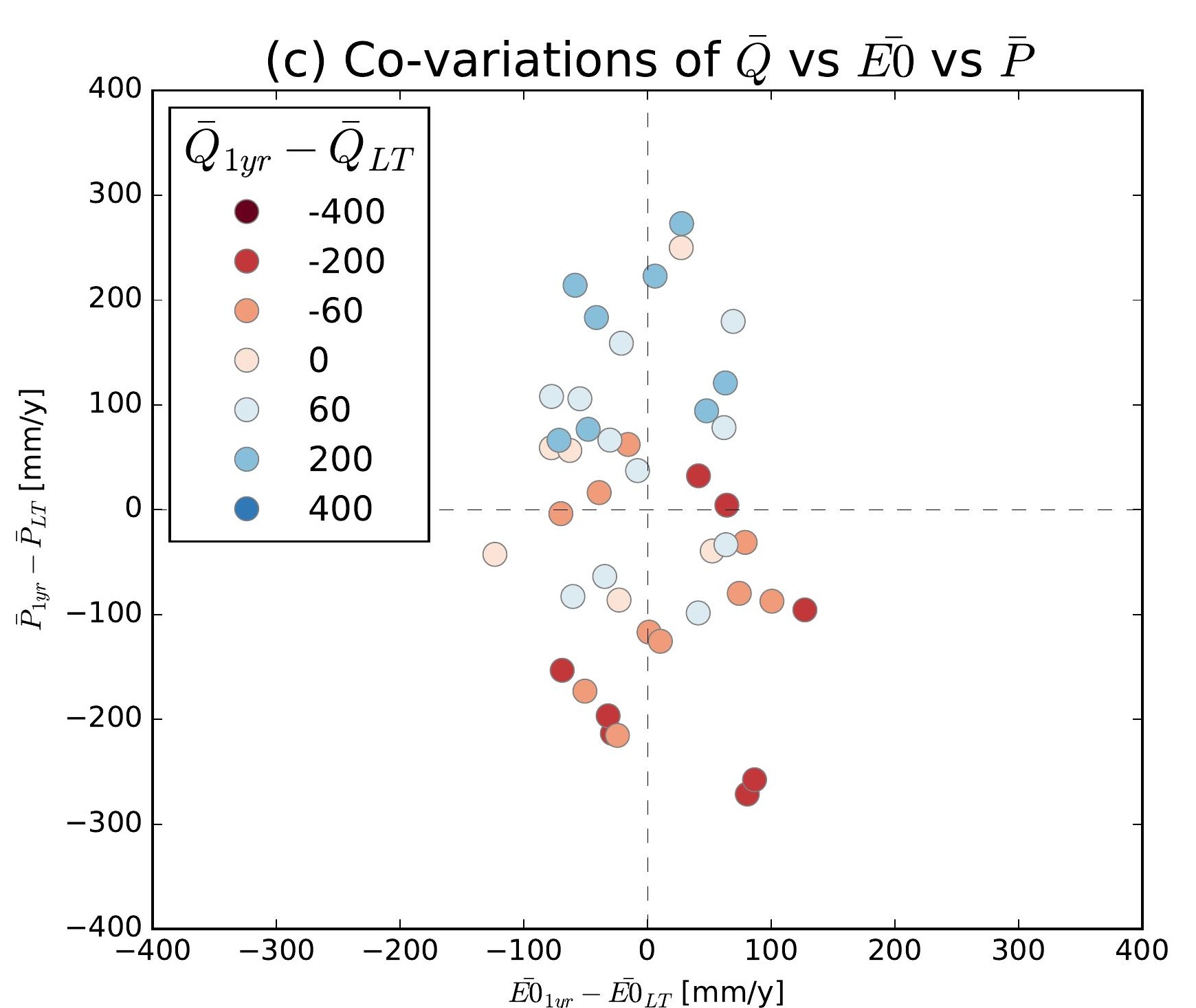
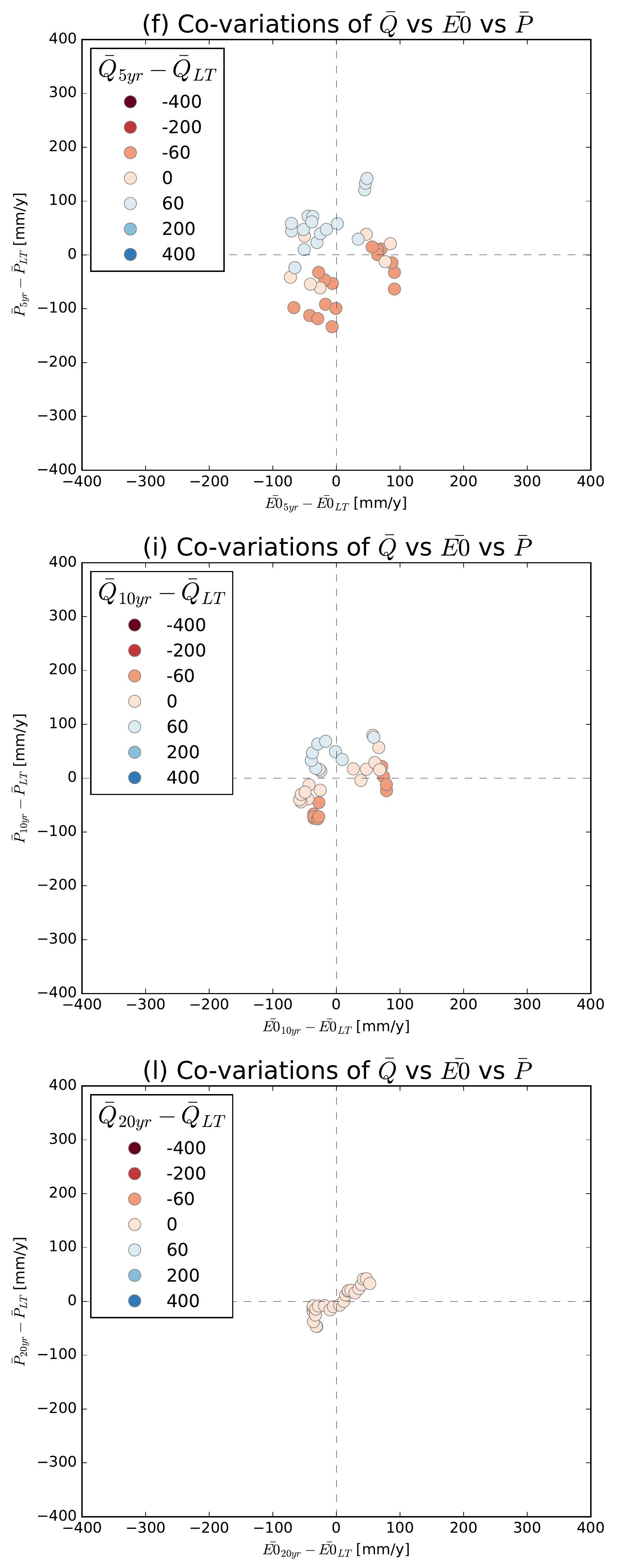

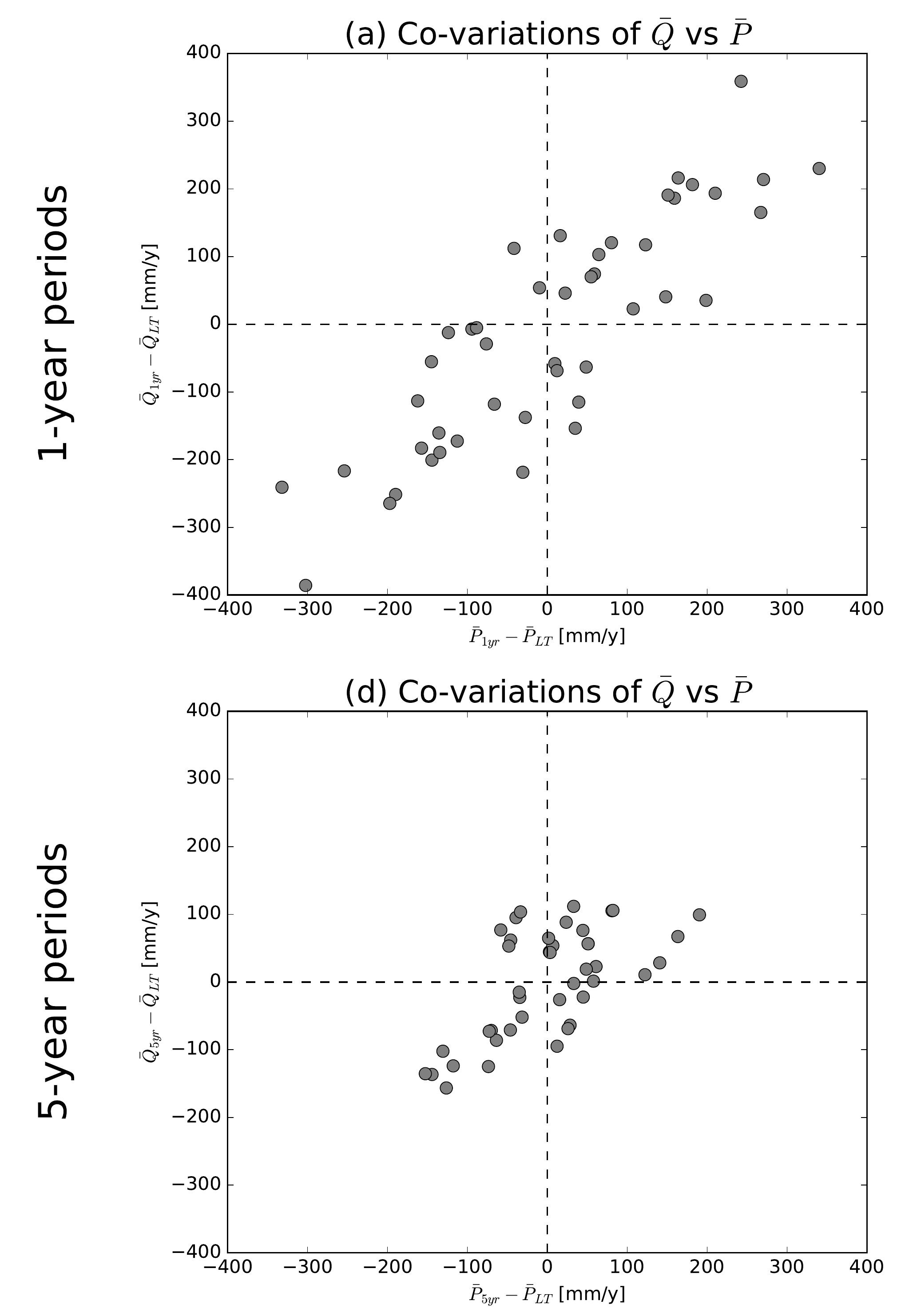

(g) Co-variations of $\bar{Q}$ vs $\bar{P}$

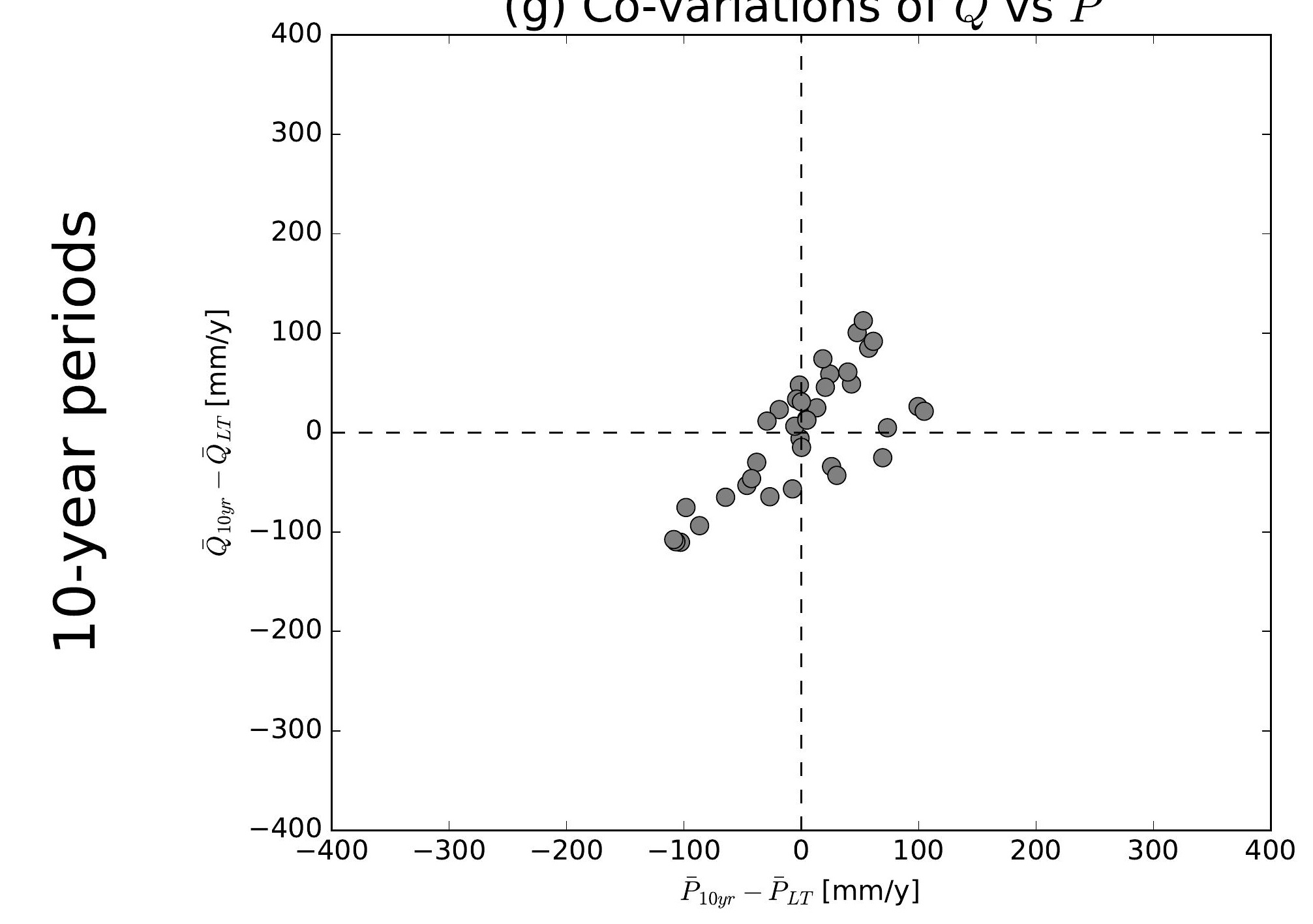

(j) Co-variations of $\bar{Q}$ vs $\bar{P}$

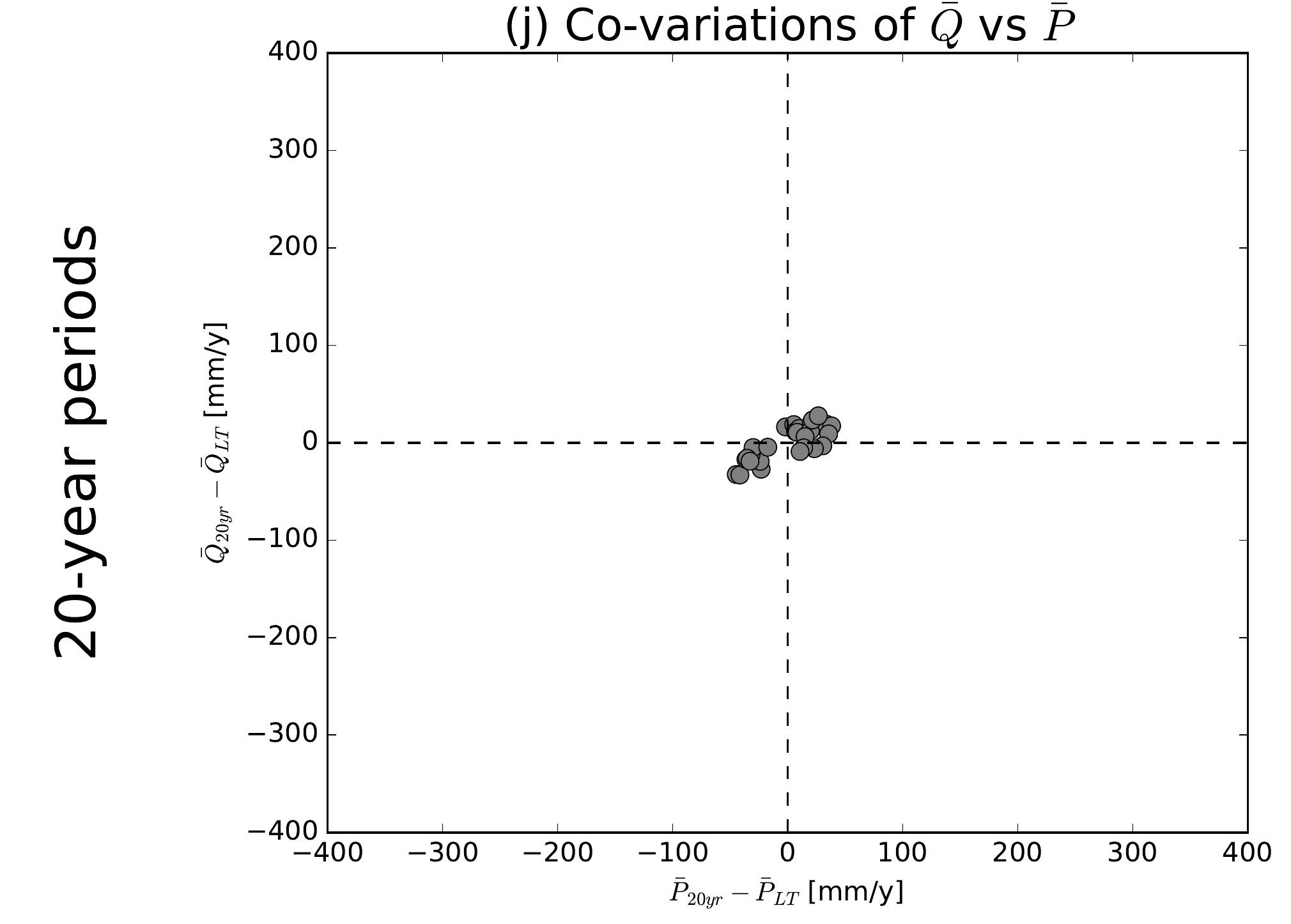

(b) Co-variations of $\bar{Q}$ vs $\overline{E 0}$

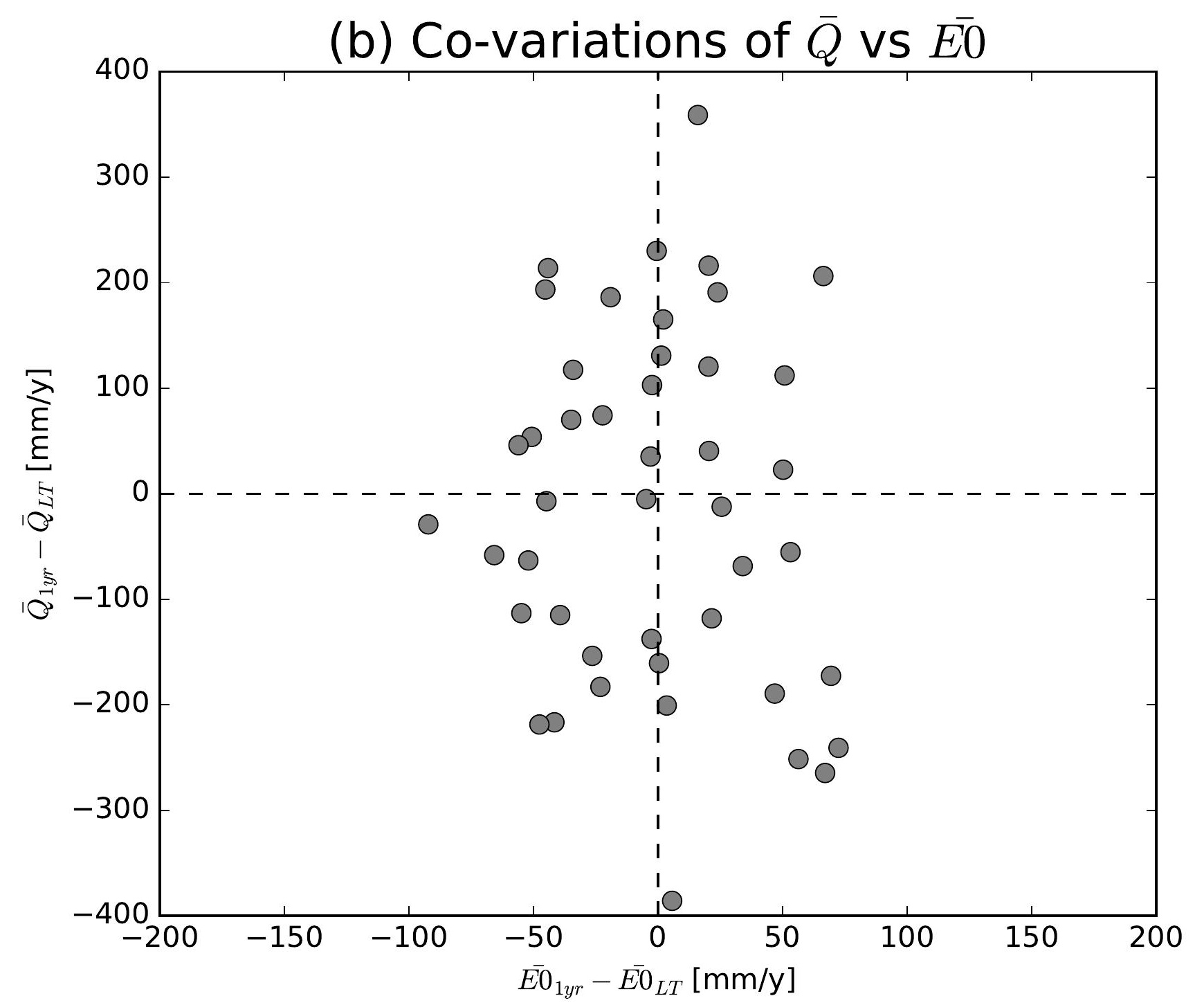

(e) Co-variations of $\bar{Q}$ vs $\overline{E 0}$

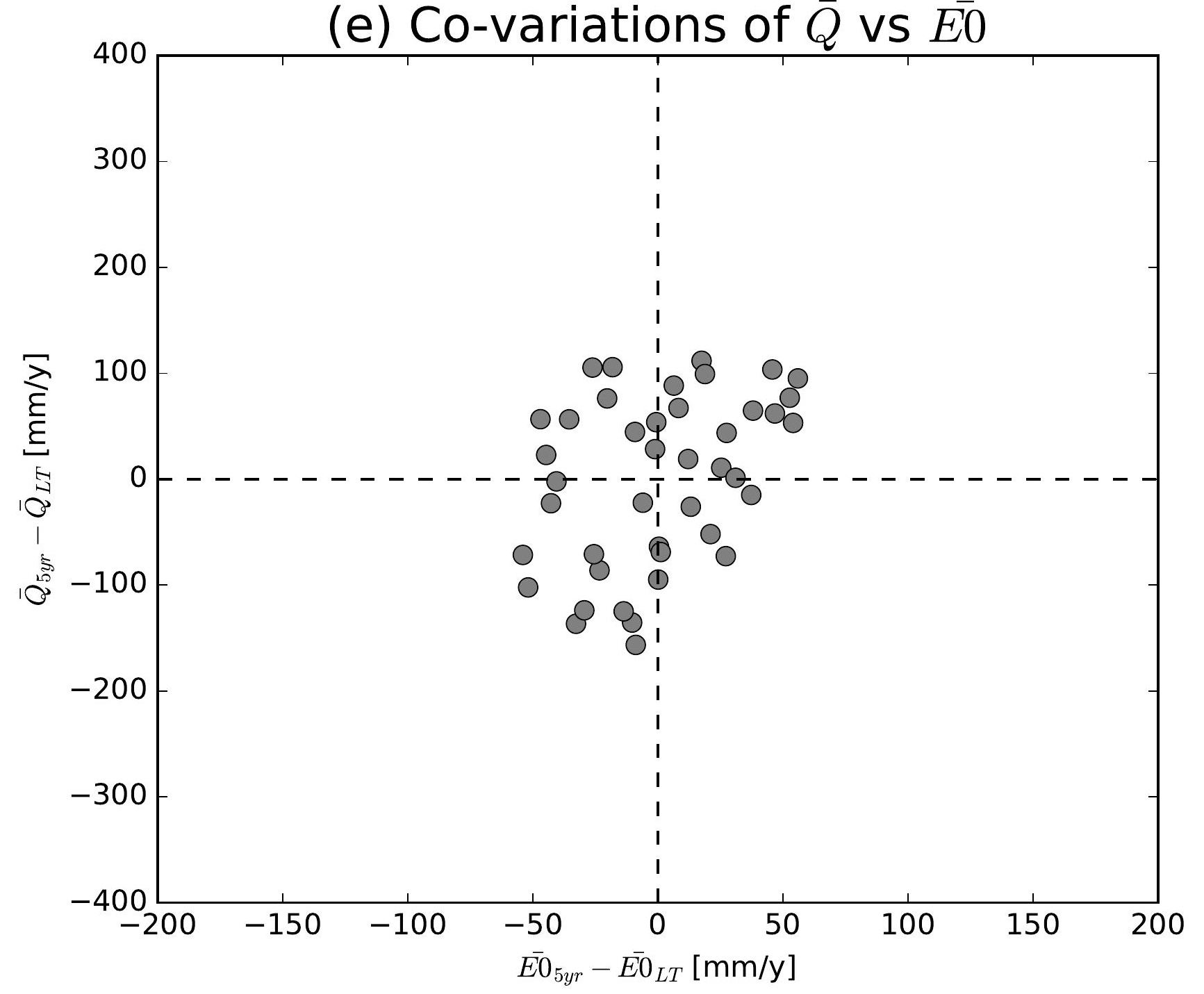

(h) Co-variations of $\bar{Q}$ vs $\overline{E 0}$

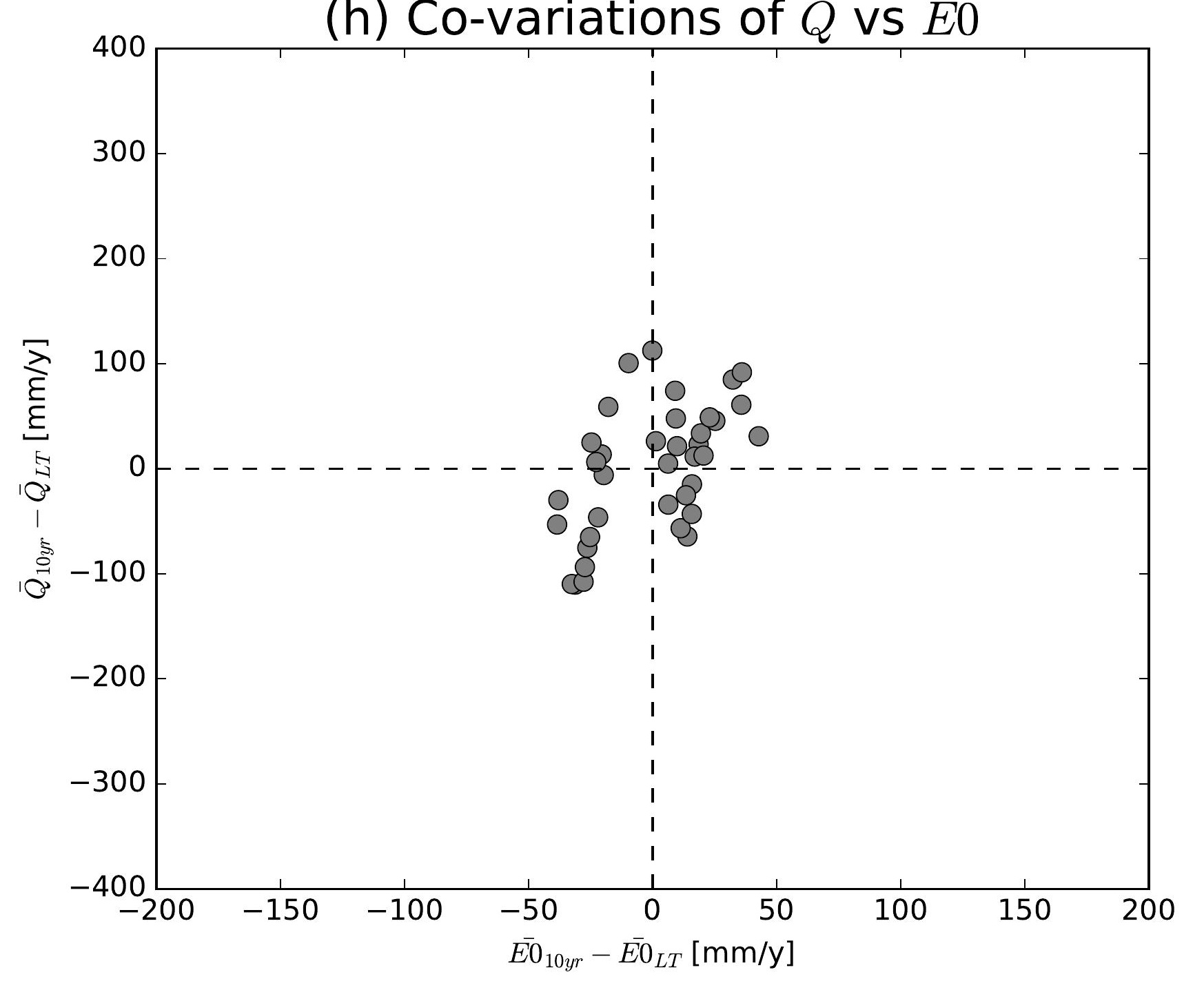

(k) Co-variations of $\bar{Q}$ vs $\overline{E 0}$

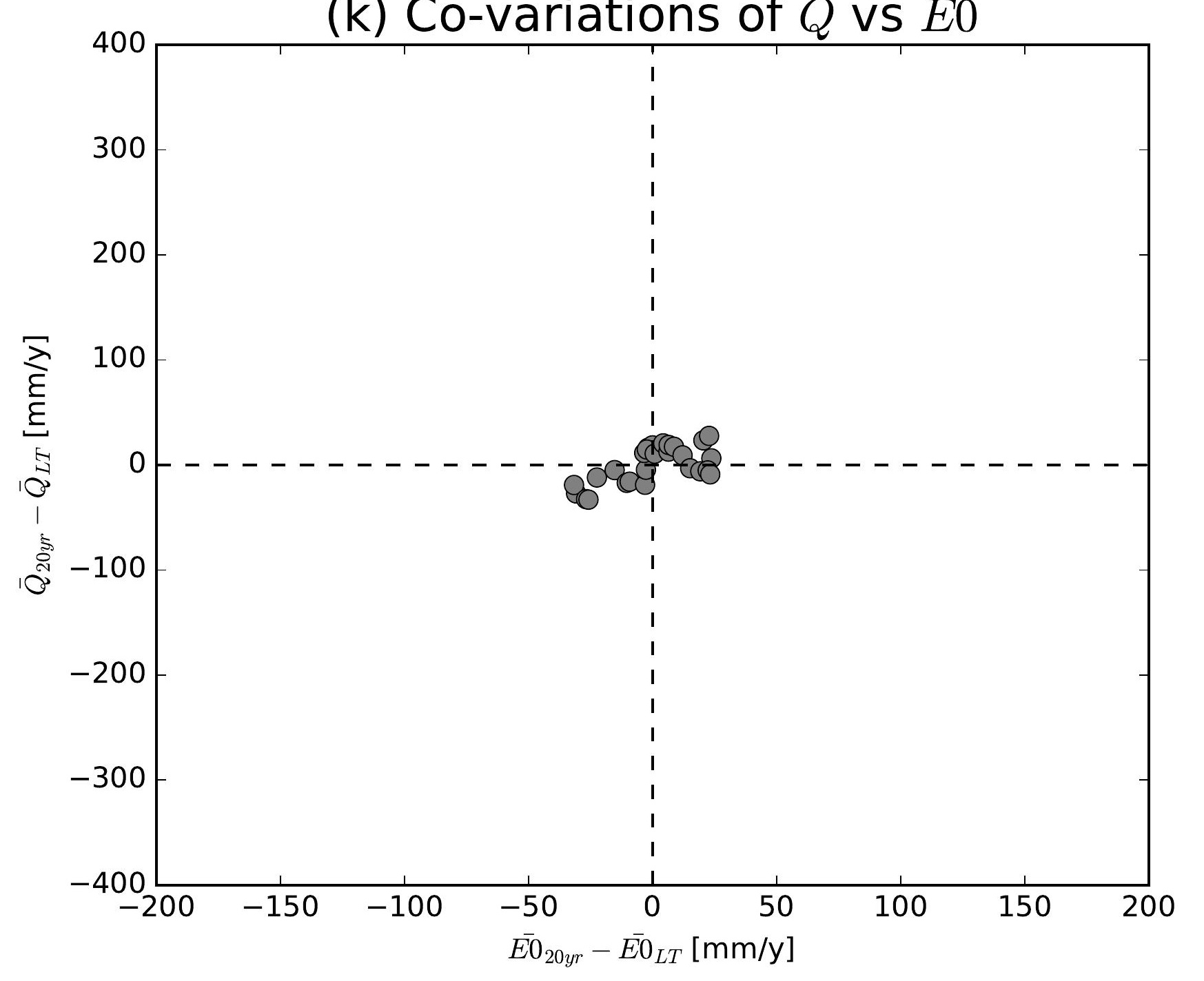

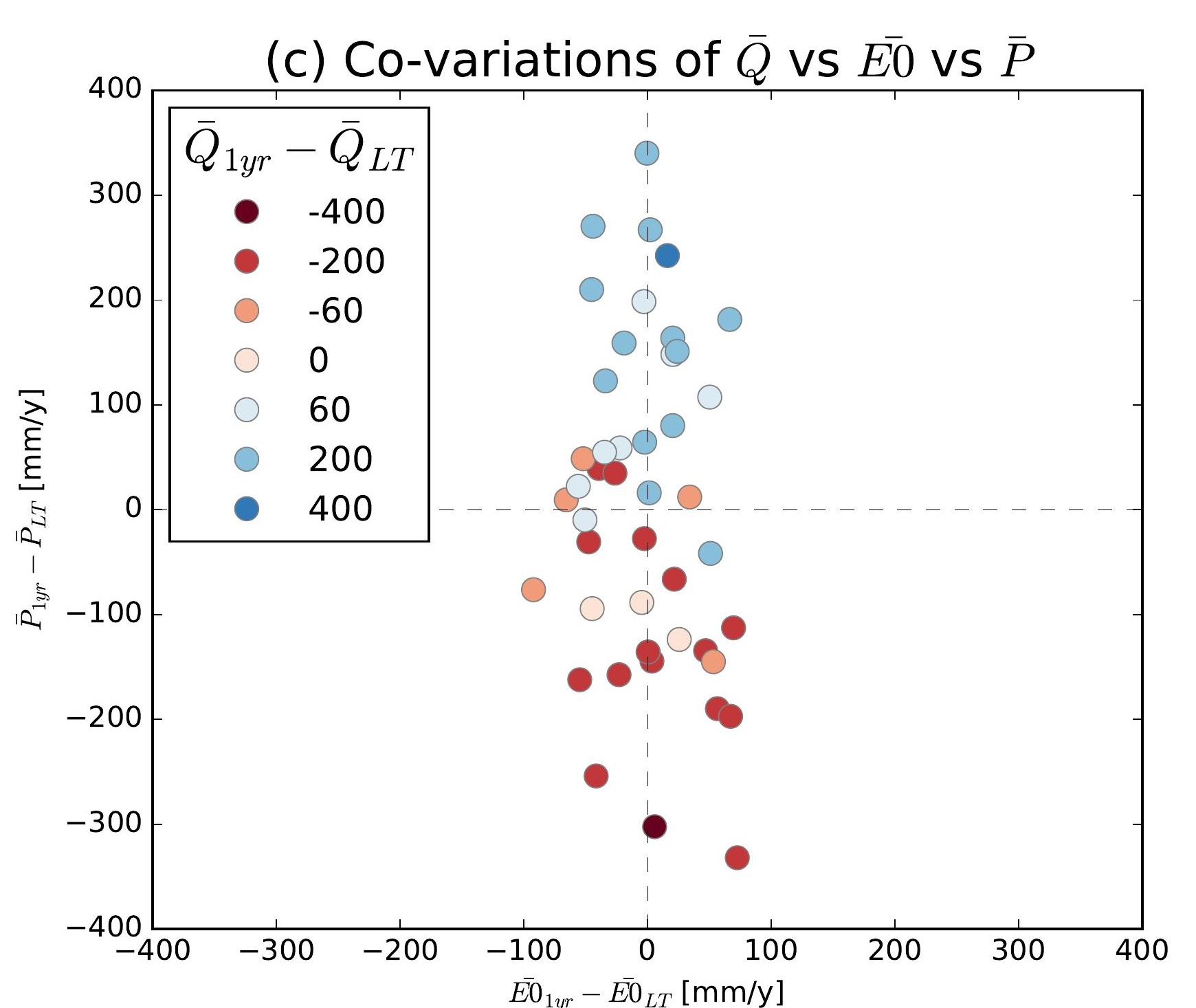
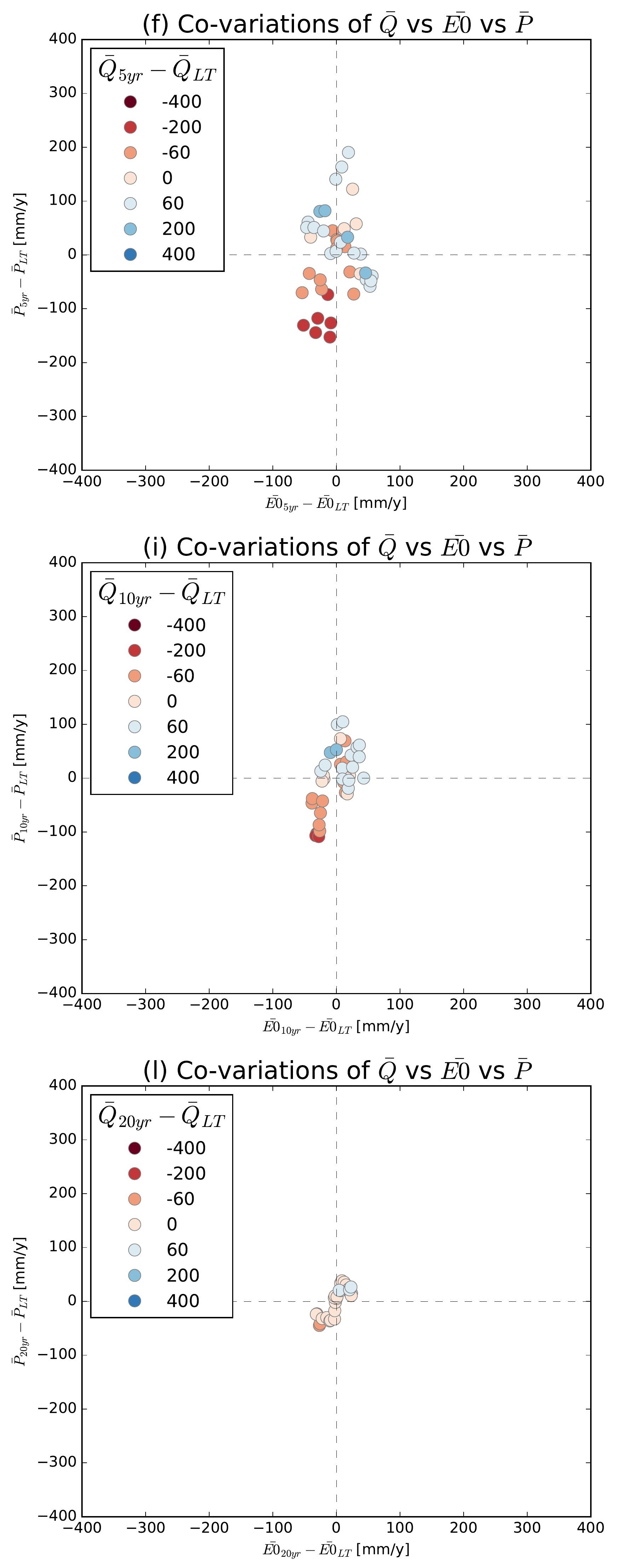

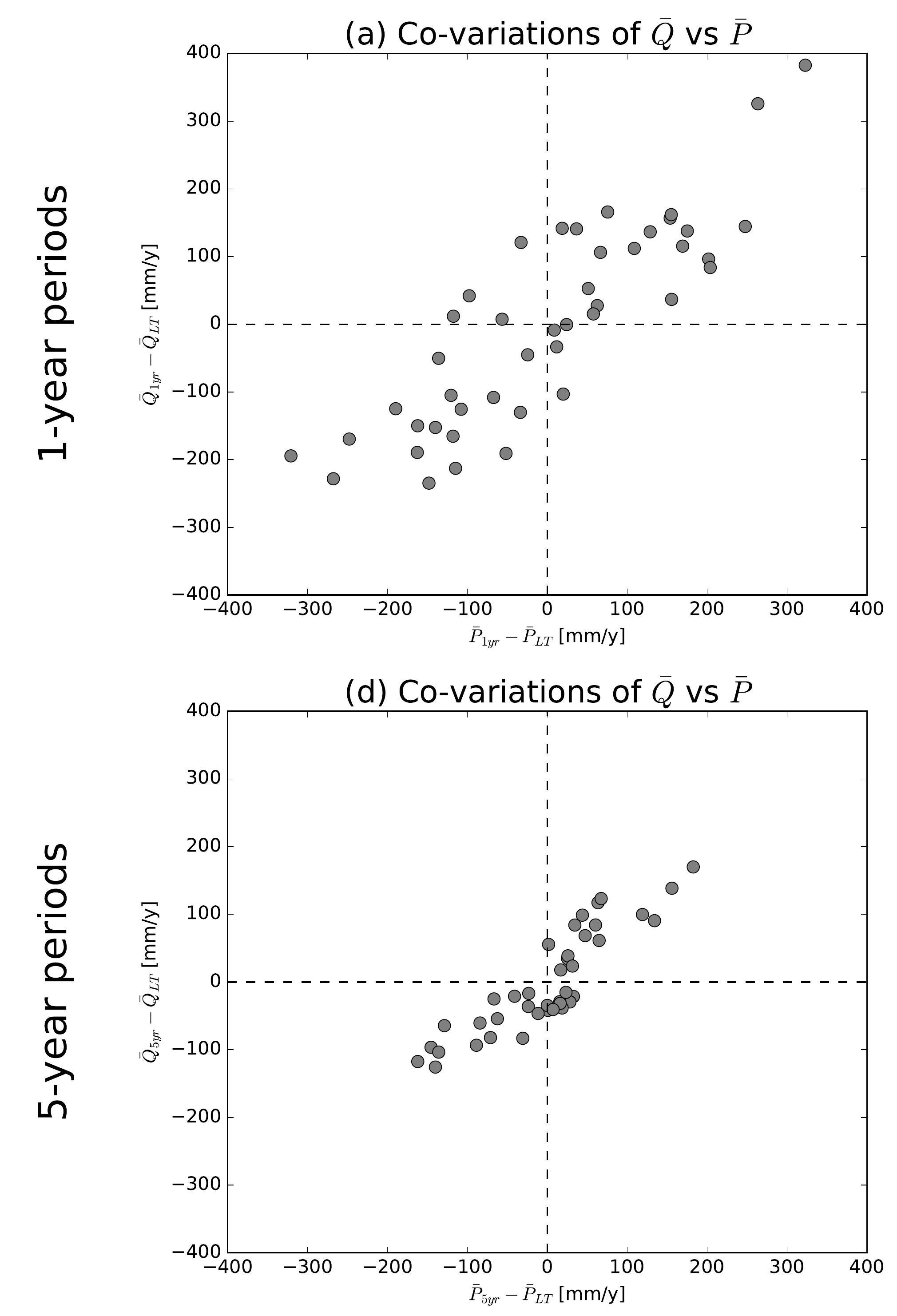

(g) Co-variations of $\bar{Q}$ vs $\bar{P}$
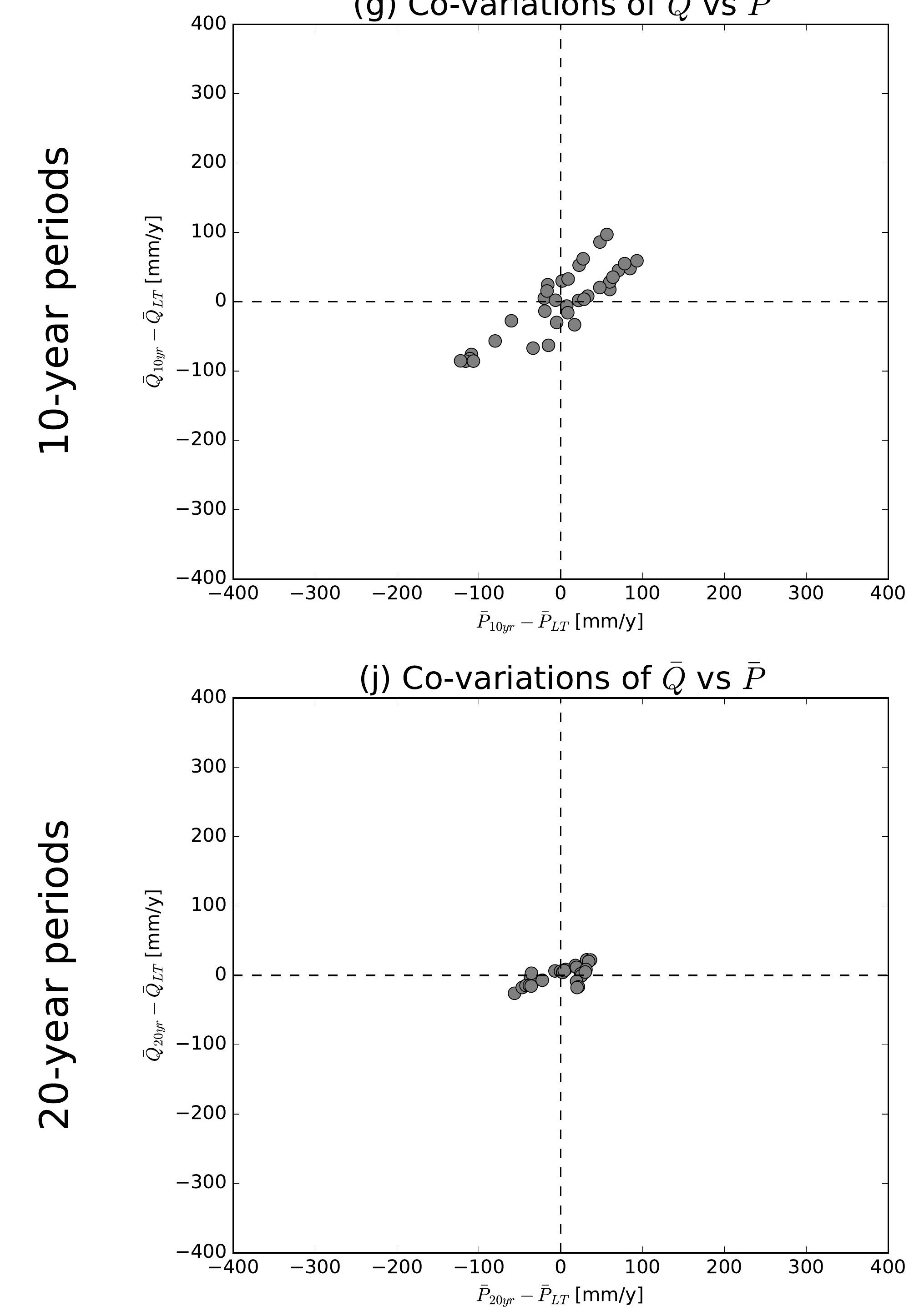

(b) Co-variations of $\bar{Q}$ vs $\overline{E 0}$

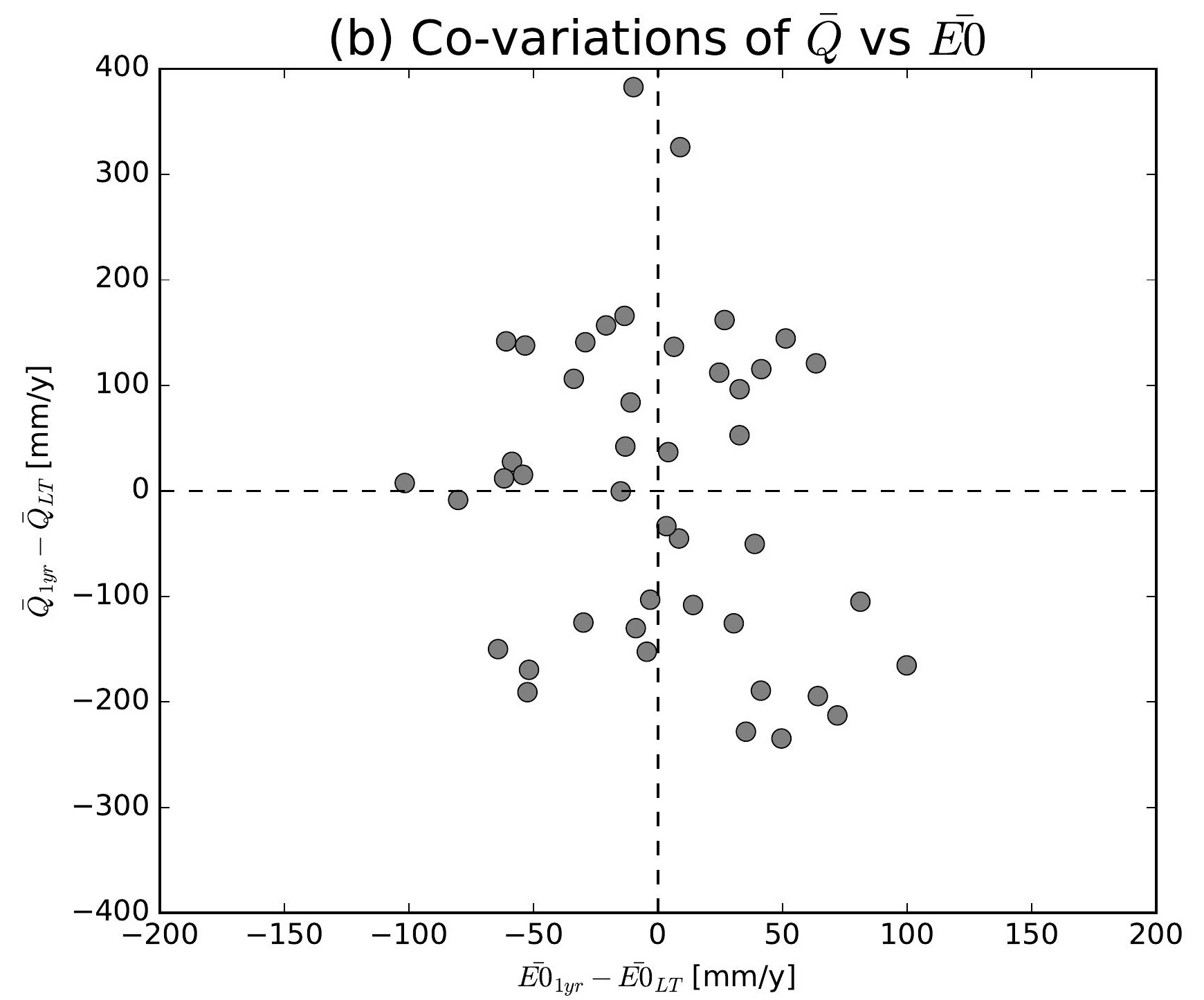

(e) Co-variations of $\bar{Q}$ vs $\overline{E 0}$

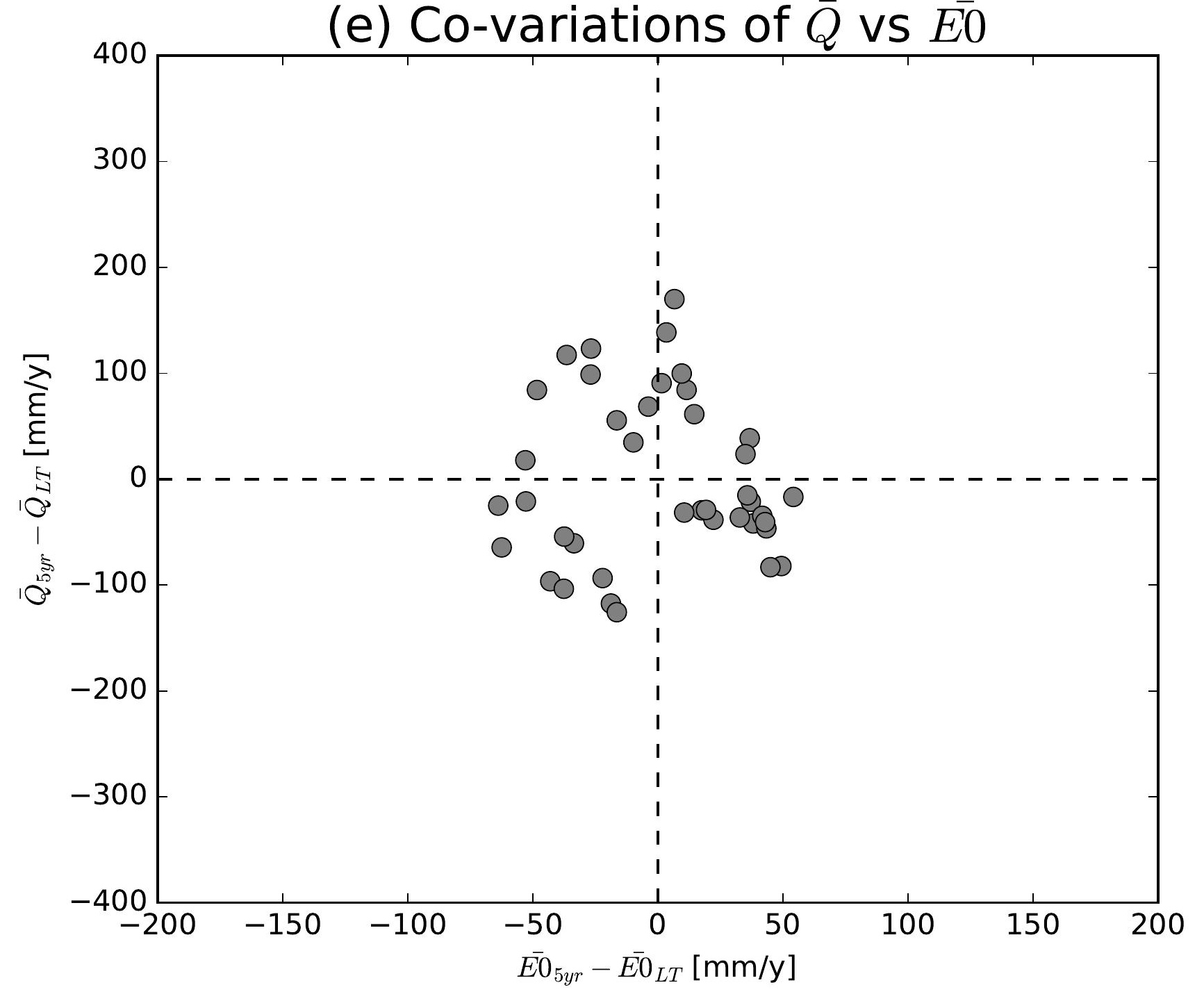

(h) Co-variations of $\bar{Q}$ vs $\overline{E 0}$

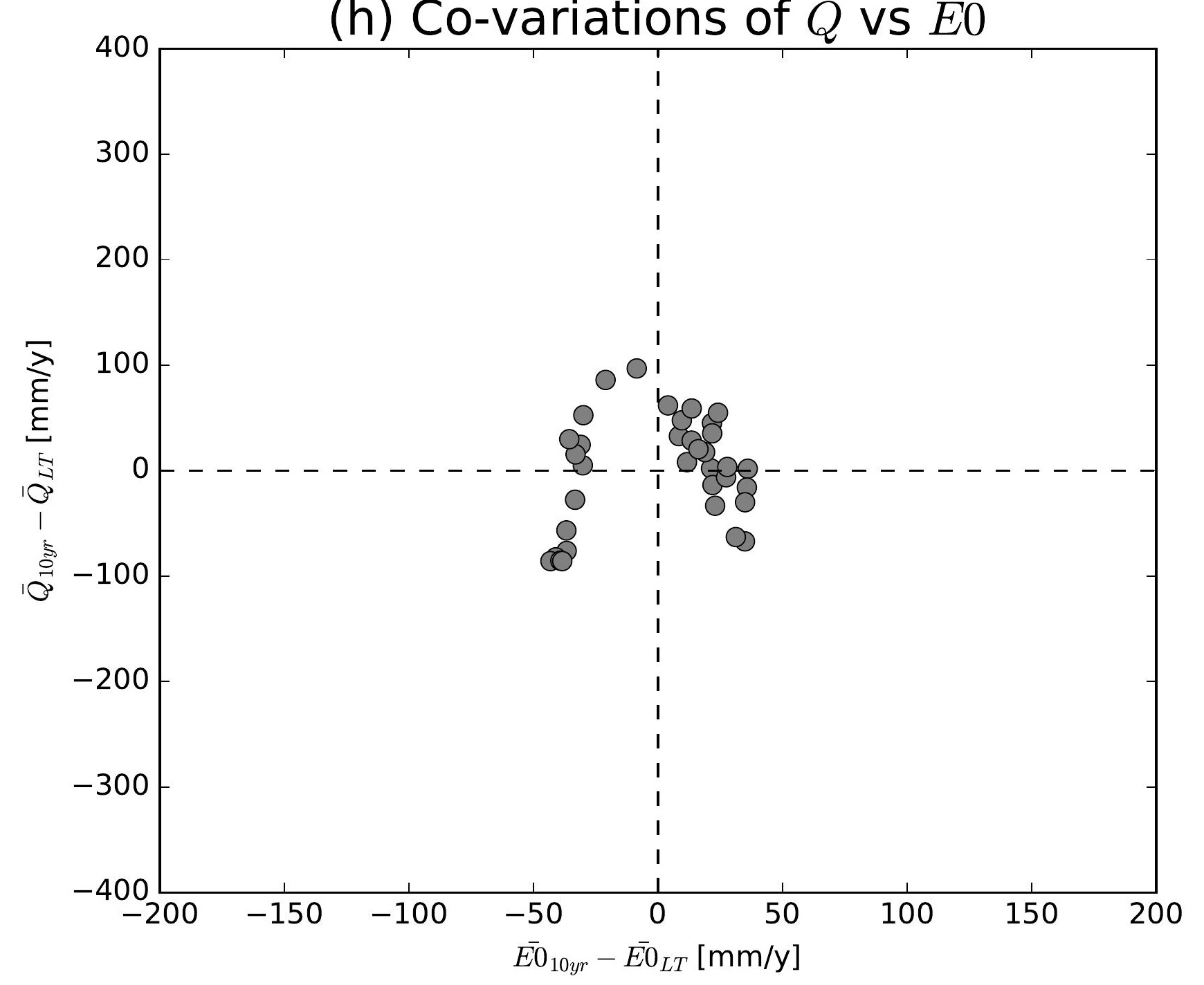

(k) Co-variations of $\bar{Q}$ vs $\overline{E 0}$

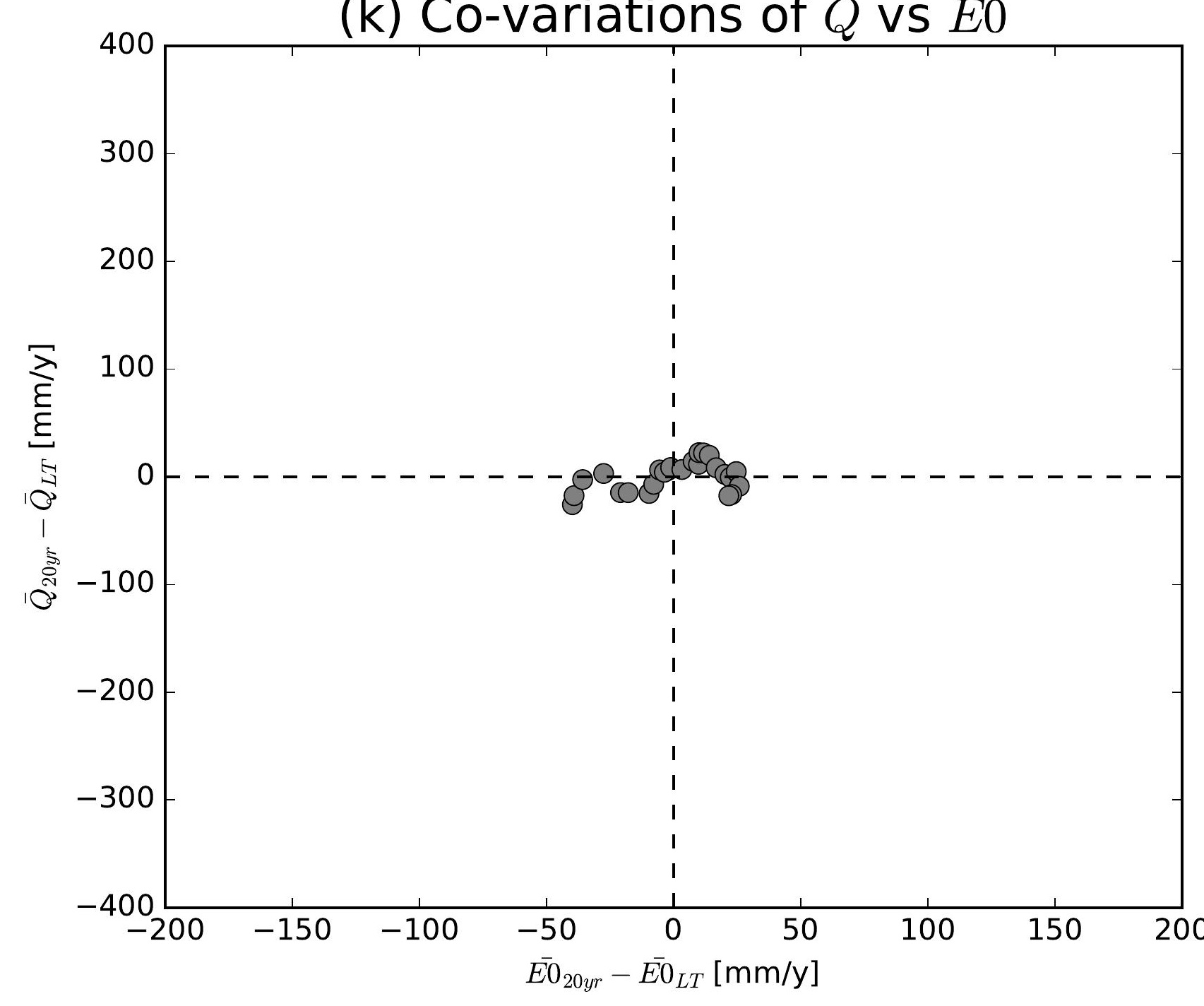

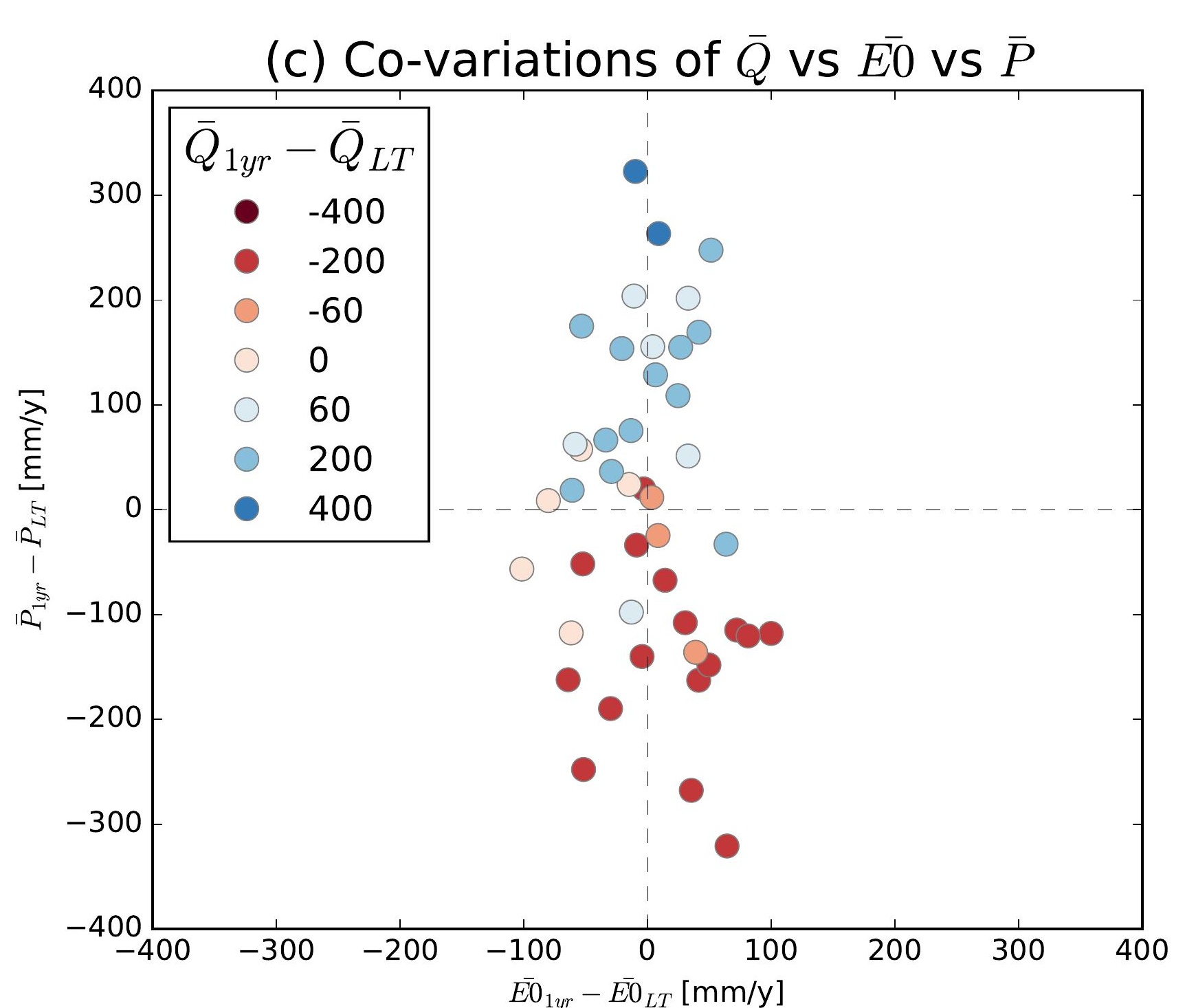
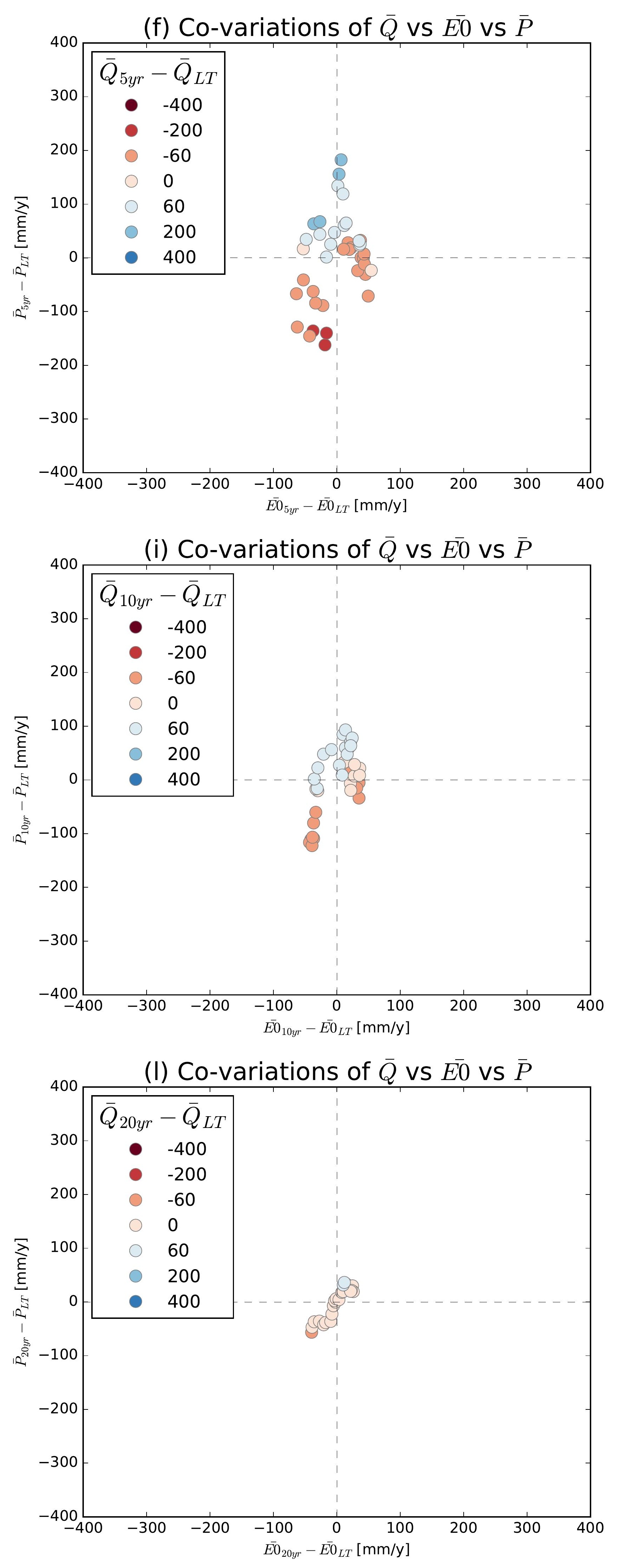

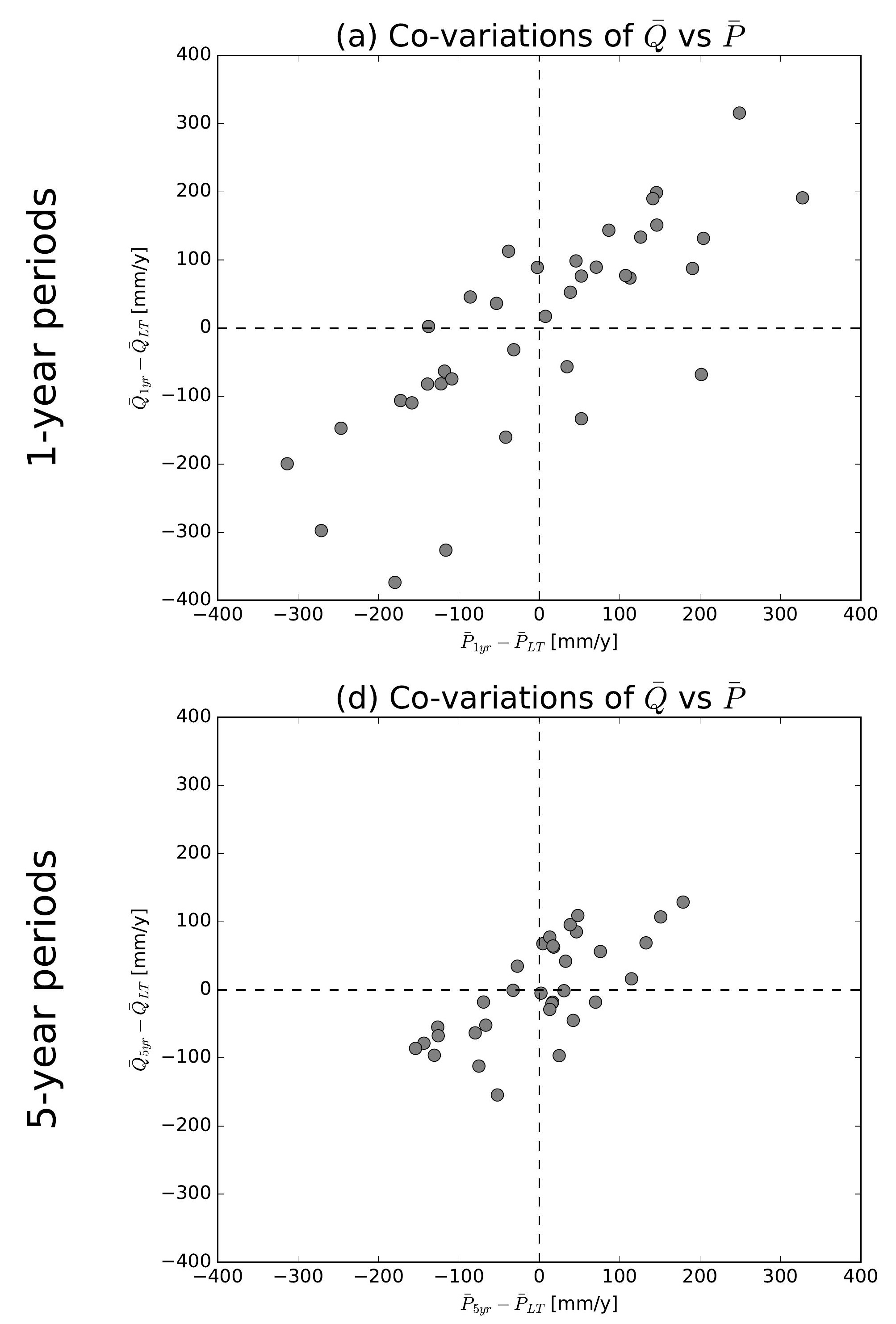

(g) Co-variations of $\bar{Q}$ vs $\bar{P}$

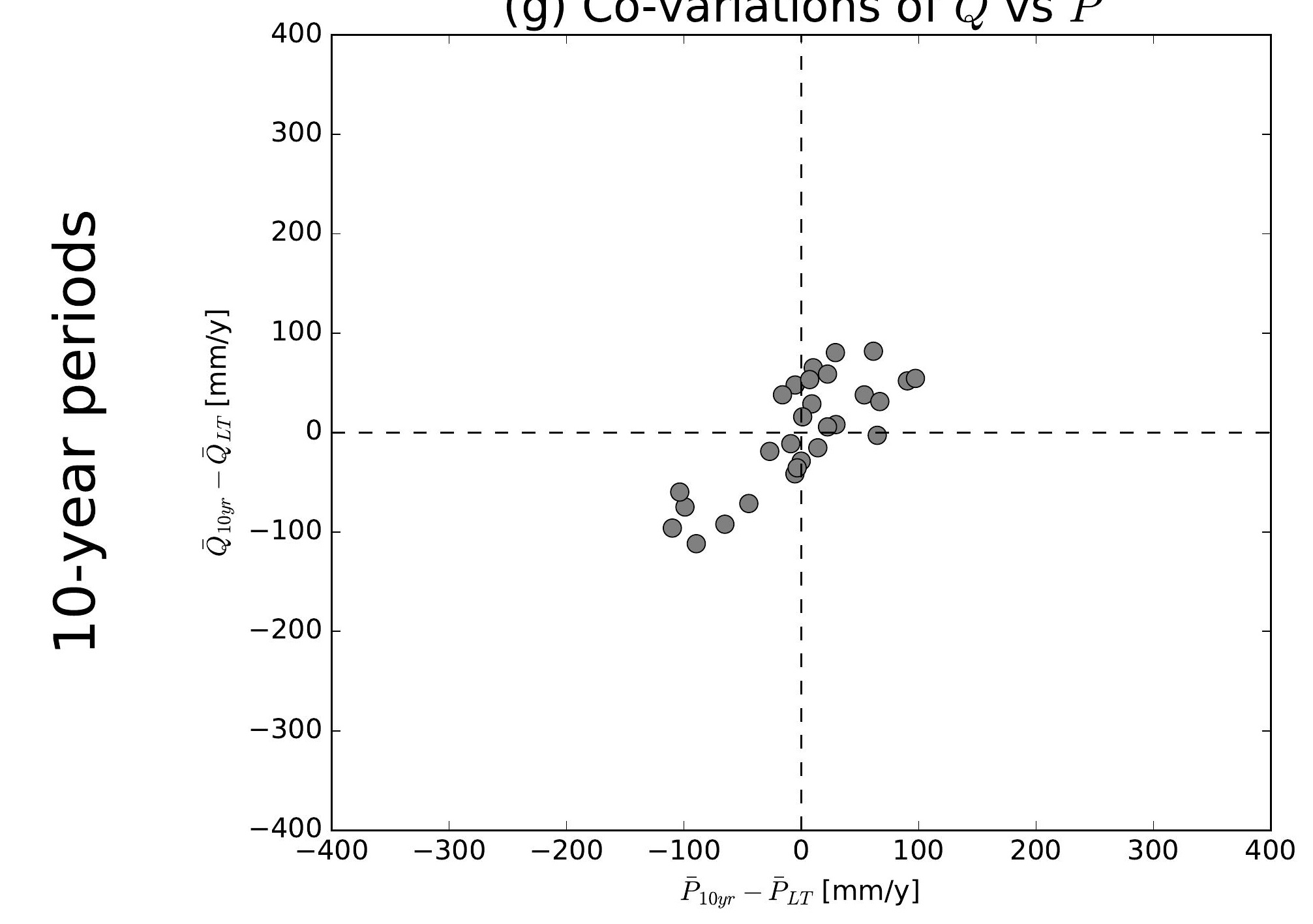

(j) Co-variations of $\bar{Q}$ vs $\bar{P}$

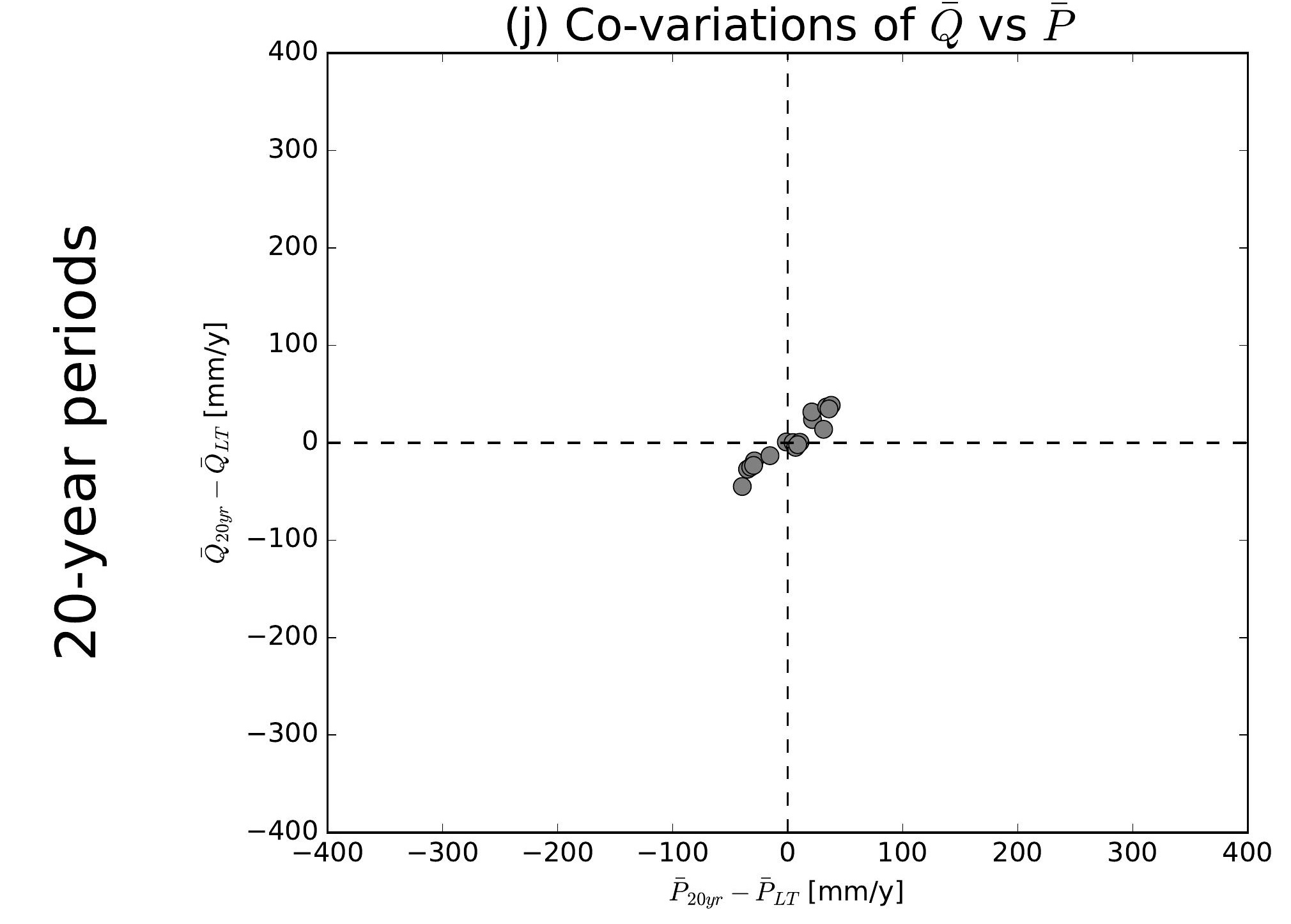

(b) Co-variations of $\bar{Q}$ vs $\overline{E 0}$

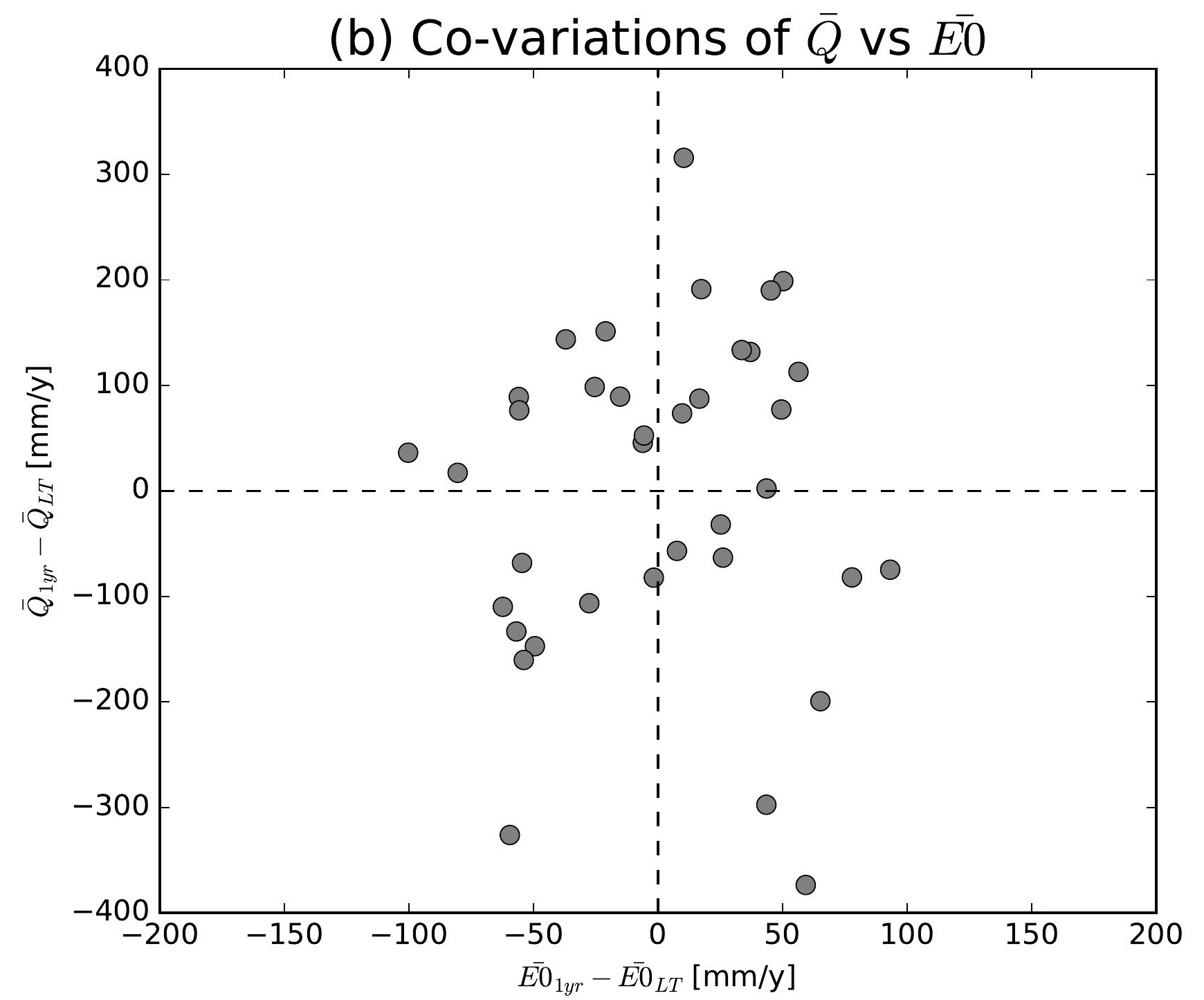

(e) Co-variations of $\bar{Q}$ vs $\overline{E 0}$

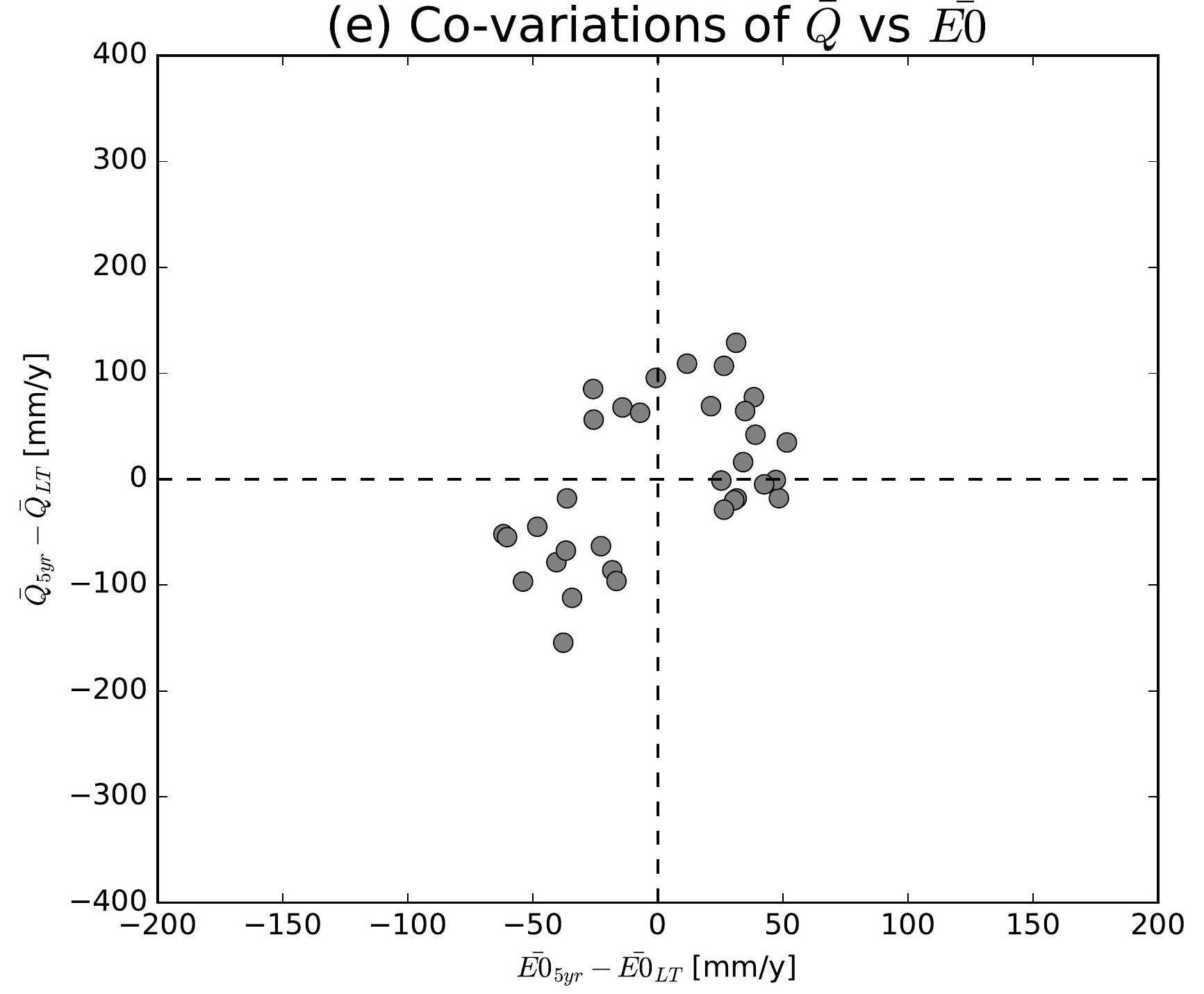

(h) Co-variations of $\bar{Q}$ vs $\overline{E 0}$

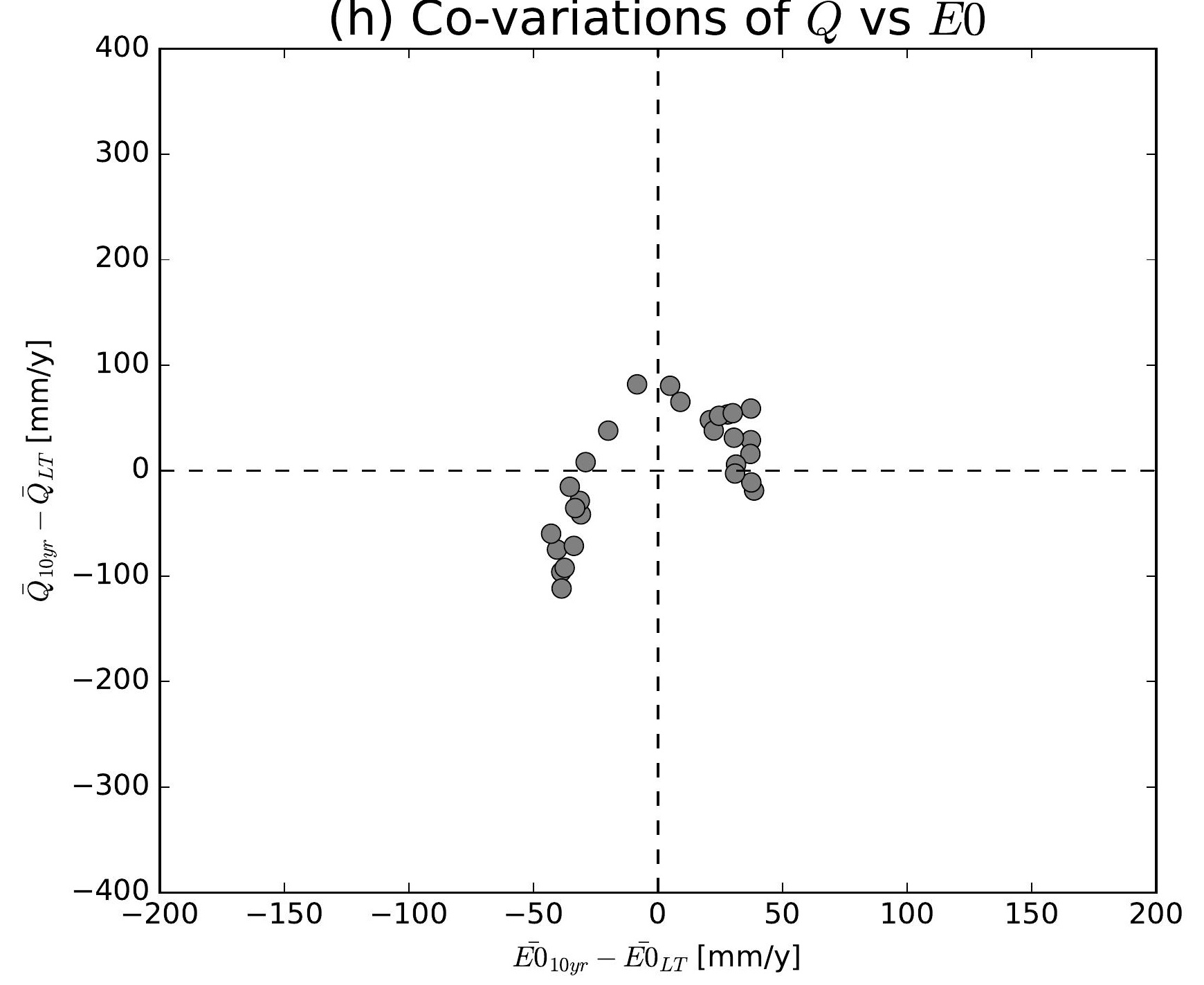

(k) Co-variations of $\bar{Q}$ vs $\overline{E 0}$

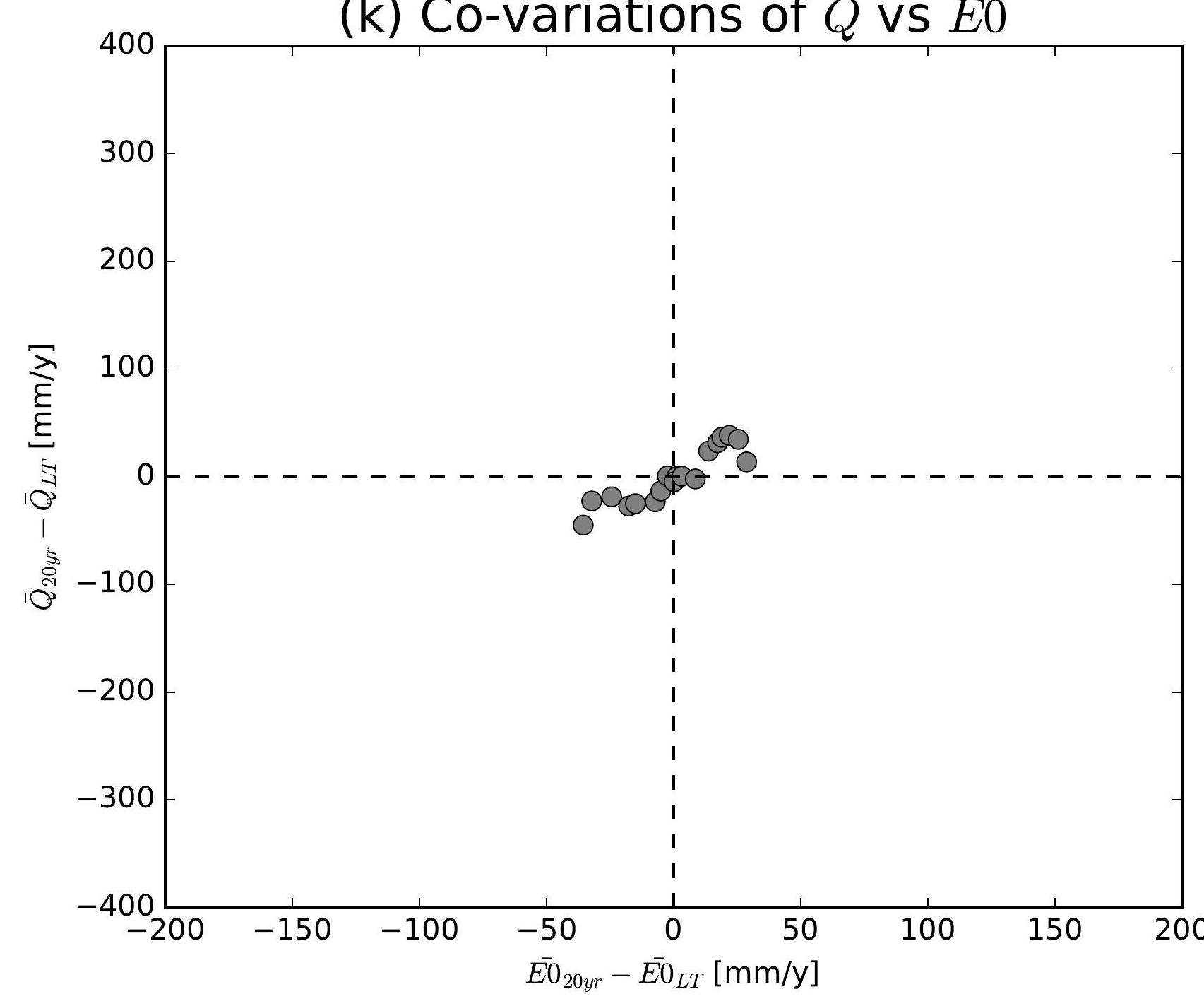

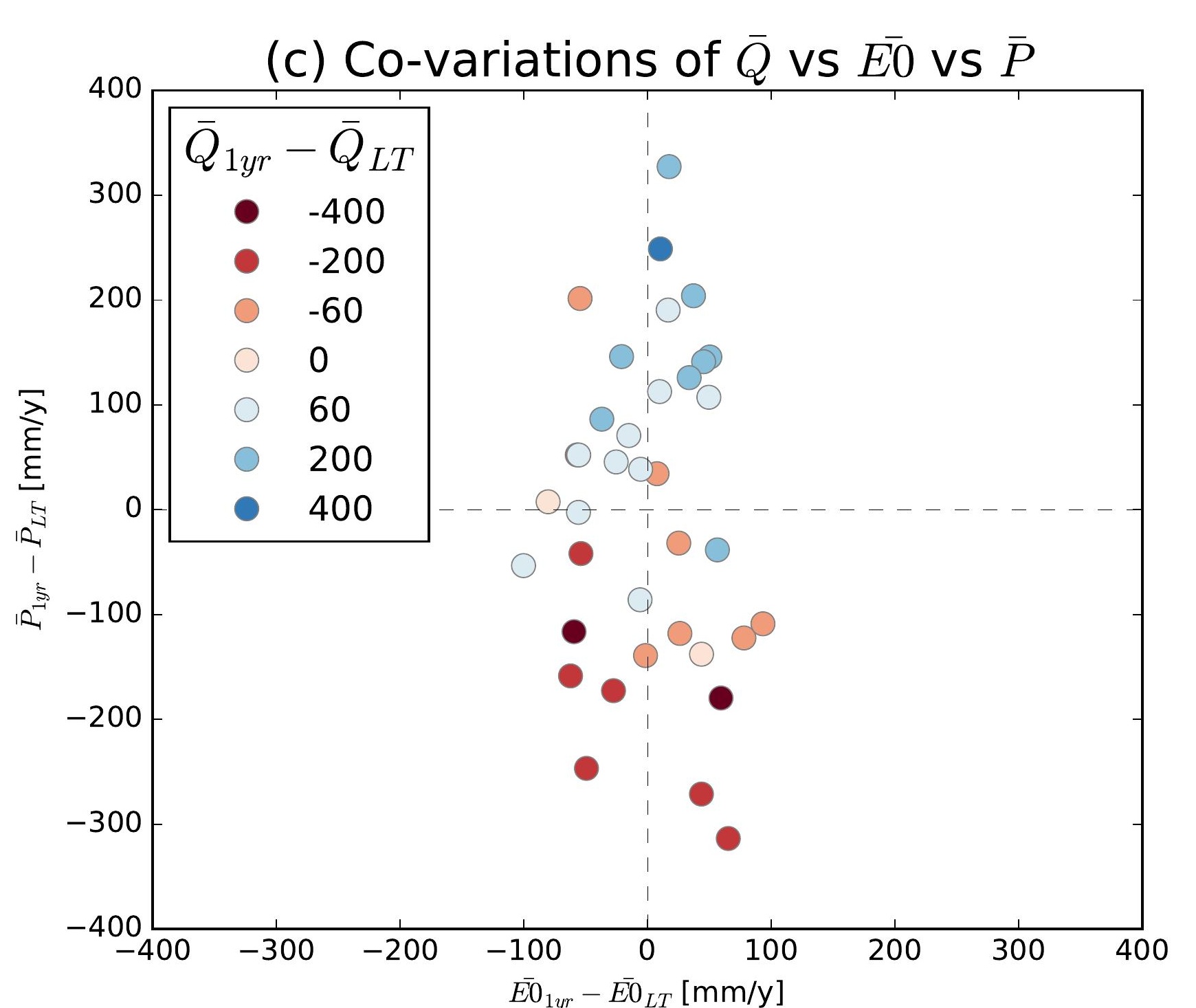
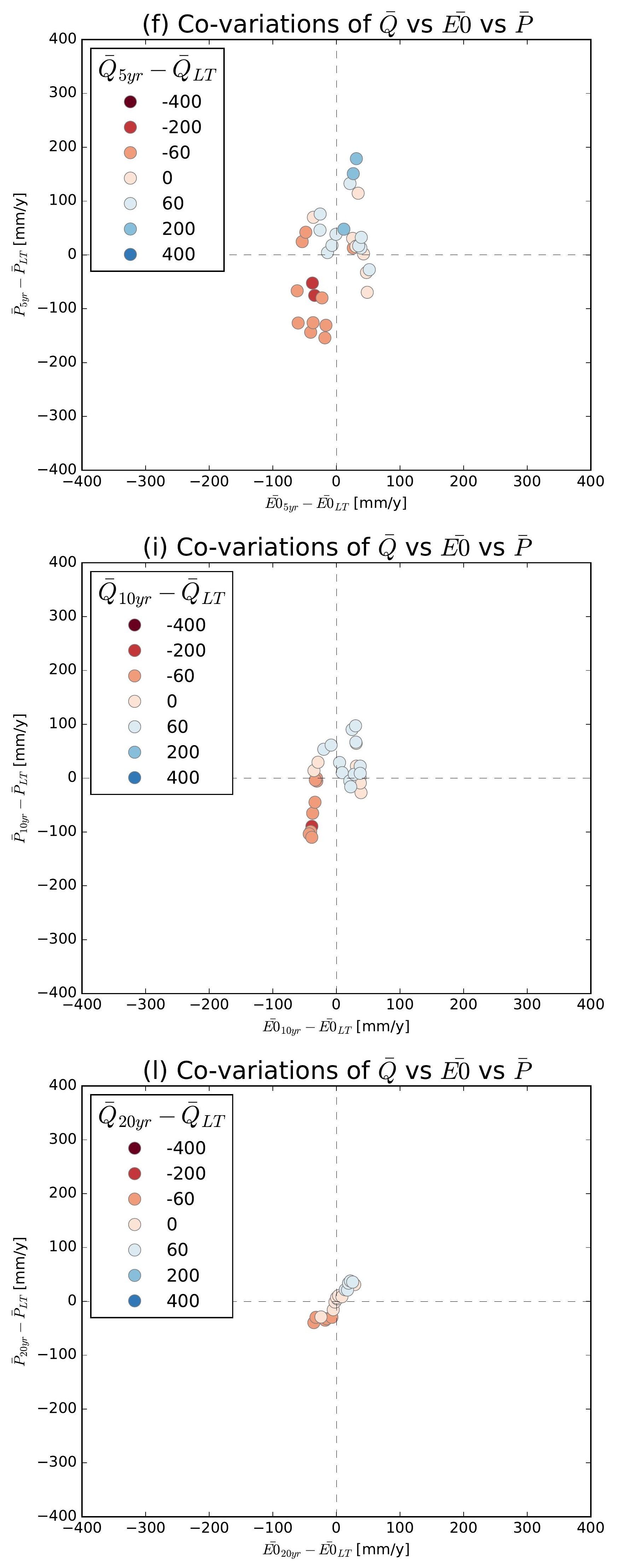

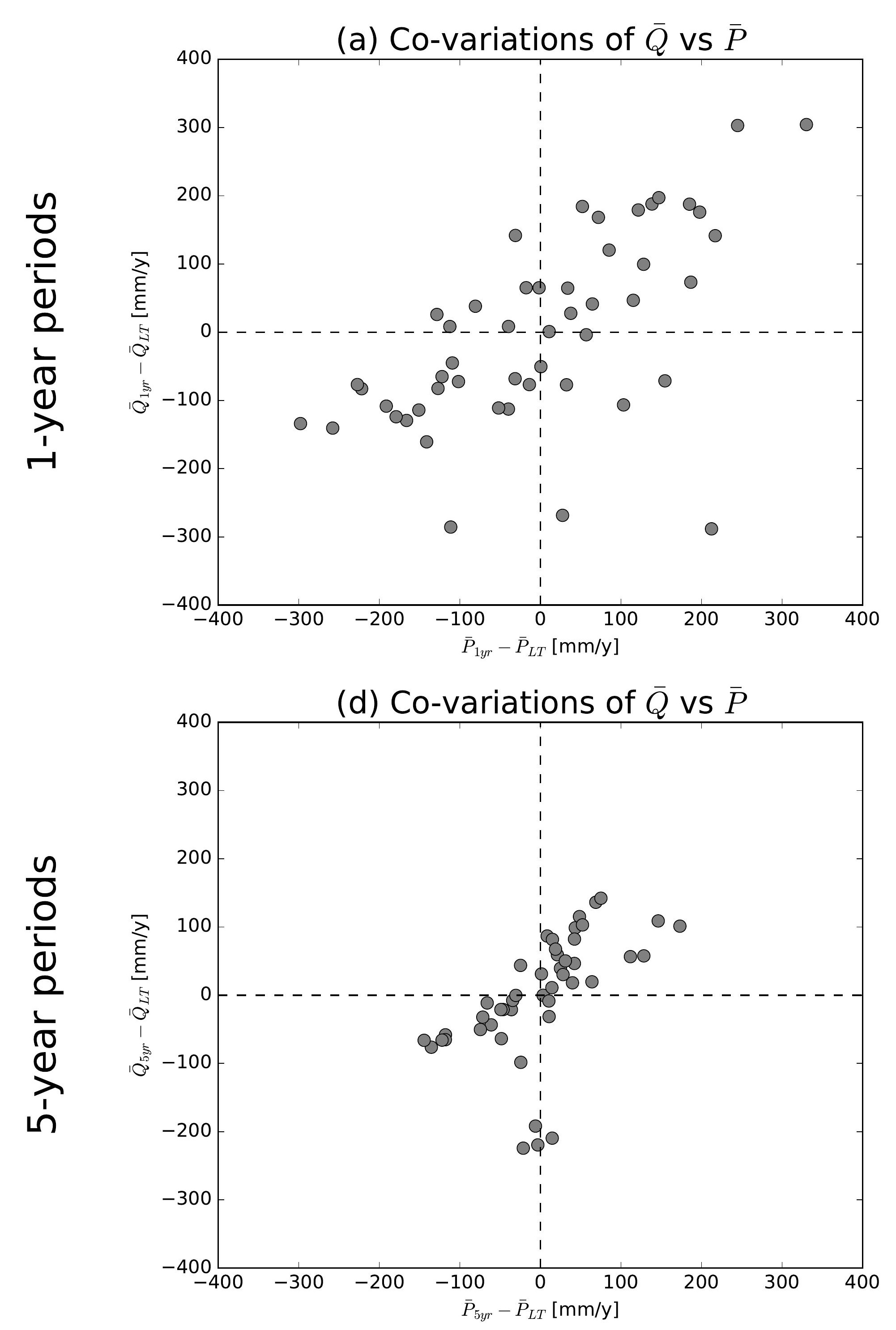

(g) Co-variations of $\bar{Q}$ vs $\bar{P}$

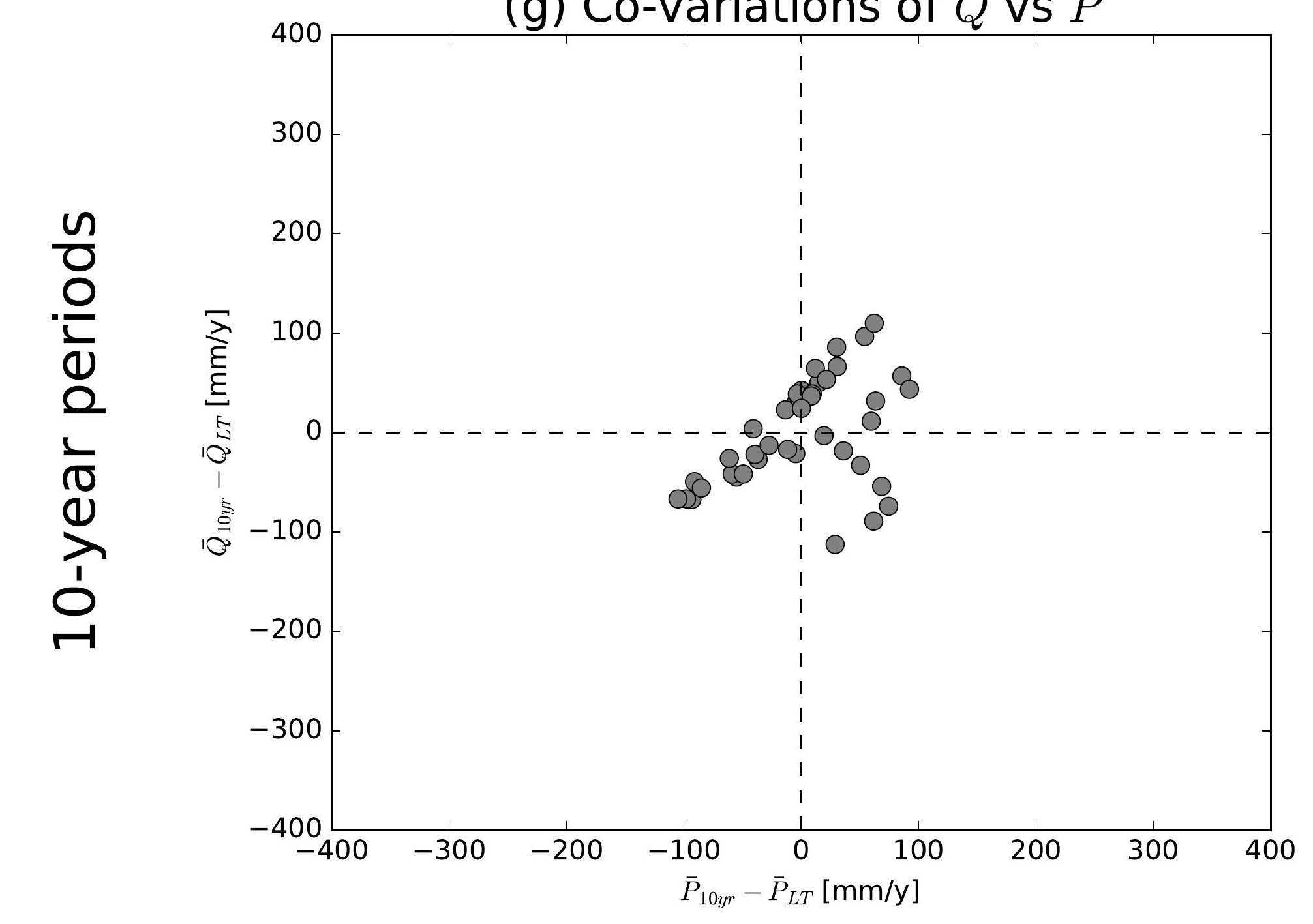

(j) Co-variations of $\bar{Q}$ vs $\bar{P}$

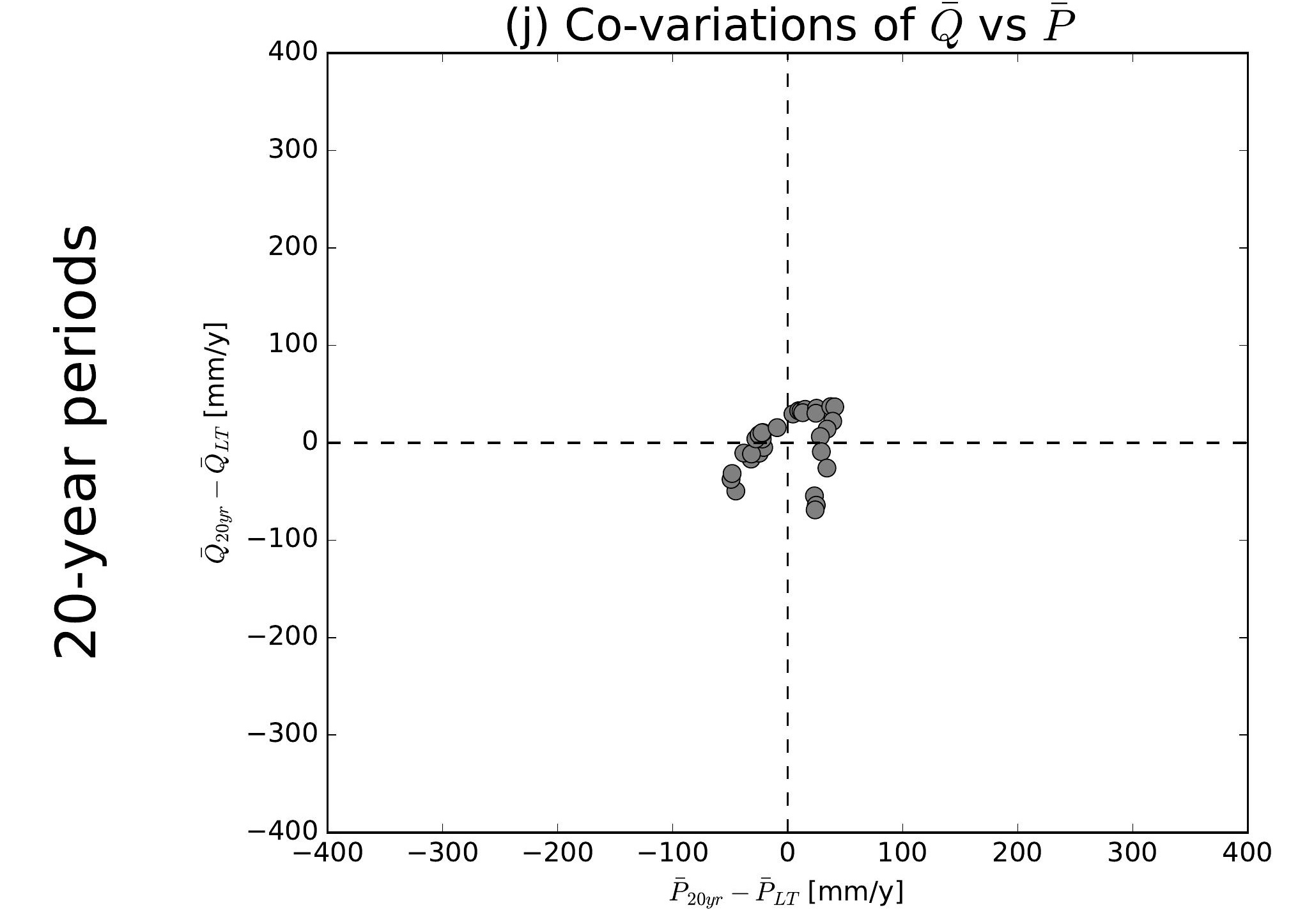

(b) Co-variations of $\bar{Q}$ vs $\overline{E 0}$

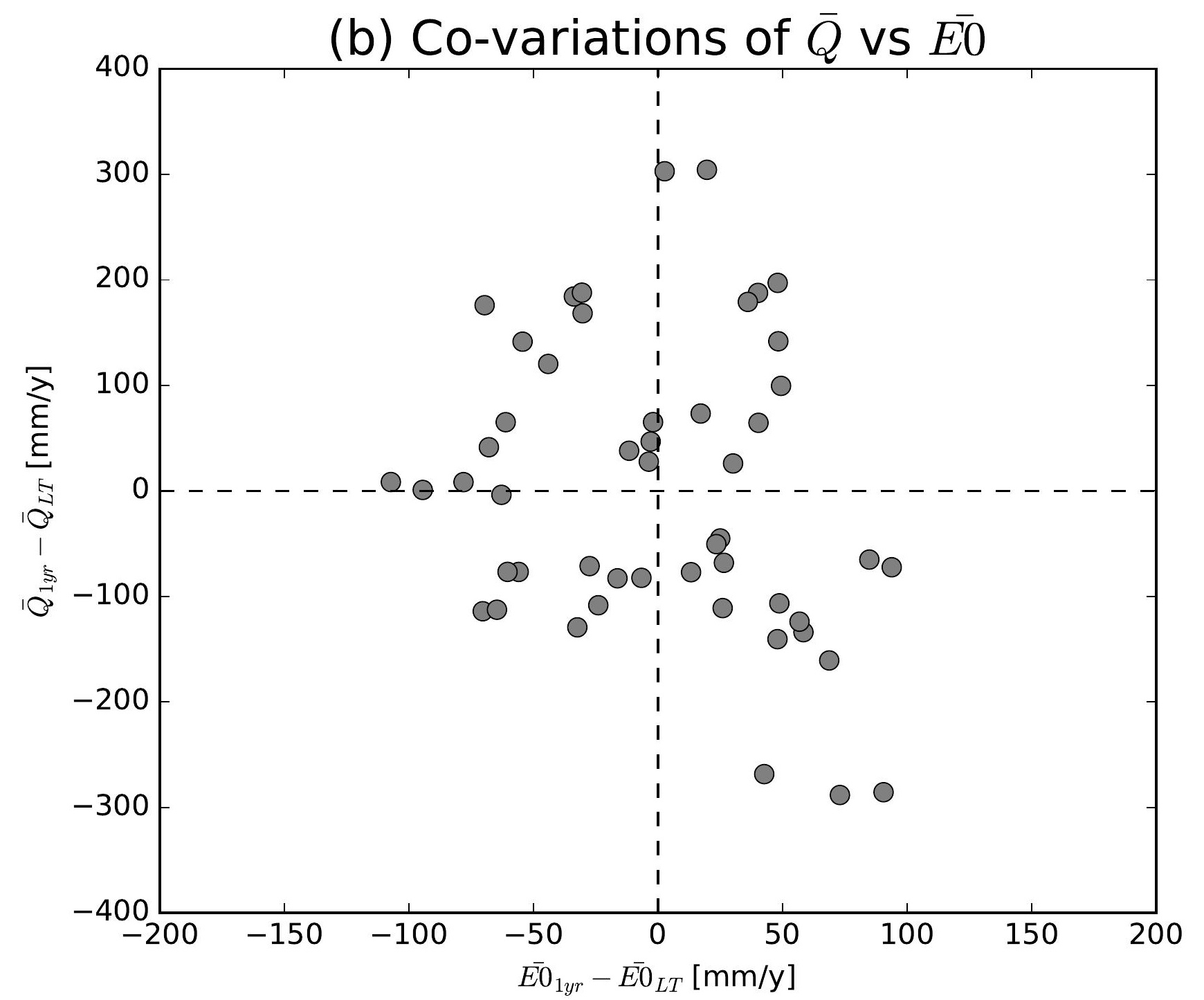

(e) Co-variations of $\bar{Q}$ vs $\overline{E 0}$

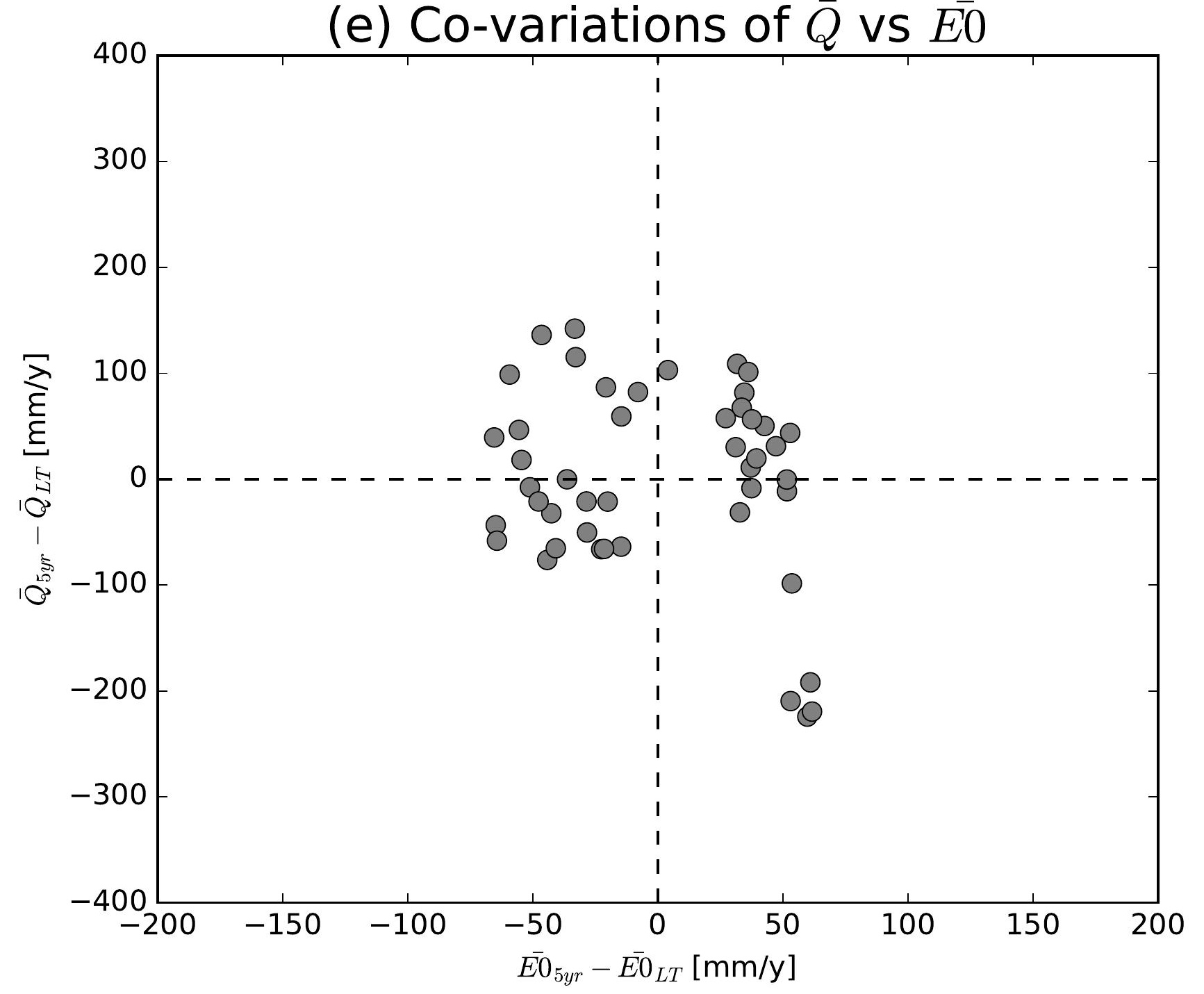

(h) Co-variations of $\bar{Q}$ vs $\overline{E 0}$

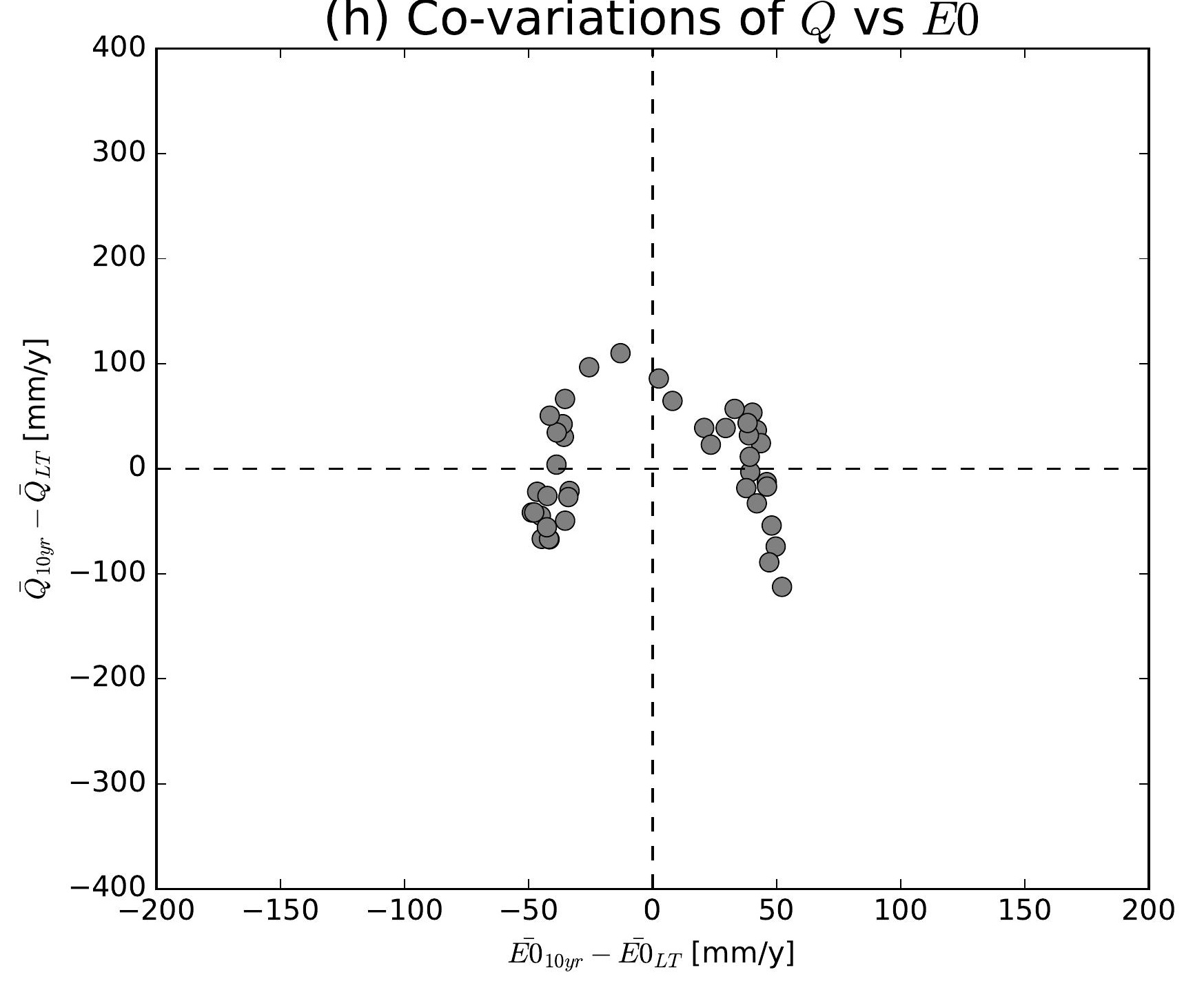

(k) Co-variations of $\bar{Q}$ vs $\overline{E 0}$

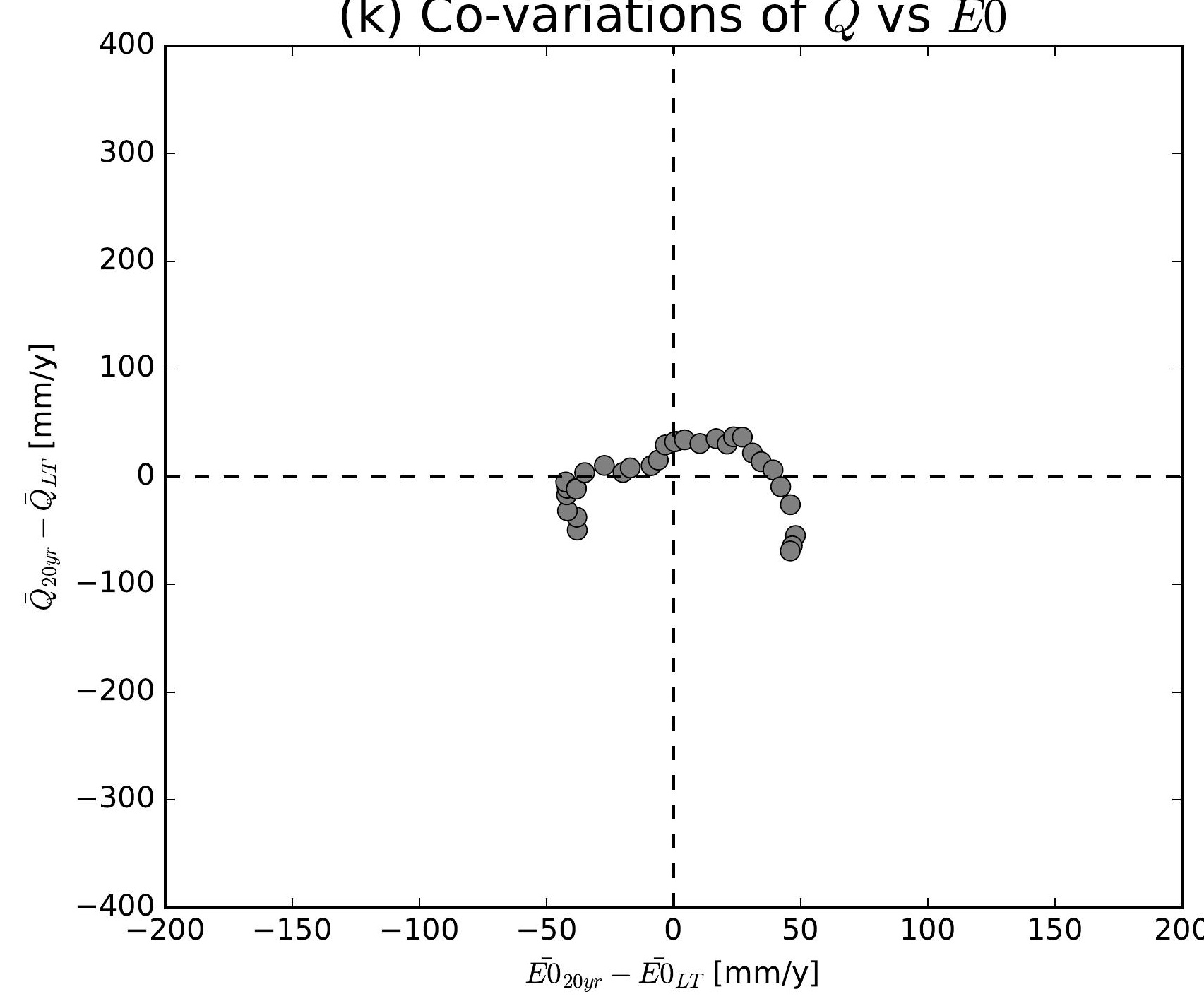

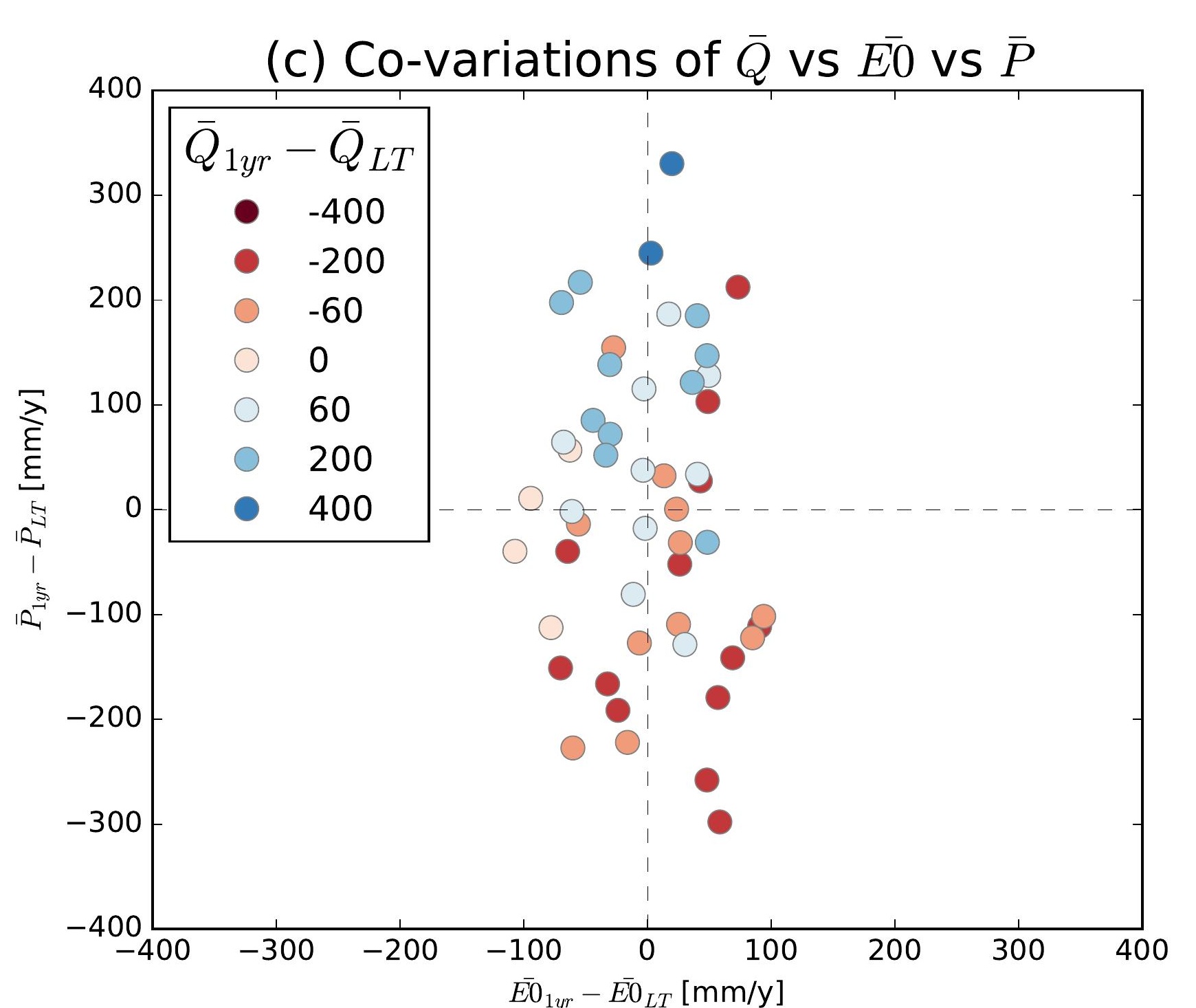
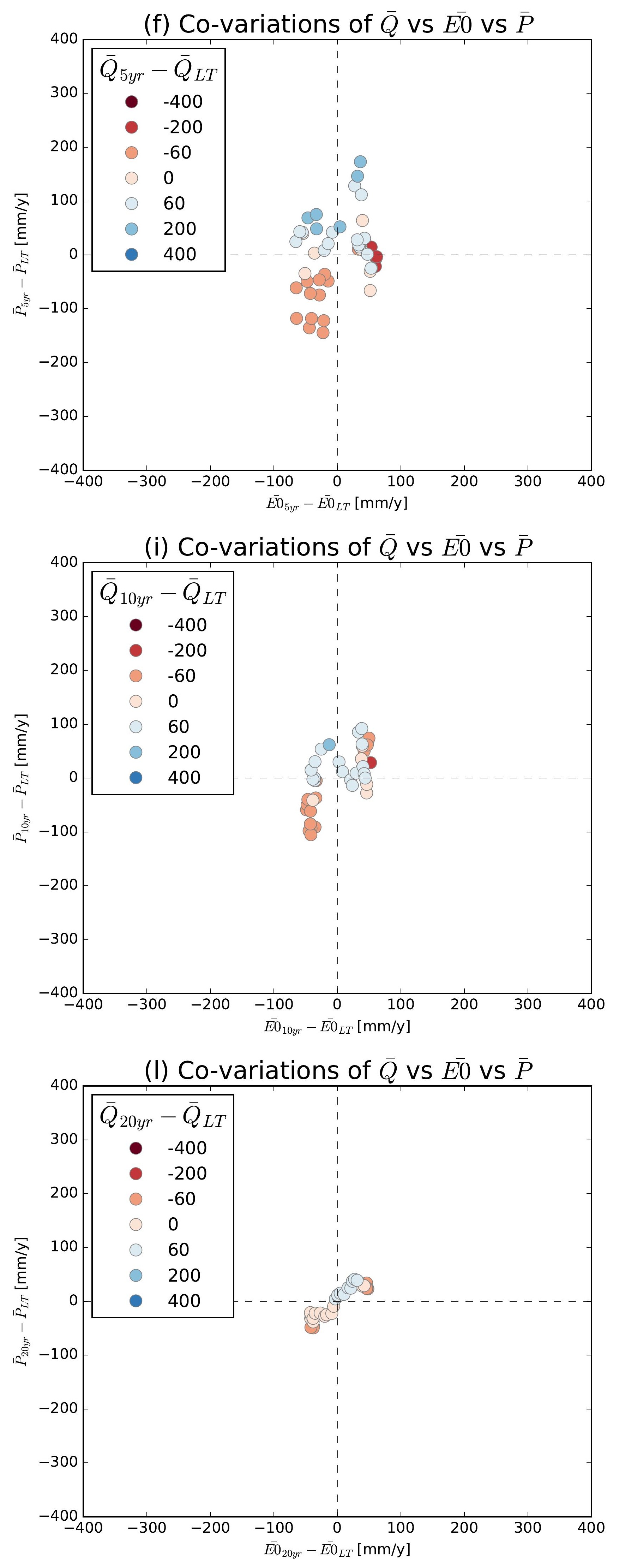

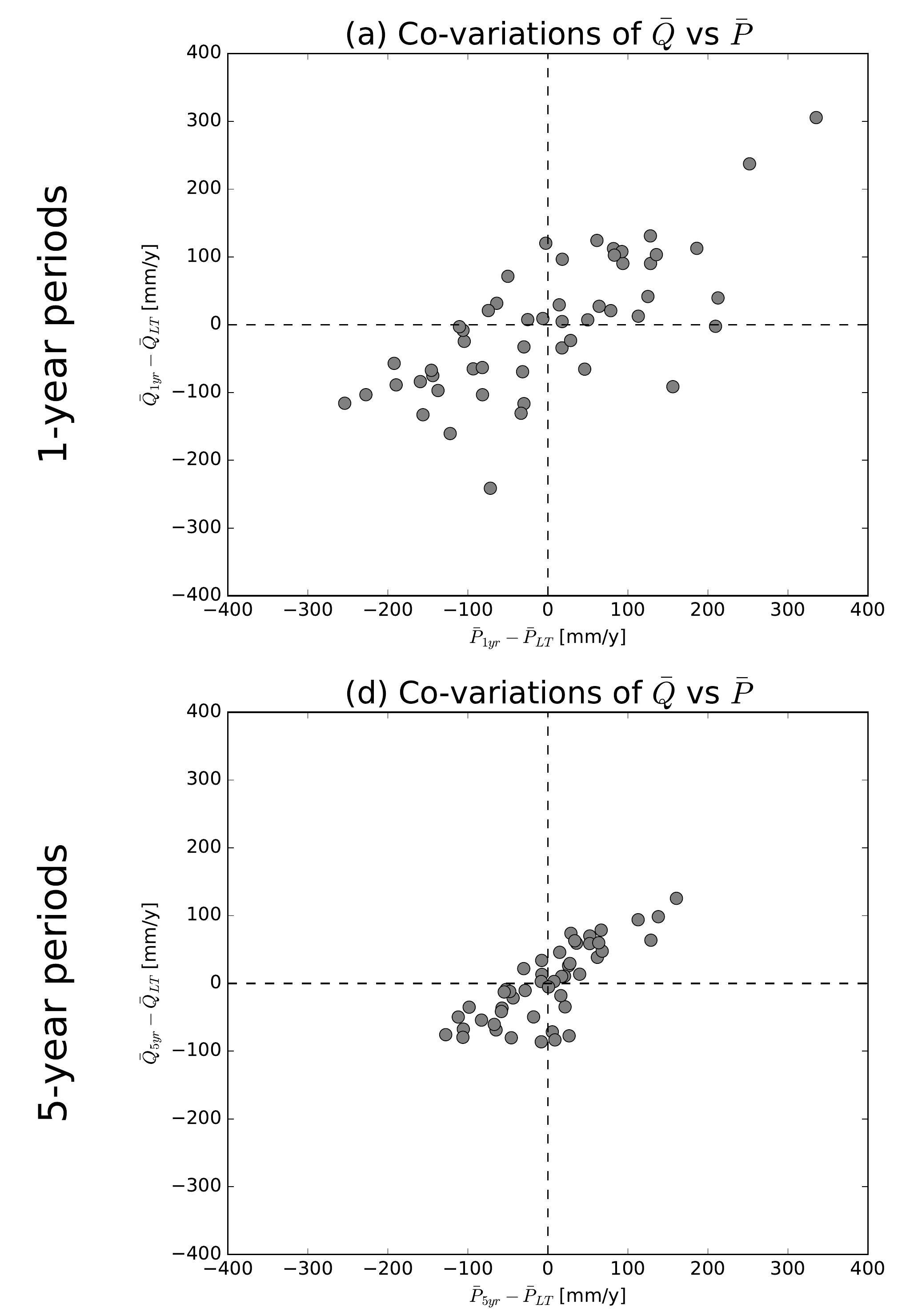

(g) Co-variations of $\bar{Q}$ vs $\bar{P}$

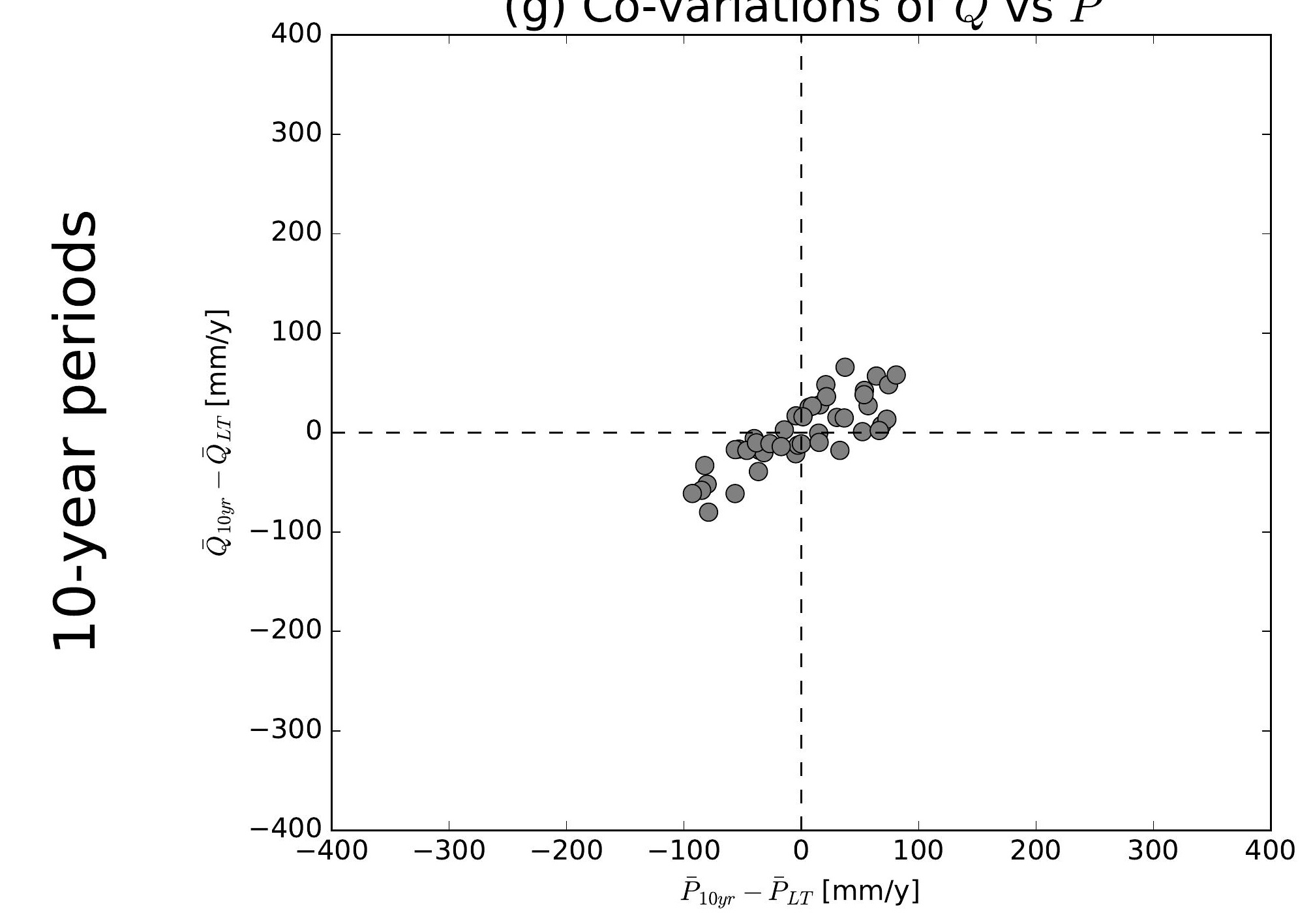

(j) Co-variations of $\bar{Q}$ vs $\bar{P}$

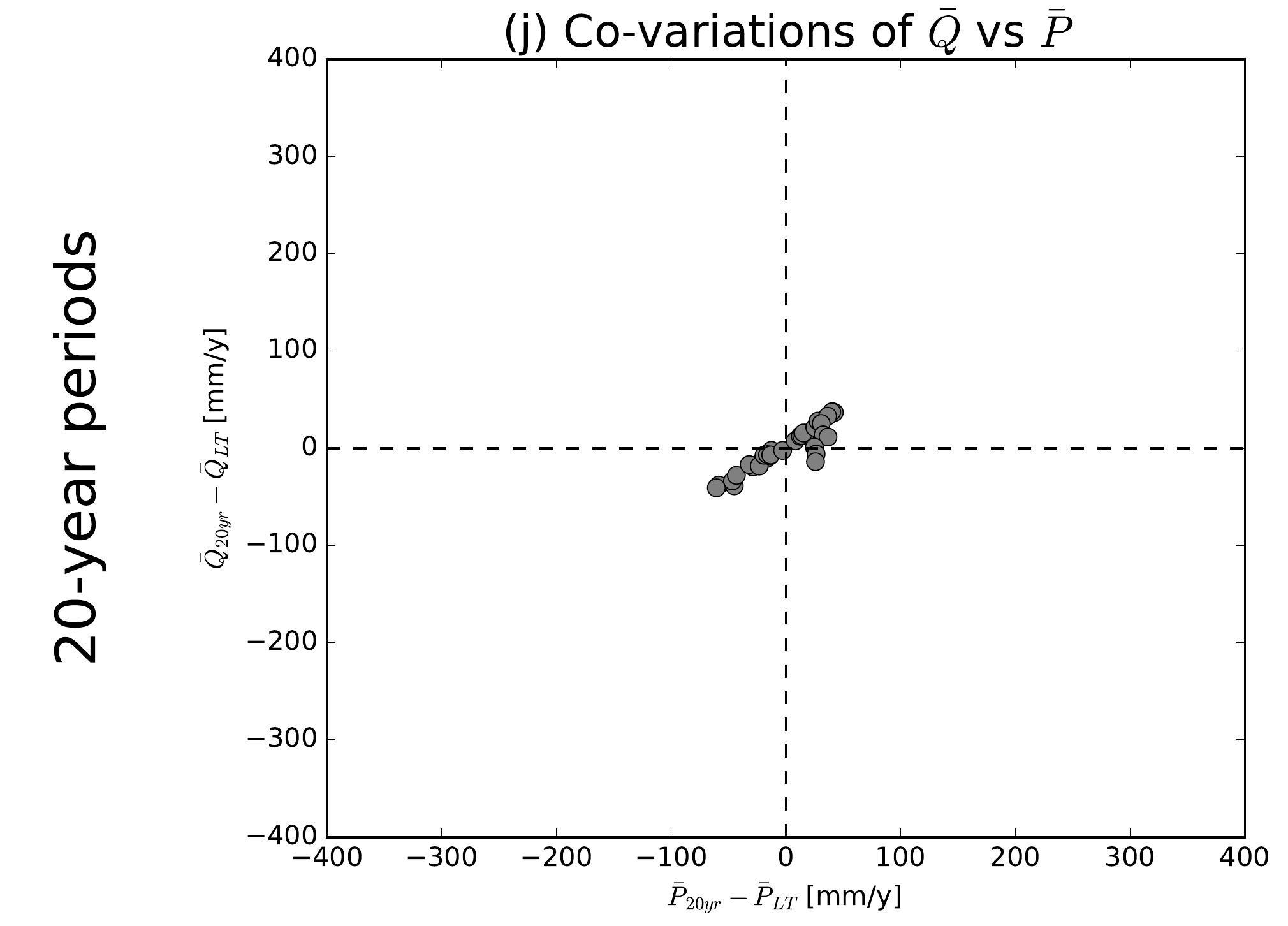

(b) Co-variations of $\bar{Q}$ vs $\overline{E 0}$

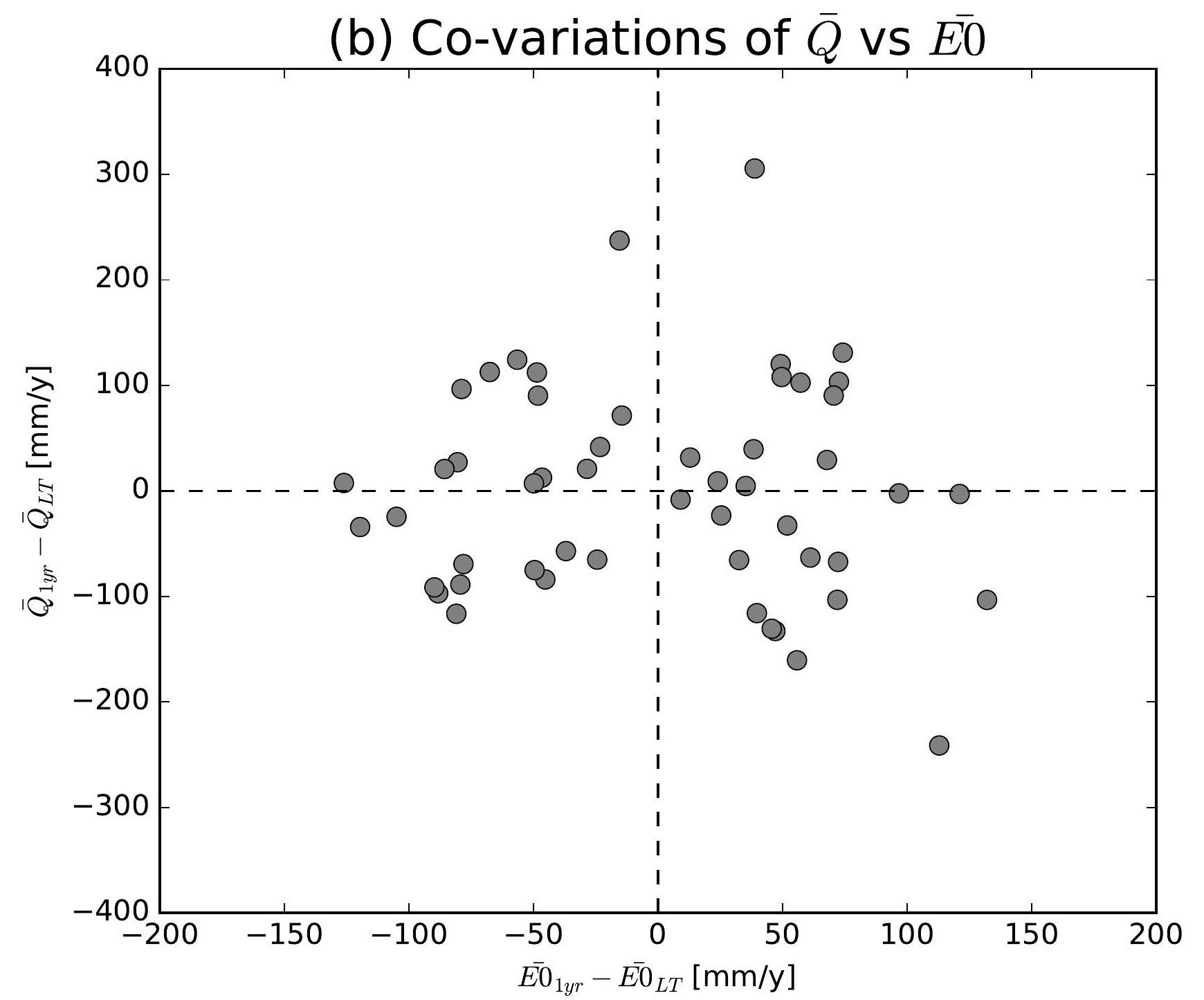

(e) Co-variations of $\bar{Q}$ vs $\overline{E 0}$

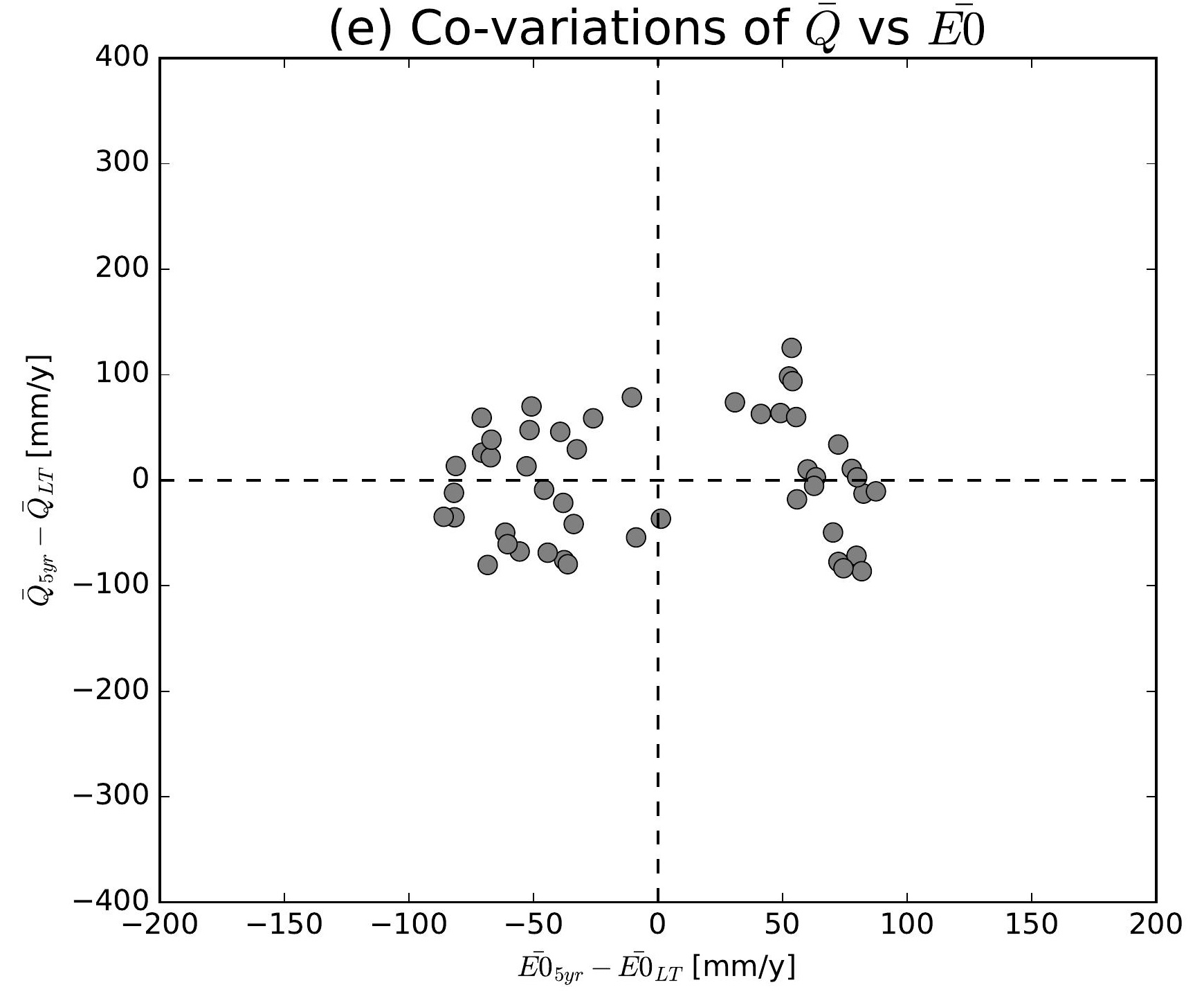

(h) Co-variations of $\bar{Q}$ vs $\overline{E 0}$

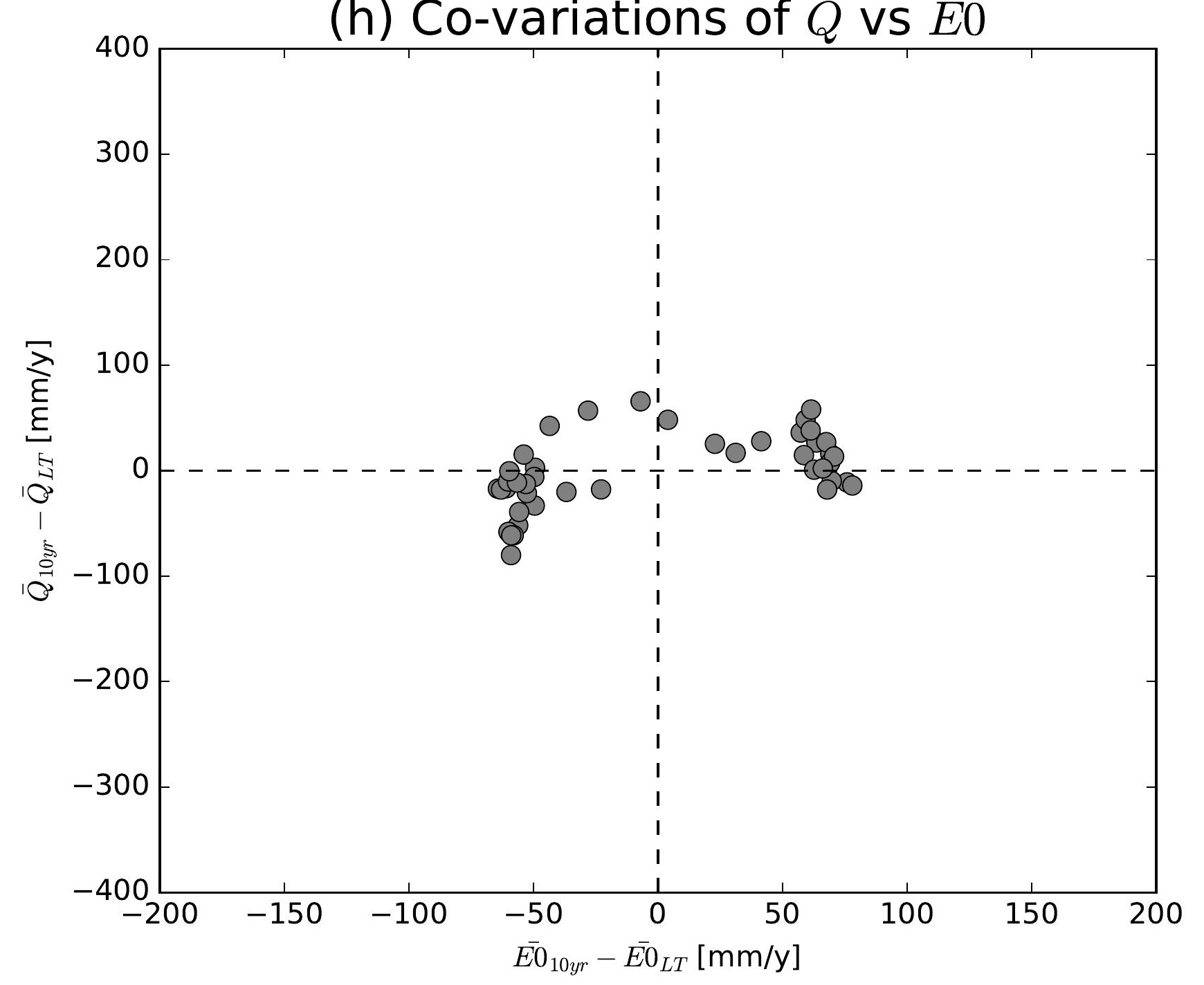

(k) Co-variations of $\bar{Q}$ vs $\overline{E 0}$

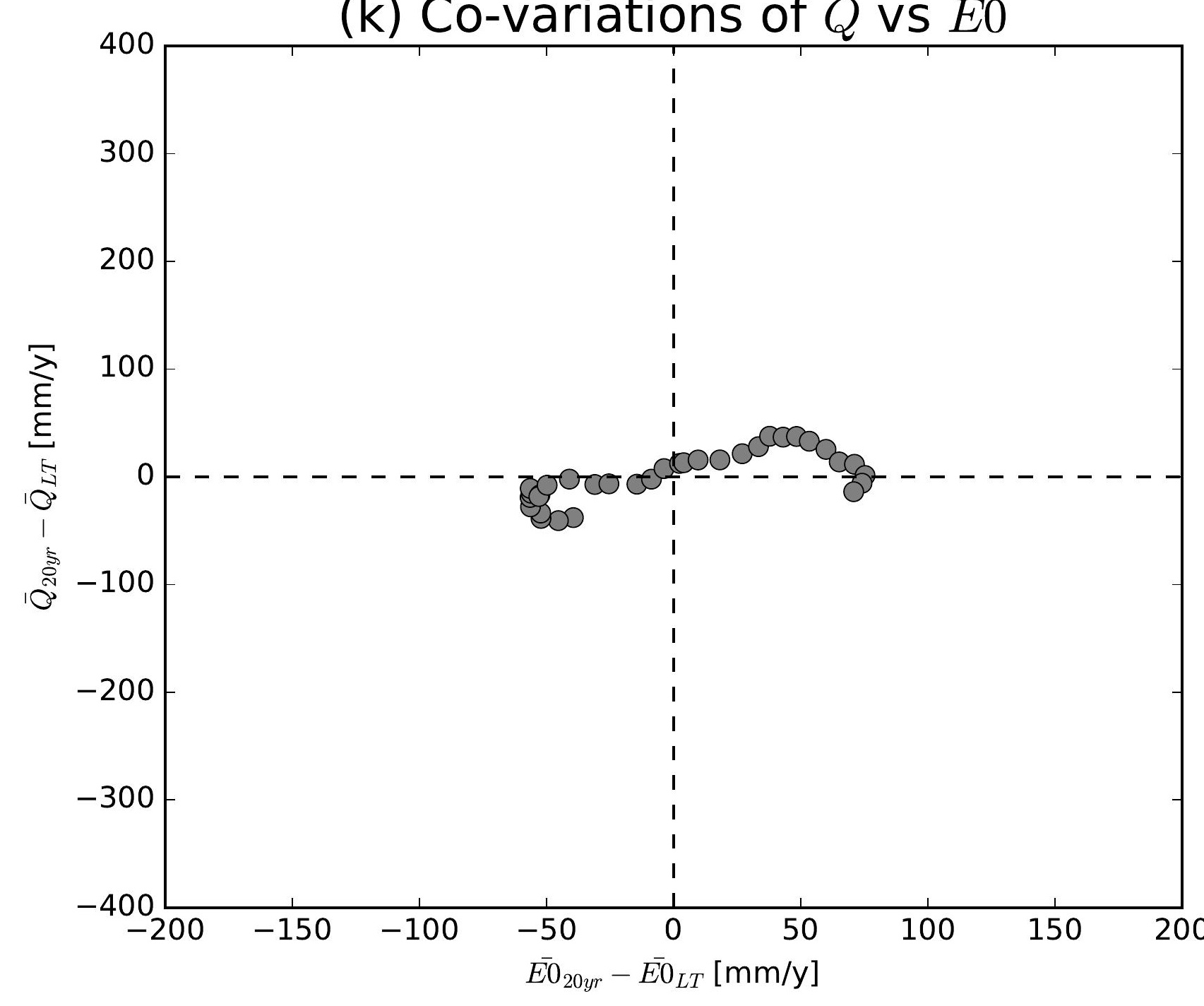

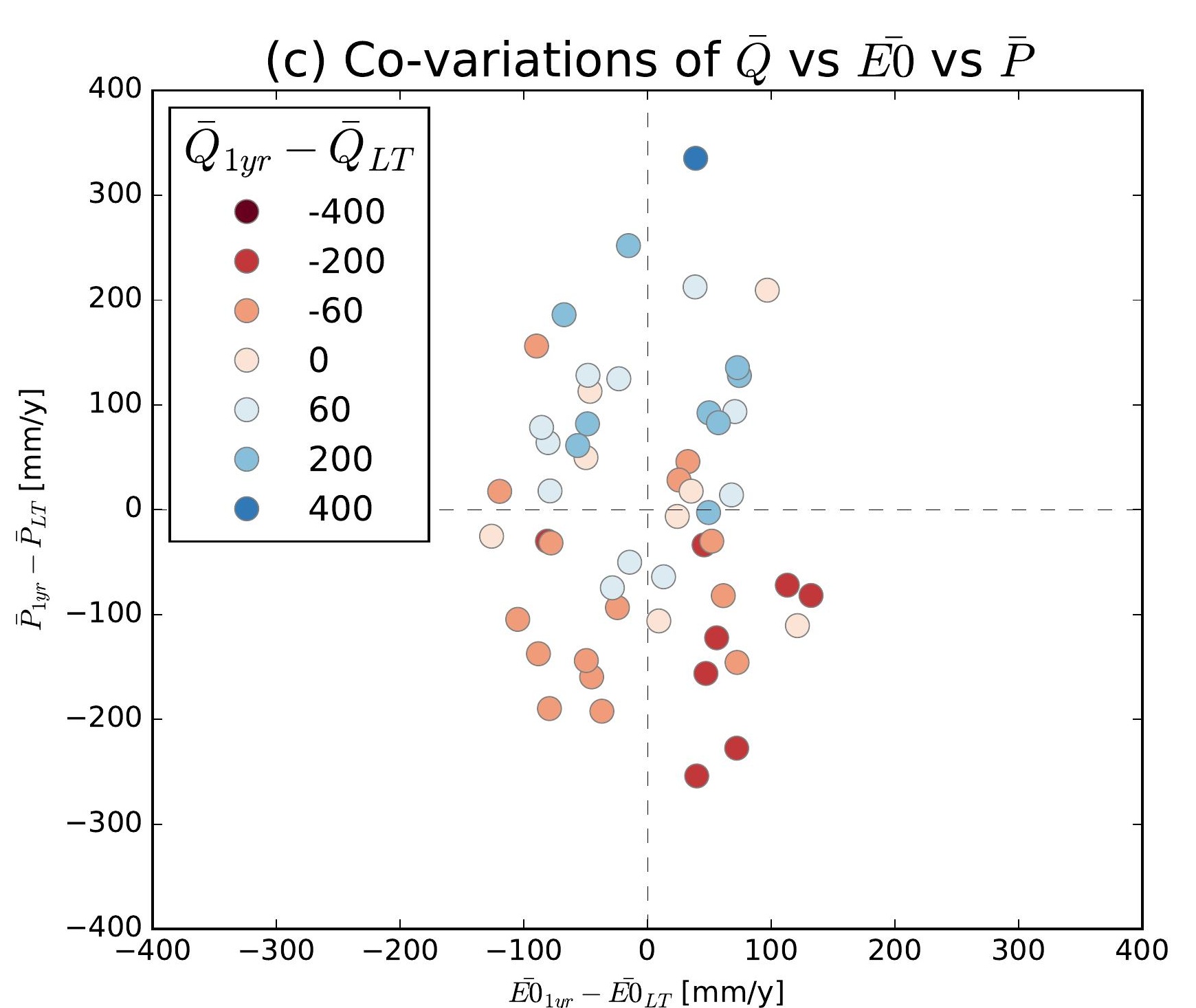
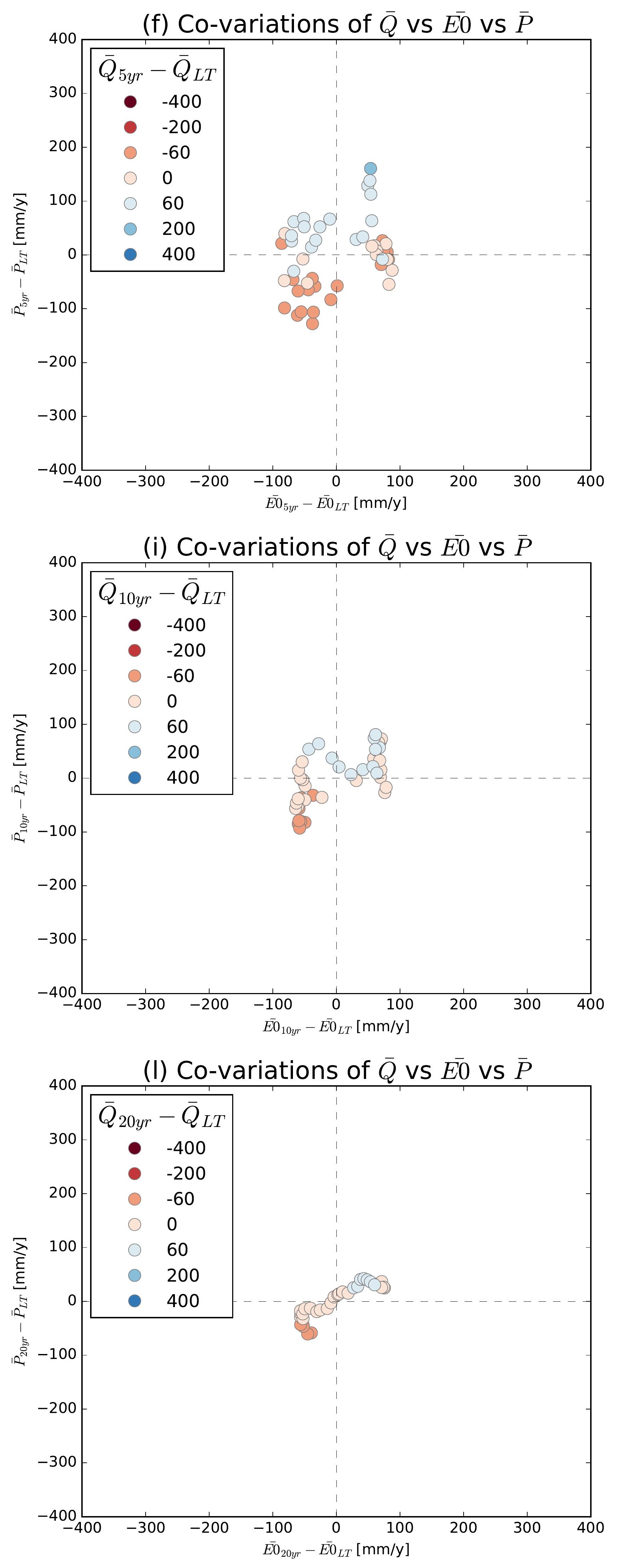

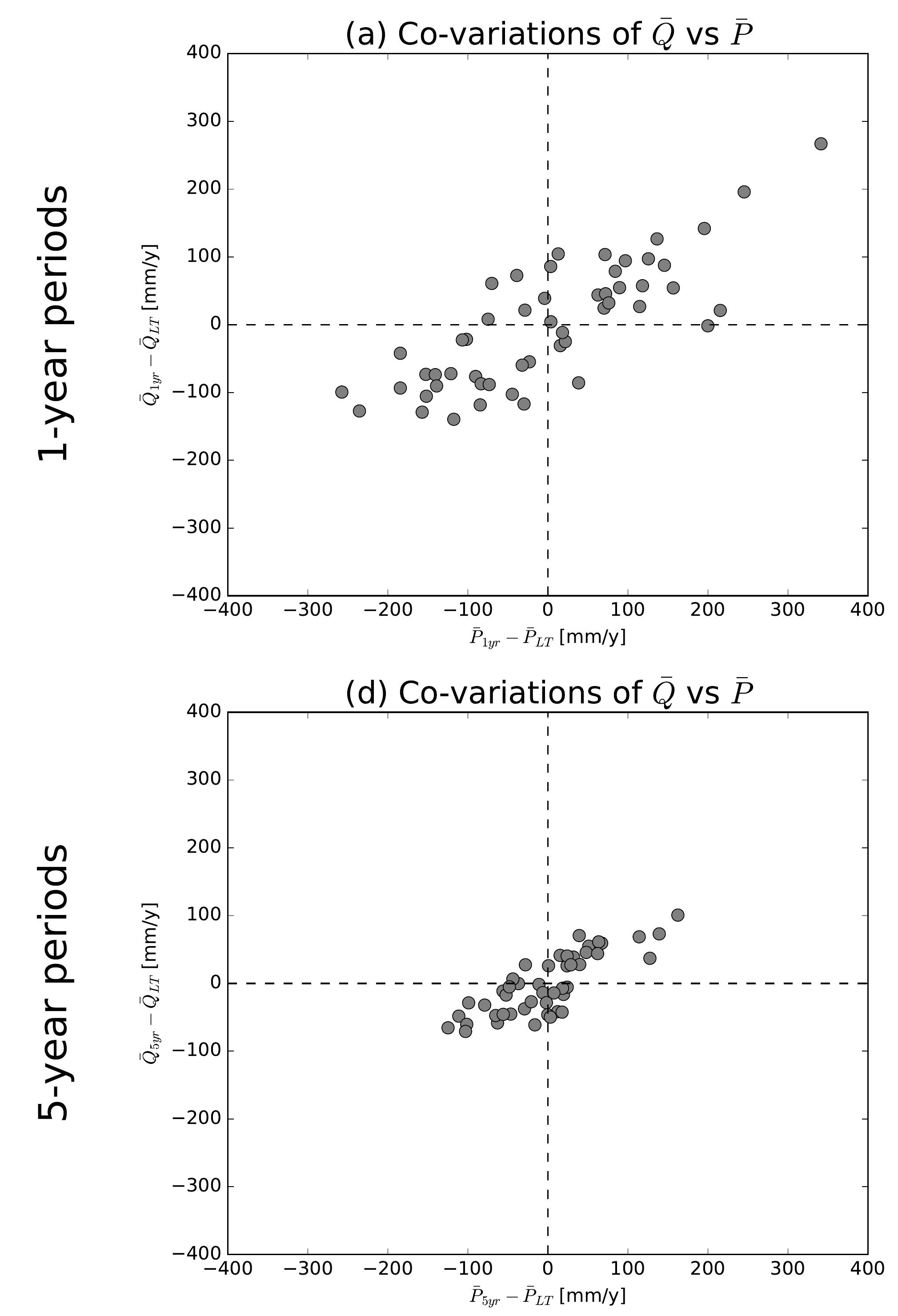

(g) Co-variations of $\bar{Q}$ vs $\bar{P}$
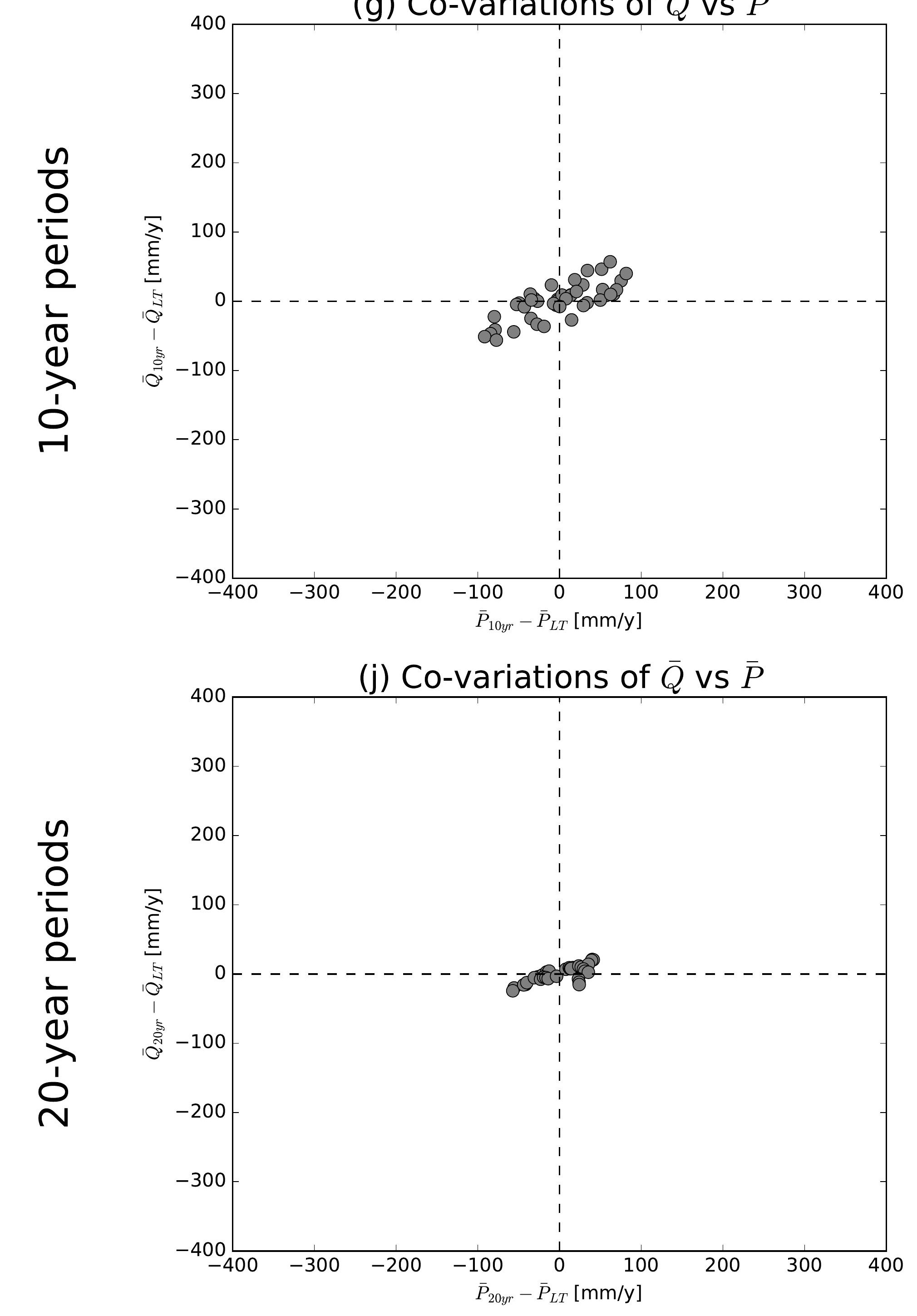

(b) Co-variations of $\bar{Q}$ vs $\overline{E 0}$

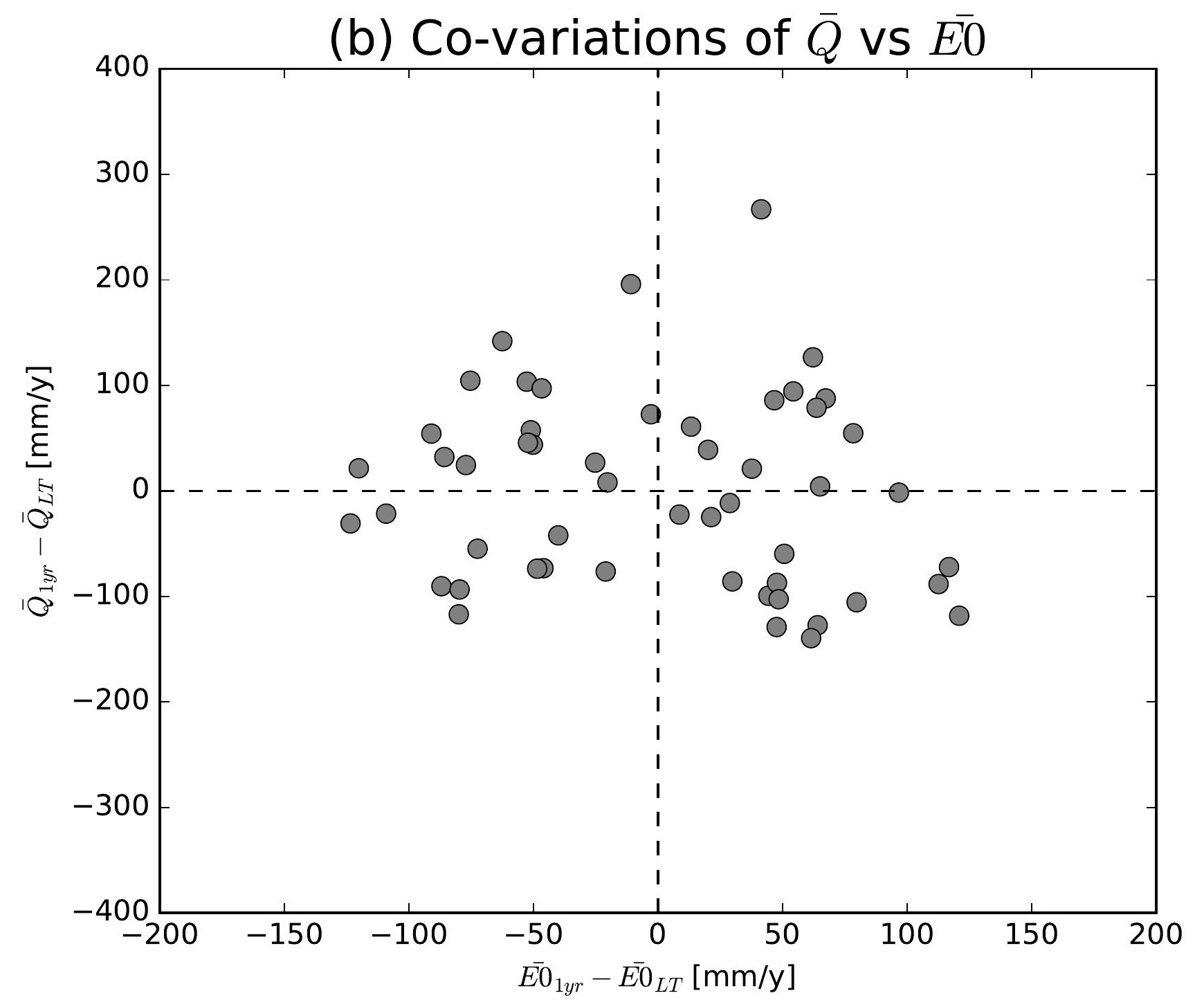

(e) Co-variations of $\bar{Q}$ vs $\overline{E 0}$

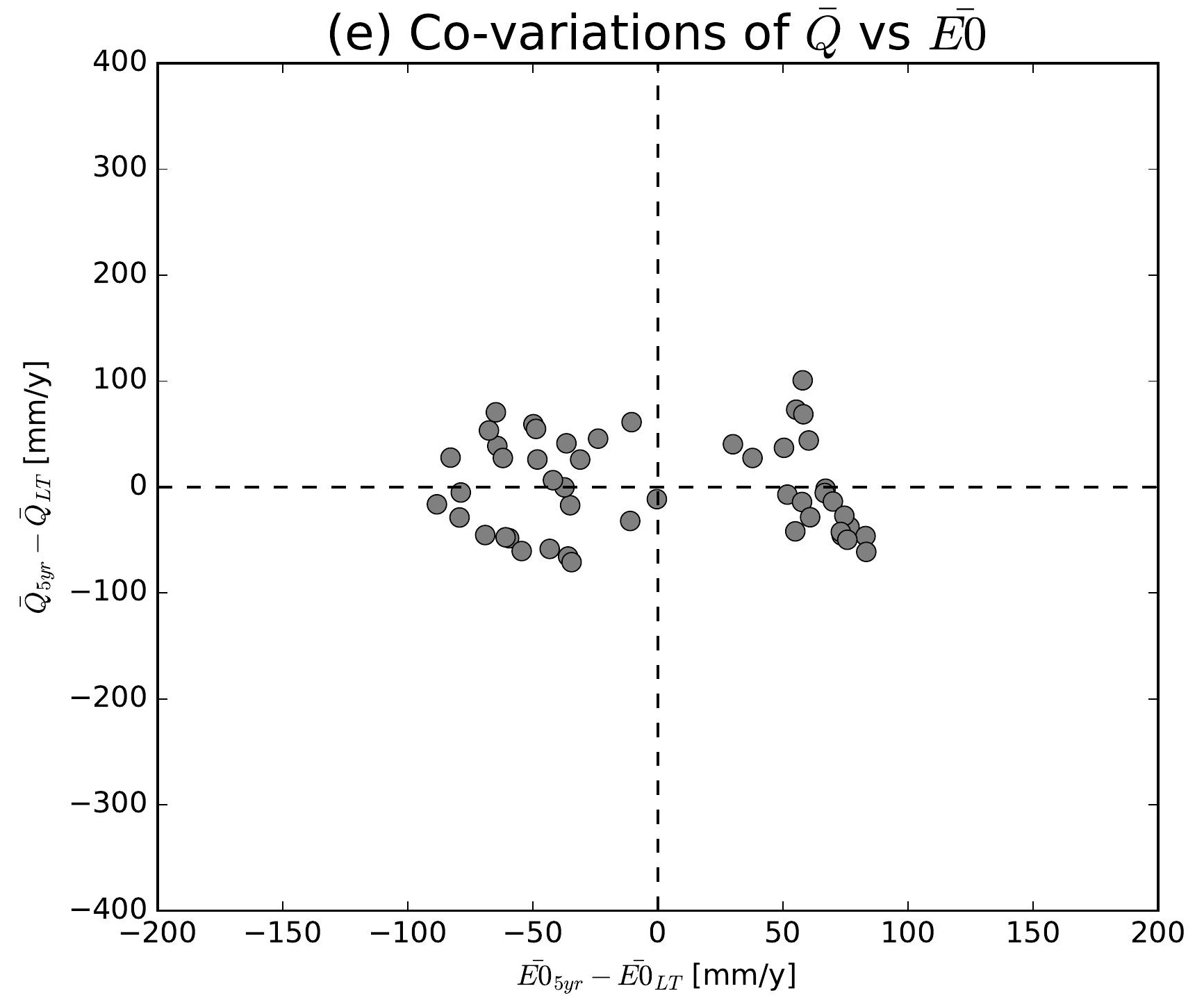

(h) Co-variations of $\bar{Q}$ vs $\overline{E 0}$

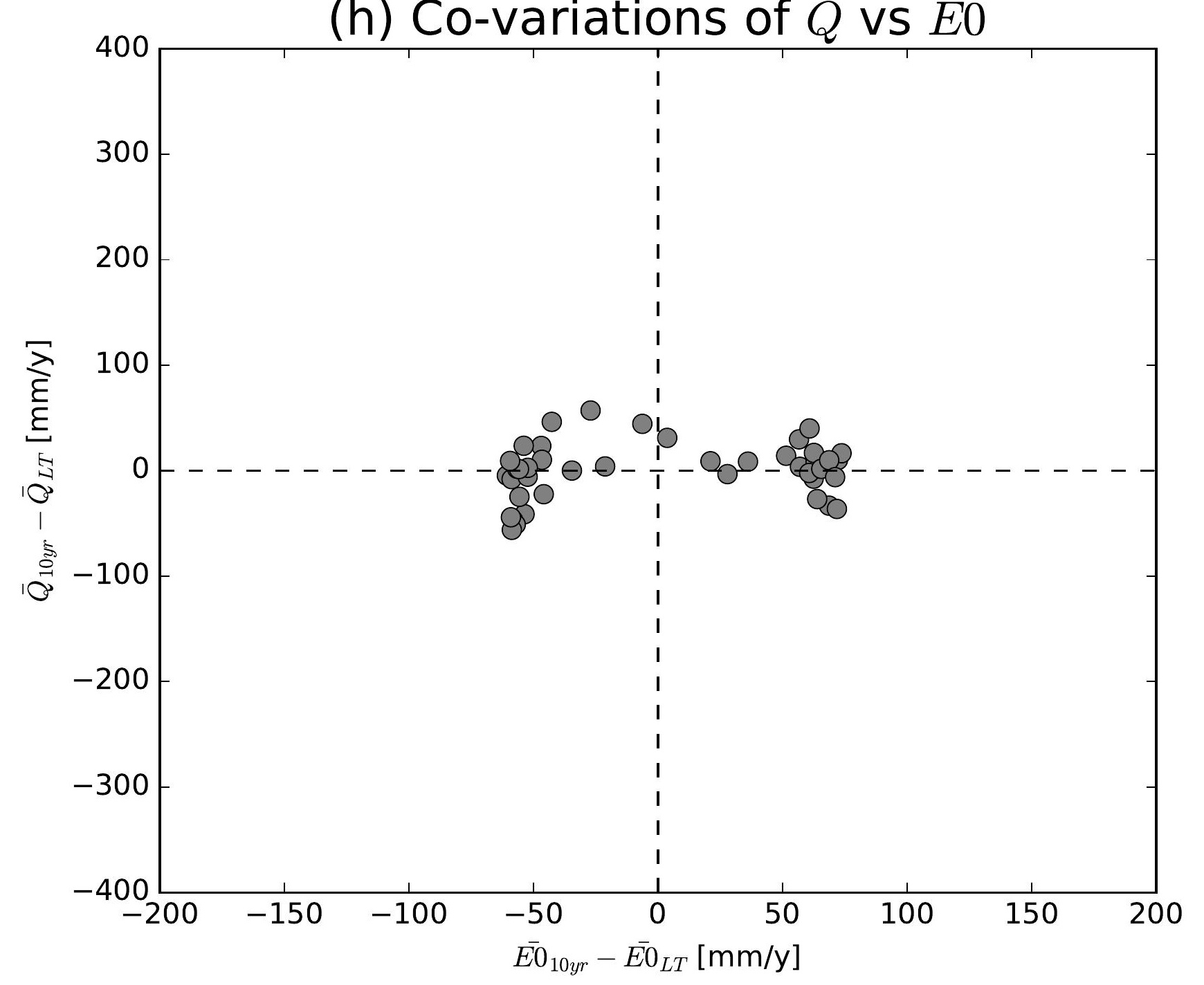

(k) Co-variations of $\bar{Q}$ vs $\overline{E 0}$

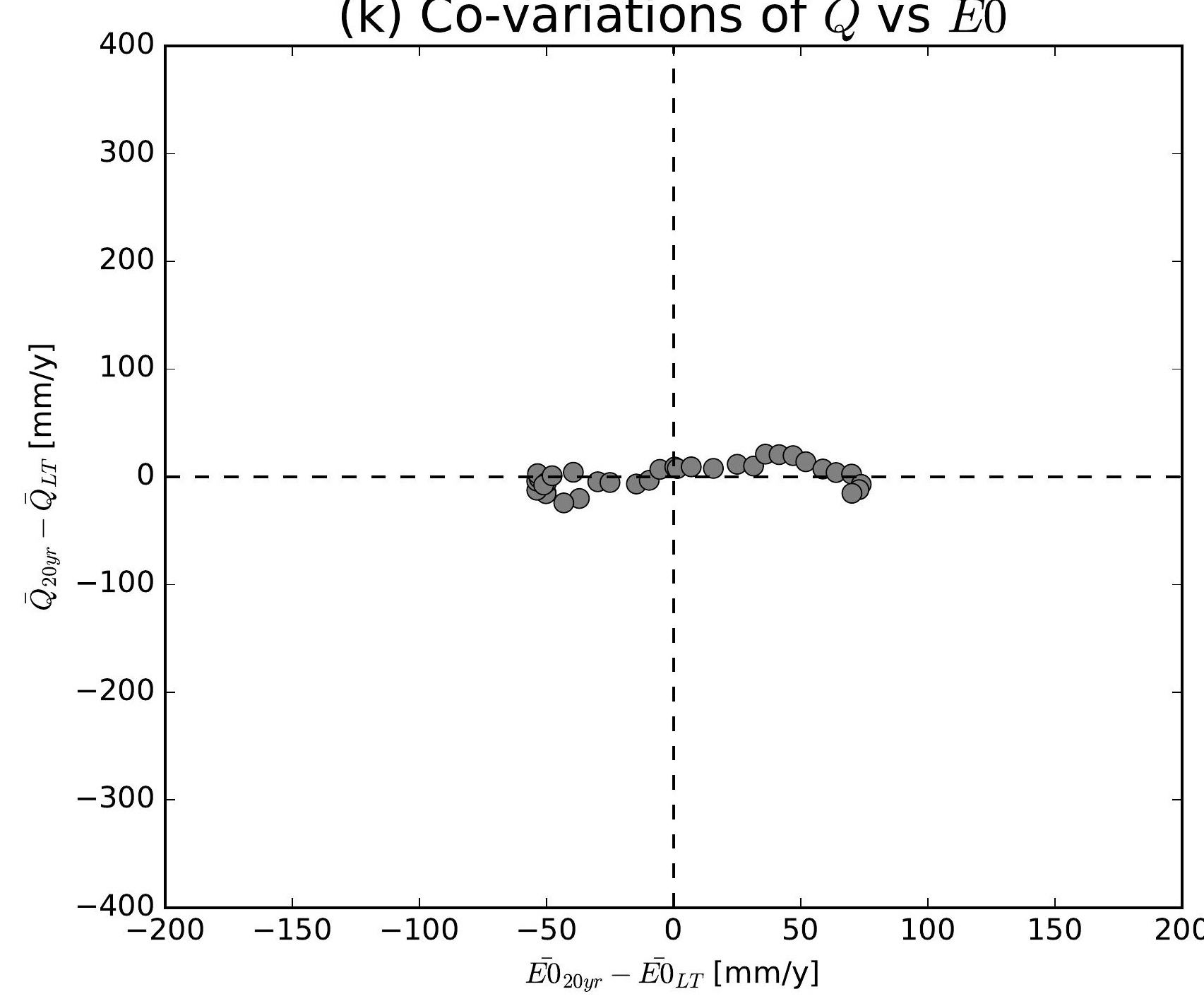

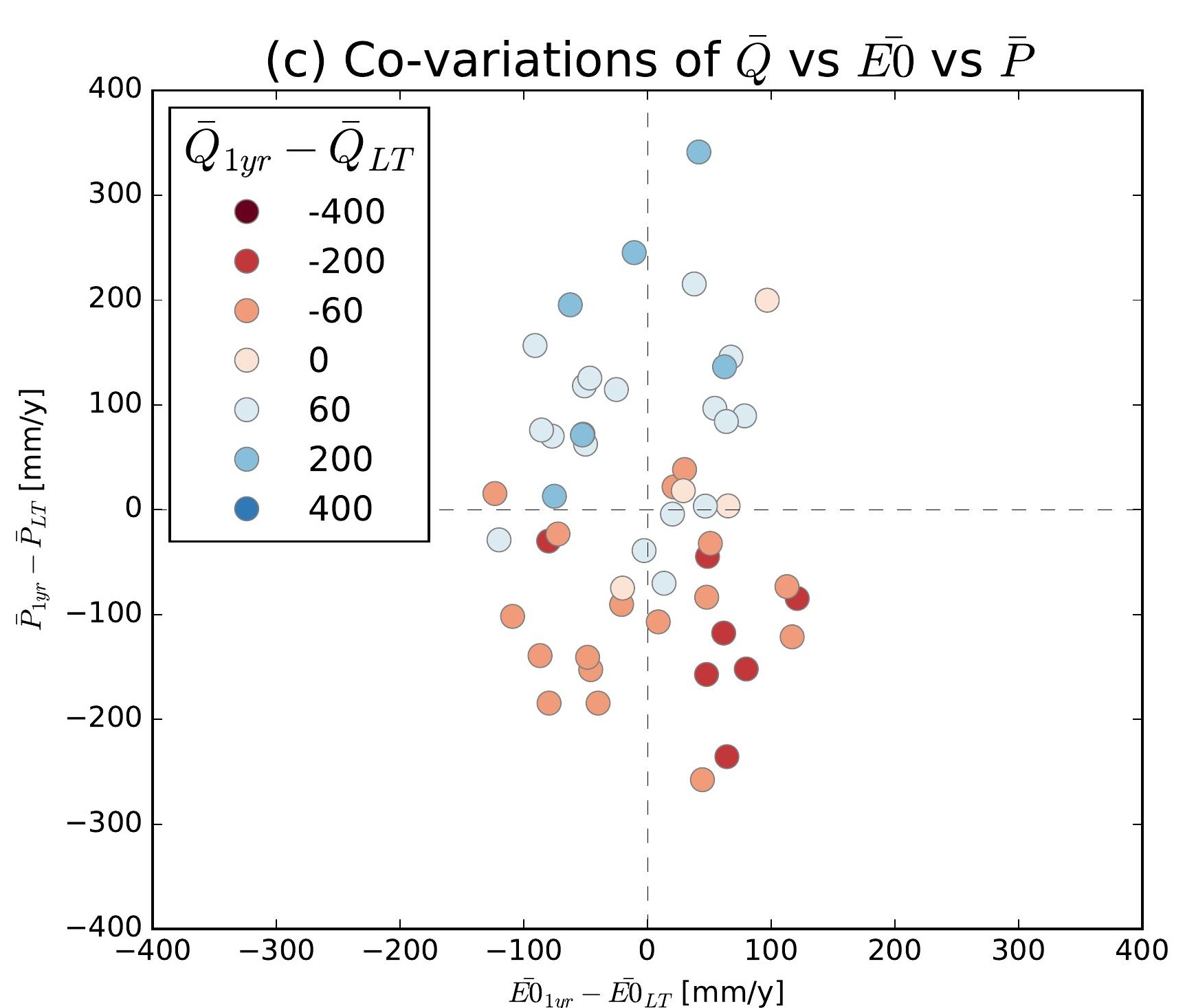
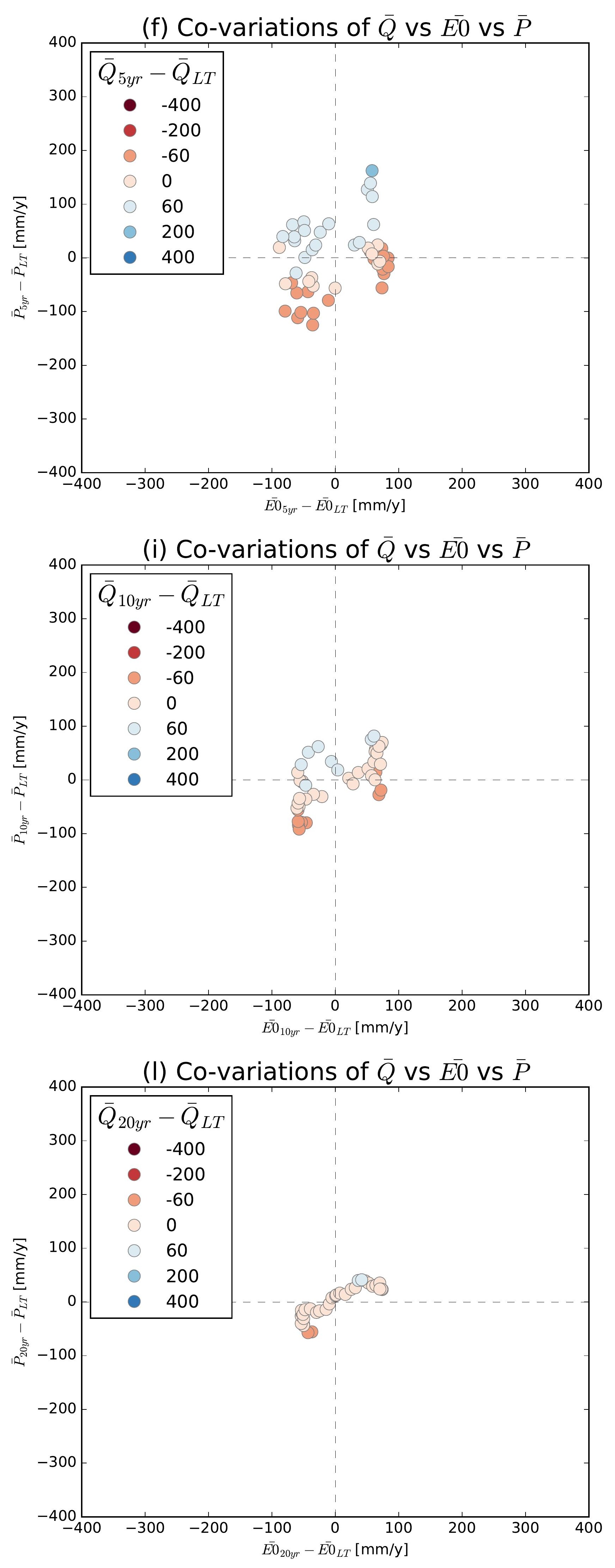

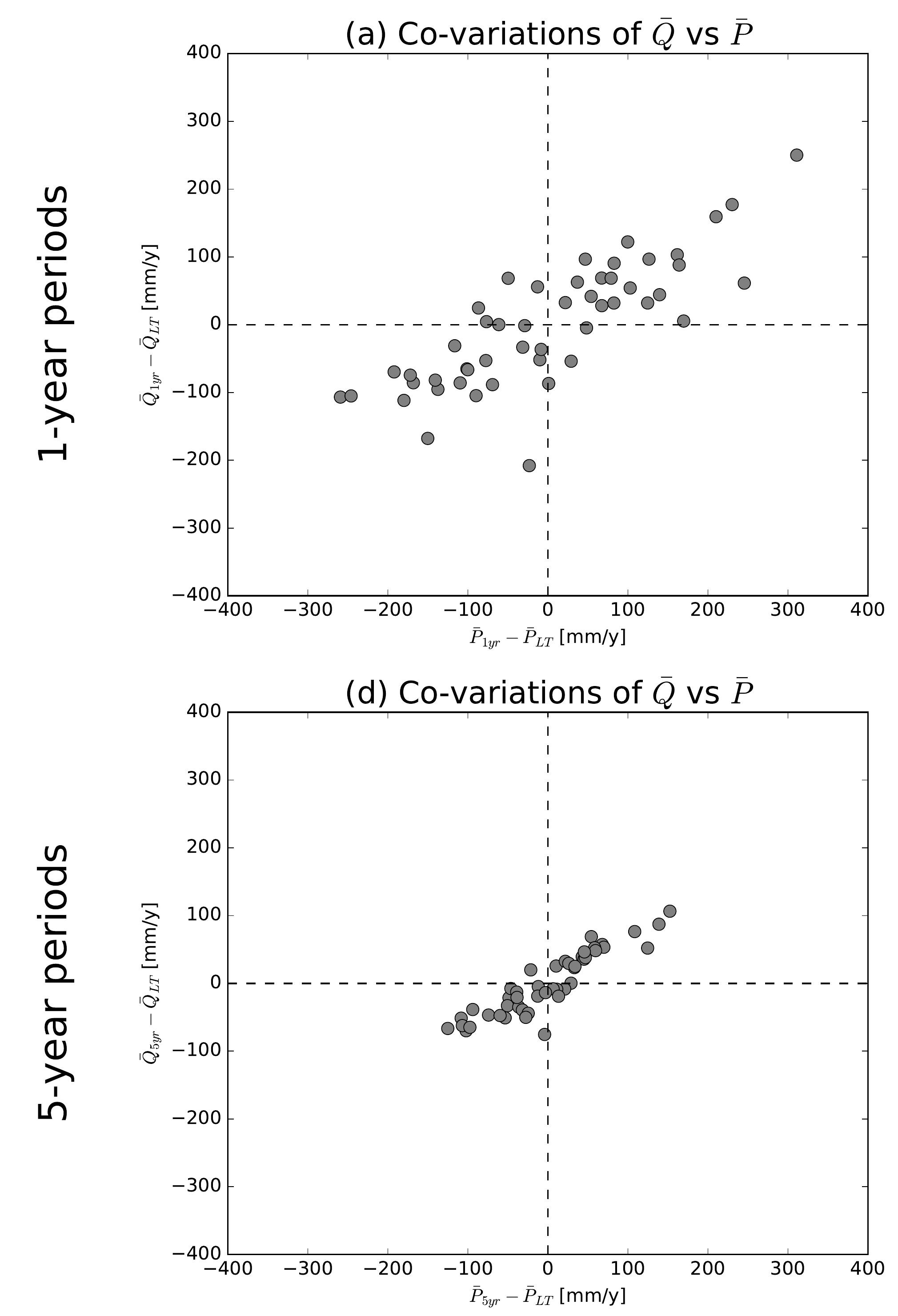

(g) Co-variations of $\bar{Q}$ vs $\bar{P}$

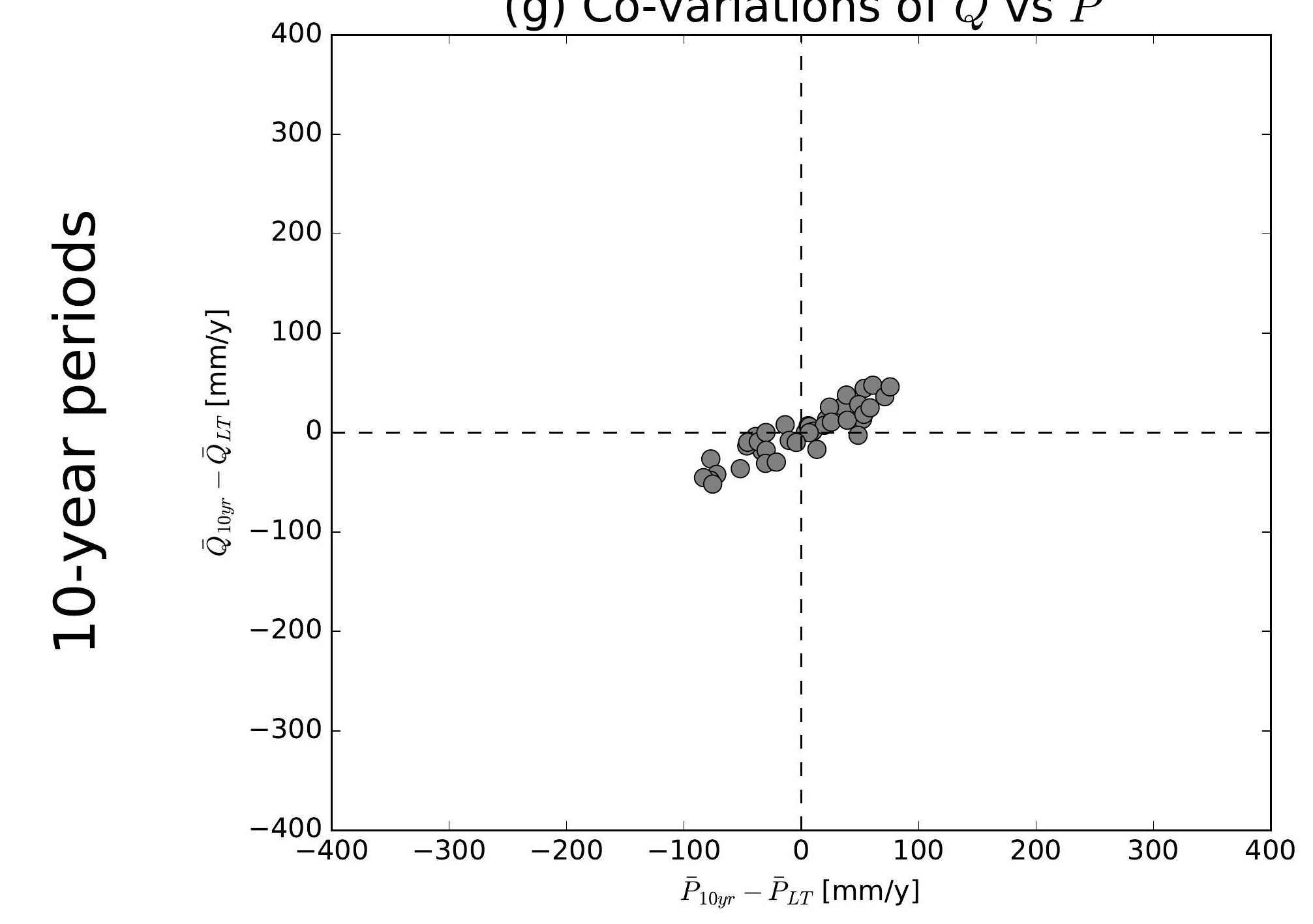

(j) Co-variations of $\bar{Q}$ vs $\bar{P}$

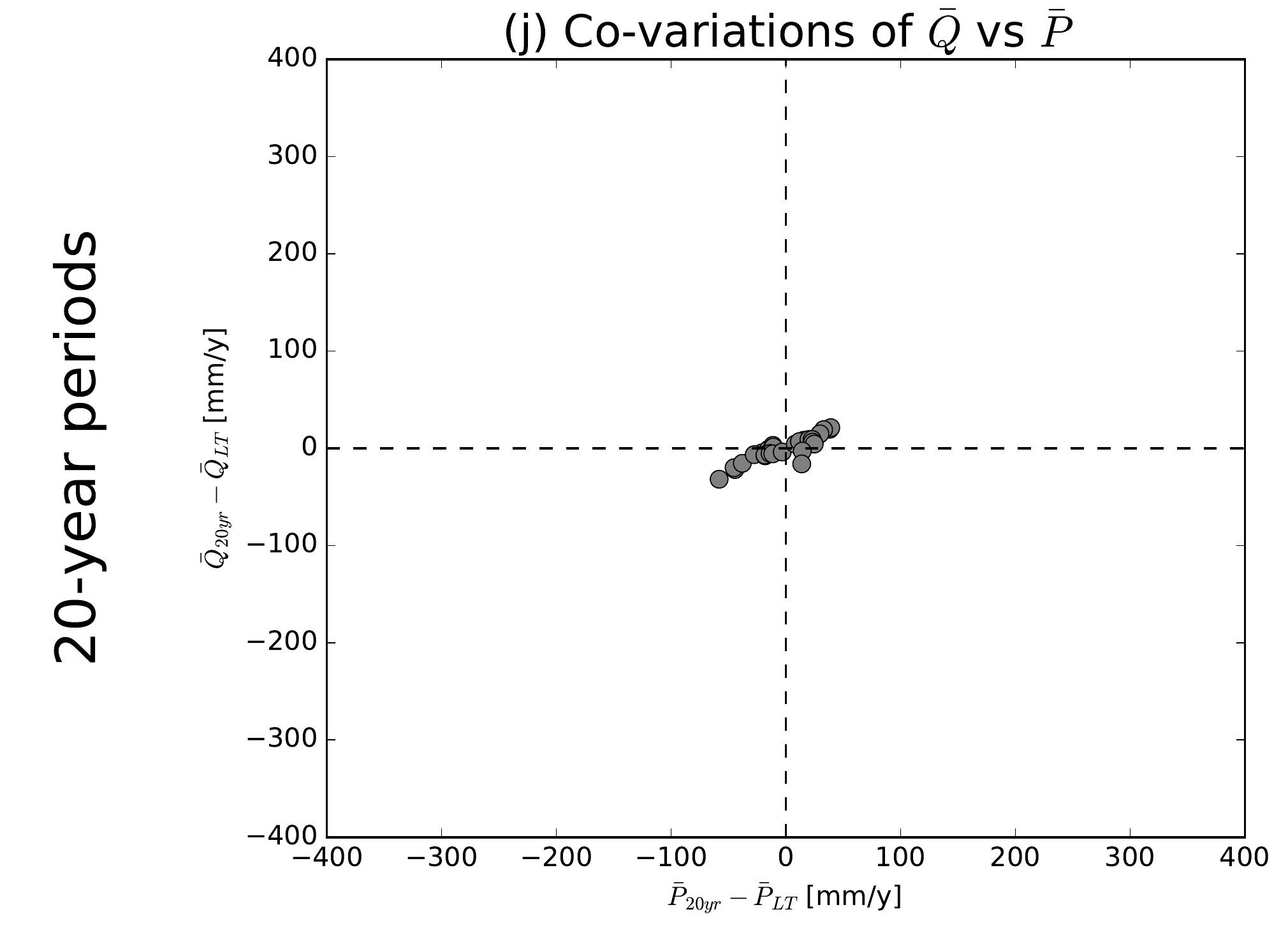

(b) Co-variations of $\bar{Q}$ vs $\overline{E 0}$

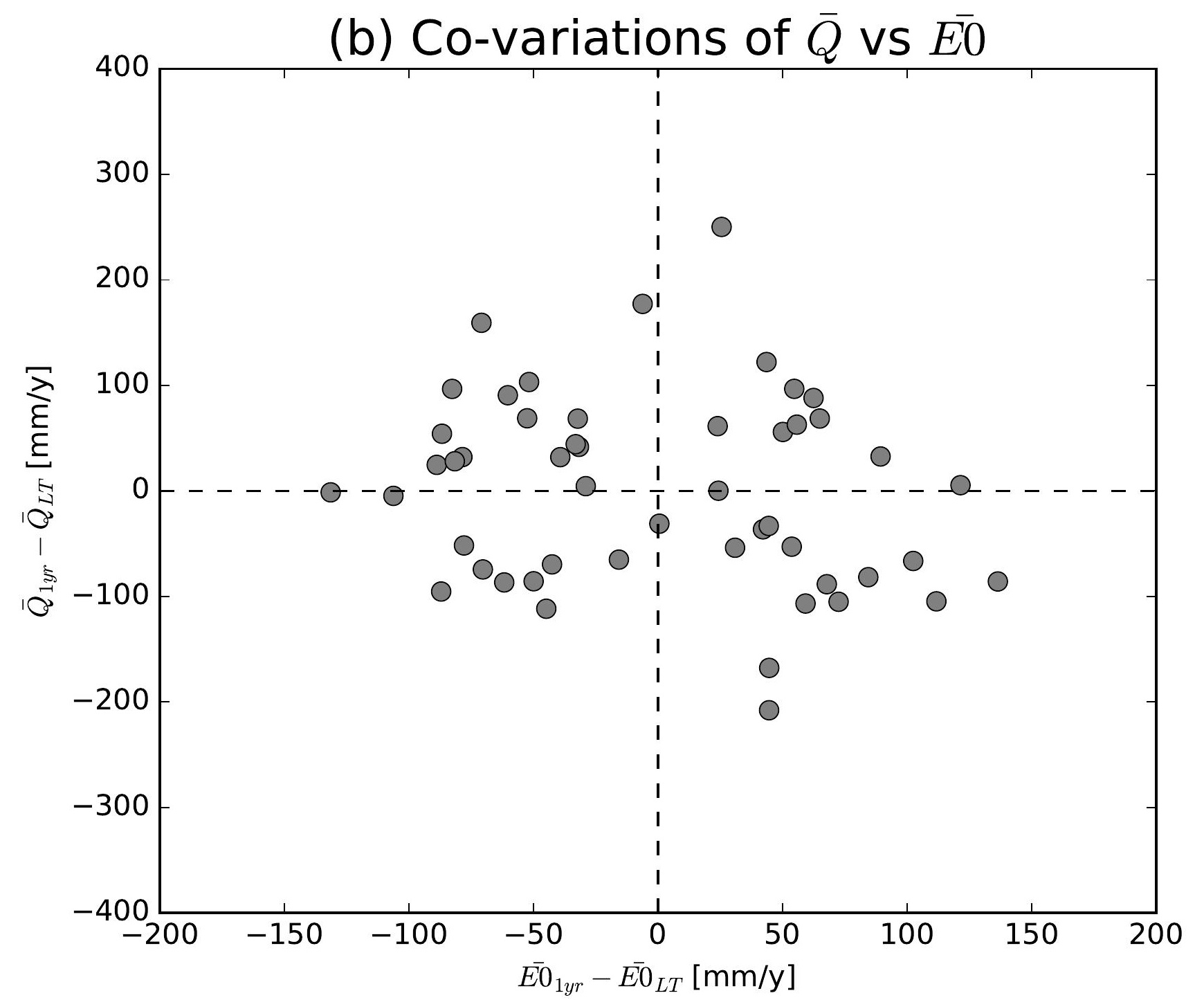

(e) Co-variations of $\bar{Q}$ vs $\overline{E 0}$

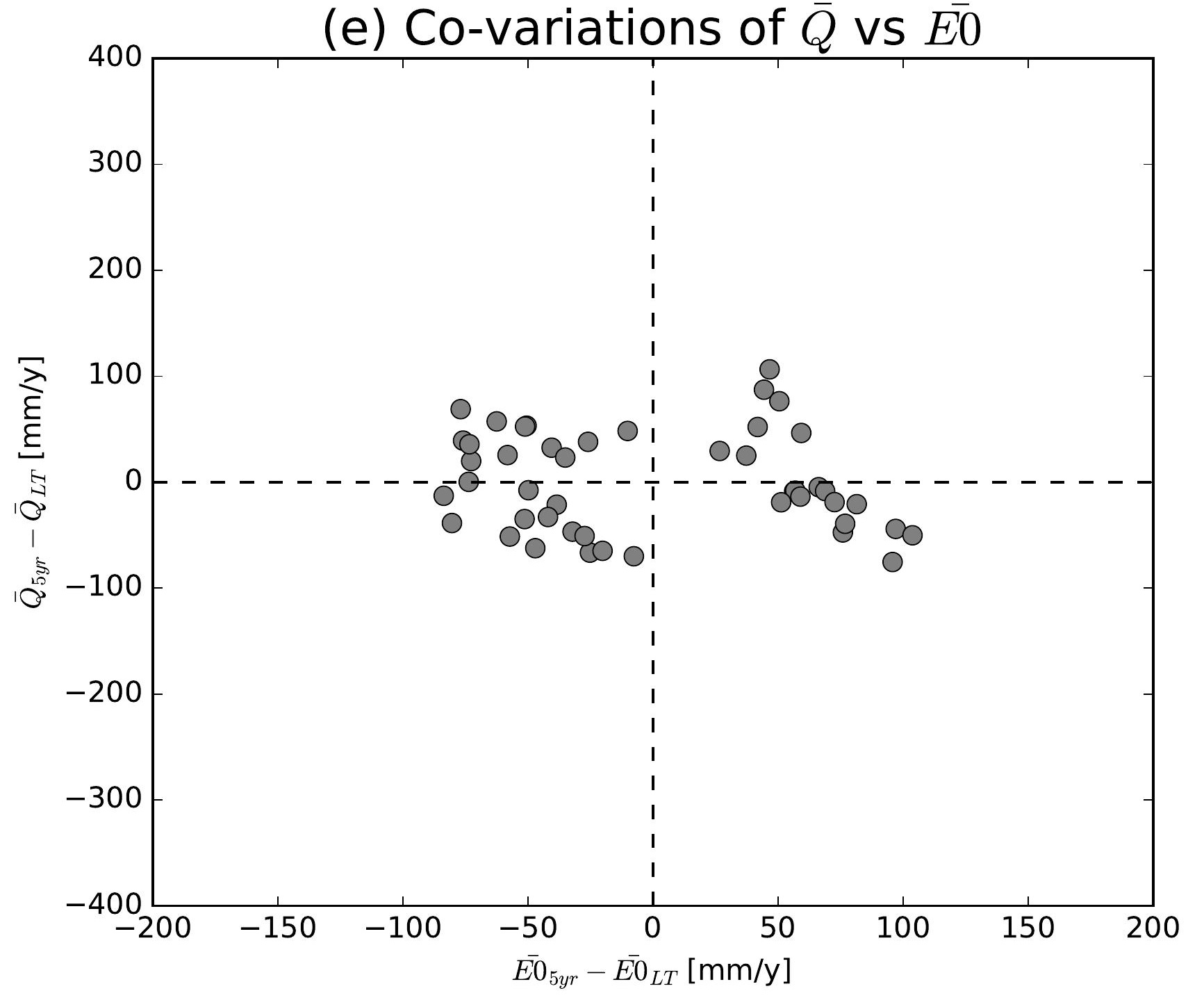

(h) Co-variations of $\bar{Q}$ vs $\overline{E 0}$

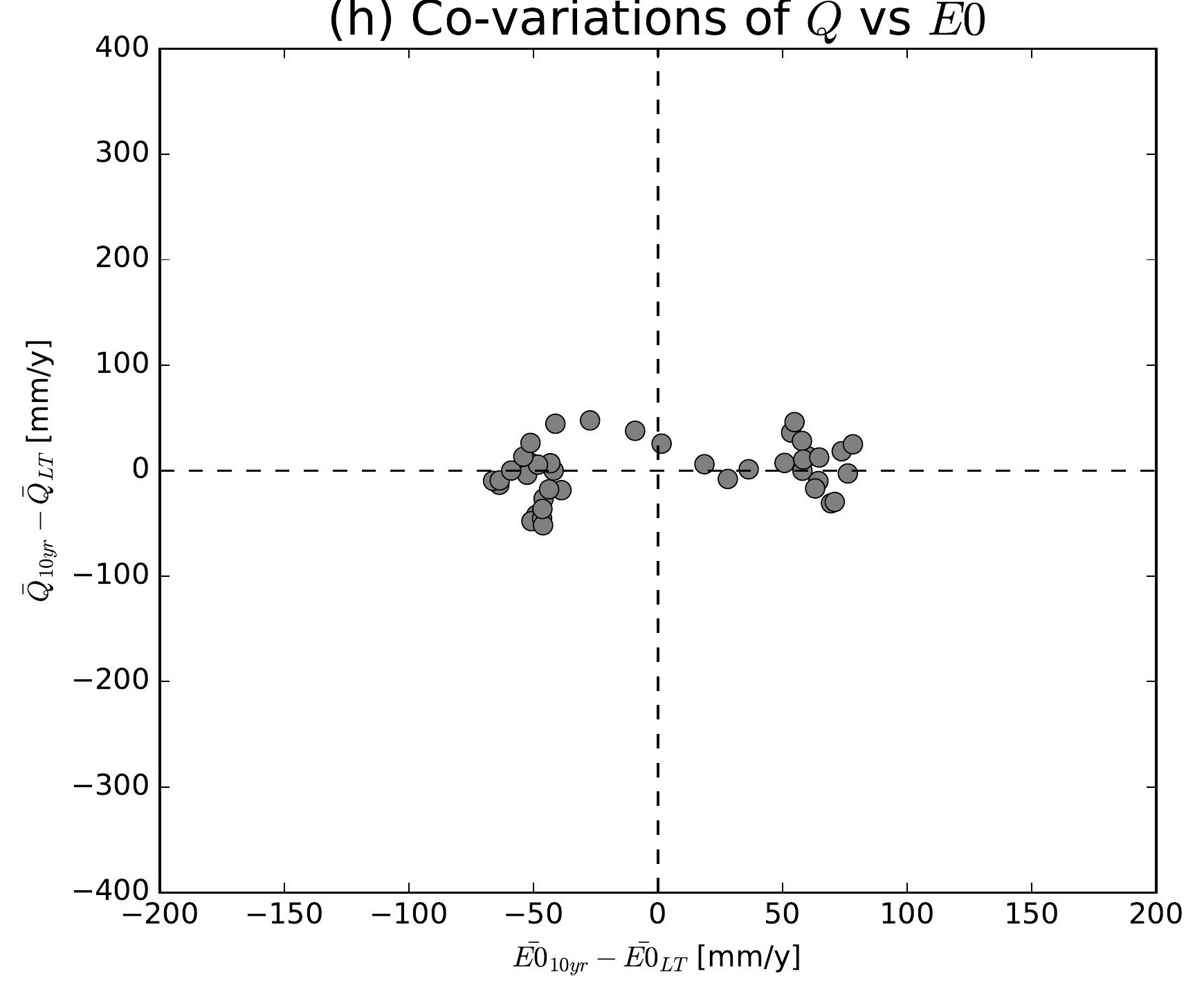

(k) Co-variations of $\bar{Q}$ vs $\overline{E 0}$

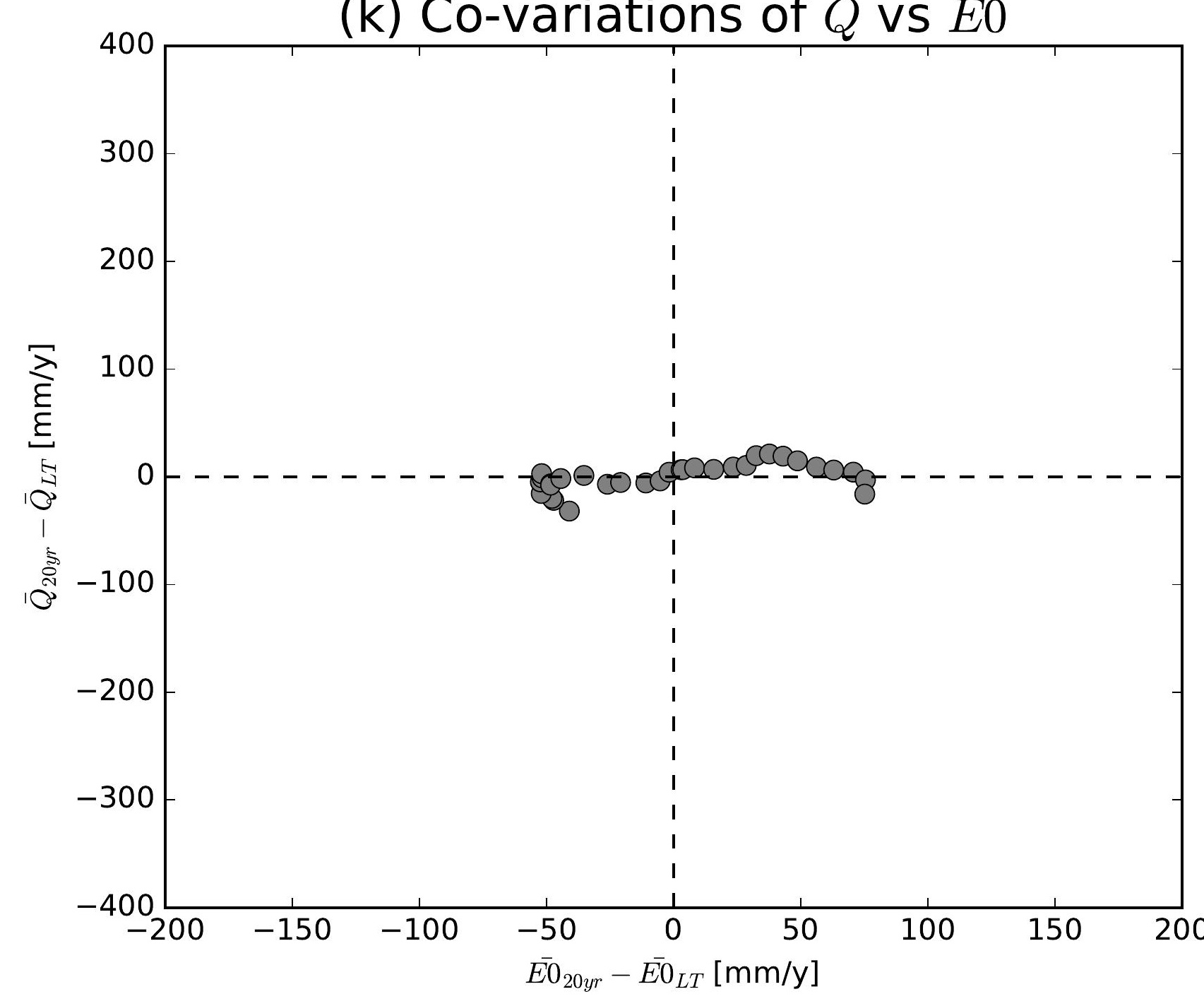

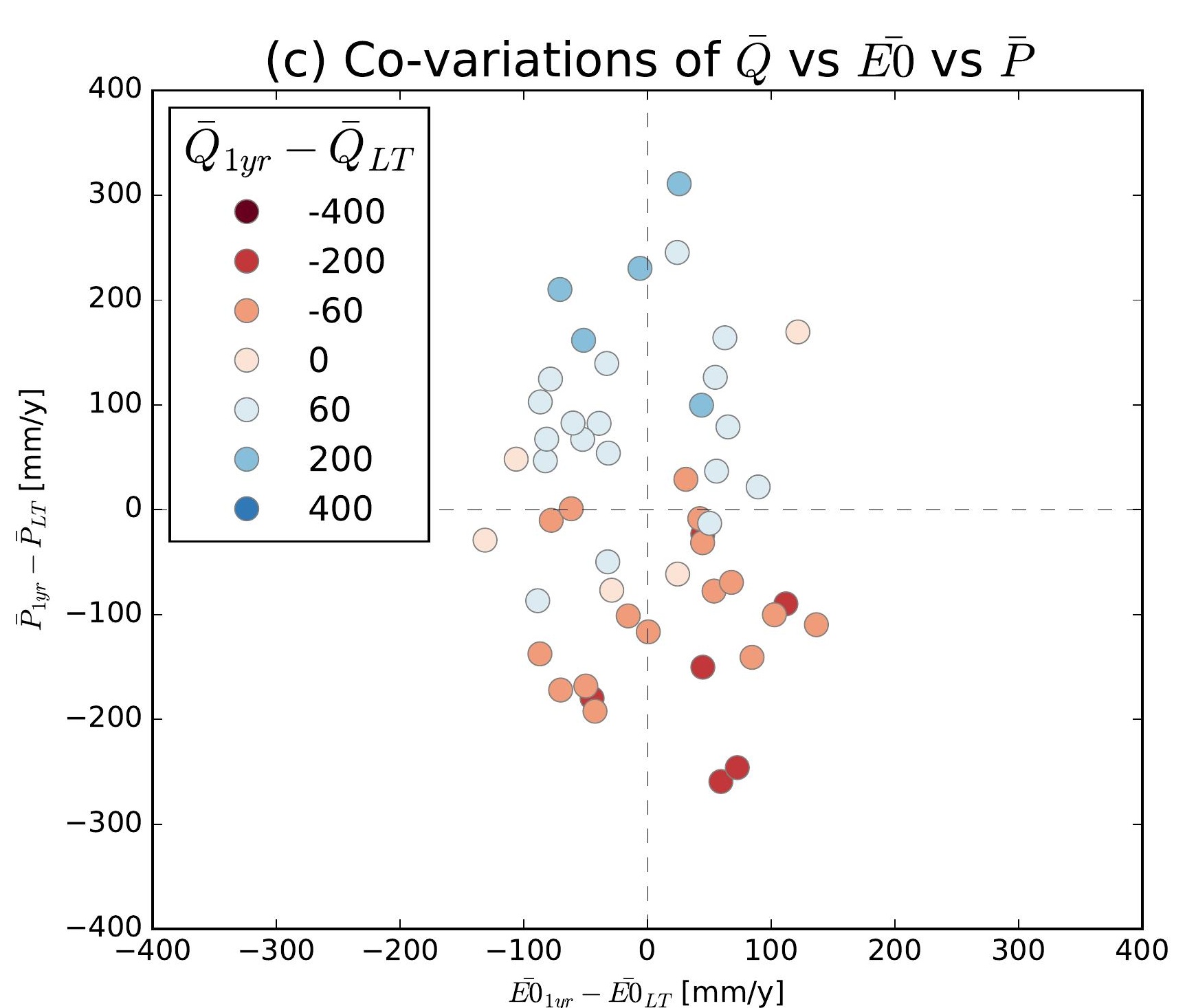
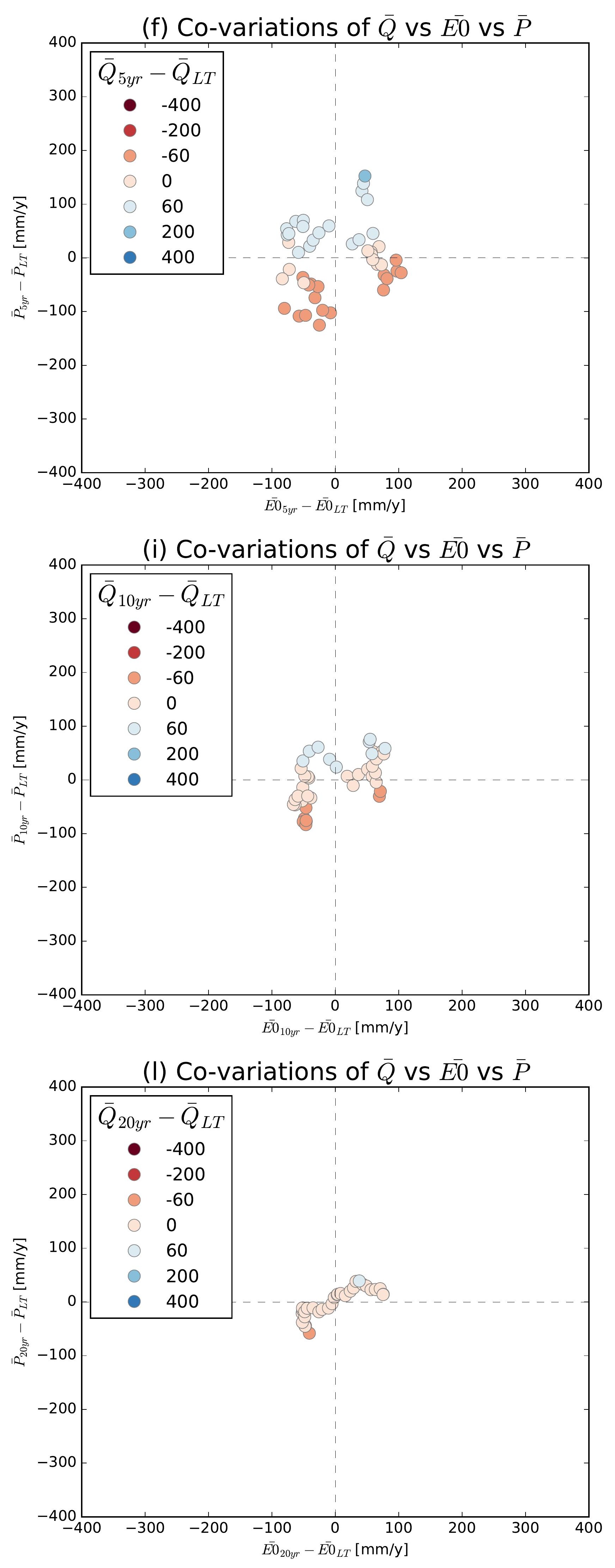

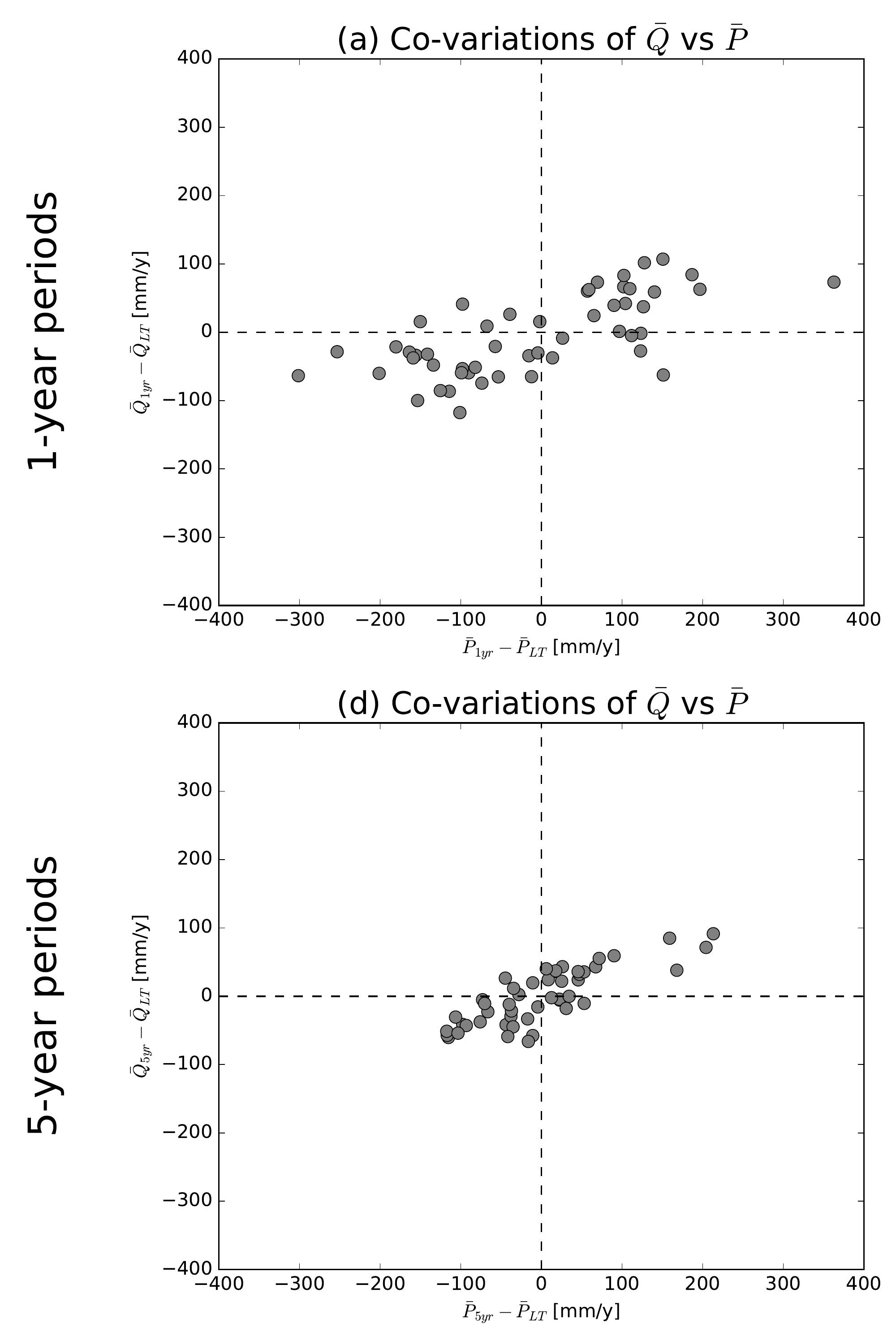

(g) Co-variations of $\bar{Q}$ vs $\bar{P}$
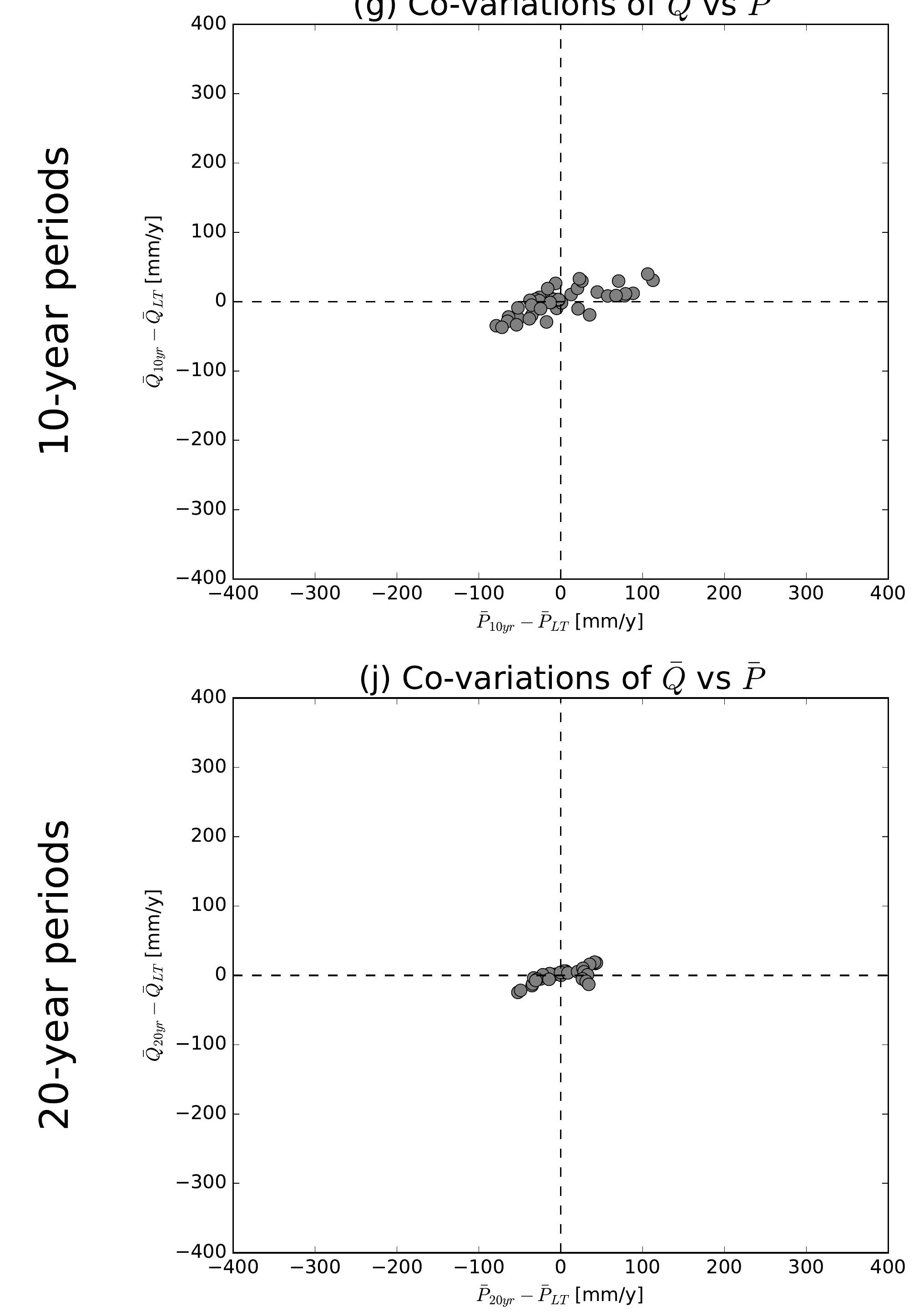

(b) Co-variations of $\bar{Q}$ vs $\overline{E 0}$

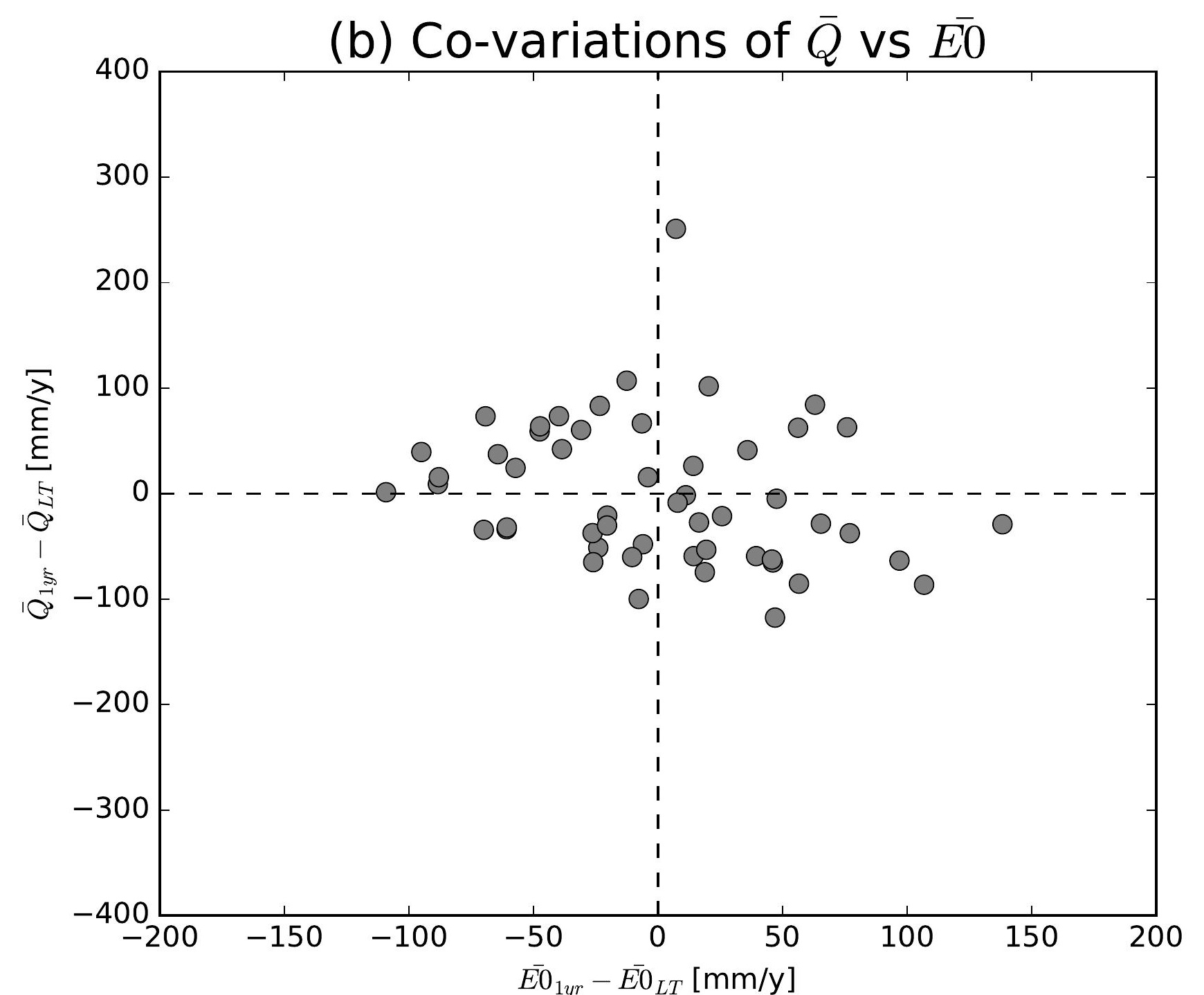

(e) Co-variations of $\bar{Q}$ vs $\overline{E 0}$

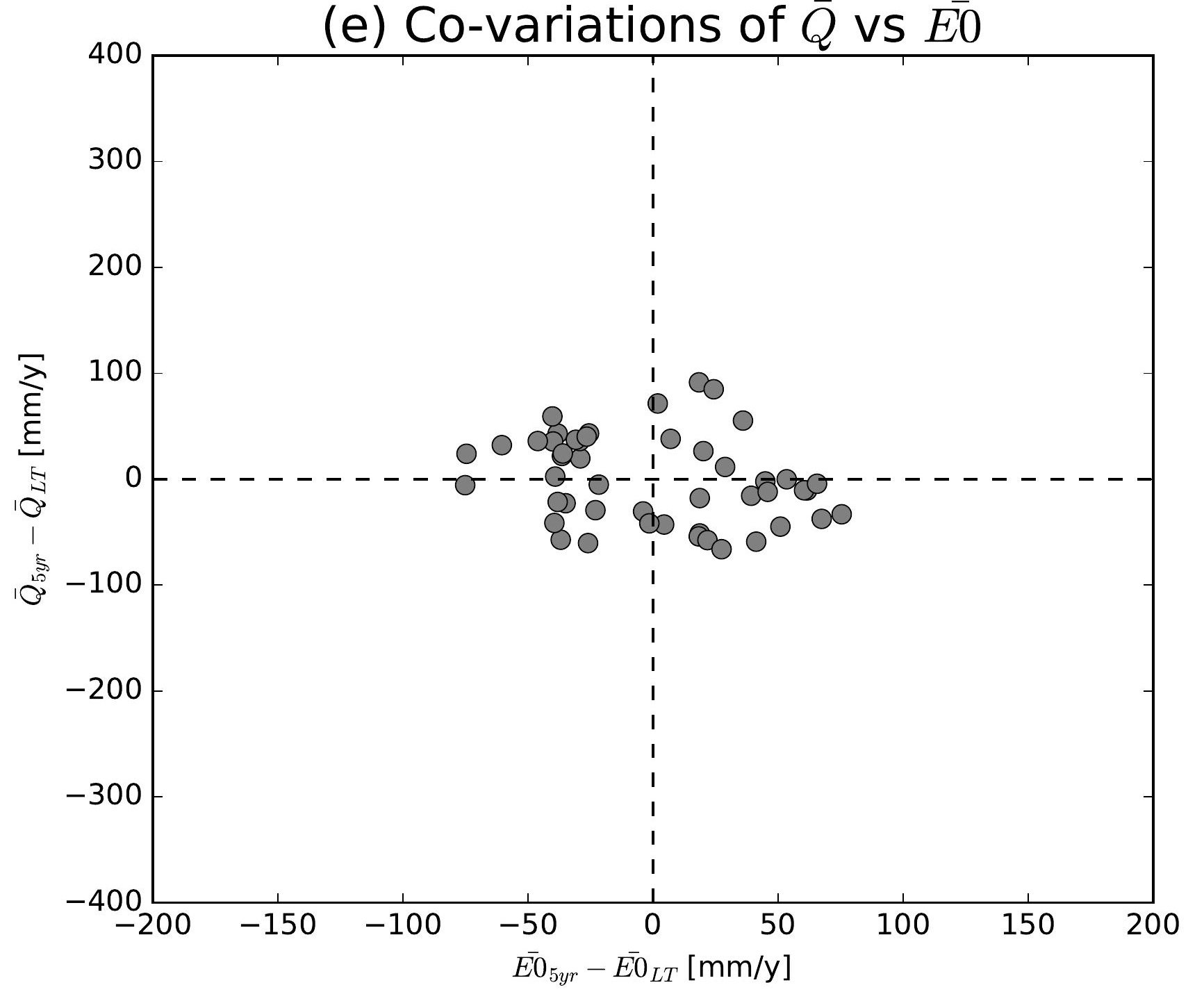

(h) Co-variations of $\bar{Q}$ vs $\overline{E 0}$

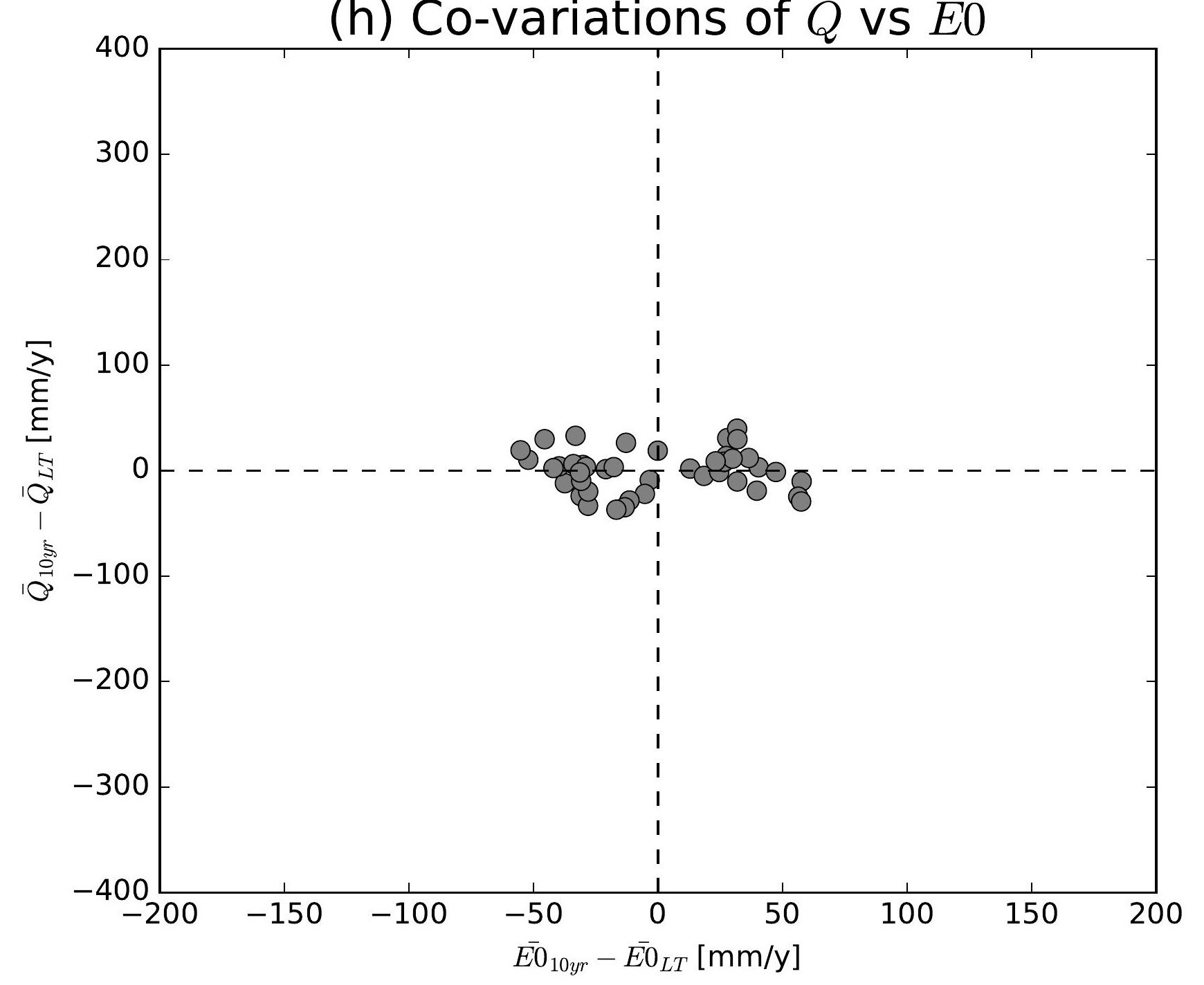

(k) Co-variations of $\bar{Q}$ vs $\overline{E 0}$

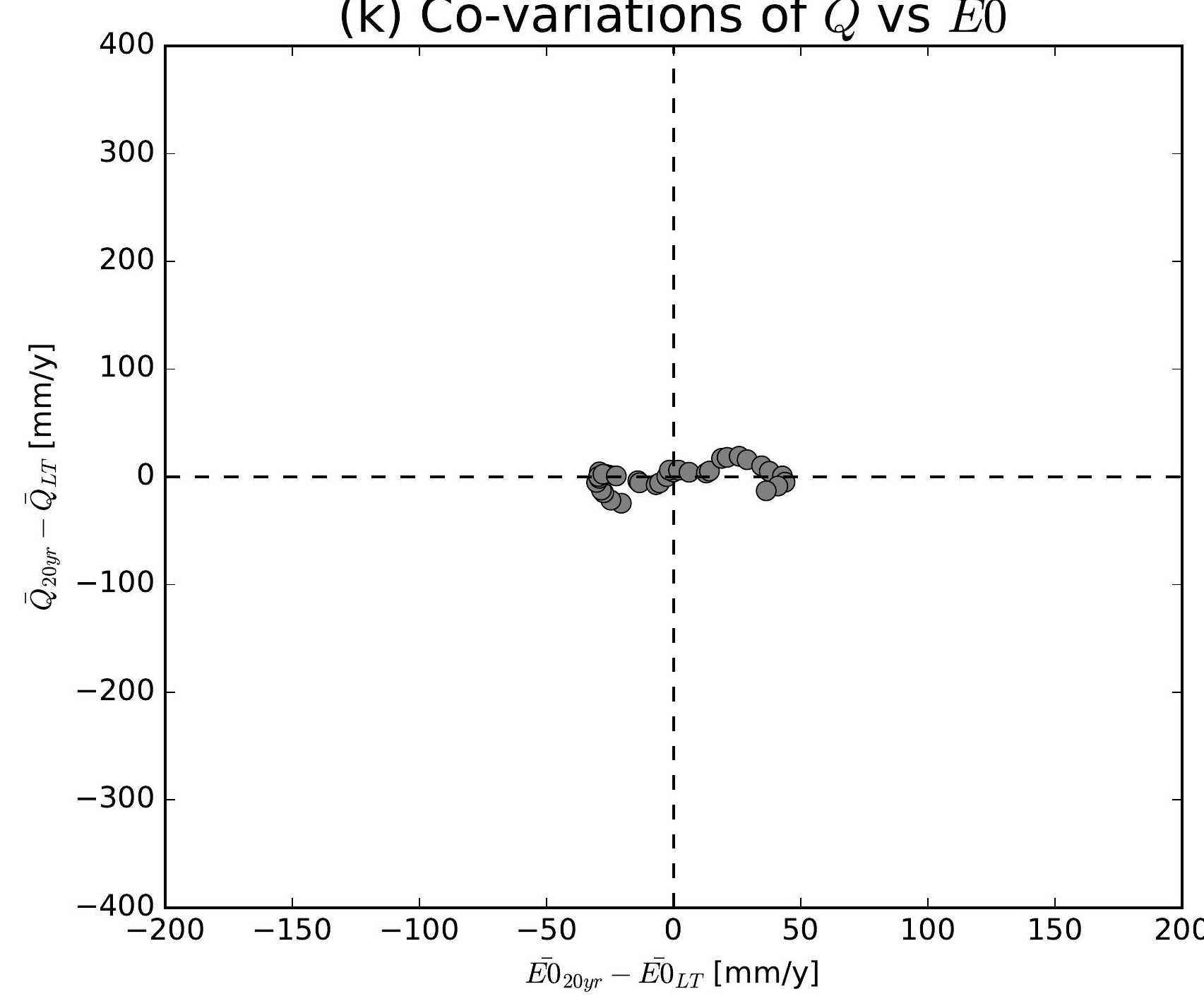

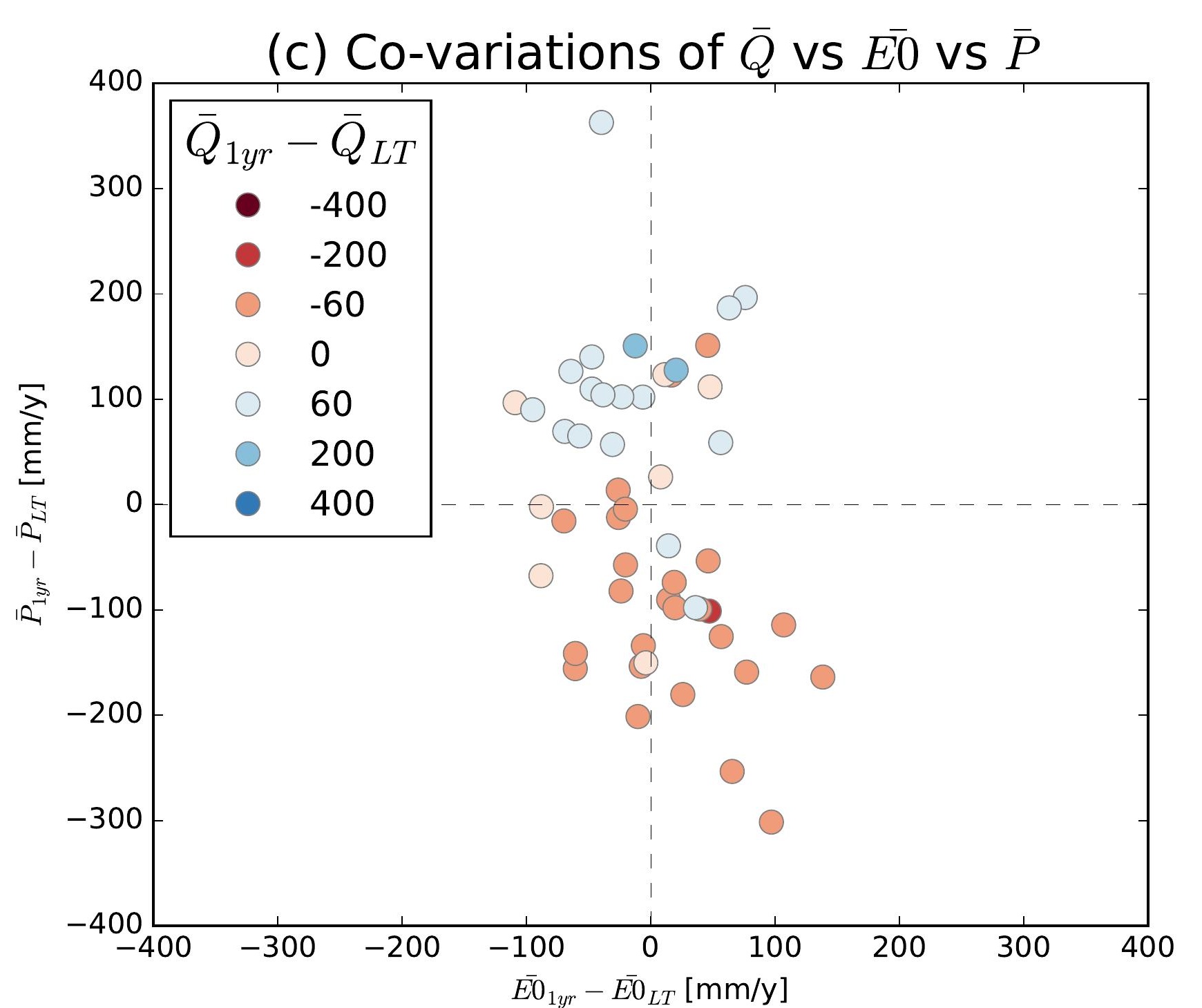
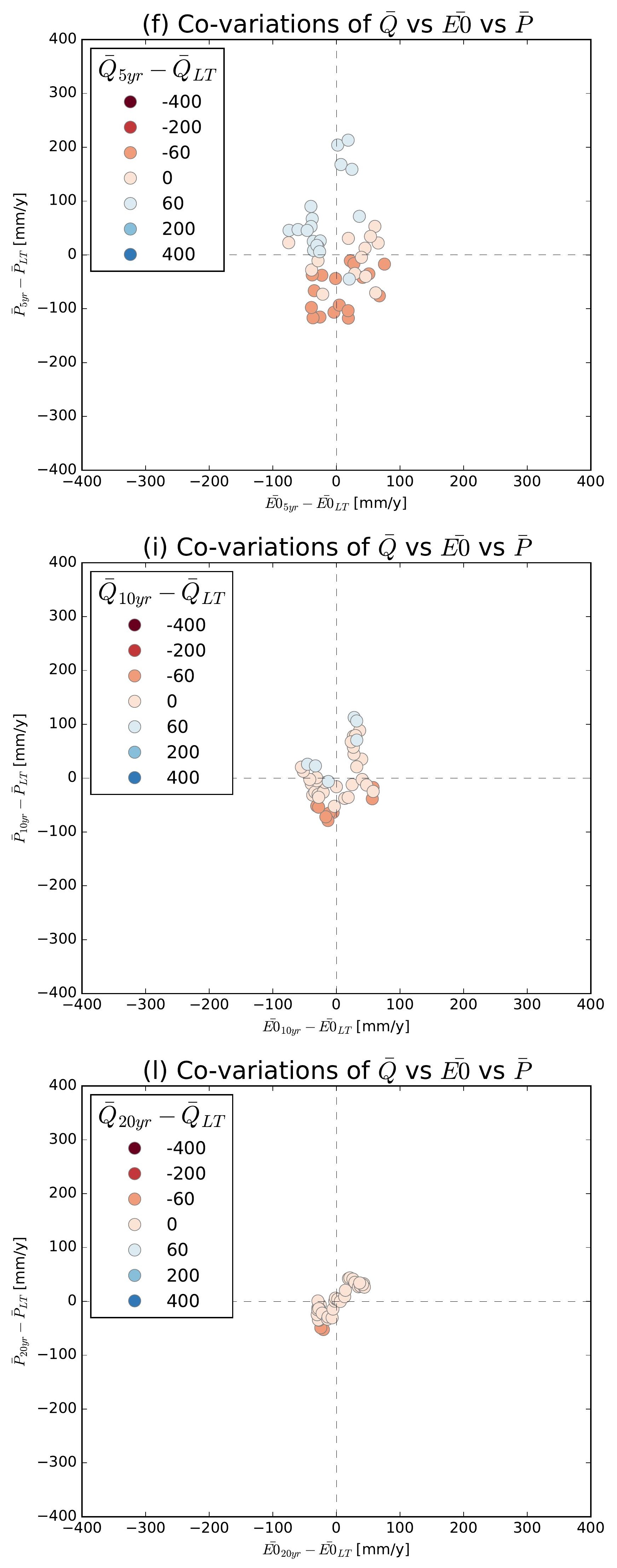

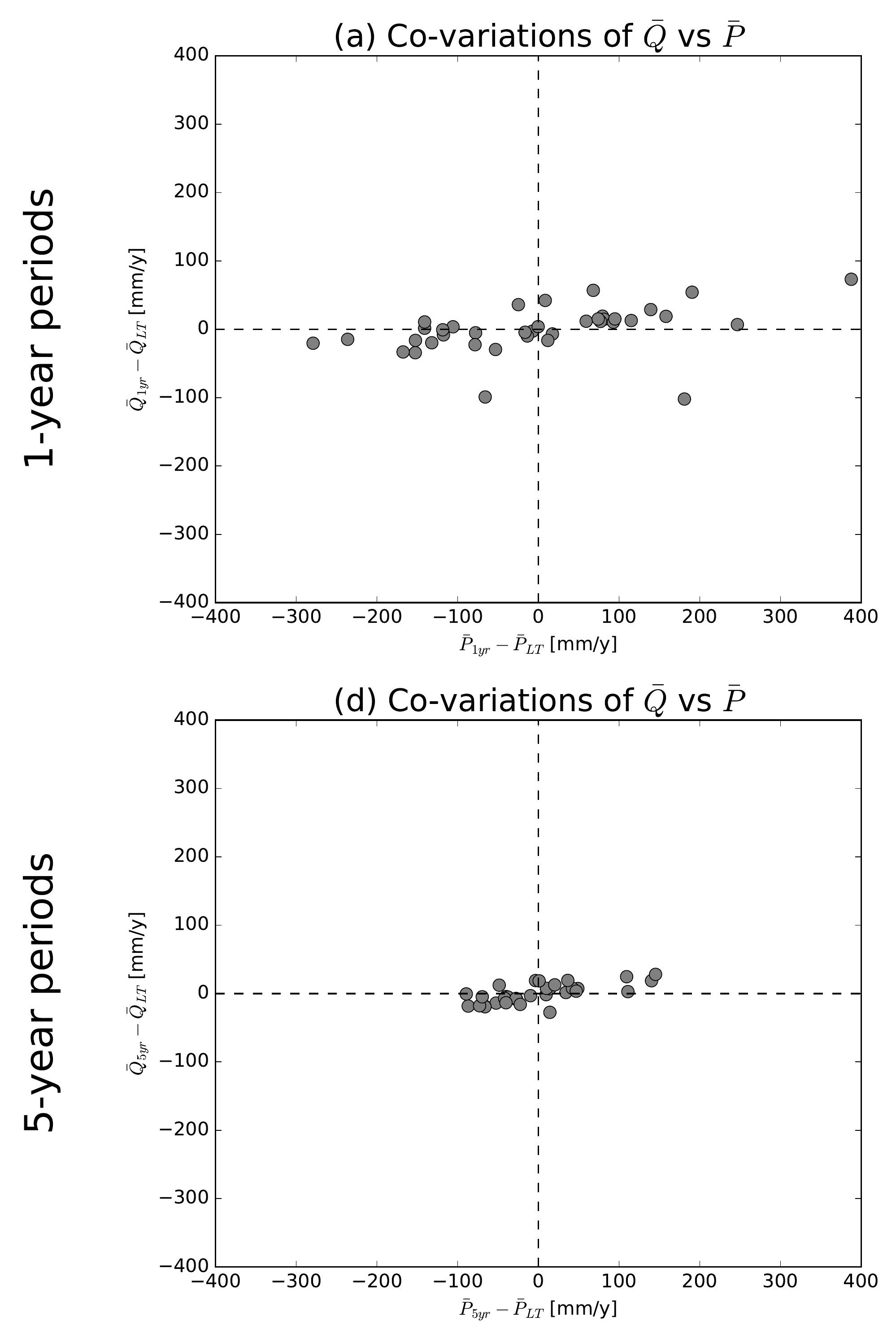

(g) Co-variations of $\bar{Q}$ vs $\bar{P}$
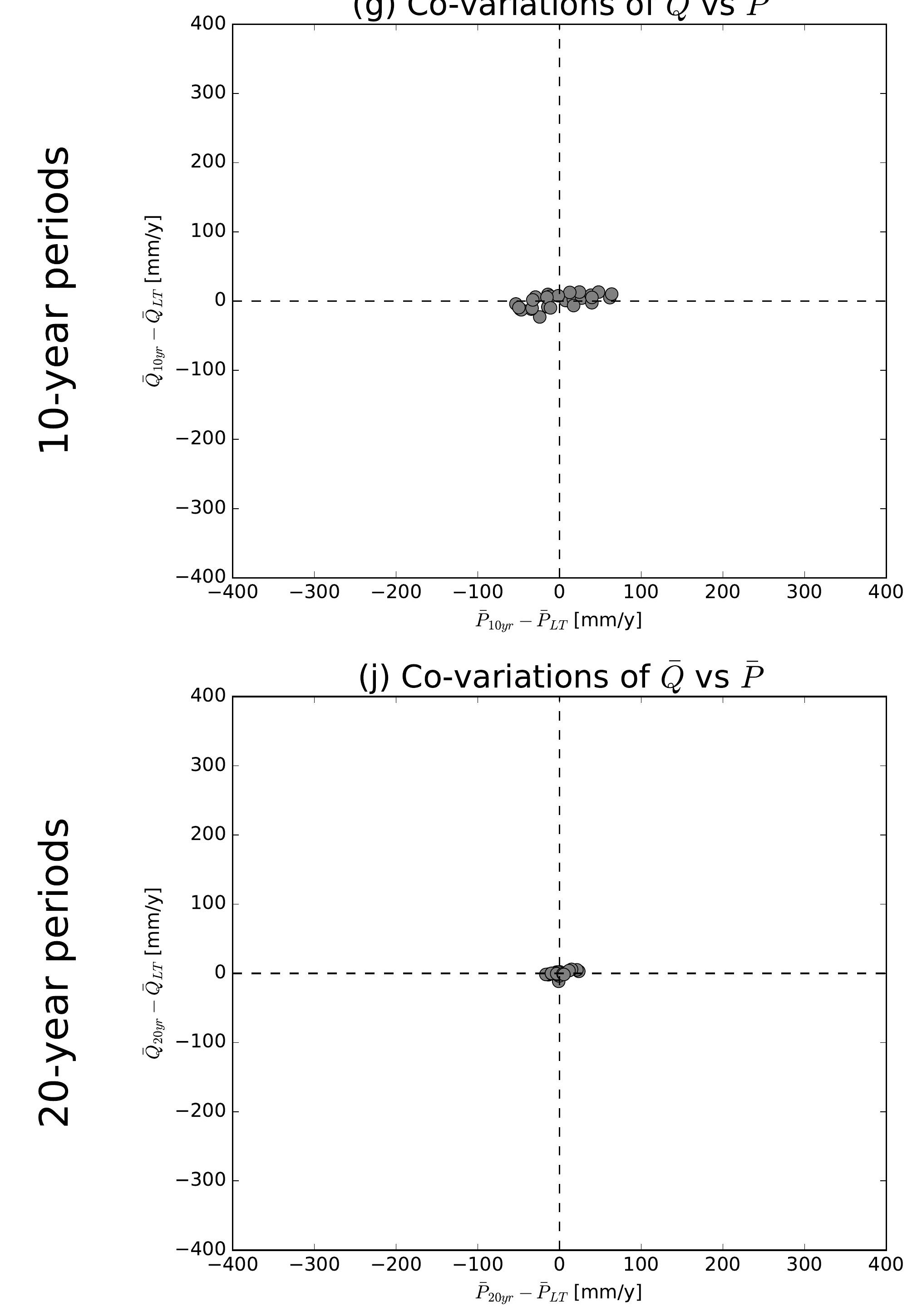

(b) Co-variations of $\bar{Q}$ vs $\overline{E 0}$

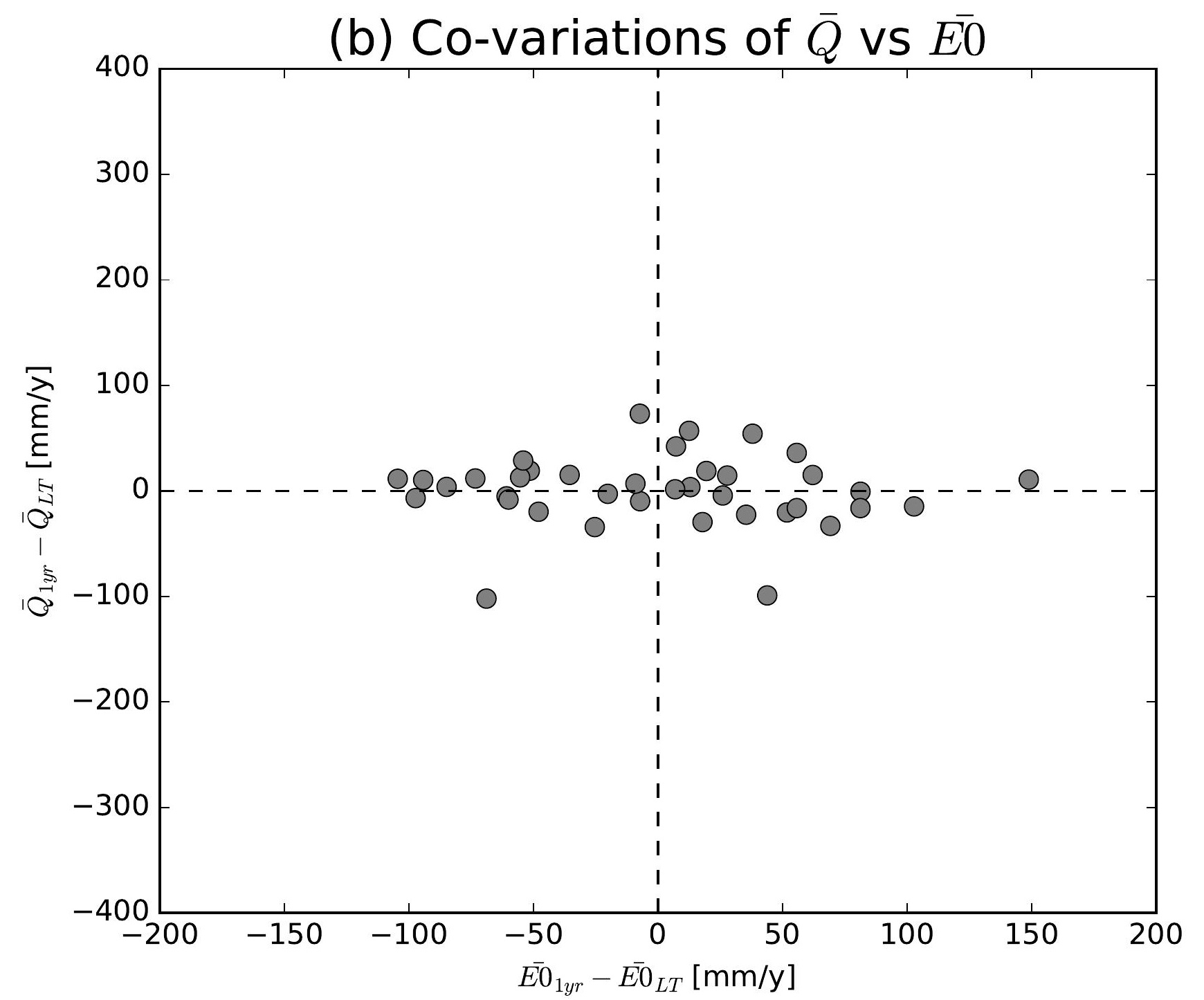

(e) Co-variations of $\bar{Q}$ vs $\overline{E 0}$

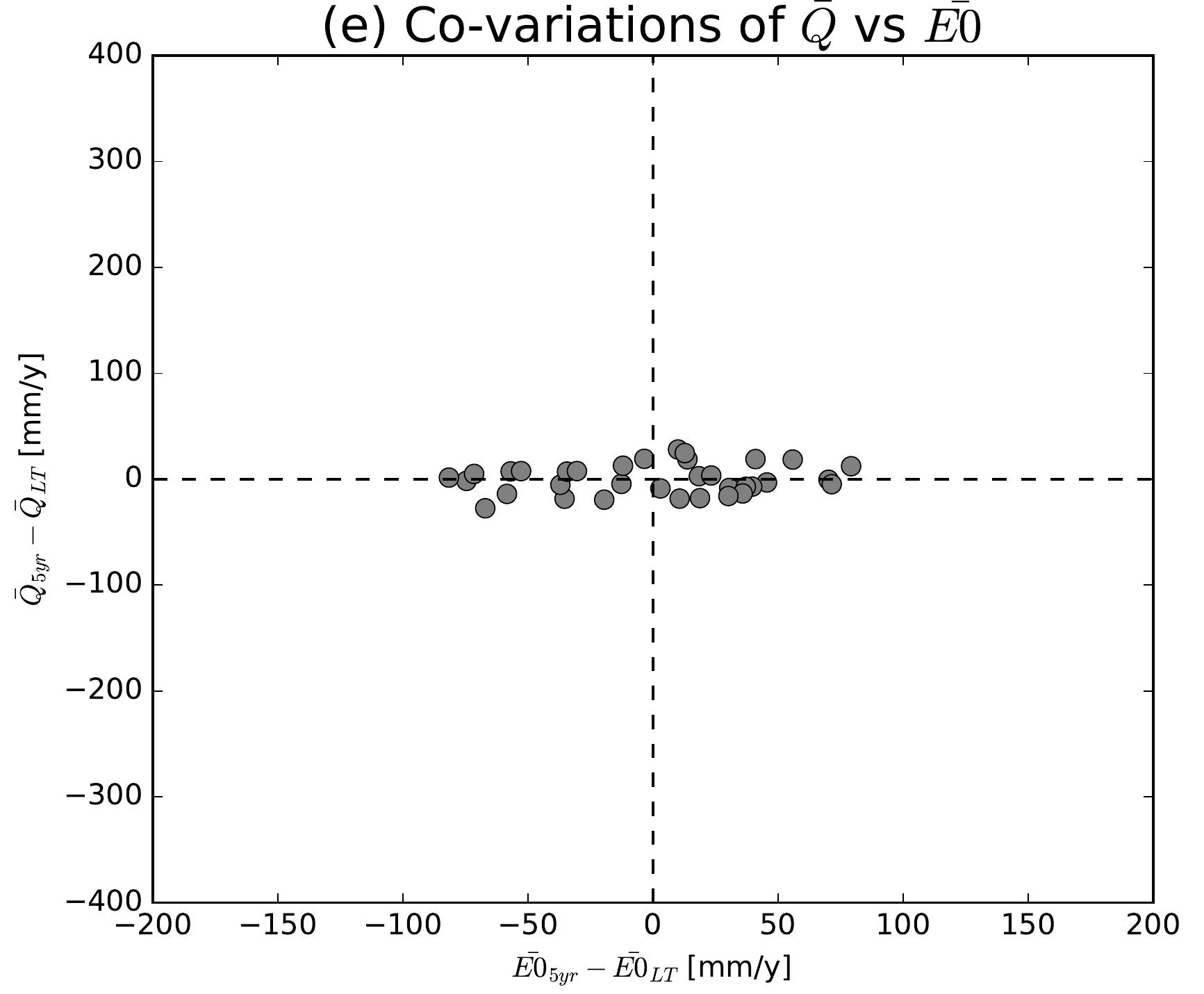

(h) Co-variations of $\bar{Q}$ vs $\overline{E 0}$

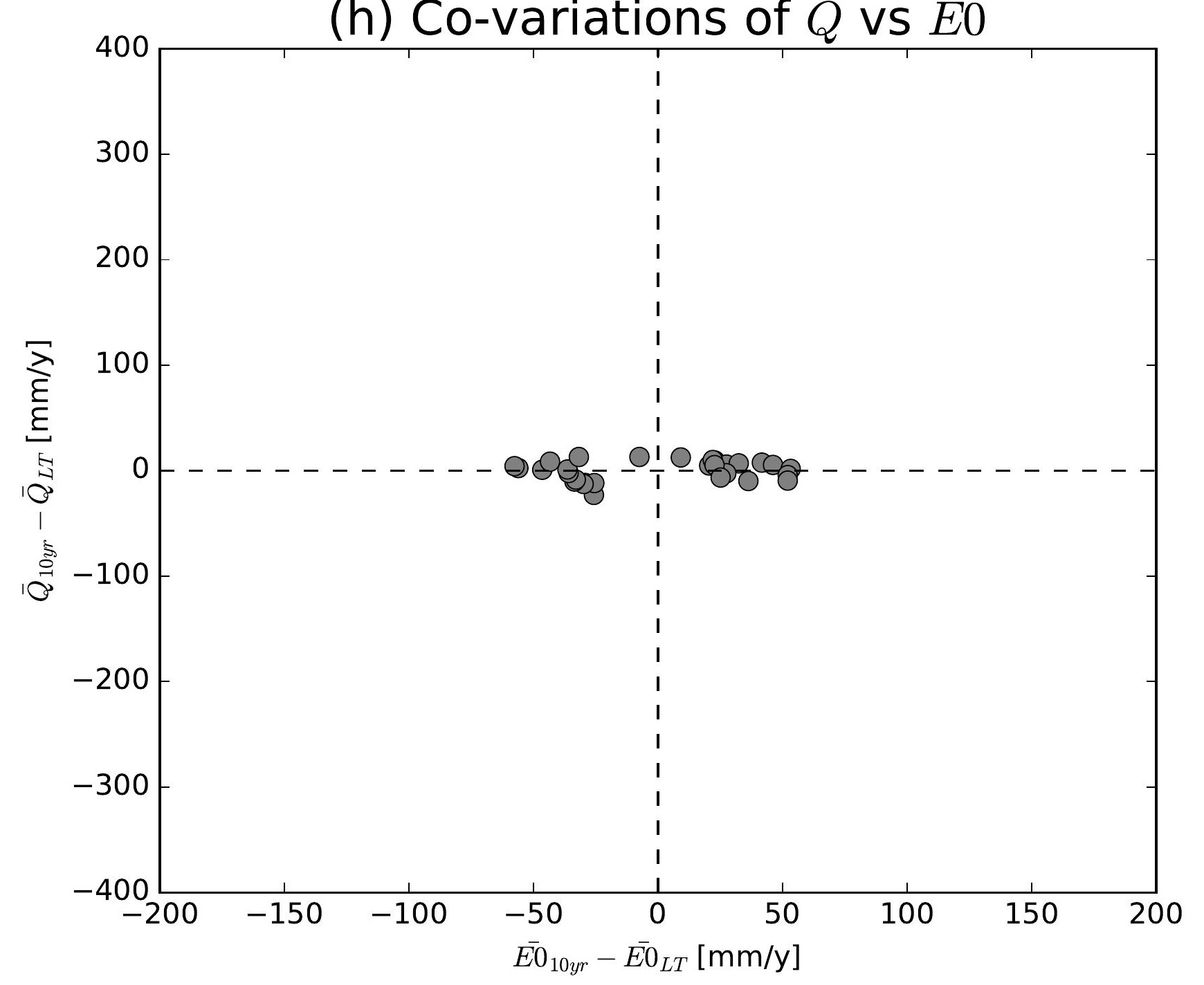

(k) Co-variations of $\bar{Q}$ vs $\overline{E 0}$

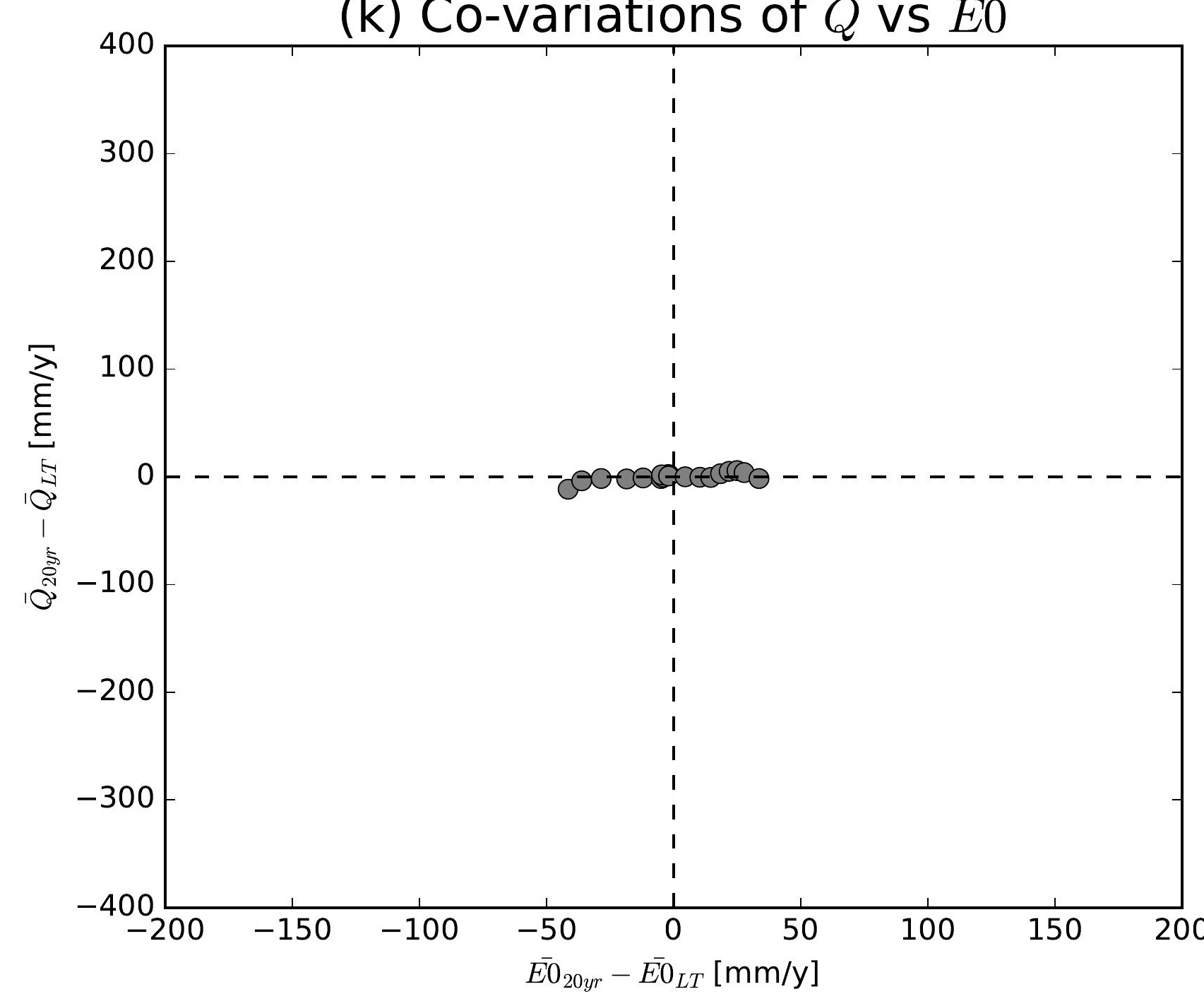

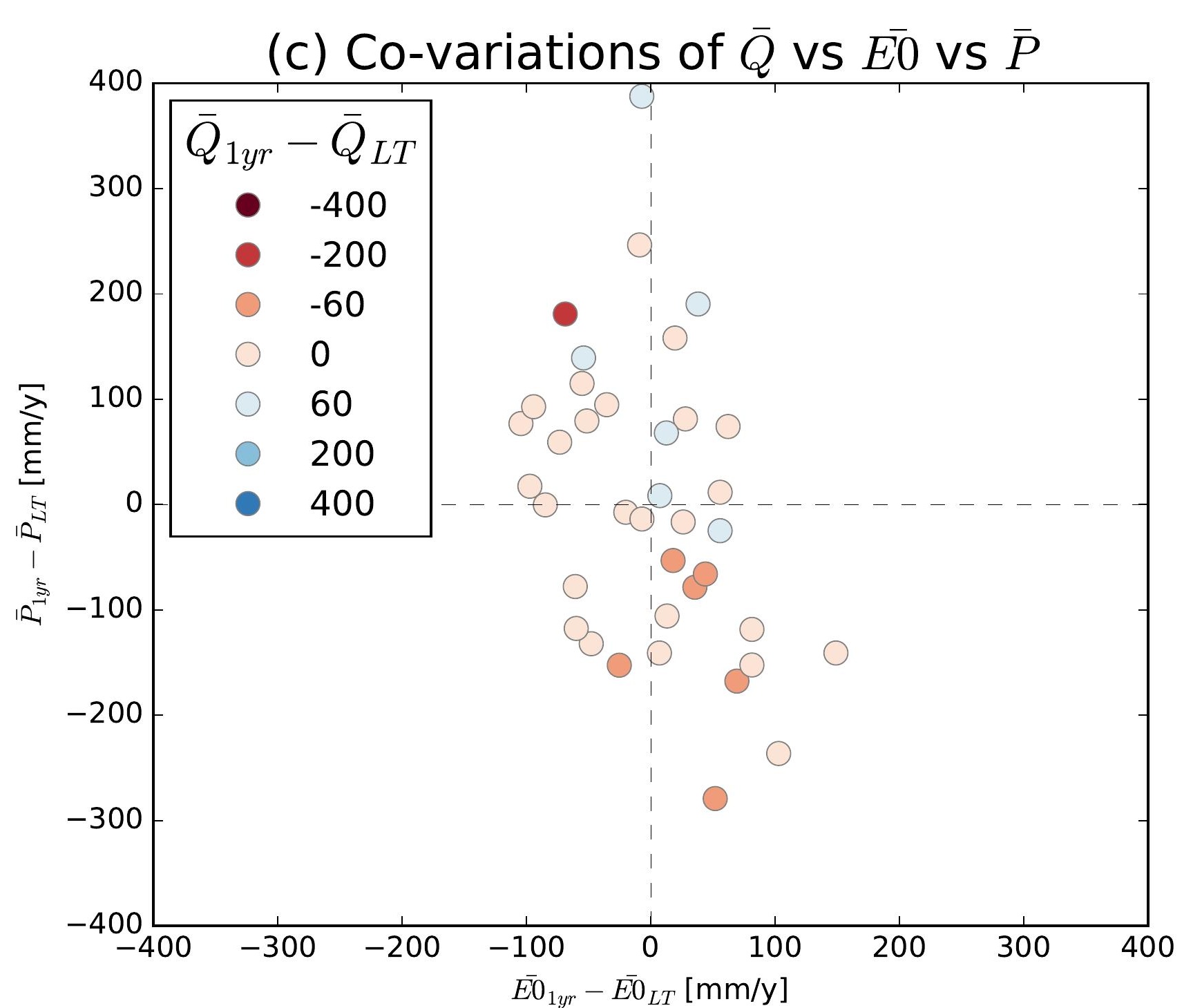
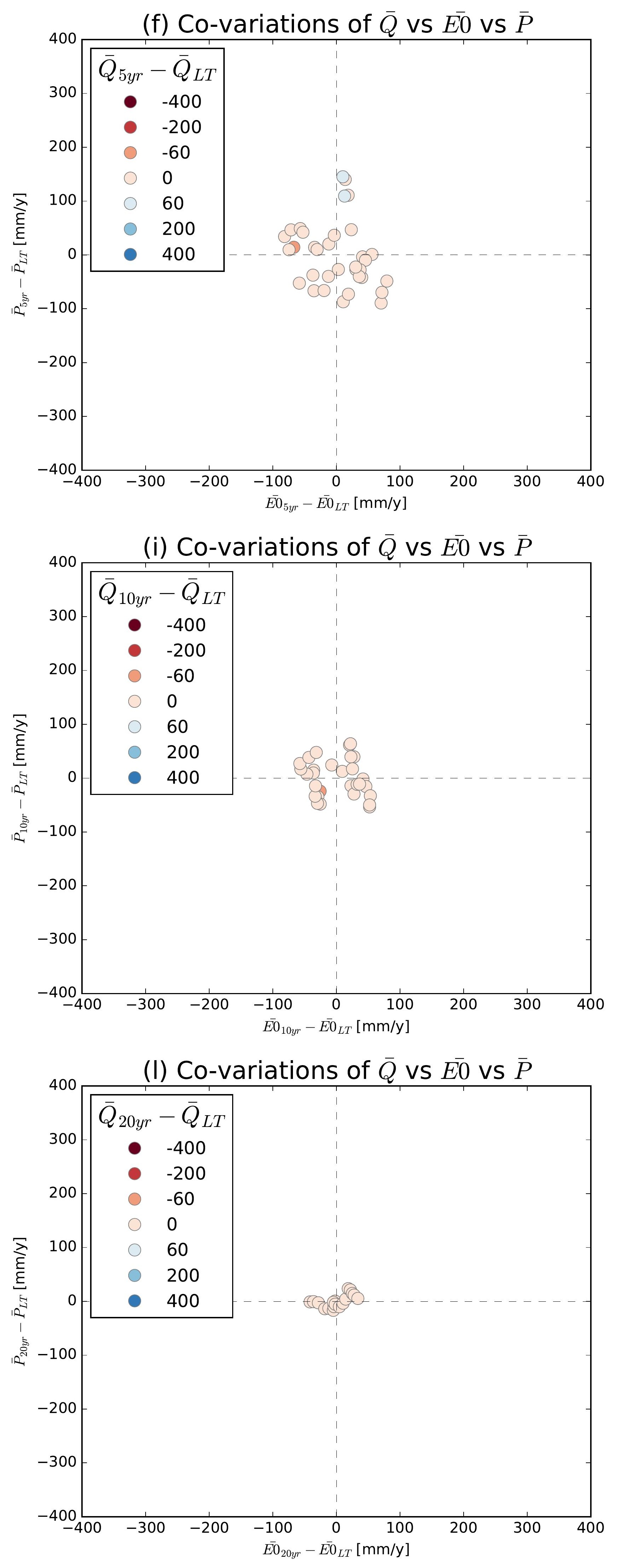

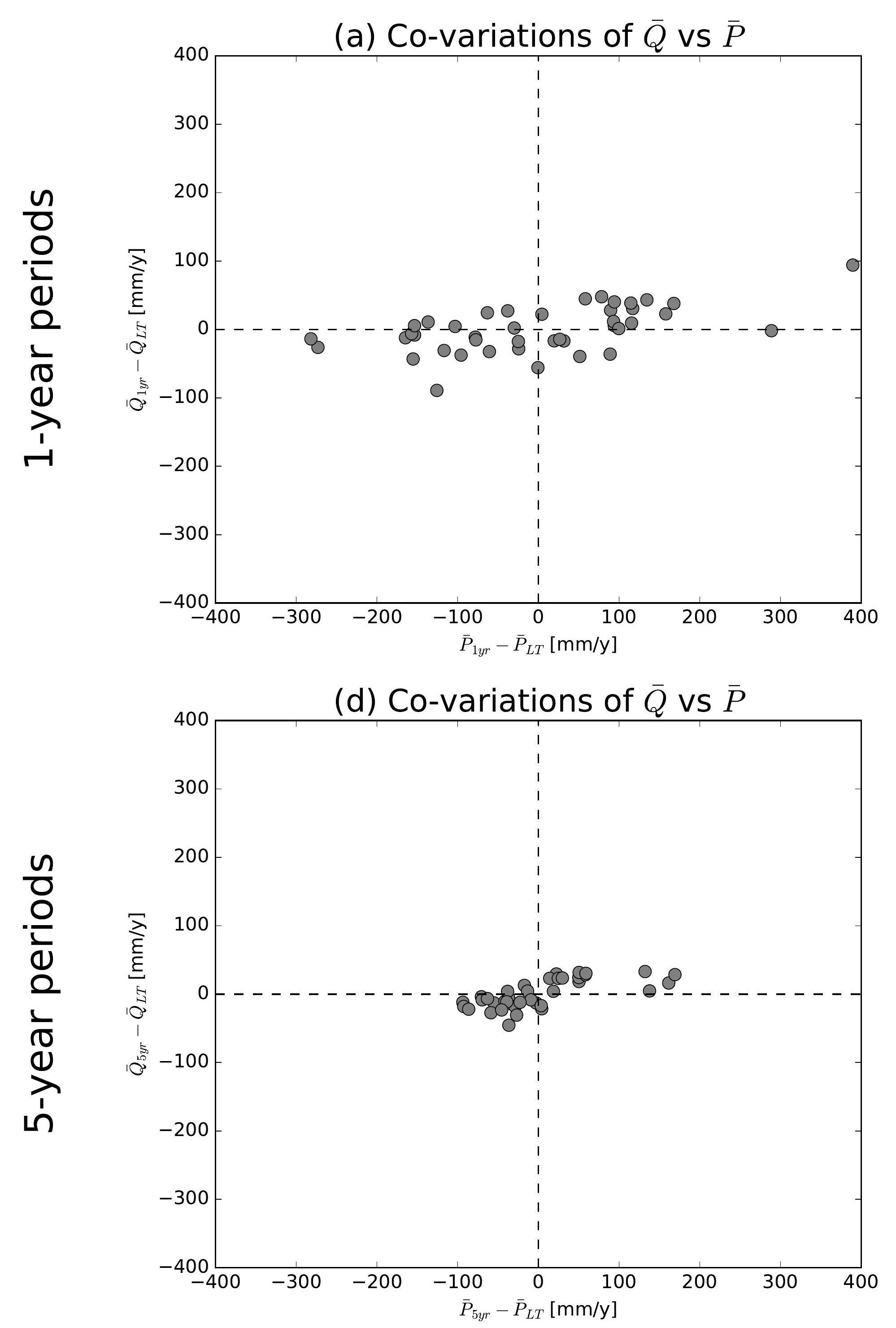

(g) Co-variations of $\bar{Q}$ vs $\bar{P}$
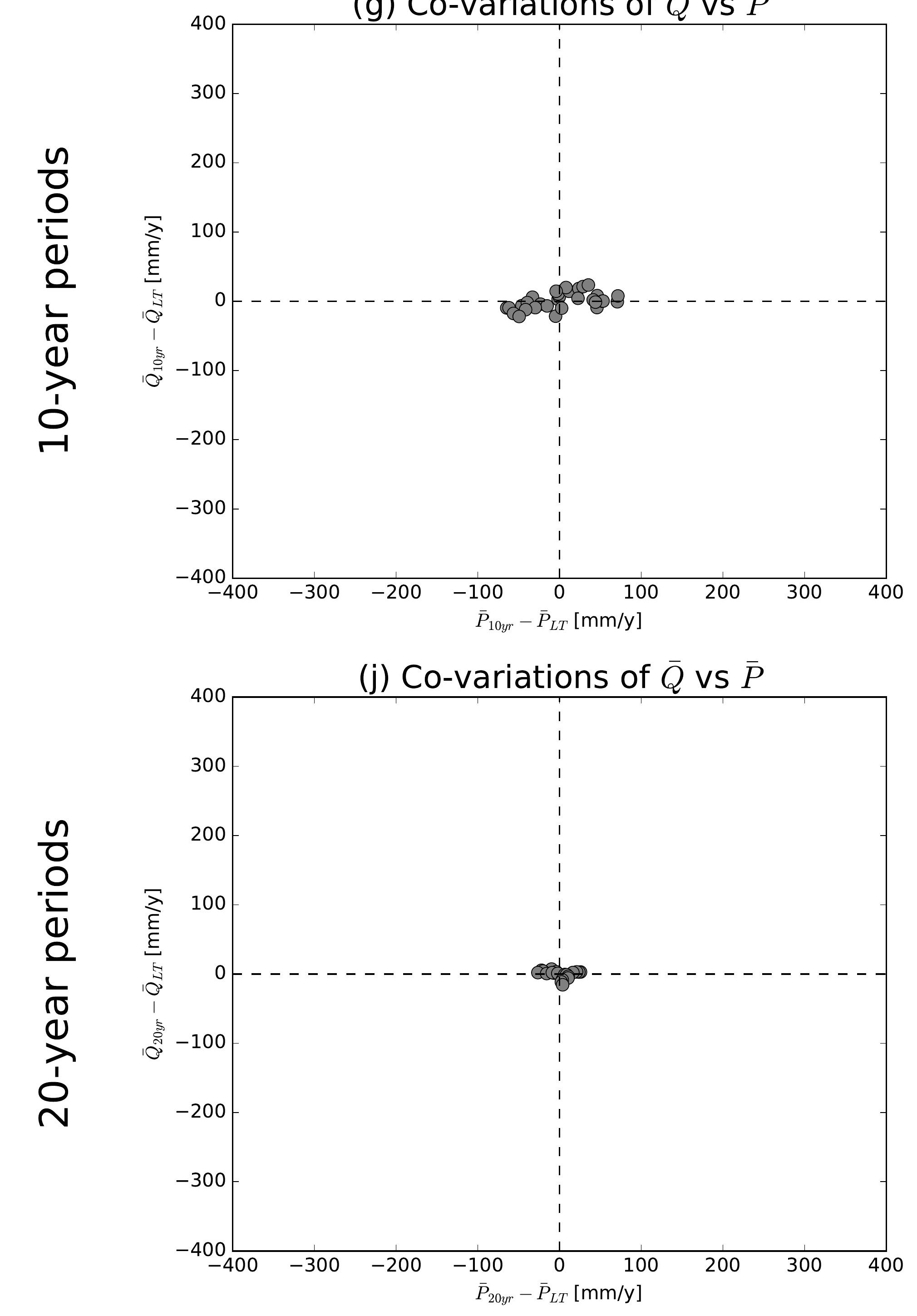

(b) Co-variations of $\bar{Q}$ vs $\overline{E 0}$

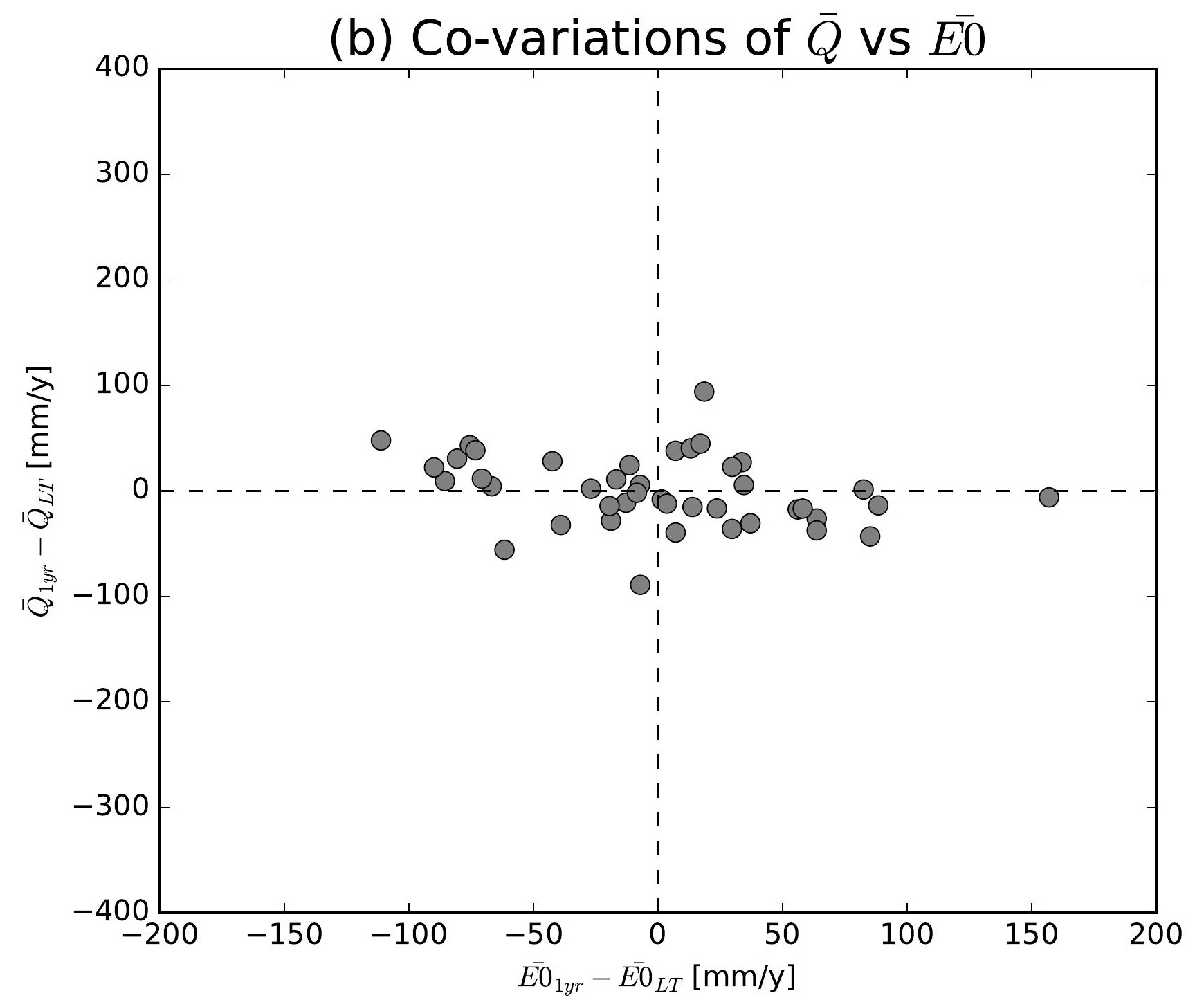

(e) Co-variations of $\bar{Q}$ vs $\overline{E 0}$

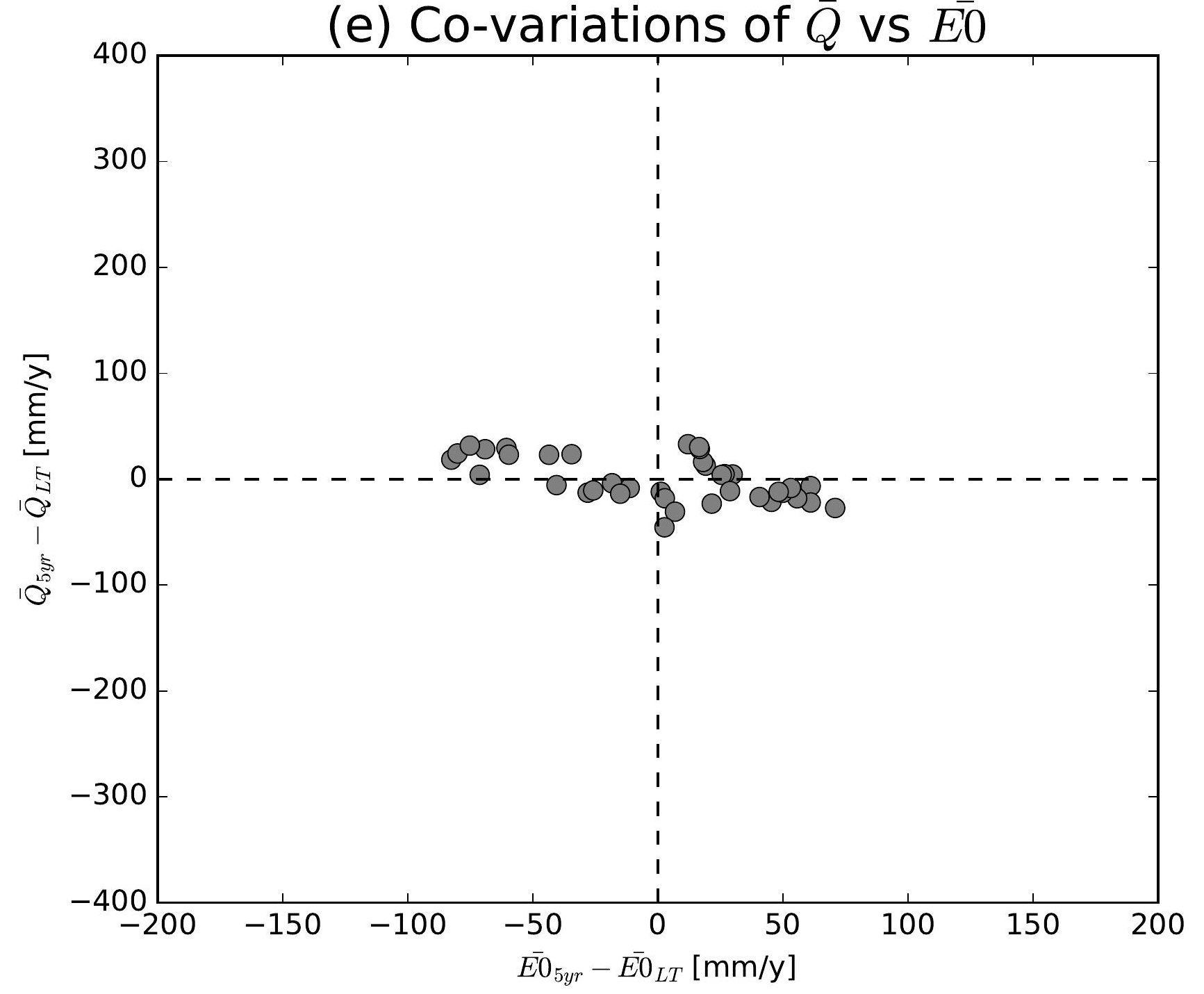

(h) Co-variations of $\bar{Q}$ vs $\overline{E 0}$

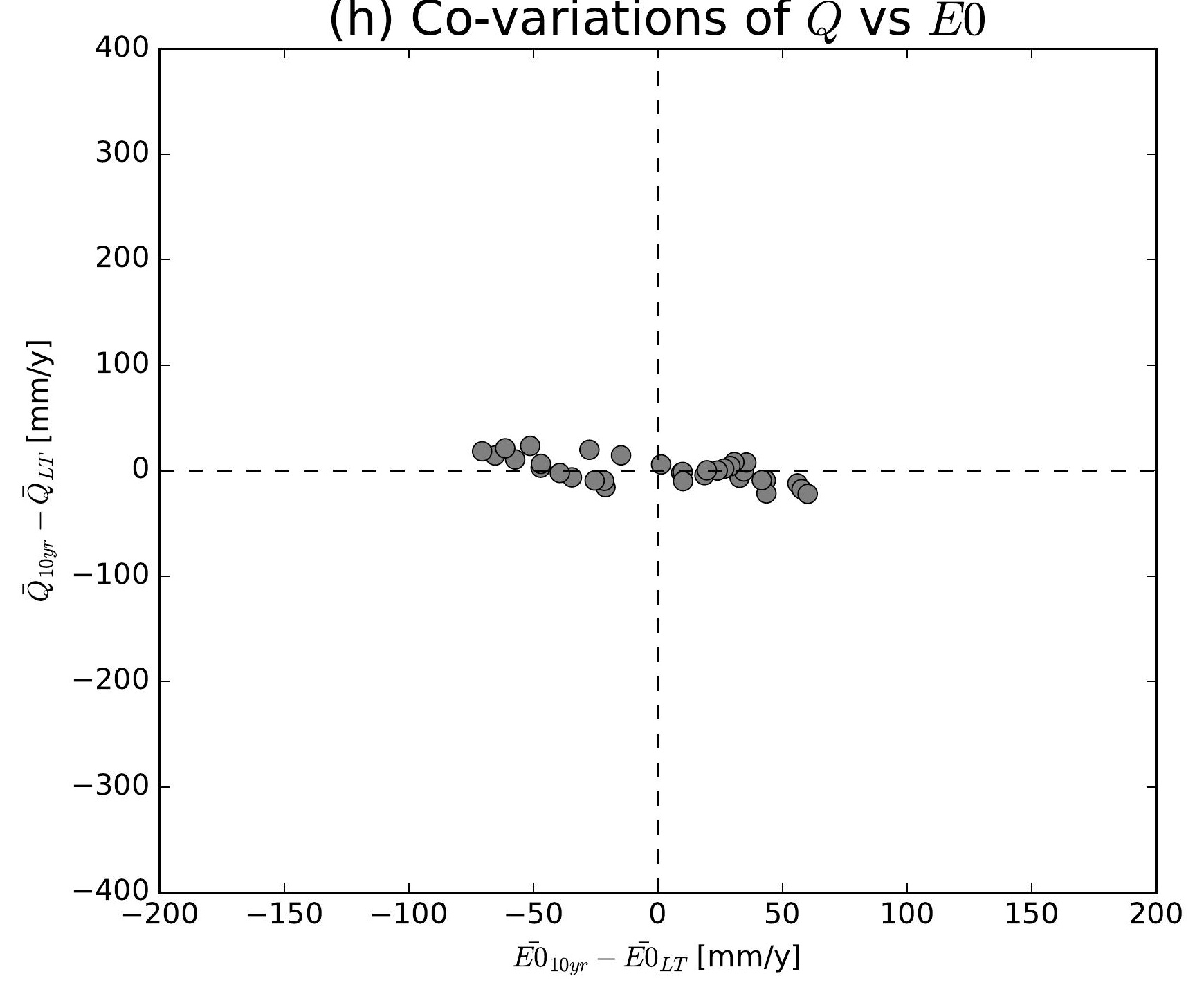

(k) Co-variations of $\bar{Q}$ vs $\overline{E 0}$

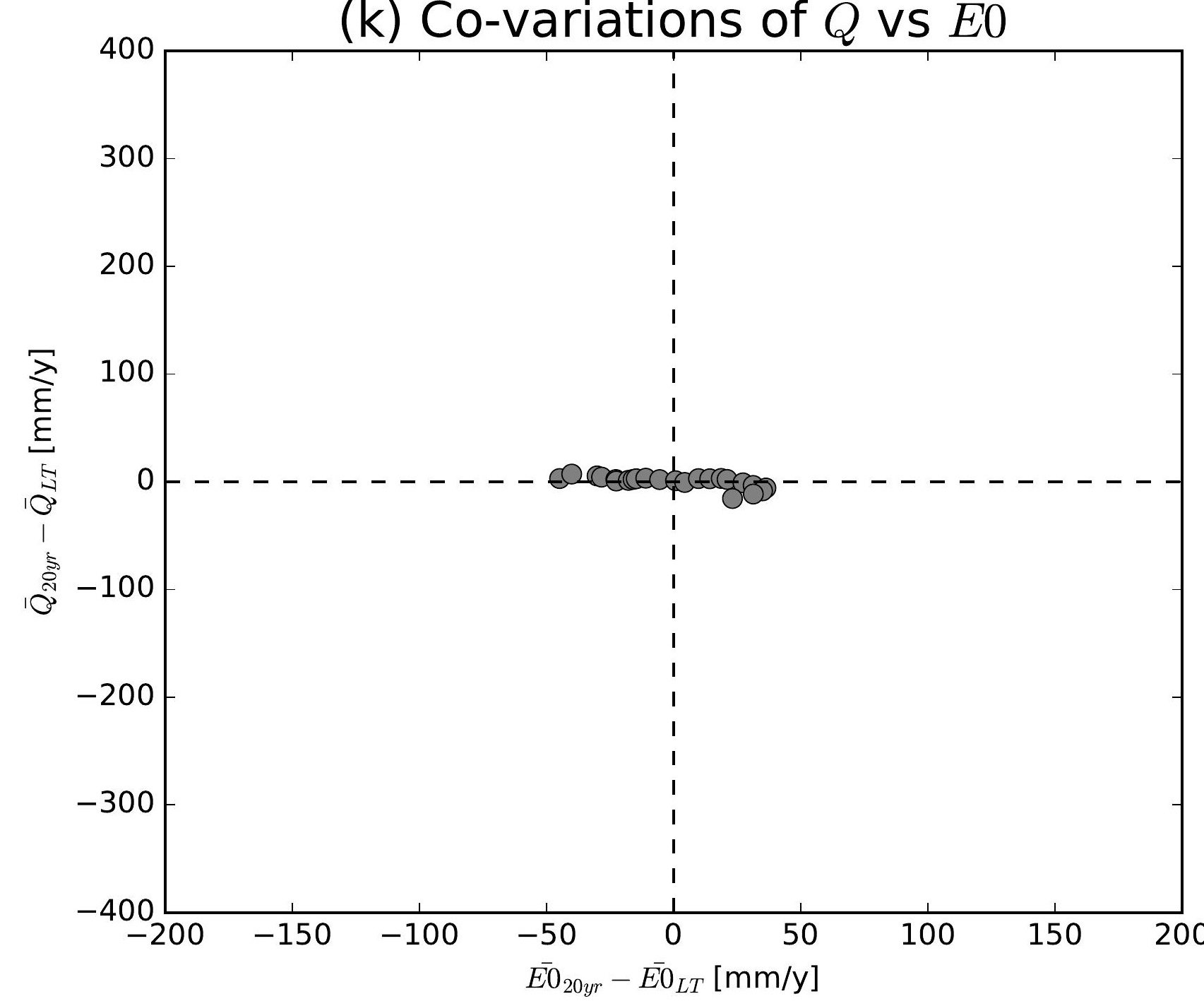

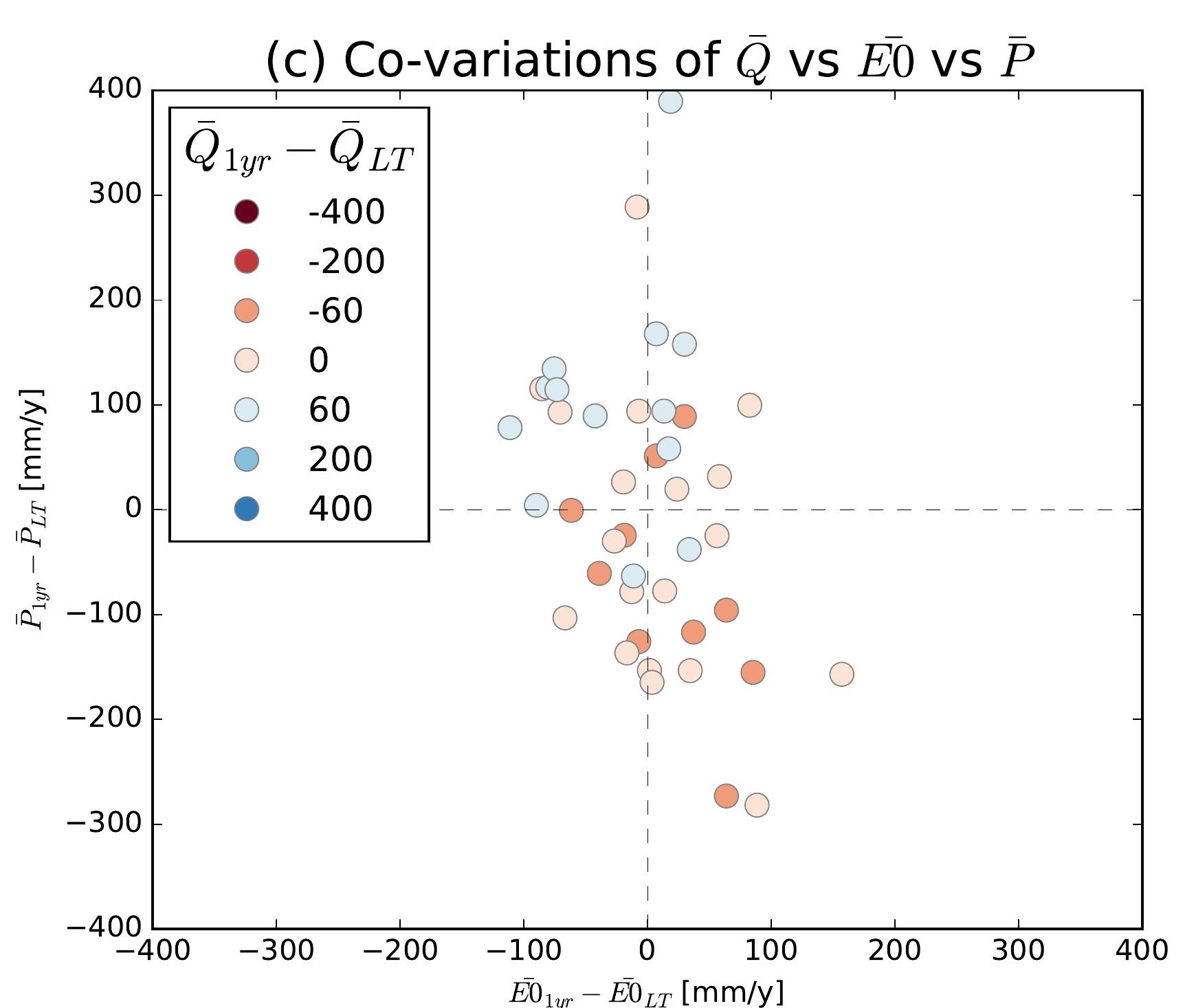
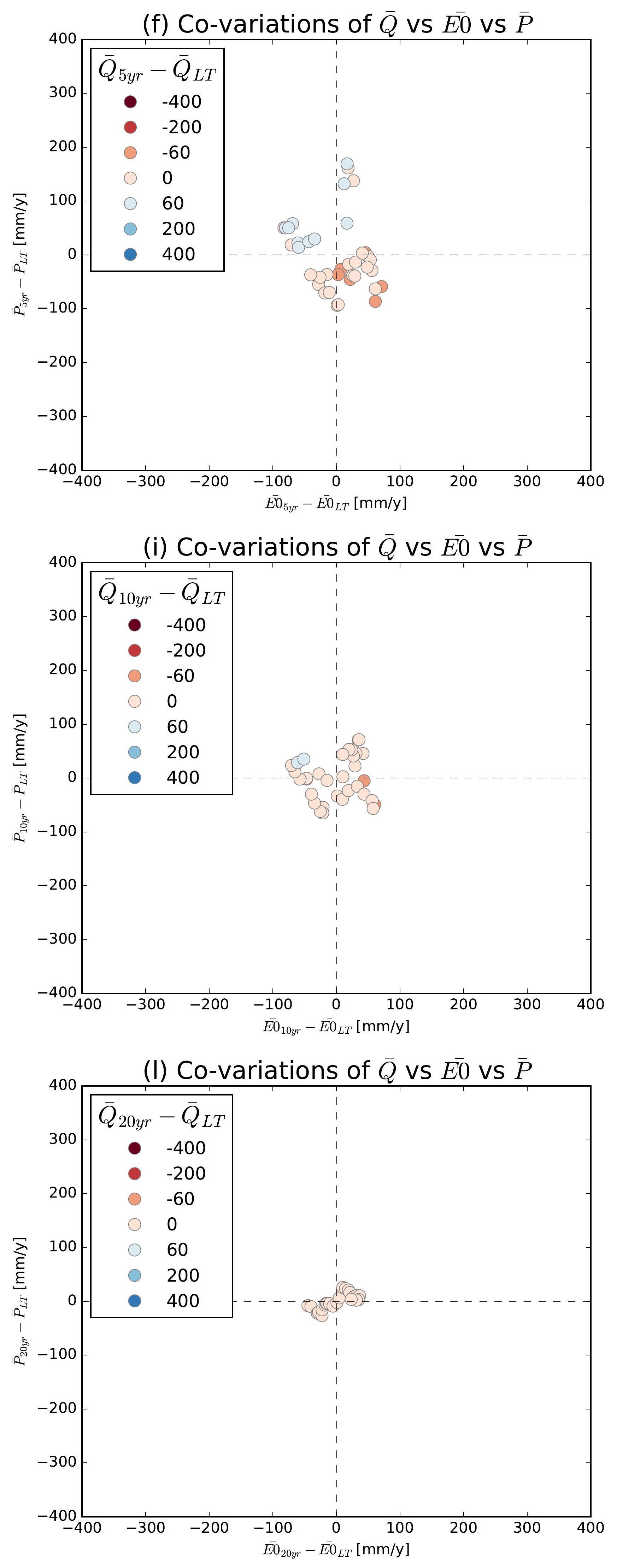

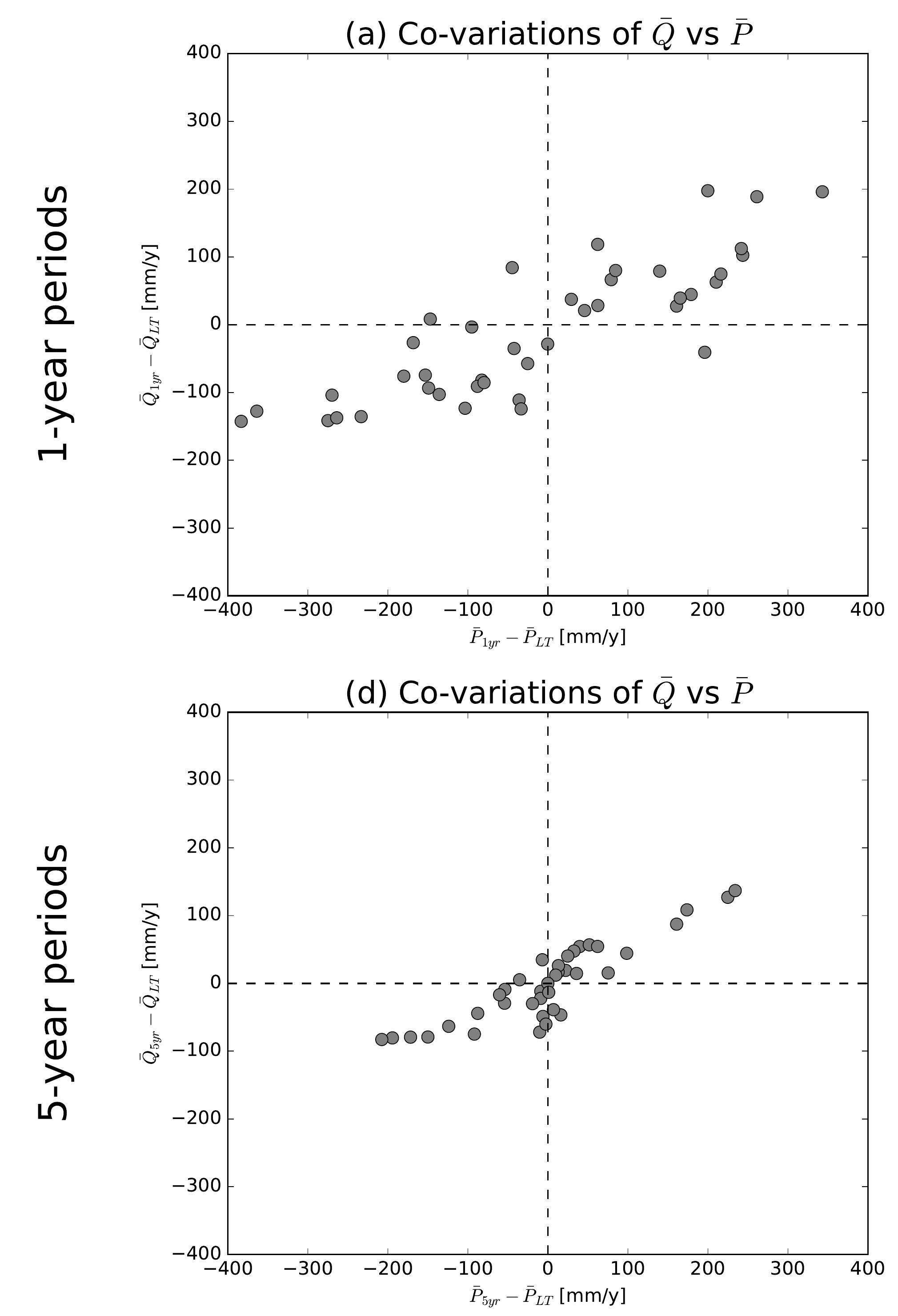

(g) Co-variations of $\bar{Q}$ vs $\bar{P}$
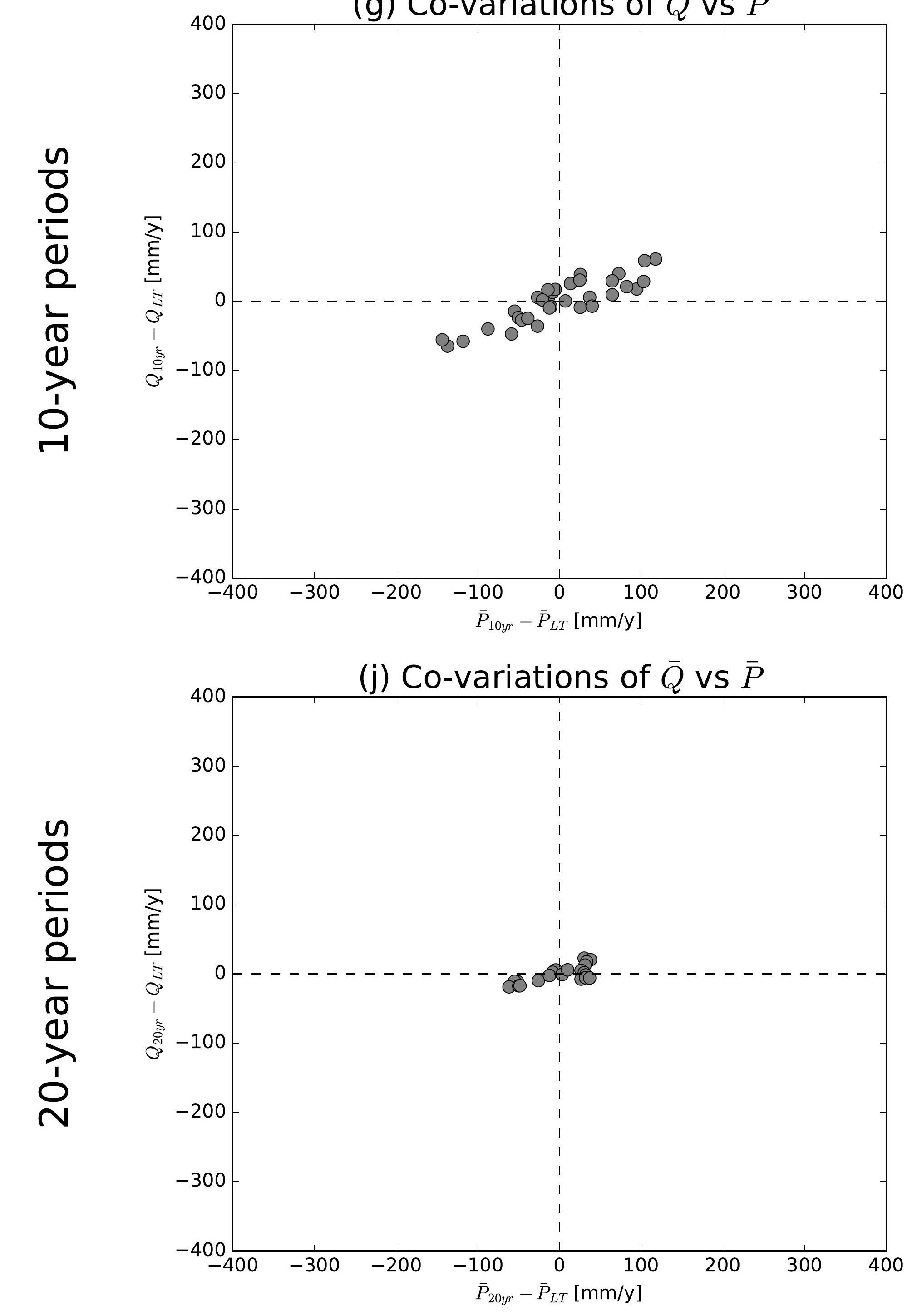

(b) Co-variations of $\bar{Q}$ vs $\overline{E 0}$

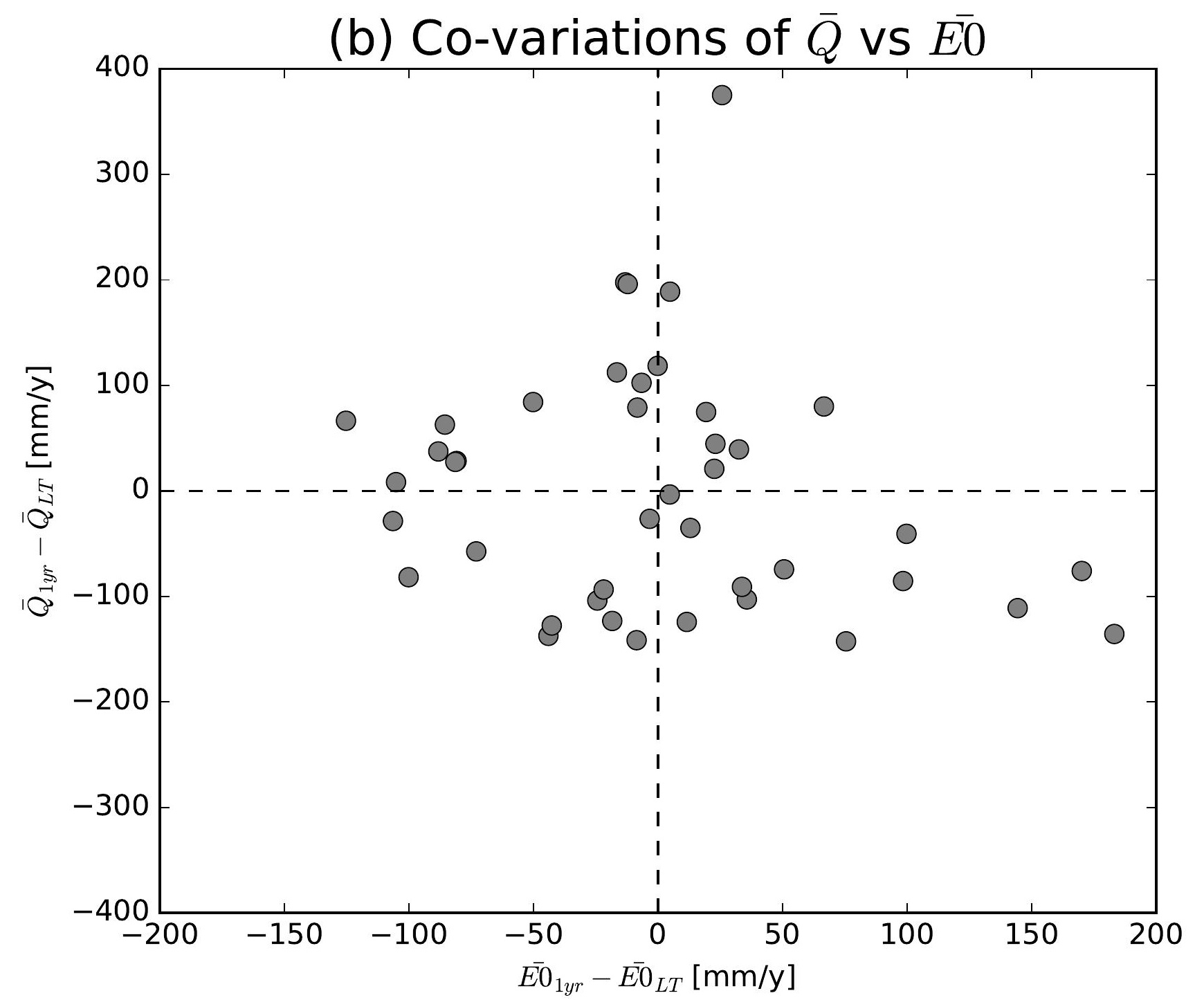

(e) Co-variations of $\bar{Q}$ vs $\overline{E 0}$

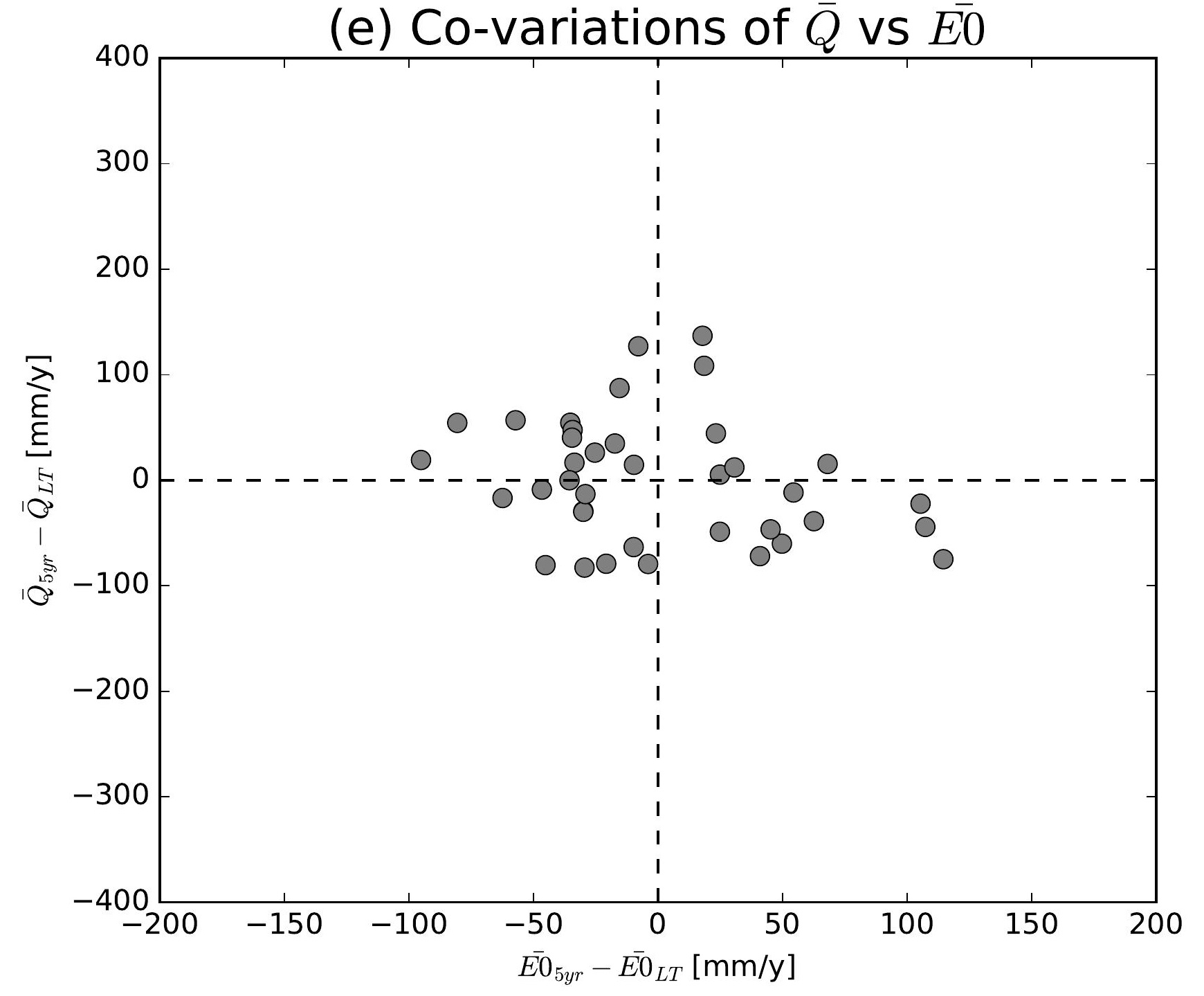

(h) Co-variations of $\bar{Q}$ vs $\overline{E 0}$

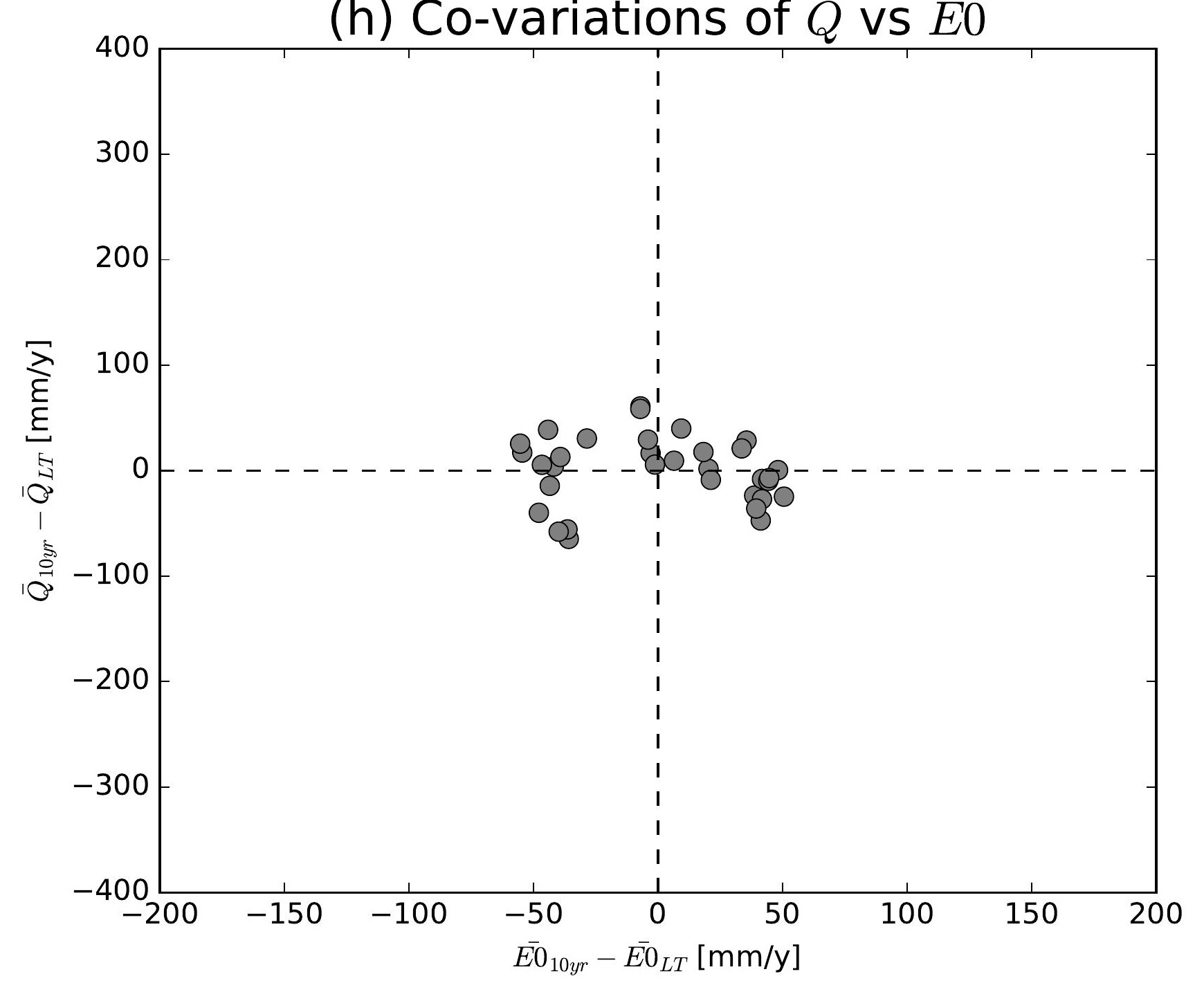

(k) Co-variations of $\bar{Q}$ vs $\overline{E 0}$

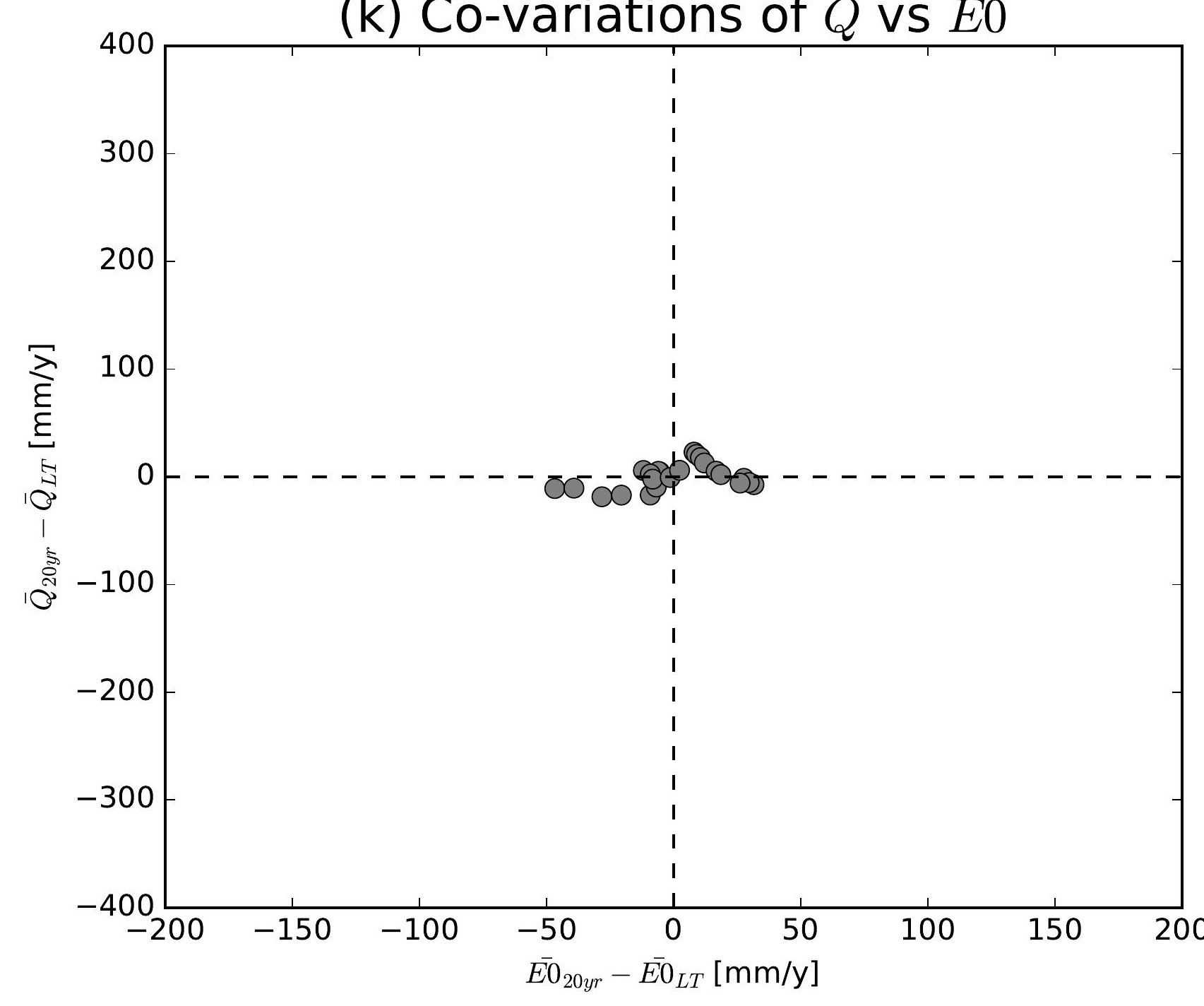

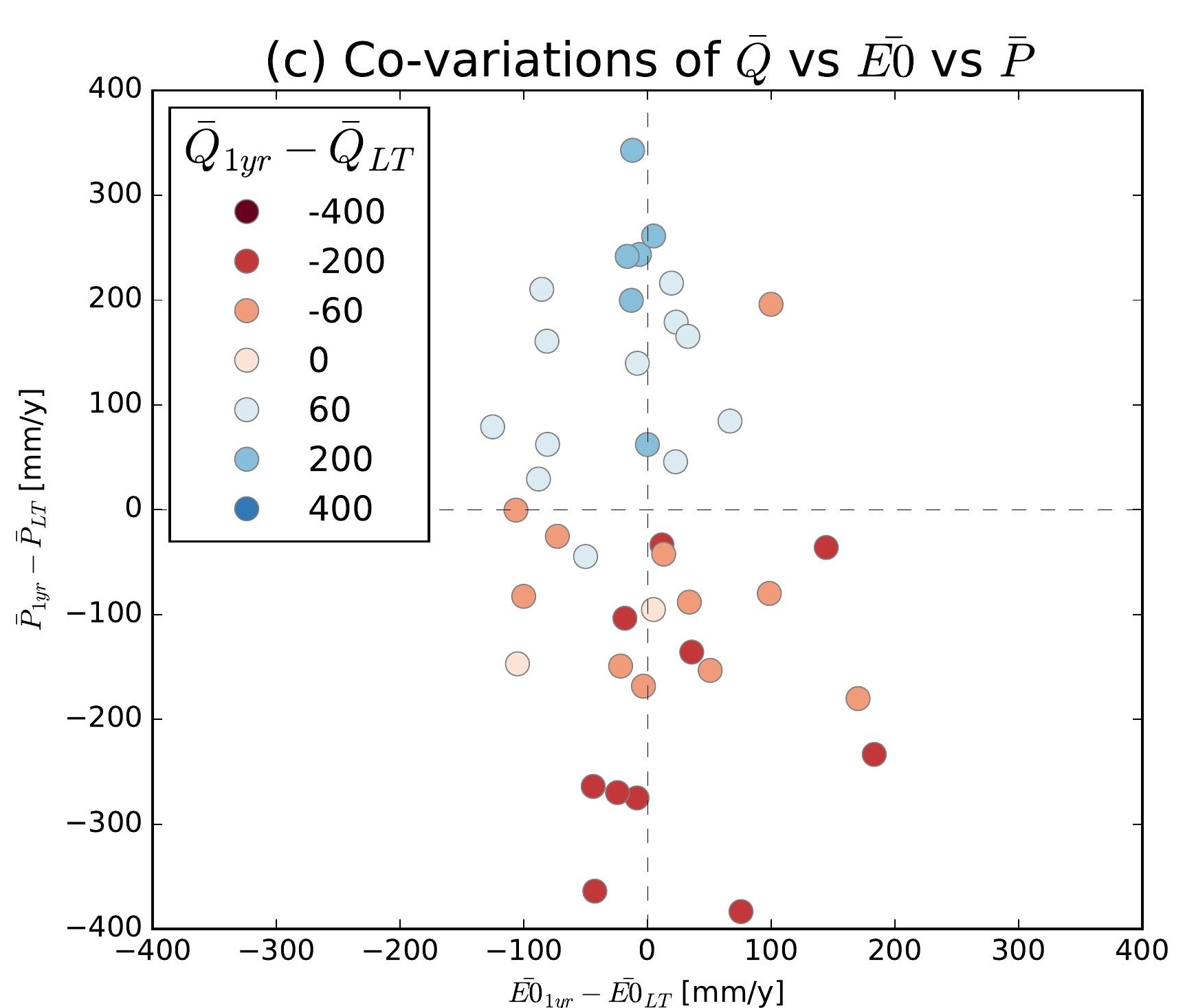
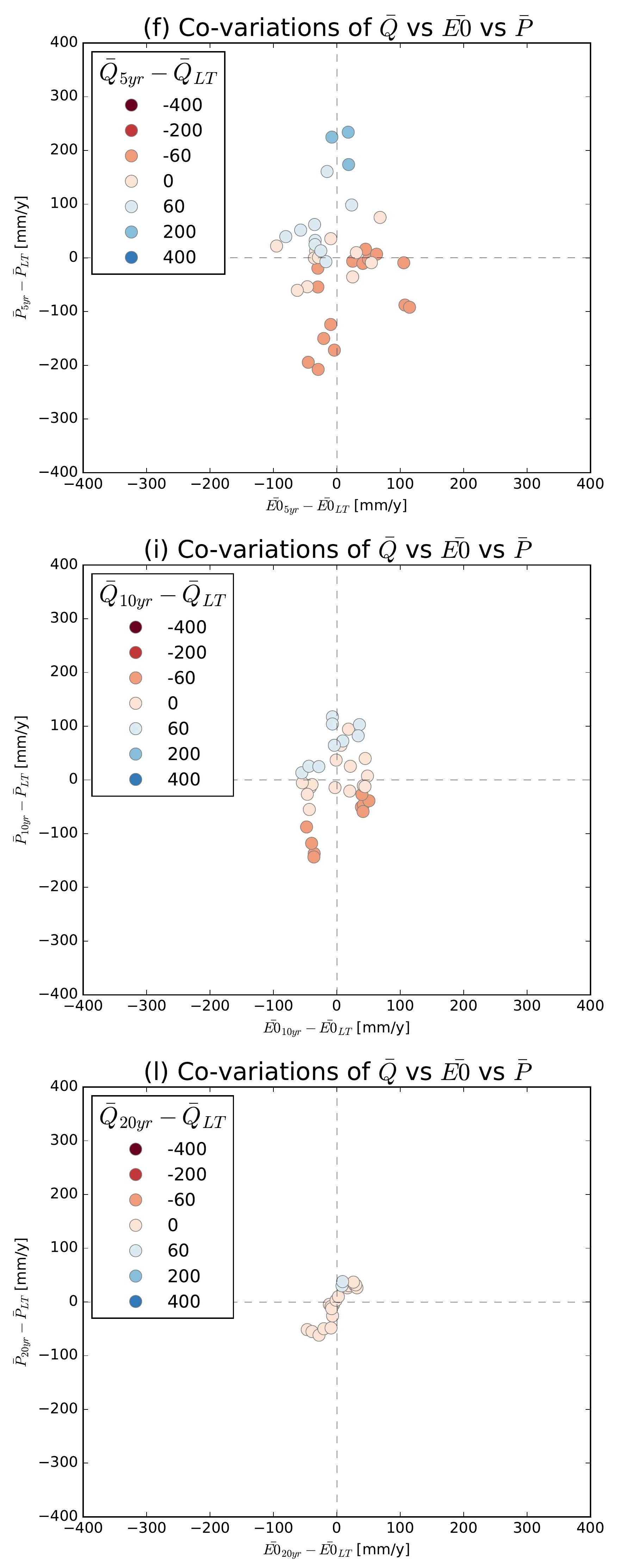

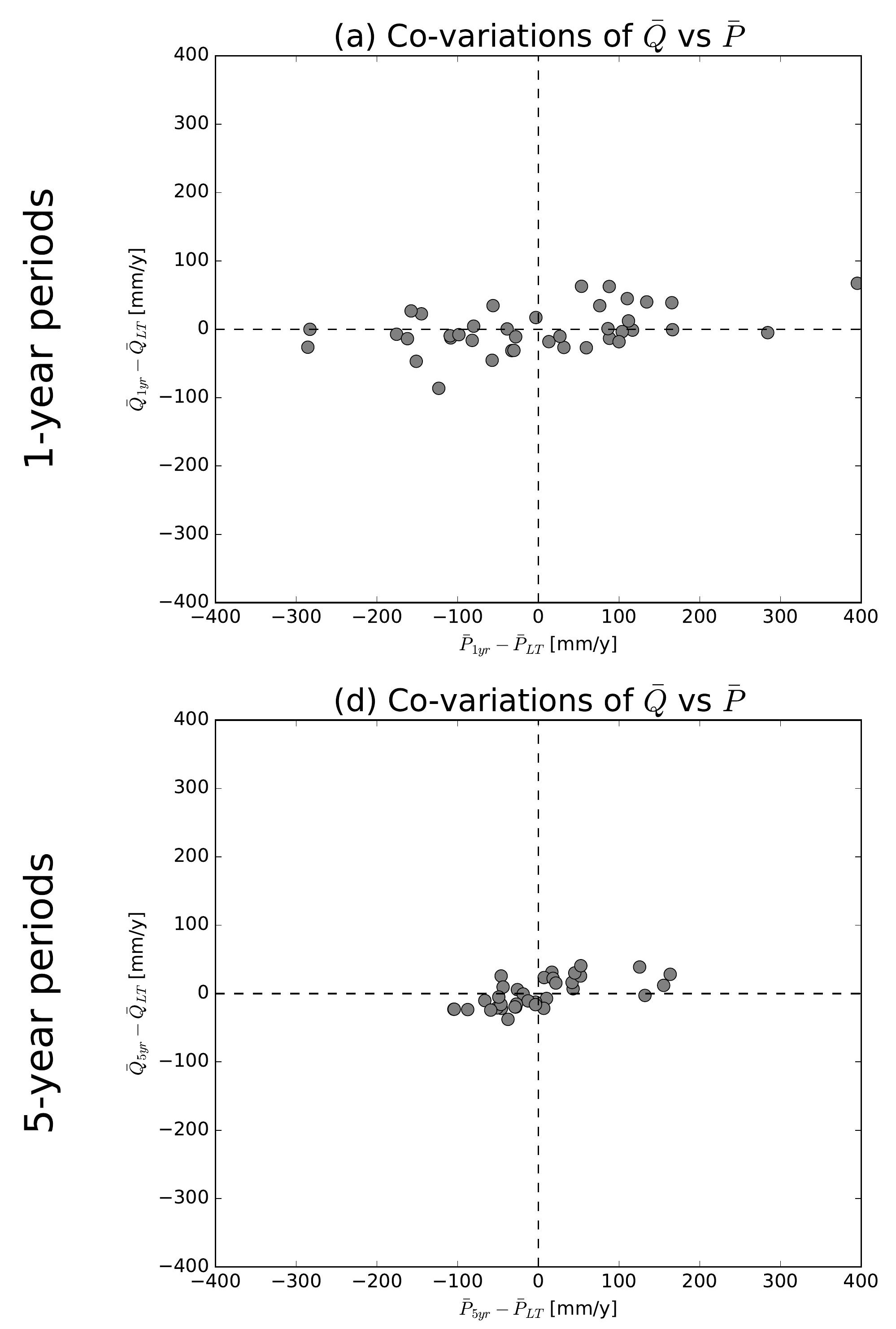

(g) Co-variations of $\bar{Q}$ vs $\bar{P}$
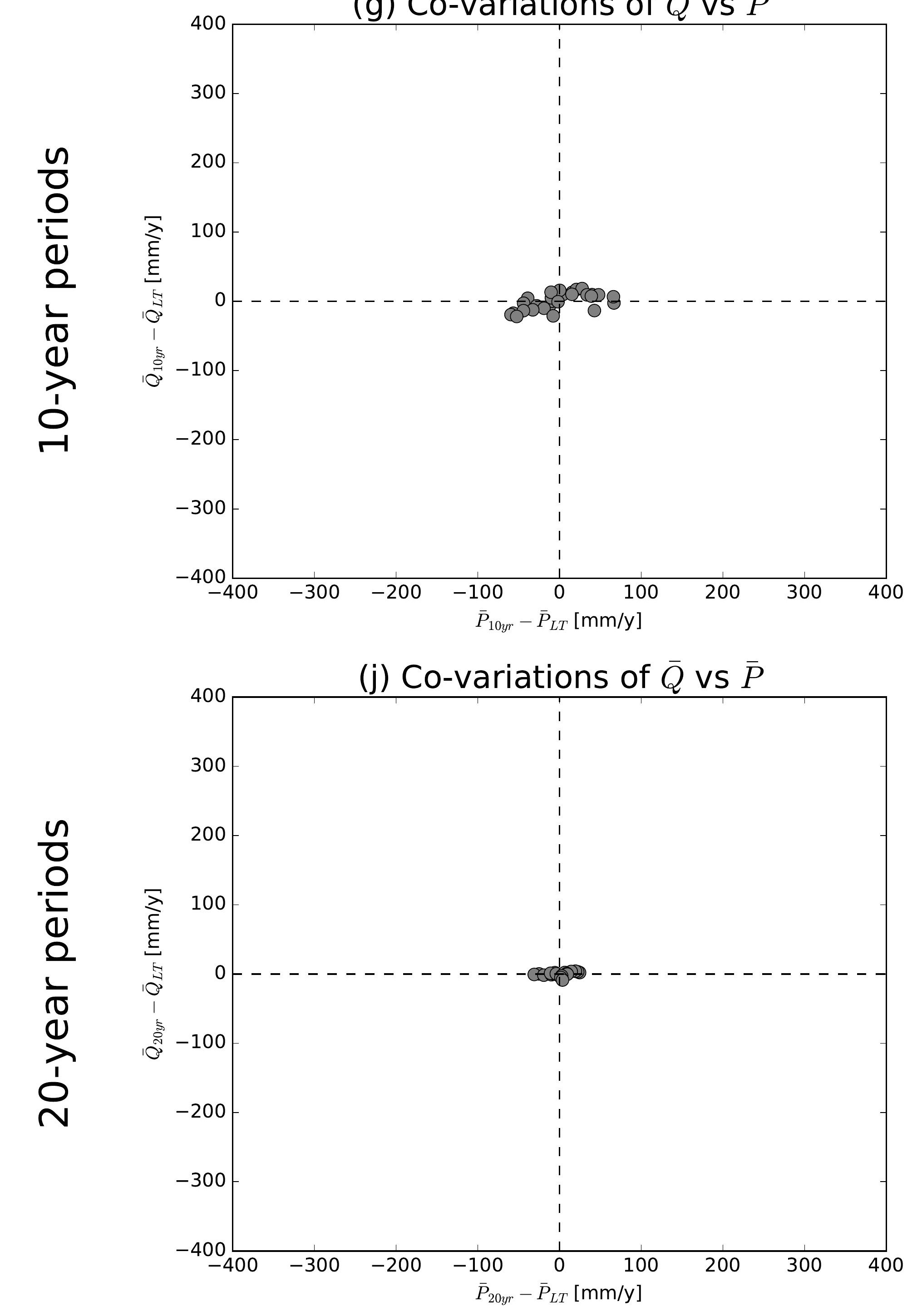

(b) Co-variations of $\bar{Q}$ vs $\overline{E 0}$

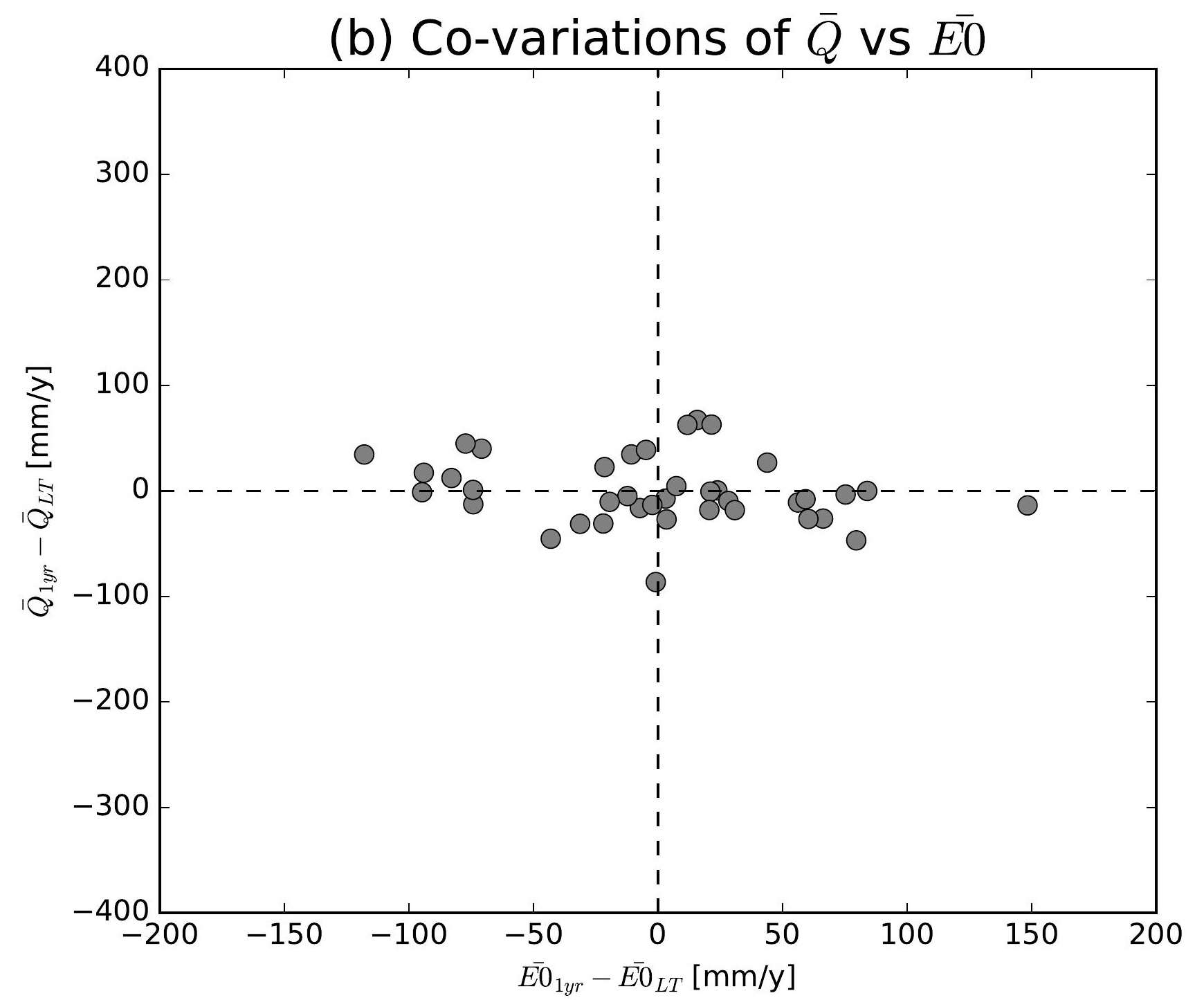

(e) Co-variations of $\bar{Q}$ vs $\overline{E 0}$

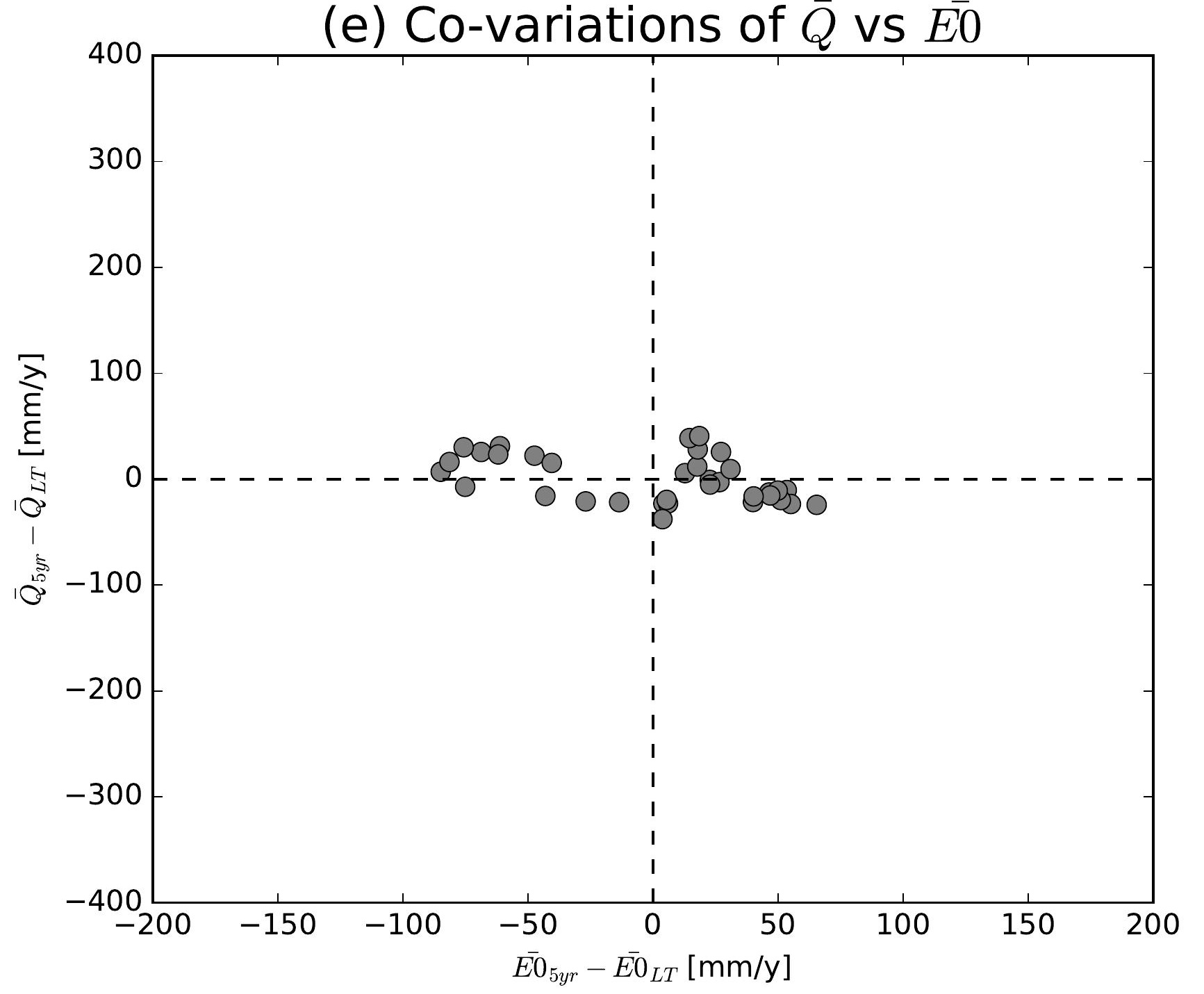

(h) Co-variations of $\bar{Q}$ vs $\overline{E 0}$

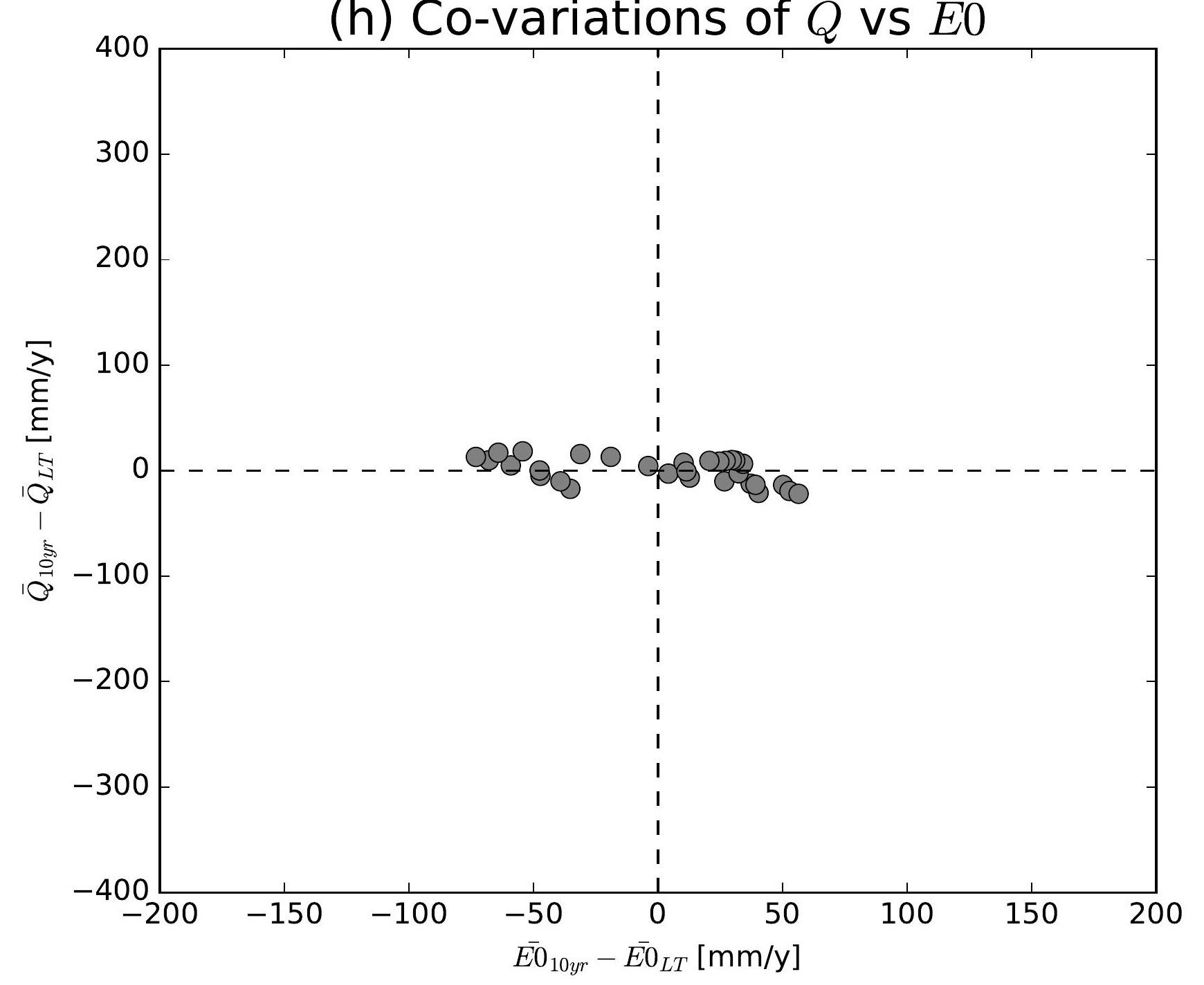

(k) Co-variations of $\bar{Q}$ vs $\overline{E 0}$

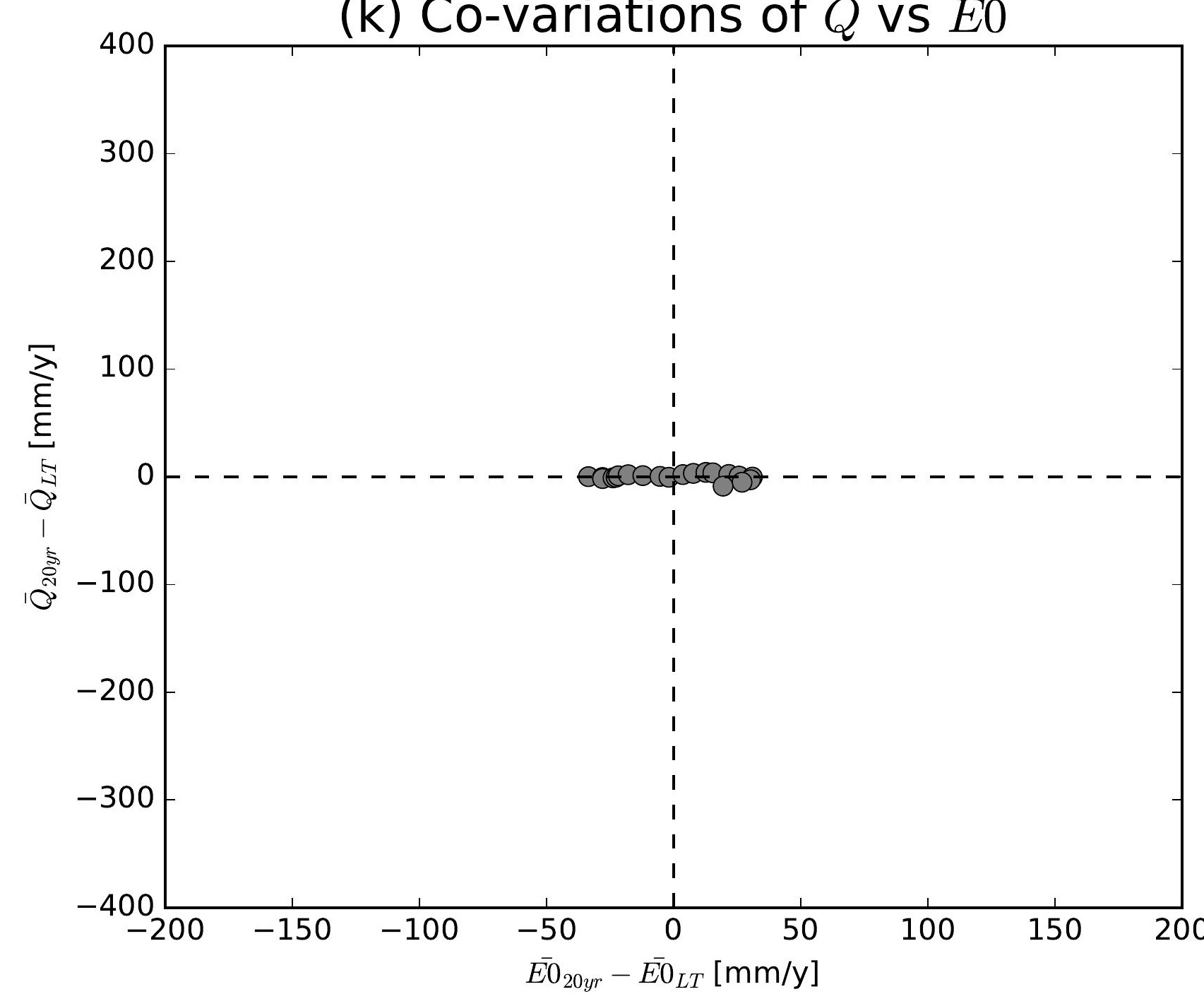

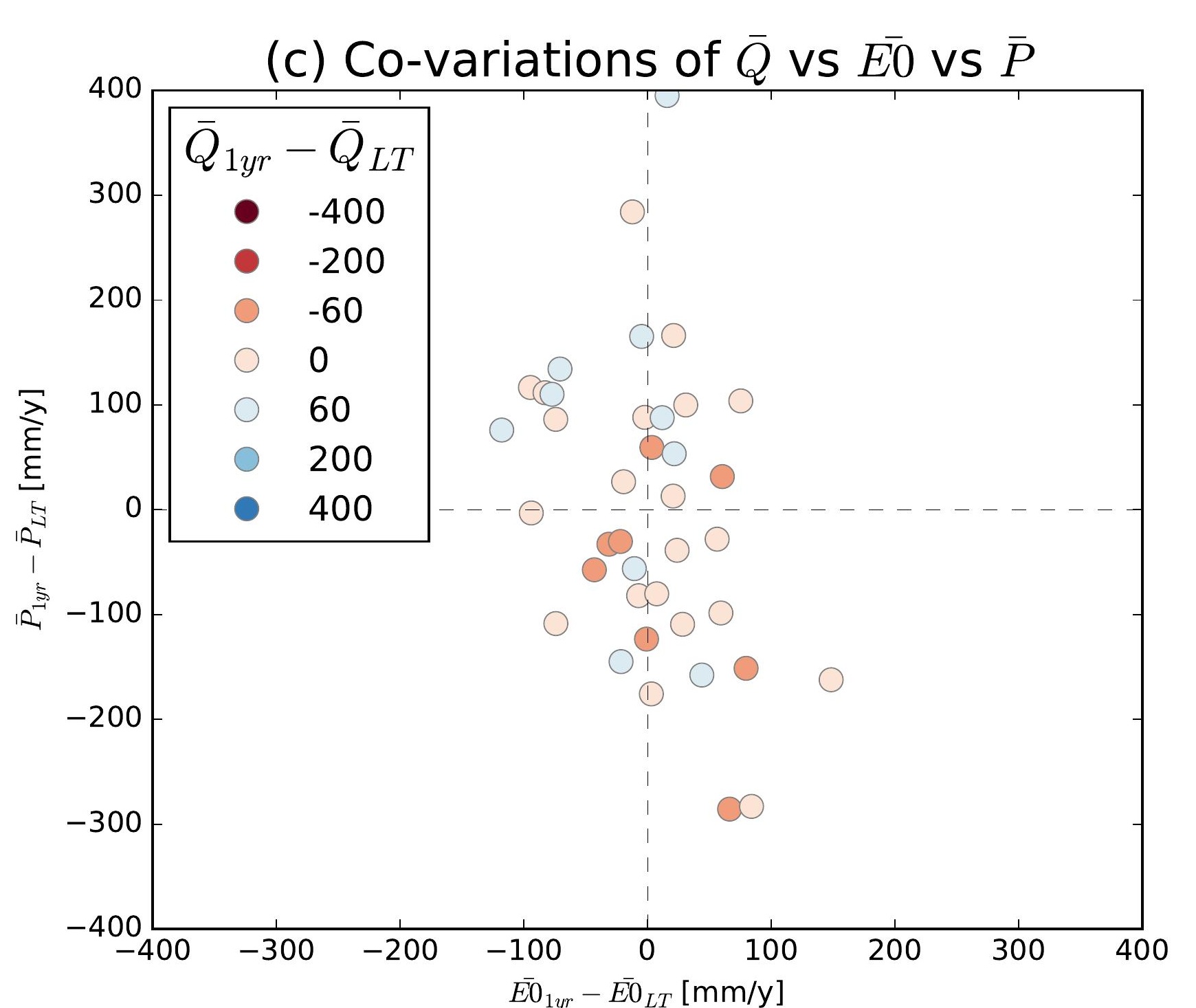
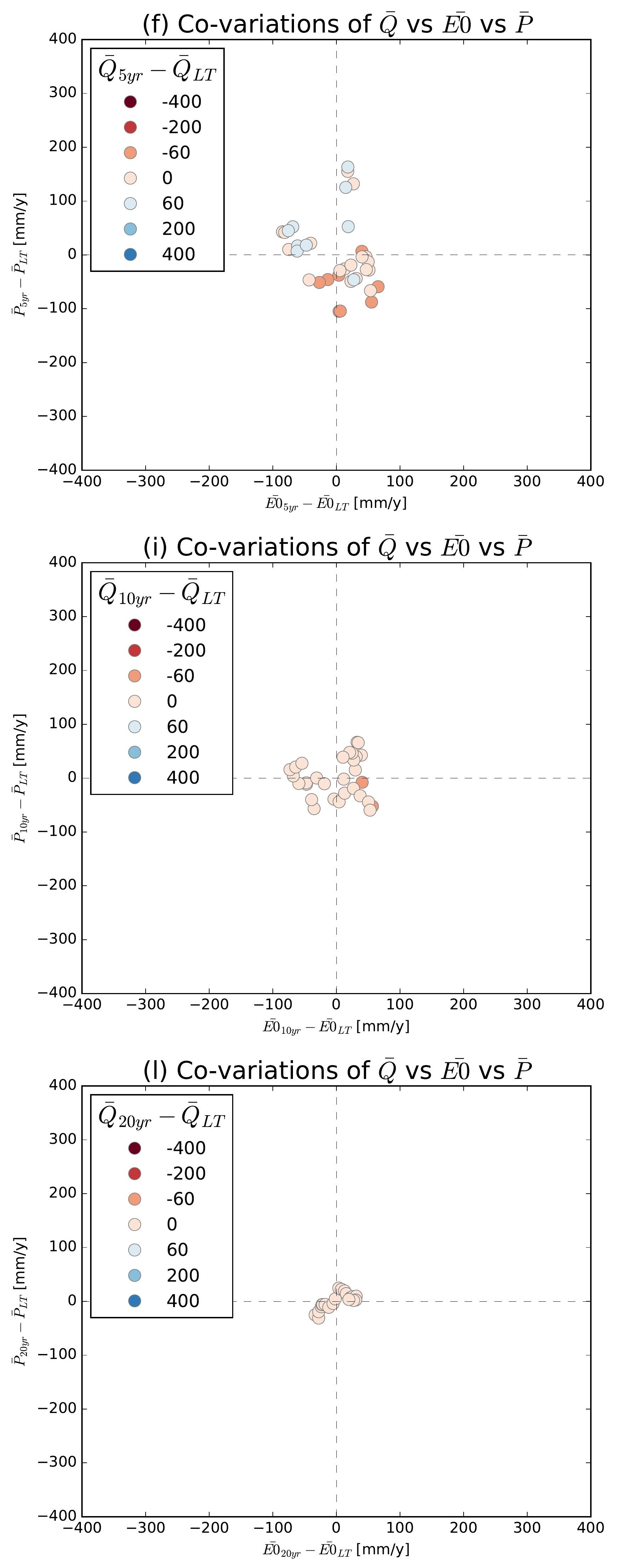

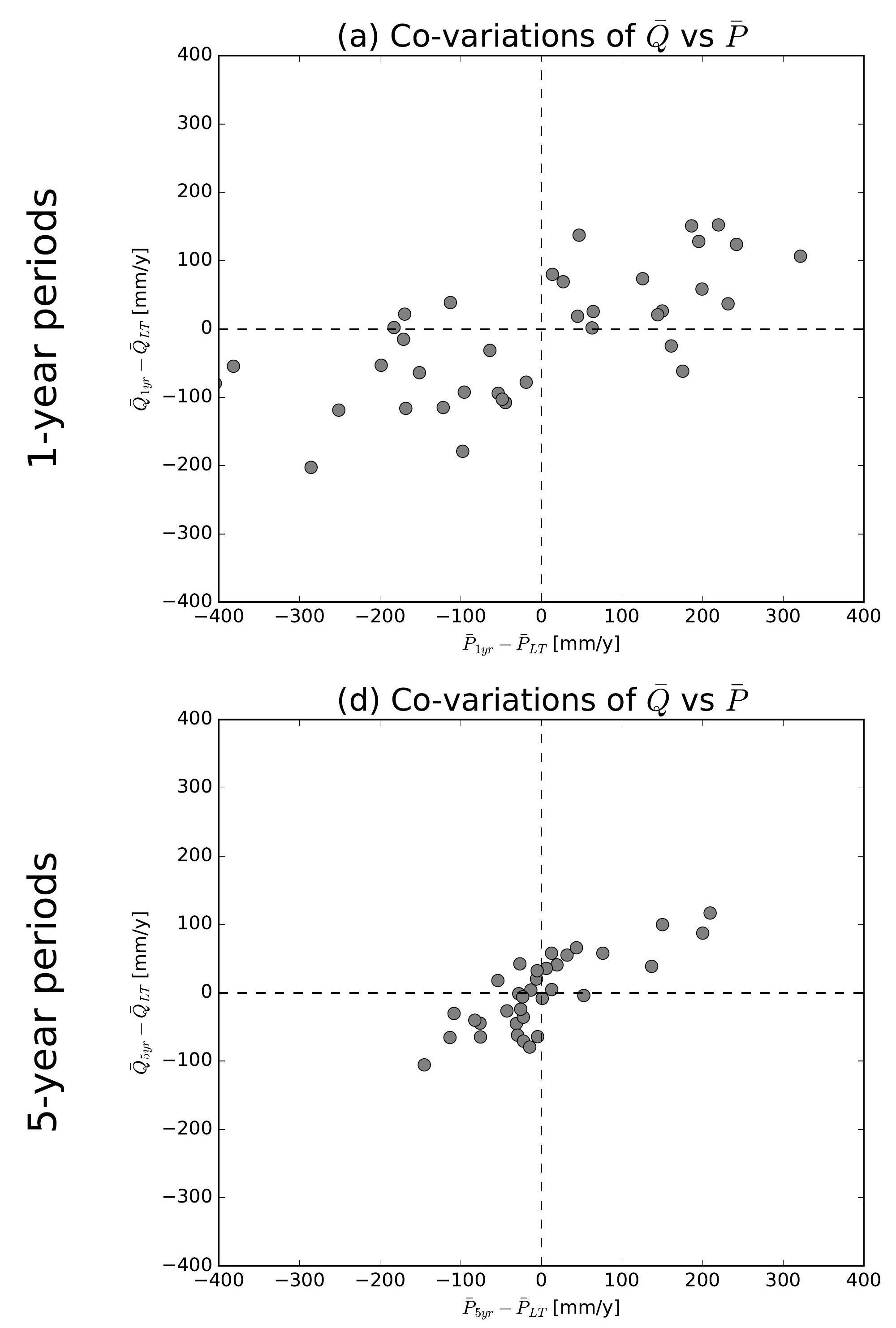

(g) Co-variations of $\bar{Q}$ vs $\bar{P}$

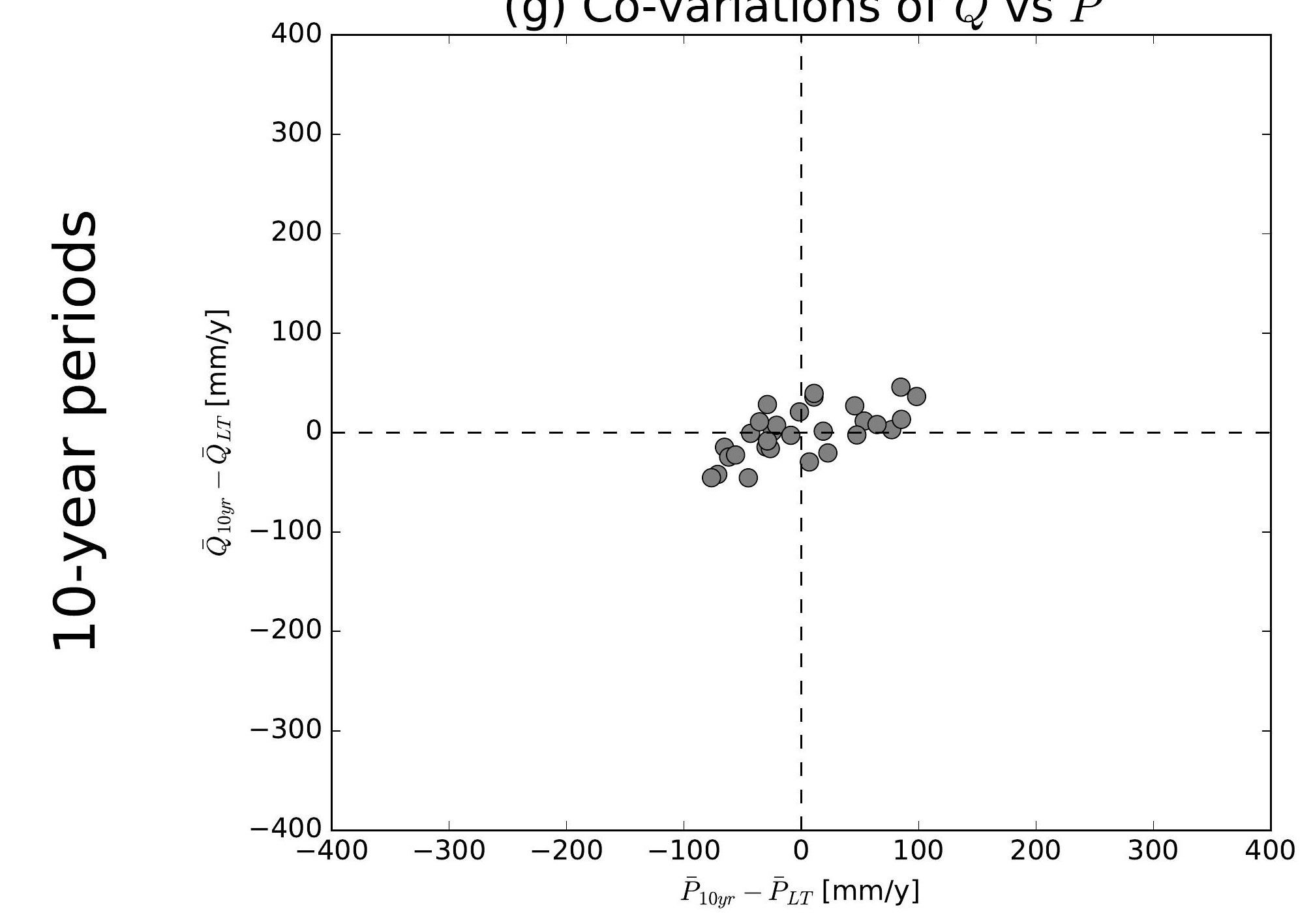

(j) Co-variations of $\bar{Q}$ vs $\bar{P}$

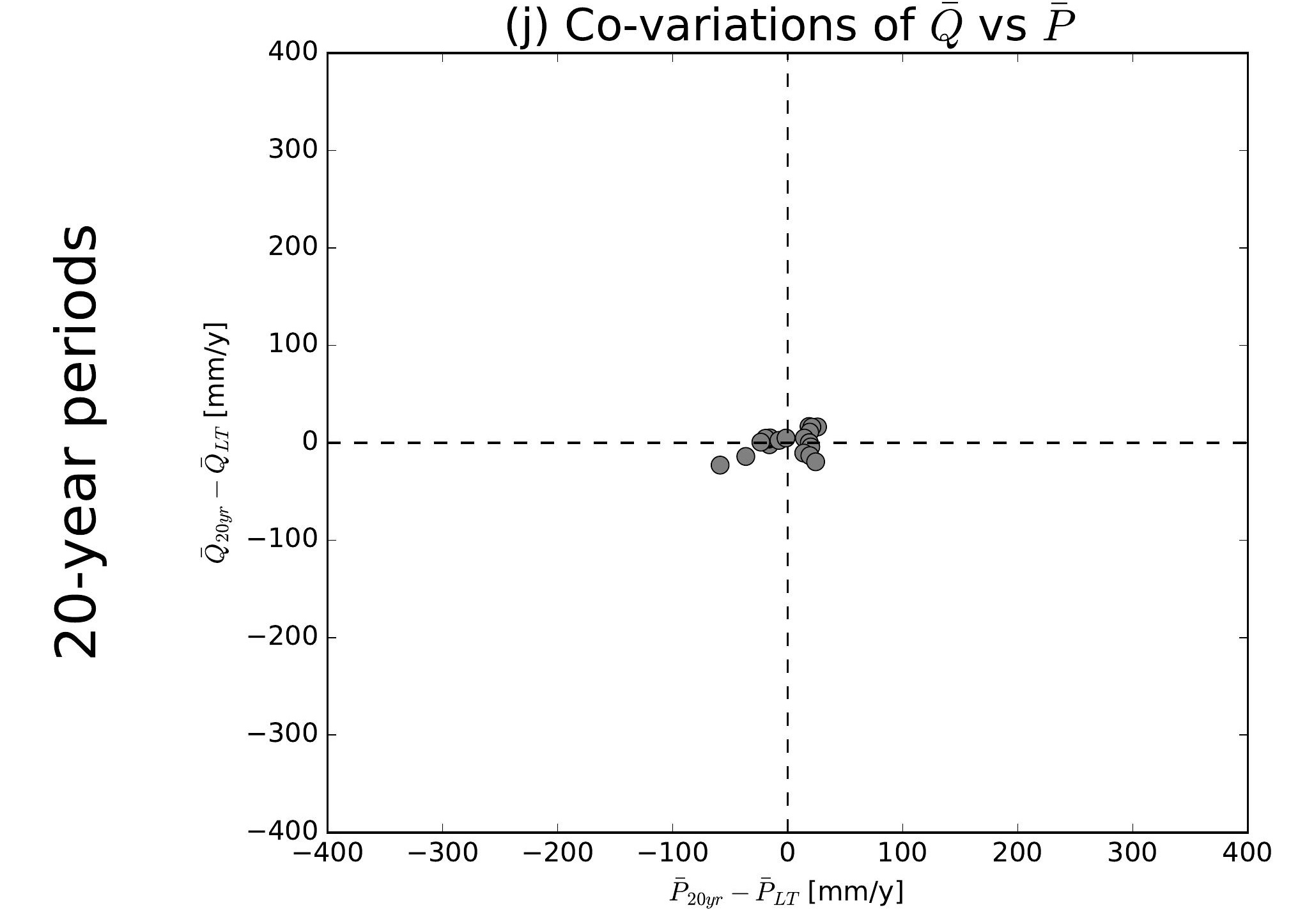

(b) Co-variations of $\bar{Q}$ vs $\overline{E 0}$

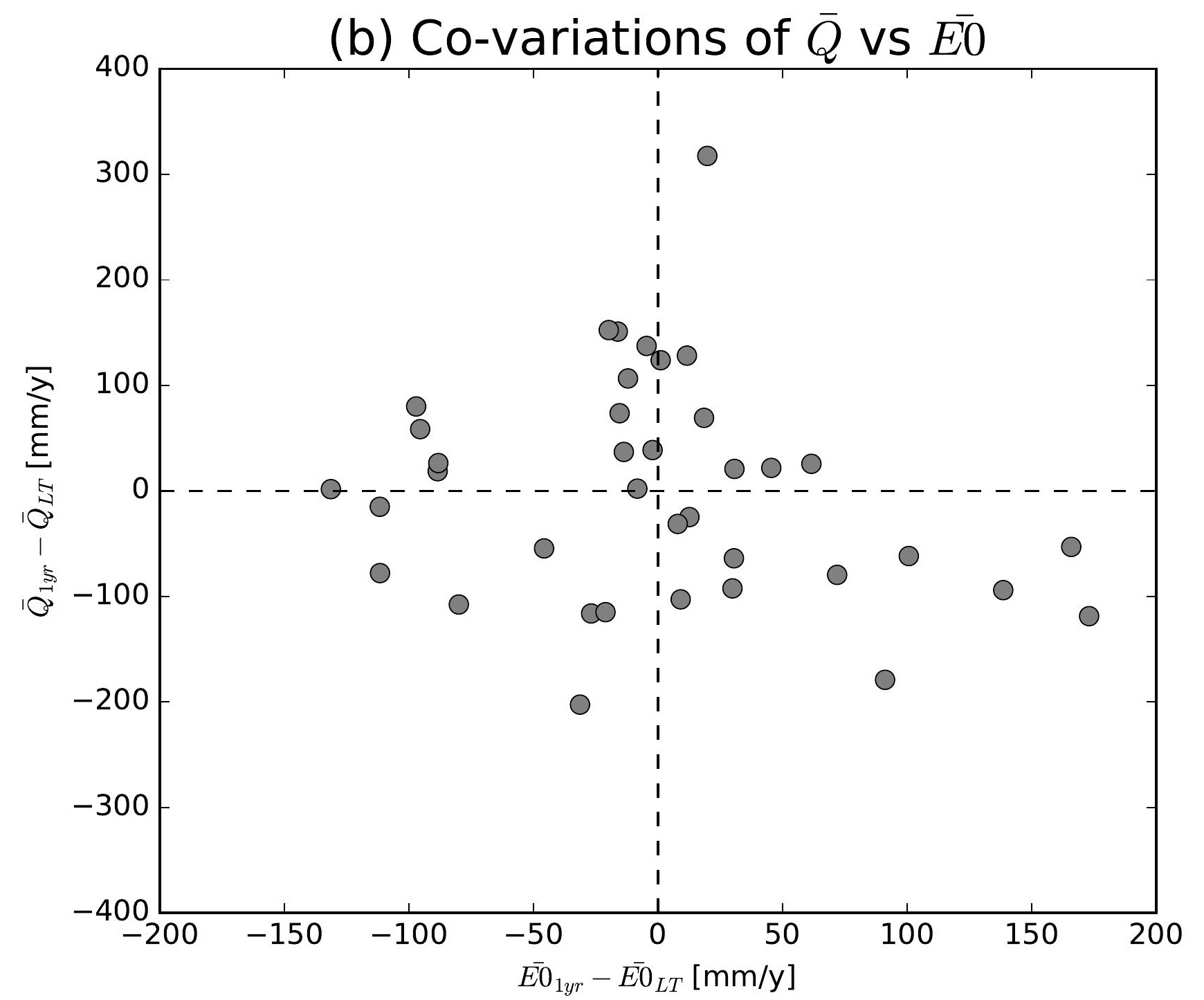

(e) Co-variations of $\bar{Q}$ vs $\overline{E 0}$

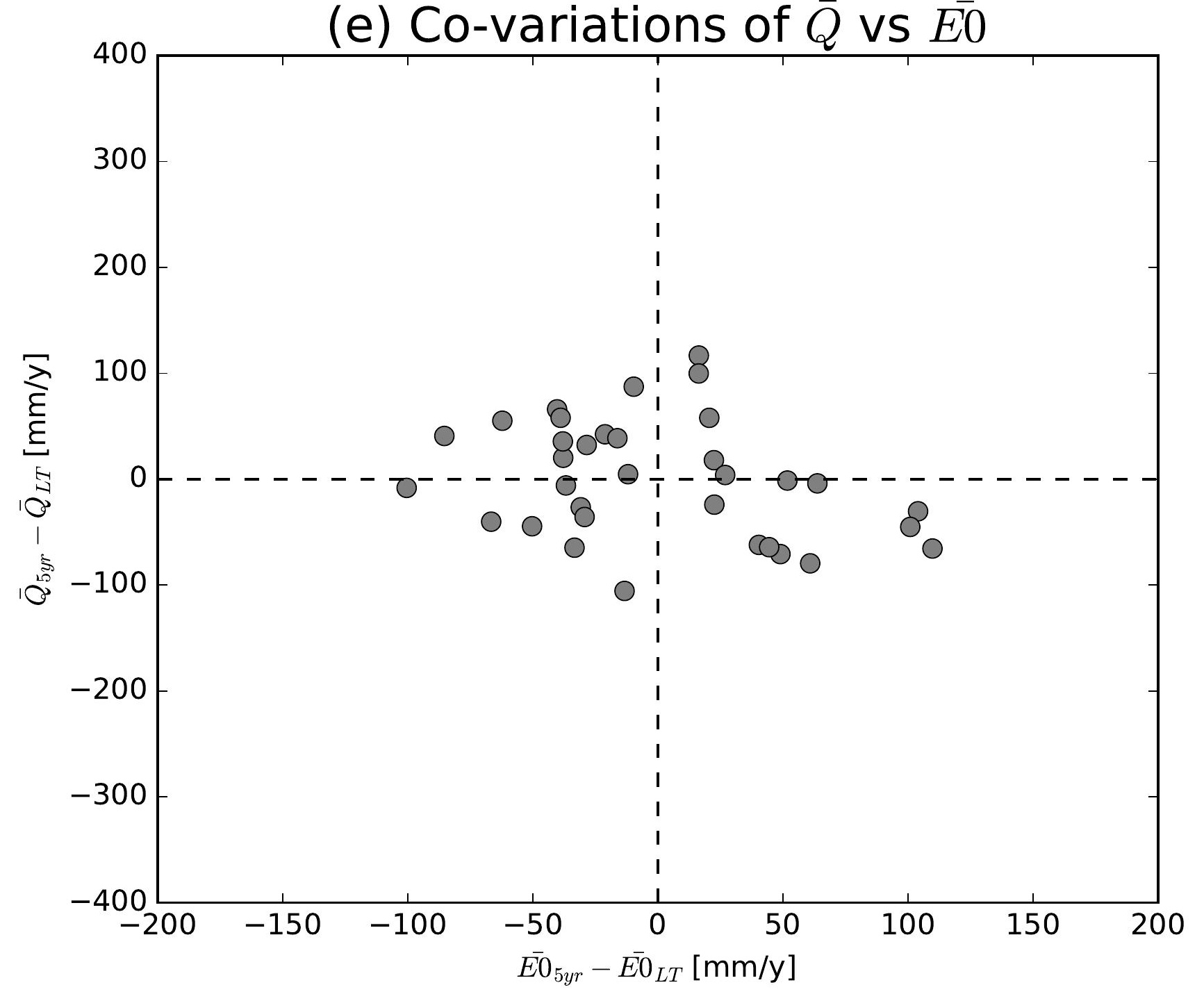

(h) Co-variations of $\bar{Q}$ vs $\overline{E 0}$

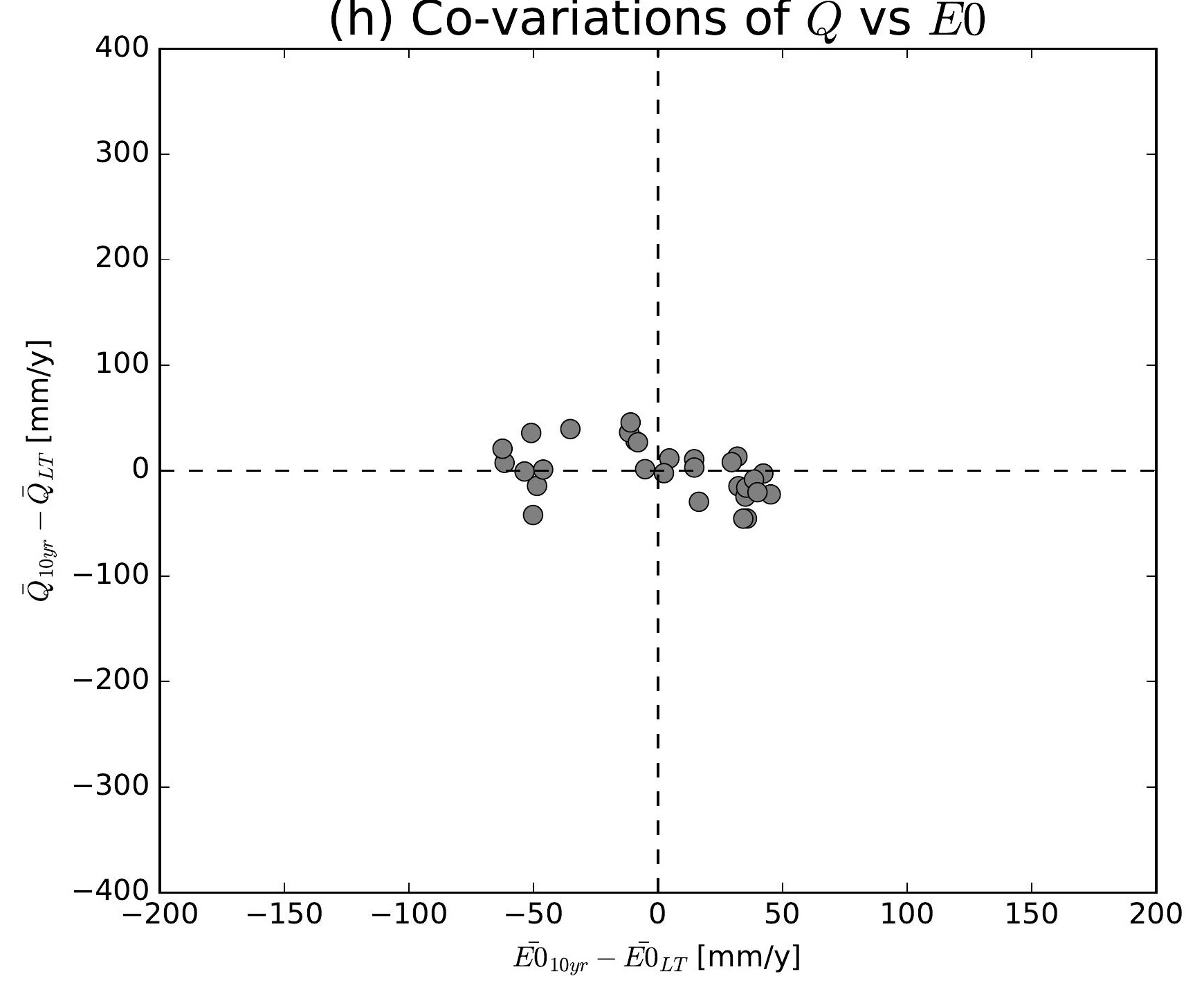

(k) Co-variations of $\bar{Q}$ vs $\overline{E 0}$

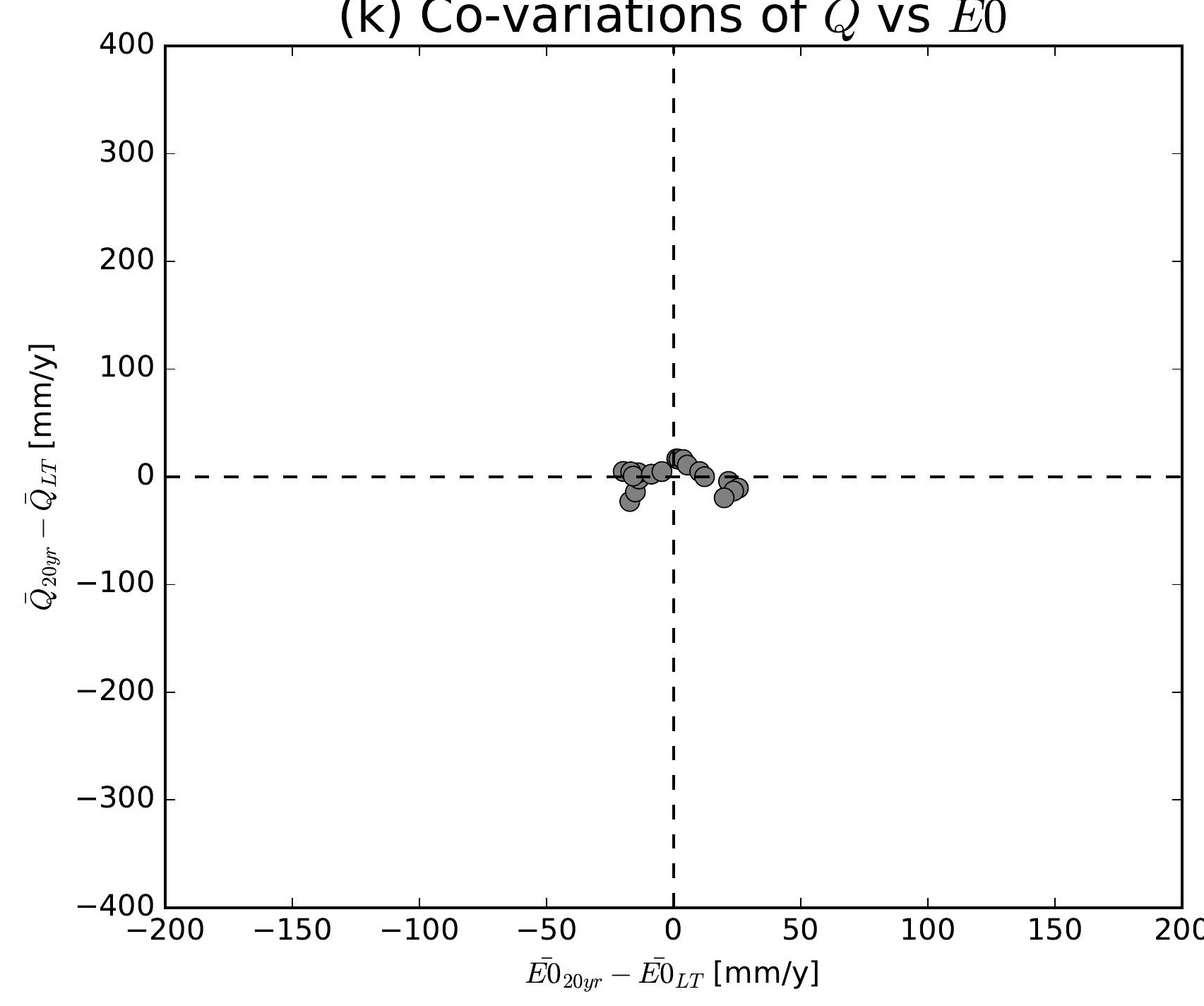

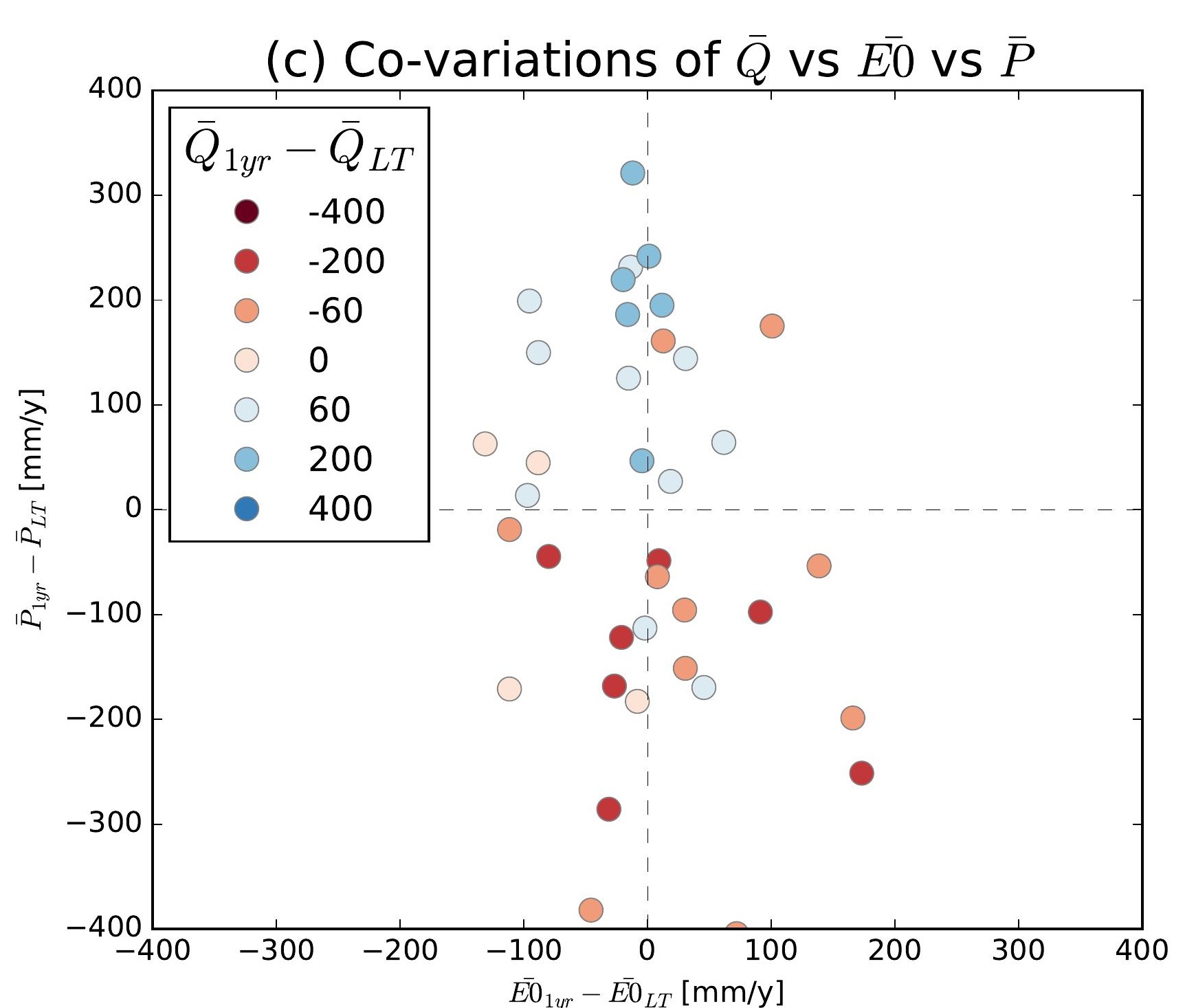
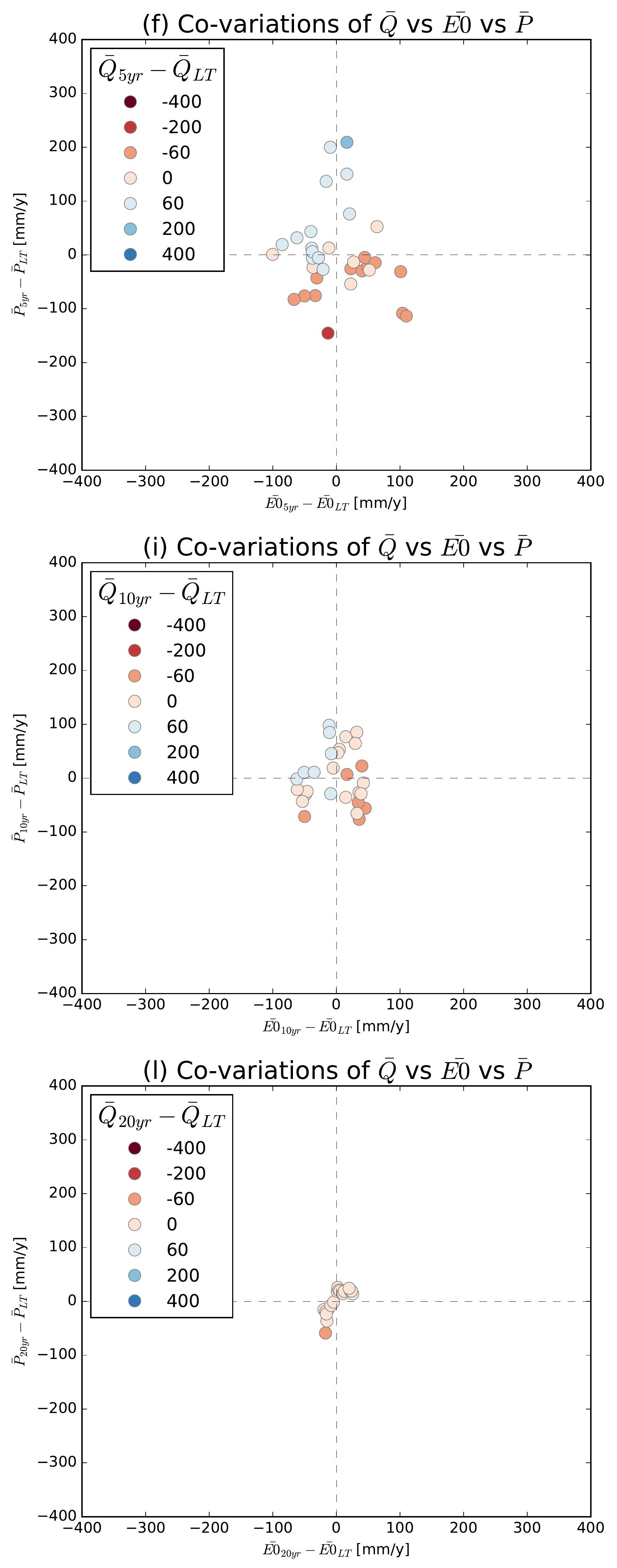

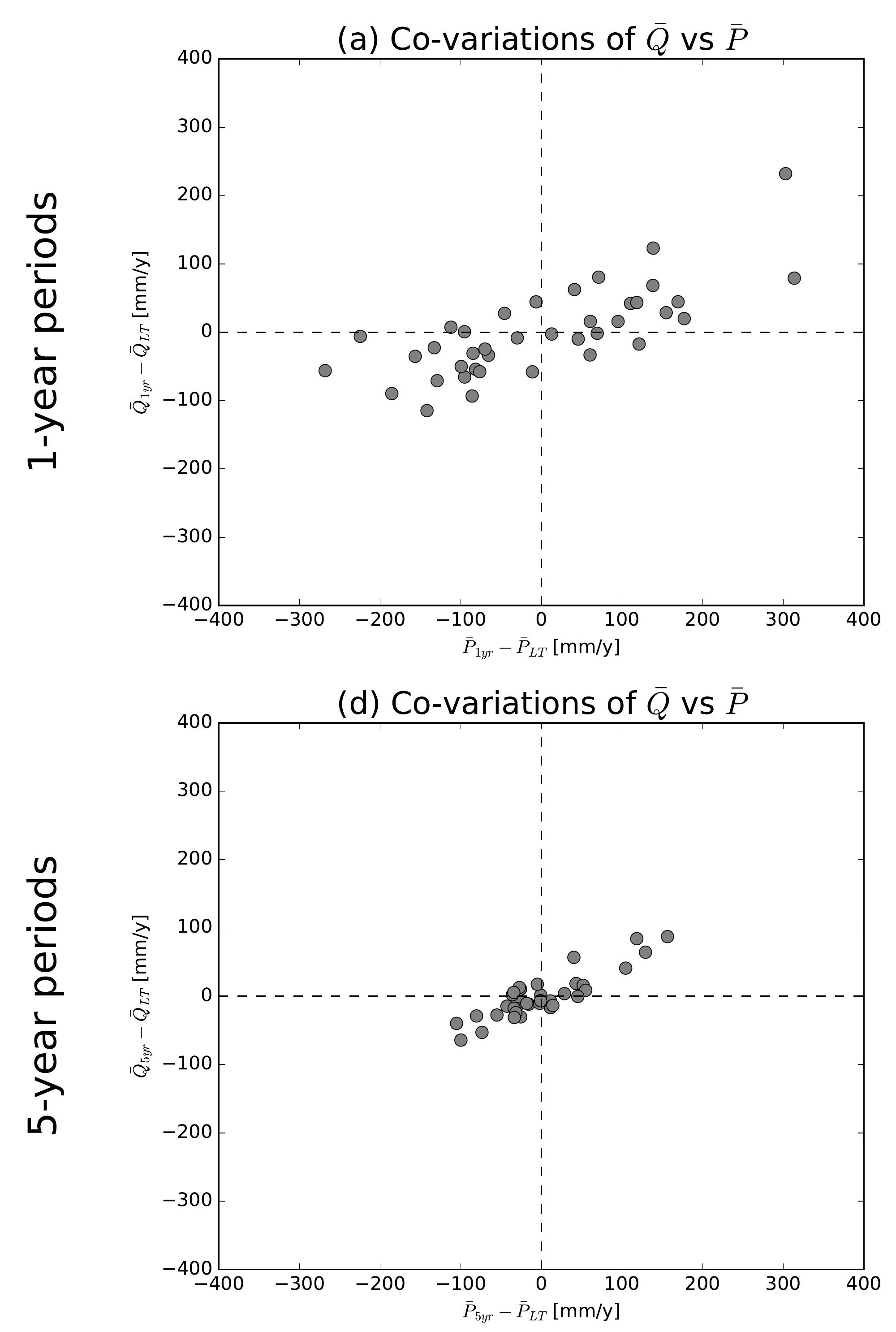

(g) Co-variations of $\bar{Q}$ vs $\bar{P}$
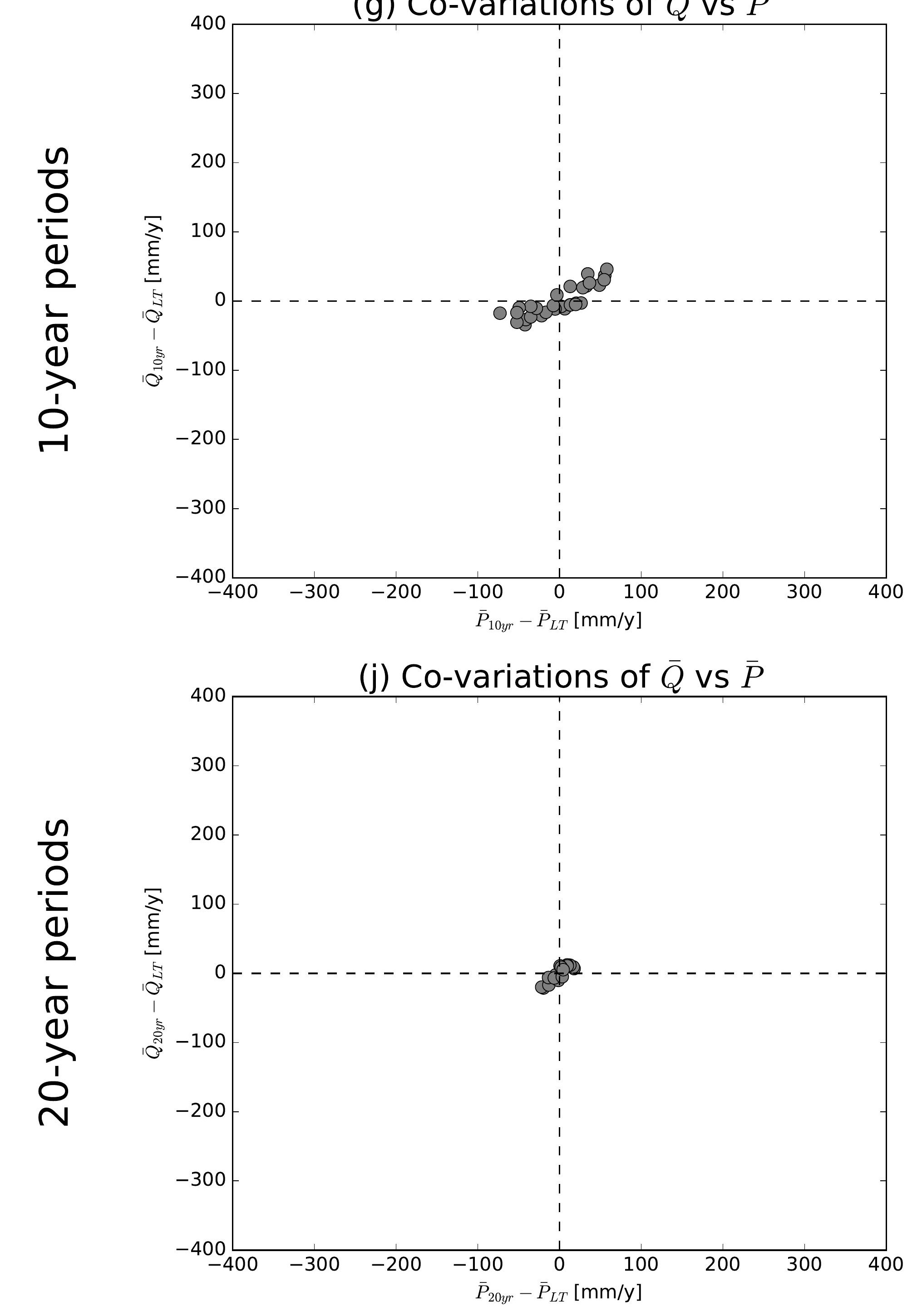

(b) Co-variations of $\bar{Q}$ vs $\overline{E 0}$

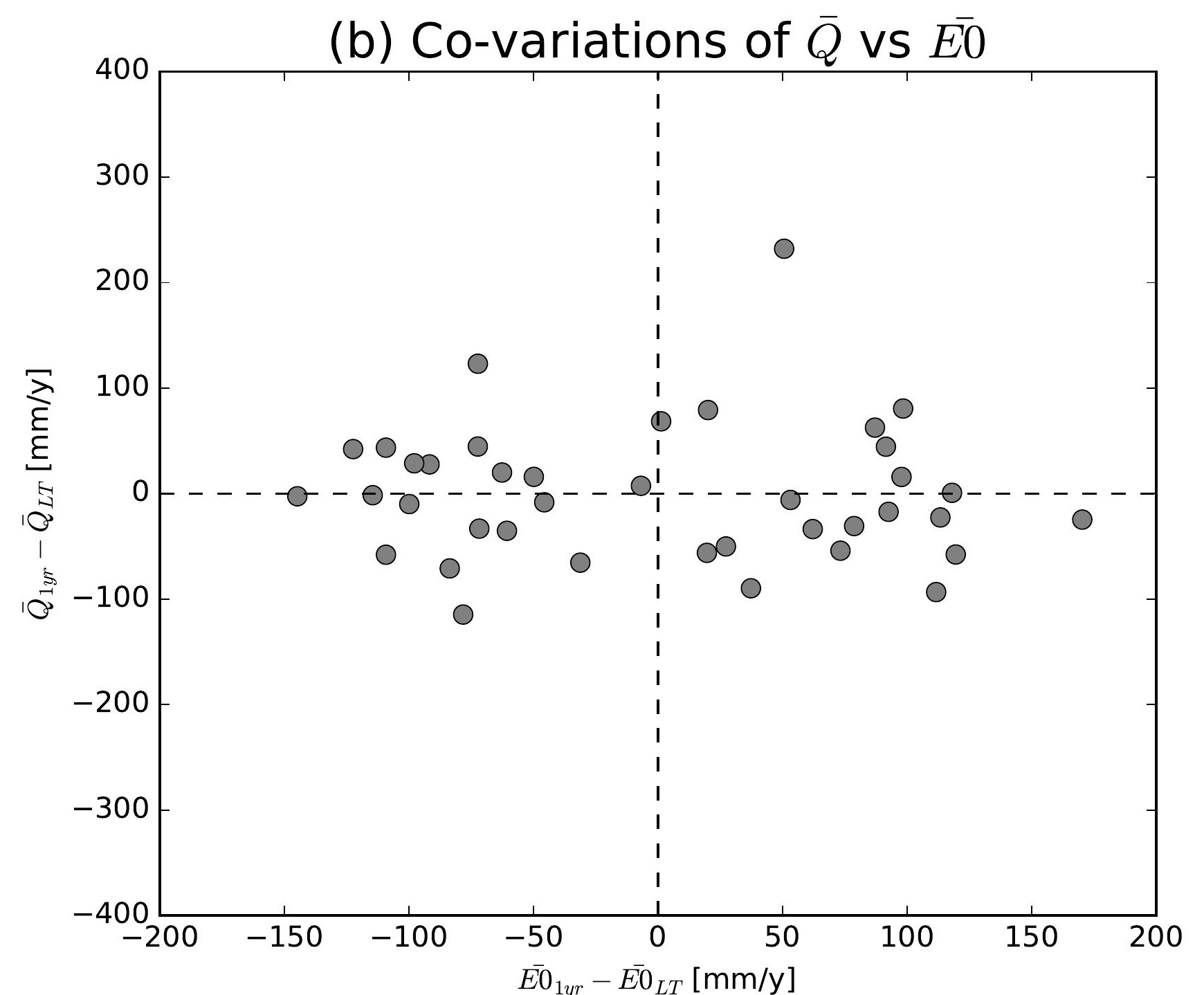

(e) Co-variations of $\bar{Q}$ vs $\overline{E 0}$

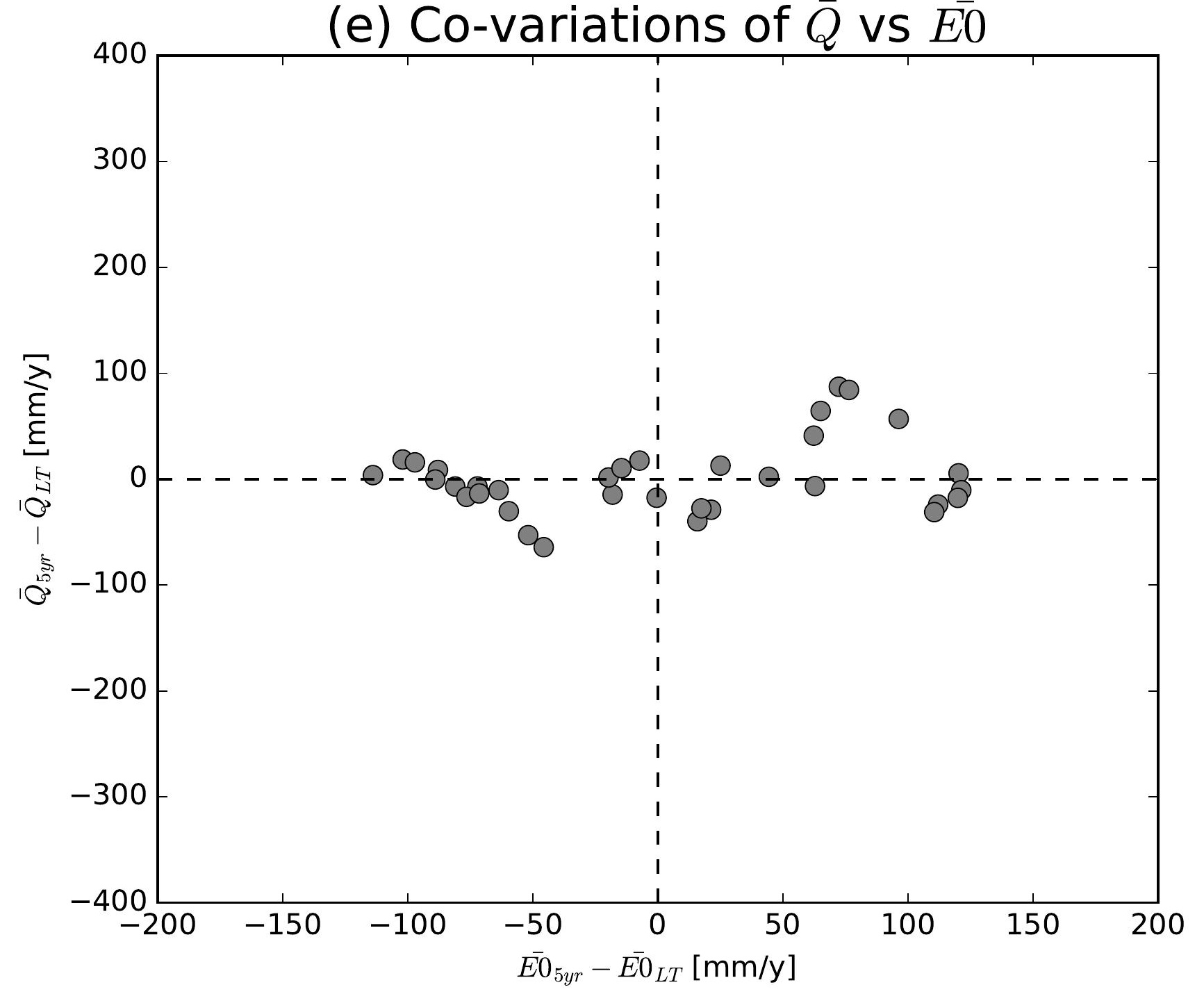

(h) Co-variations of $\bar{Q}$ vs $\overline{E 0}$

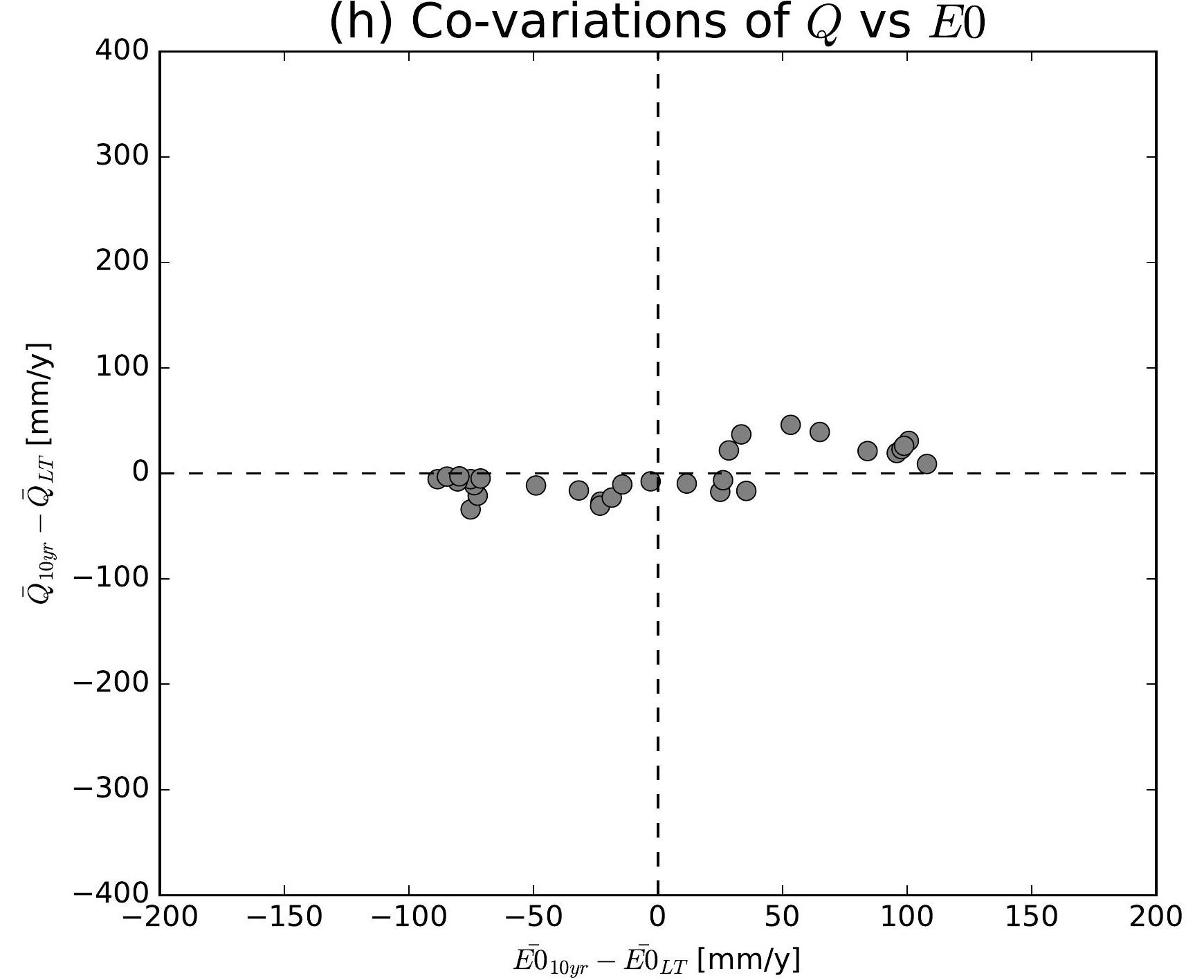

(k) Co-variations of $\bar{Q}$ vs $\overline{E 0}$

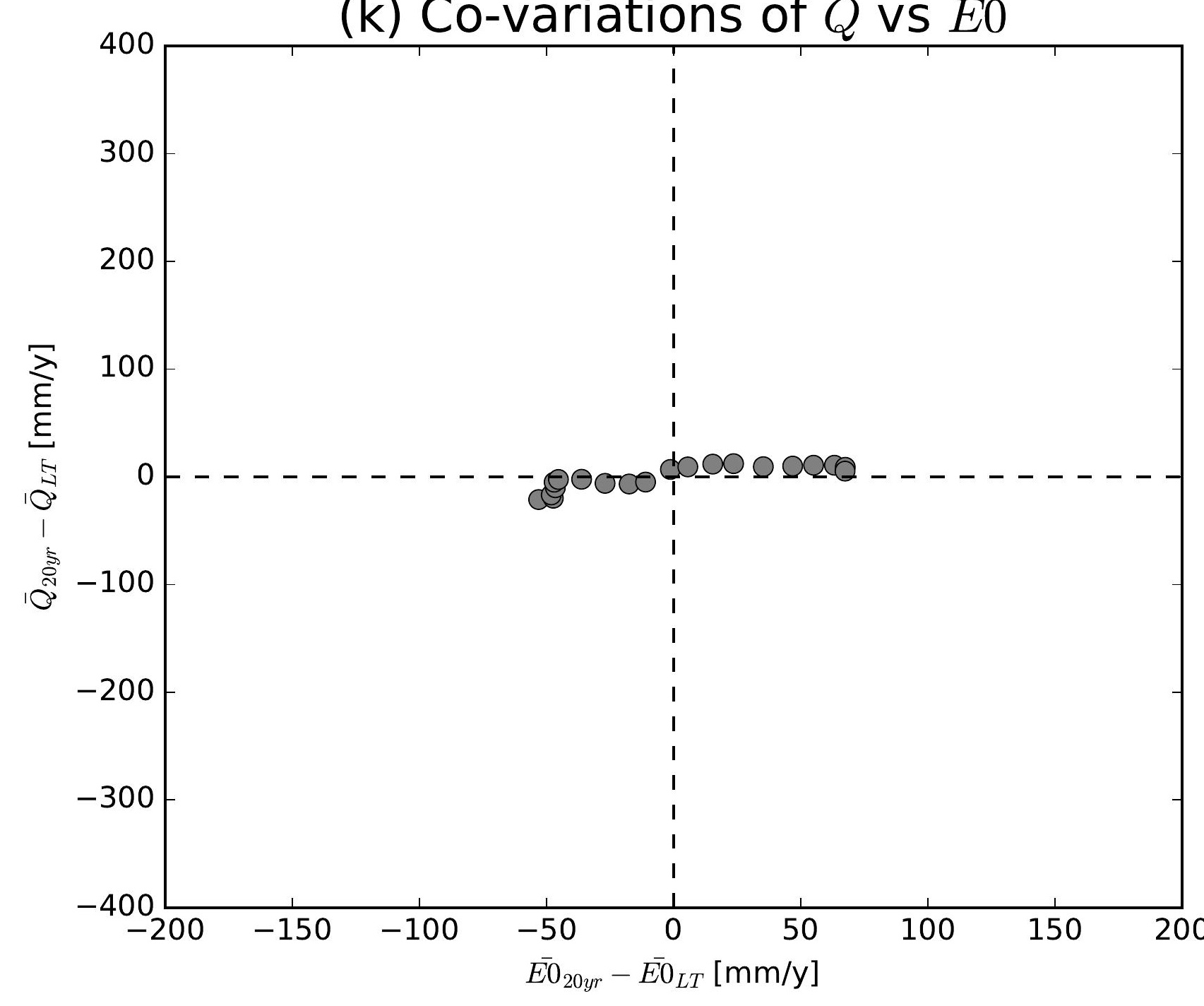

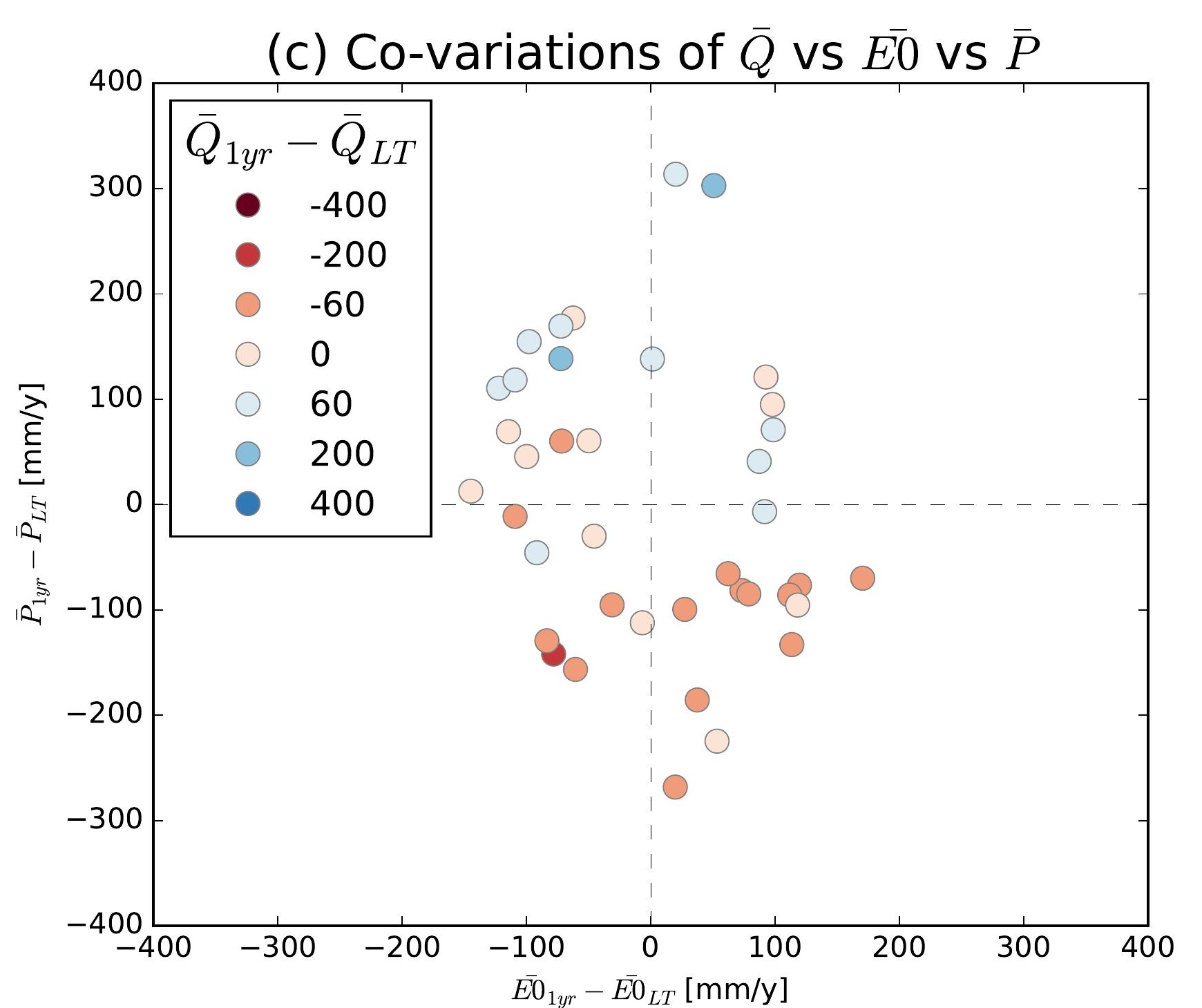
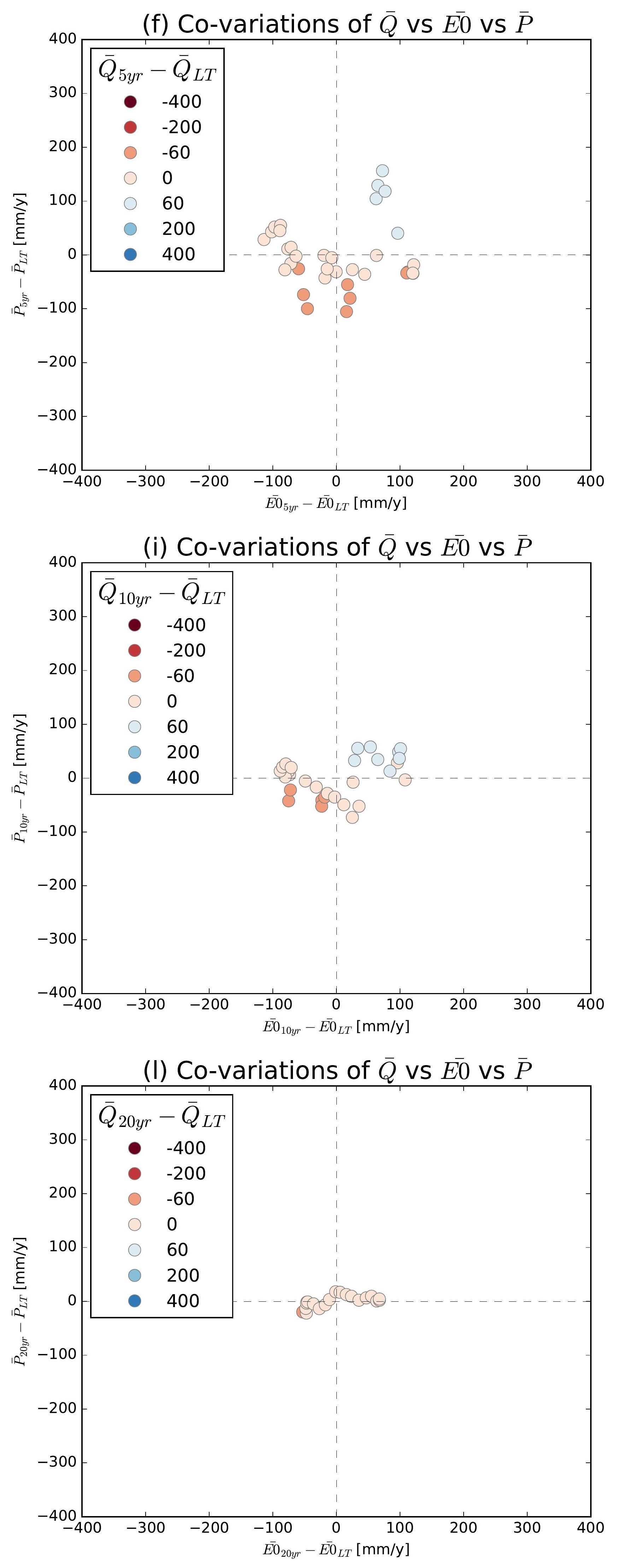

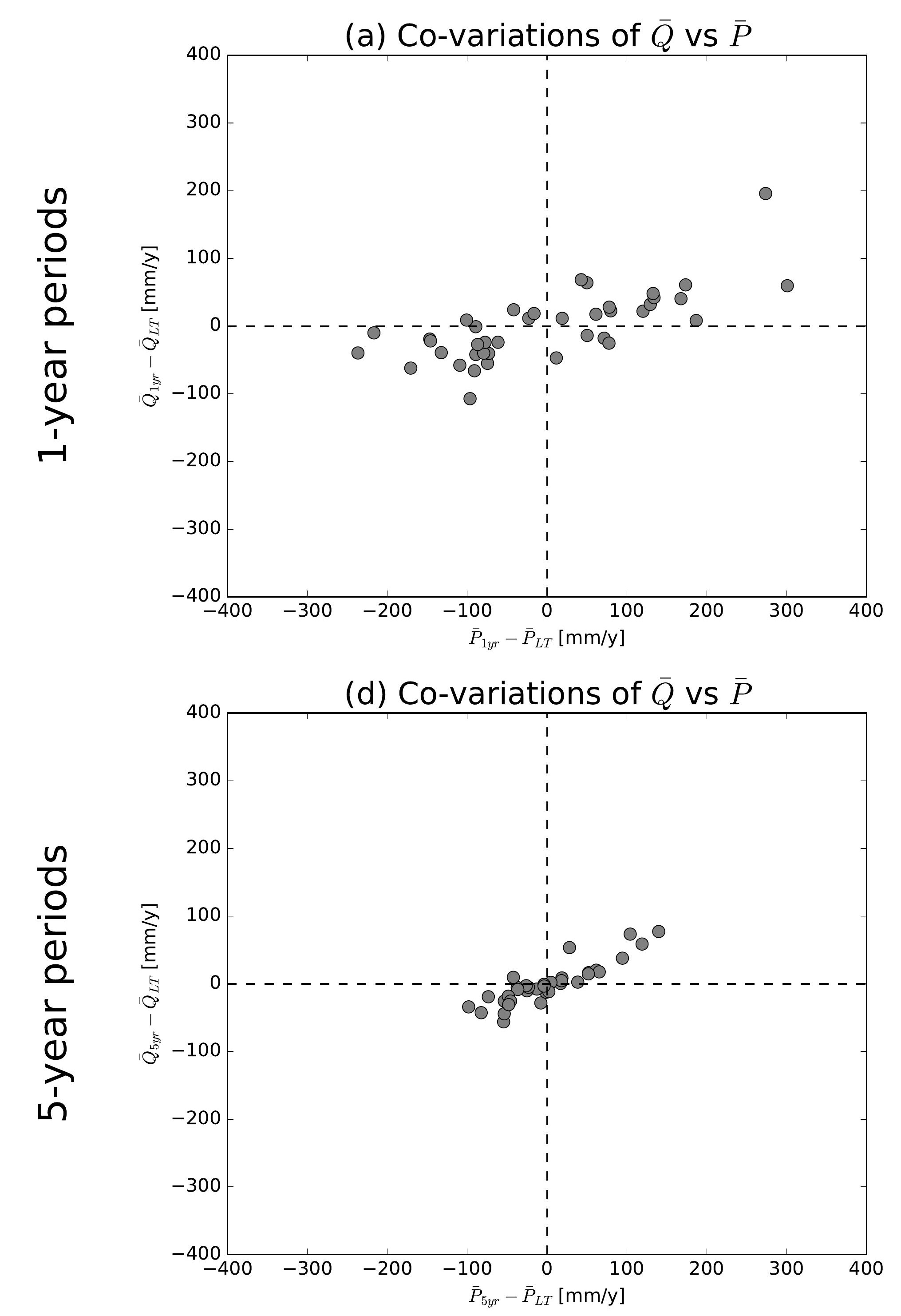

(g) Co-variations of $\bar{Q}$ vs $\bar{P}$
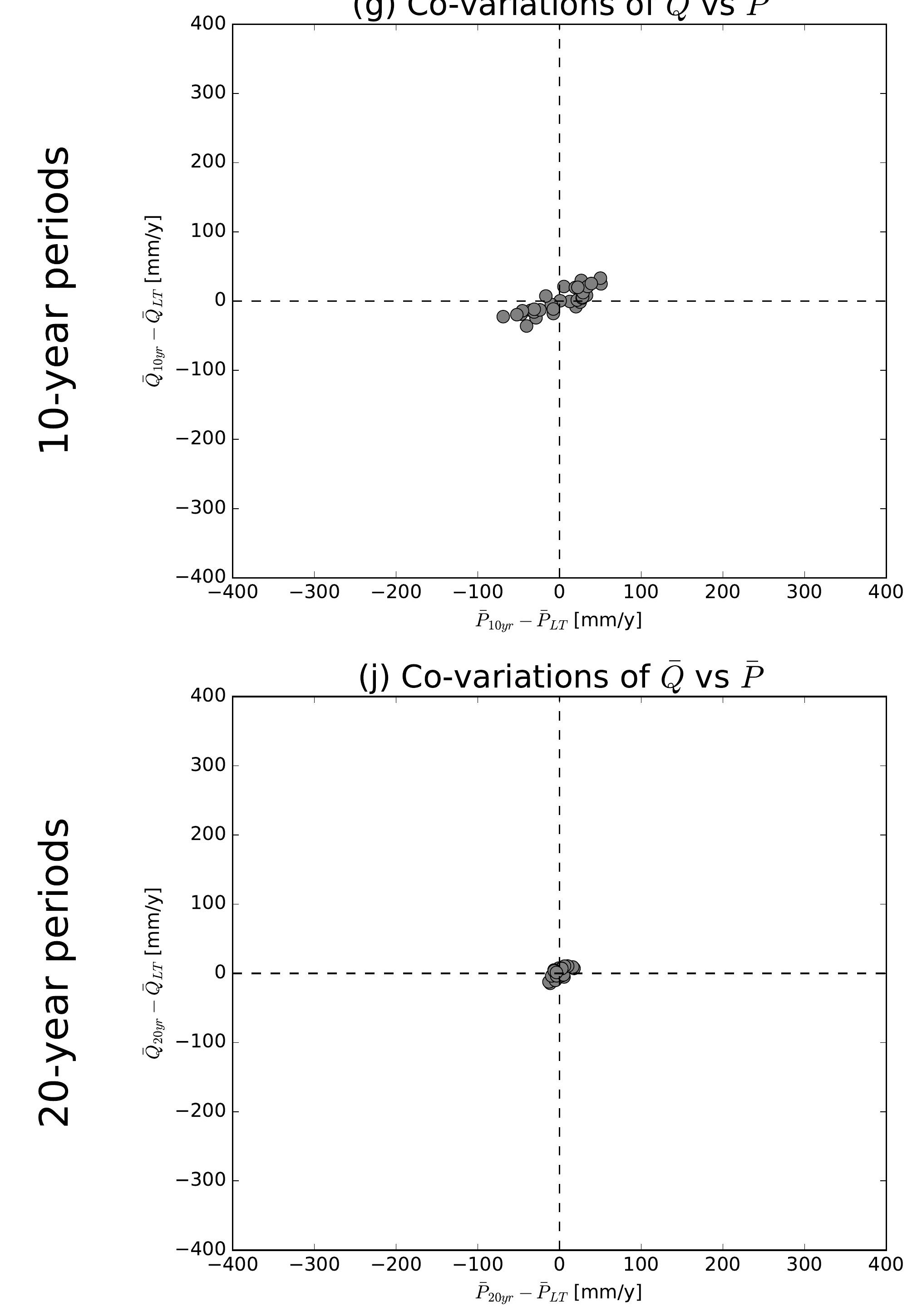

(b) Co-variations of $\bar{Q}$ vs $\overline{E 0}$

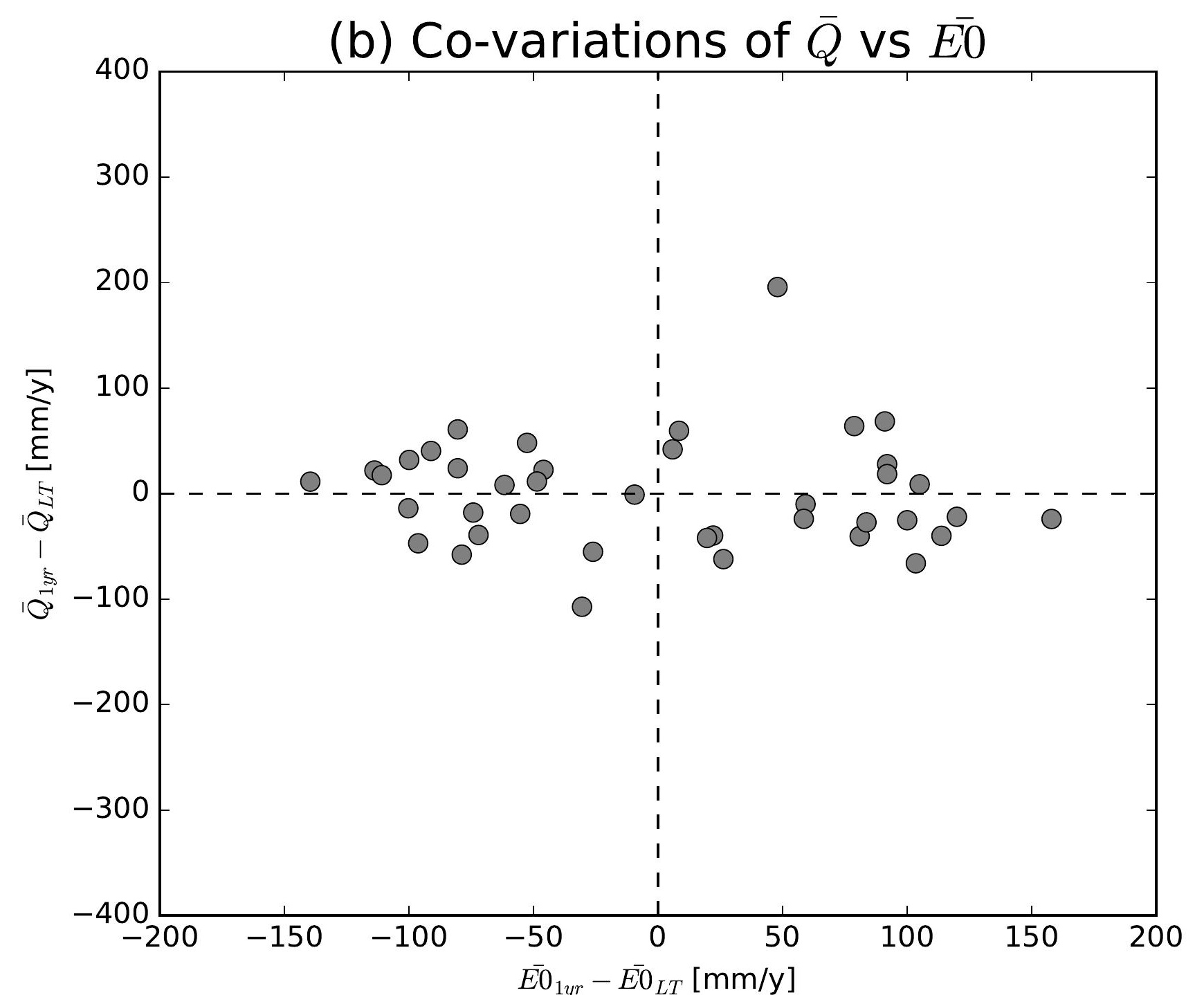

(e) Co-variations of $\bar{Q}$ vs $\overline{E 0}$

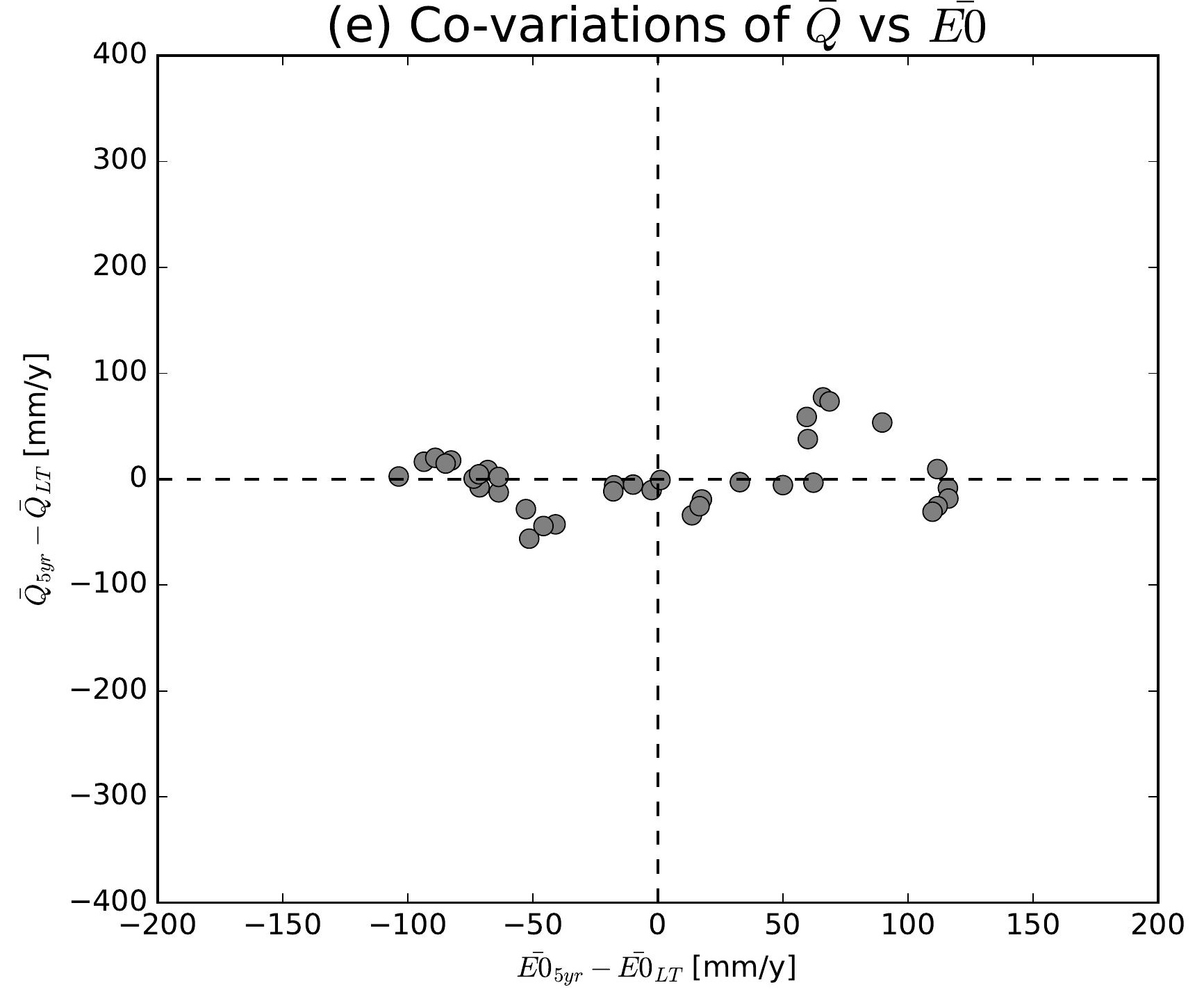

(h) Co-variations of $\bar{Q}$ vs $\overline{E 0}$

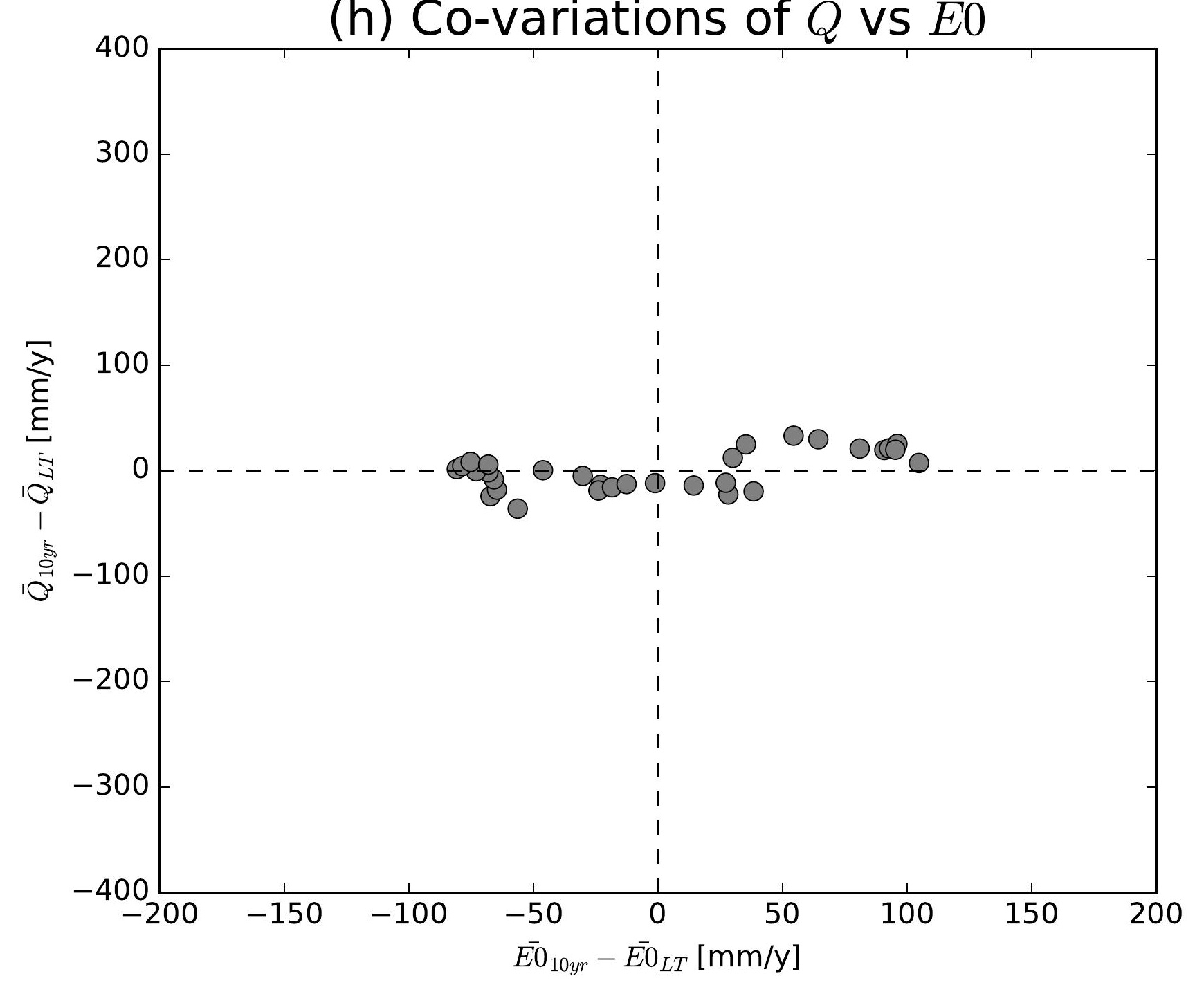

(k) Co-variations of $\bar{Q}$ vs $\overline{E 0}$

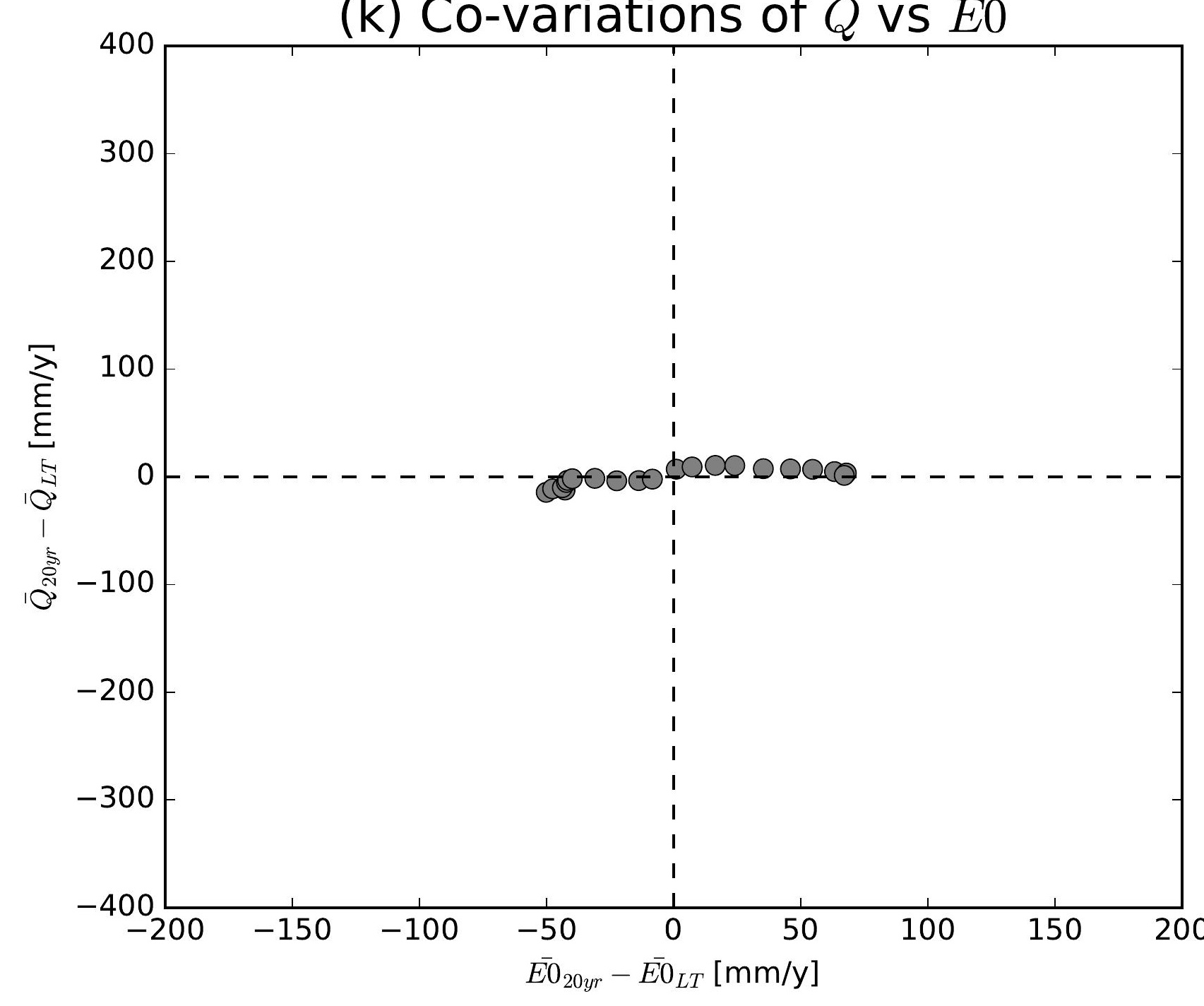

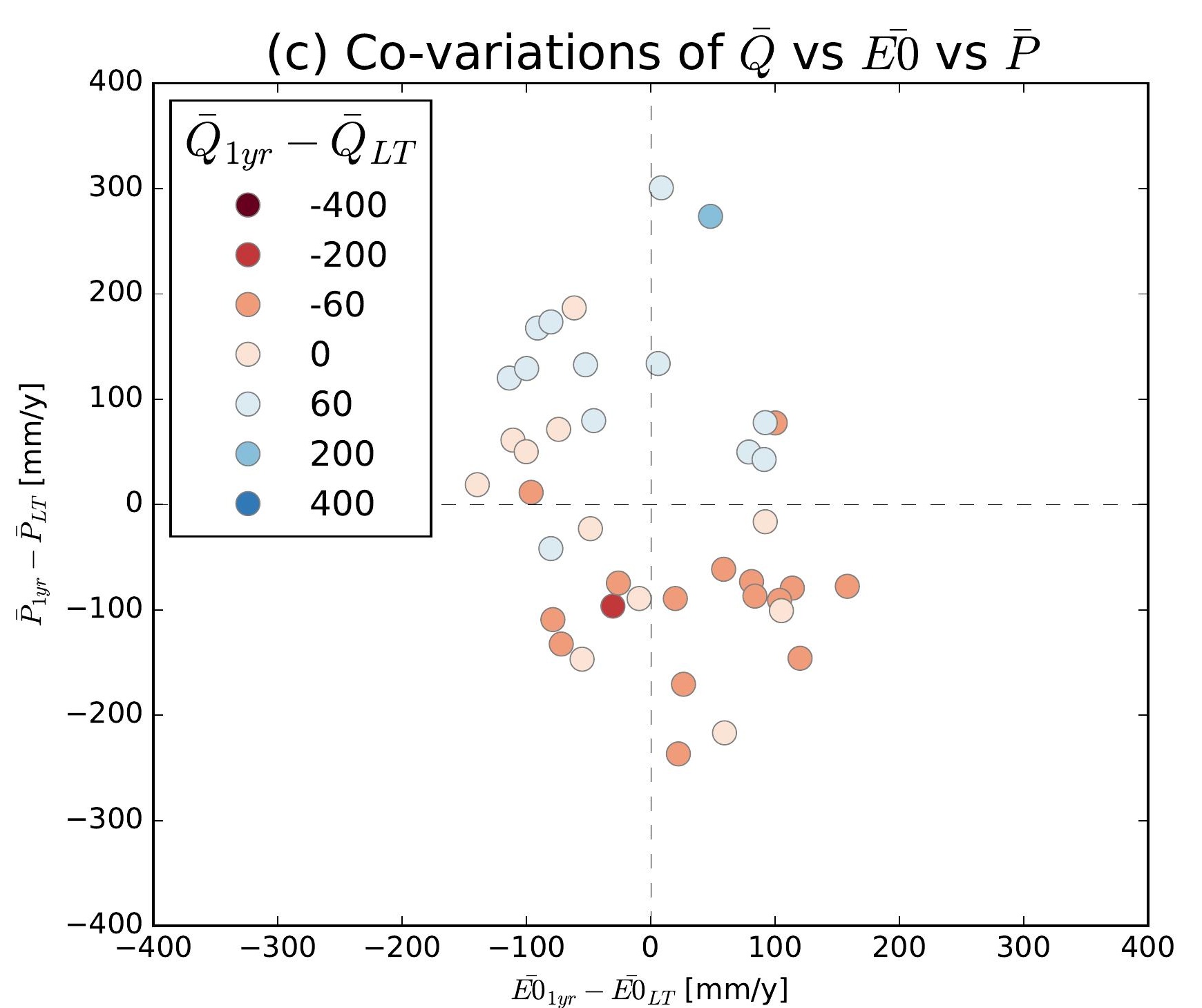
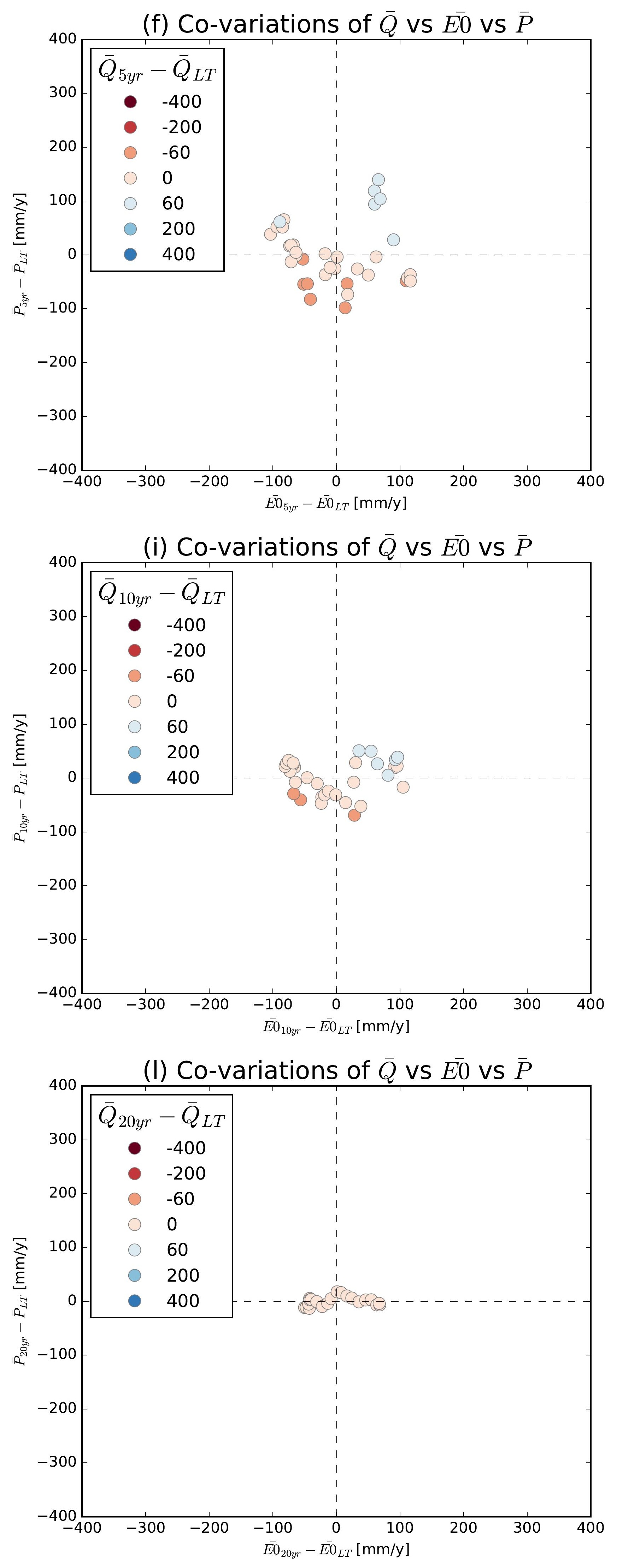

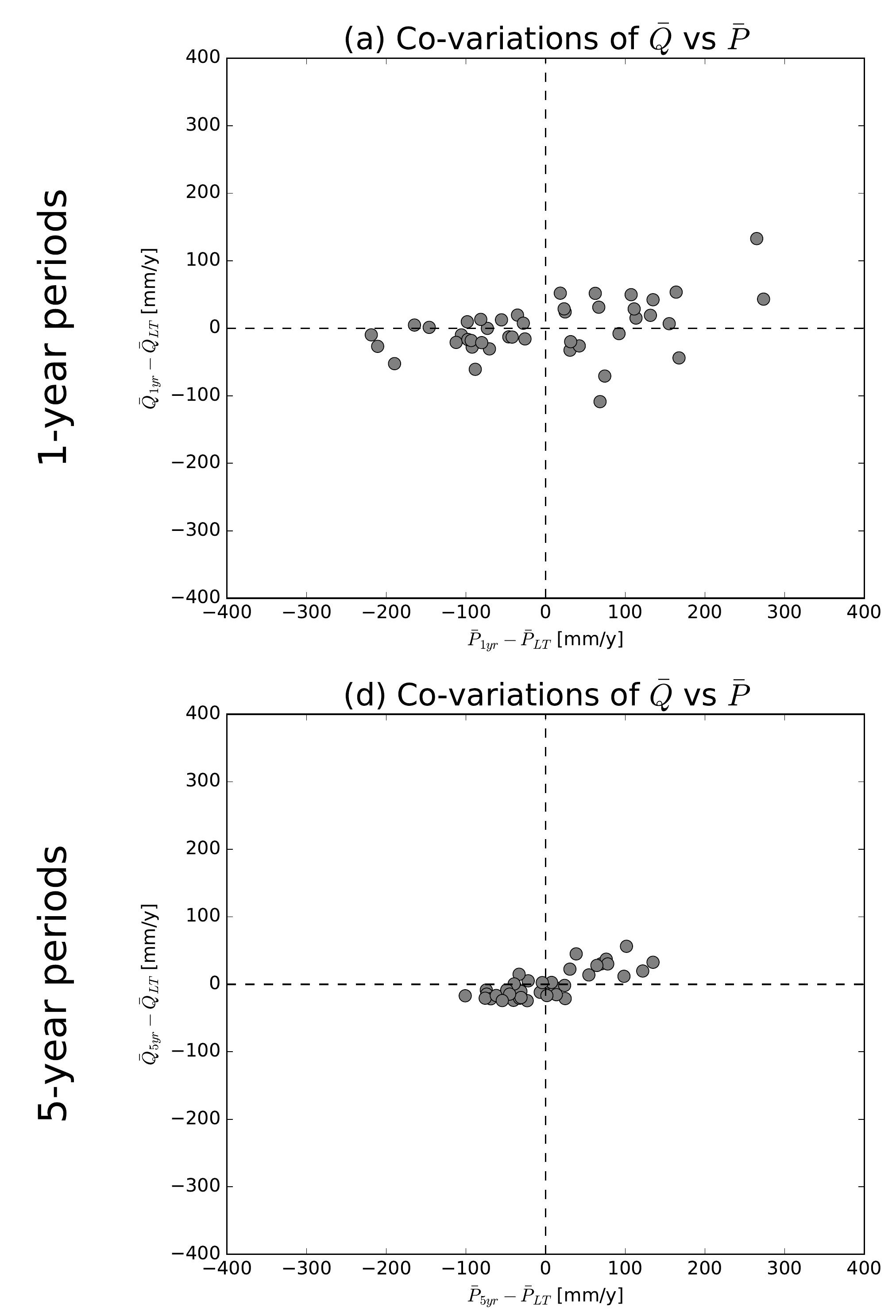

(g) Co-variations of $\bar{Q}$ vs $\bar{P}$
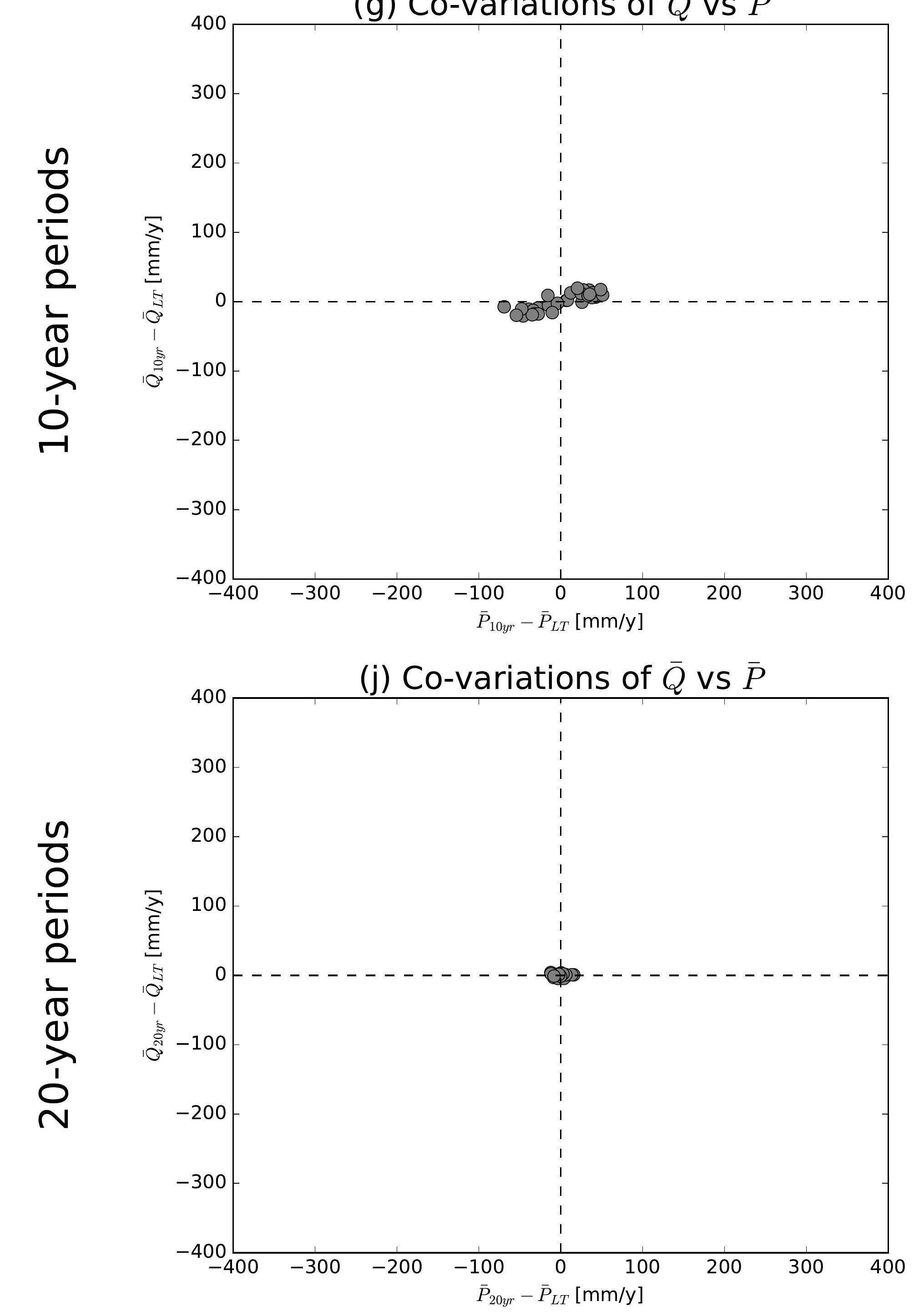

(b) Co-variations of $\bar{Q}$ vs $\overline{E 0}$

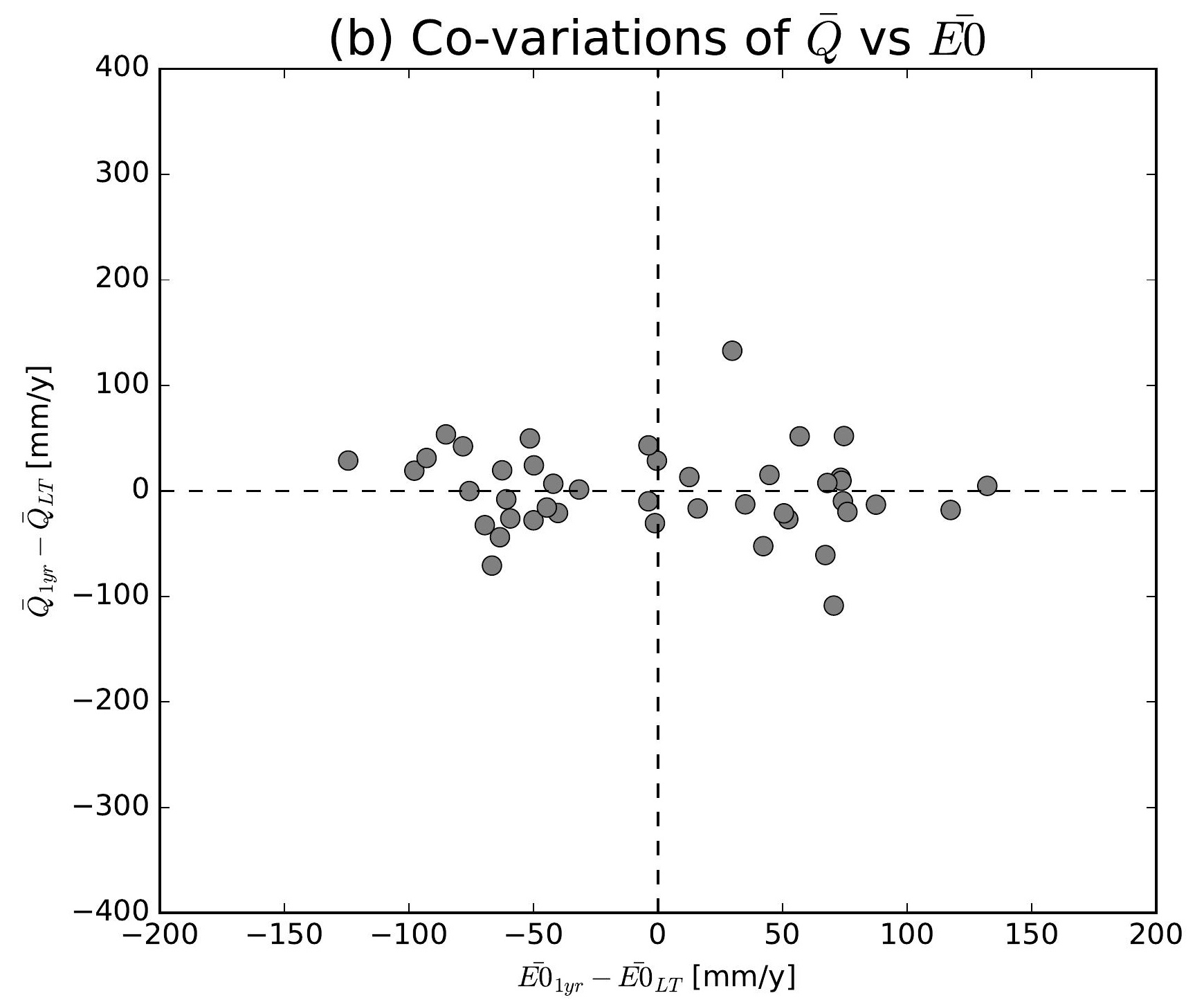

(e) Co-variations of $\bar{Q}$ vs $\overline{E 0}$

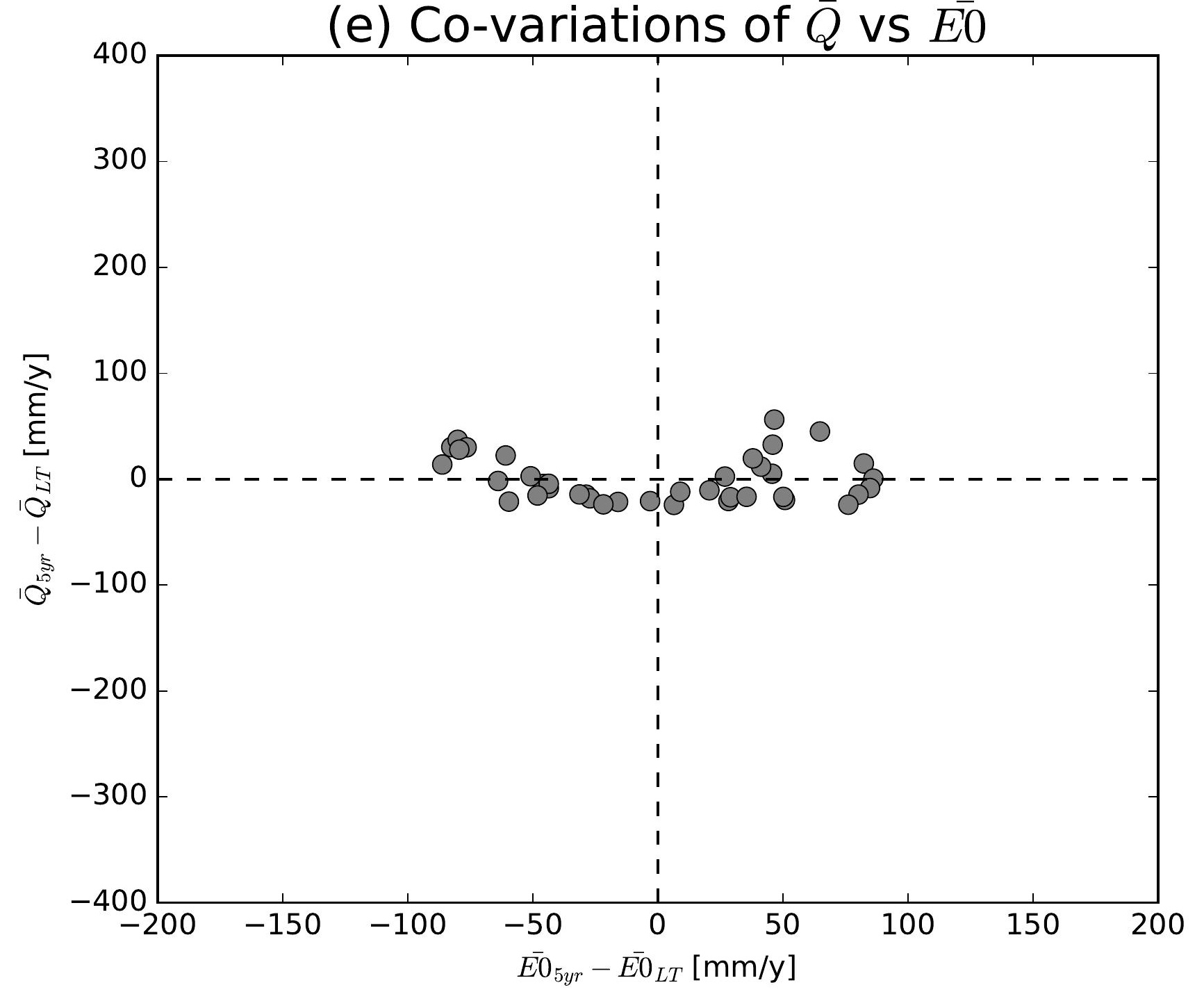

(h) Co-variations of $\bar{Q}$ vs $\overline{E 0}$

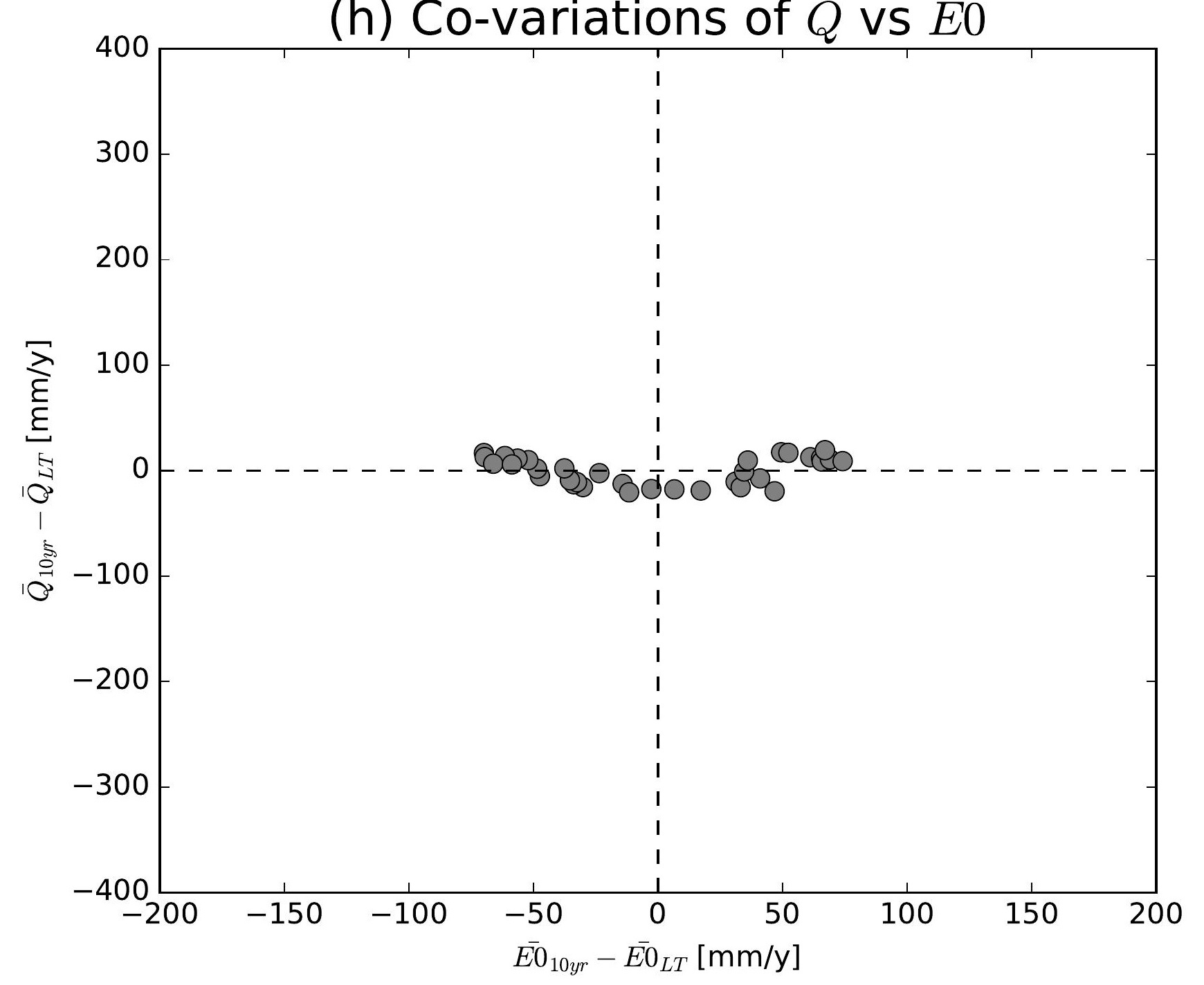

(k) Co-variations of $\bar{Q}$ vs $\overline{E 0}$

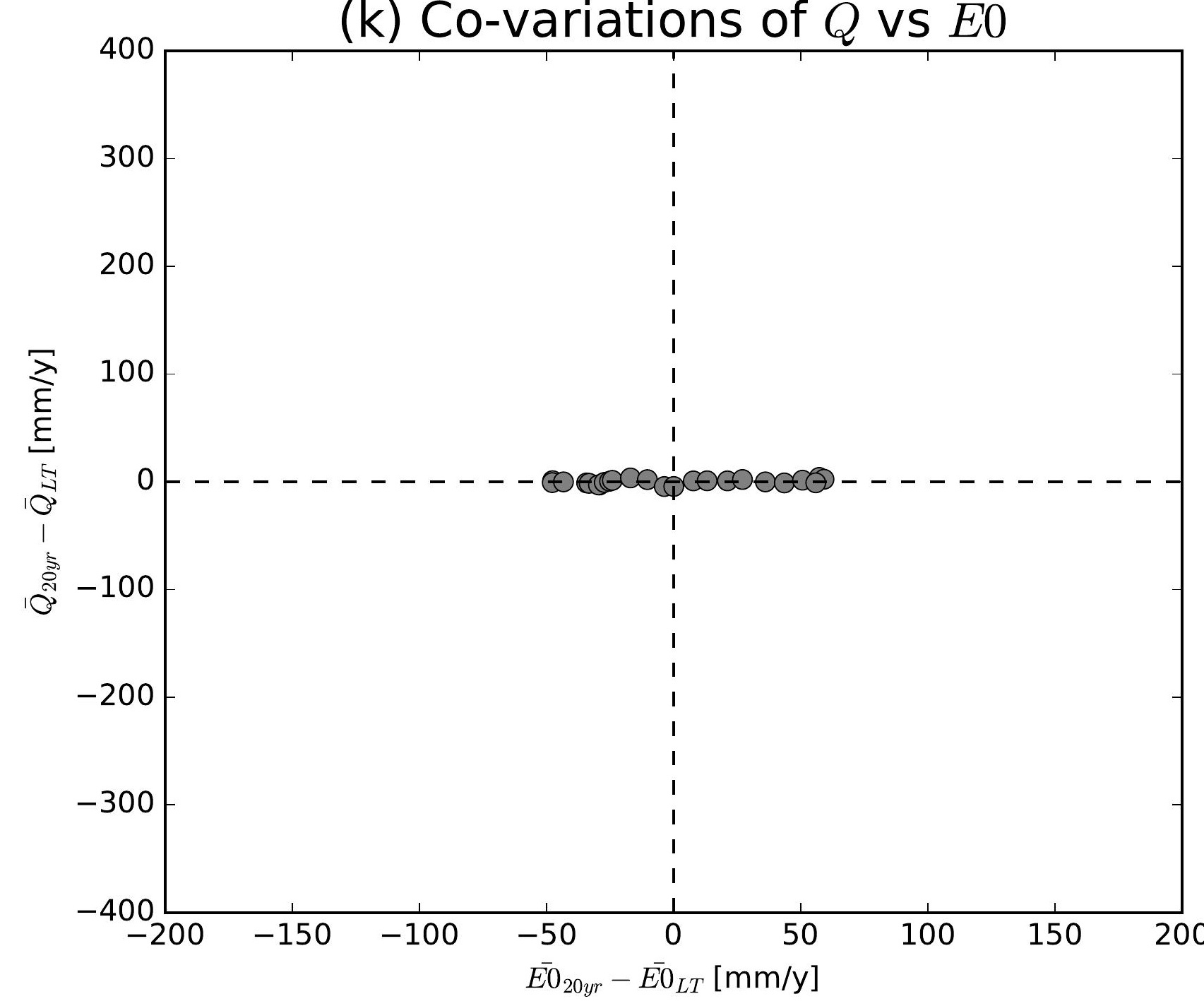

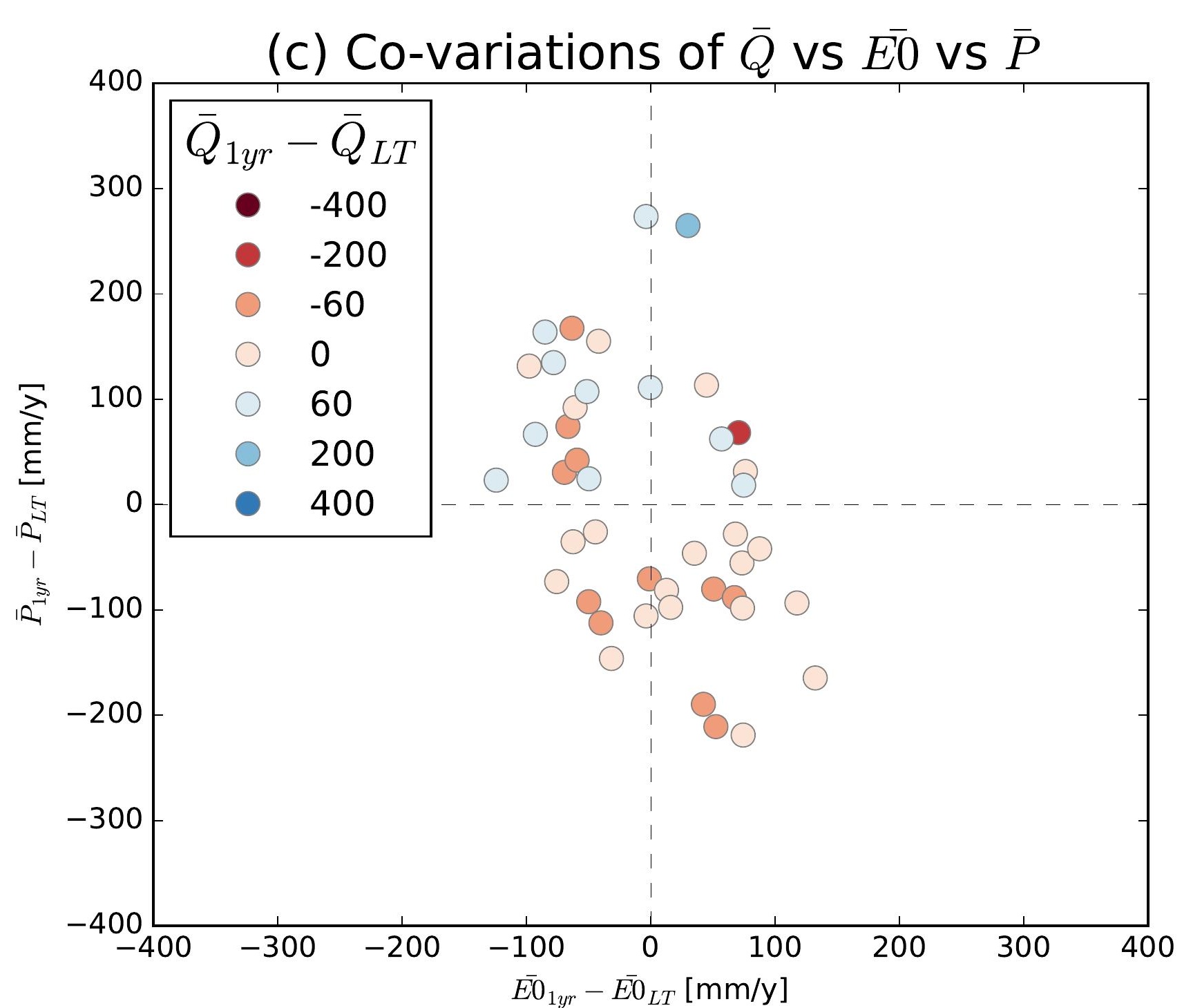
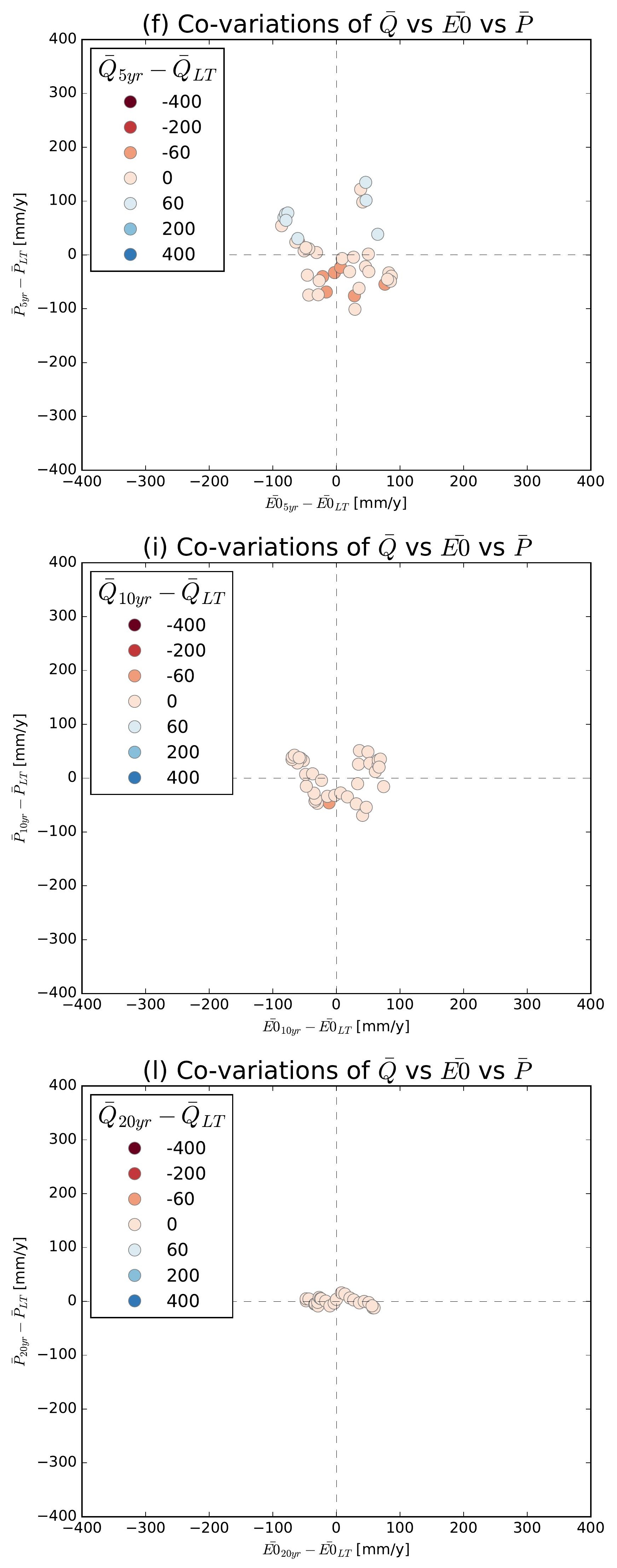

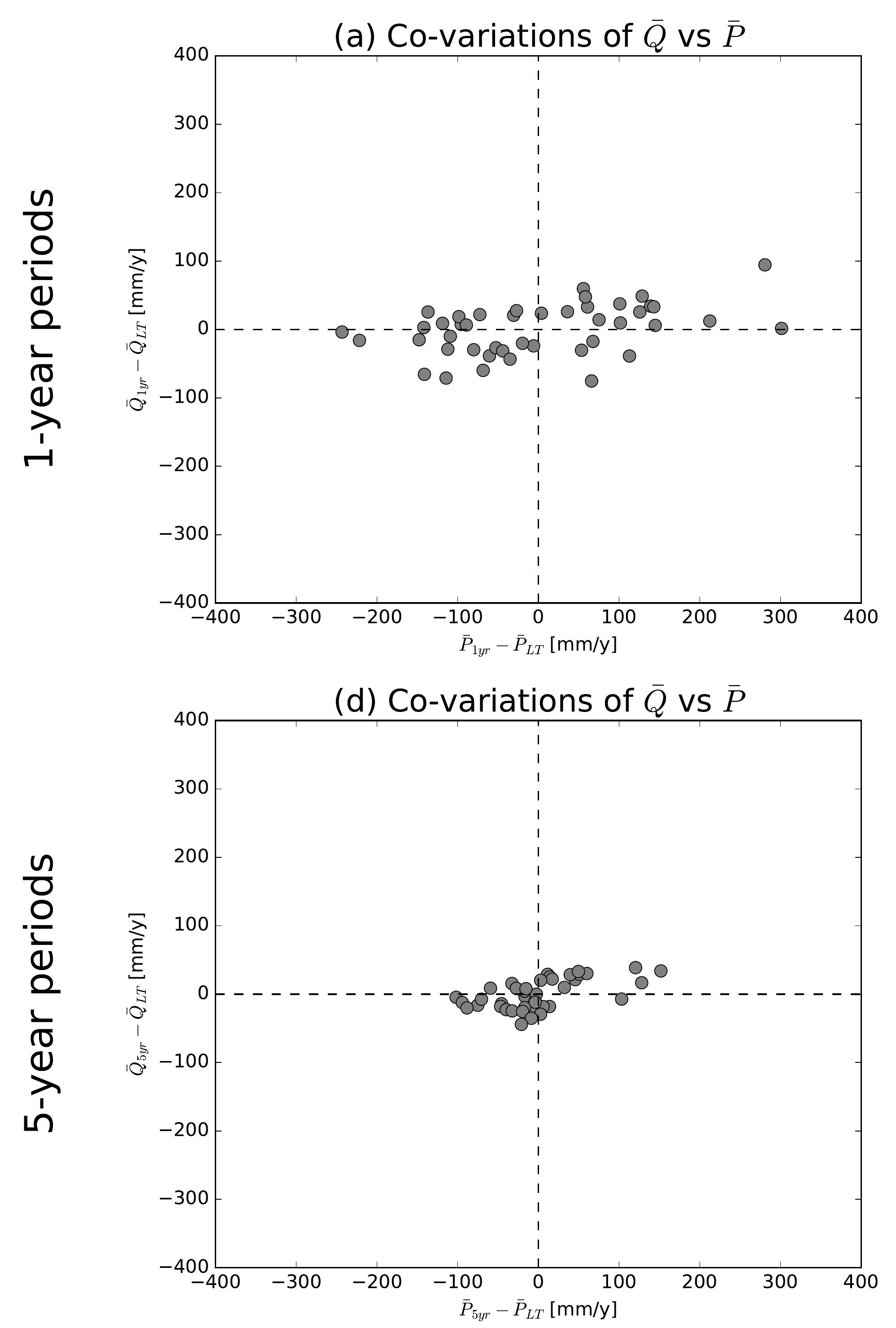

(g) Co-variations of $\bar{Q}$ vs $\bar{P}$
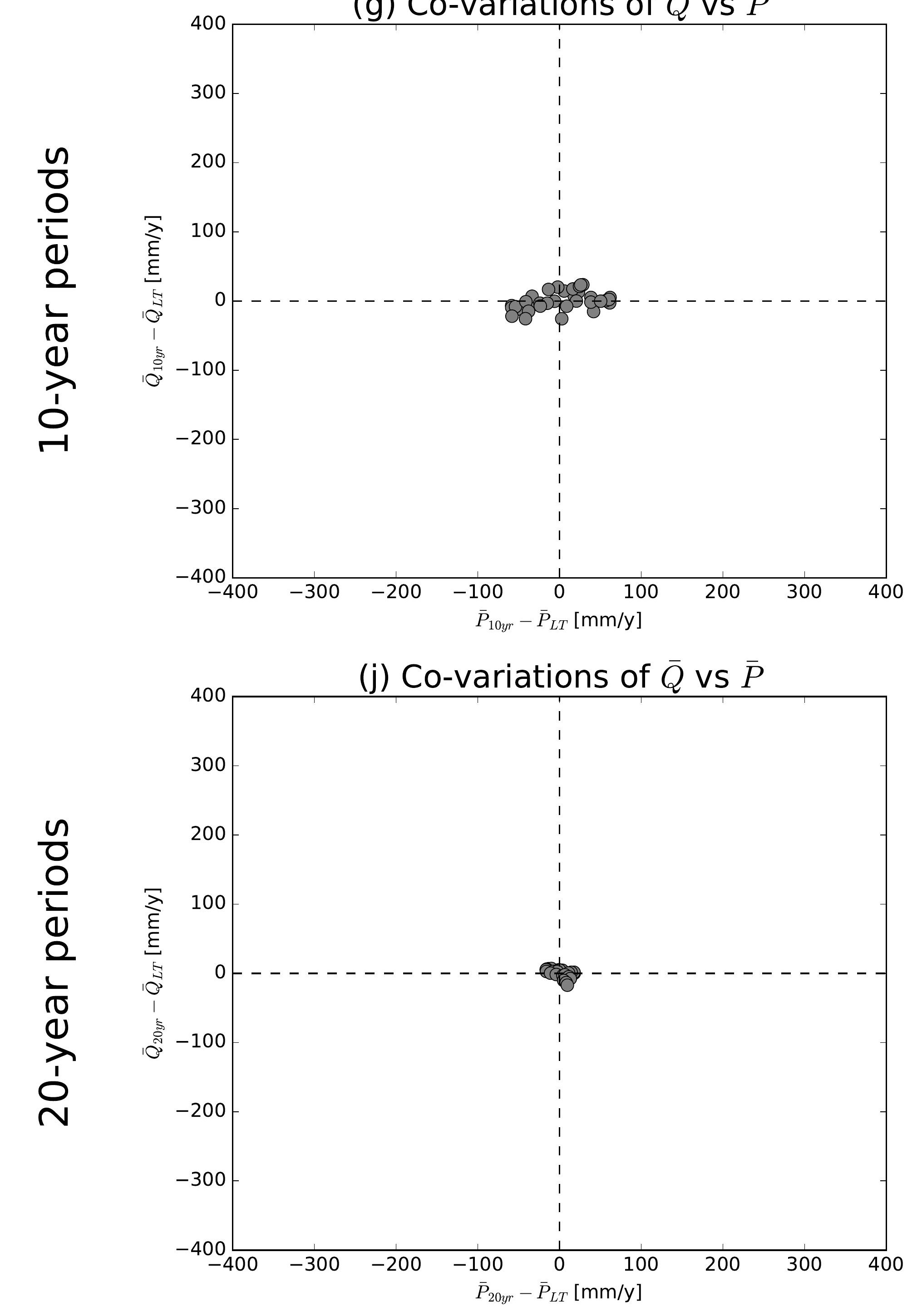

(b) Co-variations of $\bar{Q}$ vs $\overline{E 0}$

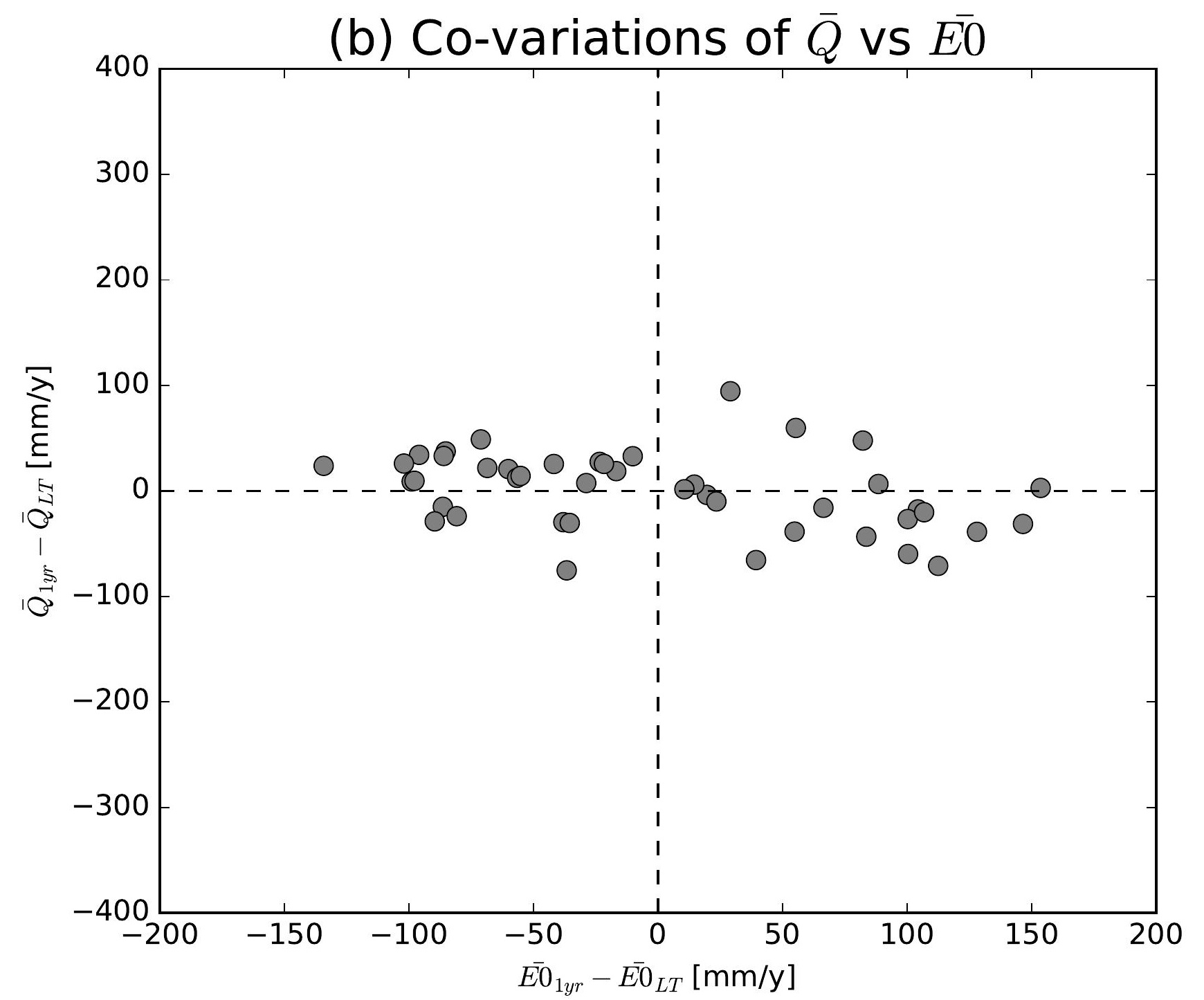

(e) Co-variations of $\bar{Q}$ vs $\overline{E 0}$

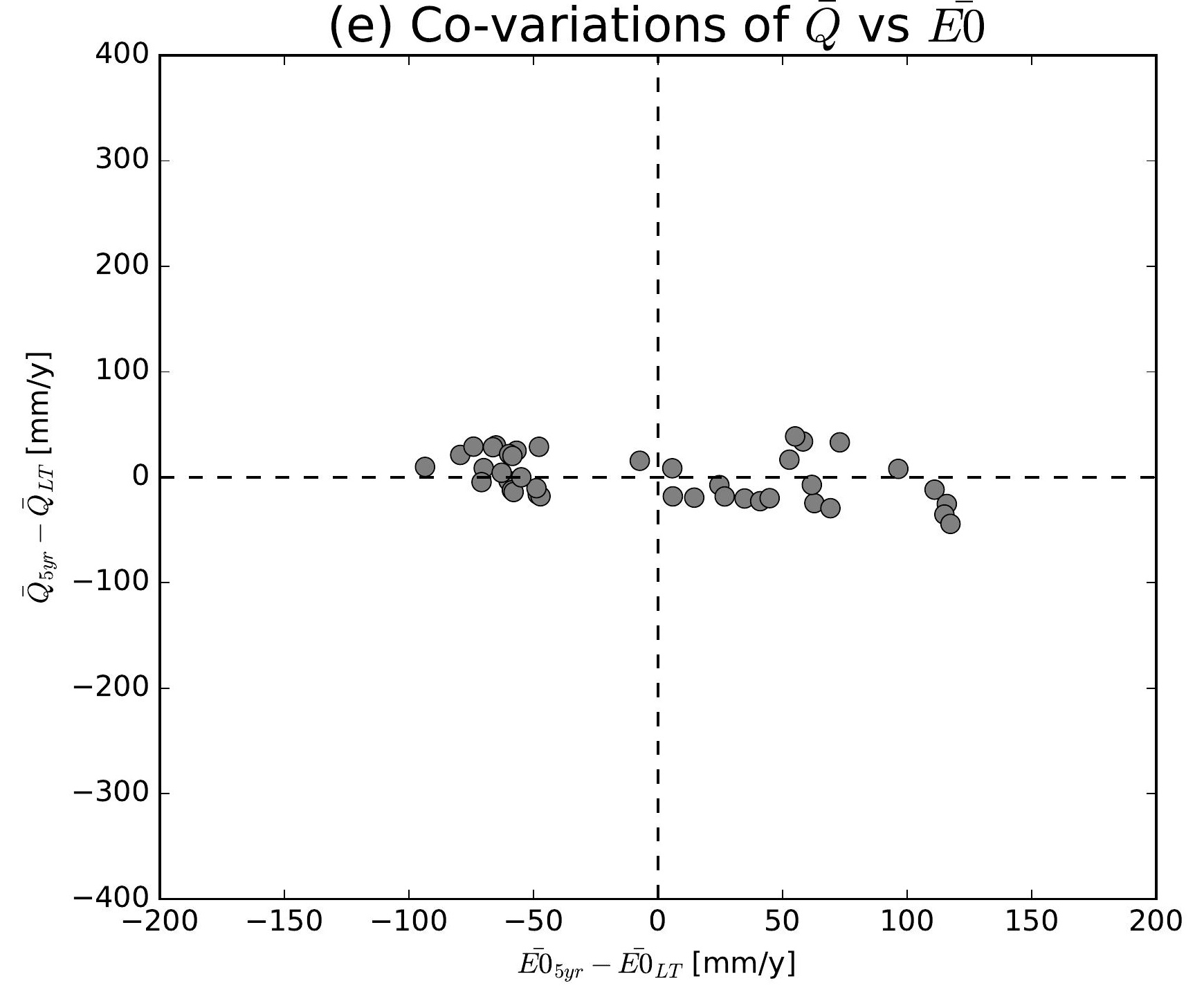

(h) Co-variations of $\bar{Q}$ vs $\overline{E 0}$

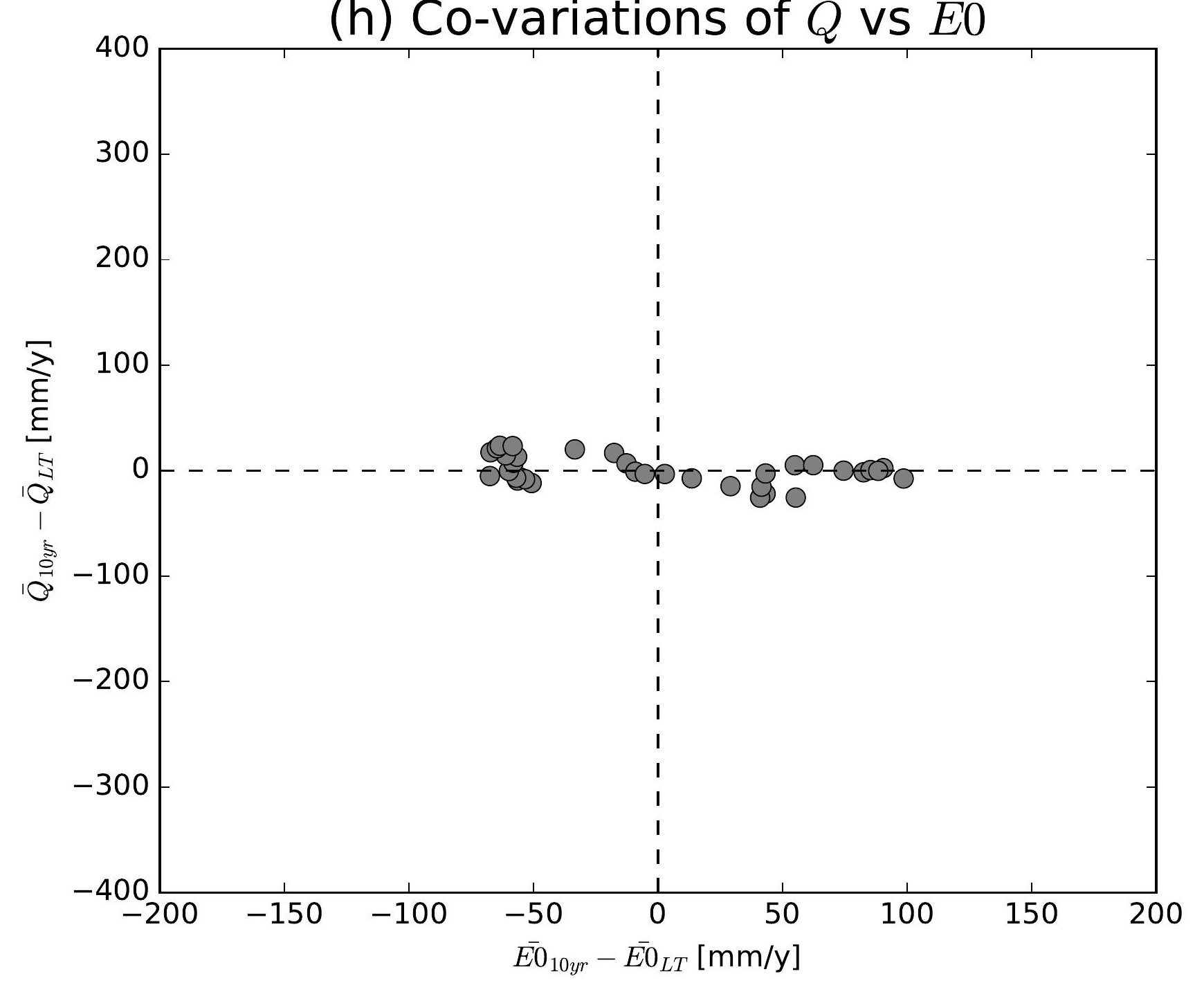

(k) Co-variations of $\bar{Q}$ vs $\overline{E 0}$

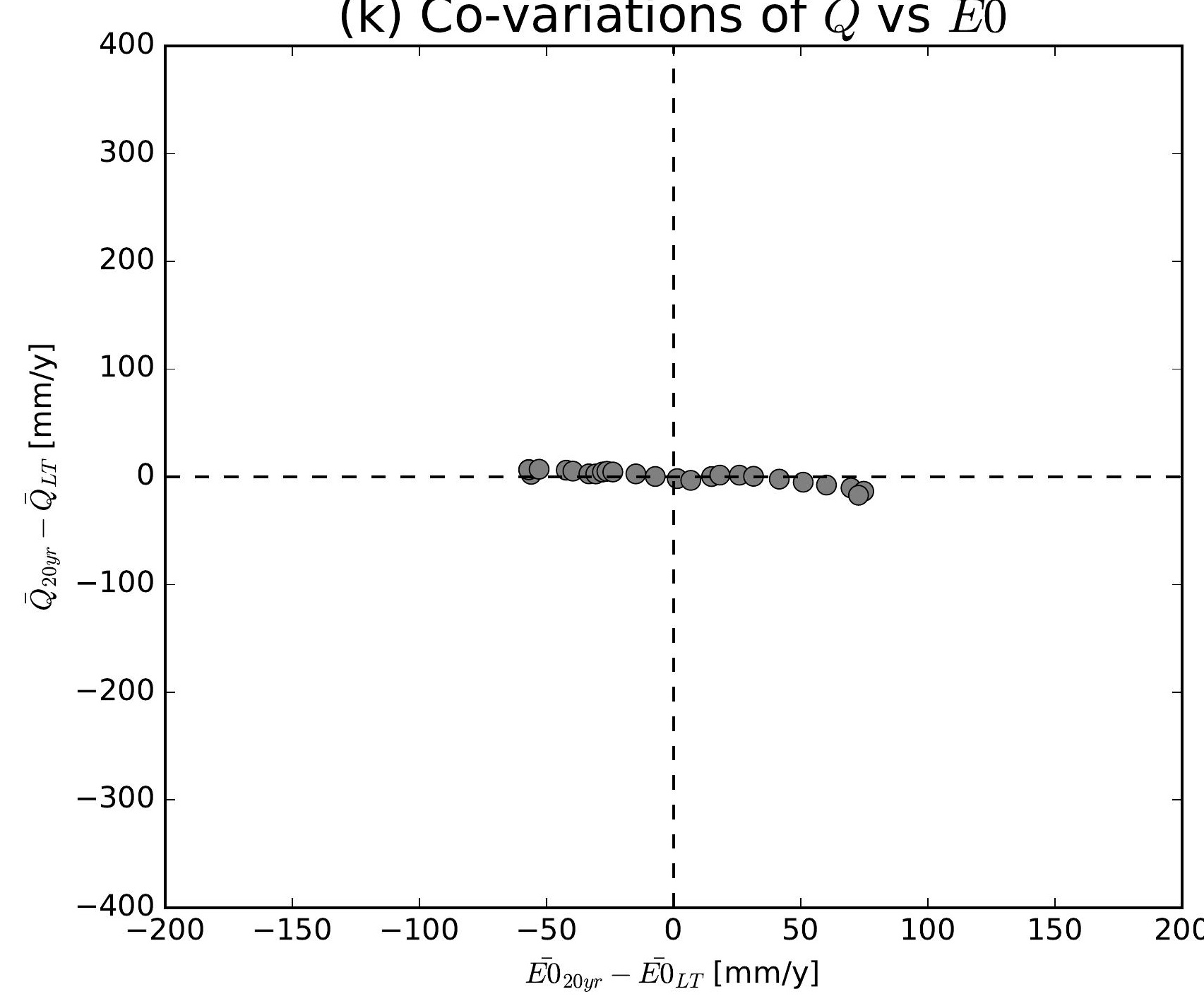

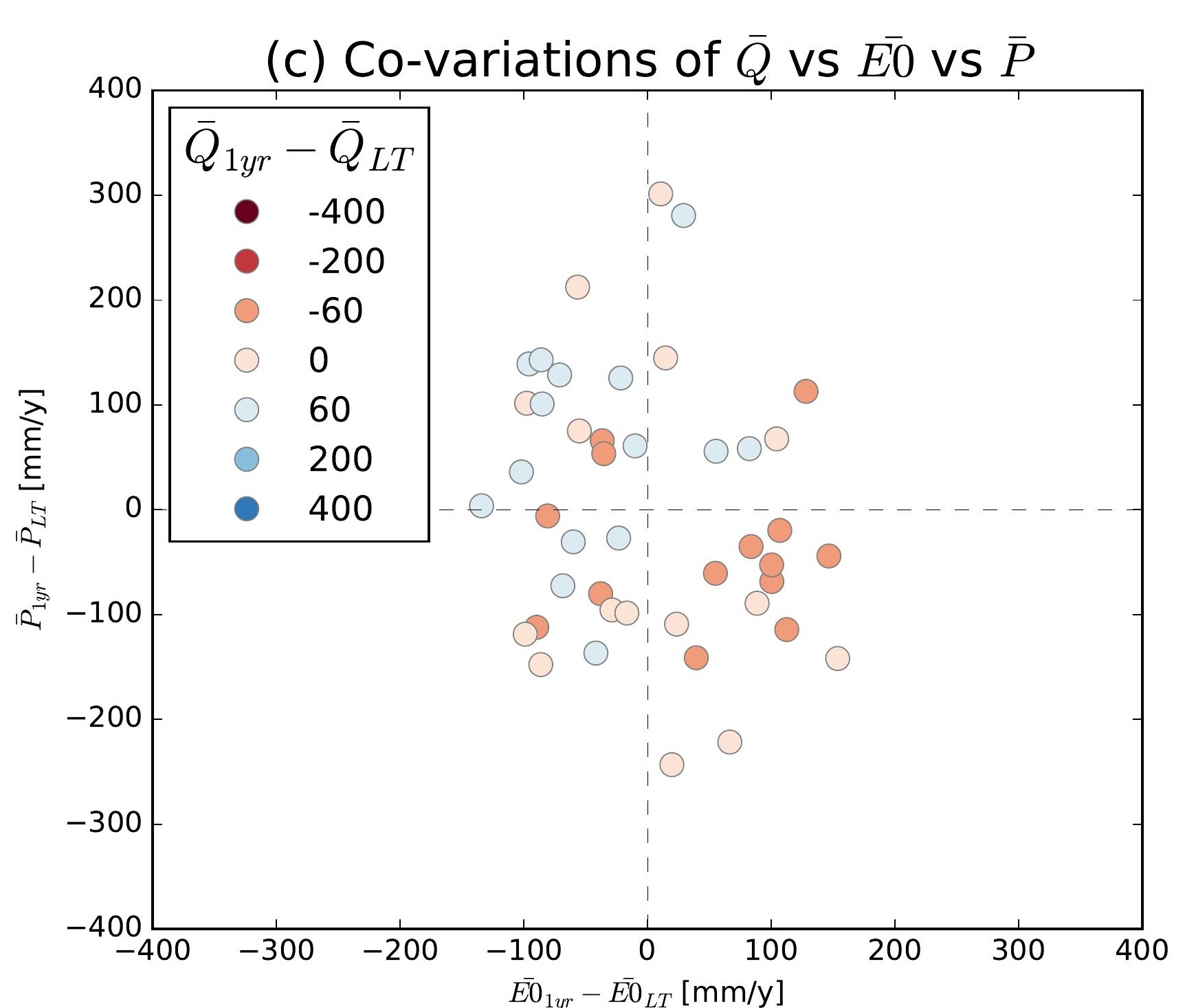
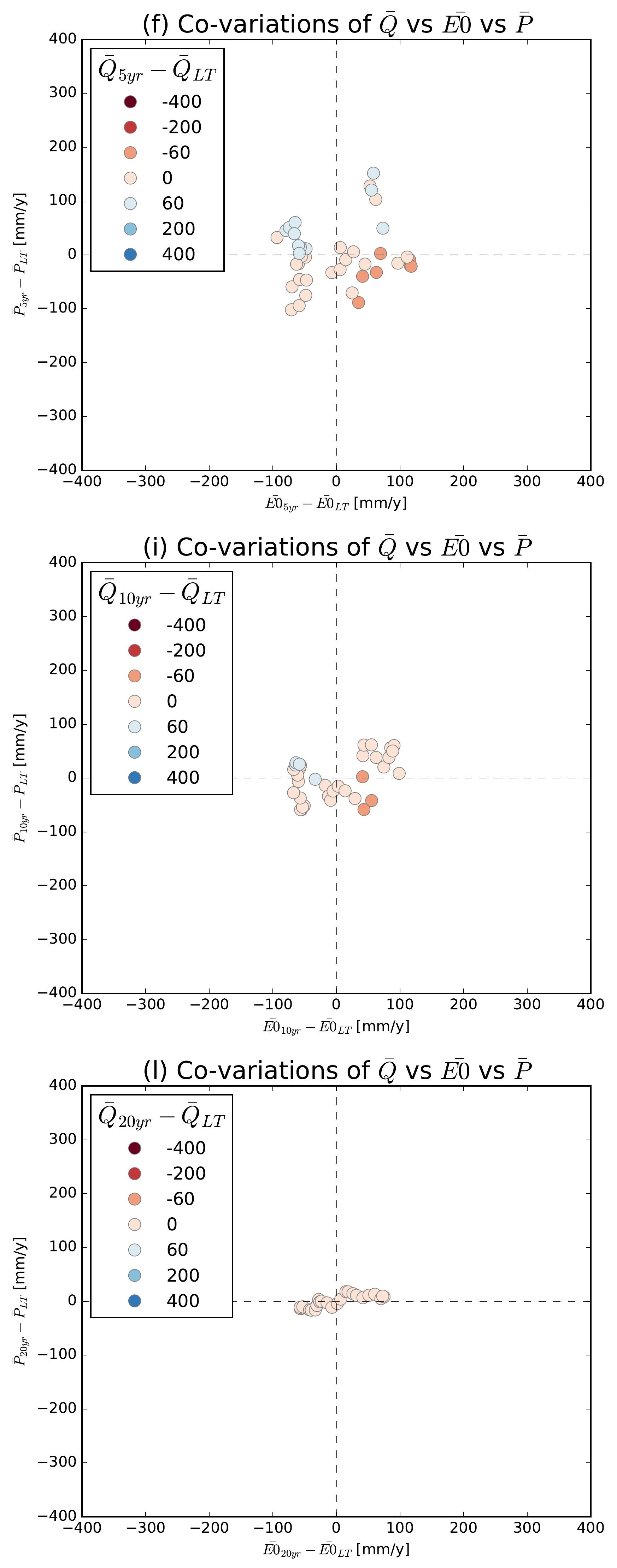

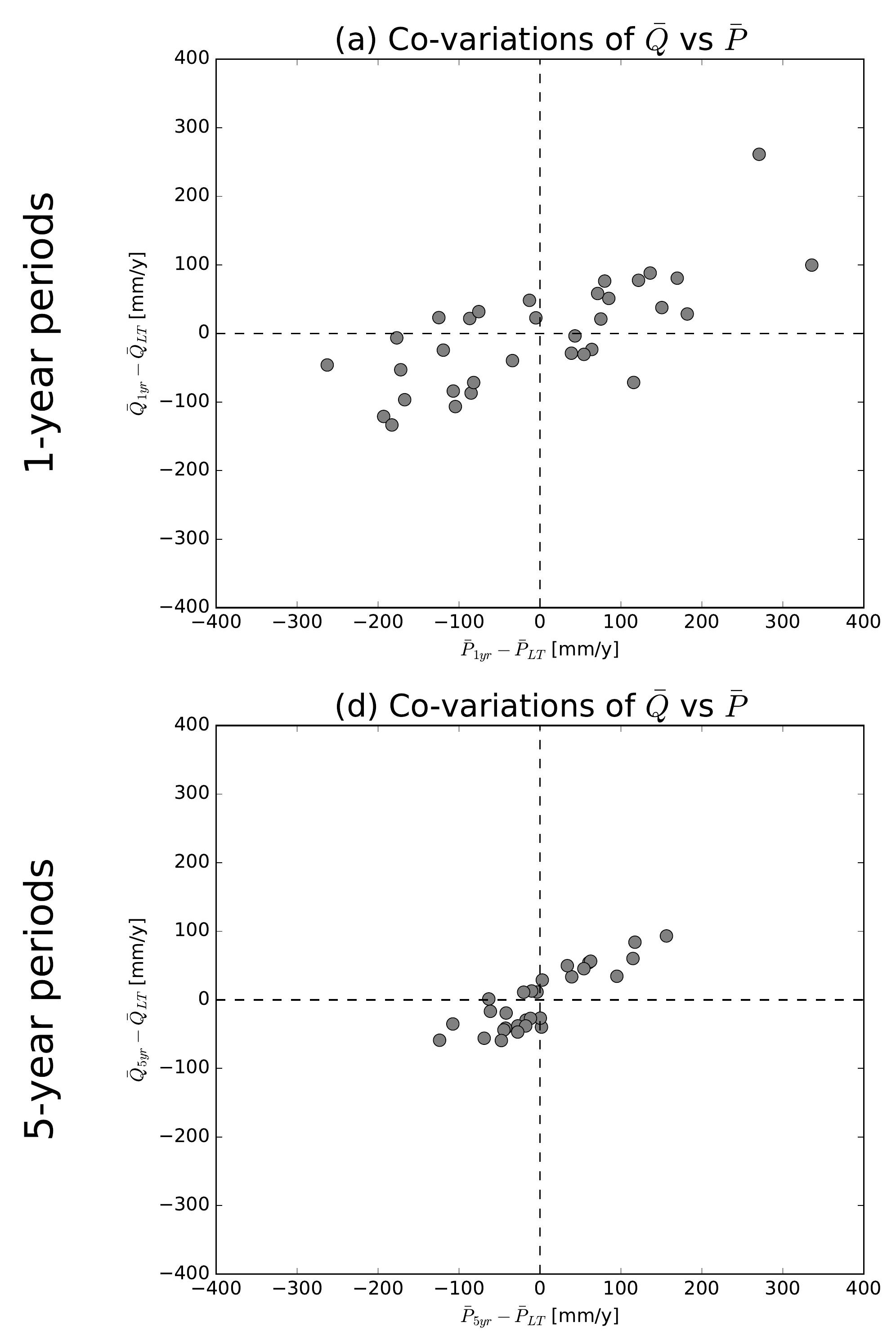

(g) Co-variations of $\bar{Q}$ vs $\bar{P}$

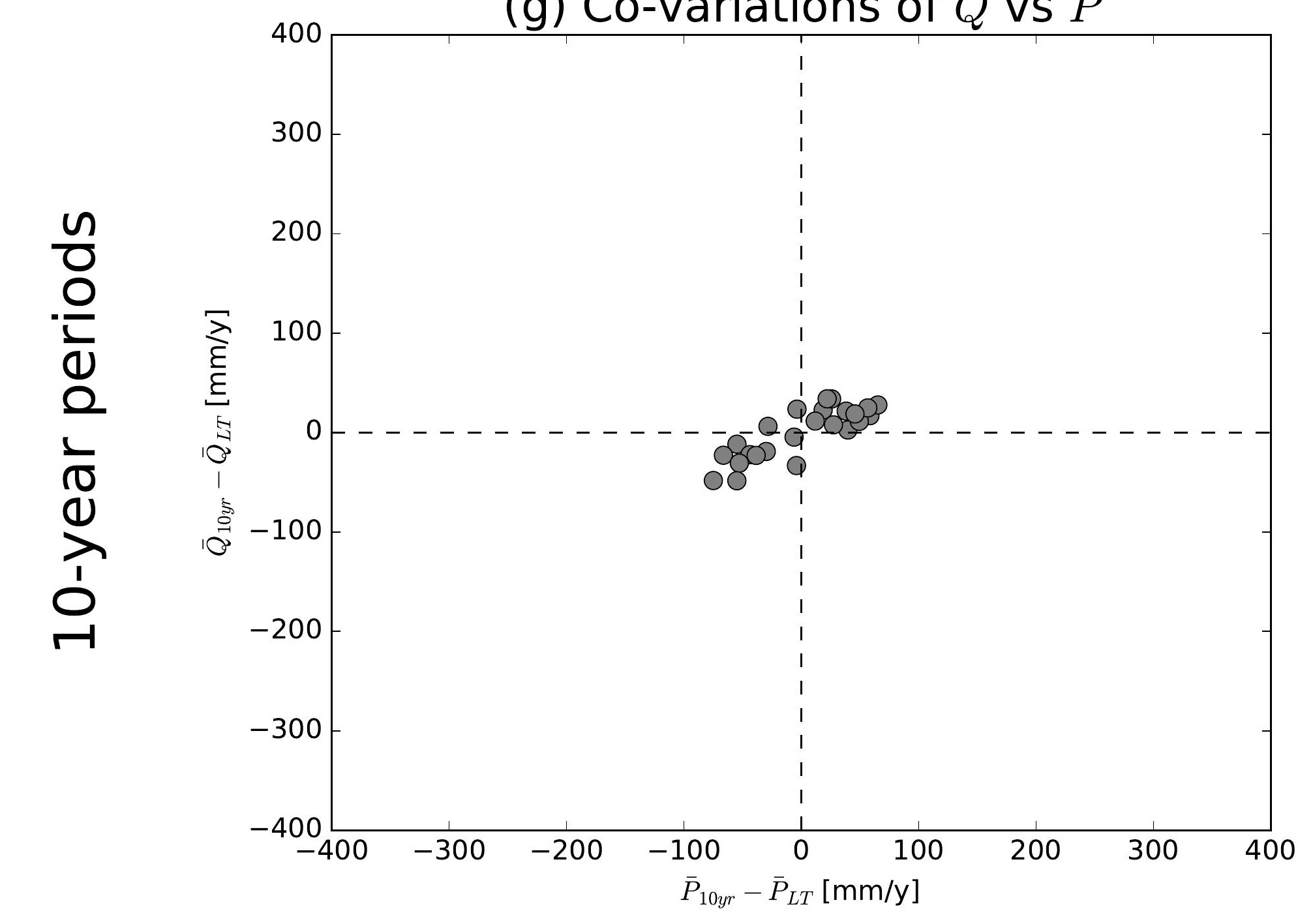

(j) Co-variations of $\bar{Q}$ vs $\bar{P}$

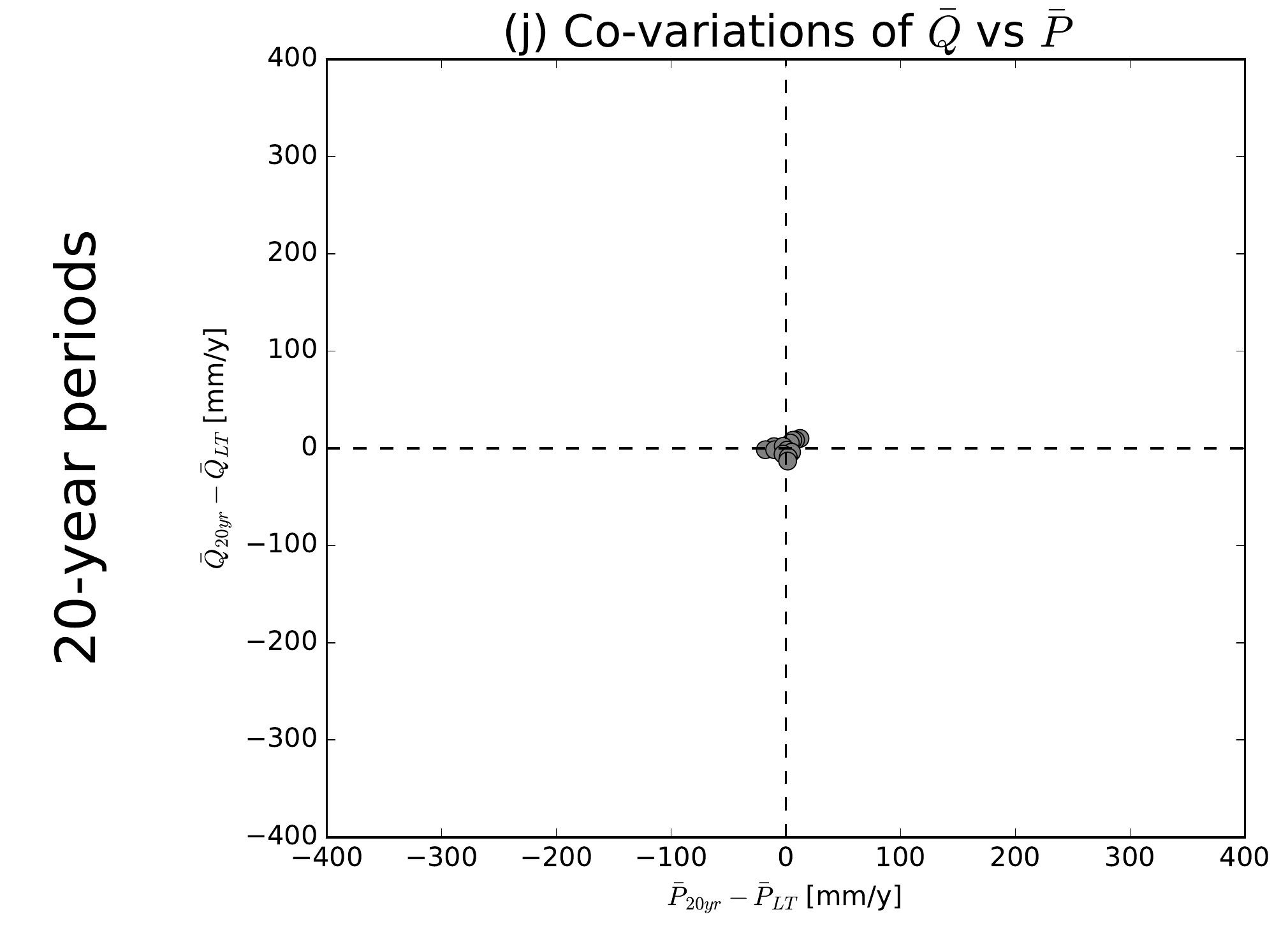

(b) Co-variations of $\bar{Q}$ vs $\overline{E 0}$

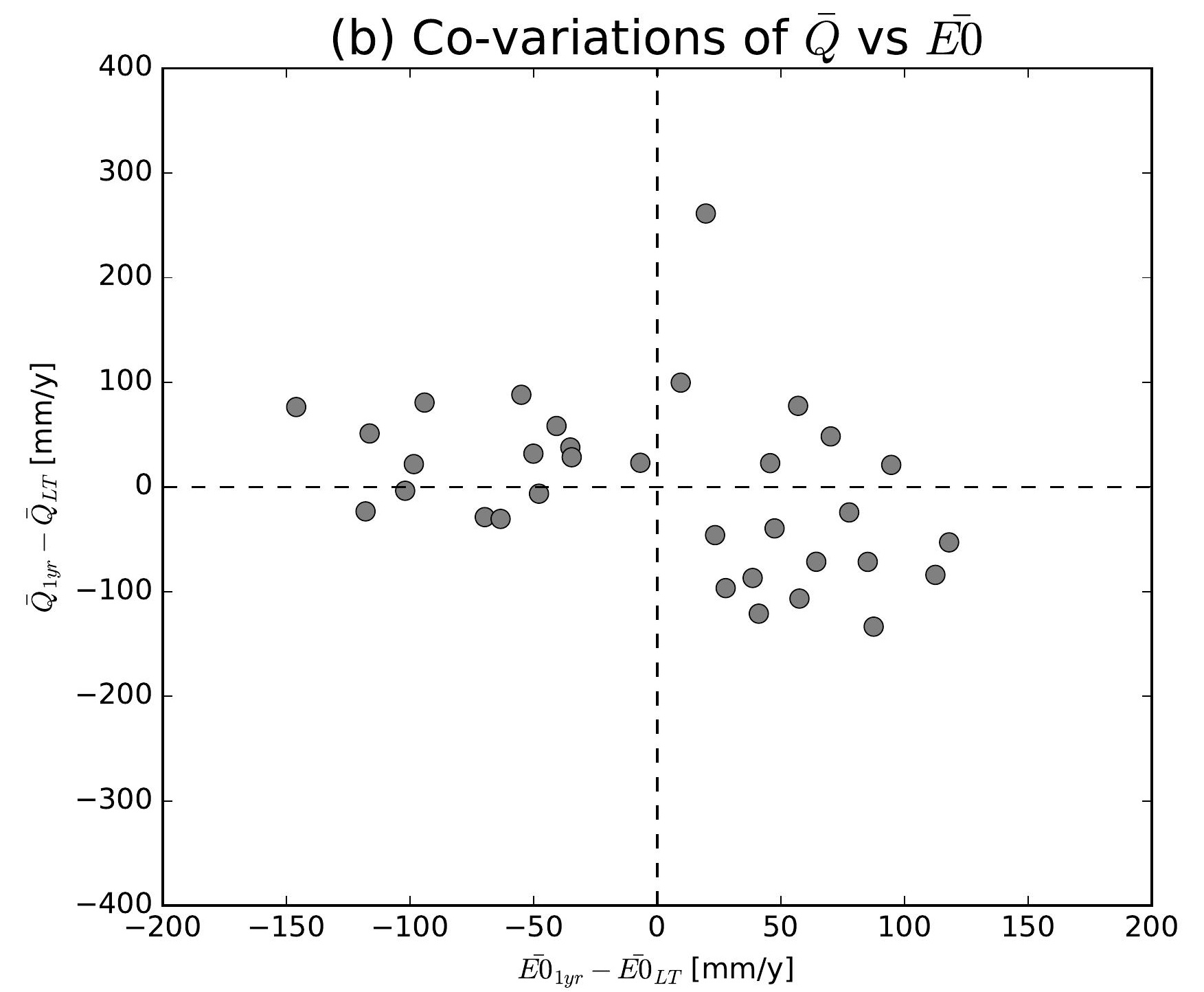

(e) Co-variations of $\bar{Q}$ vs $\overline{E 0}$

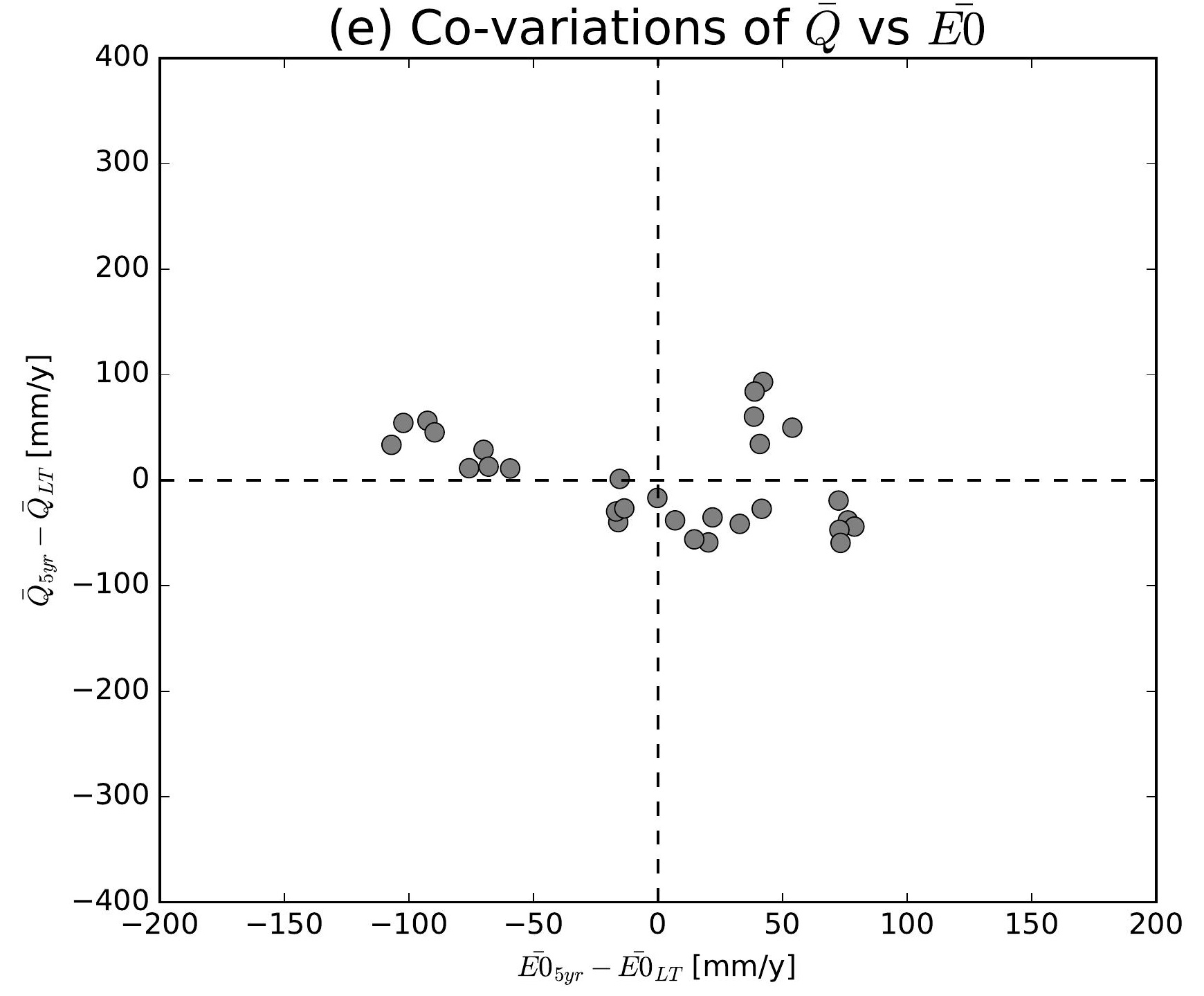

(h) Co-variations of $\bar{Q}$ vs $\overline{E 0}$

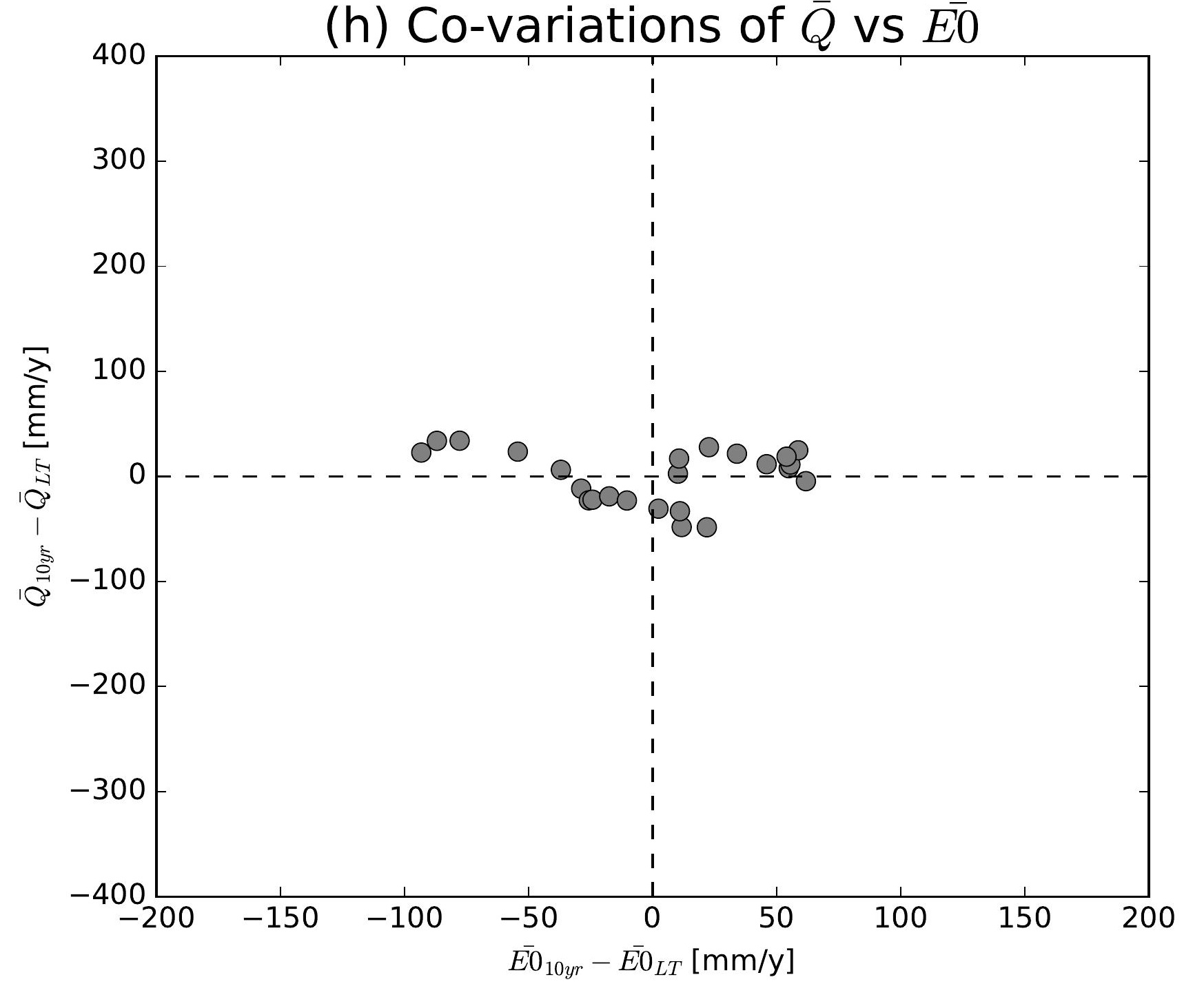

(k) Co-variations of $\bar{Q}$ vs $\overline{E 0}$

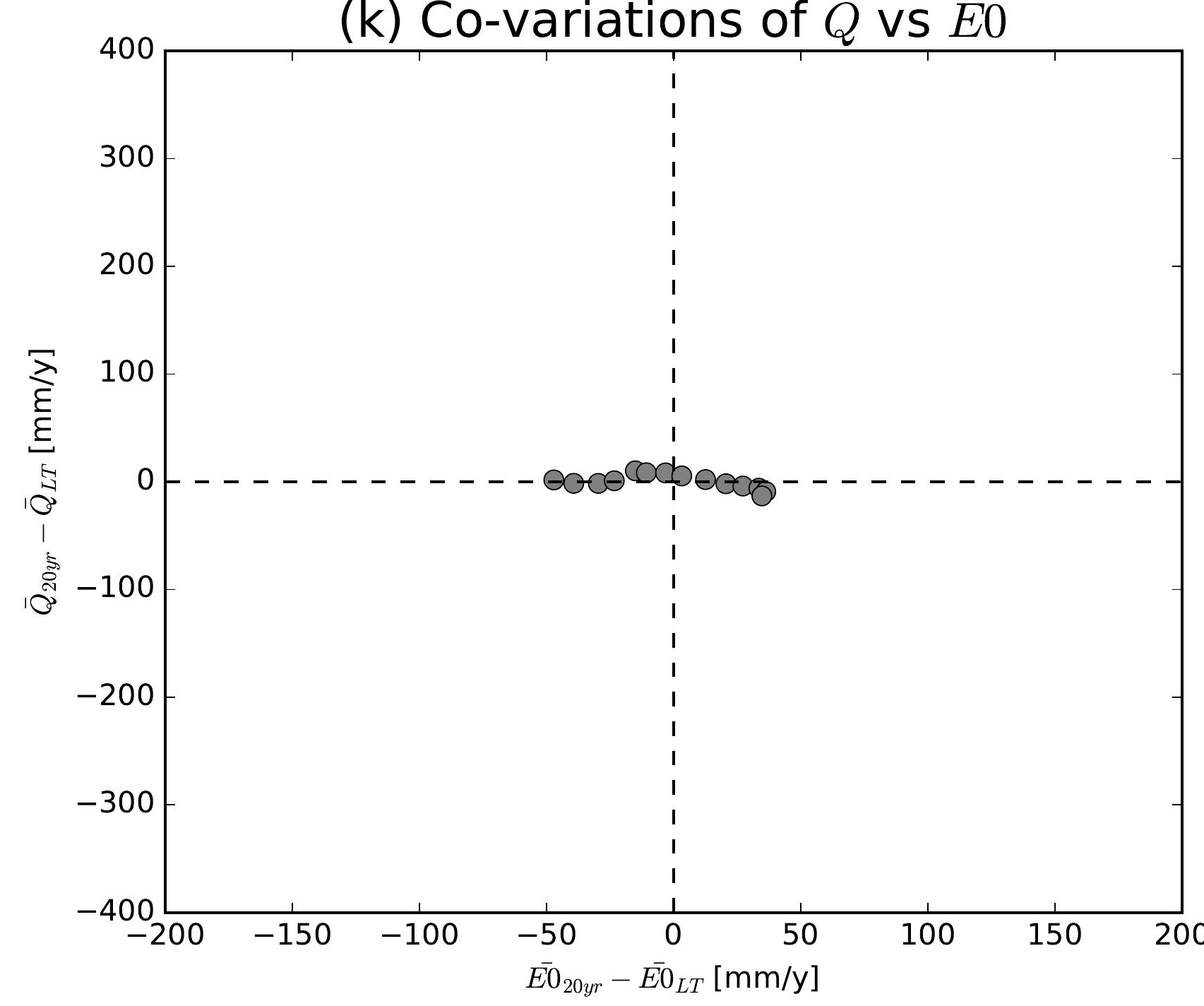

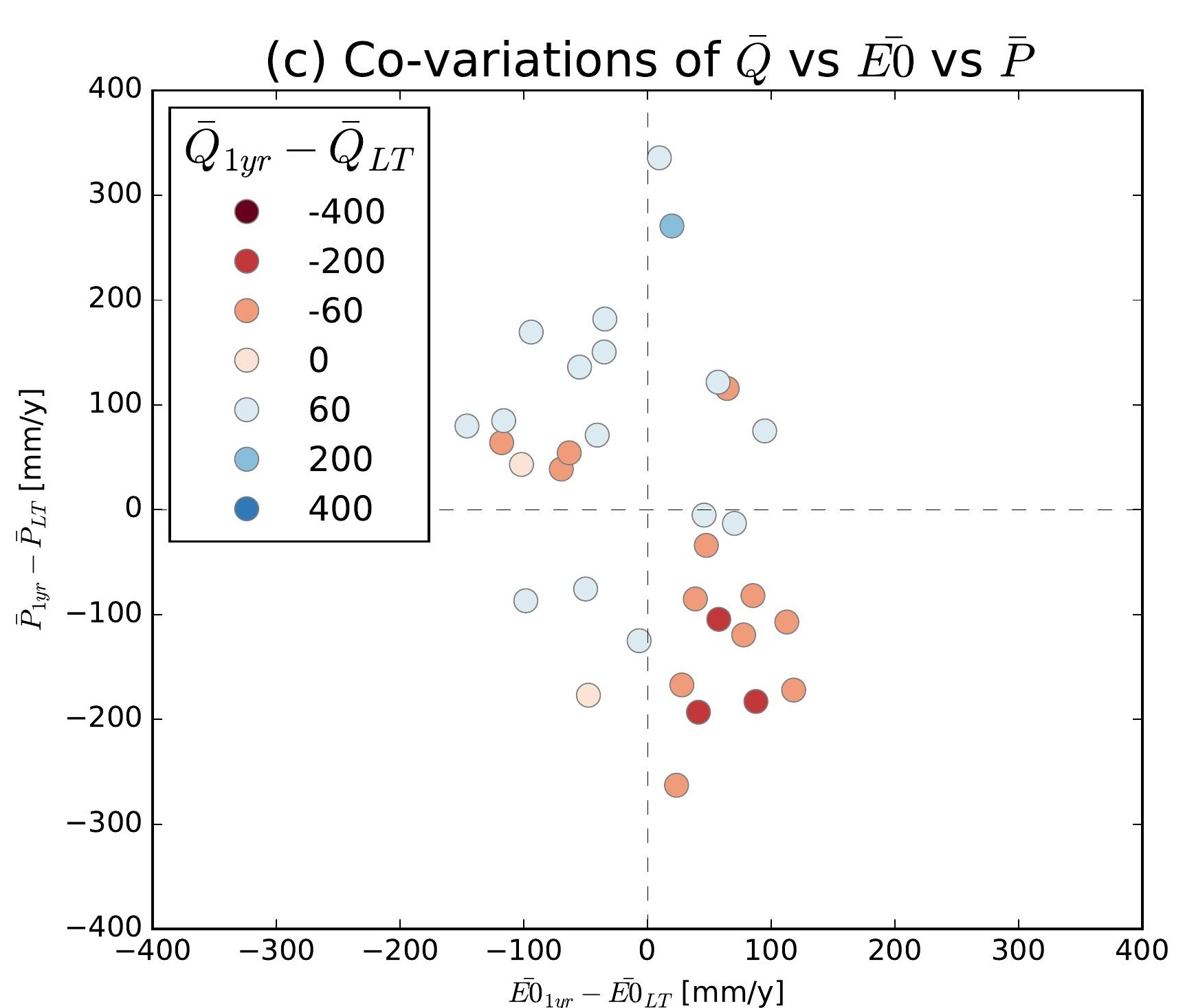
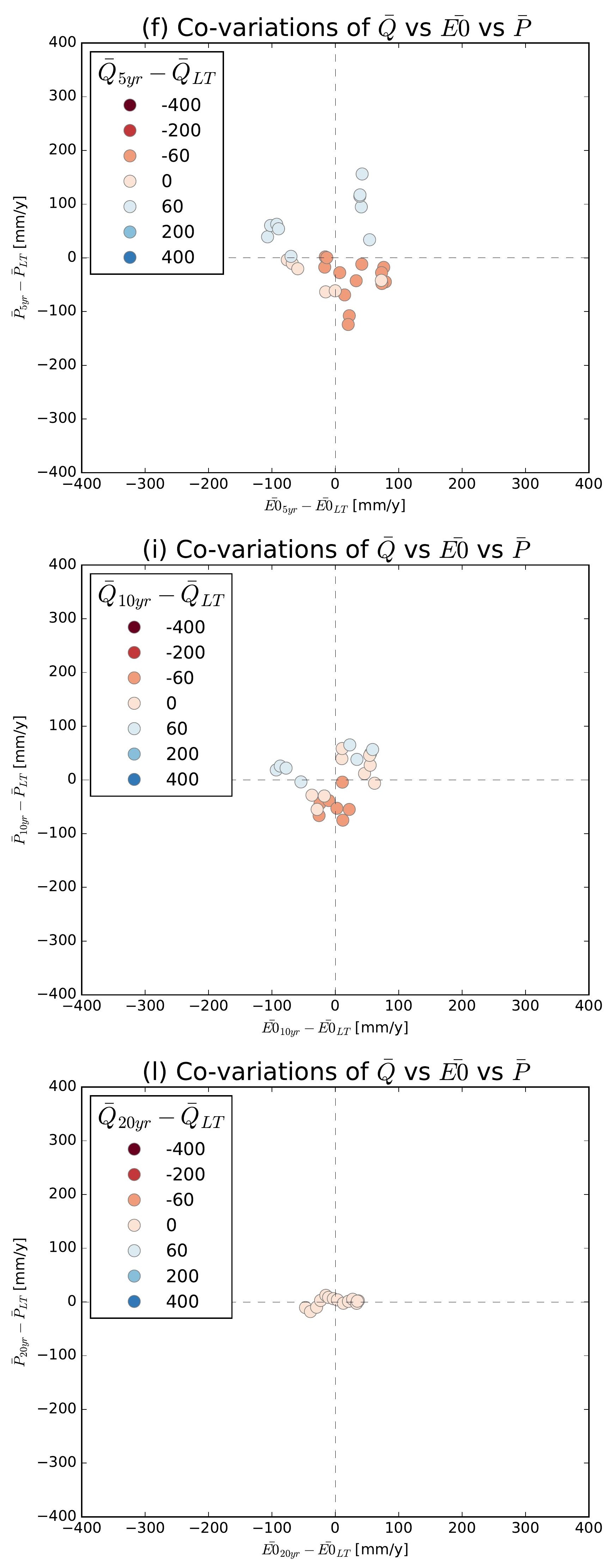

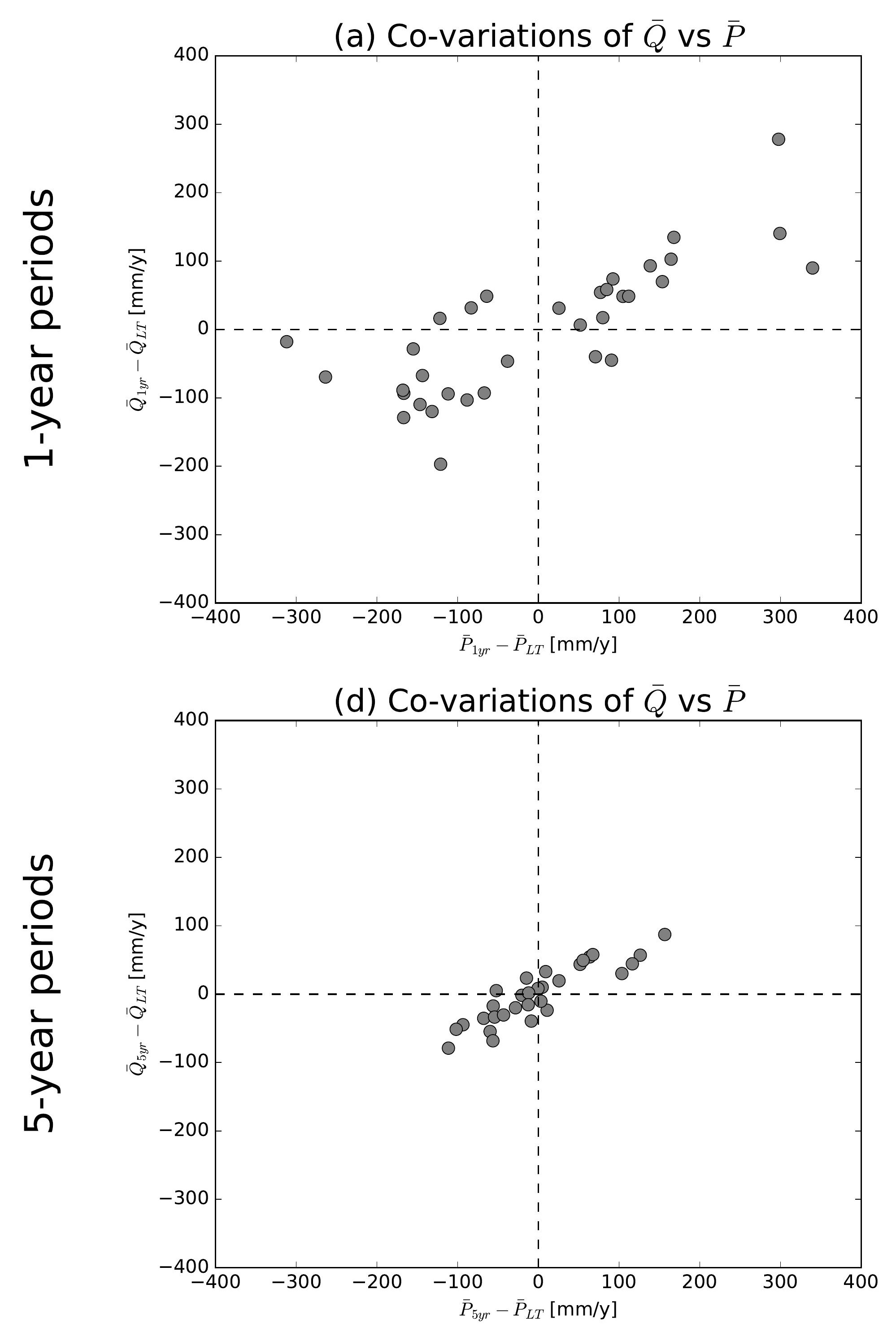

(g) Co-variations of $\bar{Q}$ vs $\bar{P}$

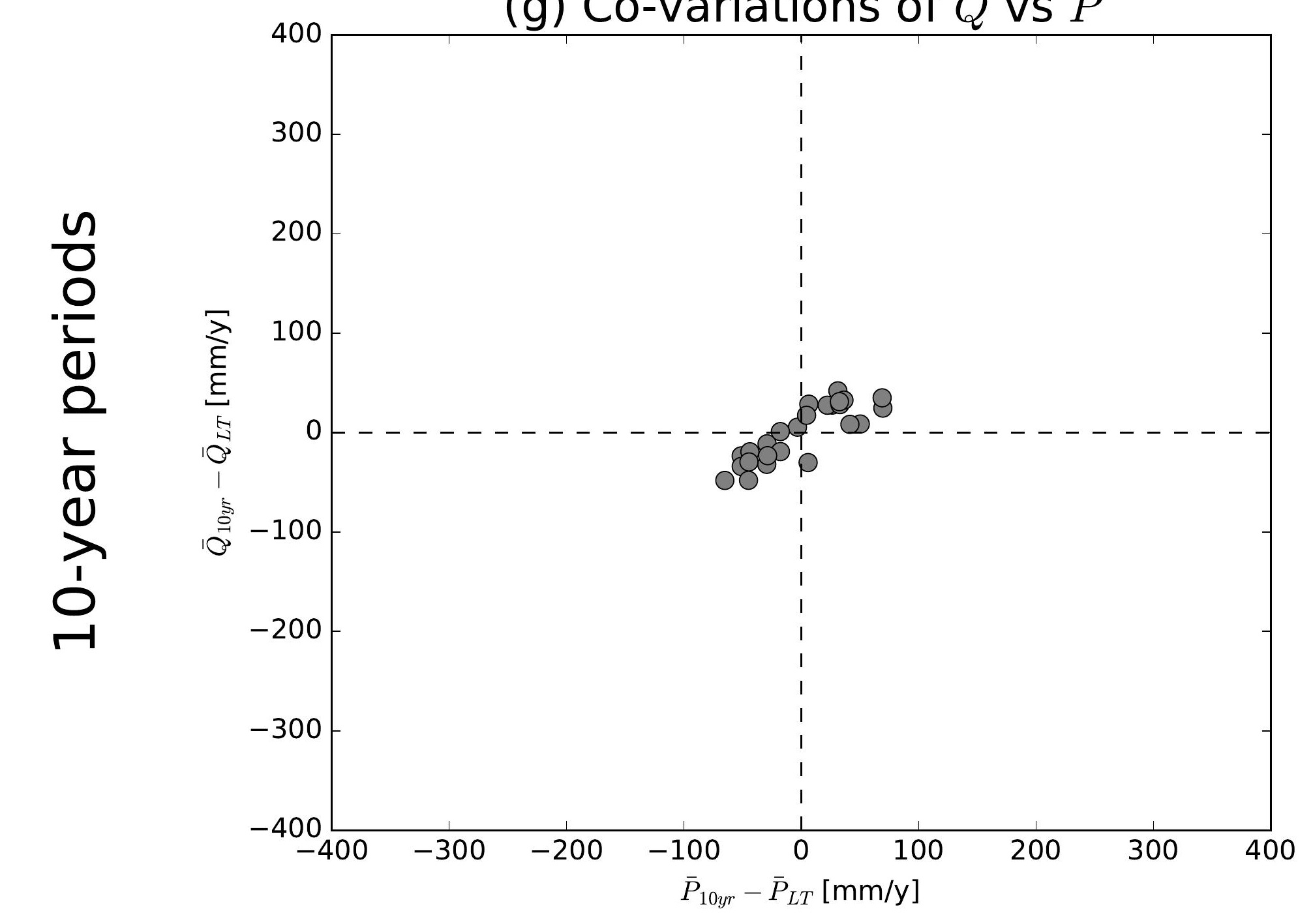

(j) Co-variations of $\bar{Q}$ vs $\bar{P}$

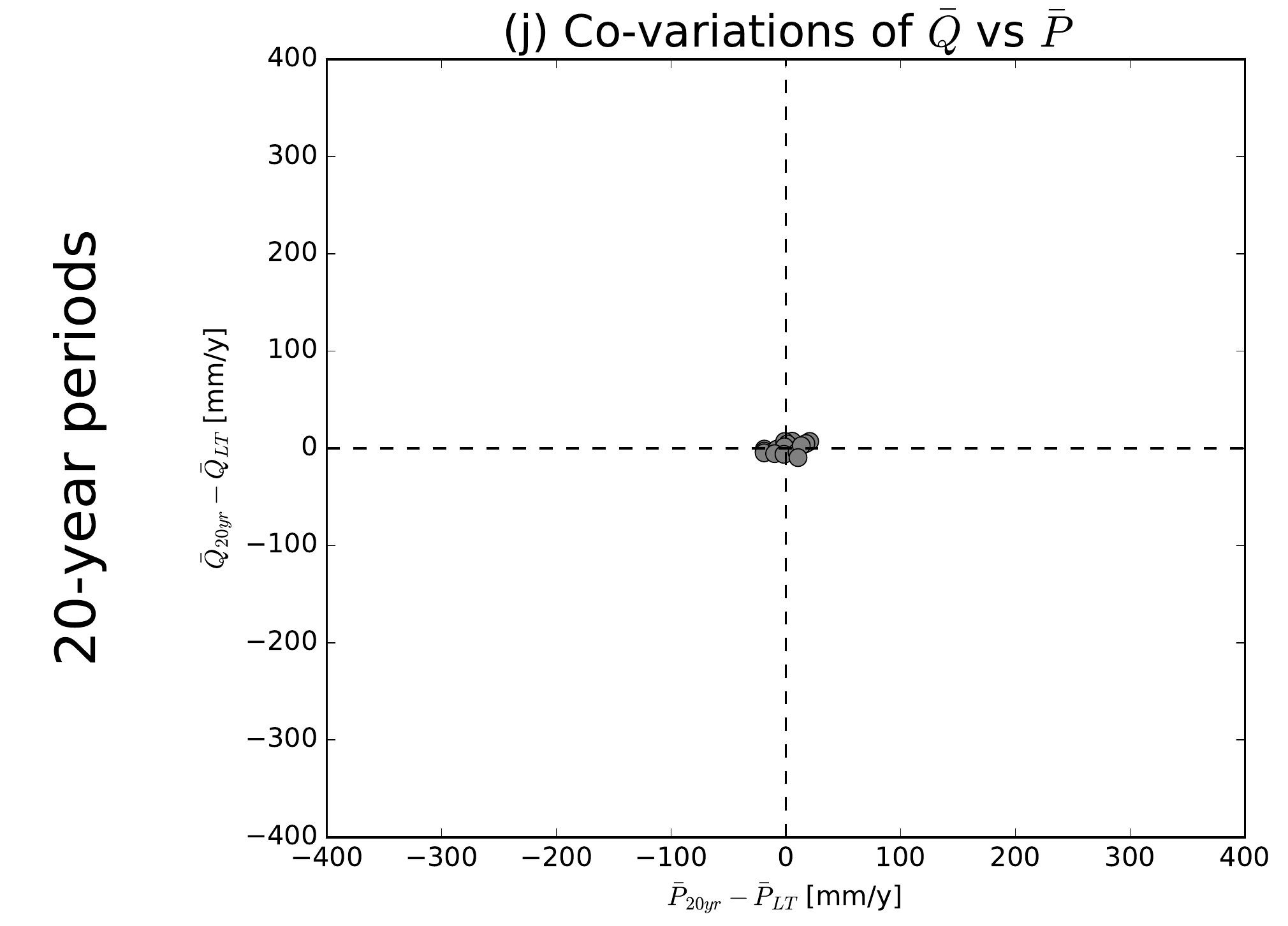

(b) Co-variations of $\bar{Q}$ vs $\overline{E 0}$

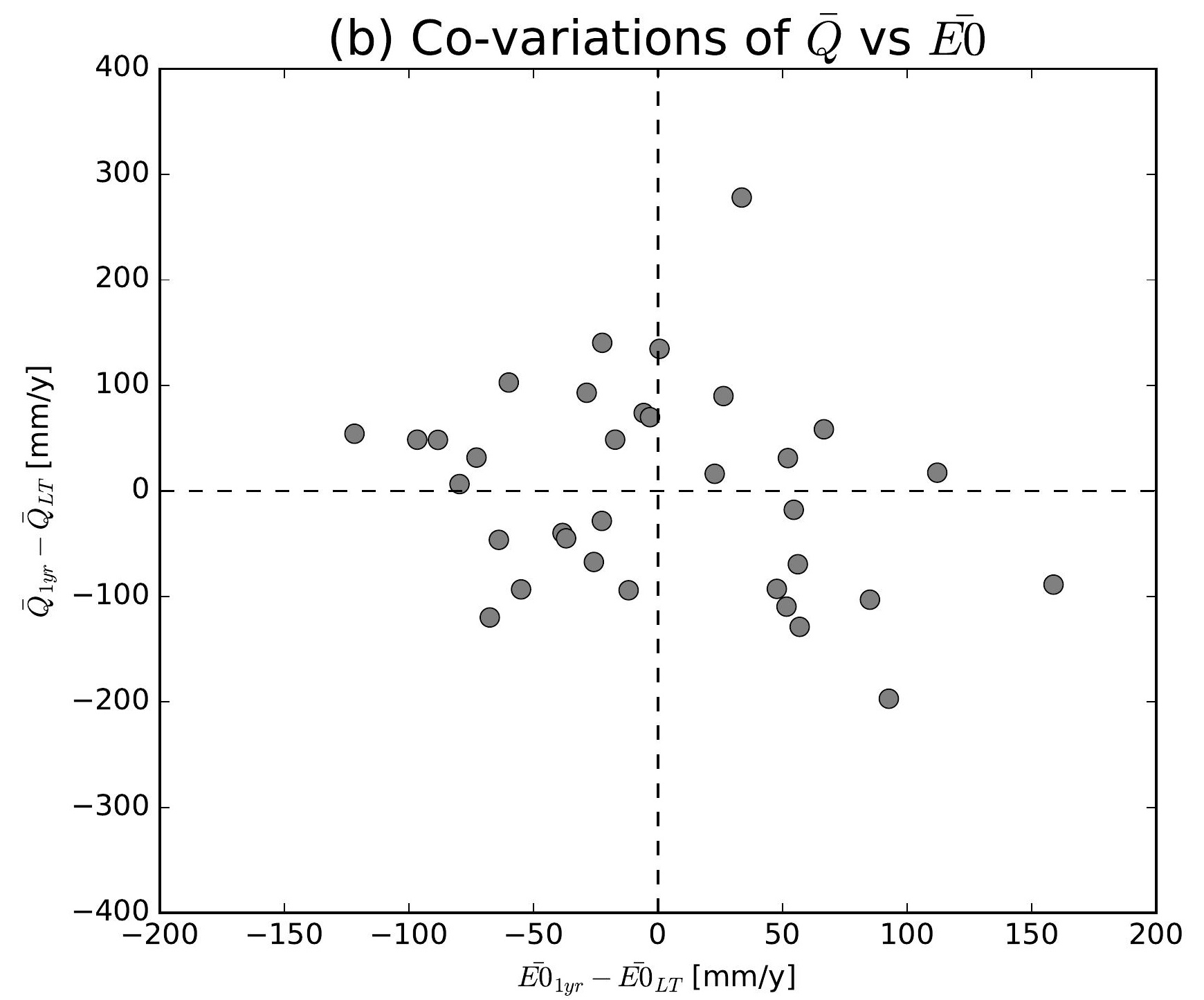

(e) Co-variations of $\bar{Q}$ vs $\overline{E 0}$

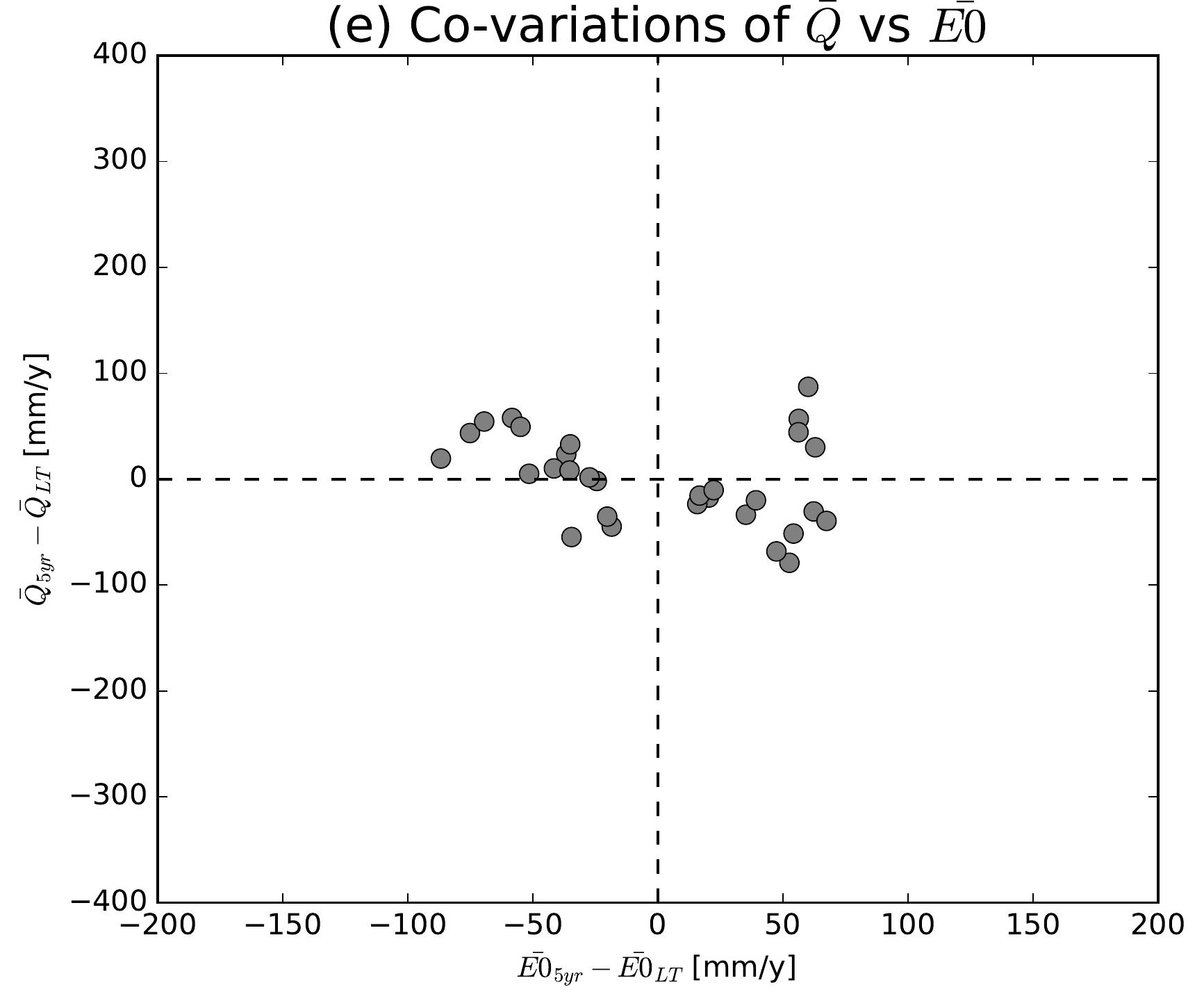

(h) Co-variations of $\bar{Q}$ vs $\overline{E 0}$

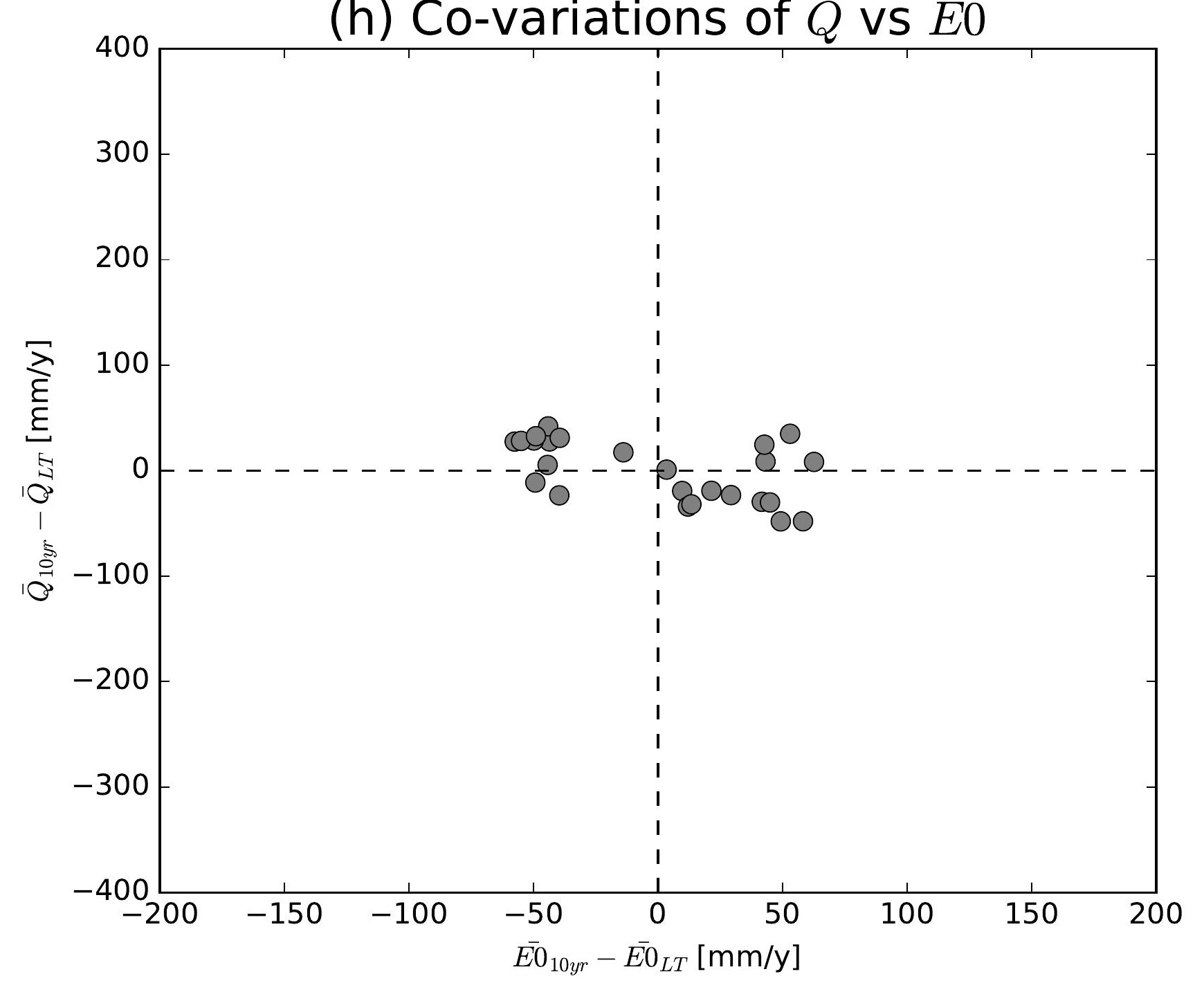

(k) Co-variations of $\bar{Q}$ vs $\overline{E 0}$

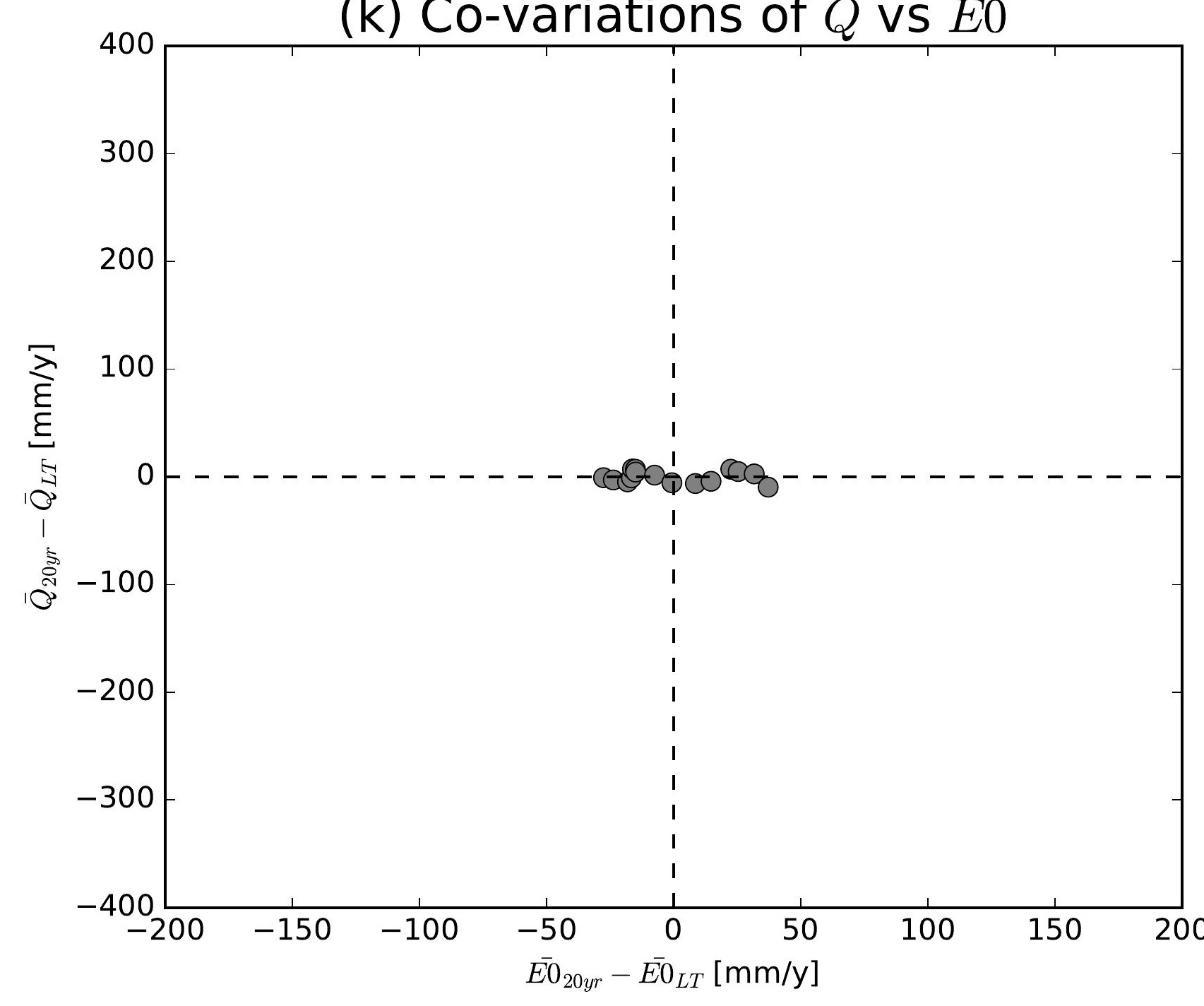

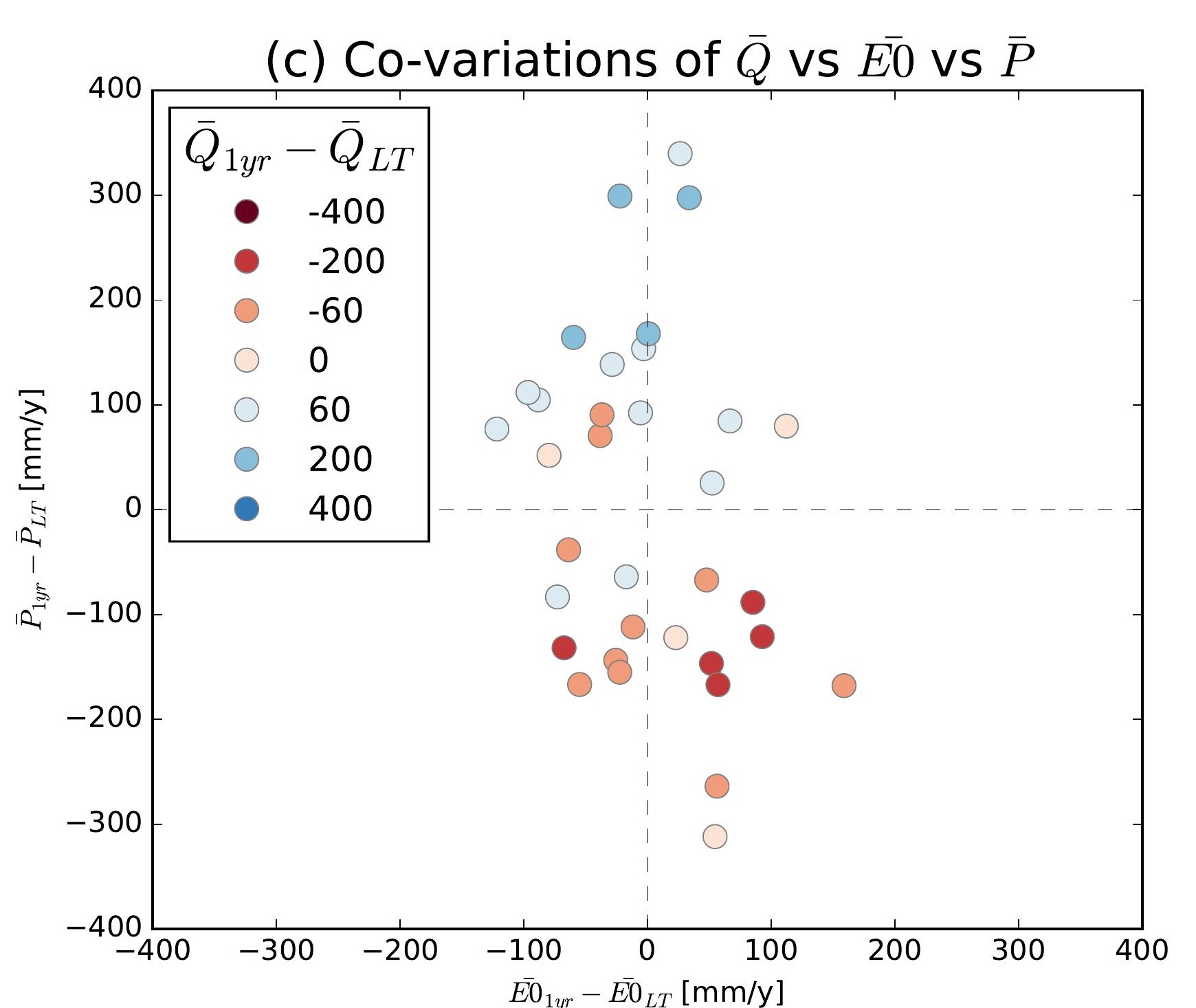
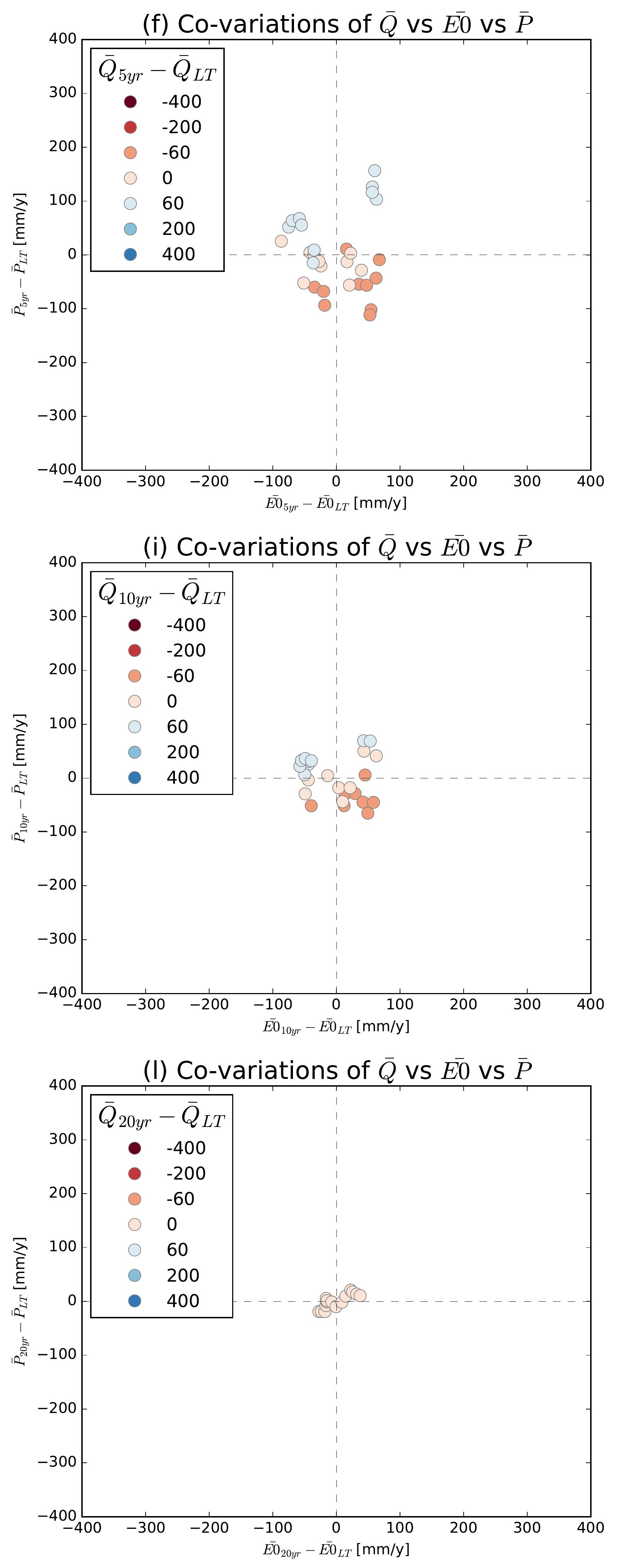

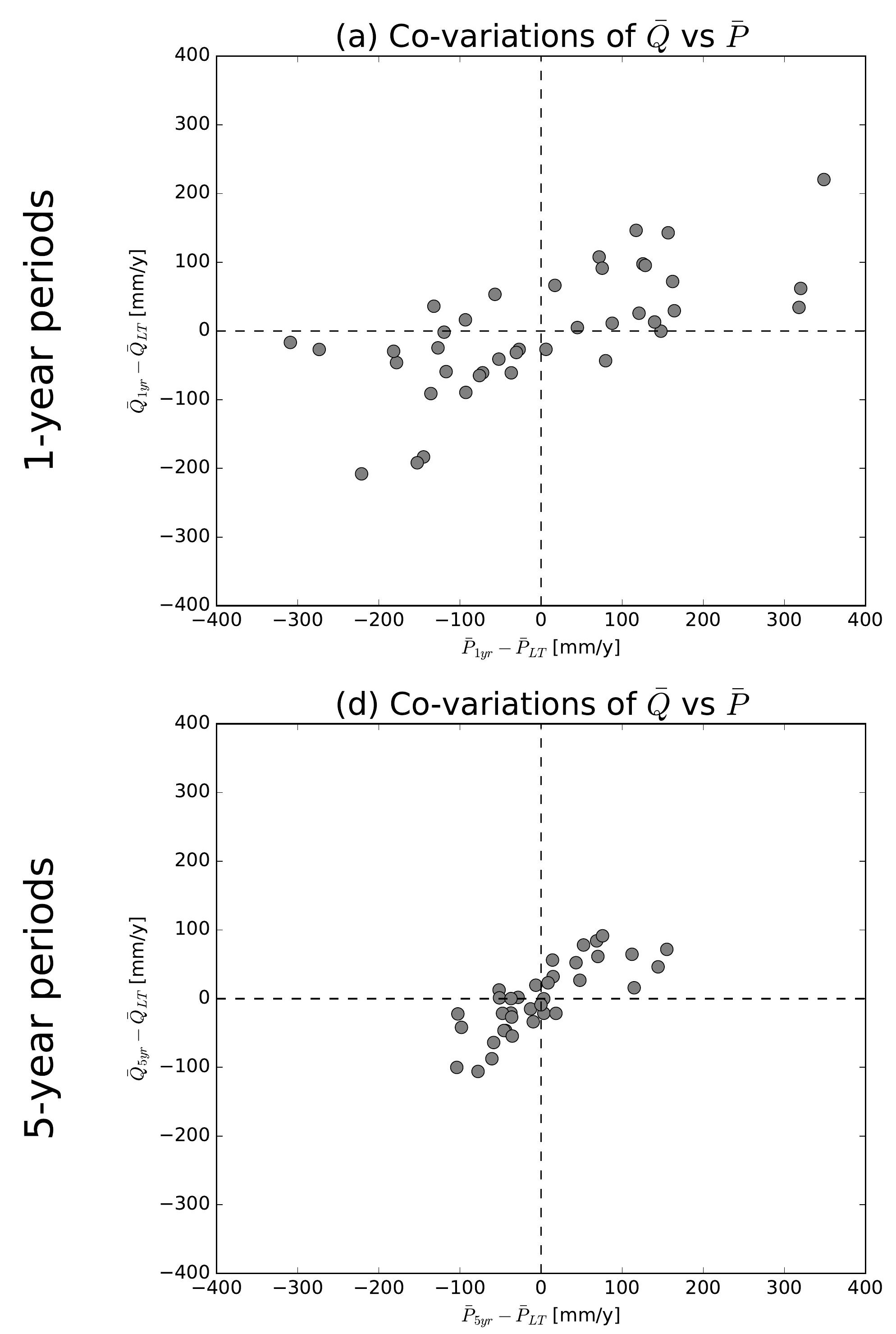

(g) Co-variations of $\bar{Q}$ vs $\bar{P}$

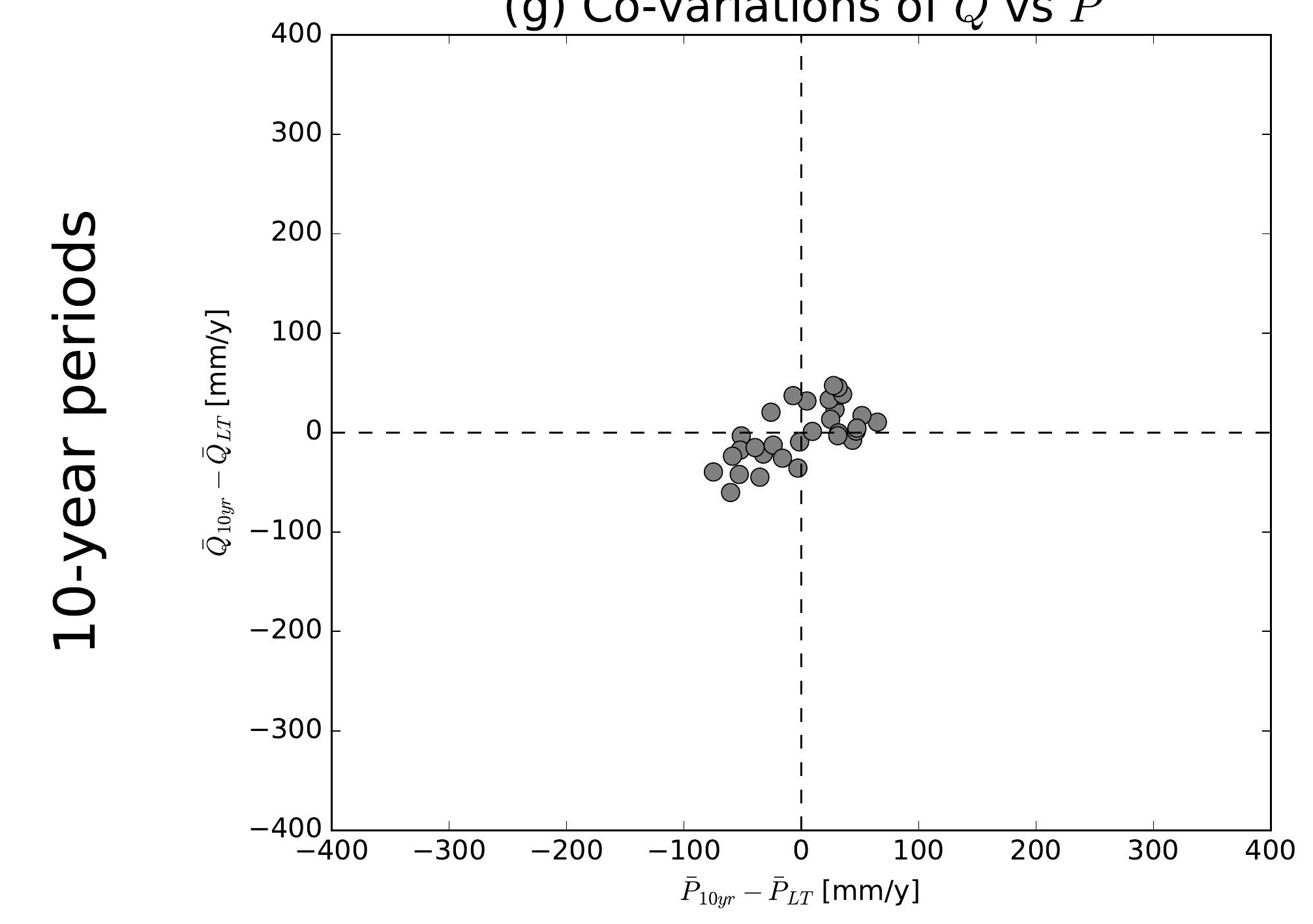

(j) Co-variations of $\bar{Q}$ vs $\bar{P}$

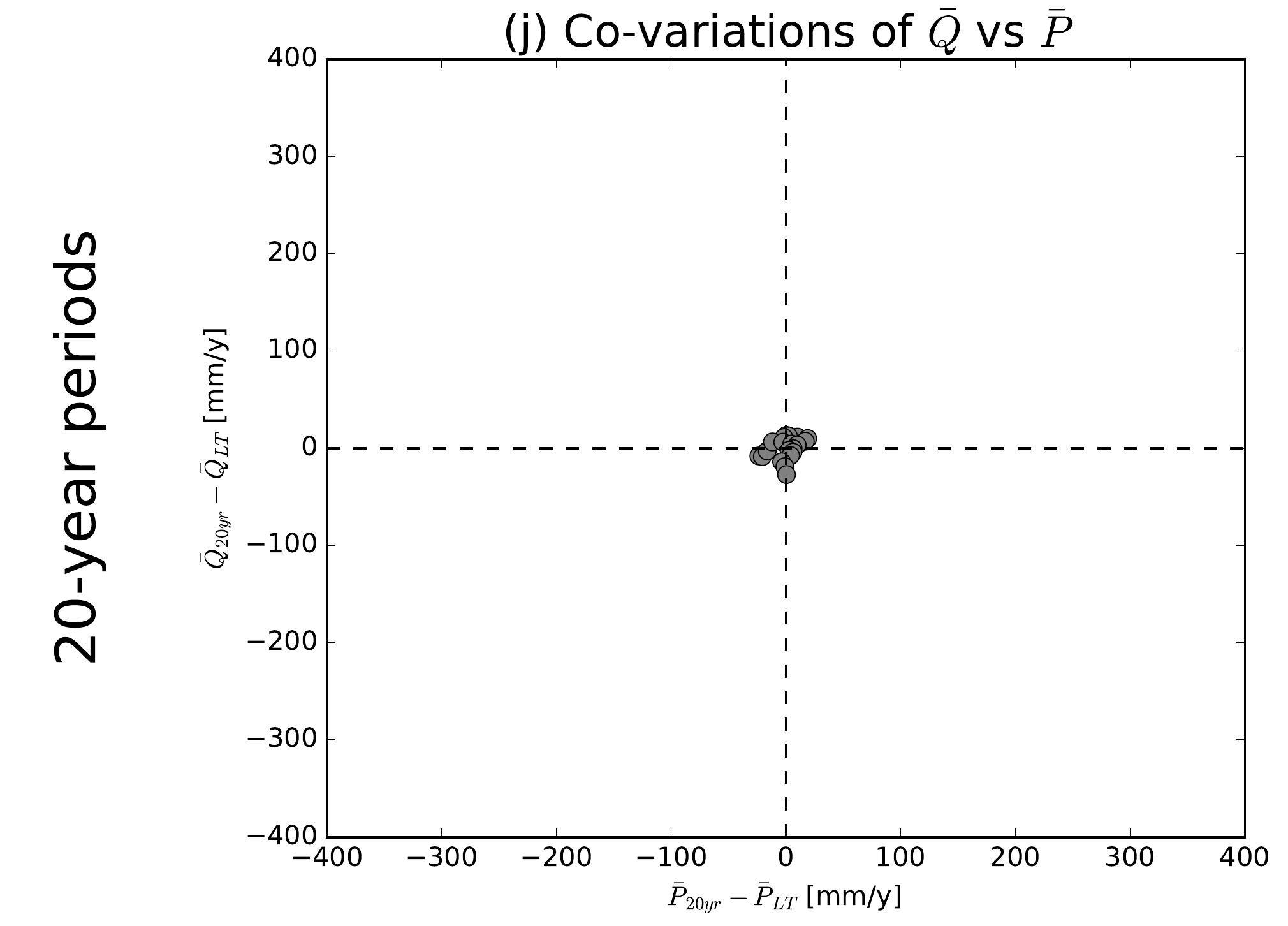

(b) Co-variations of $\bar{Q}$ vs $\overline{E 0}$

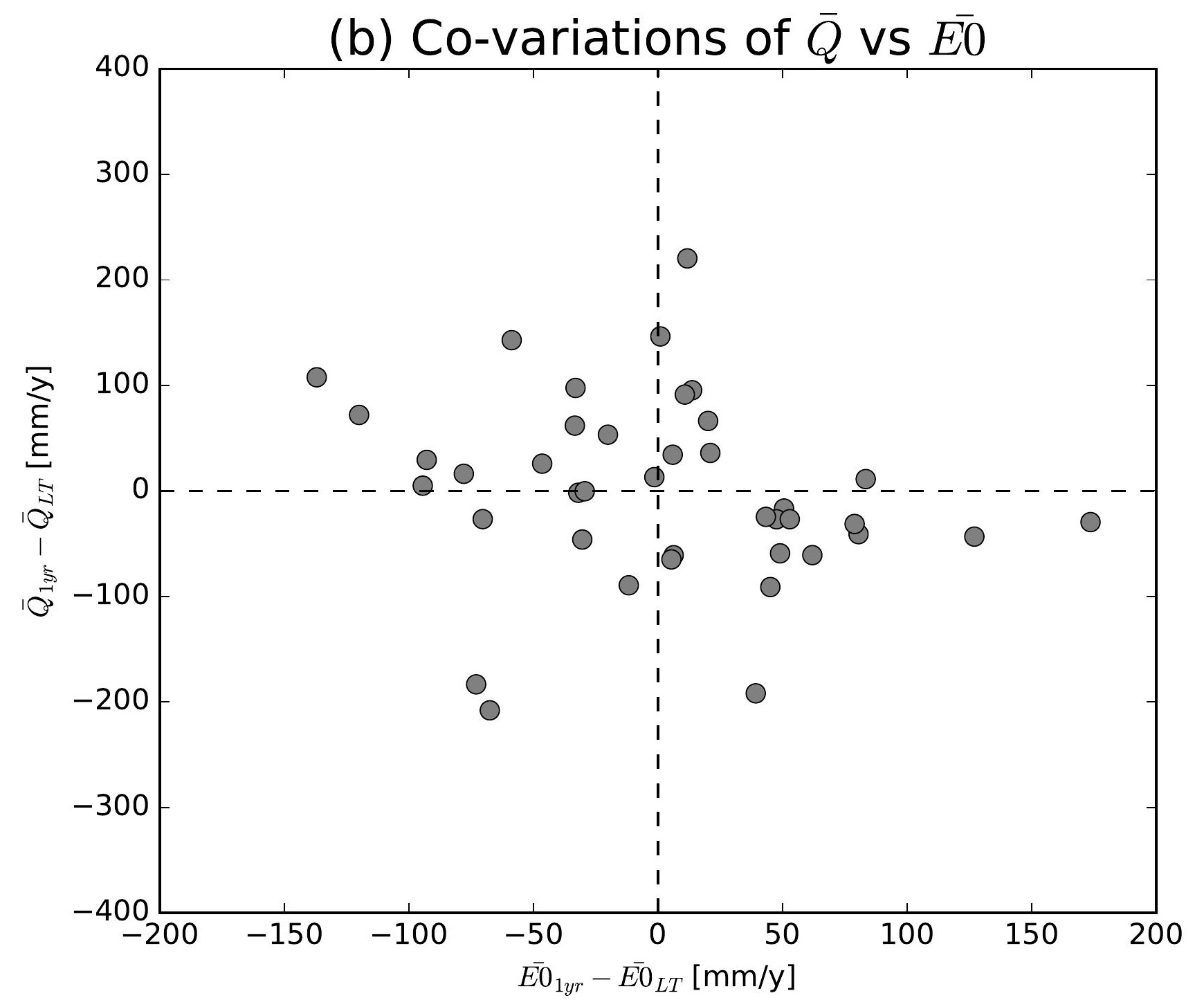

(e) Co-variations of $\bar{Q}$ vs $\overline{E 0}$

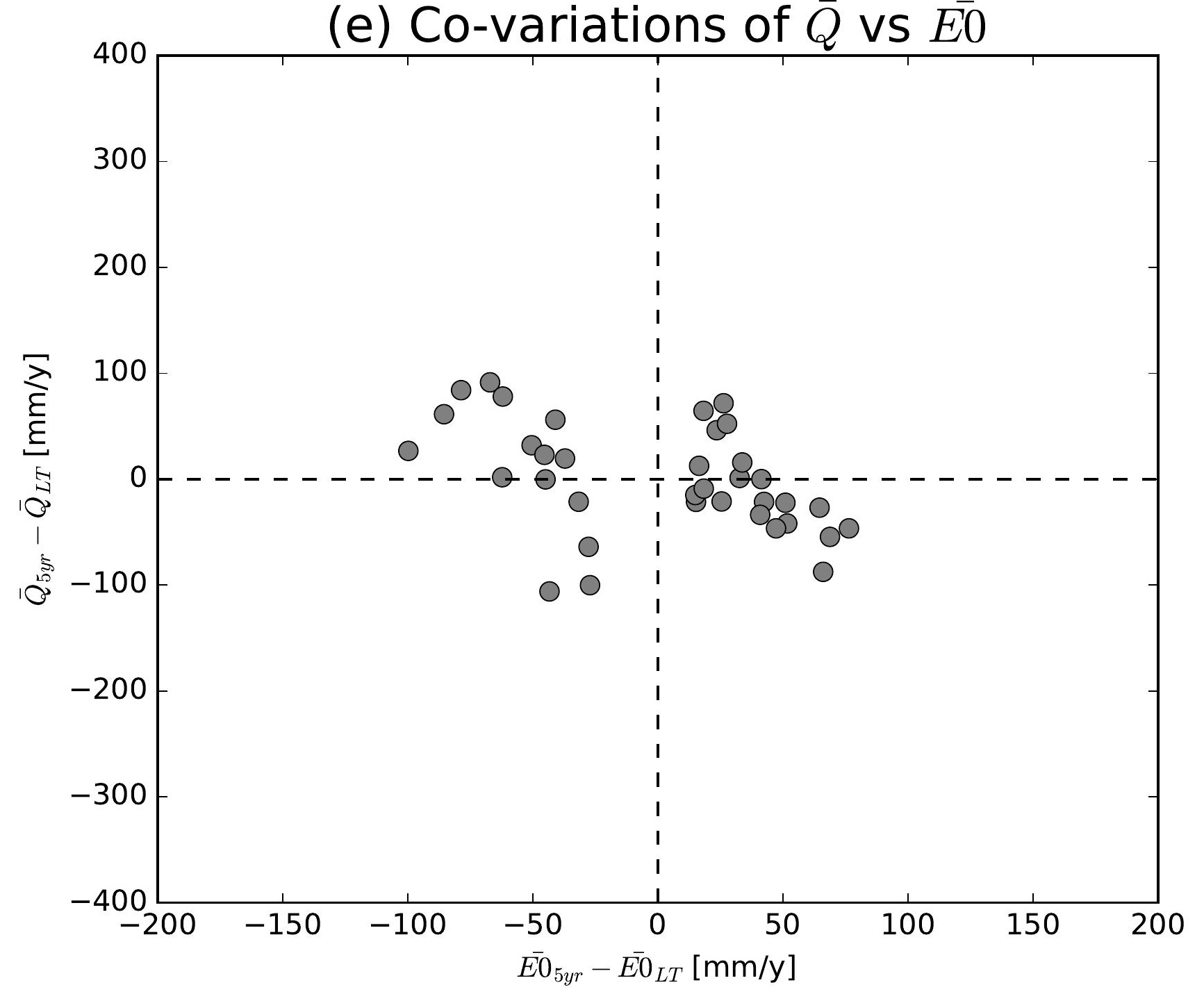

(h) Co-variations of $\bar{Q}$ vs $\overline{E 0}$

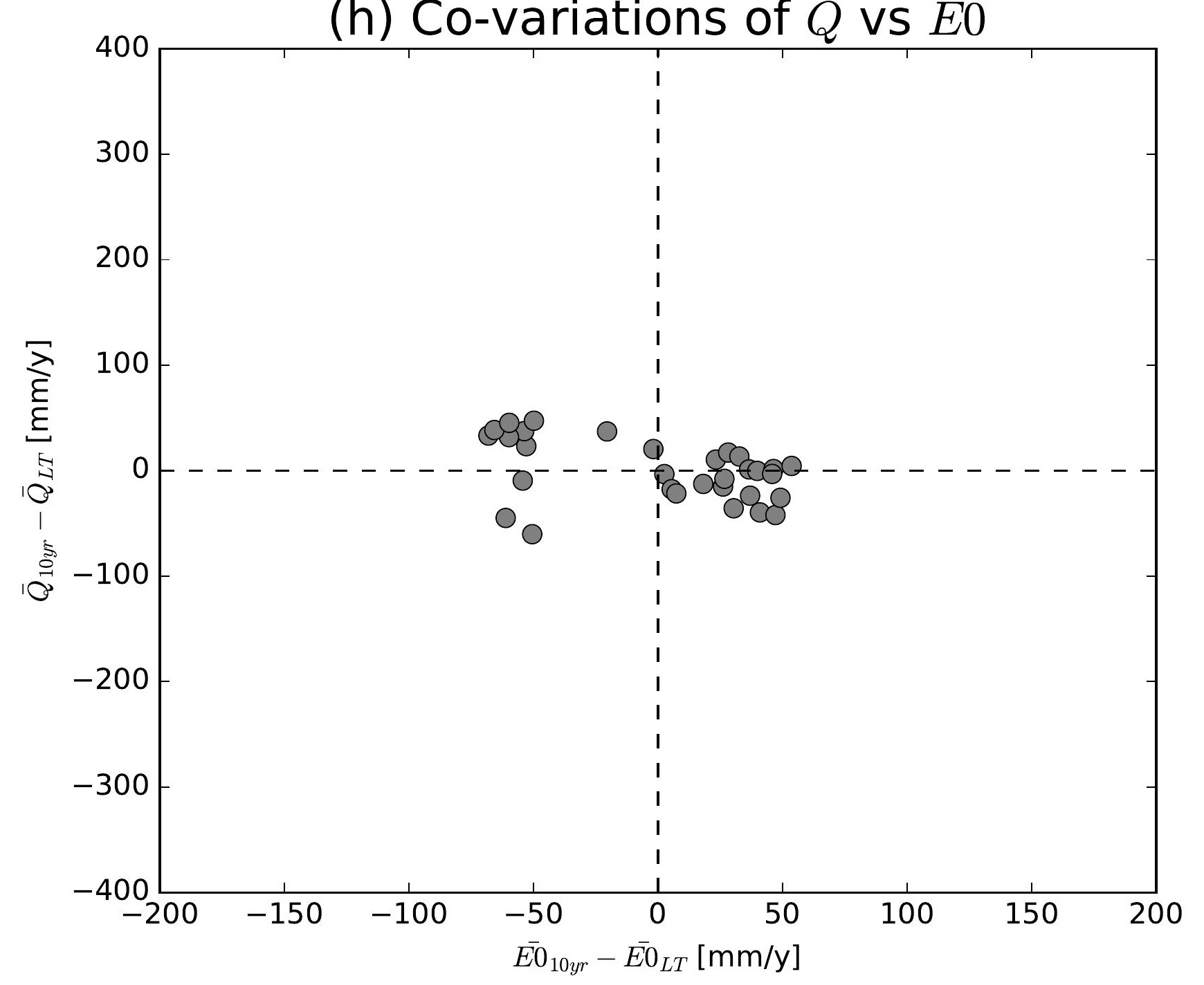

(k) Co-variations of $\bar{Q}$ vs $\overline{E 0}$

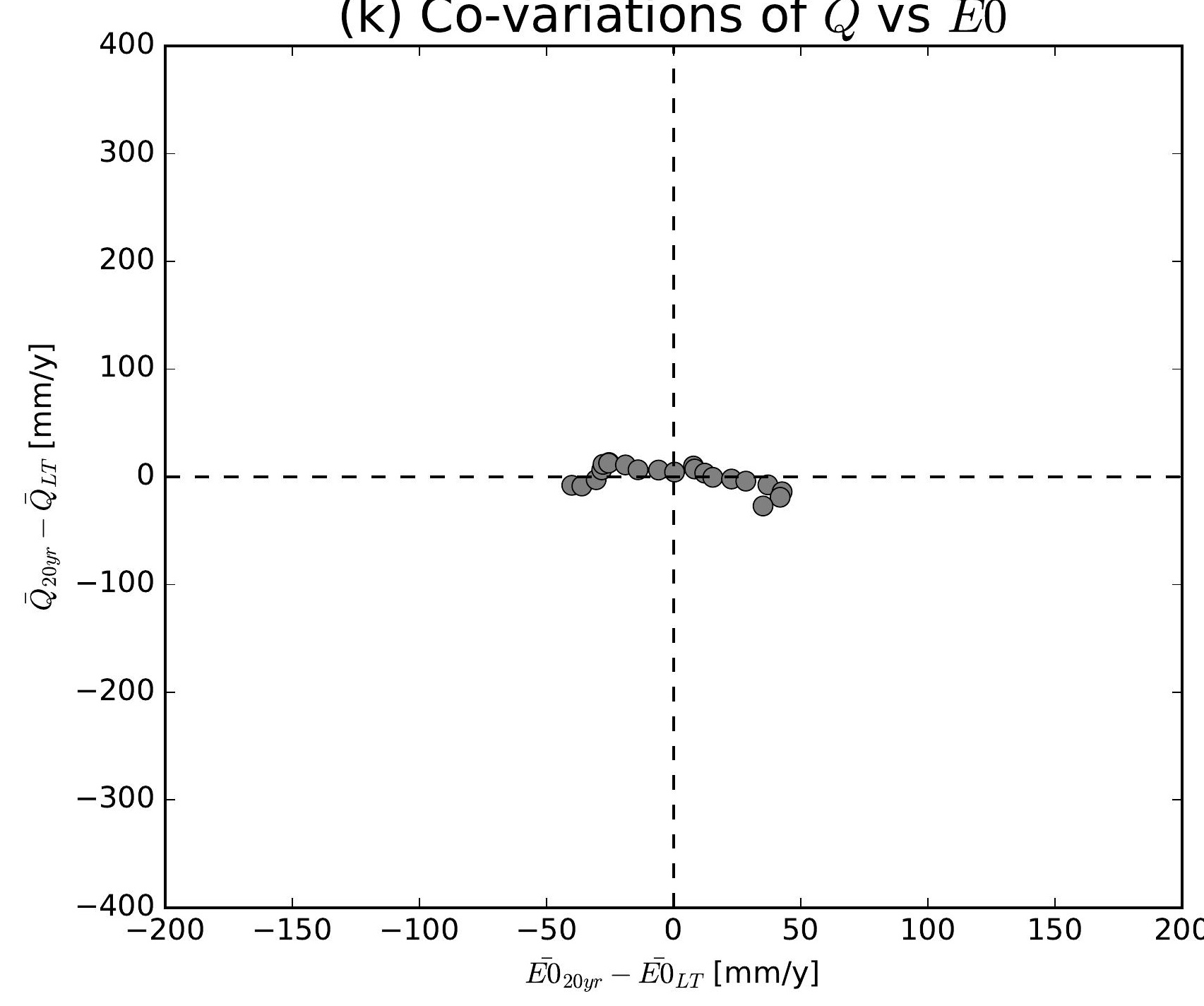

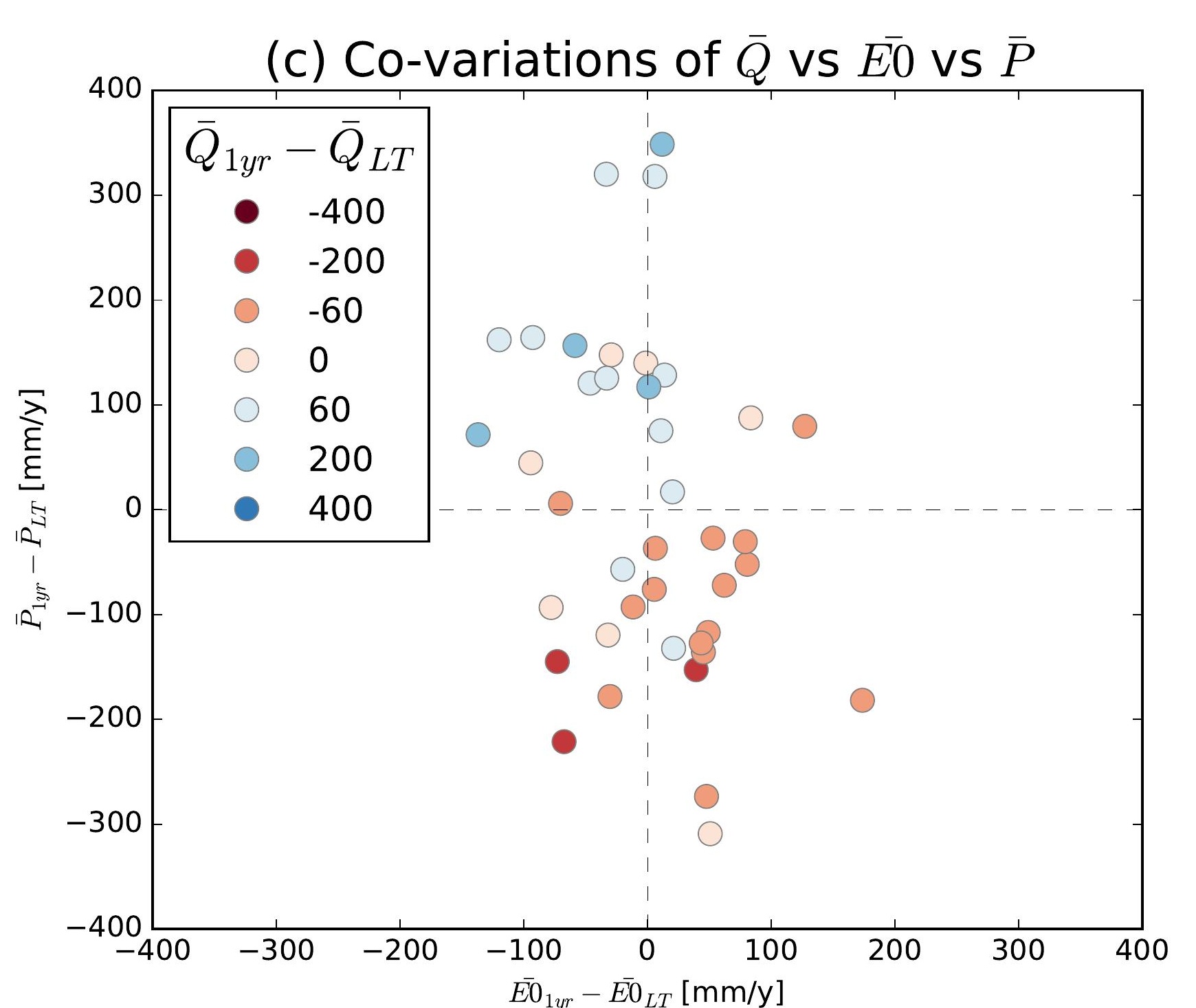
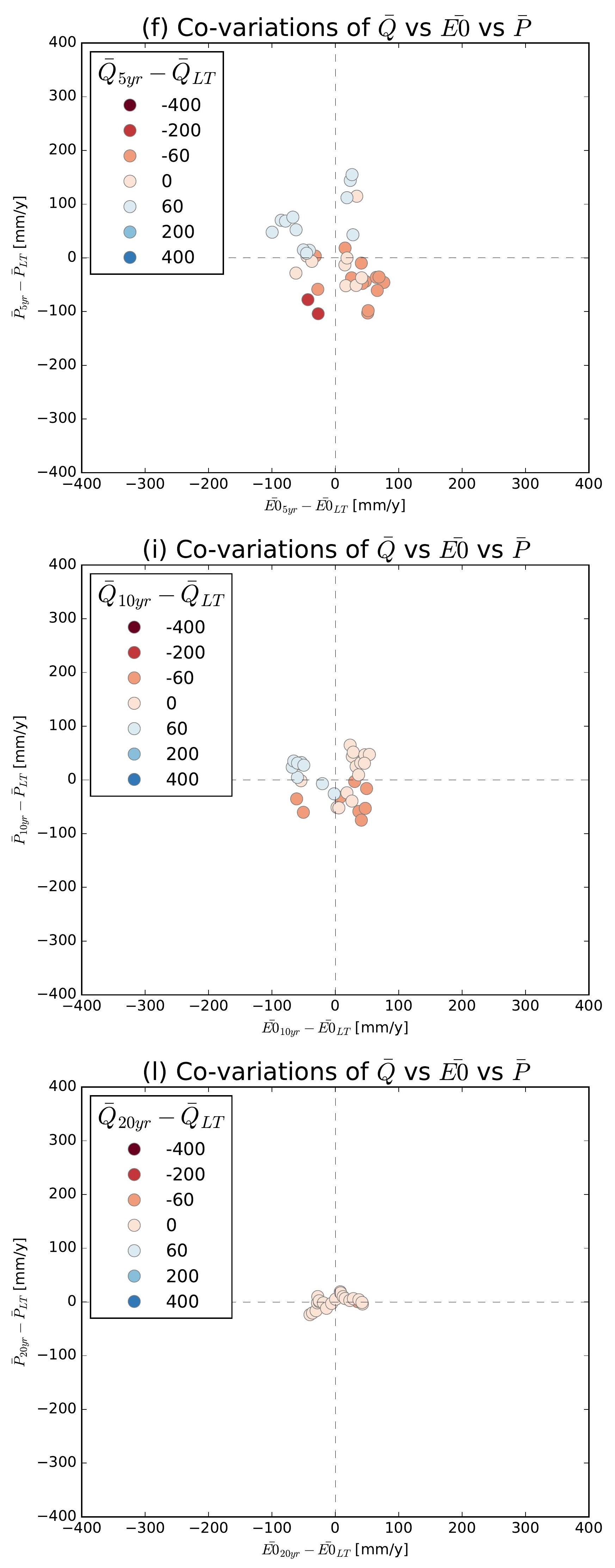

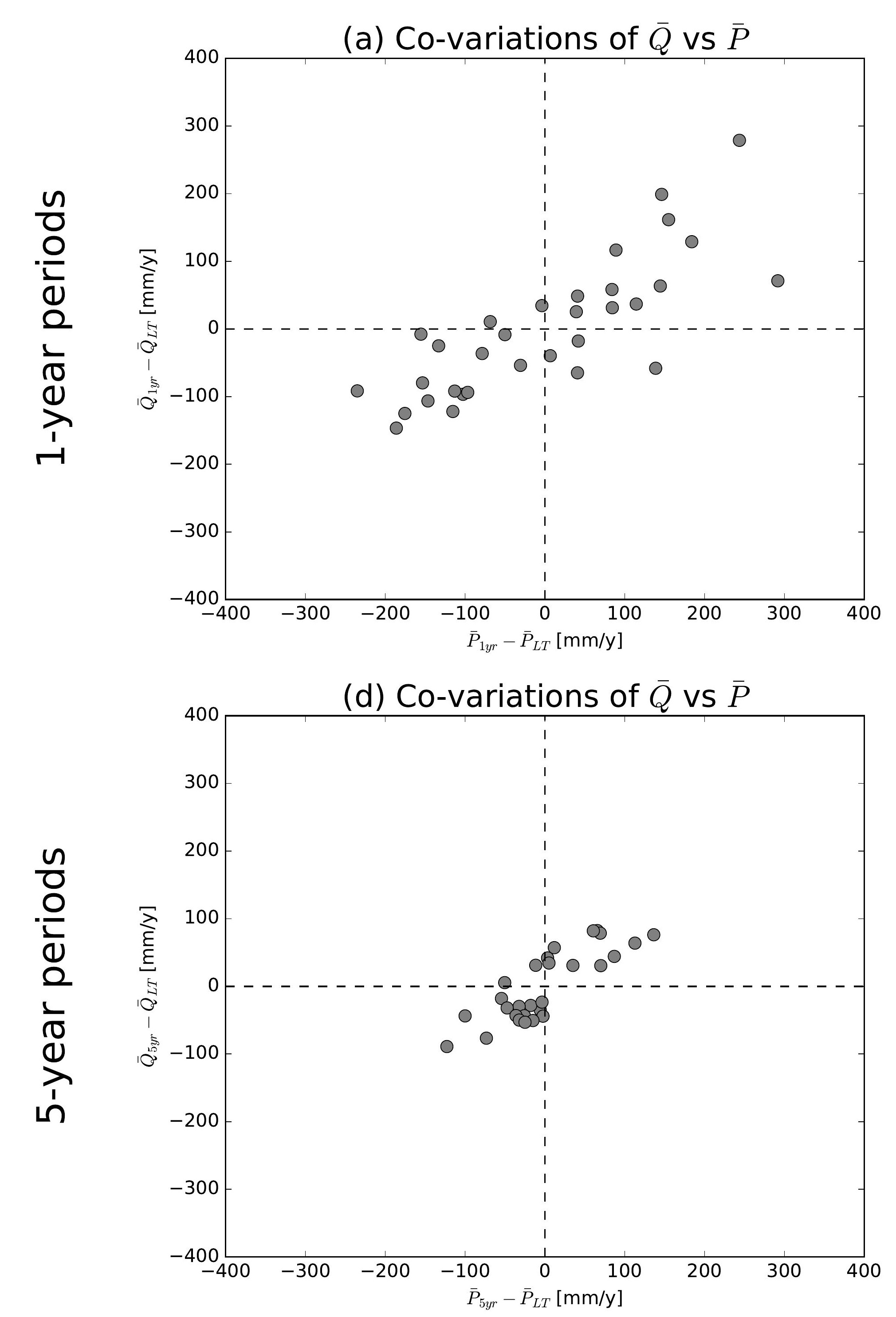

(g) Co-variations of $\bar{Q}$ vs $\bar{P}$
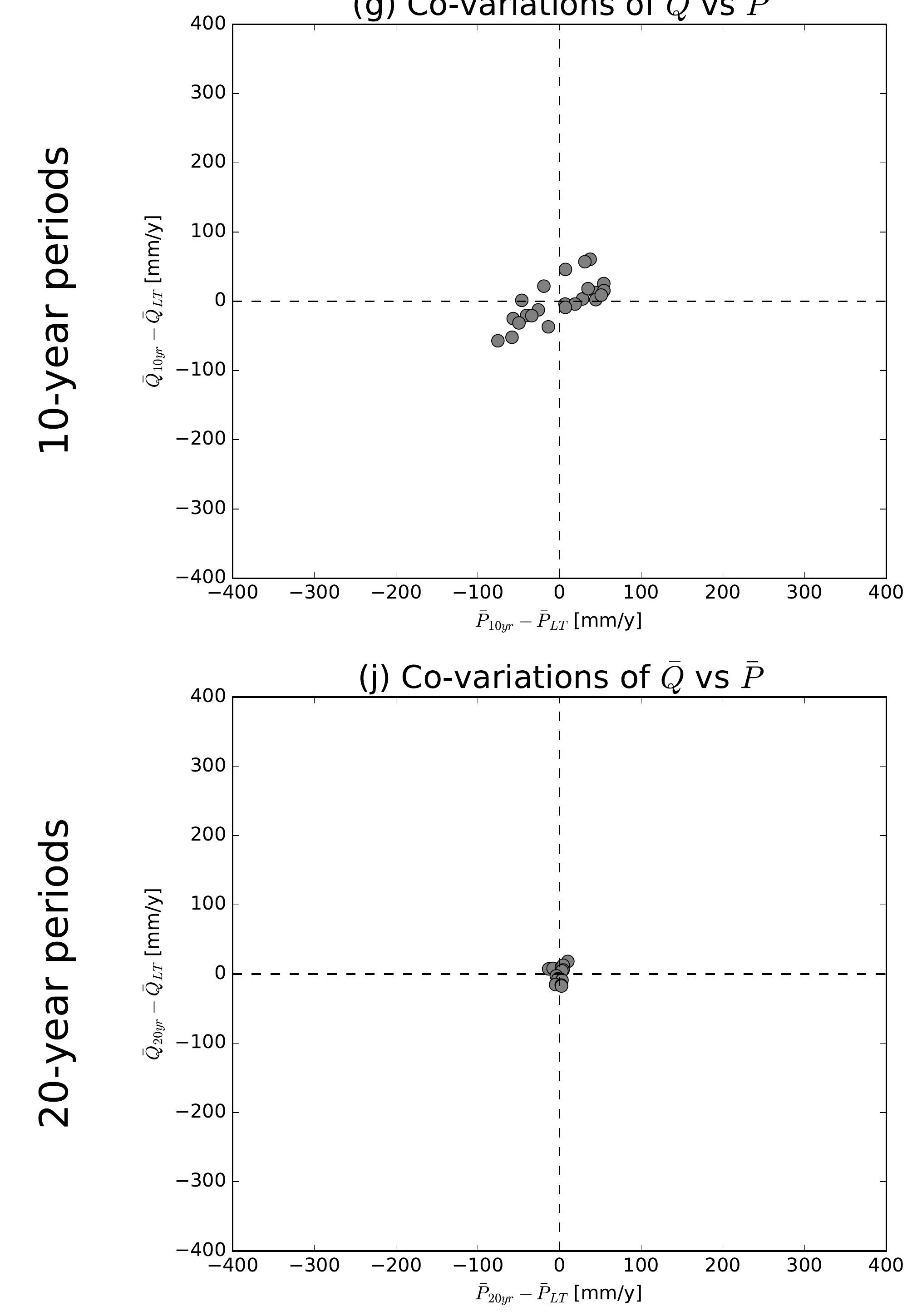

(b) Co-variations of $\bar{Q}$ vs $\overline{E 0}$

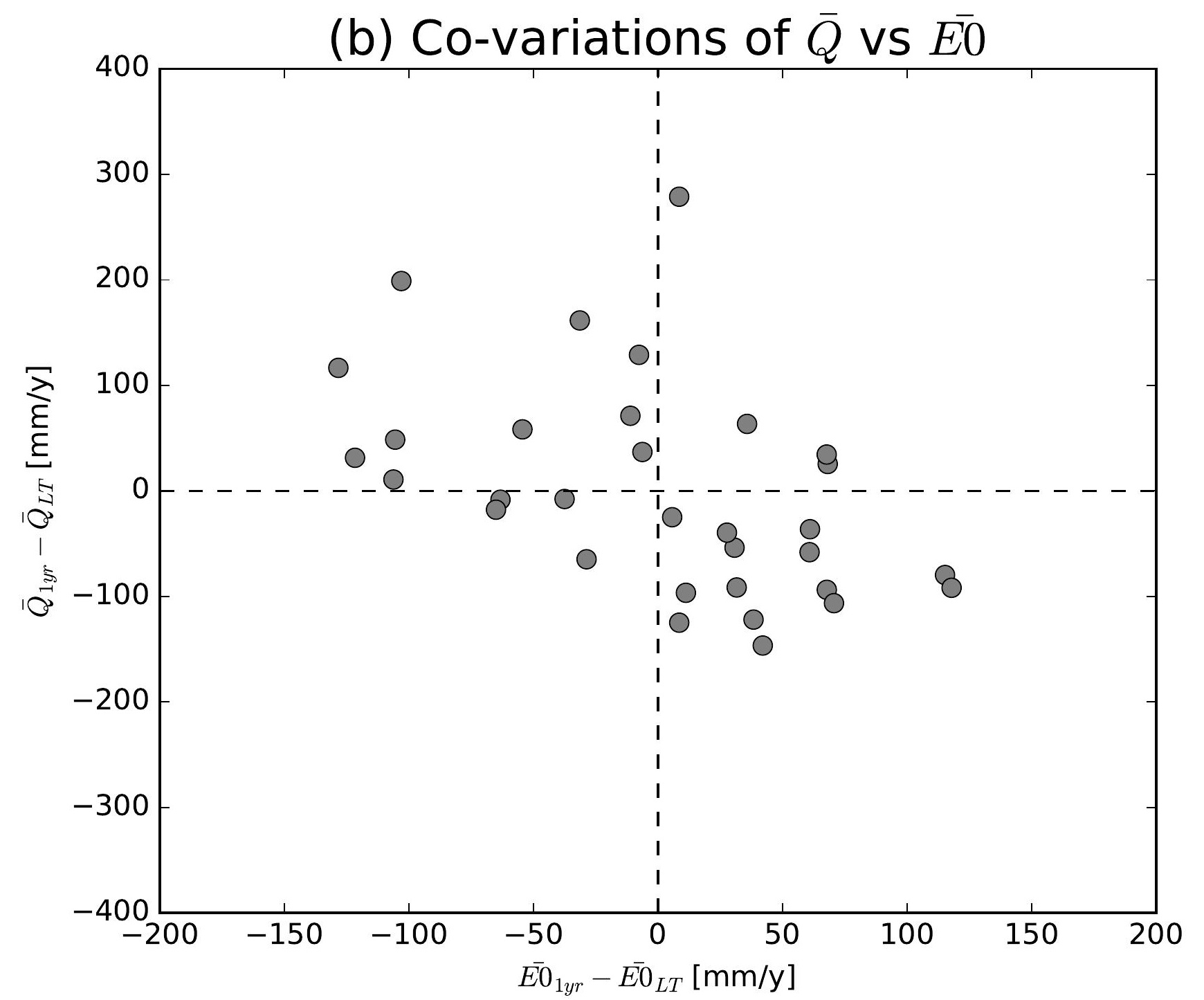

(e) Co-variations of $\bar{Q}$ vs $\overline{E 0}$

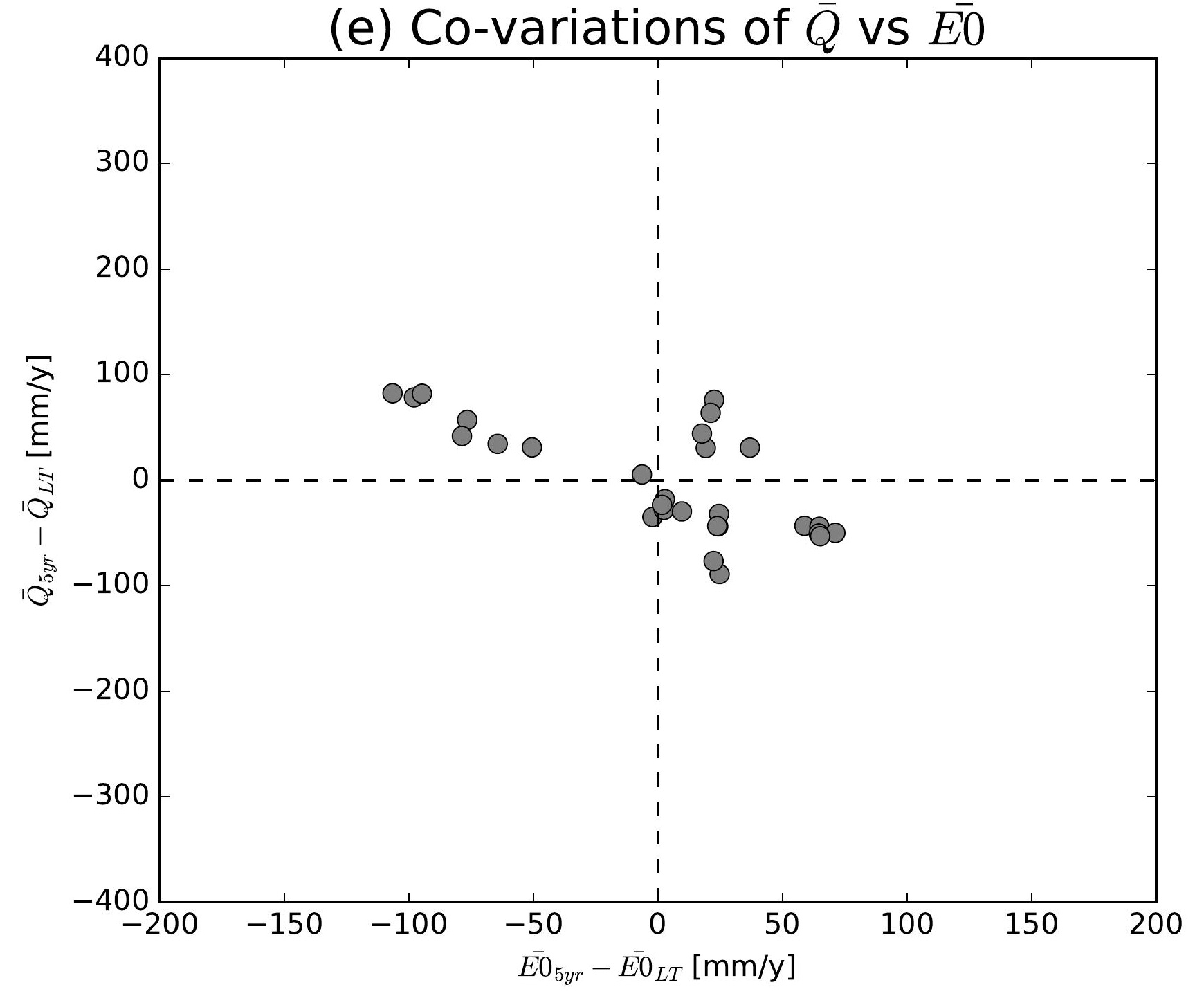

(h) Co-variations of $\bar{Q}$ vs $\overline{E 0}$

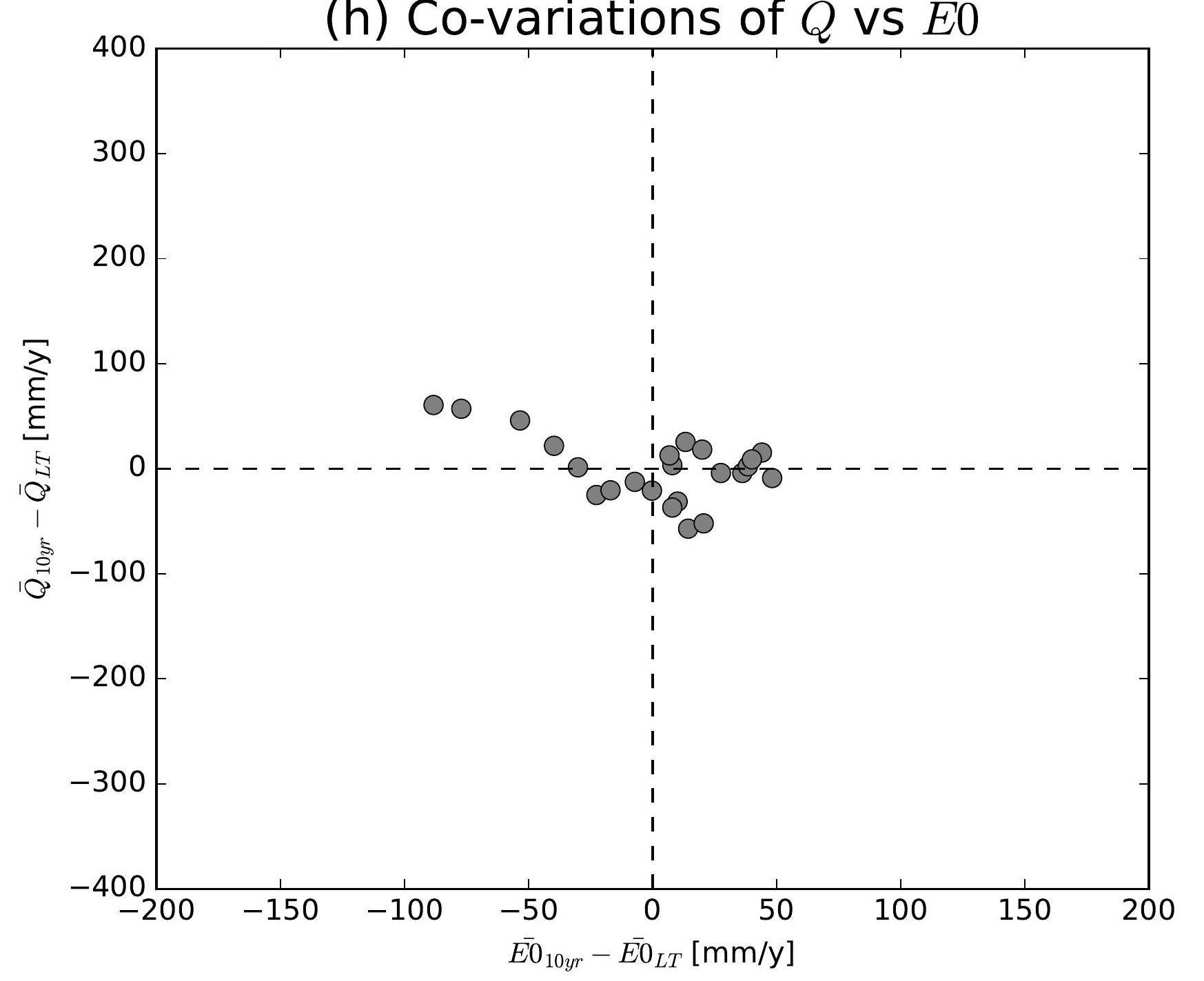

(k) Co-variations of $\bar{Q}$ vs $\overline{E 0}$

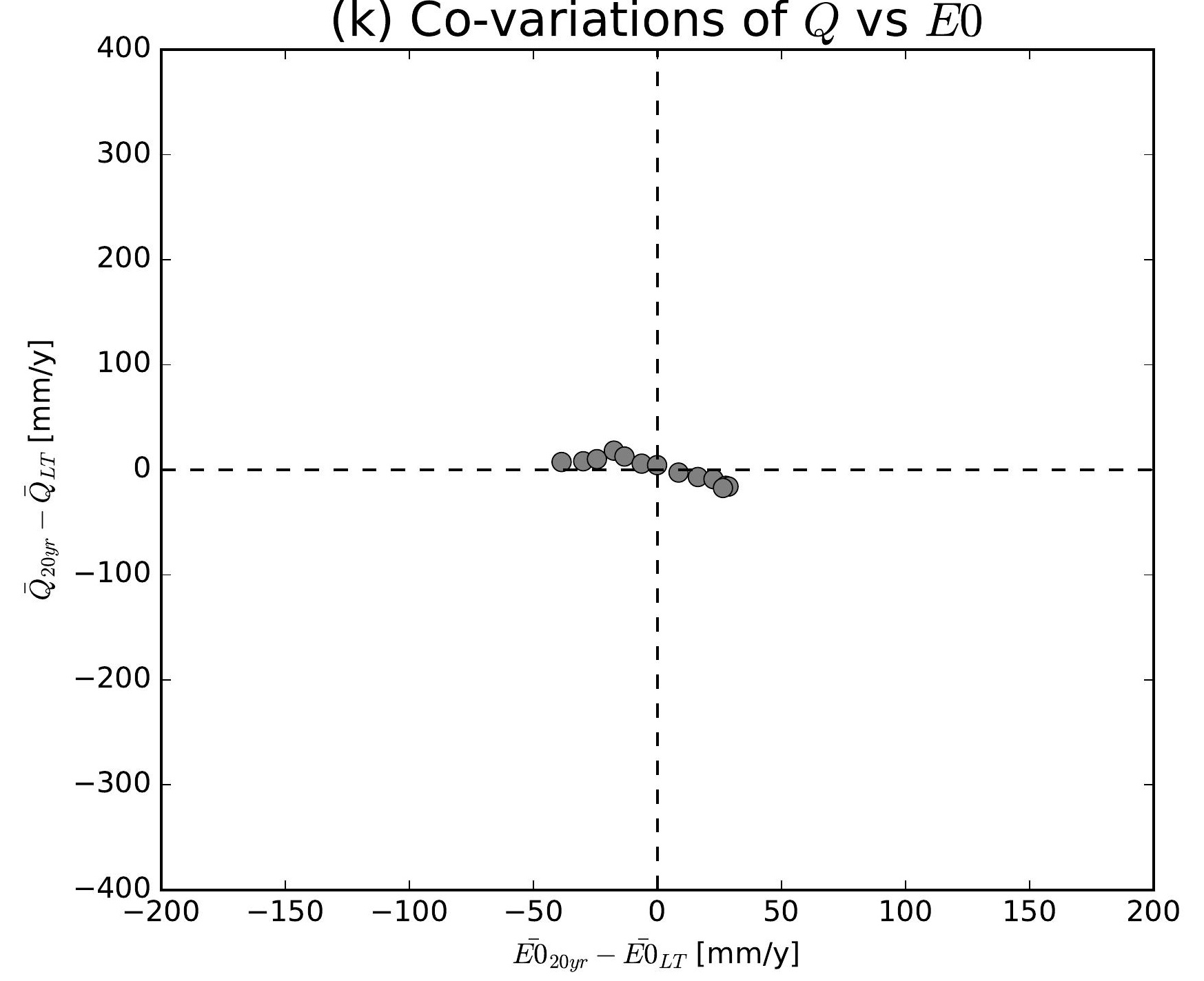

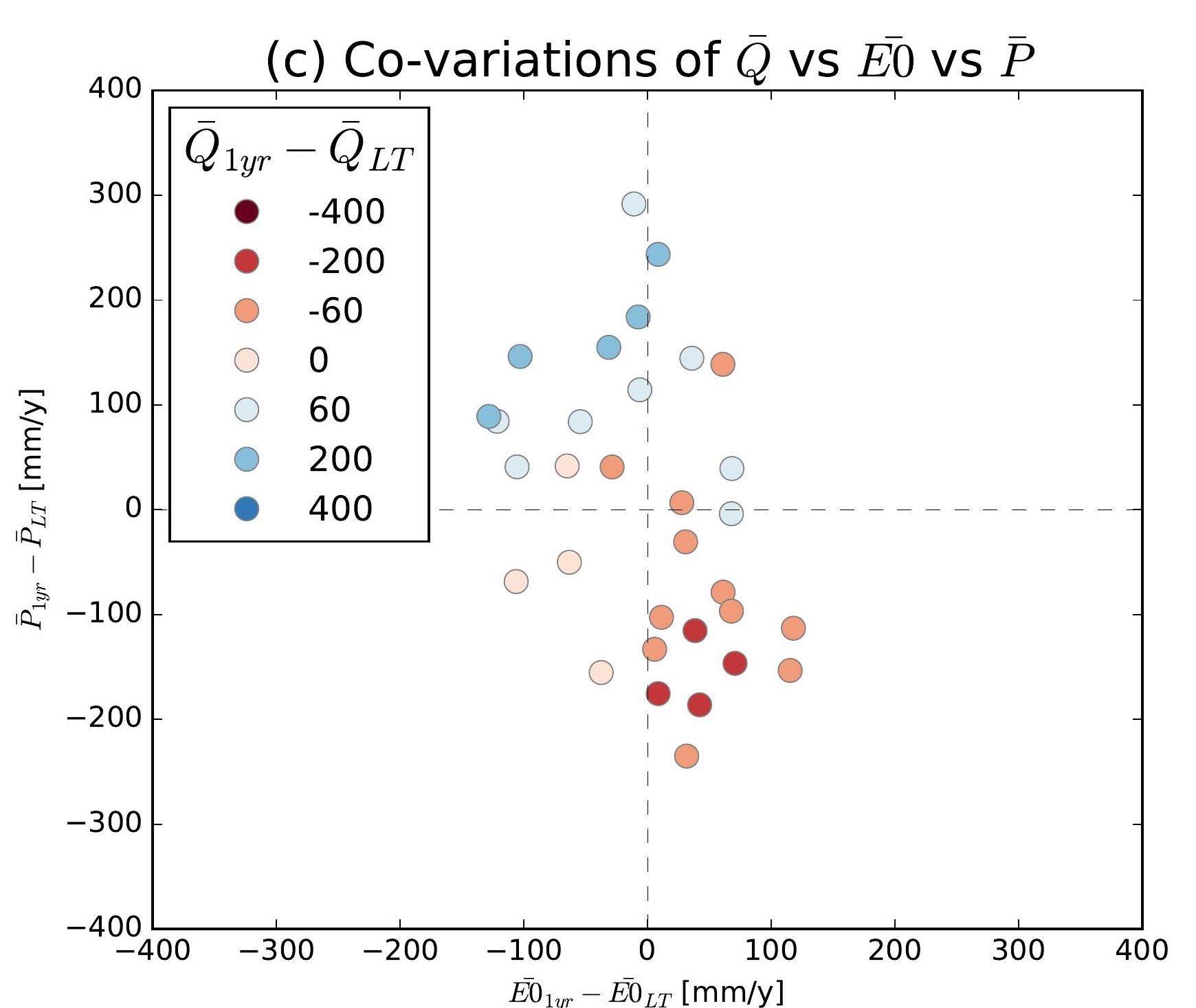
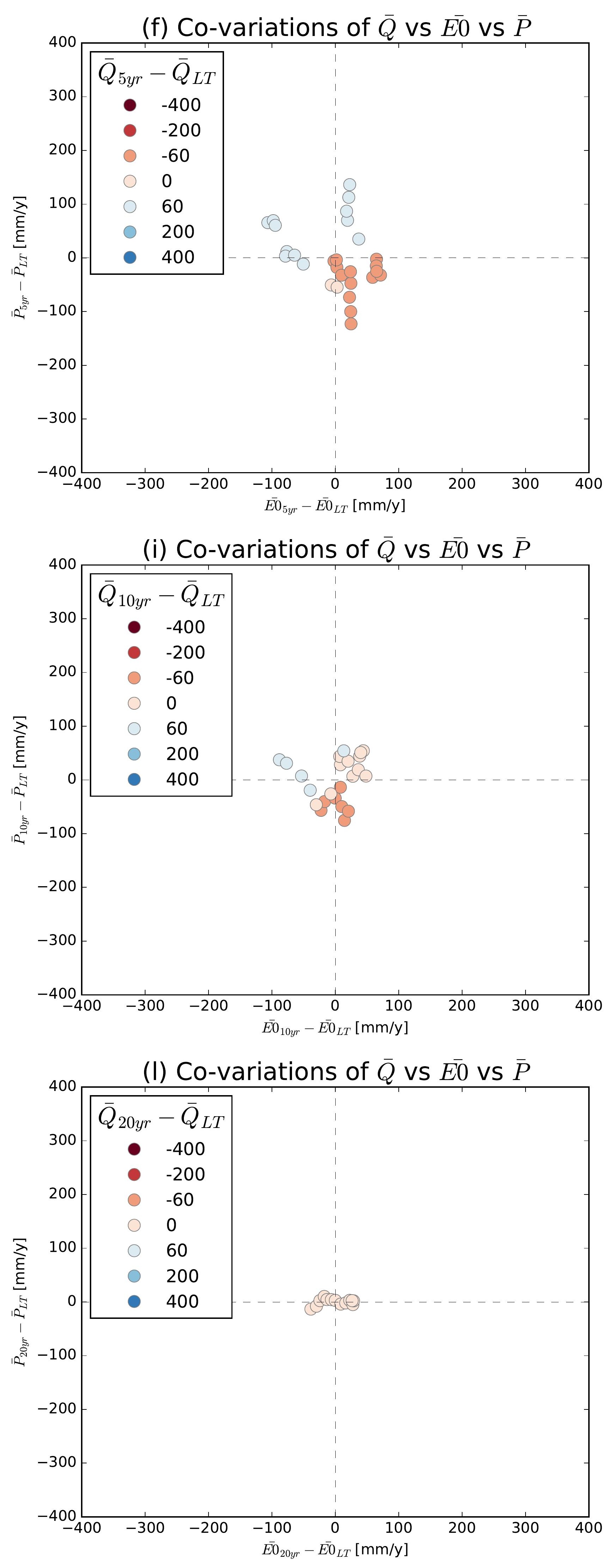

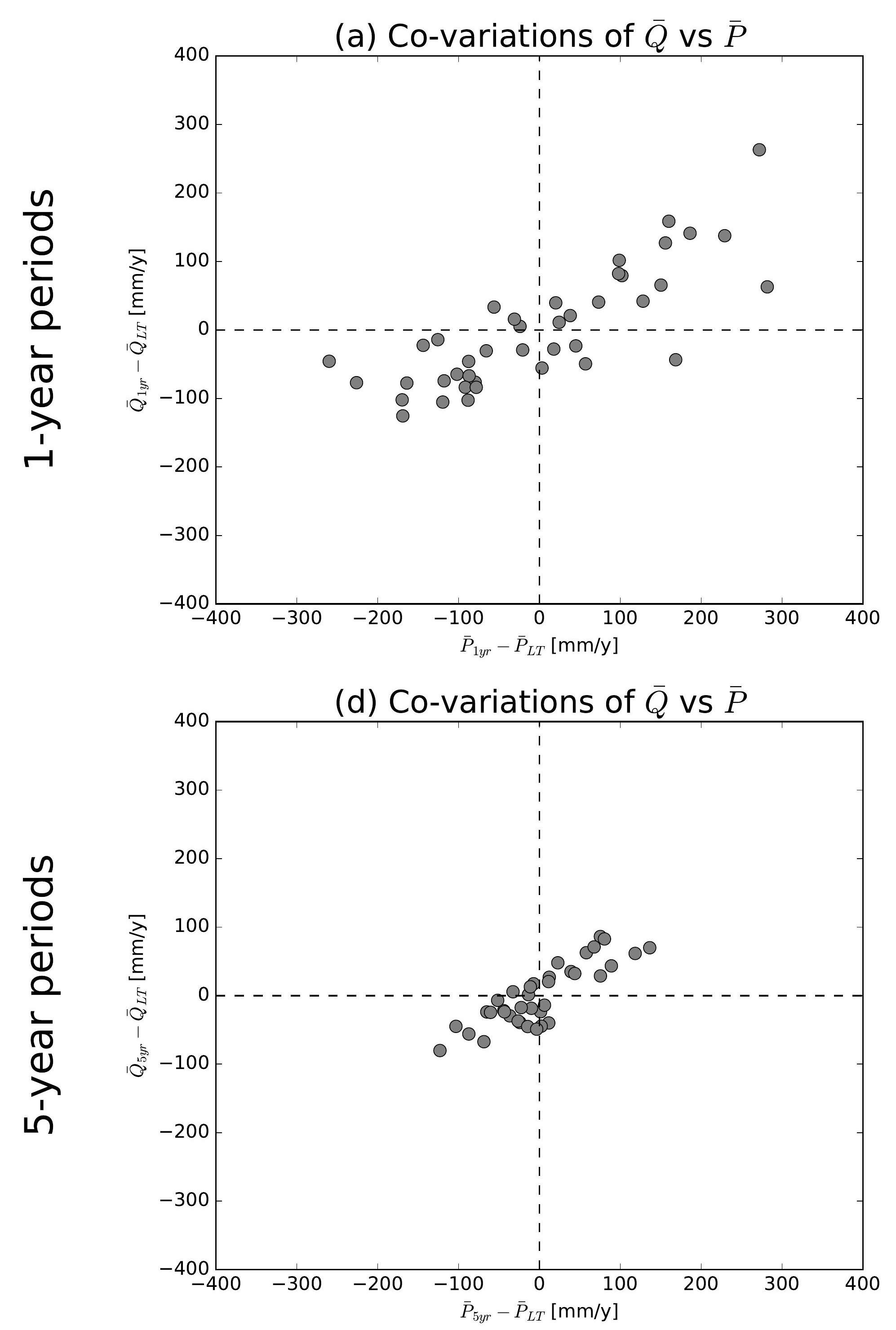

(g) Co-variations of $\bar{Q}$ vs $\bar{P}$

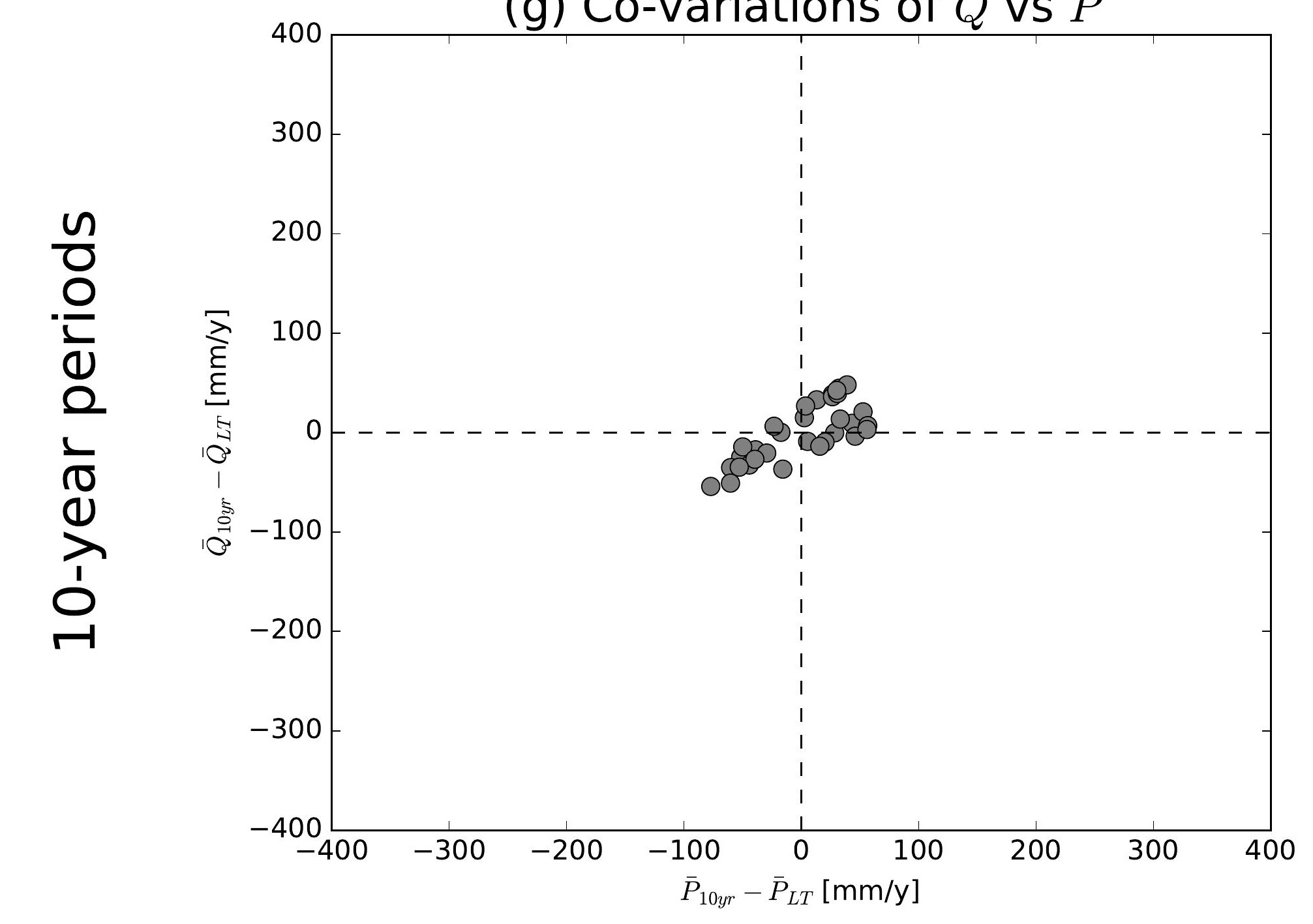

(j) Co-variations of $\bar{Q}$ vs $\bar{P}$

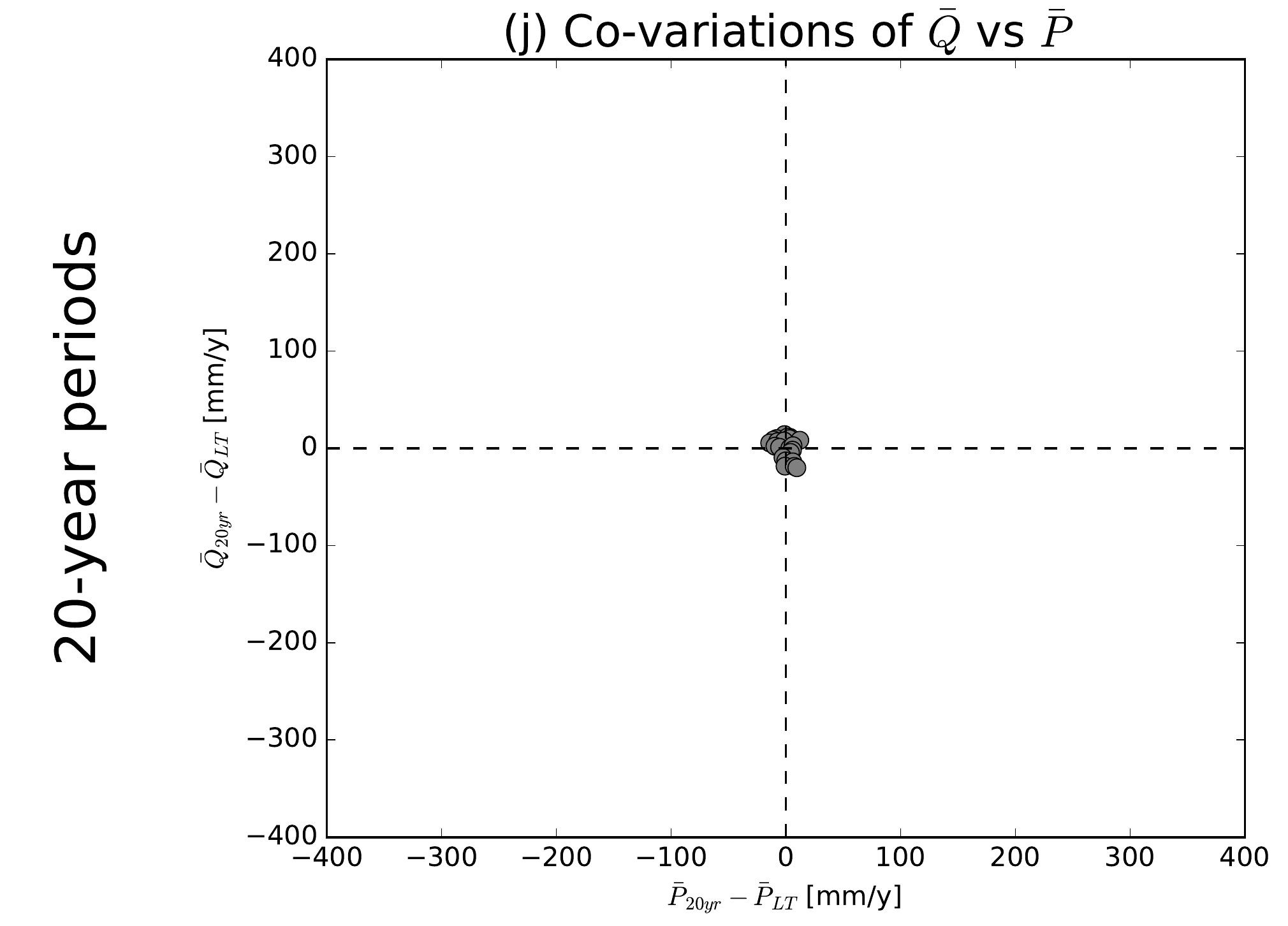

(b) Co-variations of $\bar{Q}$ vs $\overline{E 0}$

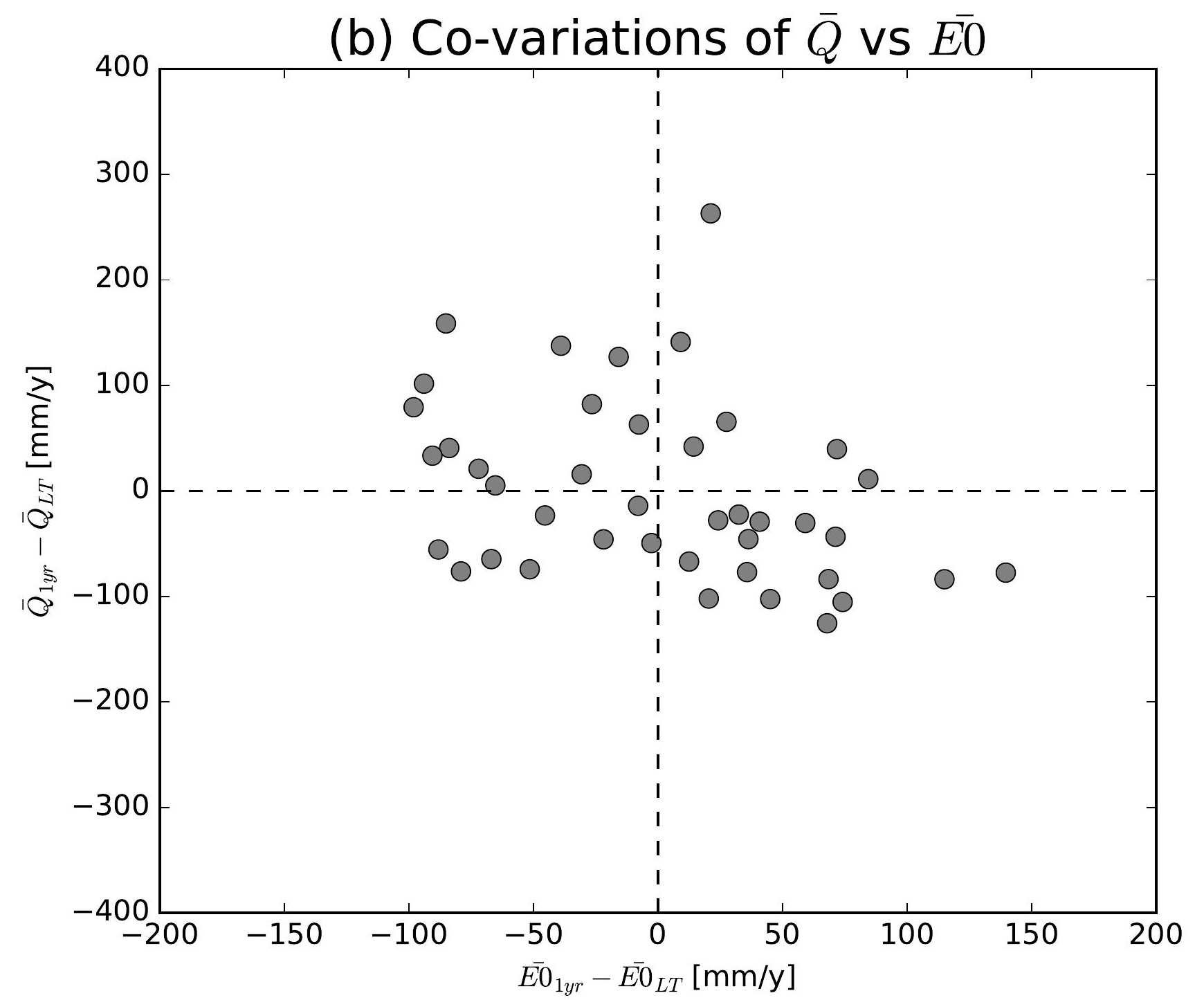

(e) Co-variations of $\bar{Q}$ vs $\overline{E 0}$

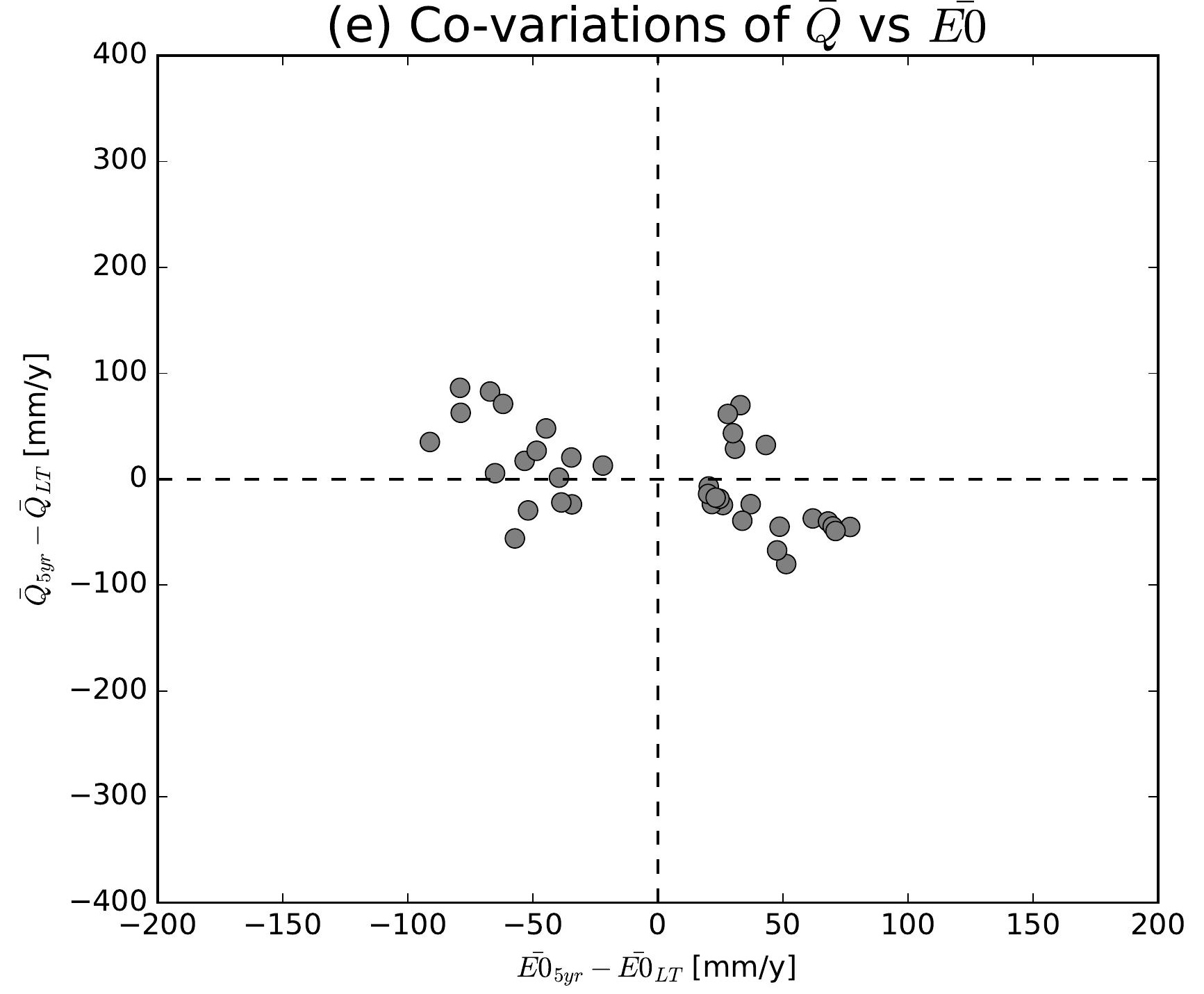

(h) Co-variations of $\bar{Q}$ vs $\overline{E 0}$

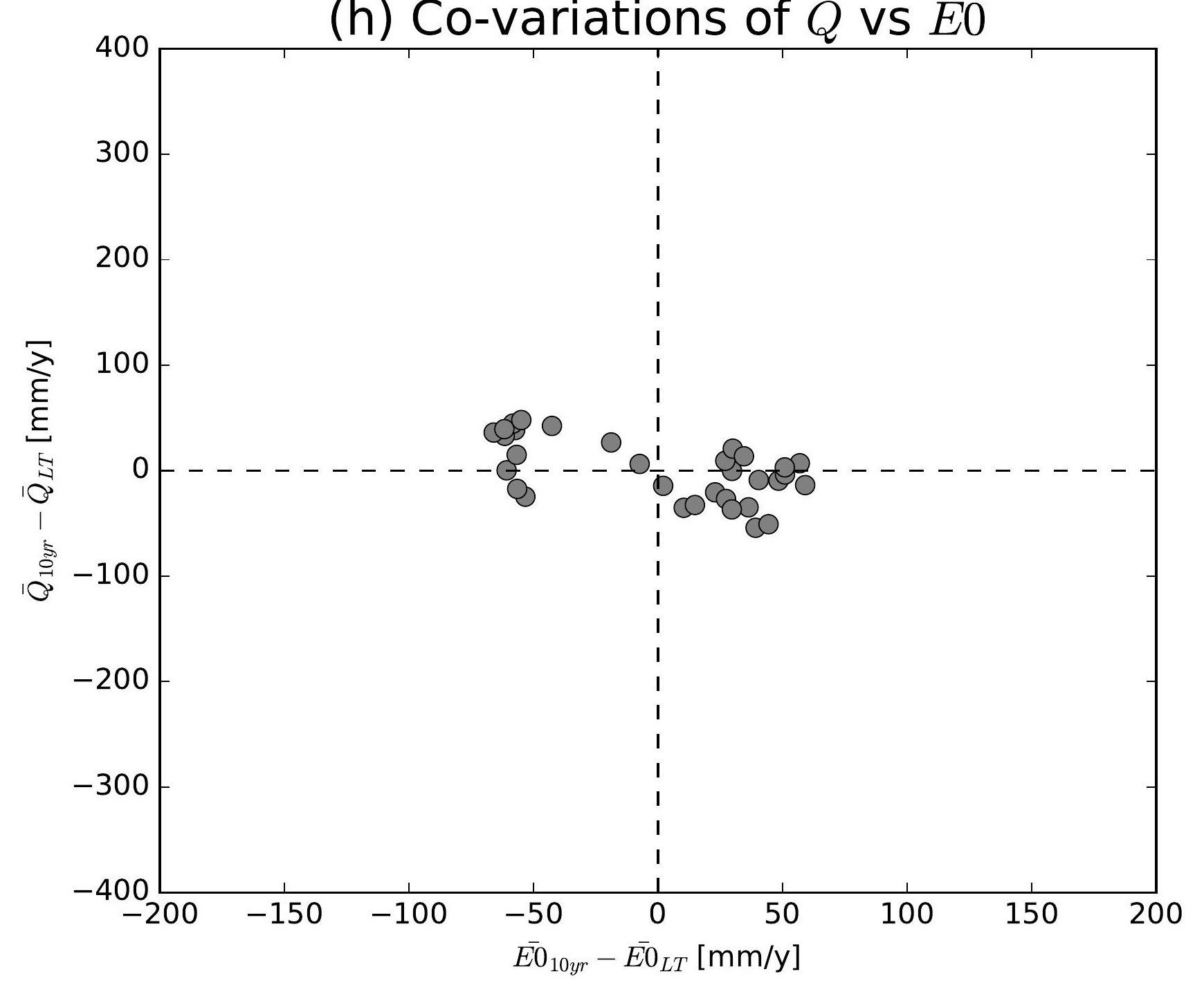

(k) Co-variations of $\bar{Q}$ vs $\overline{E 0}$

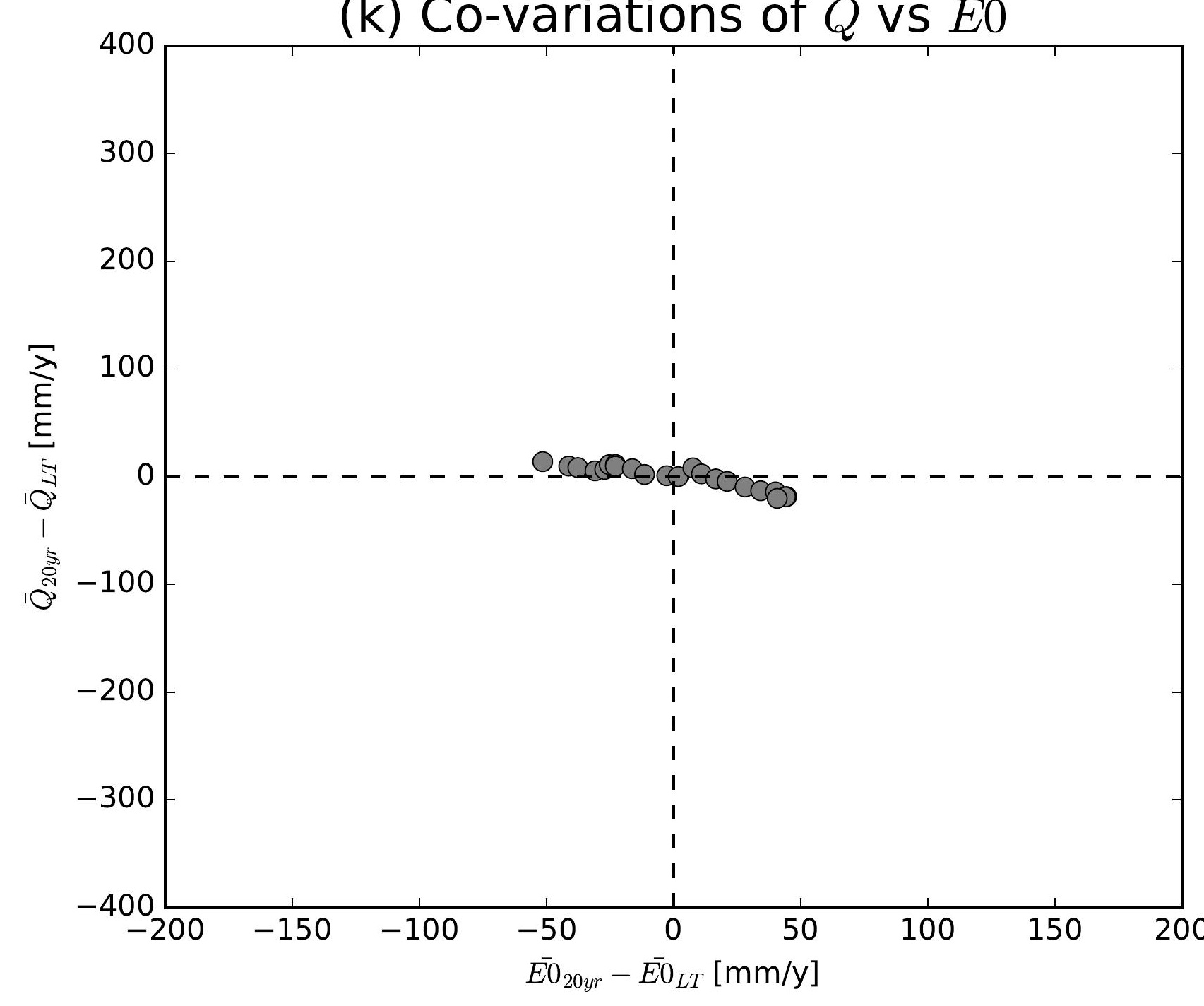

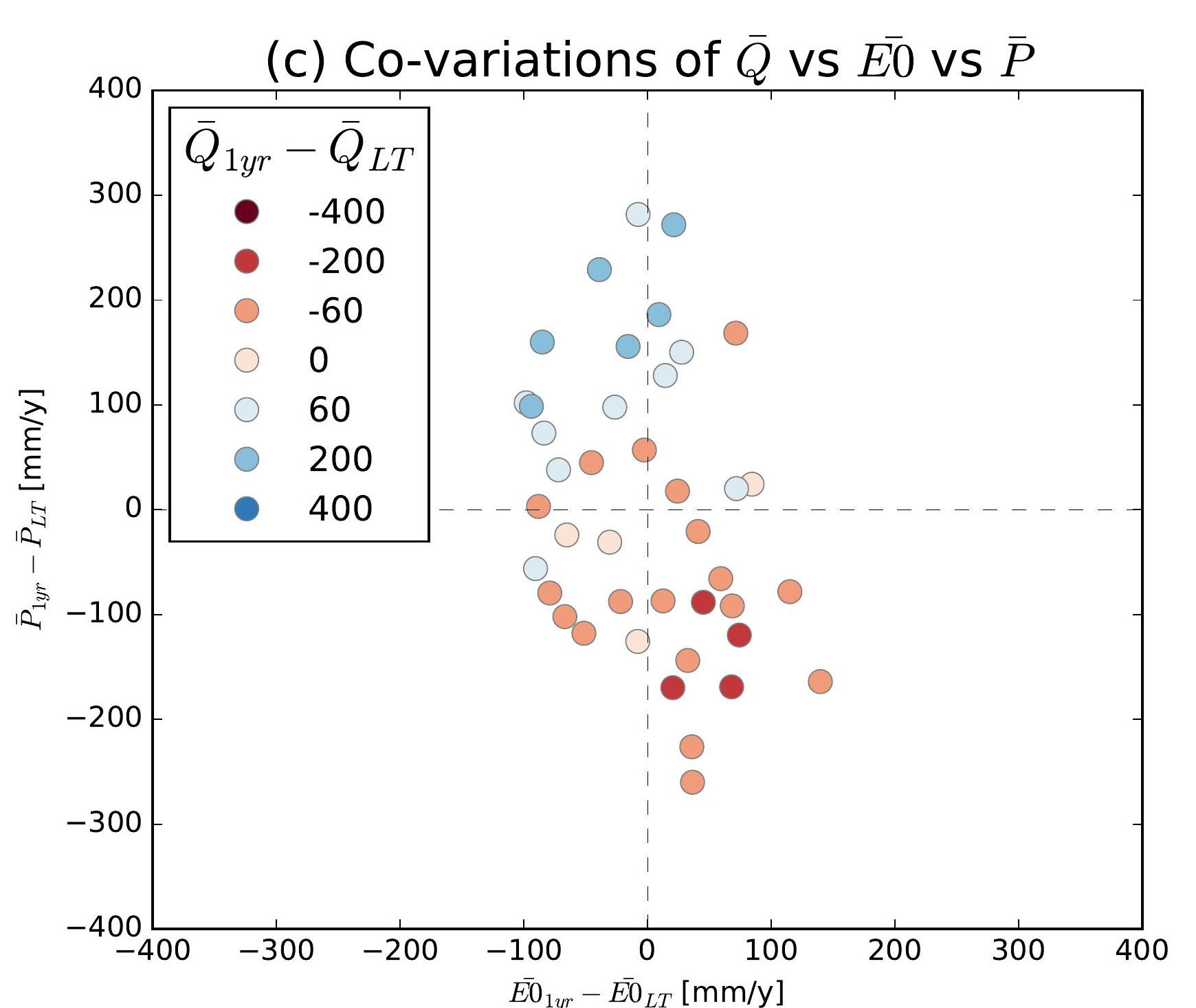
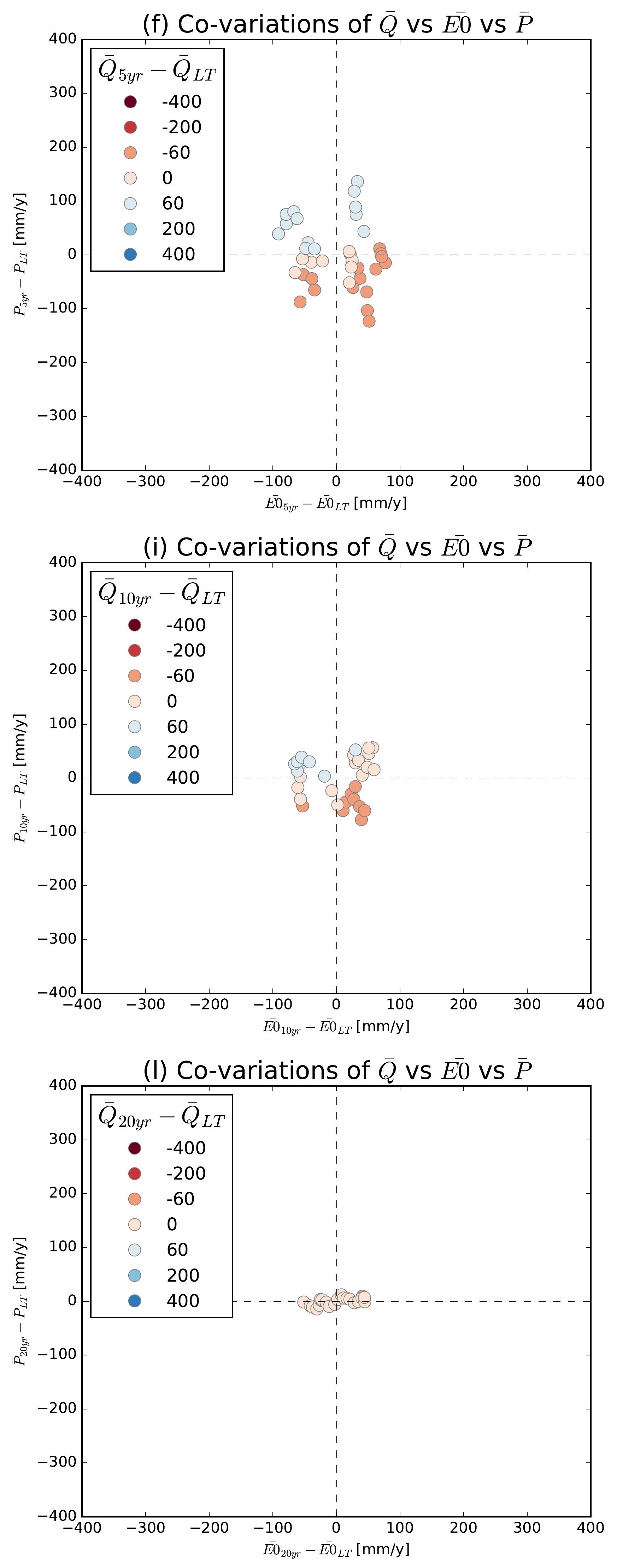

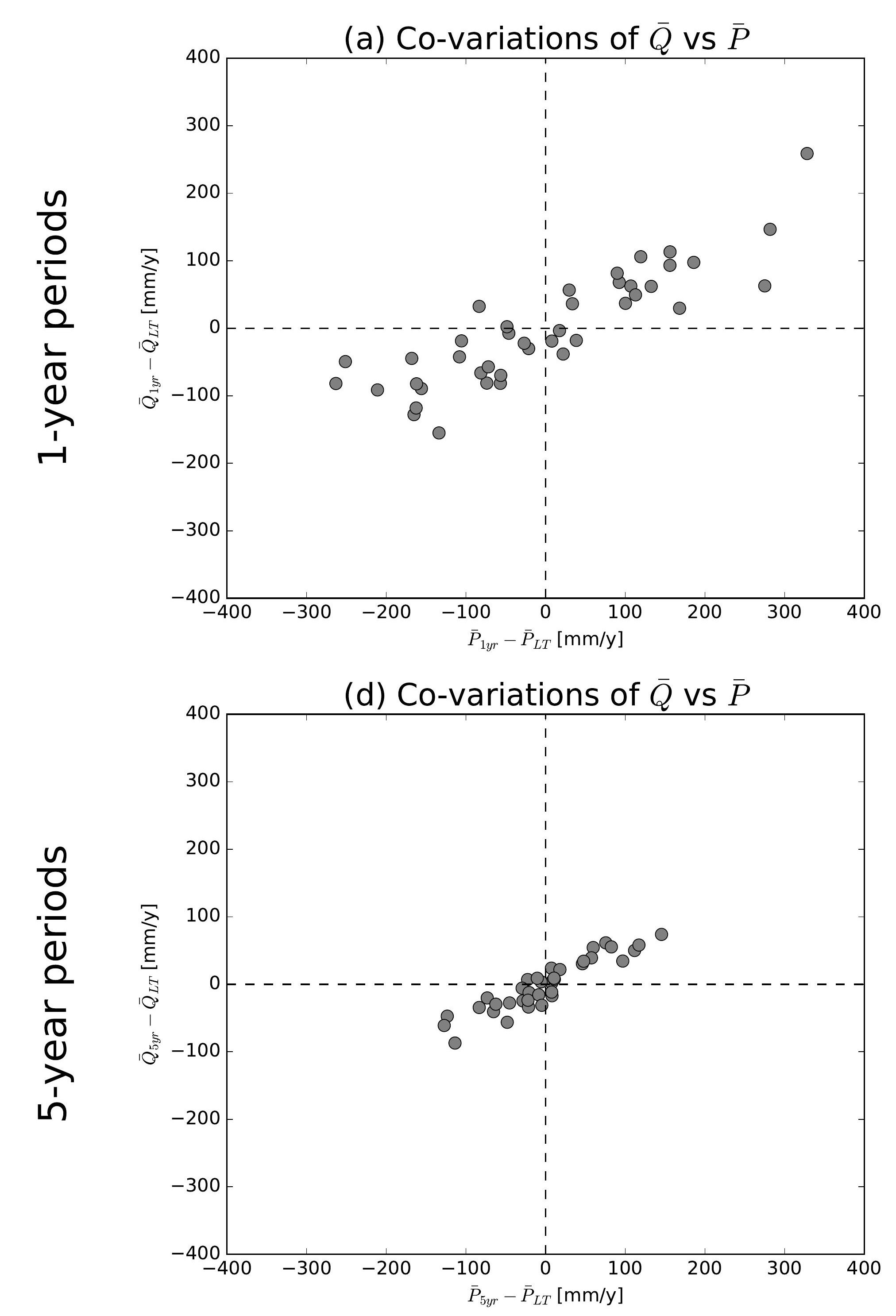

(g) Co-variations of $\bar{Q}$ vs $\bar{P}$
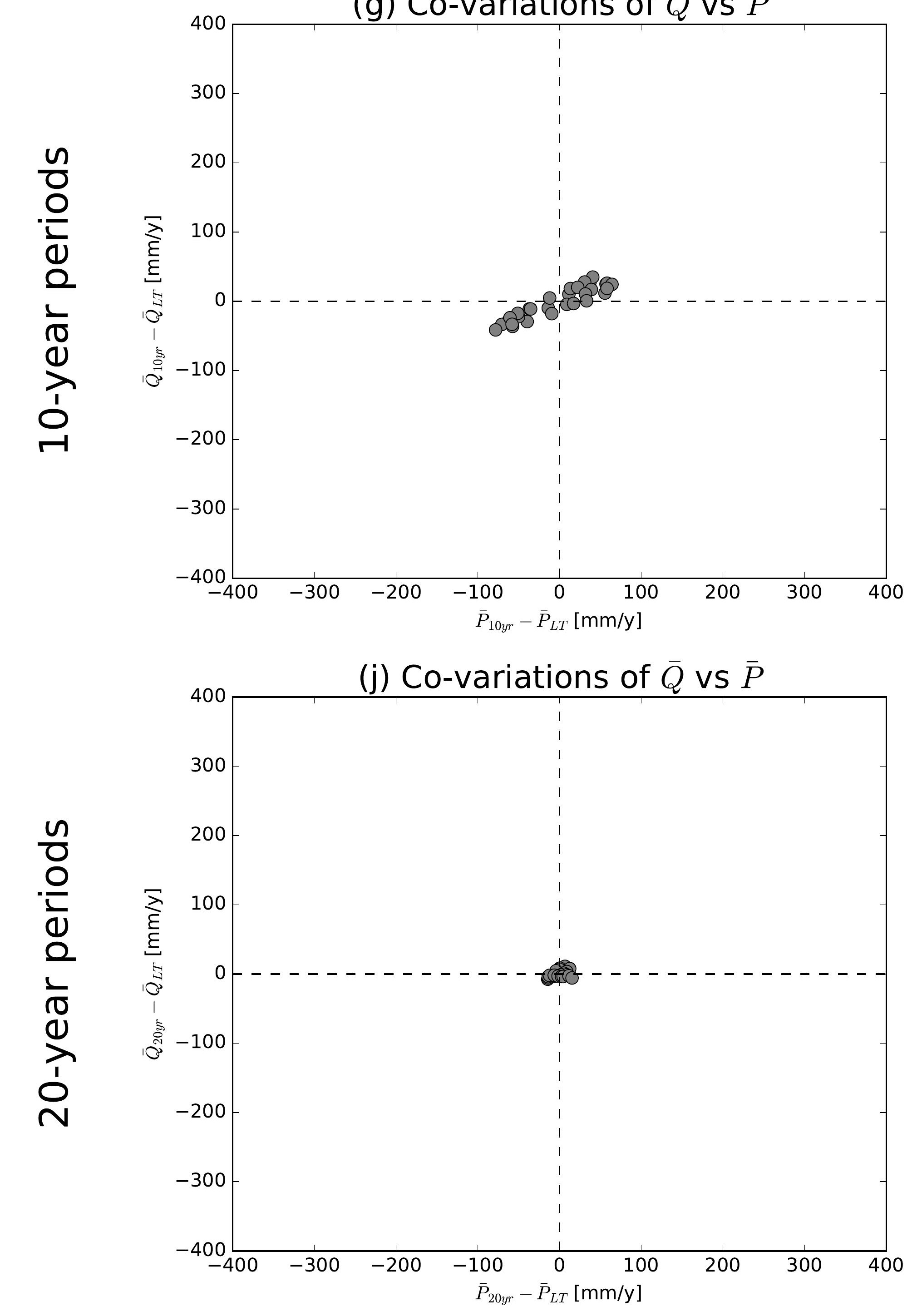

(b) Co-variations of $\bar{Q}$ vs $\overline{E 0}$

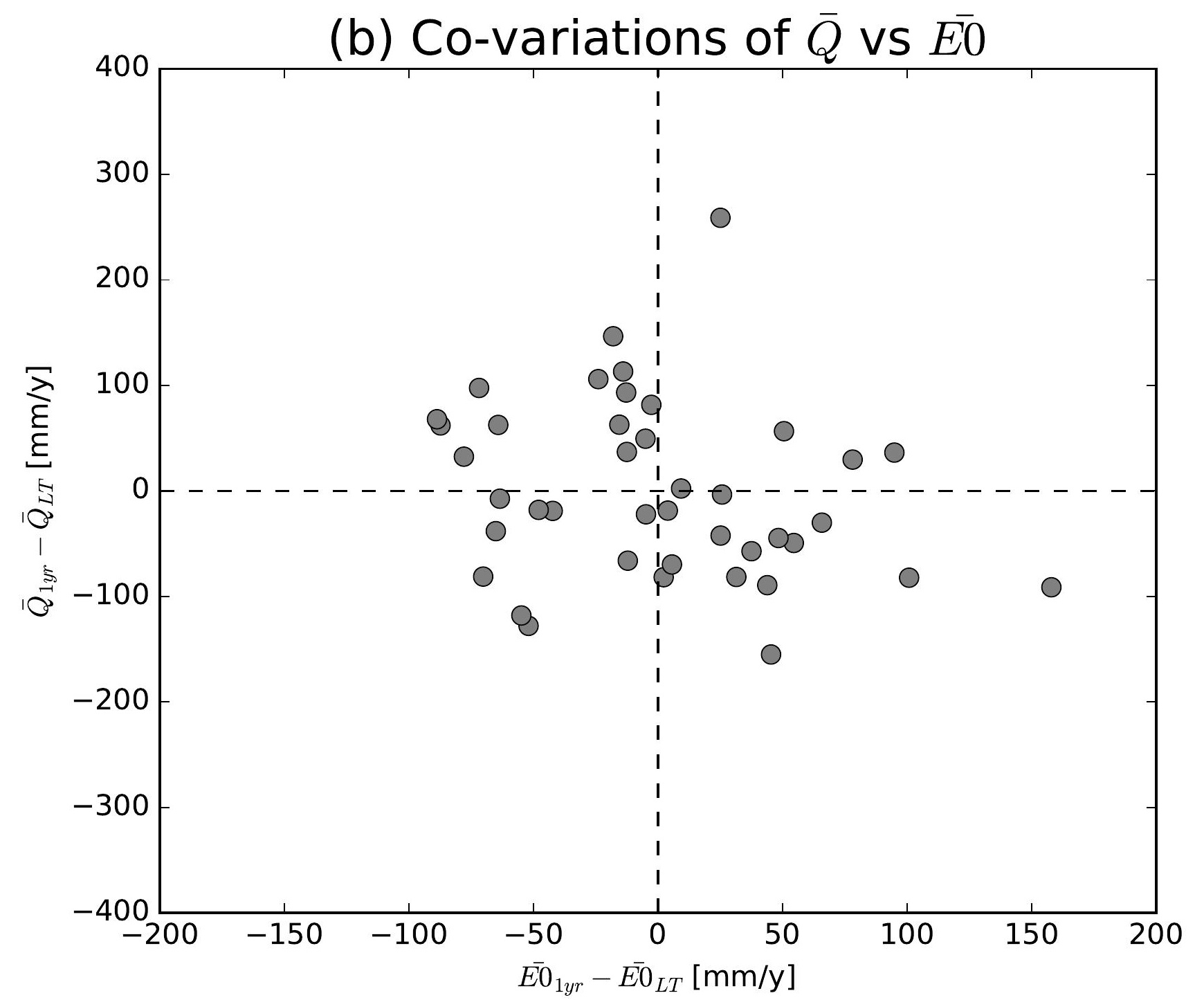

(e) Co-variations of $\bar{Q}$ vs $\overline{E 0}$

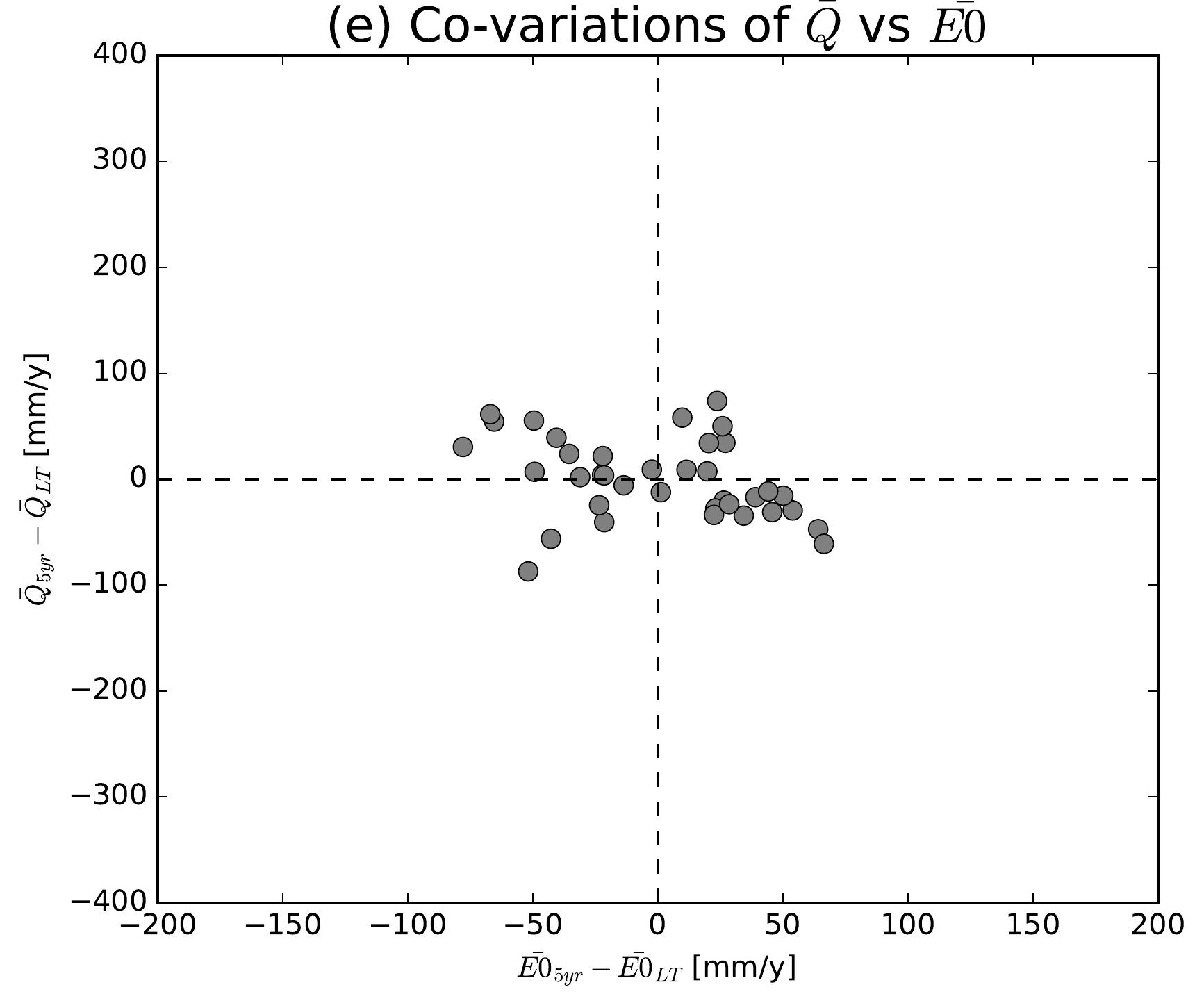

(h) Co-variations of $\bar{Q}$ vs $\overline{E 0}$

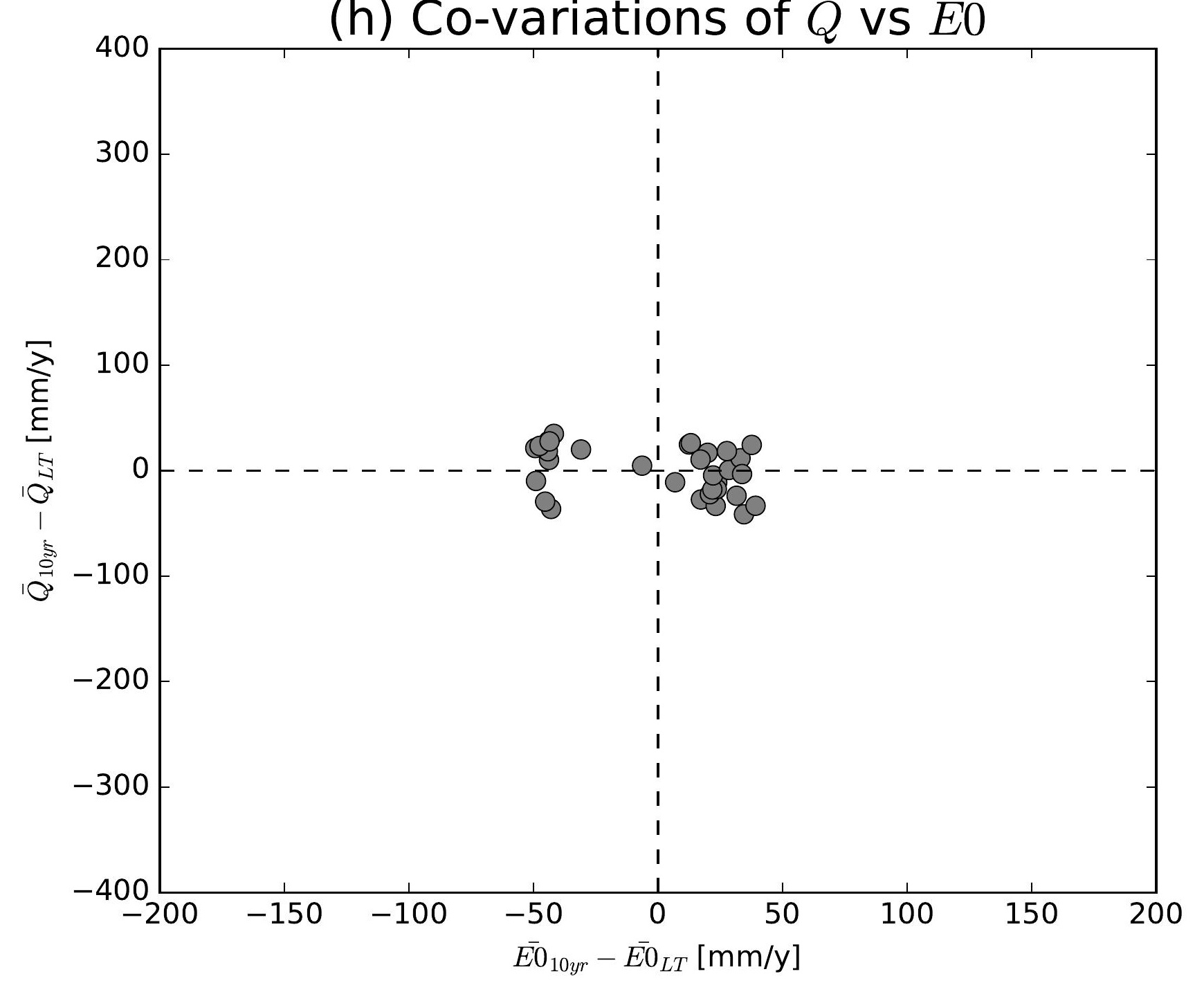

(k) Co-variations of $\bar{Q}$ vs $\overline{E 0}$

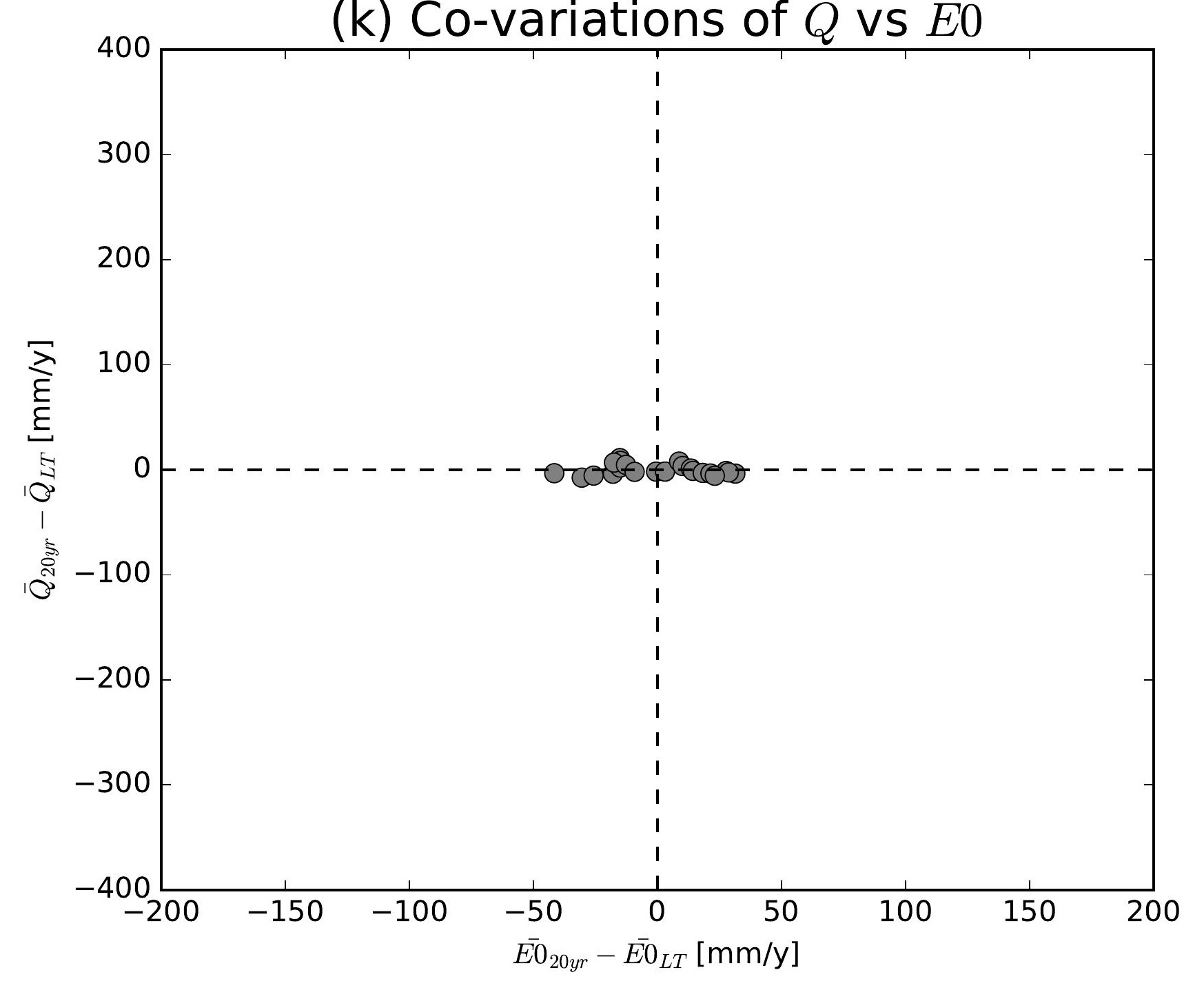

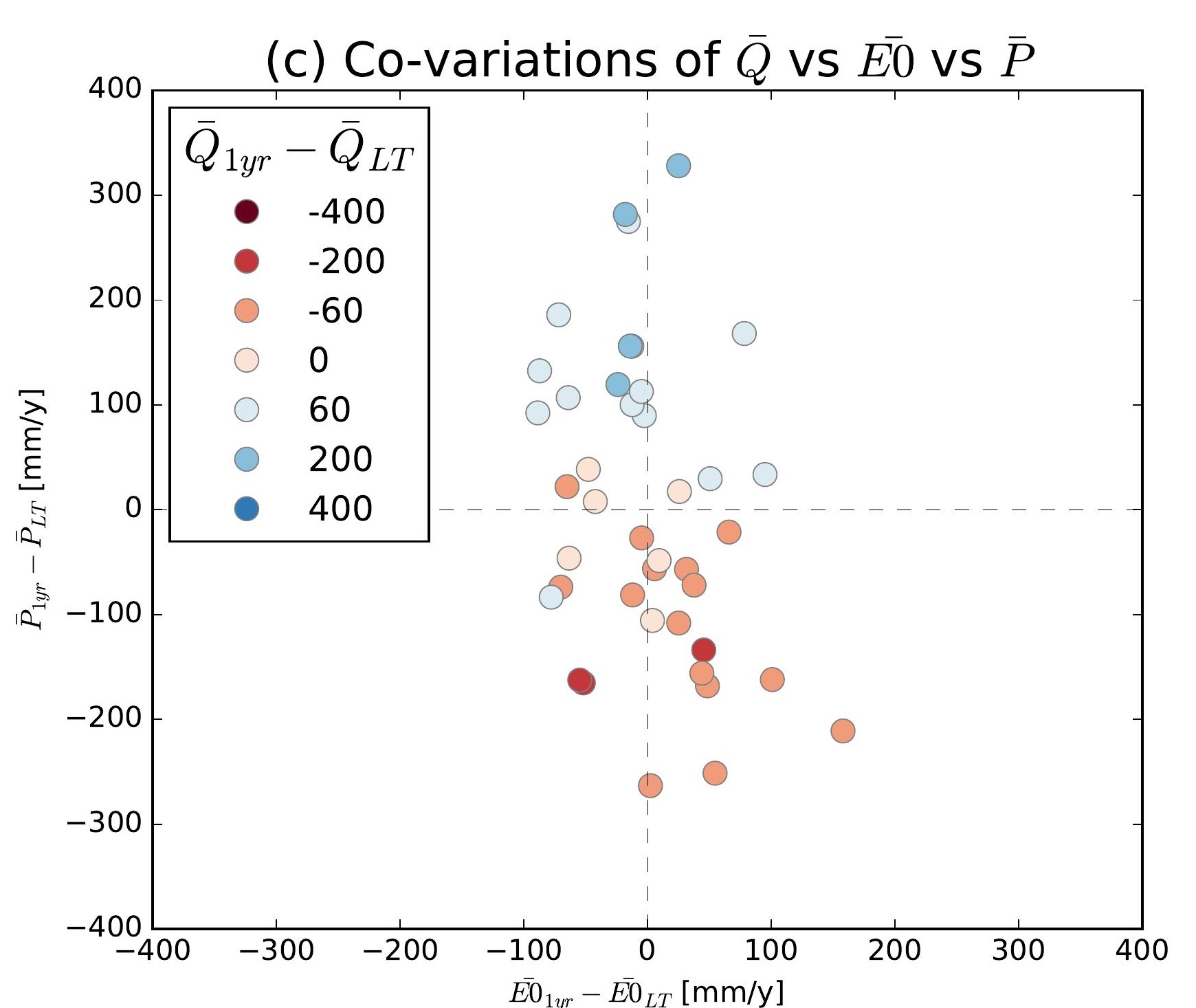
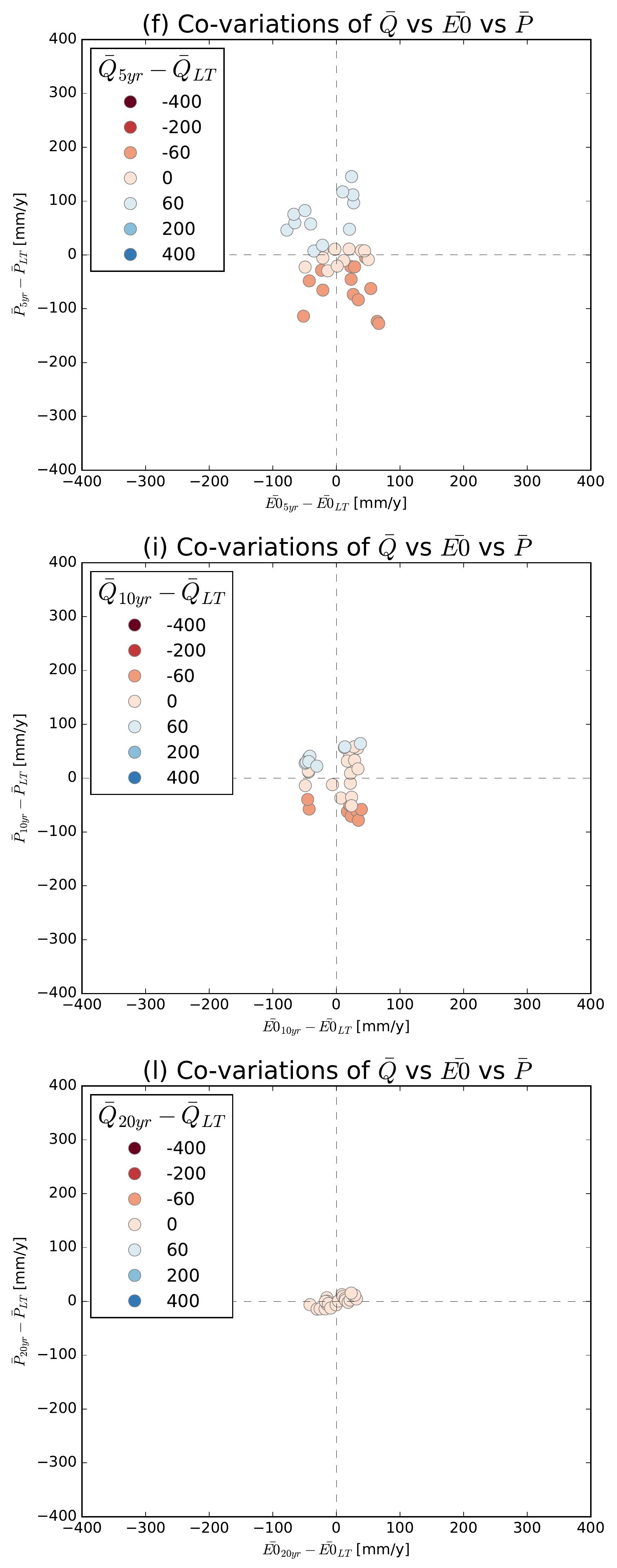

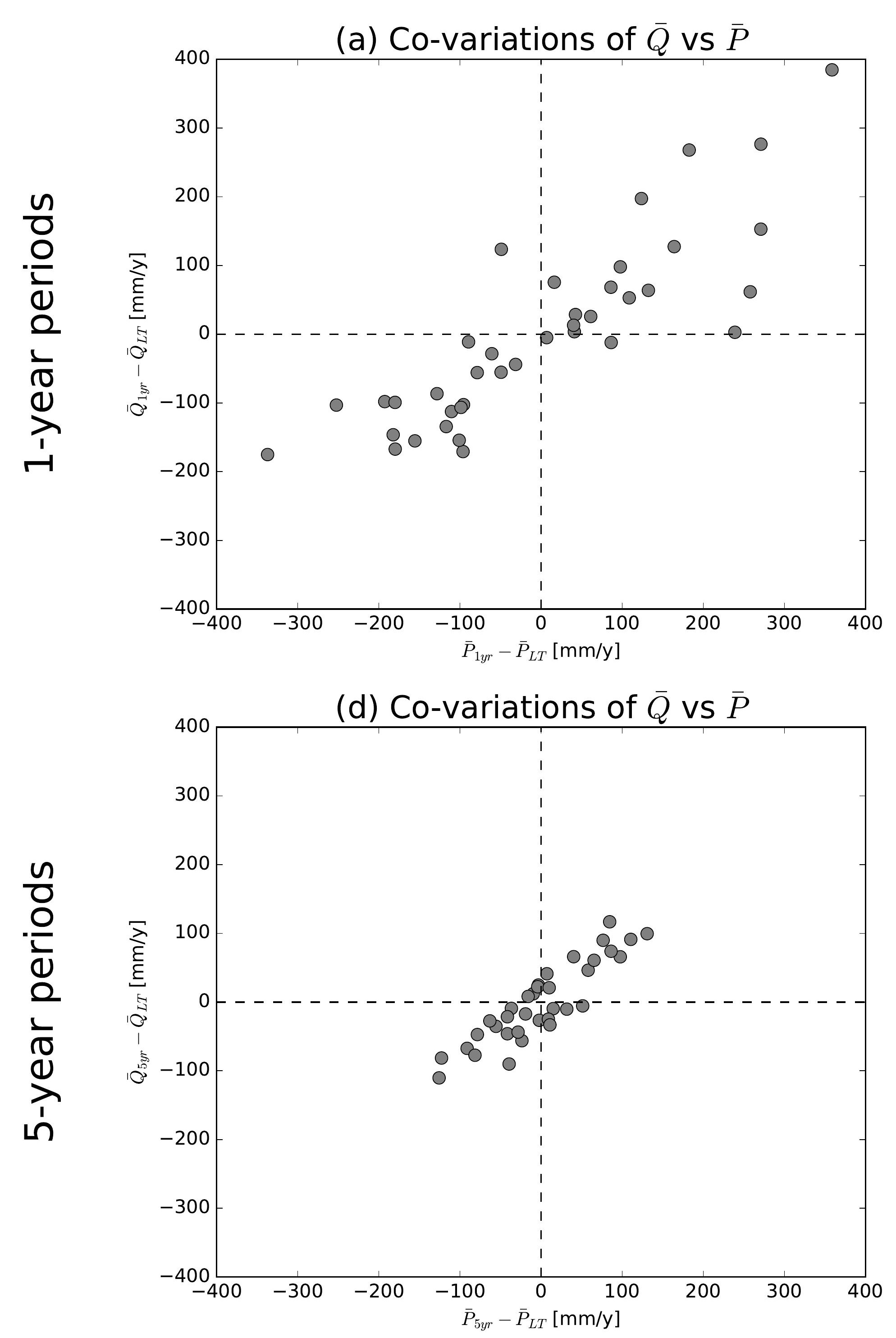

(g) Co-variations of $\bar{Q}$ vs $\bar{P}$

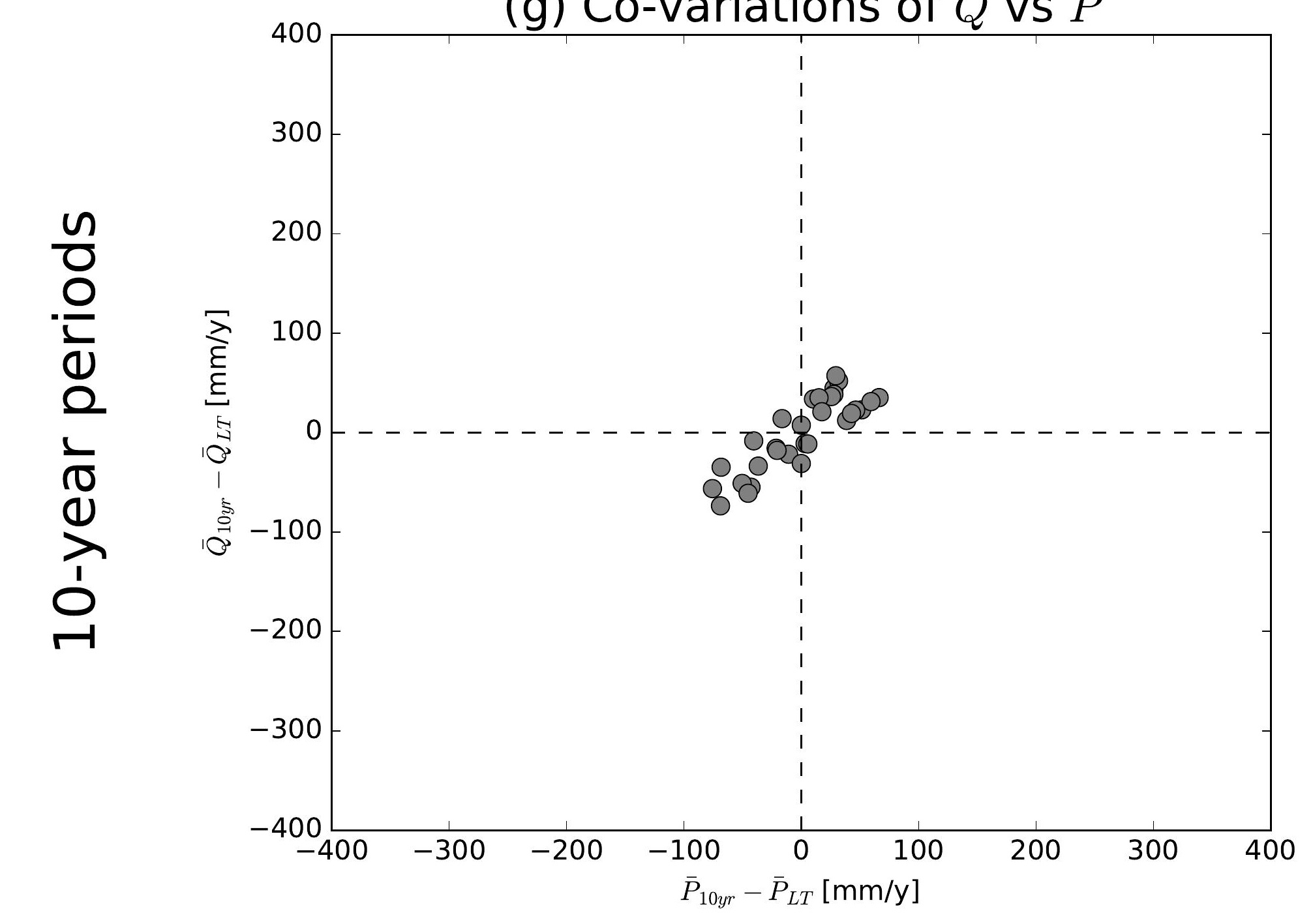

(j) Co-variations of $\bar{Q}$ vs $\bar{P}$

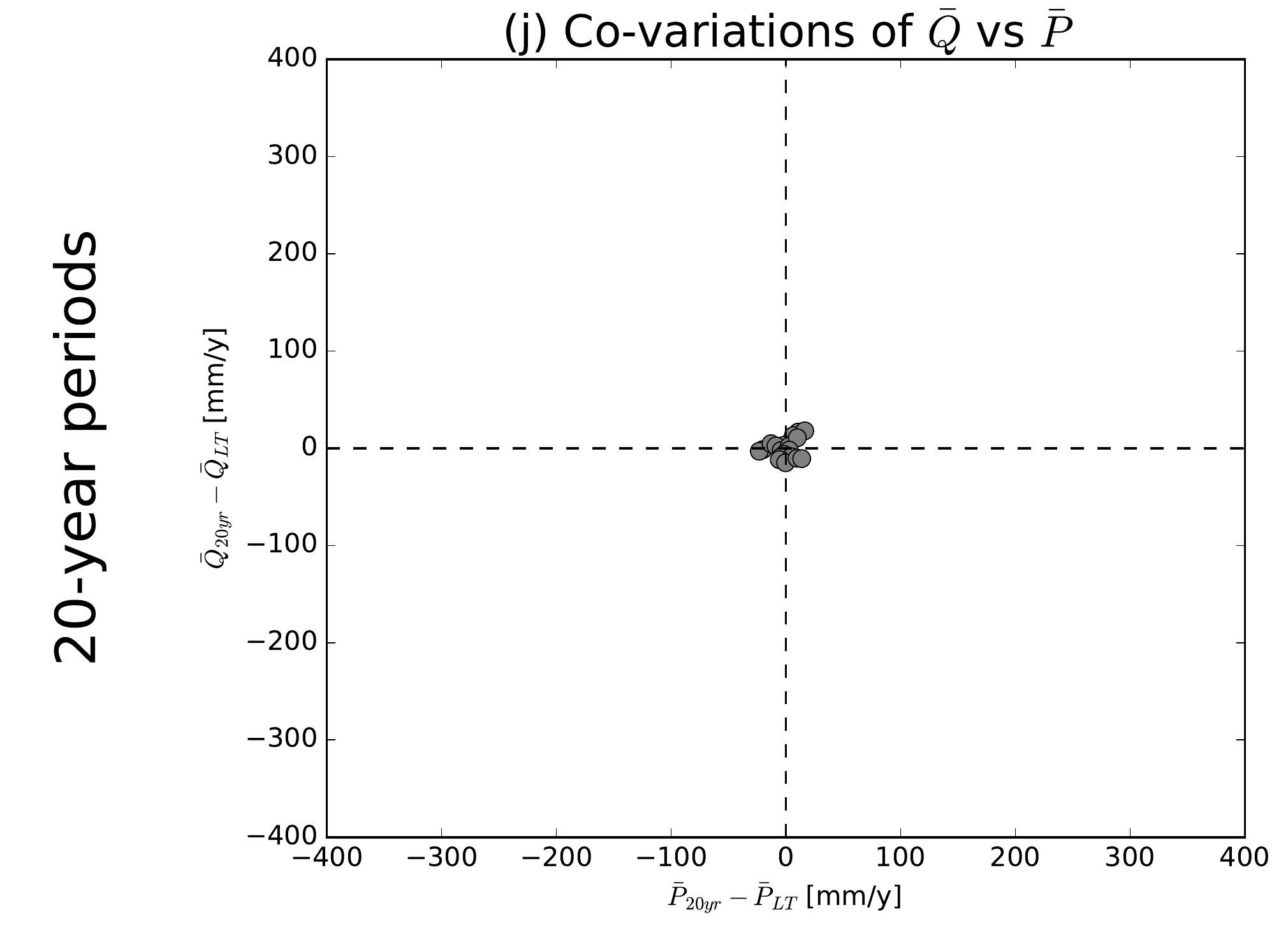

(b) Co-variations of $\bar{Q}$ vs $\overline{E 0}$

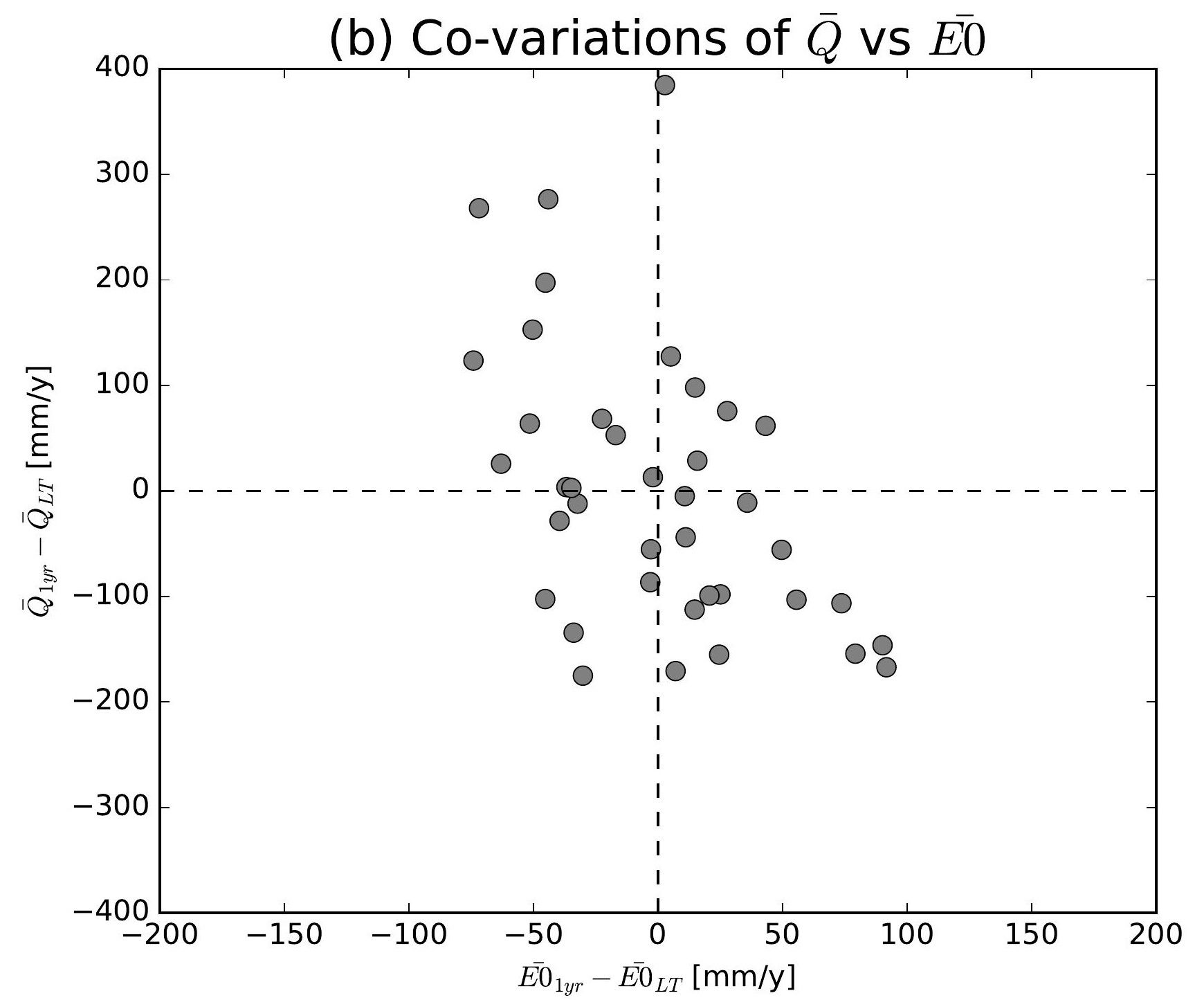

(e) Co-variations of $\bar{Q}$ vs $\overline{E 0}$

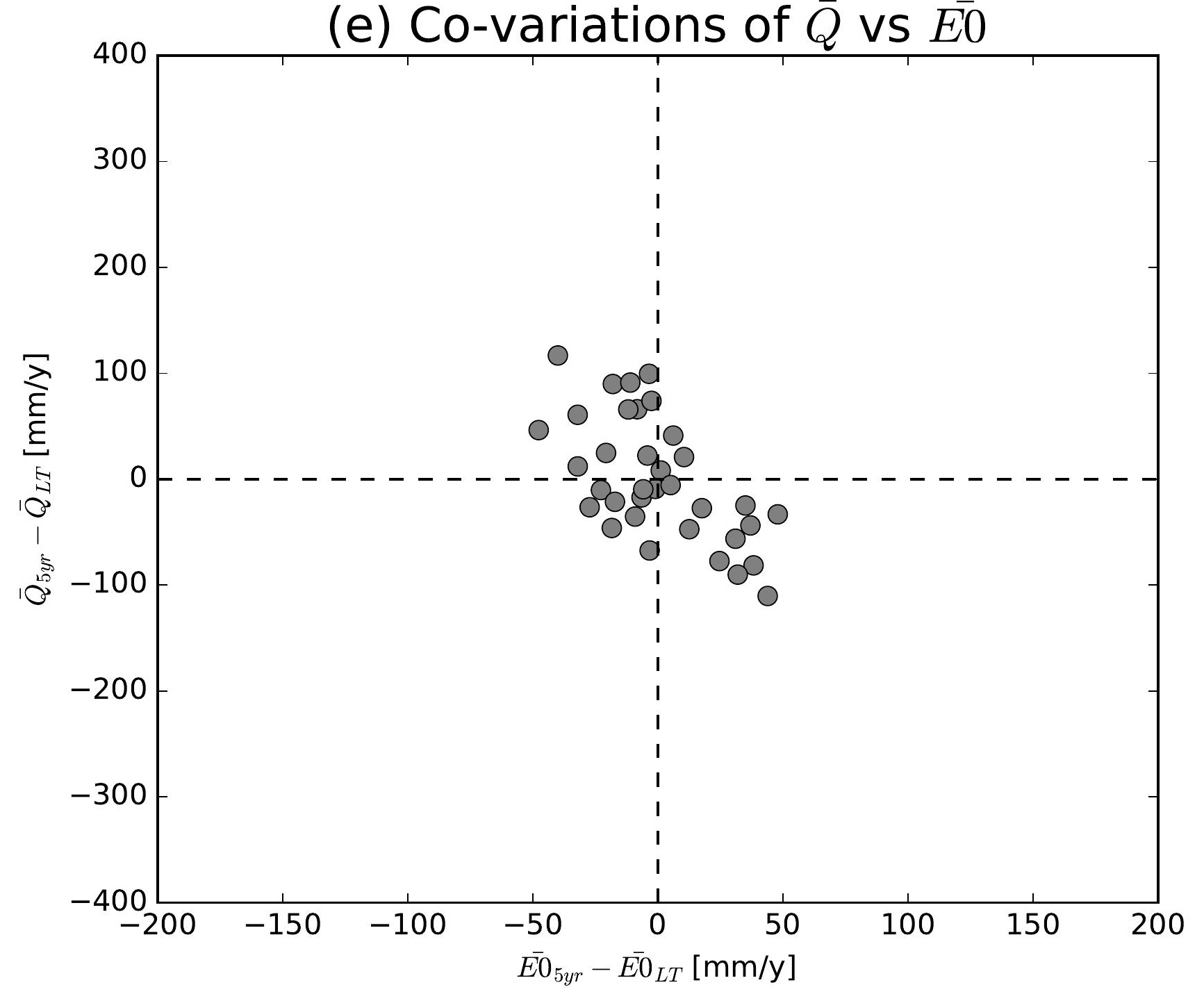

(h) Co-variations of $\bar{Q}$ vs $\overline{E 0}$

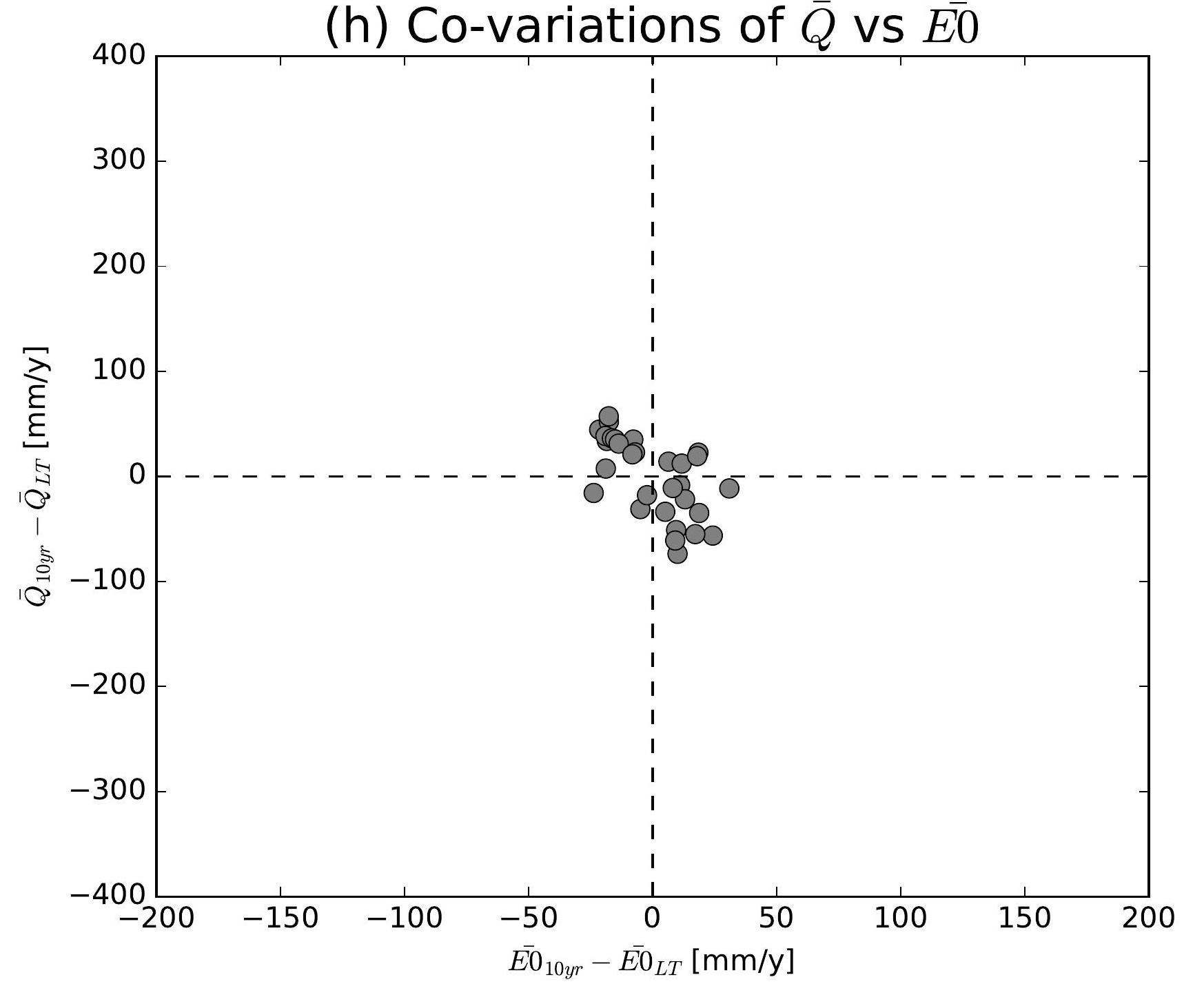

(k) Co-variations of $\bar{Q}$ vs $\overline{E 0}$

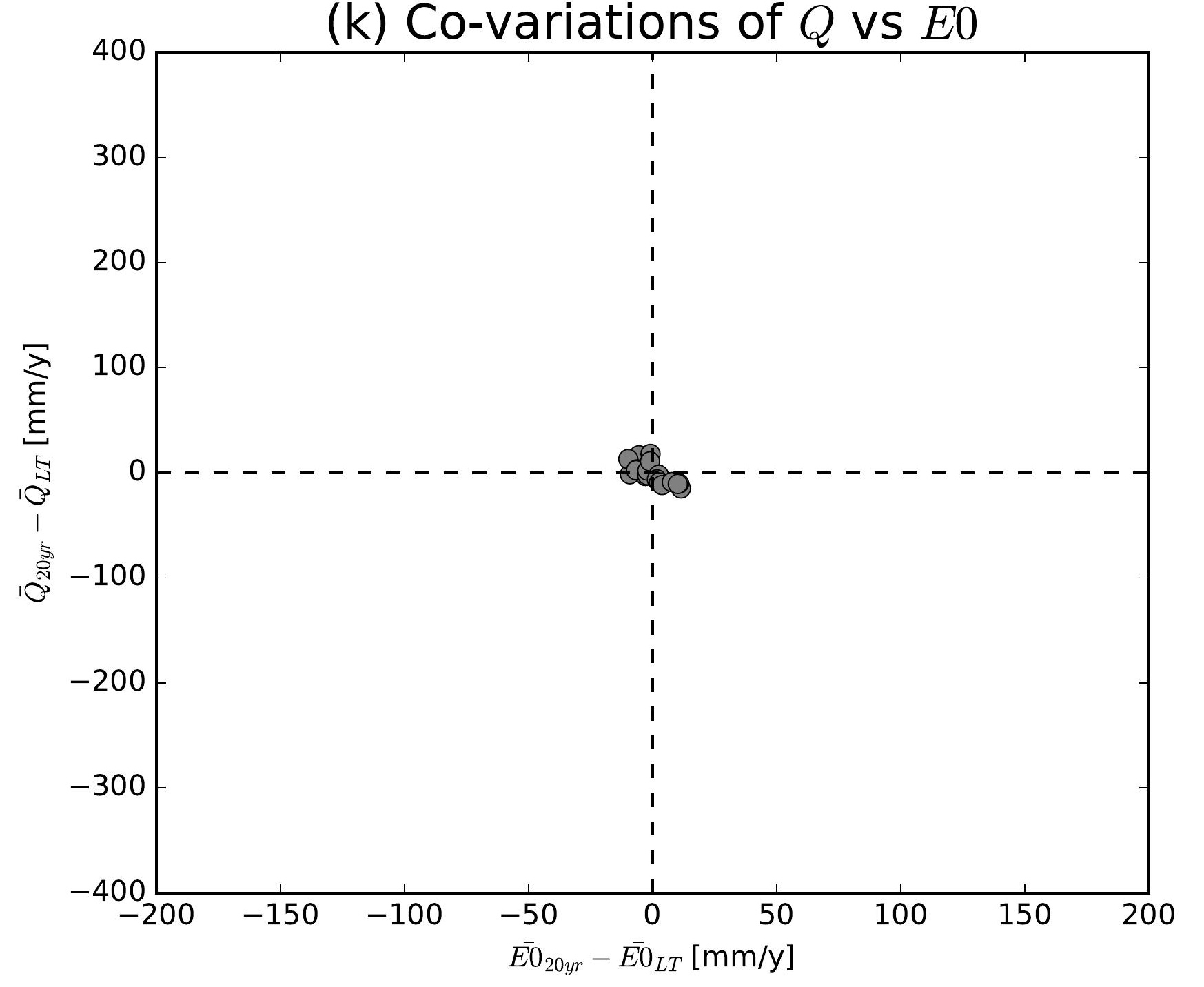

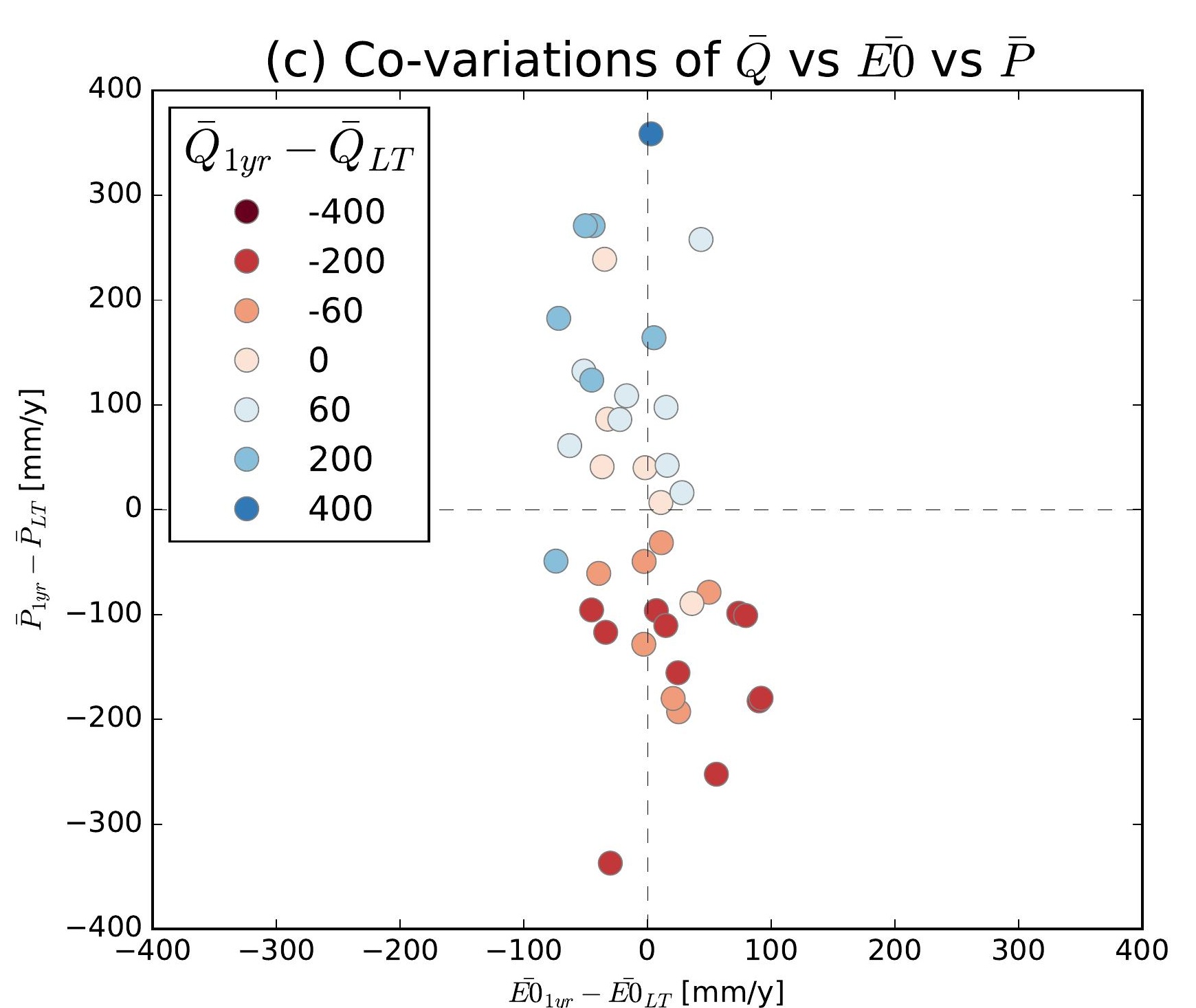
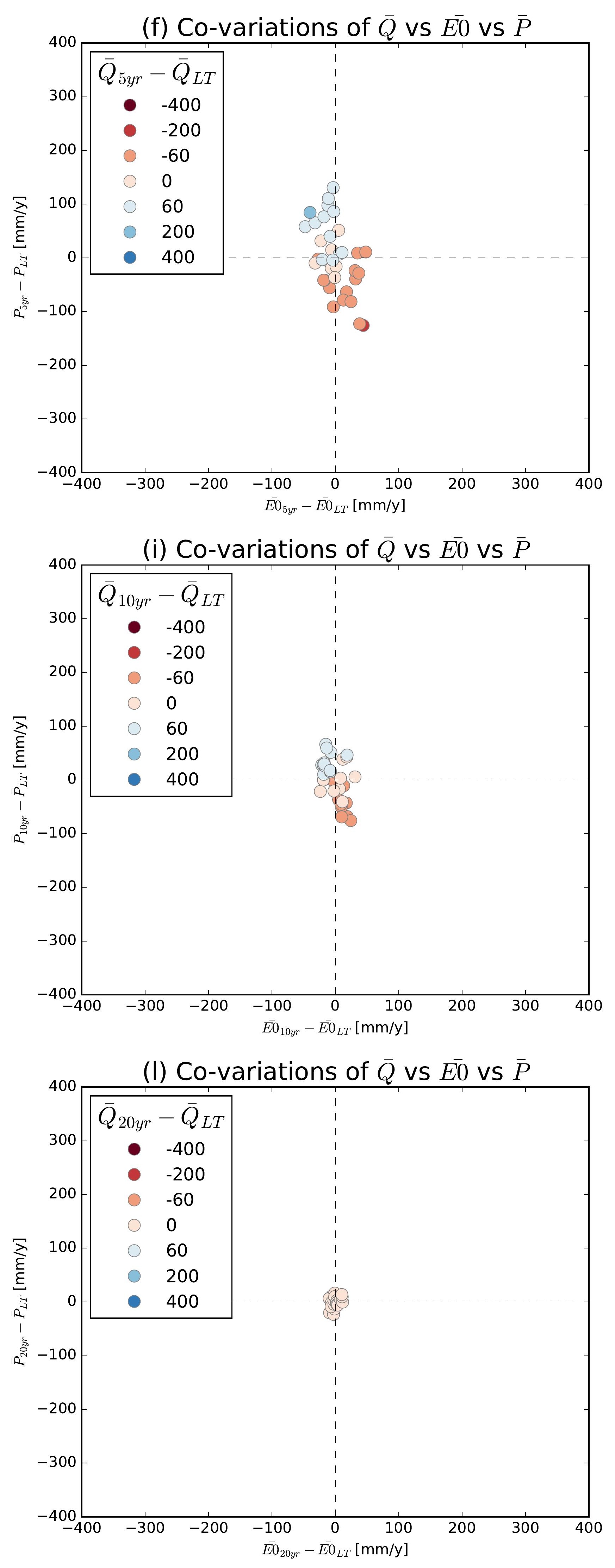

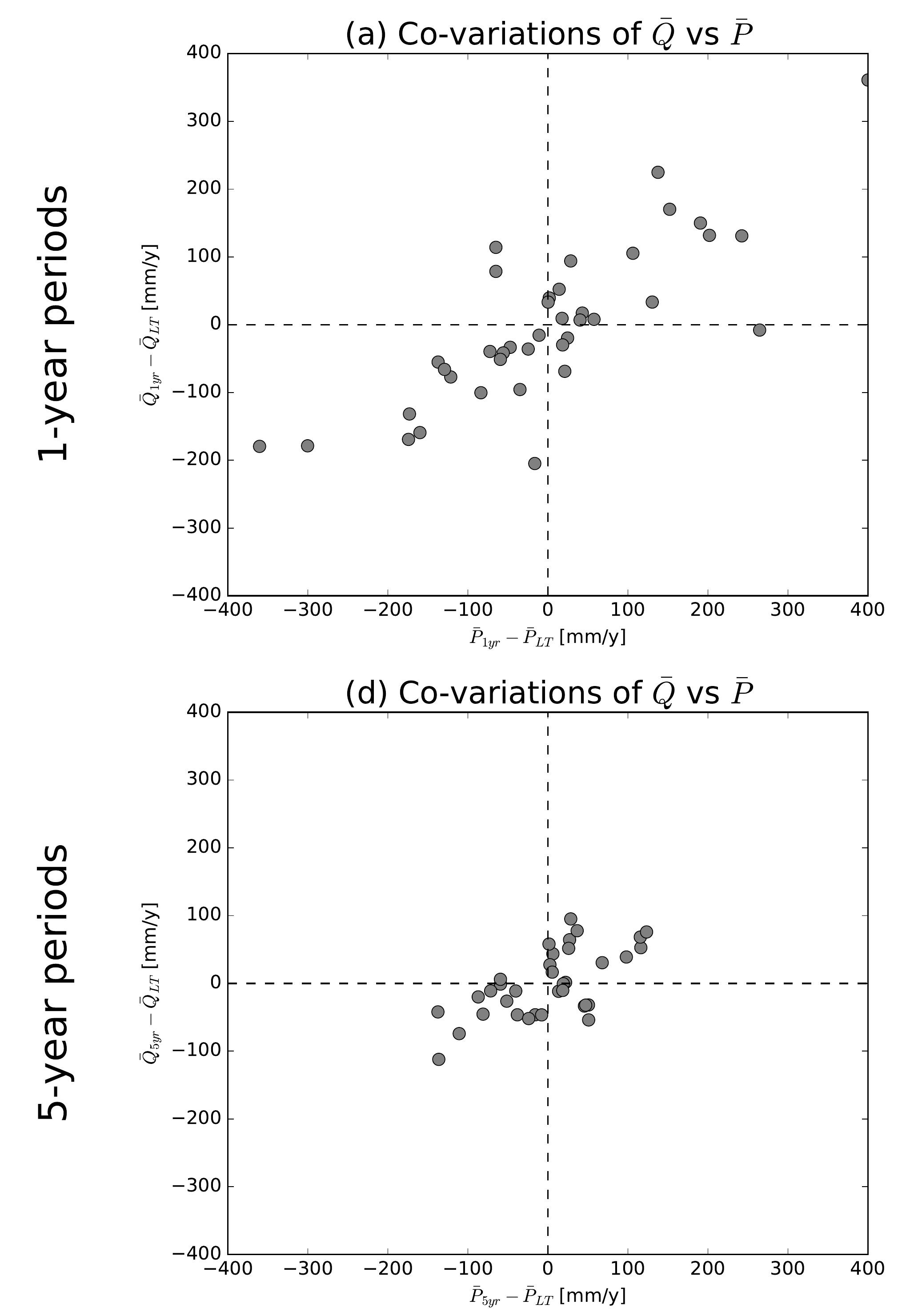

(g) Co-variations of $\bar{Q}$ vs $\bar{P}$
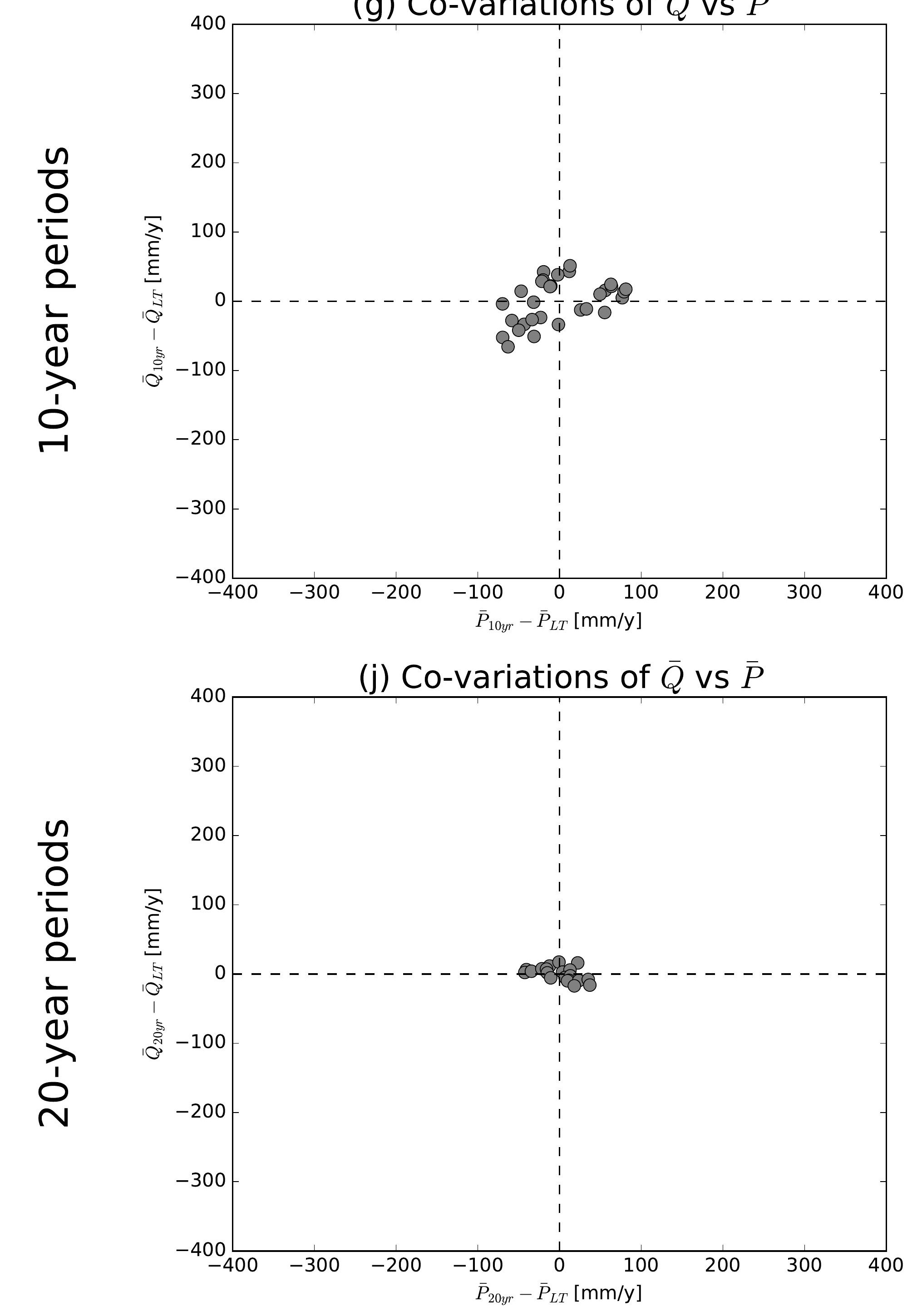

(b) Co-variations of $\bar{Q}$ vs $\overline{E 0}$

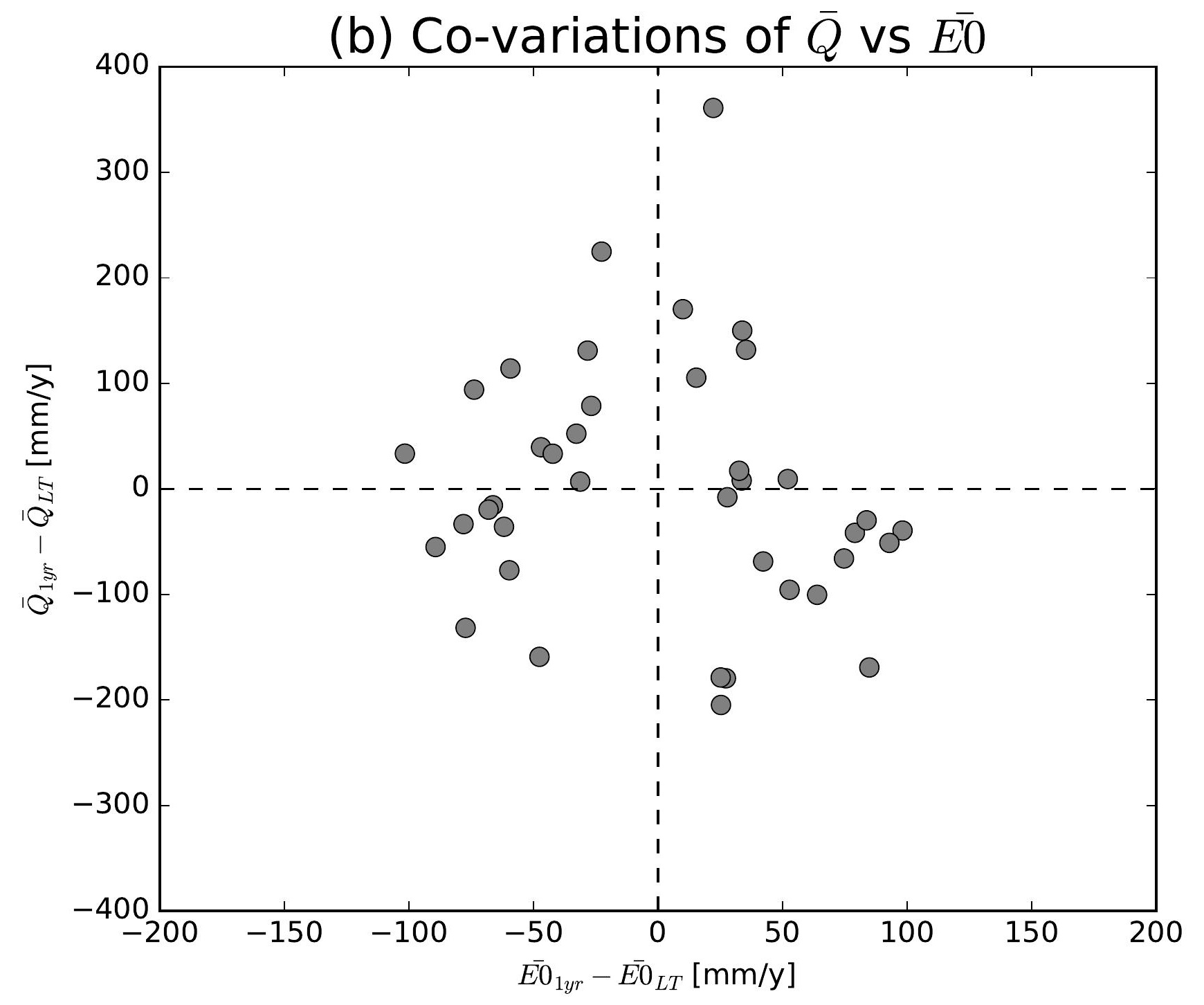

(e) Co-variations of $\bar{Q}$ vs $\overline{E 0}$

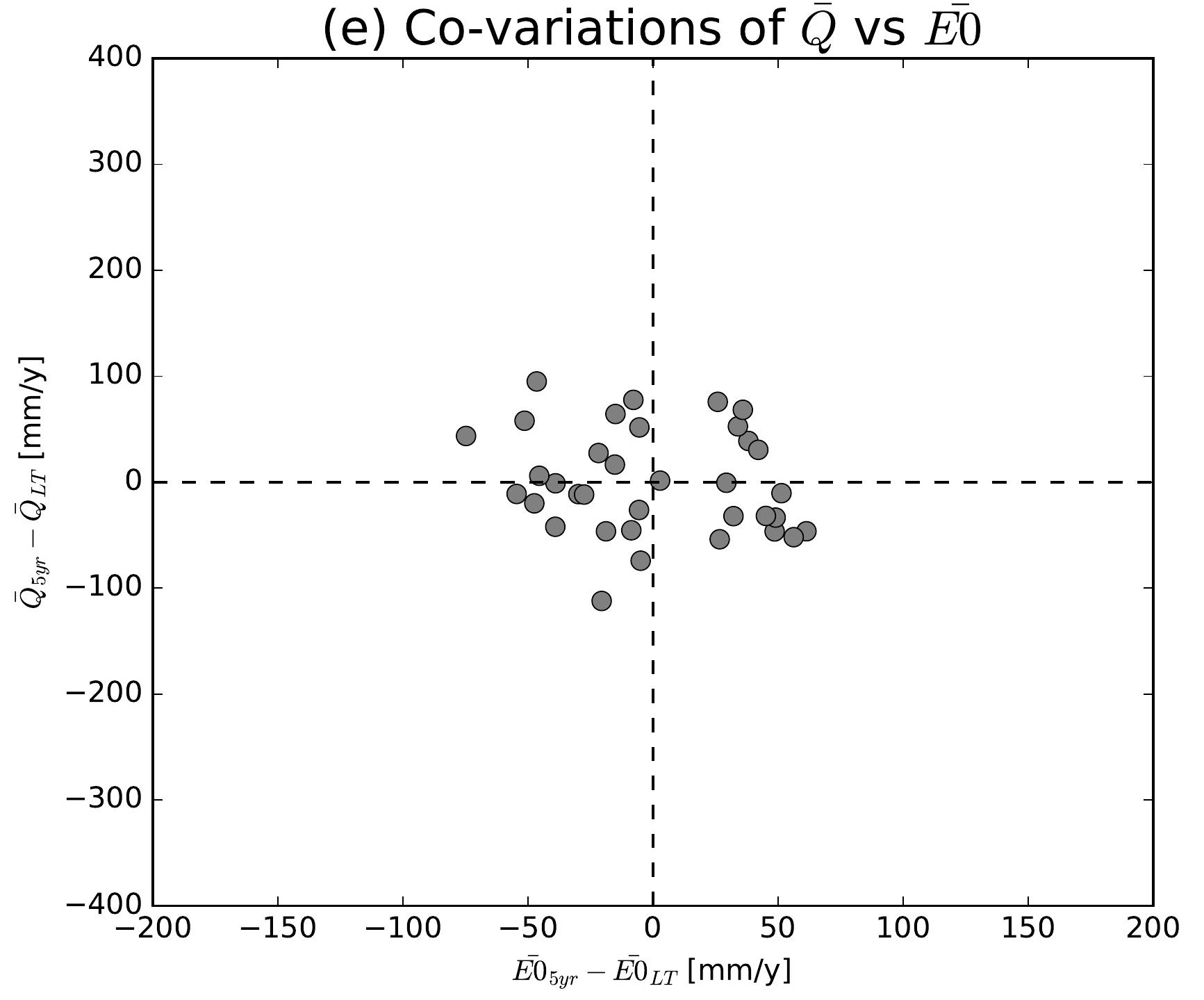

(h) Co-variations of $\bar{Q}$ vs $\overline{E 0}$

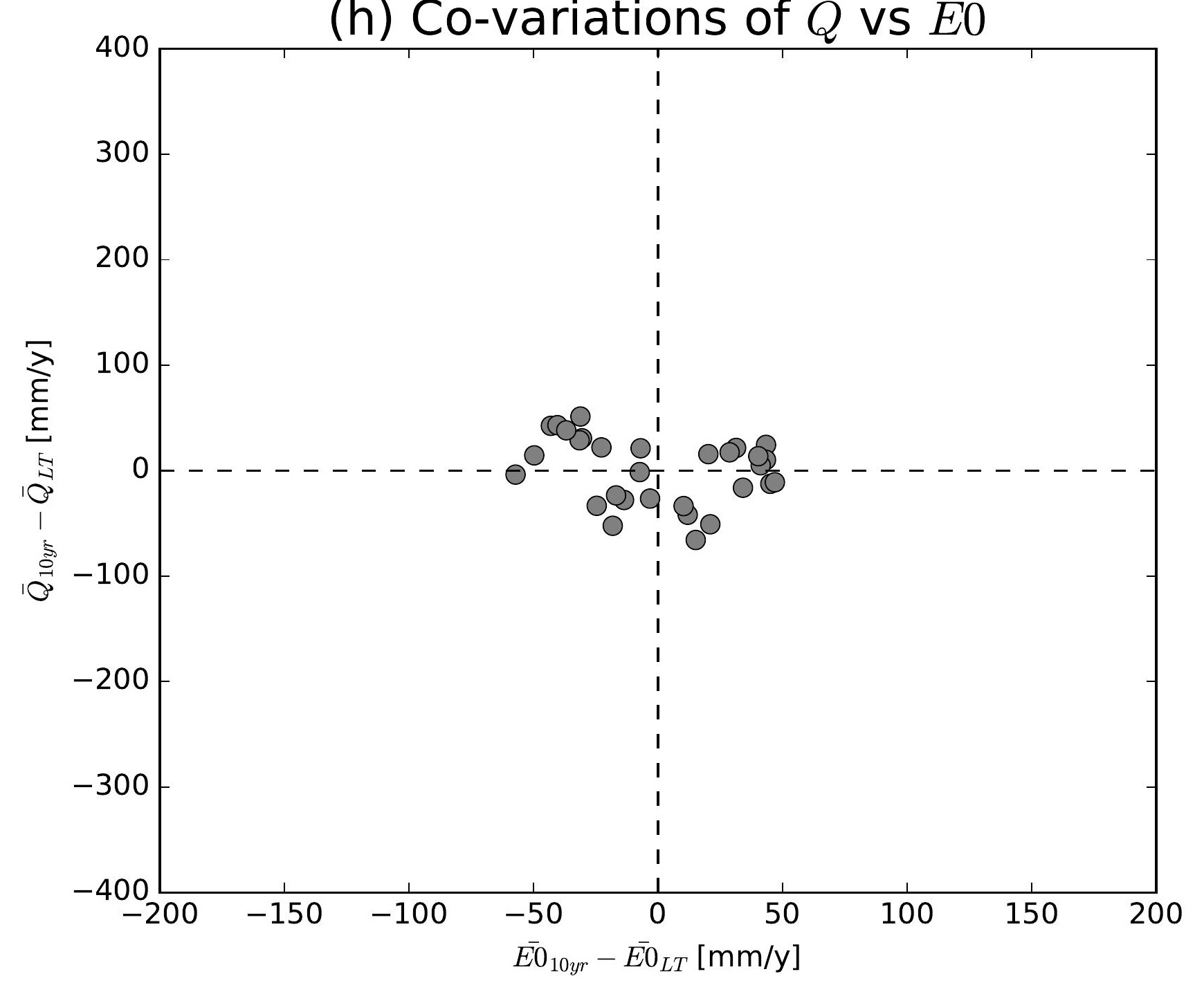

(k) Co-variations of $\bar{Q}$ vs $\overline{E 0}$

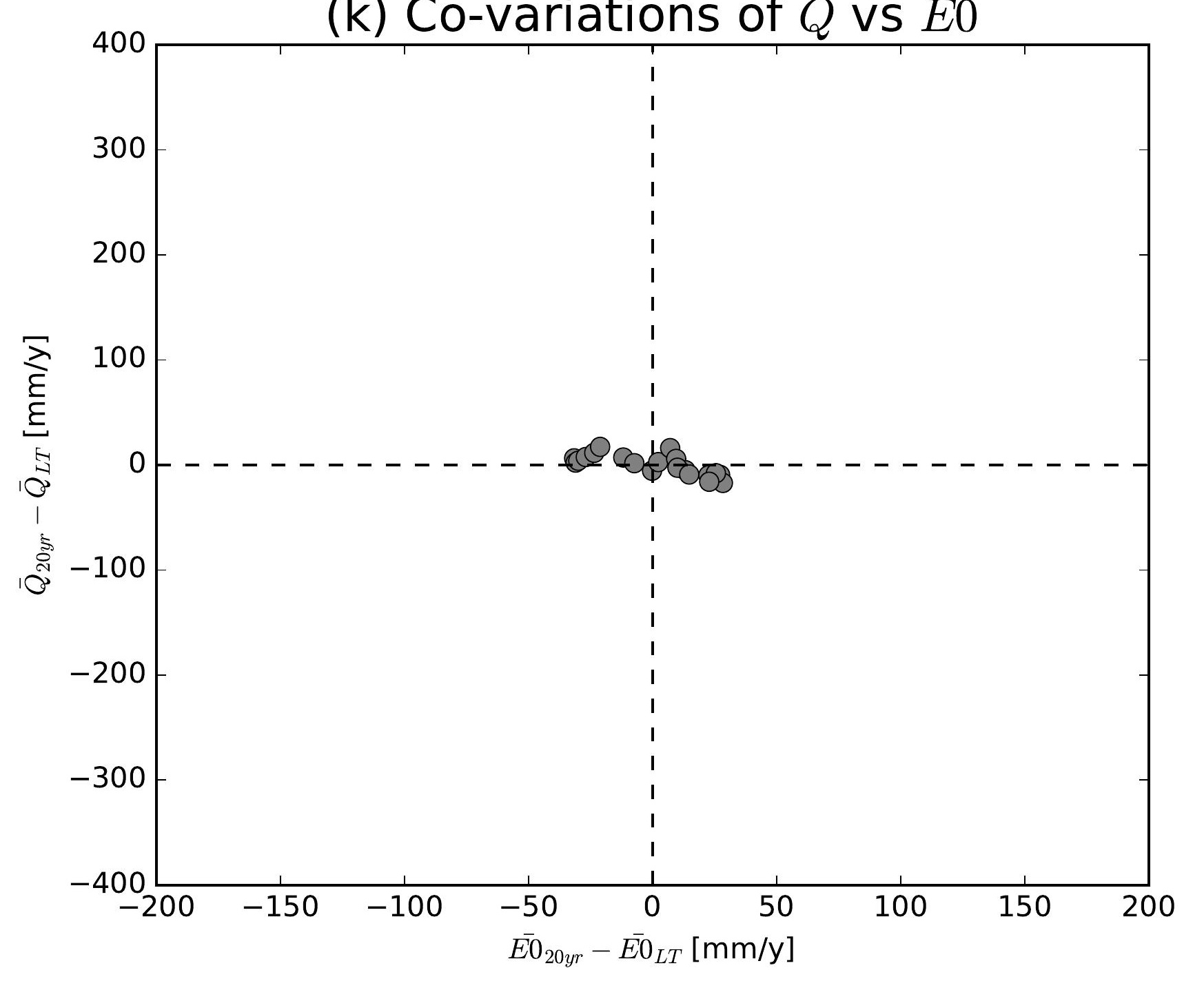

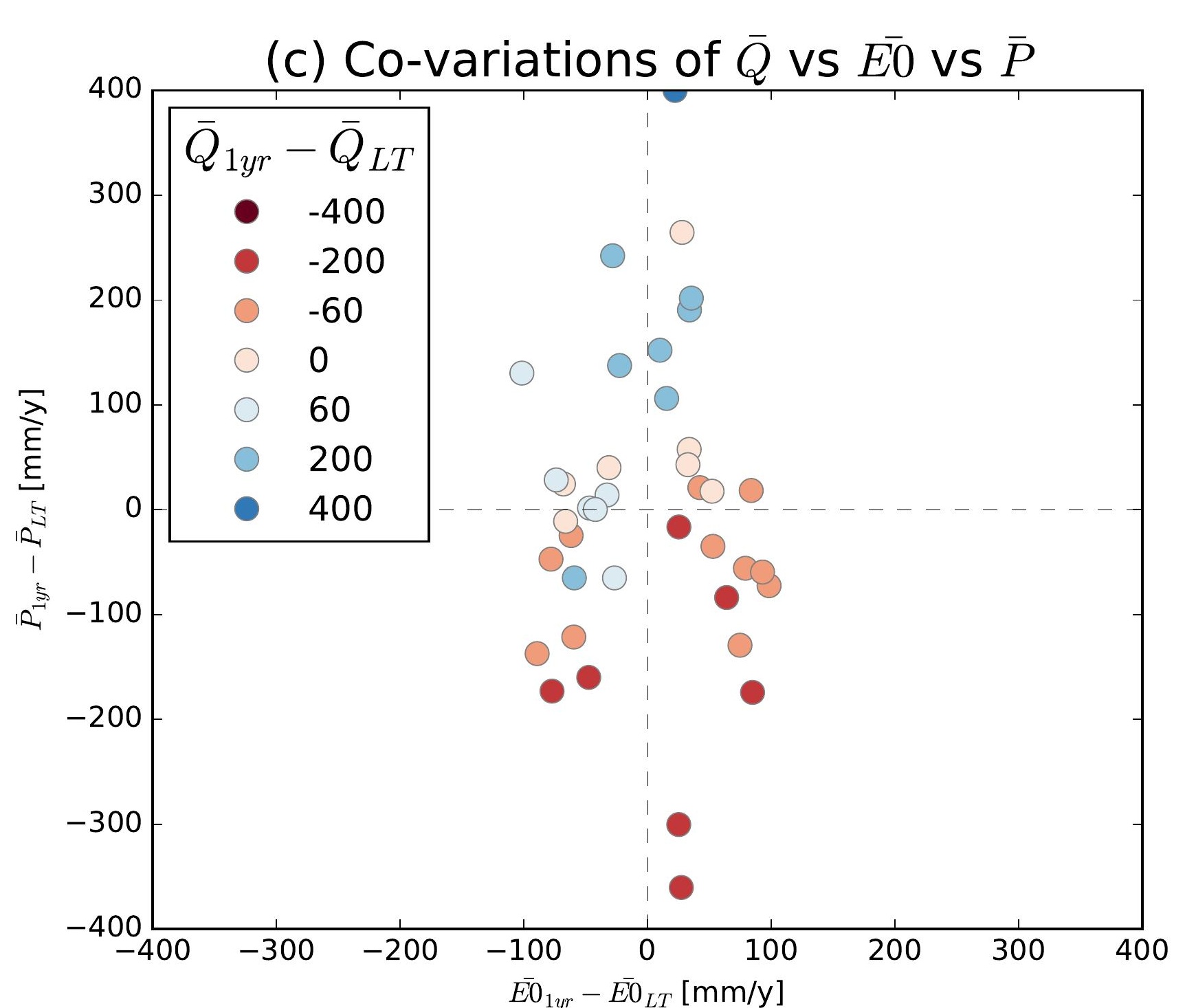
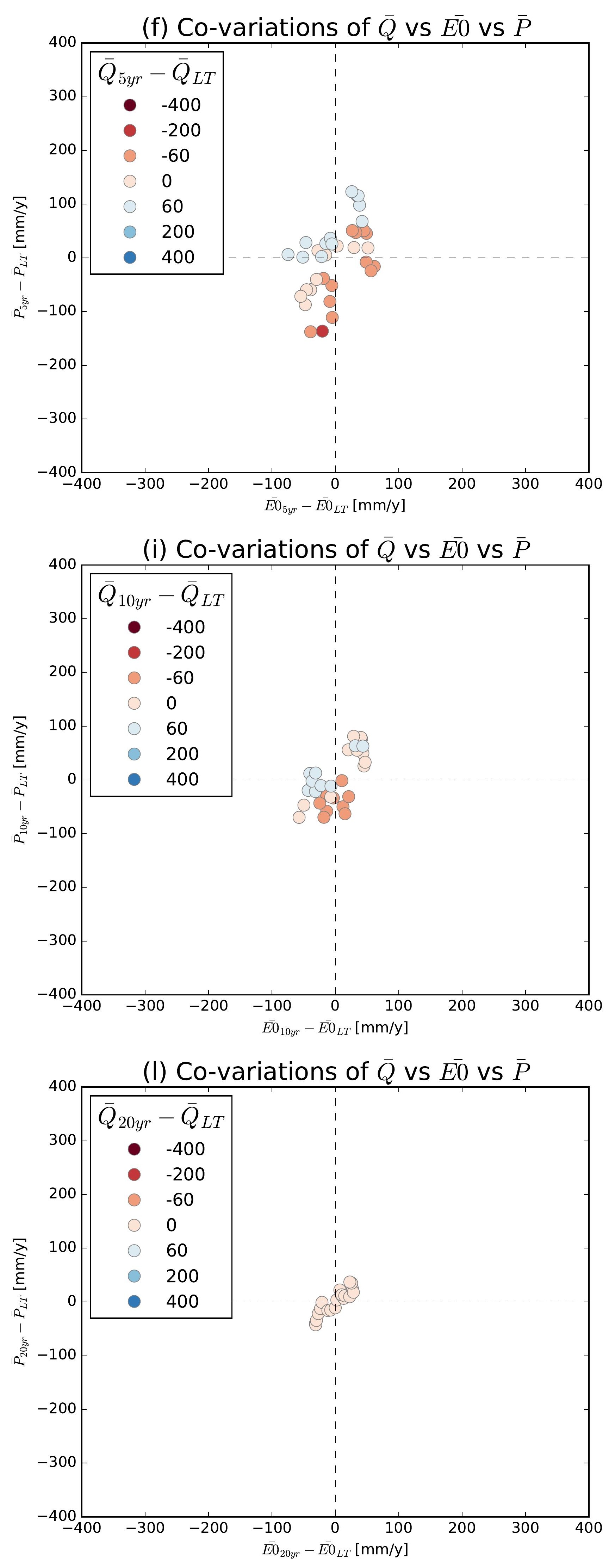

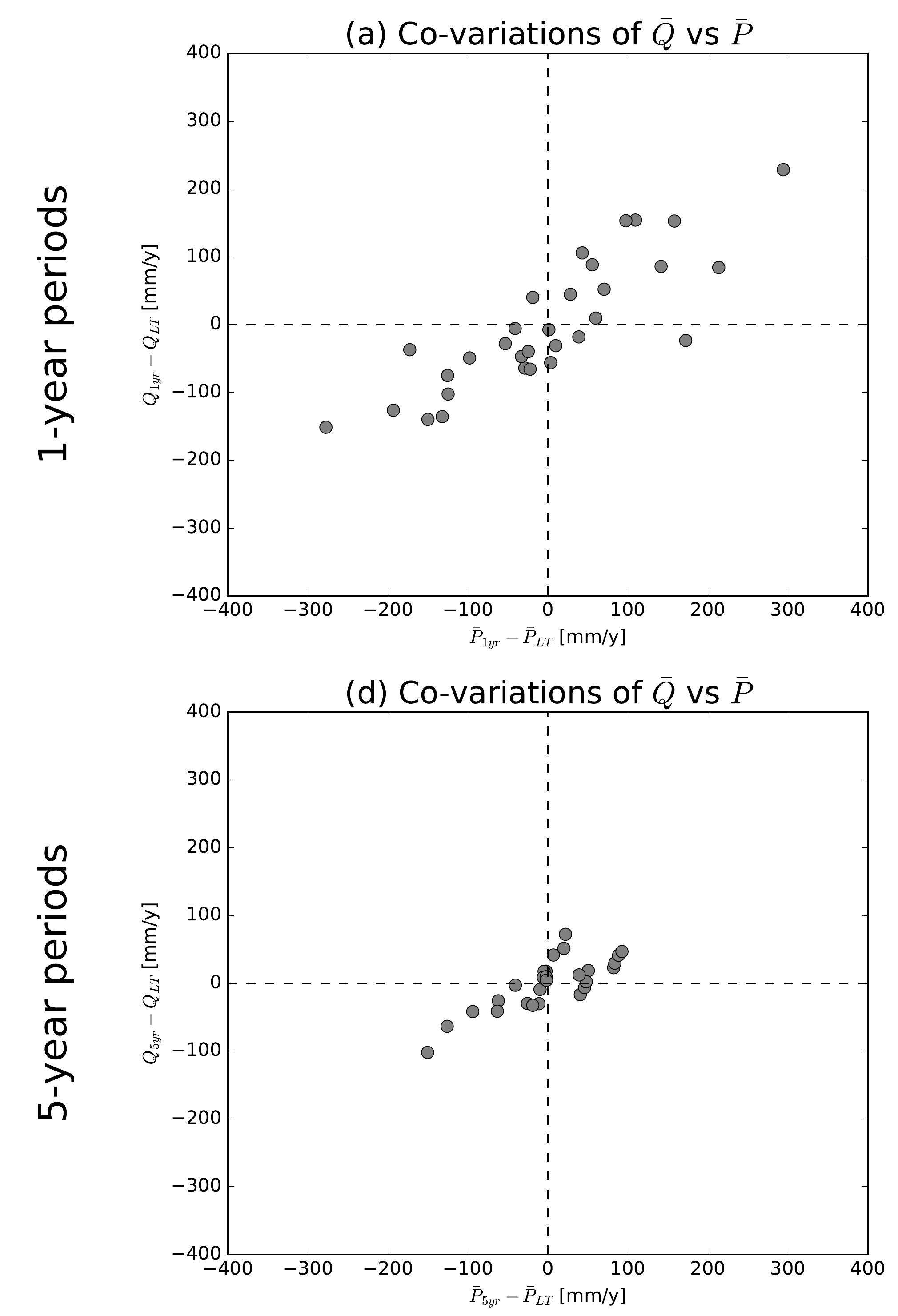

(g) Co-variations of $\bar{Q}$ vs $\bar{P}$
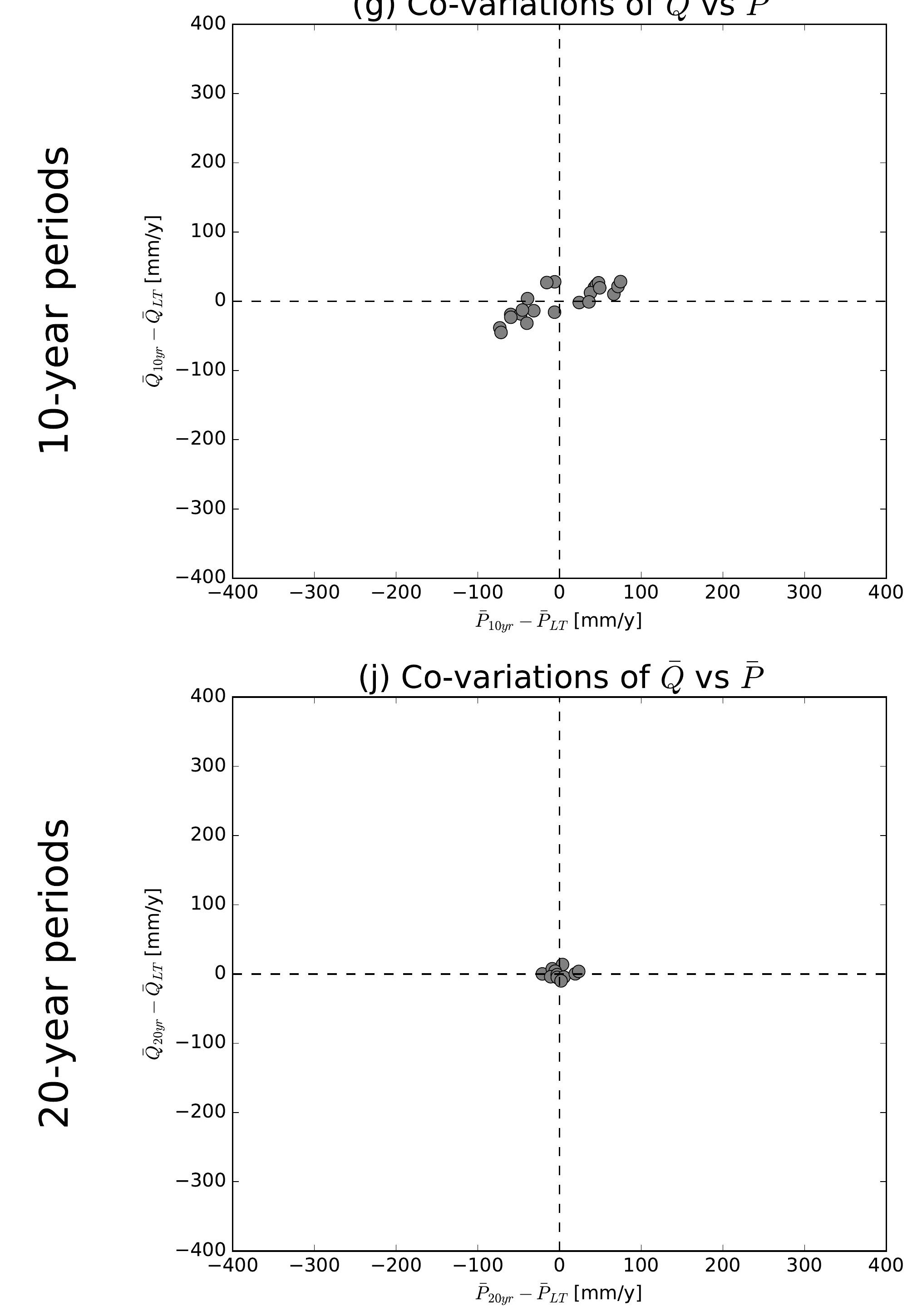

(b) Co-variations of $\bar{Q}$ vs $\overline{E 0}$

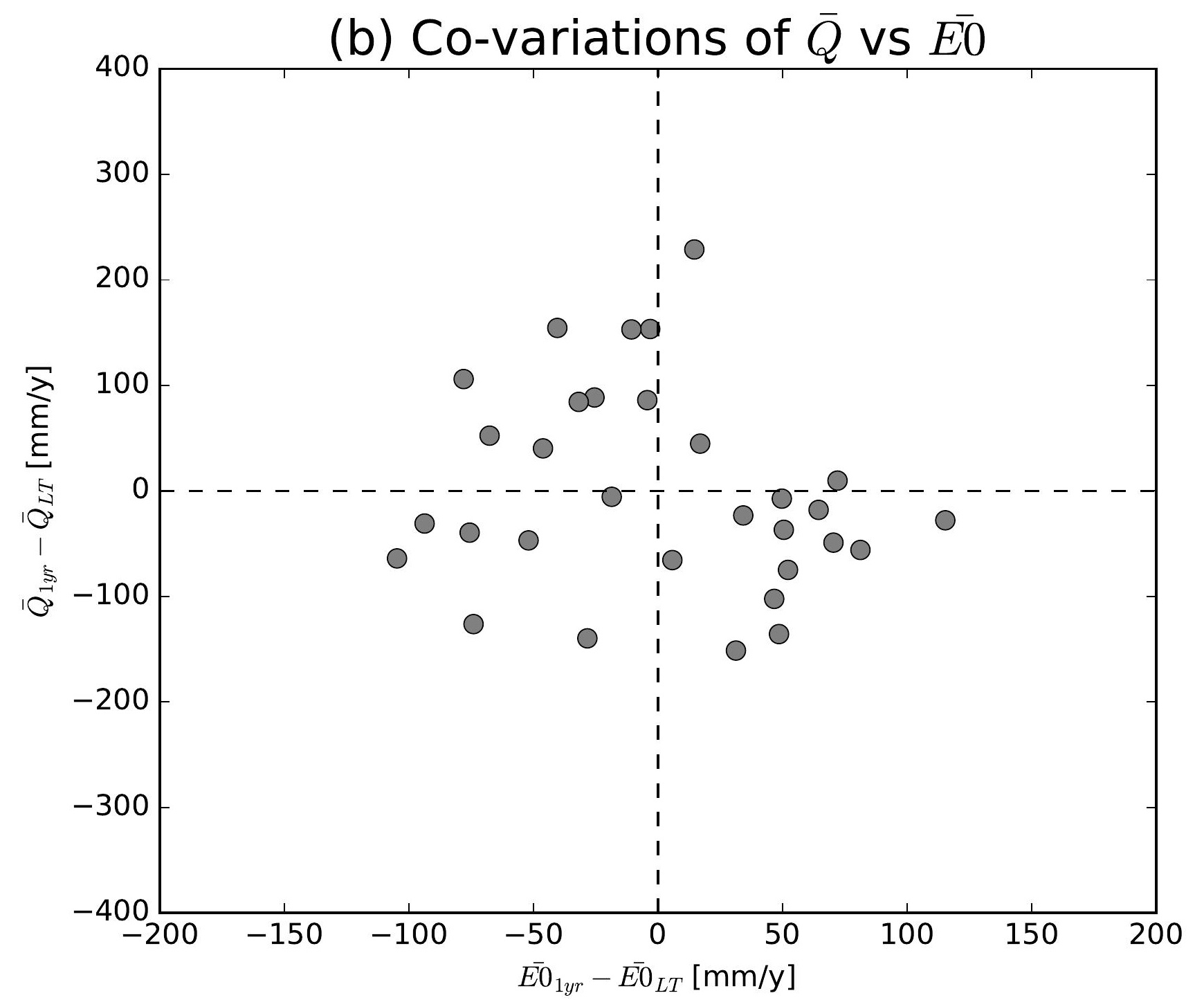

(e) Co-variations of $\bar{Q}$ vs $\overline{E 0}$

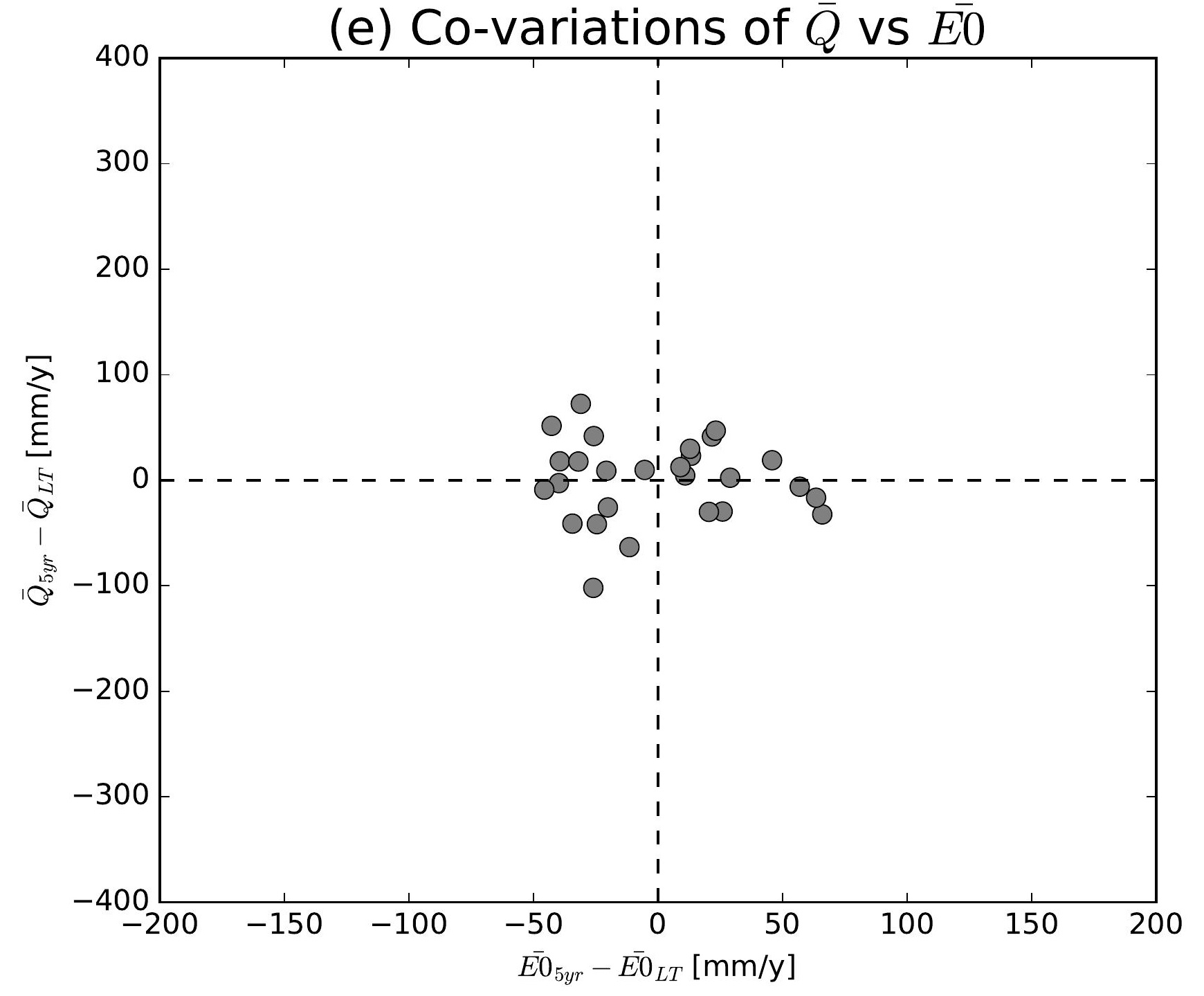

(h) Co-variations of $\bar{Q}$ vs $\overline{E 0}$

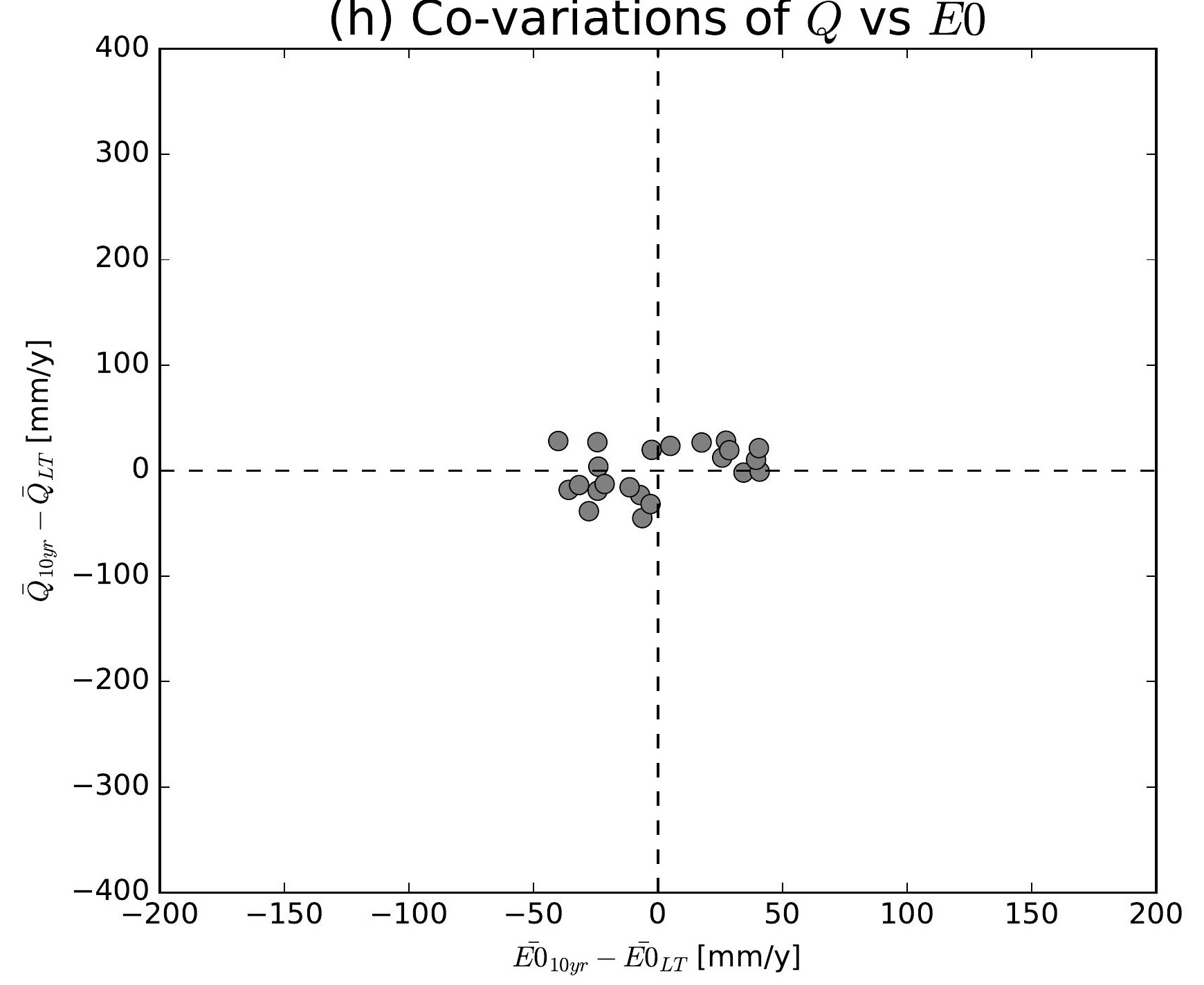

(k) Co-variations of $\bar{Q}$ vs $\overline{E 0}$

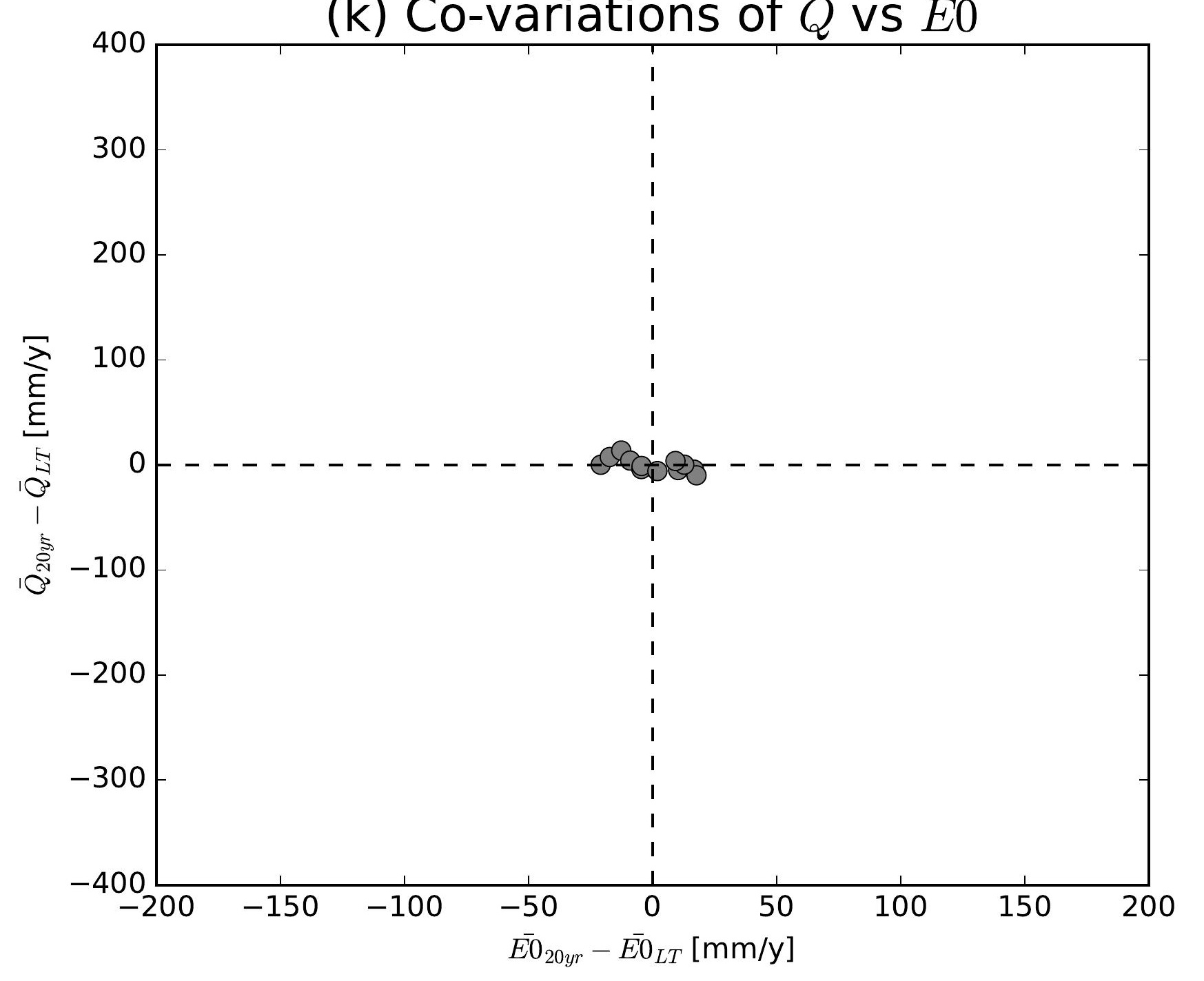

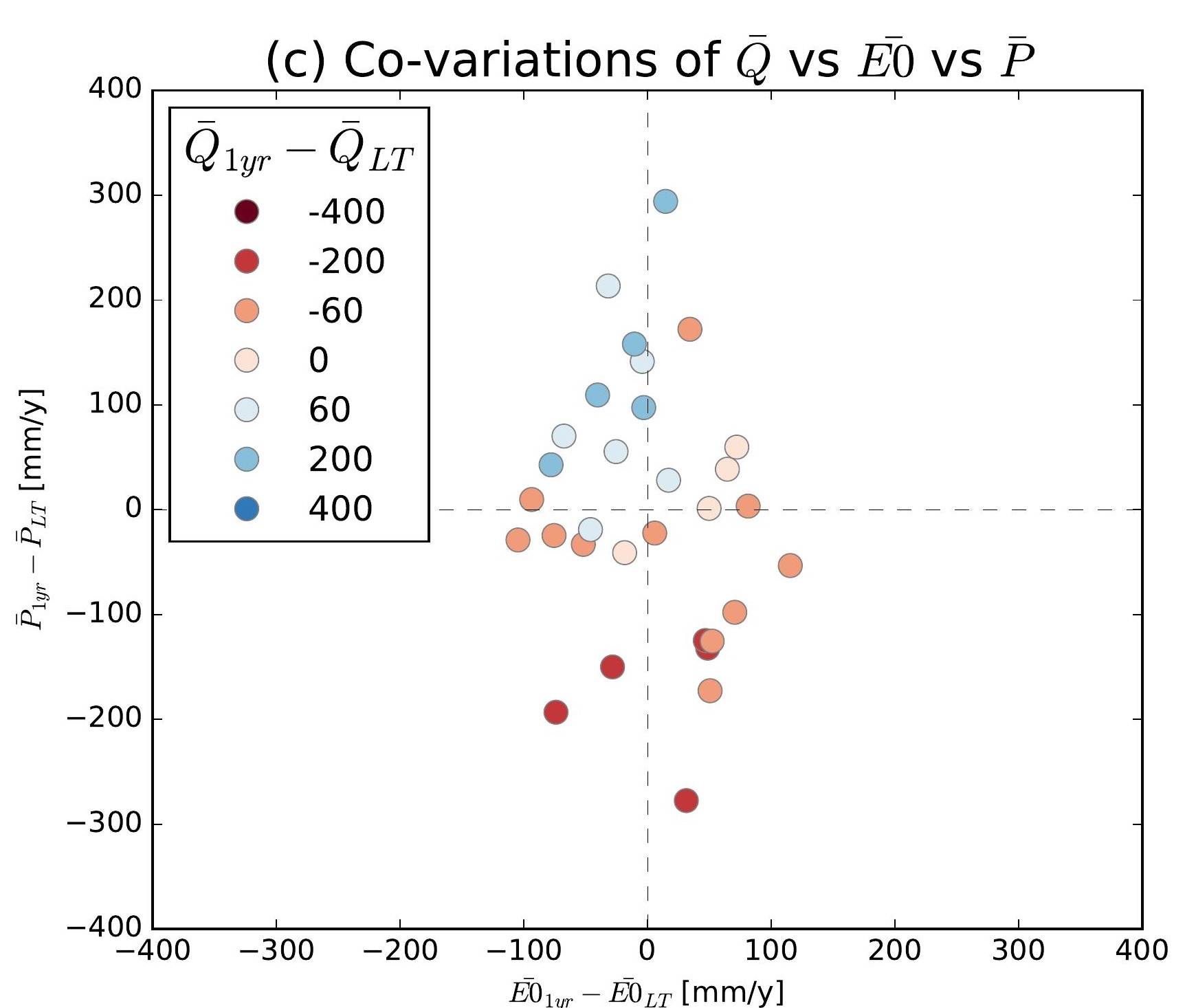
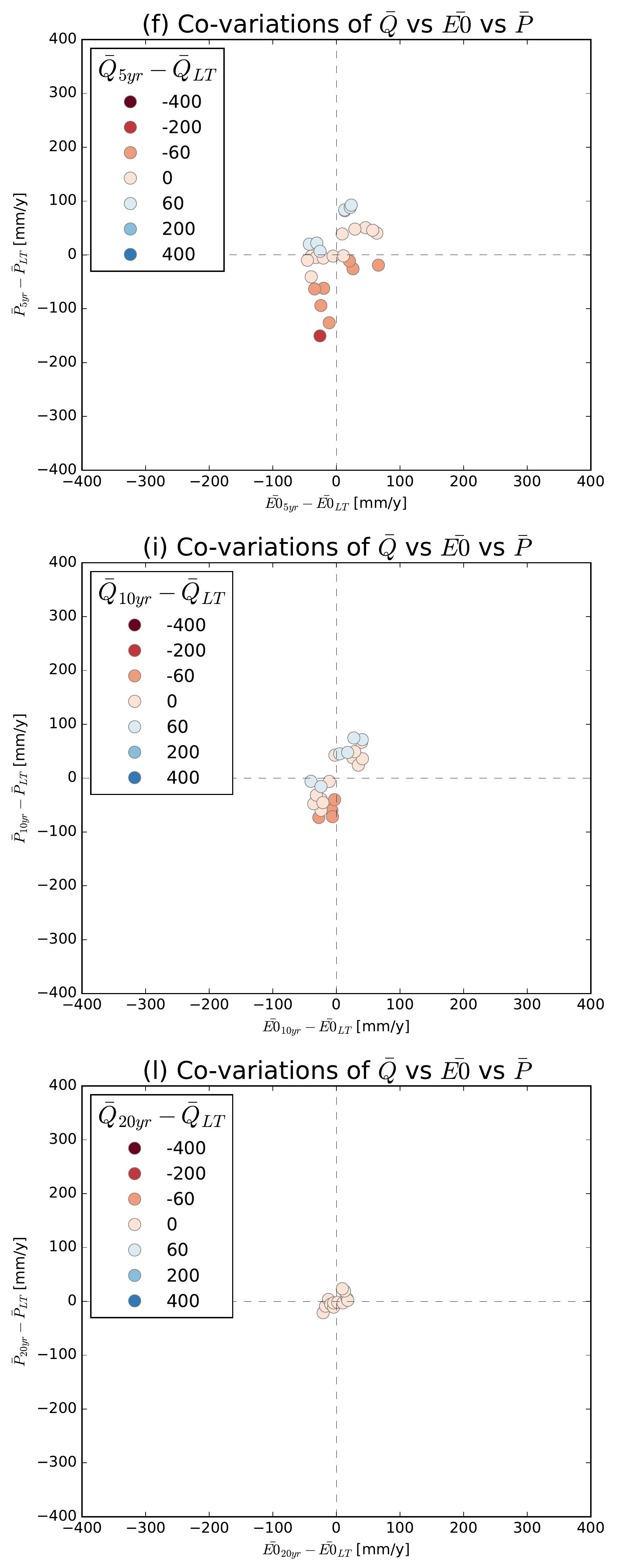

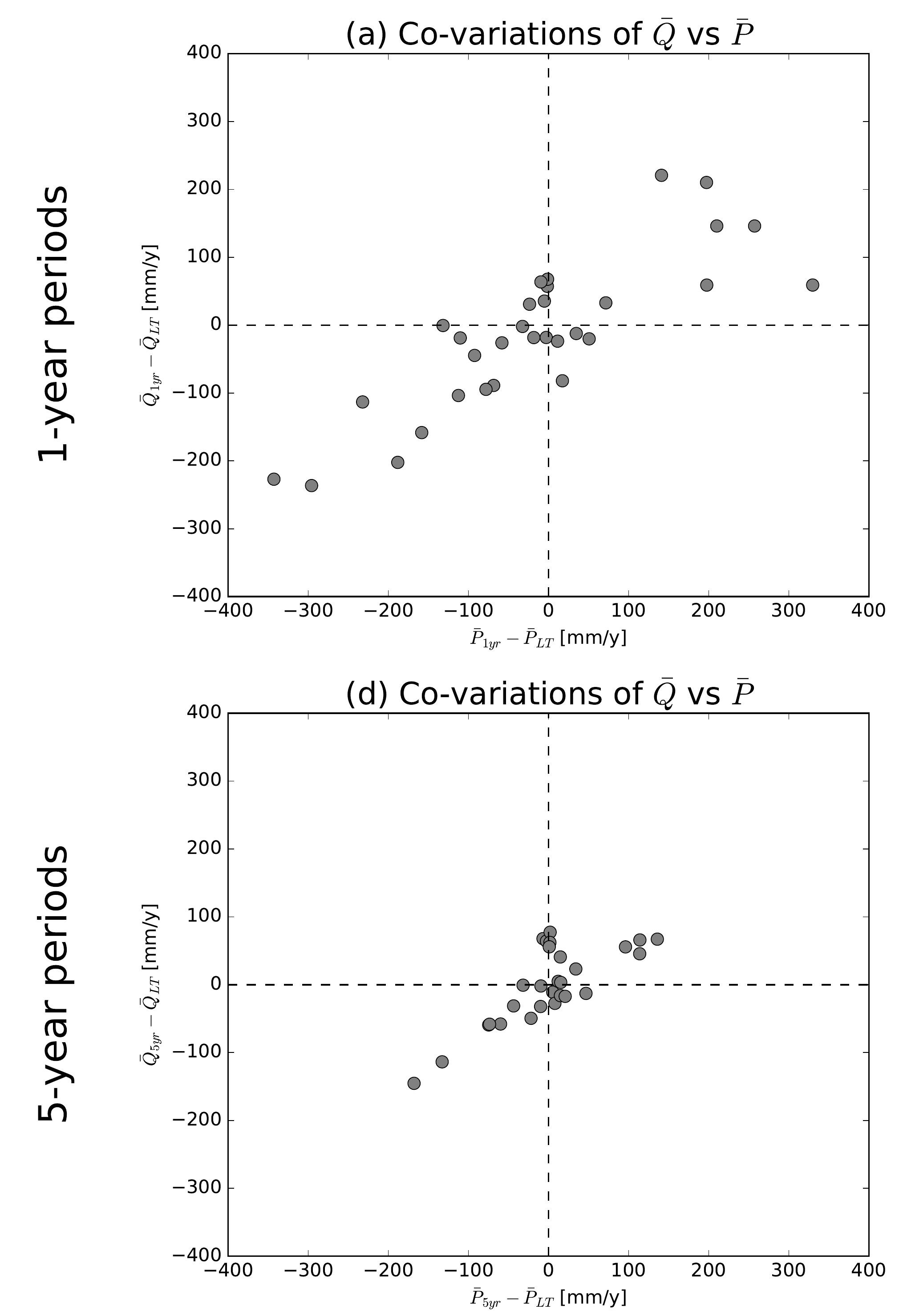

(g) Co-variations of $\bar{Q}$ vs $\bar{P}$
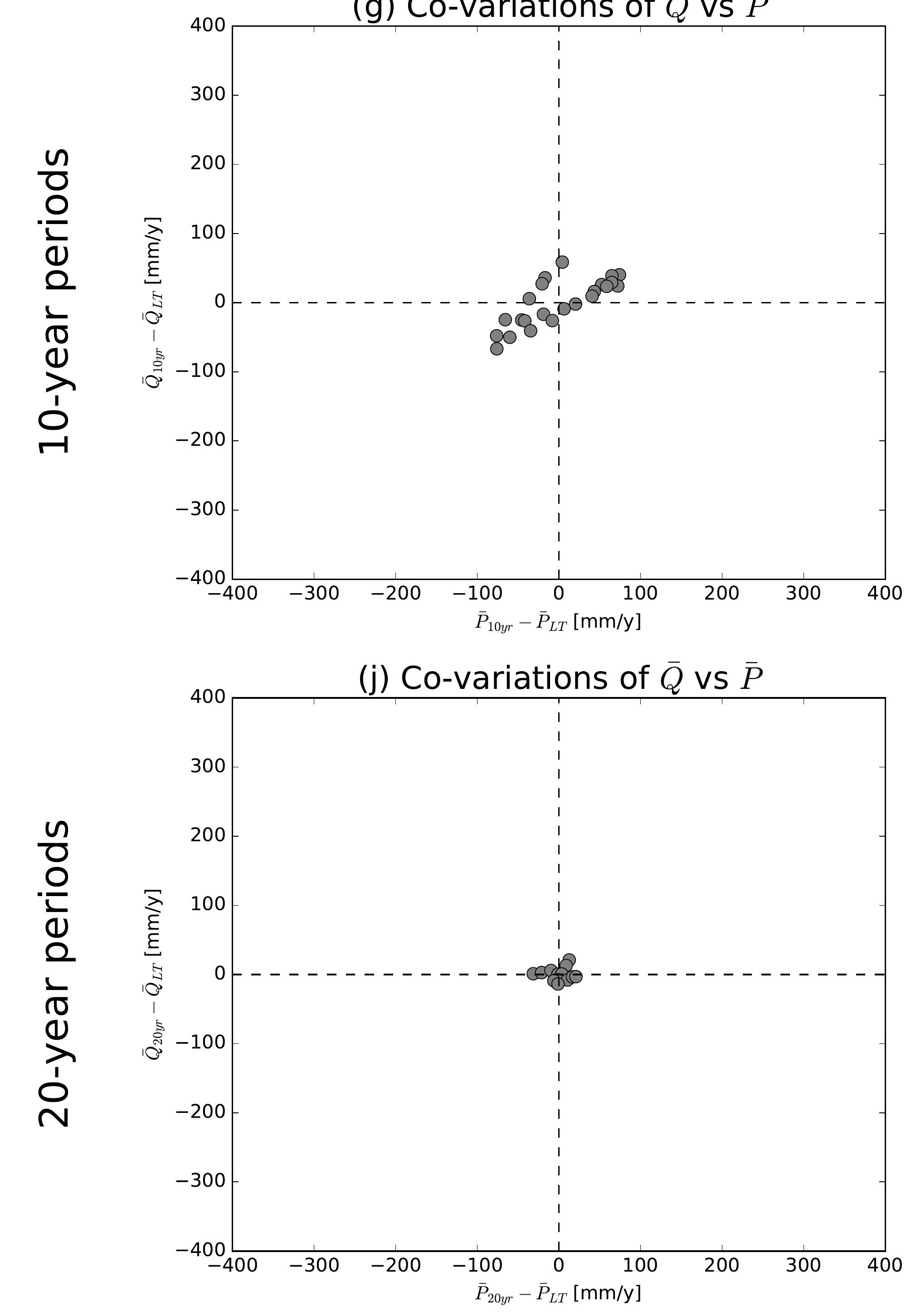

(b) Co-variations of $\bar{Q}$ vs $\overline{E 0}$

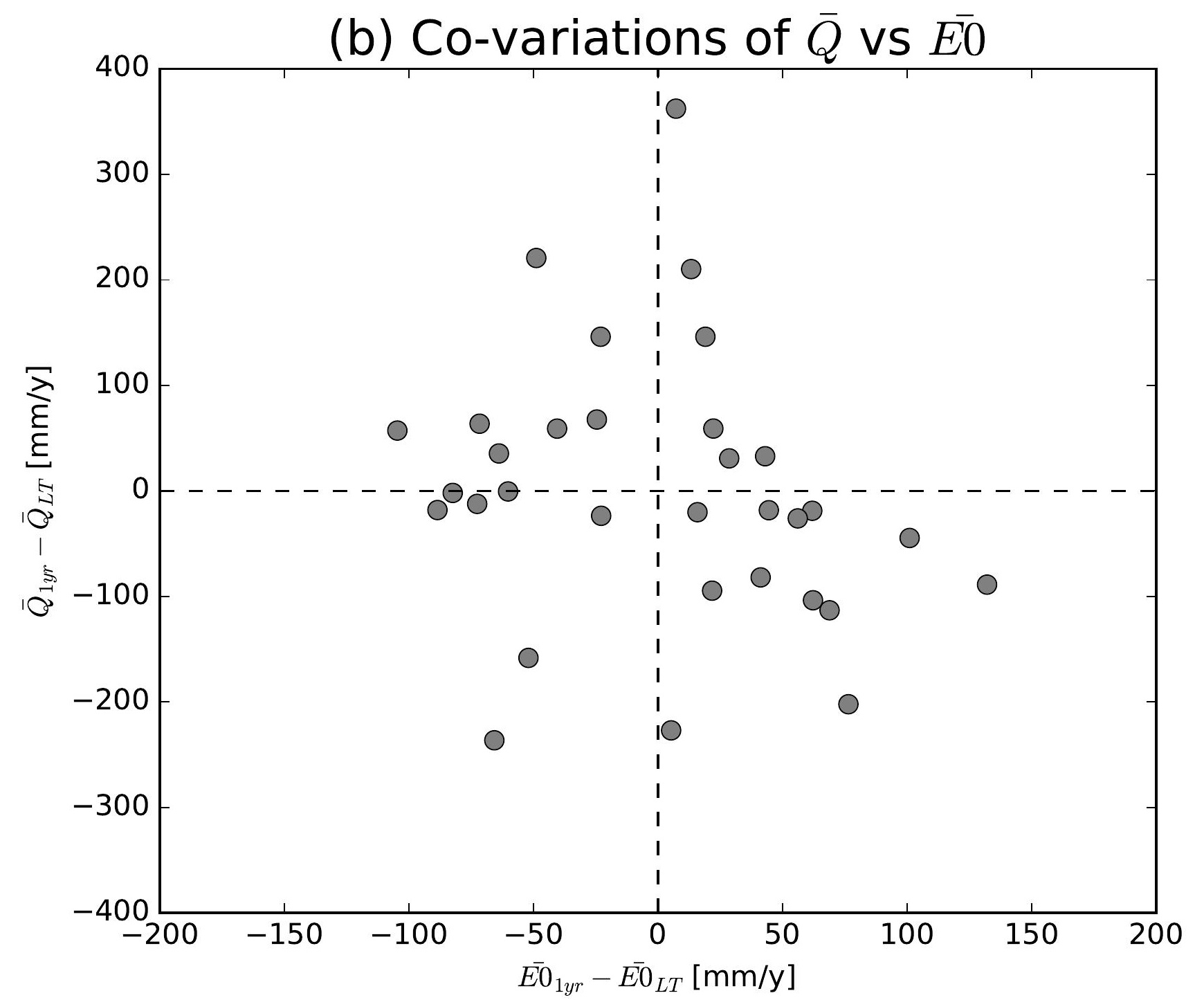

(e) Co-variations of $\bar{Q}$ vs $\overline{E 0}$

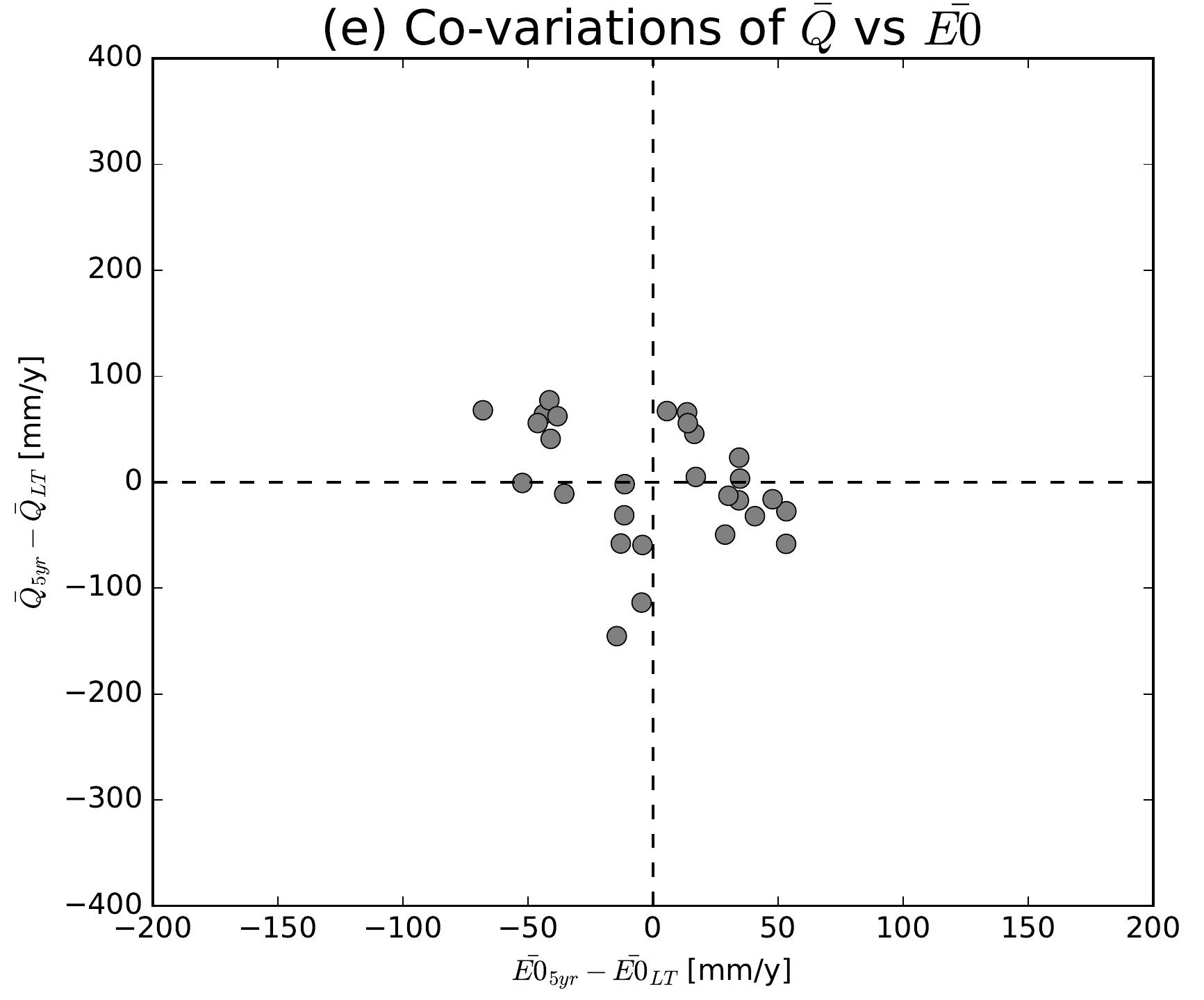

(h) Co-variations of $\bar{Q}$ vs $\overline{E 0}$

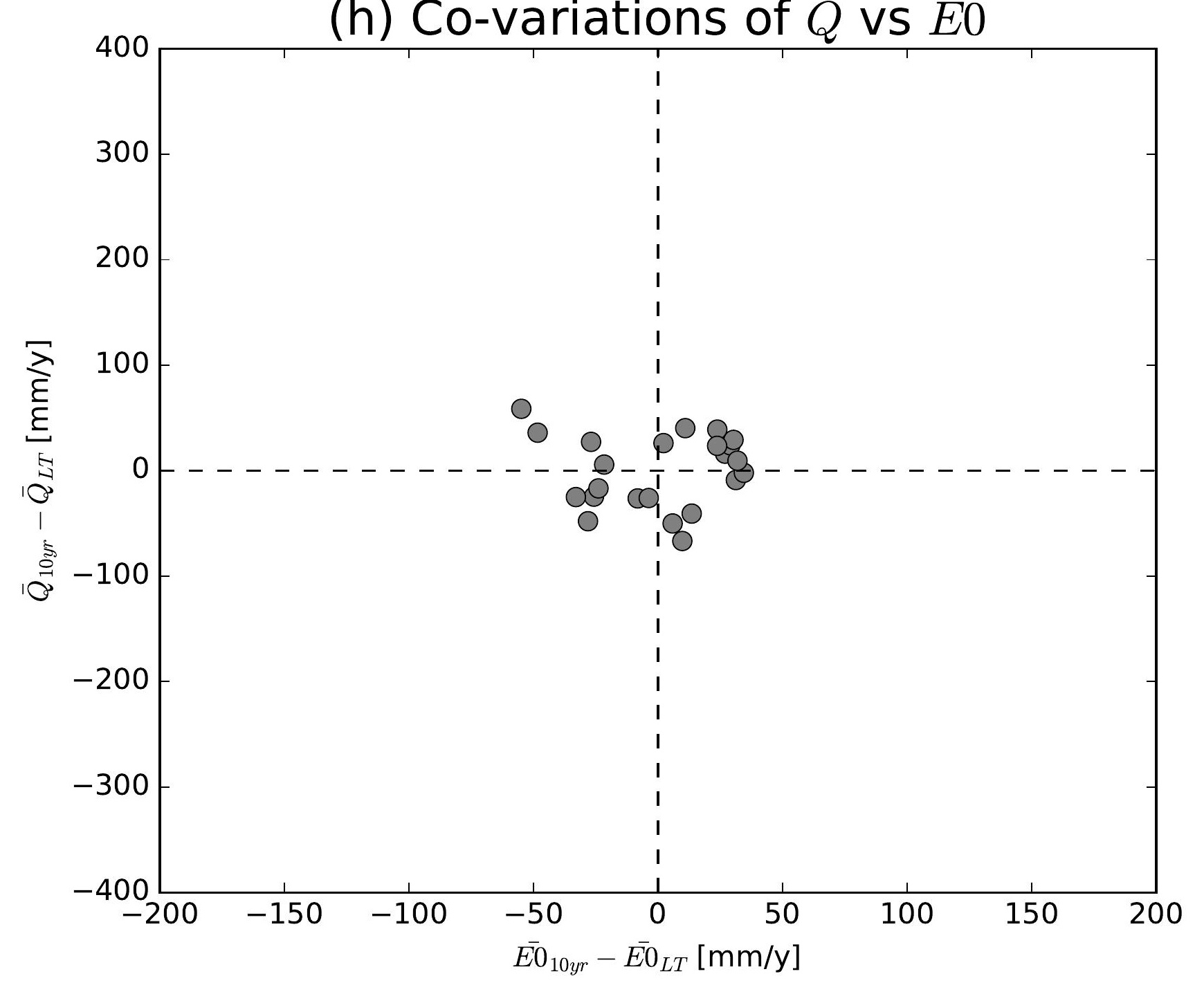

(k) Co-variations of $\bar{Q}$ vs $\overline{E 0}$

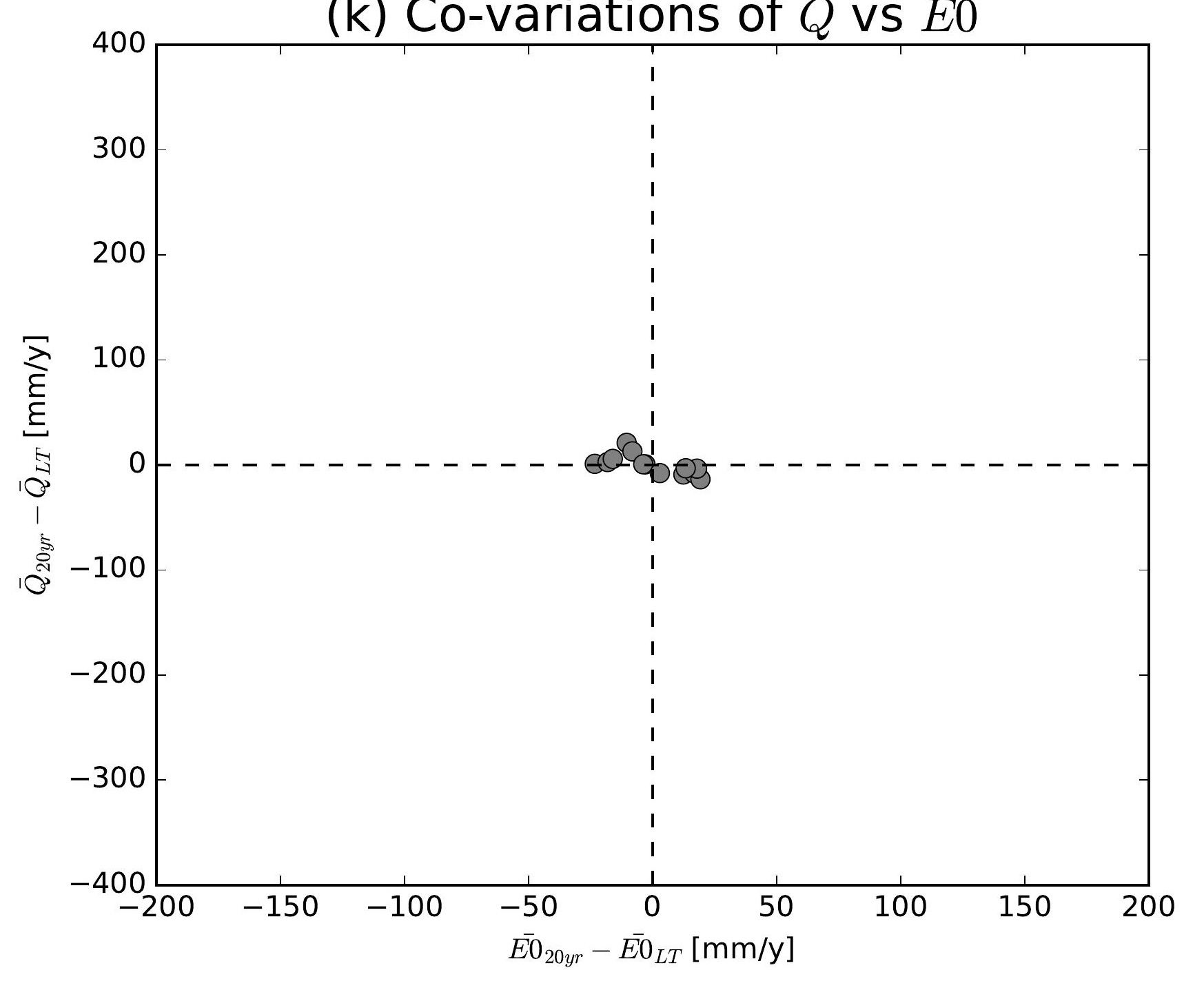

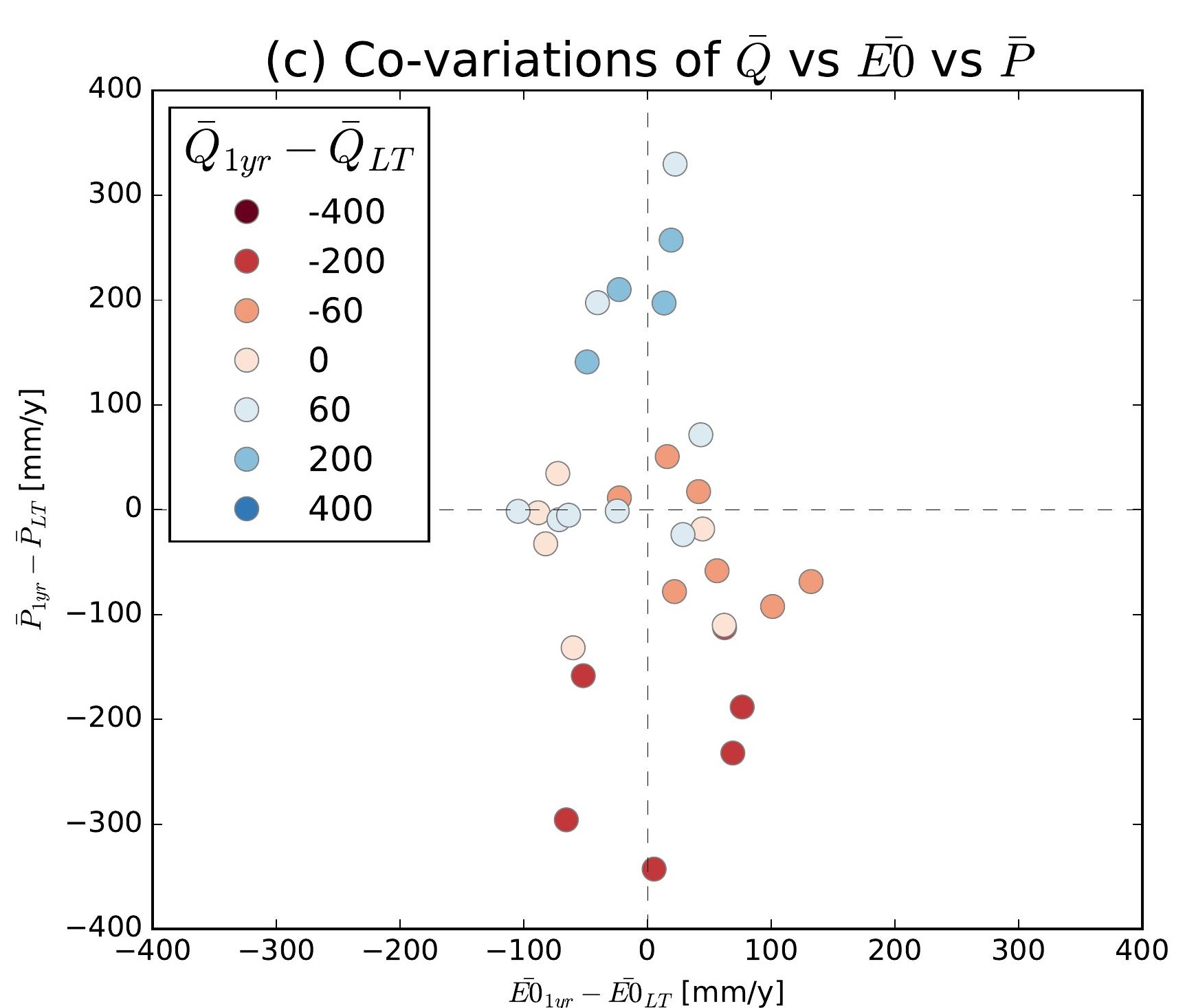
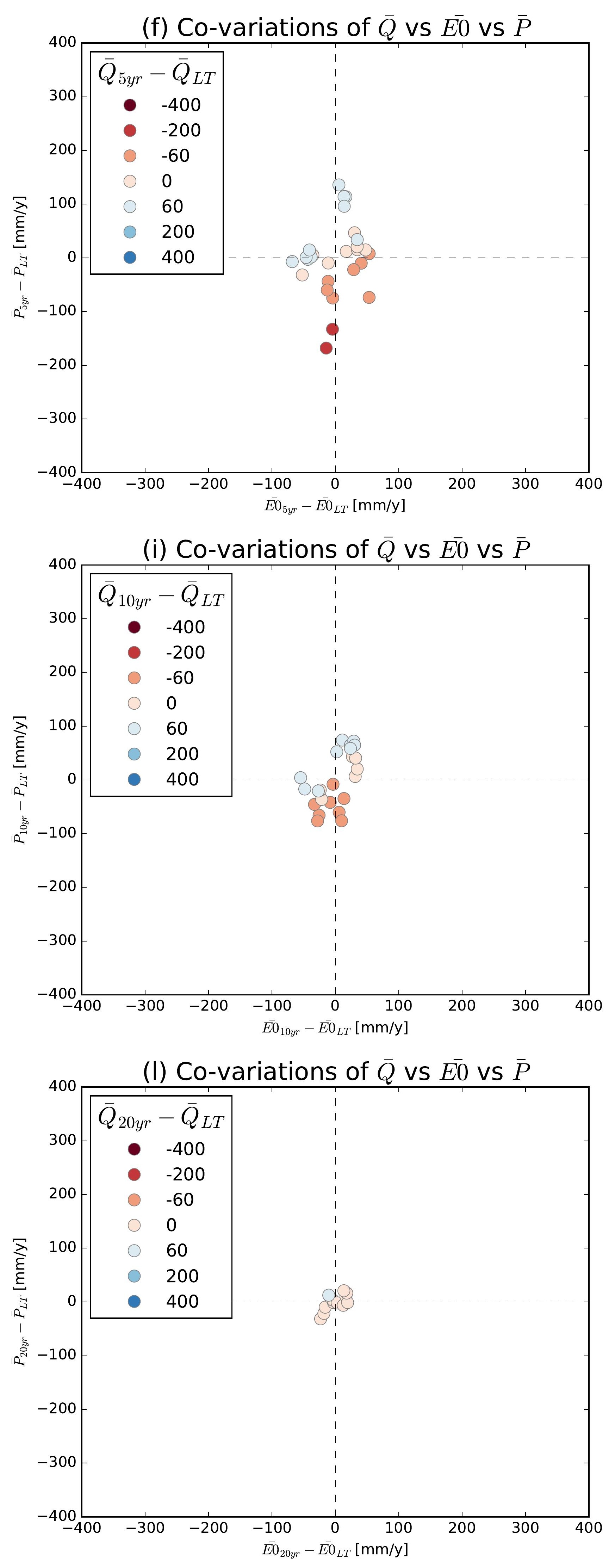

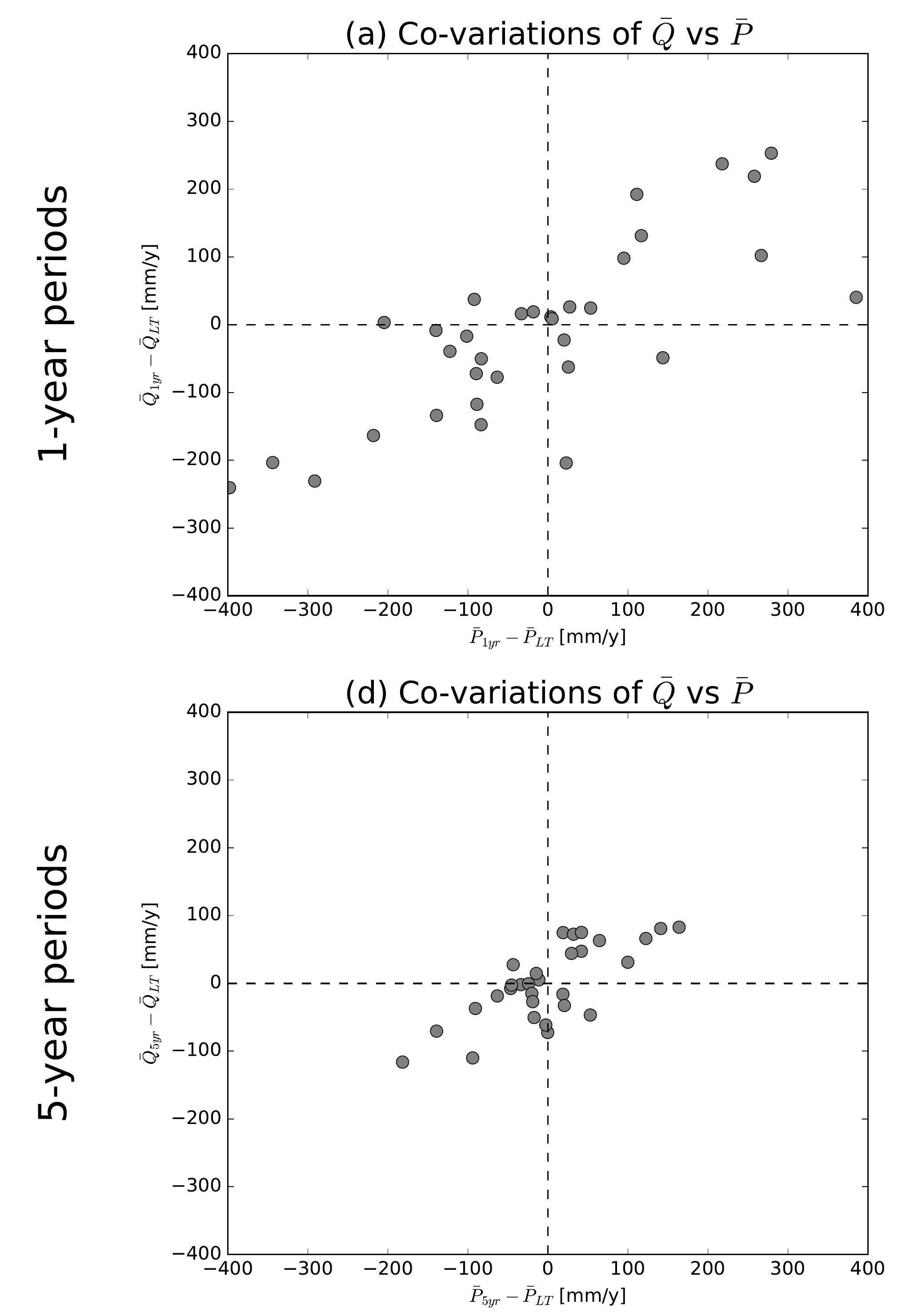

(g) Co-variations of $\bar{Q}$ vs $\bar{P}$
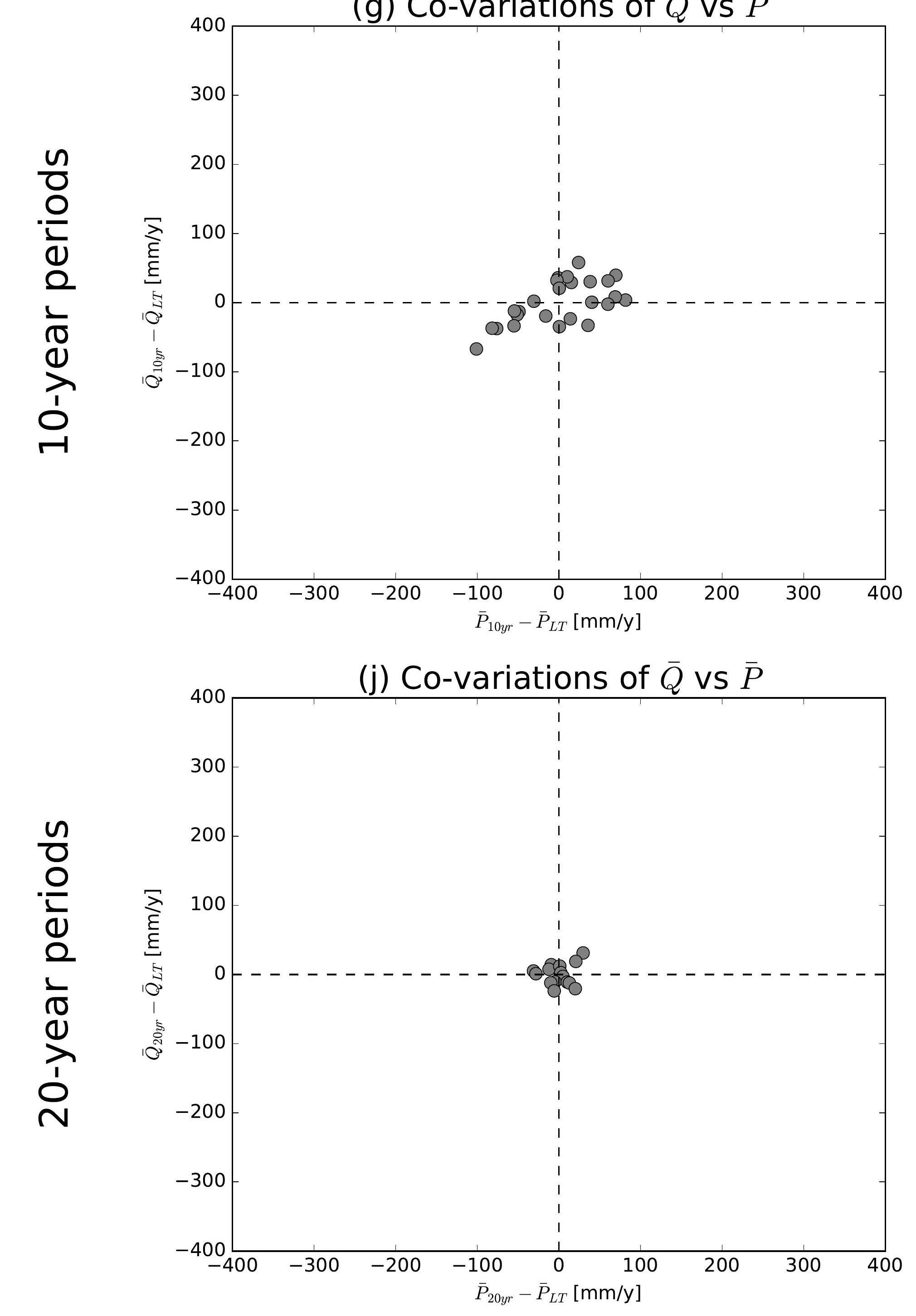

(b) Co-variations of $\bar{Q}$ vs $\overline{E 0}$

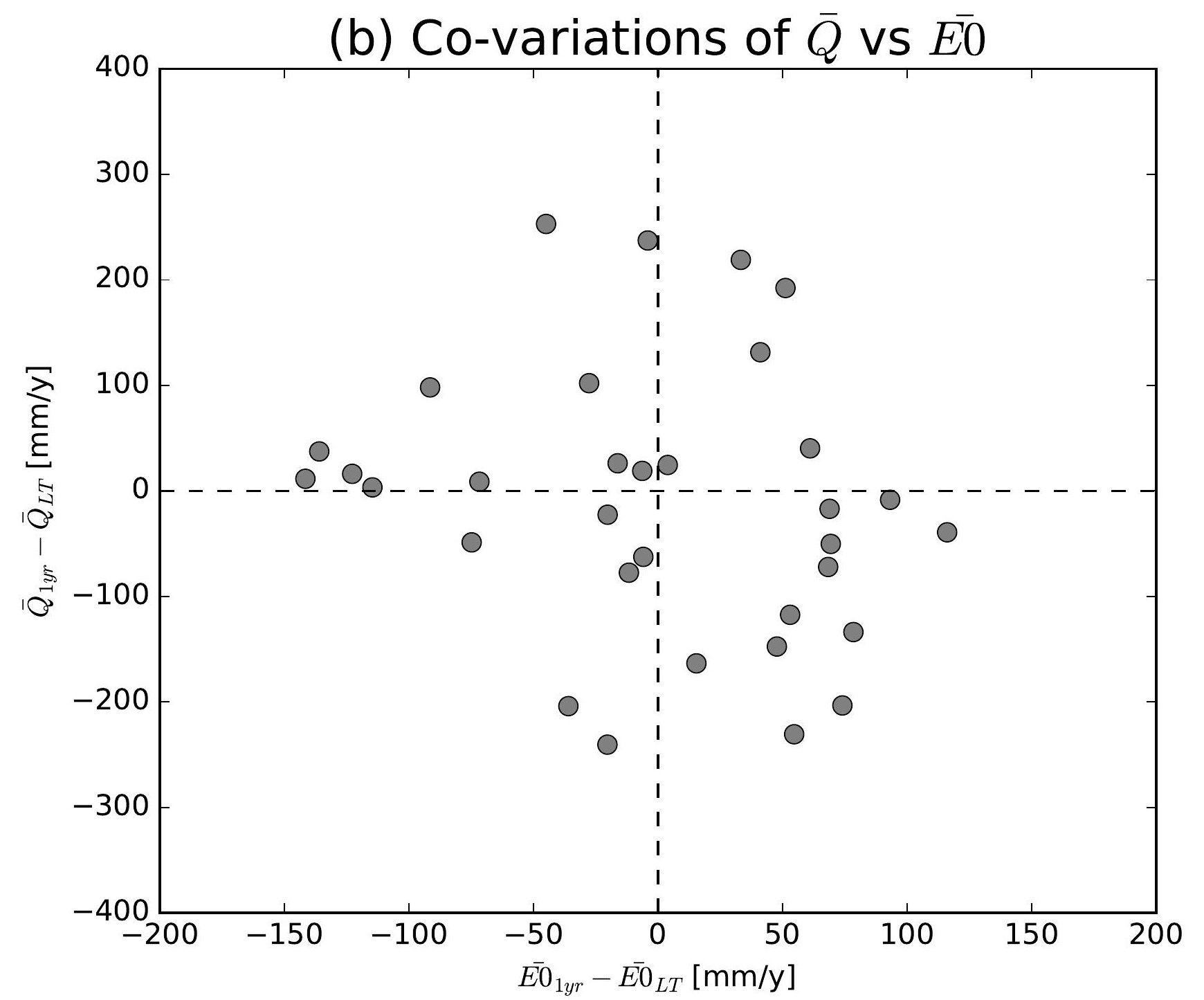

(e) Co-variations of $\bar{Q}$ vs $\overline{E 0}$

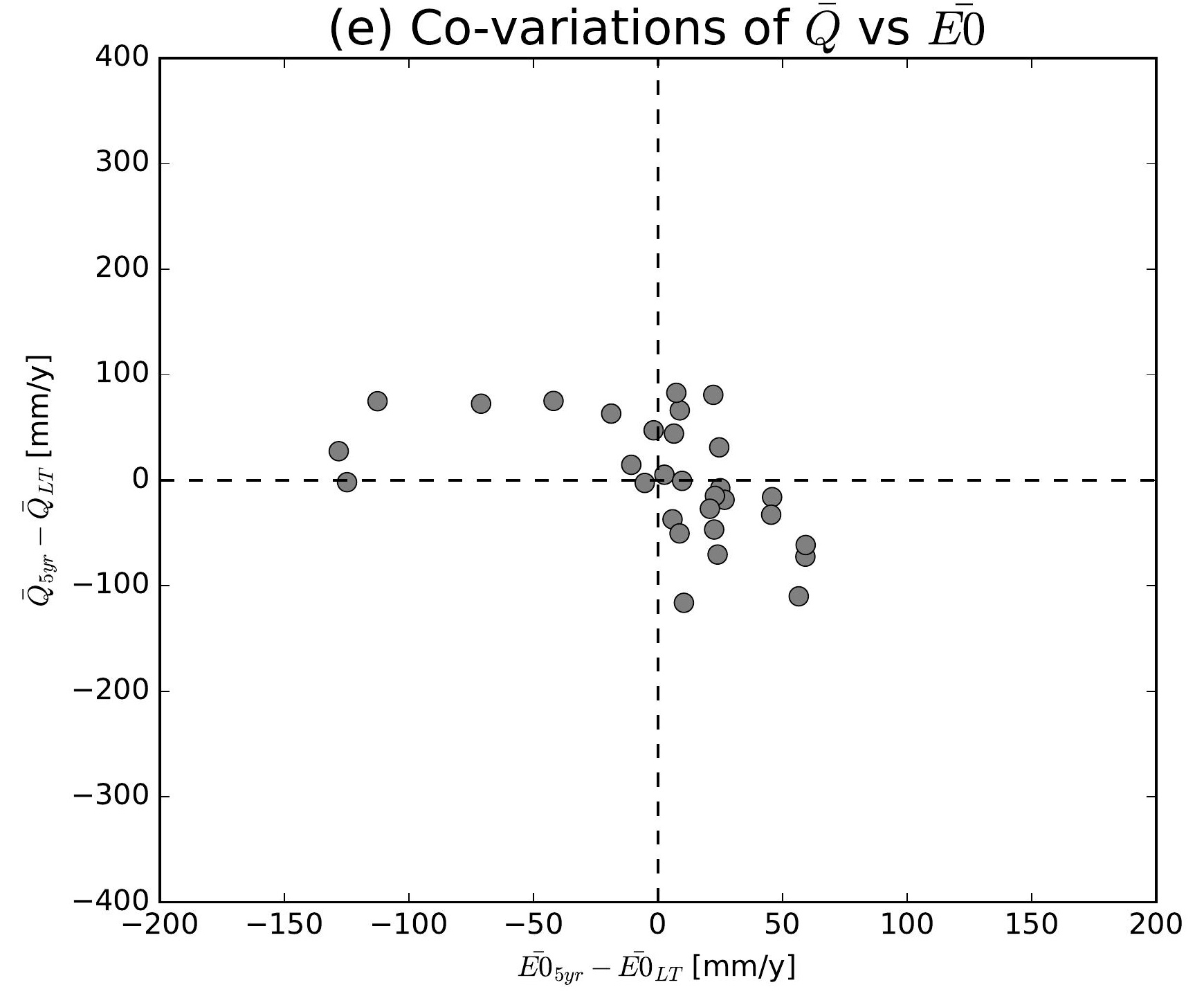

(h) Co-variations of $\bar{Q}$ vs $\overline{E 0}$

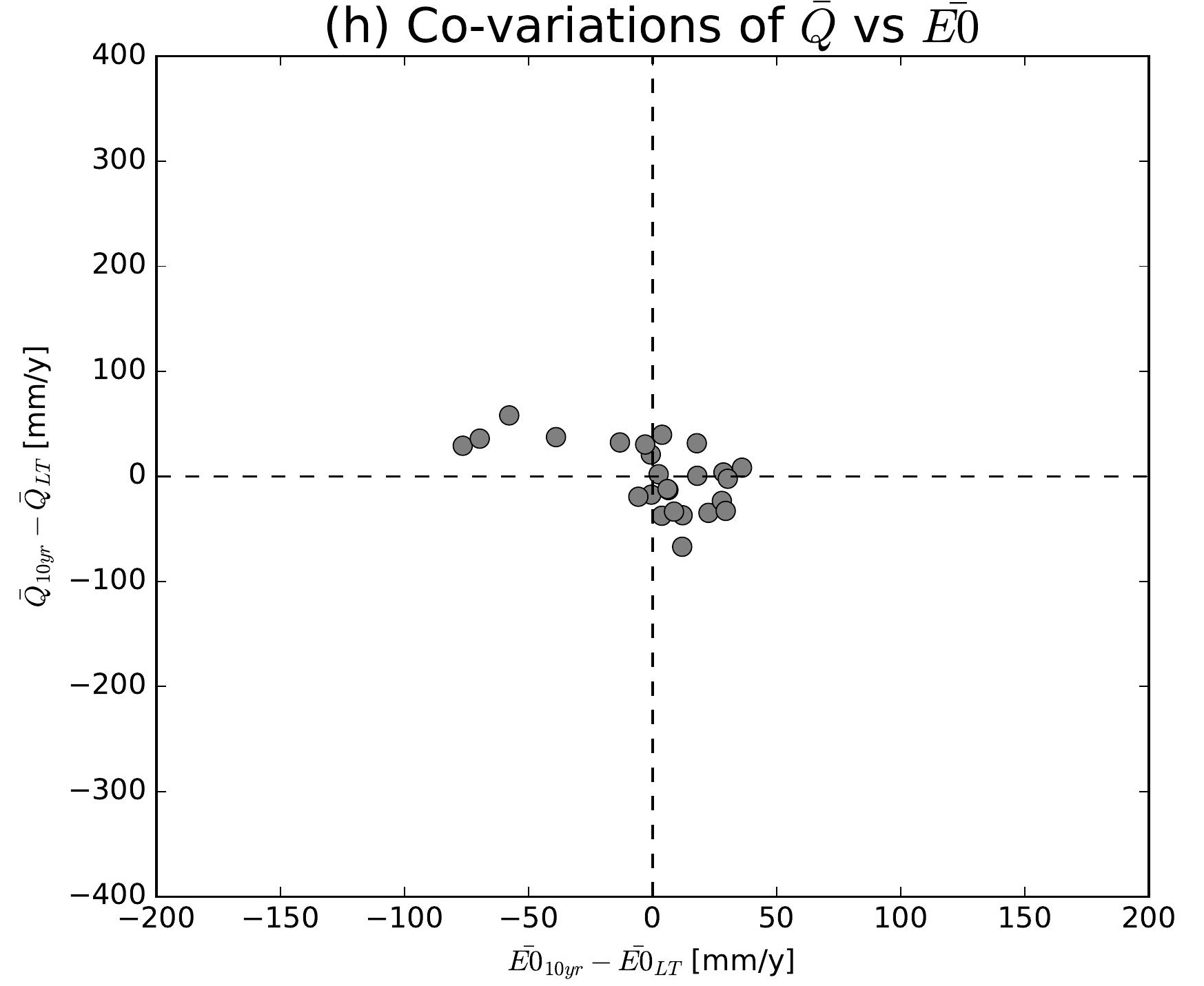

(k) Co-variations of $\bar{Q}$ vs $\overline{E 0}$

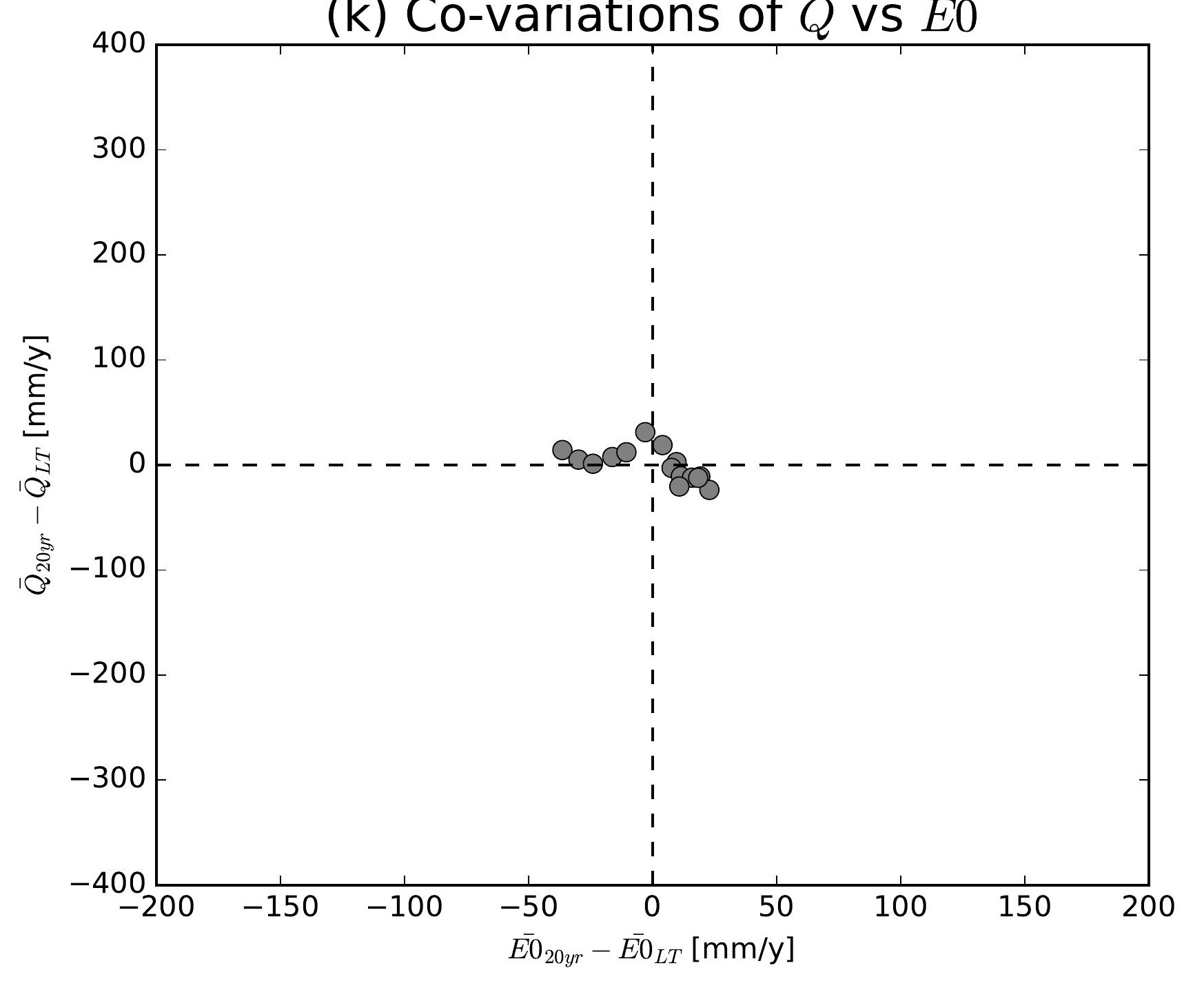

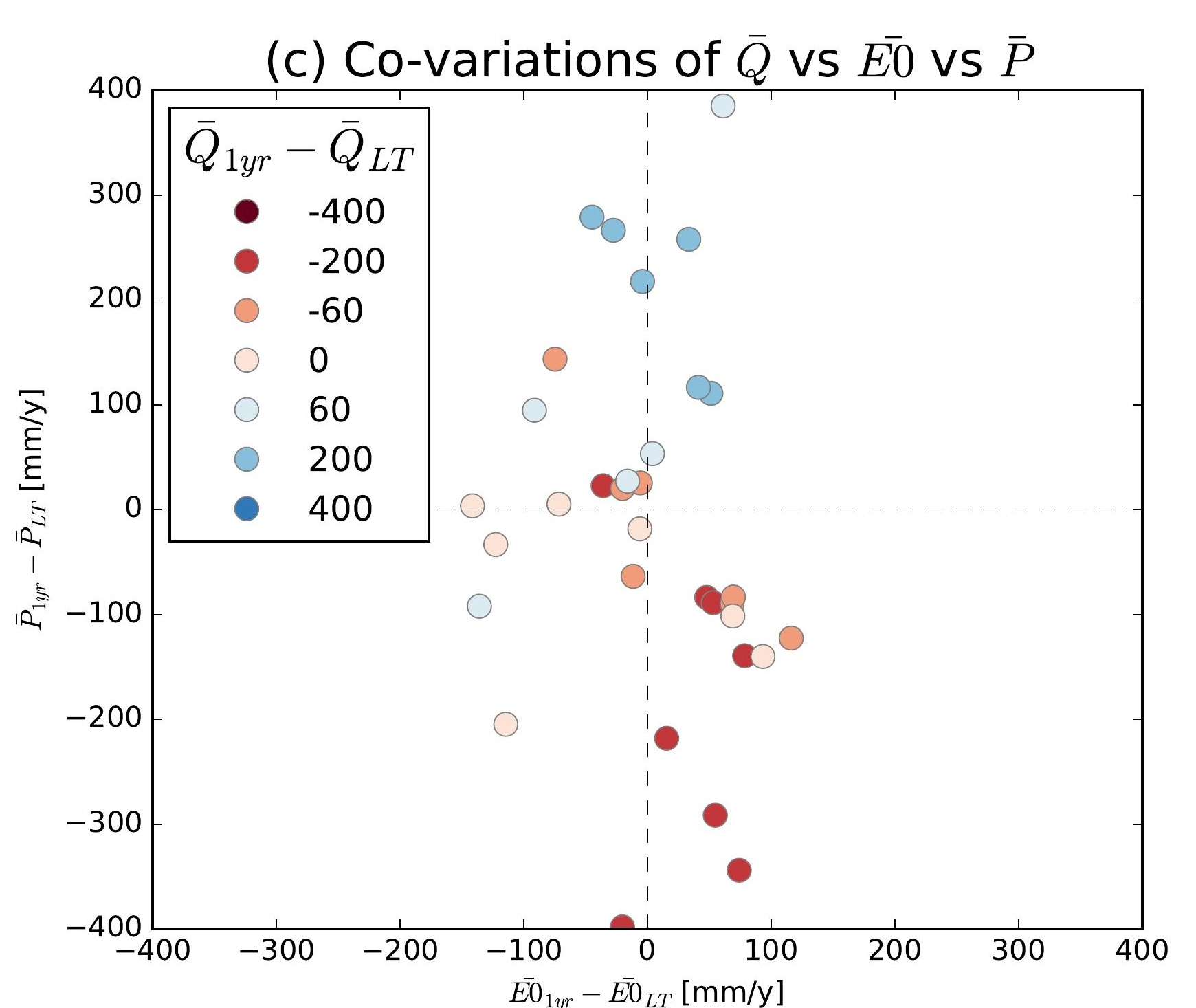
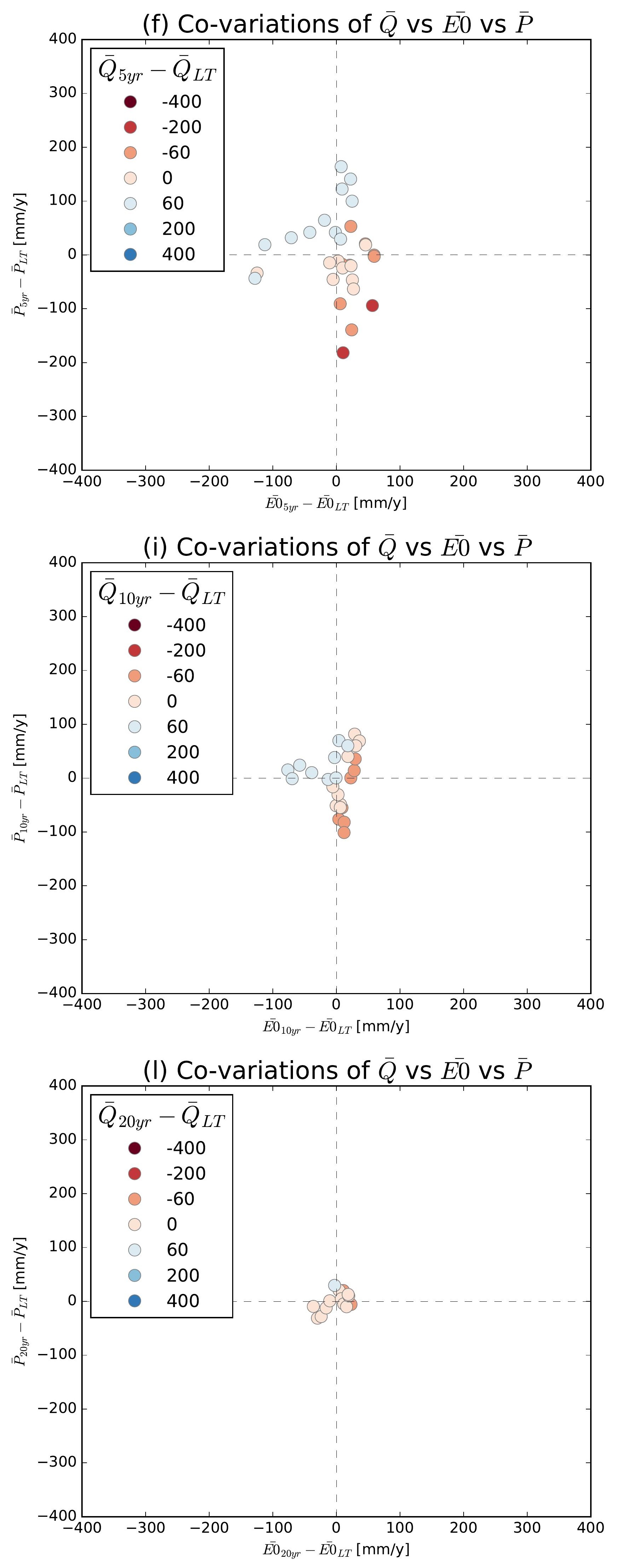

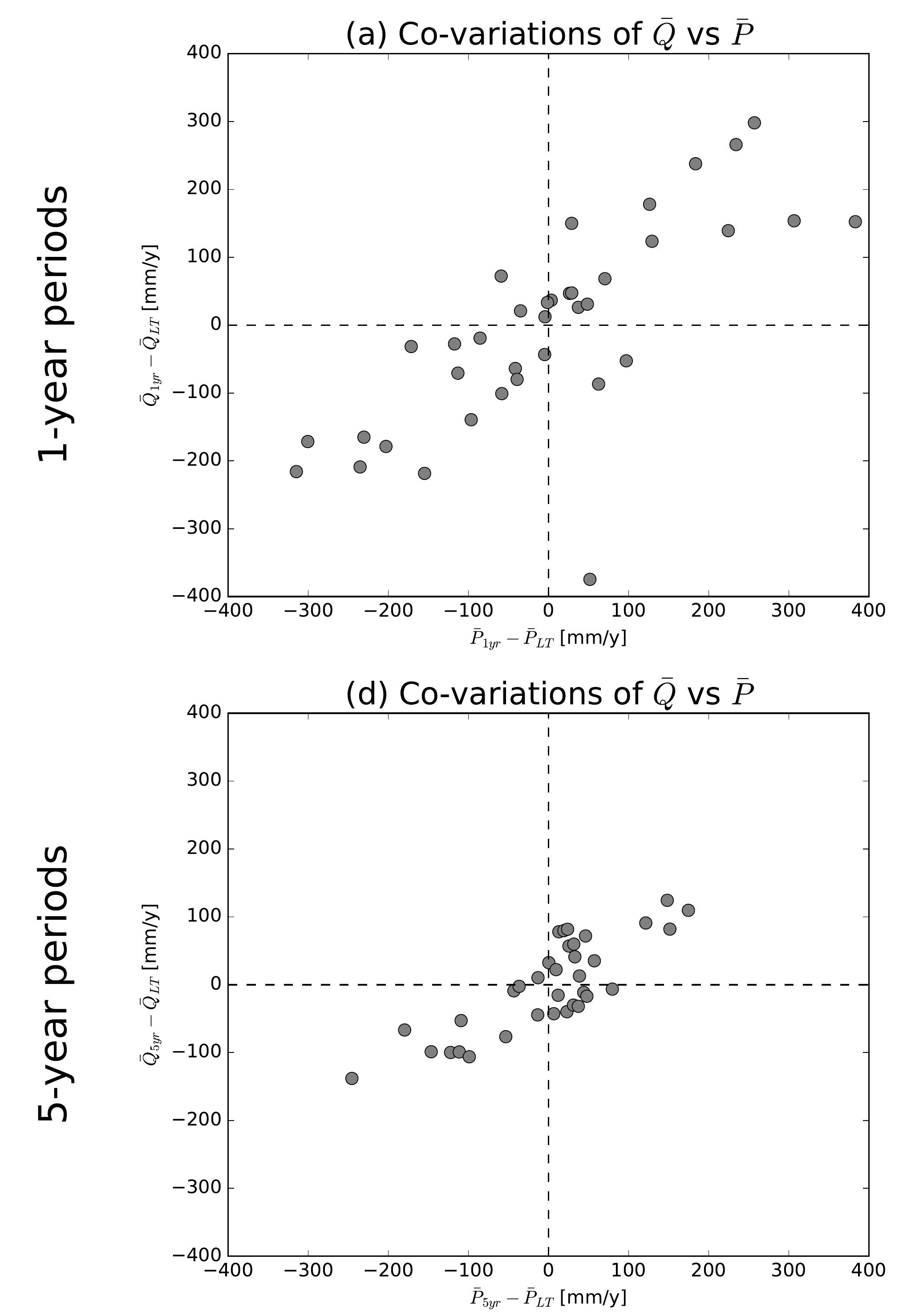

(g) Co-variations of $\bar{Q}$ vs $\bar{P}$
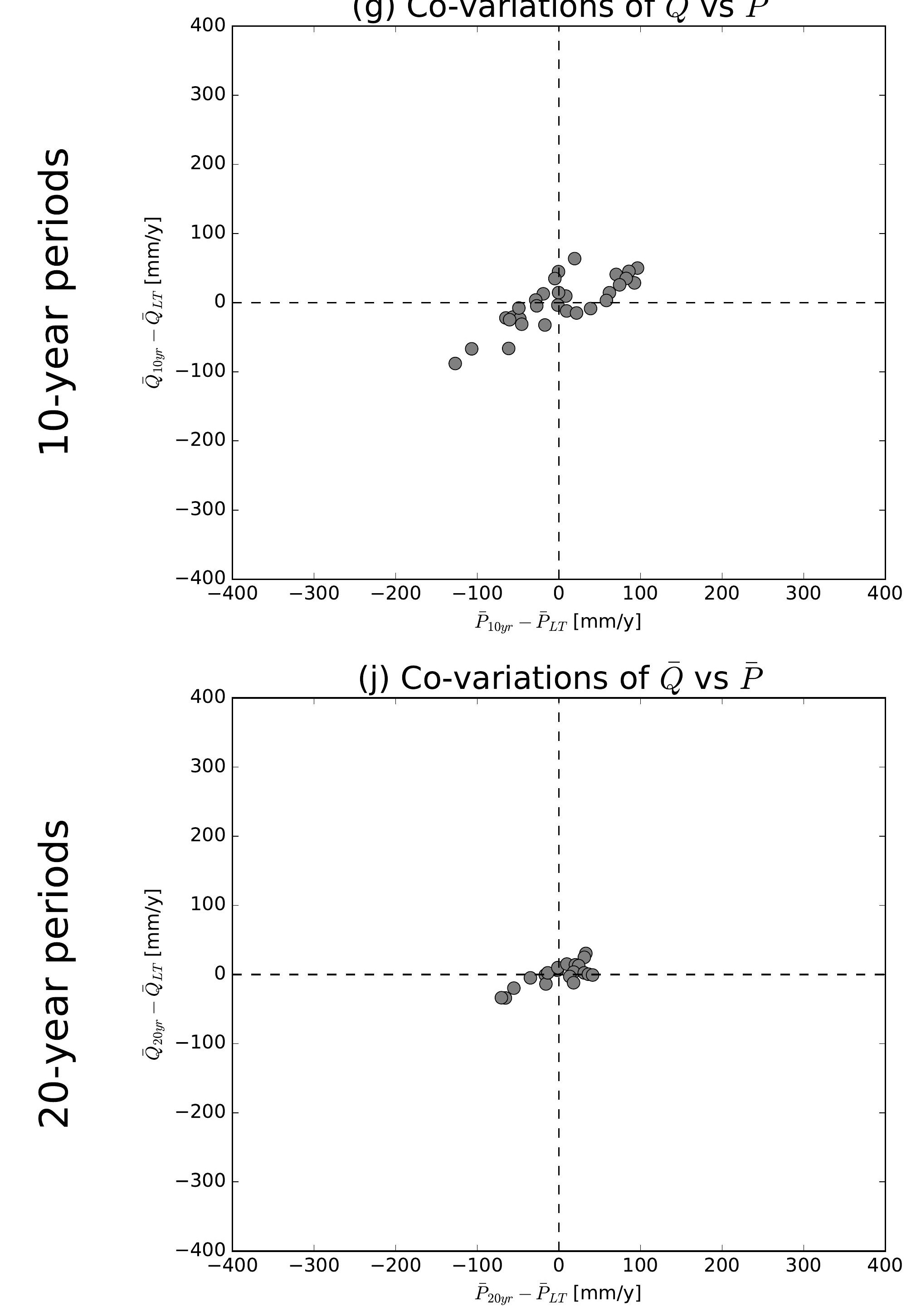

(b) Co-variations of $\bar{Q}$ vs $\overline{E 0}$

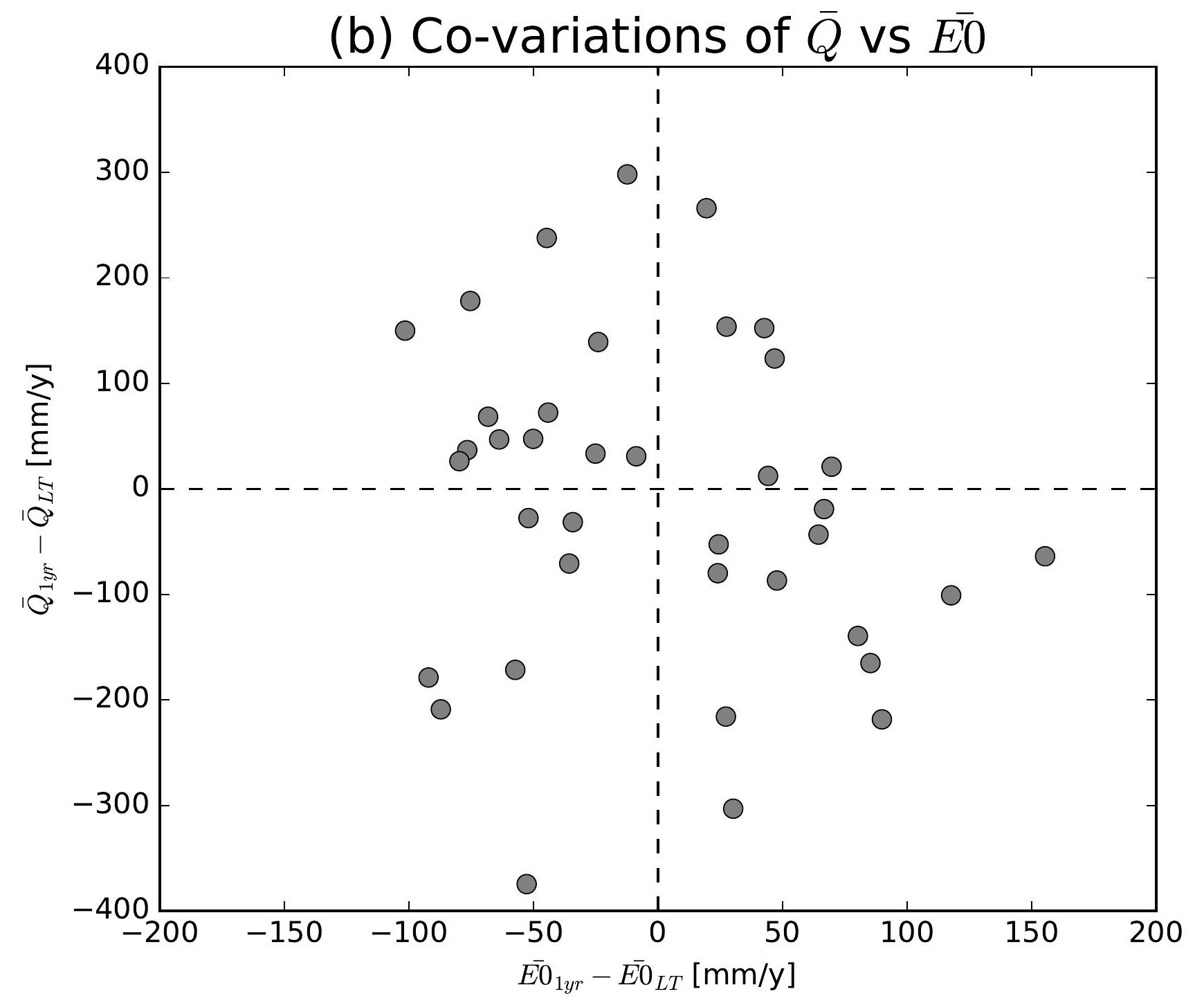

(e) Co-variations of $\bar{Q}$ vs $\overline{E 0}$

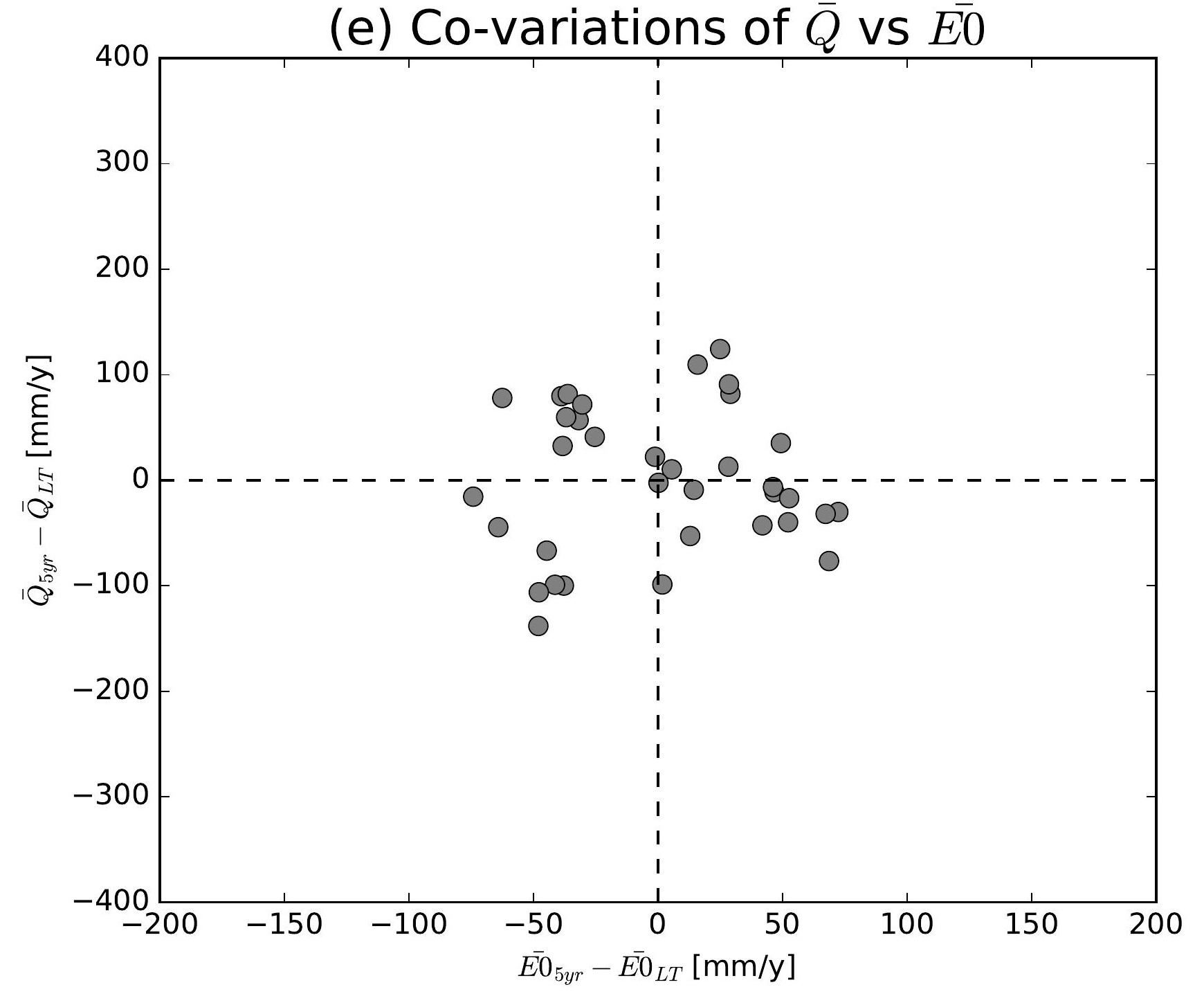

(h) Co-variations of $\bar{Q}$ vs $\overline{E 0}$

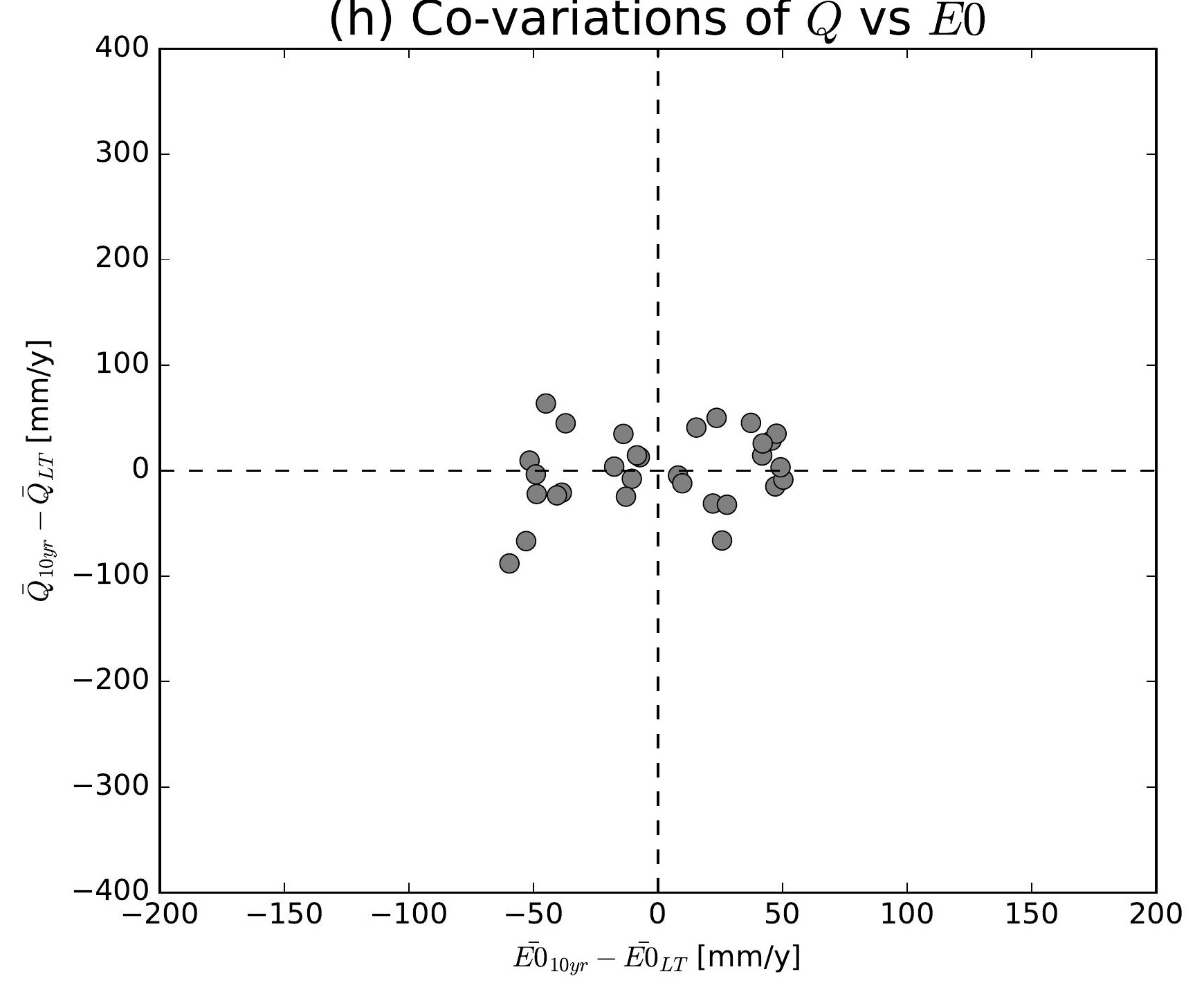

(k) Co-variations of $\bar{Q}$ vs $\overline{E 0}$

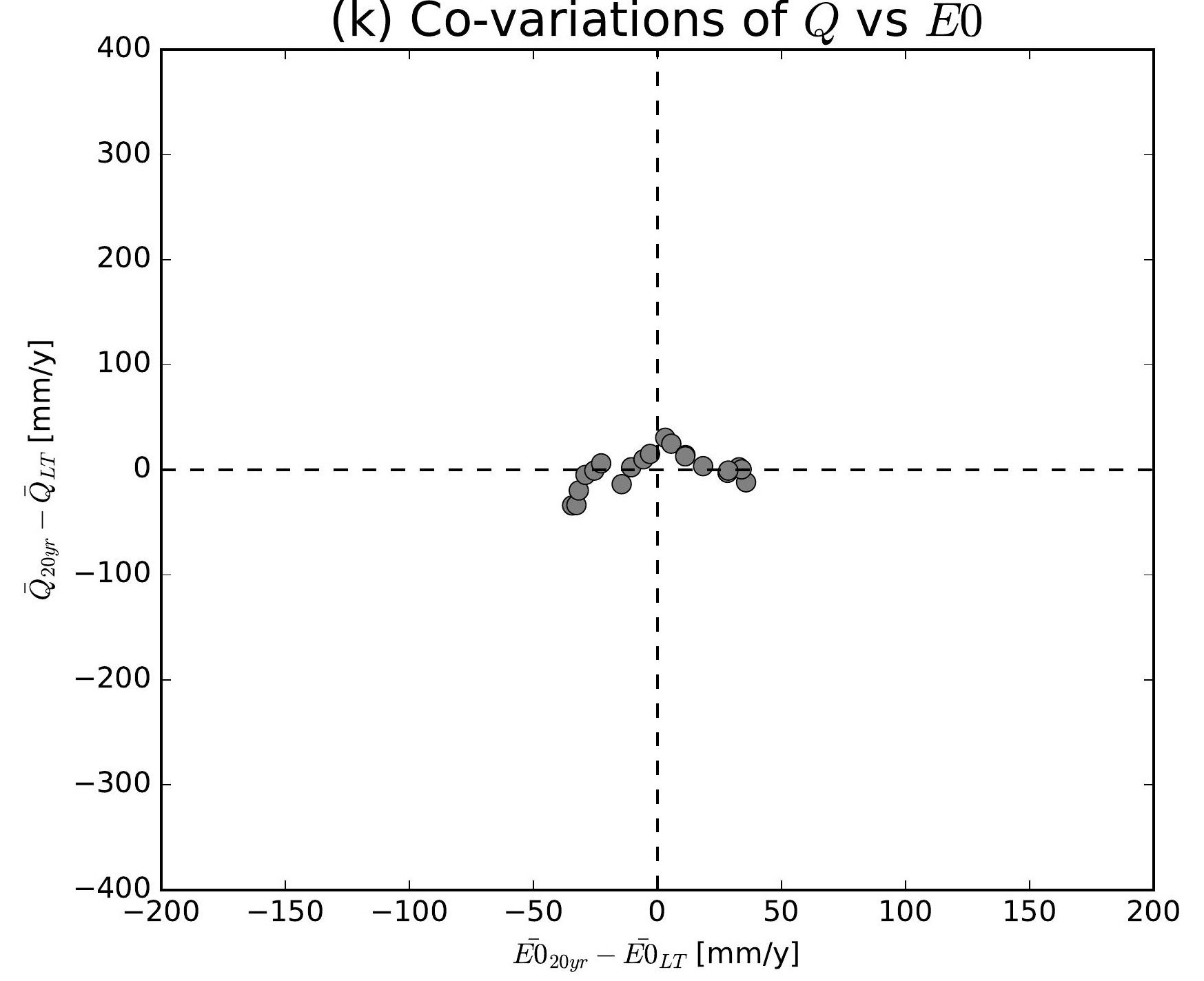

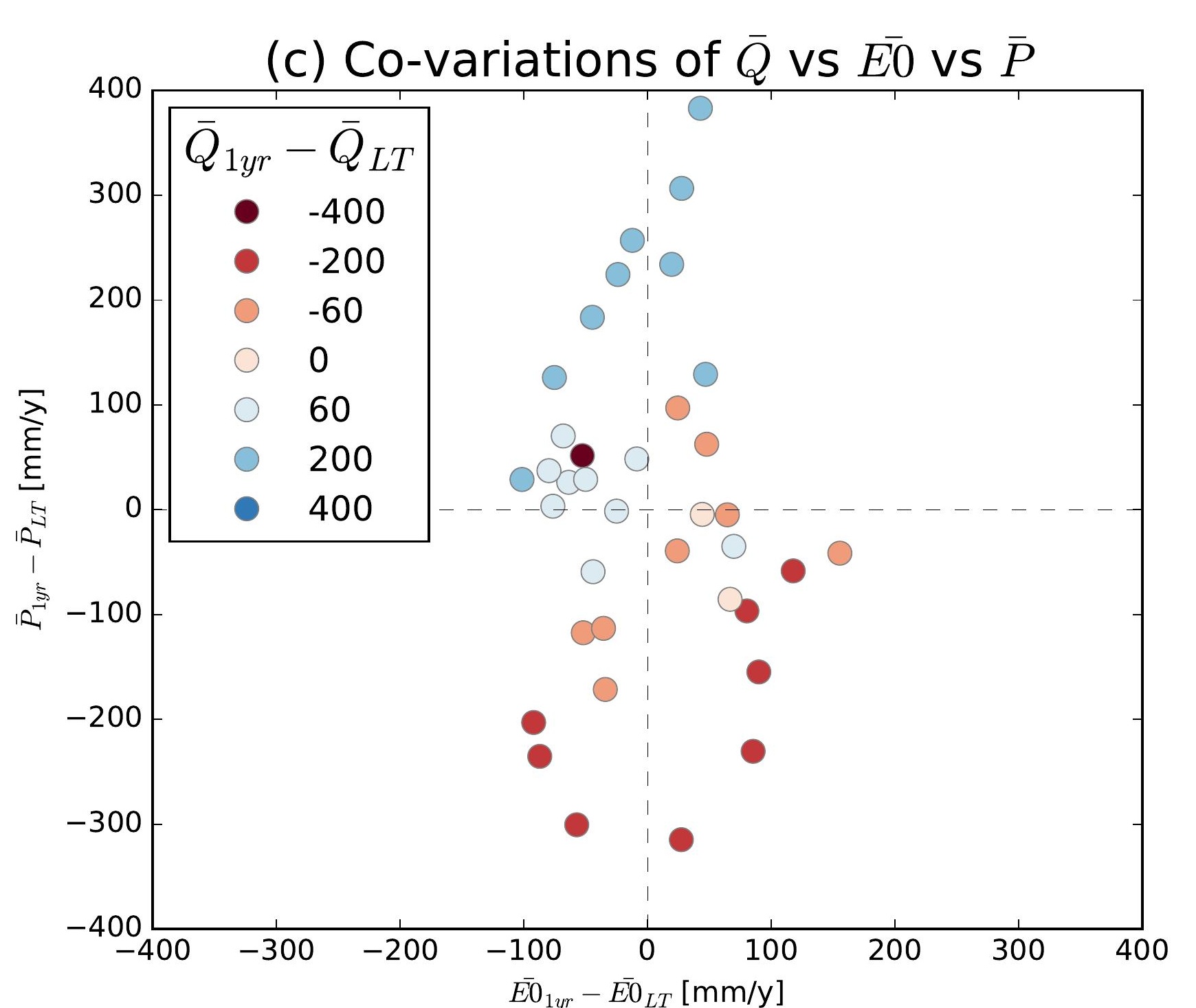
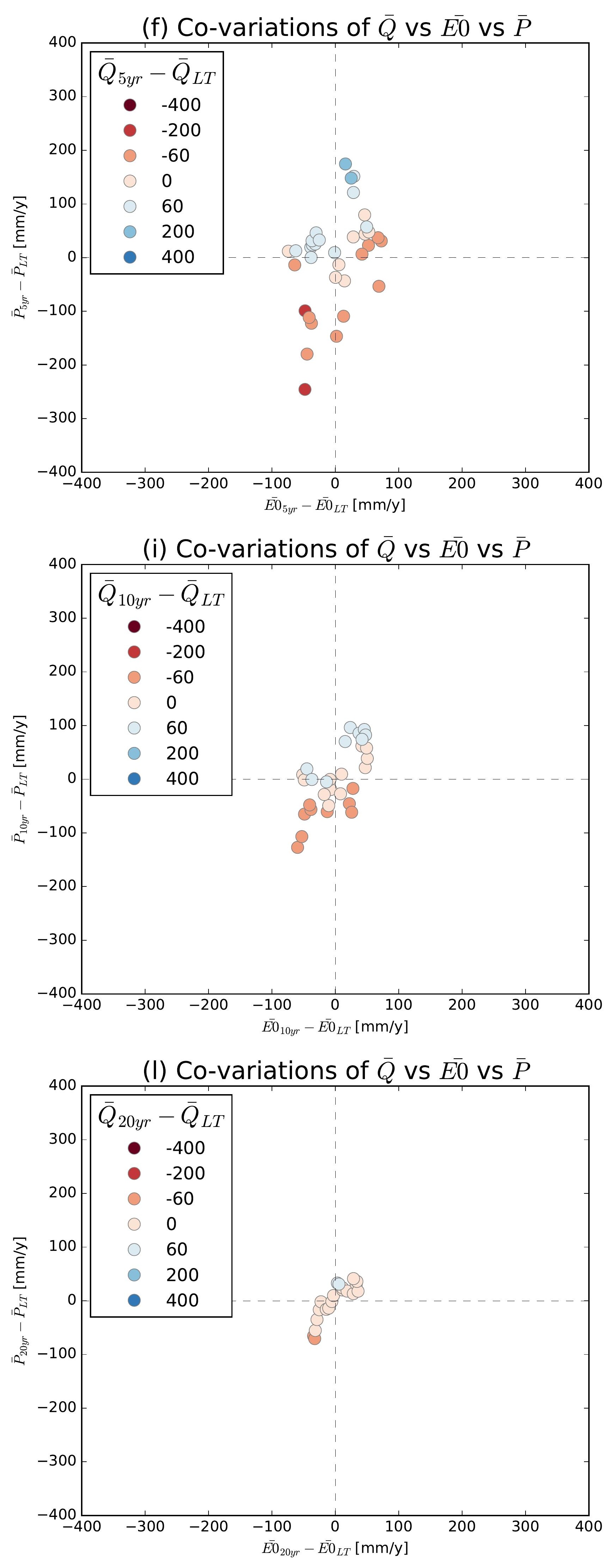

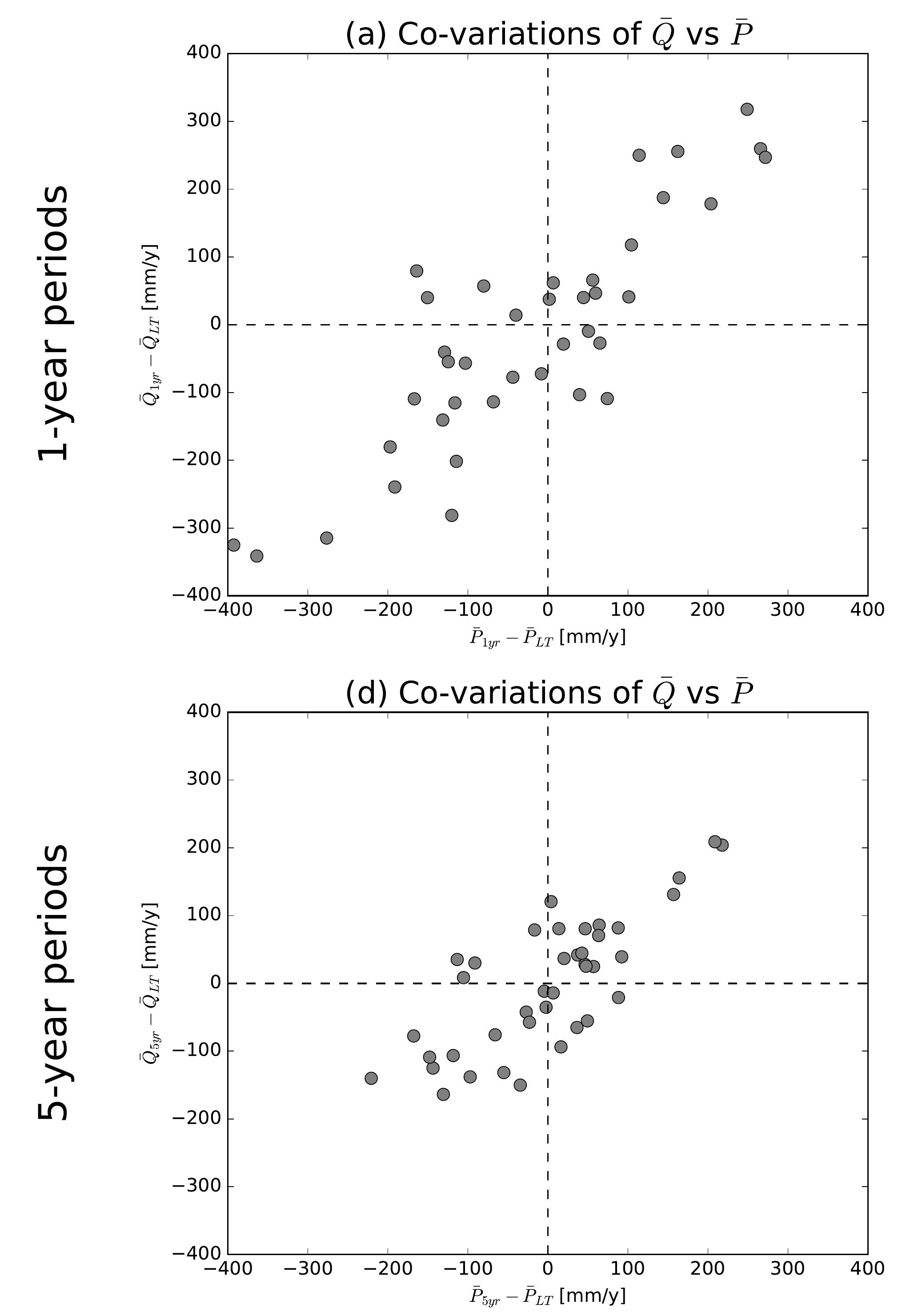

(g) Co-variations of $\bar{Q}$ vs $\bar{P}$
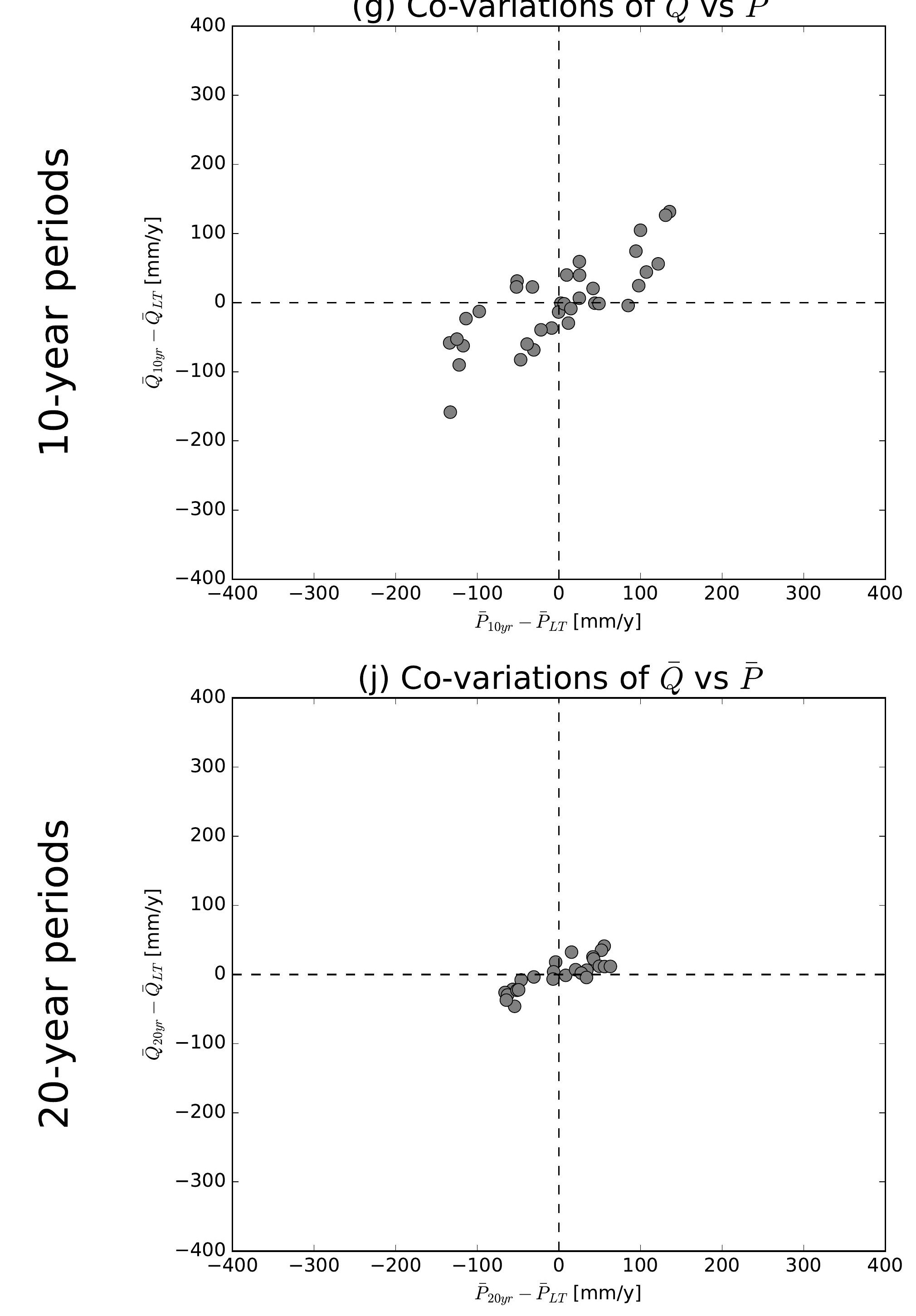

(b) Co-variations of $\bar{Q}$ vs $\overline{E 0}$

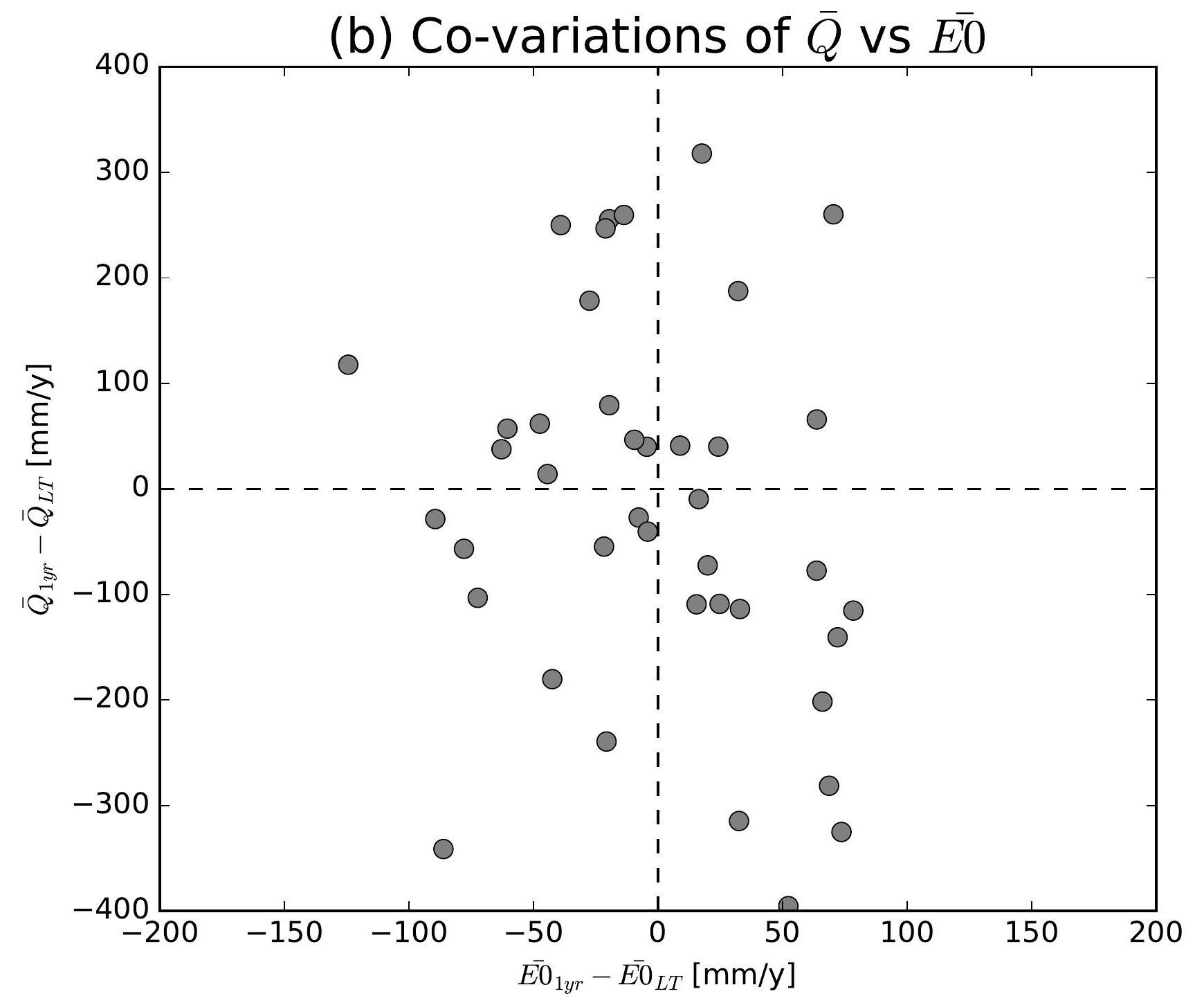

(e) Co-variations of $\bar{Q}$ vs $\overline{E 0}$

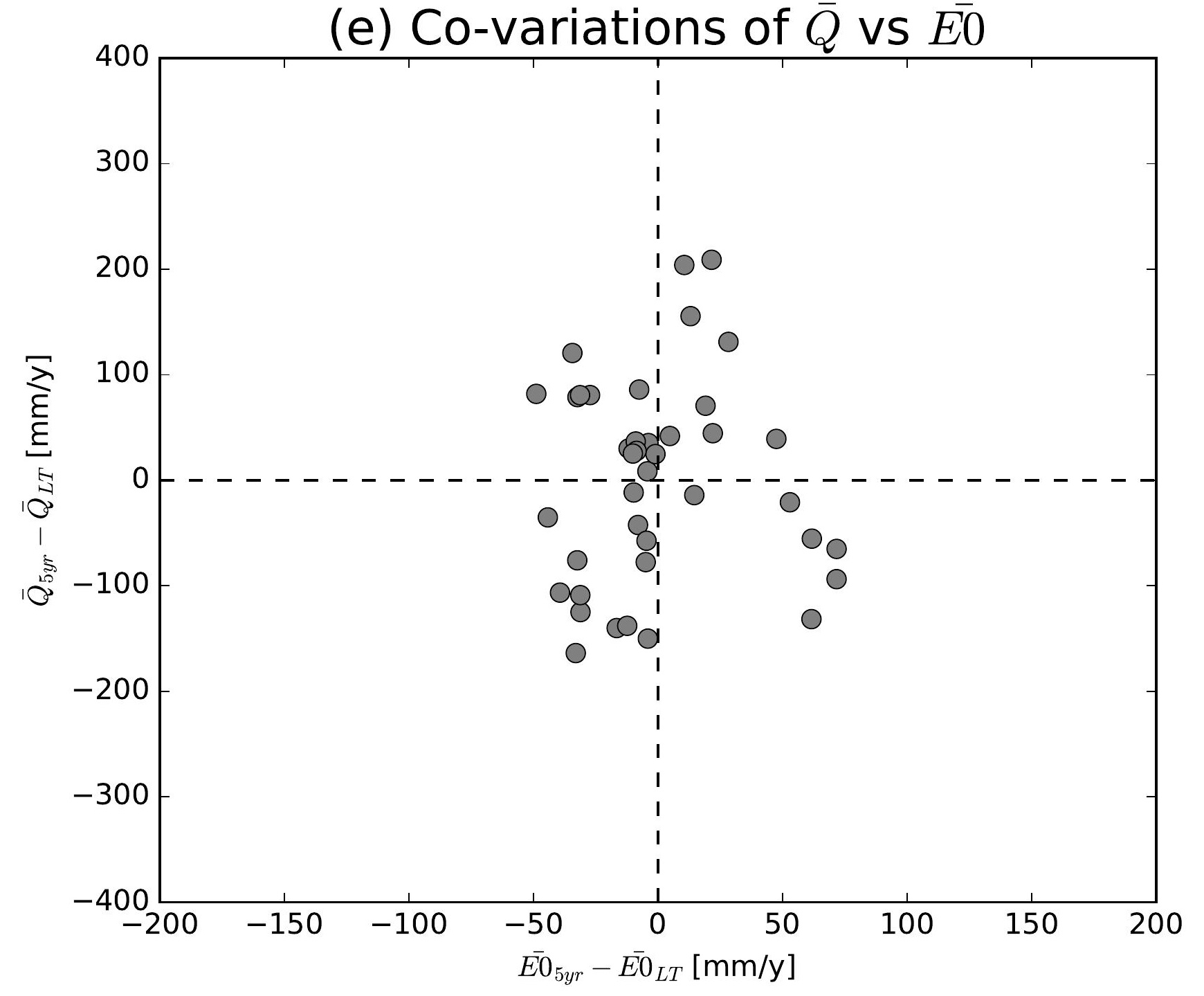

(h) Co-variations of $\bar{Q}$ vs $\overline{E 0}$

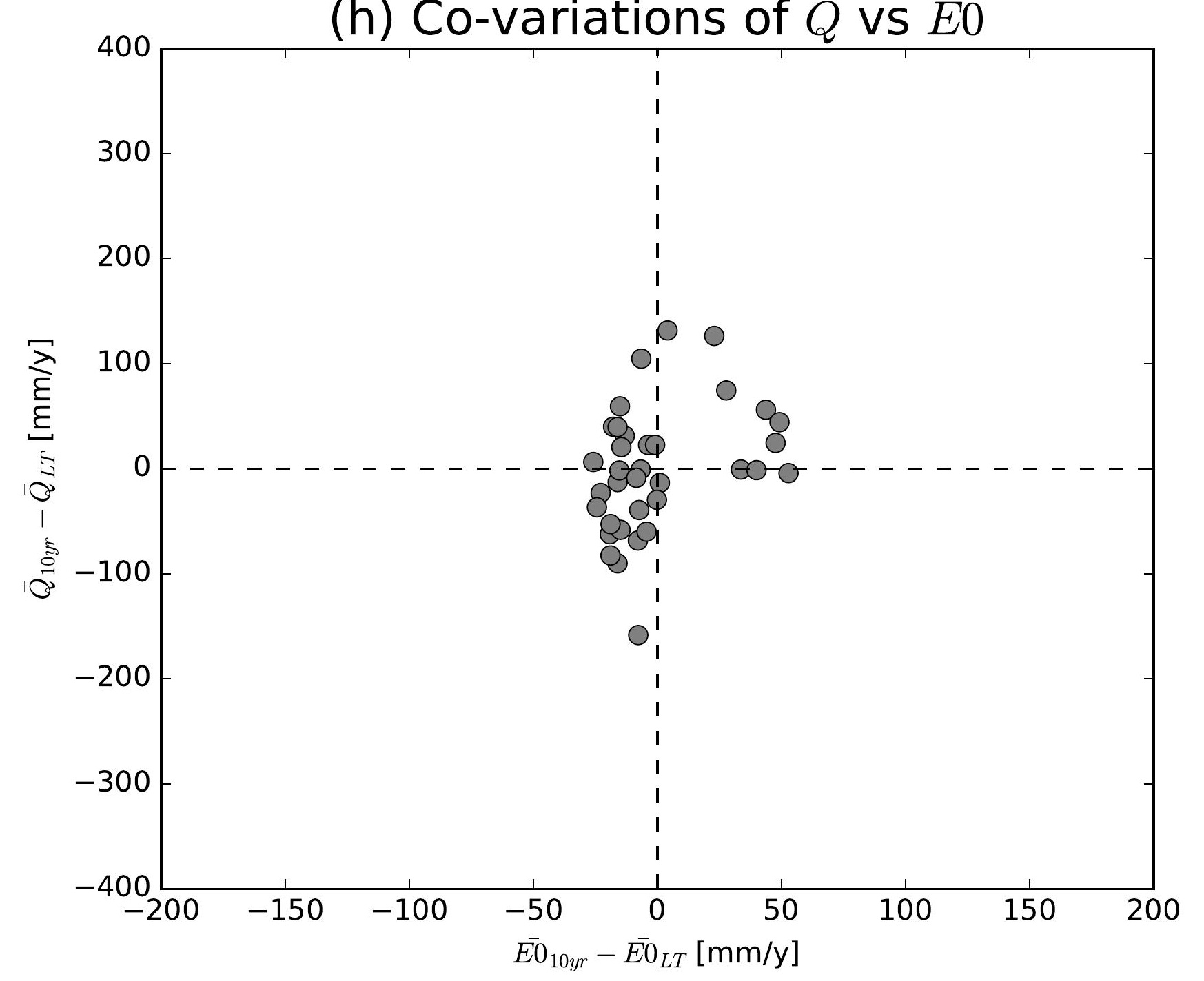

(k) Co-variations of $\bar{Q}$ vs $\overline{E 0}$

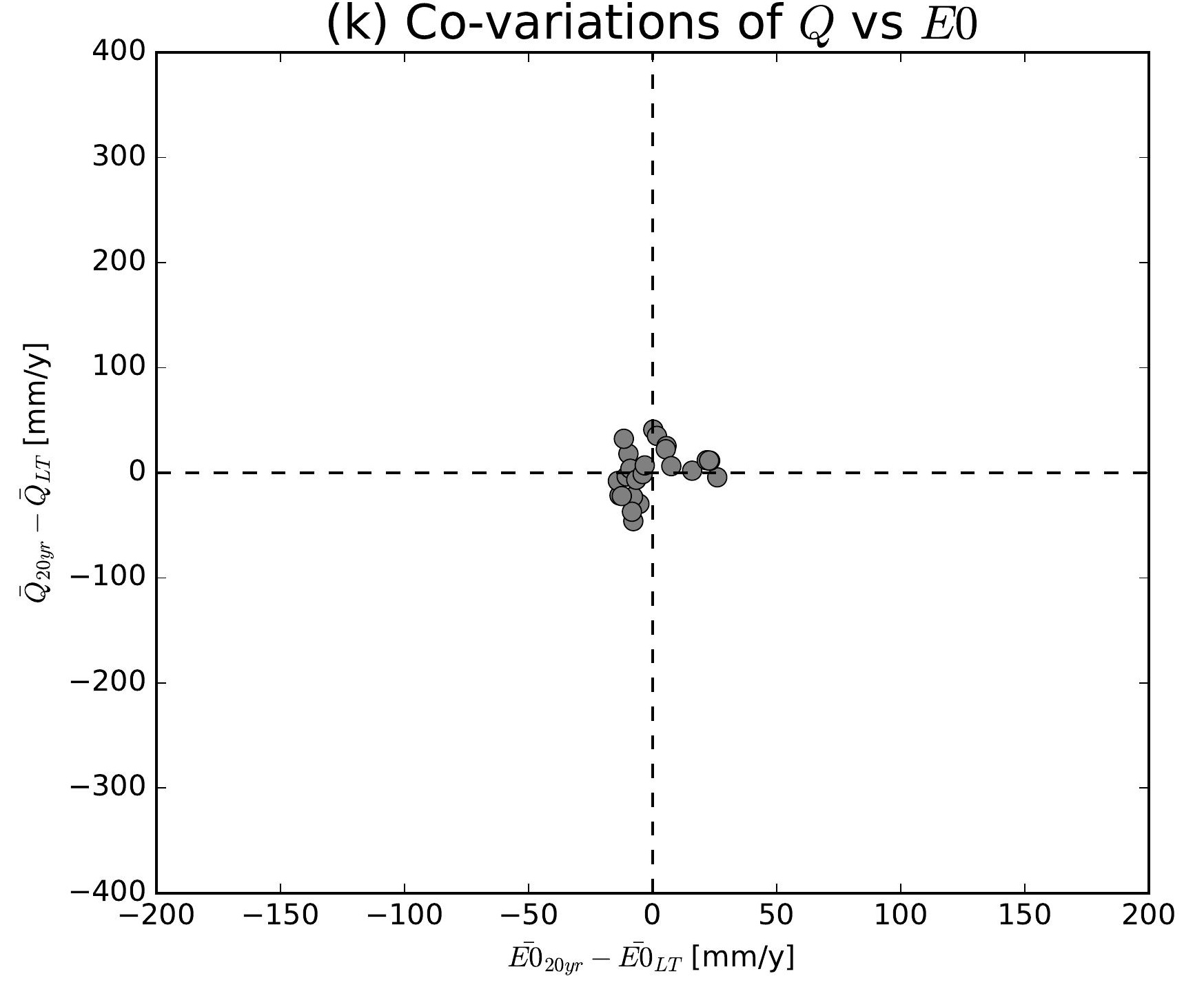

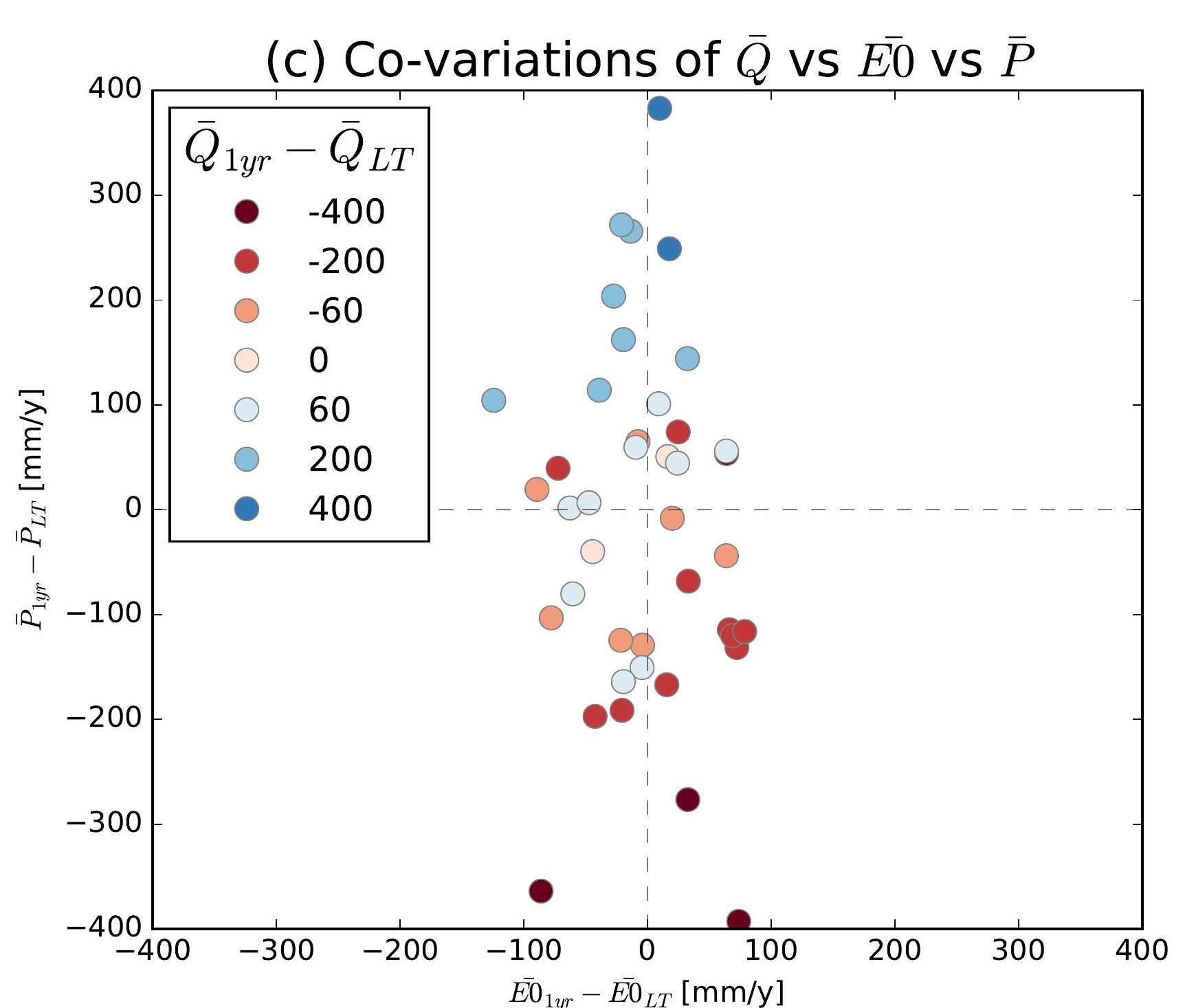
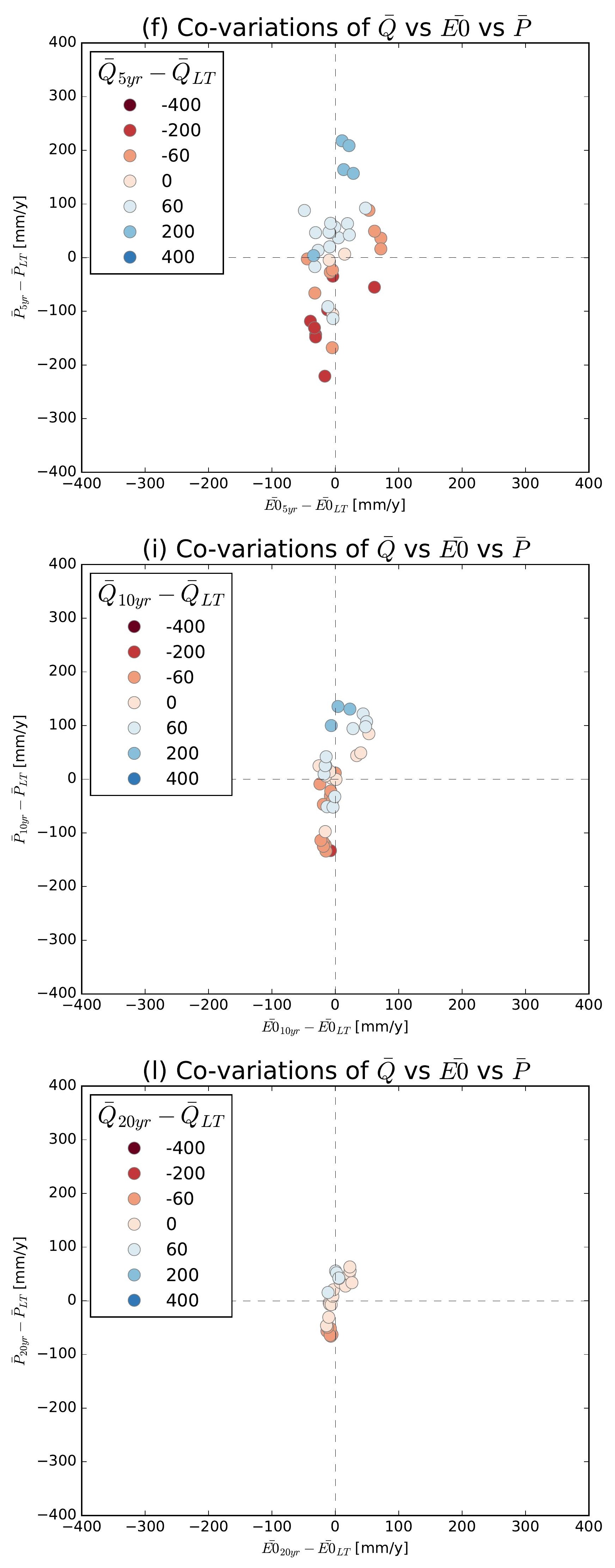

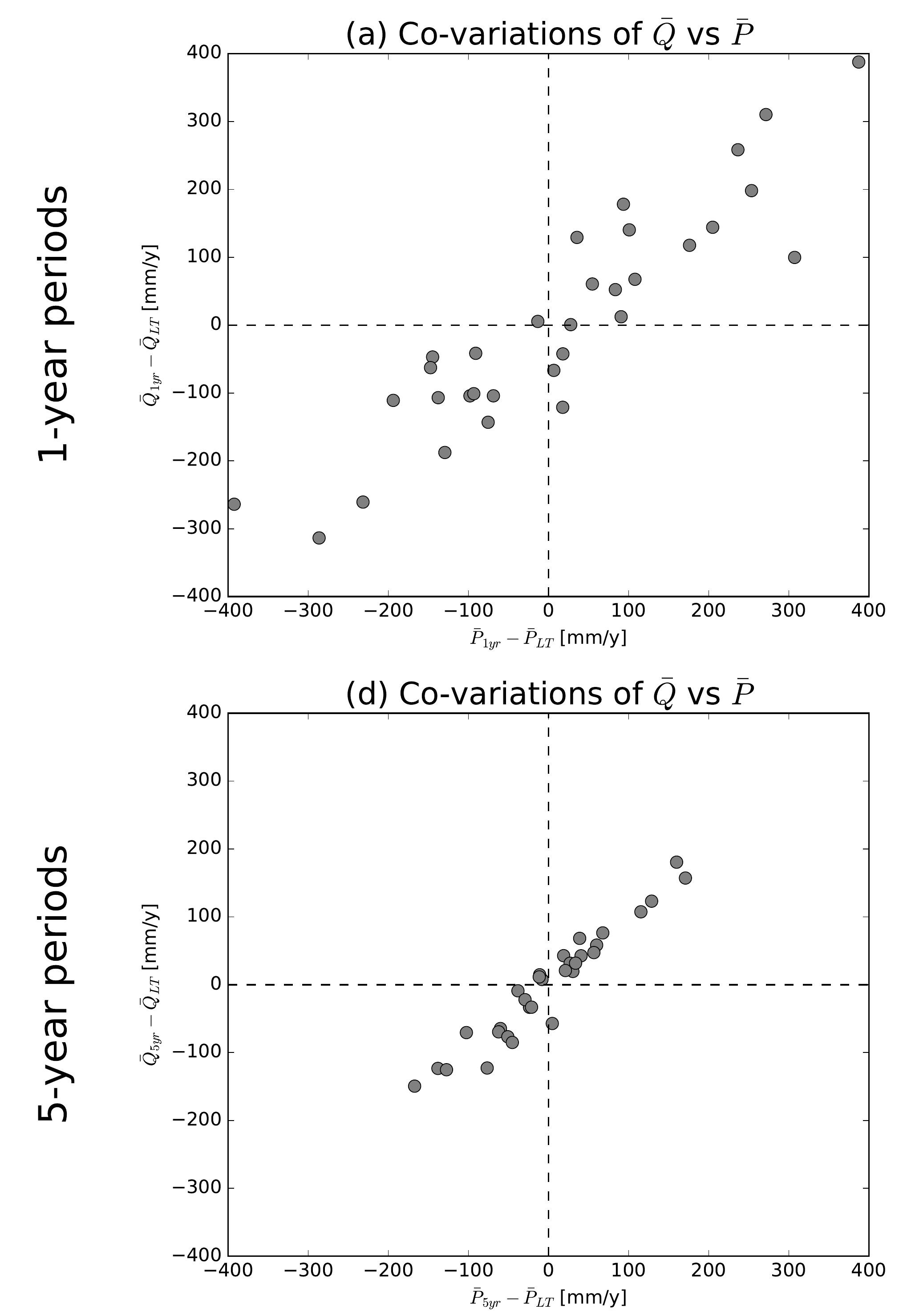

(g) Co-variations of $\bar{Q}$ vs $\bar{P}$
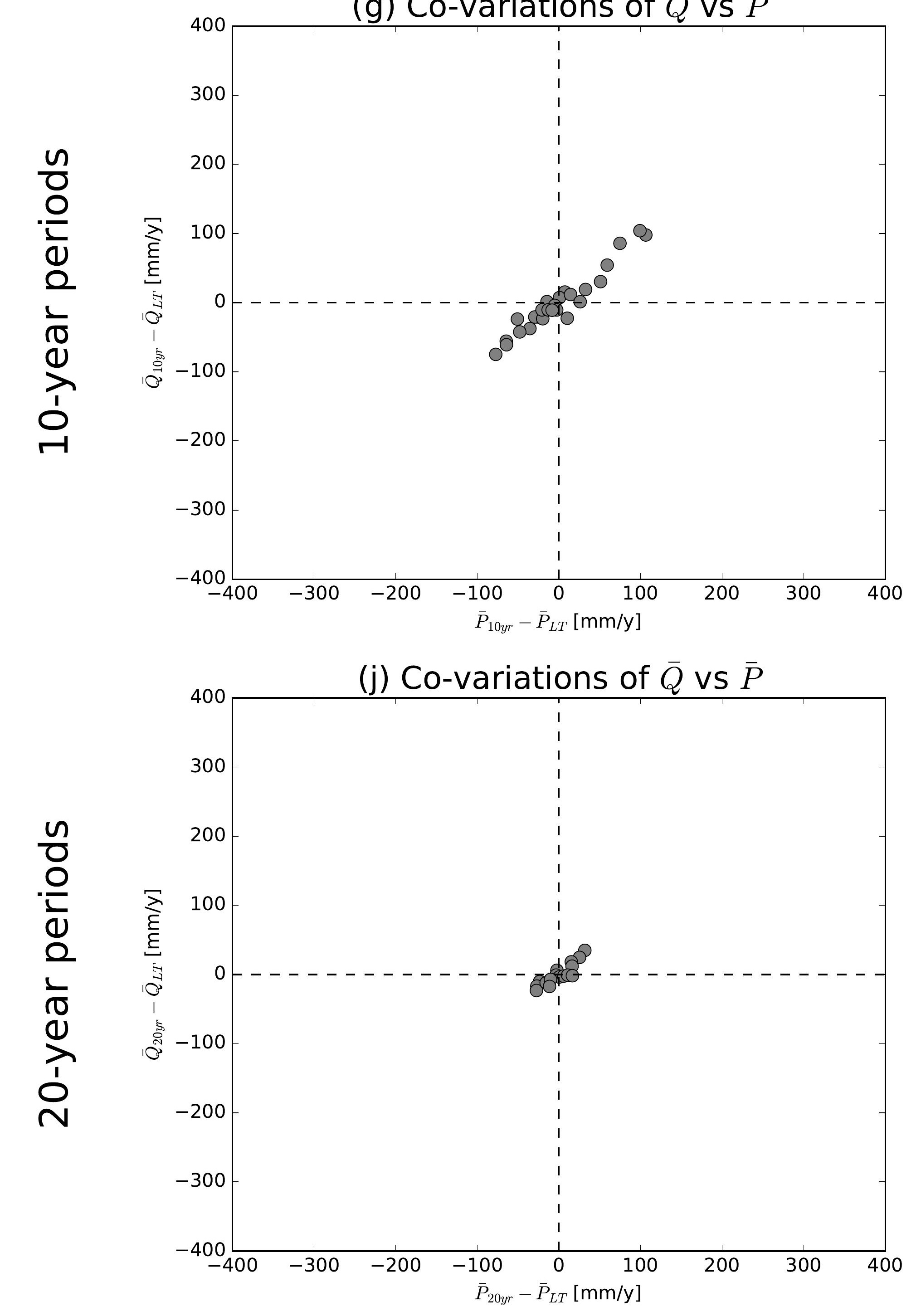

(b) Co-variations of $\bar{Q}$ vs $\overline{E 0}$

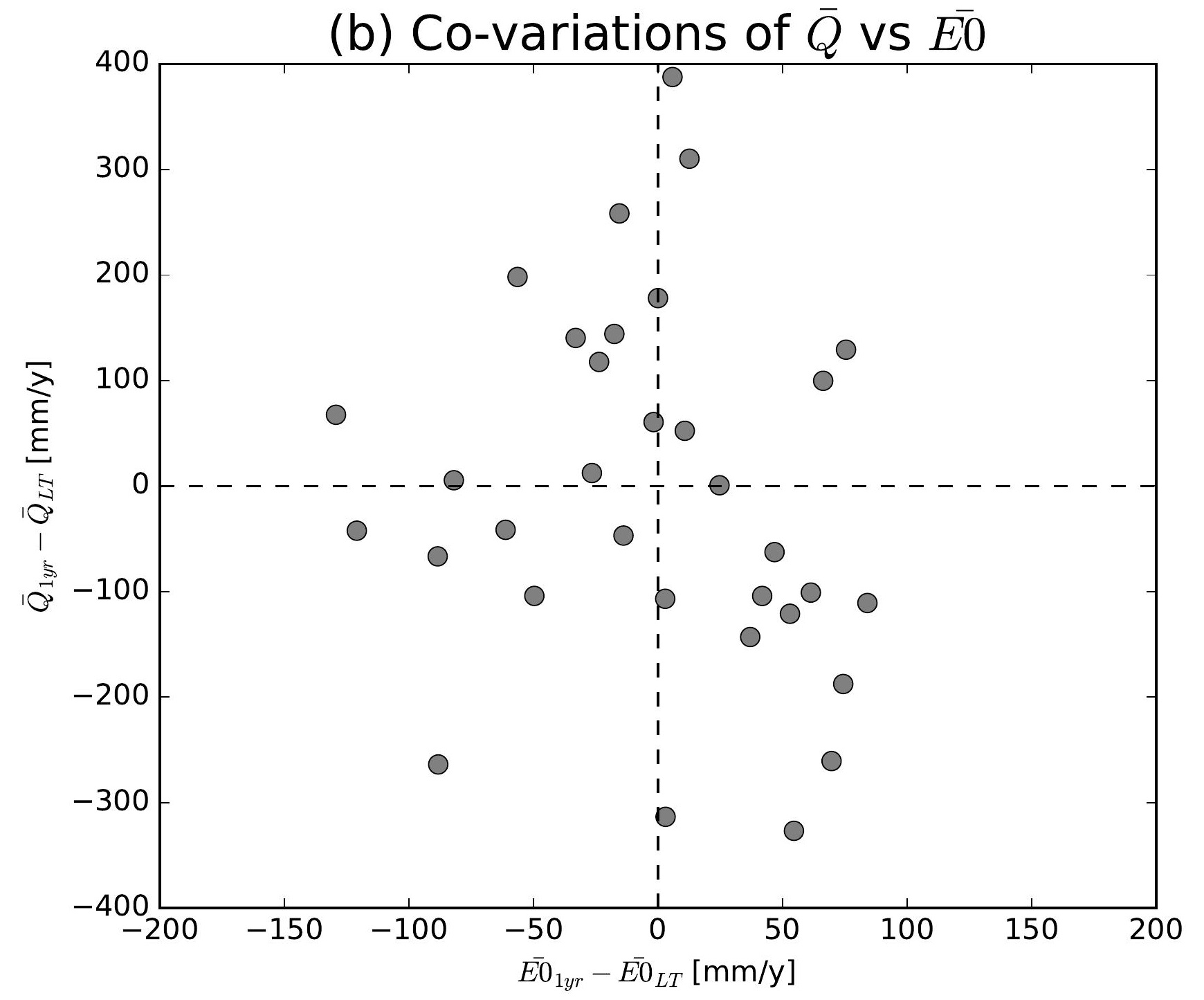

(e) Co-variations of $\bar{Q}$ vs $\overline{E 0}$

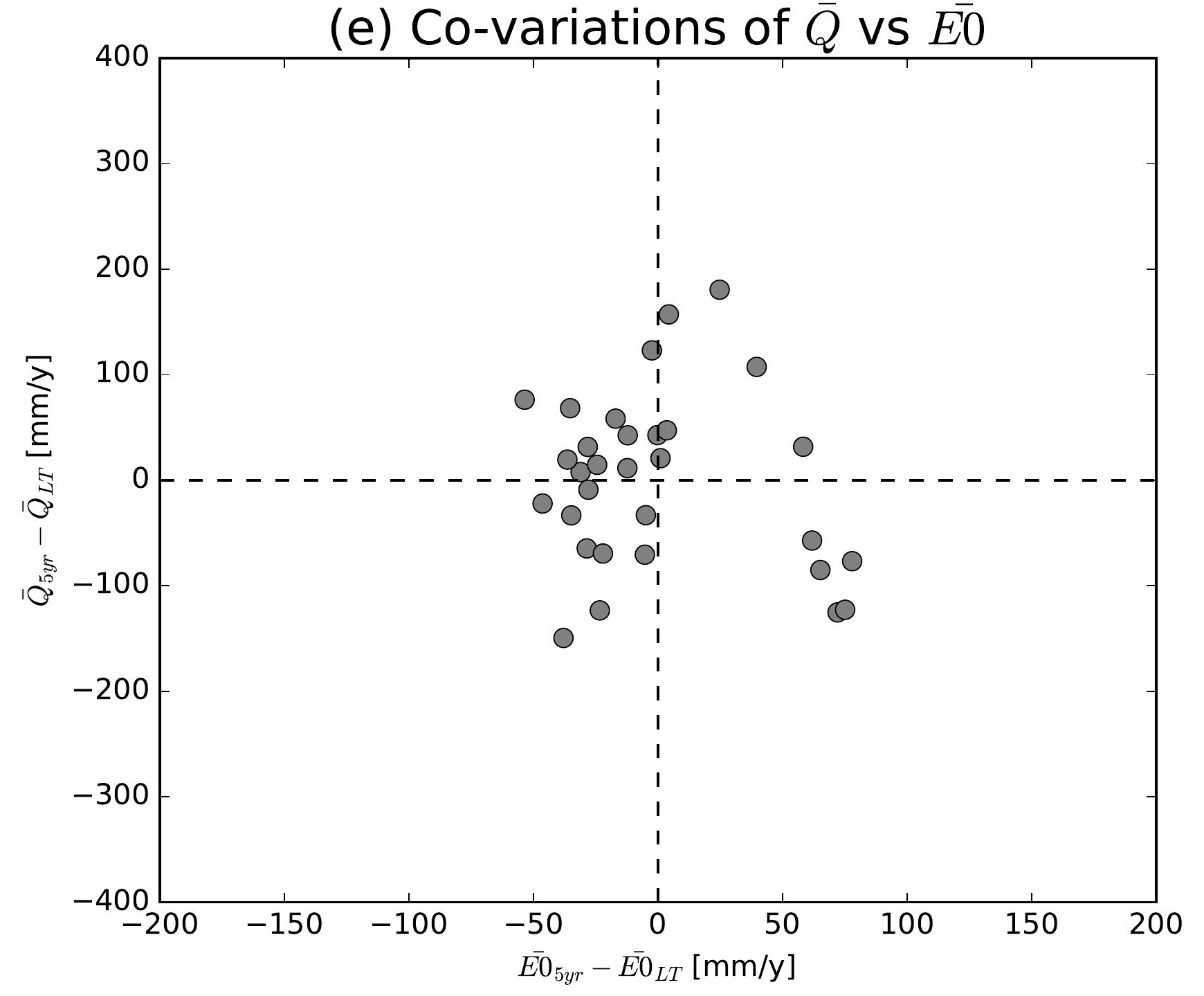

(h) Co-variations of $\bar{Q}$ vs $\overline{E 0}$

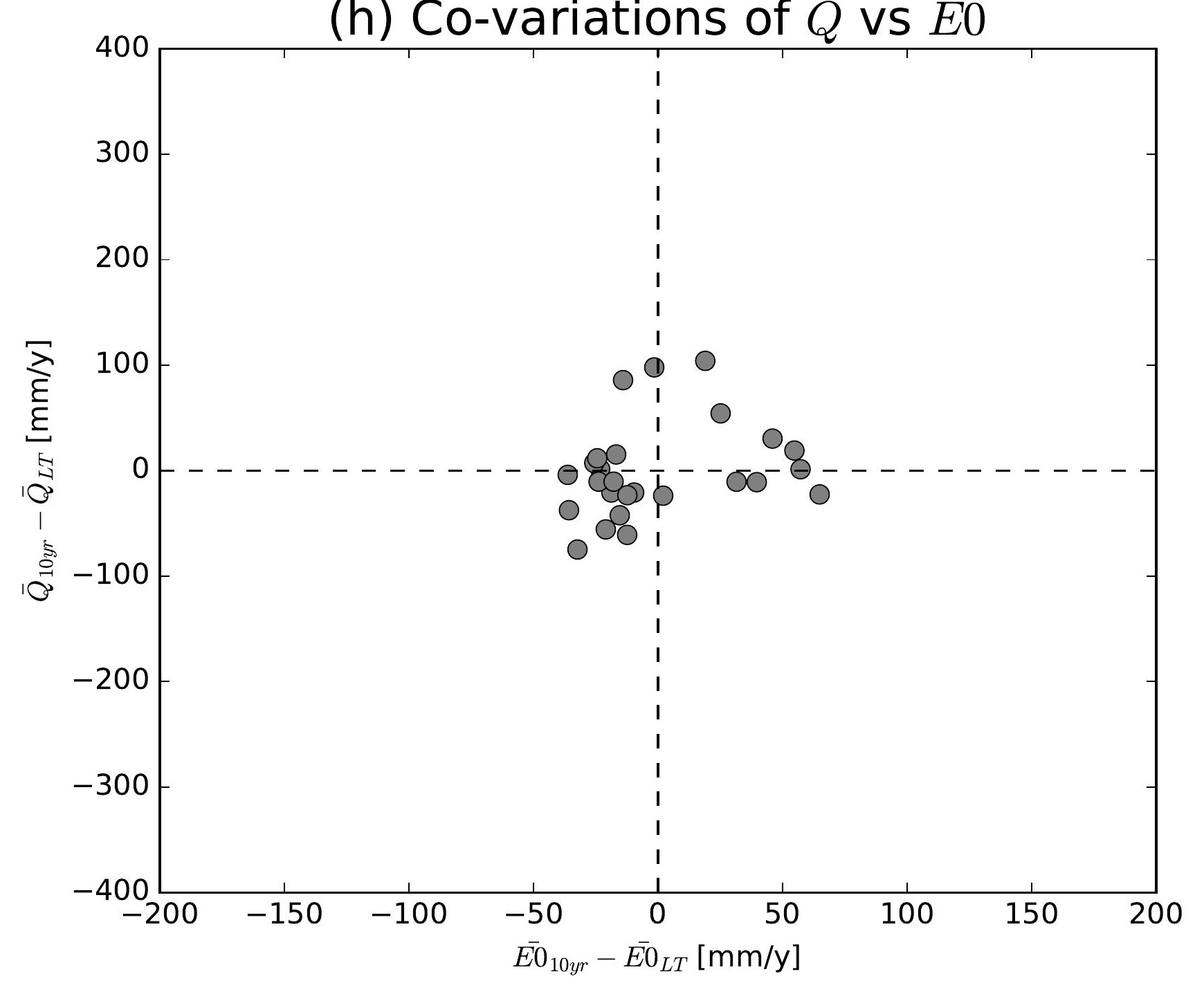

(k) Co-variations of $\bar{Q}$ vs $\overline{E 0}$

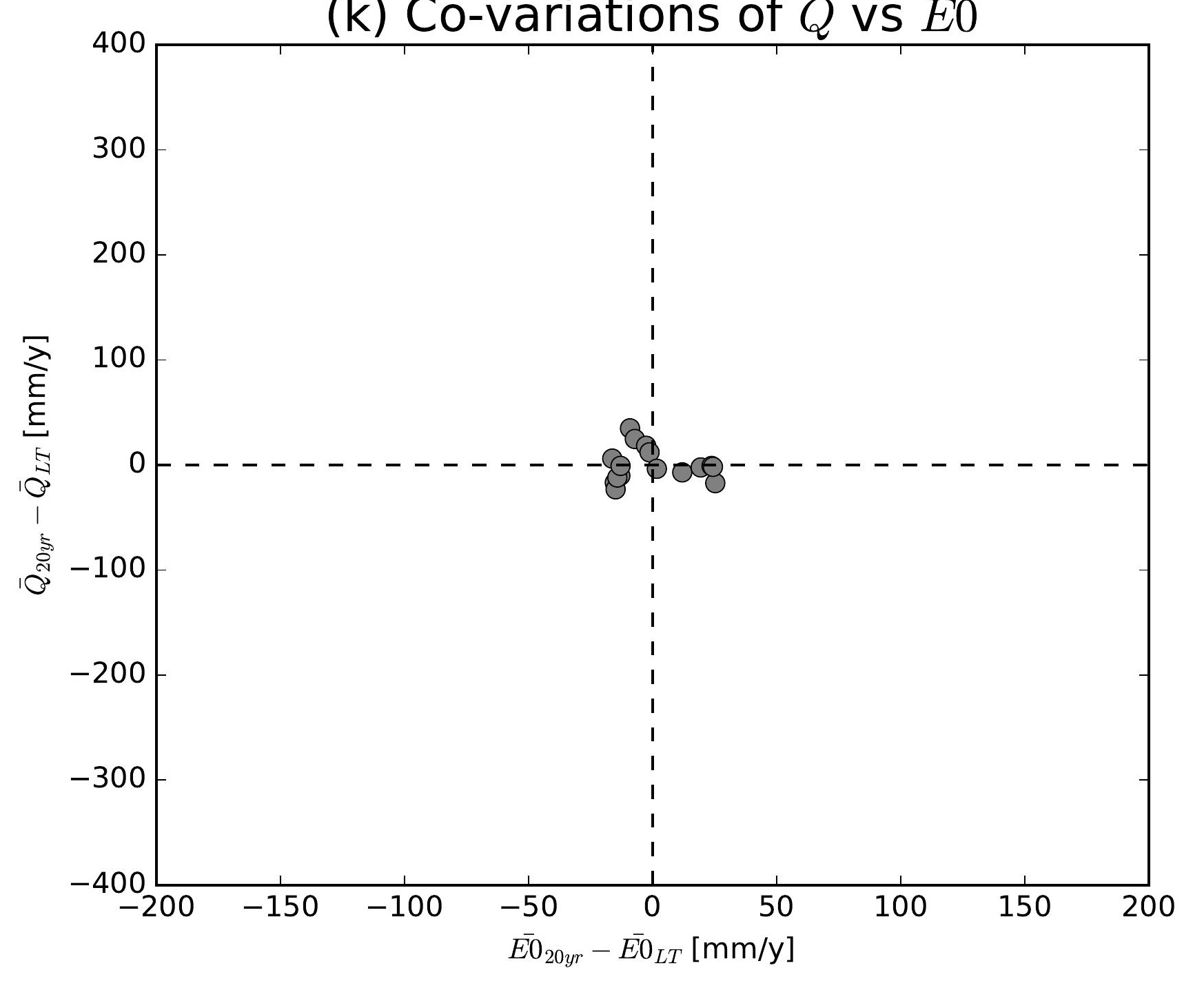

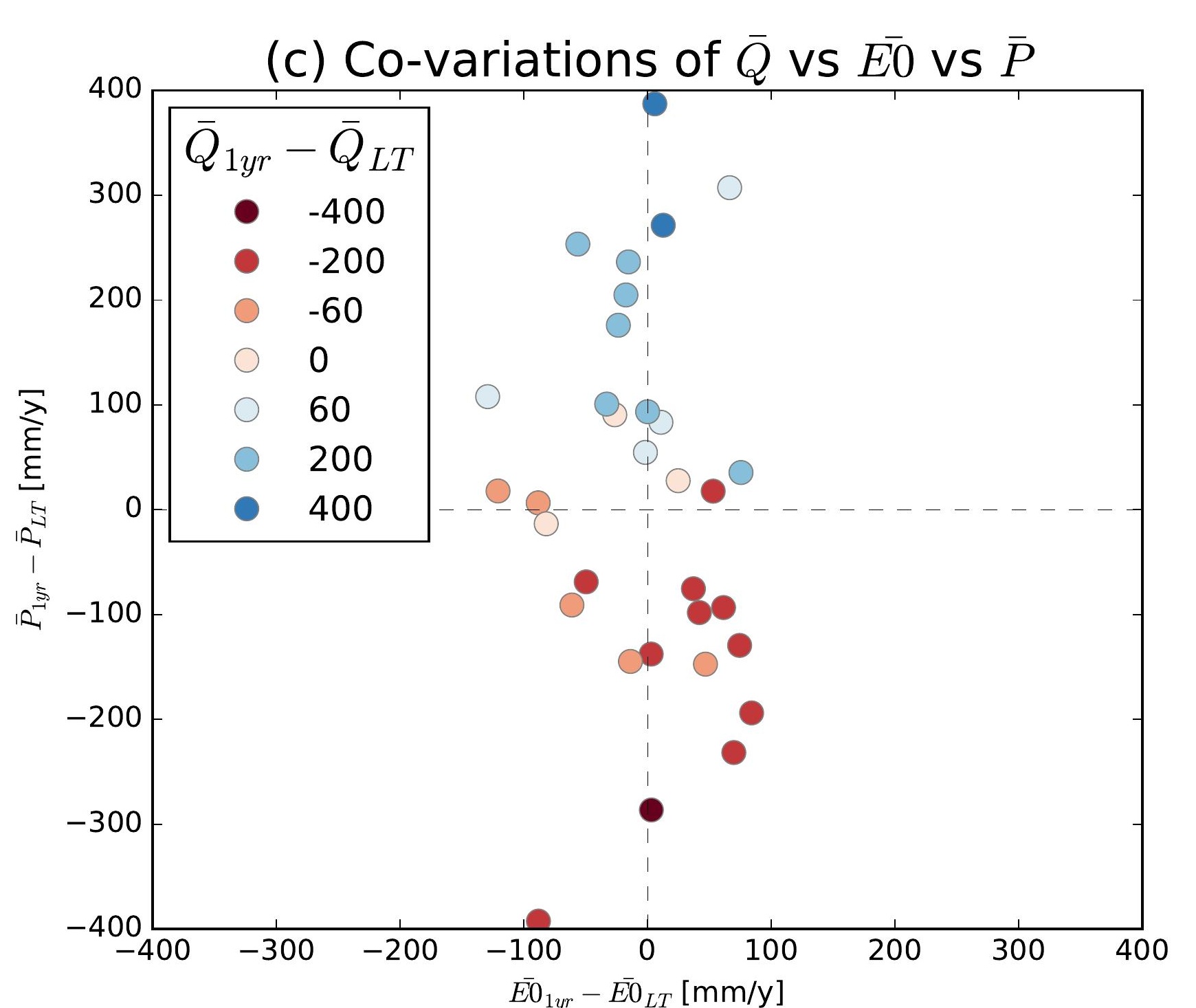
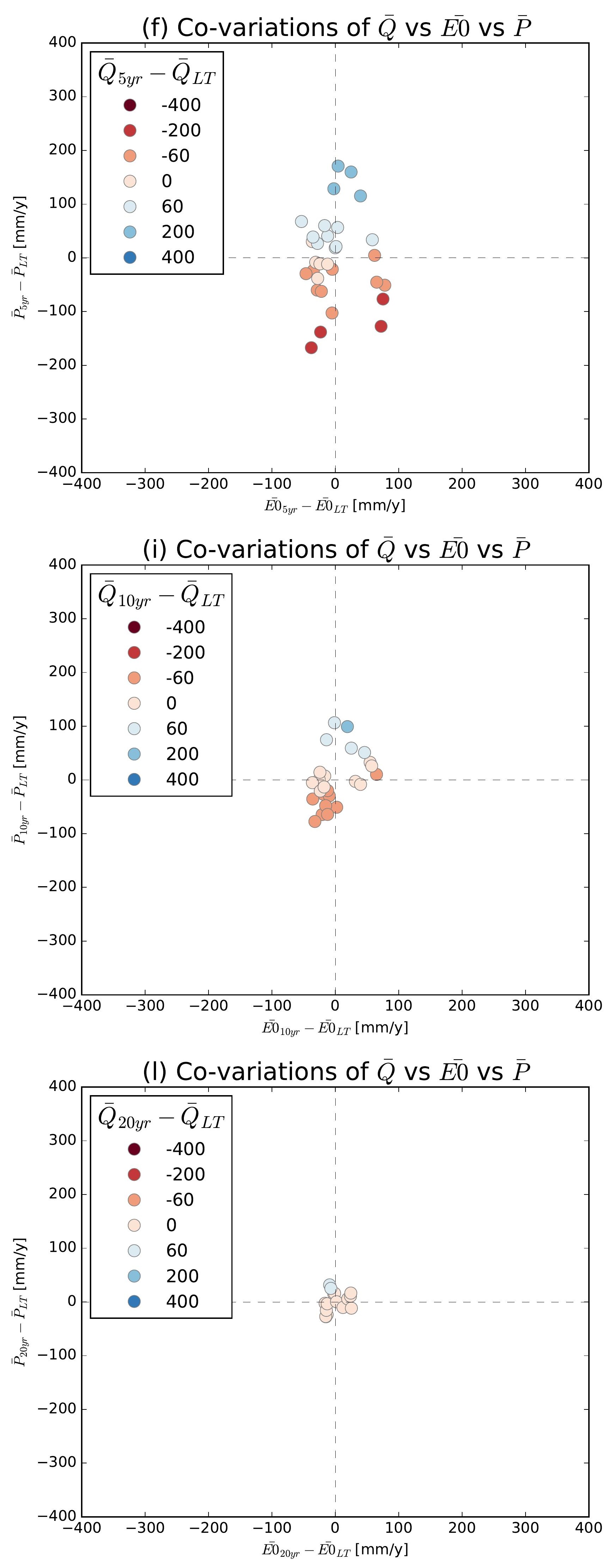

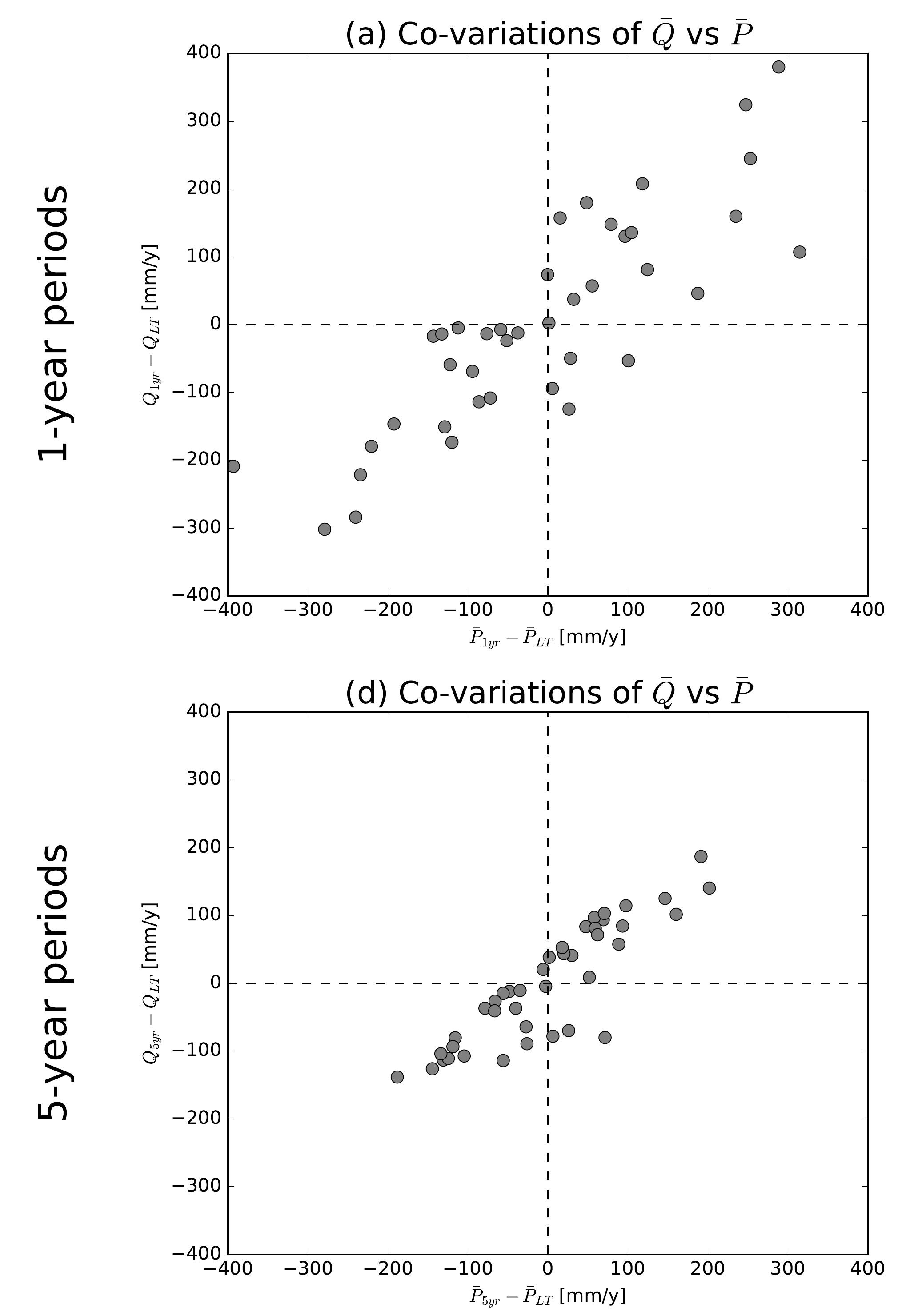

(g) Co-variations of $\bar{Q}$ vs $\bar{P}$
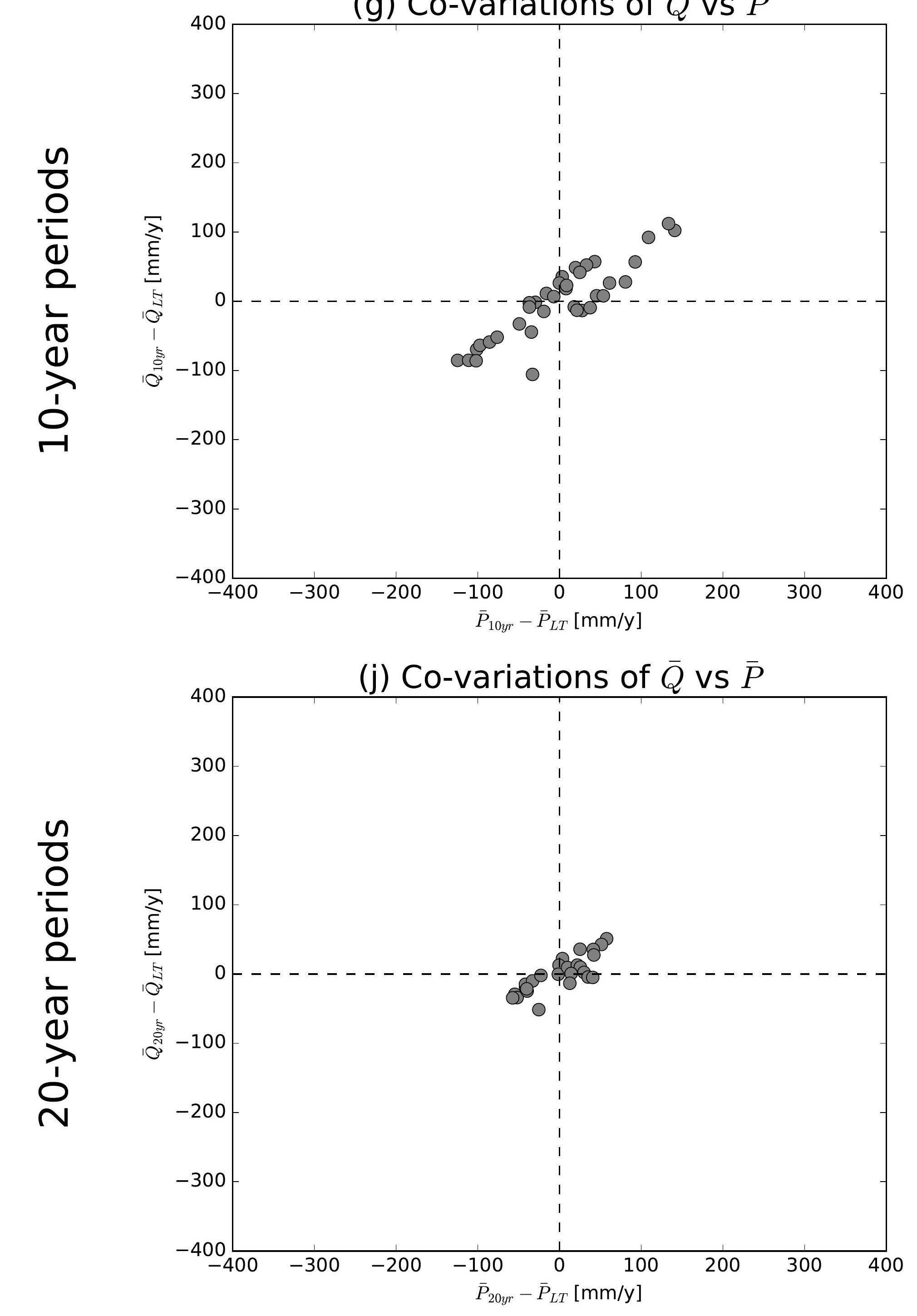

(b) Co-variations of $\bar{Q}$ vs $\overline{E 0}$

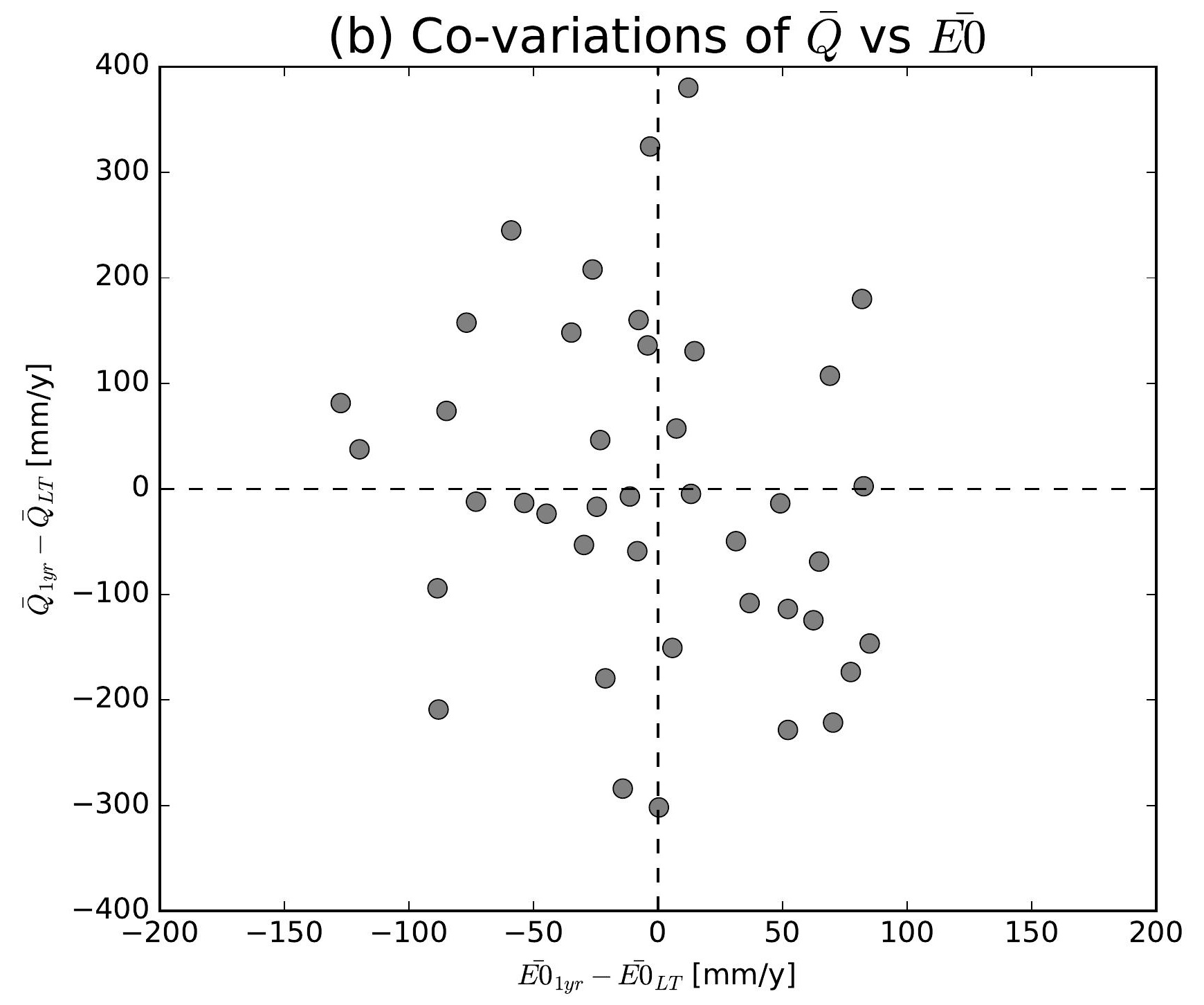

(e) Co-variations of $\bar{Q}$ vs $\overline{E 0}$

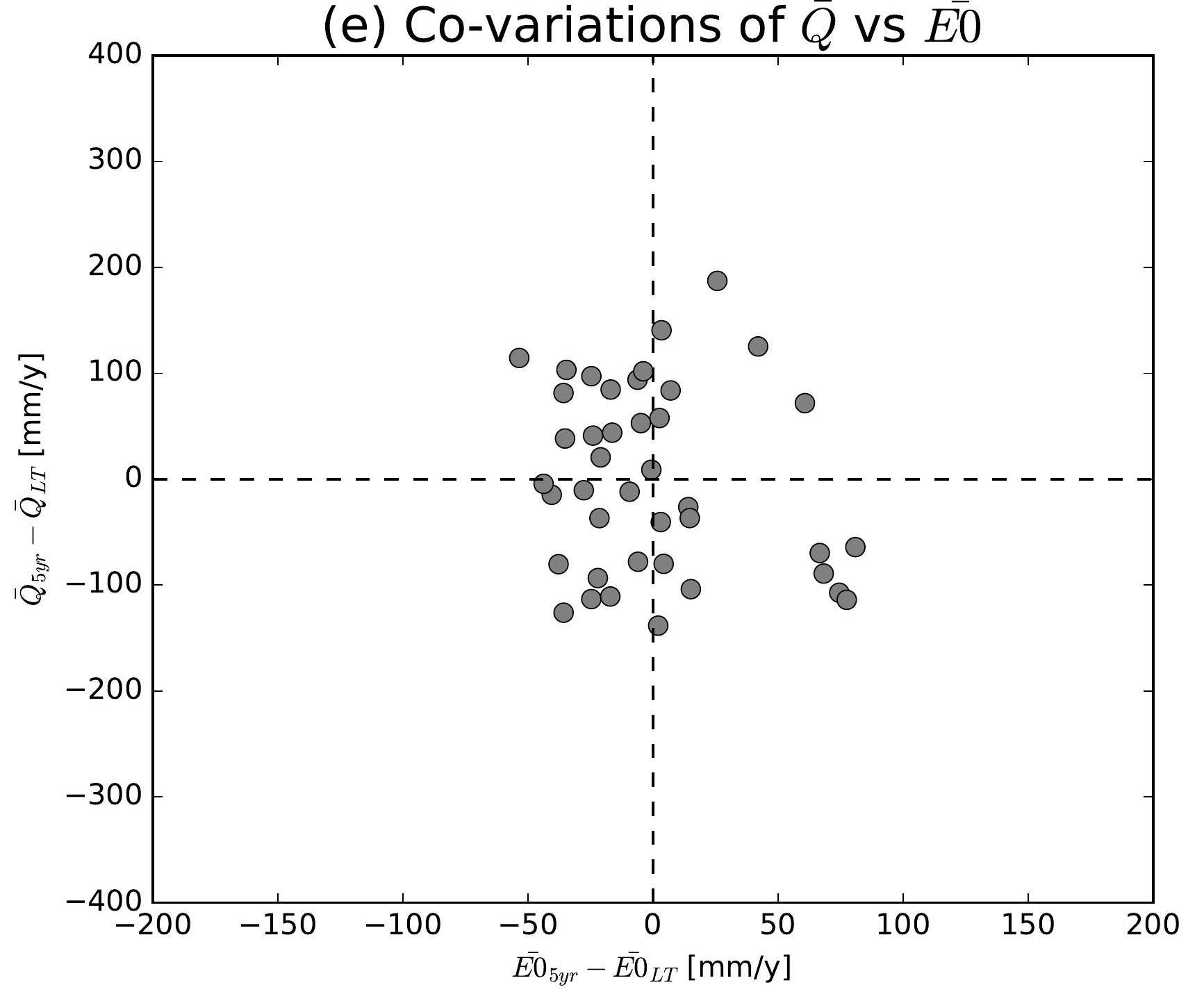

(h) Co-variations of $\bar{Q}$ vs $\overline{E 0}$

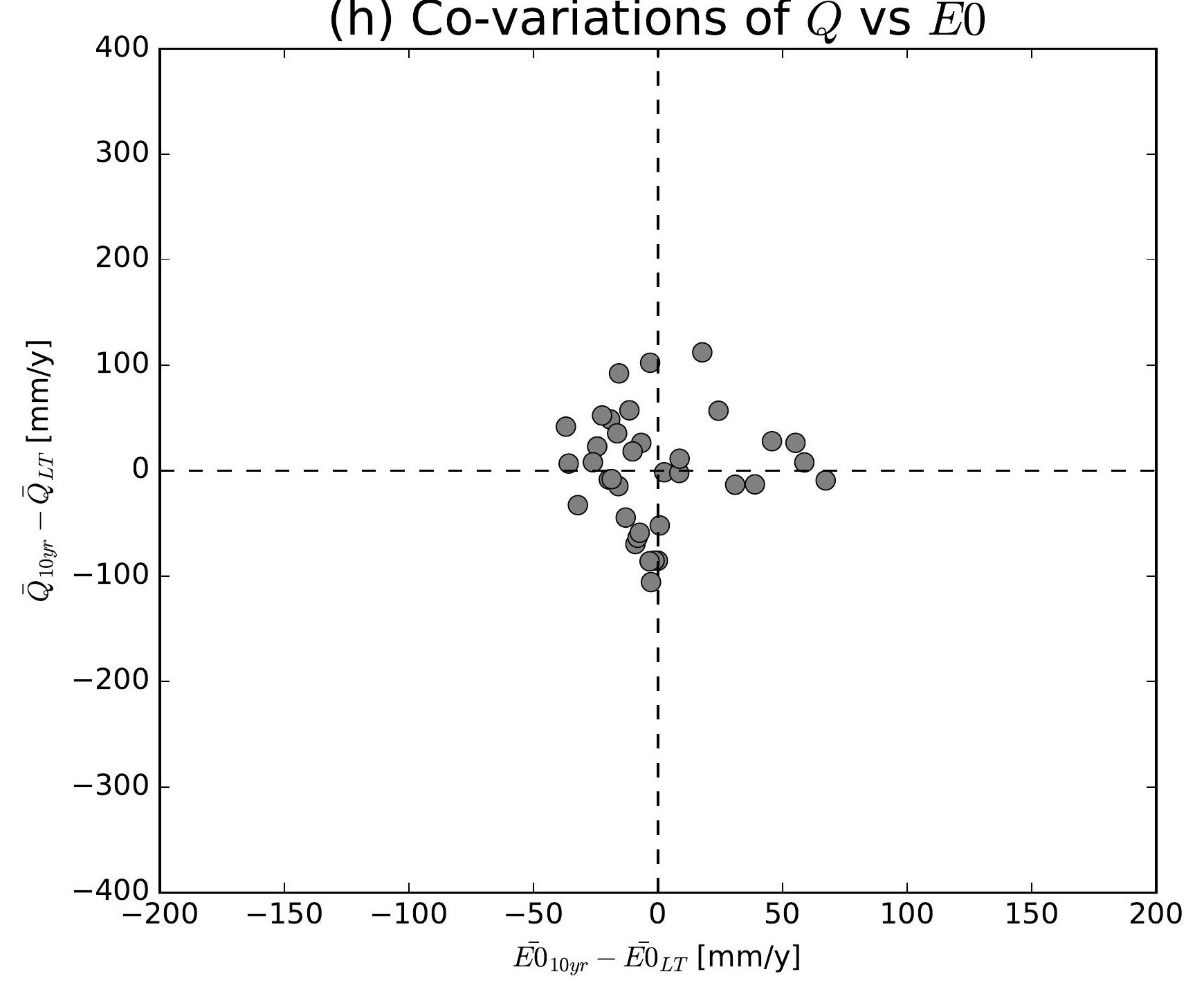

(k) Co-variations of $\bar{Q}$ vs $\overline{E 0}$

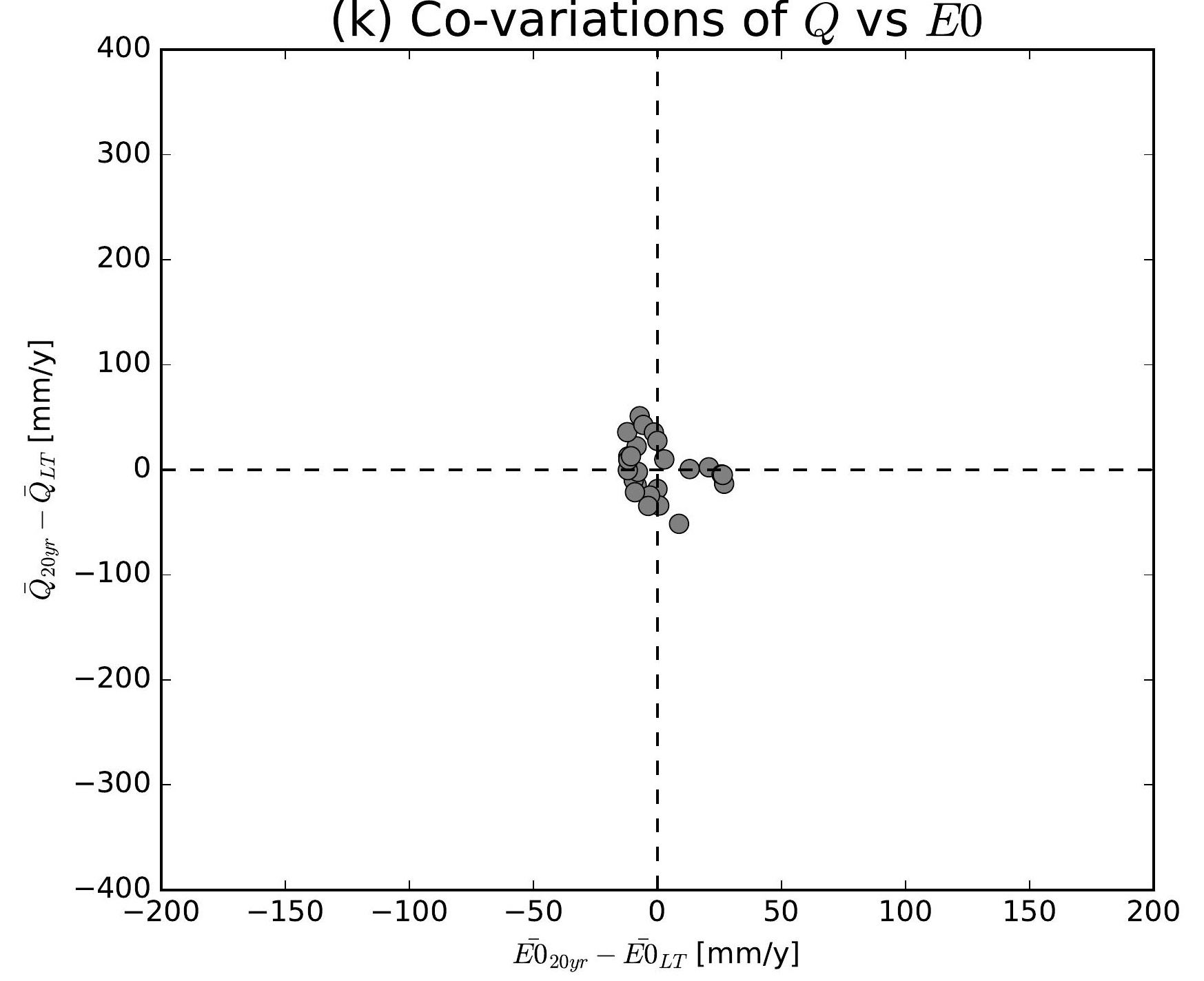

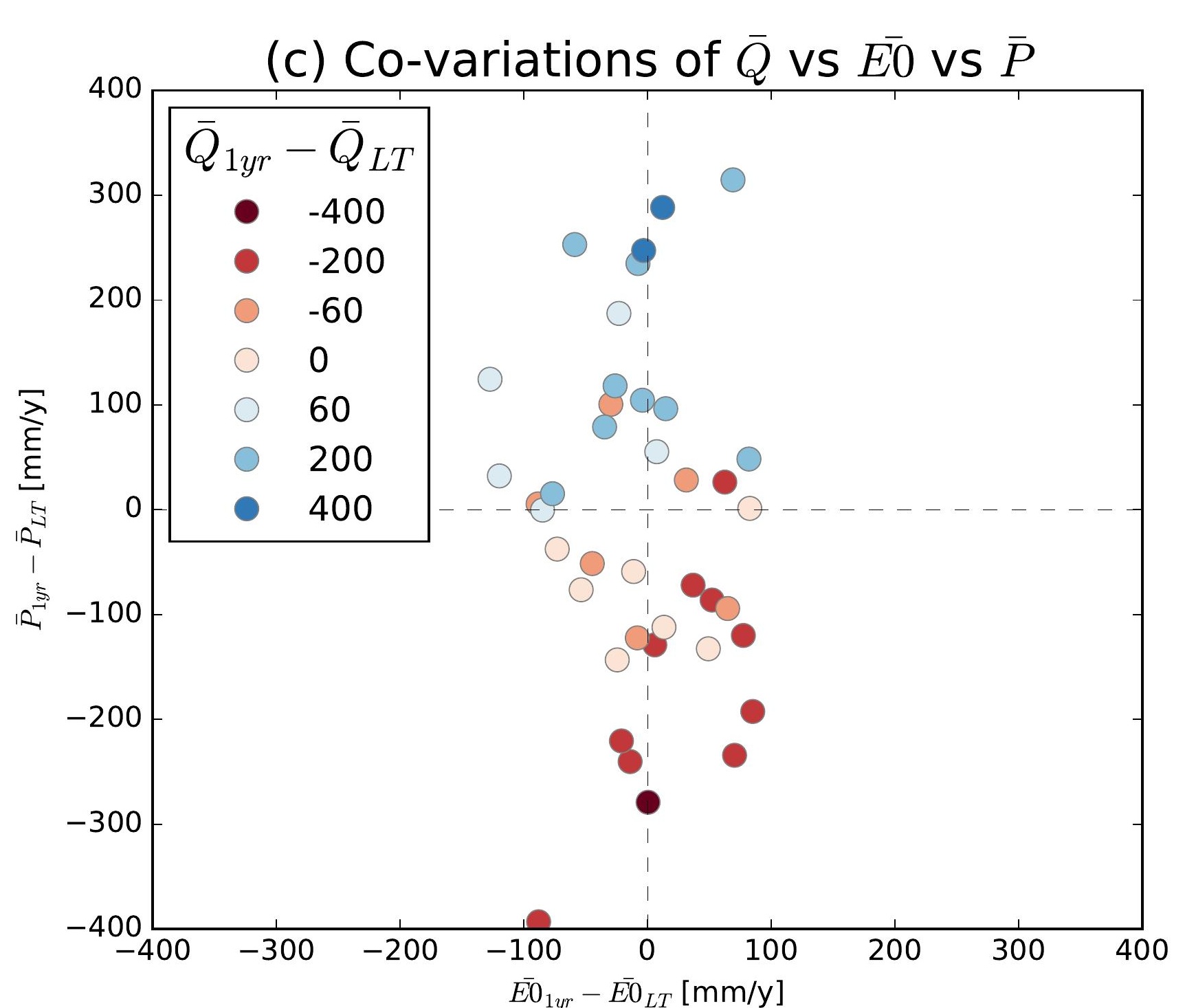
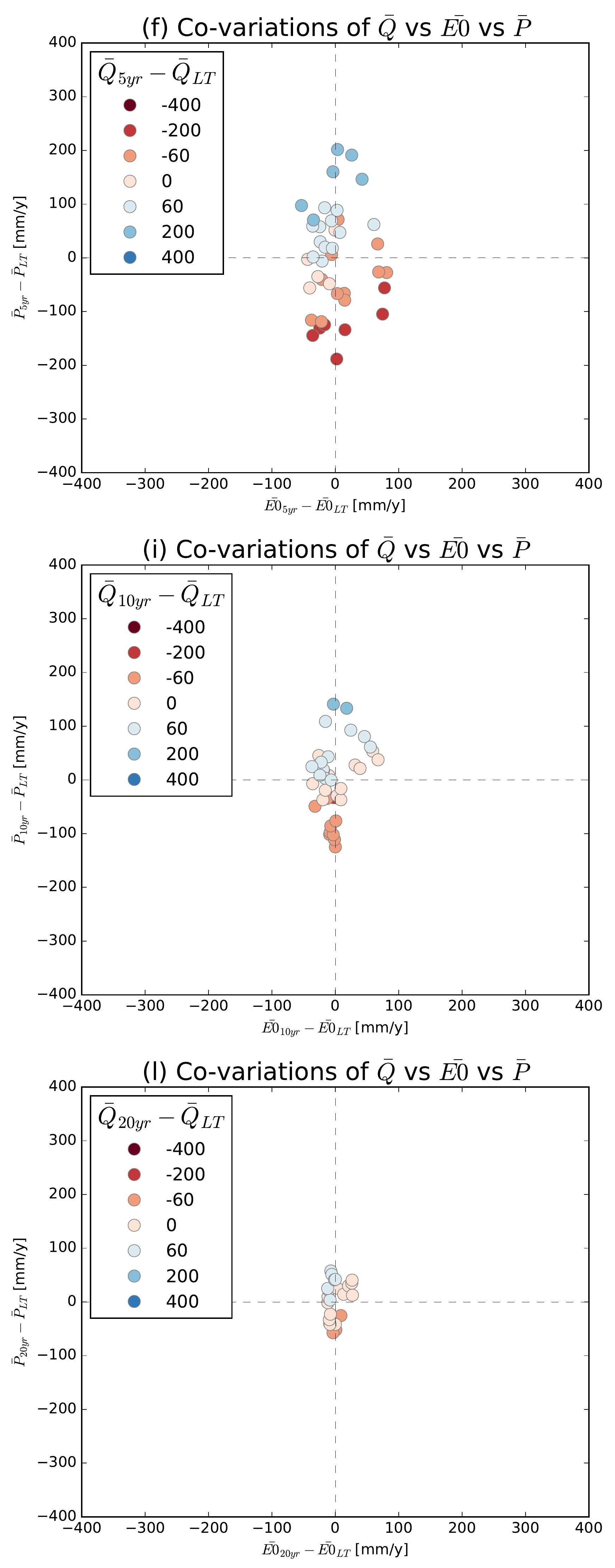

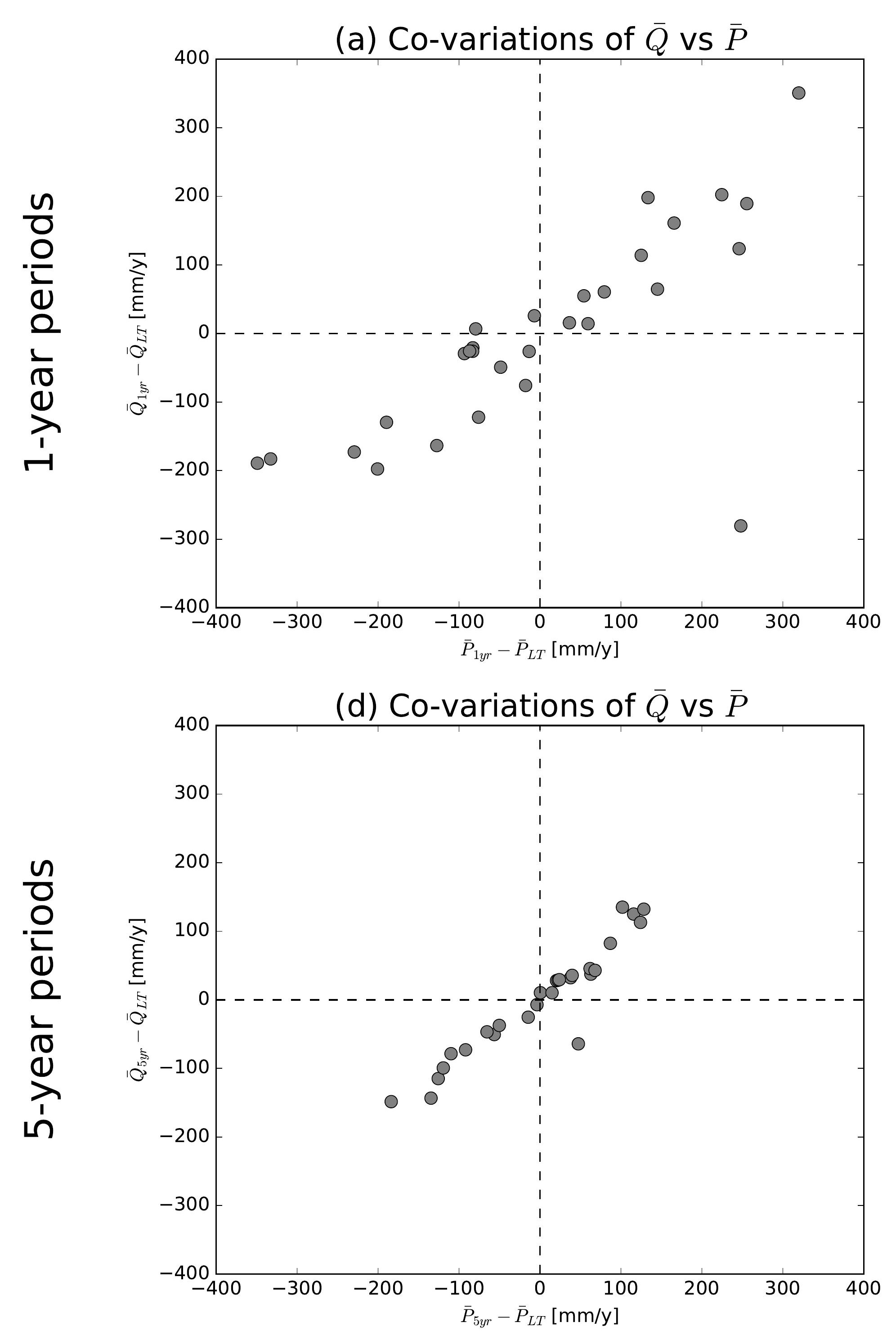

(g) Co-variations of $\bar{Q}$ vs $\bar{P}$
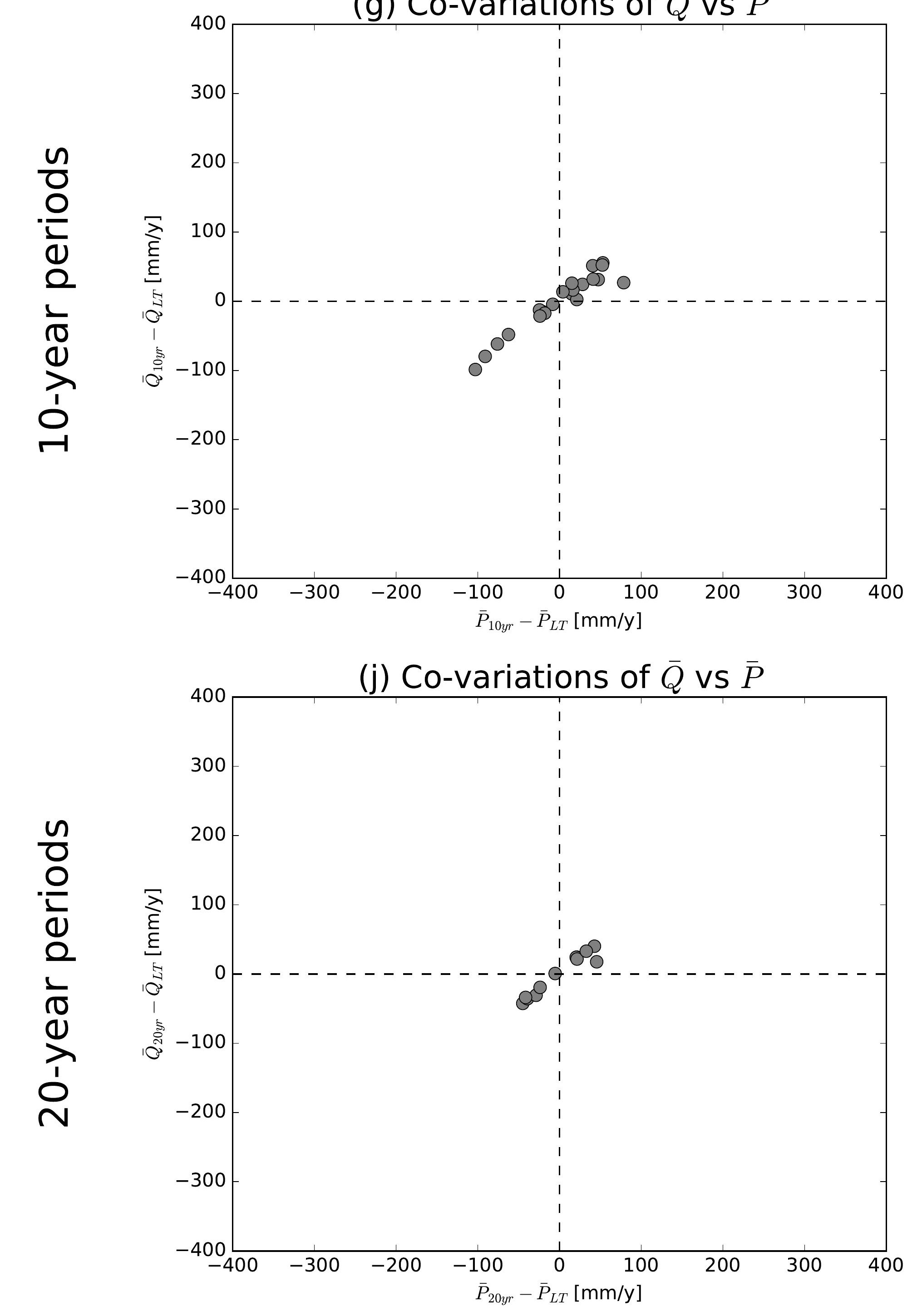

(b) Co-variations of $\bar{Q}$ vs $\overline{E 0}$

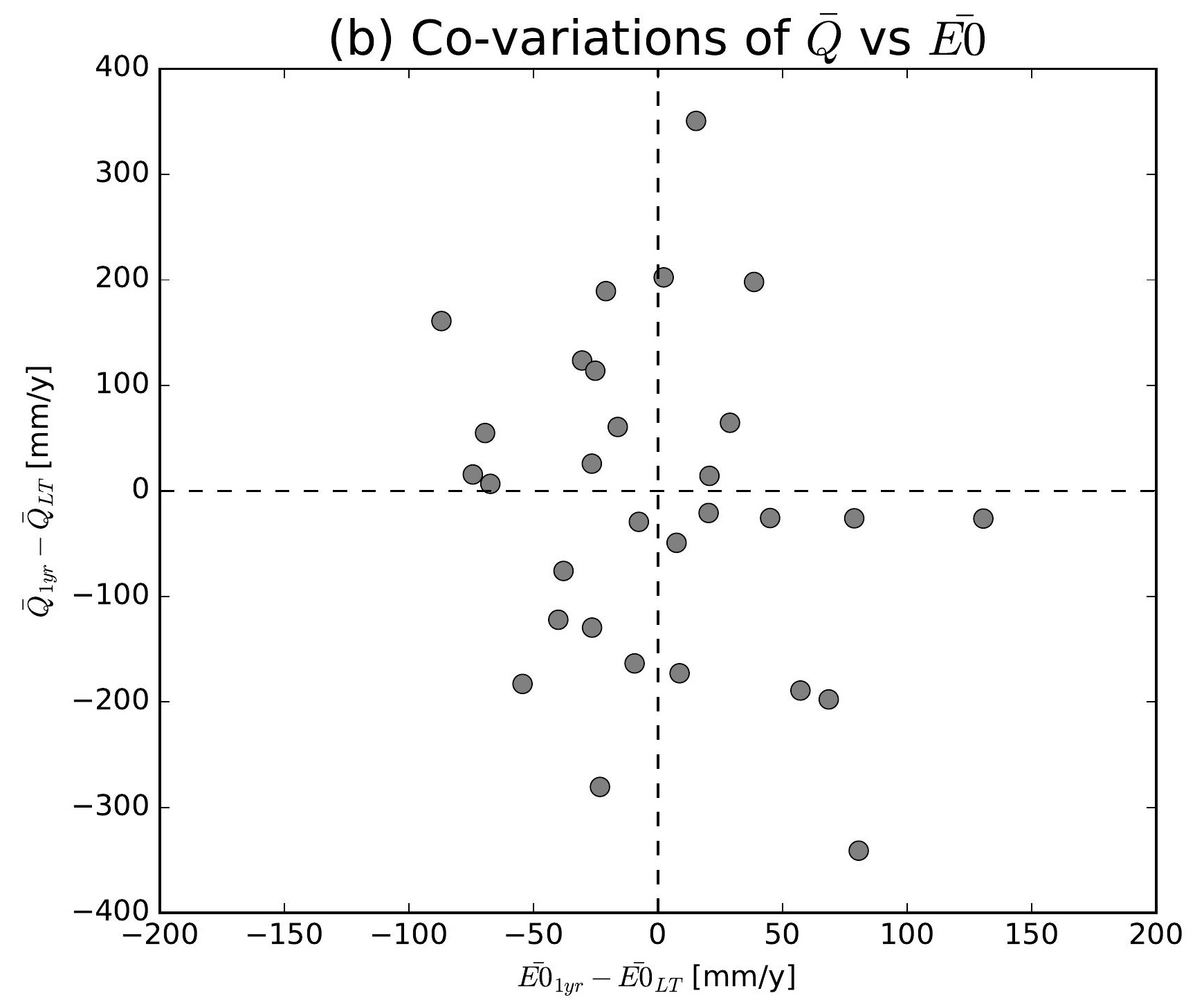

(e) Co-variations of $\bar{Q}$ vs $\overline{E 0}$

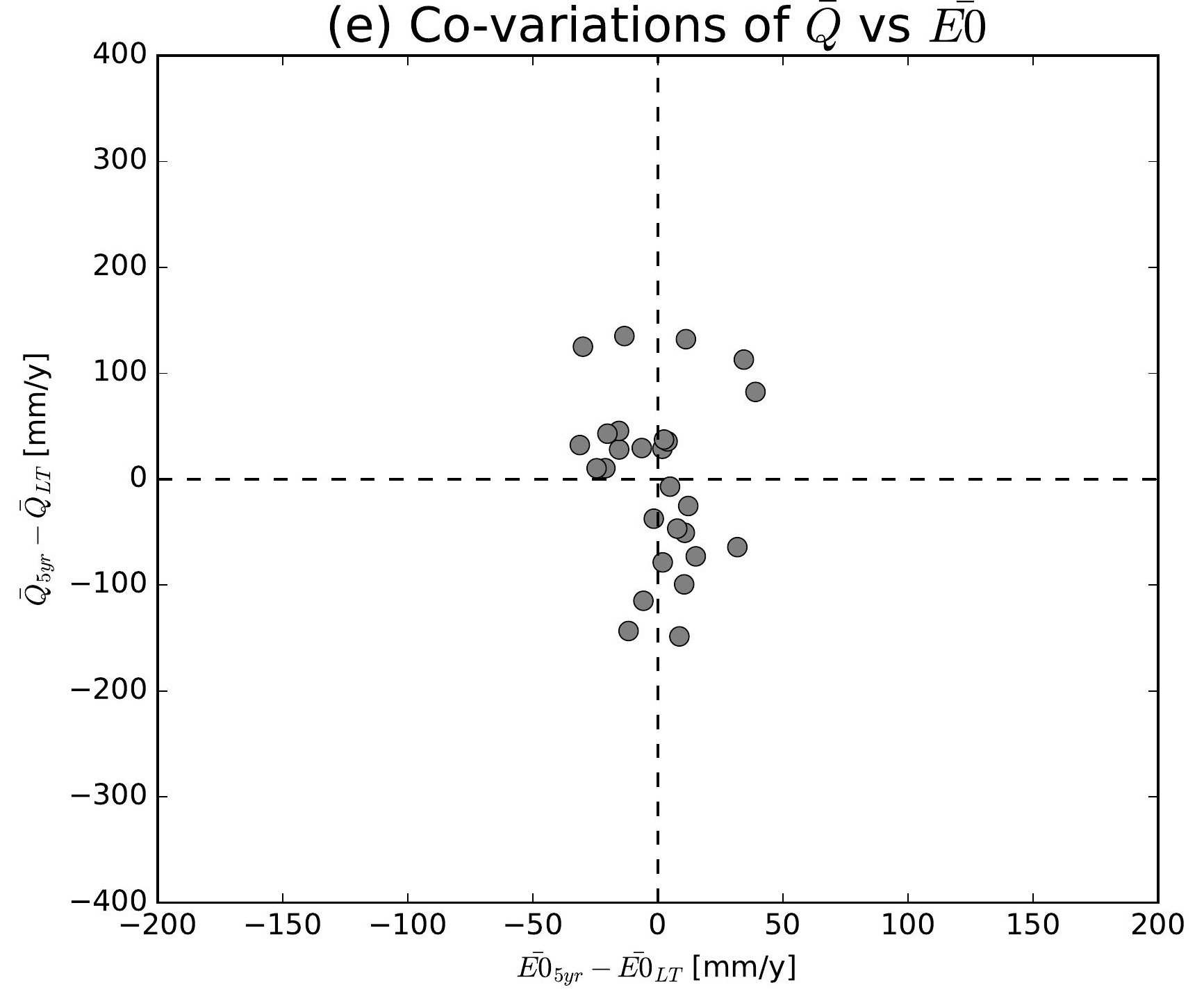

(h) Co-variations of $\bar{Q}$ vs $\overline{E 0}$

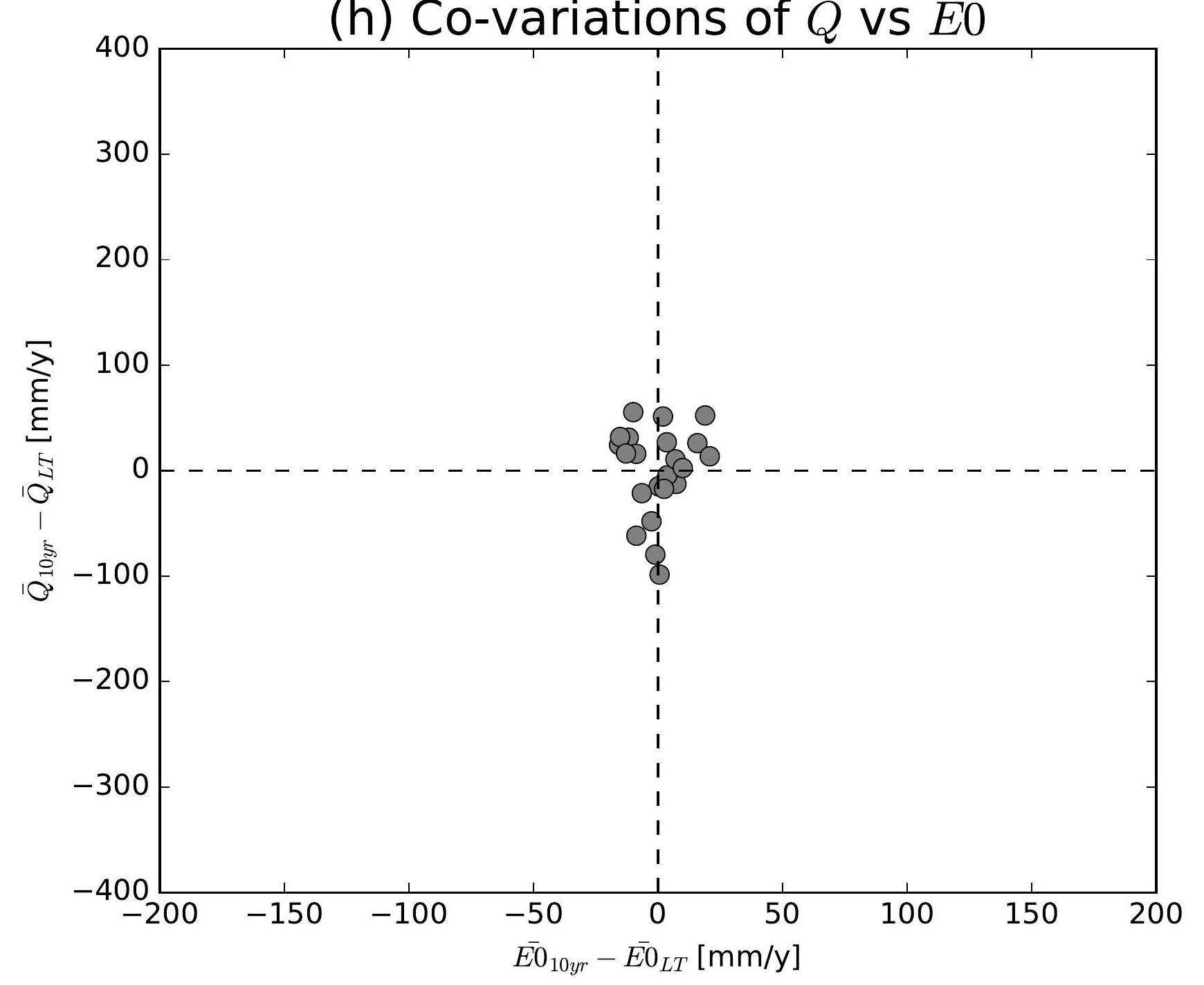

(k) Co-variations of $\bar{Q}$ vs $\overline{E 0}$

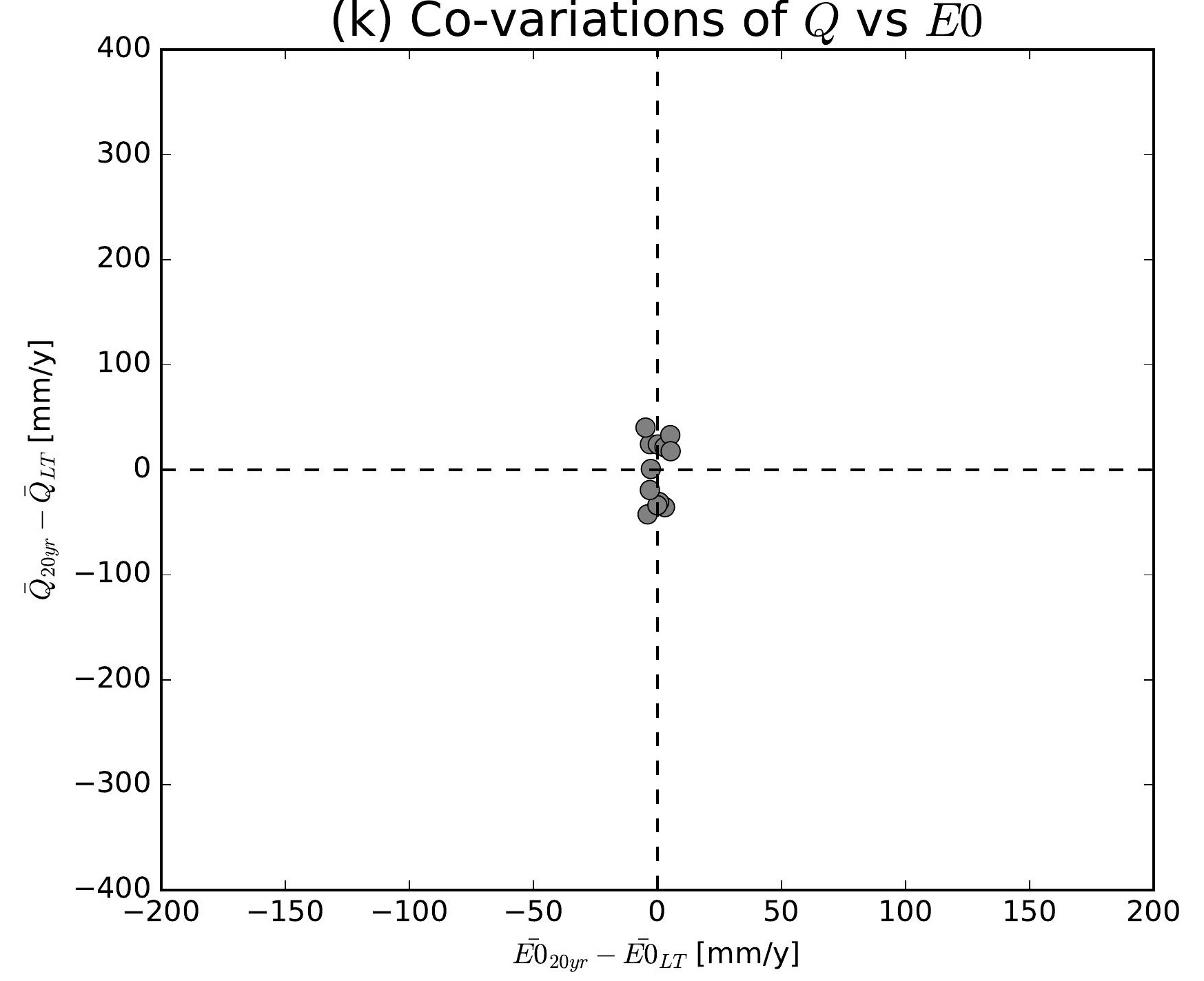

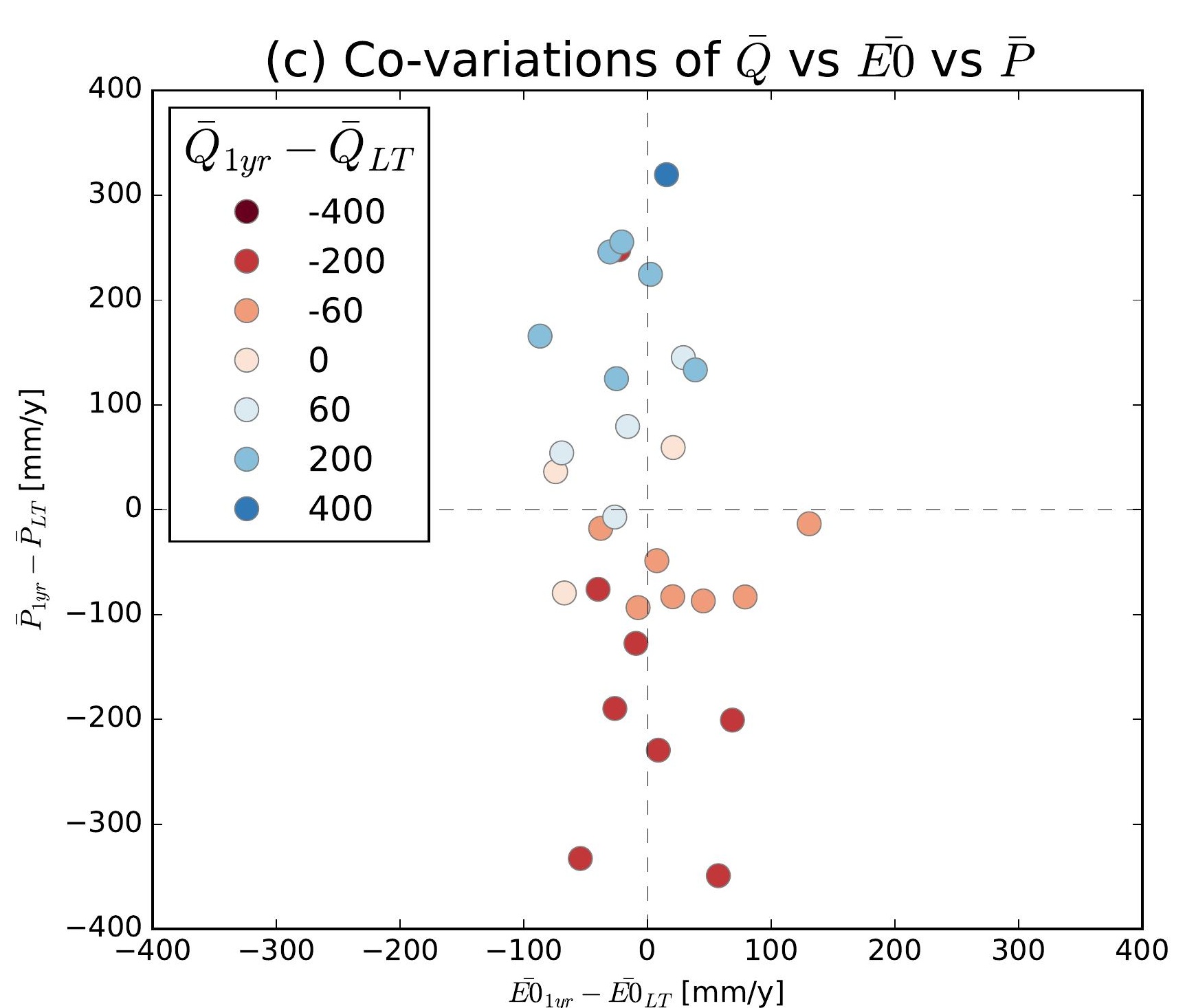
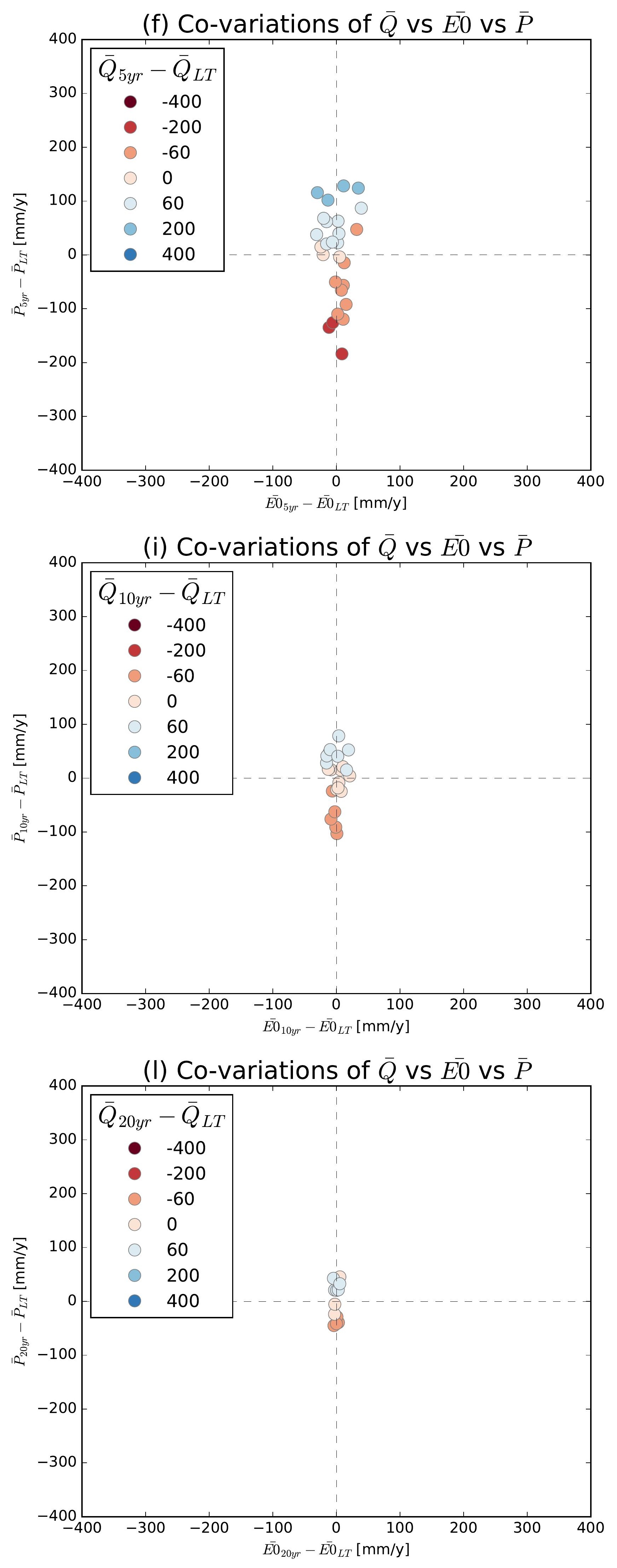

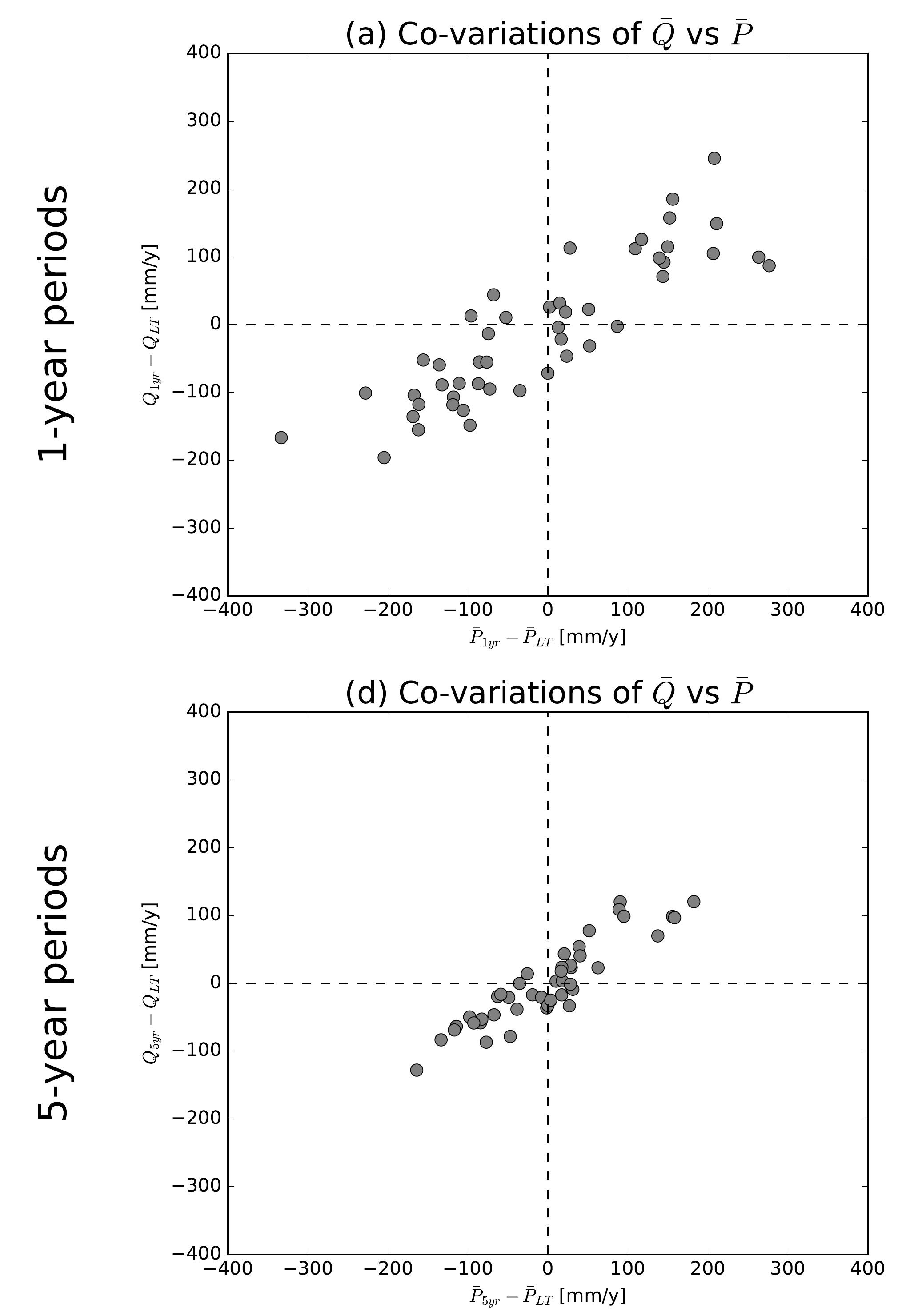

(g) Co-variations of $\bar{Q}$ vs $\bar{P}$
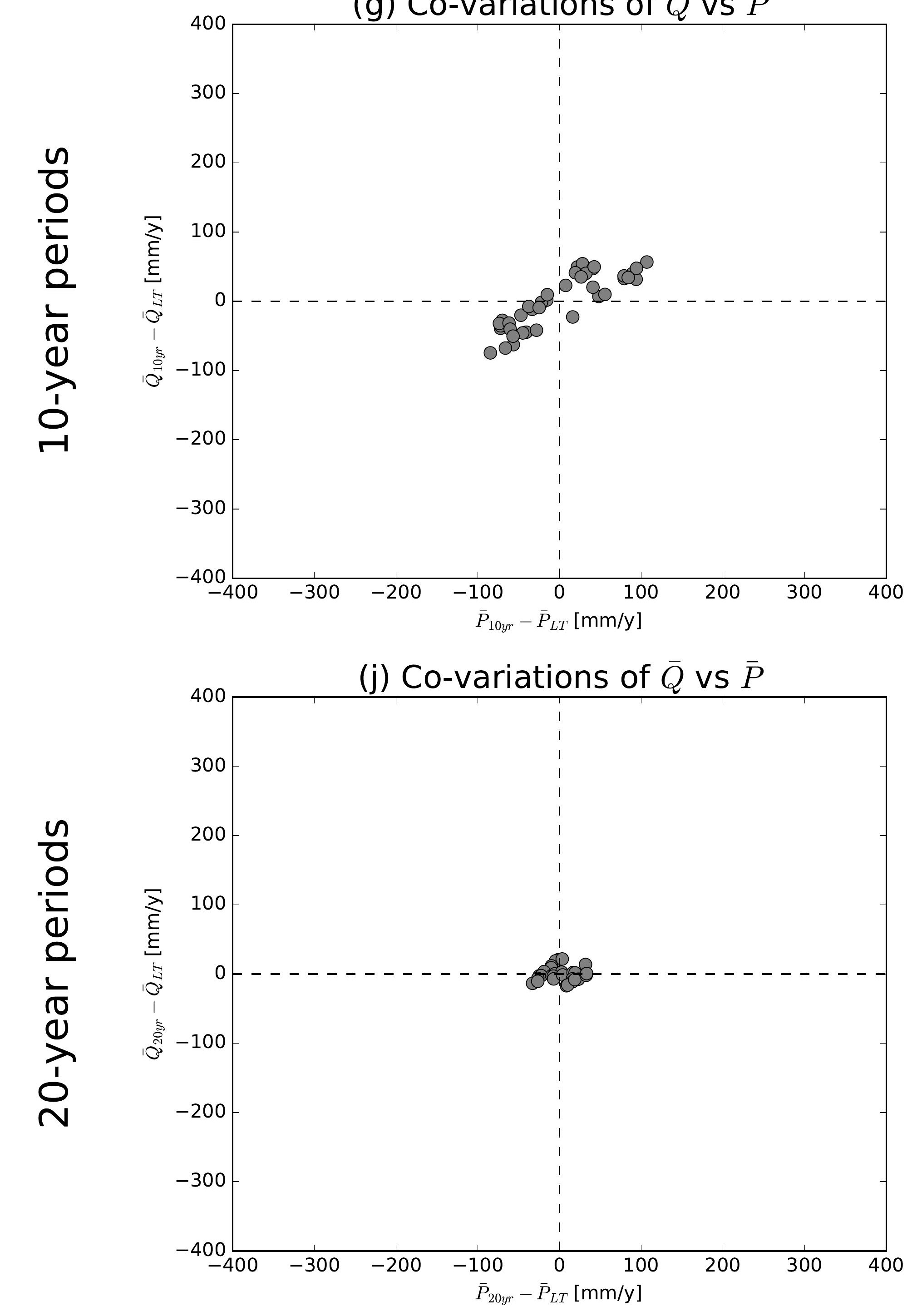

(b) Co-variations of $\bar{Q}$ vs $\overline{E 0}$

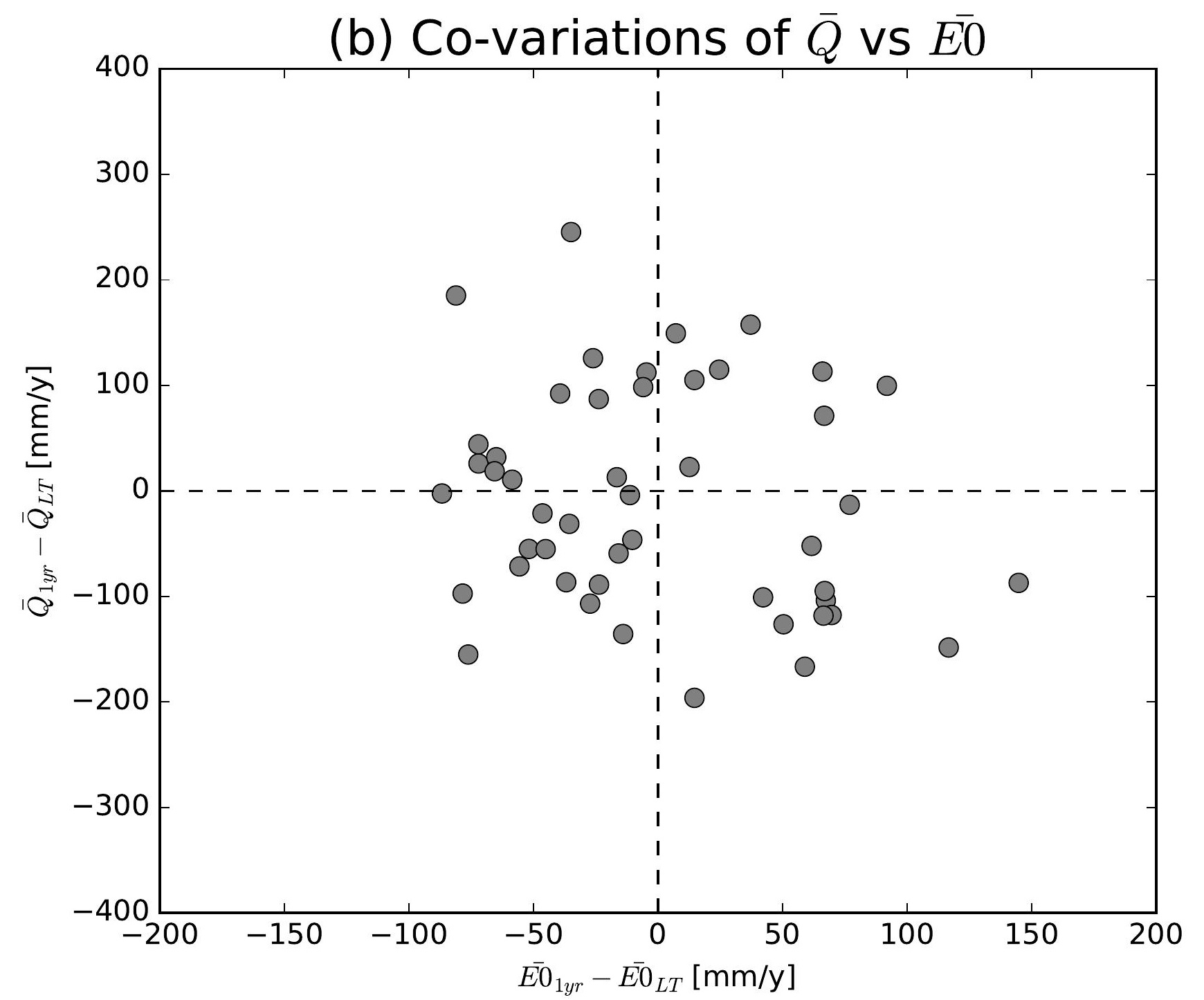

(e) Co-variations of $\bar{Q}$ vs $\overline{E 0}$

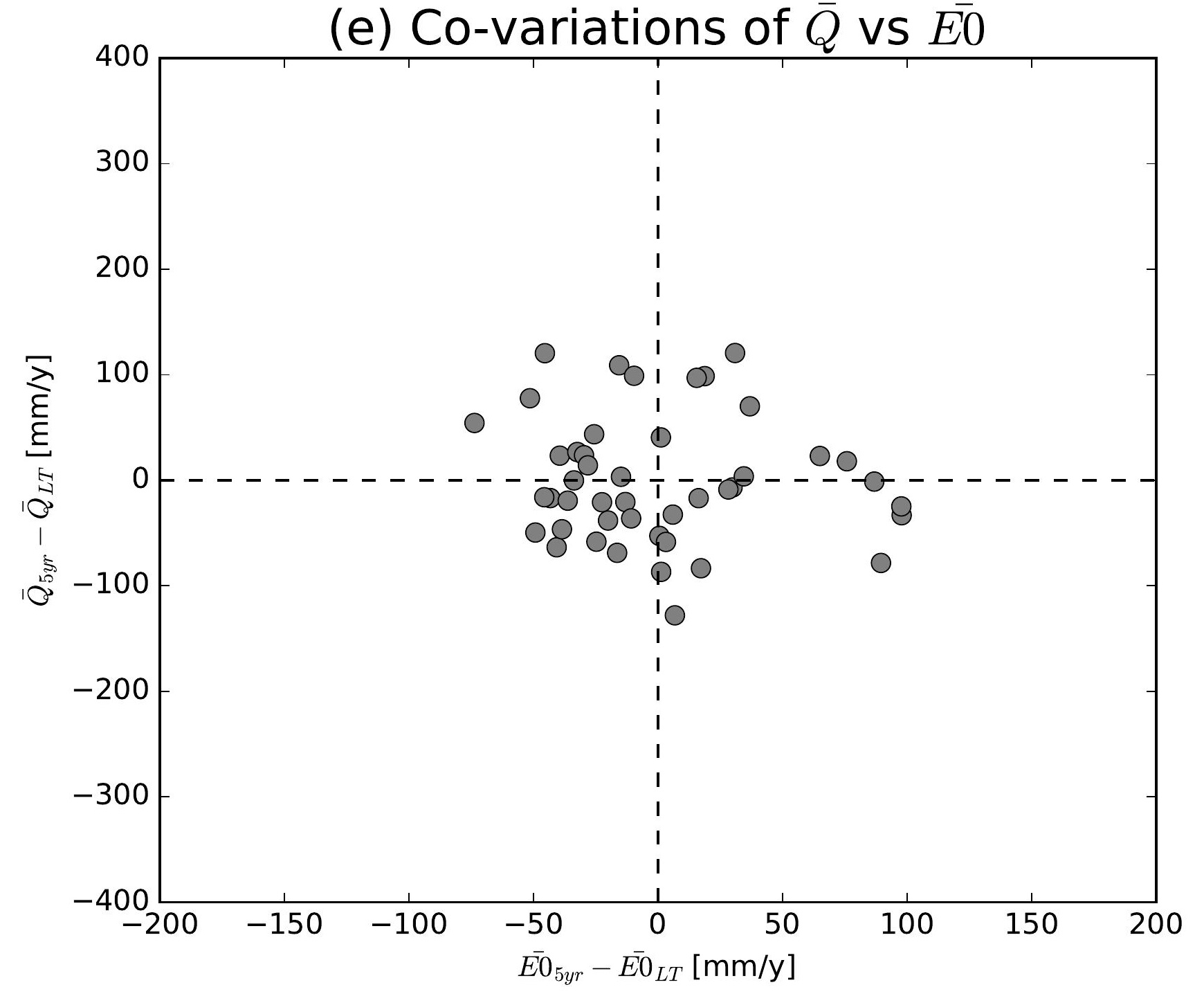

(h) Co-variations of $\bar{Q}$ vs $\overline{E 0}$

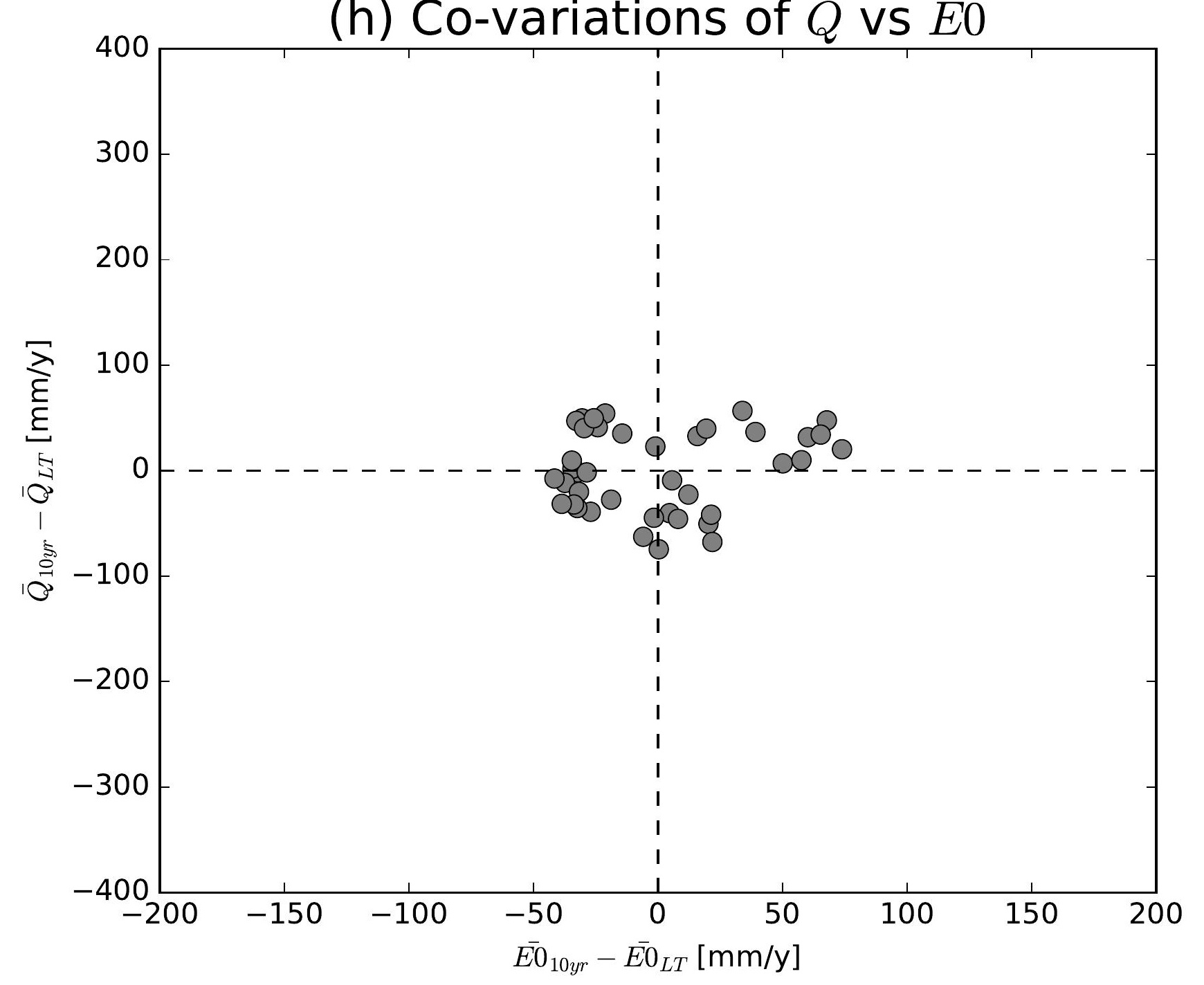

(k) Co-variations of $\bar{Q}$ vs $\overline{E 0}$

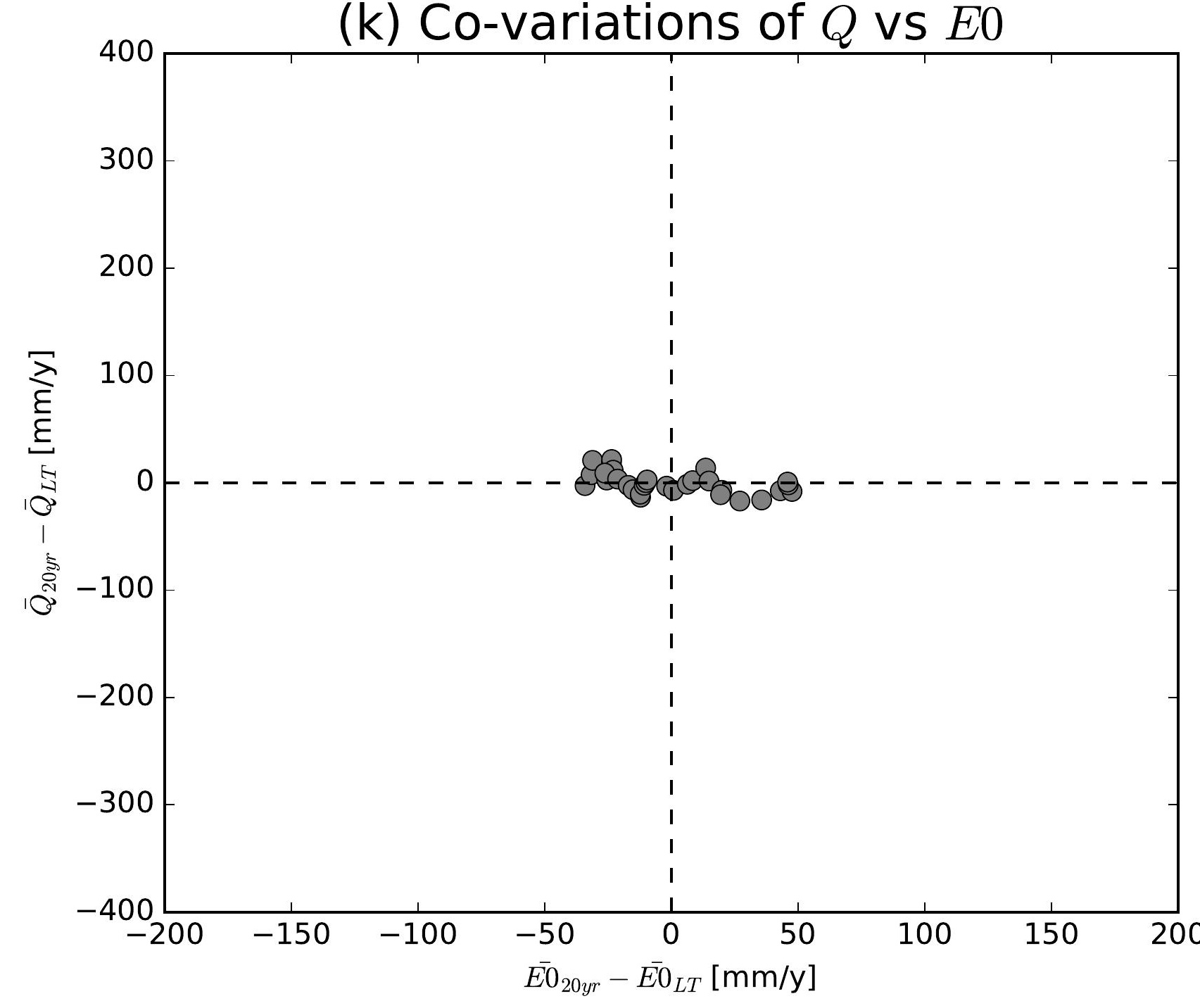

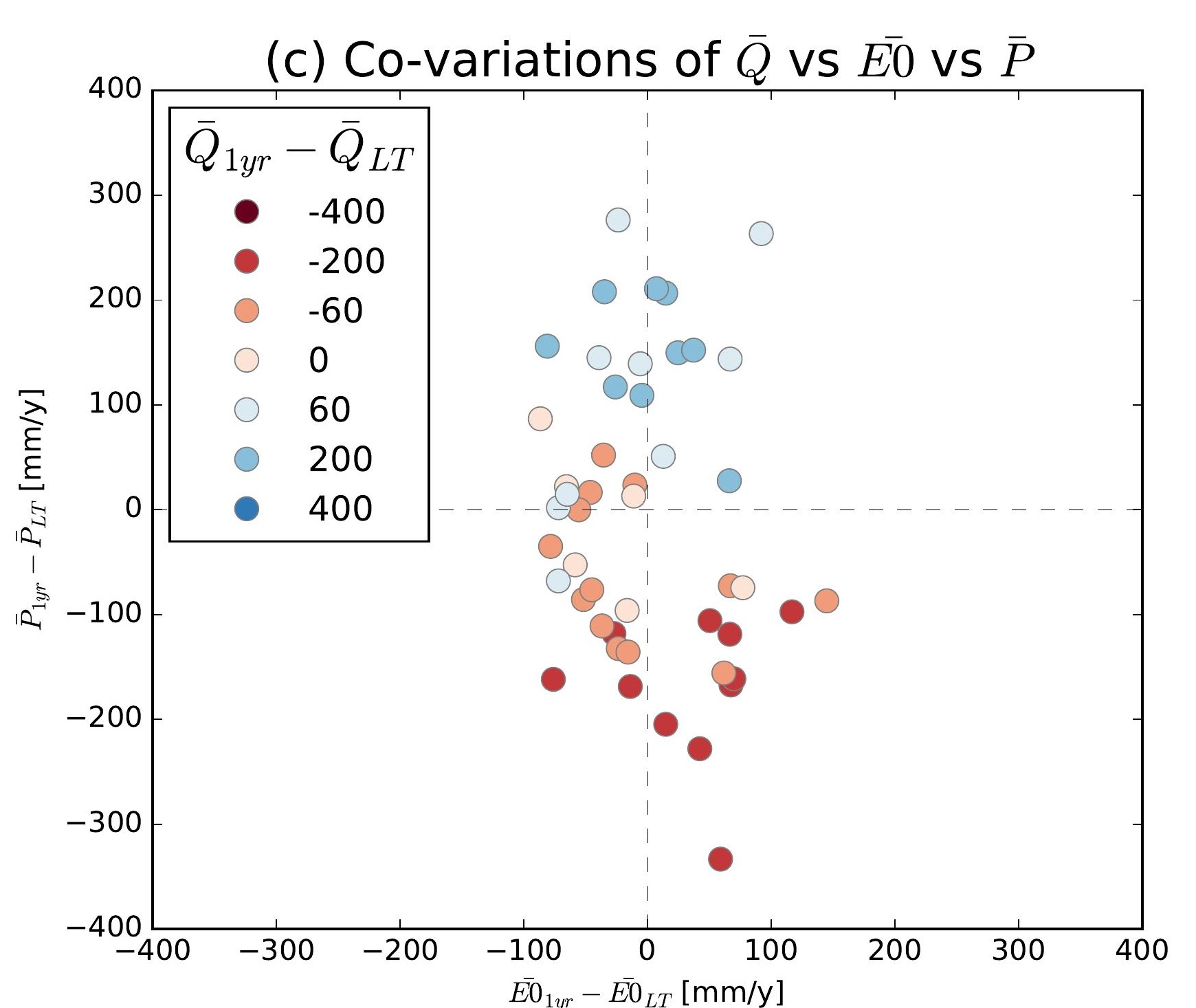
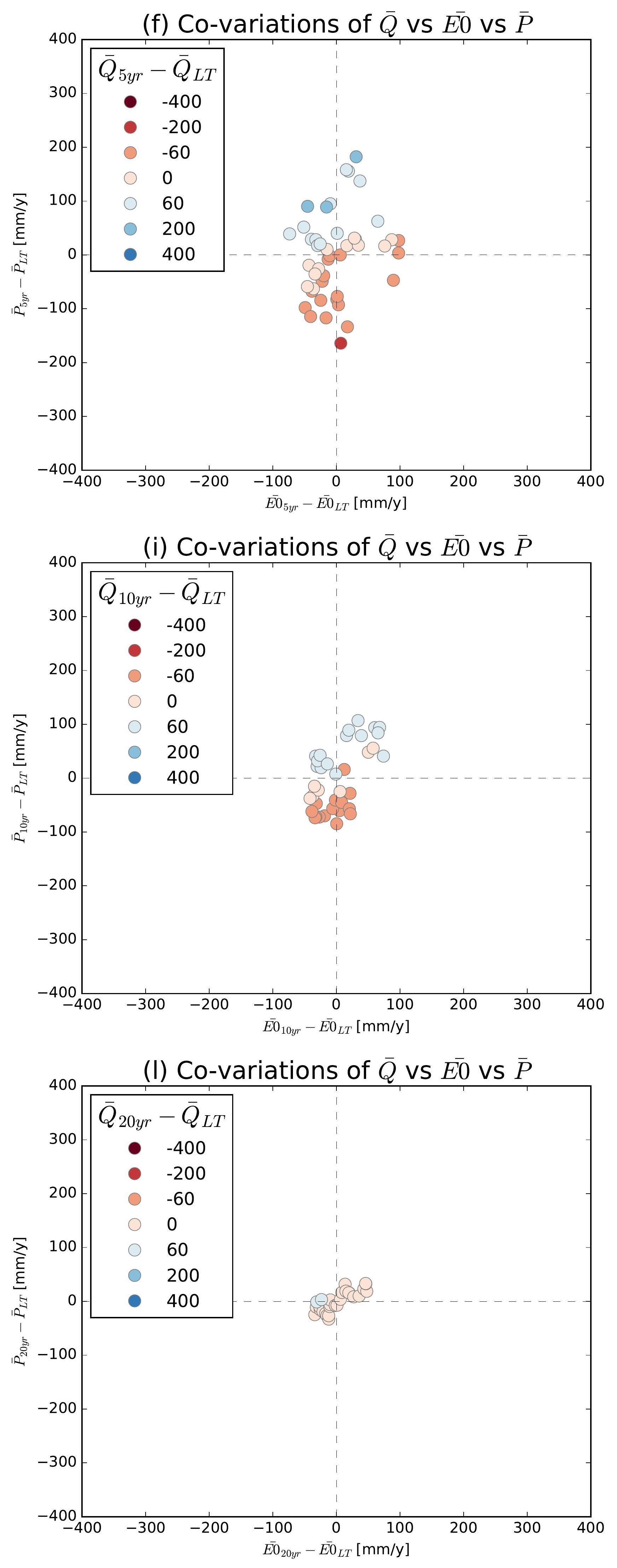

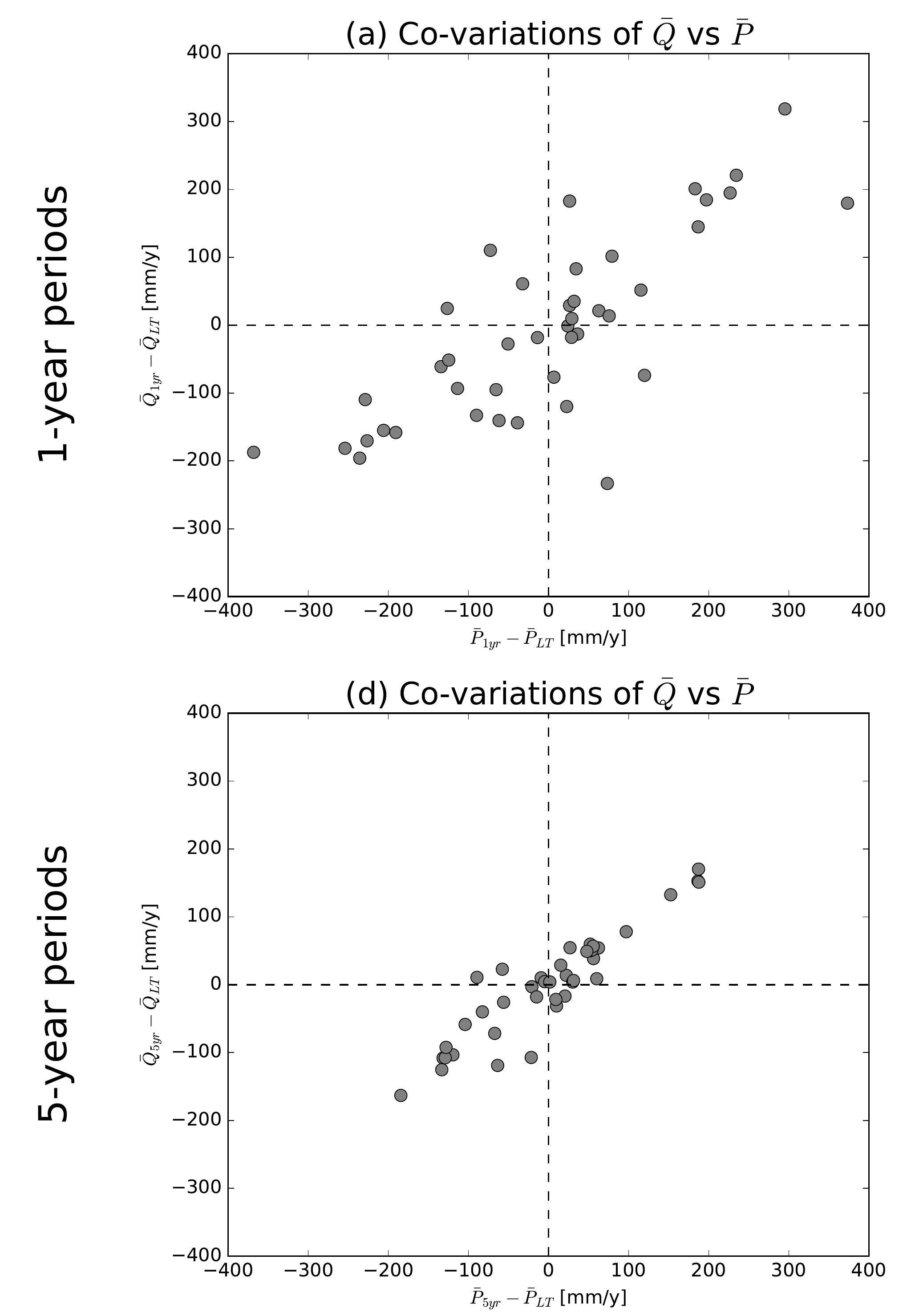

(g) Co-variations of $\bar{Q}$ vs $\bar{P}$
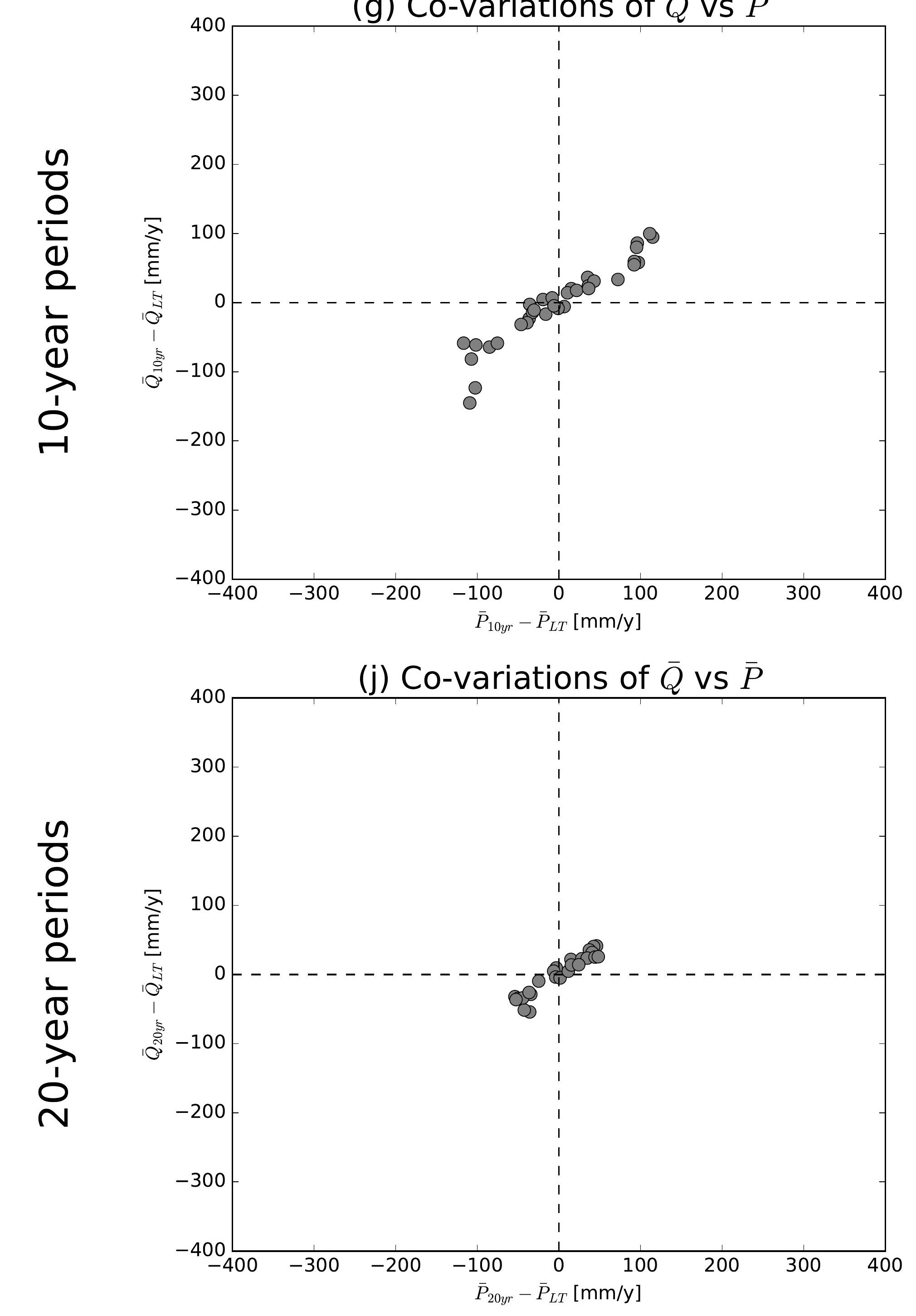

(b) Co-variations of $\bar{Q}$ vs $\overline{E 0}$

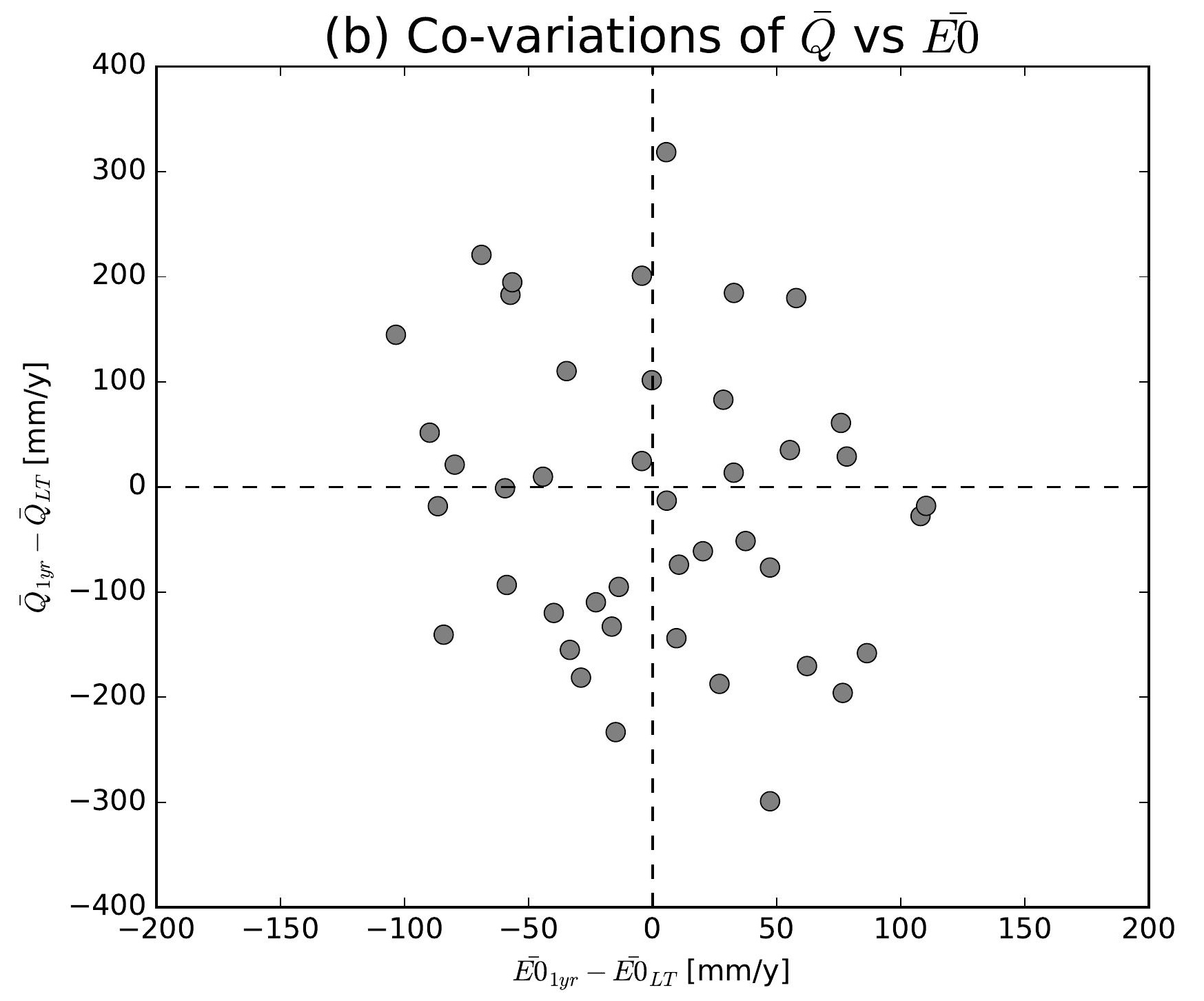

(e) Co-variations of $\bar{Q}$ vs $\overline{E 0}$

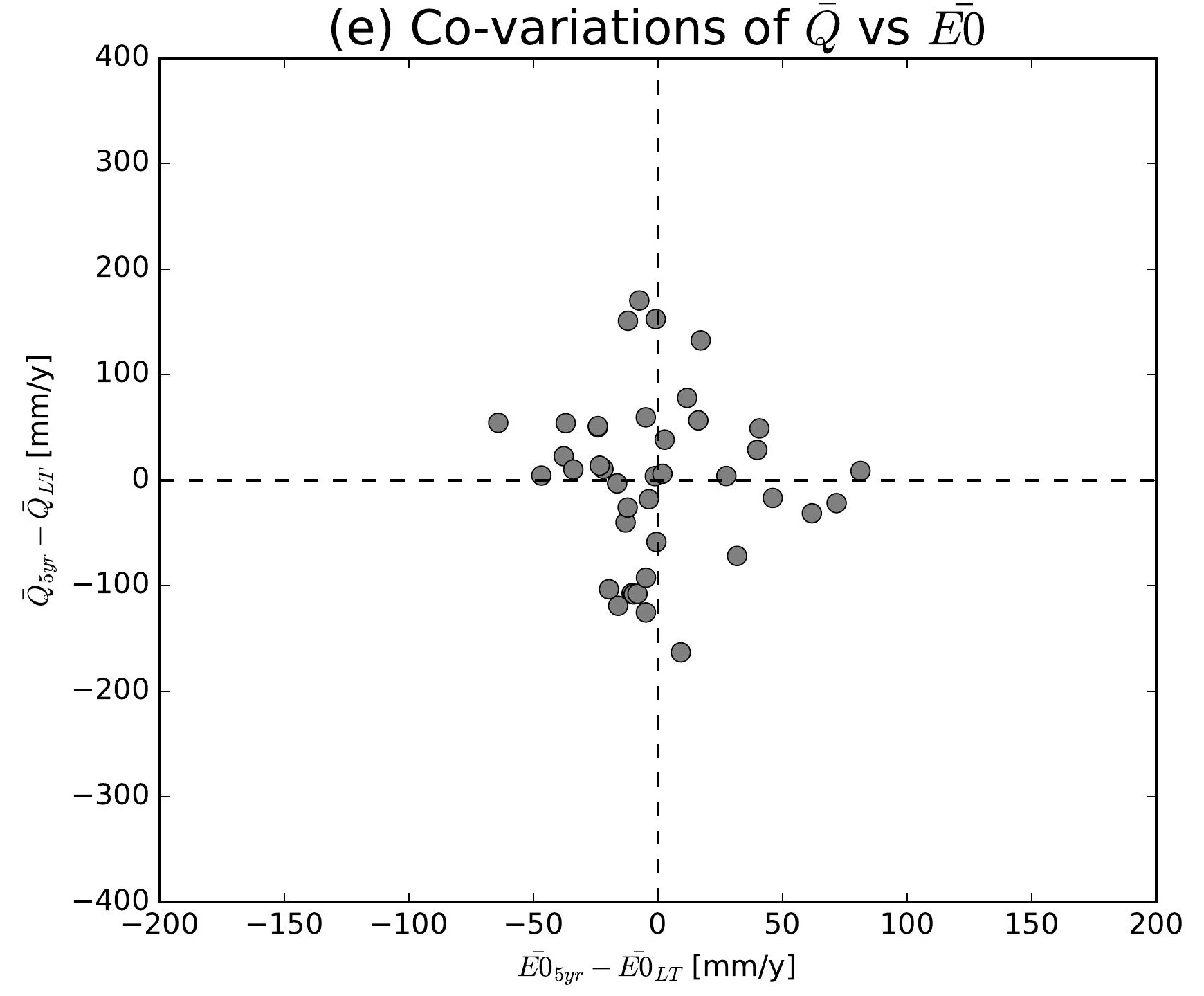

(h) Co-variations of $\bar{Q}$ vs $\overline{E 0}$

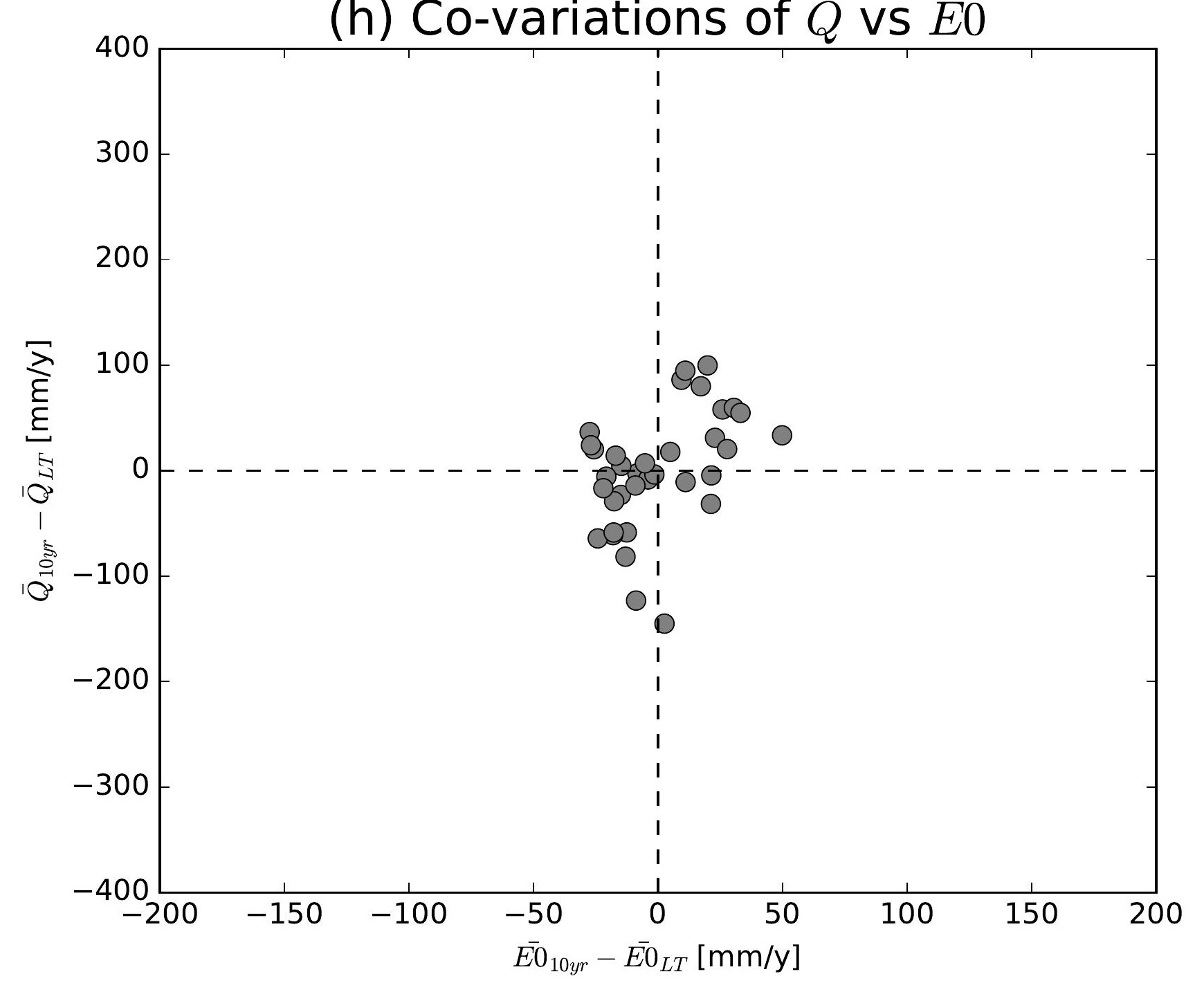

(k) Co-variations of $\bar{Q}$ vs $\overline{E 0}$

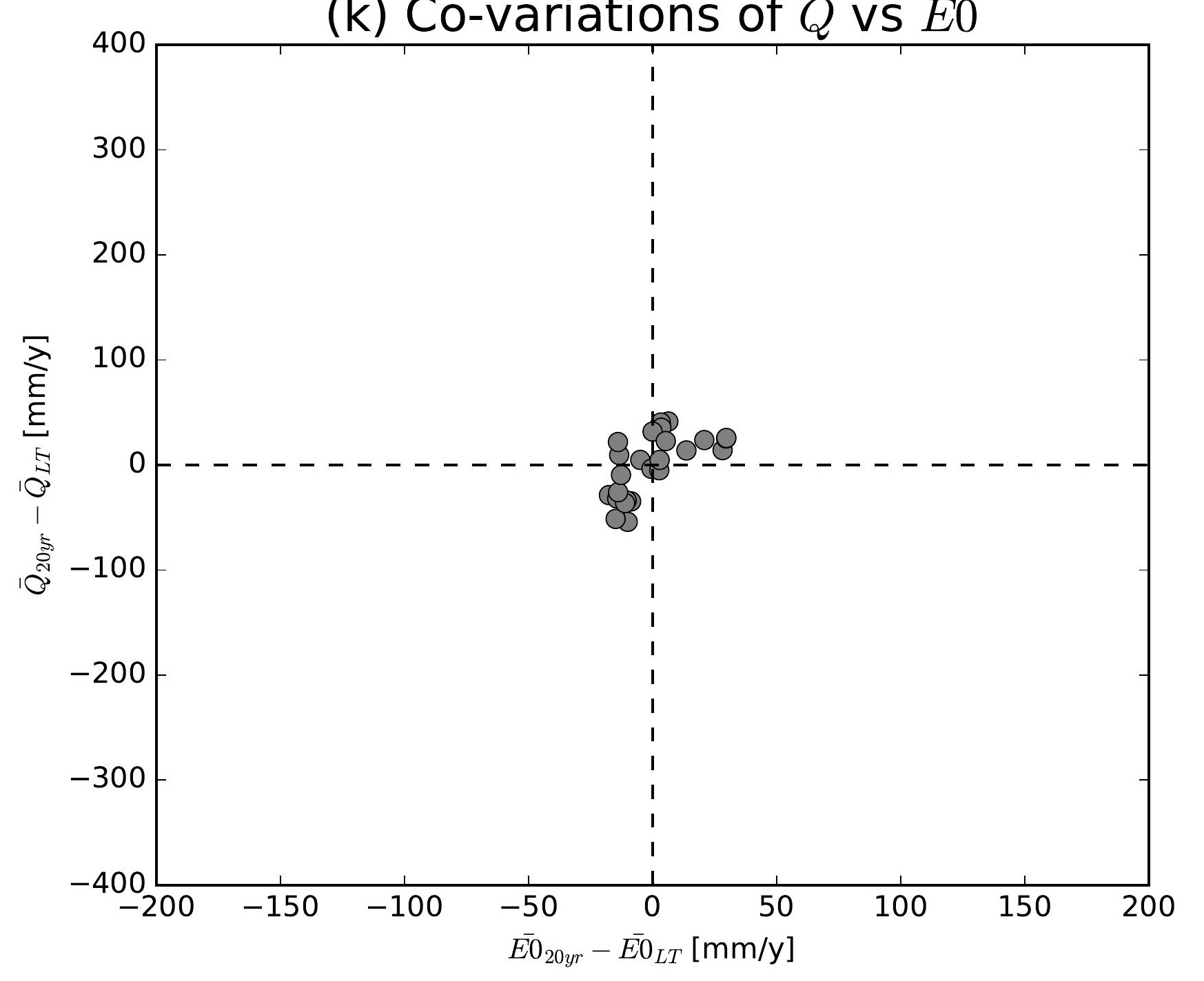

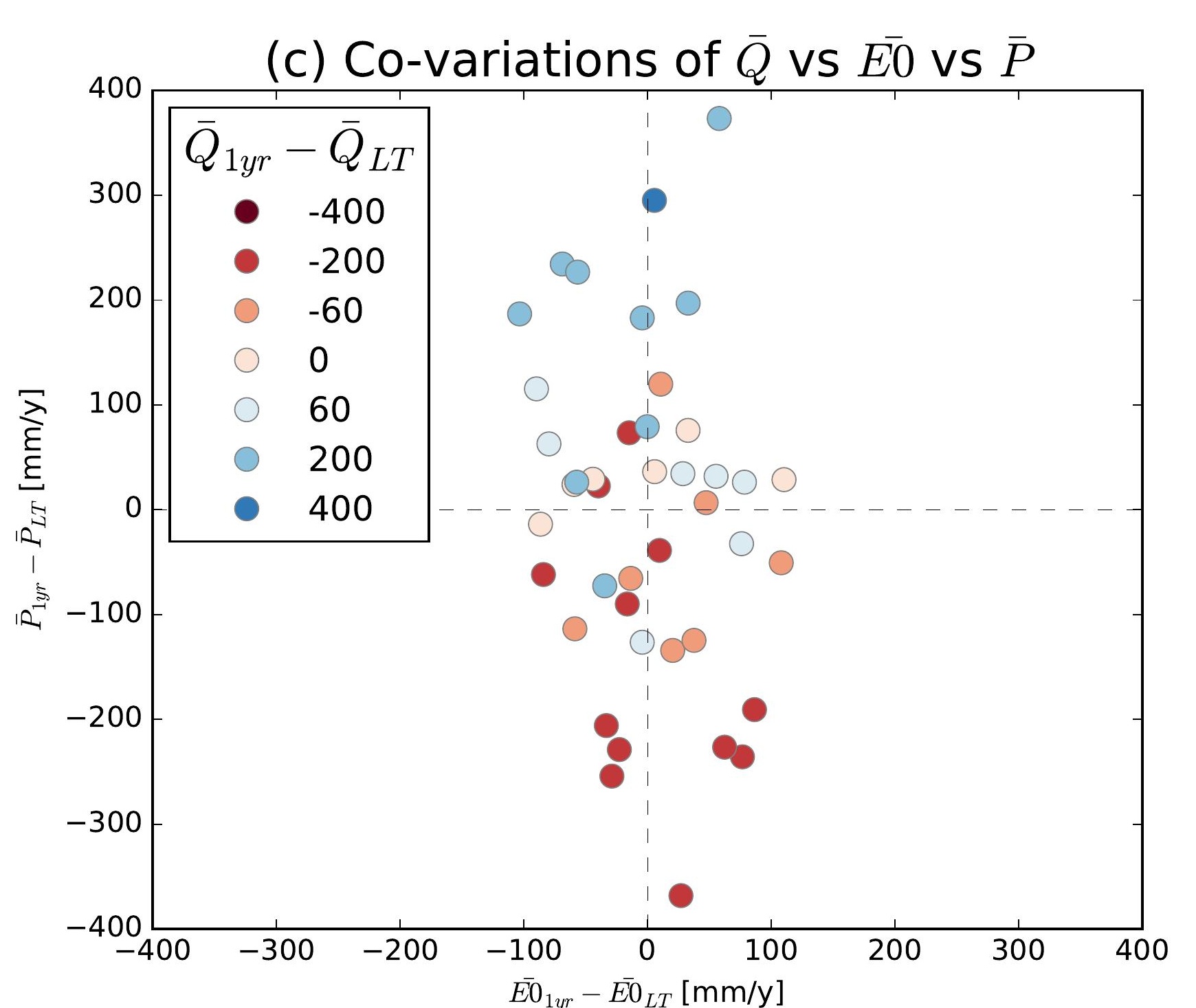
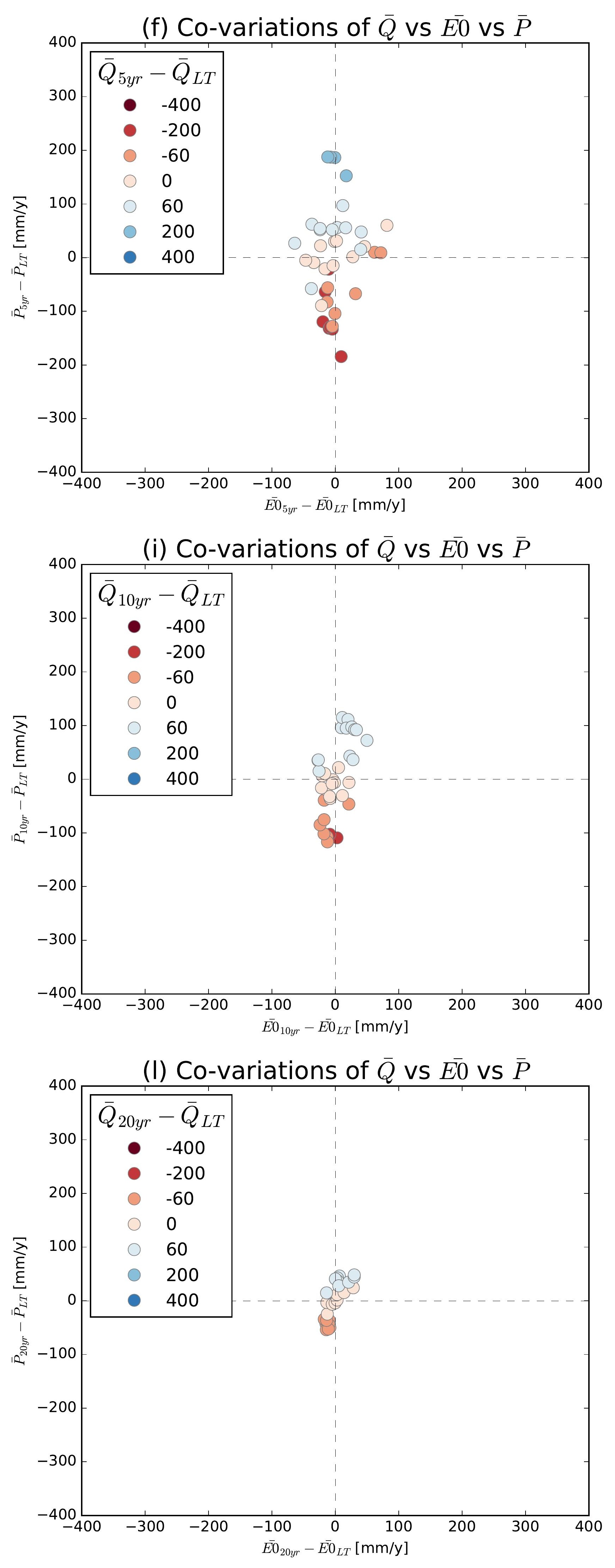

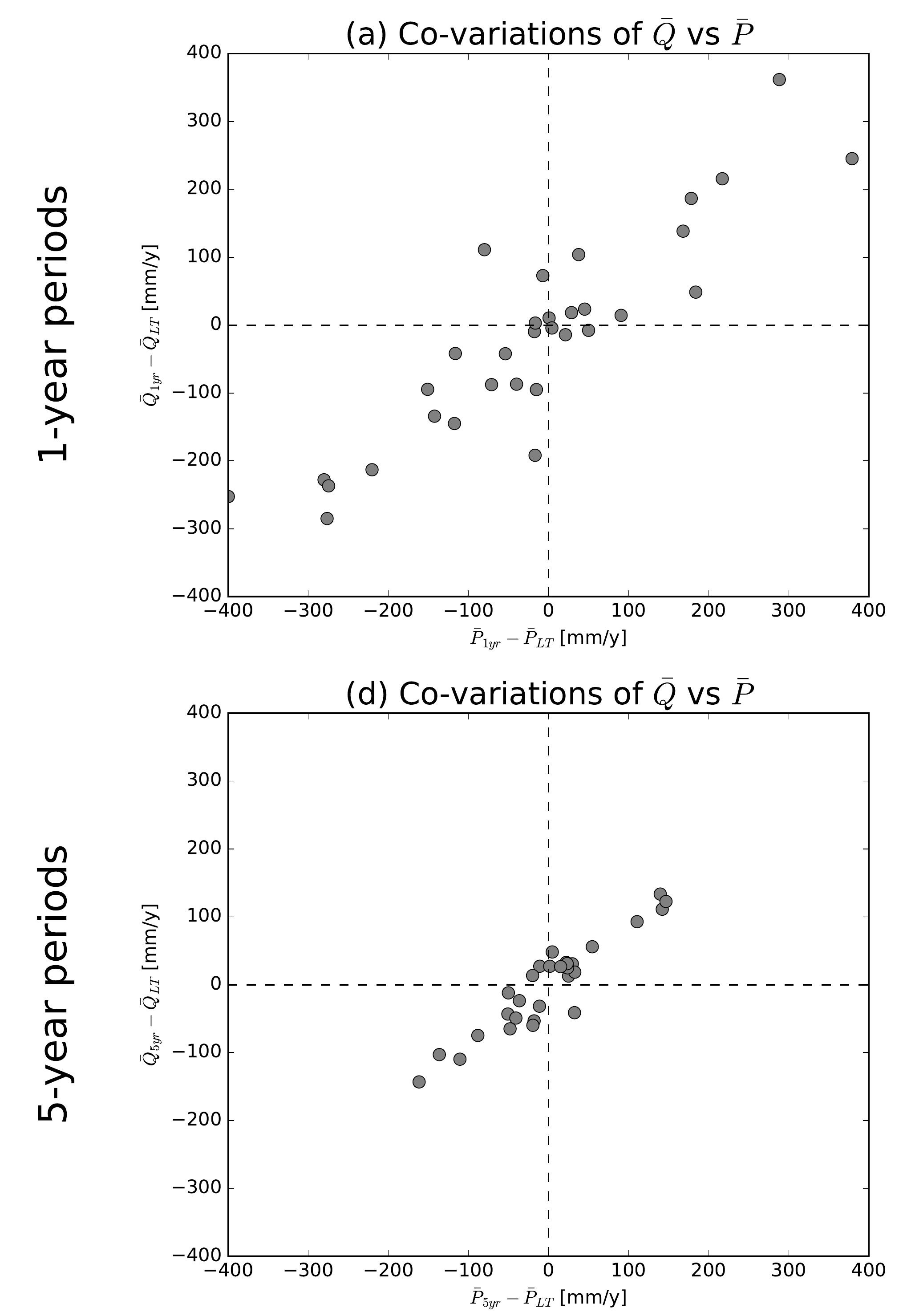

(g) Co-variations of $\bar{Q}$ vs $\bar{P}$
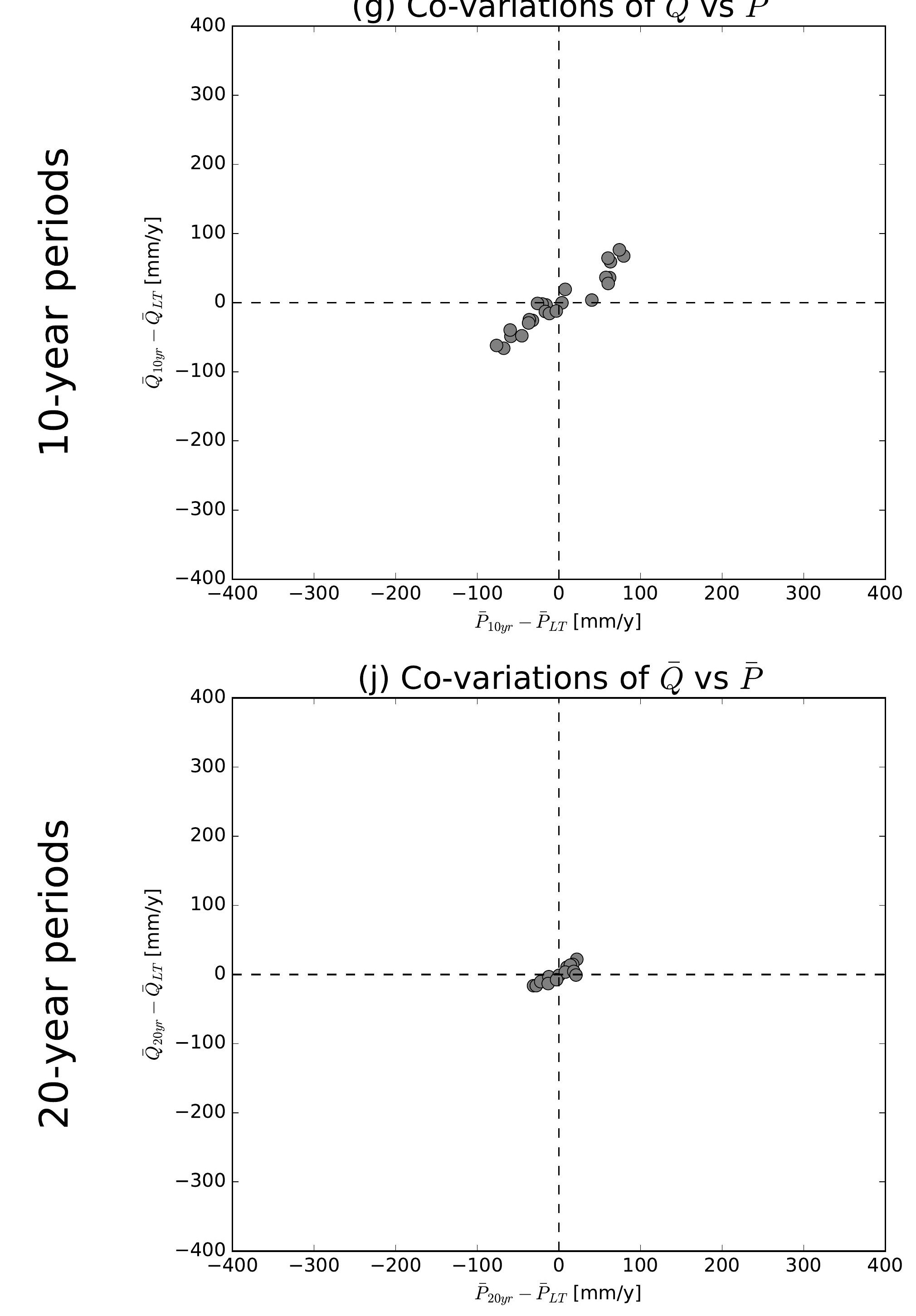

(b) Co-variations of $\bar{Q}$ vs $\overline{E 0}$

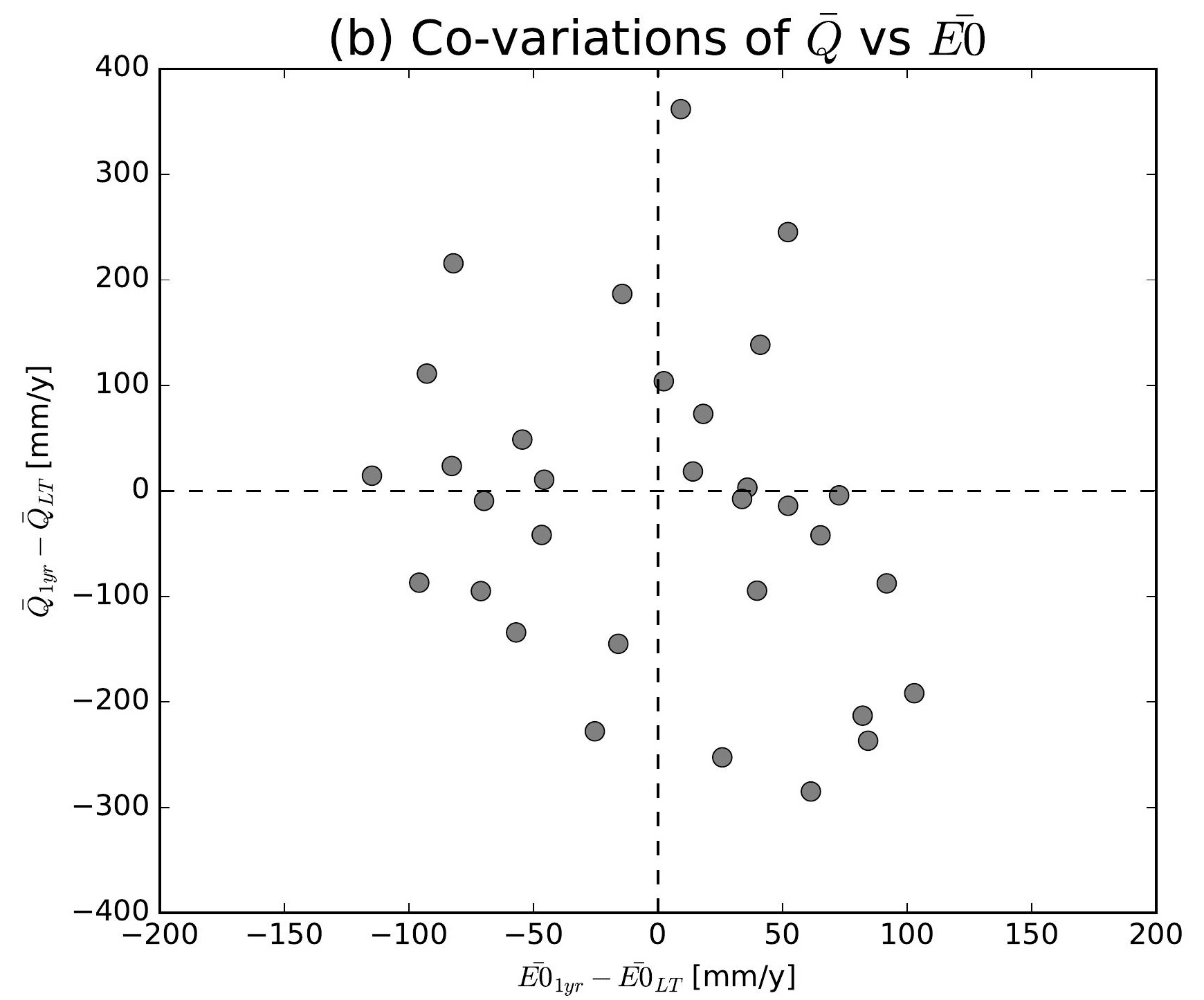

(e) Co-variations of $\bar{Q}$ vs $\overline{E 0}$

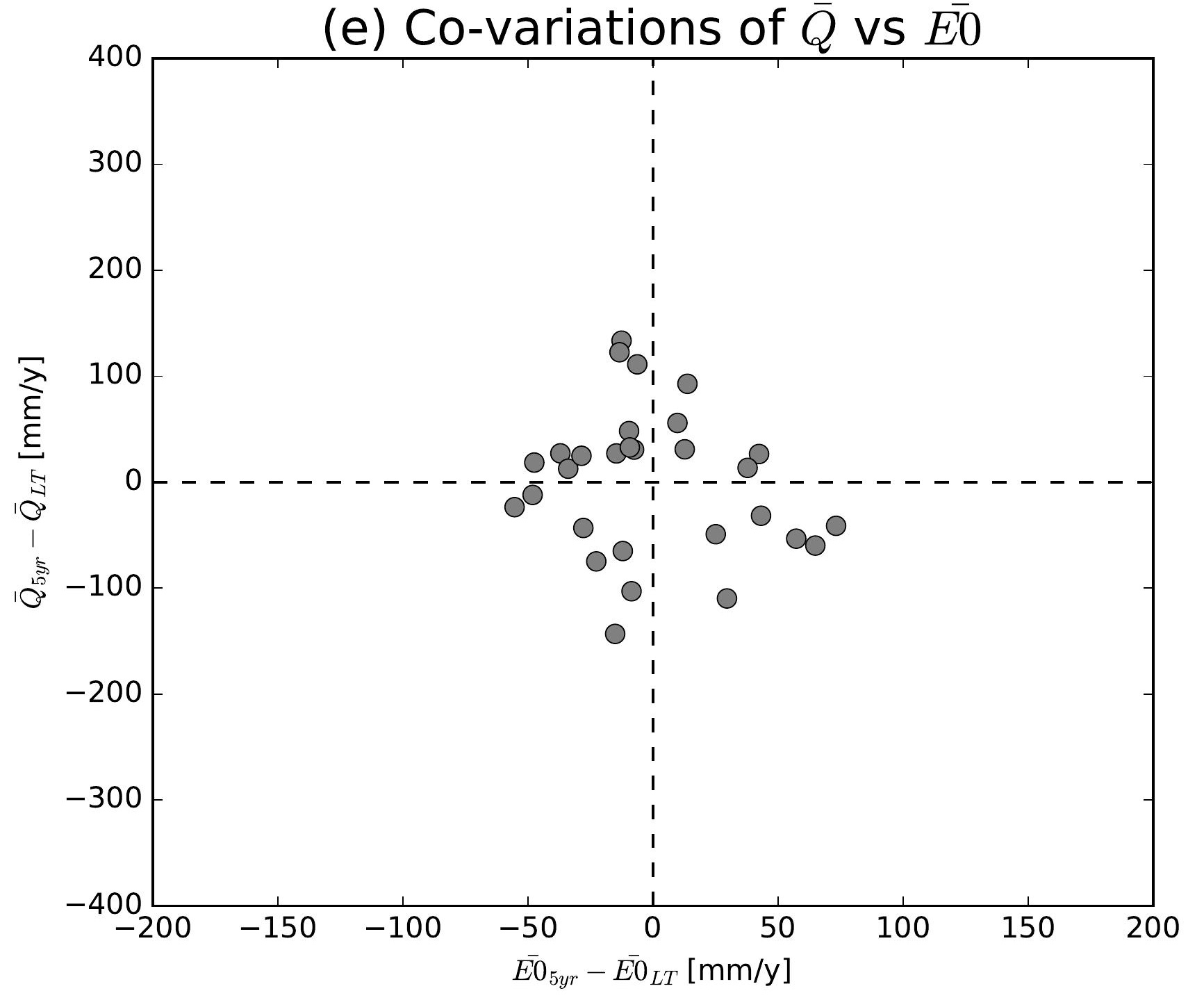

(h) Co-variations of $\bar{Q}$ vs $\overline{E 0}$

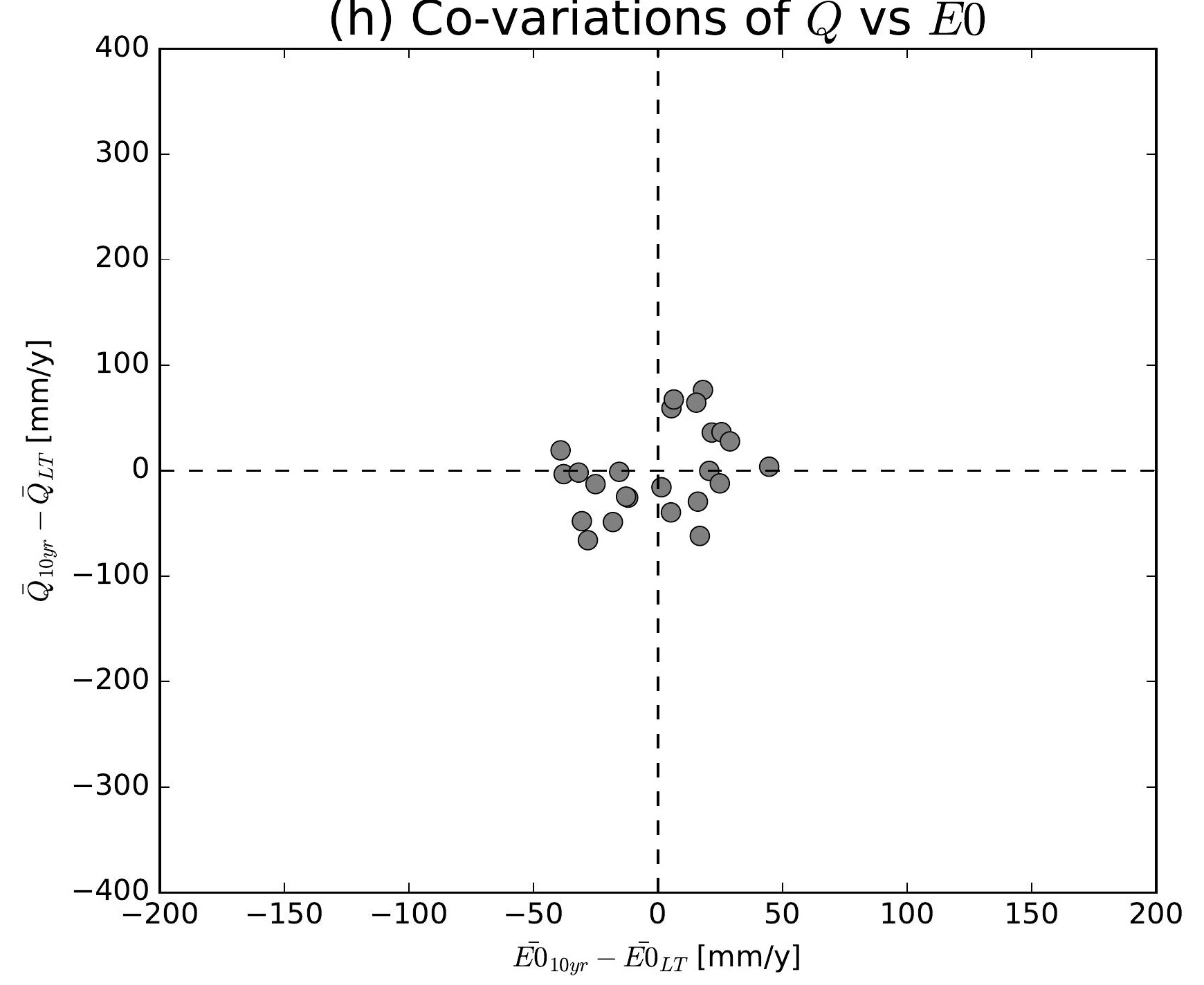

(k) Co-variations of $\bar{Q}$ vs $\overline{E 0}$

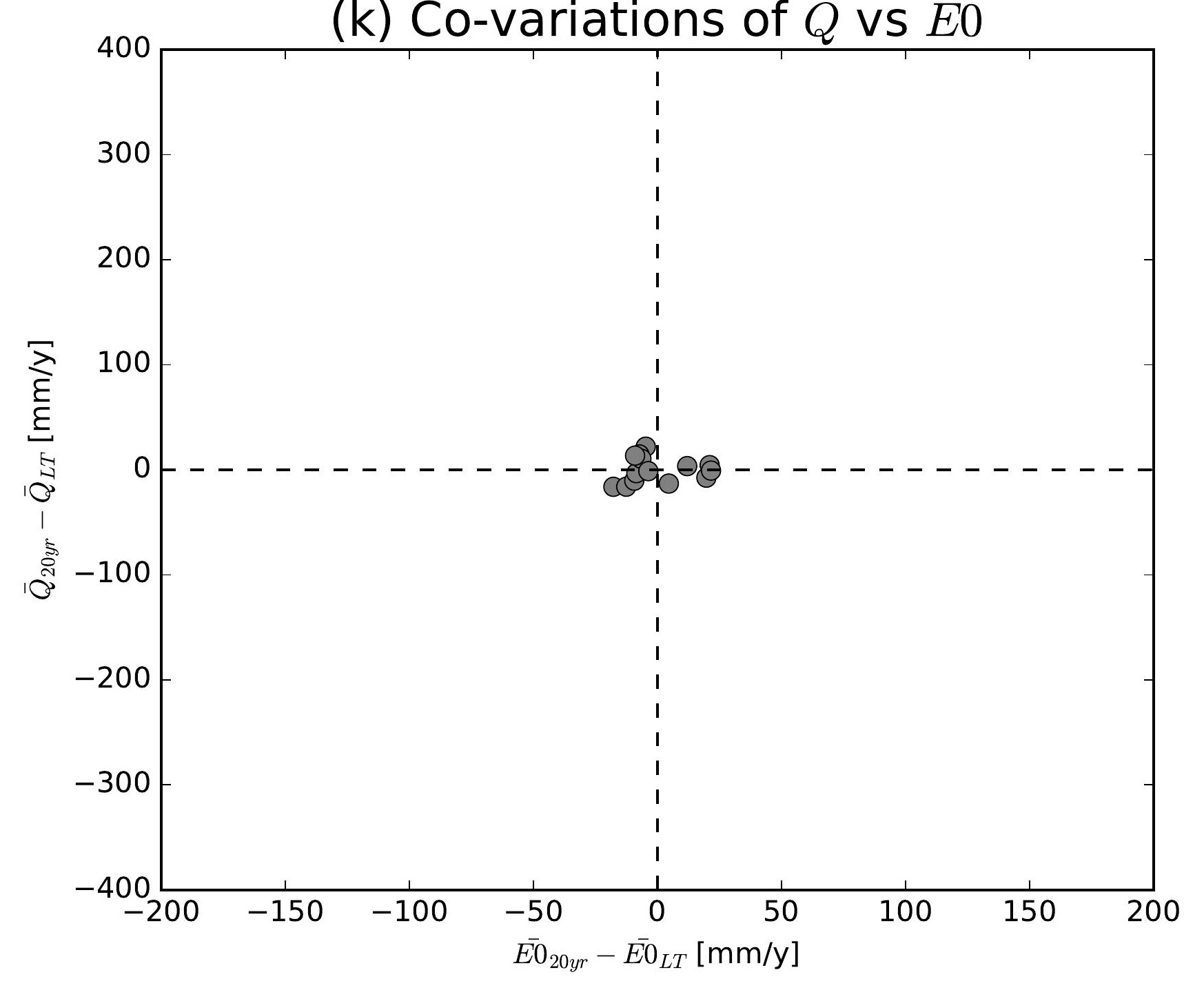

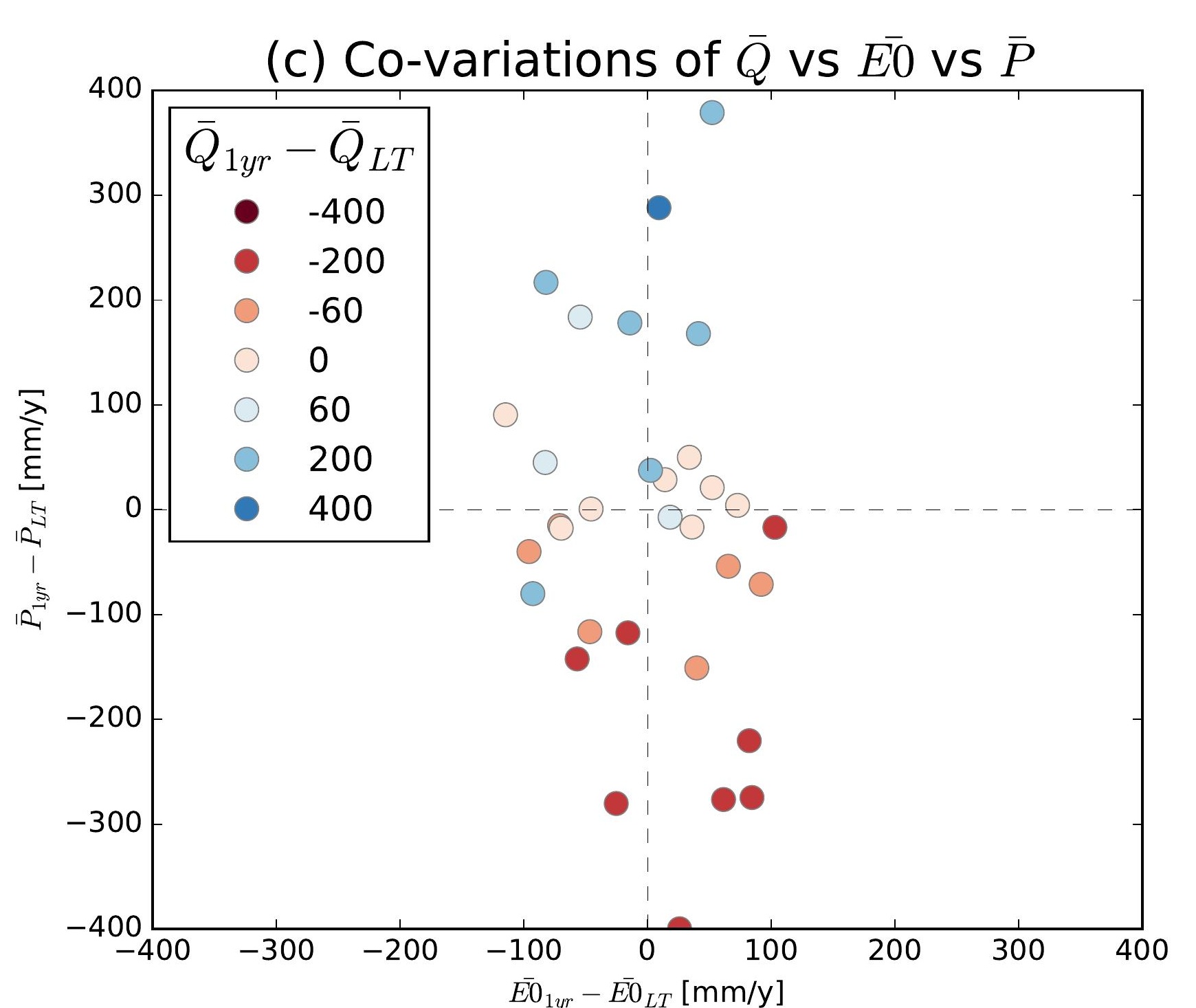
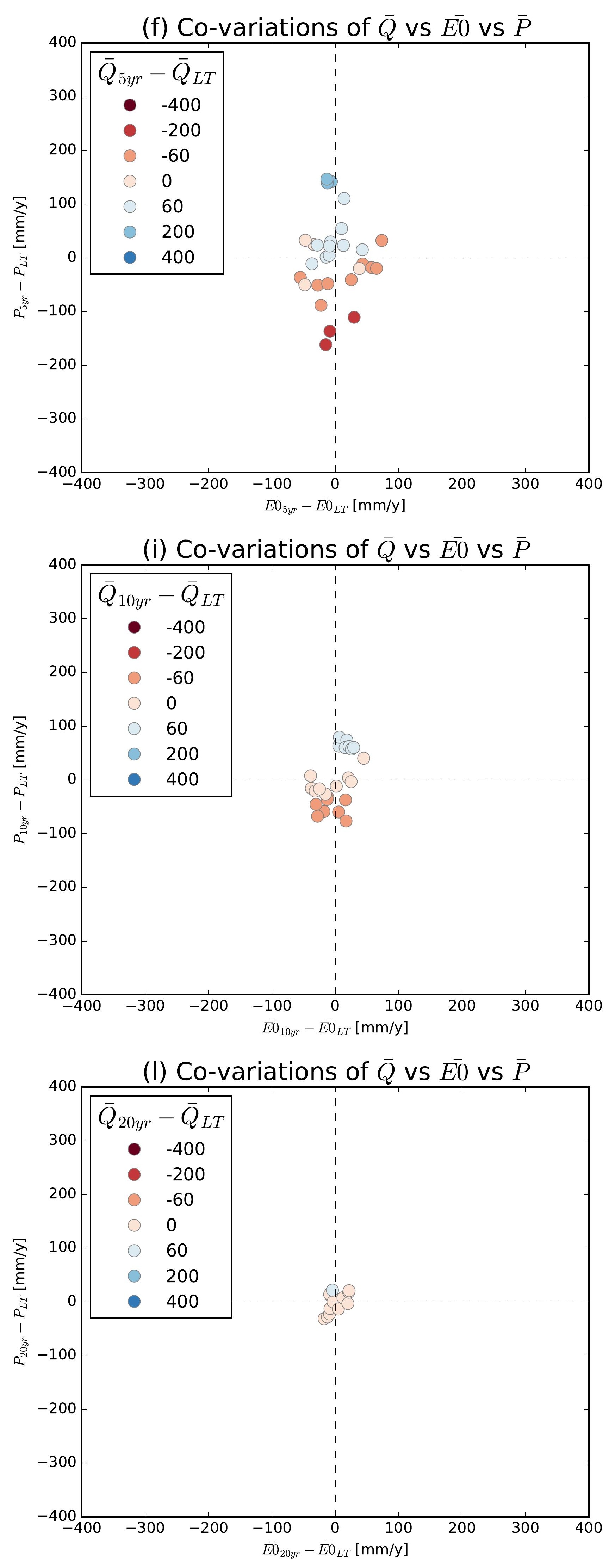

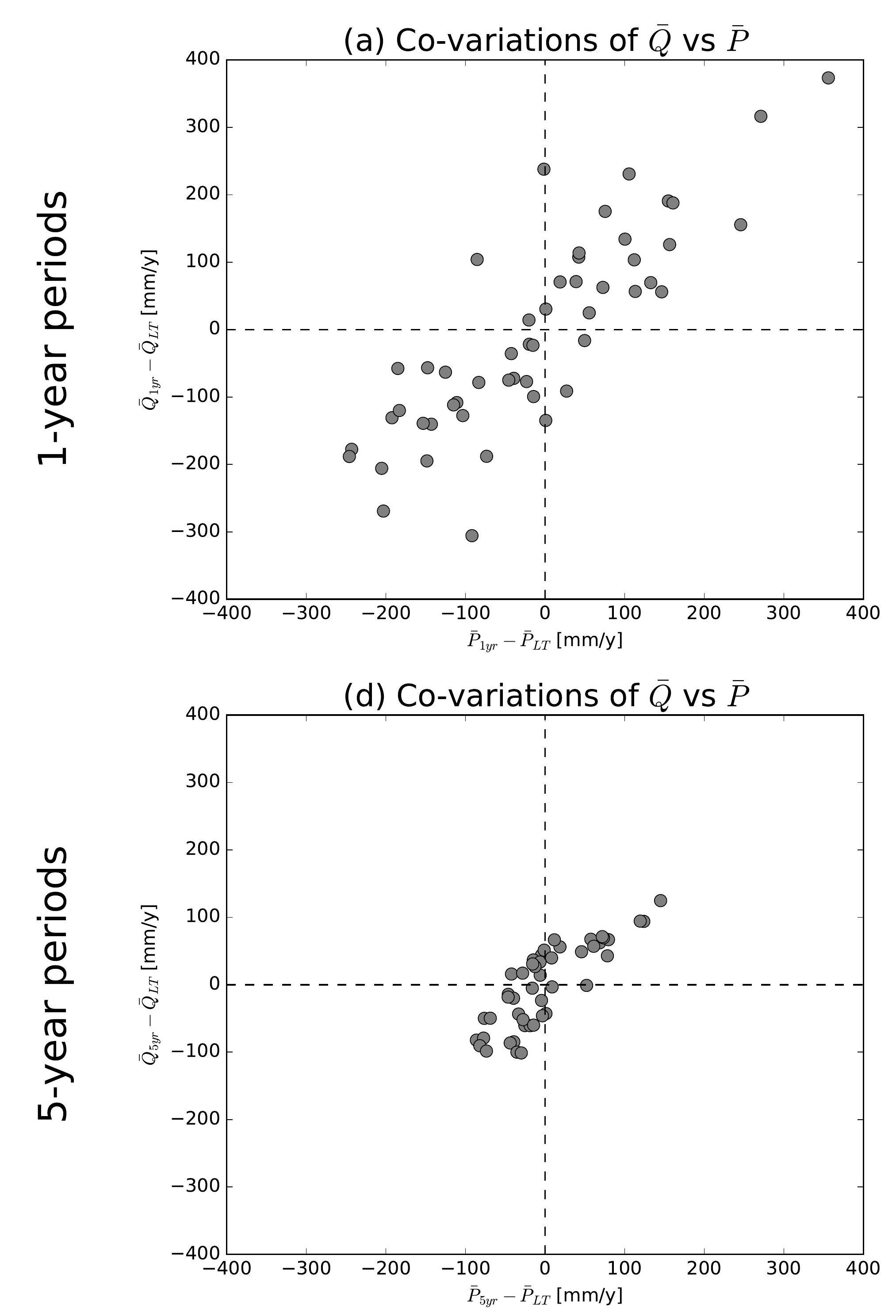

(g) Co-variations of $\bar{Q}$ vs $\bar{P}$
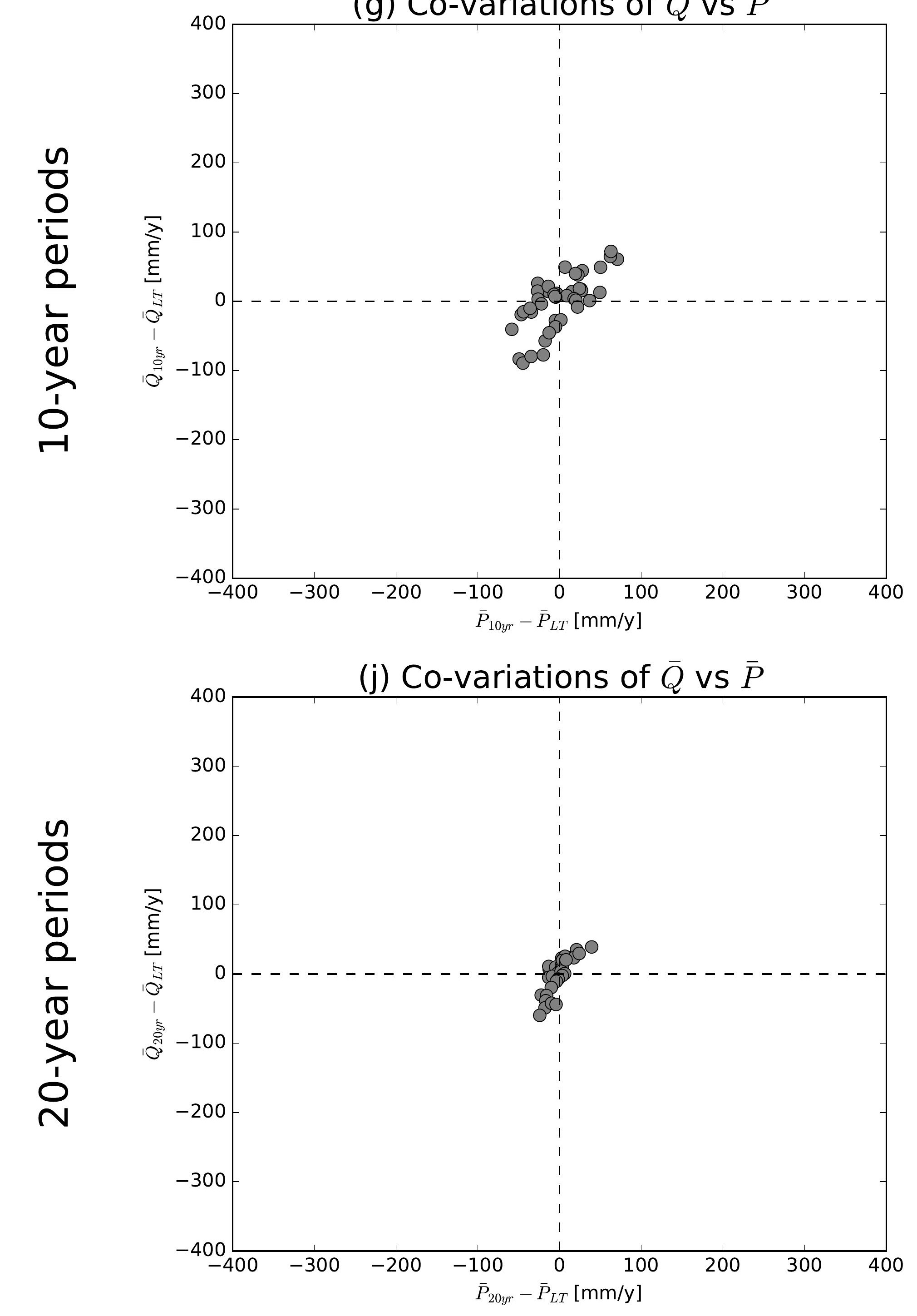

(b) Co-variations of $\bar{Q}$ vs $\overline{E 0}$

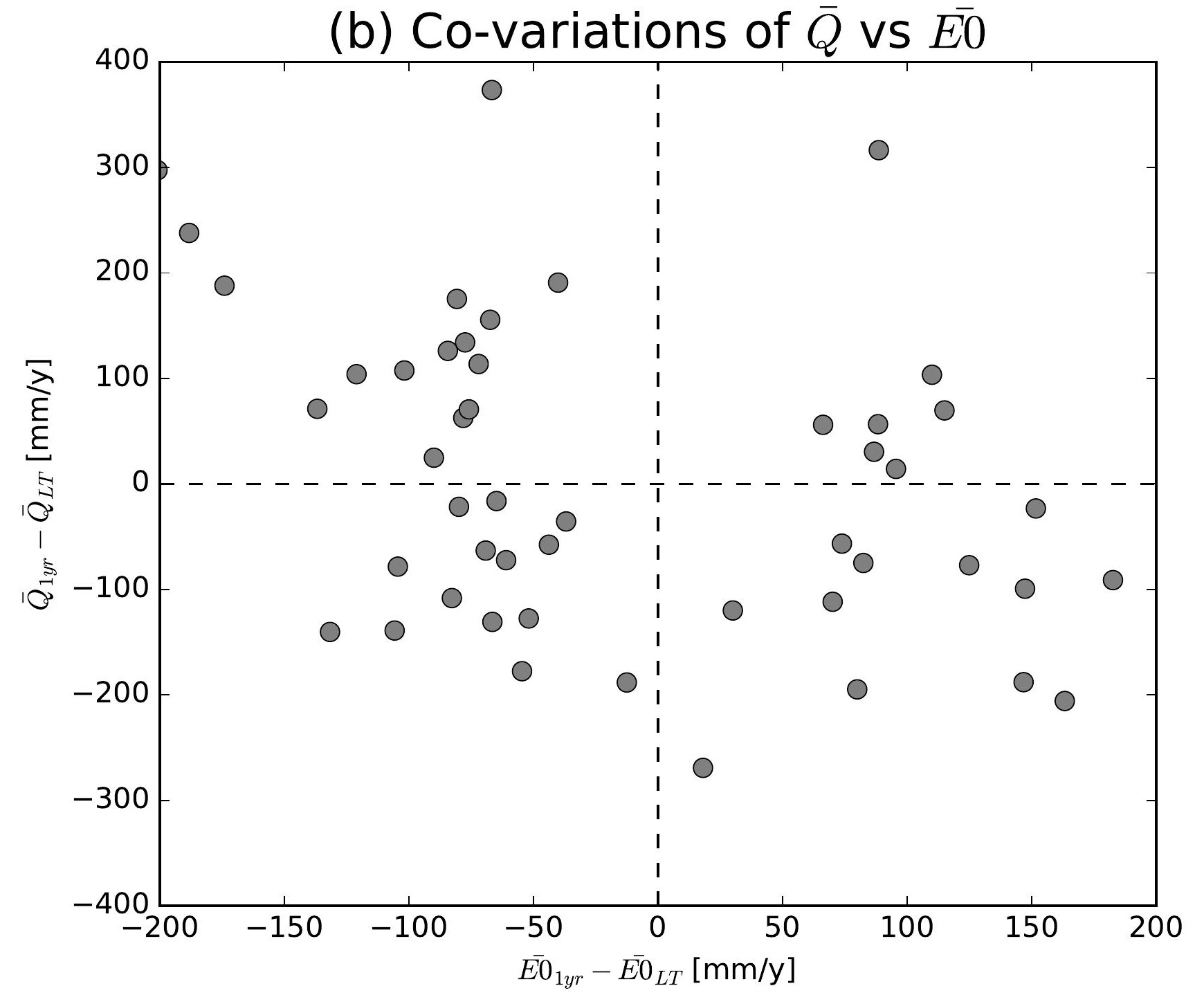

(e) Co-variations of $\bar{Q}$ vs $\overline{E 0}$

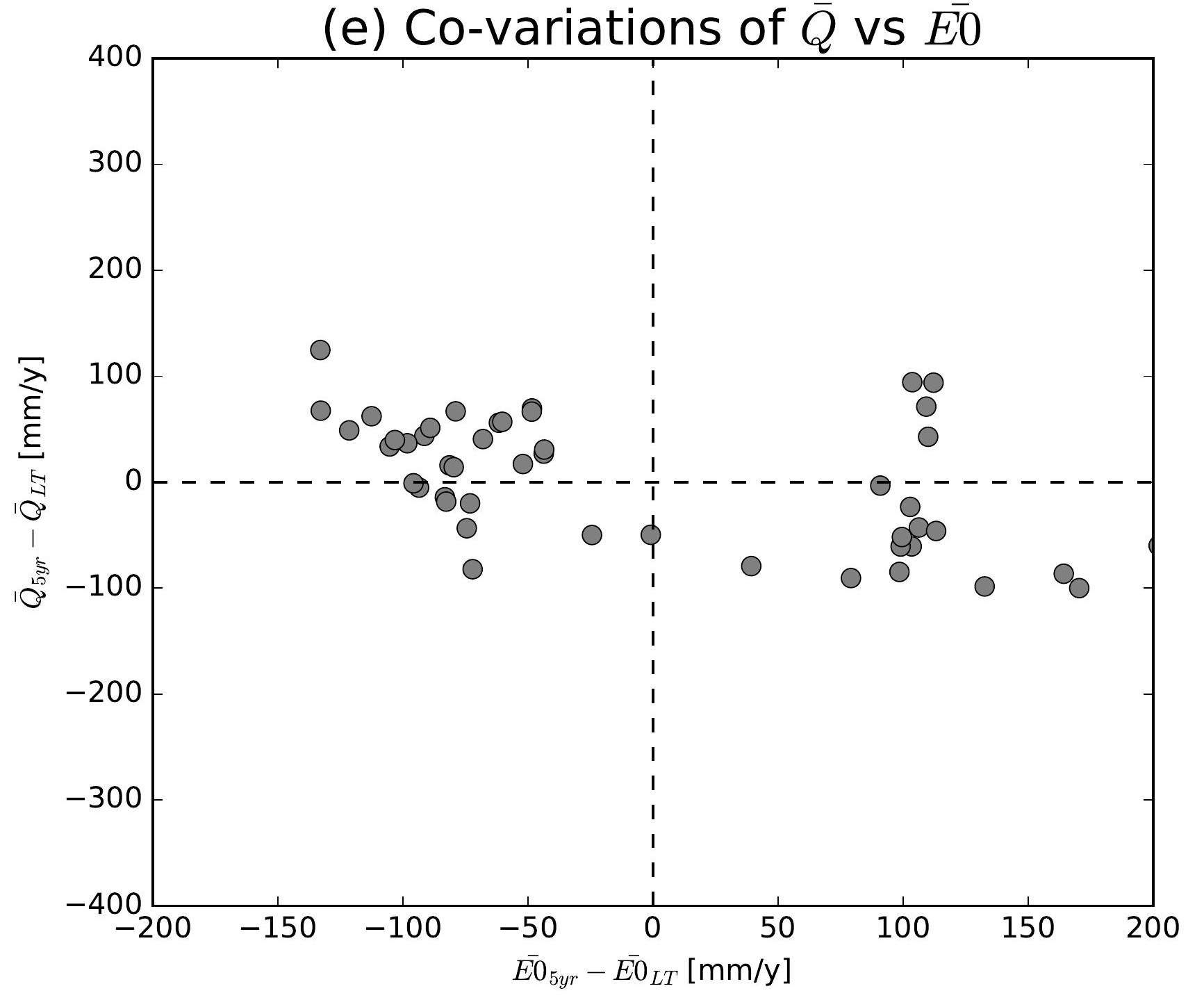

(h) Co-variations of $\bar{Q}$ vs $\overline{E 0}$

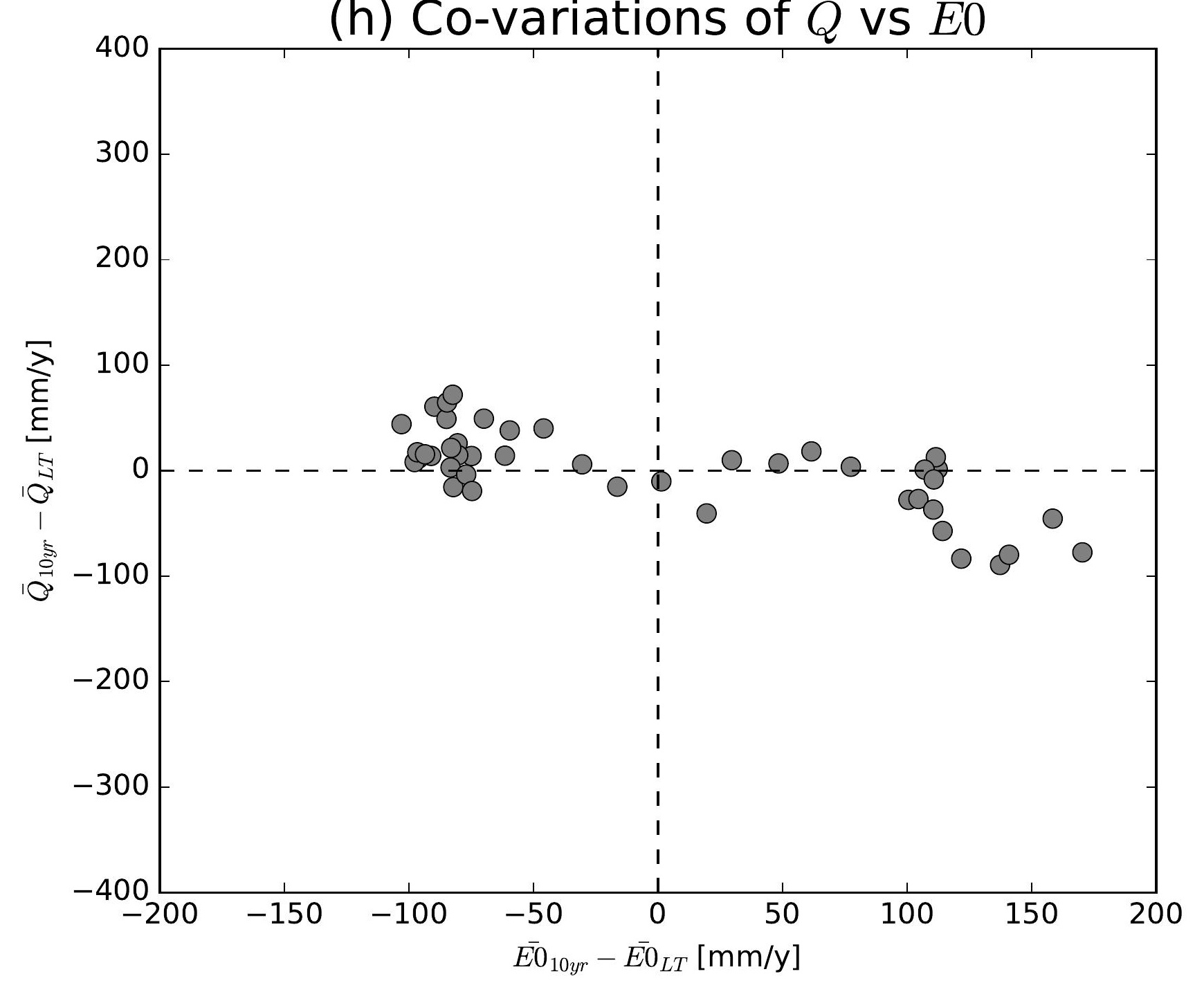

(k) Co-variations of $\bar{Q}$ vs $\overline{E 0}$

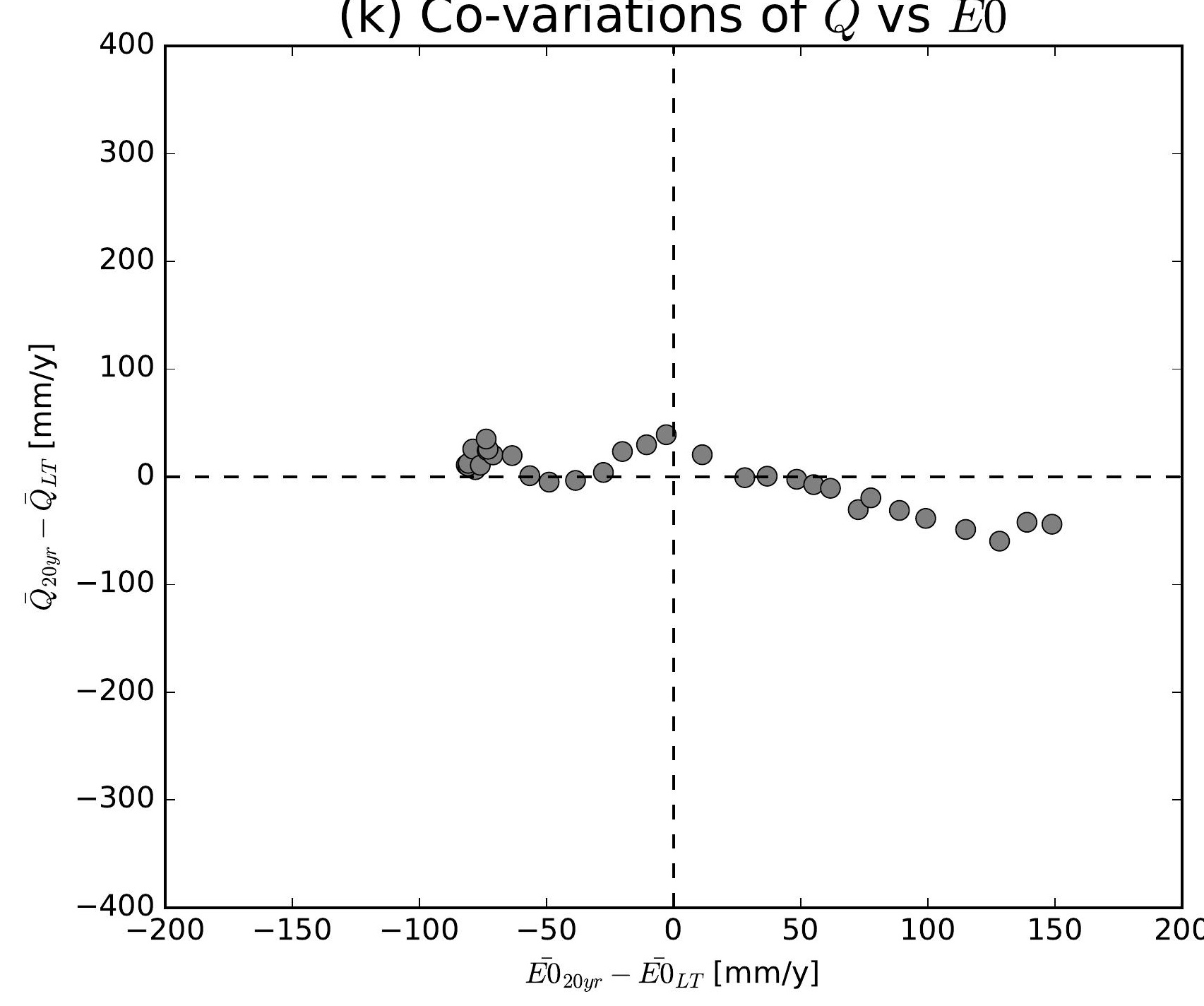

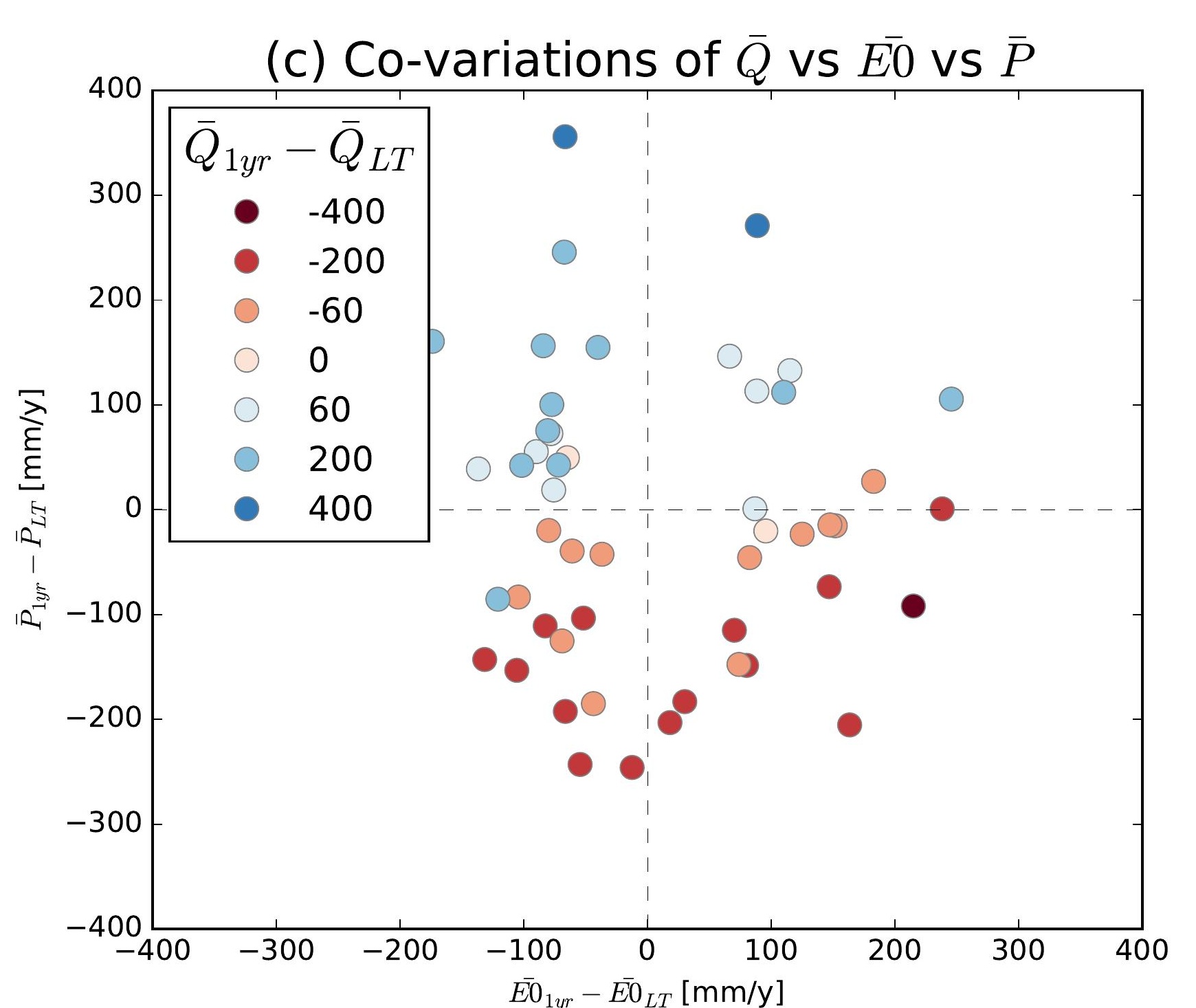
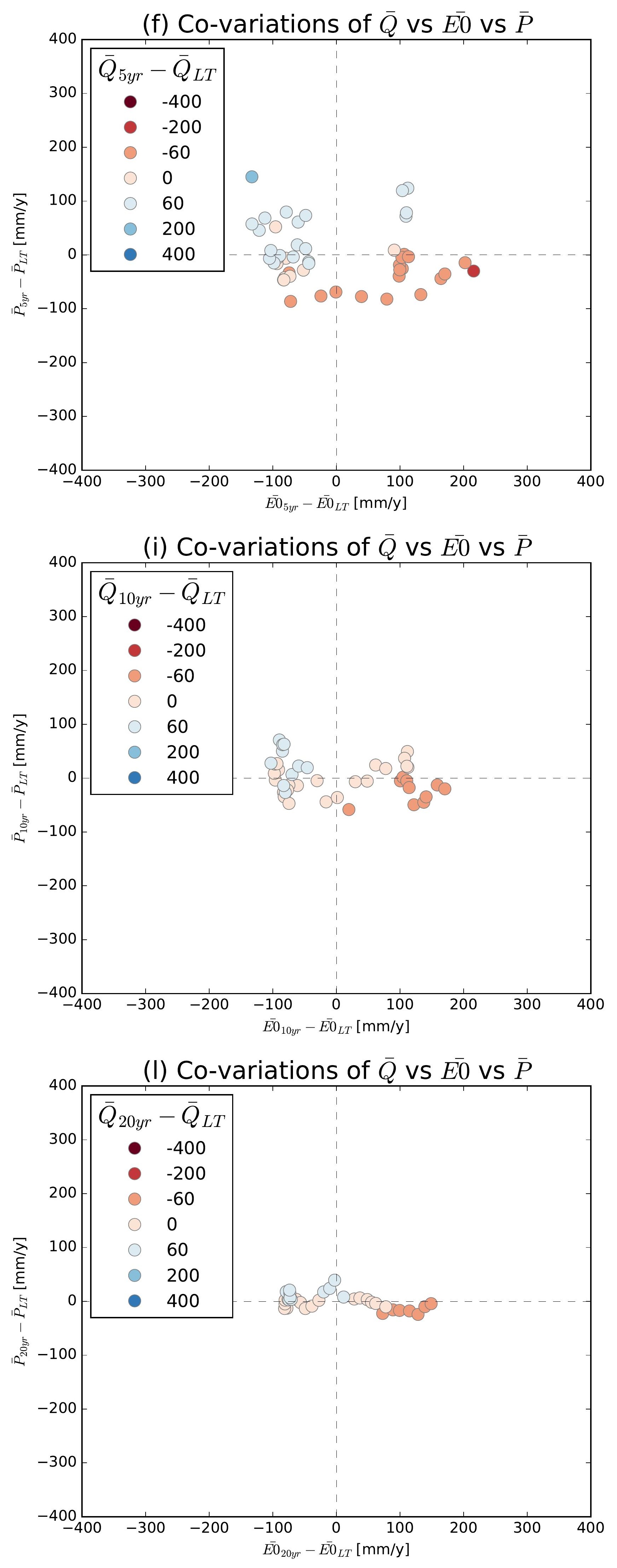

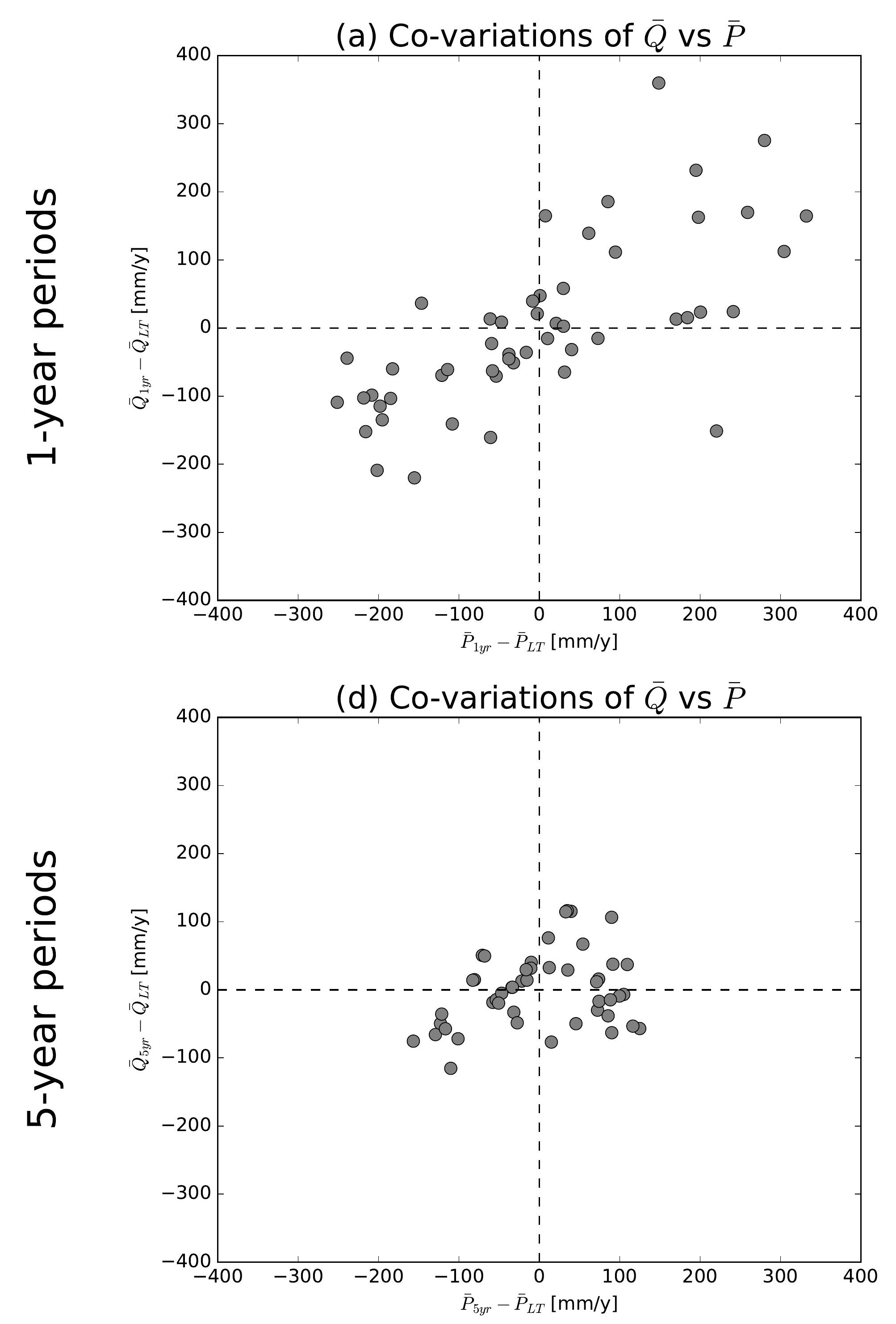

(g) Co-variations of $\bar{Q}$ vs $\bar{P}$

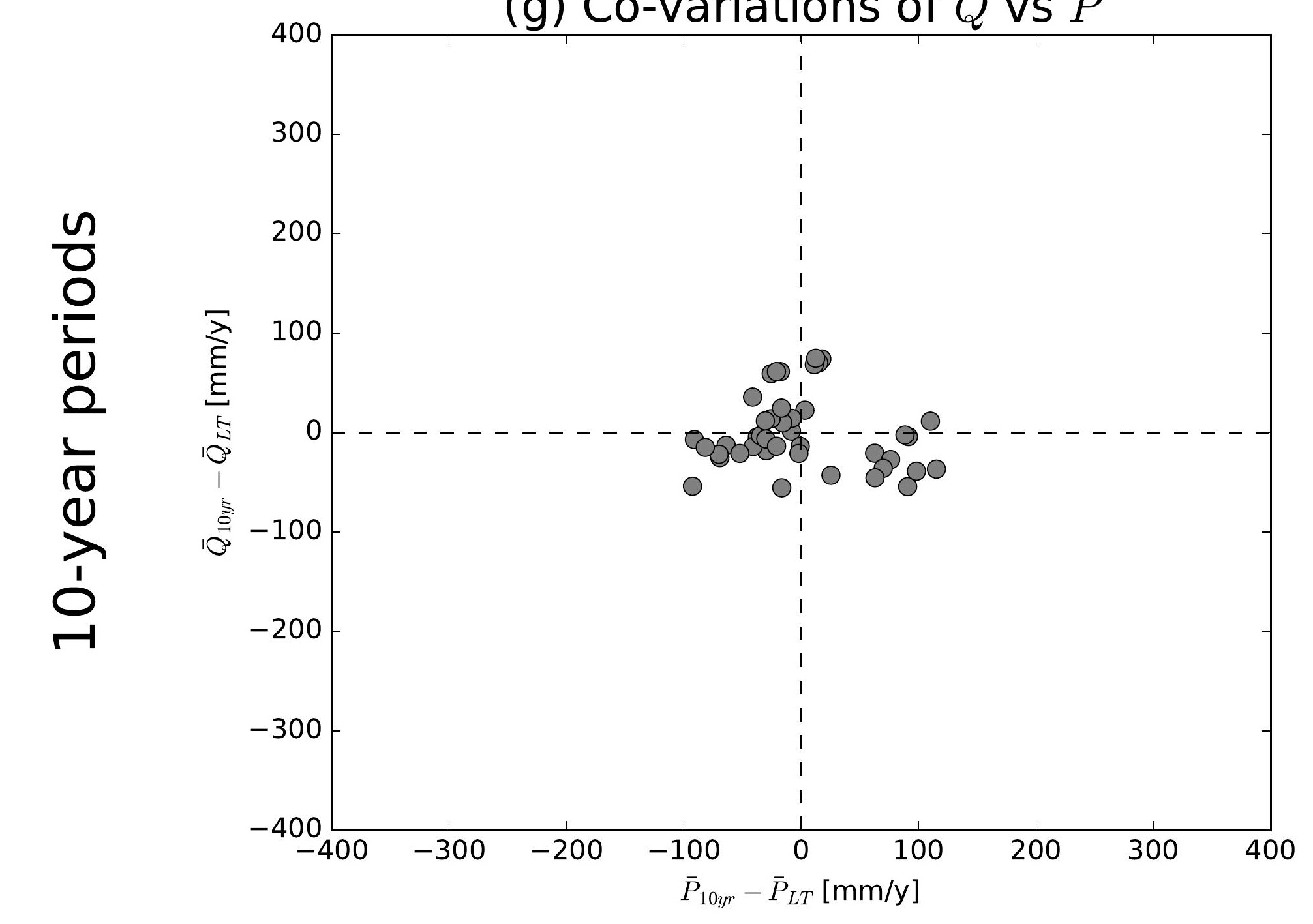

(j) Co-variations of $\bar{Q}$ vs $\bar{P}$

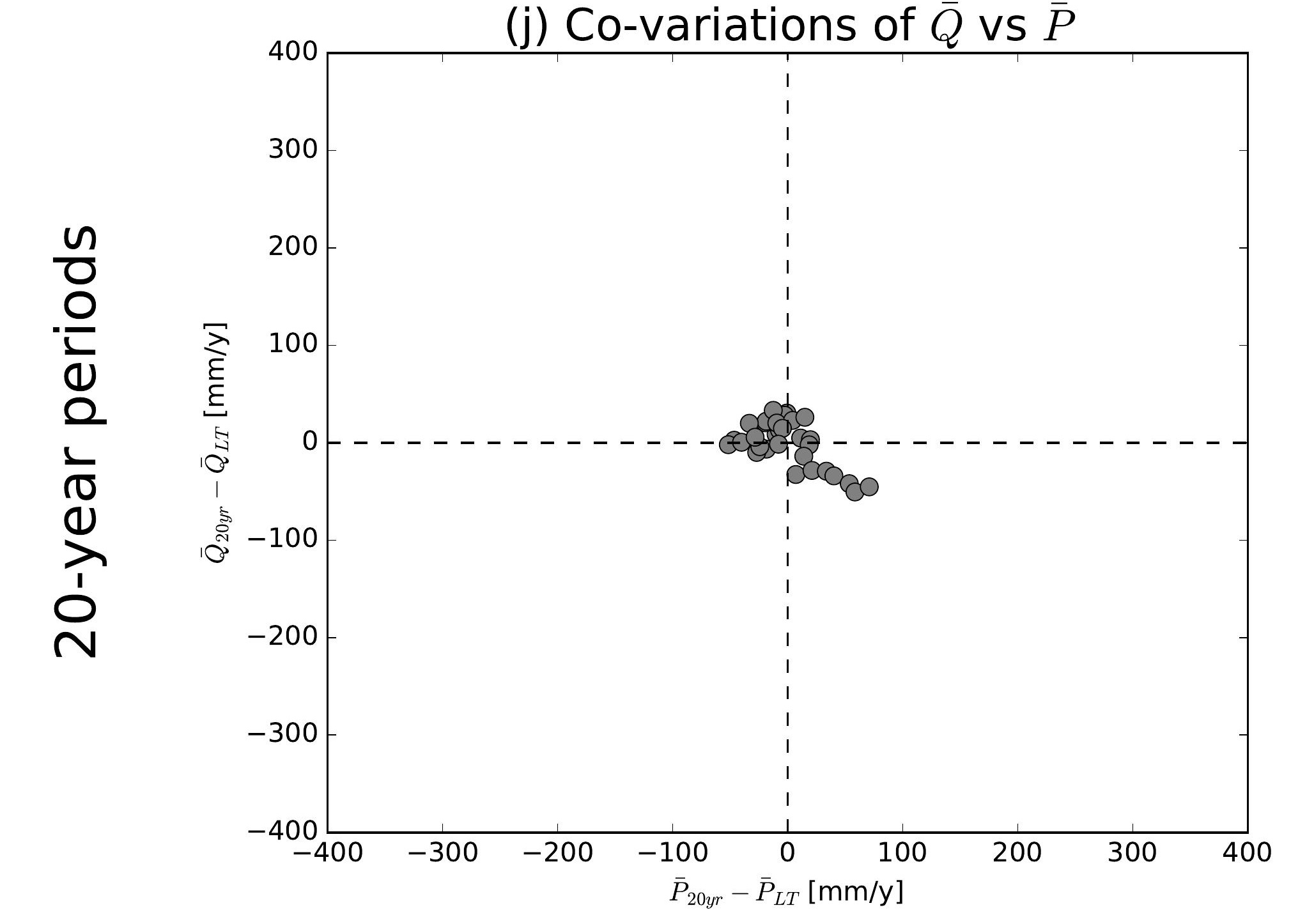

(b) Co-variations of $\bar{Q}$ vs $\overline{E 0}$

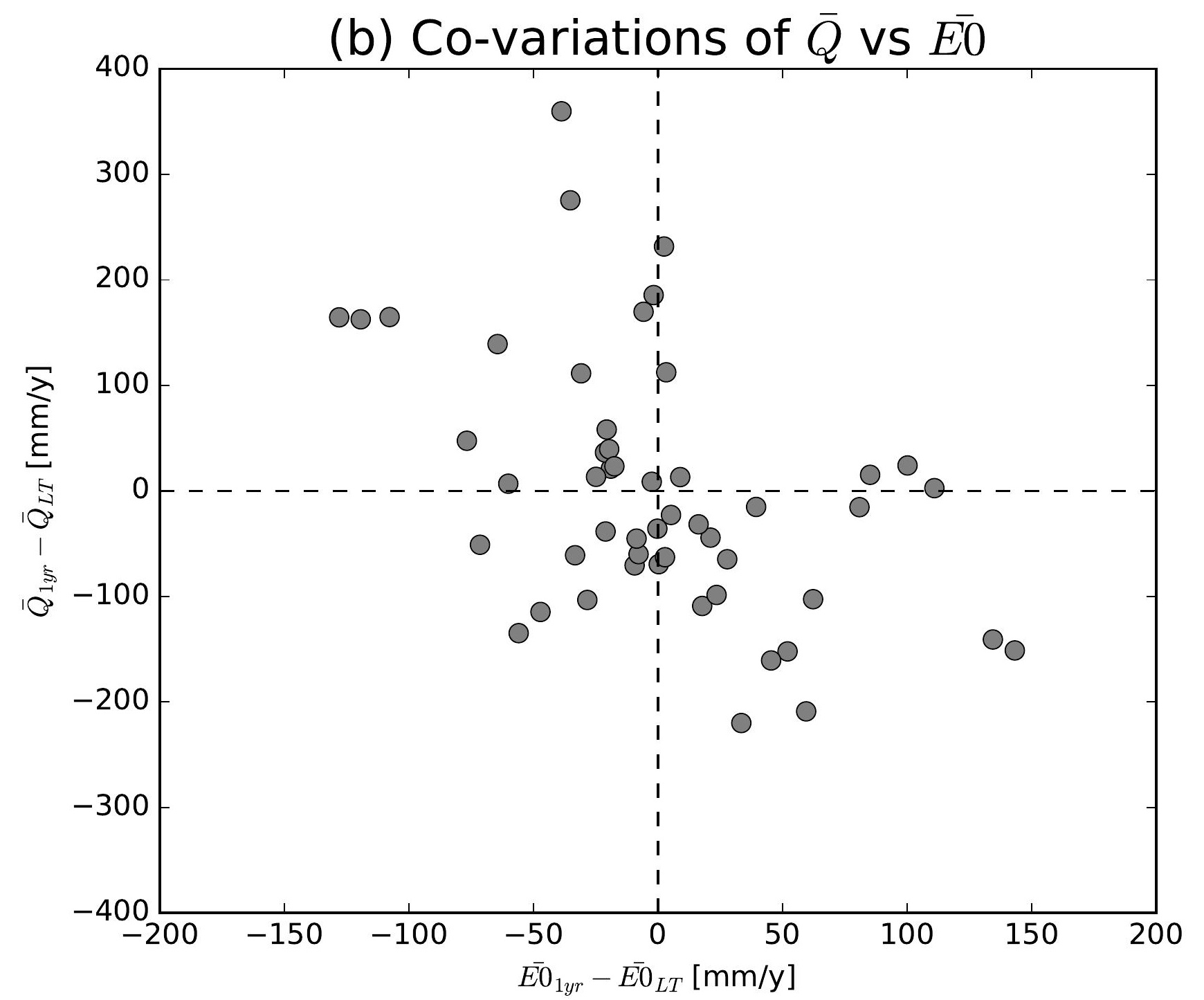

(e) Co-variations of $\bar{Q}$ vs $\overline{E 0}$

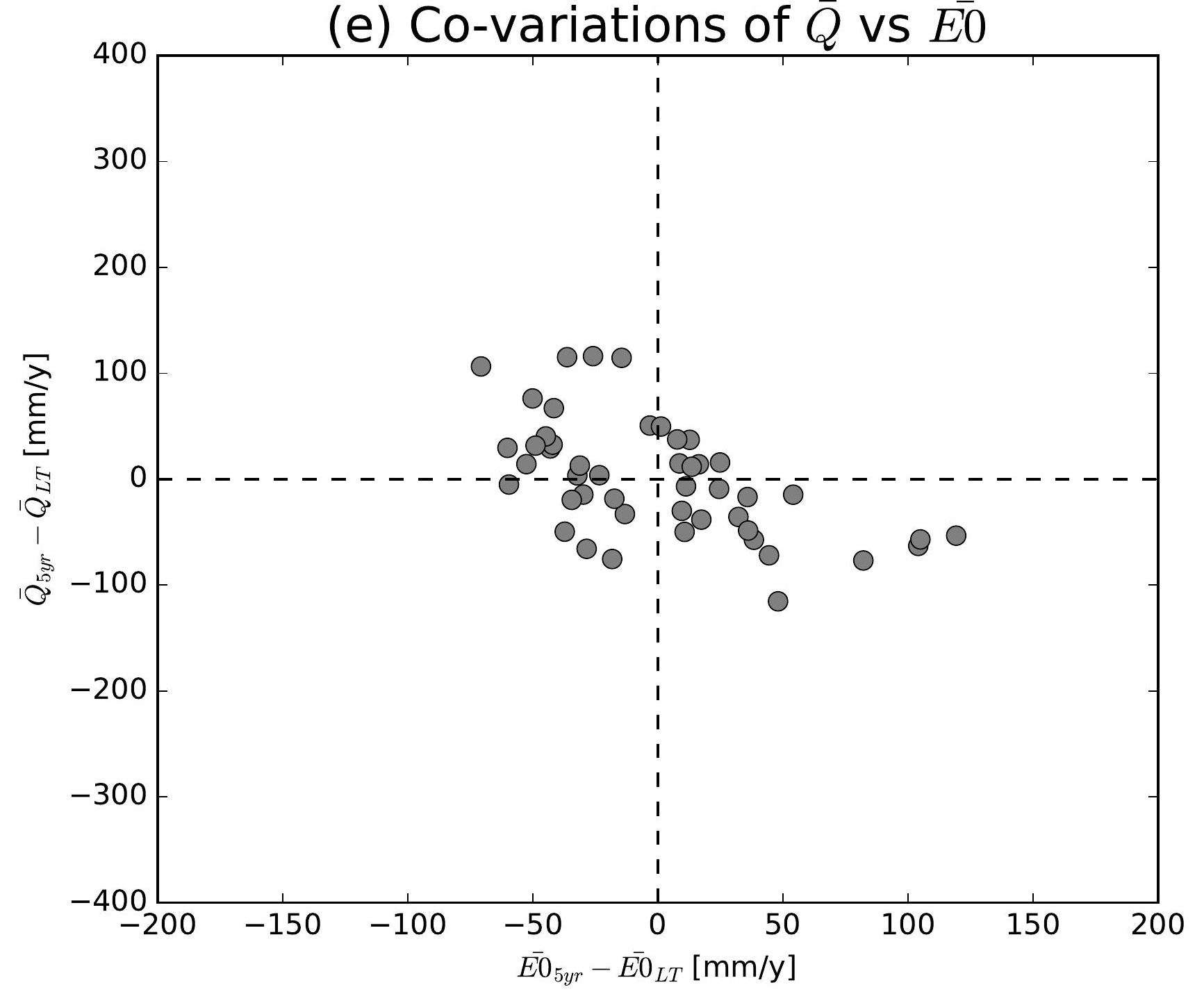

(h) Co-variations of $\bar{Q}$ vs $\overline{E 0}$

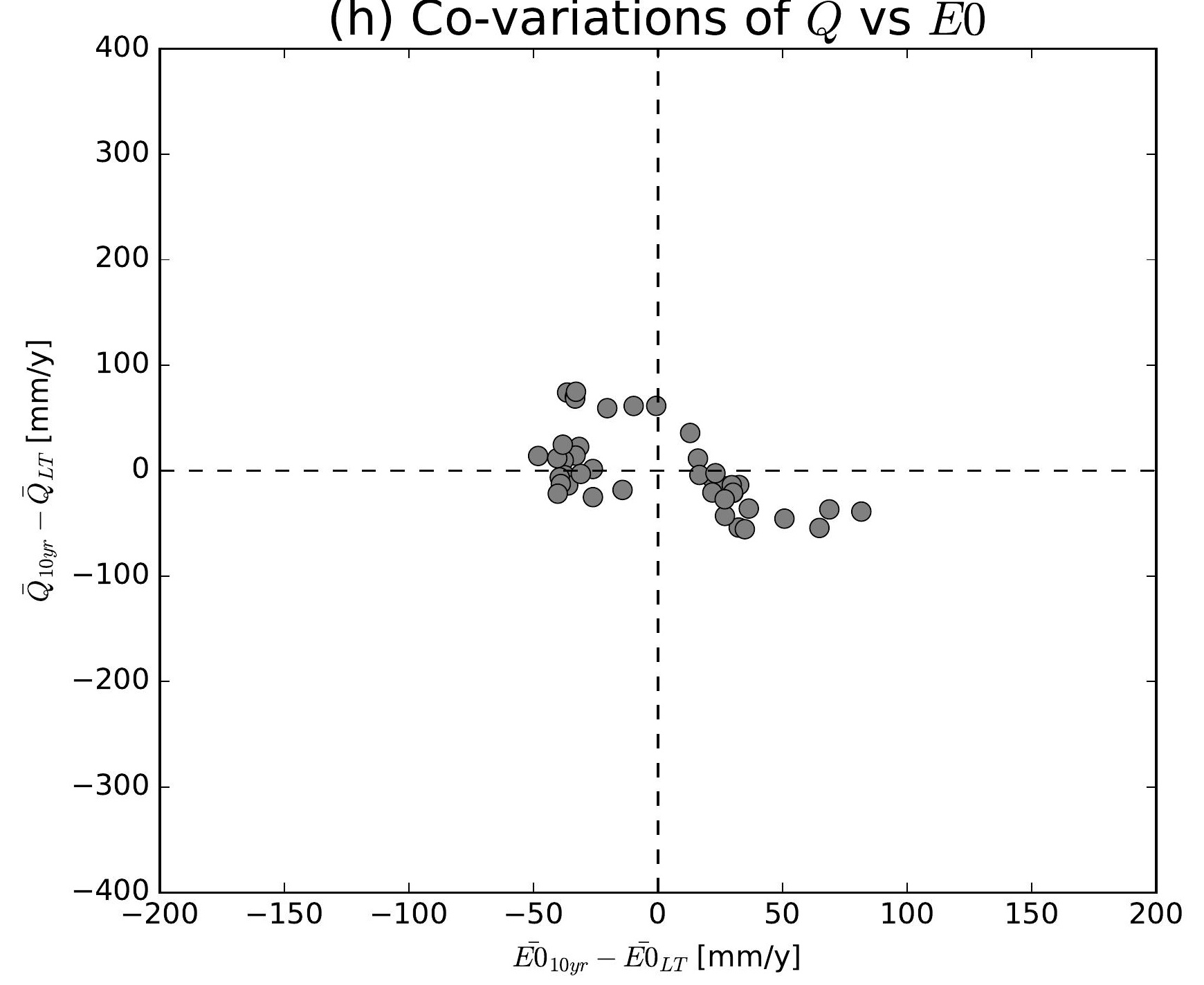

(k) Co-variations of $\bar{Q}$ vs $\overline{E 0}$

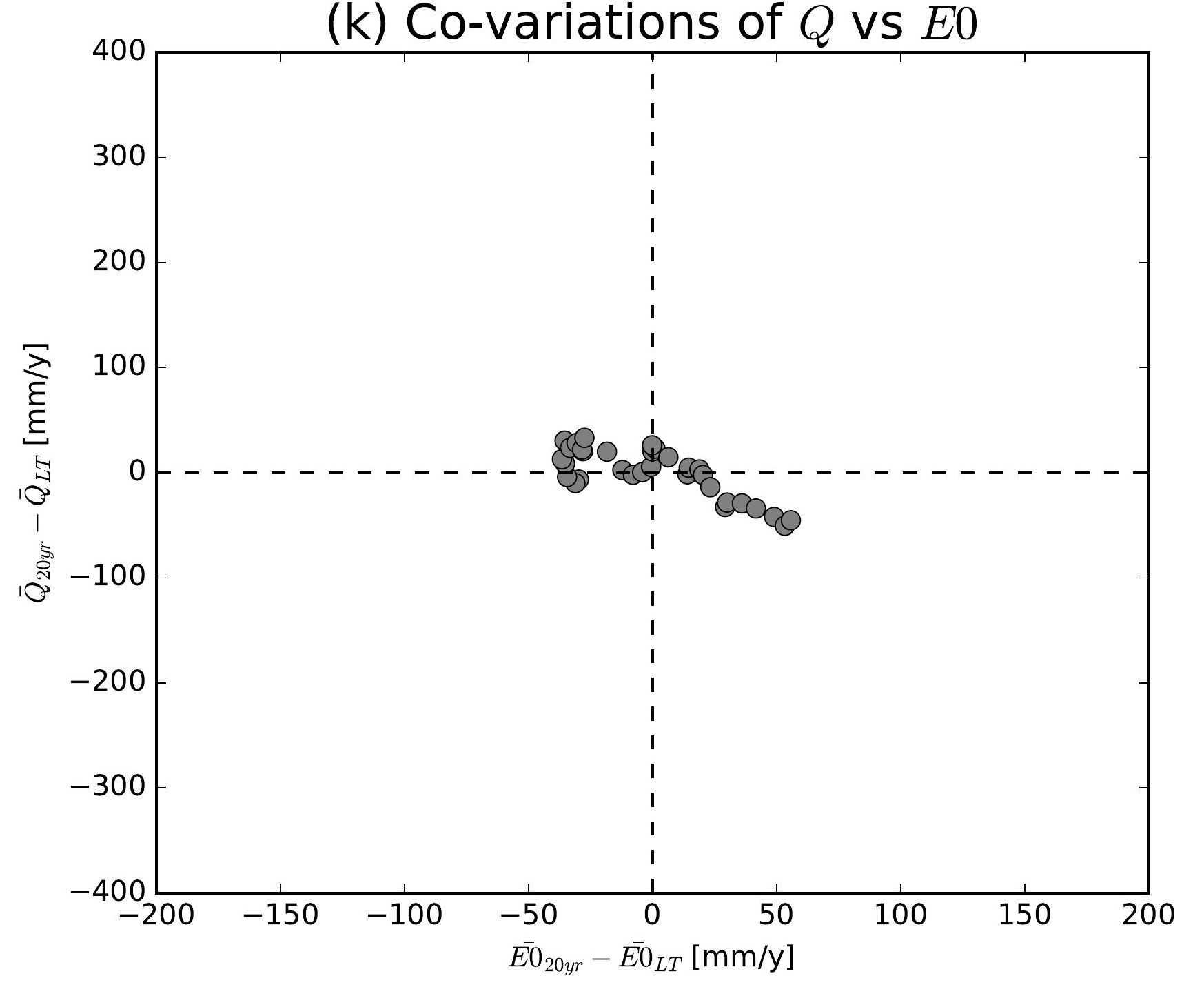

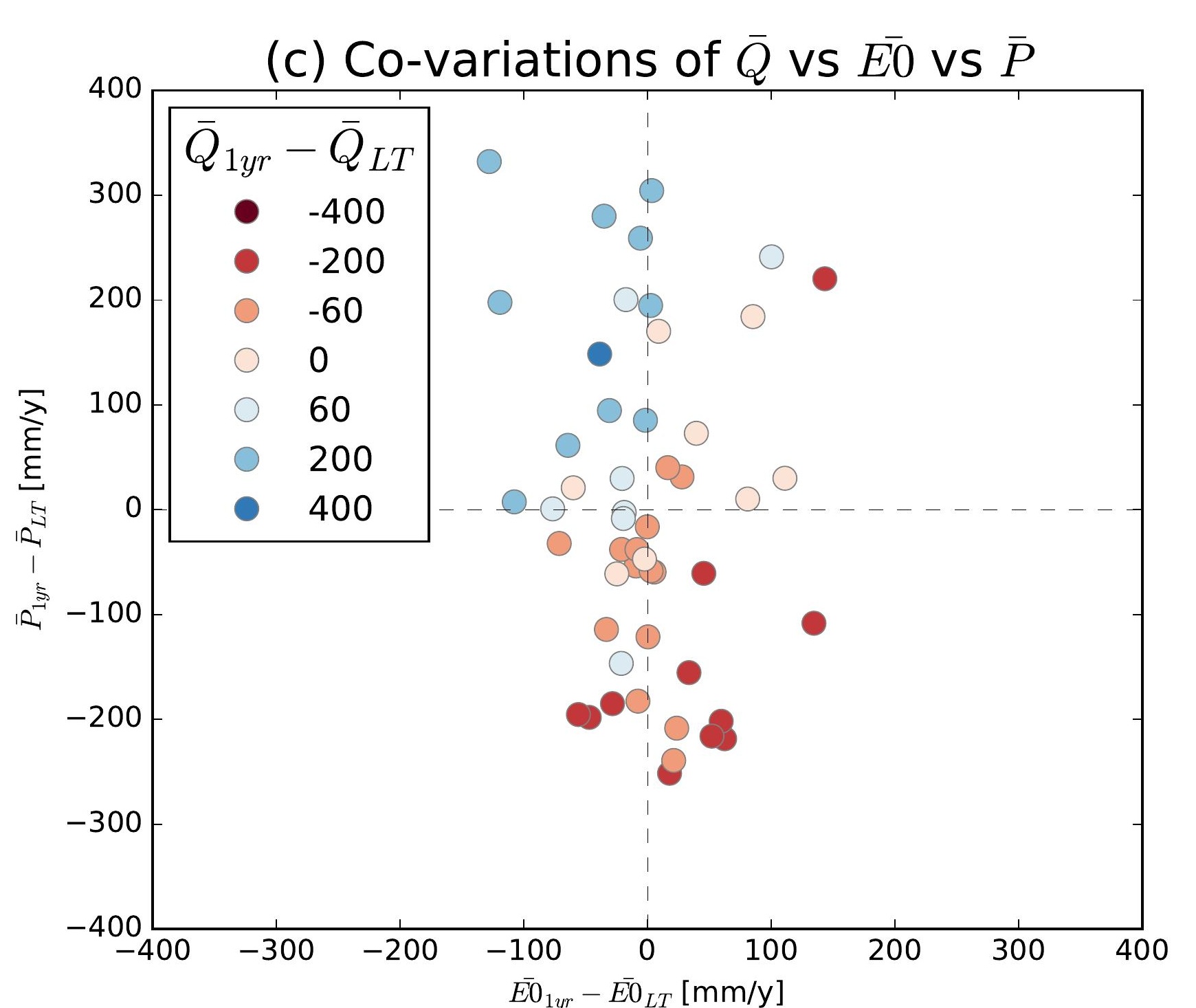
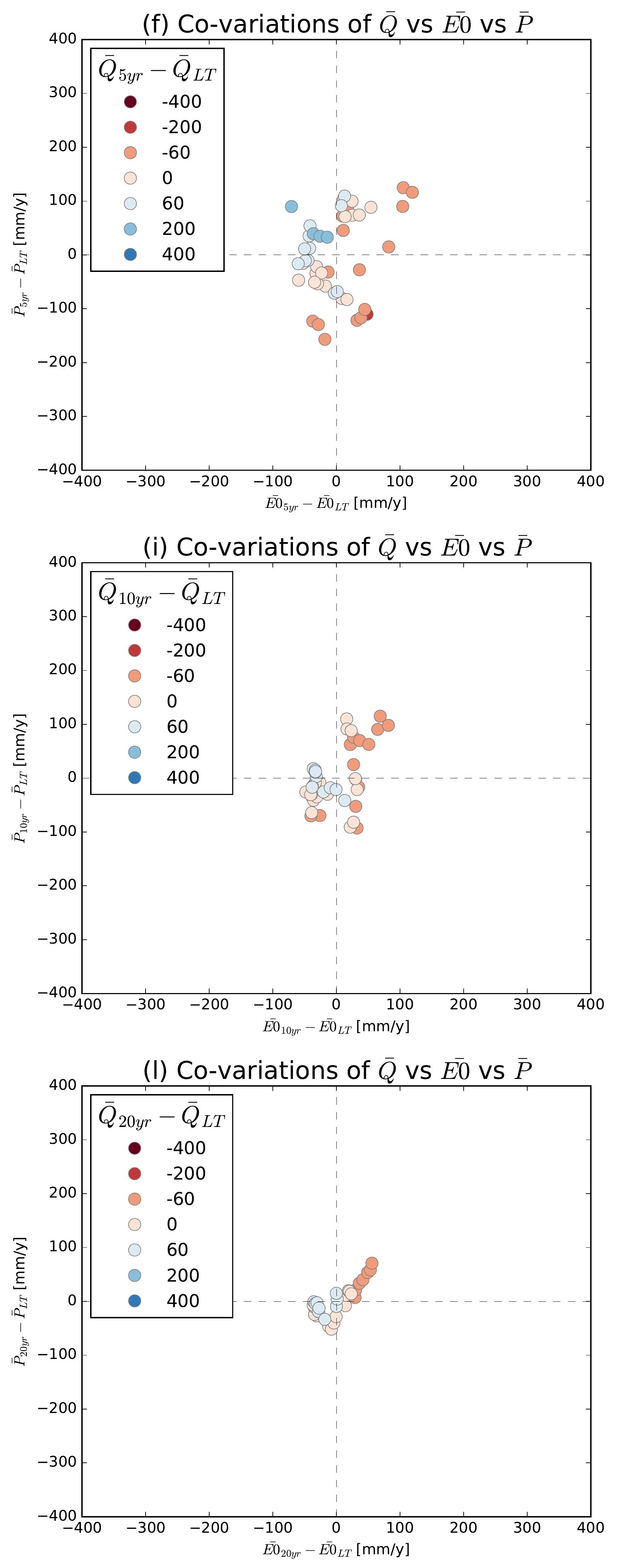

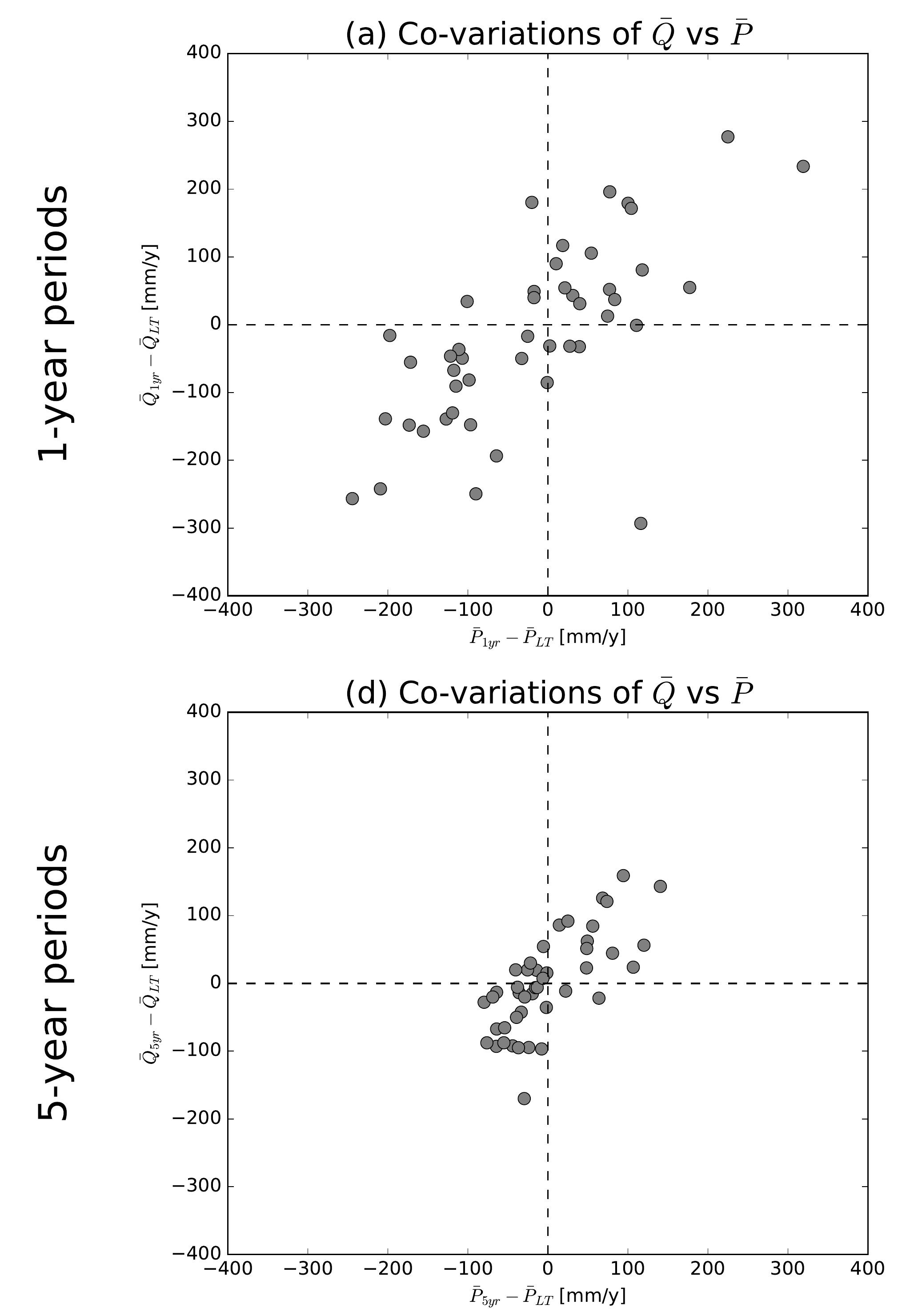

(g) Co-variations of $\bar{Q}$ vs $\bar{P}$
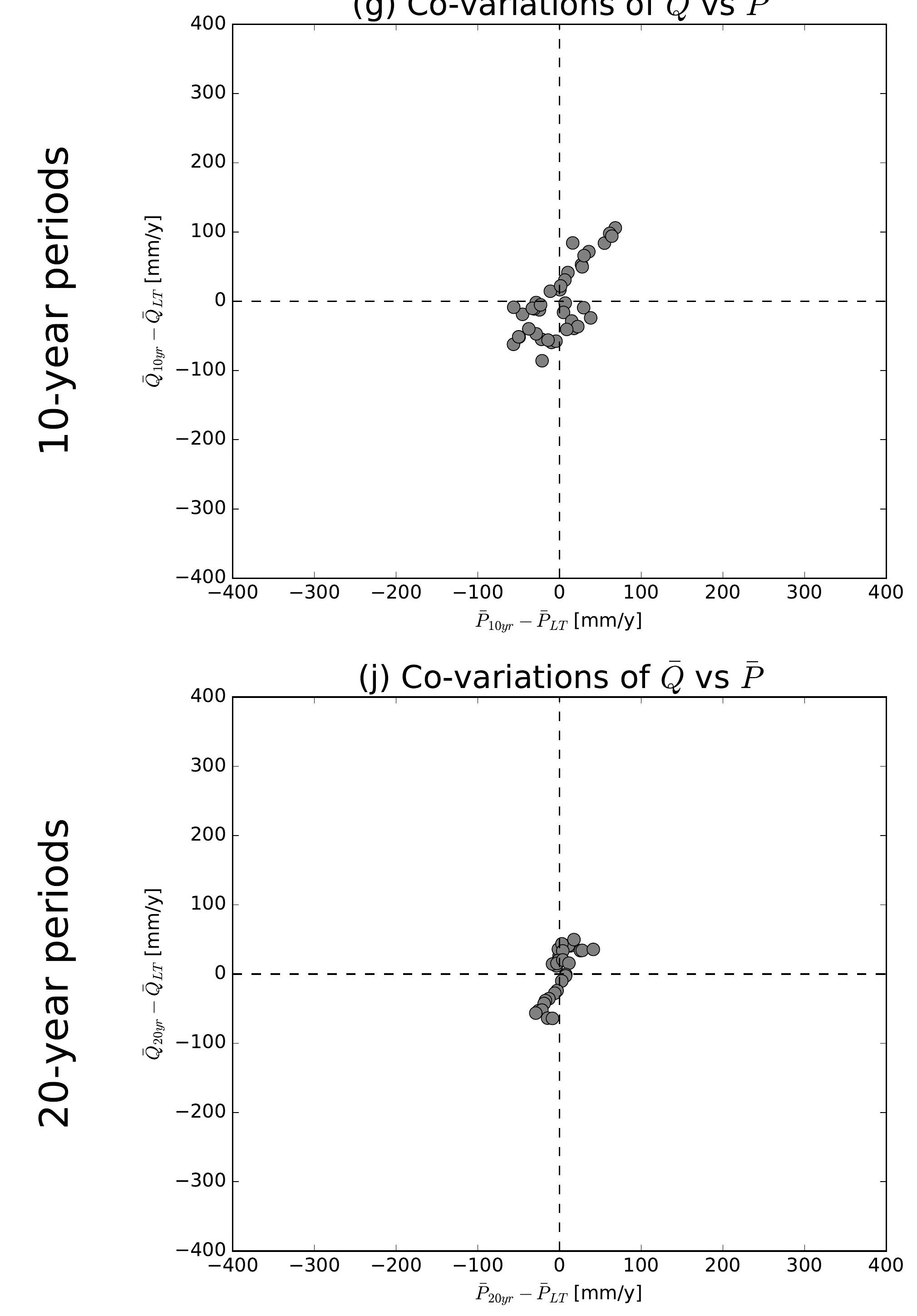

(b) Co-variations of $\bar{Q}$ vs $\overline{E 0}$

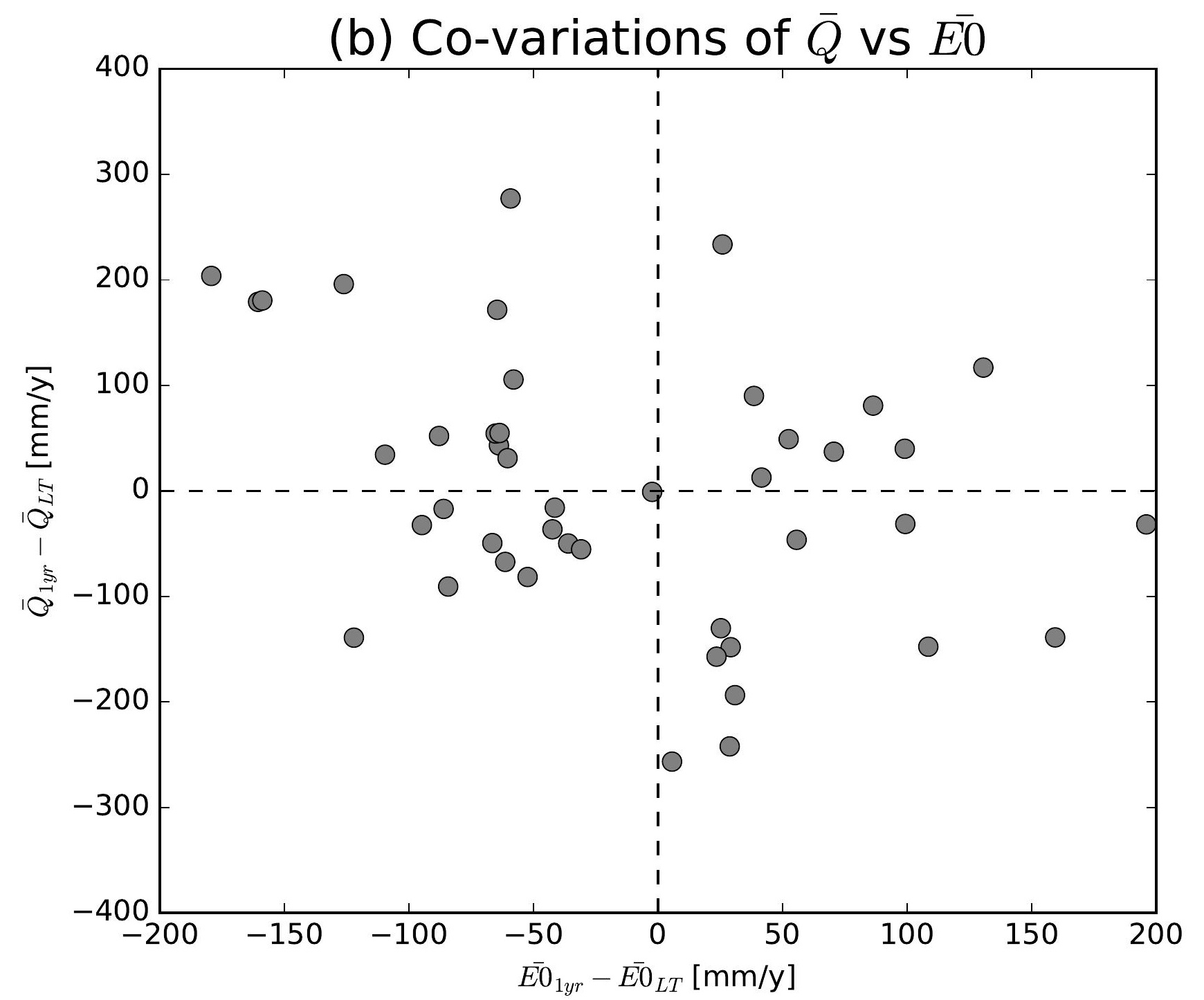

(e) Co-variations of $\bar{Q}$ vs $\overline{E 0}$

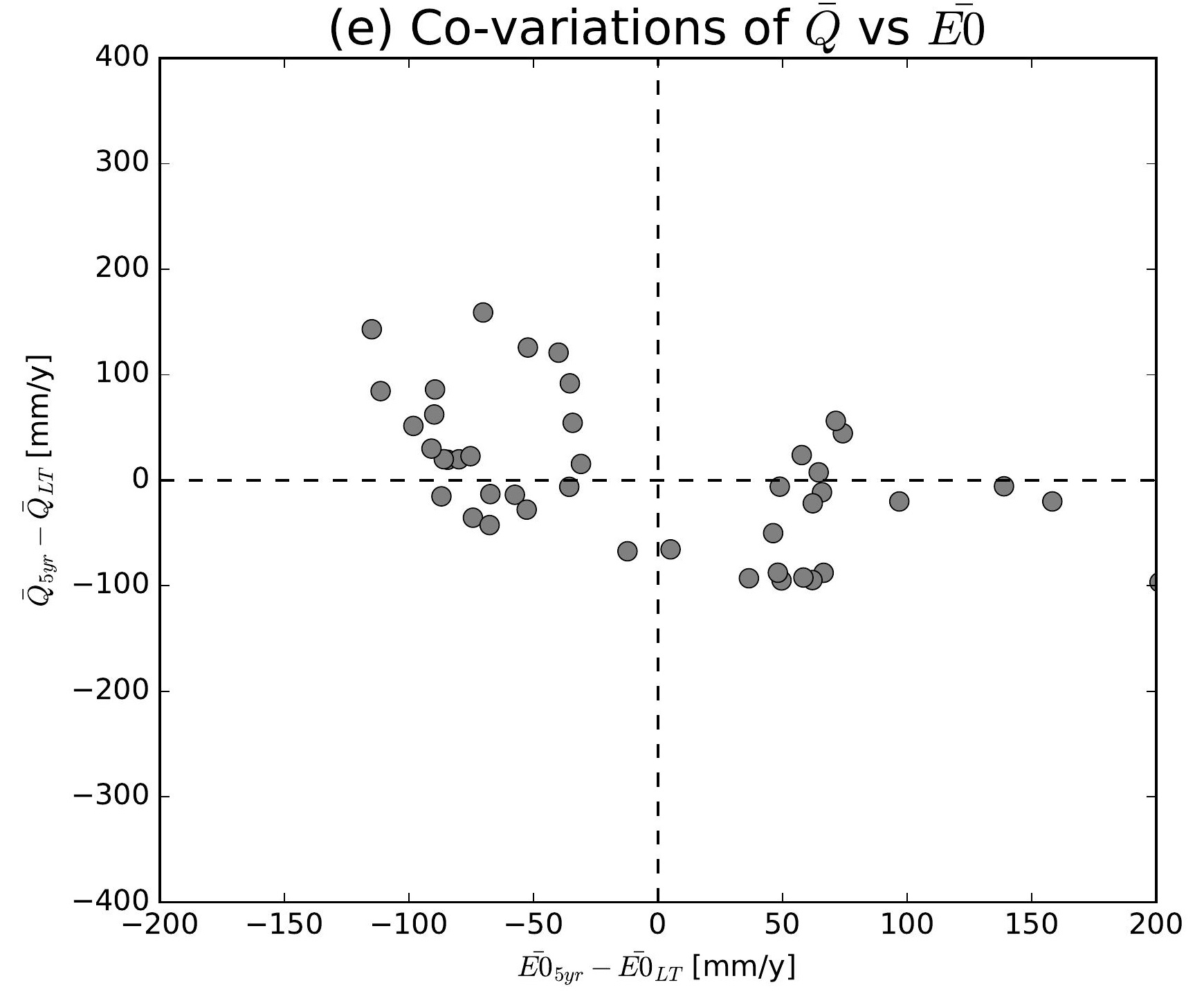

(h) Co-variations of $\bar{Q}$ vs $\overline{E 0}$

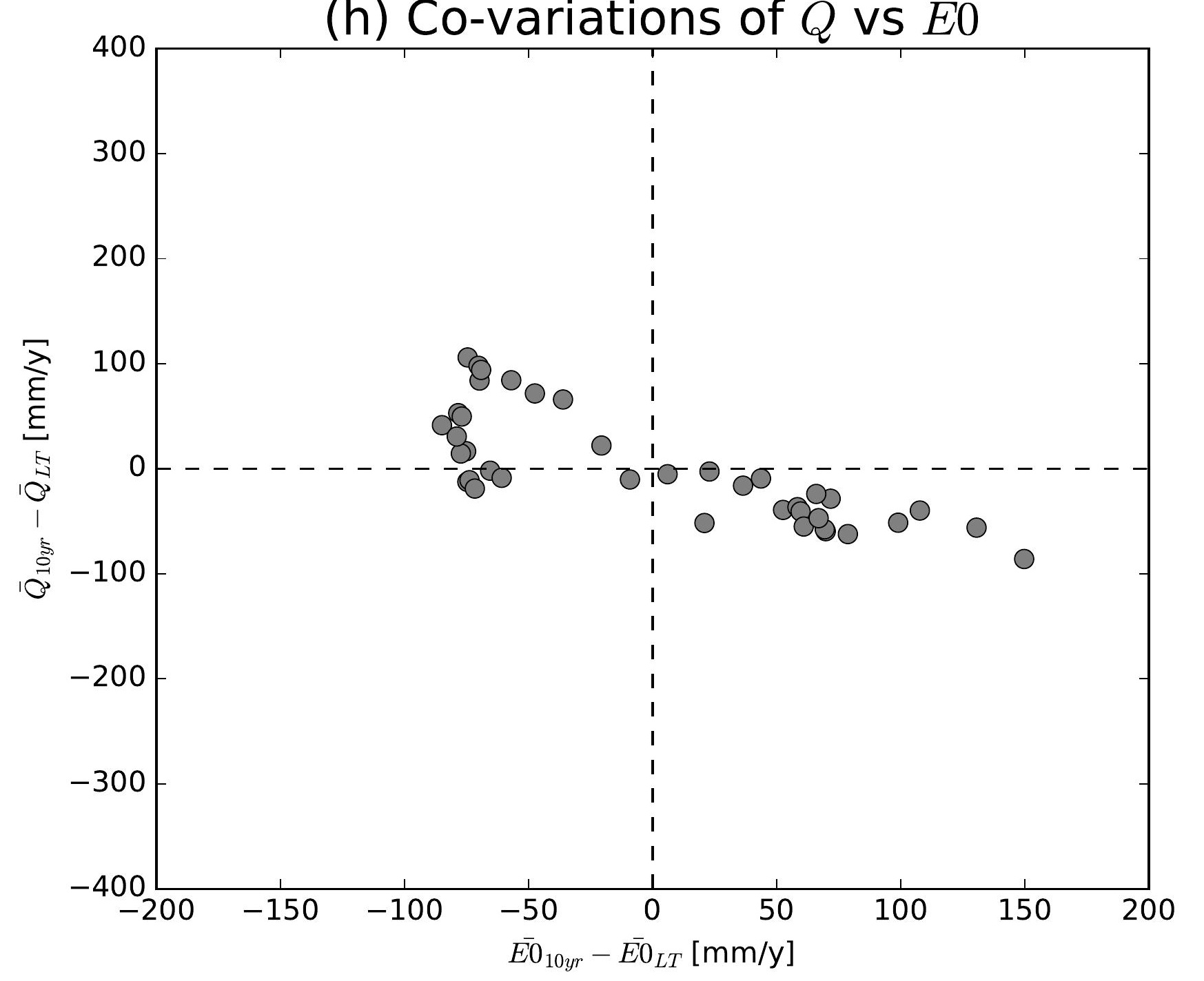

(k) Co-variations of $\bar{Q}$ vs $\overline{E 0}$

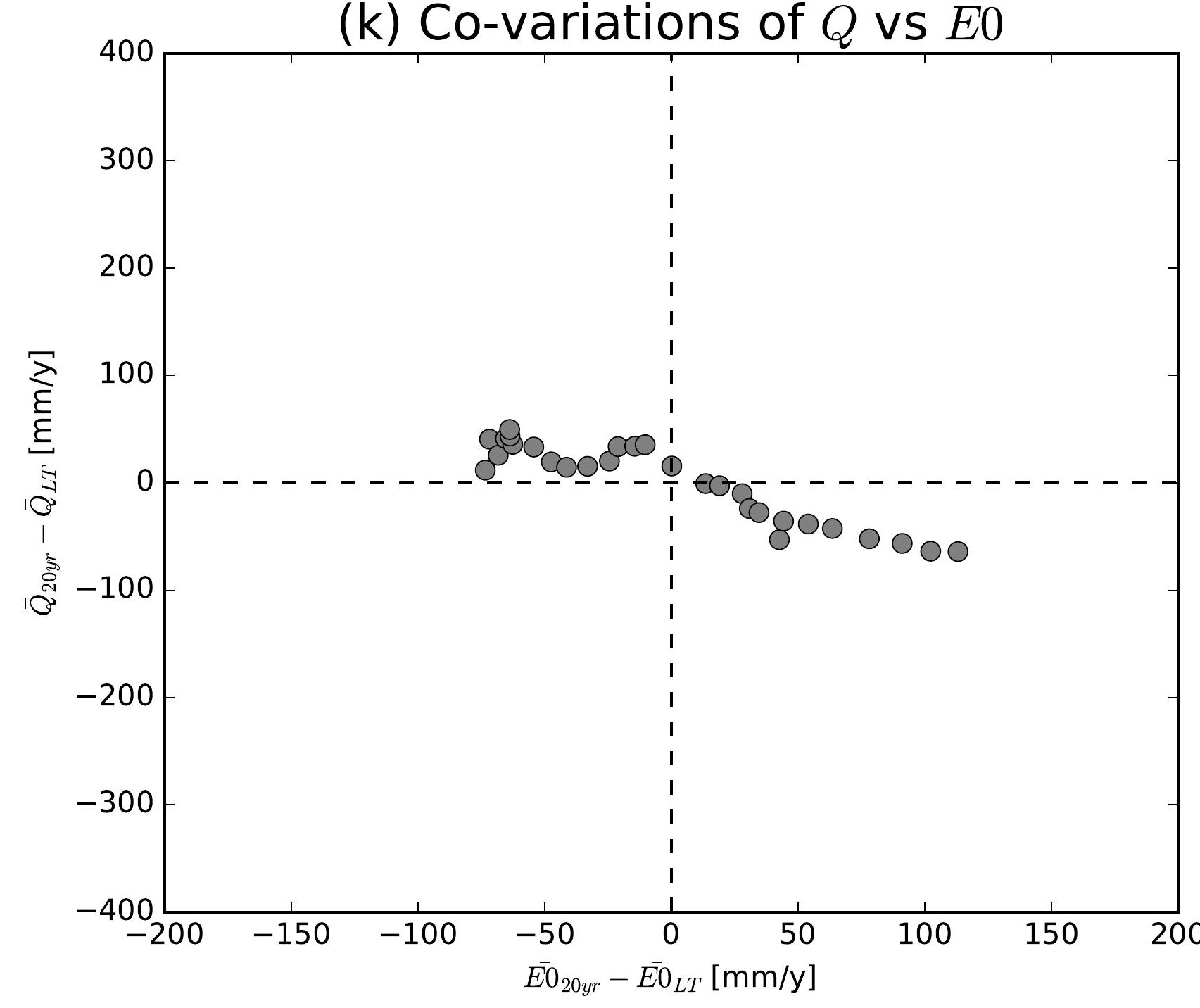

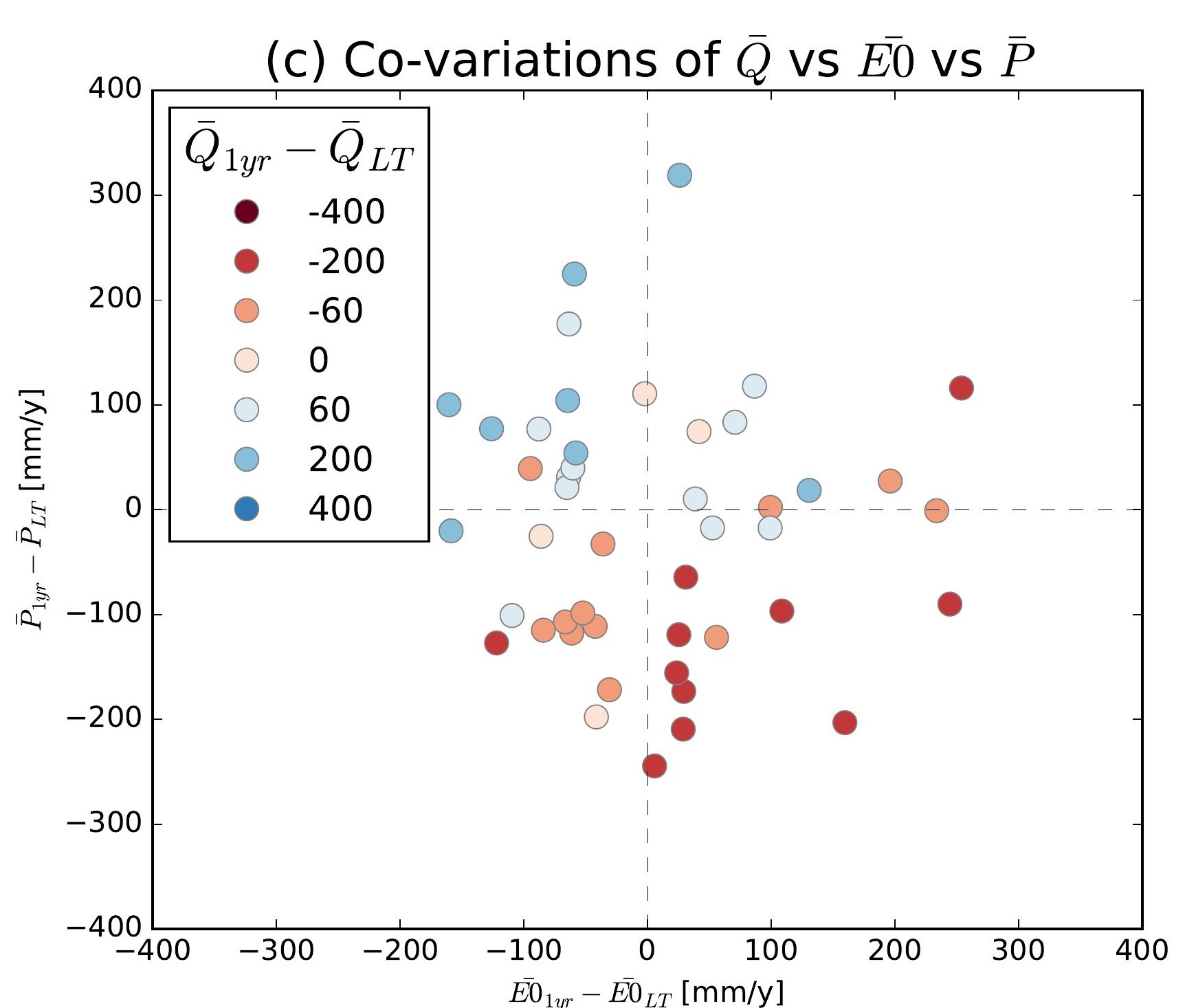
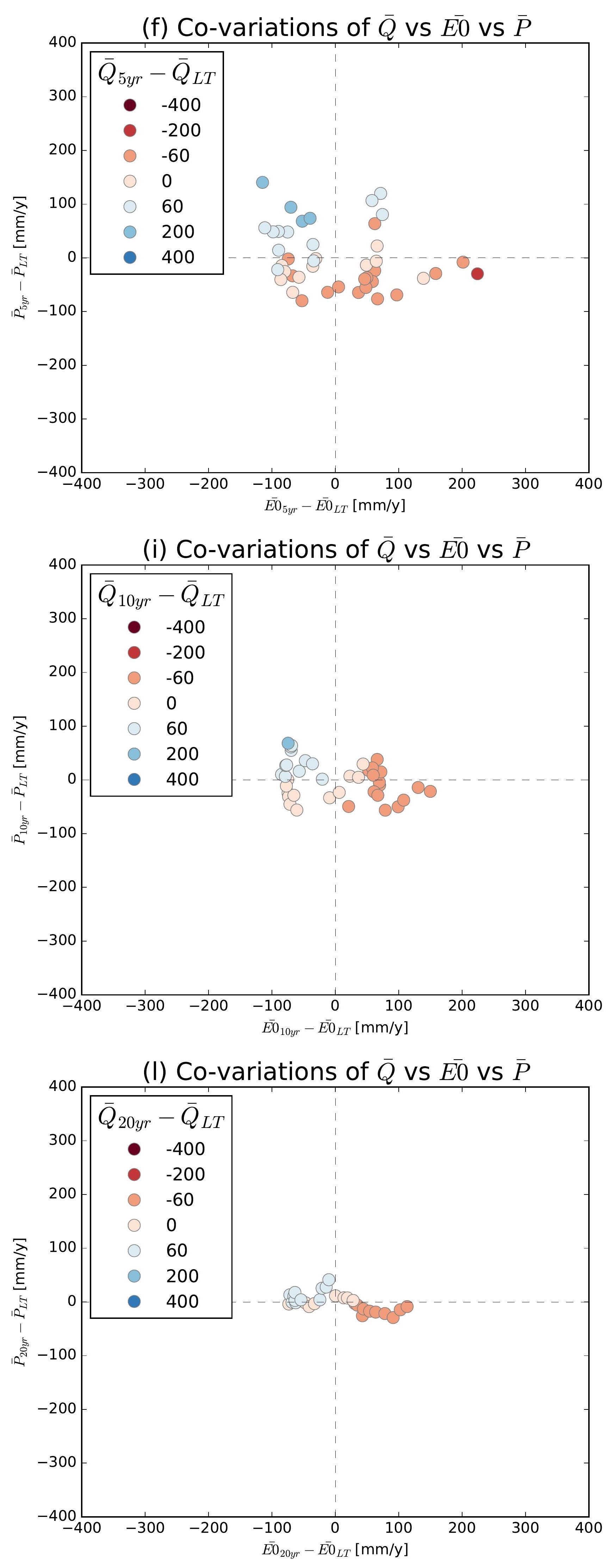

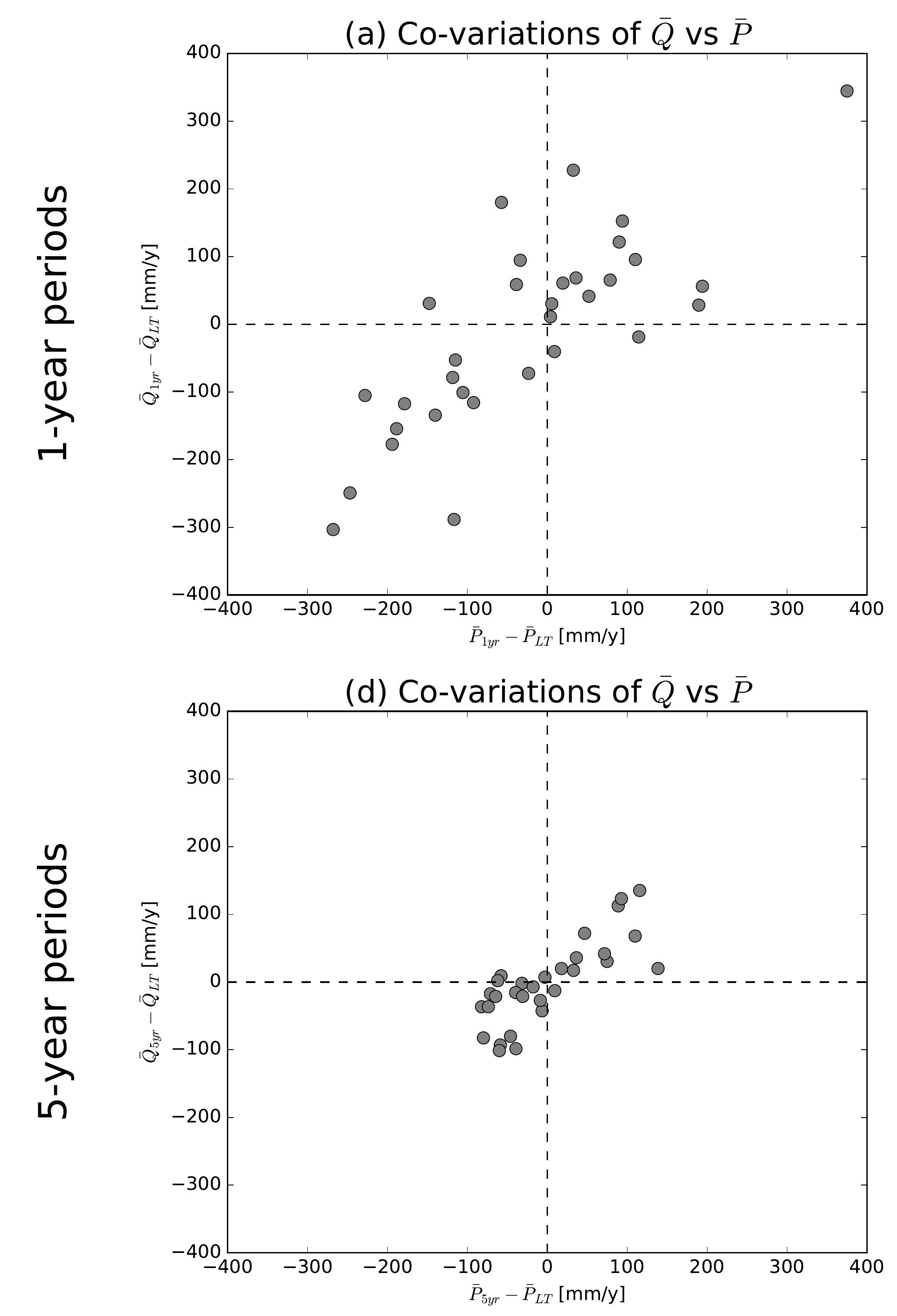

(g) Co-variations of $\bar{Q}$ vs $\bar{P}$
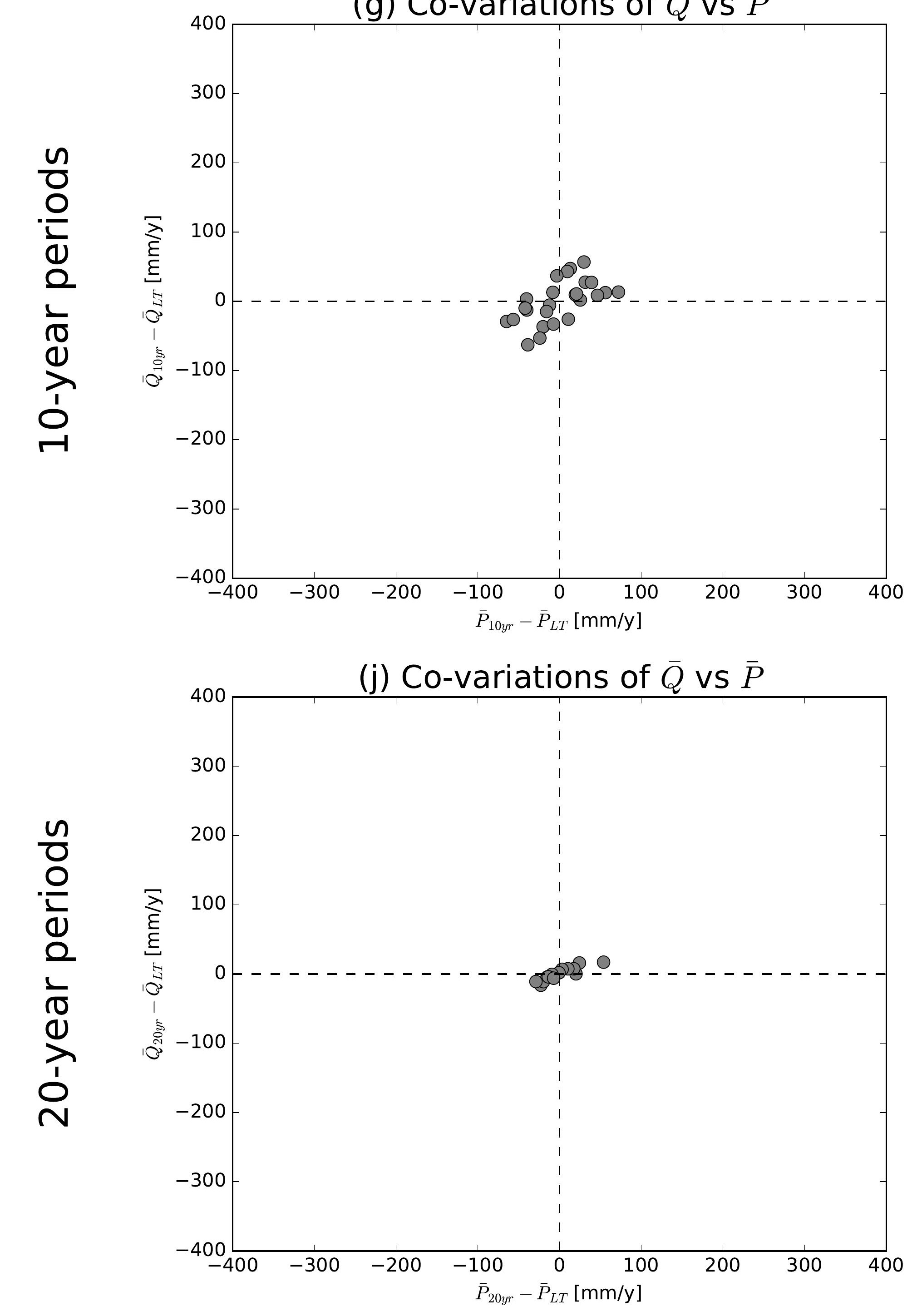

(b) Co-variations of $\bar{Q}$ vs $\overline{E 0}$

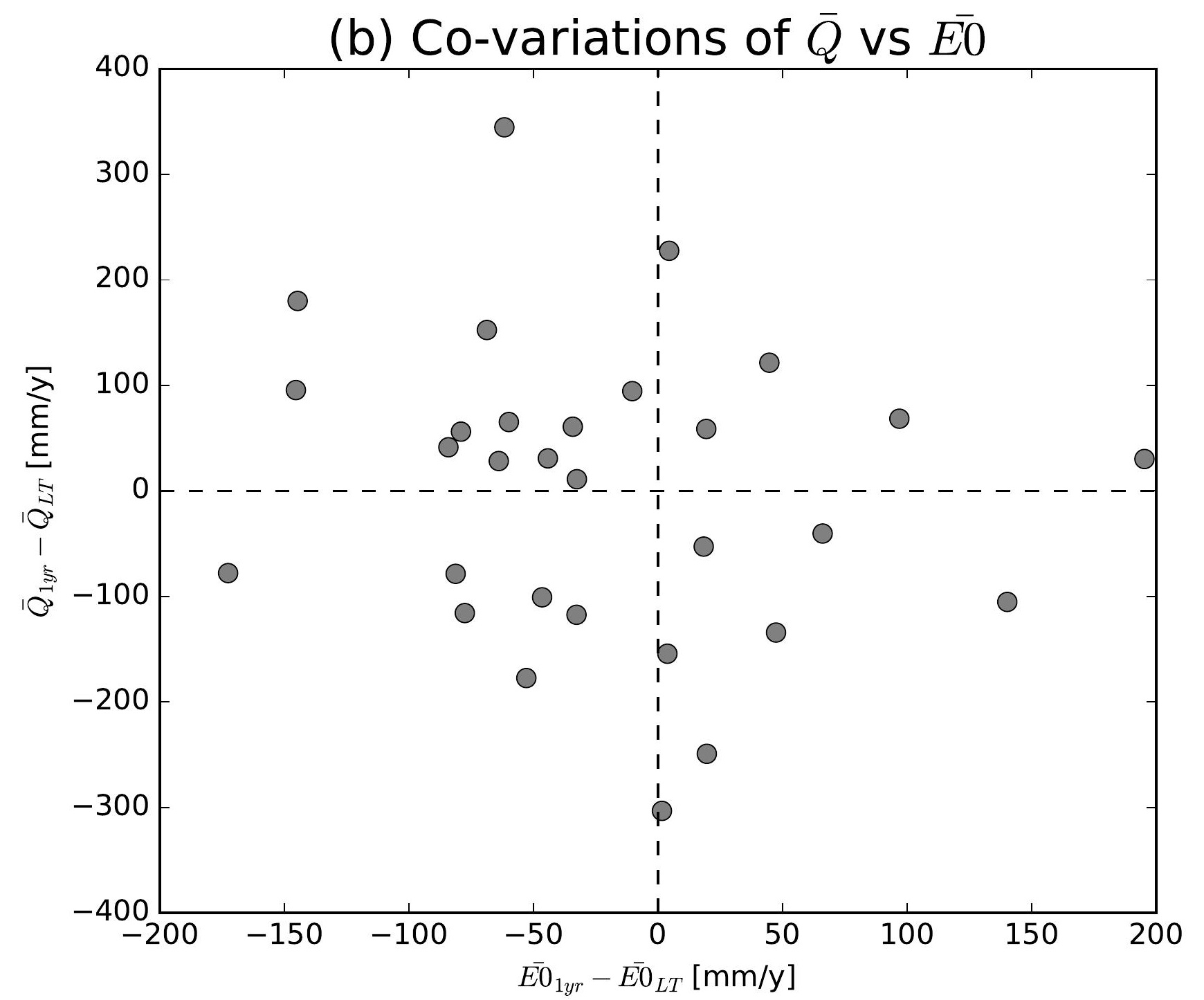

(e) Co-variations of $\bar{Q}$ vs $\overline{E 0}$

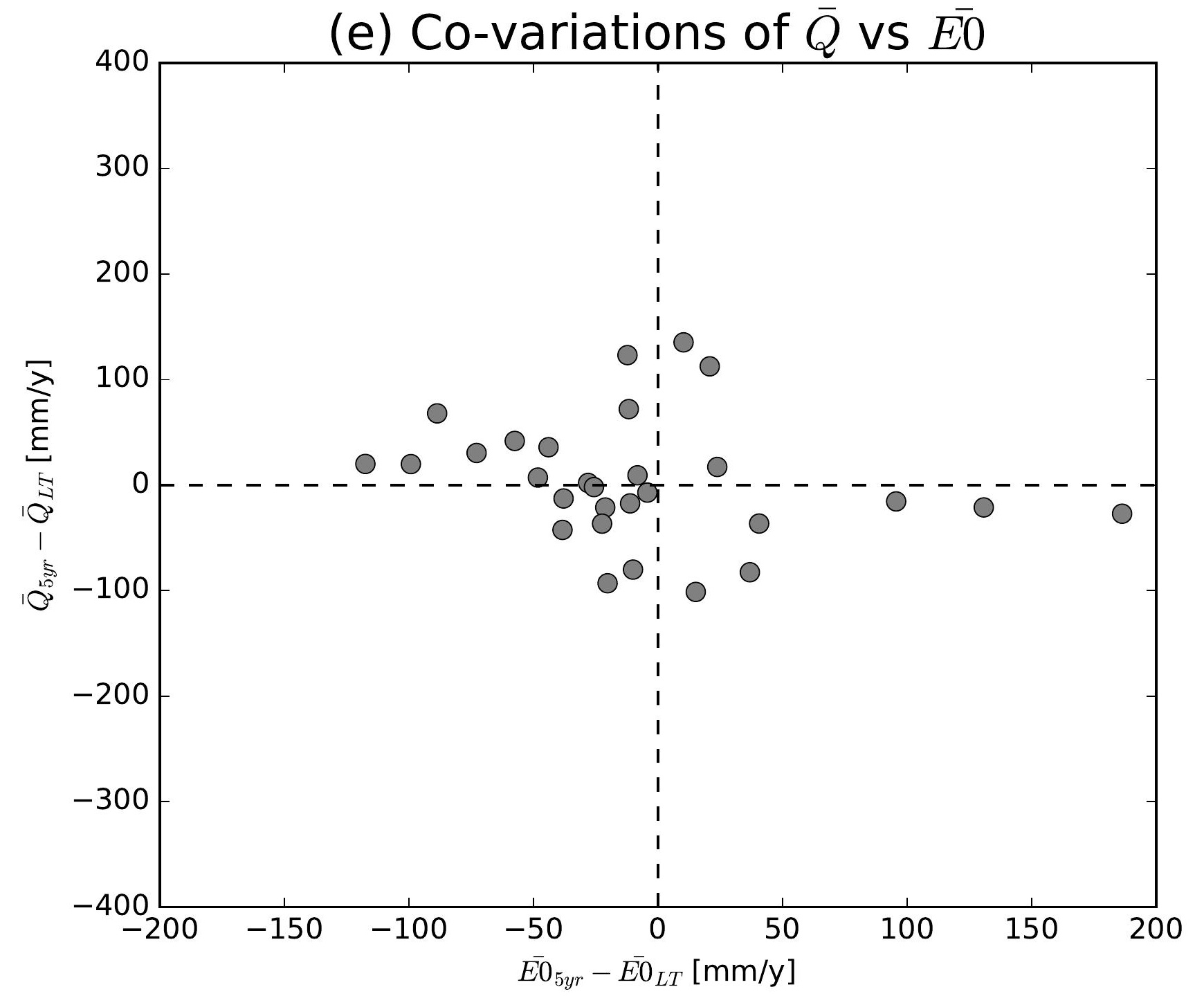

(h) Co-variations of $\bar{Q}$ vs $\overline{E 0}$

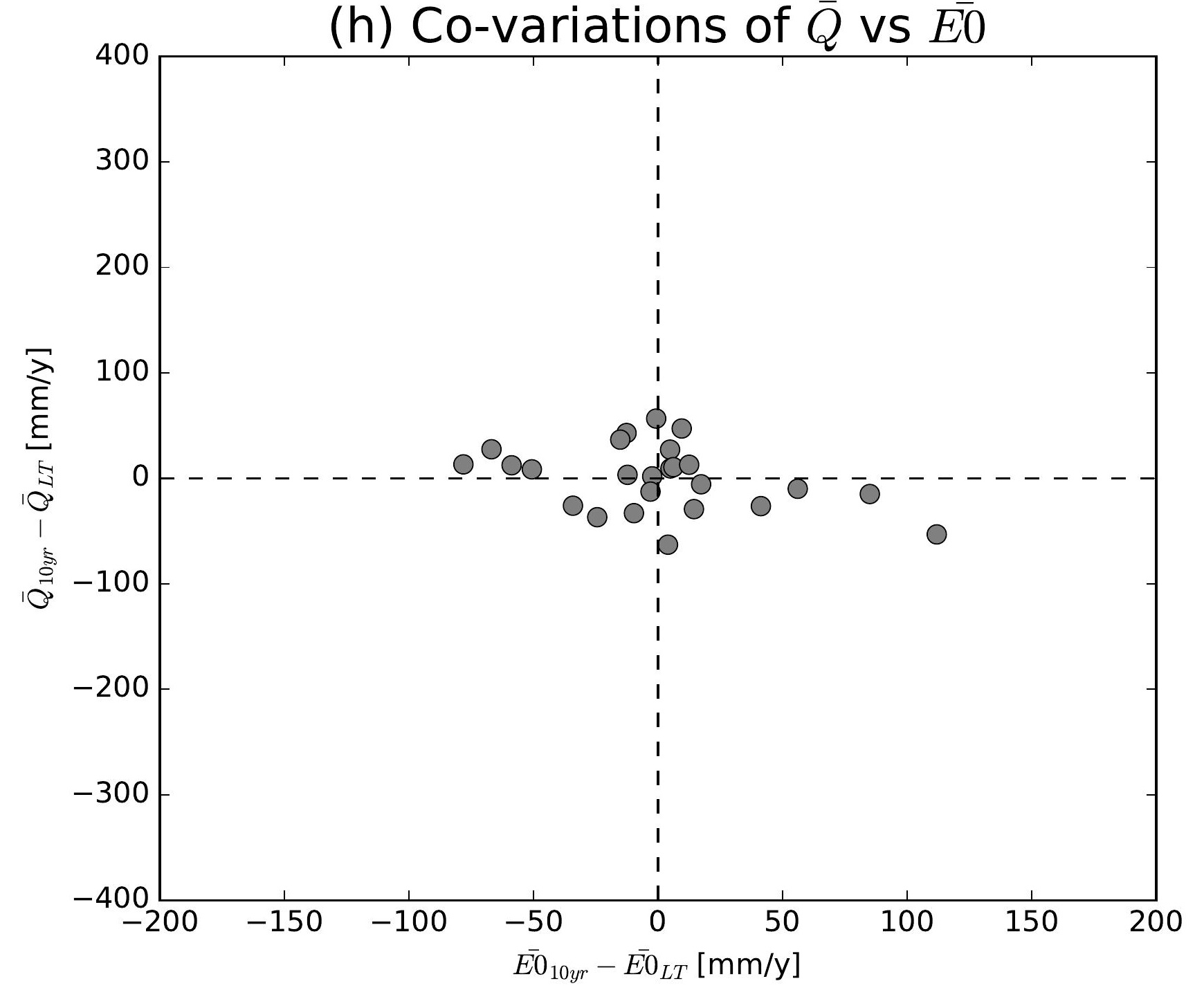

(k) Co-variations of $\bar{Q}$ vs $\overline{E 0}$

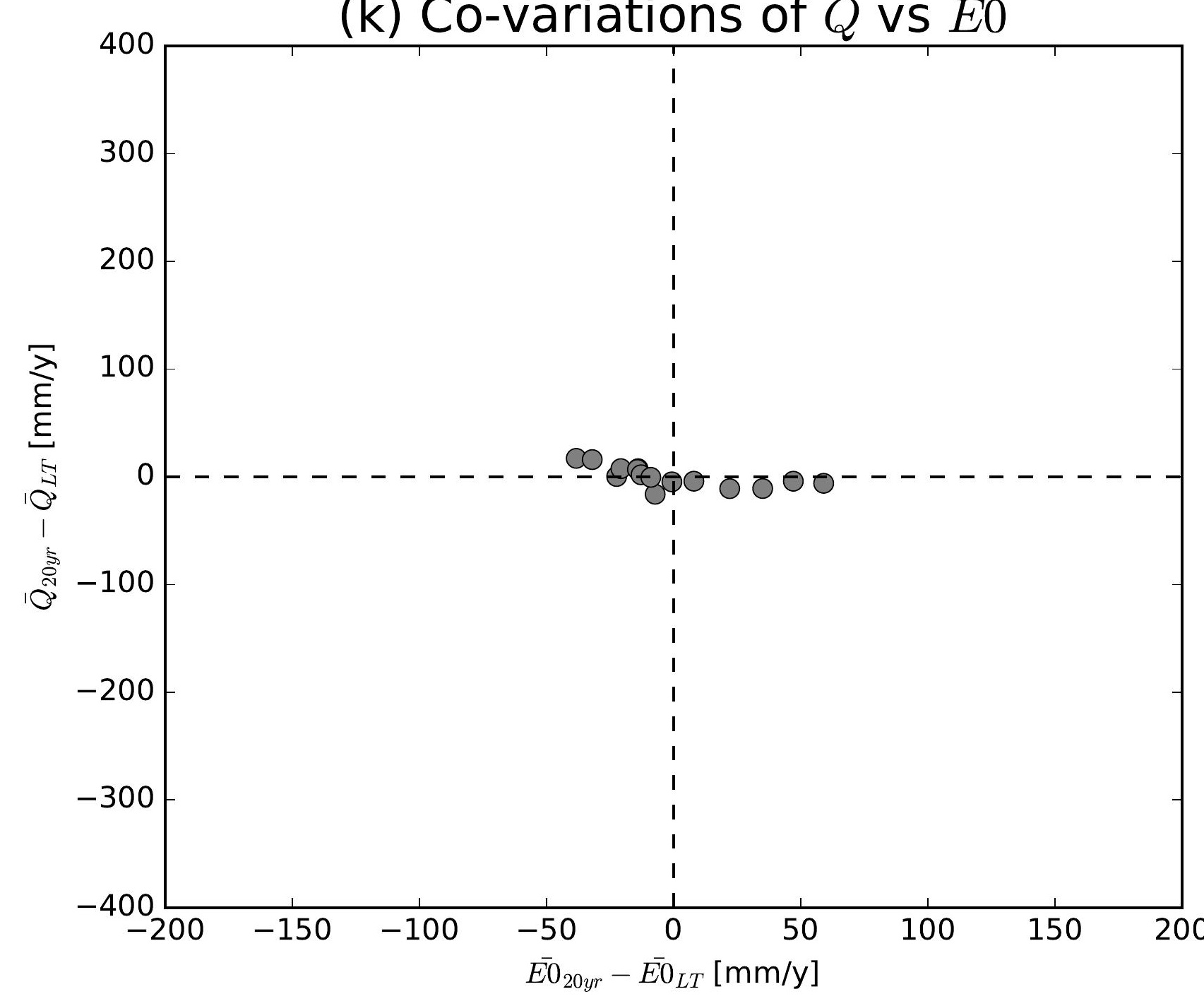

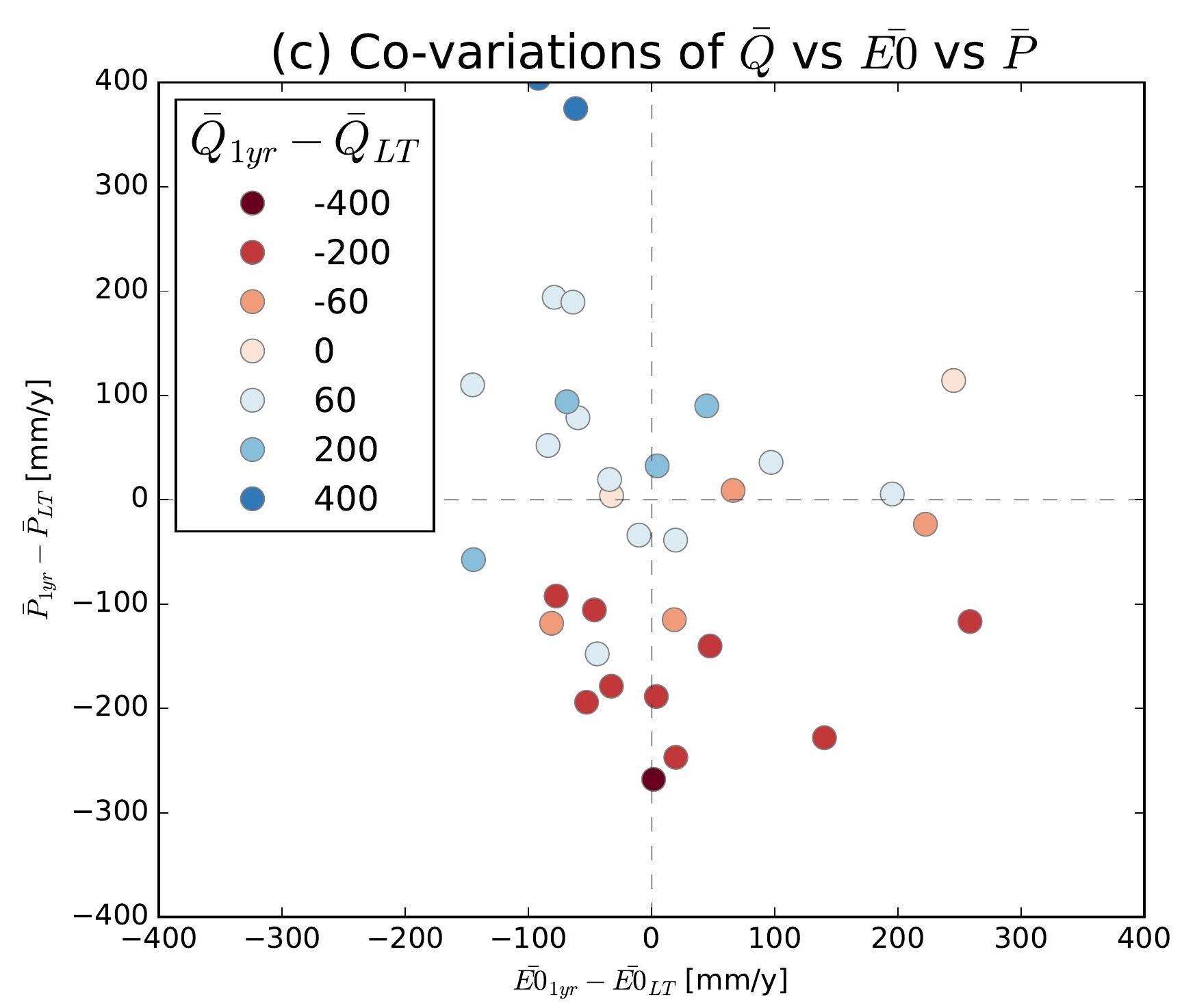
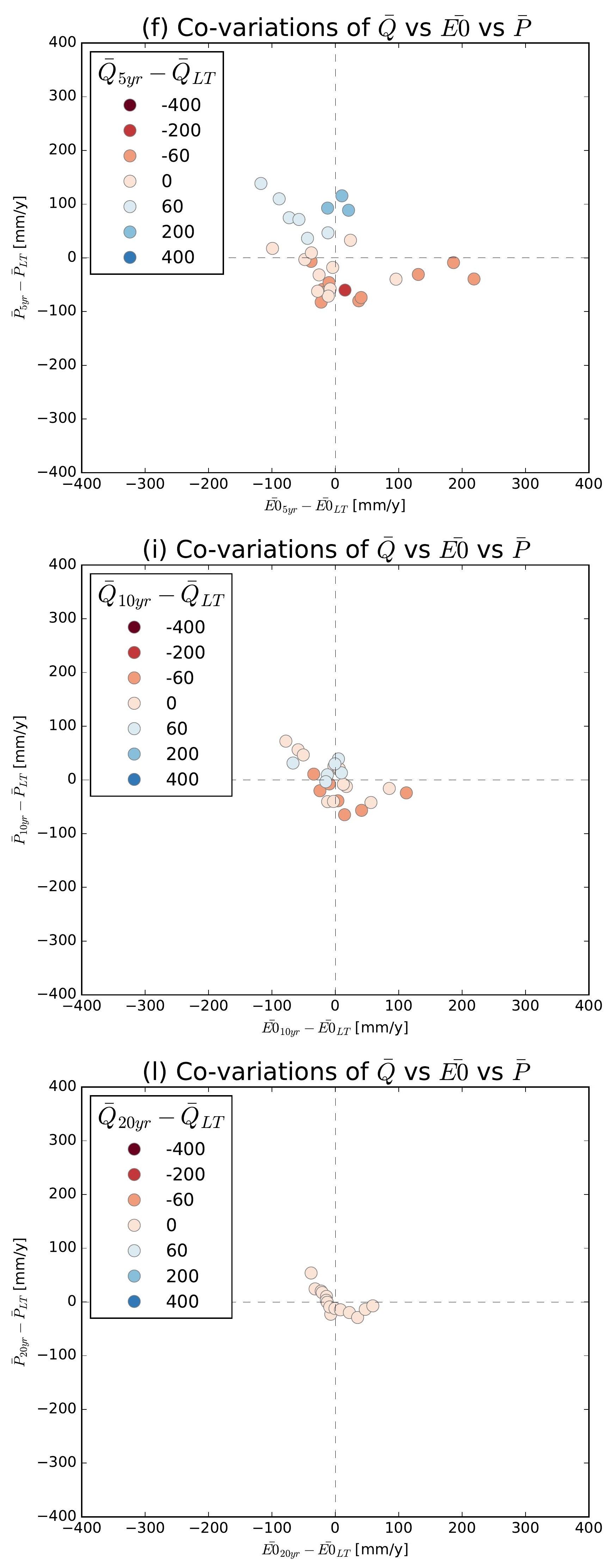

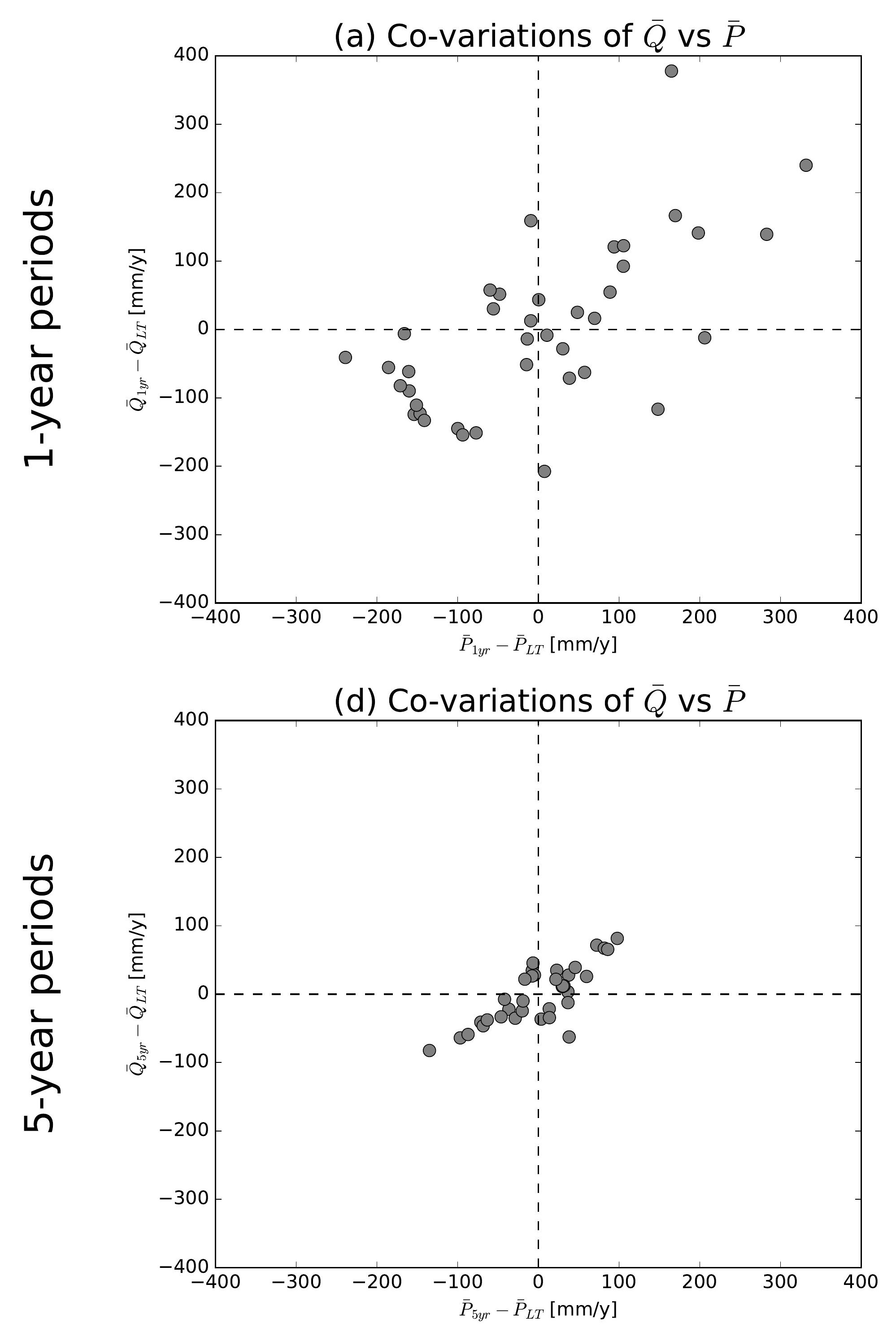

(g) Co-variations of $\bar{Q}$ vs $\bar{P}$

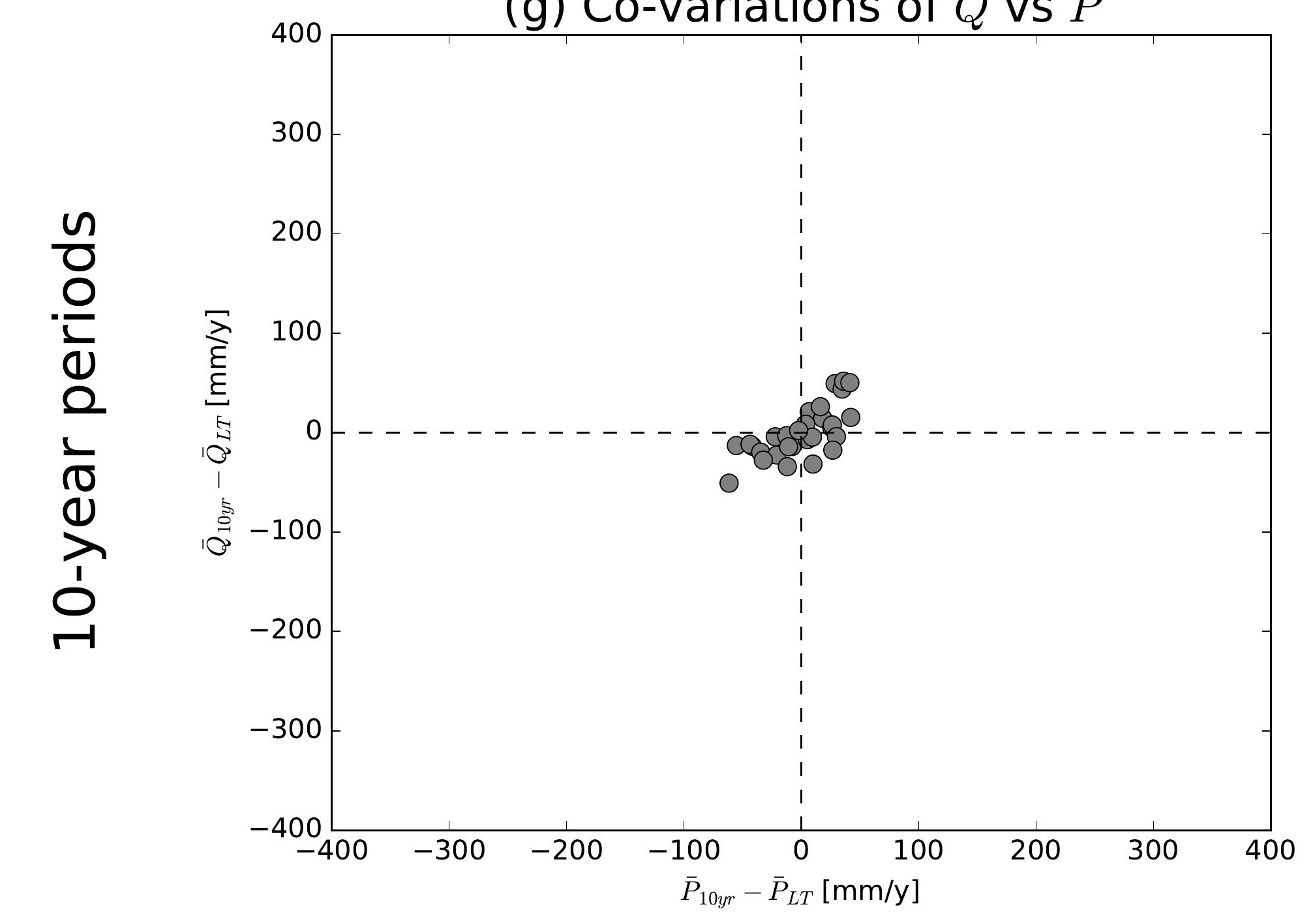

(j) Co-variations of $\bar{Q}$ vs $\bar{P}$

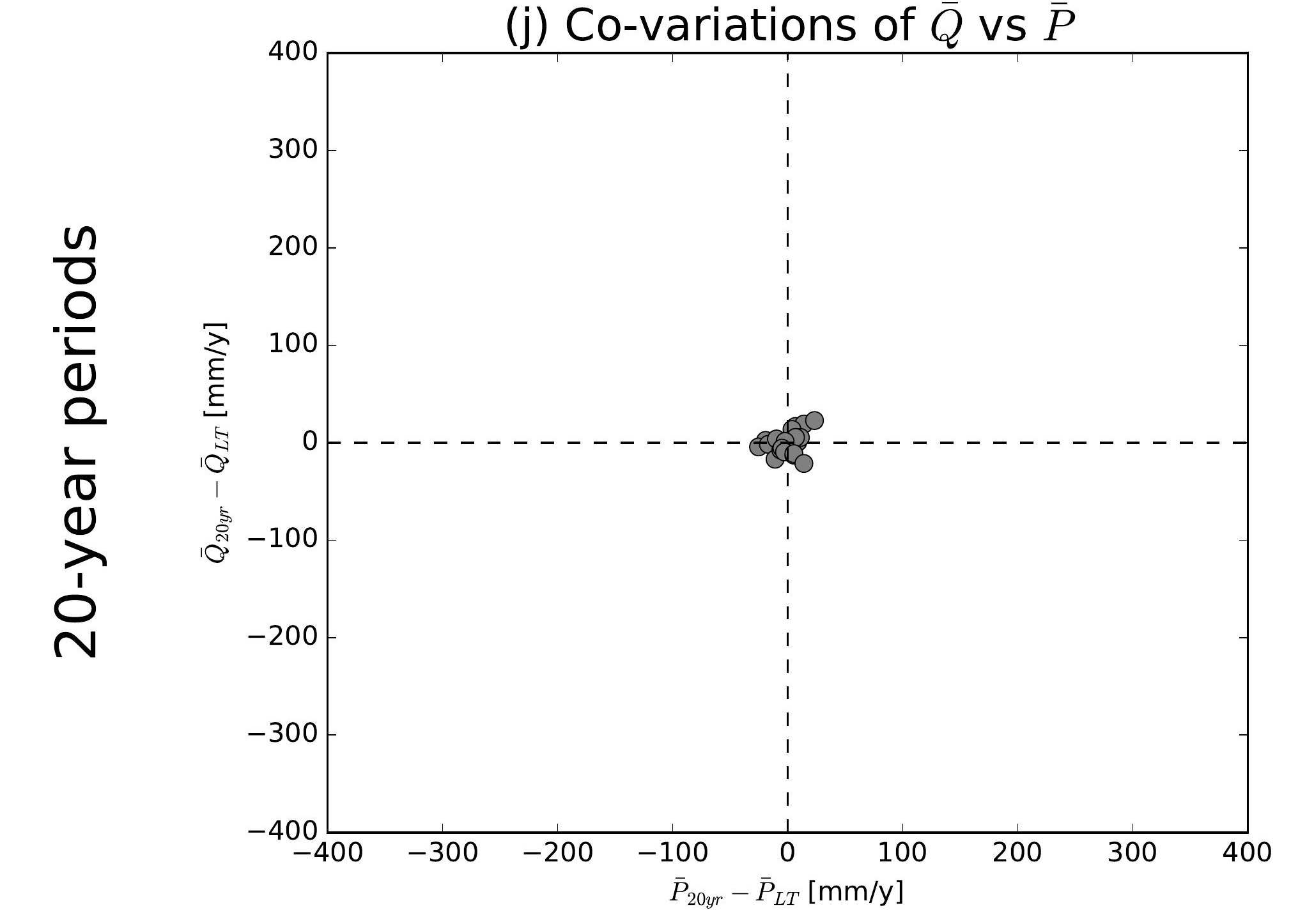

(b) Co-variations of $\bar{Q}$ vs $\overline{E 0}$

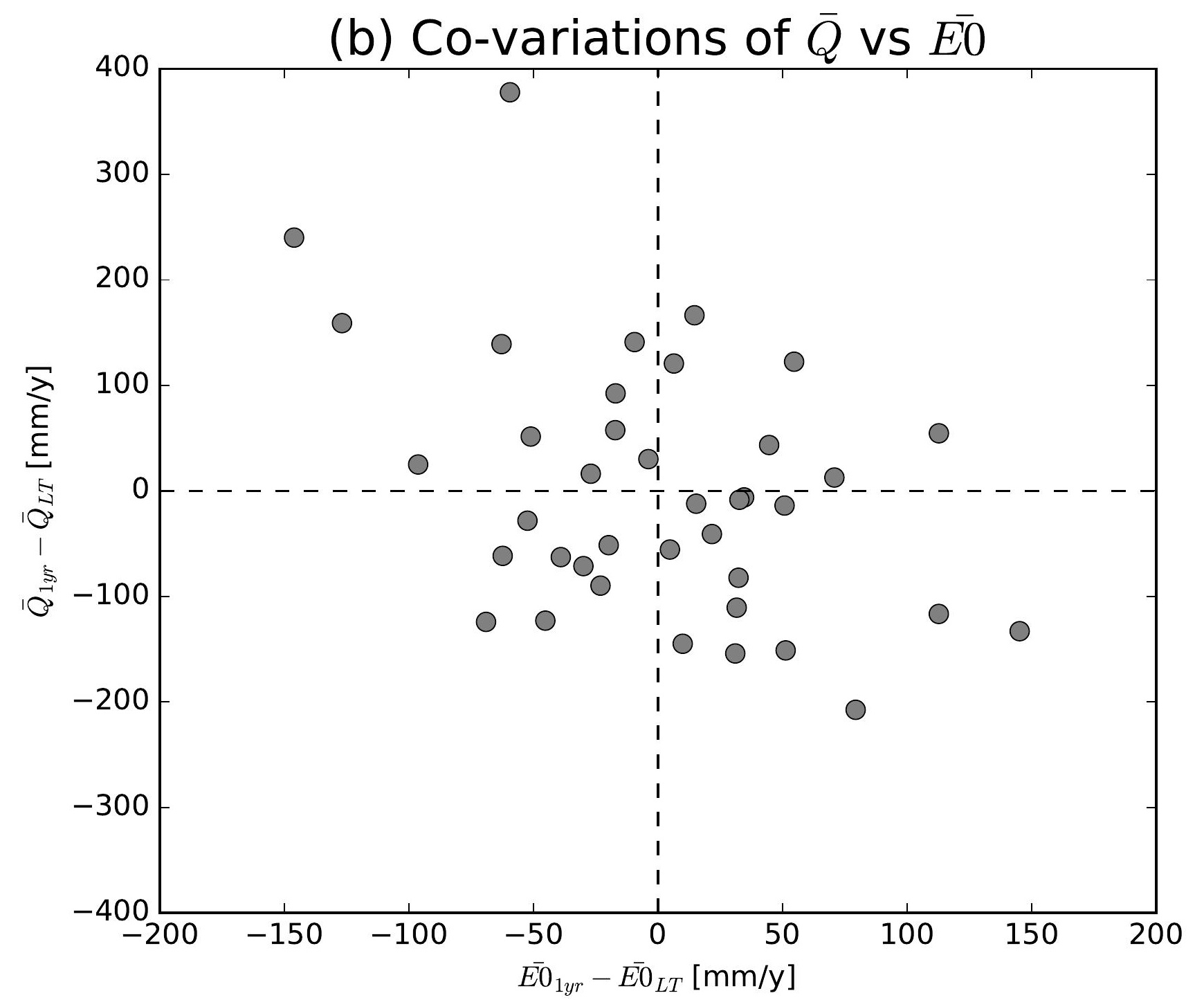

(e) Co-variations of $\bar{Q}$ vs $\overline{E 0}$

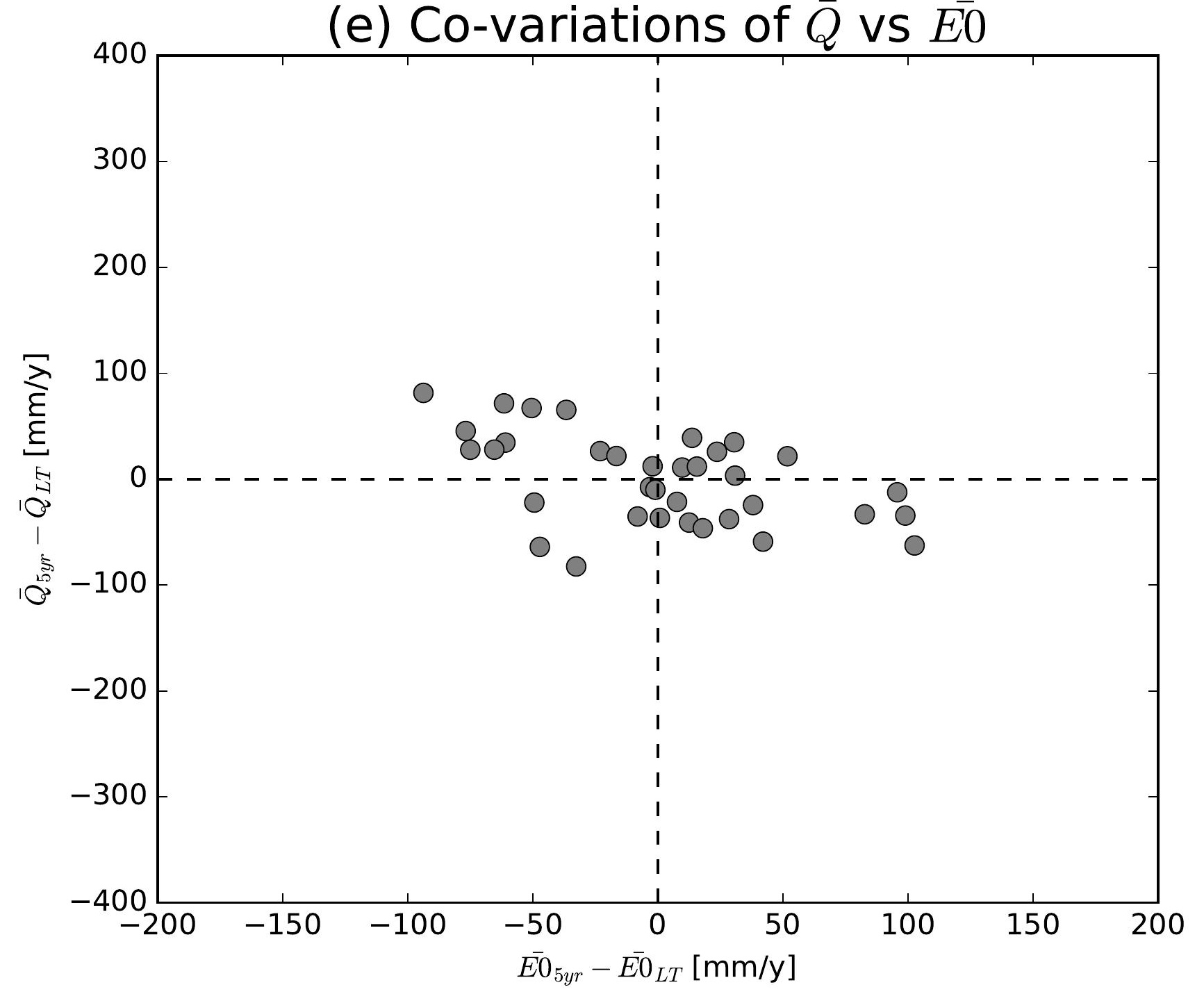

(h) Co-variations of $\bar{Q}$ vs $\overline{E 0}$

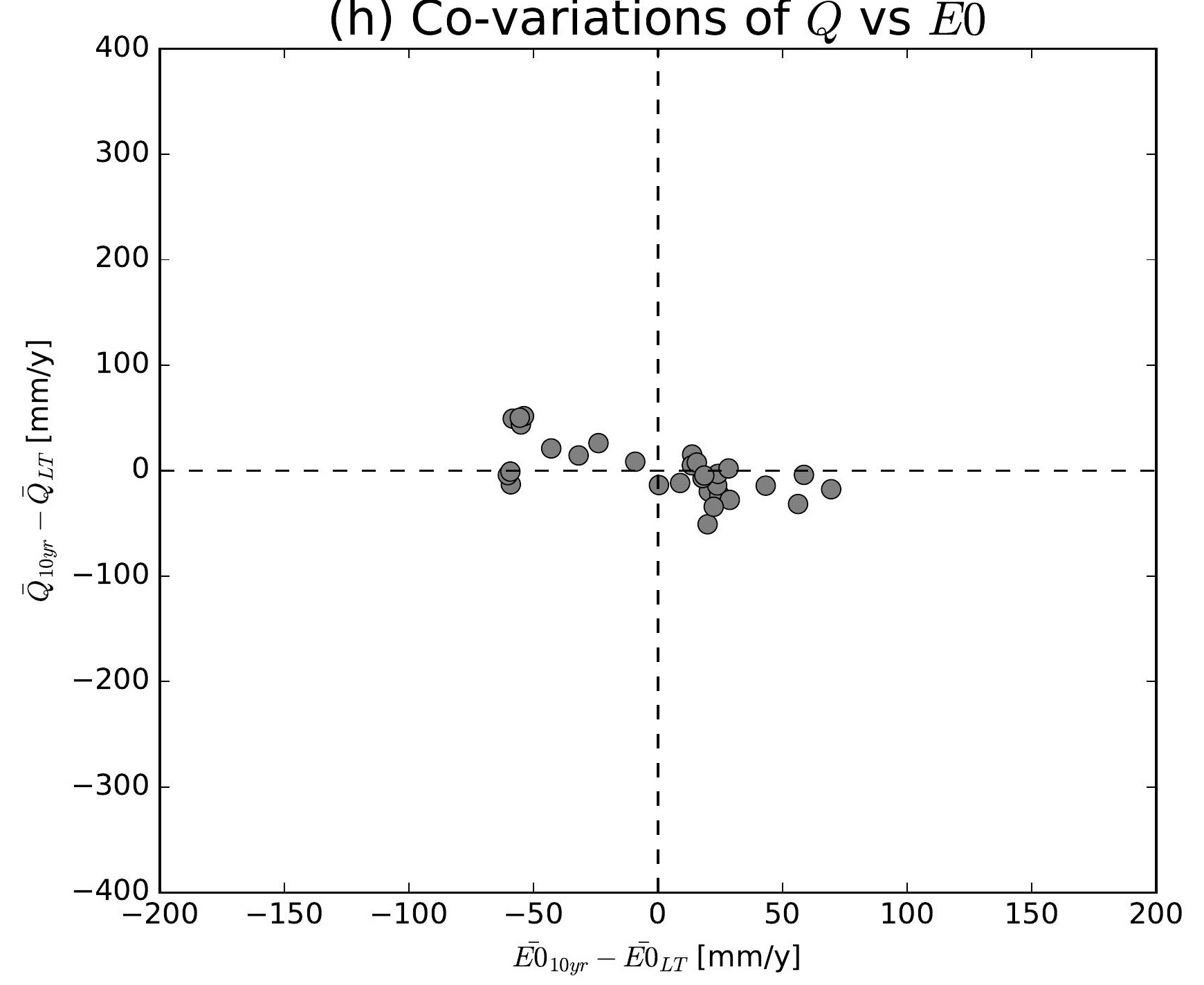

(k) Co-variations of $\bar{Q}$ vs $\overline{E 0}$

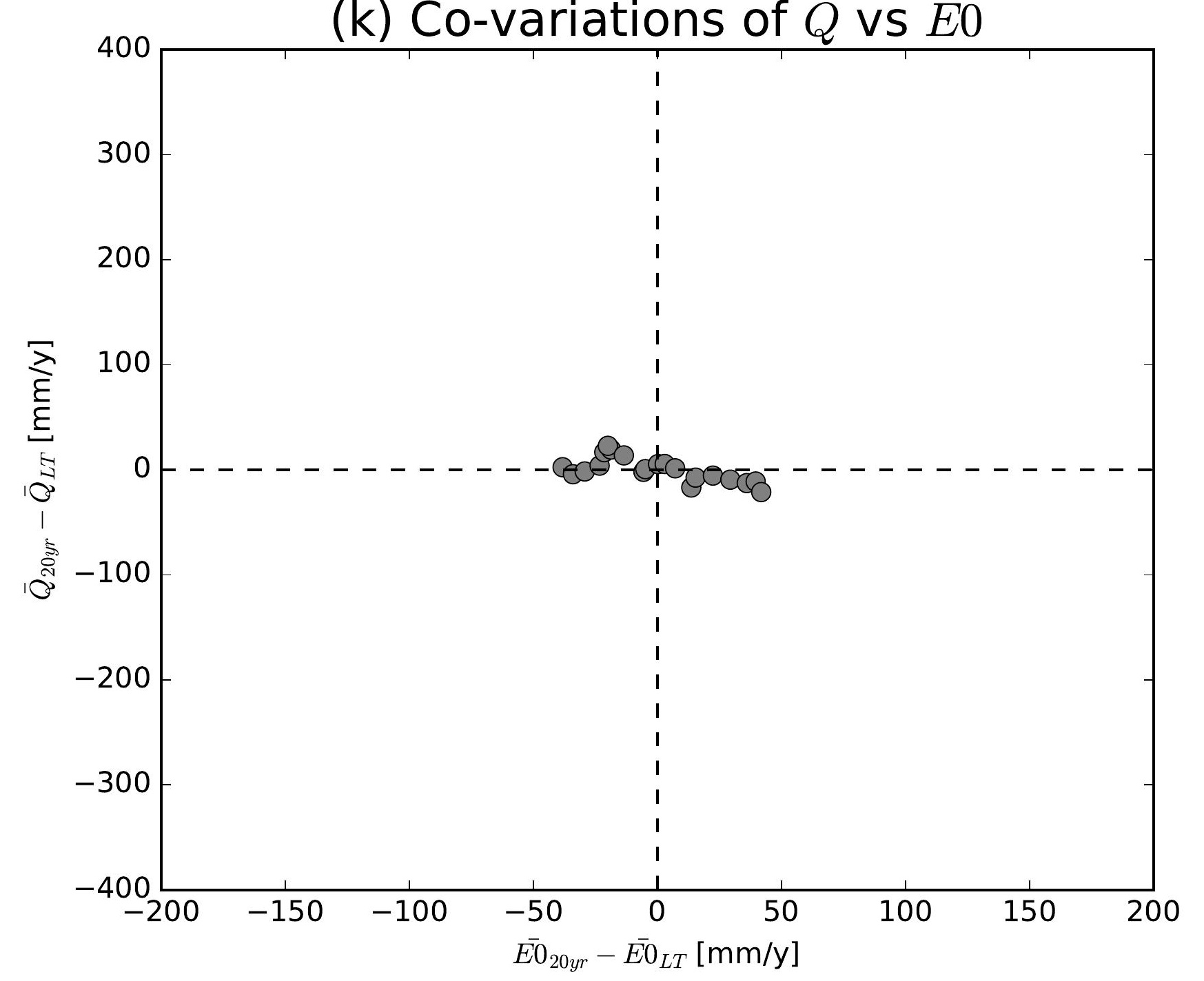

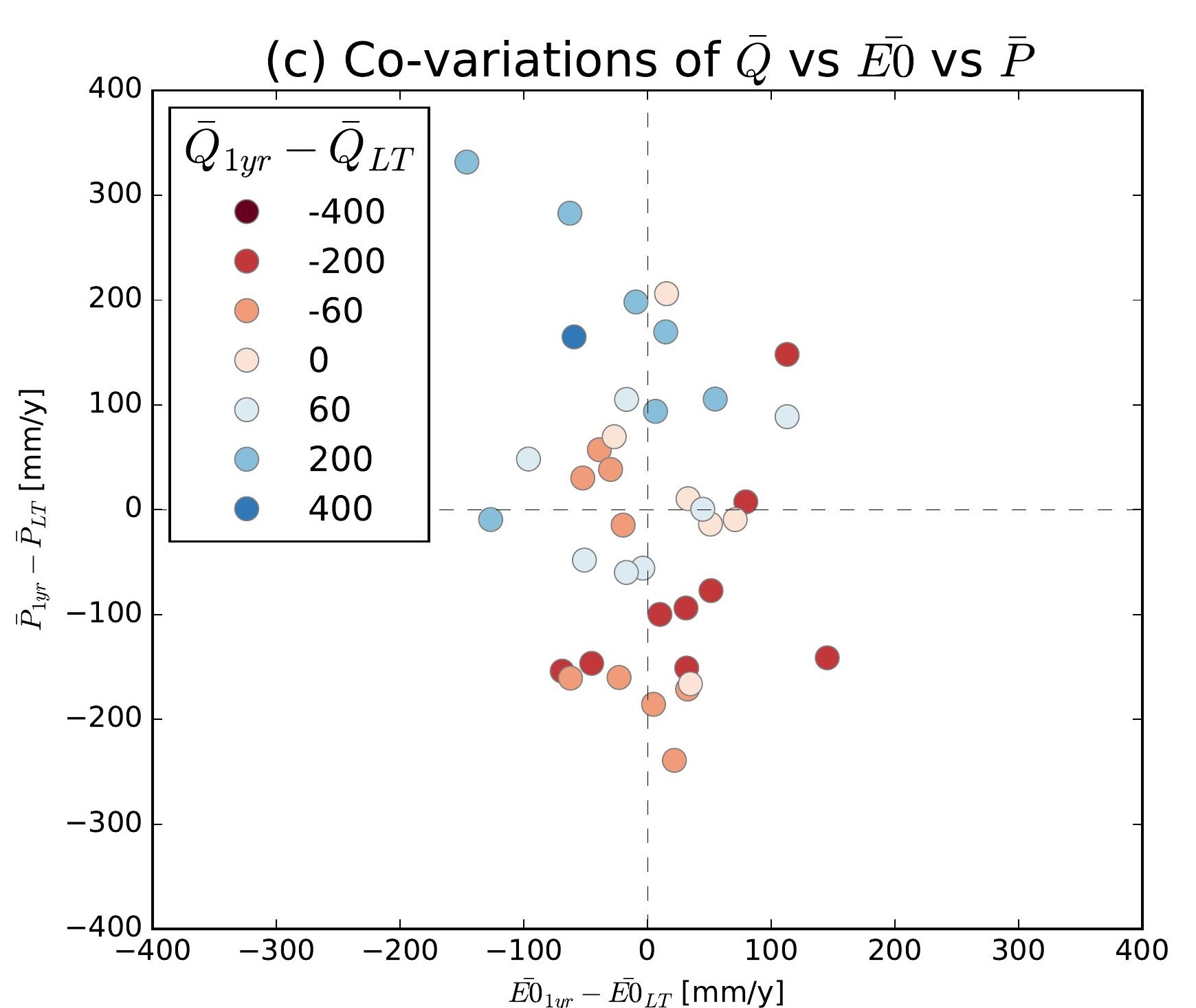
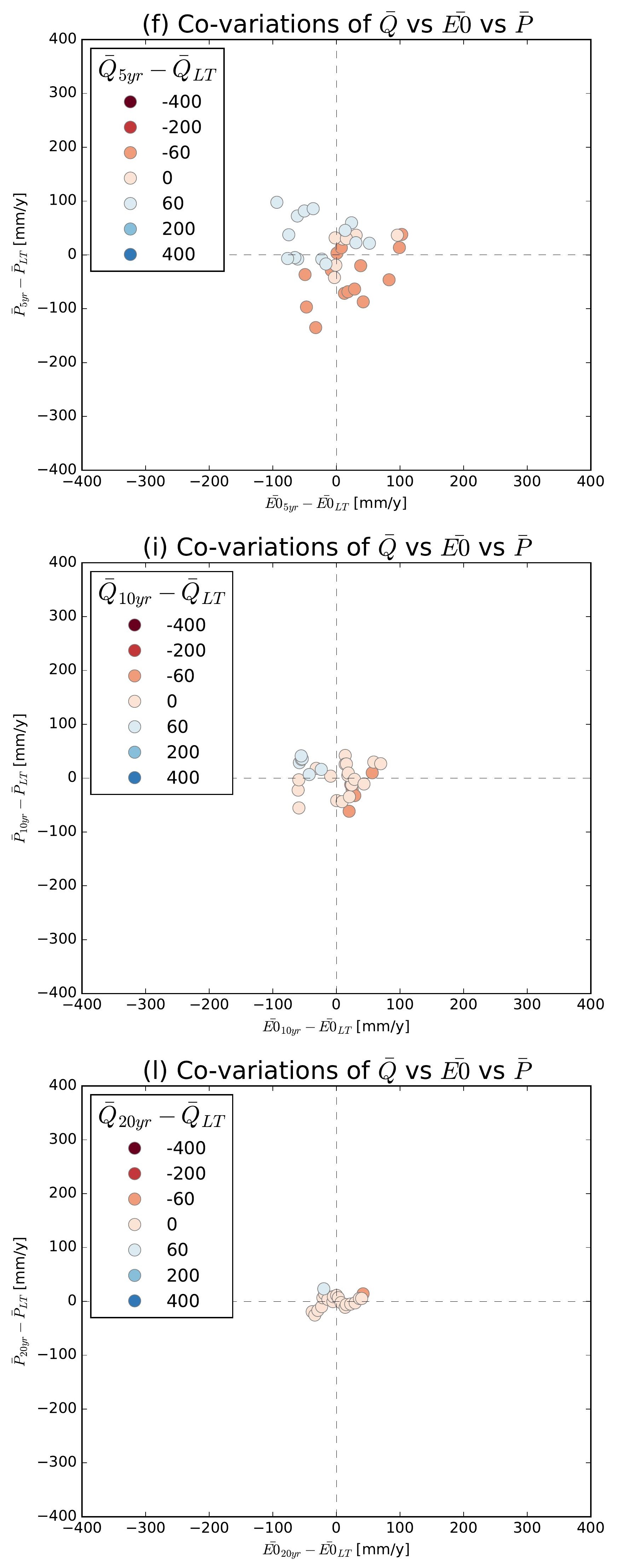

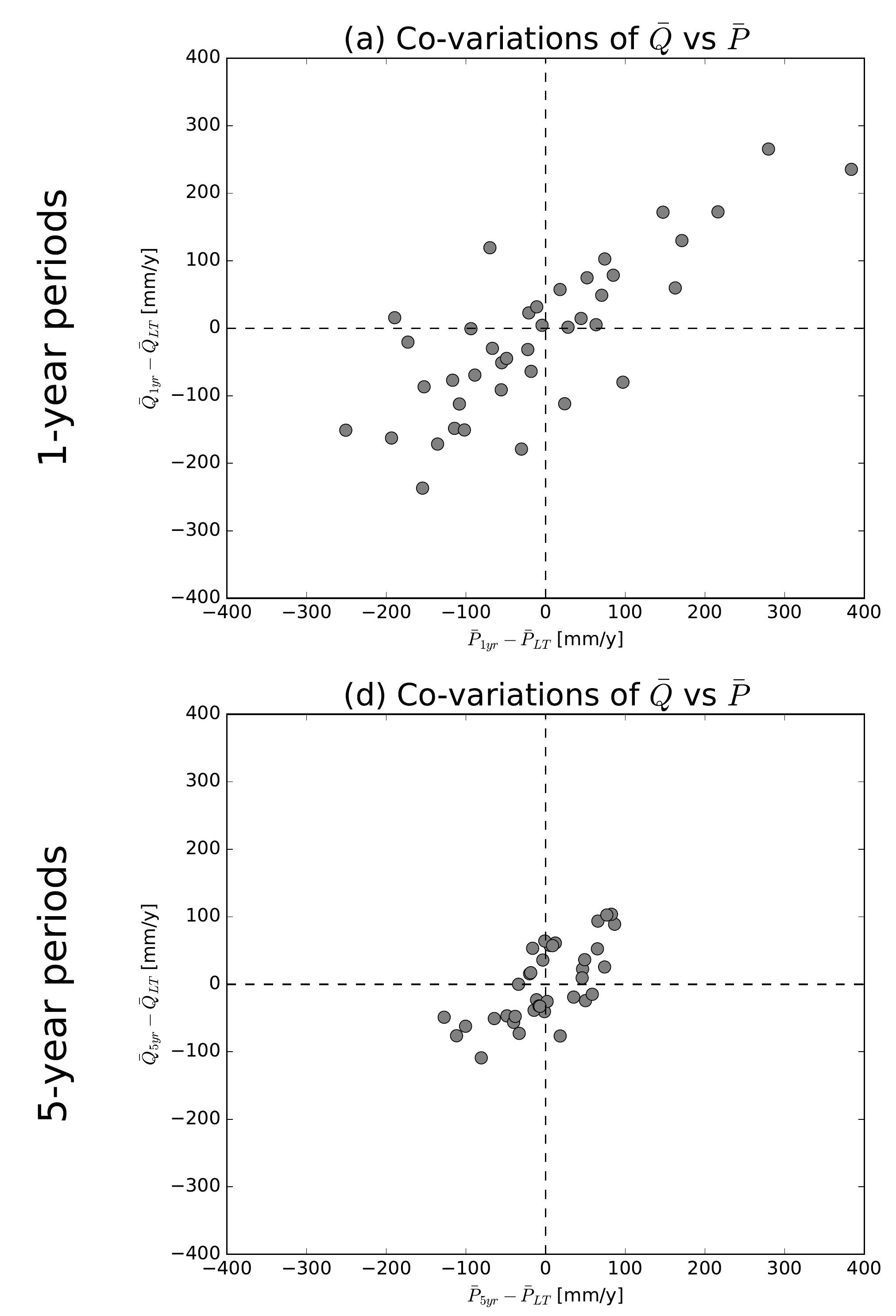

(g) Co-variations of $\bar{Q}$ vs $\bar{P}$

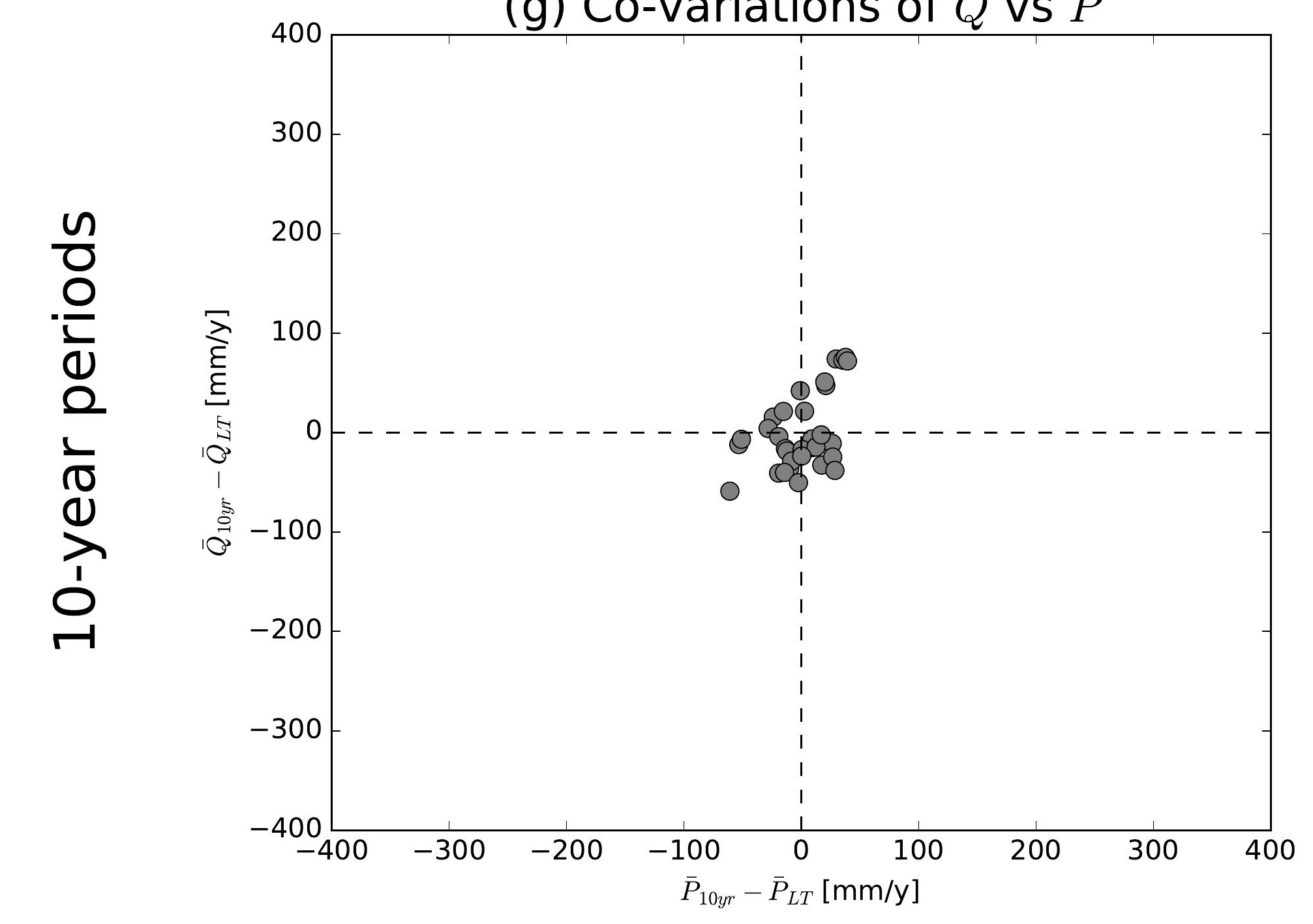

(j) Co-variations of $\bar{Q}$ vs $\bar{P}$

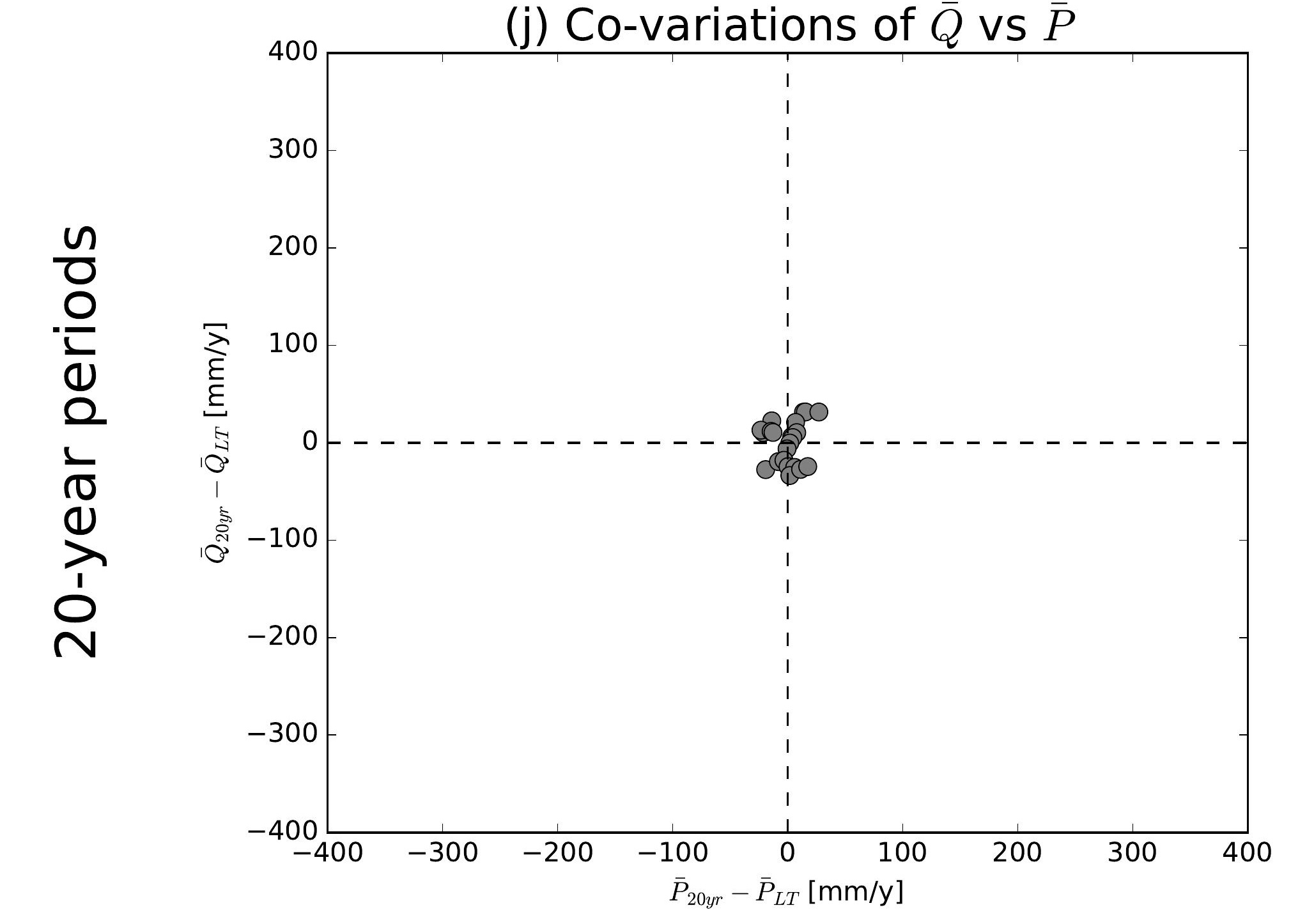

(b) Co-variations of $\bar{Q}$ vs $\overline{E 0}$

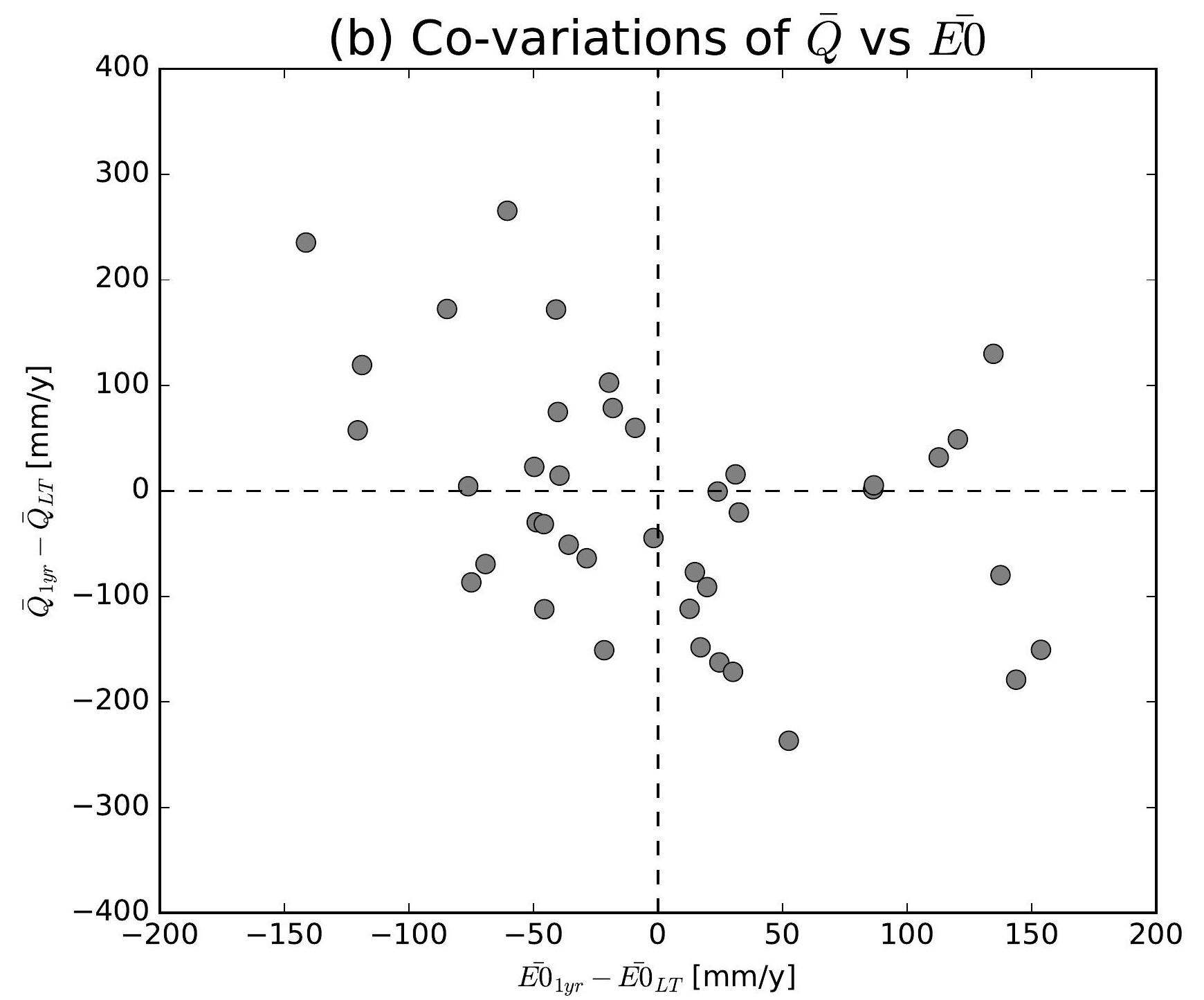

(e) Co-variations of $\bar{Q}$ vs $\overline{E 0}$

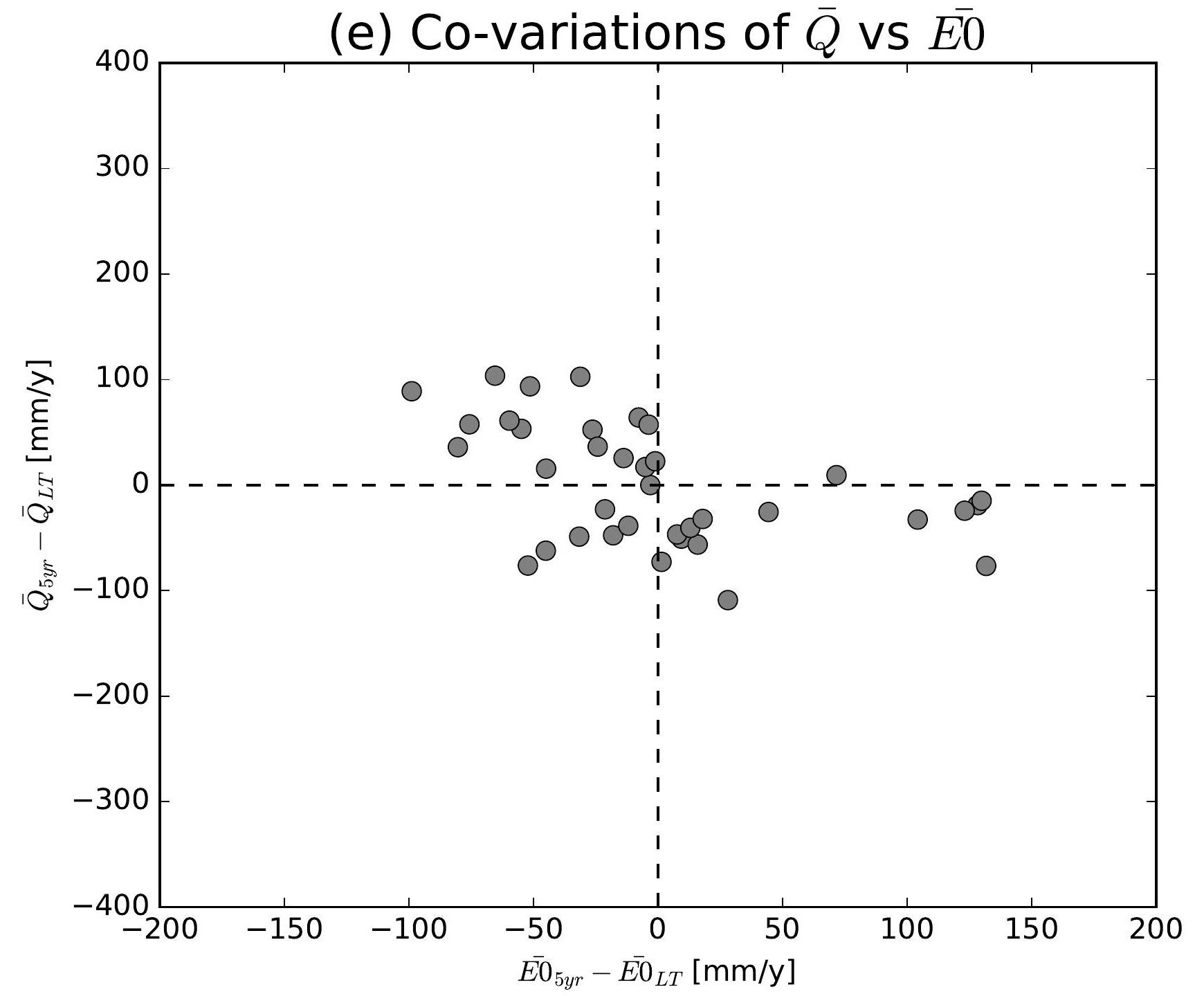

(h) Co-variations of $\bar{Q}$ vs $\overline{E 0}$

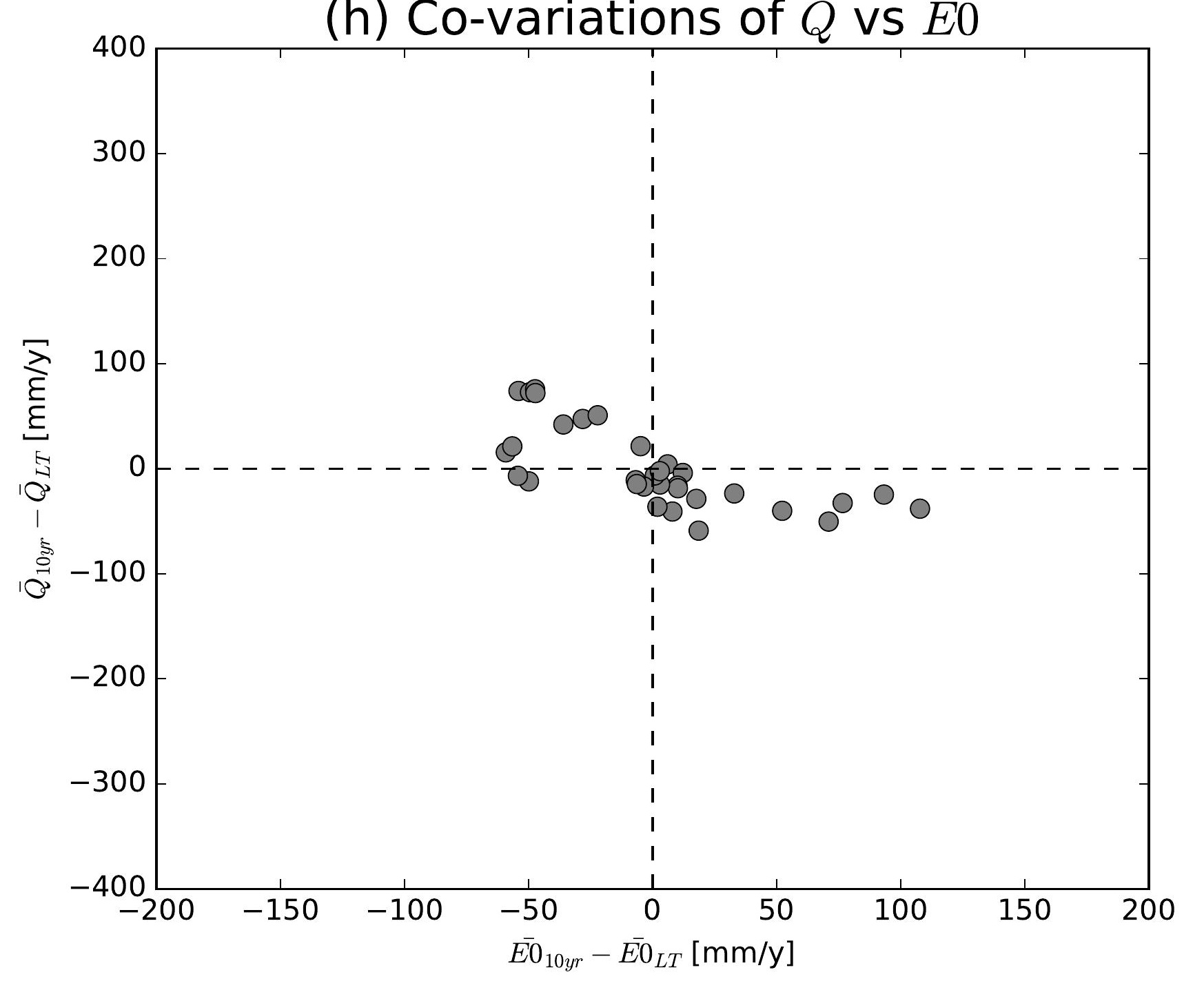

(k) Co-variations of $\bar{Q}$ vs $\overline{E 0}$

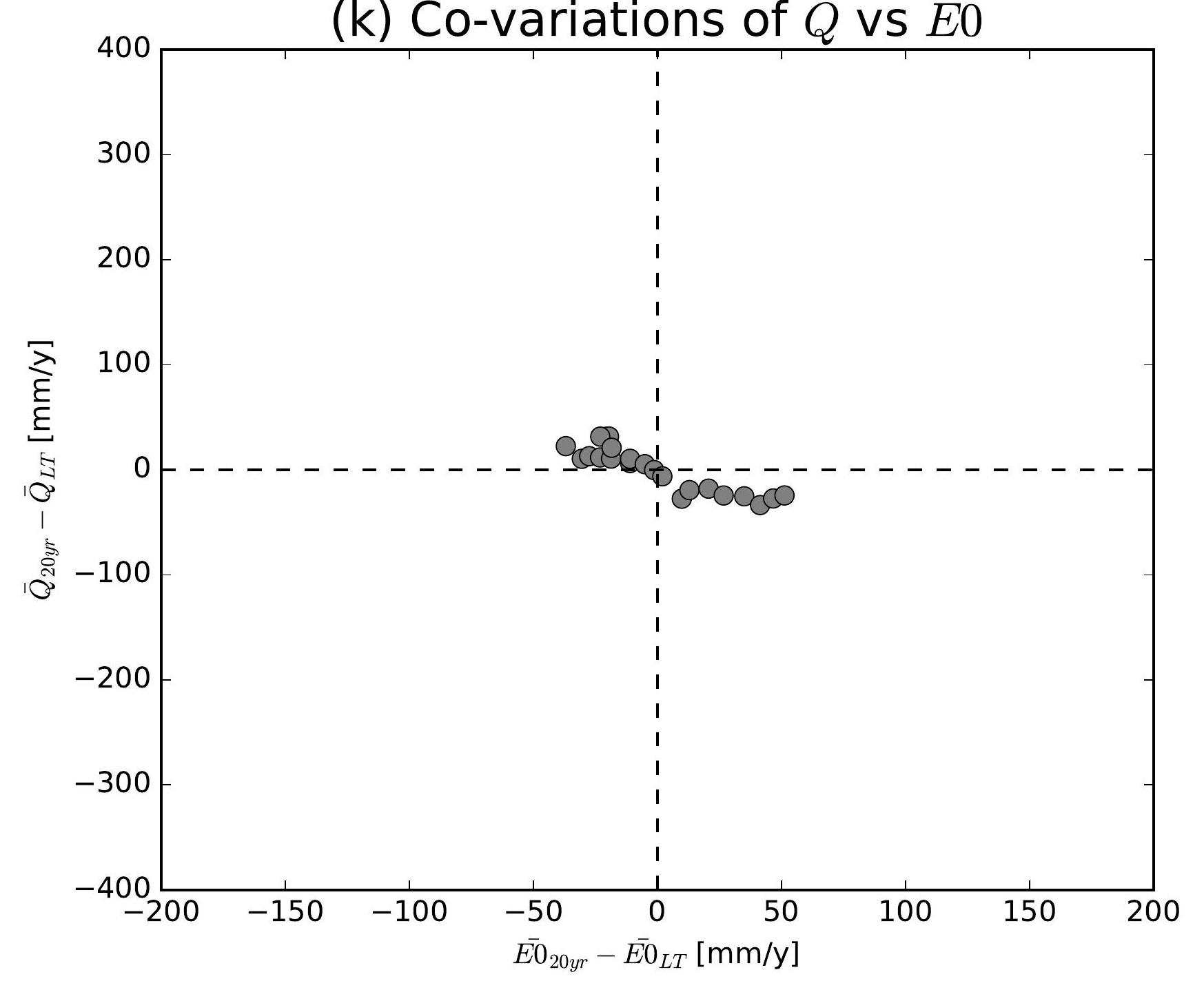

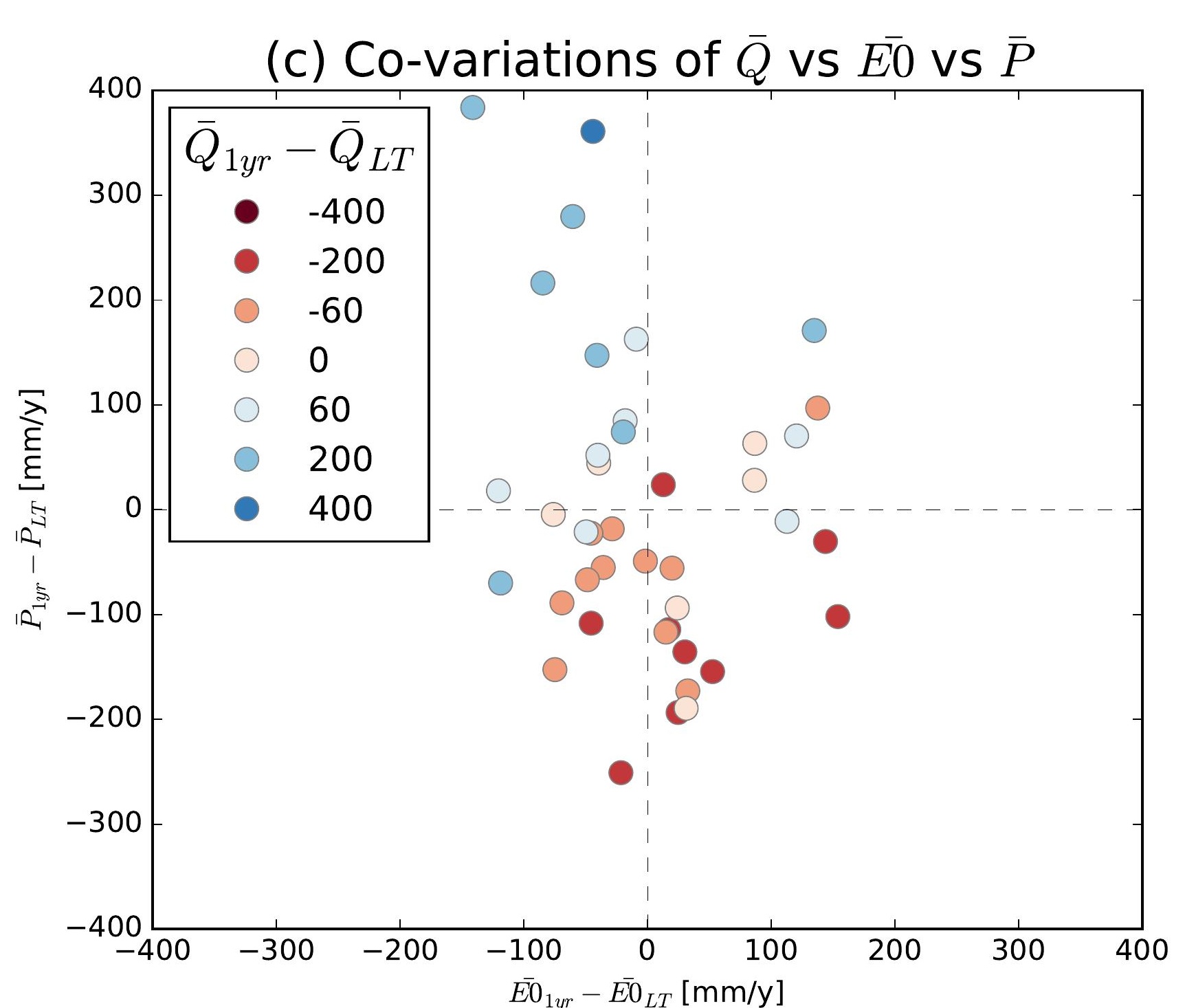
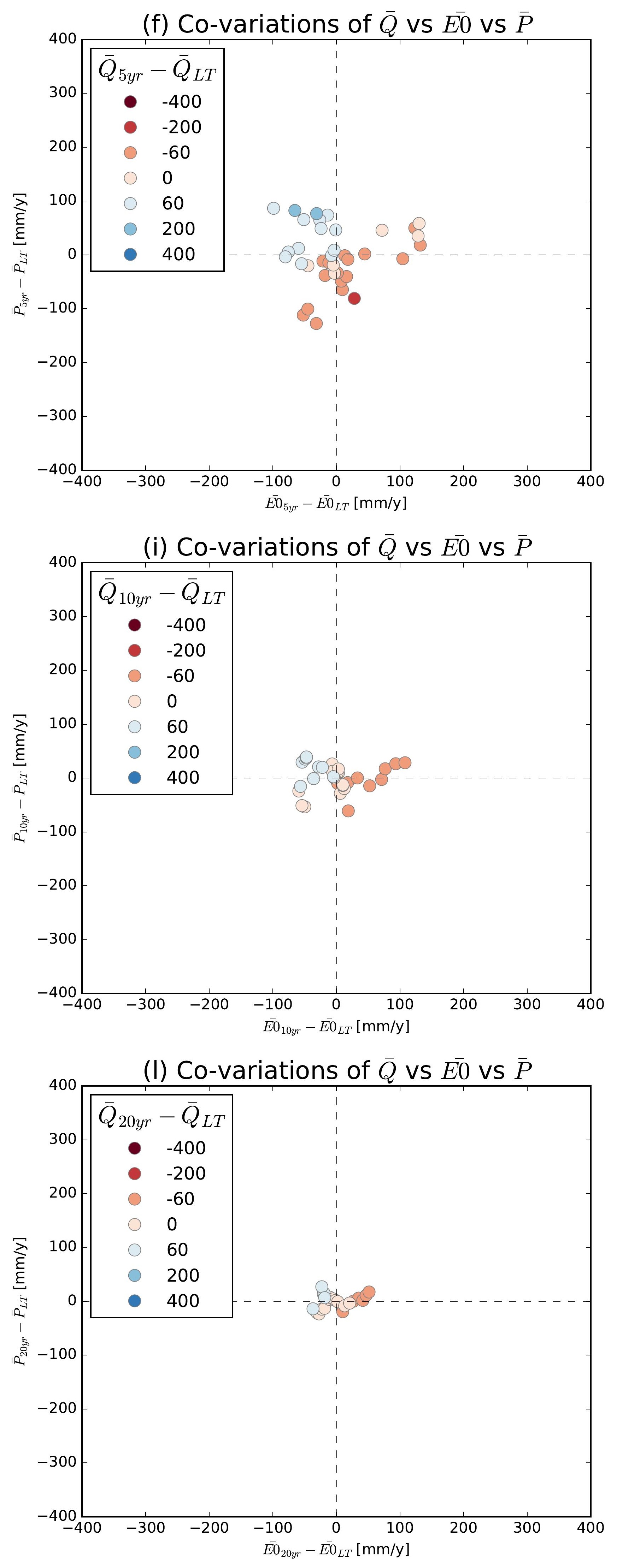

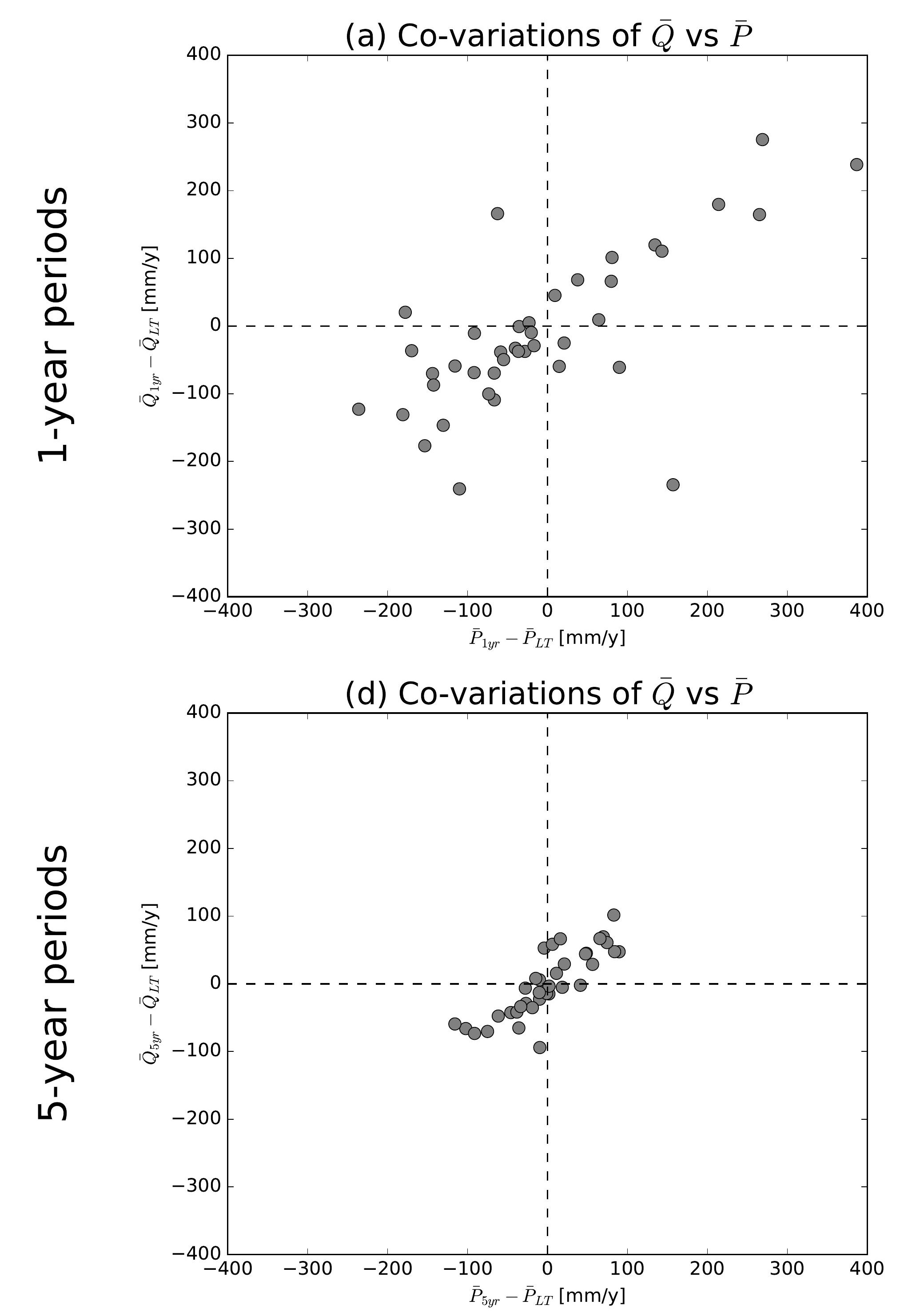

(g) Co-variations of $\bar{Q}$ vs $\bar{P}$

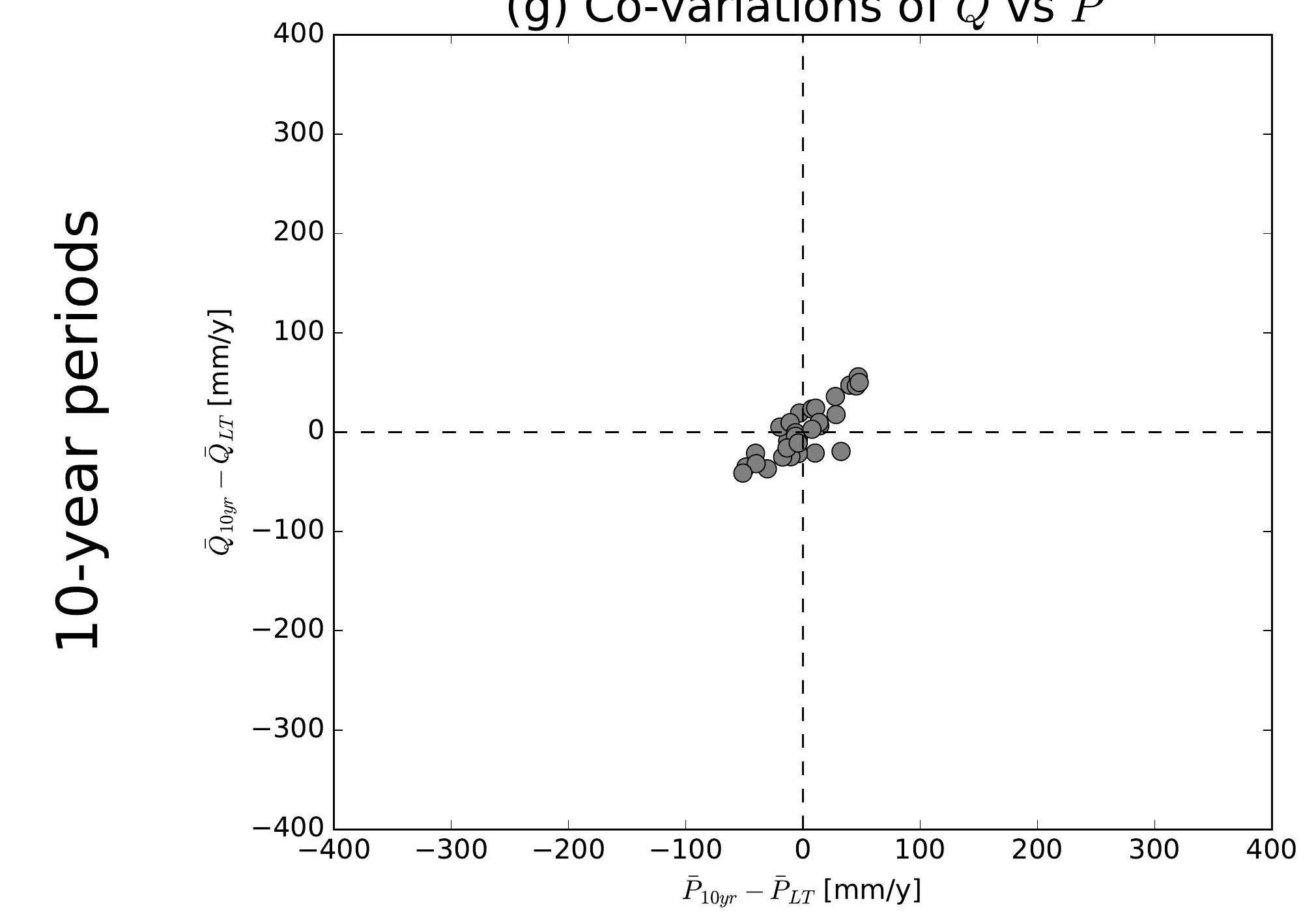

(j) Co-variations of $\bar{Q}$ vs $\bar{P}$

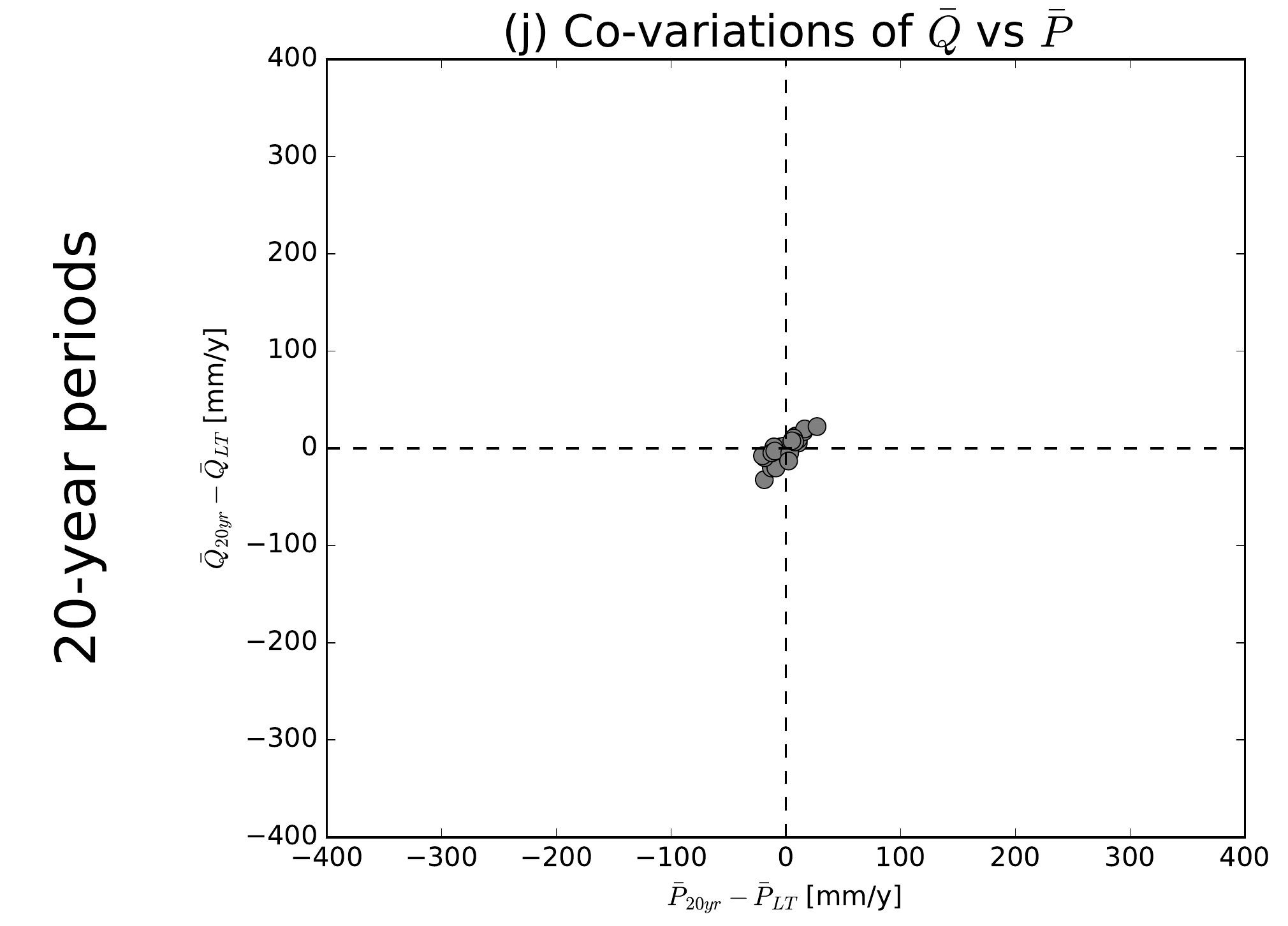

(b) Co-variations of $\bar{Q}$ vs $\overline{E 0}$

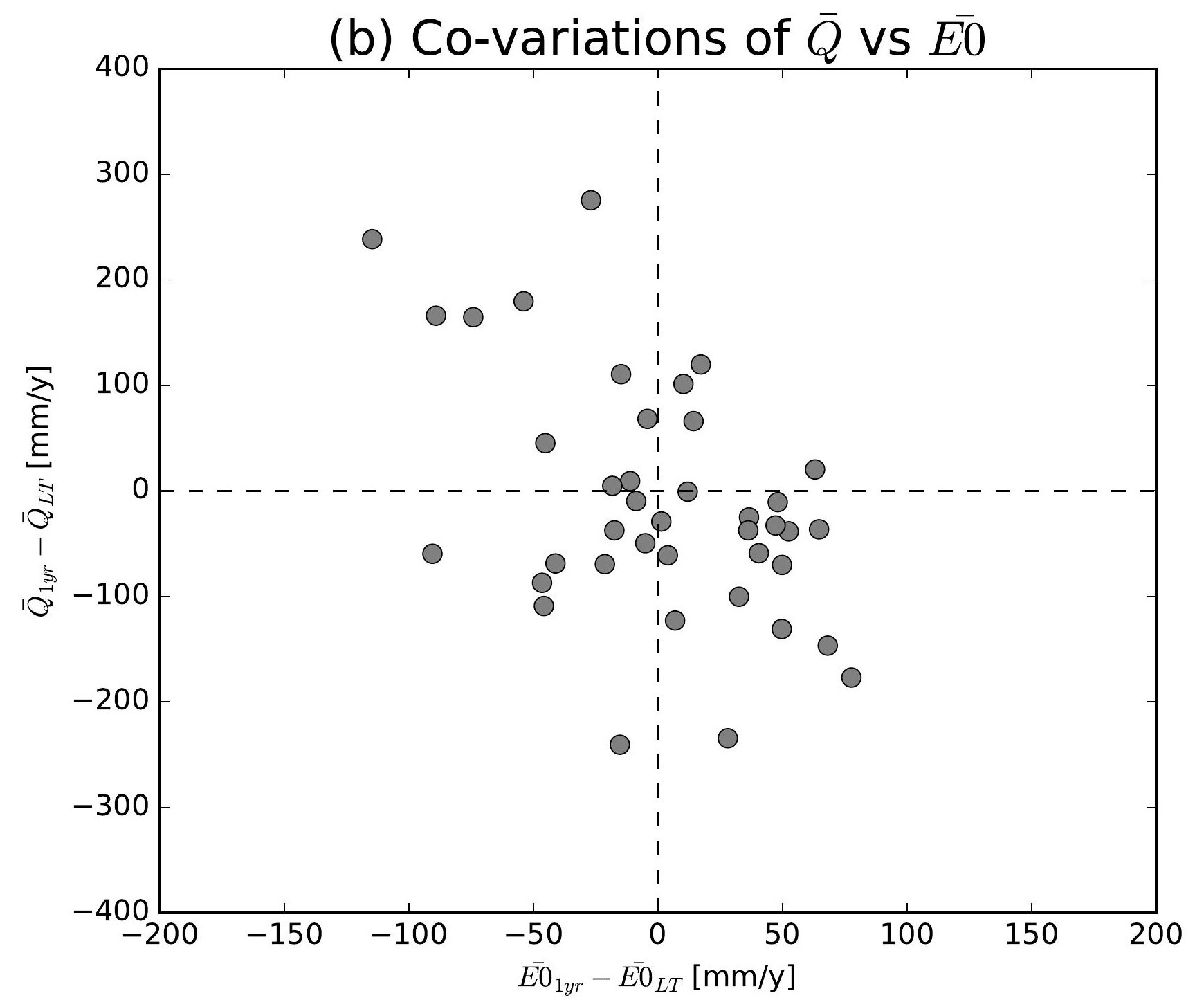

(e) Co-variations of $\bar{Q}$ vs $\overline{E 0}$

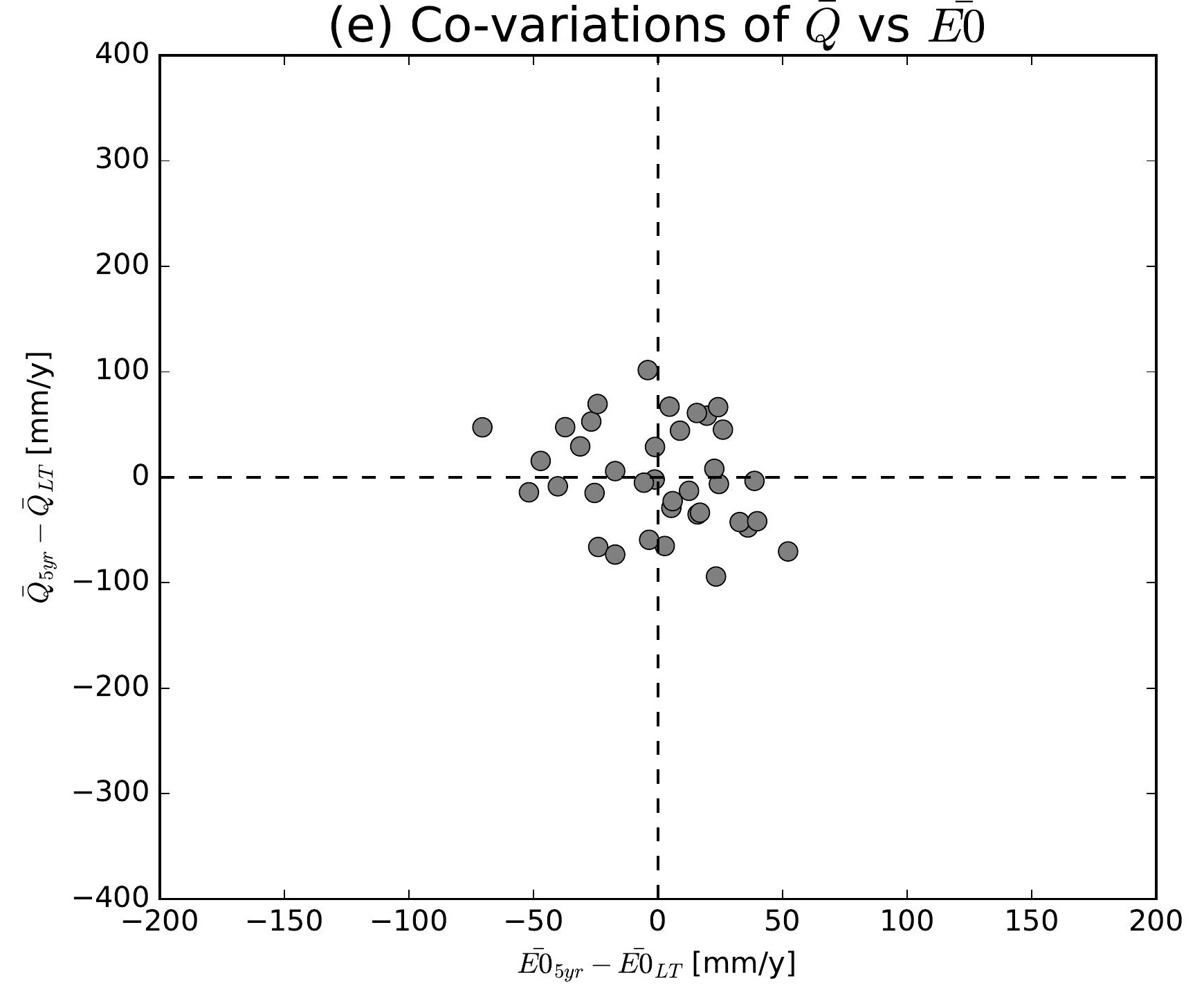

(h) Co-variations of $\bar{Q}$ vs $\overline{E 0}$

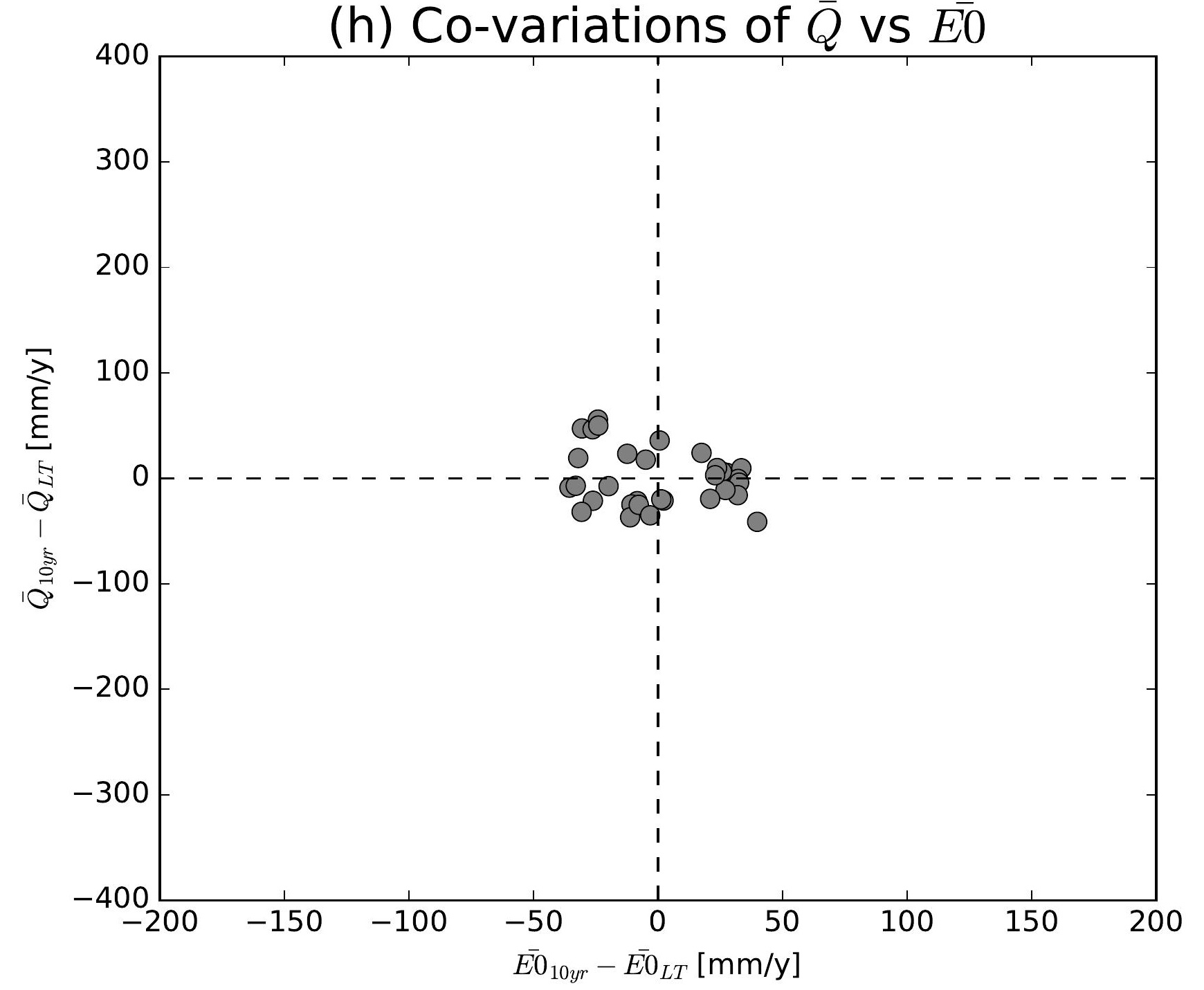

(k) Co-variations of $\bar{Q}$ vs $\overline{E 0}$

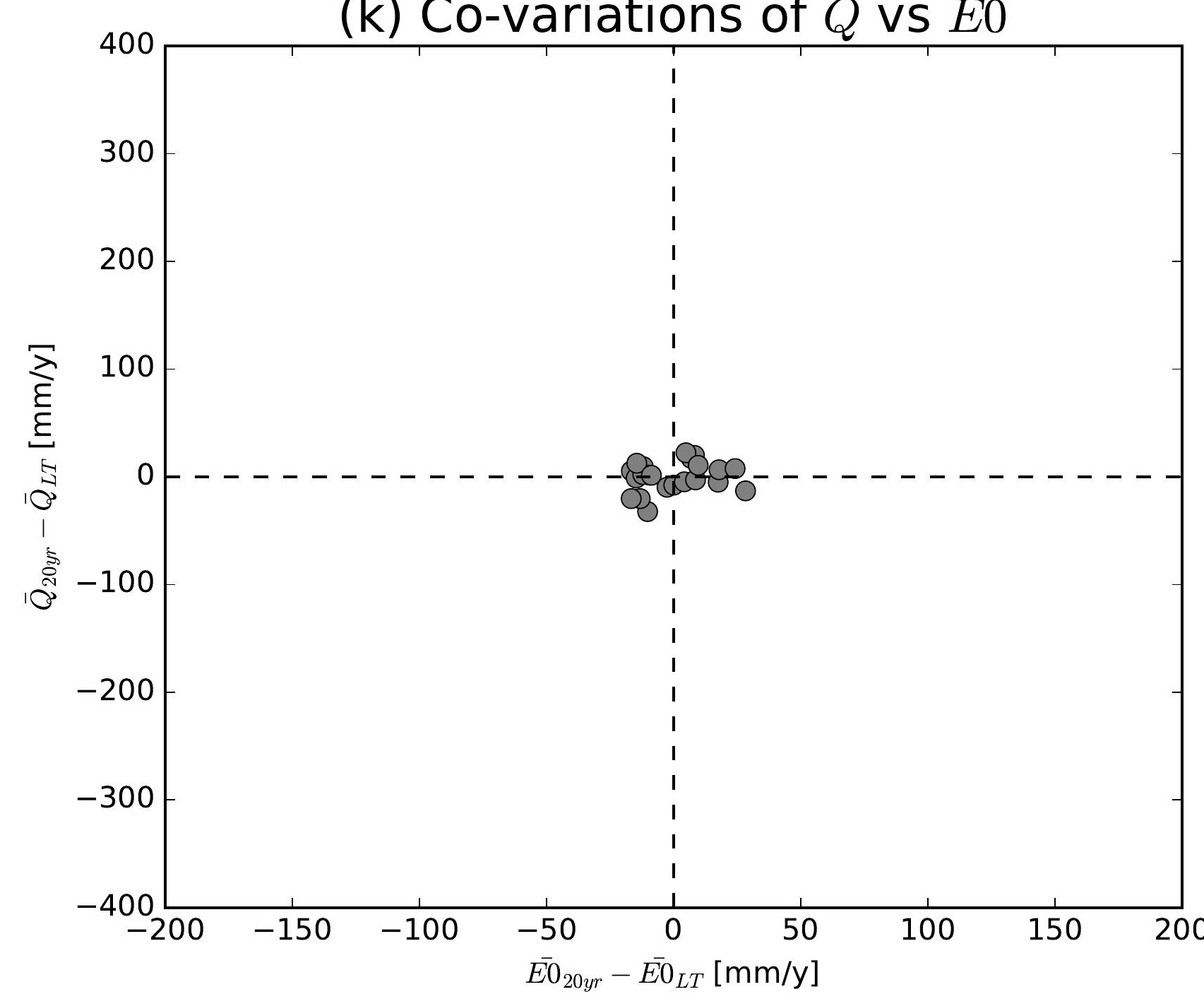

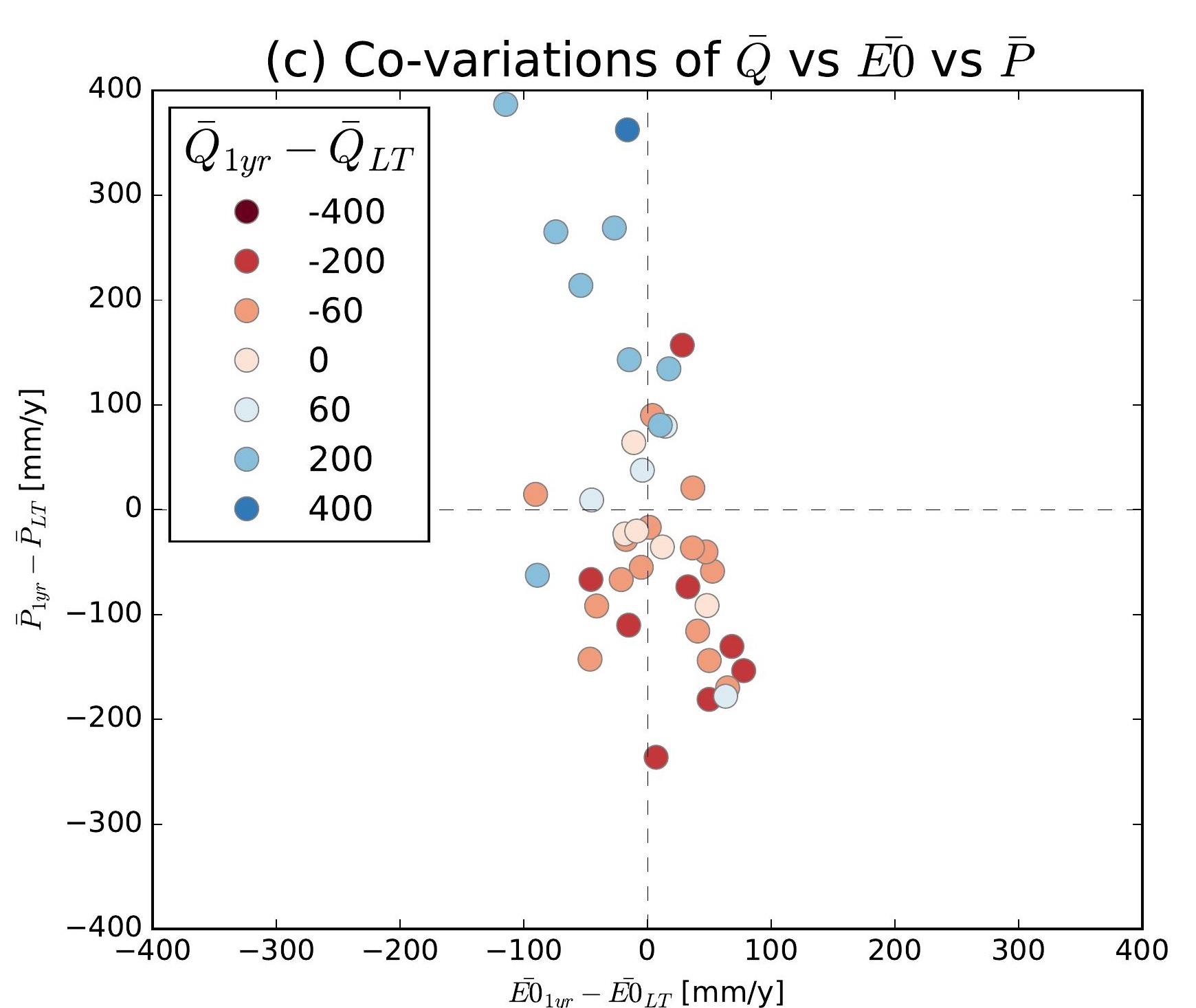
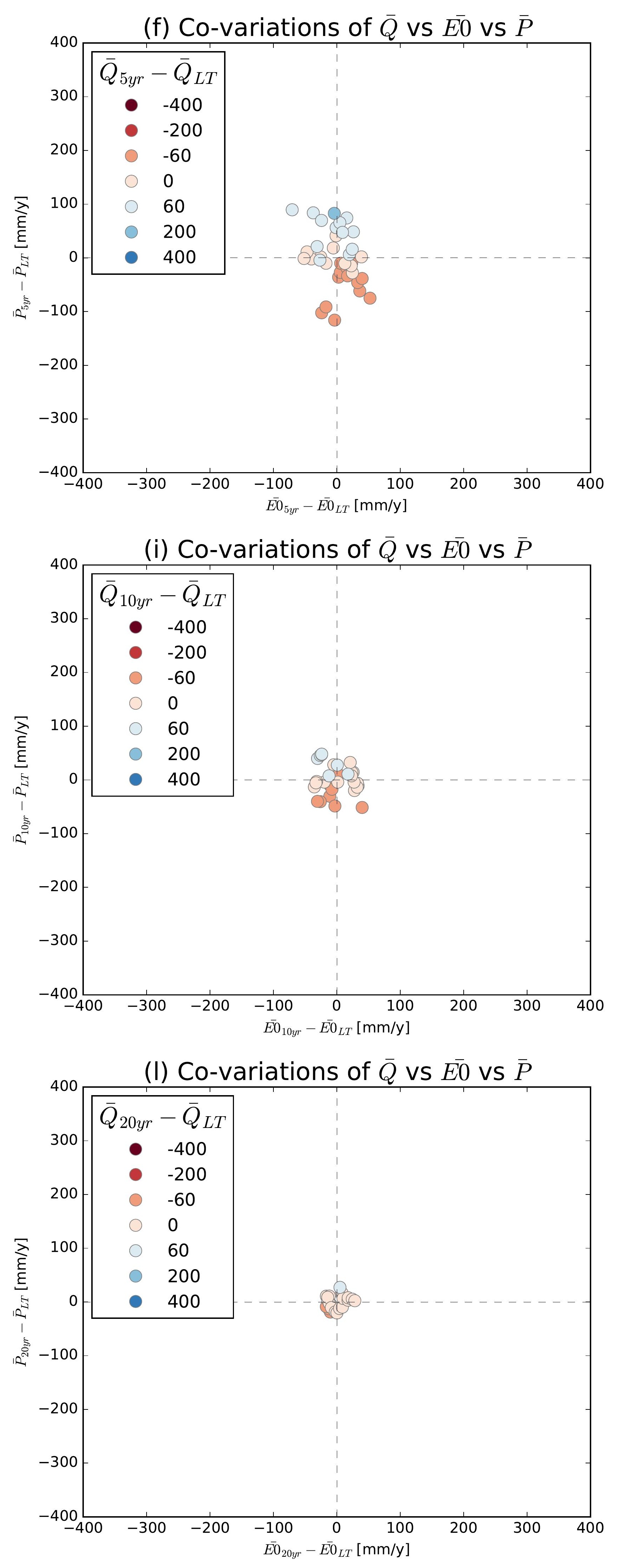

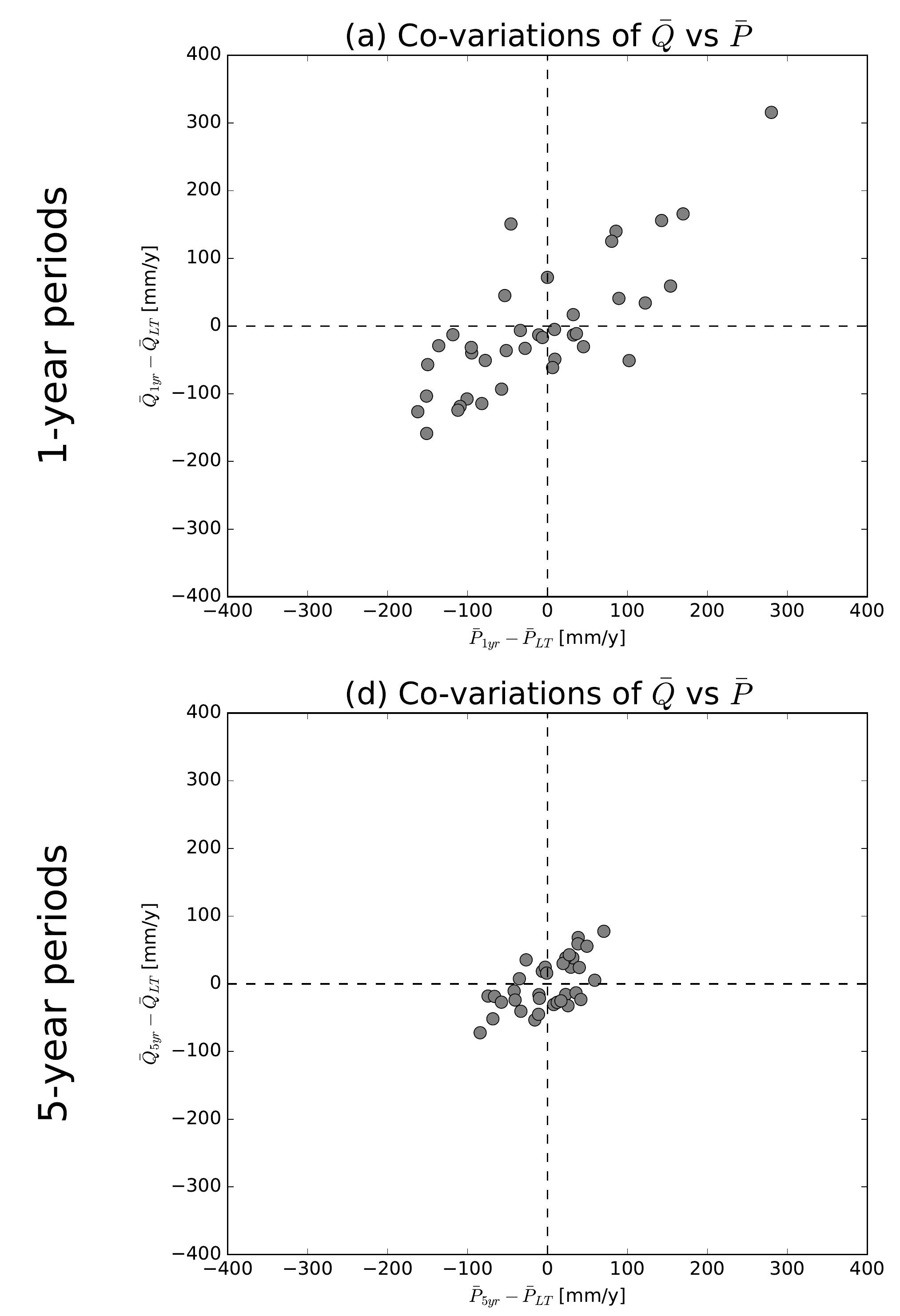

(g) Co-variations of $\bar{Q}$ vs $\bar{P}$
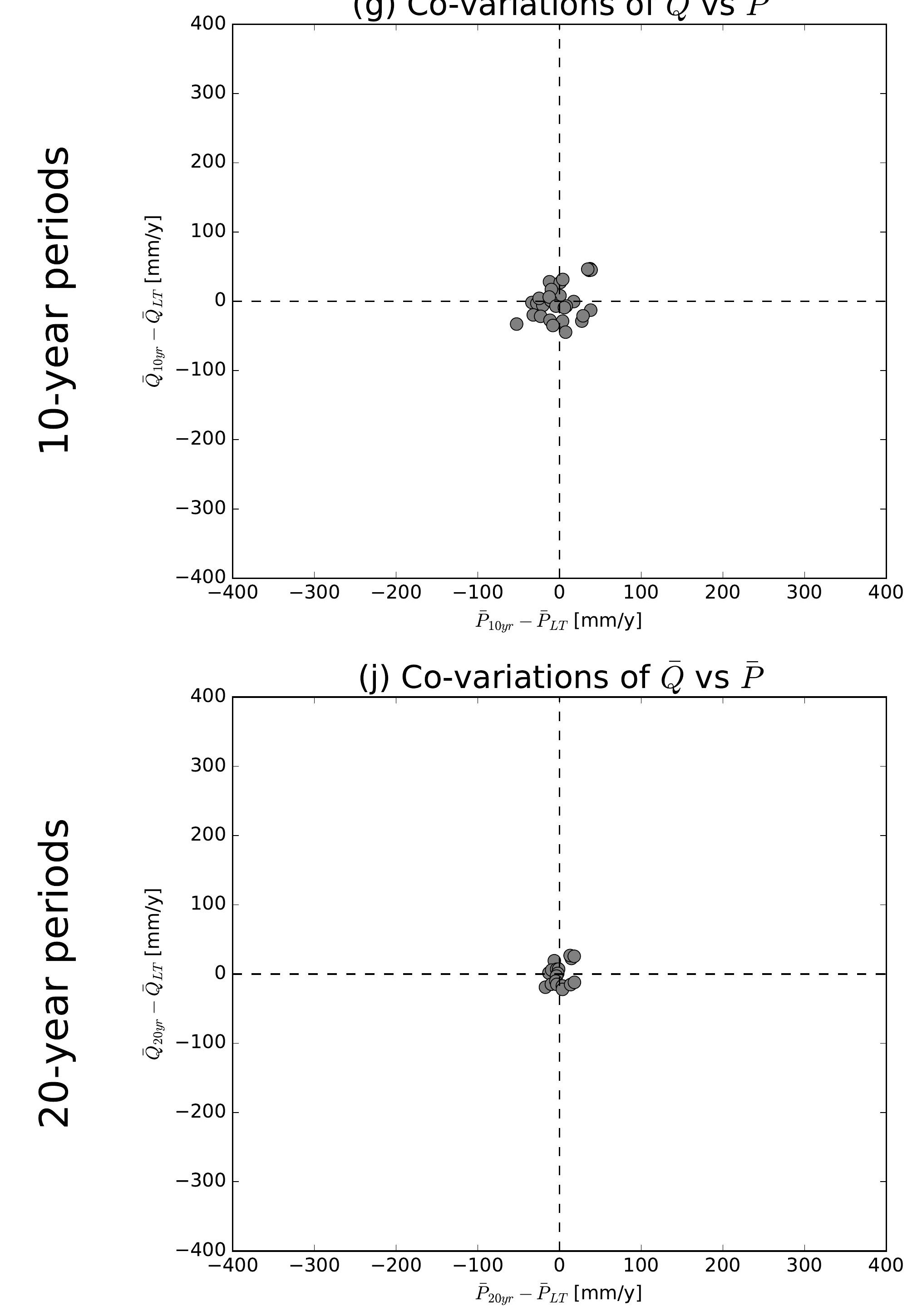

(b) Co-variations of $\bar{Q}$ vs $\overline{E 0}$

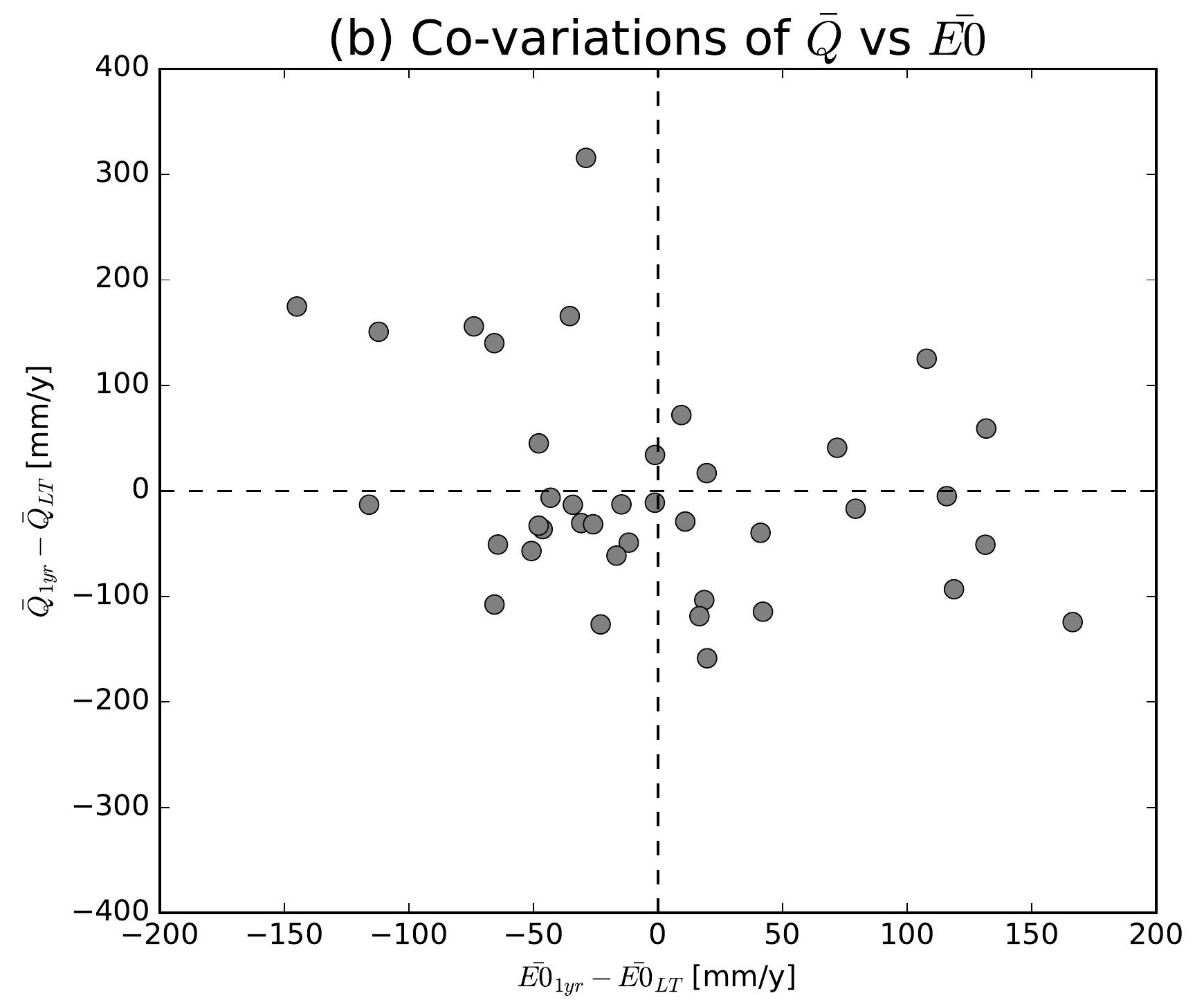

(e) Co-variations of $\bar{Q}$ vs $\overline{E 0}$

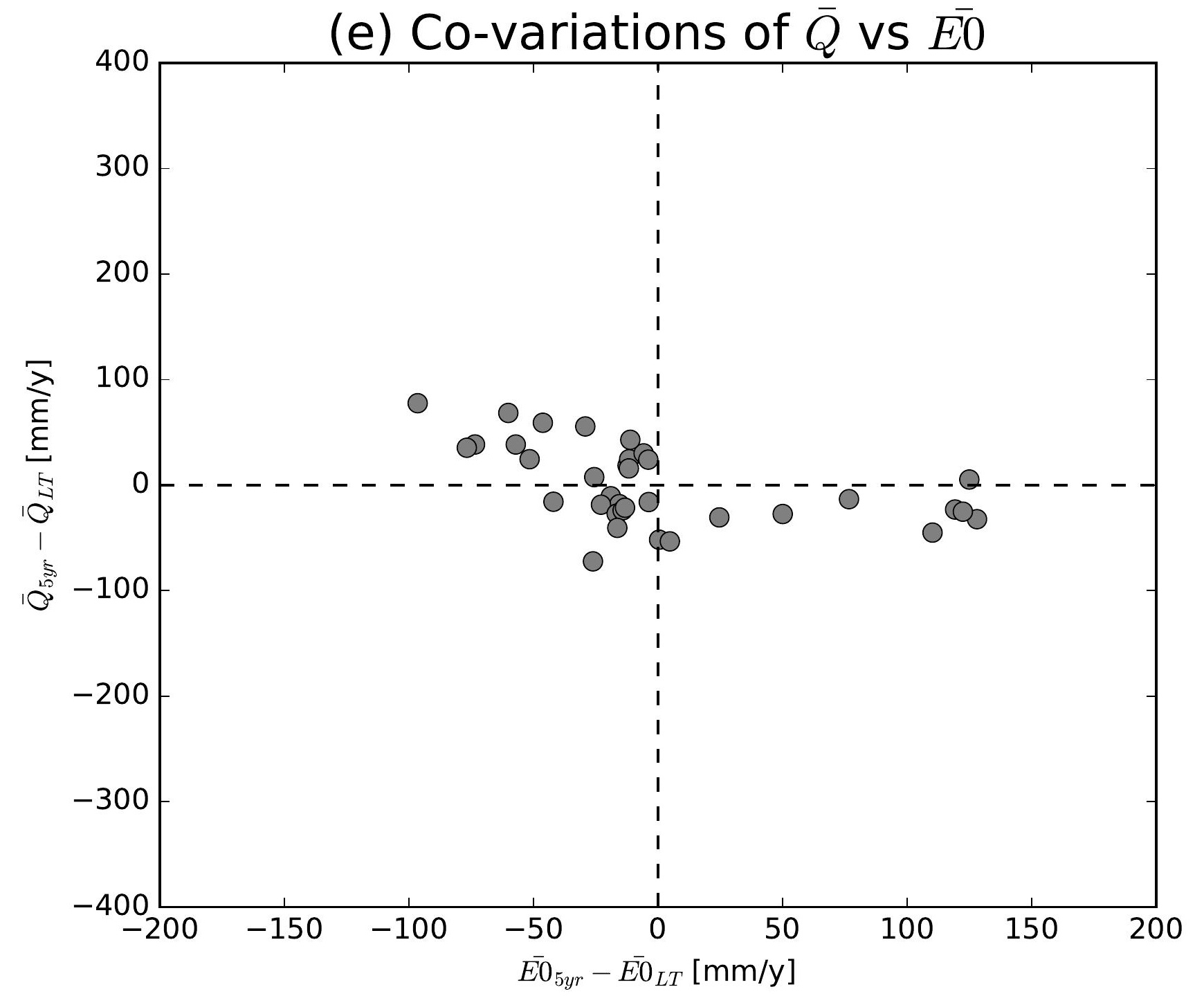

(h) Co-variations of $\bar{Q}$ vs $\overline{E 0}$

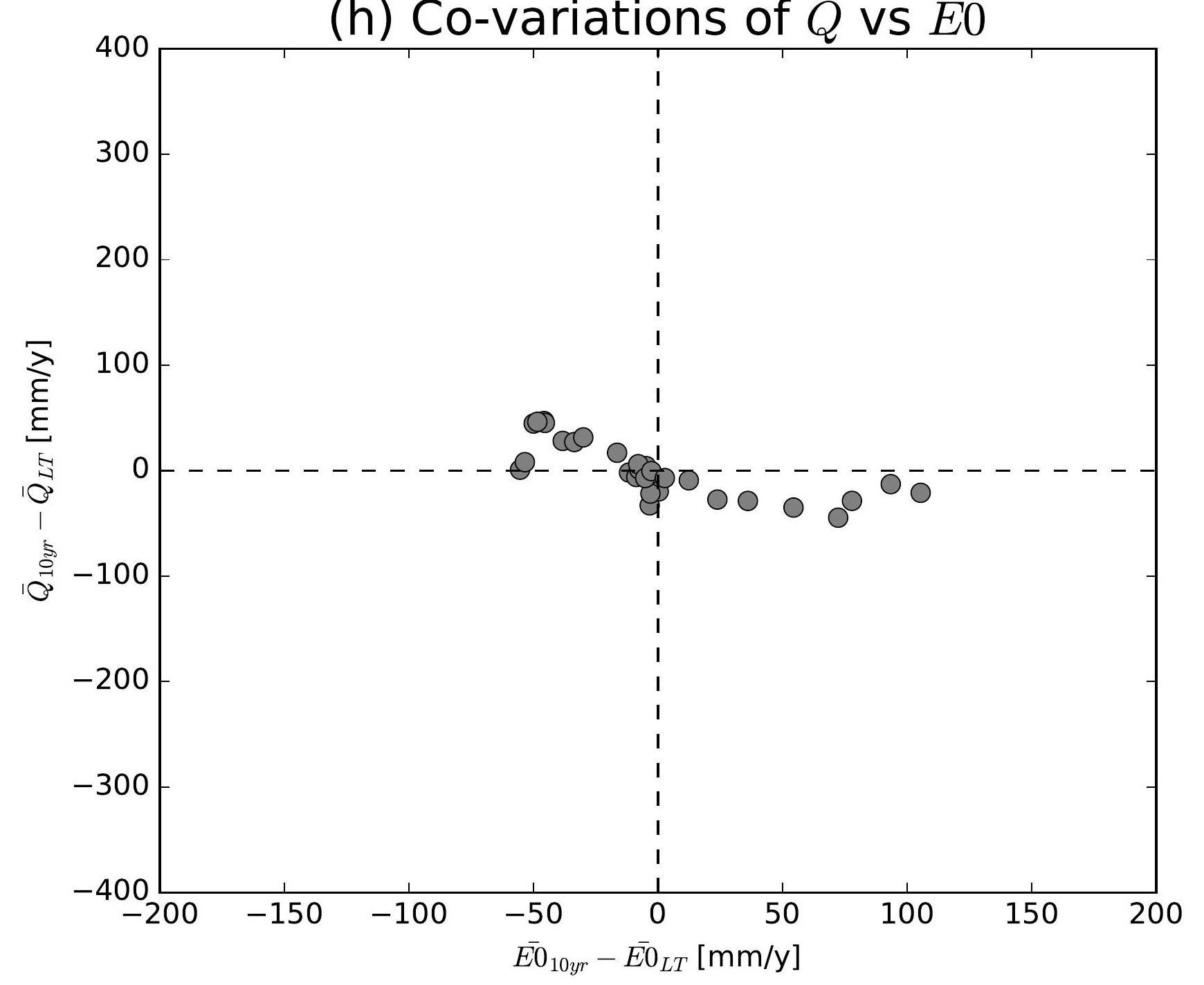

(k) Co-variations of $\bar{Q}$ vs $\overline{E 0}$

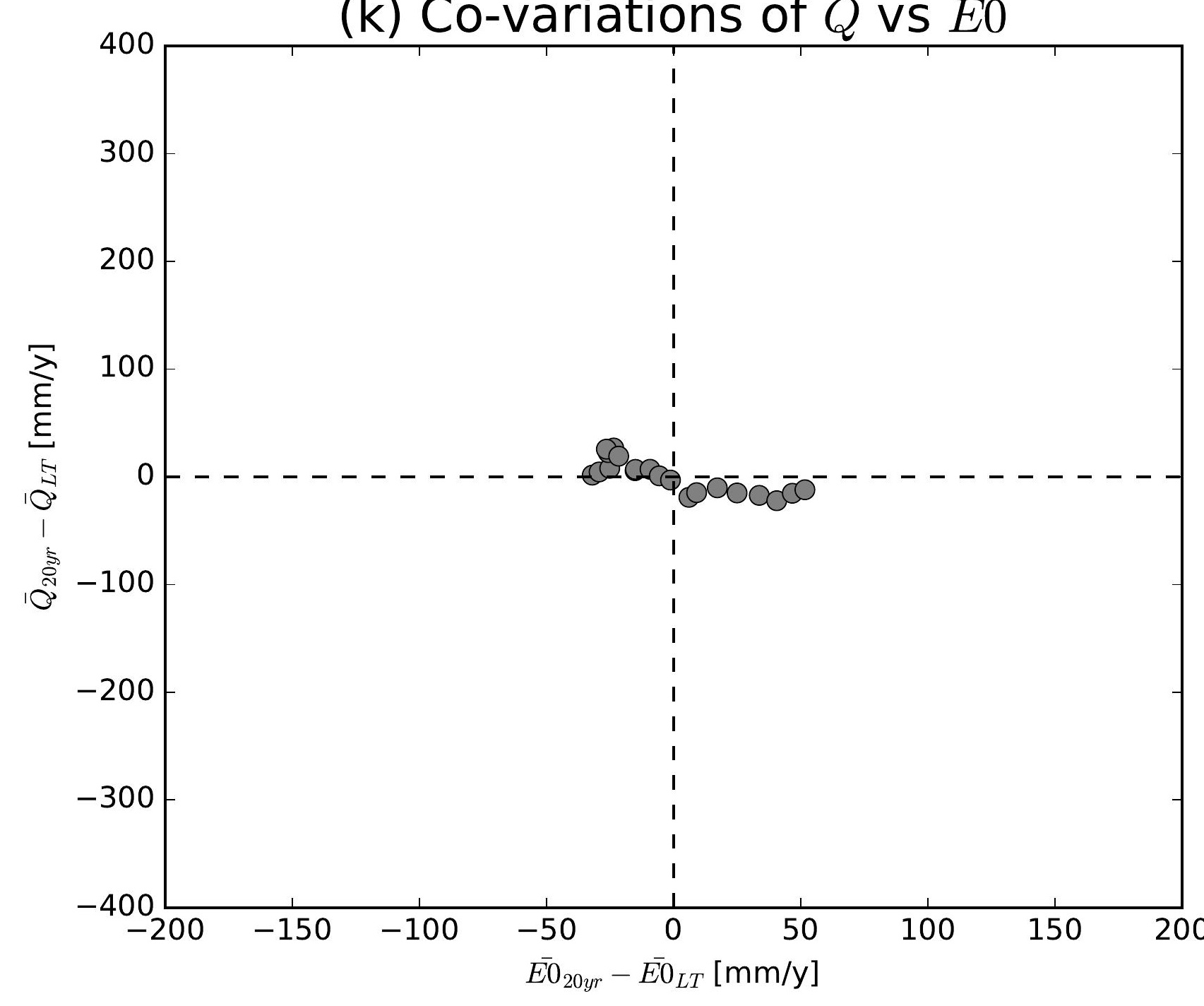

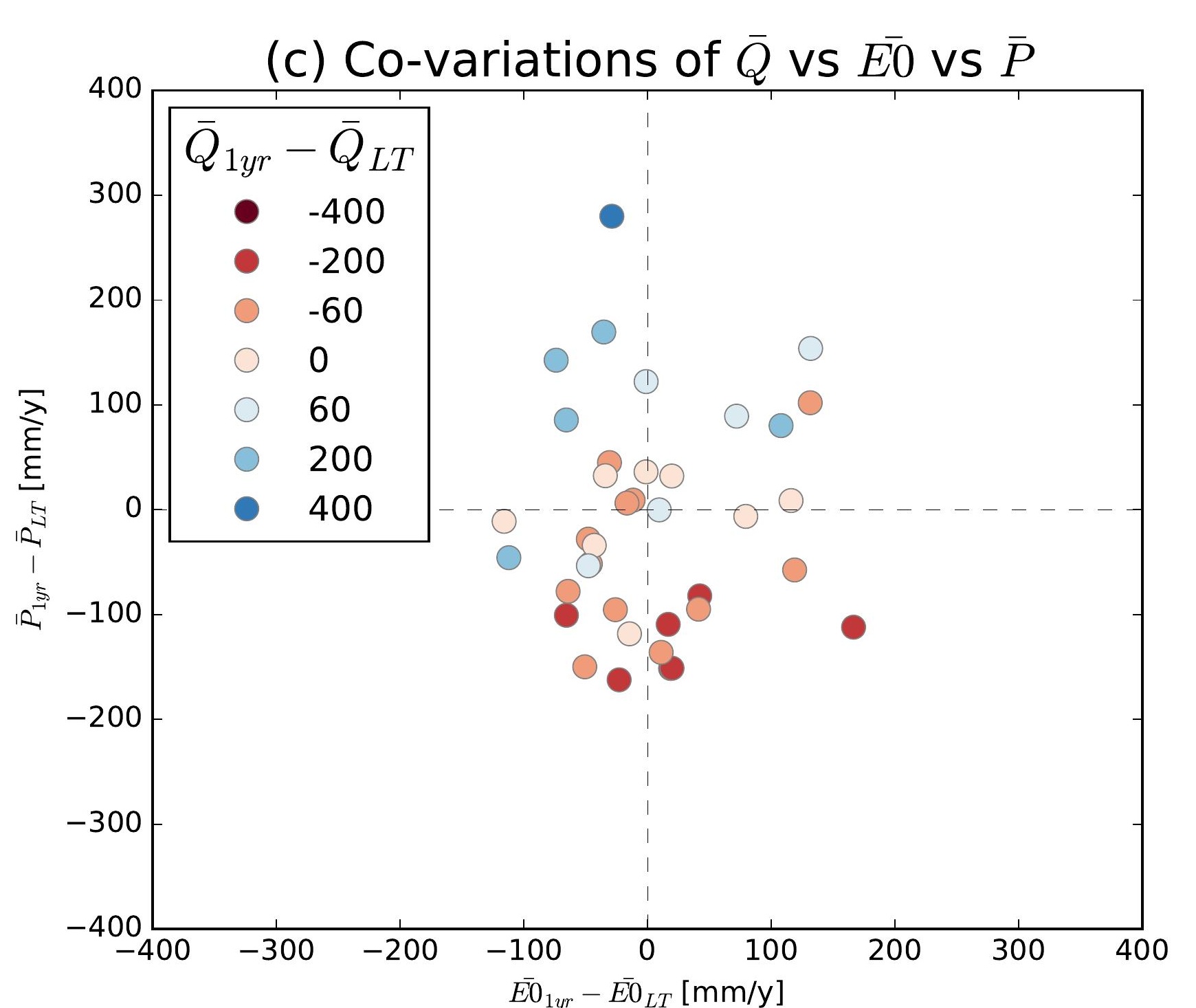
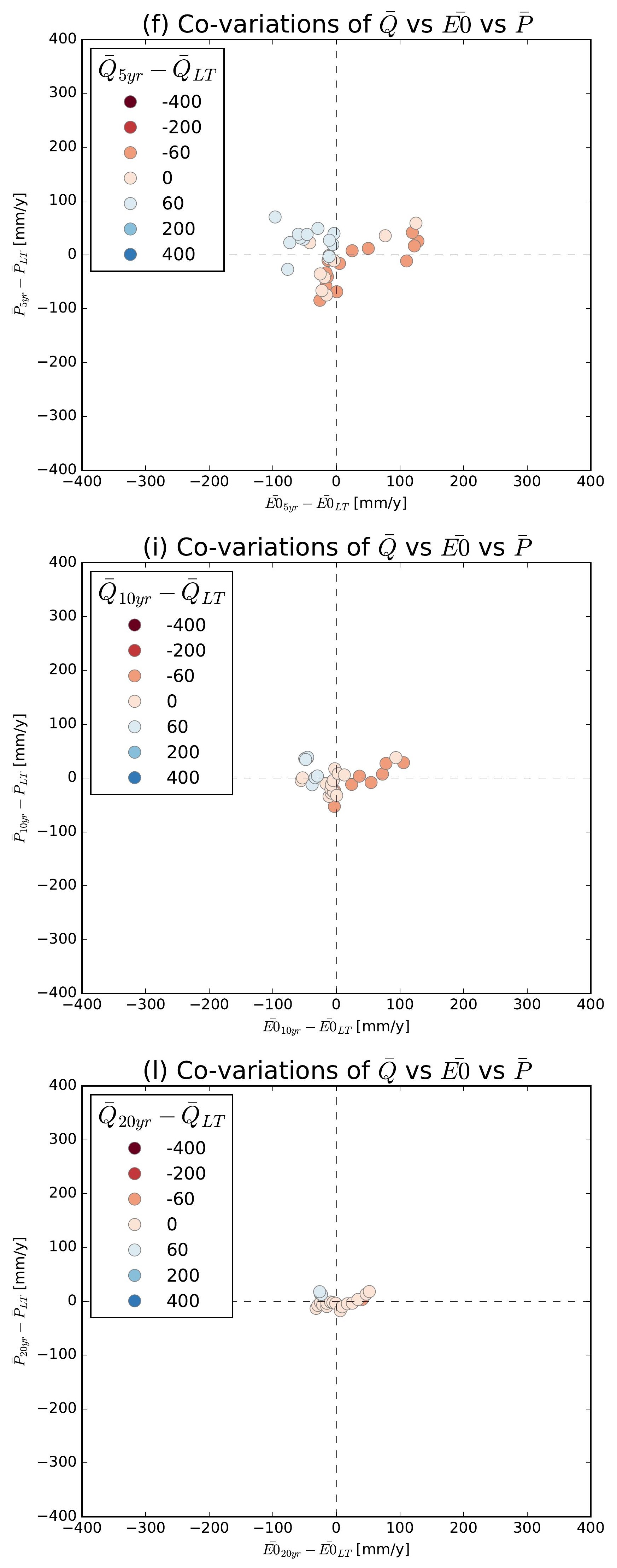

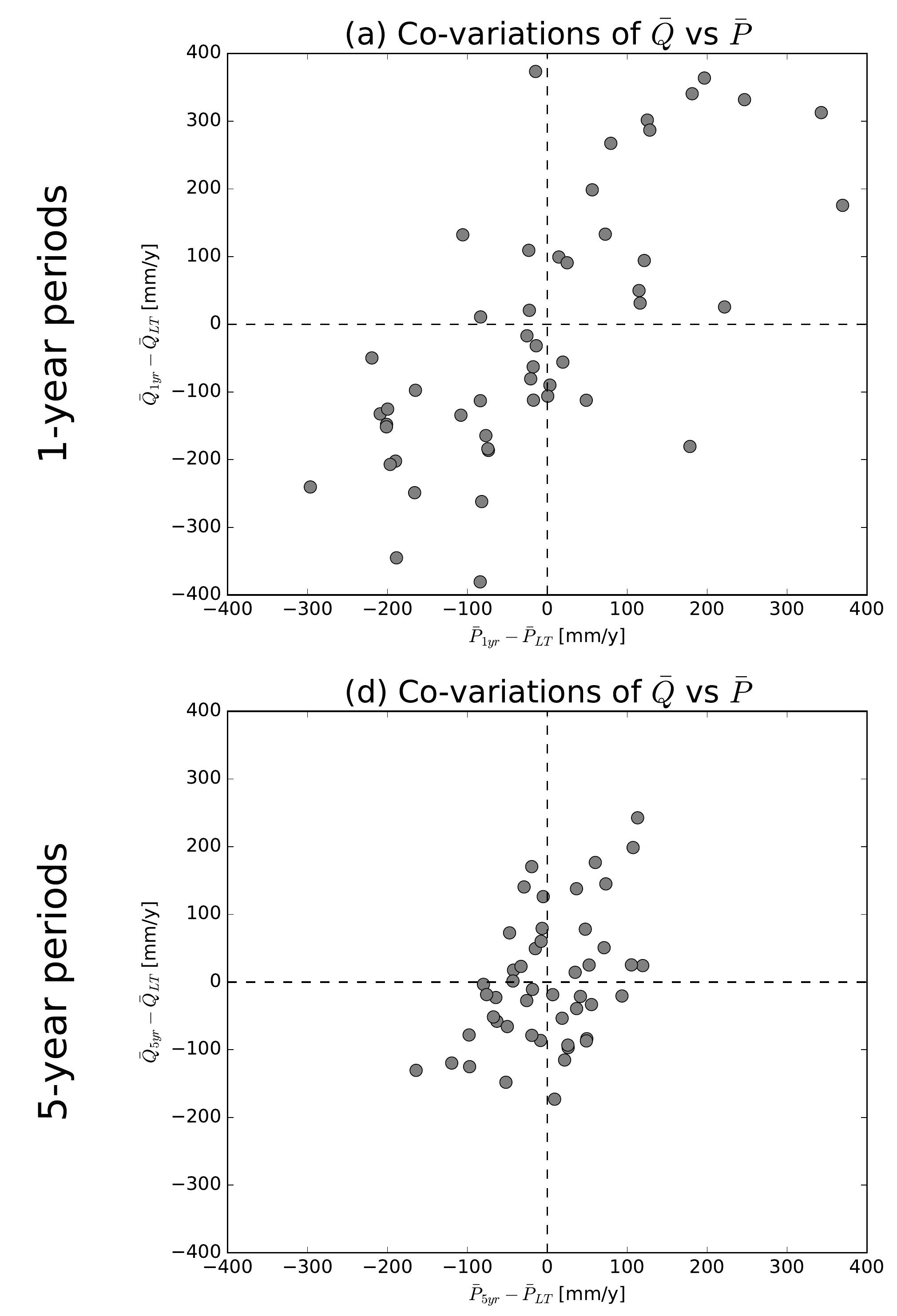

(g) Co-variations of $\bar{Q}$ vs $\bar{P}$
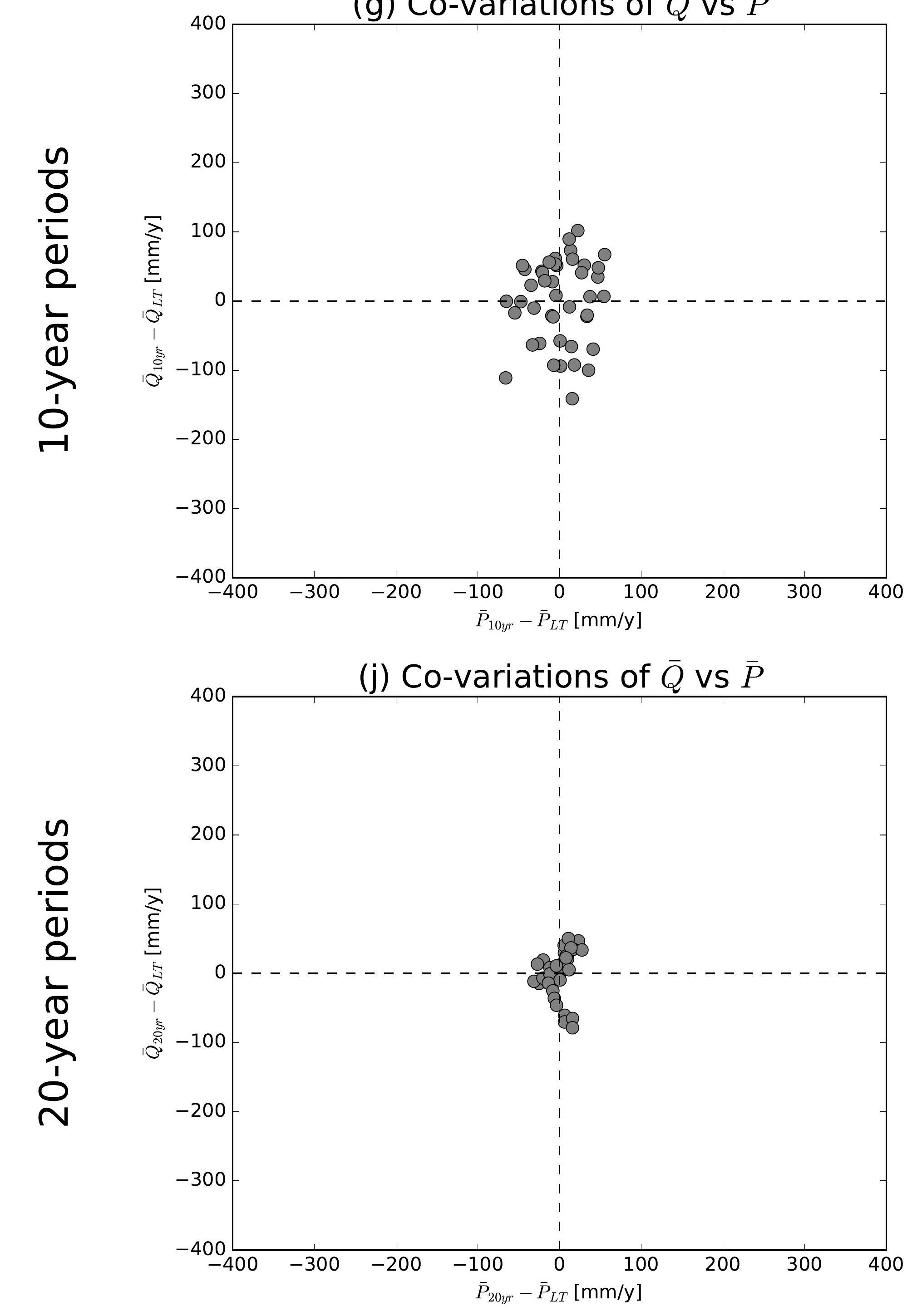

(b) Co-variations of $\bar{Q}$ vs $\overline{E 0}$

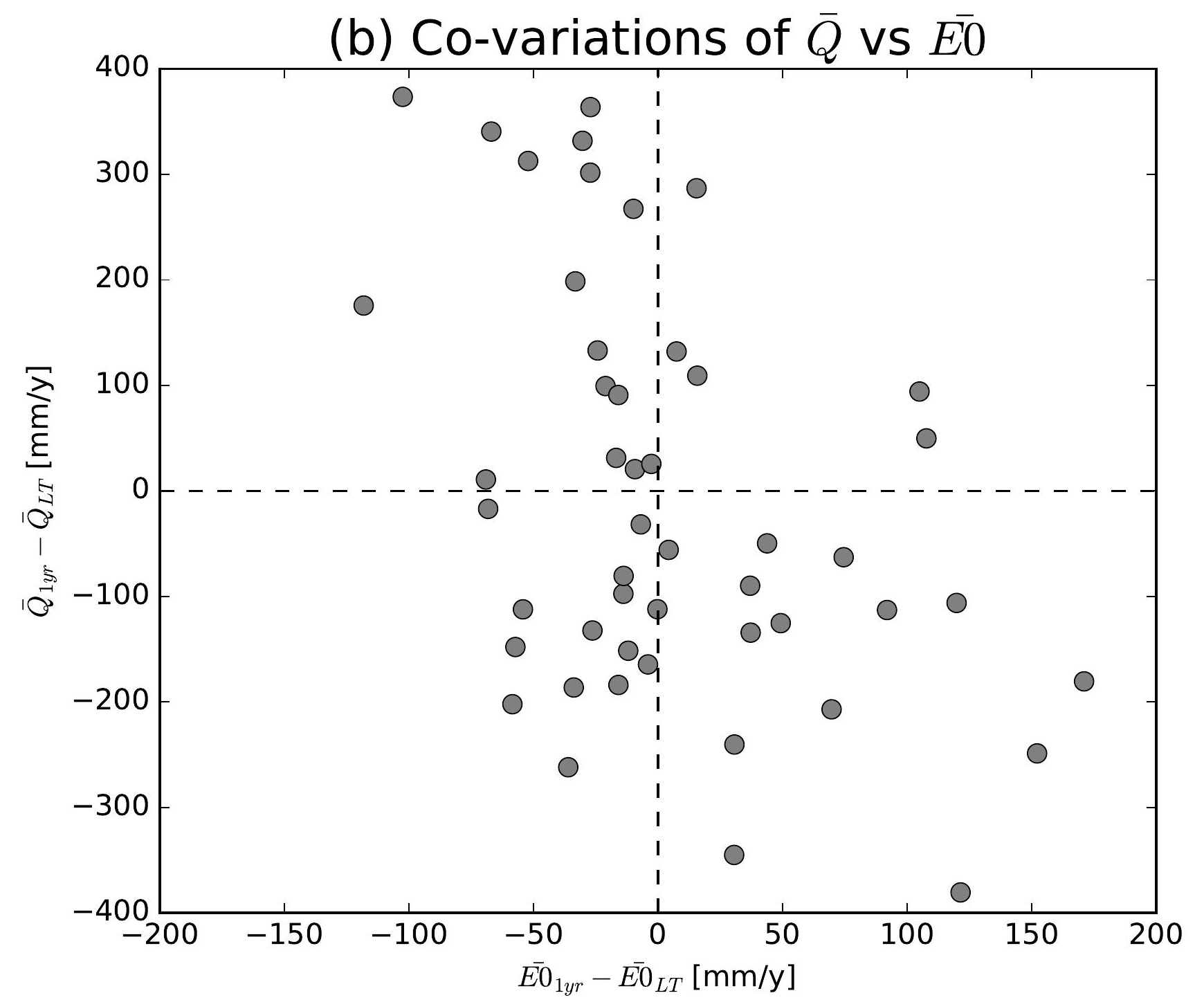

(e) Co-variations of $\bar{Q}$ vs $\overline{E 0}$

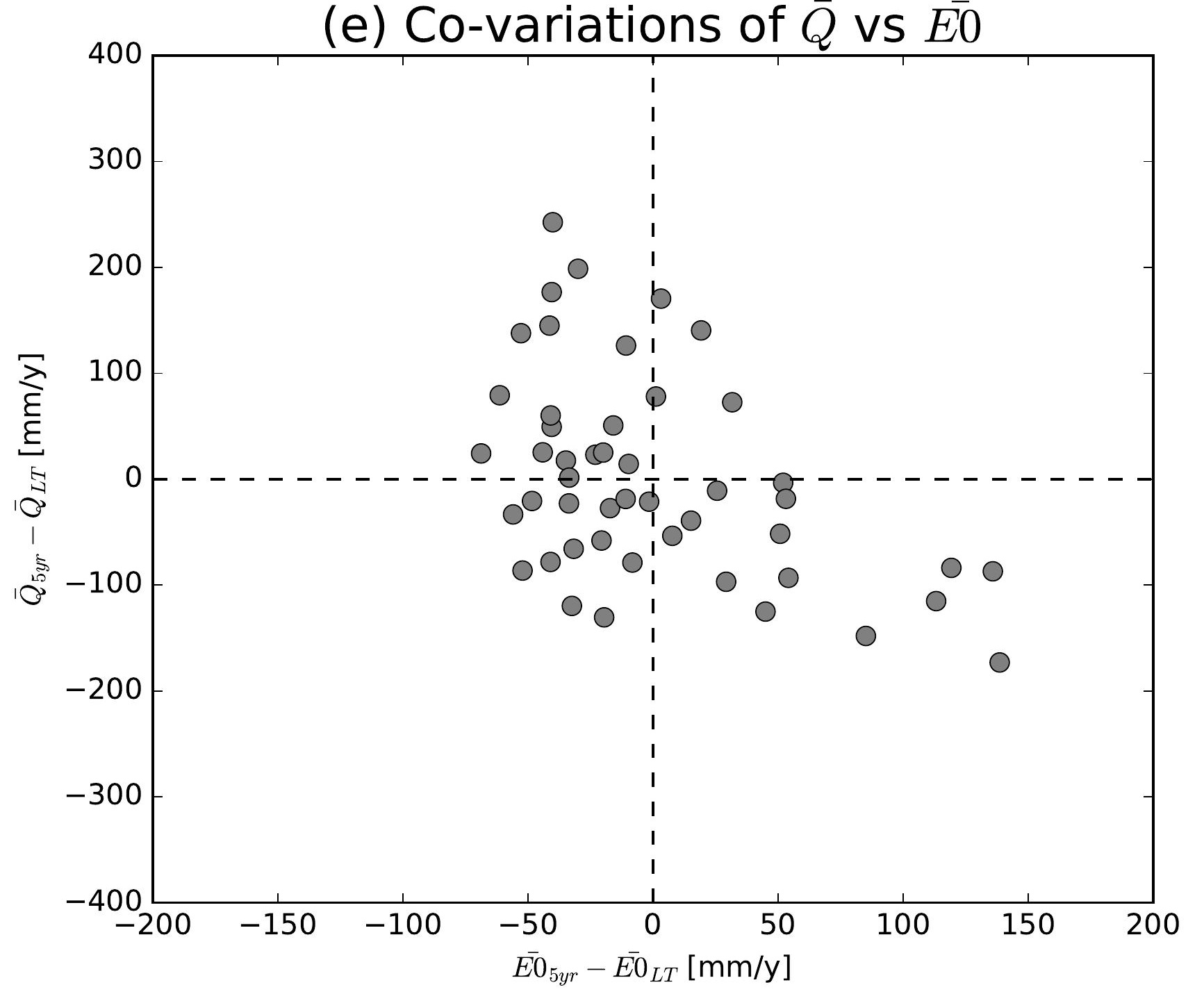

(h) Co-variations of $\bar{Q}$ vs $\overline{E 0}$

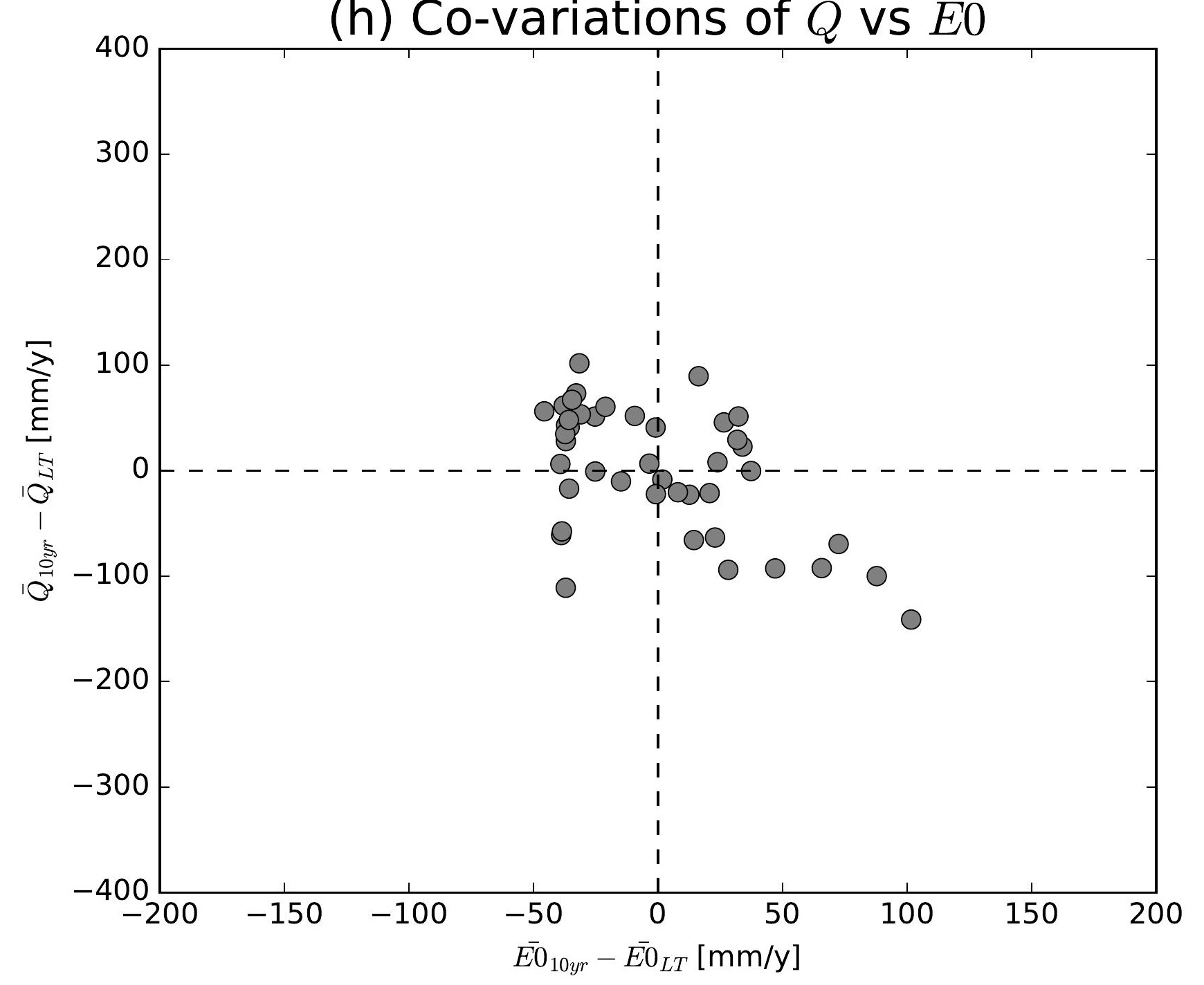

(k) Co-variations of $\bar{Q}$ vs $\overline{E 0}$

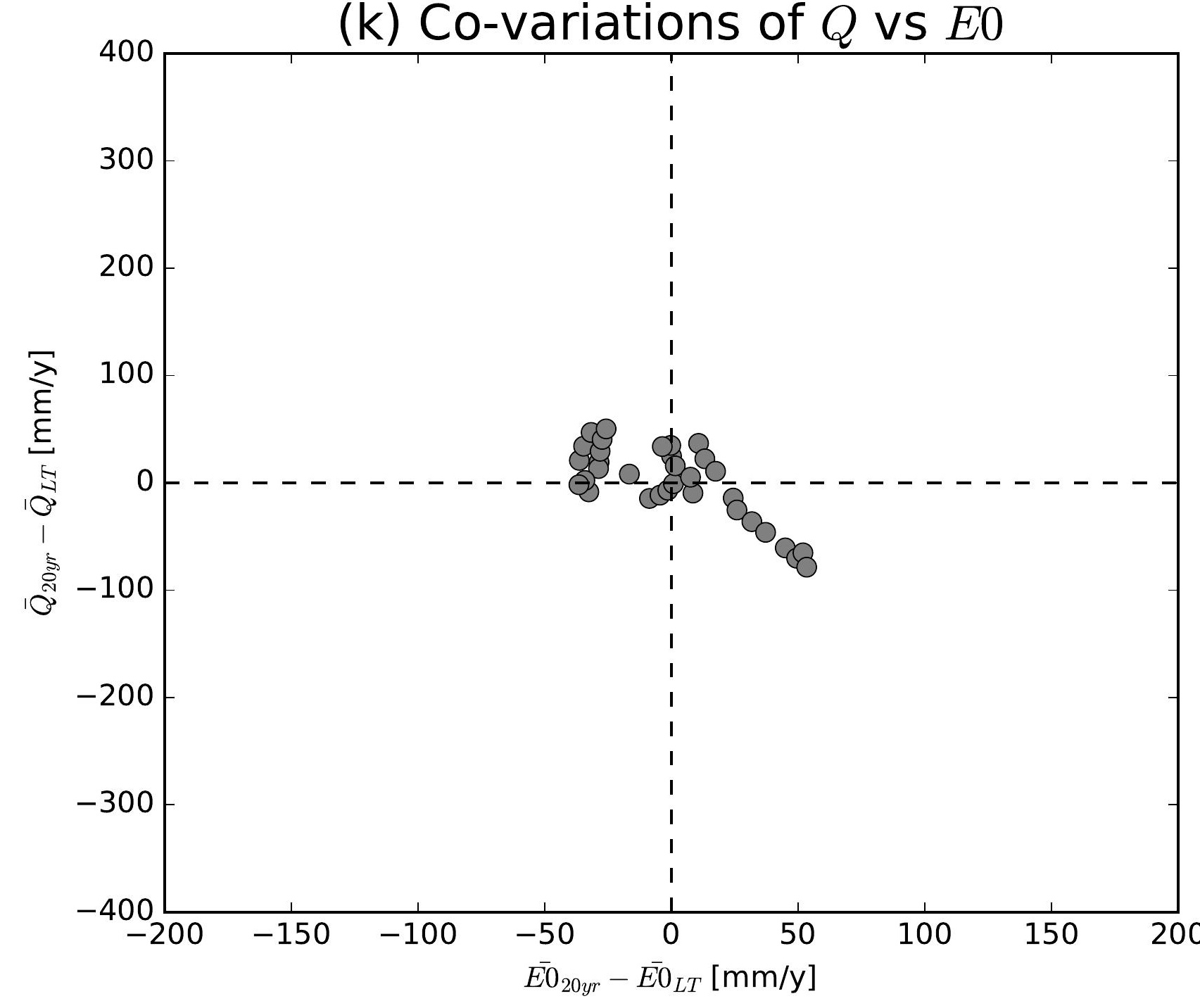

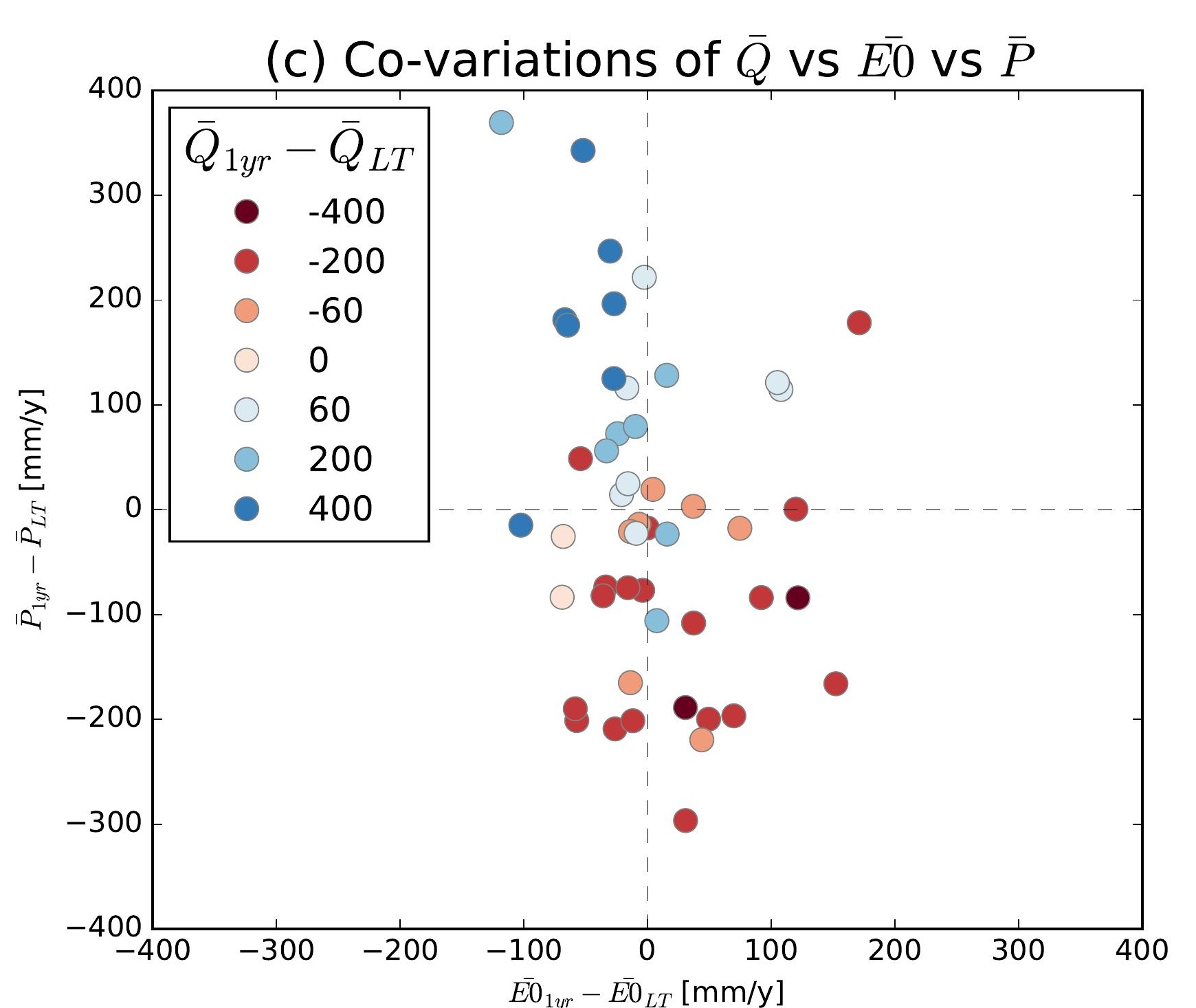
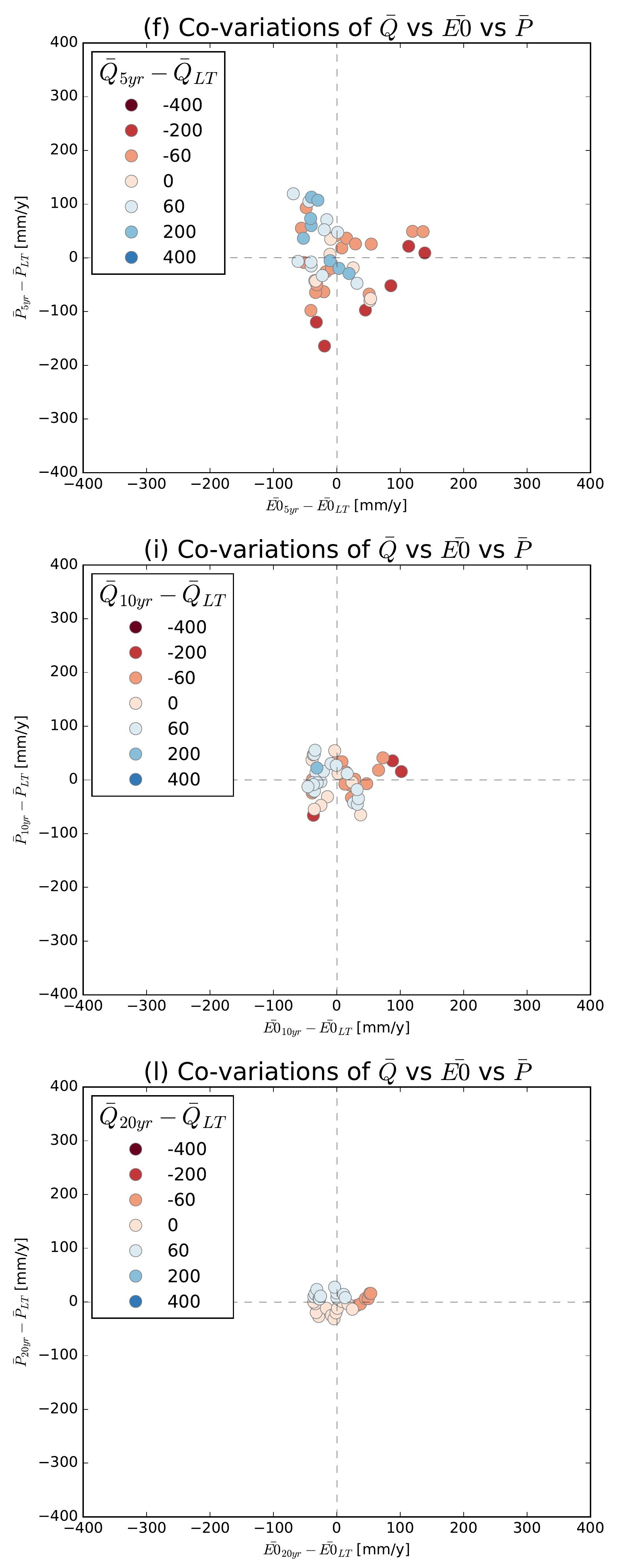

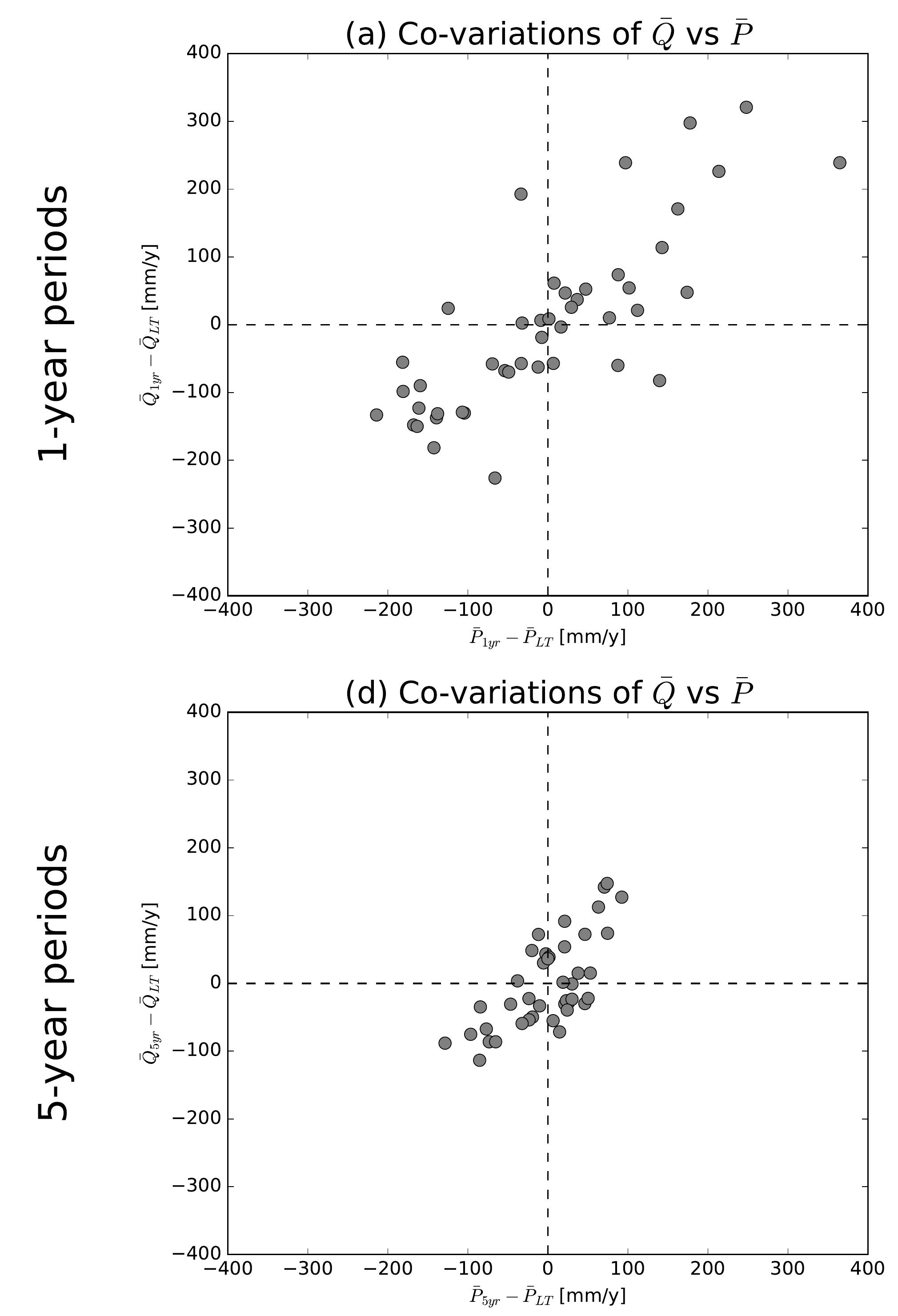

(g) Co-variations of $\bar{Q}$ vs $\bar{P}$
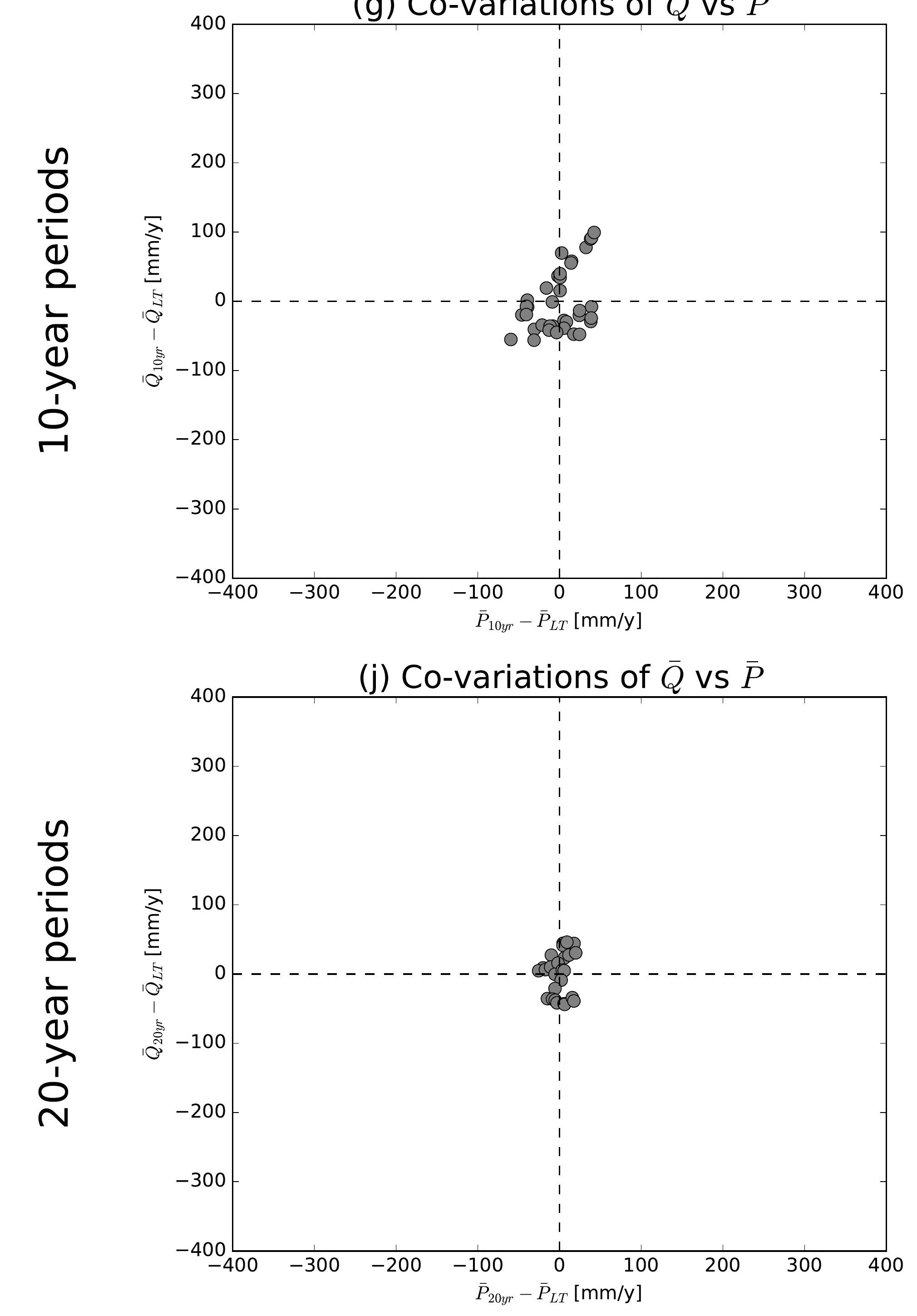

(b) Co-variations of $\bar{Q}$ vs $\overline{E 0}$

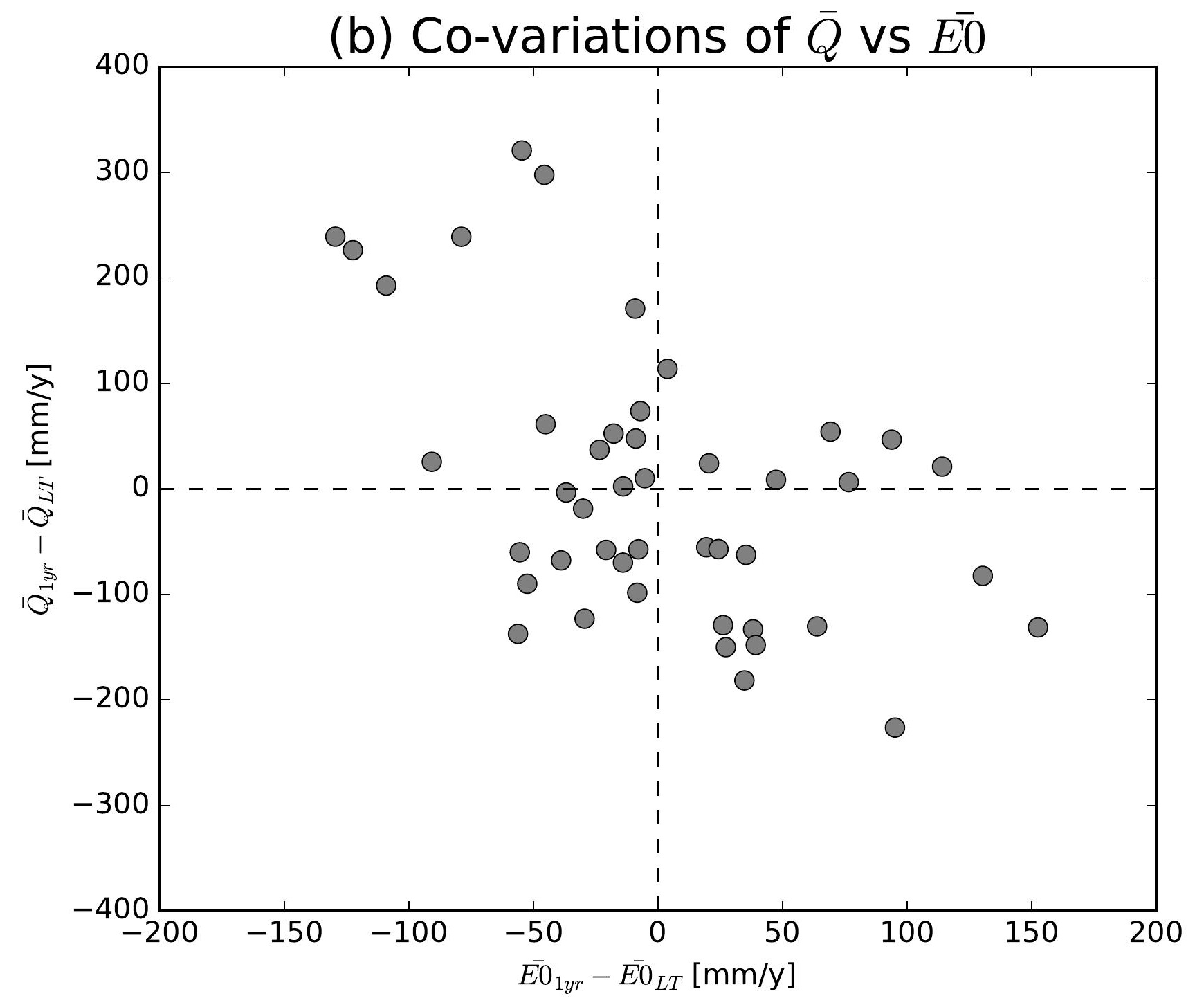

(e) Co-variations of $\bar{Q}$ vs $\overline{E 0}$

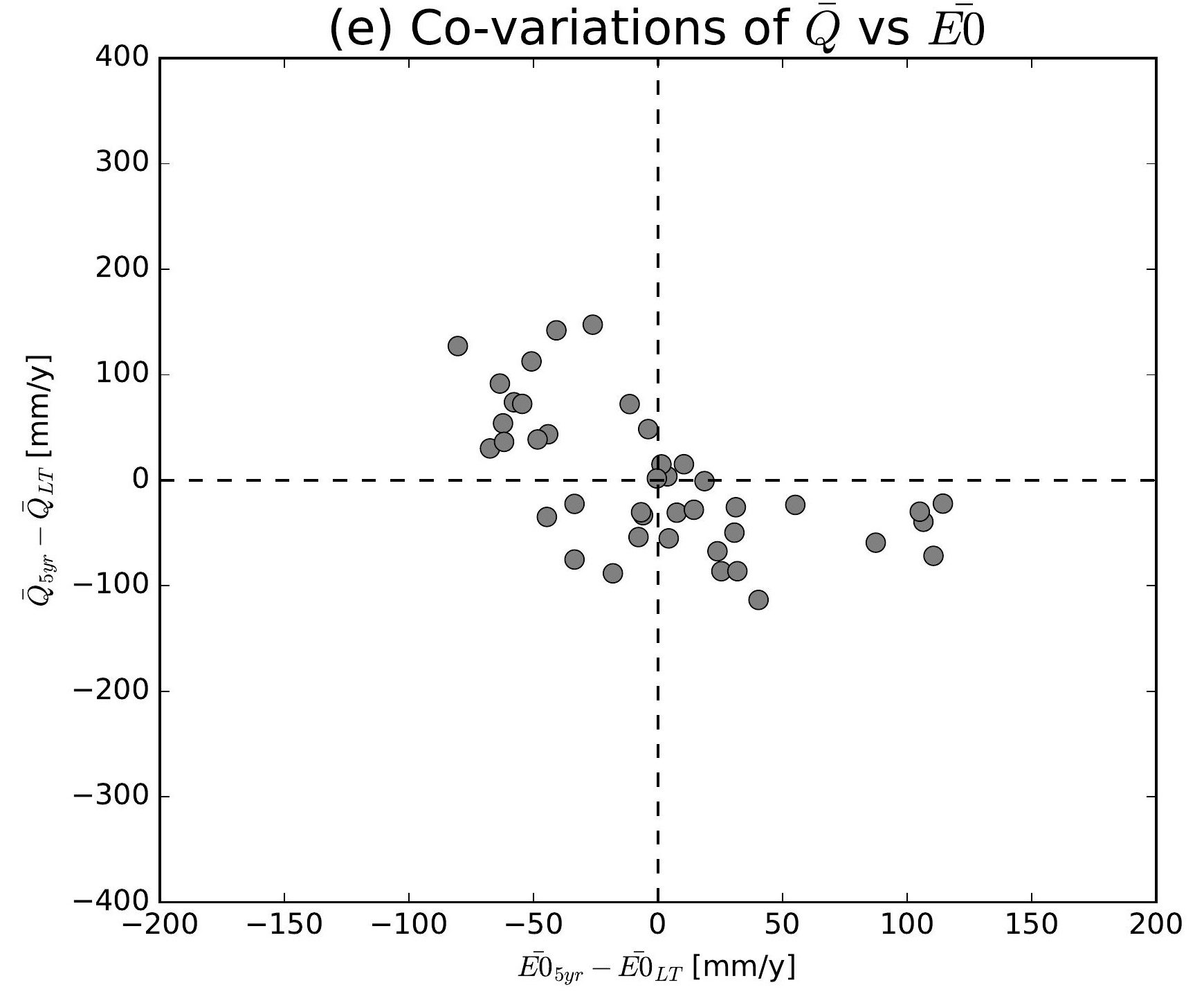

(h) Co-variations of $\bar{Q}$ vs $\overline{E 0}$

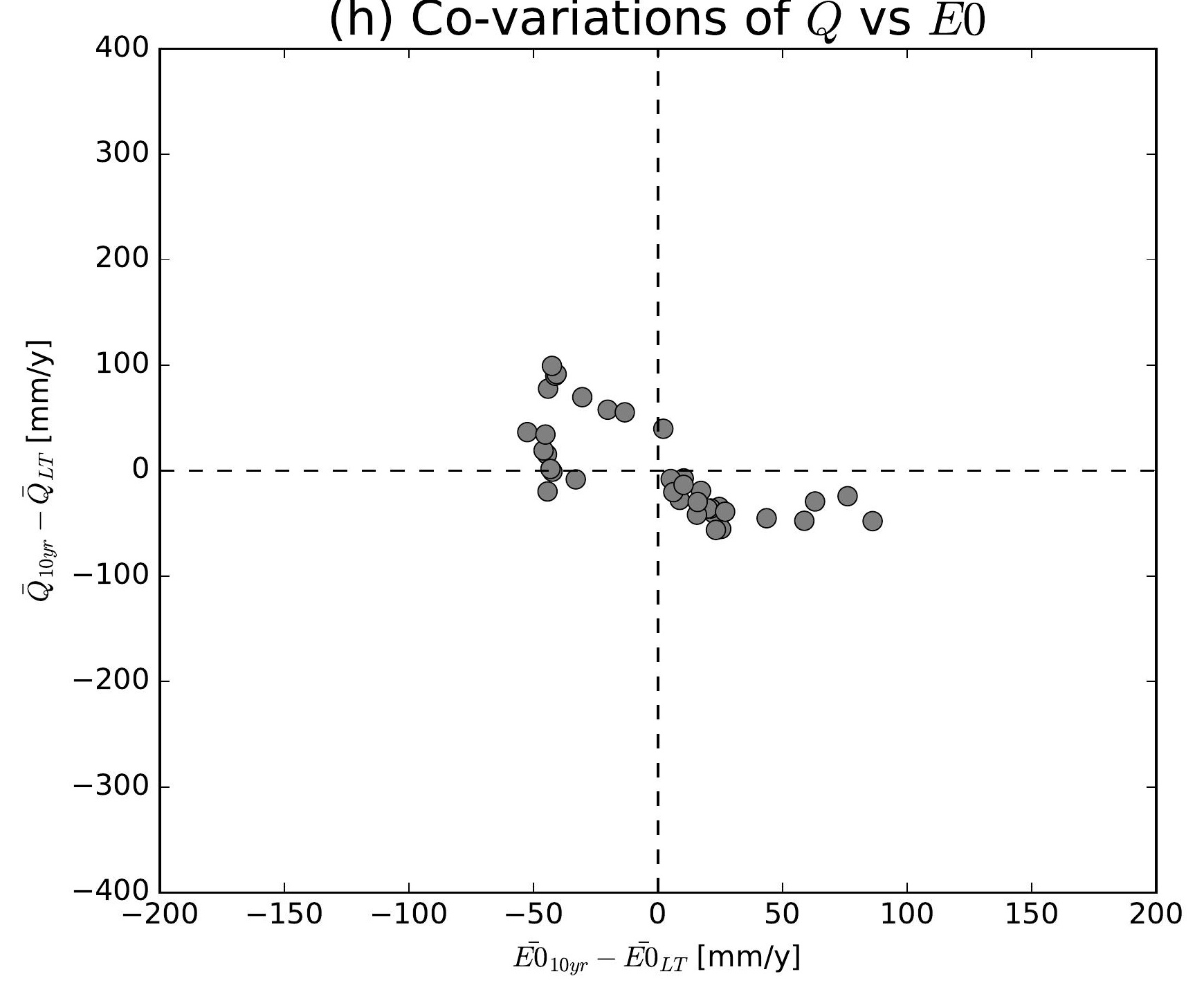

(k) Co-variations of $\bar{Q}$ vs $\overline{E 0}$

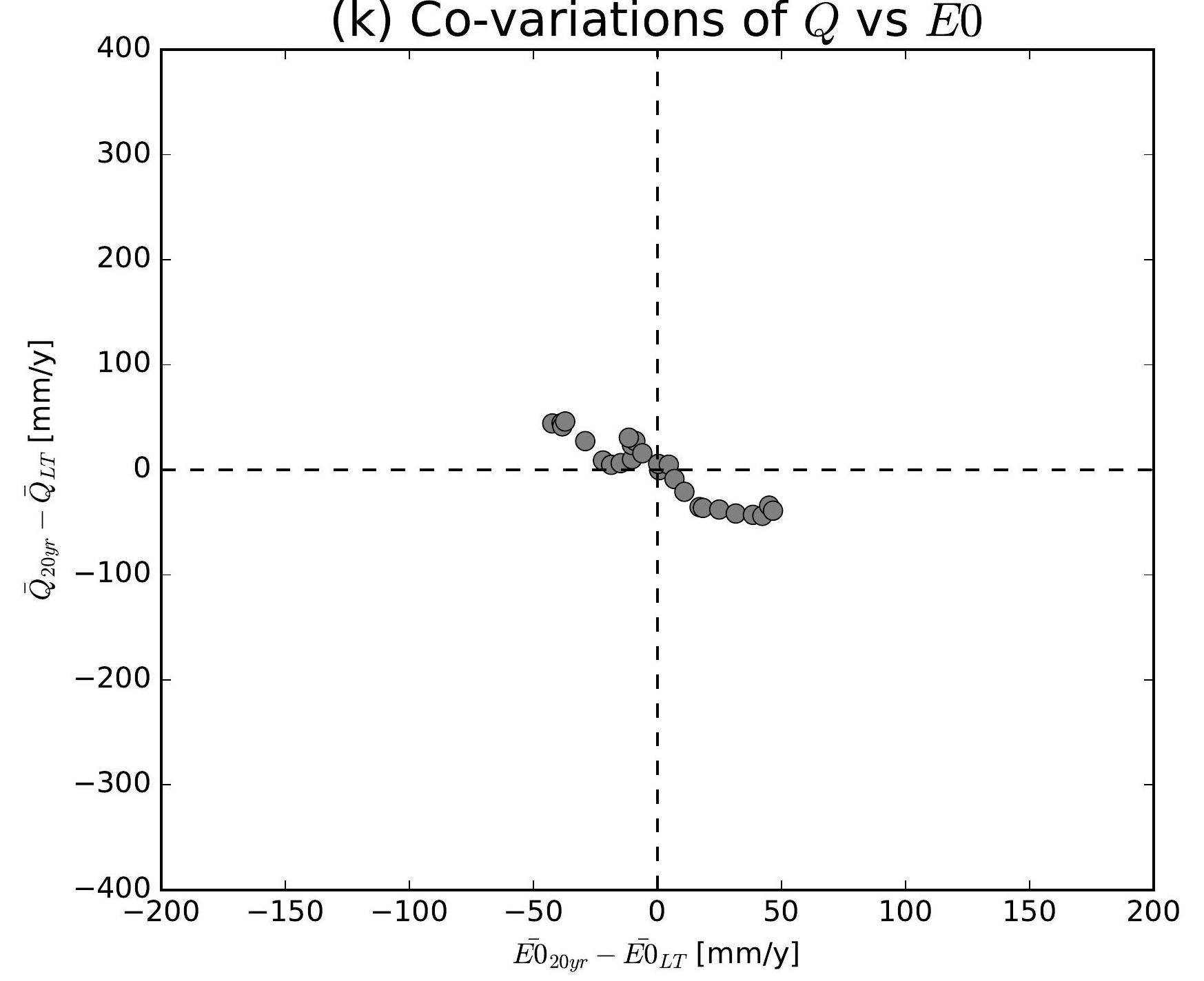

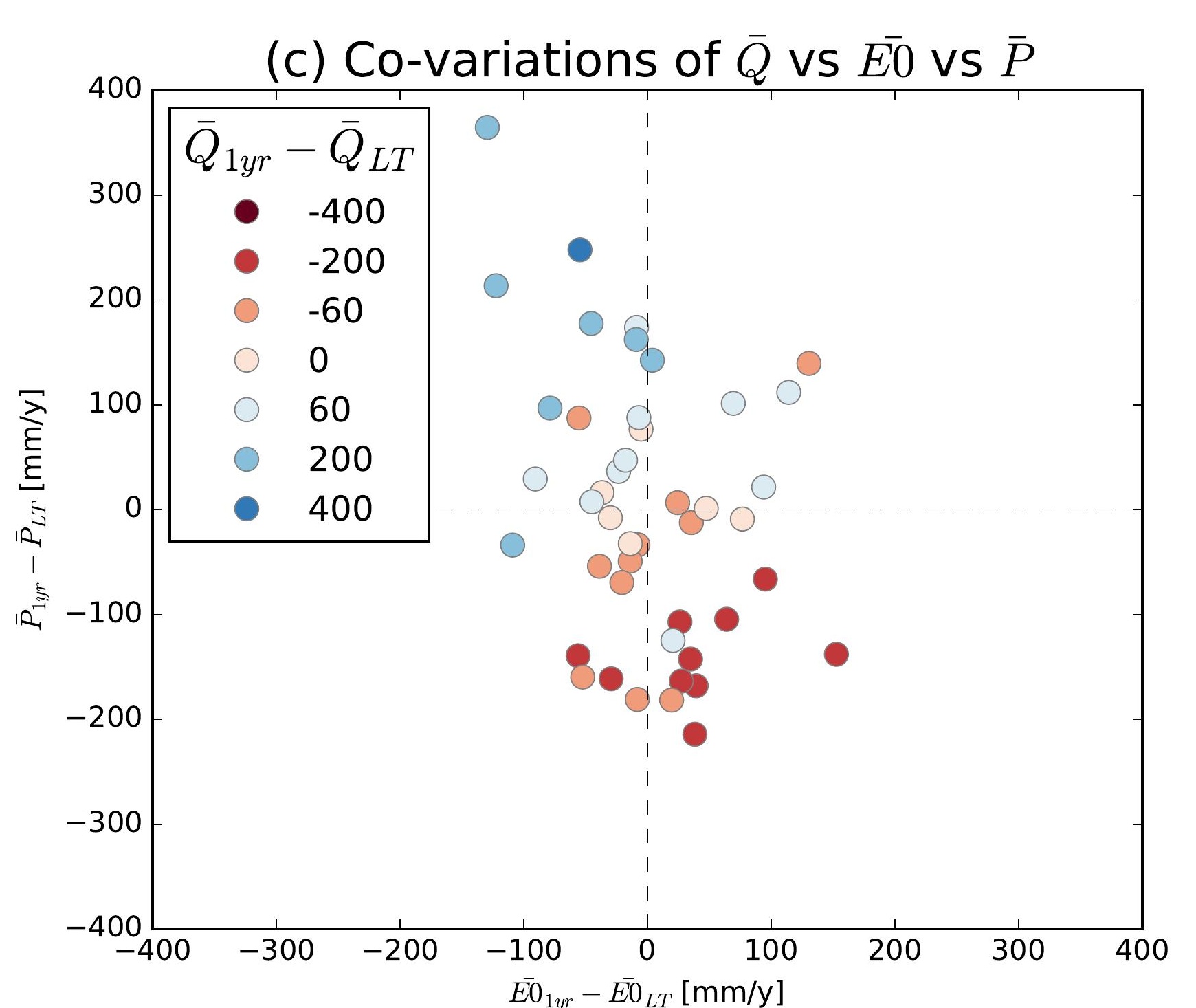
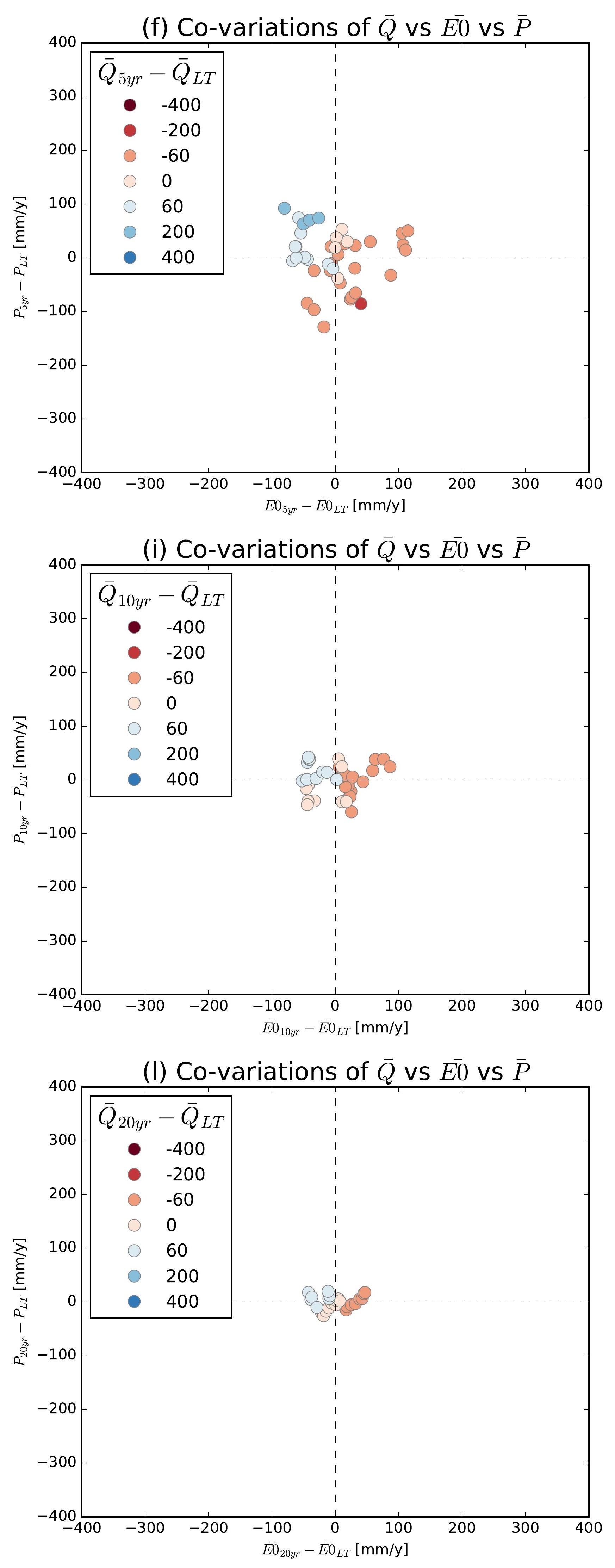

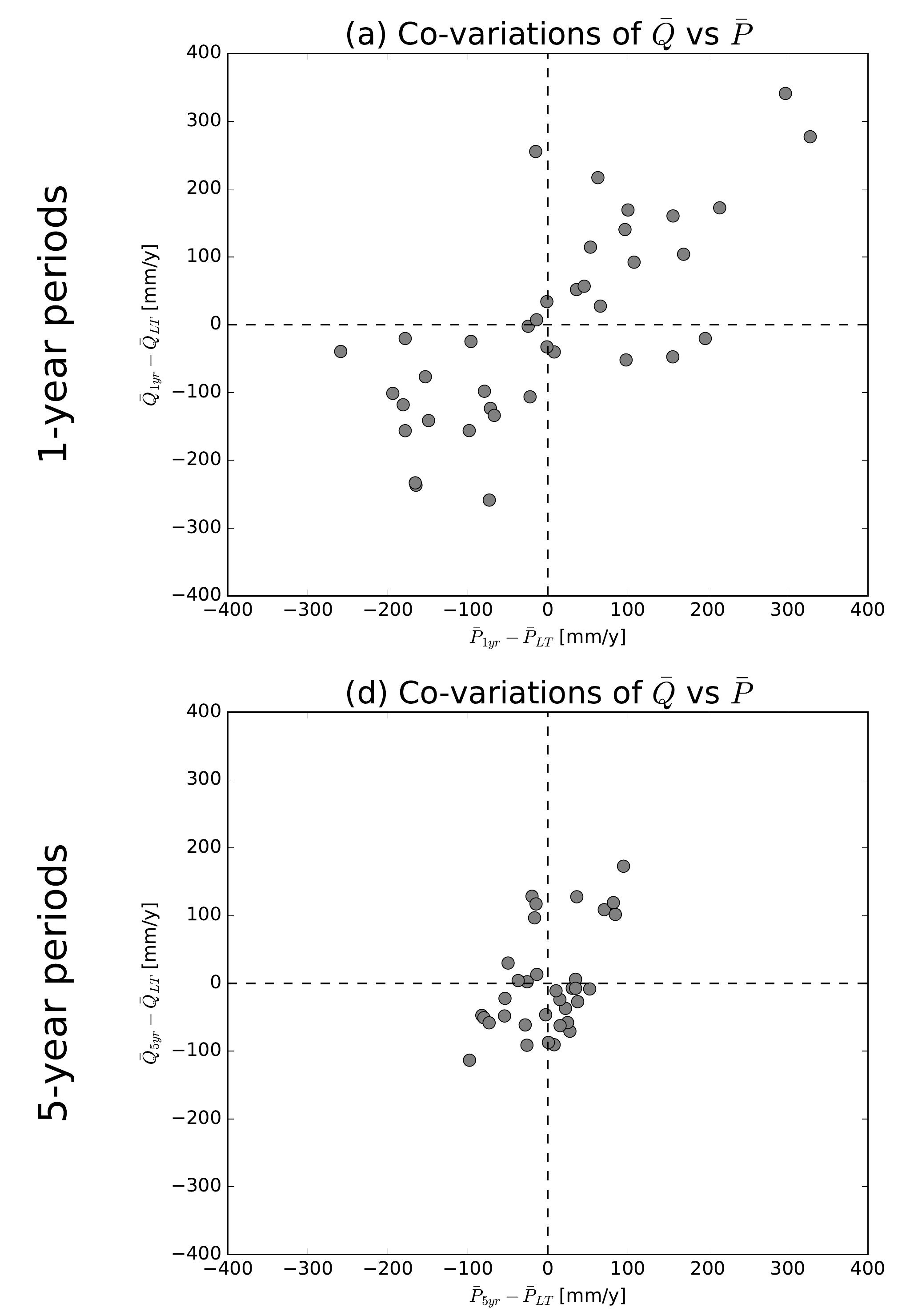

(g) Co-variations of $\bar{Q}$ vs $\bar{P}$
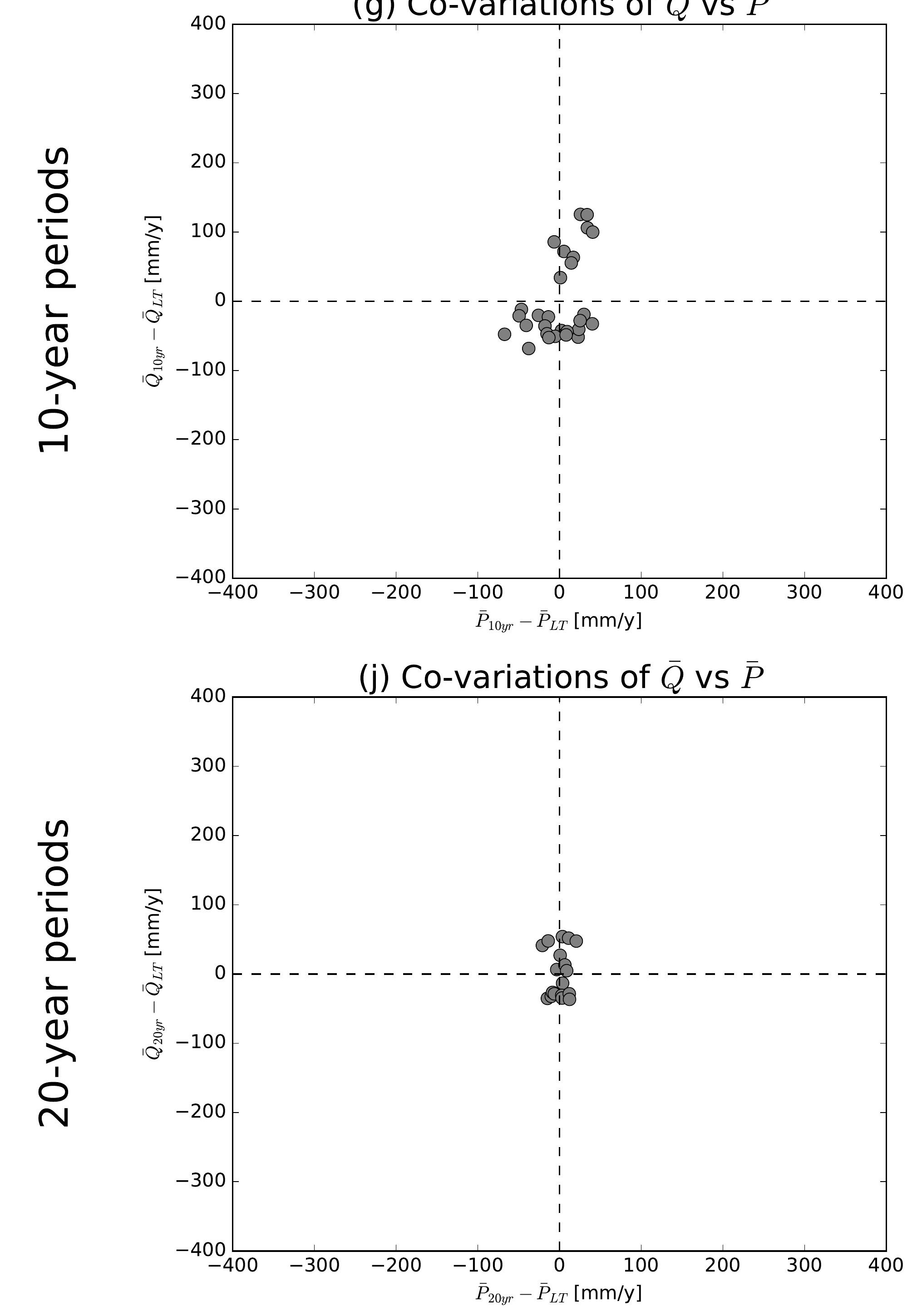

(b) Co-variations of $\bar{Q}$ vs $\overline{E 0}$

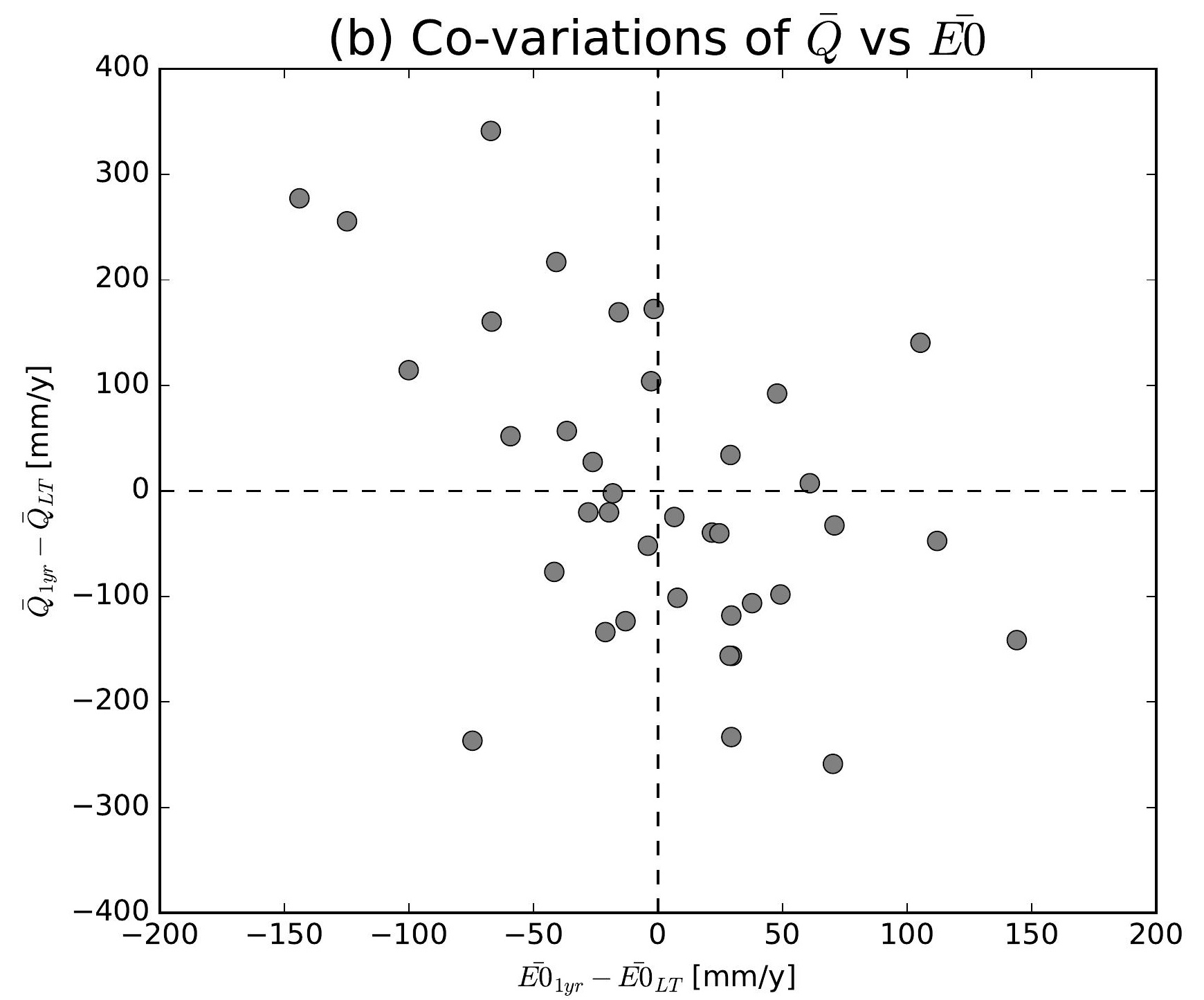

(e) Co-variations of $\bar{Q}$ vs $\overline{E 0}$

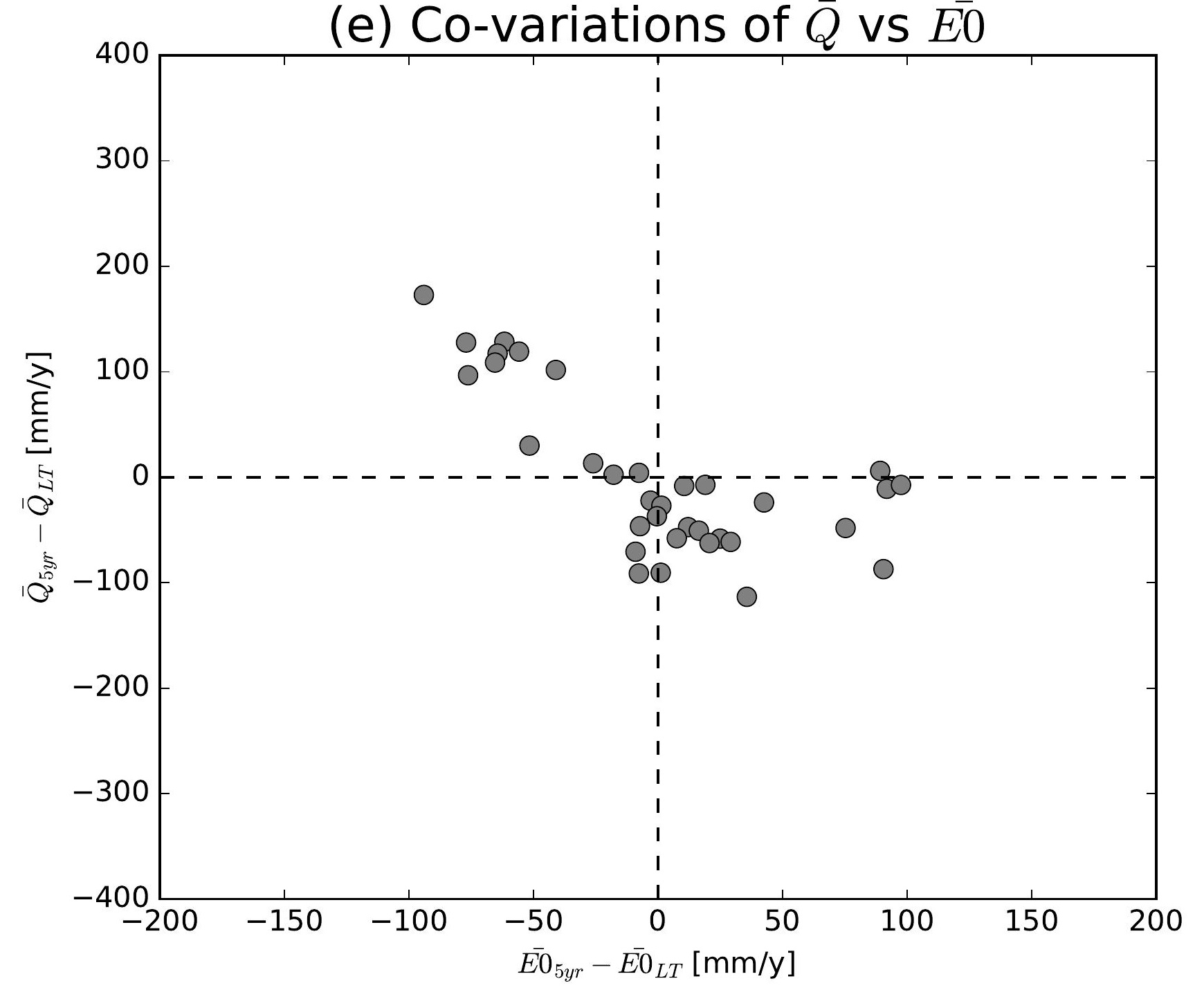

(h) Co-variations of $\bar{Q}$ vs $\overline{E 0}$

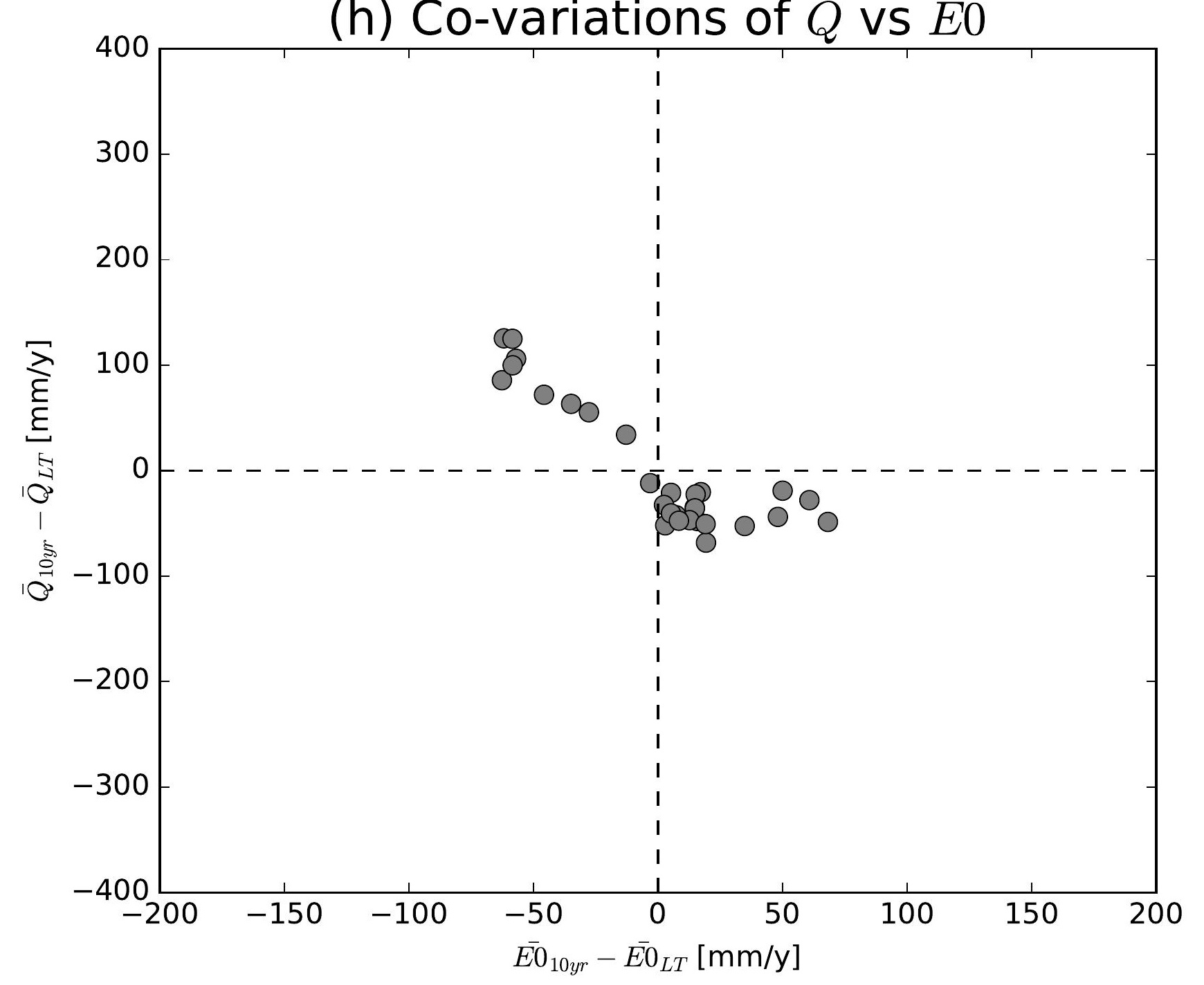

(k) Co-variations of $\bar{Q}$ vs $\overline{E 0}$

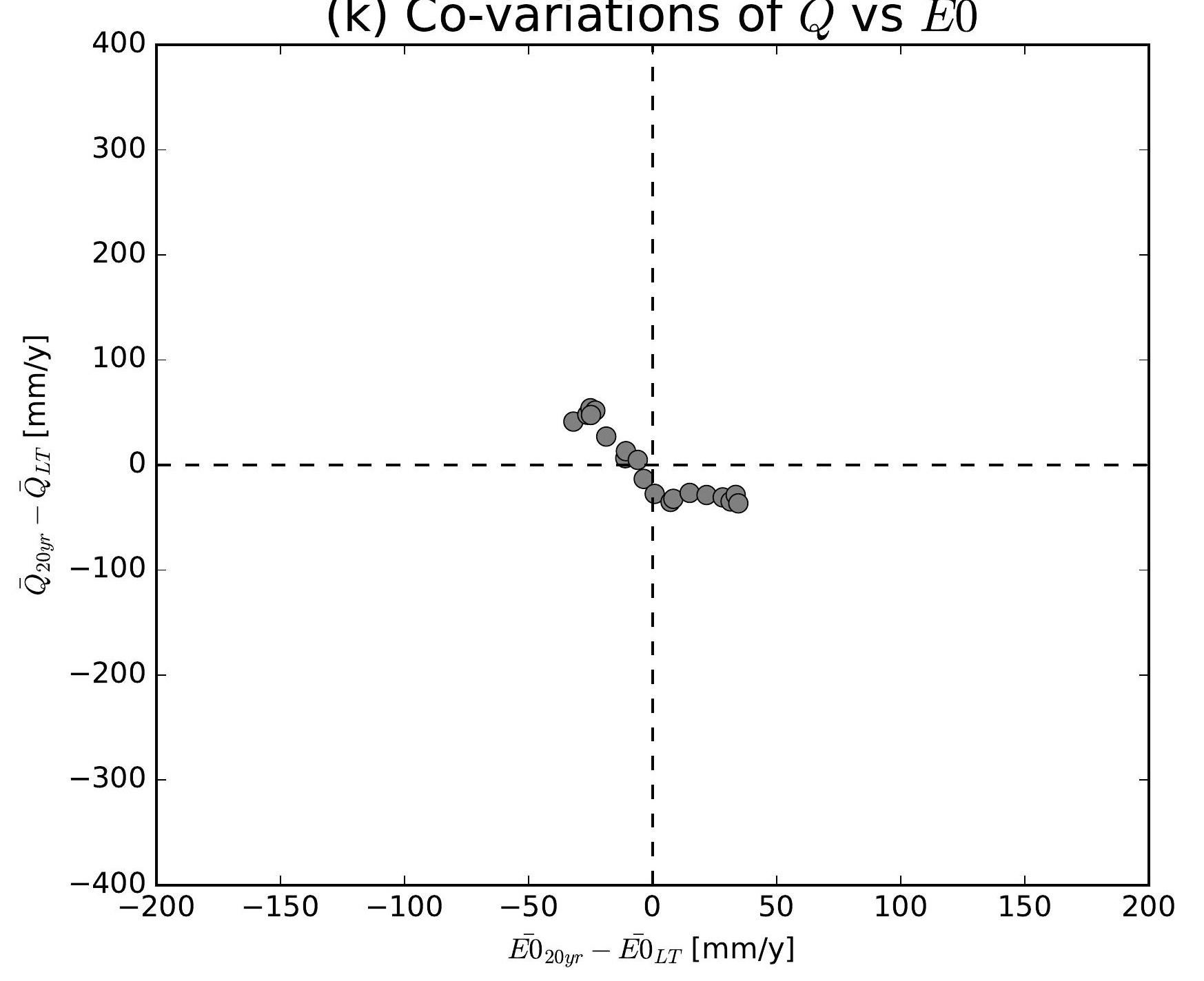

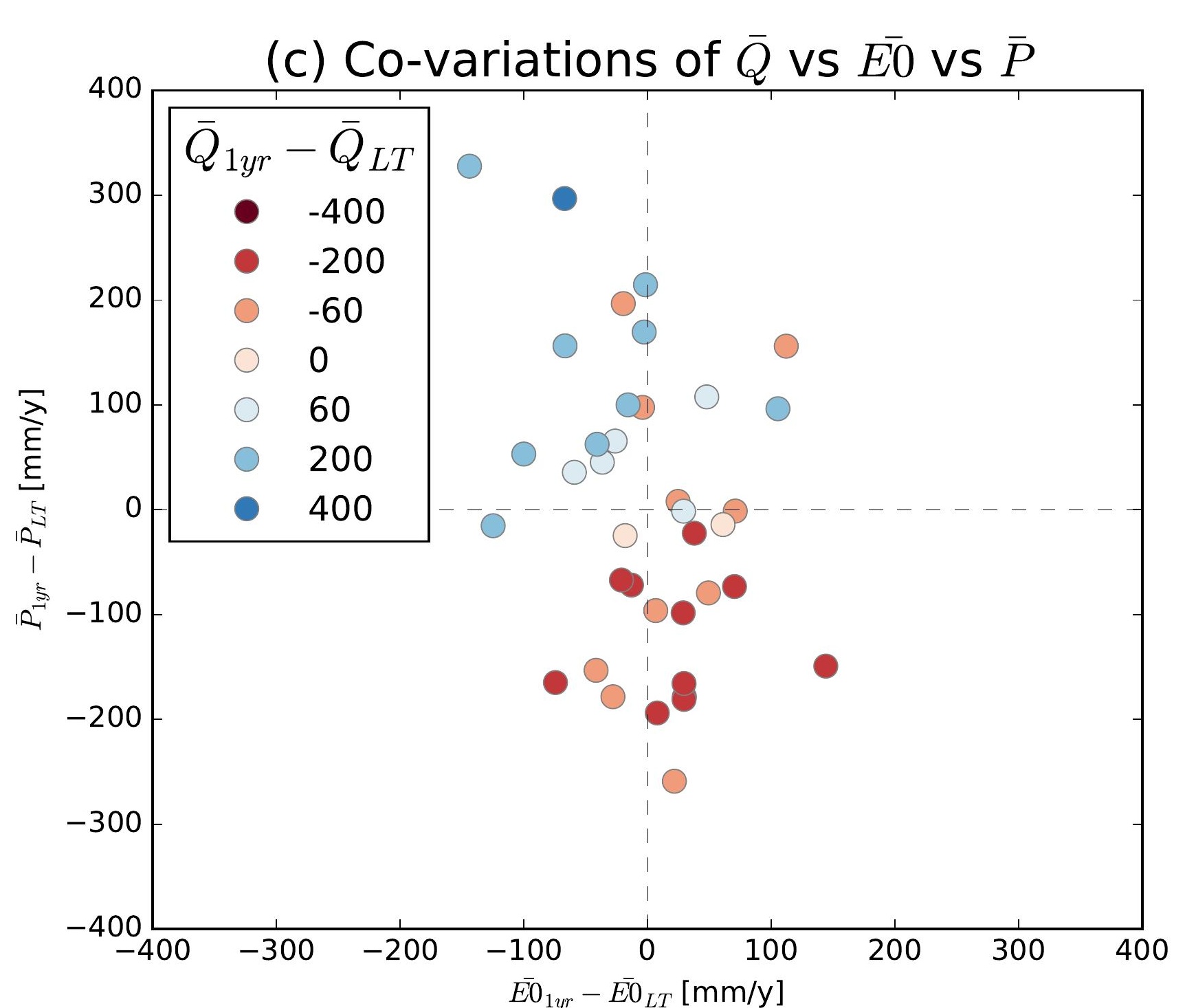
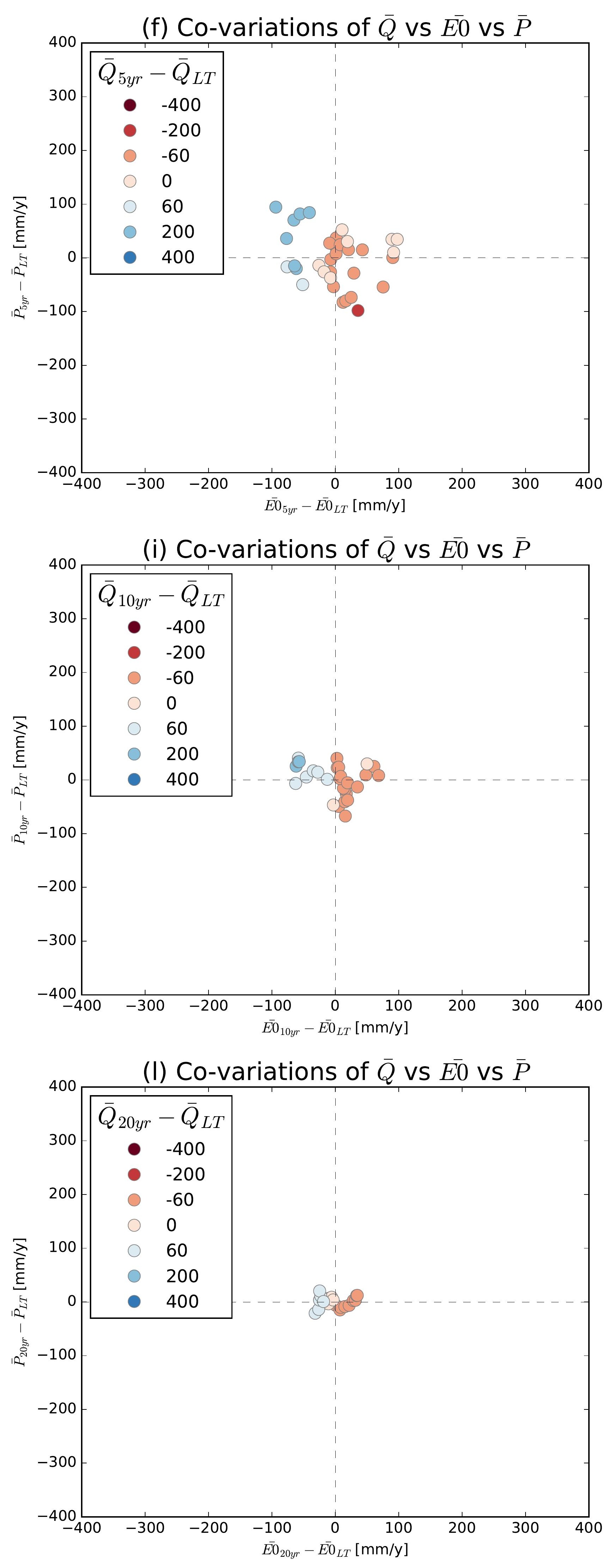

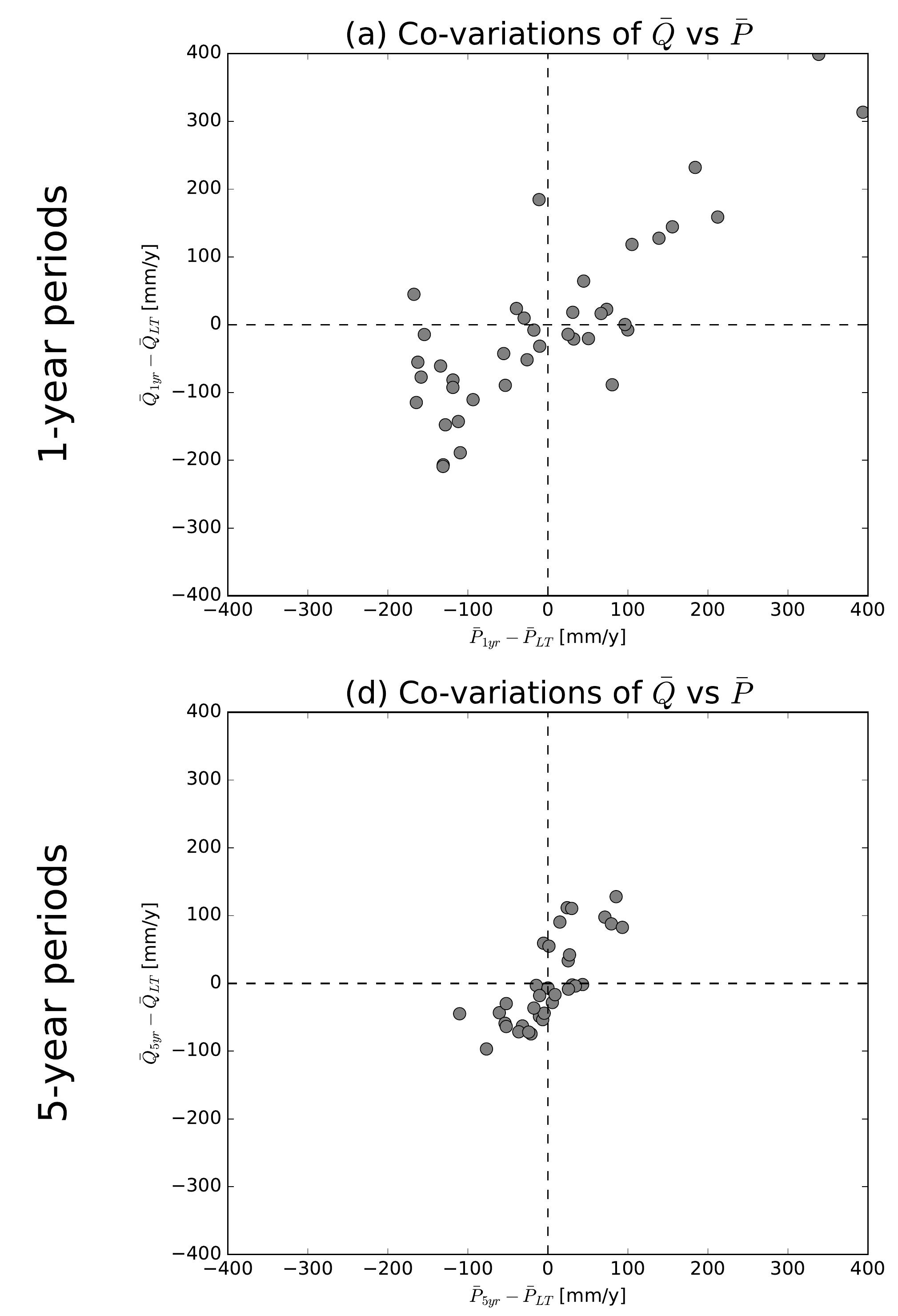

(g) Co-variations of $\bar{Q}$ vs $\bar{P}$
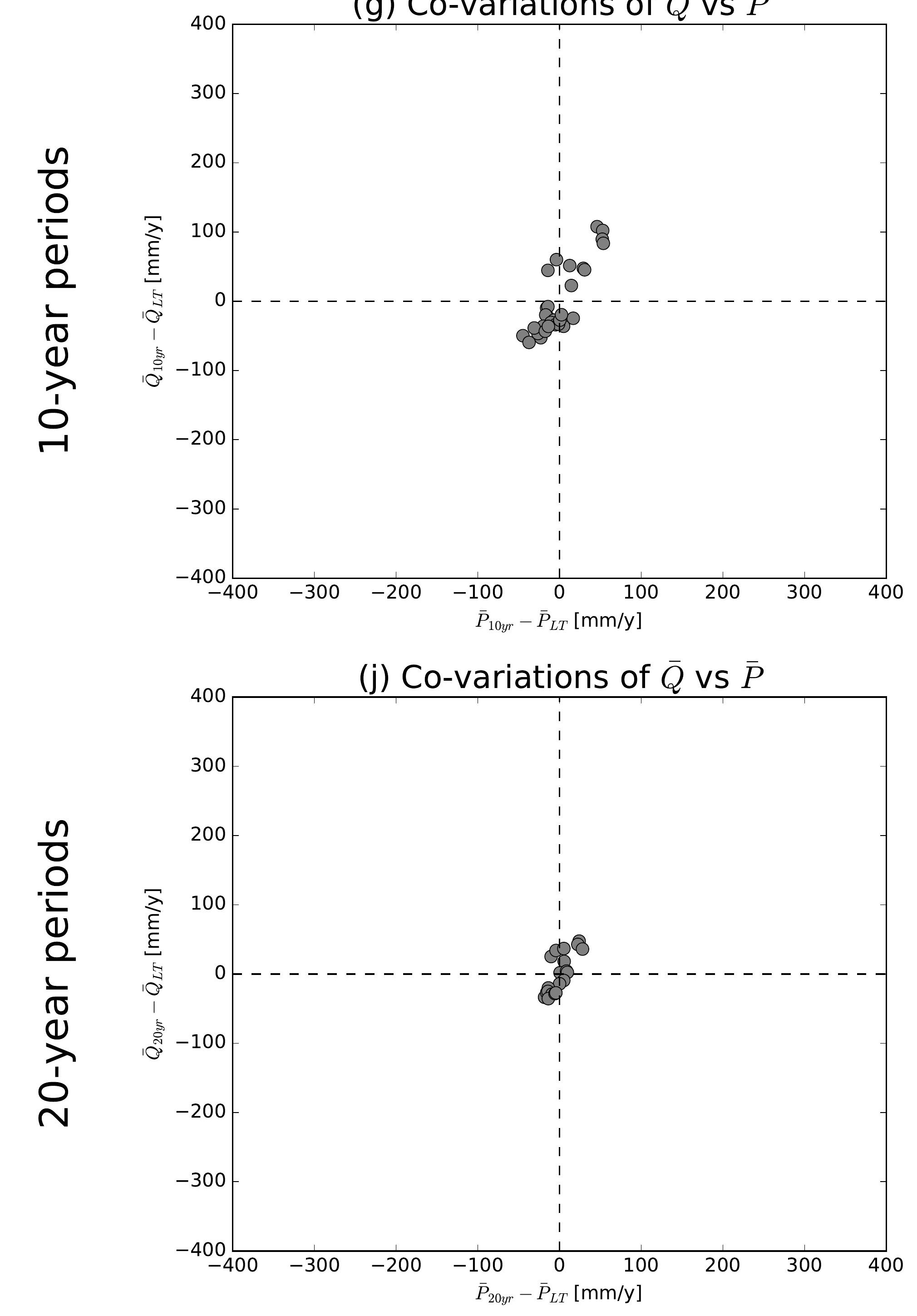

(b) Co-variations of $\bar{Q}$ vs $\overline{E 0}$

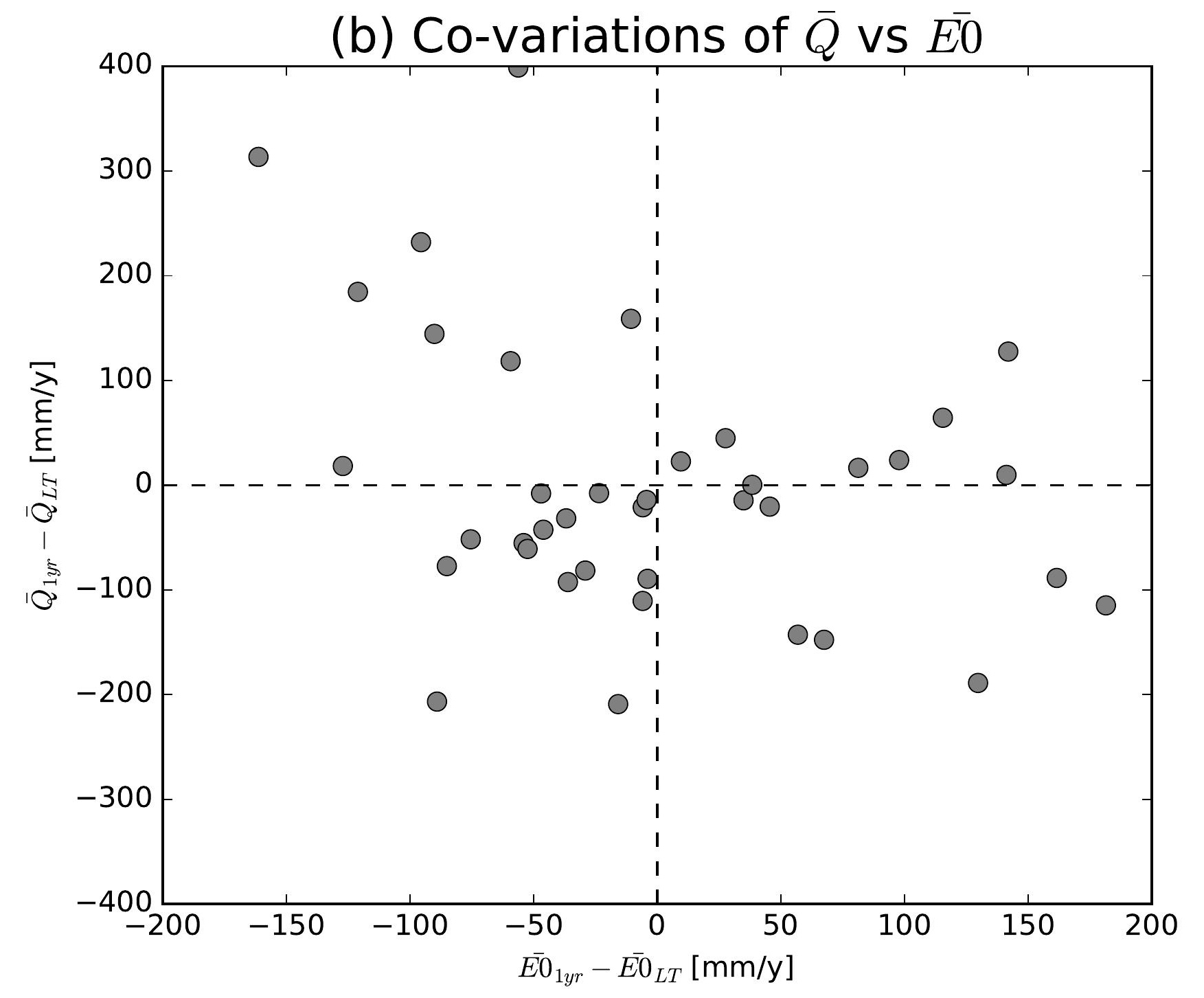

(e) Co-variations of $\bar{Q}$ vs $\overline{E 0}$

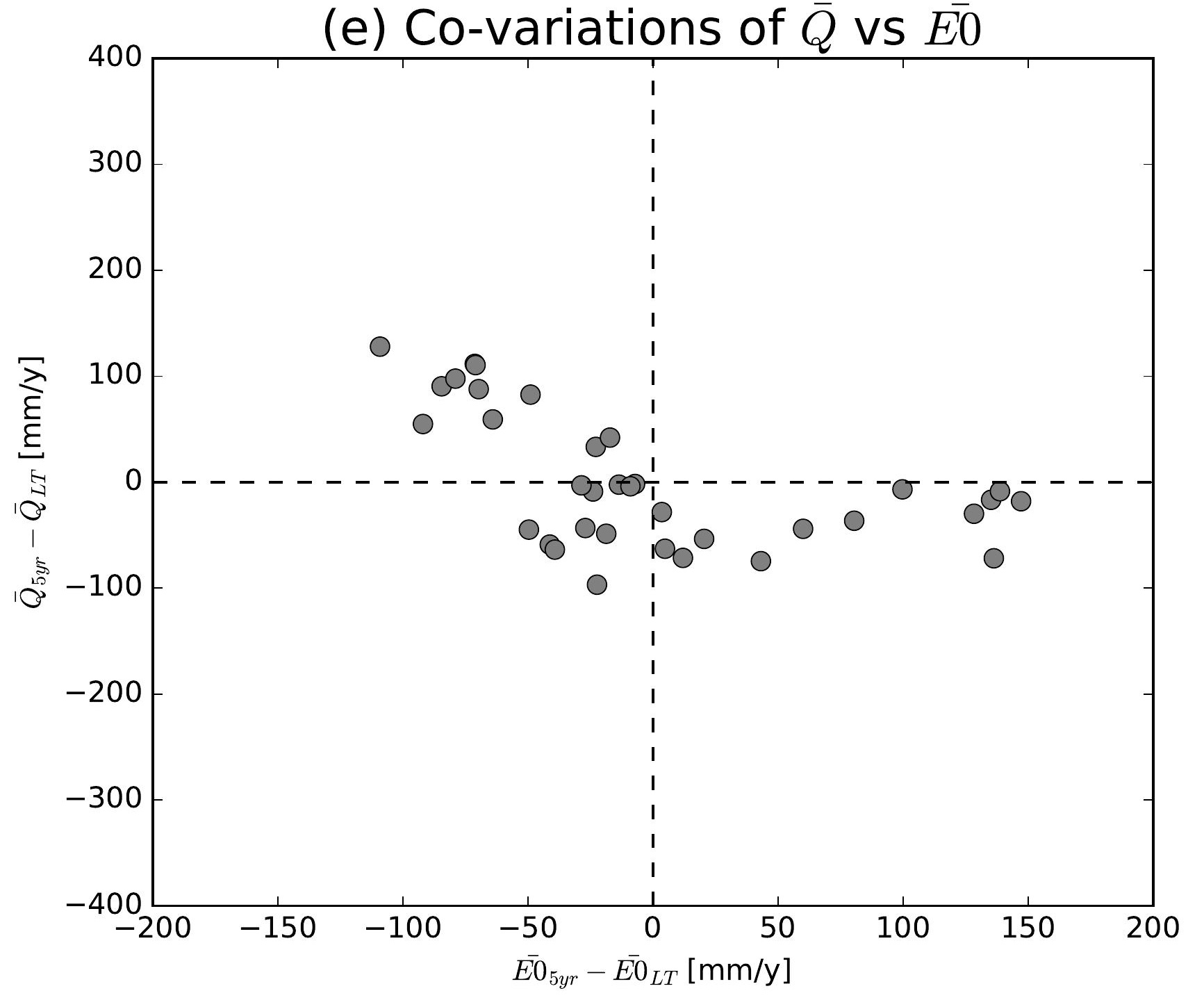

(h) Co-variations of $\bar{Q}$ vs $\overline{E 0}$

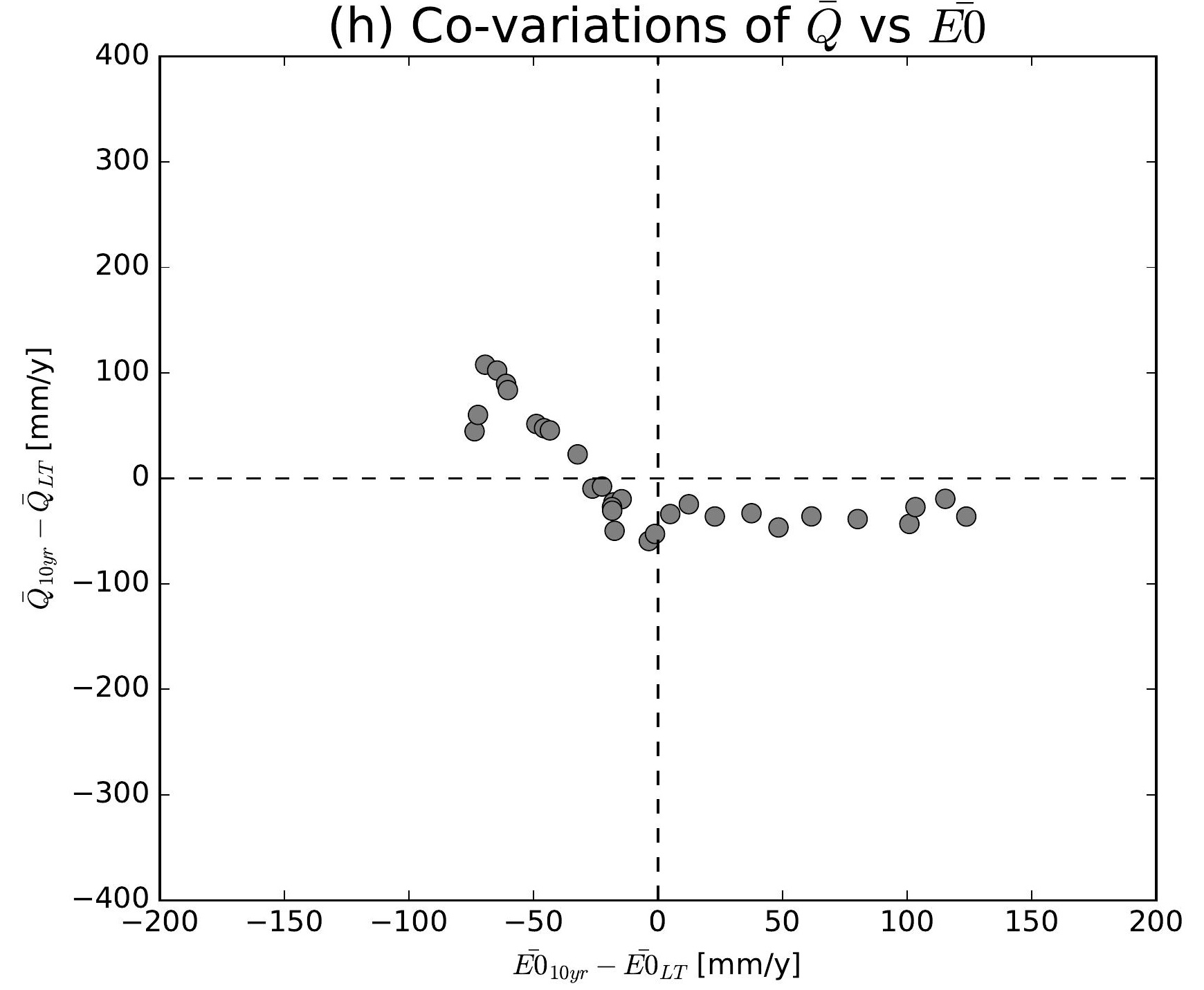

(k) Co-variations of $\bar{Q}$ vs $\overline{E 0}$

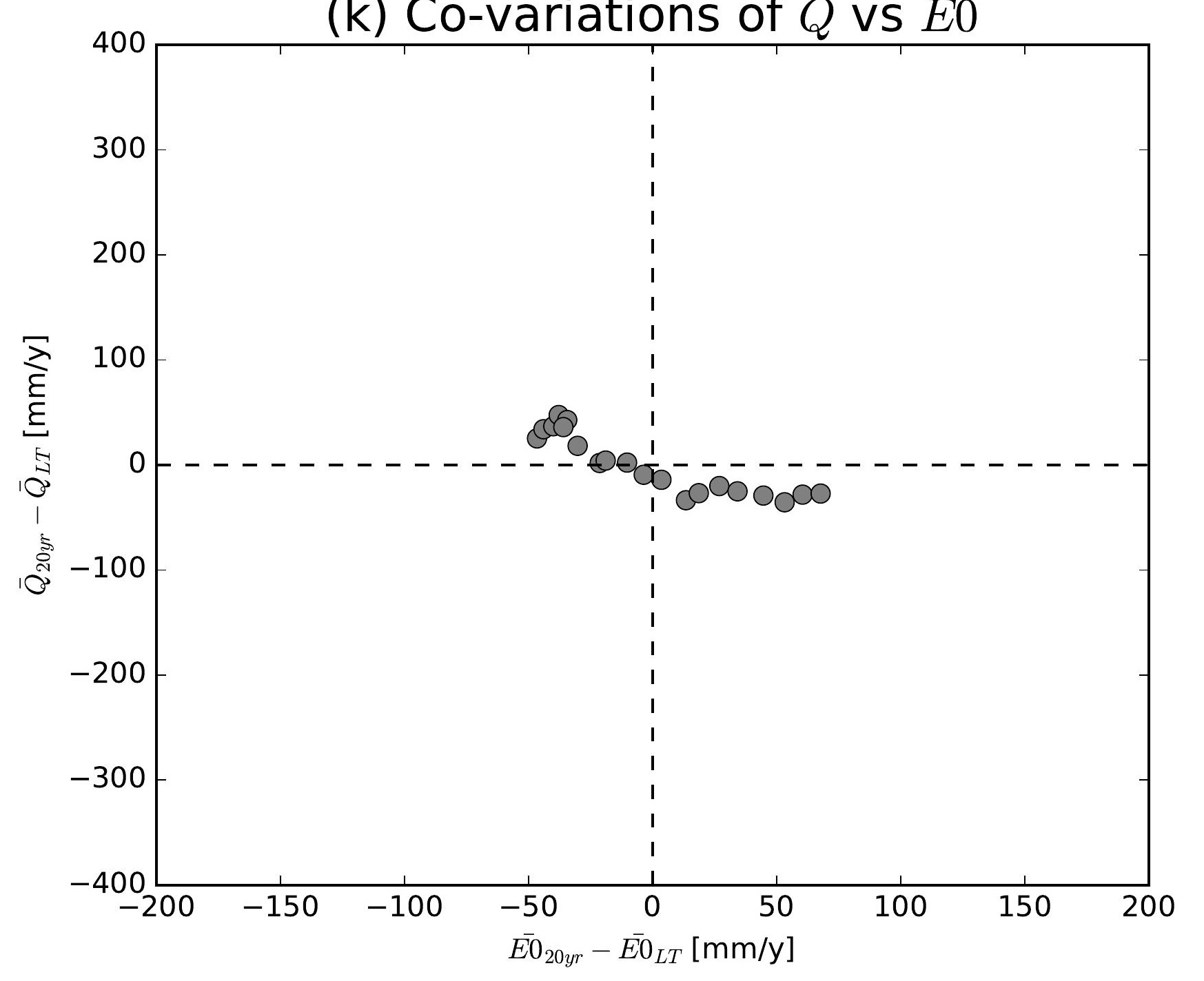

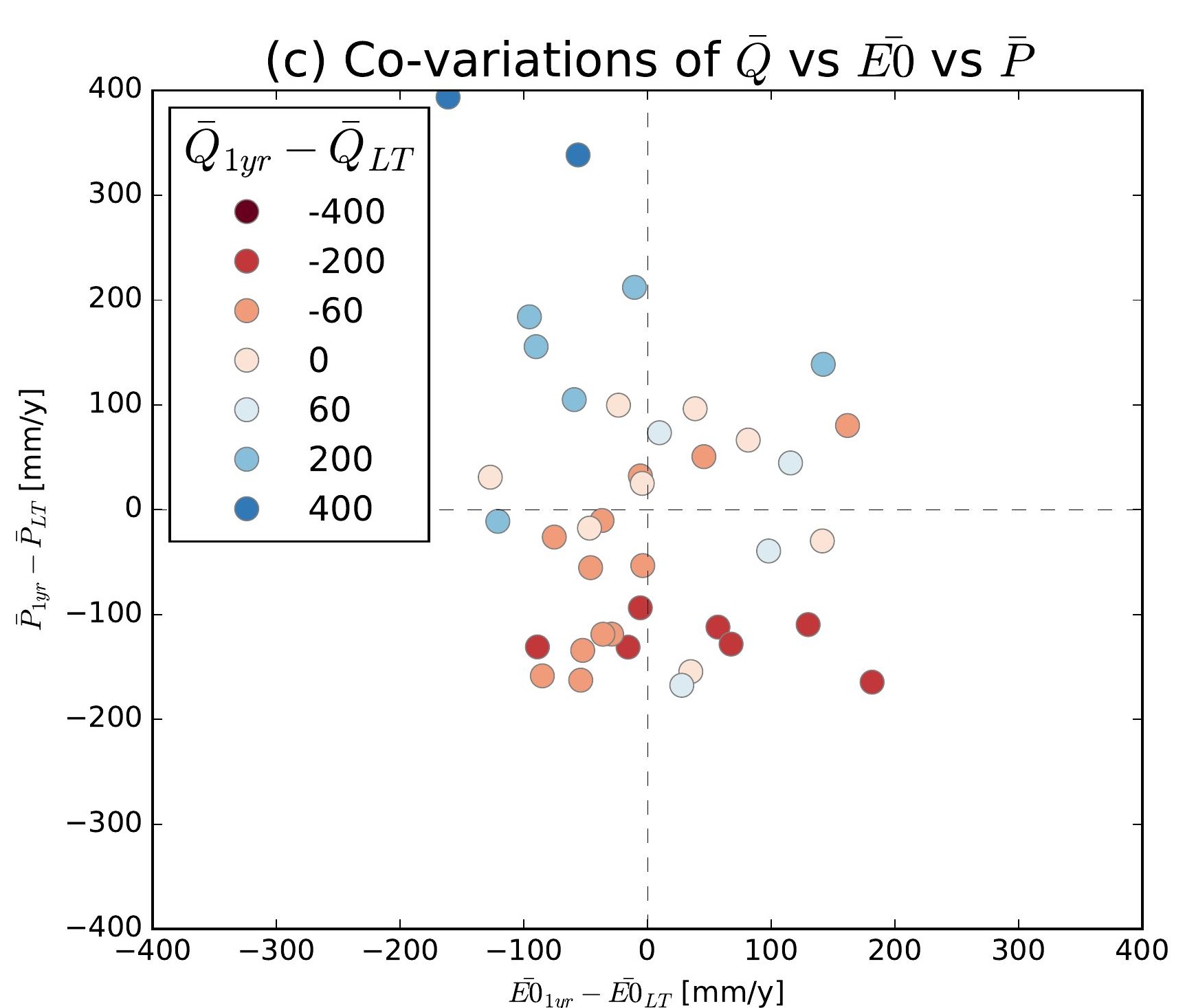
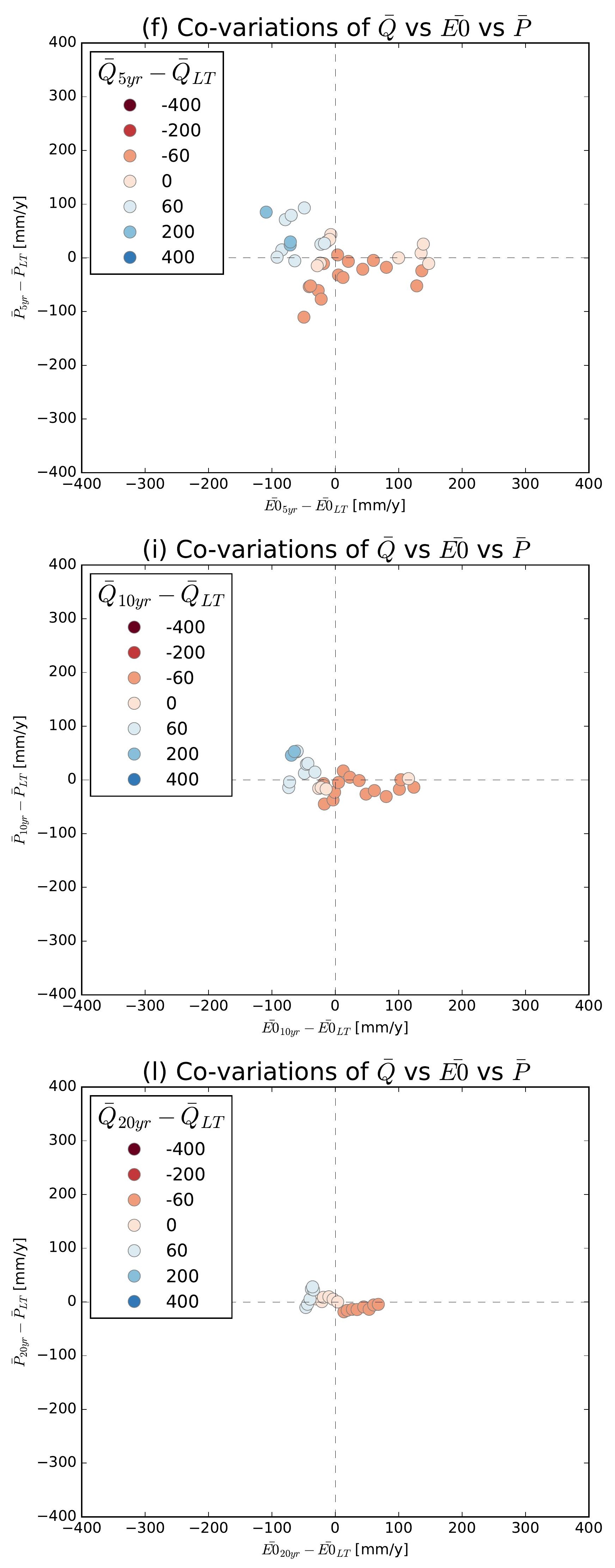

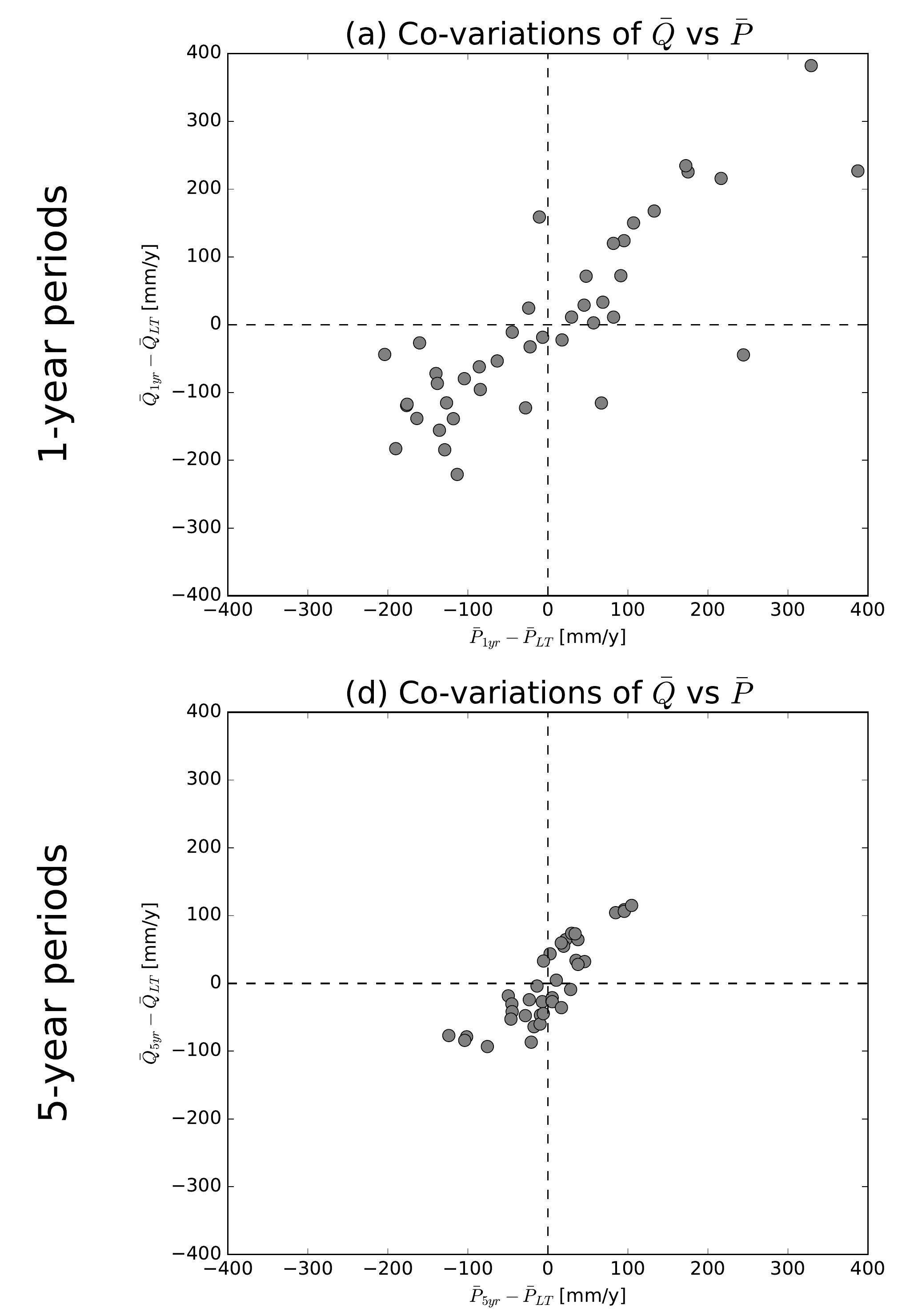

(g) Co-variations of $\bar{Q}$ vs $\bar{P}$
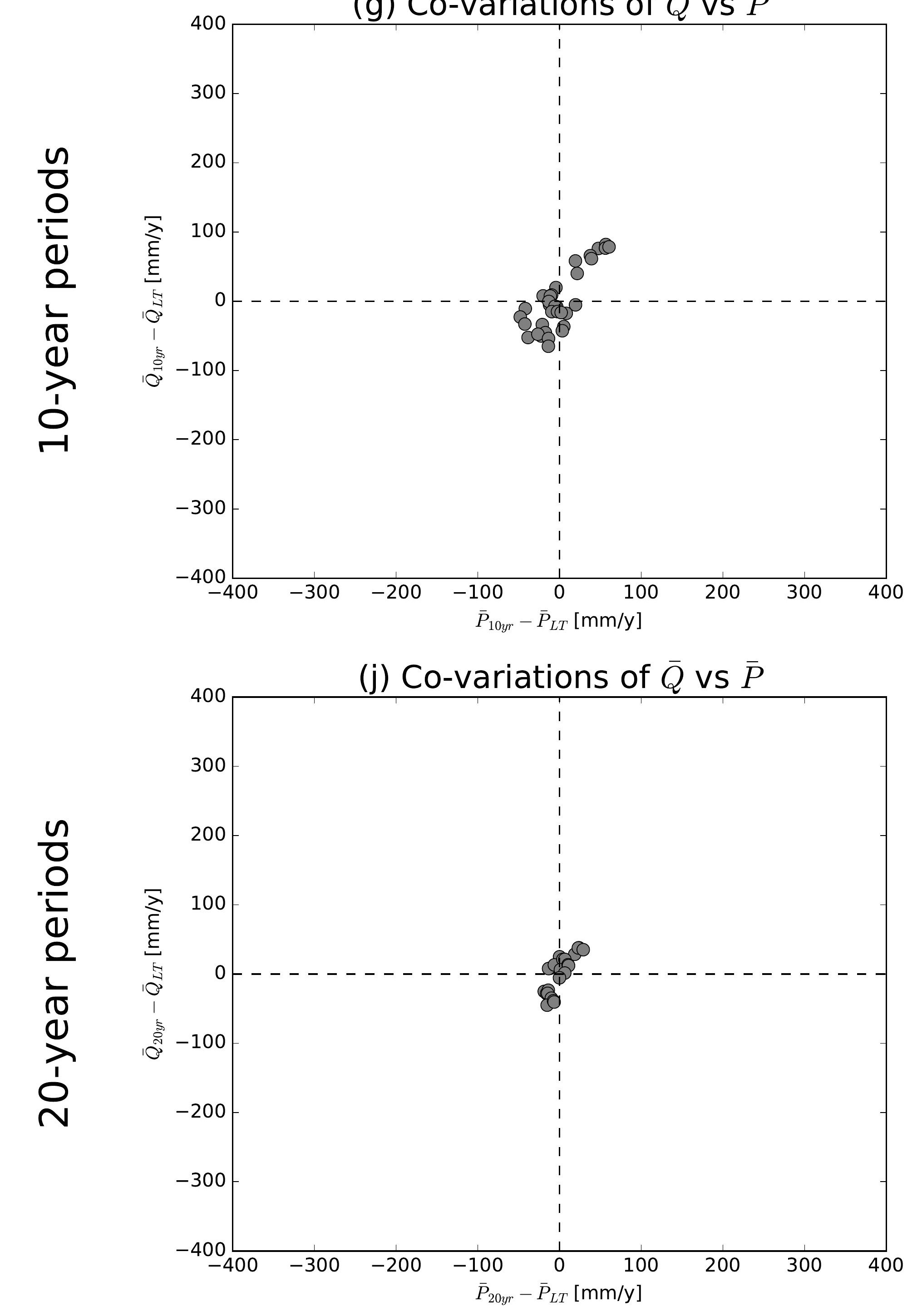

(b) Co-variations of $\bar{Q}$ vs $\overline{E 0}$

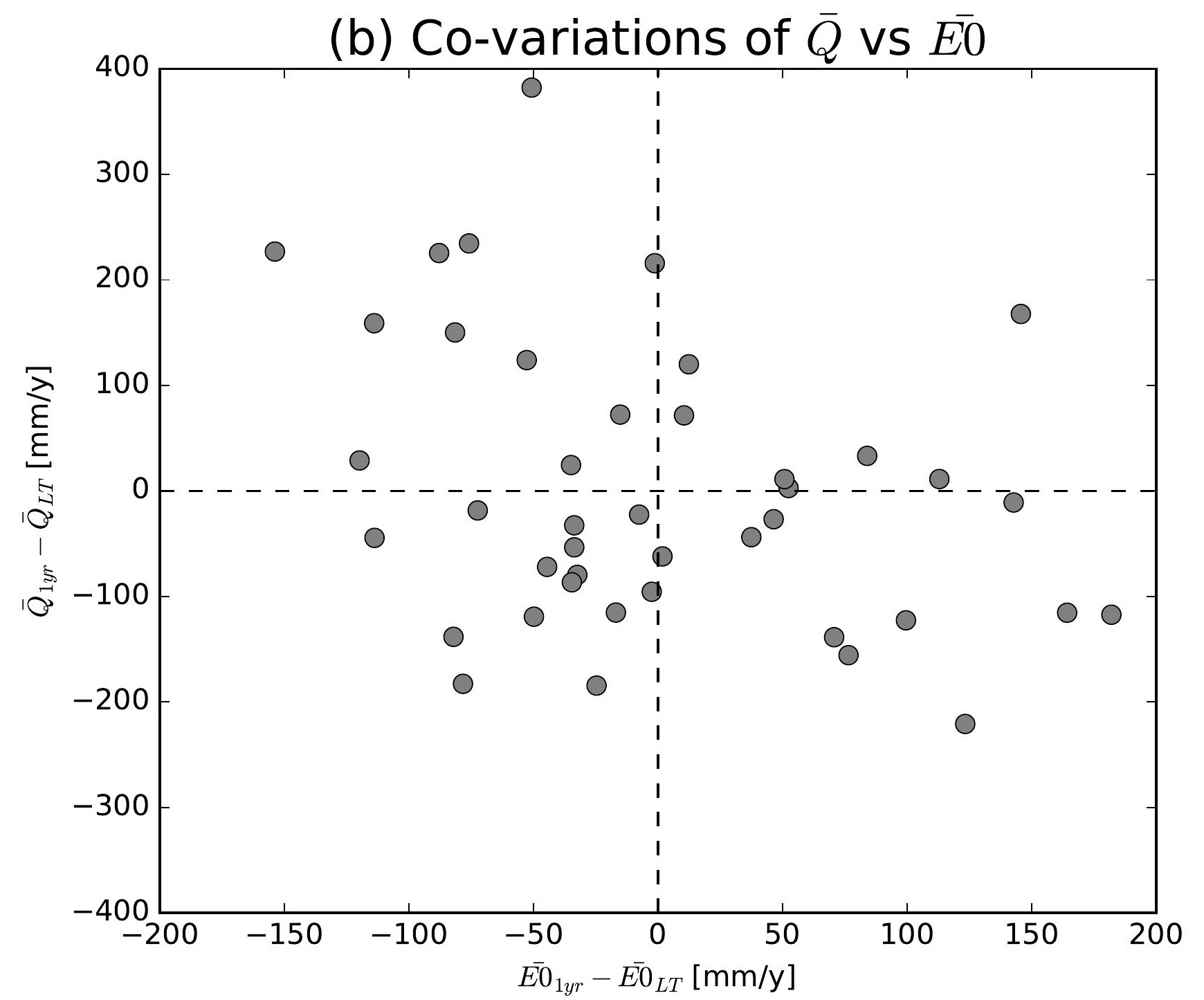

(e) Co-variations of $\bar{Q}$ vs $\overline{E 0}$

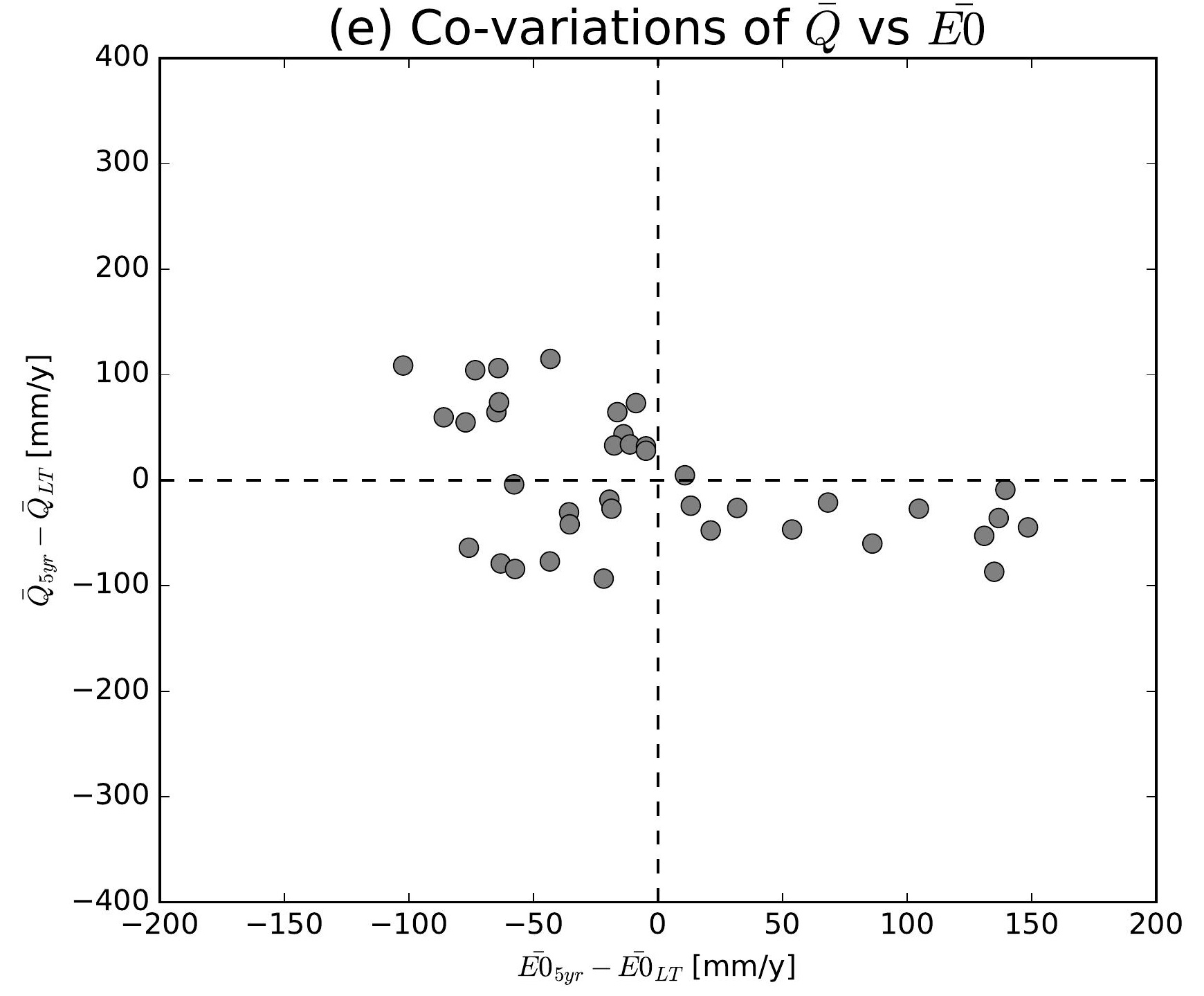

(h) Co-variations of $\bar{Q}$ vs $\overline{E 0}$

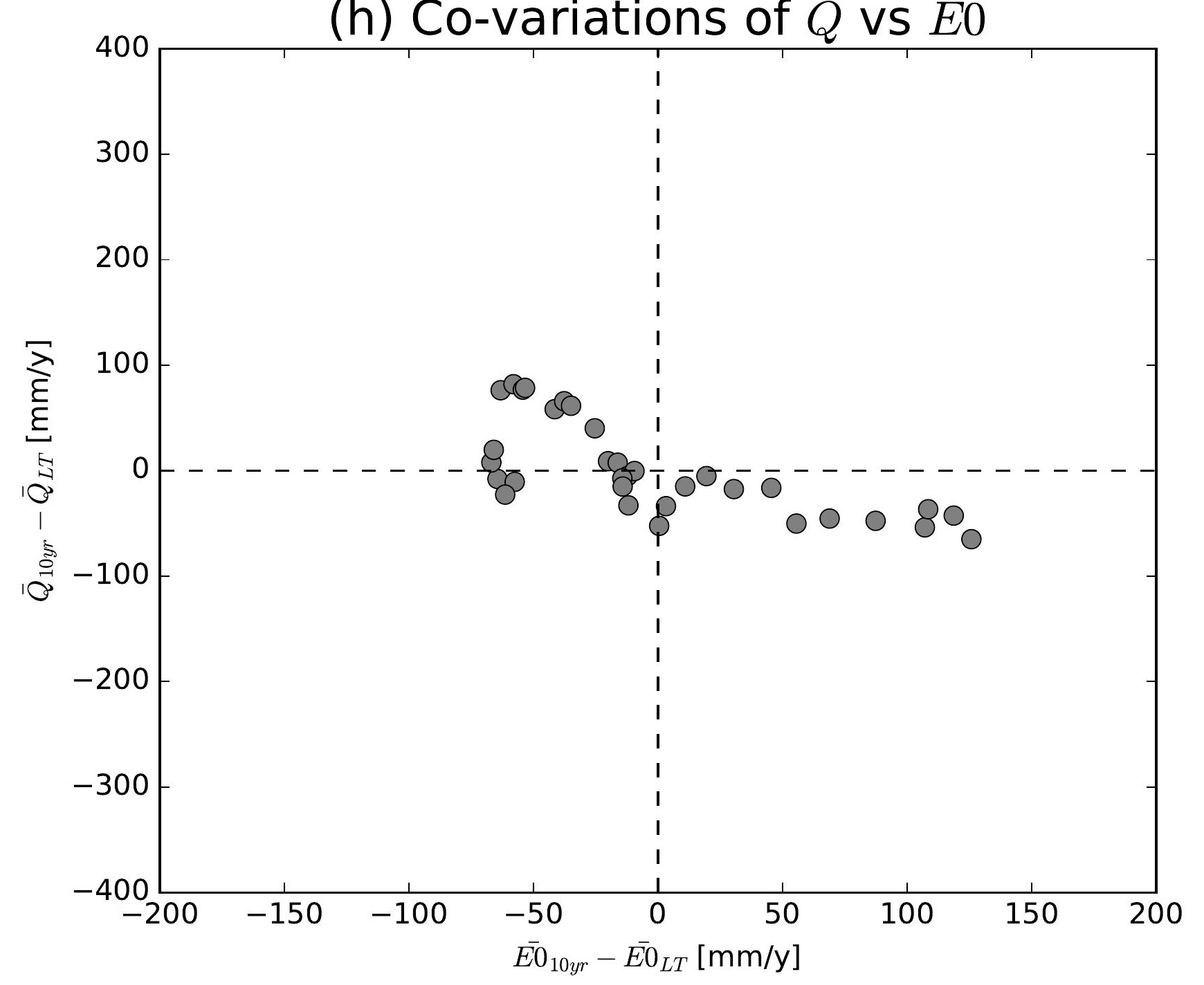

(k) Co-variations of $\bar{Q}$ vs $\overline{E 0}$

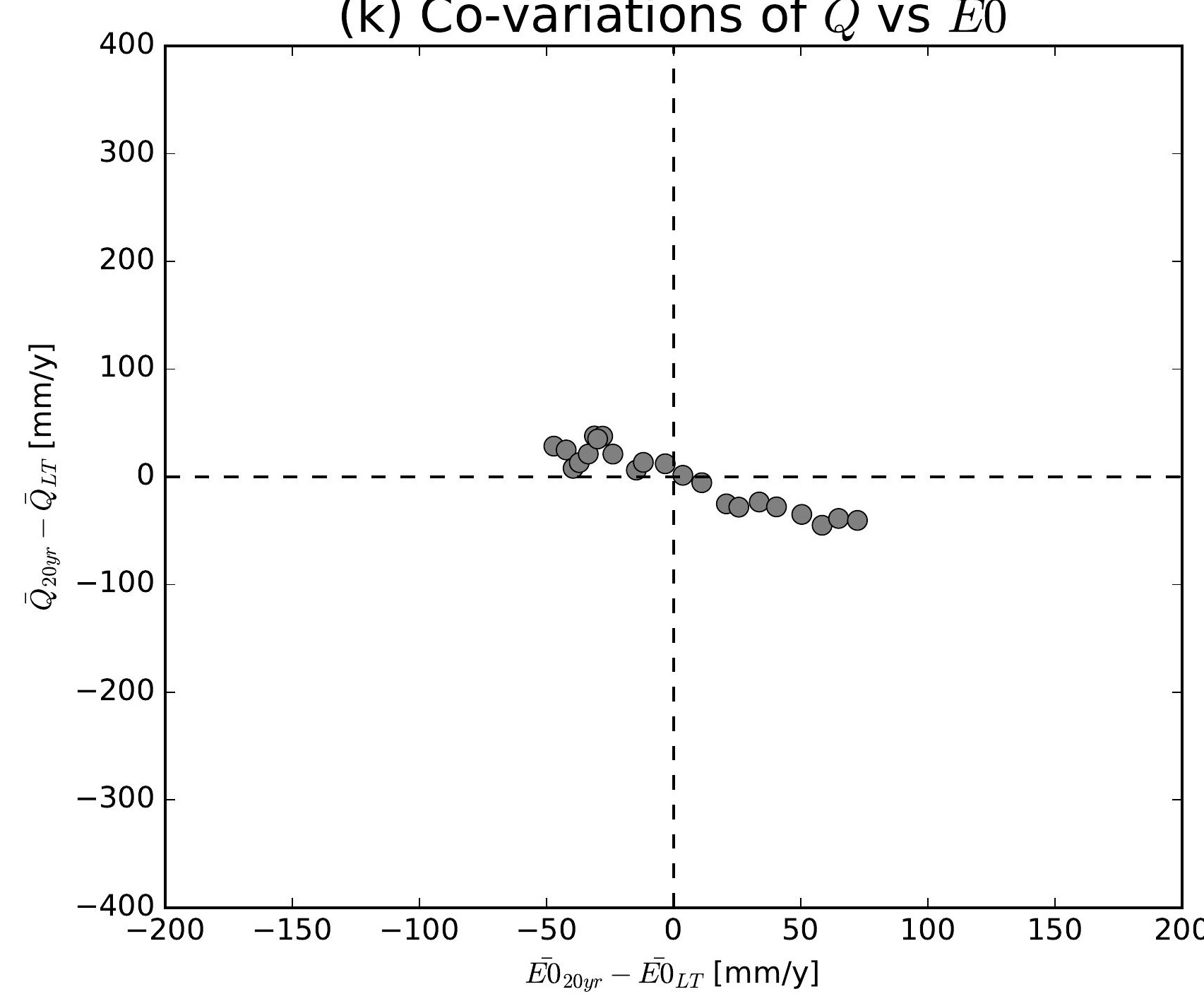

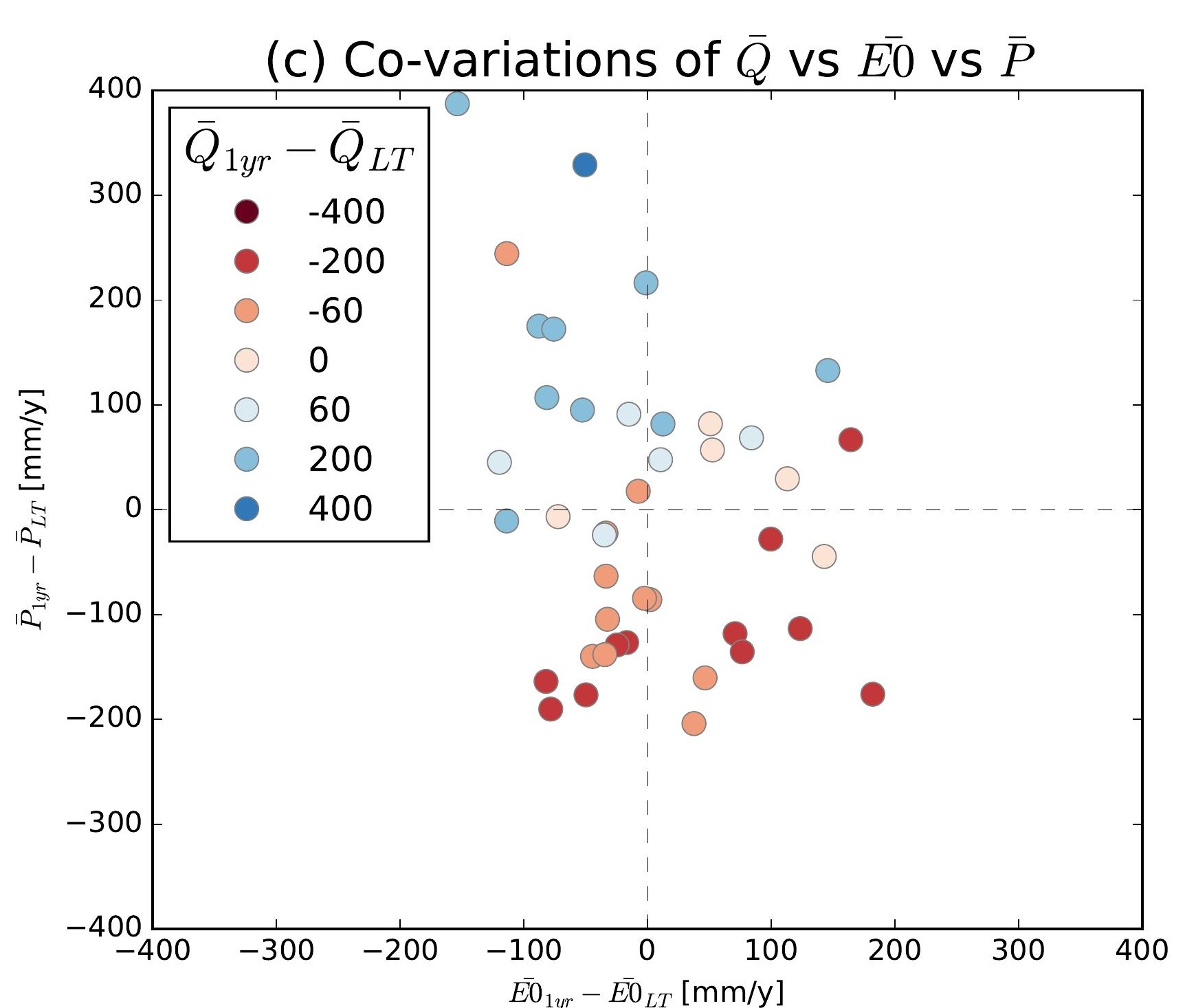
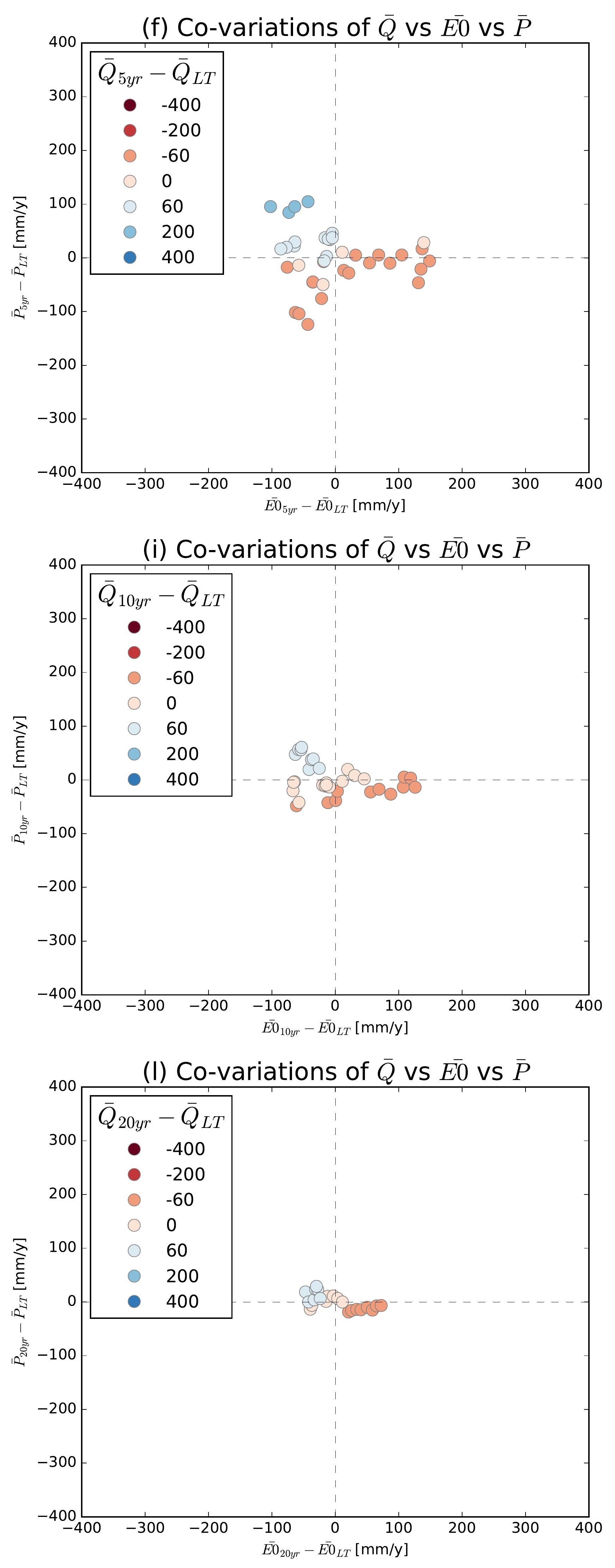

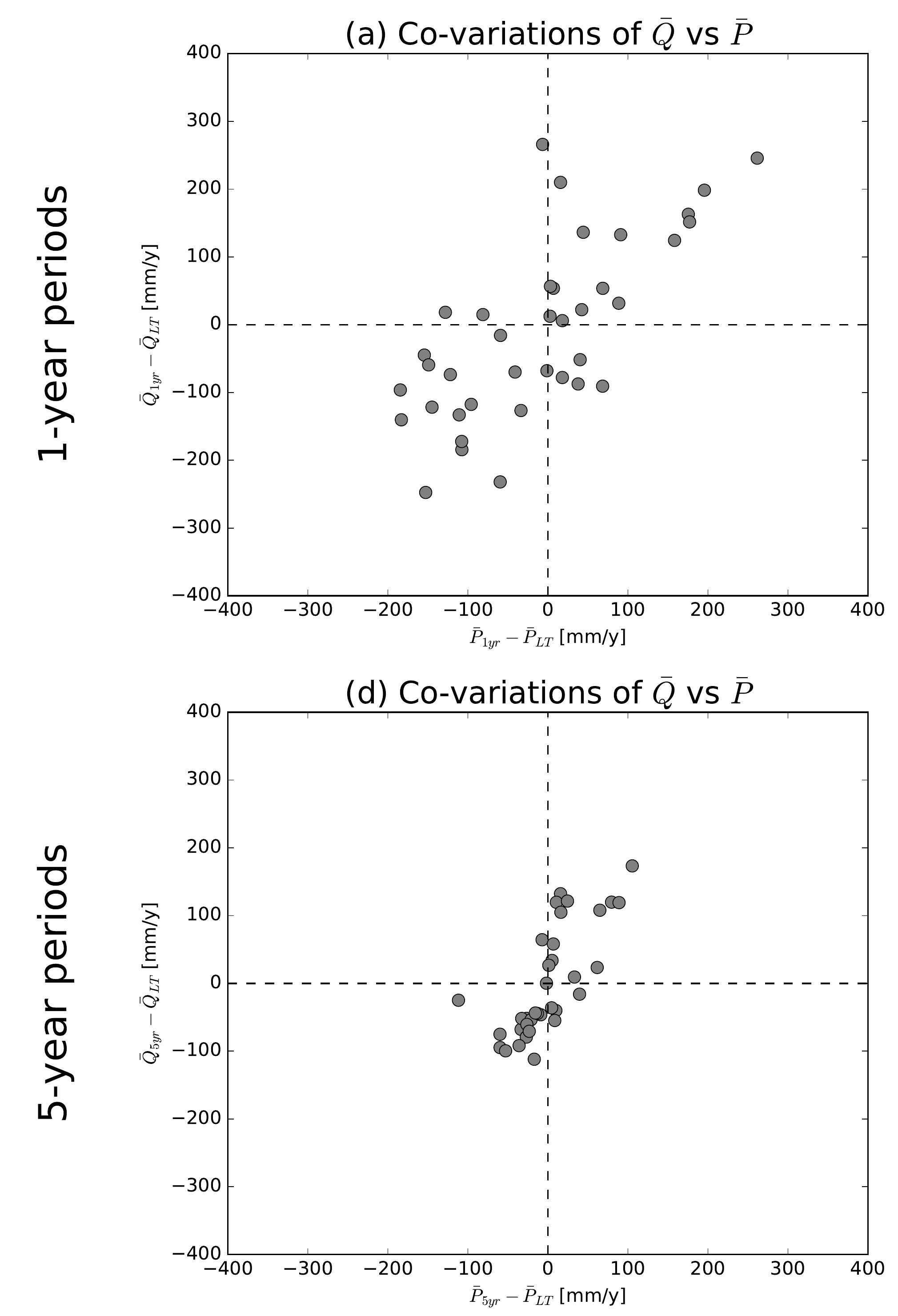

(g) Co-variations of $\bar{Q}$ vs $\bar{P}$
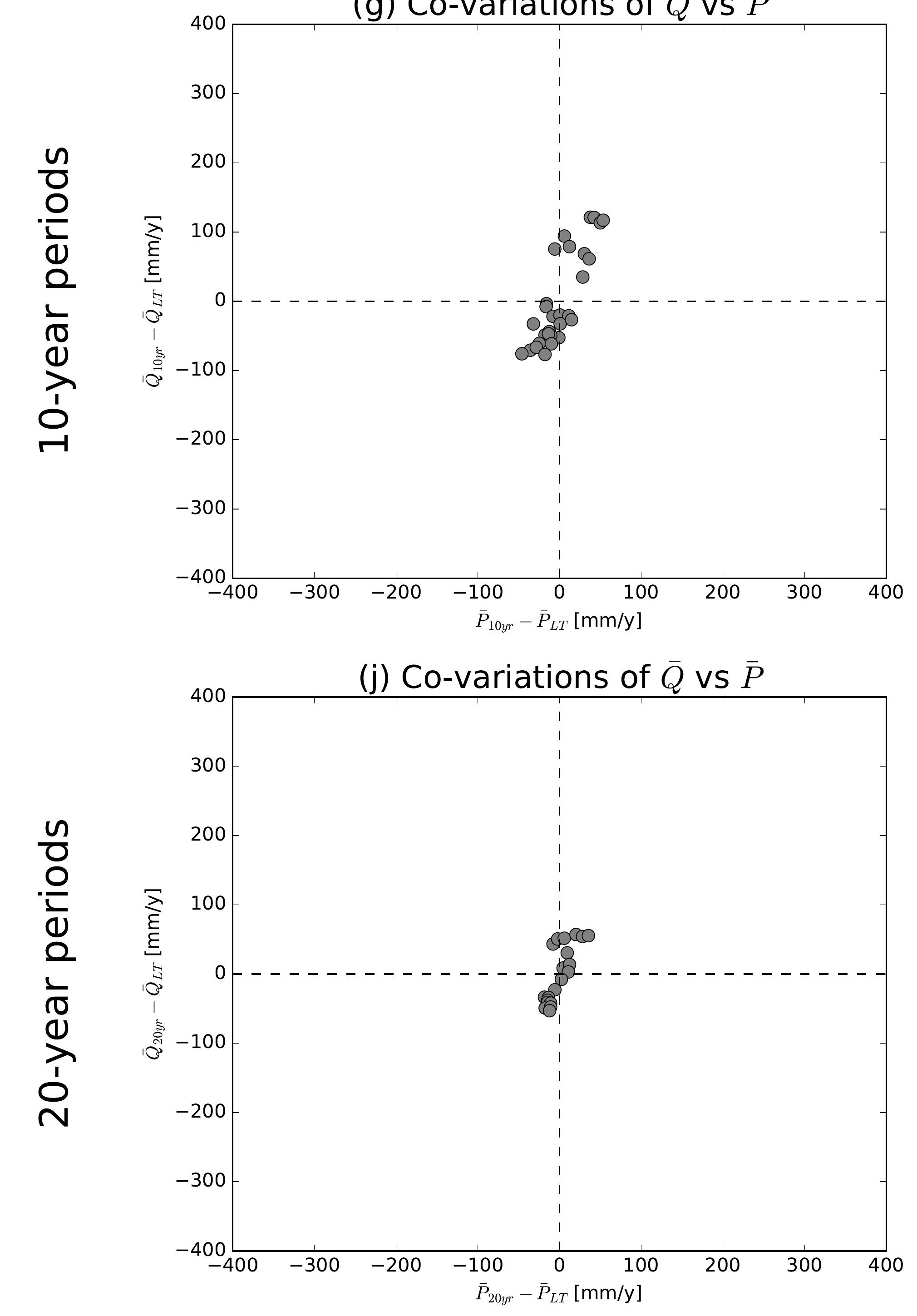

(b) Co-variations of $\bar{Q}$ vs $\overline{E 0}$

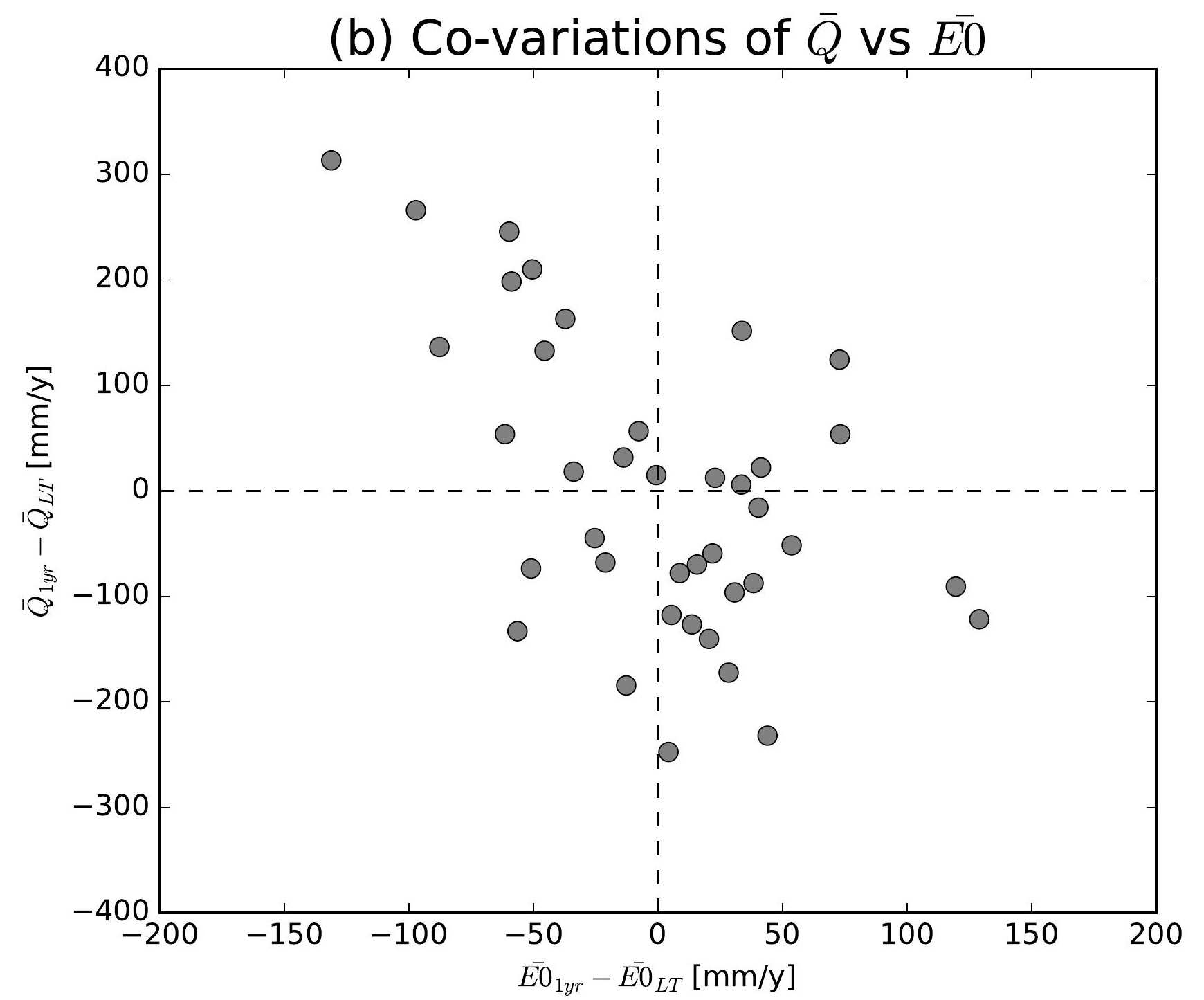

(e) Co-variations of $\bar{Q}$ vs $\overline{E 0}$

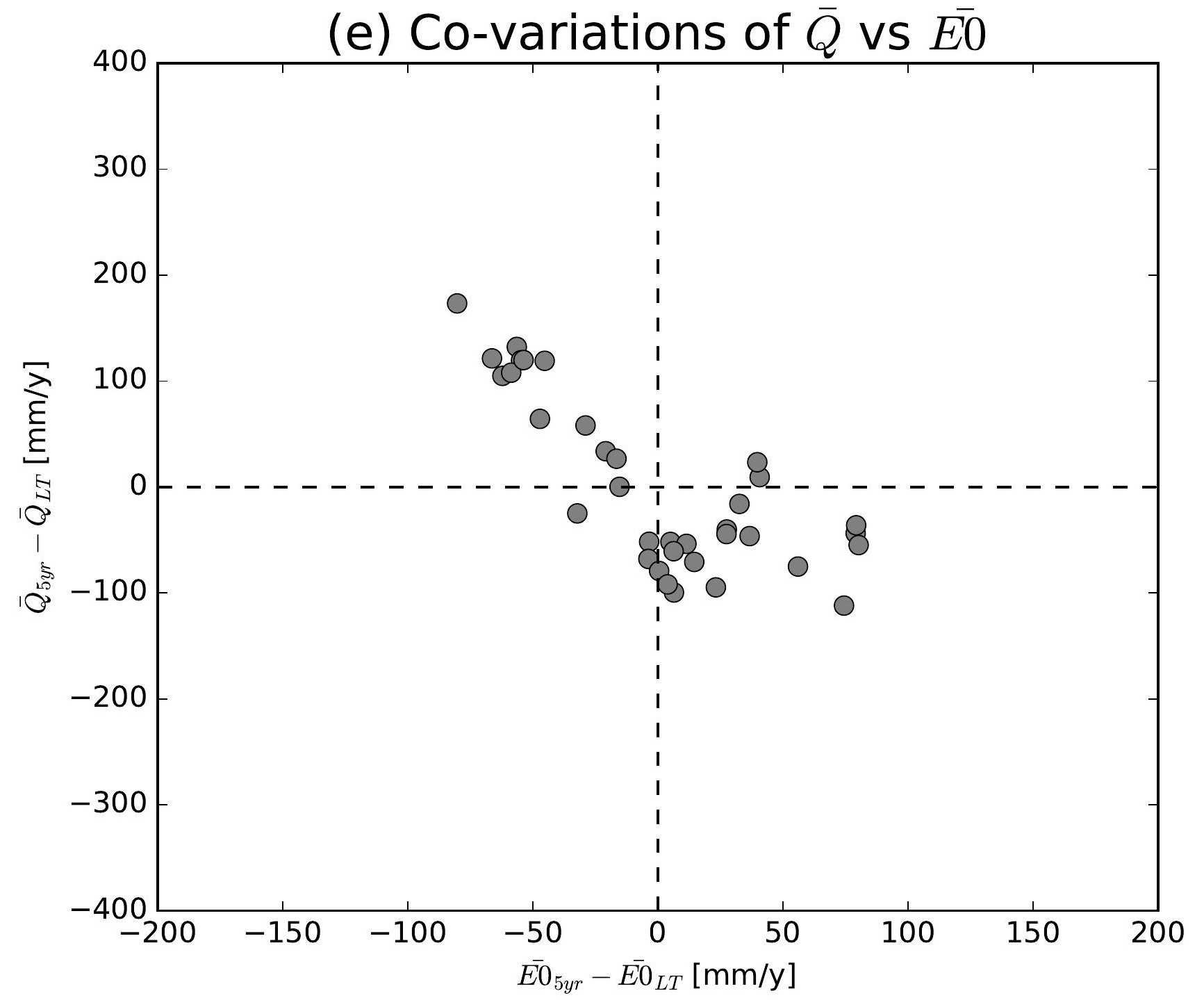

(h) Co-variations of $\bar{Q}$ vs $\overline{E 0}$

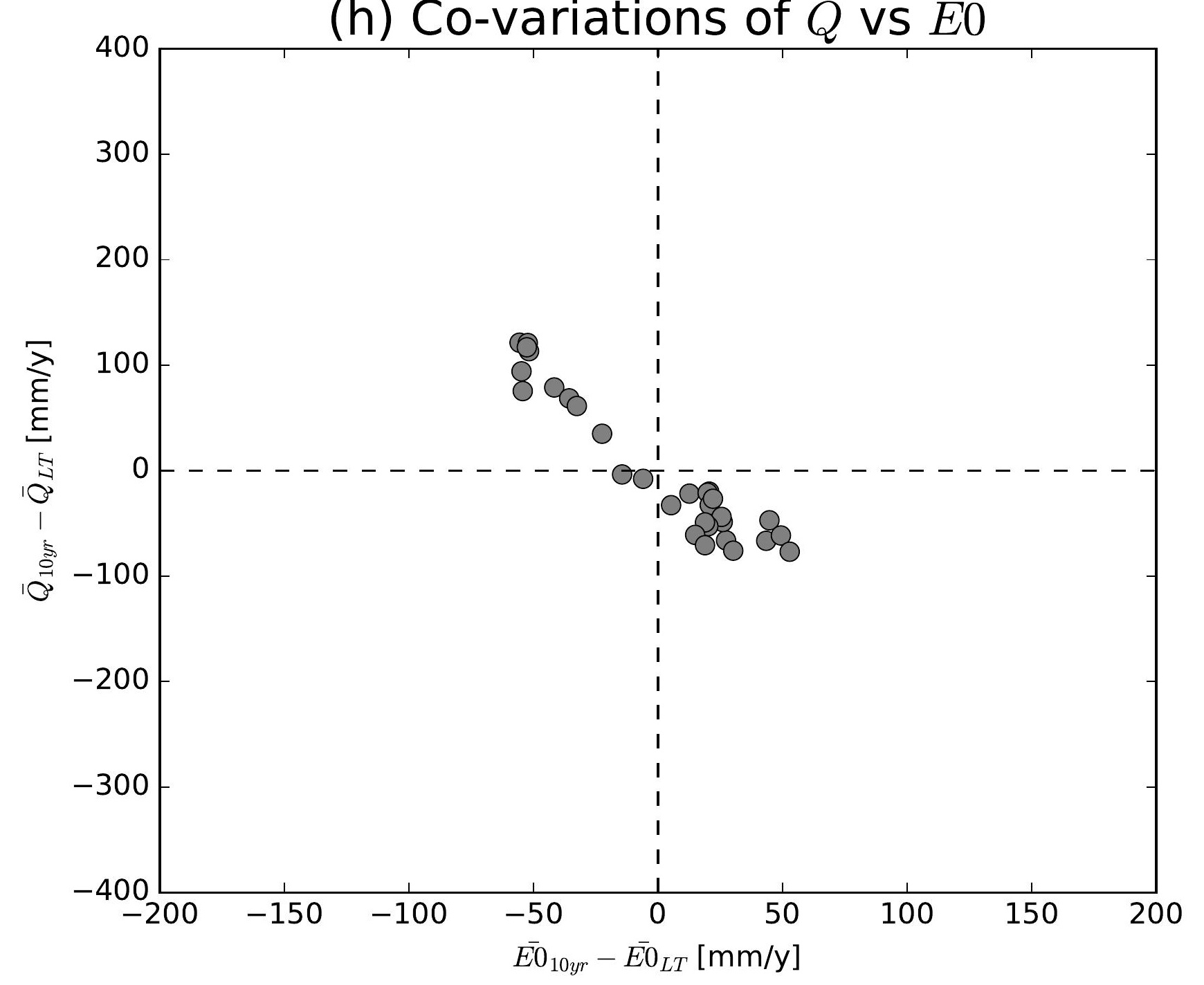

(k) Co-variations of $\bar{Q}$ vs $\overline{E 0}$

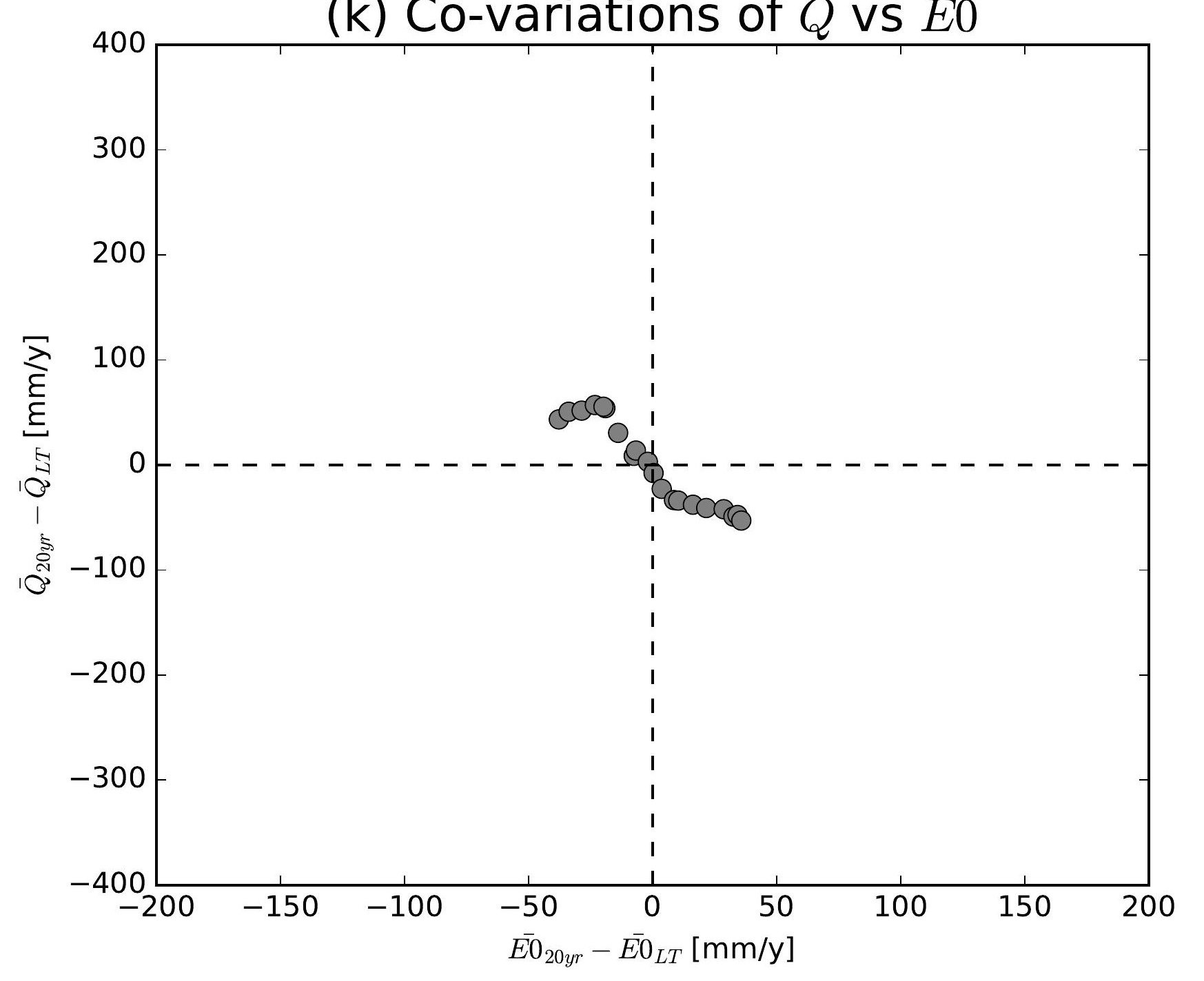

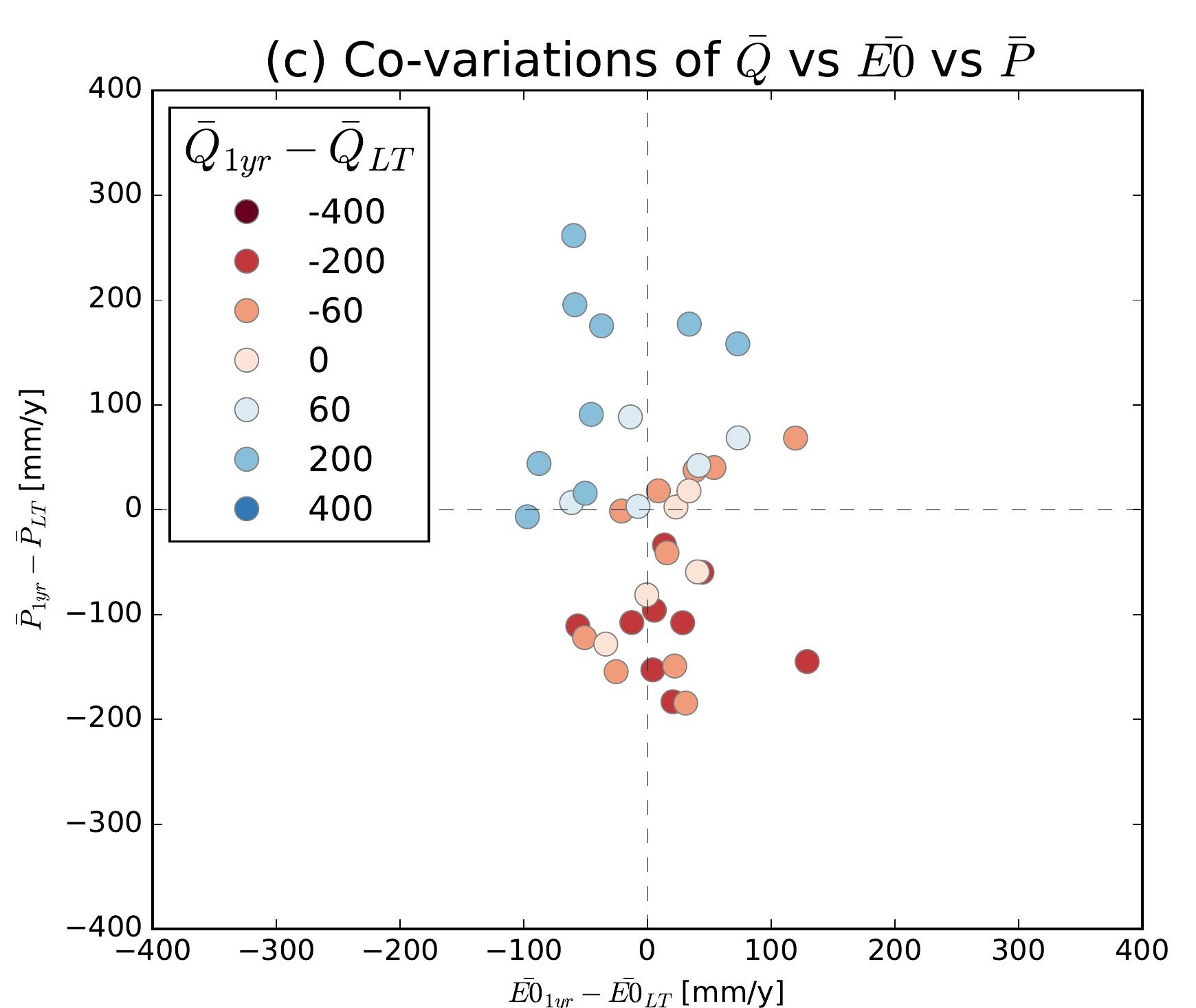
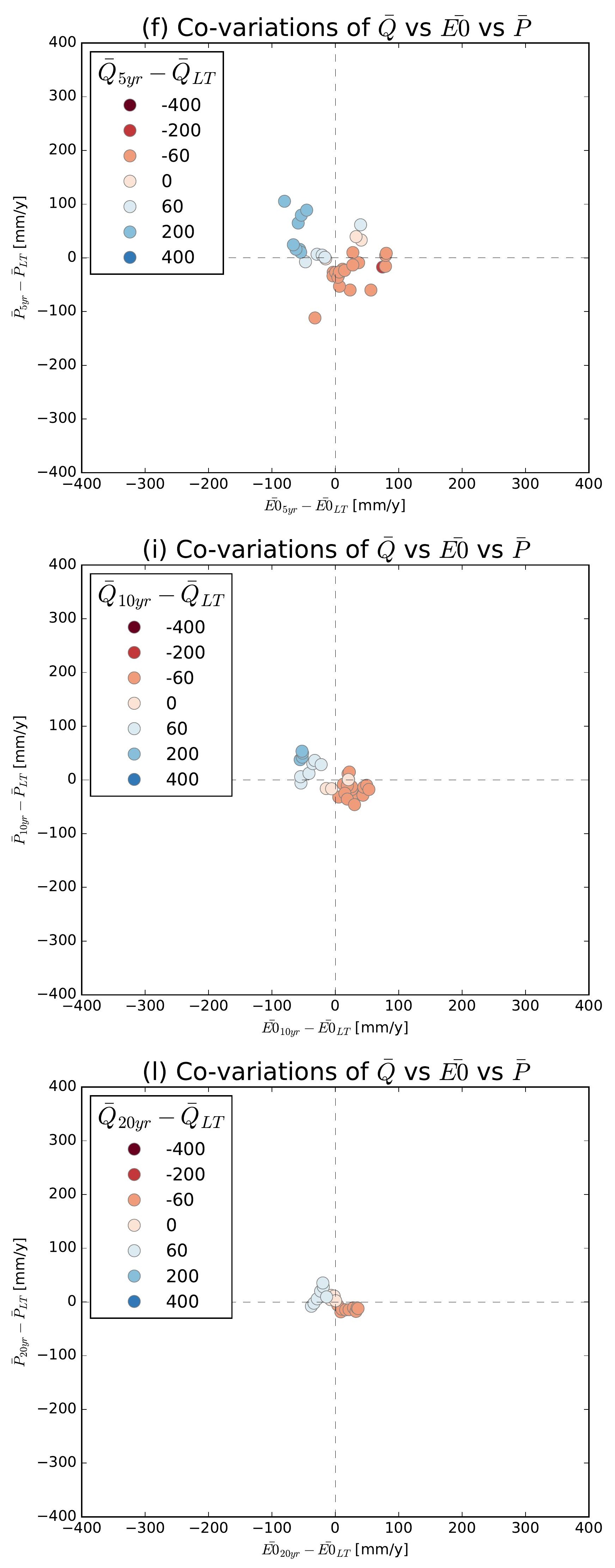

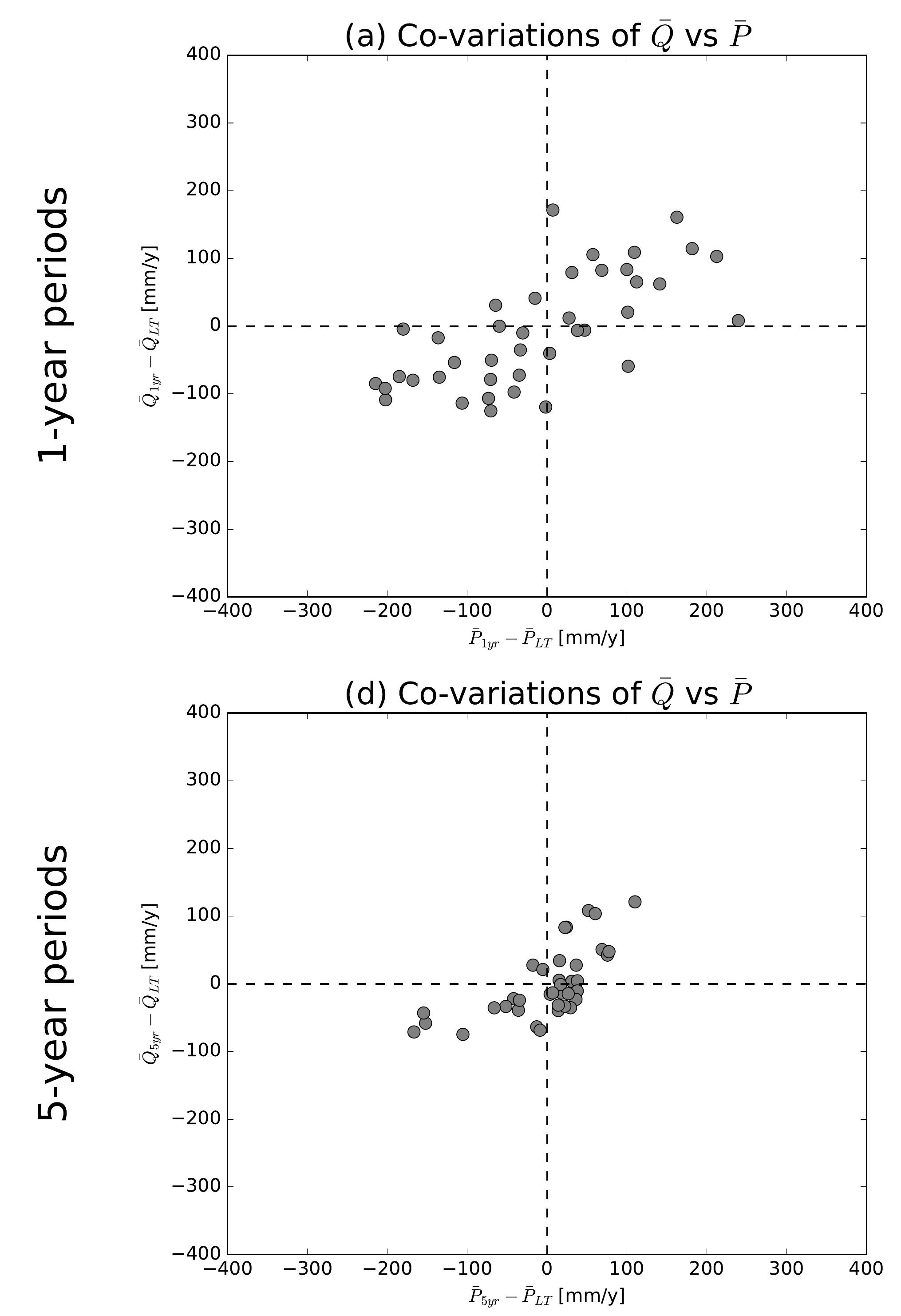

(g) Co-variations of $\bar{Q}$ vs $\bar{P}$
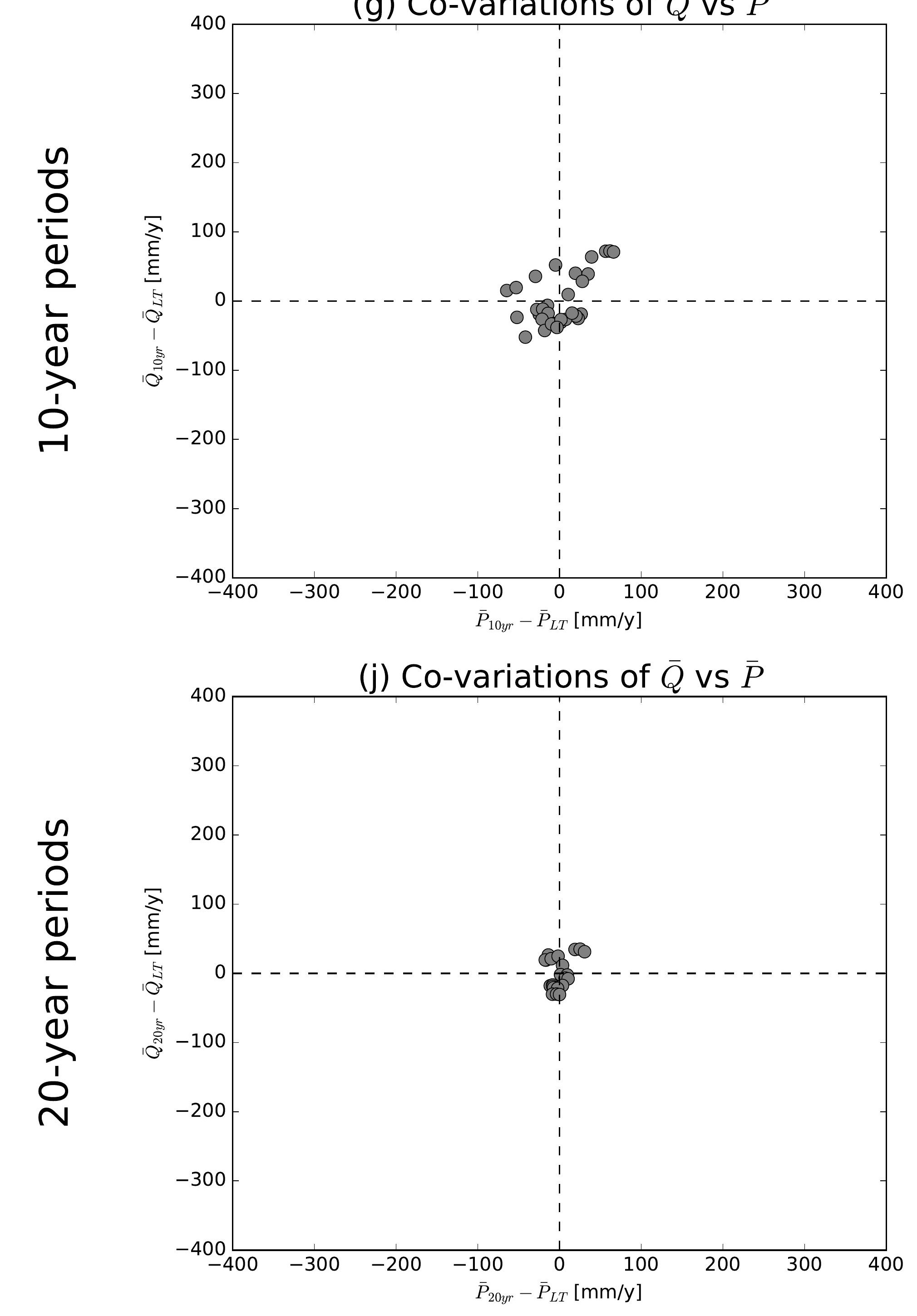

(b) Co-variations of $\bar{Q}$ vs $\overline{E 0}$

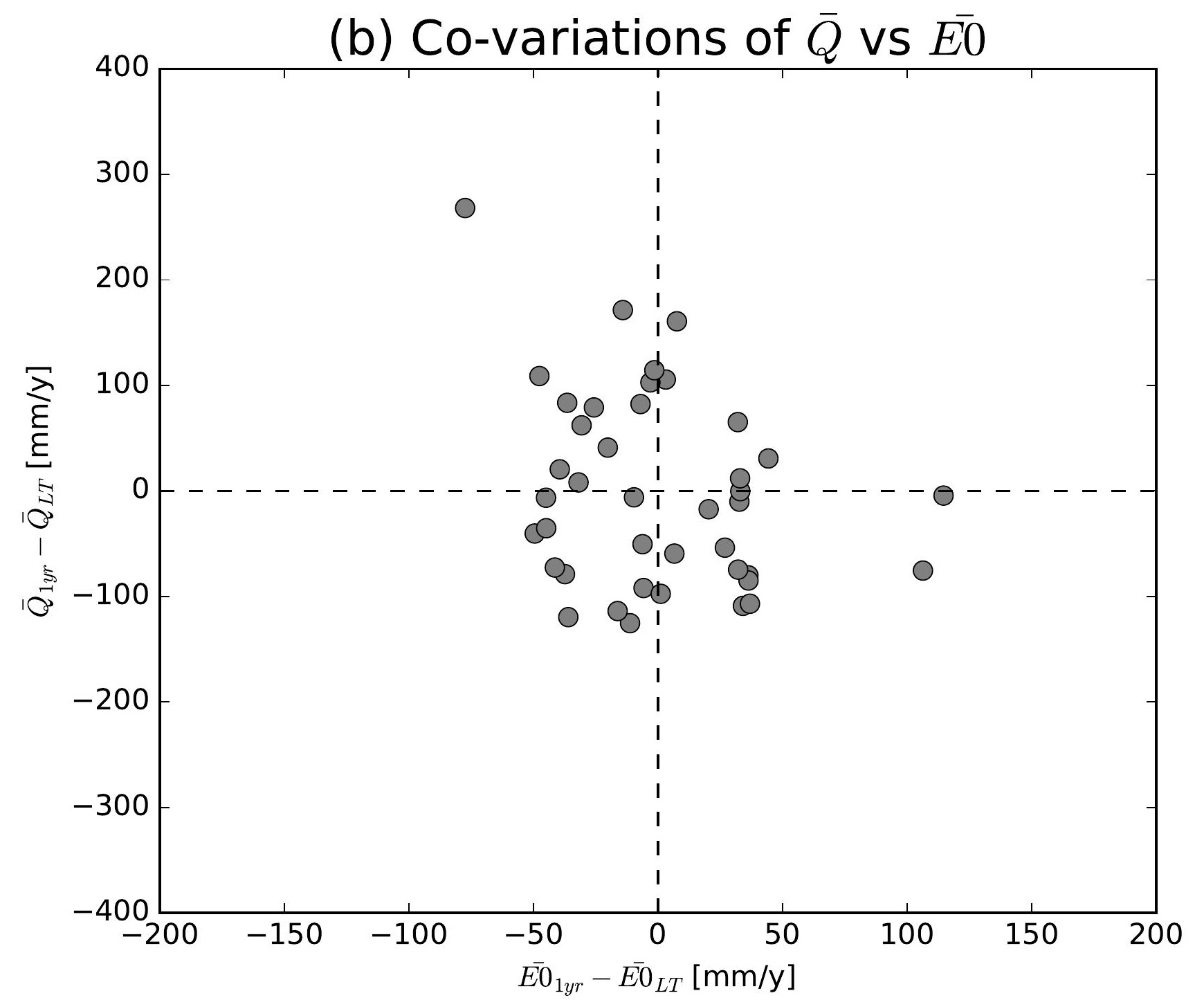

(e) Co-variations of $\bar{Q}$ vs $\overline{E 0}$

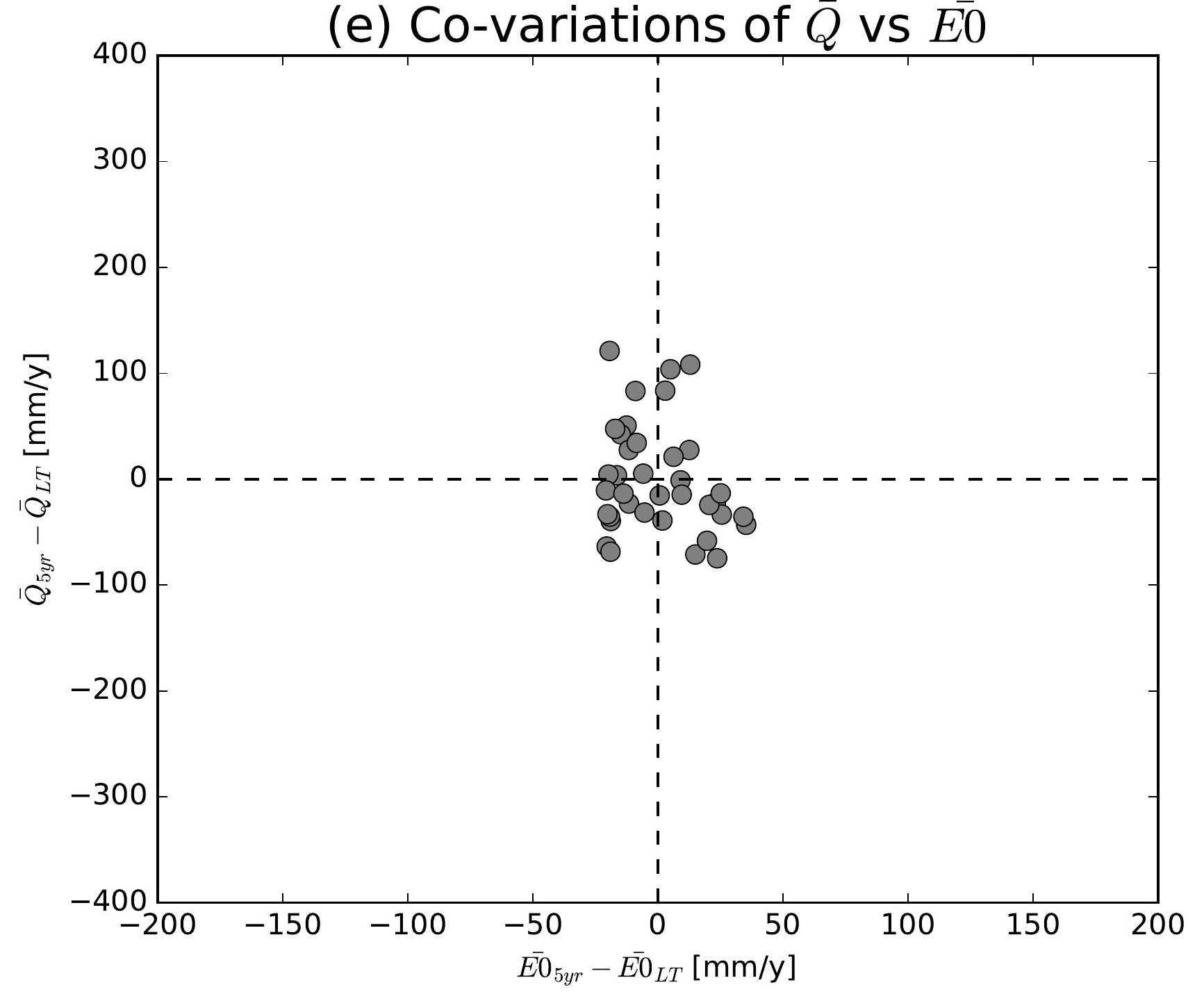

(h) Co-variations of $\bar{Q}$ vs $\overline{E 0}$

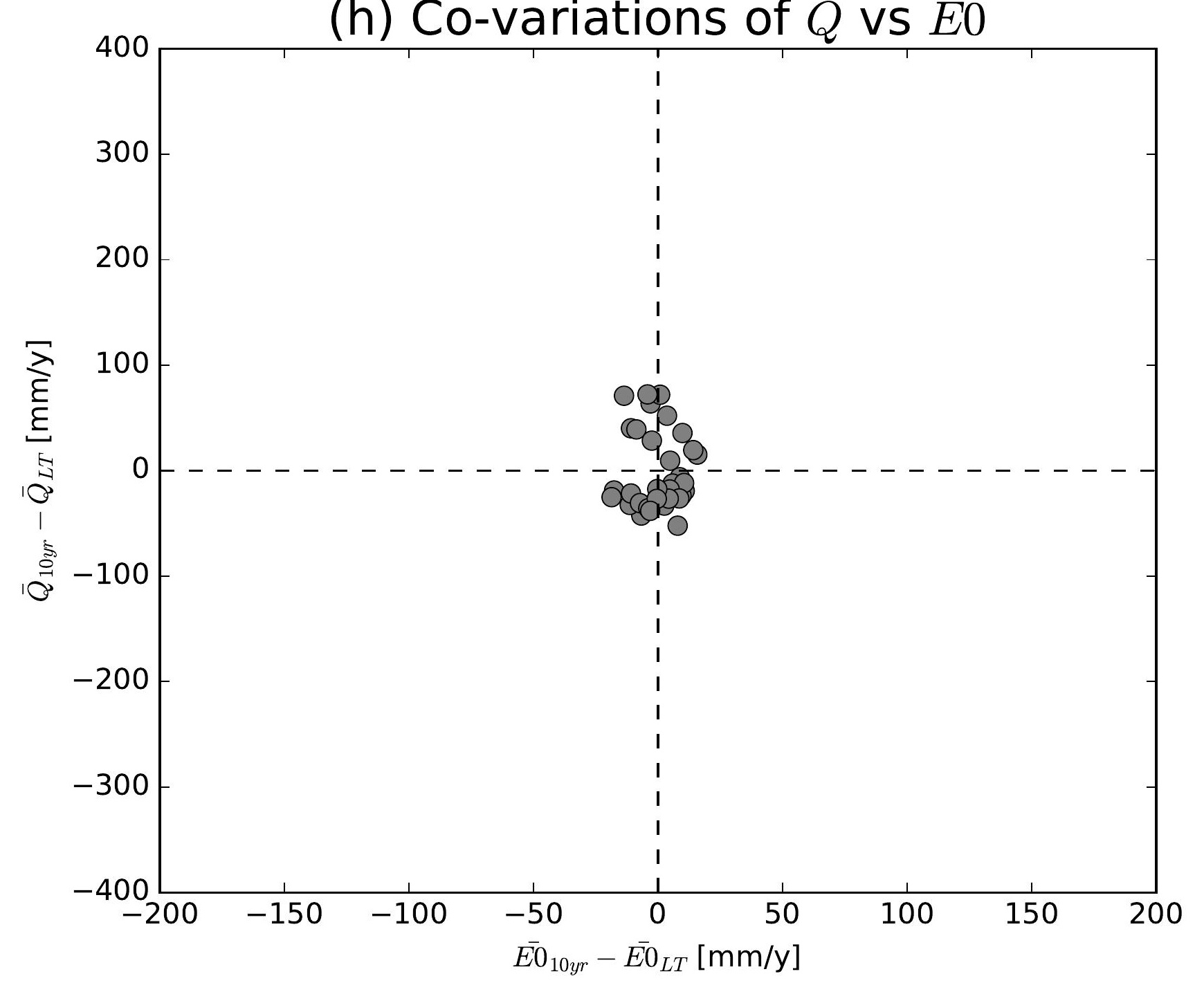

(k) Co-variations of $\bar{Q}$ vs $\overline{E 0}$

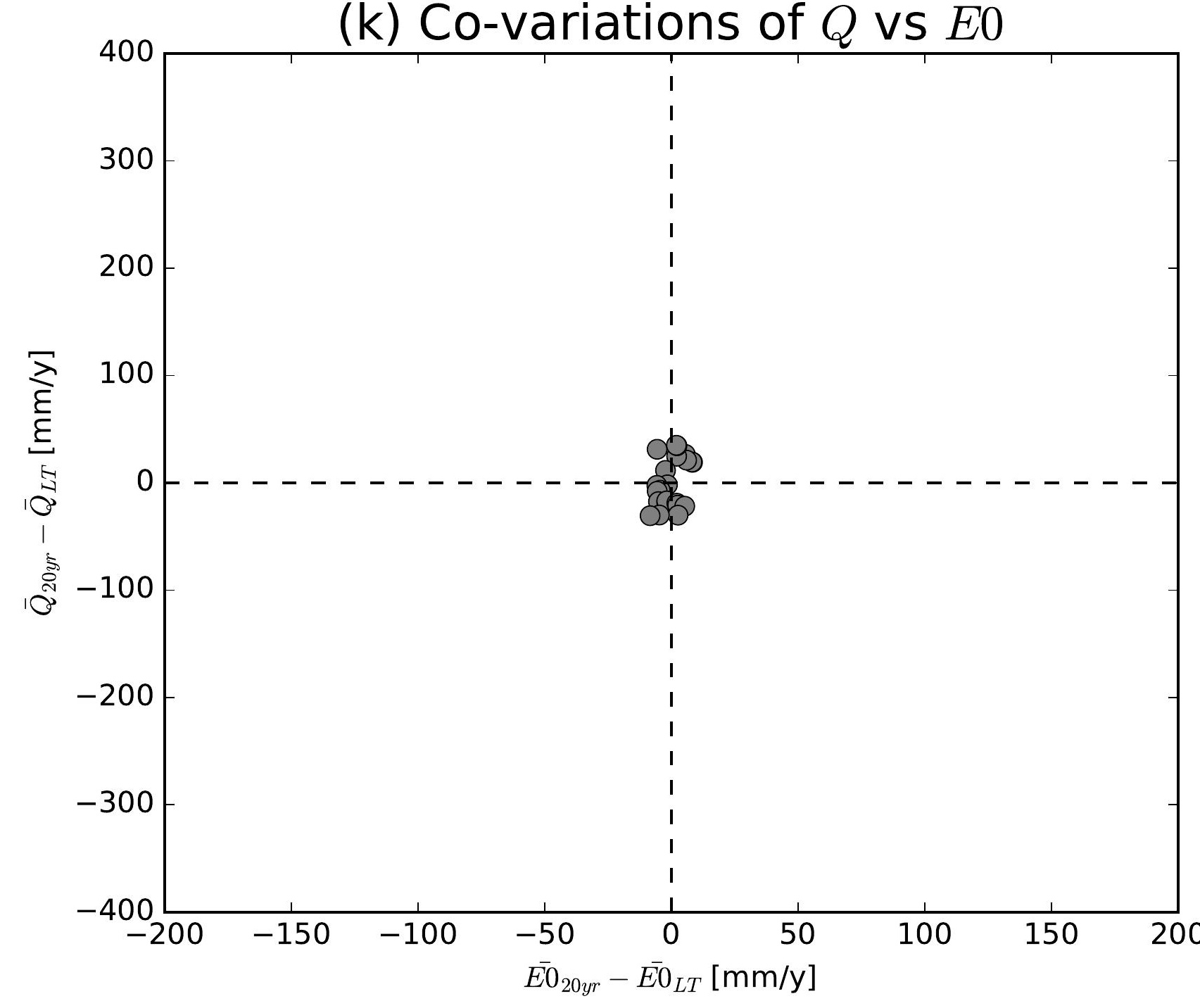

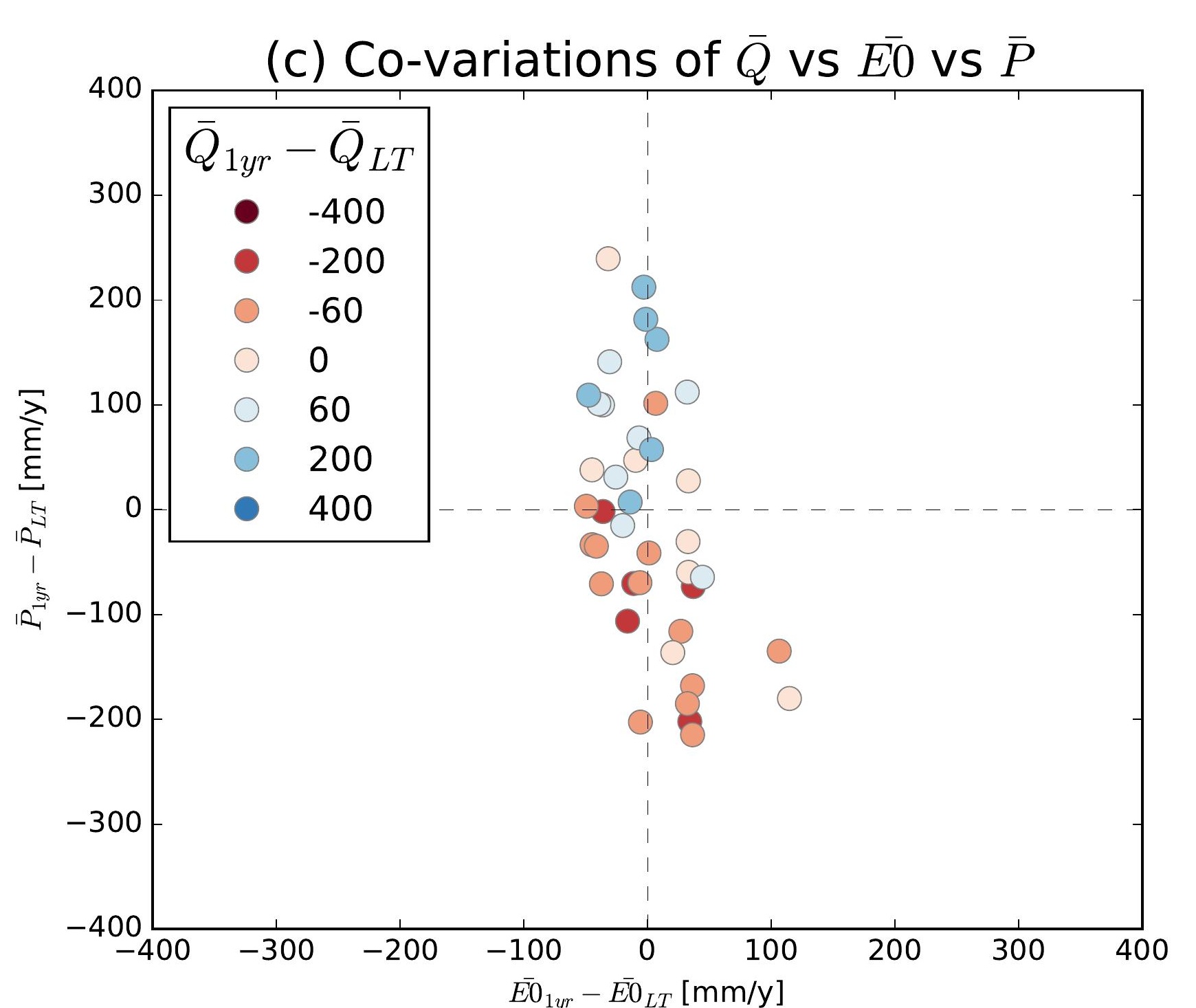
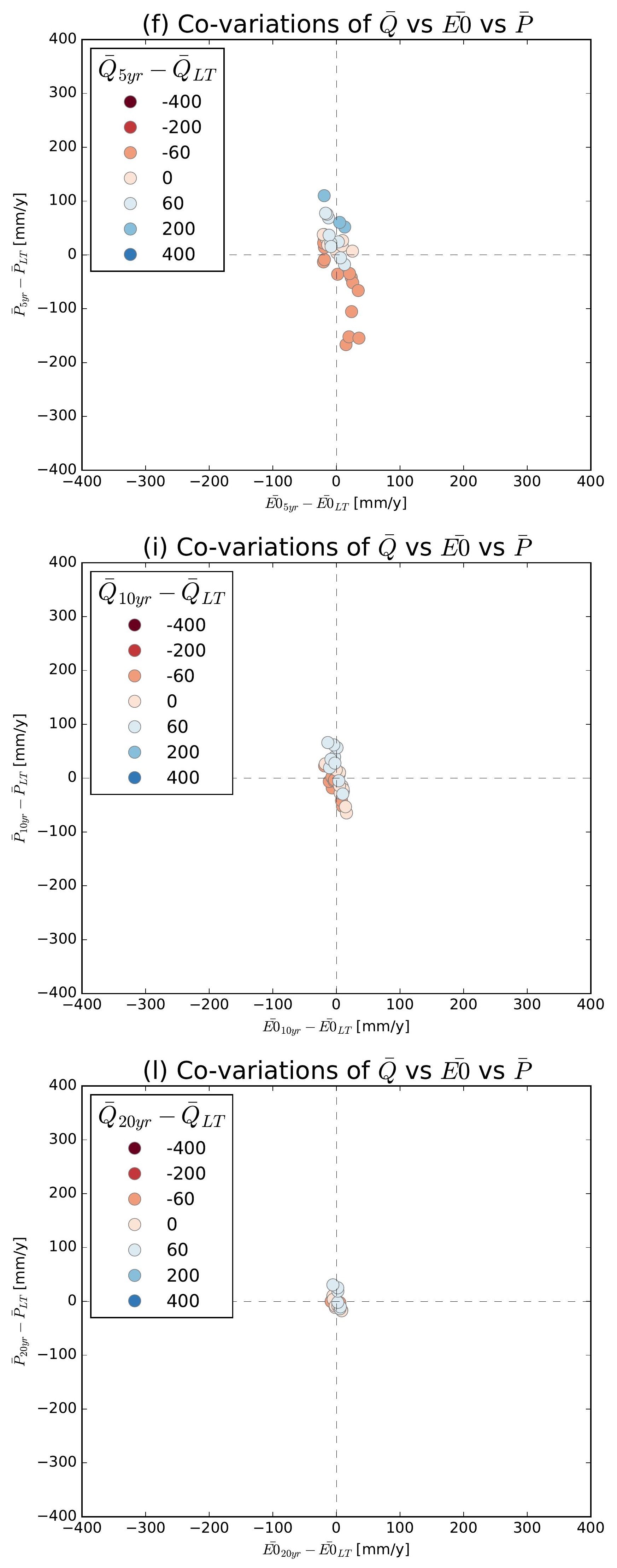

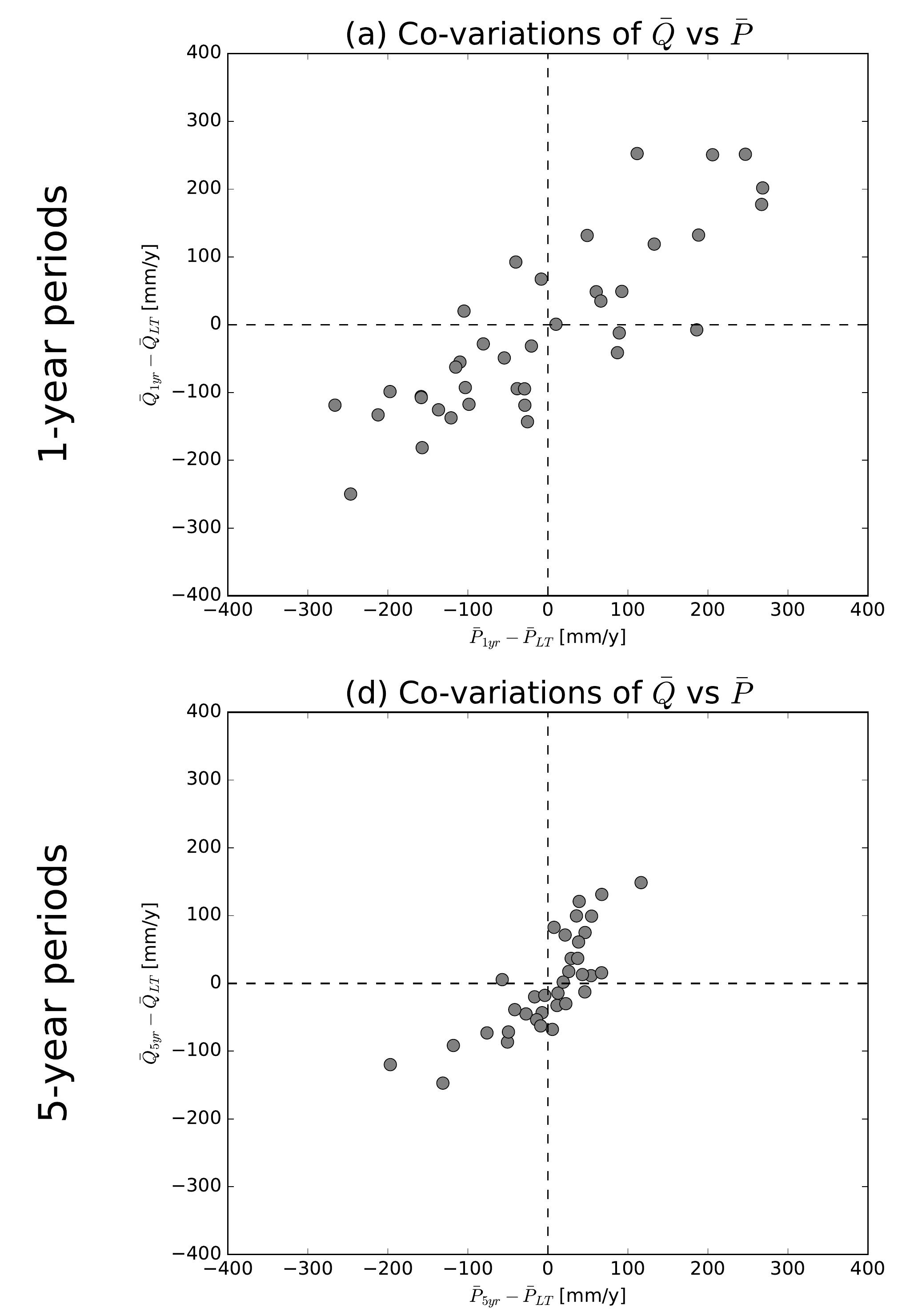

(g) Co-variations of $\bar{Q}$ vs $\bar{P}$
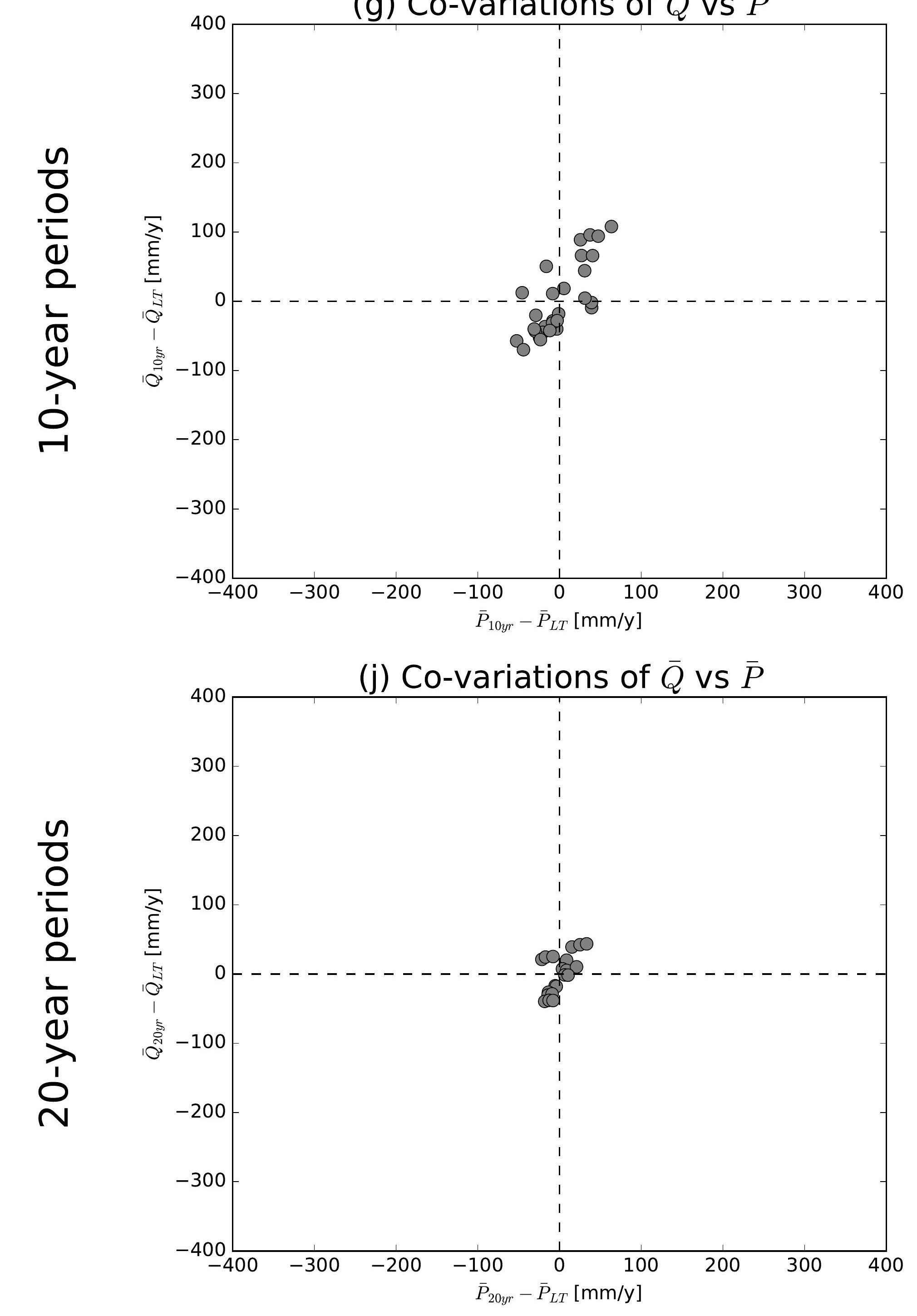

(b) Co-variations of $\bar{Q}$ vs $\overline{E 0}$

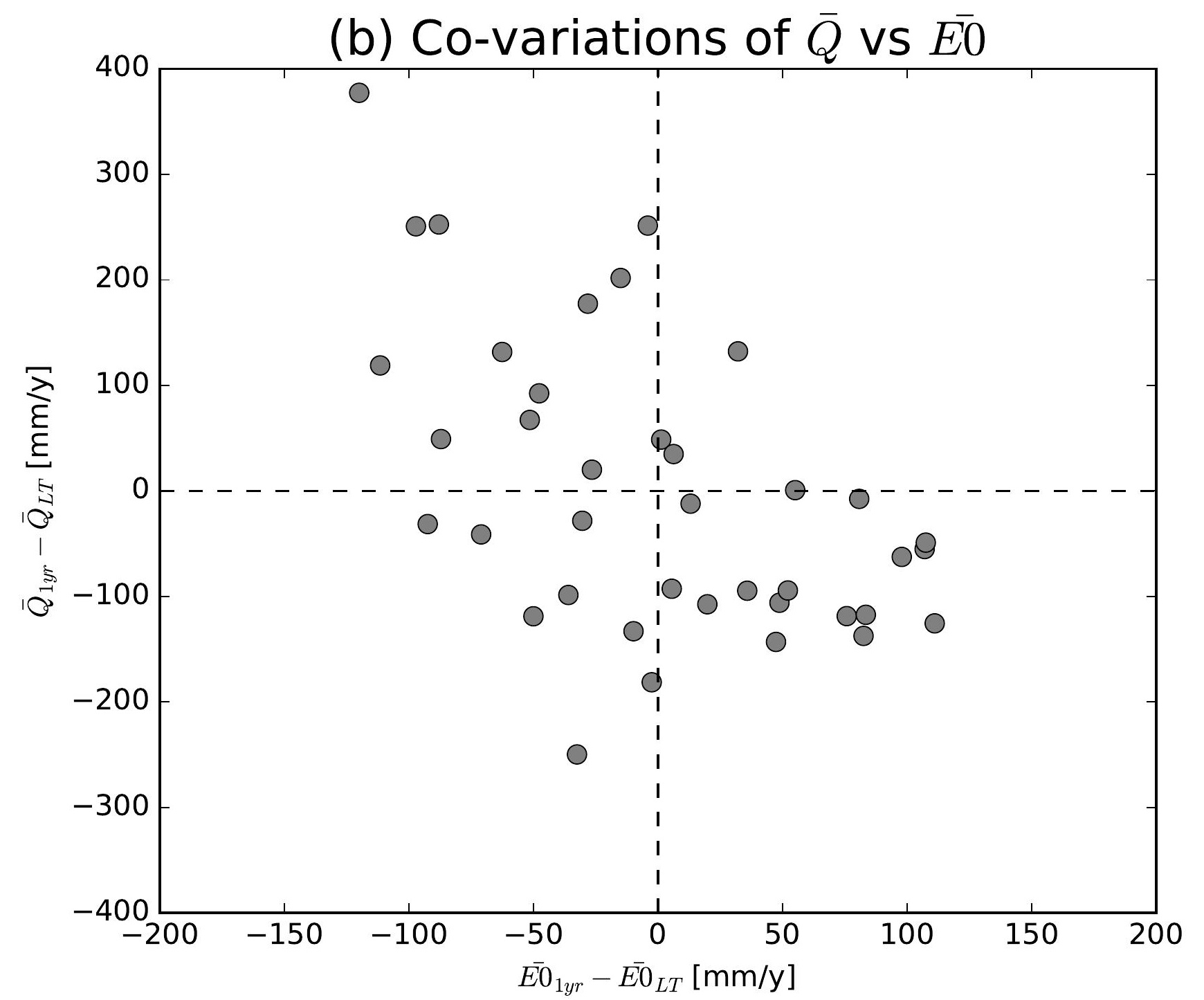

(e) Co-variations of $\bar{Q}$ vs $\overline{E 0}$

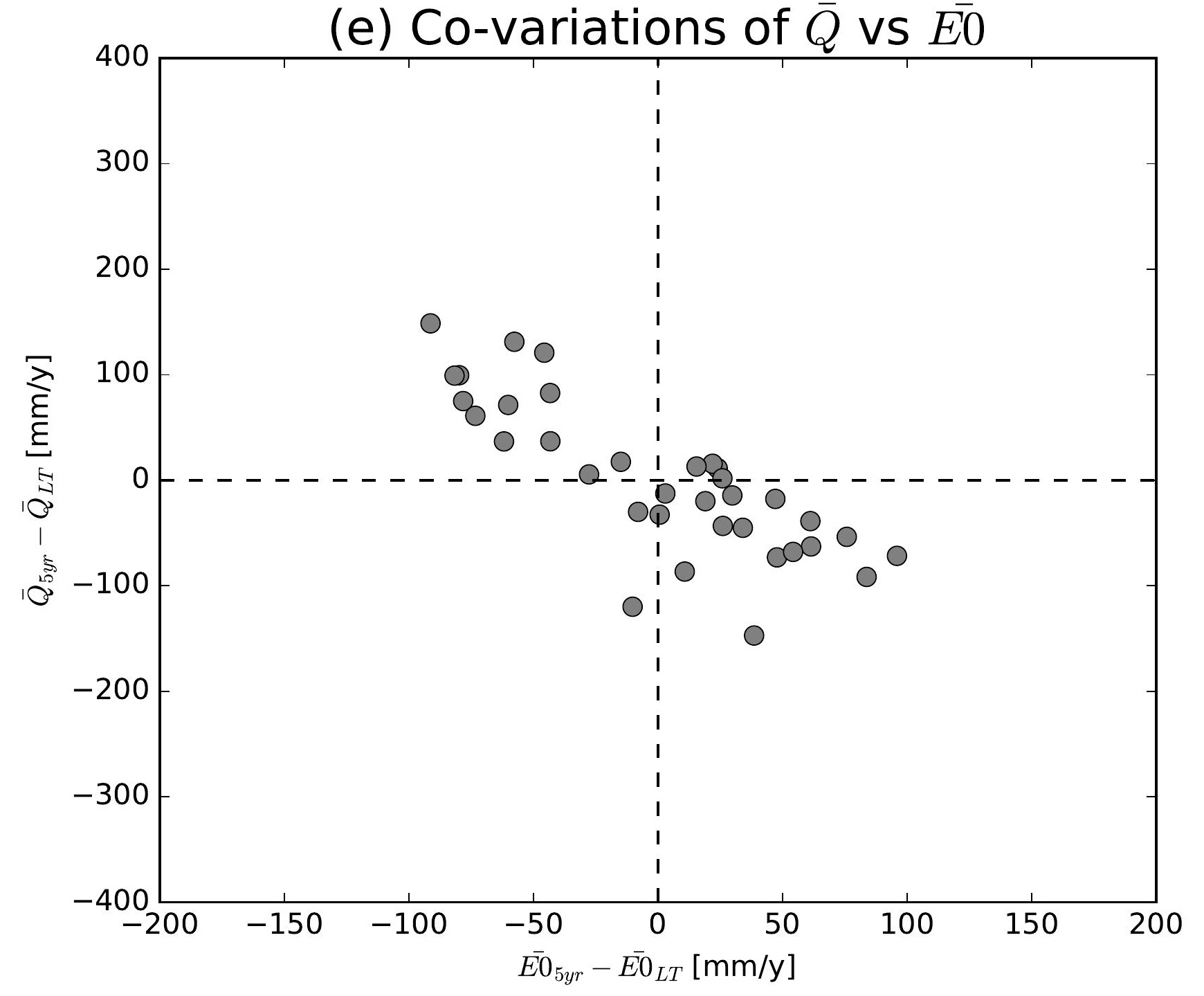

(h) Co-variations of $\bar{Q}$ vs $\overline{E 0}$

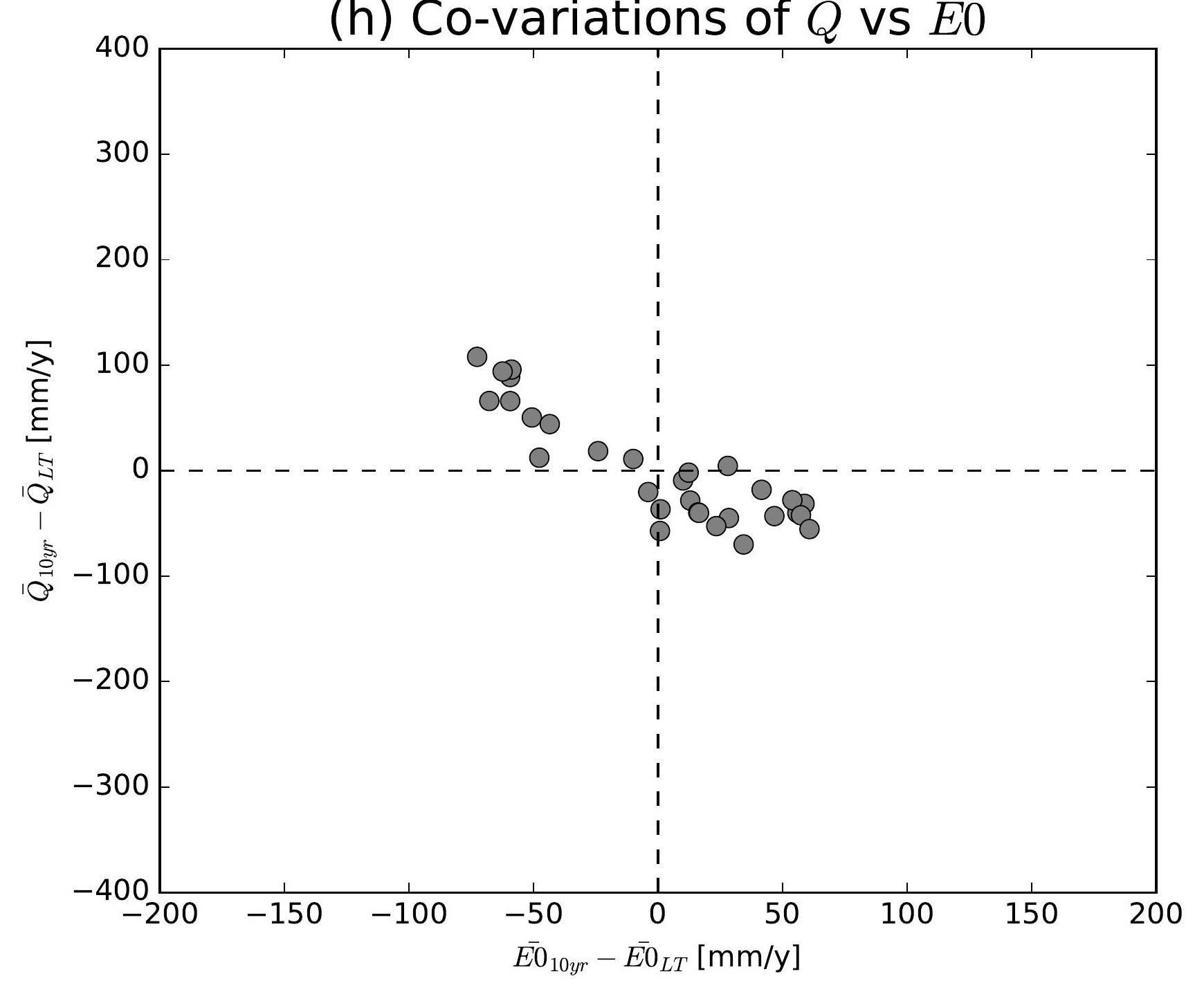

(k) Co-variations of $\bar{Q}$ vs $\overline{E 0}$

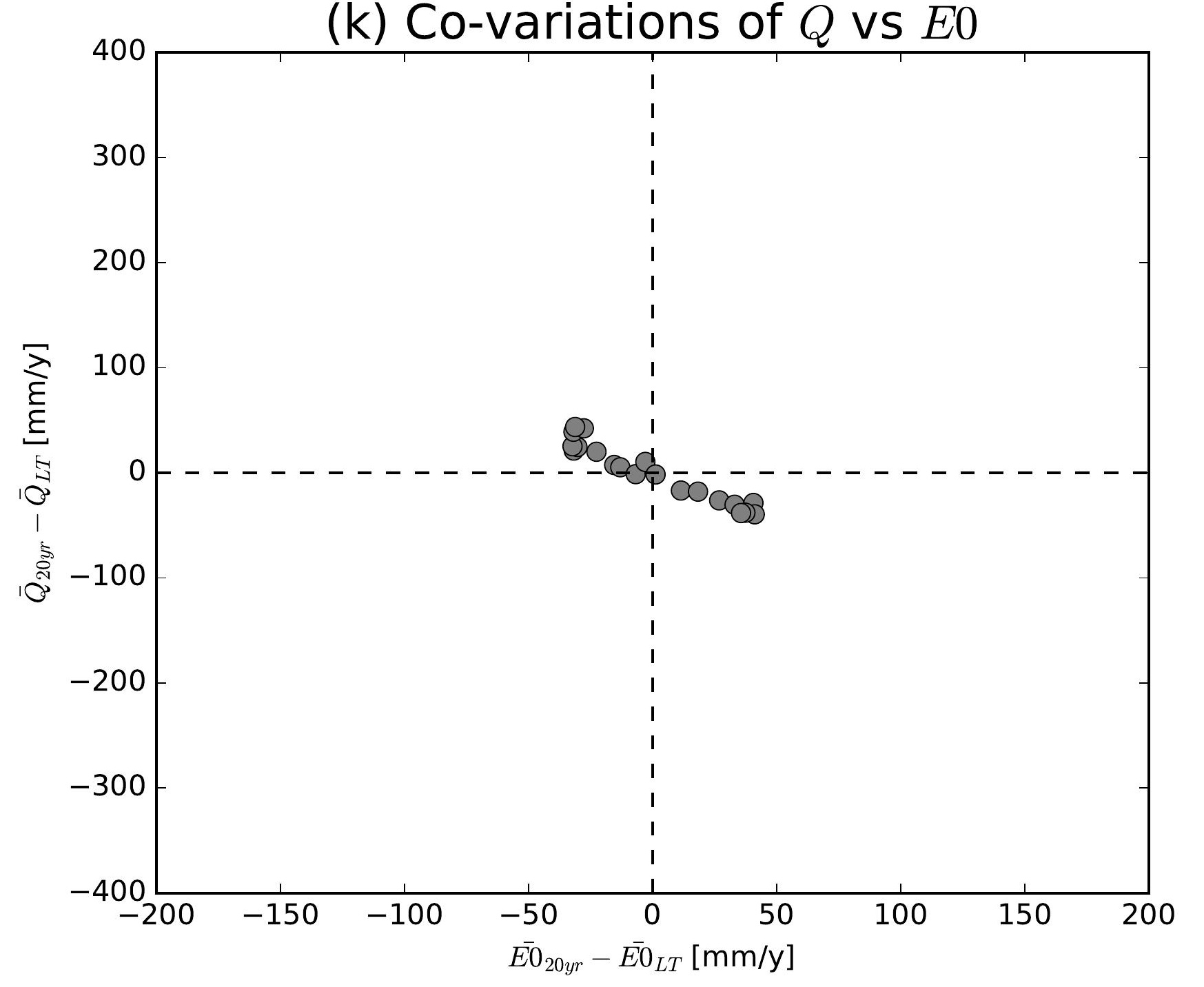

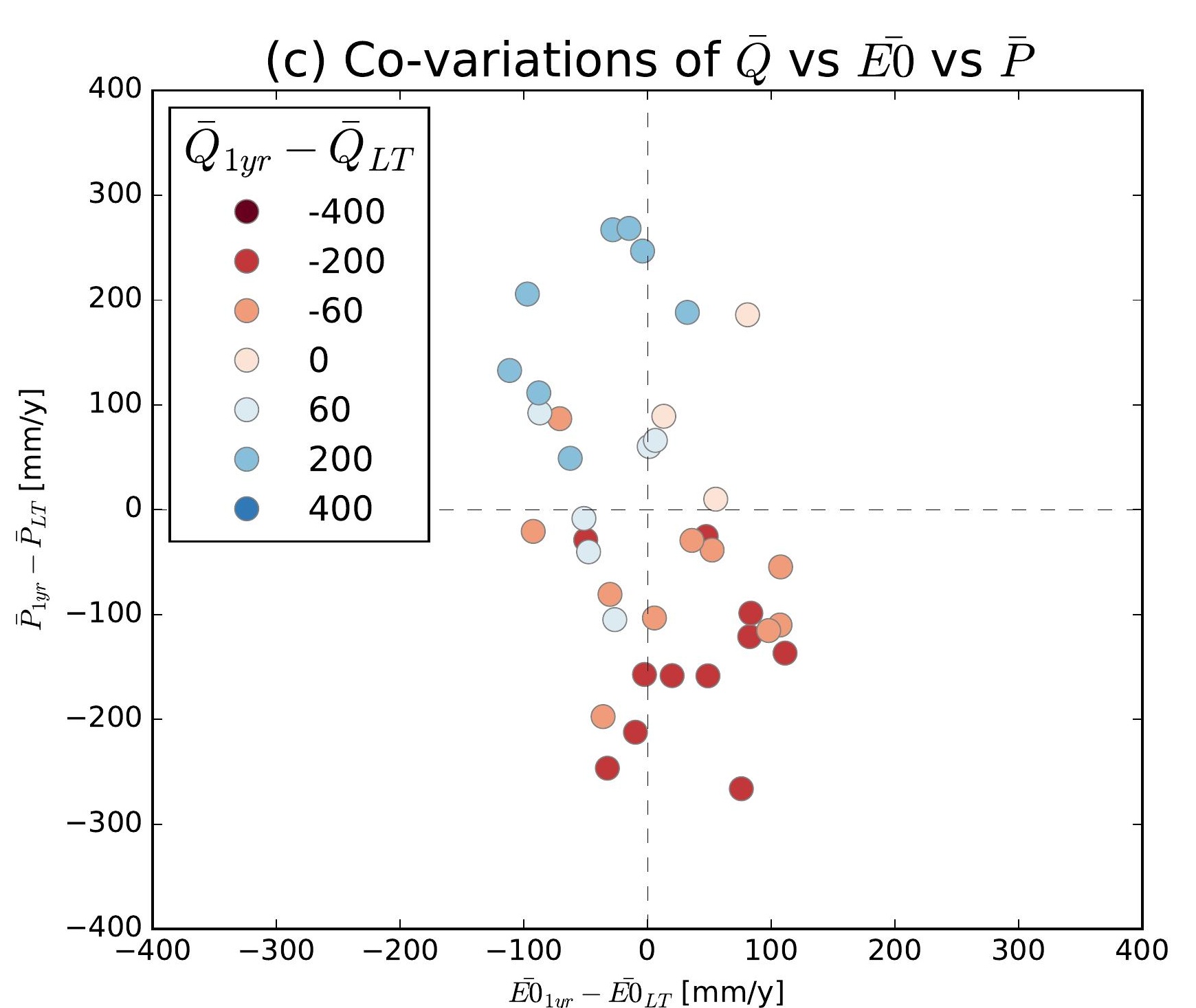
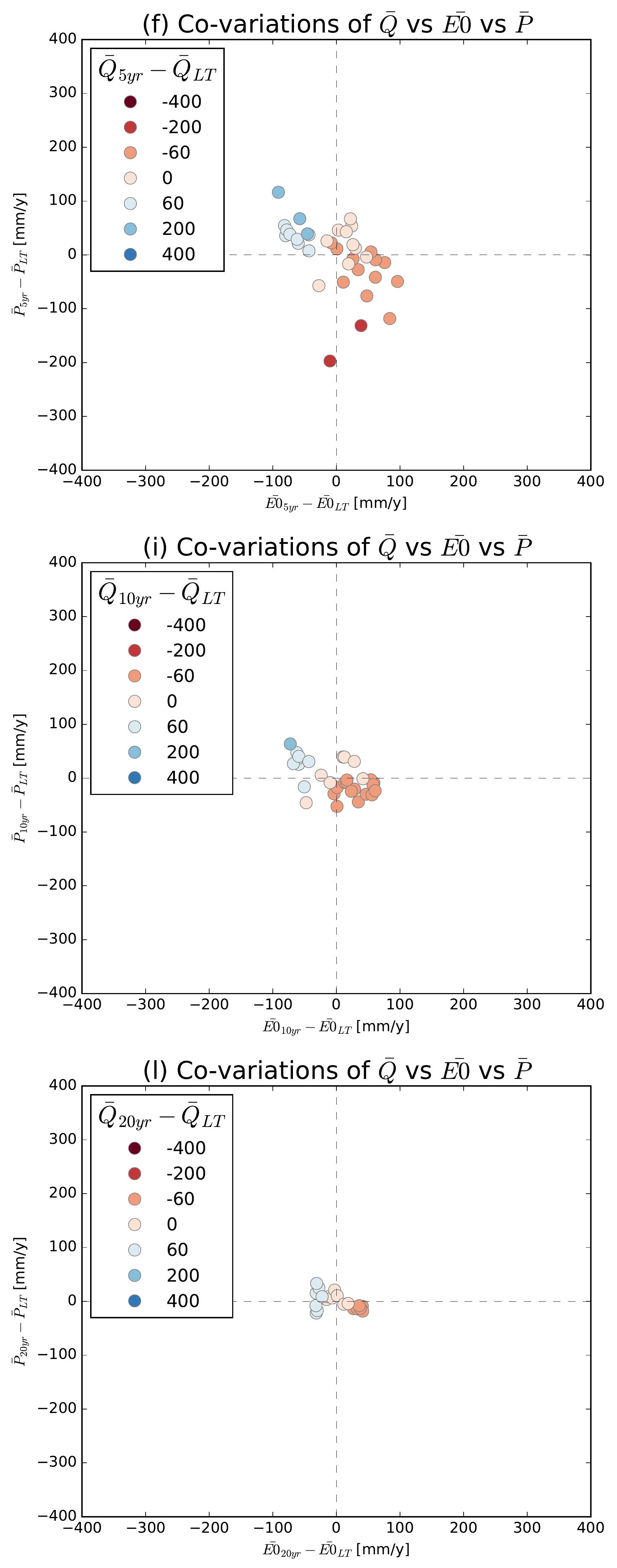

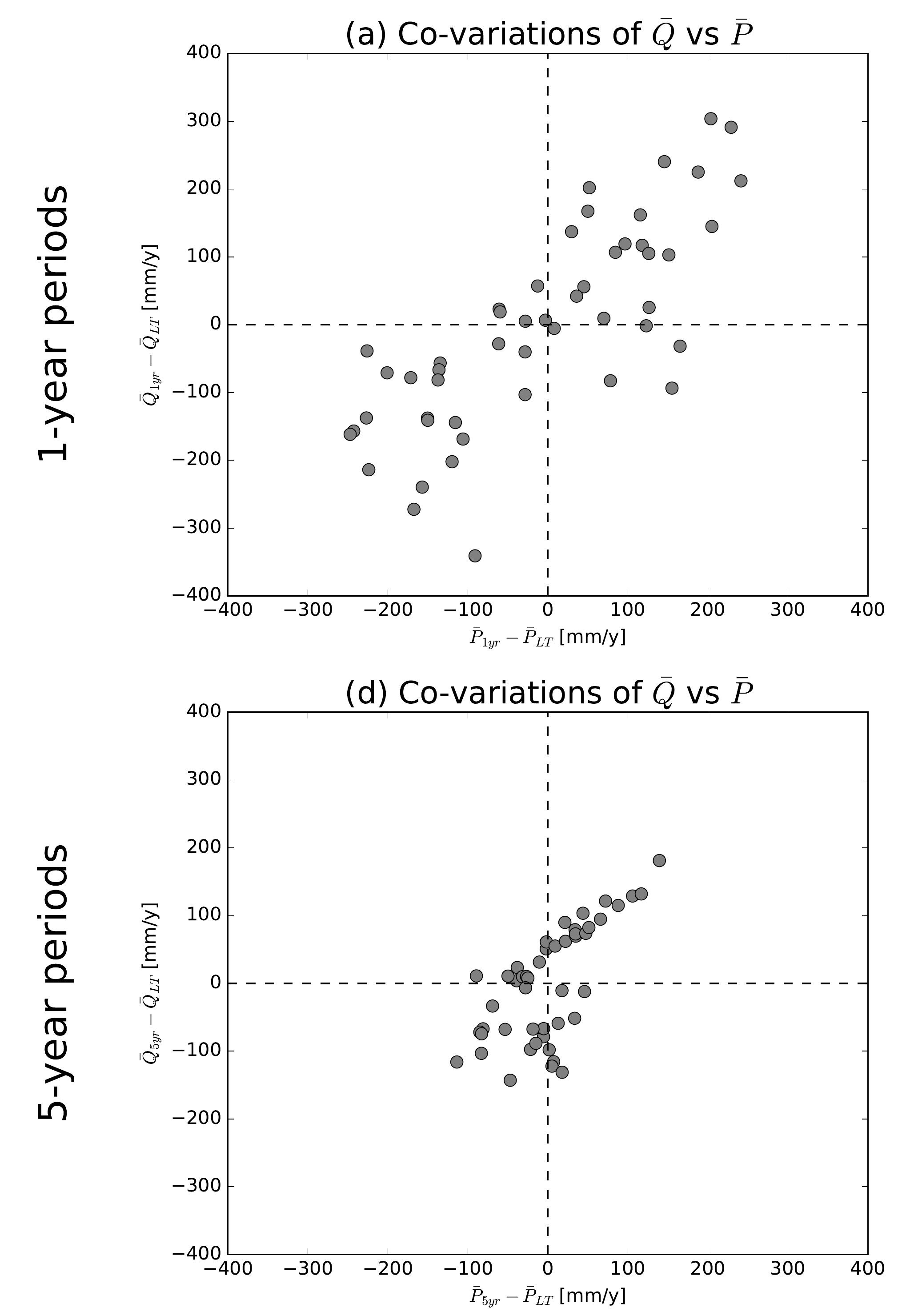

(g) Co-variations of $\bar{Q}$ vs $\bar{P}$
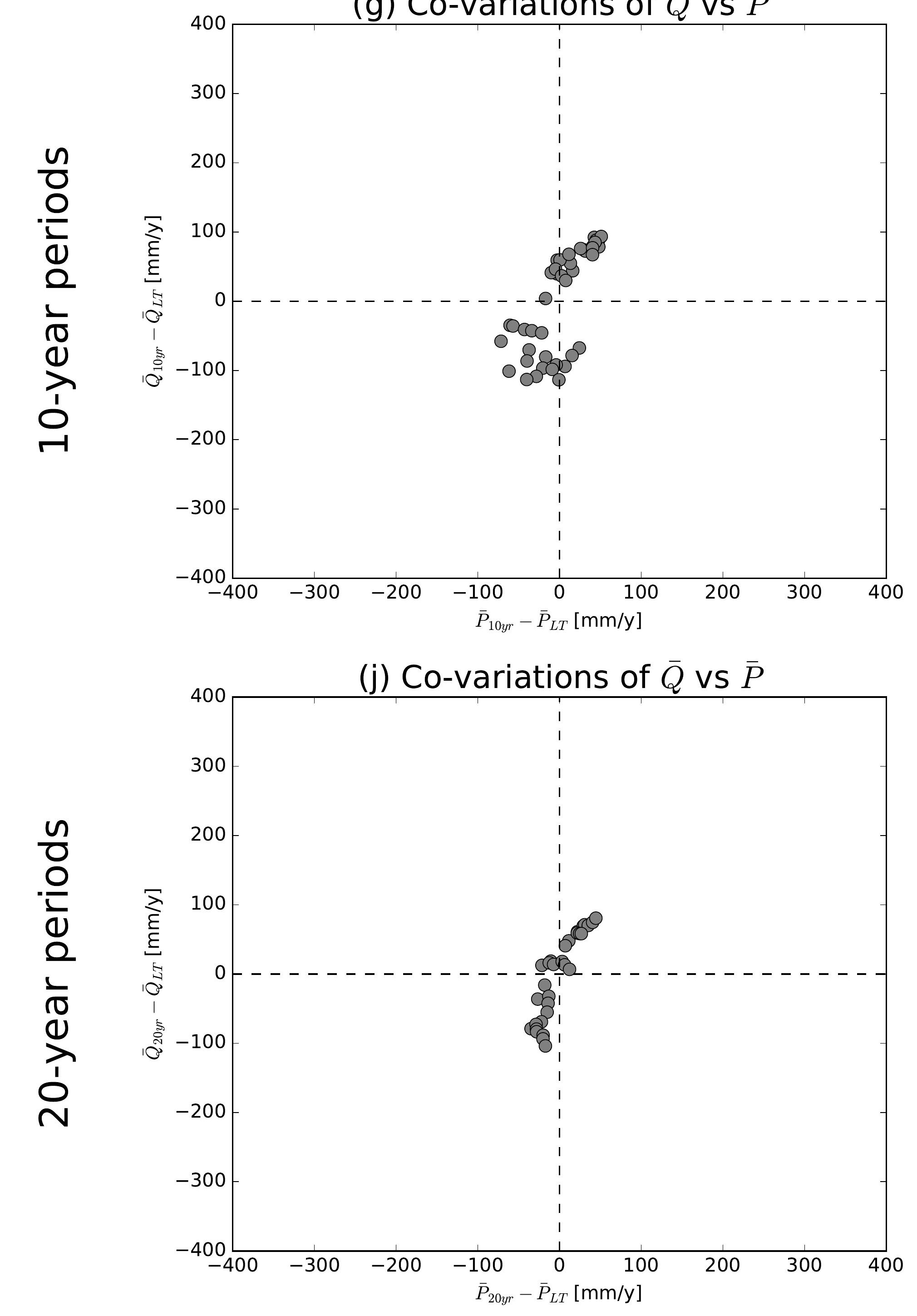

(b) Co-variations of $\bar{Q}$ vs $\overline{E 0}$

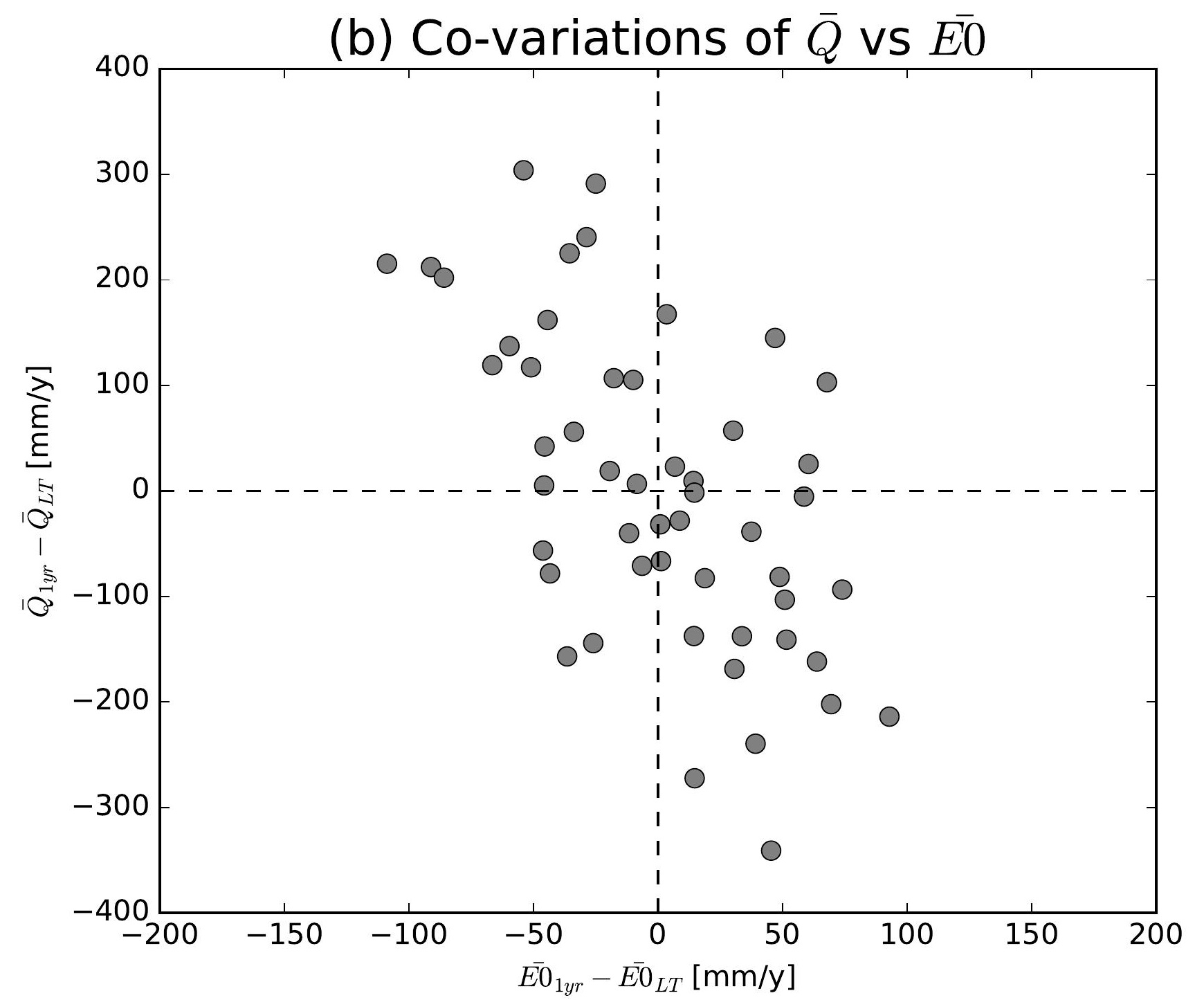

(e) Co-variations of $\bar{Q}$ vs $\overline{E 0}$

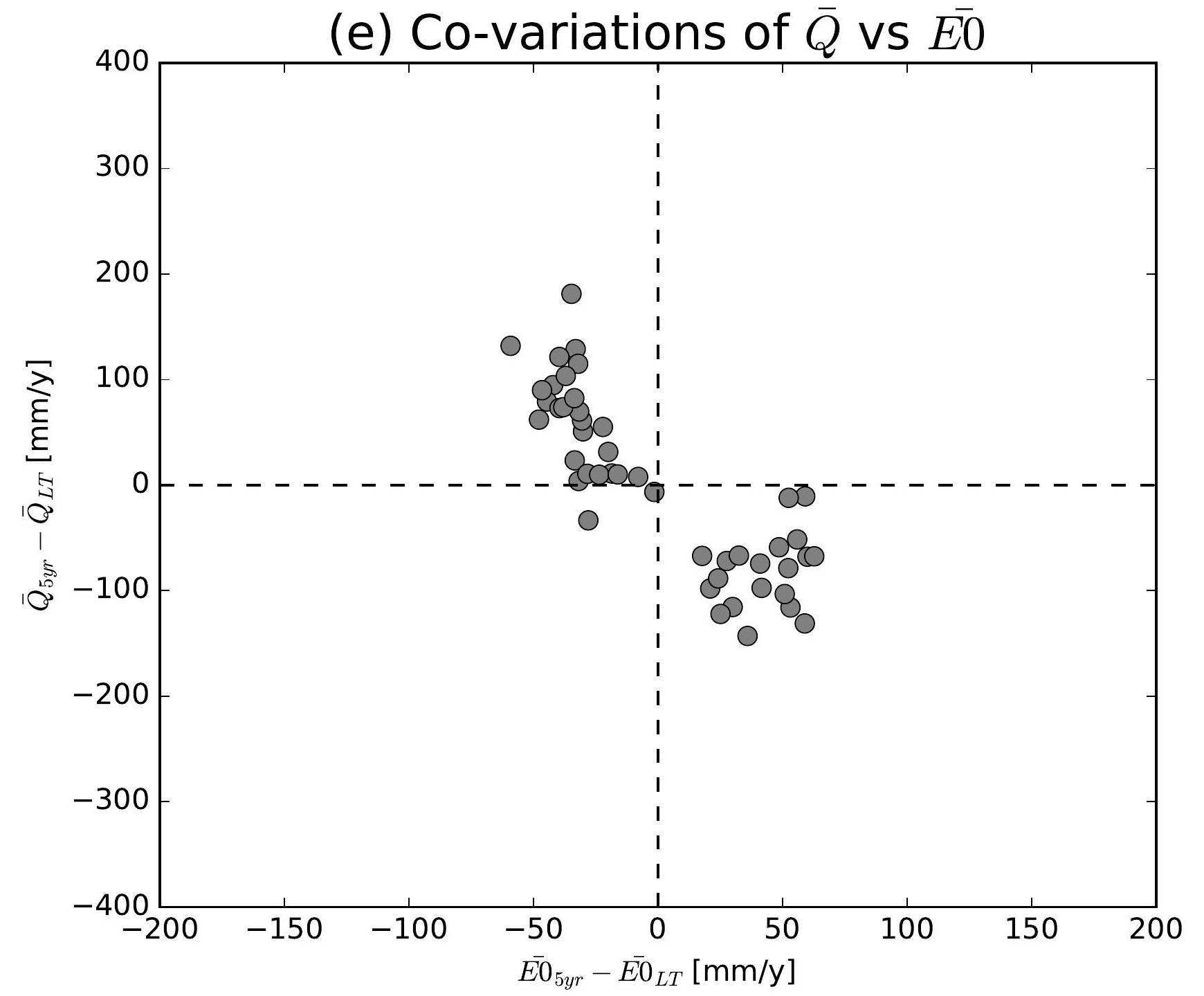

(h) Co-variations of $\bar{Q}$ vs $\overline{E 0}$

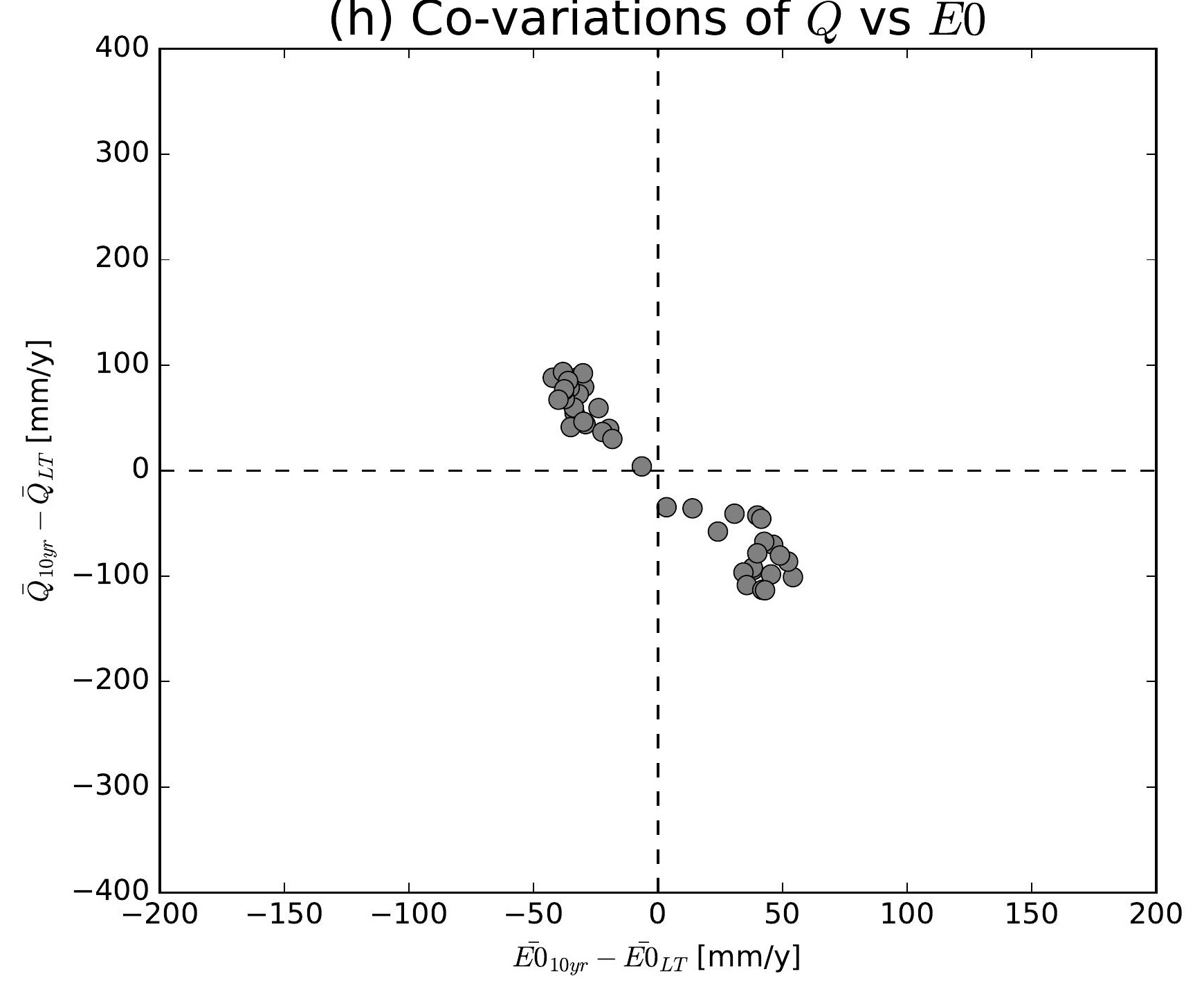

(k) Co-variations of $\bar{Q}$ vs $\overline{E 0}$

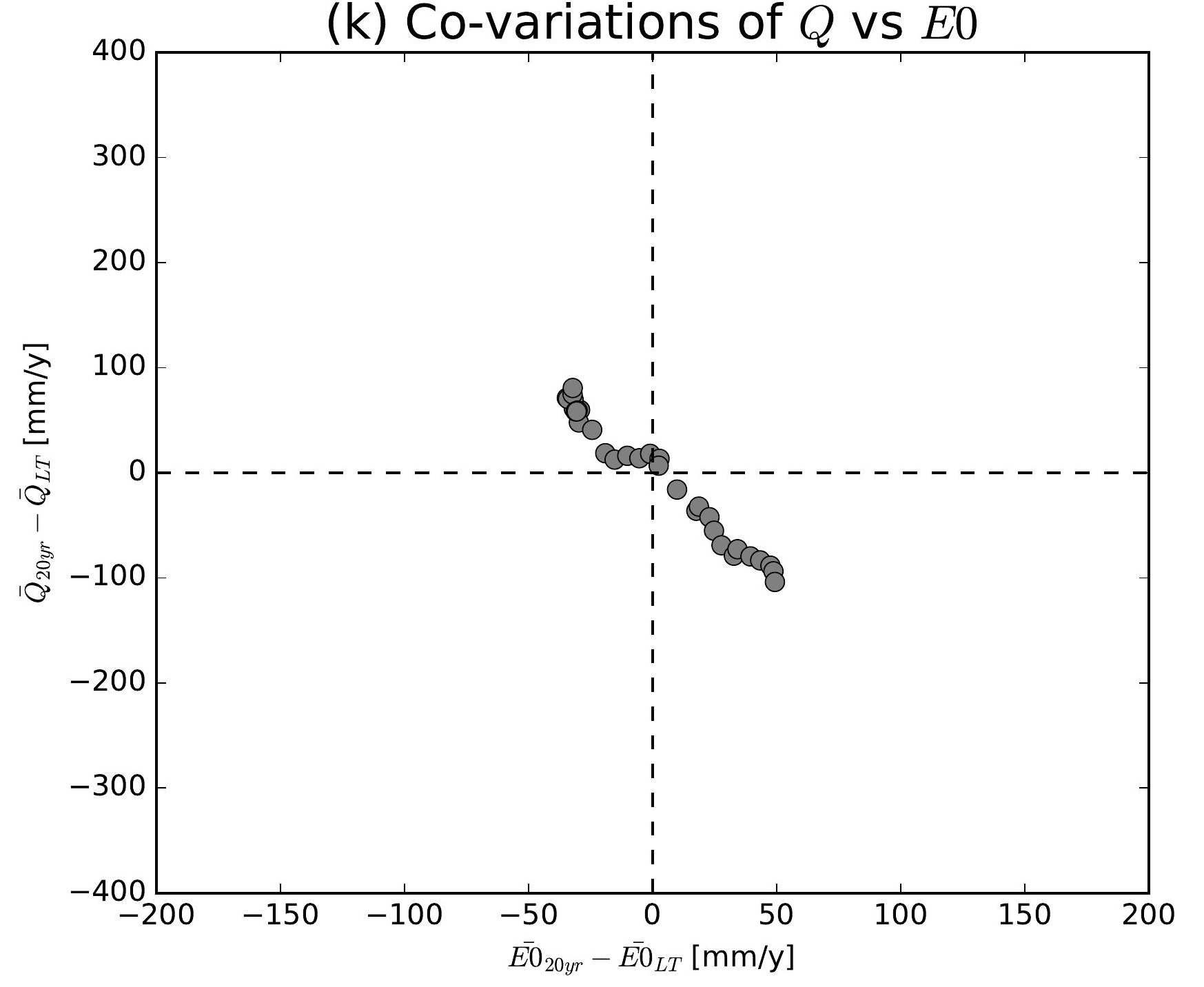

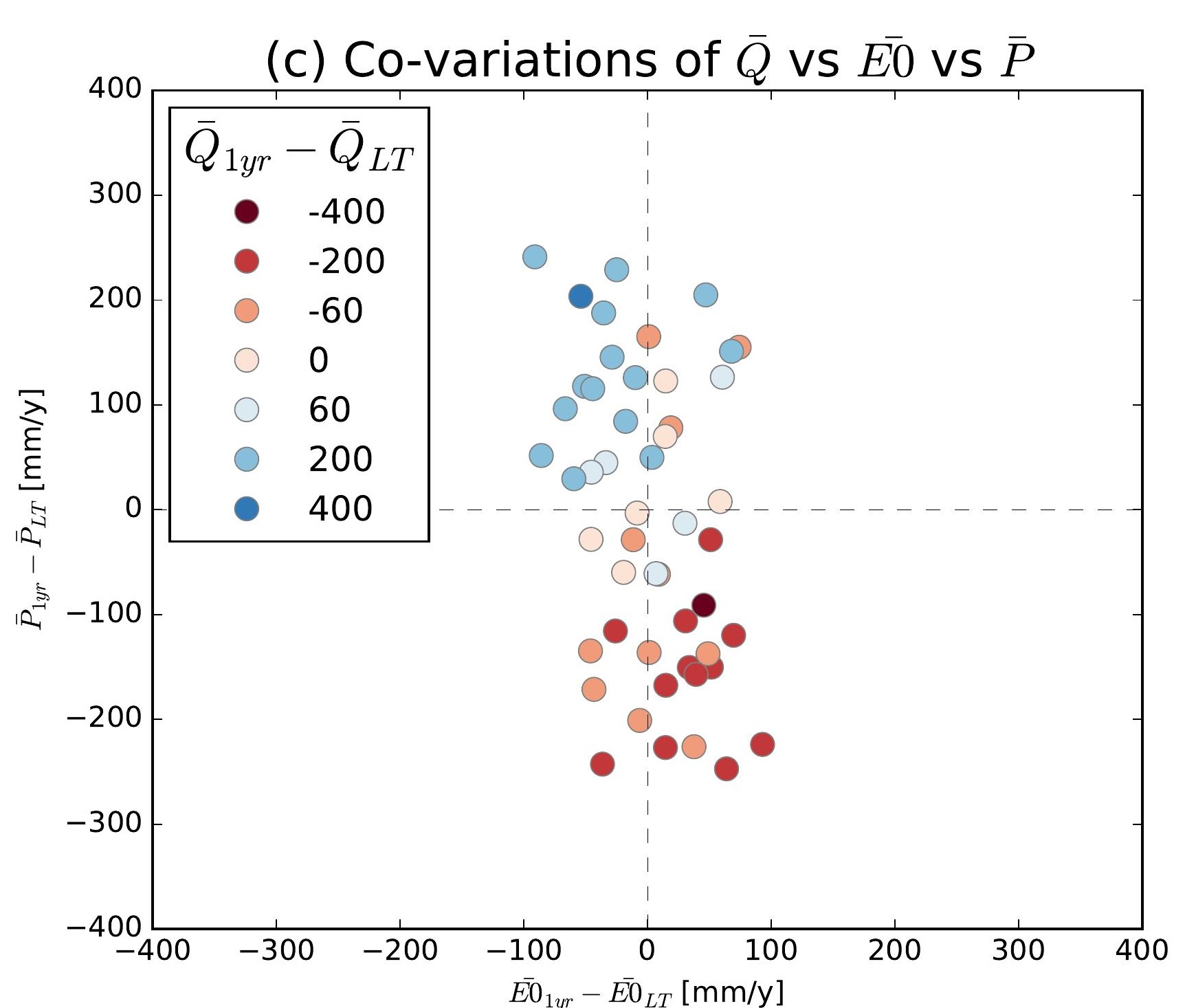
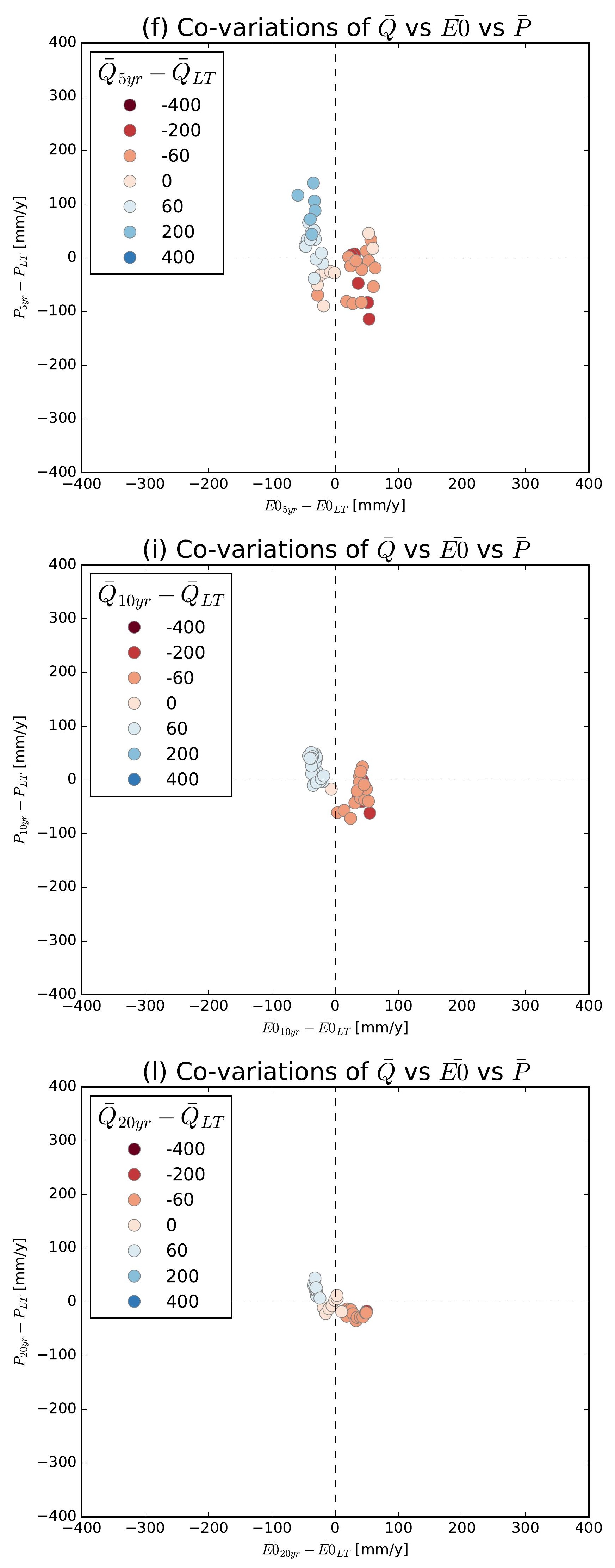

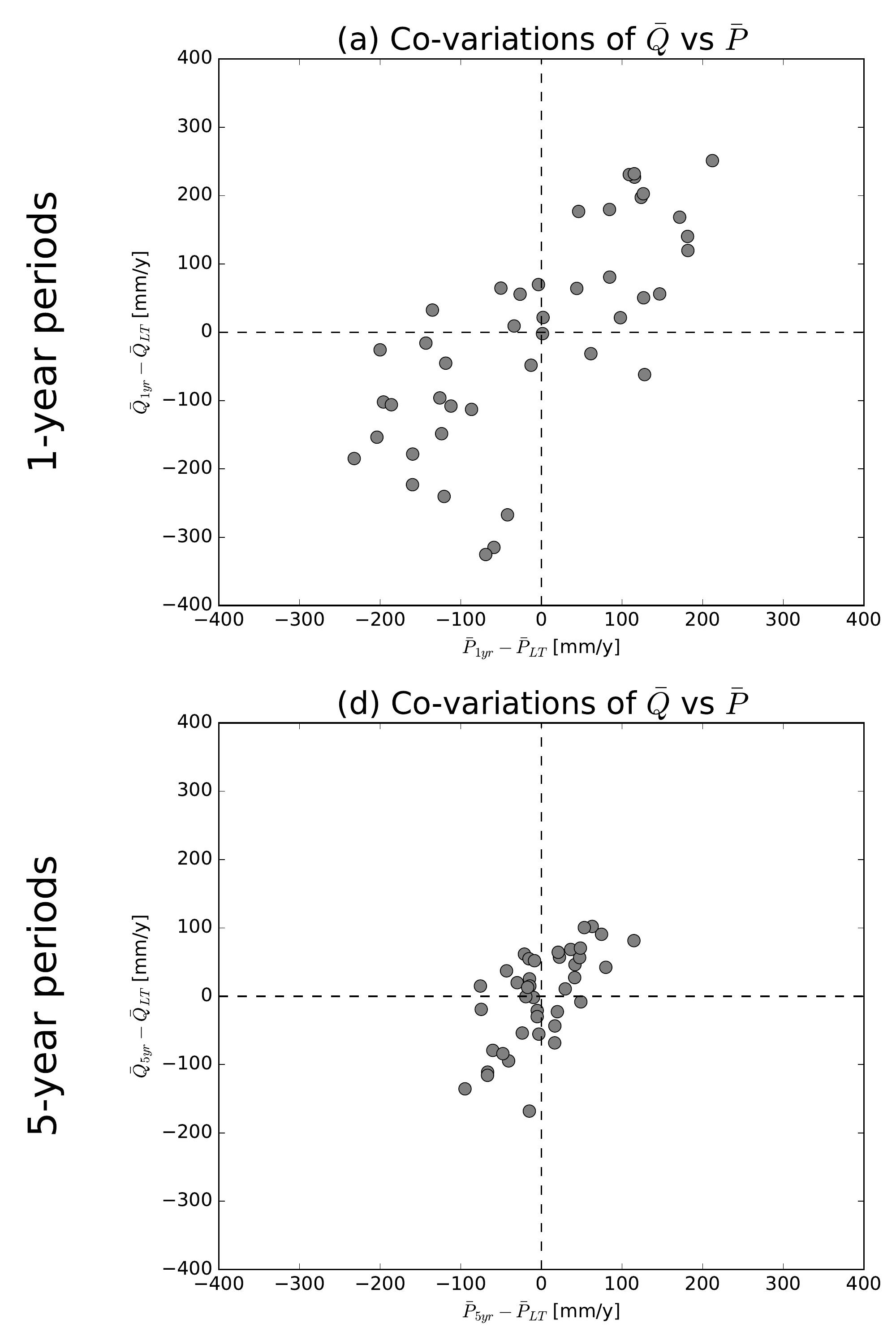

(g) Co-variations of $\bar{Q}$ vs $\bar{P}$

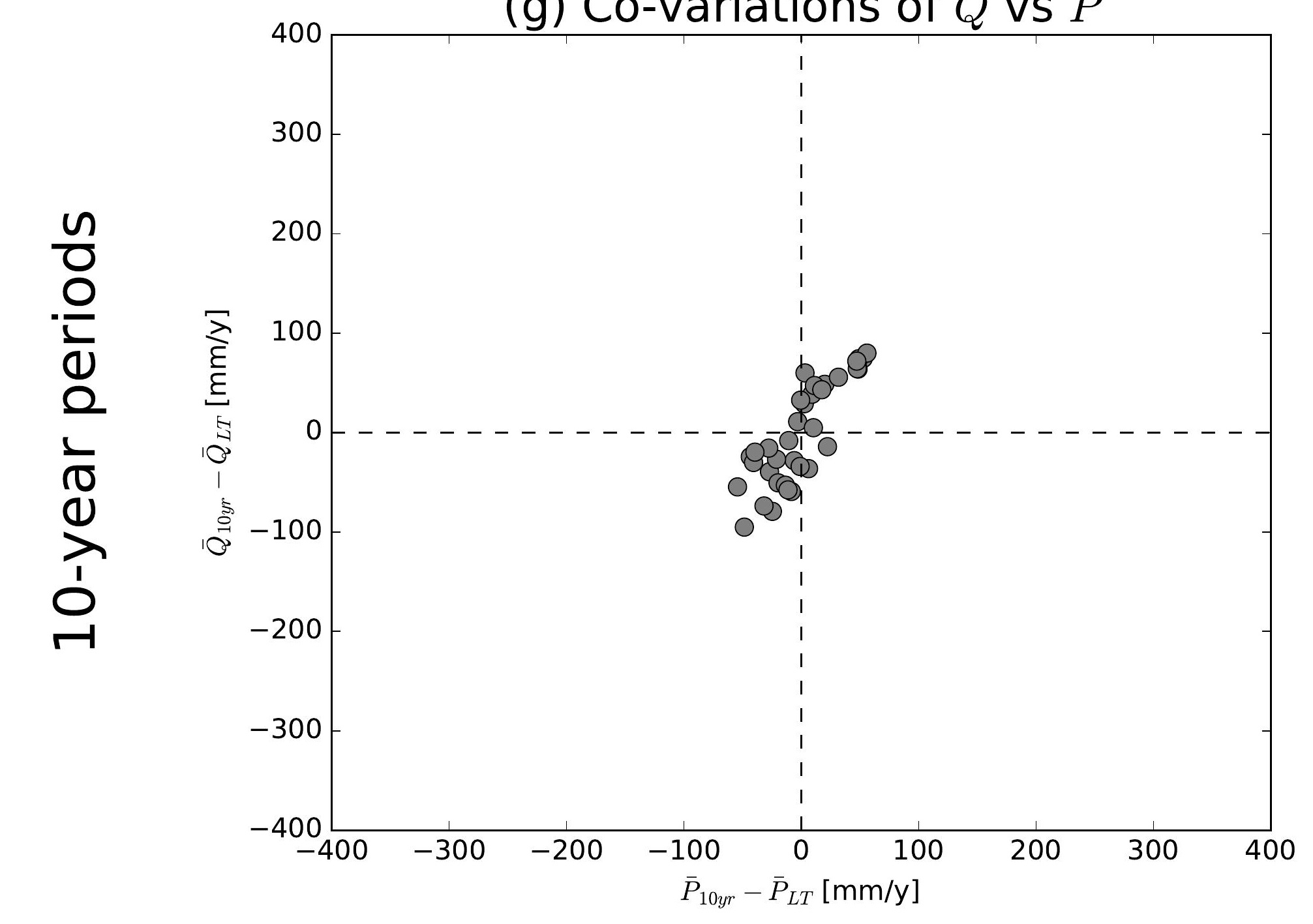

(j) Co-variations of $\bar{Q}$ vs $\bar{P}$

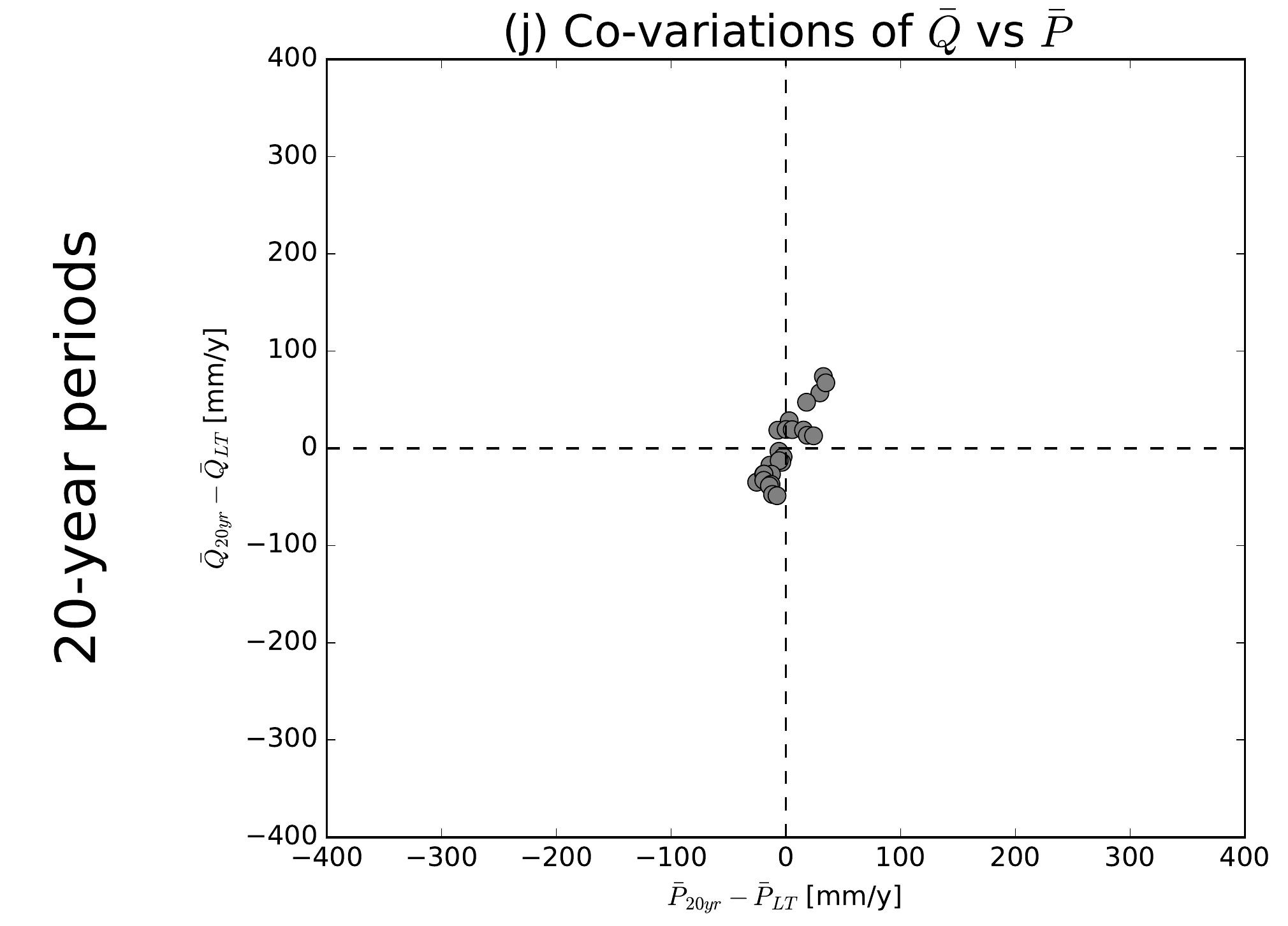

(b) Co-variations of $\bar{Q}$ vs $\overline{E 0}$

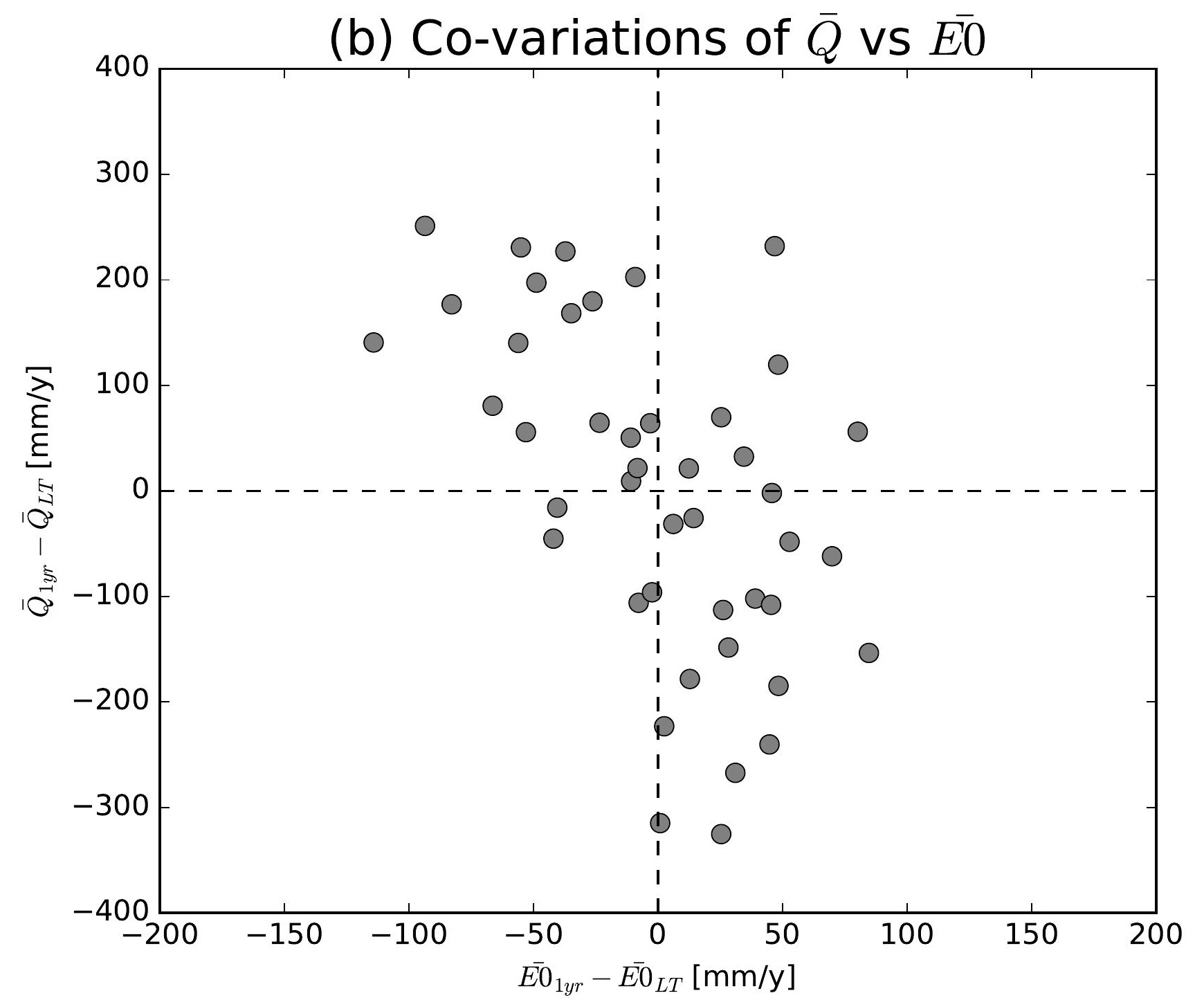

(e) Co-variations of $\bar{Q}$ vs $\overline{E 0}$

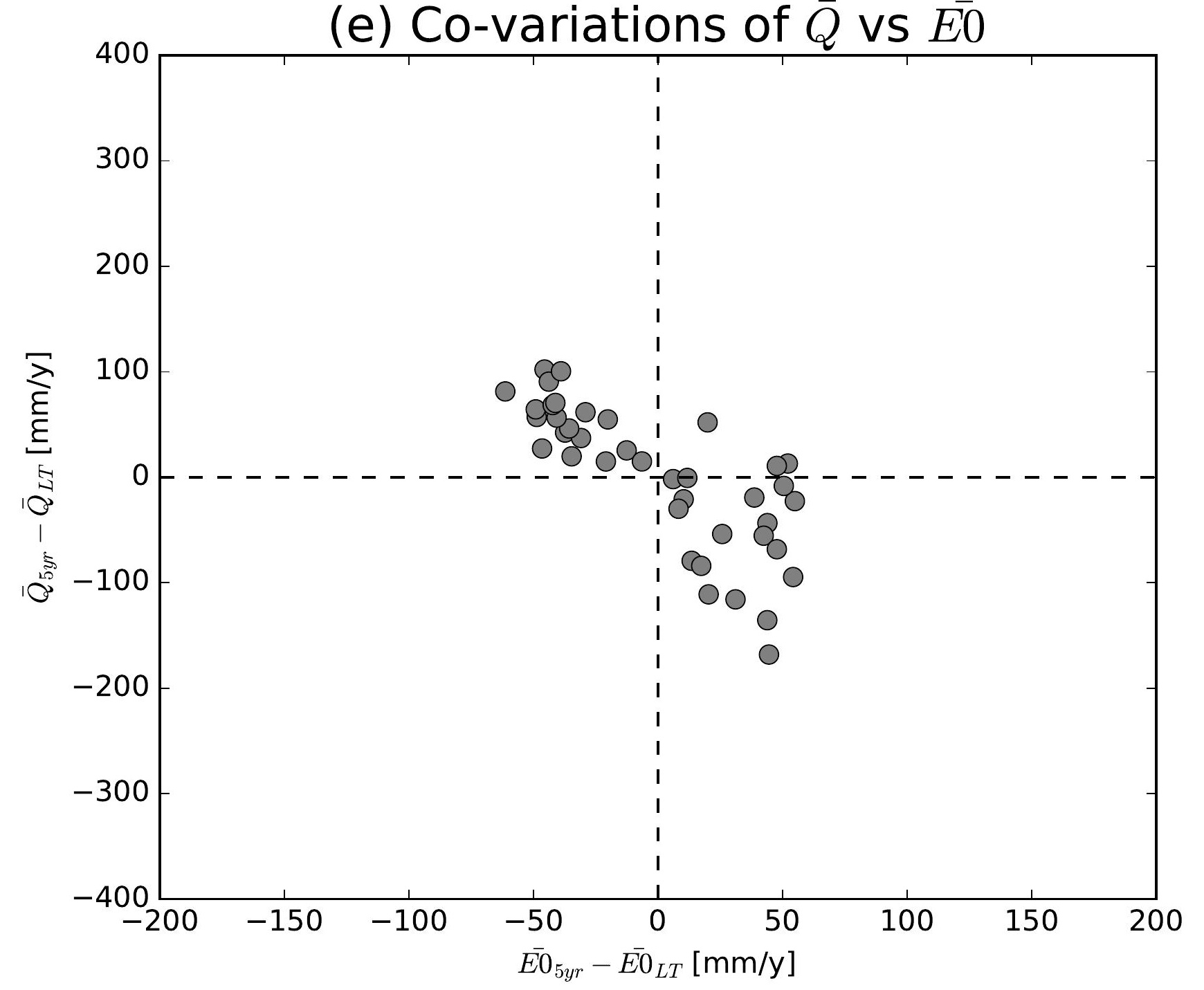

(h) Co-variations of $\bar{Q}$ vs $\overline{E 0}$

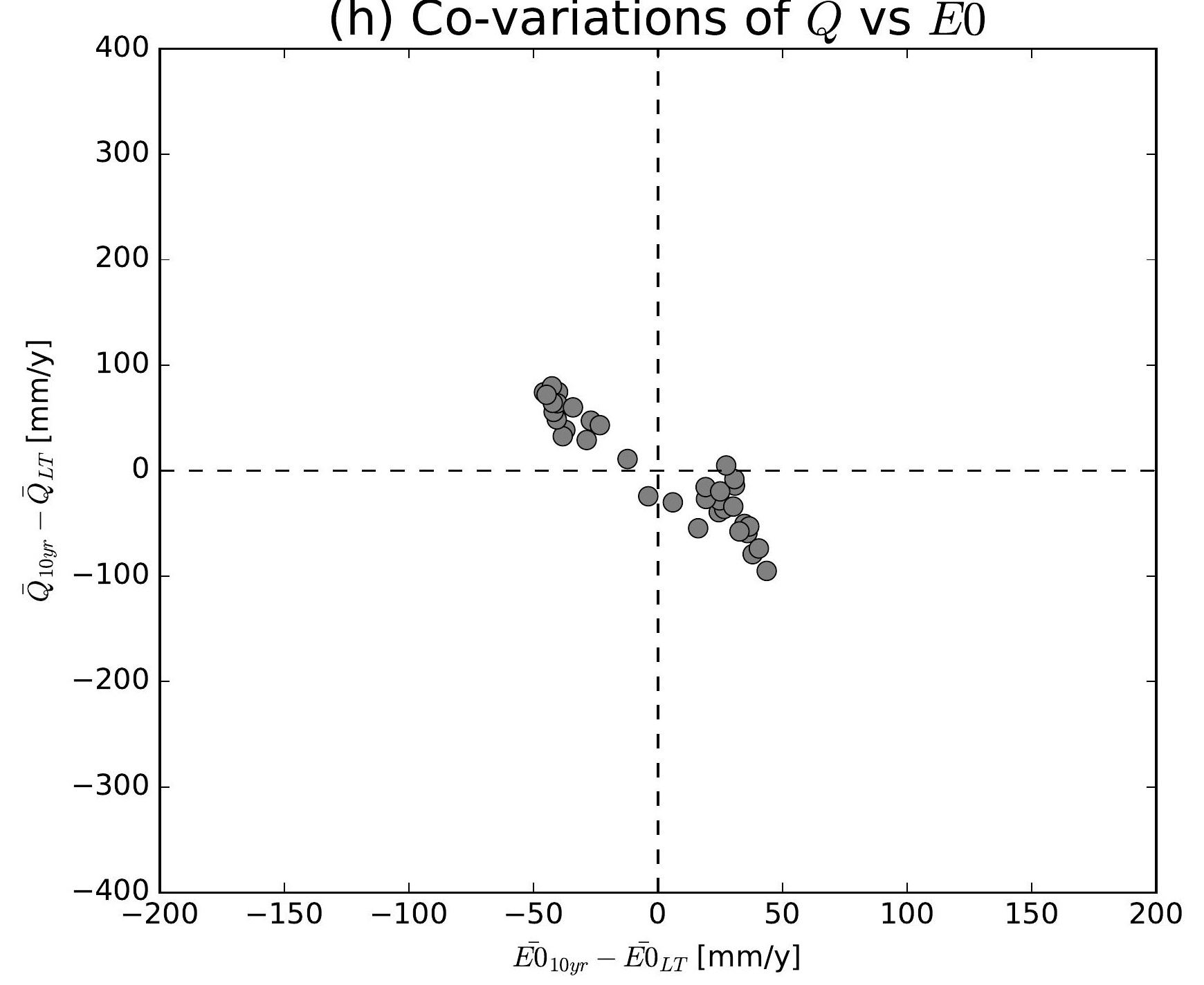

(k) Co-variations of $\bar{Q}$ vs $\overline{E 0}$

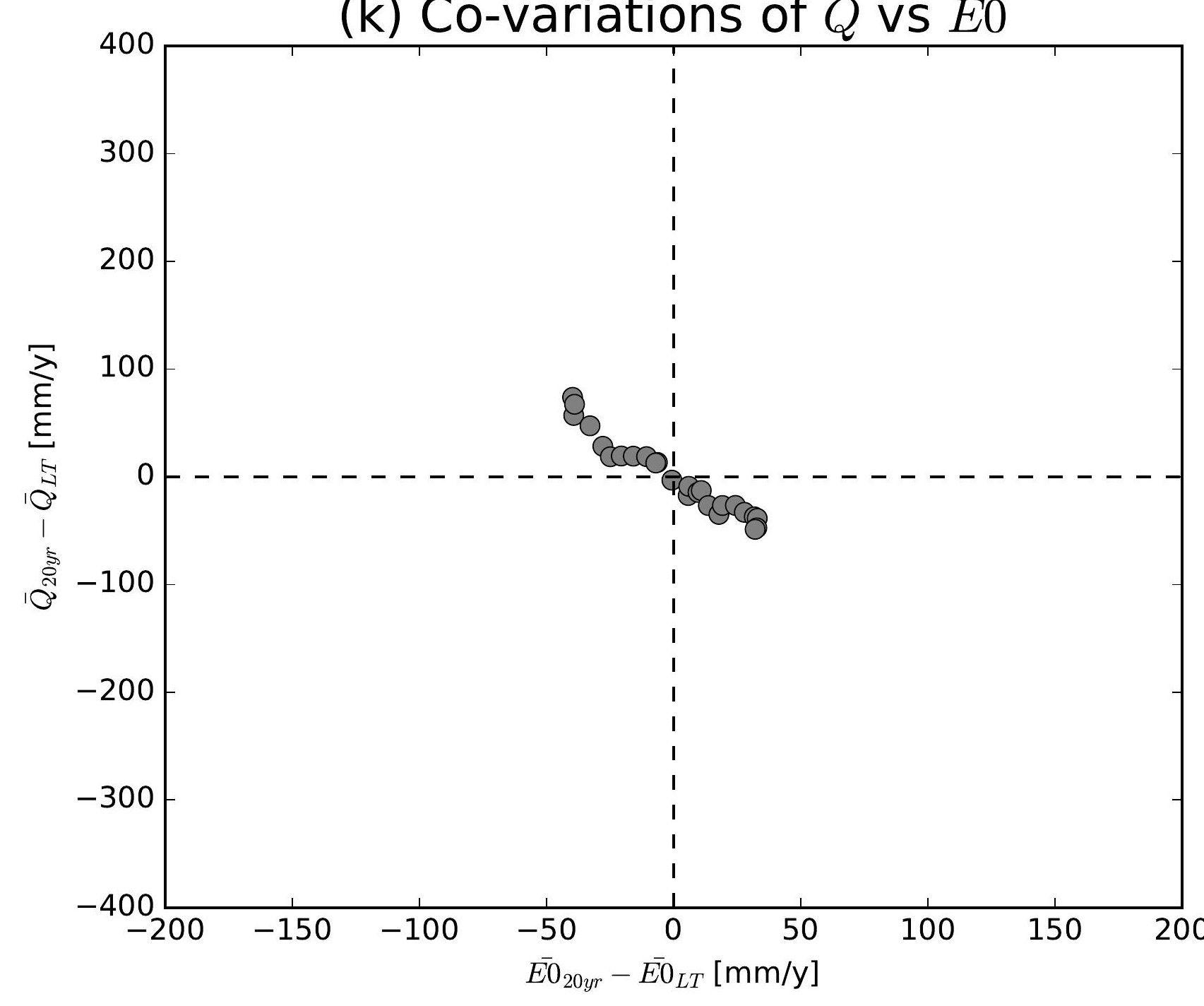

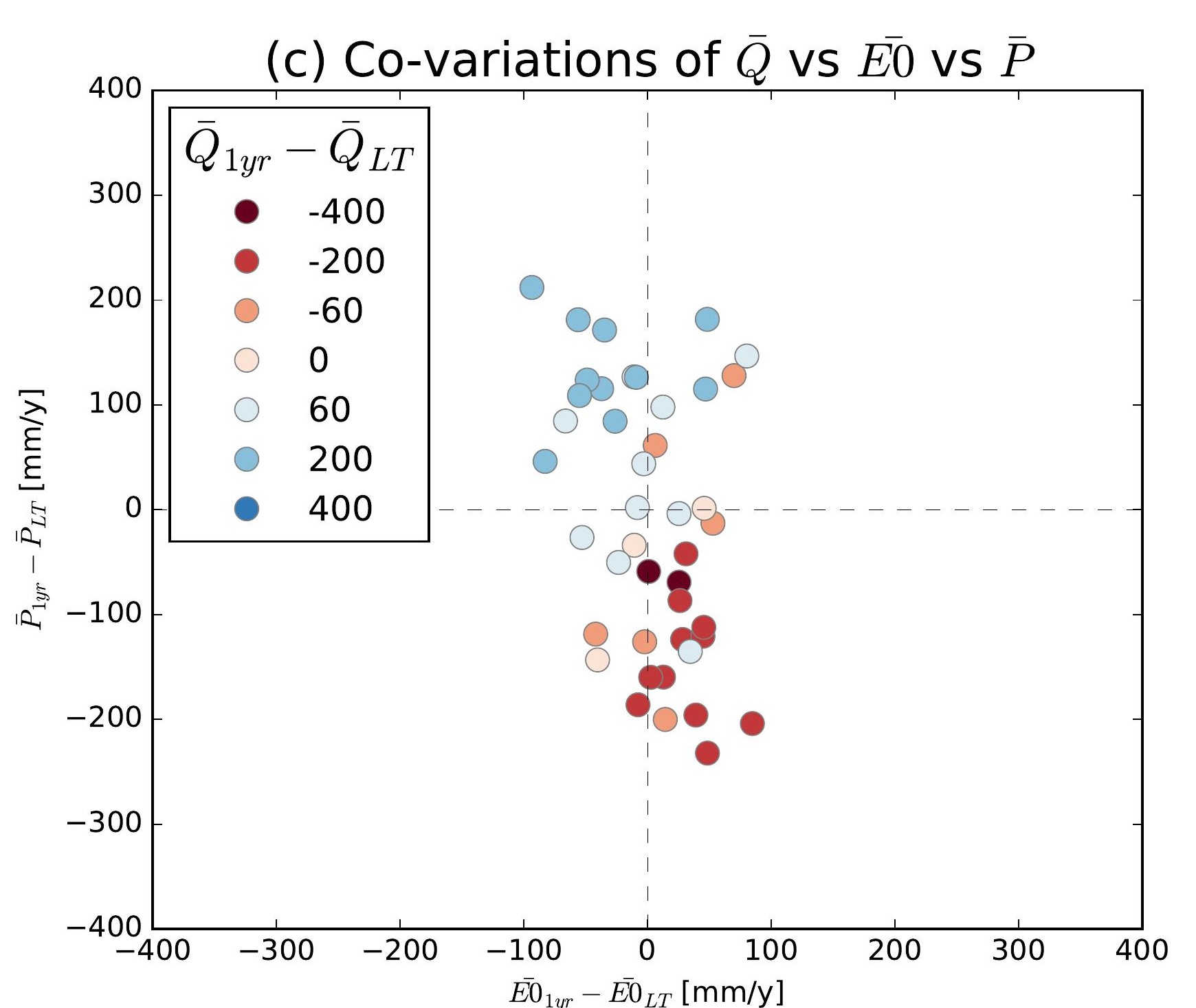
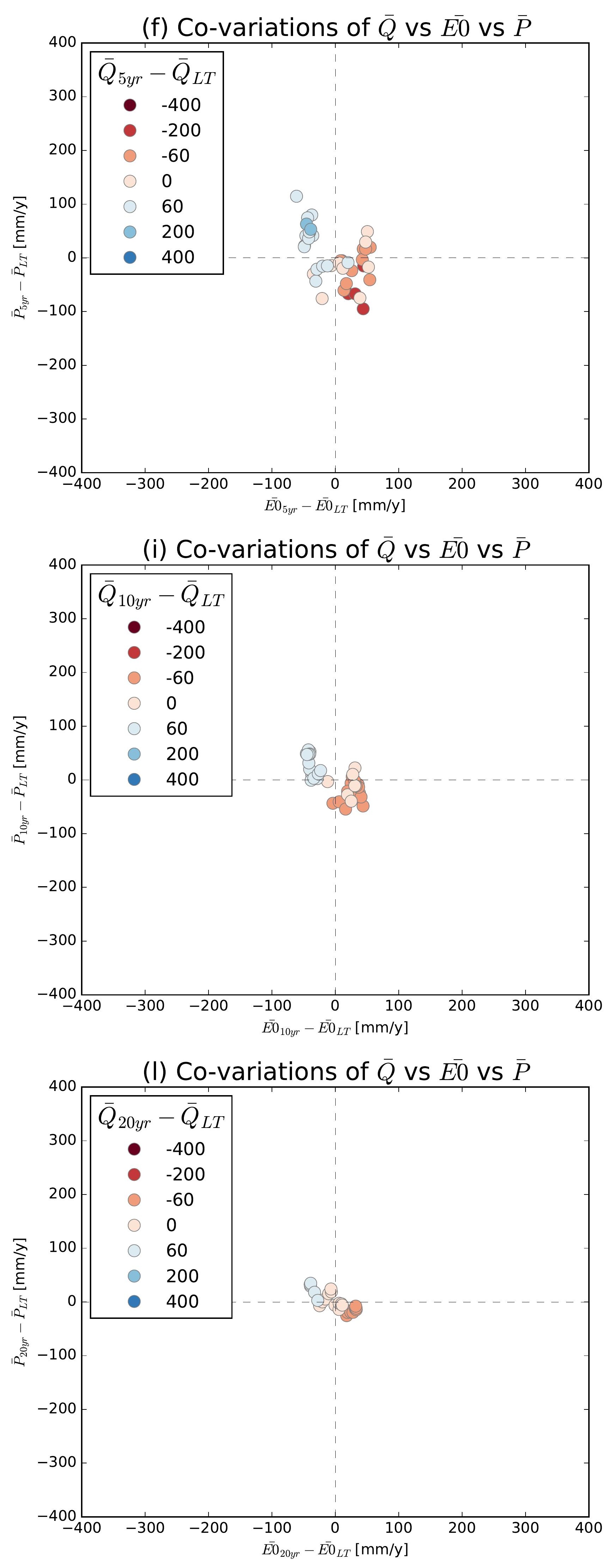

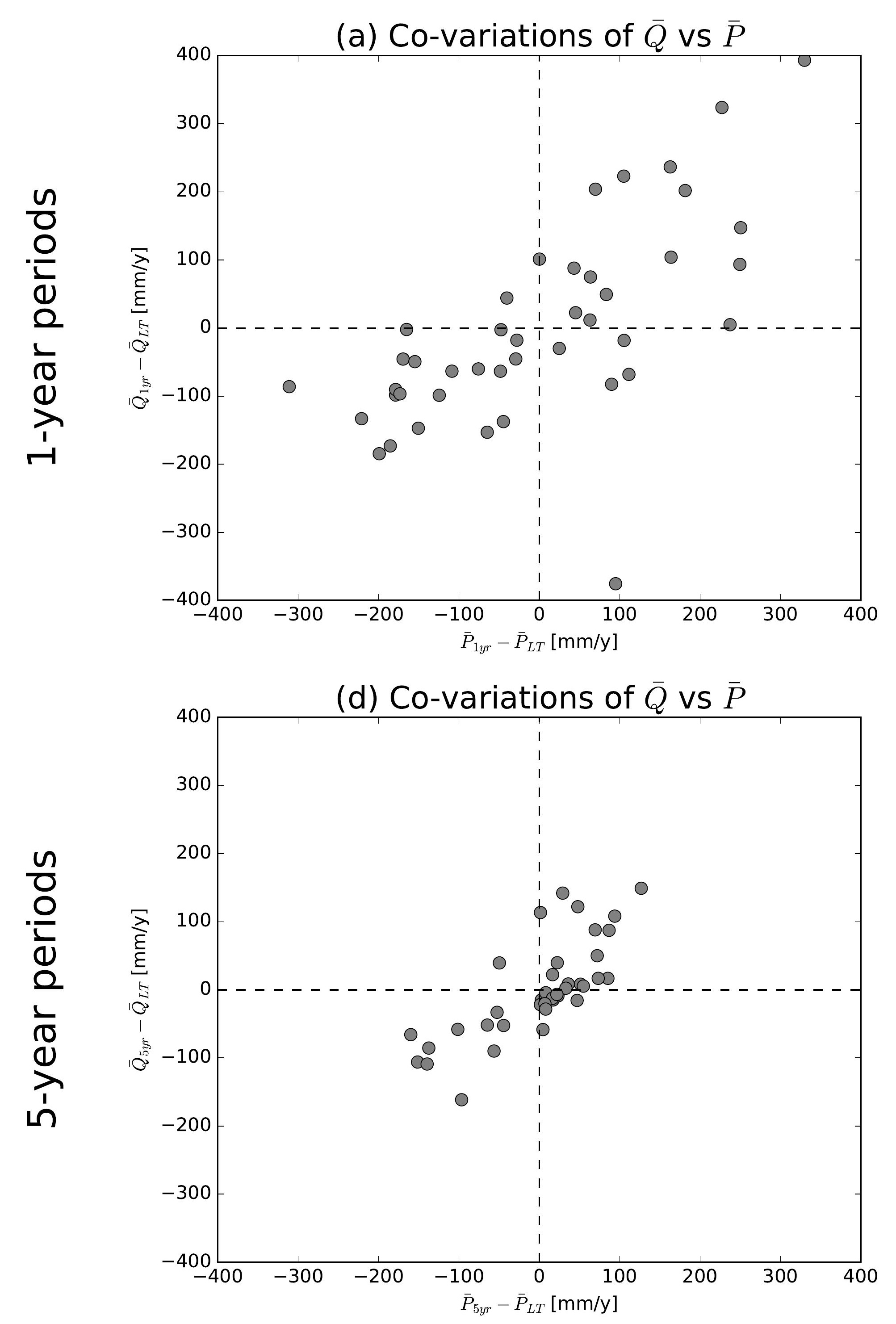

(g) Co-variations of $\bar{Q}$ vs $\bar{P}$

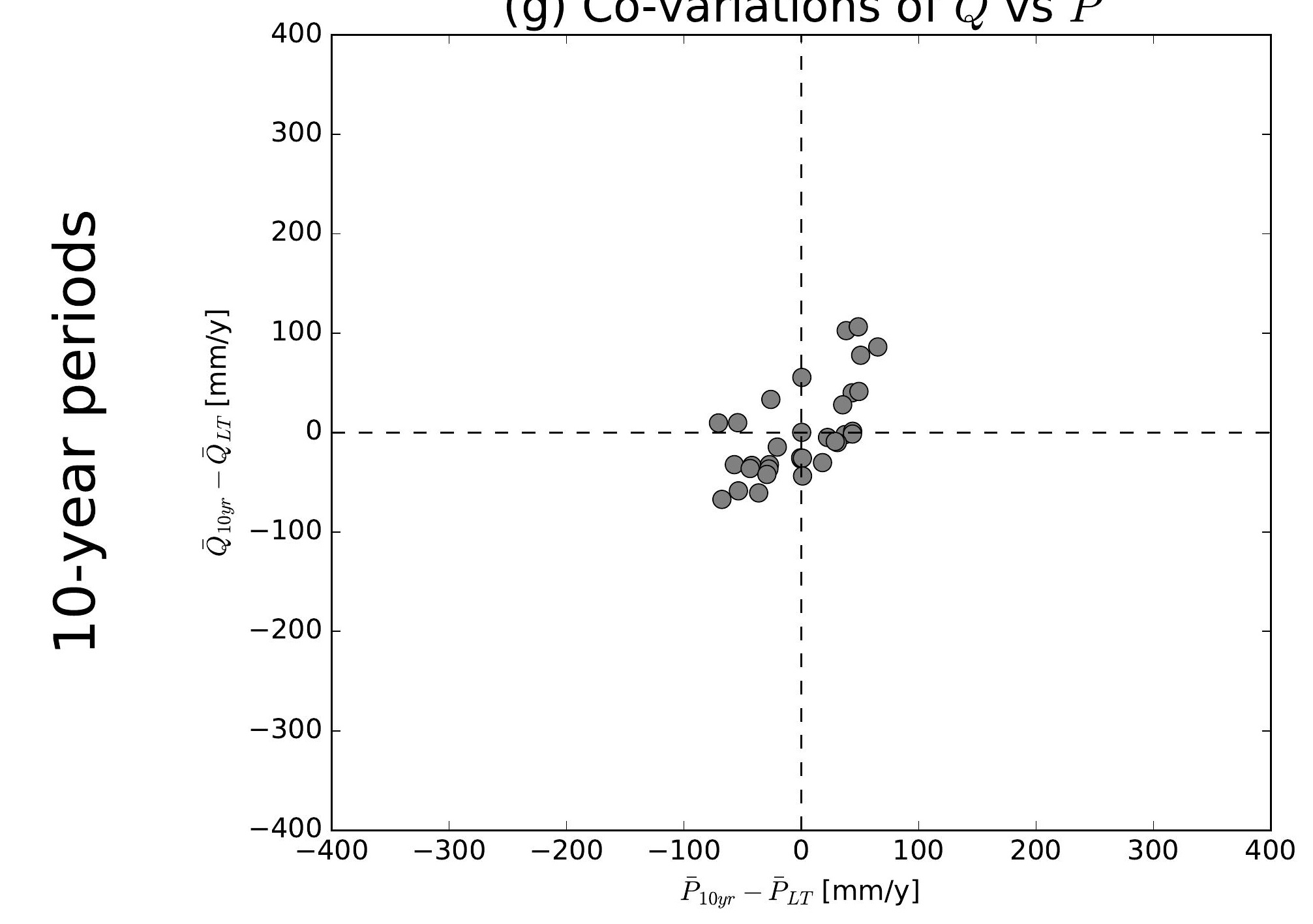

(j) Co-variations of $\bar{Q}$ vs $\bar{P}$

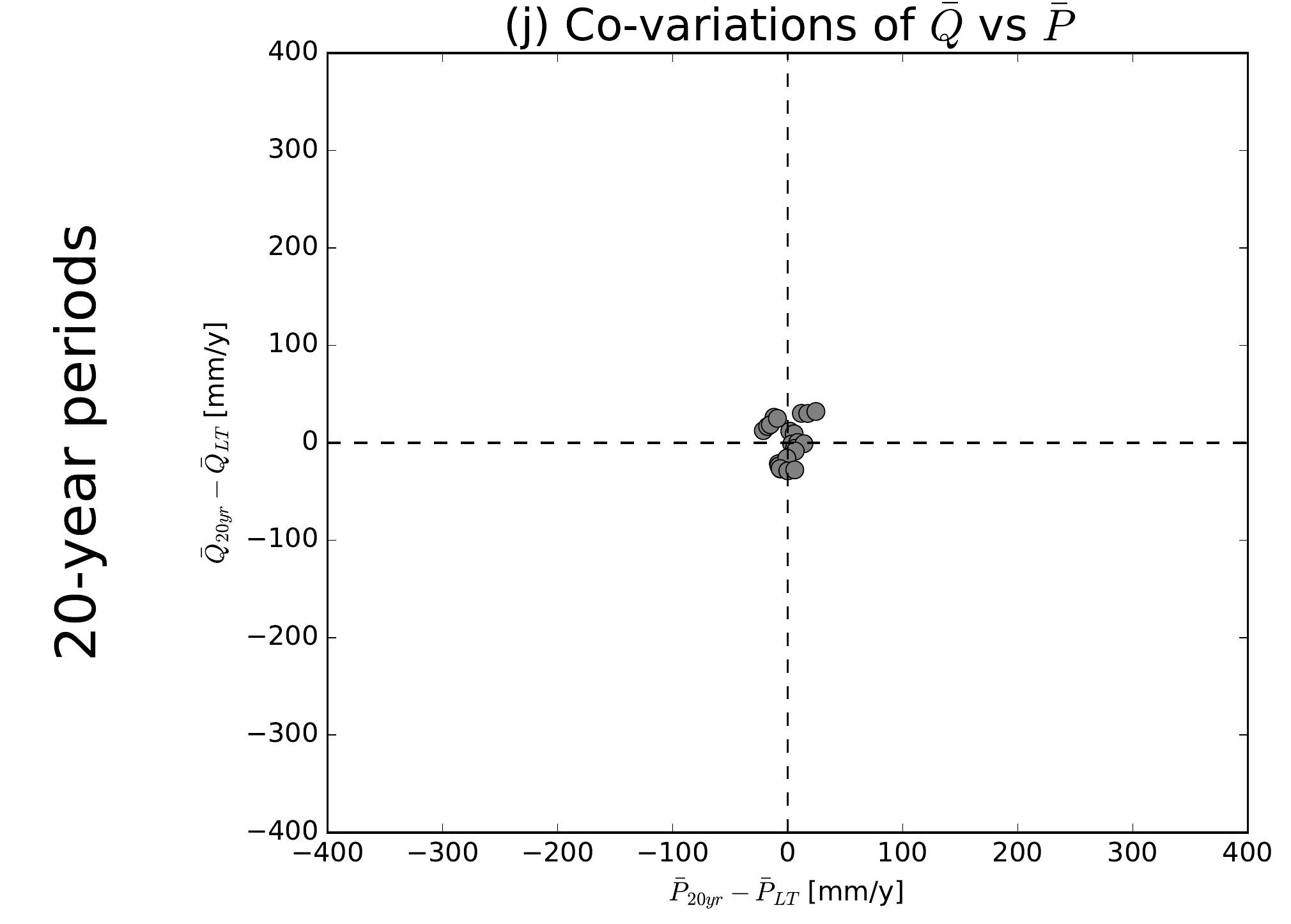

(b) Co-variations of $\bar{Q}$ vs $\overline{E 0}$

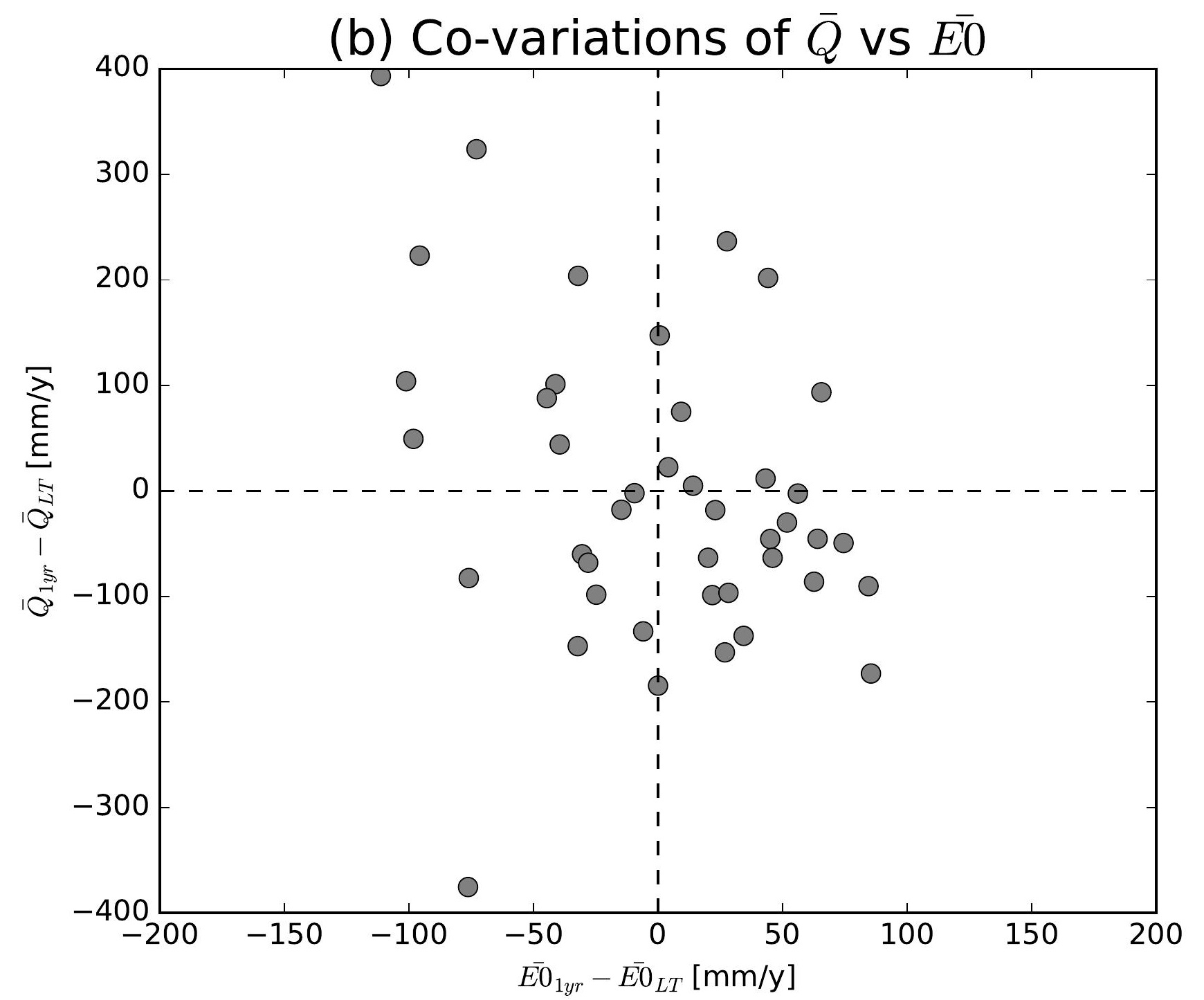

(e) Co-variations of $\bar{Q}$ vs $\overline{E 0}$

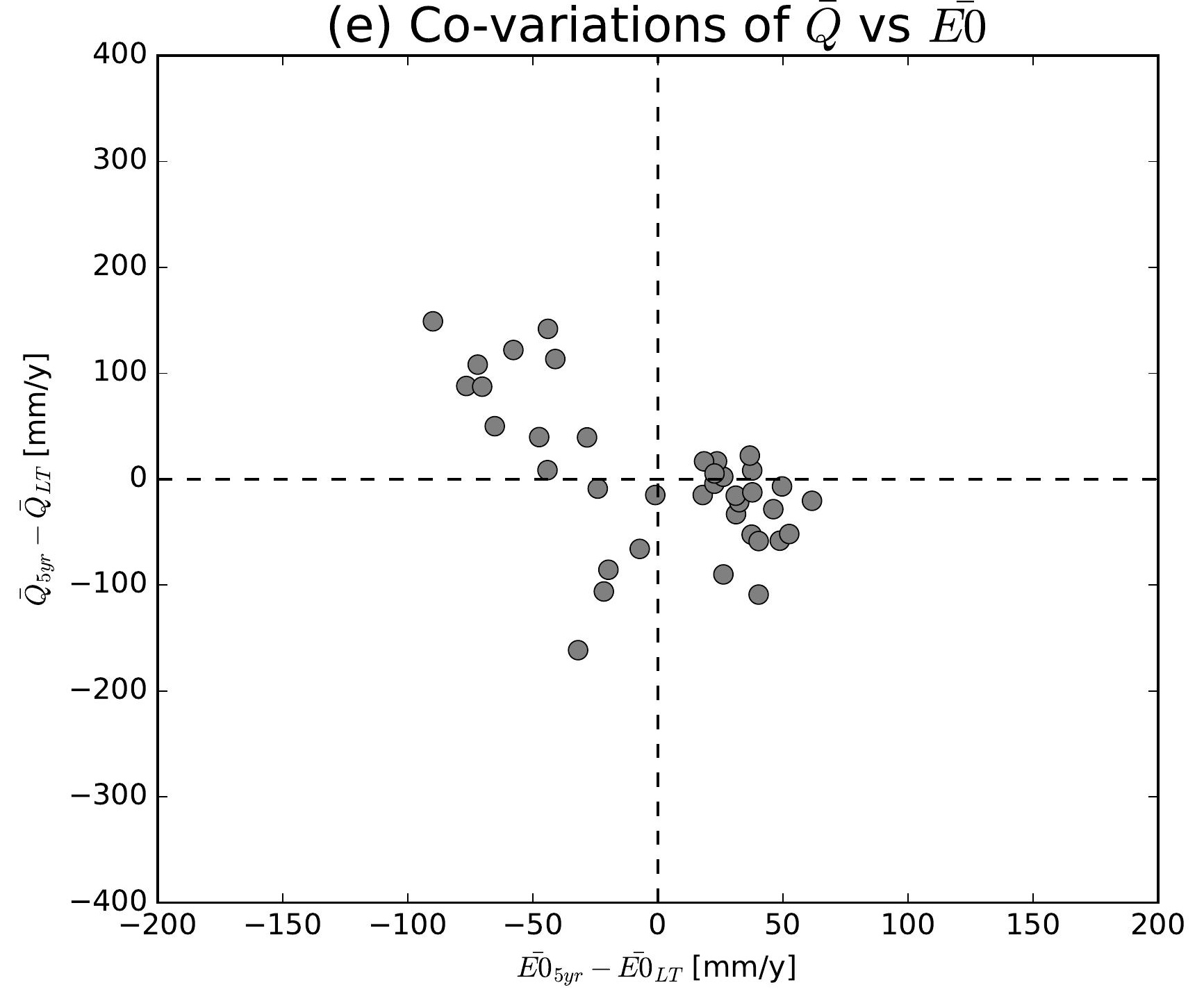

(h) Co-variations of $\bar{Q}$ vs $\overline{E 0}$

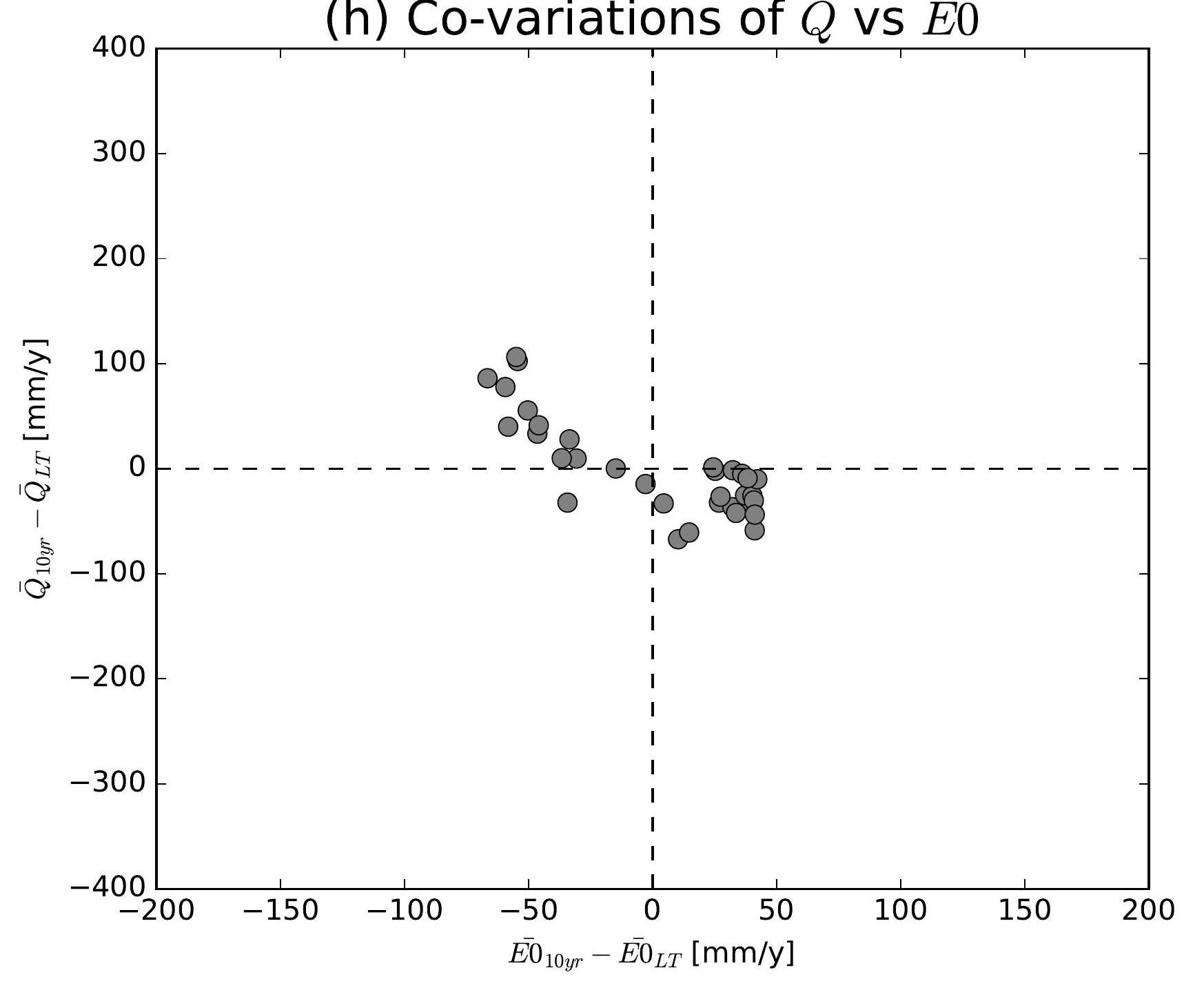

(k) Co-variations of $\bar{Q}$ vs $\overline{E 0}$

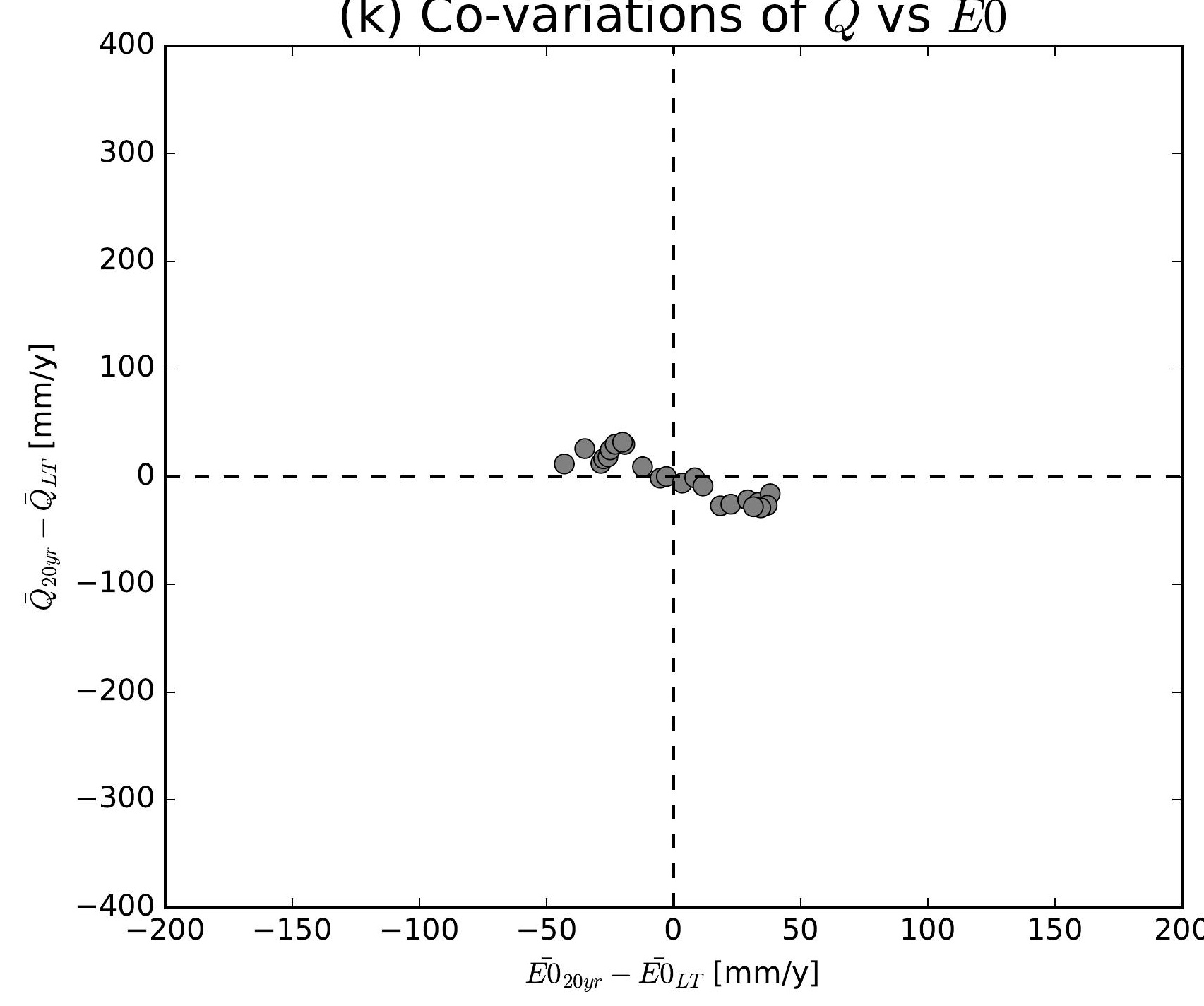

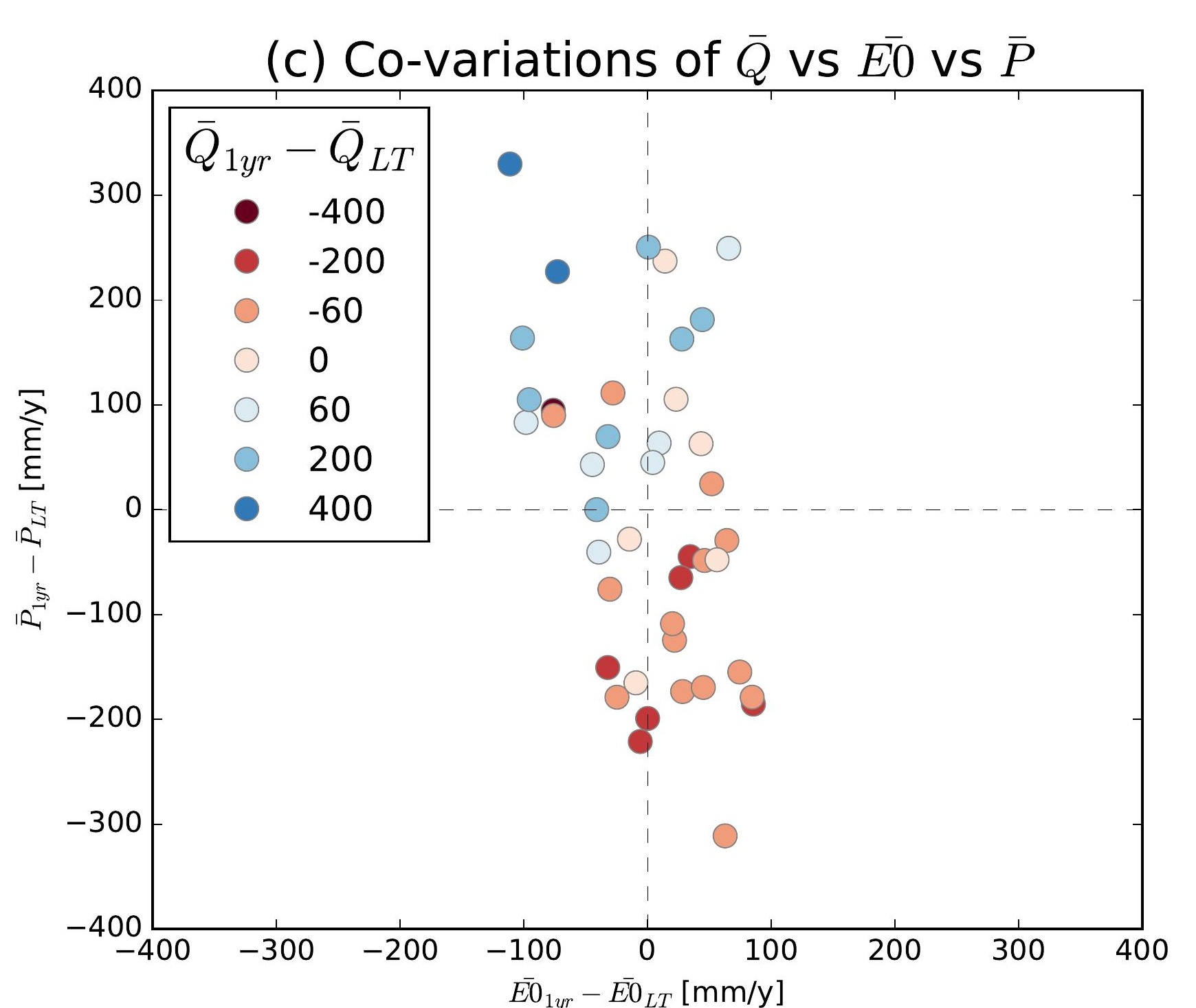
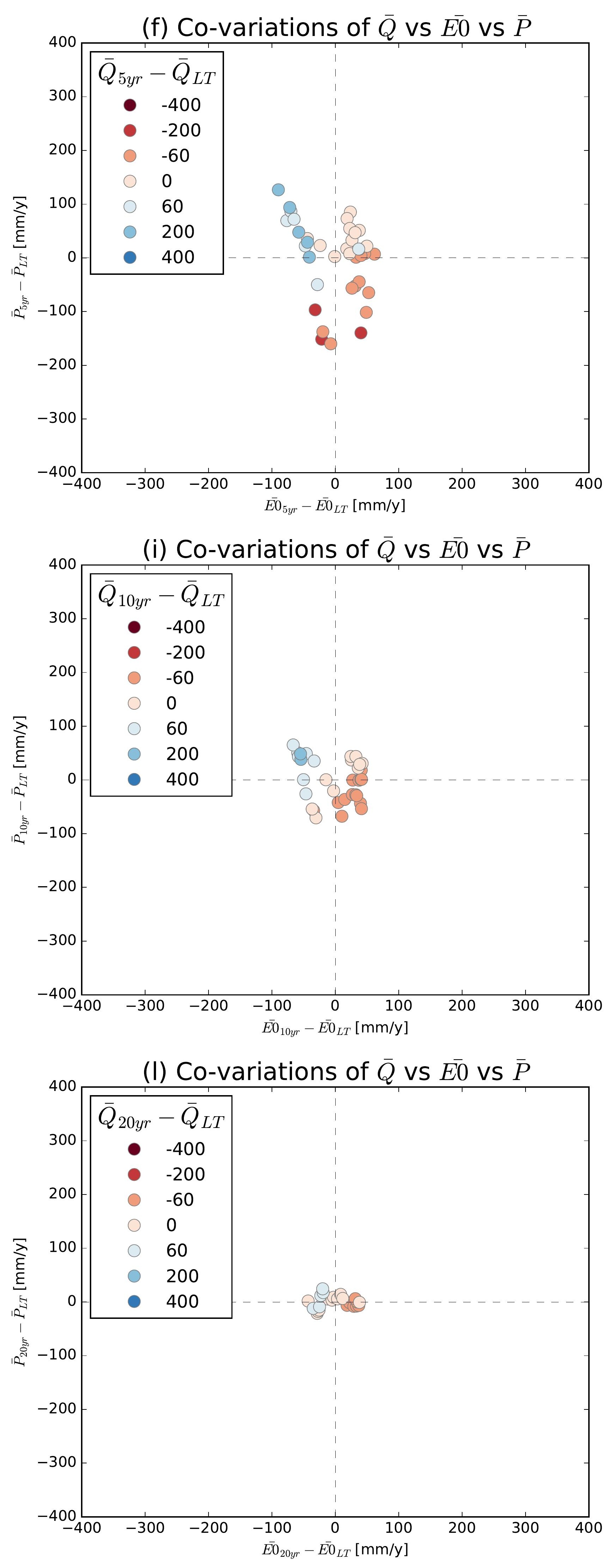

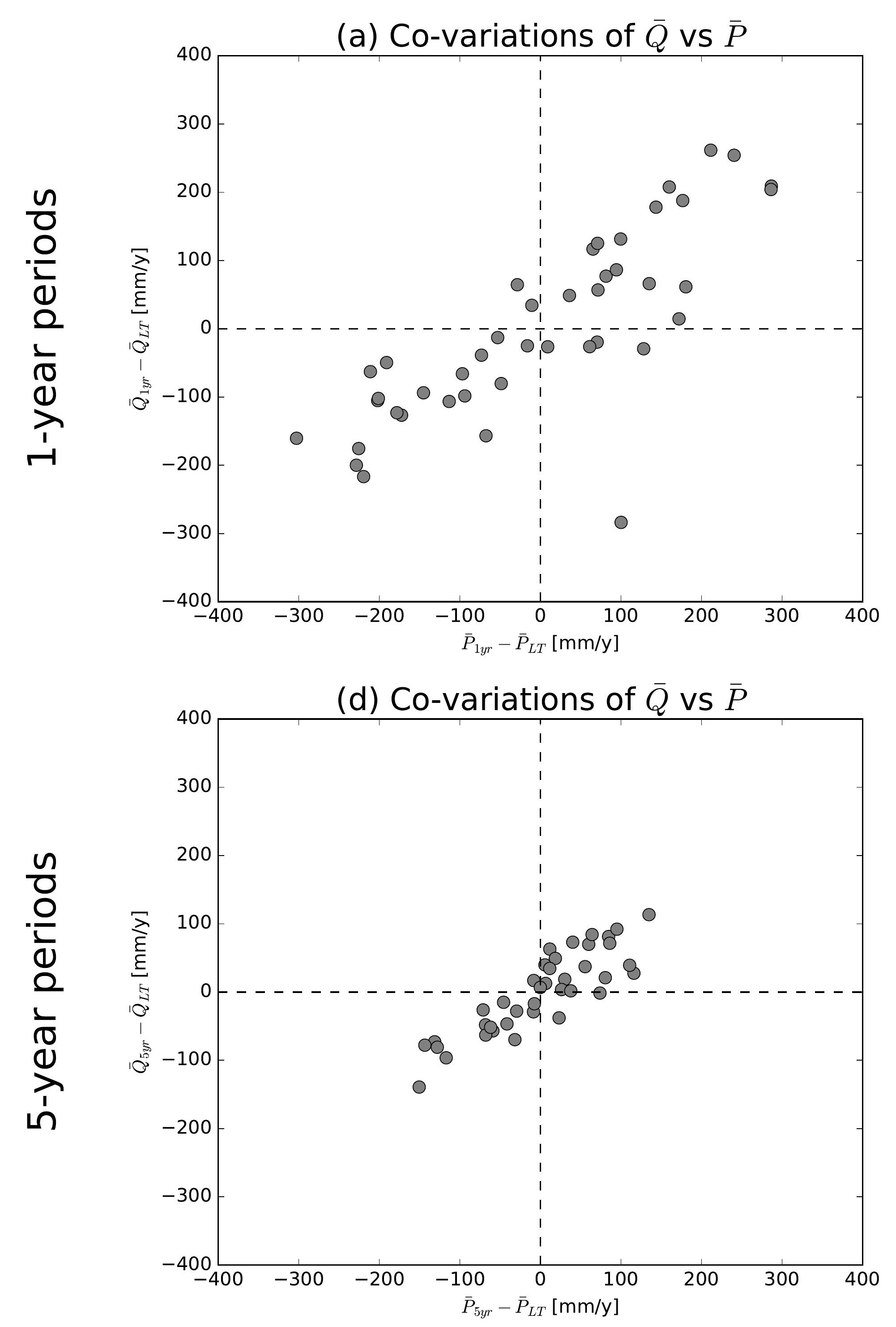

(g) Co-variations of $\bar{Q}$ vs $\bar{P}$

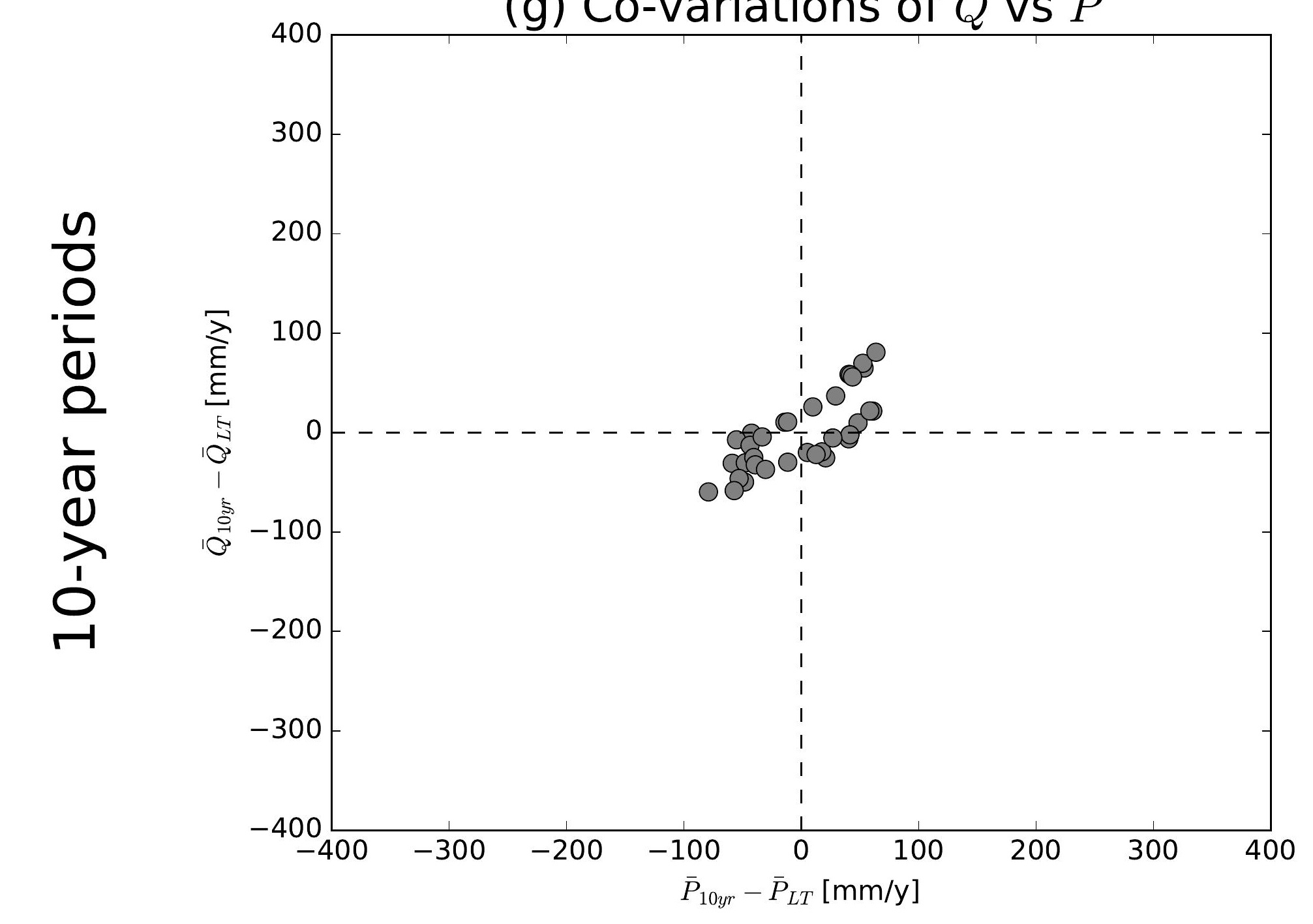

(j) Co-variations of $\bar{Q}$ vs $\bar{P}$

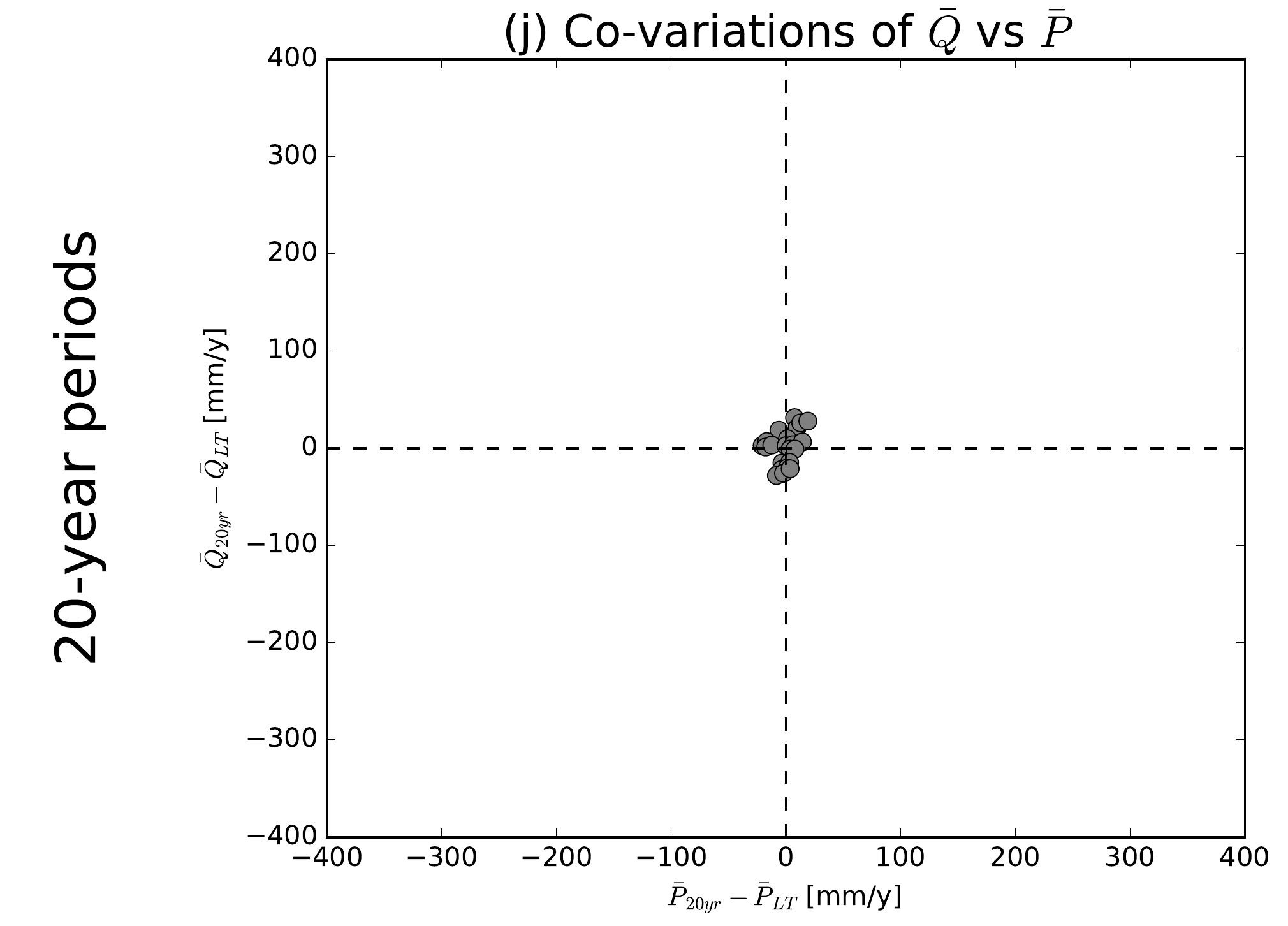

(b) Co-variations of $\bar{Q}$ vs $\overline{E 0}$

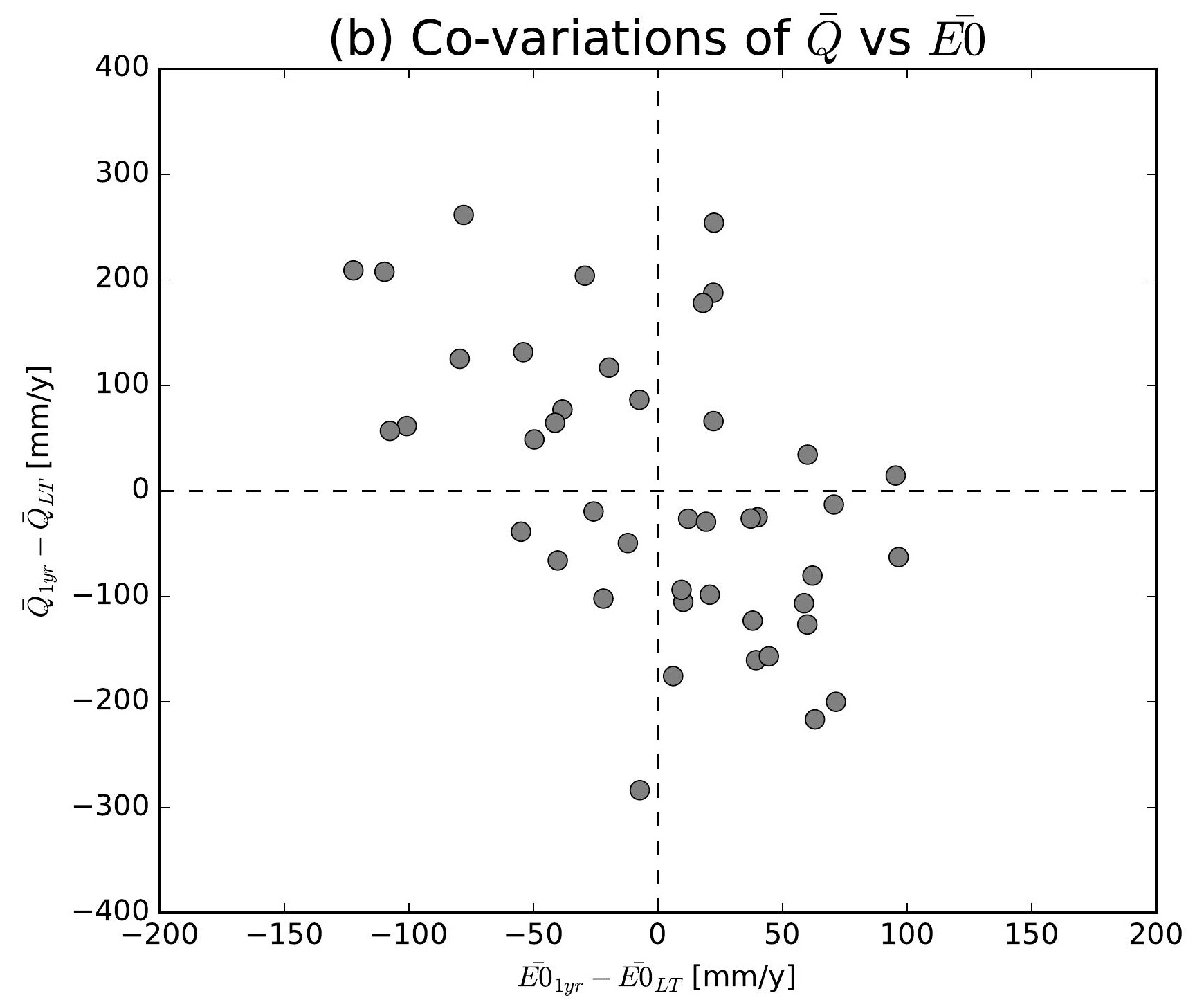

(e) Co-variations of $\bar{Q}$ vs $\overline{E 0}$

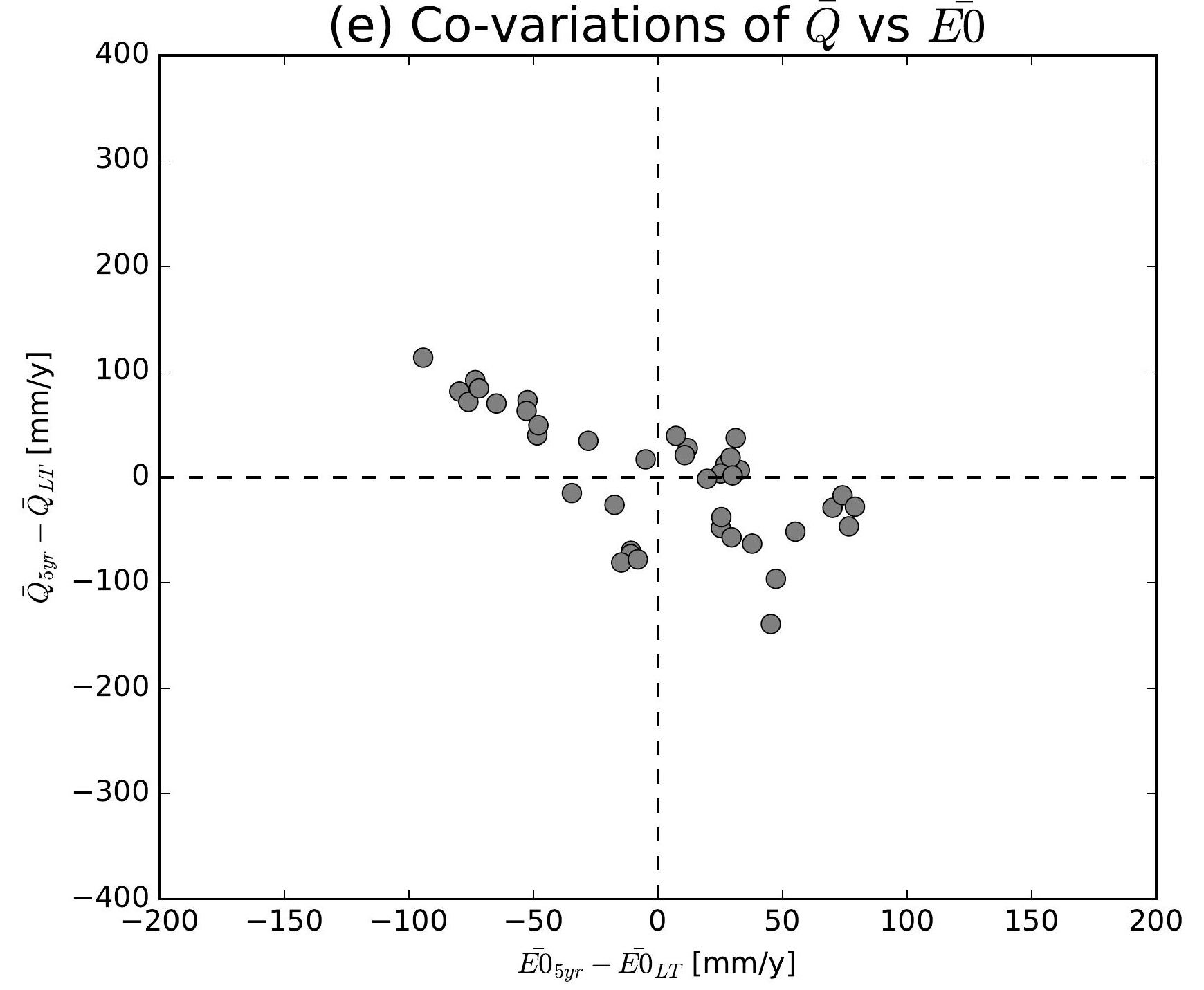

(h) Co-variations of $\bar{Q}$ vs $\overline{E 0}$

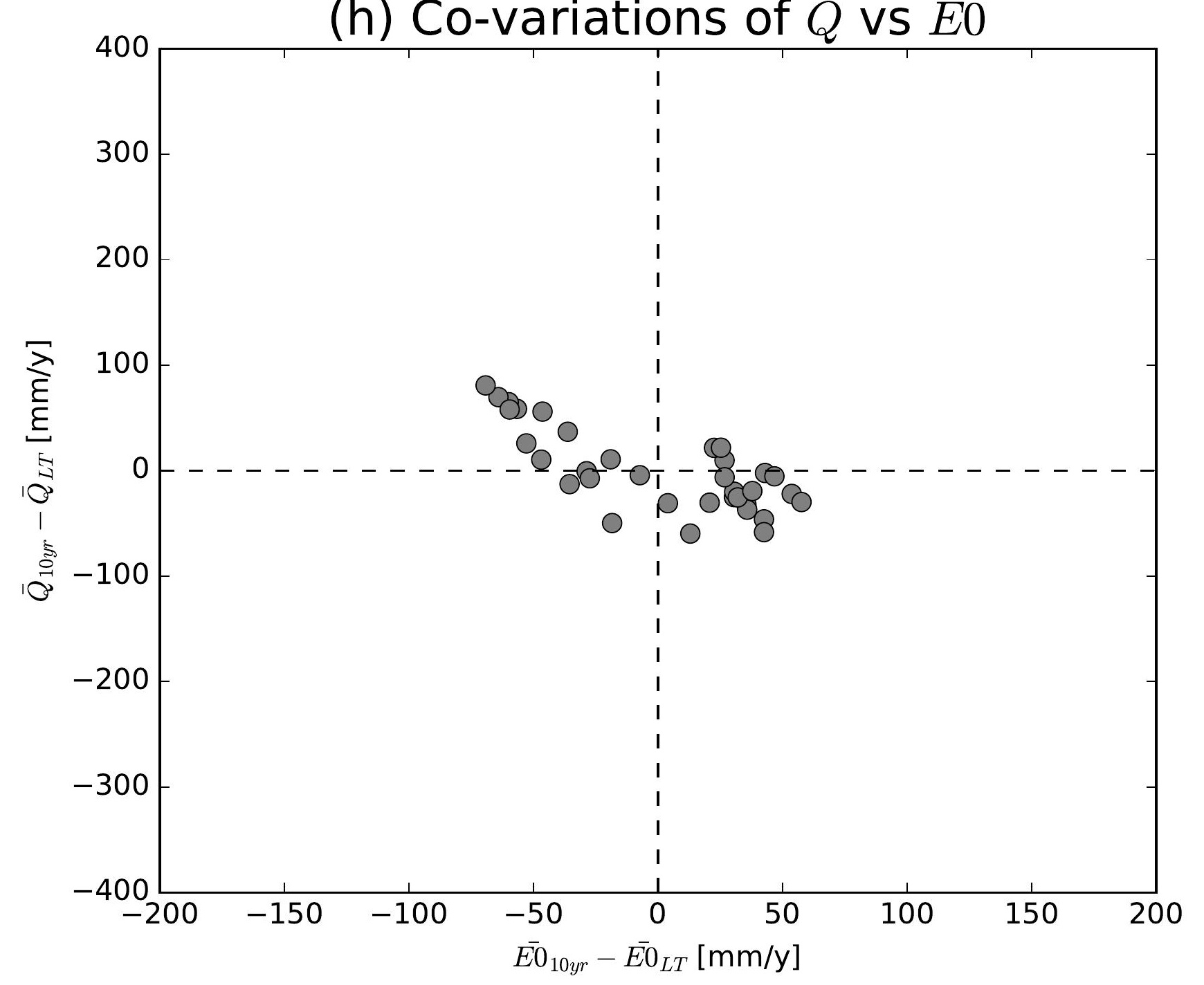

(k) Co-variations of $\bar{Q}$ vs $\overline{E 0}$

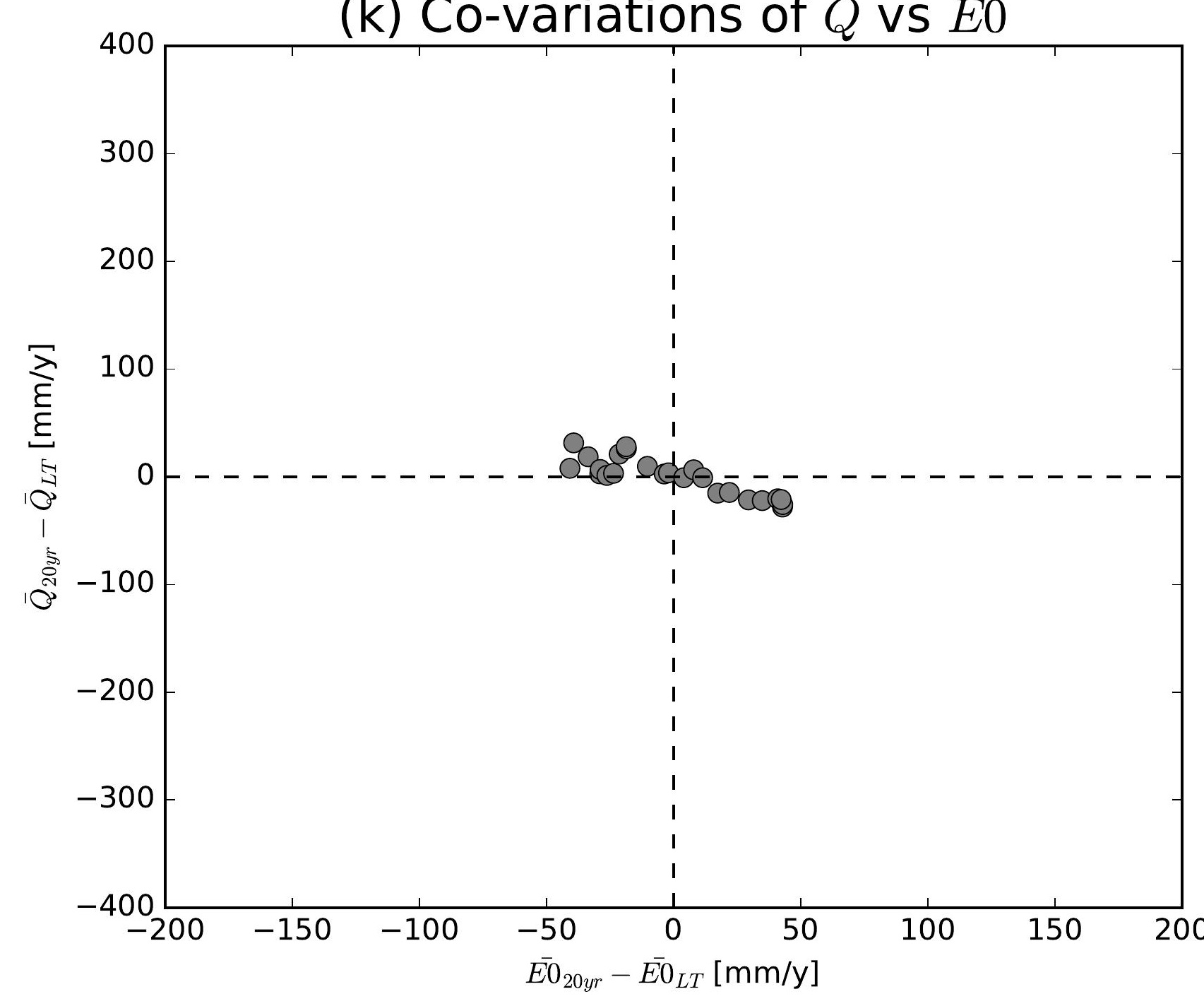

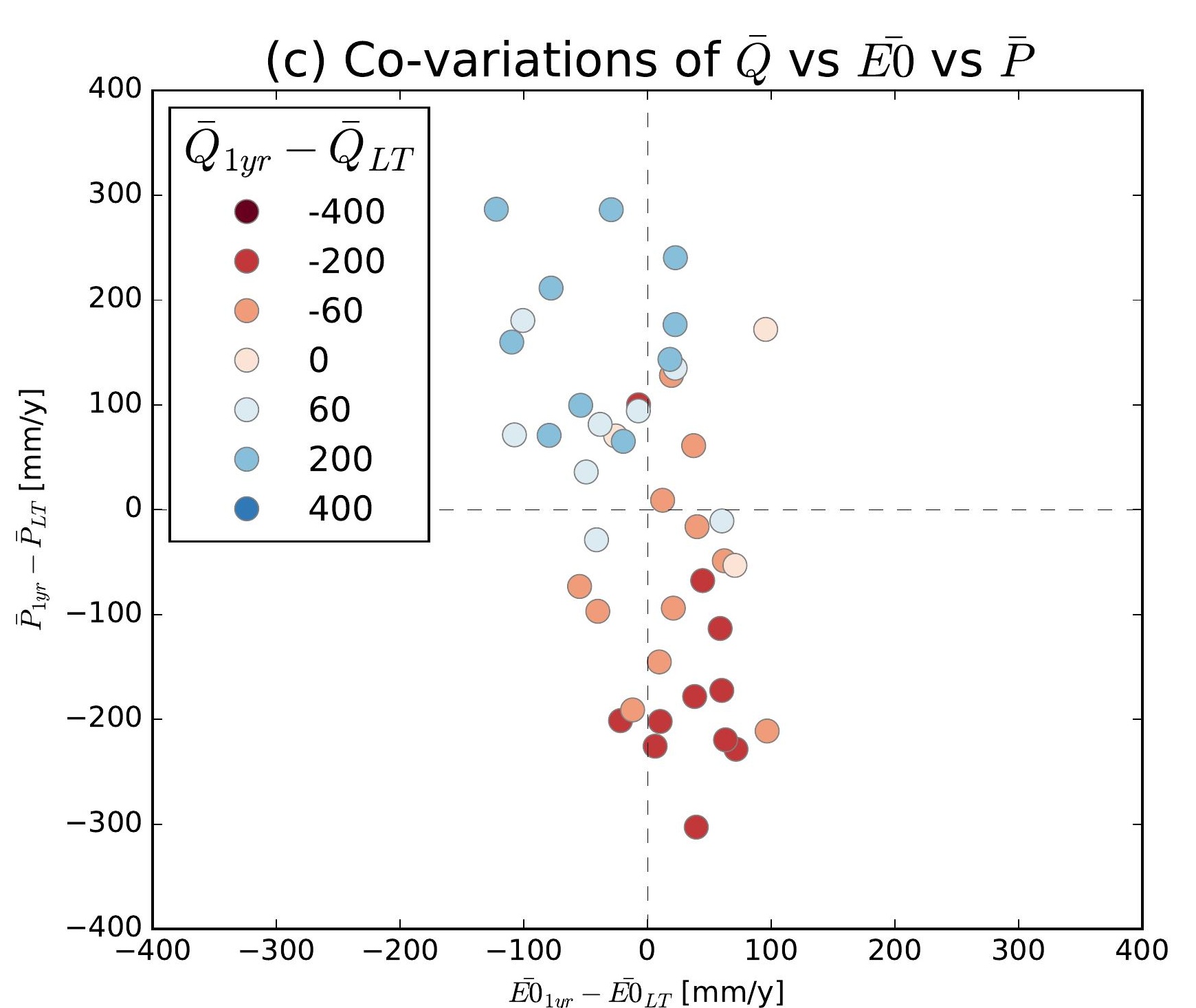
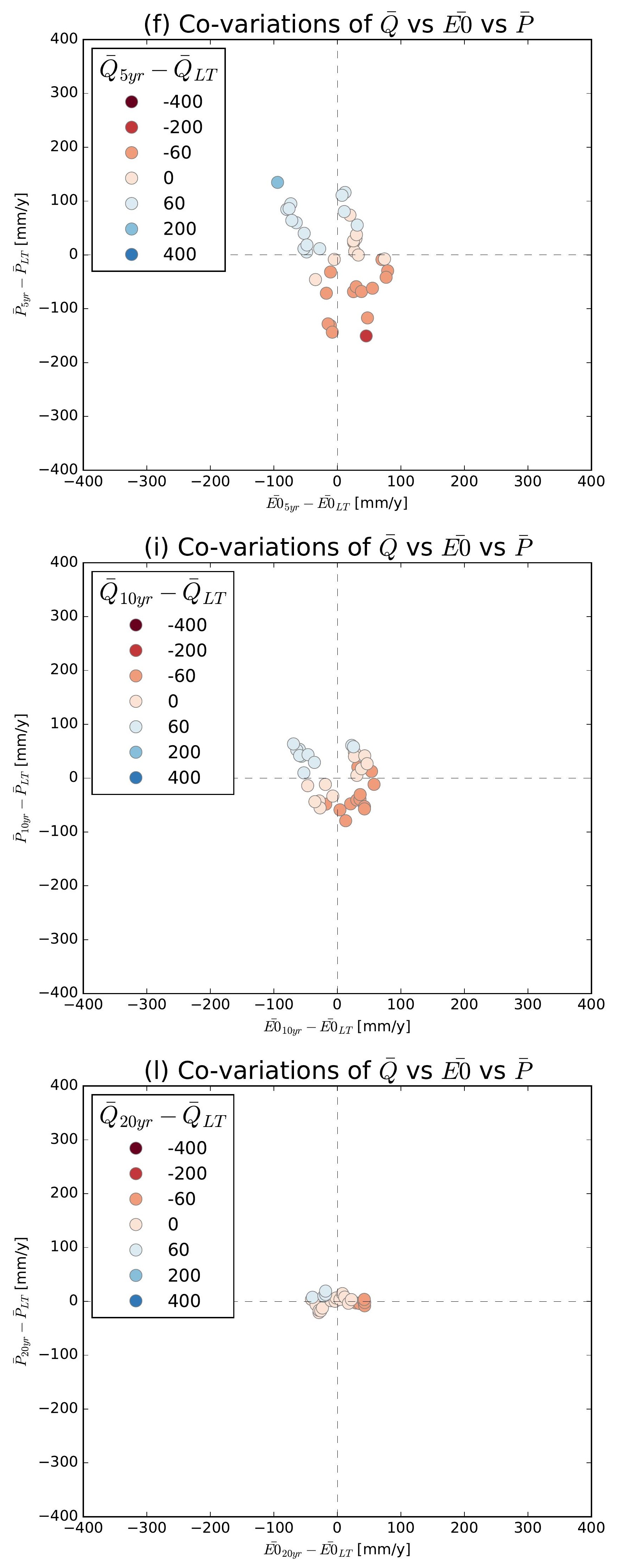

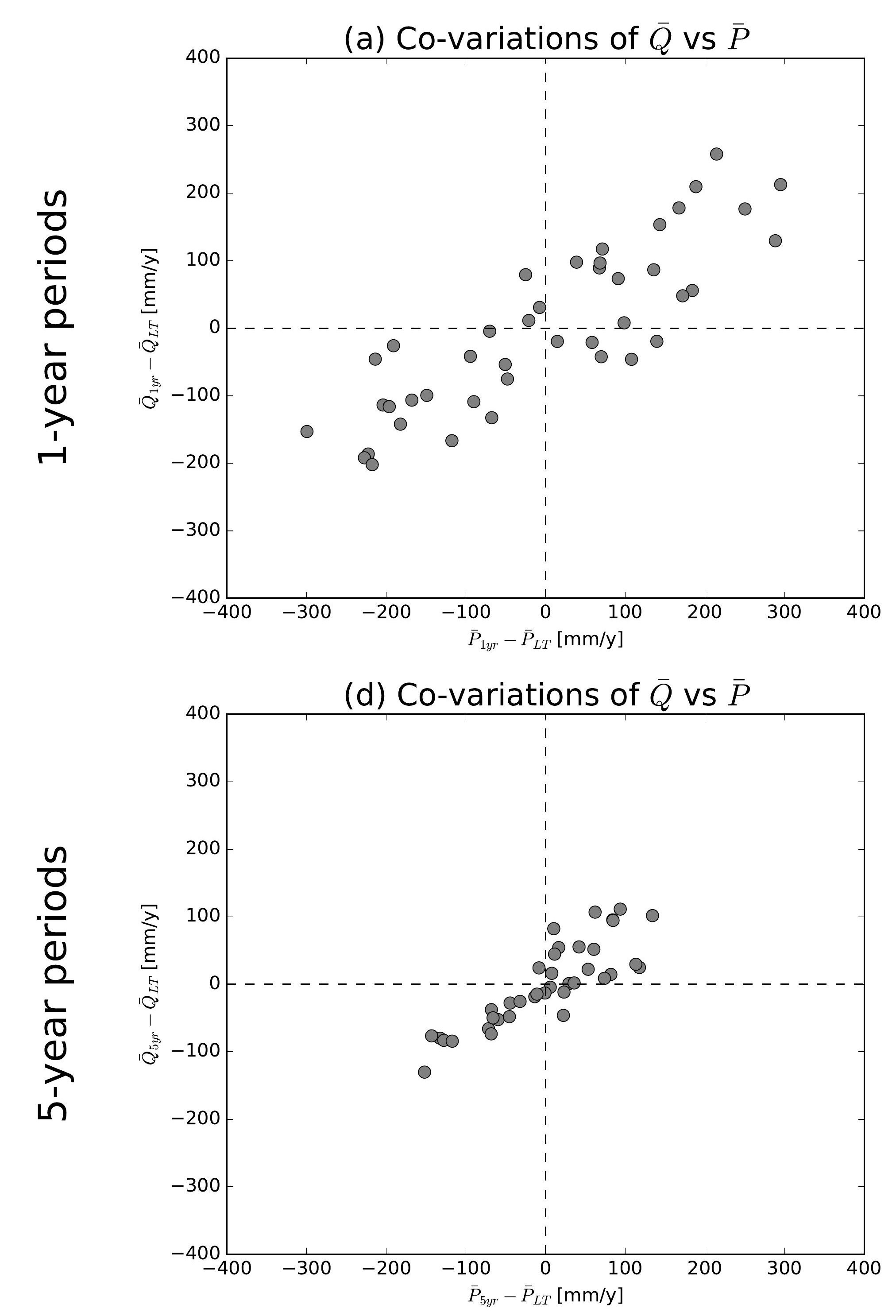

(g) Co-variations of $\bar{Q}$ vs $\bar{P}$

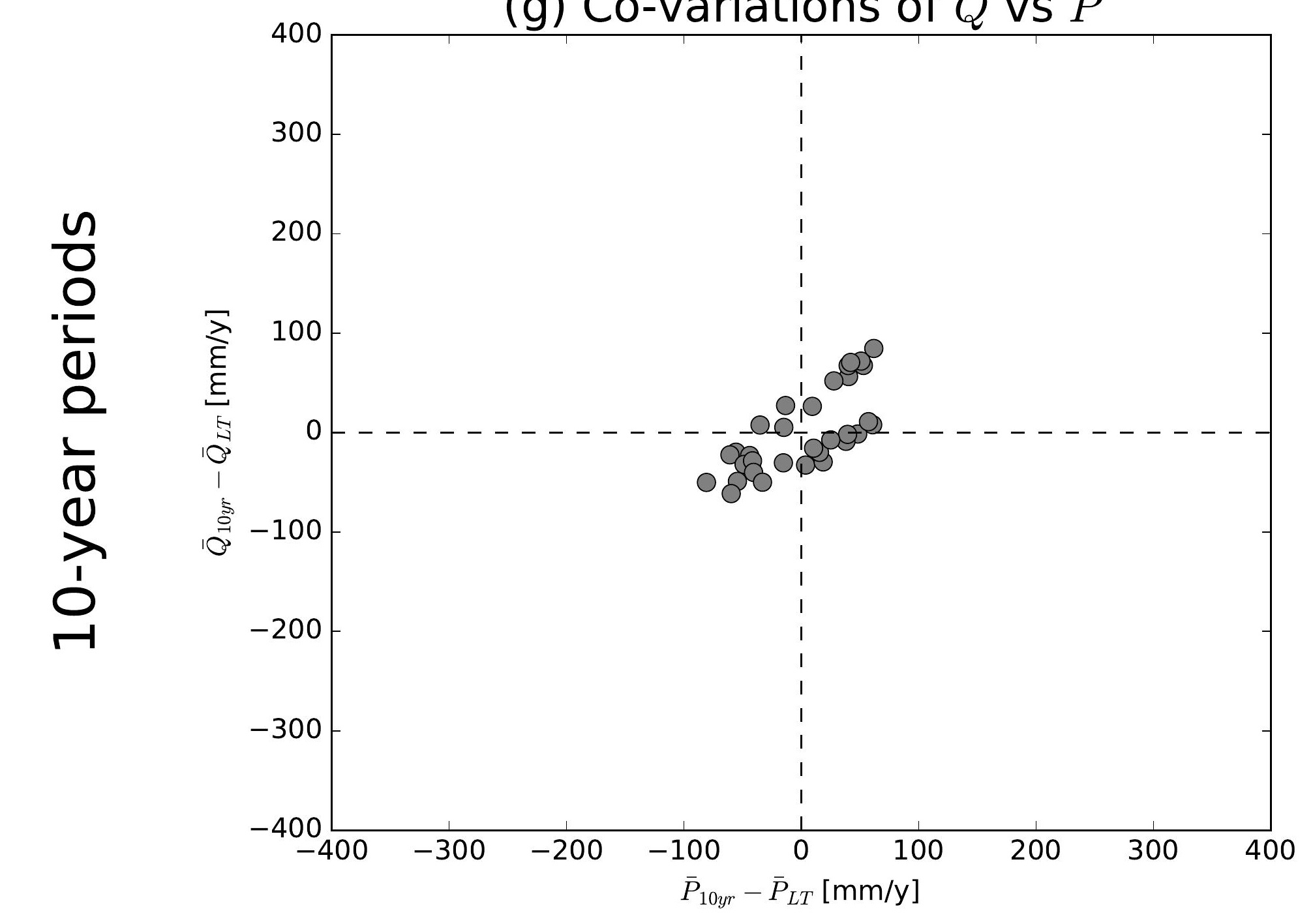

(j) Co-variations of $\bar{Q}$ vs $\bar{P}$

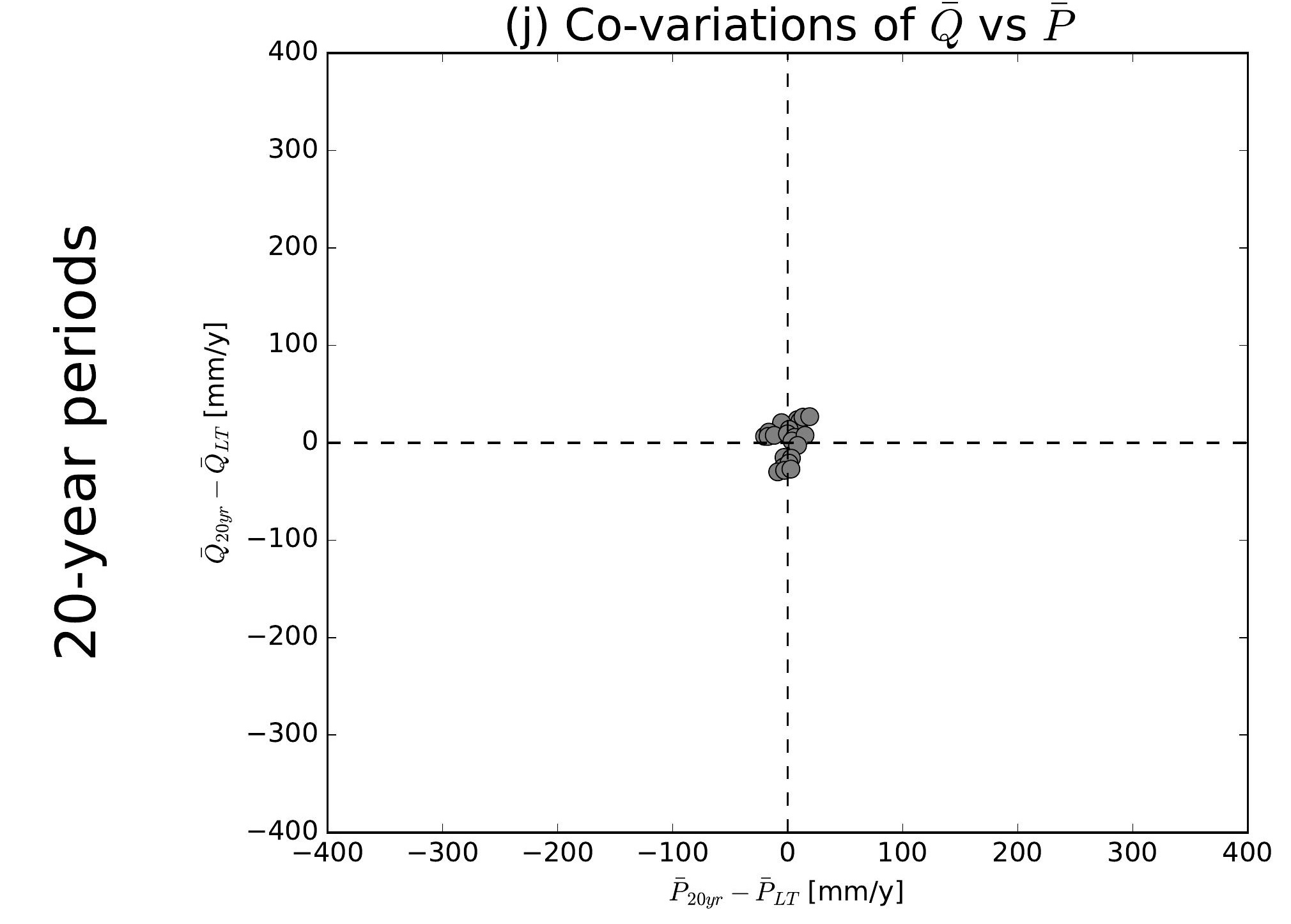

(b) Co-variations of $\bar{Q}$ vs $\overline{E 0}$

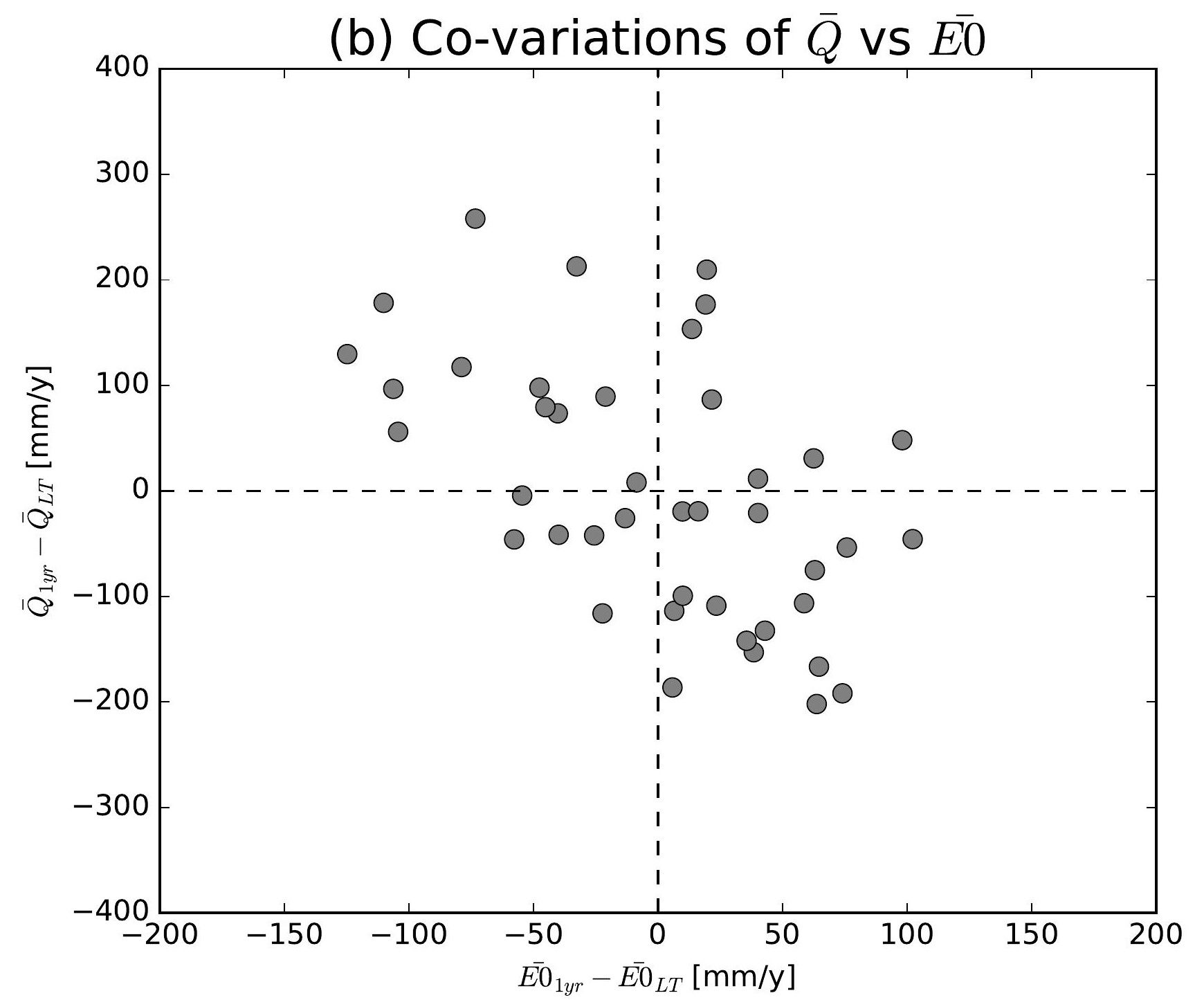

(e) Co-variations of $\bar{Q}$ vs $\overline{E 0}$

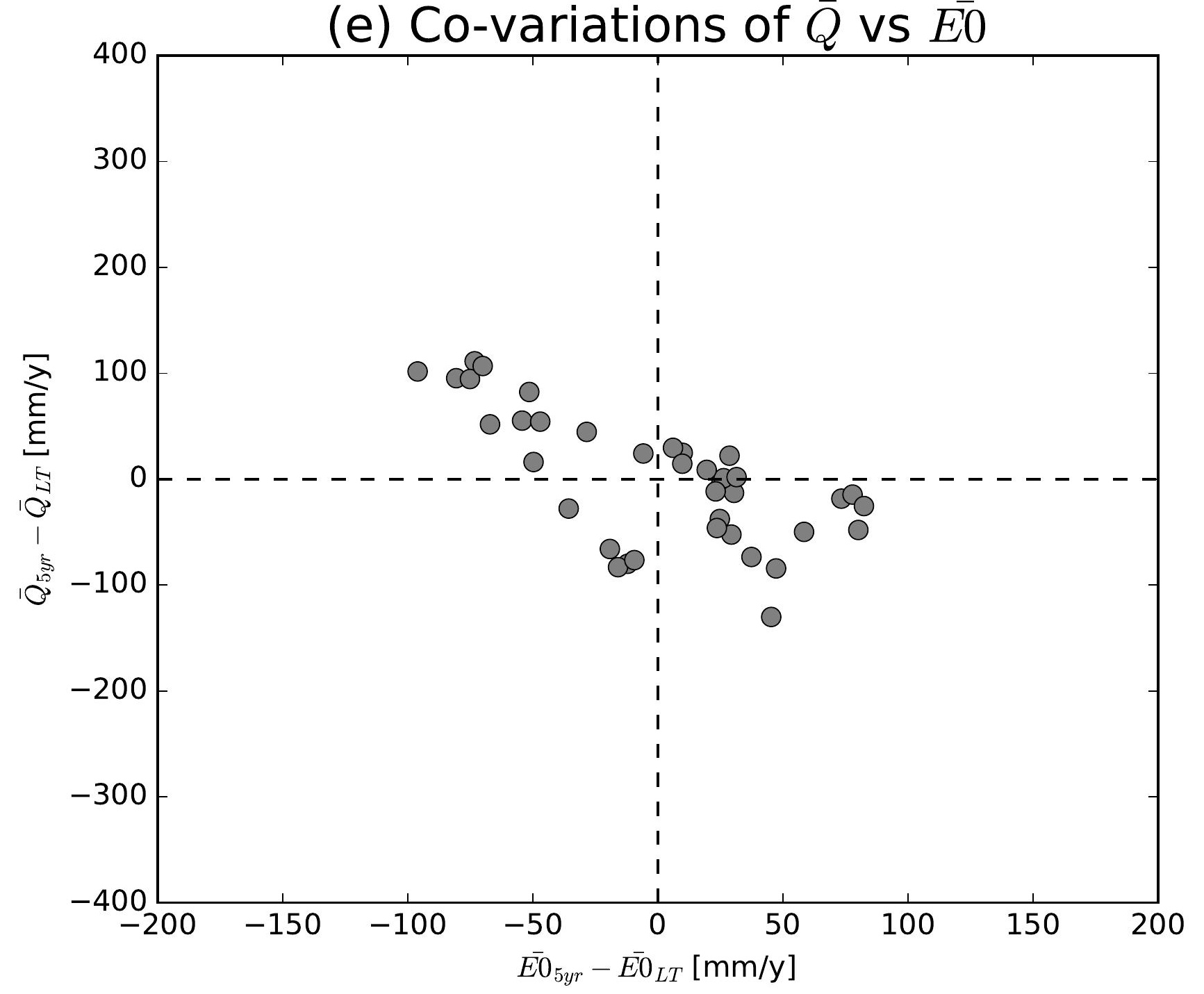

(h) Co-variations of $\bar{Q}$ vs $\overline{E 0}$

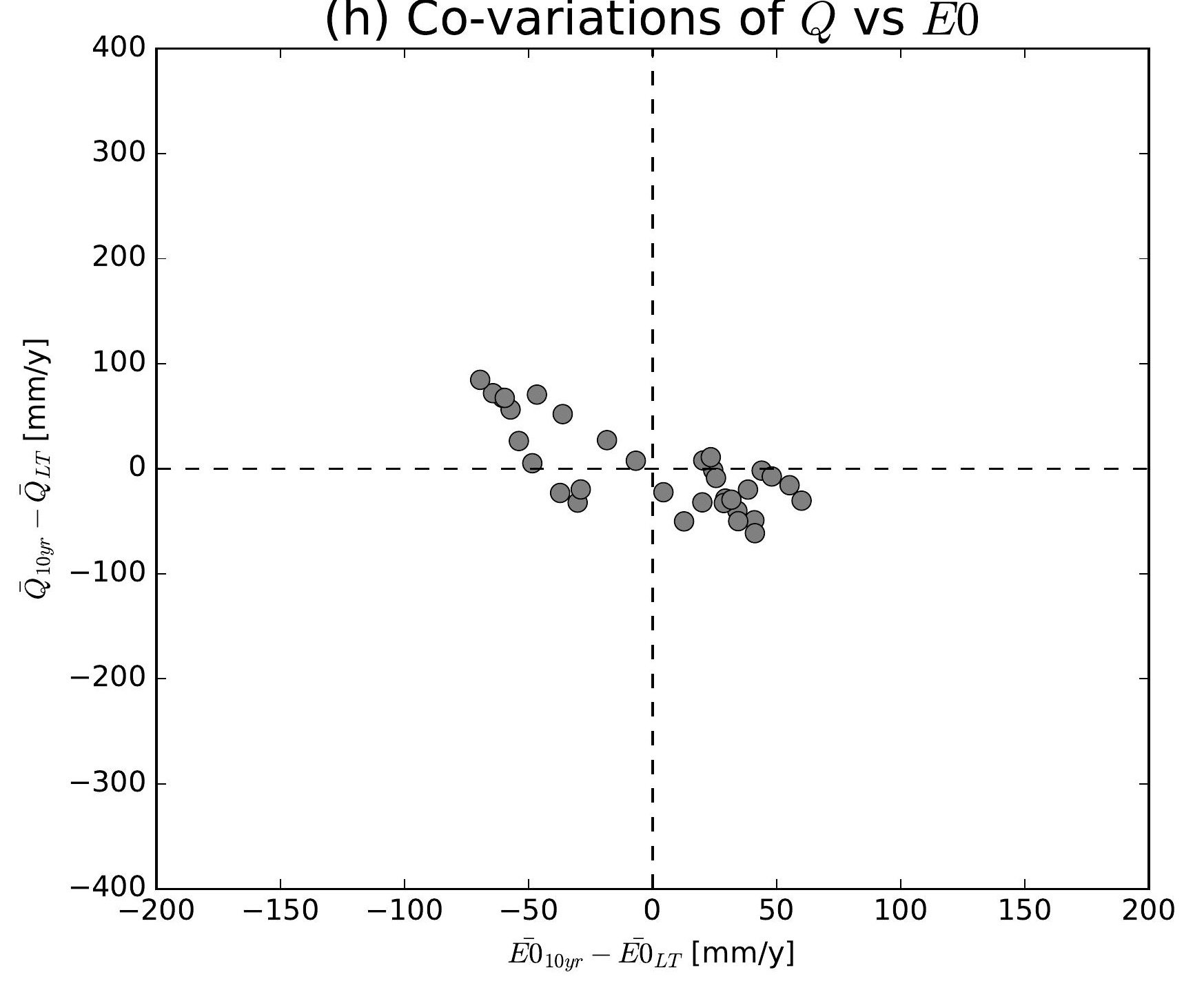

(k) Co-variations of $\bar{Q}$ vs $\overline{E 0}$

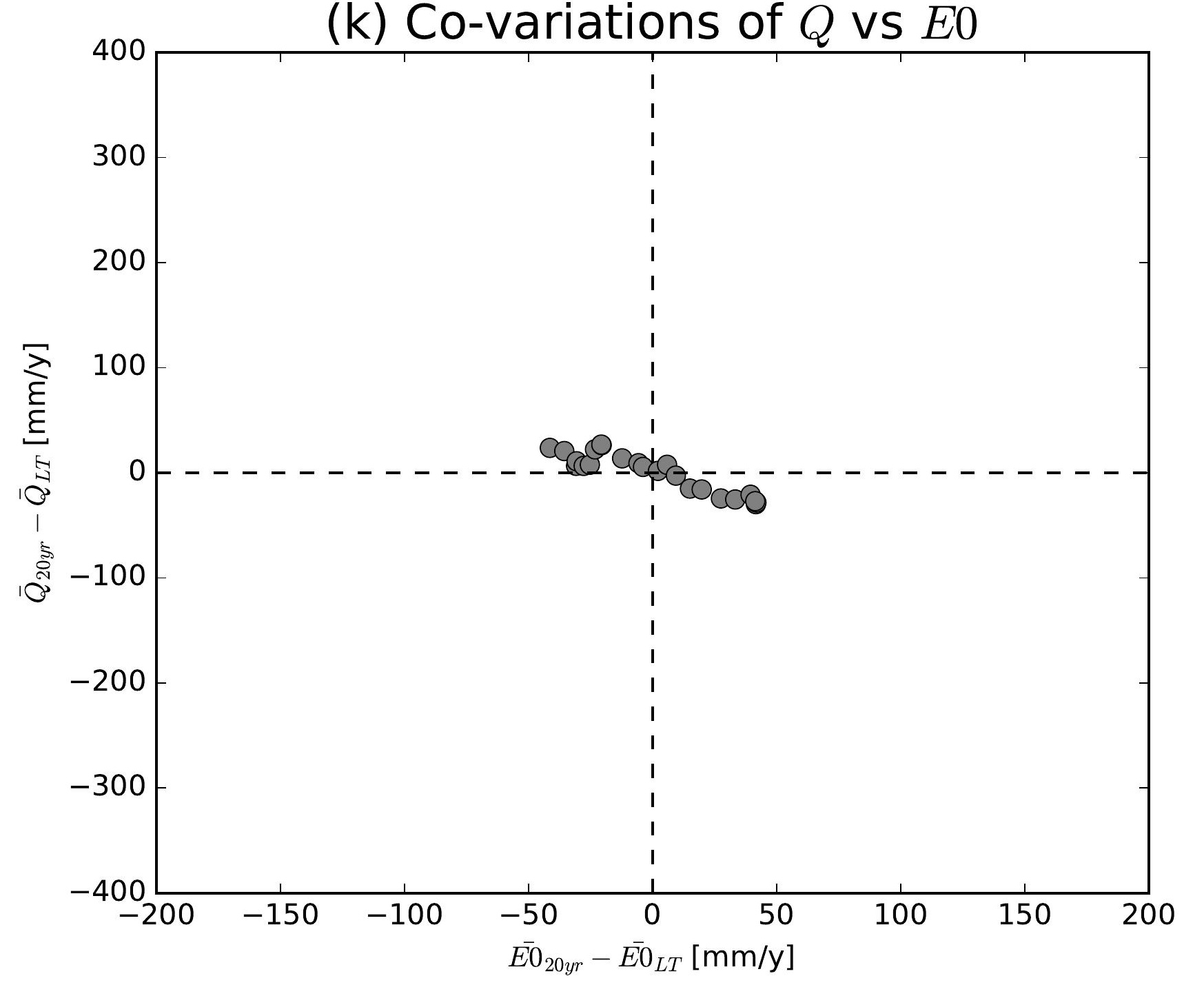

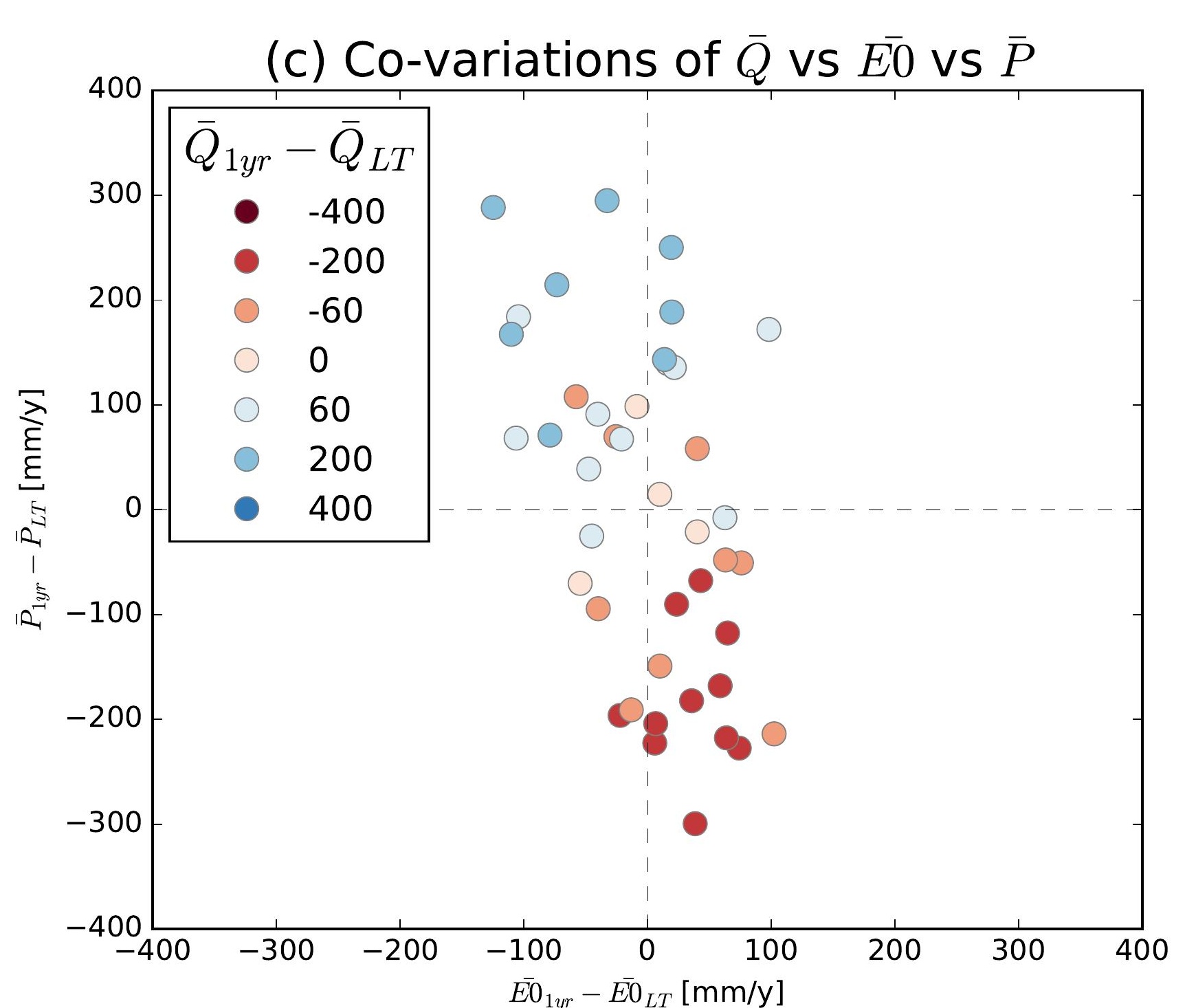
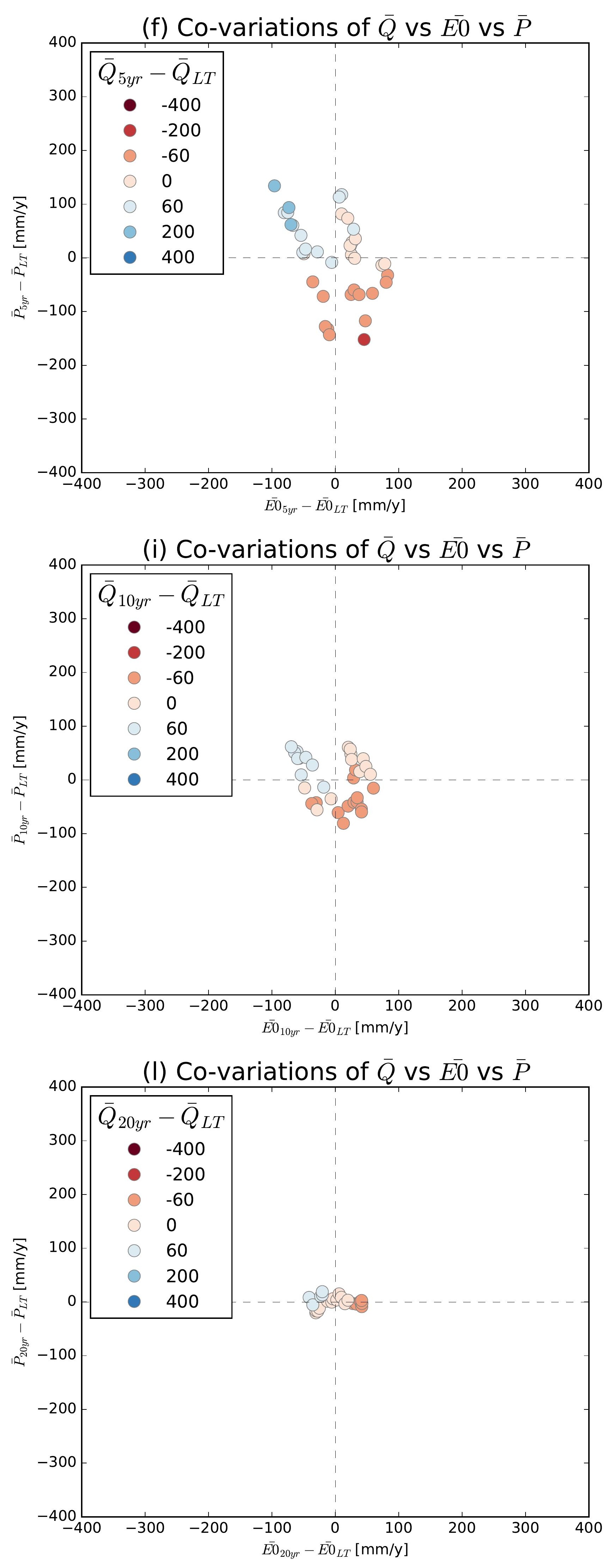

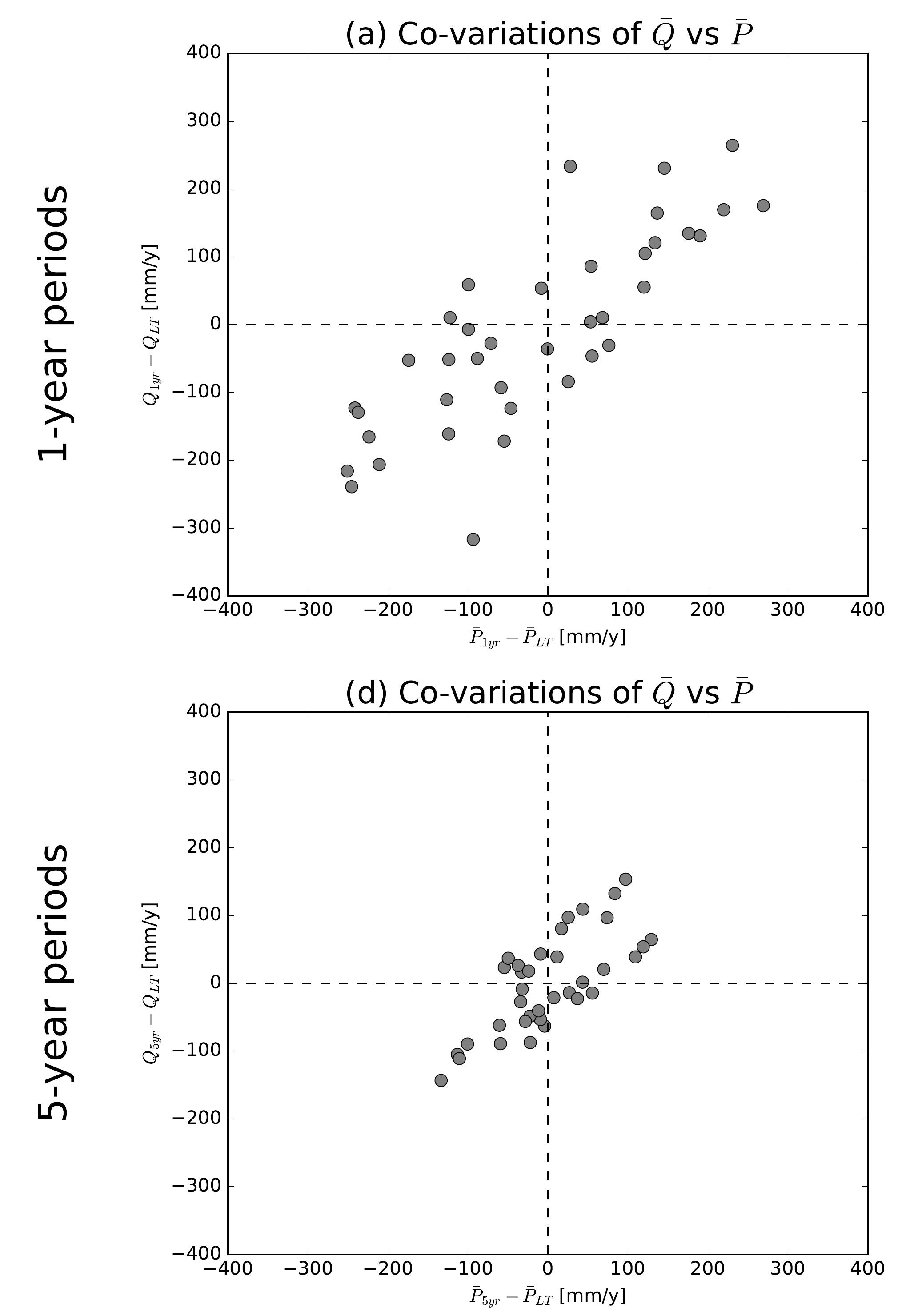

(g) Co-variations of $\bar{Q}$ vs $\bar{P}$
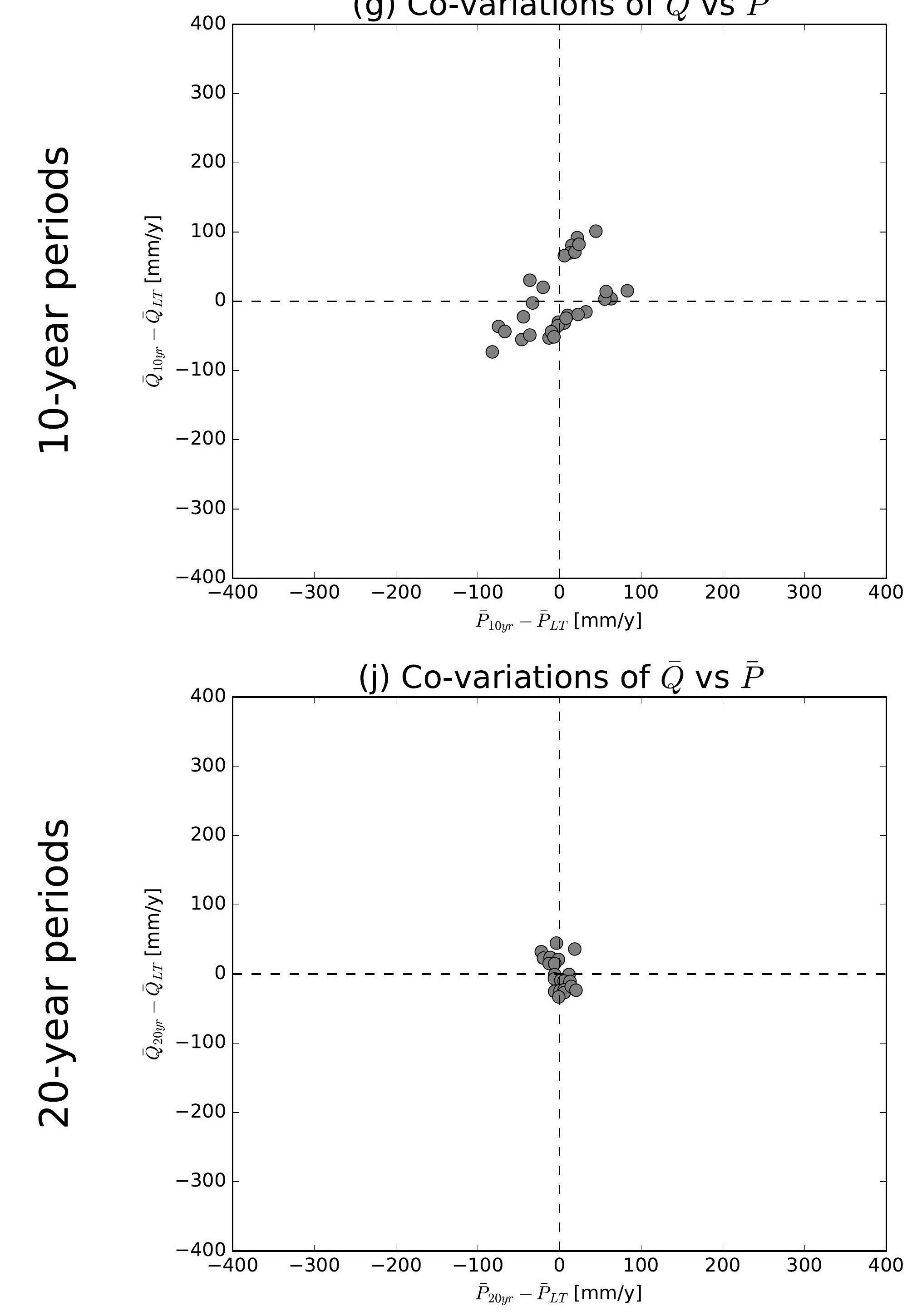

(b) Co-variations of $\bar{Q}$ vs $\overline{E 0}$

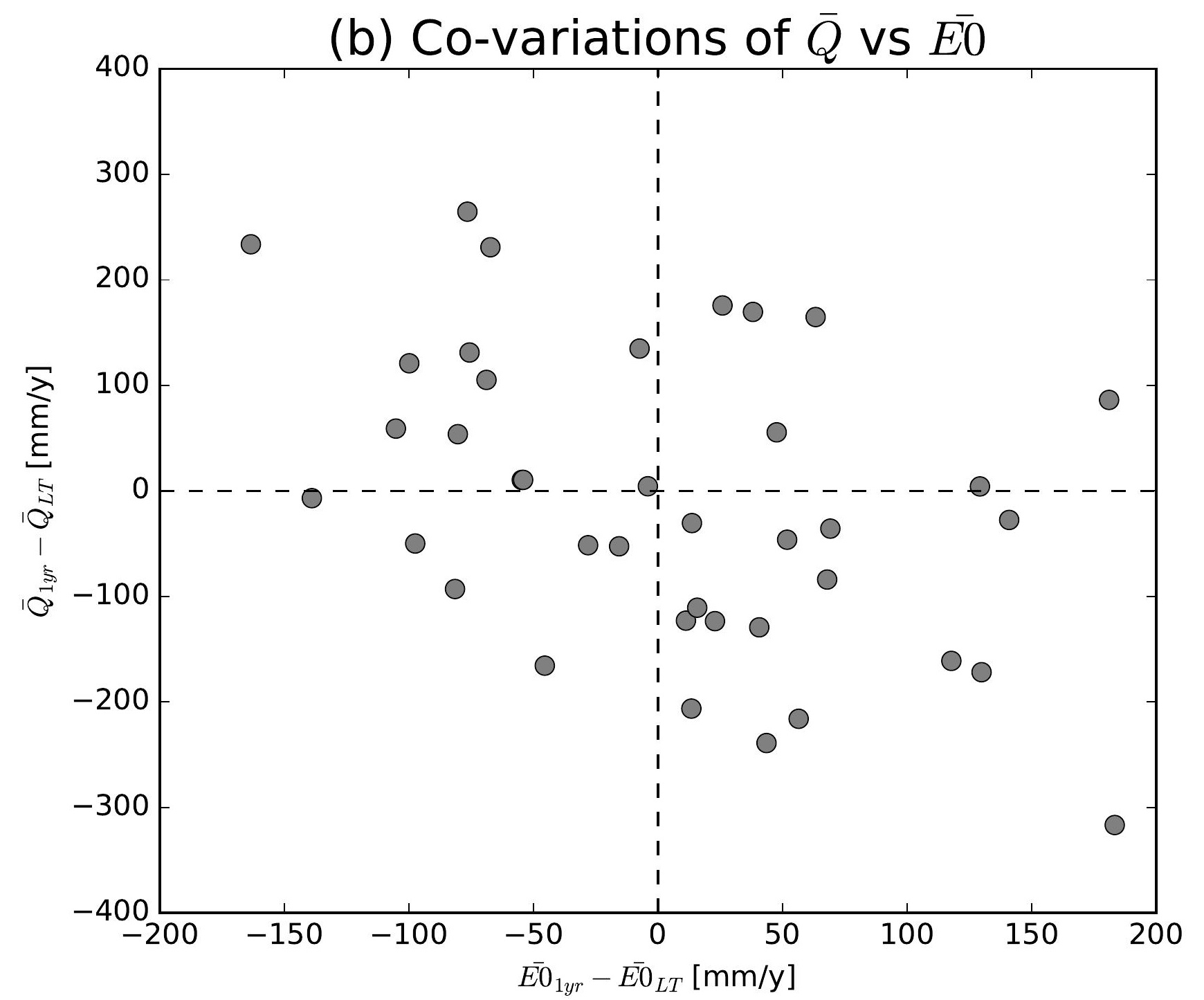

(e) Co-variations of $\bar{Q}$ vs $\overline{E 0}$

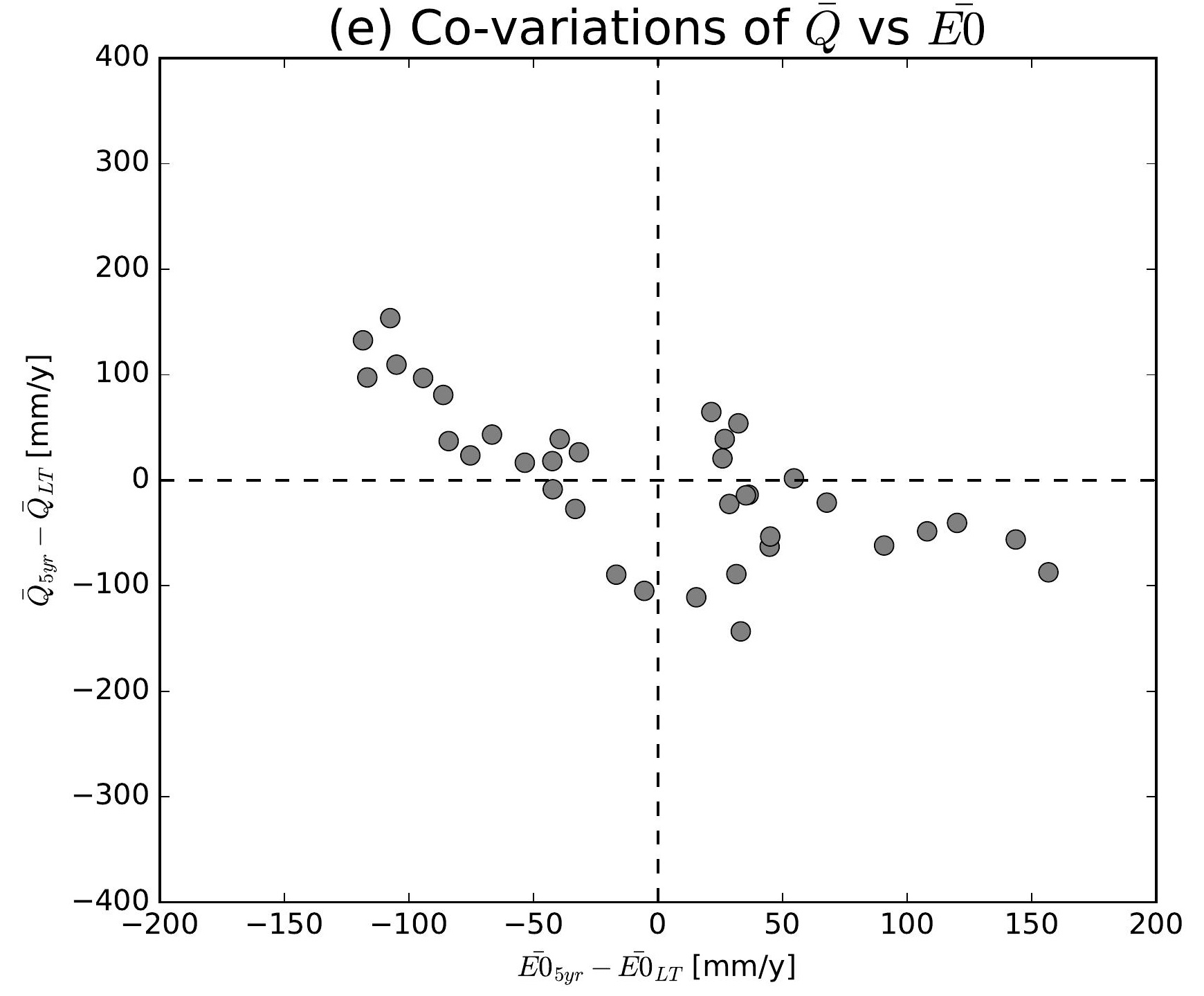

(h) Co-variations of $\bar{Q}$ vs $\overline{E 0}$

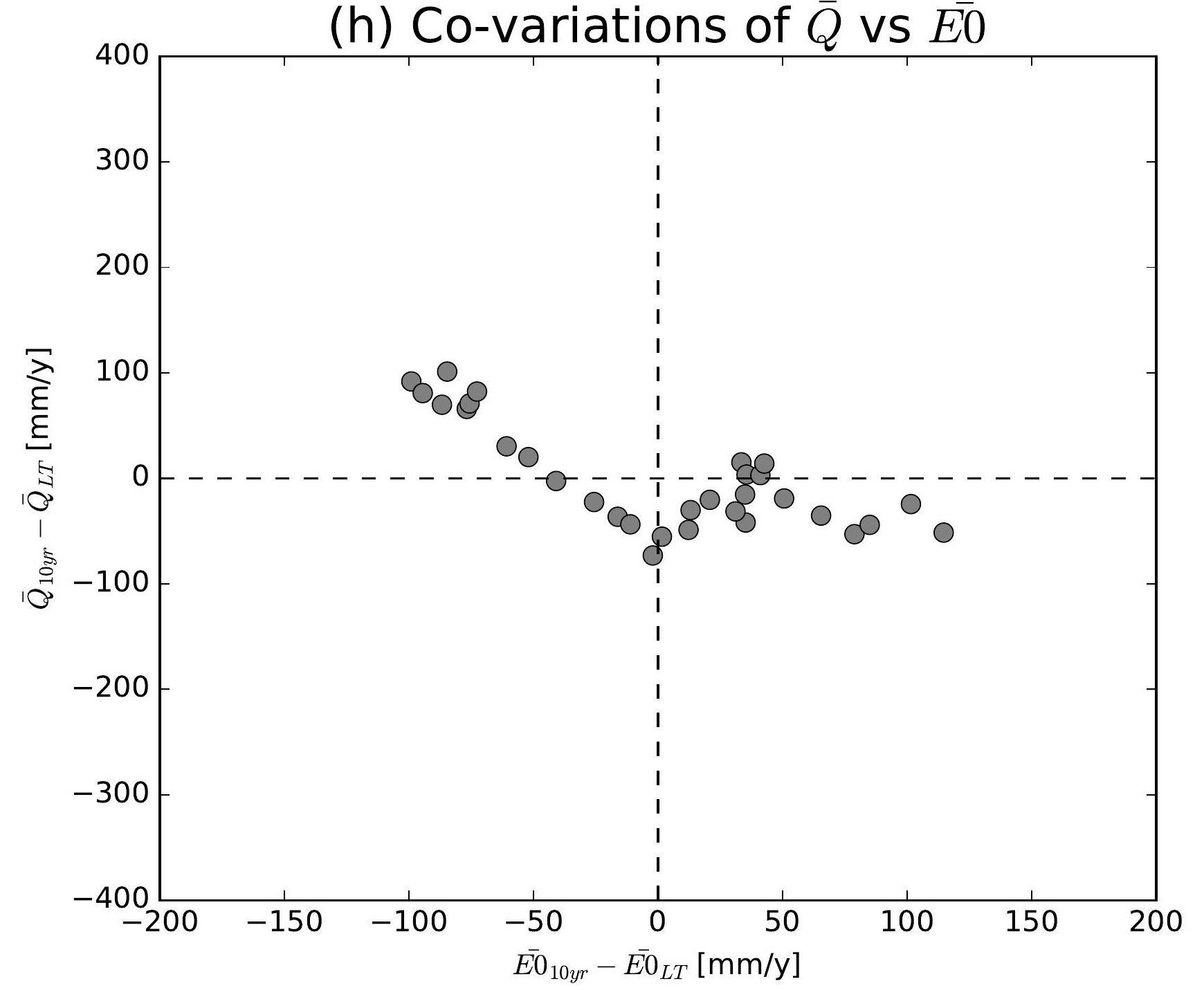

(k) Co-variations of $\bar{Q}$ vs $\overline{E 0}$

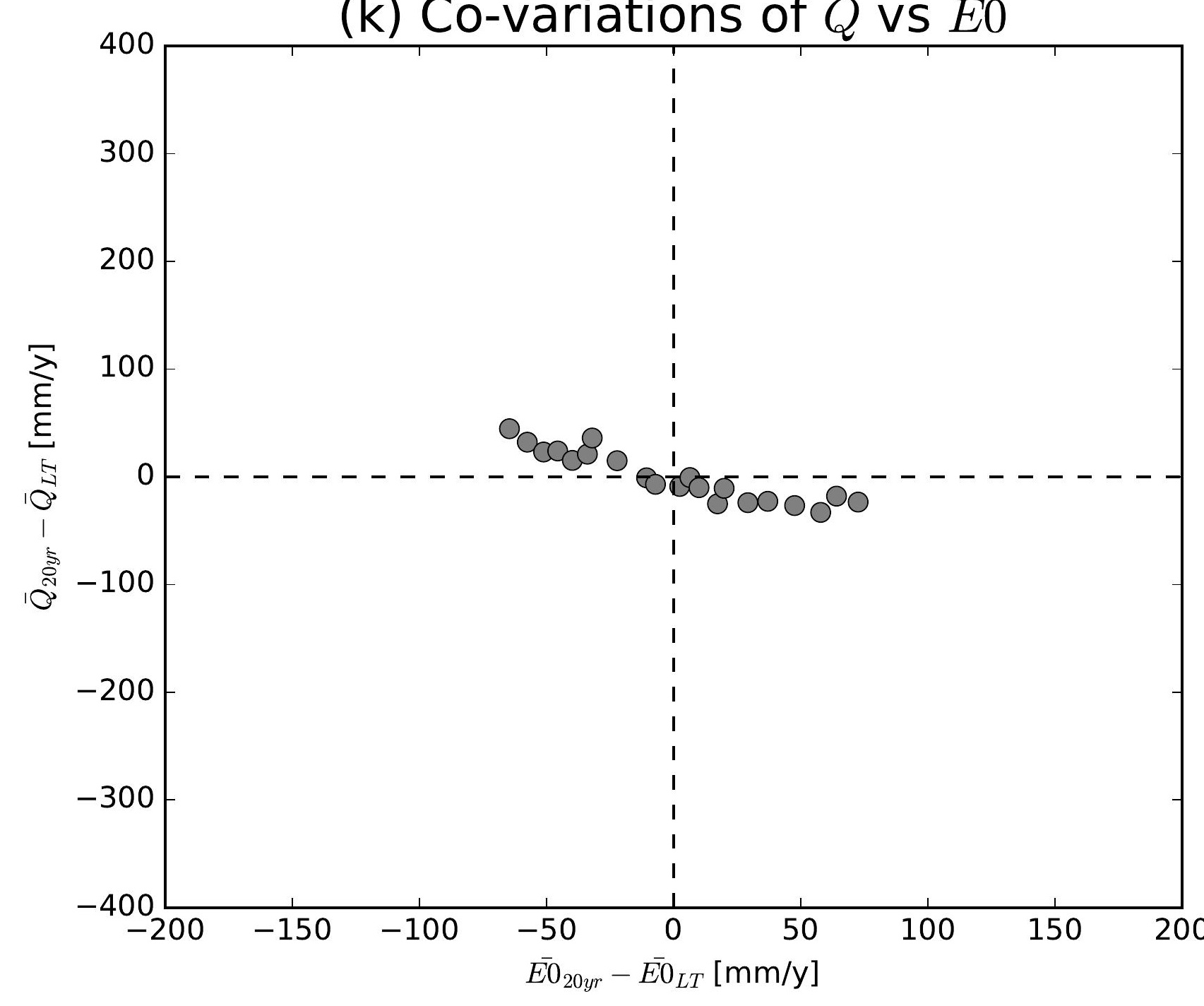

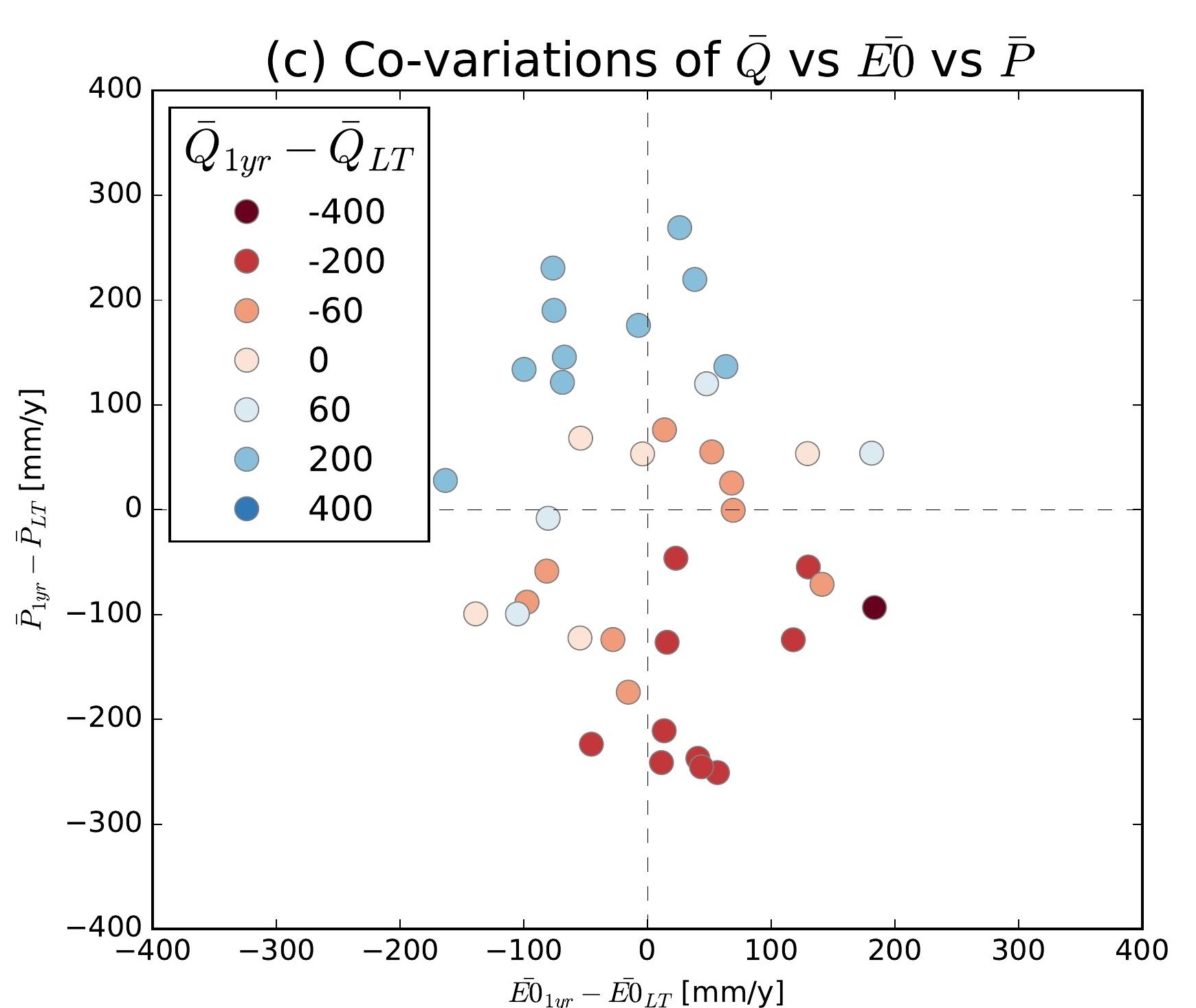
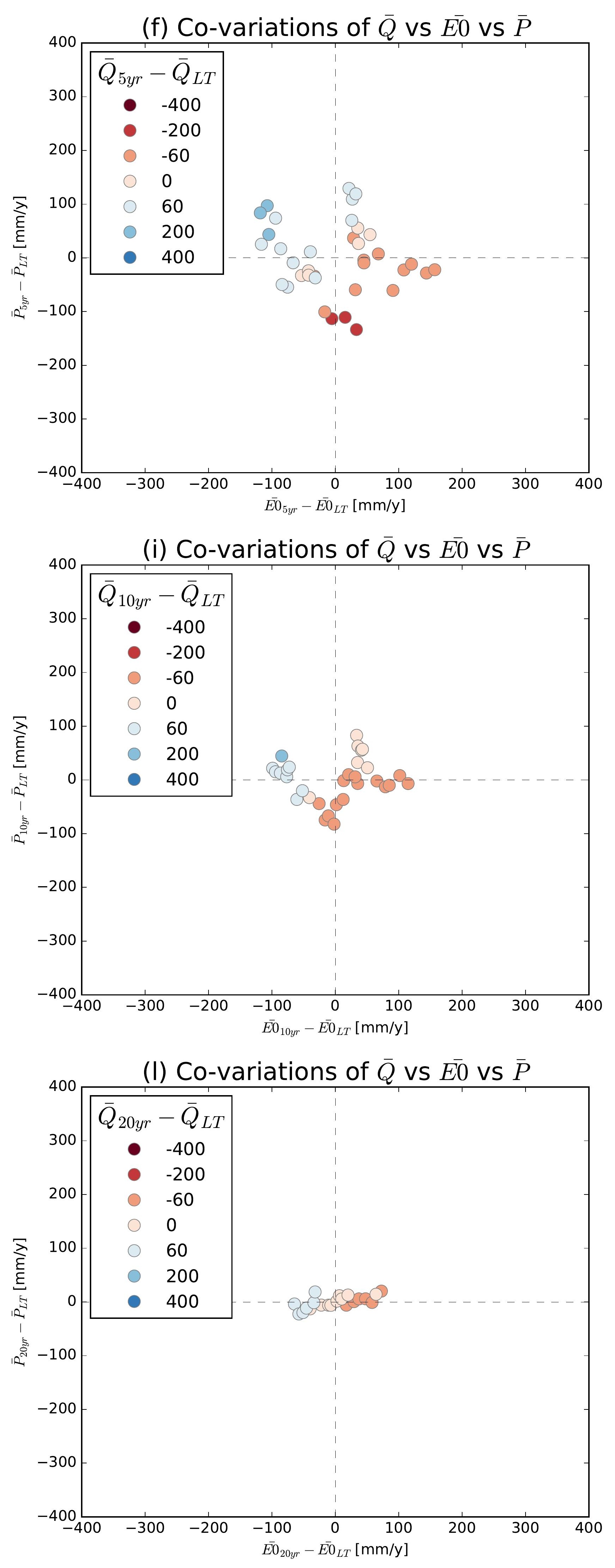

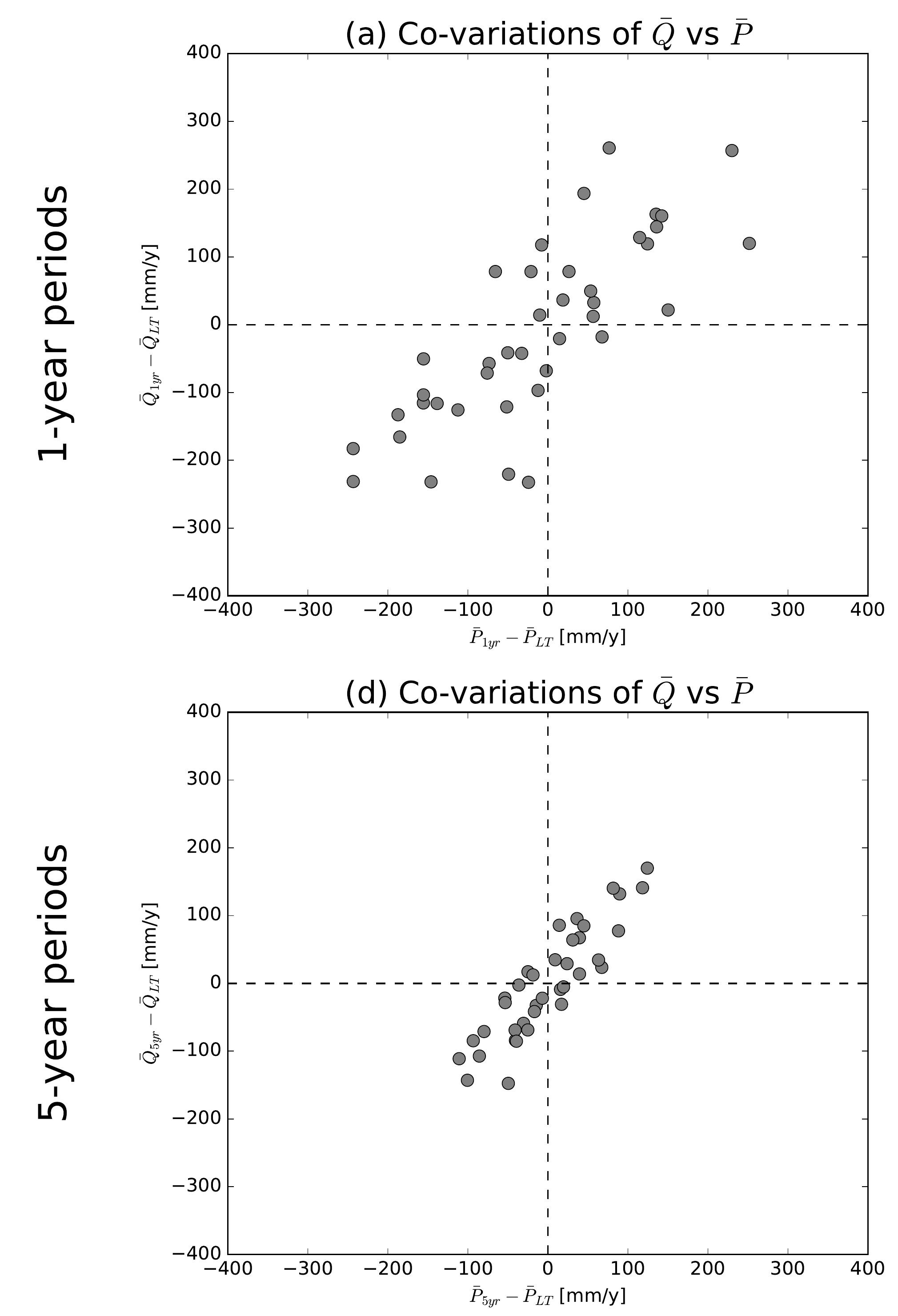

(g) Co-variations of $\bar{Q}$ vs $\bar{P}$
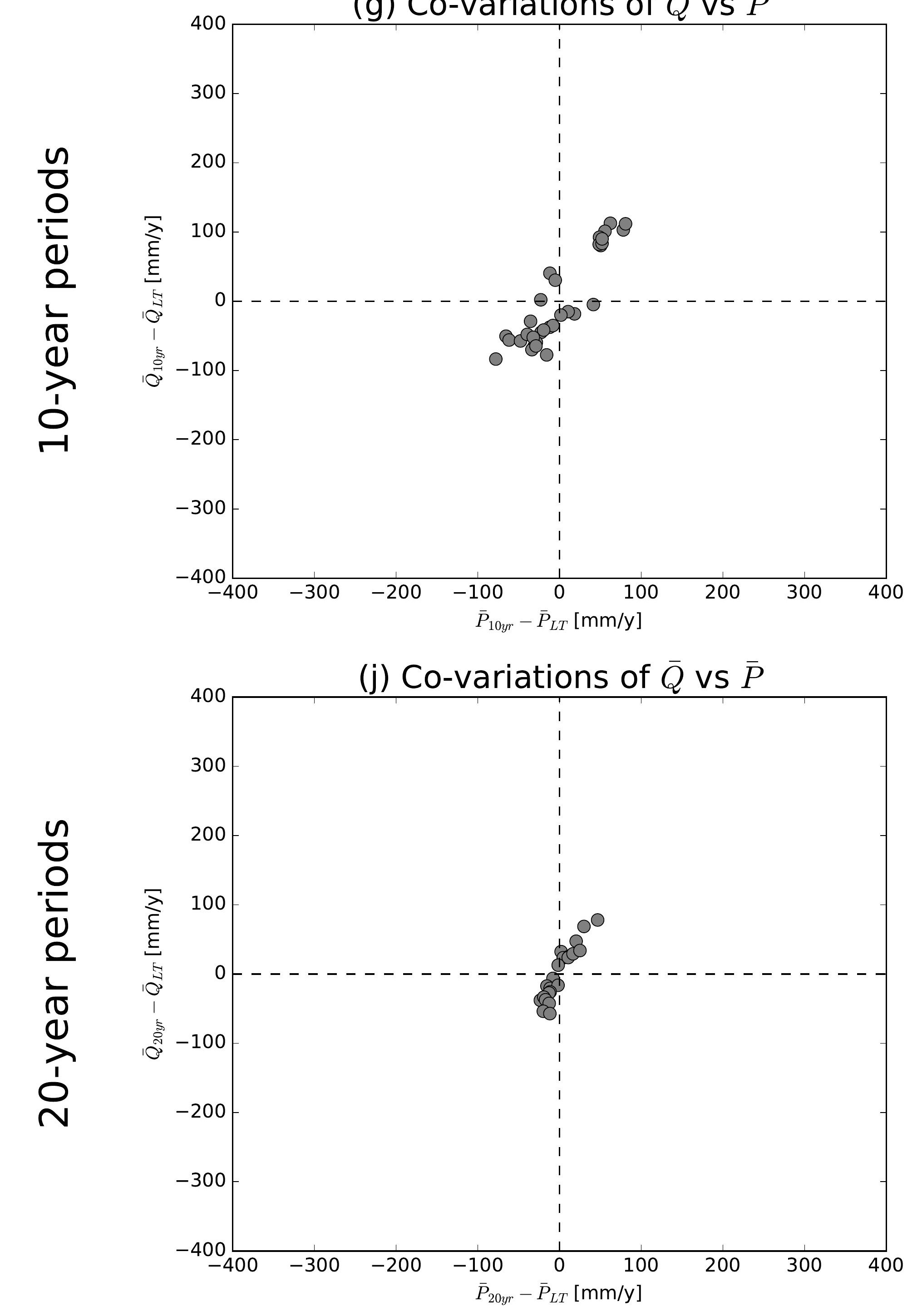

(b) Co-variations of $\bar{Q}$ vs $\overline{E 0}$

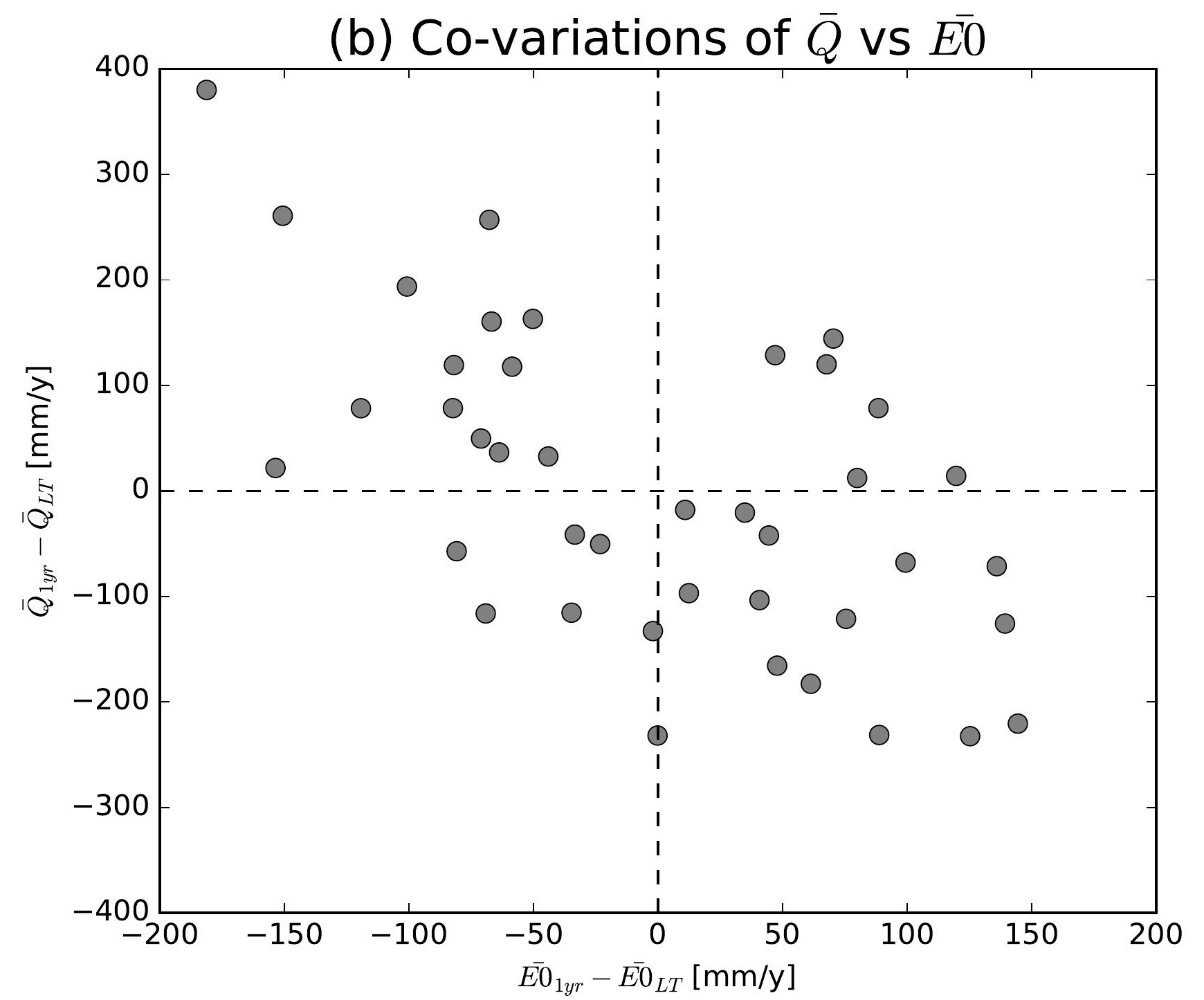

(e) Co-variations of $\bar{Q}$ vs $\overline{E 0}$

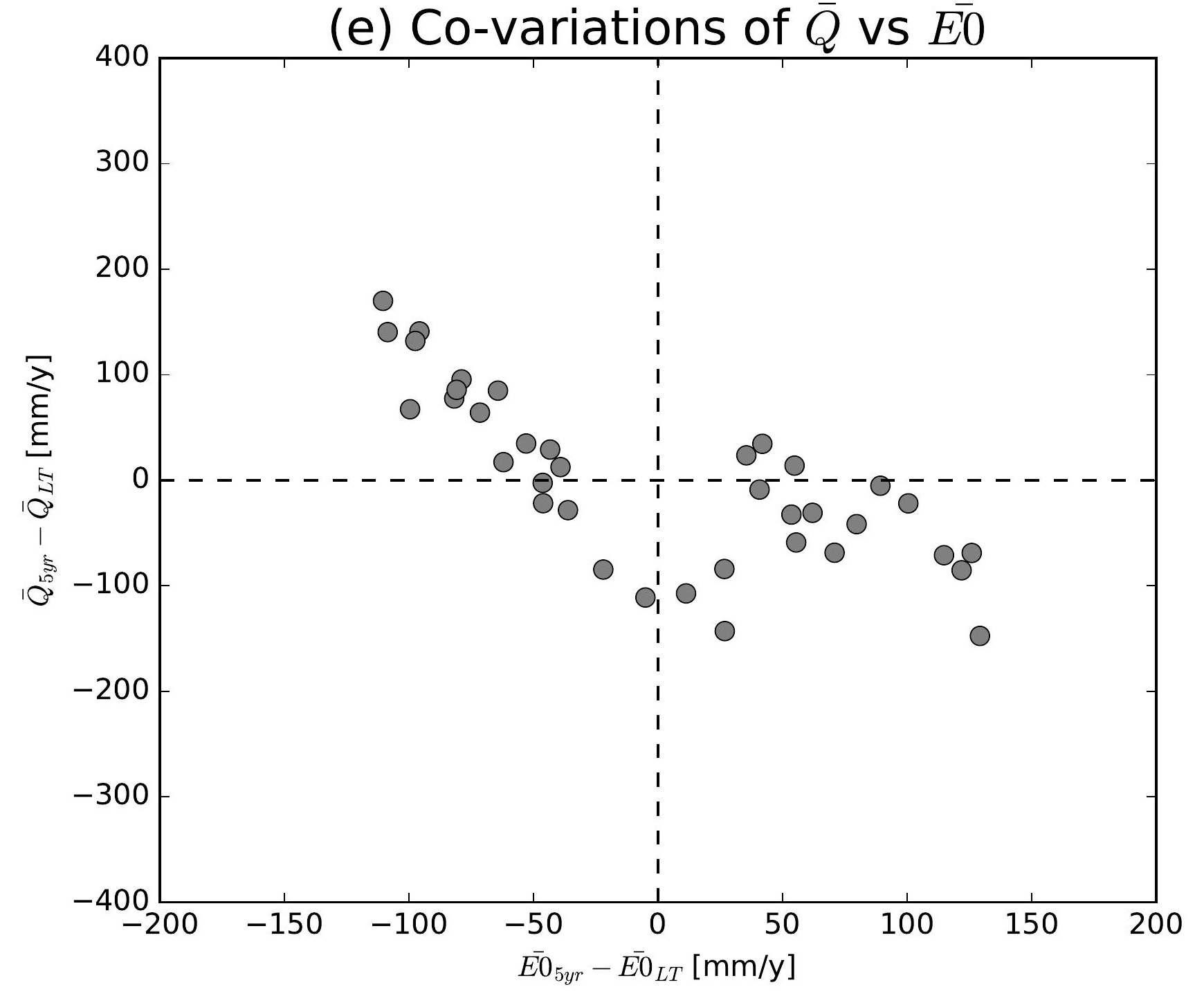

(h) Co-variations of $\bar{Q}$ vs $\overline{E 0}$

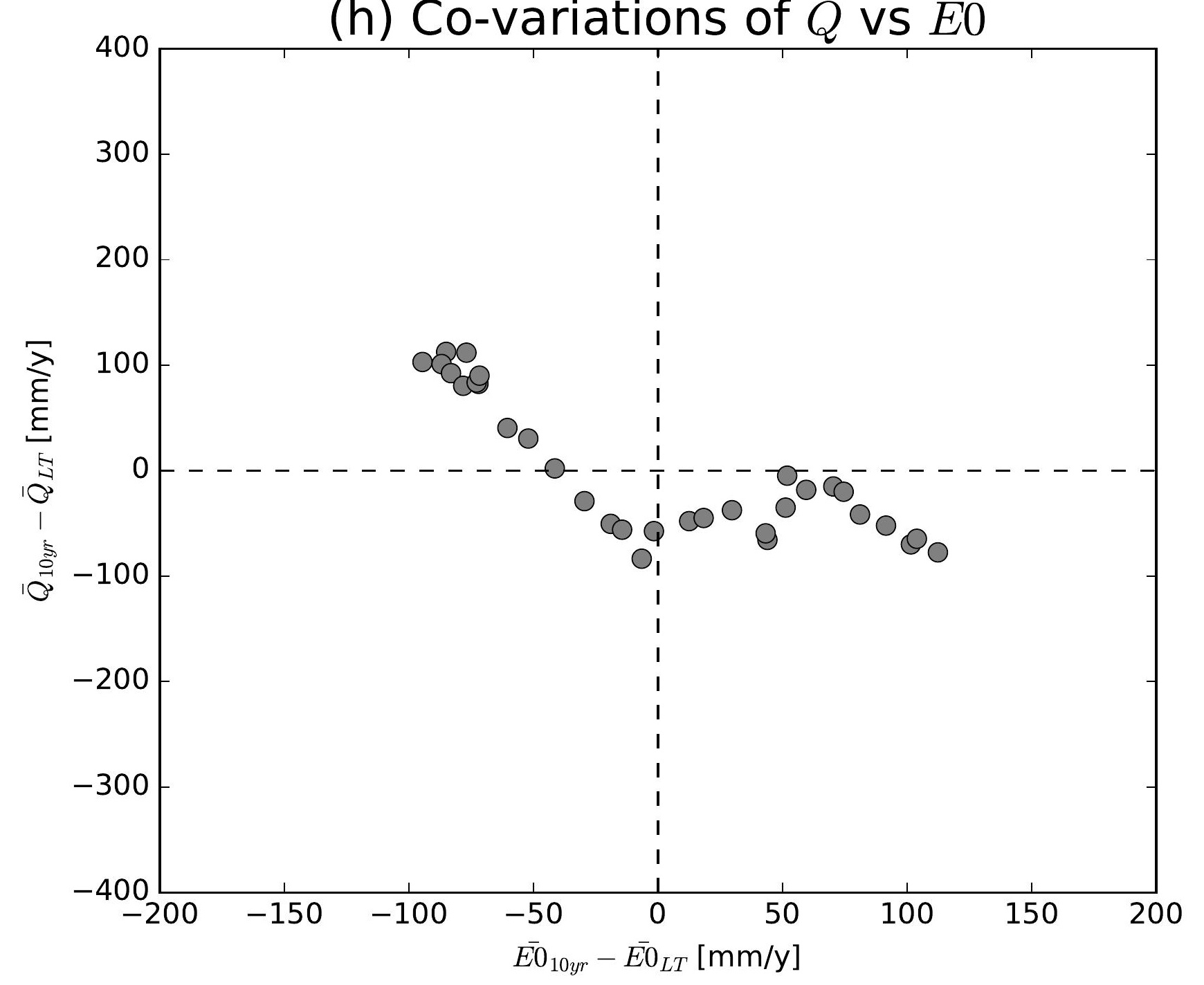

(k) Co-variations of $\bar{Q}$ vs $\overline{E 0}$

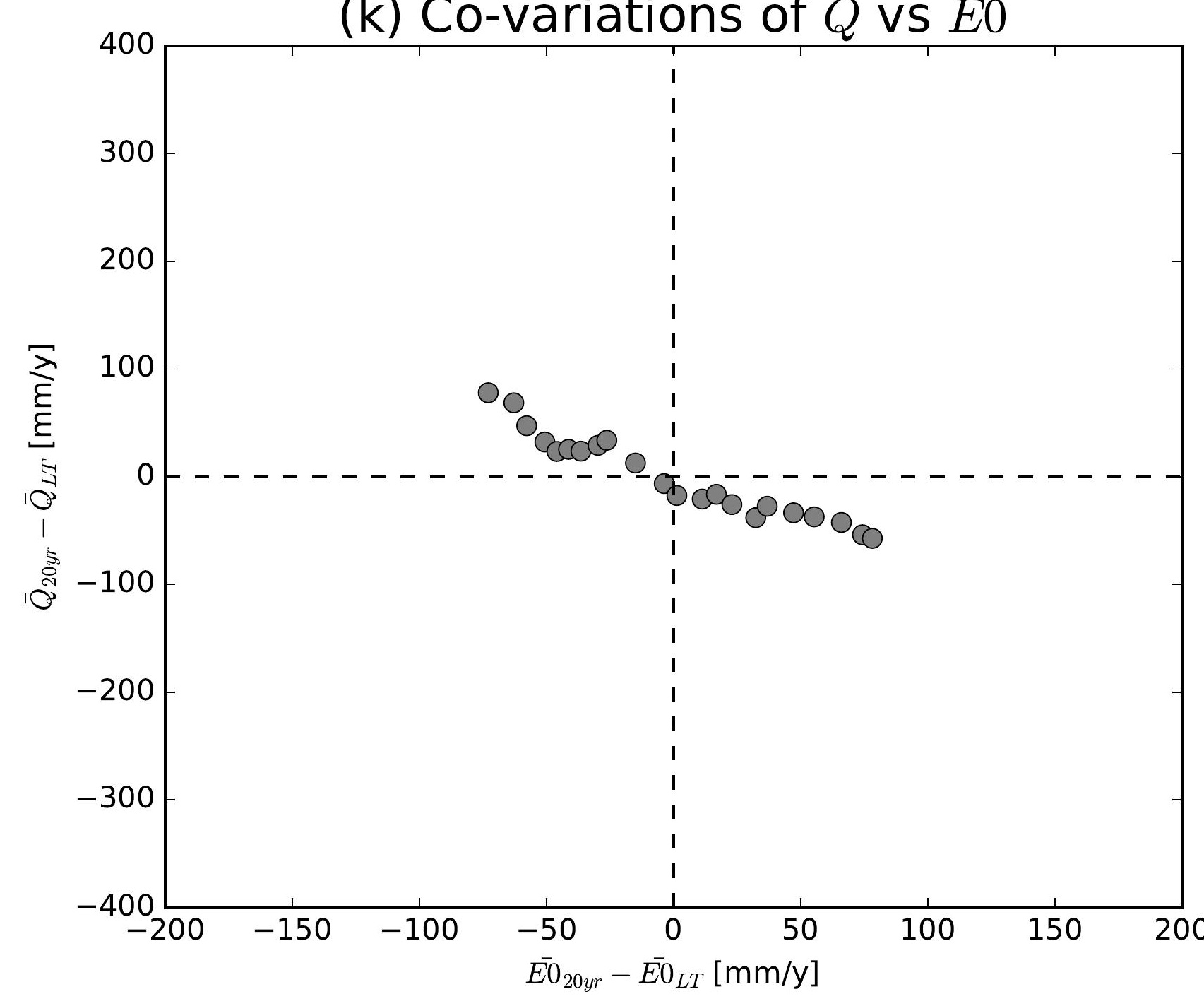

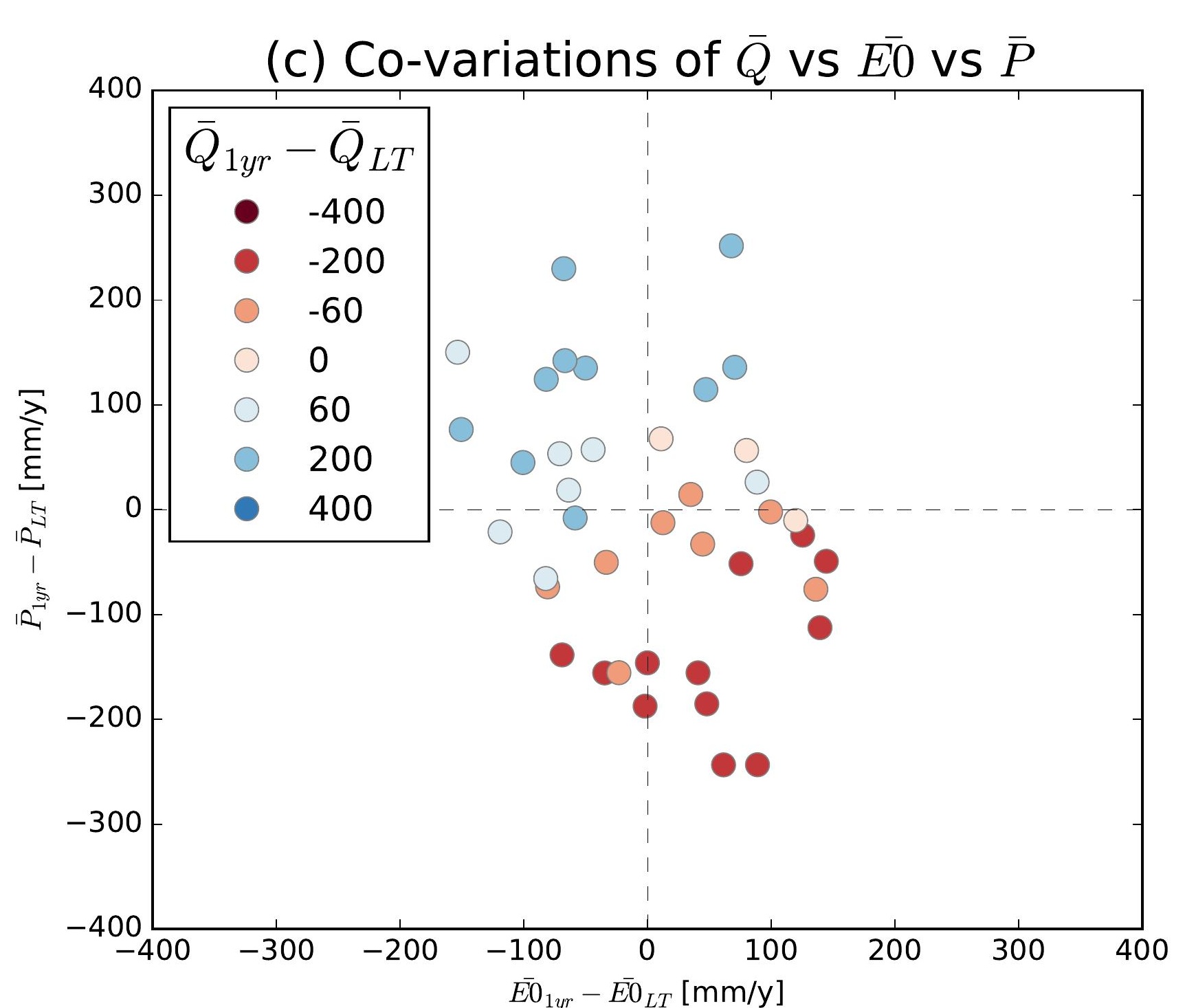
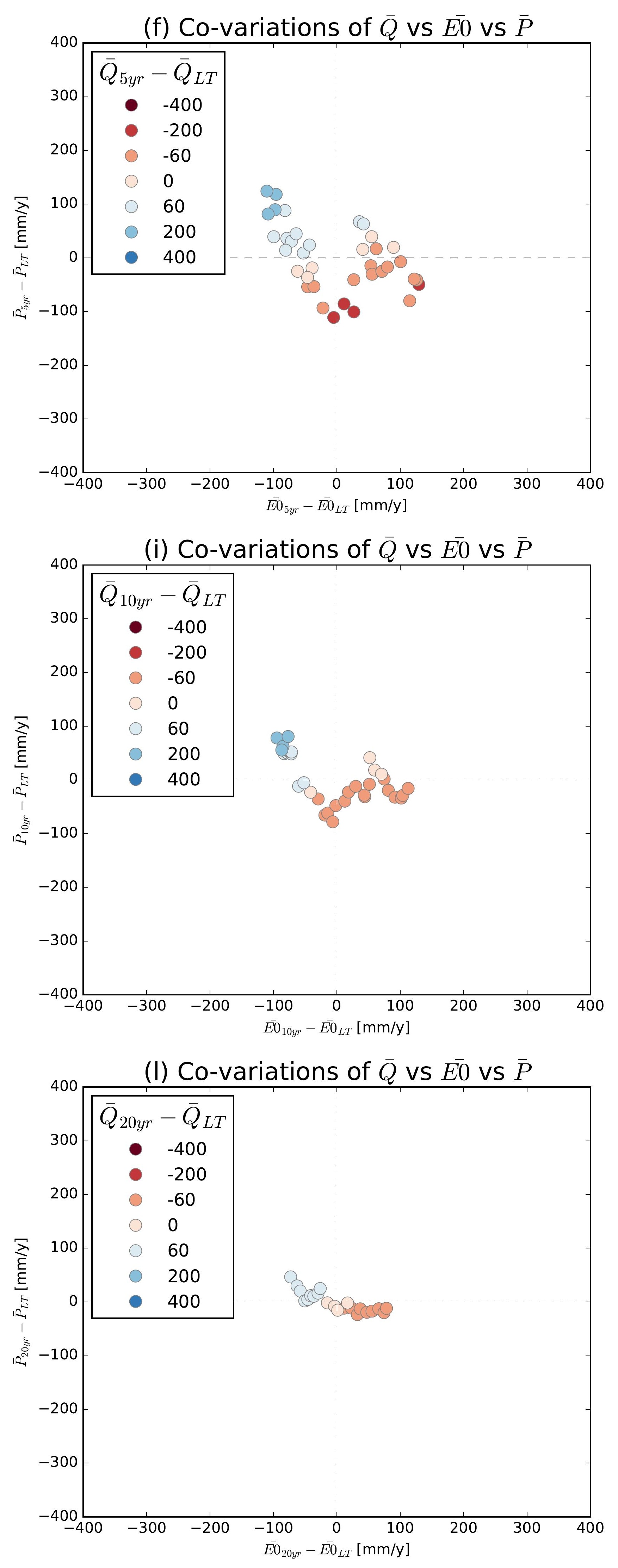

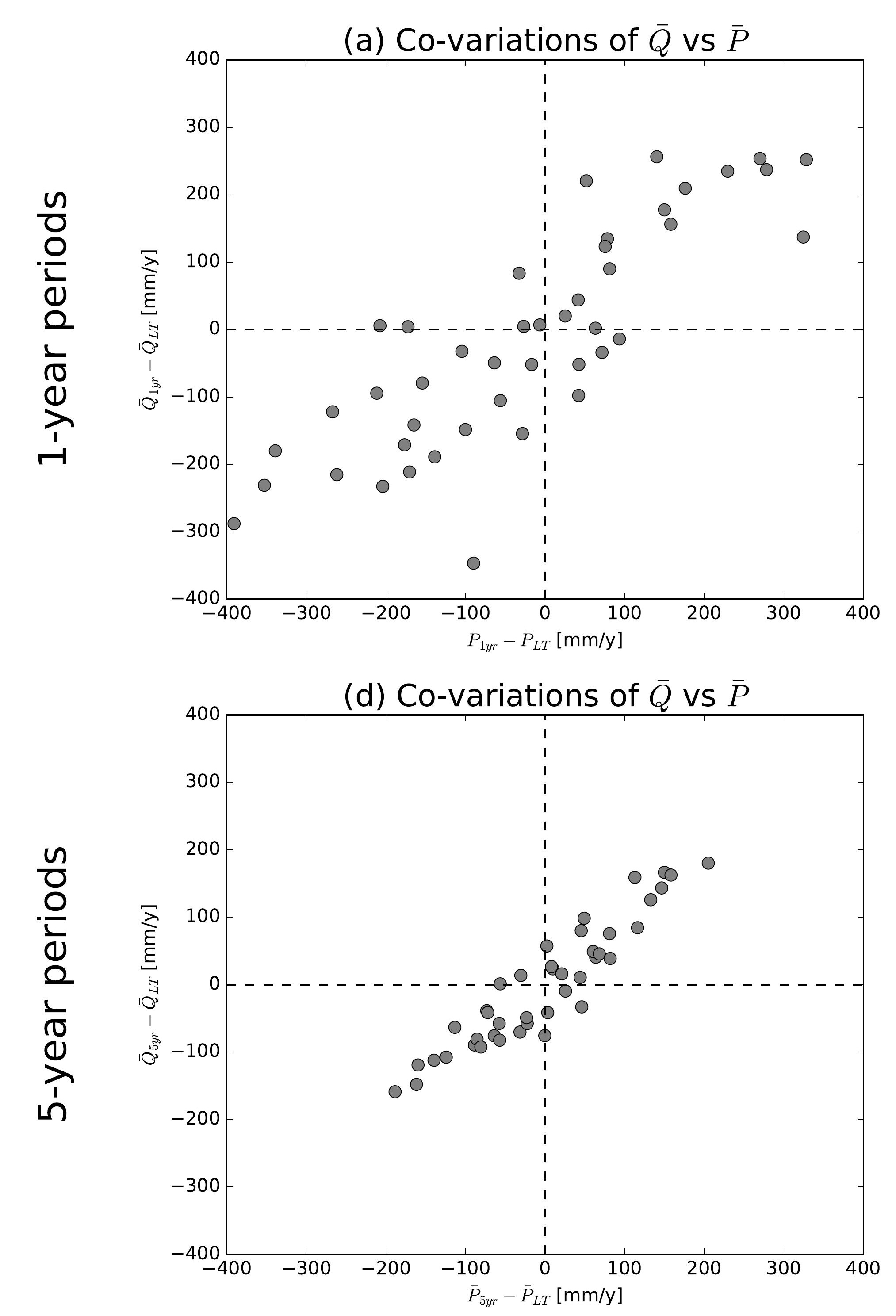

(g) Co-variations of $\bar{Q}$ vs $\bar{P}$

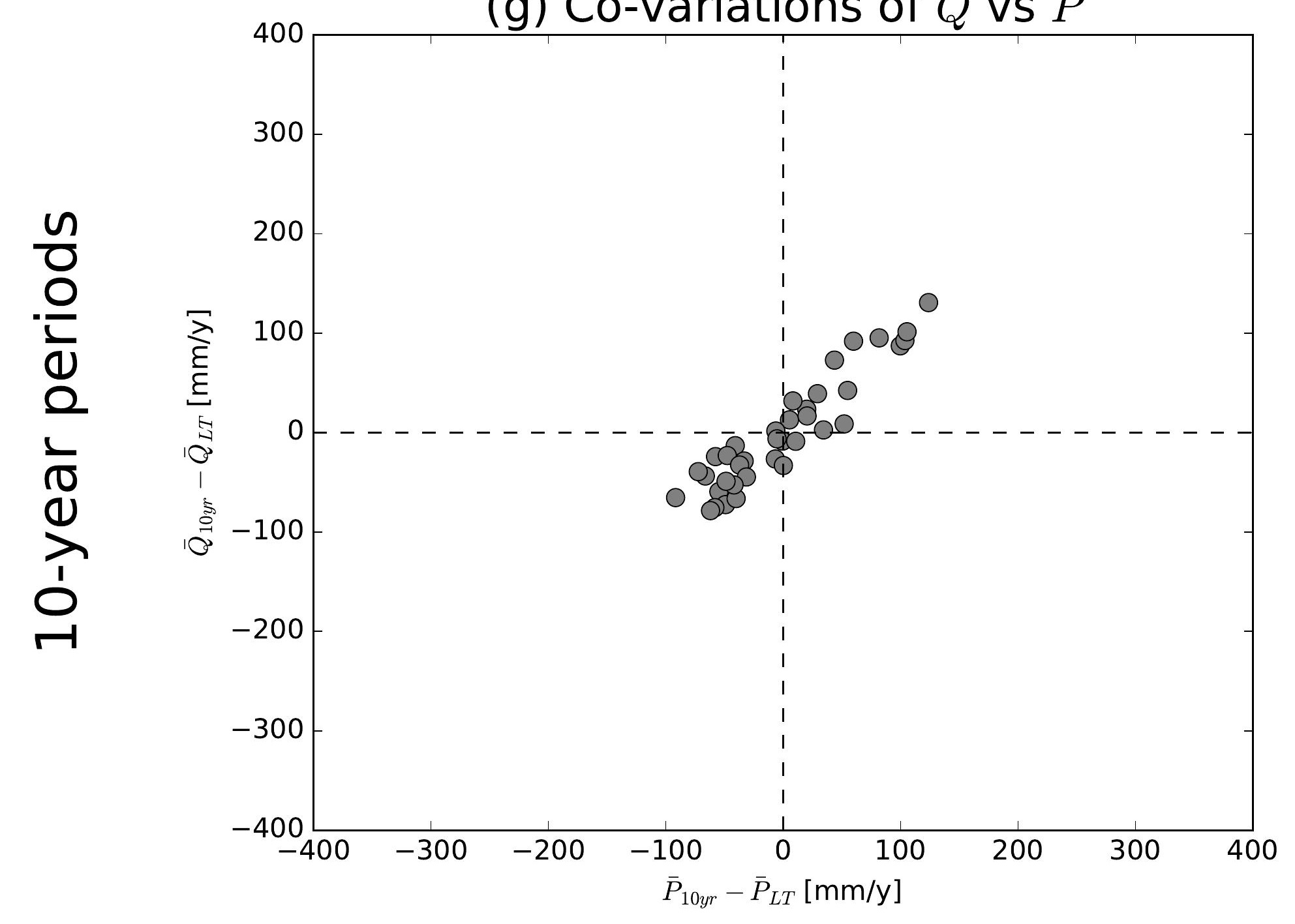

(j) Co-variations of $\bar{Q}$ vs $\bar{P}$

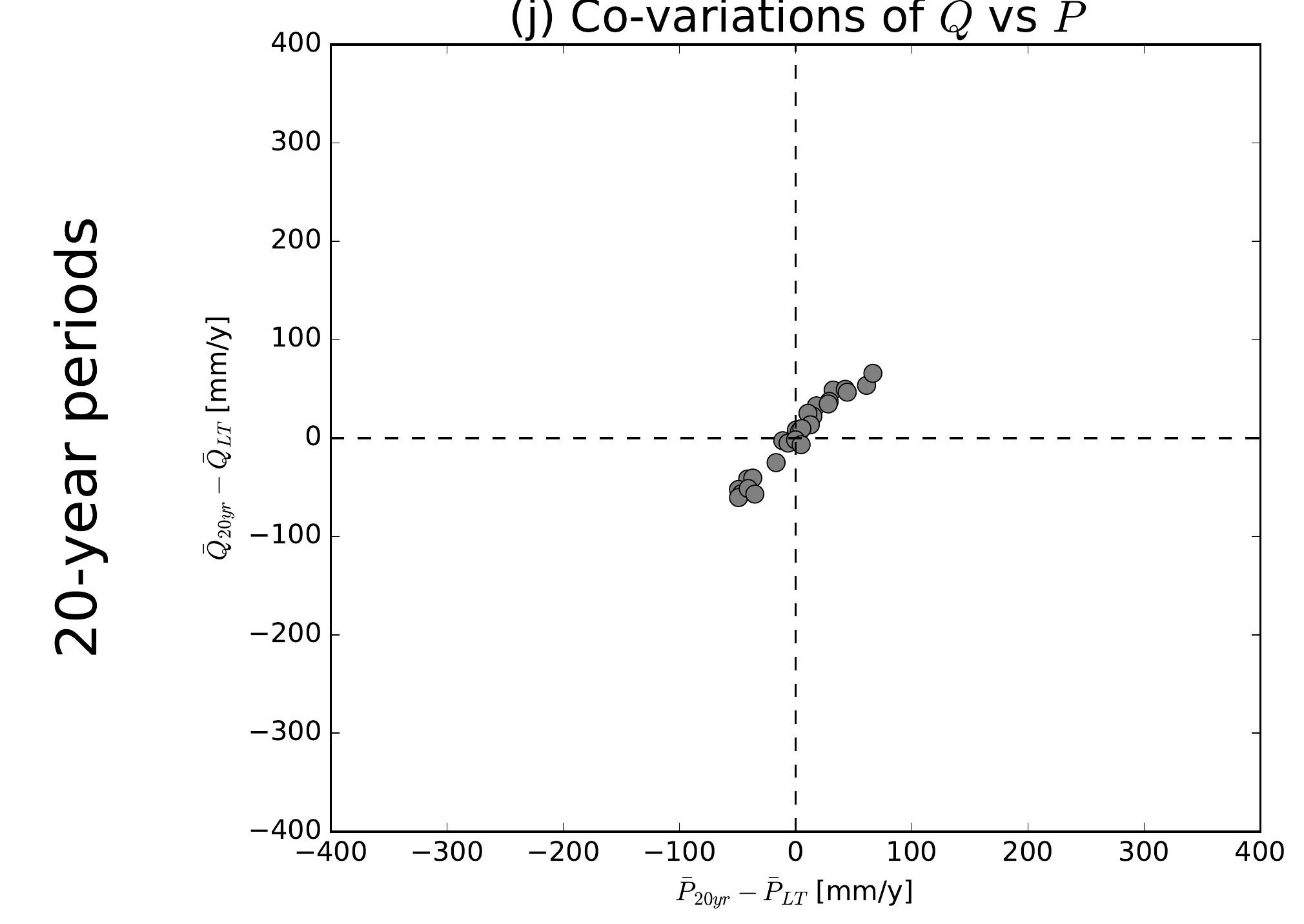

(b) Co-variations of $\bar{Q}$ vs $\overline{E 0}$

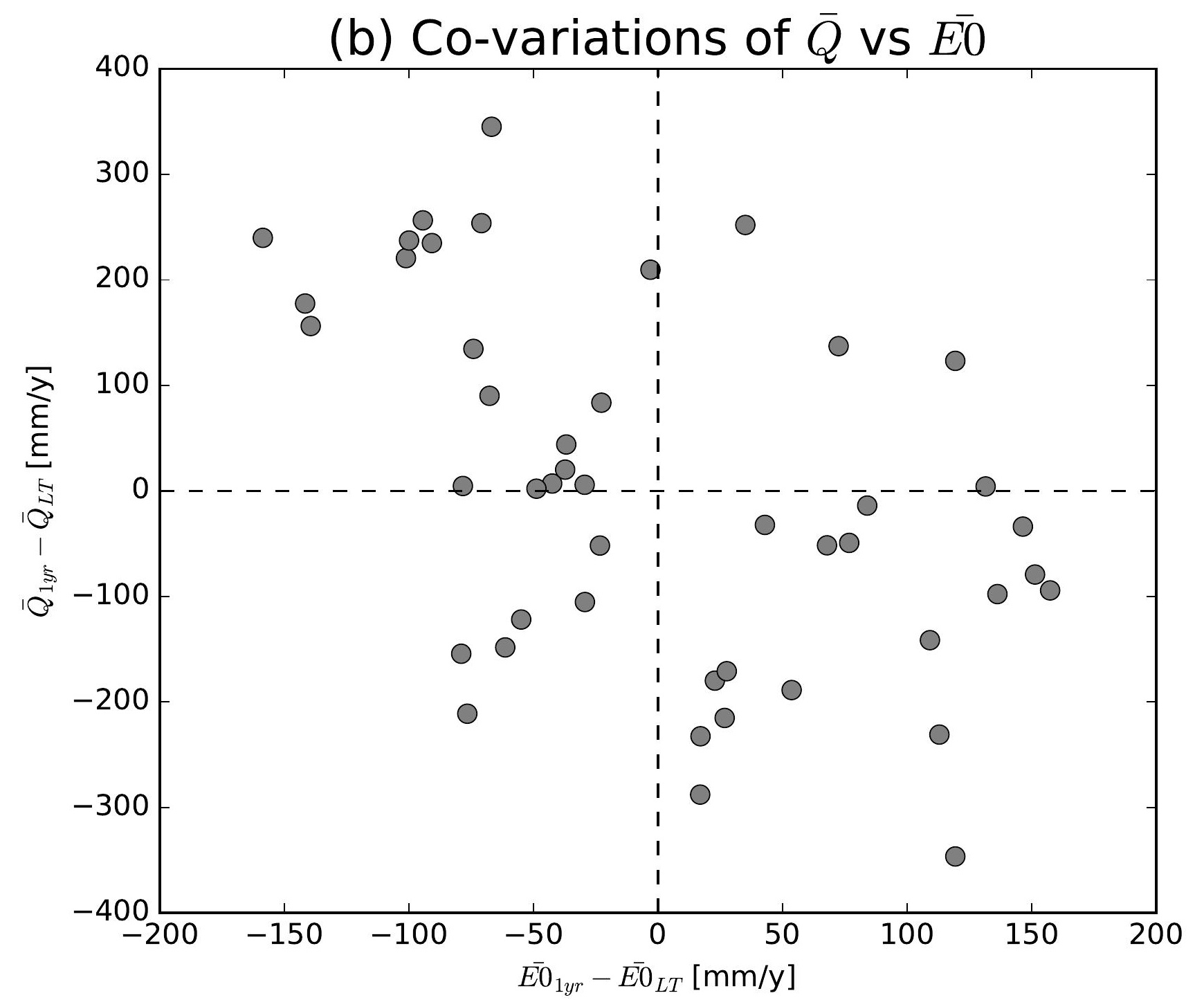

(e) Co-variations of $\bar{Q}$ vs $\overline{E 0}$

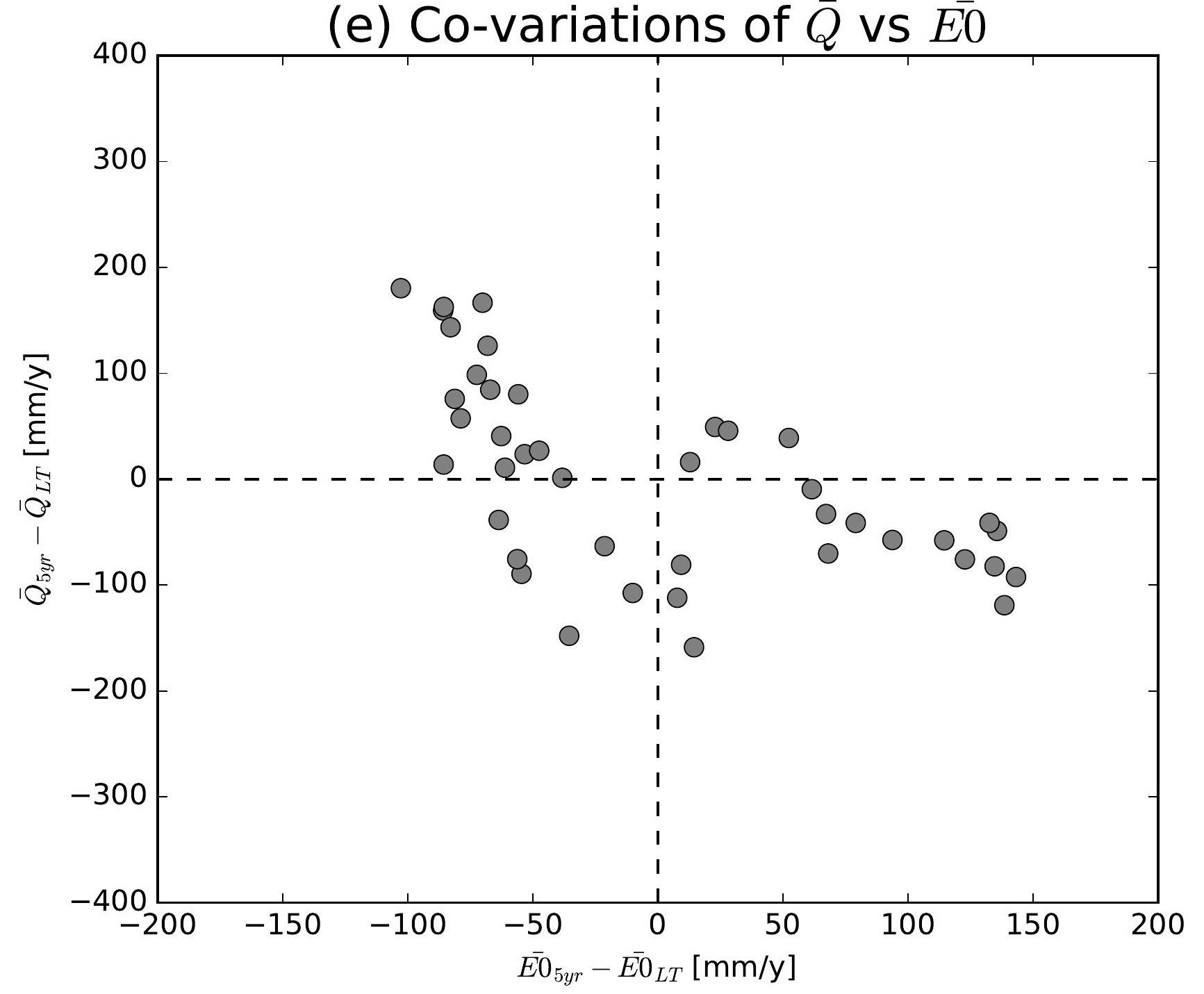

(h) Co-variations of $\bar{Q}$ vs $\overline{E 0}$

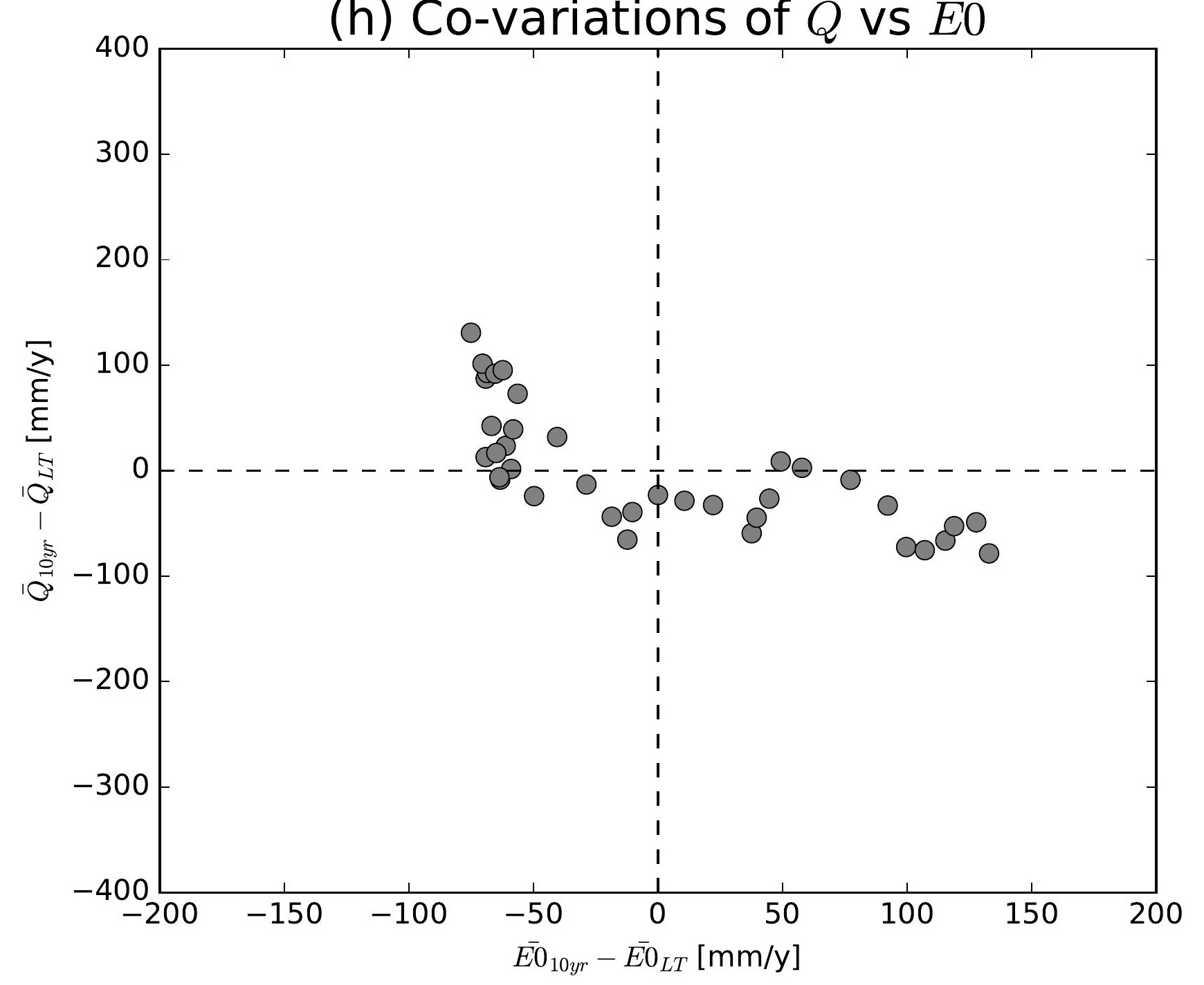

(k) Co-variations of $\bar{Q}$ vs $\overline{E 0}$

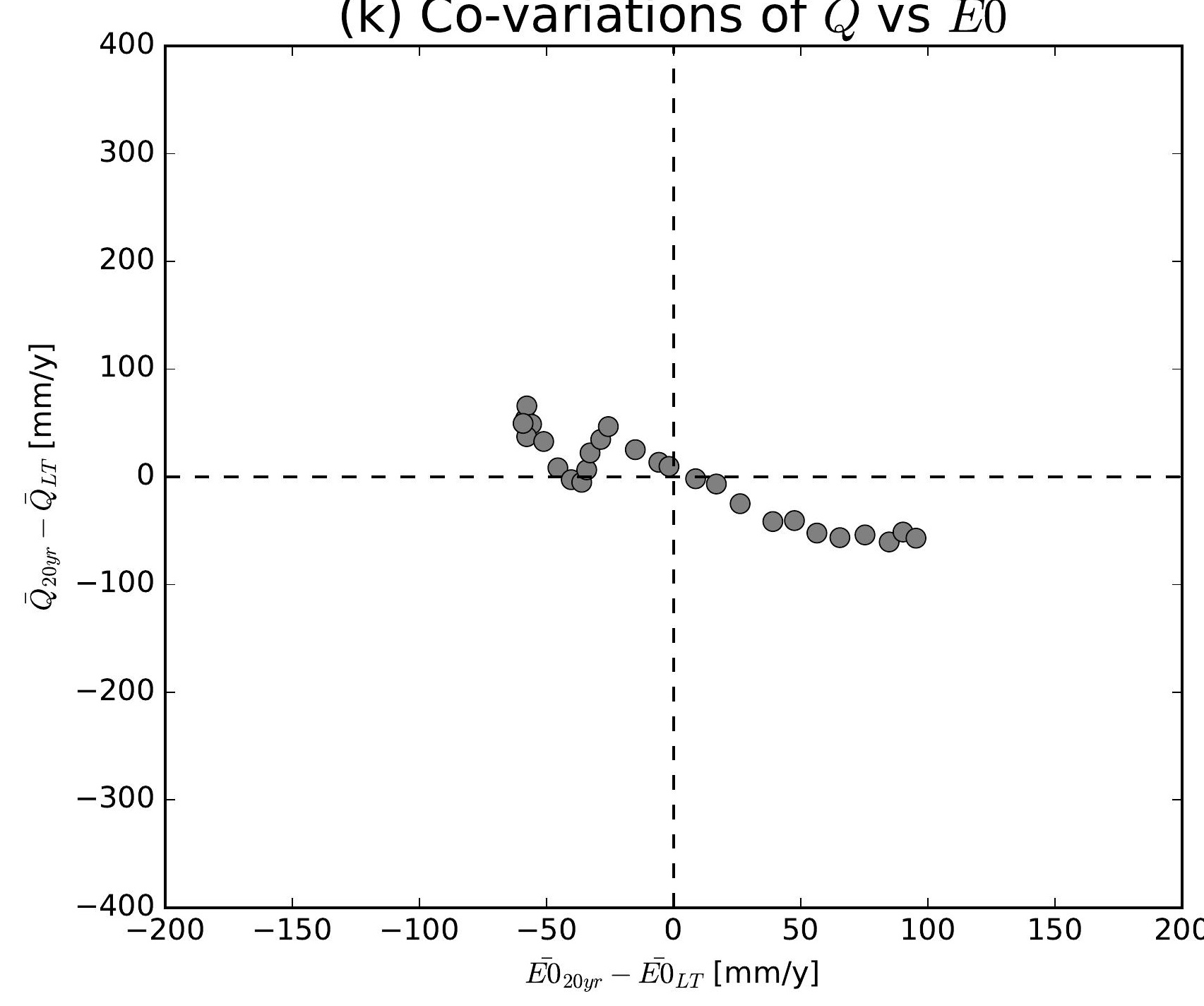

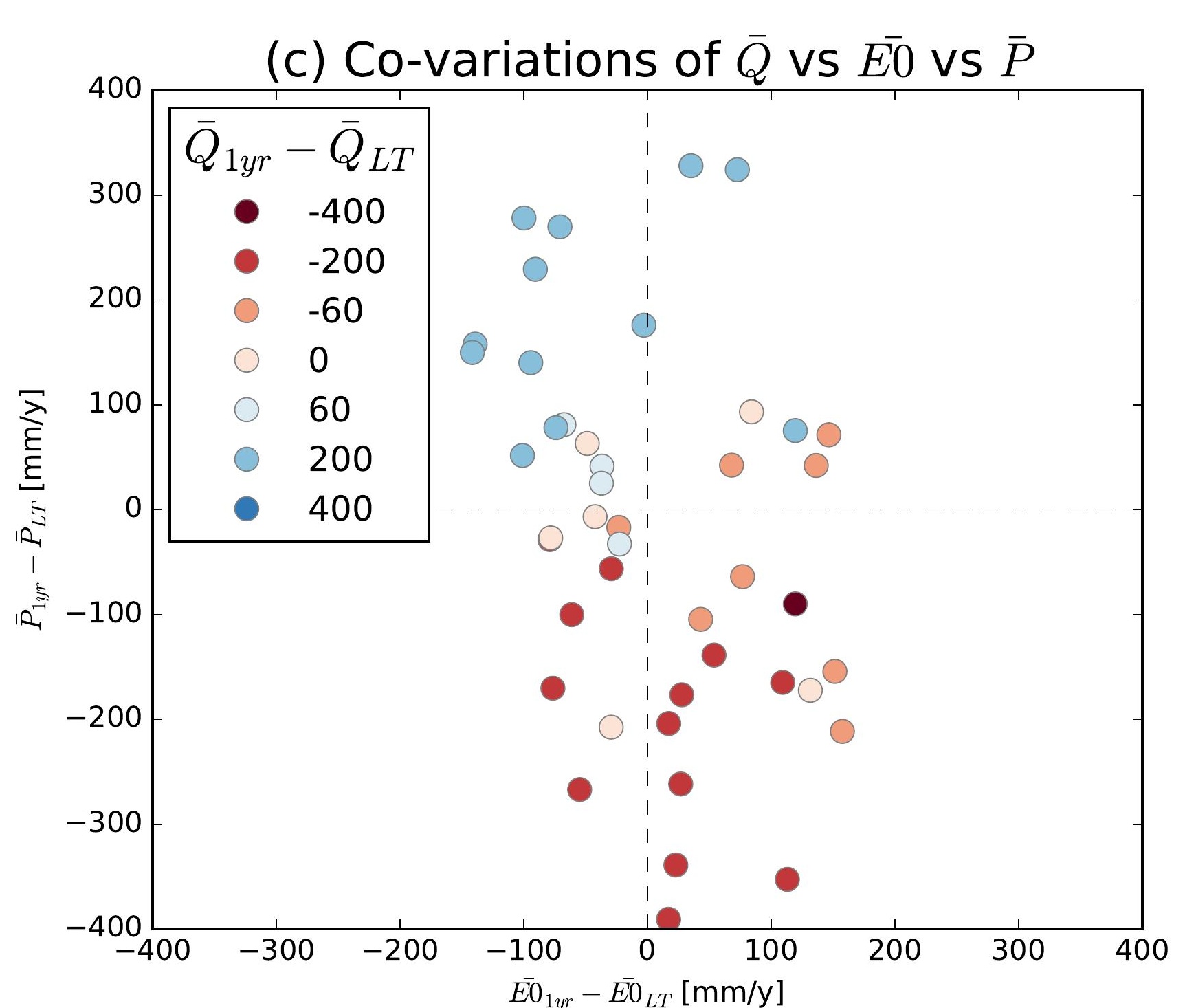
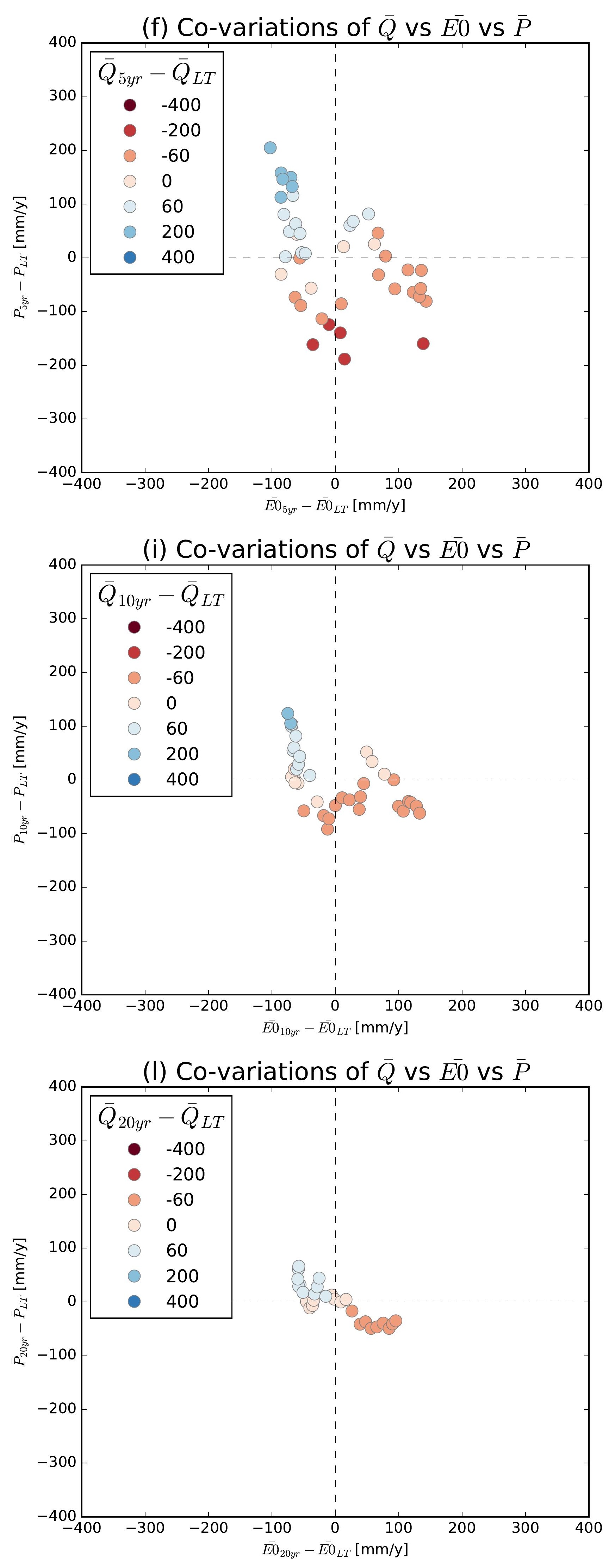

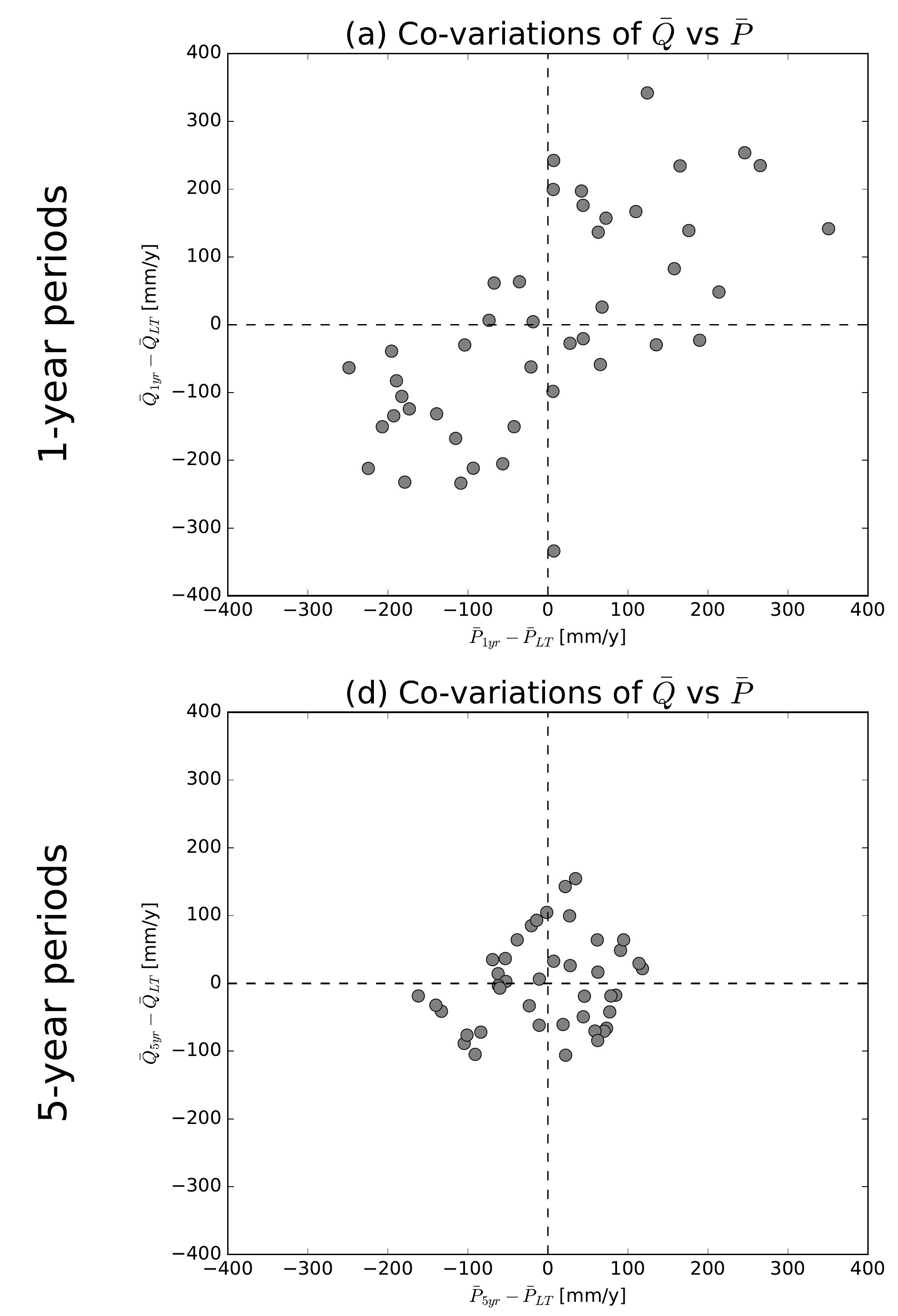

(g) Co-variations of $\bar{Q}$ vs $\bar{P}$
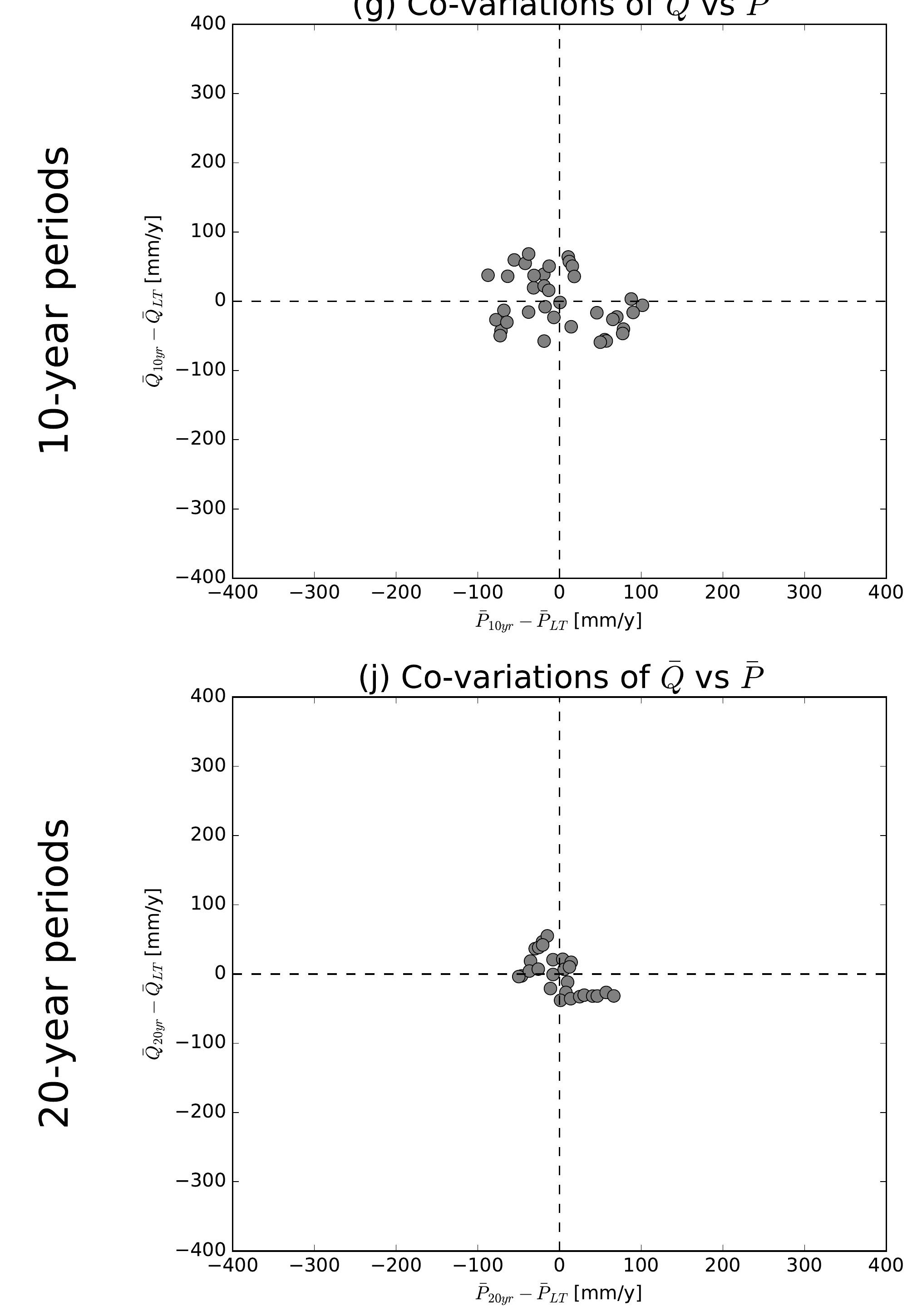

(b) Co-variations of $\bar{Q}$ vs $\overline{E 0}$

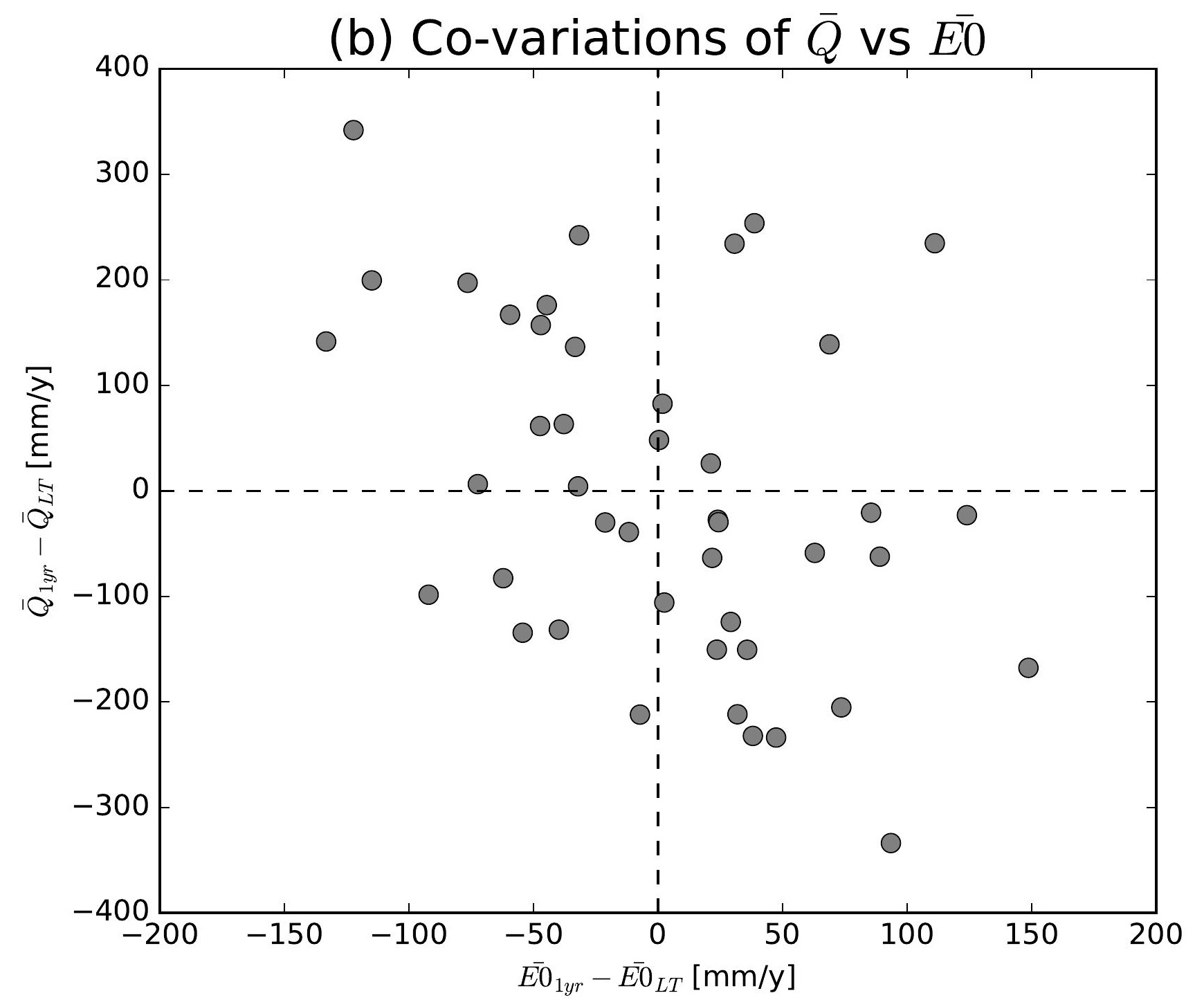

(e) Co-variations of $\bar{Q}$ vs $\overline{E 0}$

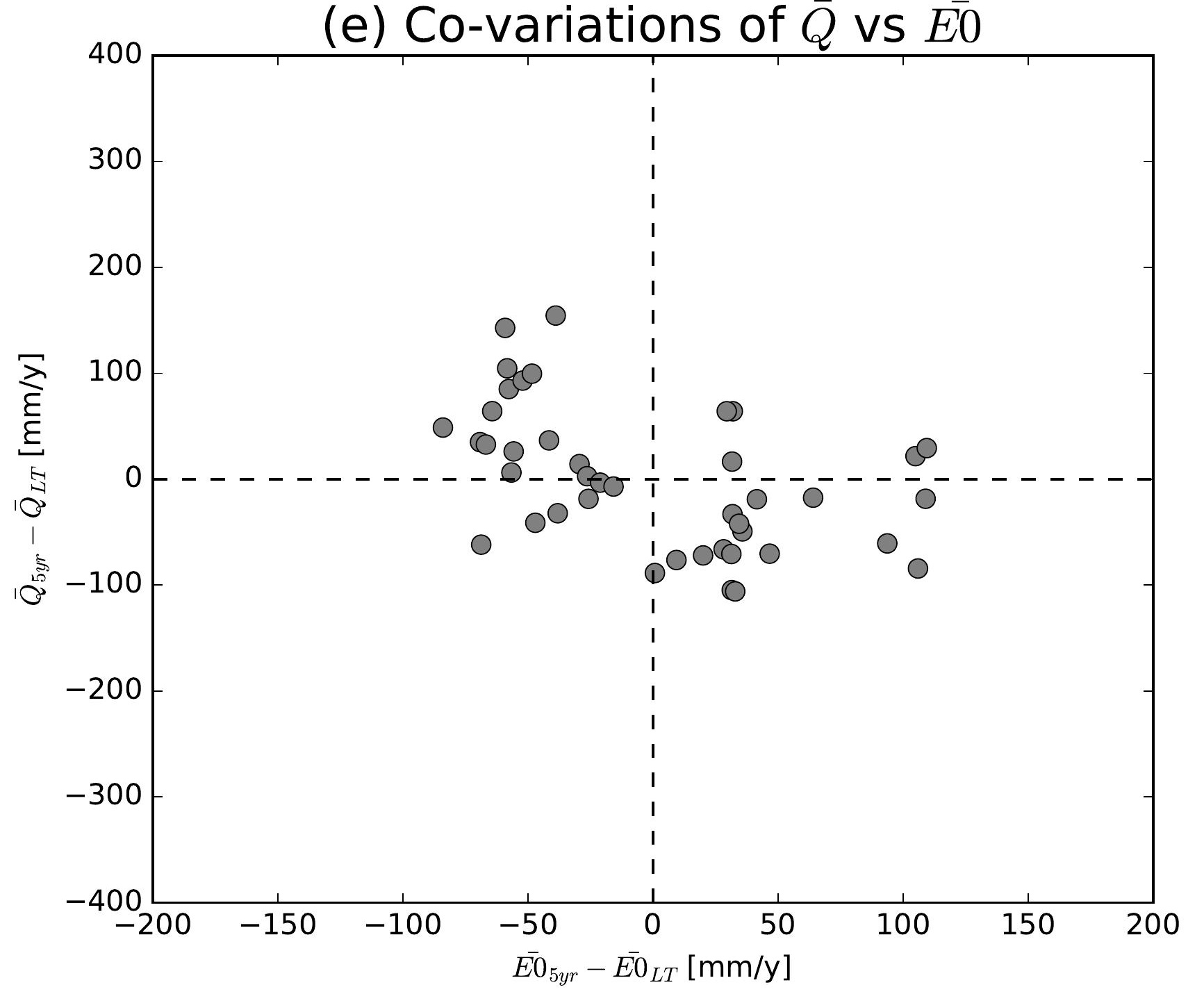

(h) Co-variations of $\bar{Q}$ vs $\overline{E 0}$

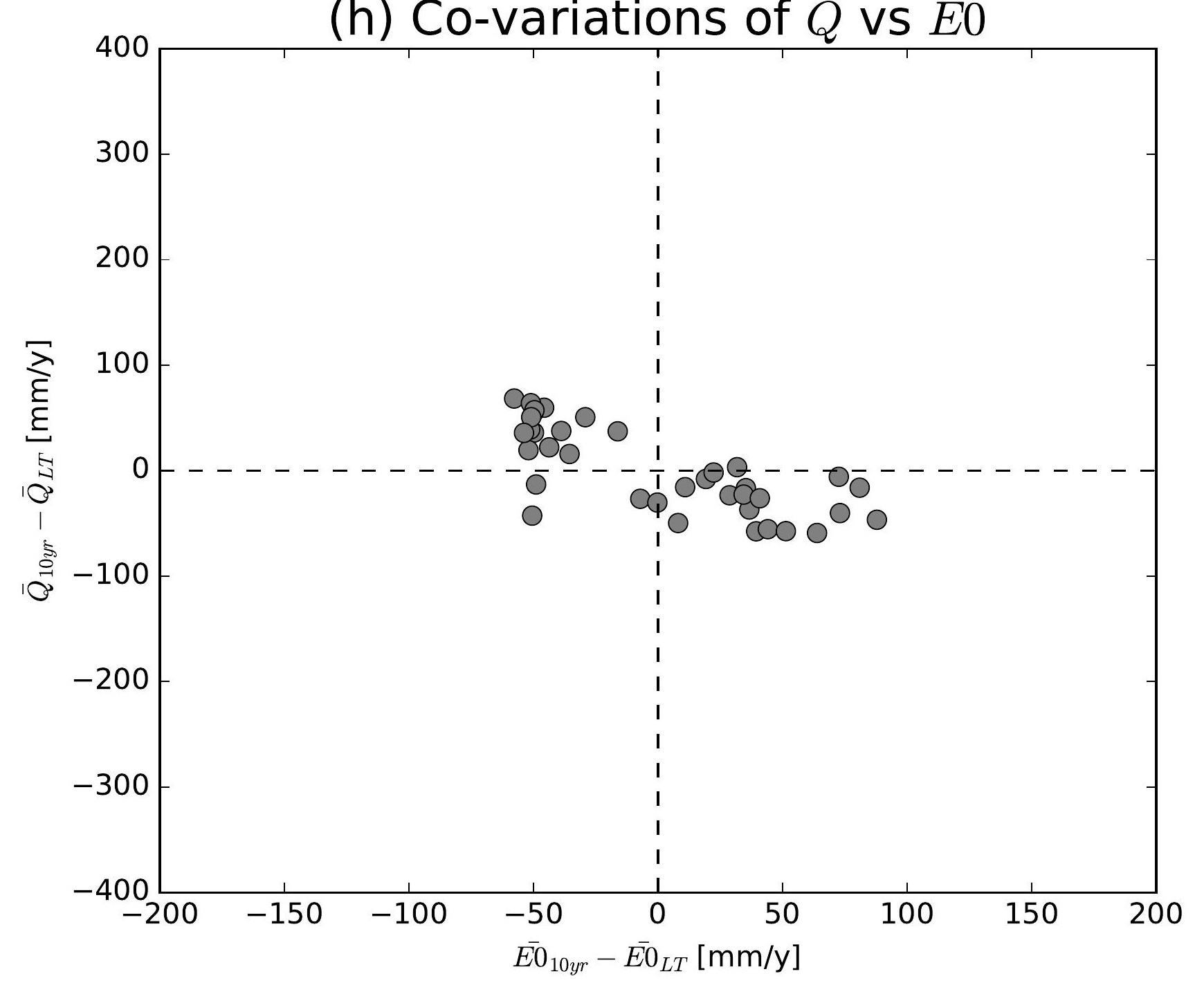

(k) Co-variations of $\bar{Q}$ vs $\overline{E 0}$

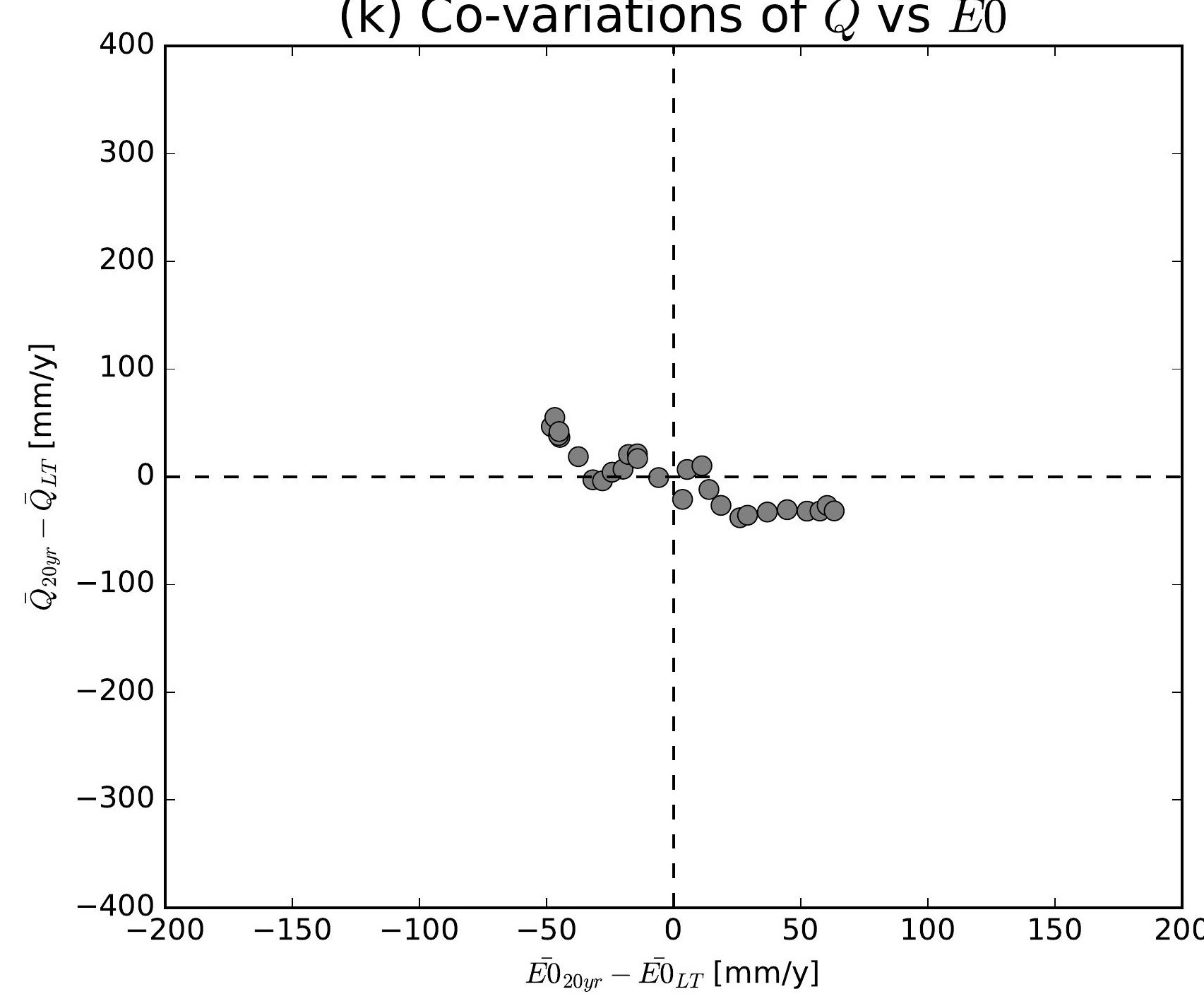

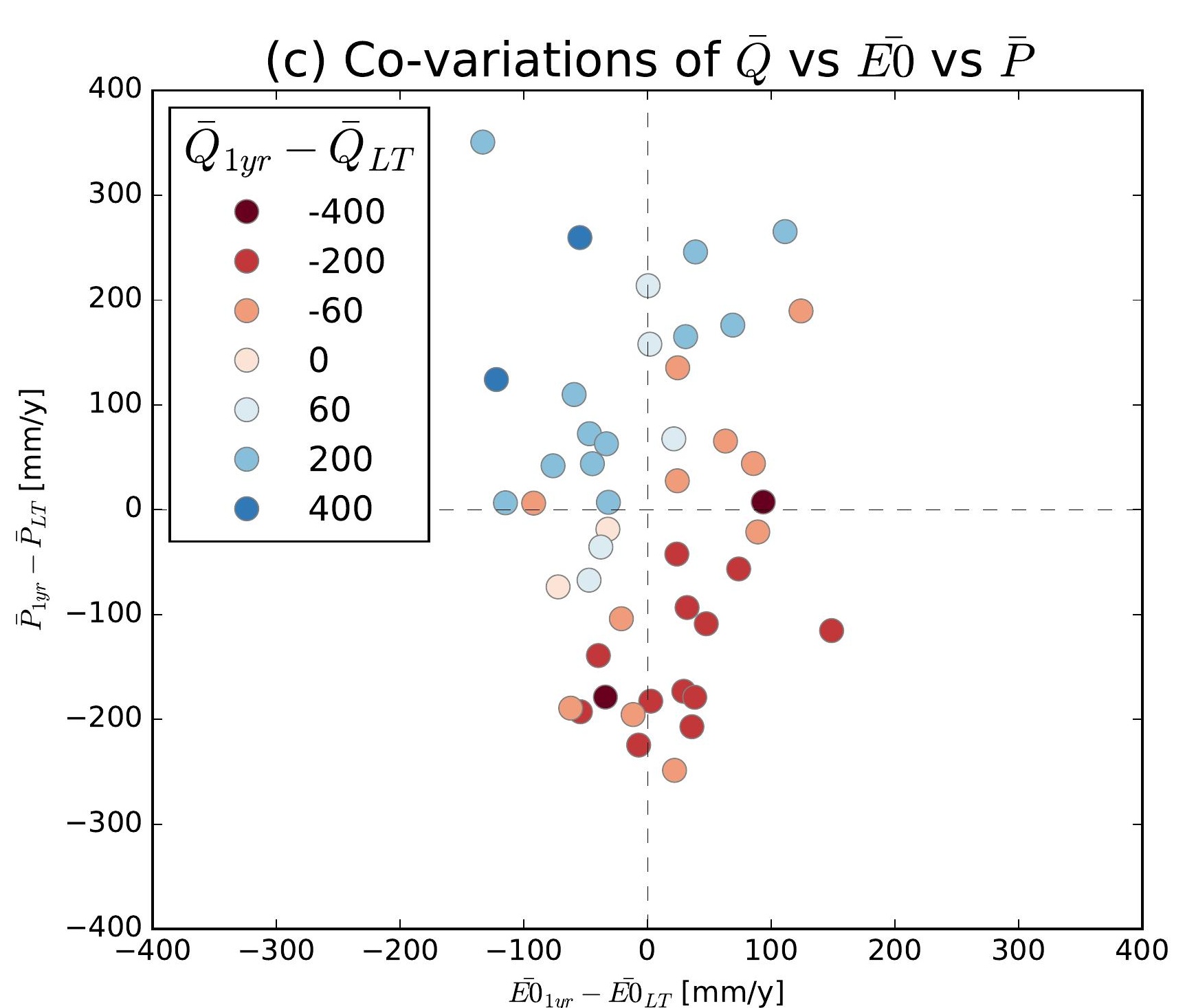
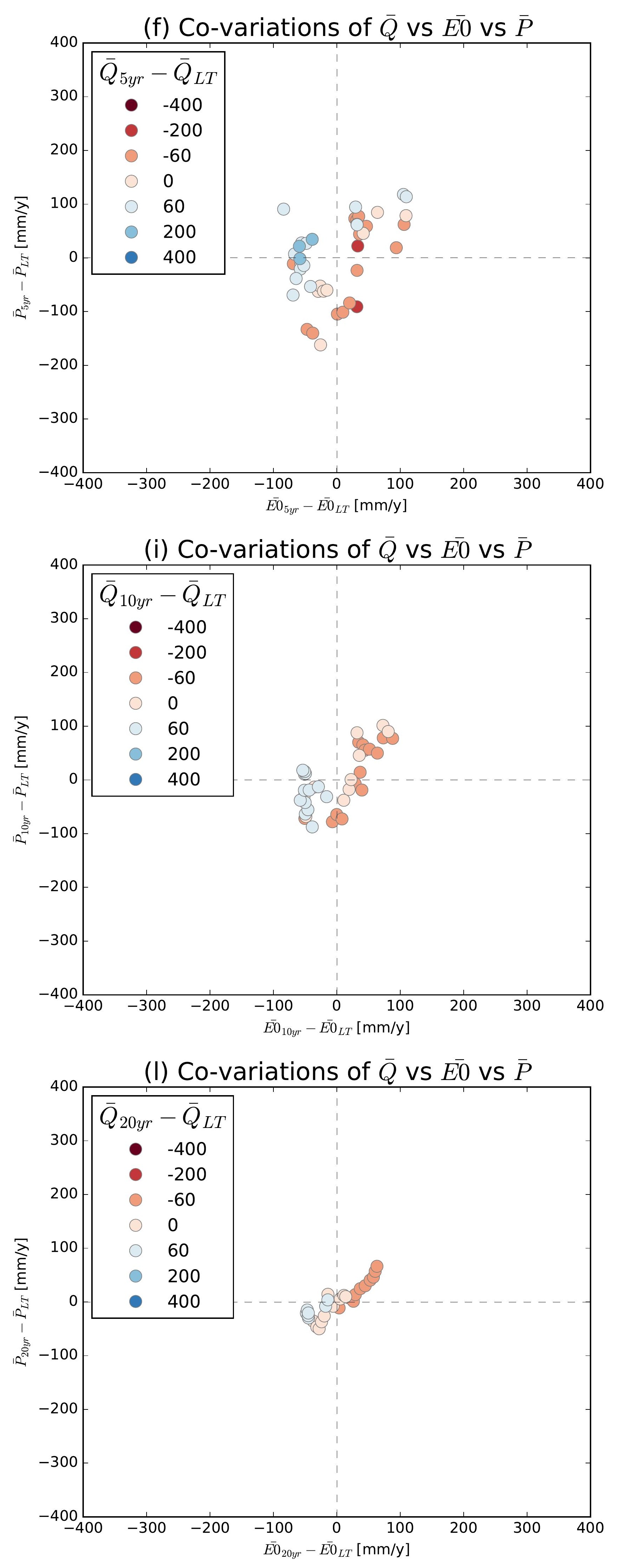

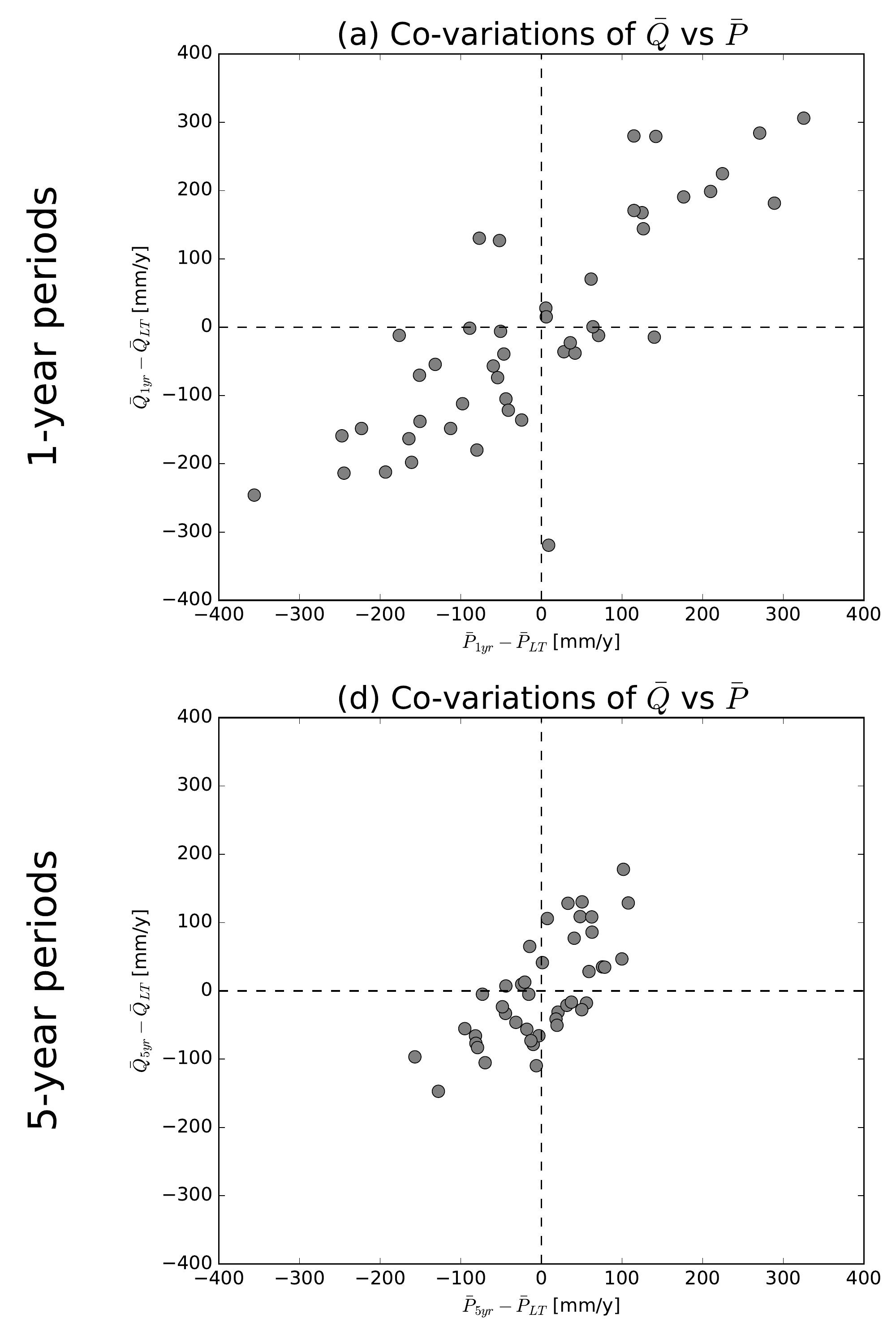

(g) Co-variations of $\bar{Q}$ vs $\bar{P}$
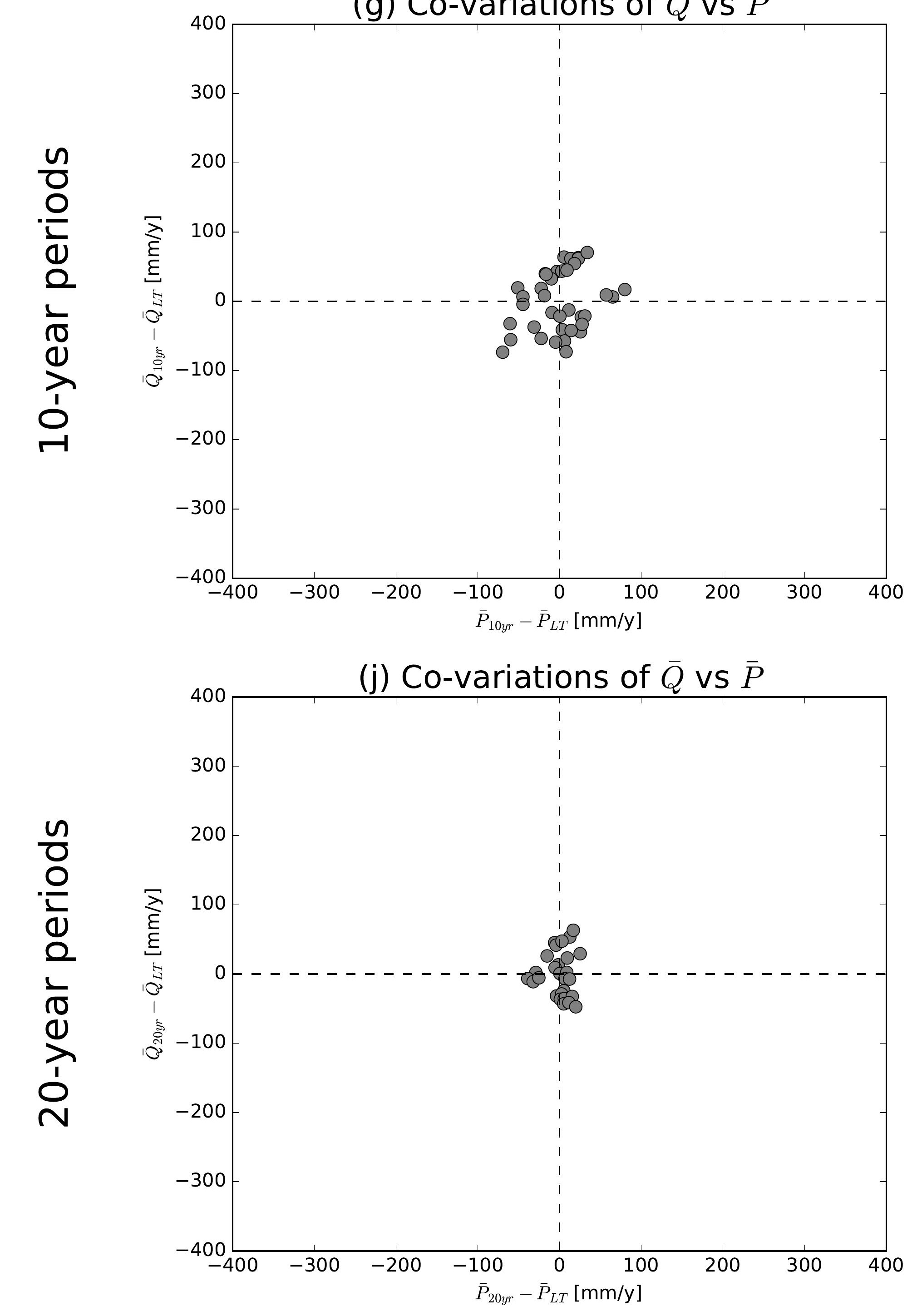

(b) Co-variations of $\bar{Q}$ vs $\overline{E 0}$

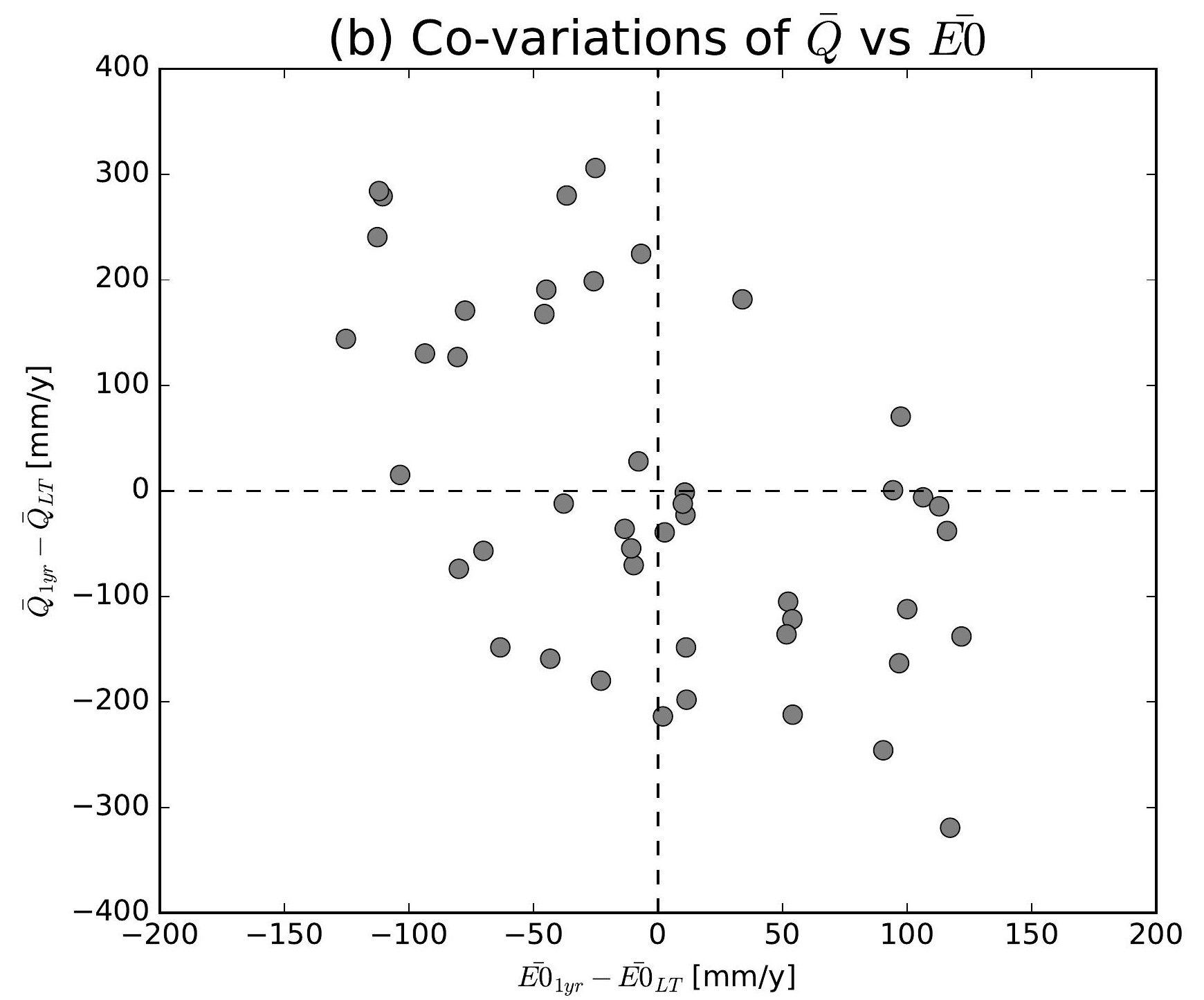

(e) Co-variations of $\bar{Q}$ vs $\overline{E 0}$

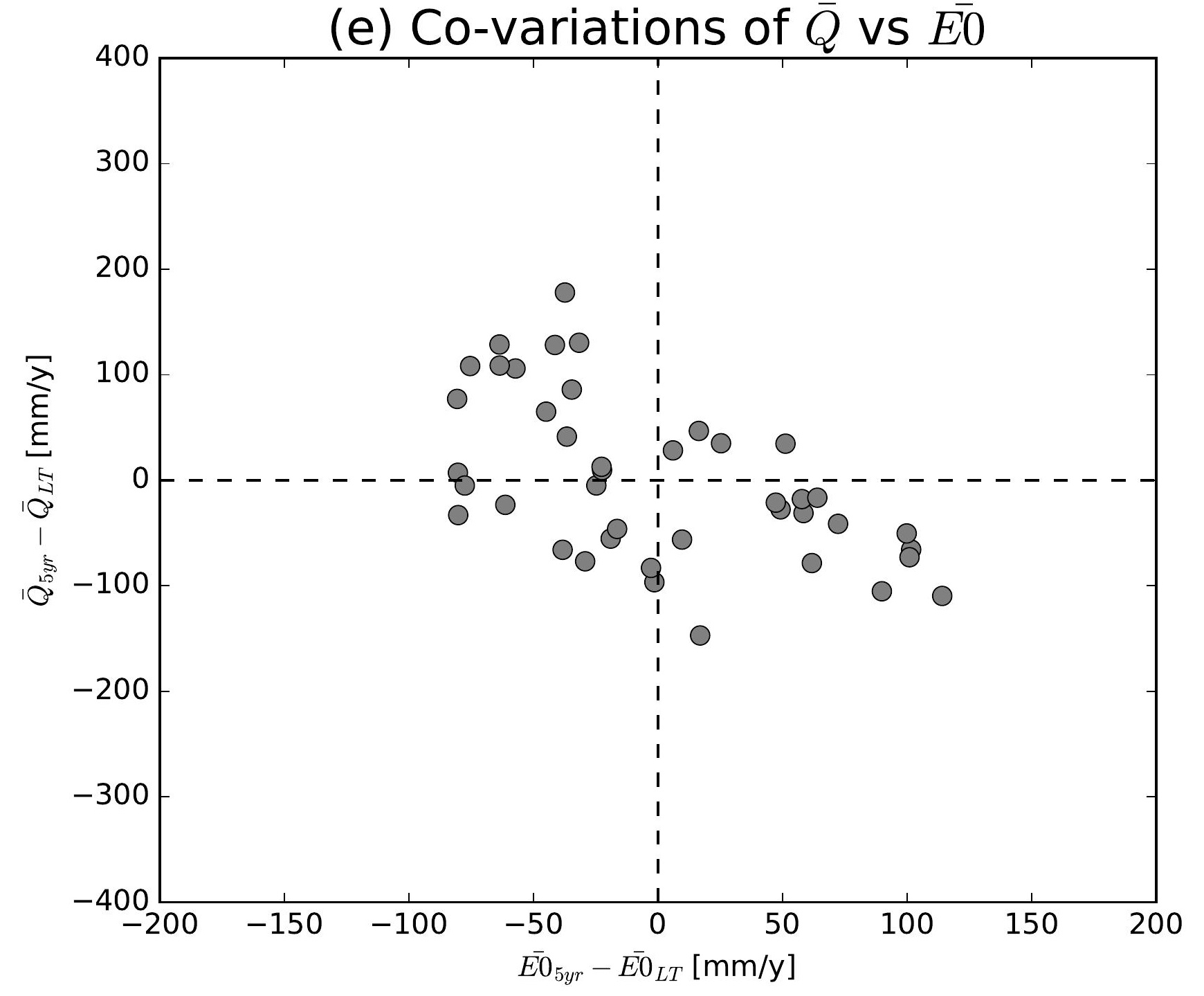

(h) Co-variations of $\bar{Q}$ vs $\overline{E 0}$

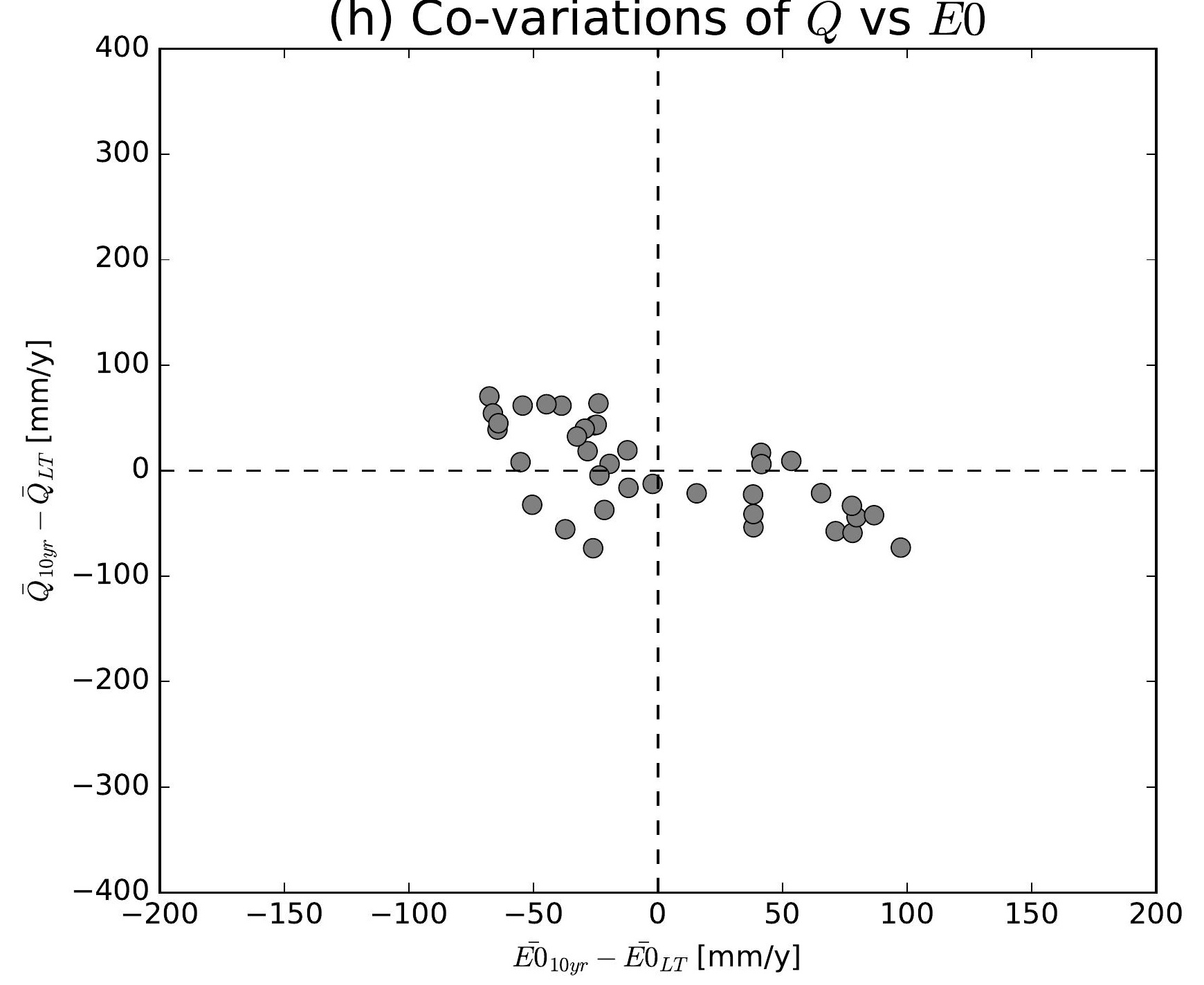

(k) Co-variations of $\bar{Q}$ vs $\overline{E 0}$

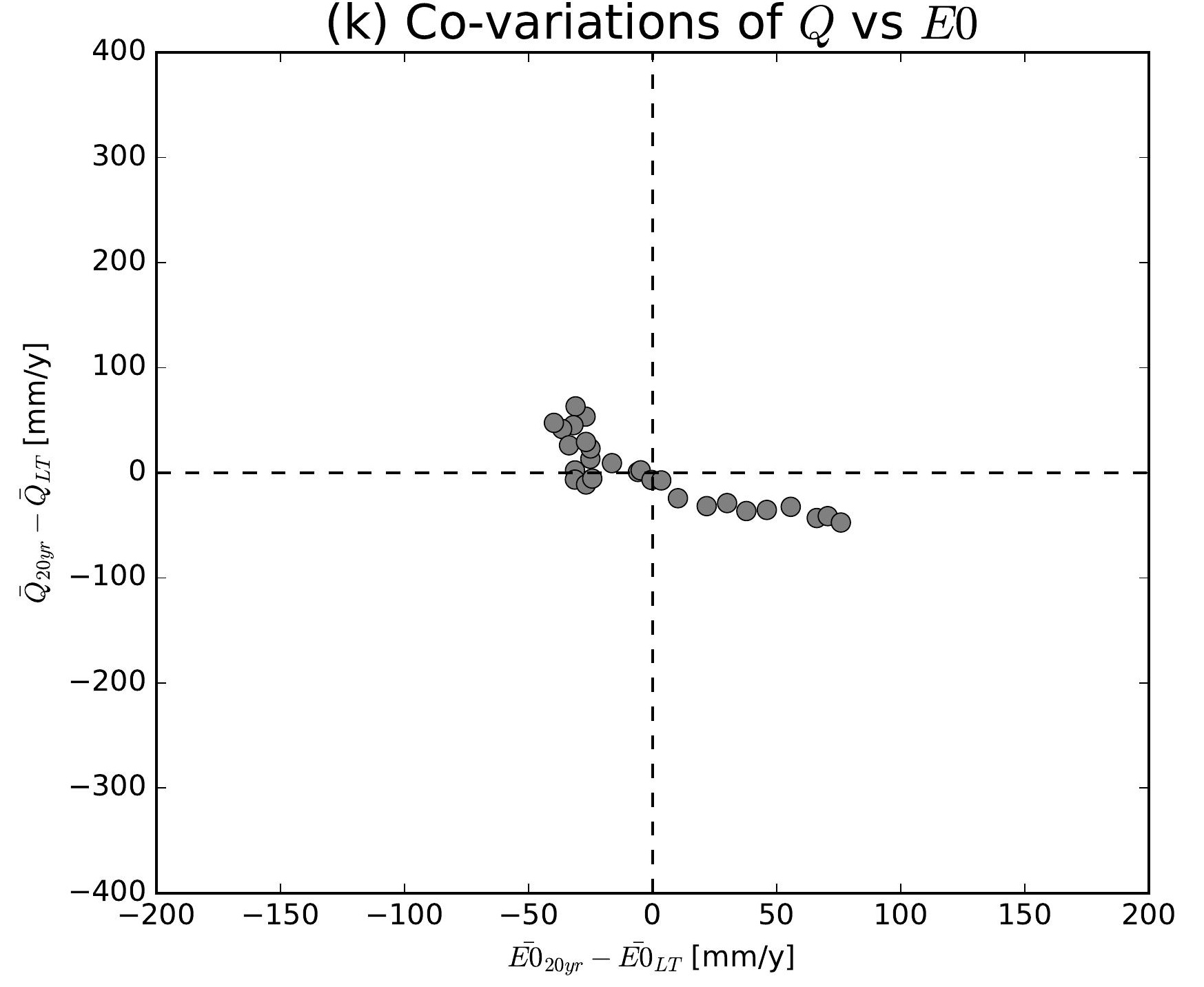

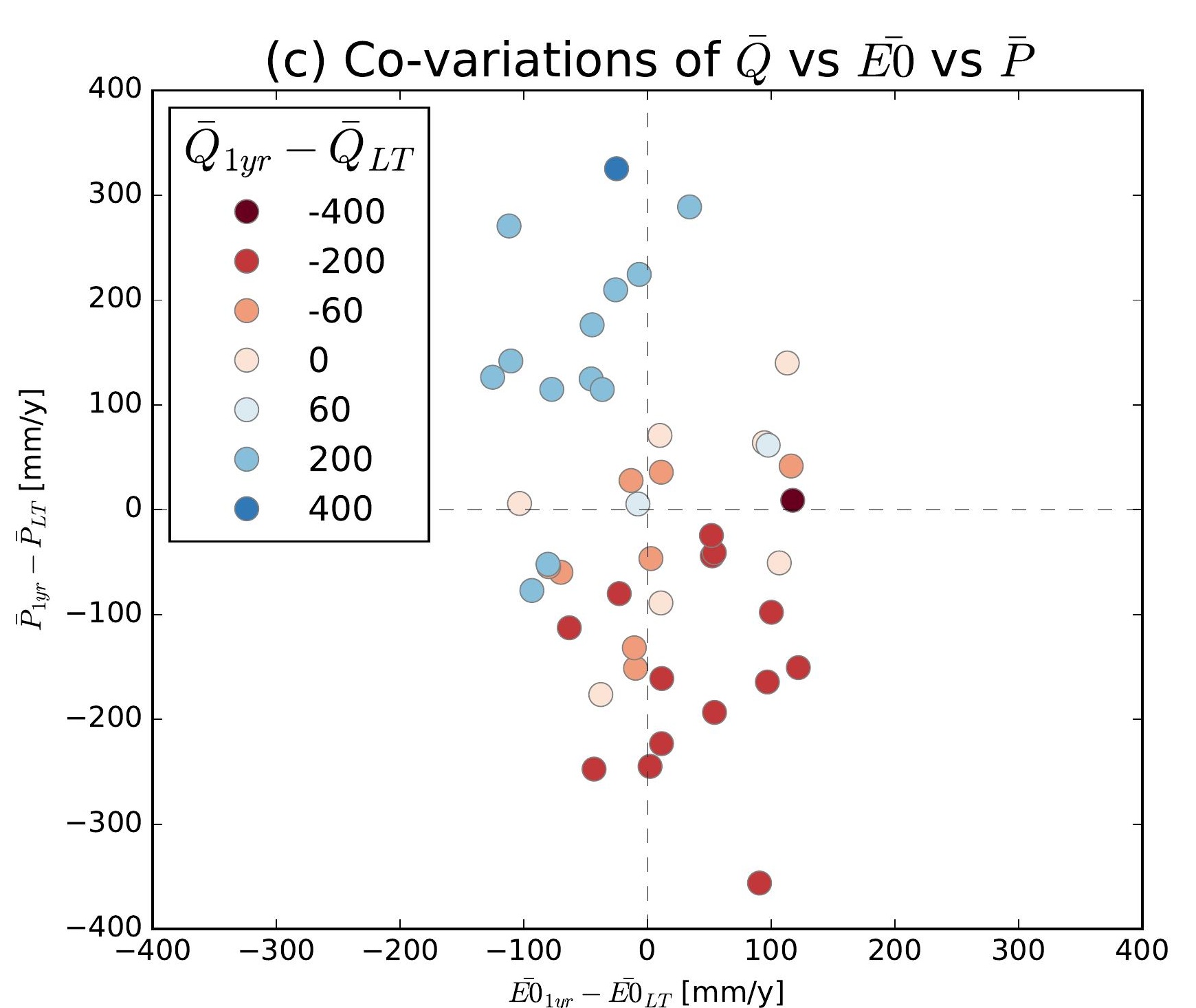
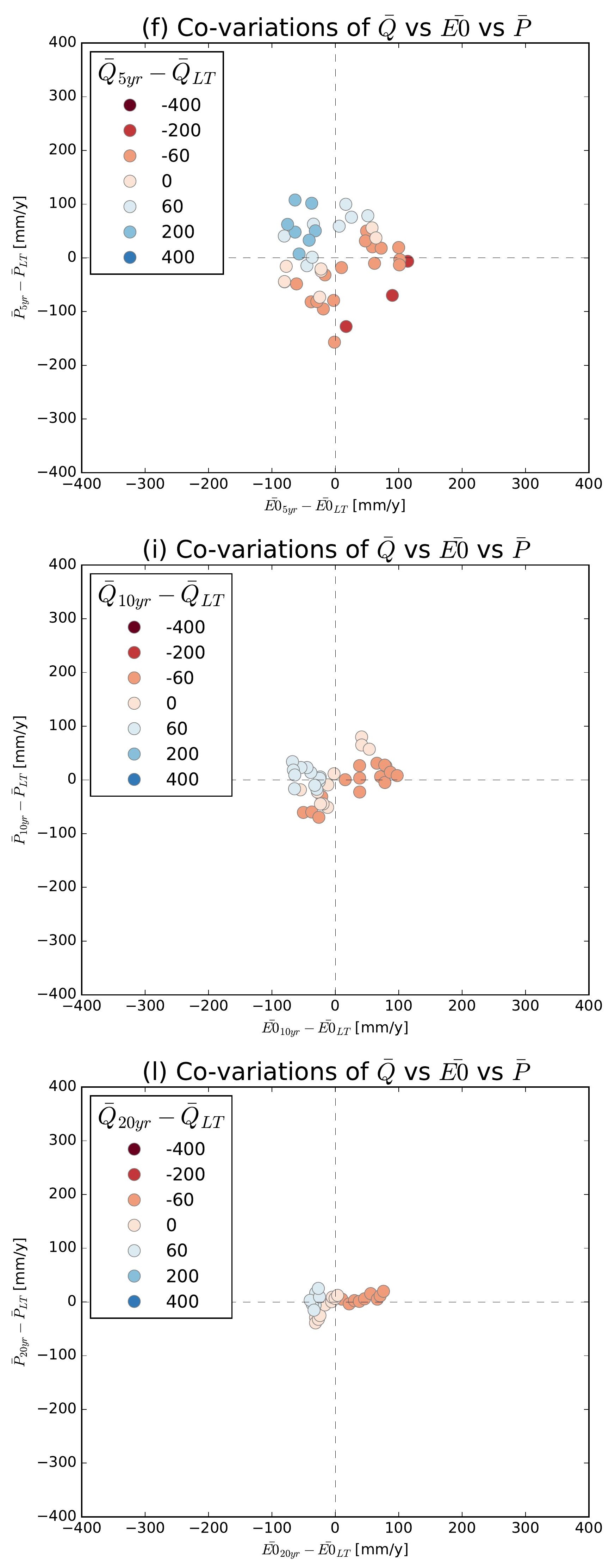

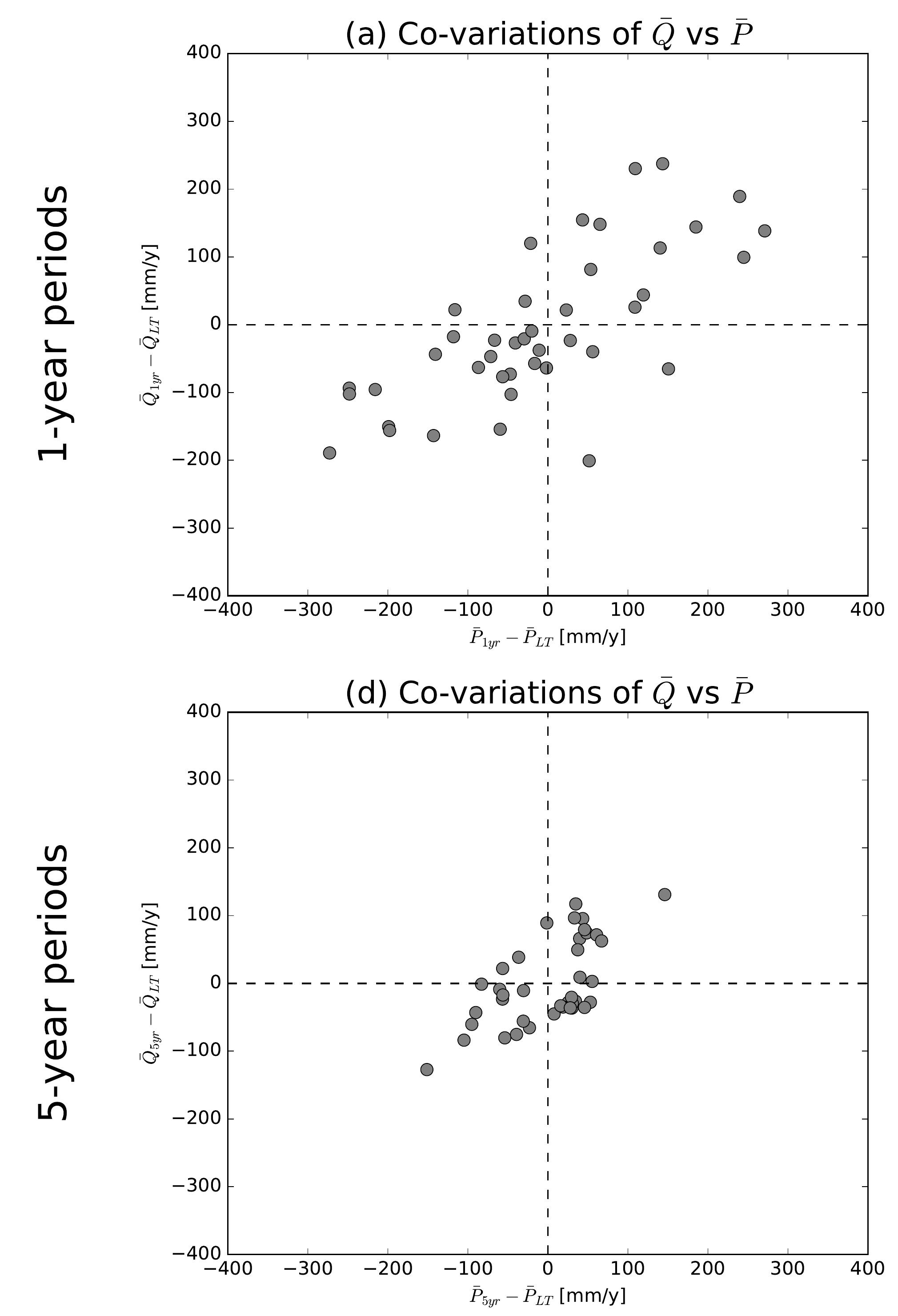

(g) Co-variations of $\bar{Q}$ vs $\bar{P}$
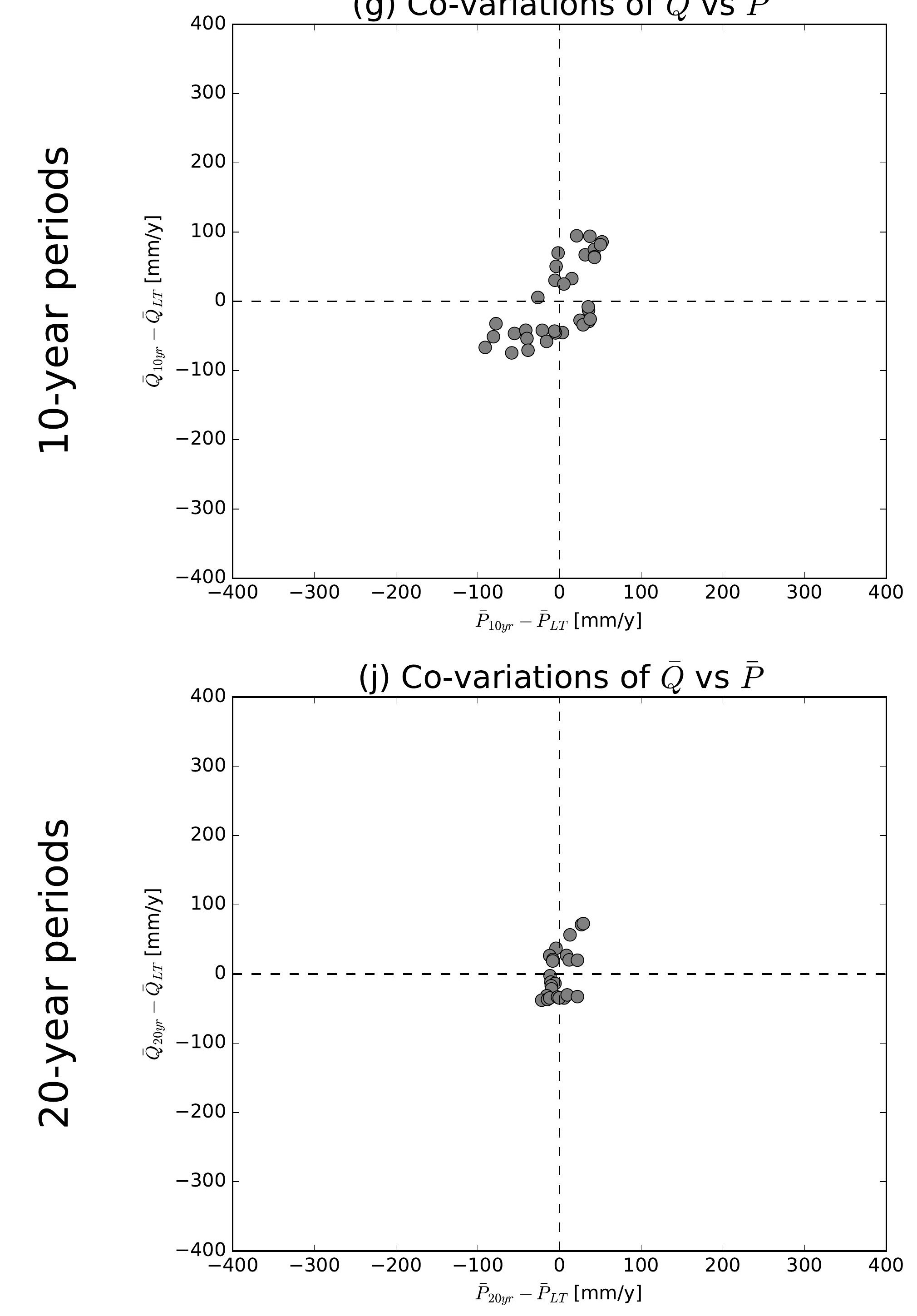

(b) Co-variations of $\bar{Q}$ vs $\overline{E 0}$

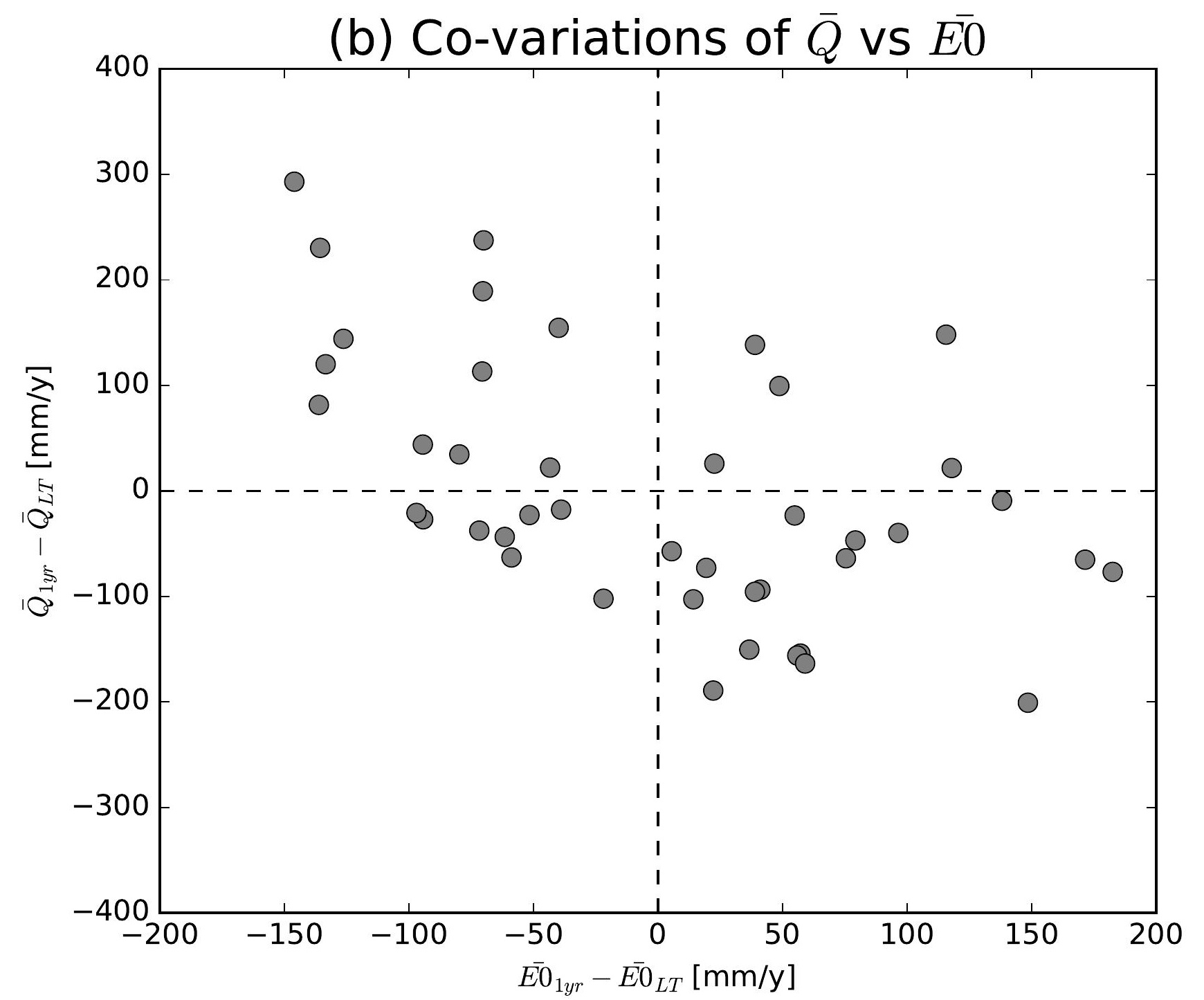

(e) Co-variations of $\bar{Q}$ vs $\overline{E 0}$

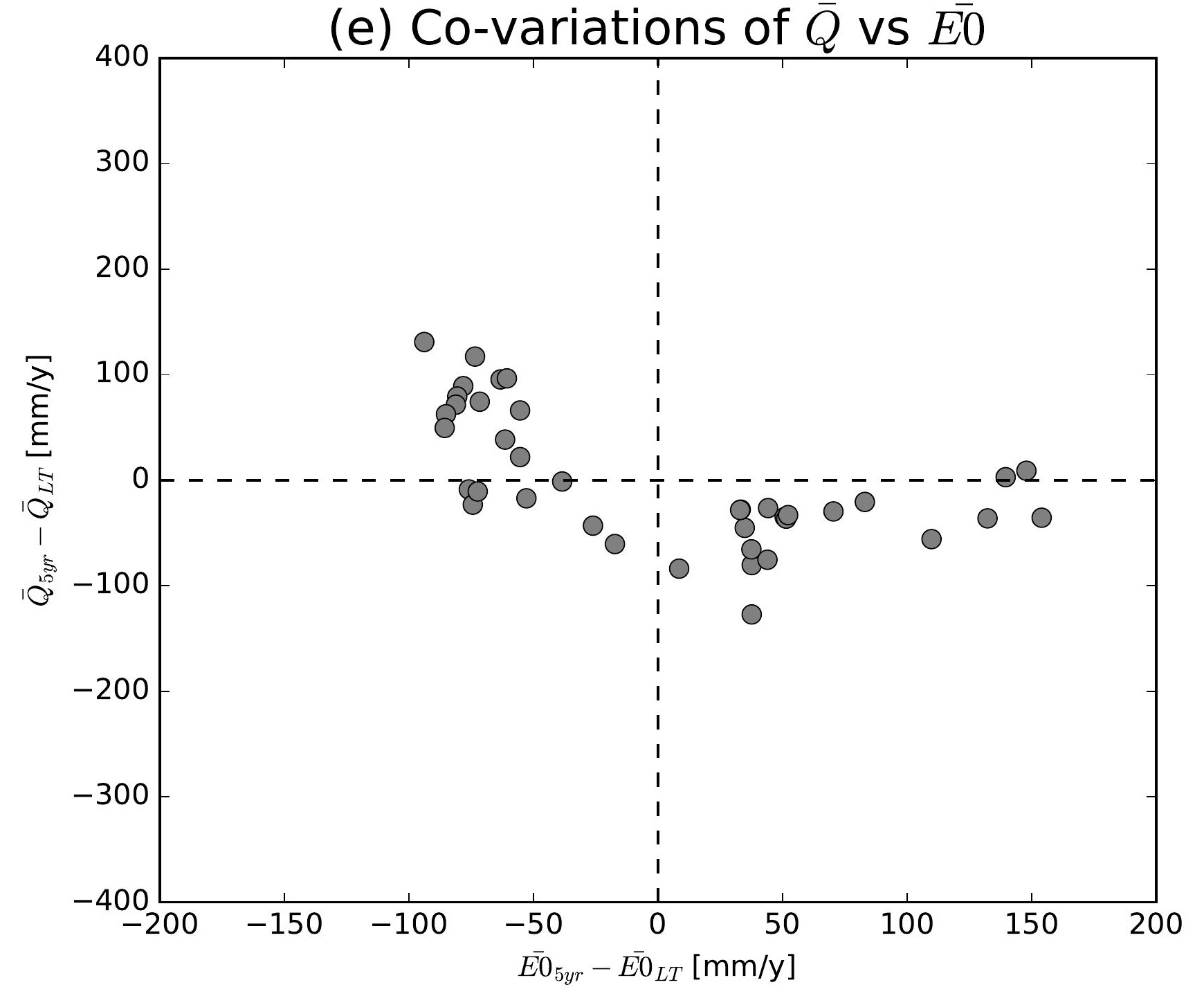

(h) Co-variations of $\bar{Q}$ vs $\overline{E 0}$

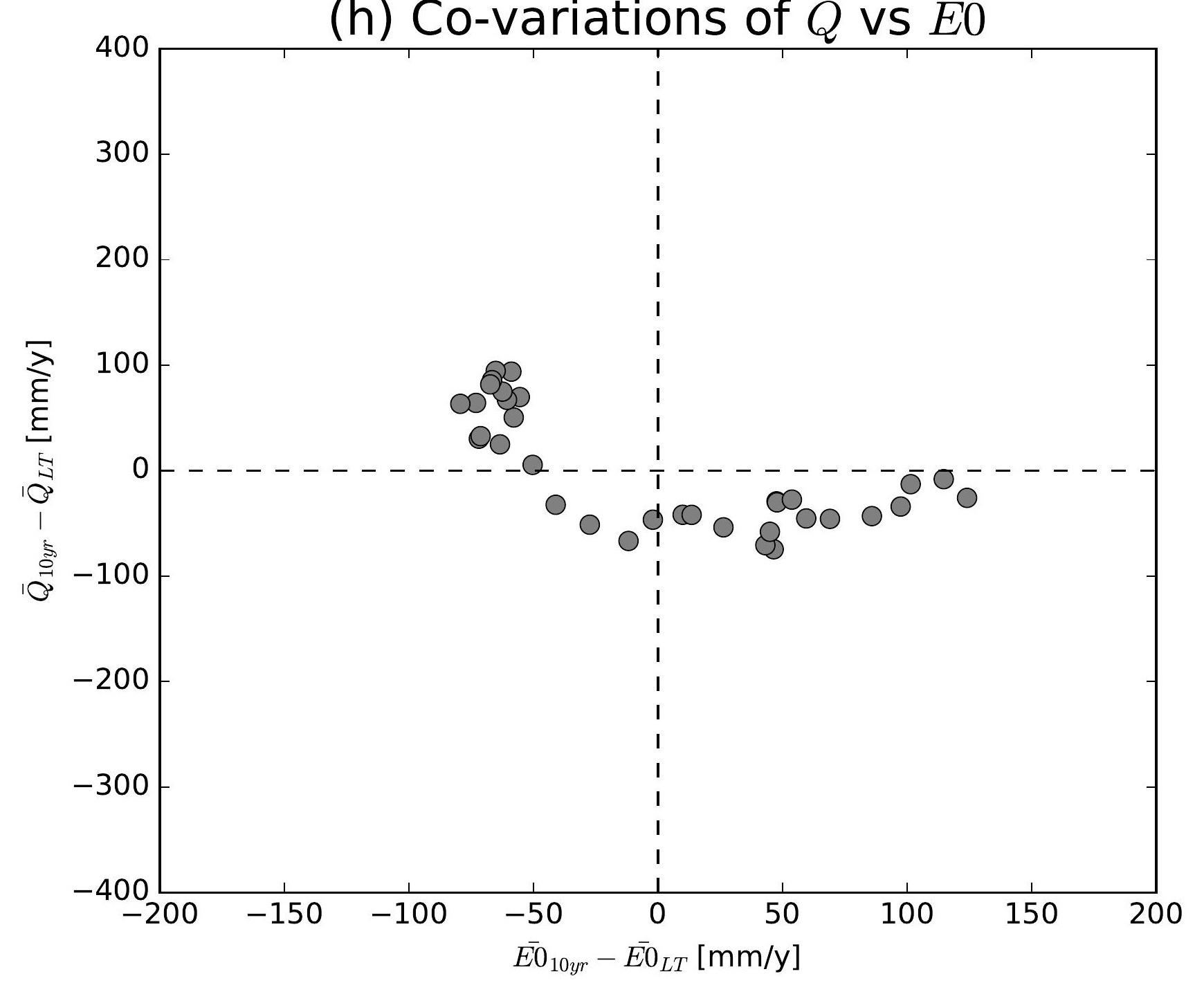

(k) Co-variations of $\bar{Q}$ vs $\overline{E 0}$

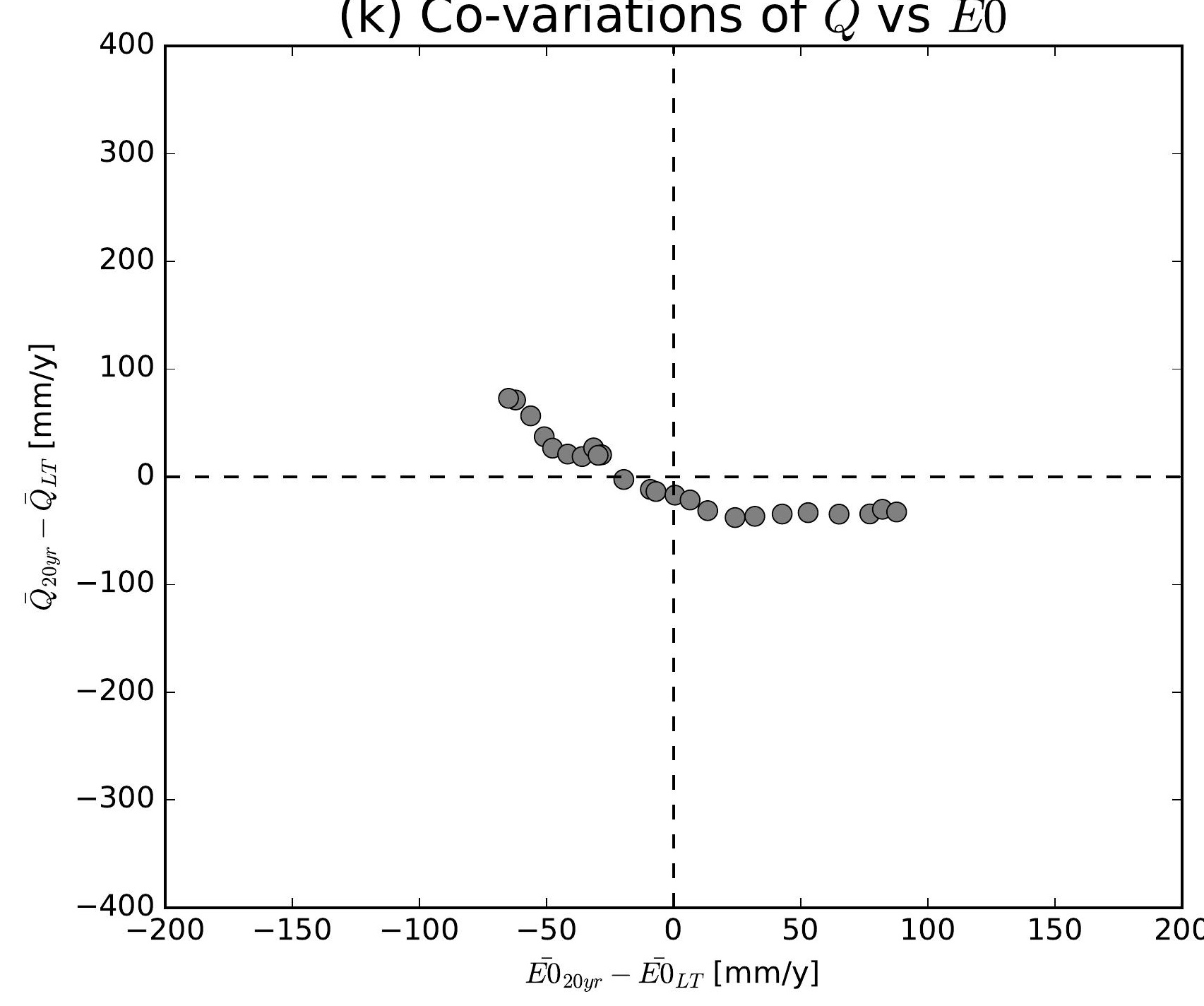

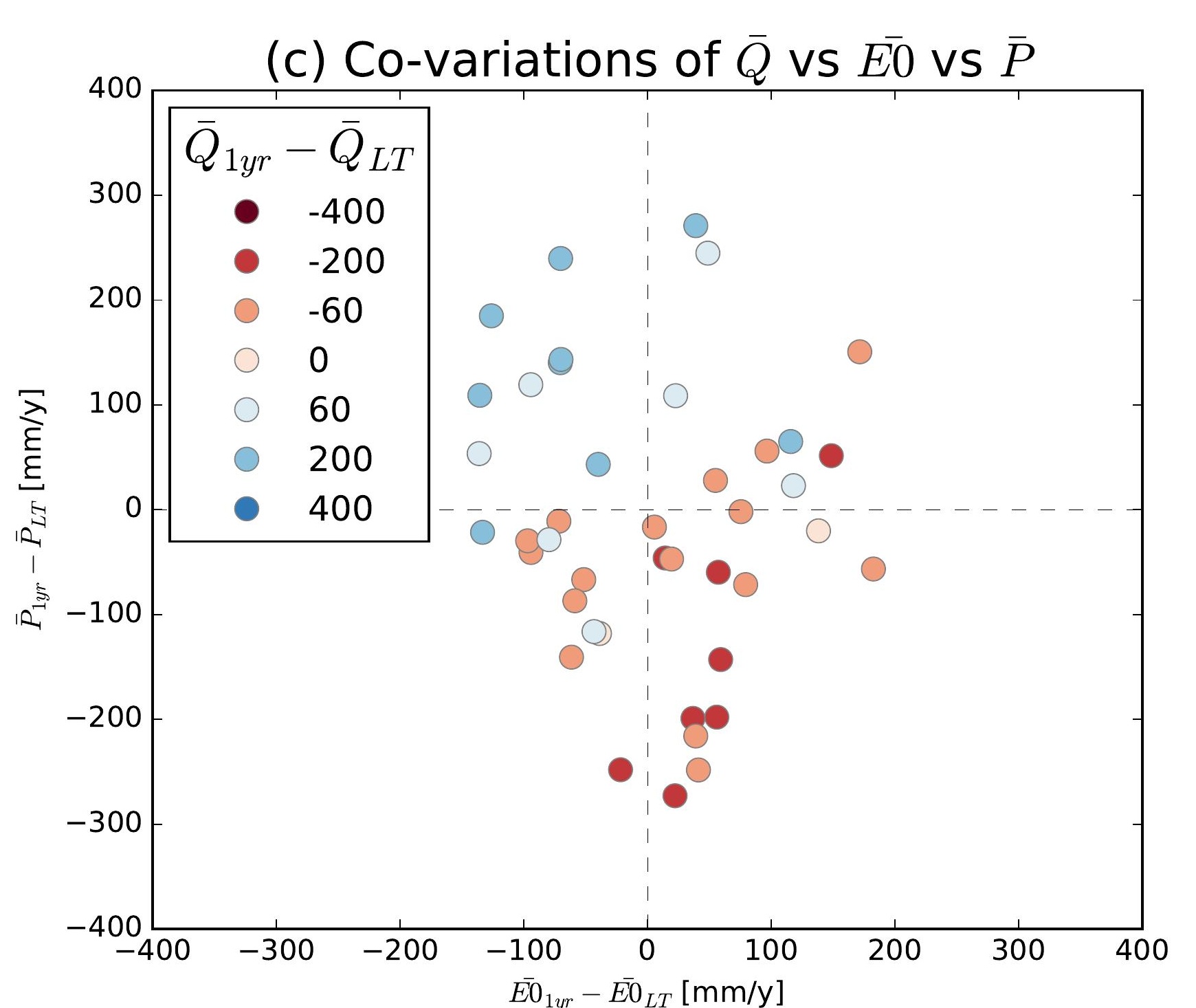
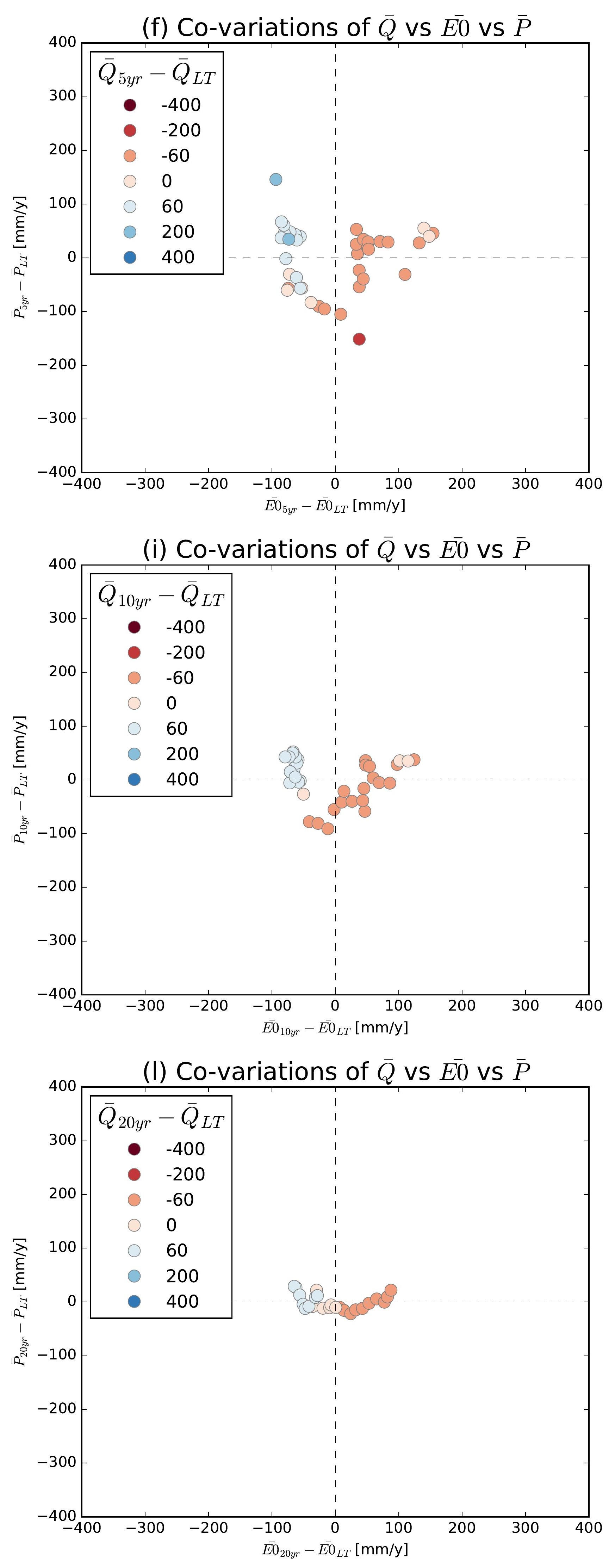

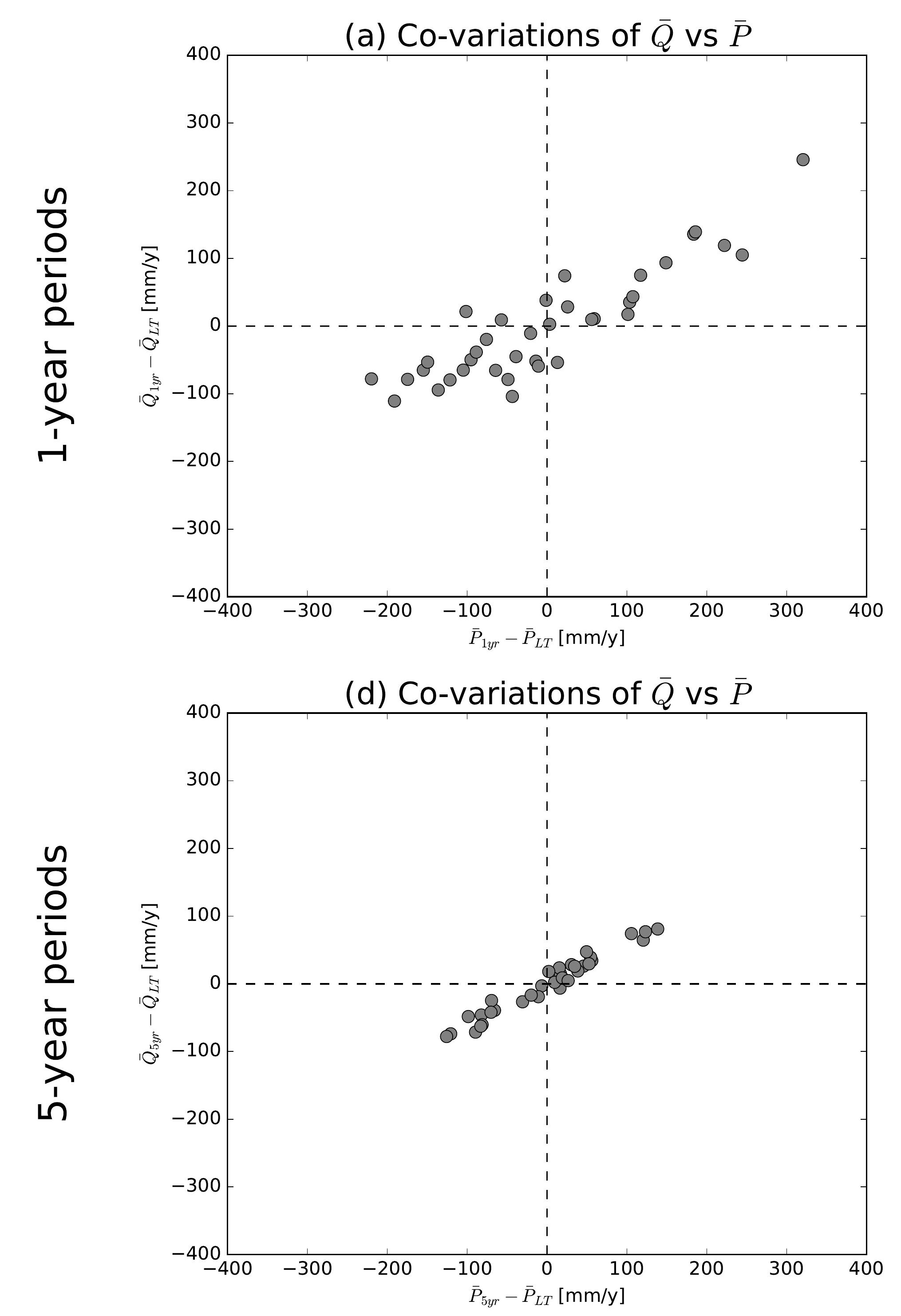

(g) Co-variations of $\bar{Q}$ vs $\bar{P}$
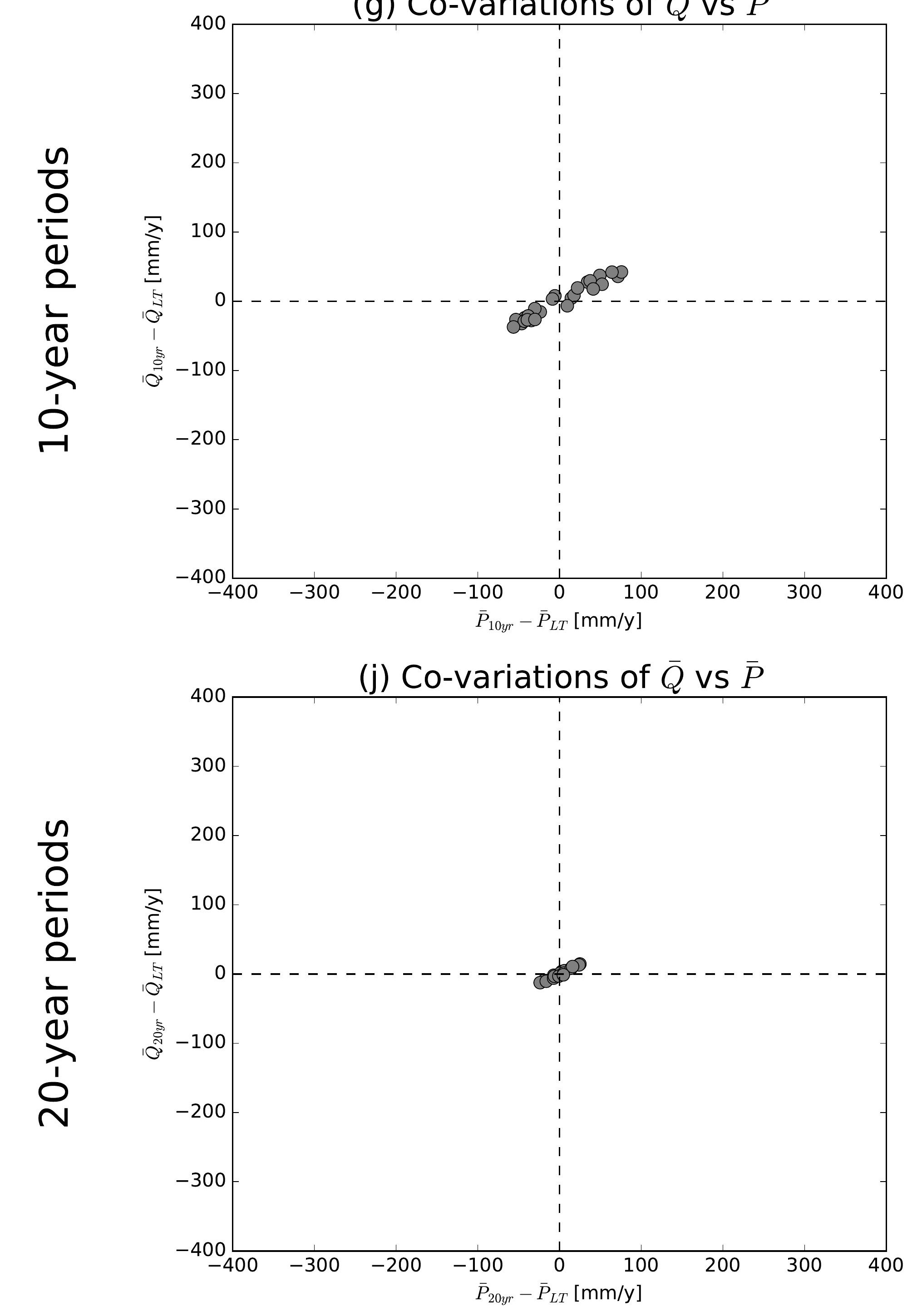

(b) Co-variations of $\bar{Q}$ vs $\overline{E 0}$

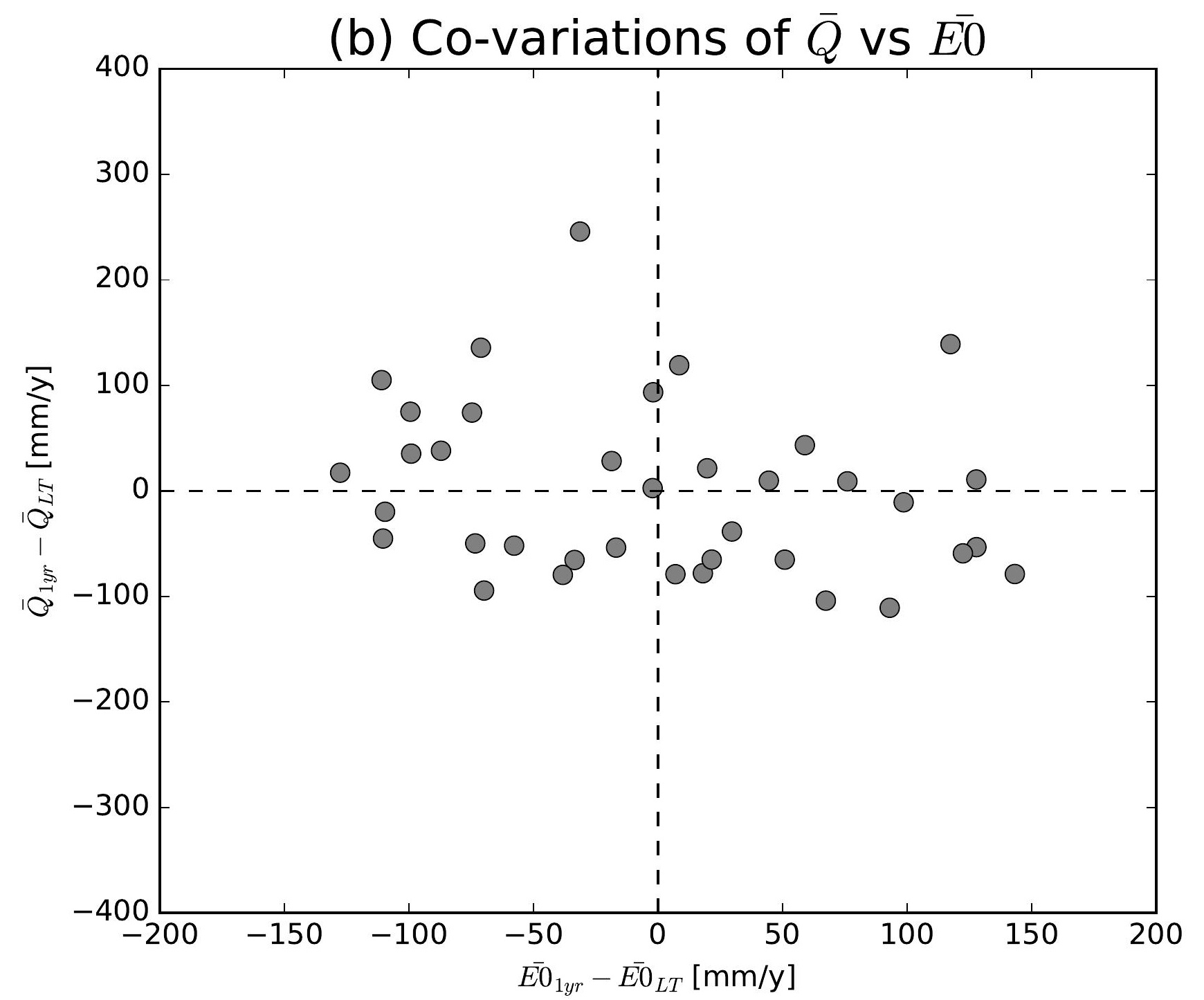

(e) Co-variations of $\bar{Q}$ vs $\overline{E 0}$

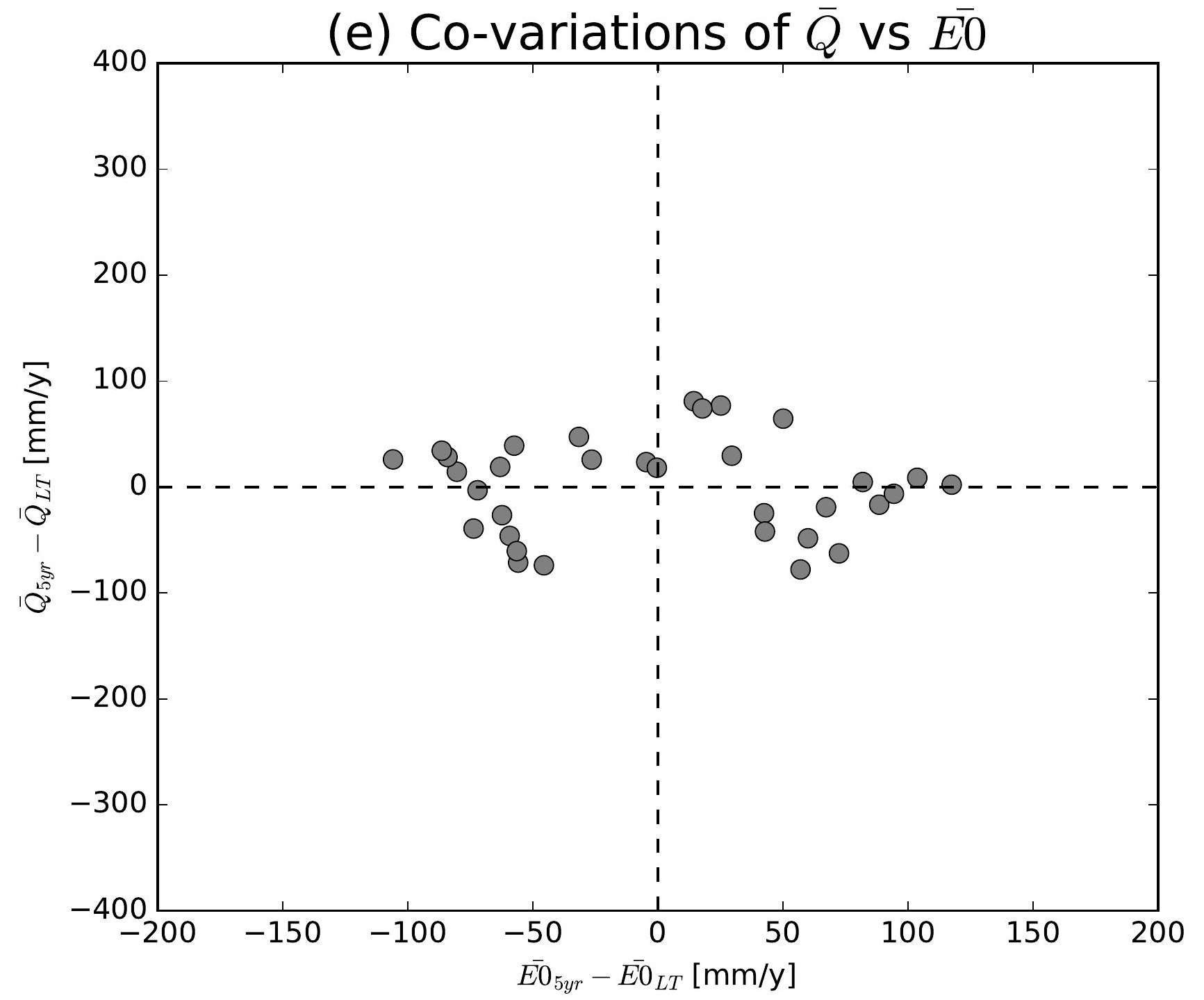

(h) Co-variations of $\bar{Q}$ vs $\overline{E 0}$

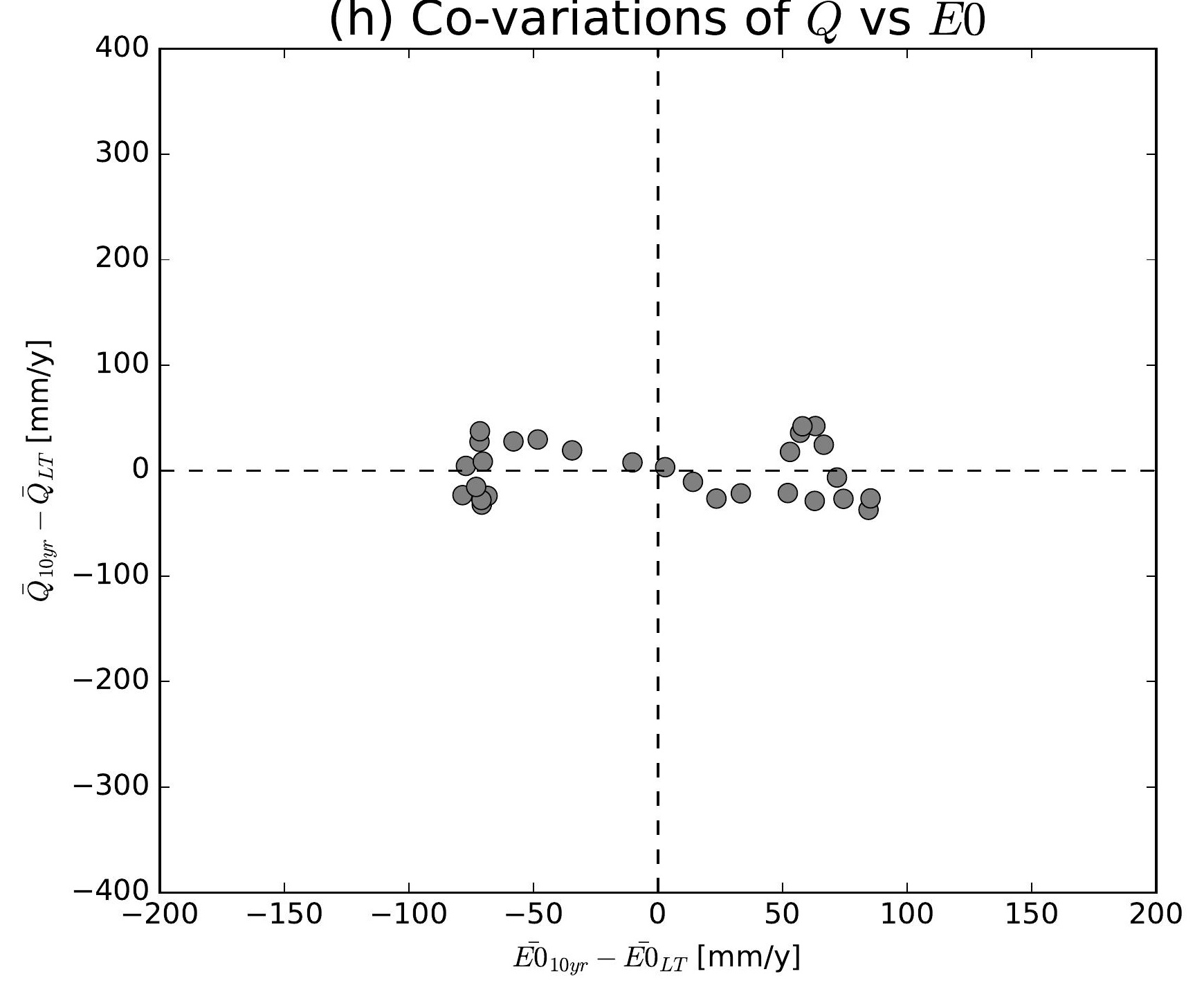

(k) Co-variations of $\bar{Q}$ vs $\overline{E 0}$

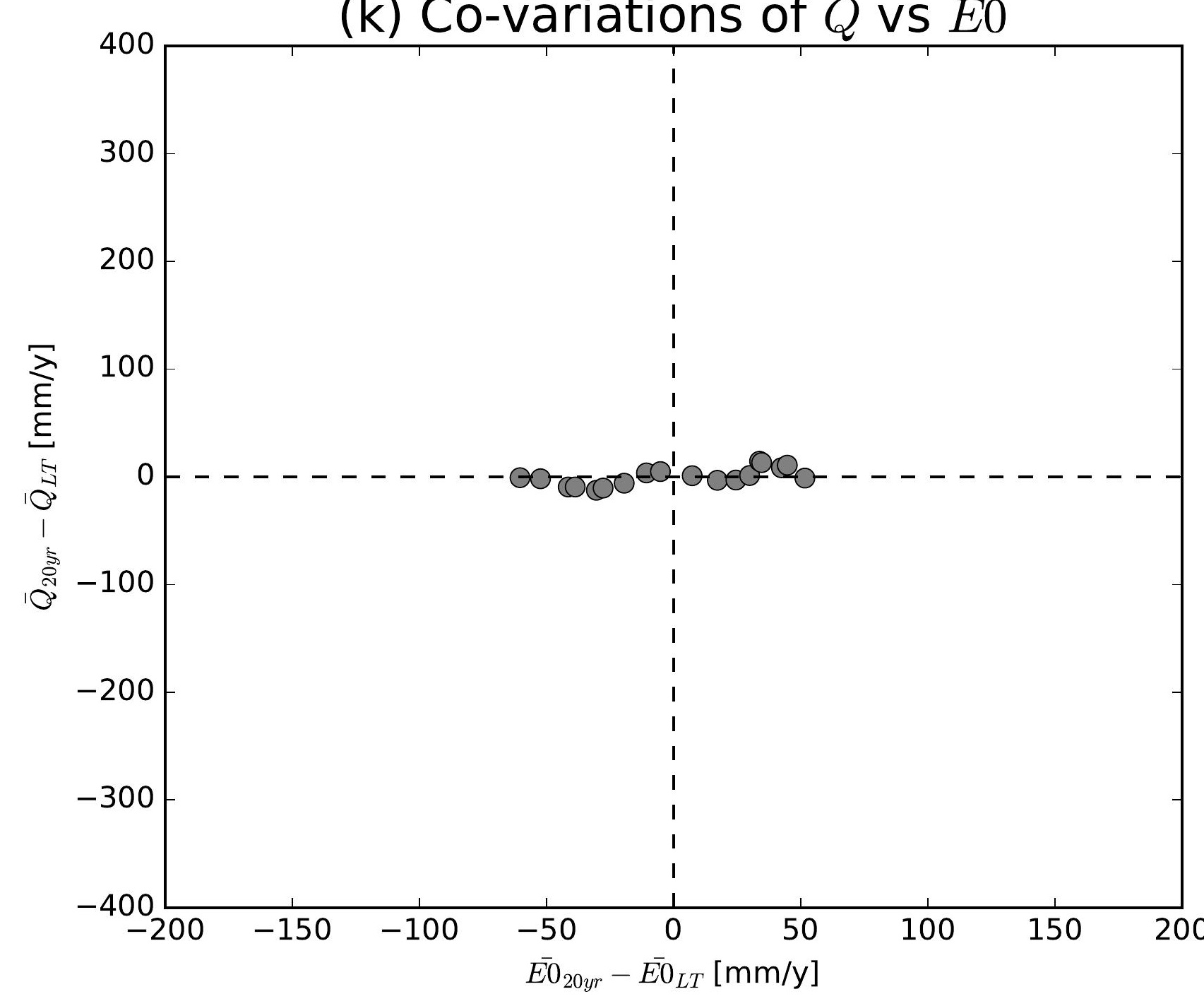

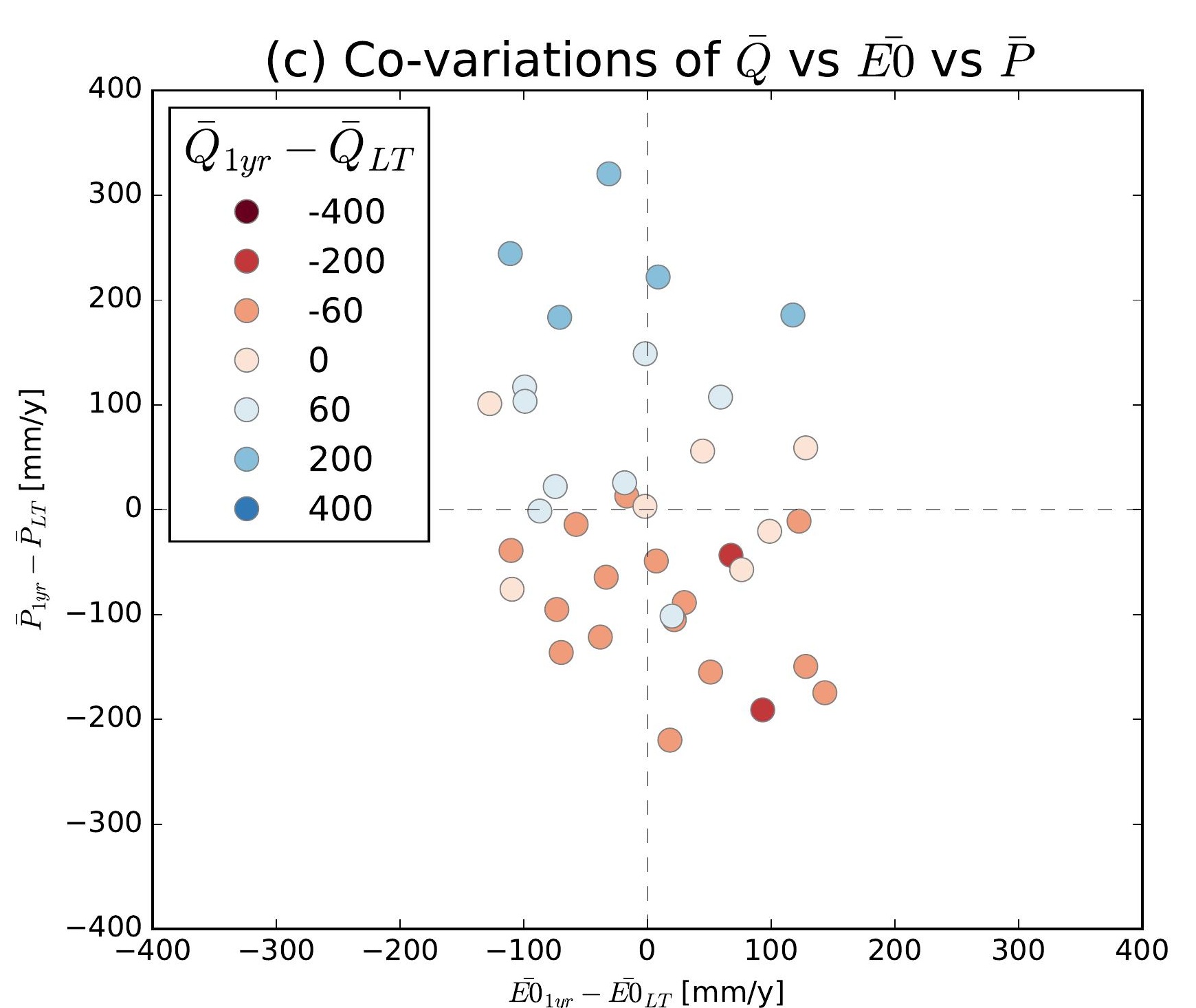
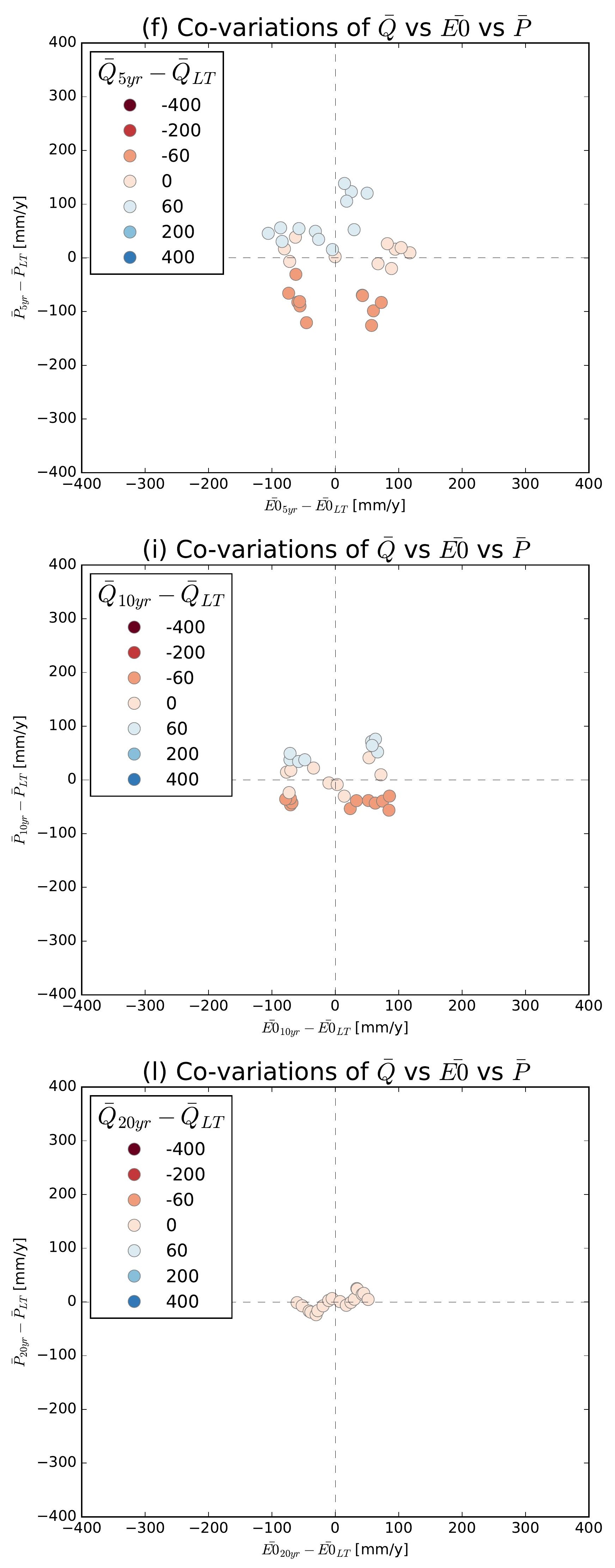

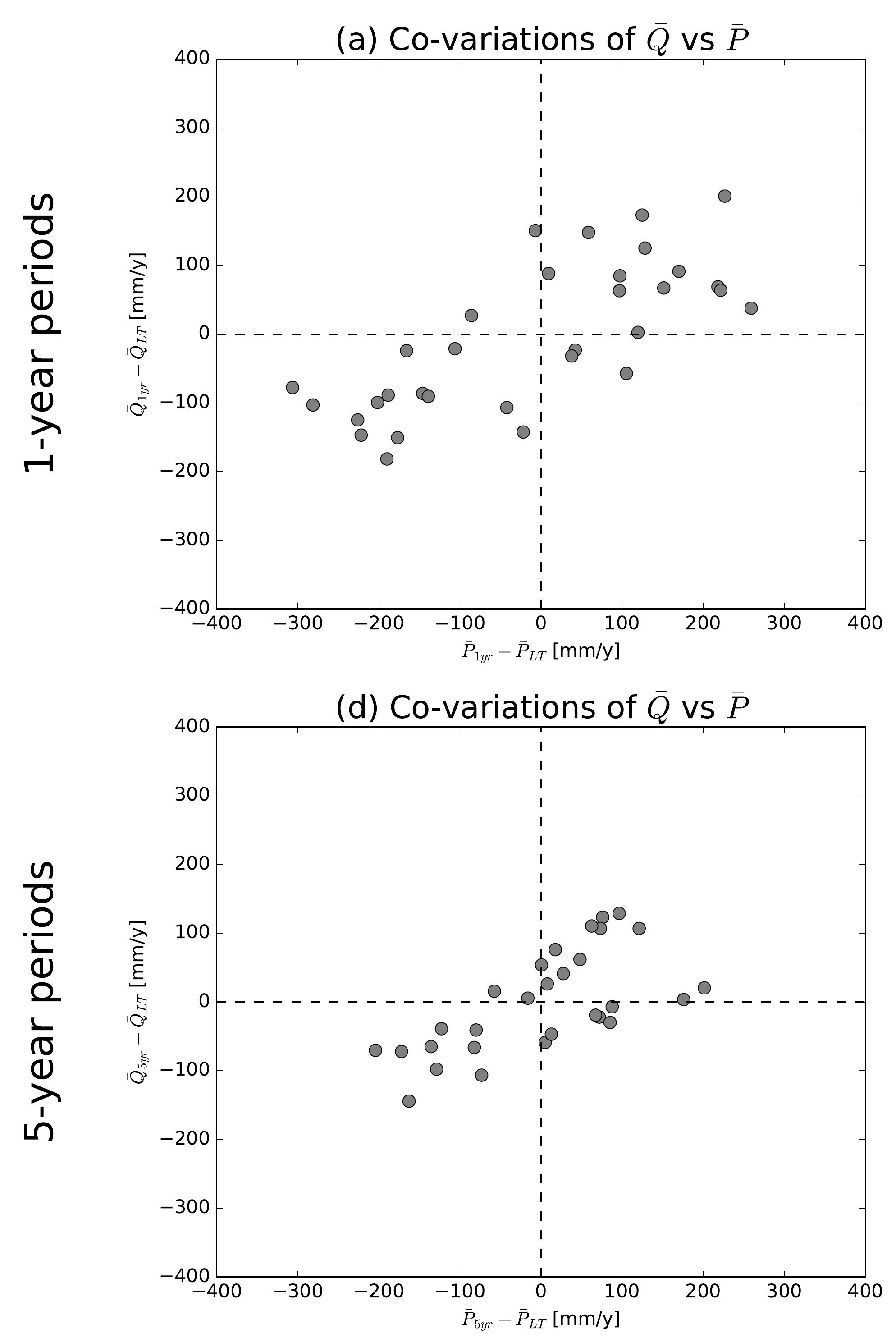

(g) Co-variations of $\bar{Q}$ vs $\bar{P}$

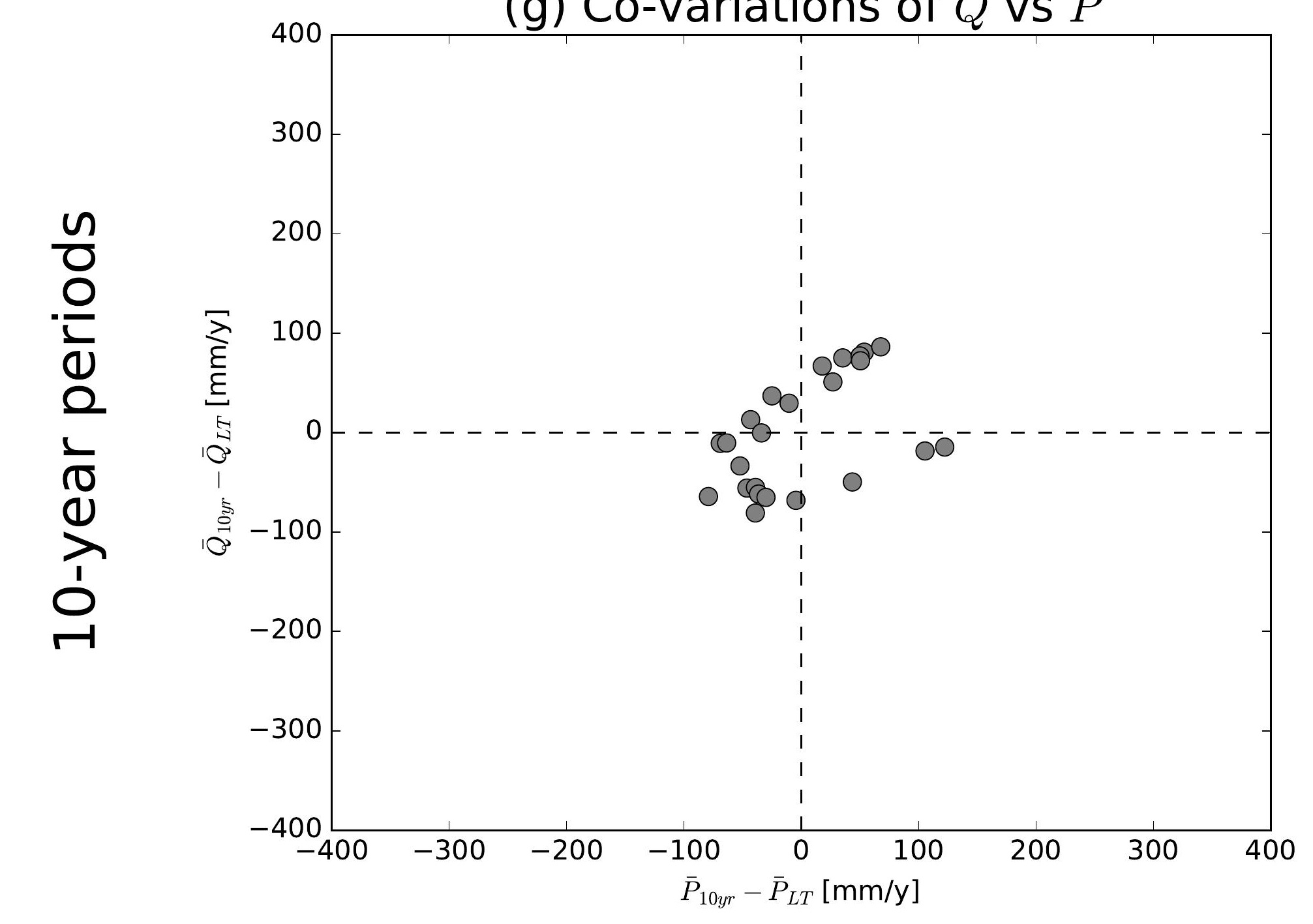

(j) Co-variations of $\bar{Q}$ vs $\bar{P}$

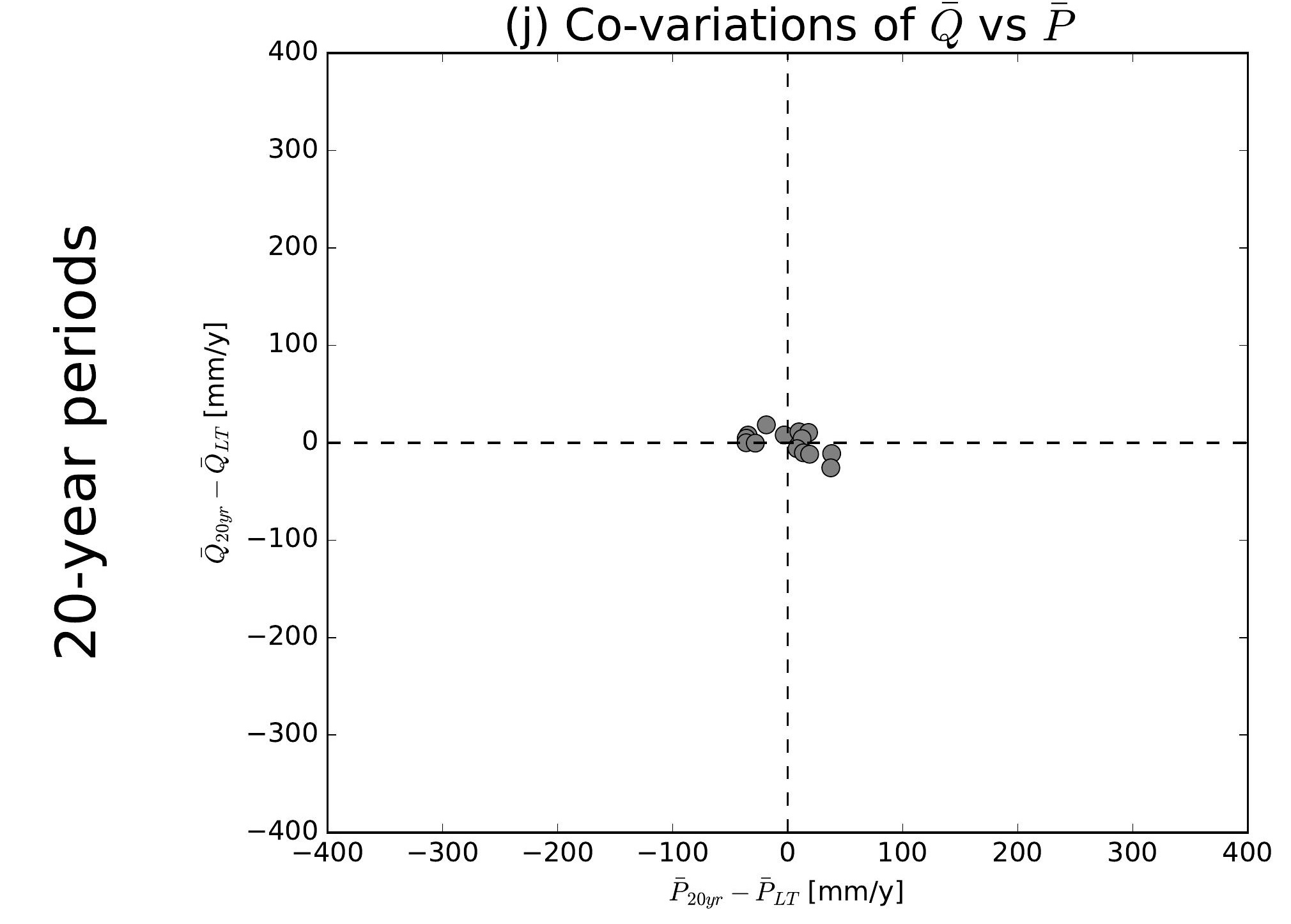

(b) Co-variations of $\bar{Q}$ vs $\overline{E 0}$

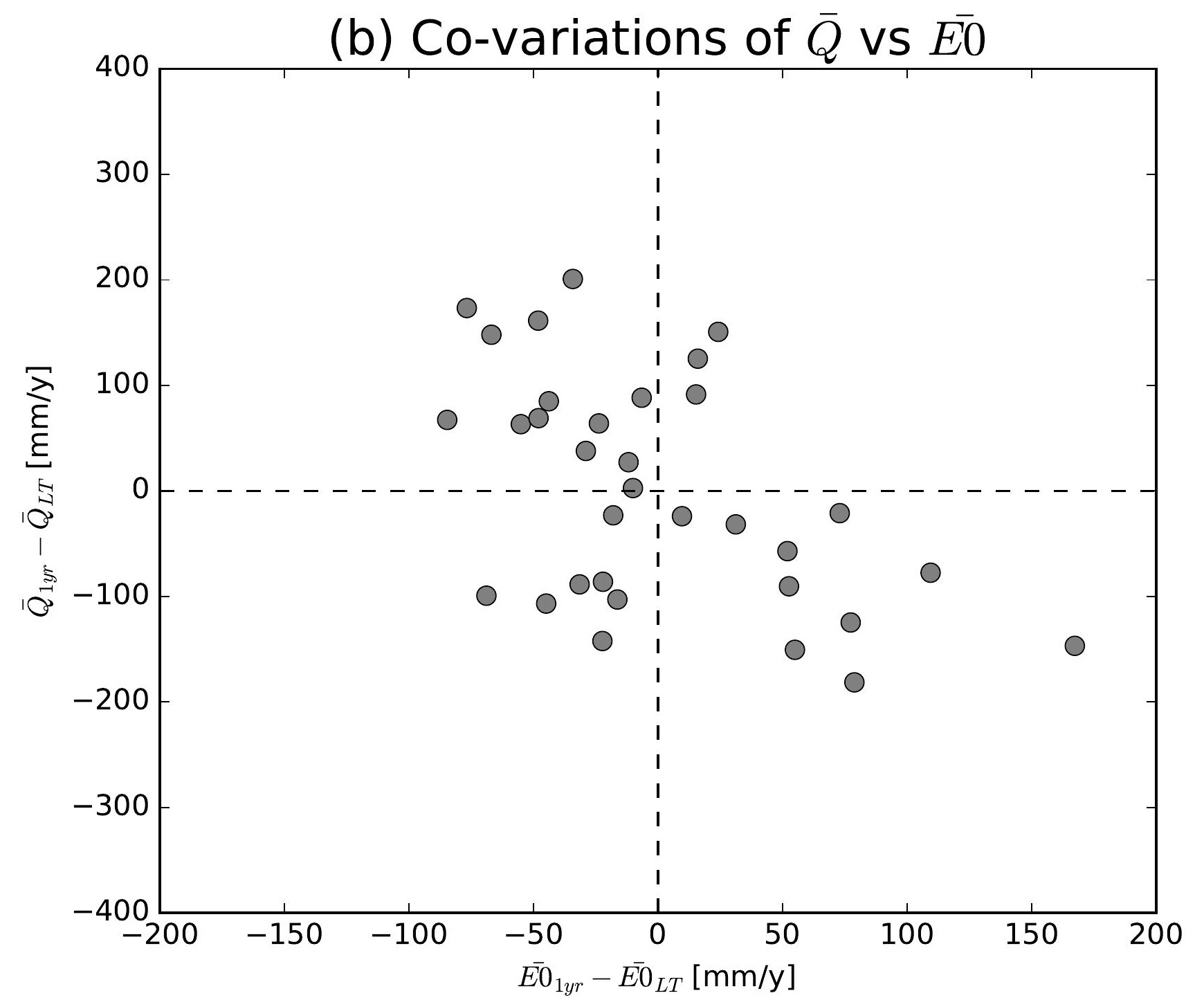

(e) Co-variations of $\bar{Q}$ vs $\overline{E 0}$

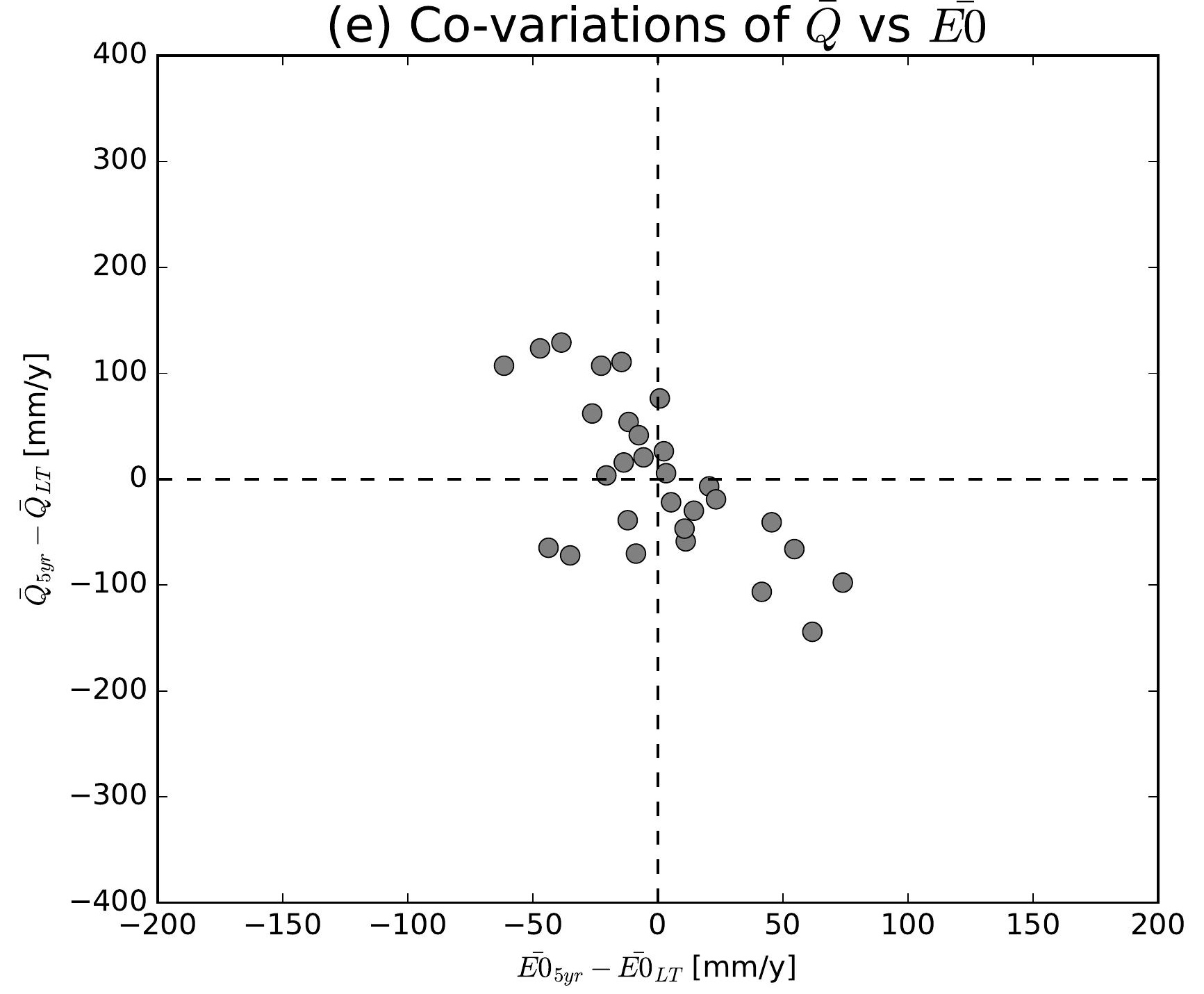

(h) Co-variations of $\bar{Q}$ vs $\overline{E 0}$

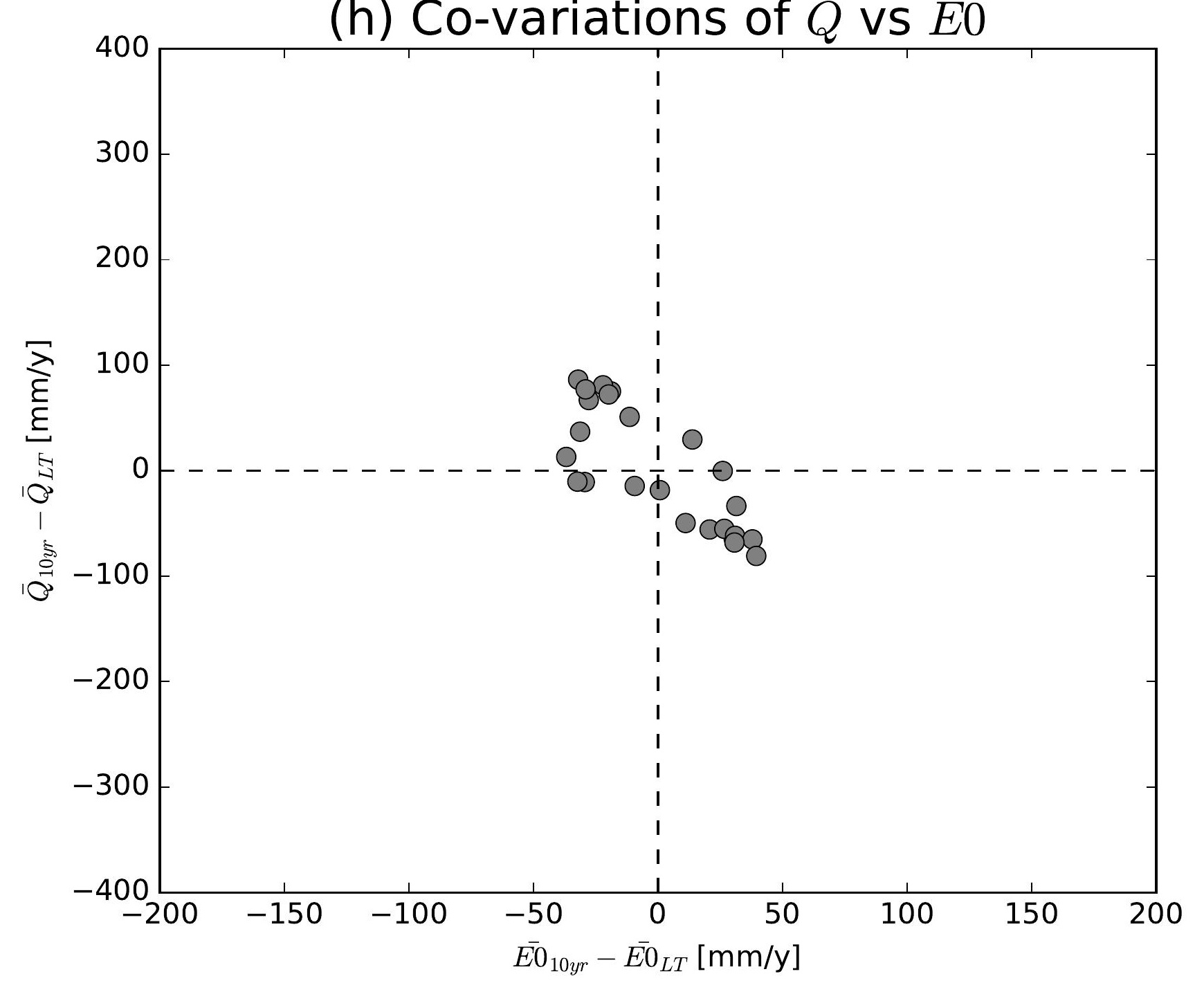

(k) Co-variations of $\bar{Q}$ vs $\overline{E 0}$

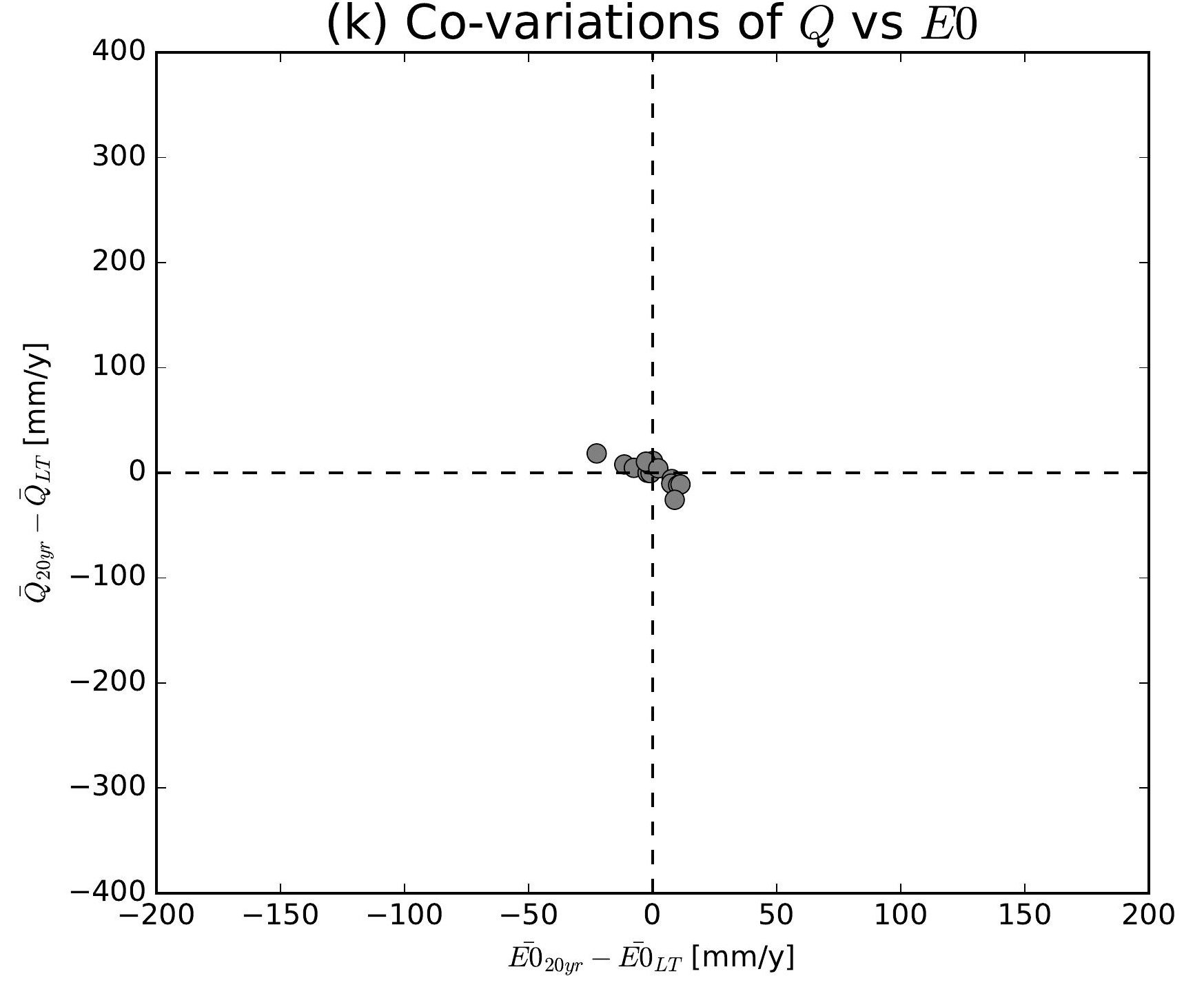

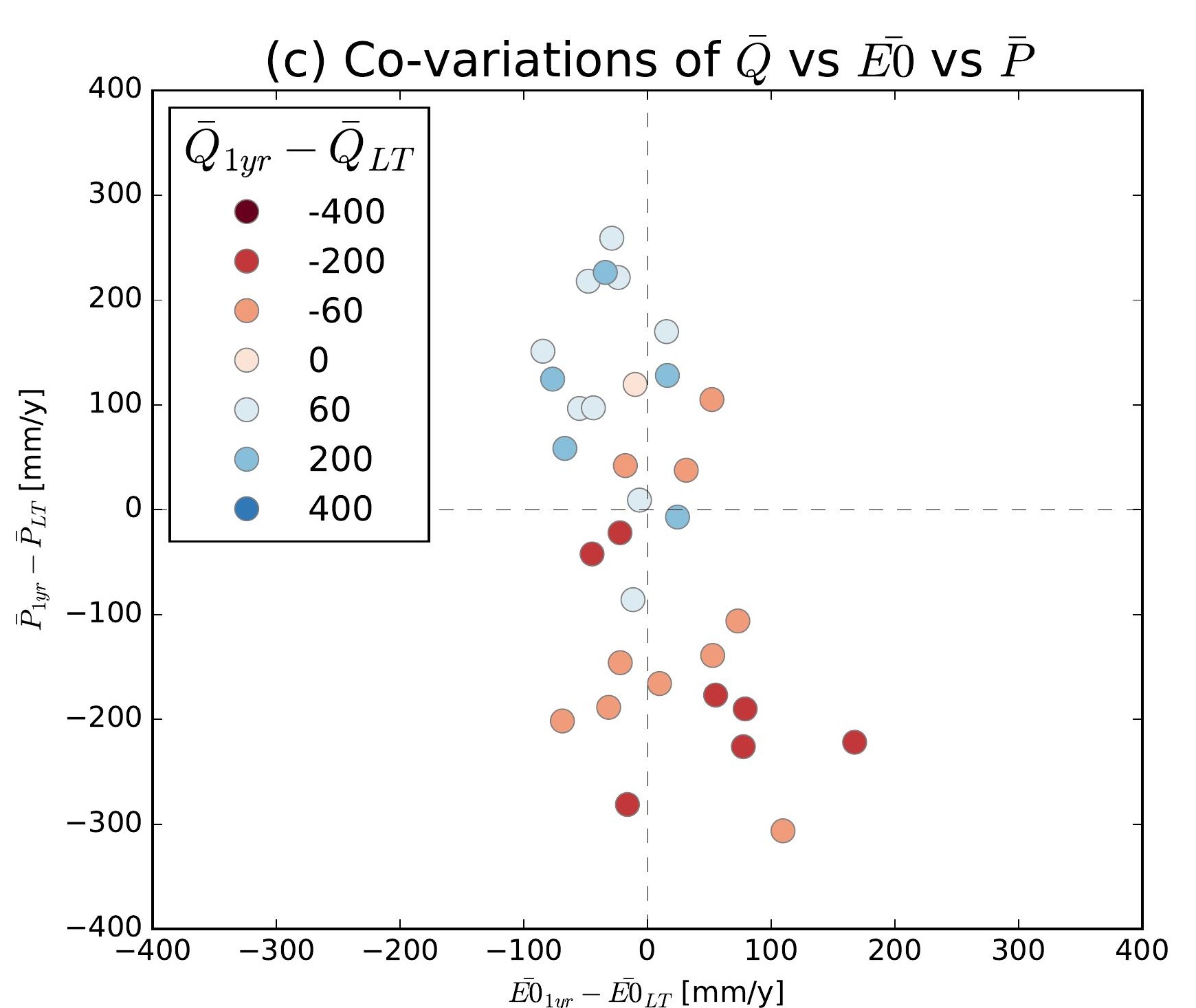
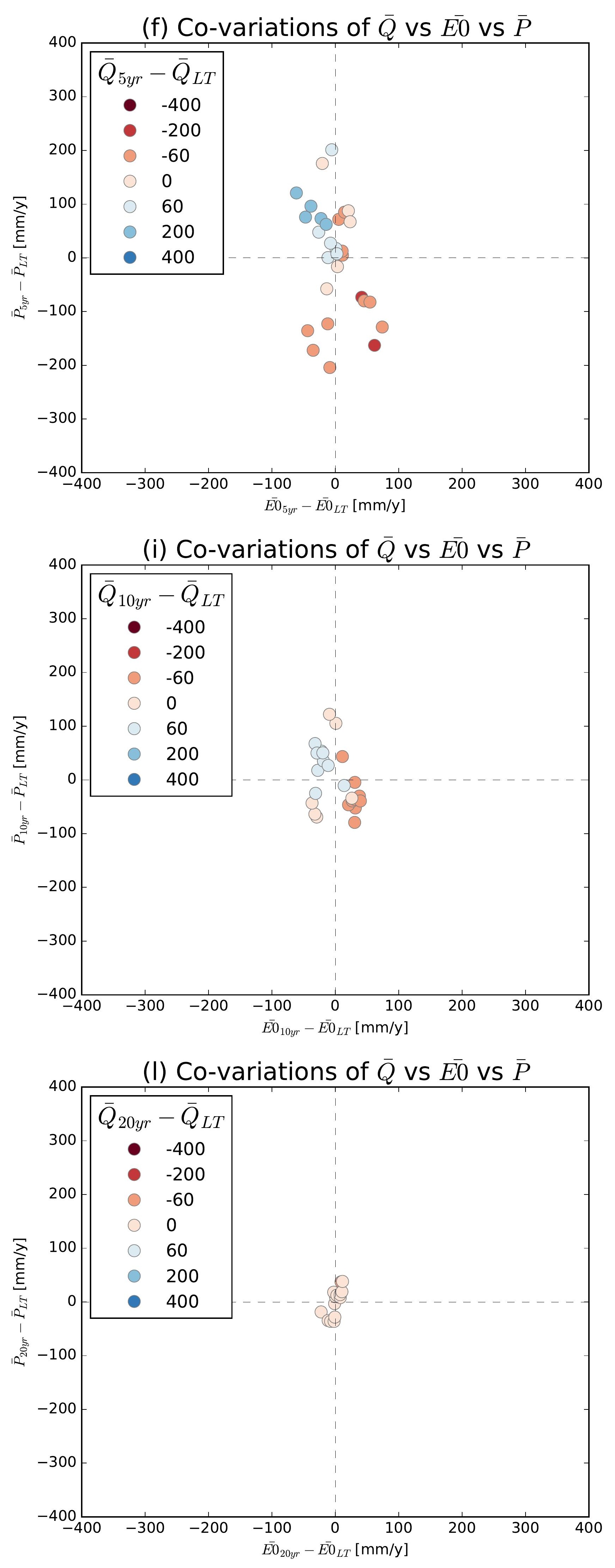

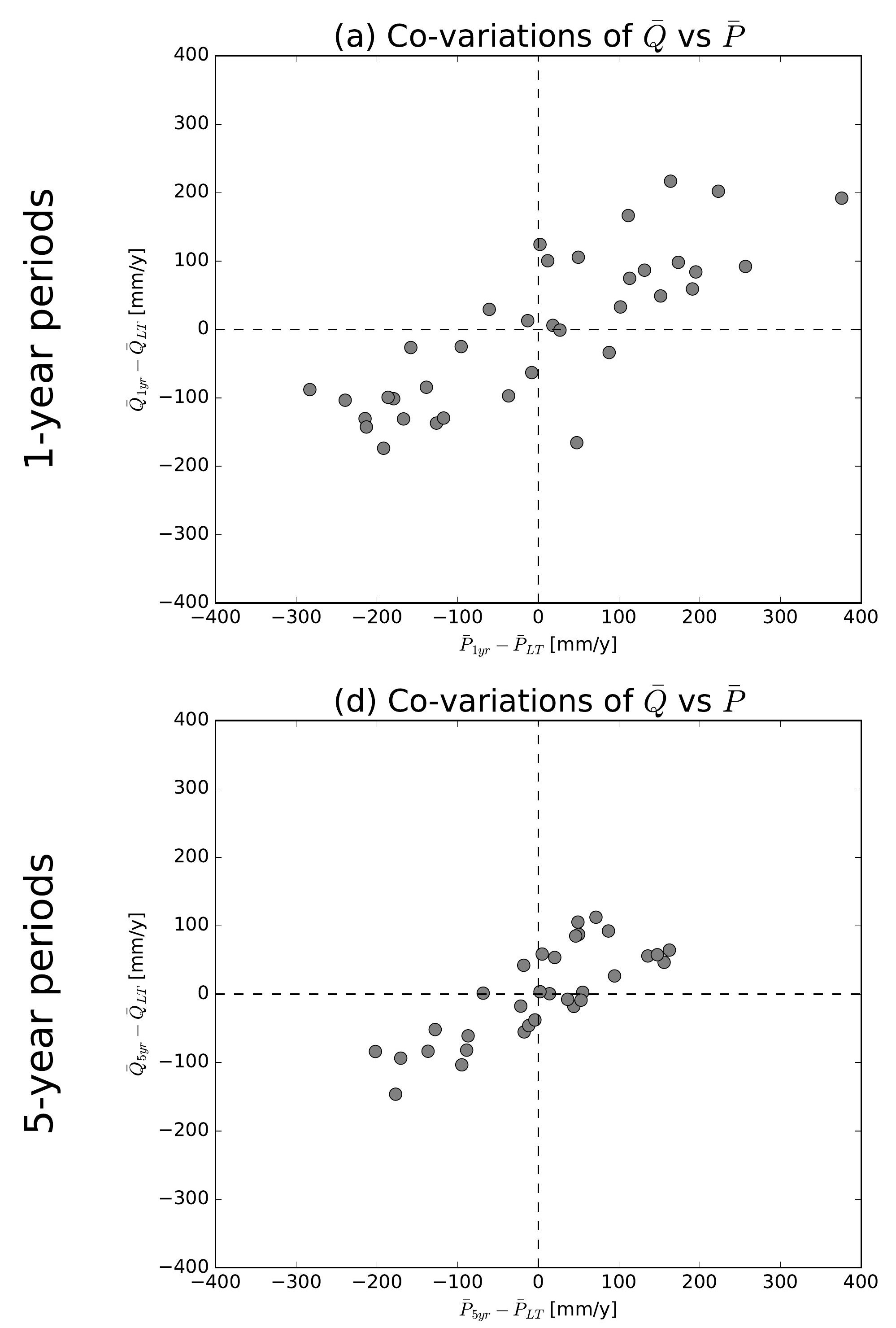

(g) Co-variations of $\bar{Q}$ vs $\bar{P}$

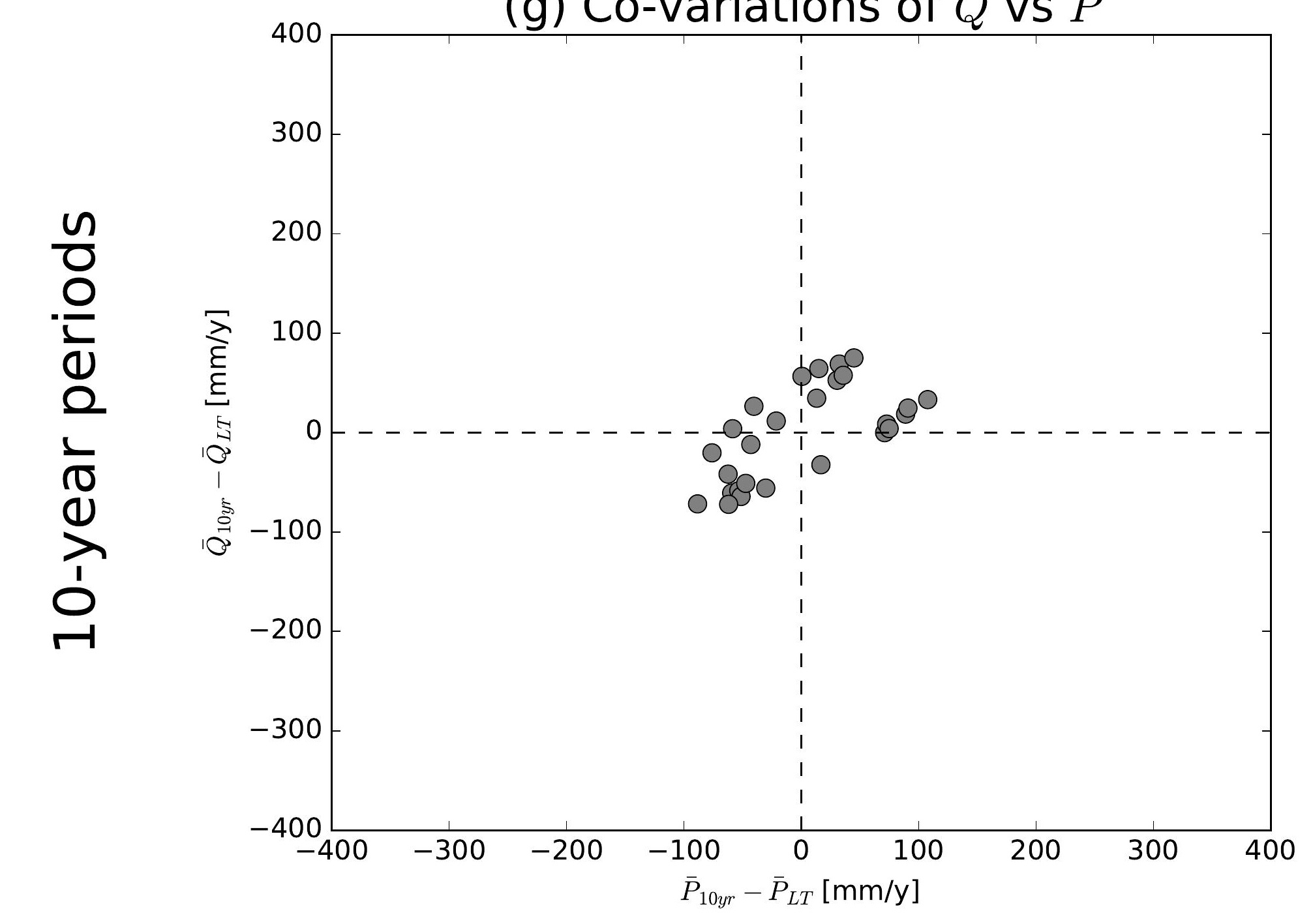

(j) Co-variations of $\bar{Q}$ vs $\bar{P}$

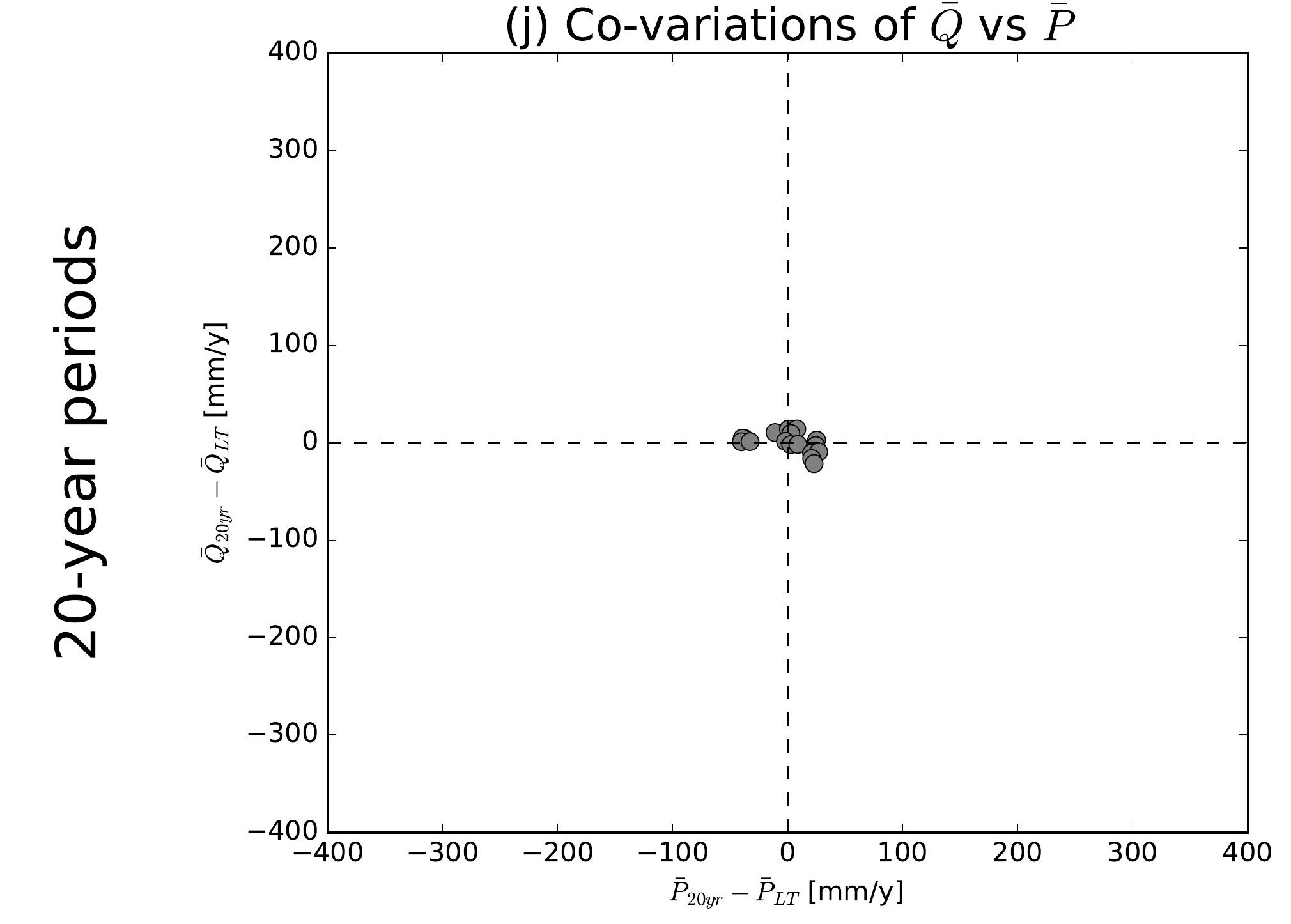

(b) Co-variations of $\bar{Q}$ vs $\overline{E 0}$

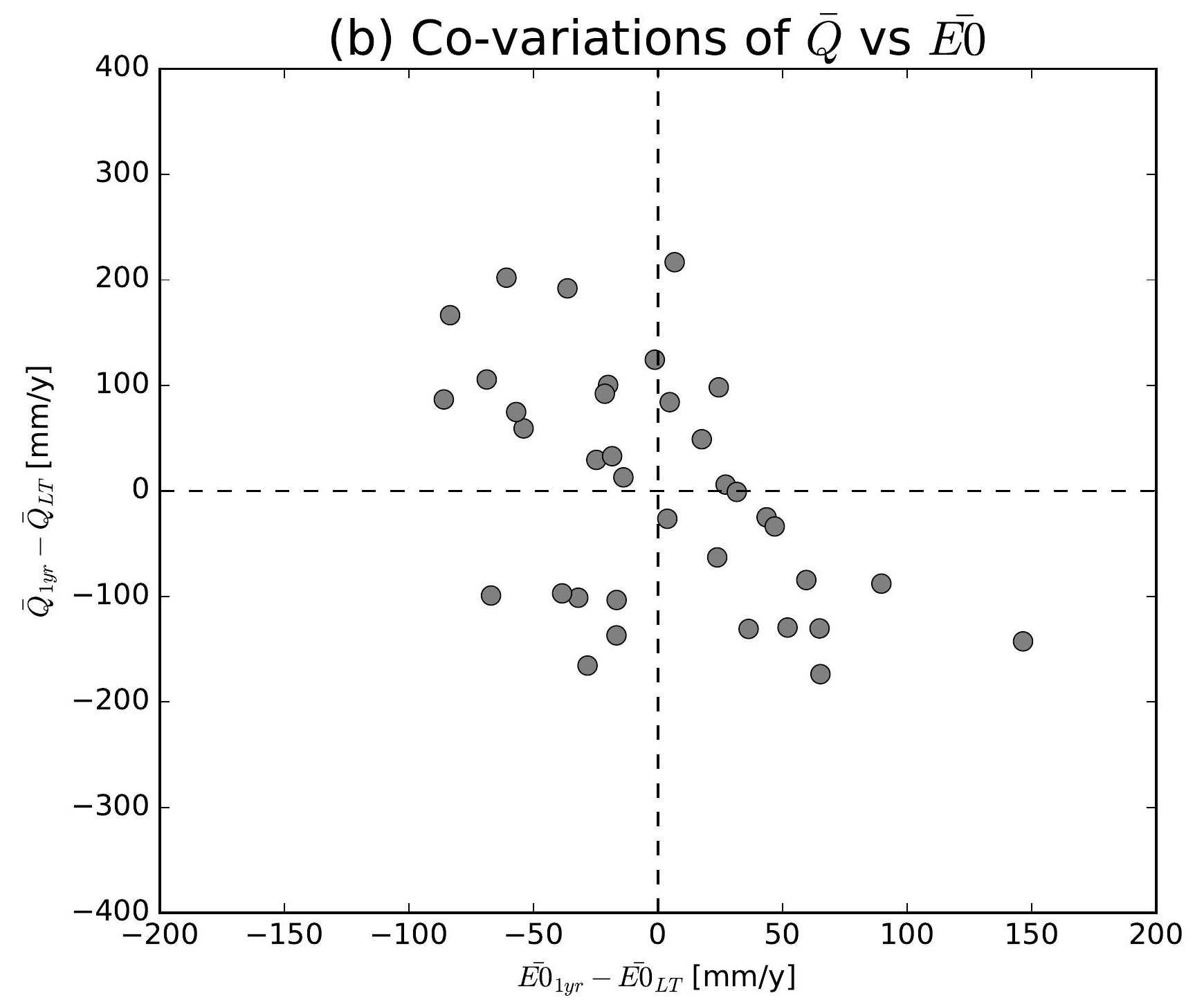

(e) Co-variations of $\bar{Q}$ vs $\overline{E 0}$

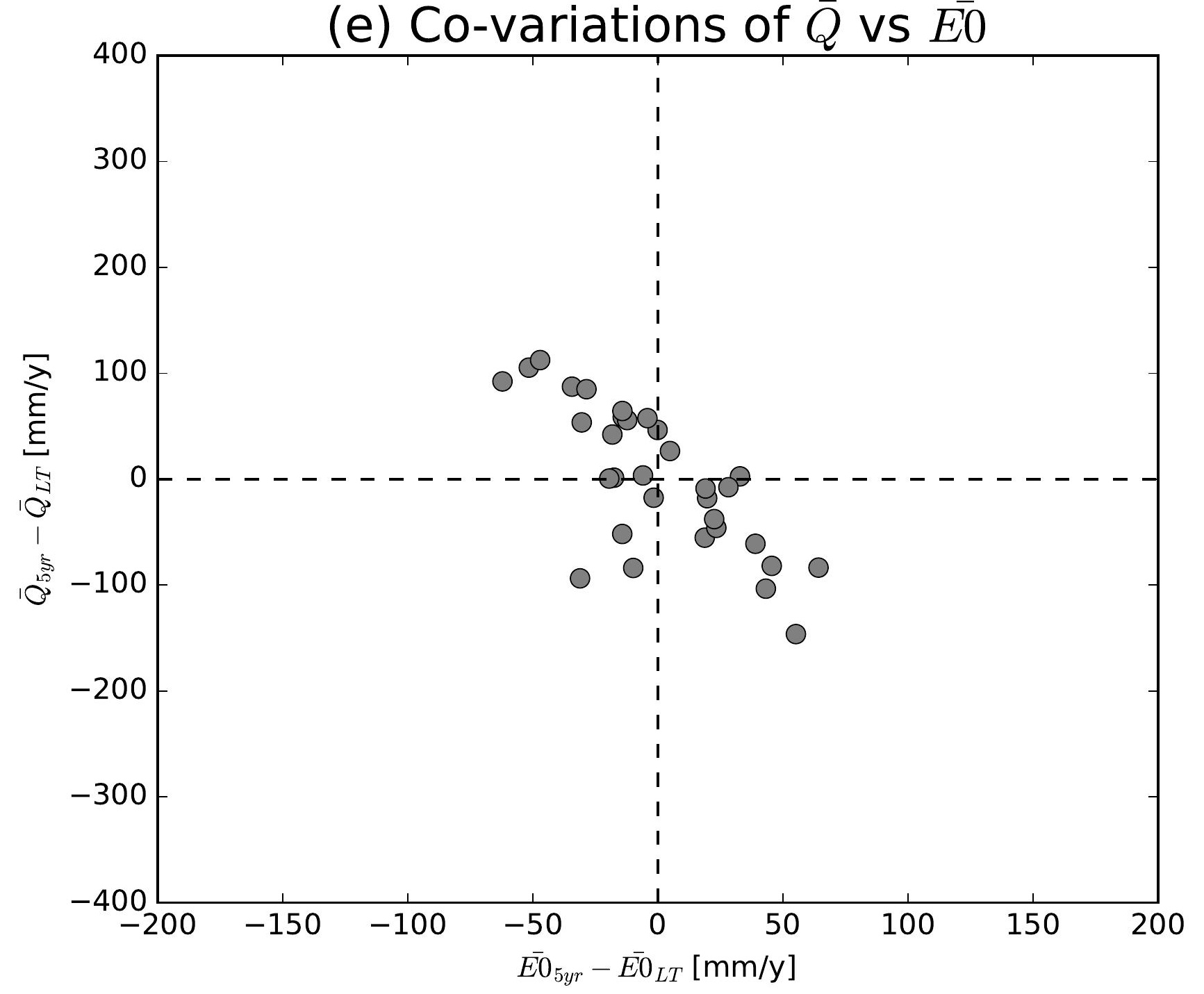

(h) Co-variations of $\bar{Q}$ vs $\overline{E 0}$

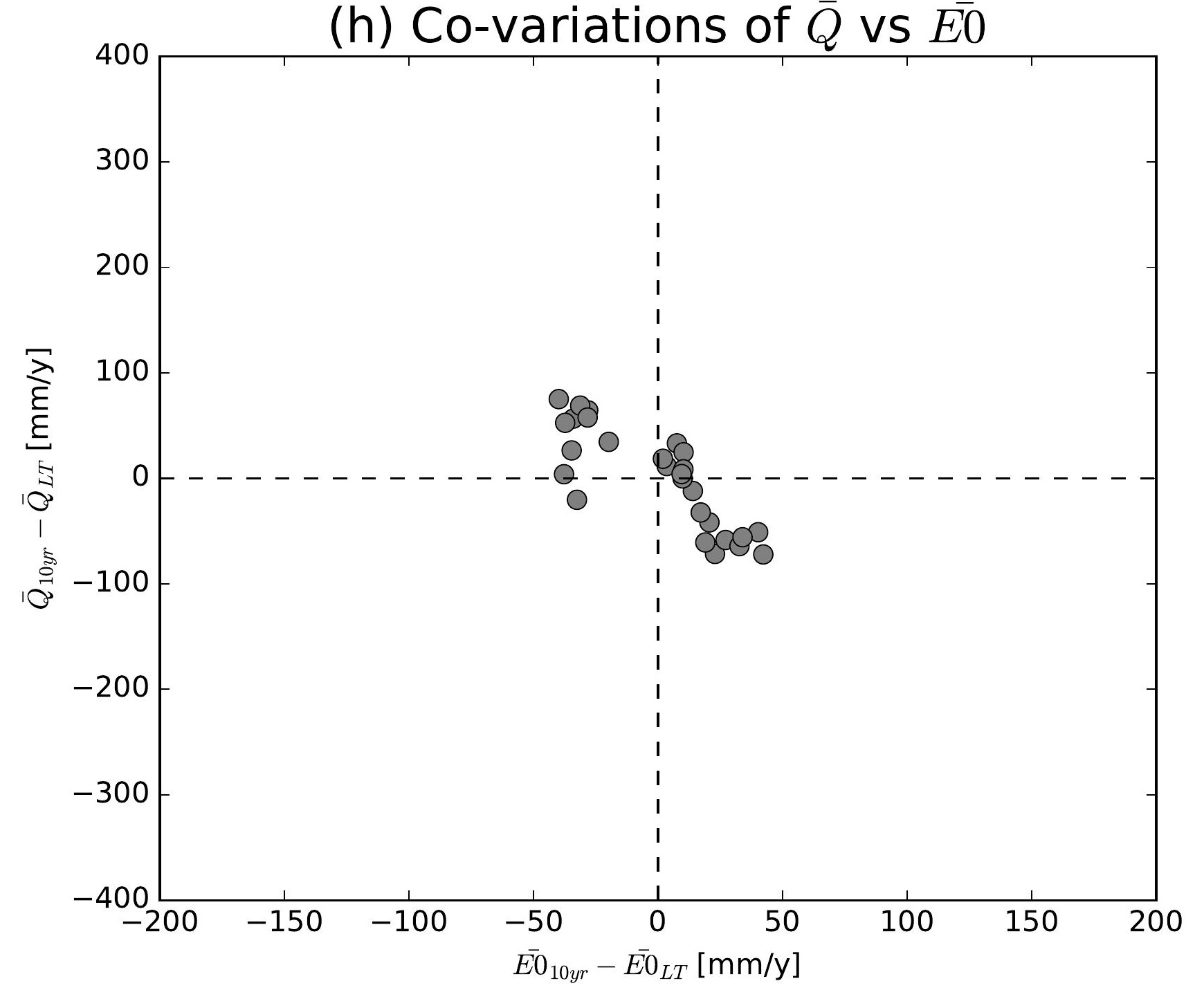

(k) Co-variations of $\bar{Q}$ vs $\overline{E 0}$

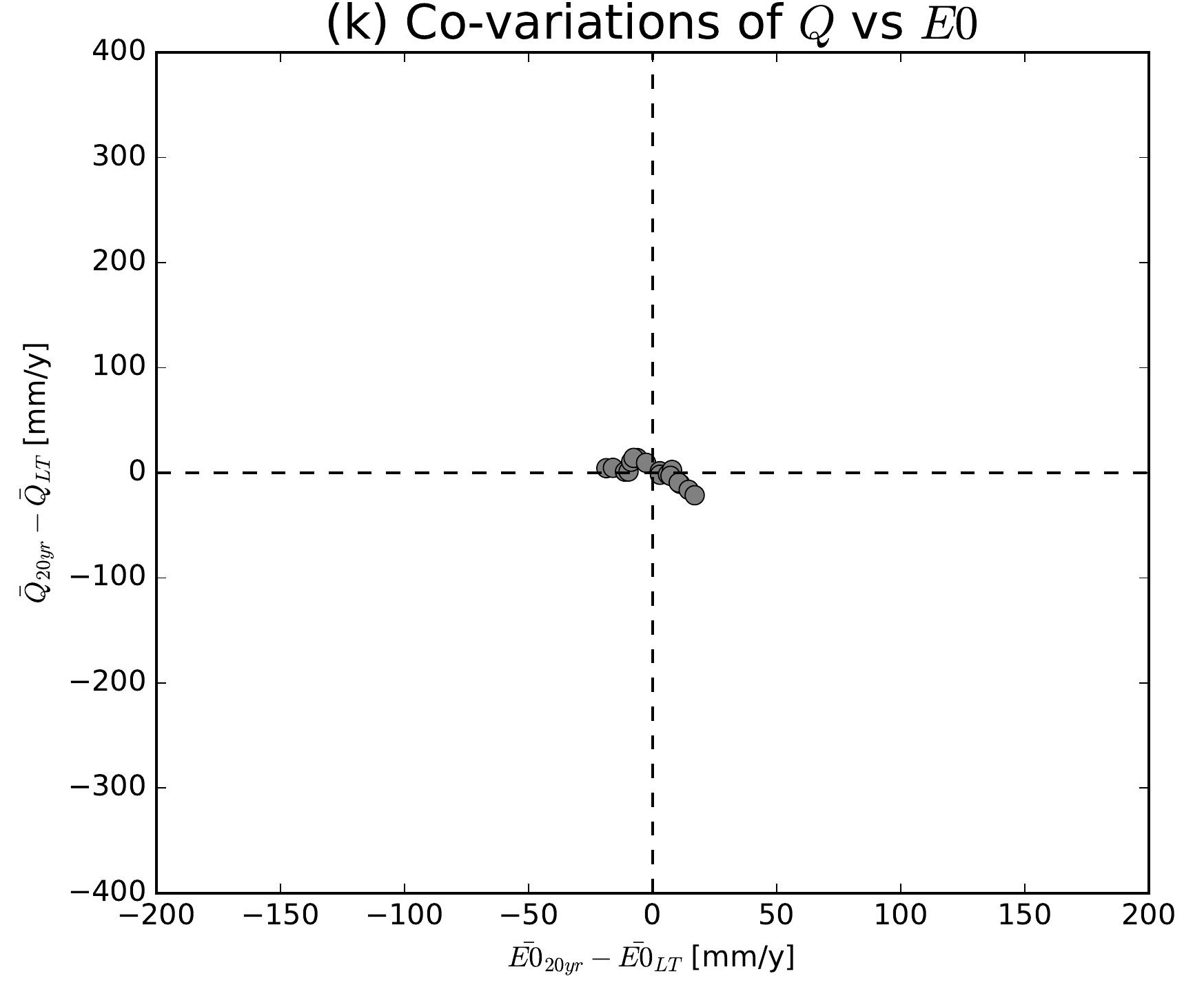

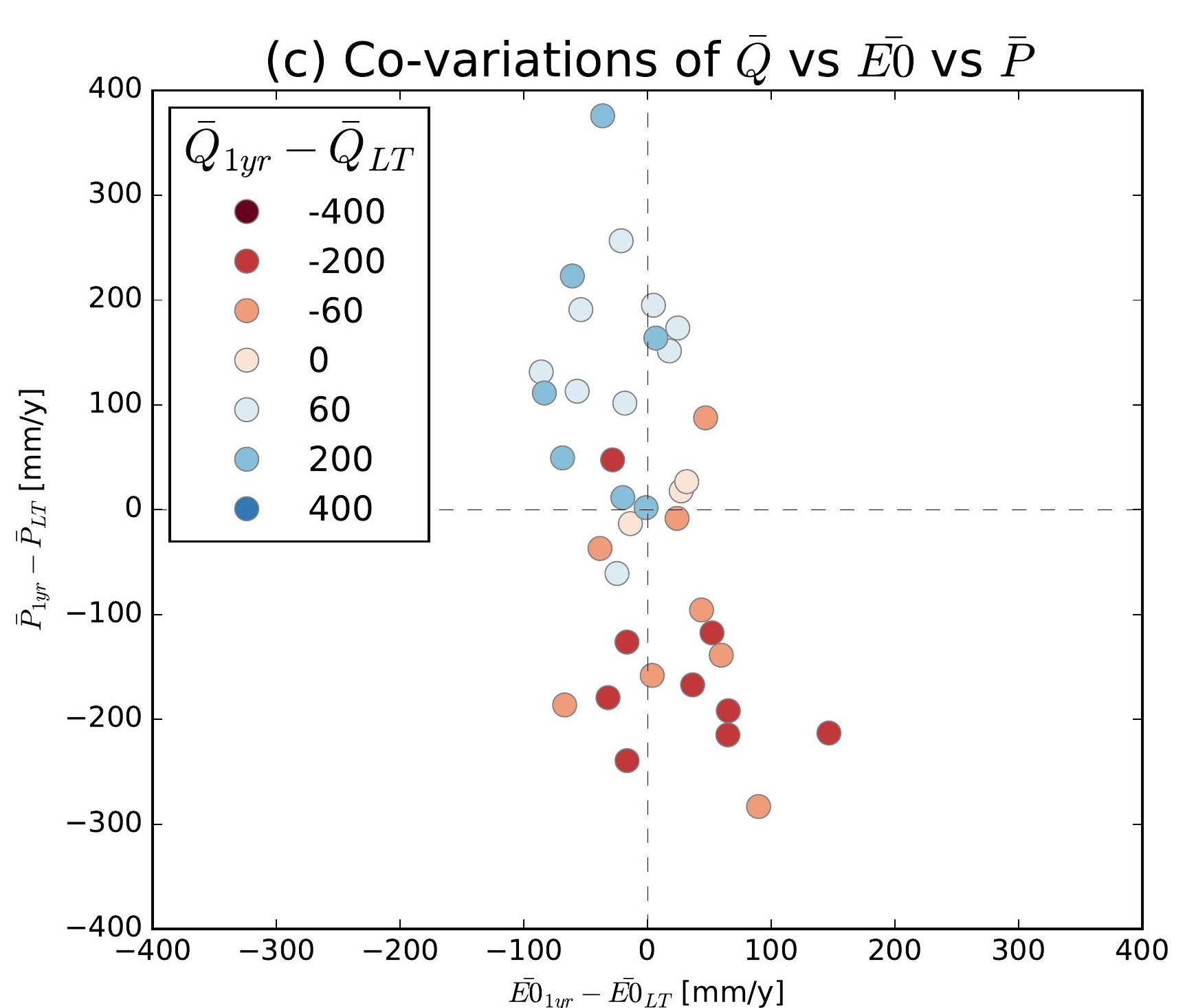
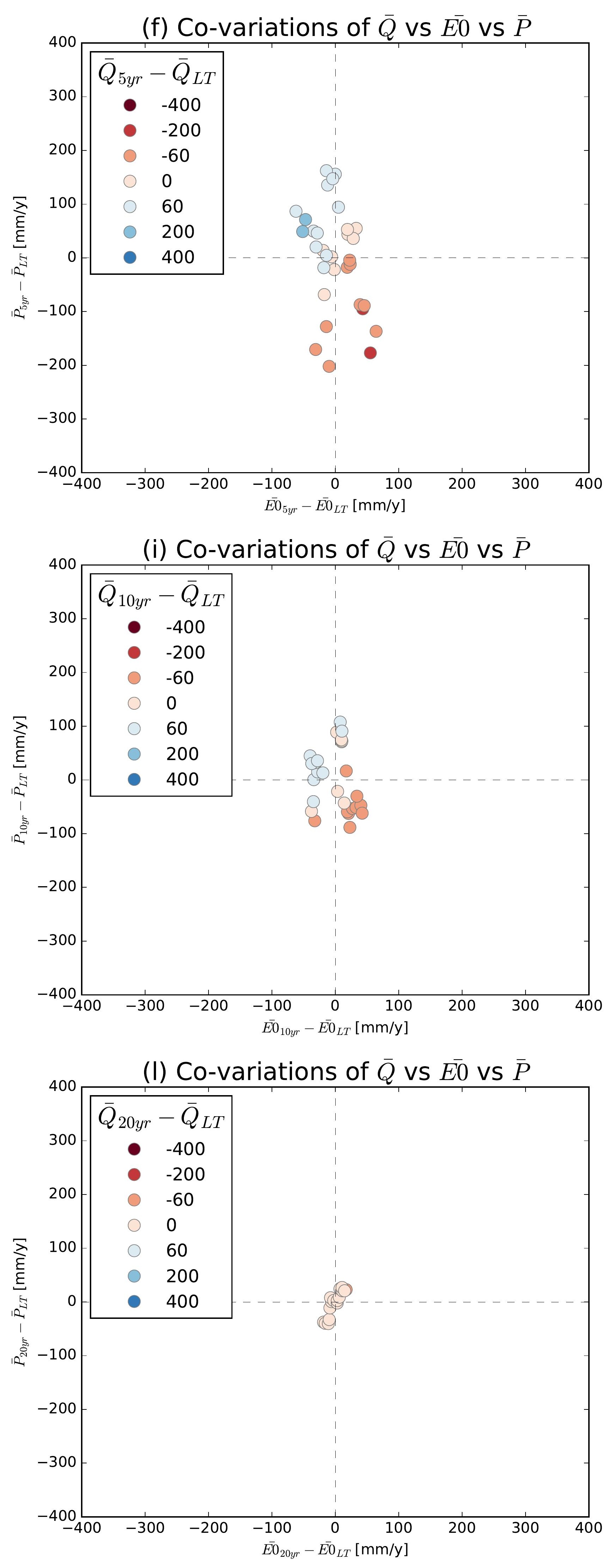

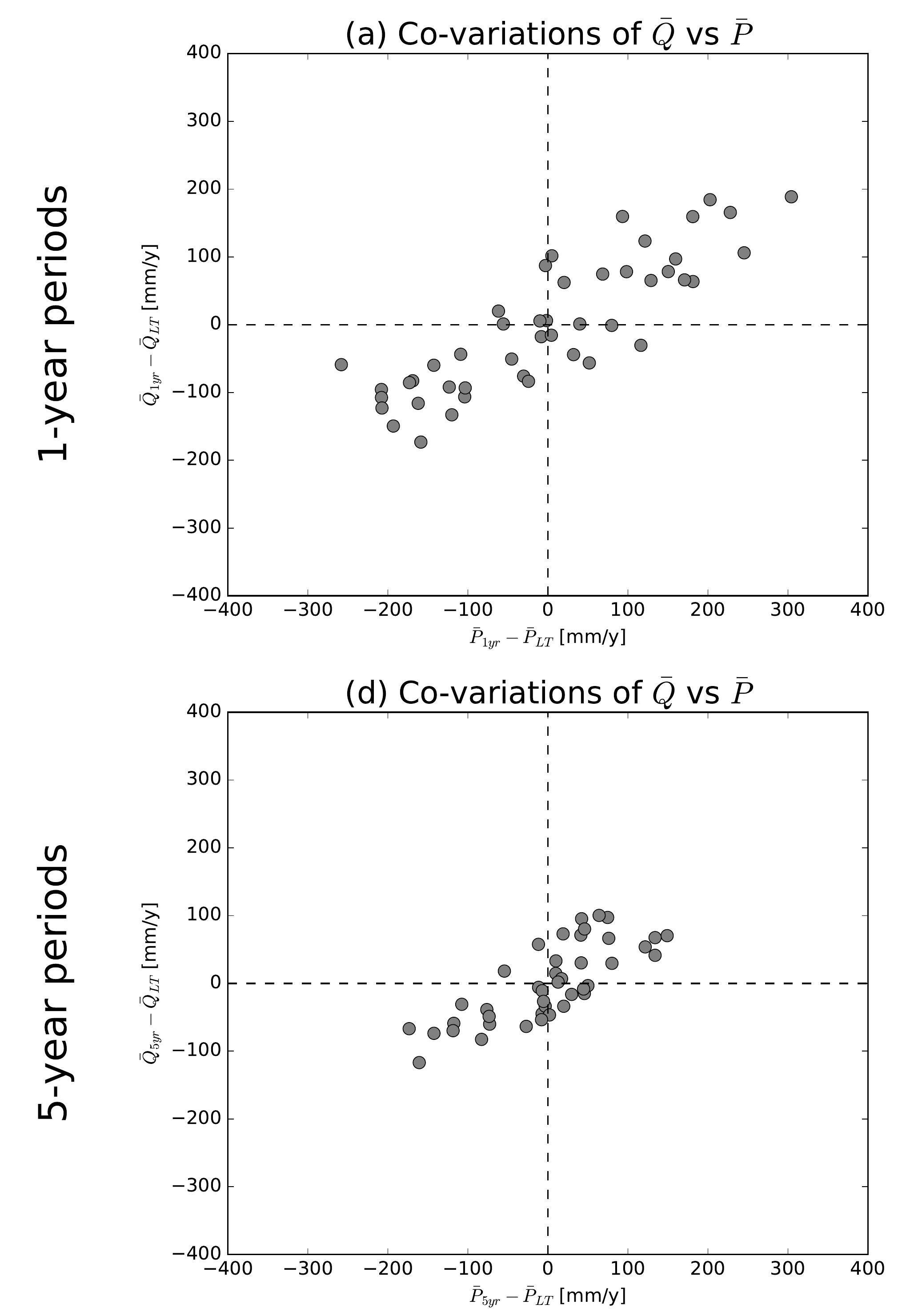

(g) Co-variations of $\bar{Q}$ vs $\bar{P}$
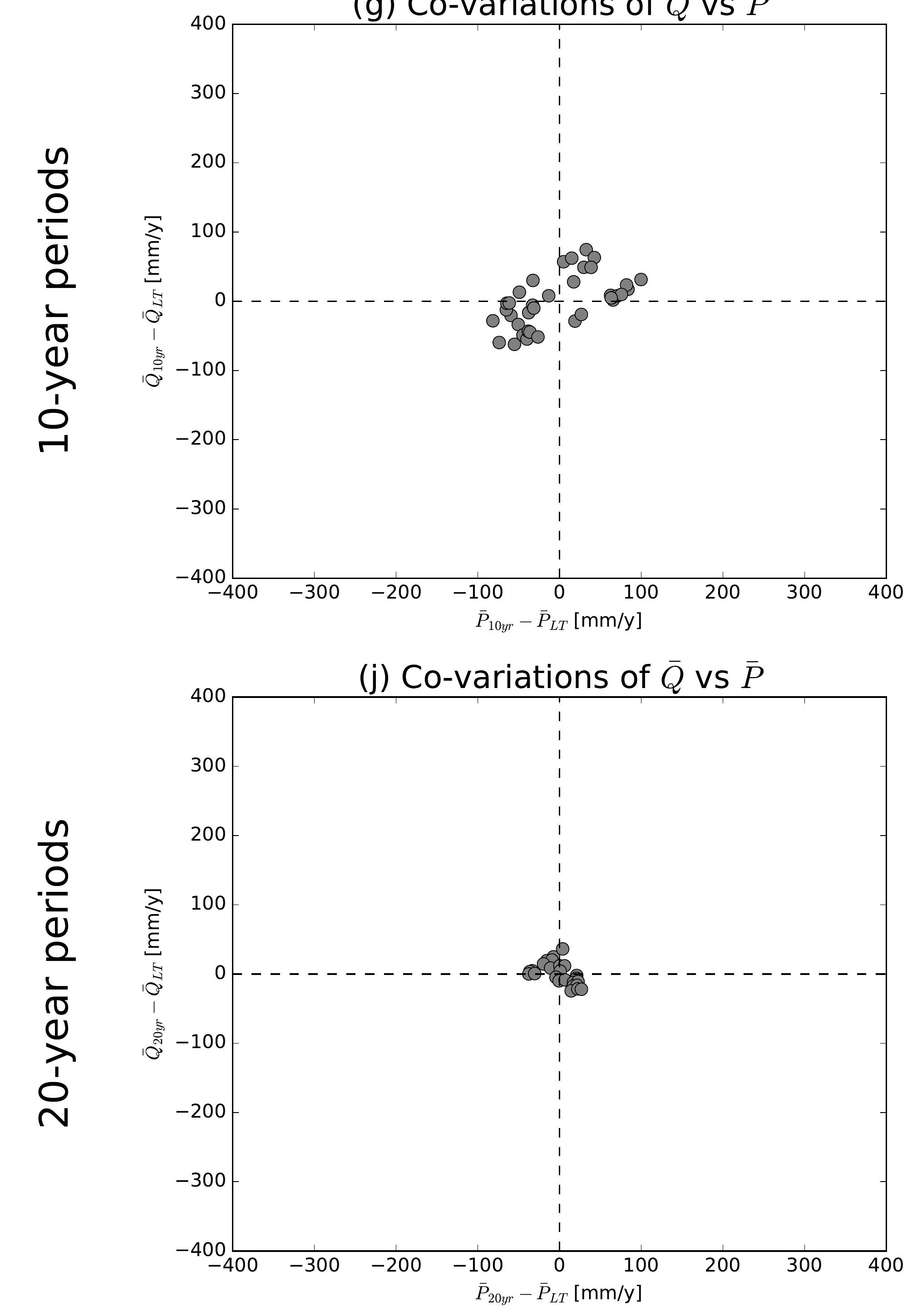

(b) Co-variations of $\bar{Q}$ vs $\overline{E 0}$

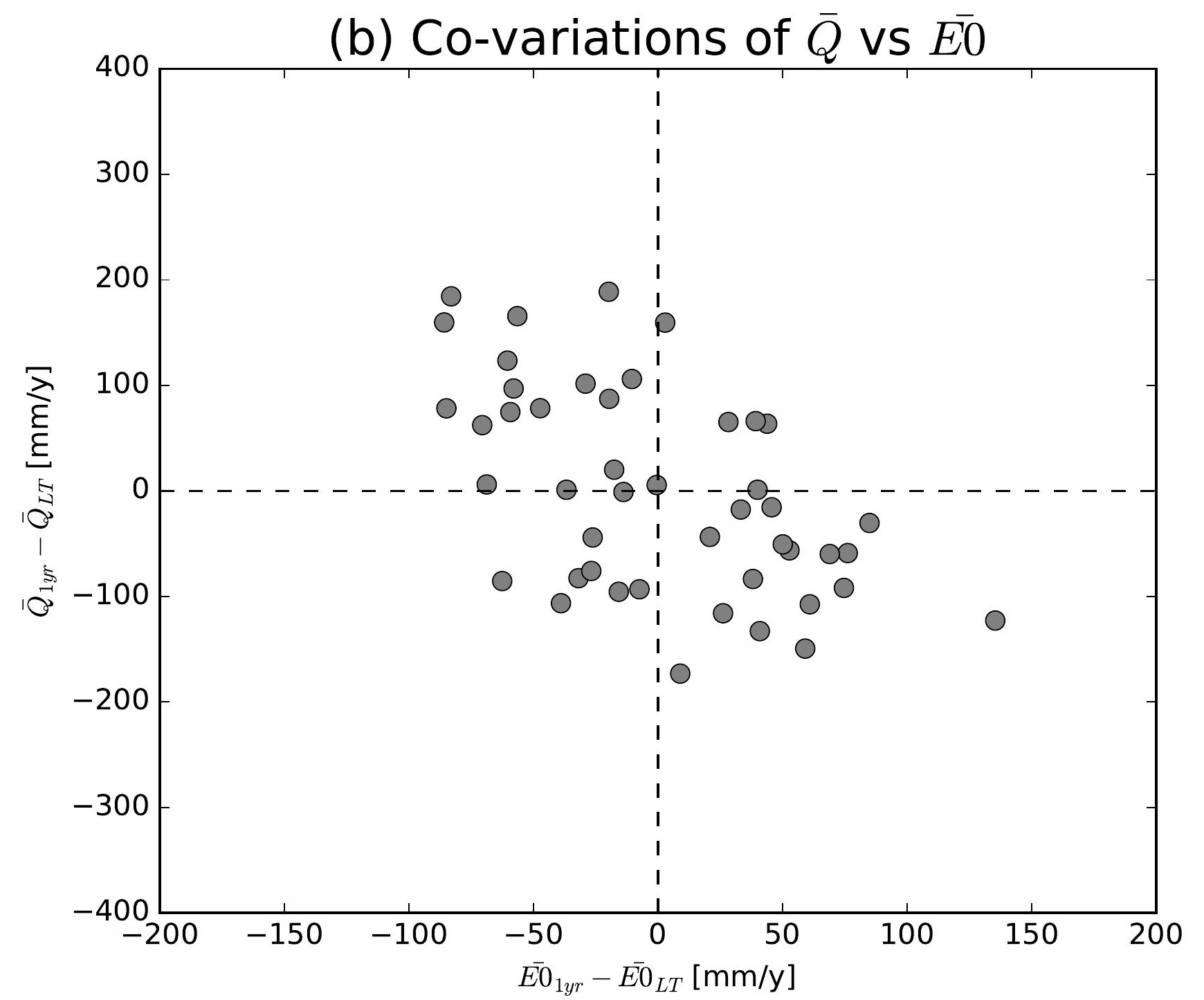

(e) Co-variations of $\bar{Q}$ vs $\overline{E 0}$

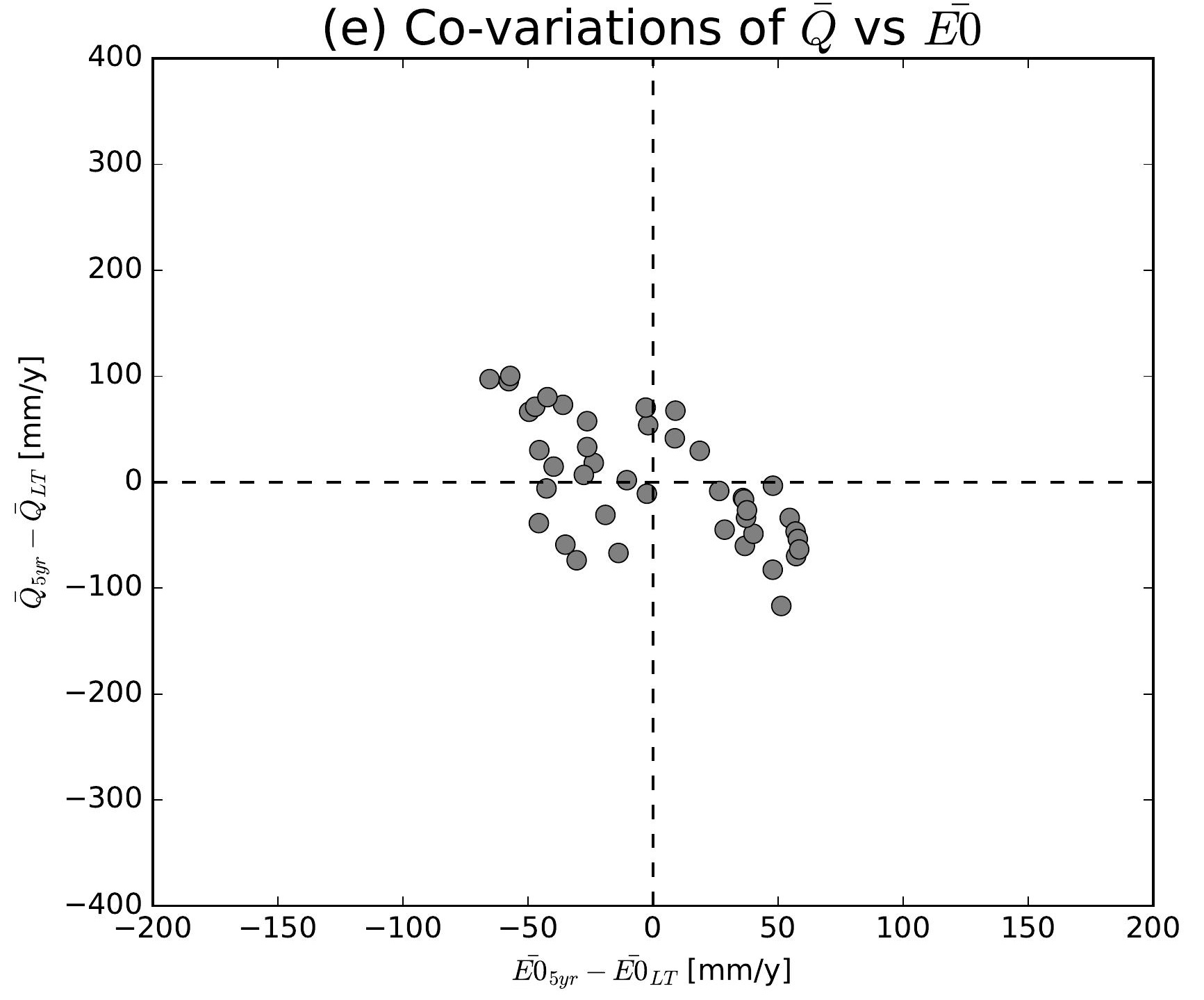

(h) Co-variations of $\bar{Q}$ vs $\overline{E 0}$

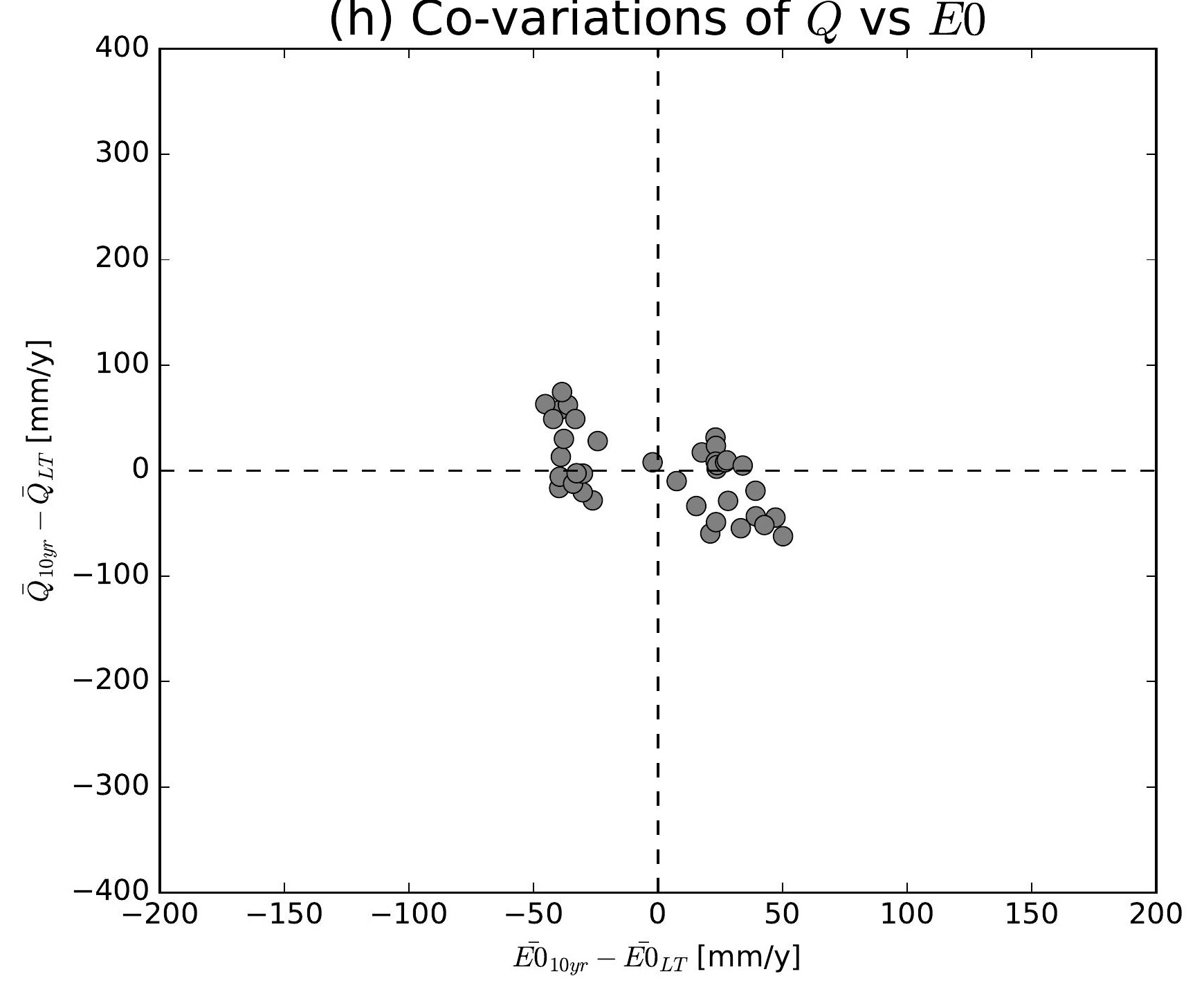

(k) Co-variations of $\bar{Q}$ vs $\overline{E 0}$

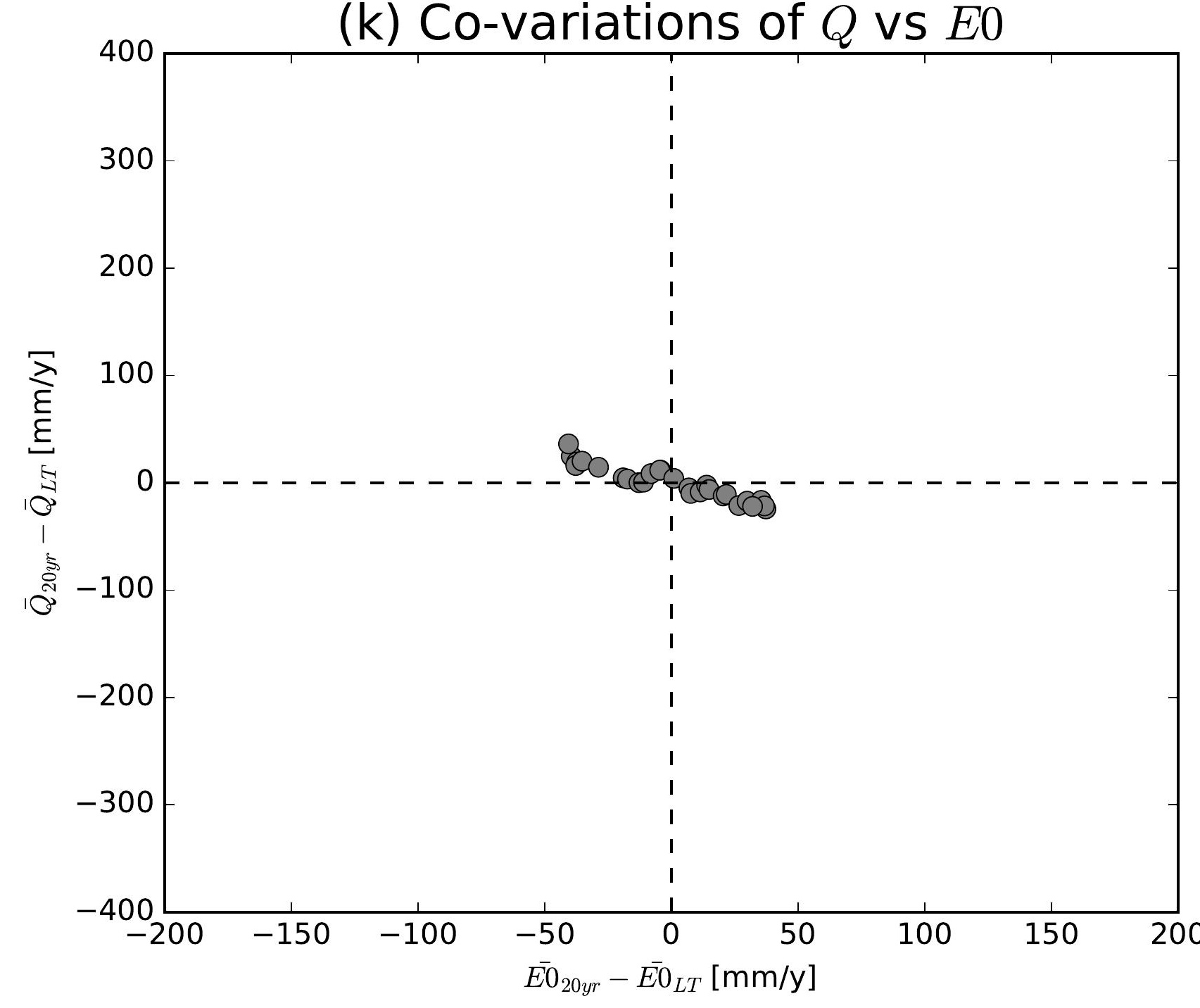

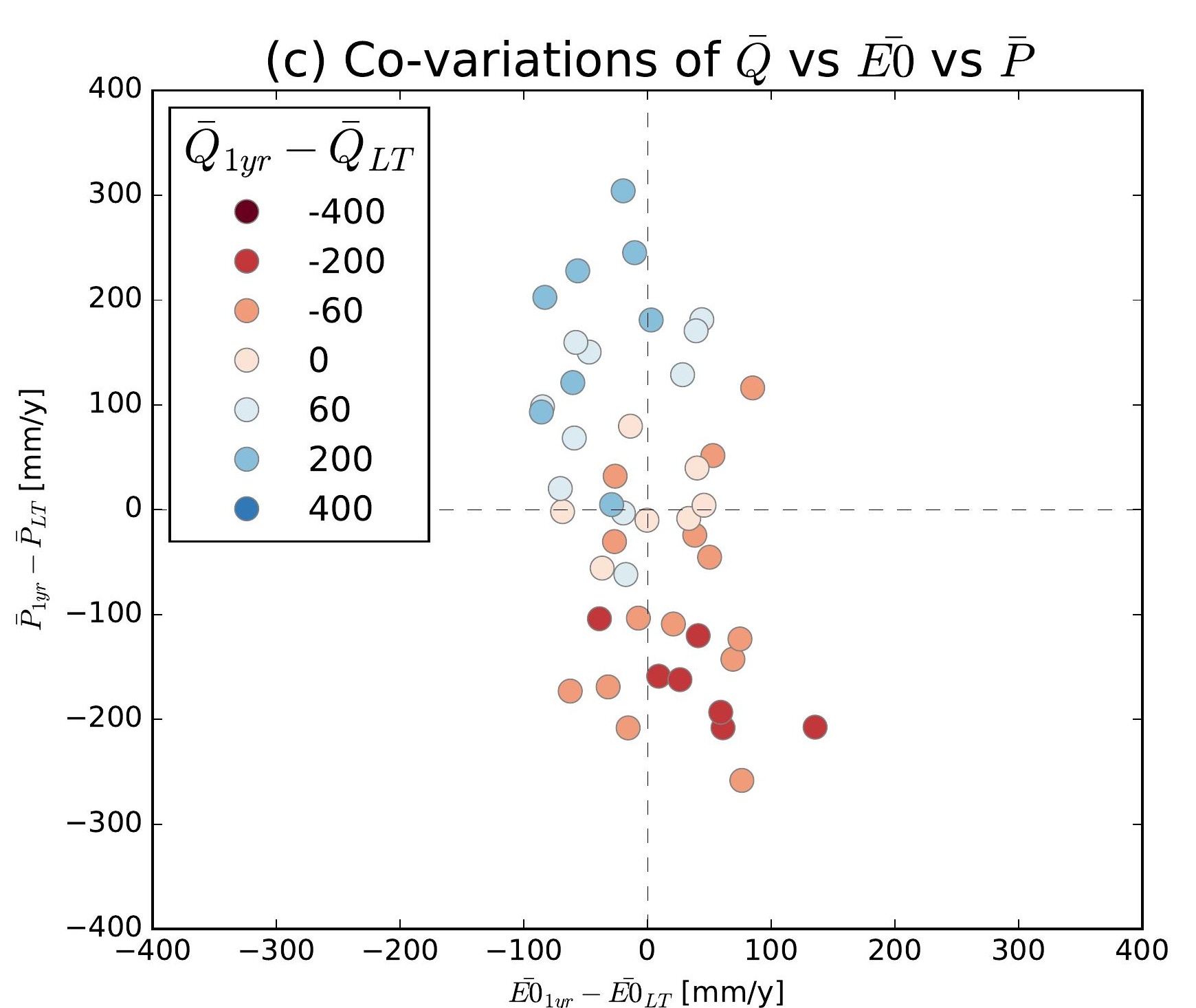
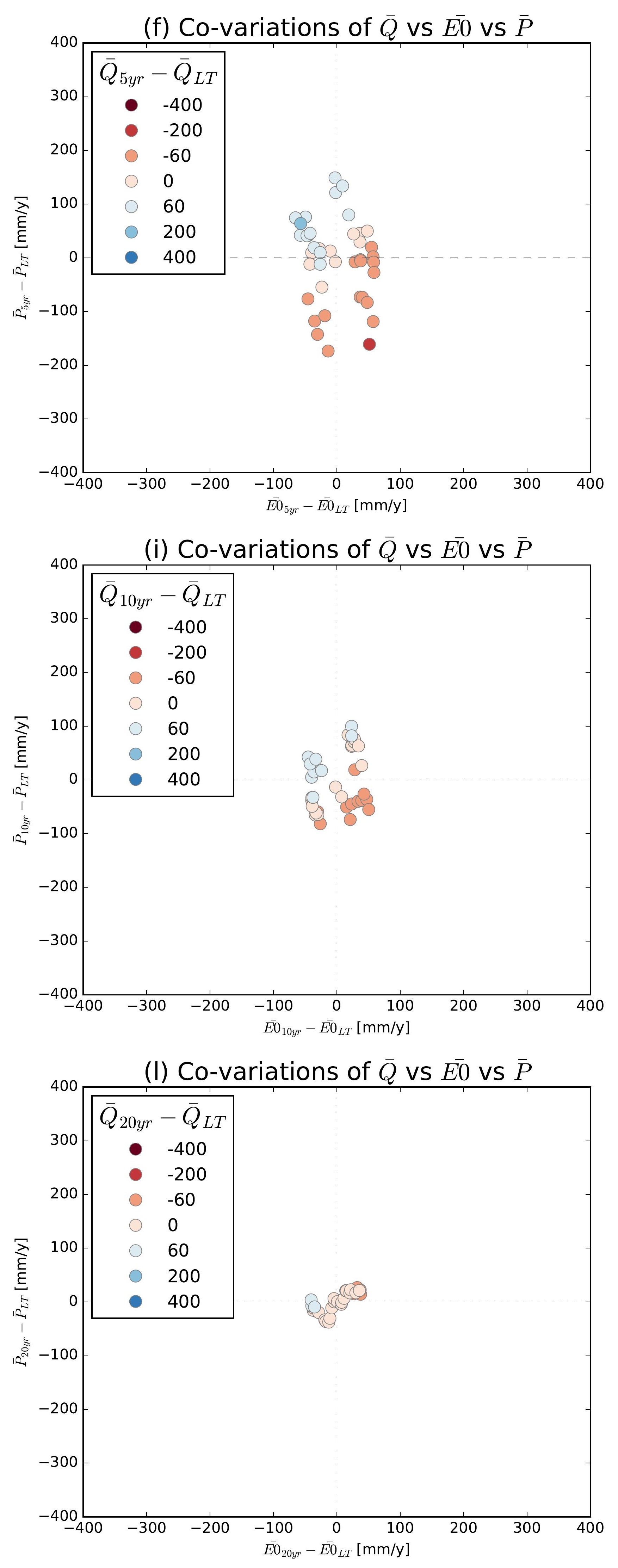

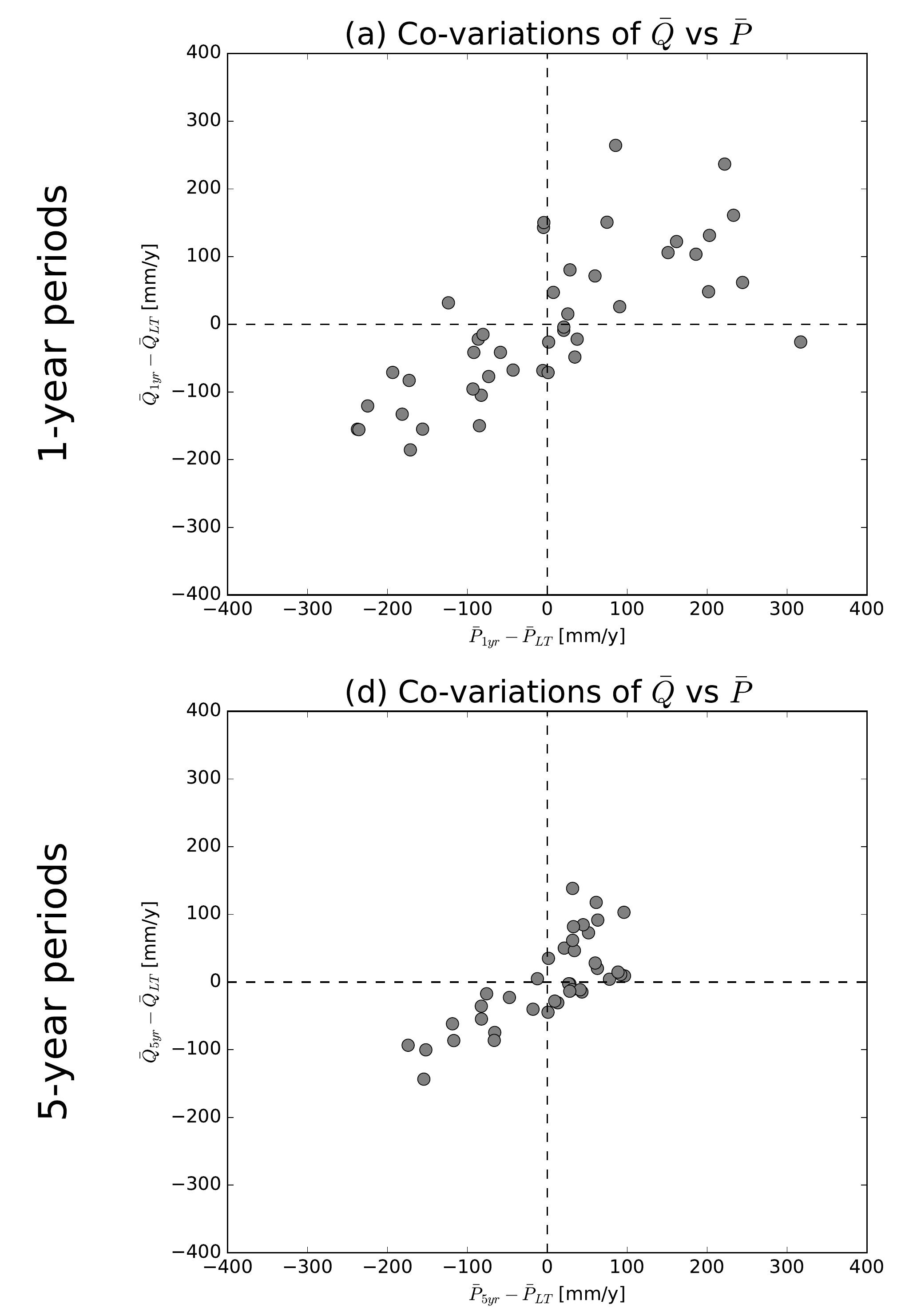

(g) Co-variations of $\bar{Q}$ vs $\bar{P}$
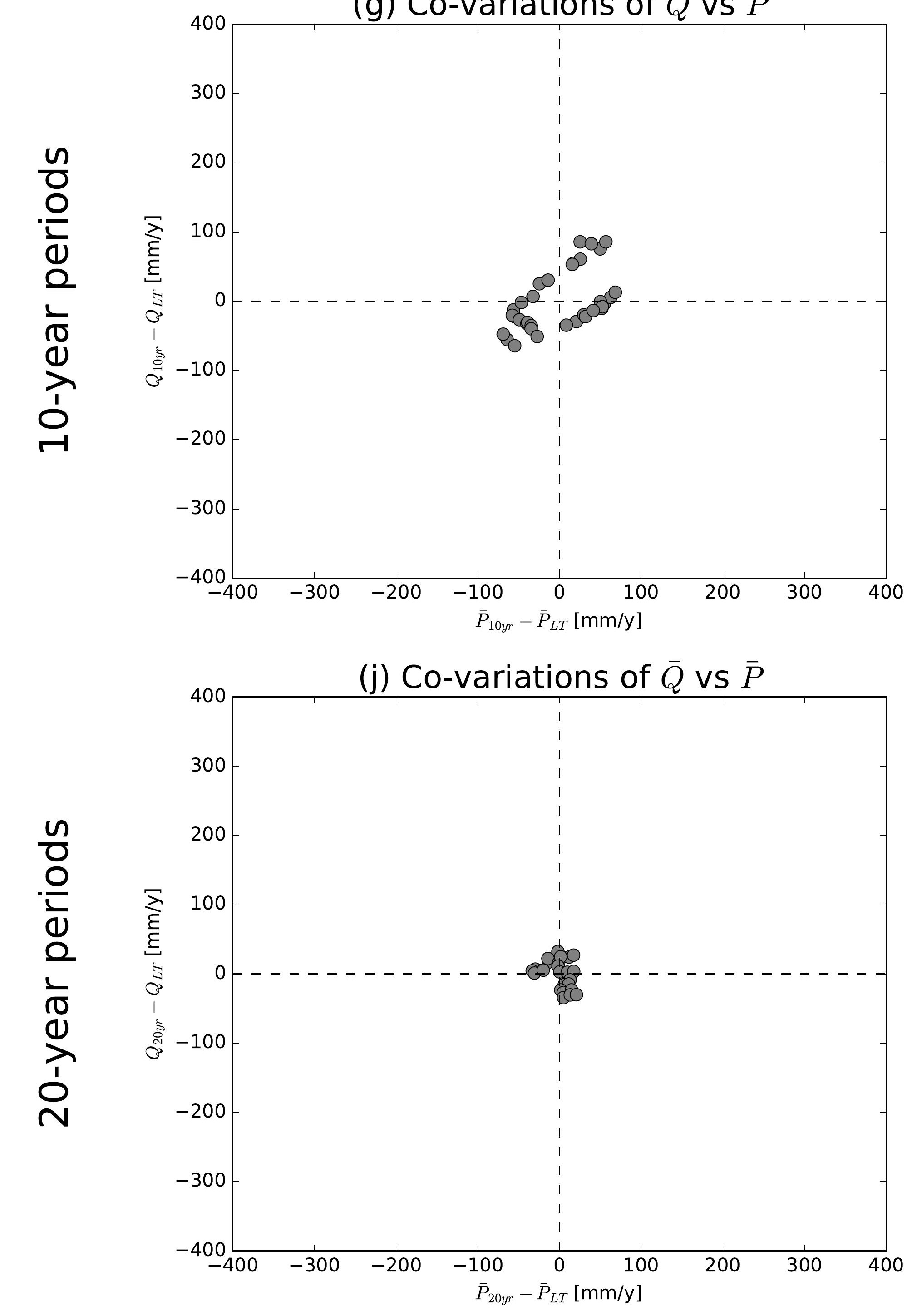

(b) Co-variations of $\bar{Q}$ vs $\overline{E 0}$

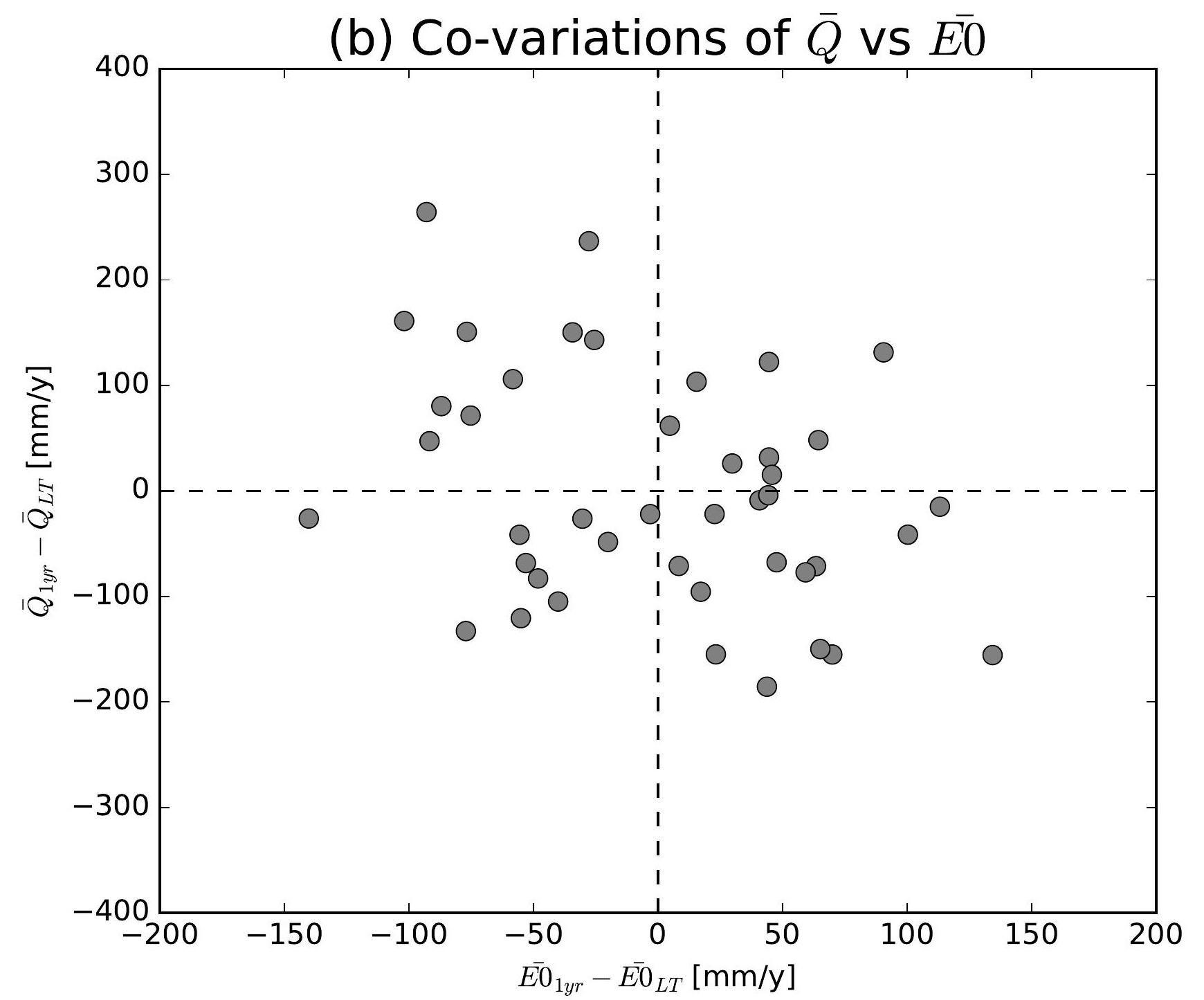

(e) Co-variations of $\bar{Q}$ vs $\overline{E 0}$

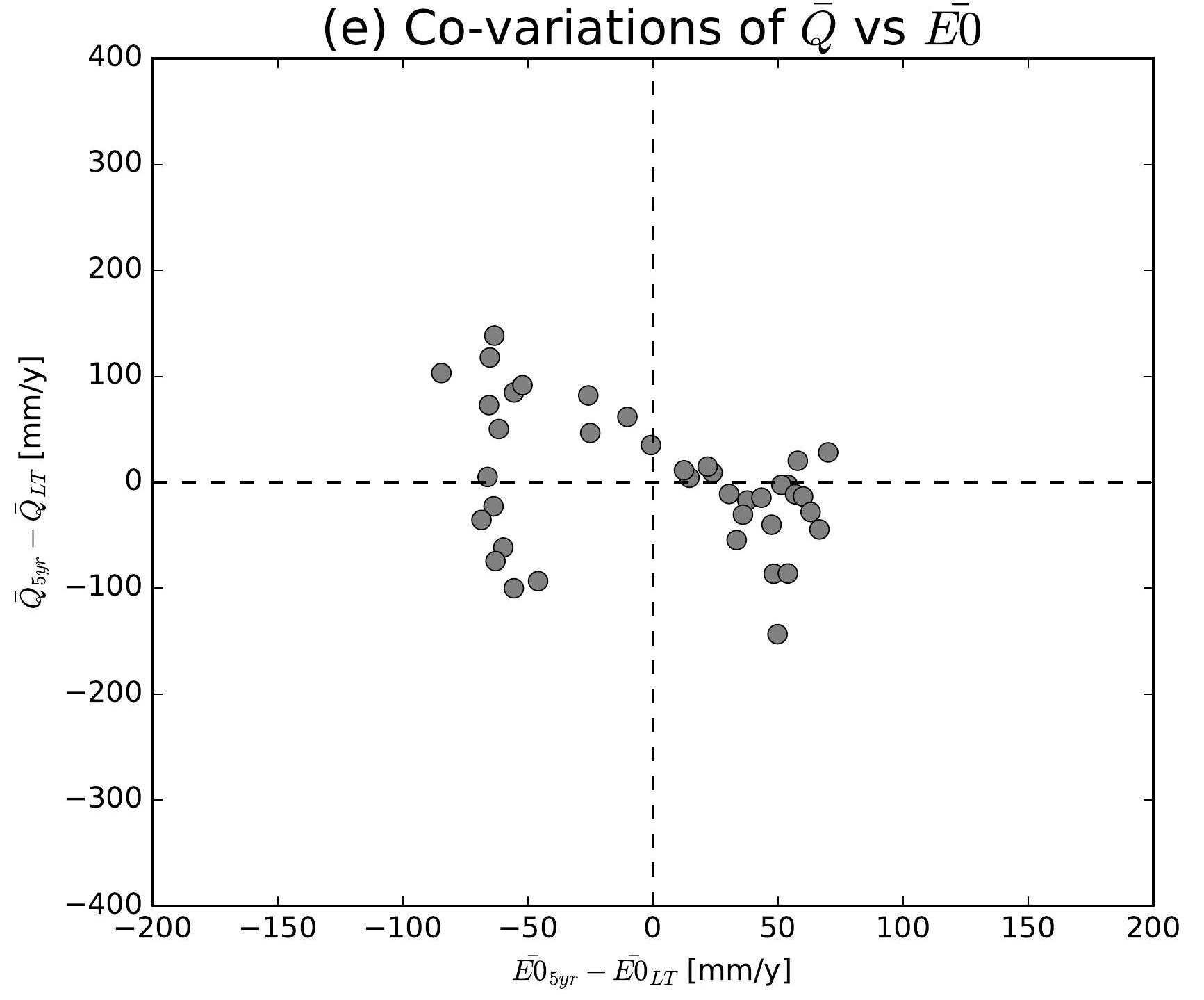

(h) Co-variations of $\bar{Q}$ vs $\overline{E 0}$

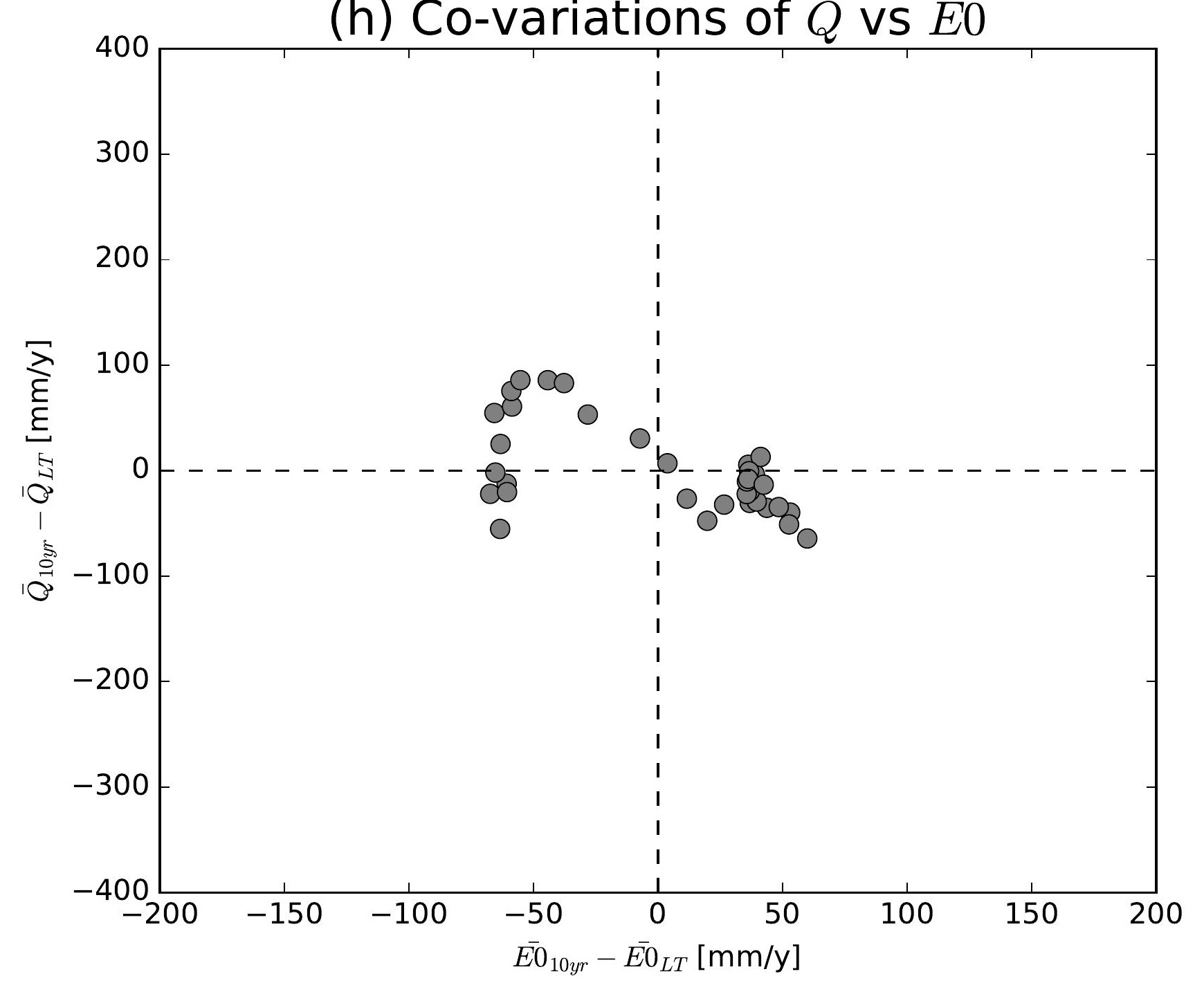

(k) Co-variations of $\bar{Q}$ vs $\overline{E 0}$

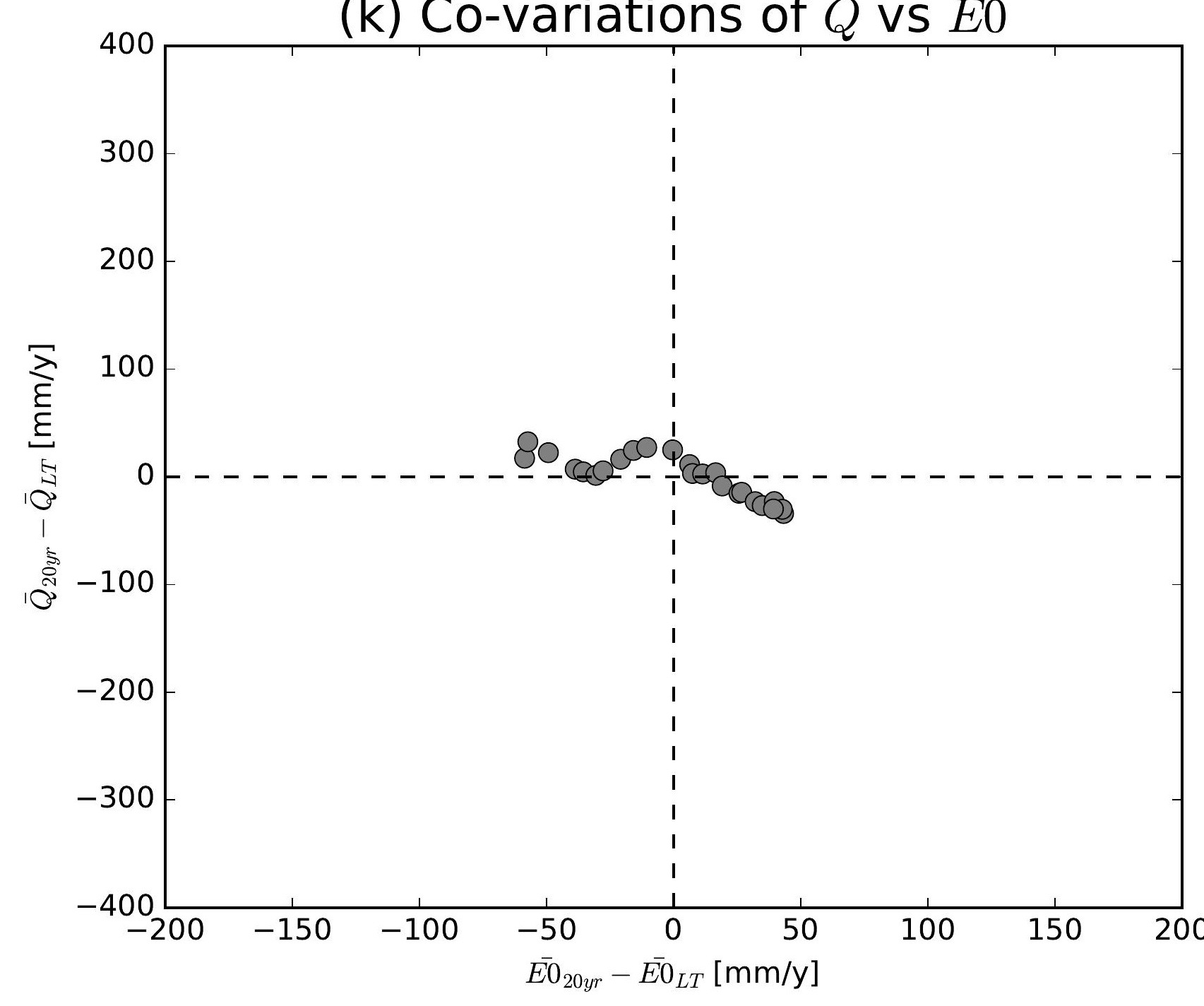

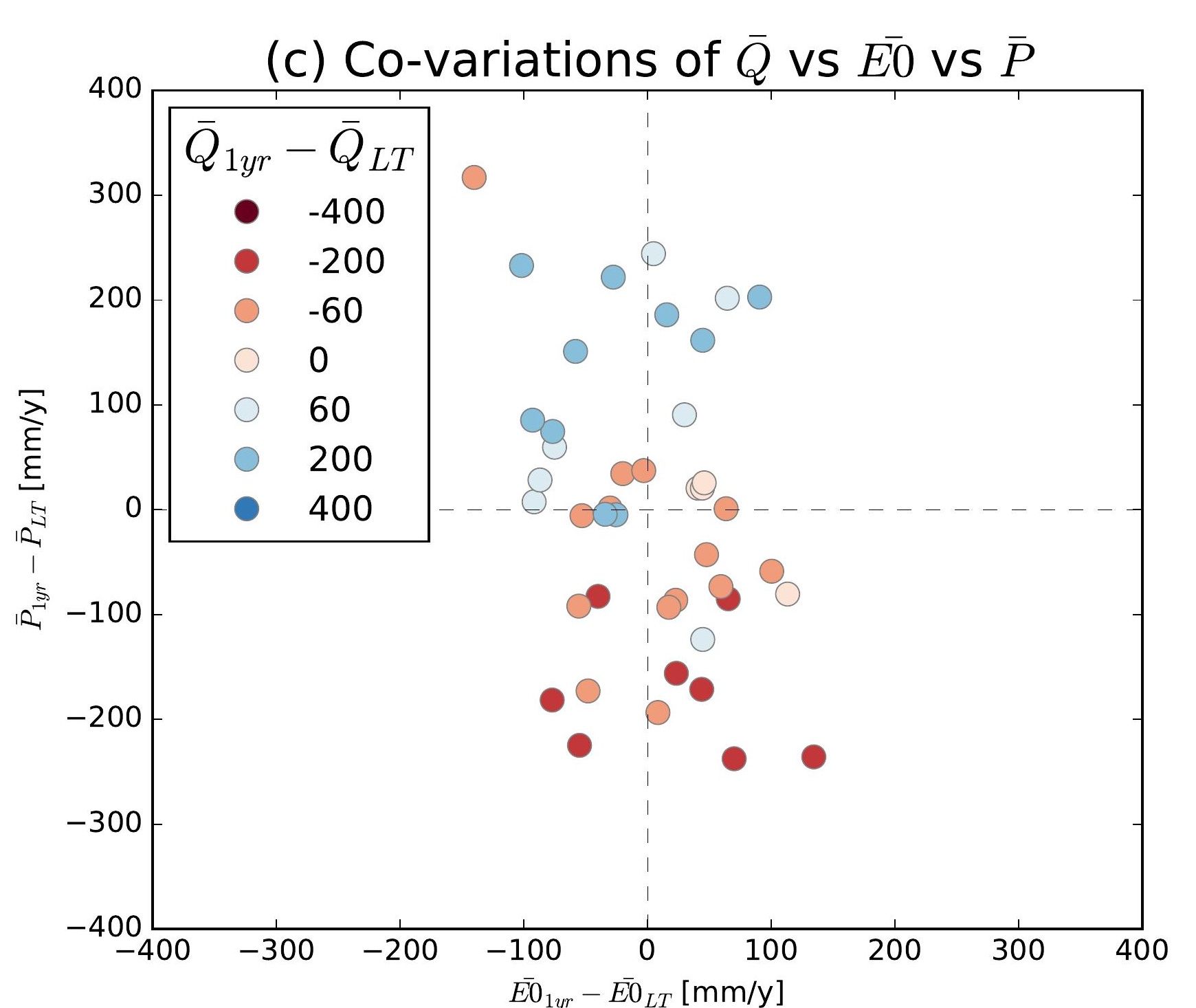
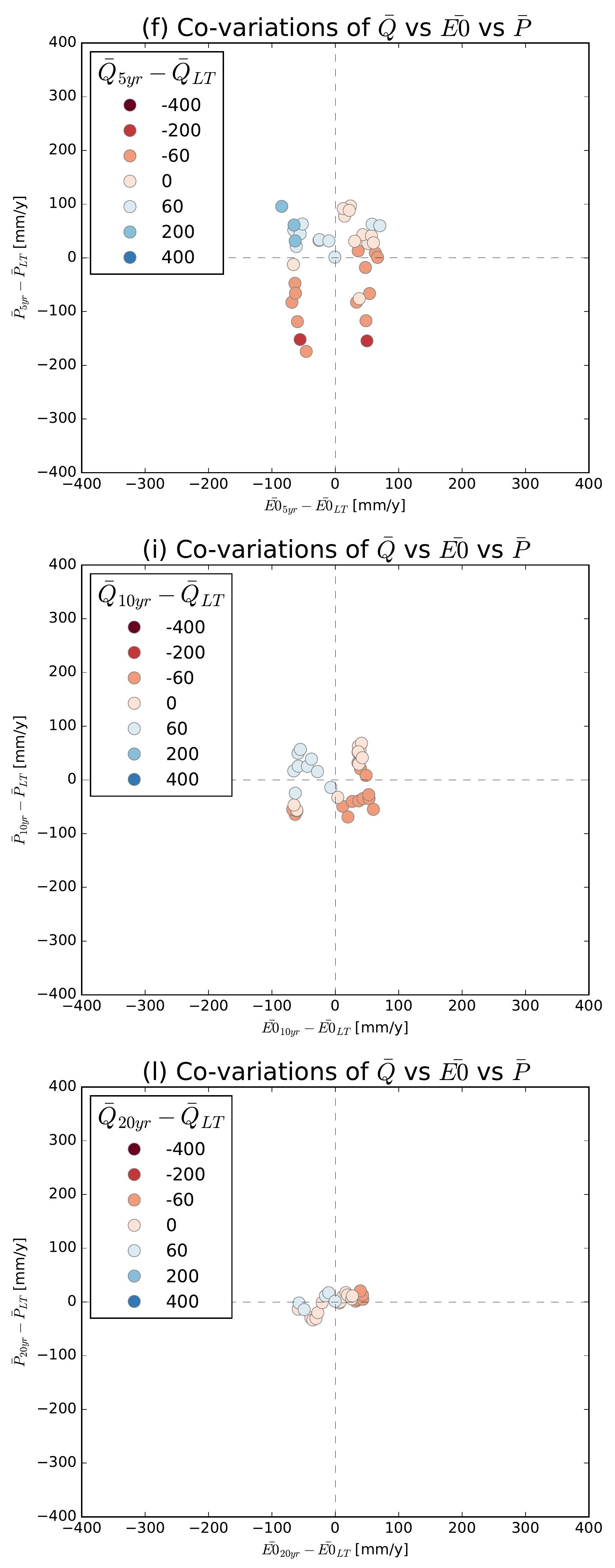

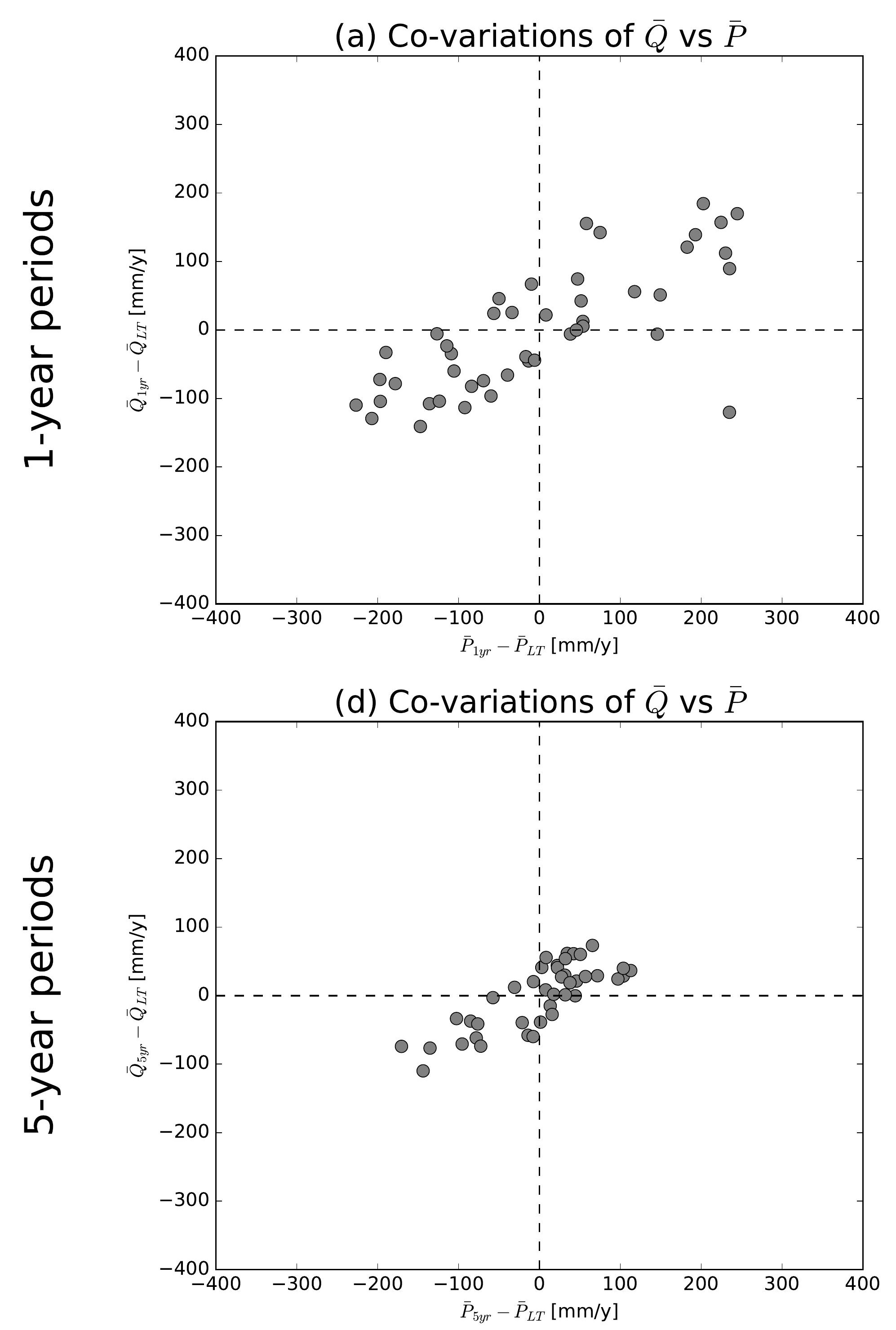

(g) Co-variations of $\bar{Q}$ vs $\bar{P}$

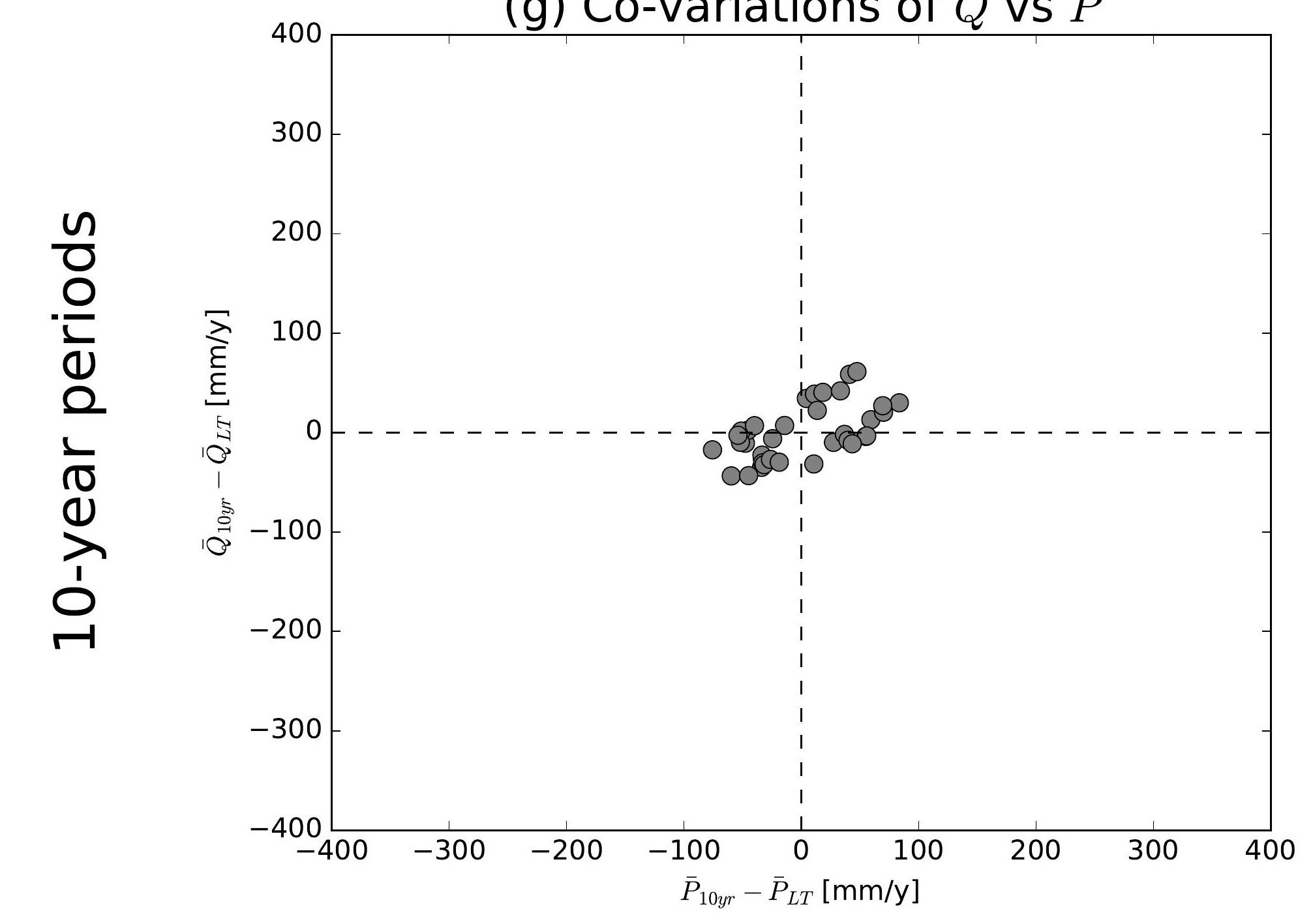

(j) Co-variations of $\bar{Q}$ vs $\bar{P}$

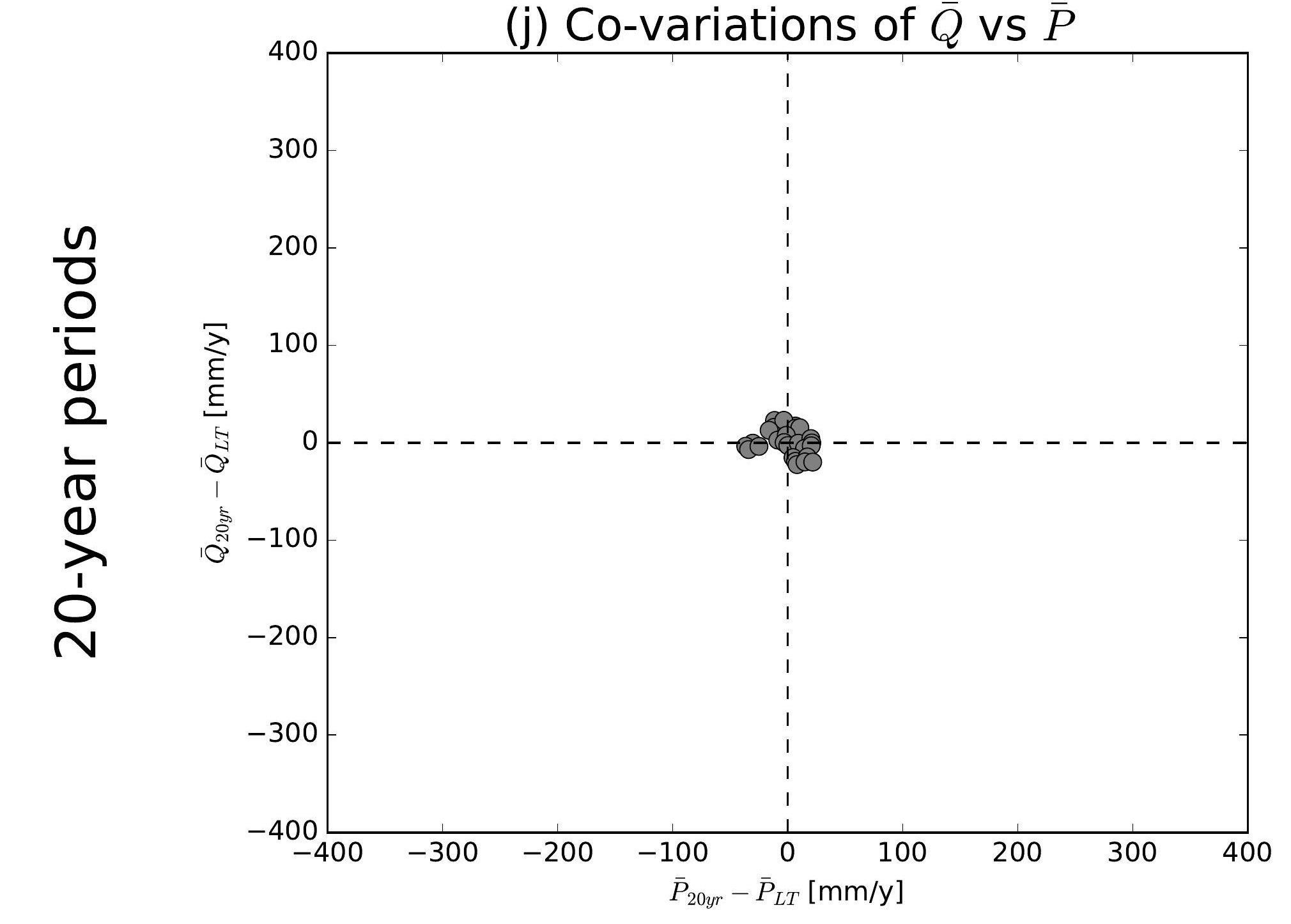

(b) Co-variations of $\bar{Q}$ vs $\overline{E 0}$

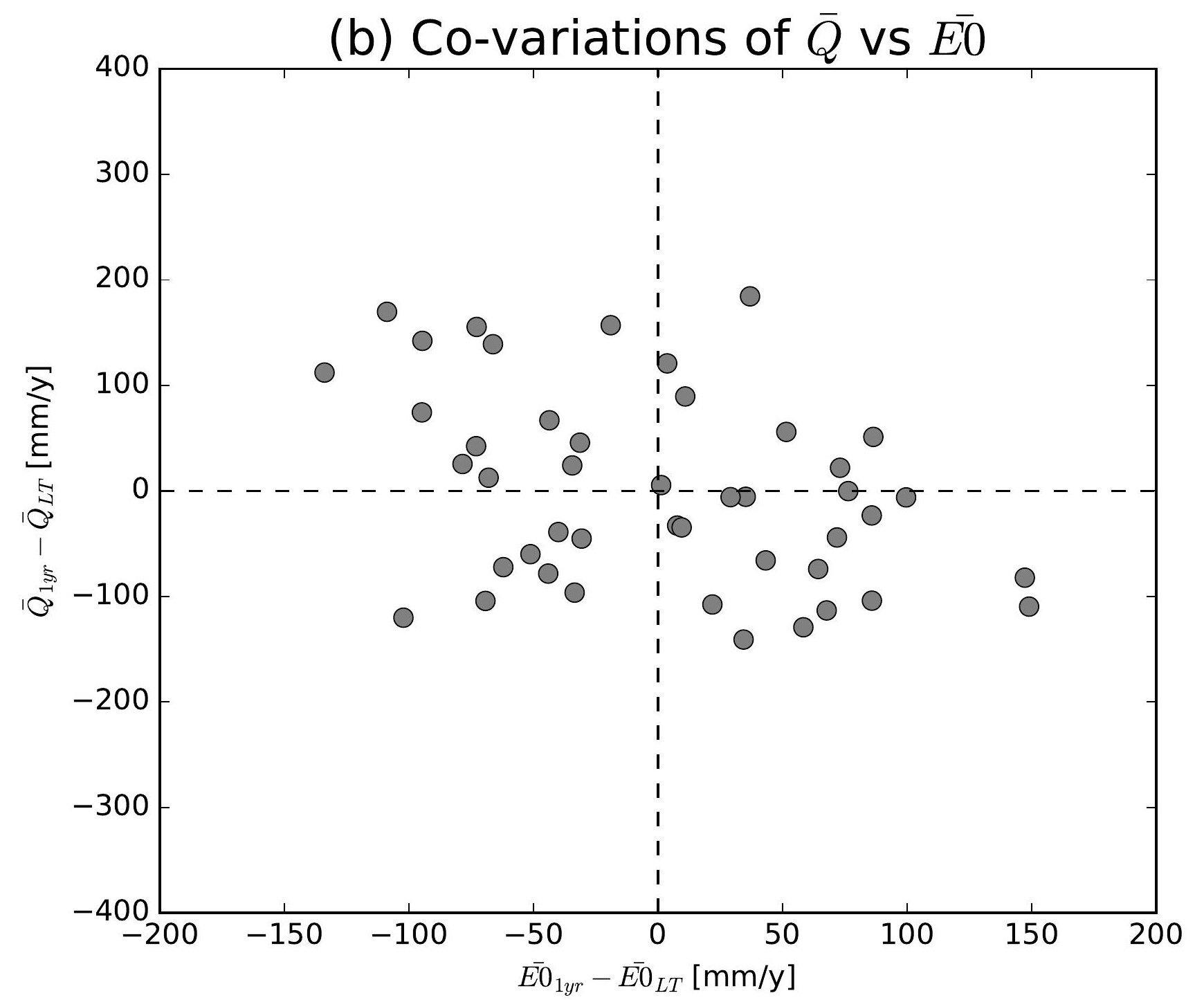

(e) Co-variations of $\bar{Q}$ vs $\overline{E 0}$

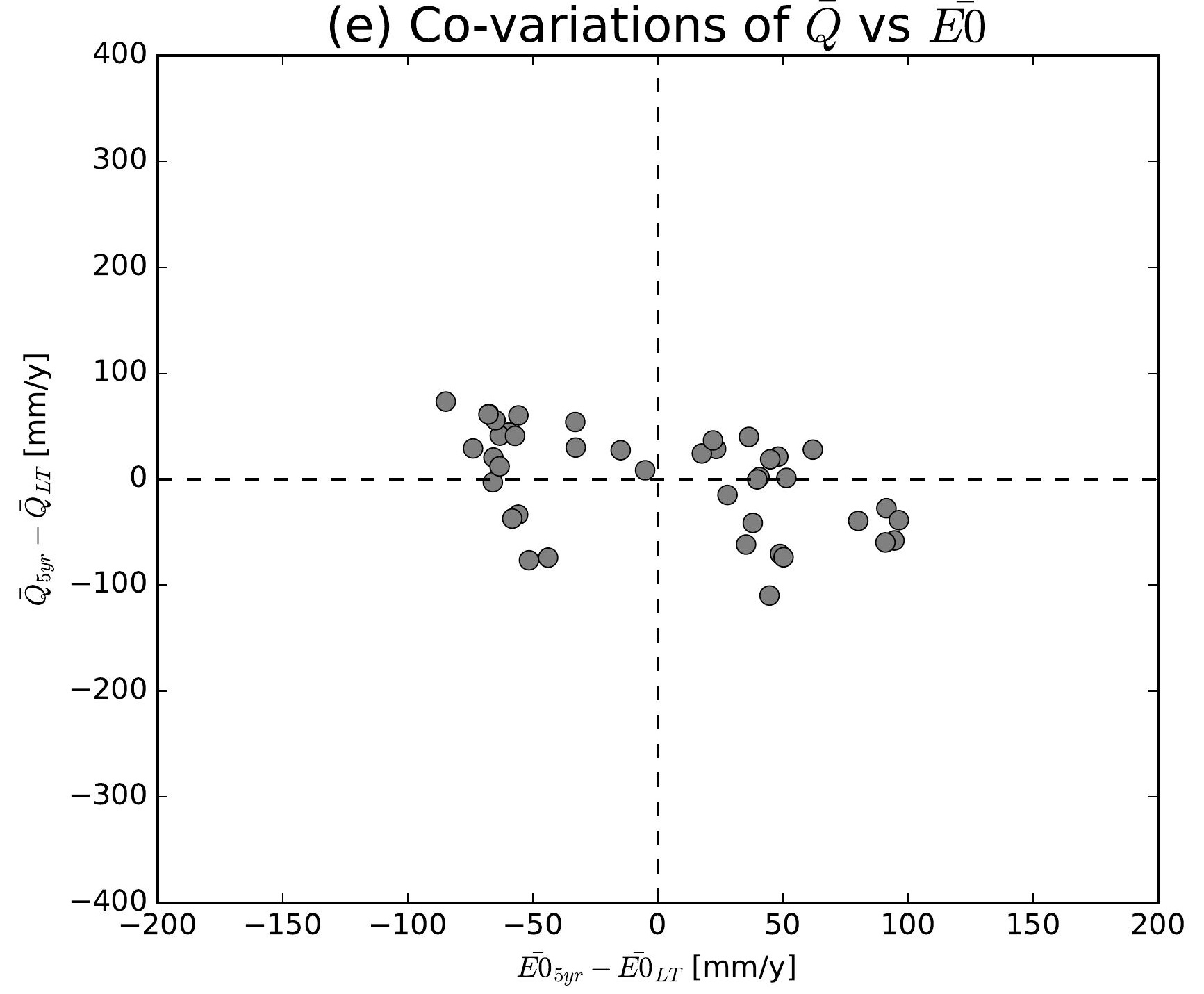

(h) Co-variations of $\bar{Q}$ vs $\overline{E 0}$

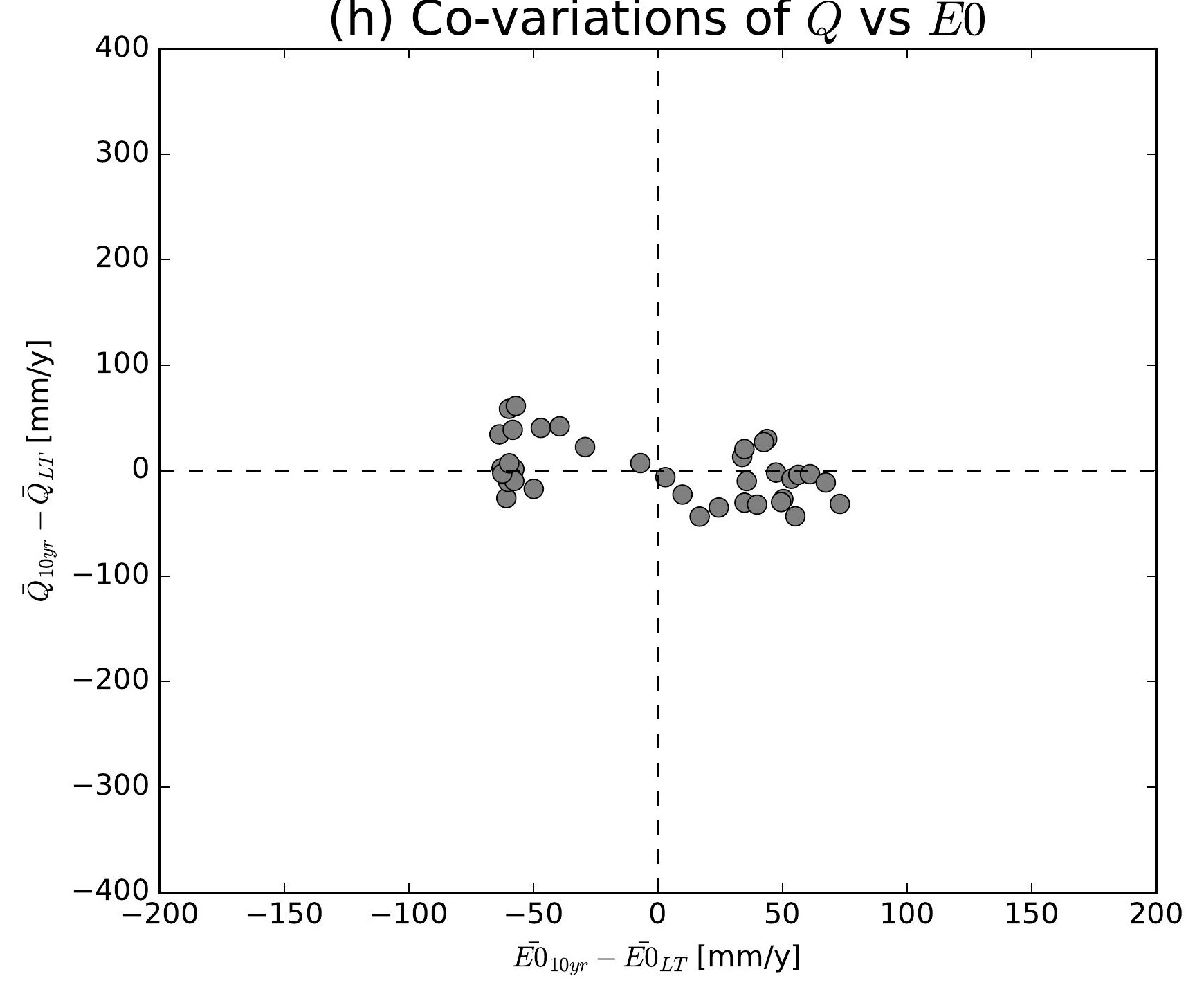

(k) Co-variations of $\bar{Q}$ vs $\overline{E 0}$

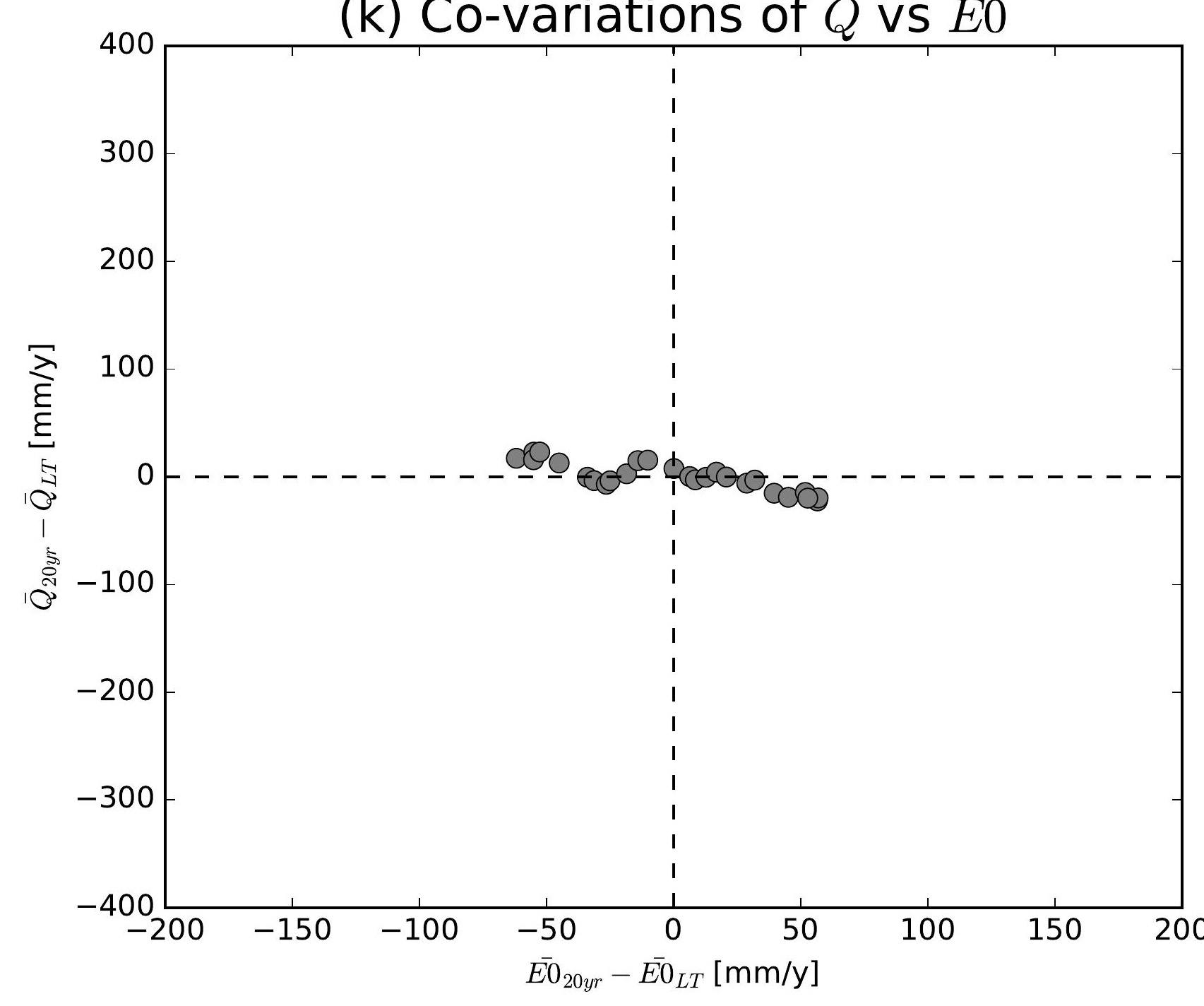

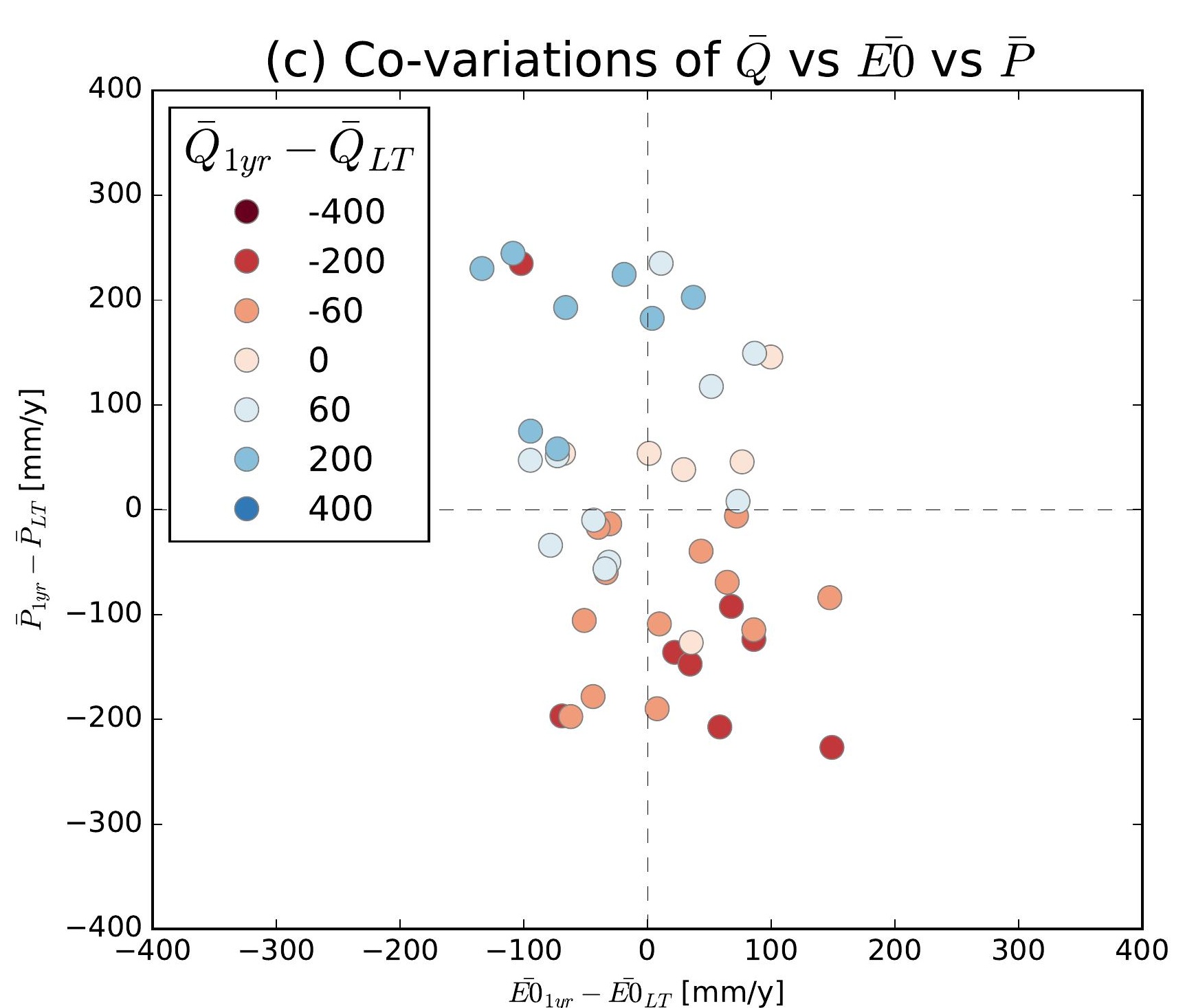
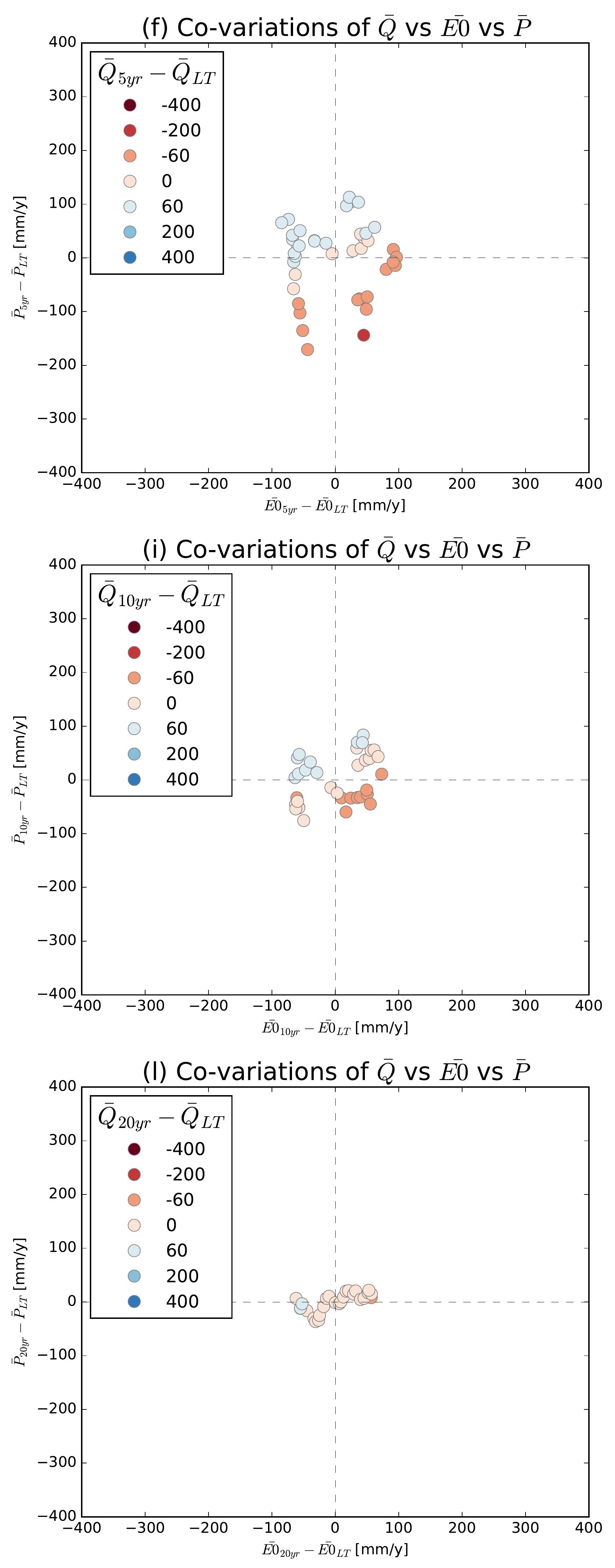

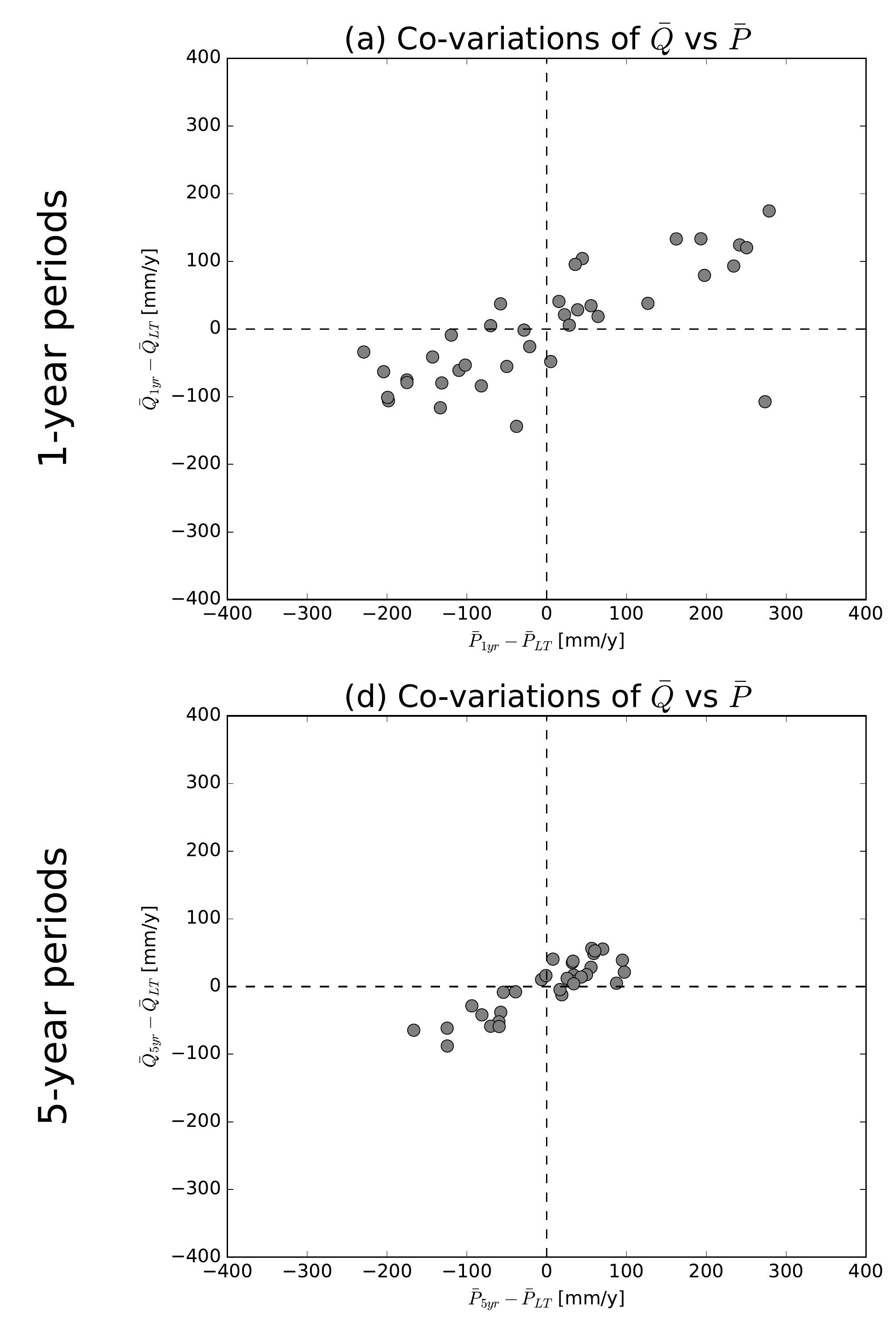

(g) Co-variations of $\bar{Q}$ vs $\bar{P}$

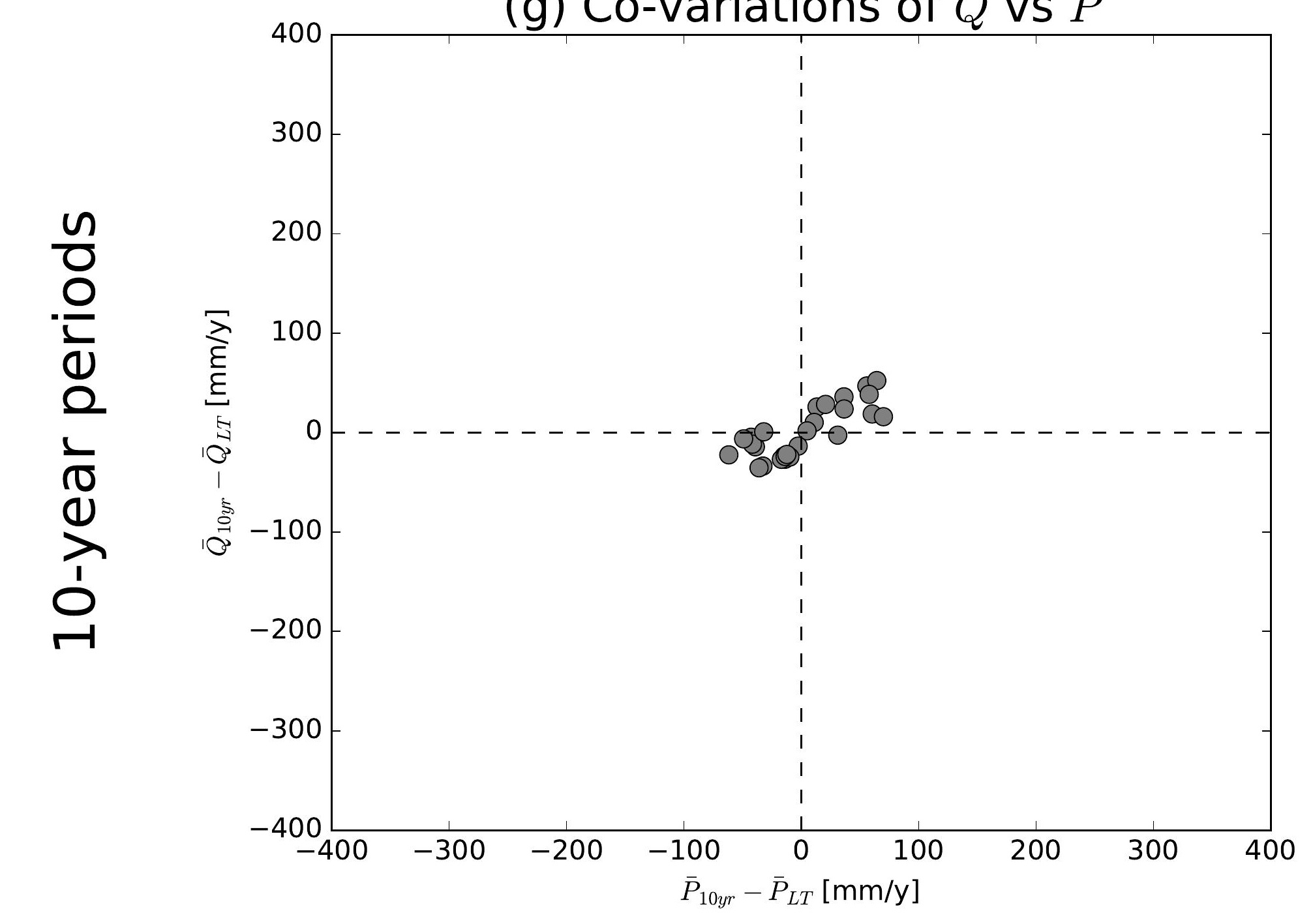

(j) Co-variations of $\bar{Q}$ vs $\bar{P}$

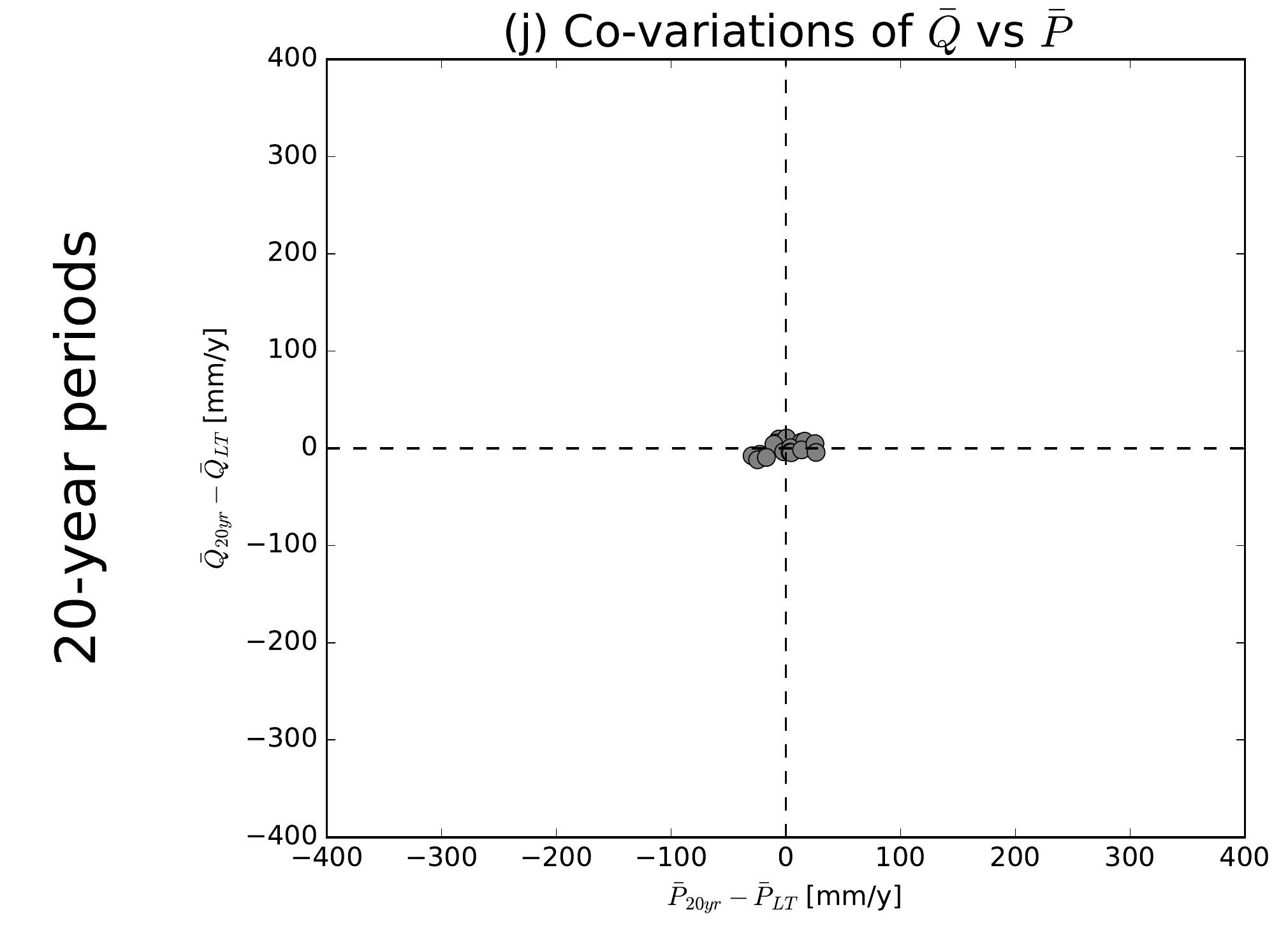

(b) Co-variations of $\bar{Q}$ vs $\overline{E 0}$

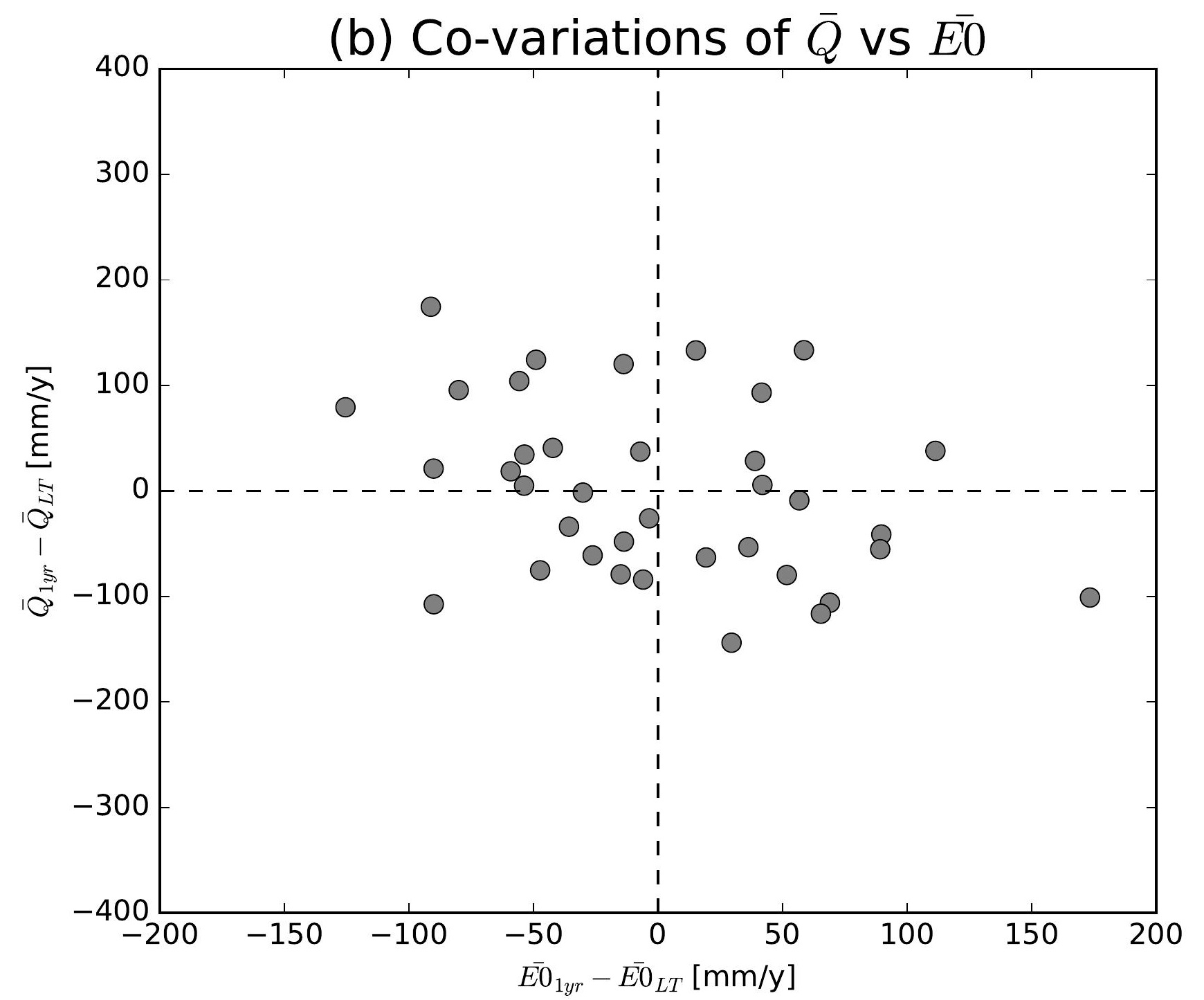

(e) Co-variations of $\bar{Q}$ vs $\overline{E 0}$

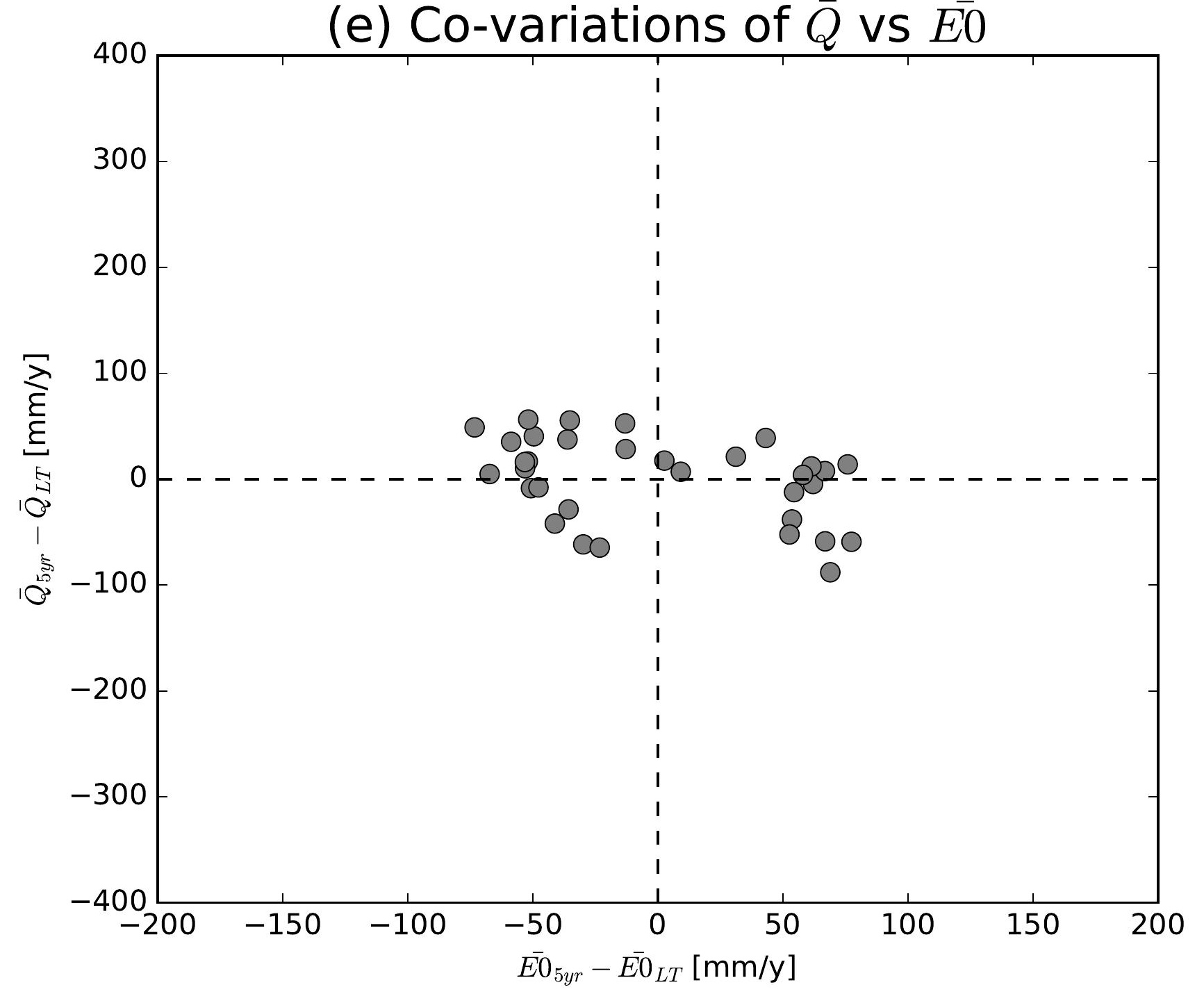

(h) Co-variations of $\bar{Q}$ vs $\overline{E 0}$

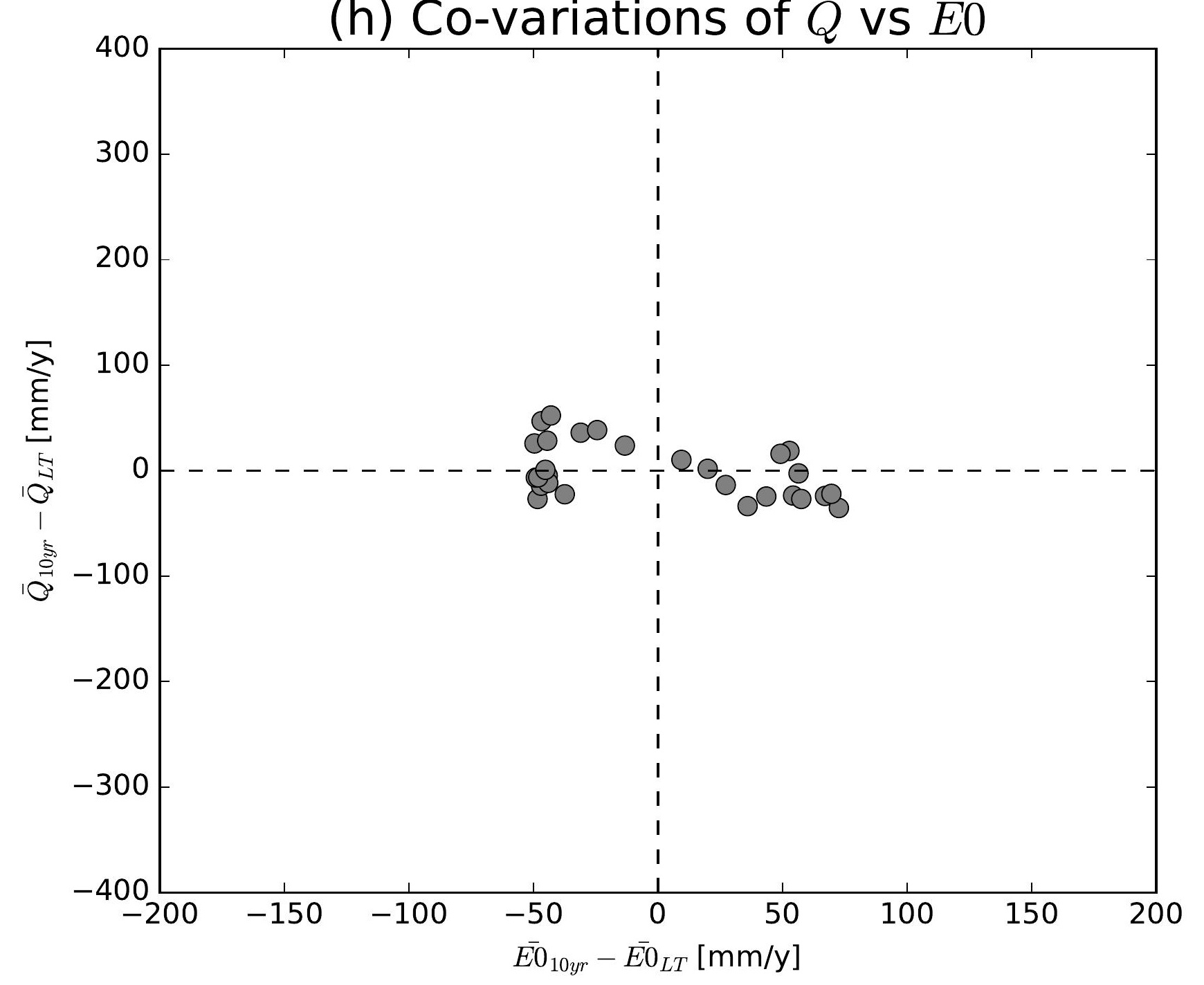

(k) Co-variations of $\bar{Q}$ vs $\overline{E 0}$

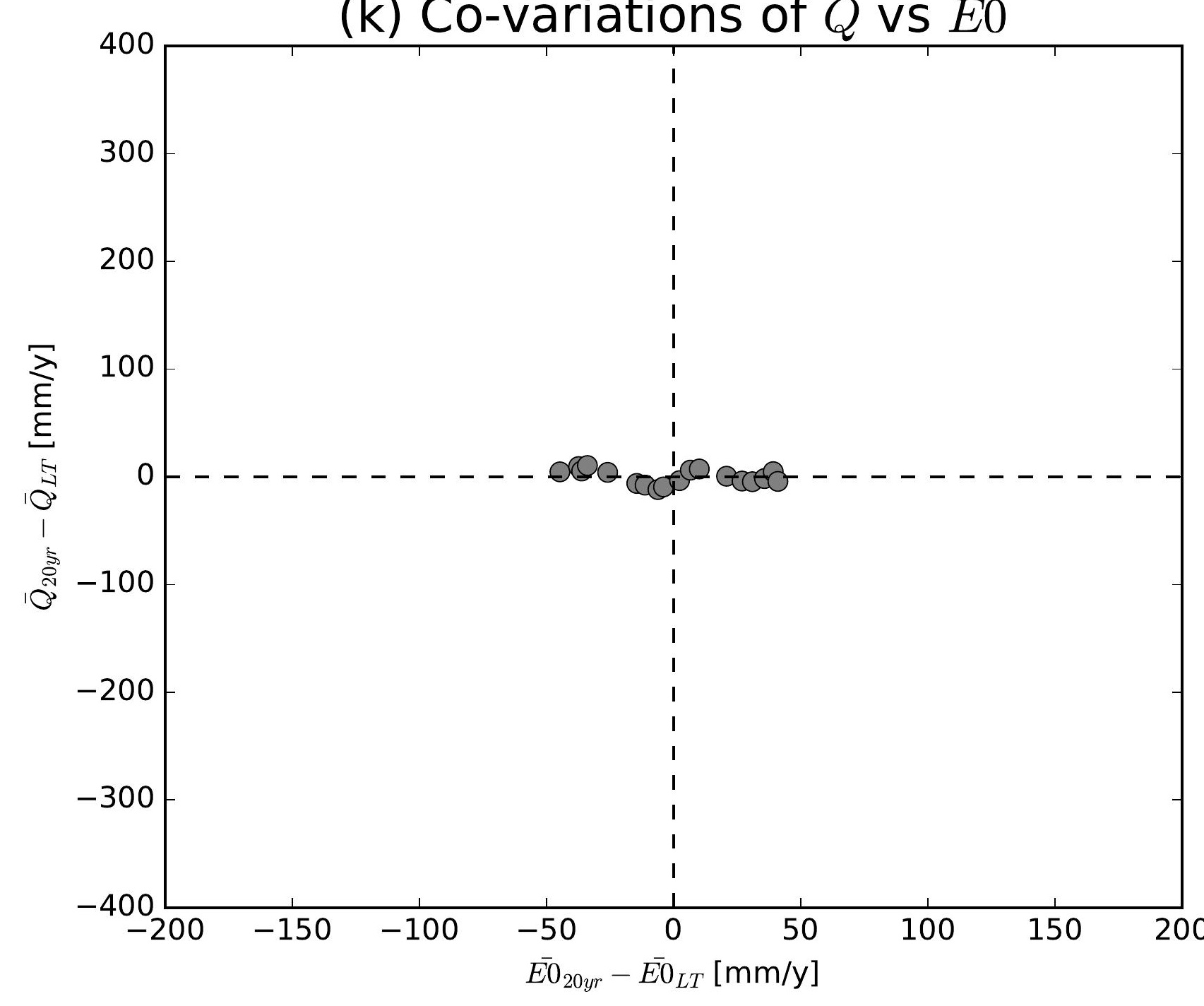

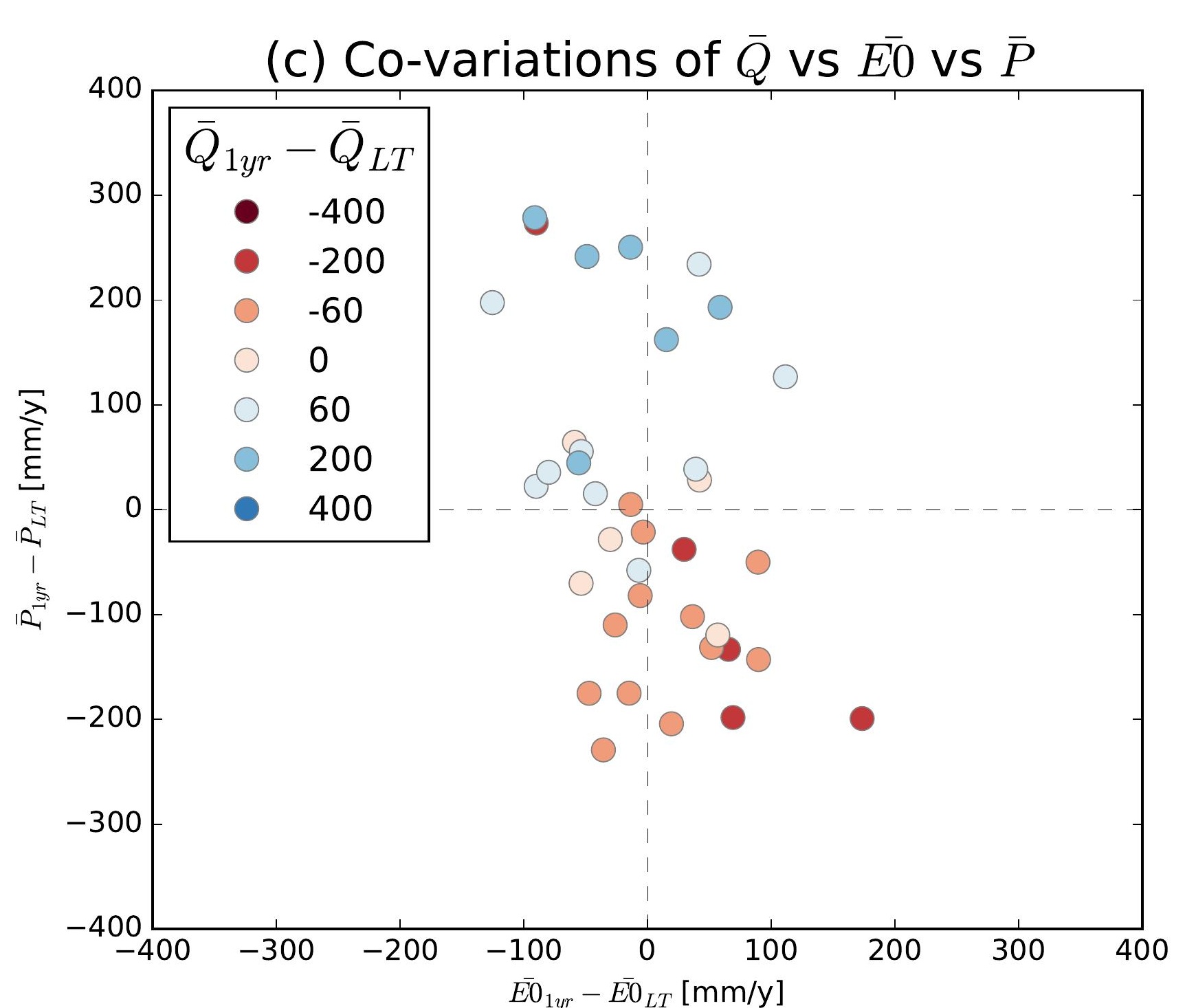
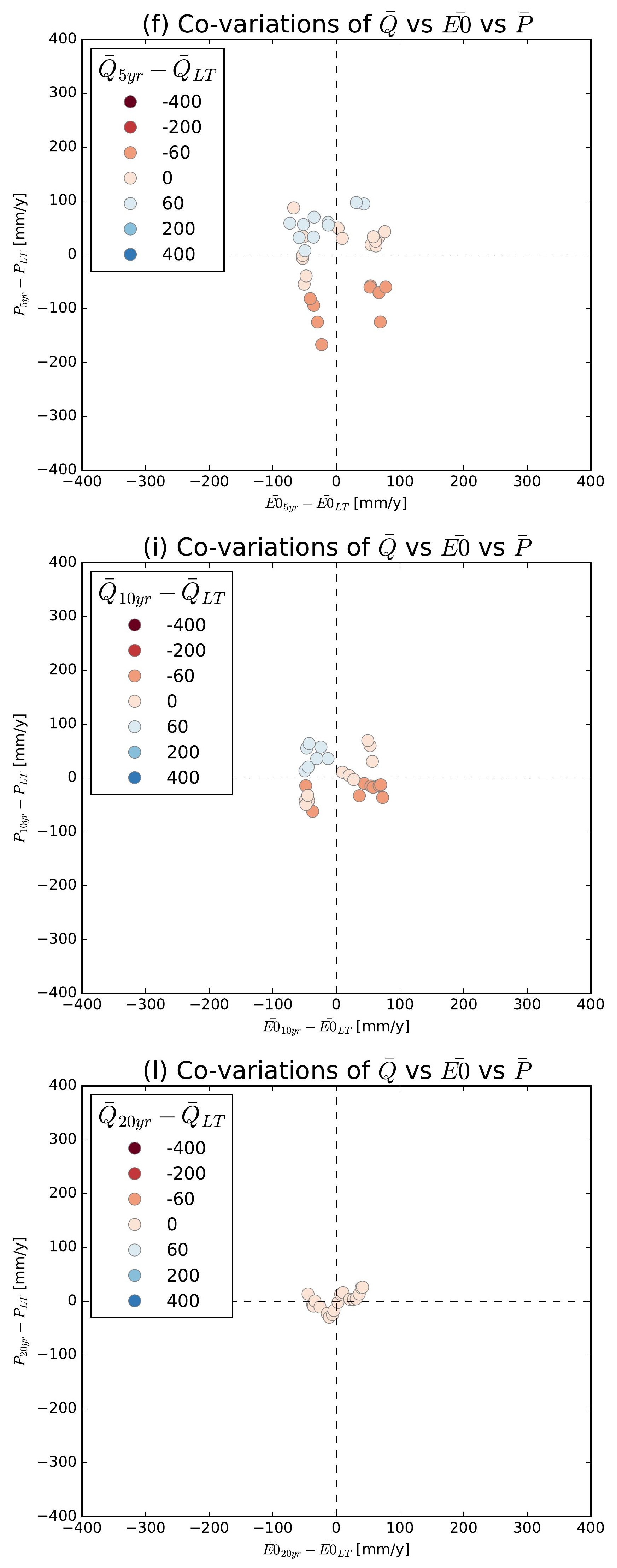

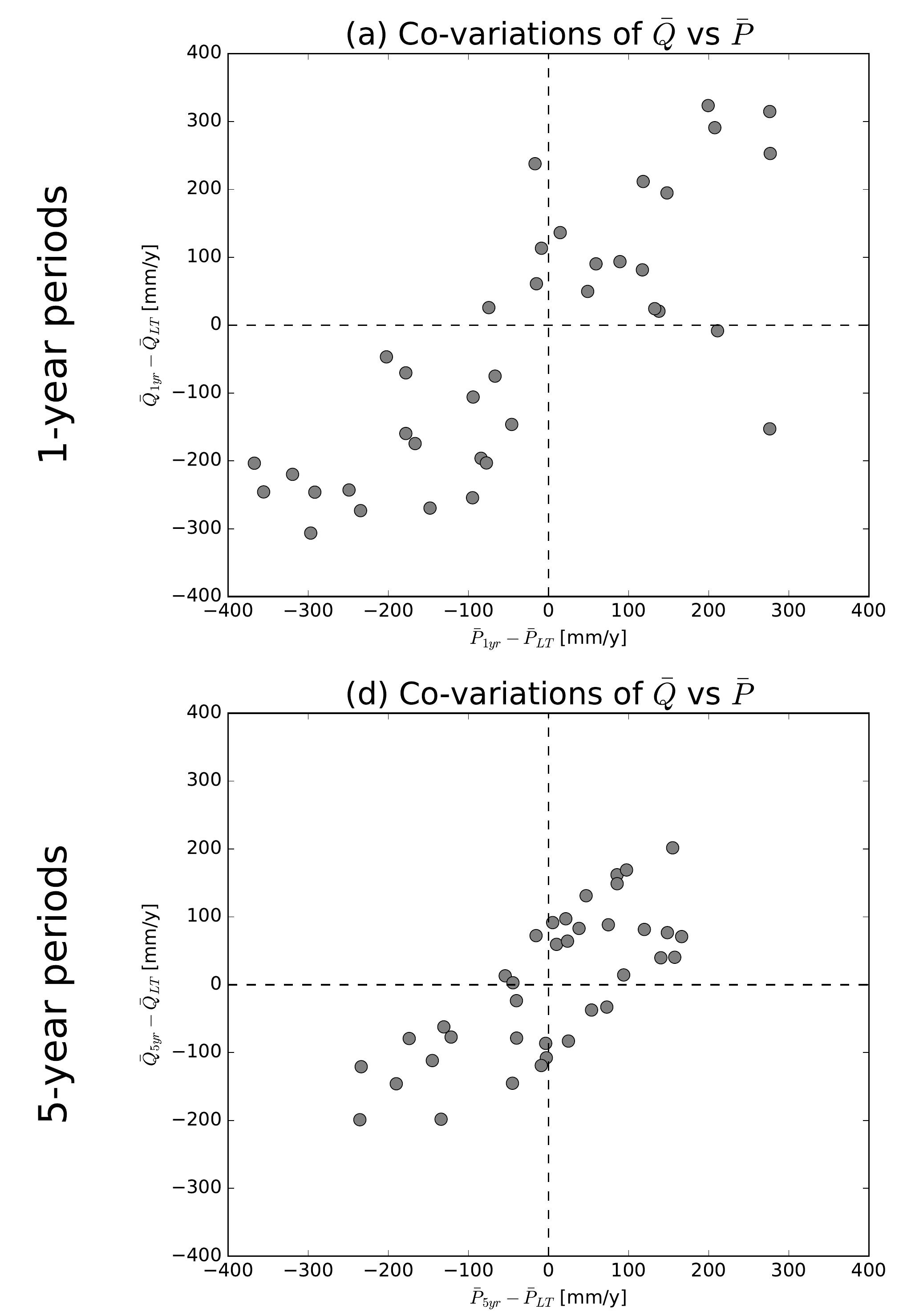

(g) Co-variations of $\bar{Q}$ vs $\bar{P}$
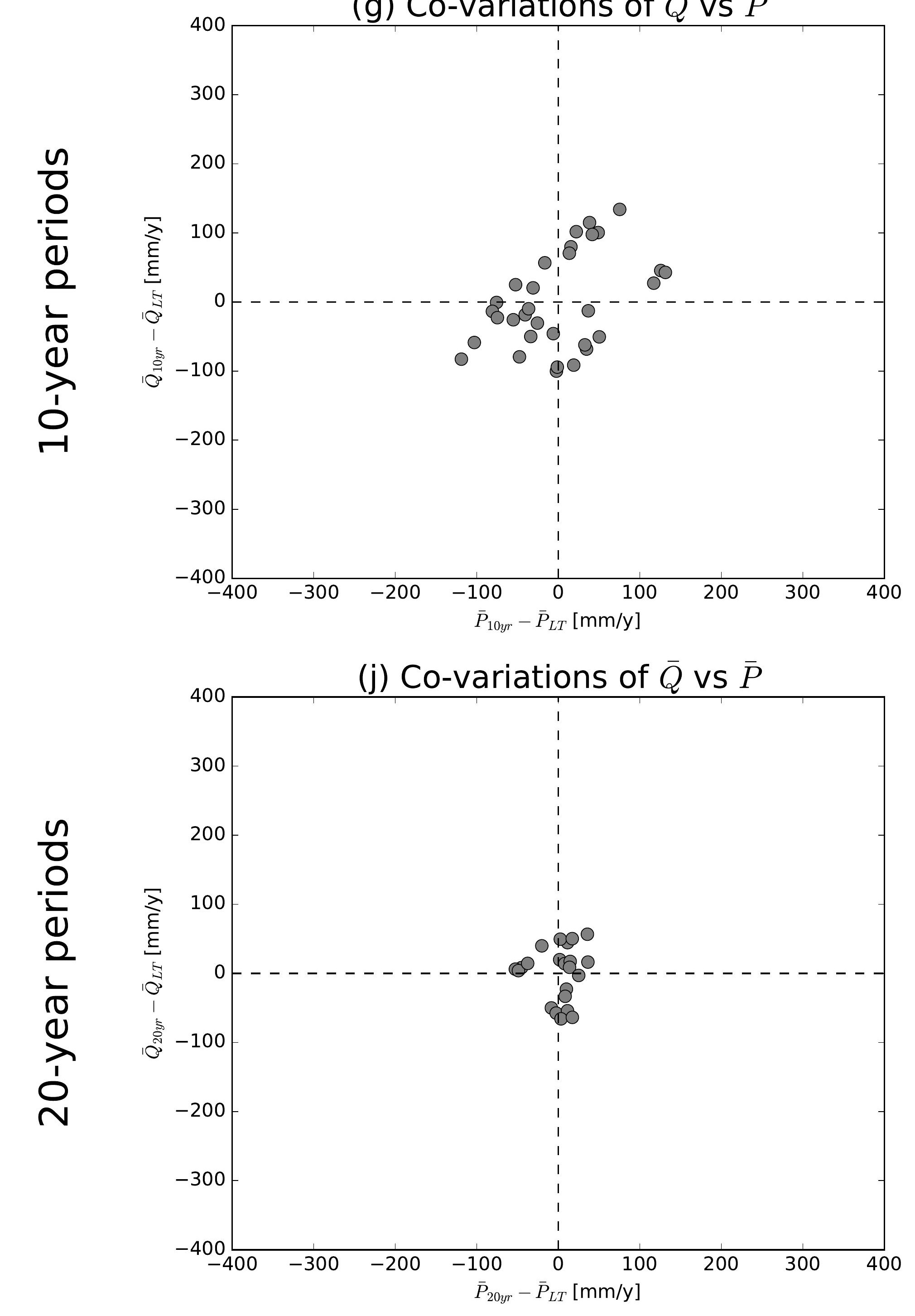

(b) Co-variations of $\bar{Q}$ vs $\overline{E 0}$

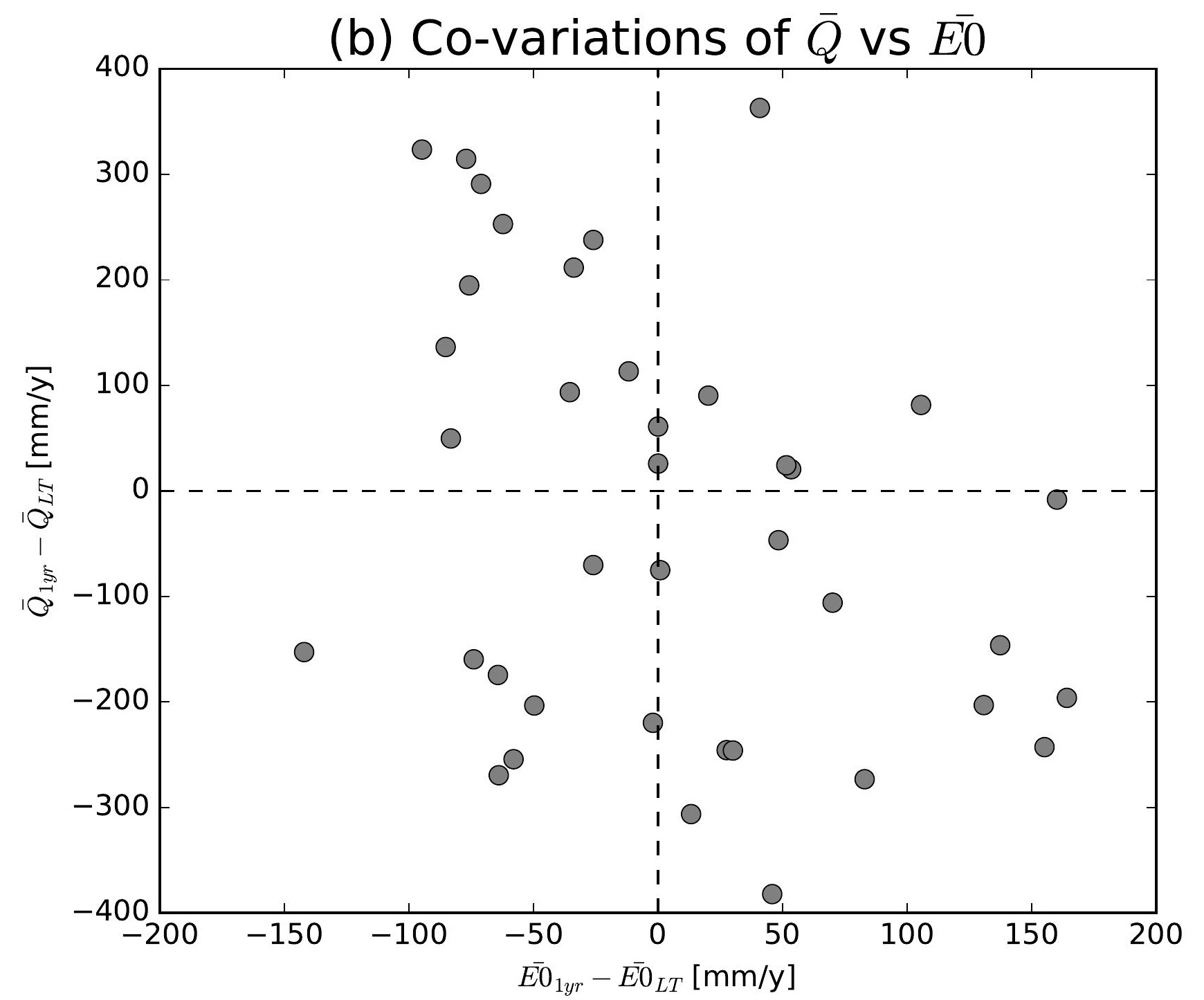

(e) Co-variations of $\bar{Q}$ vs $\overline{E 0}$

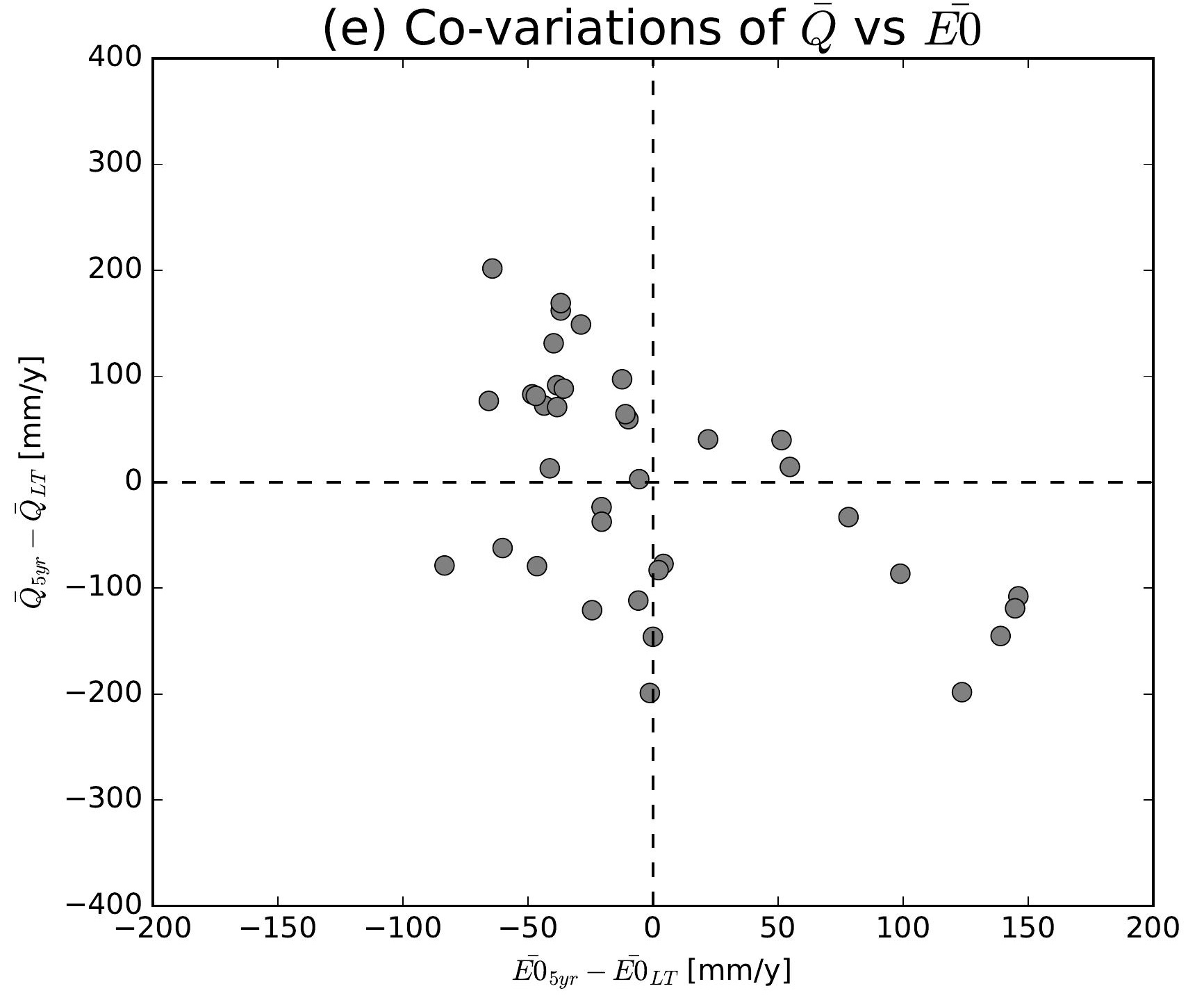

(h) Co-variations of $\bar{Q}$ vs $\overline{E 0}$

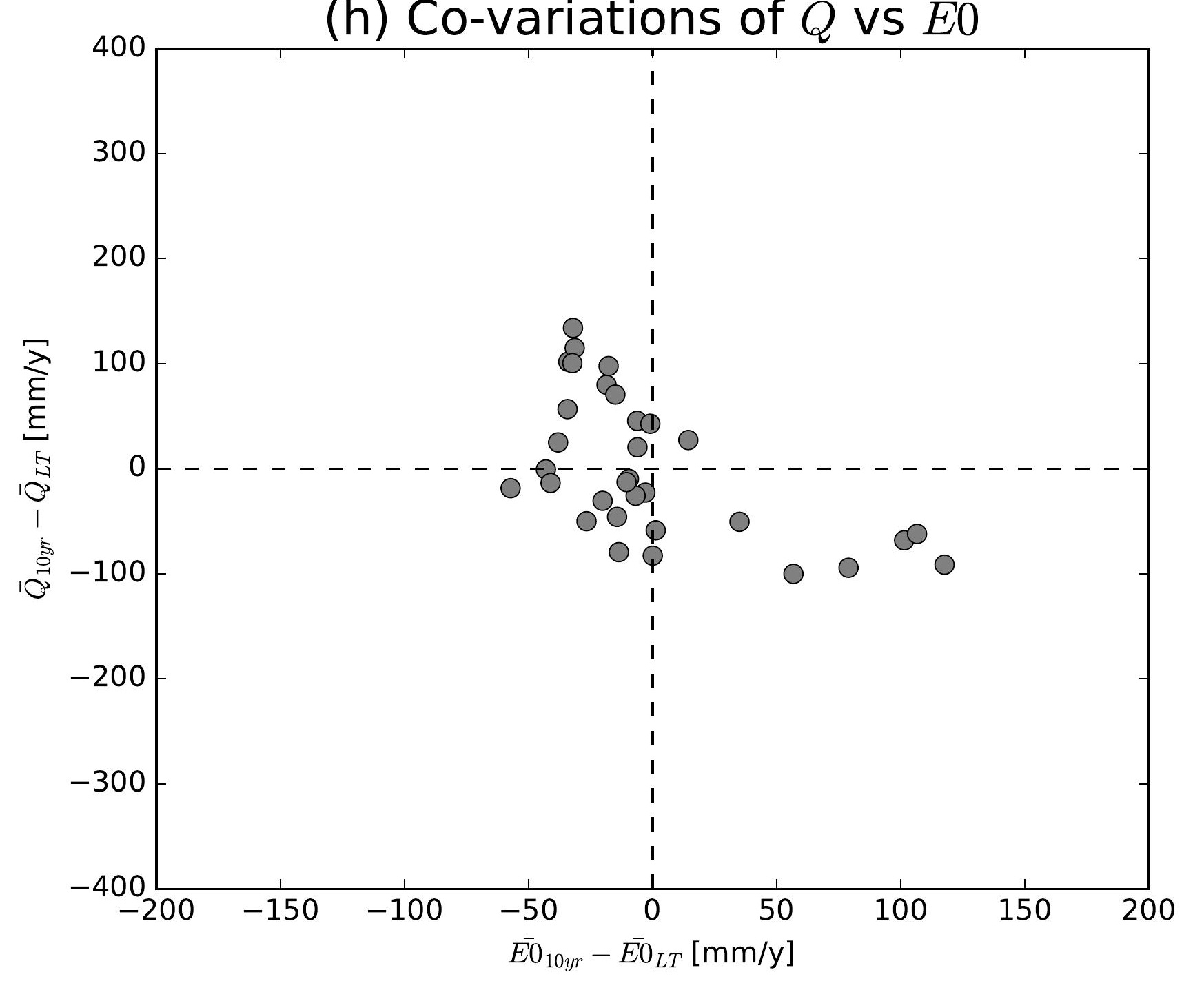

(k) Co-variations of $\bar{Q}$ vs $\overline{E 0}$

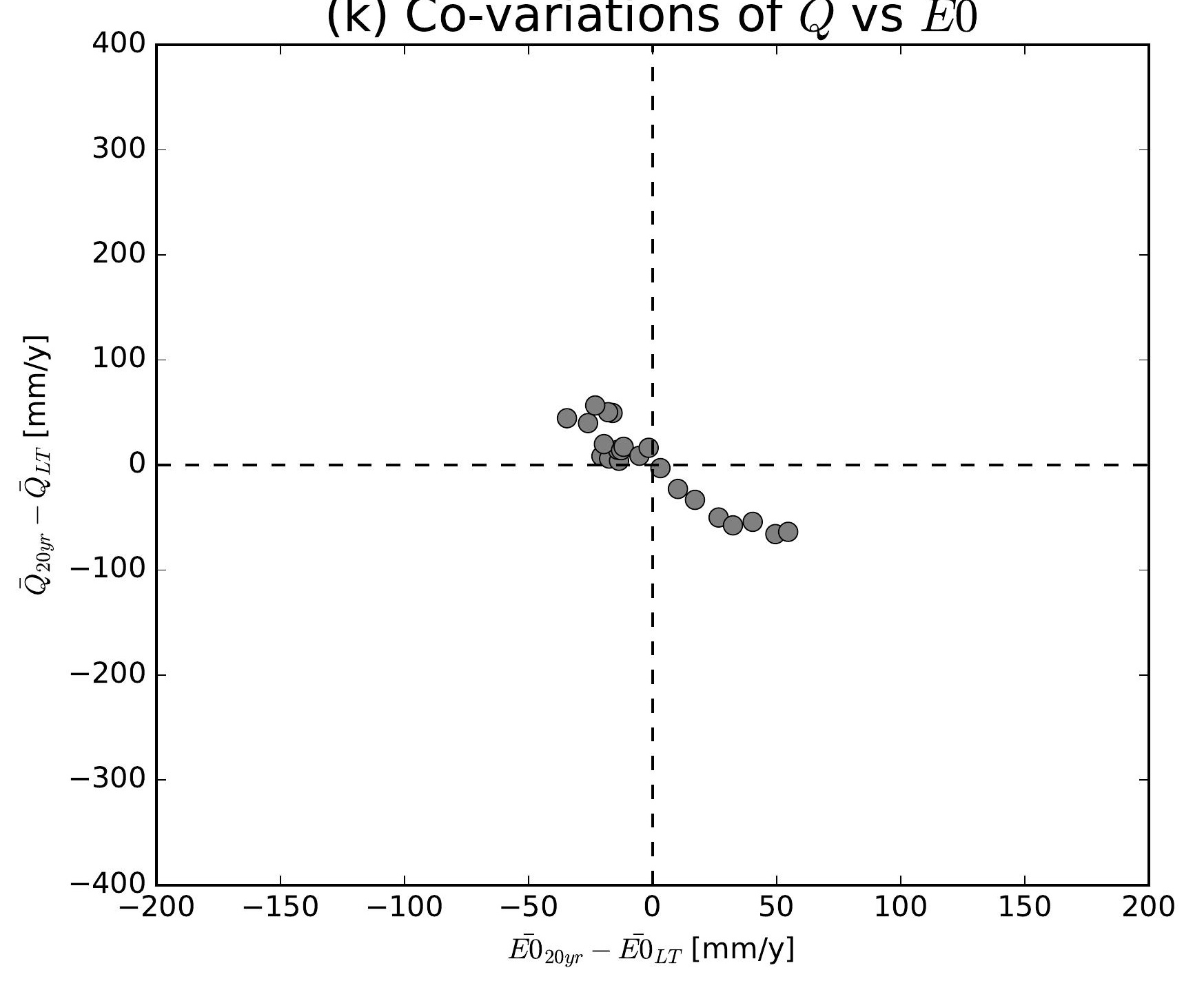

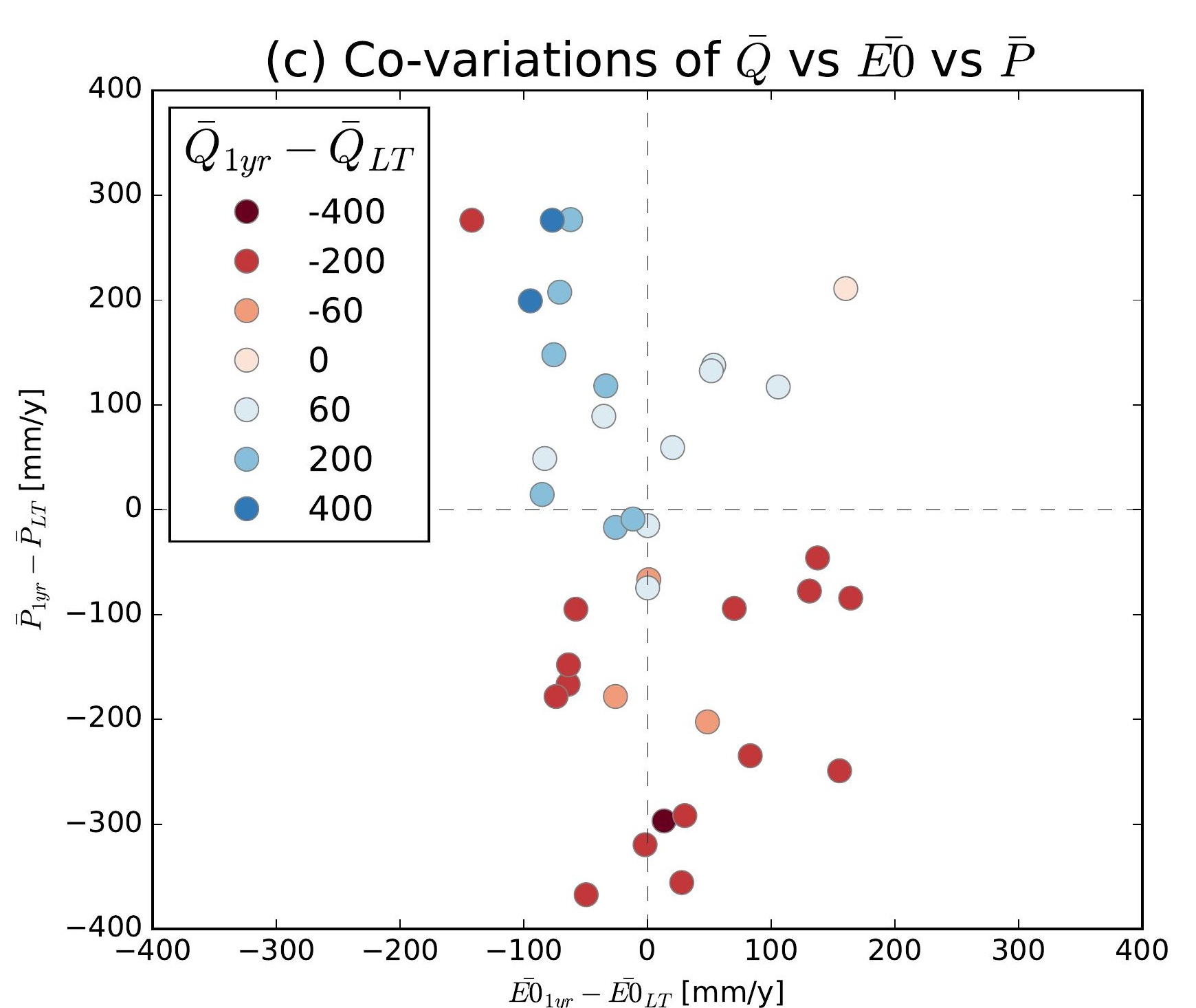
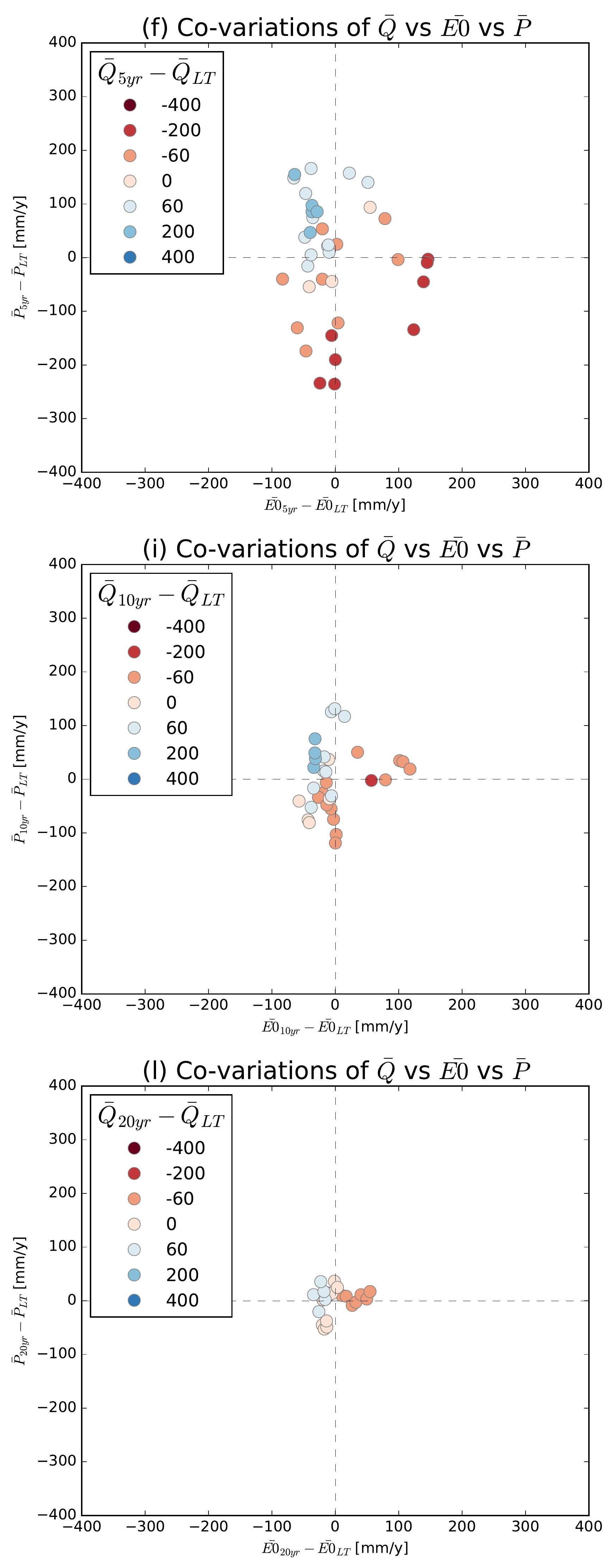

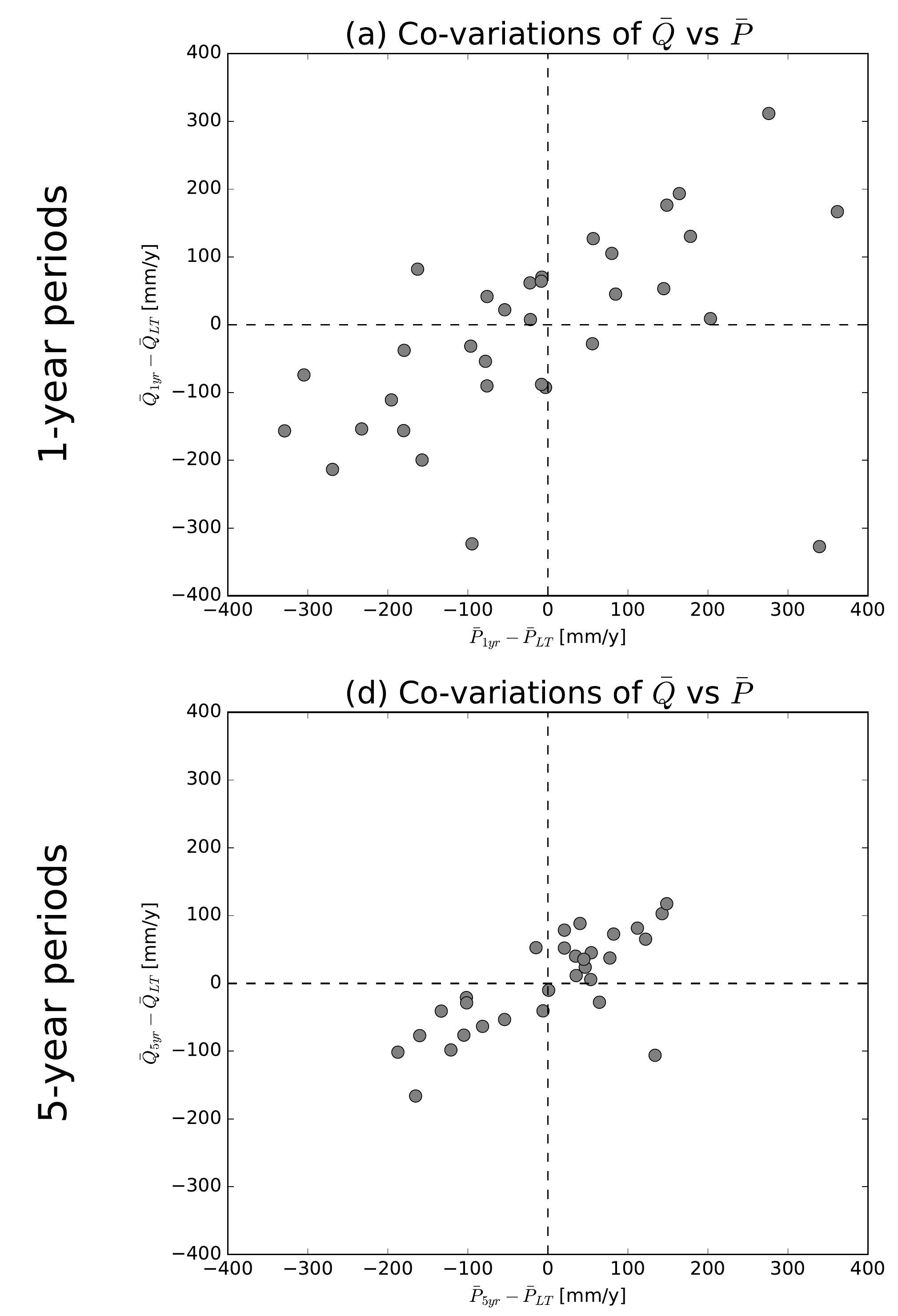

(g) Co-variations of $\bar{Q}$ vs $\bar{P}$
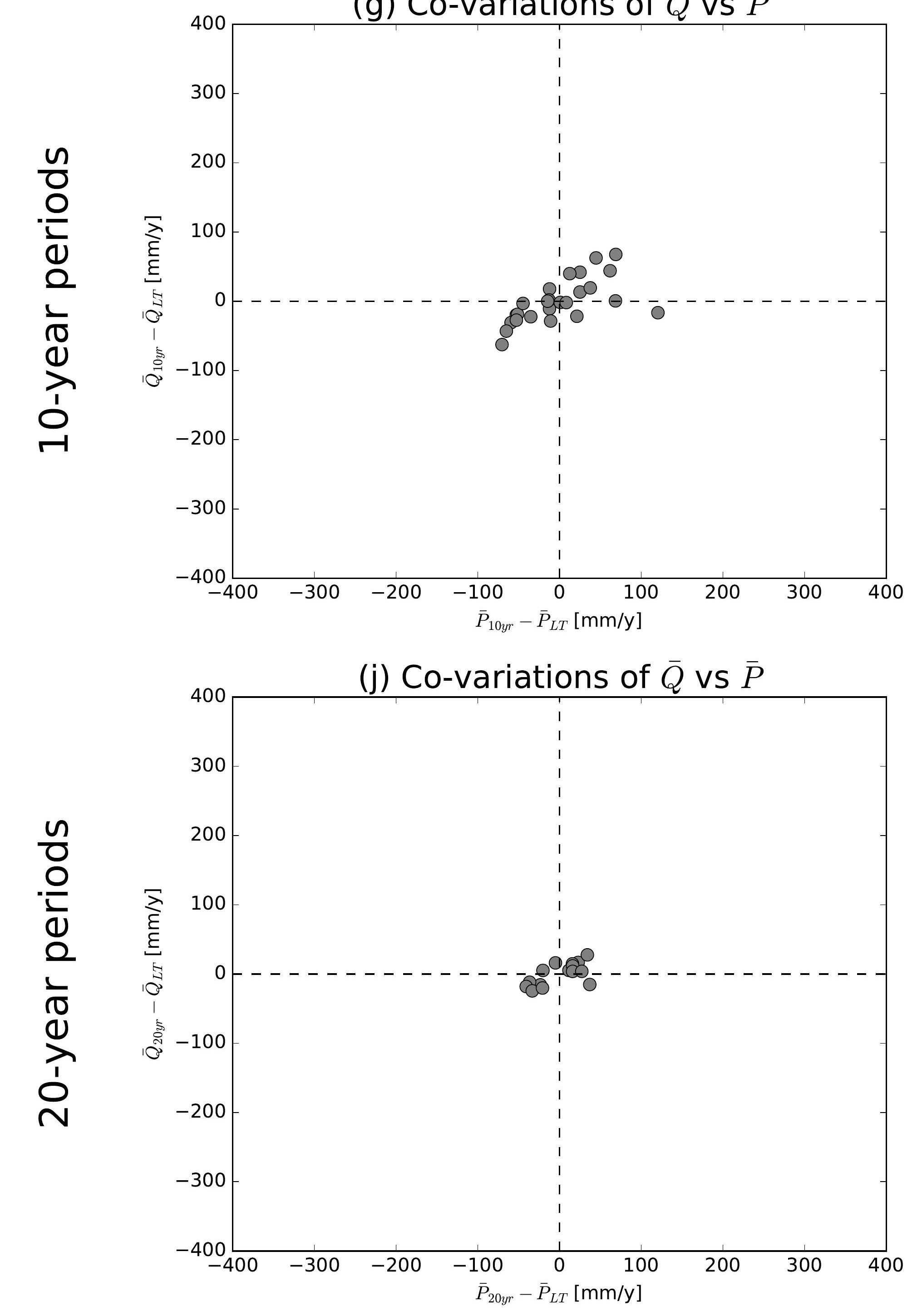

(b) Co-variations of $\bar{Q}$ vs $\overline{E 0}$

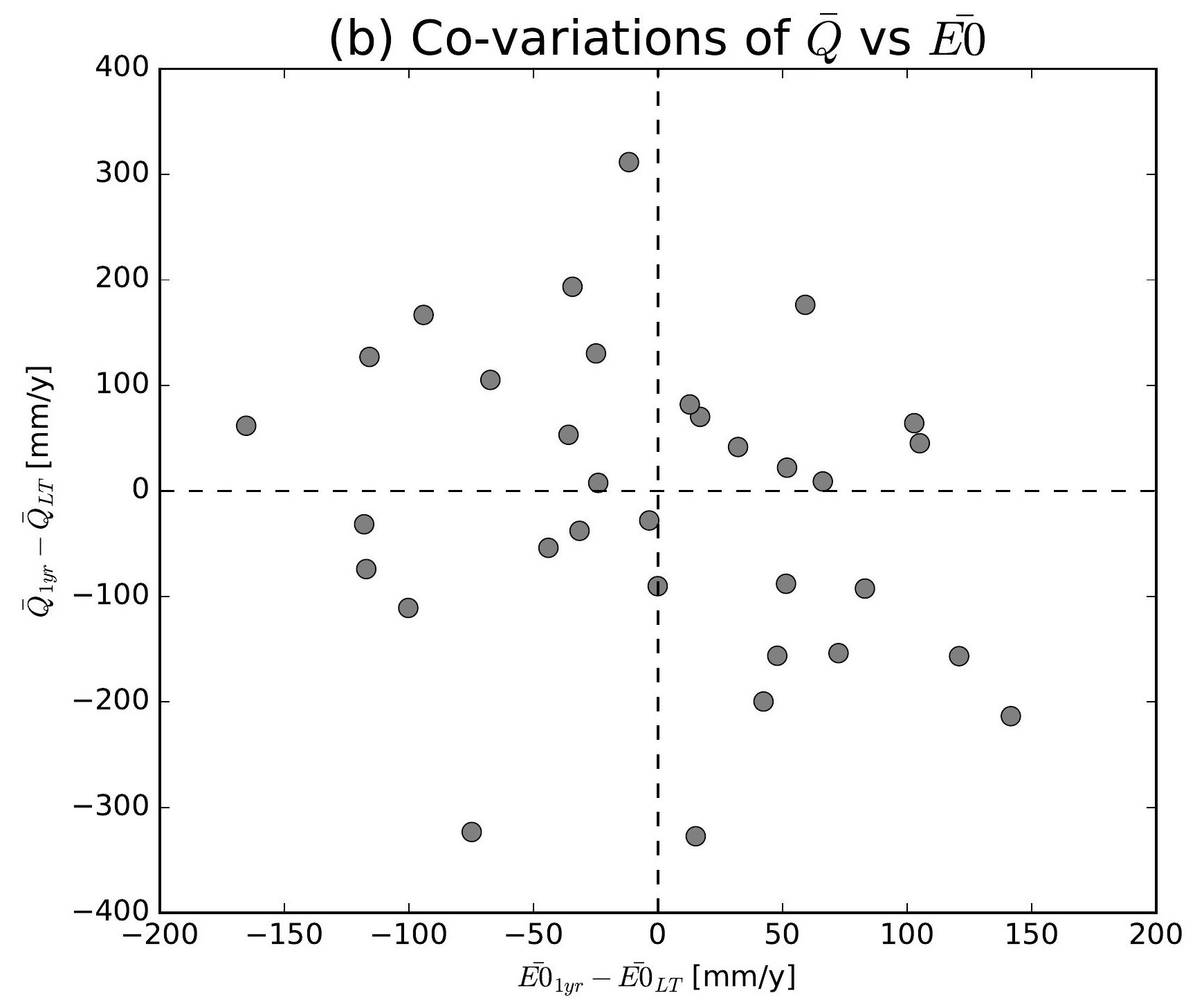

(e) Co-variations of $\bar{Q}$ vs $\overline{E 0}$

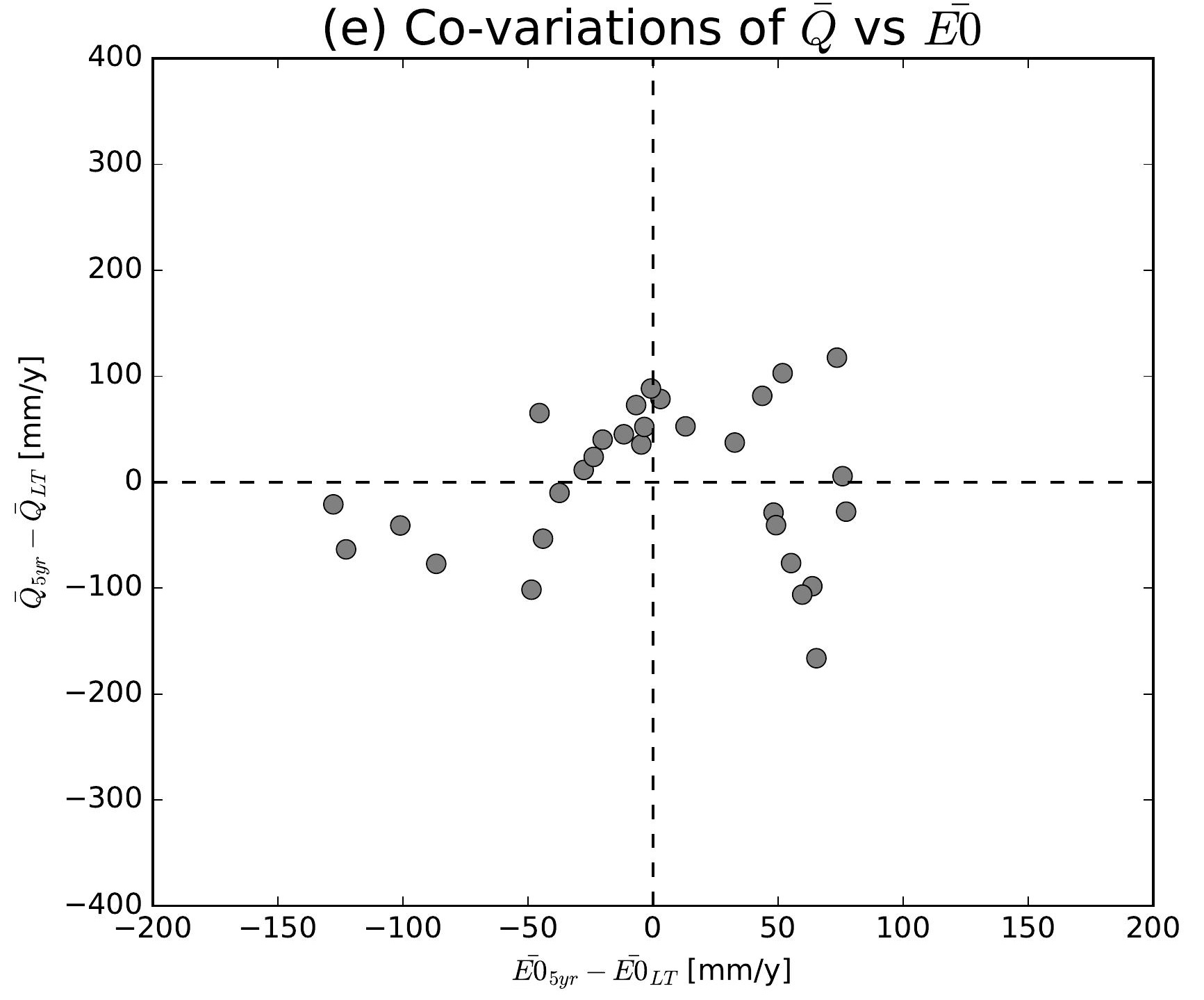

(h) Co-variations of $\bar{Q}$ vs $\overline{E 0}$

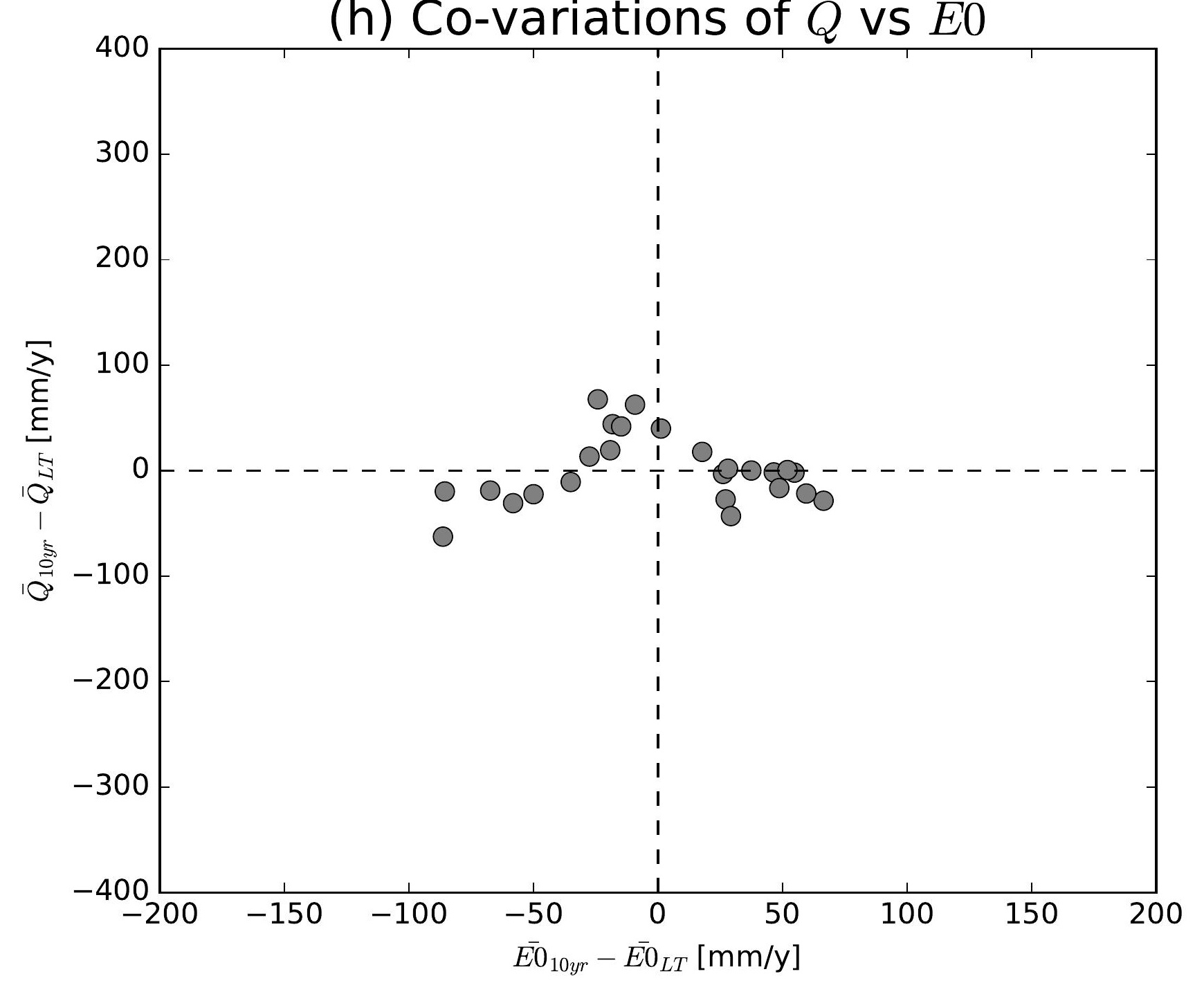

(k) Co-variations of $\bar{Q}$ vs $\overline{E 0}$

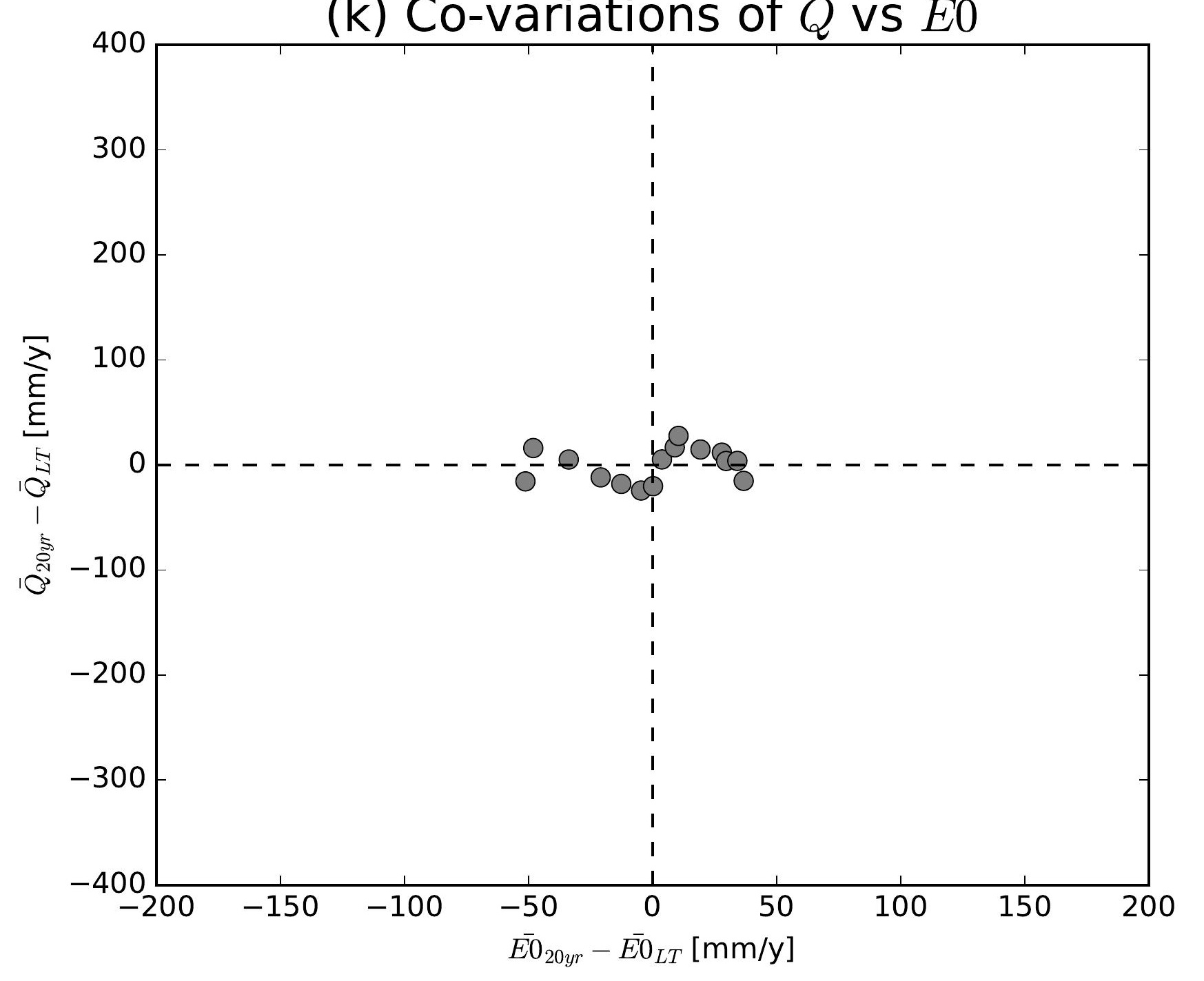

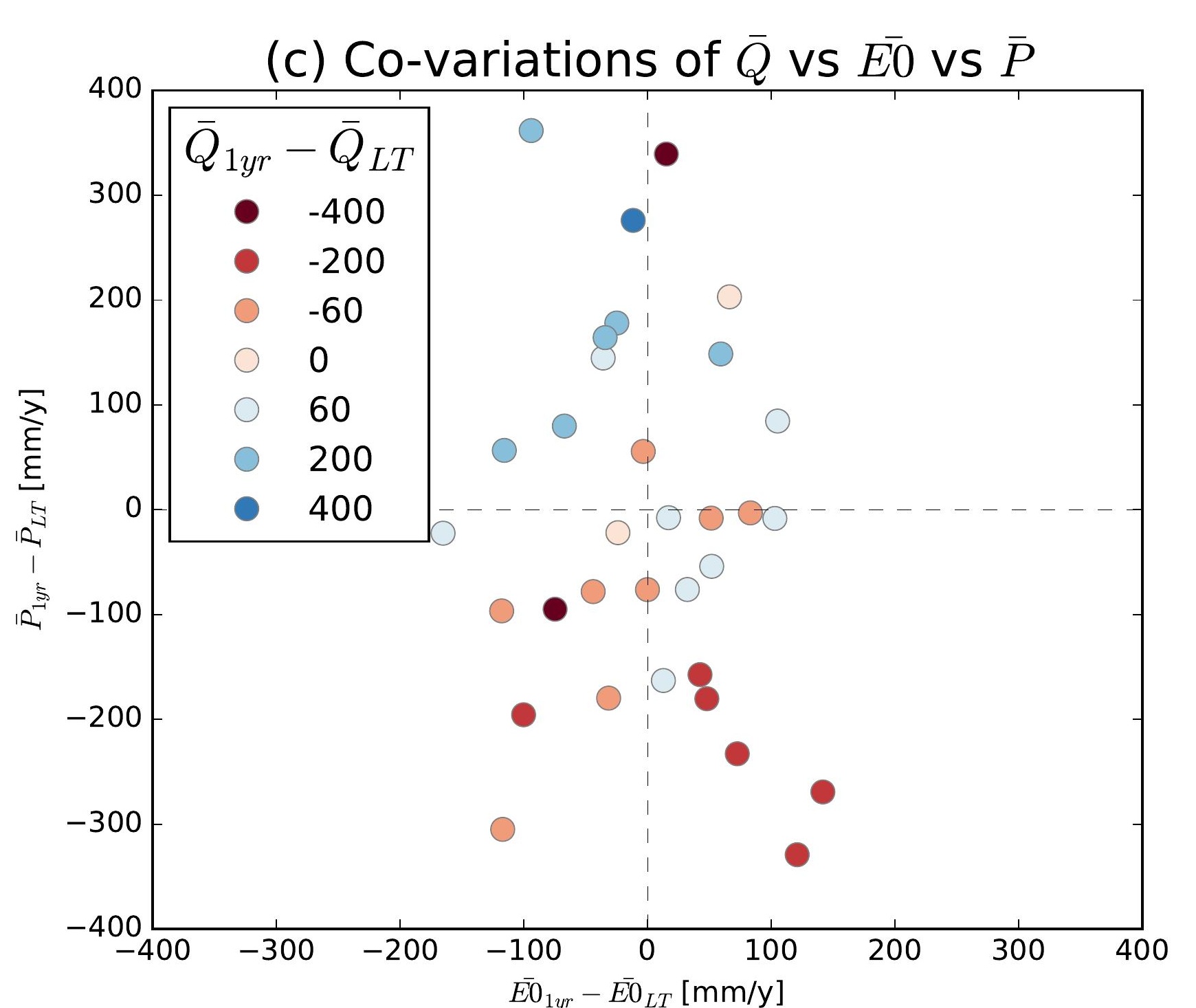
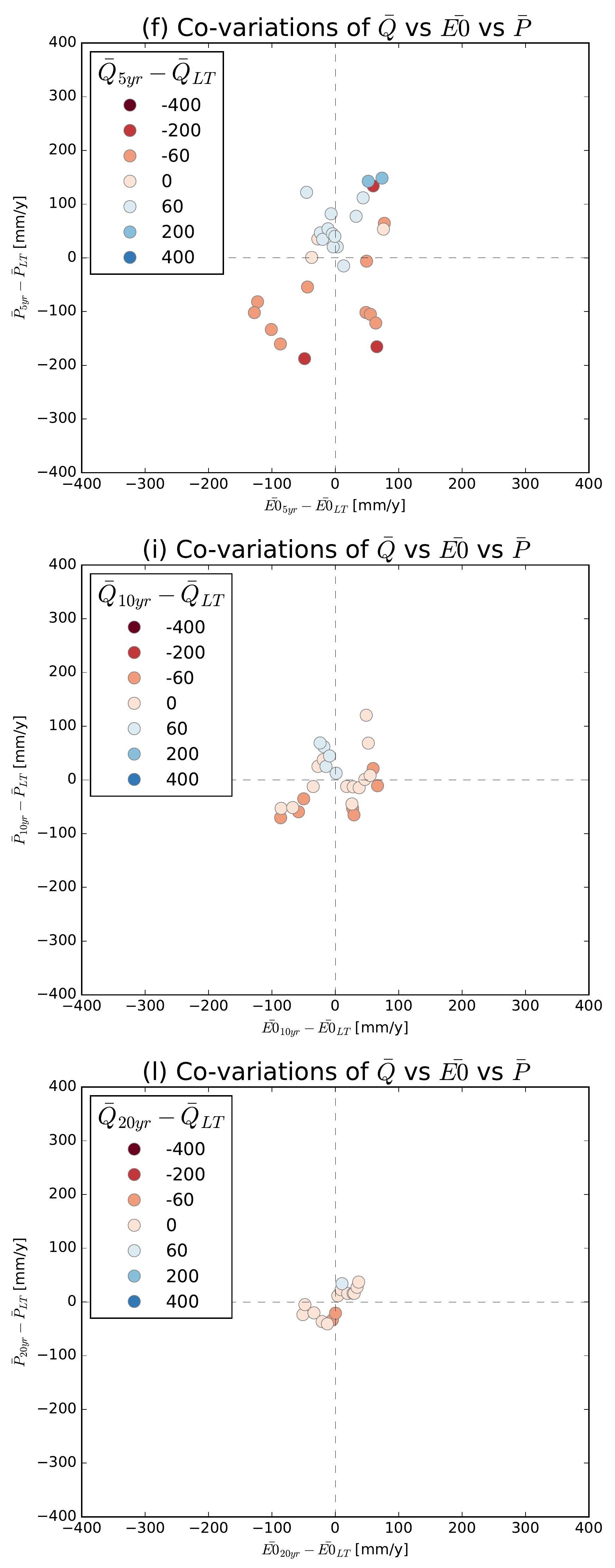

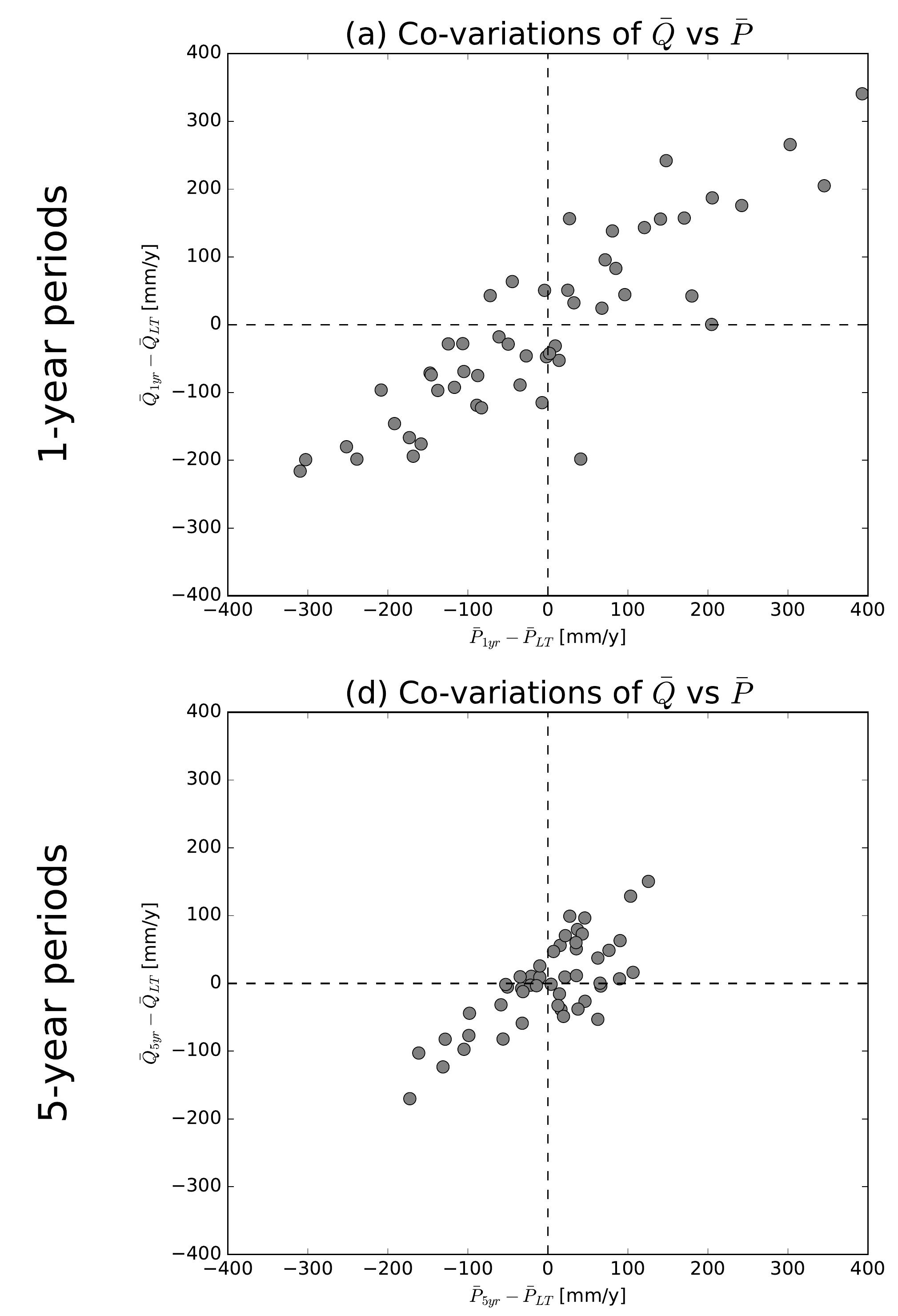

(g) Co-variations of $\bar{Q}$ vs $\bar{P}$
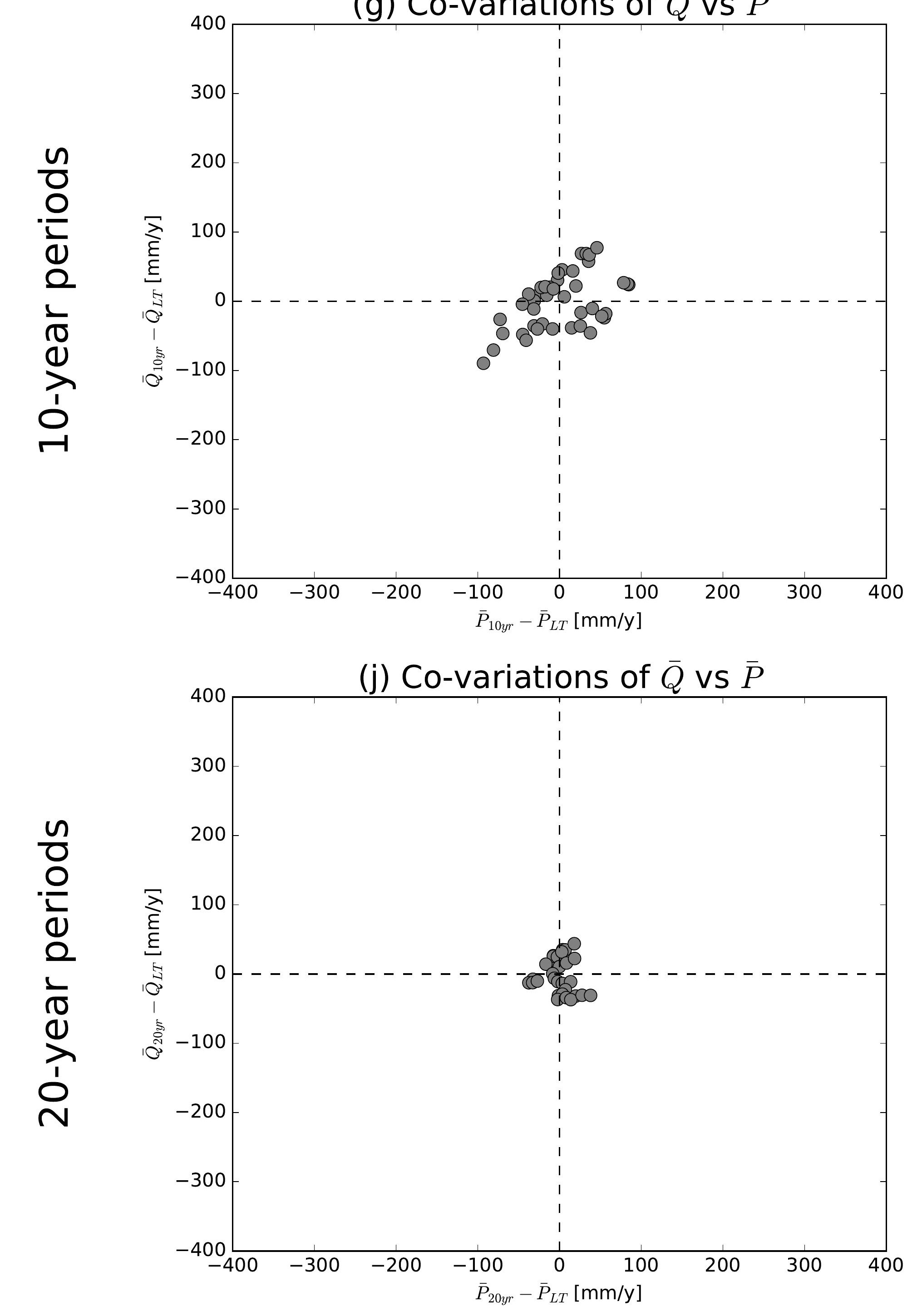

(b) Co-variations of $\bar{Q}$ vs $\overline{E 0}$

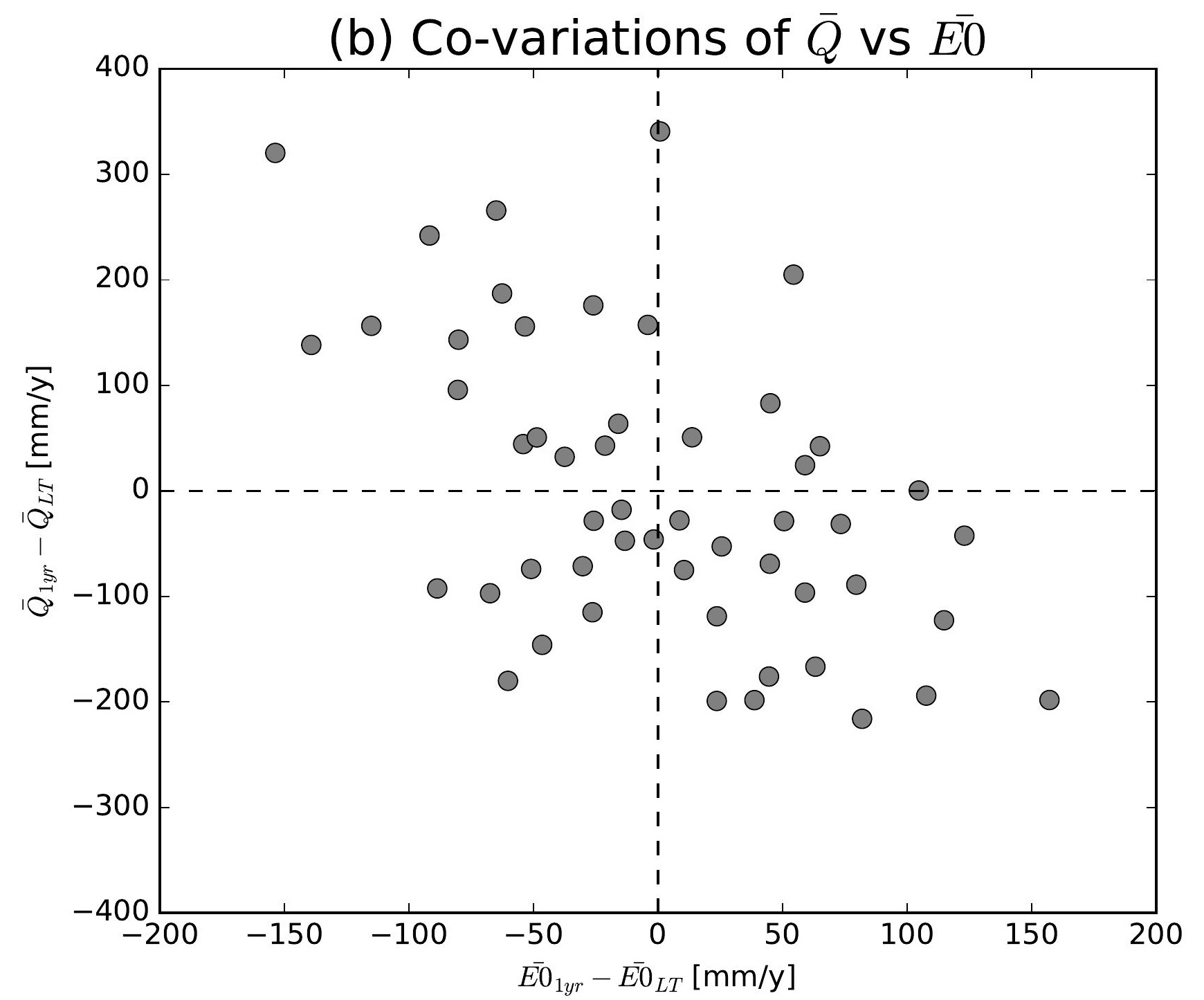

(e) Co-variations of $\bar{Q}$ vs $\overline{E 0}$

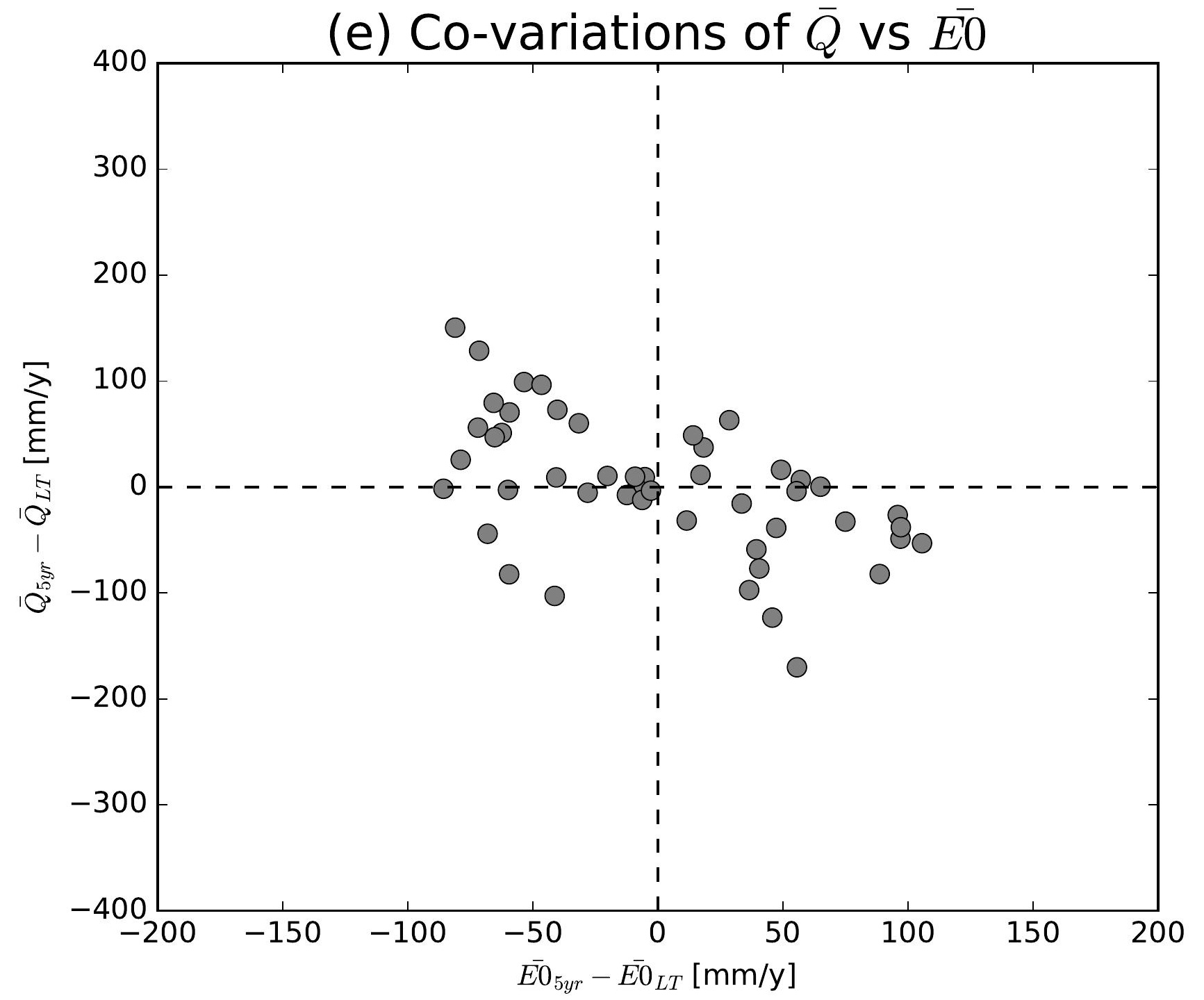

(h) Co-variations of $\bar{Q}$ vs $\overline{E 0}$

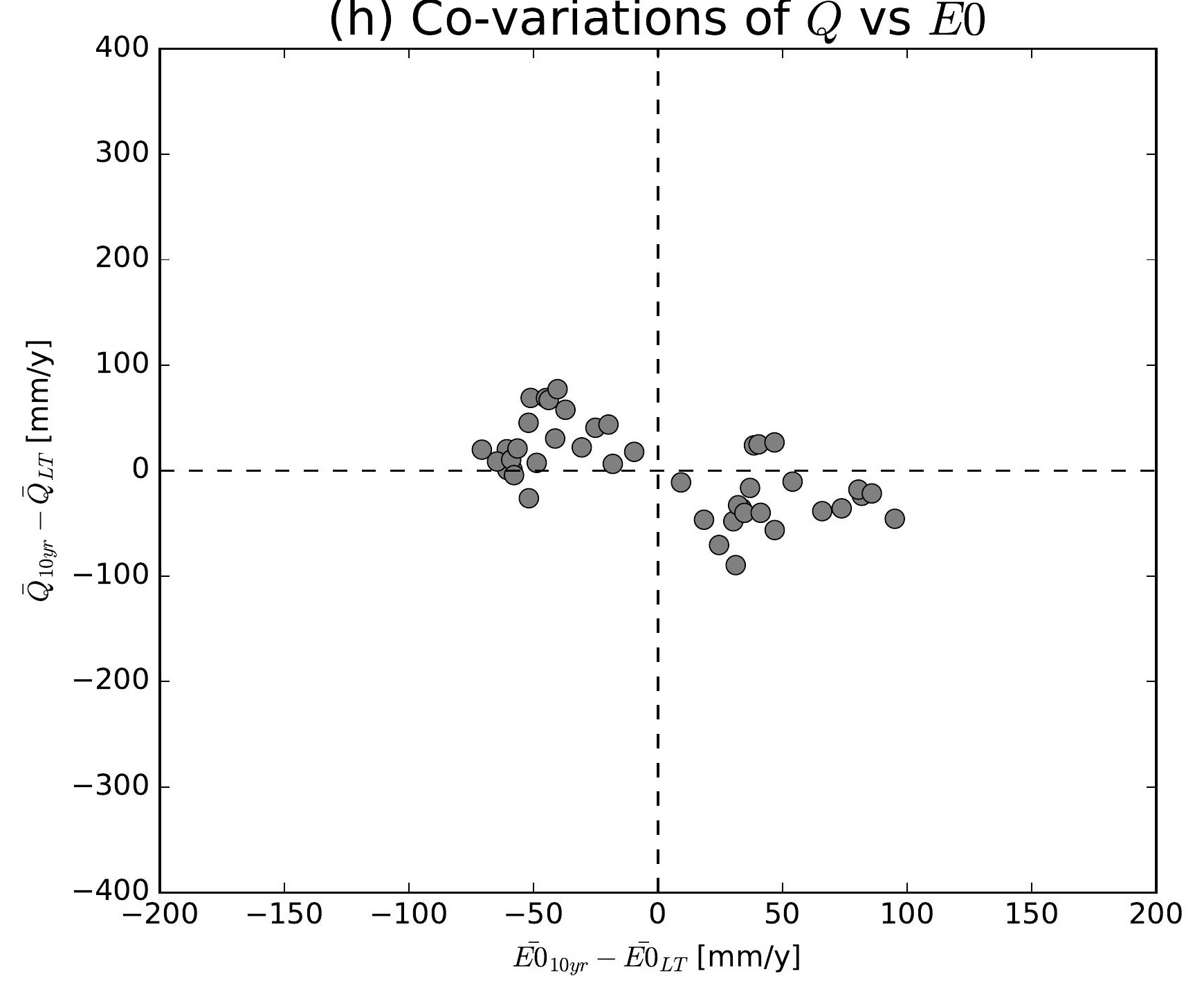

(k) Co-variations of $\bar{Q}$ vs $\overline{E 0}$

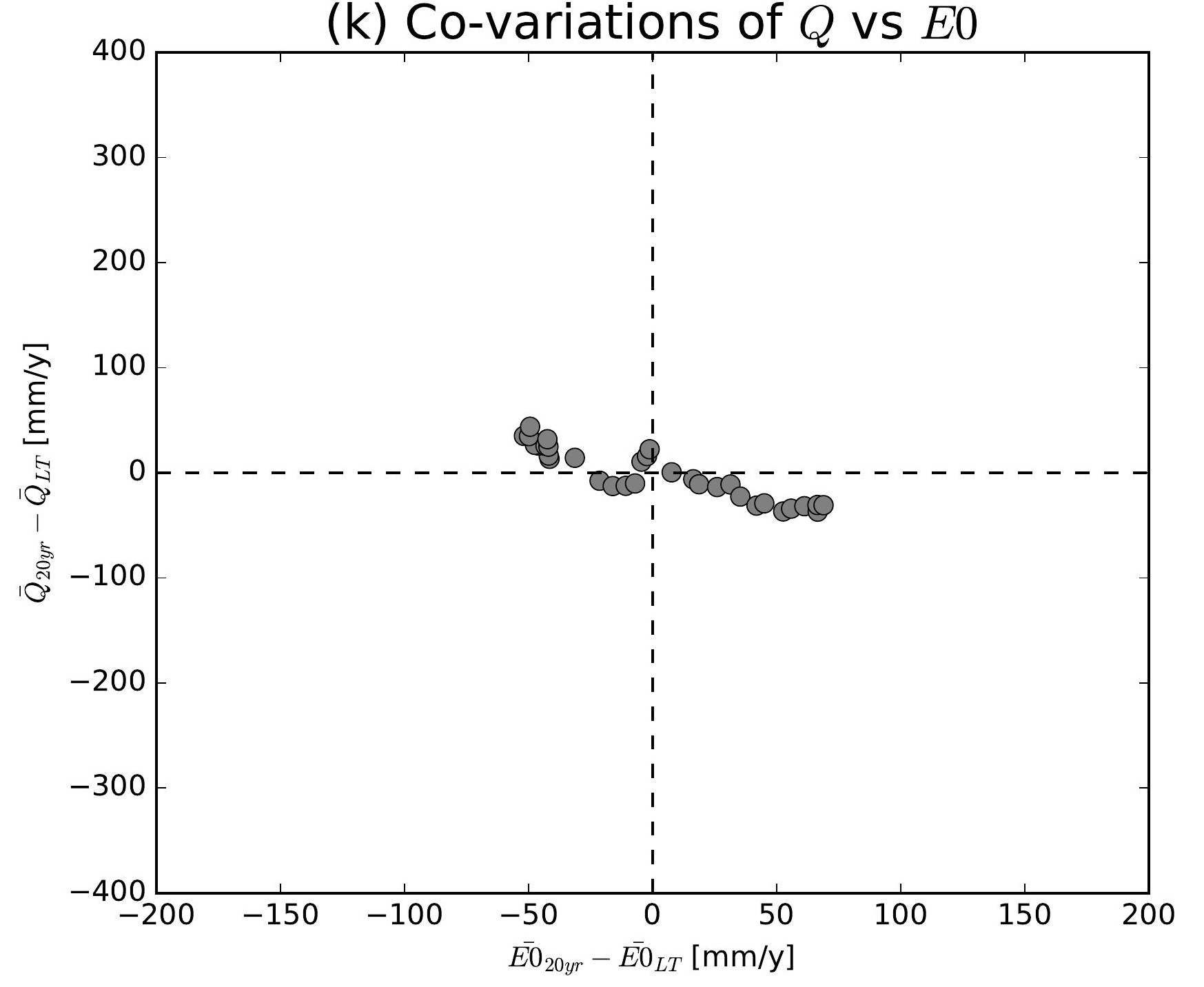

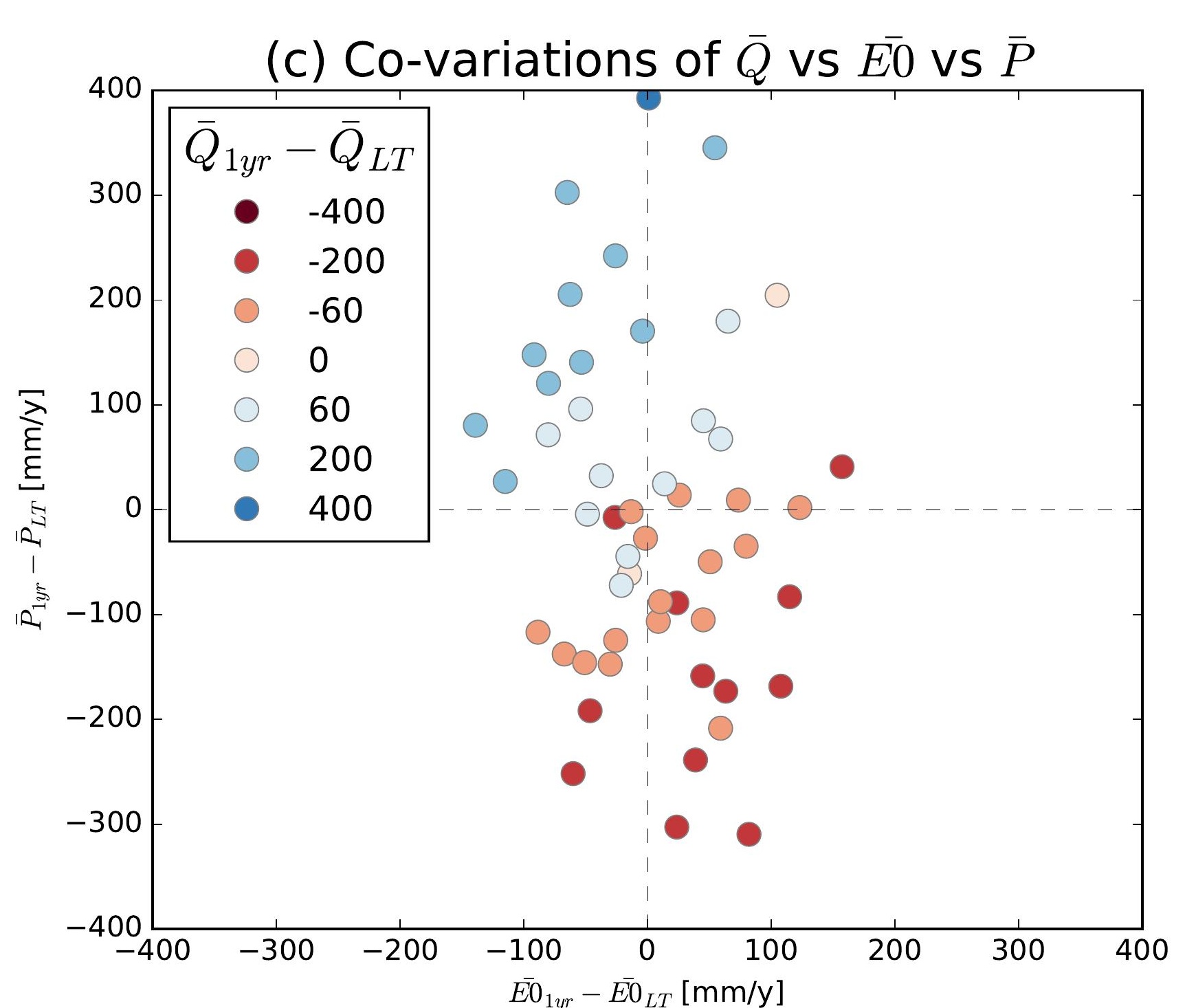
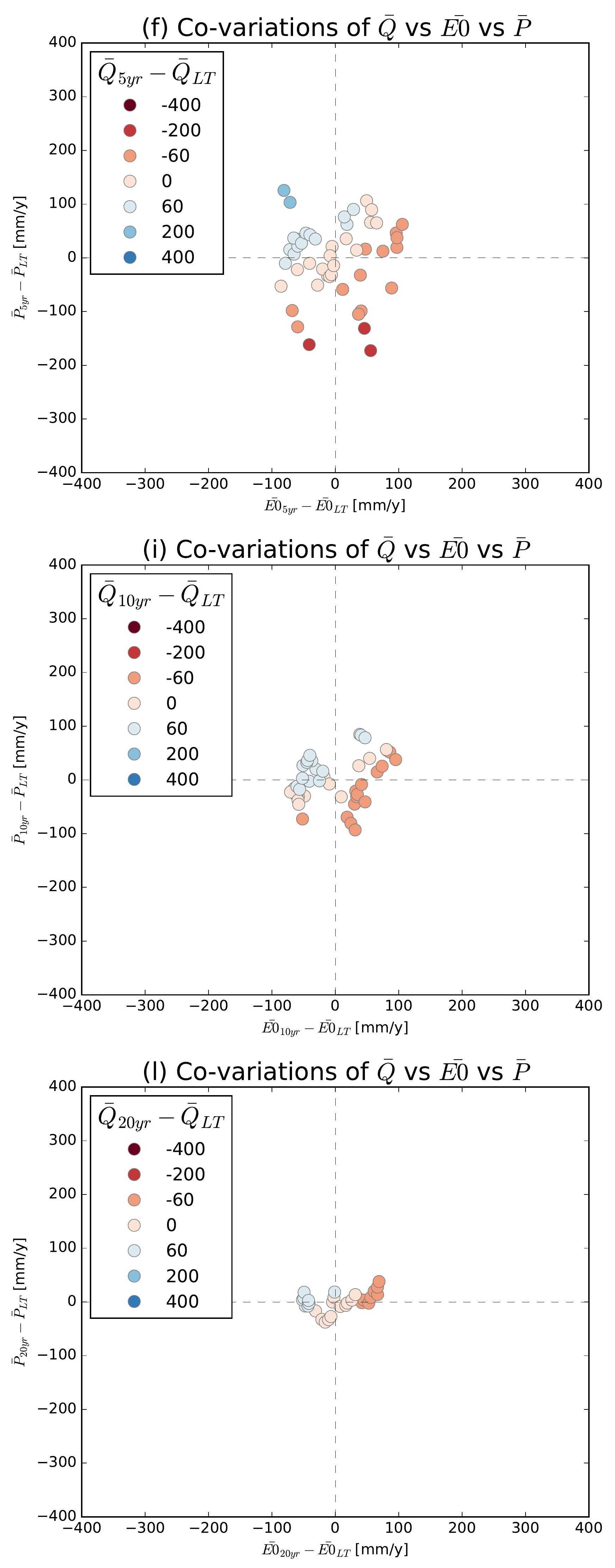

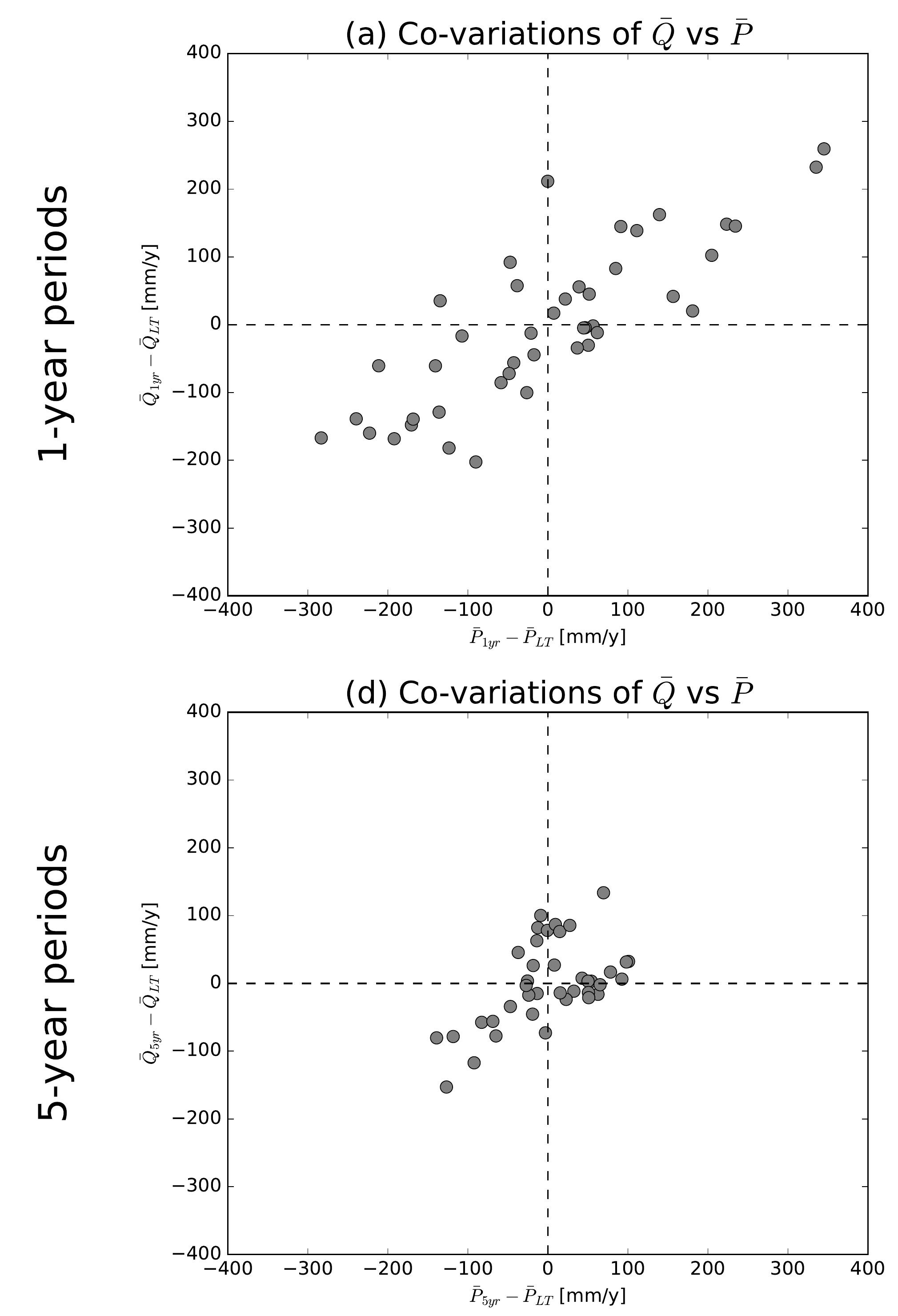

(g) Co-variations of $\bar{Q}$ vs $\bar{P}$
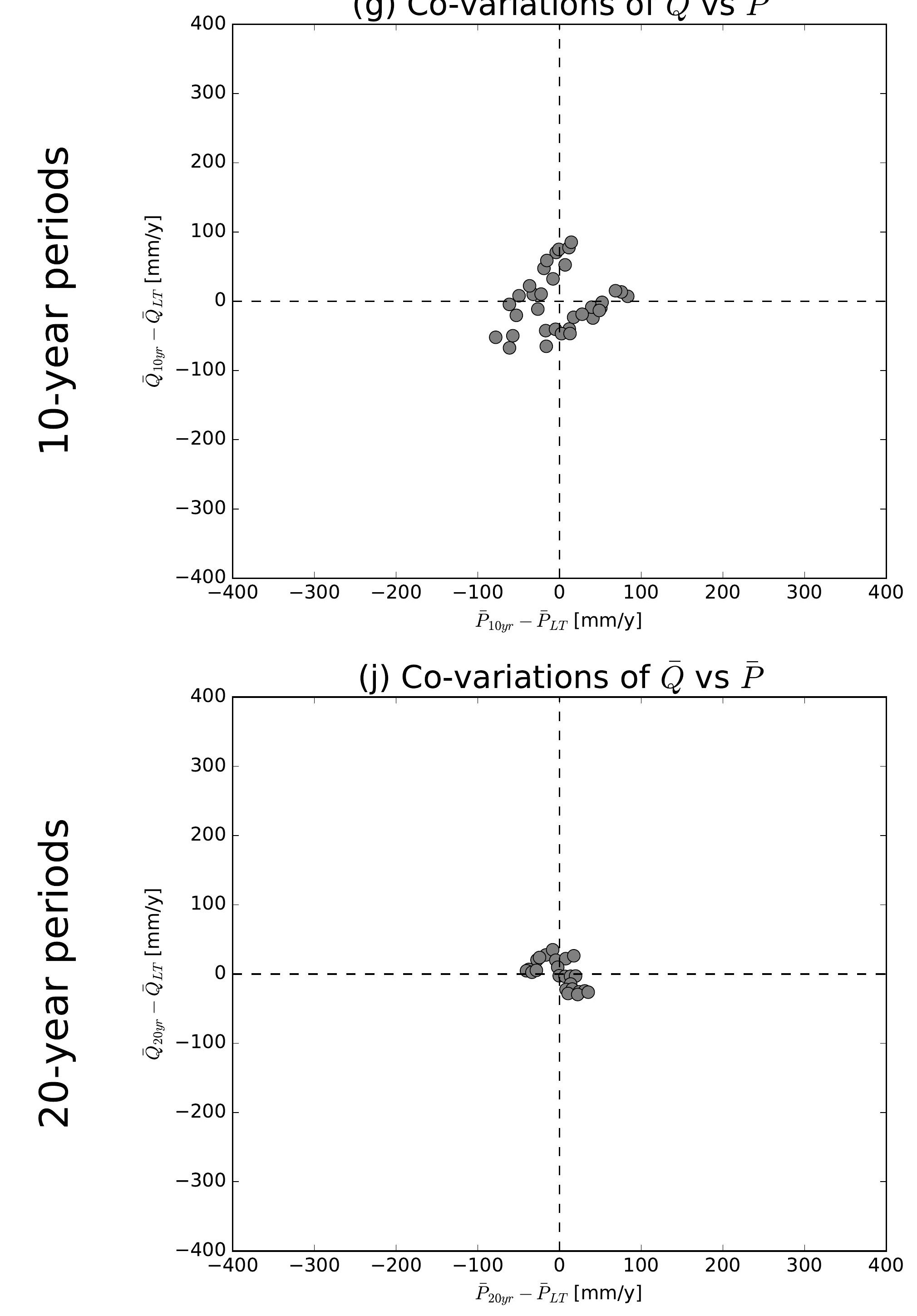

(b) Co-variations of $\bar{Q}$ vs $\overline{E 0}$

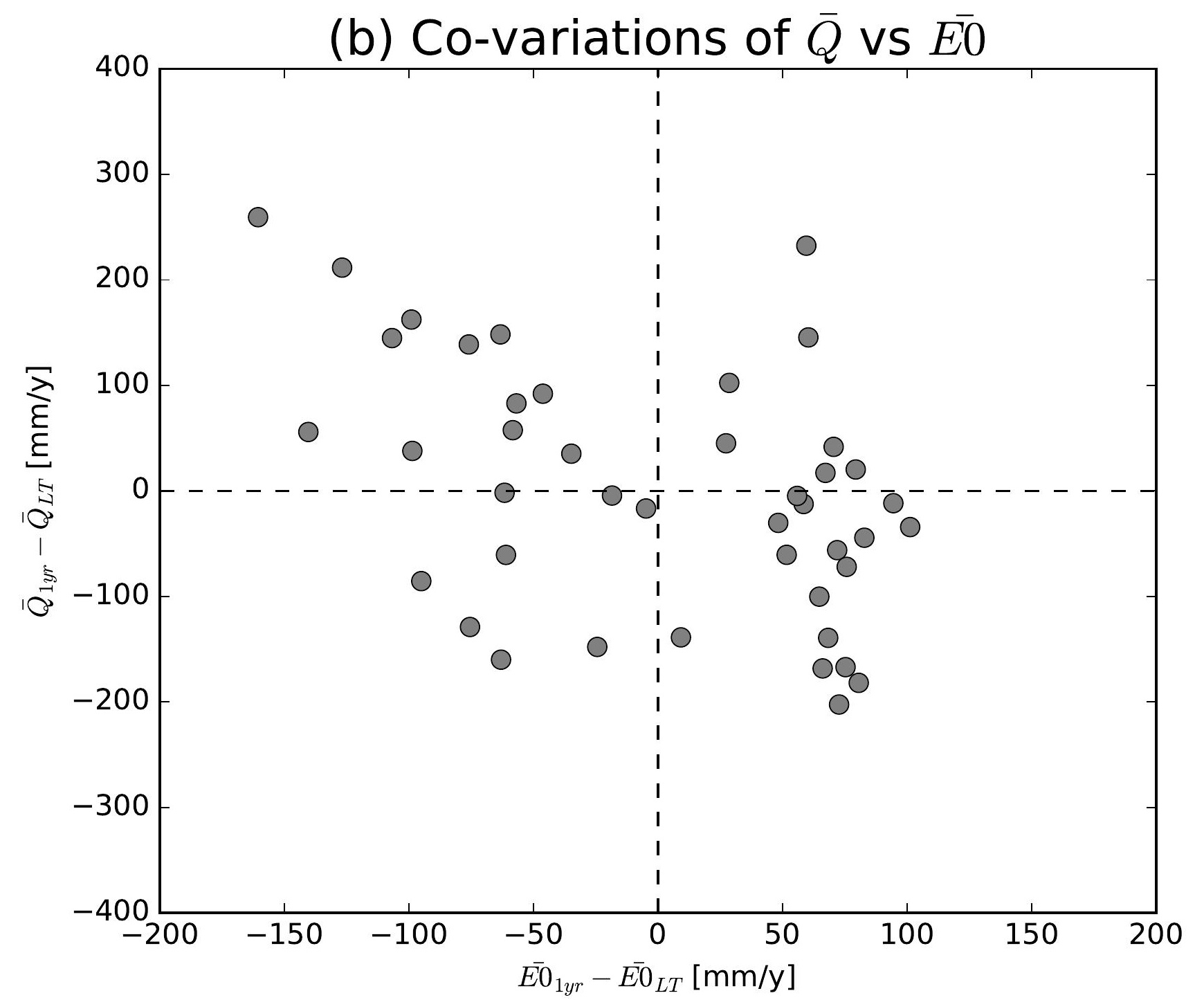

(e) Co-variations of $\bar{Q}$ vs $\overline{E 0}$

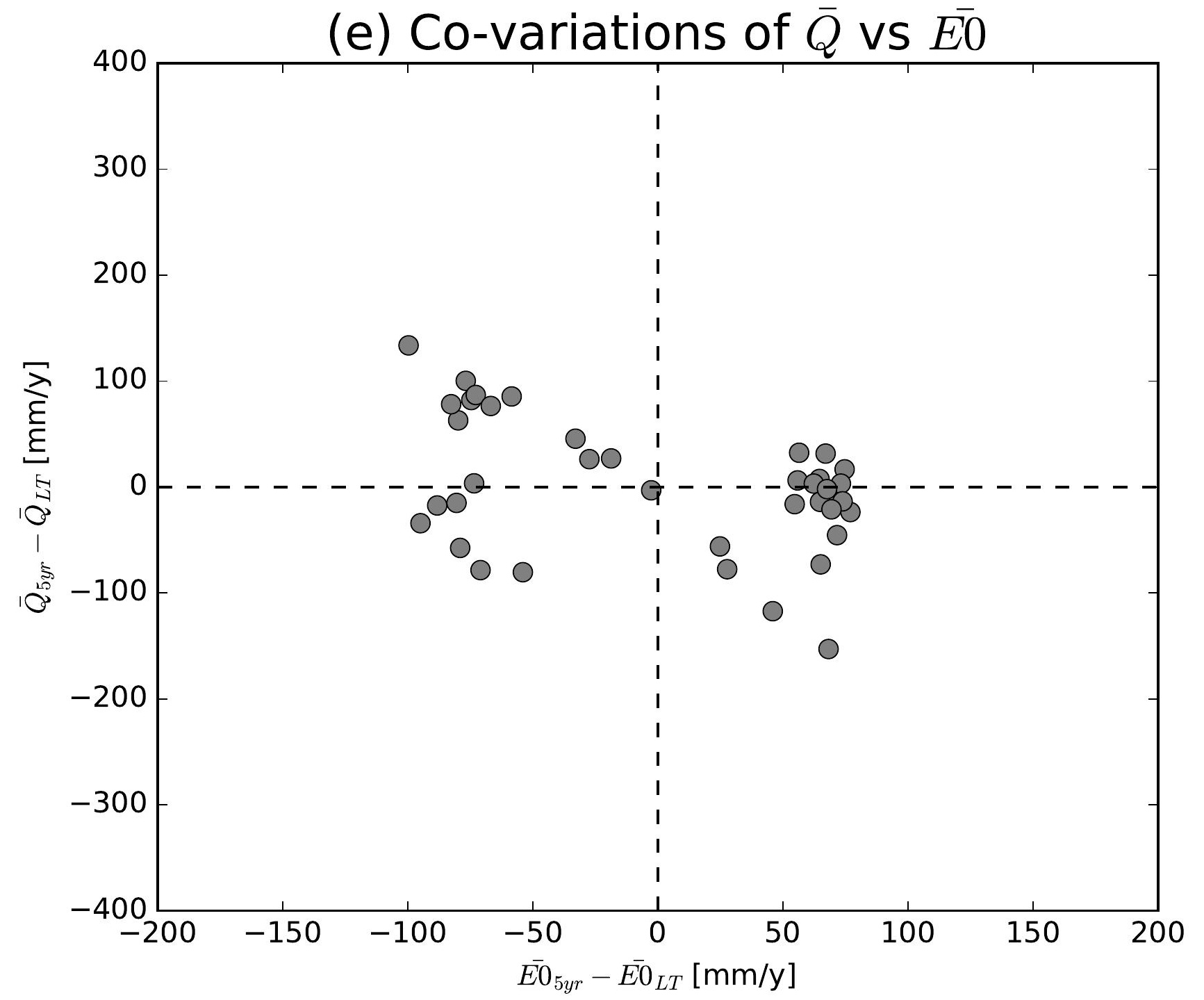

(h) Co-variations of $\bar{Q}$ vs $\overline{E 0}$

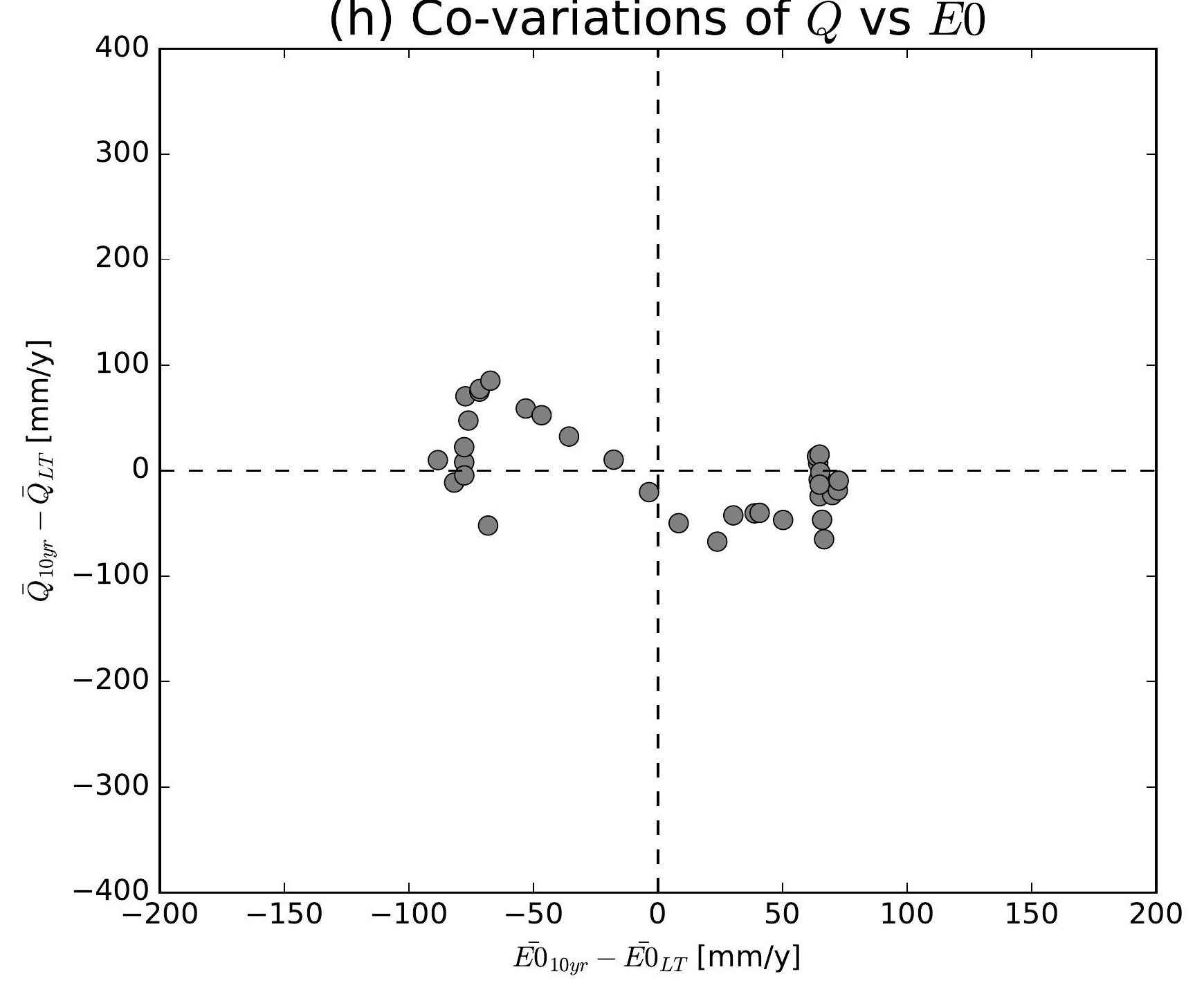

(k) Co-variations of $\bar{Q}$ vs $\overline{E 0}$

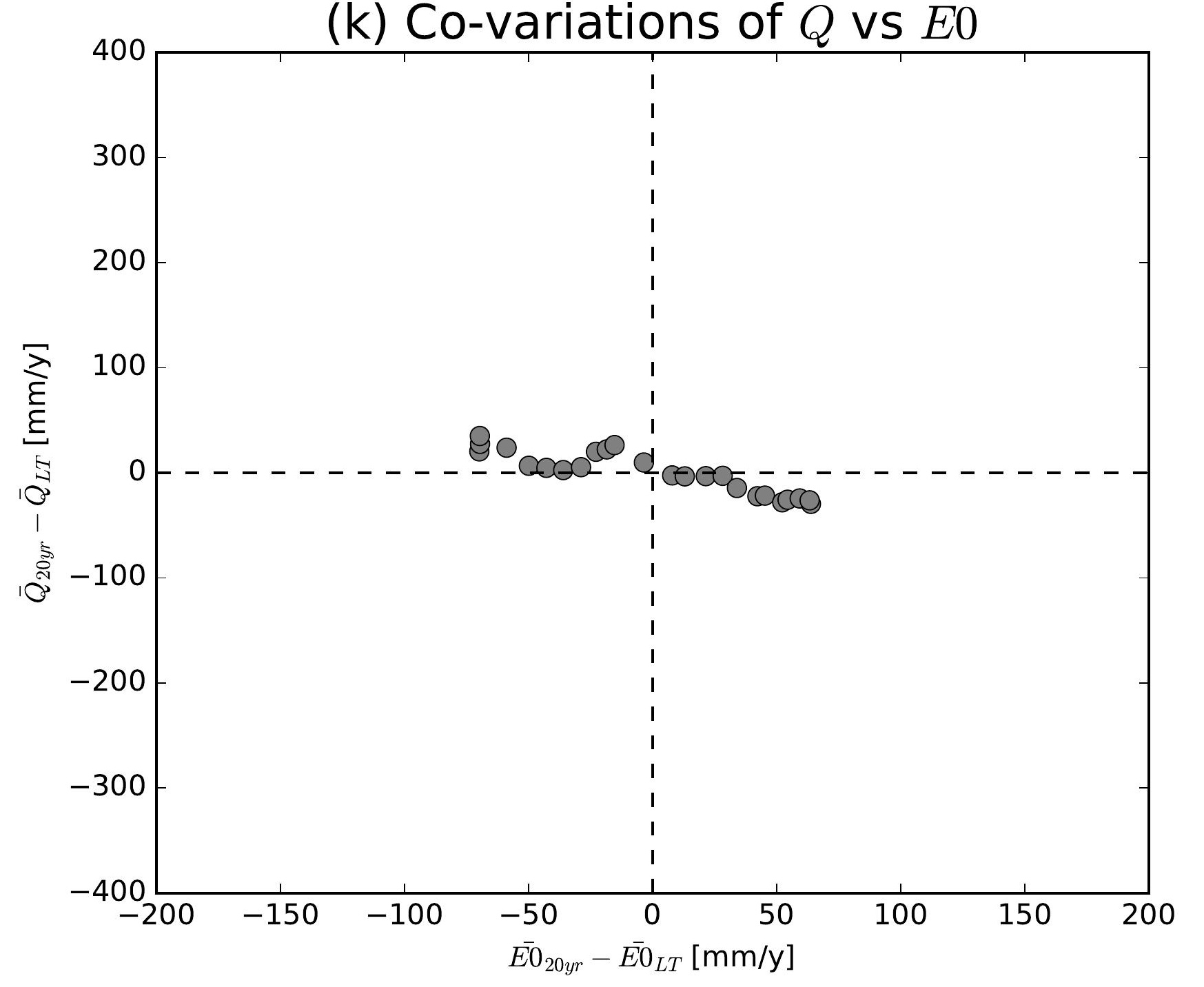

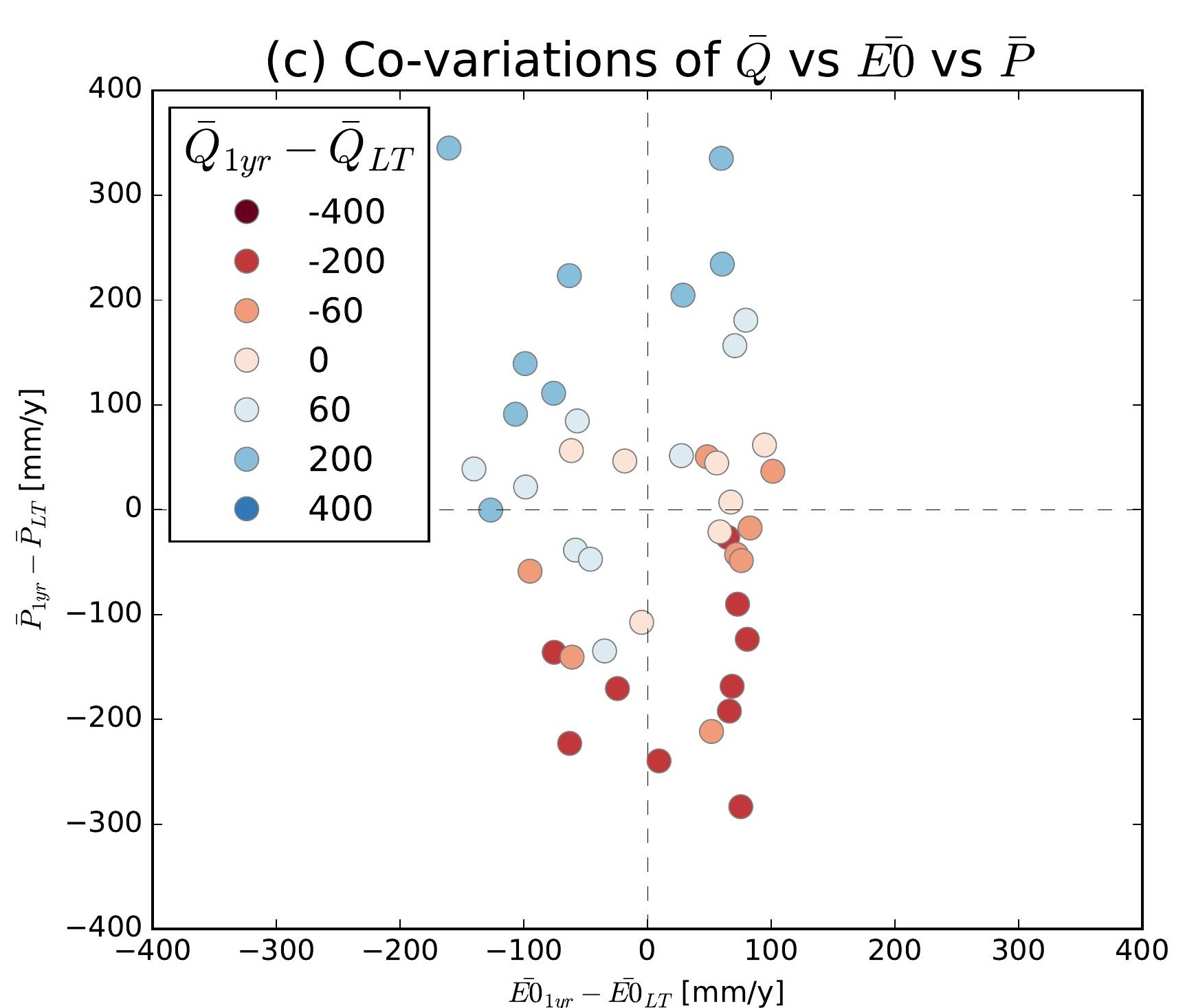
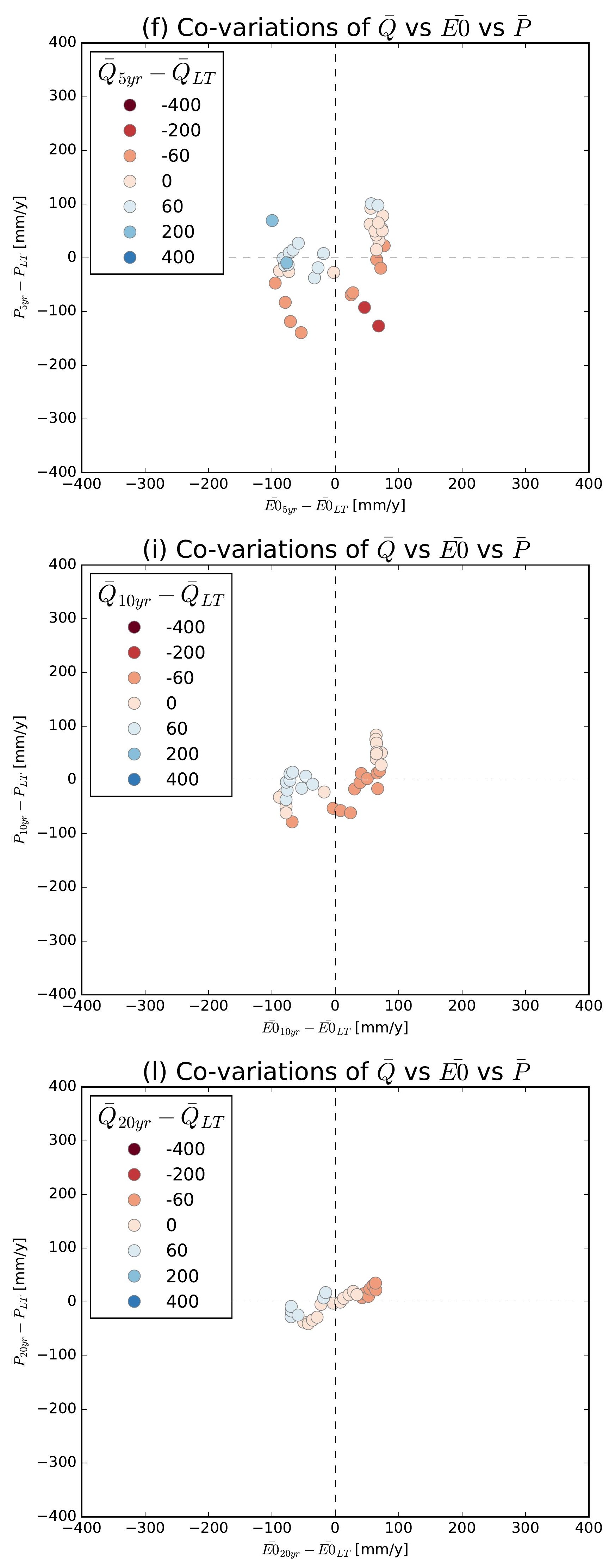

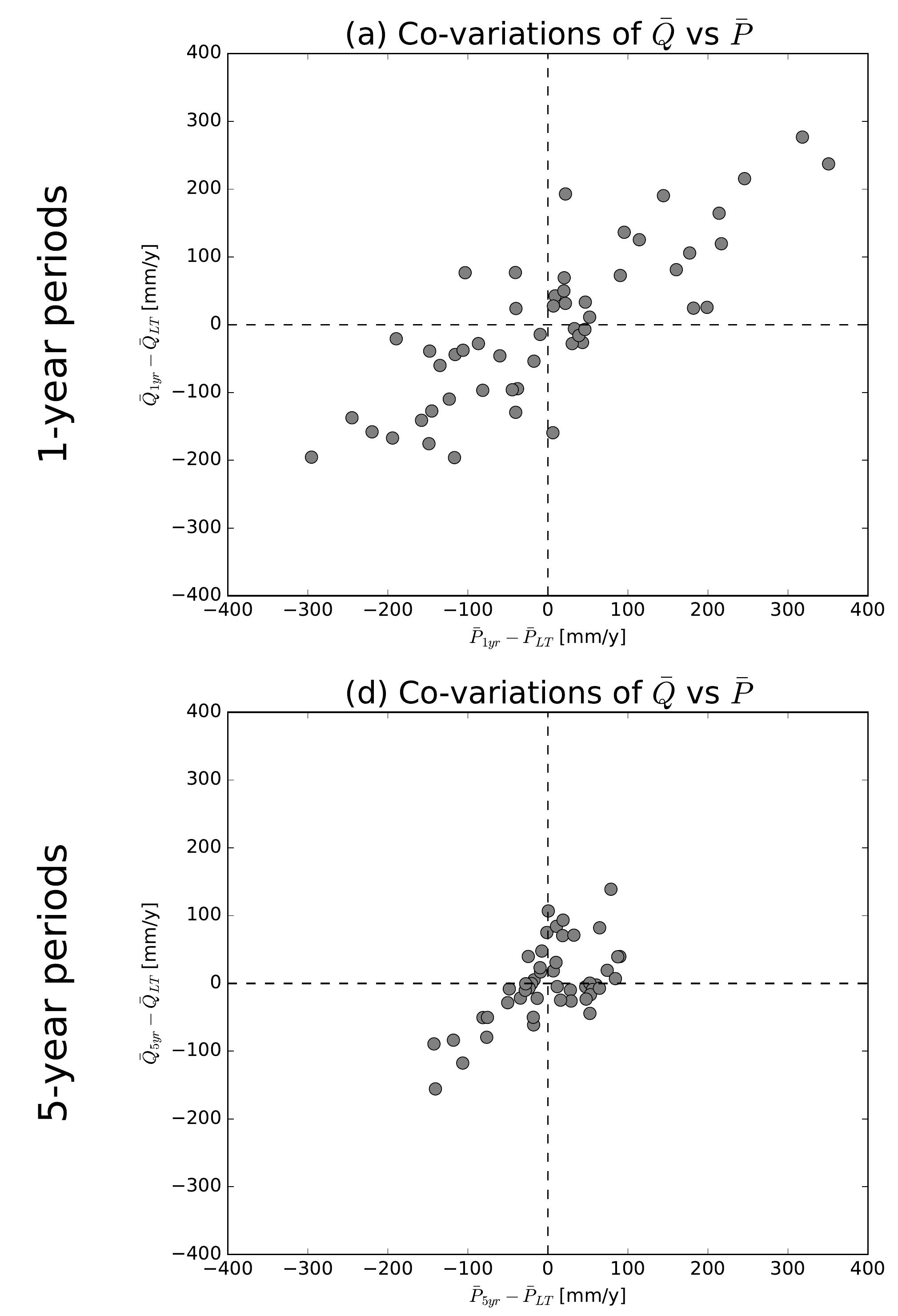

(g) Co-variations of $\bar{Q}$ vs $\bar{P}$
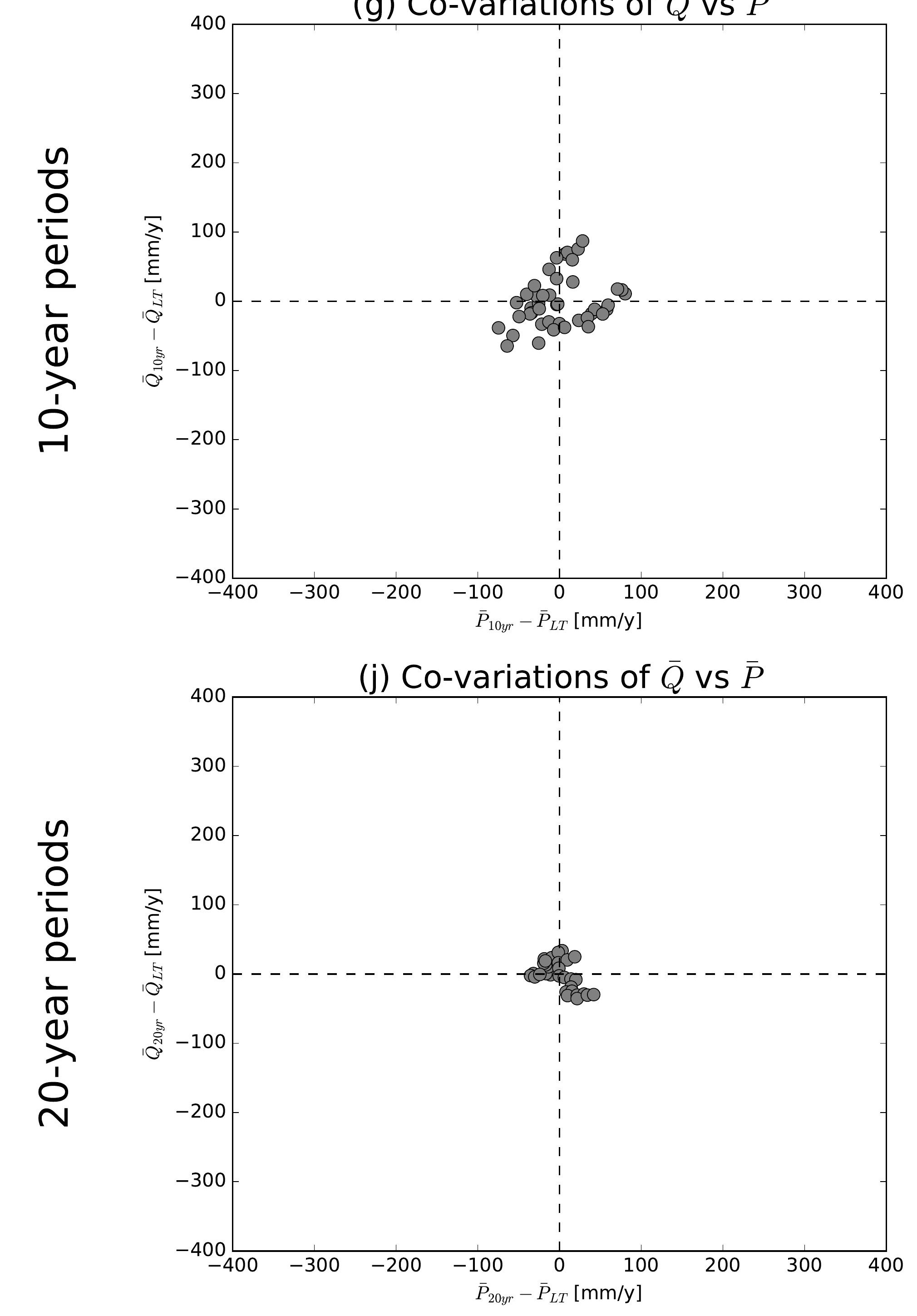

(b) Co-variations of $\bar{Q}$ vs $\overline{E 0}$

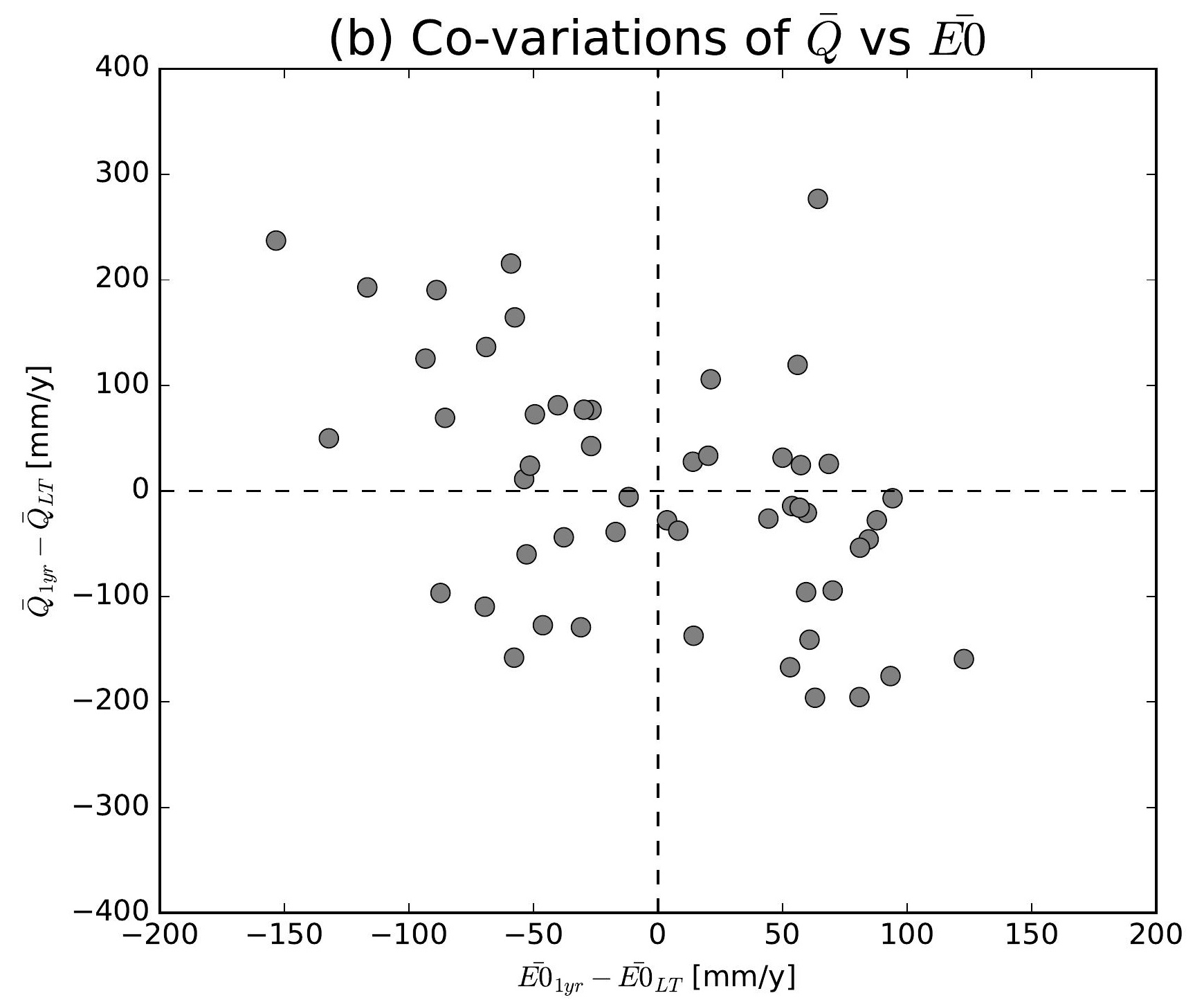

(e) Co-variations of $\bar{Q}$ vs $\overline{E 0}$

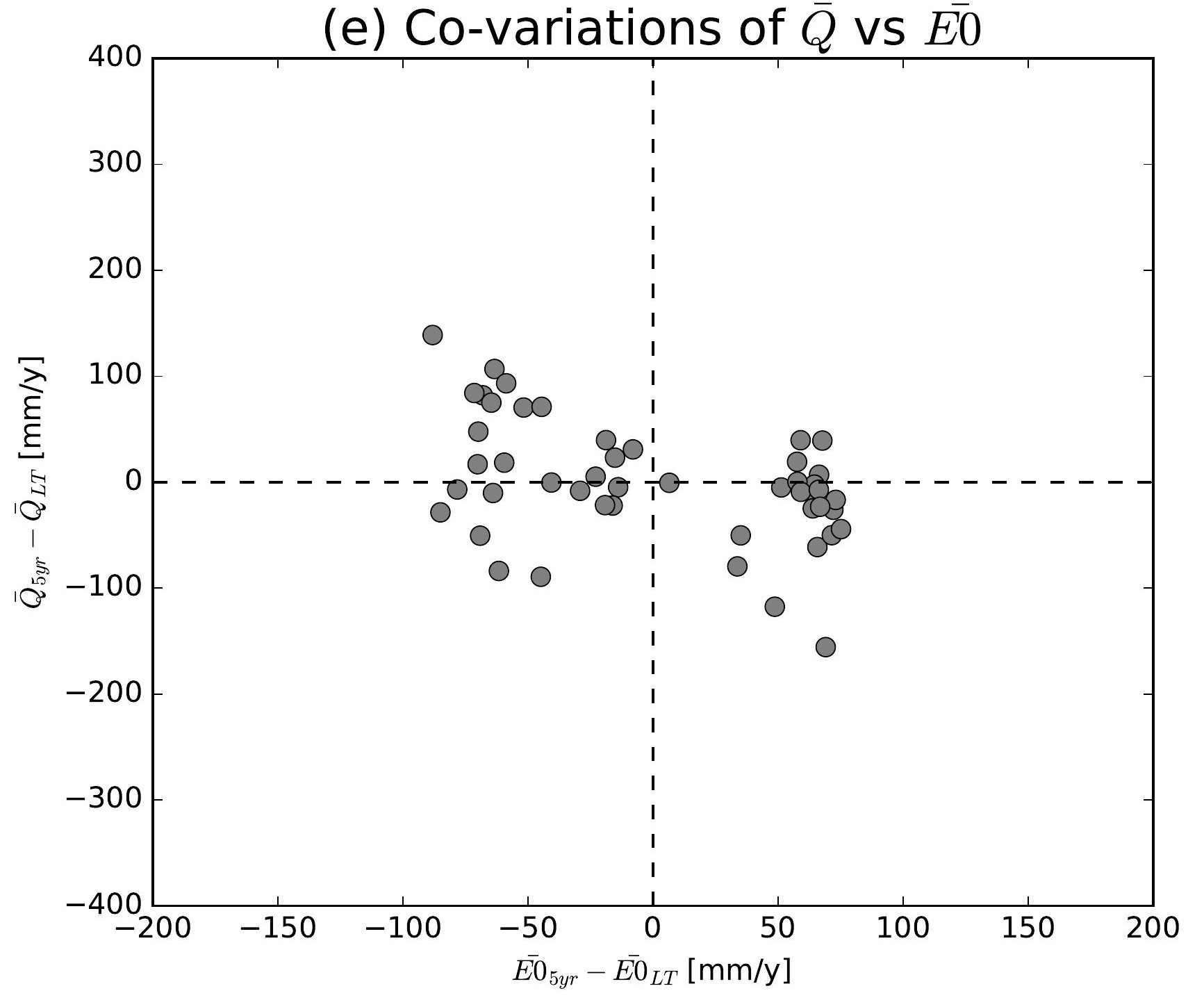

(h) Co-variations of $\bar{Q}$ vs $\overline{E 0}$

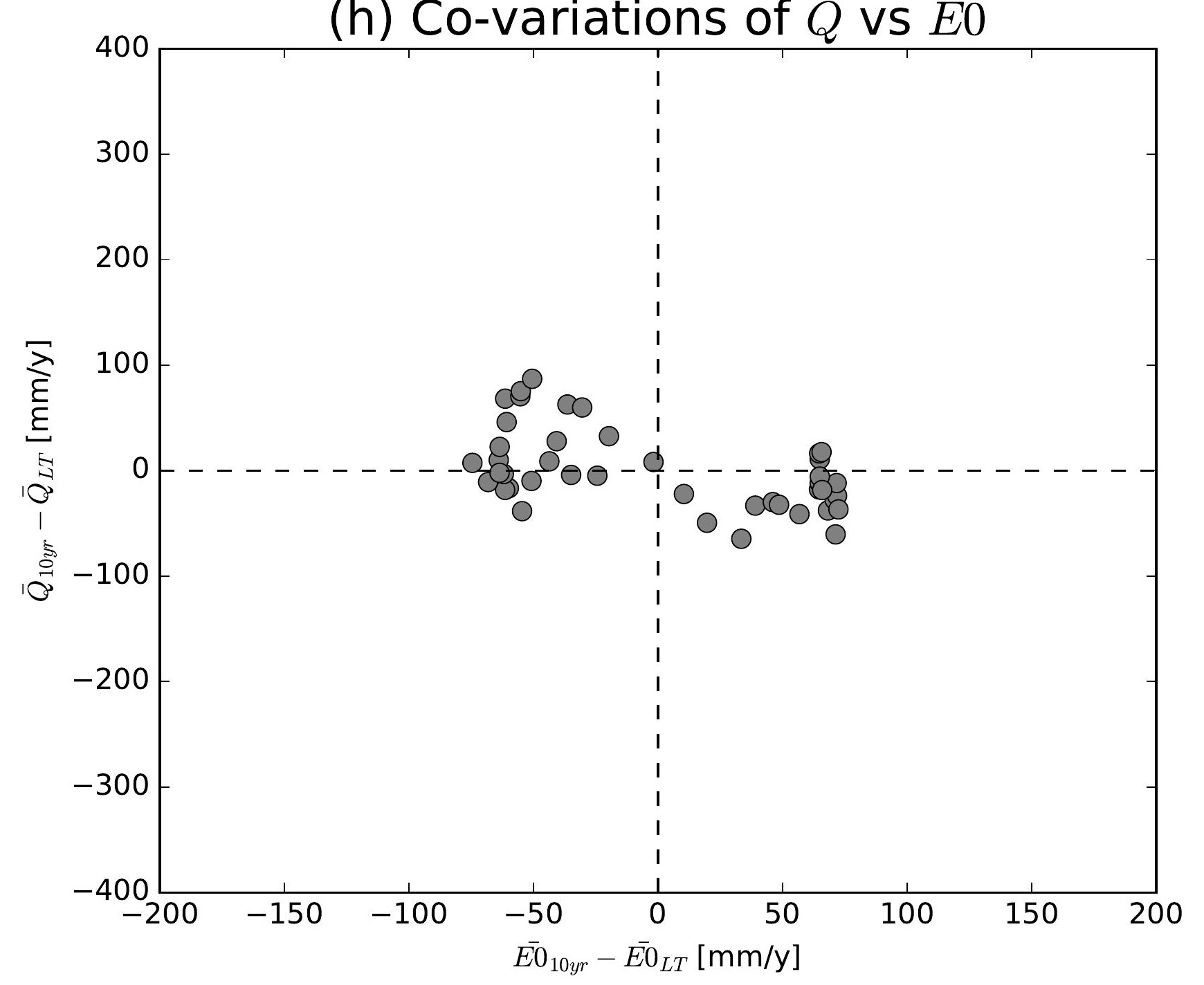

(k) Co-variations of $\bar{Q}$ vs $\overline{E 0}$

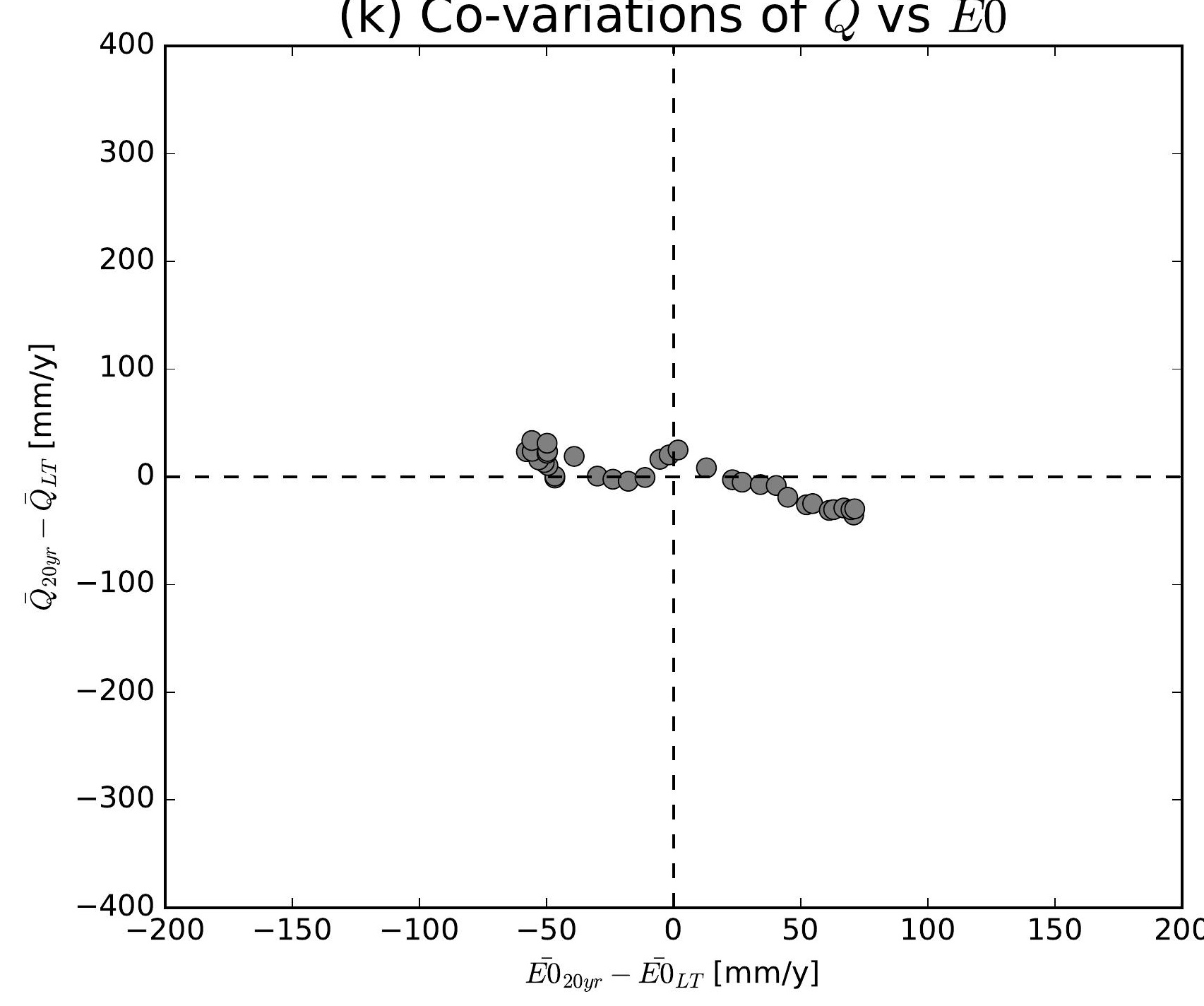

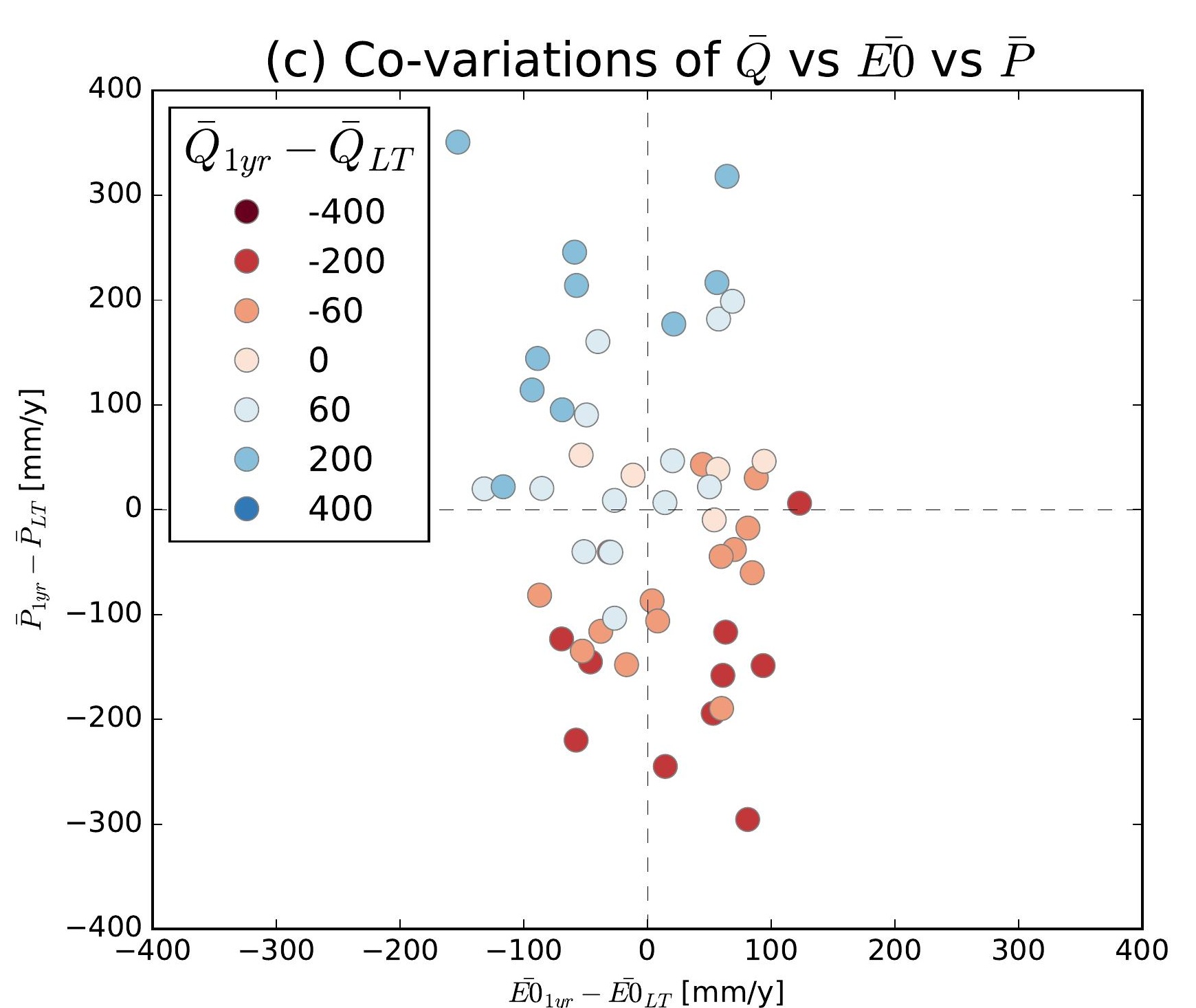
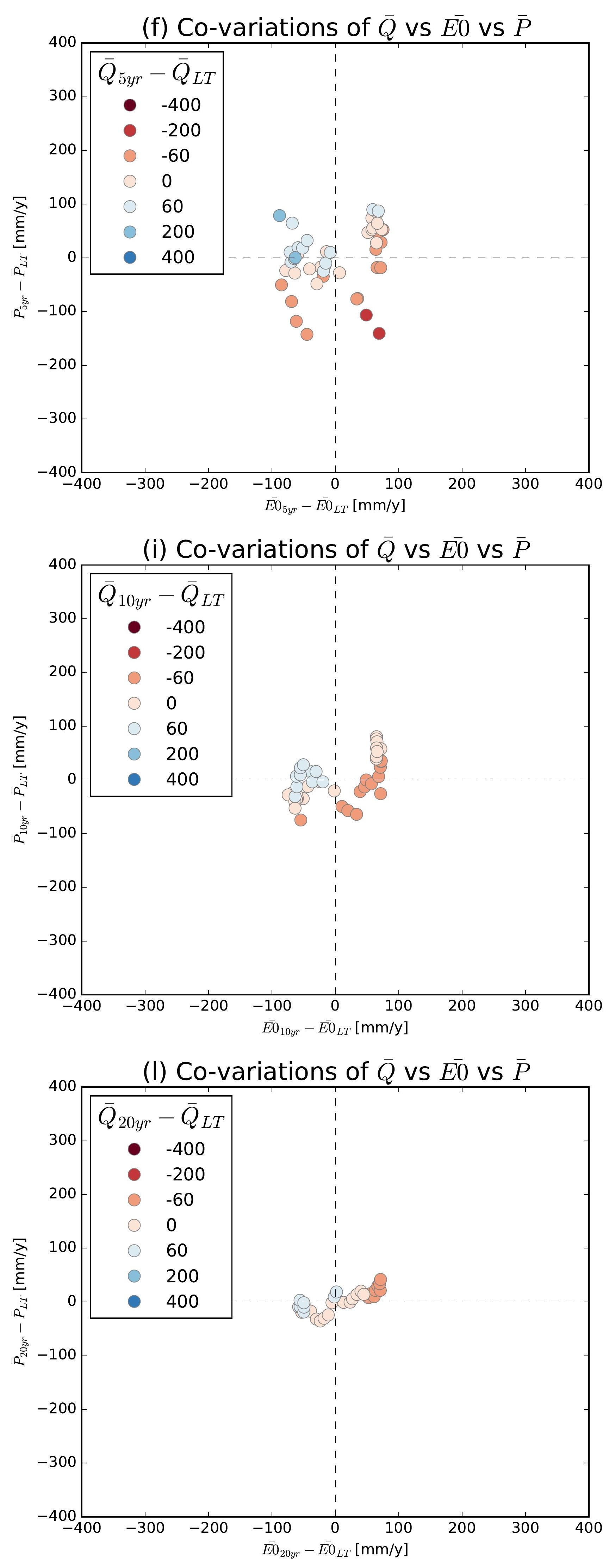

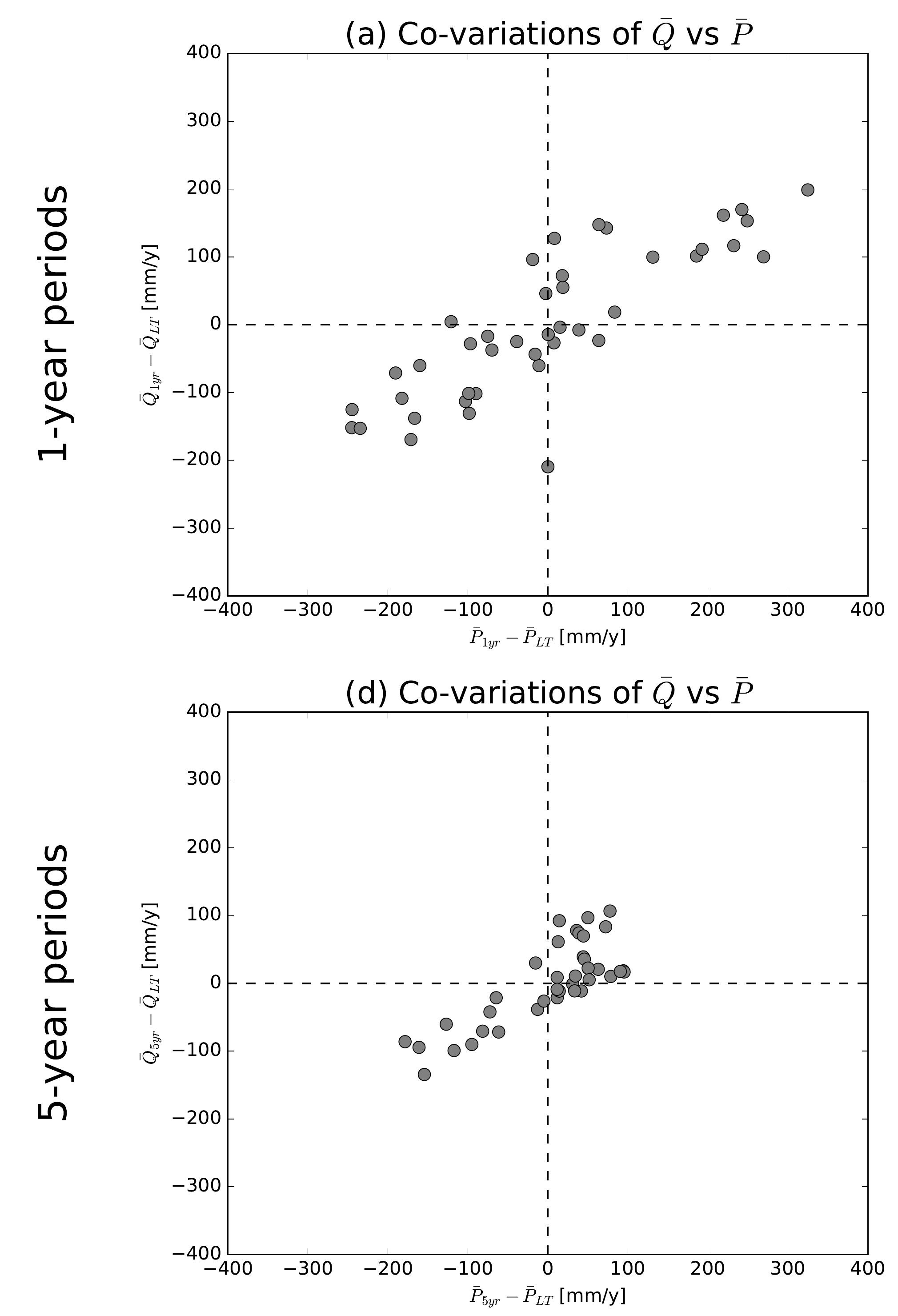

(g) Co-variations of $\bar{Q}$ vs $\bar{P}$
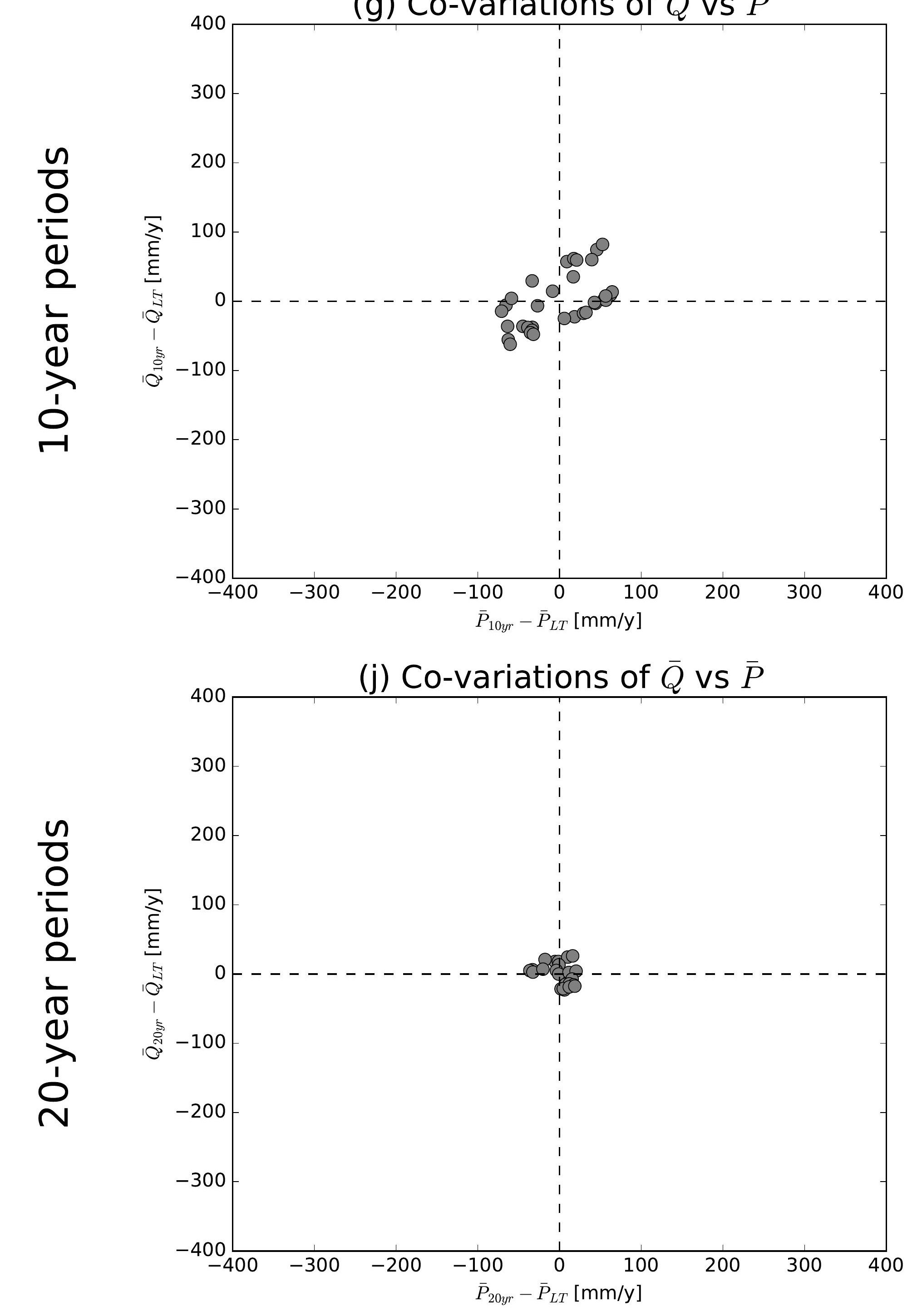

(b) Co-variations of $\bar{Q}$ vs $\overline{E 0}$

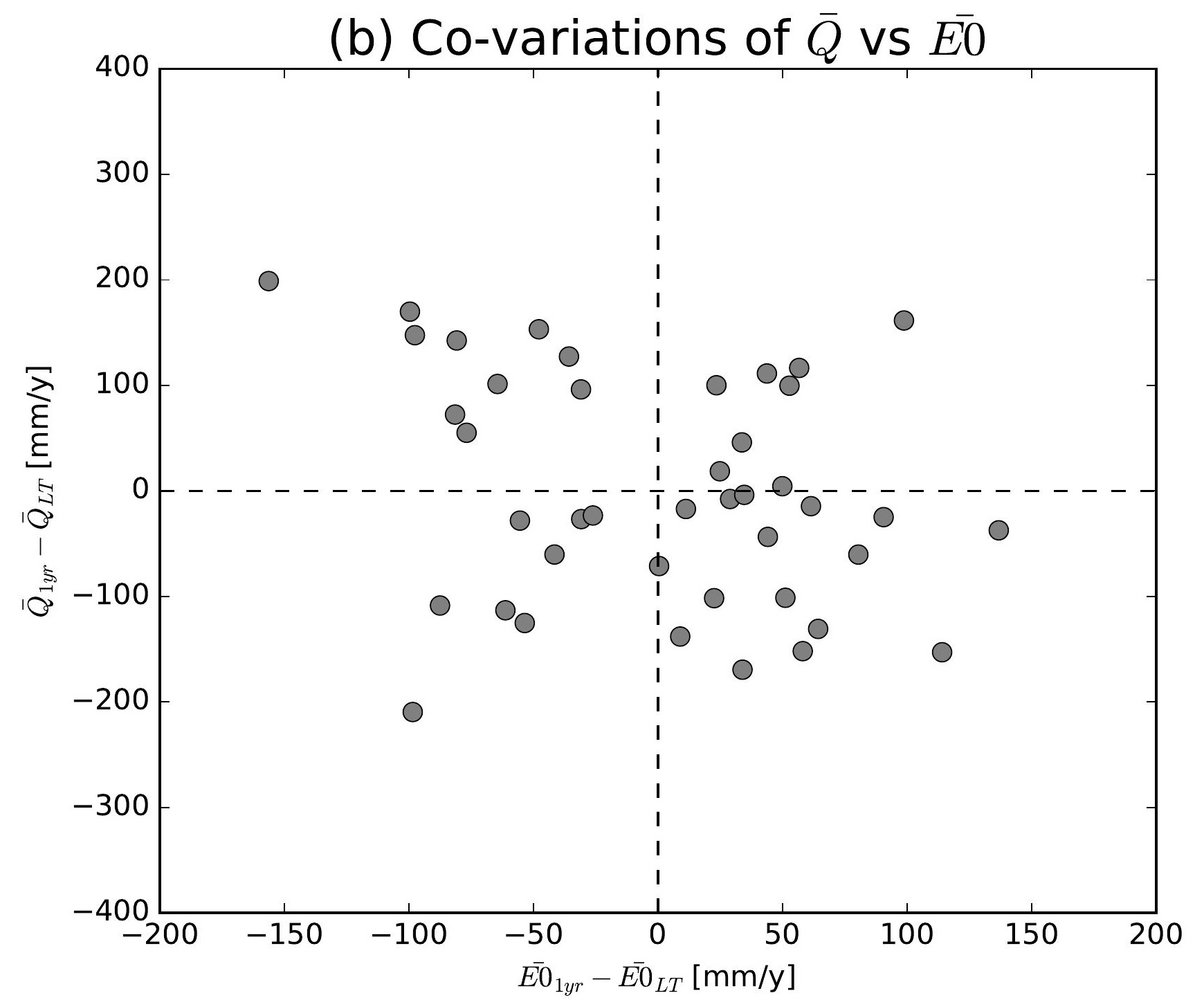

(e) Co-variations of $\bar{Q}$ vs $\overline{E 0}$

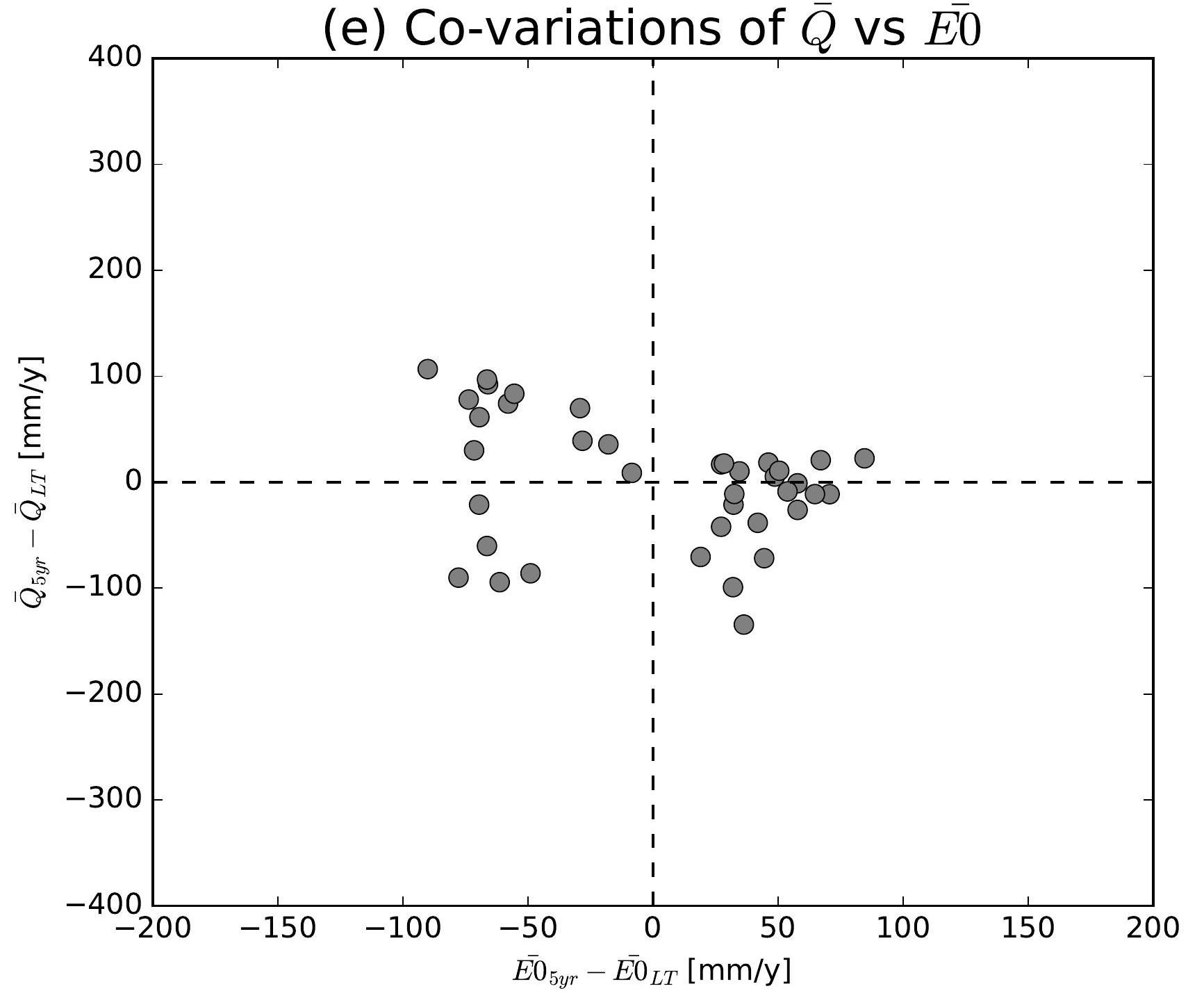

(h) Co-variations of $\bar{Q}$ vs $\overline{E 0}$

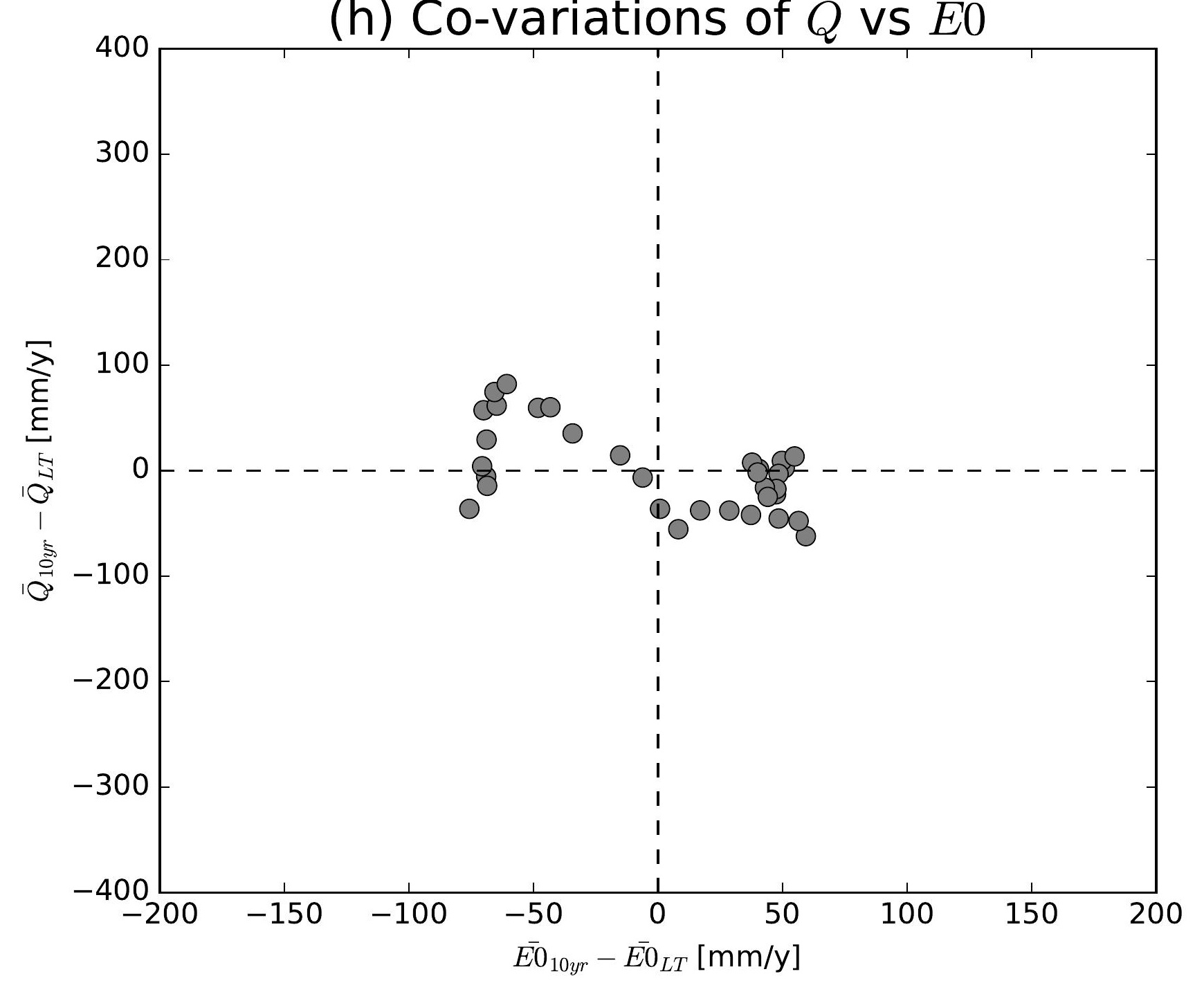

(k) Co-variations of $\bar{Q}$ vs $\overline{E 0}$

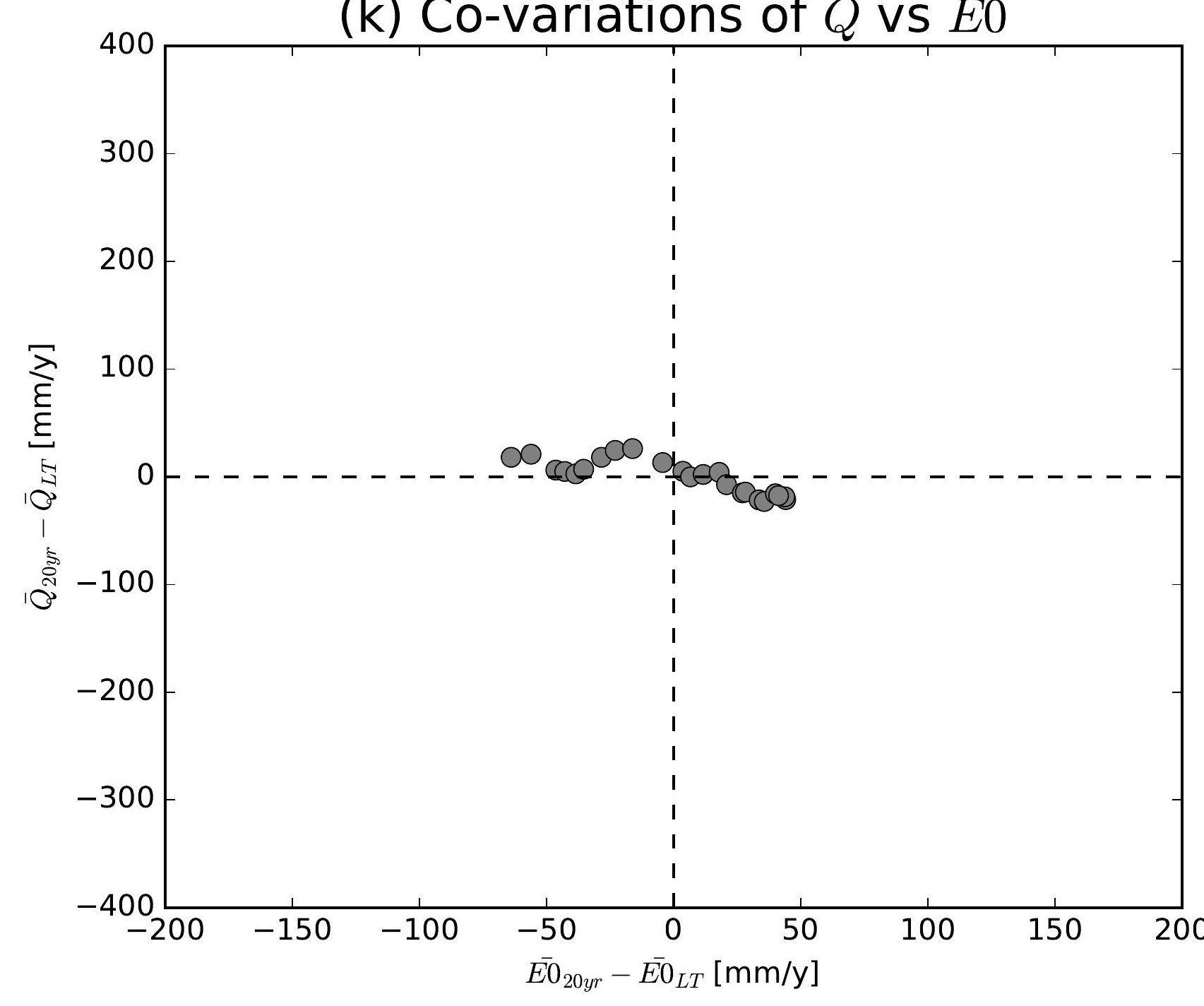

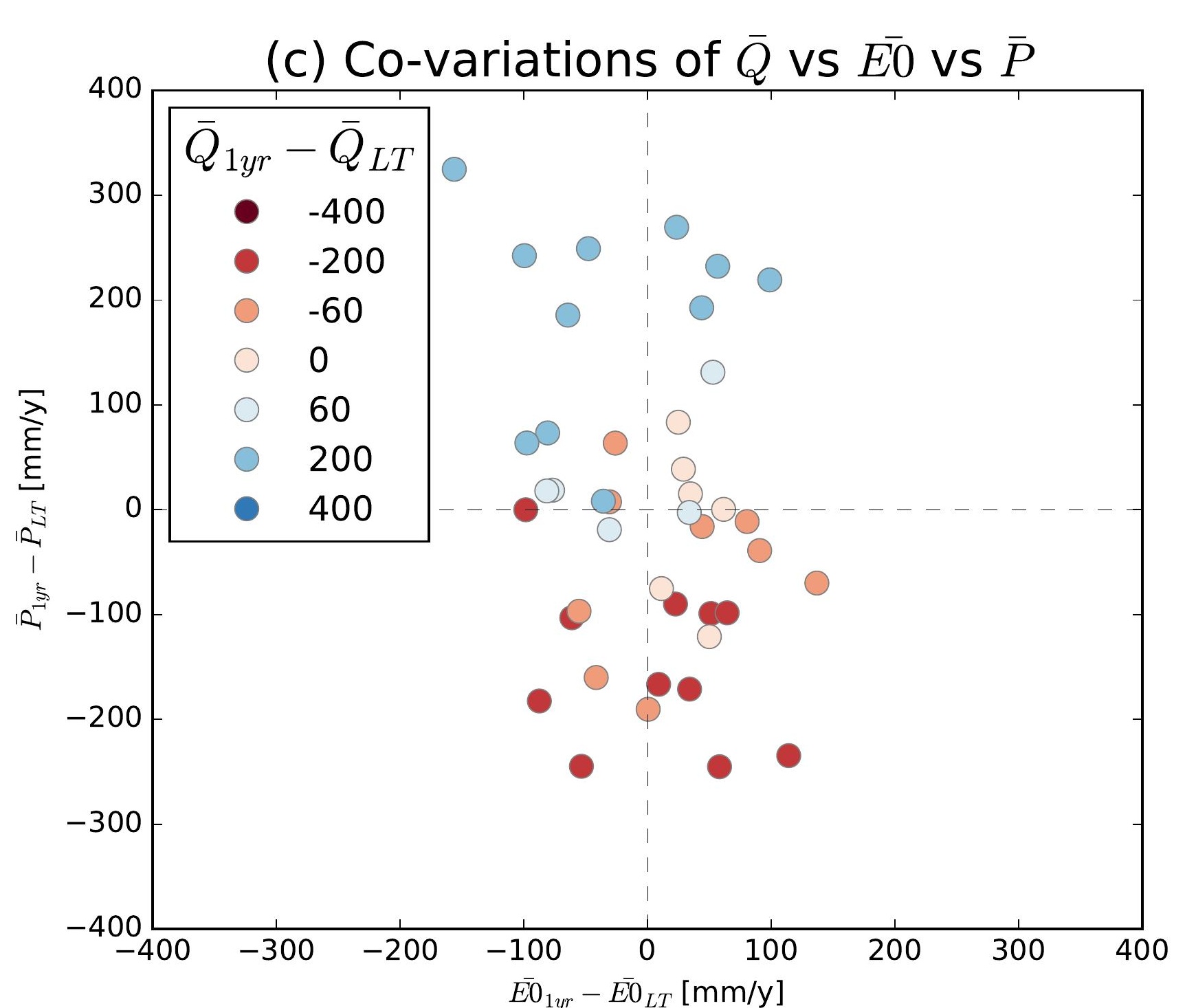
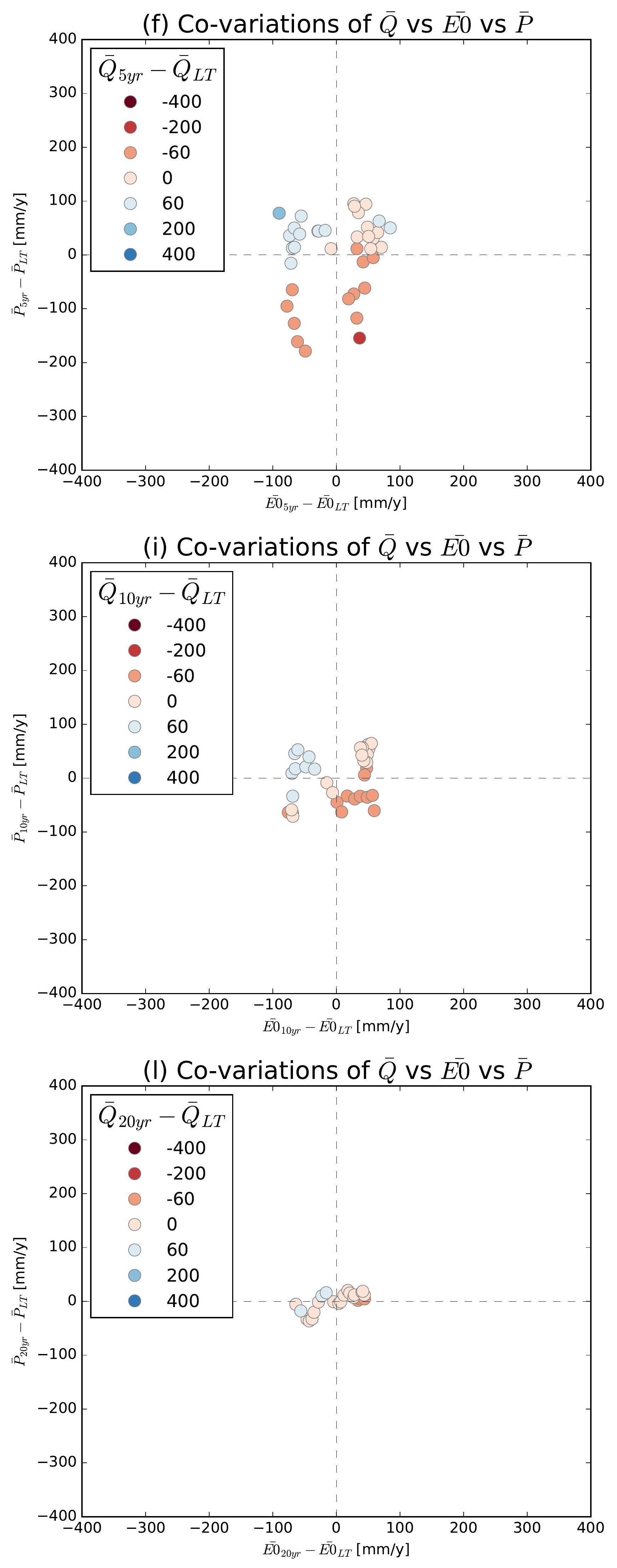

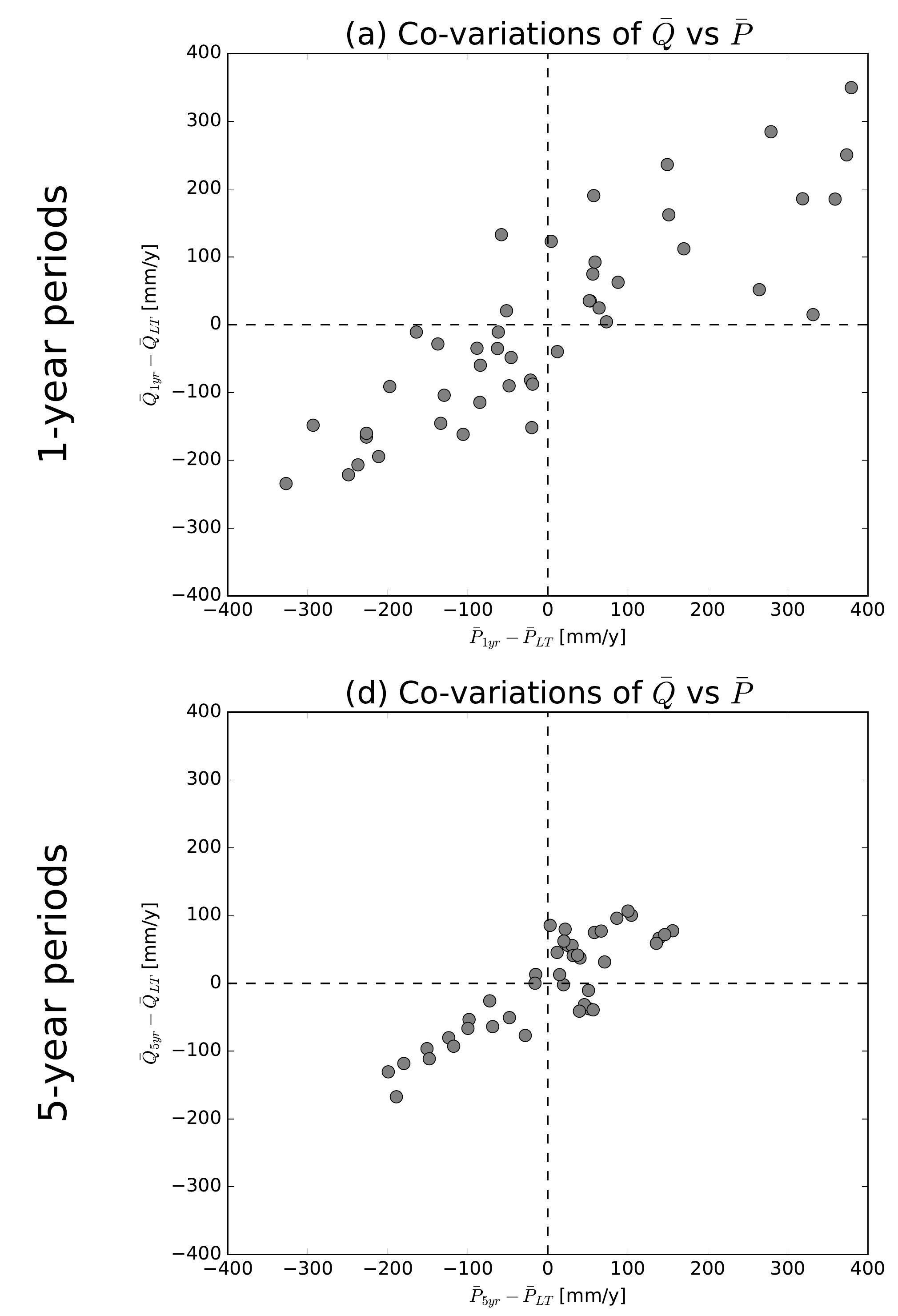

(g) Co-variations of $\bar{Q}$ vs $\bar{P}$
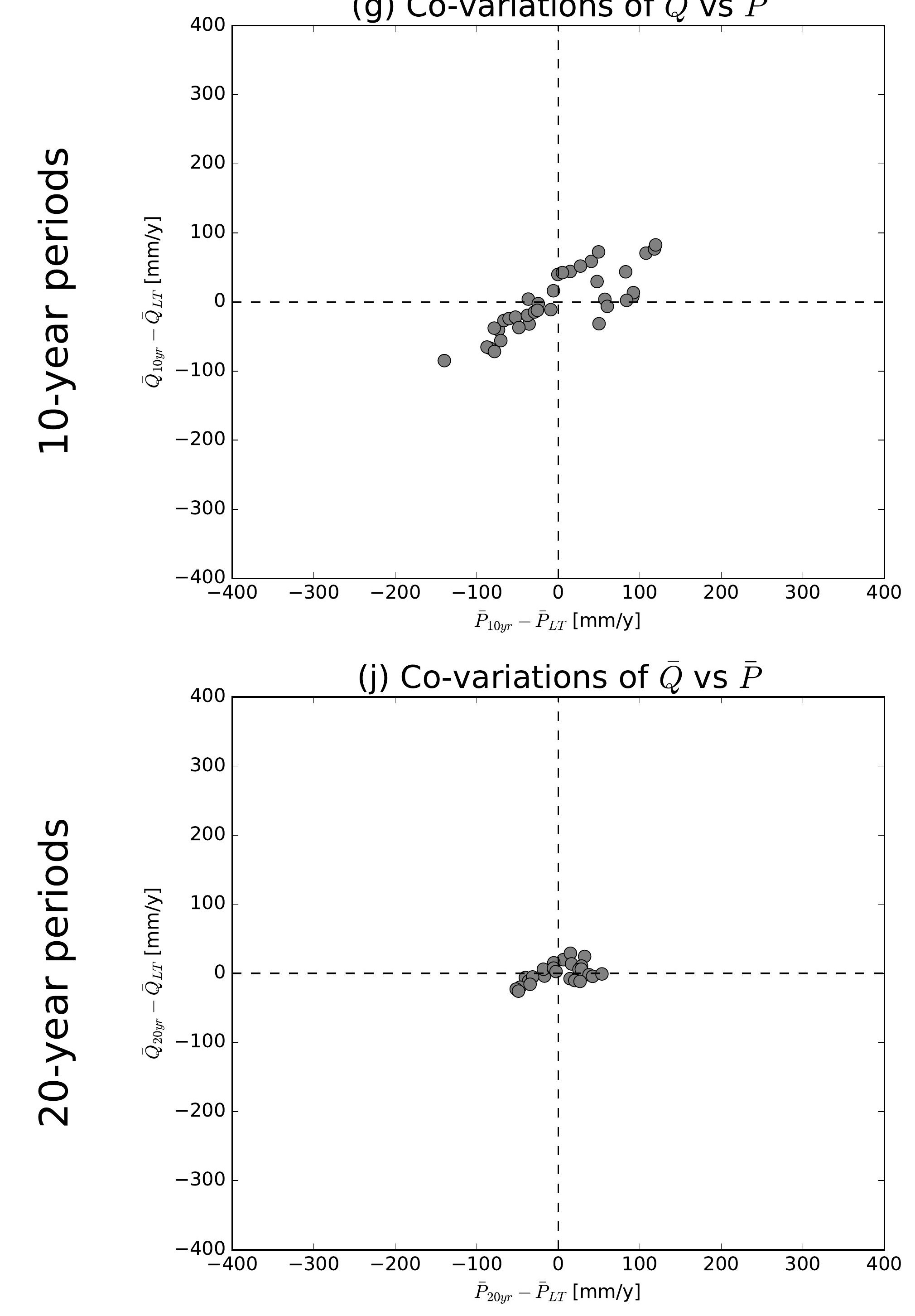

(b) Co-variations of $\bar{Q}$ vs $\overline{E 0}$

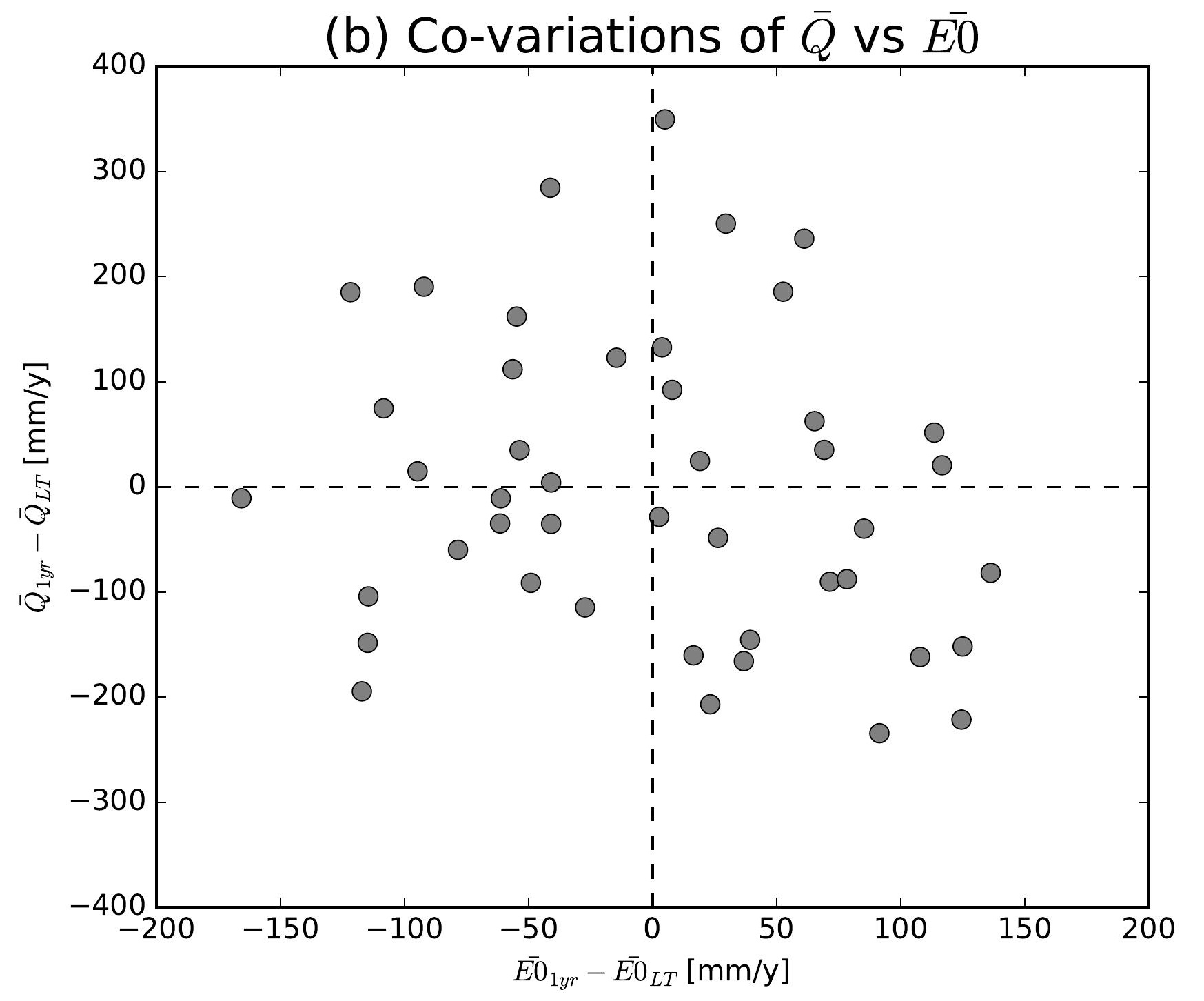

(e) Co-variations of $\bar{Q}$ vs $\overline{E 0}$

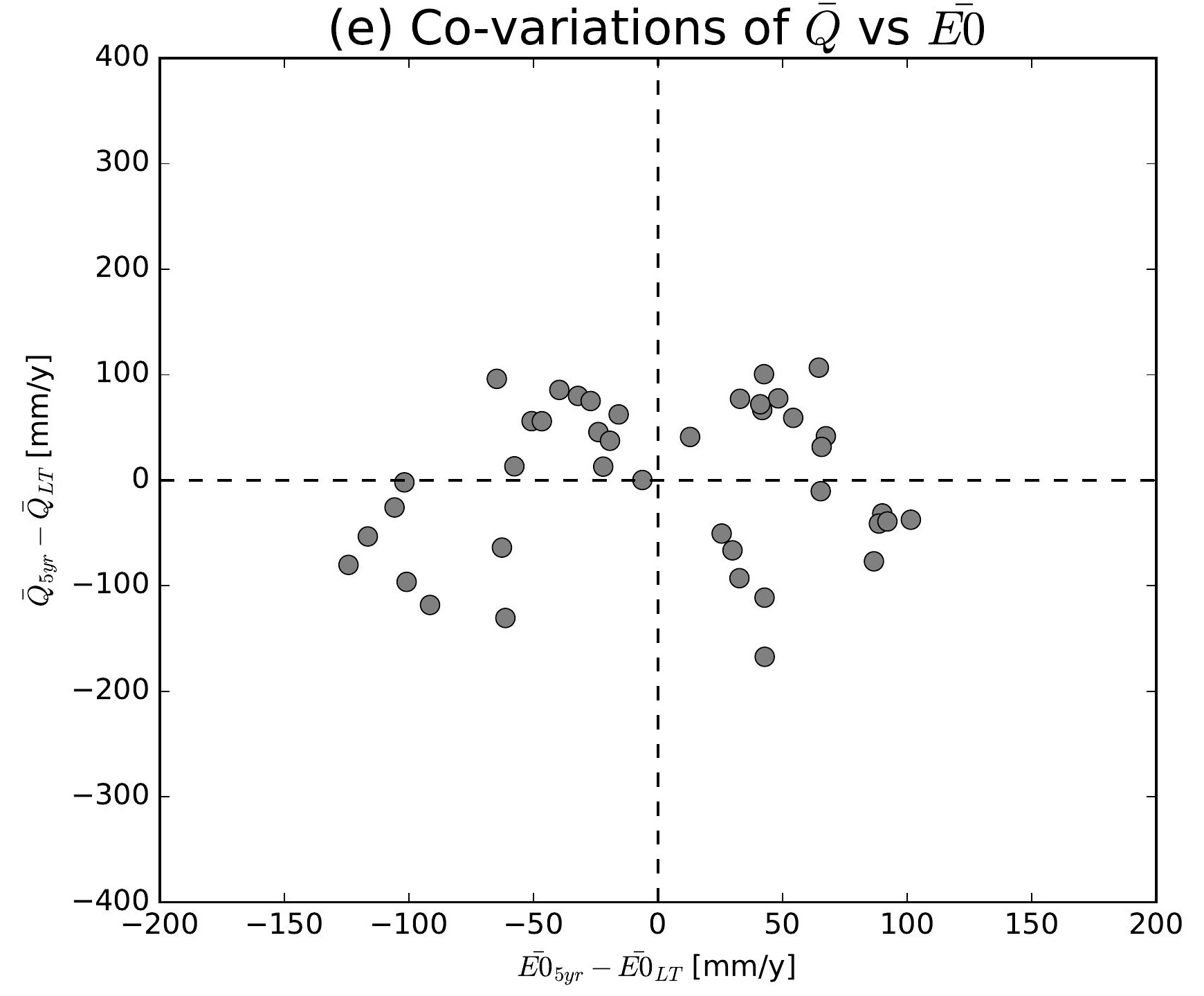

(h) Co-variations of $\bar{Q}$ vs $\overline{E 0}$

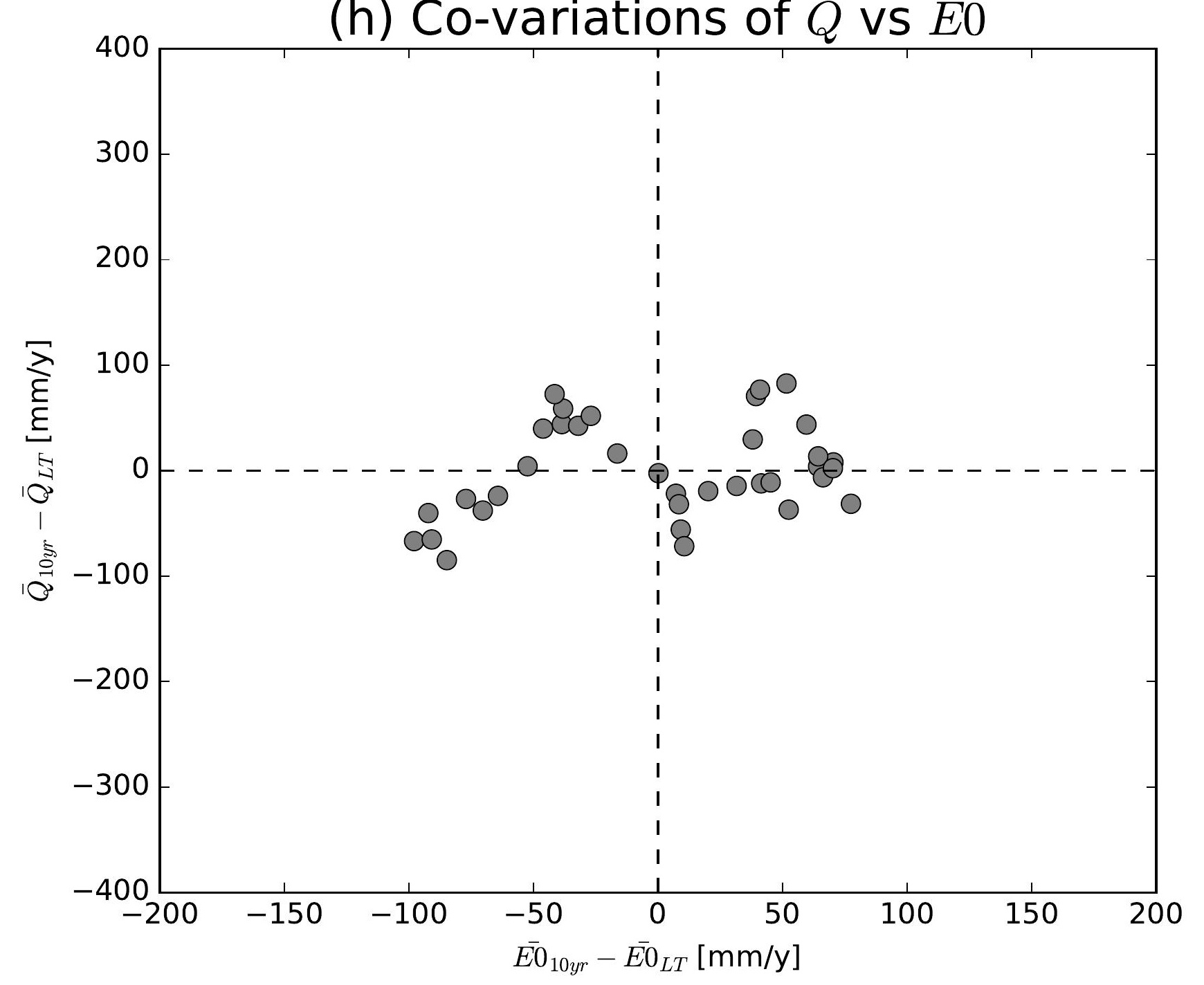

(k) Co-variations of $\bar{Q}$ vs $\overline{E 0}$

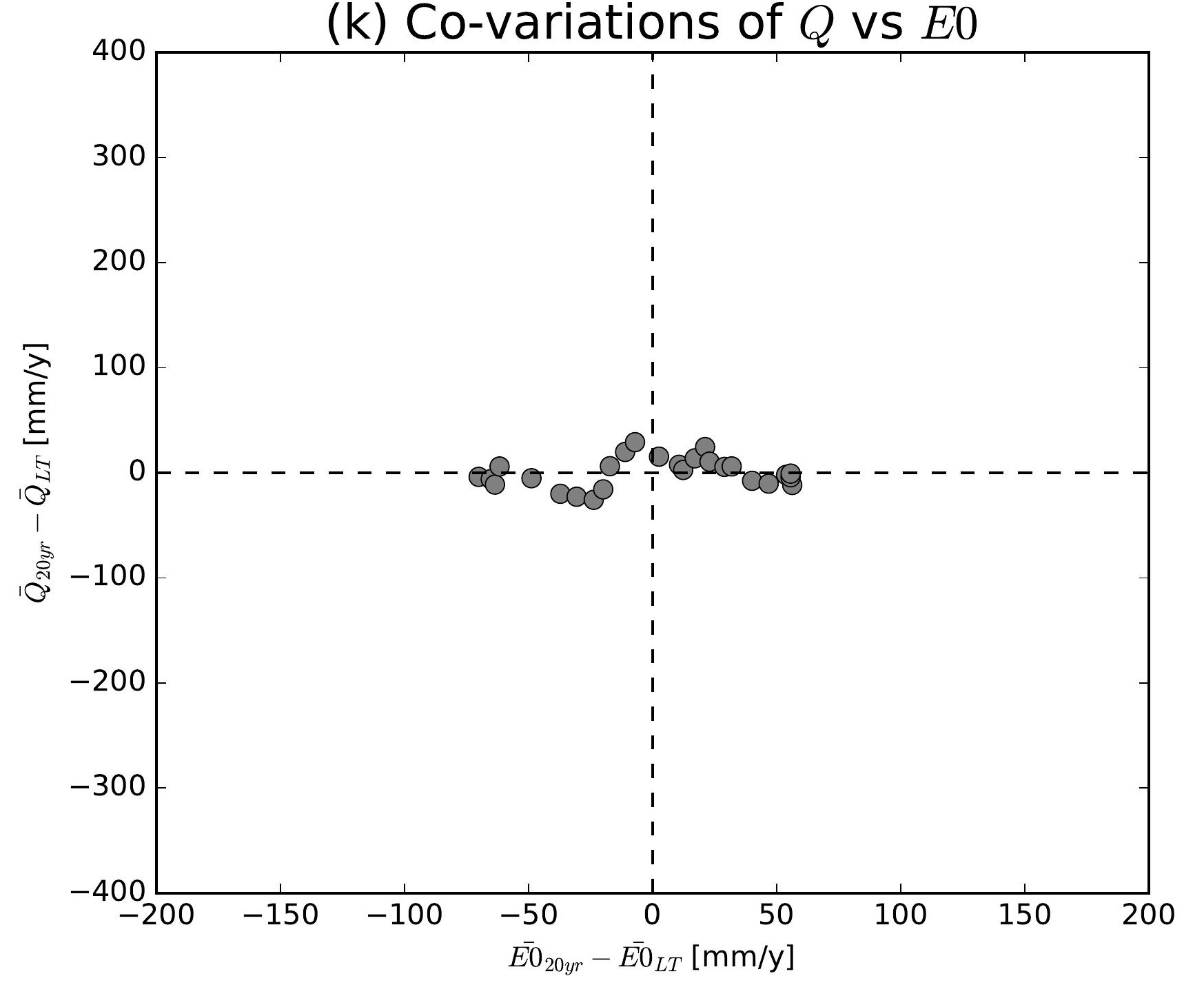

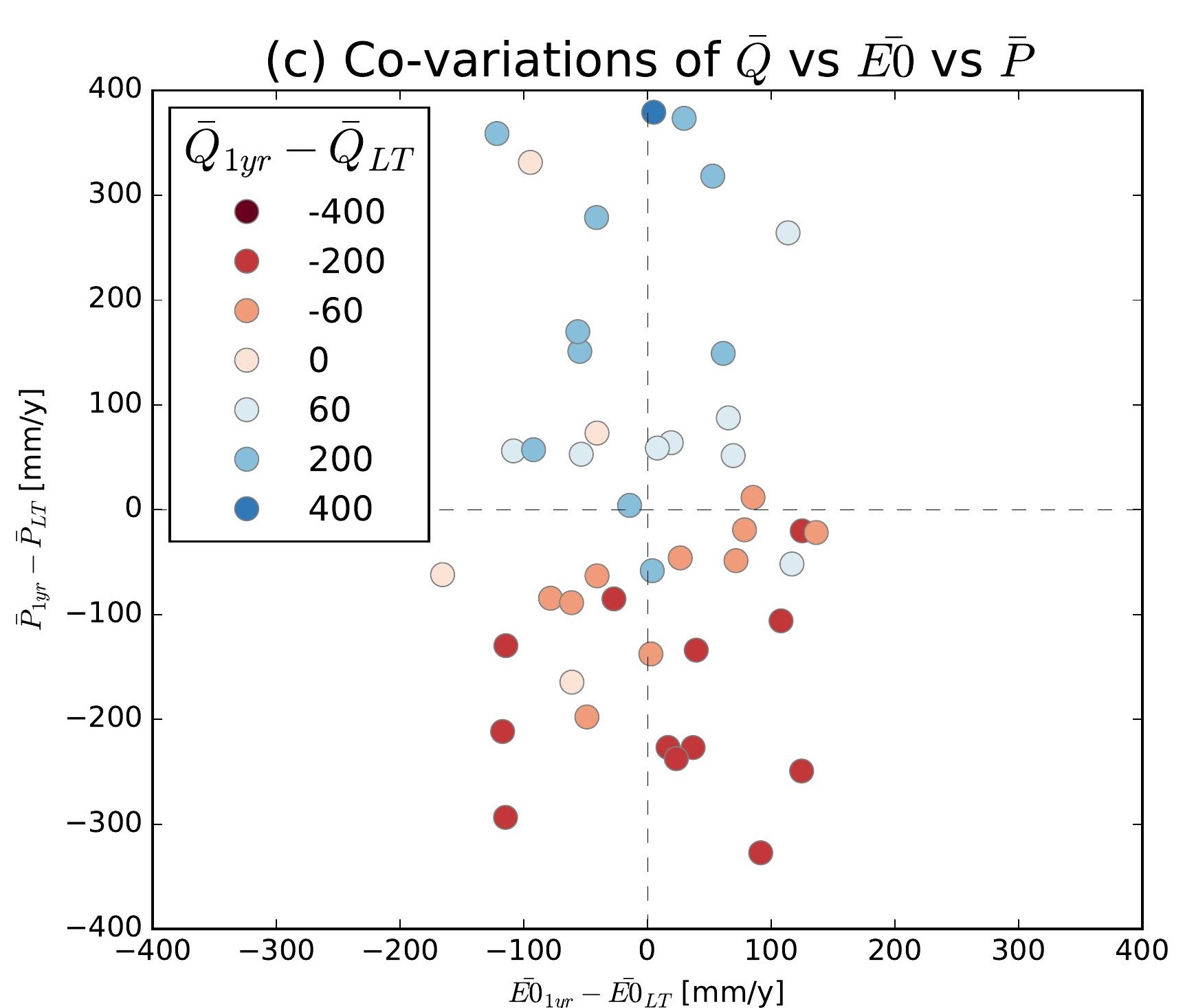
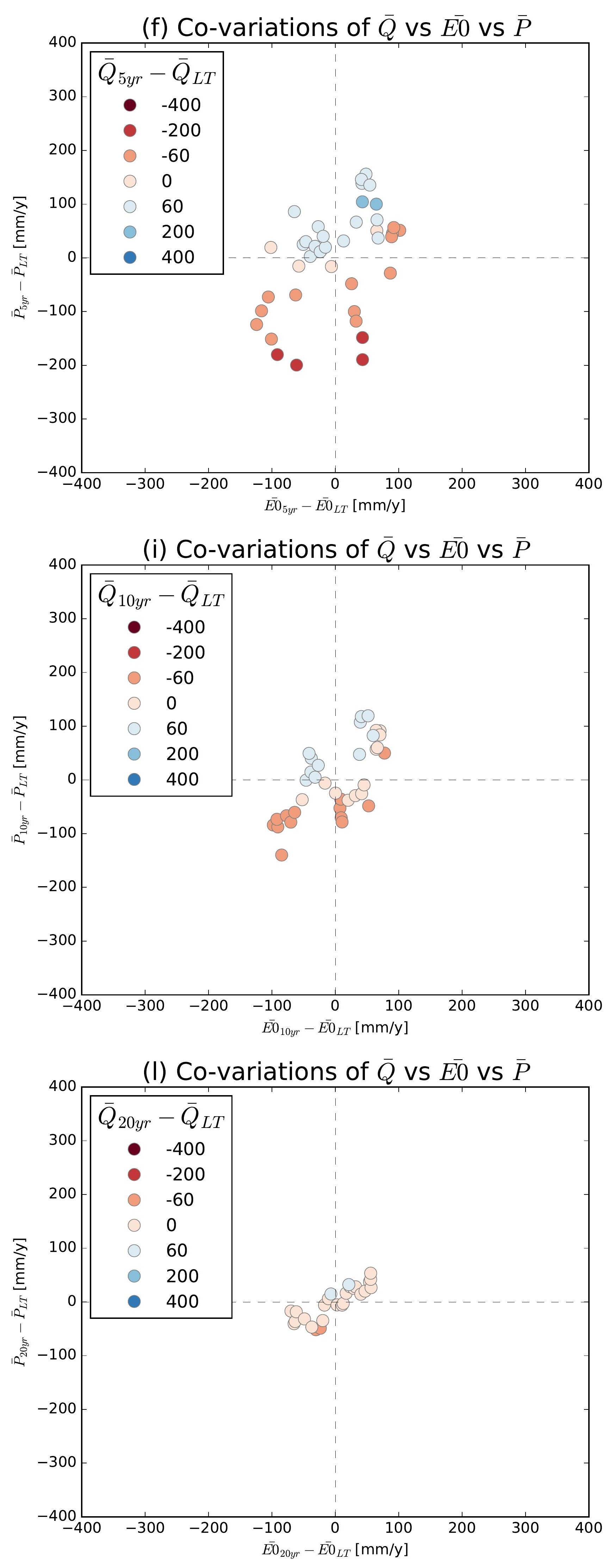

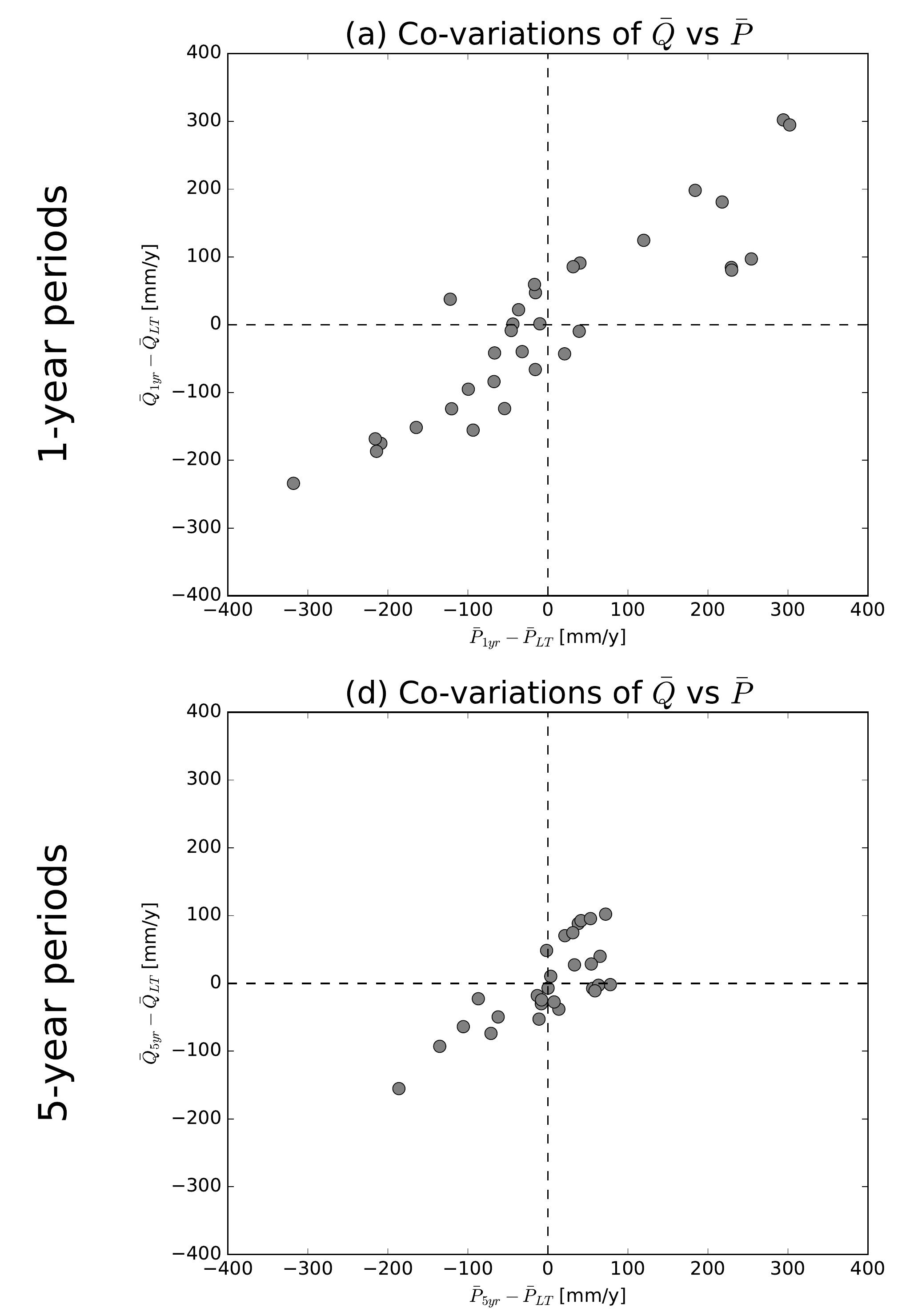

(g) Co-variations of $\bar{Q}$ vs $\bar{P}$
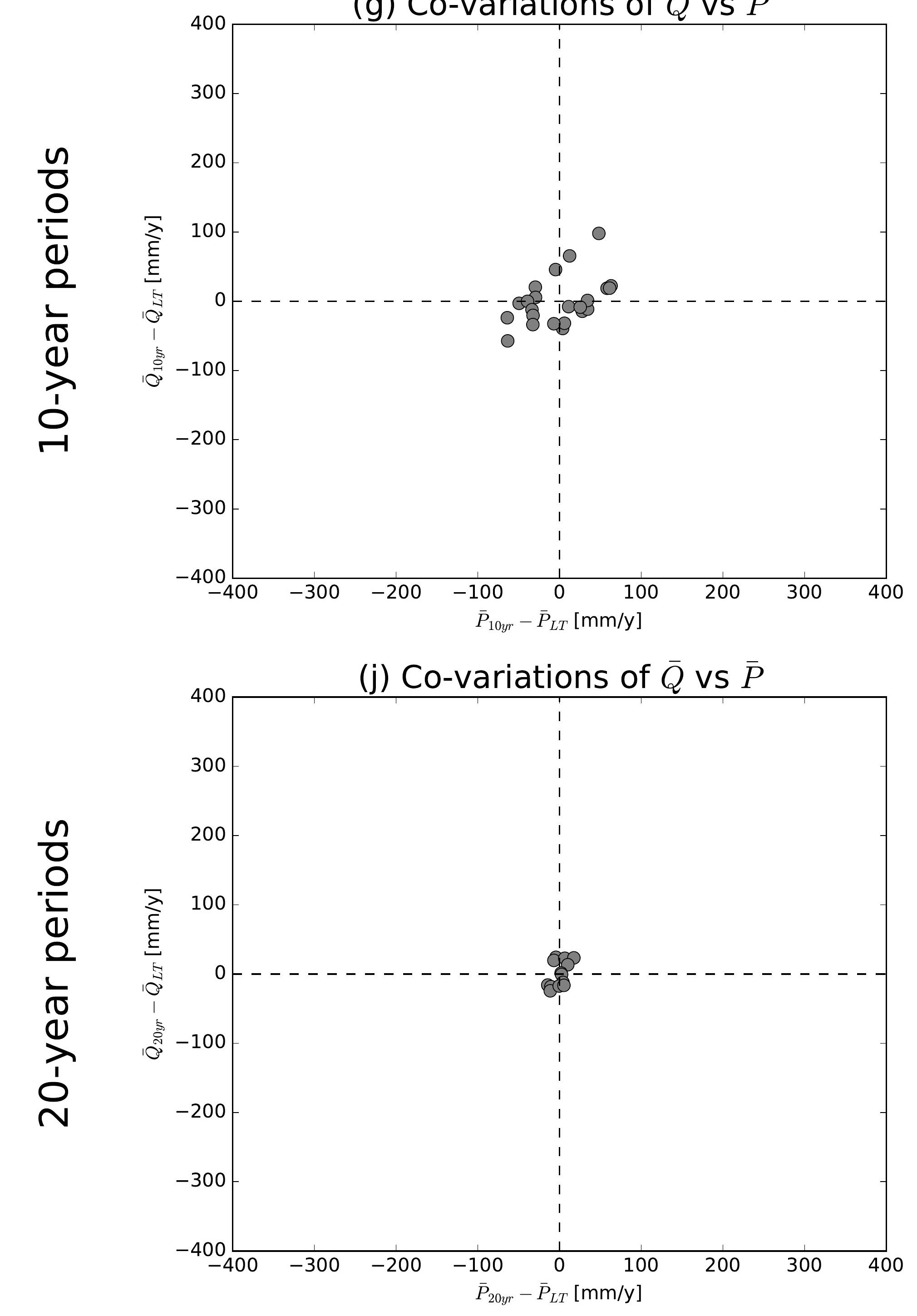

(b) Co-variations of $\bar{Q}$ vs $\overline{E 0}$

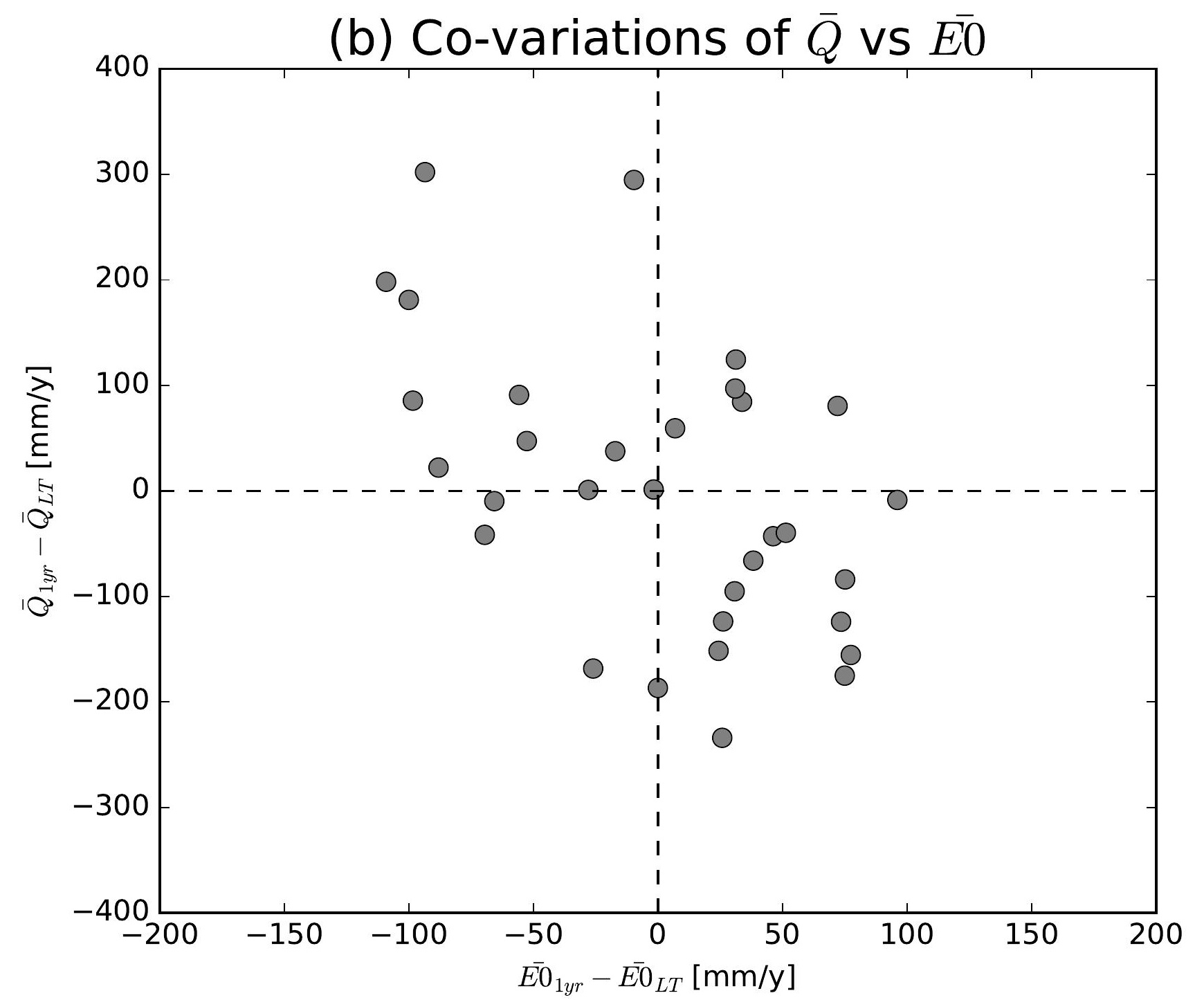

(e) Co-variations of $\bar{Q}$ vs $\overline{E 0}$

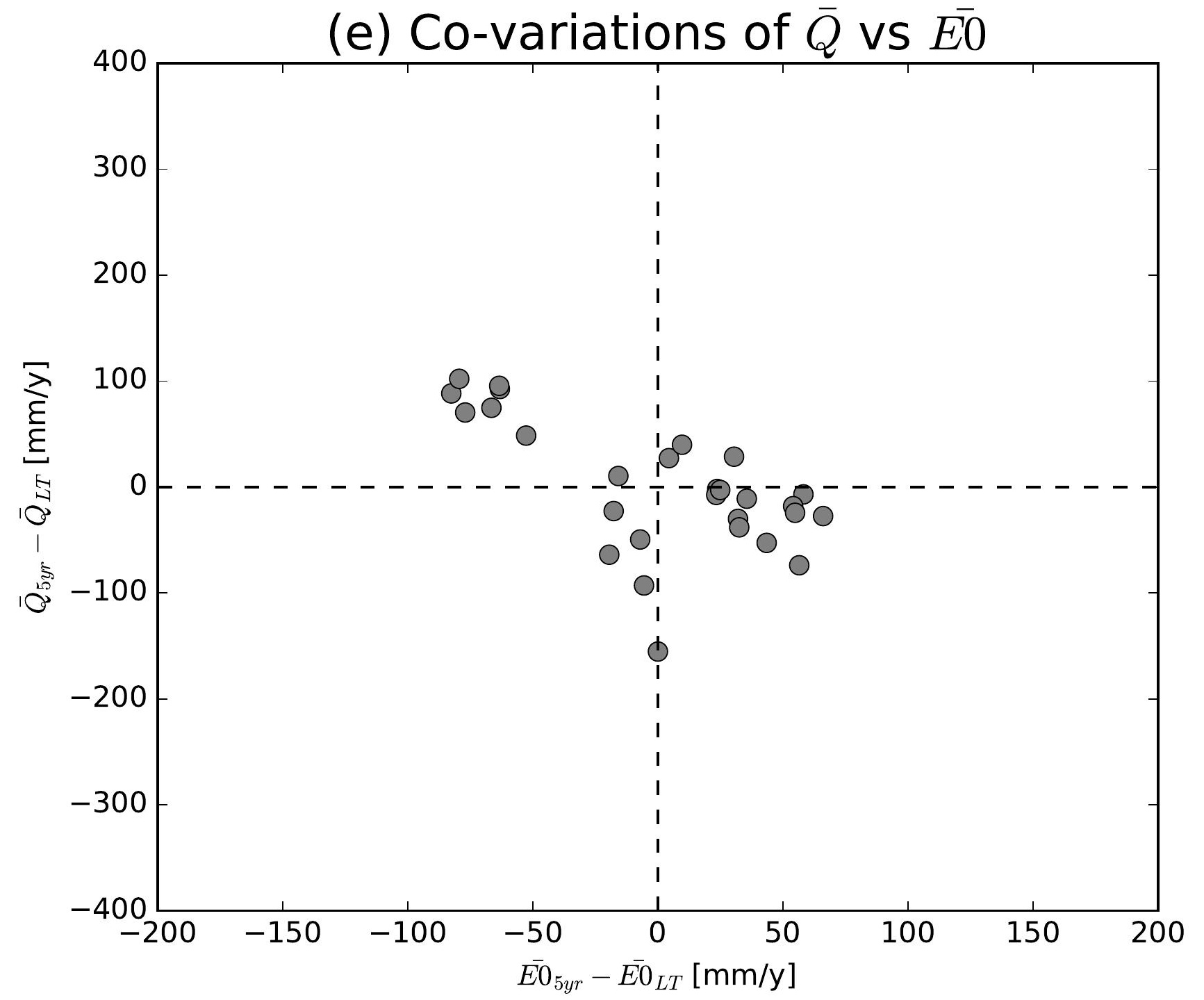

(h) Co-variations of $\bar{Q}$ vs $\overline{E 0}$

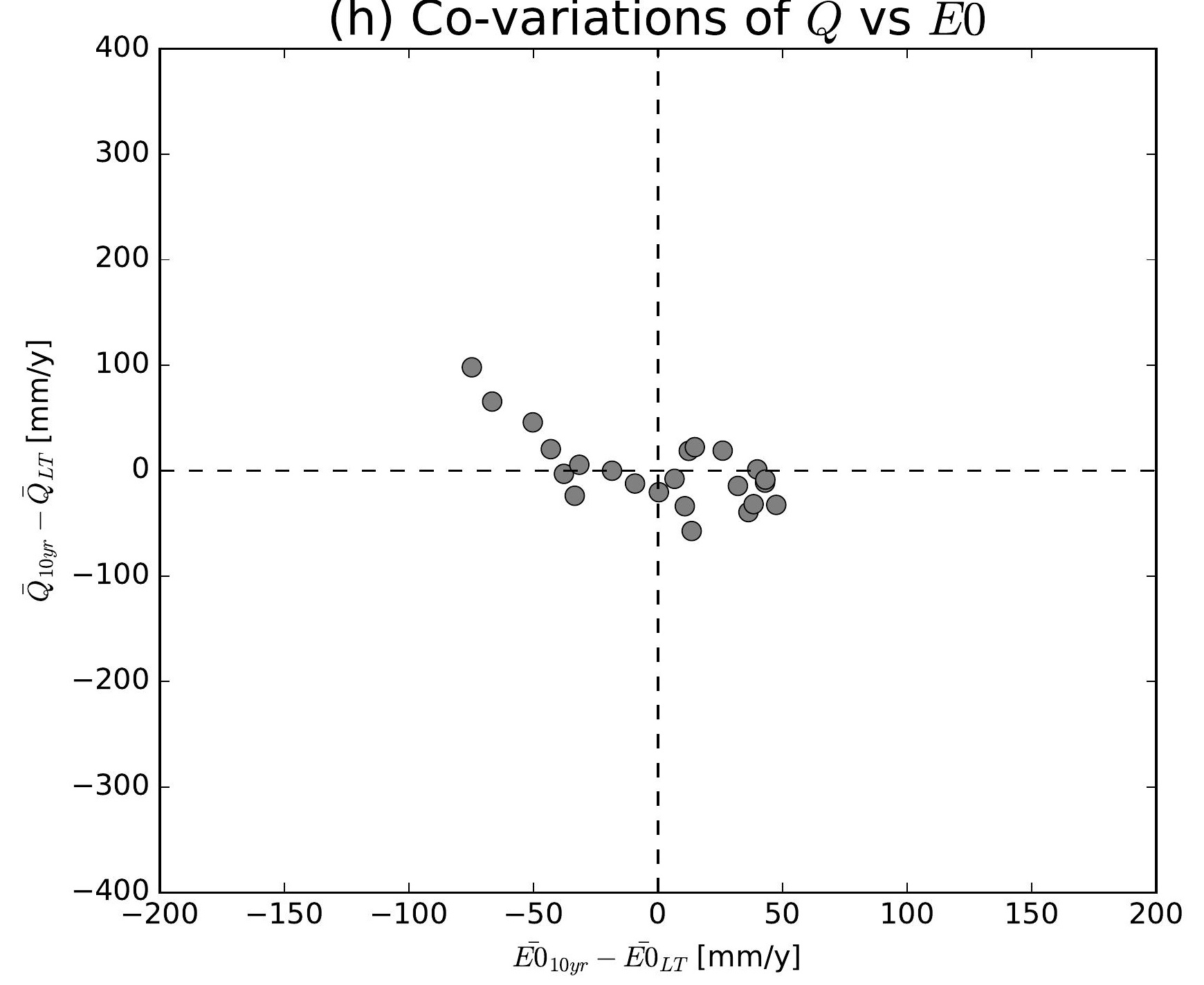

(k) Co-variations of $\bar{Q}$ vs $\overline{E 0}$

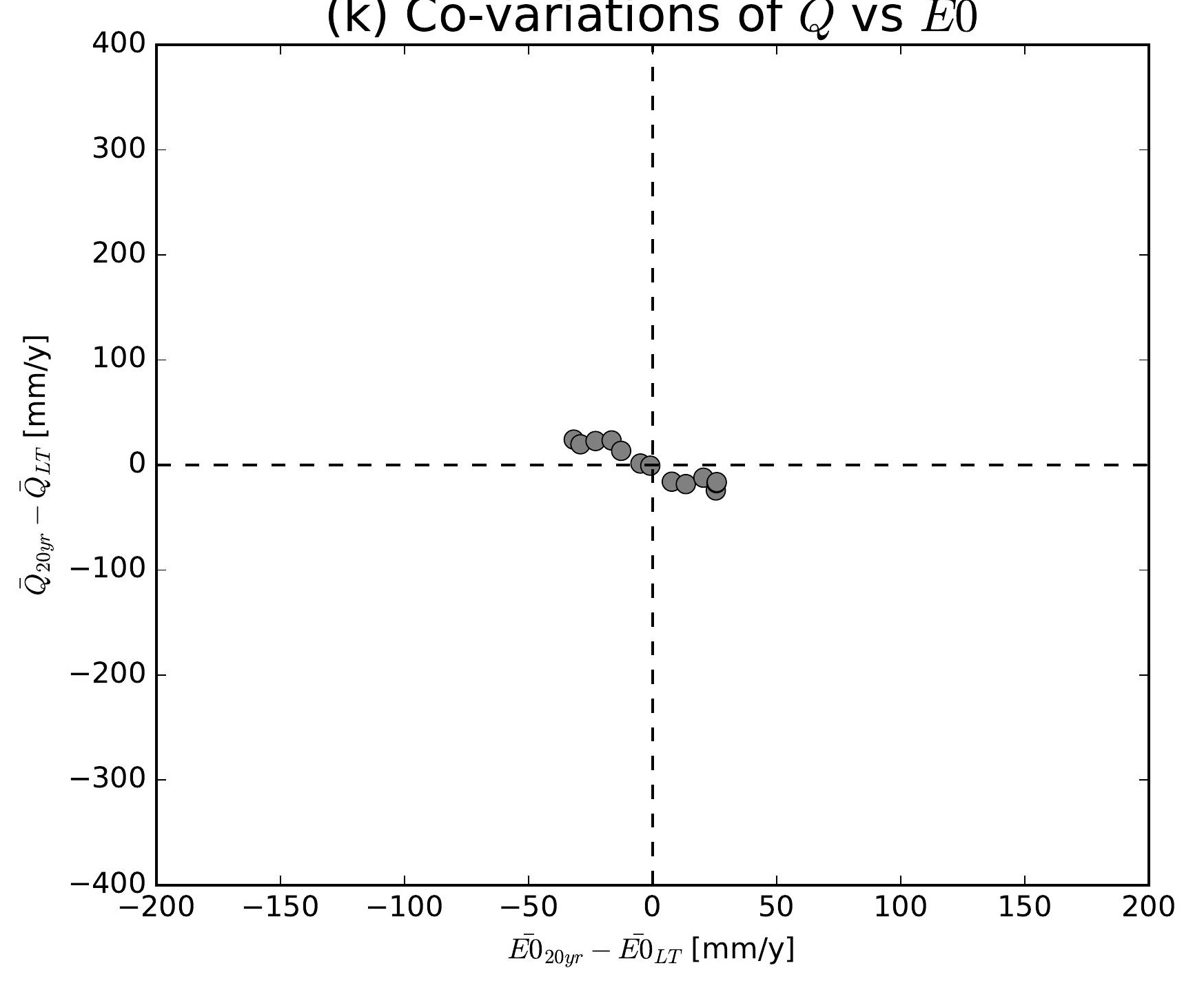

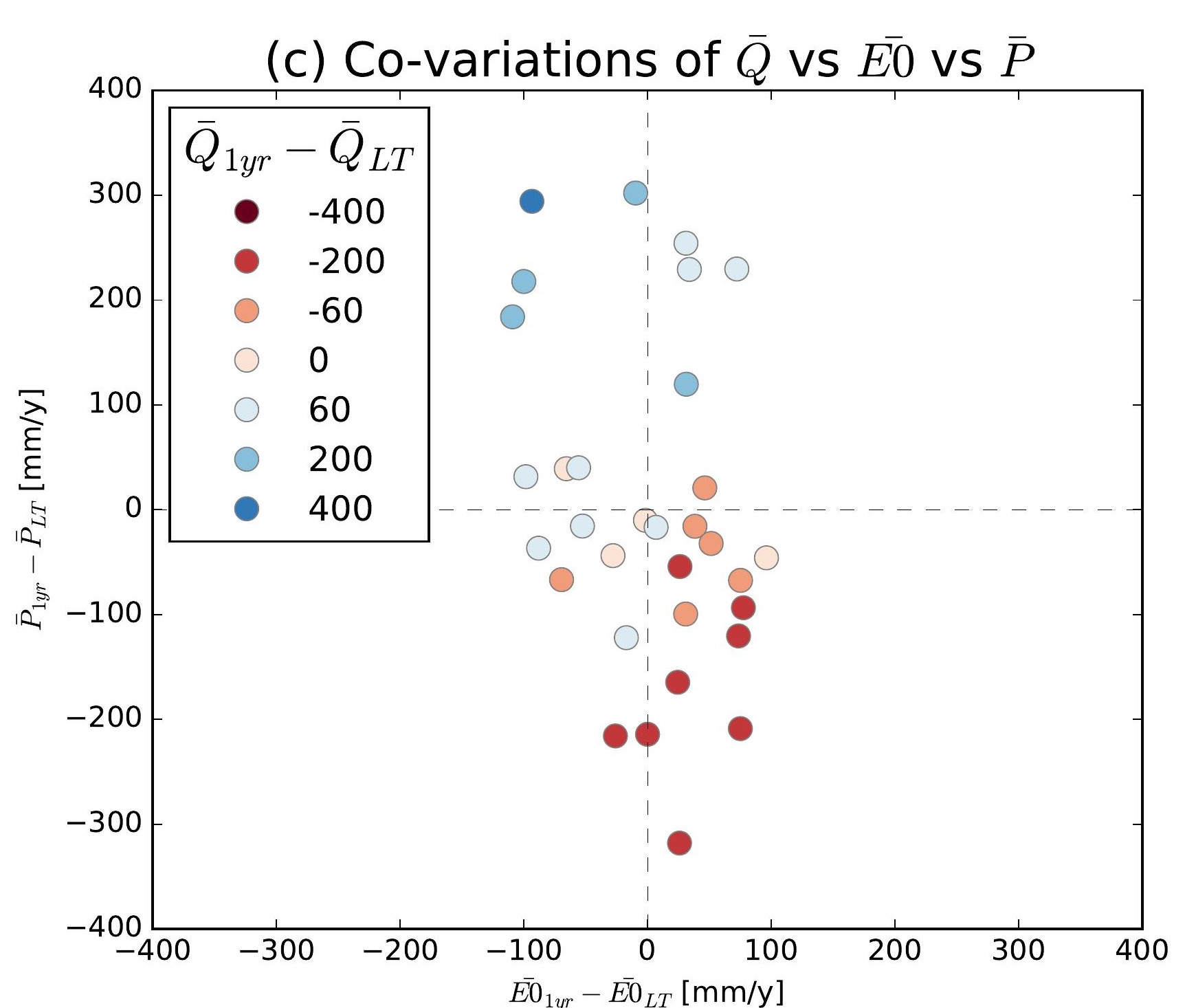
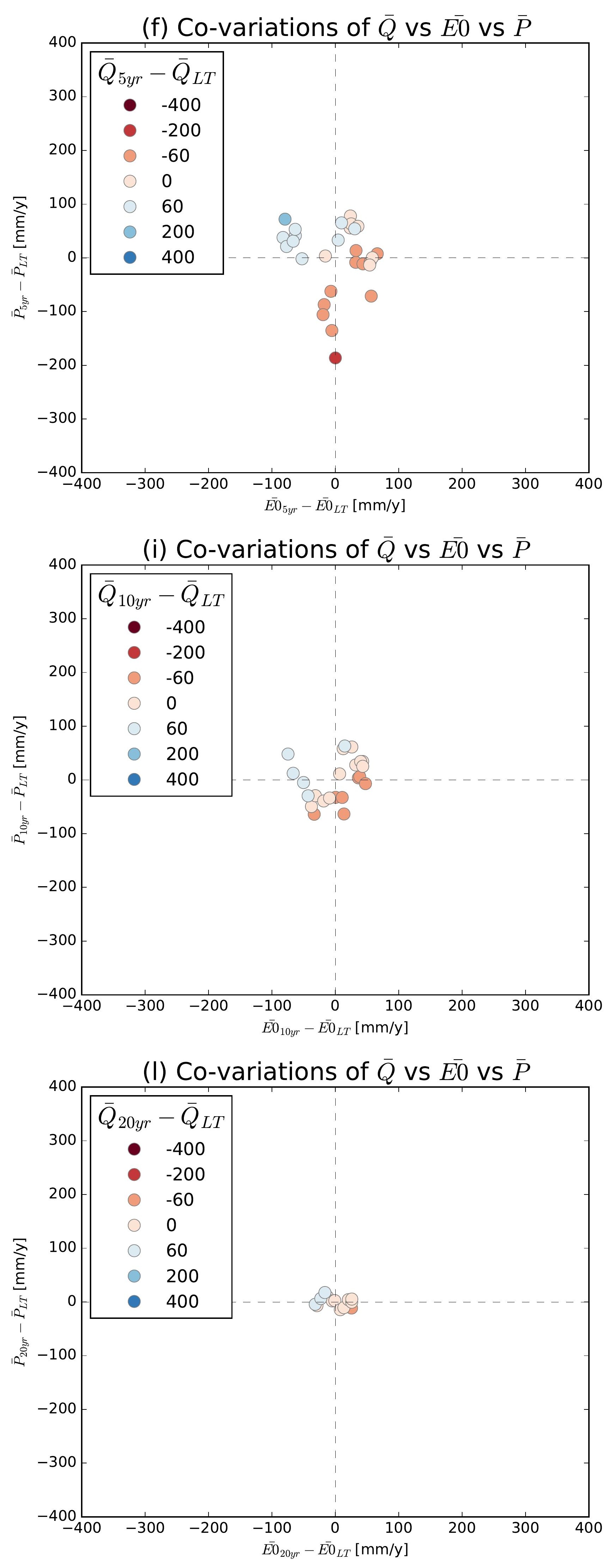

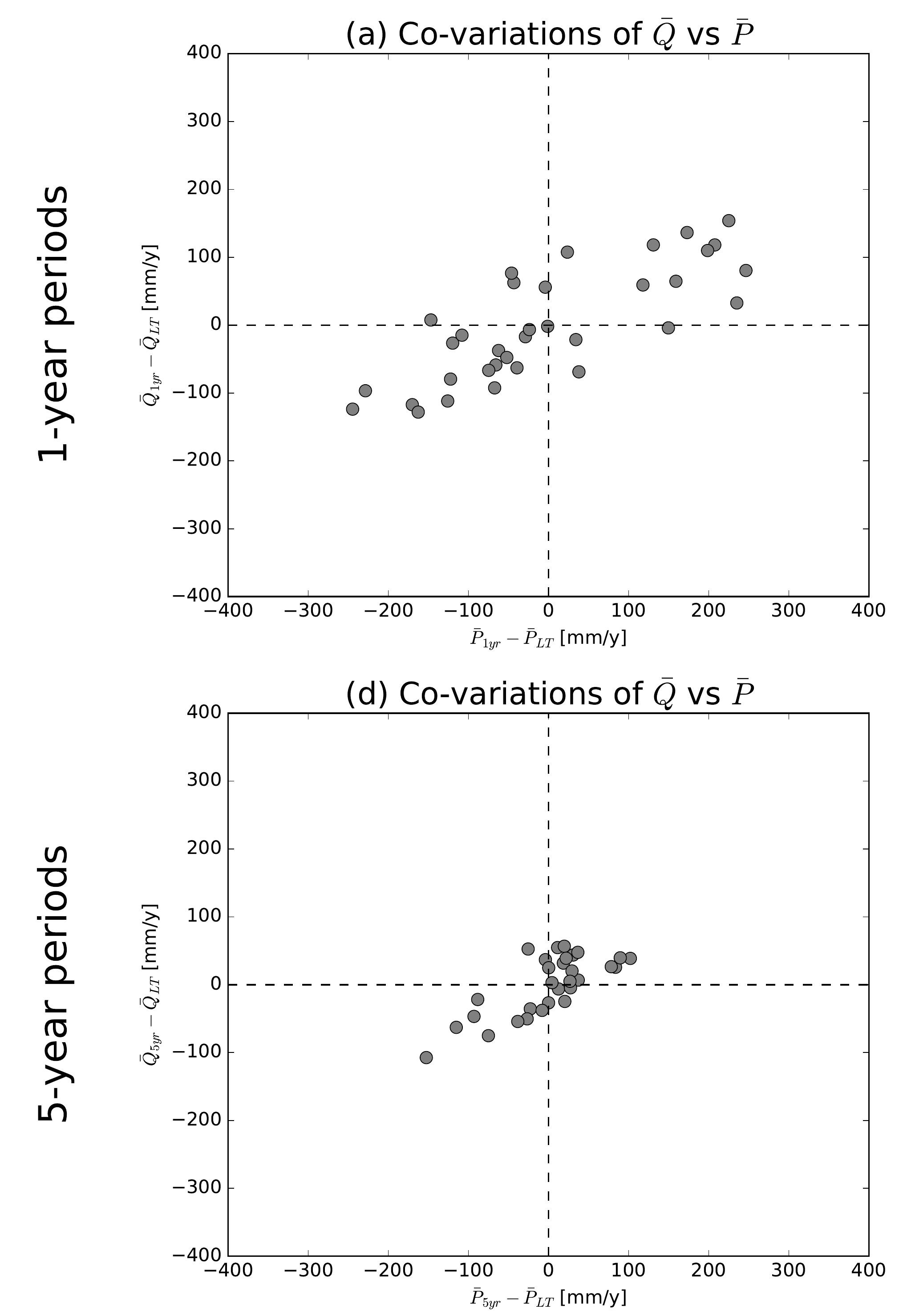

(g) Co-variations of $\bar{Q}$ vs $\bar{P}$
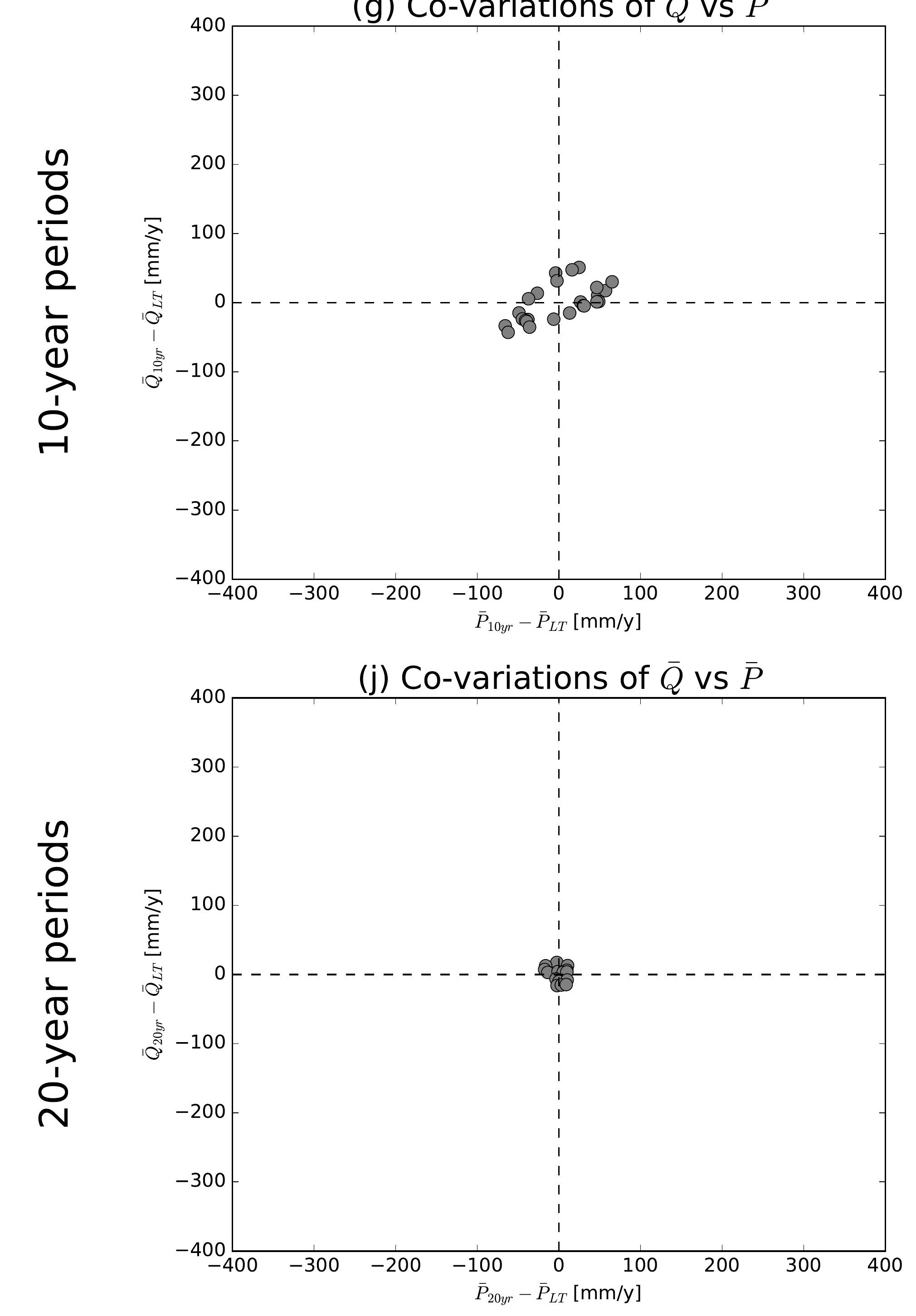

(b) Co-variations of $\bar{Q}$ vs $\overline{E 0}$

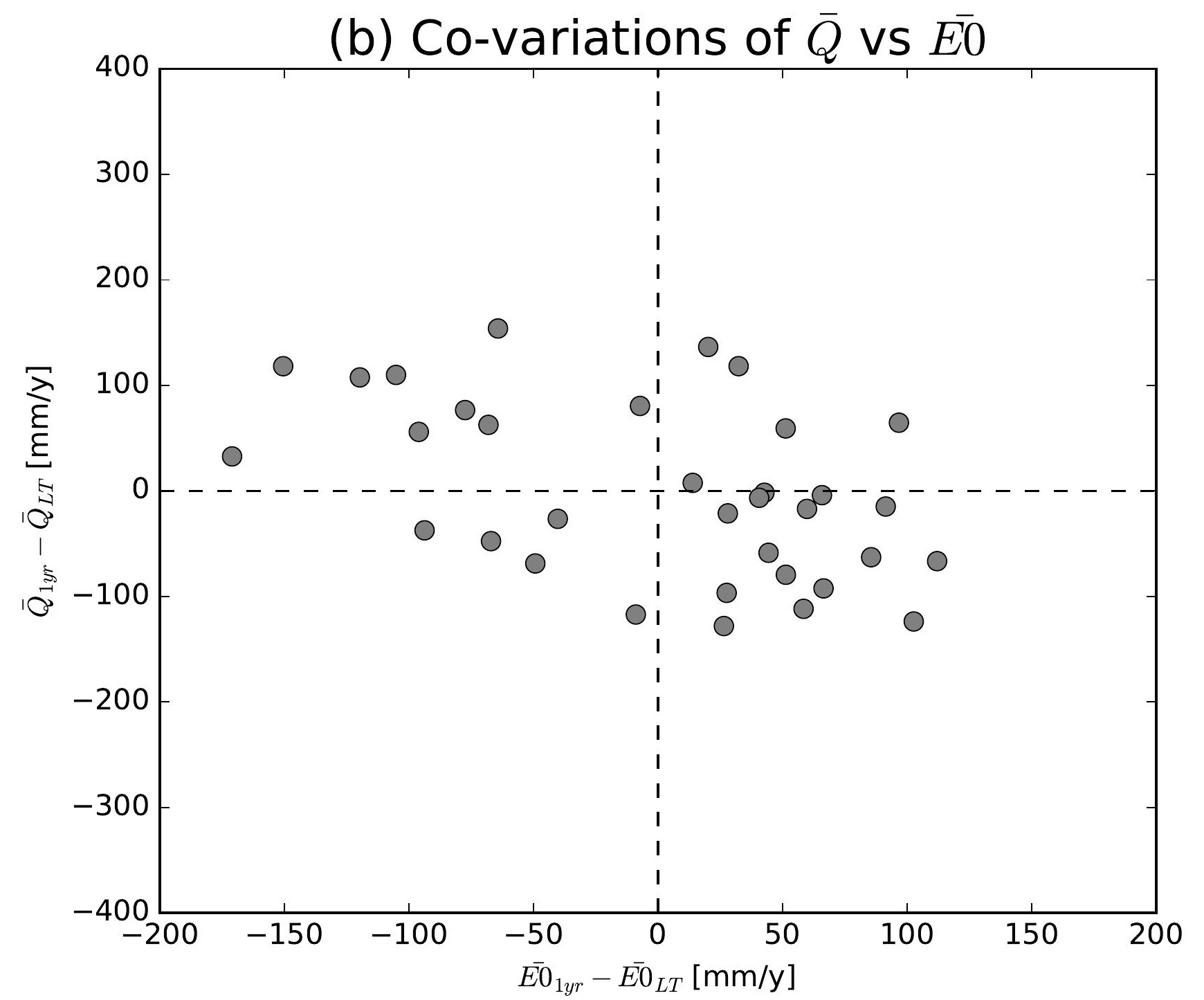

(e) Co-variations of $\bar{Q}$ vs $\overline{E 0}$

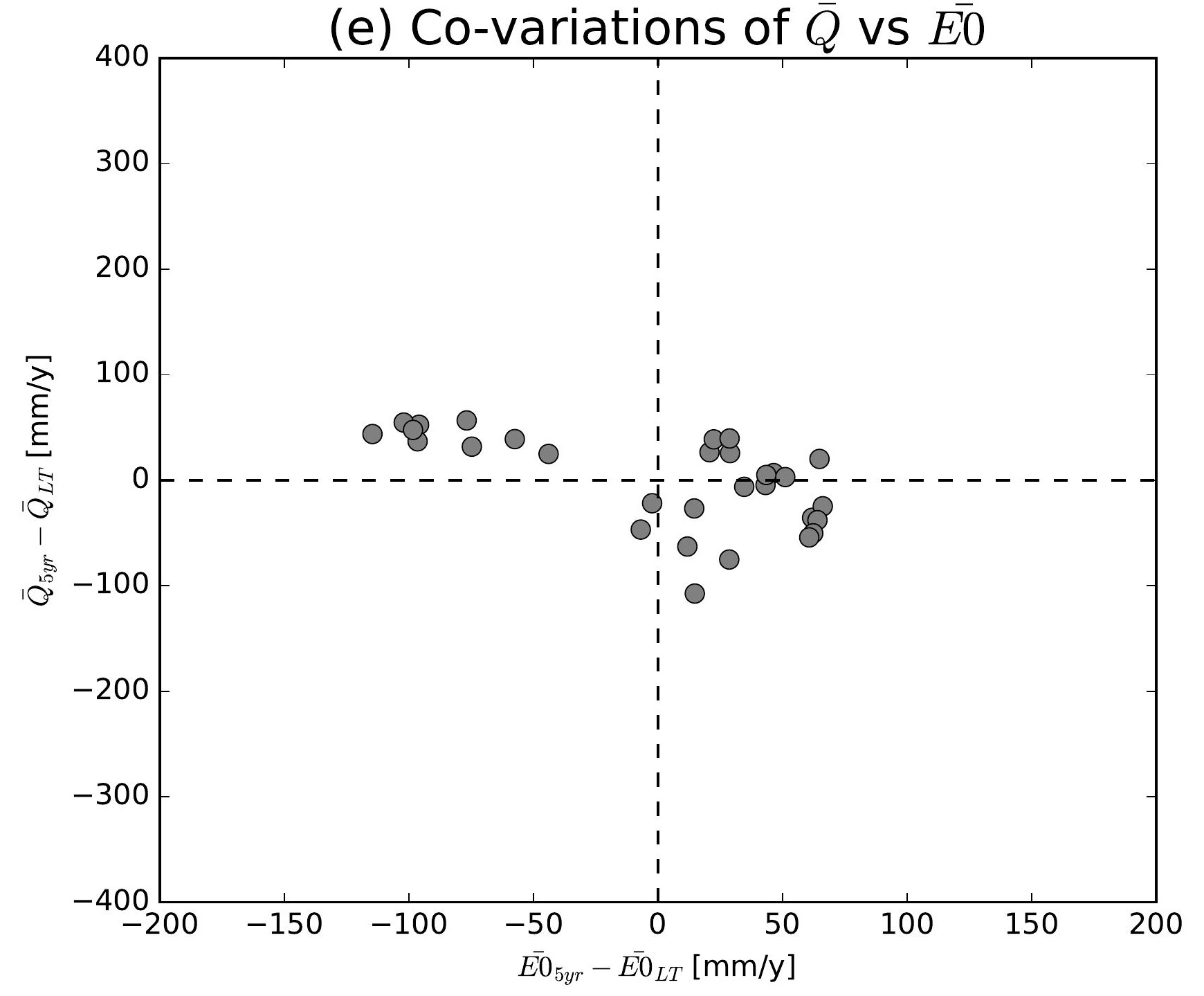

(h) Co-variations of $\bar{Q}$ vs $\overline{E 0}$

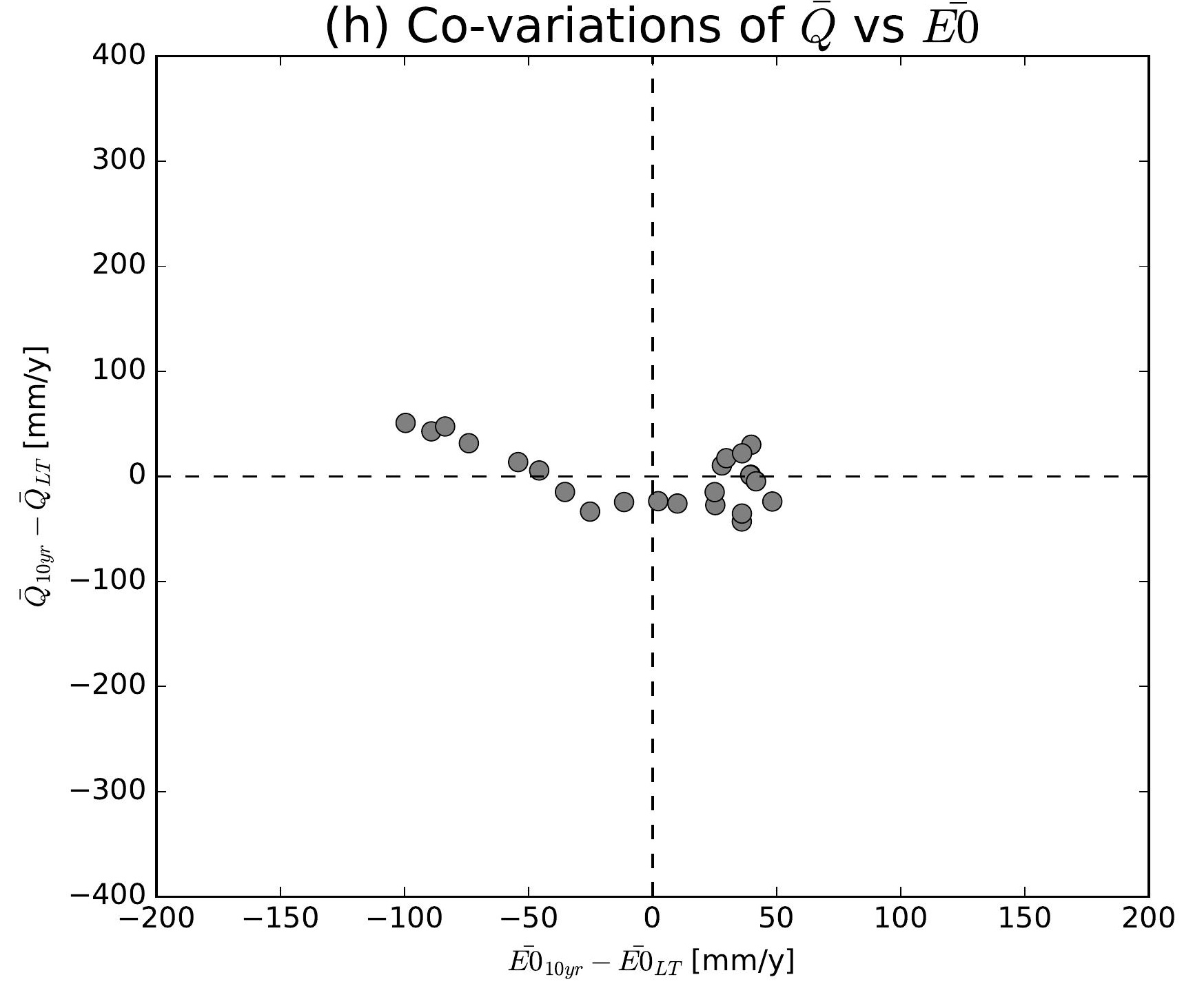

(k) Co-variations of $\bar{Q}$ vs $\overline{E 0}$

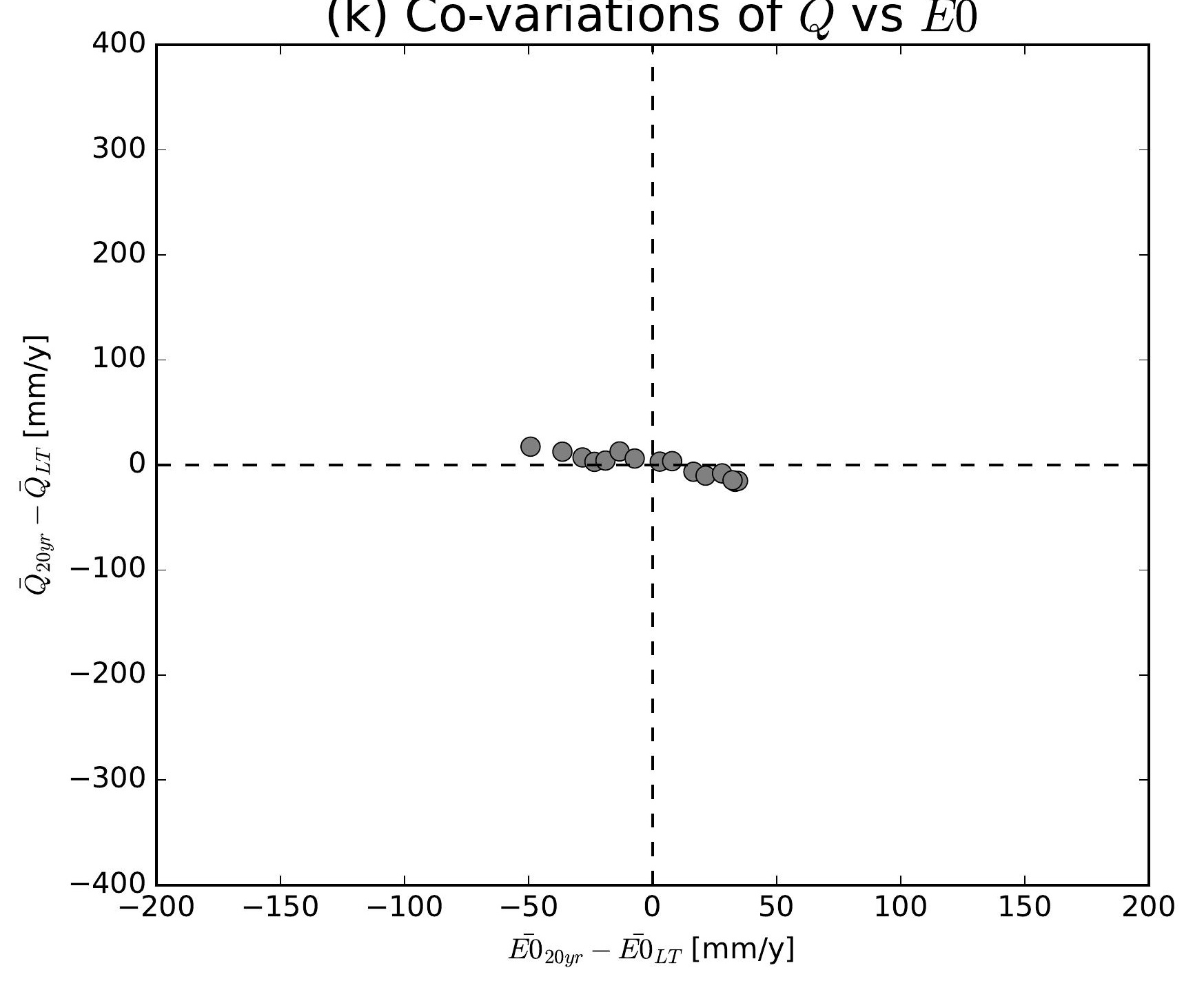

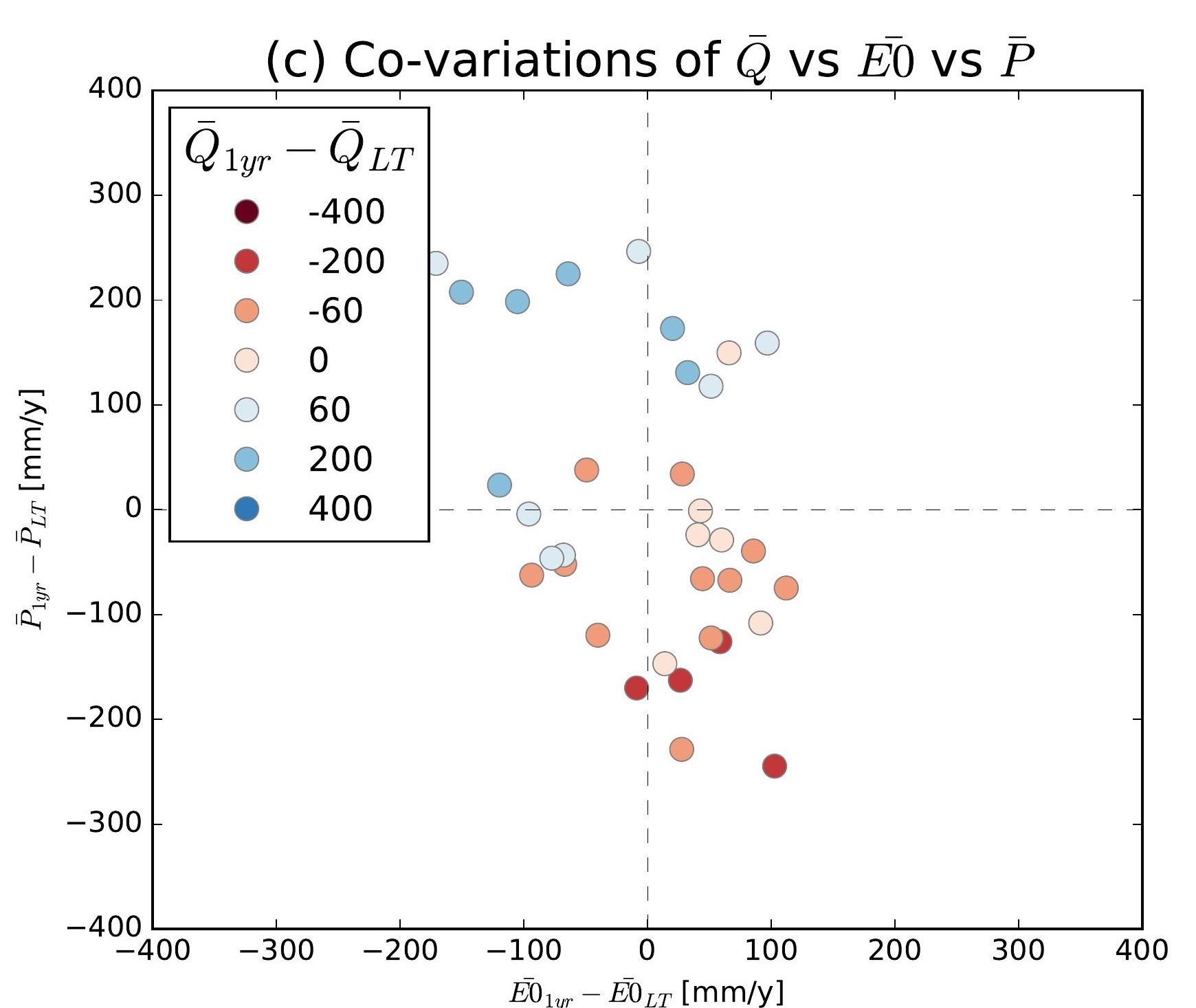
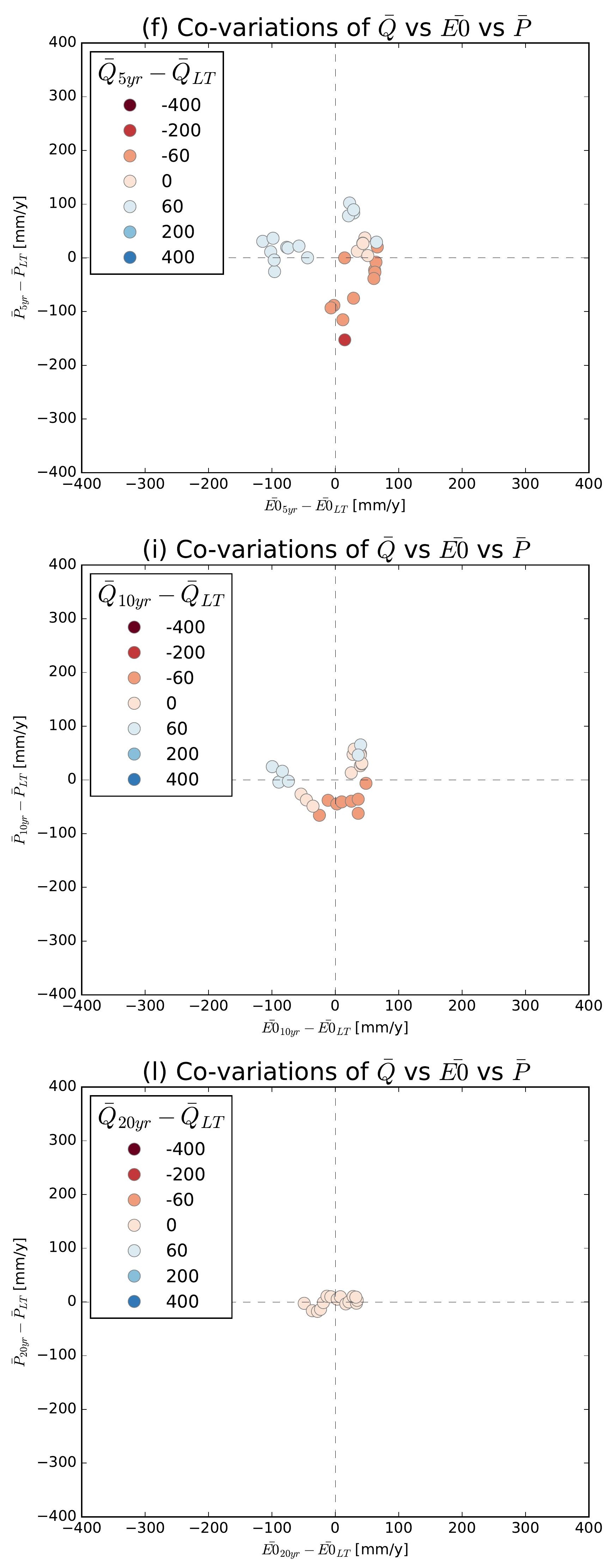

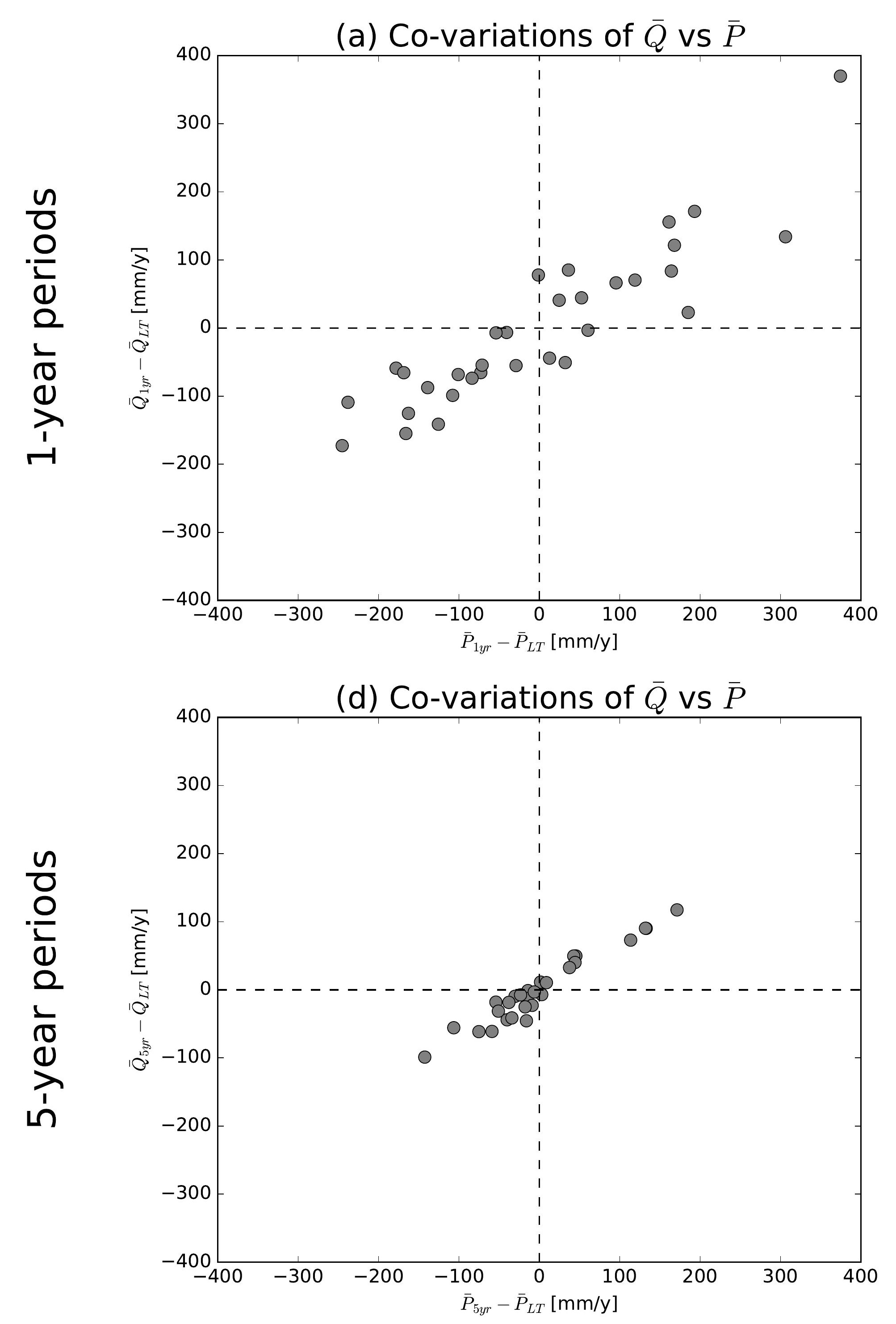

(g) Co-variations of $\bar{Q}$ vs $\bar{P}$
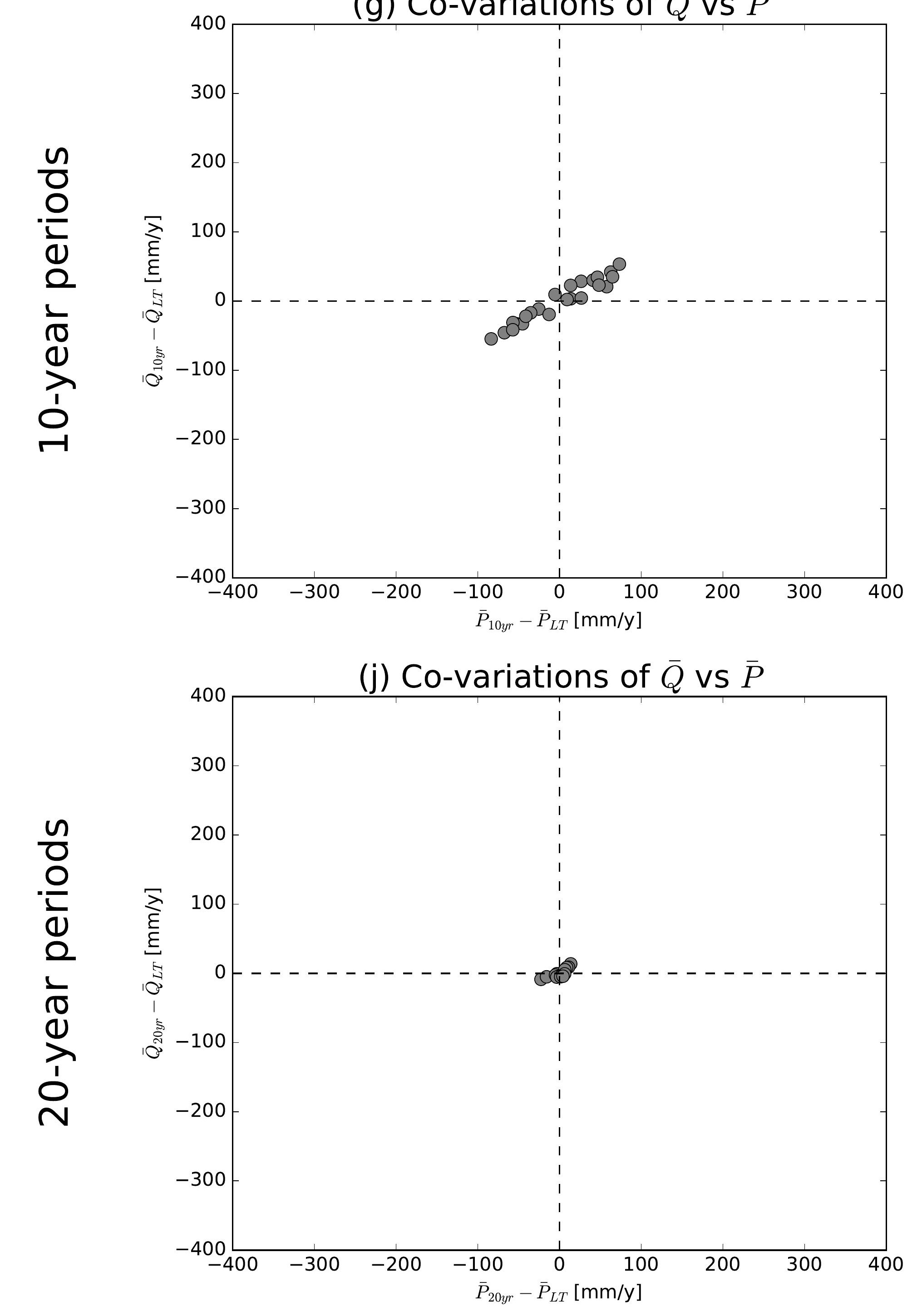

(b) Co-variations of $\bar{Q}$ vs $\overline{E 0}$

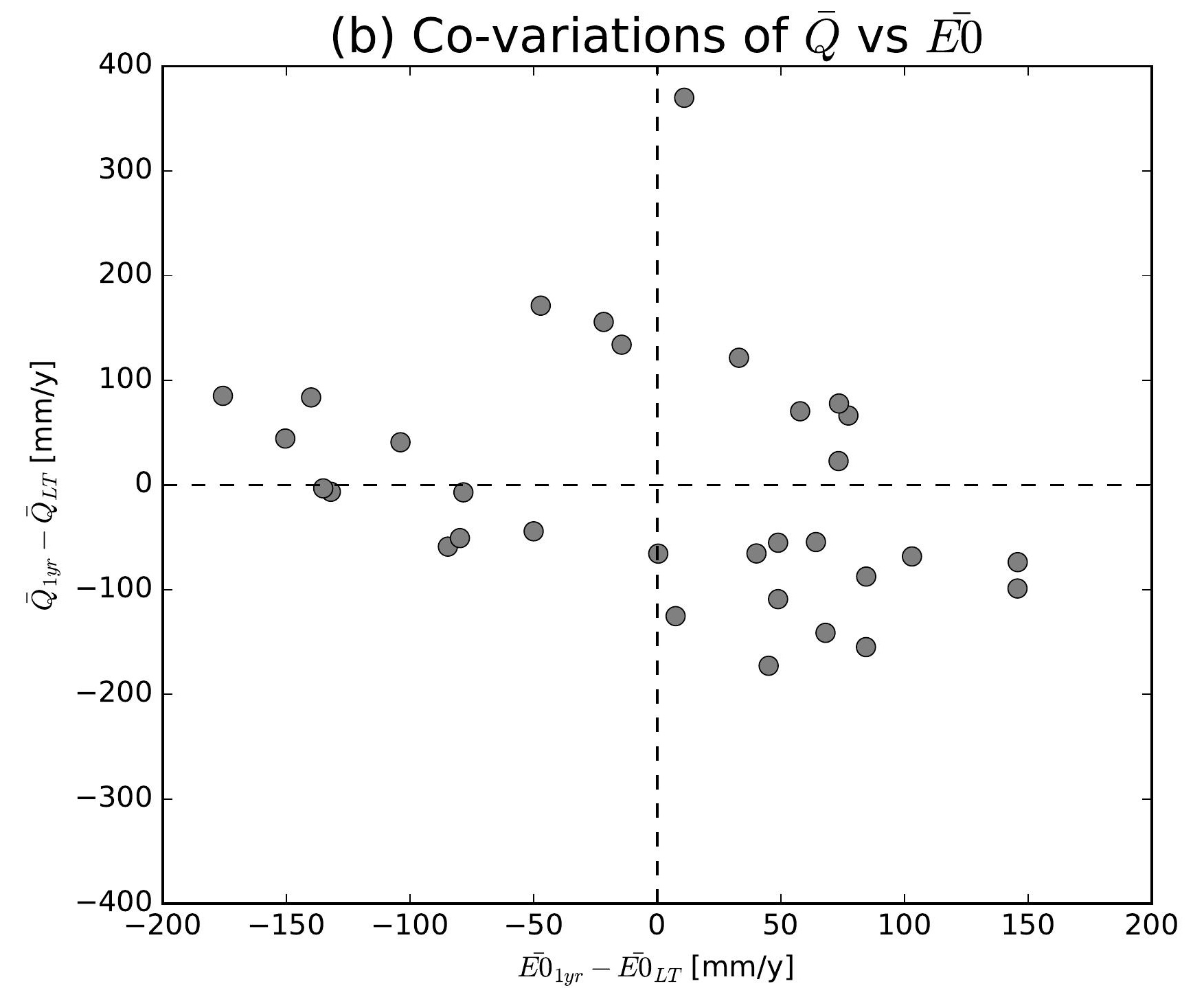

(e) Co-variations of $\bar{Q}$ vs $\overline{E 0}$

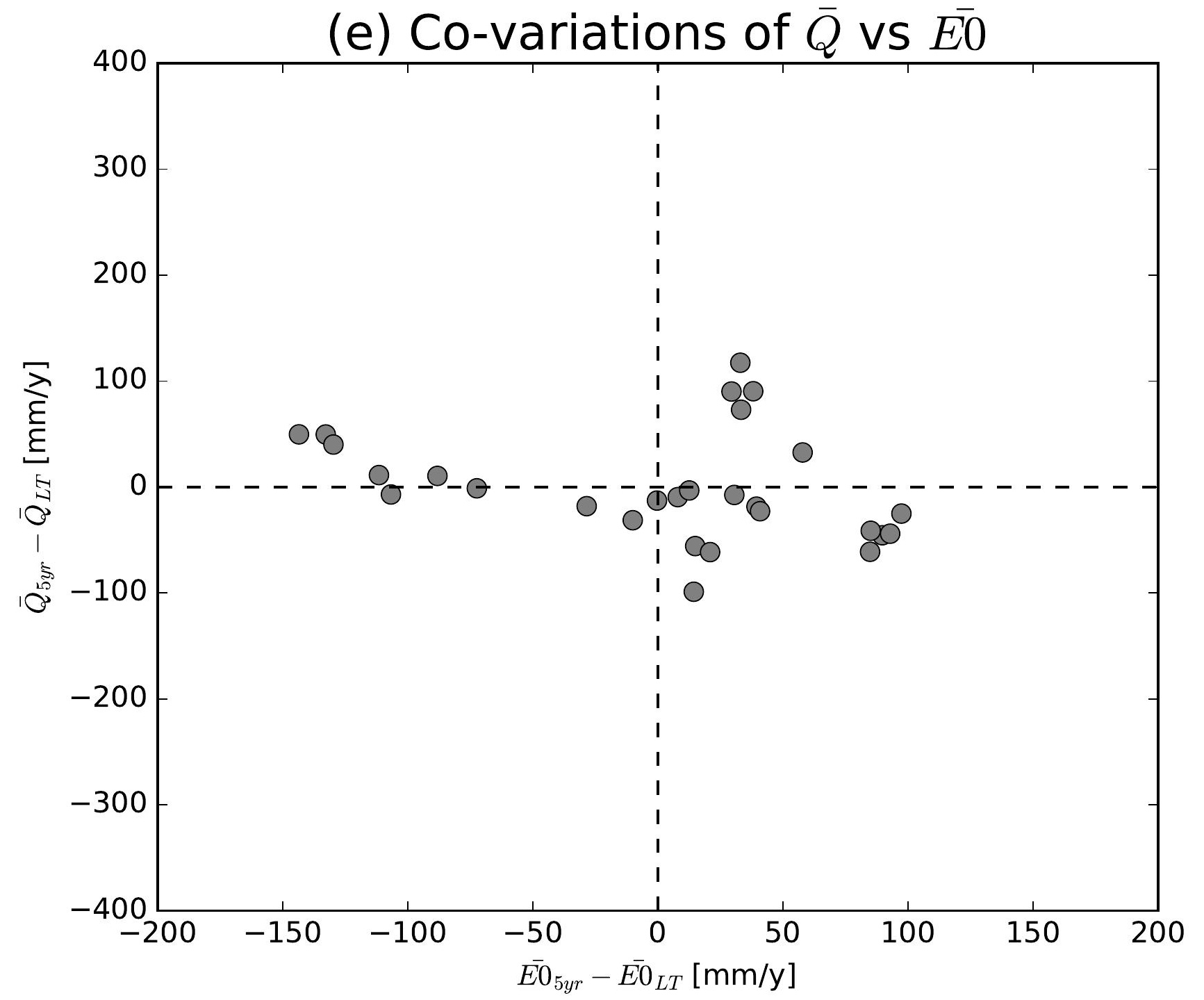

(h) Co-variations of $\bar{Q}$ vs $\overline{E 0}$

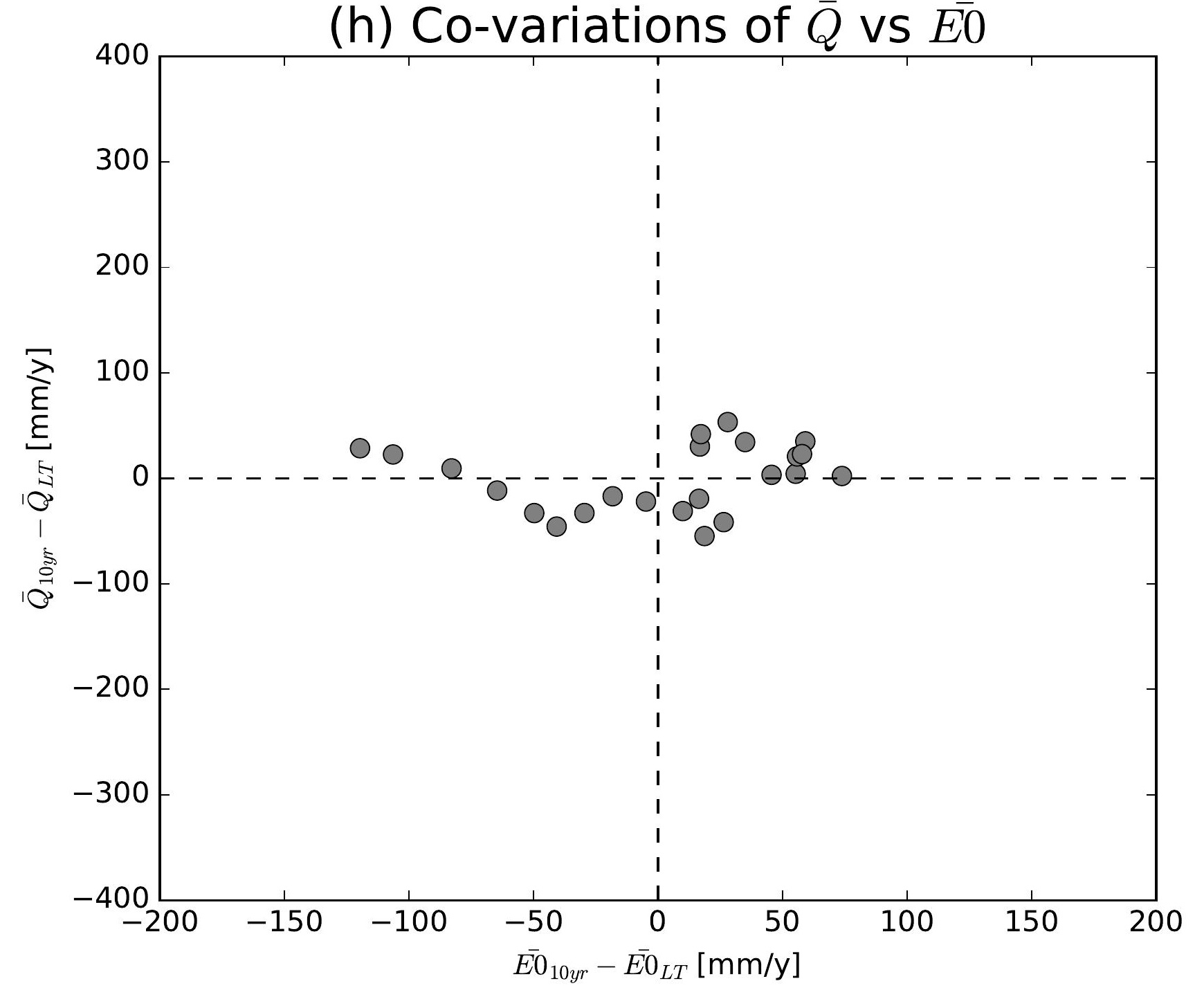

(k) Co-variations of $\bar{Q}$ vs $\overline{E 0}$

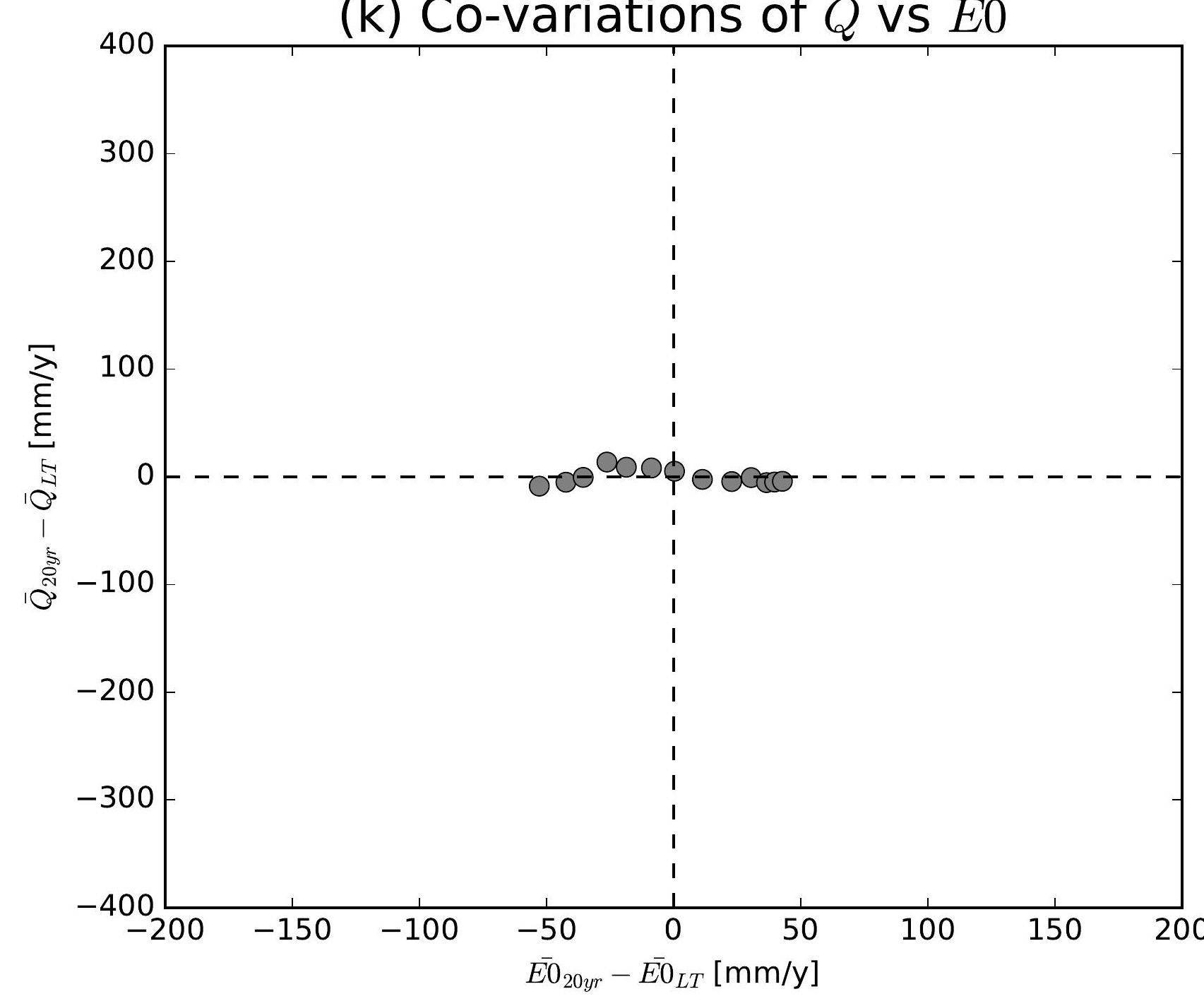

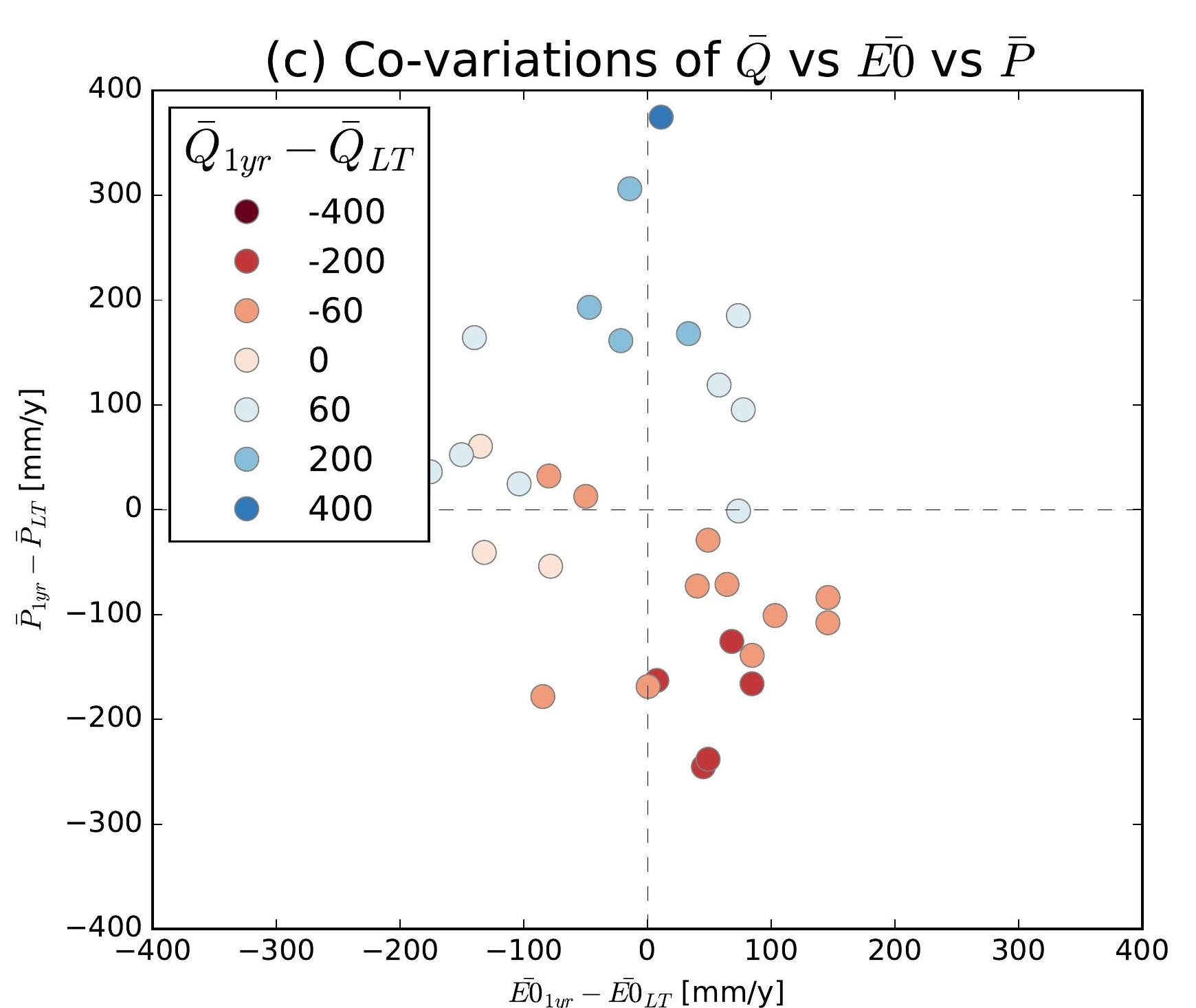
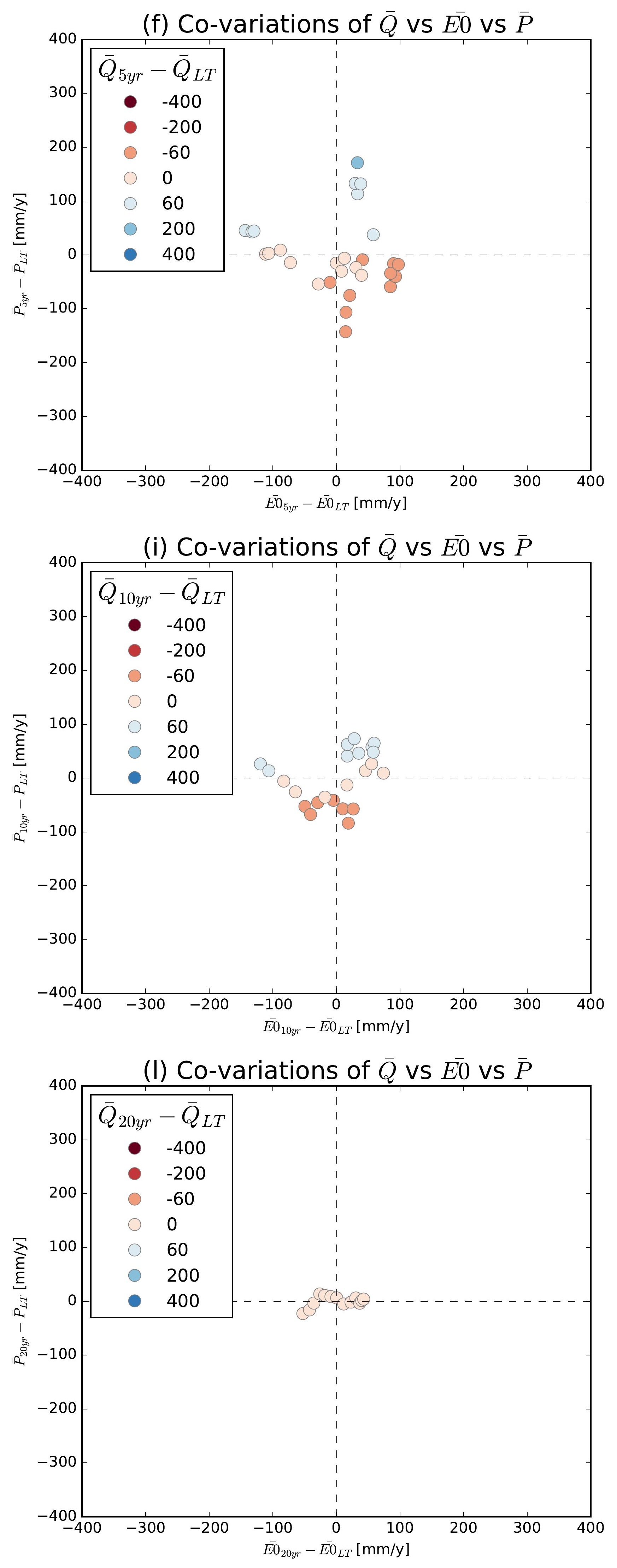

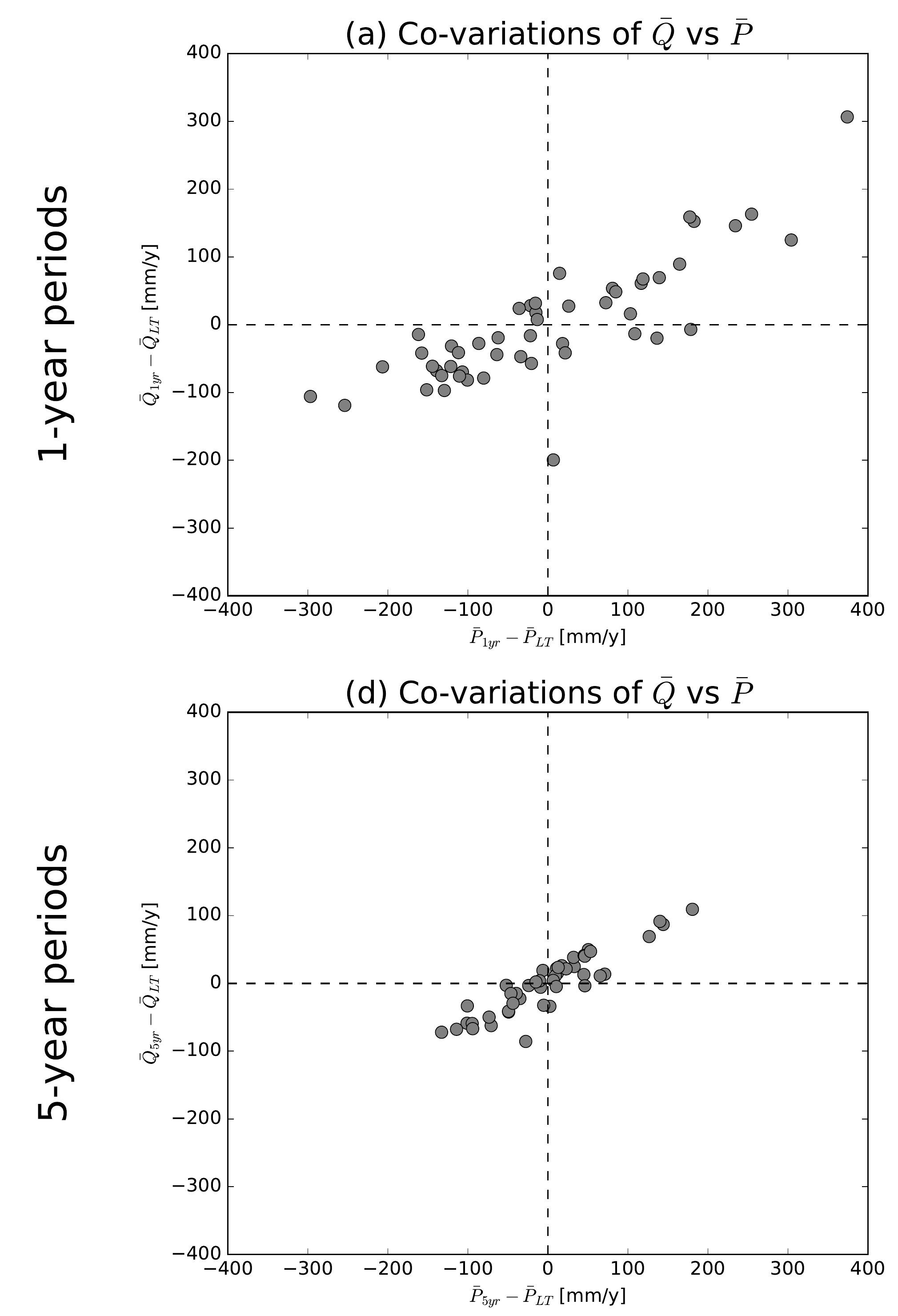

(g) Co-variations of $\bar{Q}$ vs $\bar{P}$
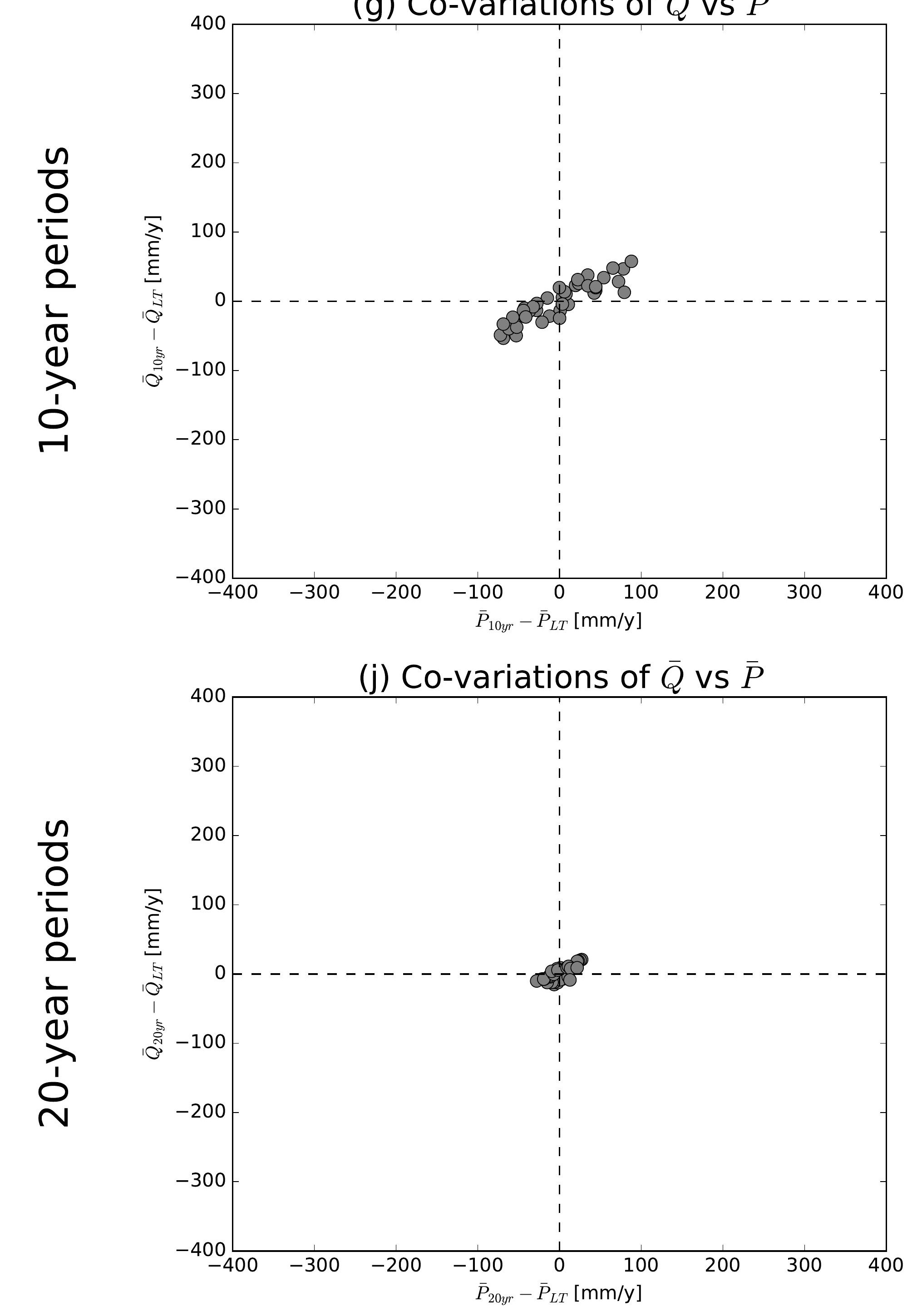

(b) Co-variations of $\bar{Q}$ vs $\overline{E 0}$

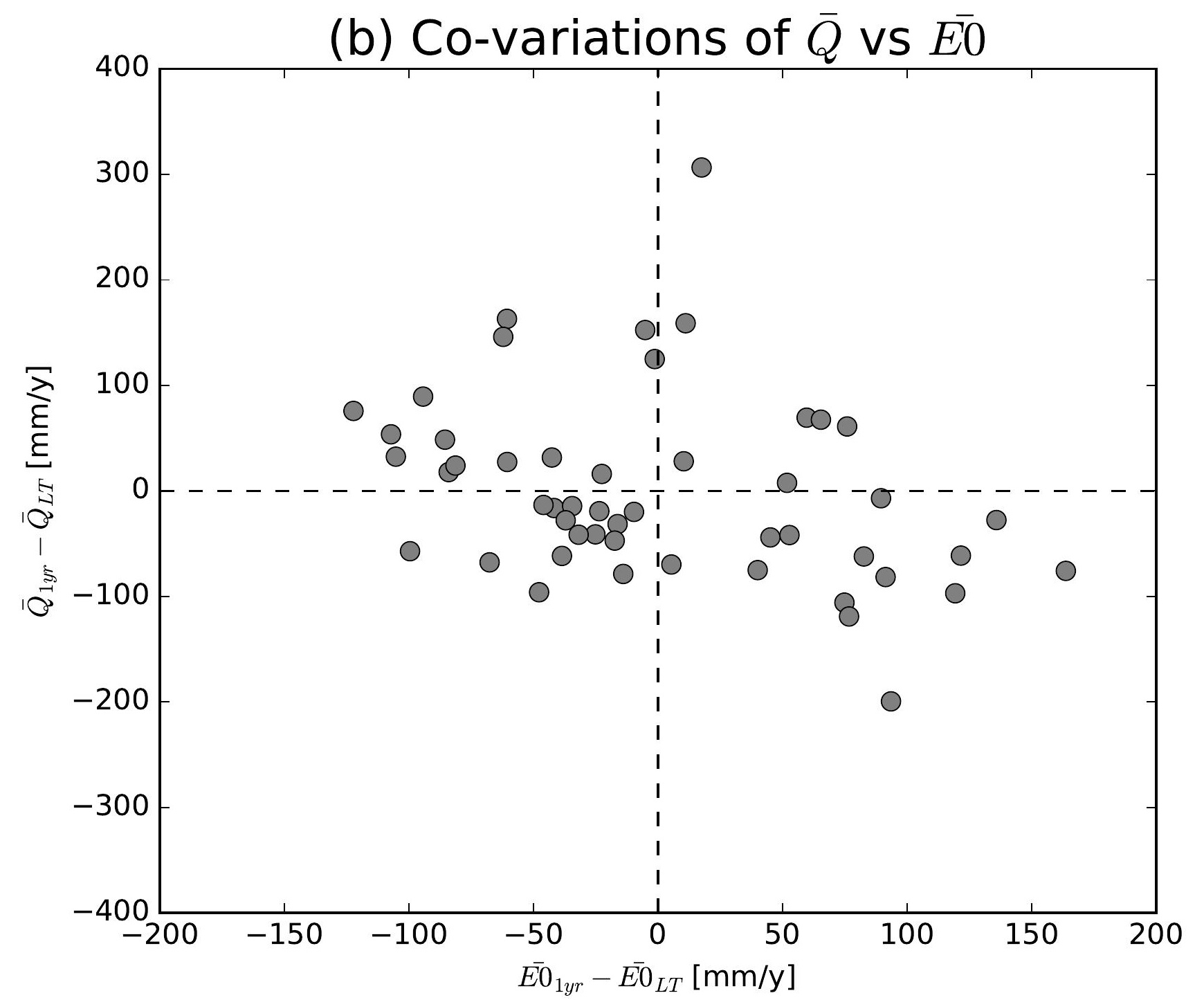

(e) Co-variations of $\bar{Q}$ vs $\overline{E 0}$

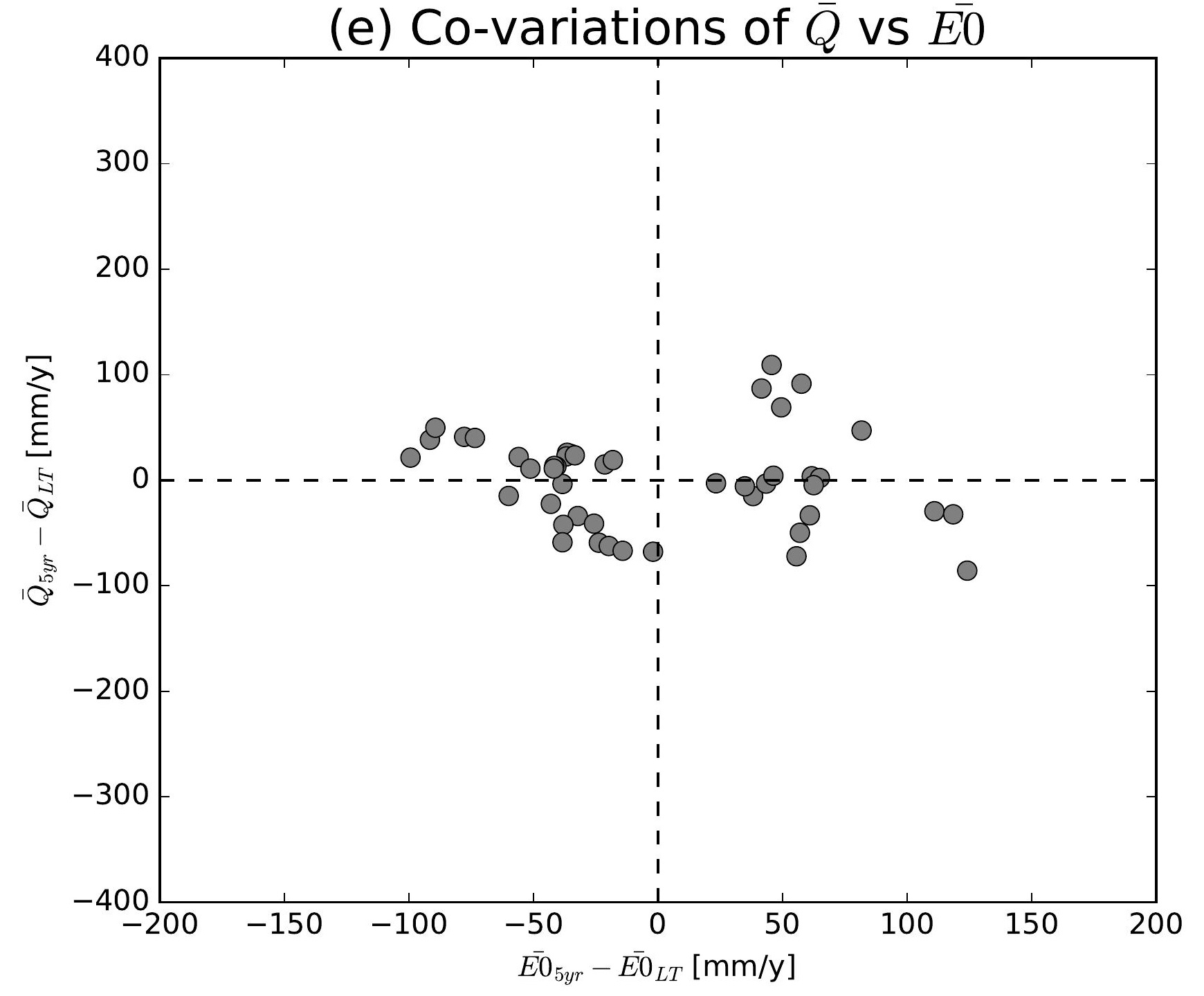

(h) Co-variations of $\bar{Q}$ vs $\overline{E 0}$

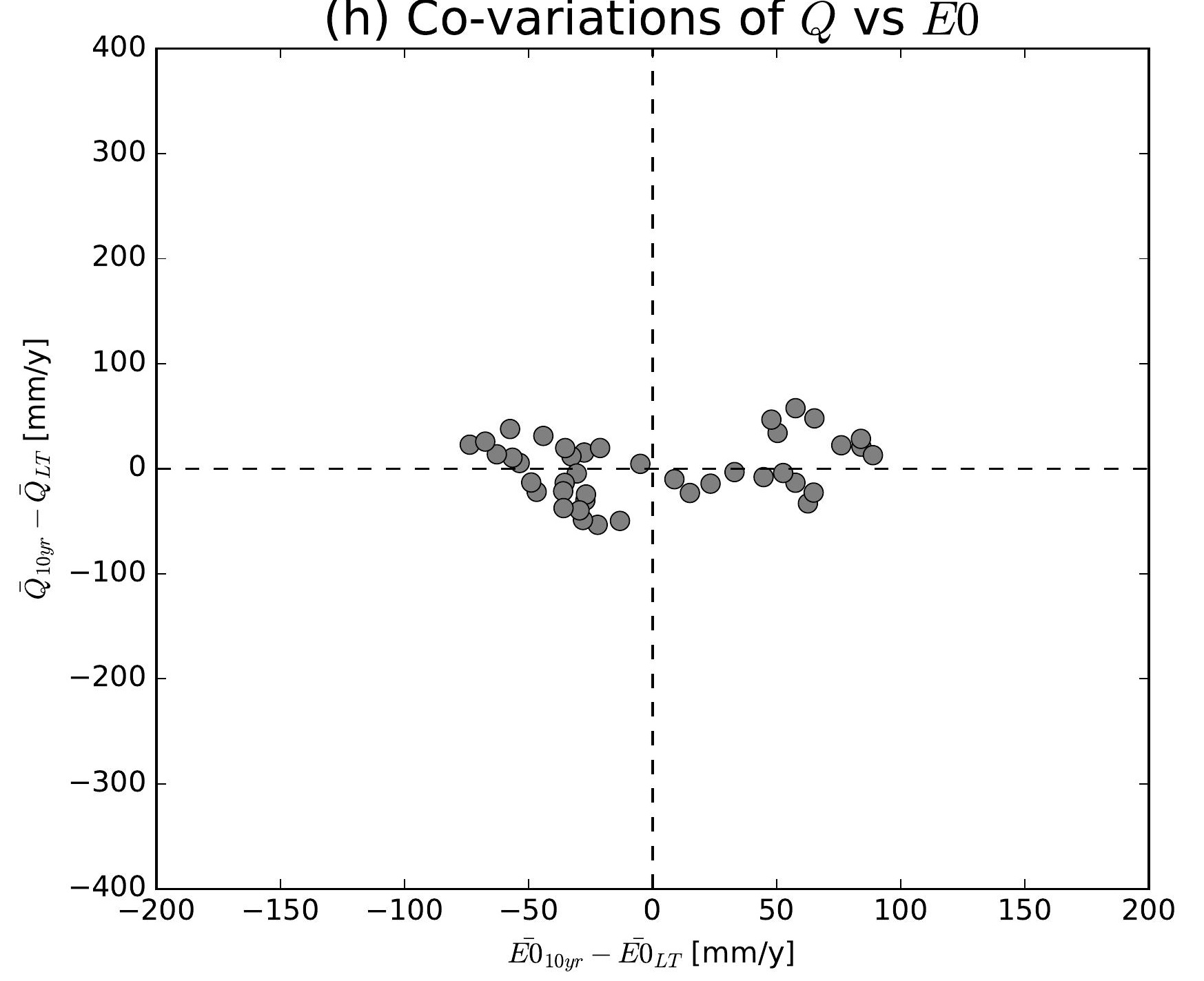

(k) Co-variations of $\bar{Q}$ vs $\overline{E 0}$

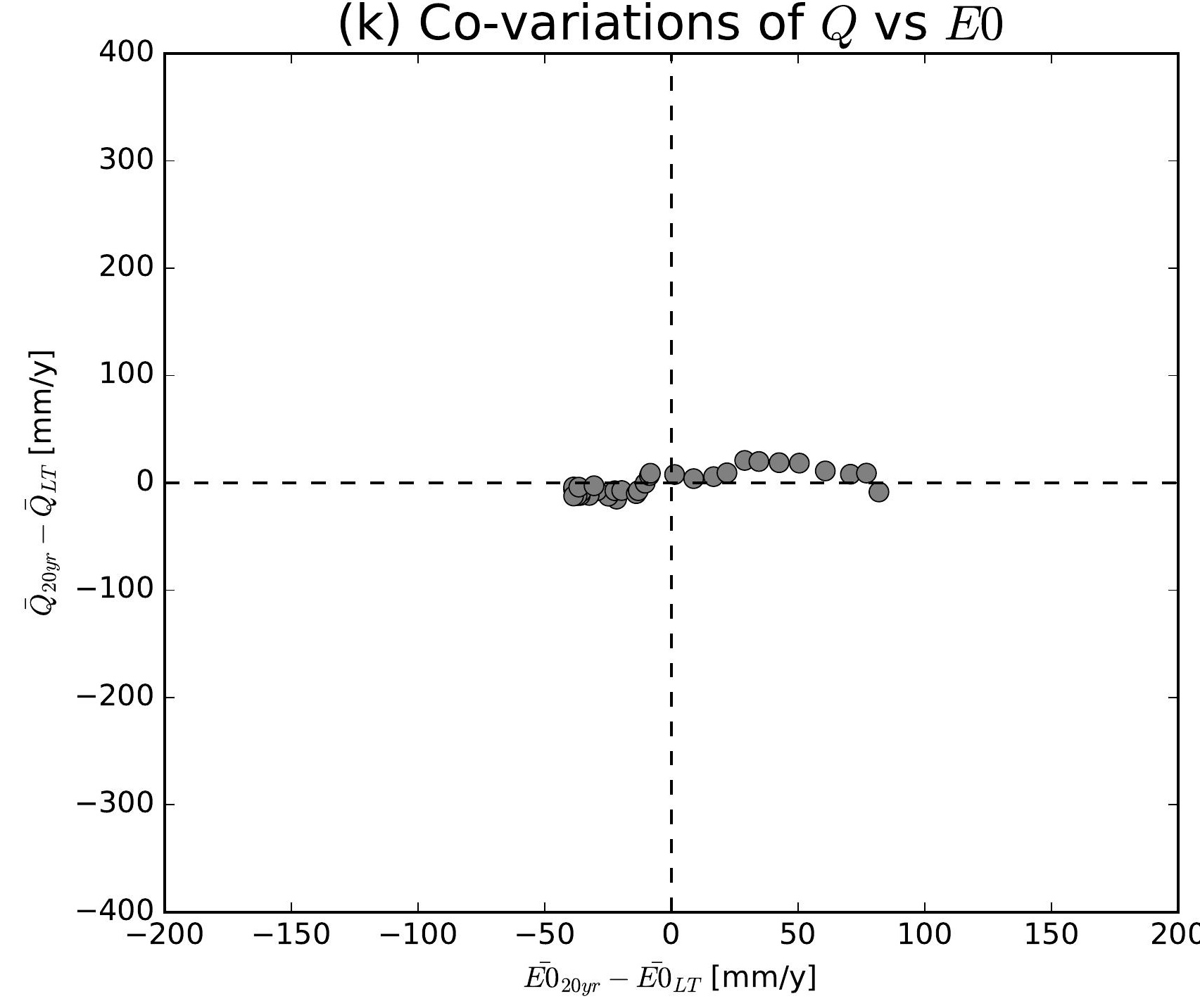

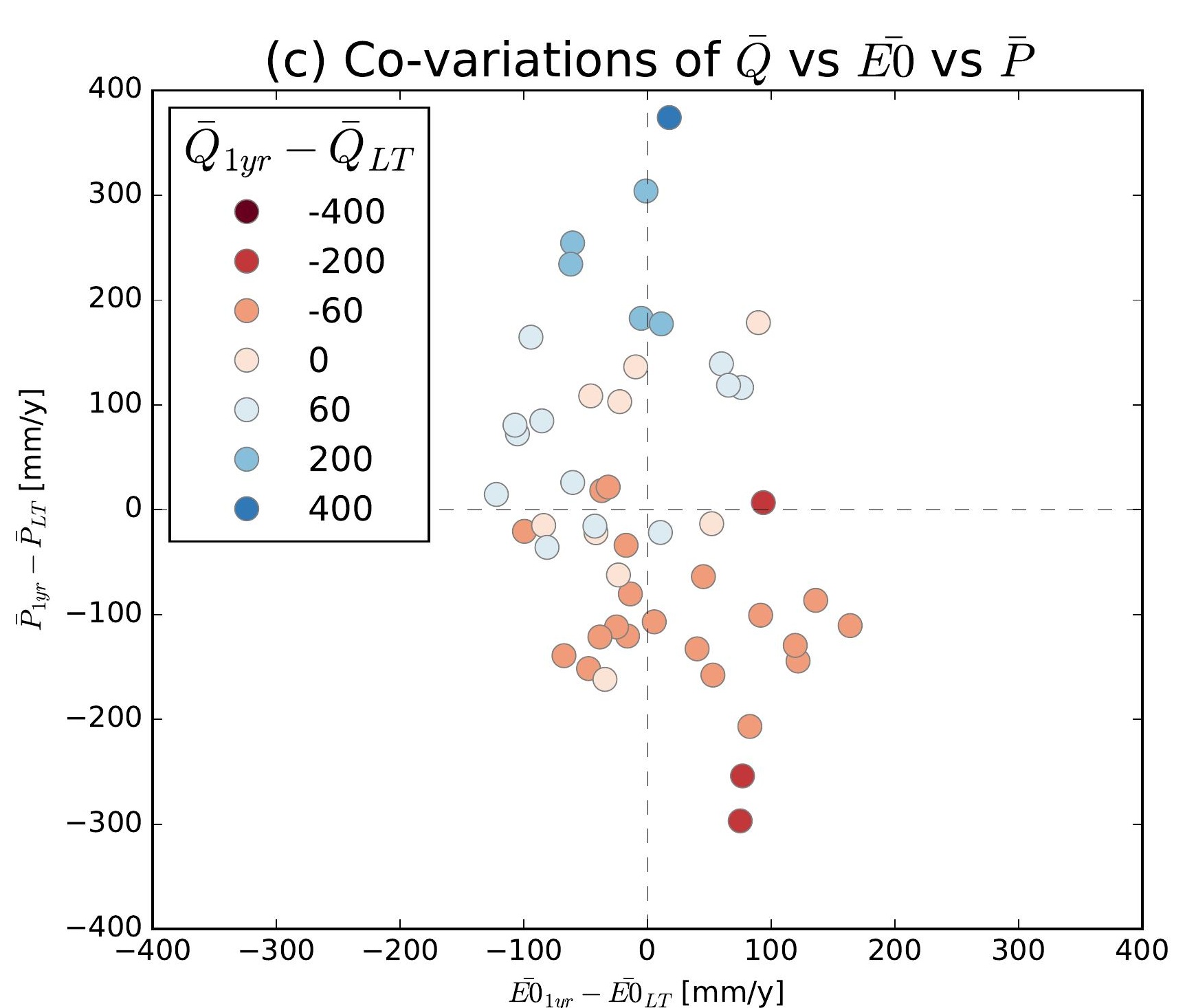
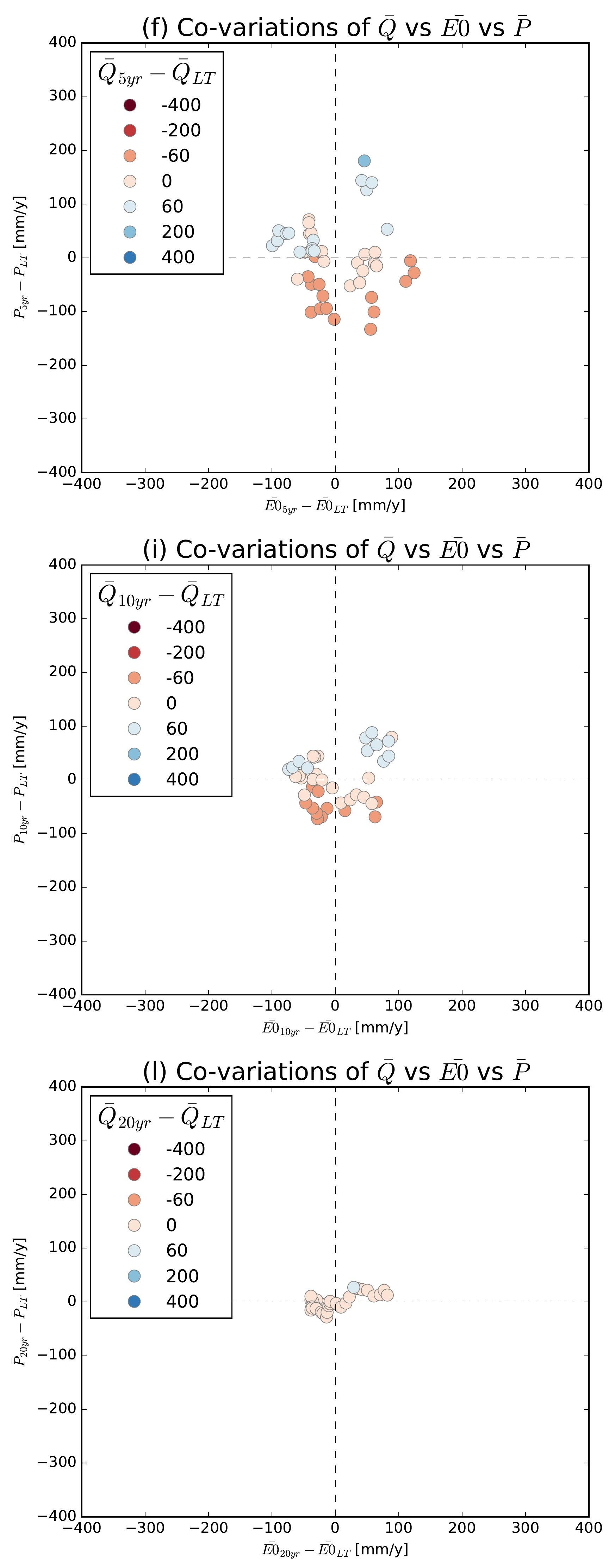

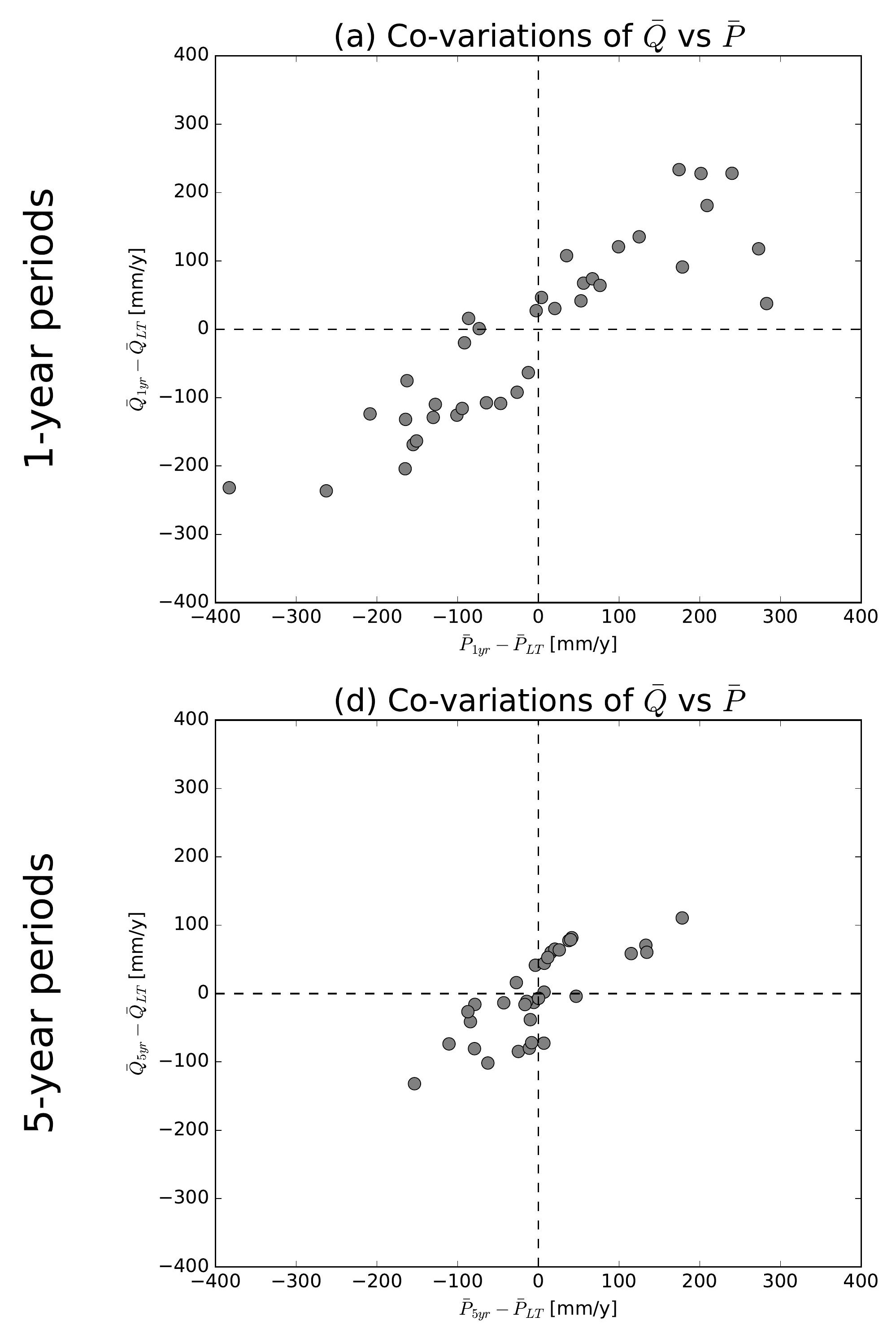

(g) Co-variations of $\bar{Q}$ vs $\bar{P}$
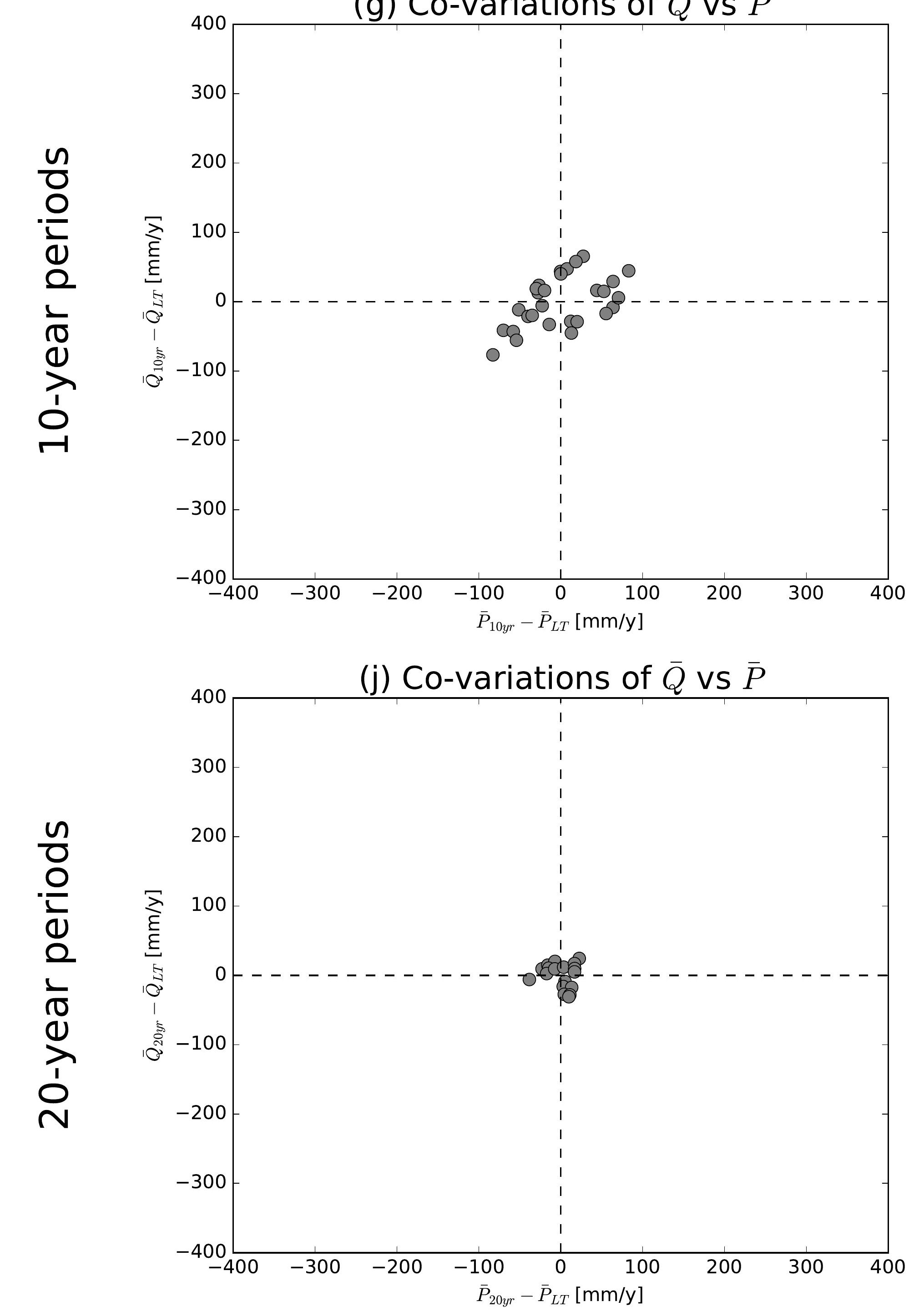

(b) Co-variations of $\bar{Q}$ vs $\overline{E 0}$

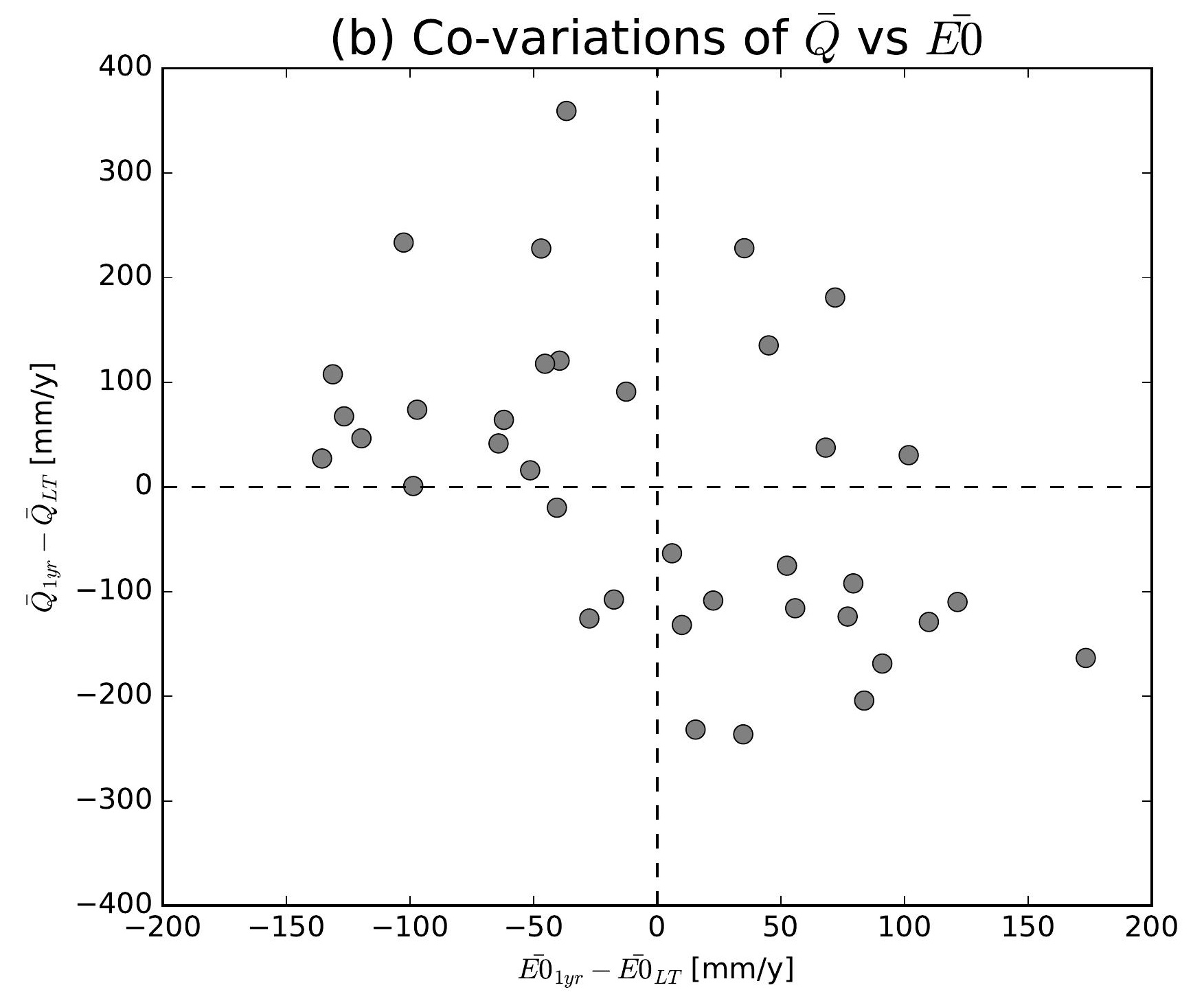

(e) Co-variations of $\bar{Q}$ vs $\overline{E 0}$

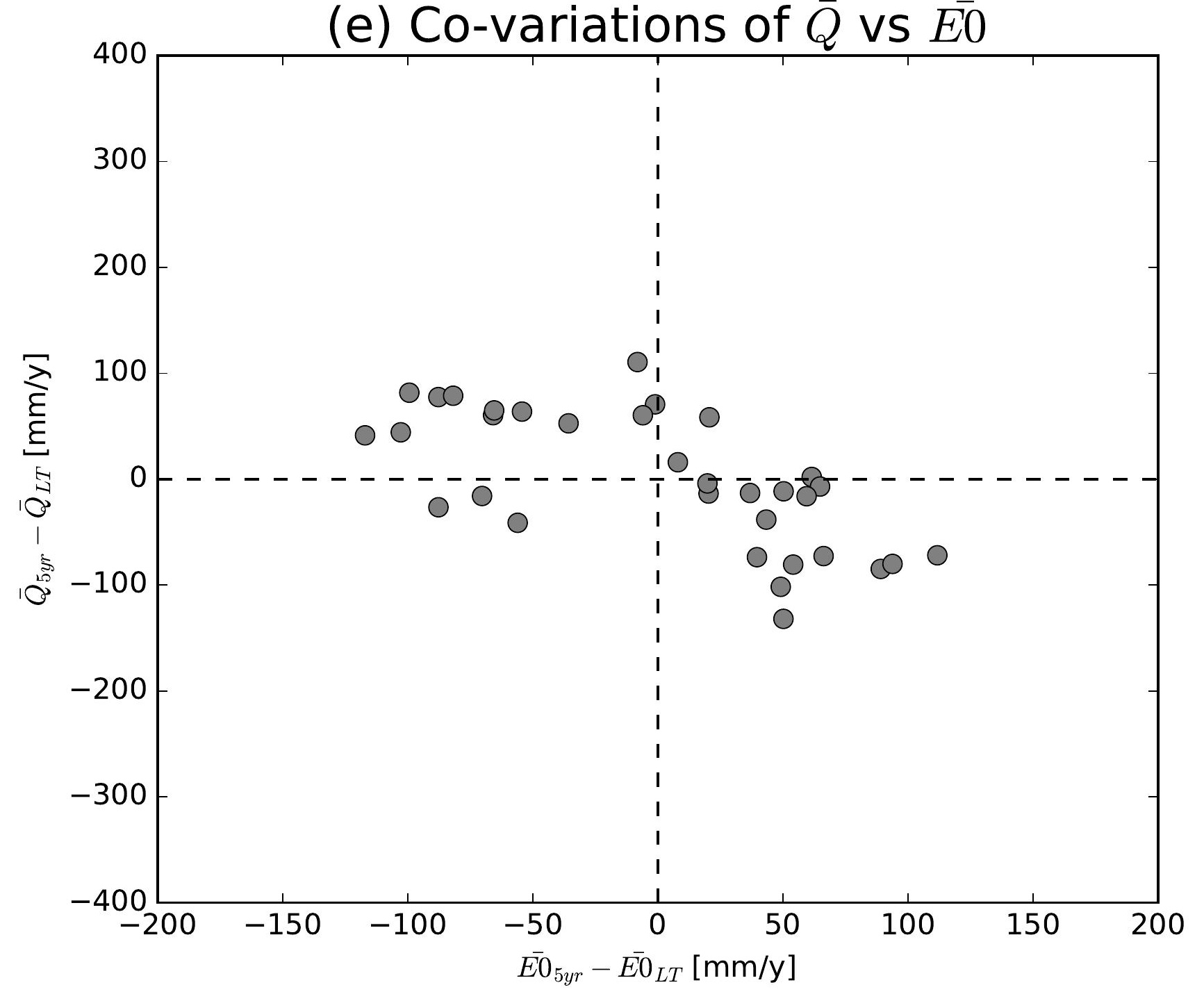

(h) Co-variations of $\bar{Q}$ vs $\overline{E 0}$

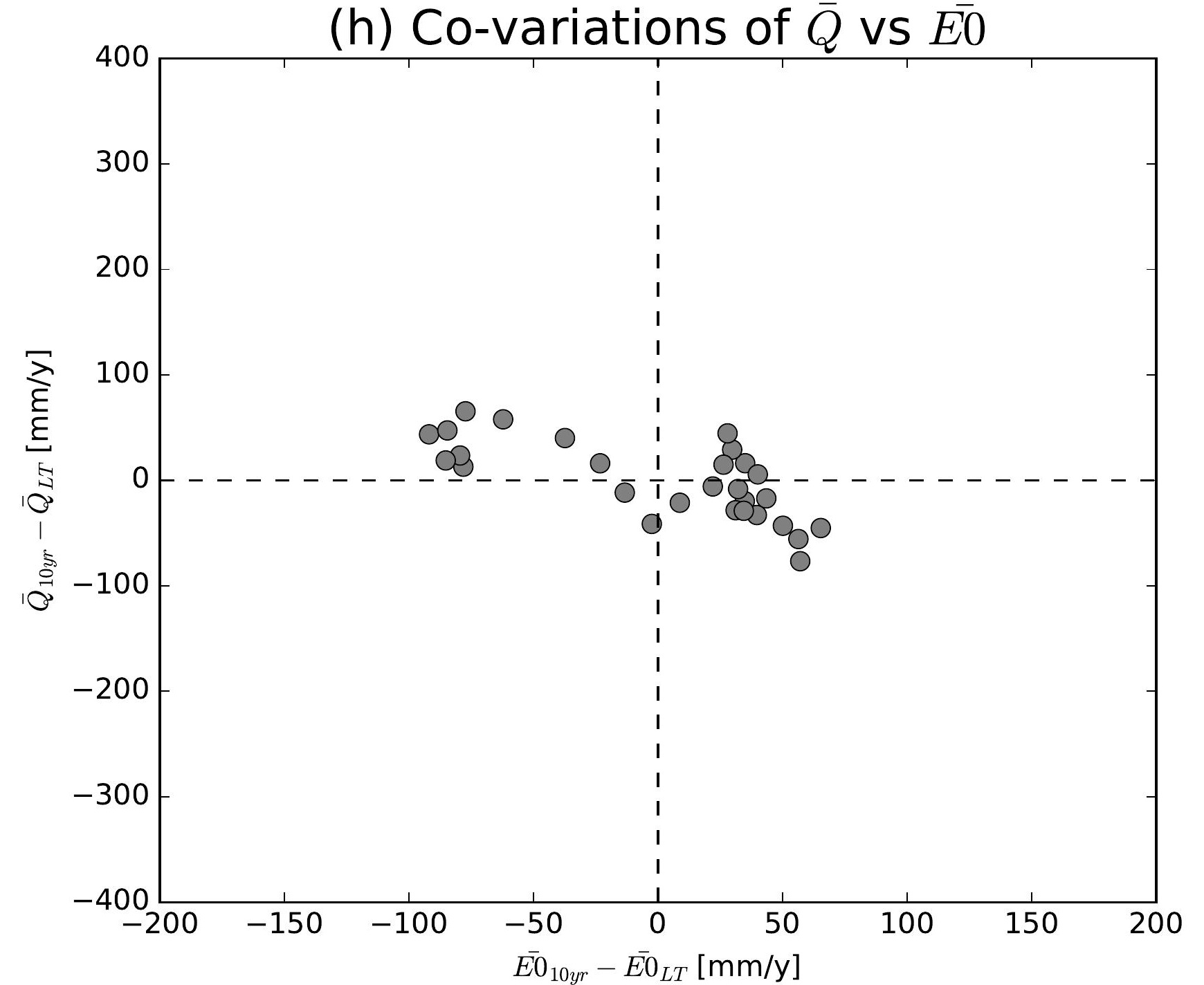

(k) Co-variations of $\bar{Q}$ vs $\overline{E 0}$

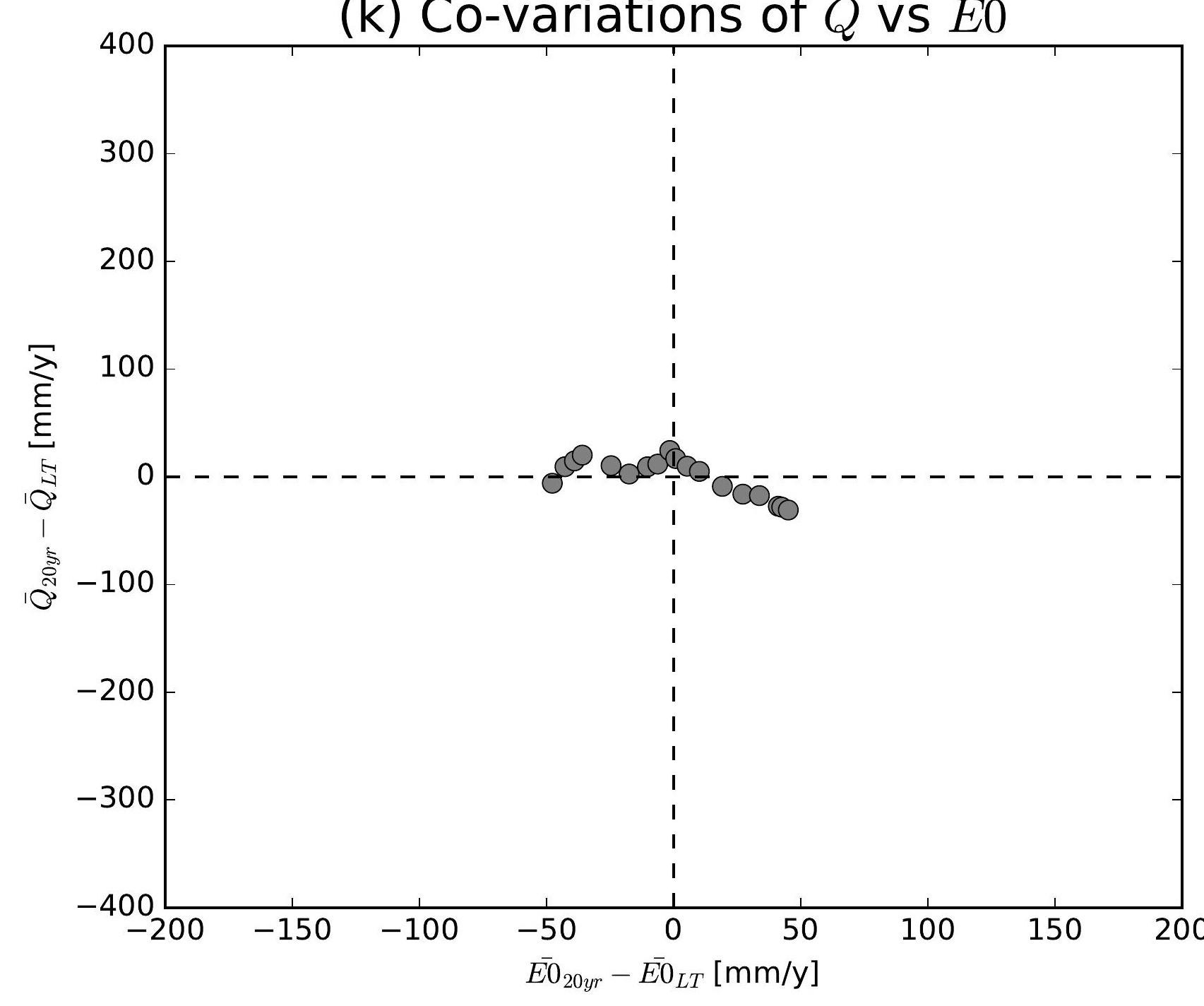

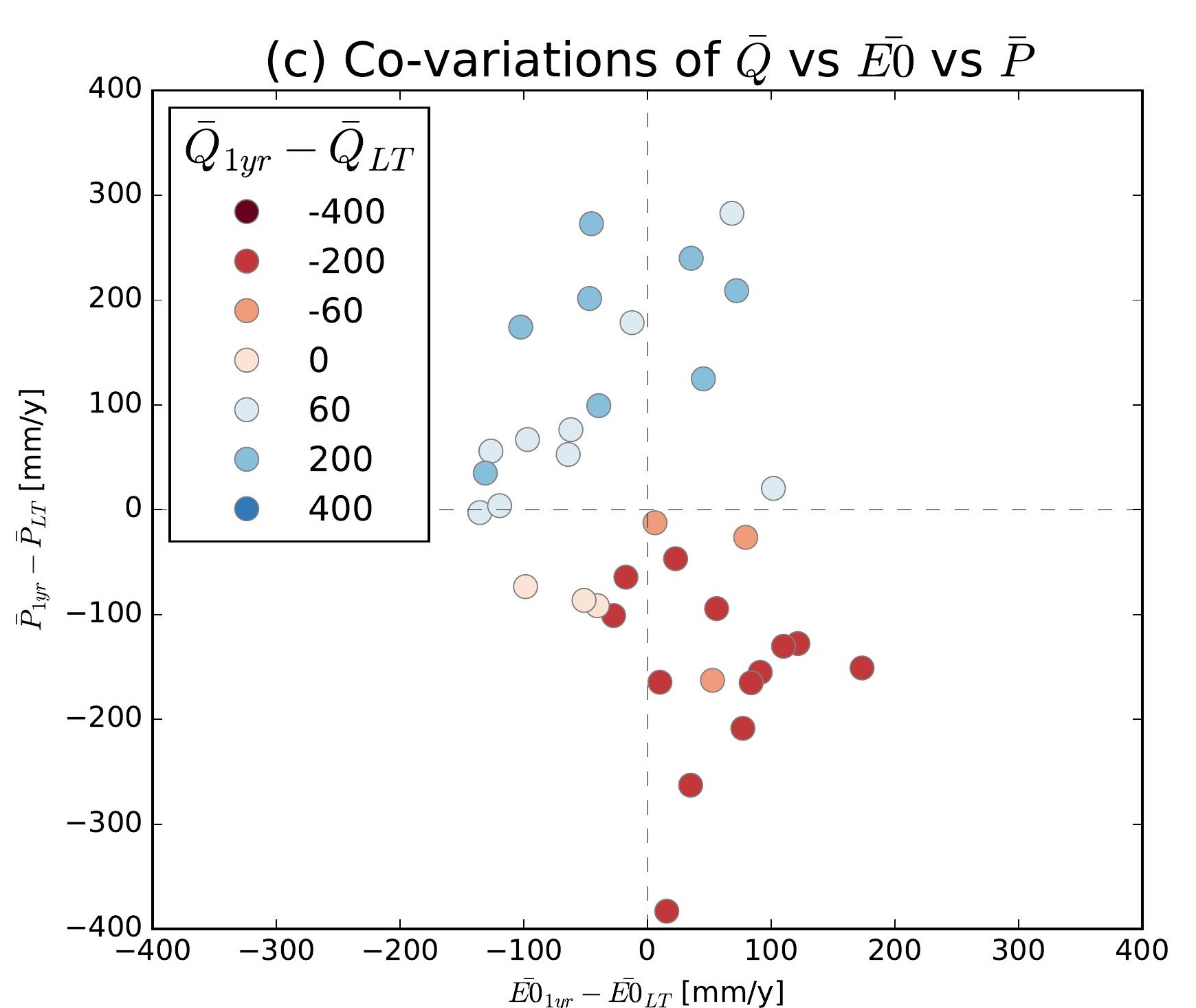
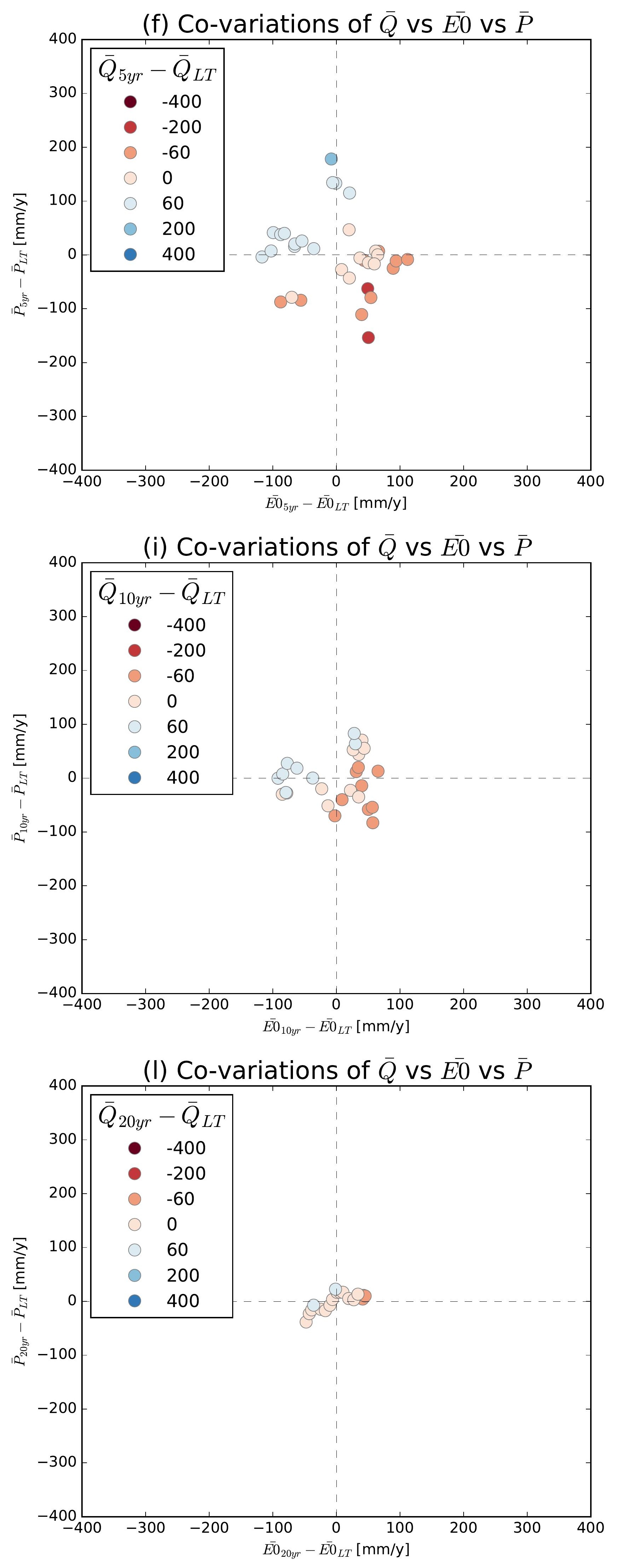

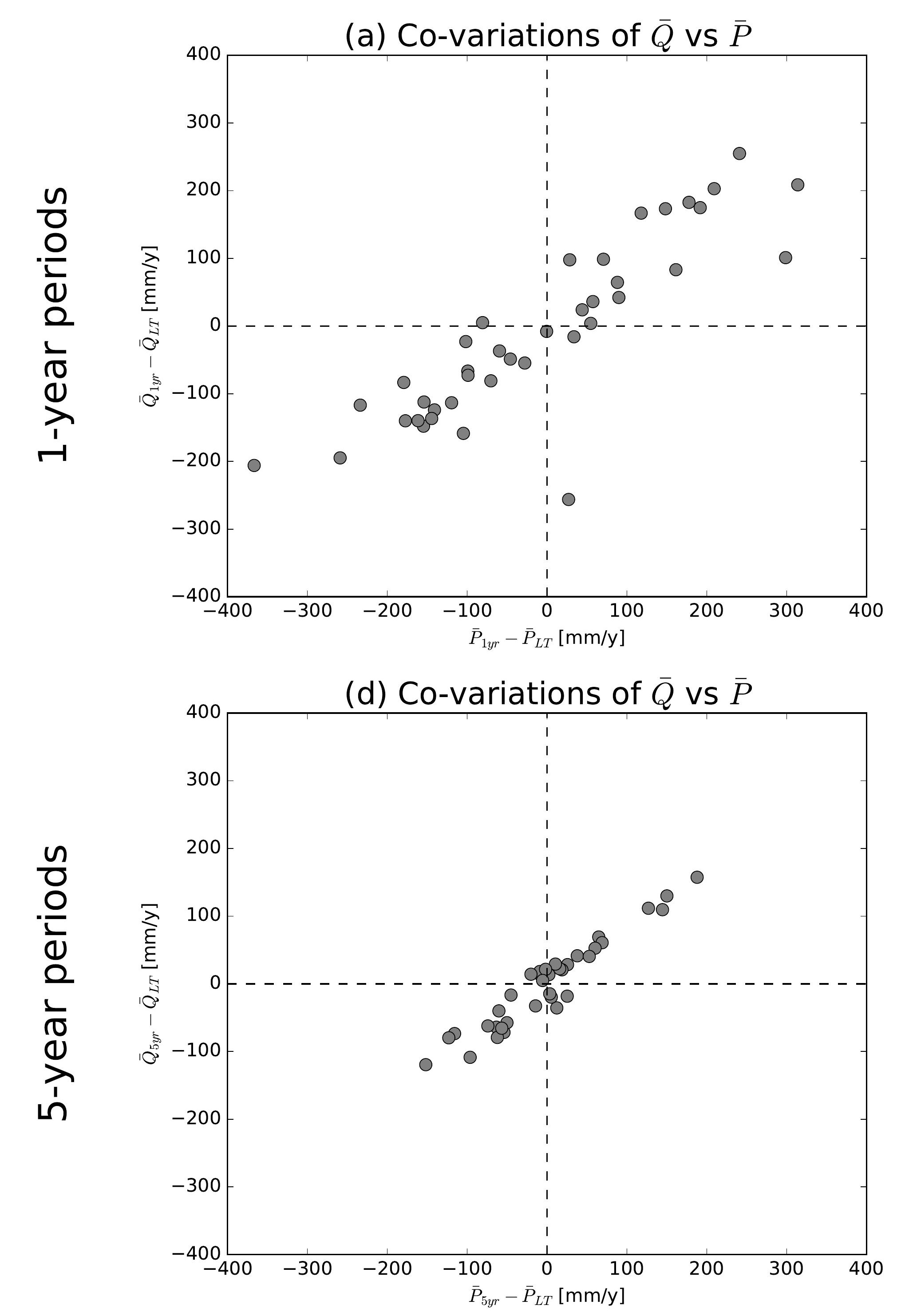

(g) Co-variations of $\bar{Q}$ vs $\bar{P}$
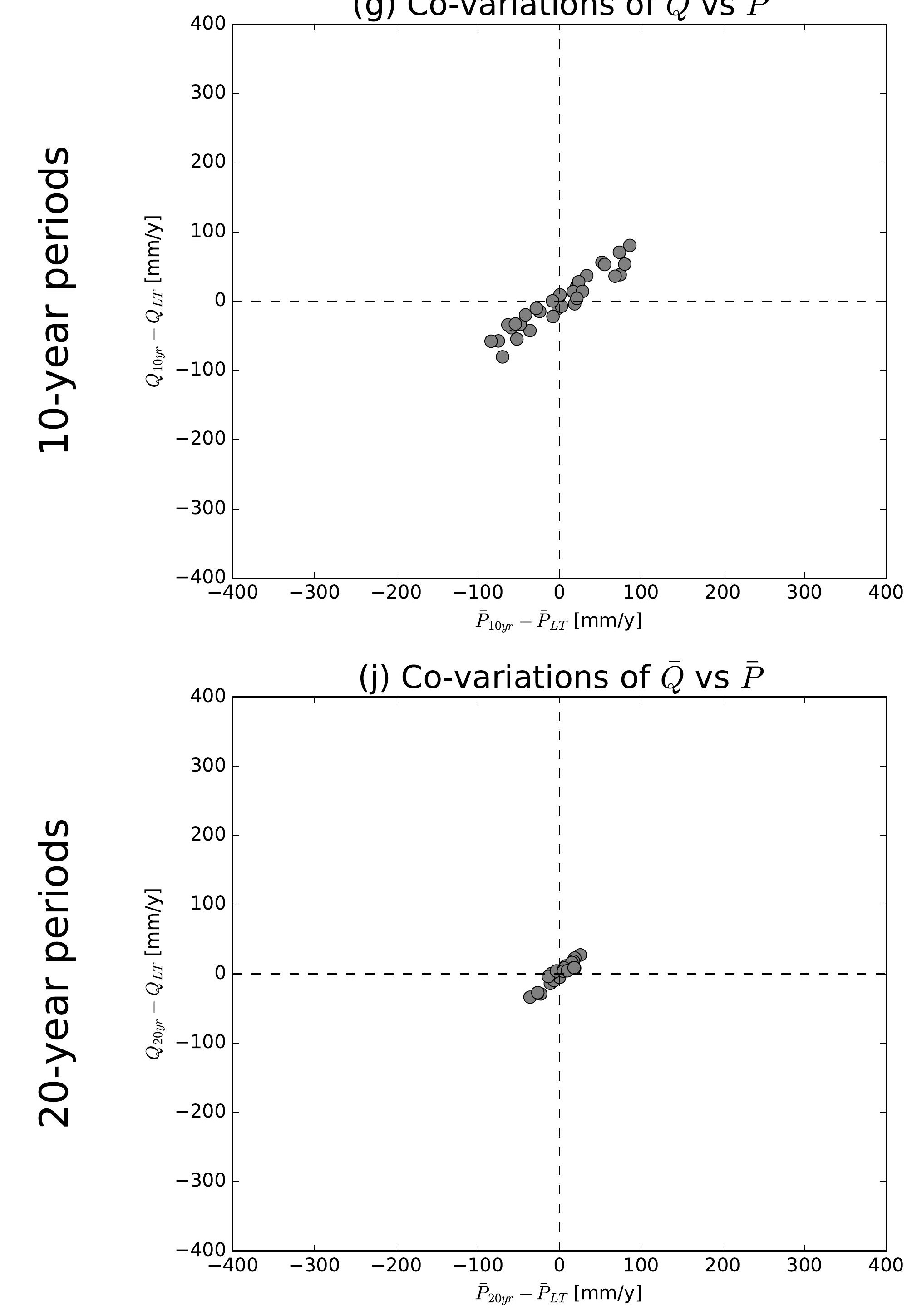

(b) Co-variations of $\bar{Q}$ vs $\overline{E 0}$

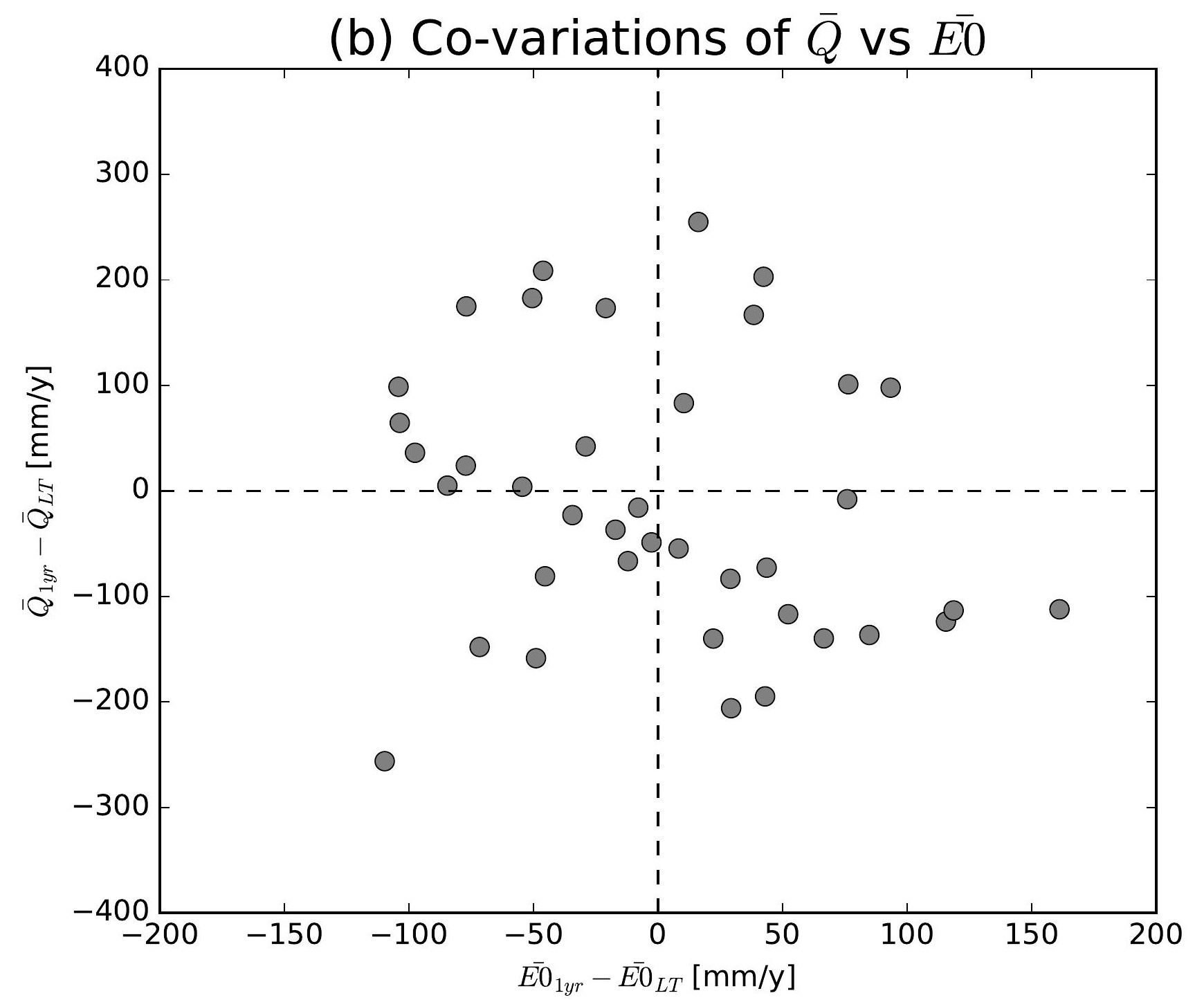

(e) Co-variations of $\bar{Q}$ vs $\overline{E 0}$

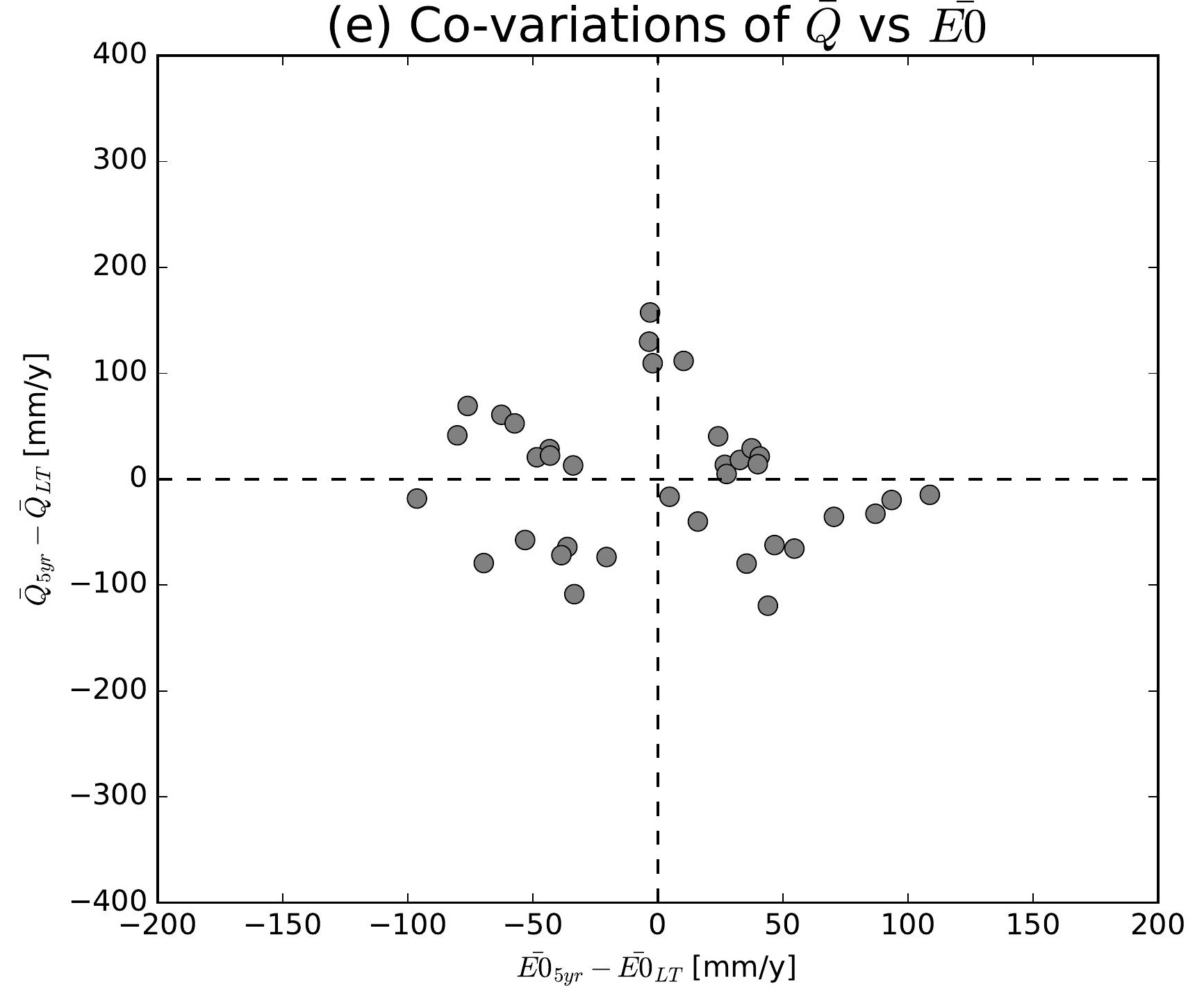

(h) Co-variations of $\bar{Q}$ vs $\overline{E 0}$

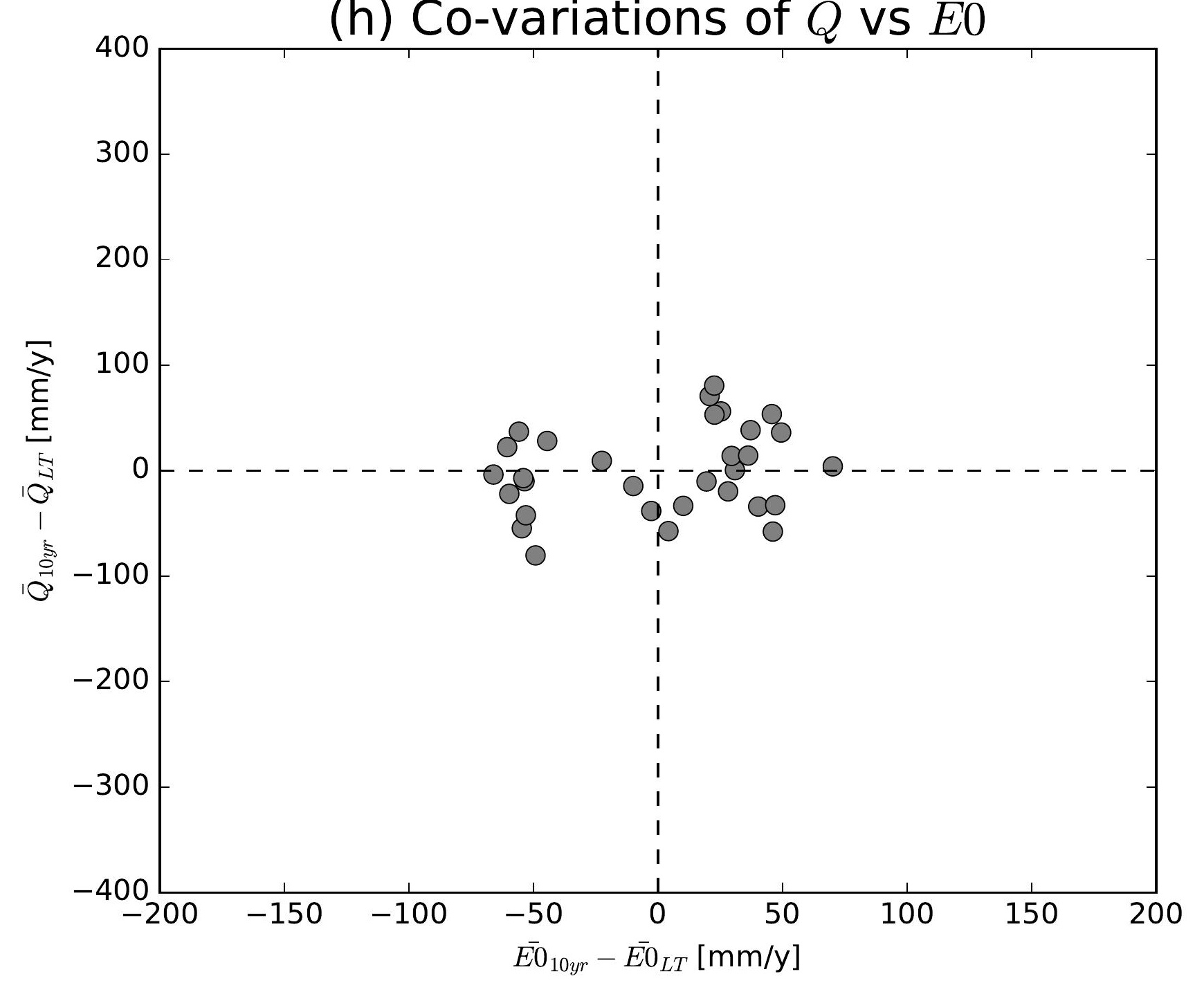

(k) Co-variations of $\bar{Q}$ vs $\overline{E 0}$

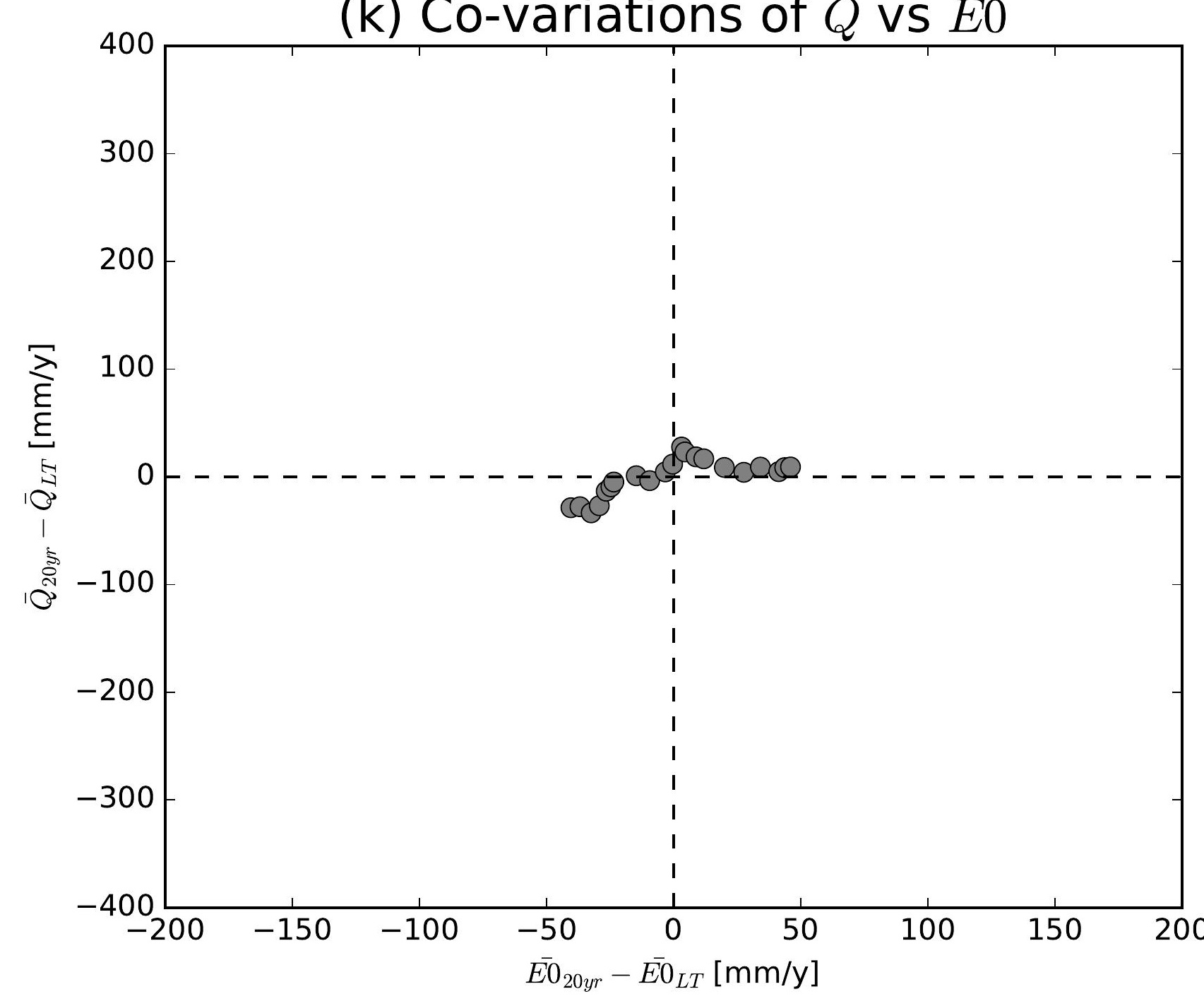

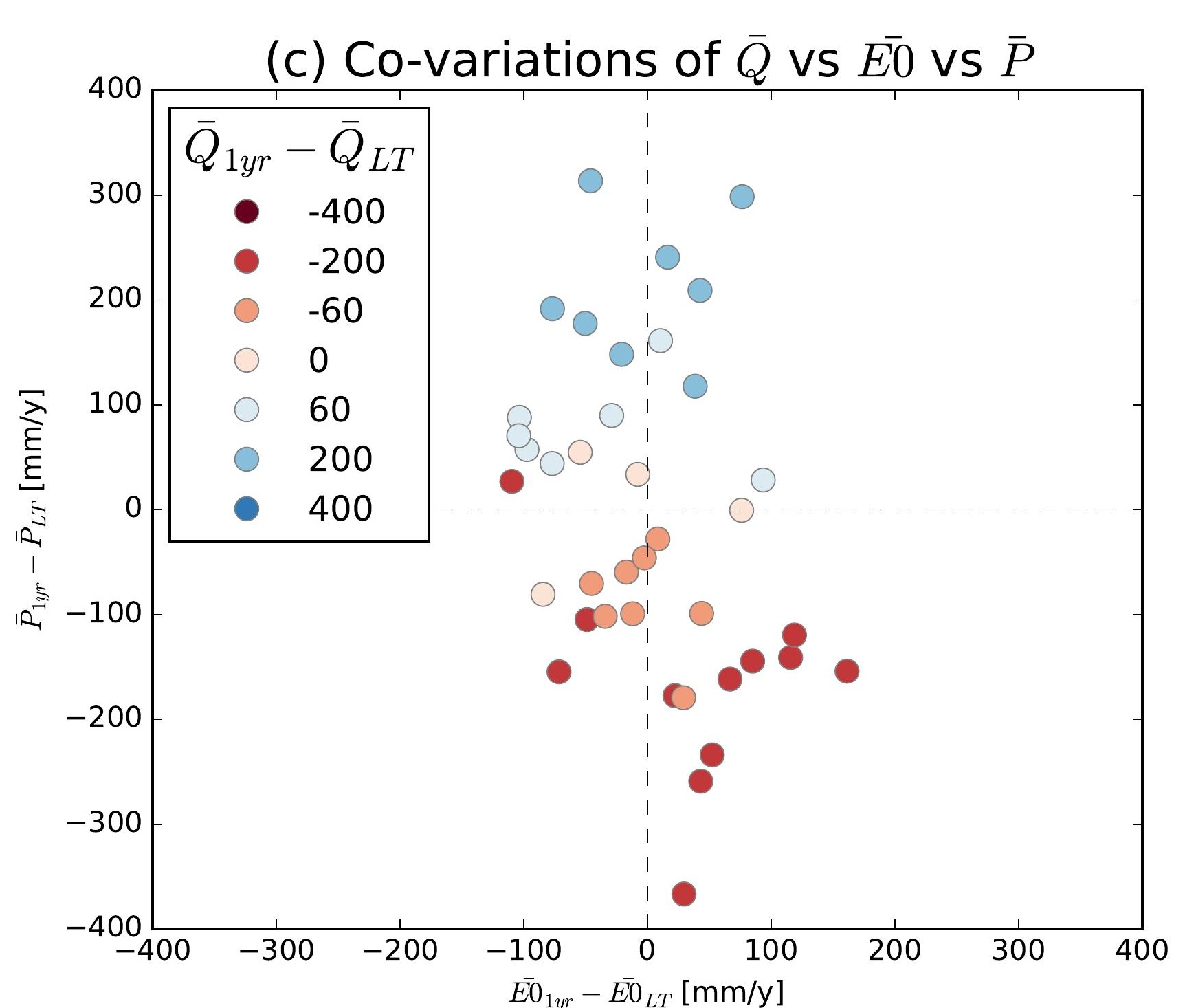
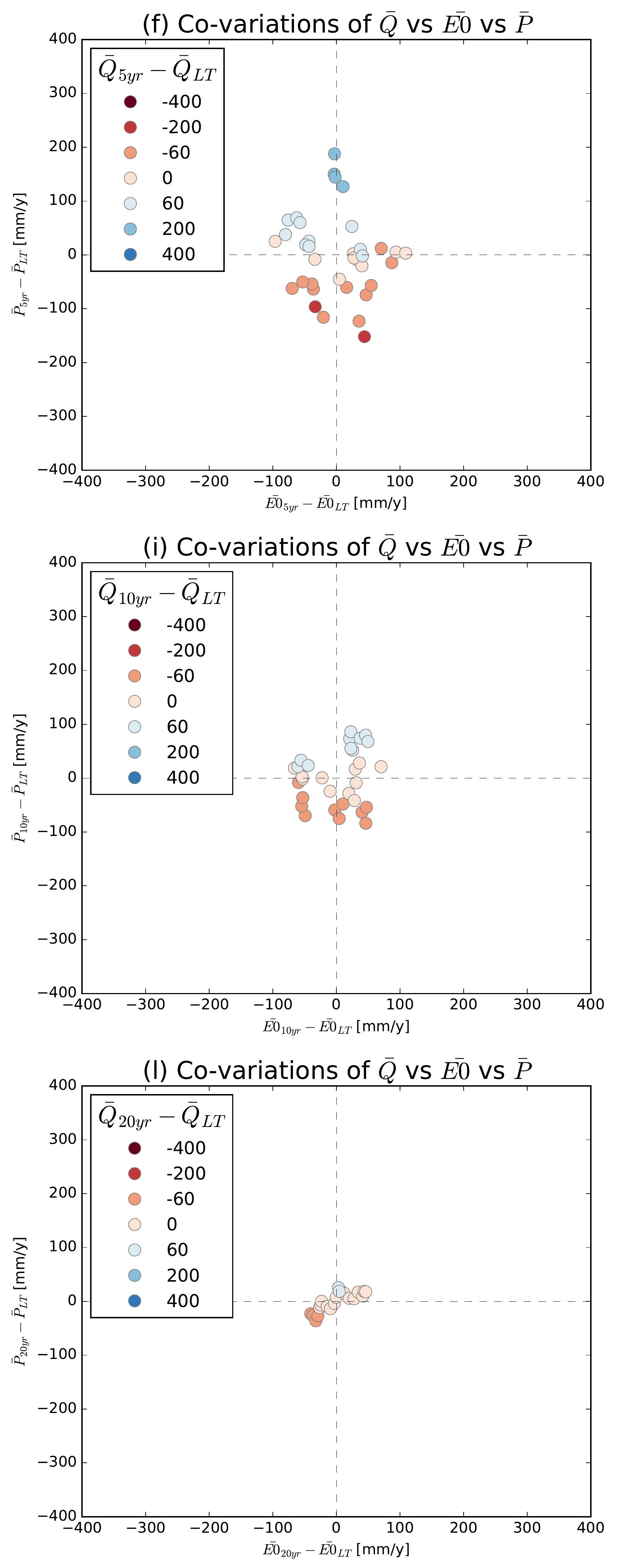

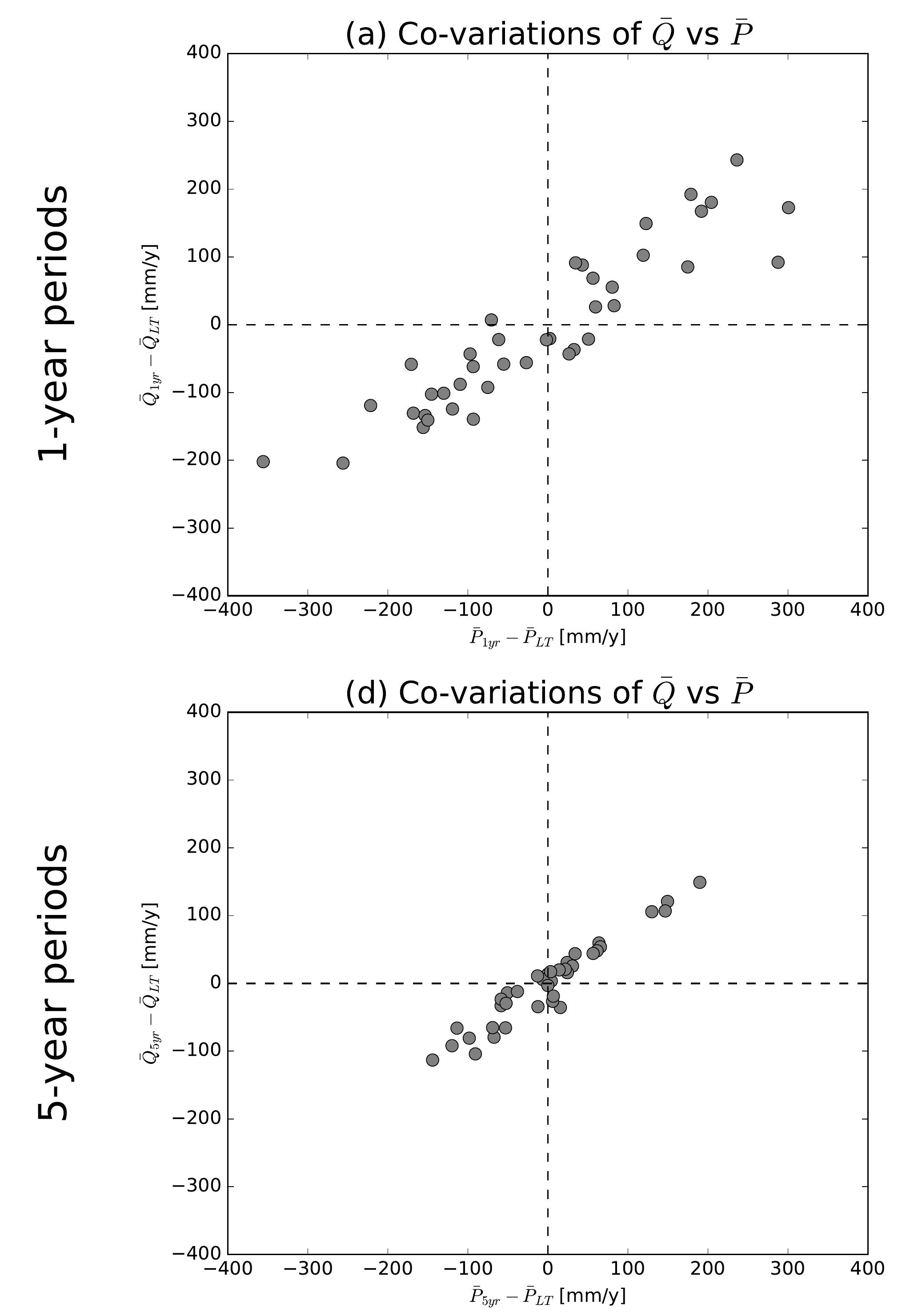

(g) Co-variations of $\bar{Q}$ vs $\bar{P}$
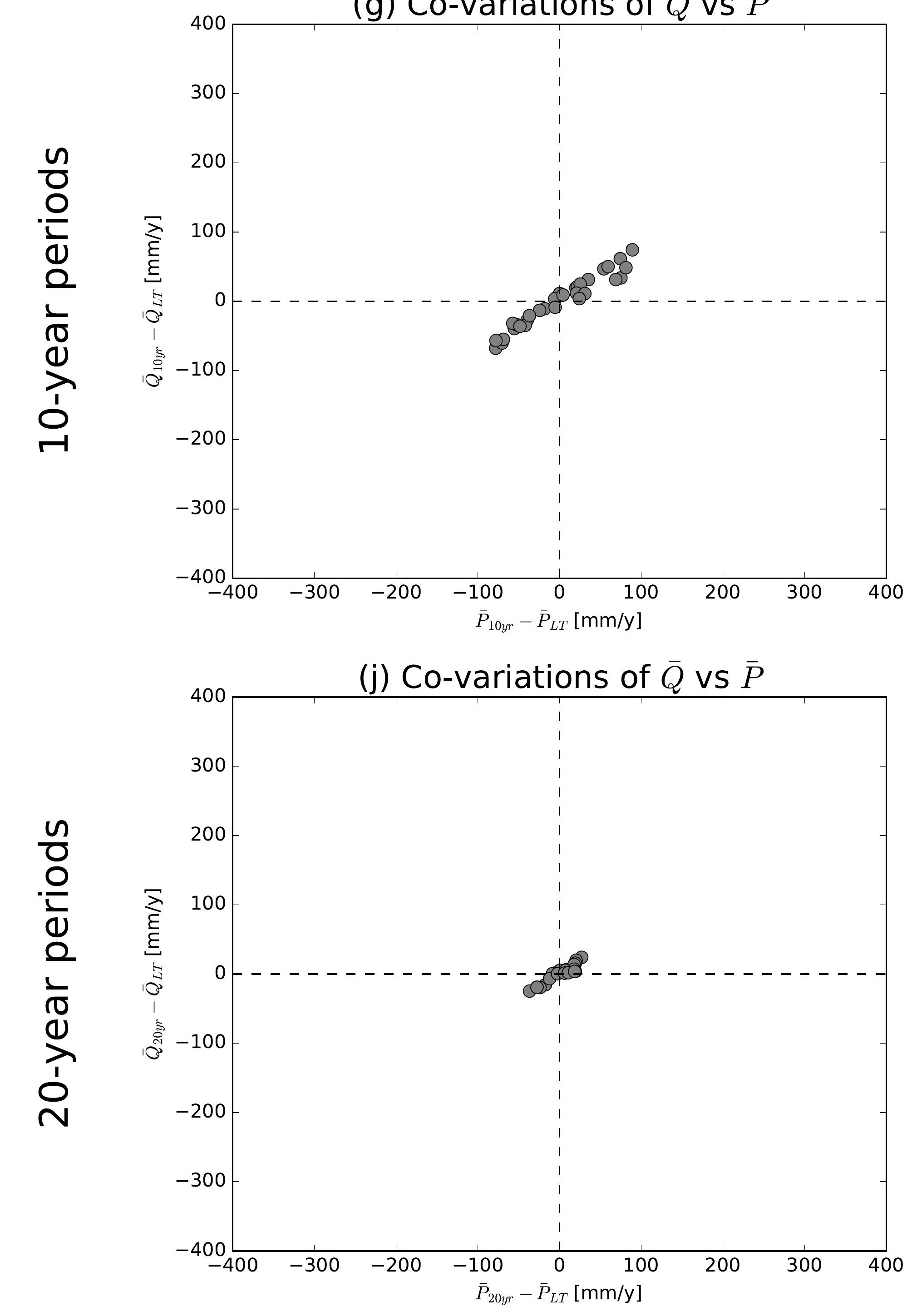

(b) Co-variations of $\bar{Q}$ vs $\overline{E 0}$

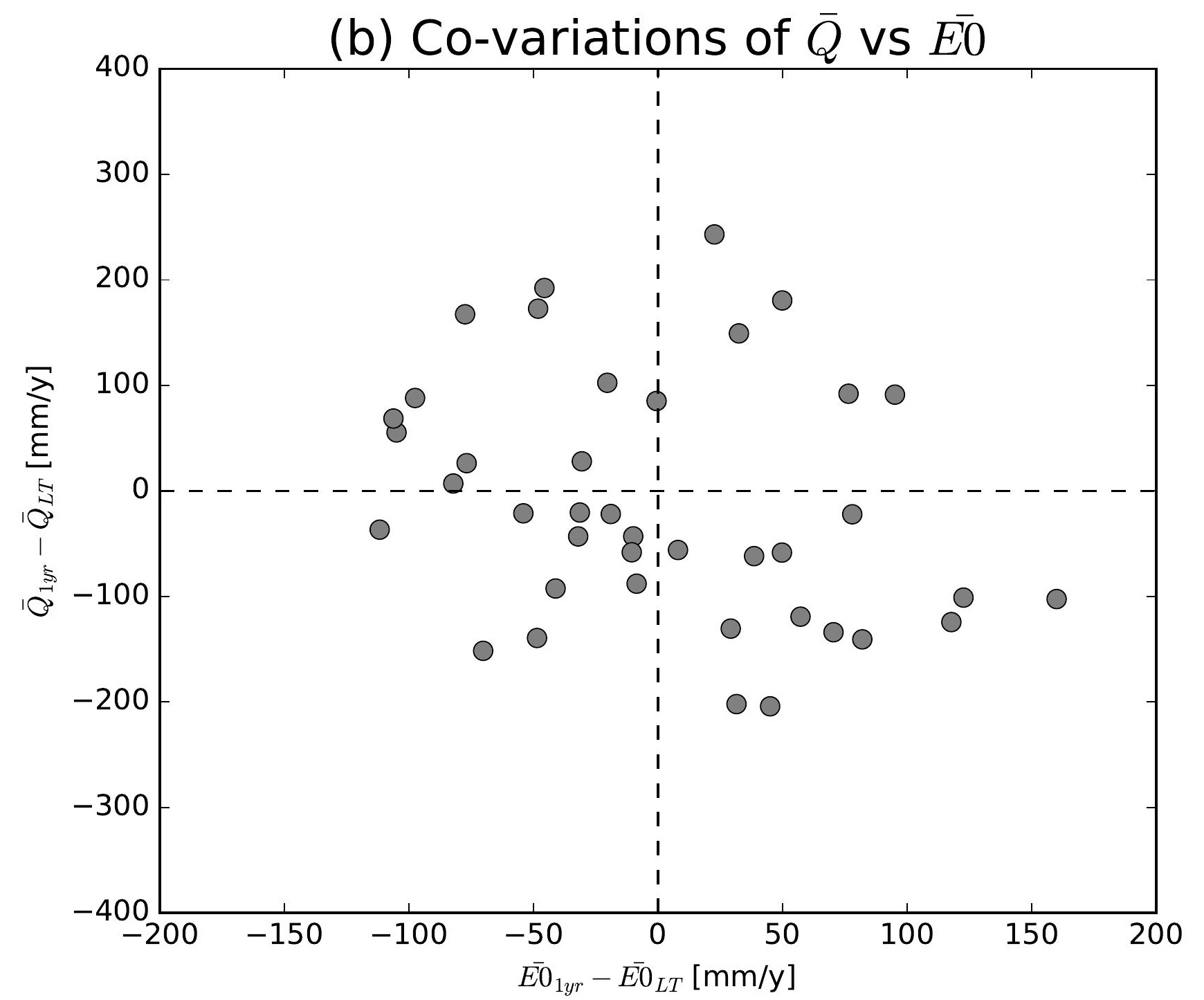

(e) Co-variations of $\bar{Q}$ vs $\overline{E 0}$

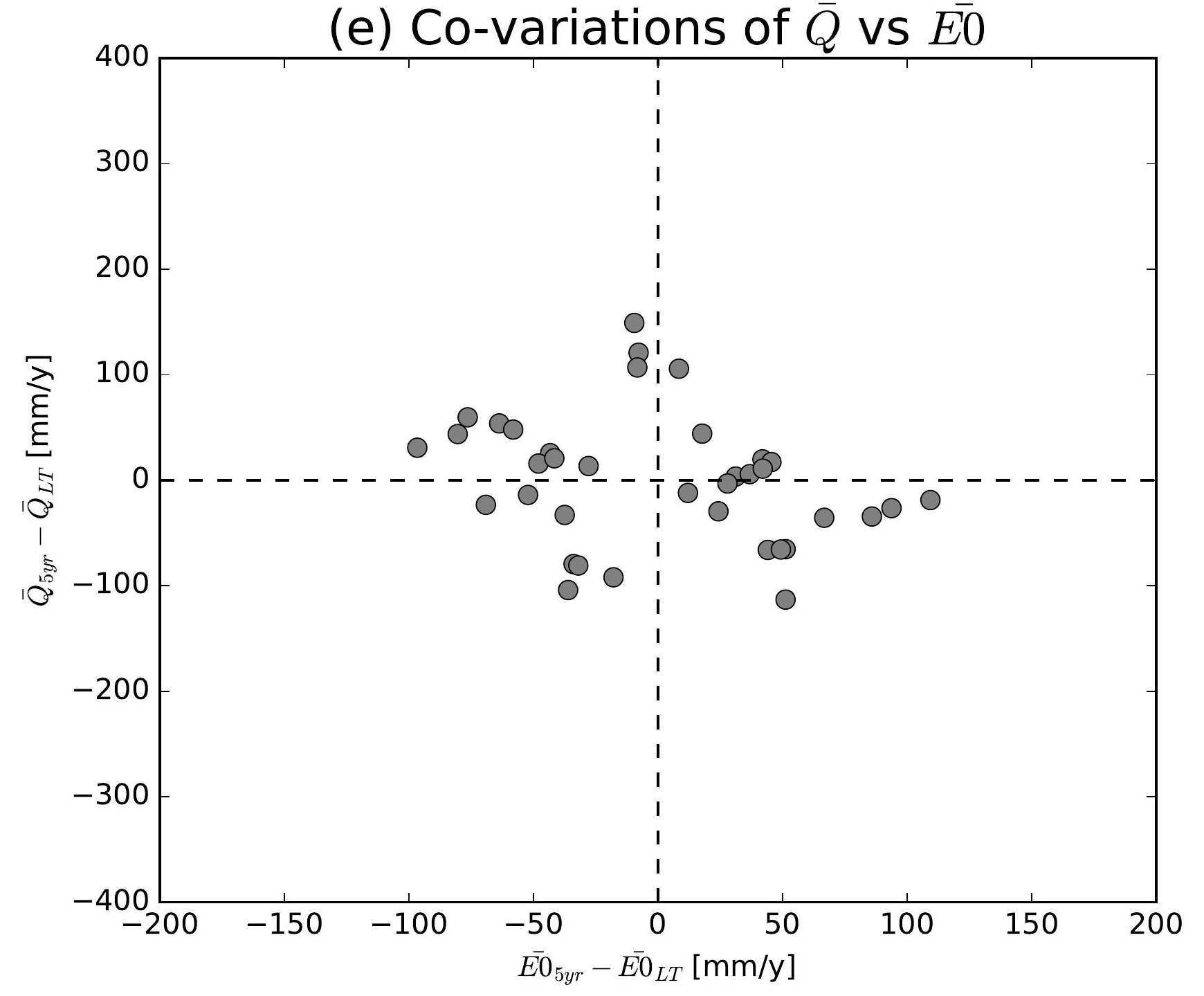

(h) Co-variations of $\bar{Q}$ vs $\overline{E 0}$

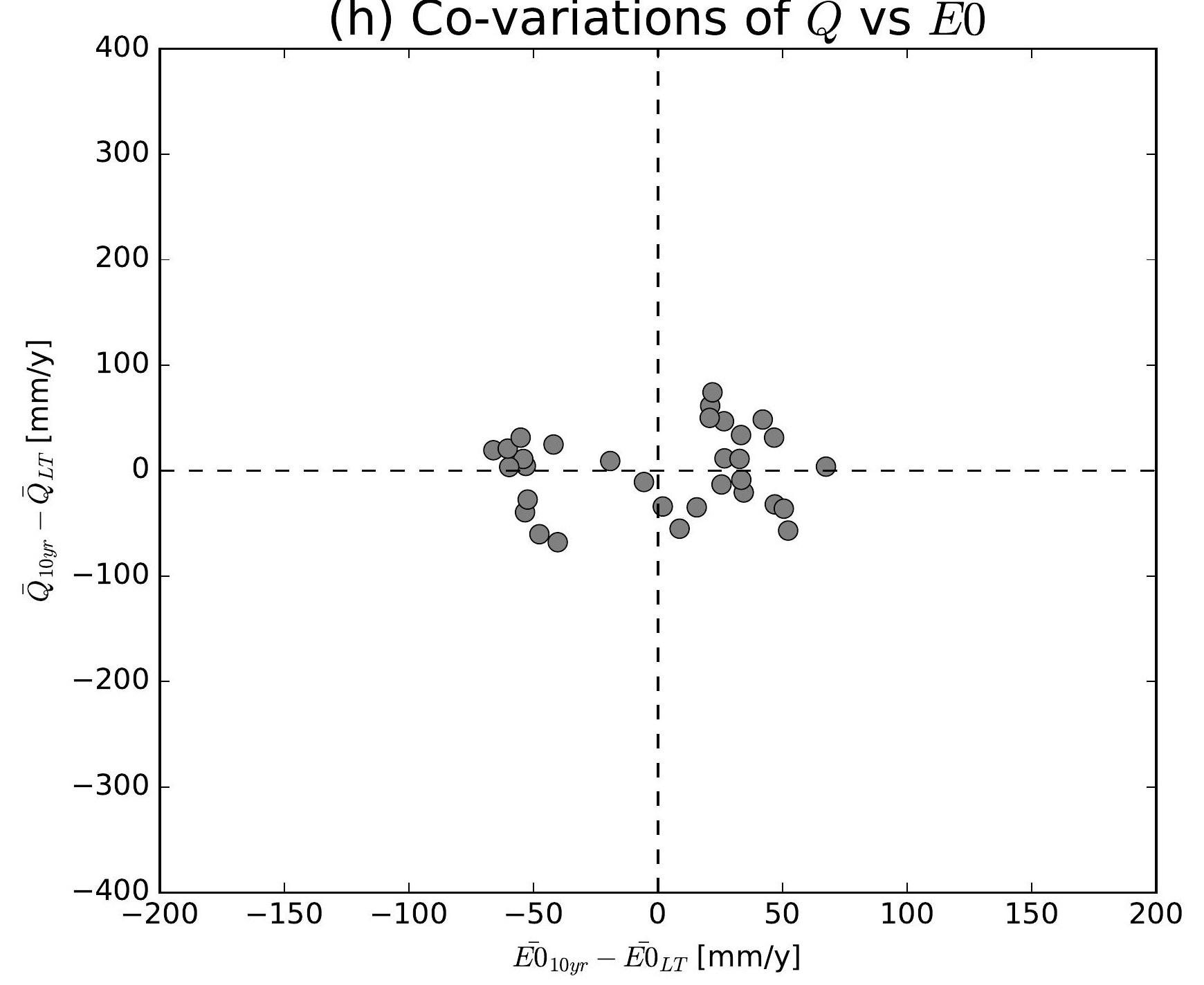

(k) Co-variations of $\bar{Q}$ vs $\overline{E 0}$

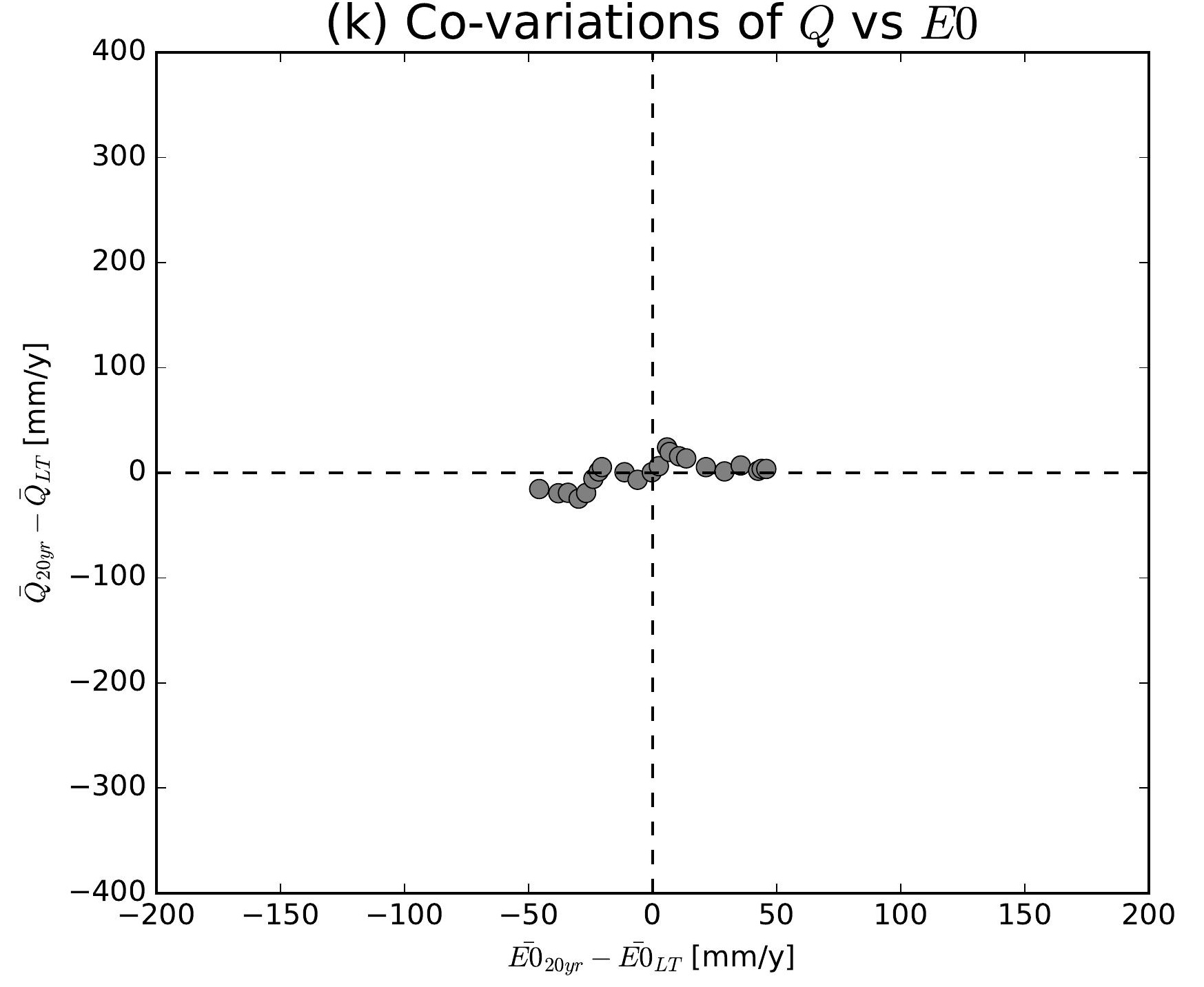

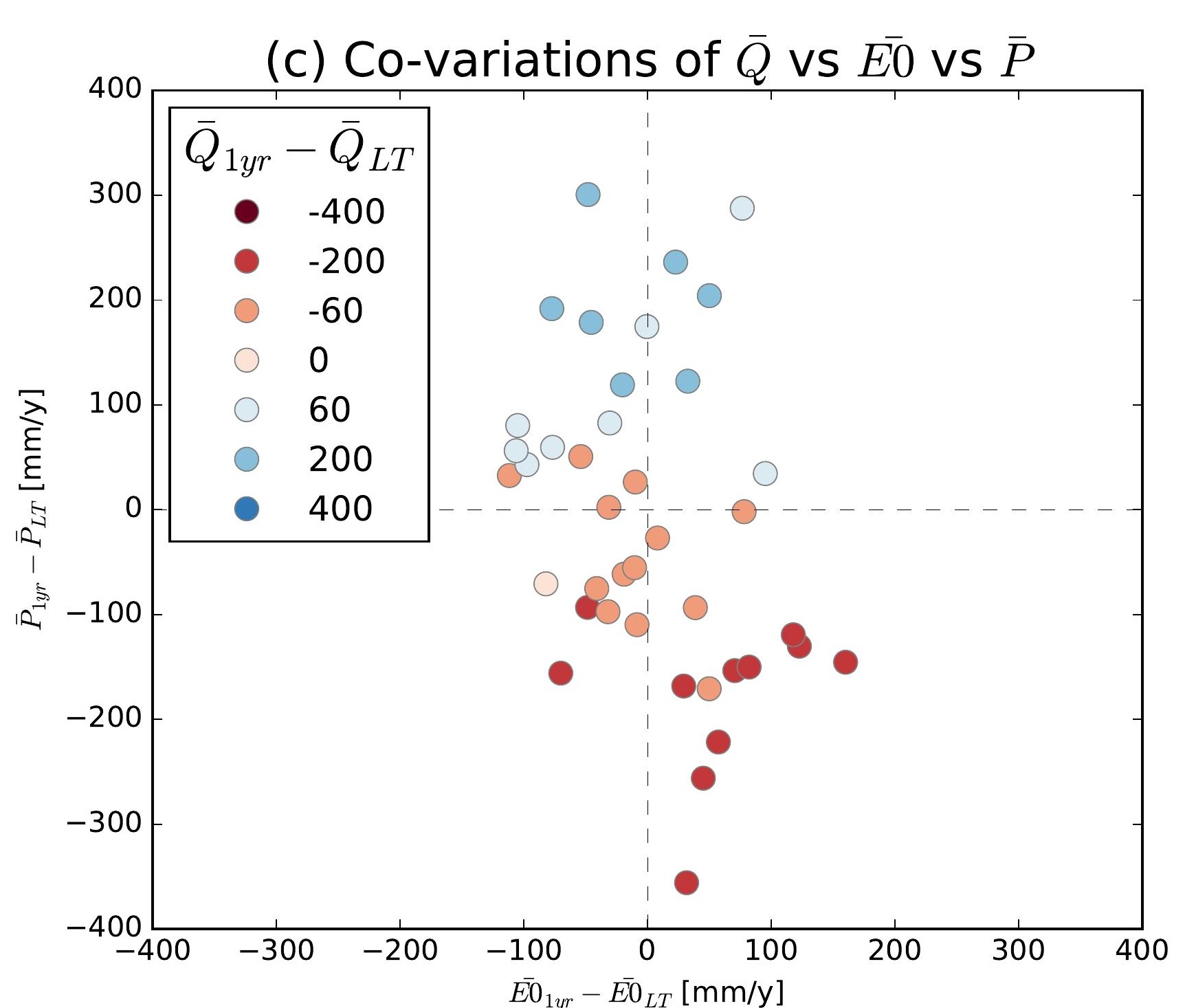
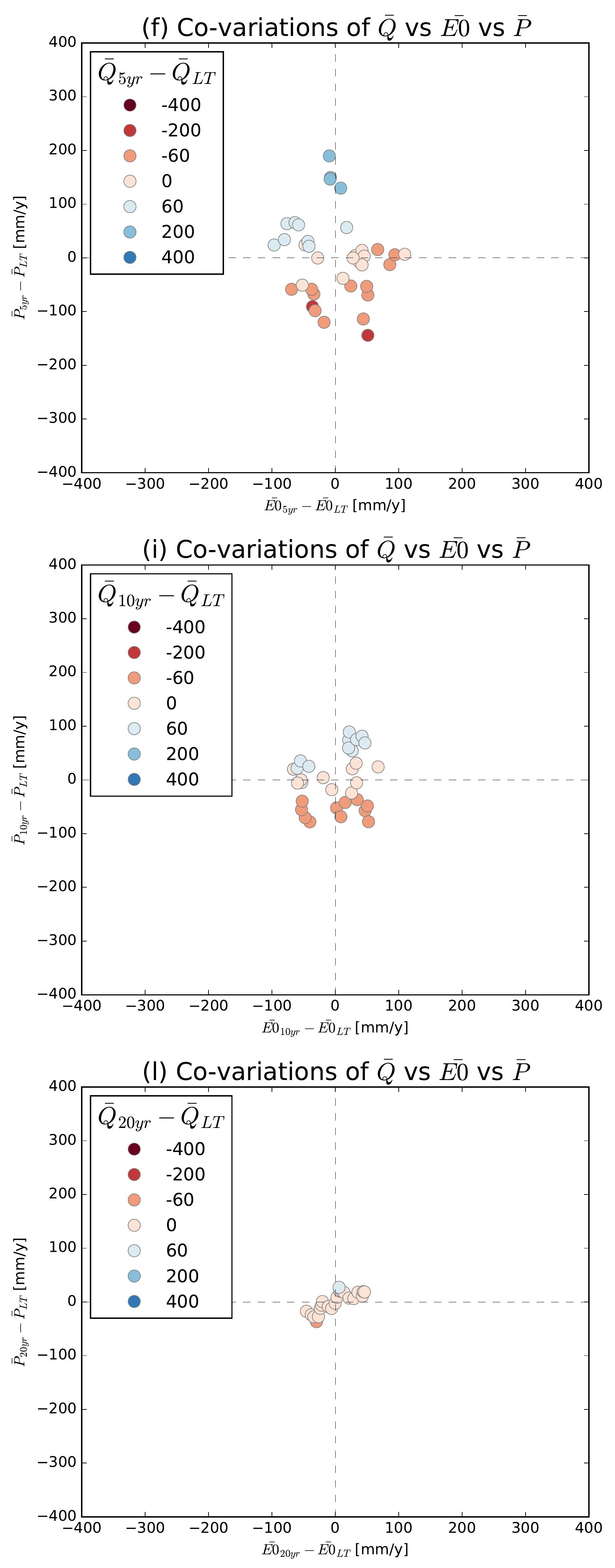

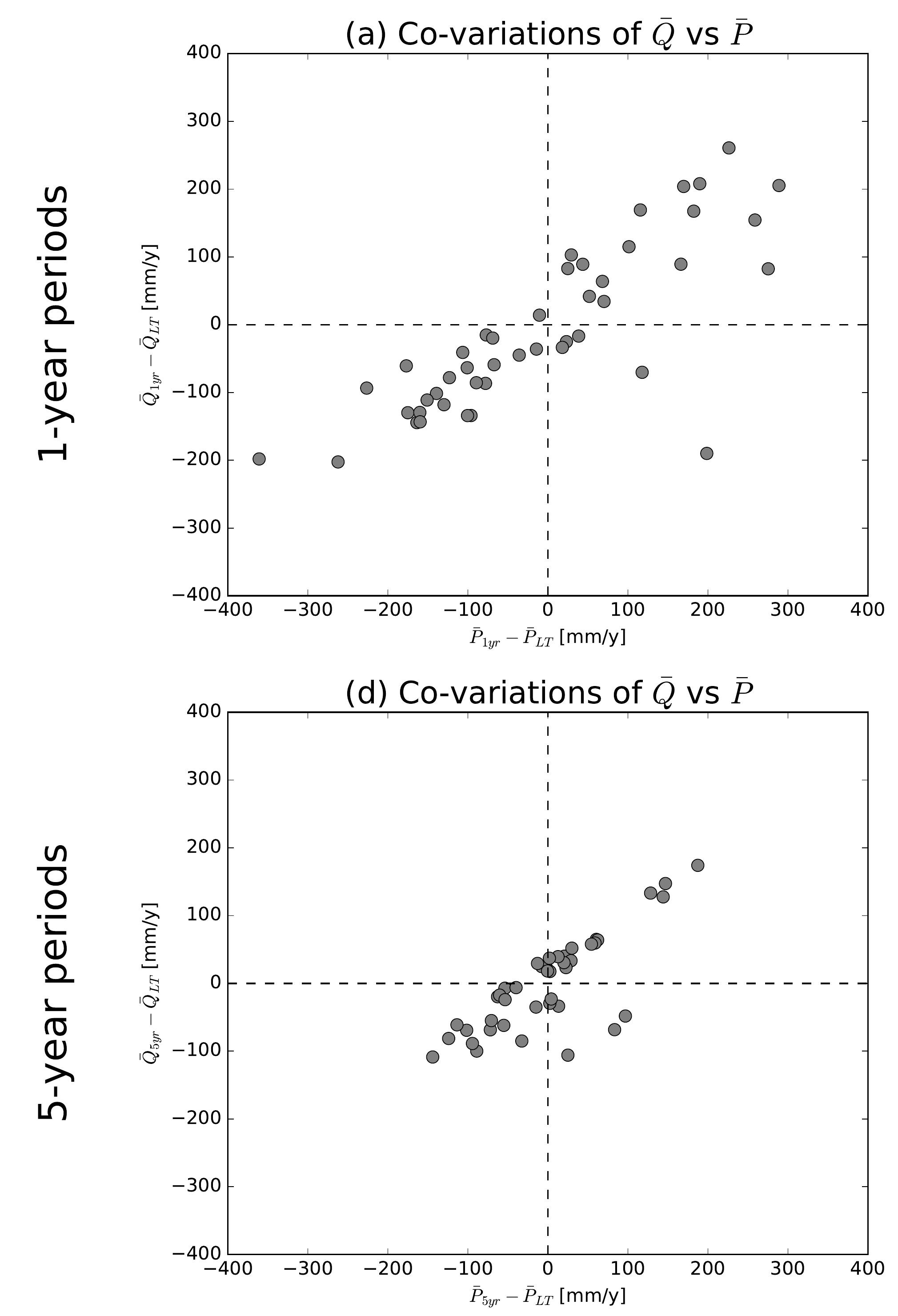

(g) Co-variations of $\bar{Q}$ vs $\bar{P}$
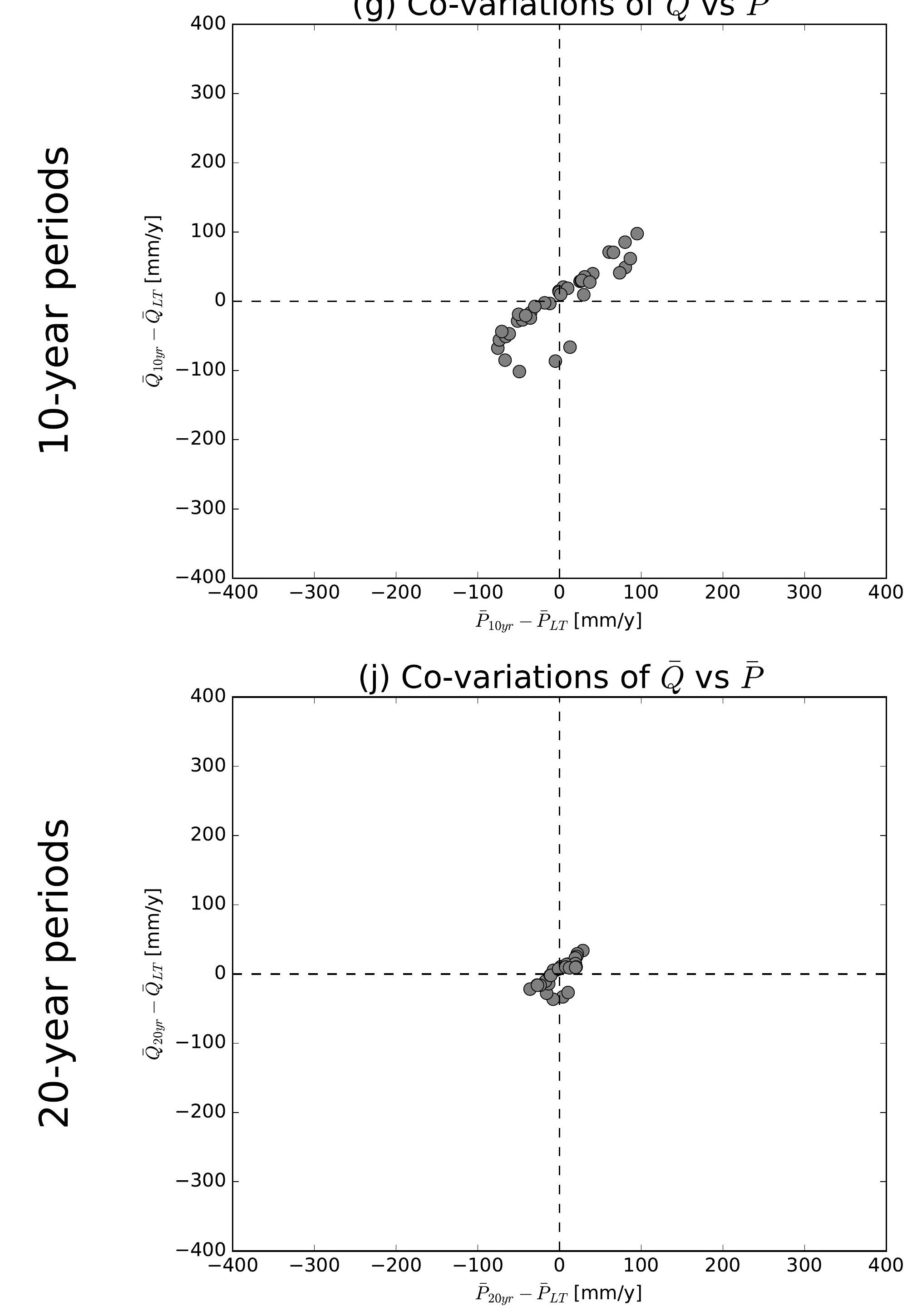

(b) Co-variations of $\bar{Q}$ vs $\overline{E 0}$

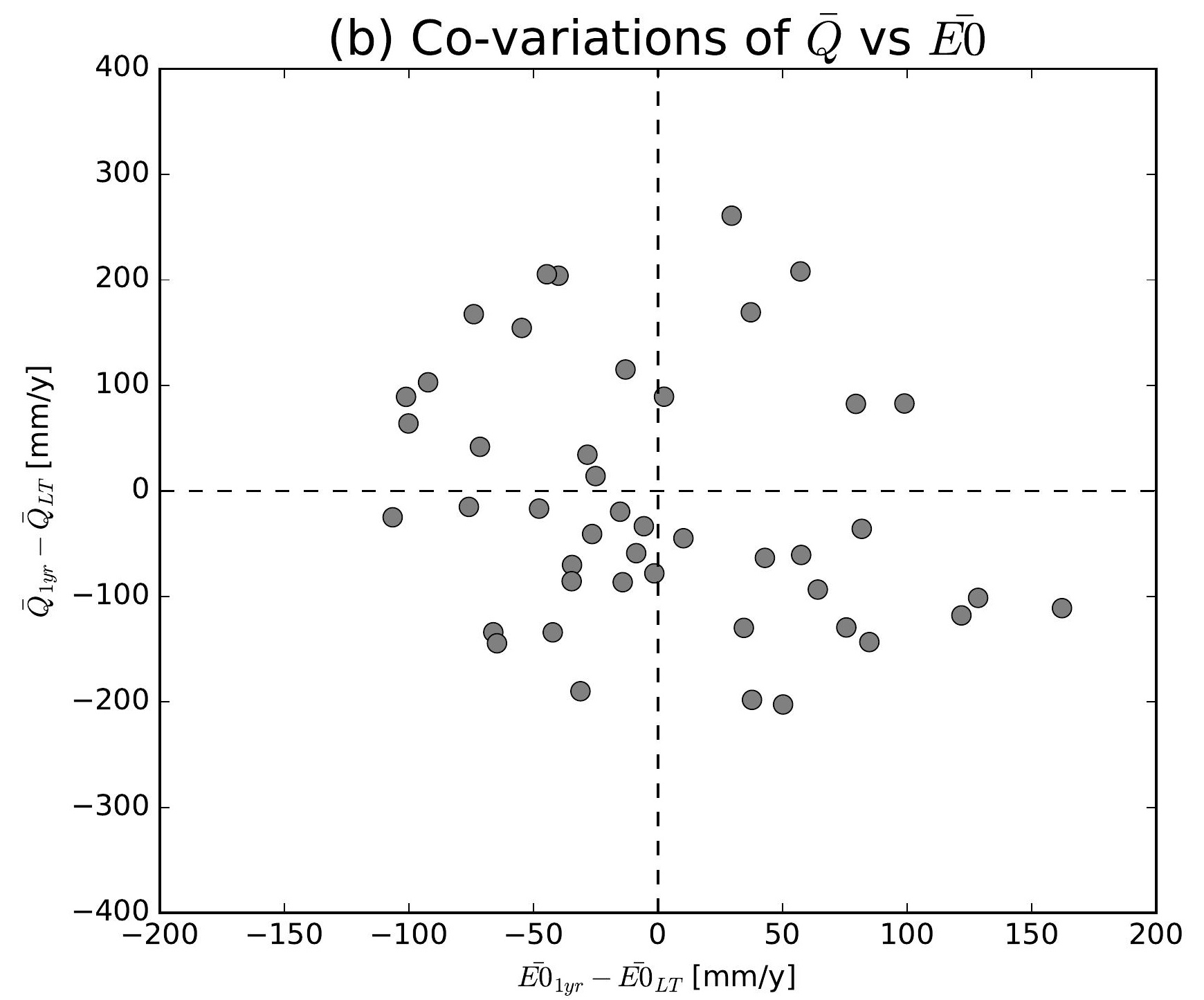

(e) Co-variations of $\bar{Q}$ vs $\overline{E 0}$

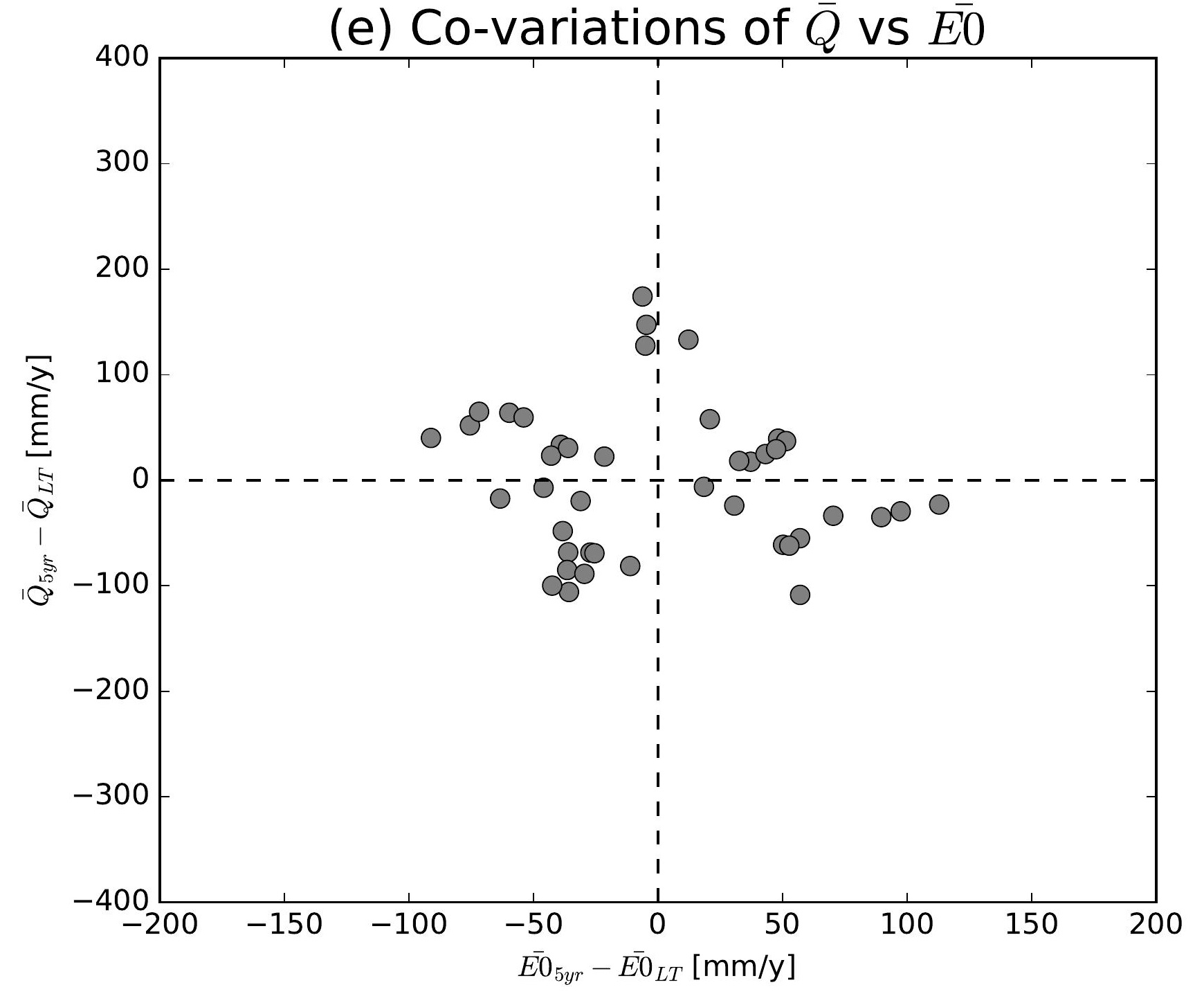

(h) Co-variations of $\bar{Q}$ vs $\overline{E 0}$

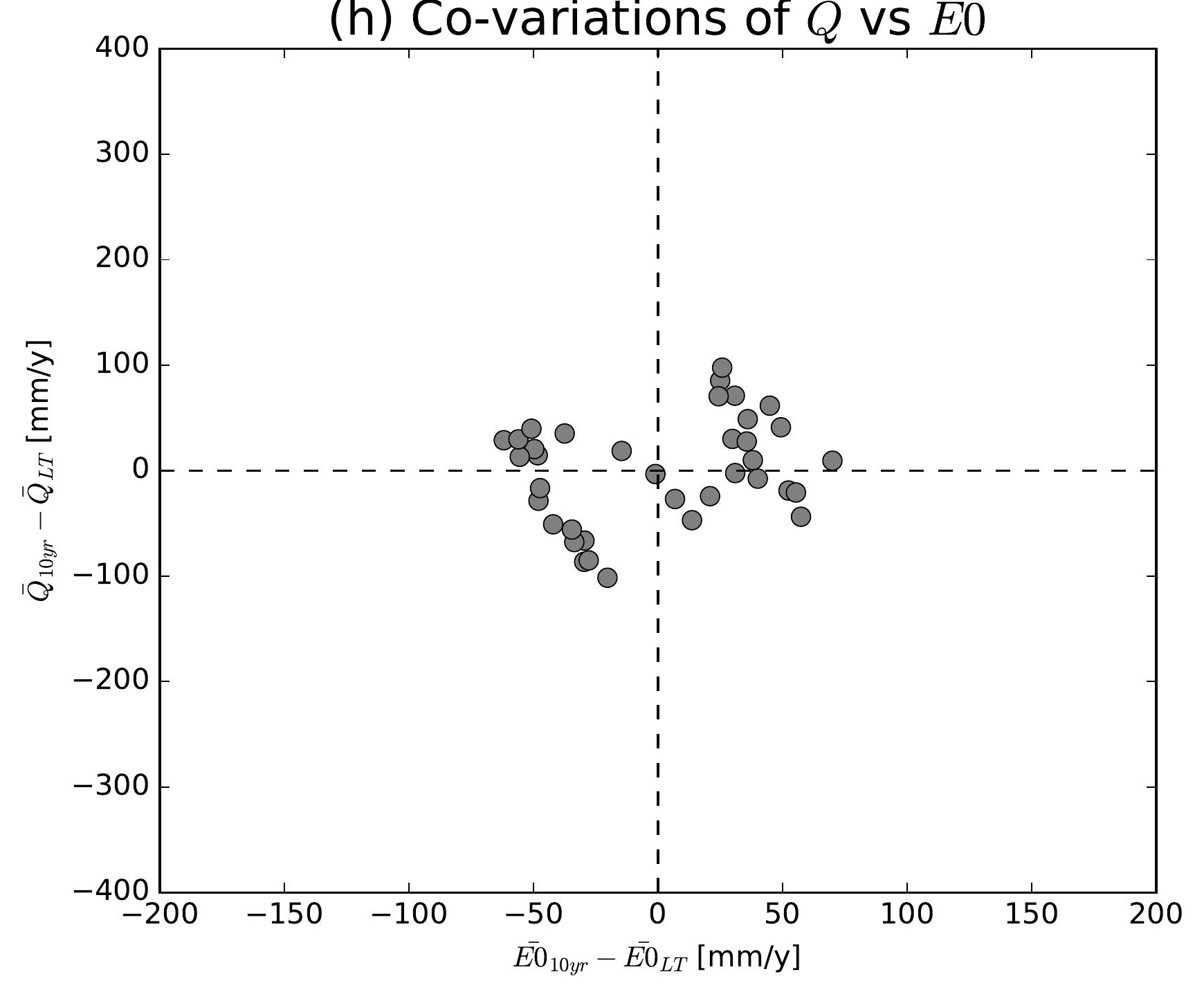

(k) Co-variations of $\bar{Q}$ vs $\overline{E 0}$

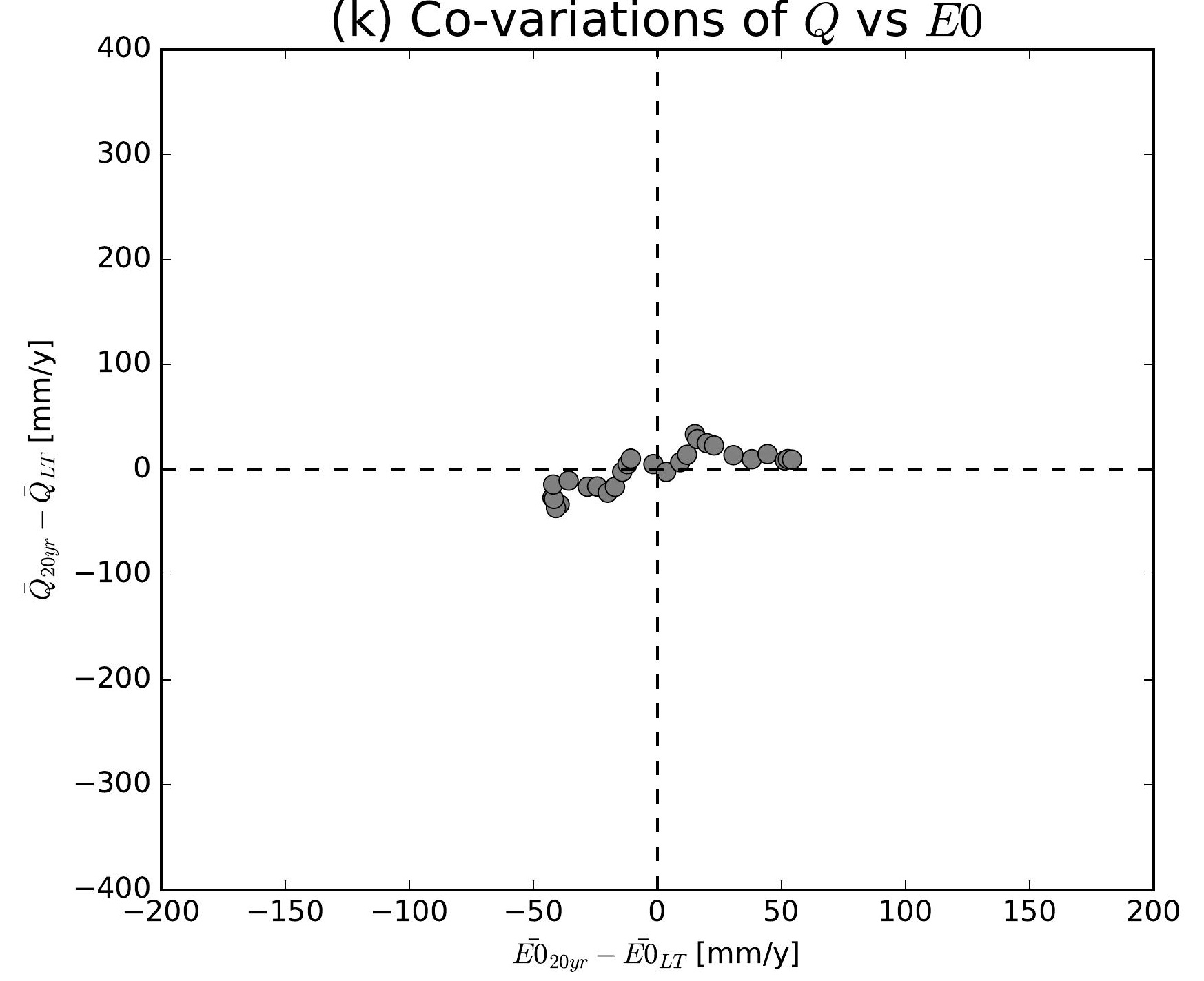

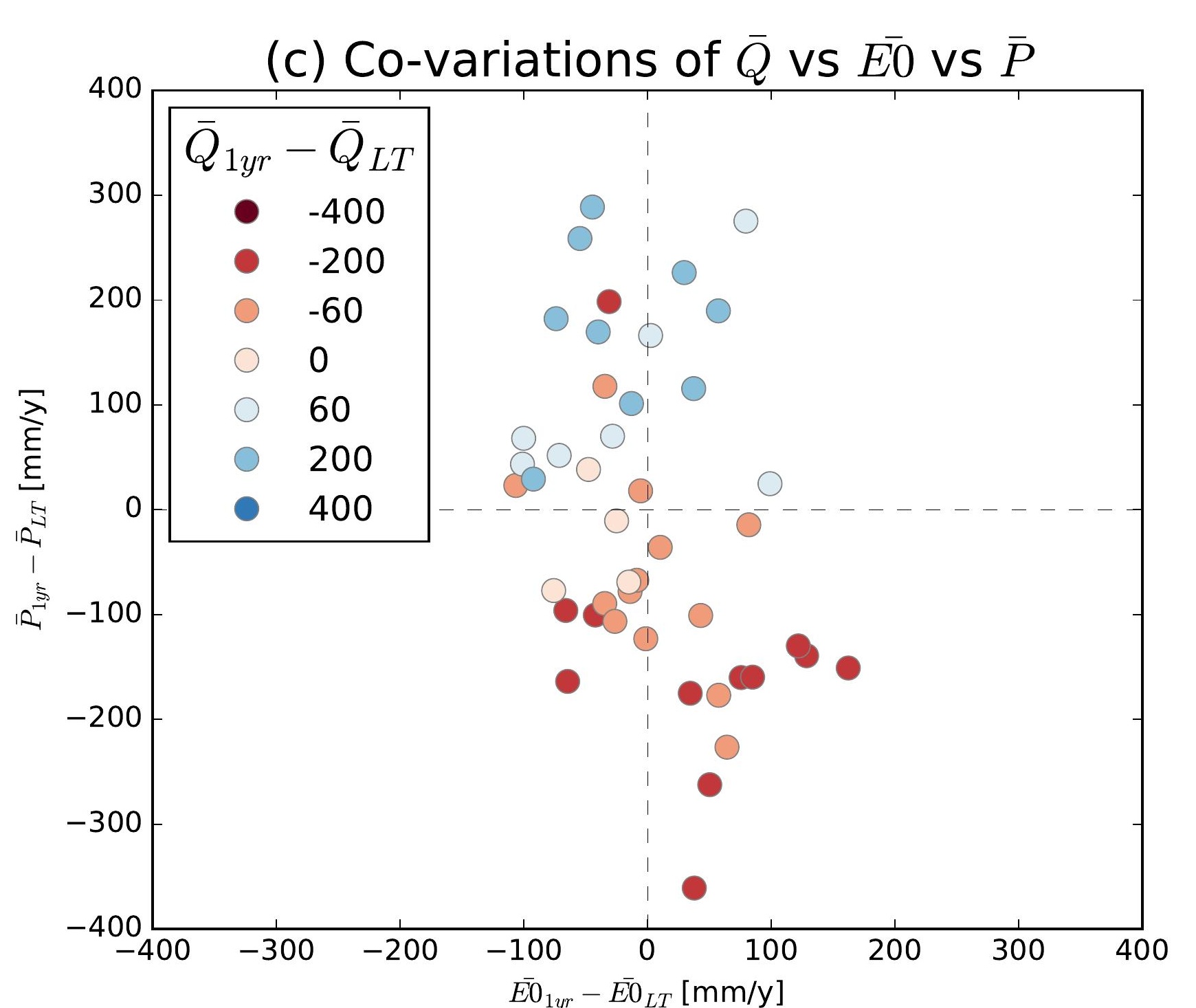
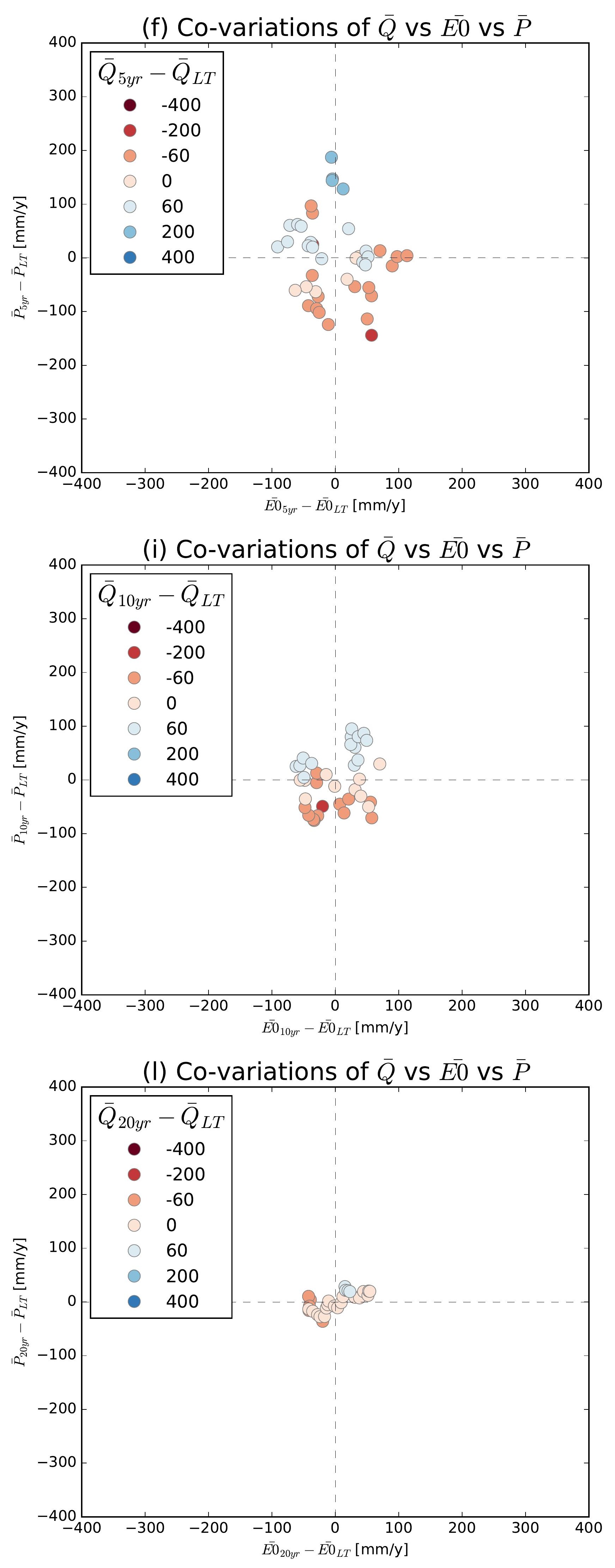

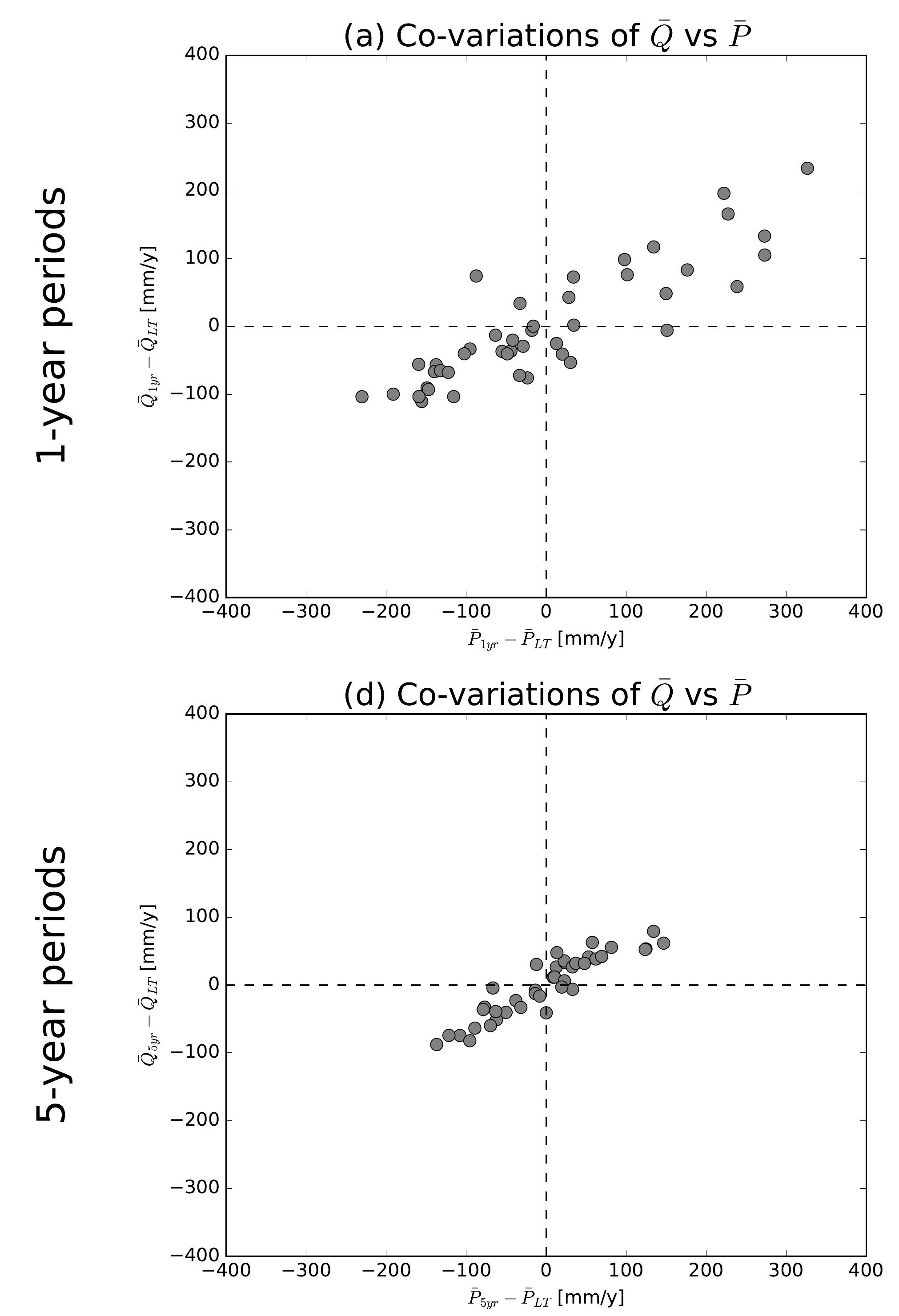

(g) Co-variations of $\bar{Q}$ vs $\bar{P}$
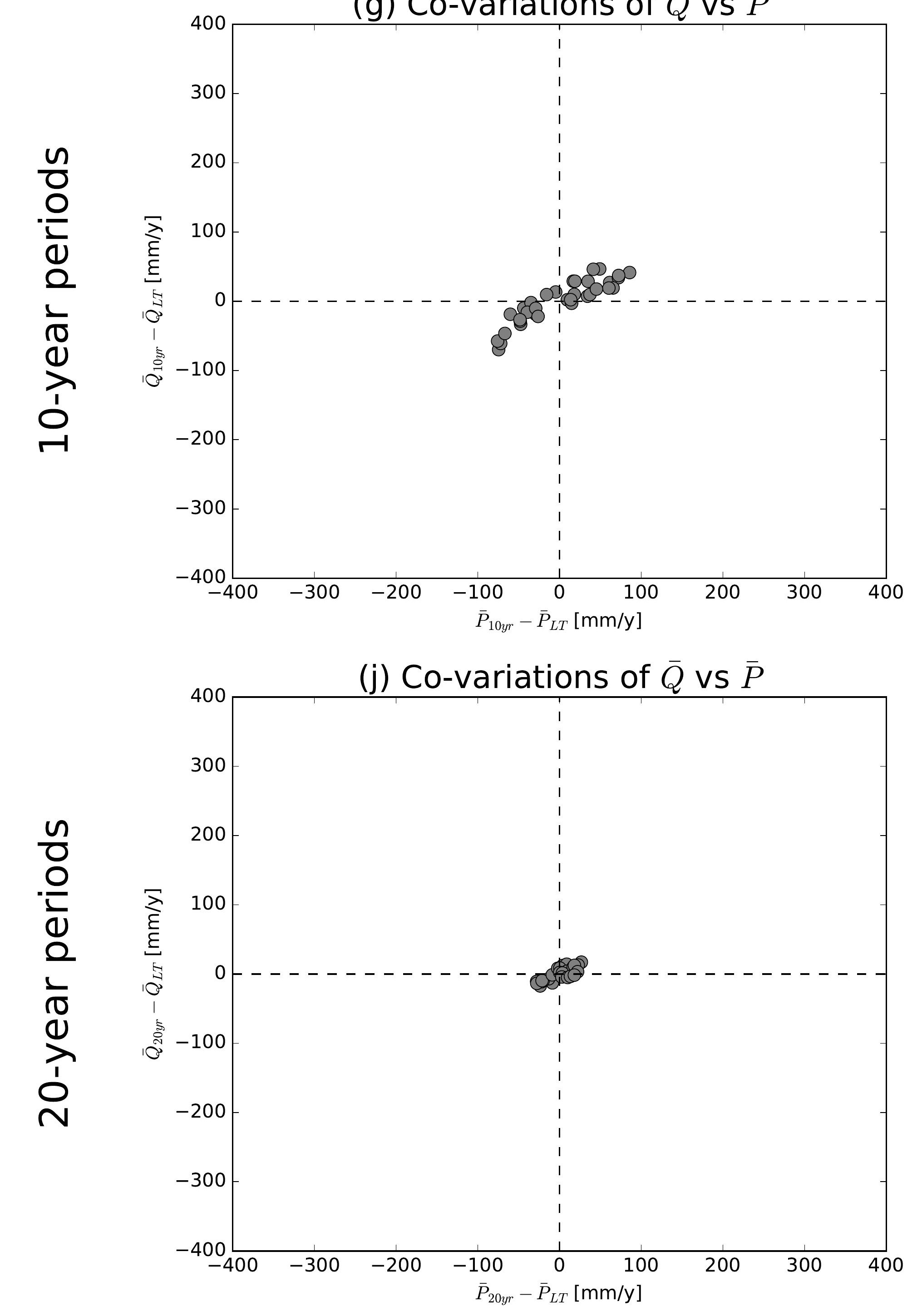

(b) Co-variations of $\bar{Q}$ vs $\overline{E 0}$

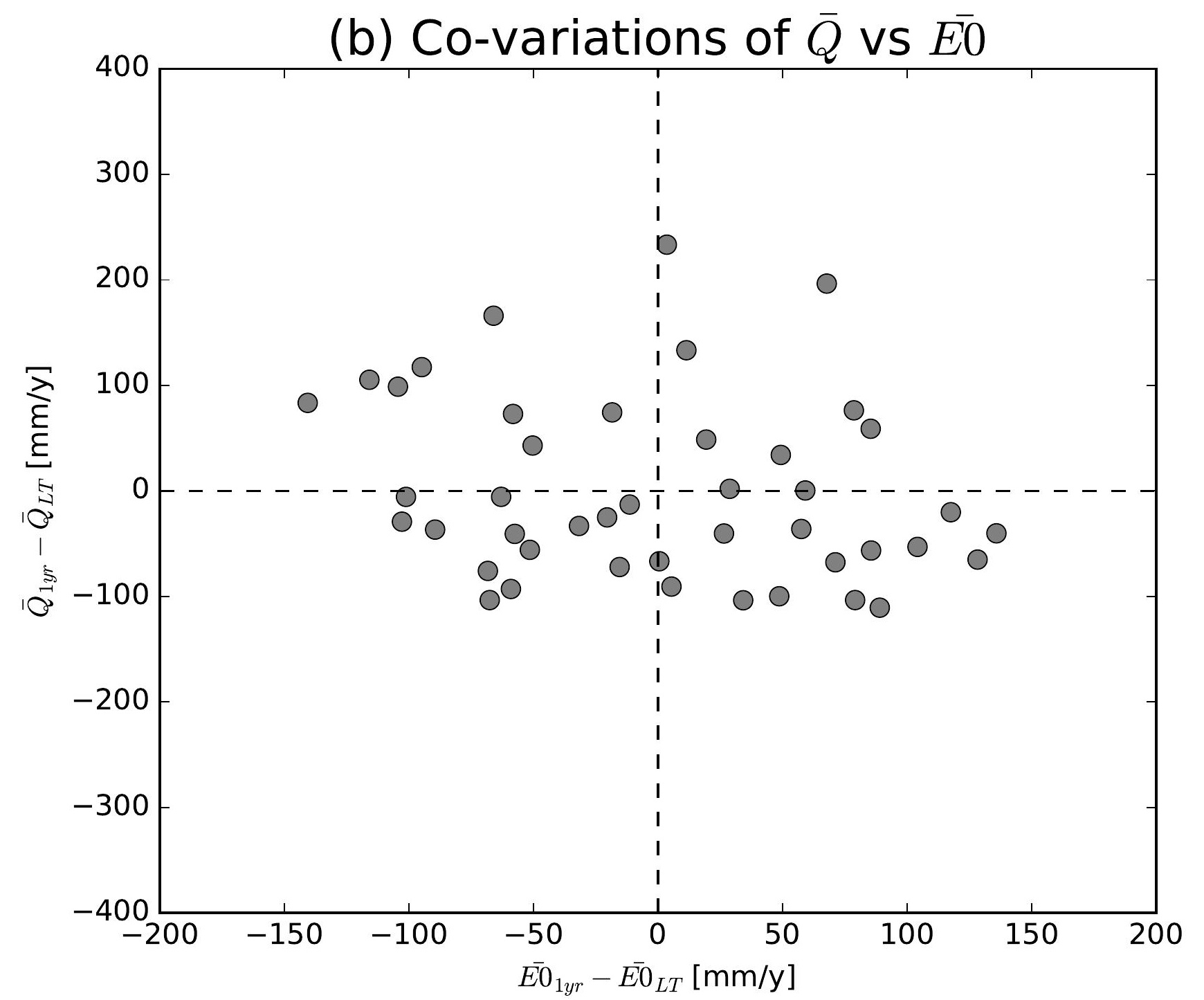

(e) Co-variations of $\bar{Q}$ vs $\overline{E 0}$

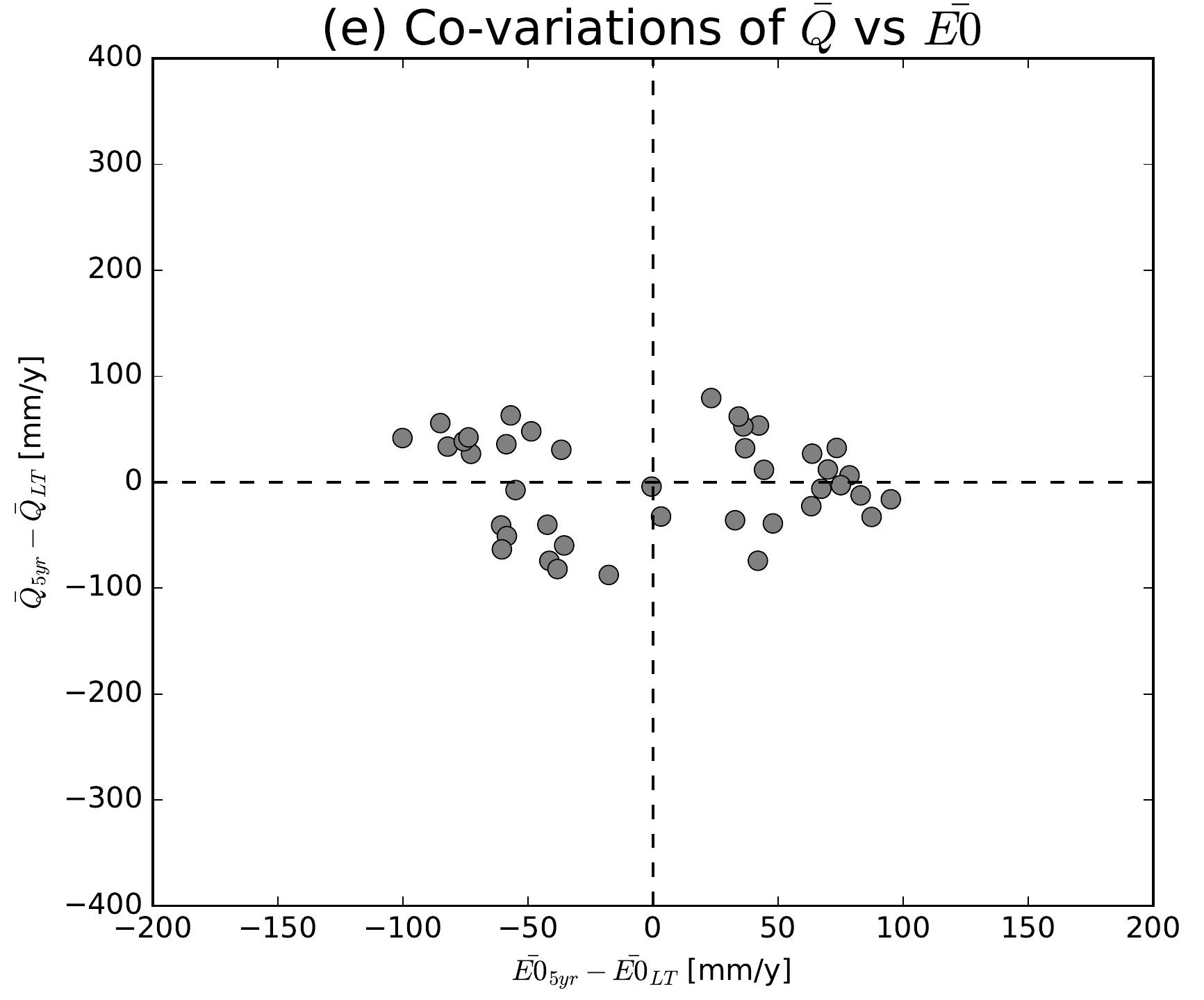

(h) Co-variations of $\bar{Q}$ vs $\overline{E 0}$

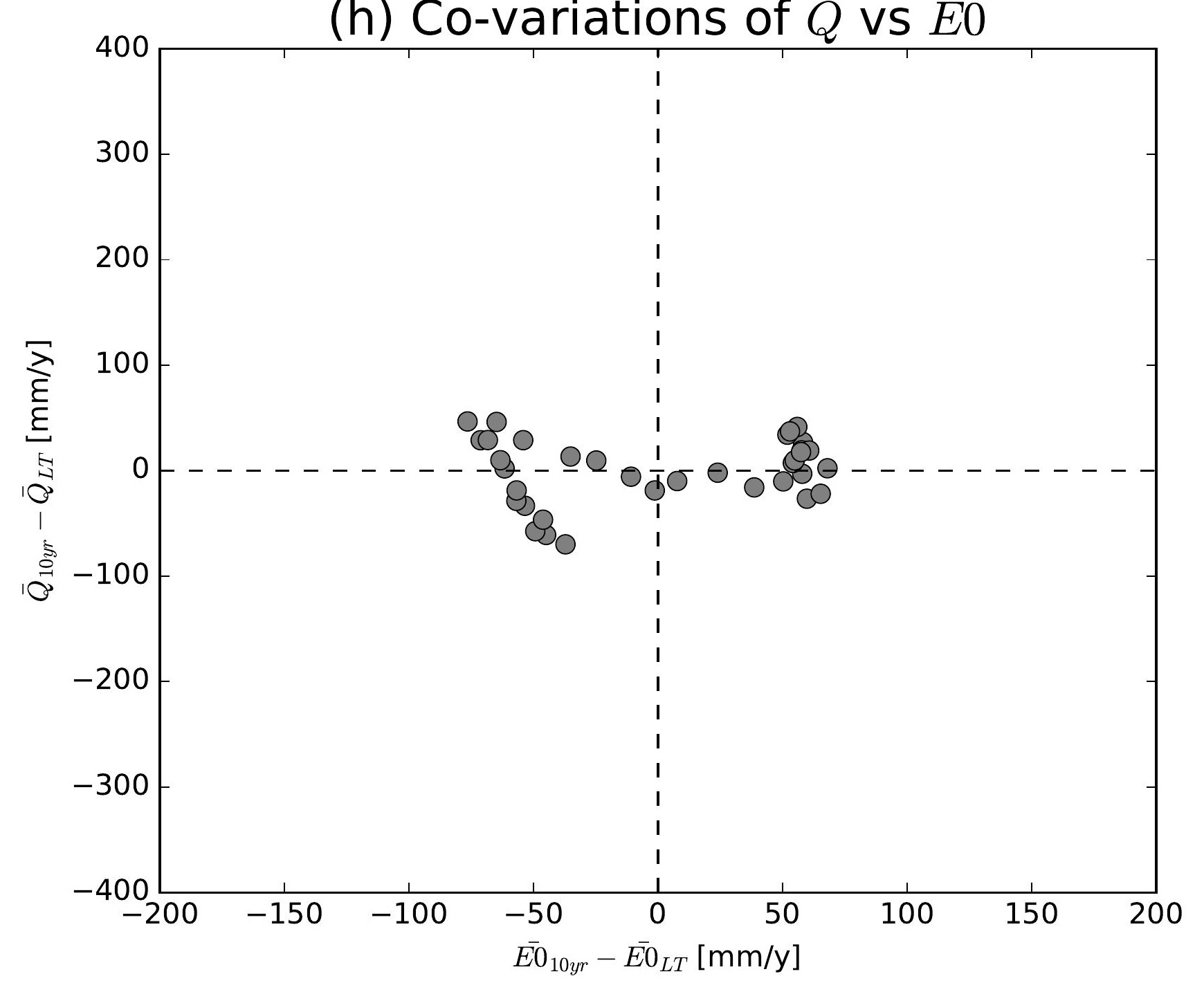

(k) Co-variations of $\bar{Q}$ vs $\overline{E 0}$

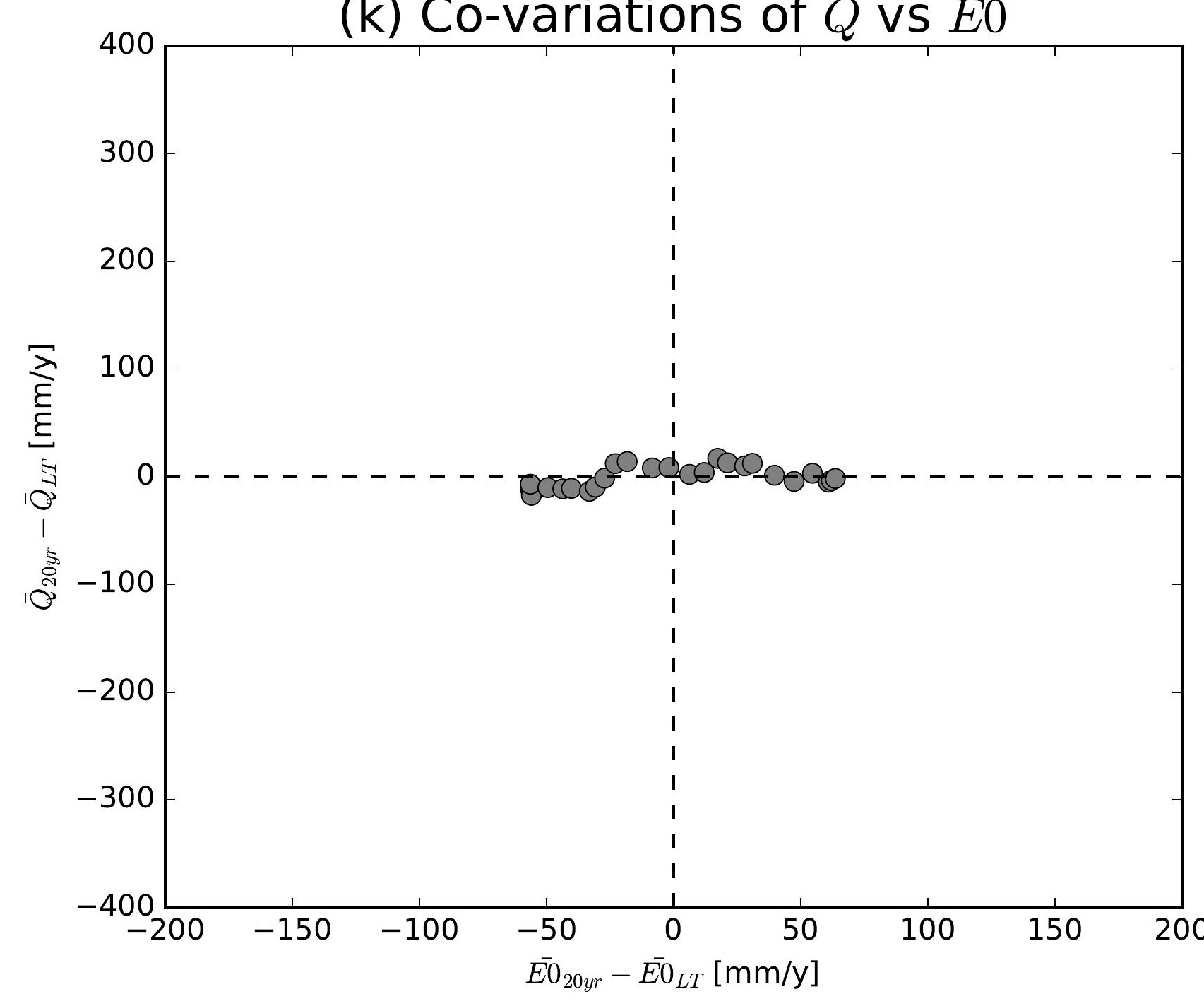

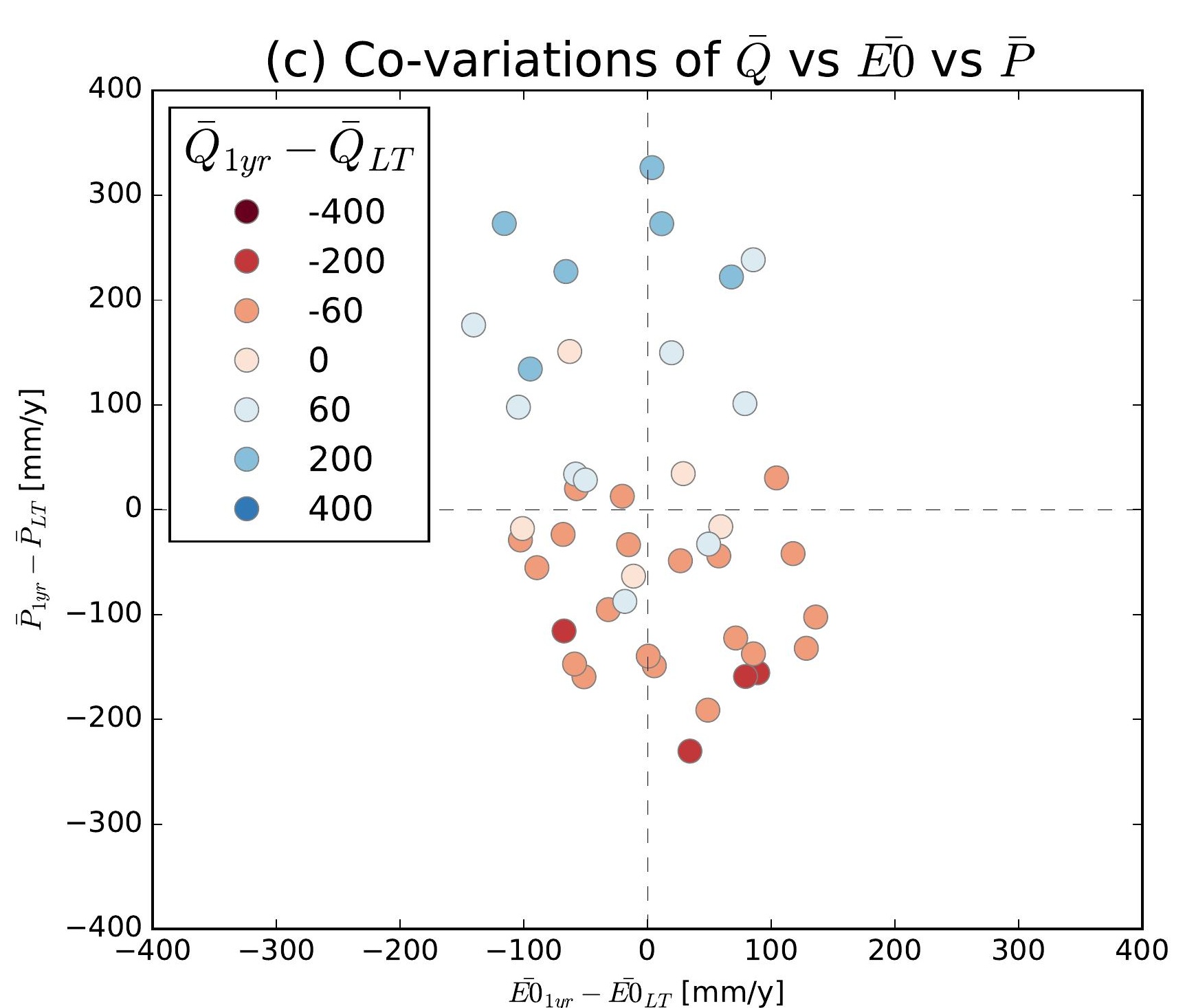
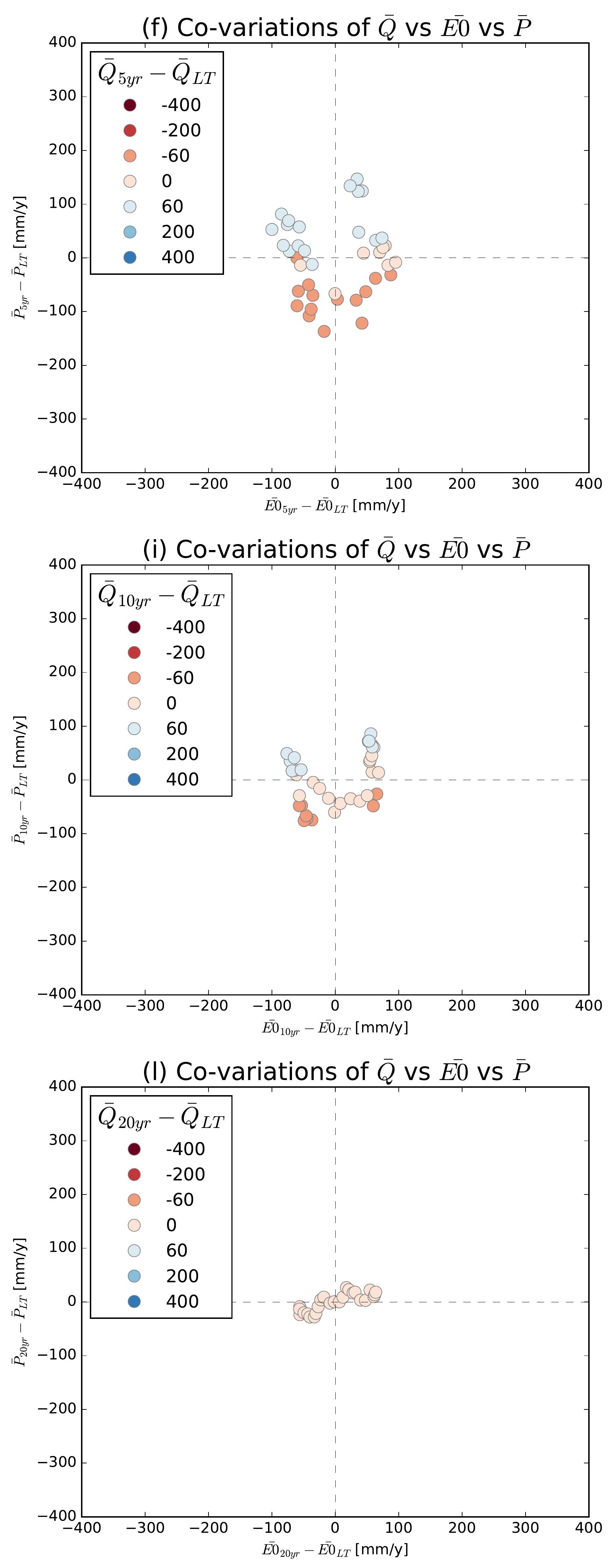

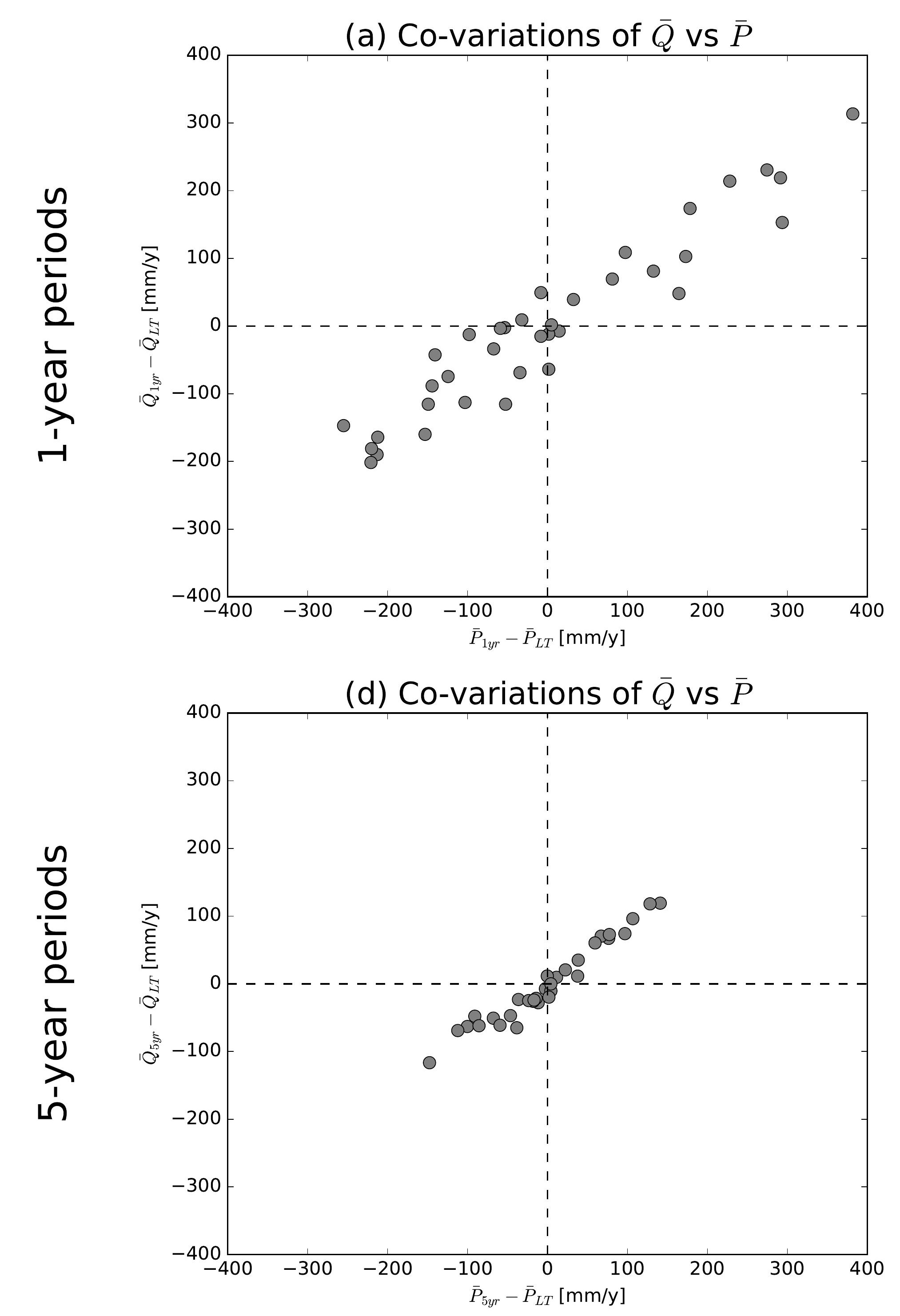

(g) Co-variations of $\bar{Q}$ vs $\bar{P}$
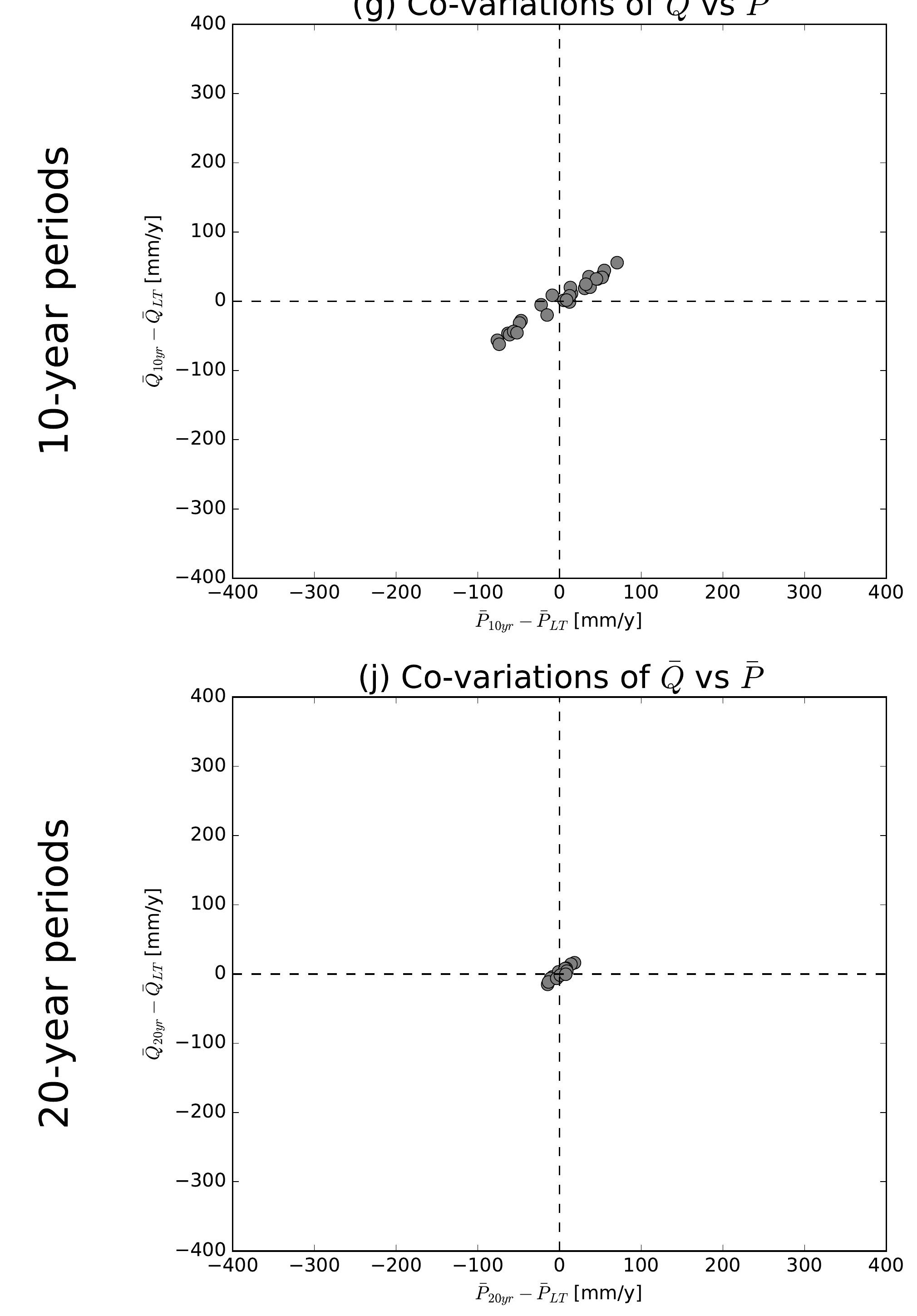

(b) Co-variations of $\bar{Q}$ vs $\overline{E 0}$

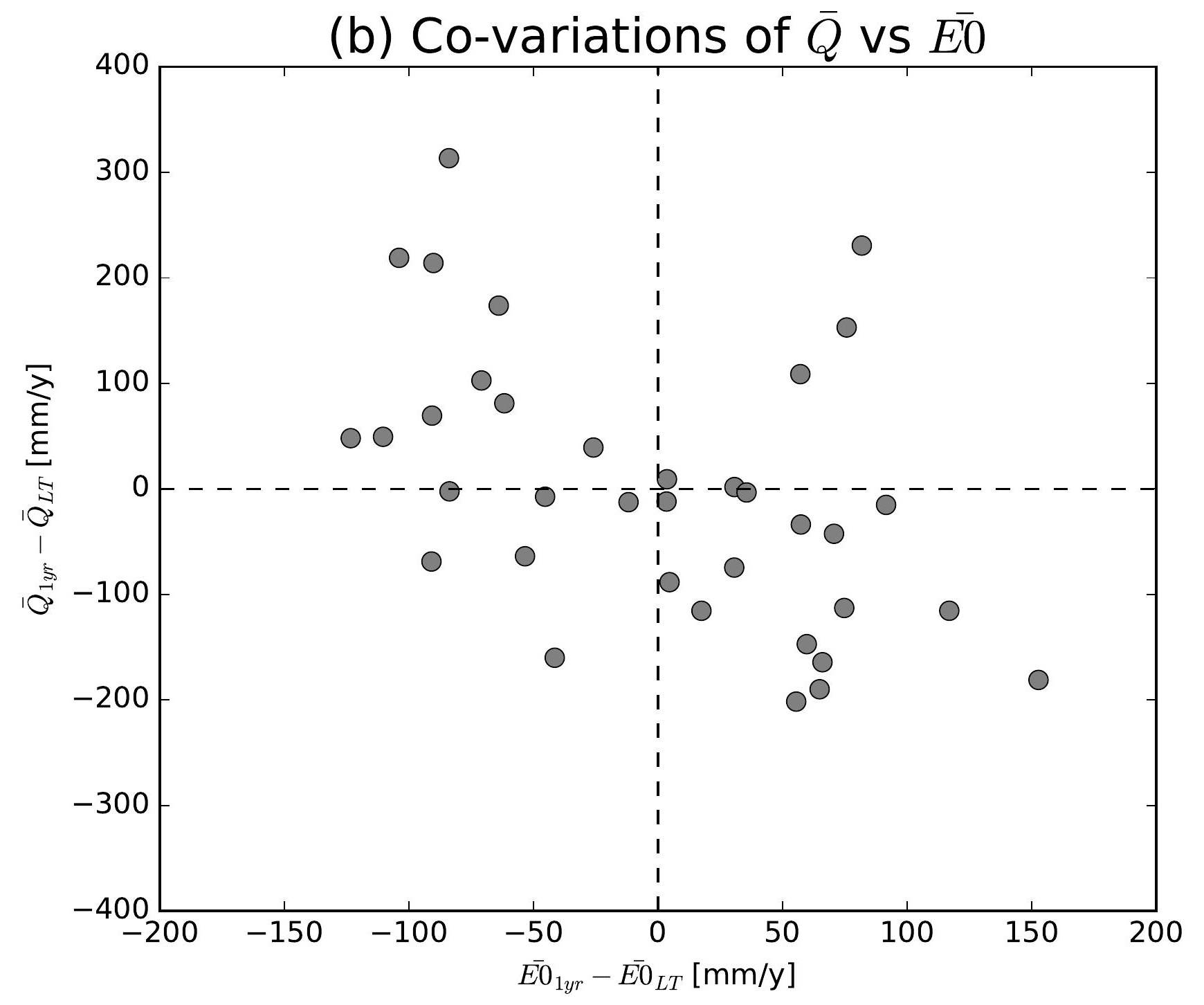

(e) Co-variations of $\bar{Q}$ vs $\overline{E 0}$

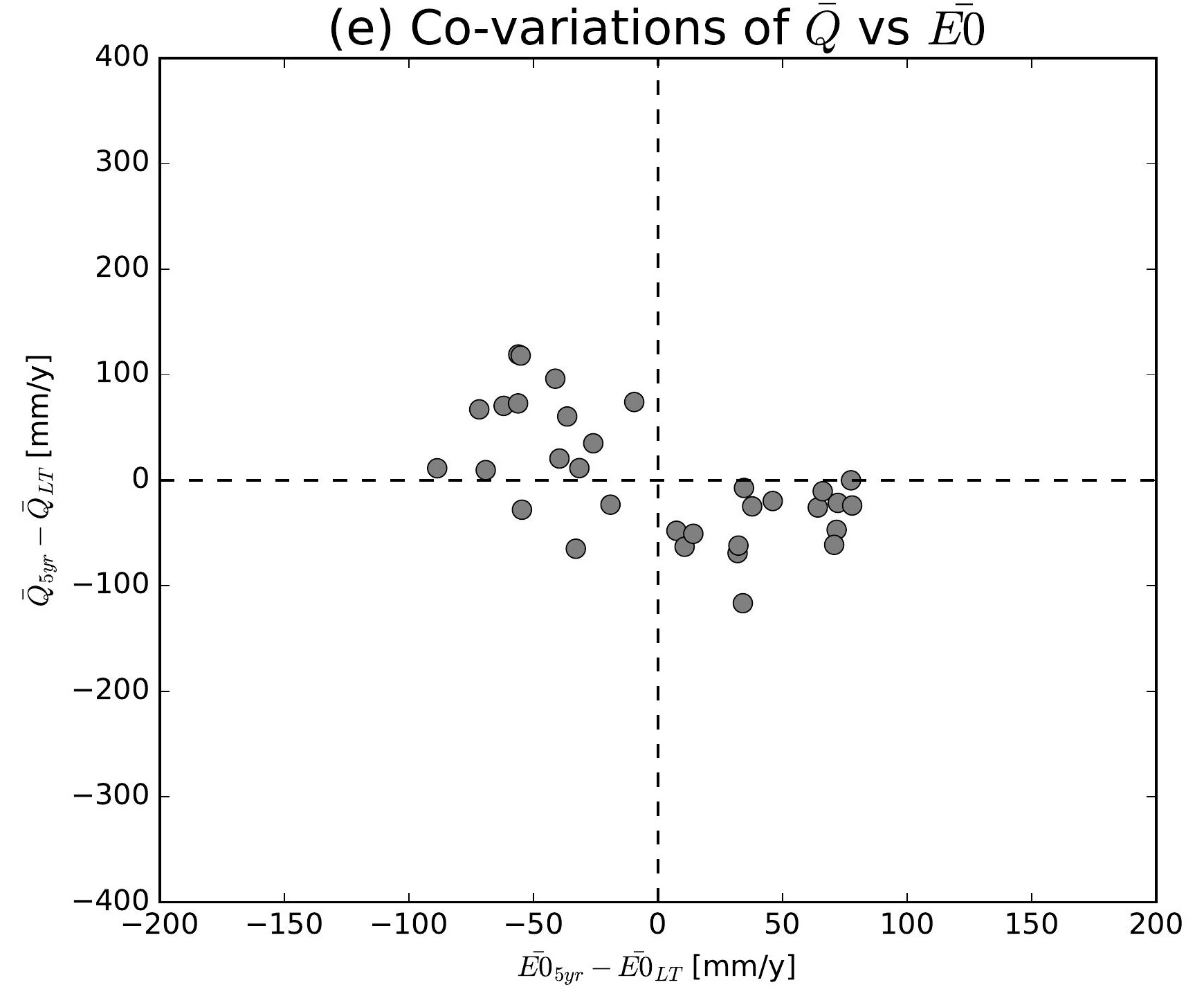

(h) Co-variations of $\bar{Q}$ vs $\overline{E 0}$

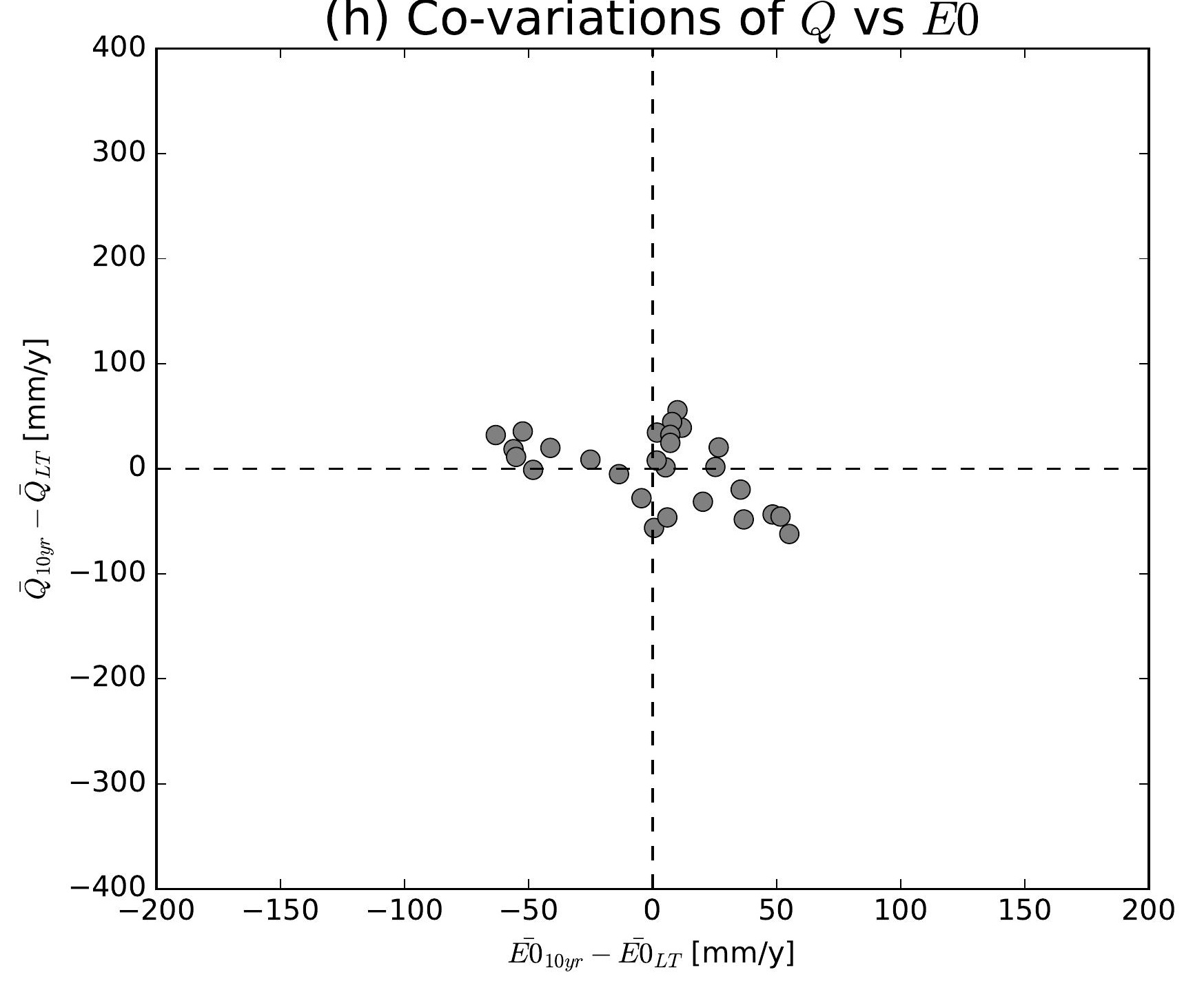

(k) Co-variations of $\bar{Q}$ vs $\overline{E 0}$

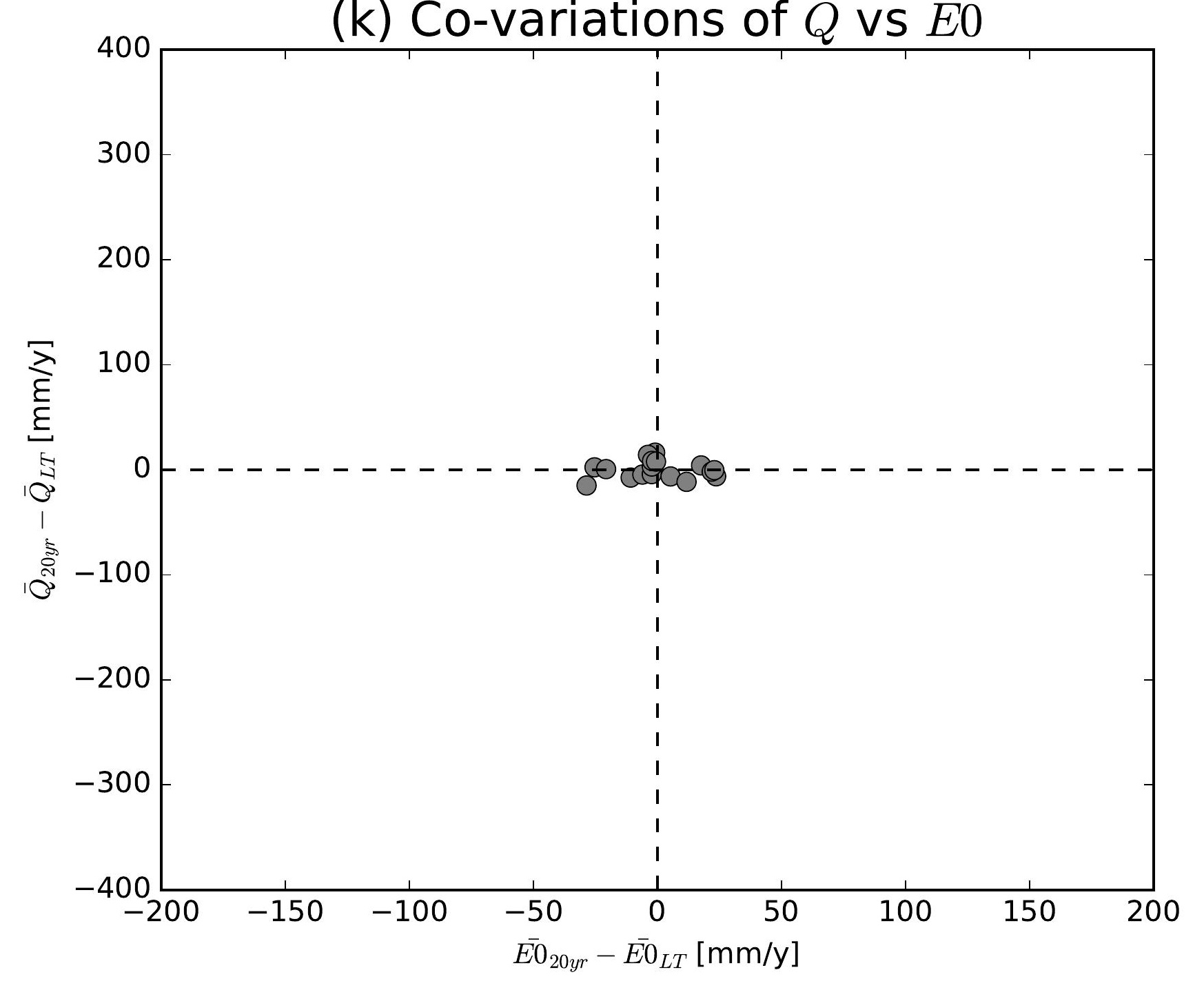

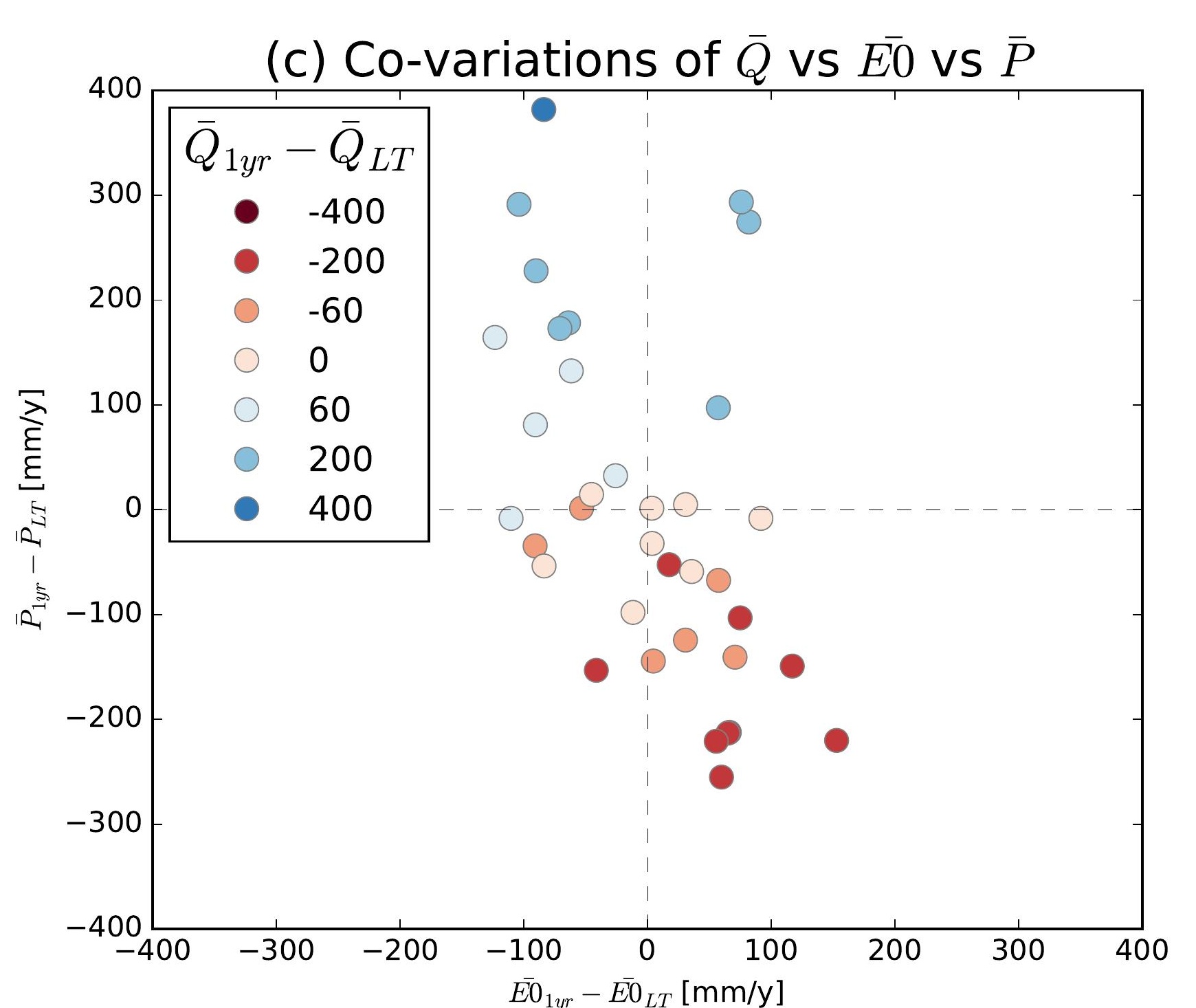
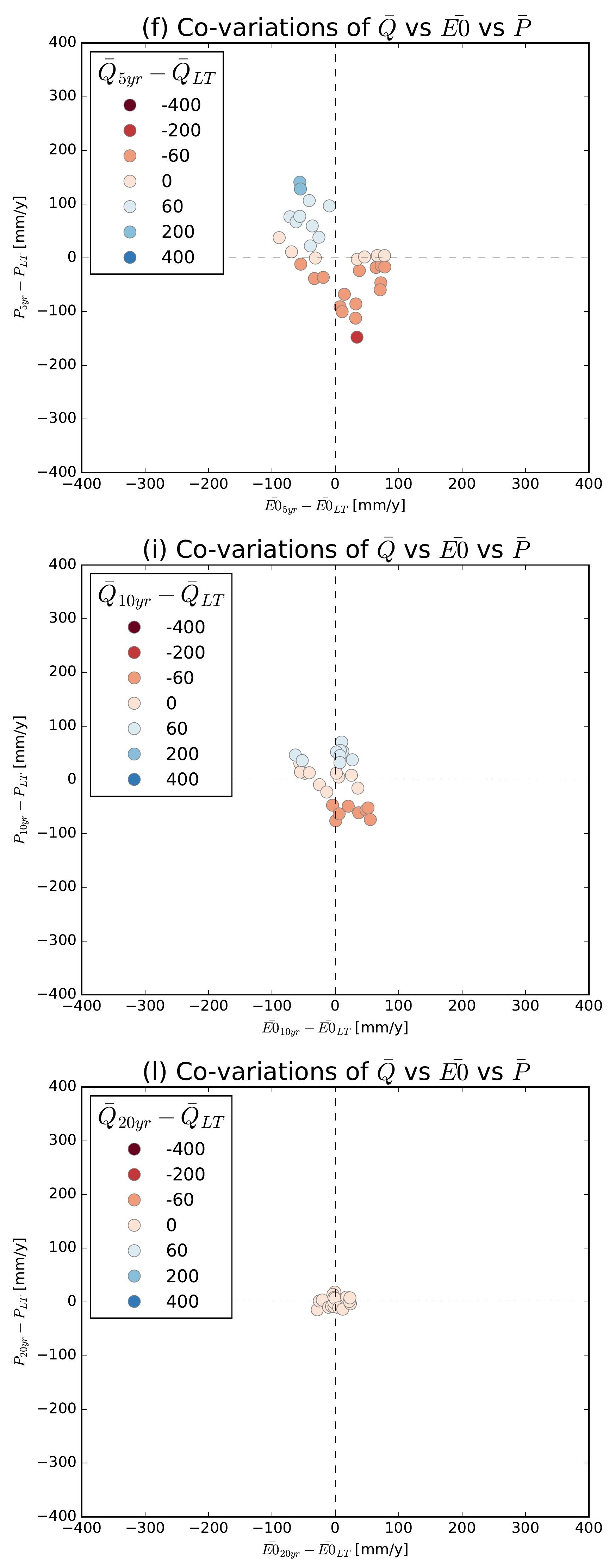

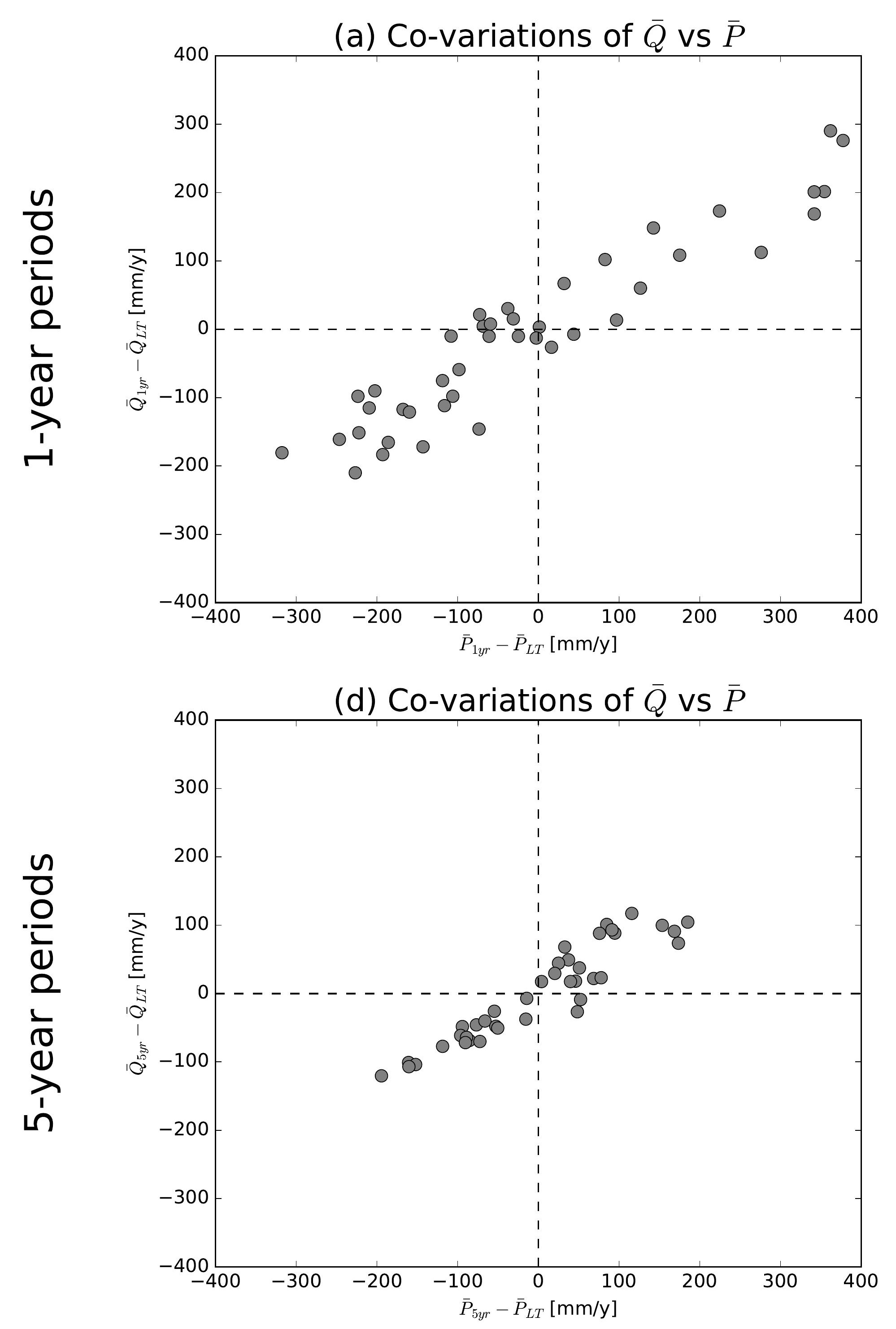

(g) Co-variations of $\bar{Q}$ vs $\bar{P}$

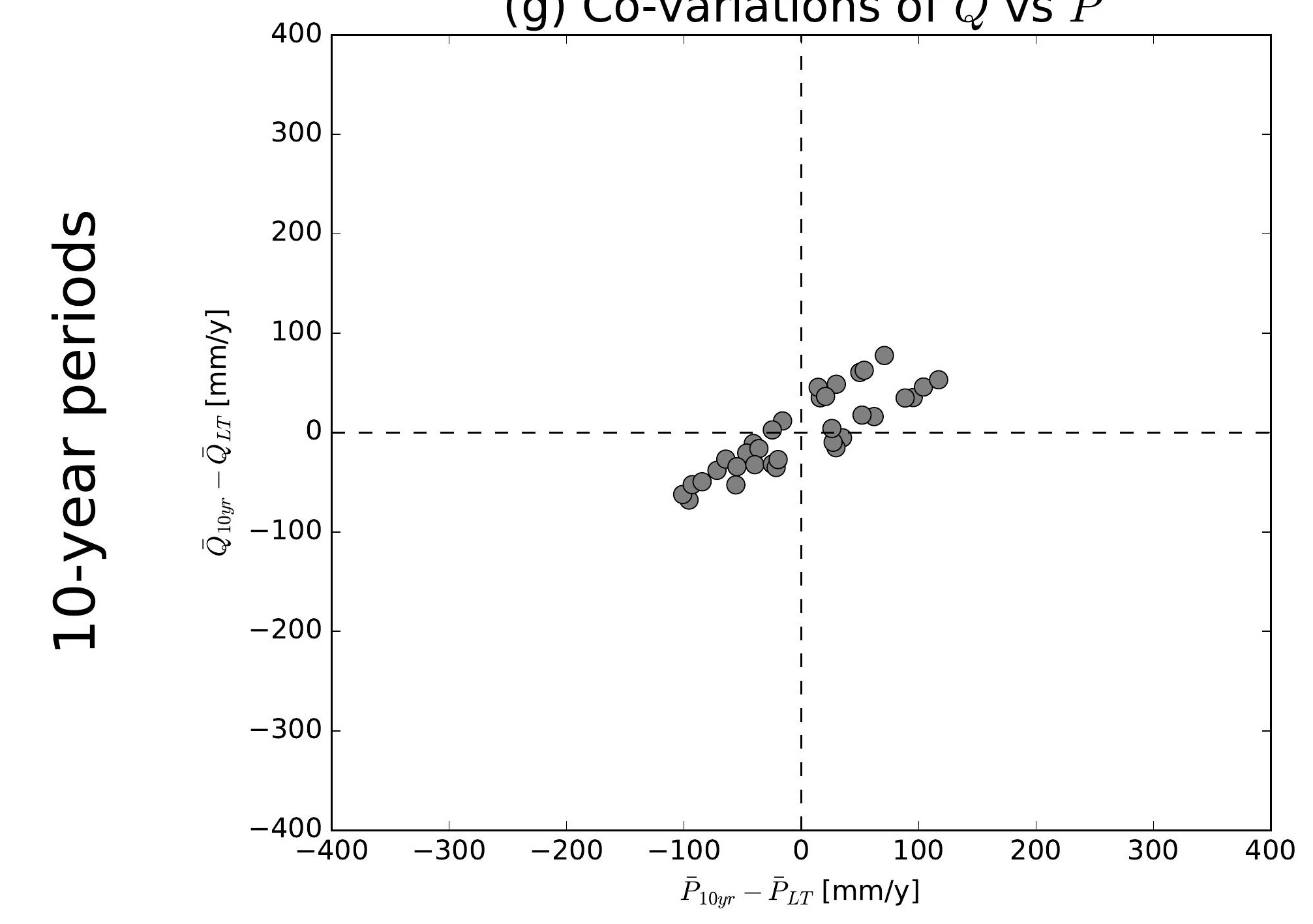

(j) Co-variations of $\bar{Q}$ vs $\bar{P}$

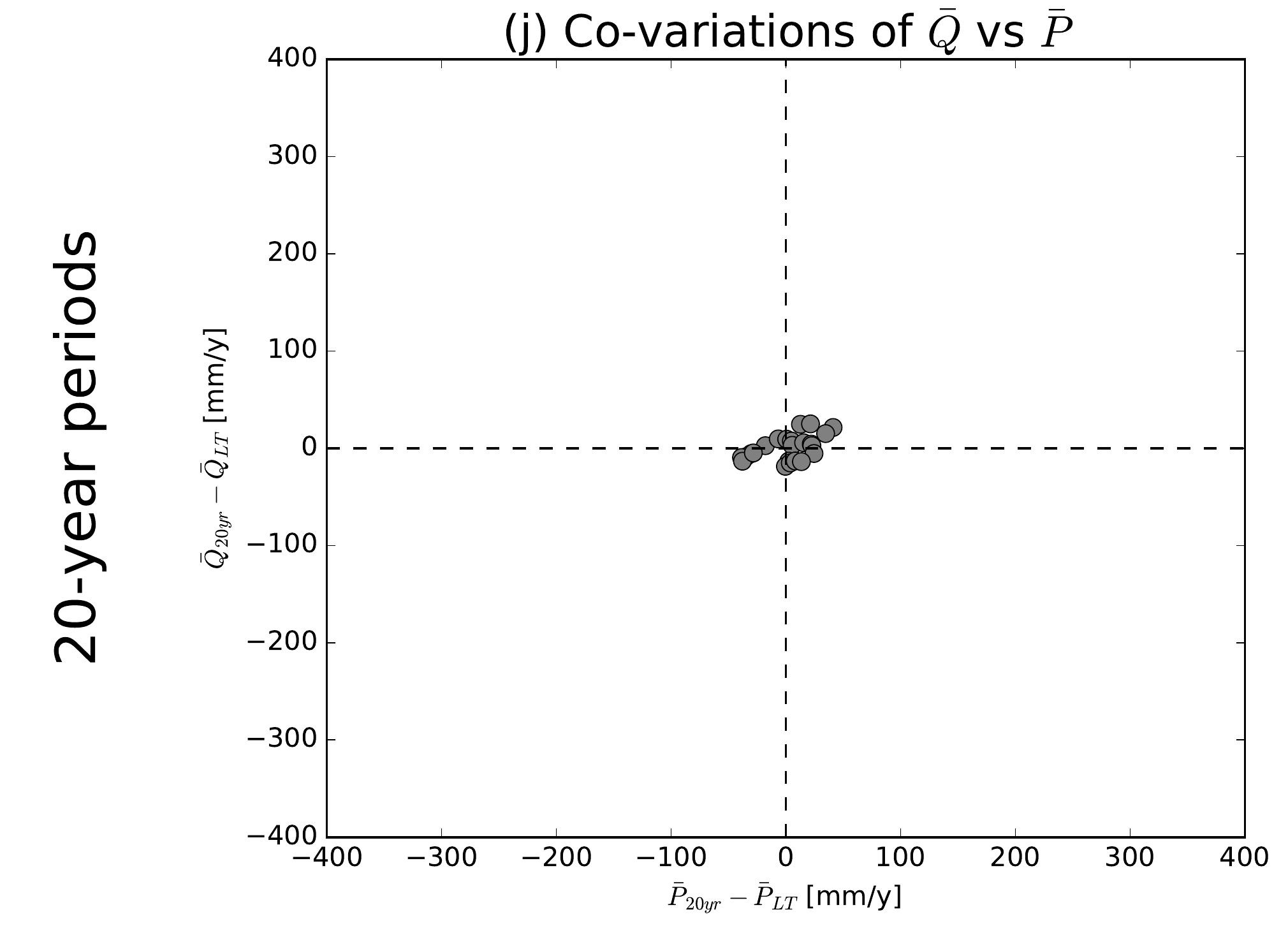

(b) Co-variations of $\bar{Q}$ vs $\overline{E 0}$

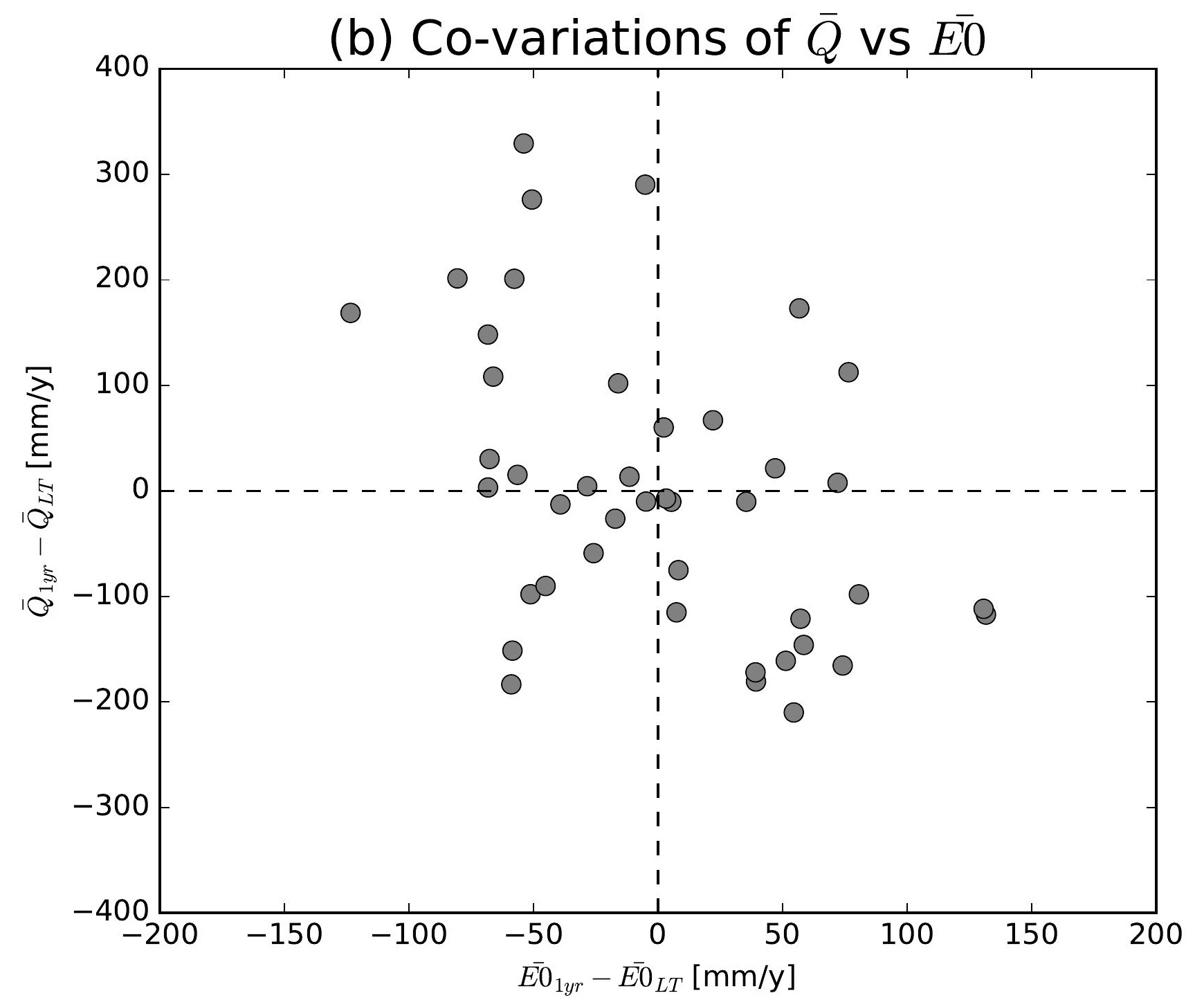

(e) Co-variations of $\bar{Q}$ vs $\overline{E 0}$

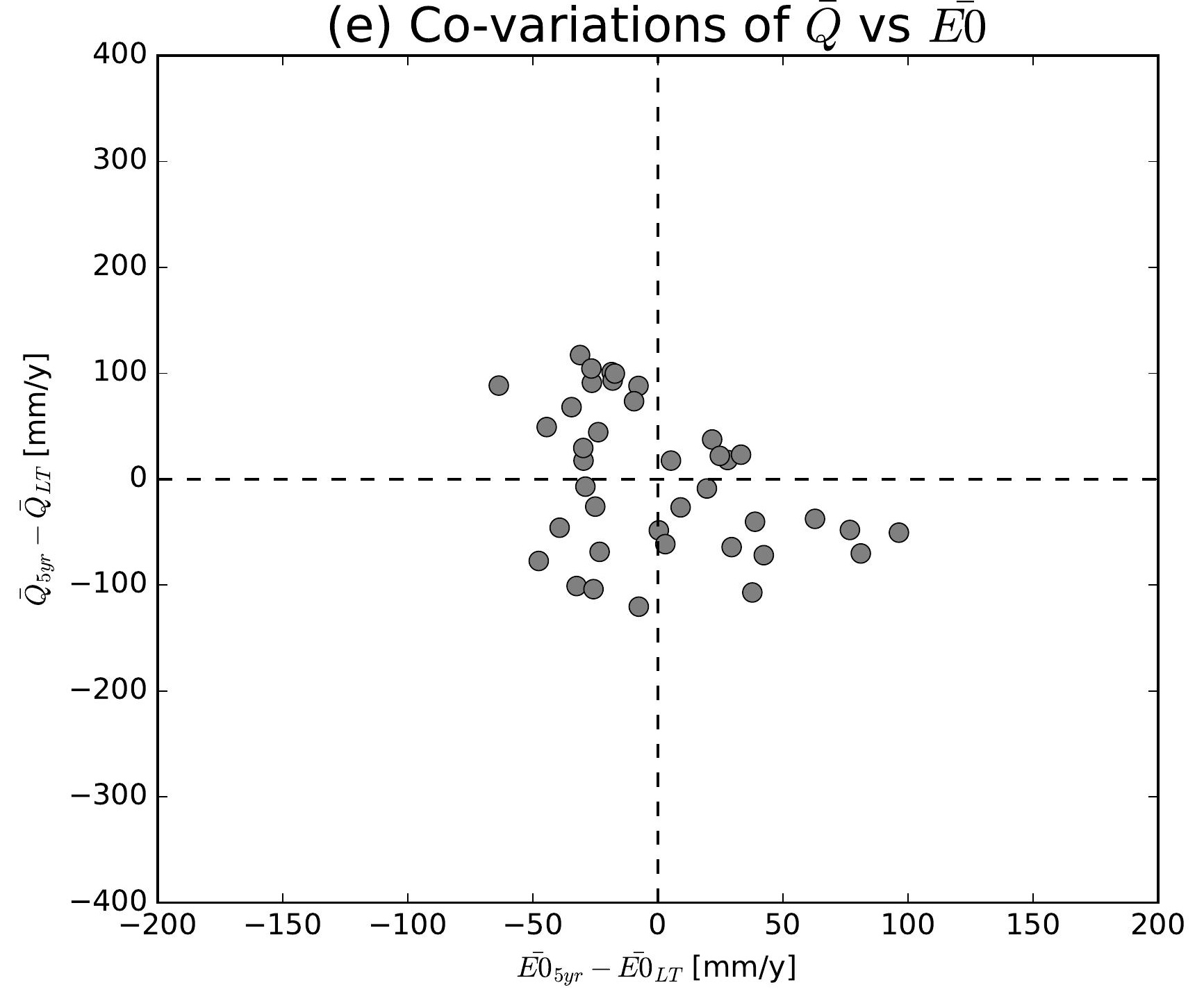

(h) Co-variations of $\bar{Q}$ vs $\overline{E 0}$

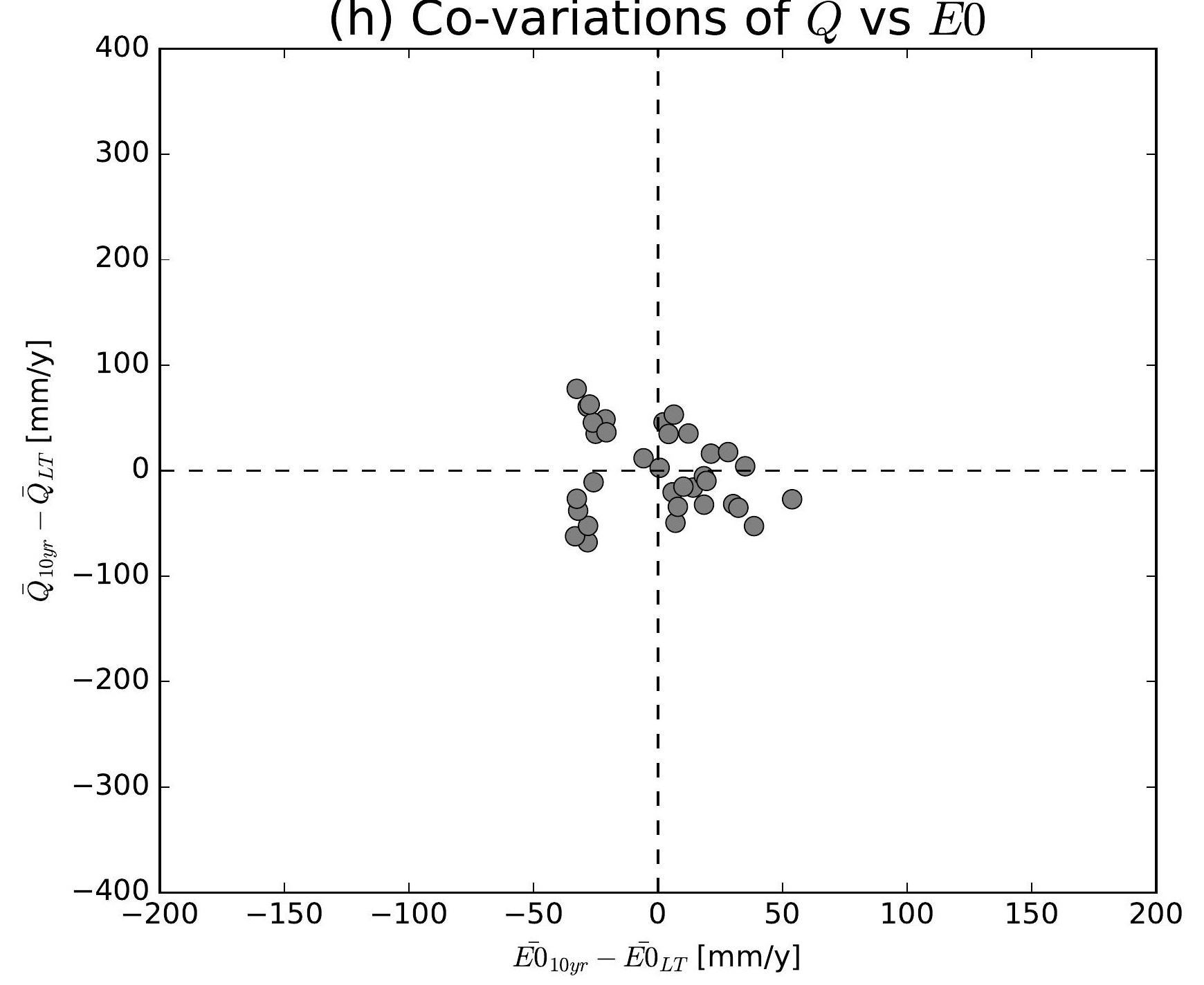

(k) Co-variations of $\bar{Q}$ vs $\overline{E 0}$

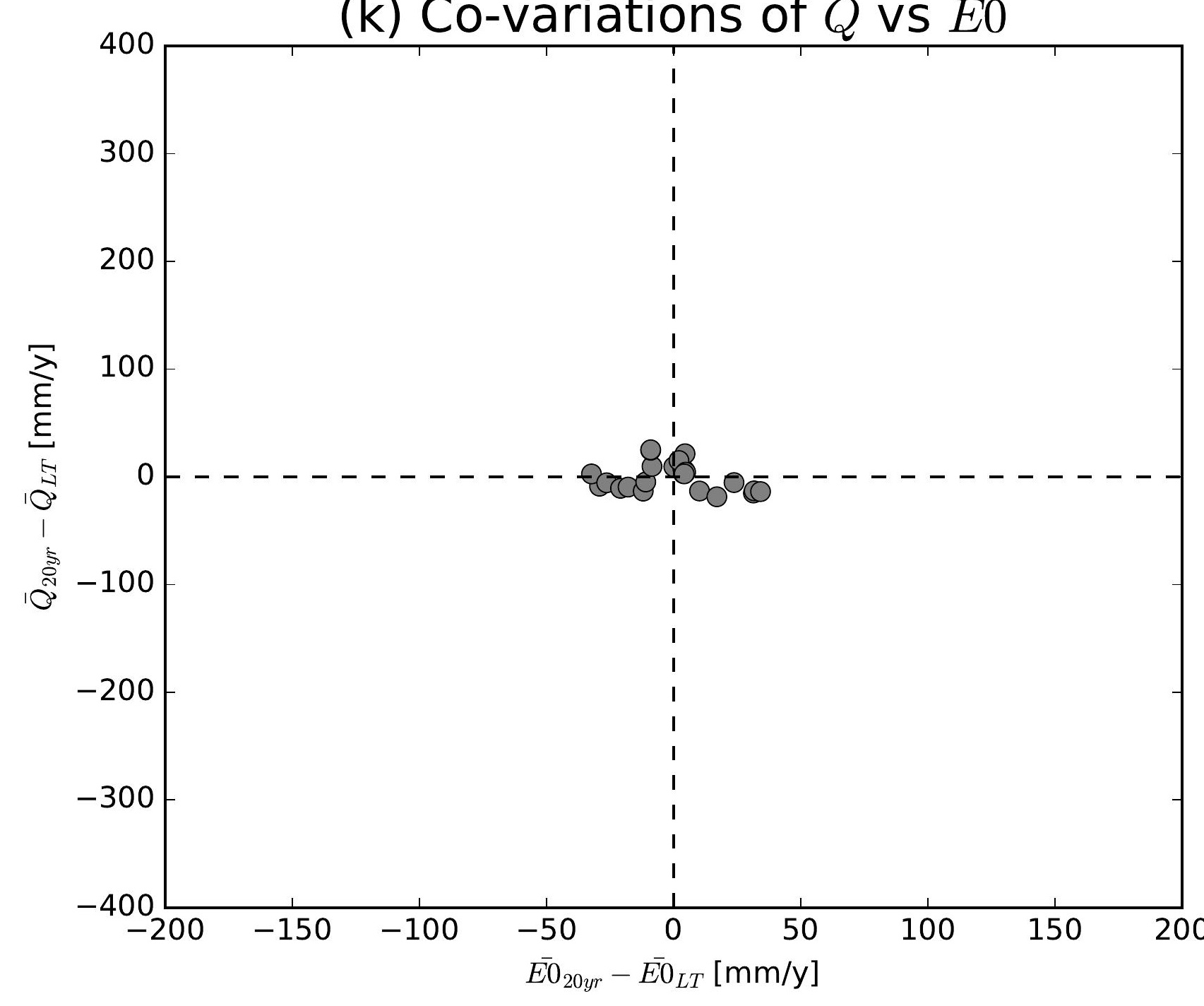

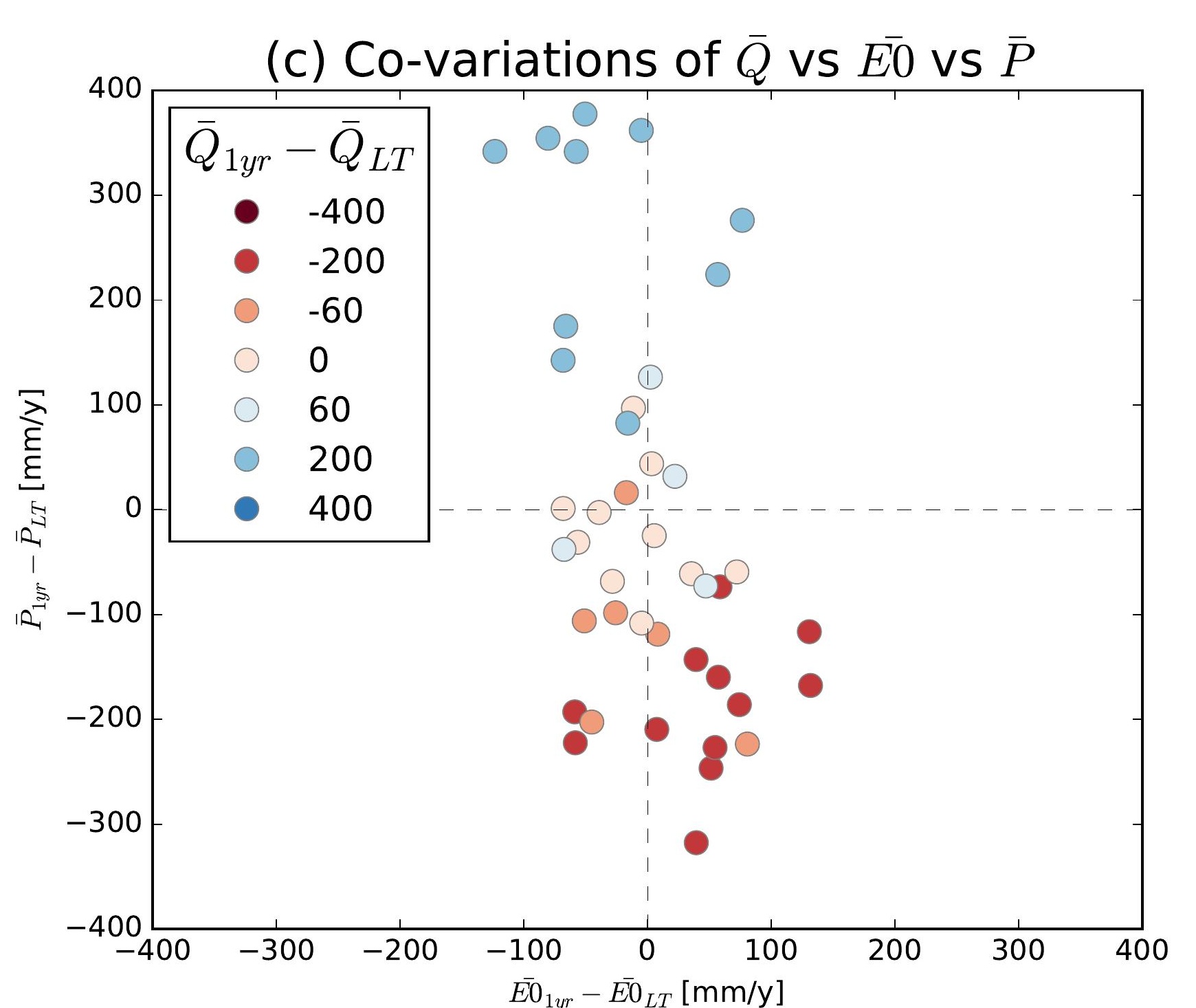
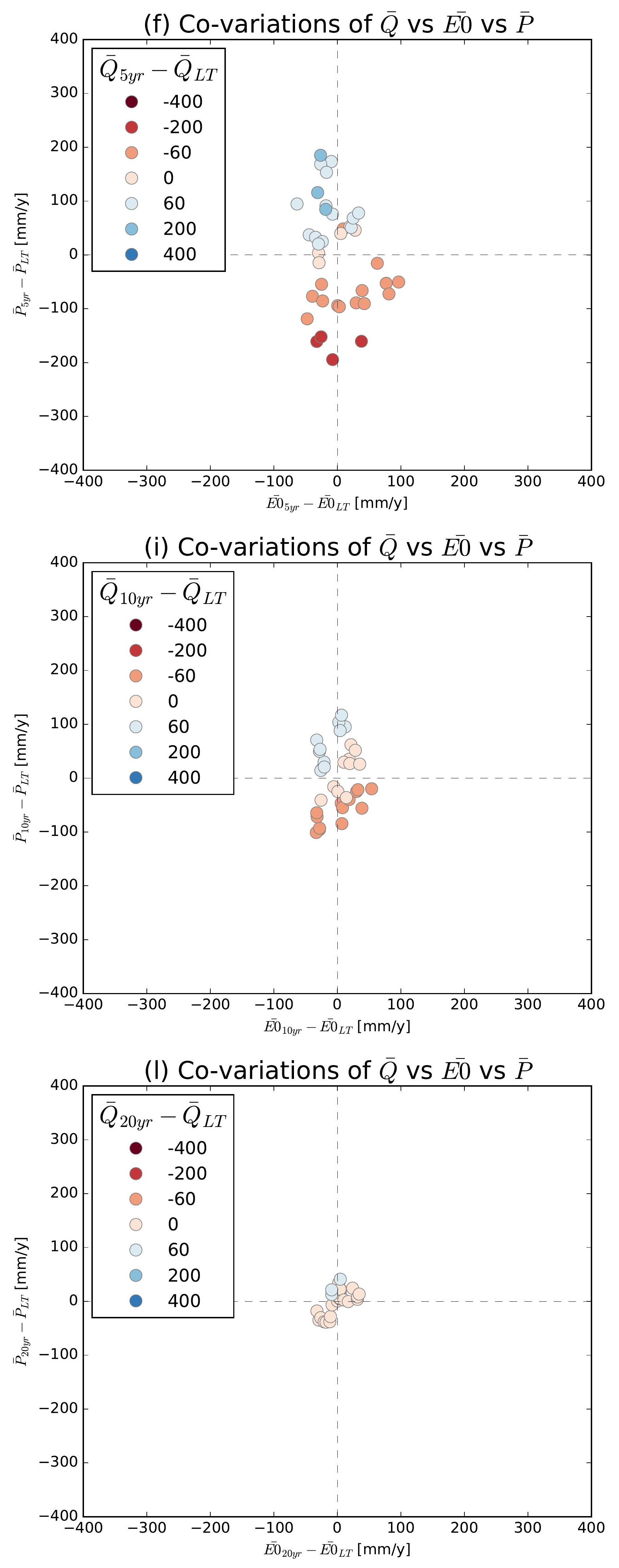

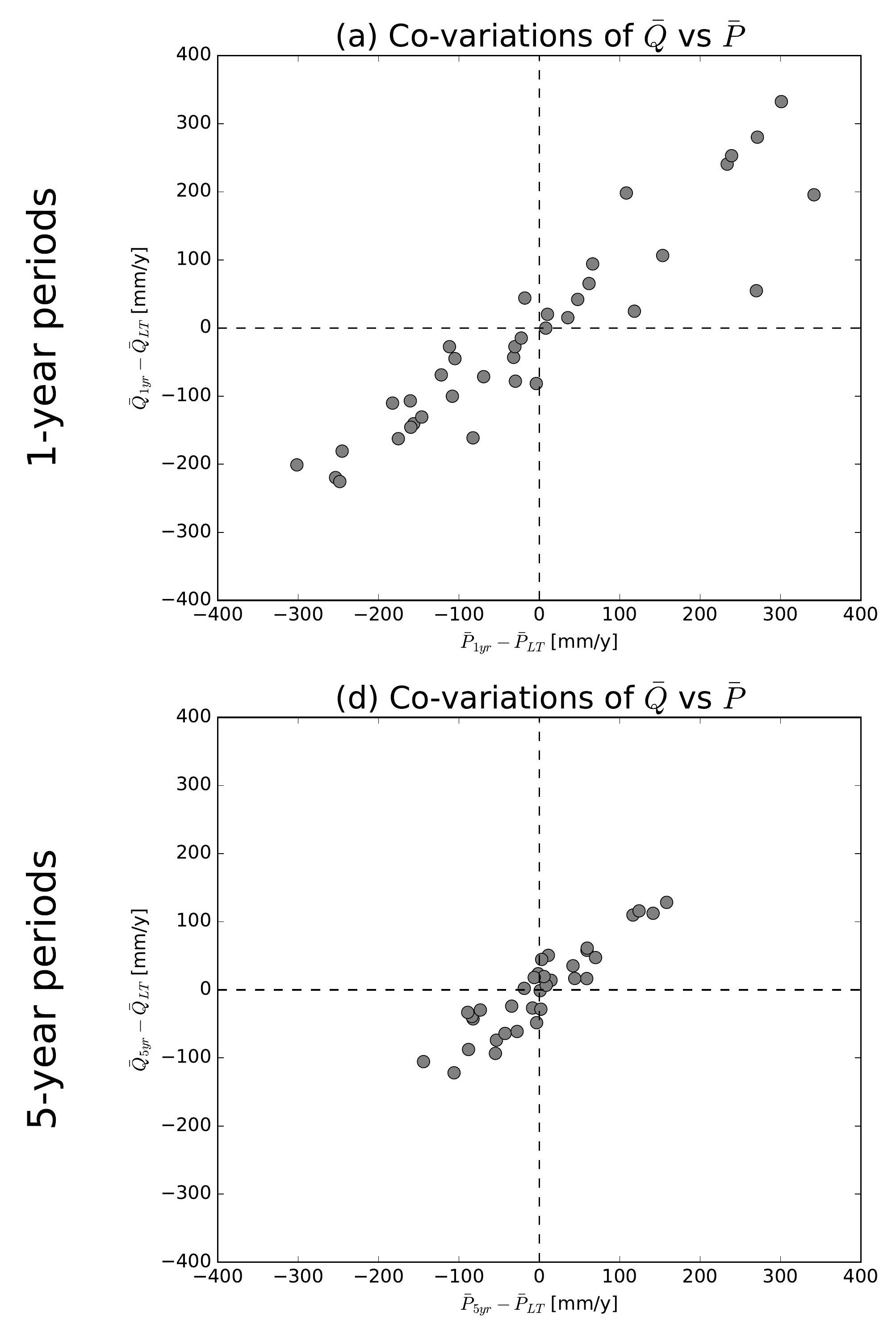

(g) Co-variations of $\bar{Q}$ vs $\bar{P}$

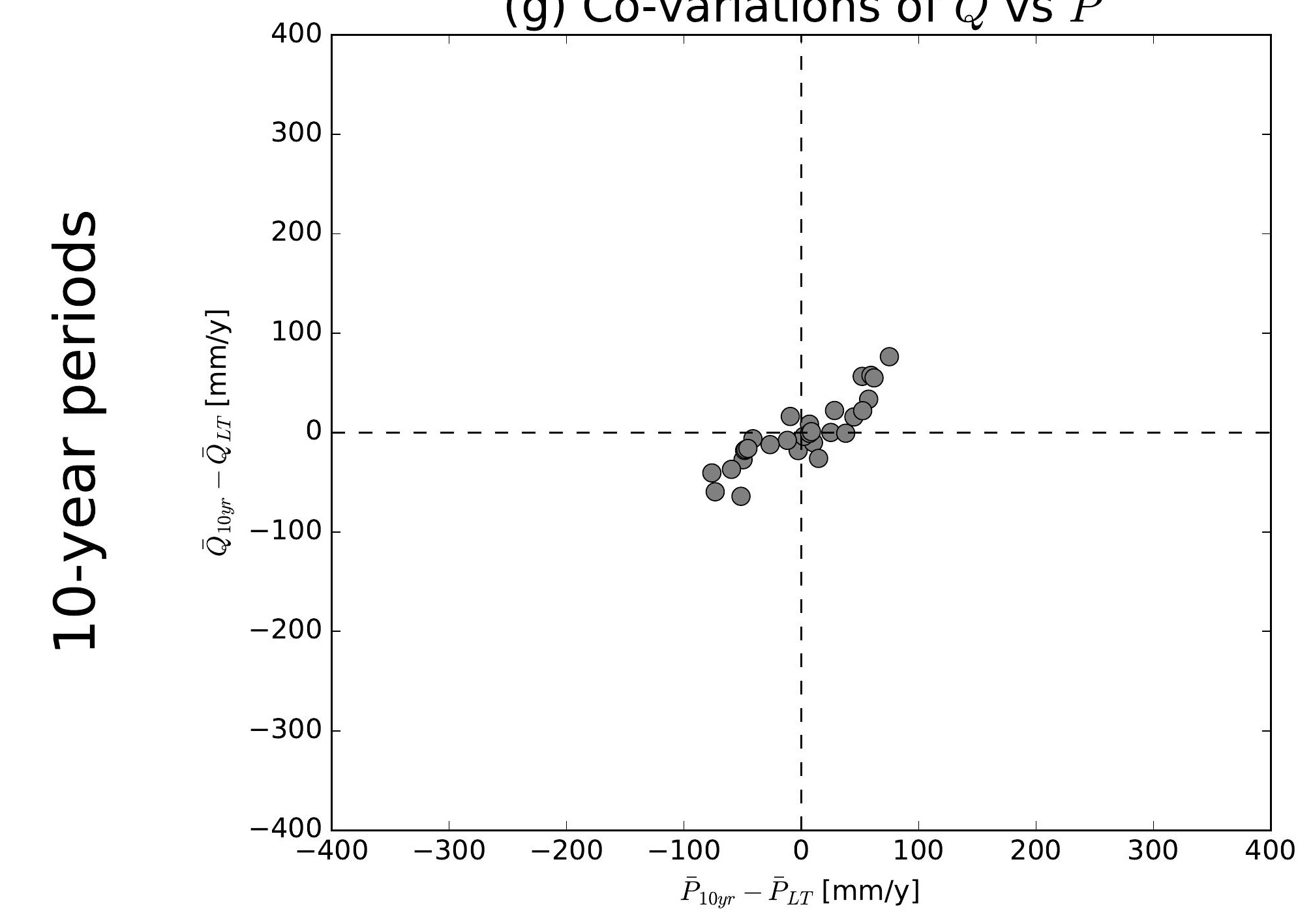

(j) Co-variations of $\bar{Q}$ vs $\bar{P}$

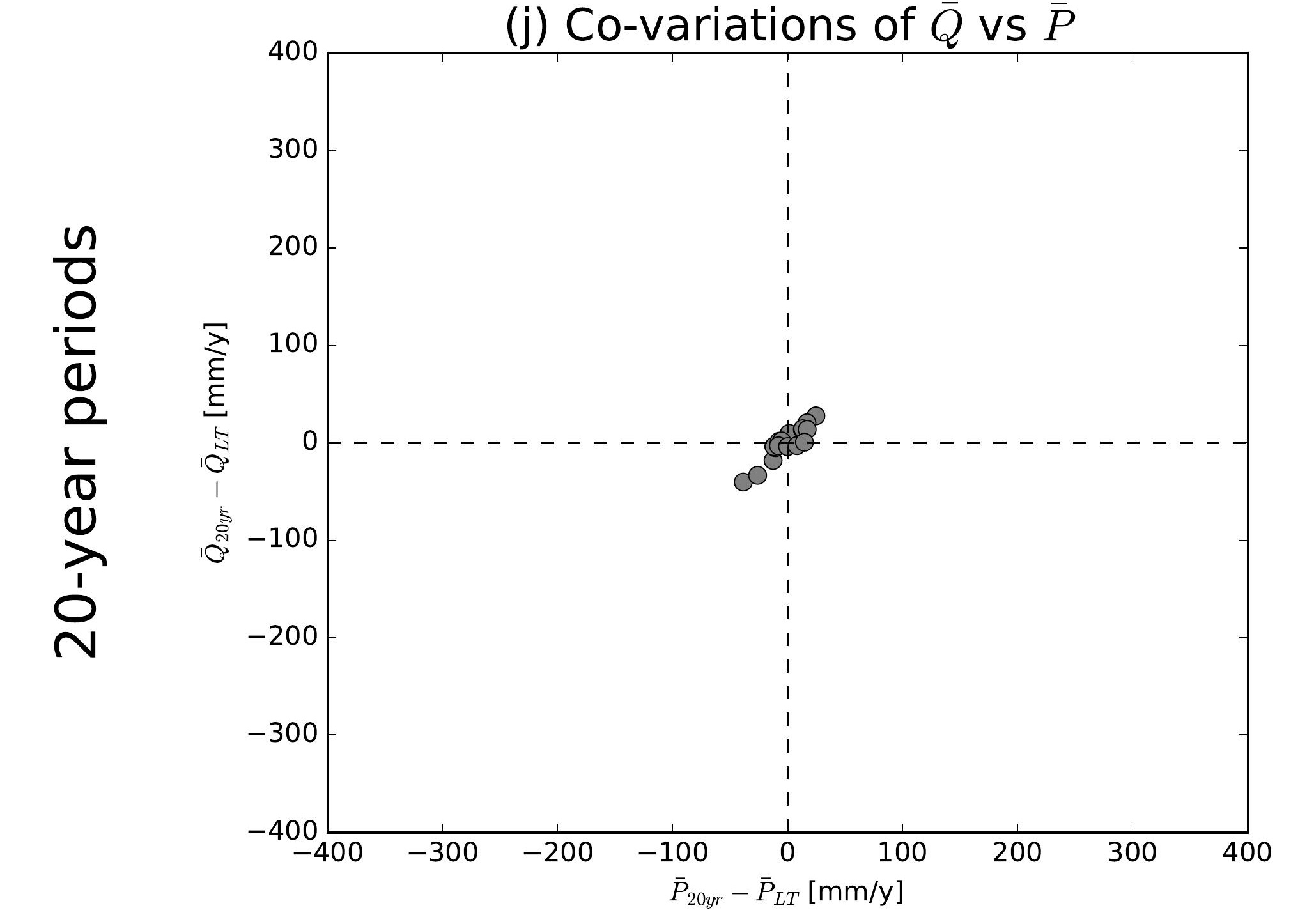

(b) Co-variations of $\bar{Q}$ vs $\overline{E 0}$

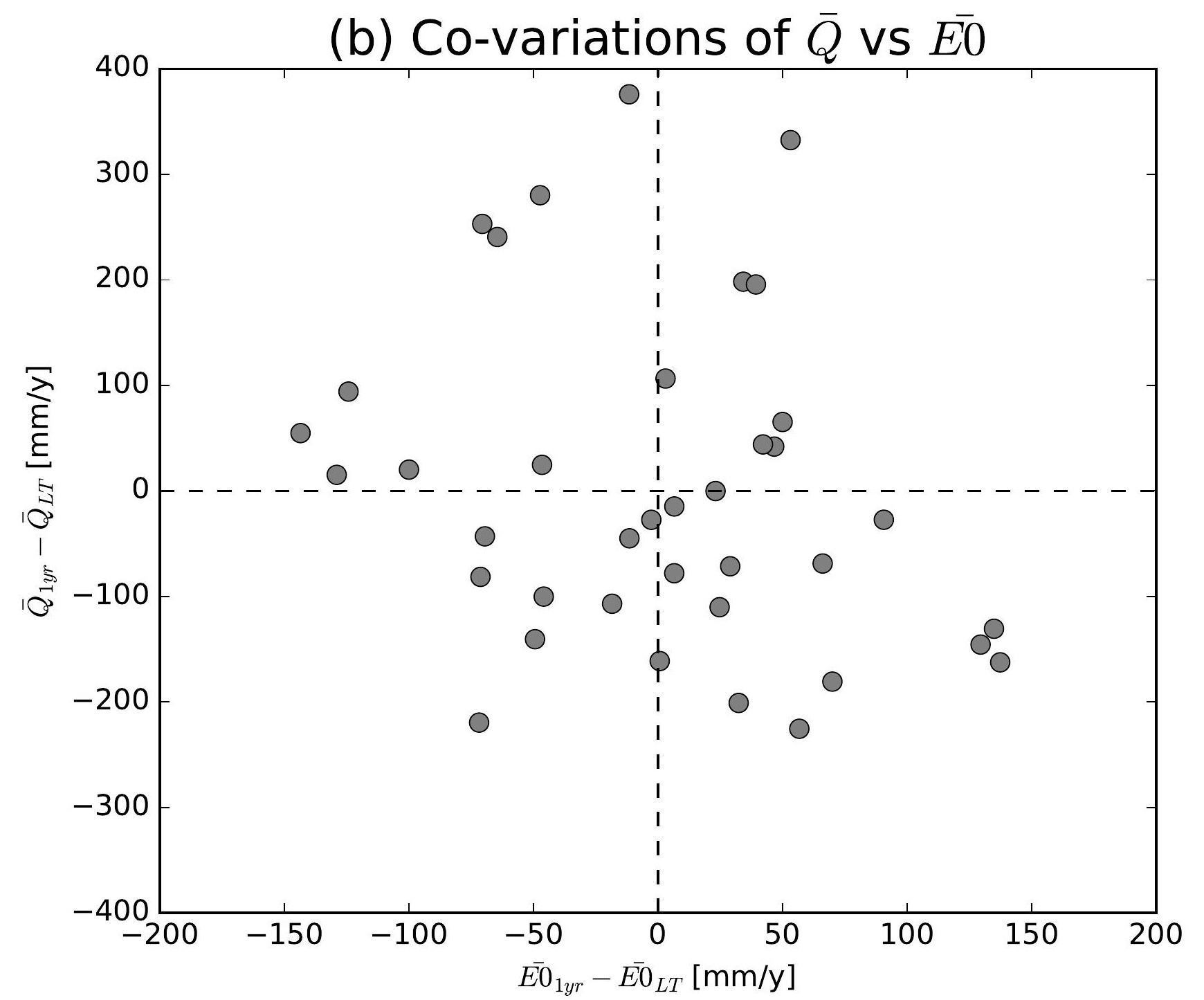

(e) Co-variations of $\bar{Q}$ vs $\overline{E 0}$

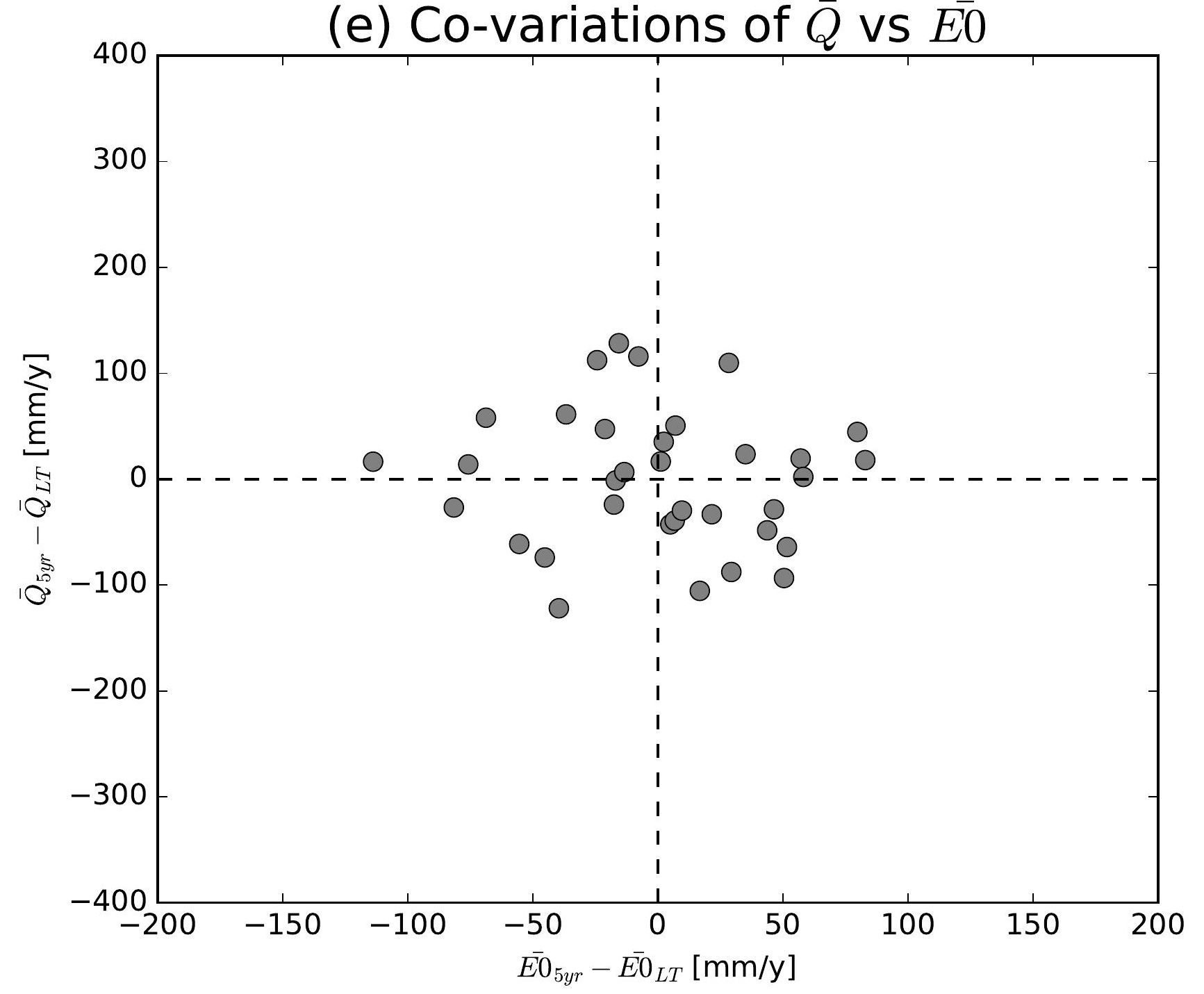

(h) Co-variations of $\bar{Q}$ vs $\overline{E 0}$

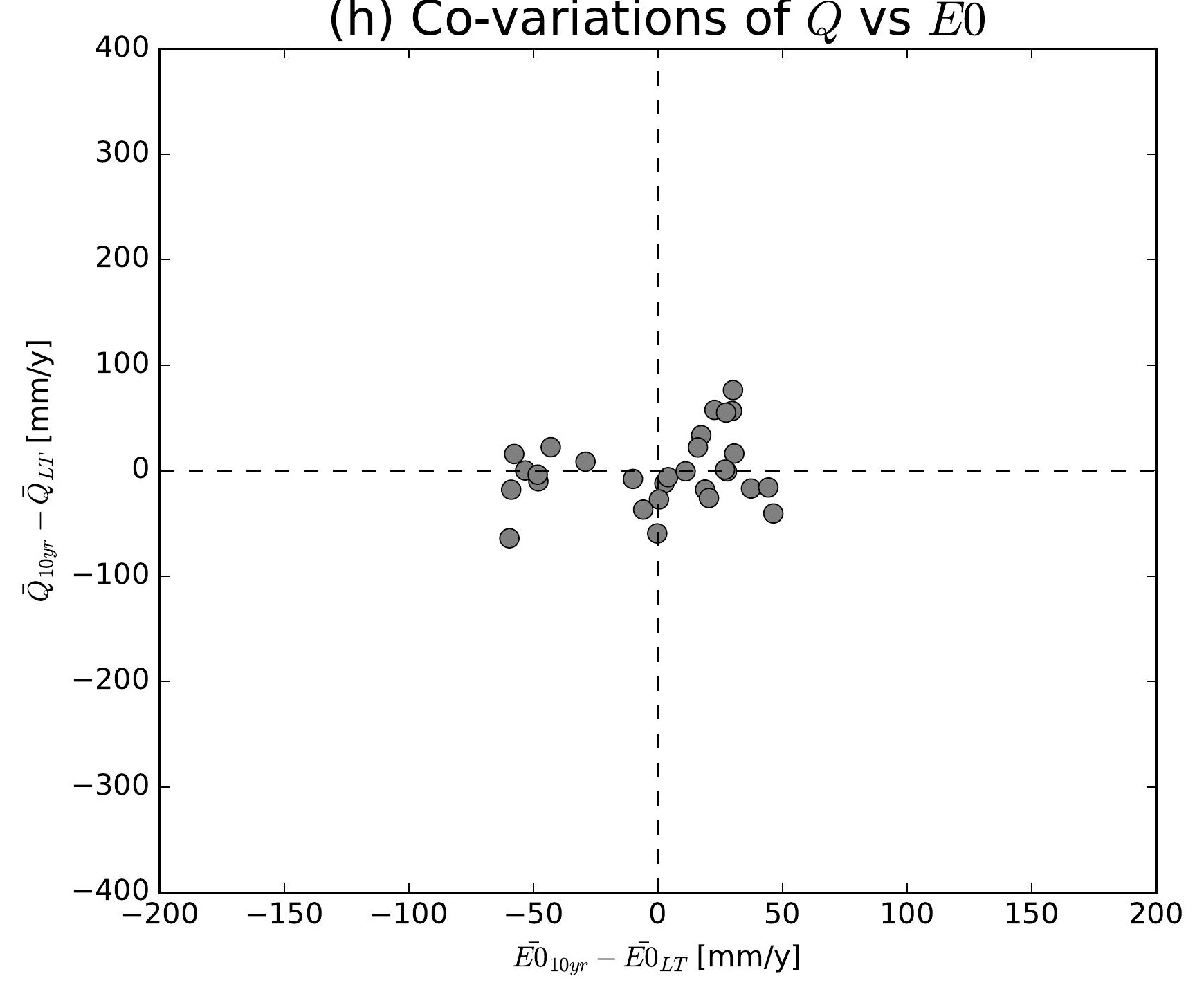

(k) Co-variations of $\bar{Q}$ vs $\overline{E 0}$

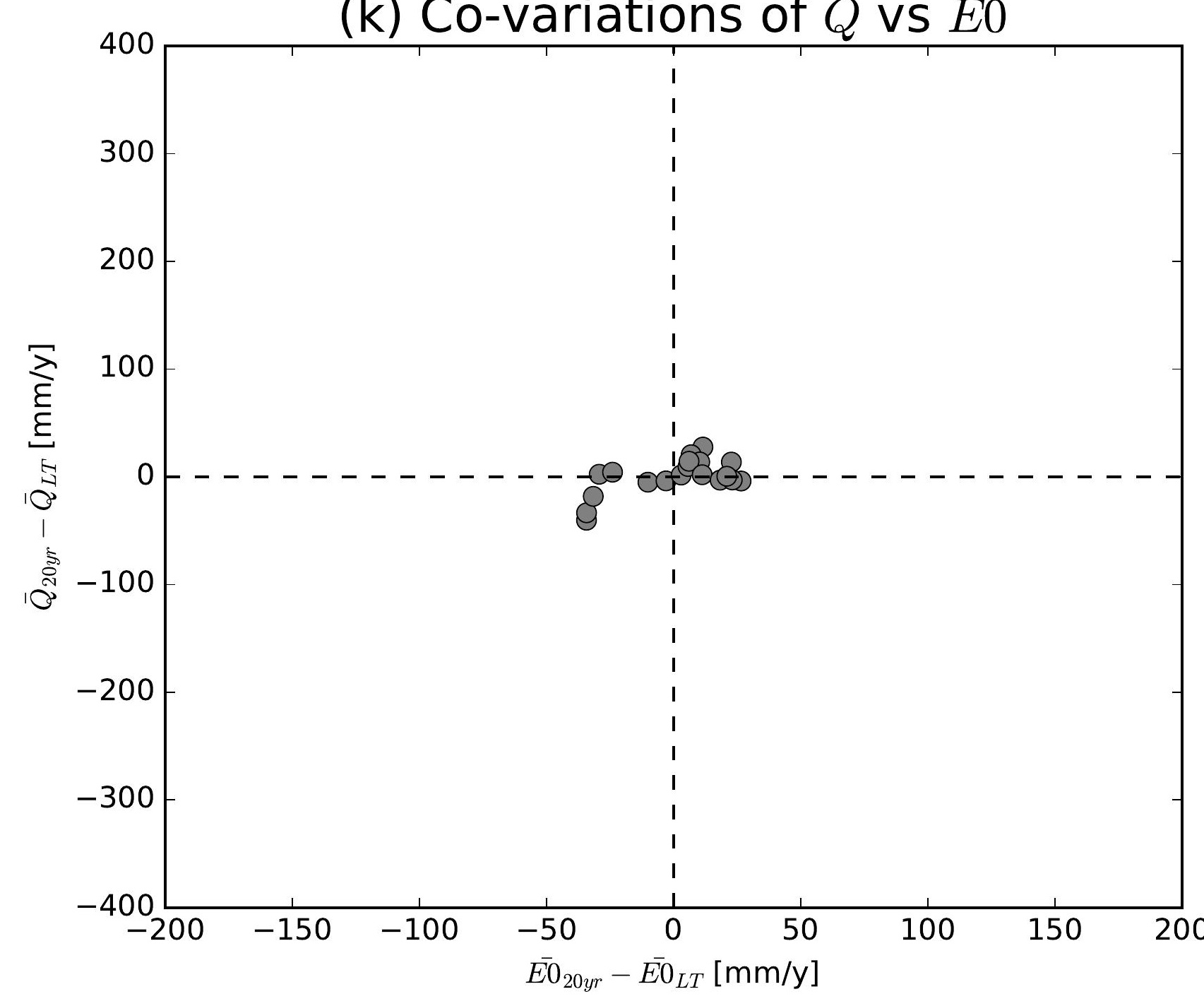

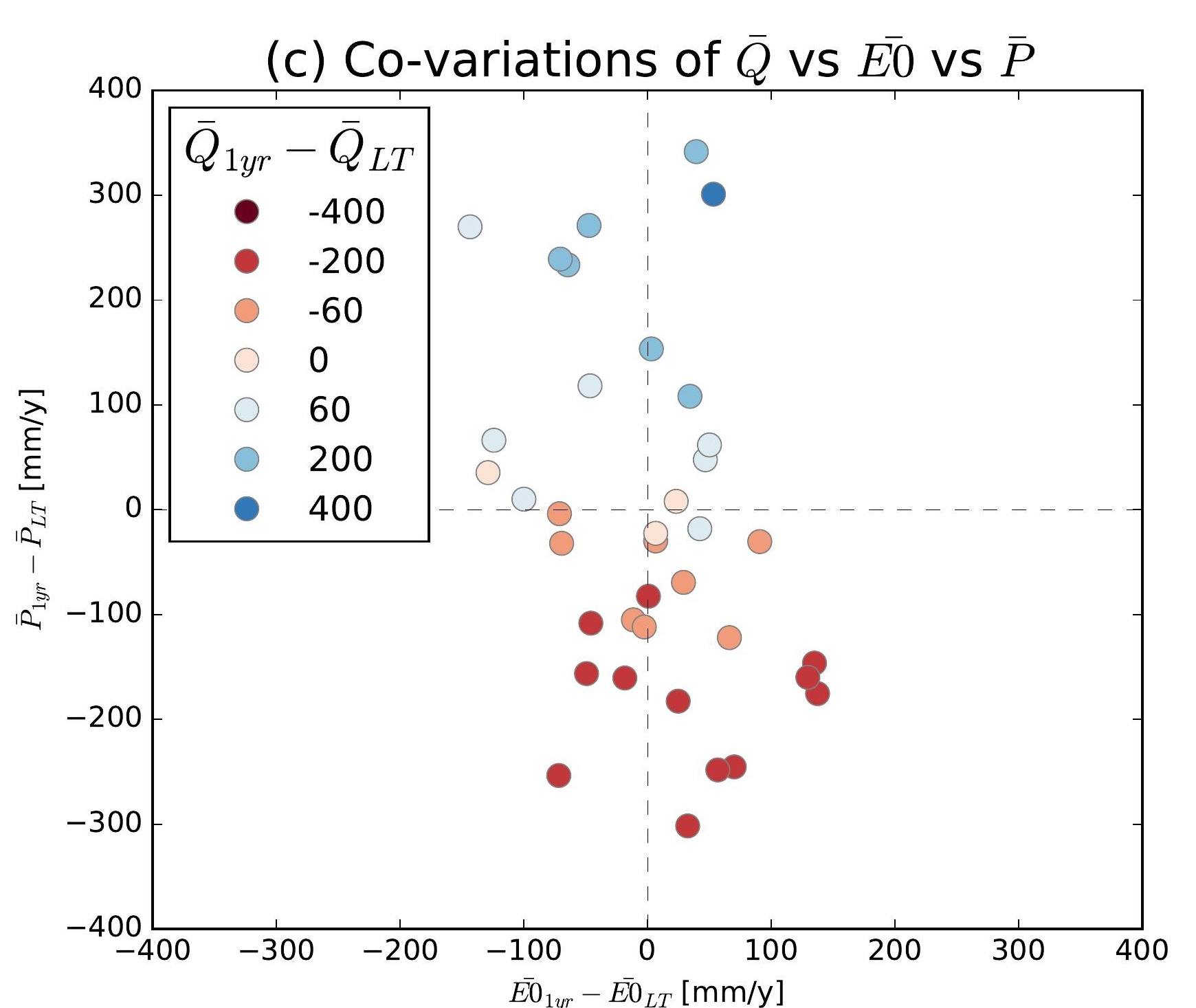
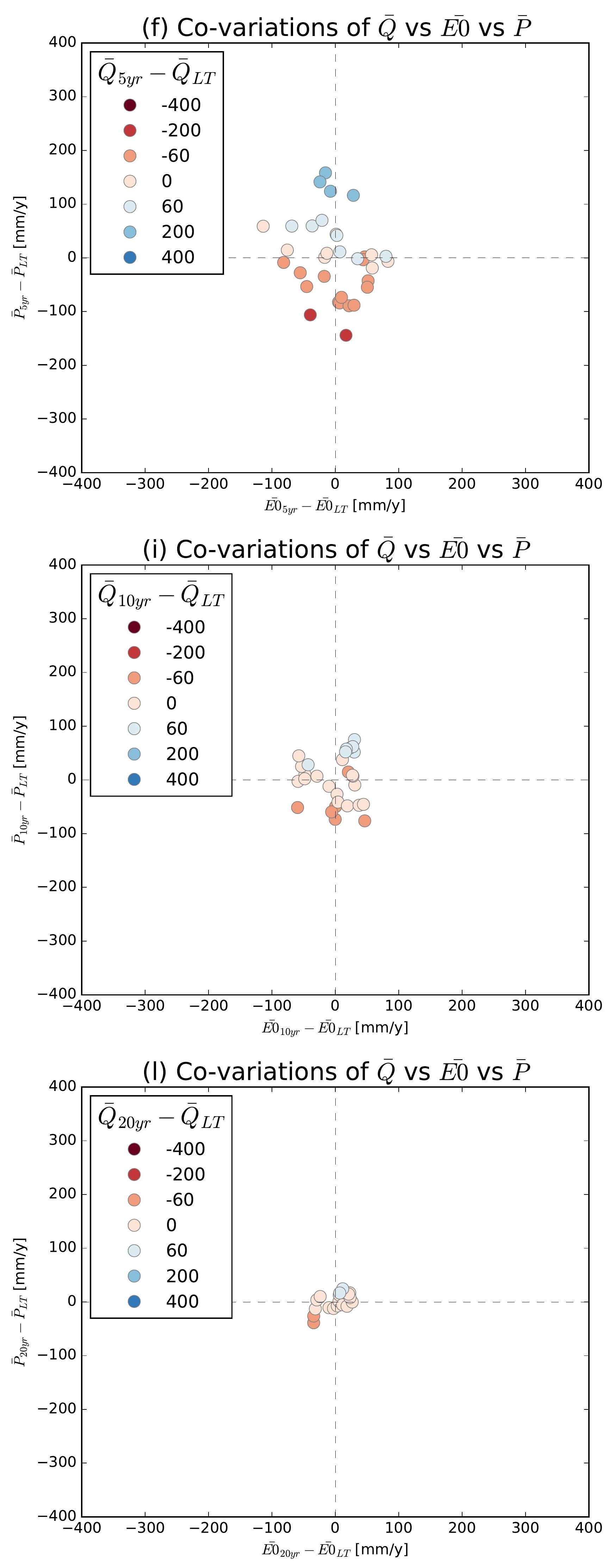

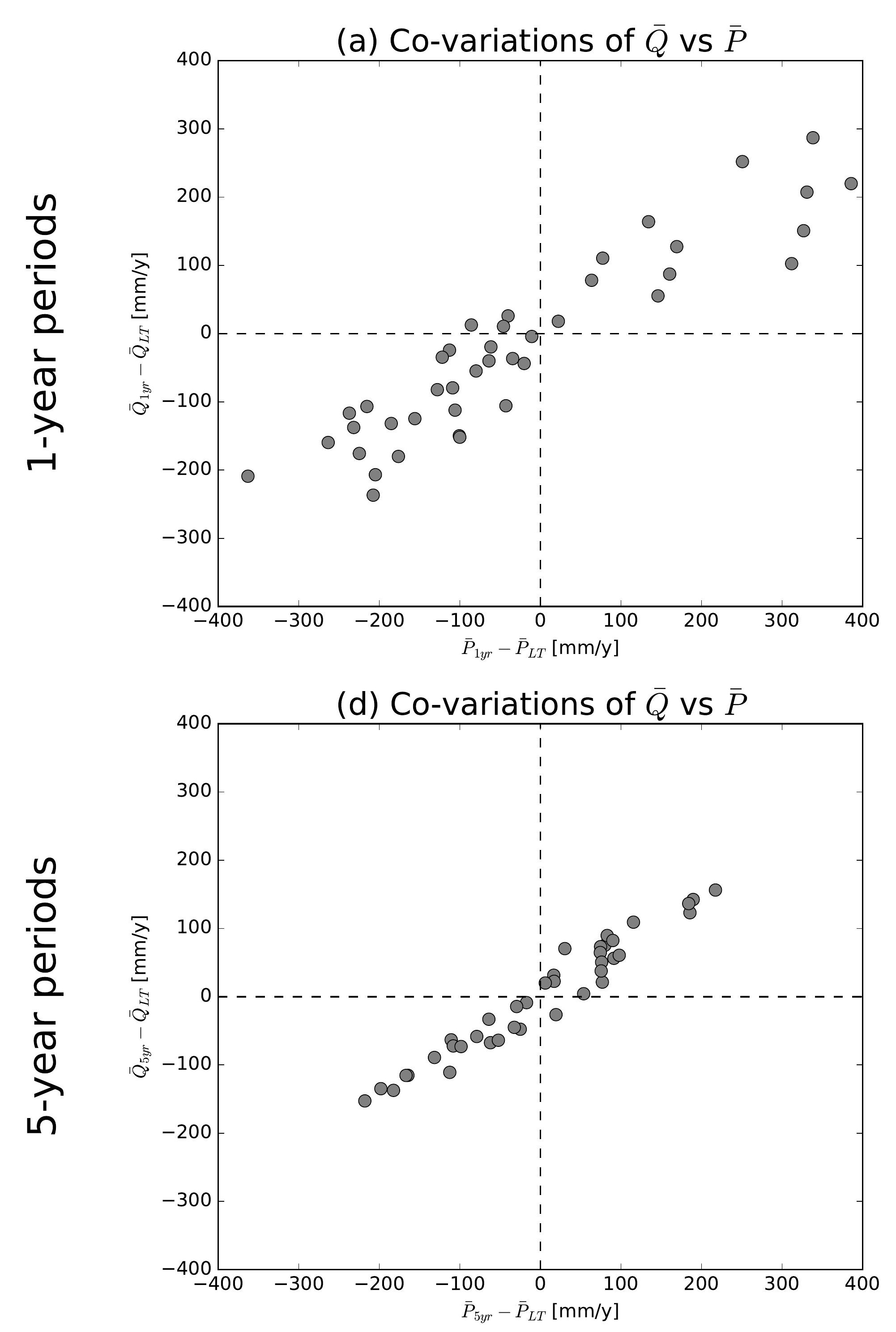

(g) Co-variations of $\bar{Q}$ vs $\bar{P}$

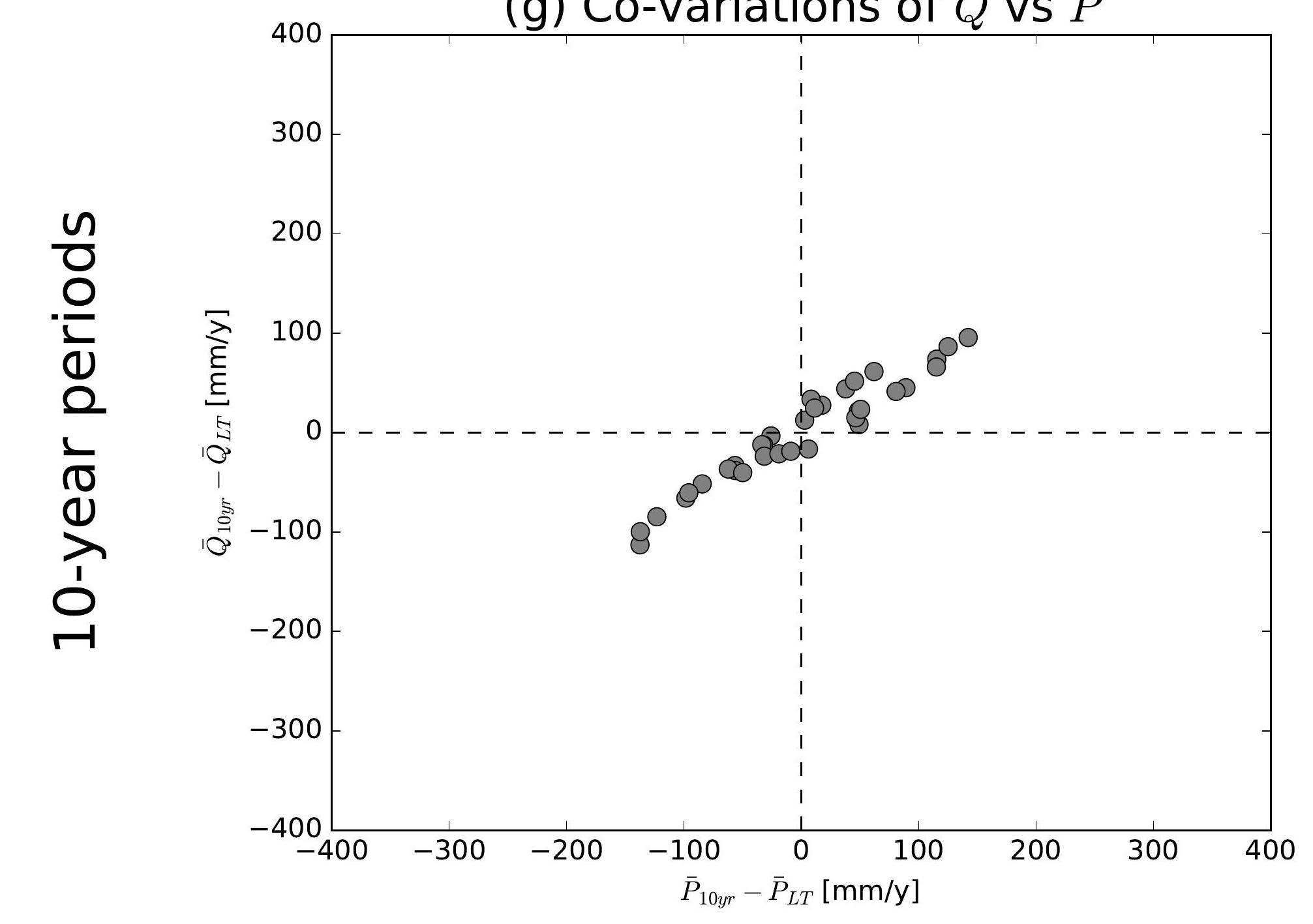

(j) Co-variations of $\bar{Q}$ vs $\bar{P}$

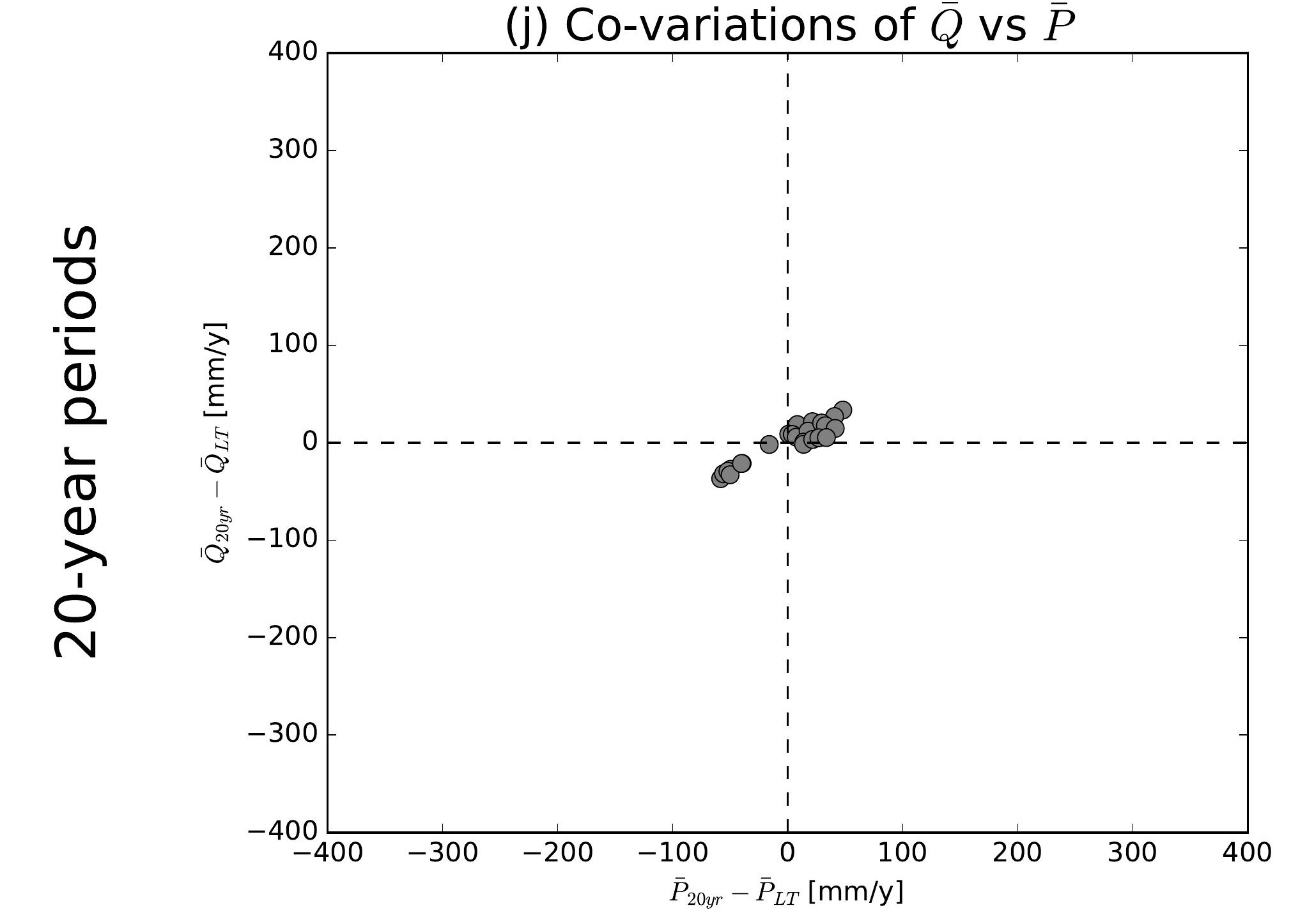

(b) Co-variations of $\bar{Q}$ vs $\overline{E 0}$

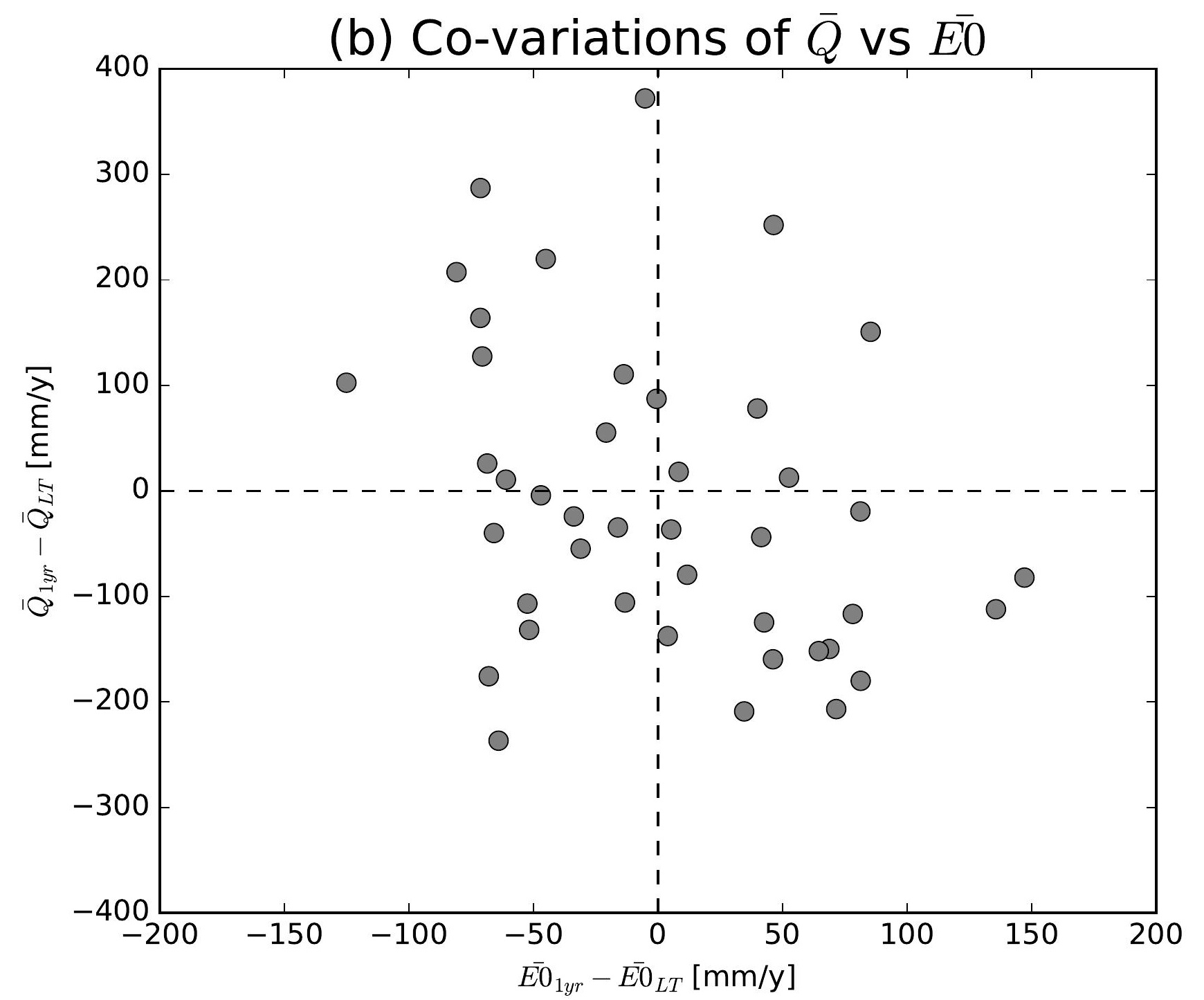

(e) Co-variations of $\bar{Q}$ vs $\overline{E 0}$

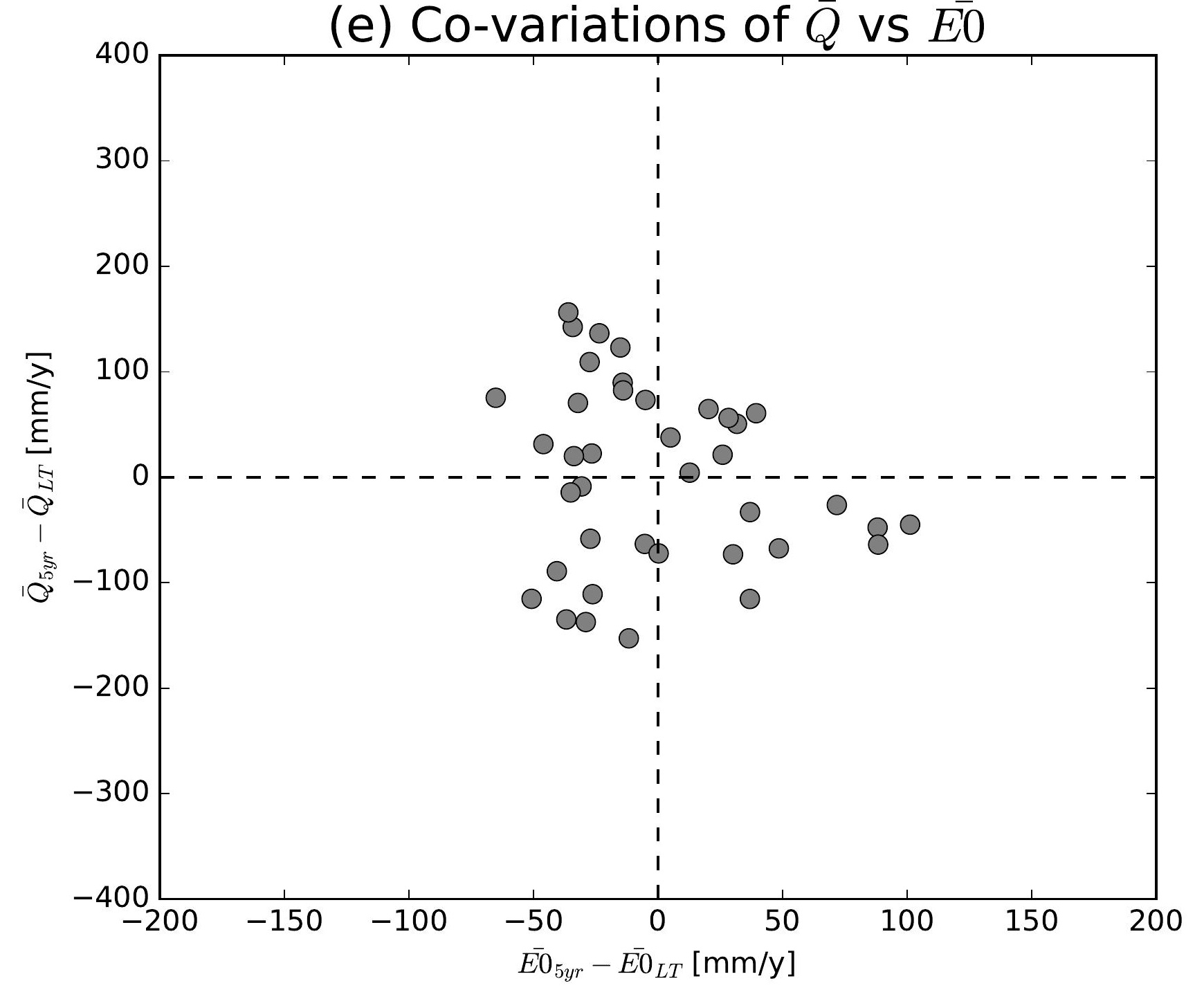

(h) Co-variations of $\bar{Q}$ vs $\overline{E 0}$

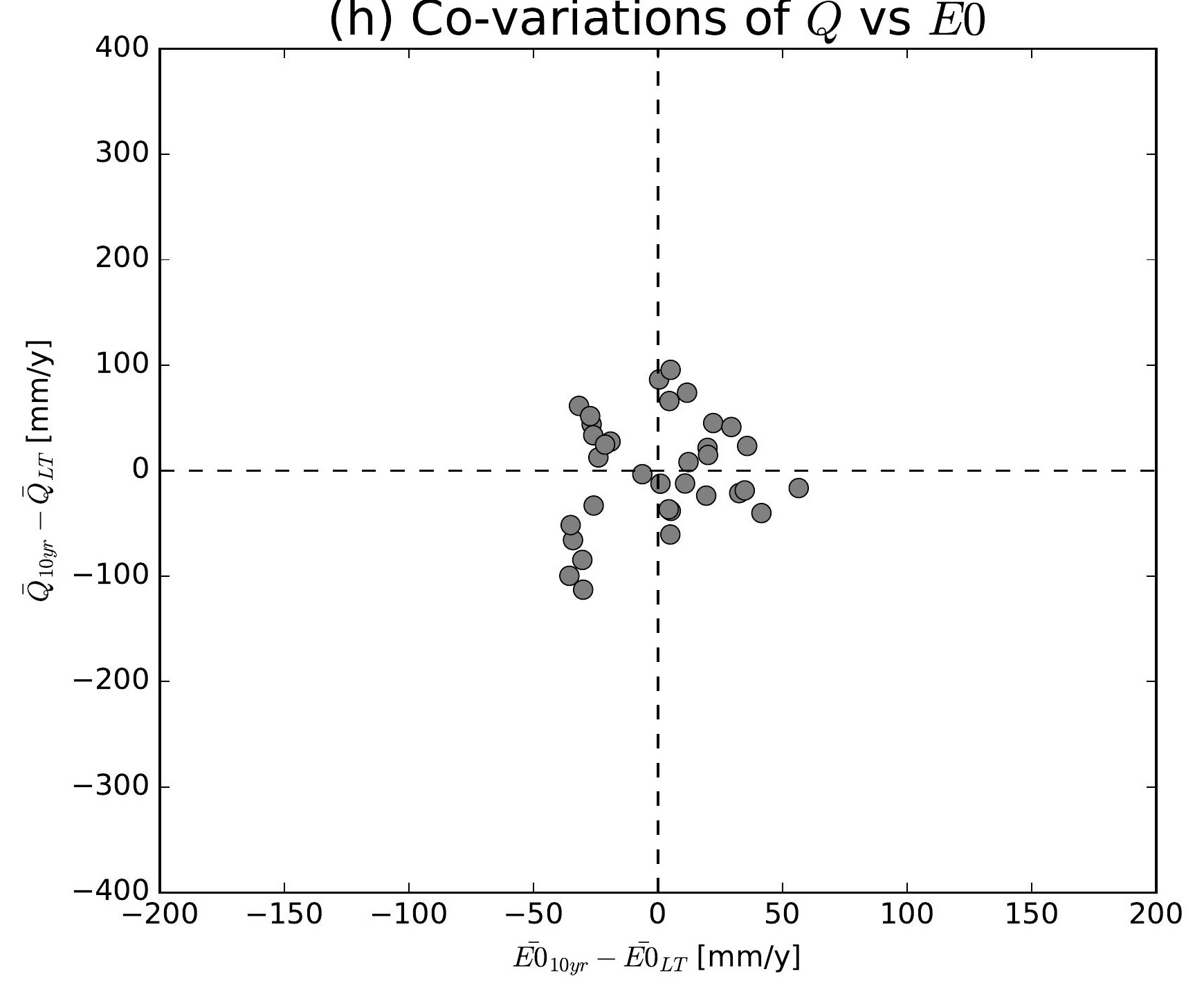

(k) Co-variations of $\bar{Q}$ vs $\overline{E 0}$

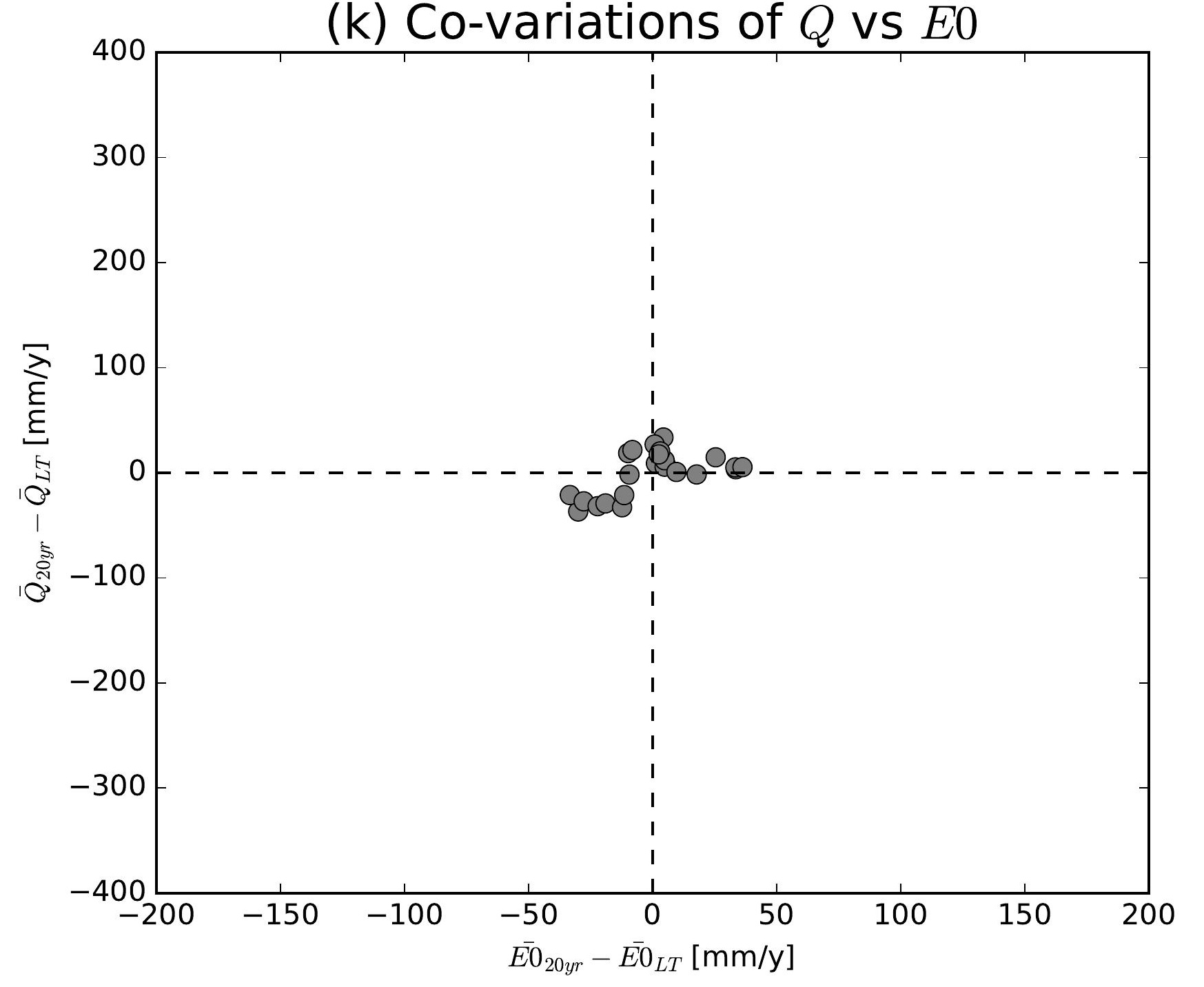

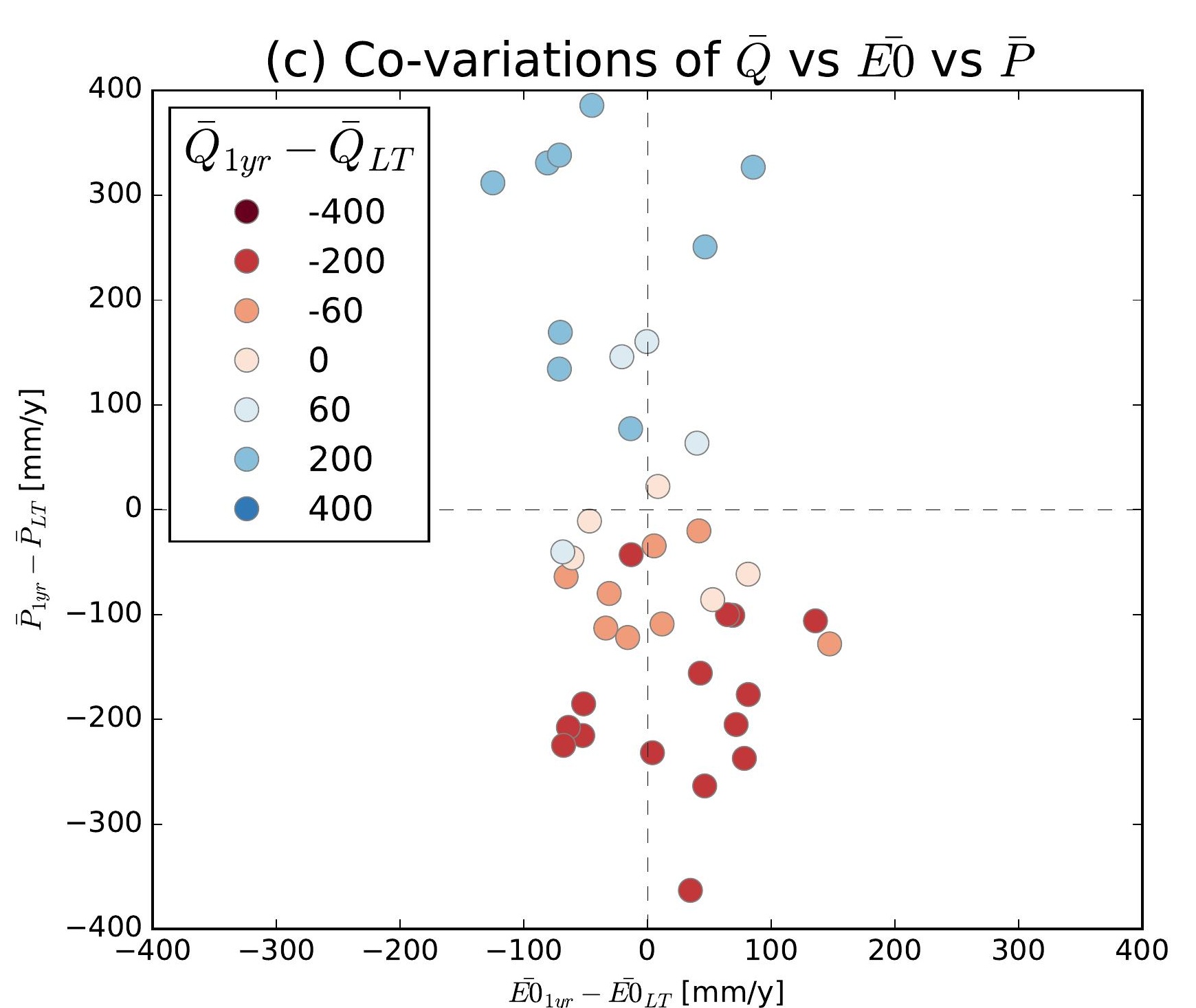
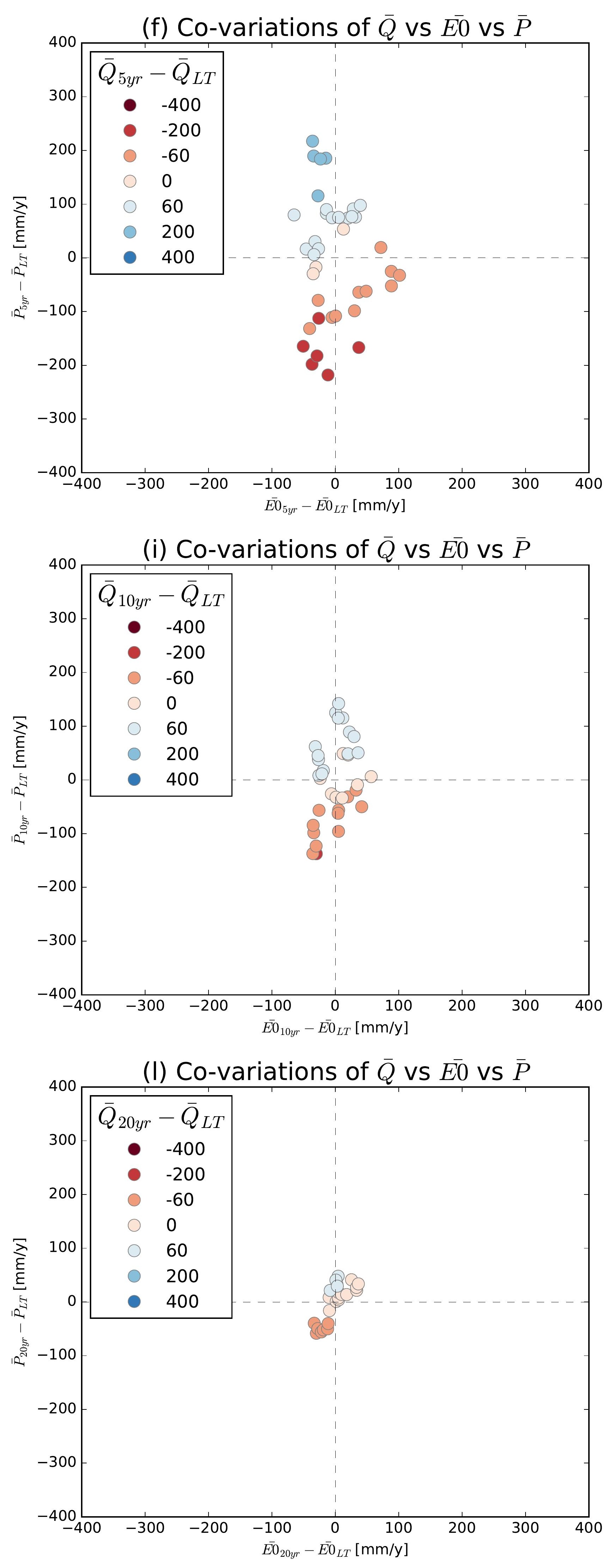

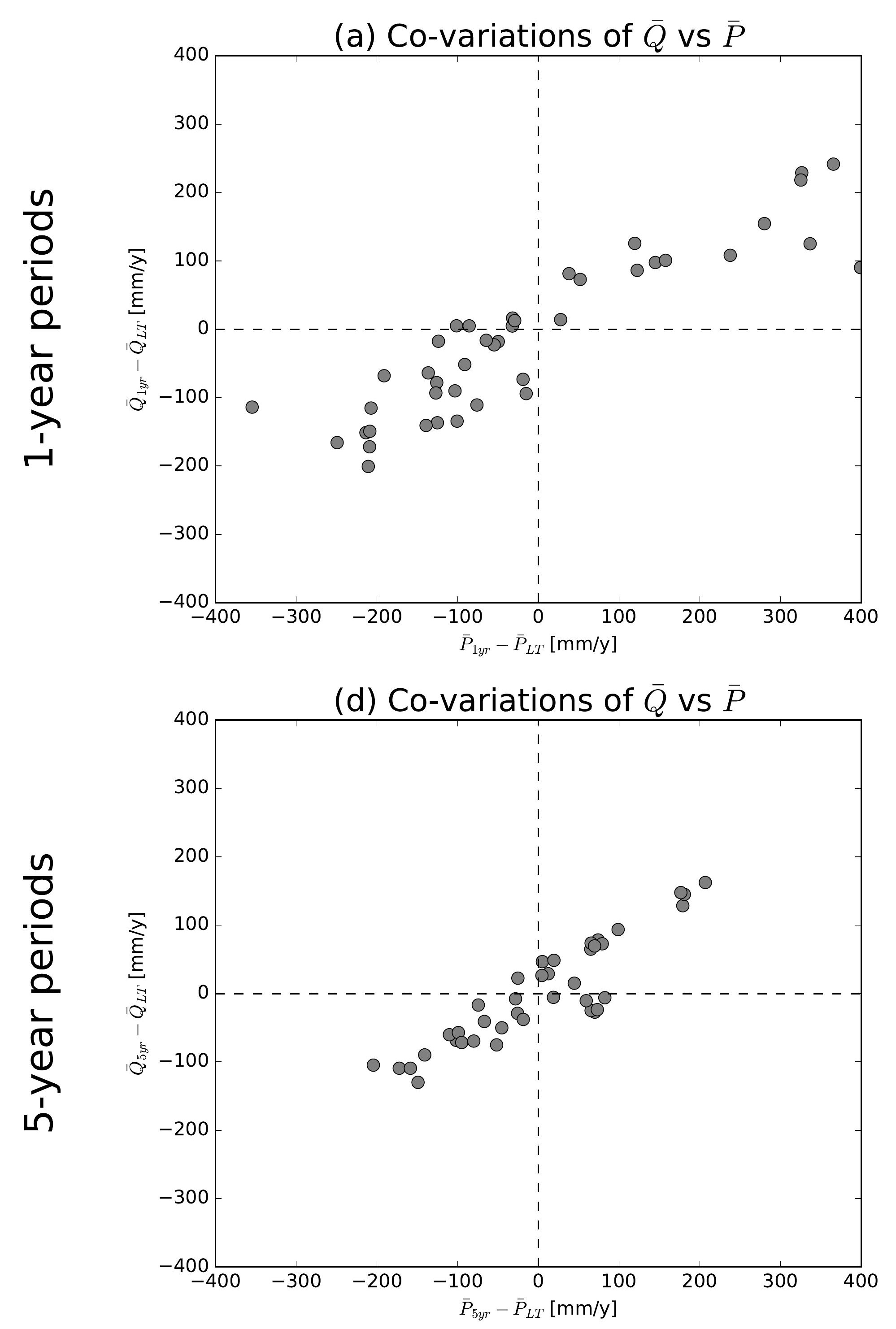

(g) Co-variations of $\bar{Q}$ vs $\bar{P}$

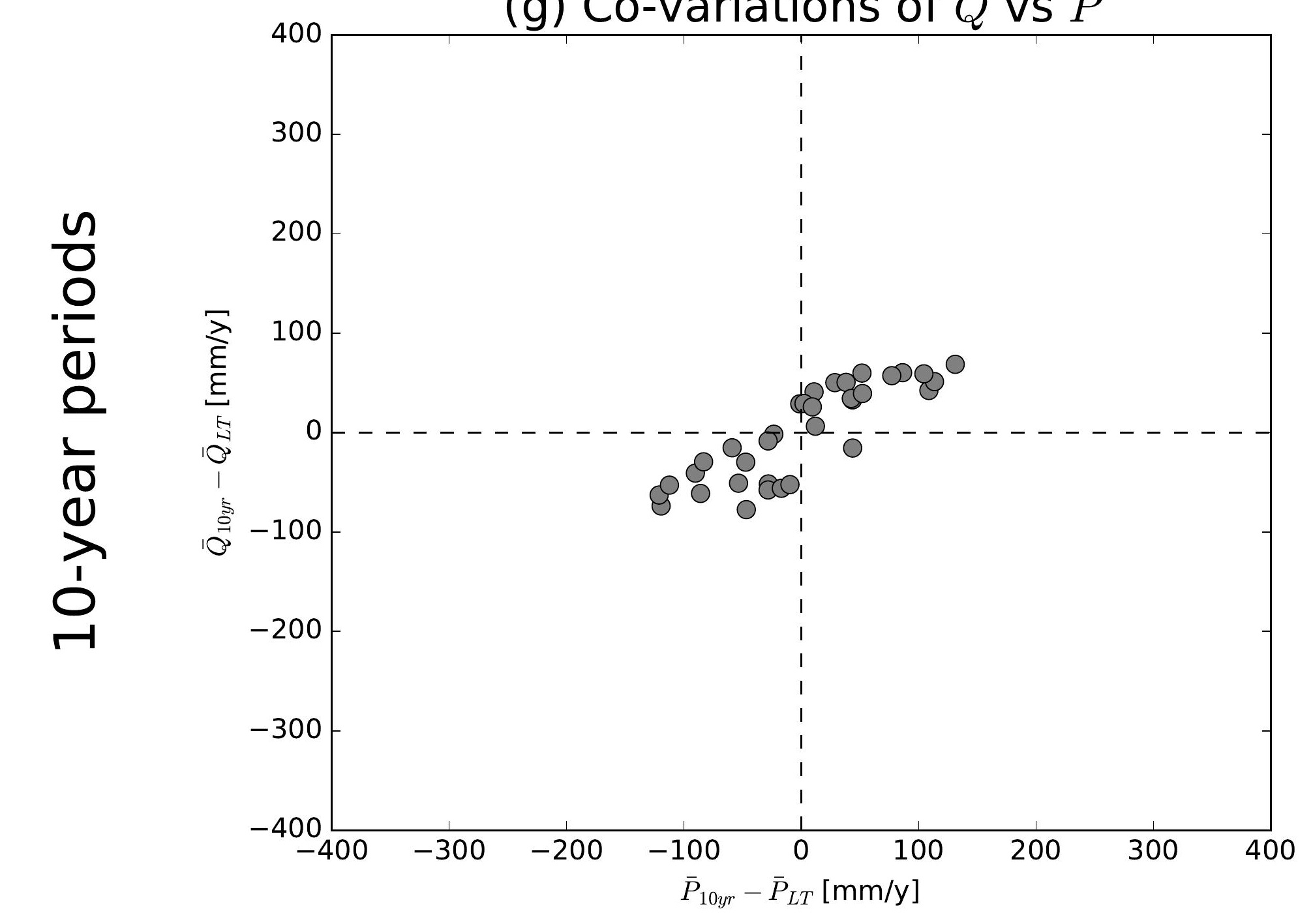

(j) Co-variations of $\bar{Q}$ vs $\bar{P}$

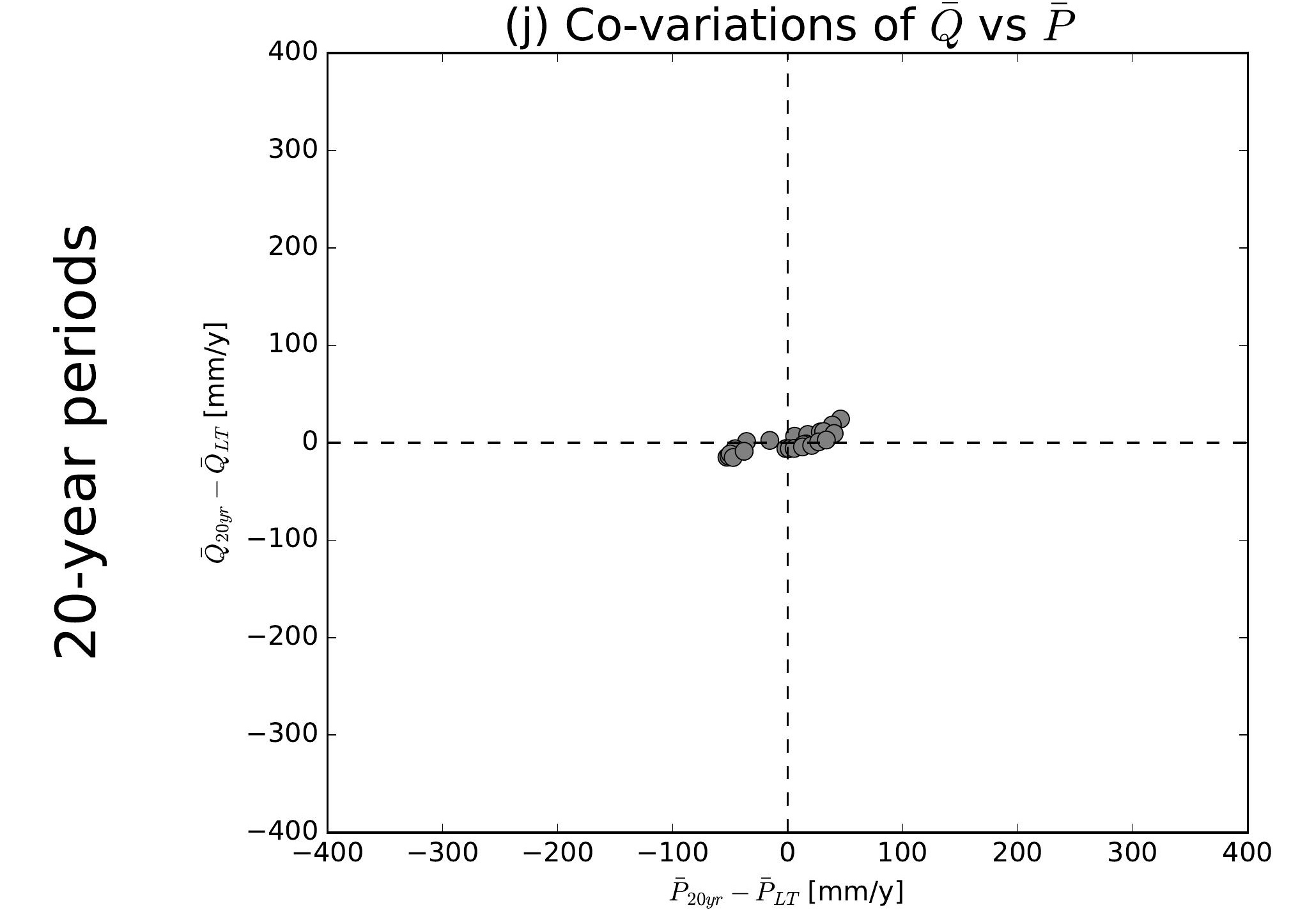

(b) Co-variations of $\bar{Q}$ vs $\overline{E 0}$

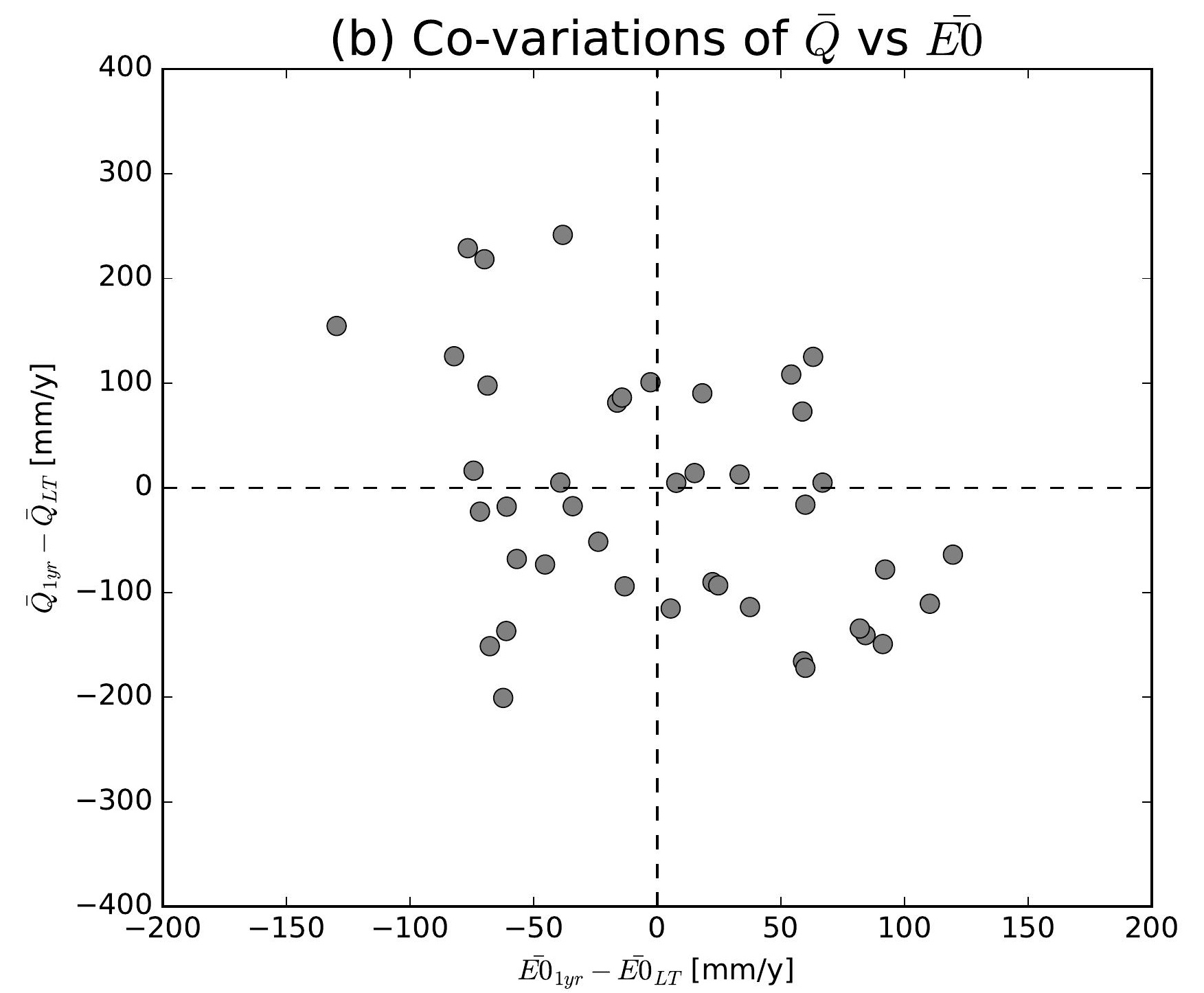

(e) Co-variations of $\bar{Q}$ vs $\overline{E 0}$

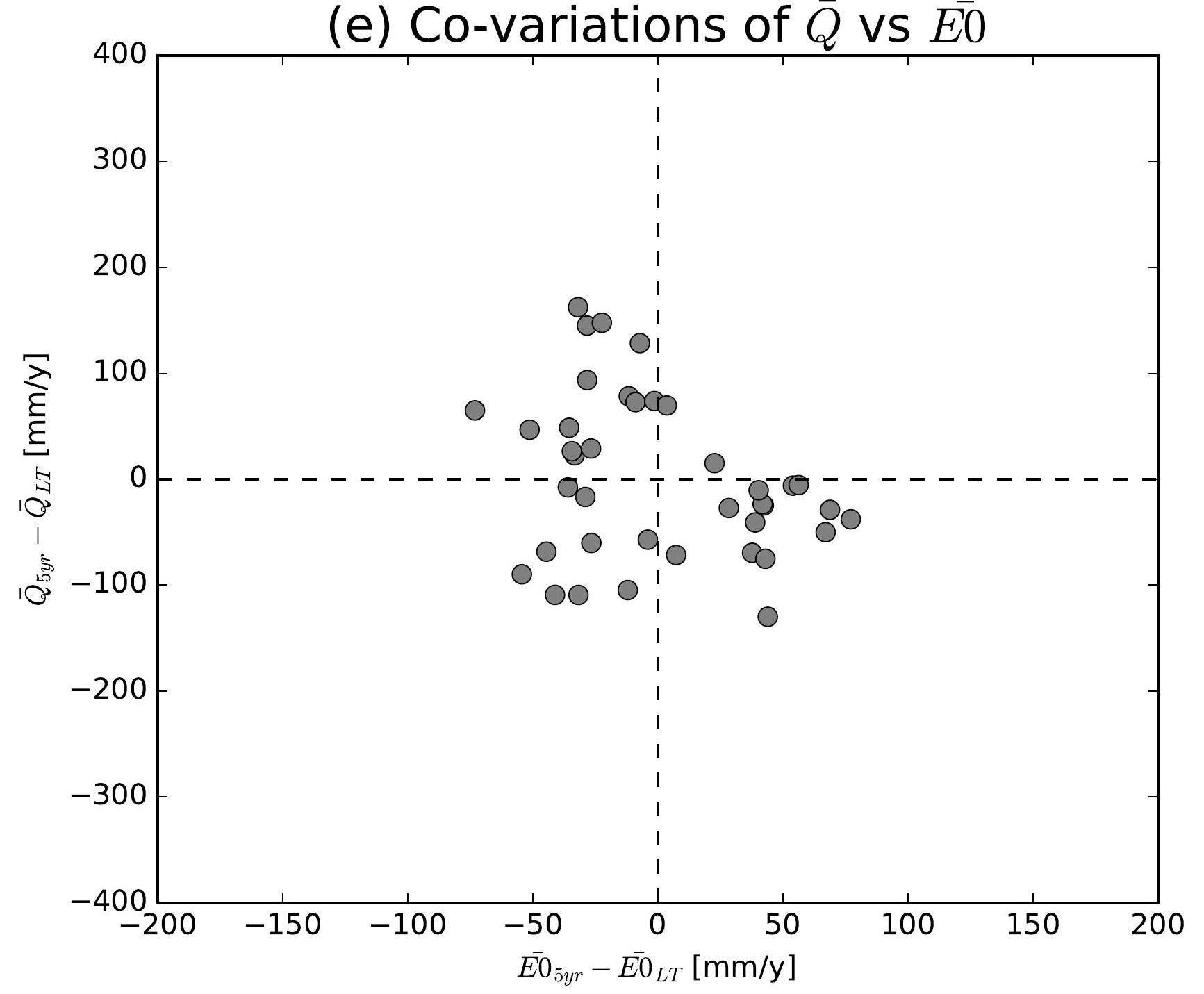

(h) Co-variations of $\bar{Q}$ vs $\overline{E 0}$

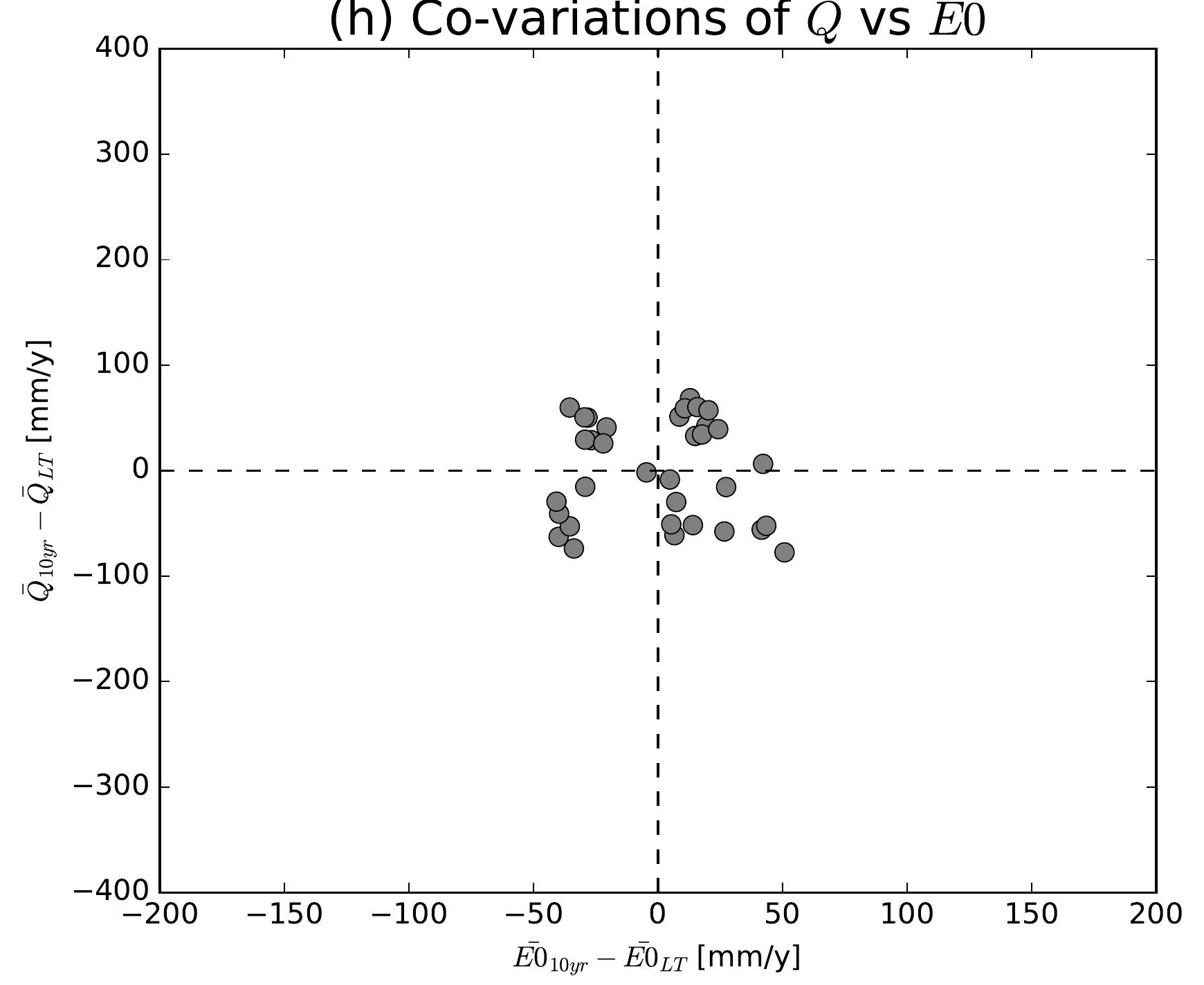

(k) Co-variations of $\bar{Q}$ vs $\overline{E 0}$

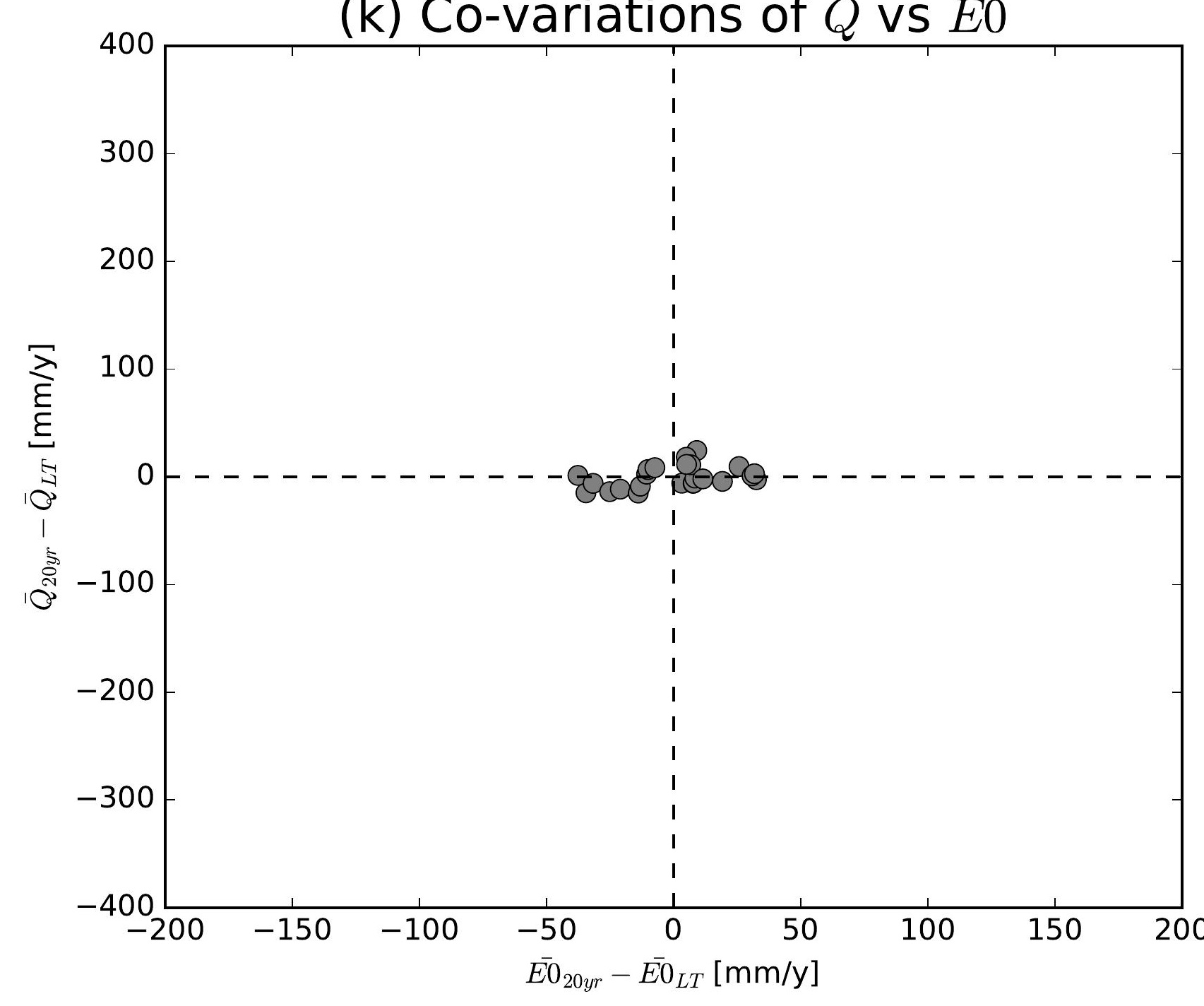

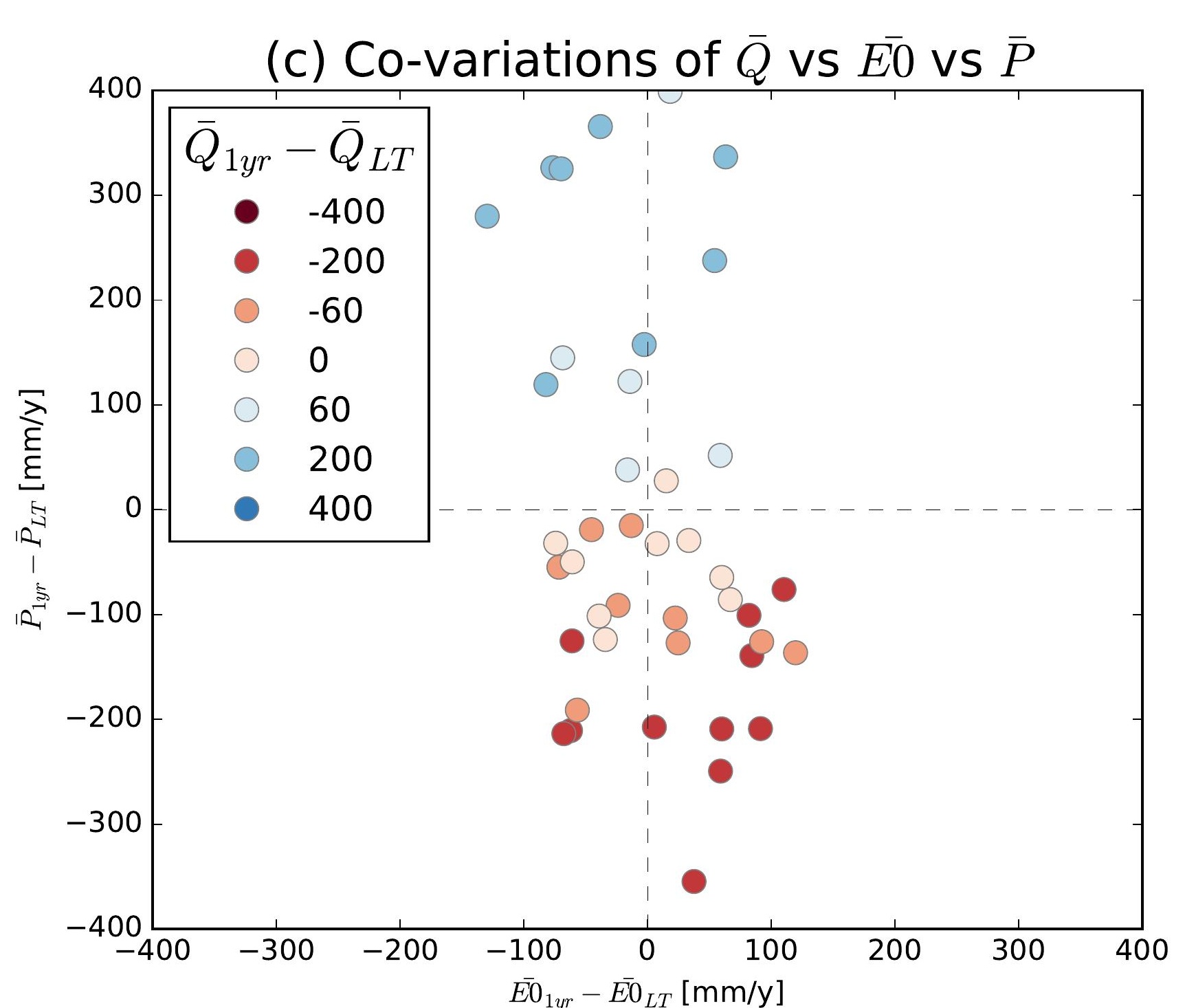
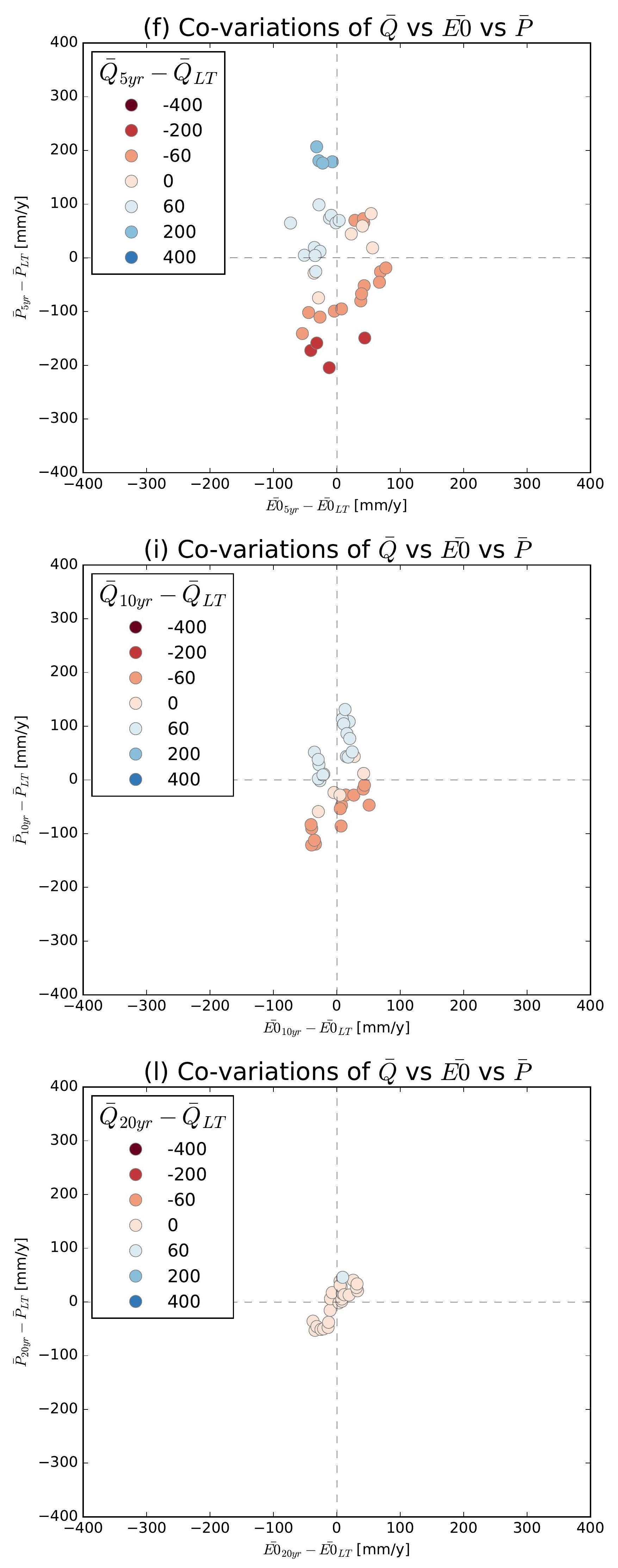


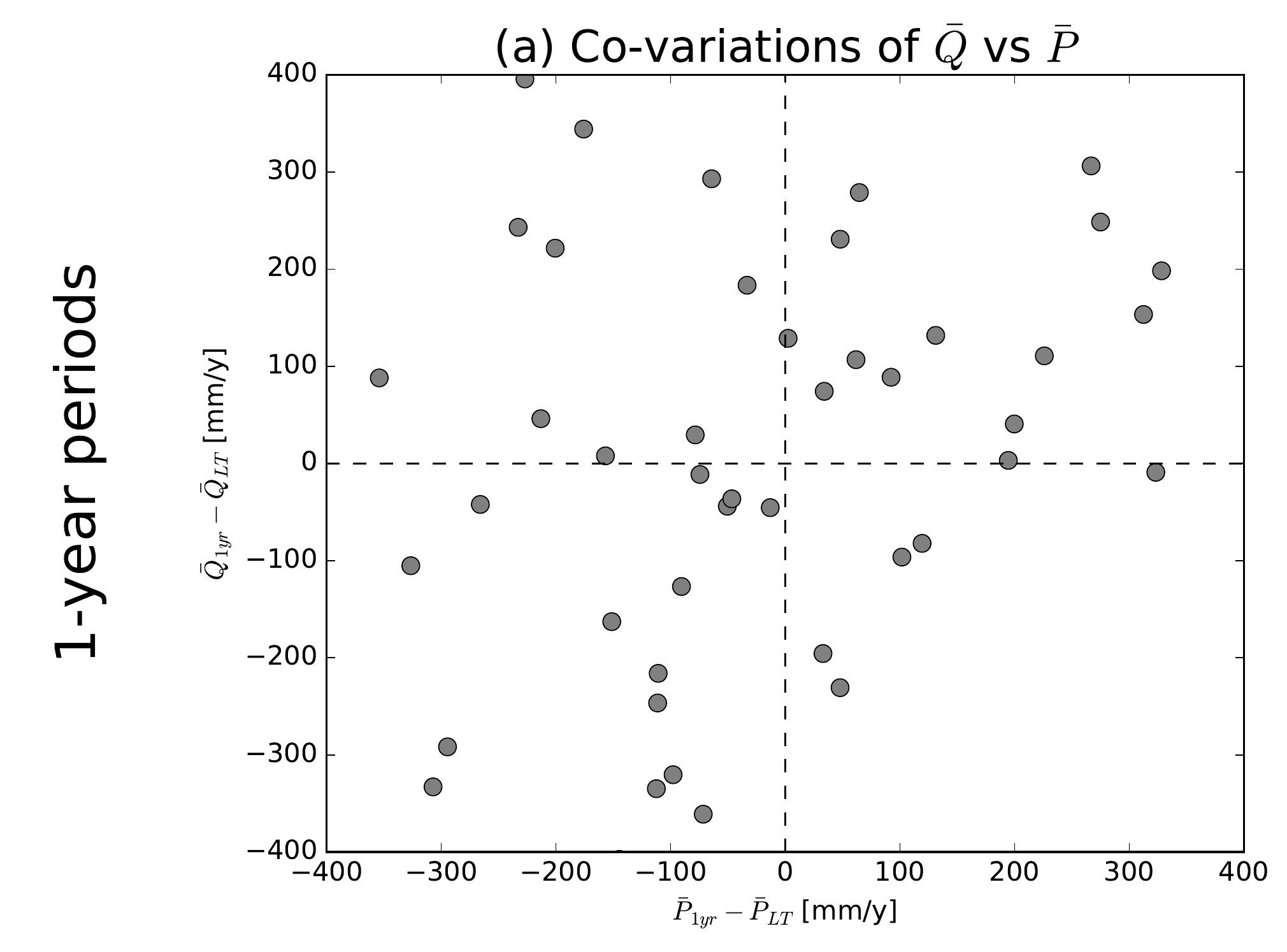

(d) Co-variations of $\bar{Q}$ vs $\bar{P}$

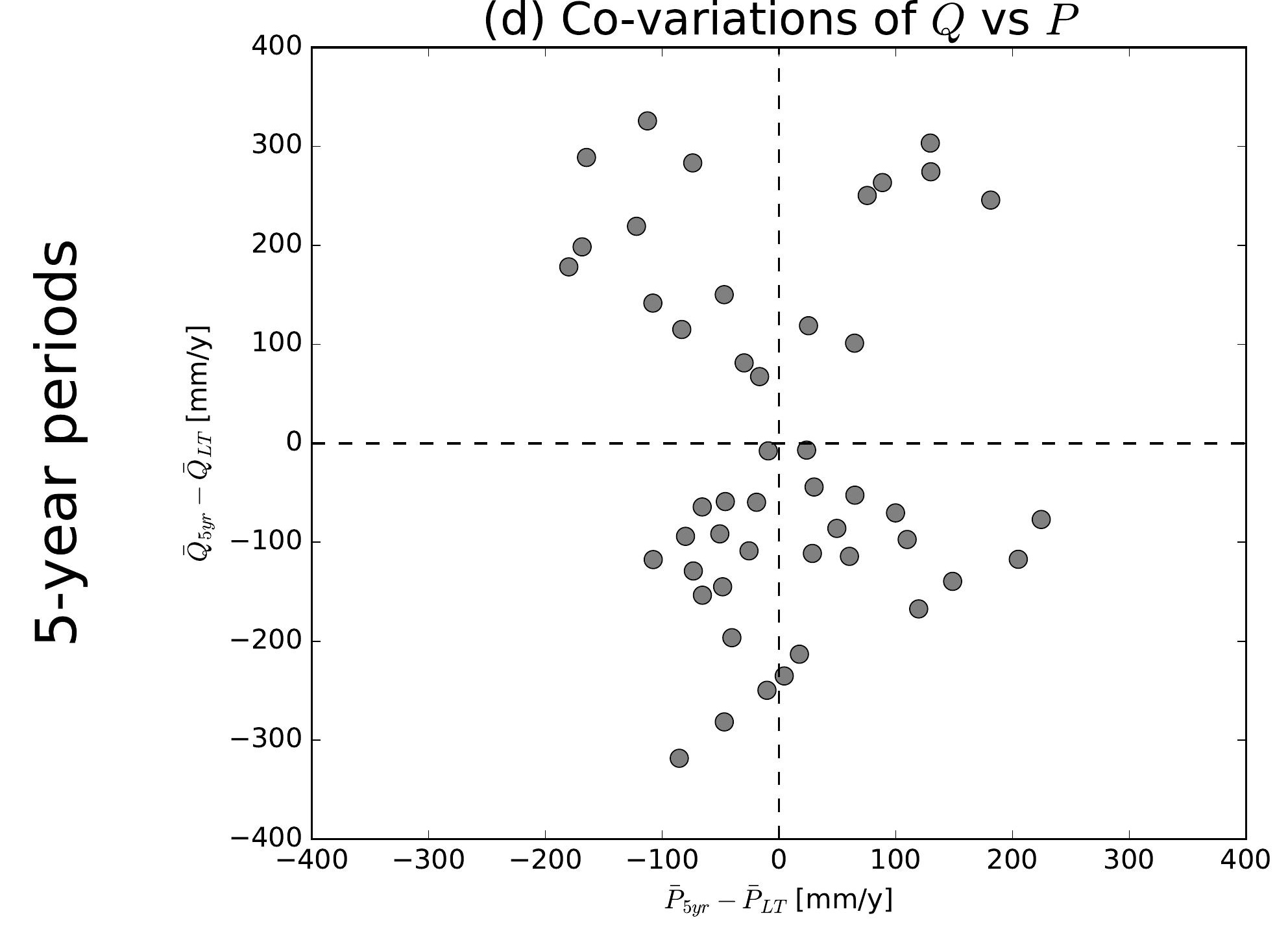

(g) Co-variations of $\bar{Q}$ vs $\bar{P}$
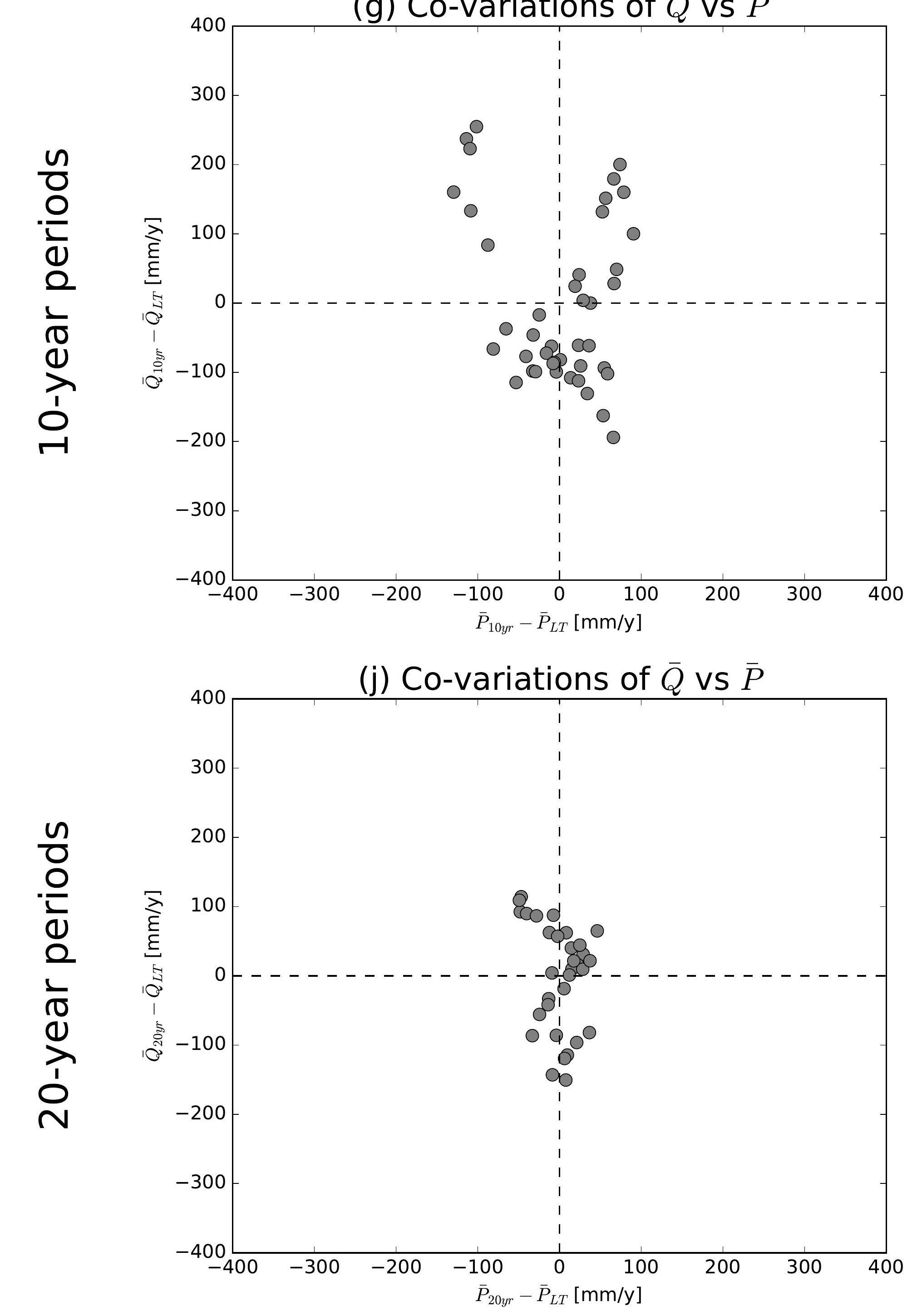

(b) Co-variations of $\bar{Q}$ vs $\overline{E 0}$

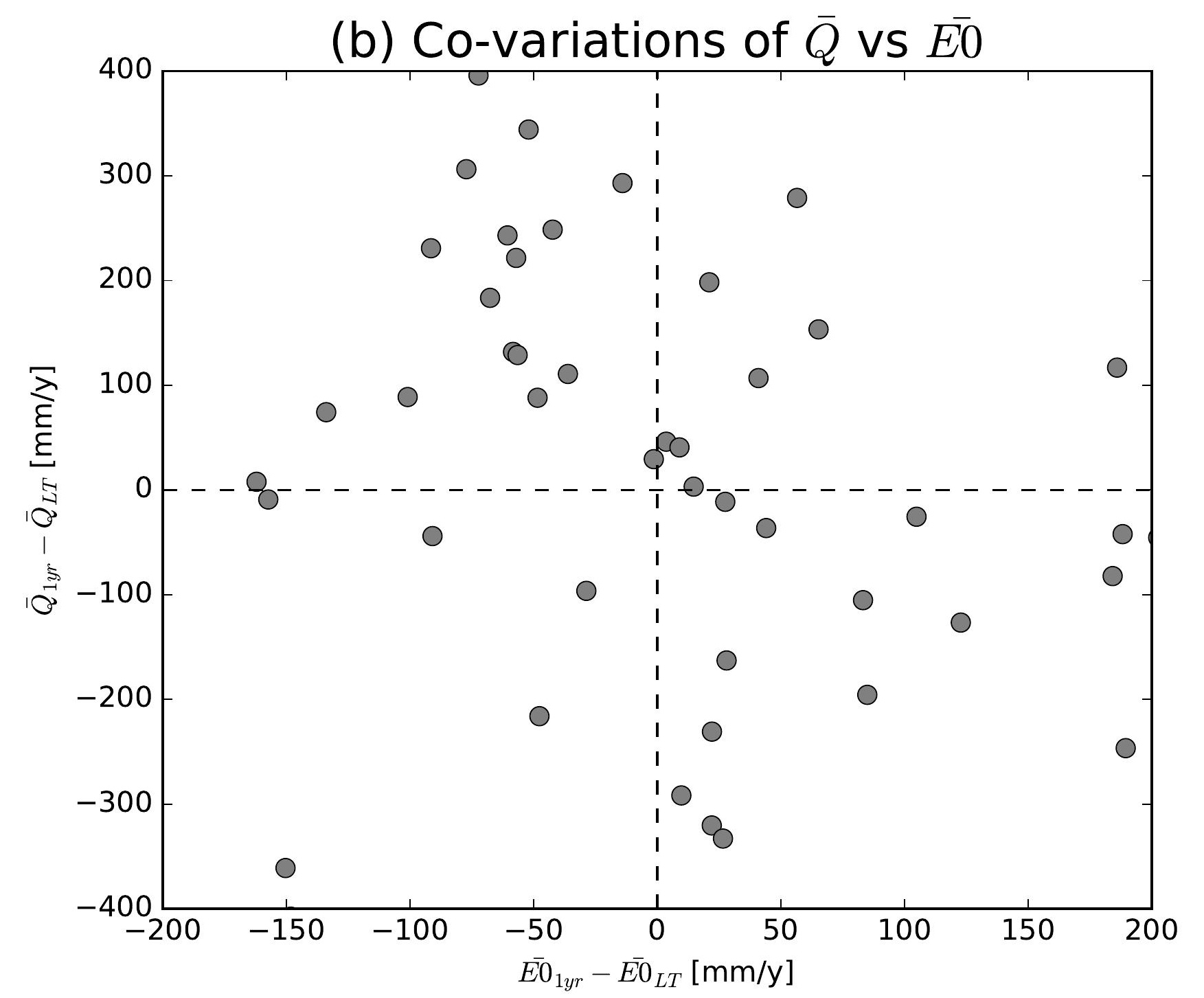

(e) Co-variations of $\bar{Q}$ vs $\overline{E 0}$

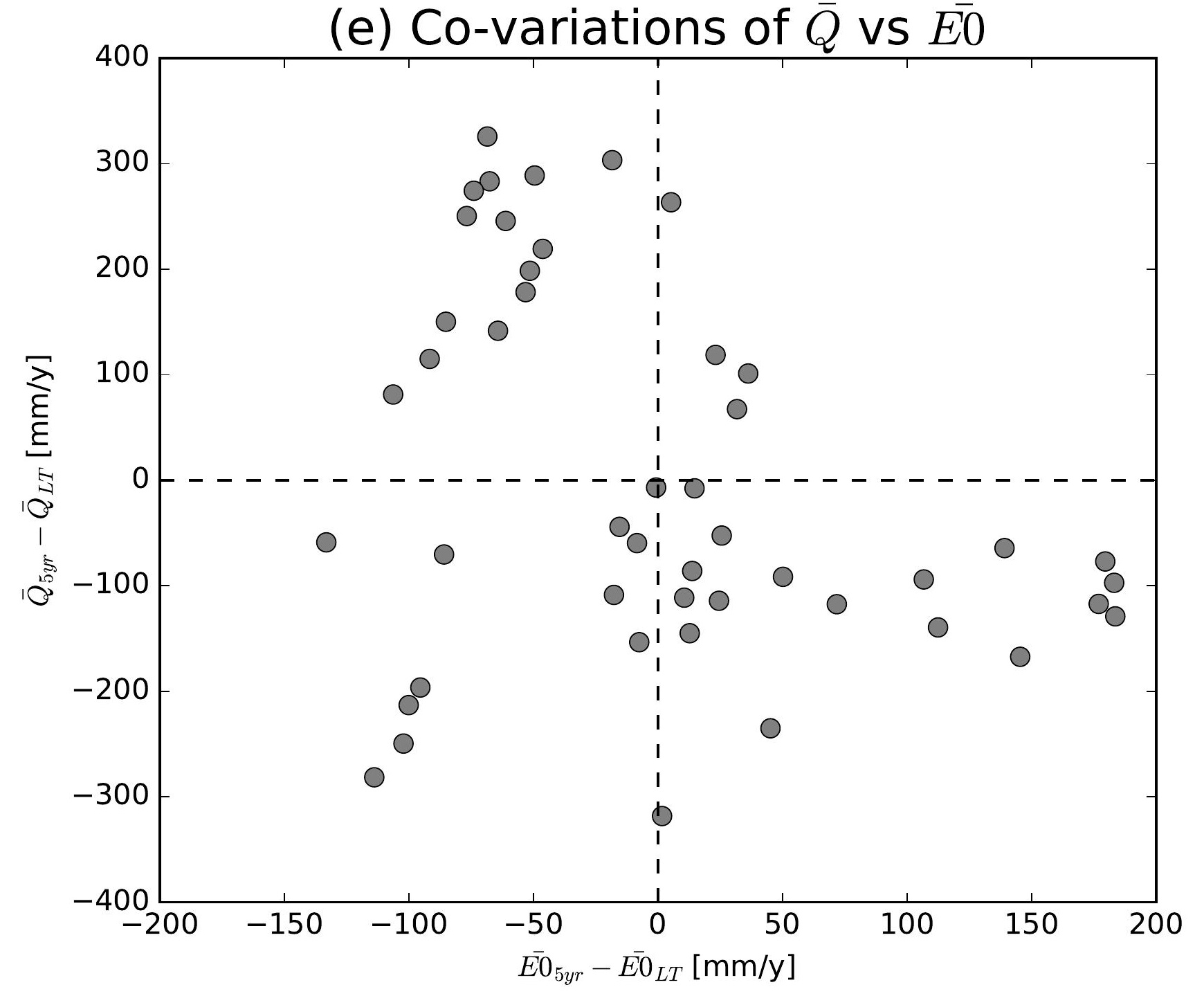

(h) Co-variations of $\bar{Q}$ vs $\overline{E 0}$

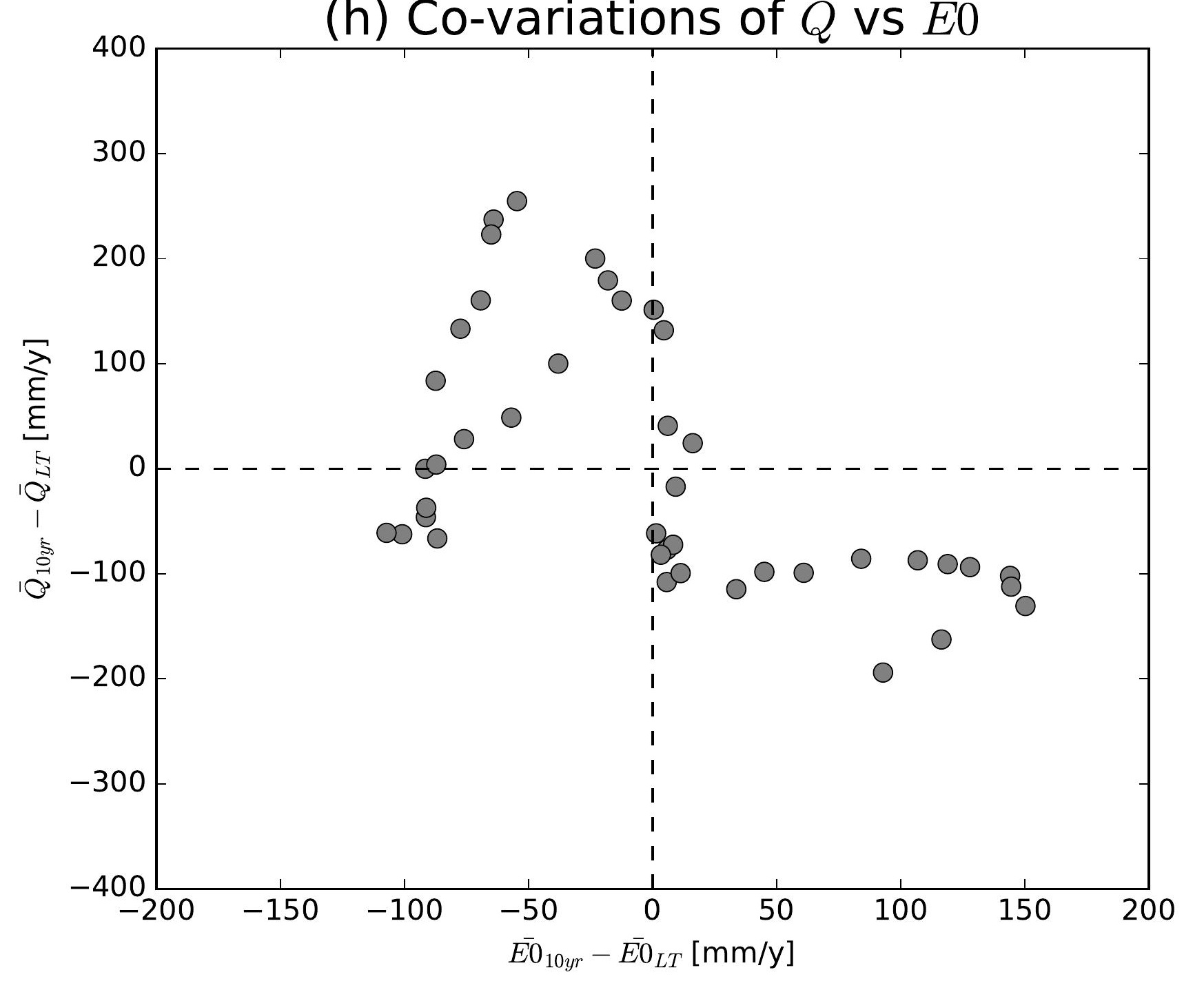

(k) Co-variations of $\bar{Q}$ vs $\overline{E 0}$

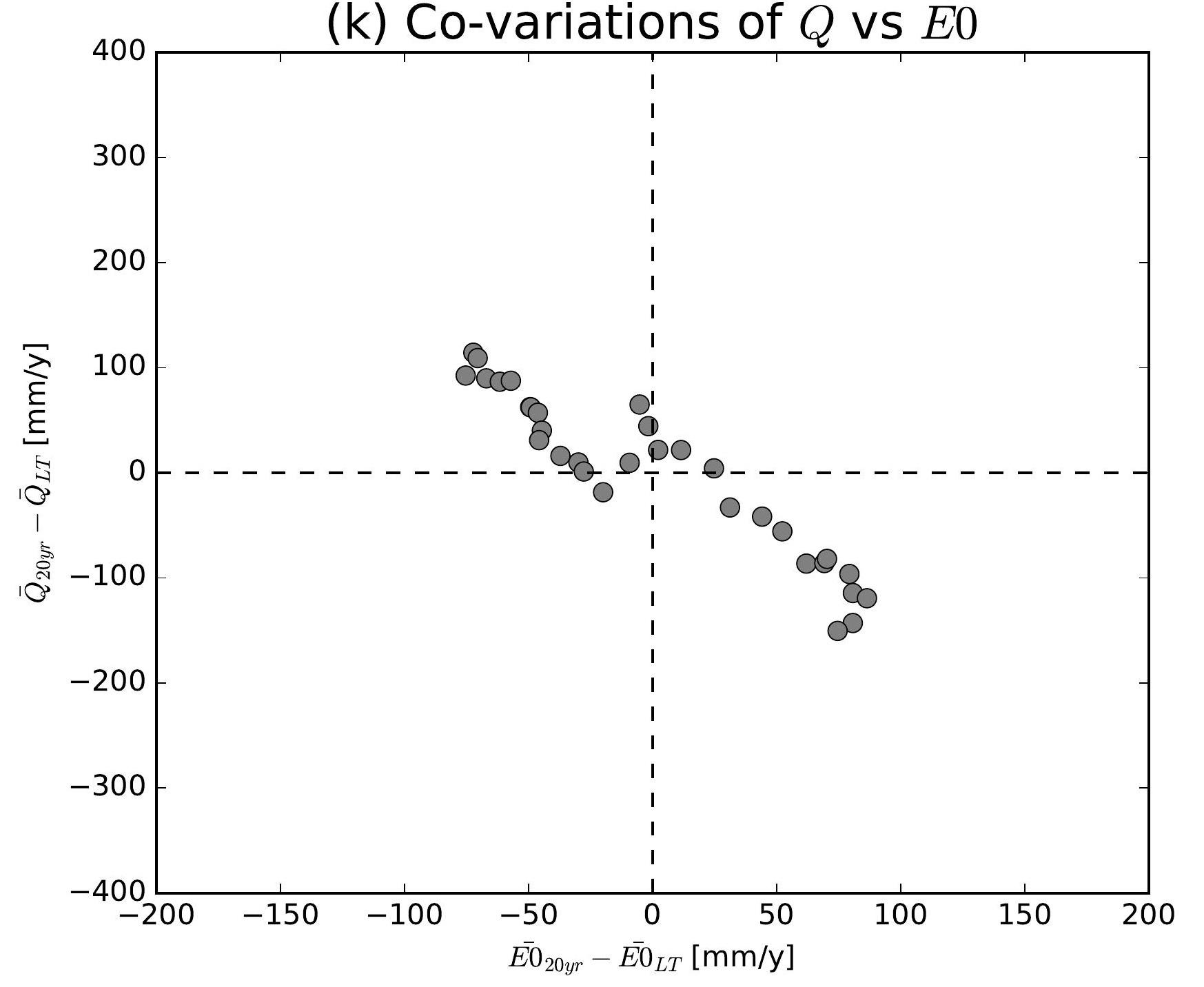

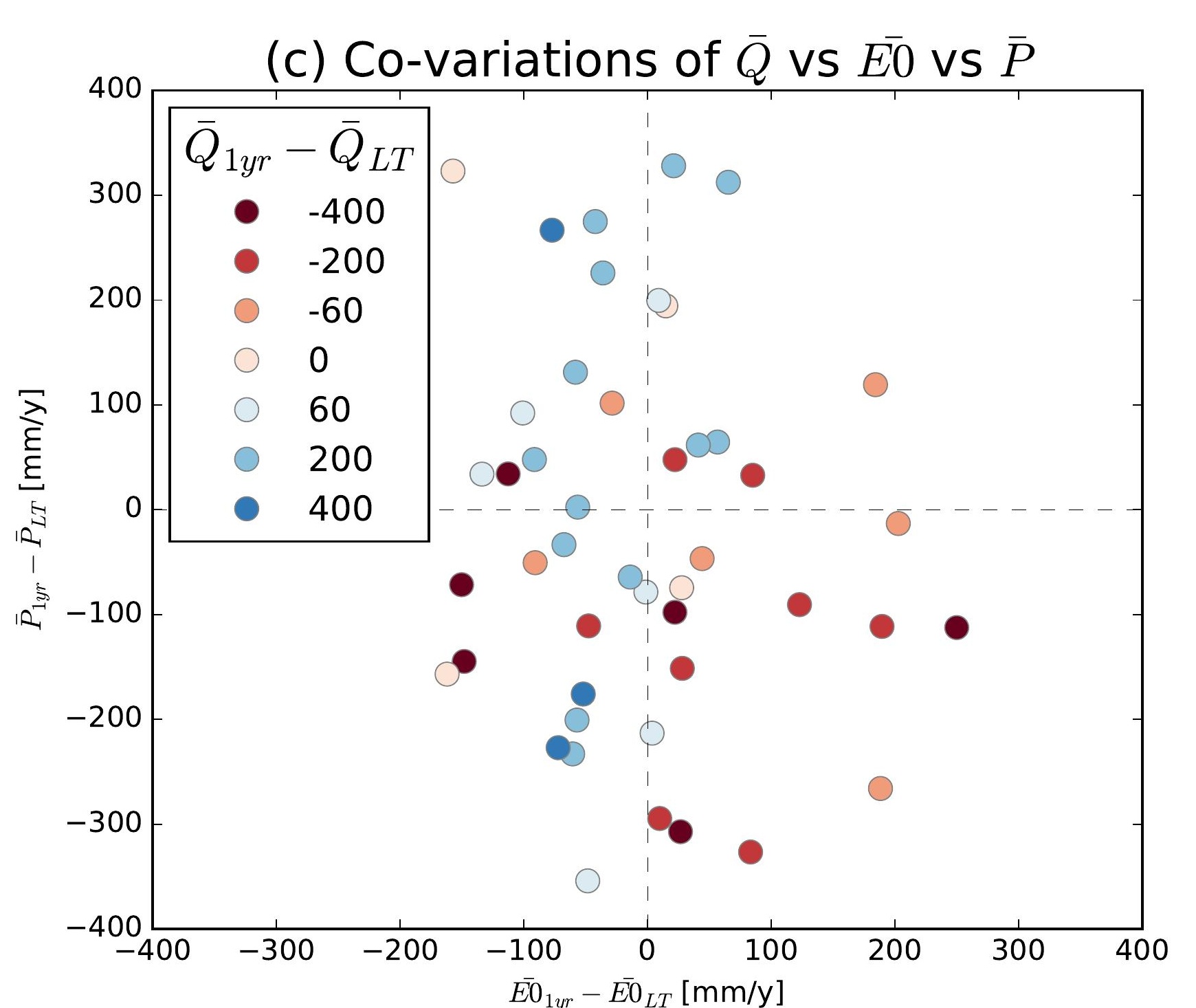
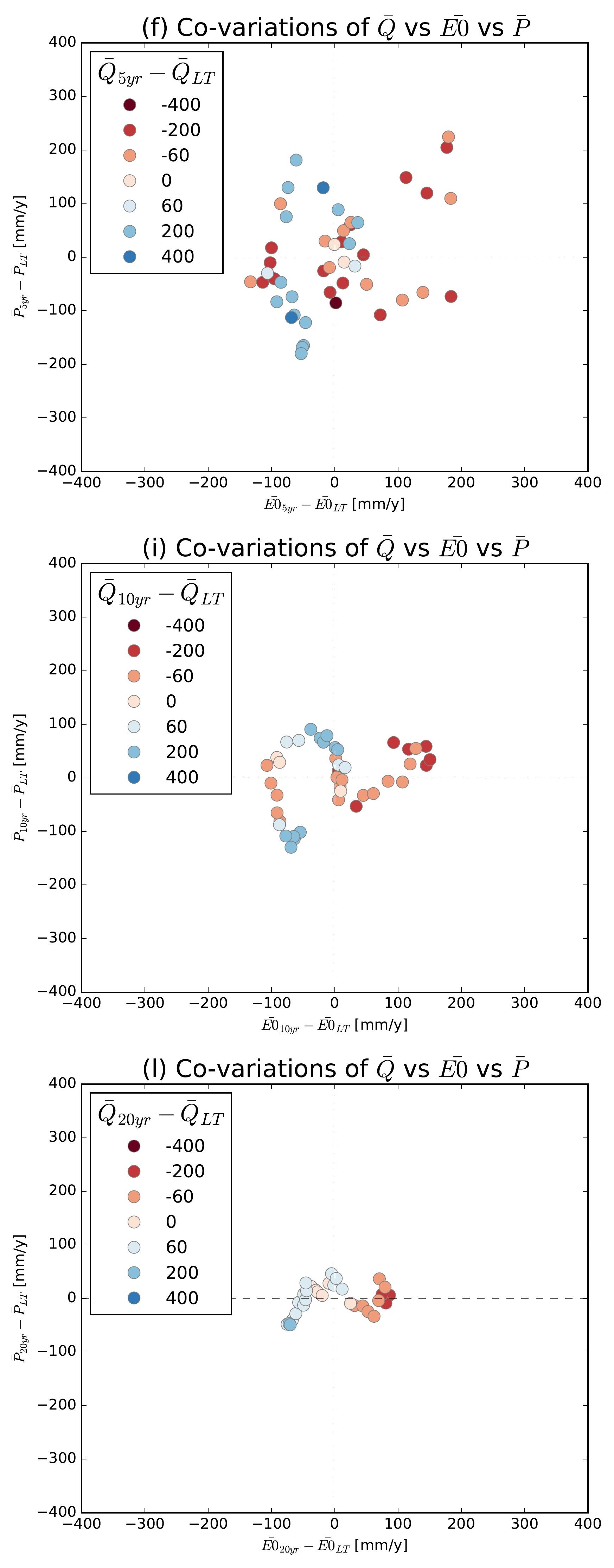

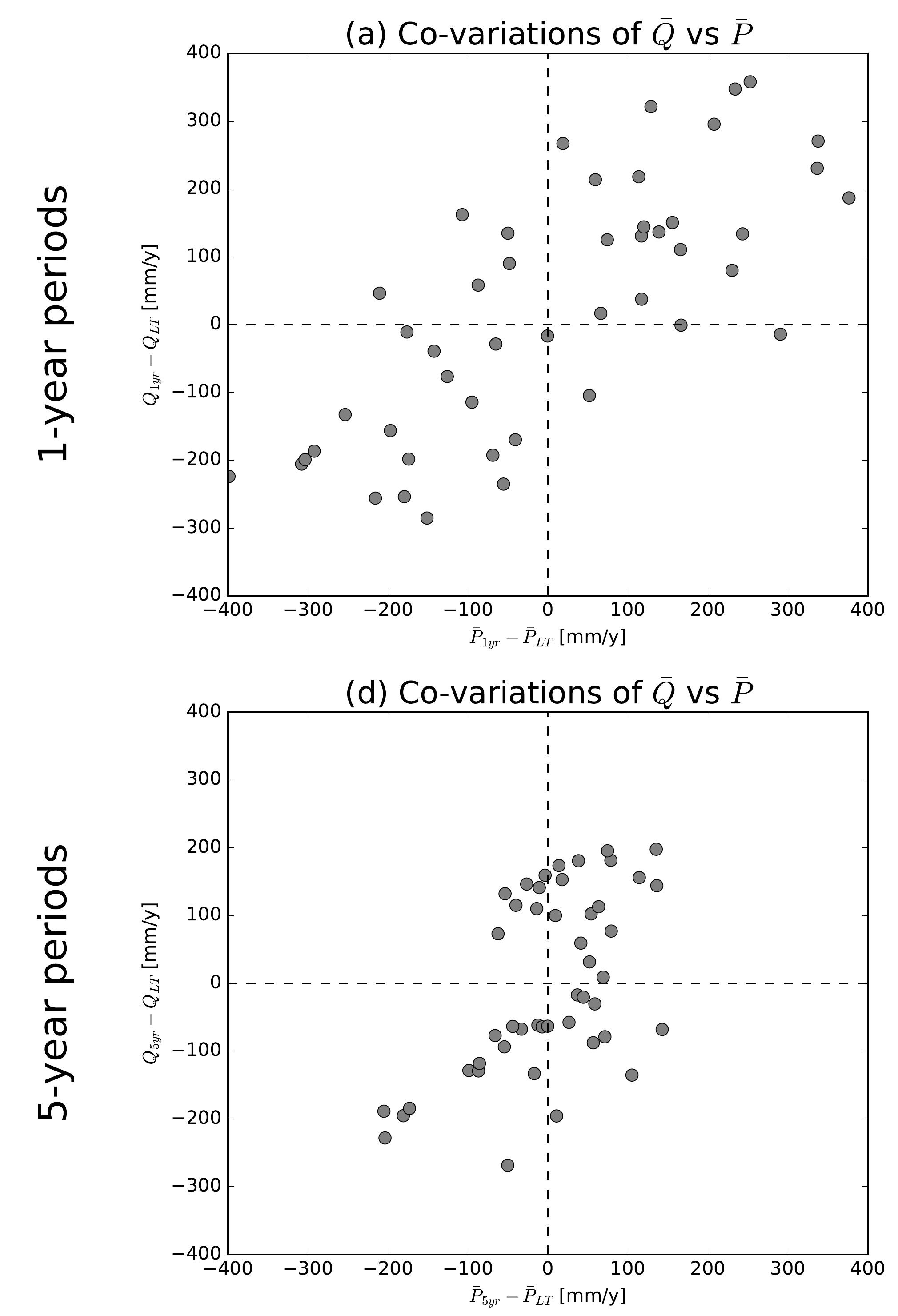

(g) Co-variations of $\bar{Q}$ vs $\bar{P}$
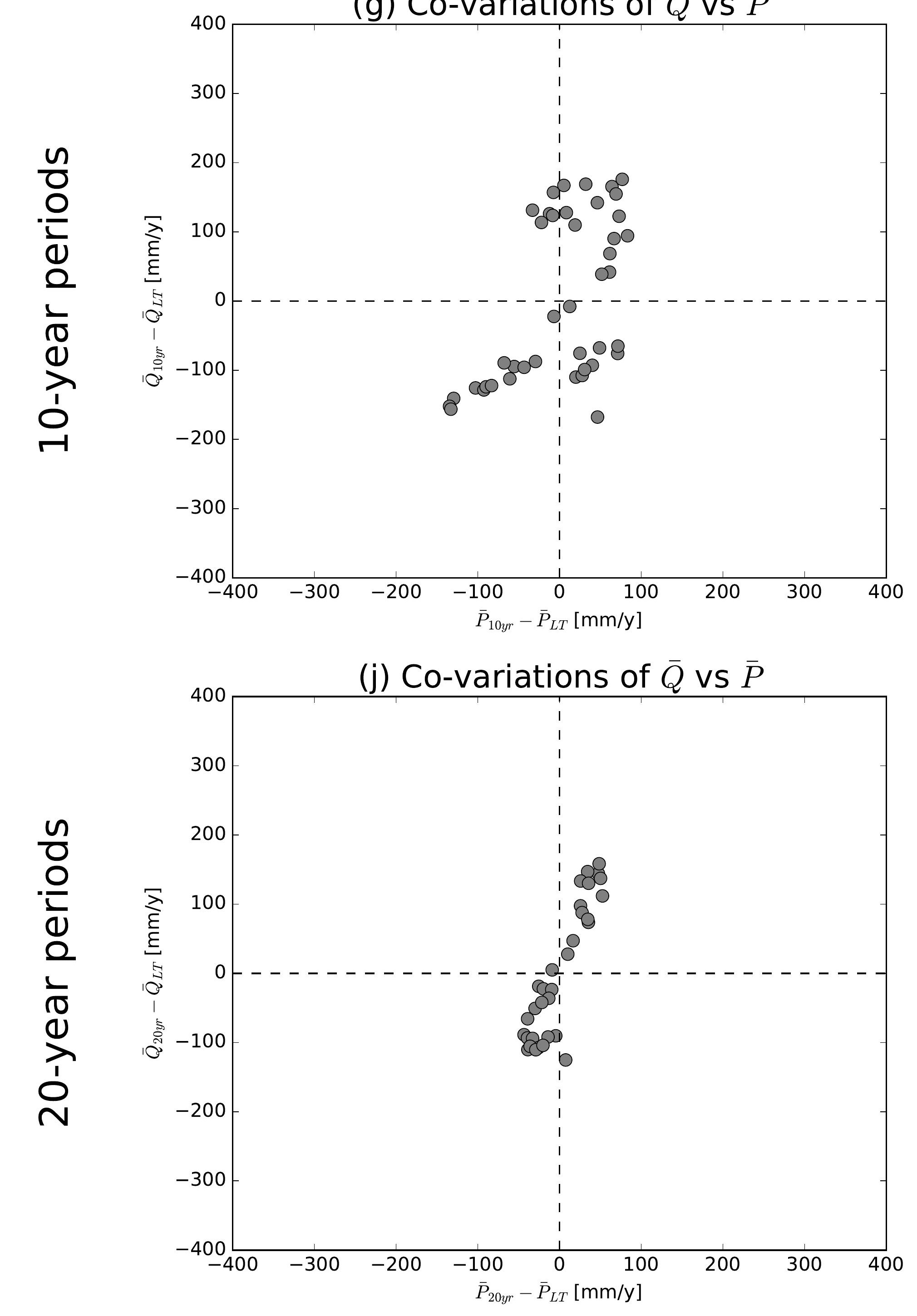

(b) Co-variations of $\bar{Q}$ vs $\overline{E 0}$

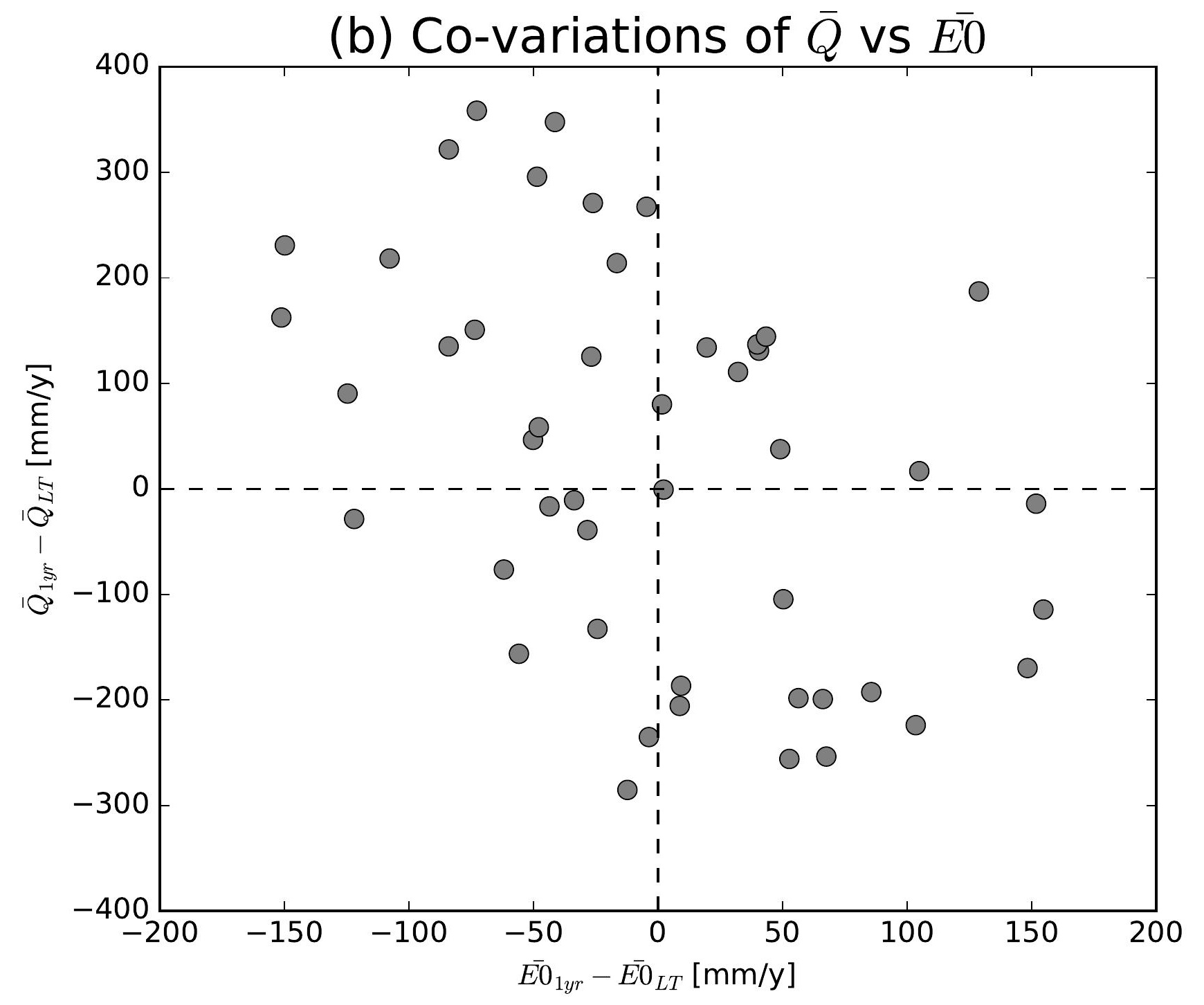

(e) Co-variations of $\bar{Q}$ vs $\overline{E 0}$

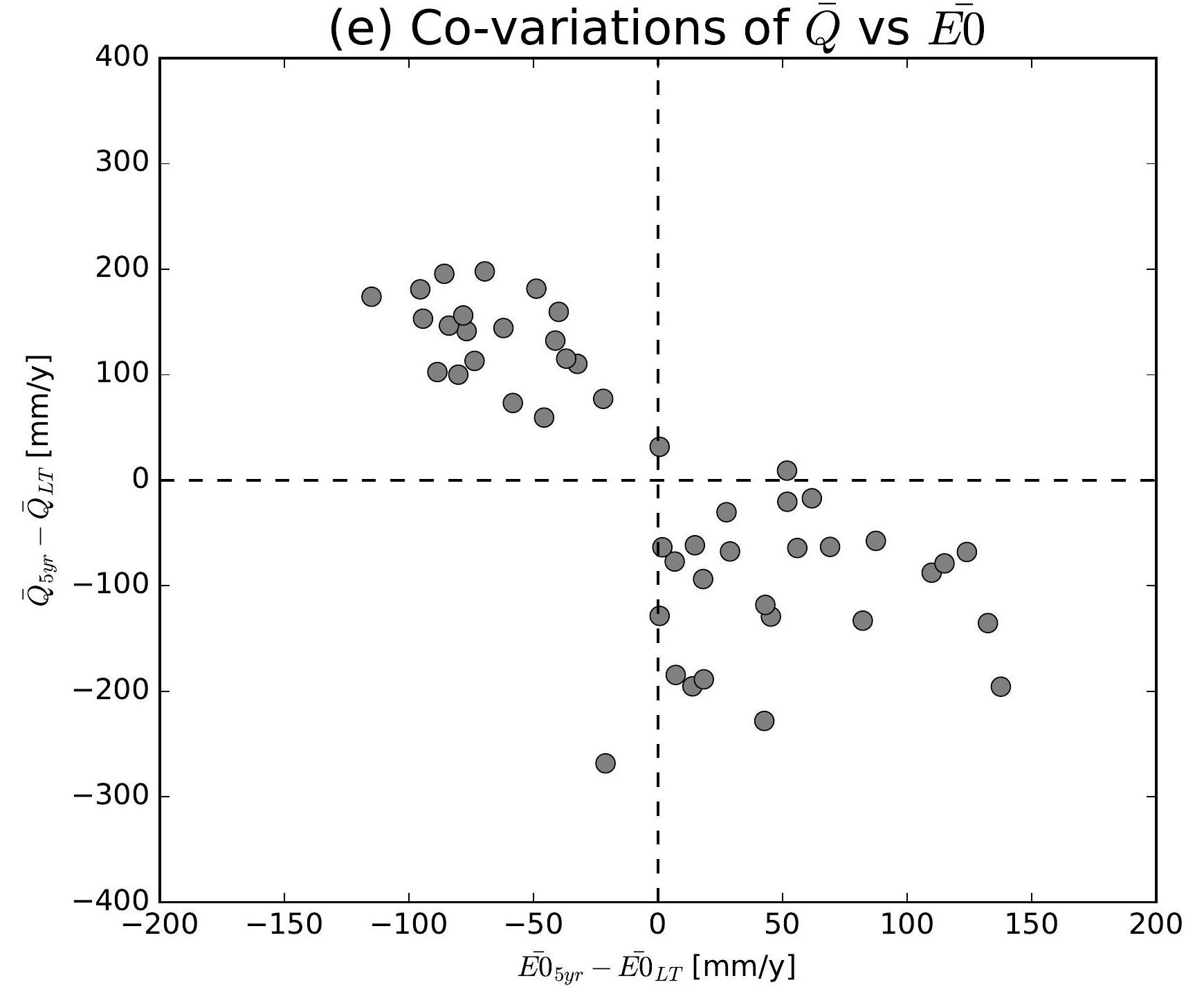

(h) Co-variations of $\bar{Q}$ vs $\overline{E 0}$

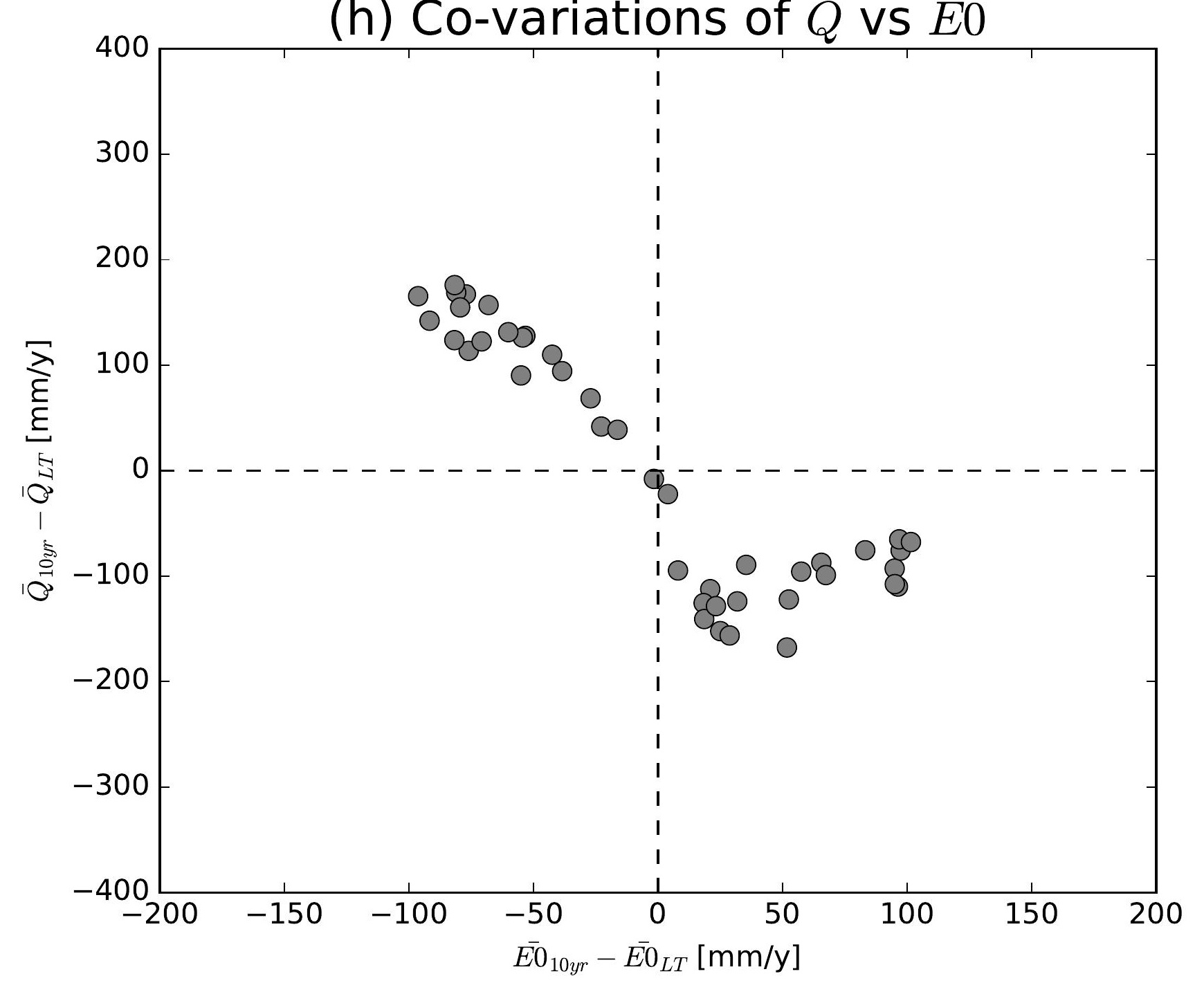

(k) Co-variations of $\bar{Q}$ vs $\overline{E 0}$

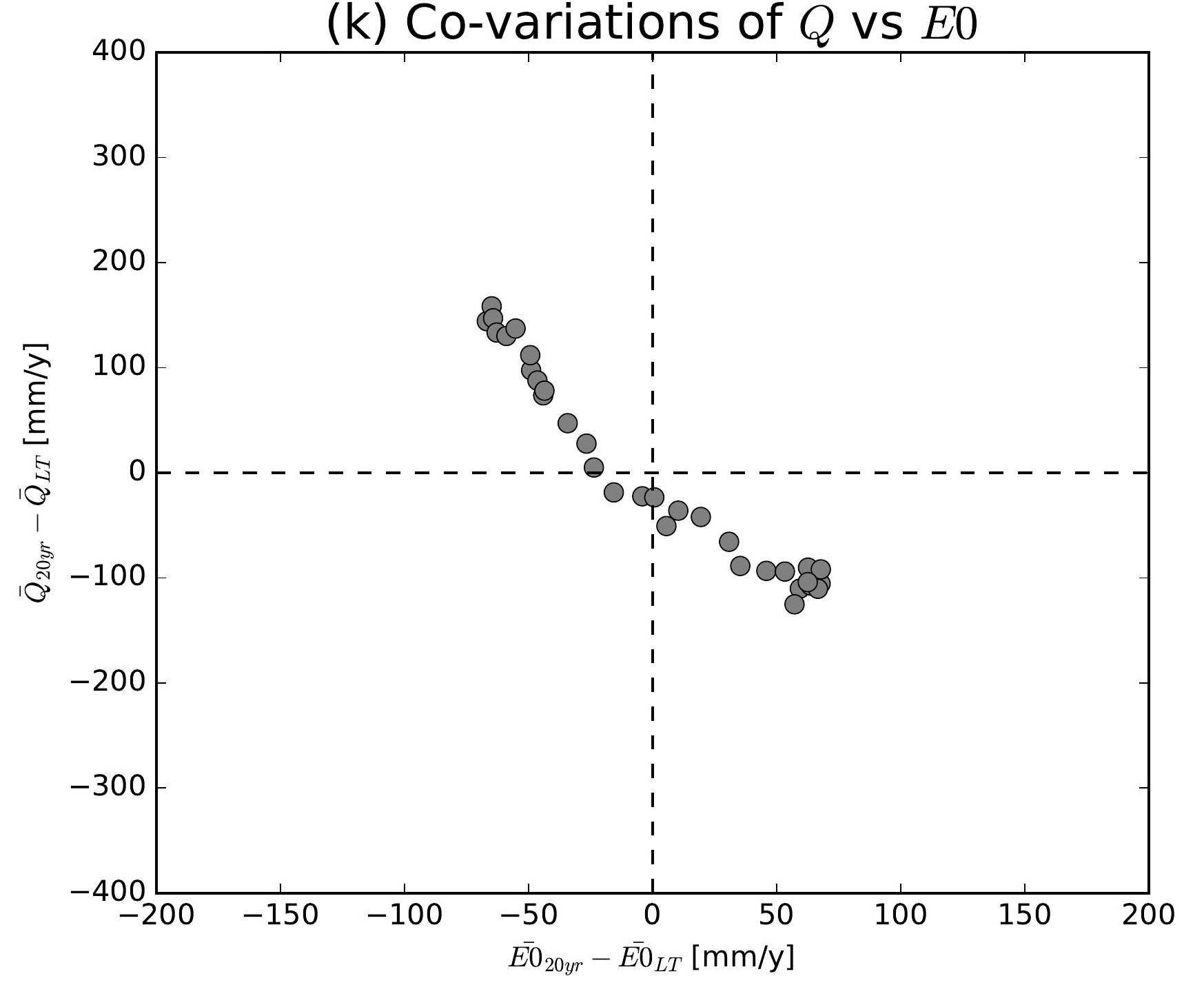

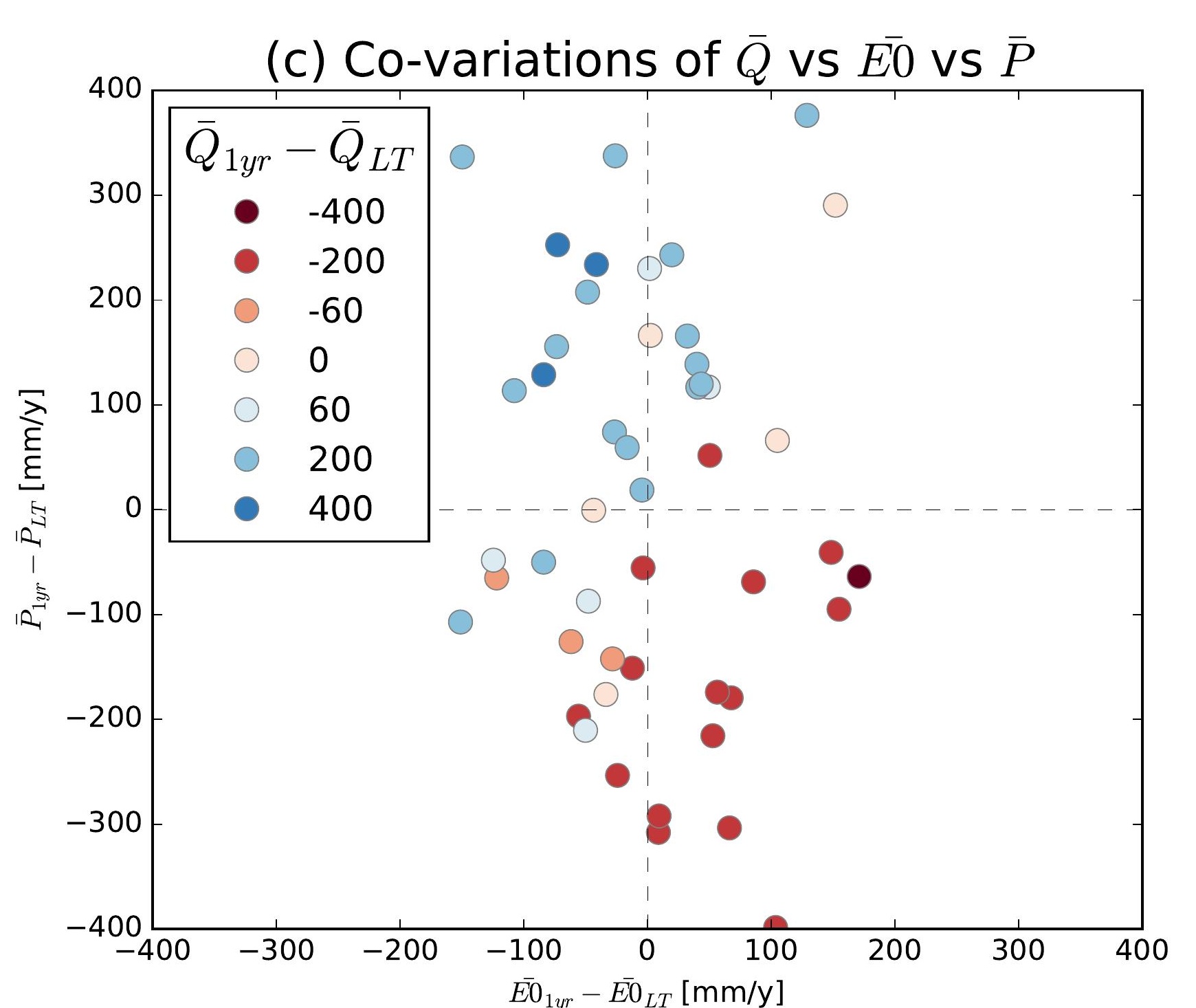
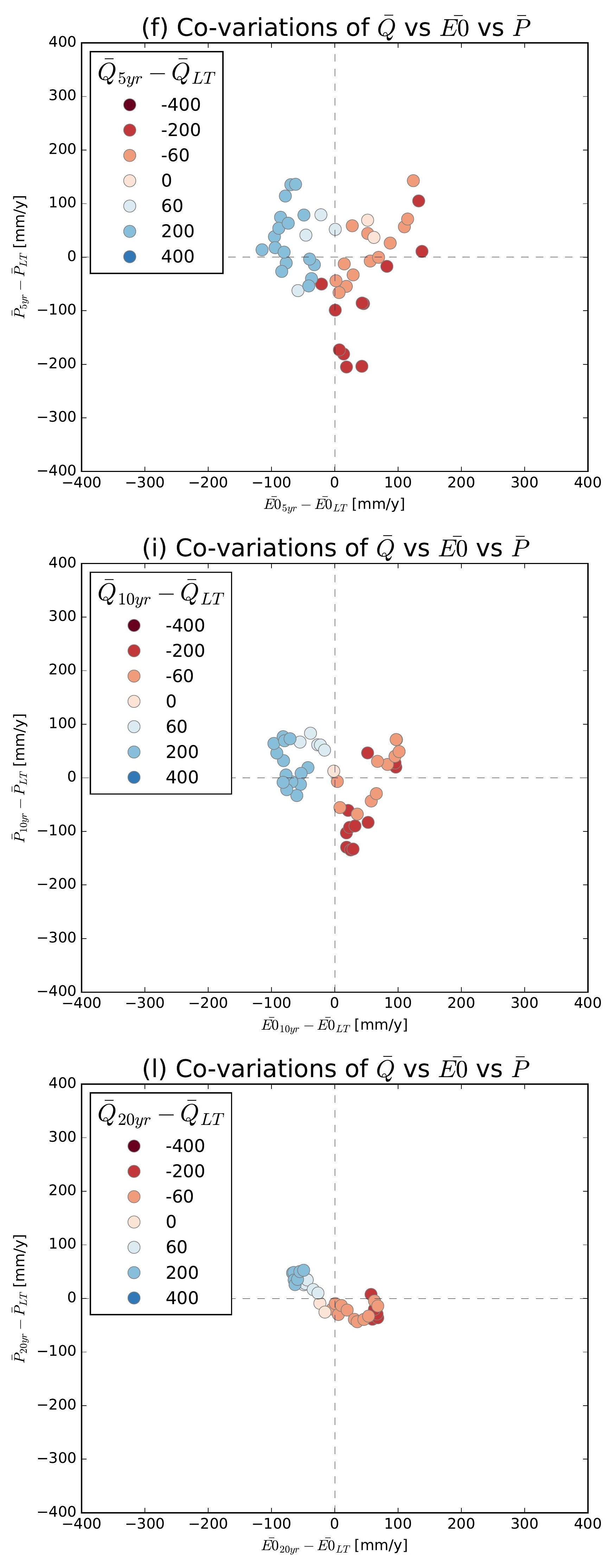

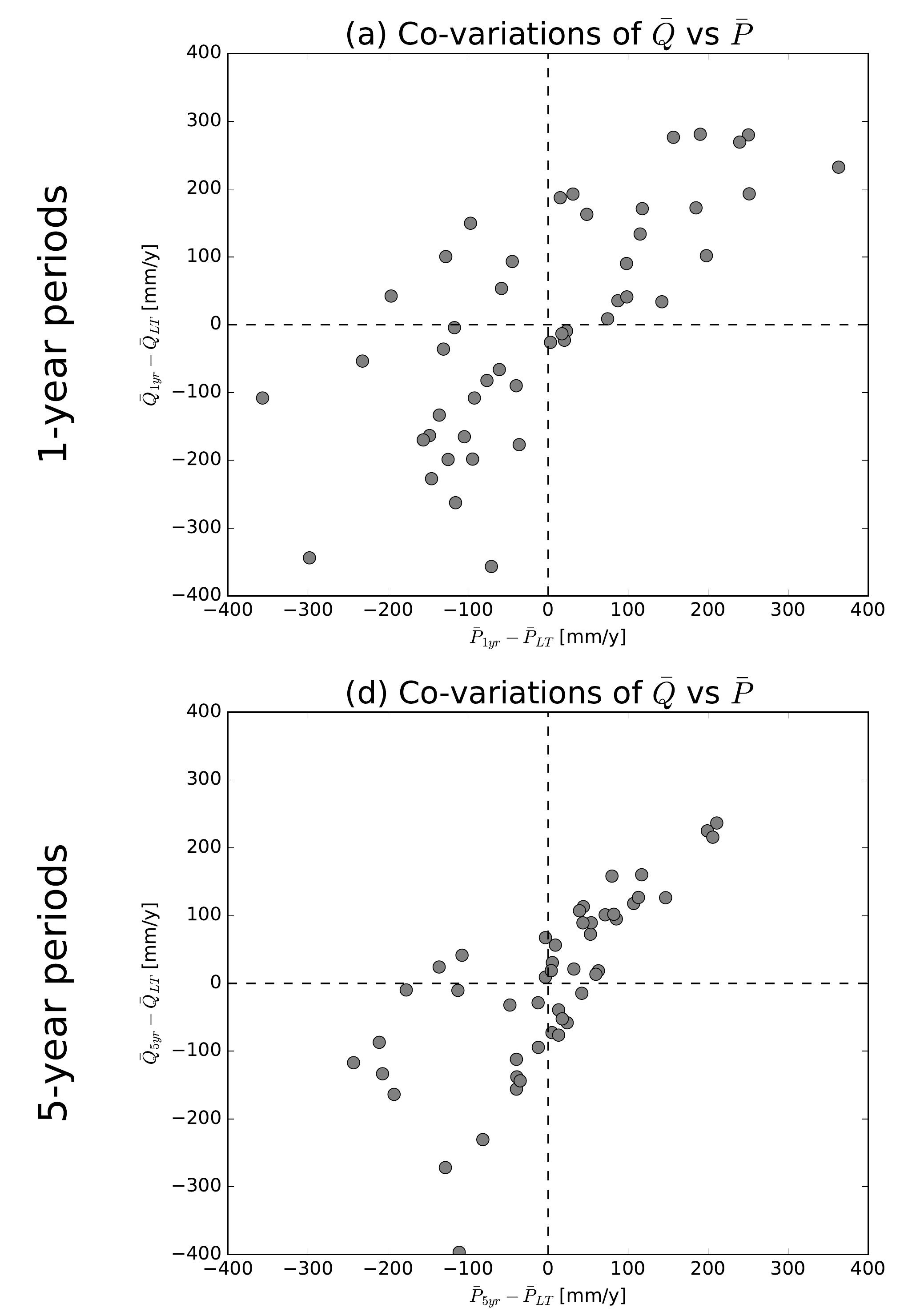

(g) Co-variations of $\bar{Q}$ vs $\bar{P}$

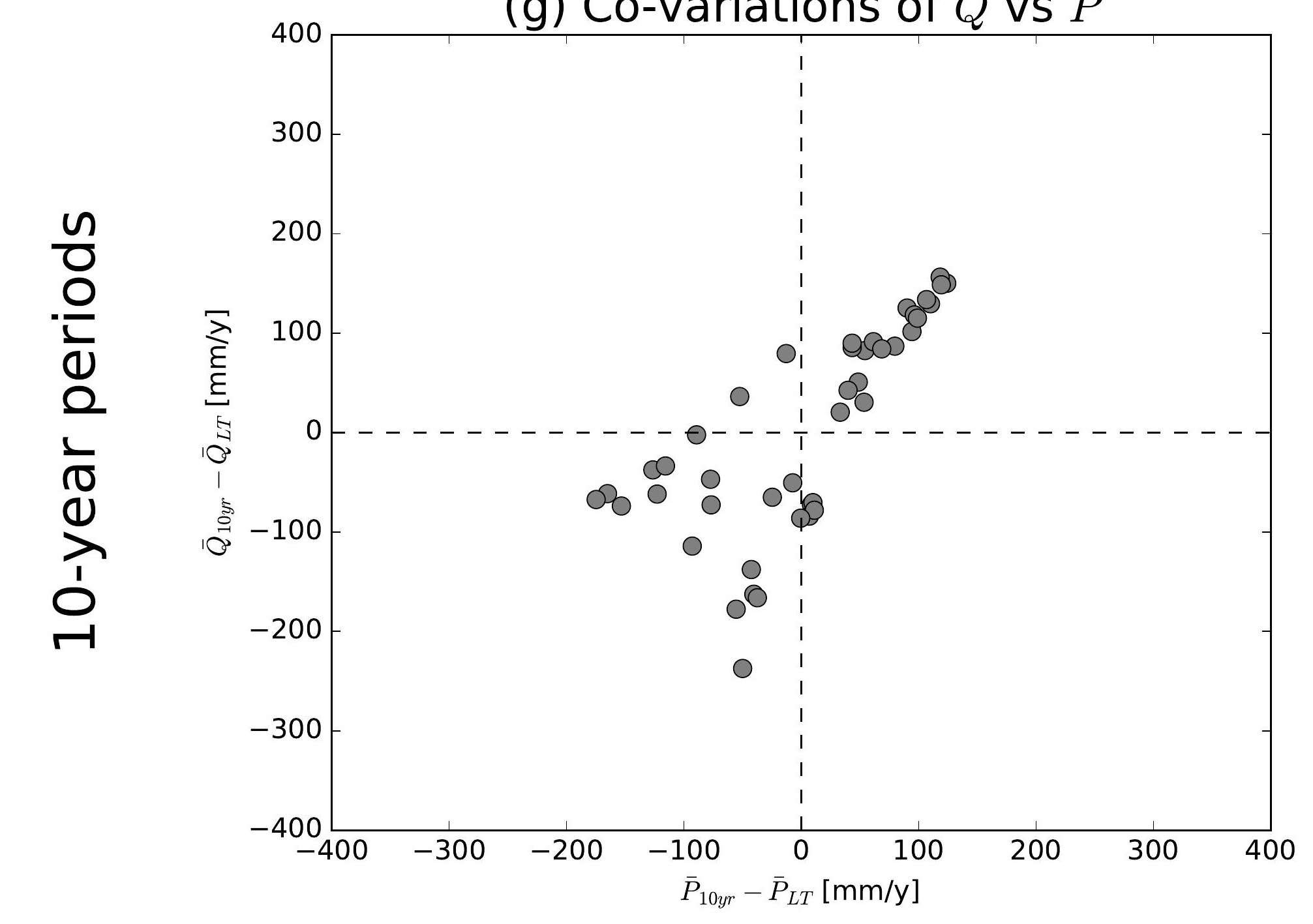

(j) Co-variations of $\bar{Q}$ vs $\bar{P}$

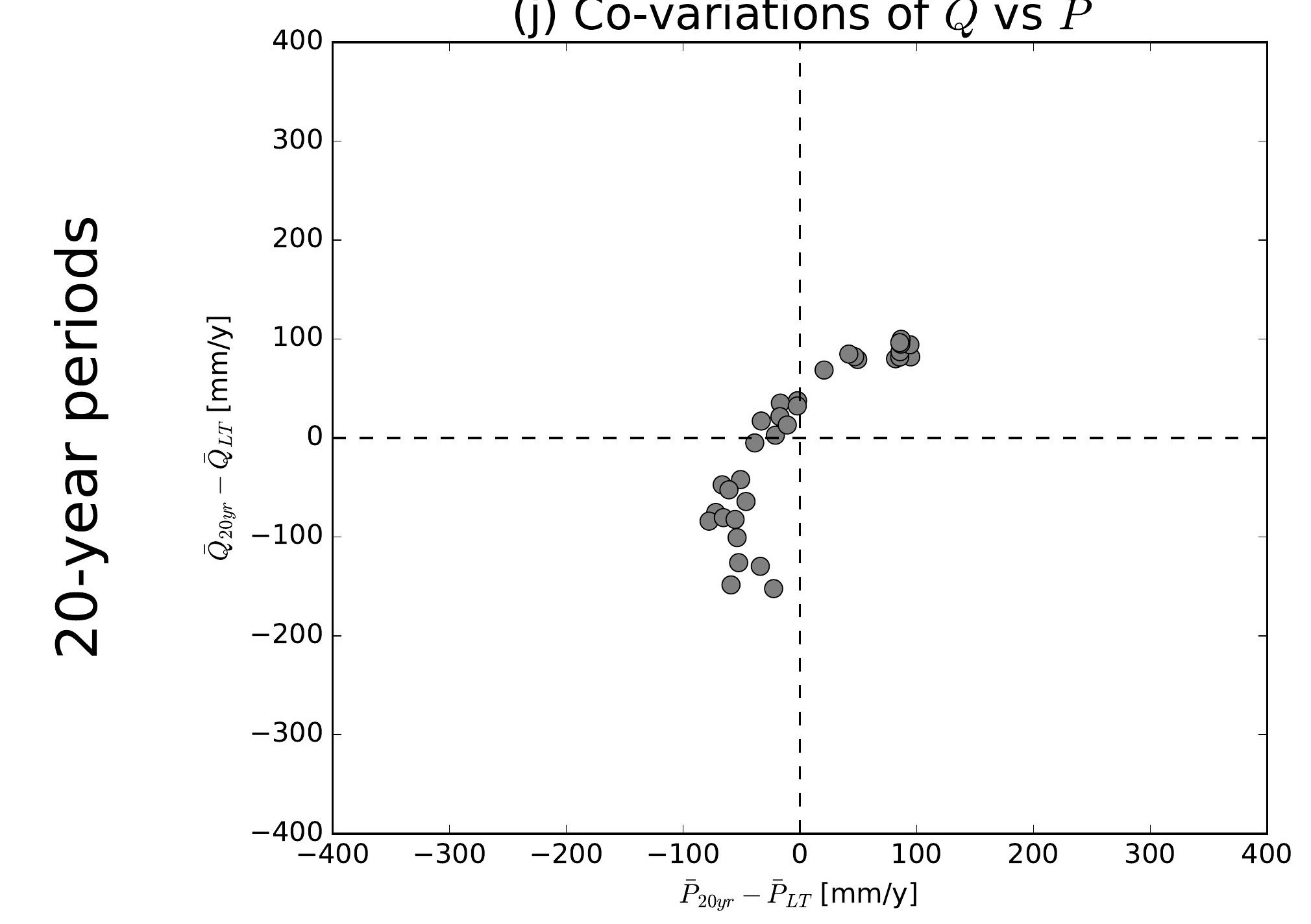

(b) Co-variations of $\bar{Q}$ vs $\overline{E 0}$

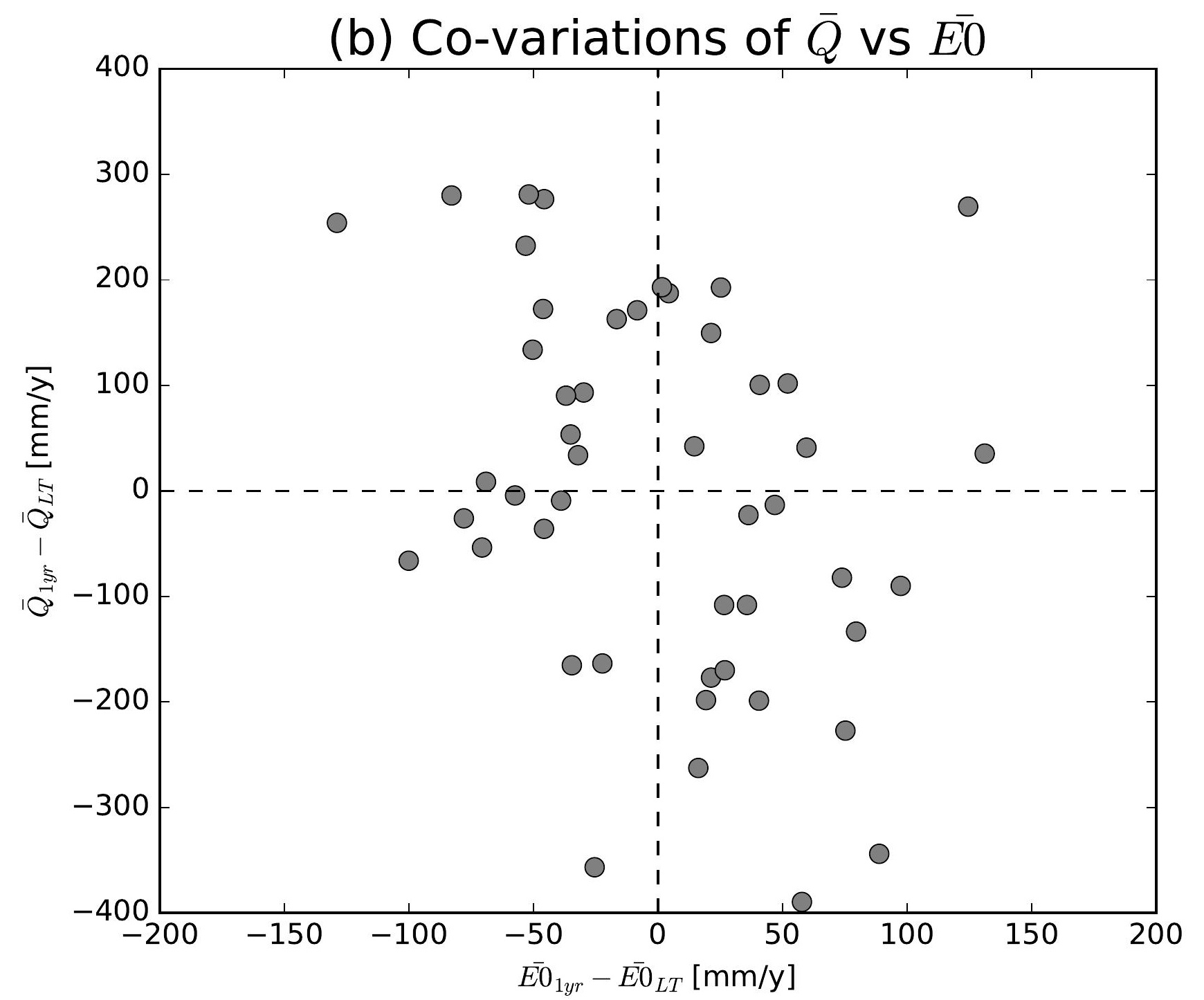

(e) Co-variations of $\bar{Q}$ vs $\overline{E 0}$

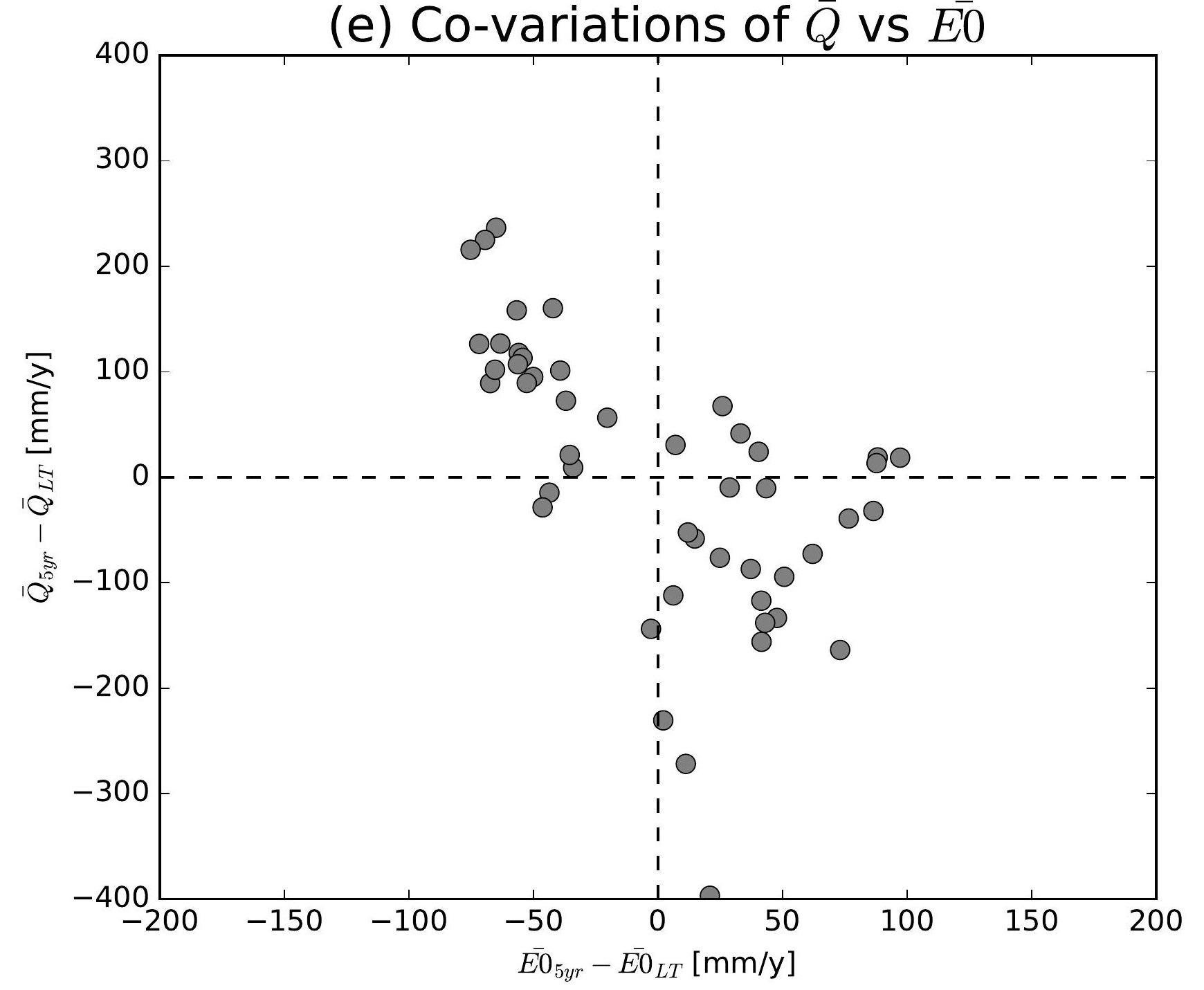

(h) Co-variations of $\bar{Q}$ vs $\overline{E 0}$

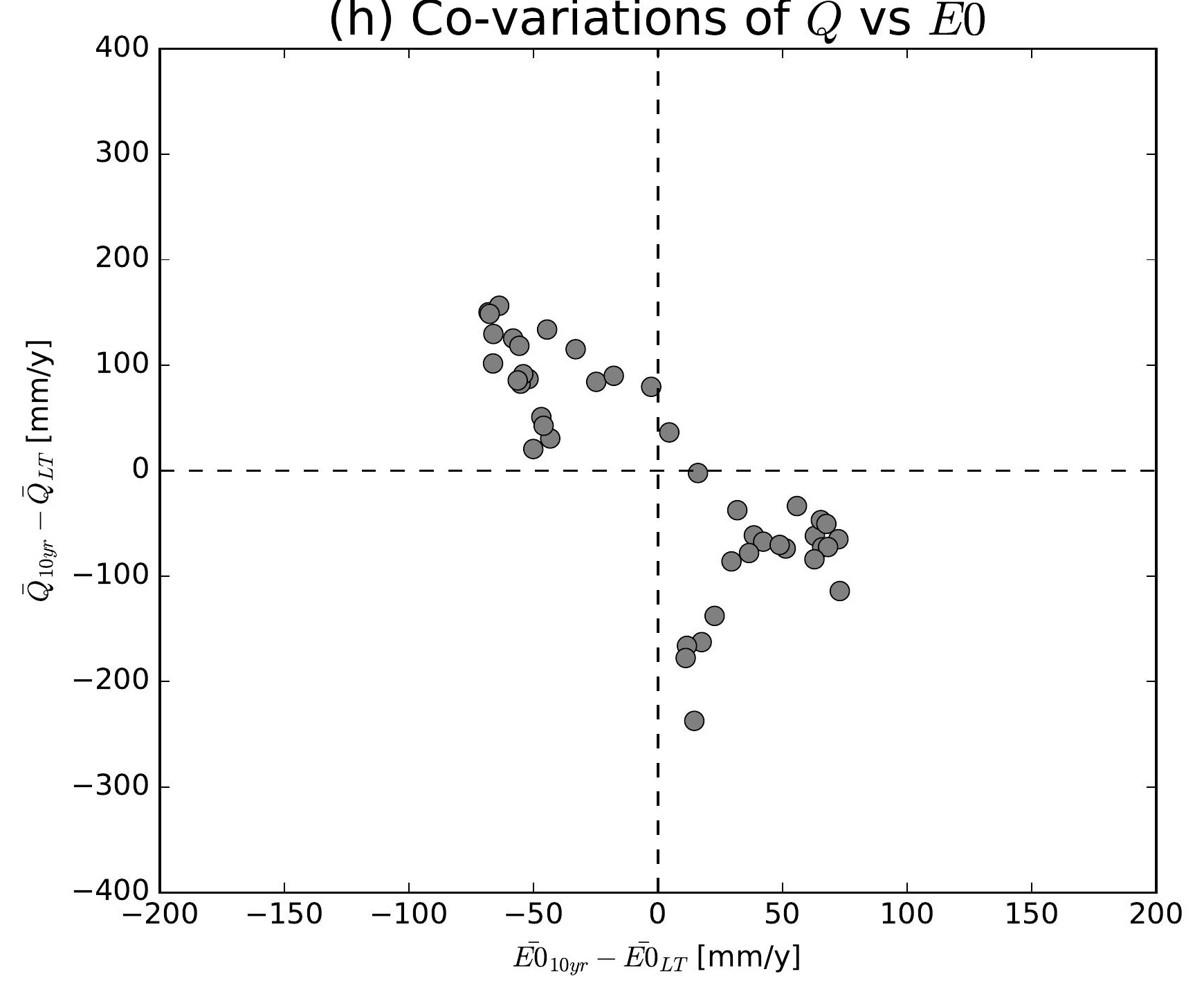

(k) Co-variations of $\bar{Q}$ vs $\overline{E 0}$

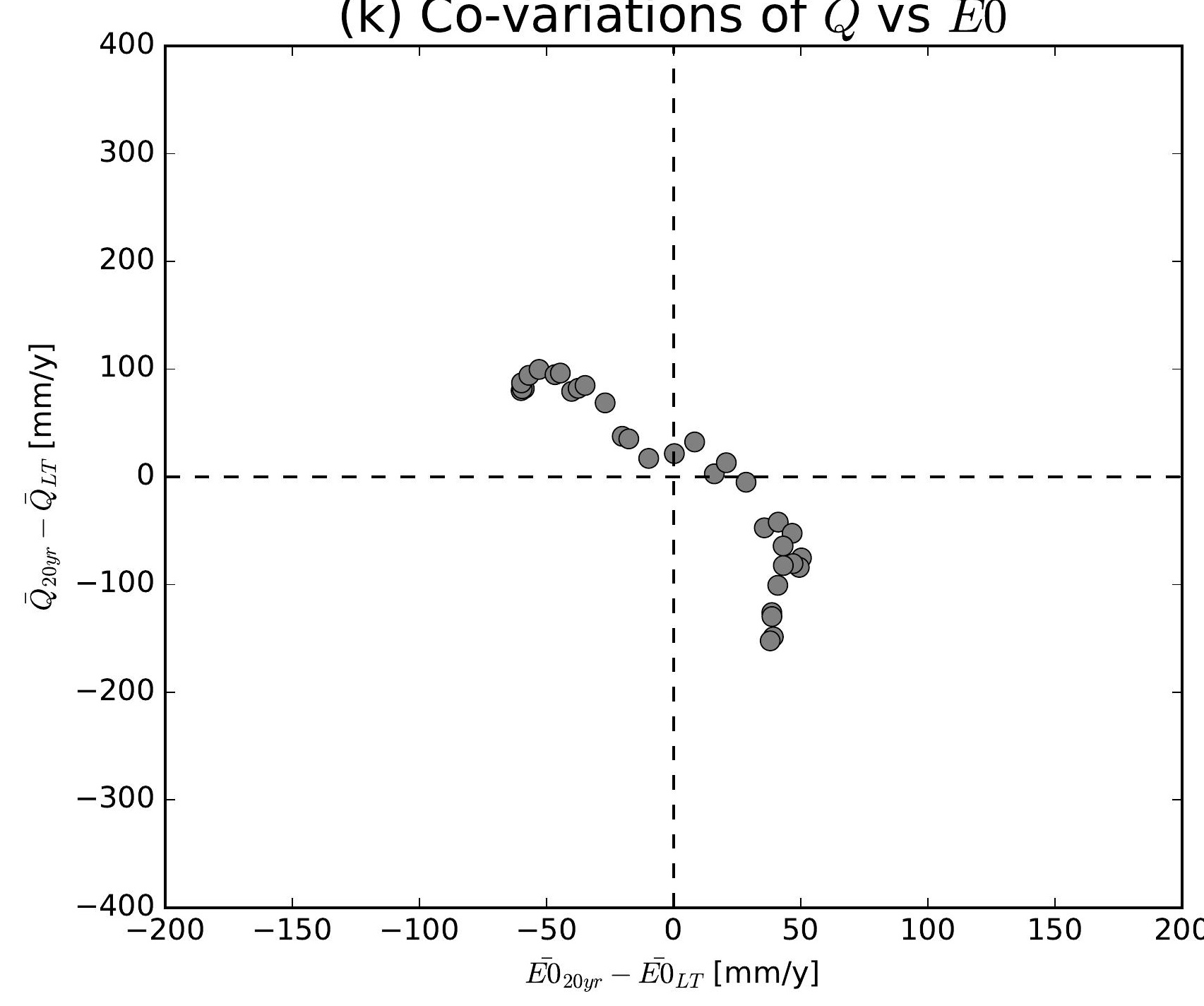

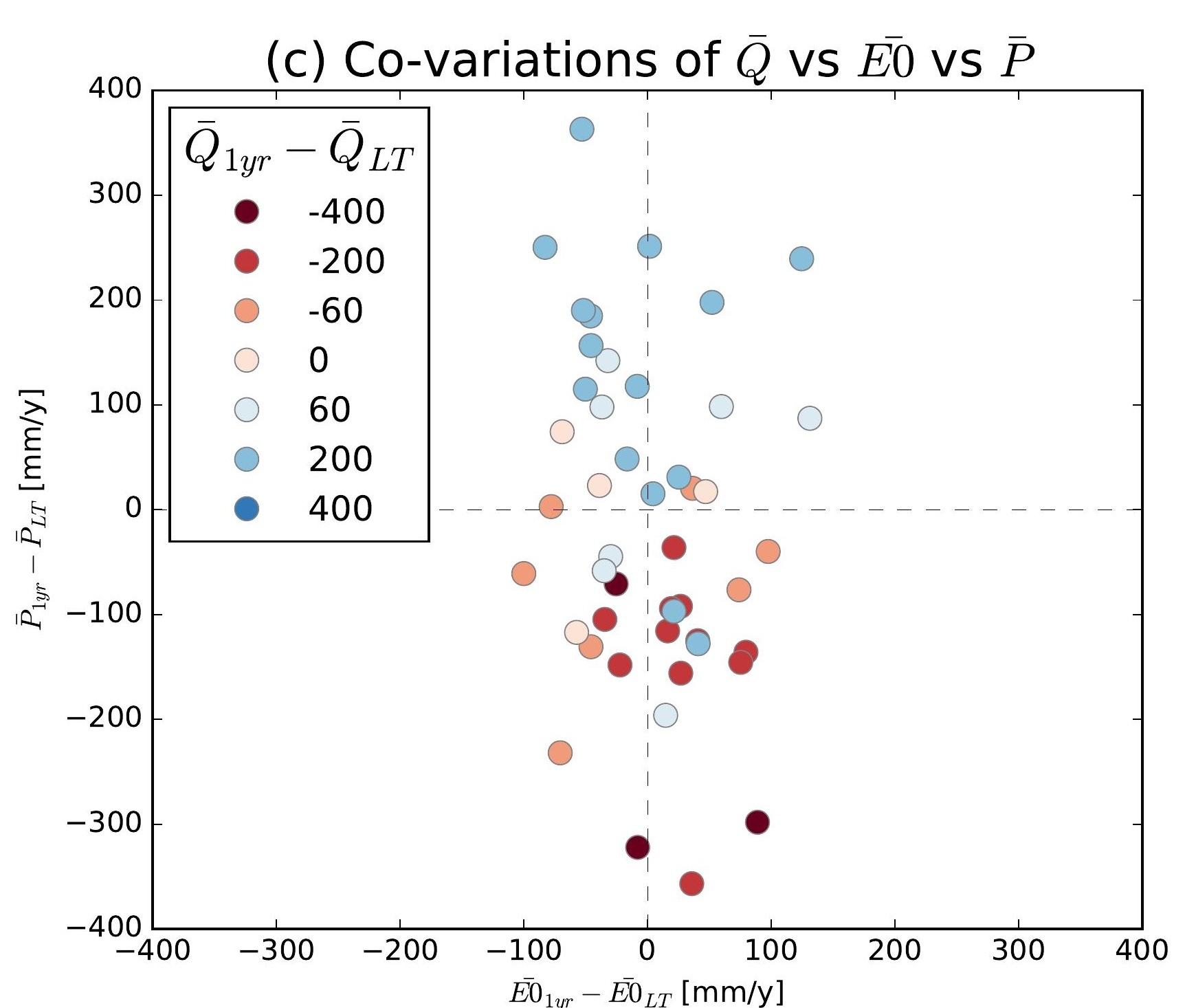
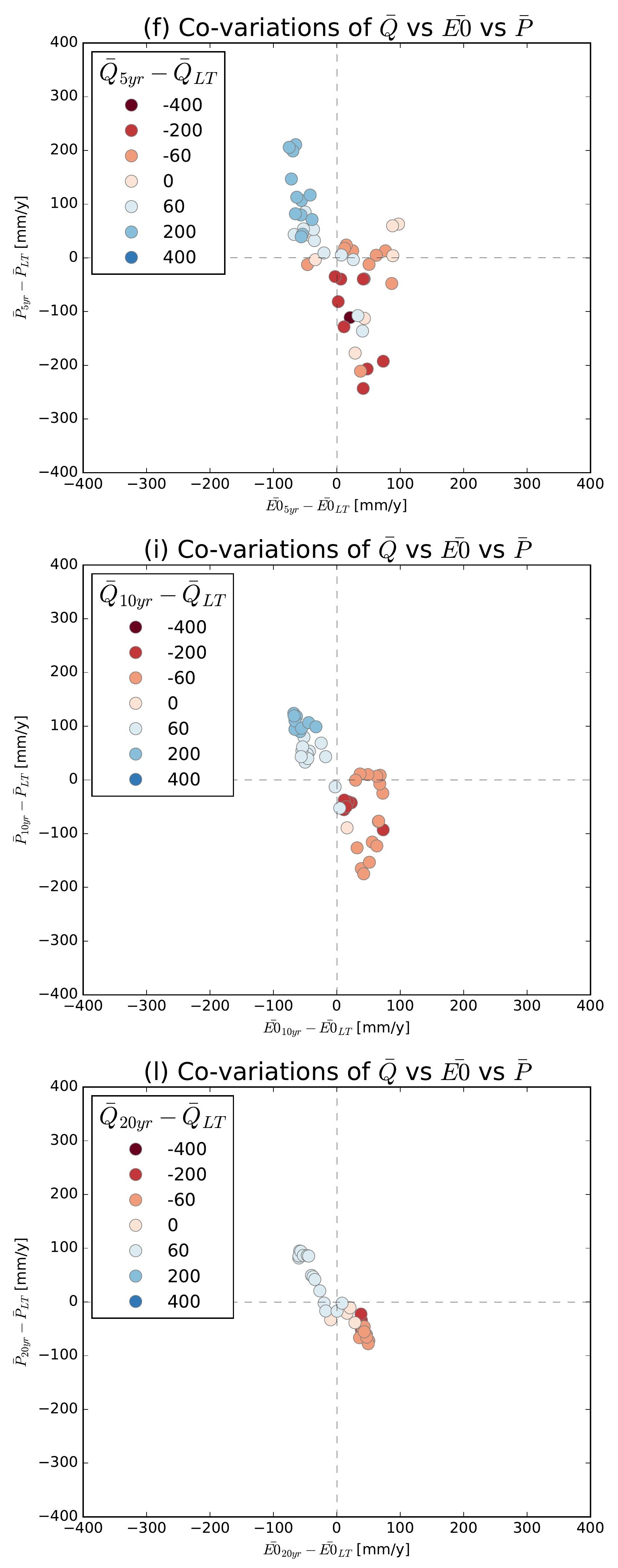

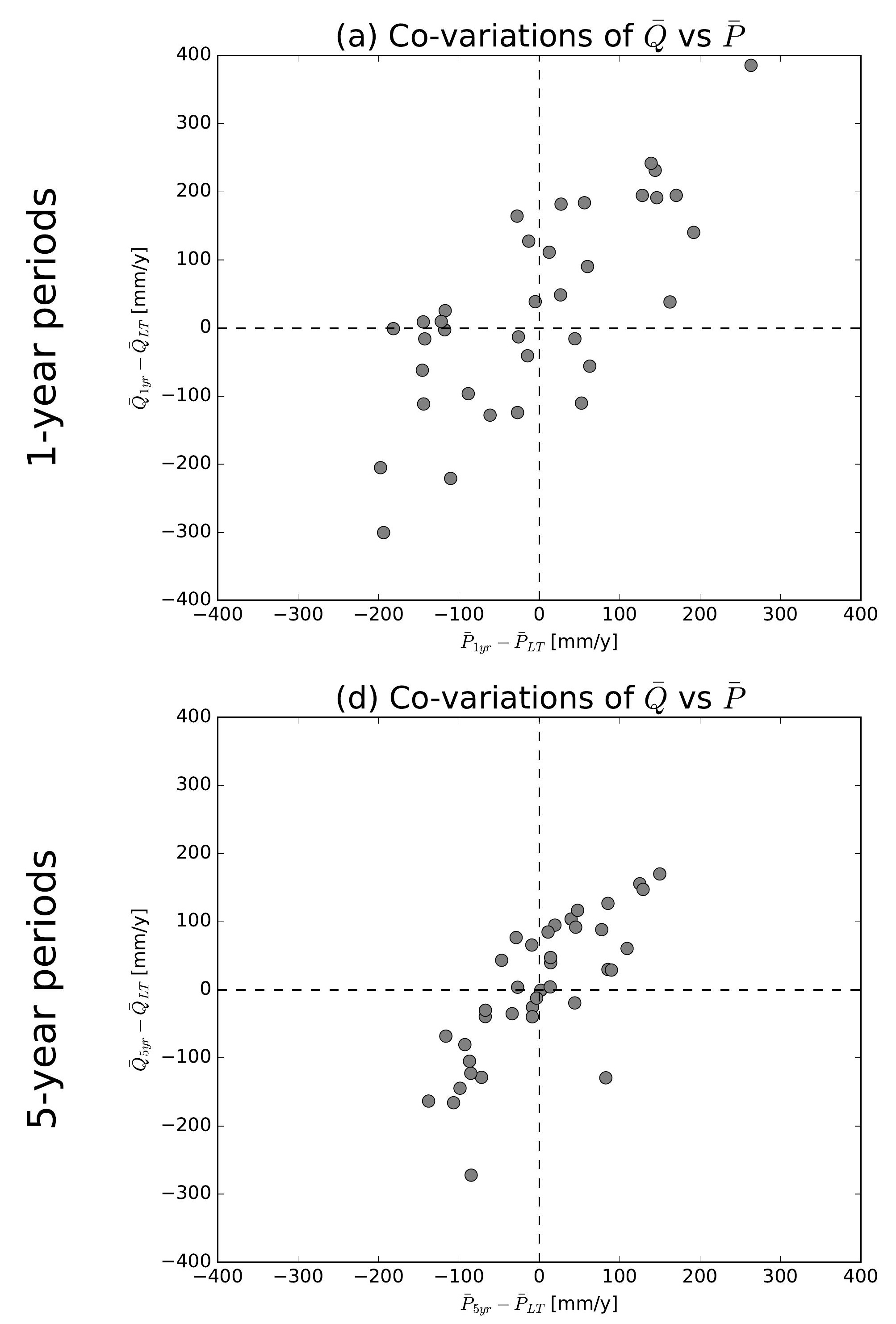

(g) Co-variations of $\bar{Q}$ vs $\bar{P}$

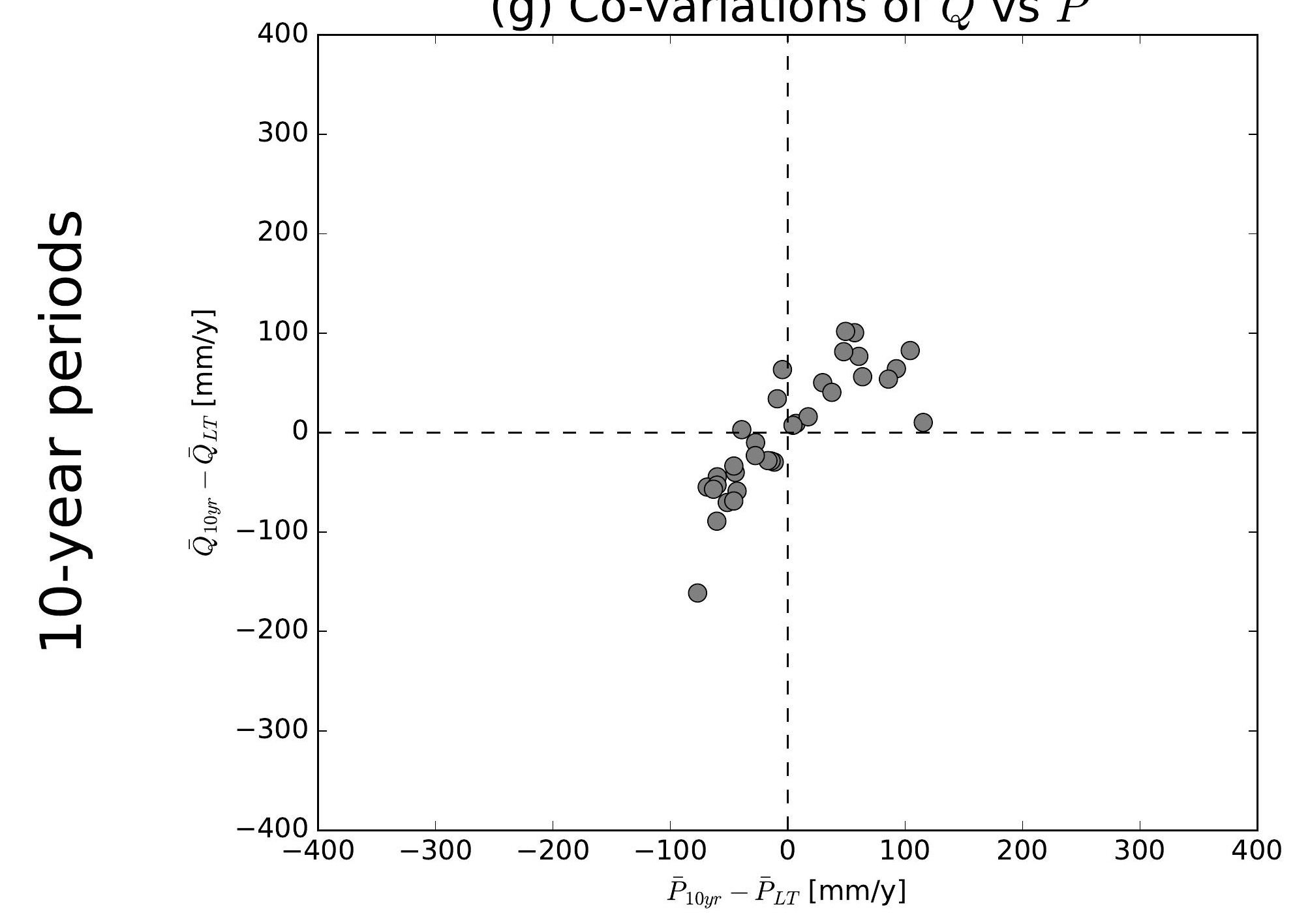

(j) Co-variations of $\bar{Q}$ vs $\bar{P}$

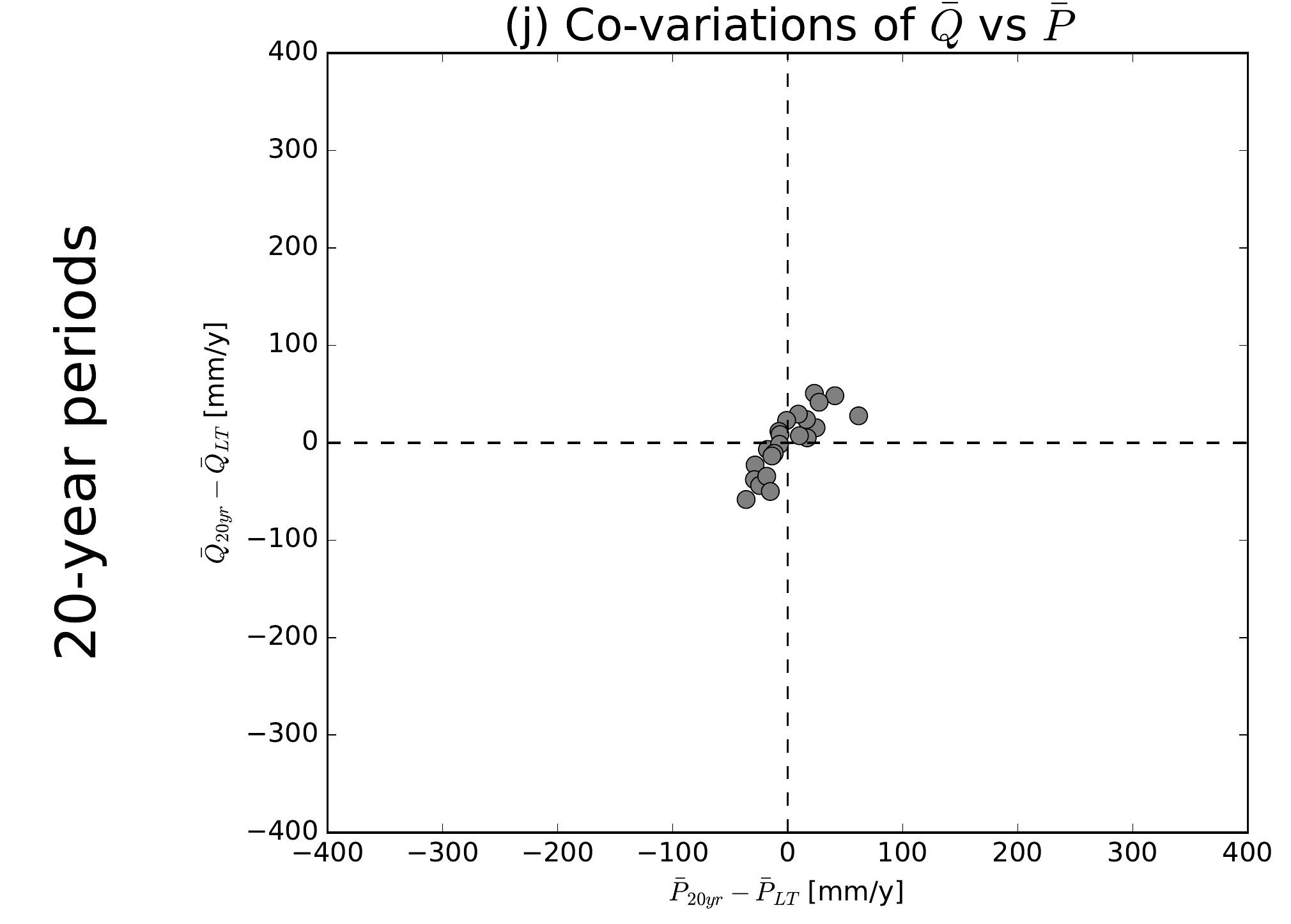

(b) Co-variations of $\bar{Q}$ vs $\overline{E 0}$

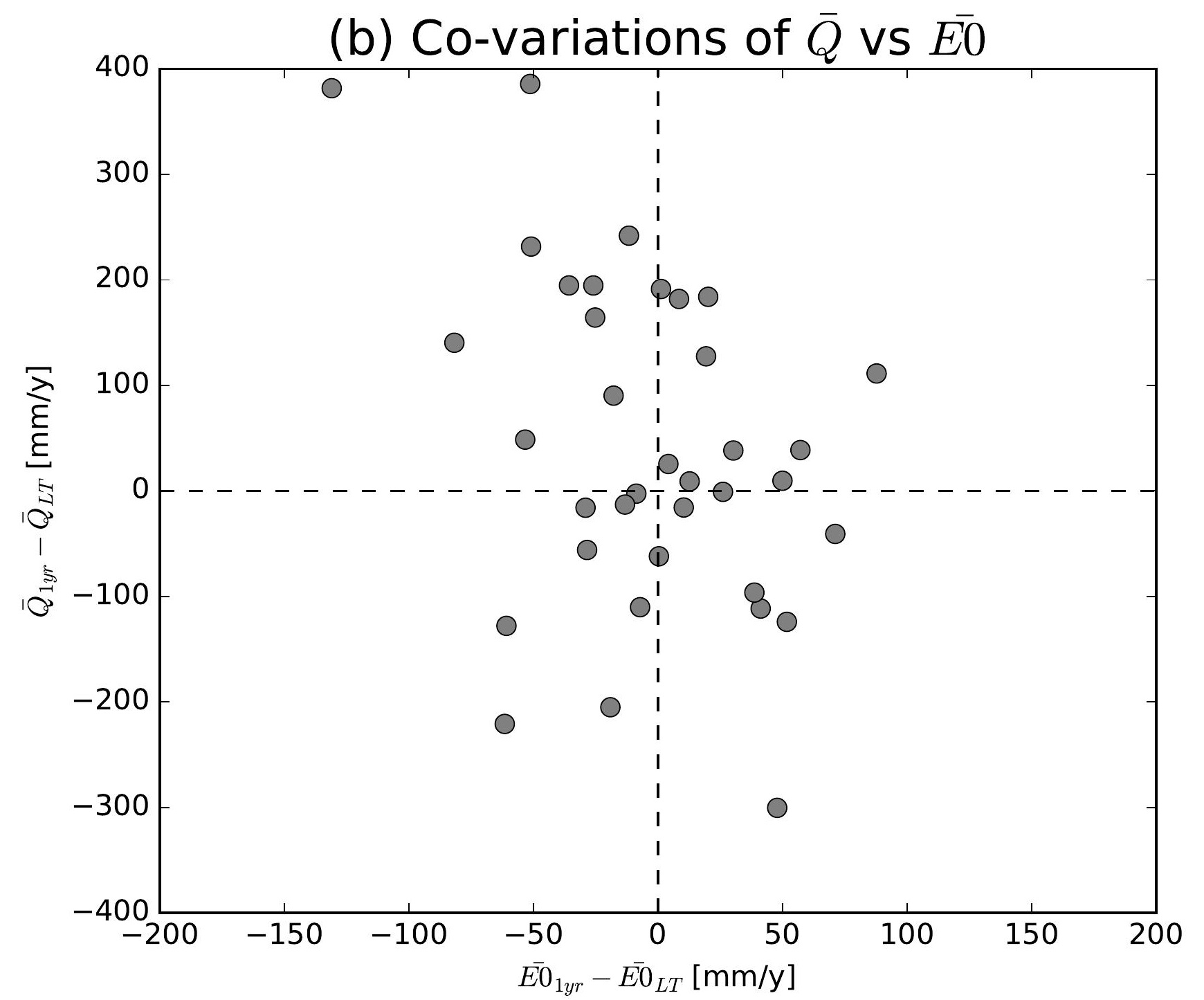

(e) Co-variations of $\bar{Q}$ vs $\overline{E 0}$

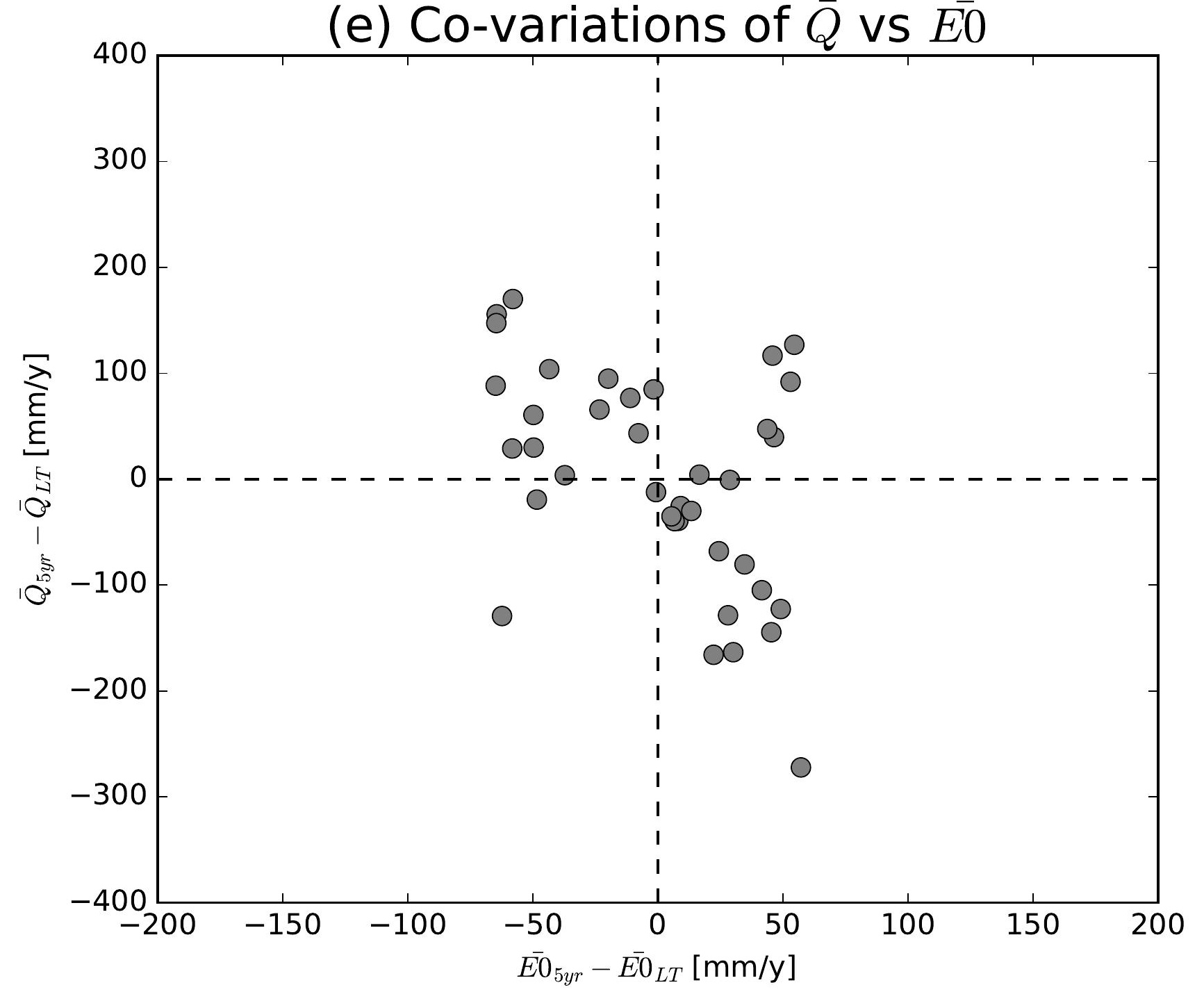

(h) Co-variations of $\bar{Q}$ vs $\overline{E 0}$

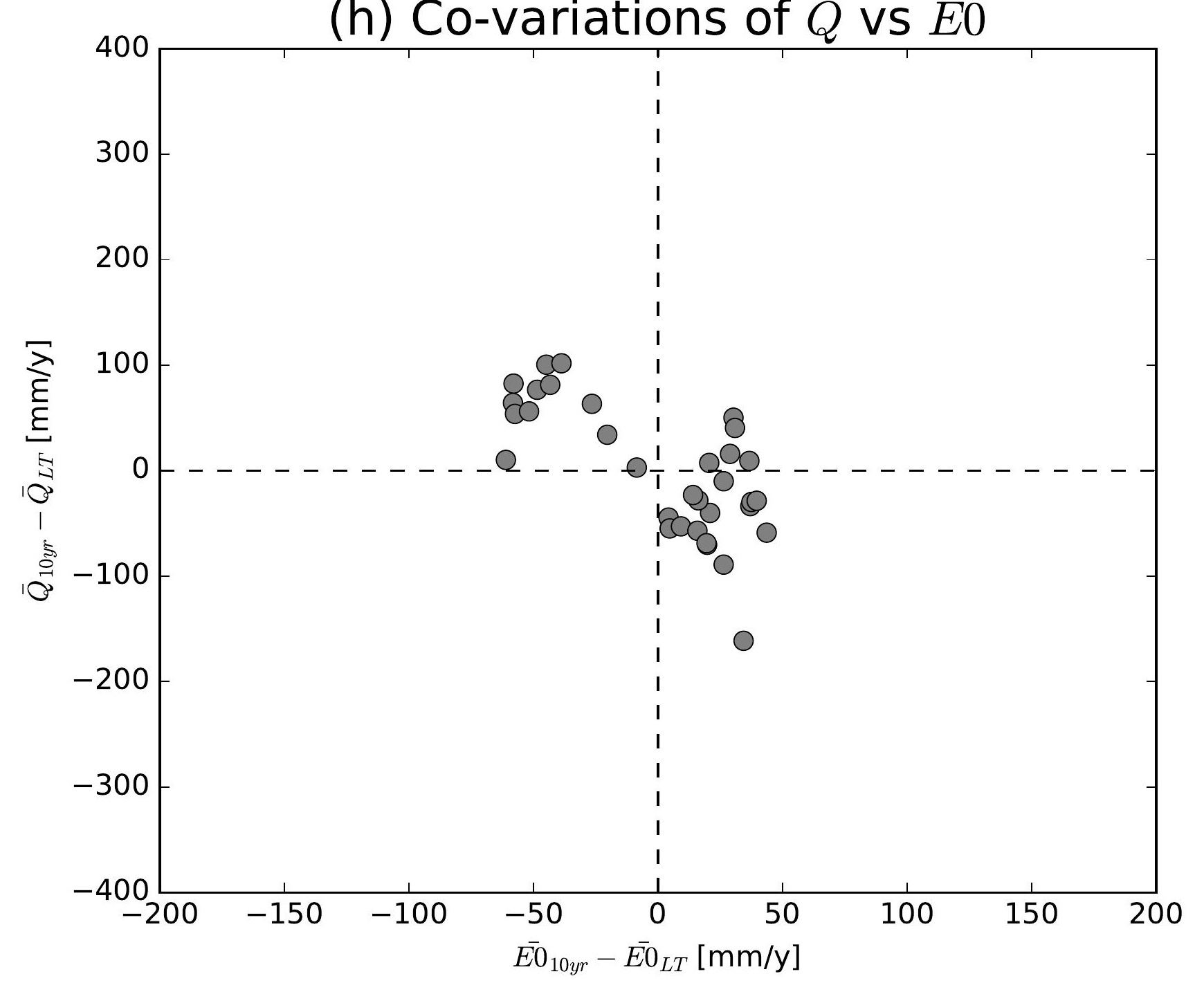

(k) Co-variations of $\bar{Q}$ vs $\overline{E 0}$

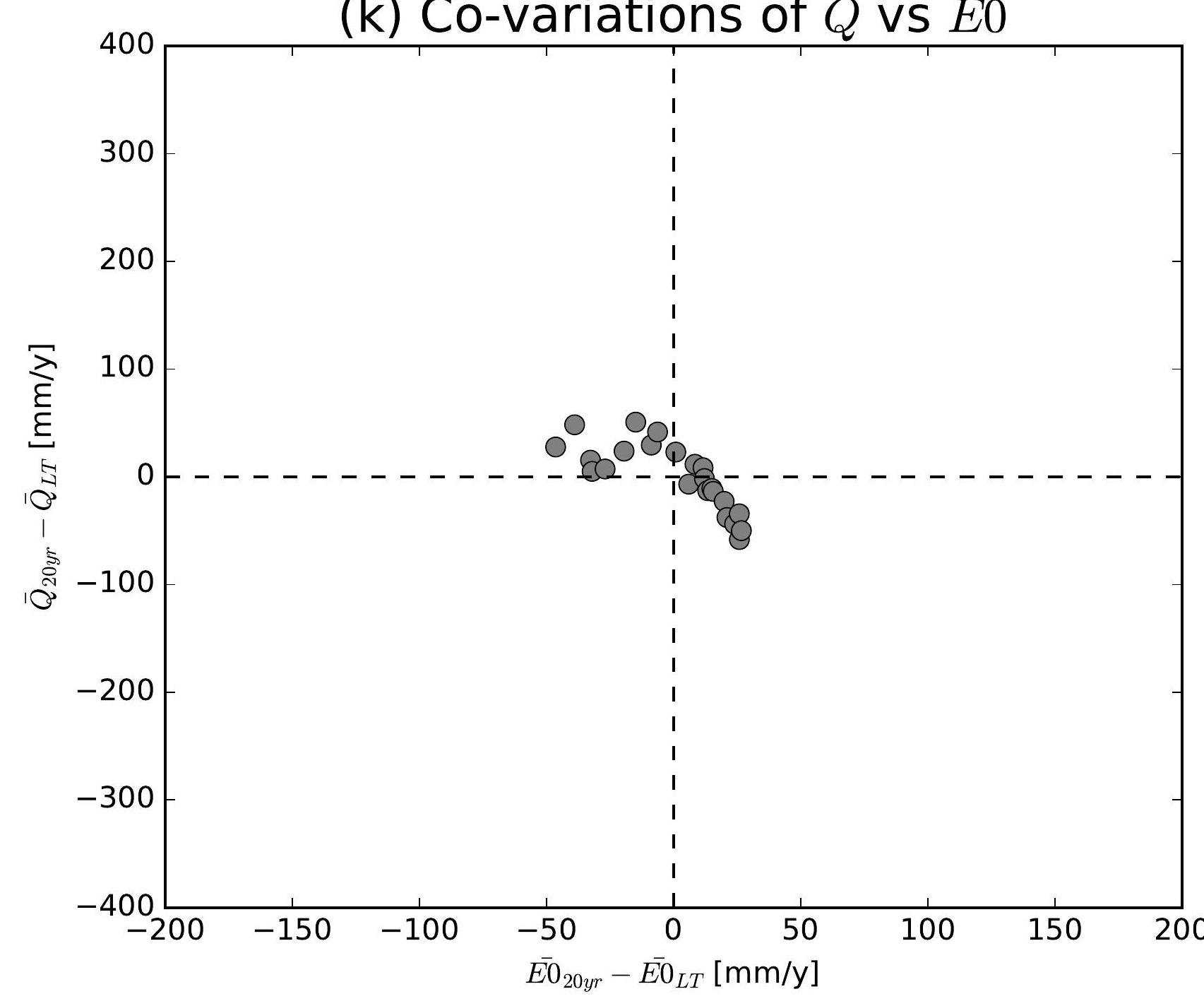

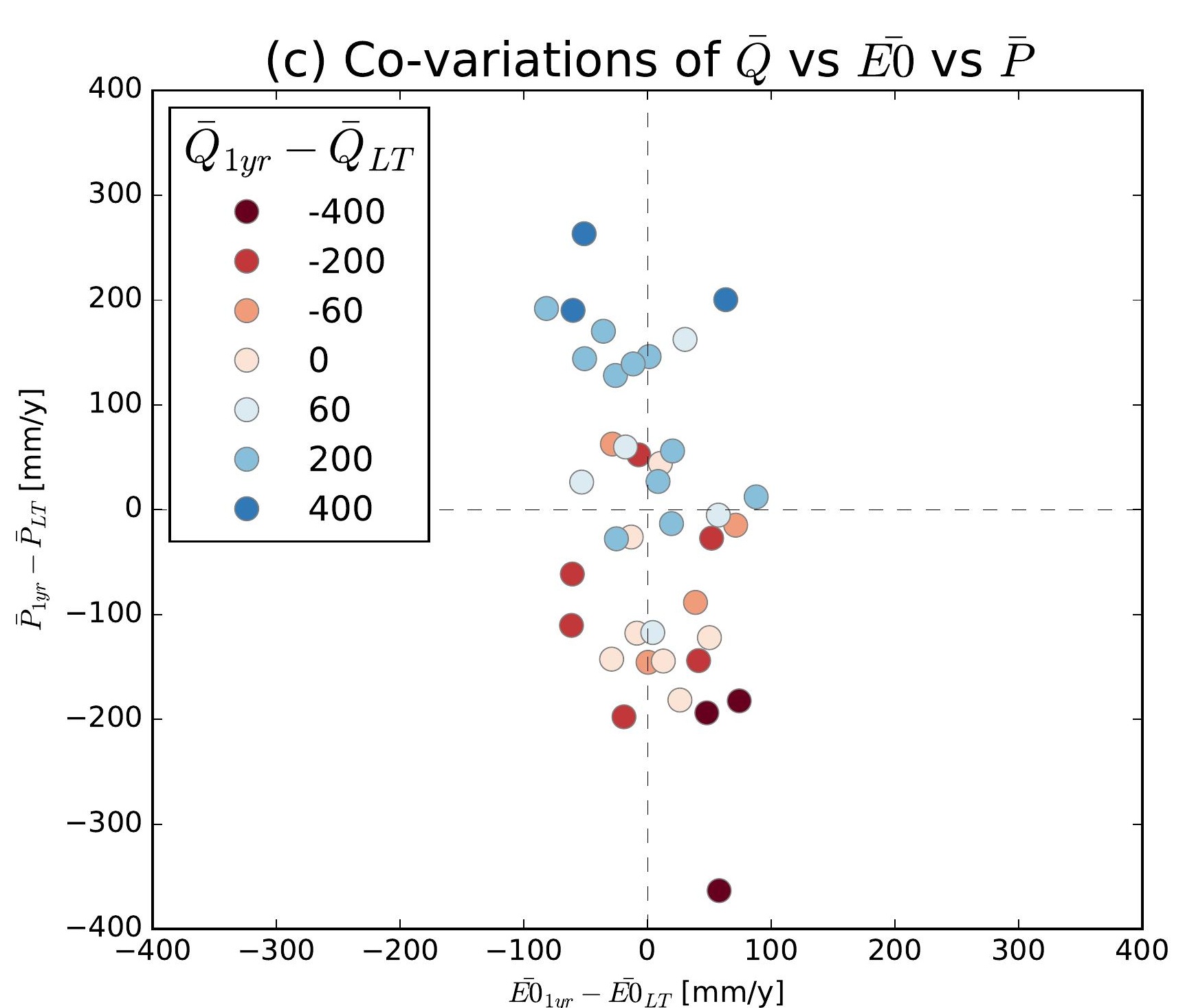
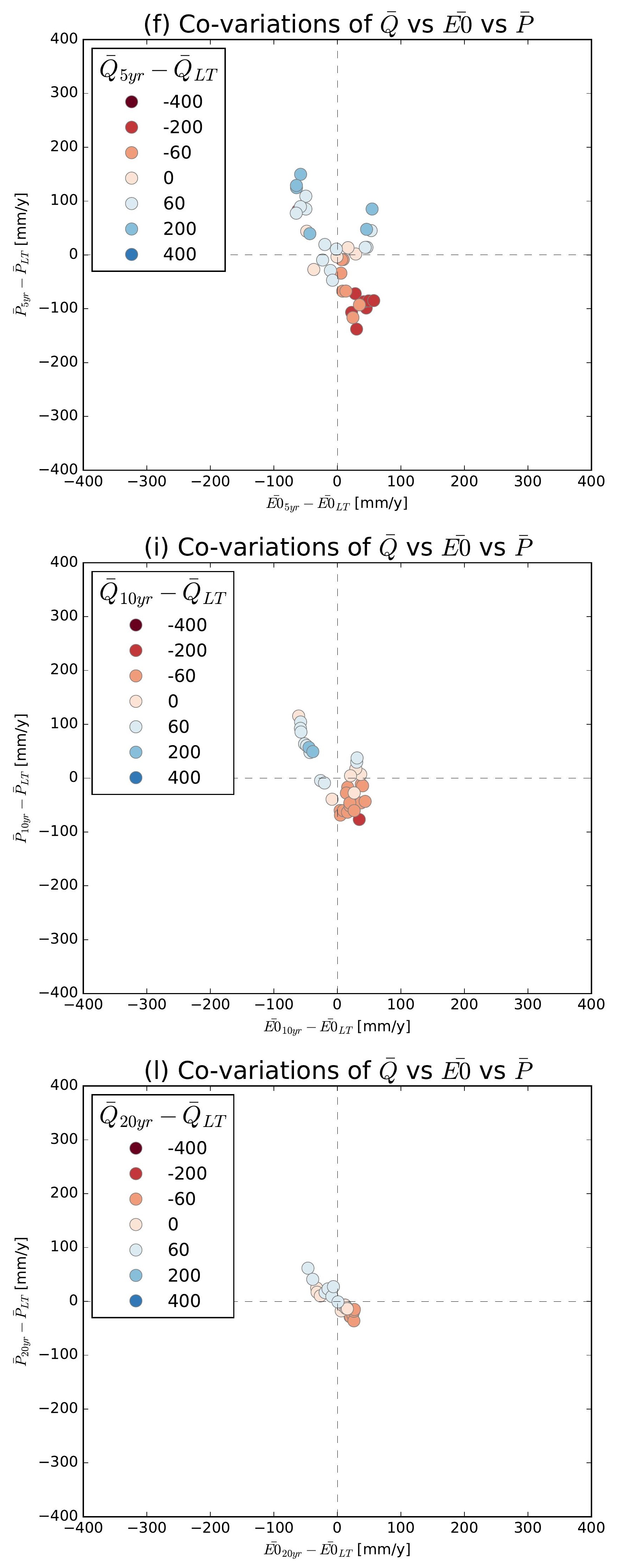

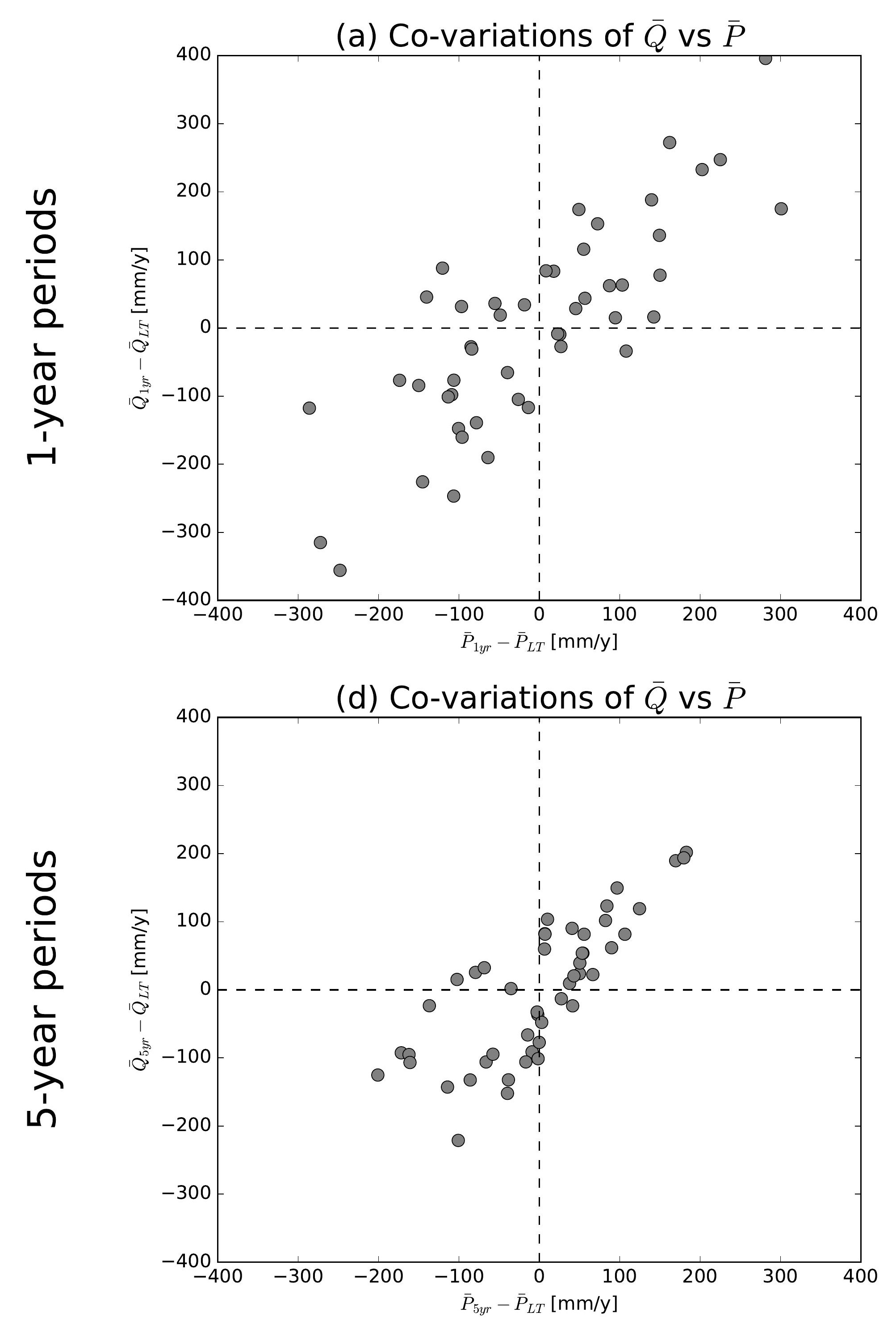

(g) Co-variations of $\bar{Q}$ vs $\bar{P}$

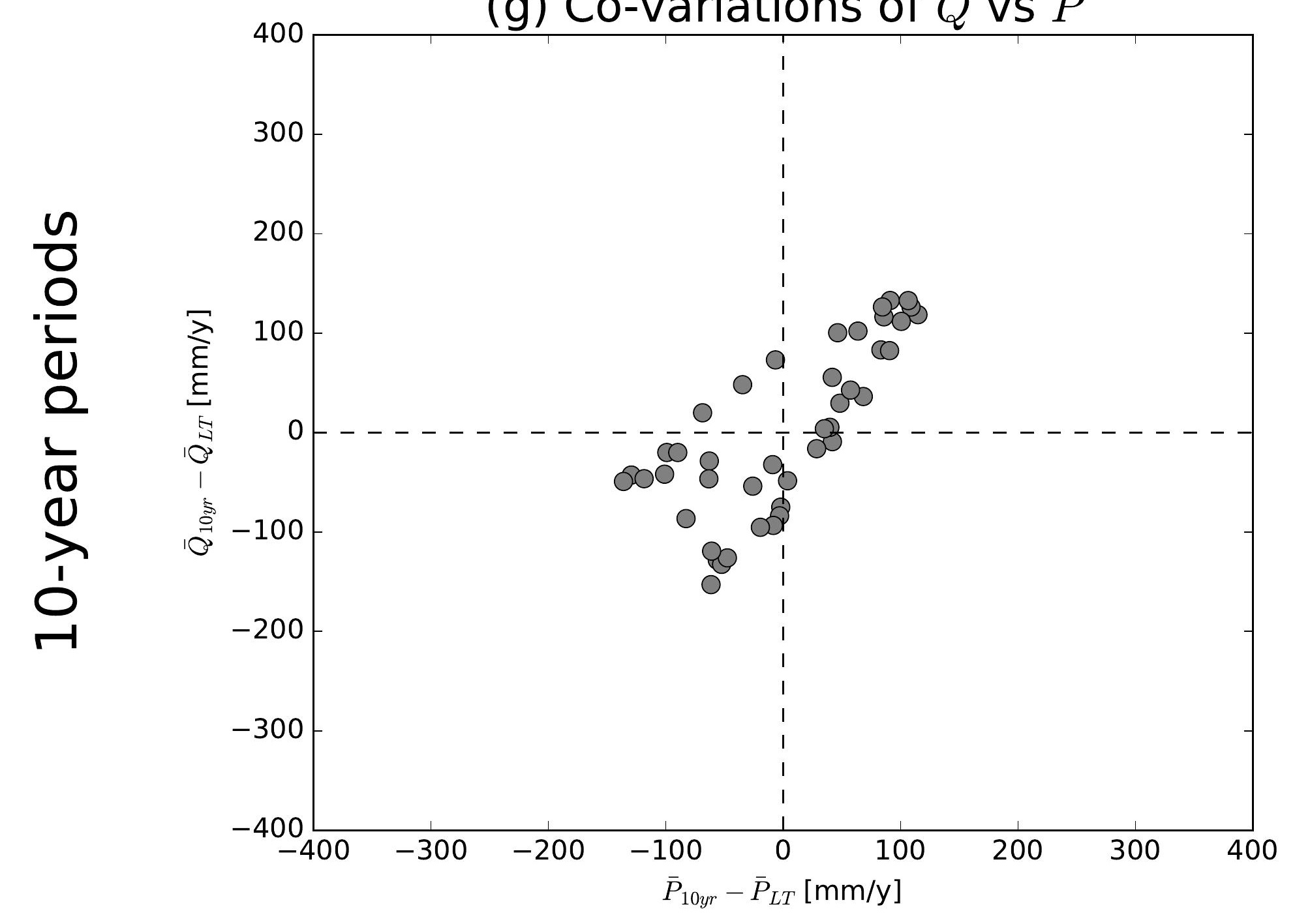

(j) Co-variations of $\bar{Q}$ vs $\bar{P}$

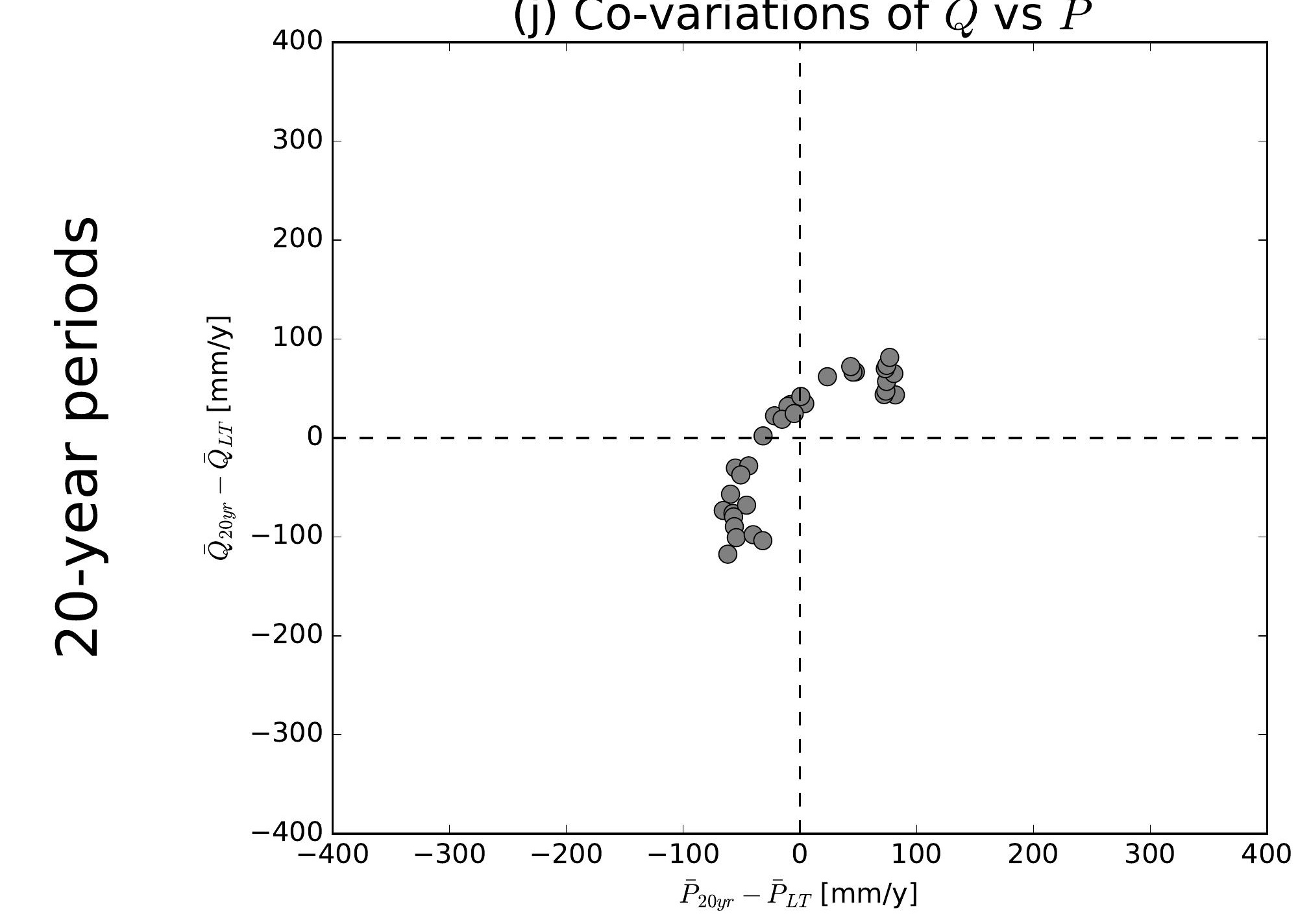

(b) Co-variations of $\bar{Q}$ vs $\overline{E 0}$

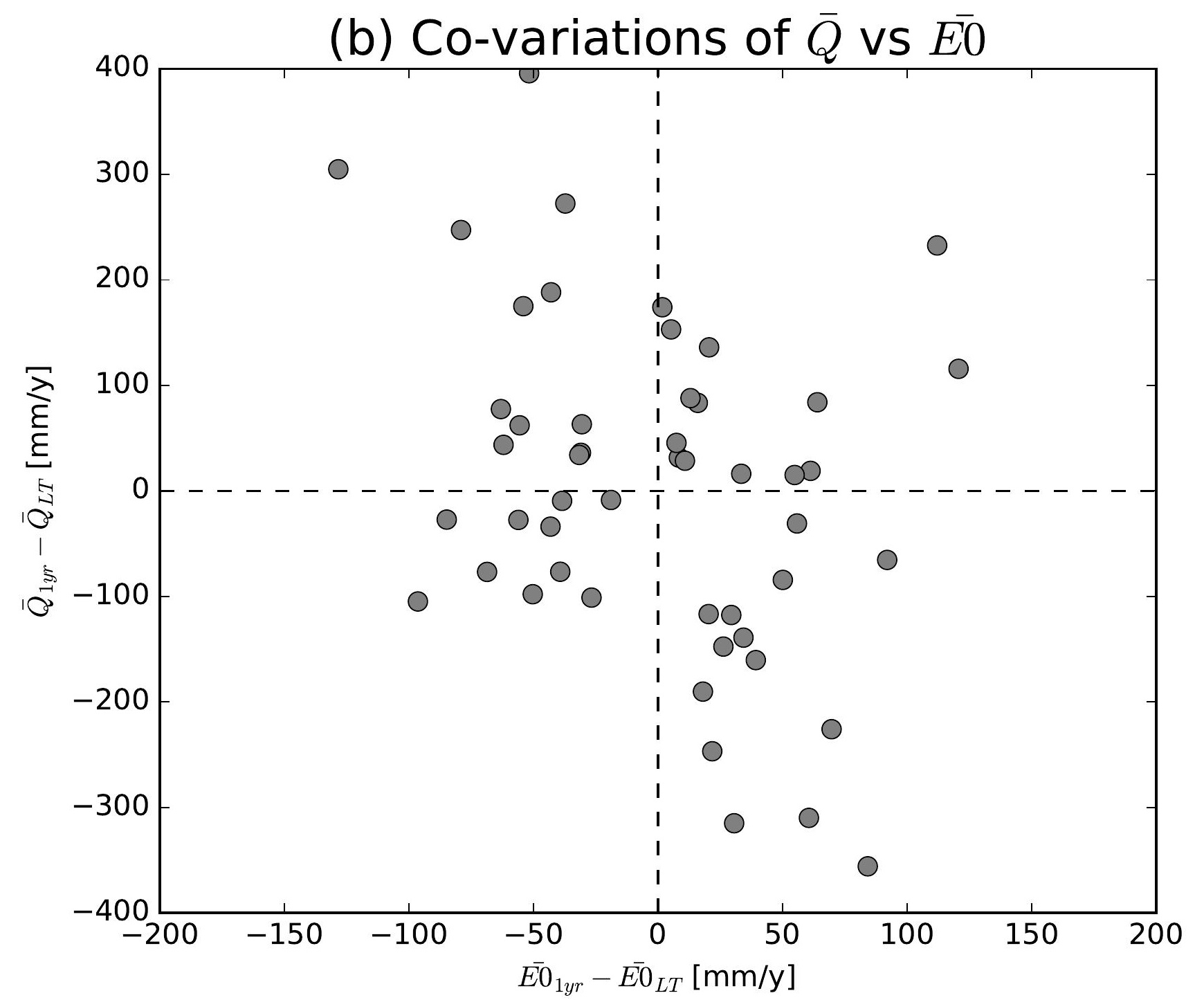

(e) Co-variations of $\bar{Q}$ vs $\overline{E 0}$

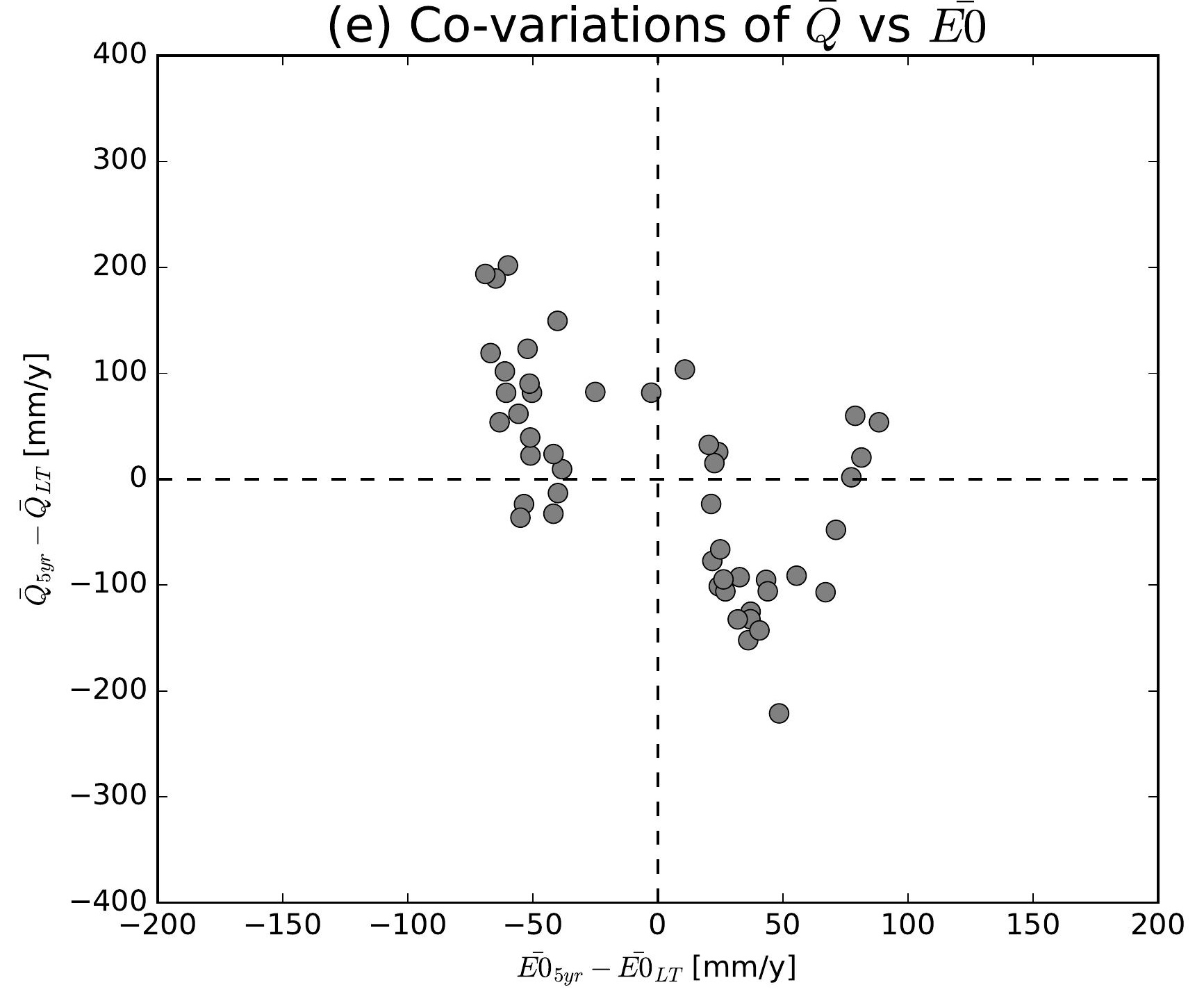

(h) Co-variations of $\bar{Q}$ vs $\overline{E 0}$

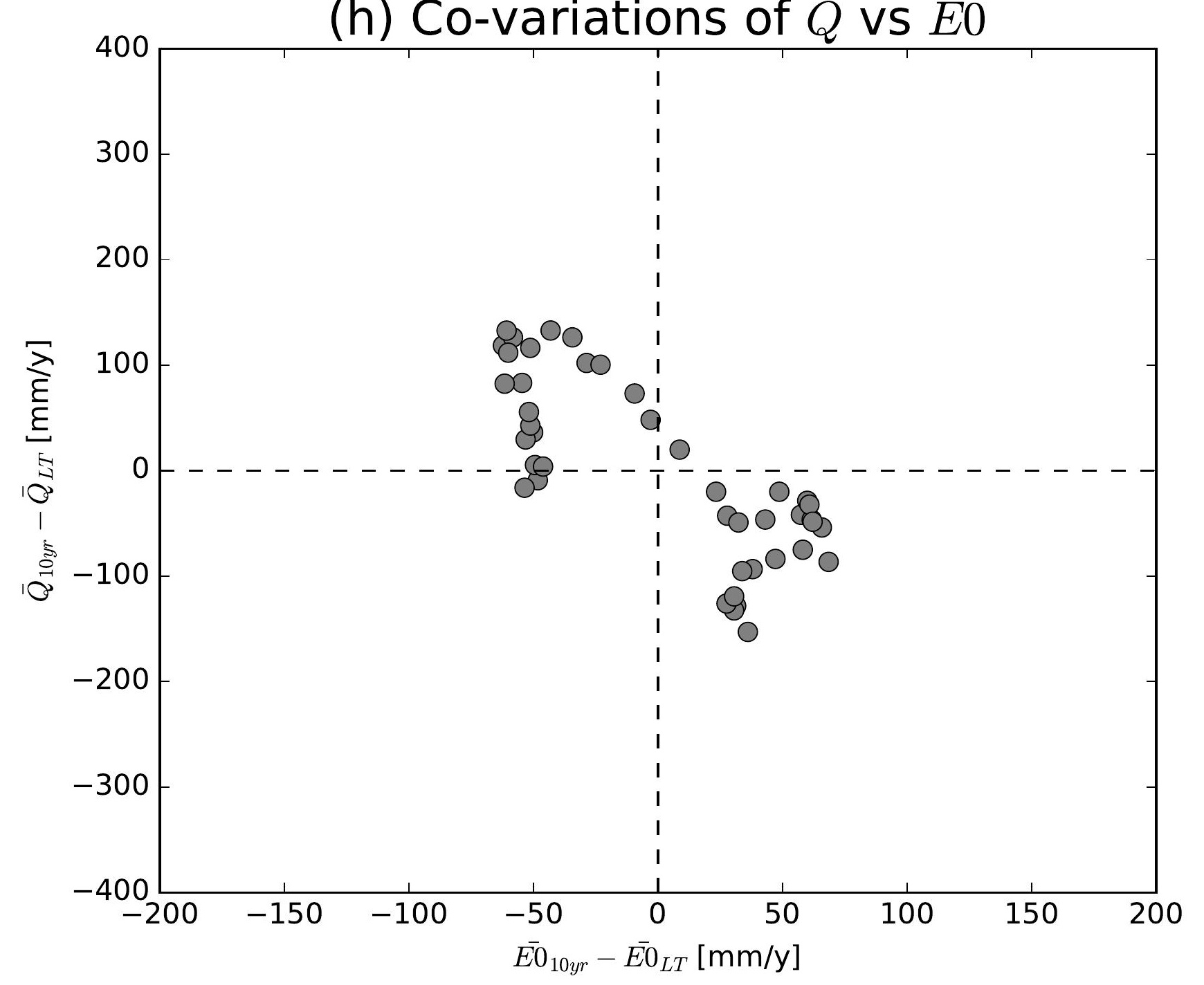

(k) Co-variations of $\bar{Q}$ vs $\overline{E 0}$

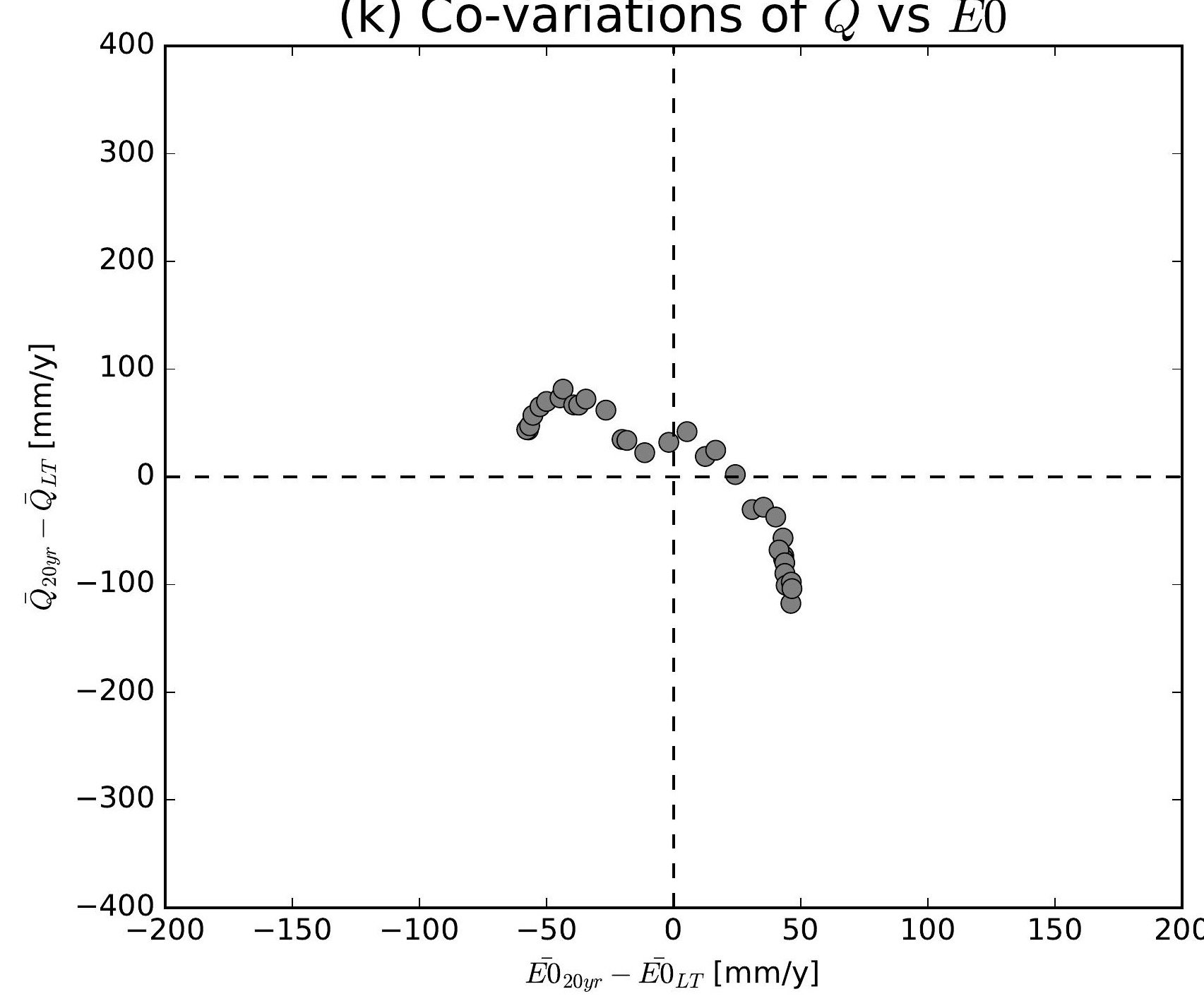

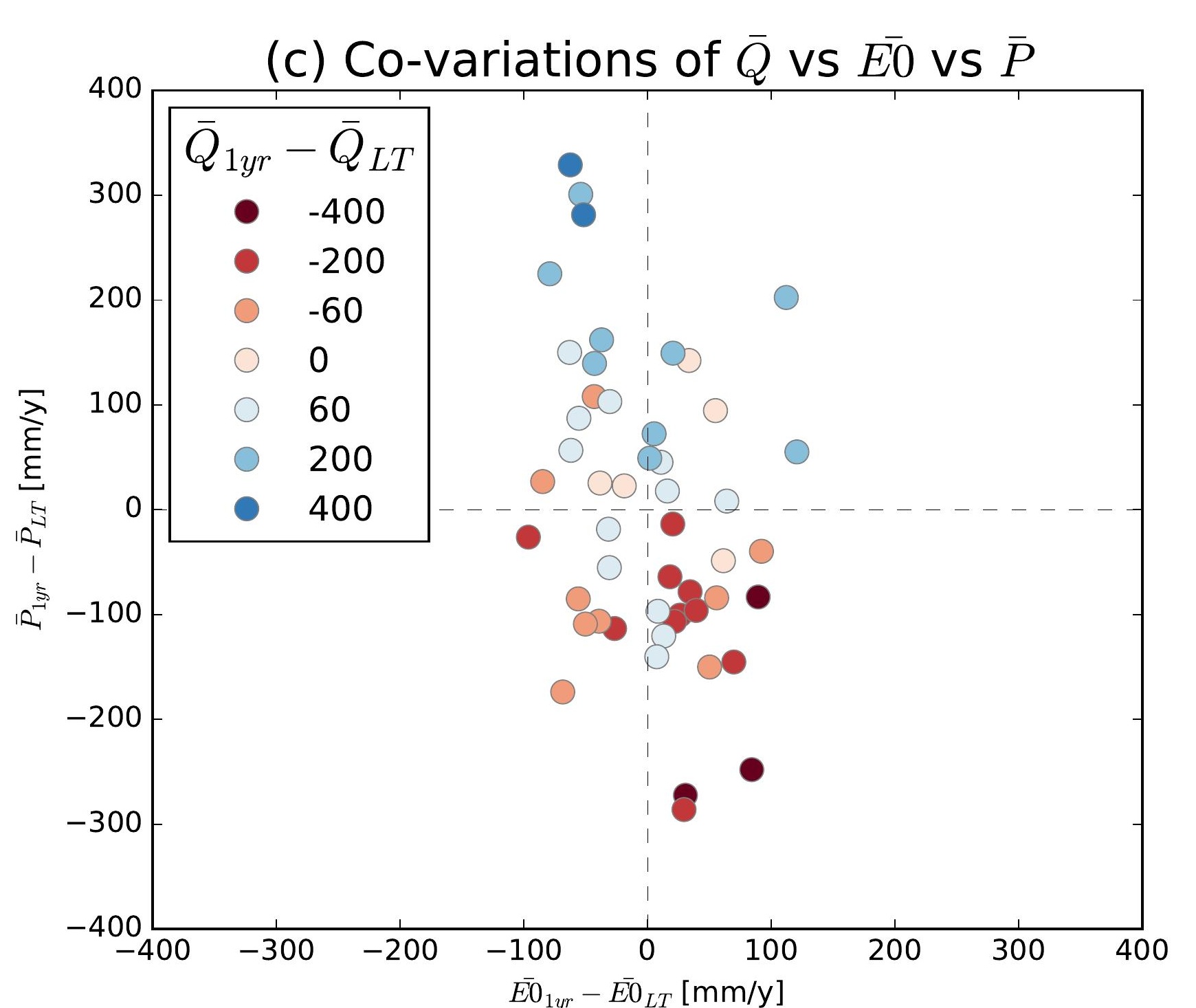
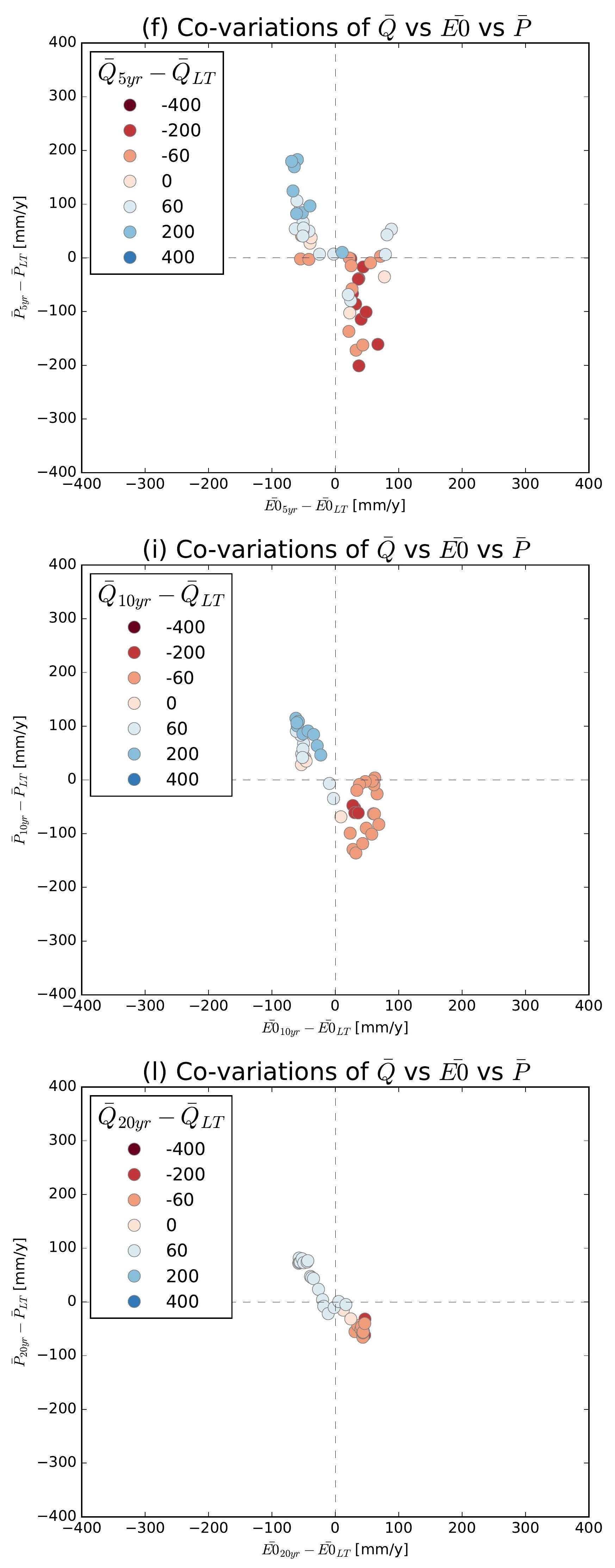

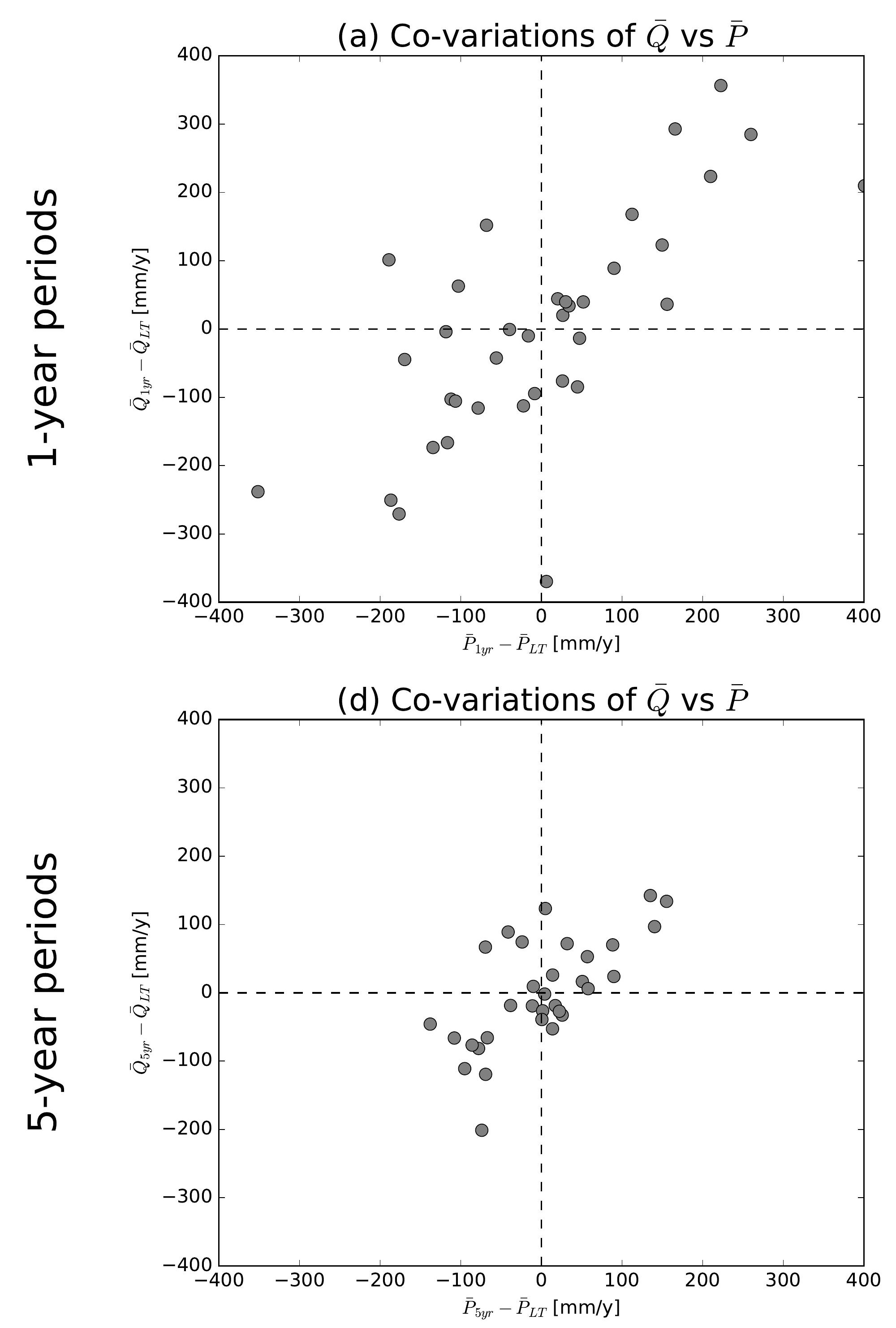

(g) Co-variations of $\bar{Q}$ vs $\bar{P}$

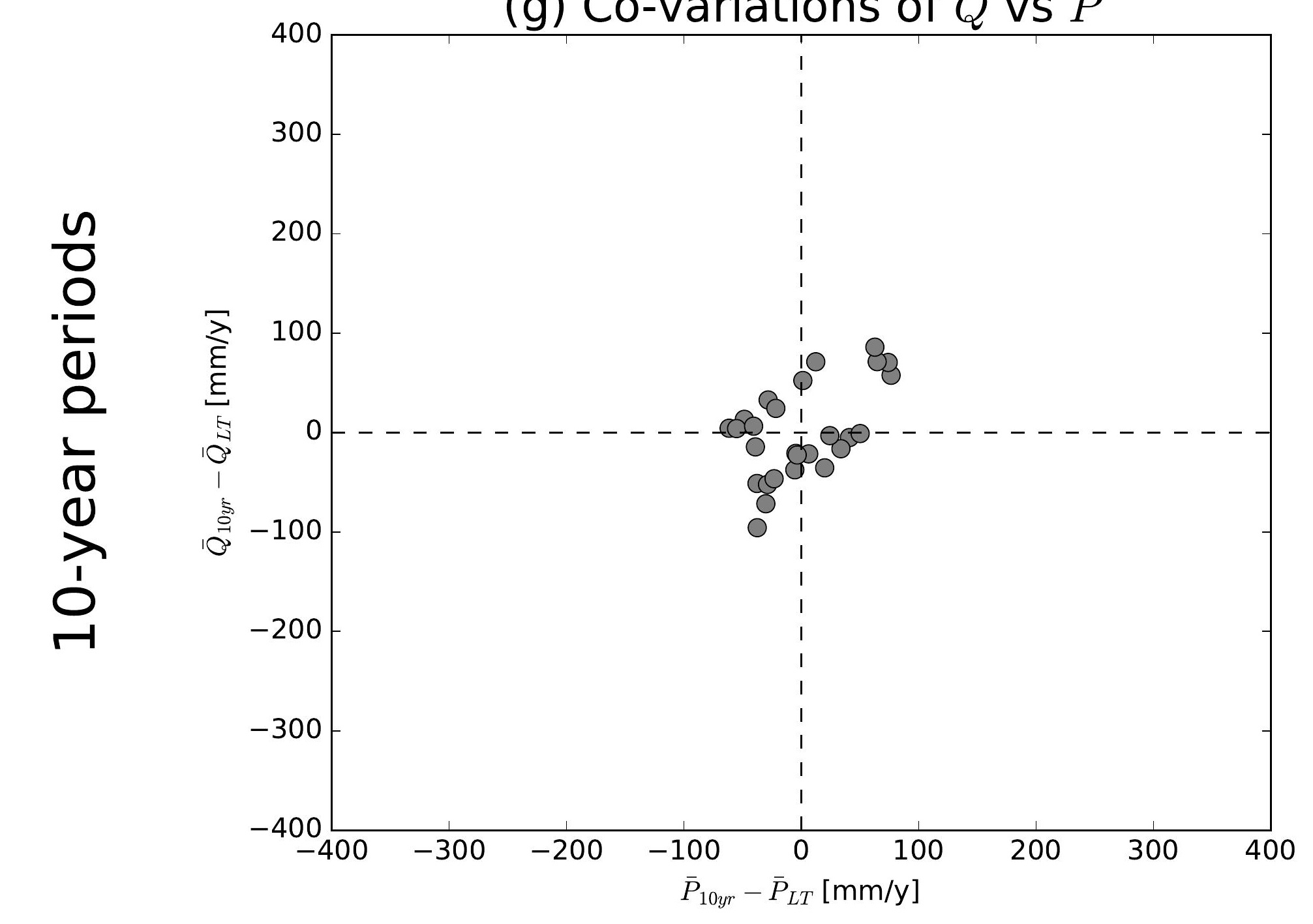

(j) Co-variations of $\bar{Q}$ vs $\bar{P}$

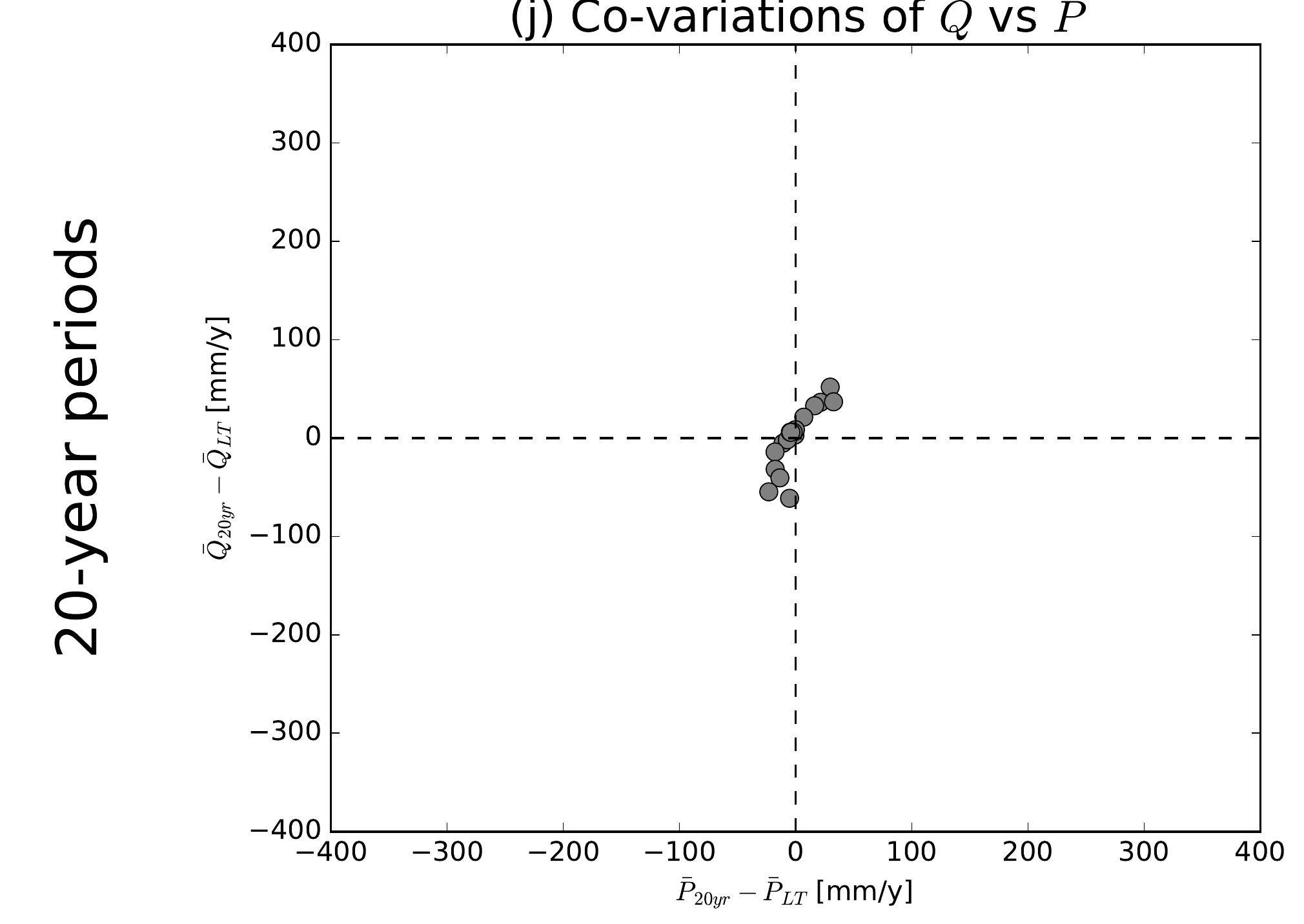

(b) Co-variations of $\bar{Q}$ vs $\overline{E 0}$

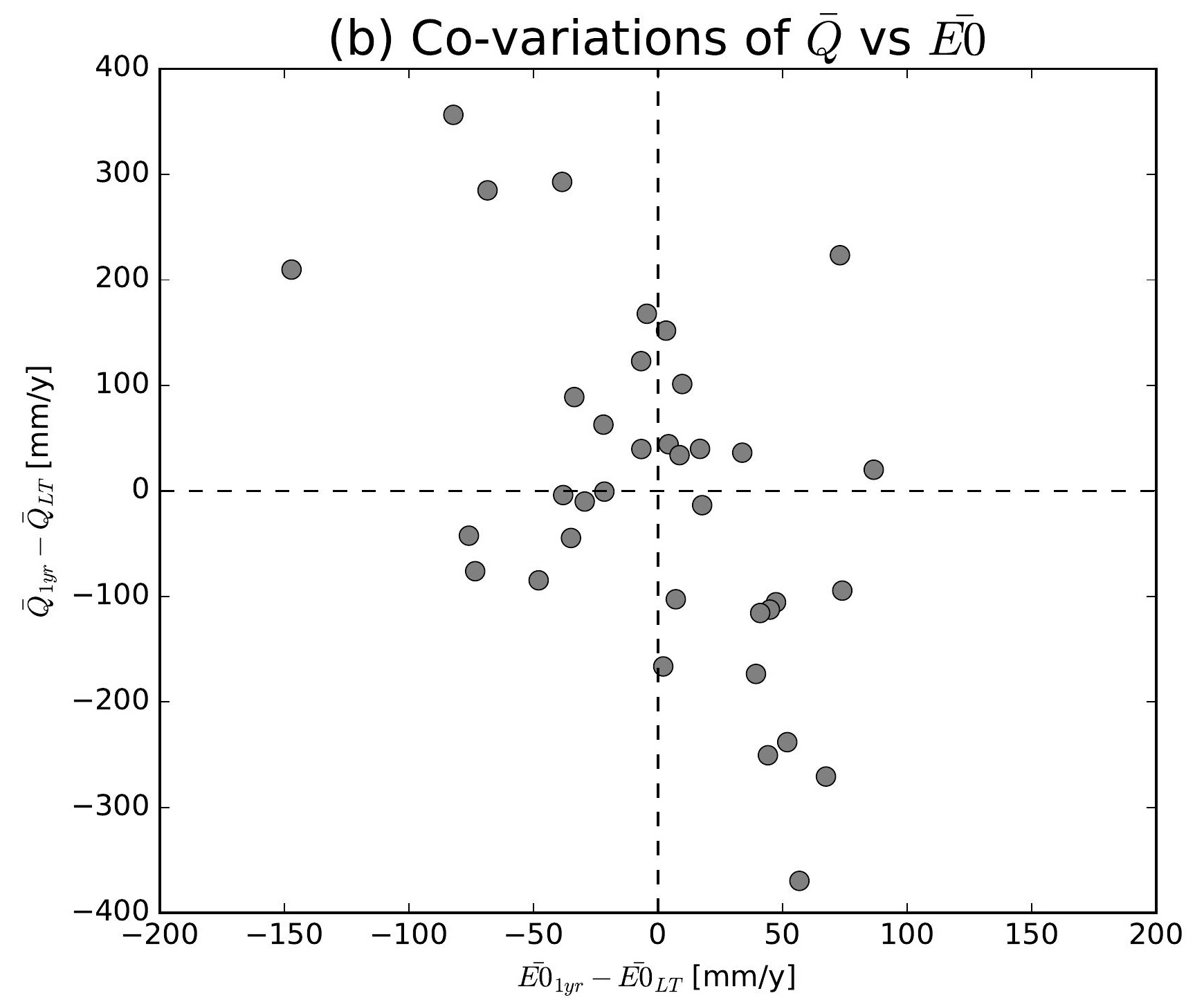

(e) Co-variations of $\bar{Q}$ vs $\overline{E 0}$

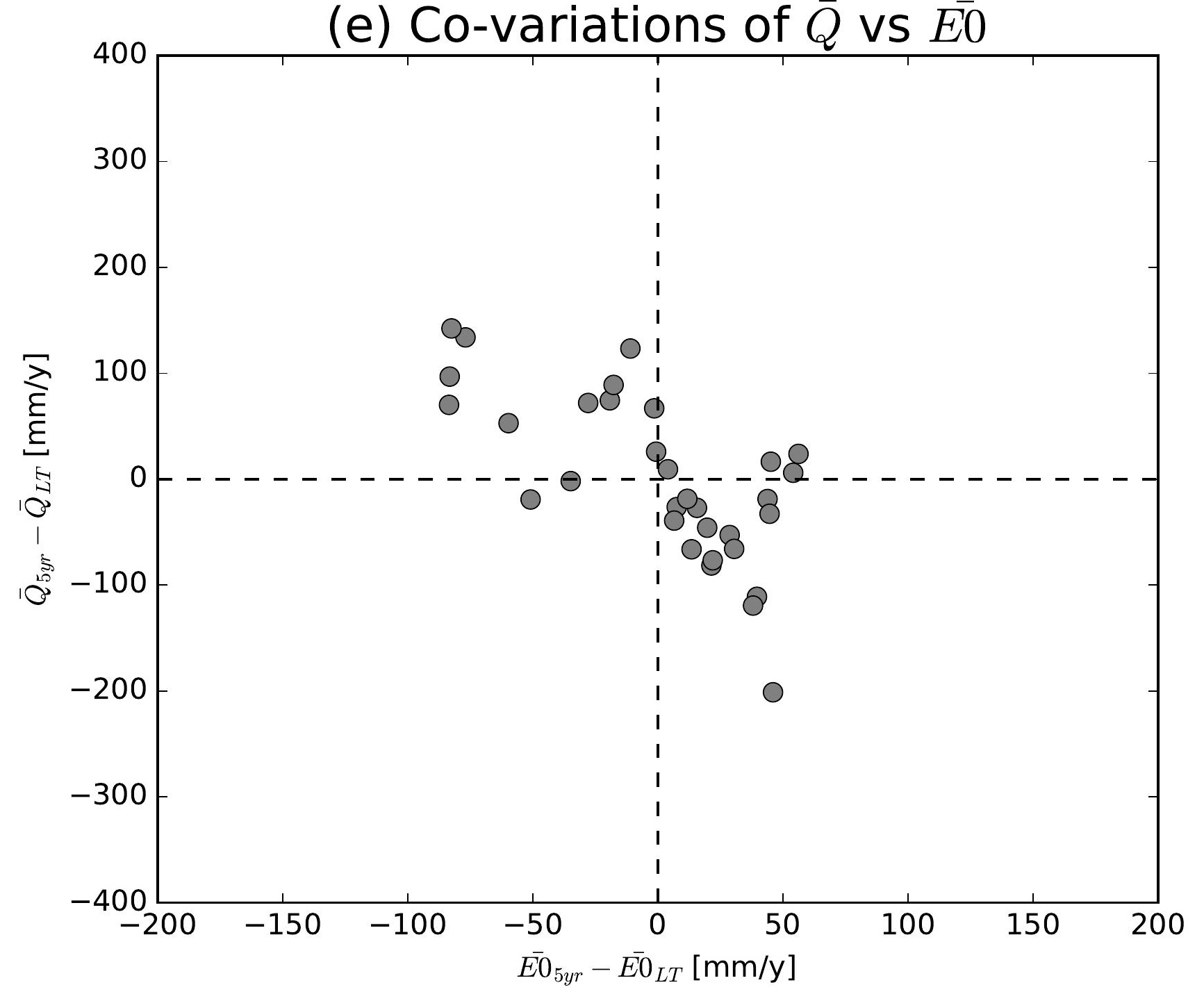

(h) Co-variations of $\bar{Q}$ vs $\overline{E 0}$

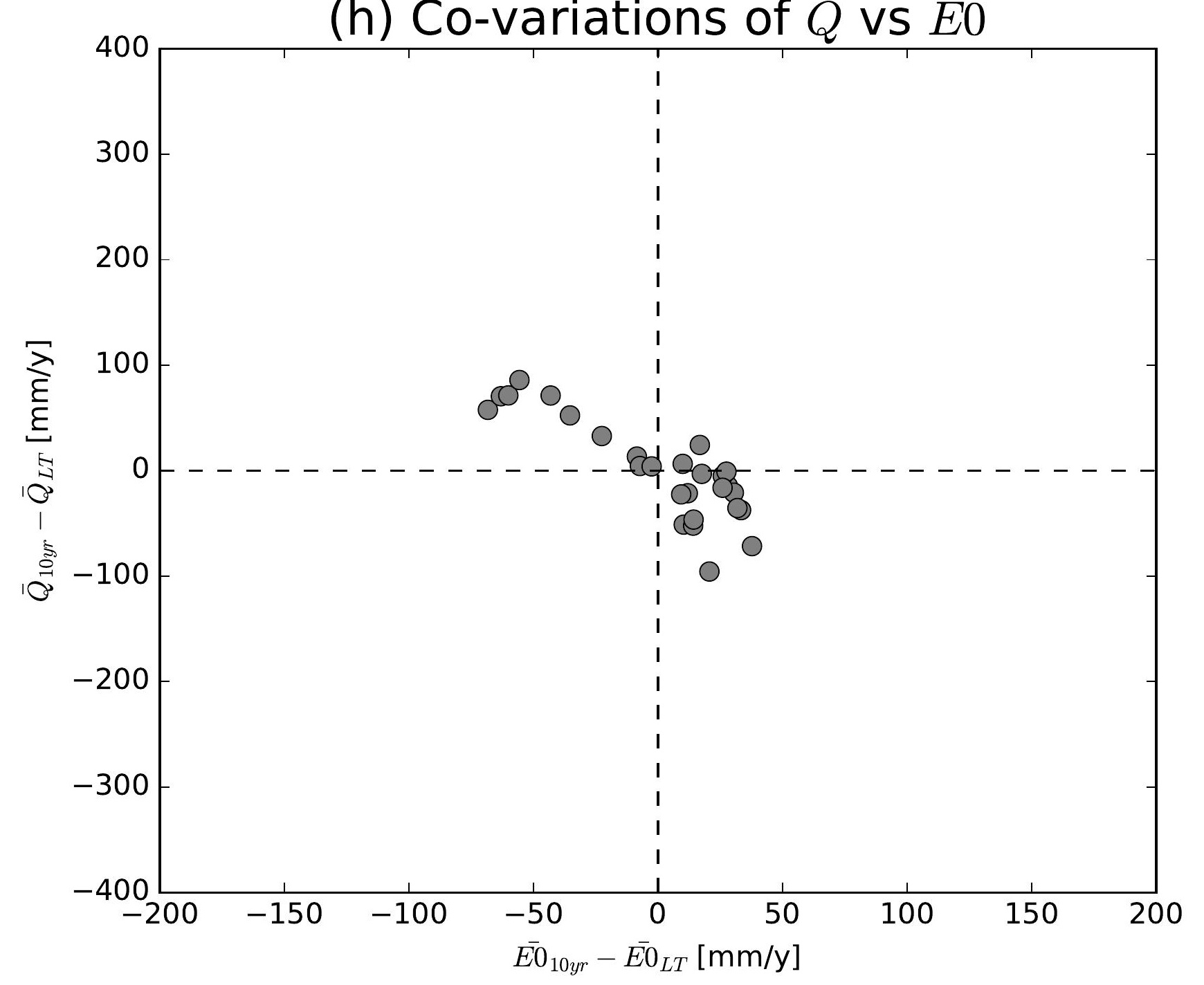

(k) Co-variations of $\bar{Q}$ vs $\overline{E 0}$

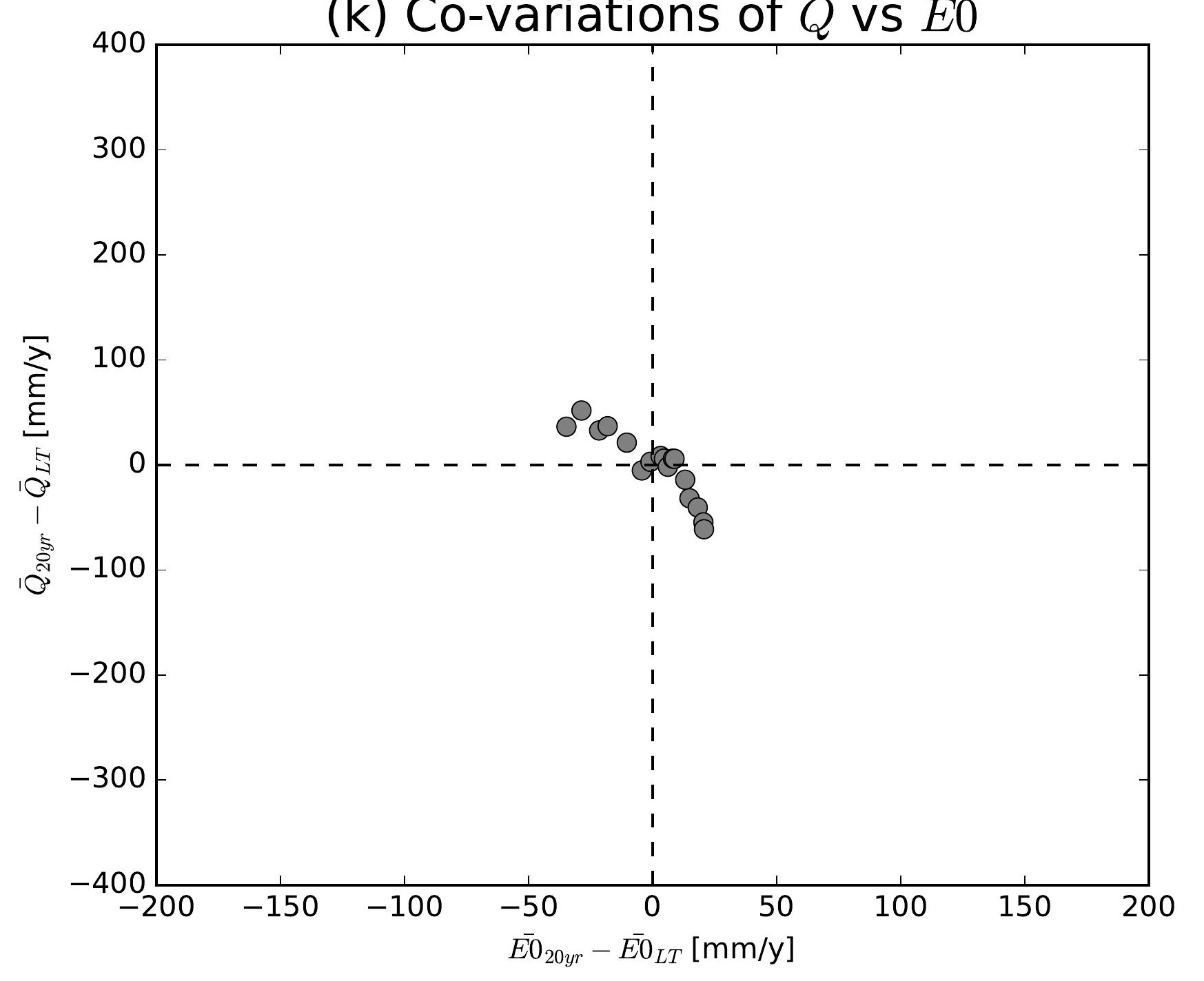

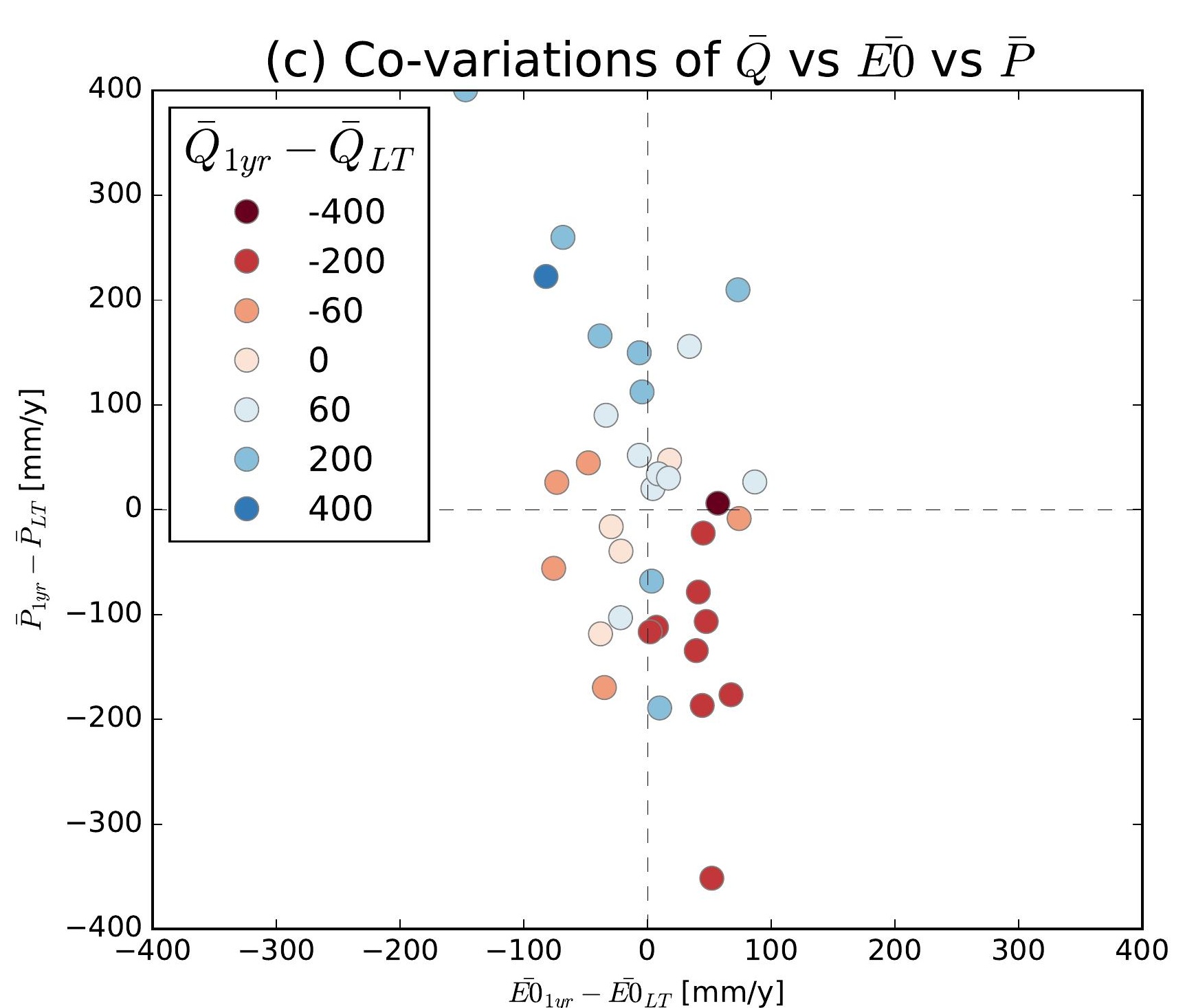
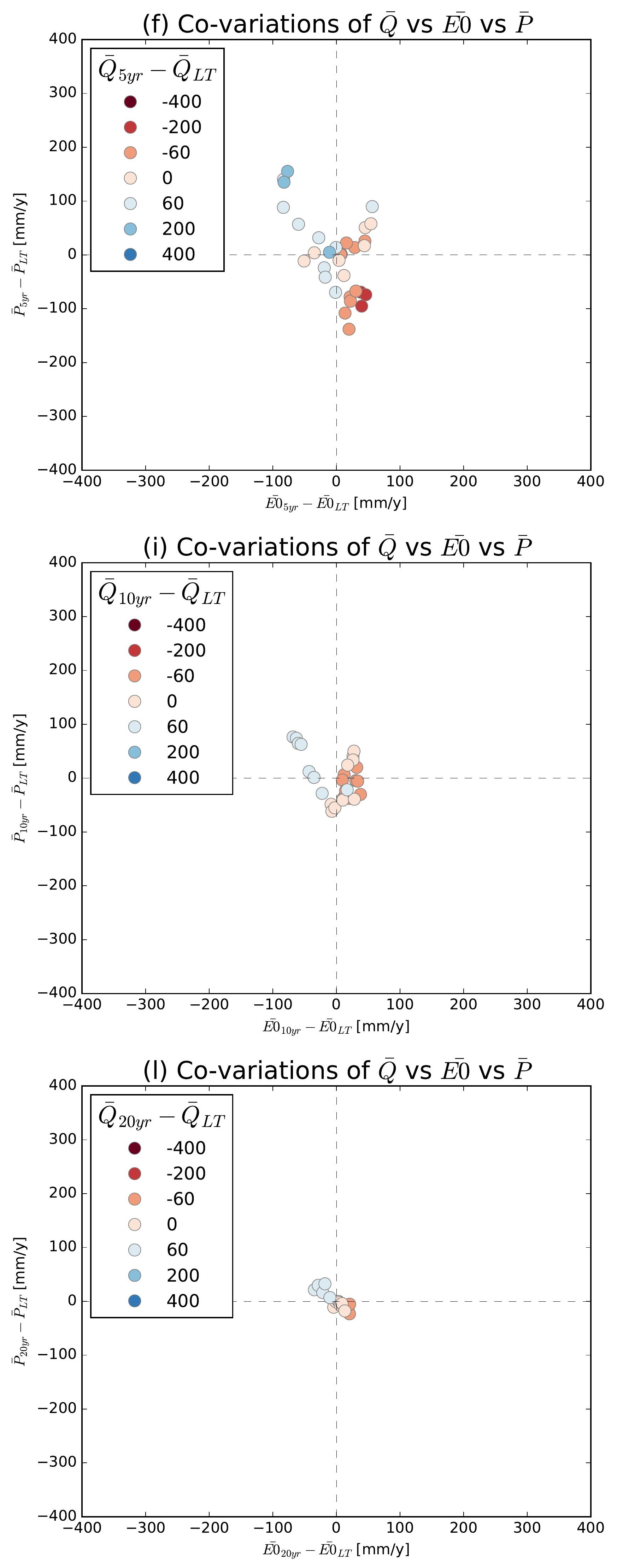

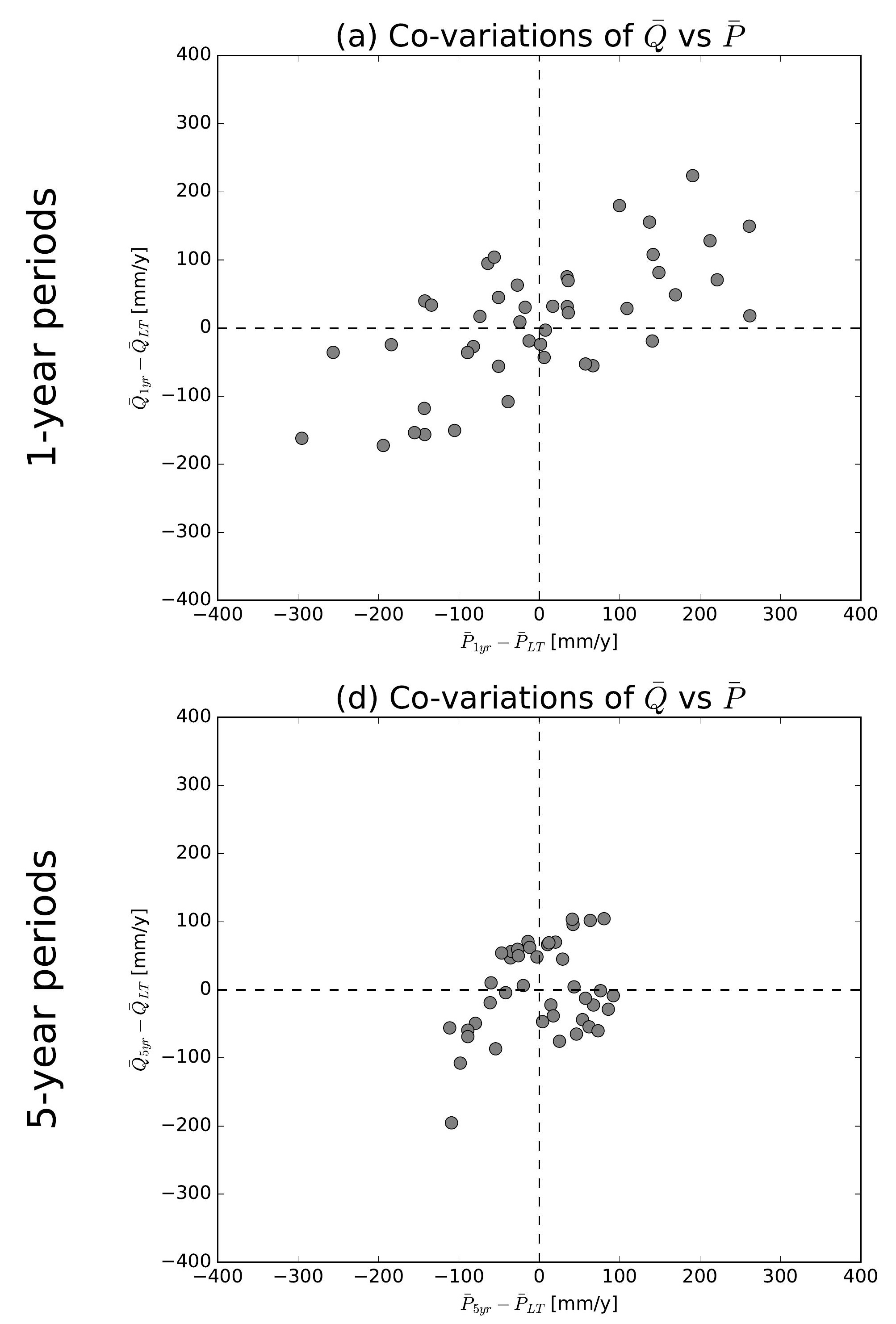

(g) Co-variations of $\bar{Q}$ vs $\bar{P}$

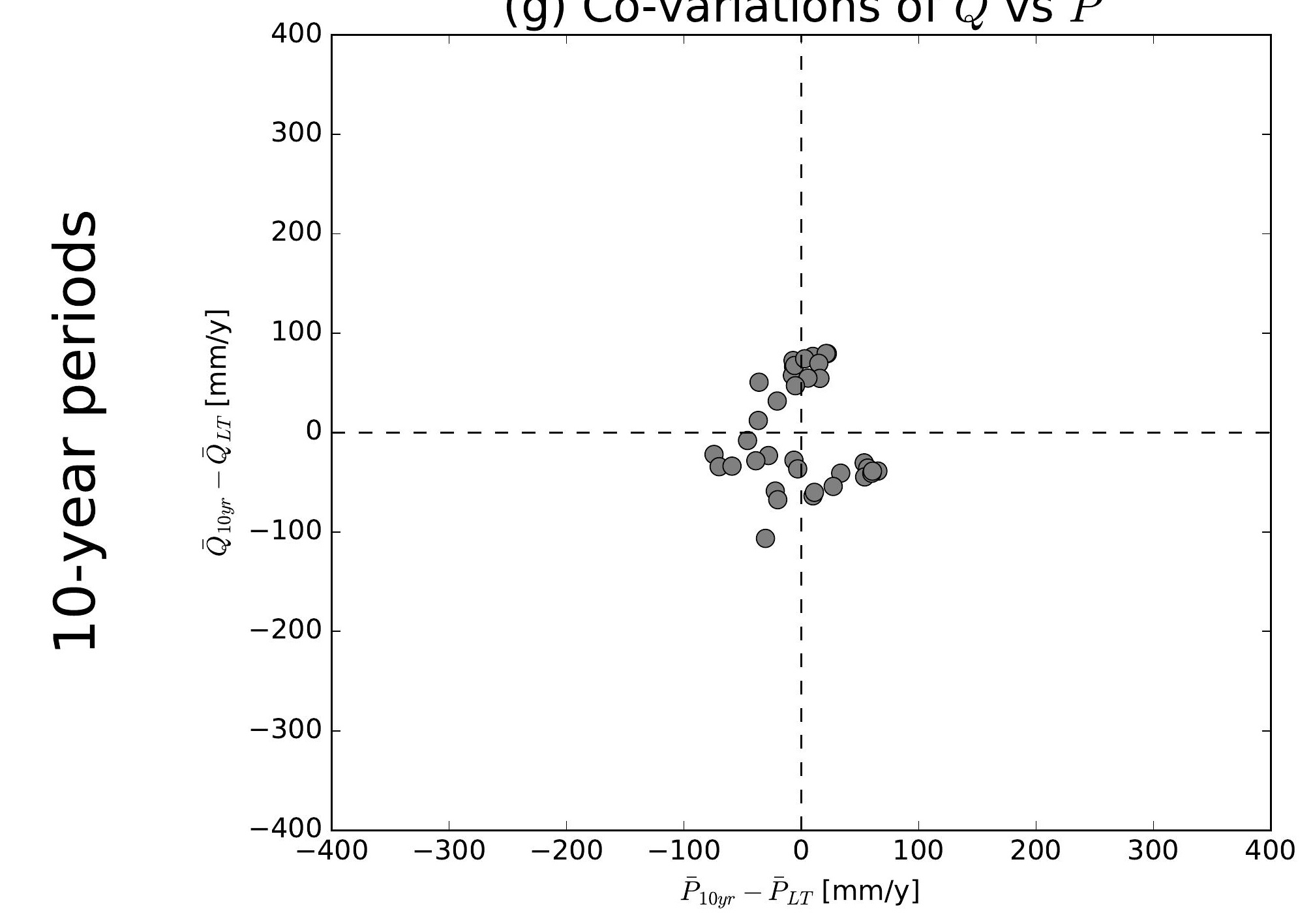

(j) Co-variations of $\bar{Q}$ vs $\bar{P}$

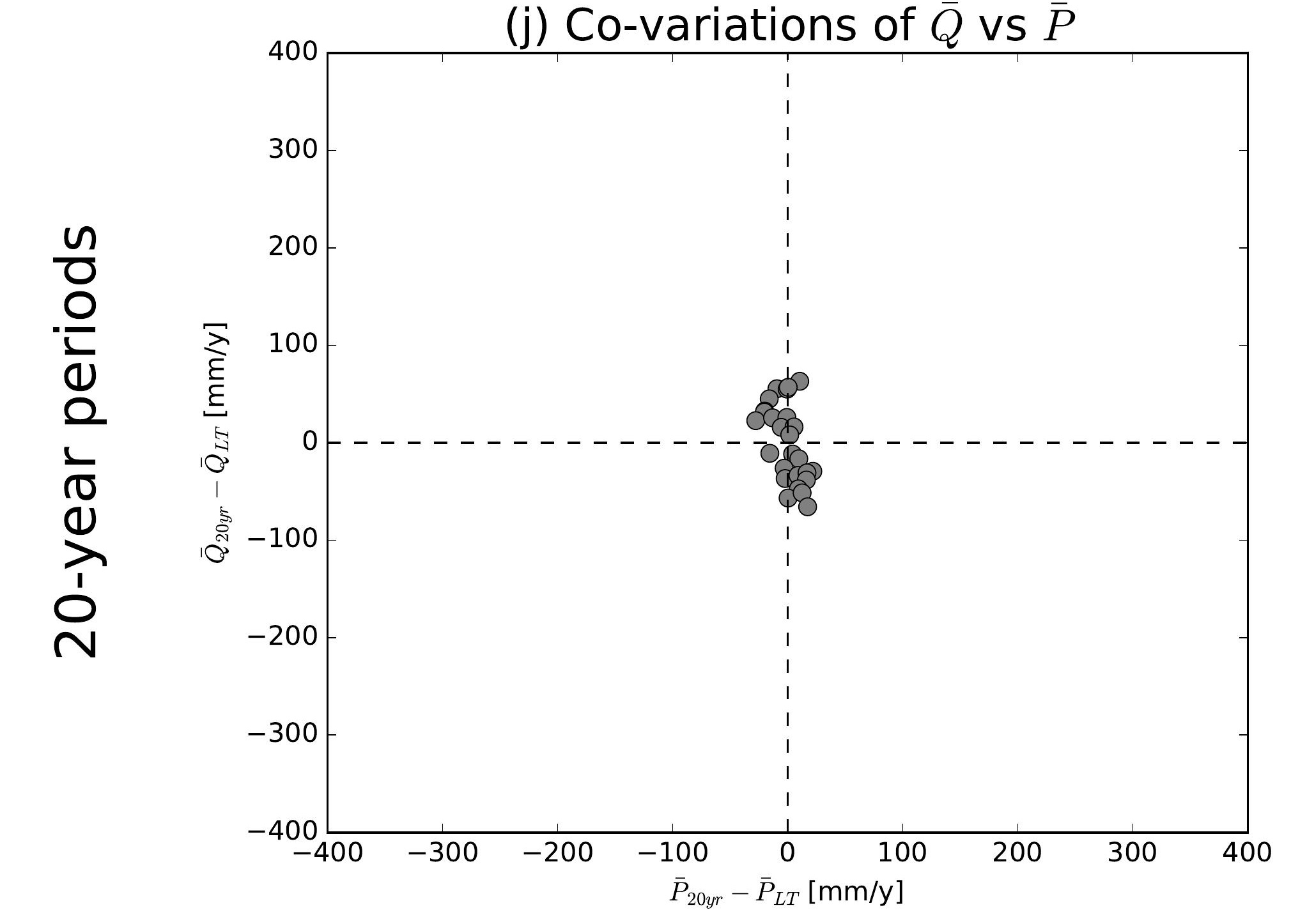

(b) Co-variations of $\bar{Q}$ vs $\overline{E 0}$

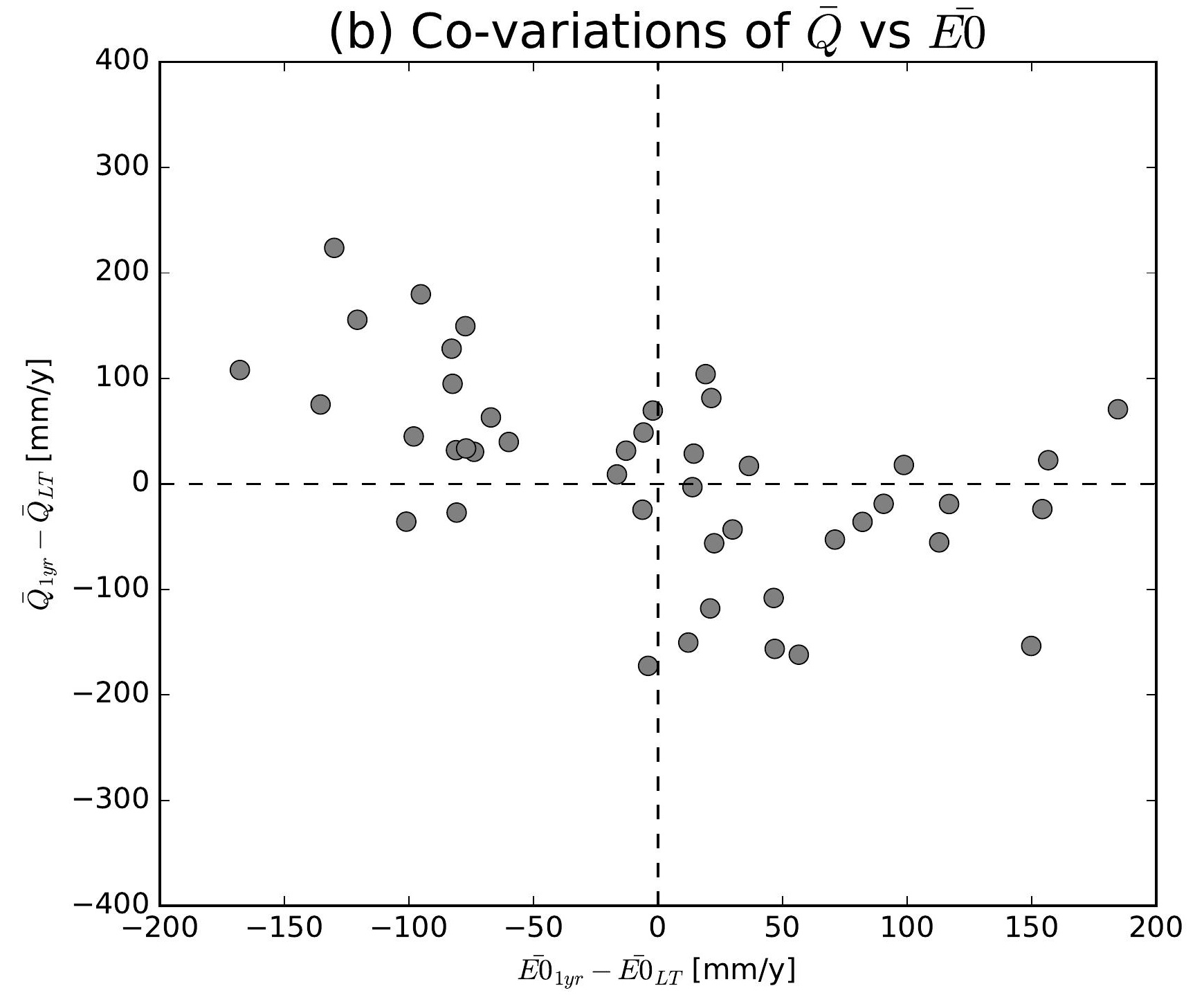

(e) Co-variations of $\bar{Q}$ vs $\overline{E 0}$

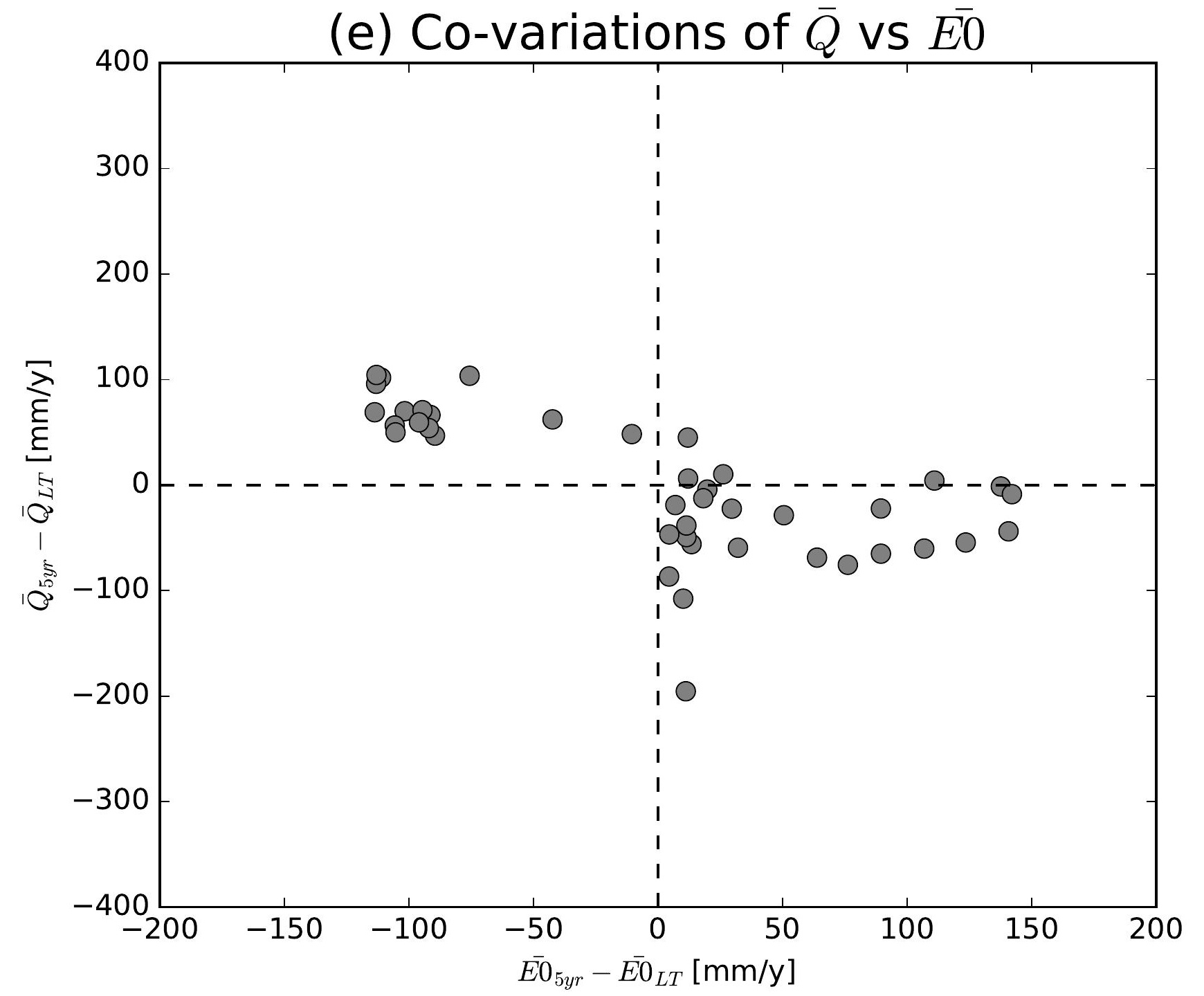

(h) Co-variations of $\bar{Q}$ vs $\overline{E 0}$

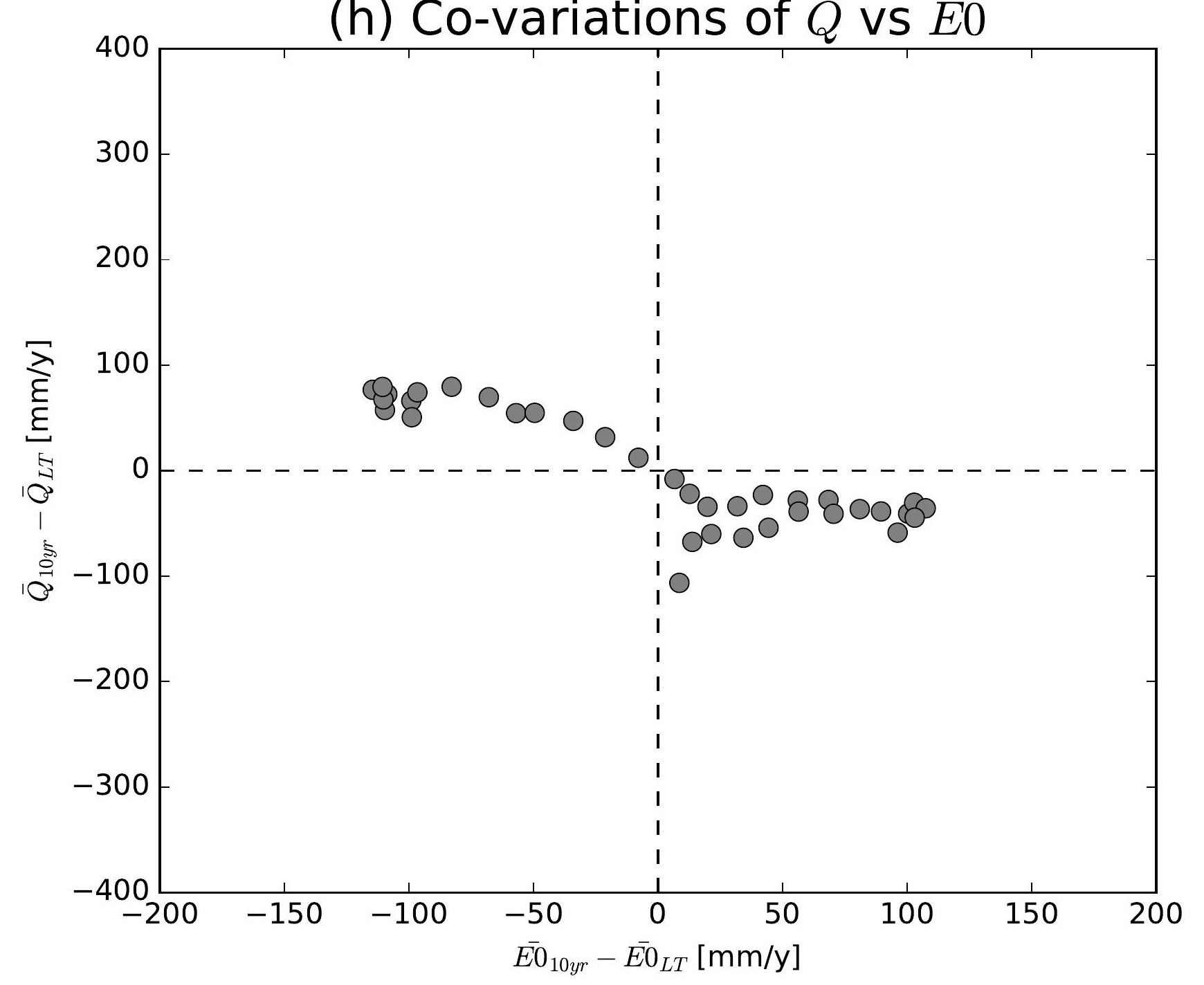

(k) Co-variations of $\bar{Q}$ vs $\overline{E 0}$

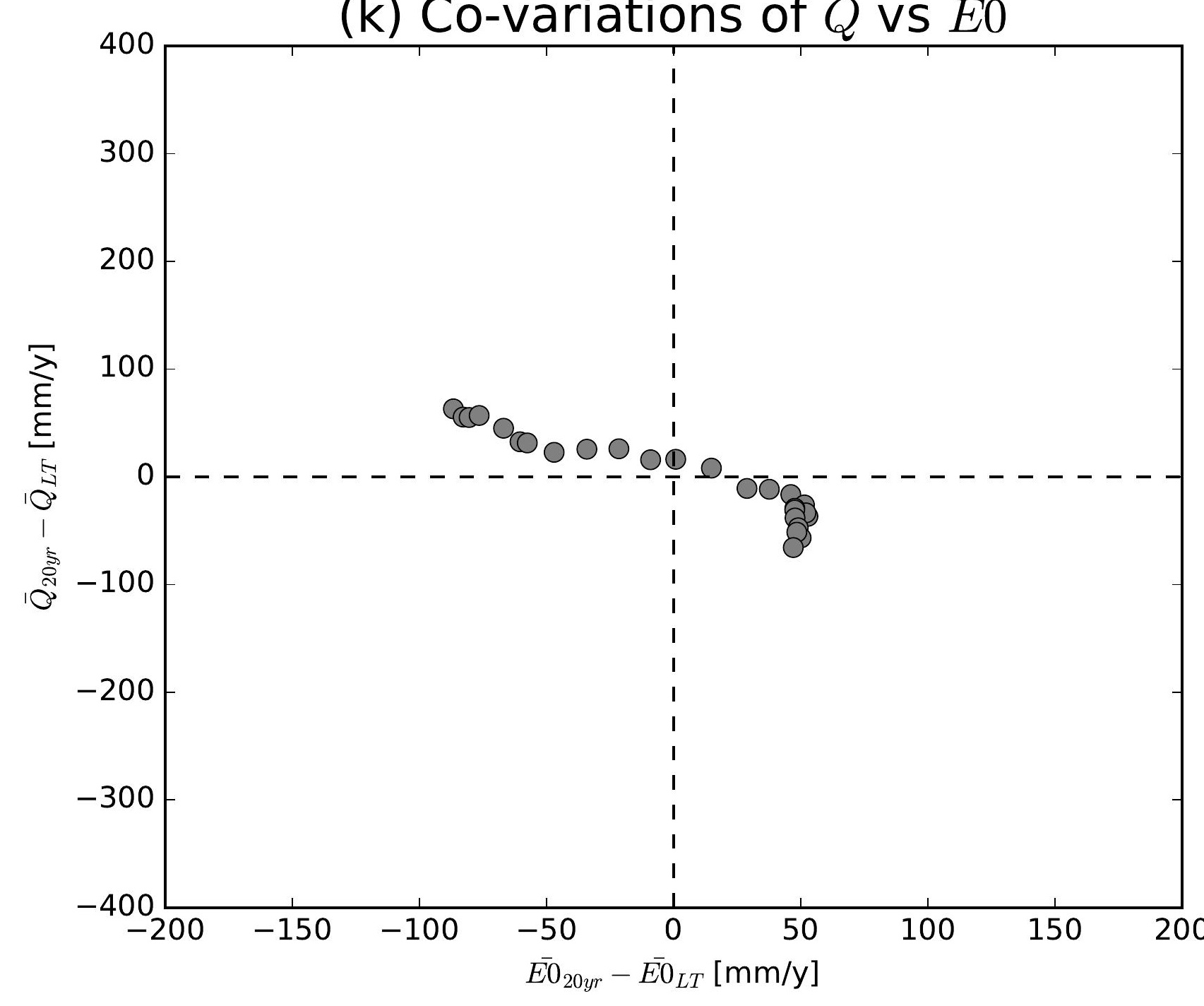

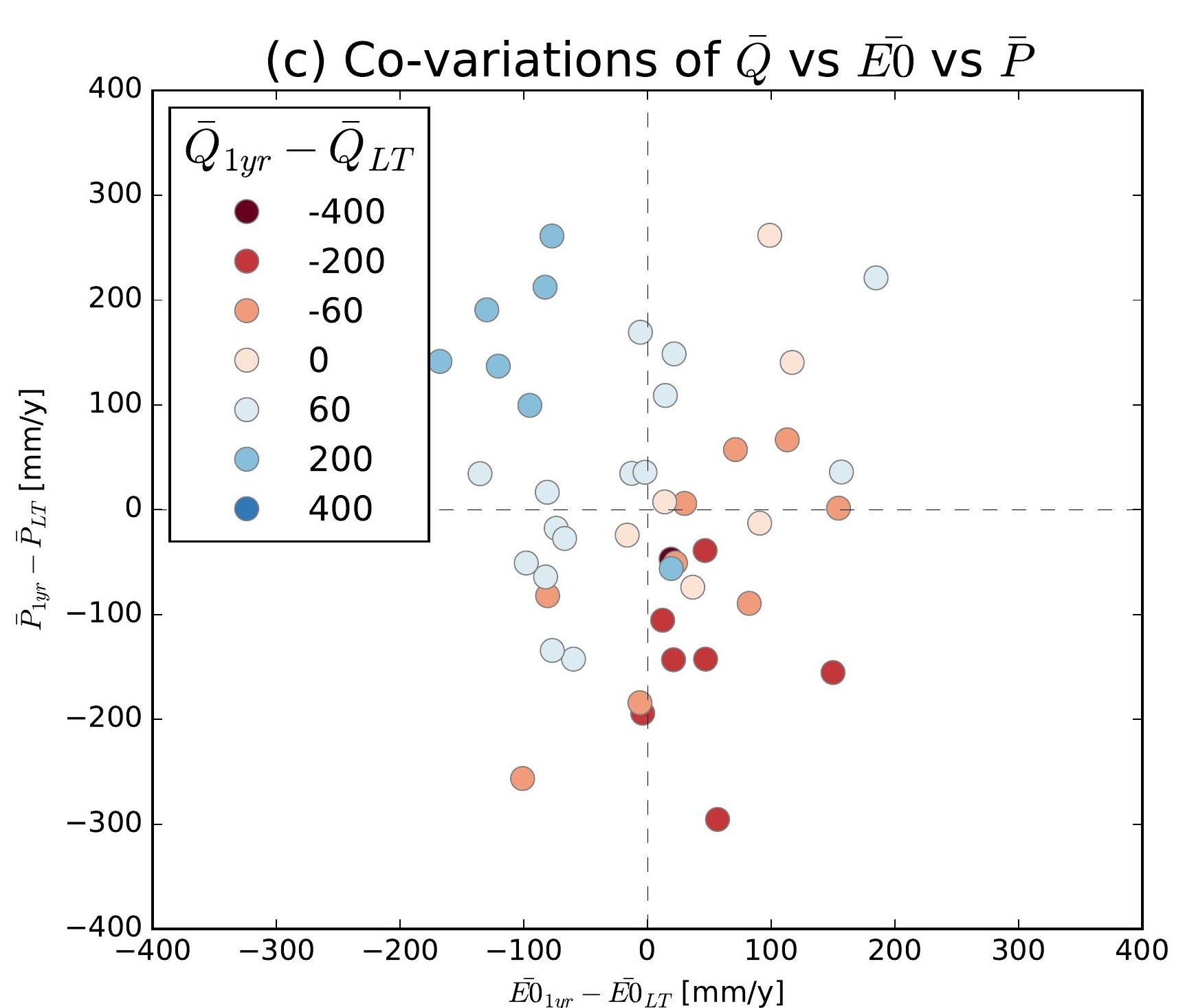
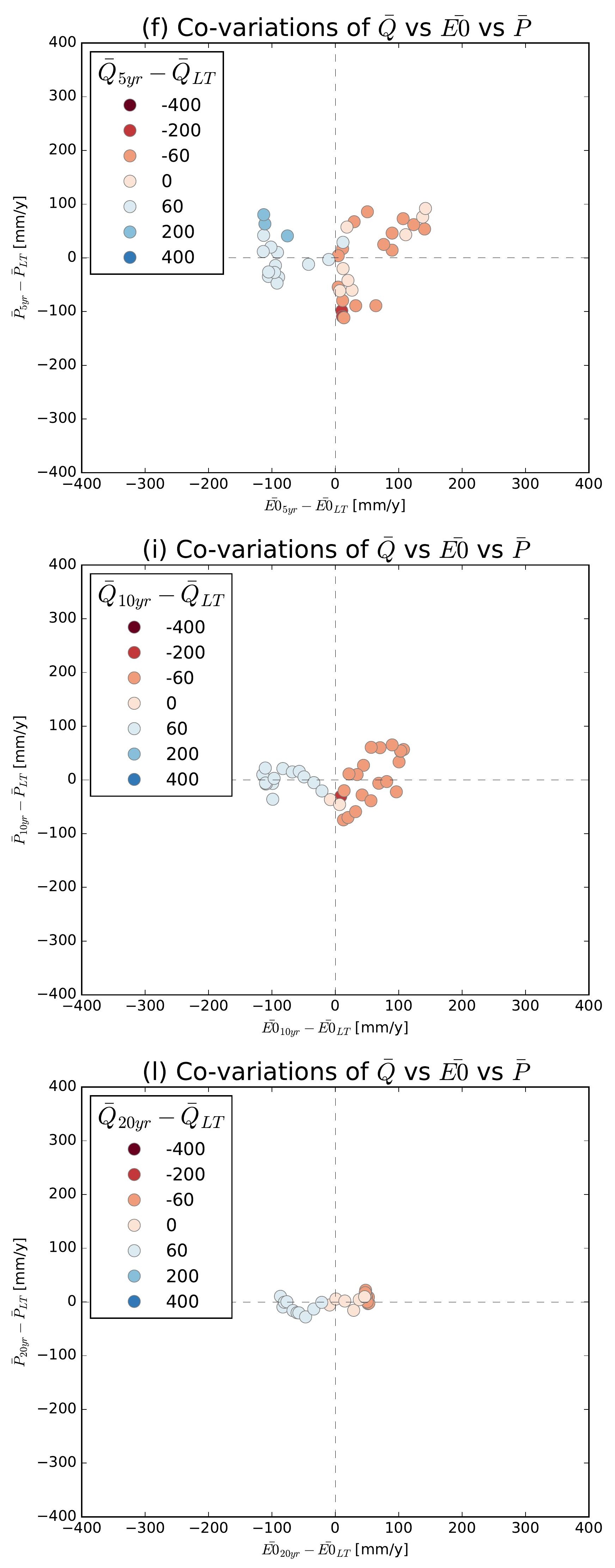

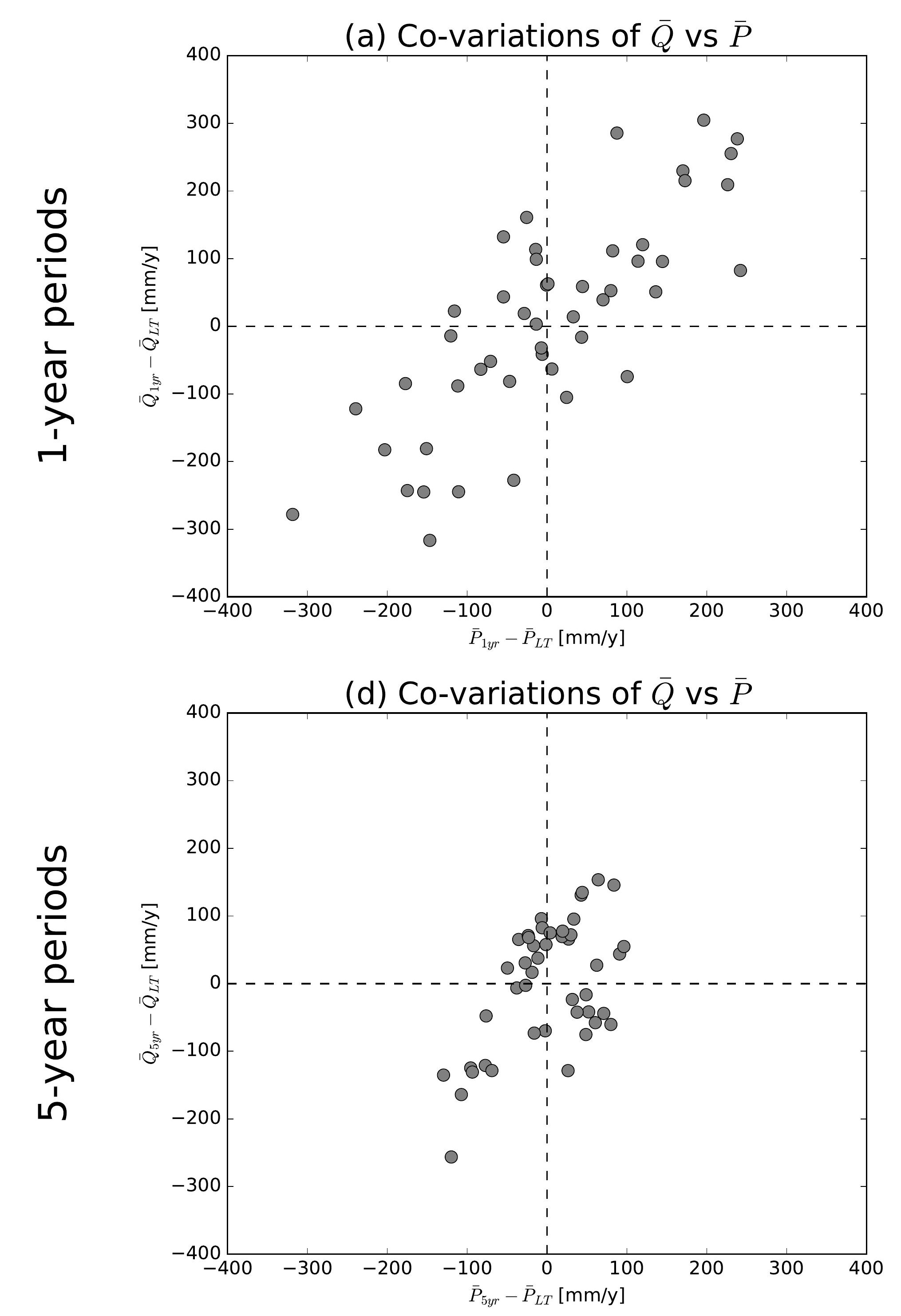

(g) Co-variations of $\bar{Q}$ vs $\bar{P}$
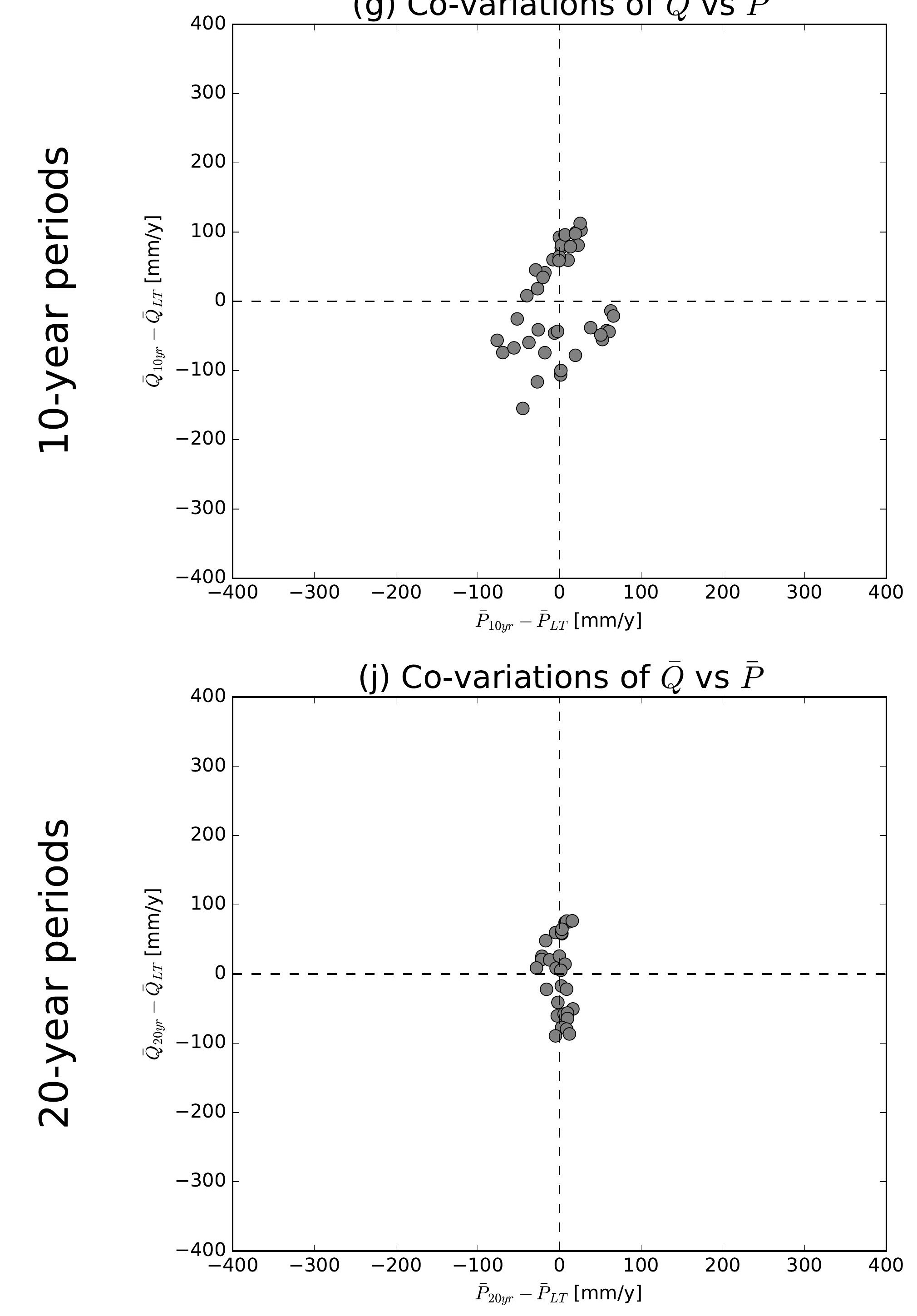

(b) Co-variations of $\bar{Q}$ vs $\overline{E 0}$

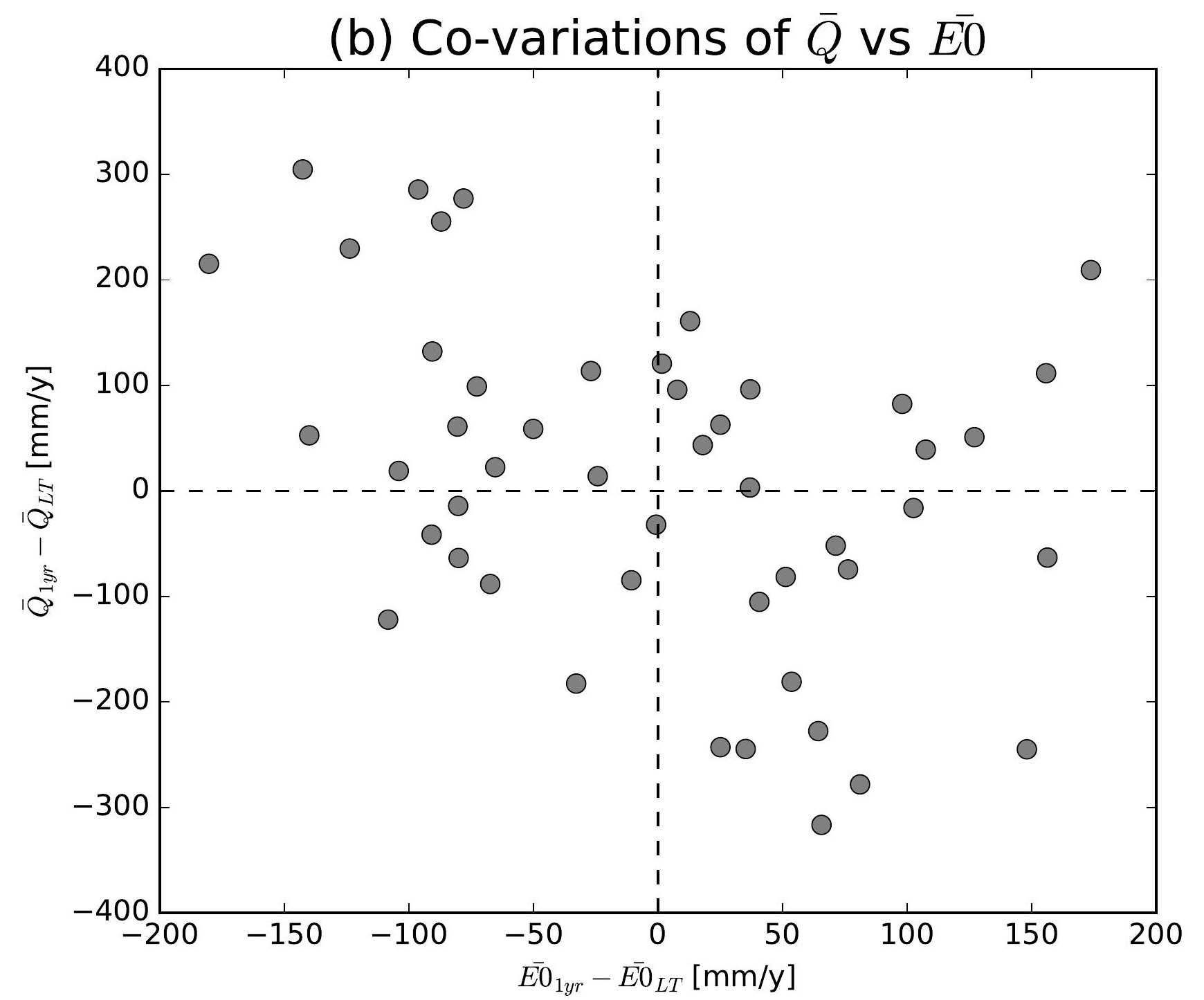

(e) Co-variations of $\bar{Q}$ vs $\overline{E 0}$

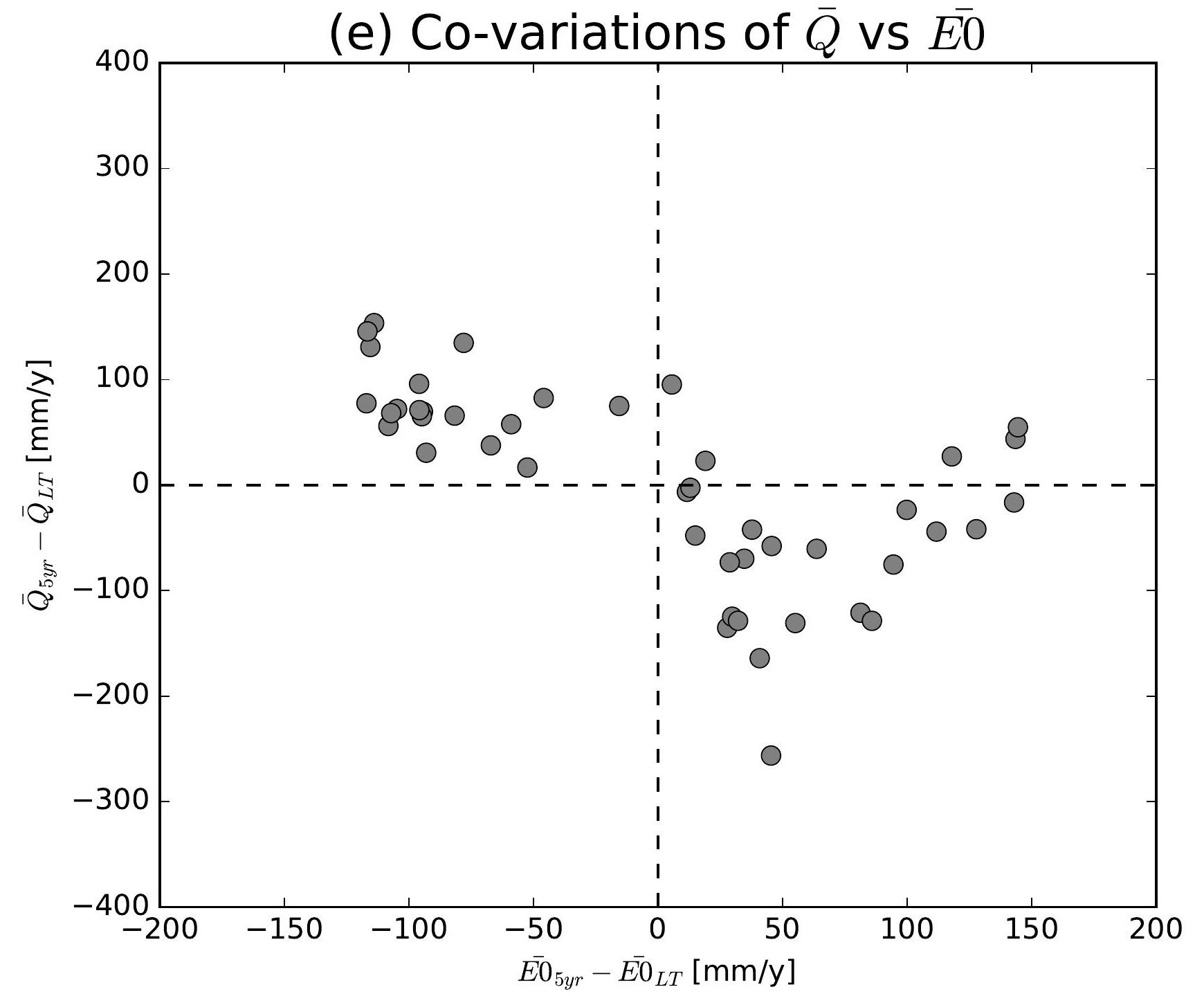

(h) Co-variations of $\bar{Q}$ vs $\overline{E 0}$

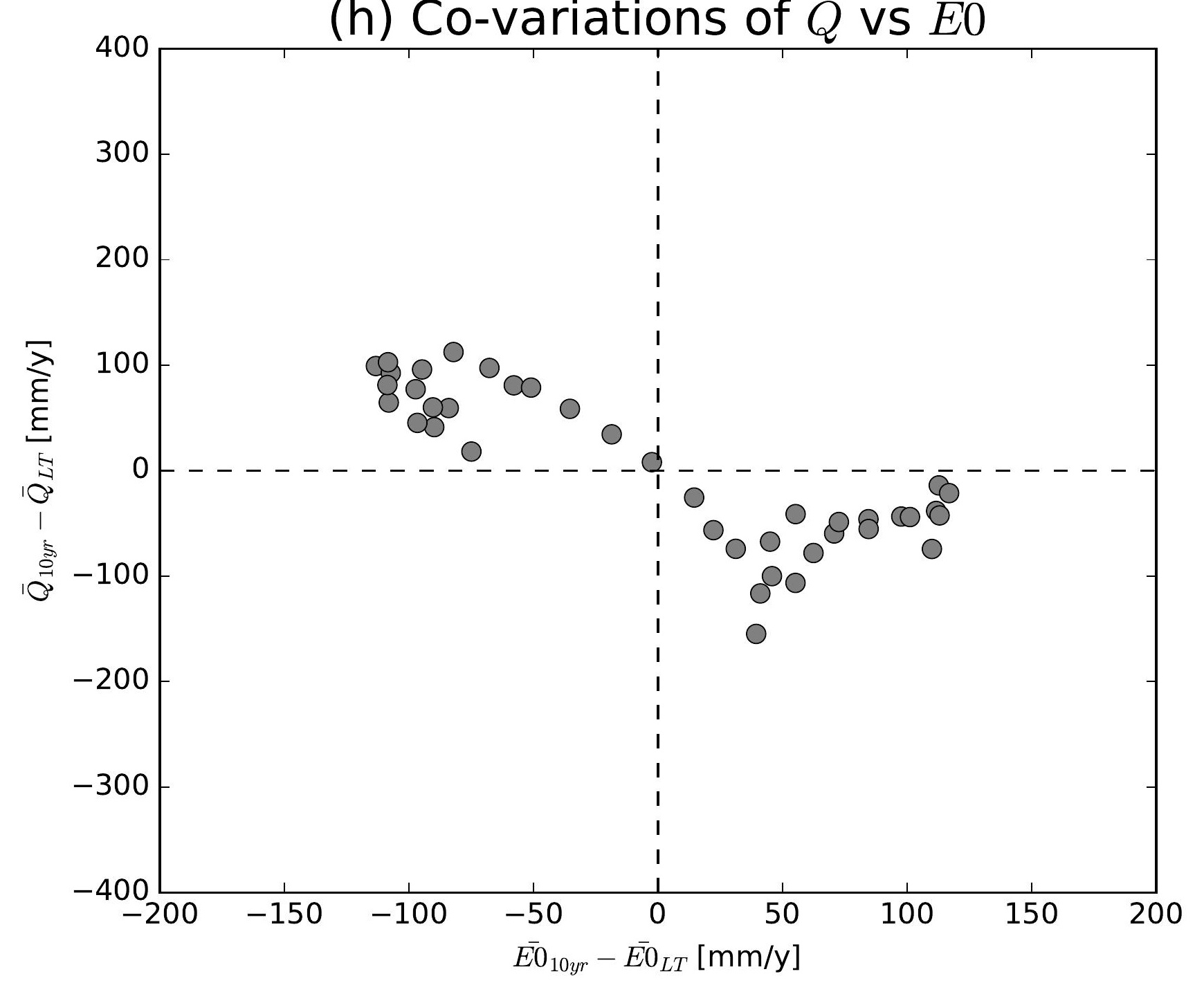

(k) Co-variations of $\bar{Q}$ vs $\overline{E 0}$

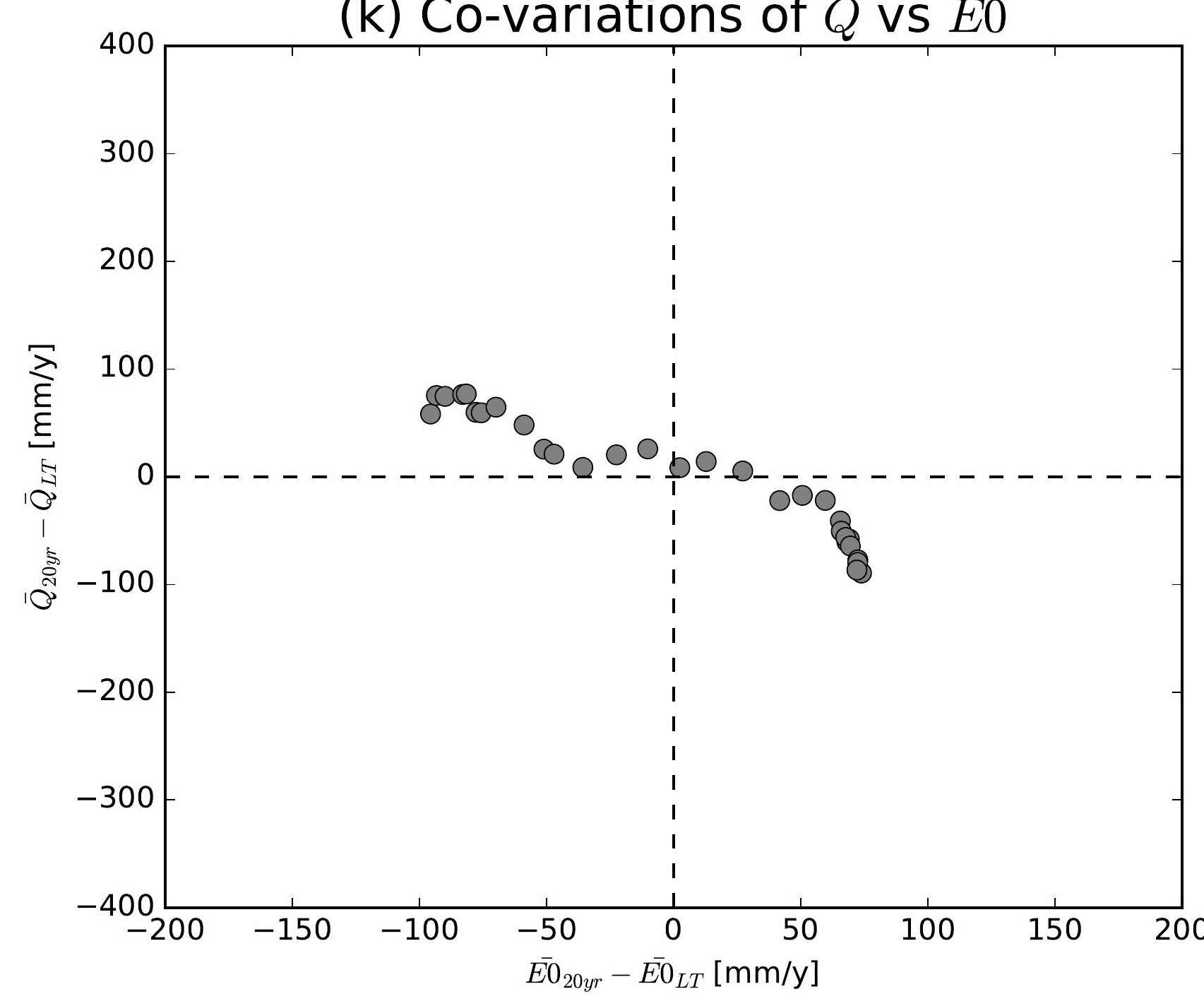

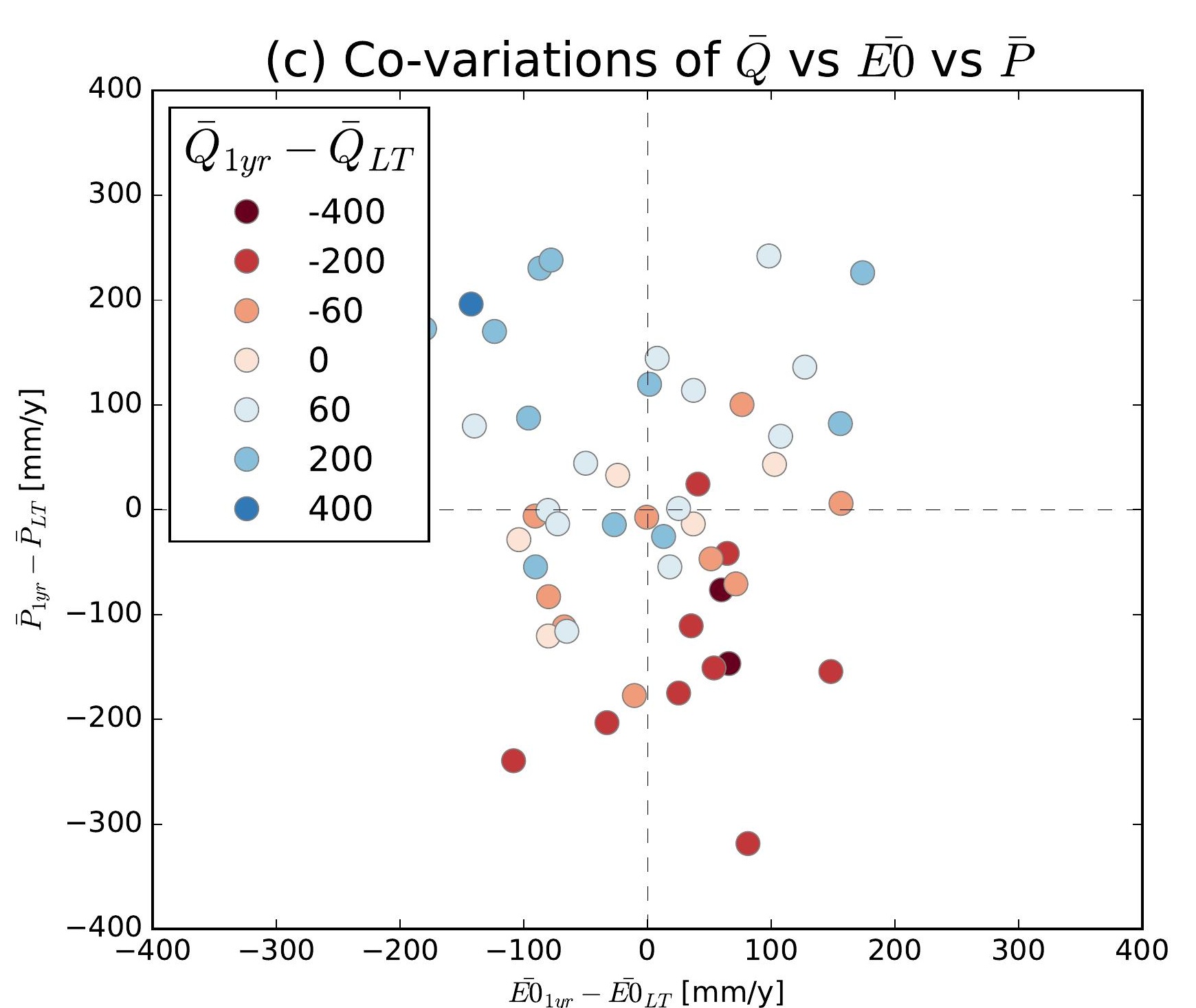
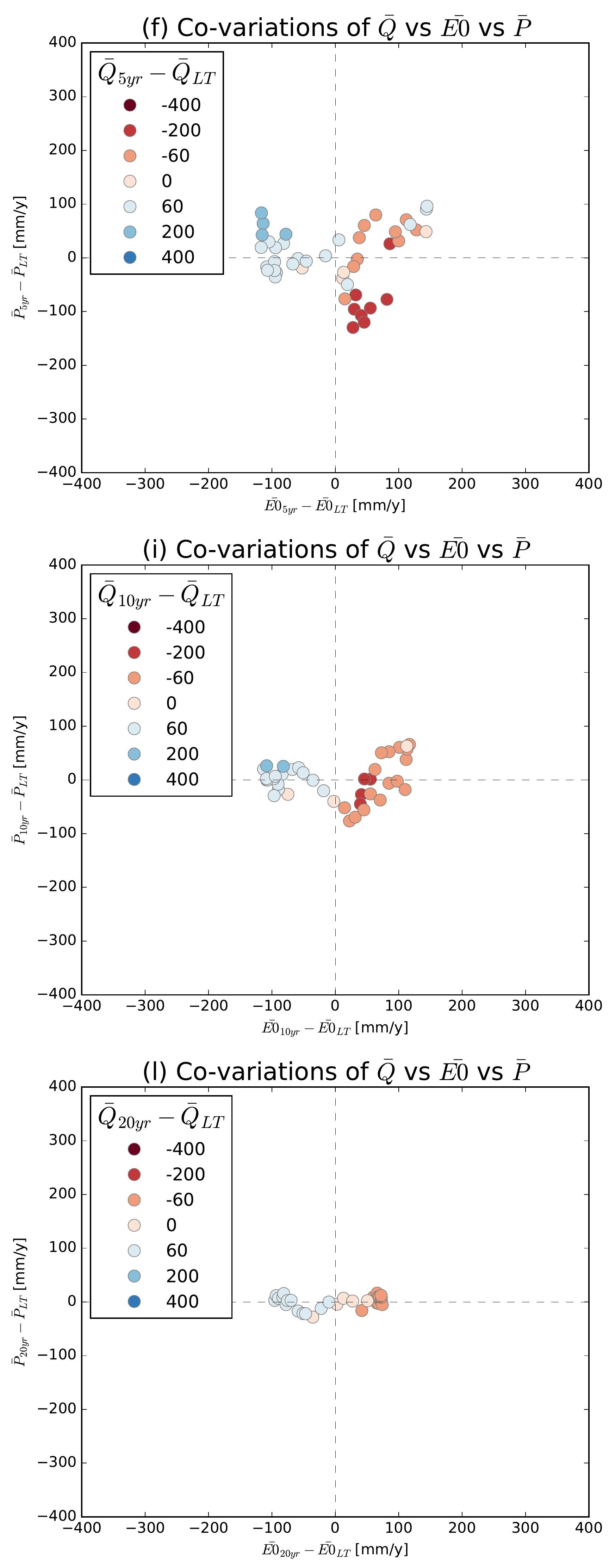

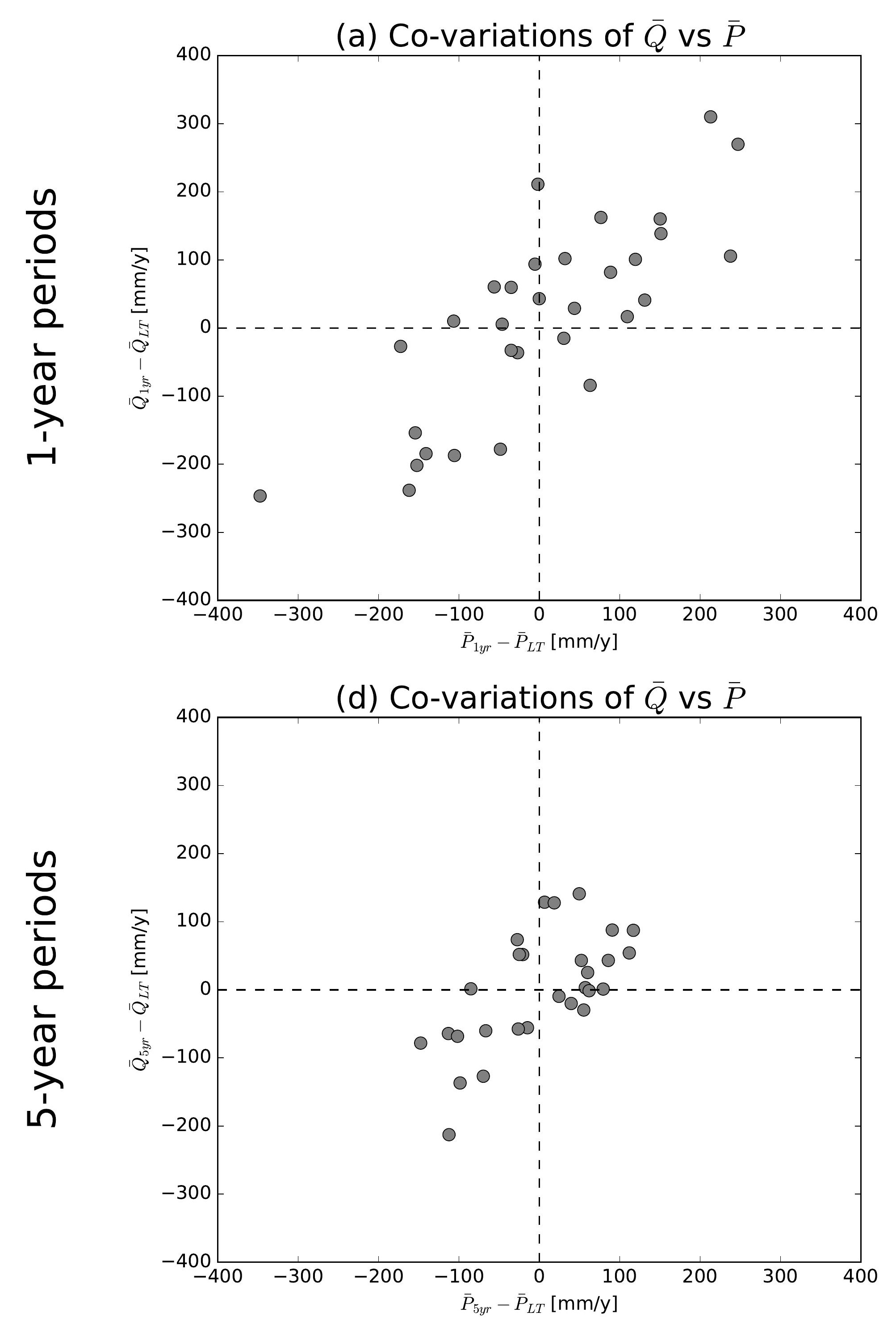

(g) Co-variations of $\bar{Q}$ vs $\bar{P}$

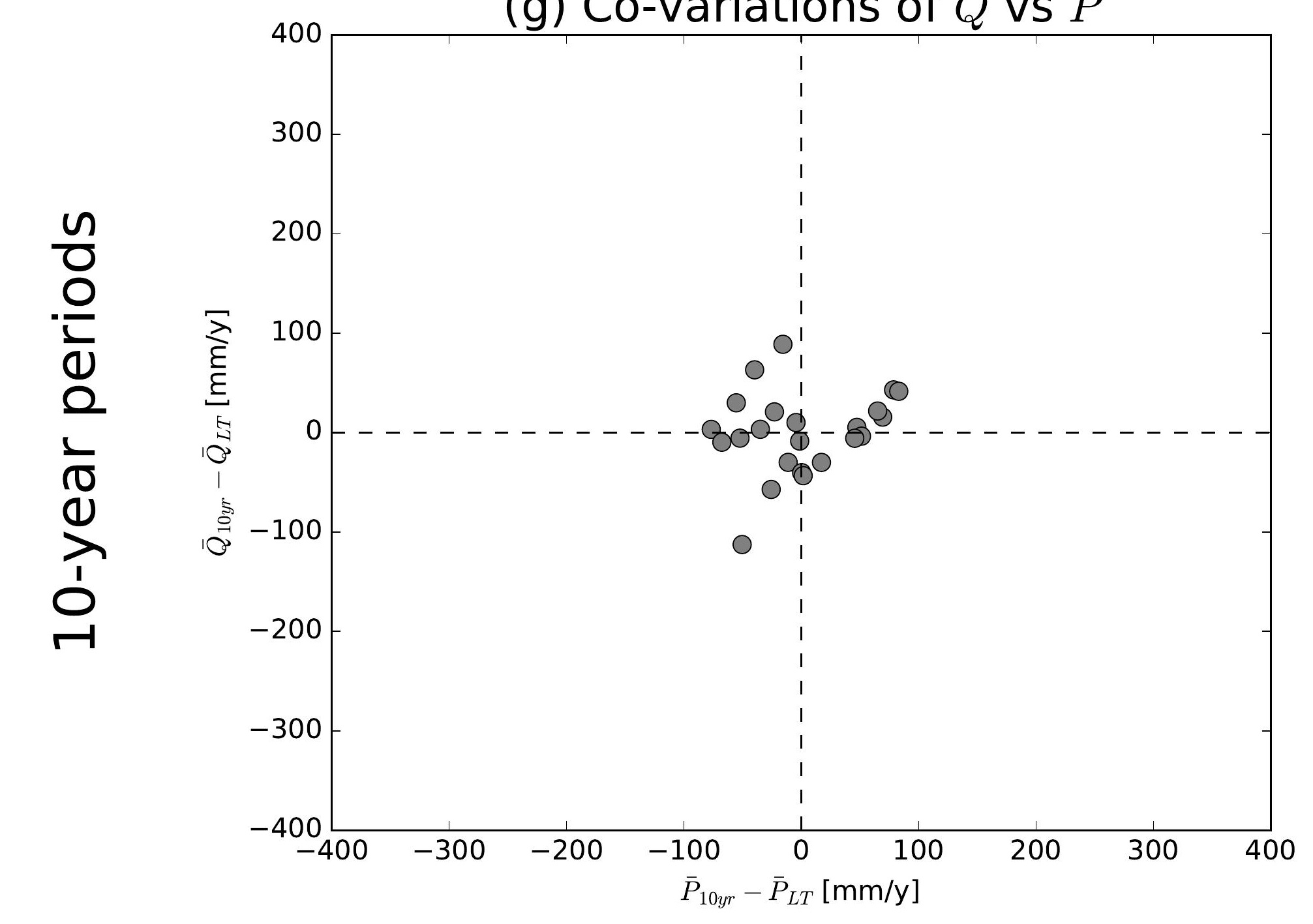

(j) Co-variations of $\bar{Q}$ vs $\bar{P}$

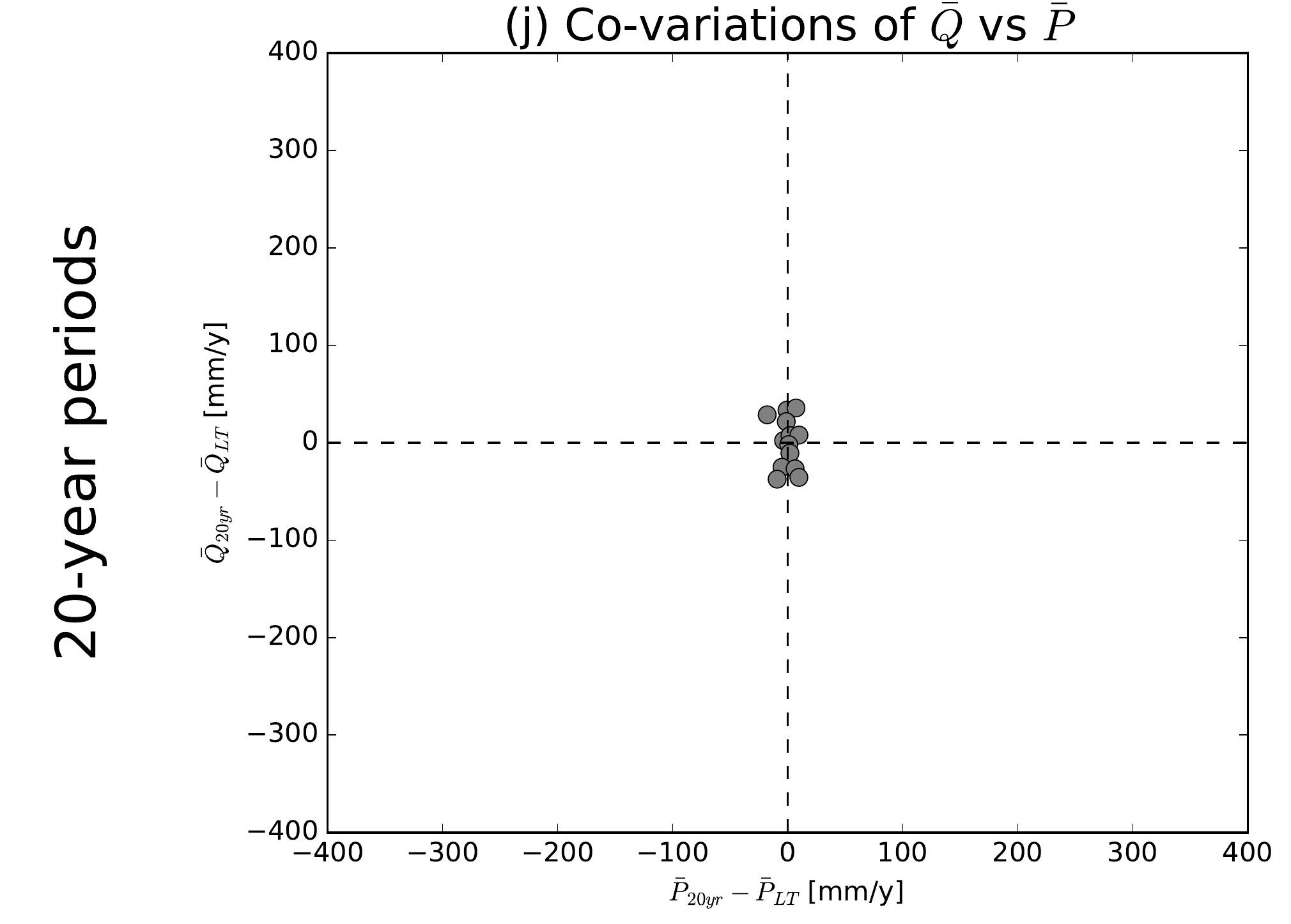

(b) Co-variations of $\bar{Q}$ vs $\overline{E 0}$

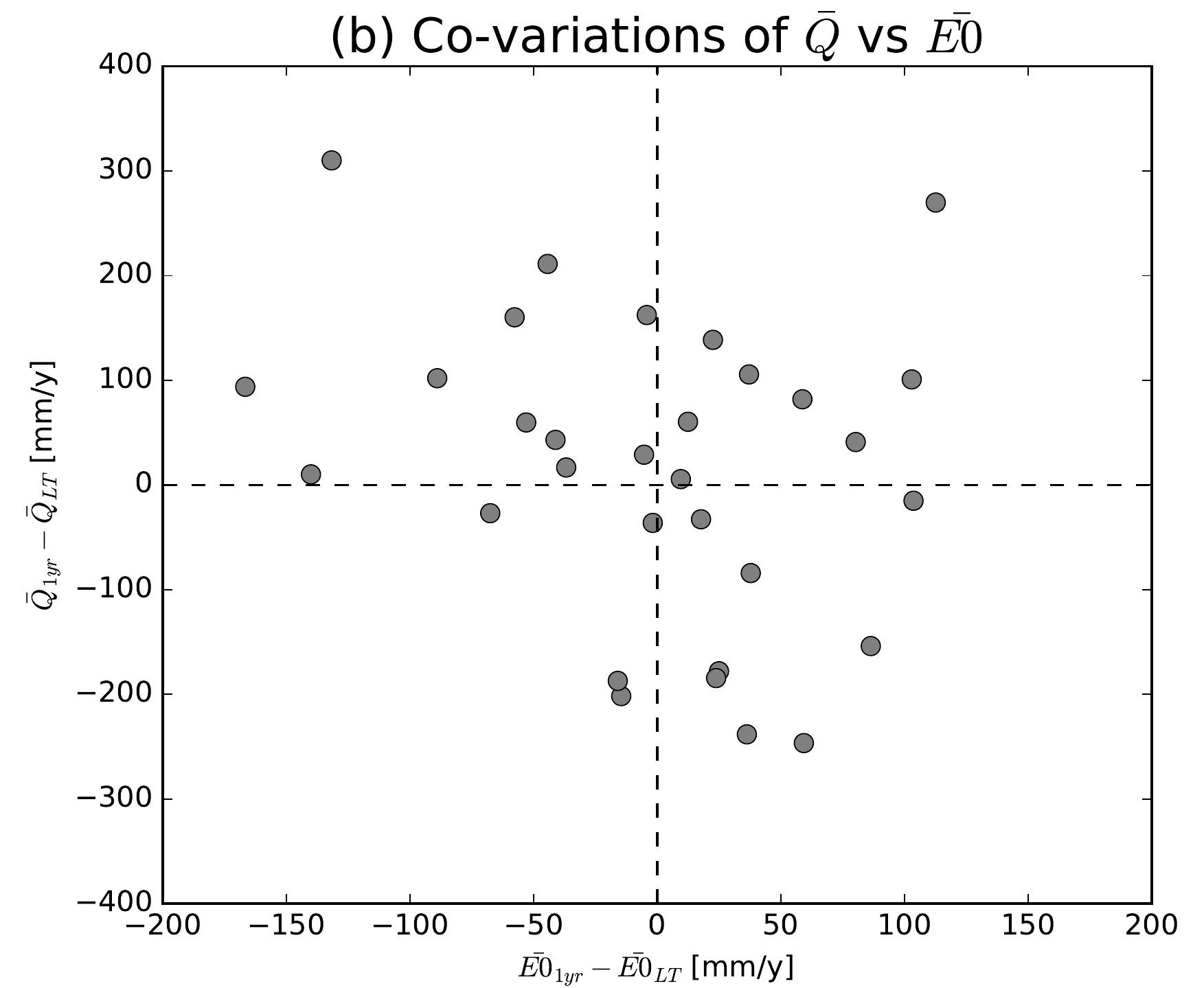

(e) Co-variations of $\bar{Q}$ vs $\overline{E 0}$

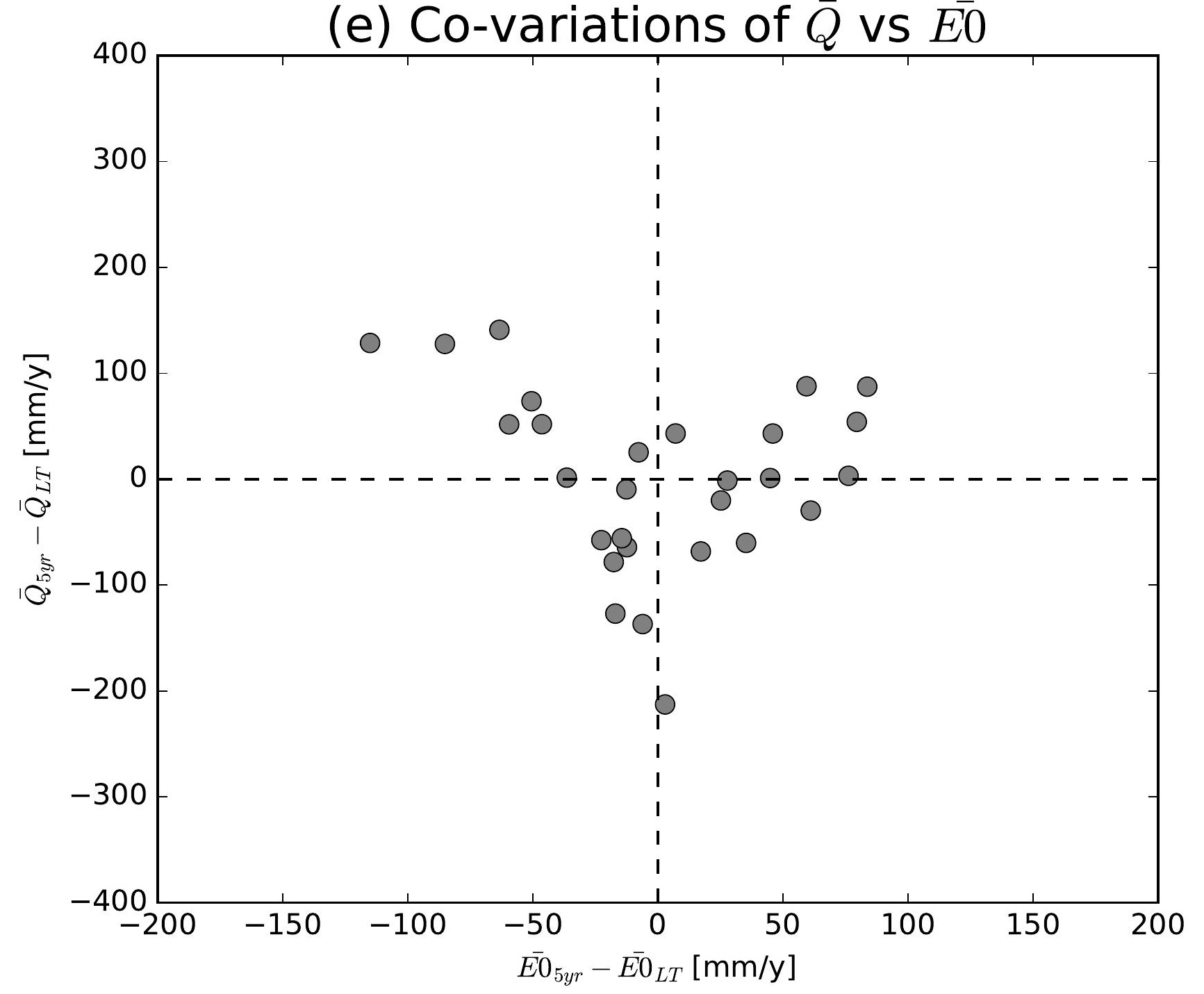

(h) Co-variations of $\bar{Q}$ vs $\overline{E 0}$

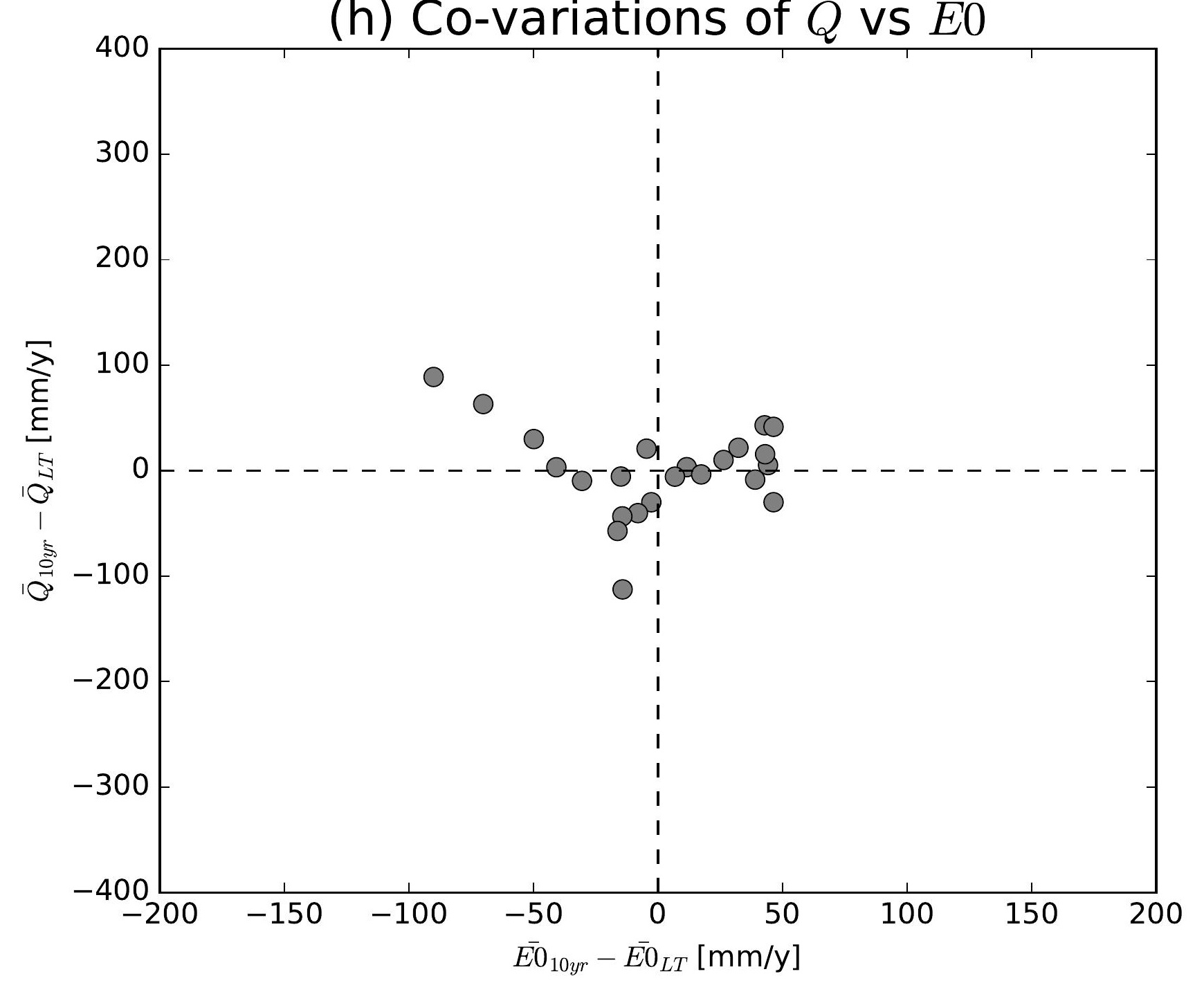

(k) Co-variations of $\bar{Q}$ vs $\overline{E 0}$

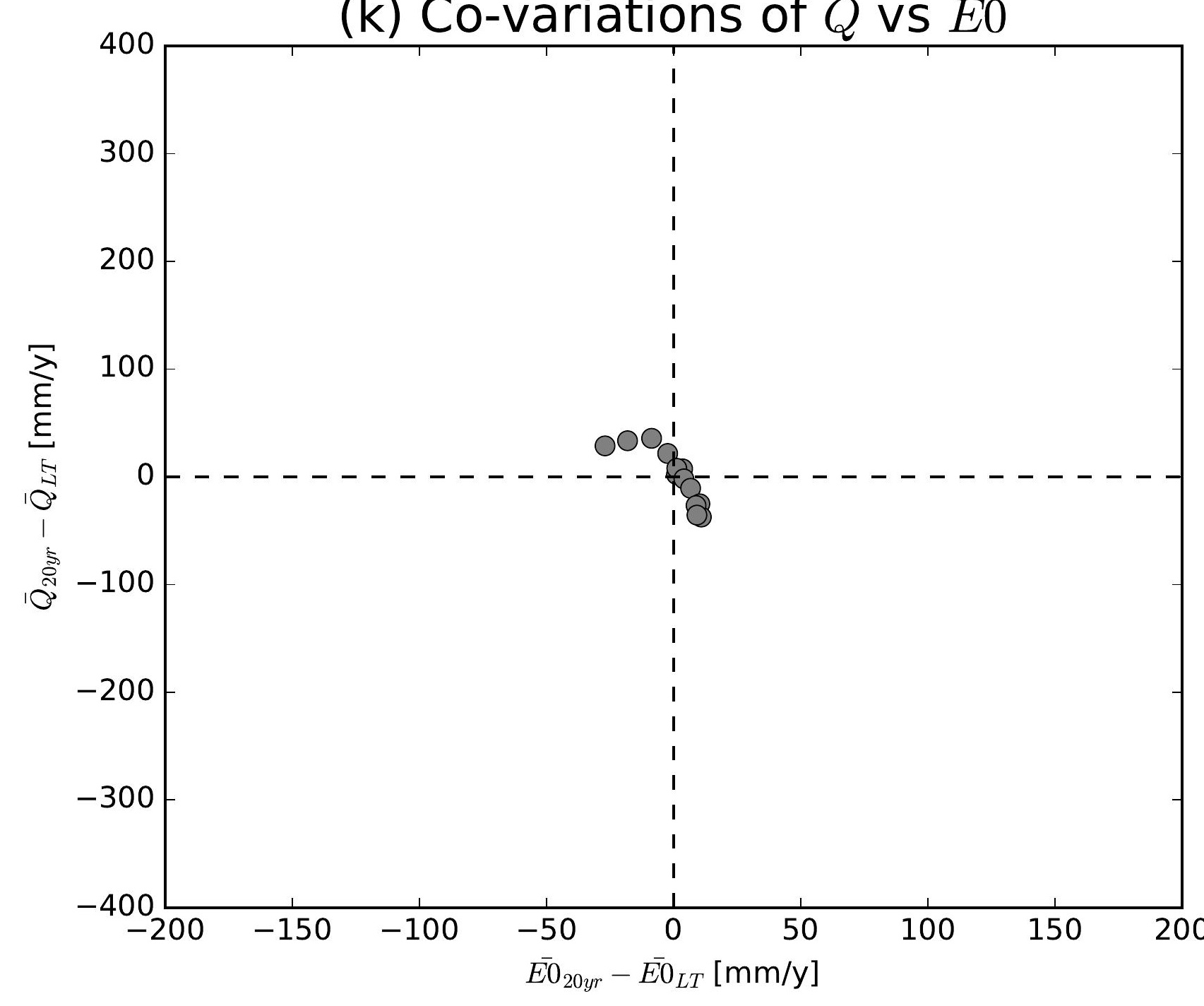

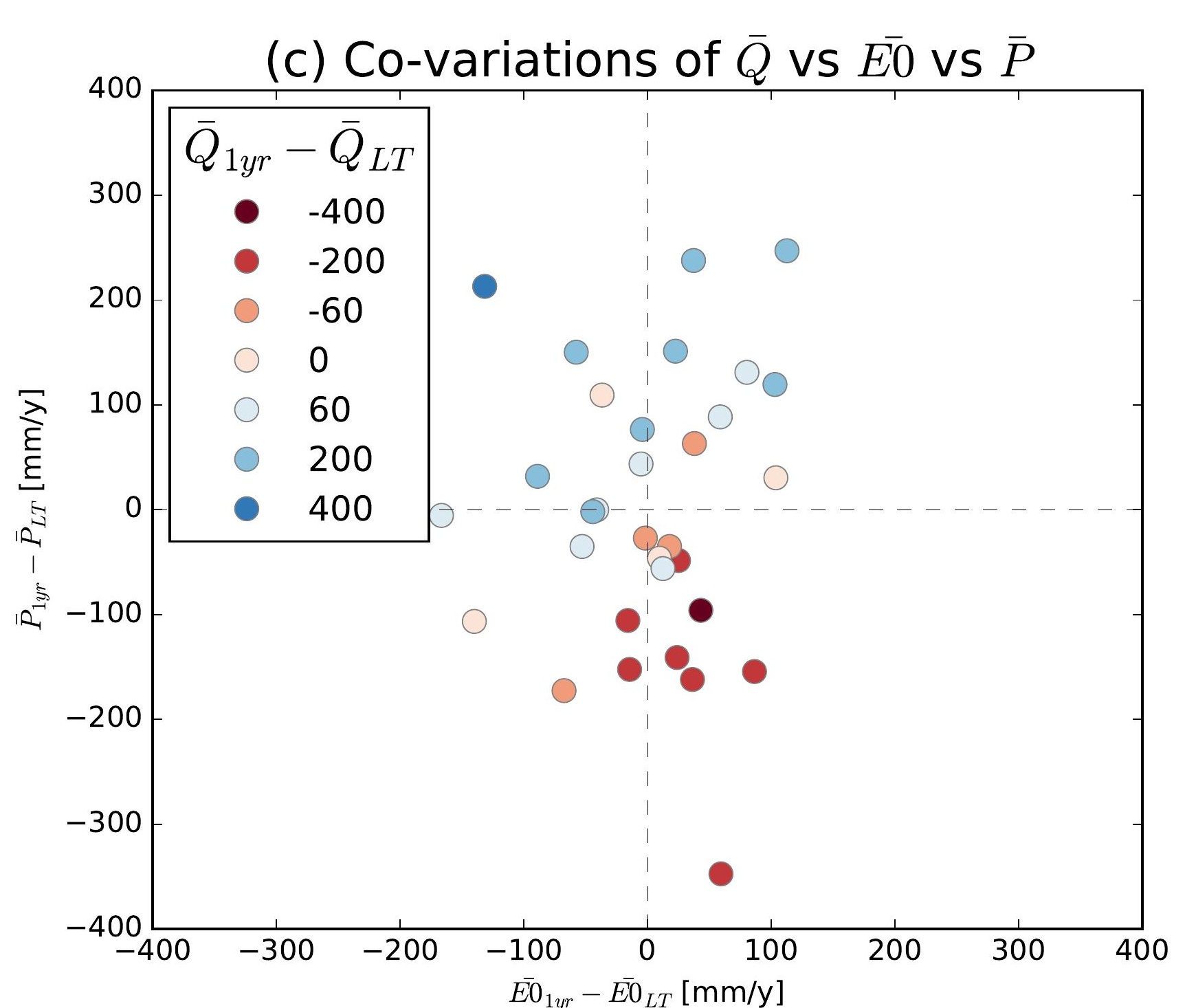
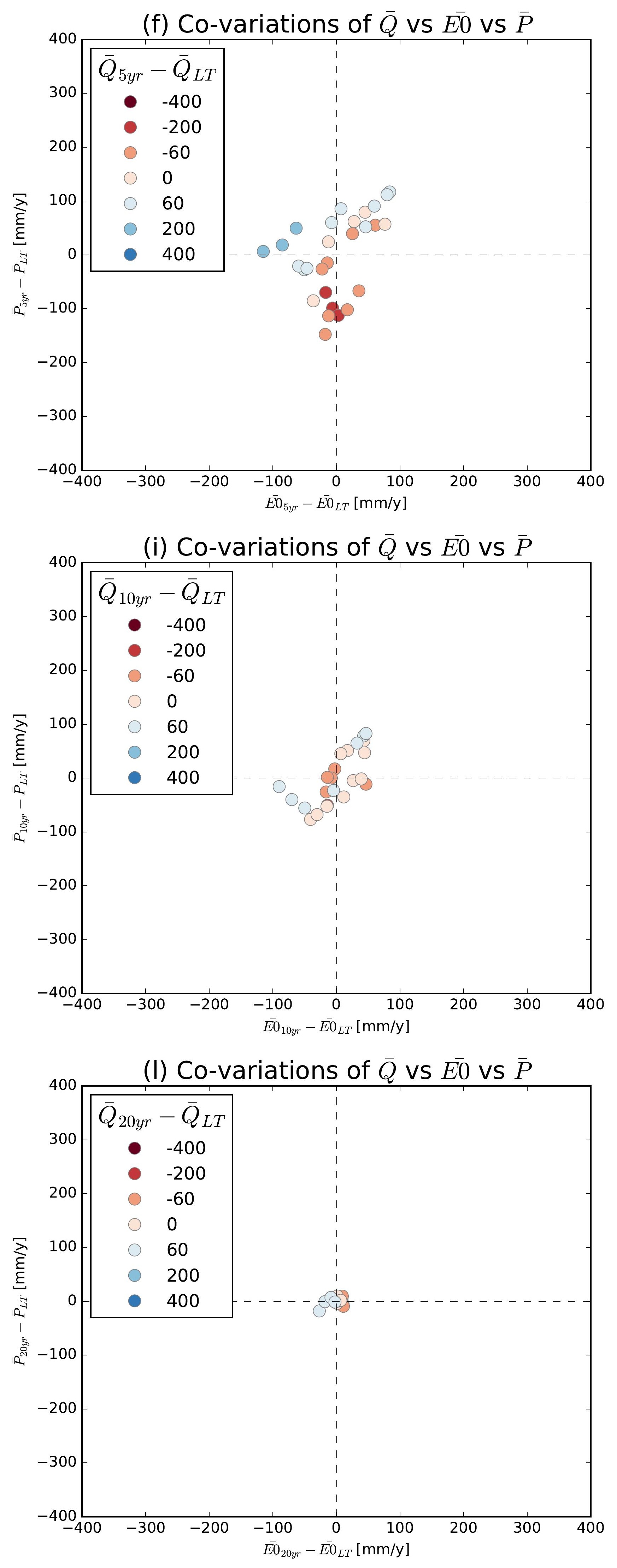

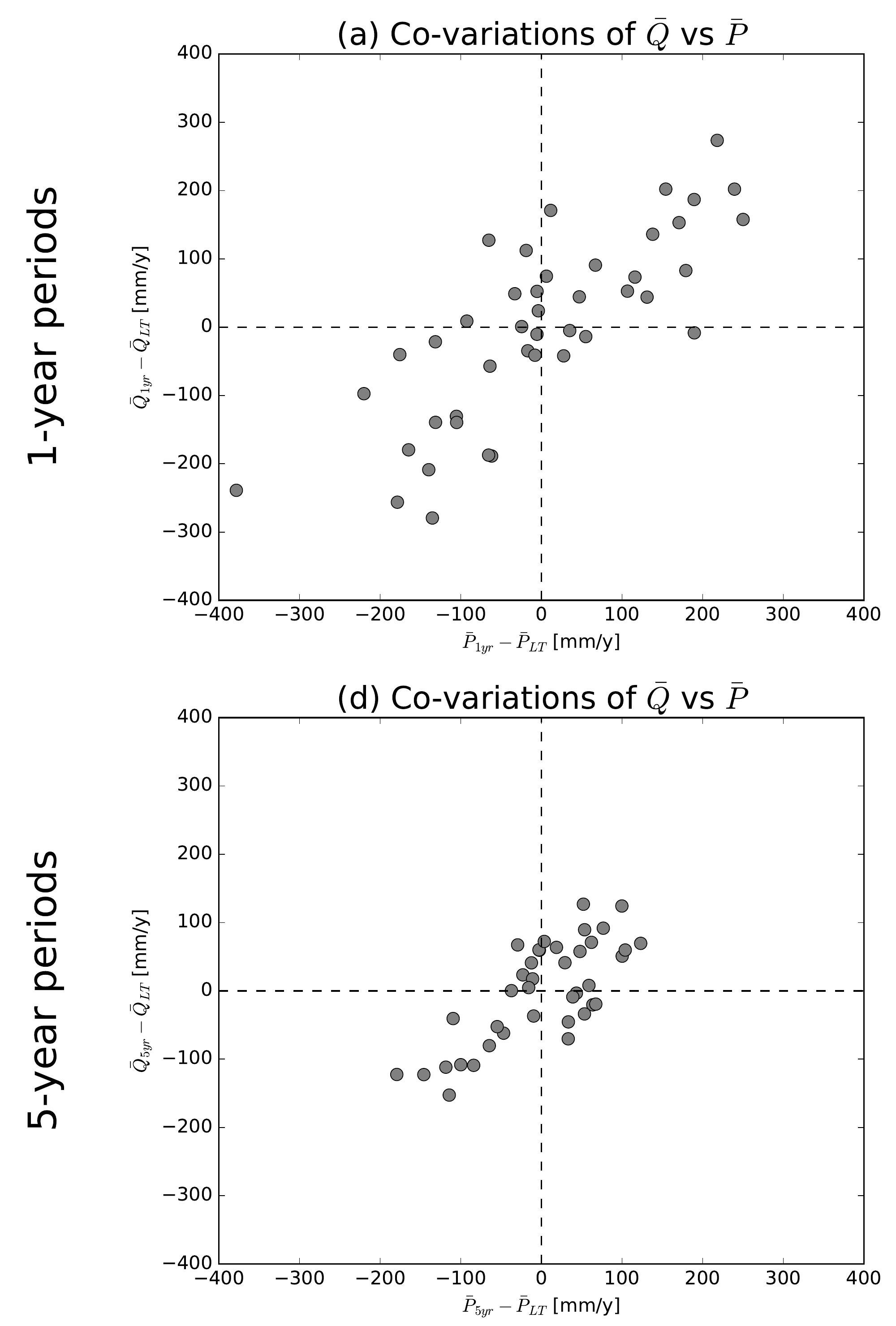

(g) Co-variations of $\bar{Q}$ vs $\bar{P}$
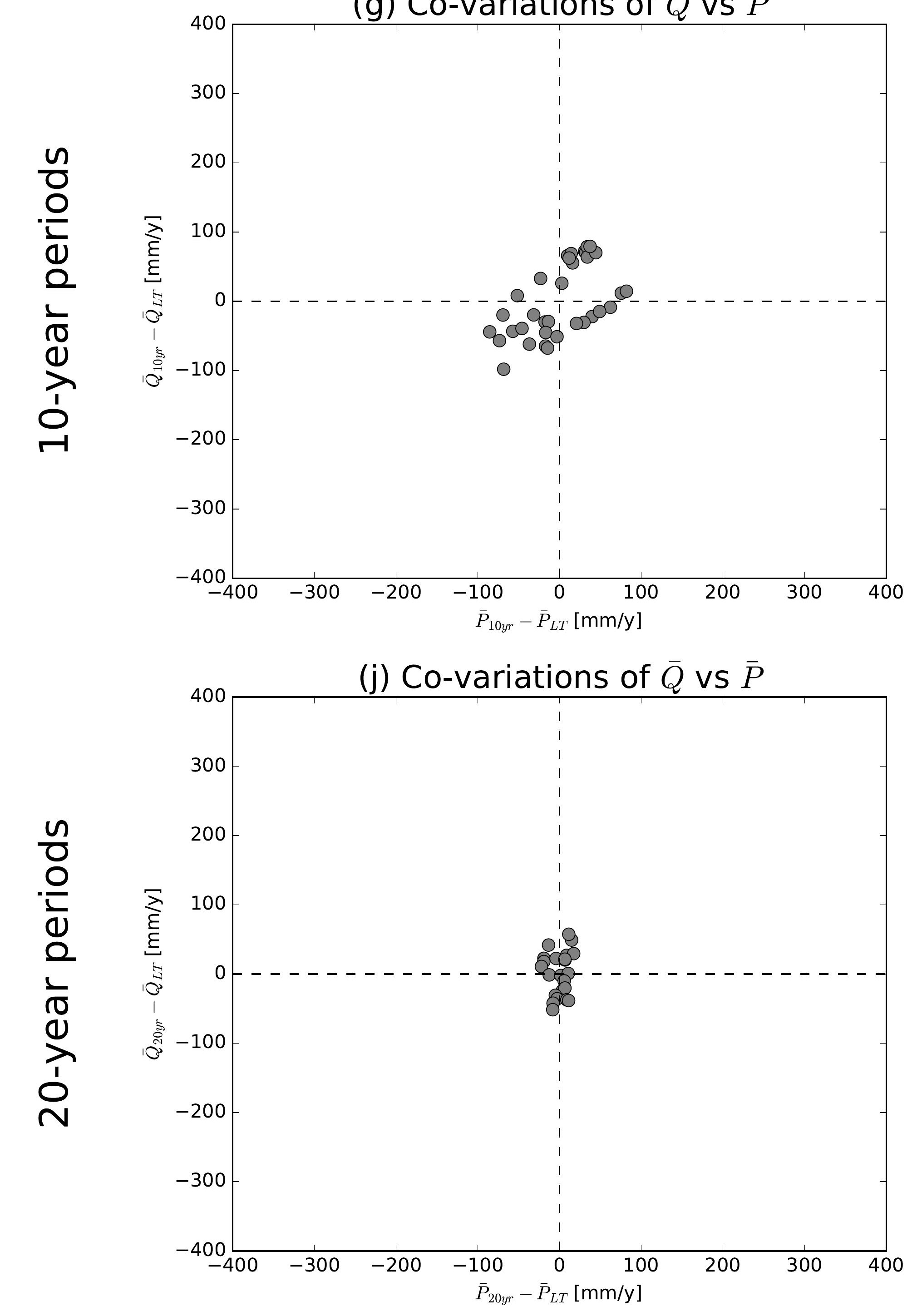

(b) Co-variations of $\bar{Q}$ vs $\overline{E 0}$

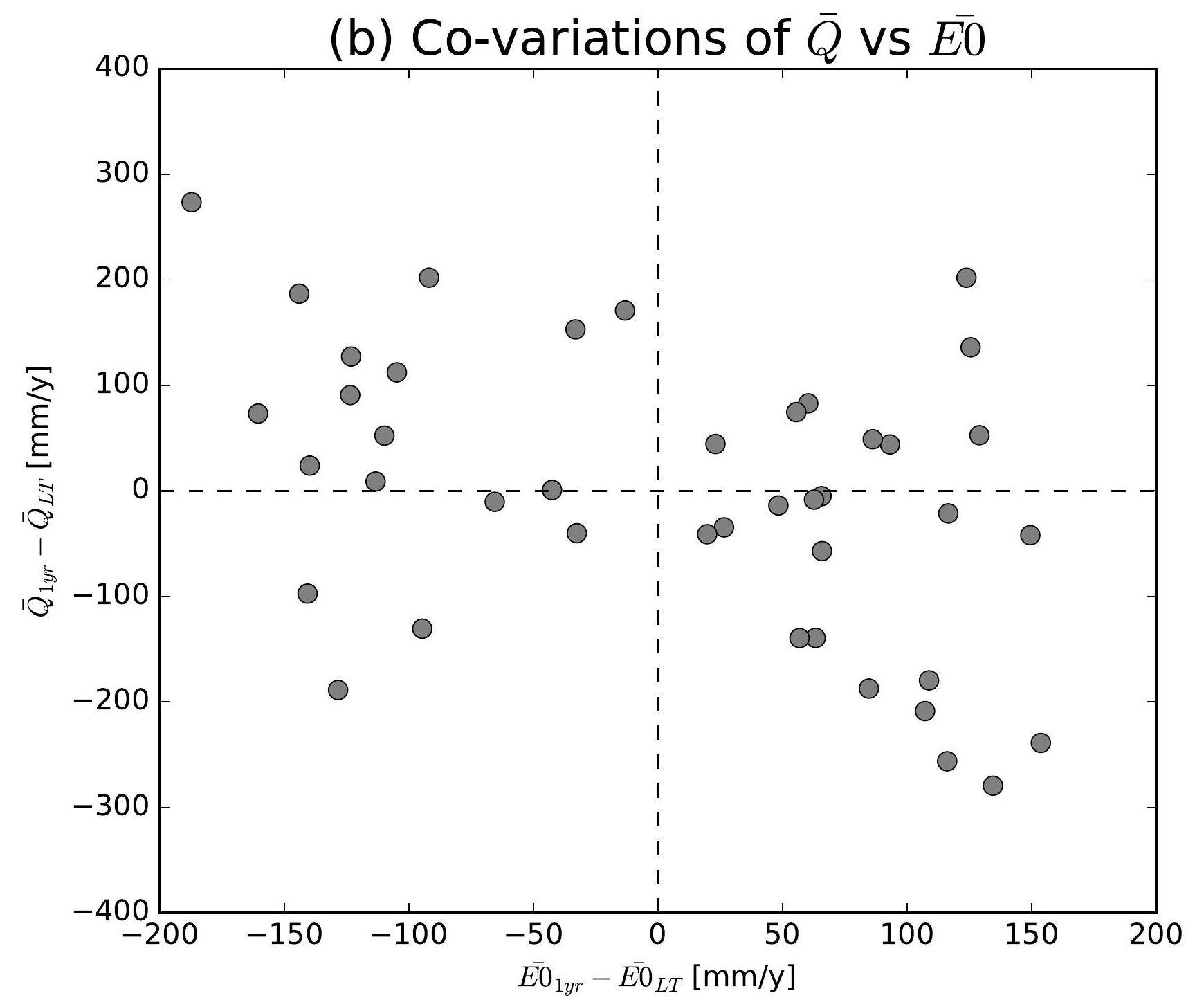

(e) Co-variations of $\bar{Q}$ vs $\overline{E 0}$

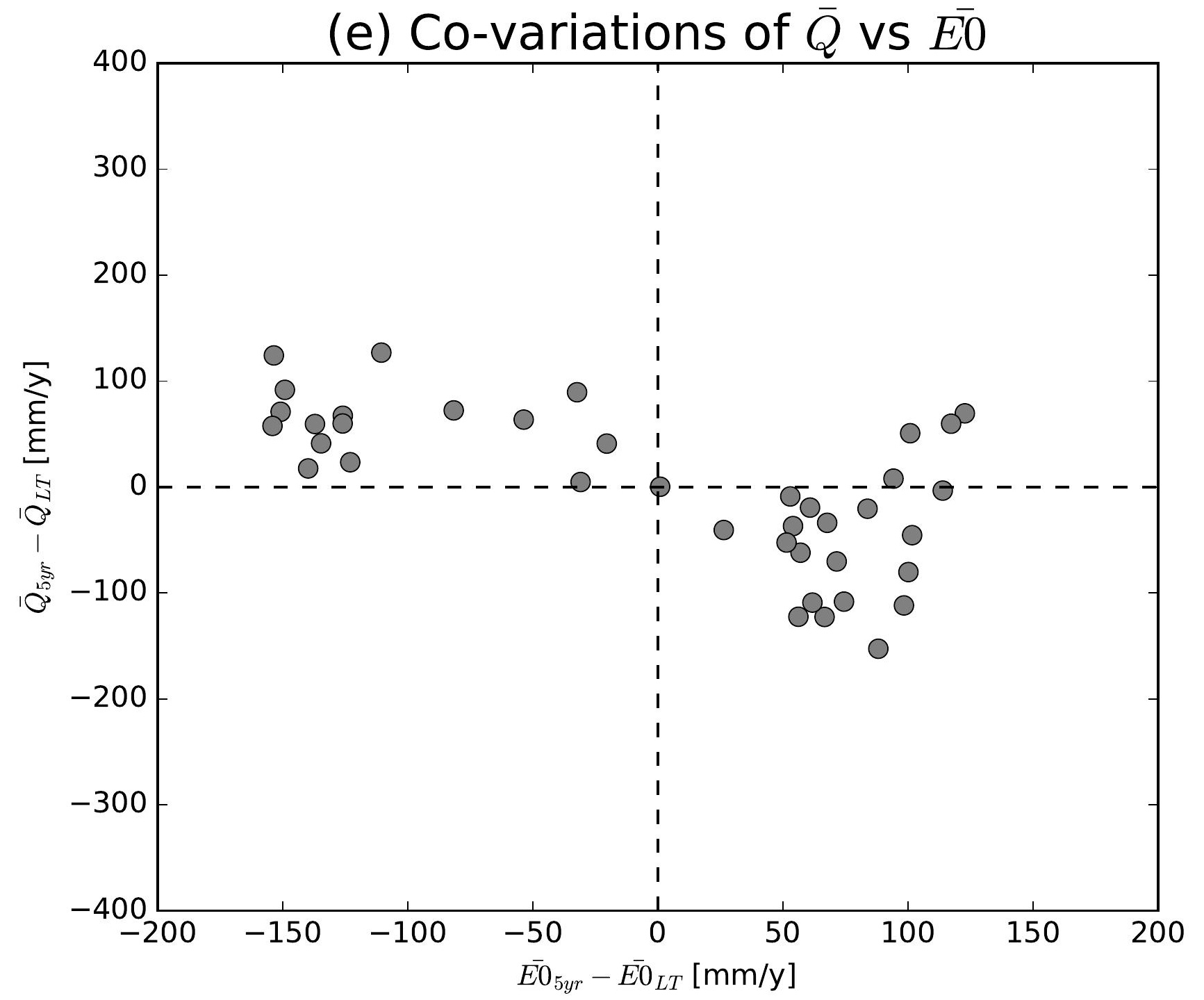

(h) Co-variations of $\bar{Q}$ vs $\overline{E 0}$

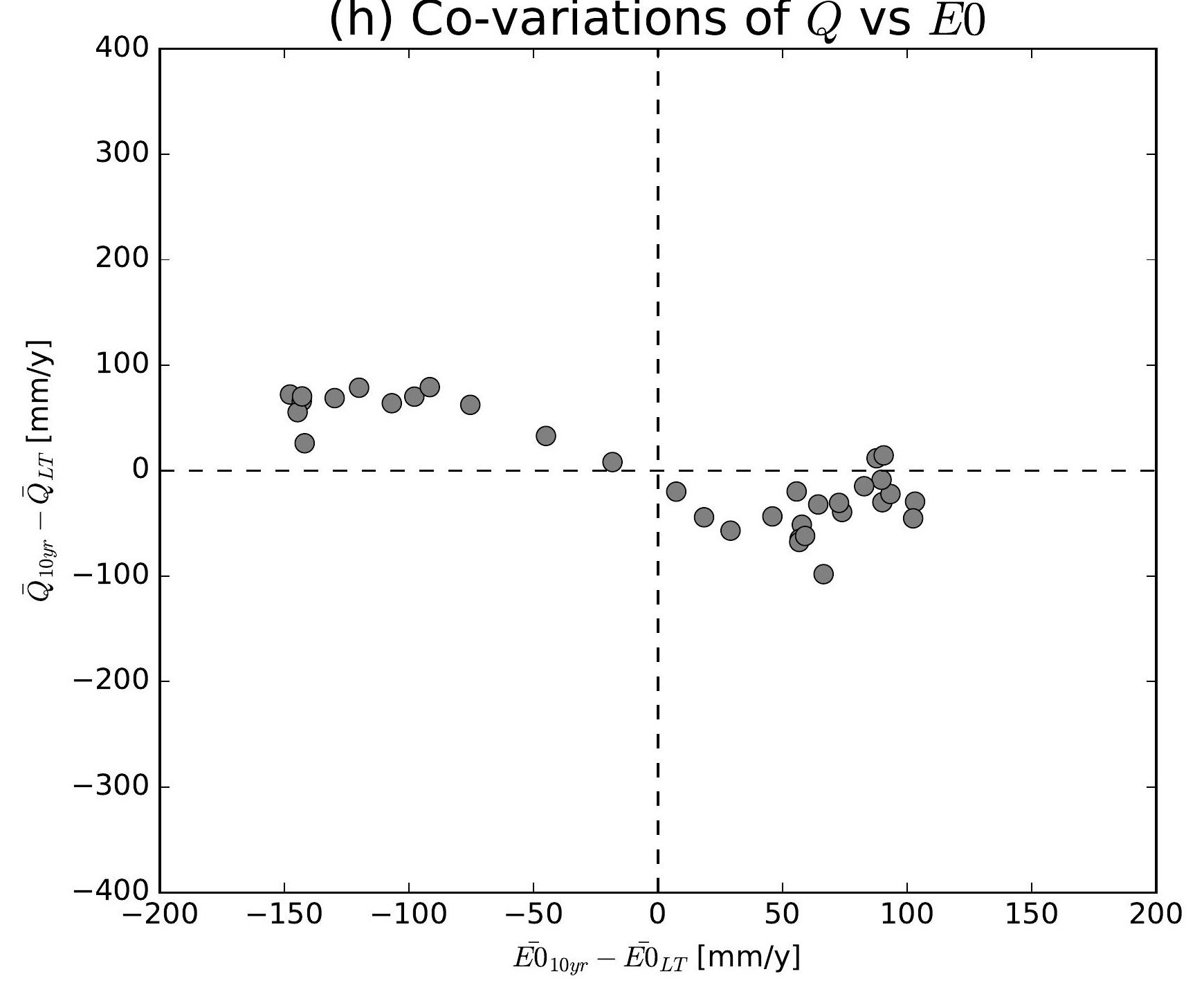

(k) Co-variations of $\bar{Q}$ vs $\overline{E 0}$

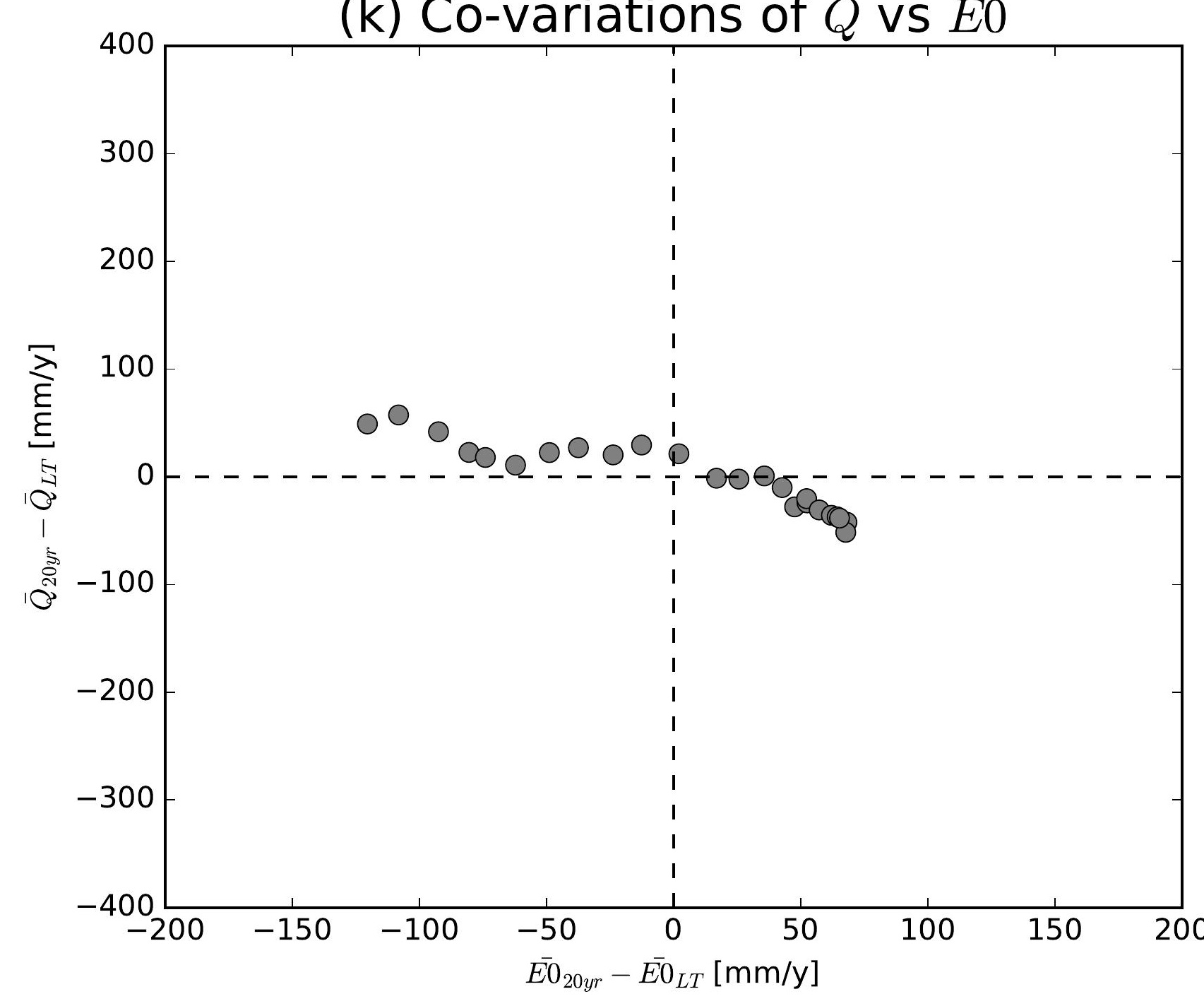

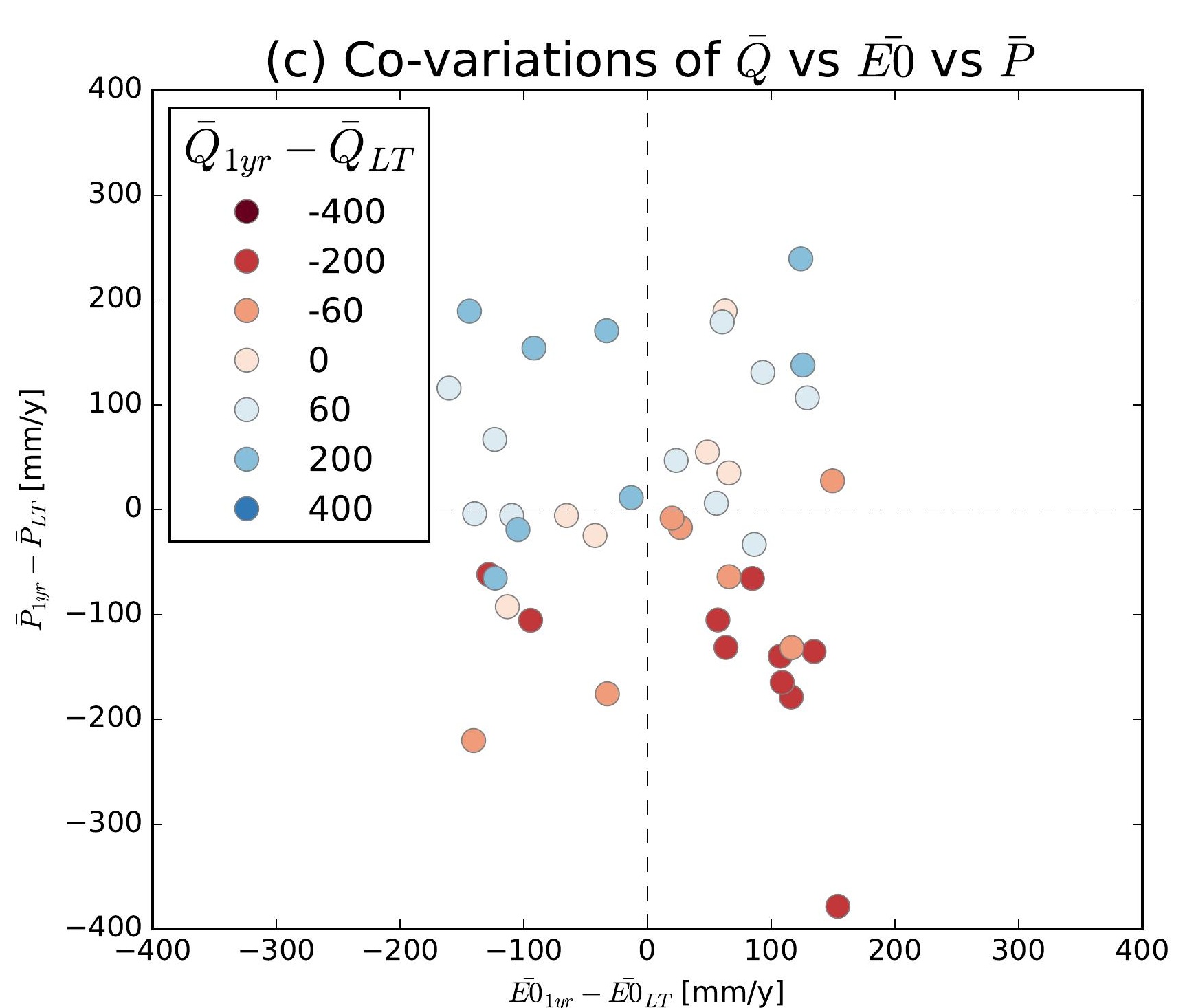
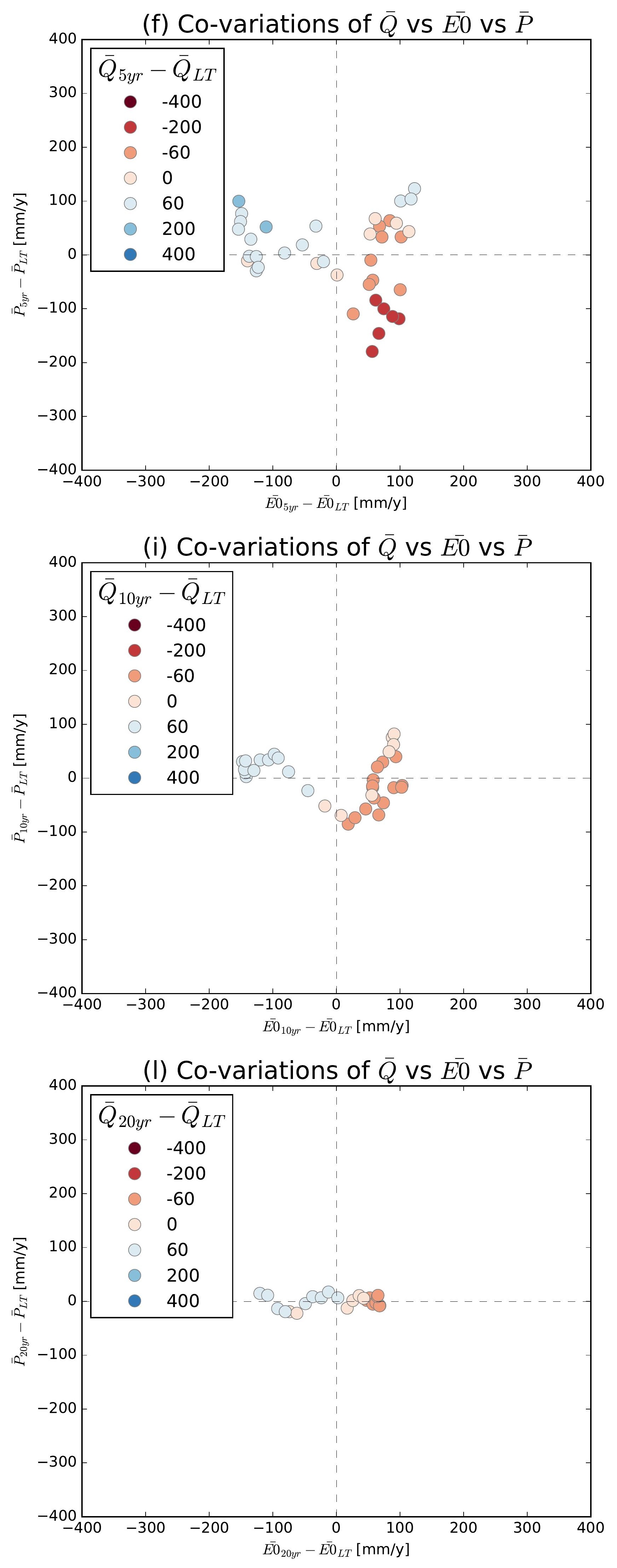

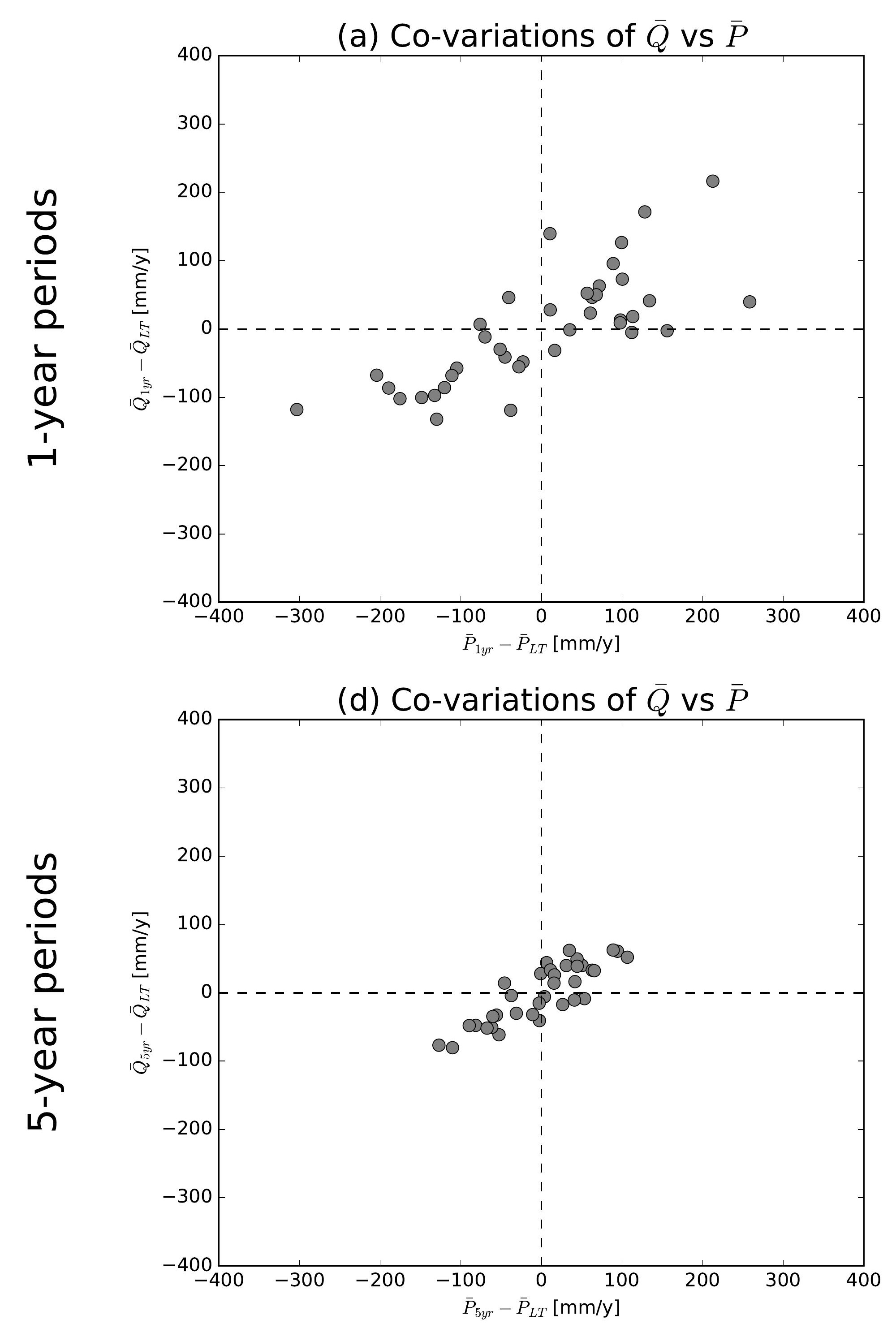

(g) Co-variations of $\bar{Q}$ vs $\bar{P}$
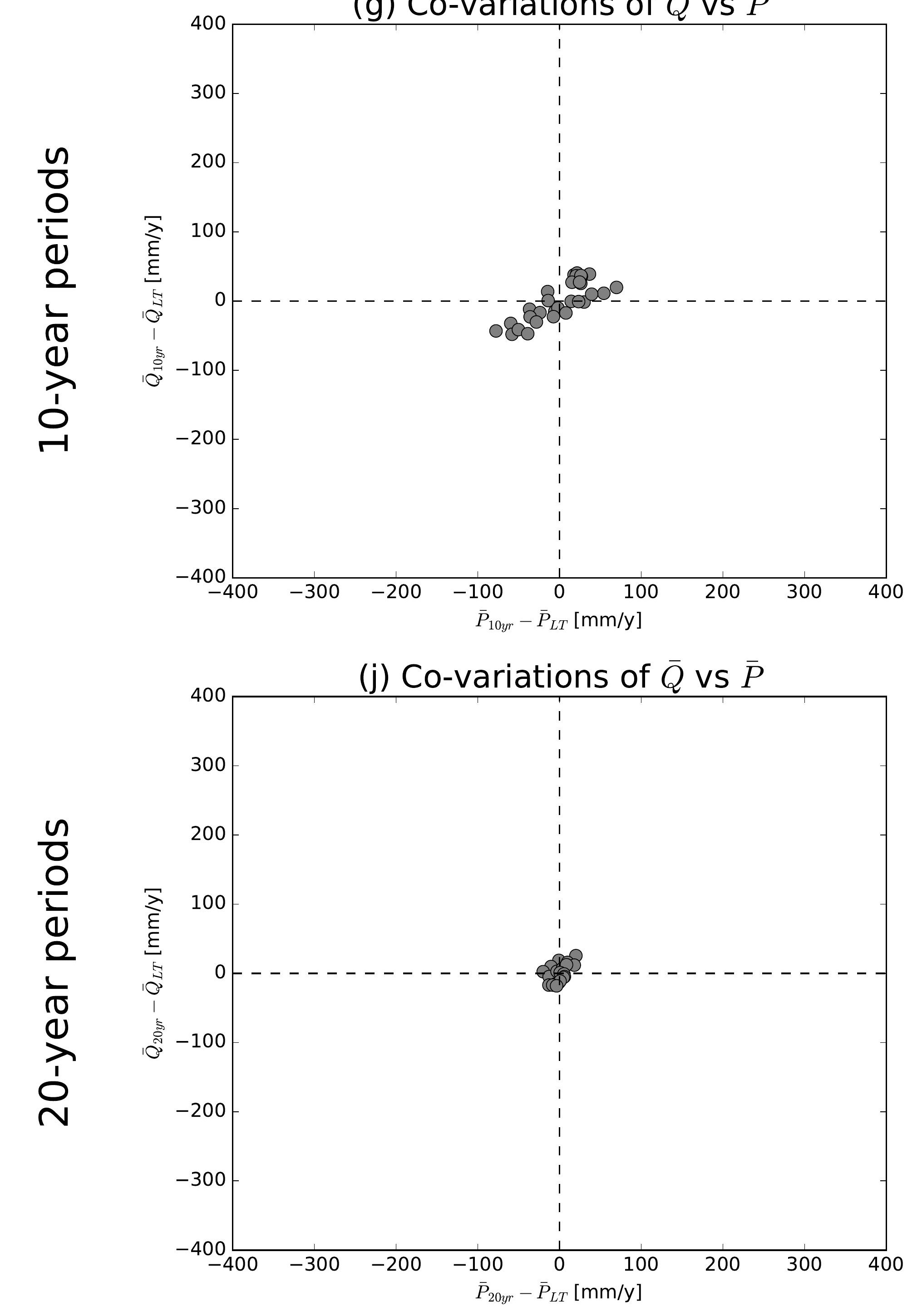

(b) Co-variations of $\bar{Q}$ vs $\overline{E 0}$

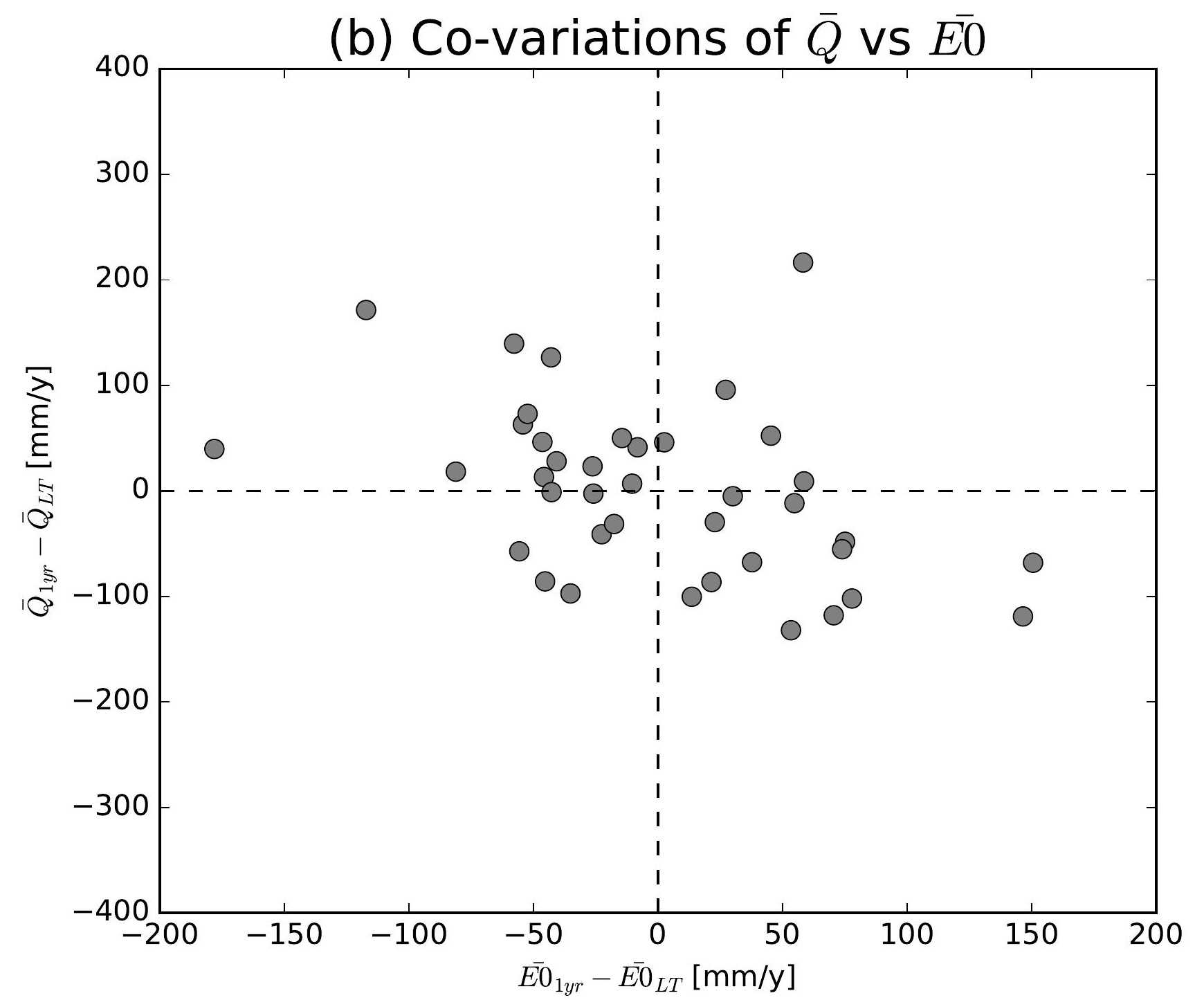

(e) Co-variations of $\bar{Q}$ vs $\overline{E 0}$

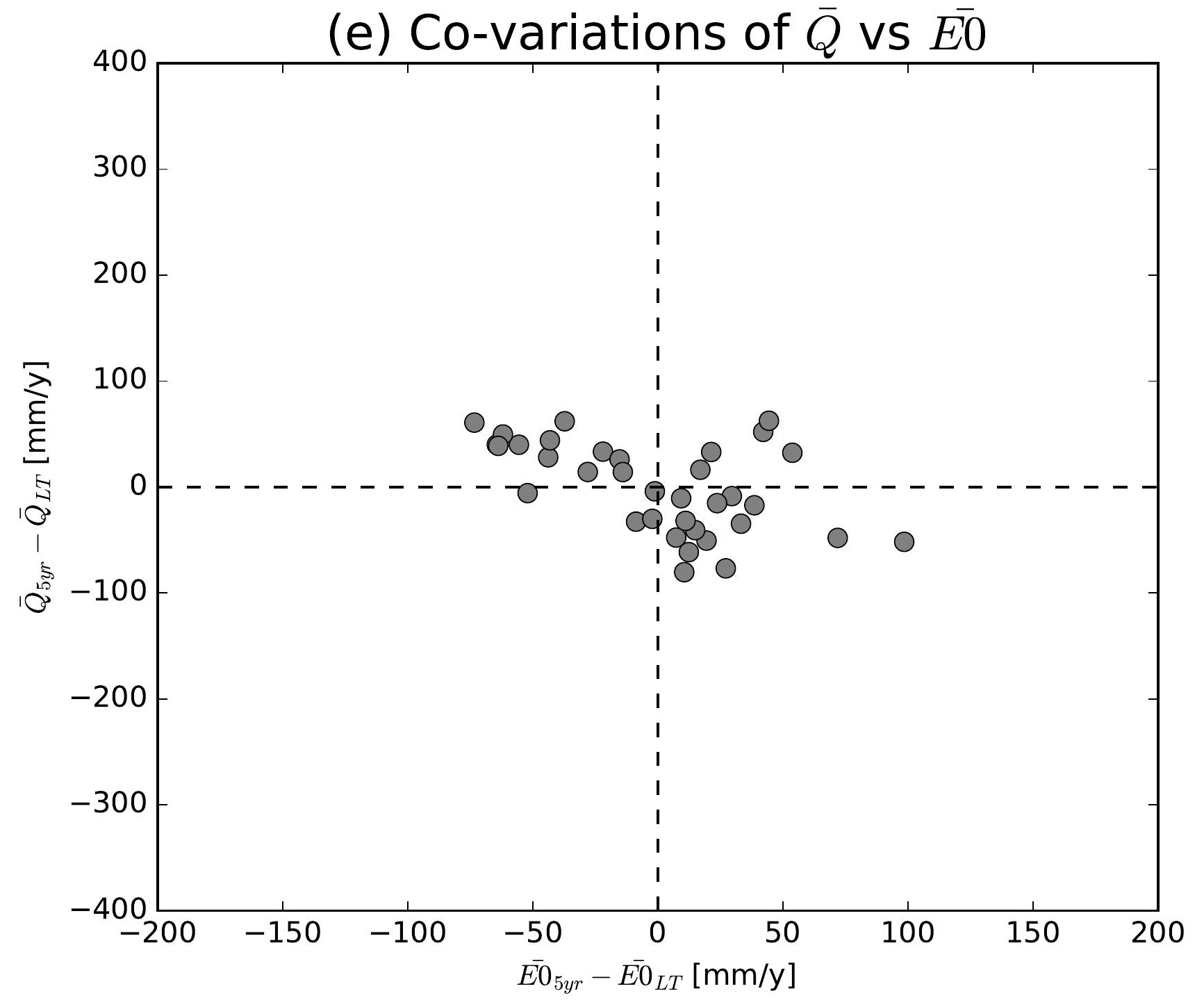

(h) Co-variations of $\bar{Q}$ vs $\overline{E 0}$

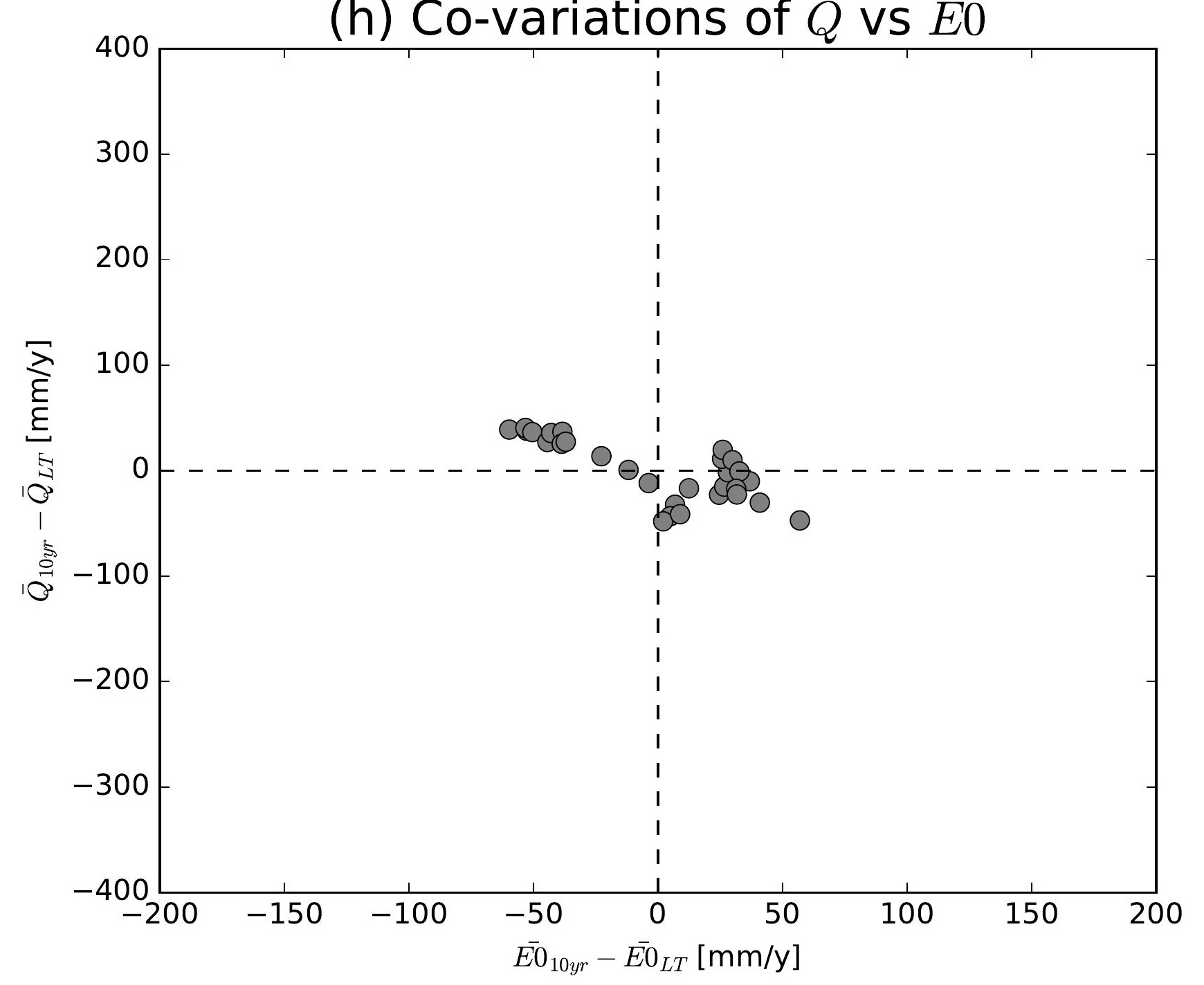

(k) Co-variations of $\bar{Q}$ vs $\overline{E 0}$

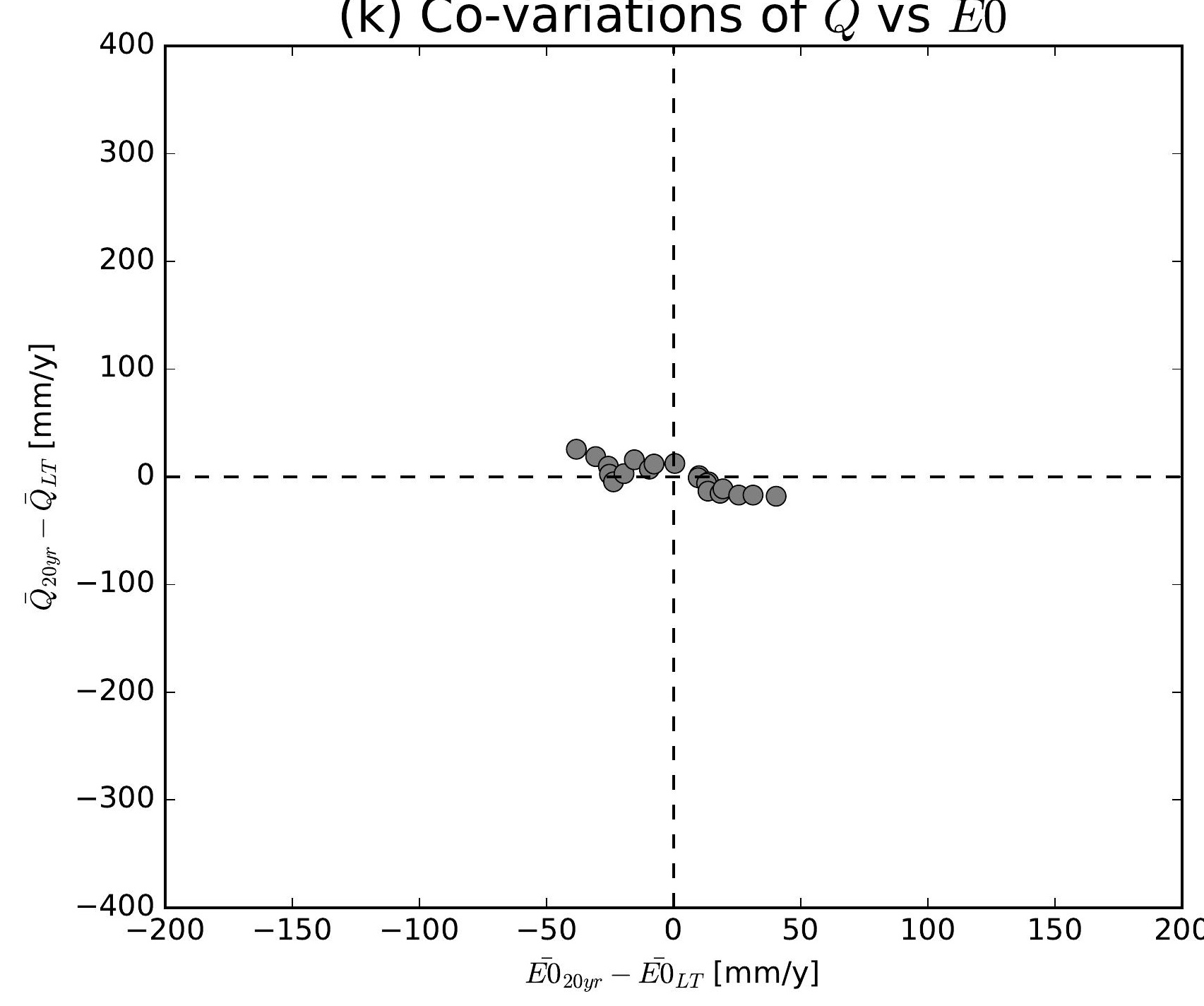

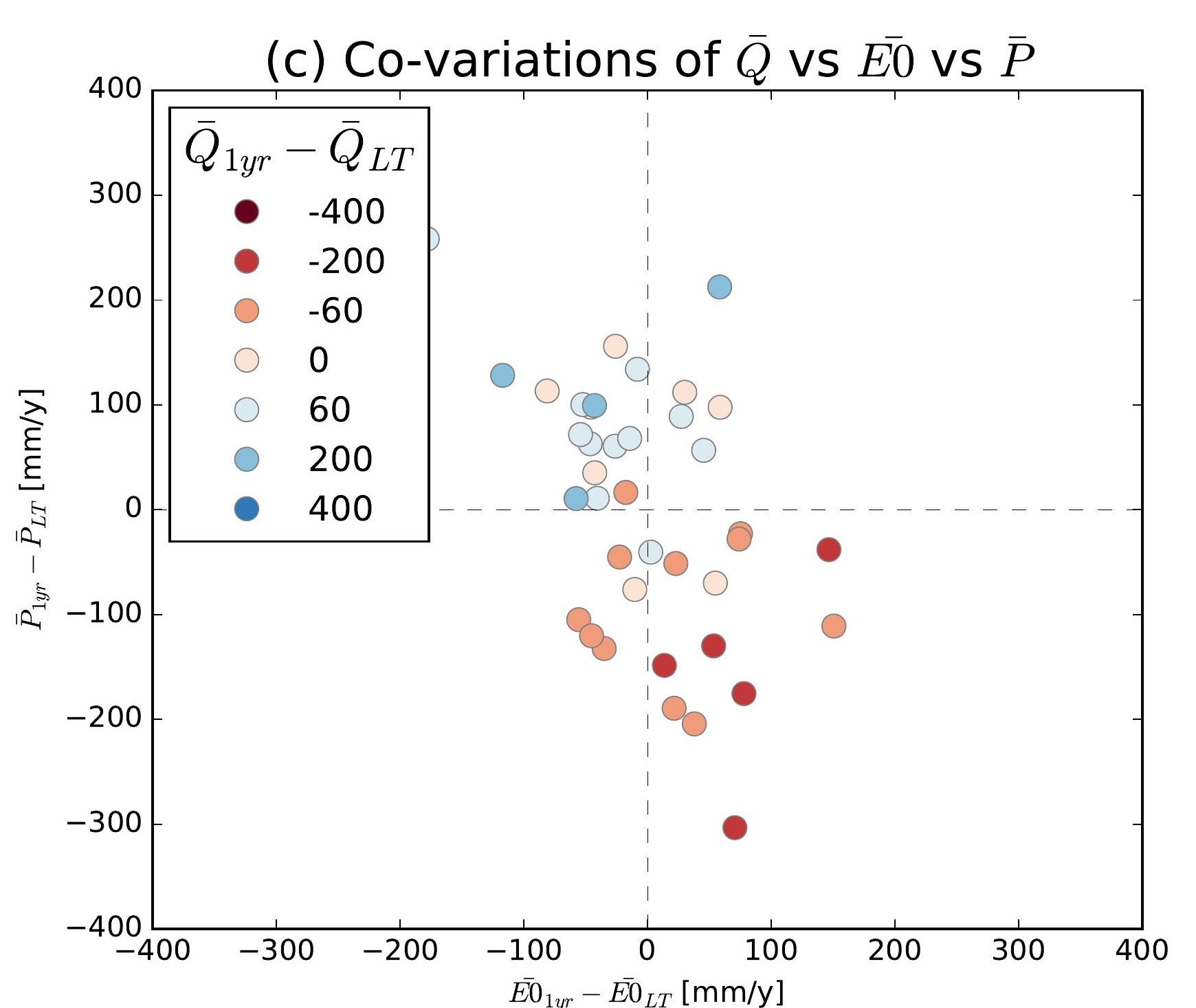
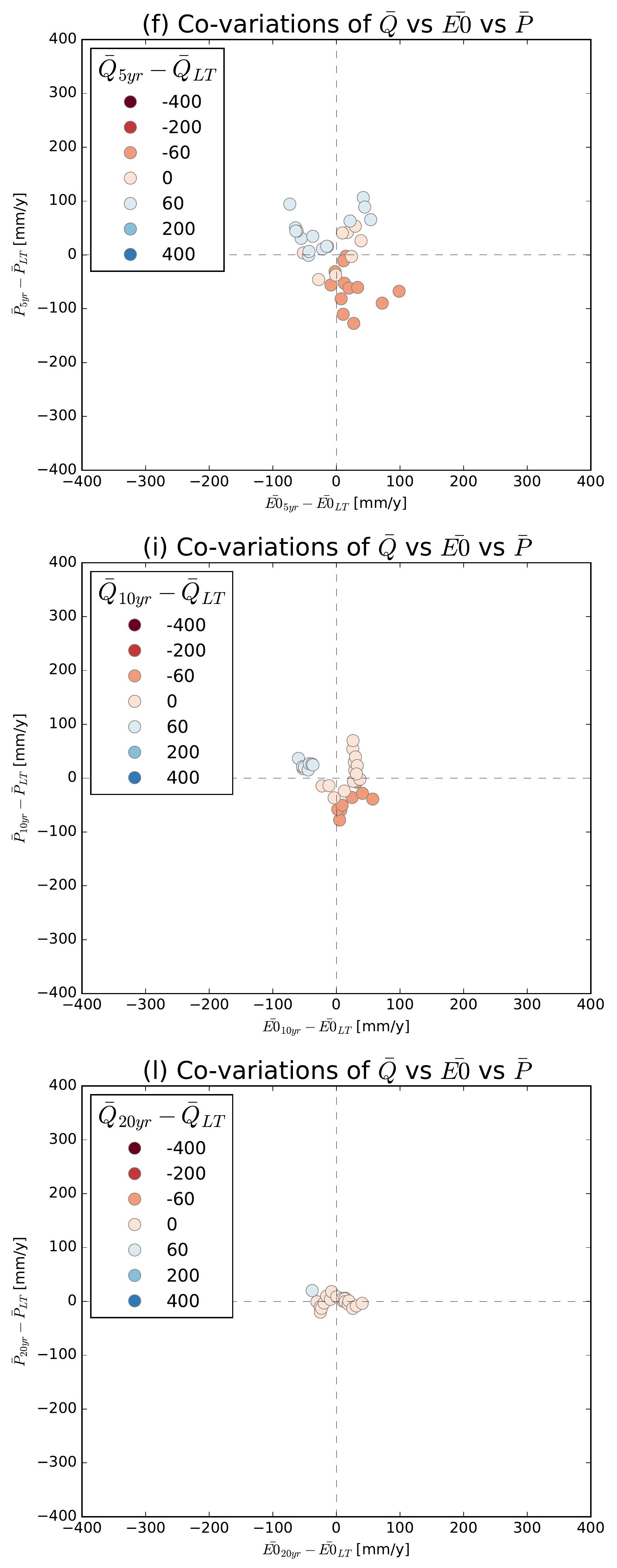

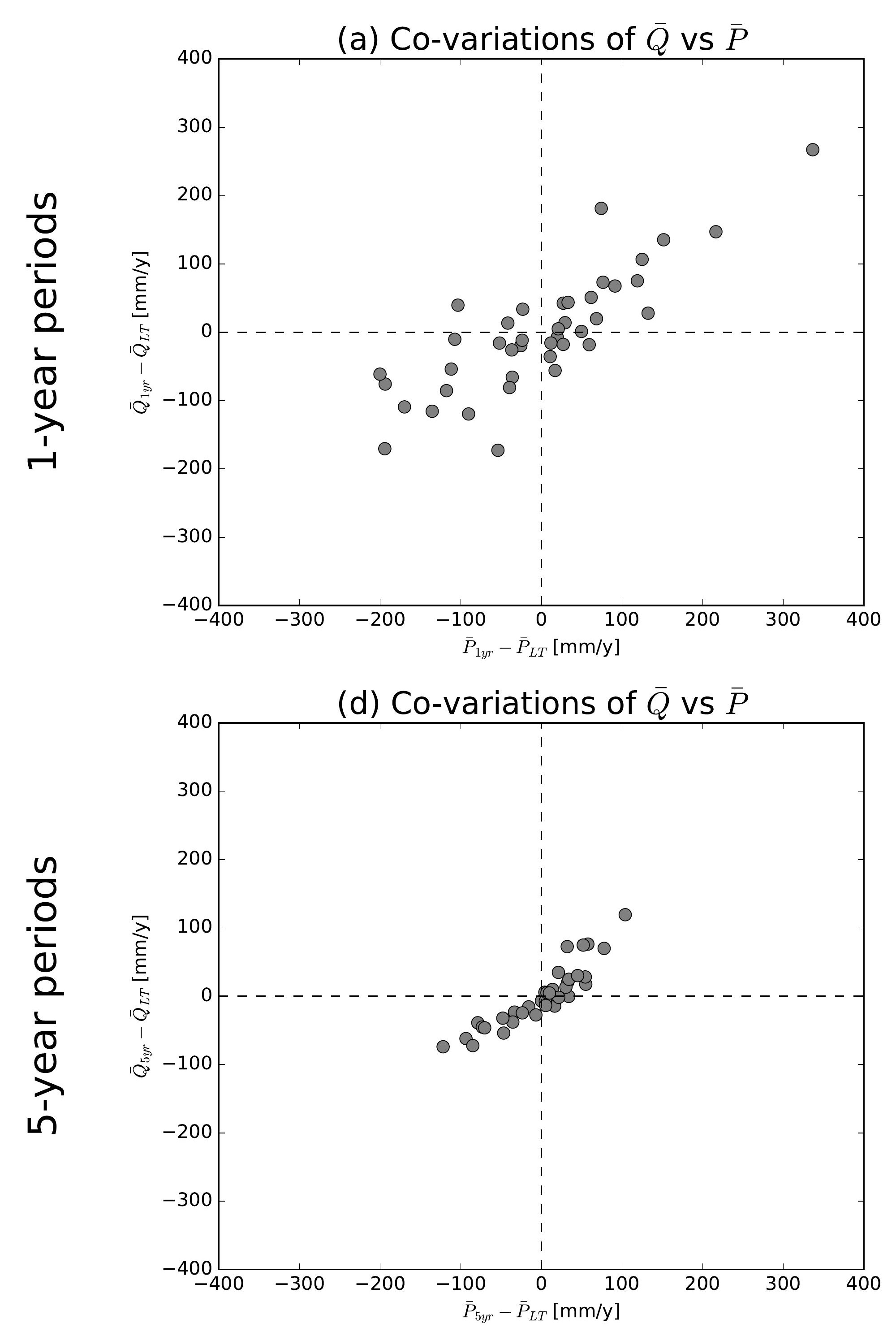

(g) Co-variations of $\bar{Q}$ vs $\bar{P}$
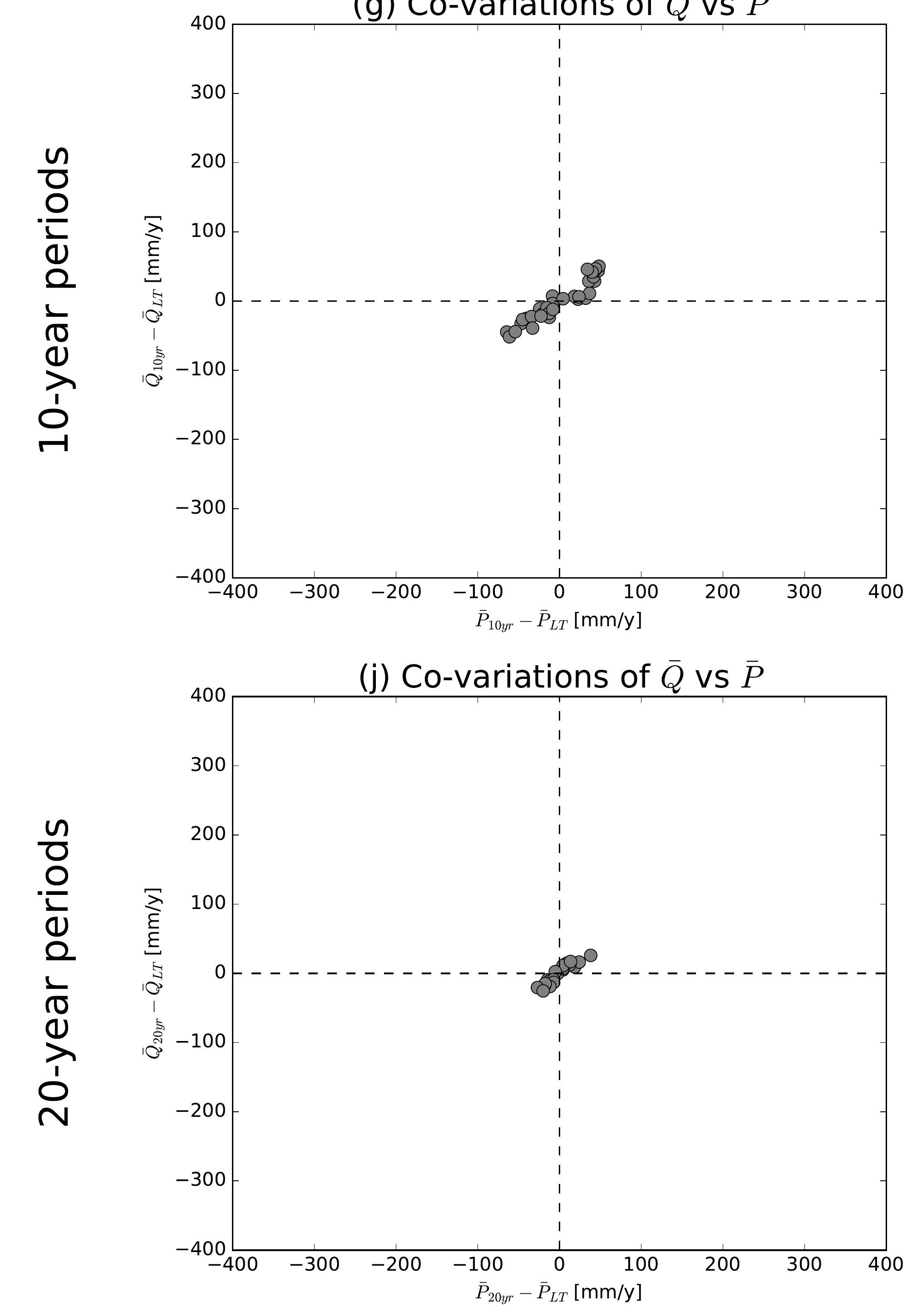

(b) Co-variations of $\bar{Q}$ vs $\overline{E 0}$

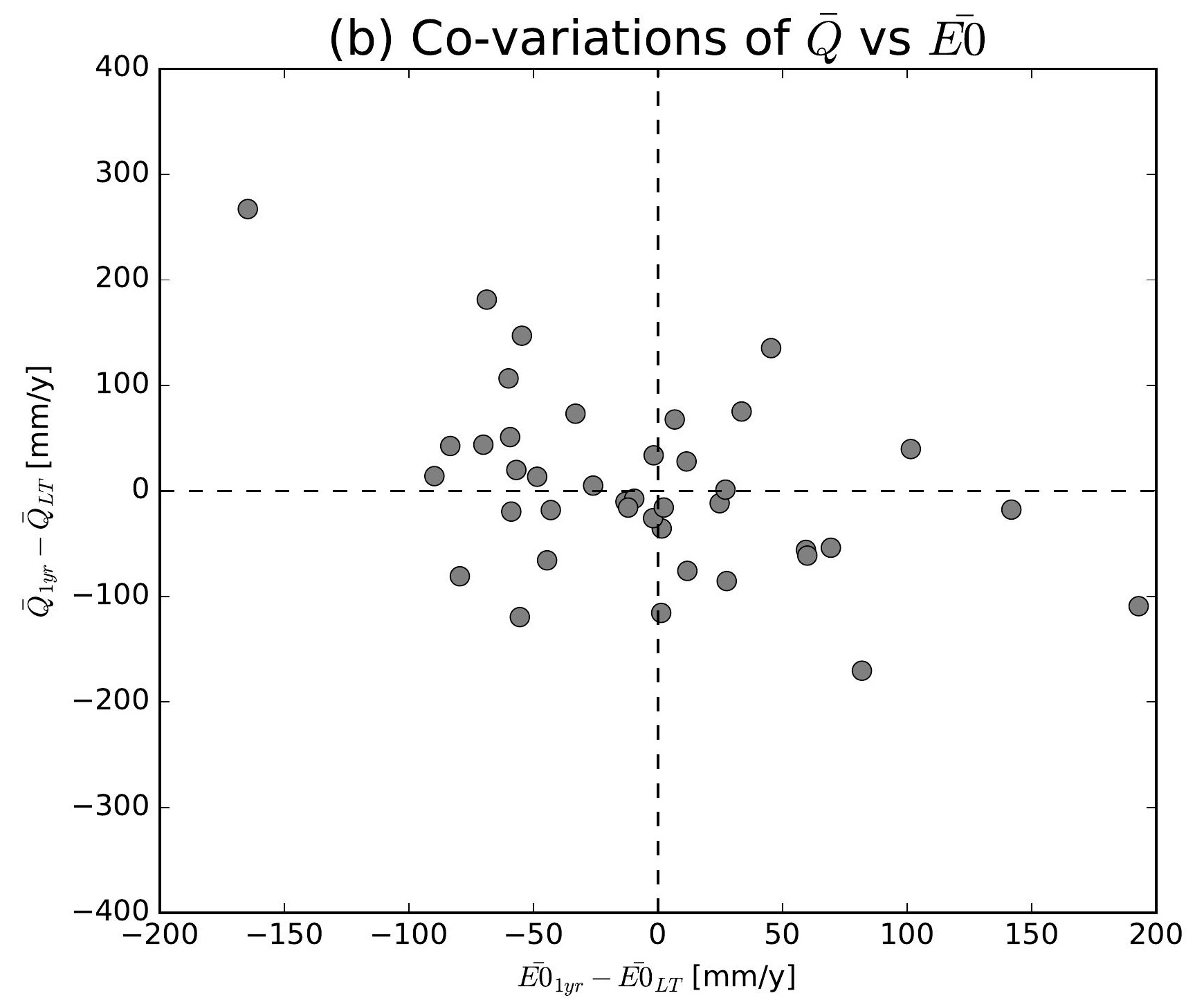

(e) Co-variations of $\bar{Q}$ vs $\overline{E 0}$

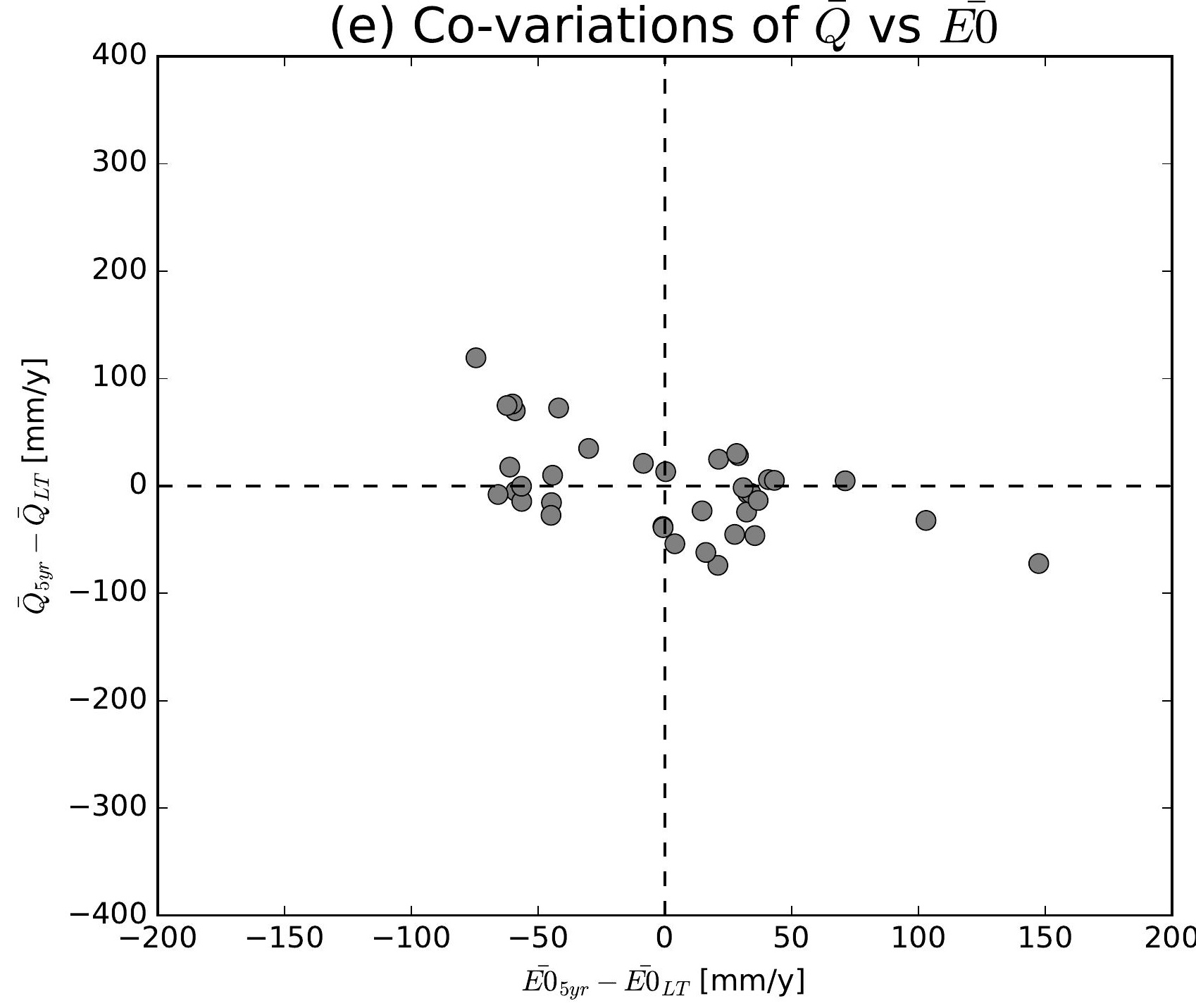

(h) Co-variations of $\bar{Q}$ vs $\overline{E 0}$

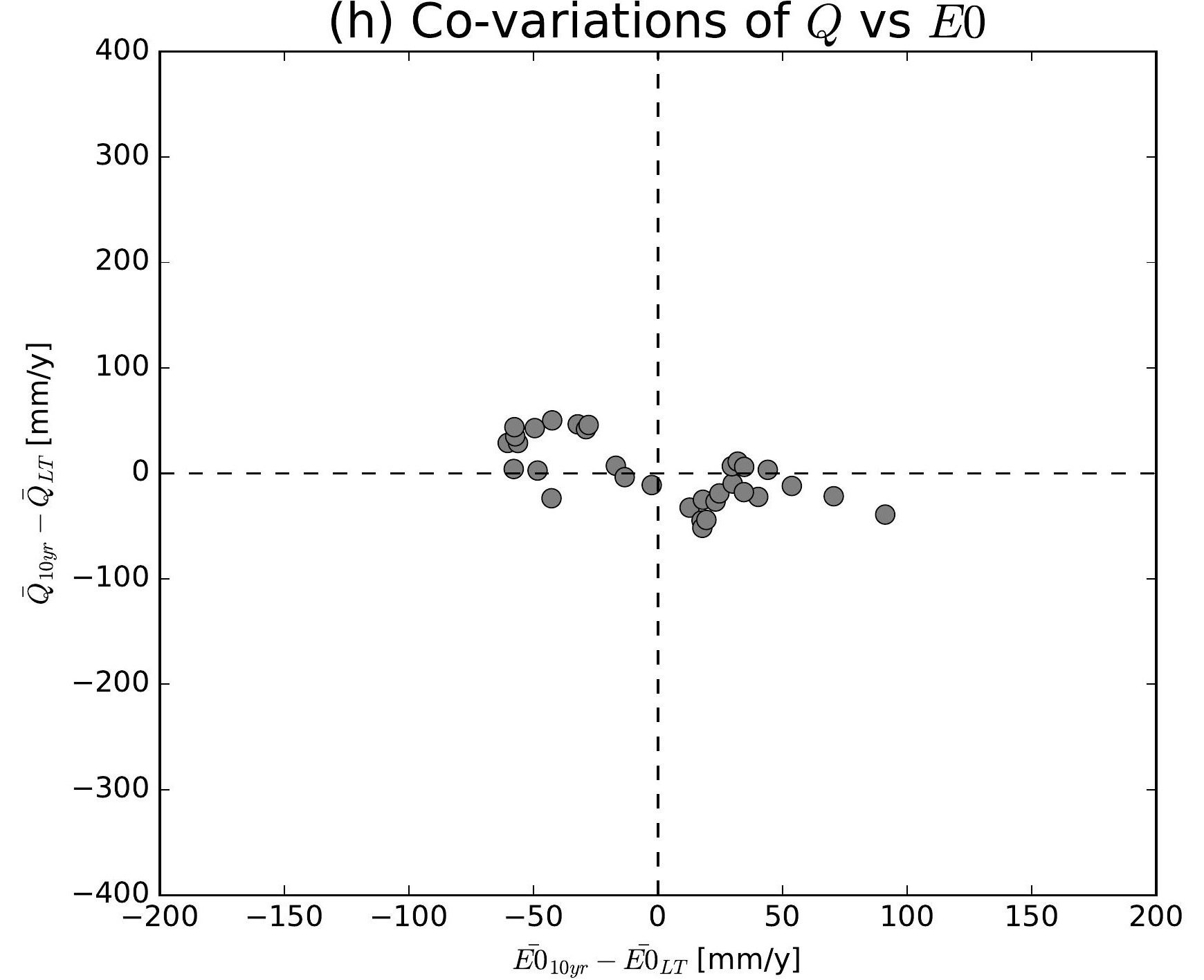

(k) Co-variations of $\bar{Q}$ vs $\overline{E 0}$

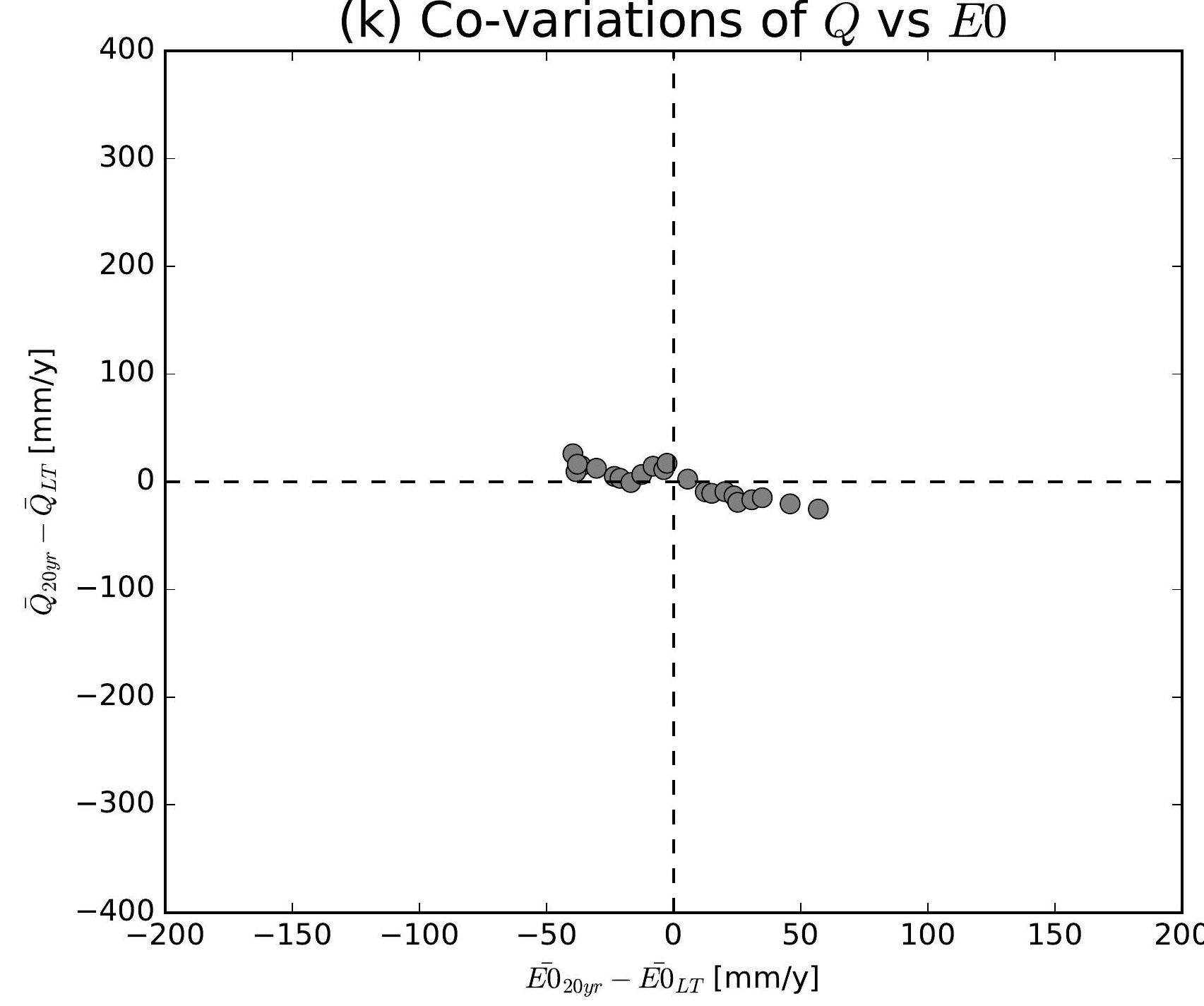

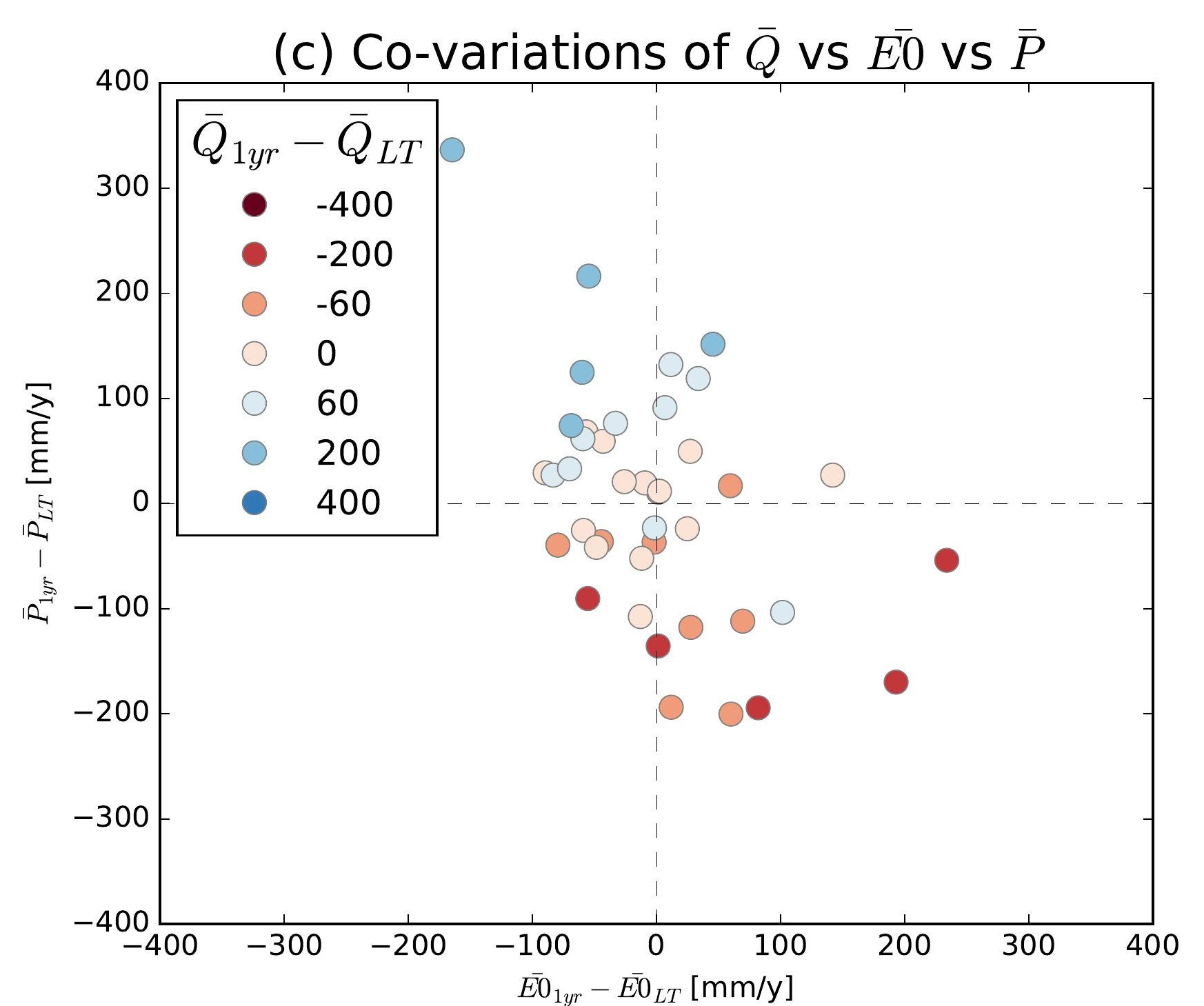
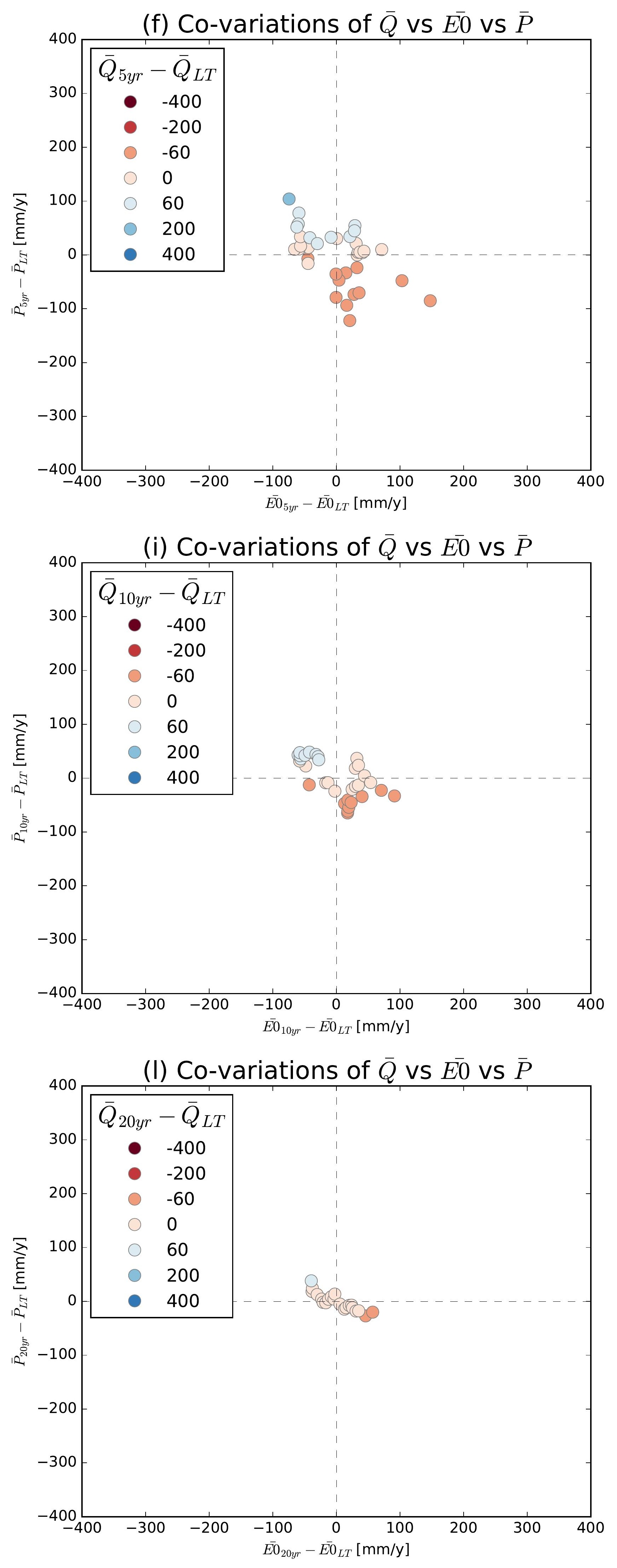

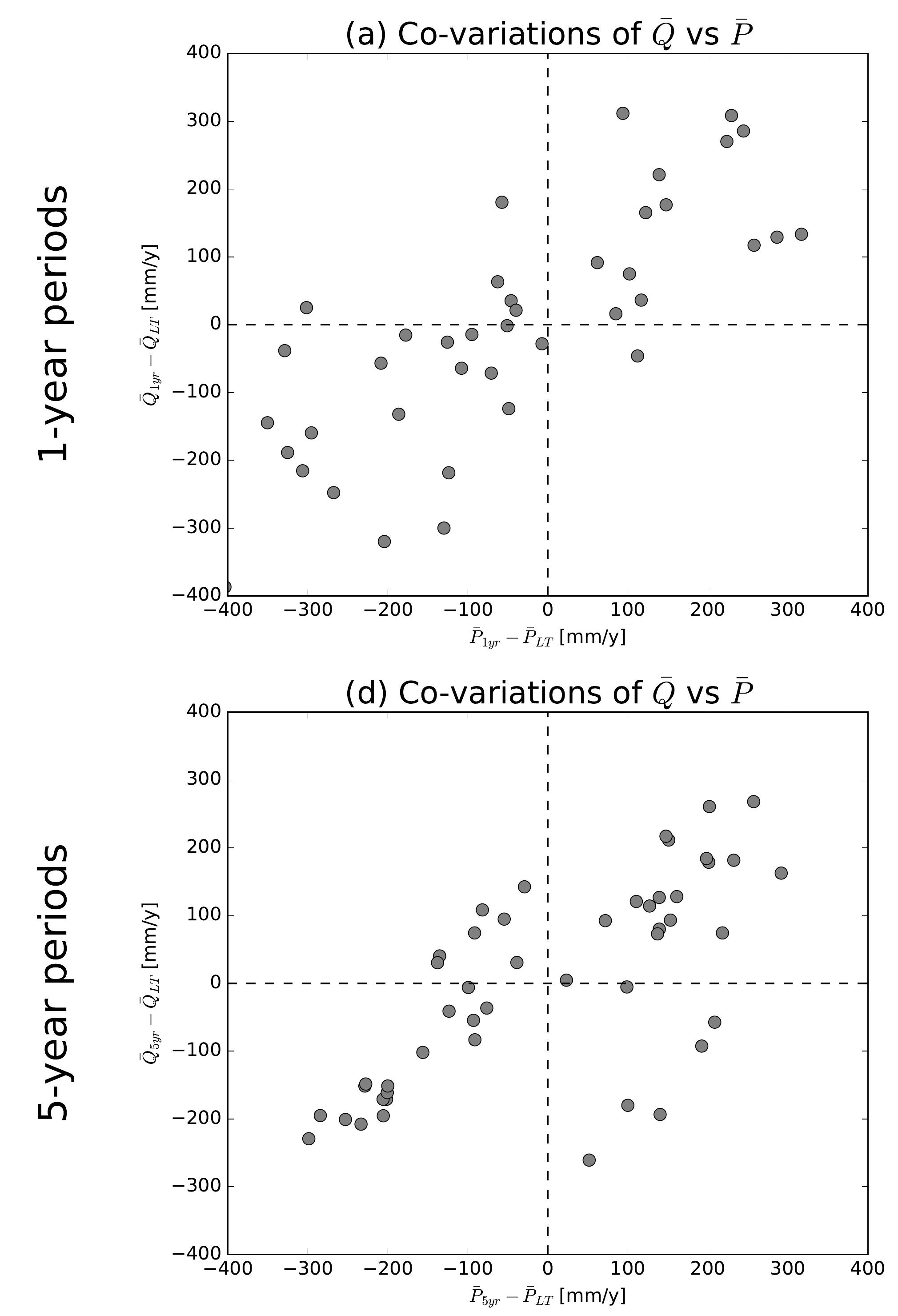

(g) Co-variations of $\bar{Q}$ vs $\bar{P}$

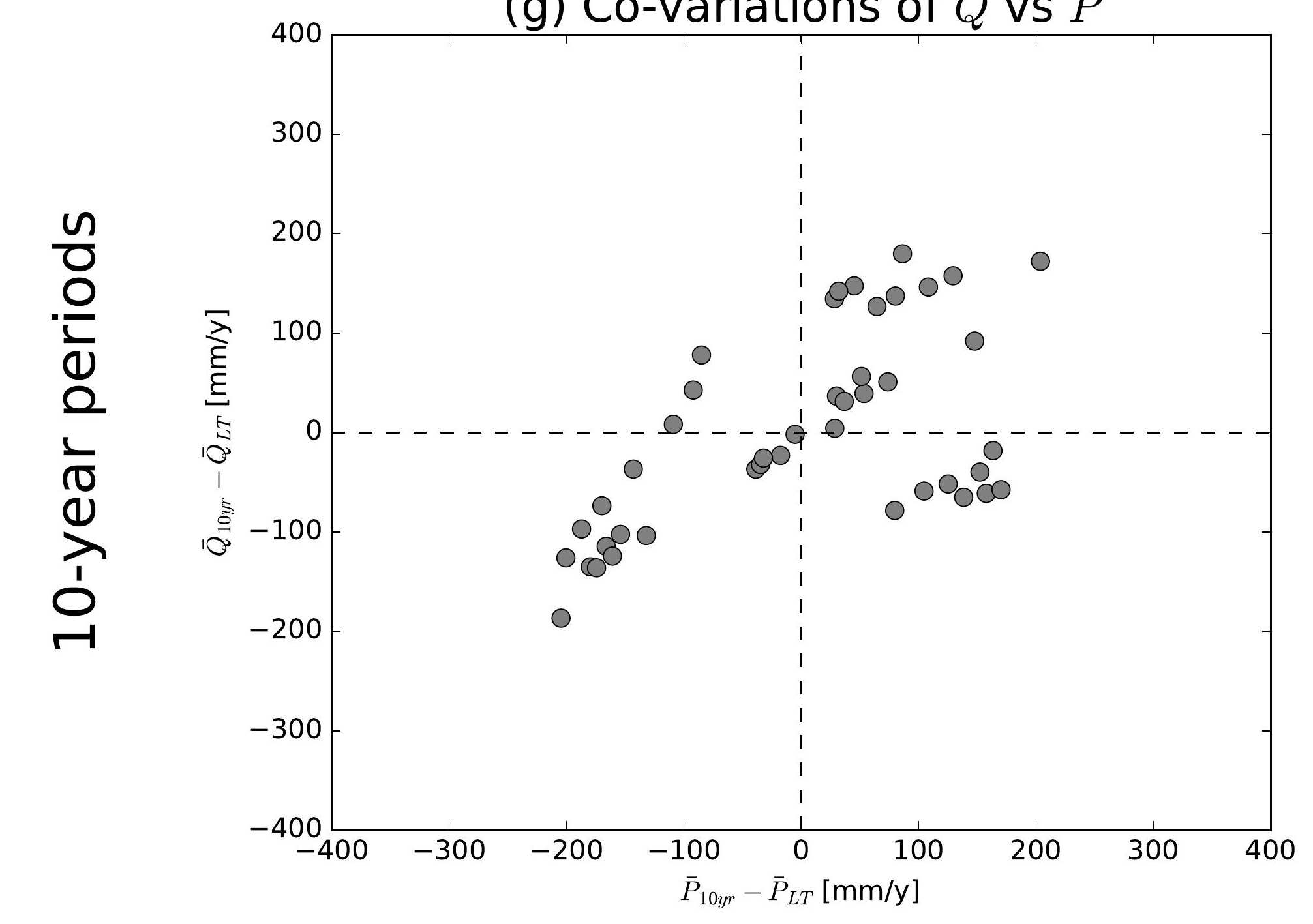

(j) Co-variations of $\bar{Q}$ vs $\bar{P}$

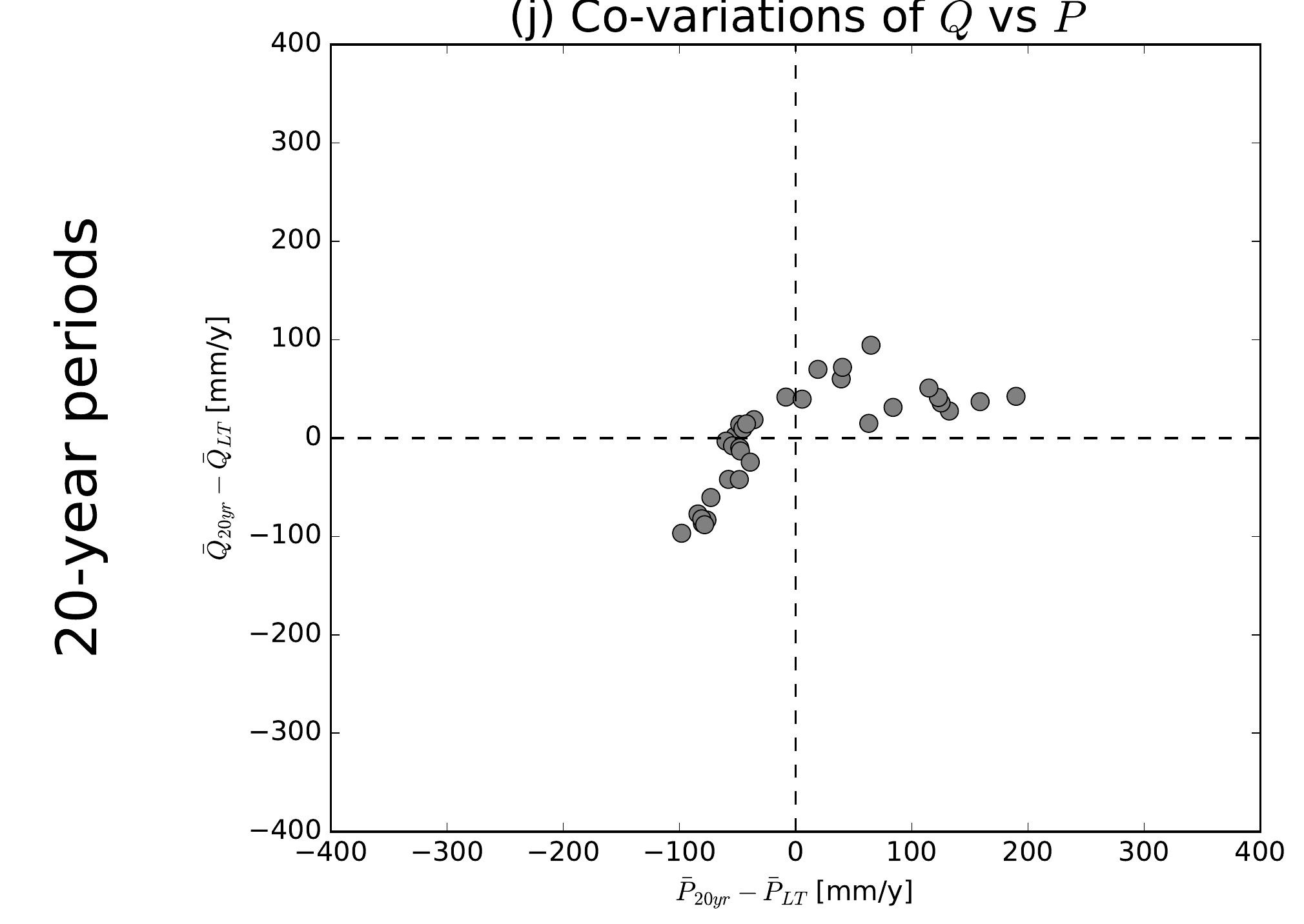

(b) Co-variations of $\bar{Q}$ vs $\overline{E 0}$

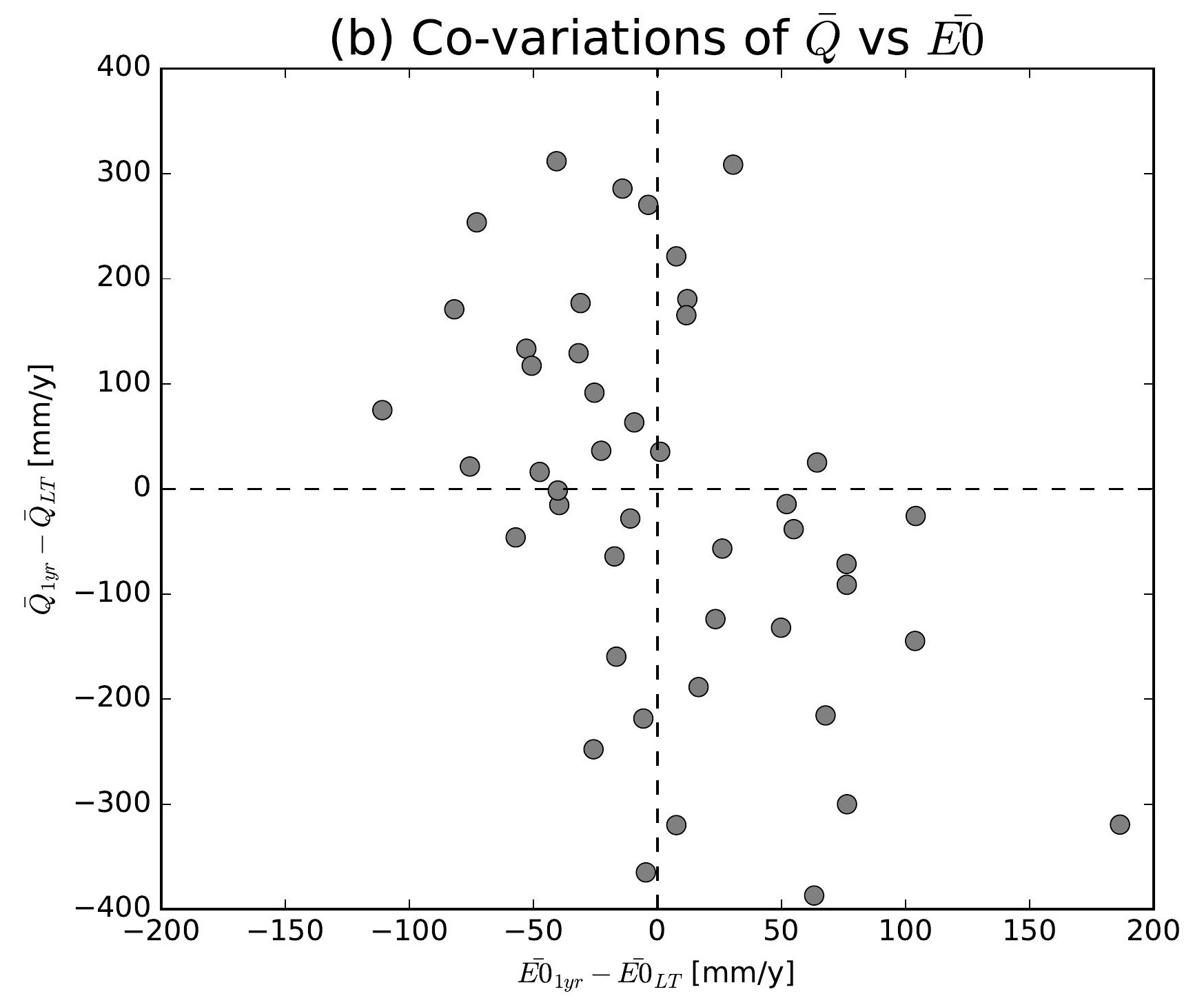

(e) Co-variations of $\bar{Q}$ vs $\overline{E 0}$

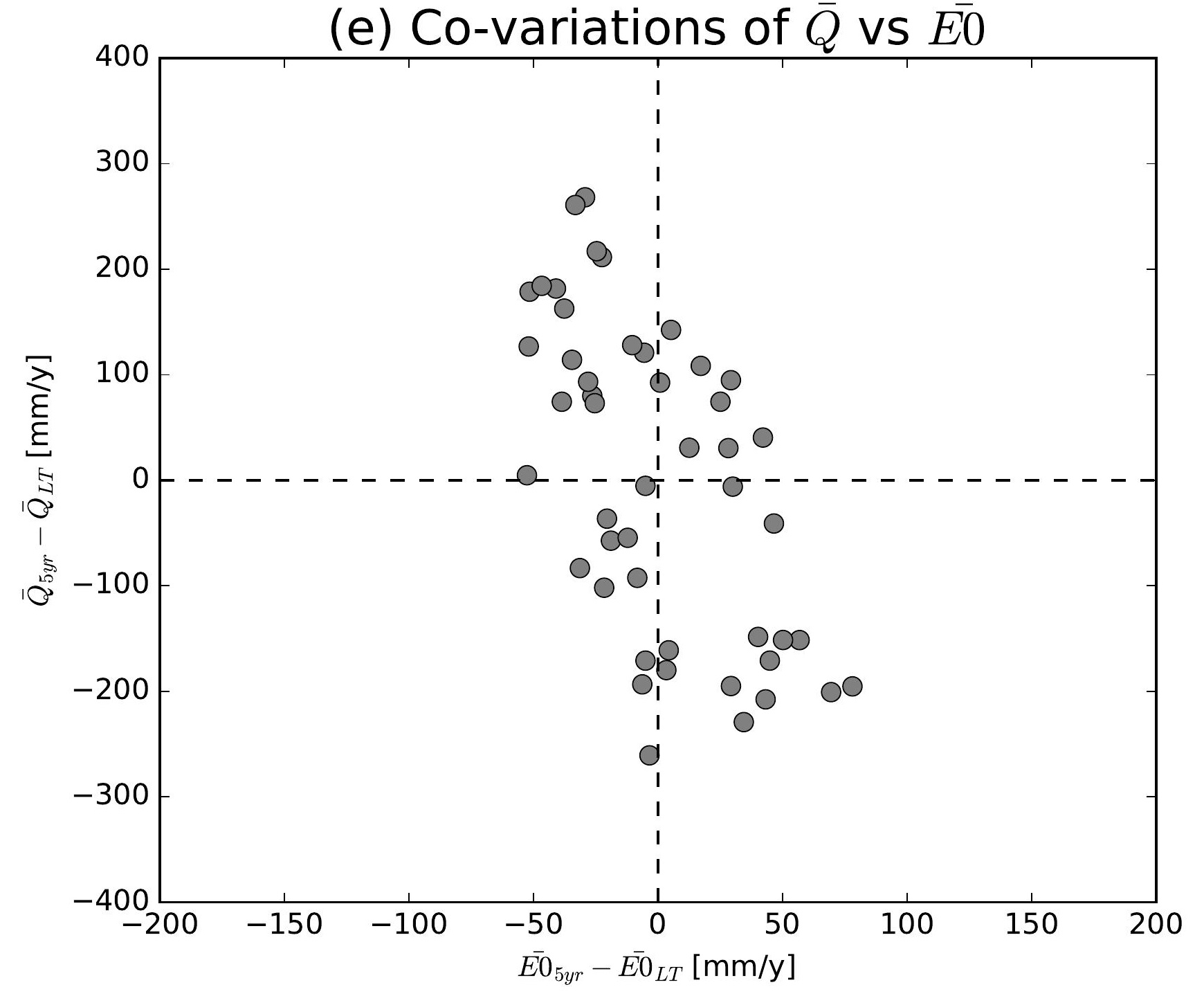

(h) Co-variations of $\bar{Q}$ vs $\overline{E 0}$

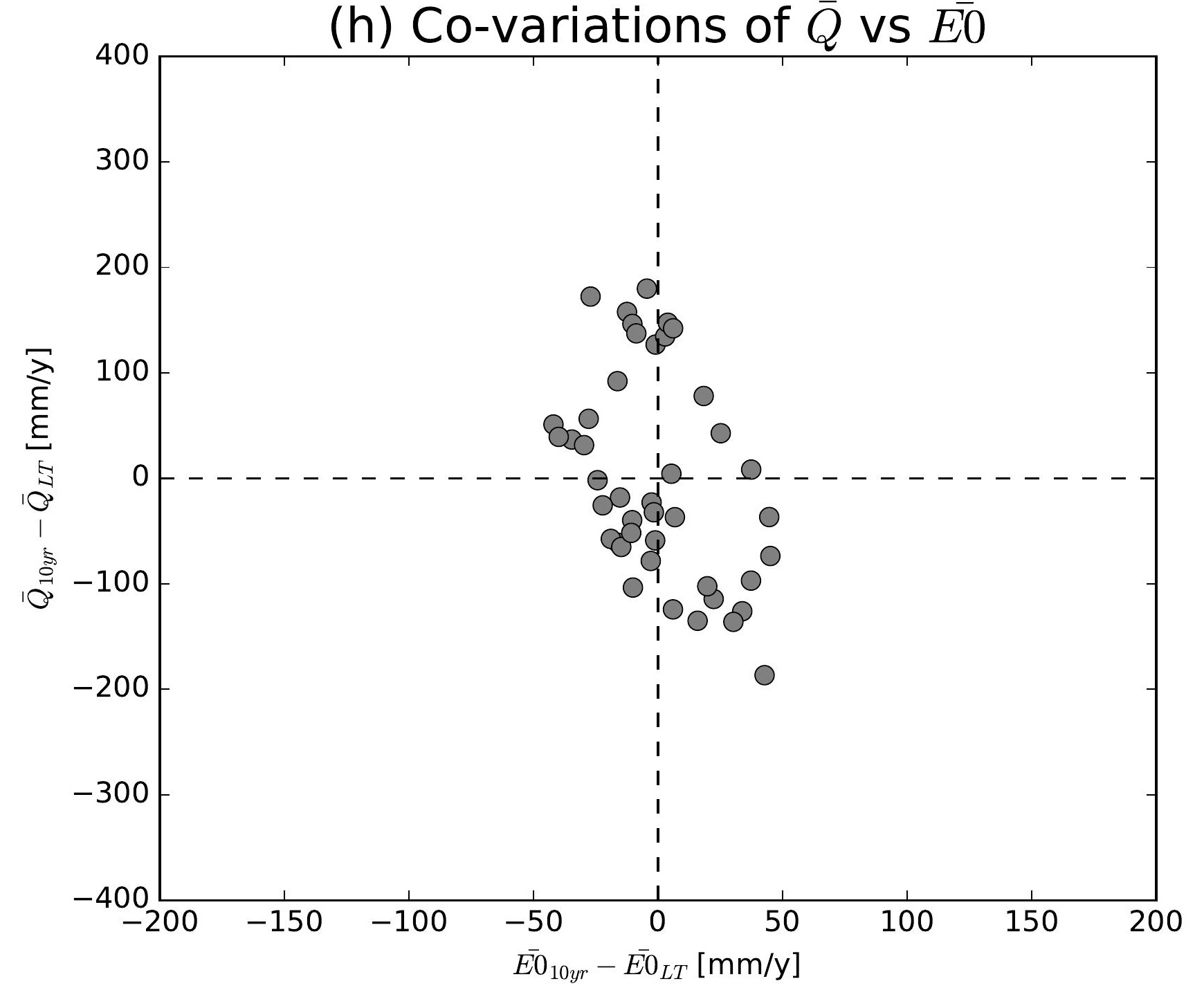

(k) Co-variations of $\bar{Q}$ vs $\overline{E 0}$

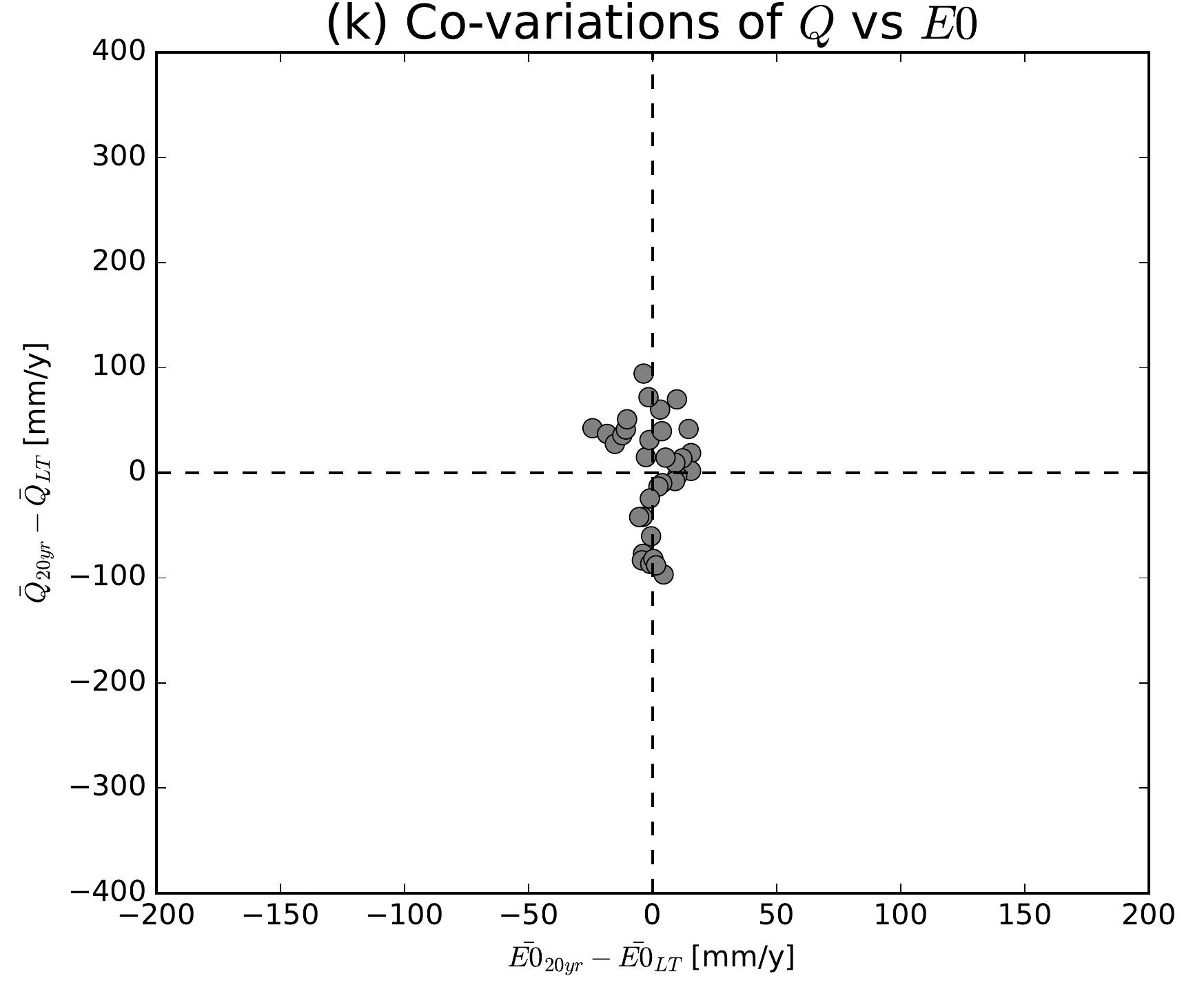

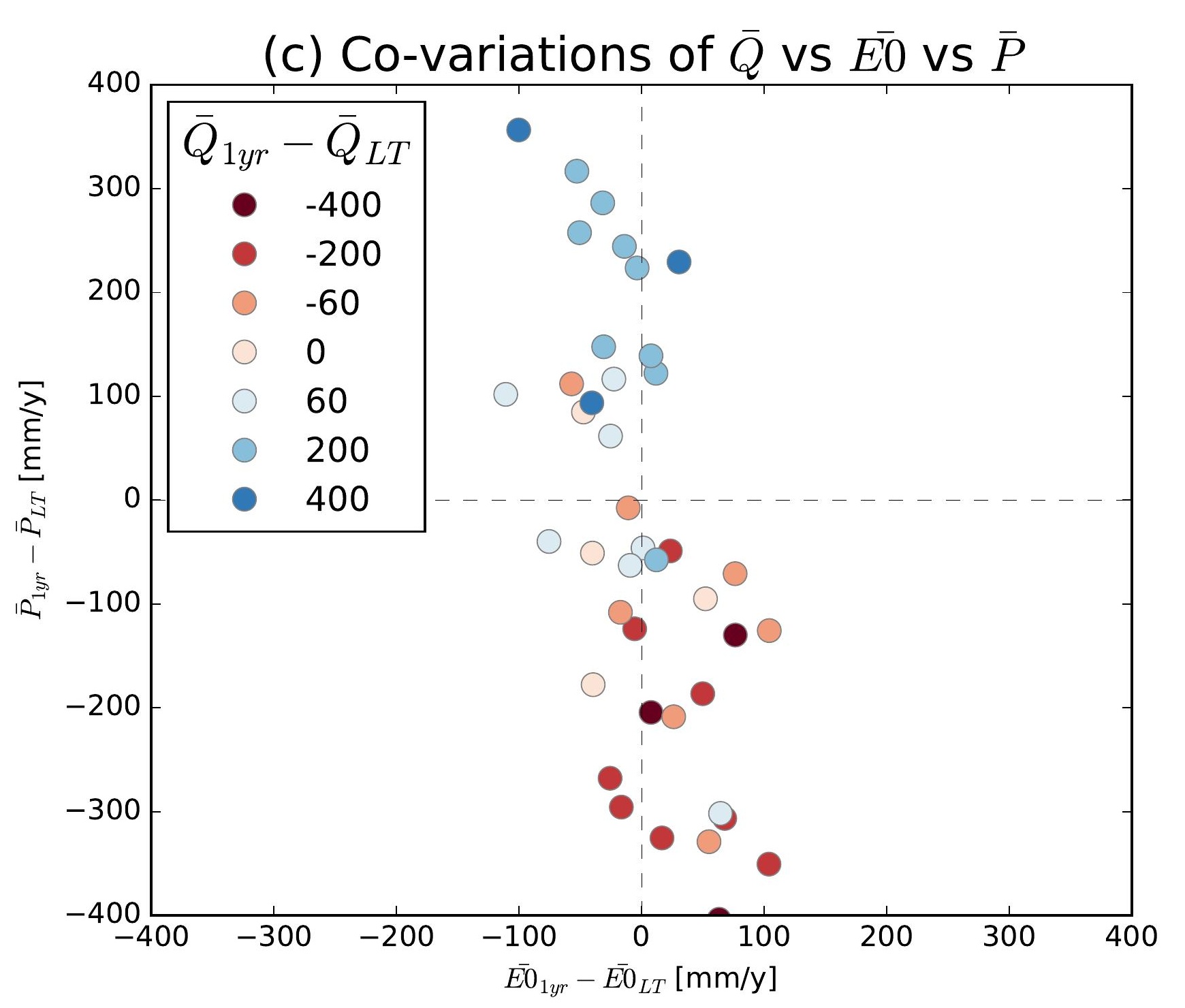
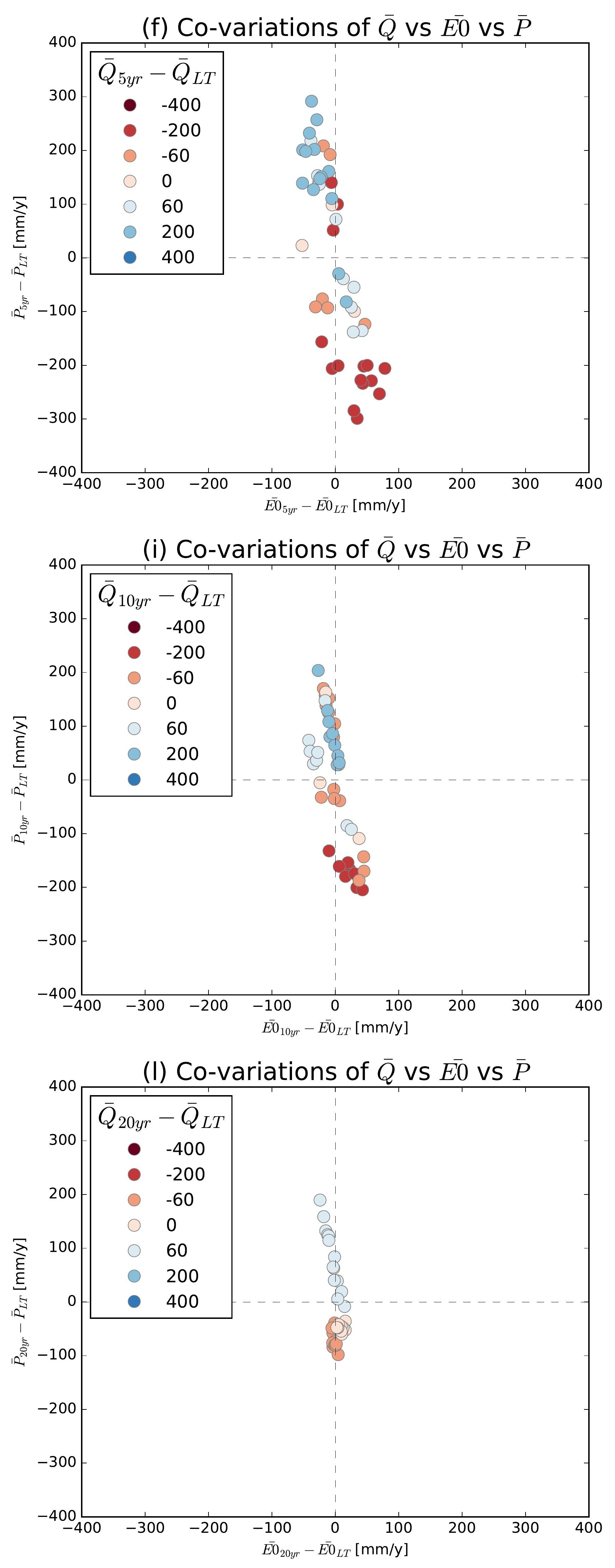

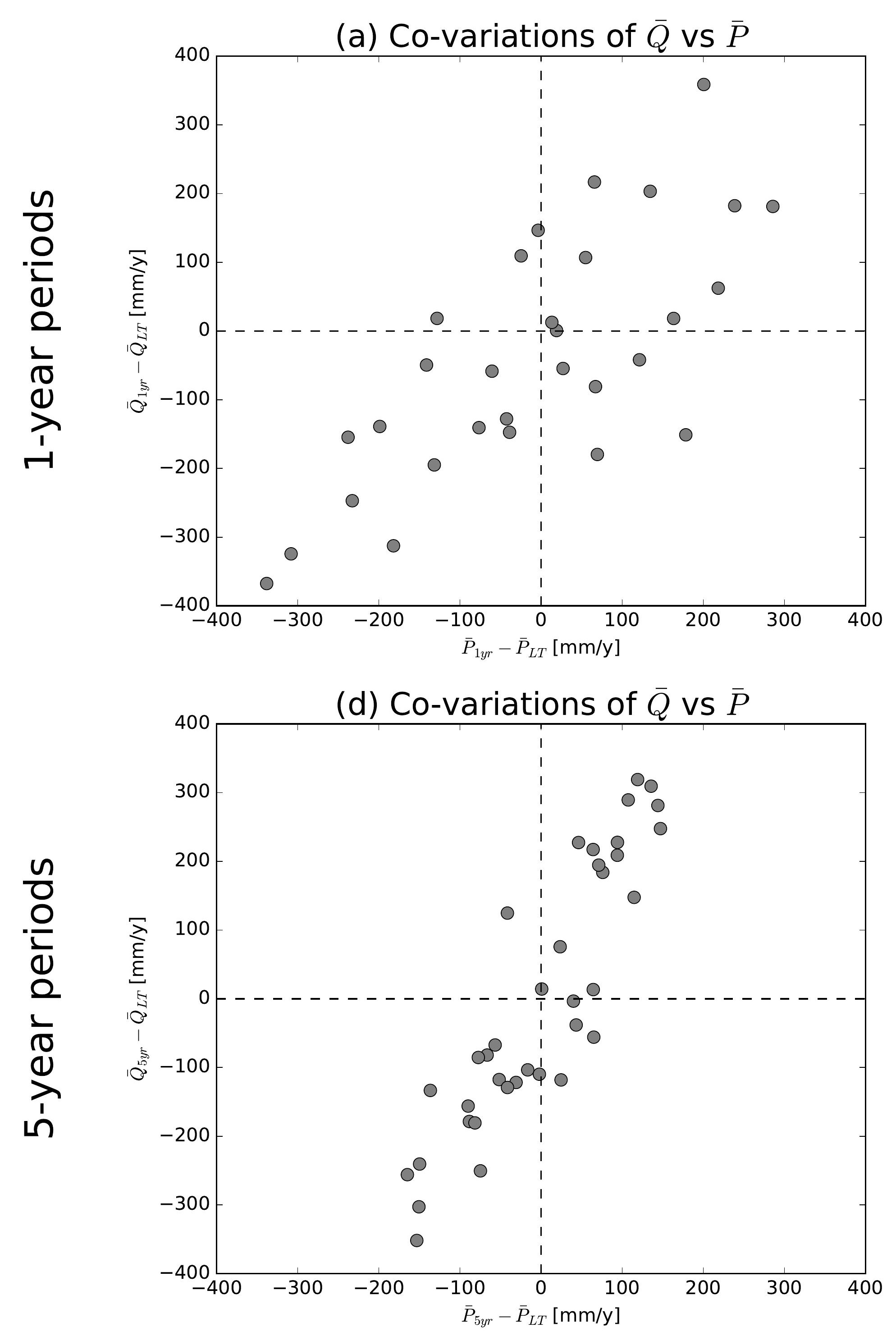

(g) Co-variations of $\bar{Q}$ vs $\bar{P}$
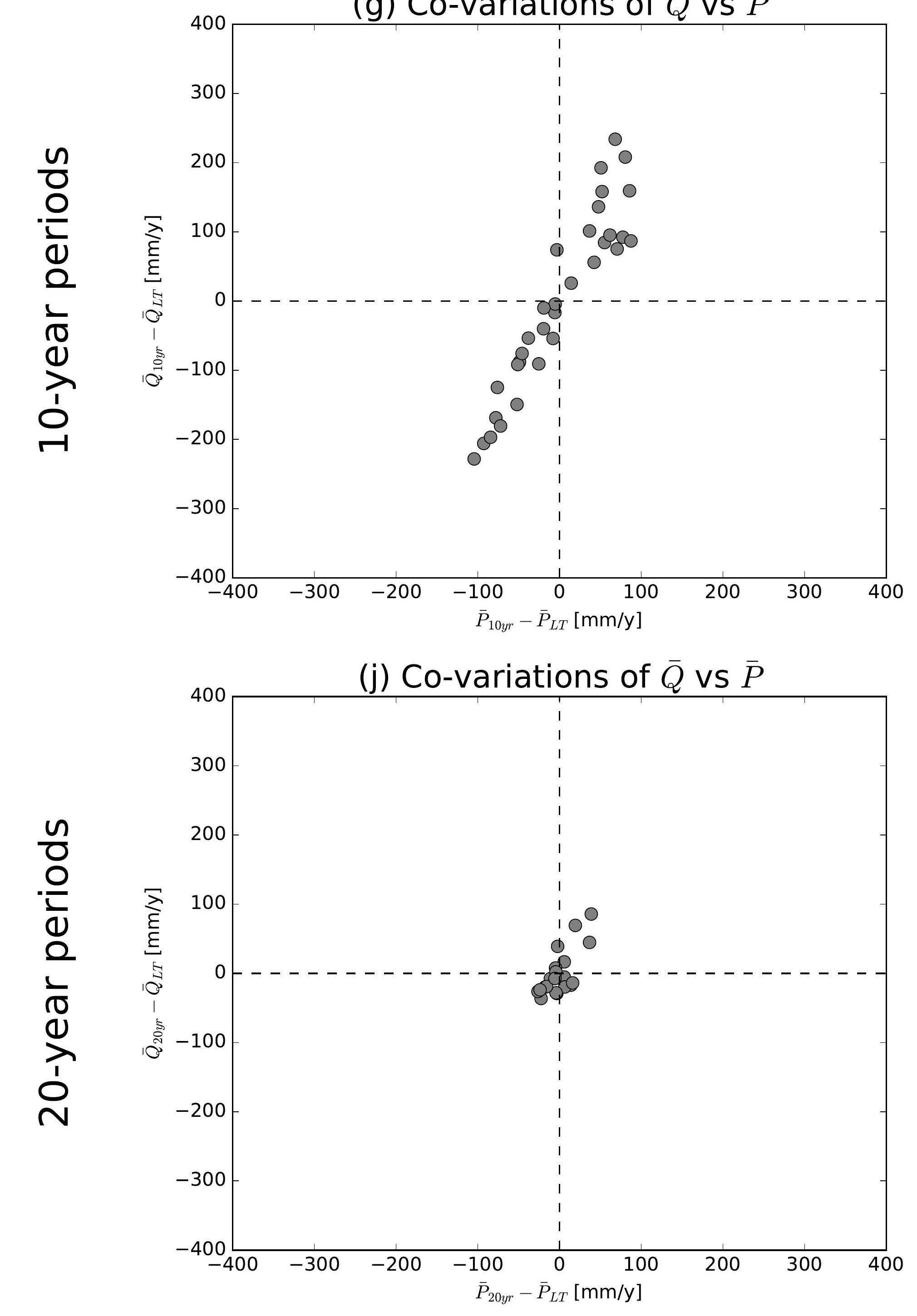

(b) Co-variations of $\bar{Q}$ vs $\overline{E 0}$

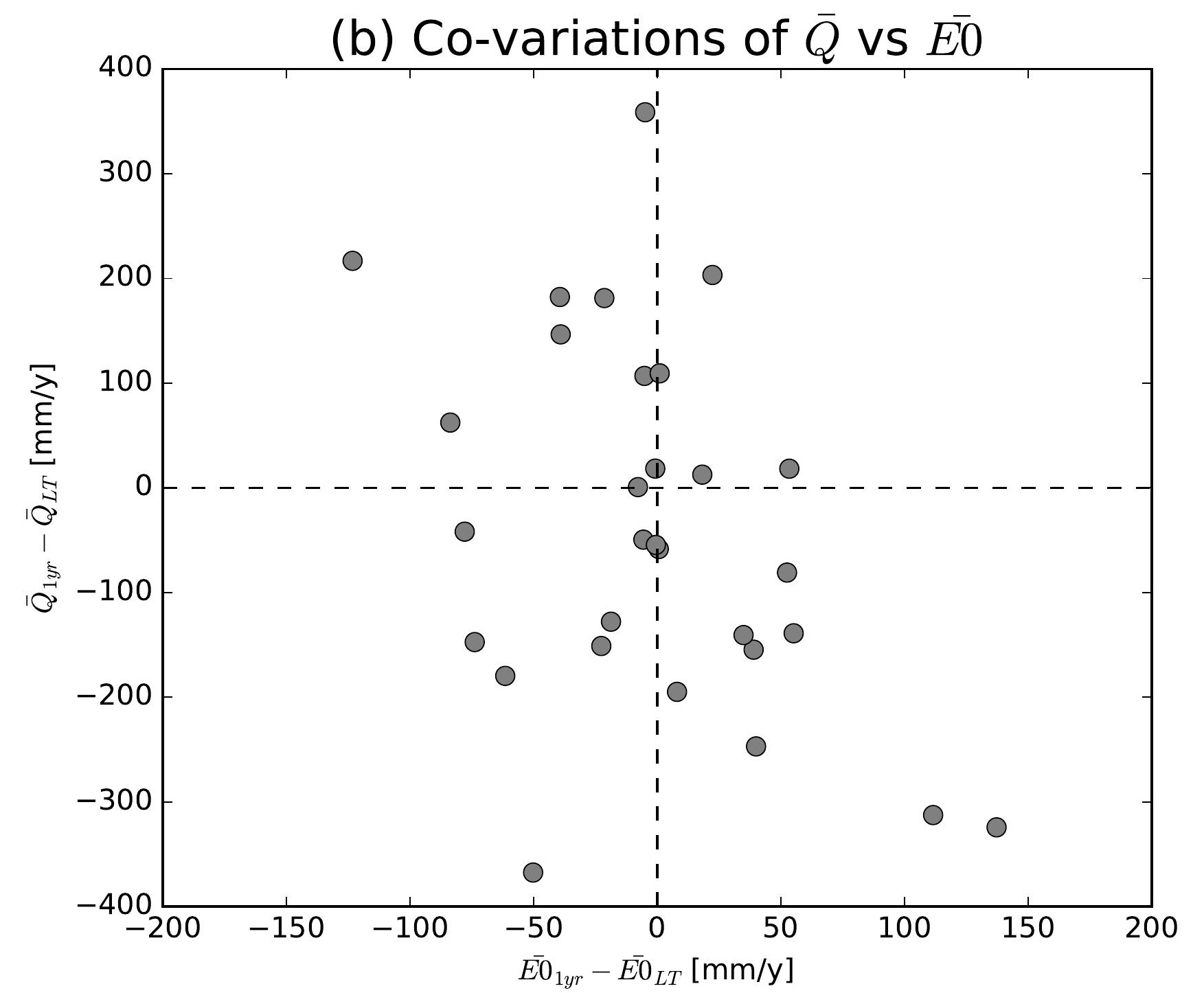

(e) Co-variations of $\bar{Q}$ vs $\overline{E 0}$

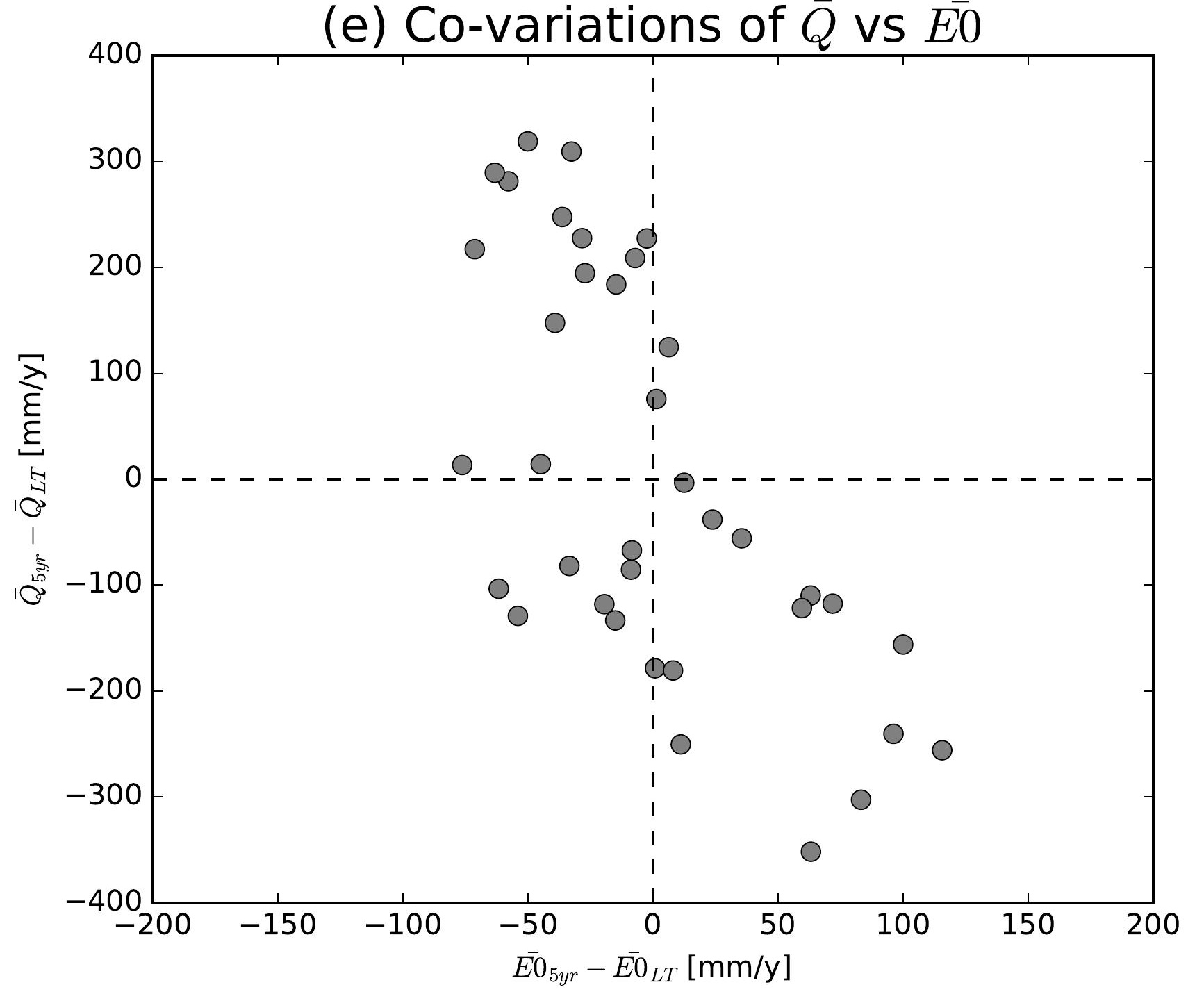

(h) Co-variations of $\bar{Q}$ vs $\overline{E 0}$

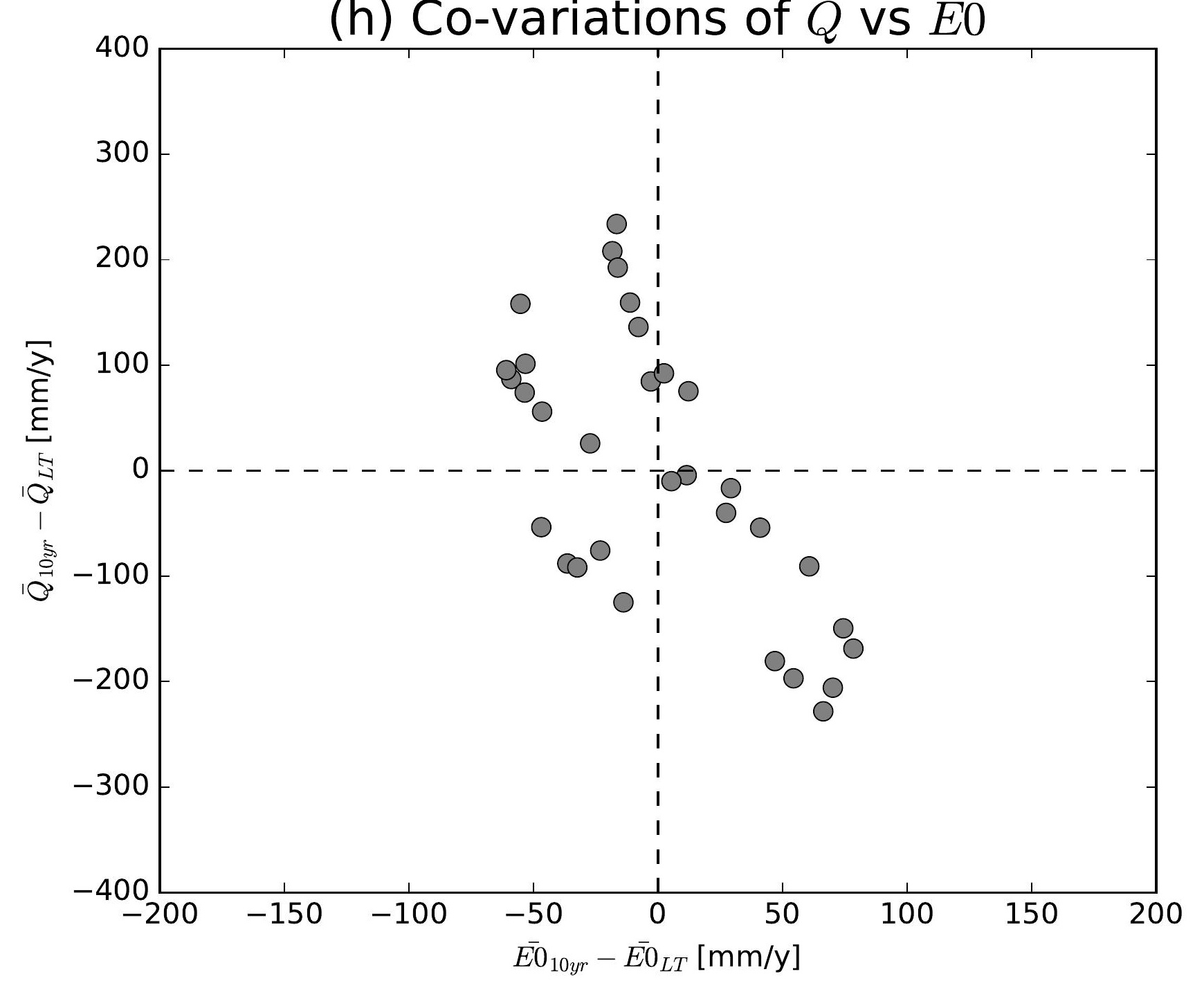

(k) Co-variations of $\bar{Q}$ vs $\overline{E 0}$

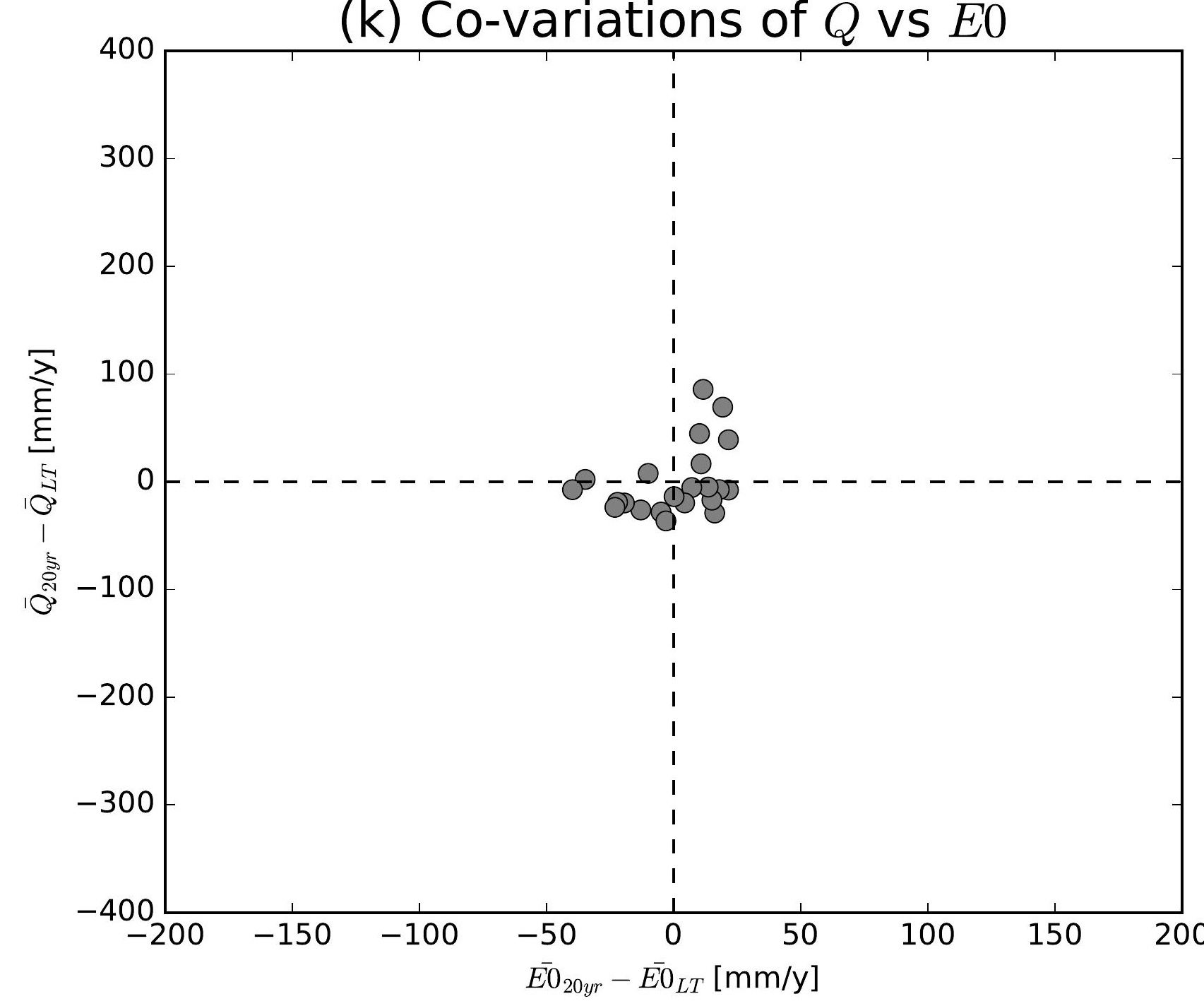

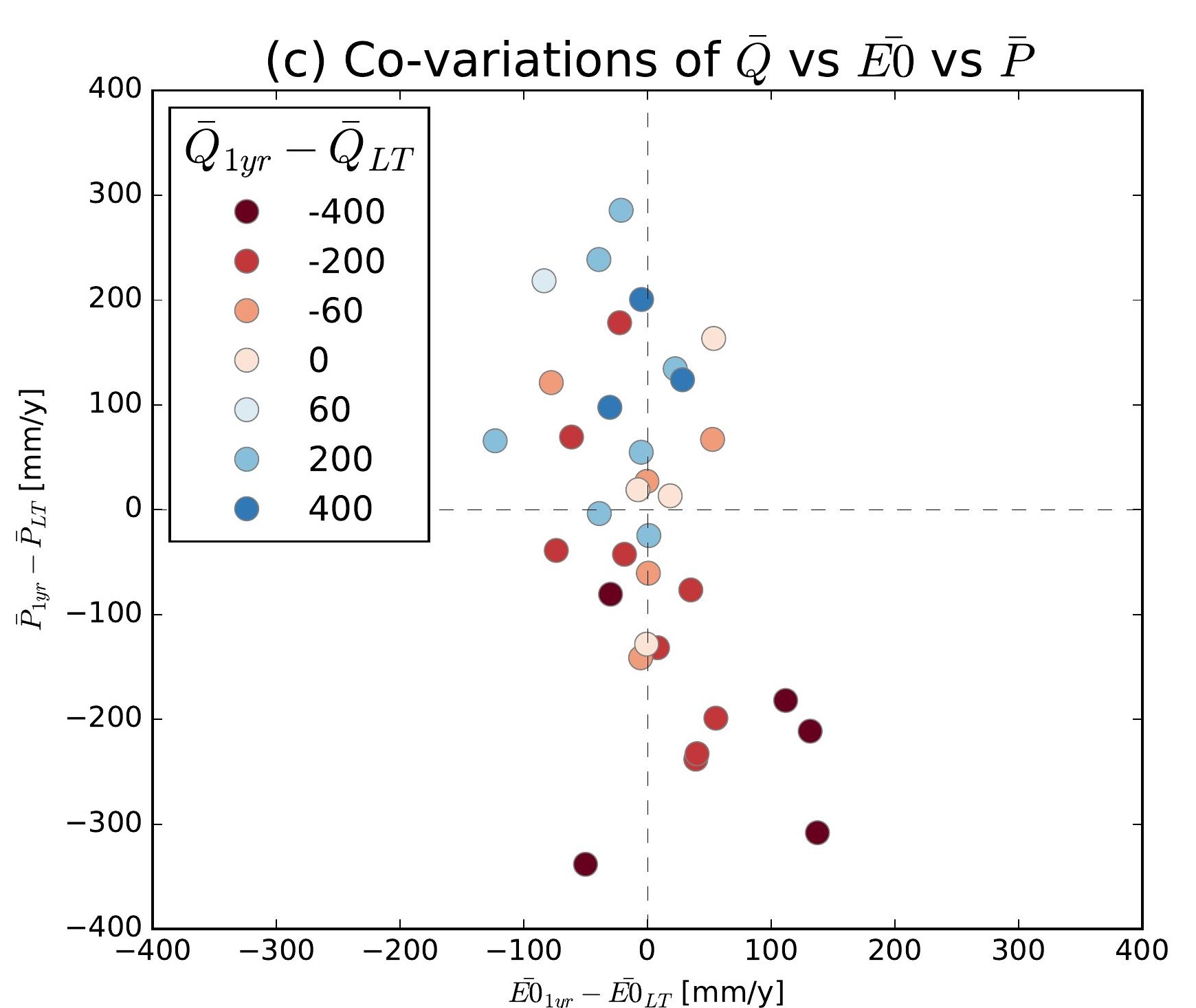
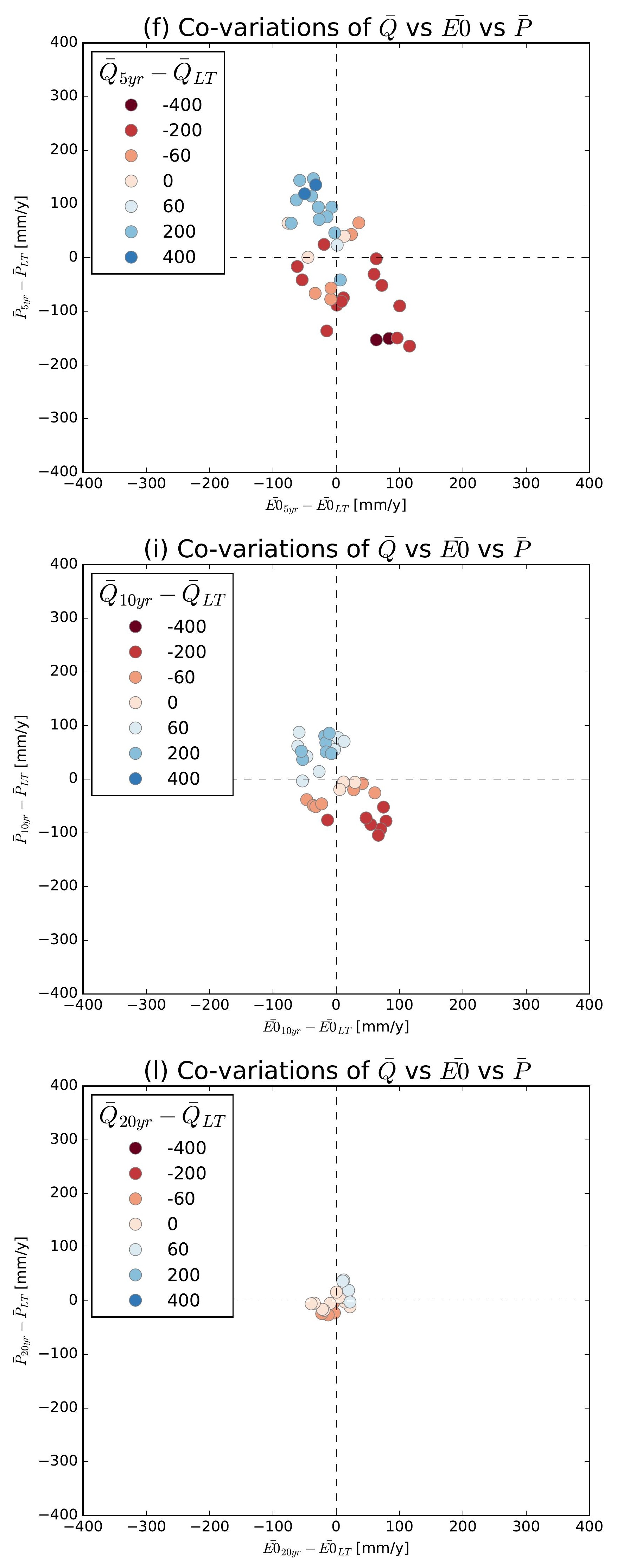

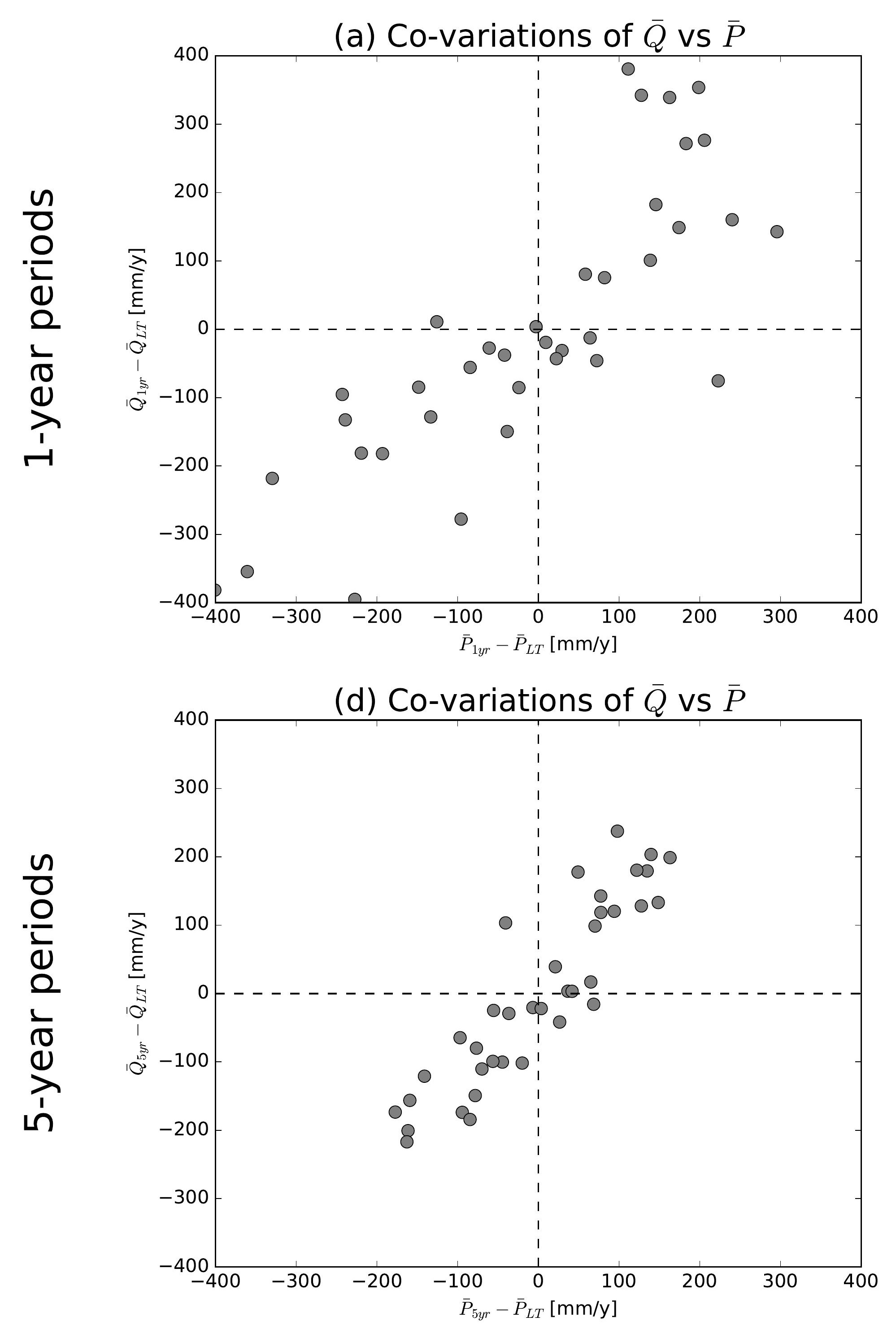

(g) Co-variations of $\bar{Q}$ vs $\bar{P}$

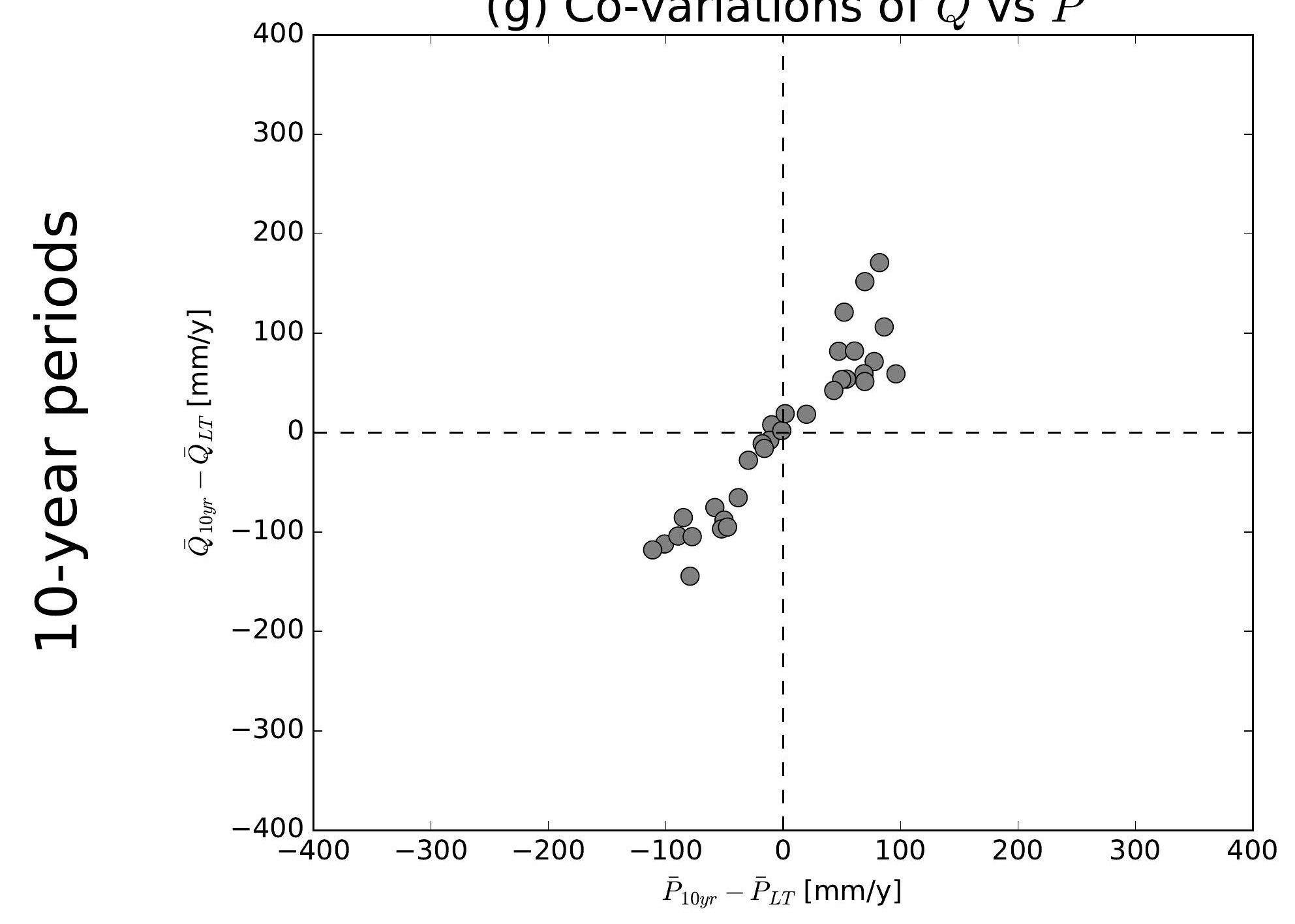

(j) Co-variations of $\bar{Q}$ vs $\bar{P}$

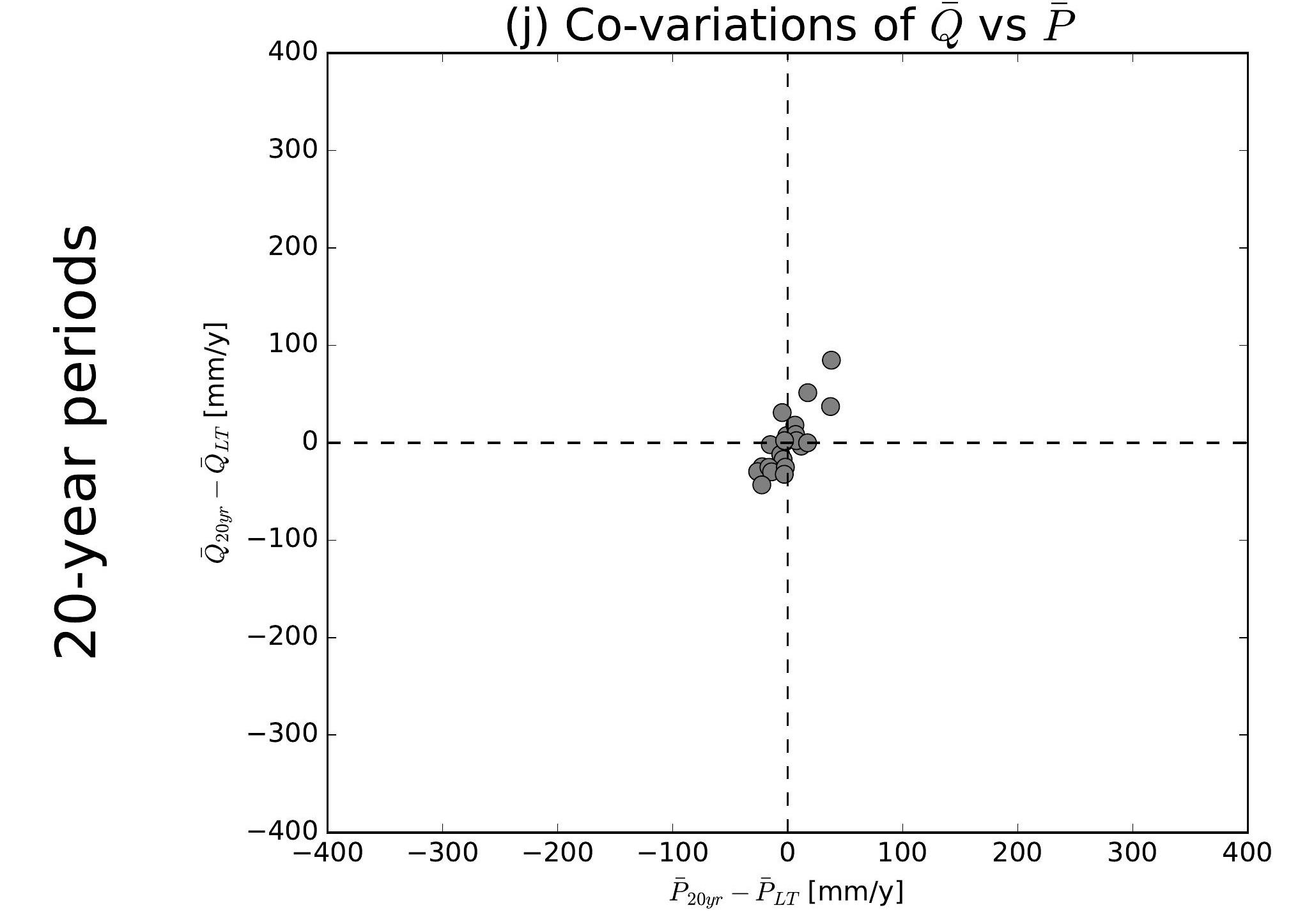

(b) Co-variations of $\bar{Q}$ vs $\overline{E 0}$

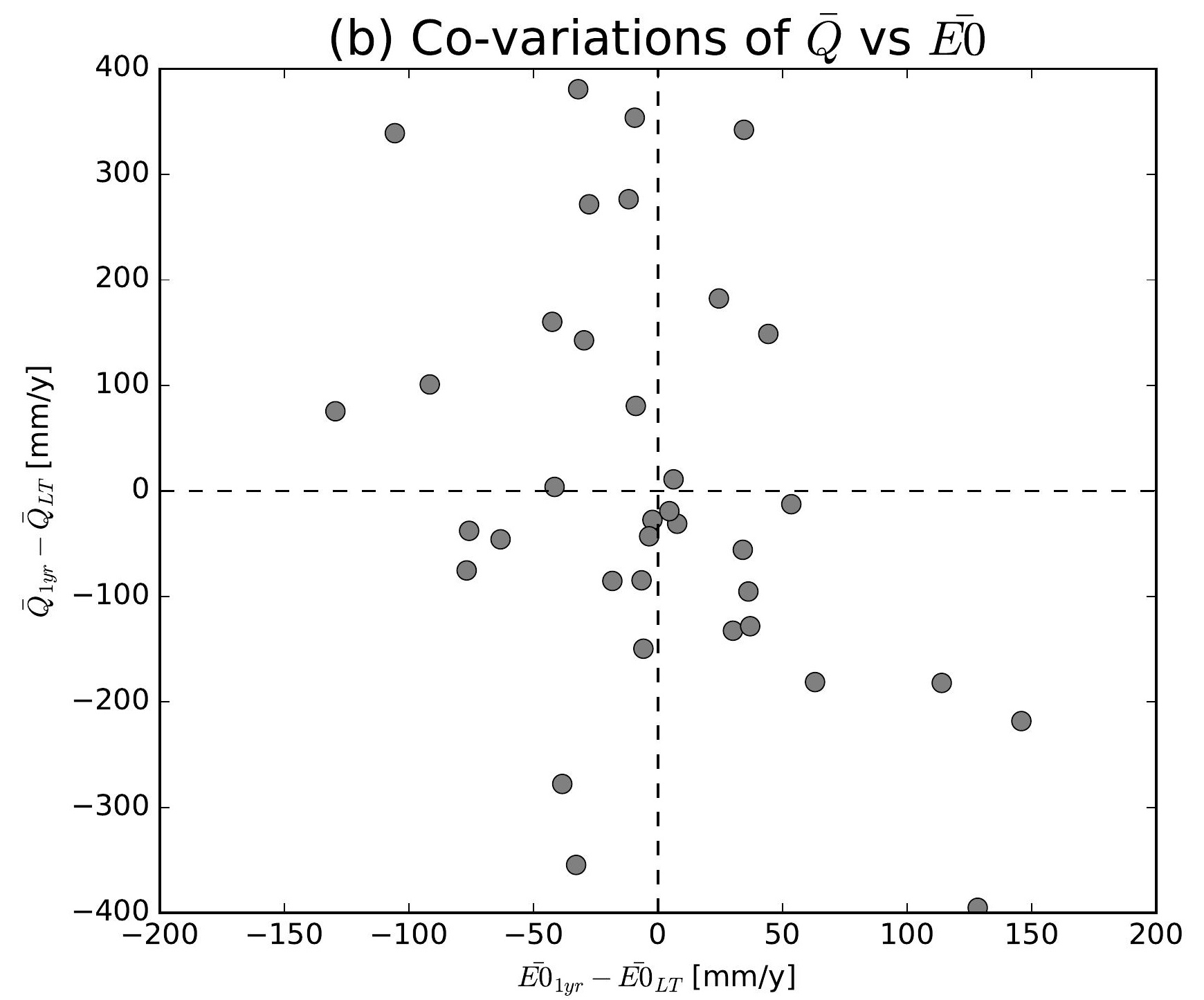

(e) Co-variations of $\bar{Q}$ vs $\overline{E 0}$

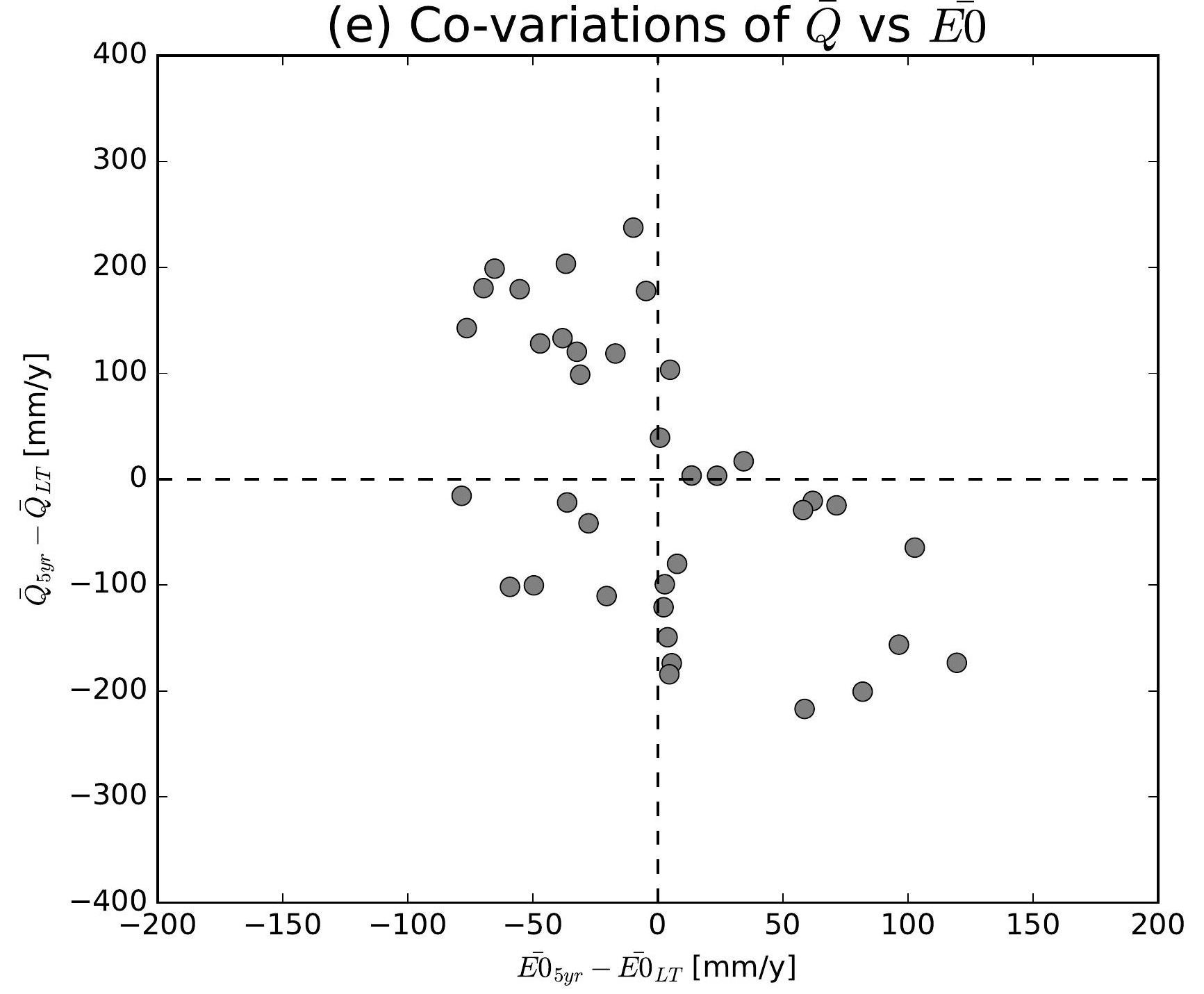

(h) Co-variations of $\bar{Q}$ vs $\overline{E 0}$

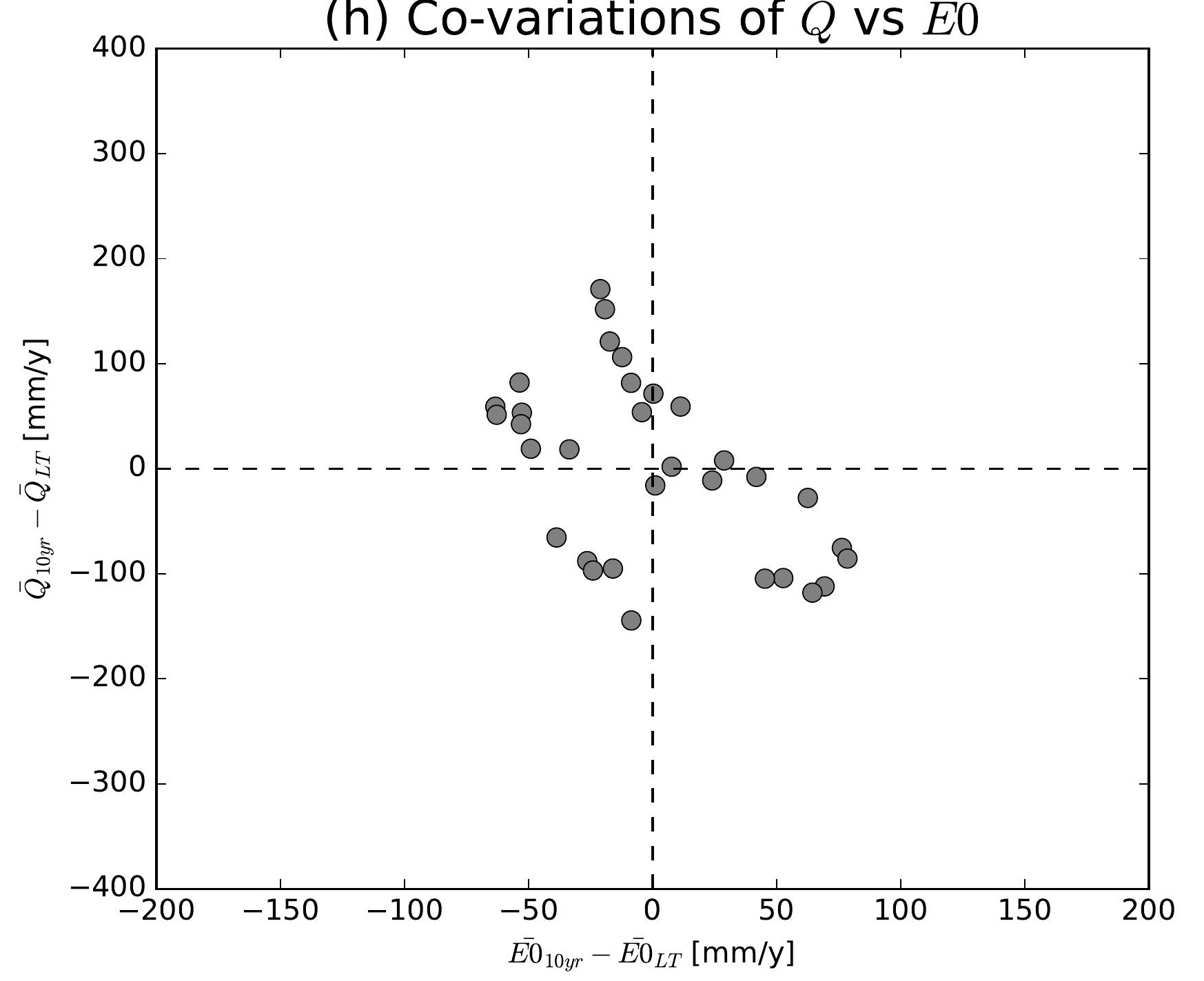

(k) Co-variations of $\bar{Q}$ vs $\overline{E 0}$

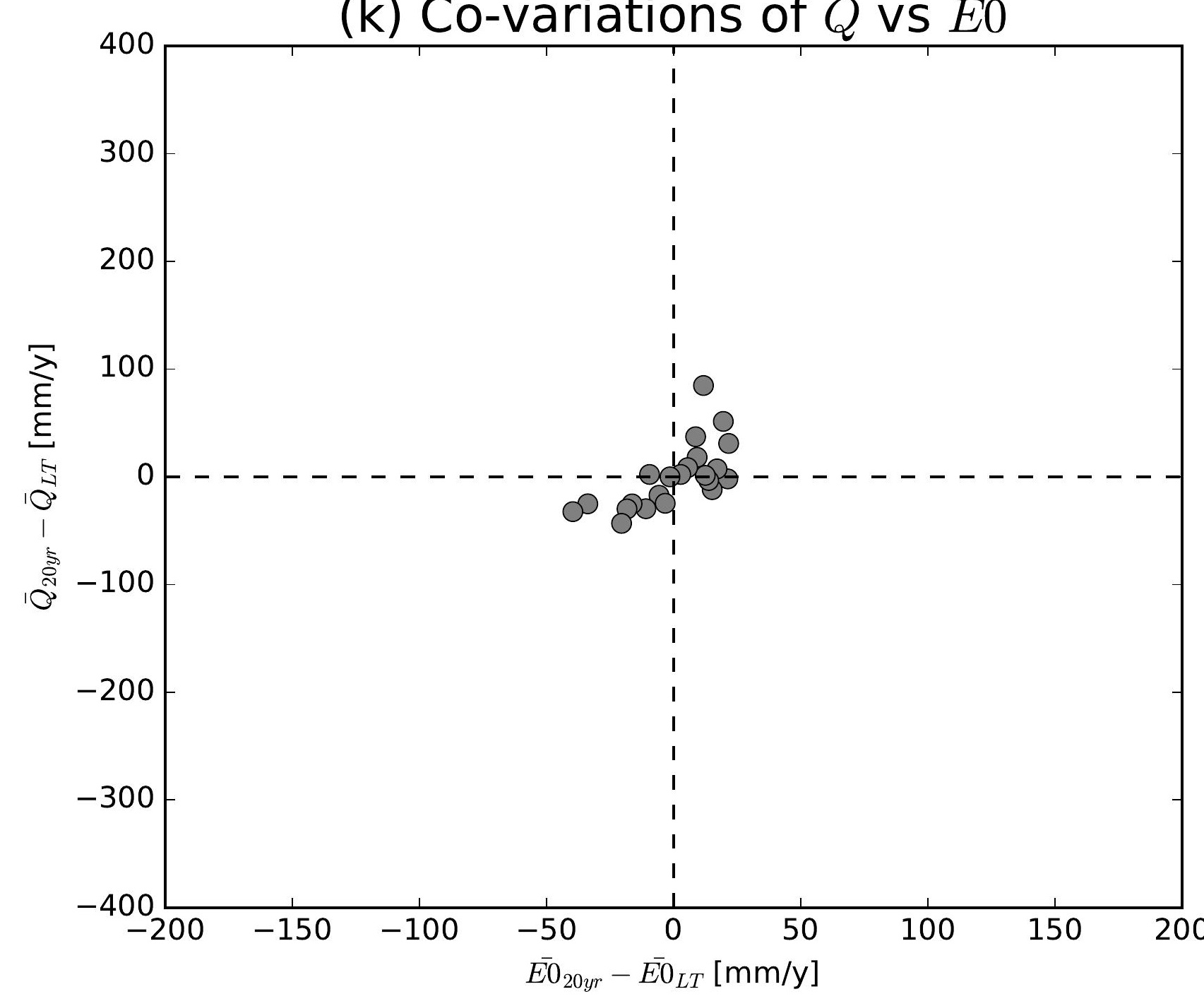

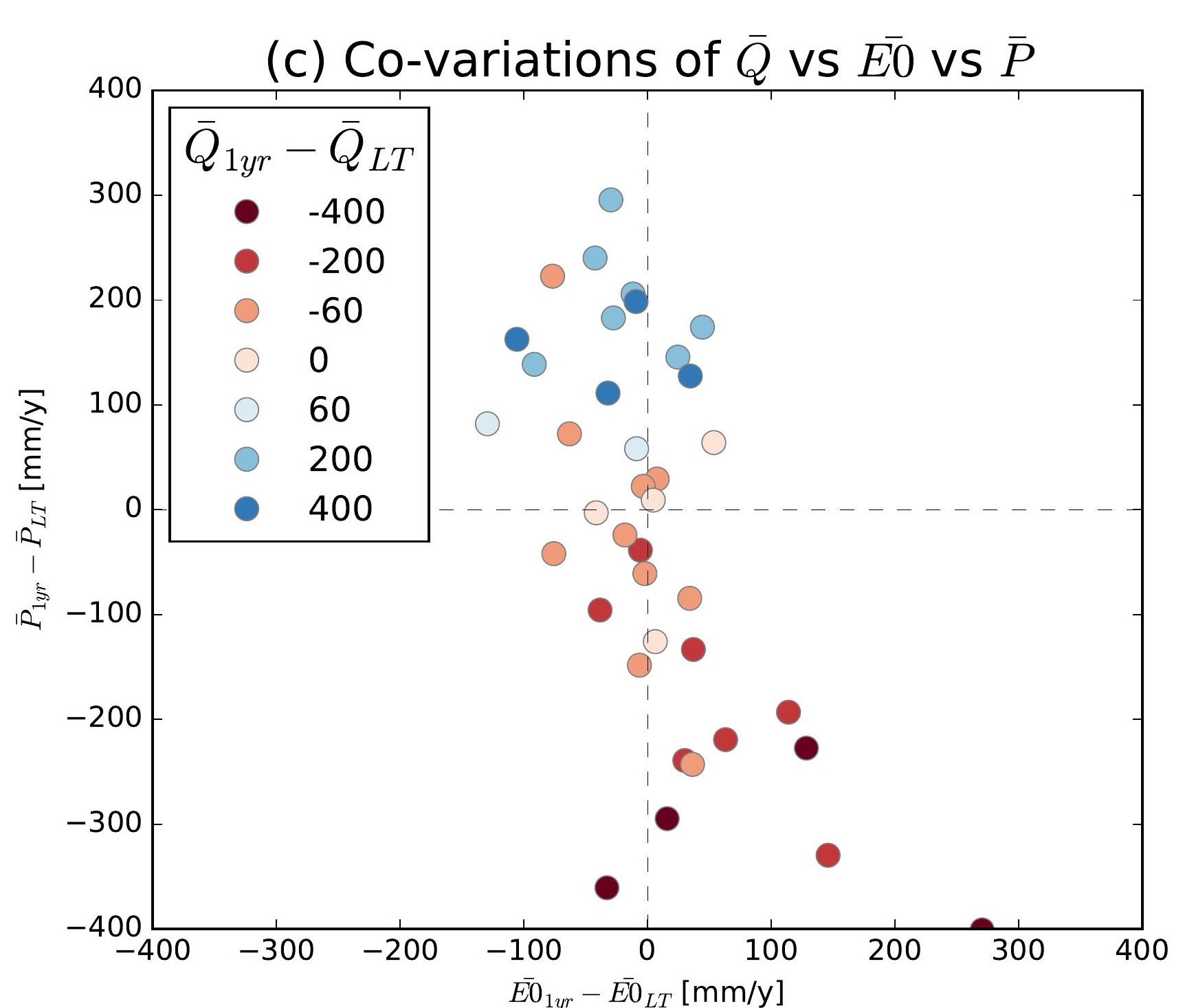
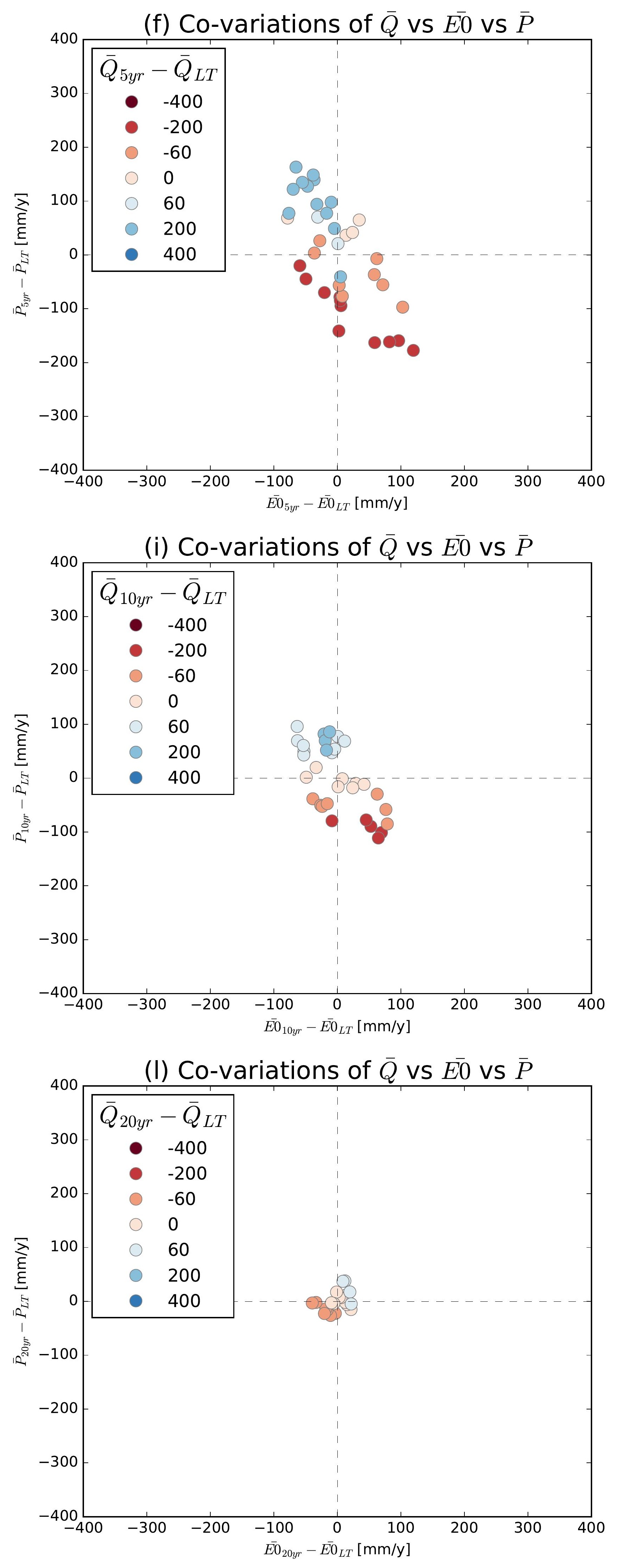

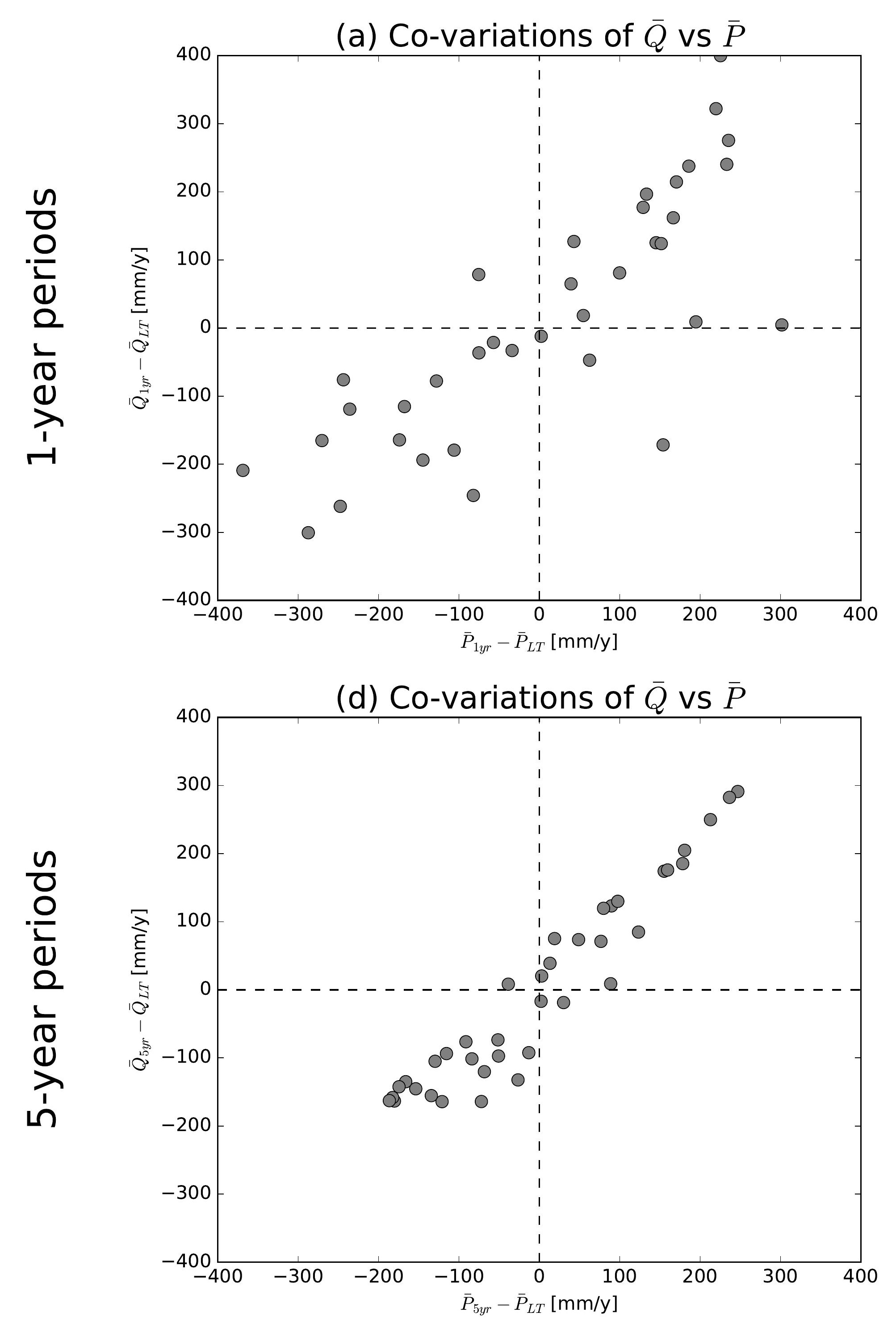

(g) Co-variations of $\bar{Q}$ vs $\bar{P}$

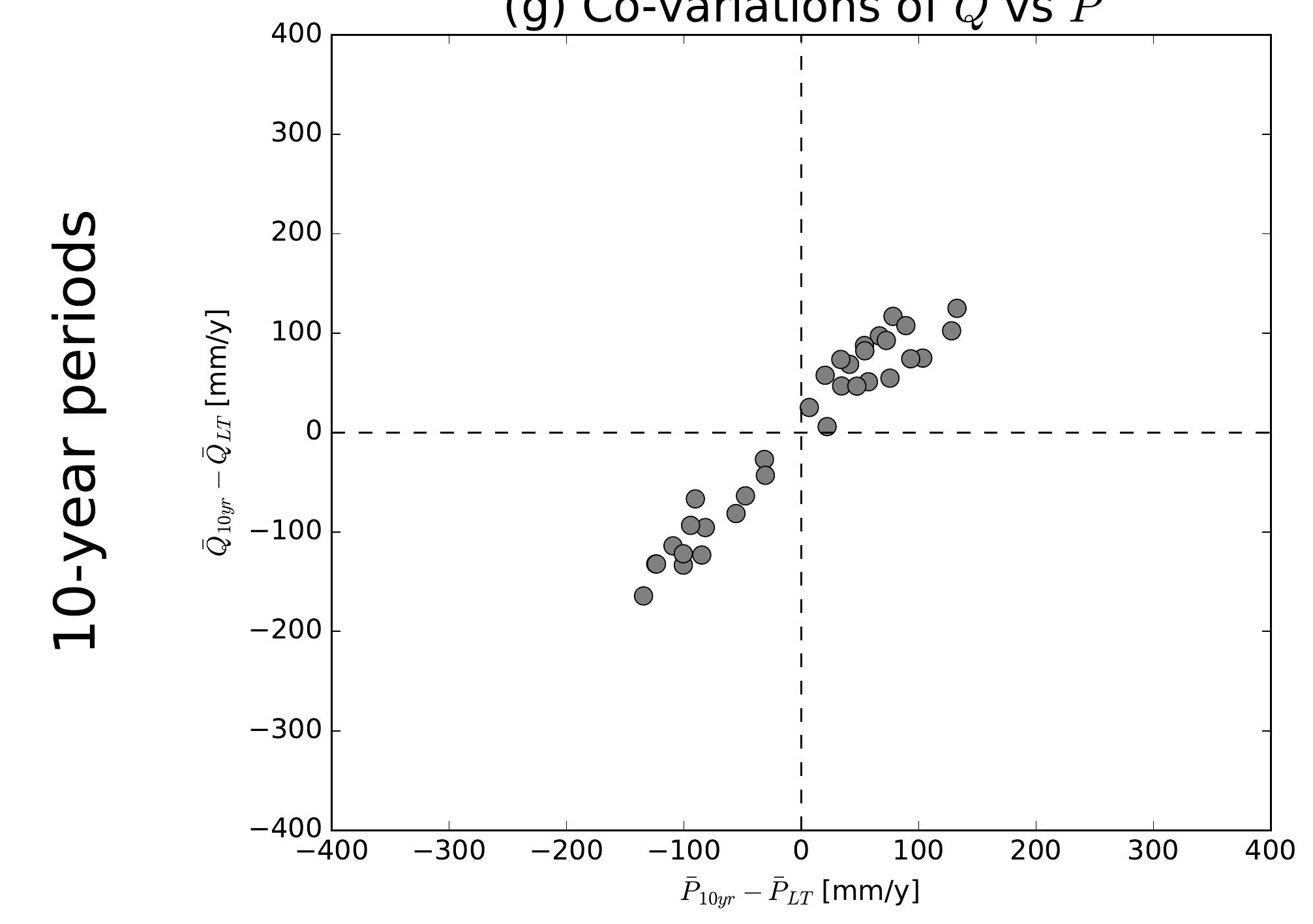

(j) Co-variations of $\bar{Q}$ vs $\bar{P}$

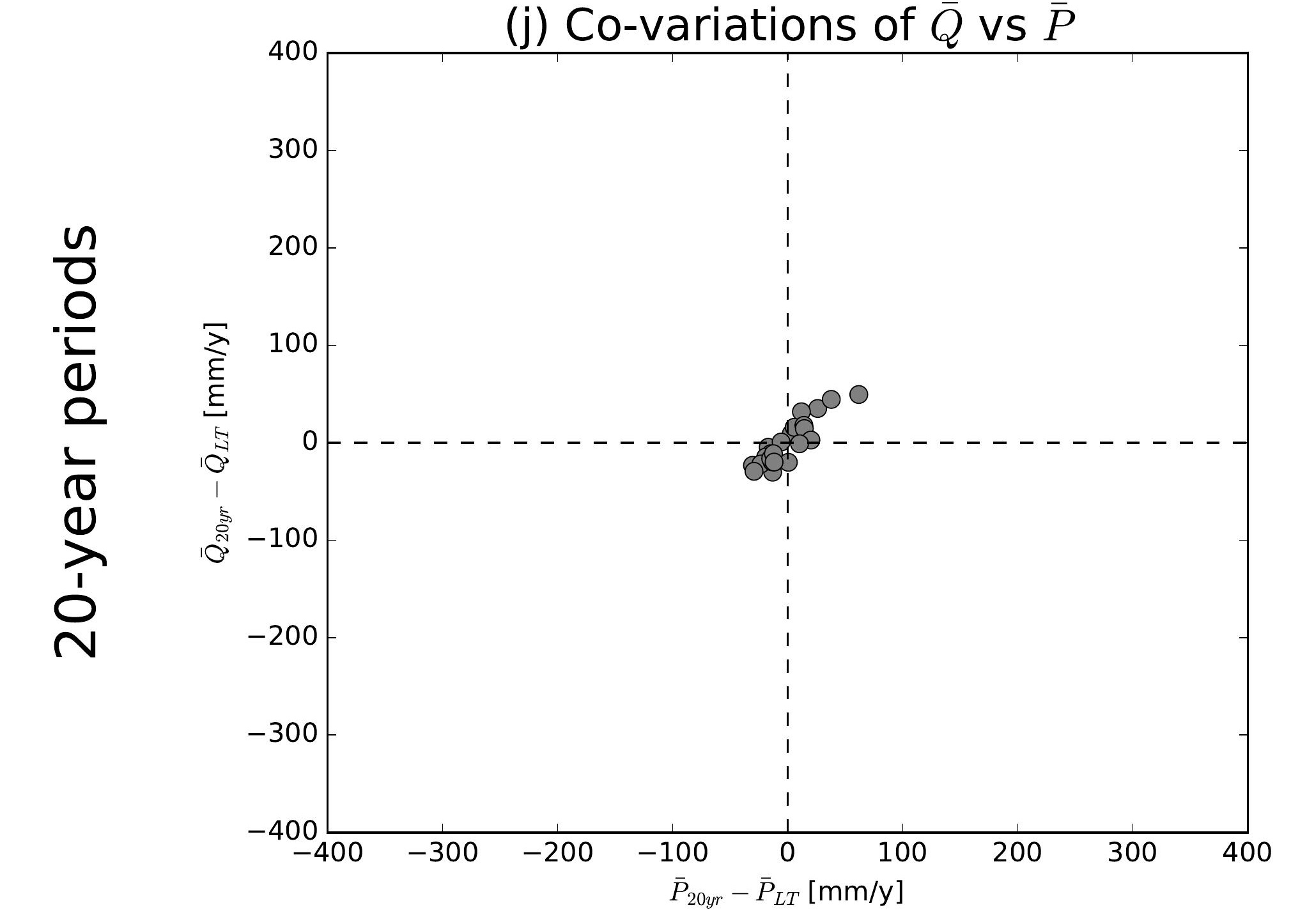

(b) Co-variations of $\bar{Q}$ vs $\overline{E 0}$

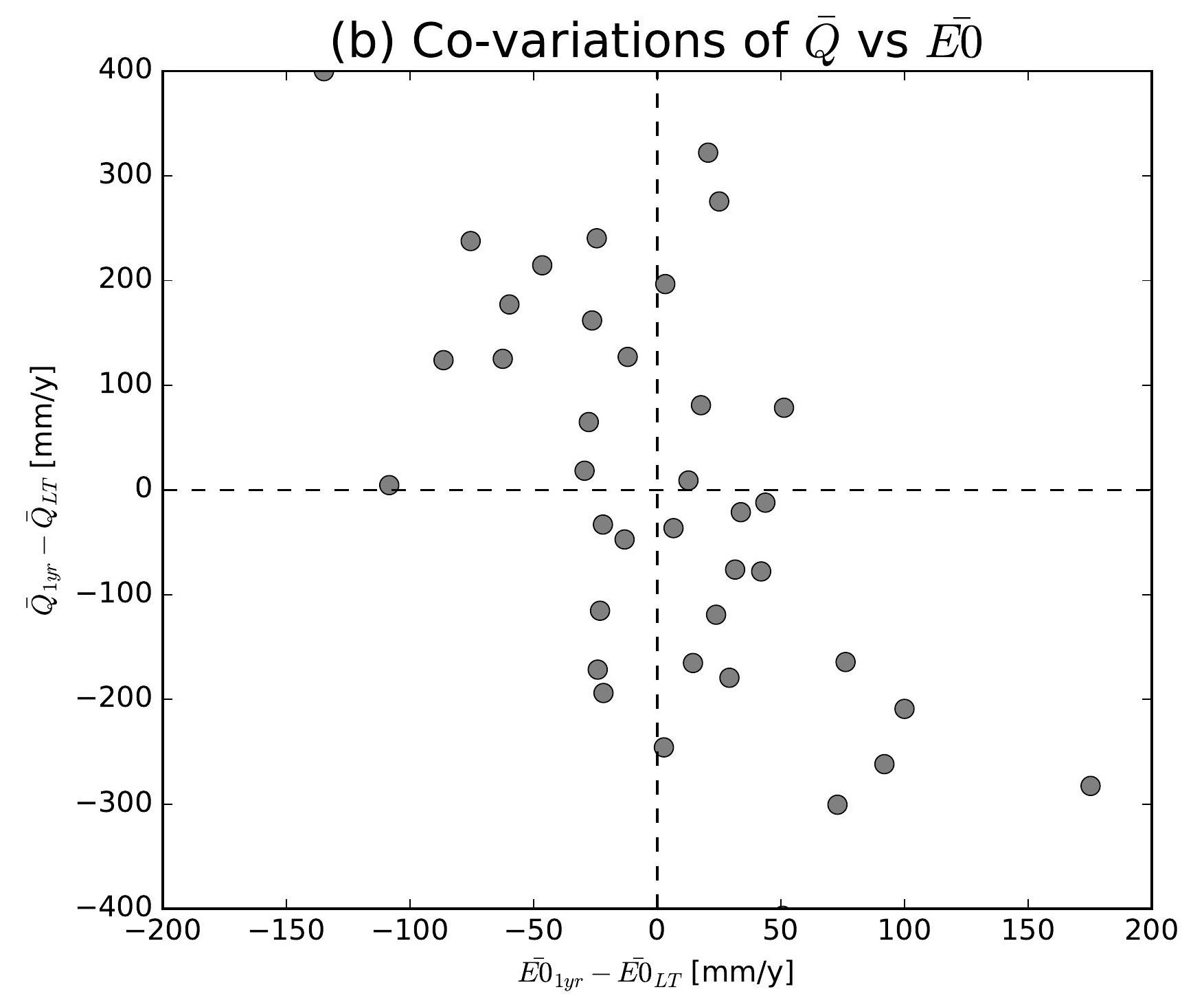

(e) Co-variations of $\bar{Q}$ vs $\overline{E 0}$

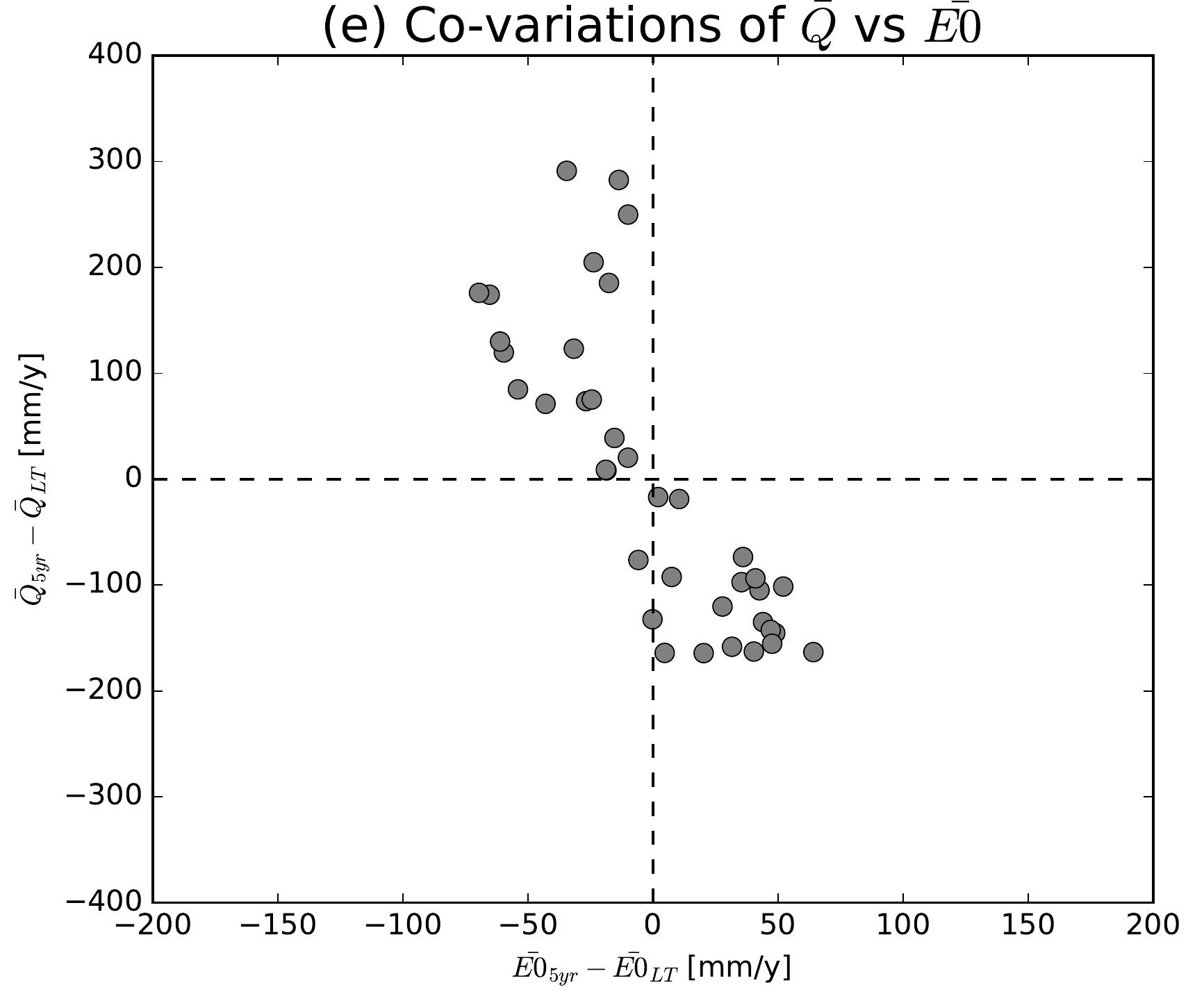

(h) Co-variations of $\bar{Q}$ vs $\overline{E 0}$

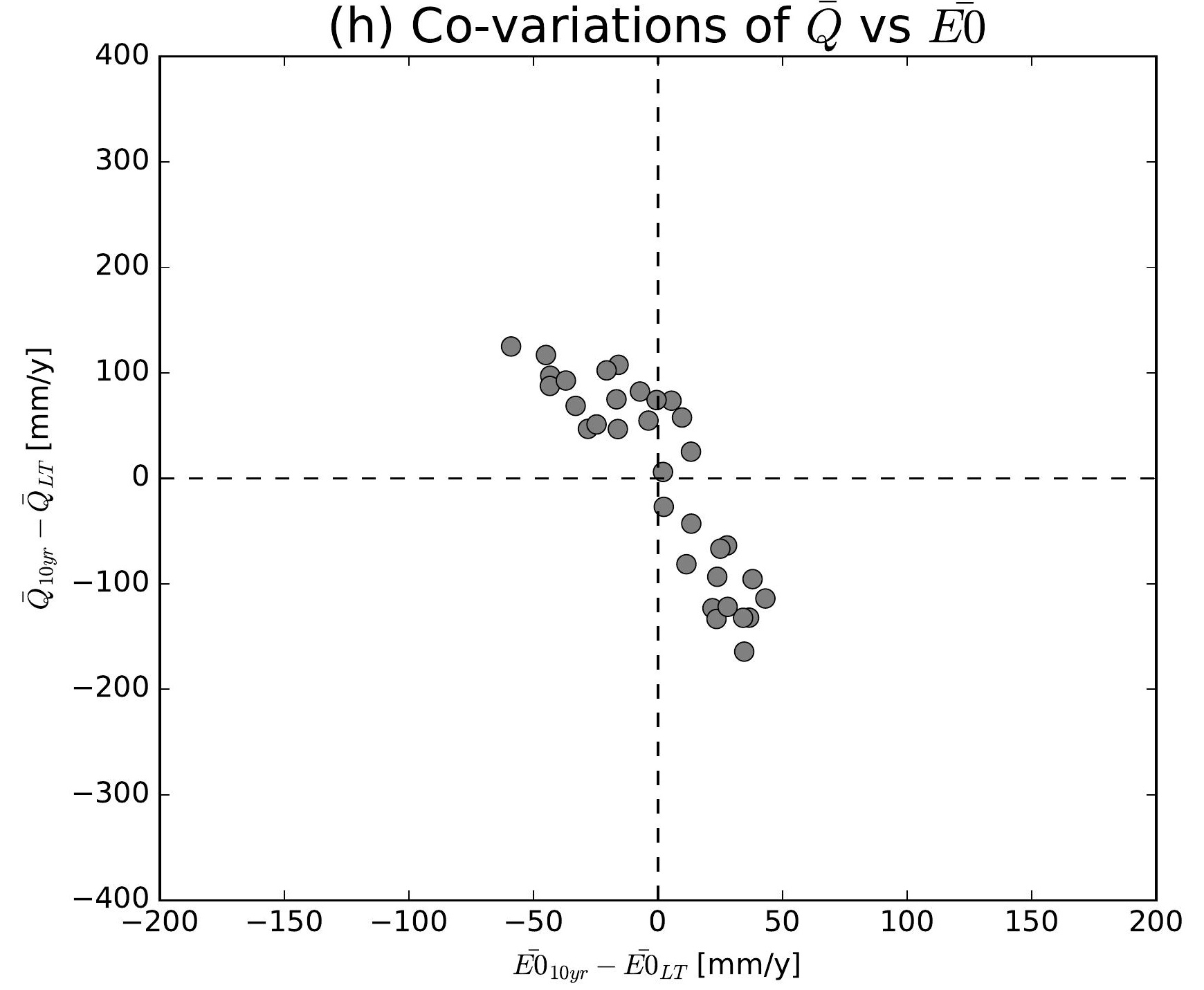

(k) Co-variations of $\bar{Q}$ vs $\overline{E 0}$

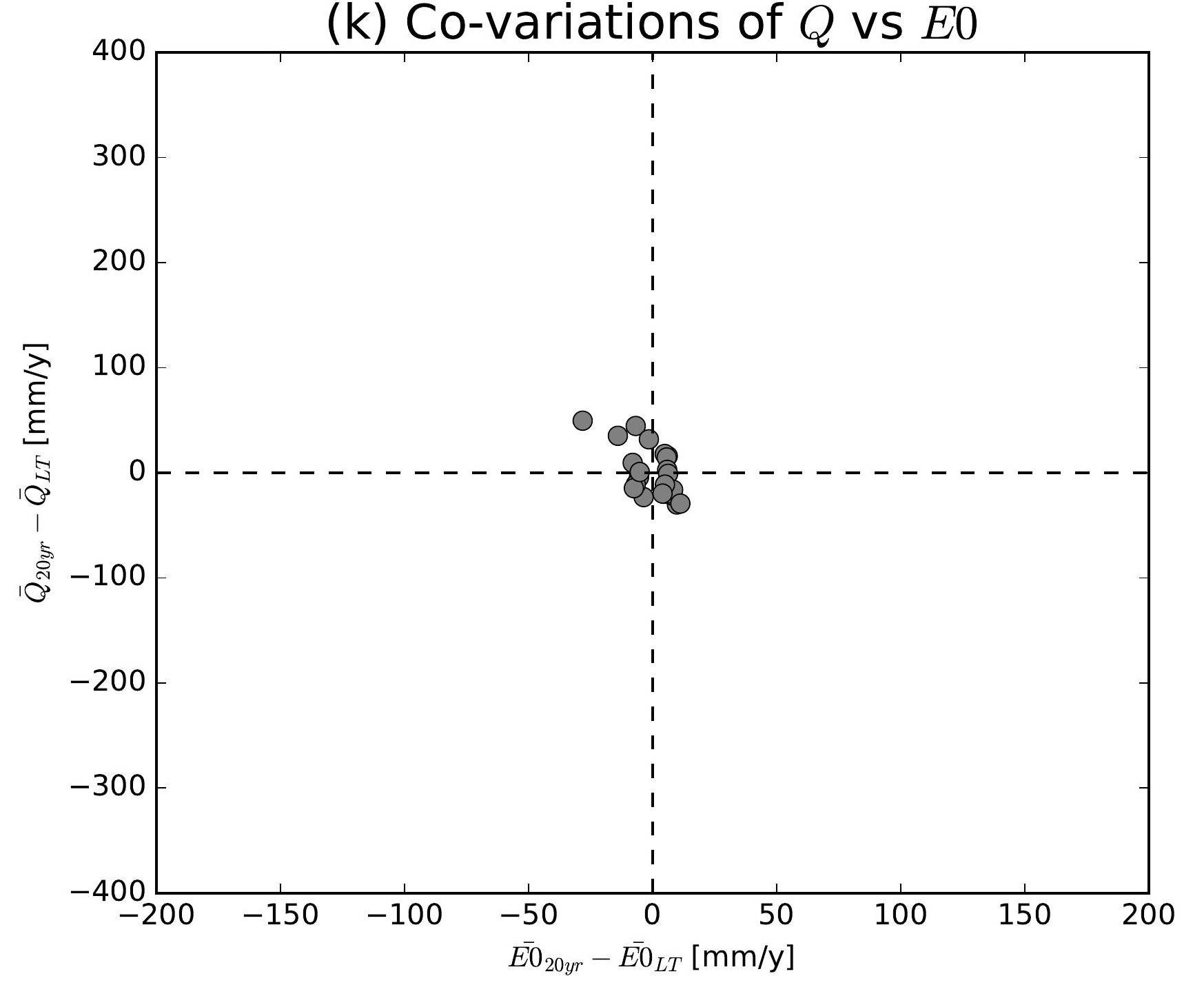

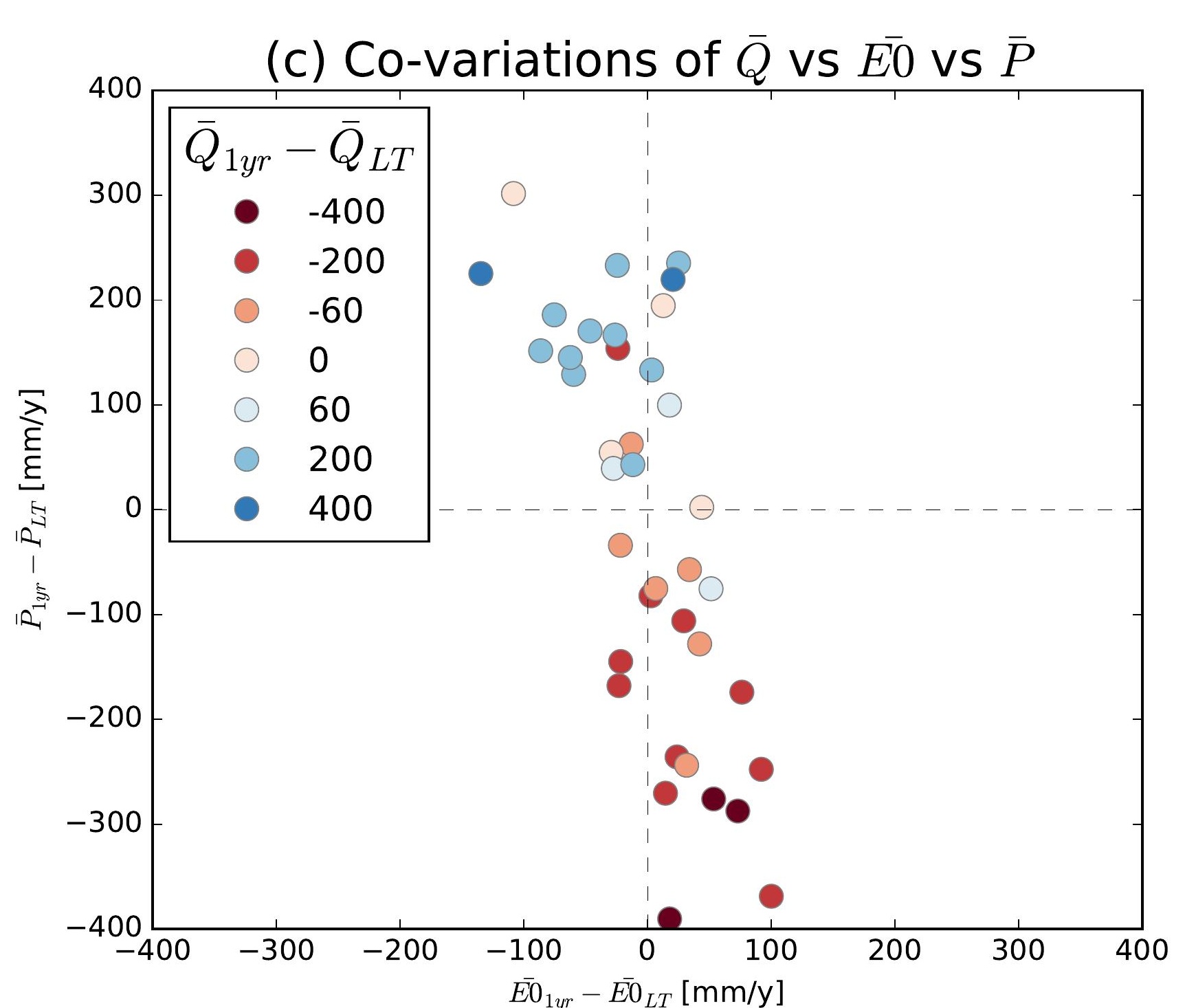
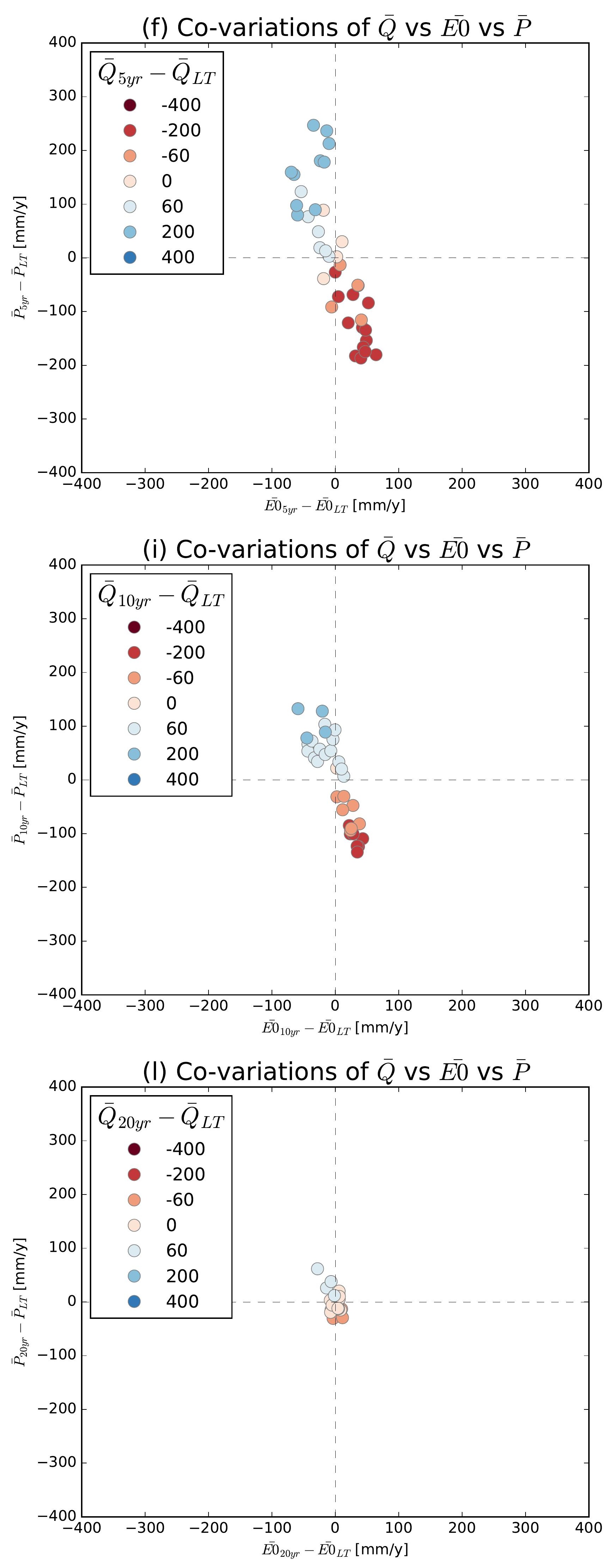

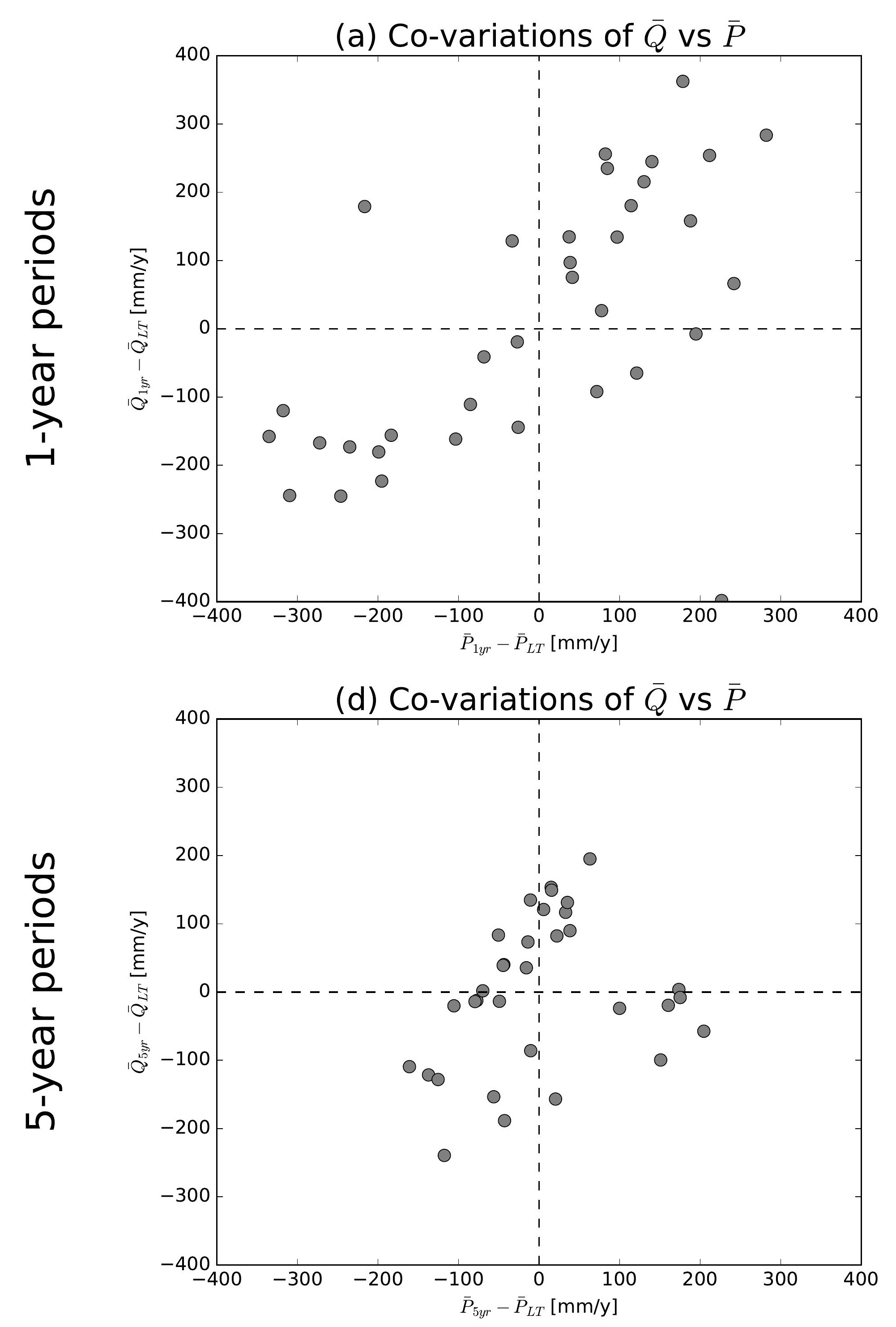

(g) Co-variations of $\bar{Q}$ vs $\bar{P}$

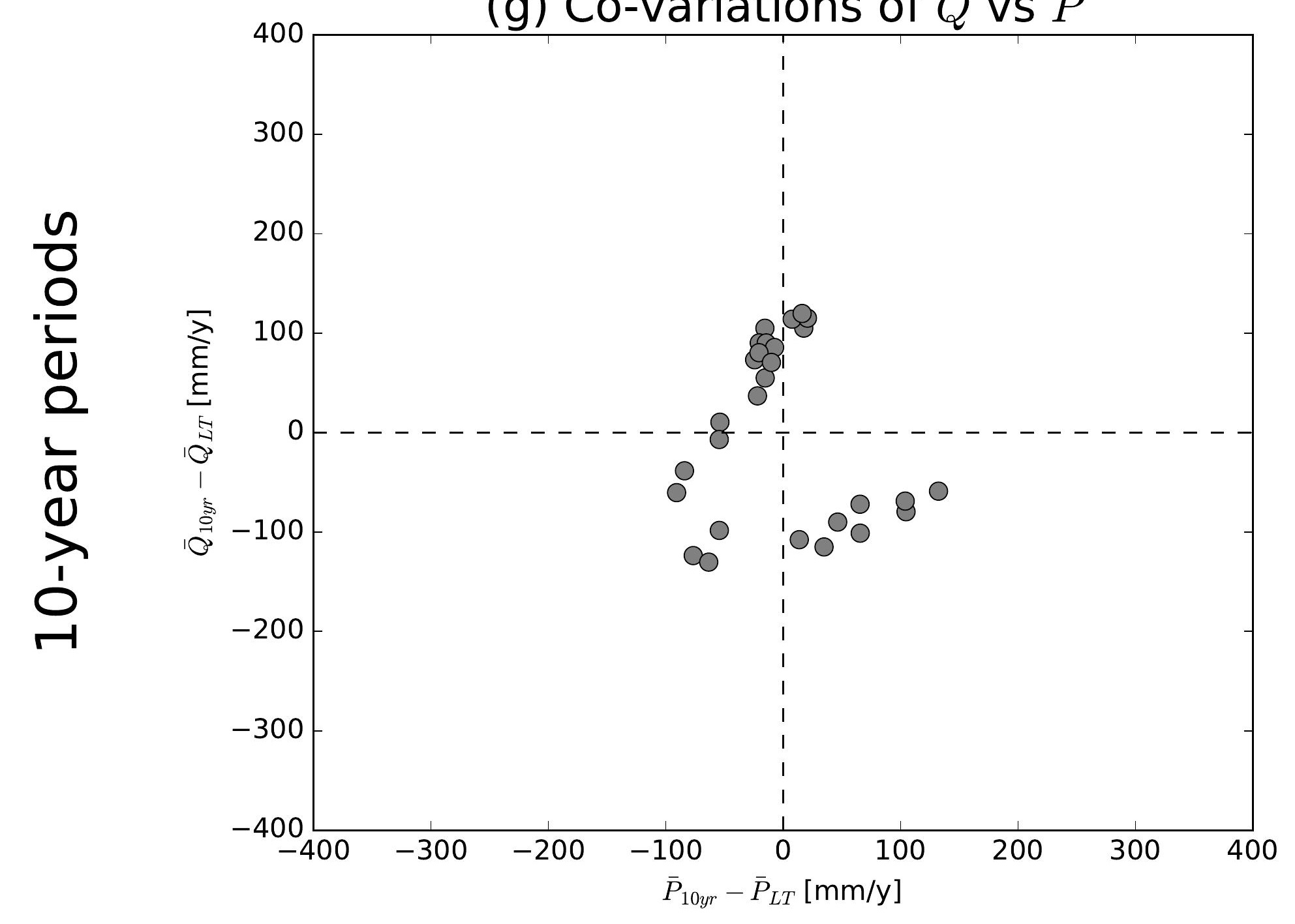

(j) Co-variations of $\bar{Q}$ vs $\bar{P}$

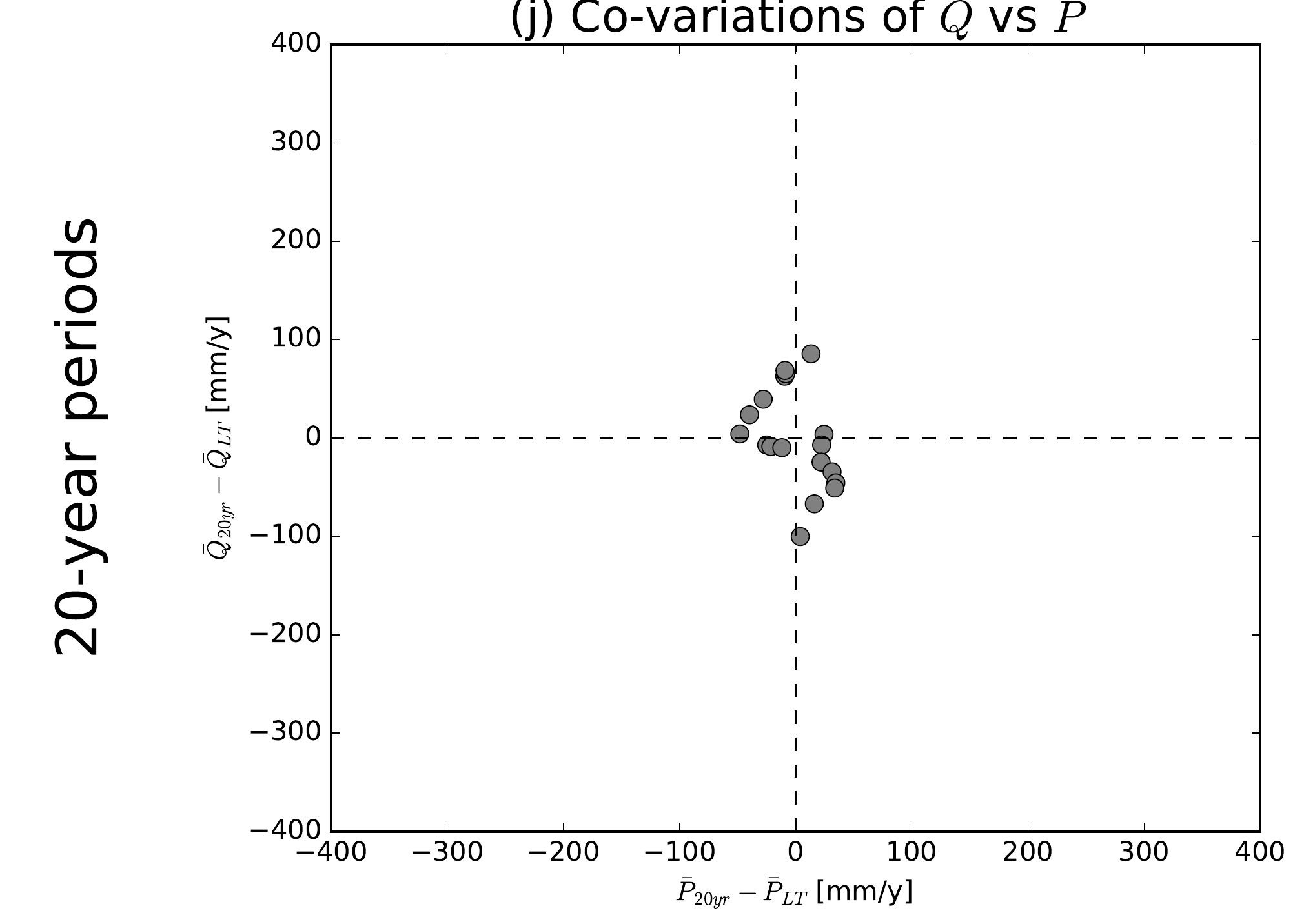

(b) Co-variations of $\bar{Q}$ vs $\overline{E 0}$

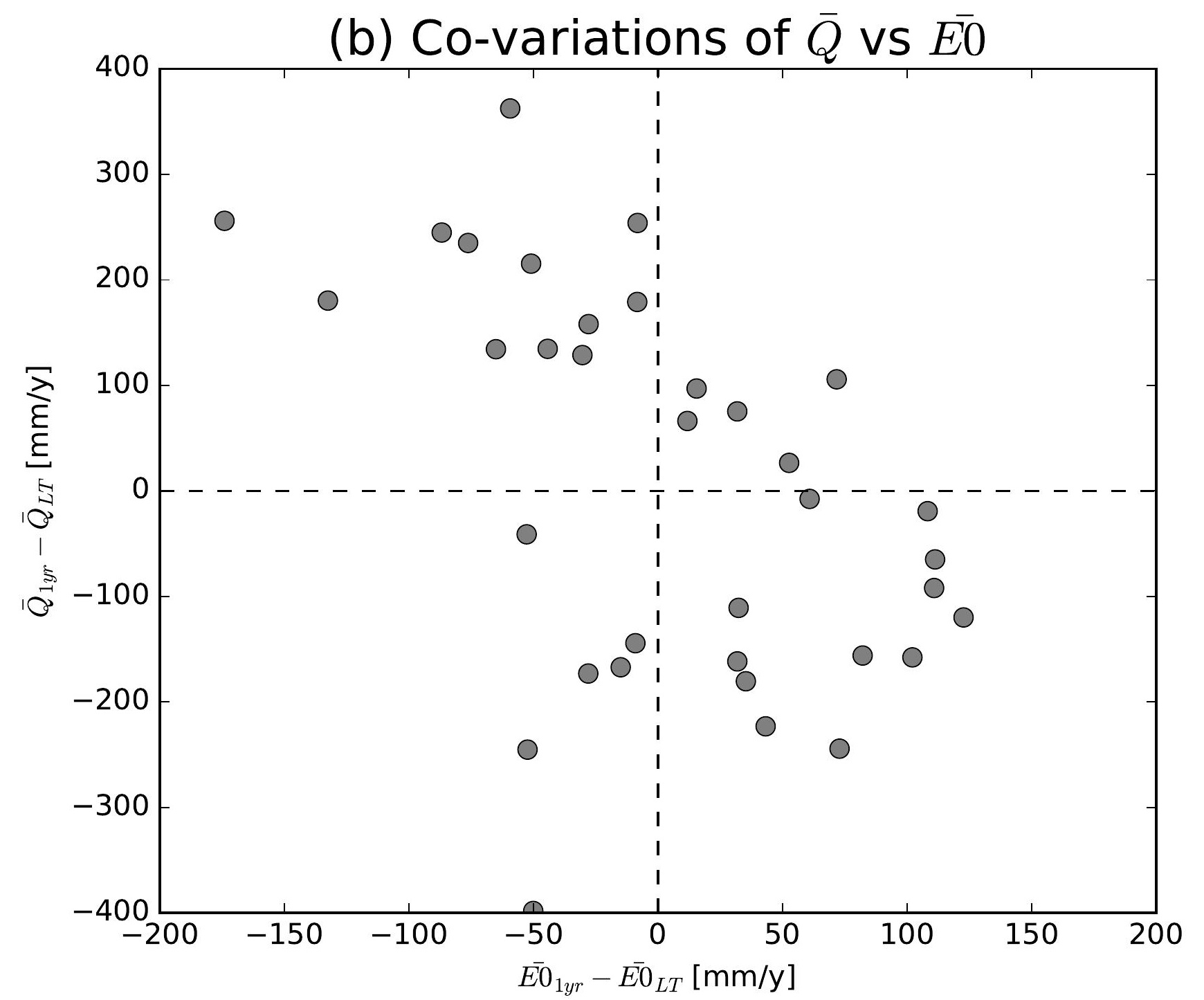

(e) Co-variations of $\bar{Q}$ vs $\overline{E 0}$

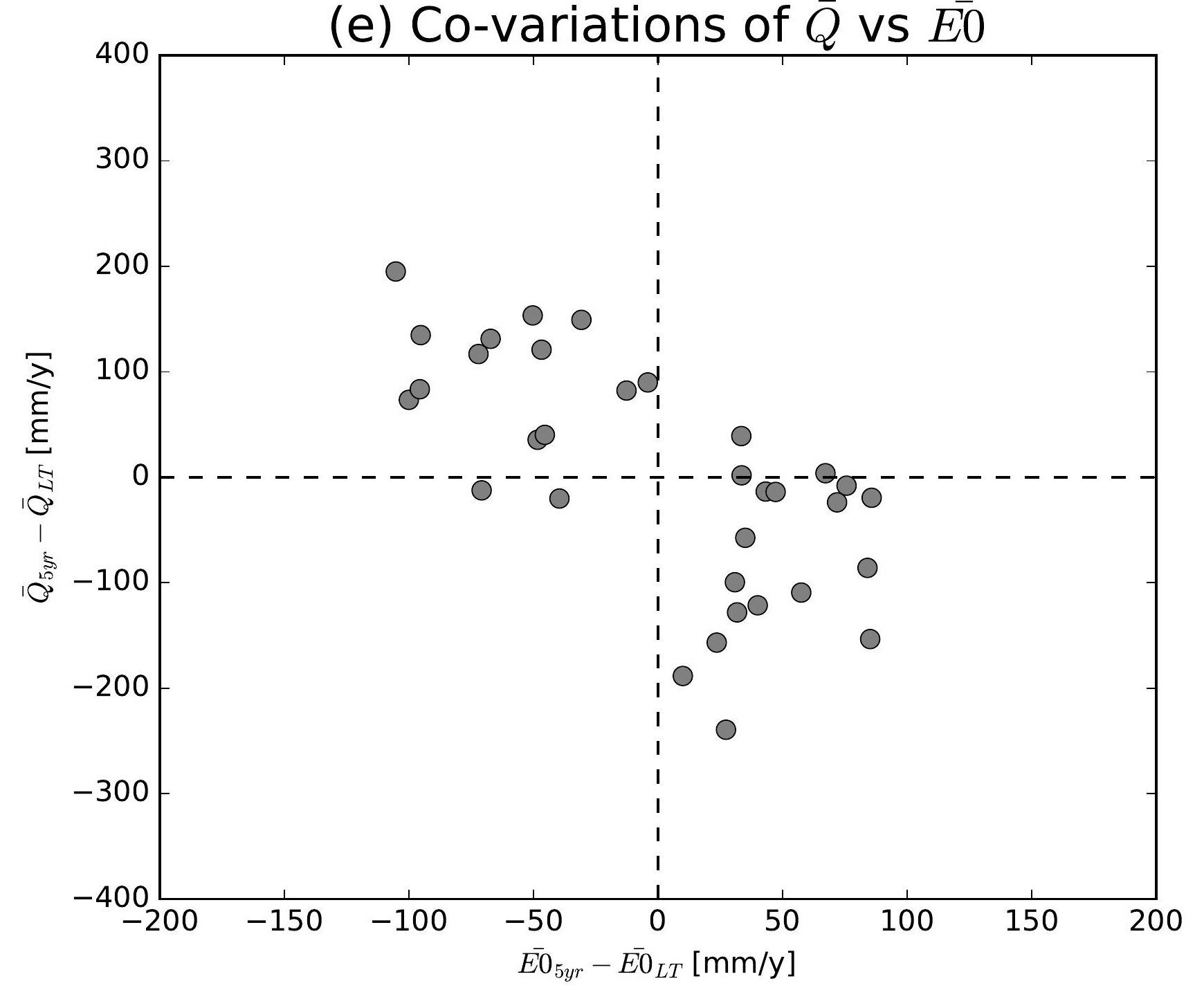

(h) Co-variations of $\bar{Q}$ vs $\overline{E 0}$

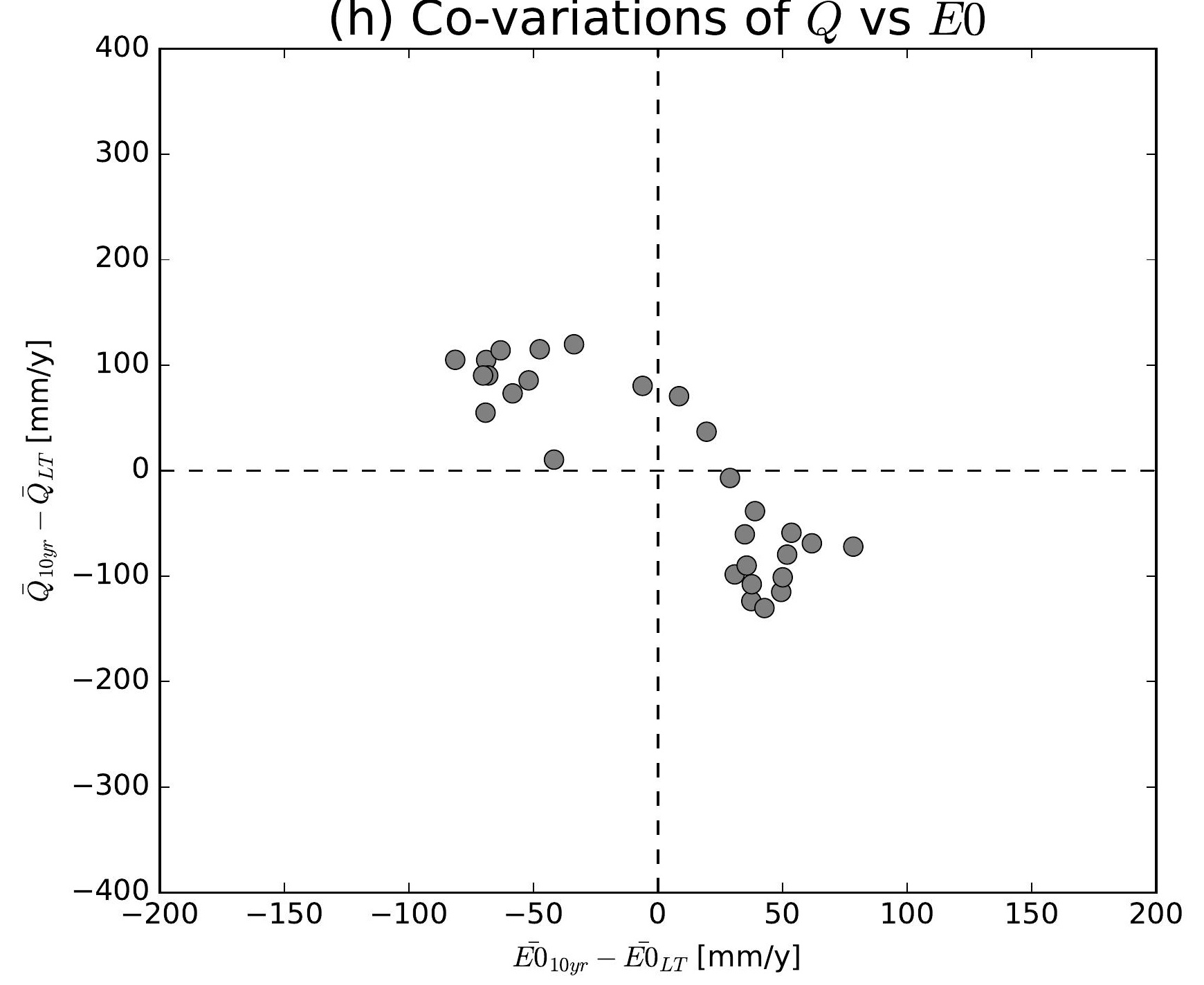

(k) Co-variations of $\bar{Q}$ vs $\overline{E 0}$

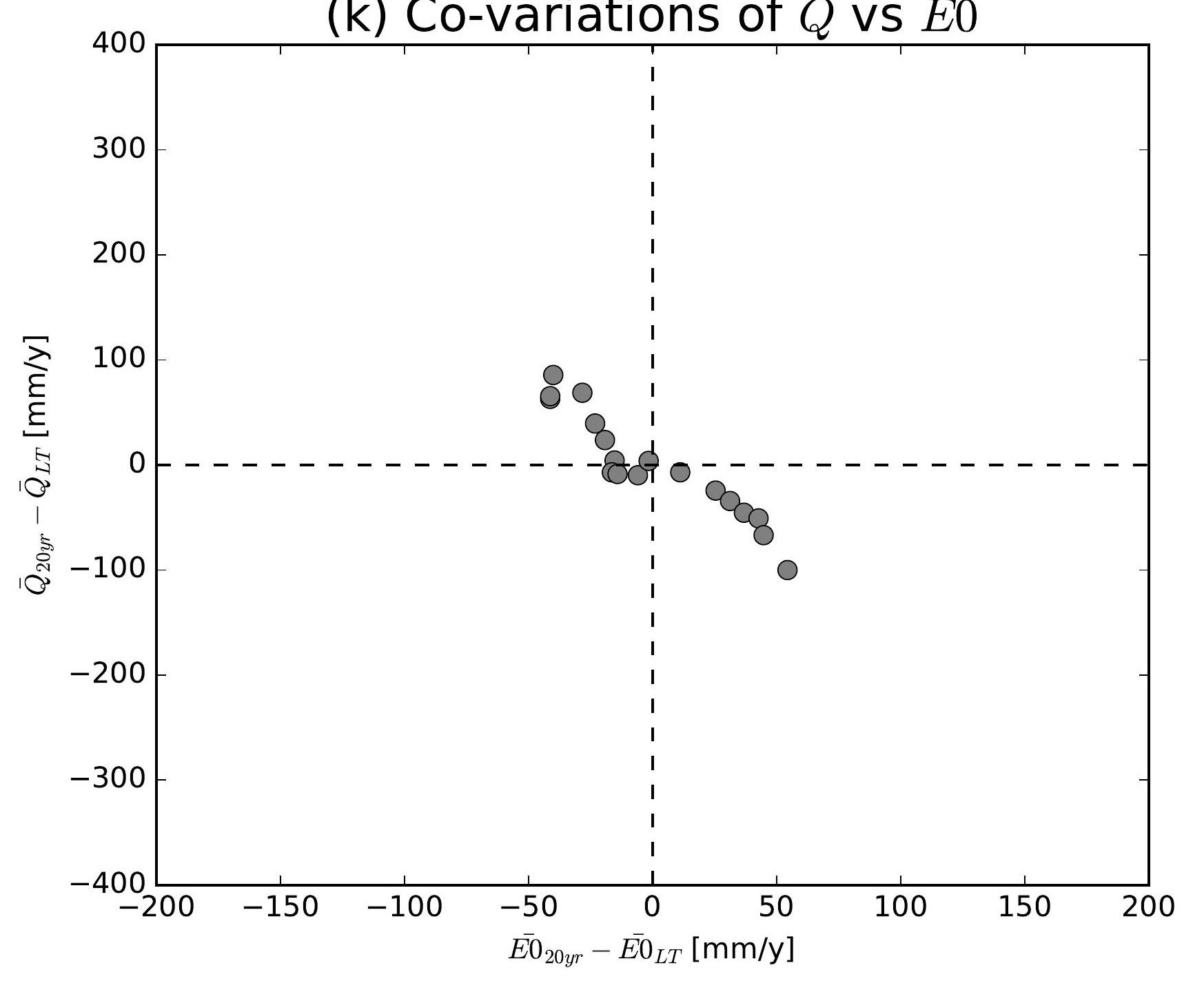

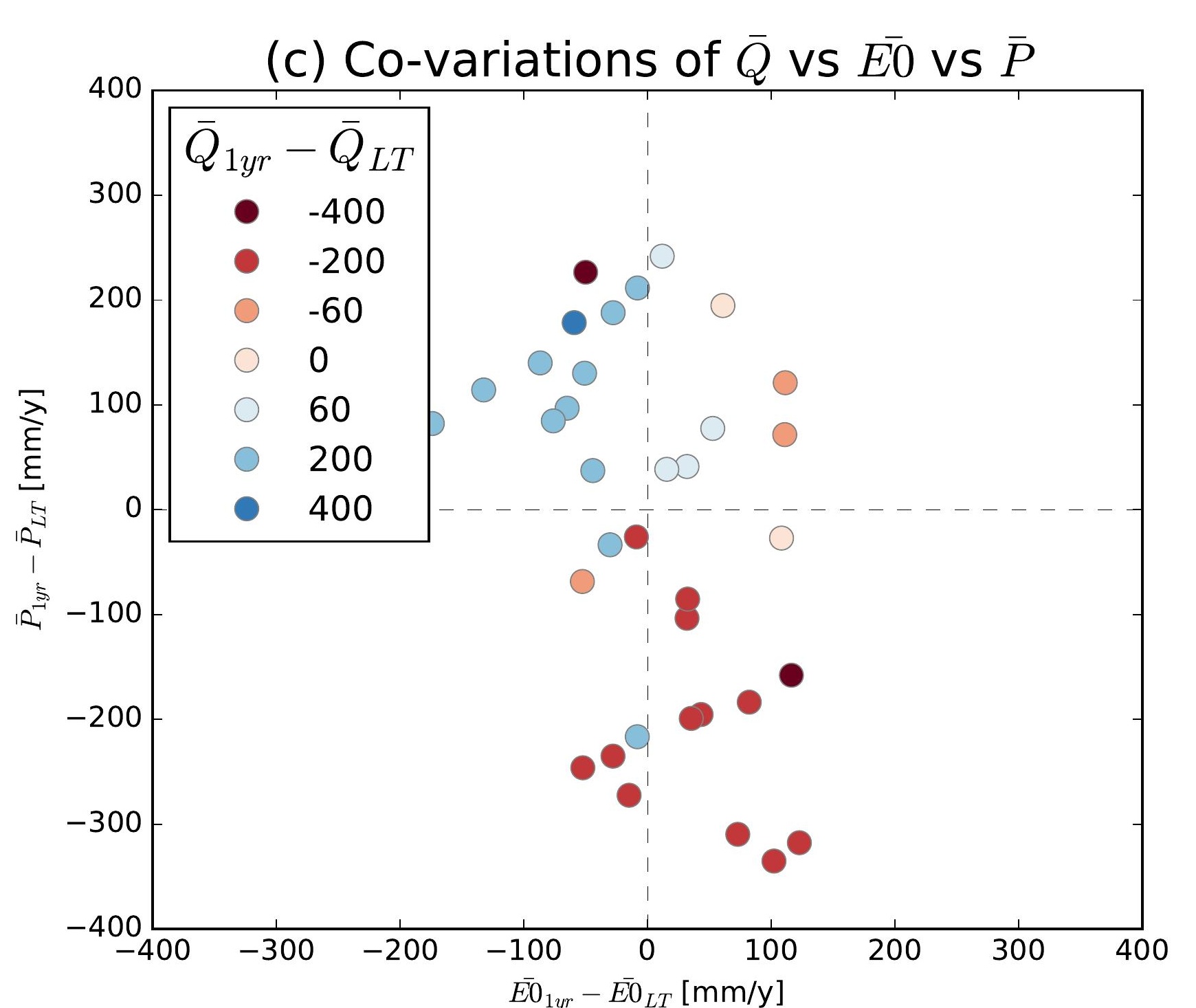
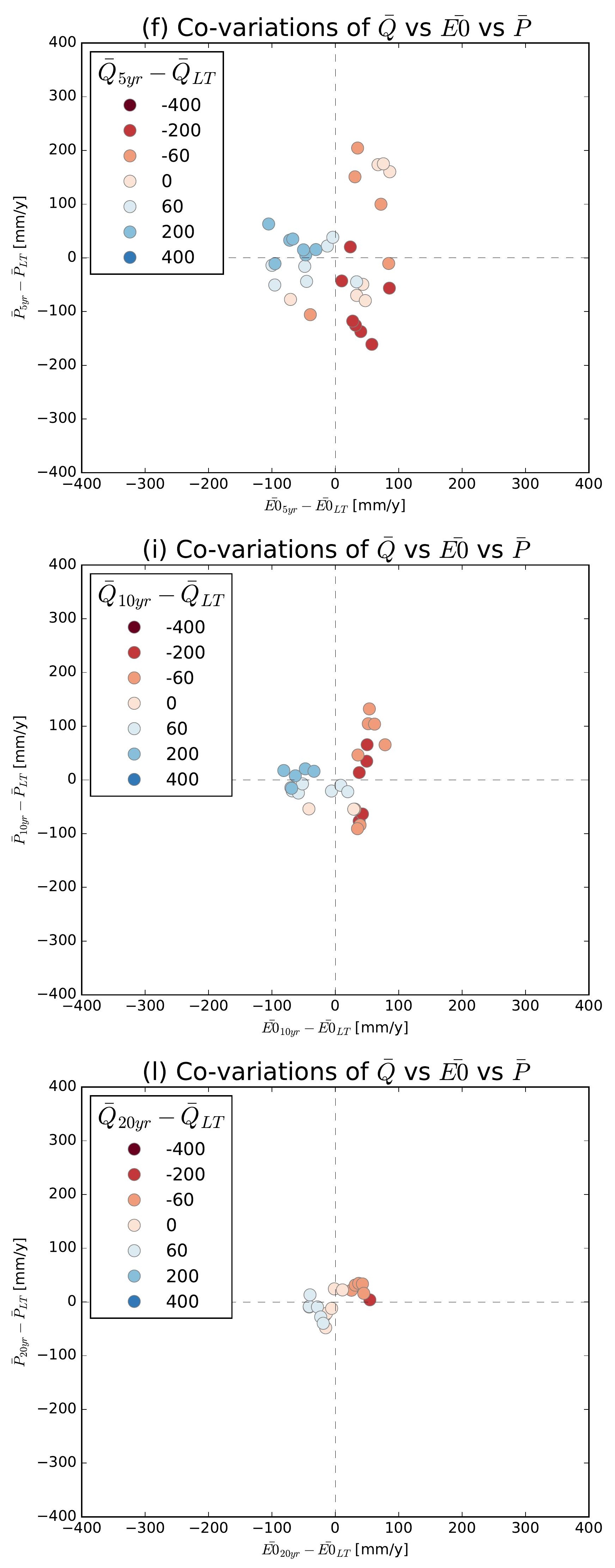

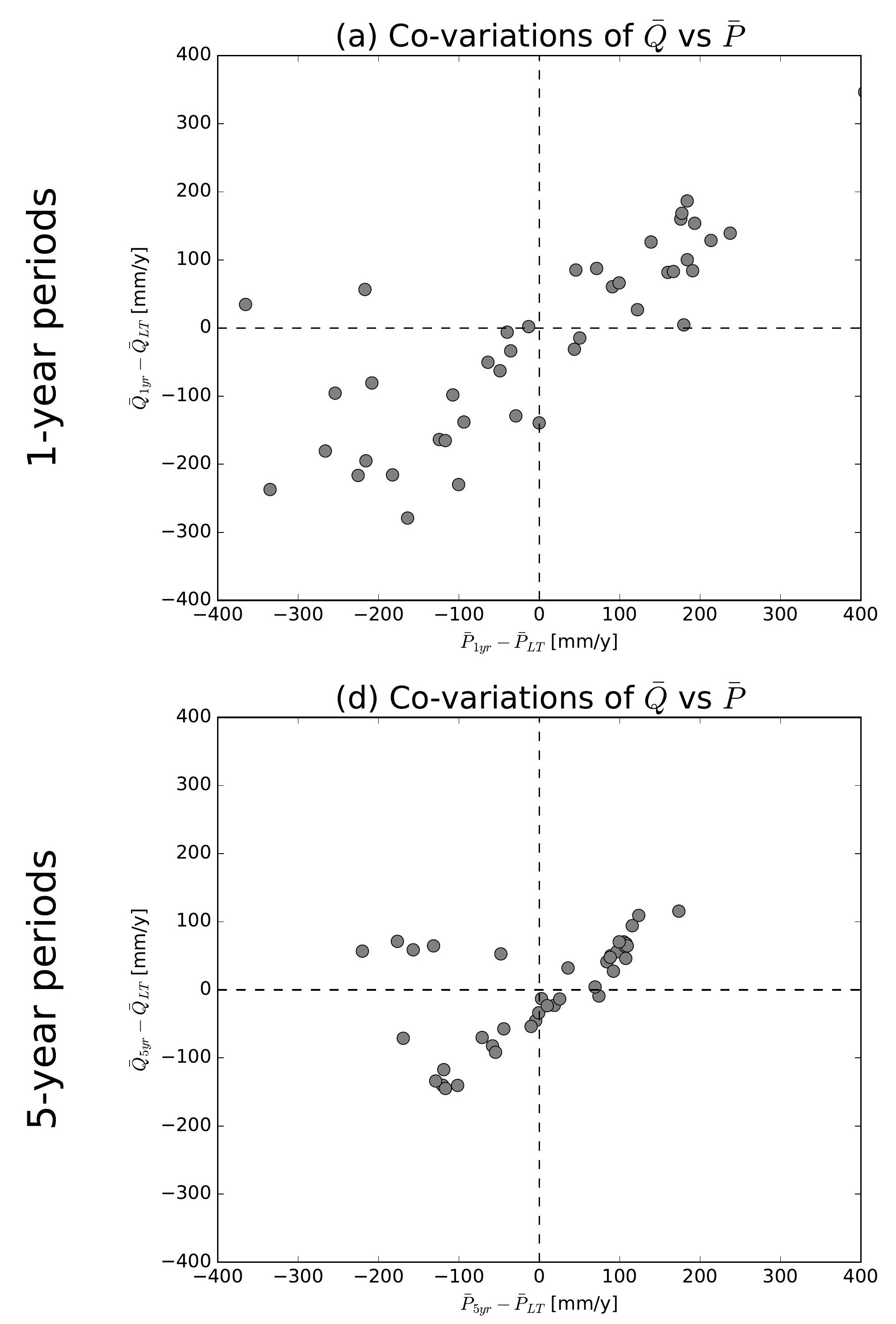

(g) Co-variations of $\bar{Q}$ vs $\bar{P}$

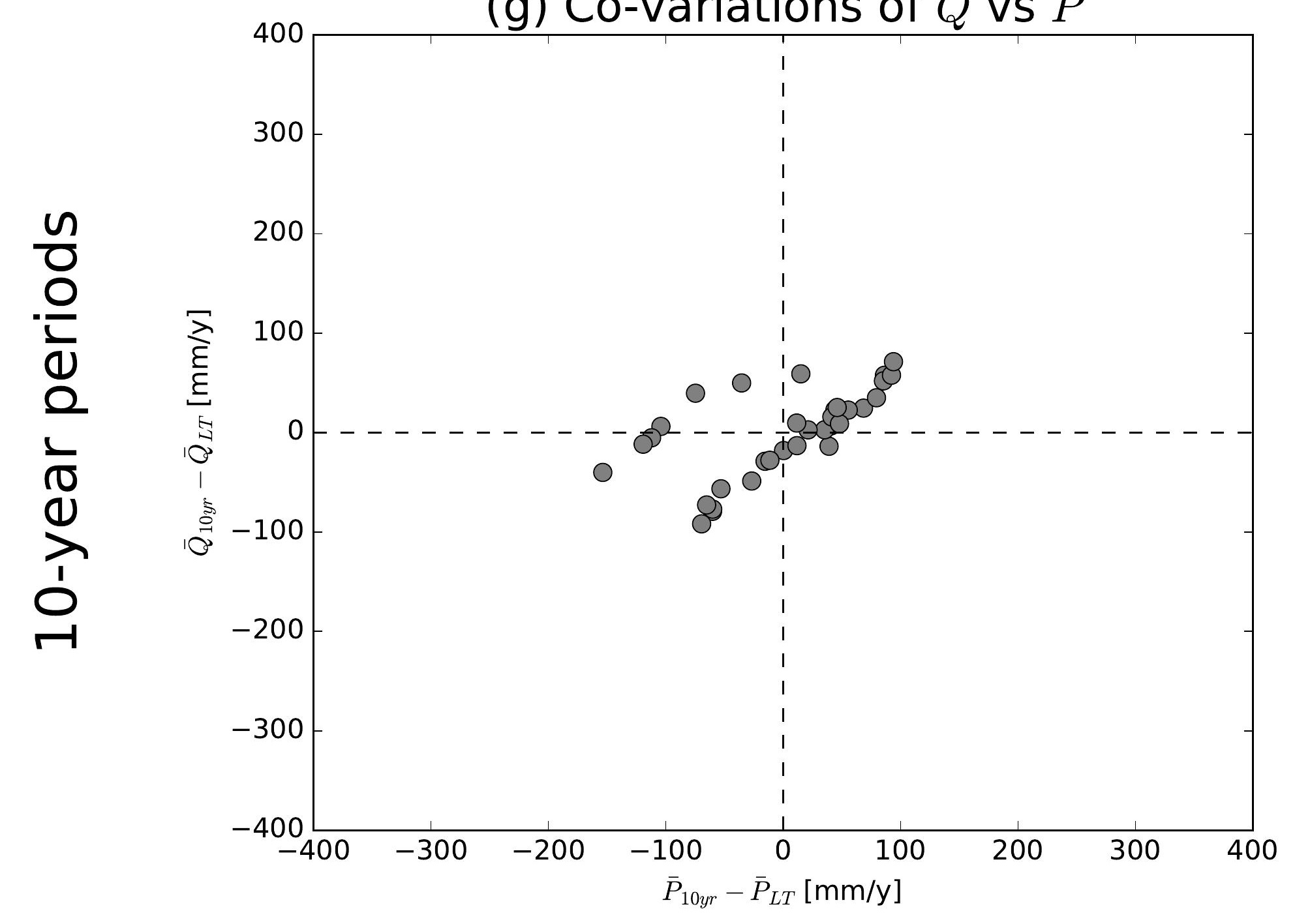

(j) Co-variations of $\bar{Q}$ vs $\bar{P}$

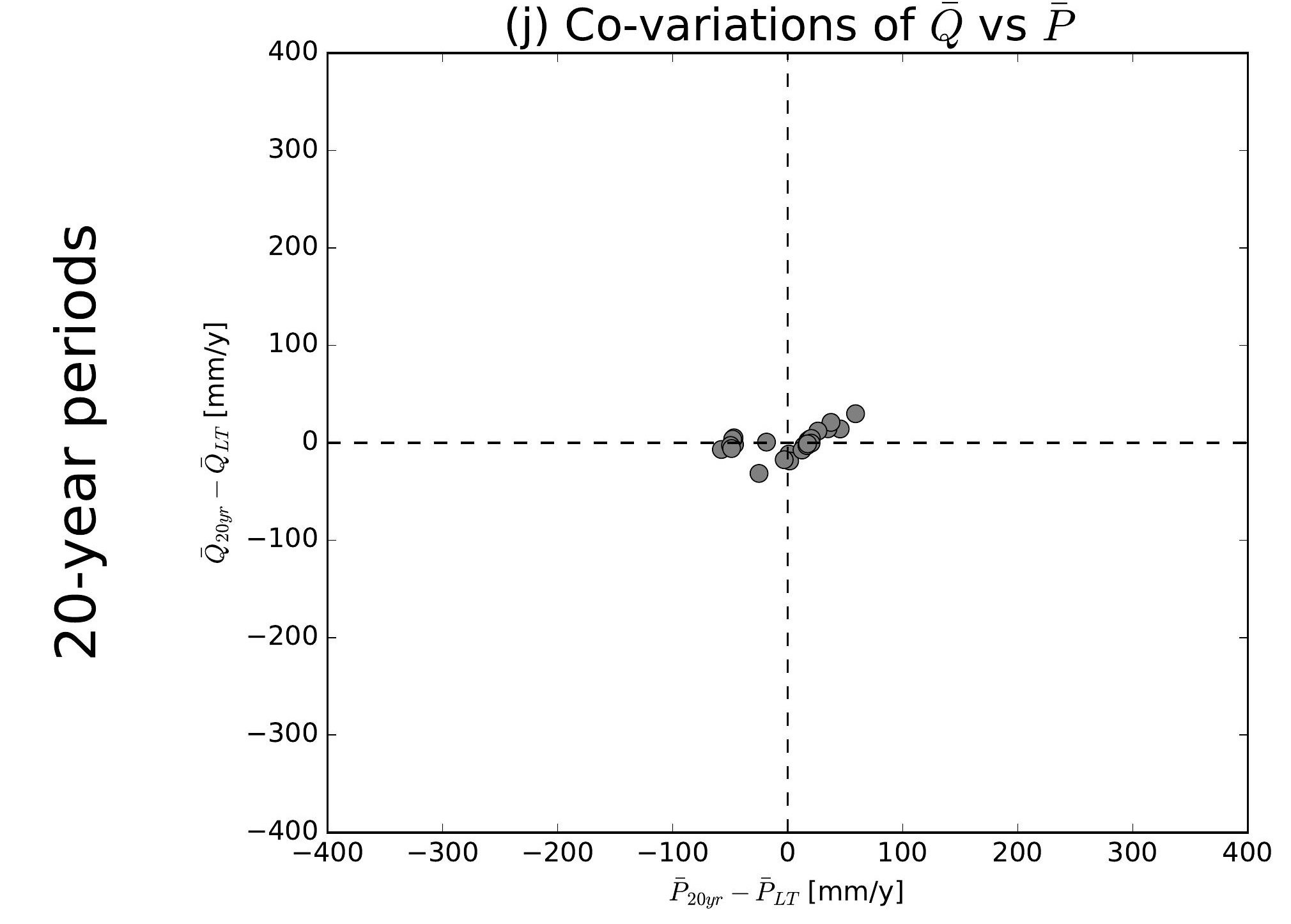

(b) Co-variations of $\bar{Q}$ vs $\overline{E 0}$

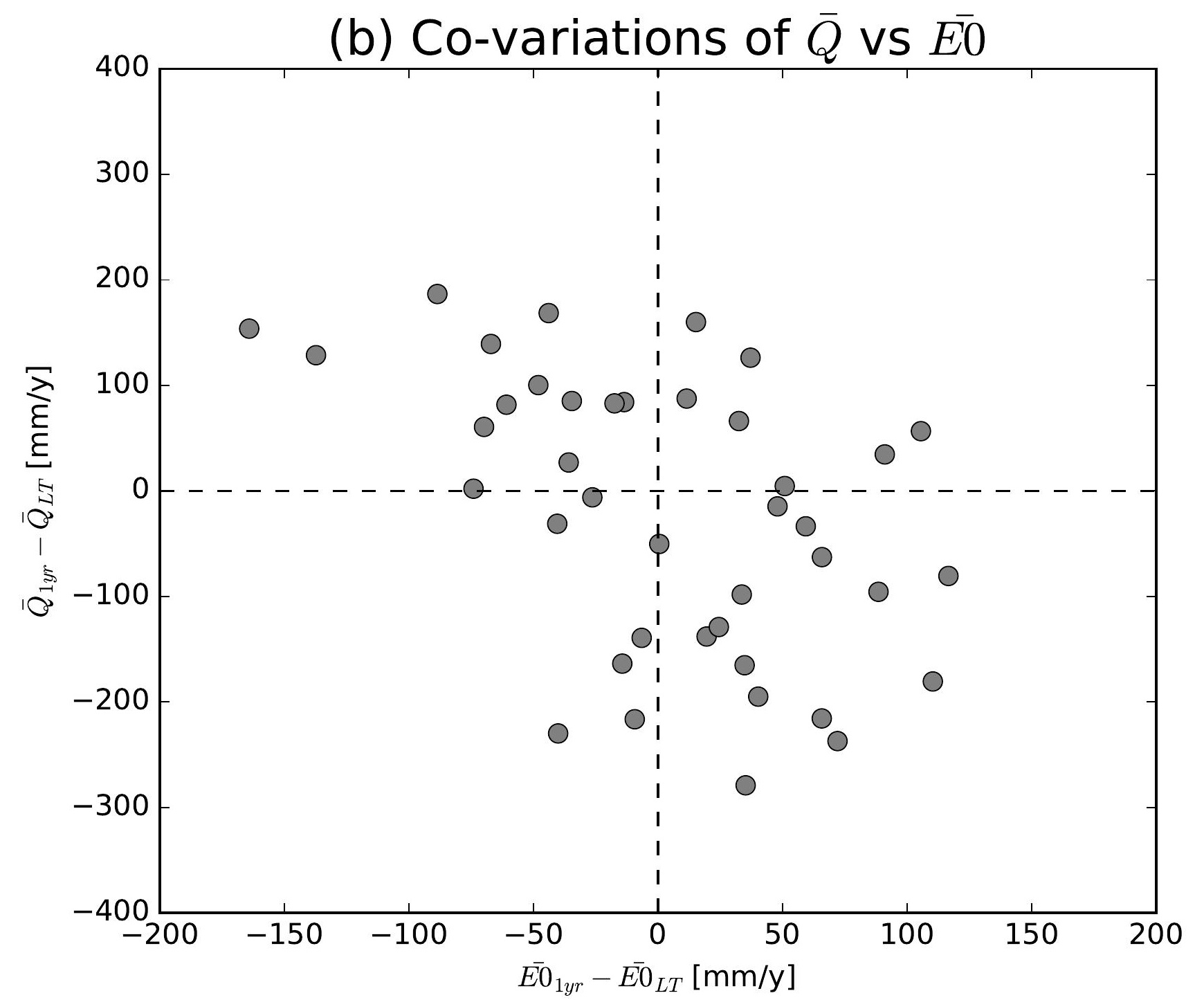

(e) Co-variations of $\bar{Q}$ vs $\overline{E 0}$

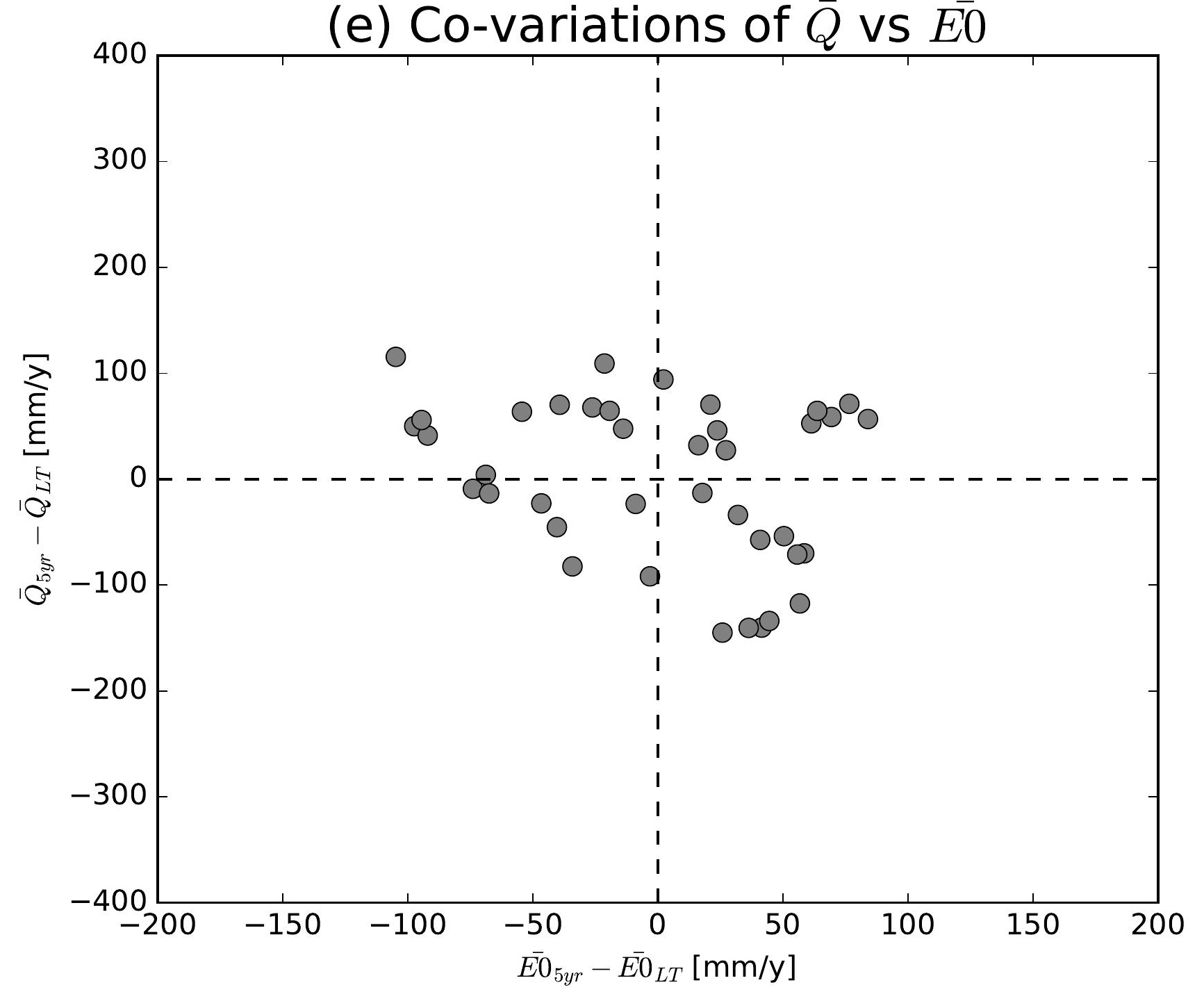

(h) Co-variations of $\bar{Q}$ vs $\overline{E 0}$

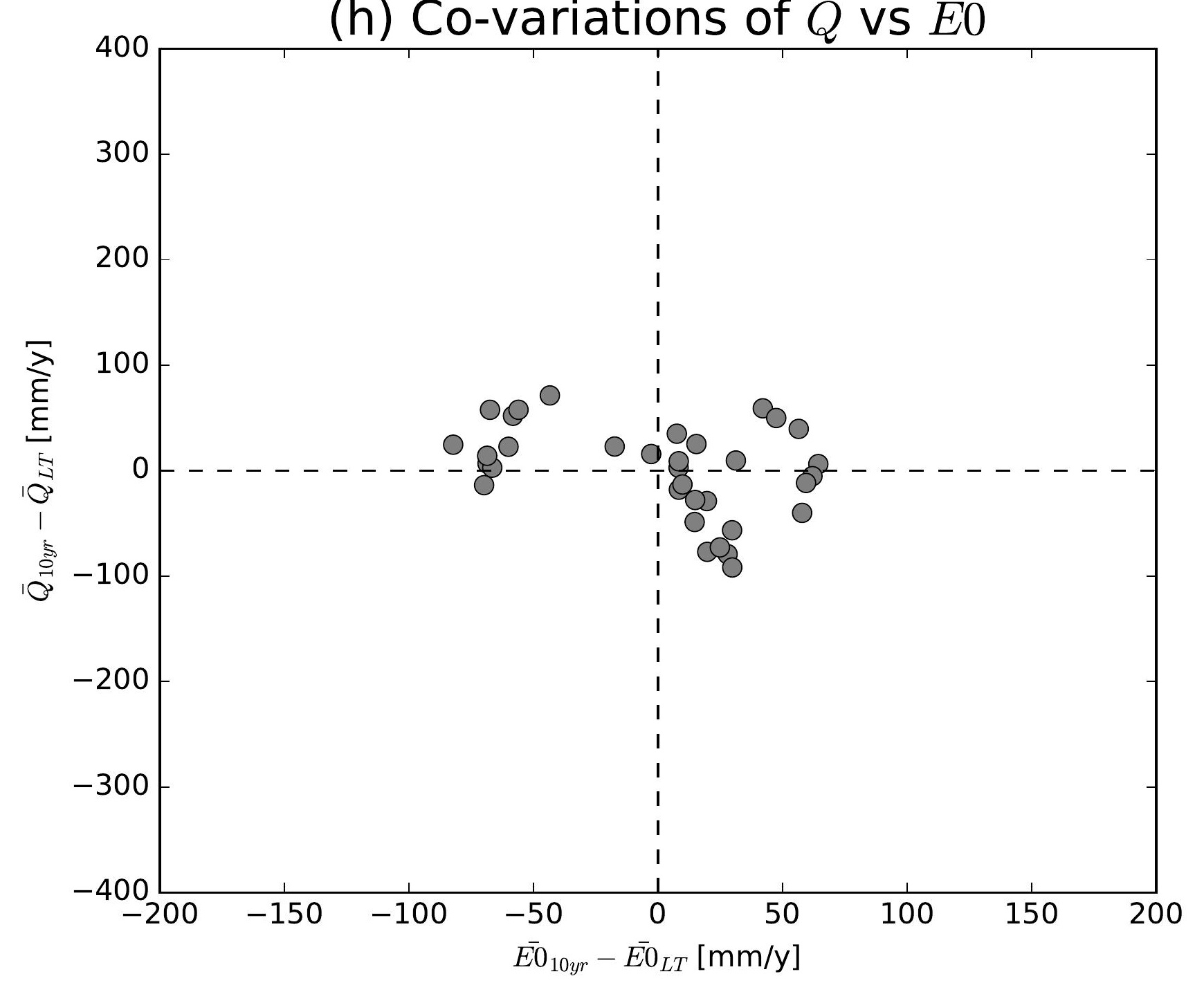

(k) Co-variations of $\bar{Q}$ vs $\overline{E 0}$

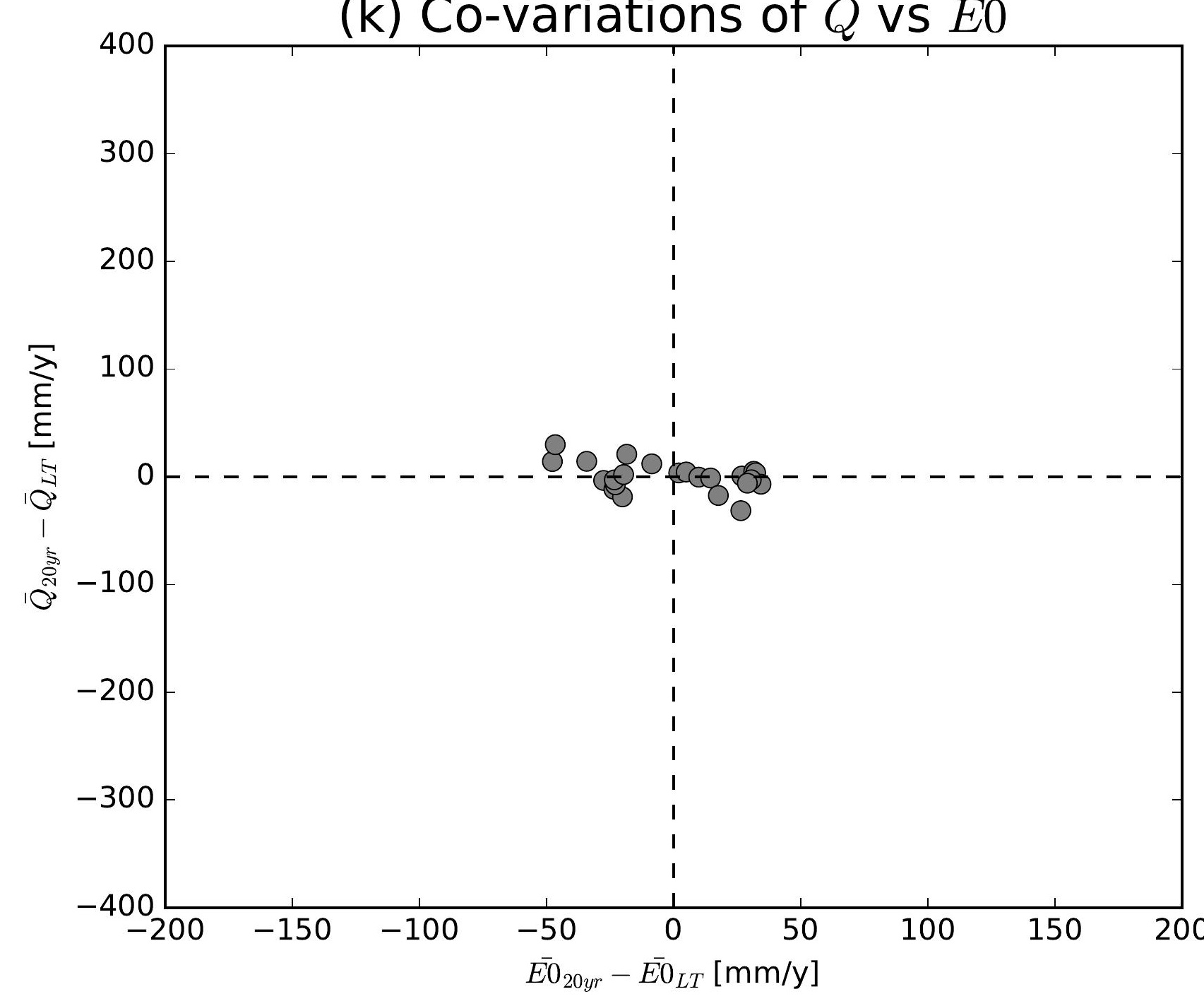

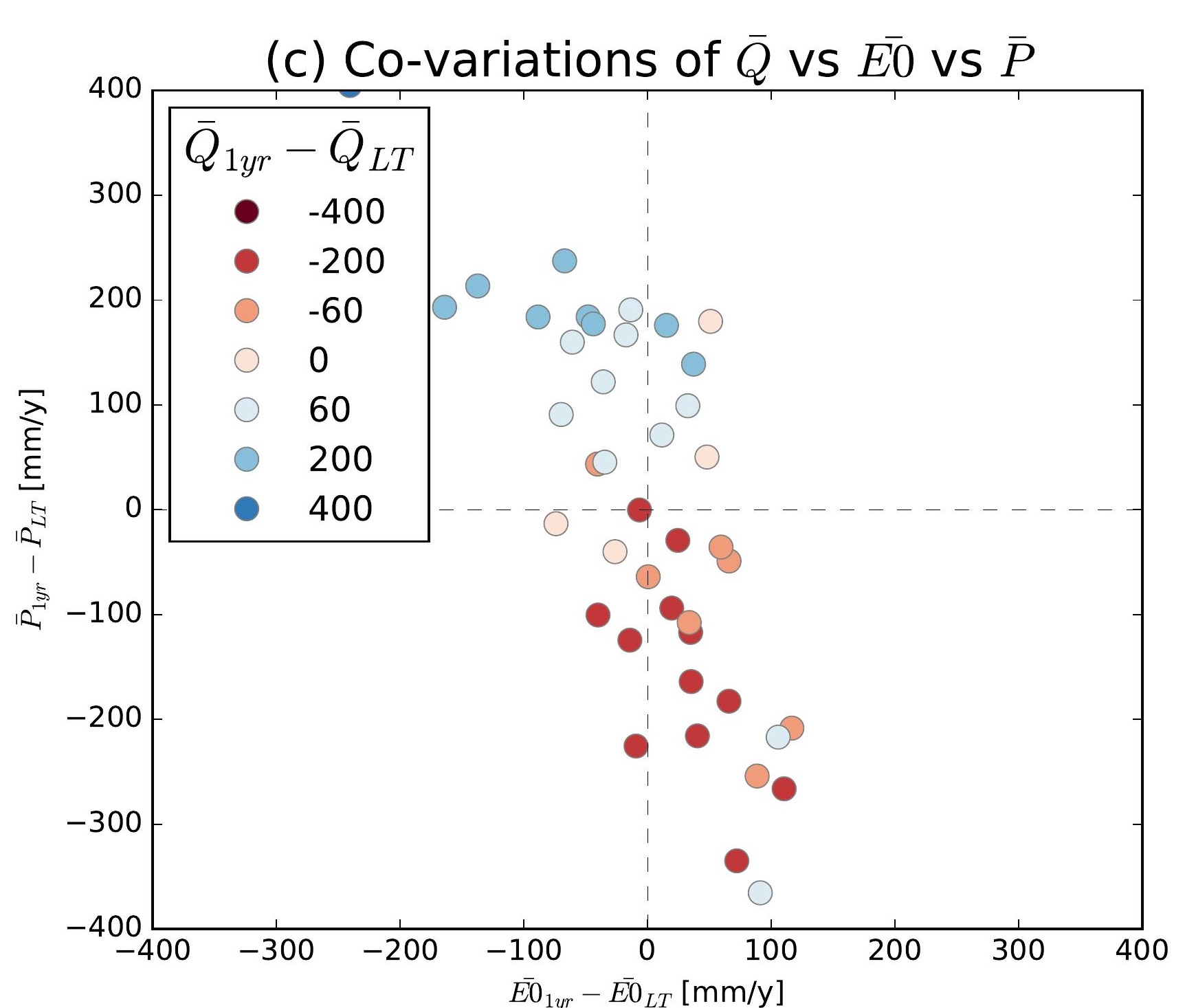
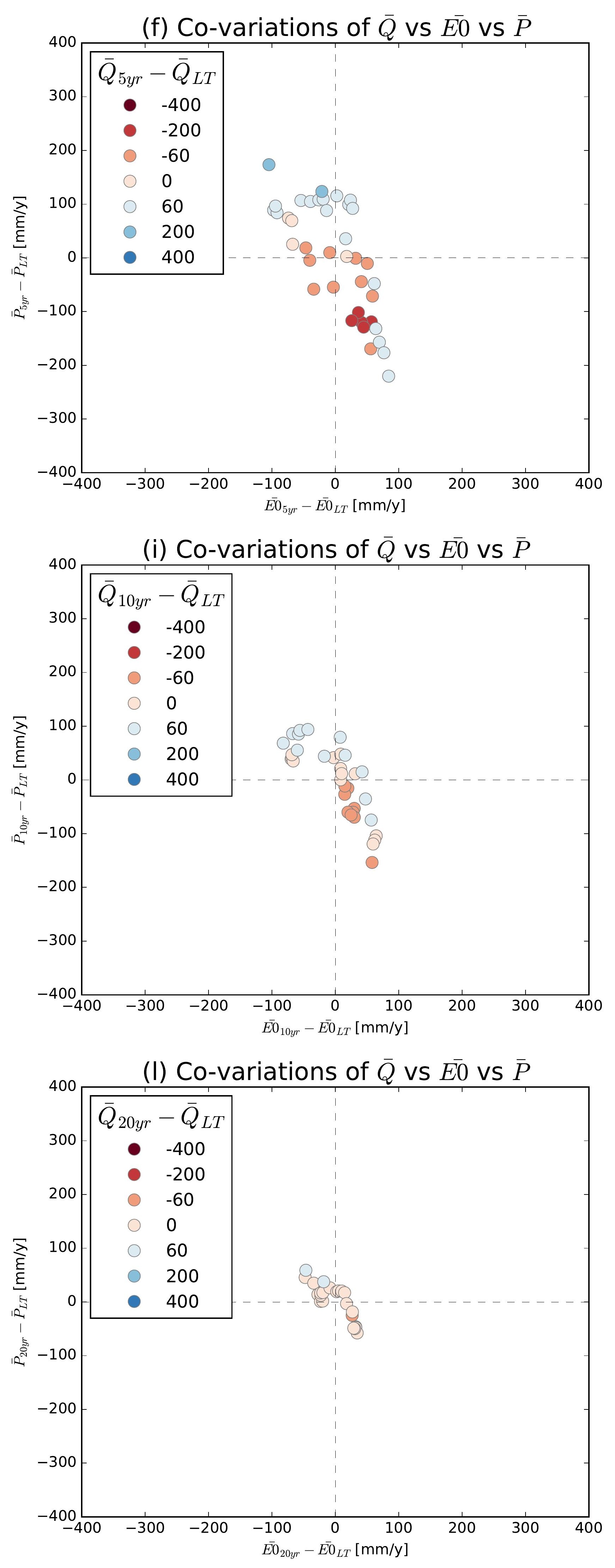

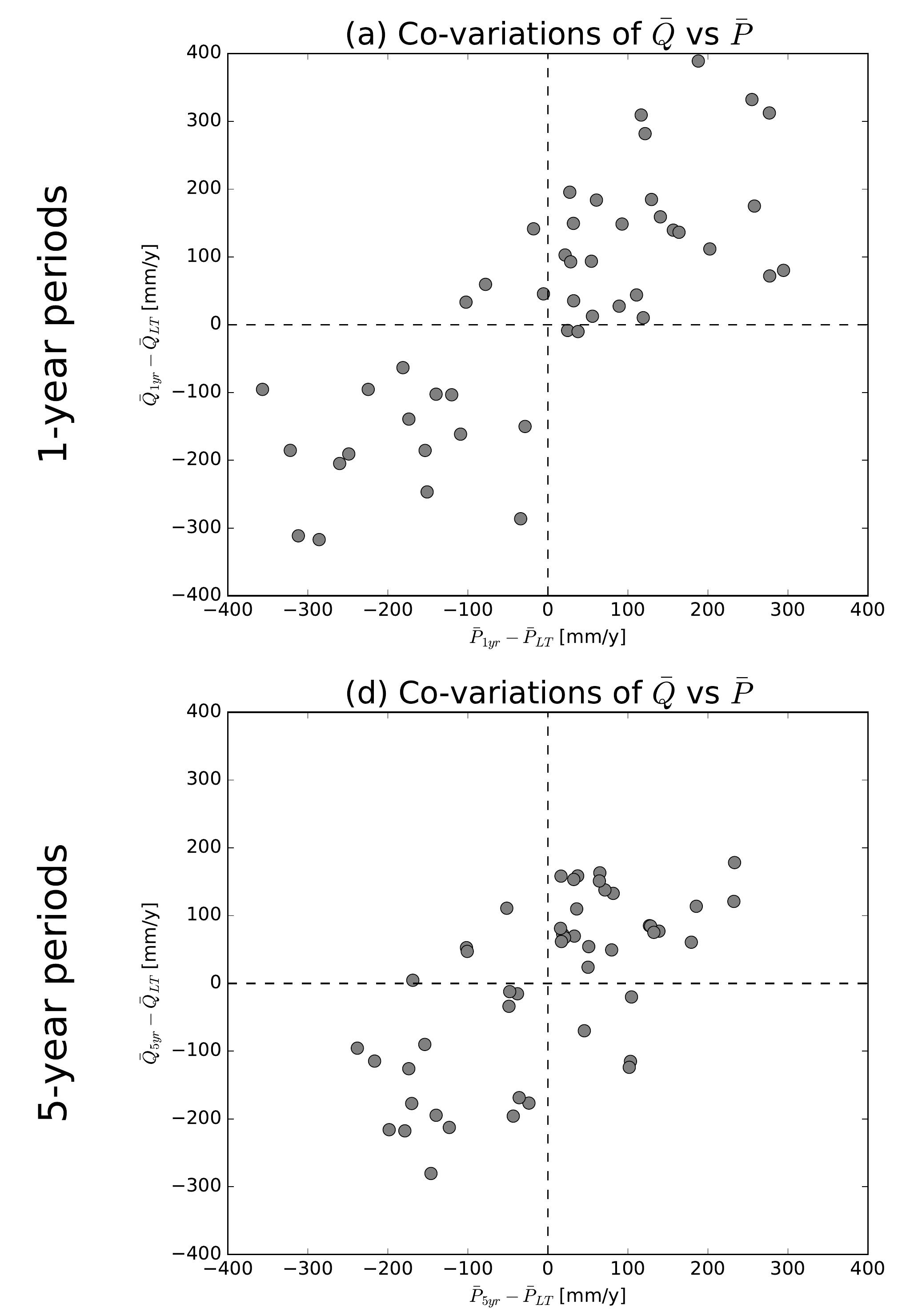

(g) Co-variations of $\bar{Q}$ vs $\bar{P}$
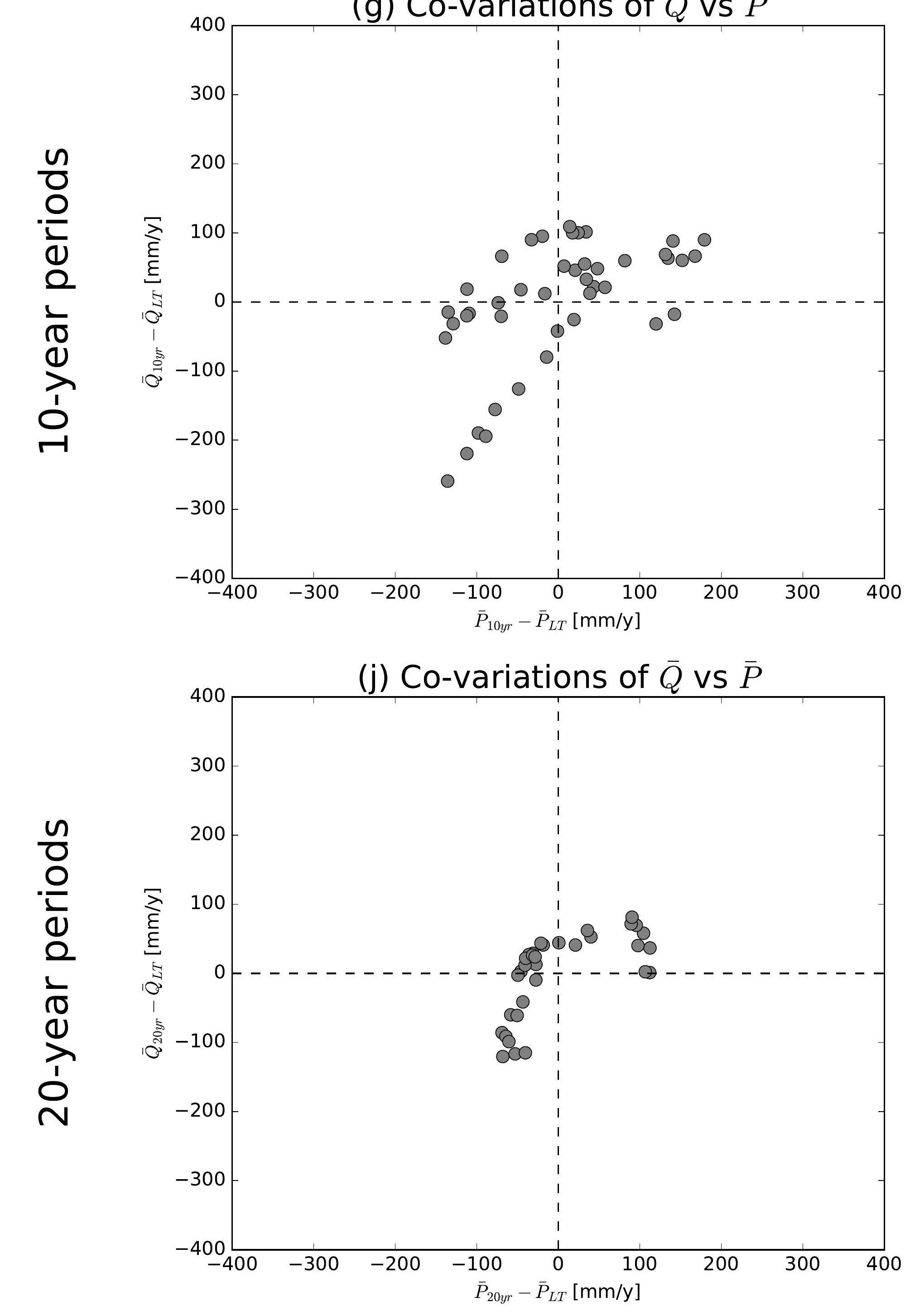

(b) Co-variations of $\bar{Q}$ vs $\overline{E 0}$

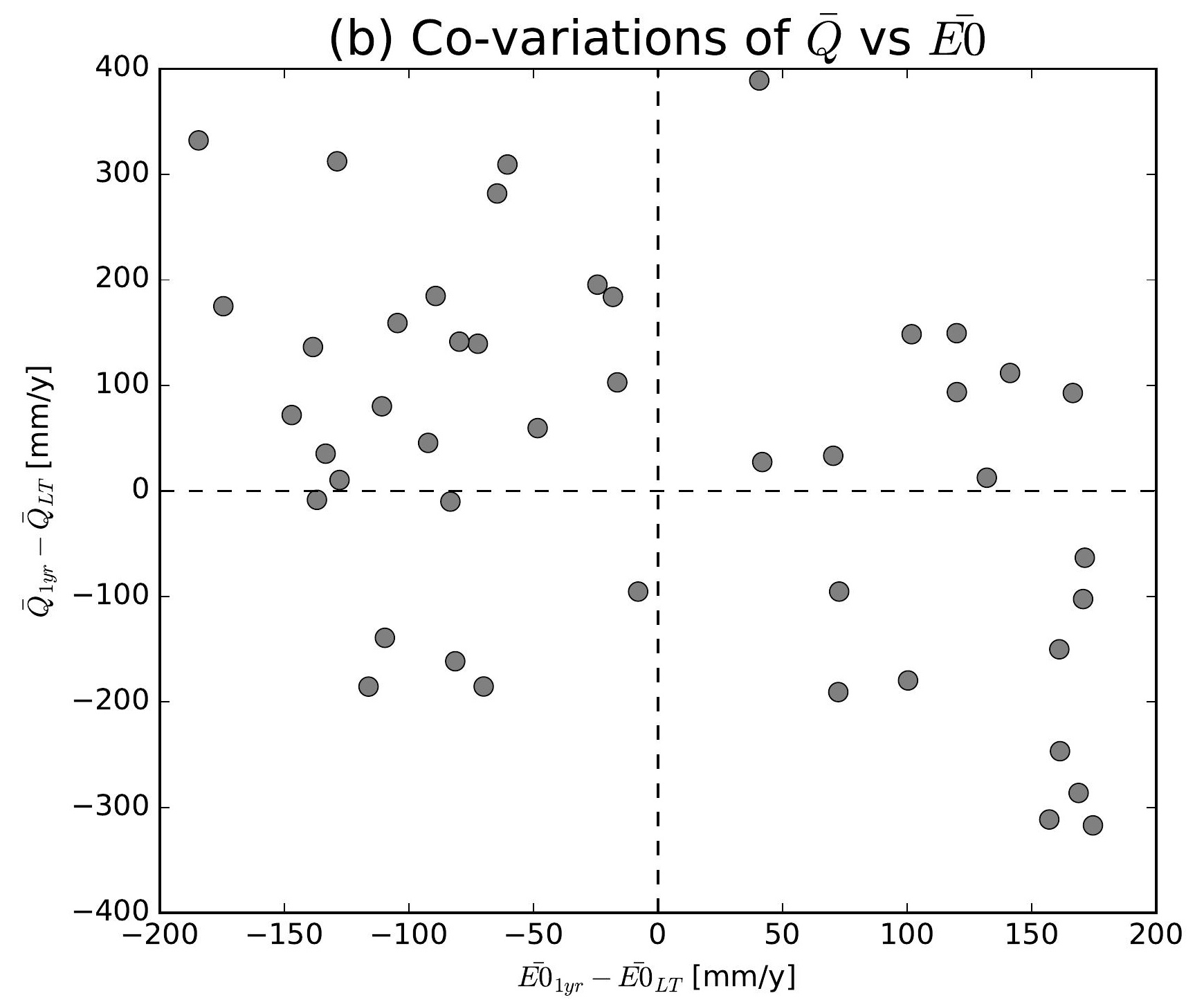

(e) Co-variations of $\bar{Q}$ vs $\overline{E 0}$

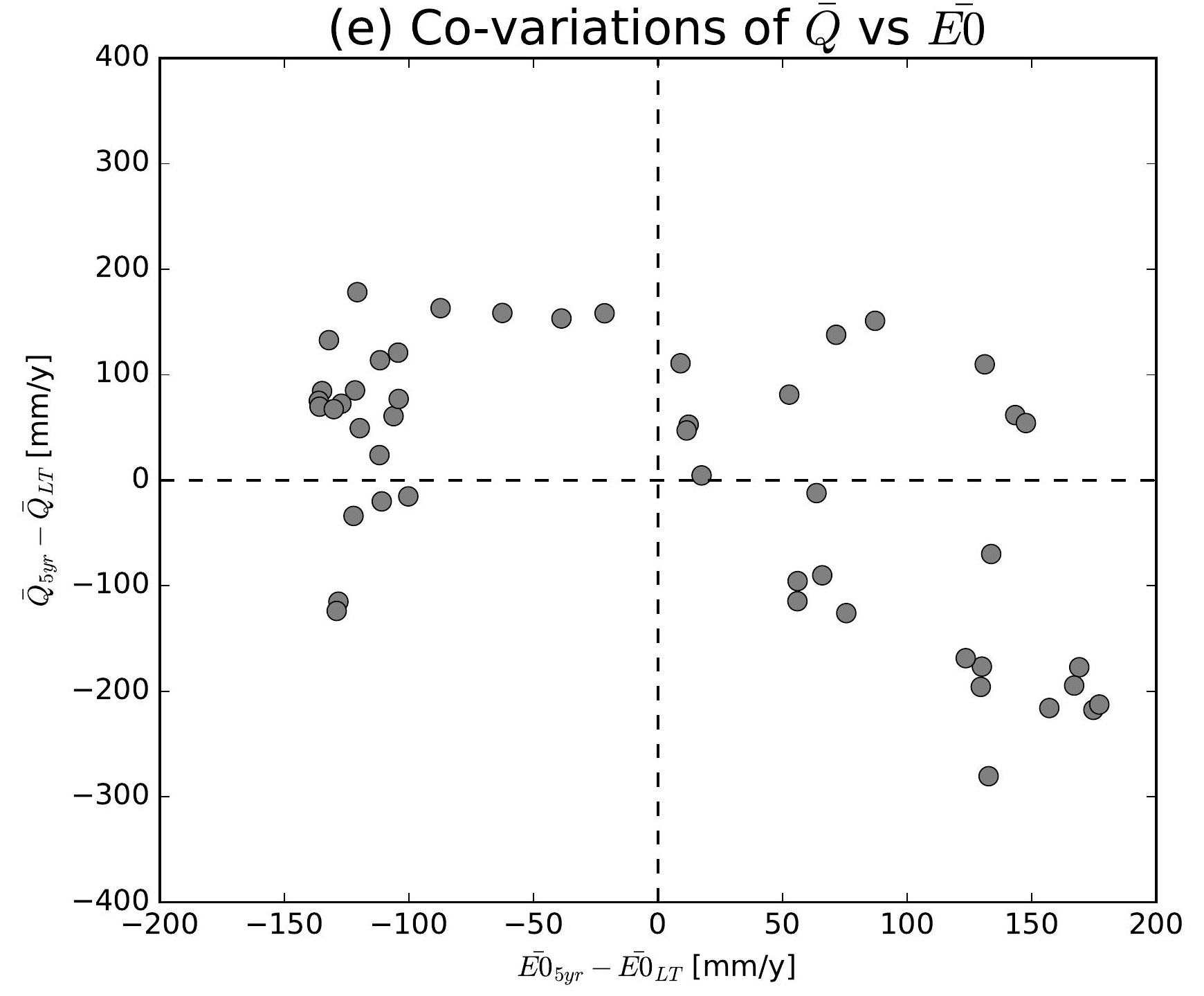

(h) Co-variations of $\bar{Q}$ vs $\overline{E 0}$

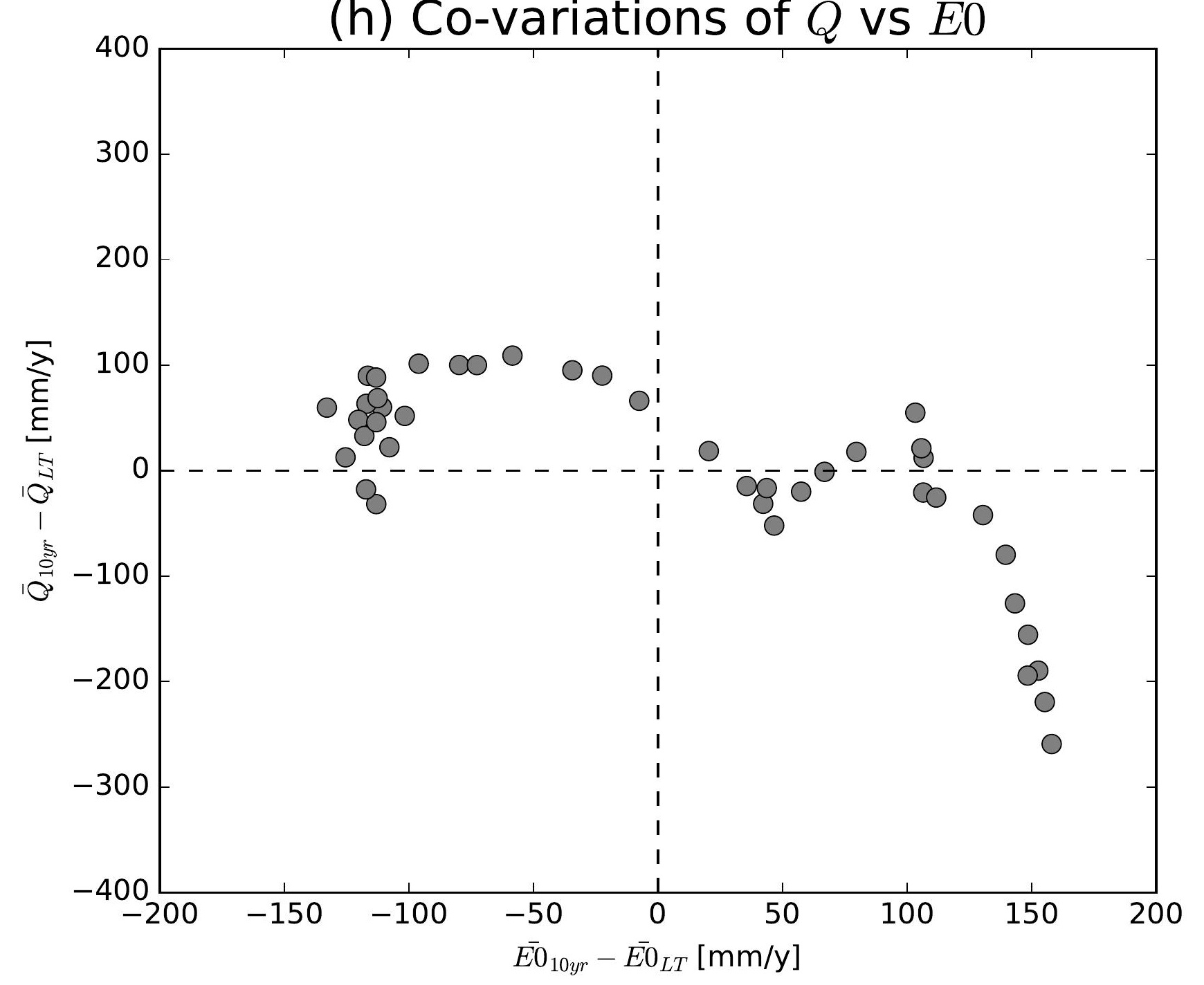

(k) Co-variations of $\bar{Q}$ vs $\overline{E 0}$

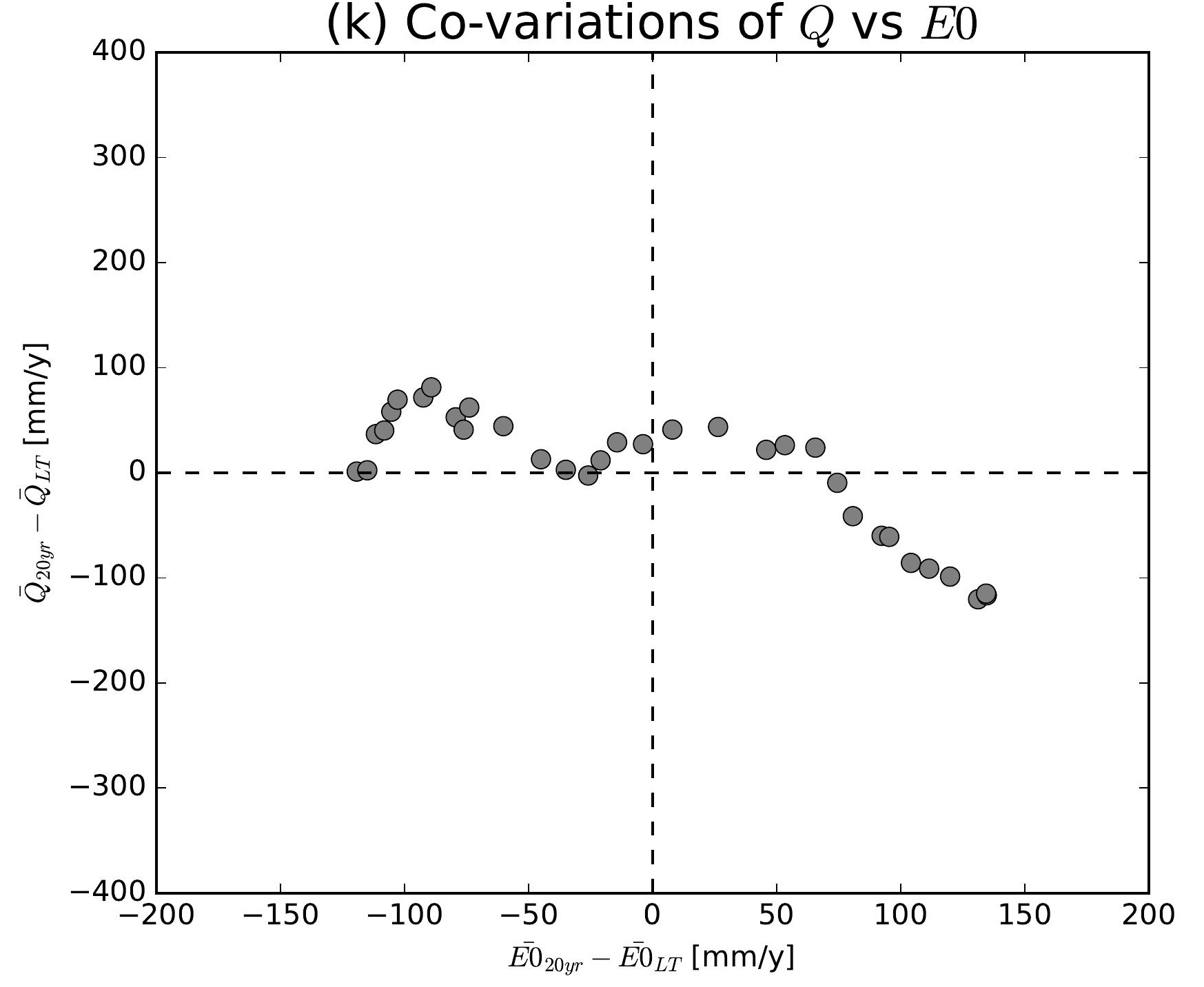

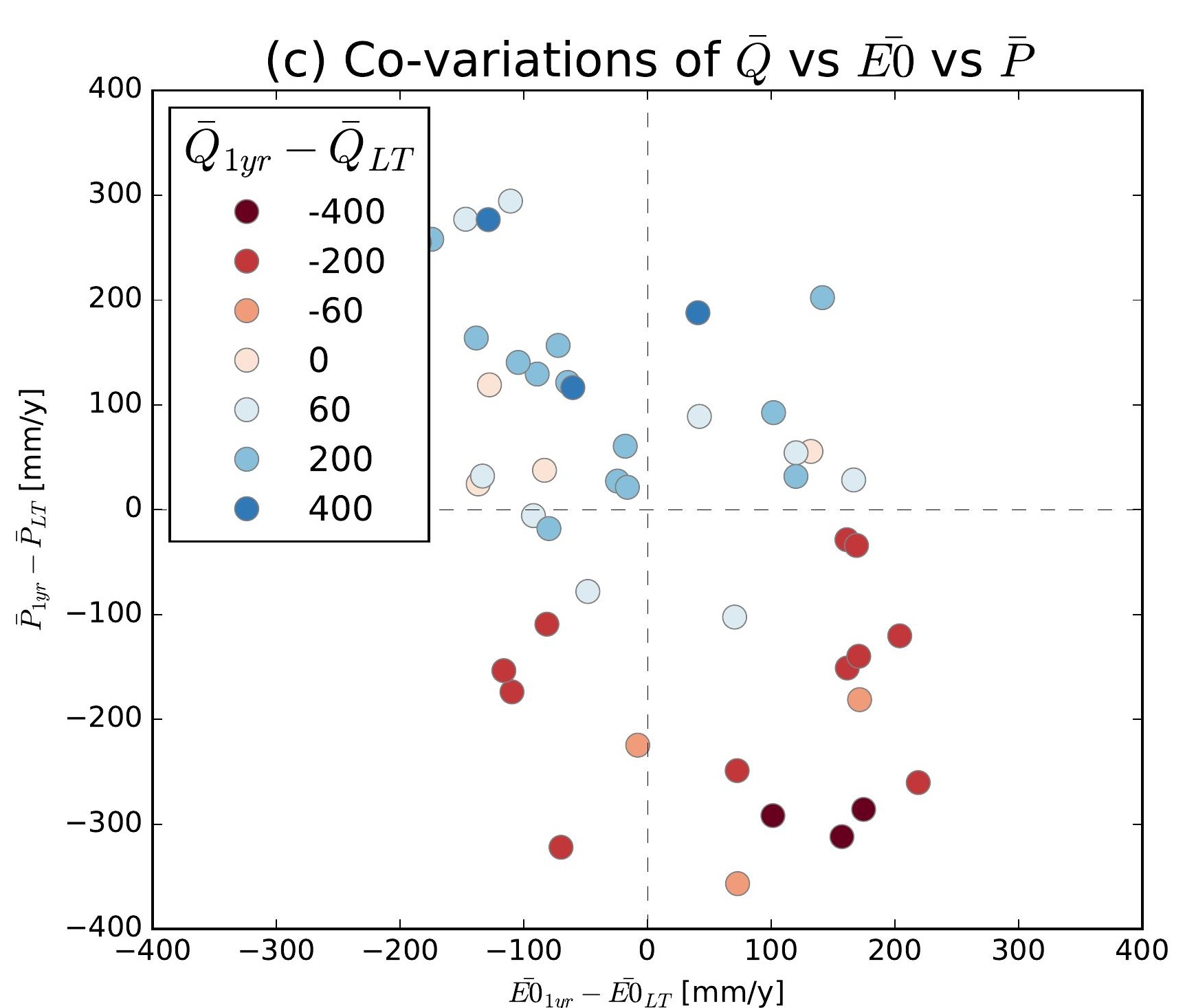
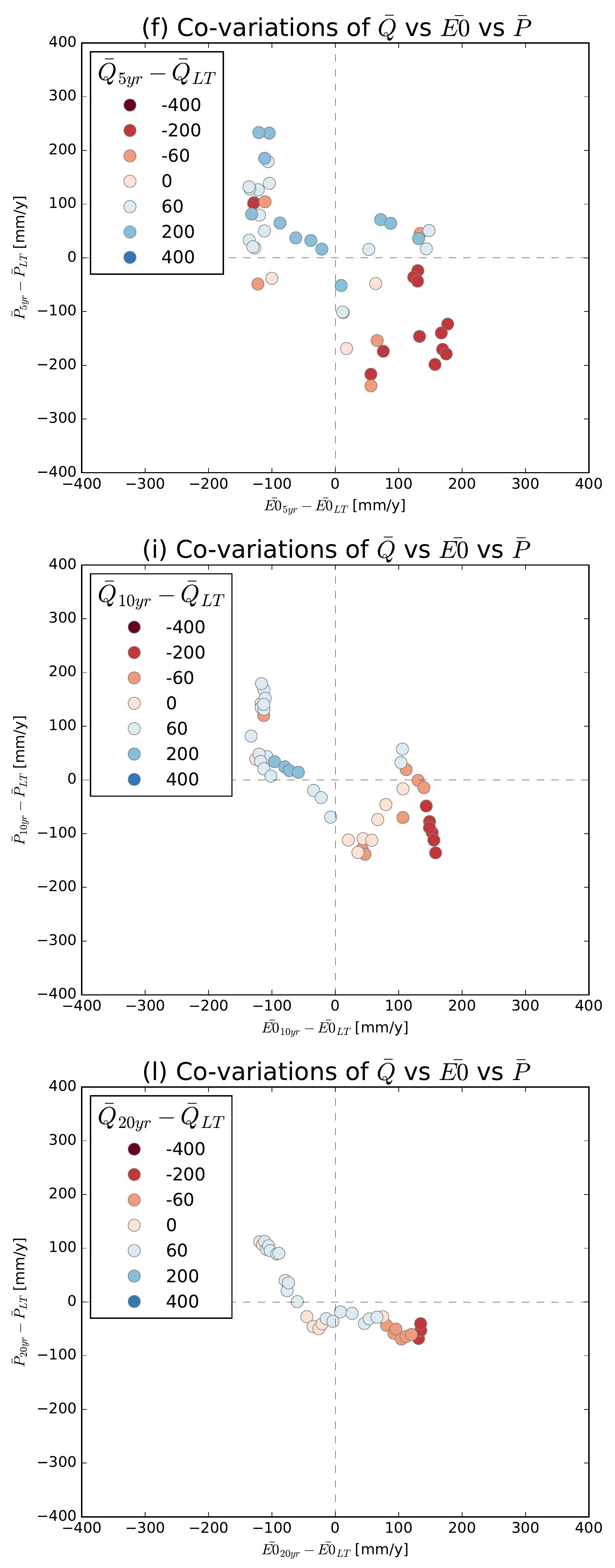

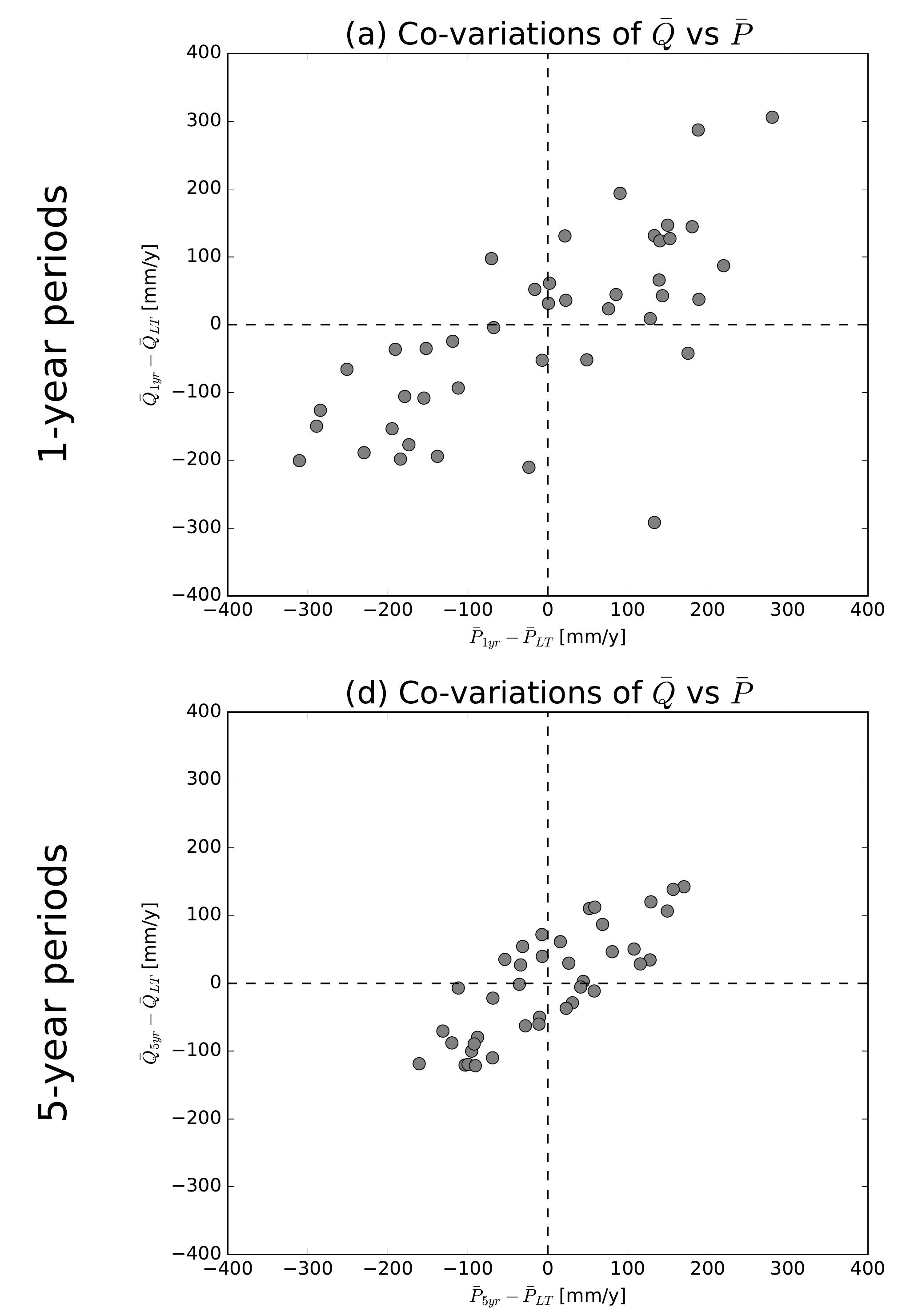

(g) Co-variations of $\bar{Q}$ vs $\bar{P}$
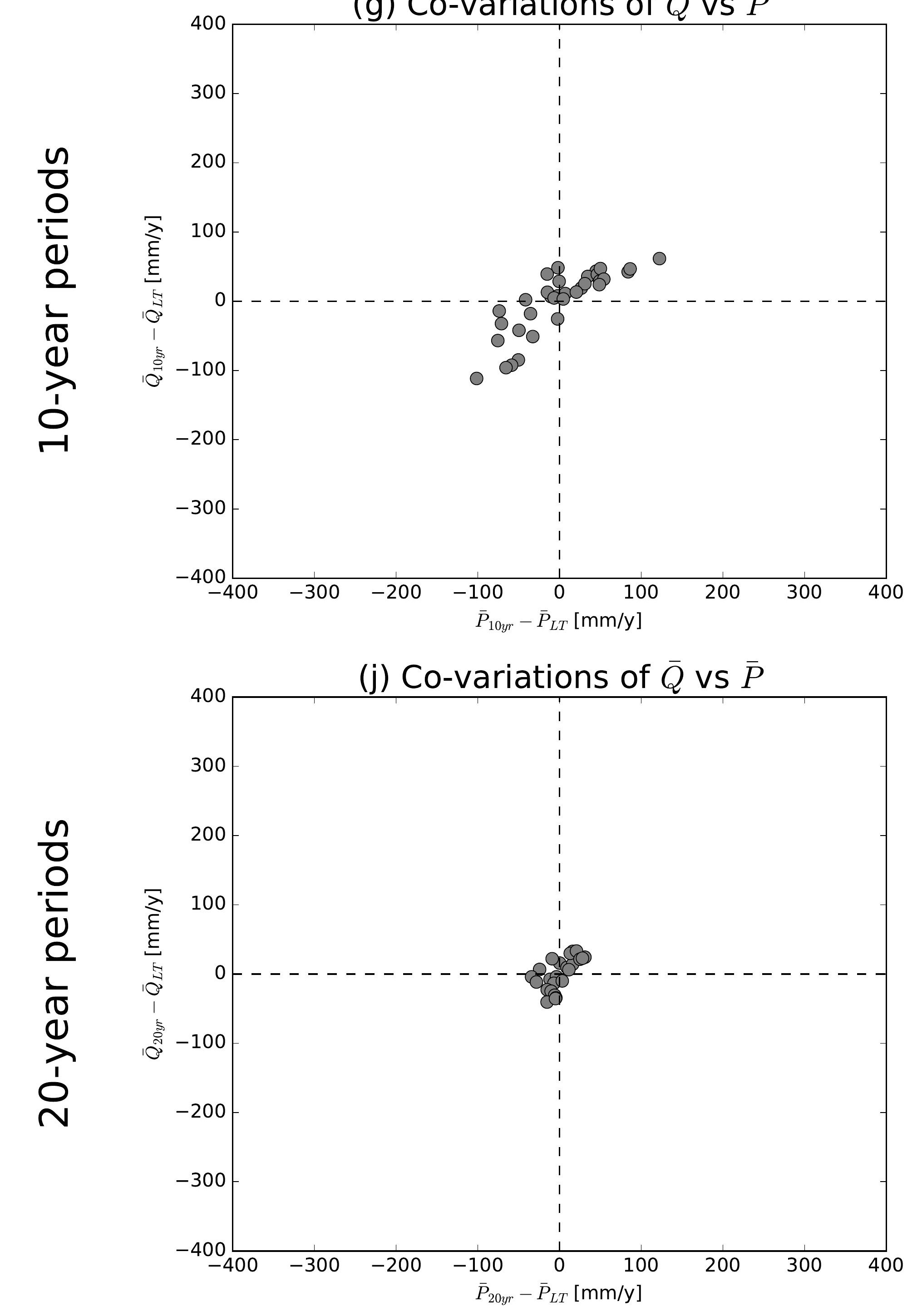

(b) Co-variations of $\bar{Q}$ vs $\overline{E 0}$

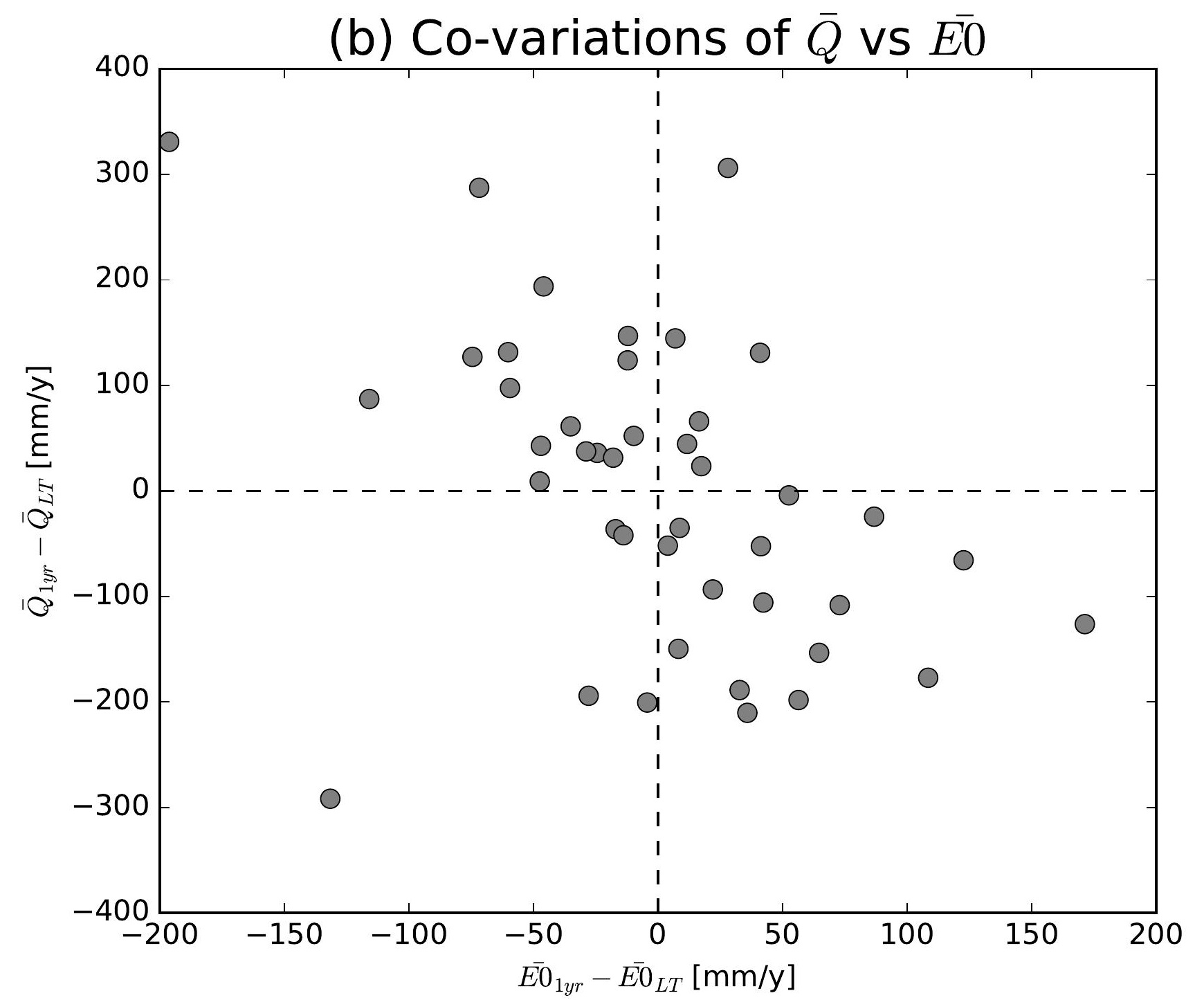

(e) Co-variations of $\bar{Q}$ vs $\overline{E 0}$

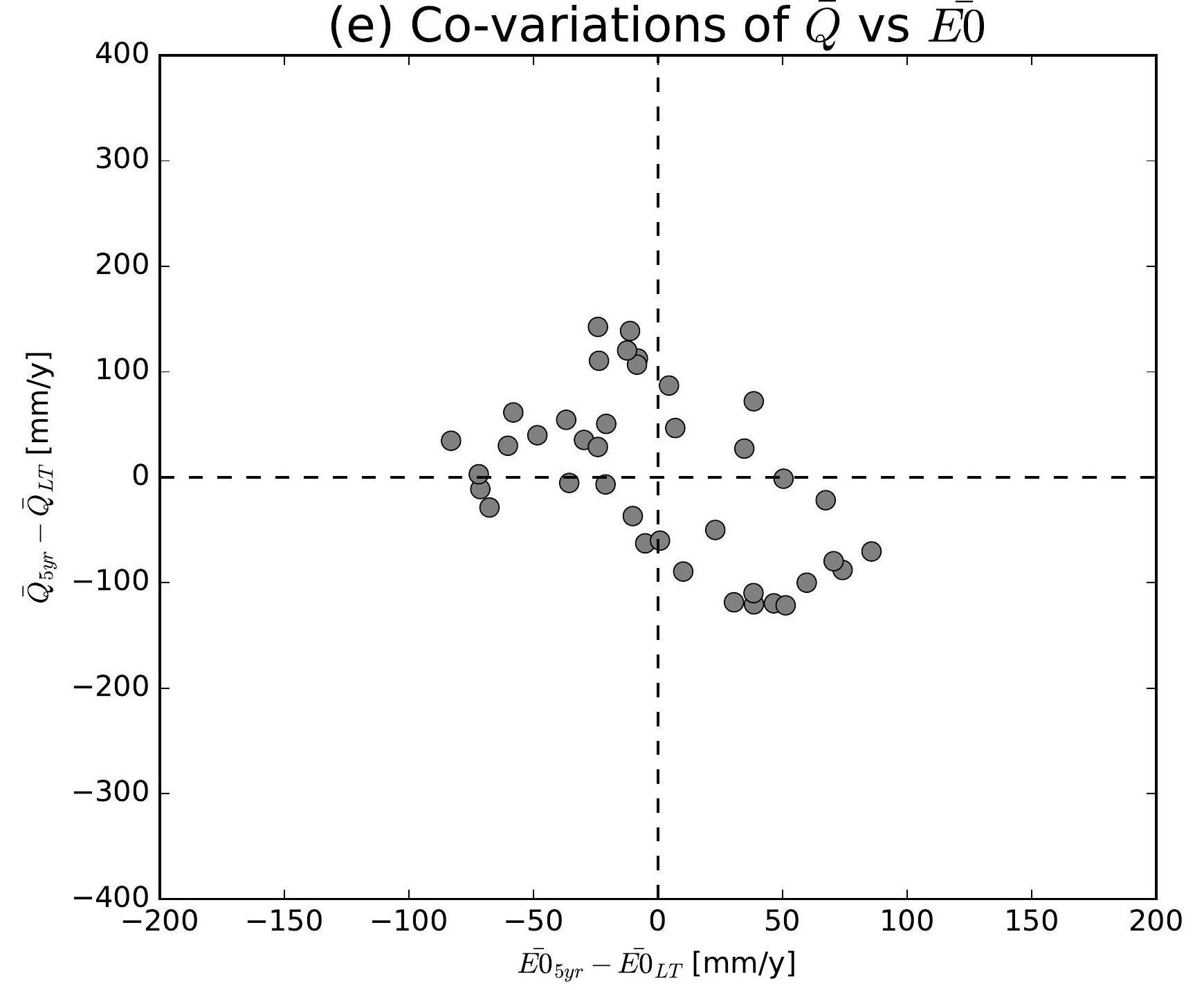

(h) Co-variations of $\bar{Q}$ vs $\overline{E 0}$

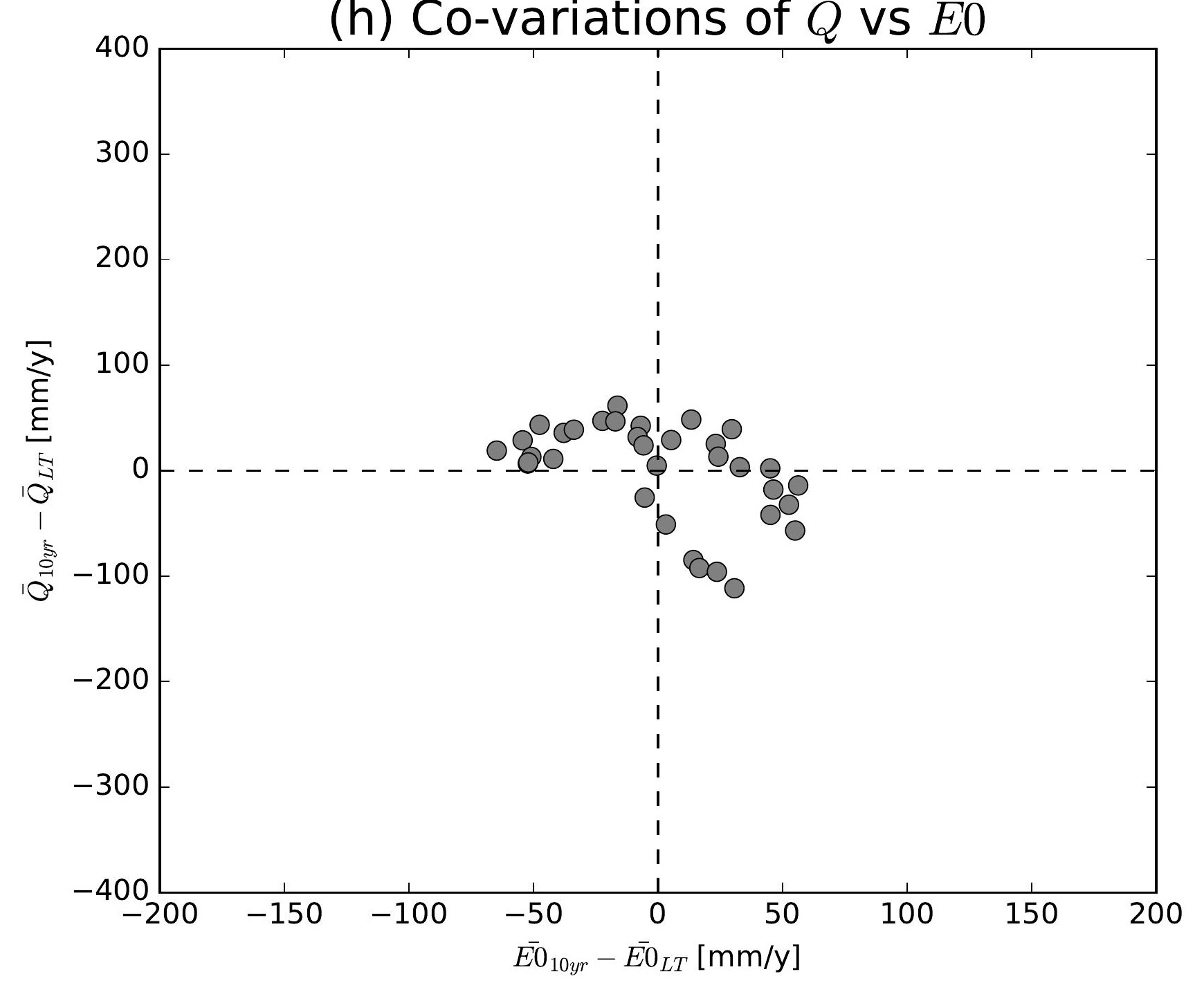

(k) Co-variations of $\bar{Q}$ vs $\overline{E 0}$

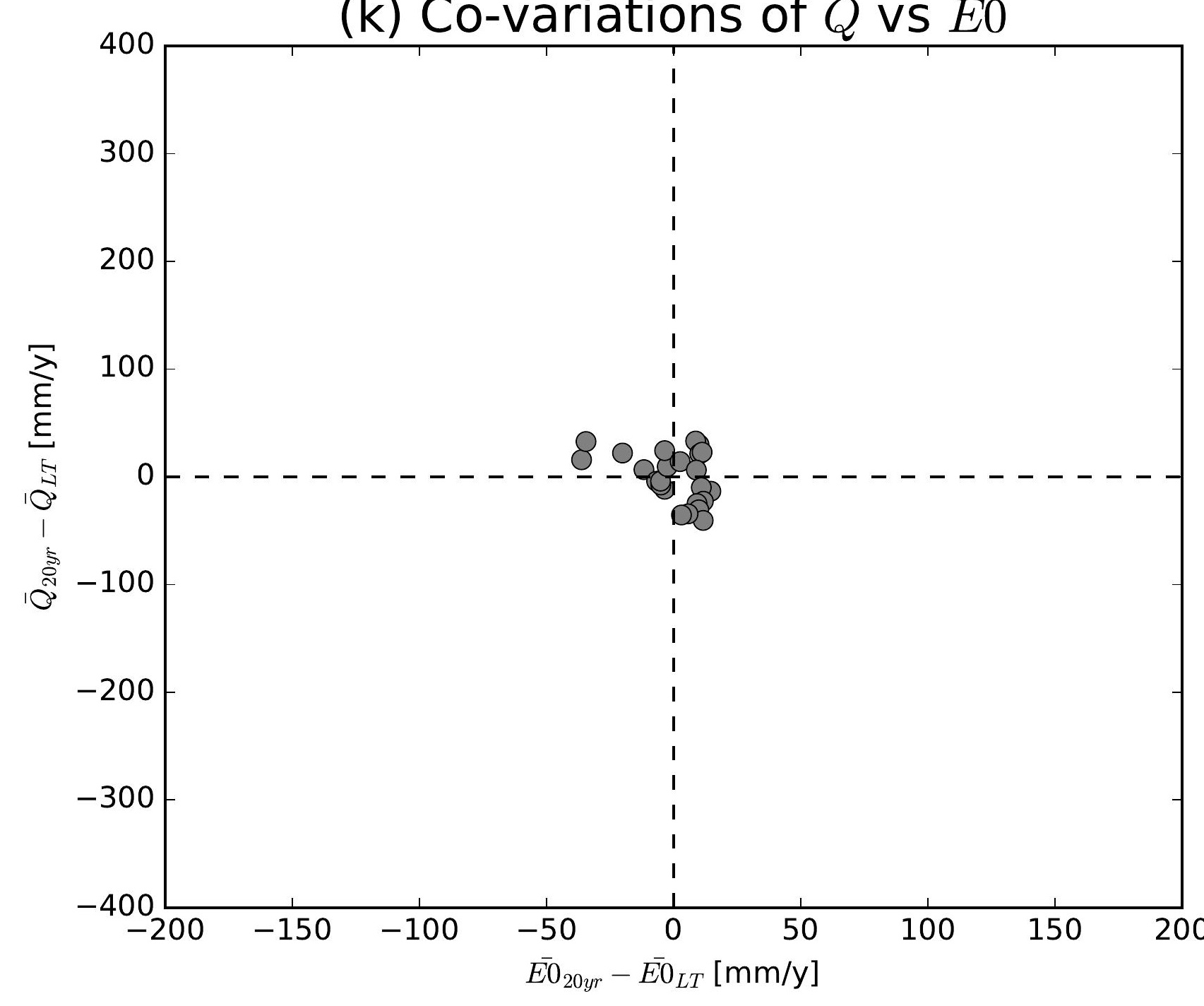

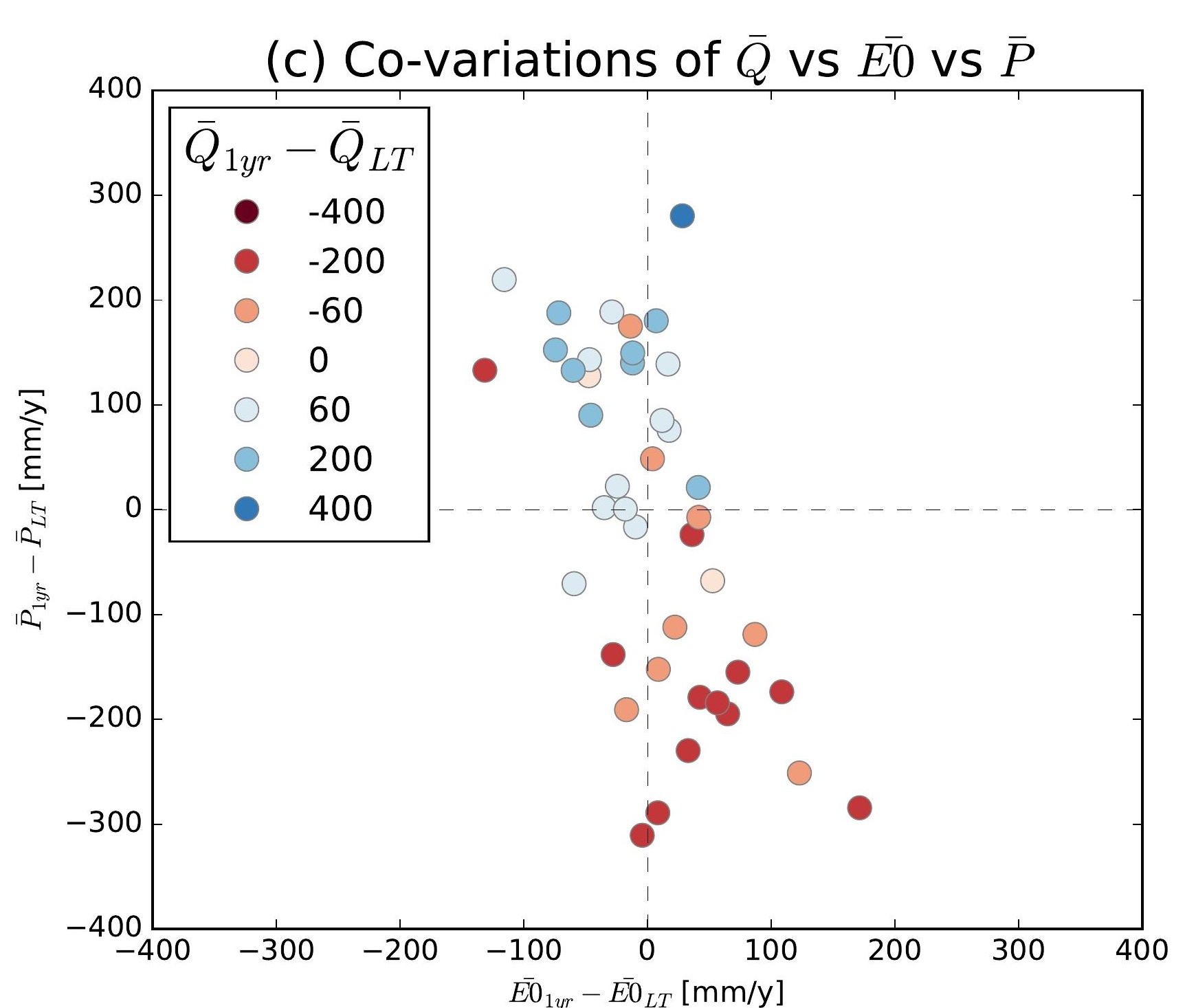
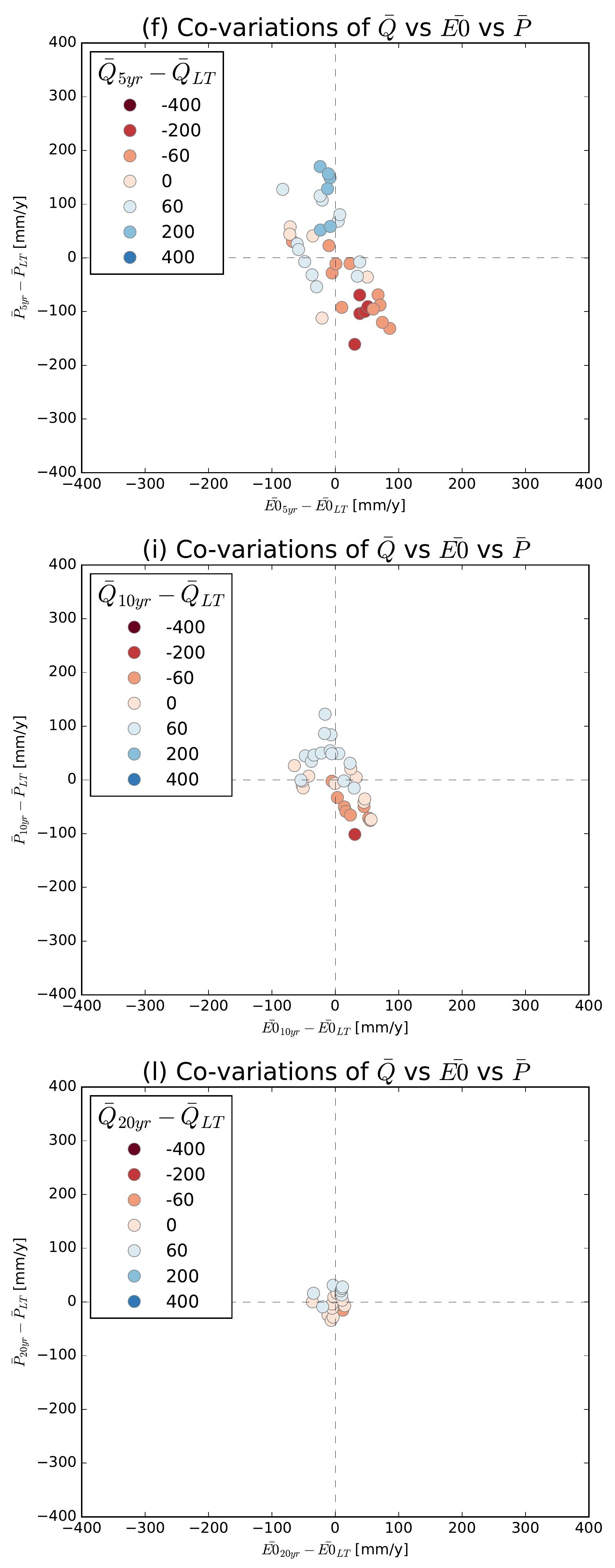

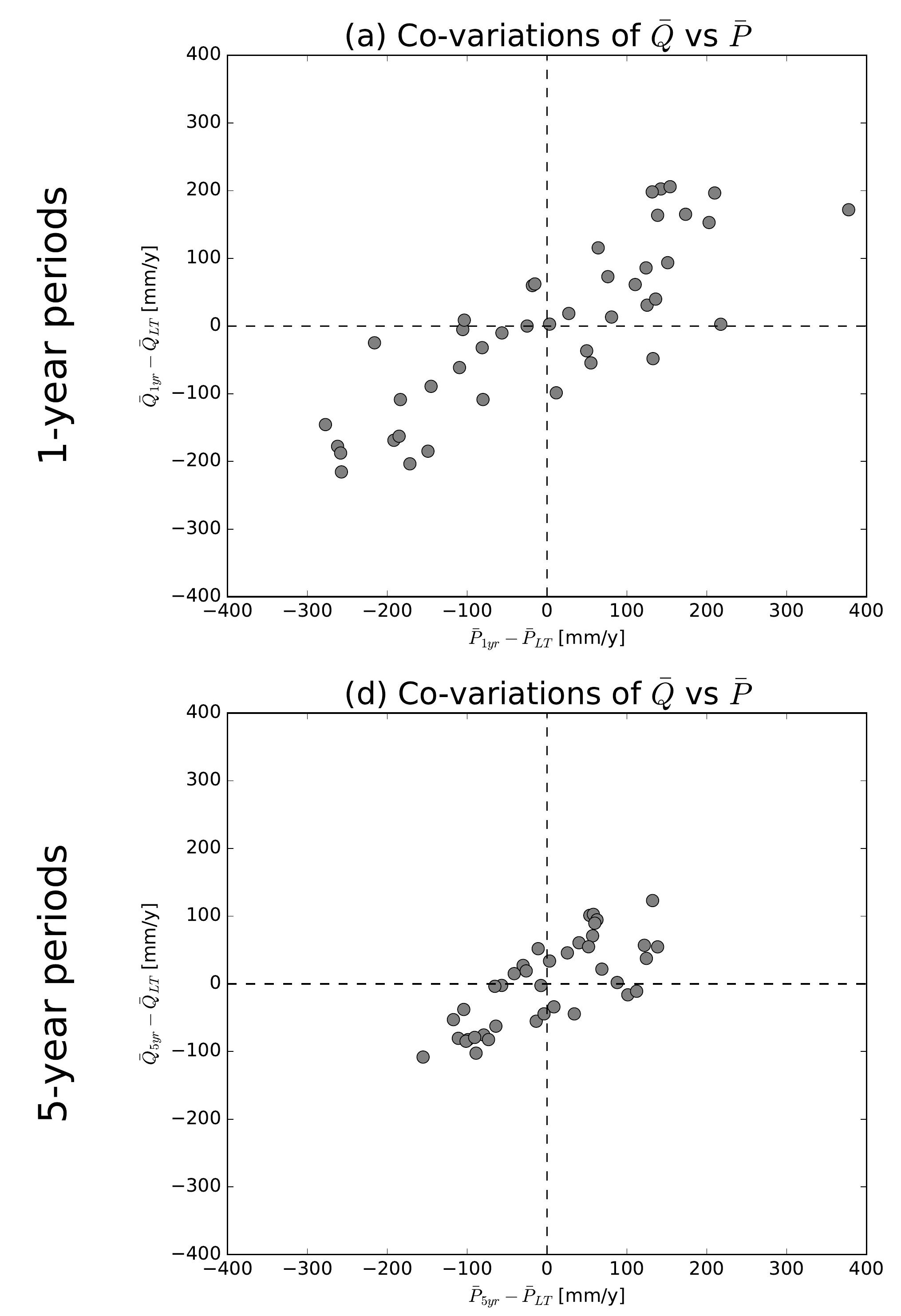

(g) Co-variations of $\bar{Q}$ vs $\bar{P}$
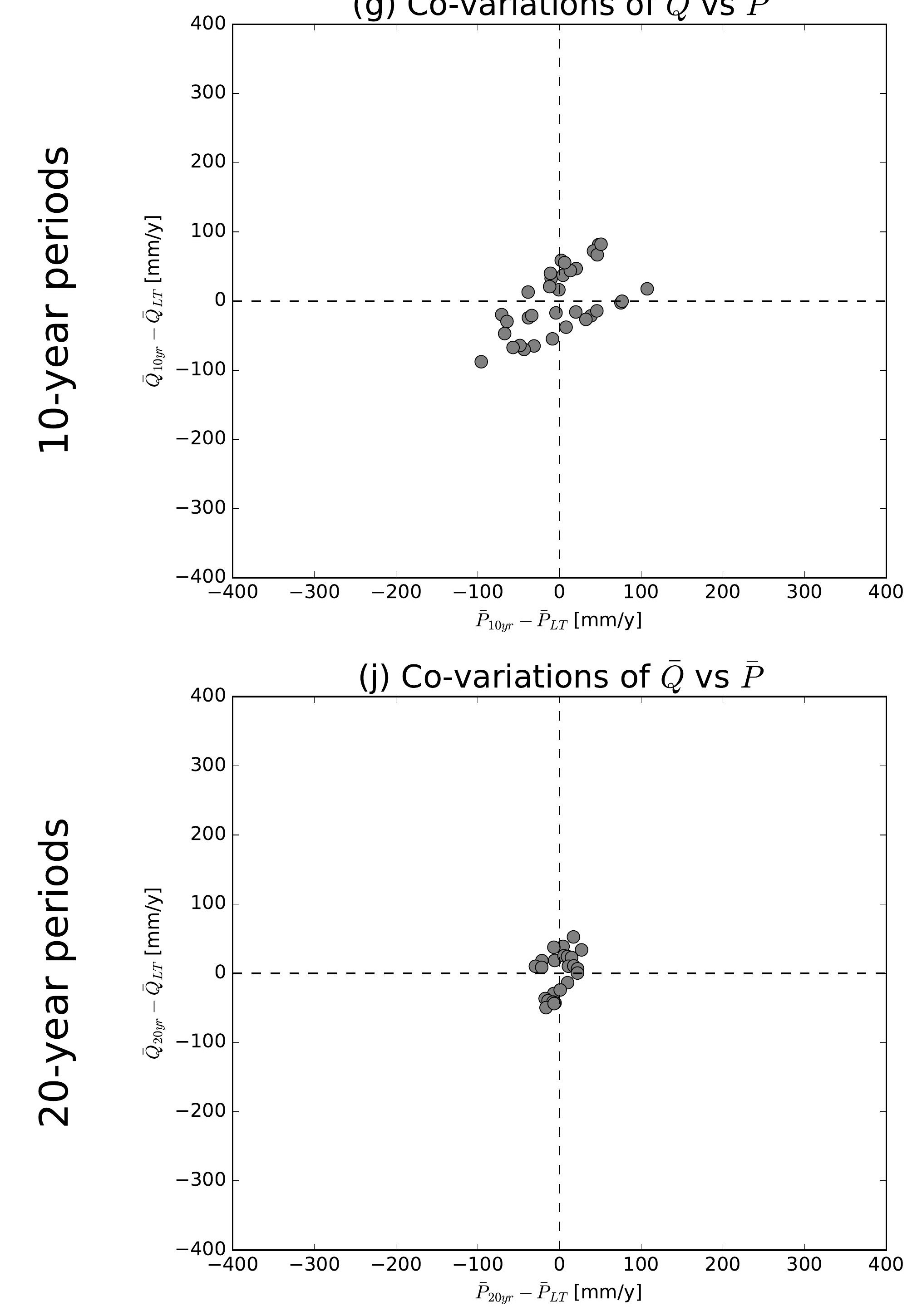

(b) Co-variations of $\bar{Q}$ vs $\overline{E 0}$

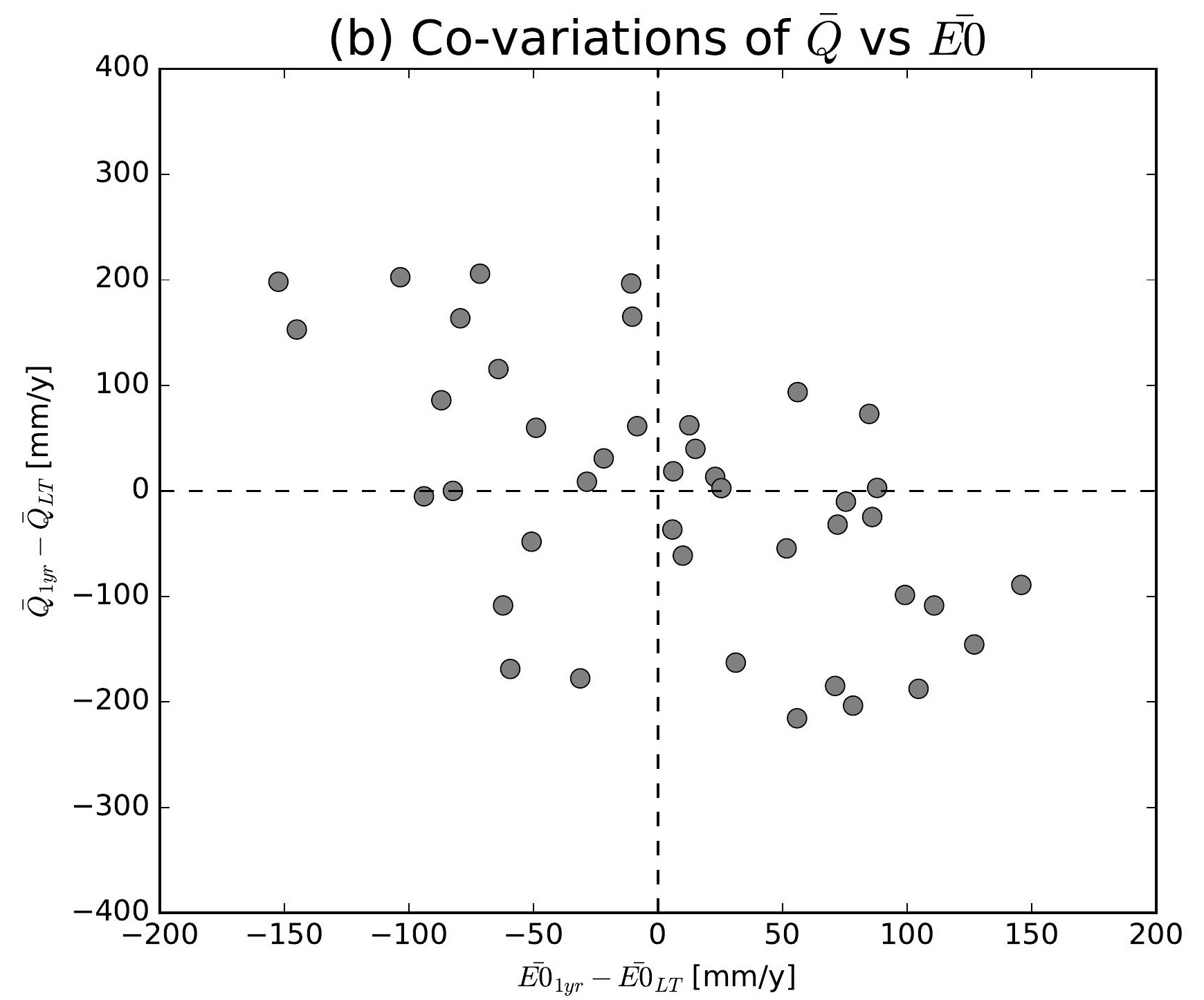

(e) Co-variations of $\bar{Q}$ vs $\overline{E 0}$

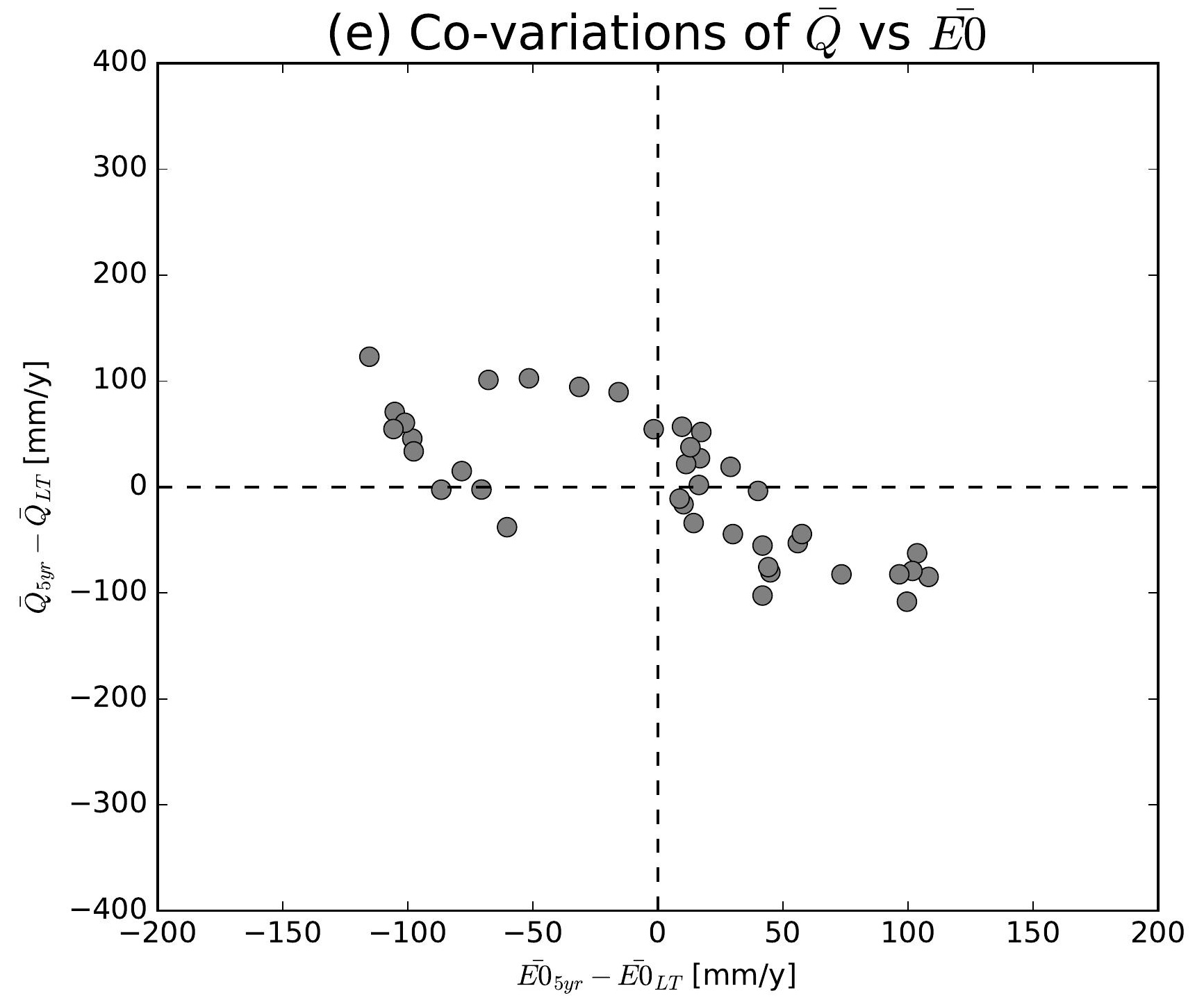

(h) Co-variations of $\bar{Q}$ vs $\overline{E 0}$

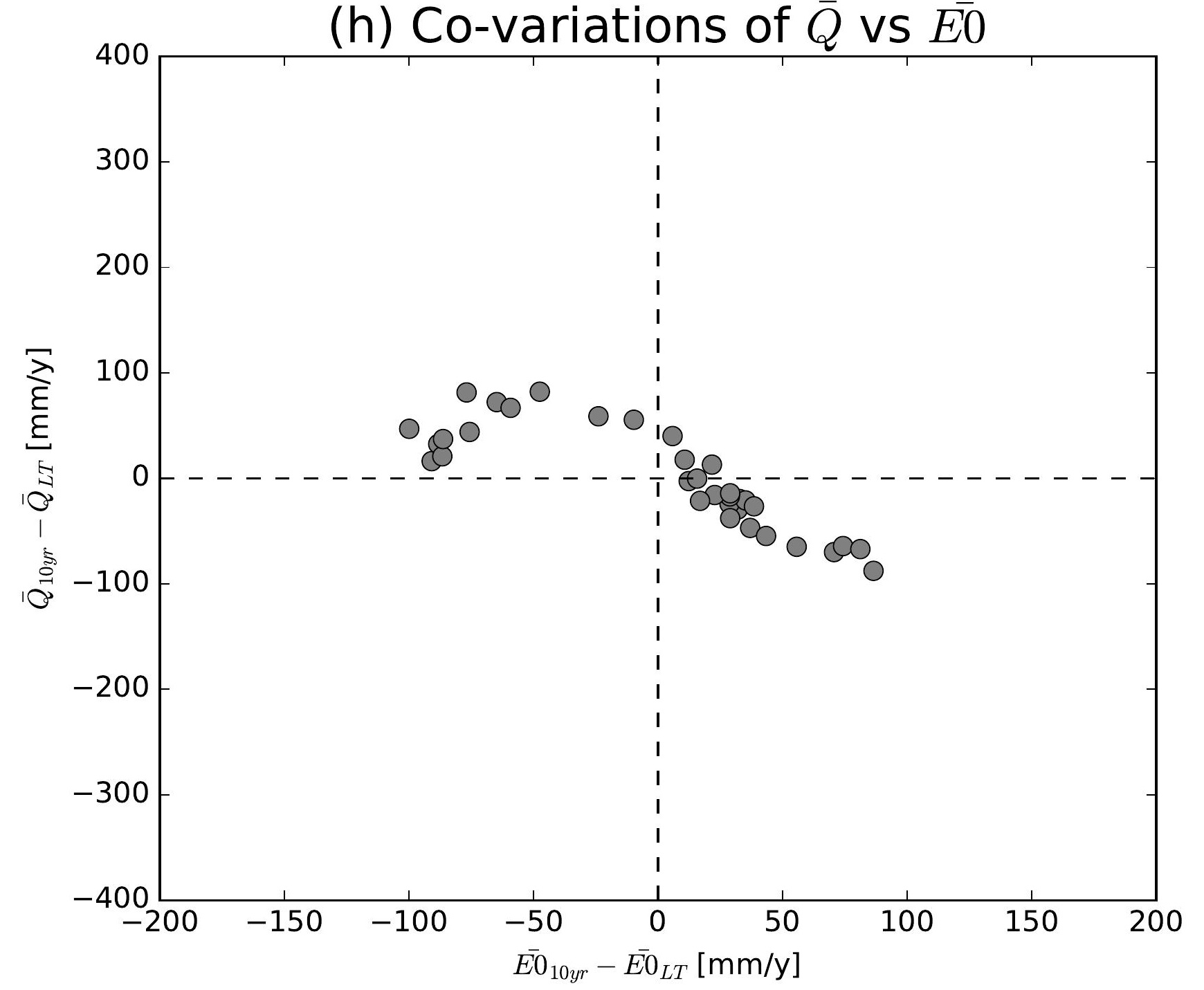

(k) Co-variations of $\bar{Q}$ vs $\overline{E 0}$

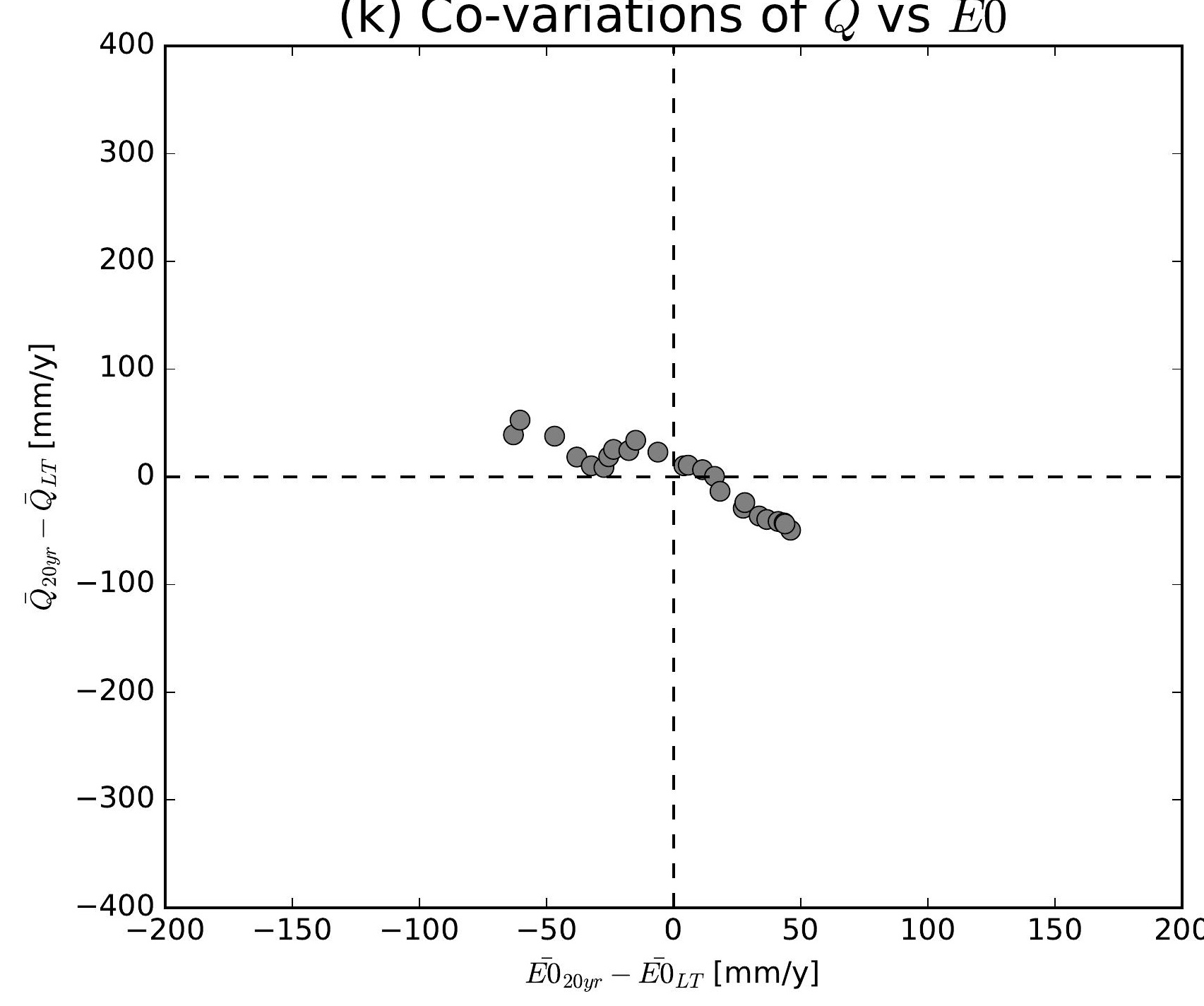

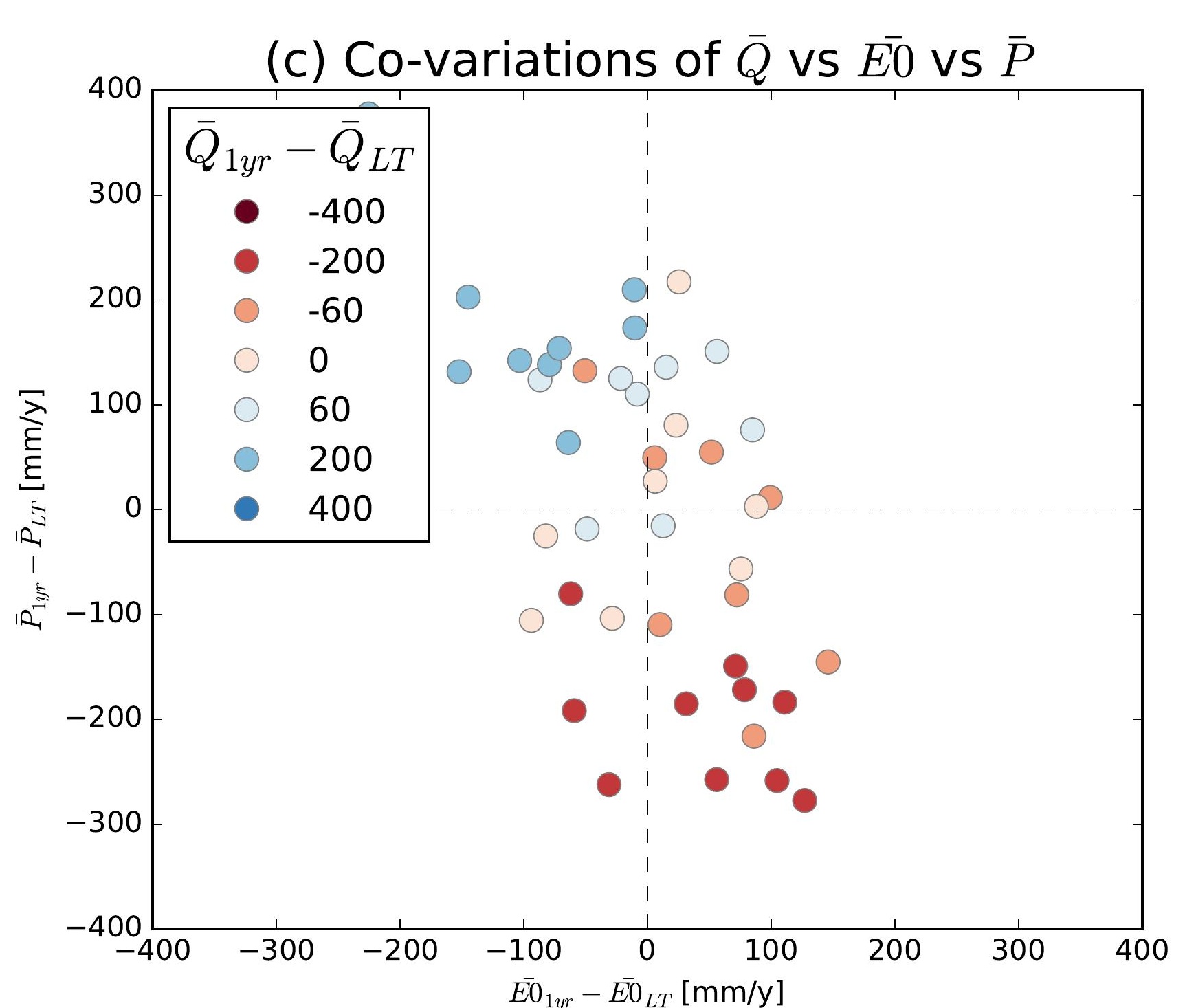
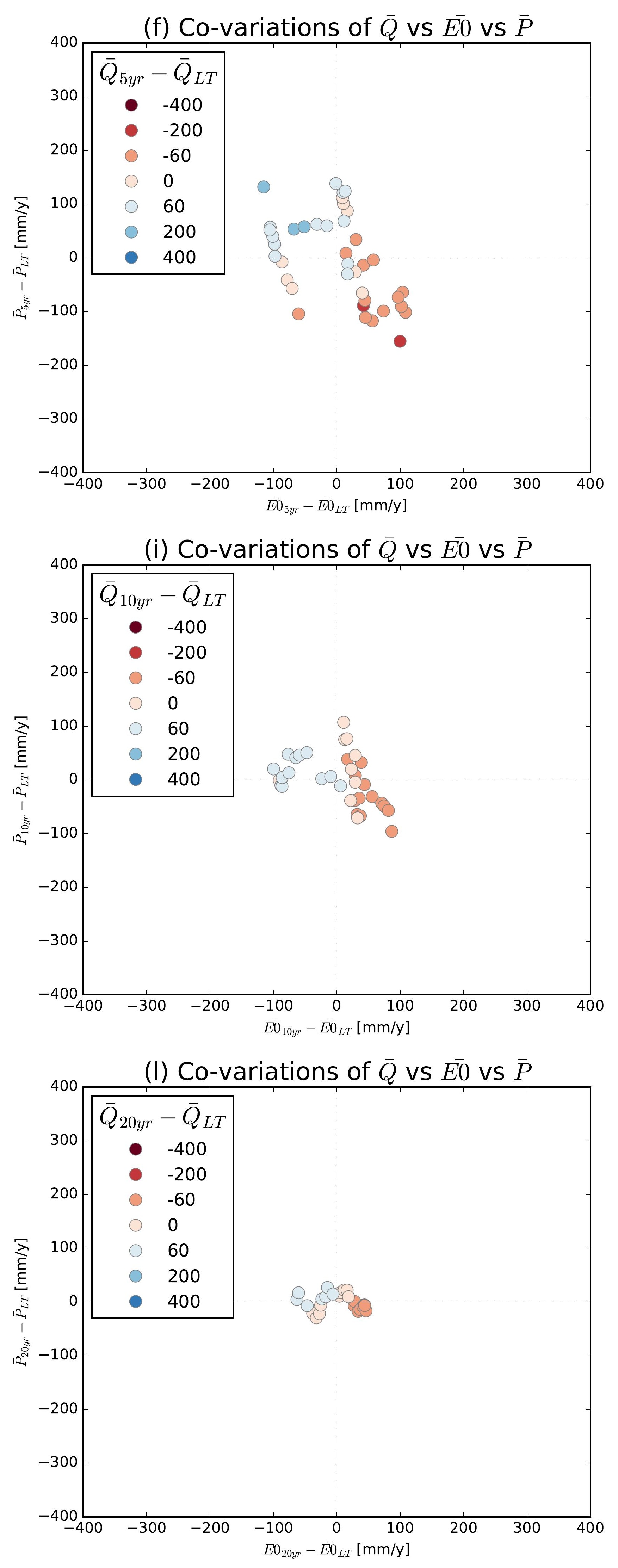

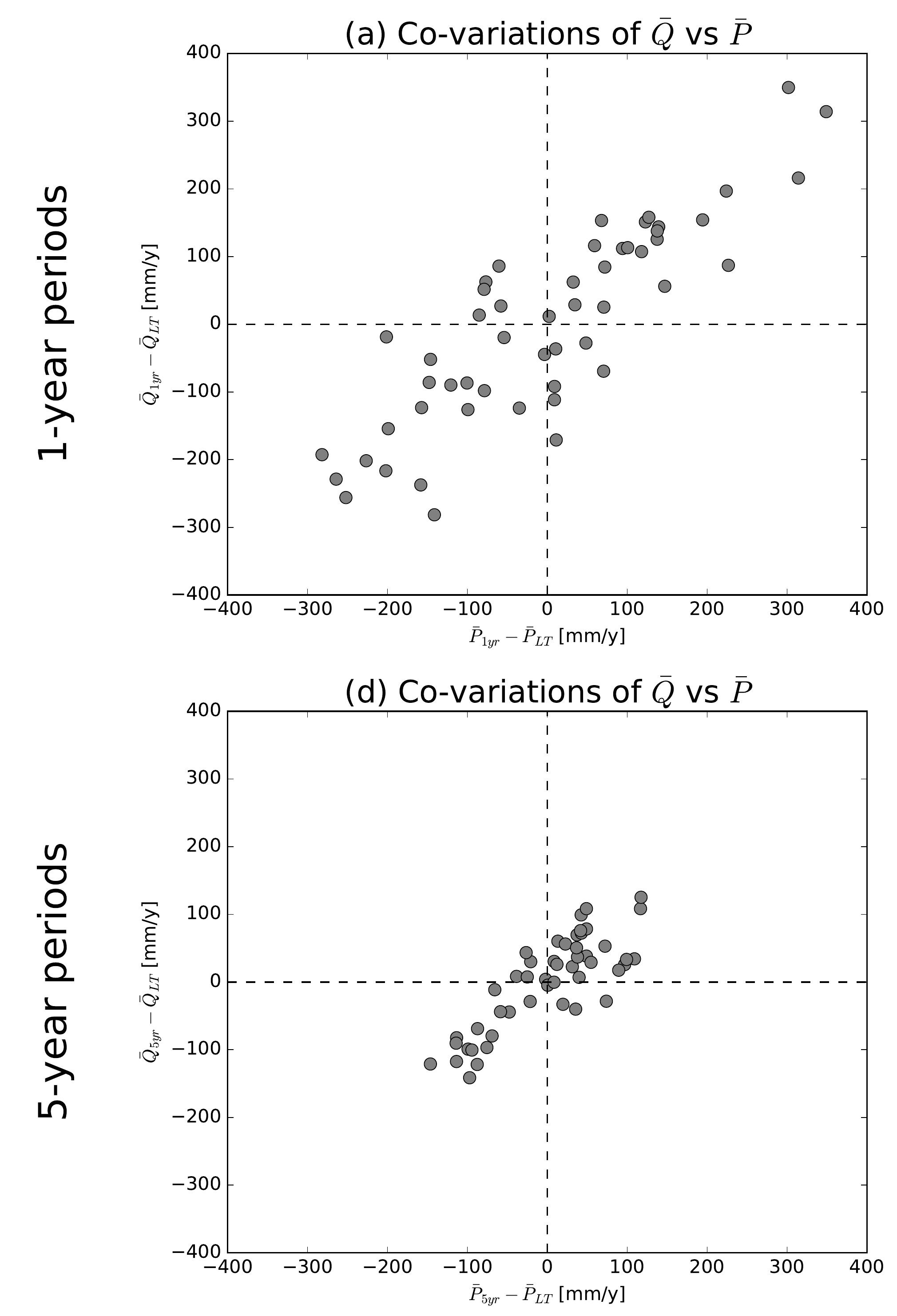

(g) Co-variations of $\bar{Q}$ vs $\bar{P}$
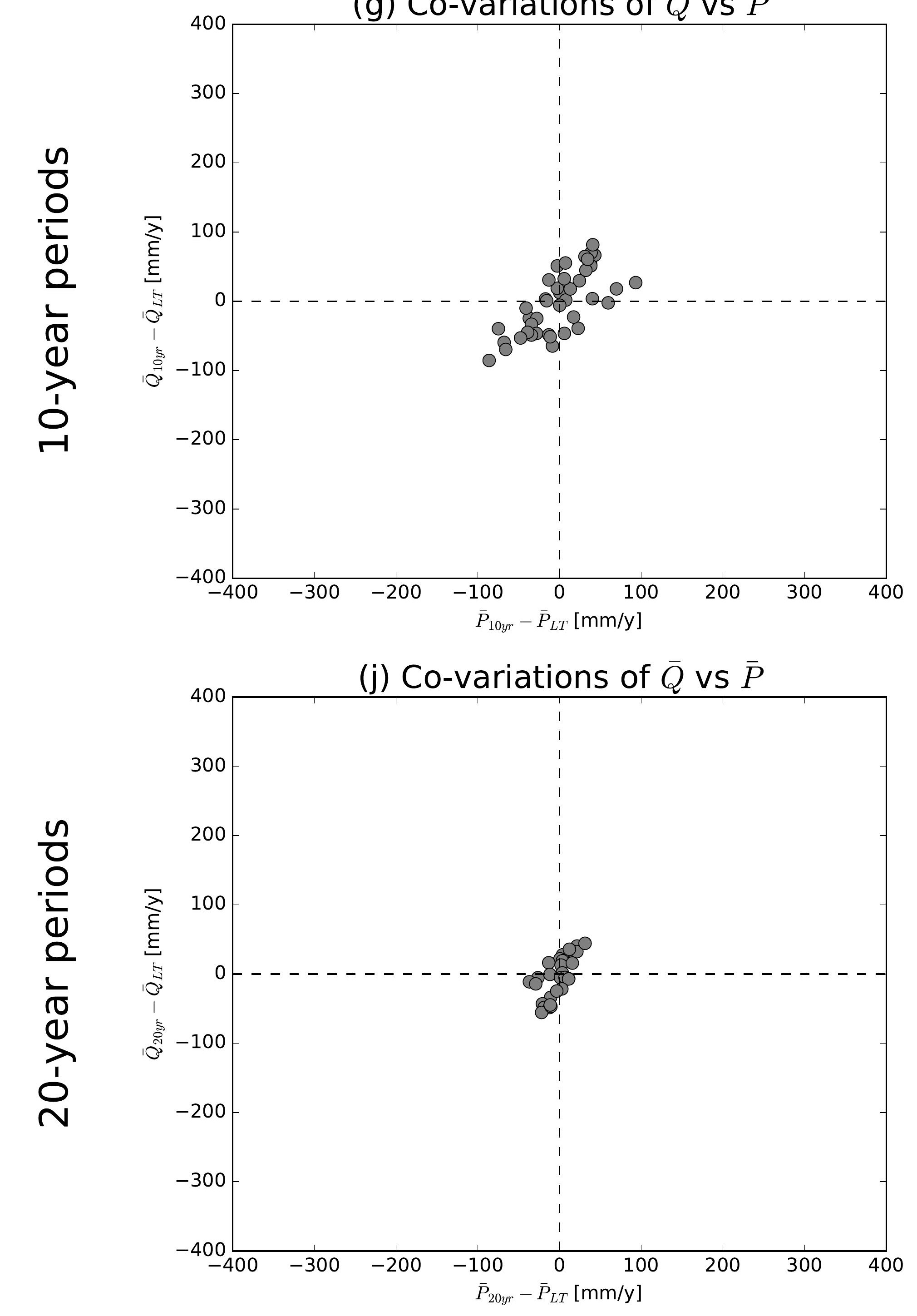

(b) Co-variations of $\bar{Q}$ vs $\overline{E 0}$

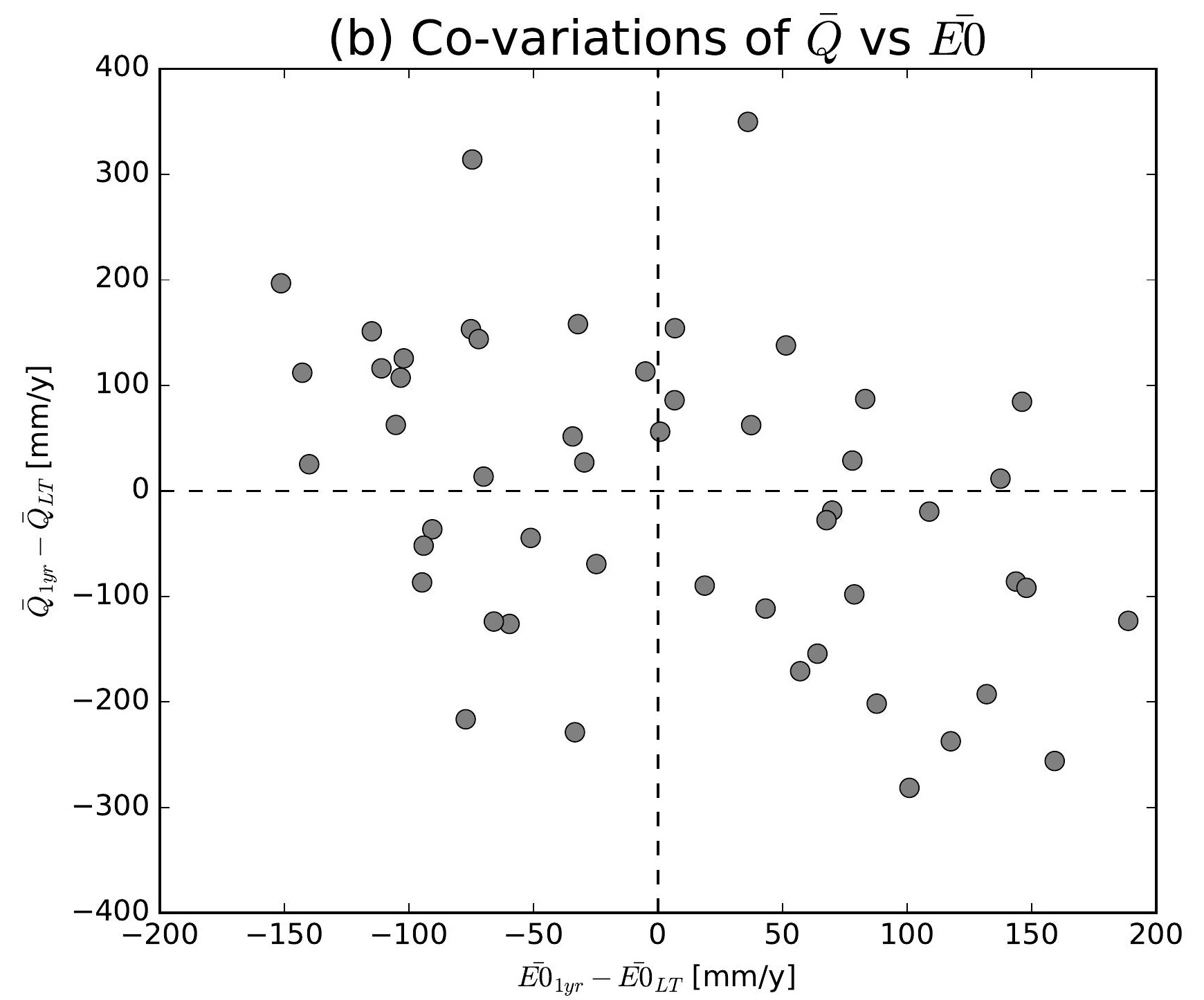

(e) Co-variations of $\bar{Q}$ vs $\overline{E 0}$

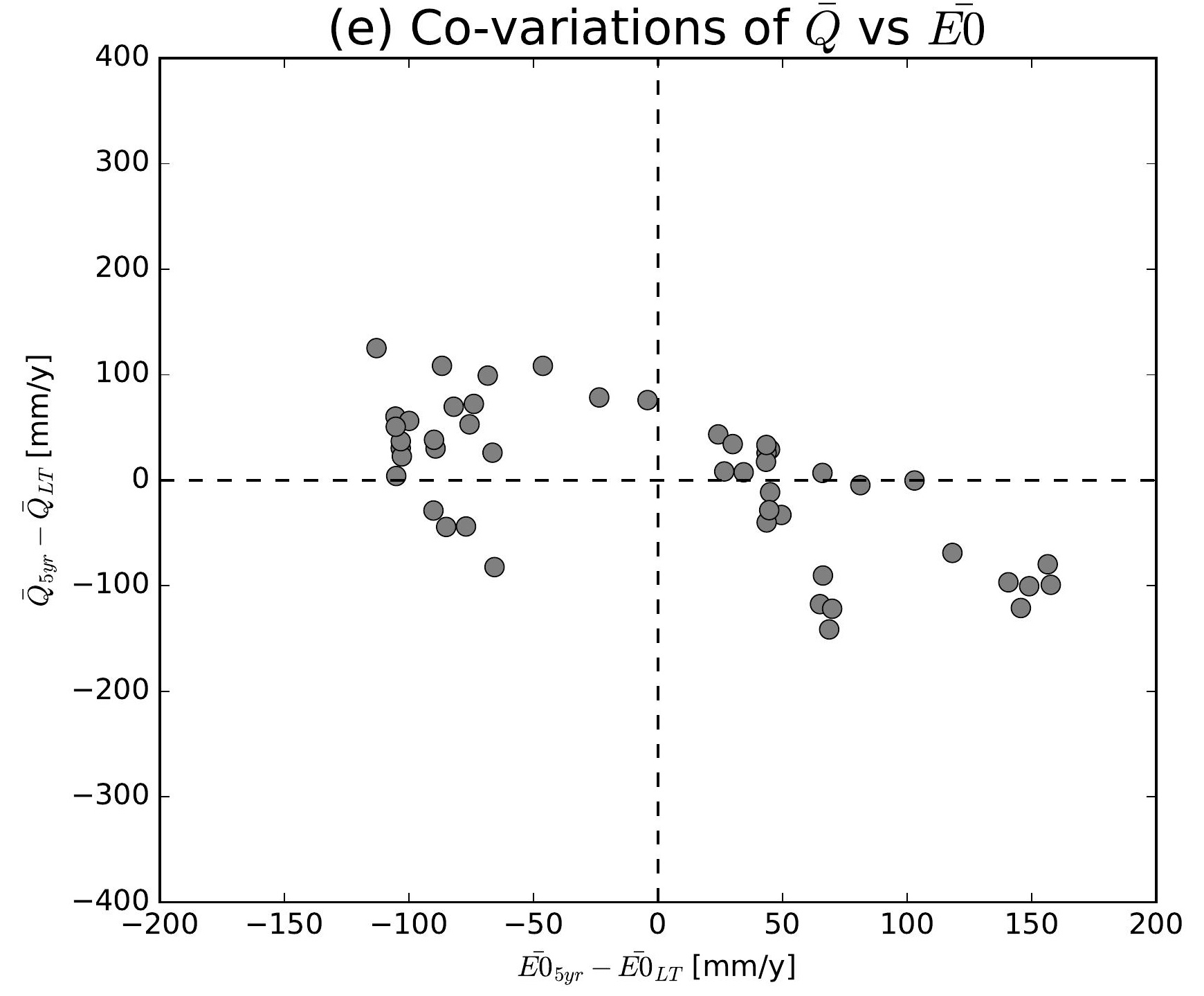

(h) Co-variations of $\bar{Q}$ vs $\overline{E 0}$

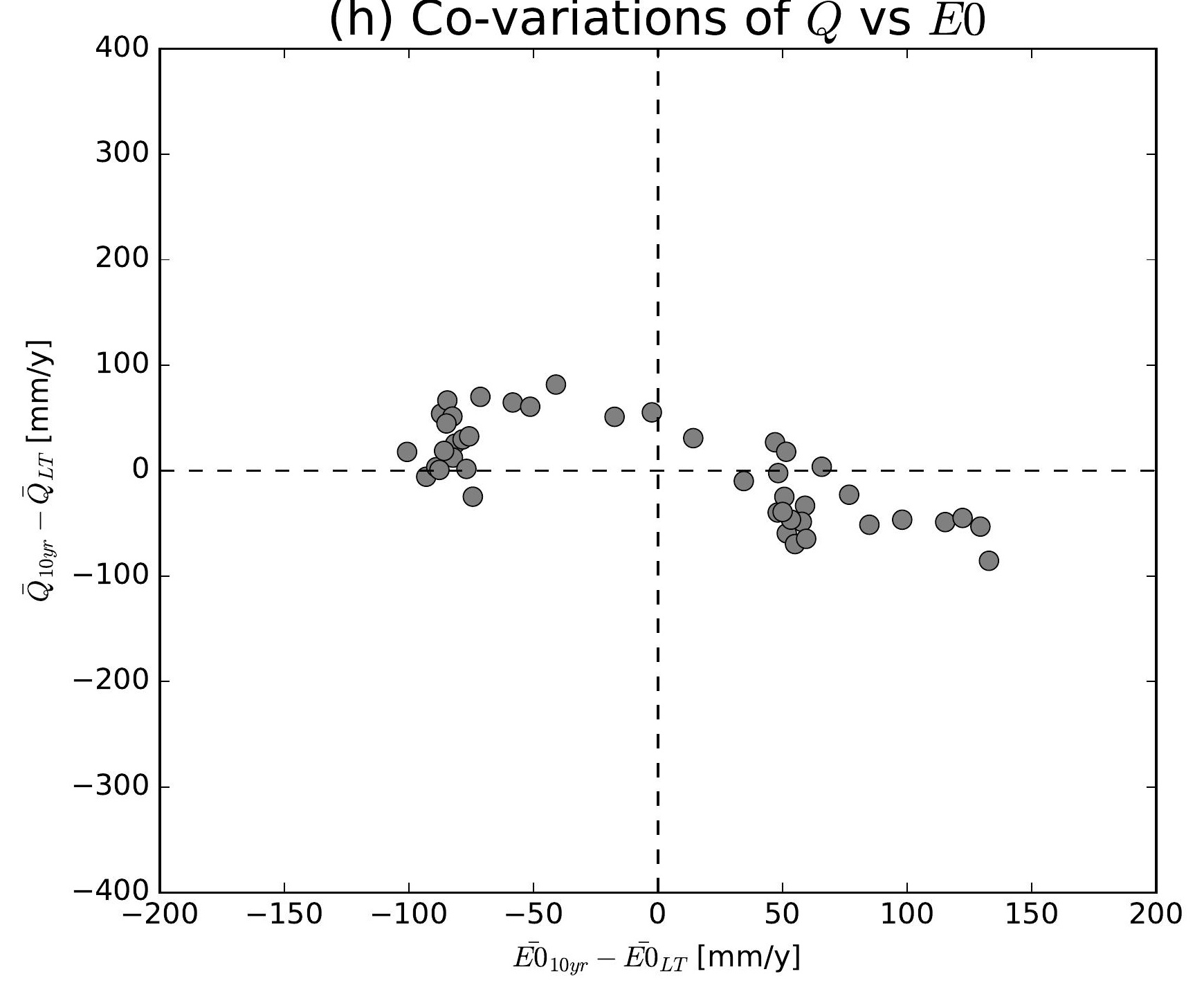

(k) Co-variations of $\bar{Q}$ vs $\overline{E 0}$

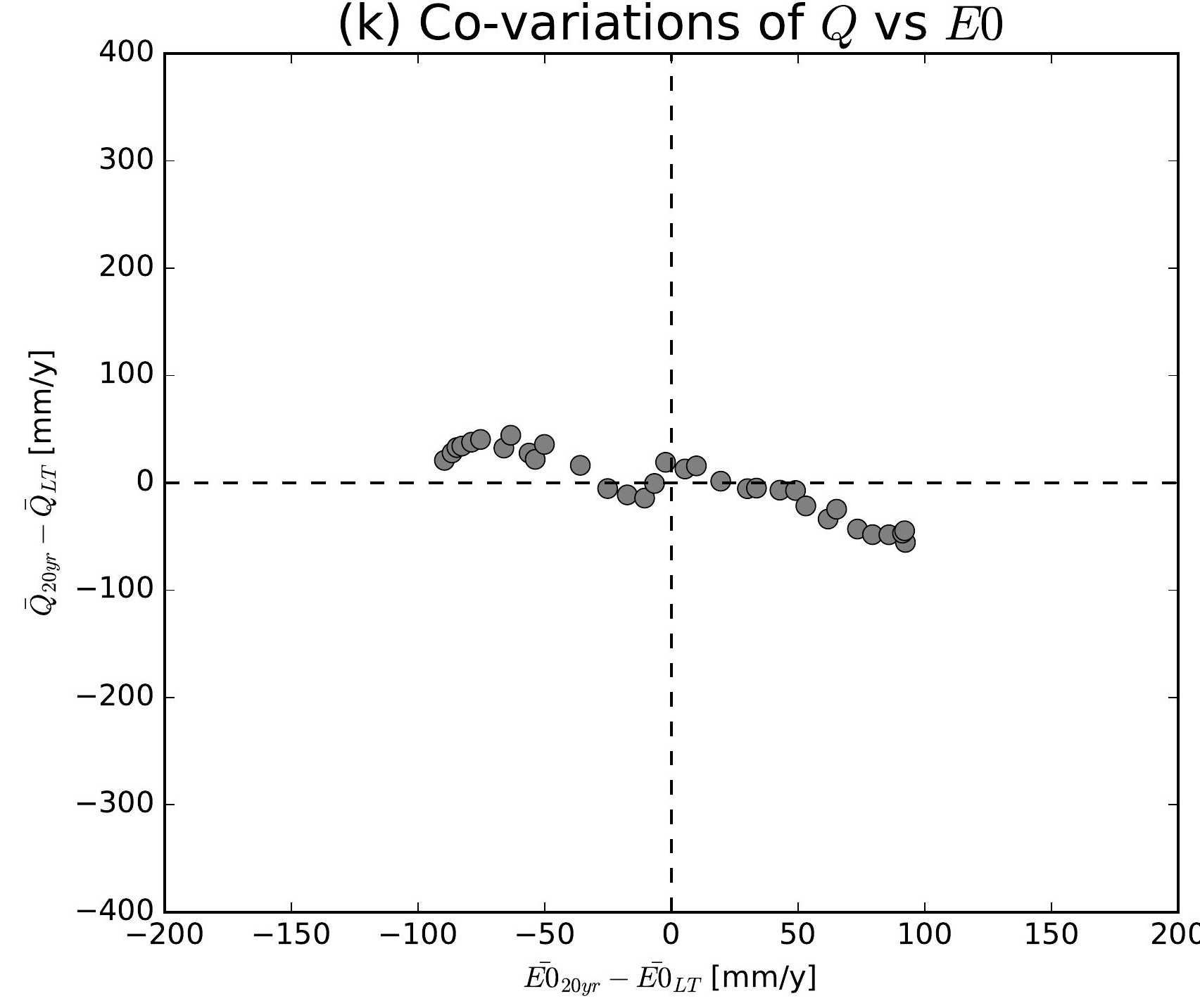

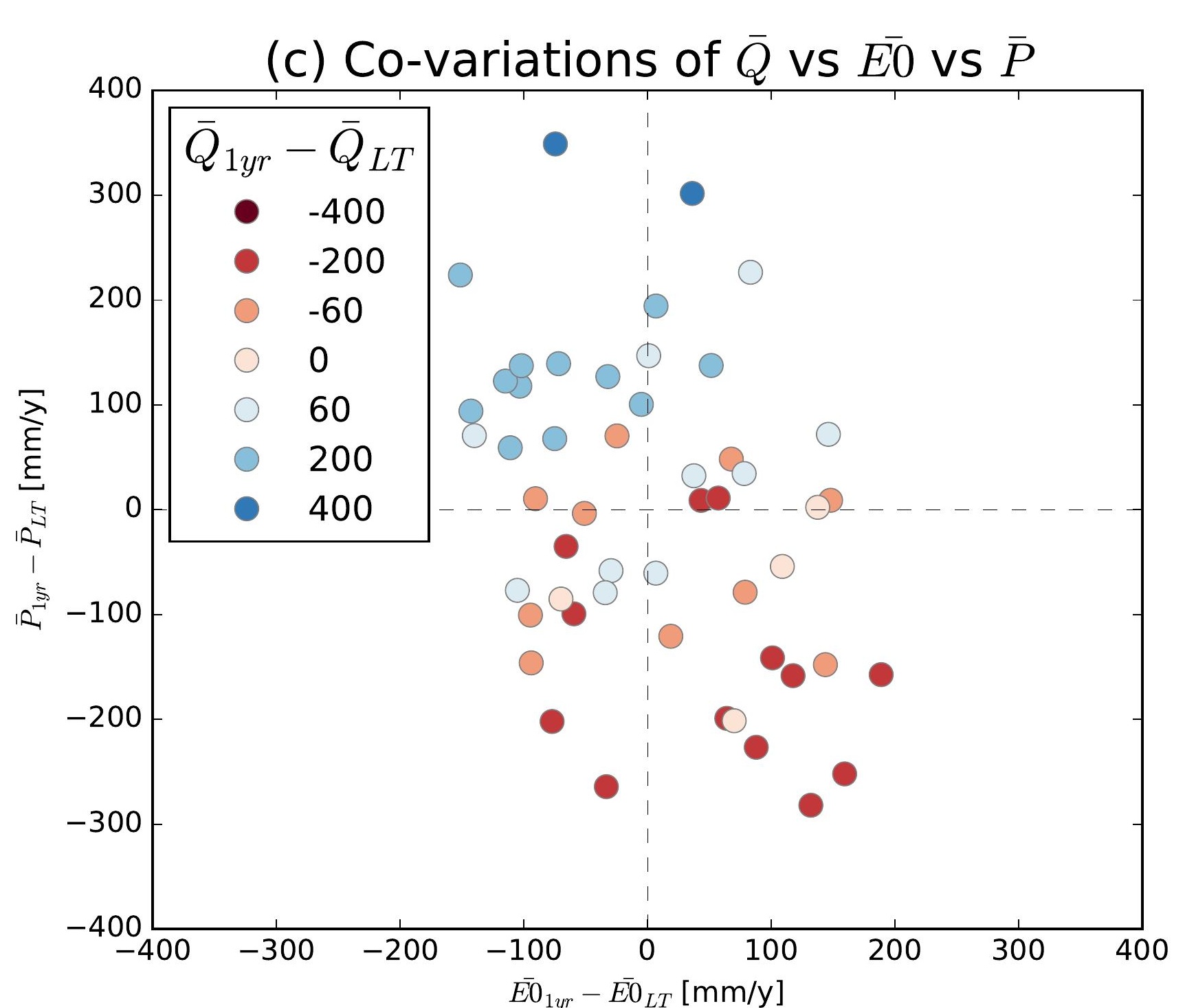
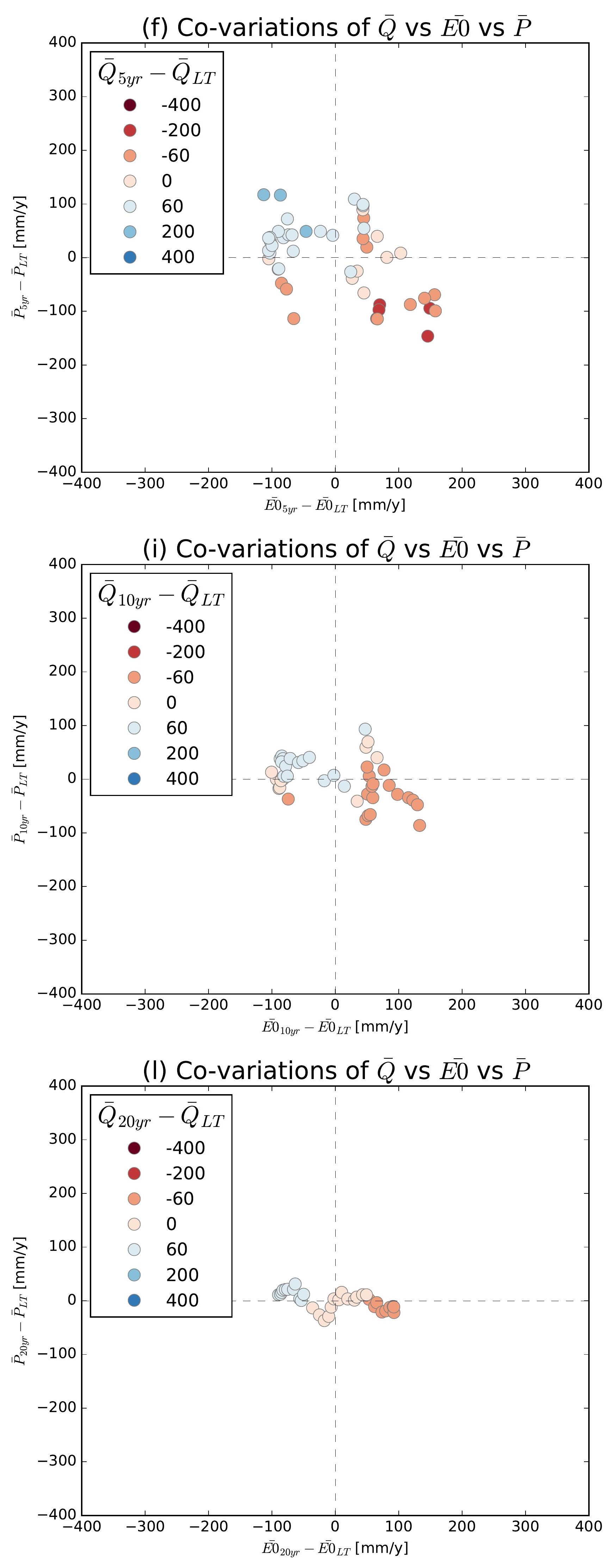

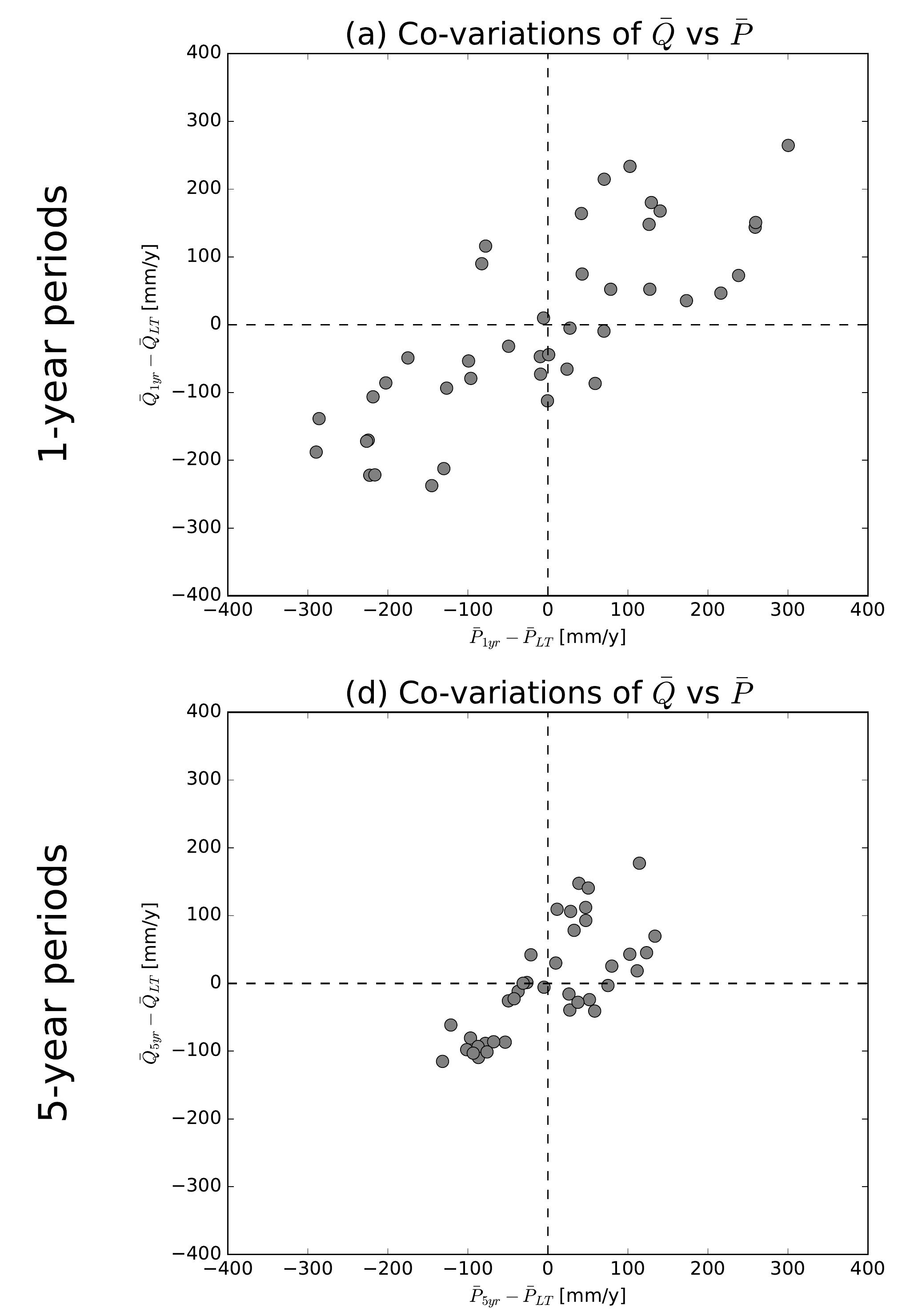

(g) Co-variations of $\bar{Q}$ vs $\bar{P}$
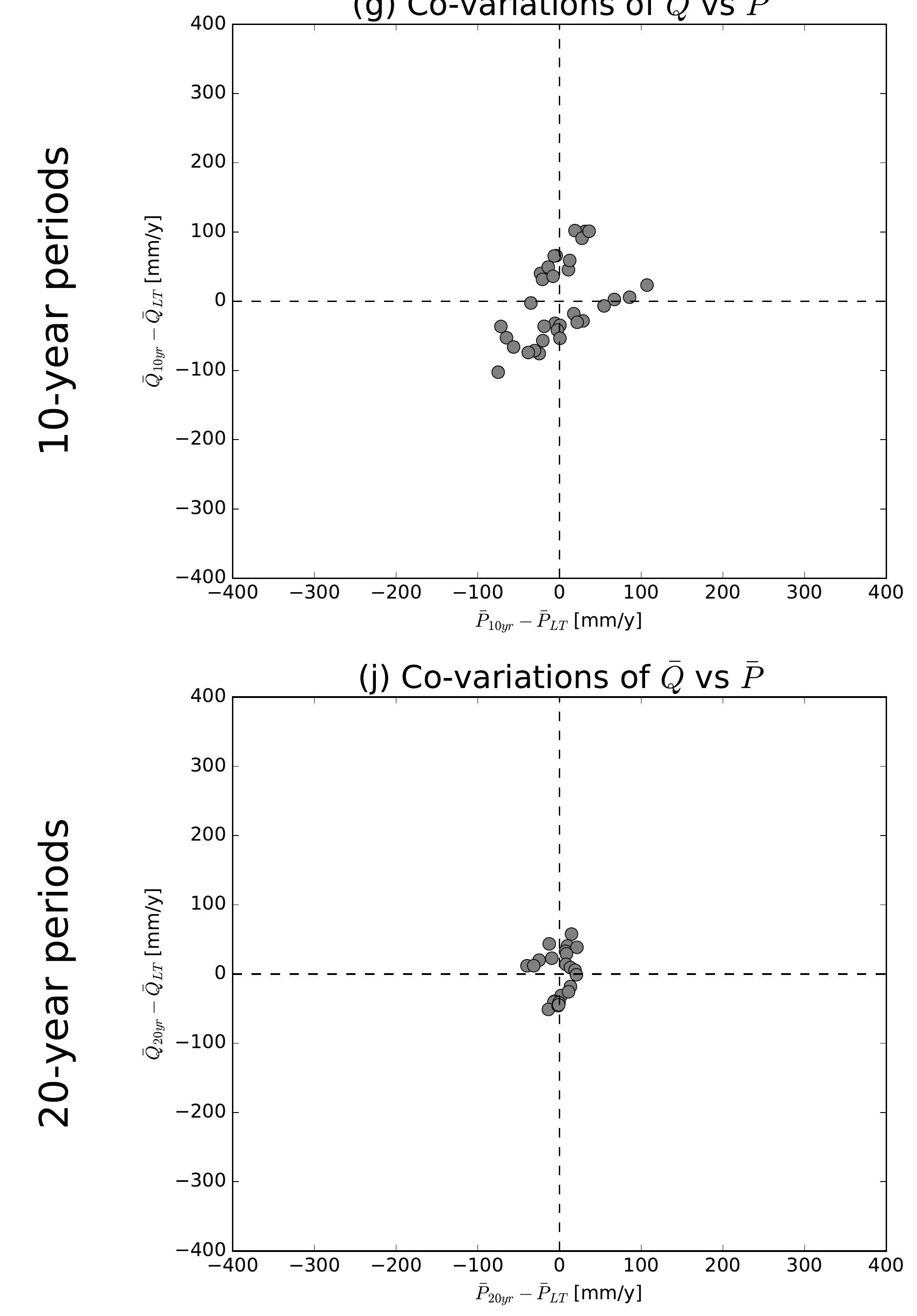

(b) Co-variations of $\bar{Q}$ vs $\overline{E 0}$

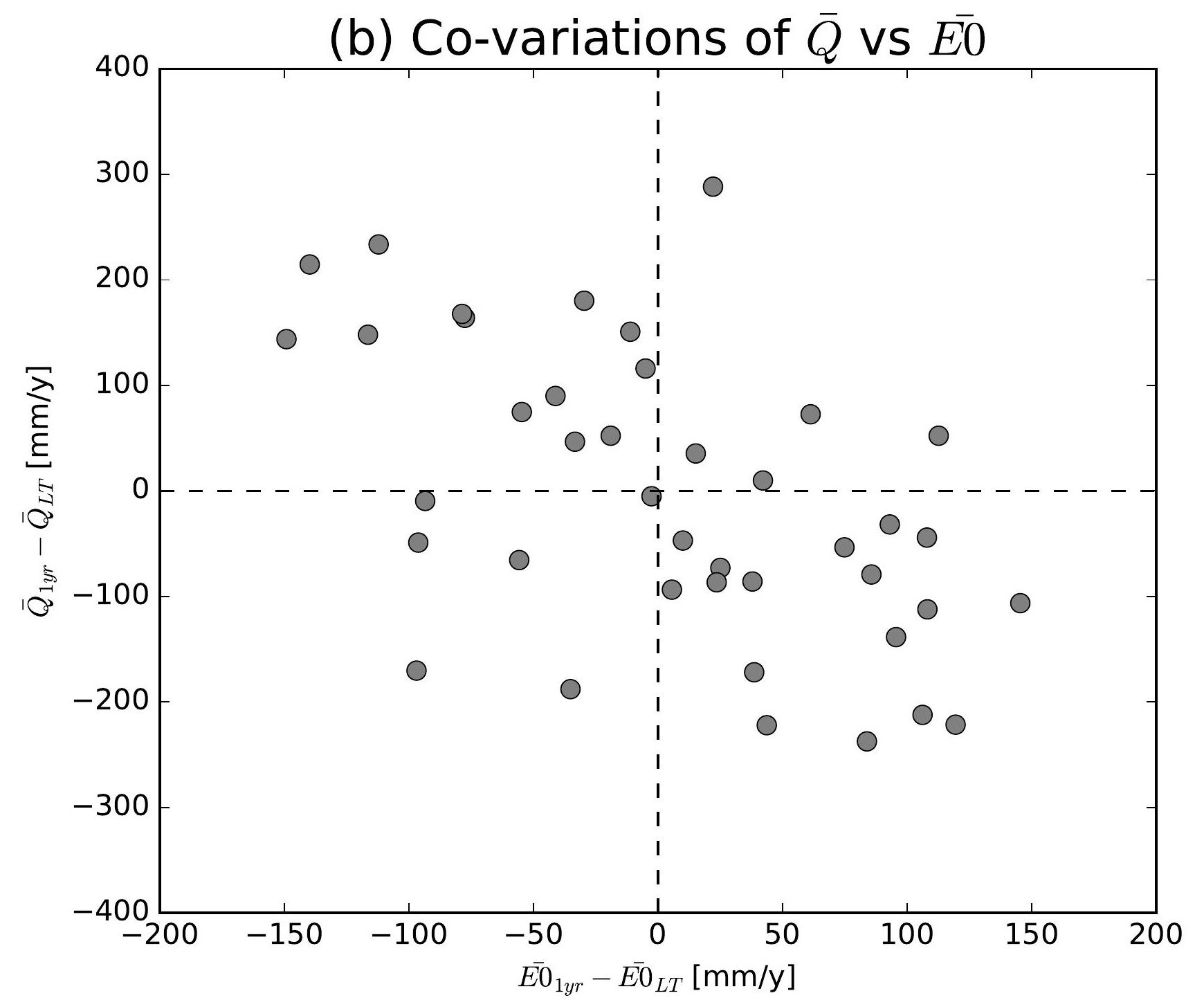

(e) Co-variations of $\bar{Q}$ vs $\overline{E 0}$

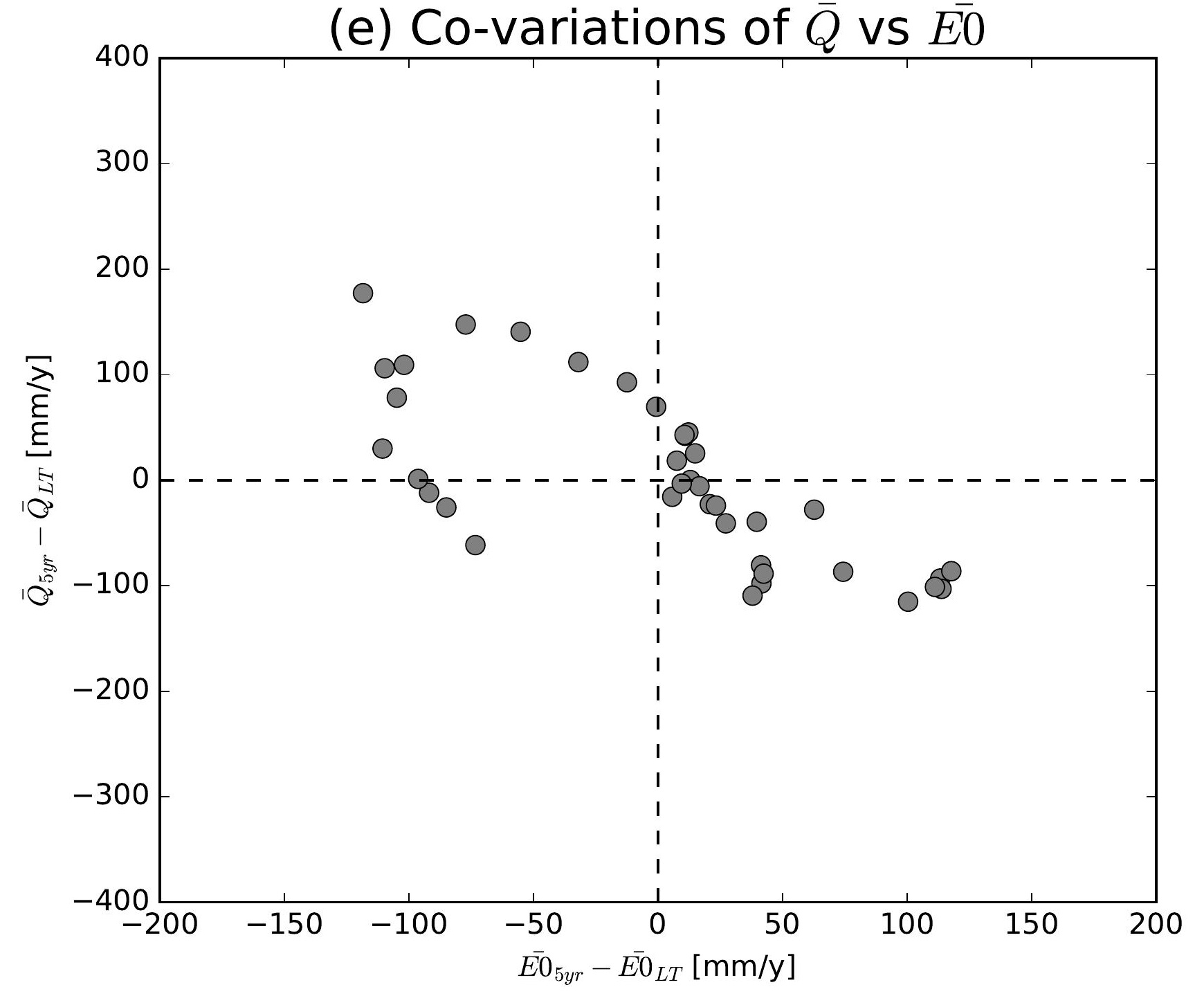

(h) Co-variations of $\bar{Q}$ vs $\overline{E 0}$

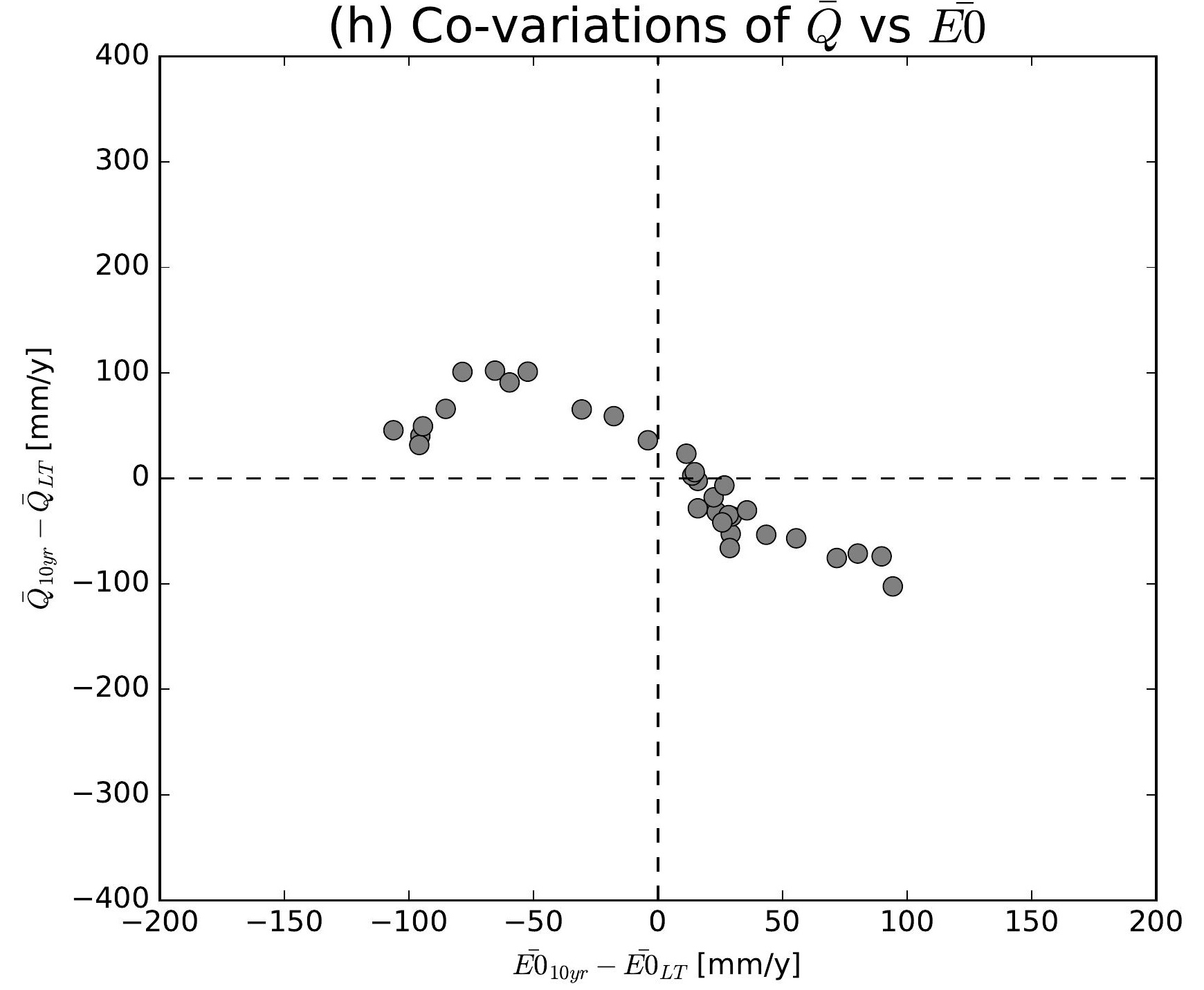

(k) Co-variations of $\bar{Q}$ vs $\overline{E 0}$

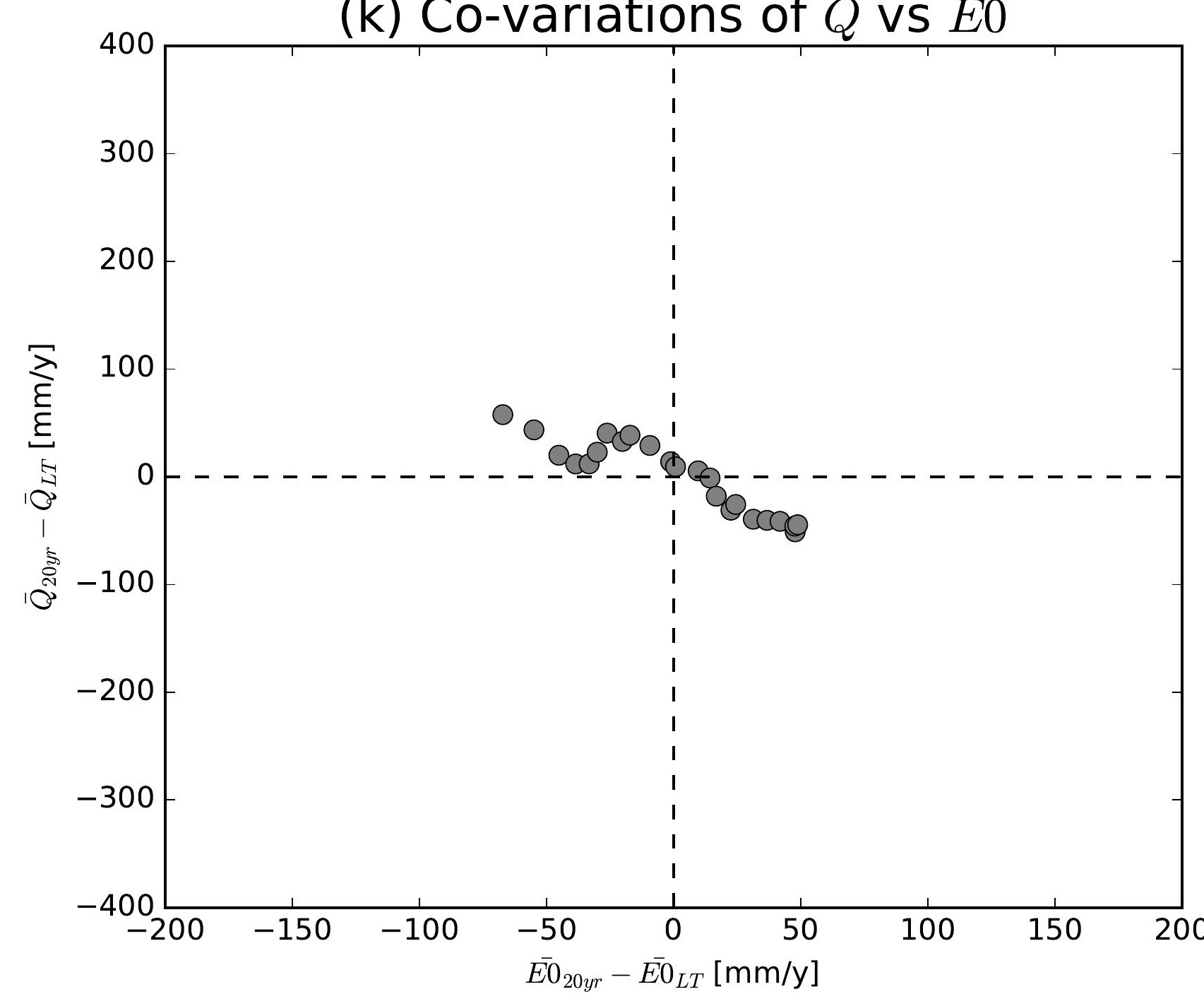

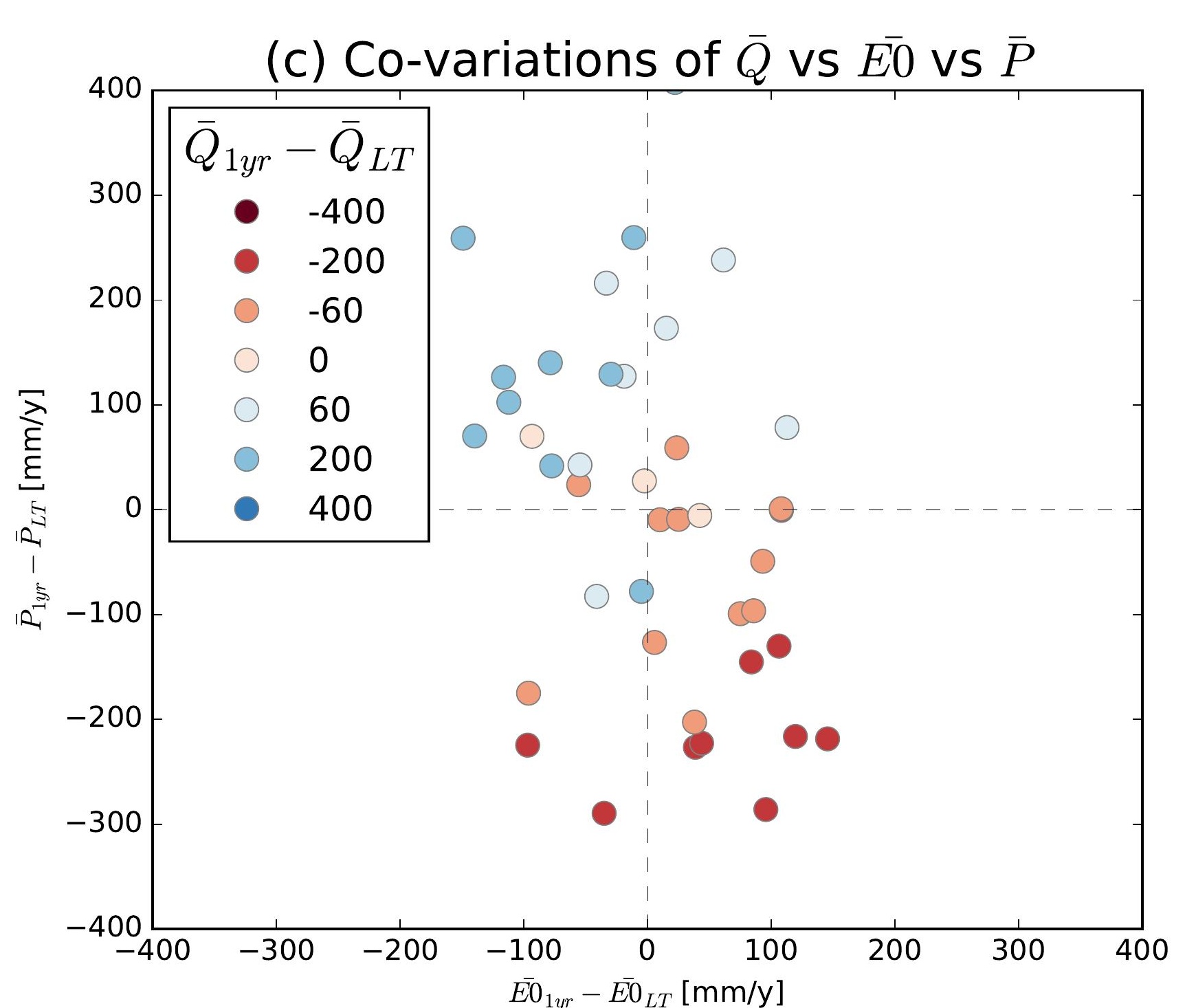
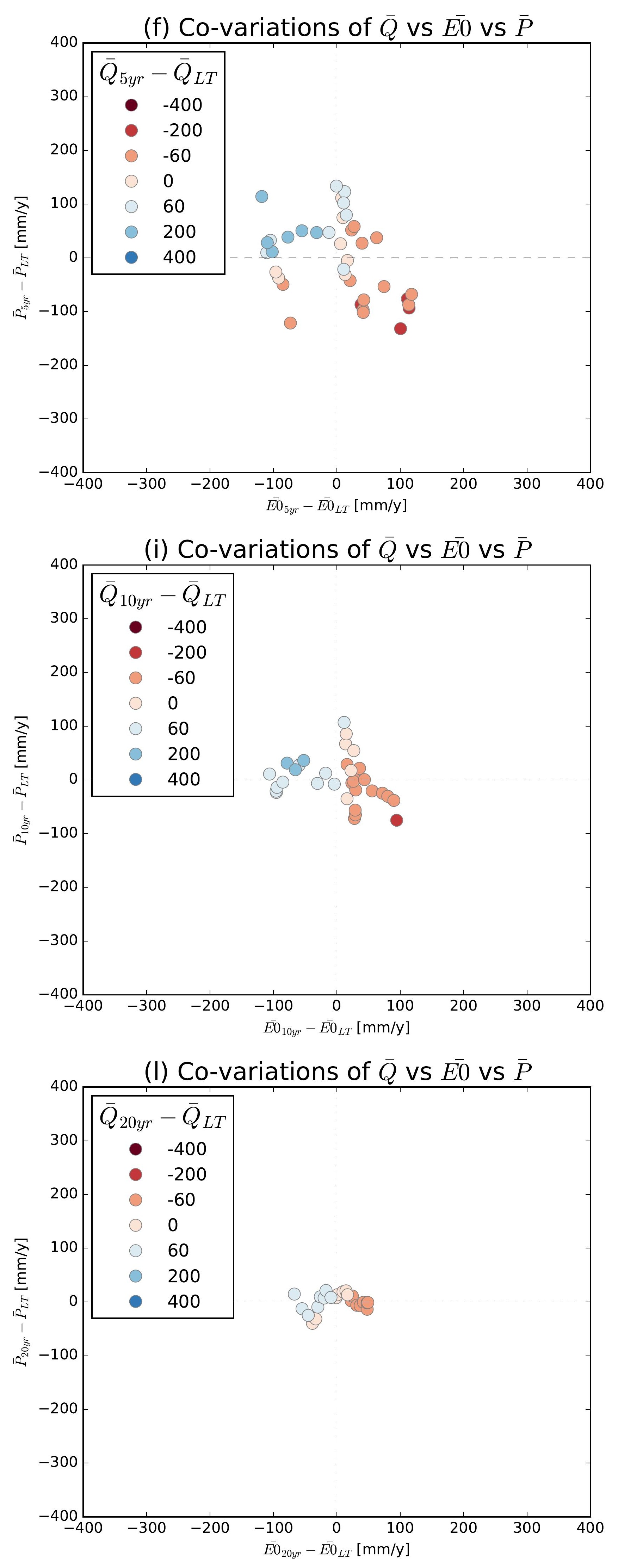

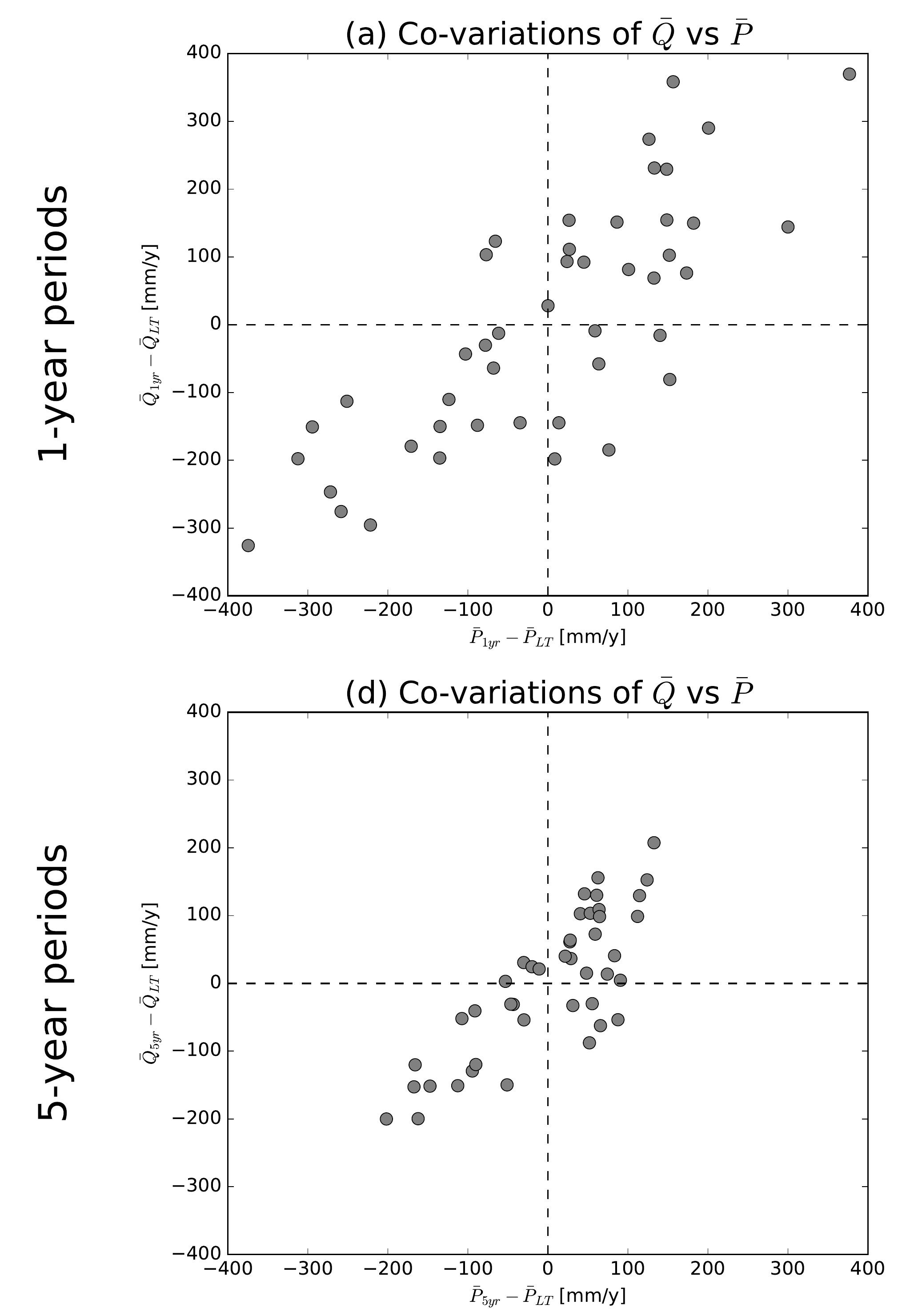

(g) Co-variations of $\bar{Q}$ vs $\bar{P}$
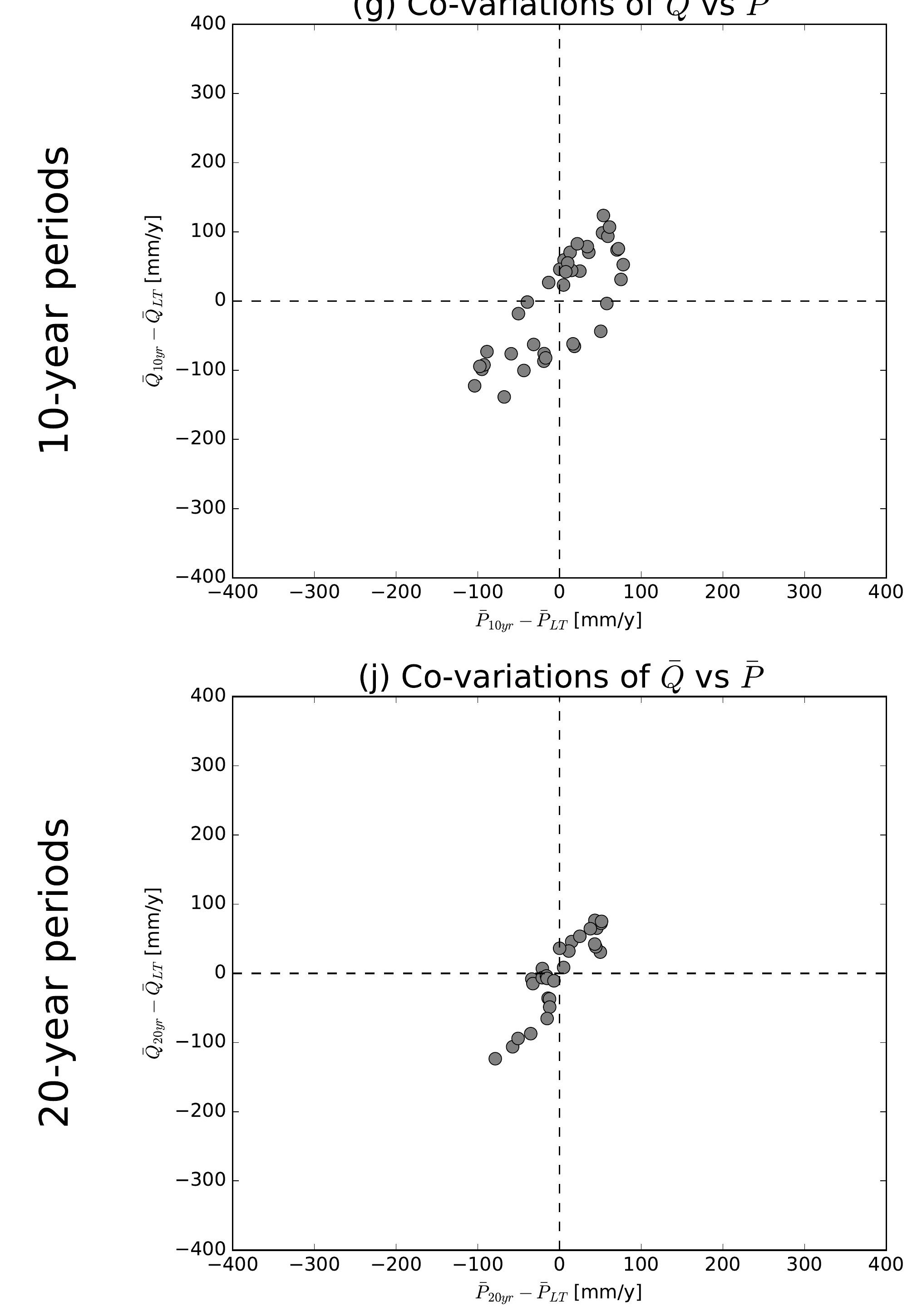

(b) Co-variations of $\bar{Q}$ vs $\overline{E 0}$

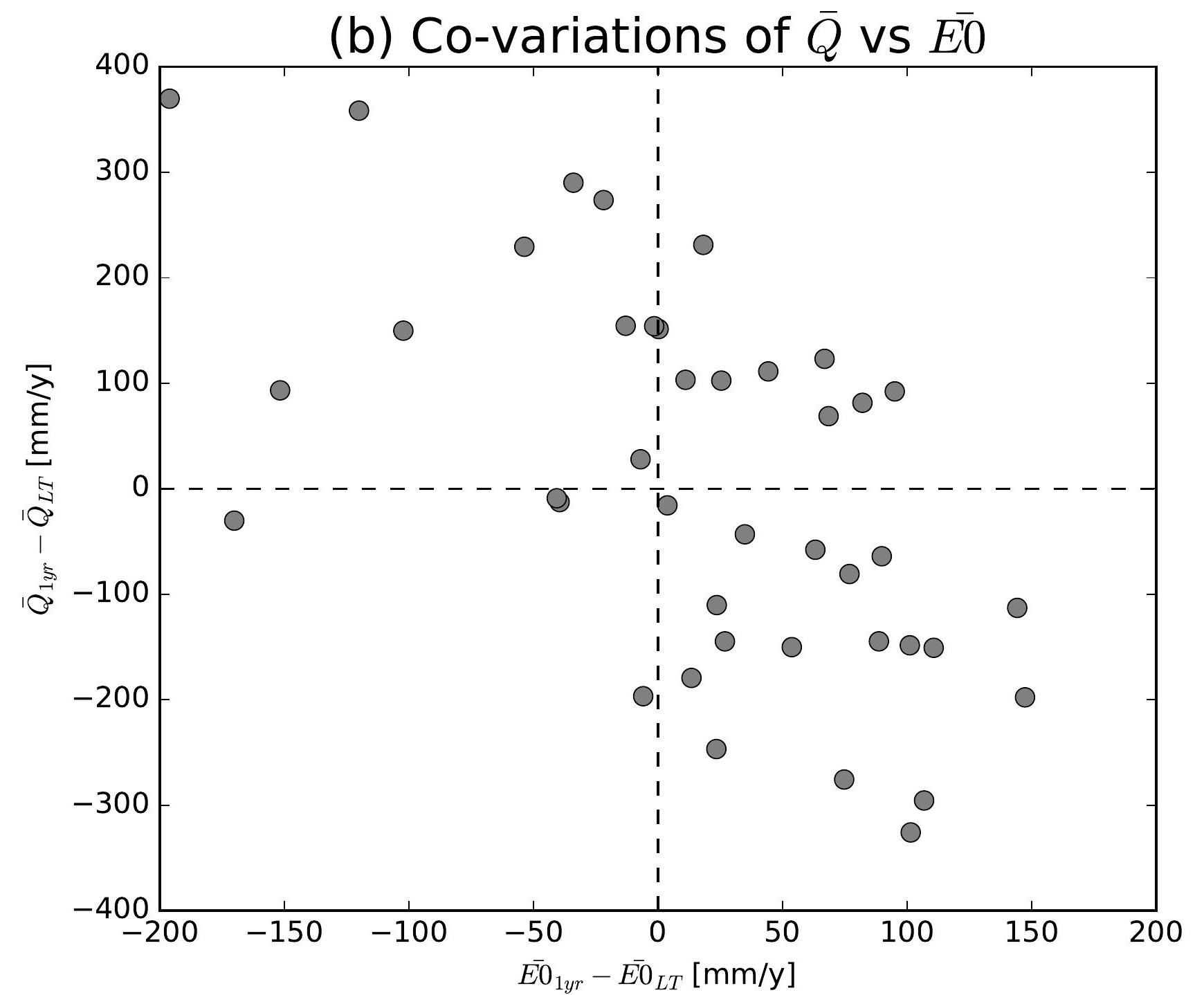

(e) Co-variations of $\bar{Q}$ vs $\overline{E 0}$

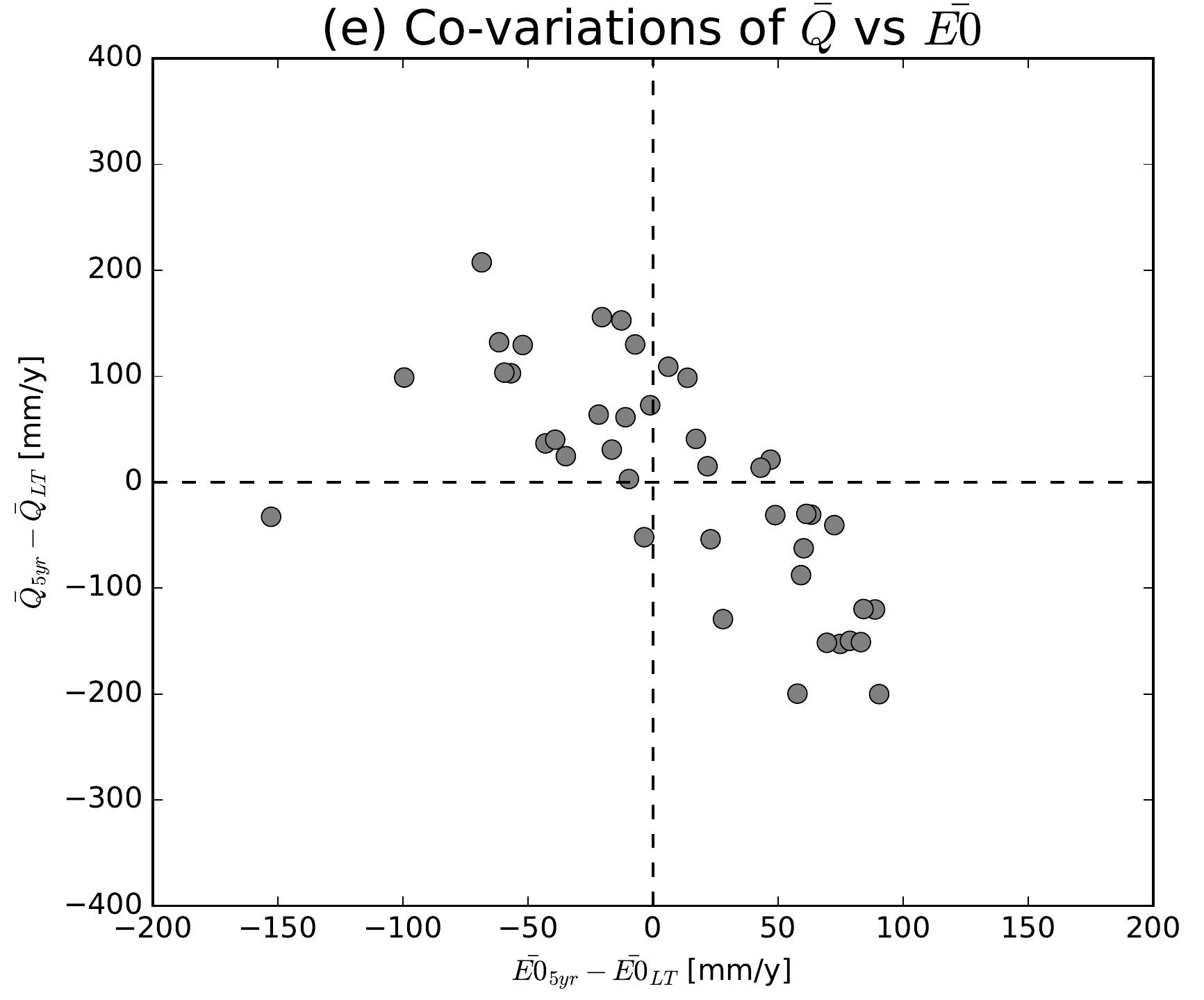

(h) Co-variations of $\bar{Q}$ vs $\overline{E 0}$

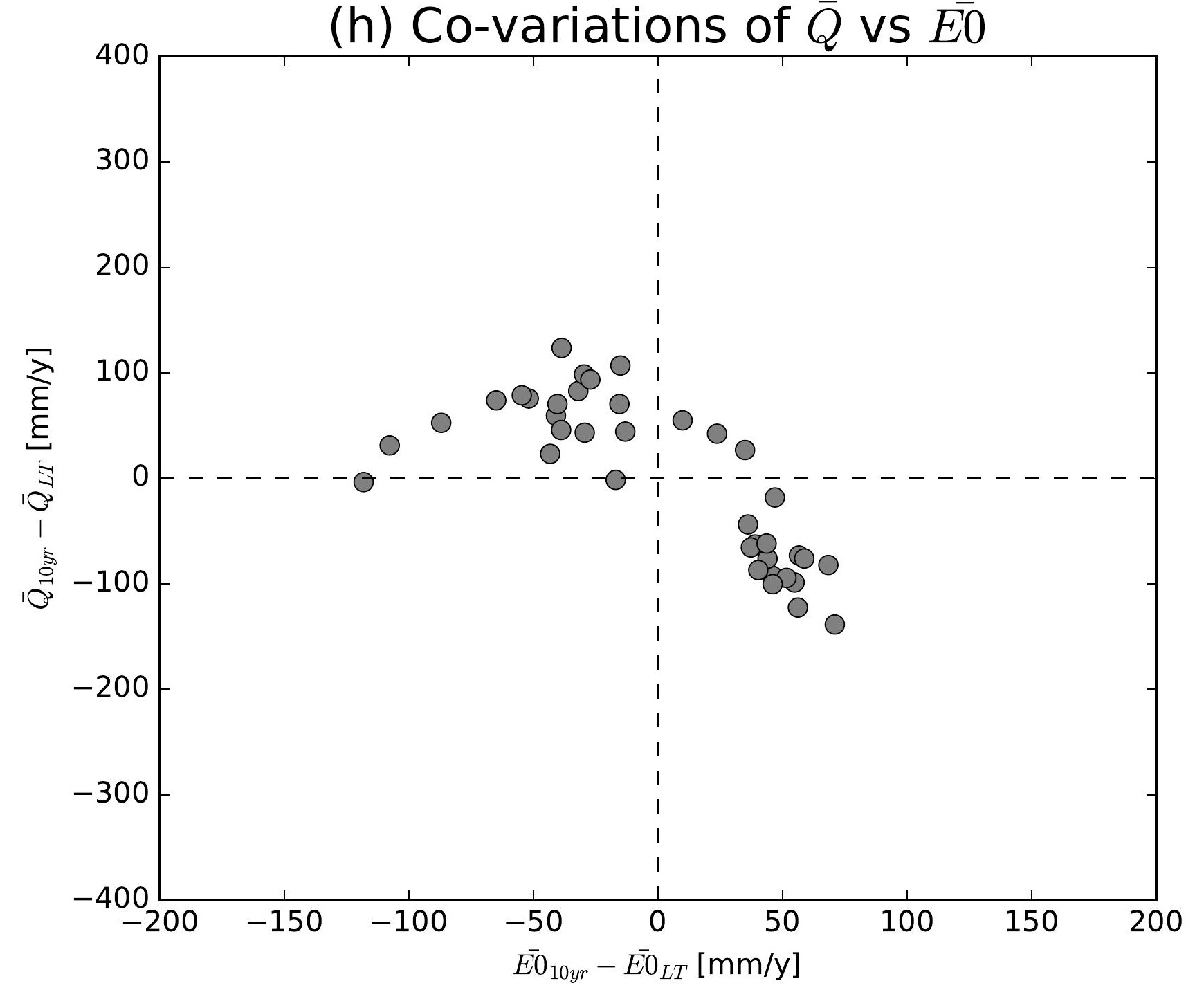

(k) Co-variations of $\bar{Q}$ vs $\overline{E 0}$

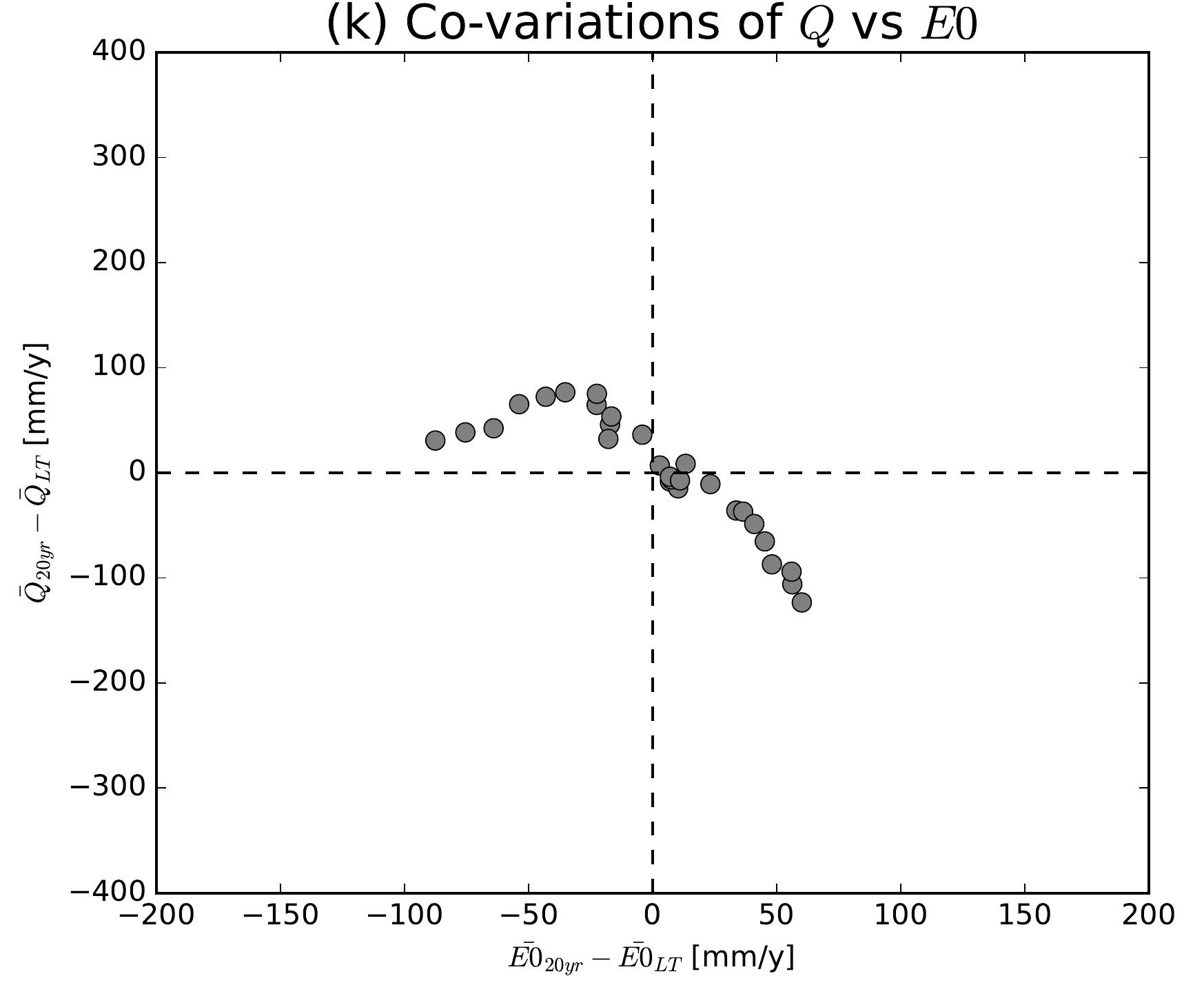

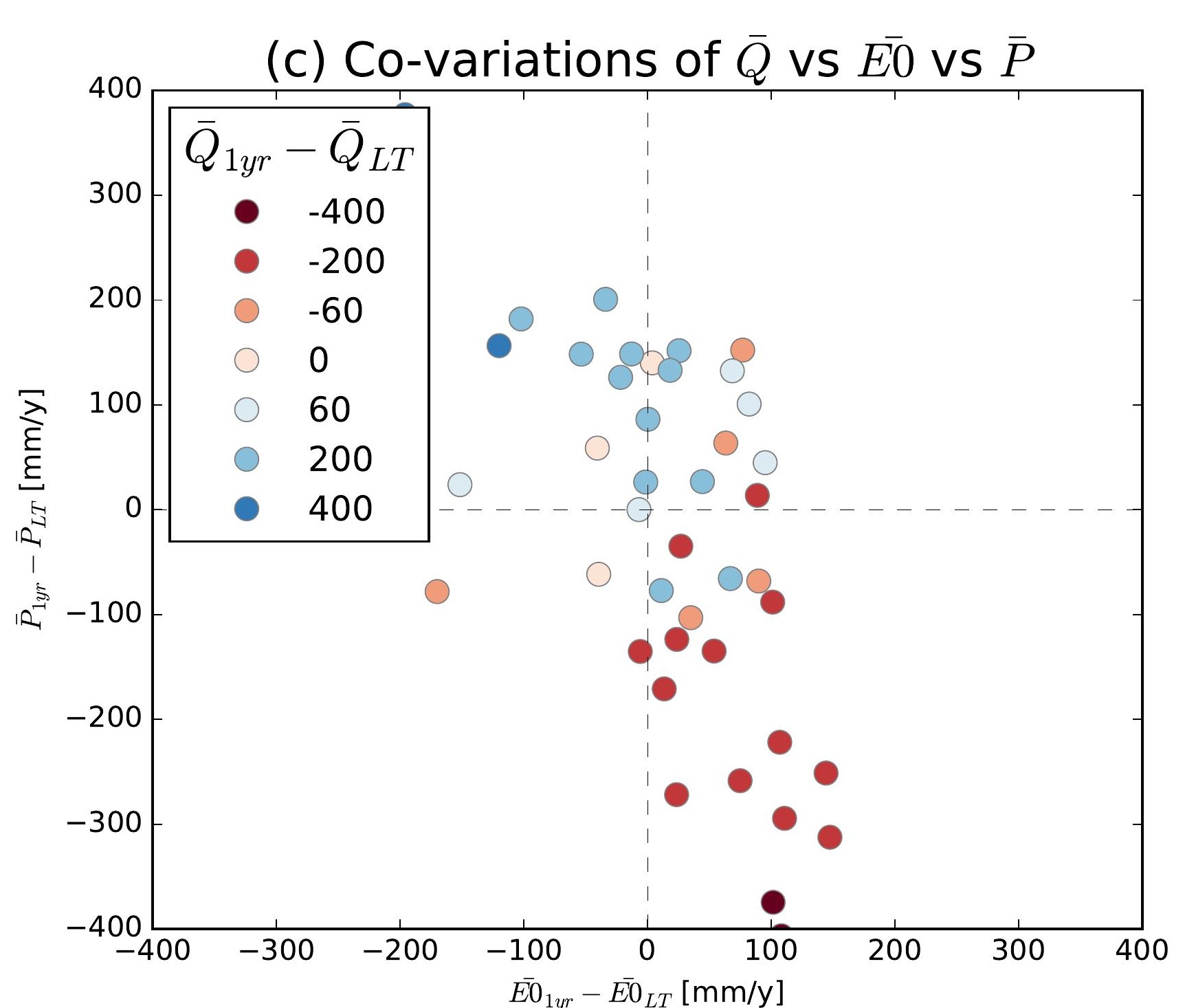
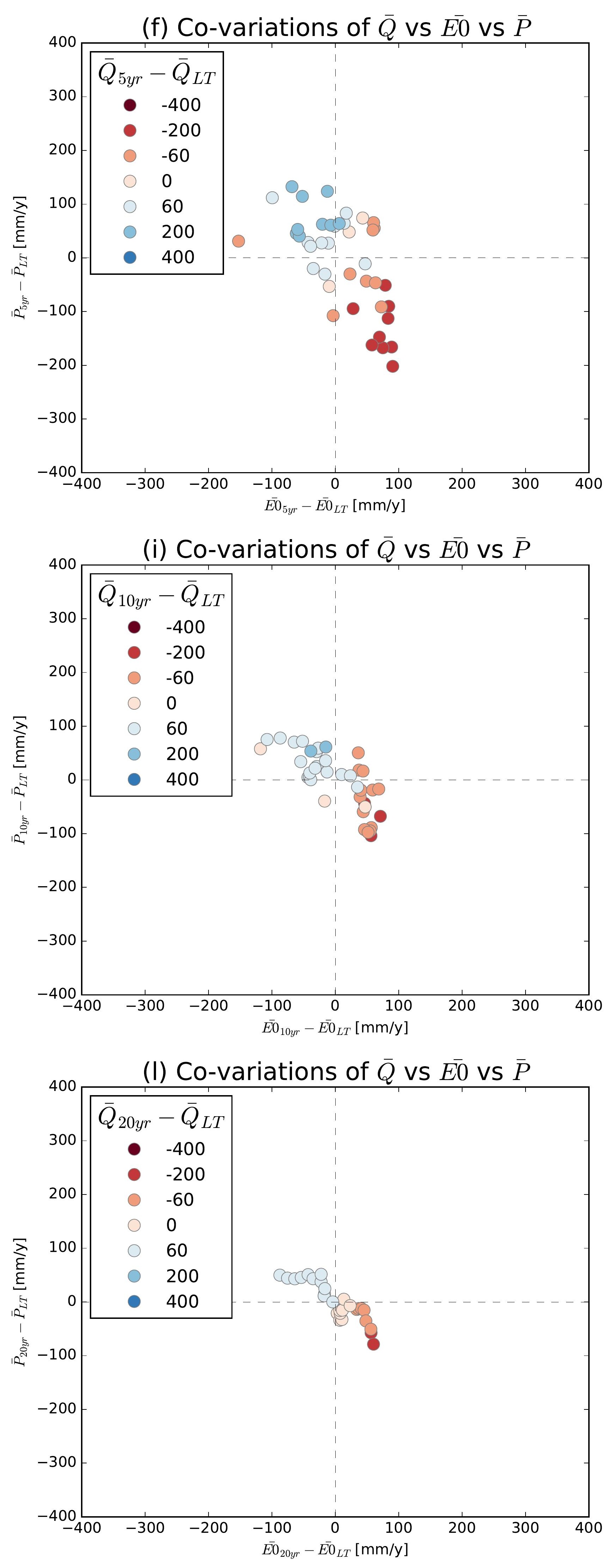

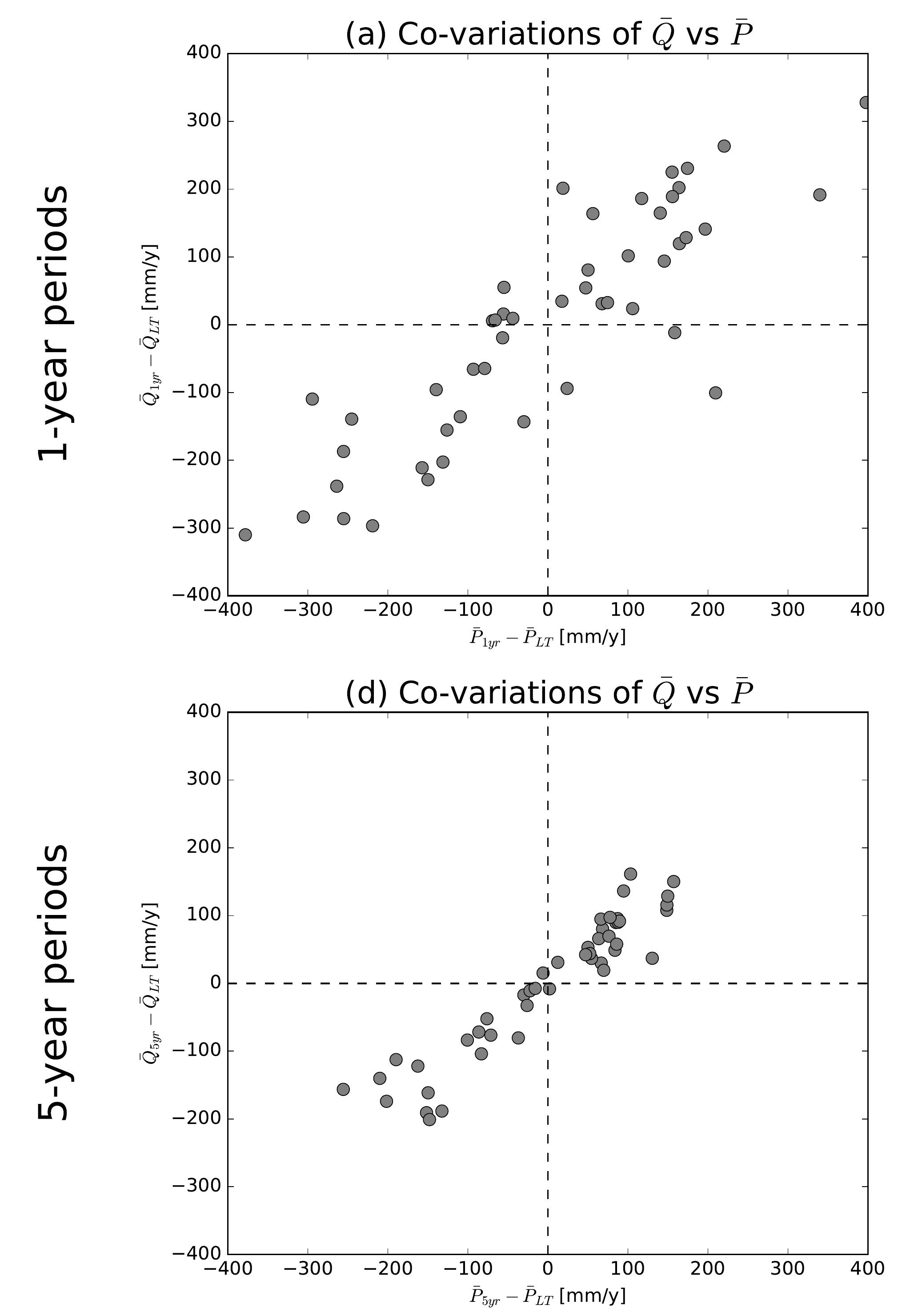

(g) Co-variations of $\bar{Q}$ vs $\bar{P}$
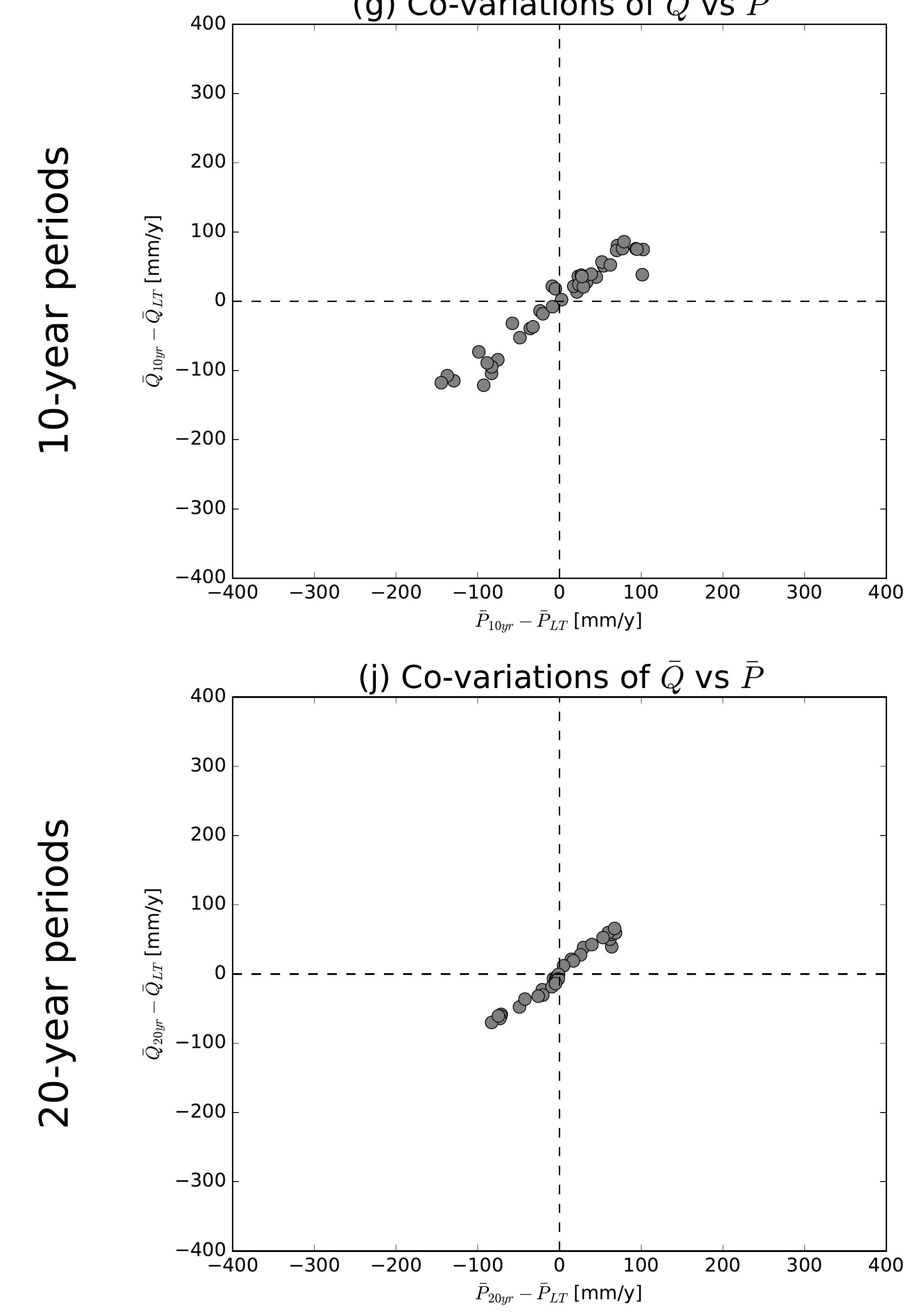

(b) Co-variations of $\bar{Q}$ vs $\overline{E 0}$

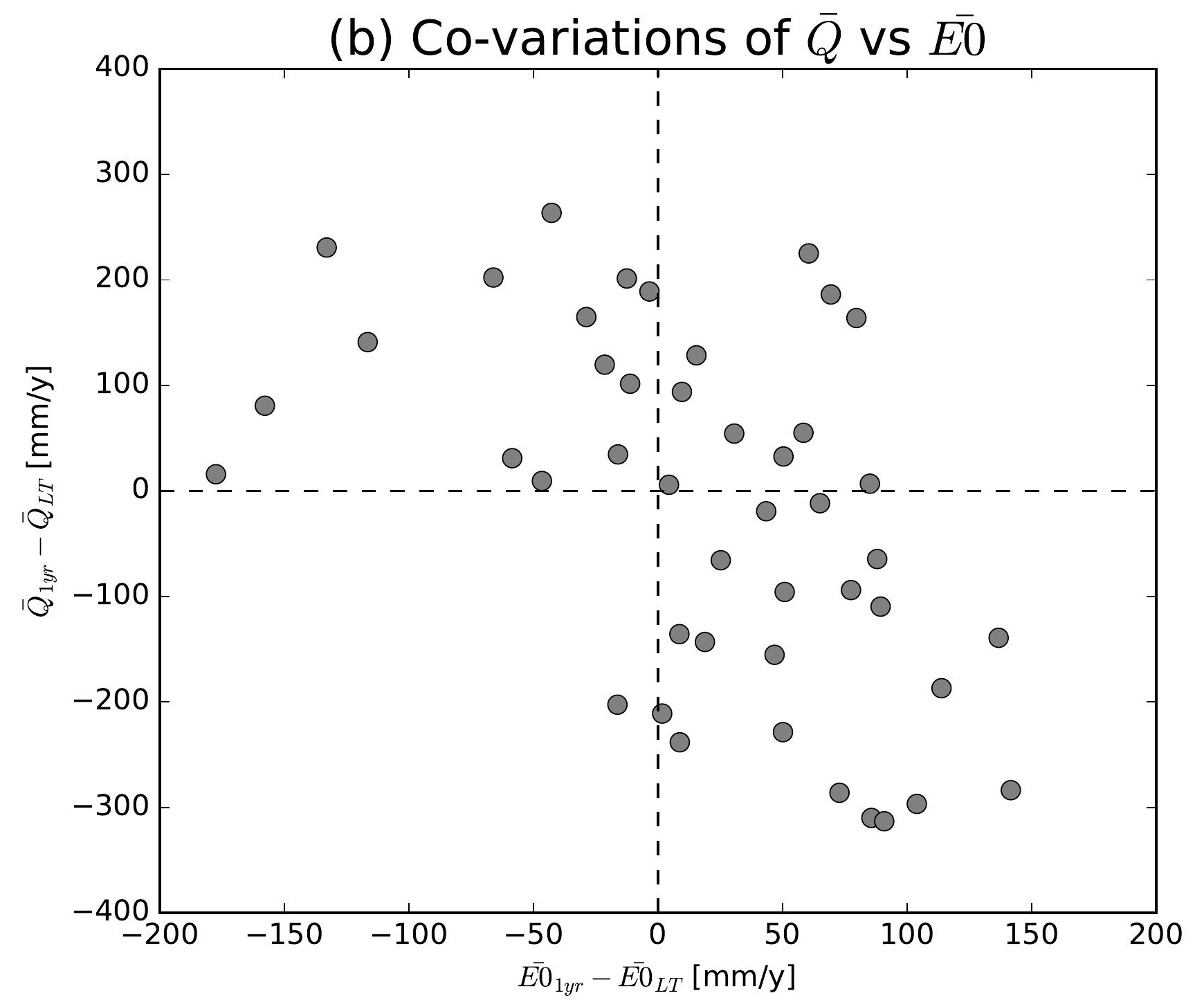

(e) Co-variations of $\bar{Q}$ vs $\overline{E 0}$

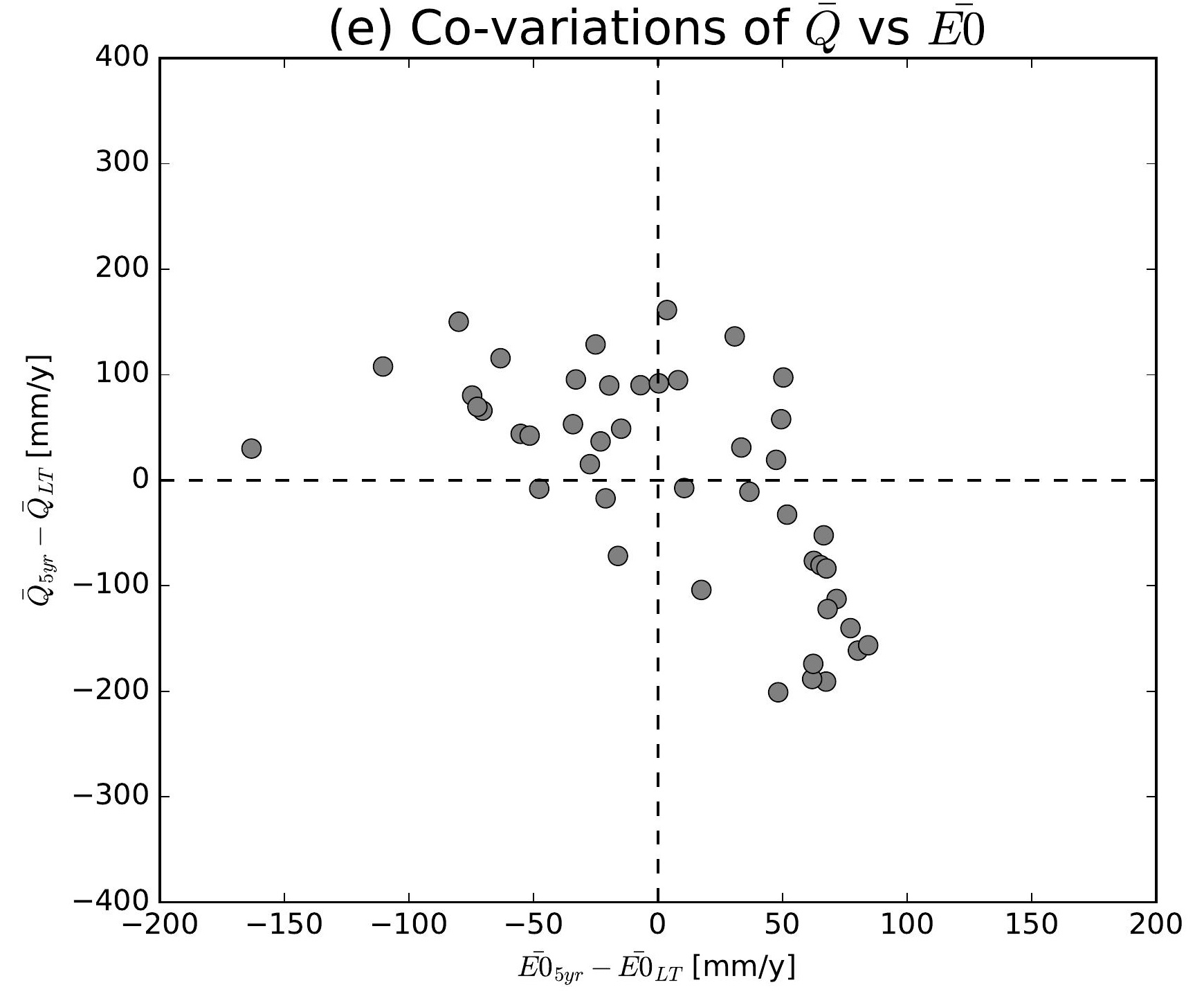

(h) Co-variations of $\bar{Q}$ vs $\overline{E 0}$

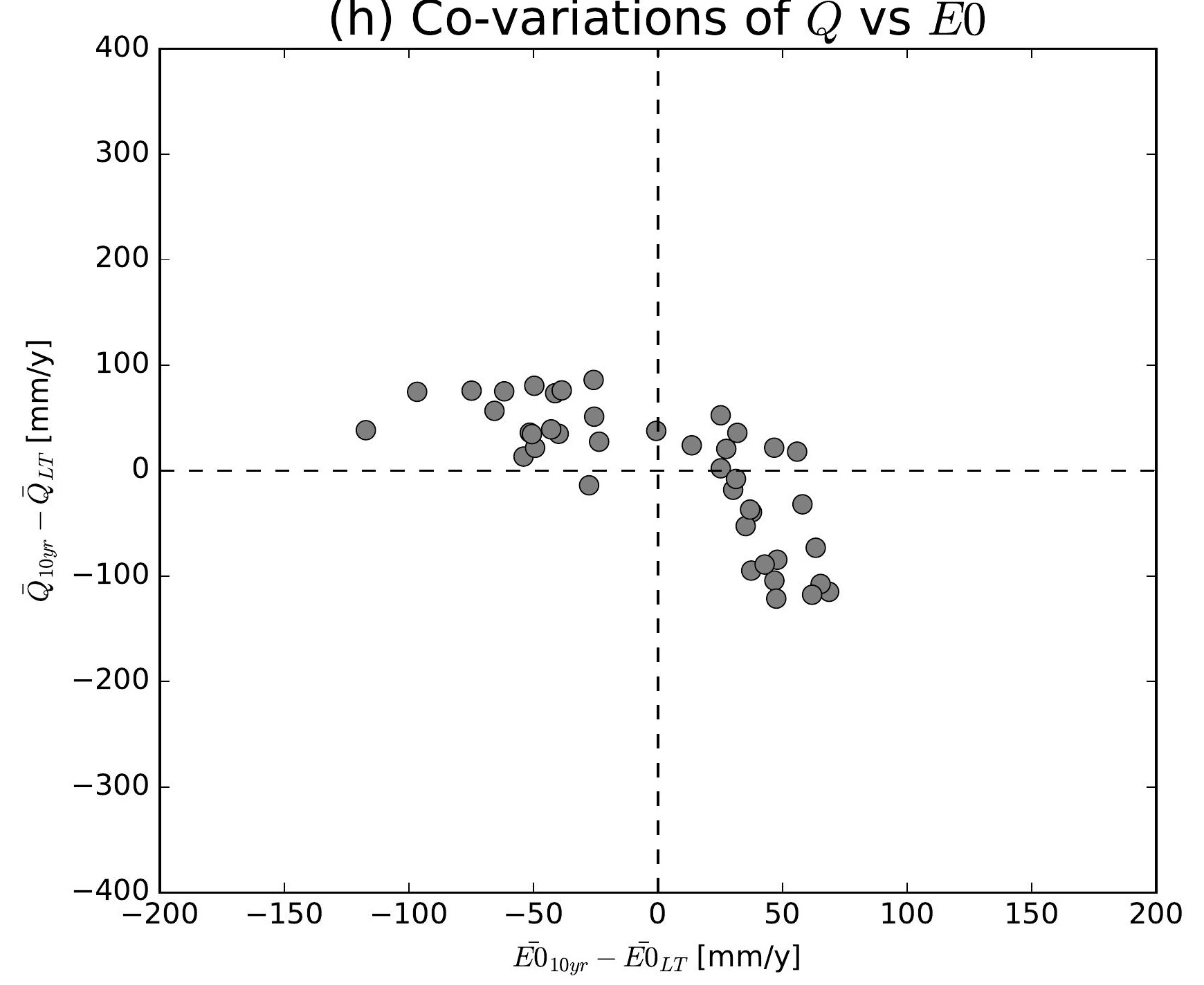

(k) Co-variations of $\bar{Q}$ vs $\overline{E 0}$

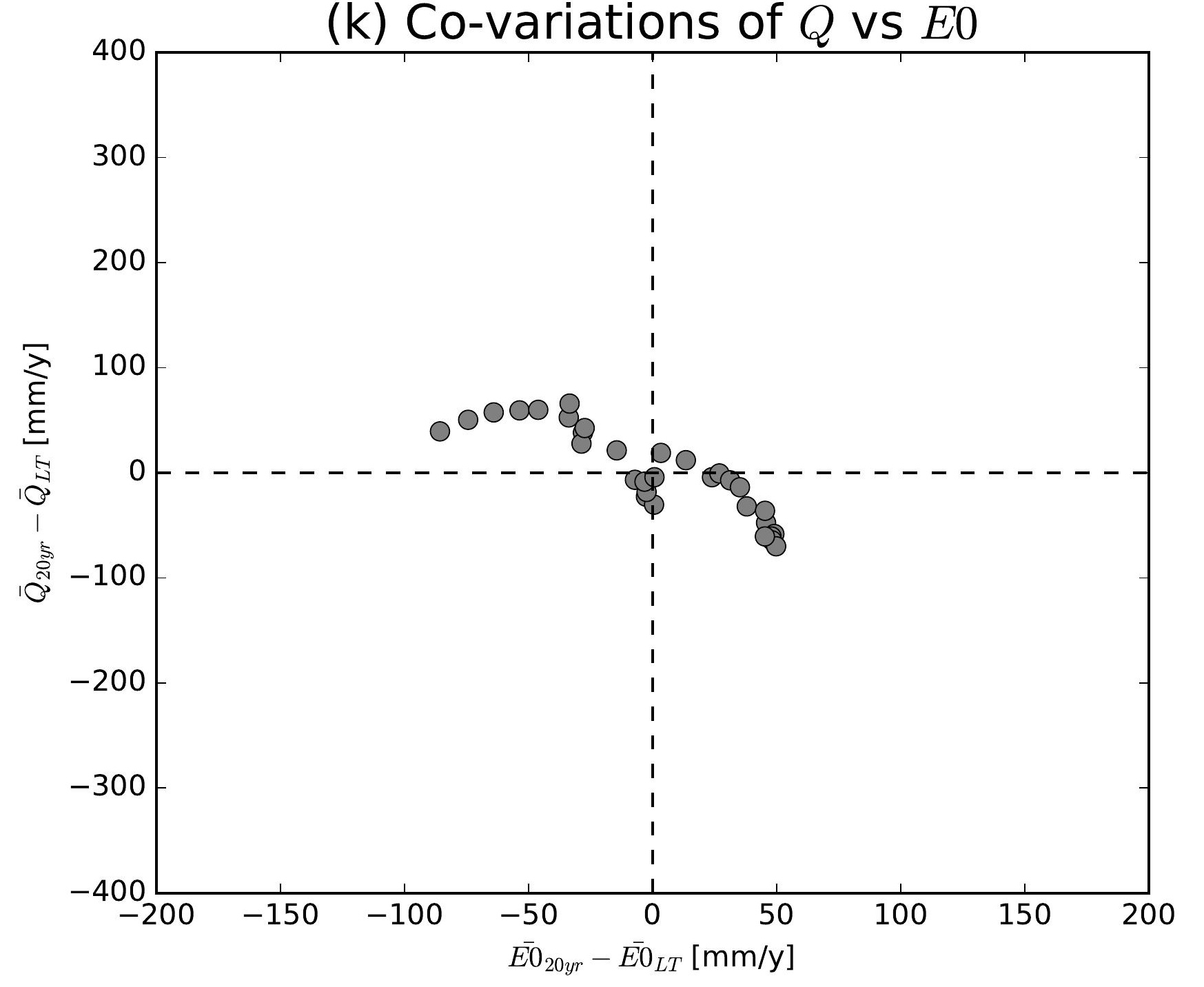

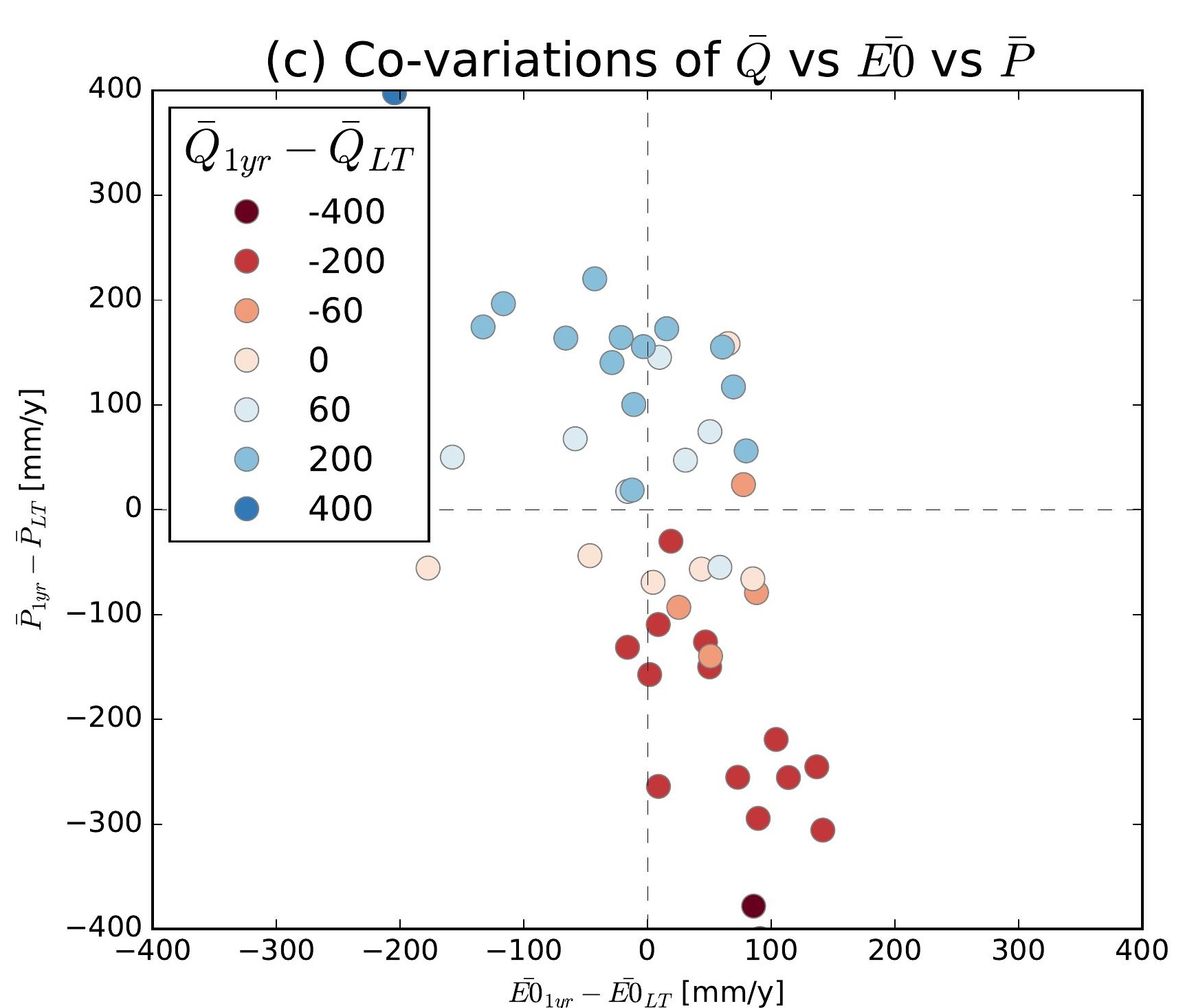
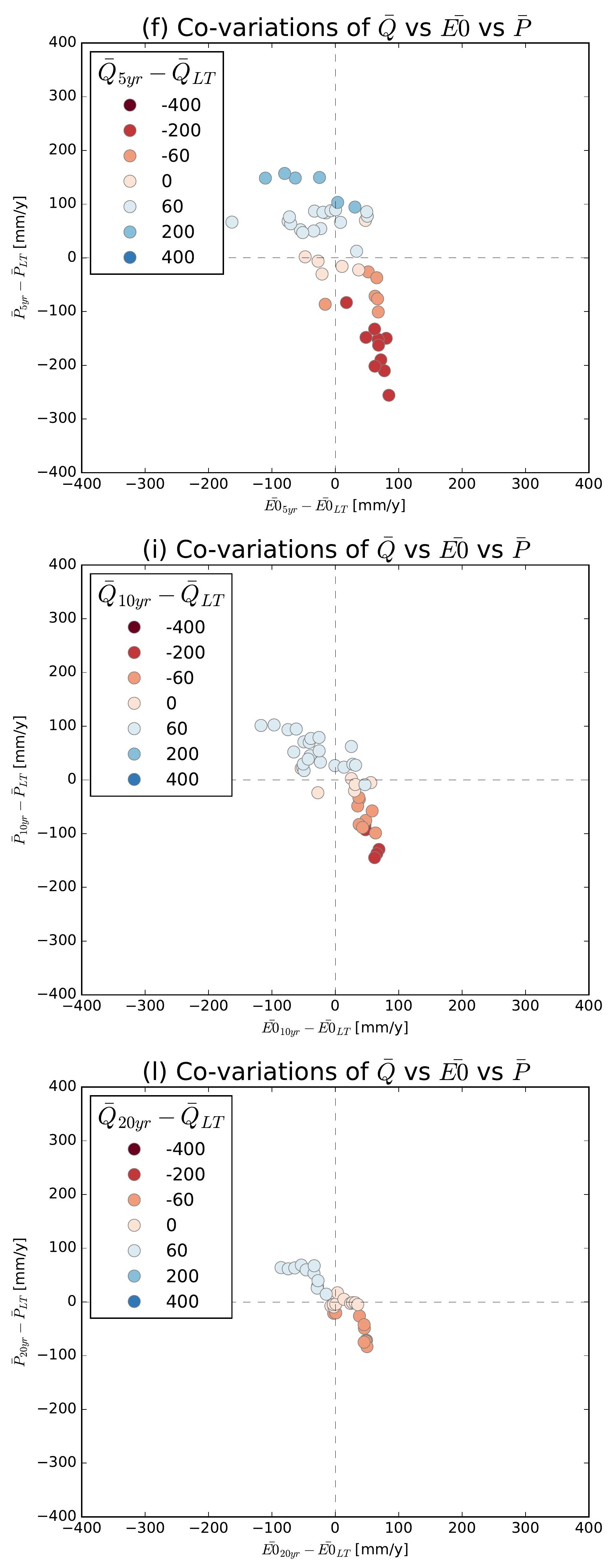

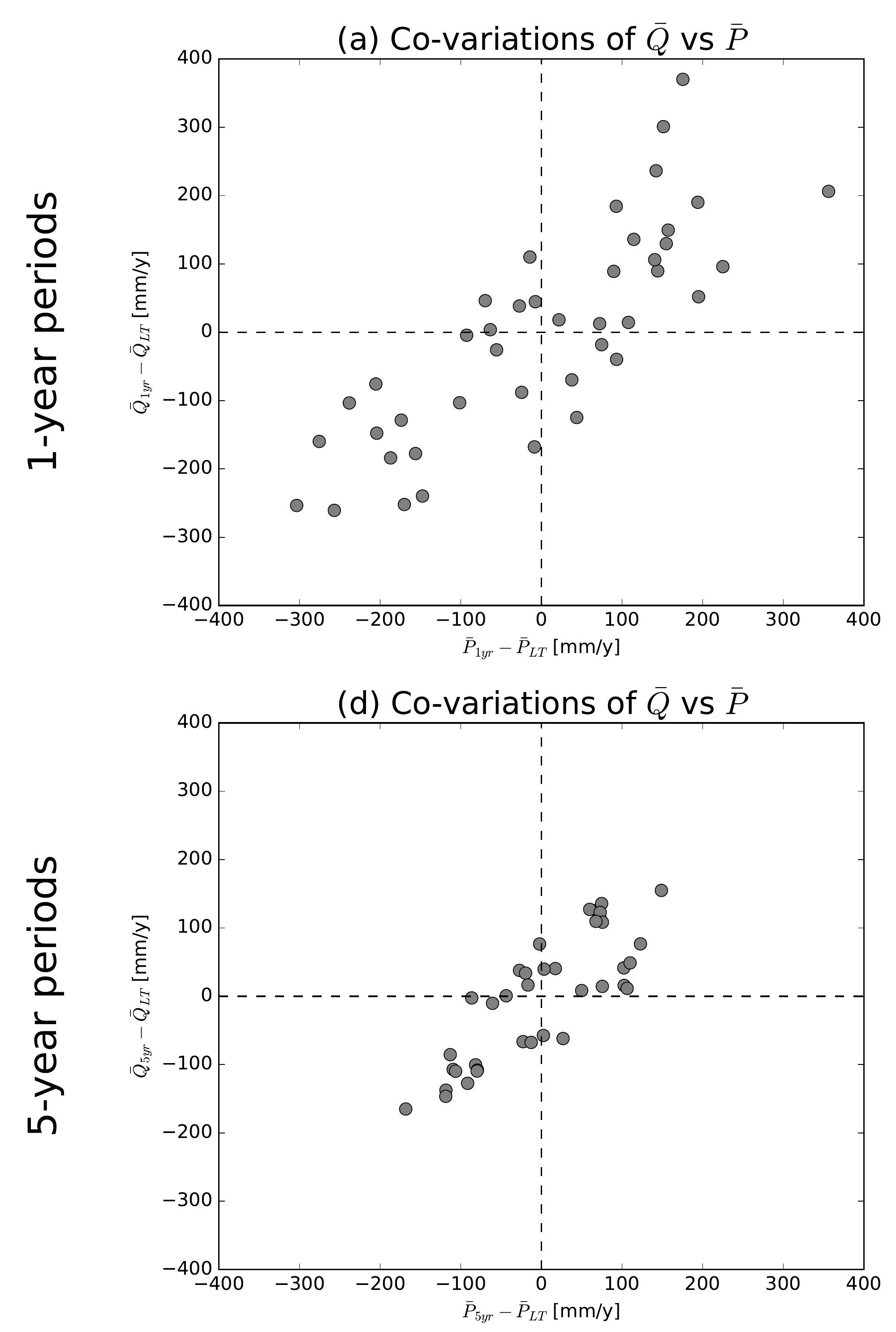

(g) Co-variations of $\bar{Q}$ vs $\bar{P}$

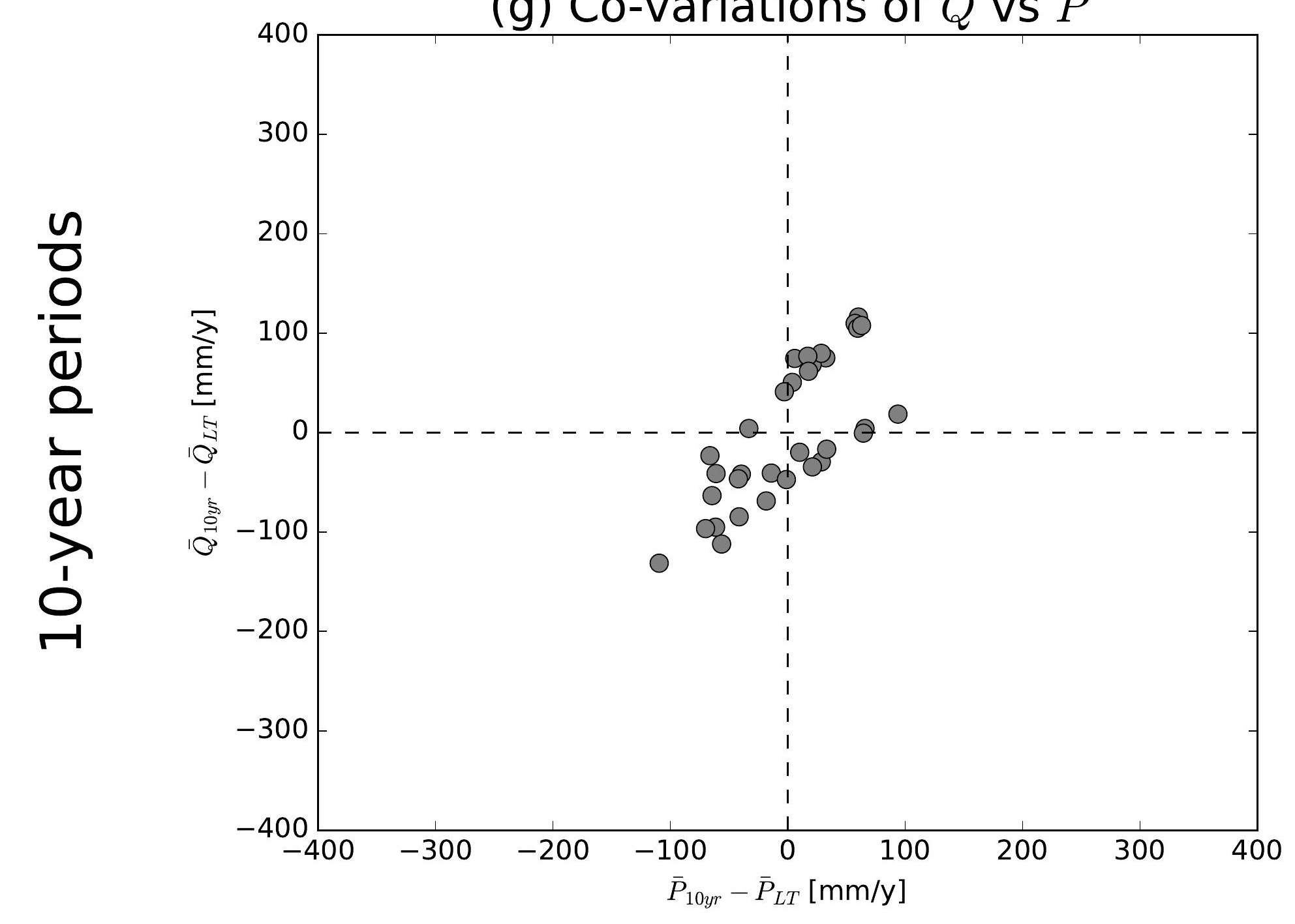

(j) Co-variations of $\bar{Q}$ vs $\bar{P}$

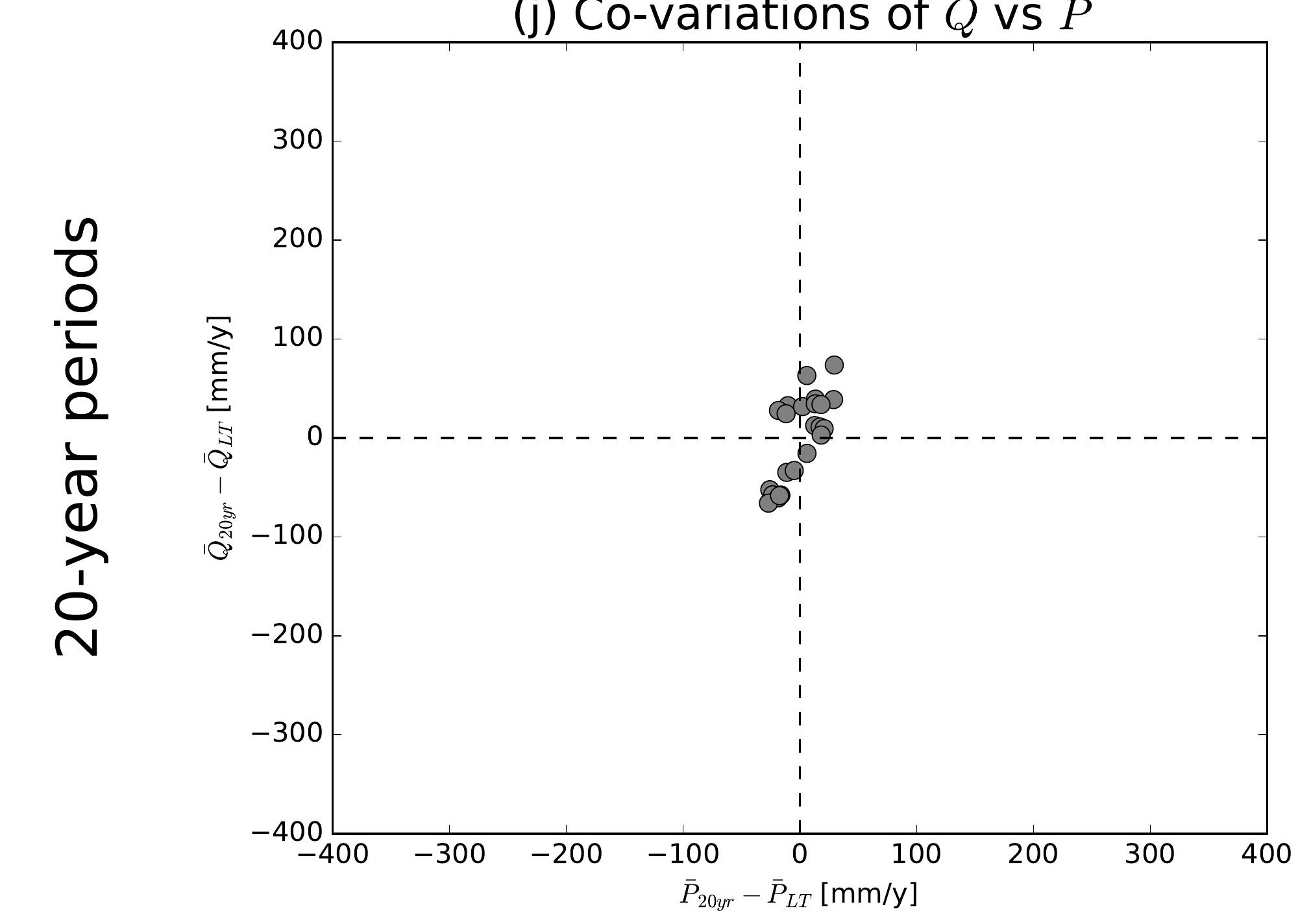

(b) Co-variations of $\bar{Q}$ vs $\overline{E 0}$

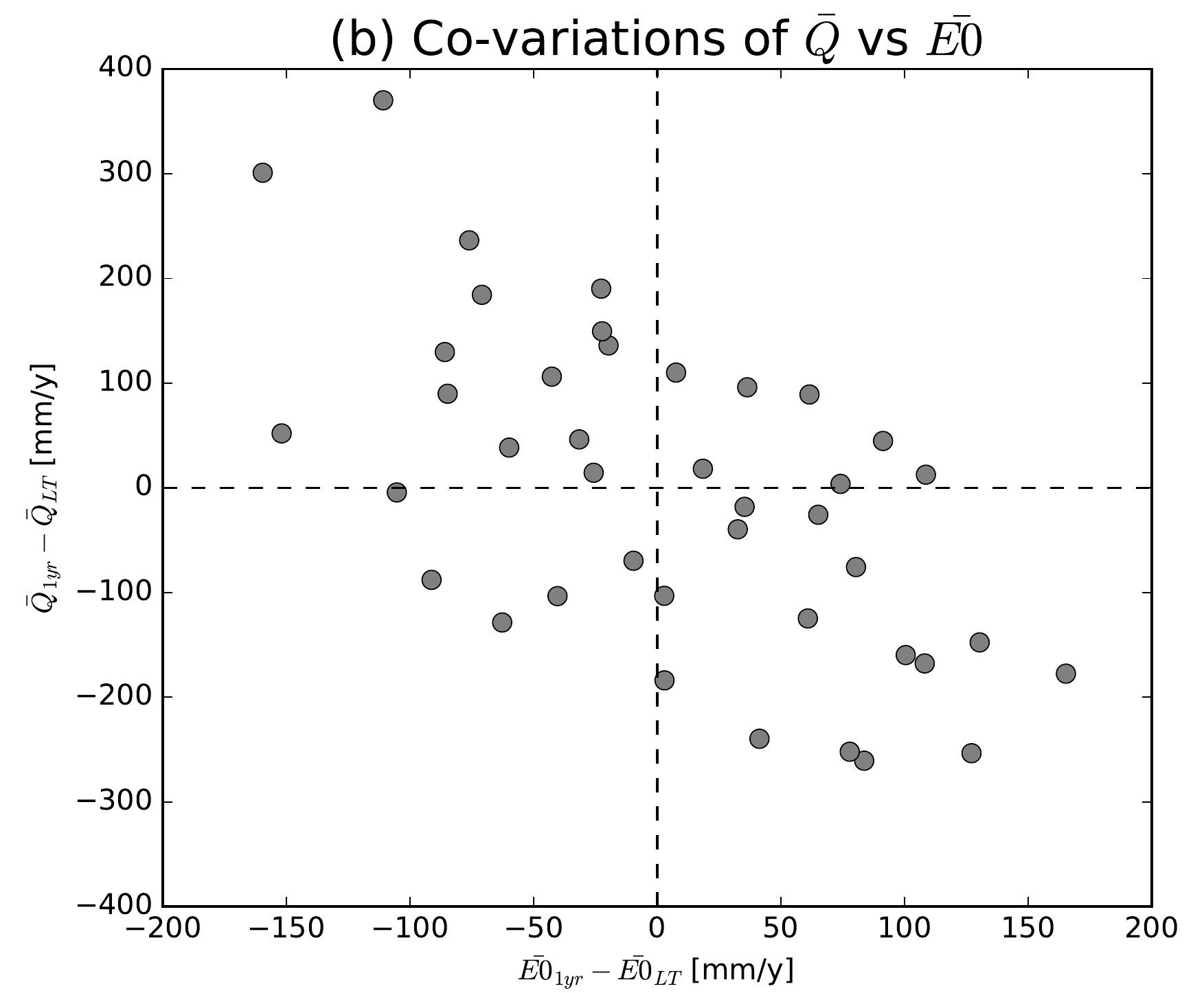

(e) Co-variations of $\bar{Q}$ vs $\overline{E 0}$

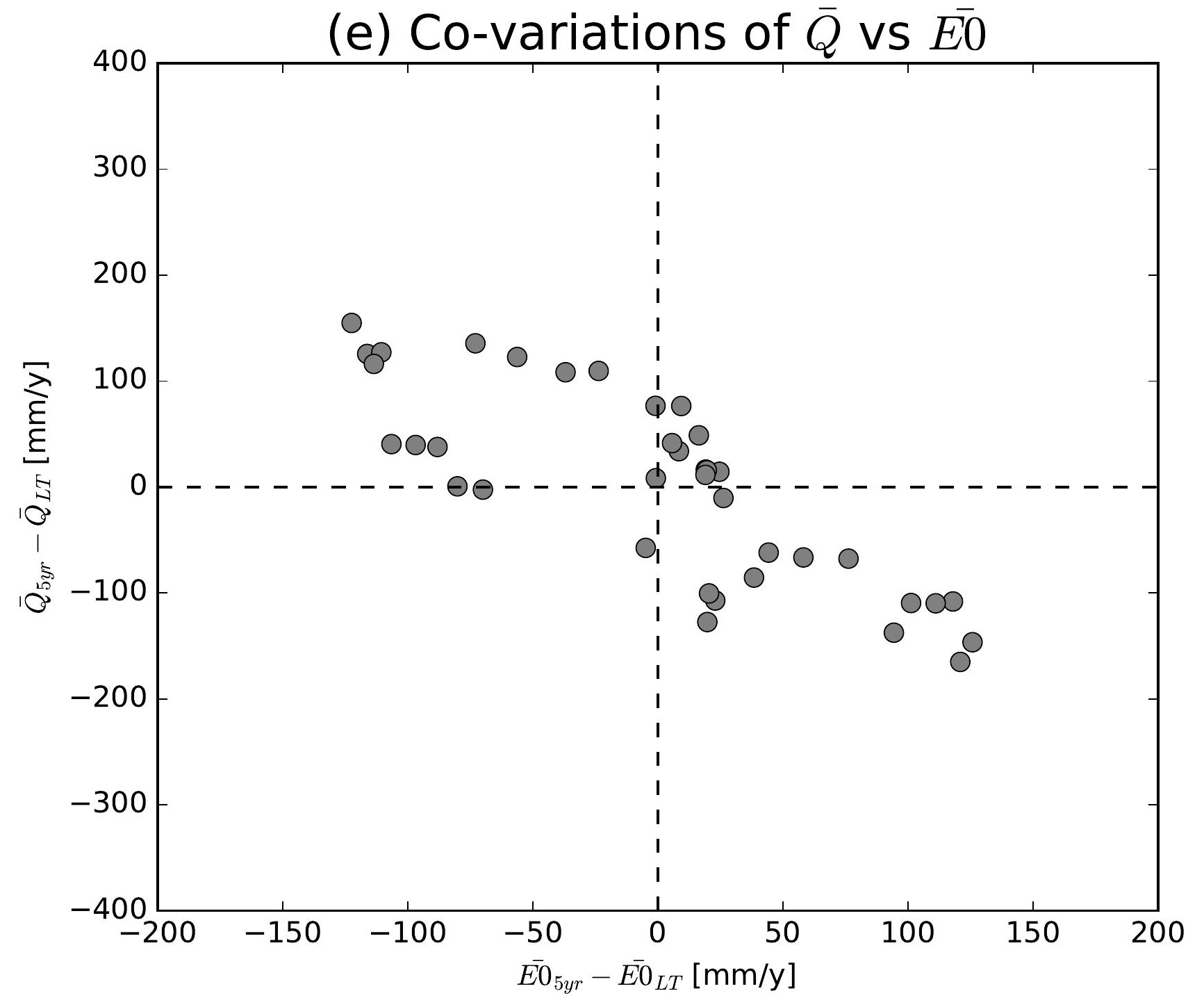

(h) Co-variations of $\bar{Q}$ vs $\overline{E 0}$

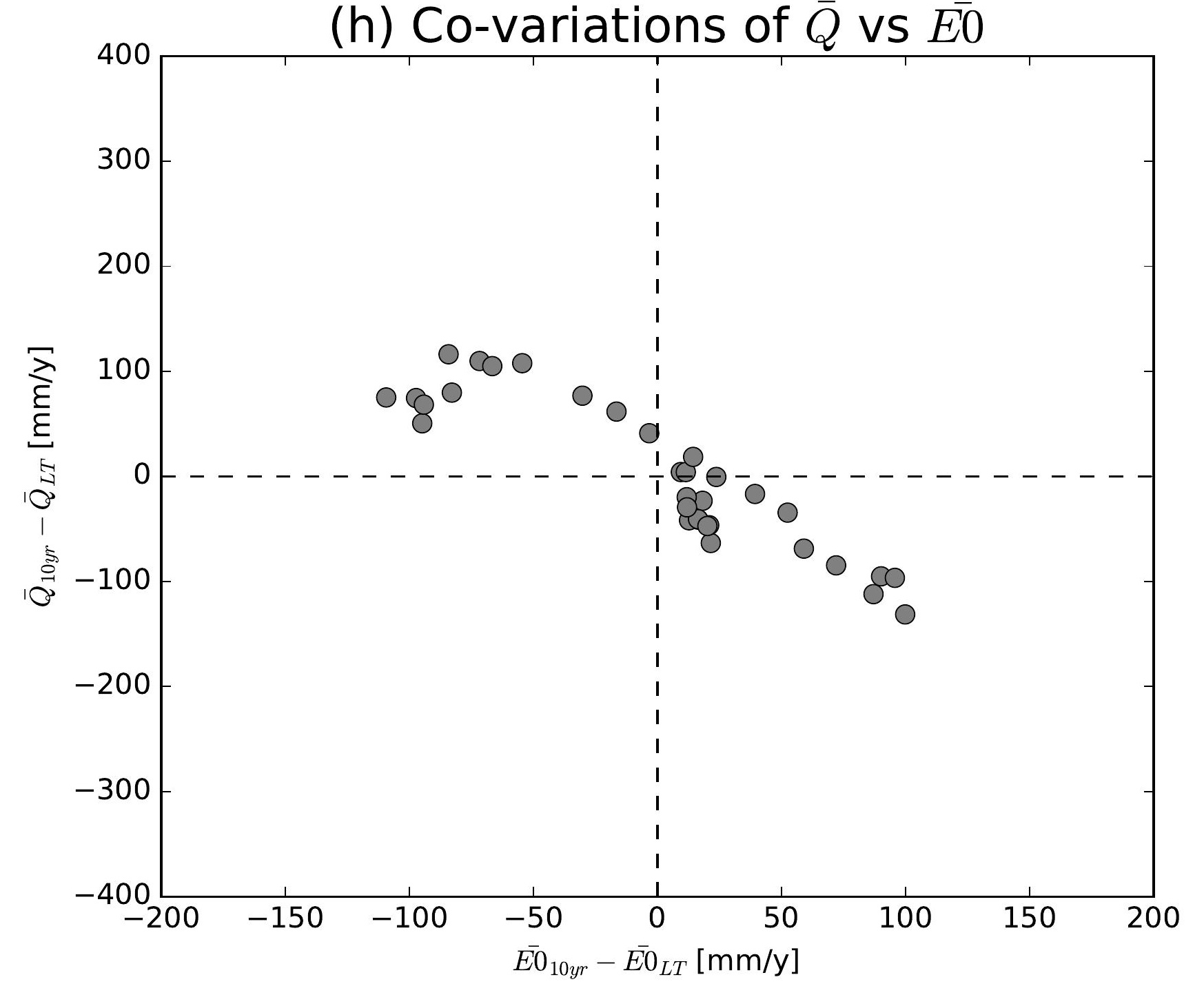

(k) Co-variations of $\bar{Q}$ vs $\overline{E 0}$

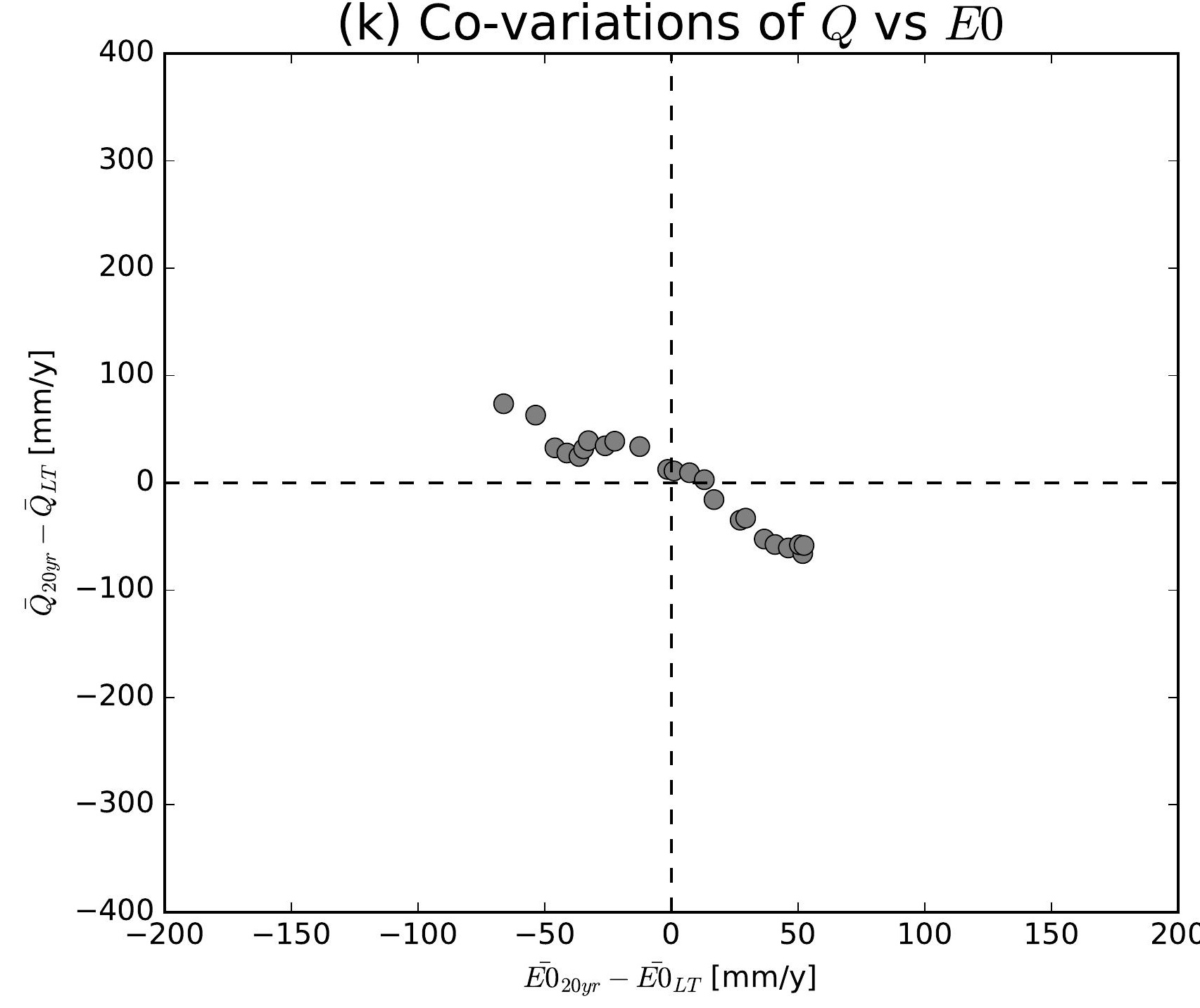

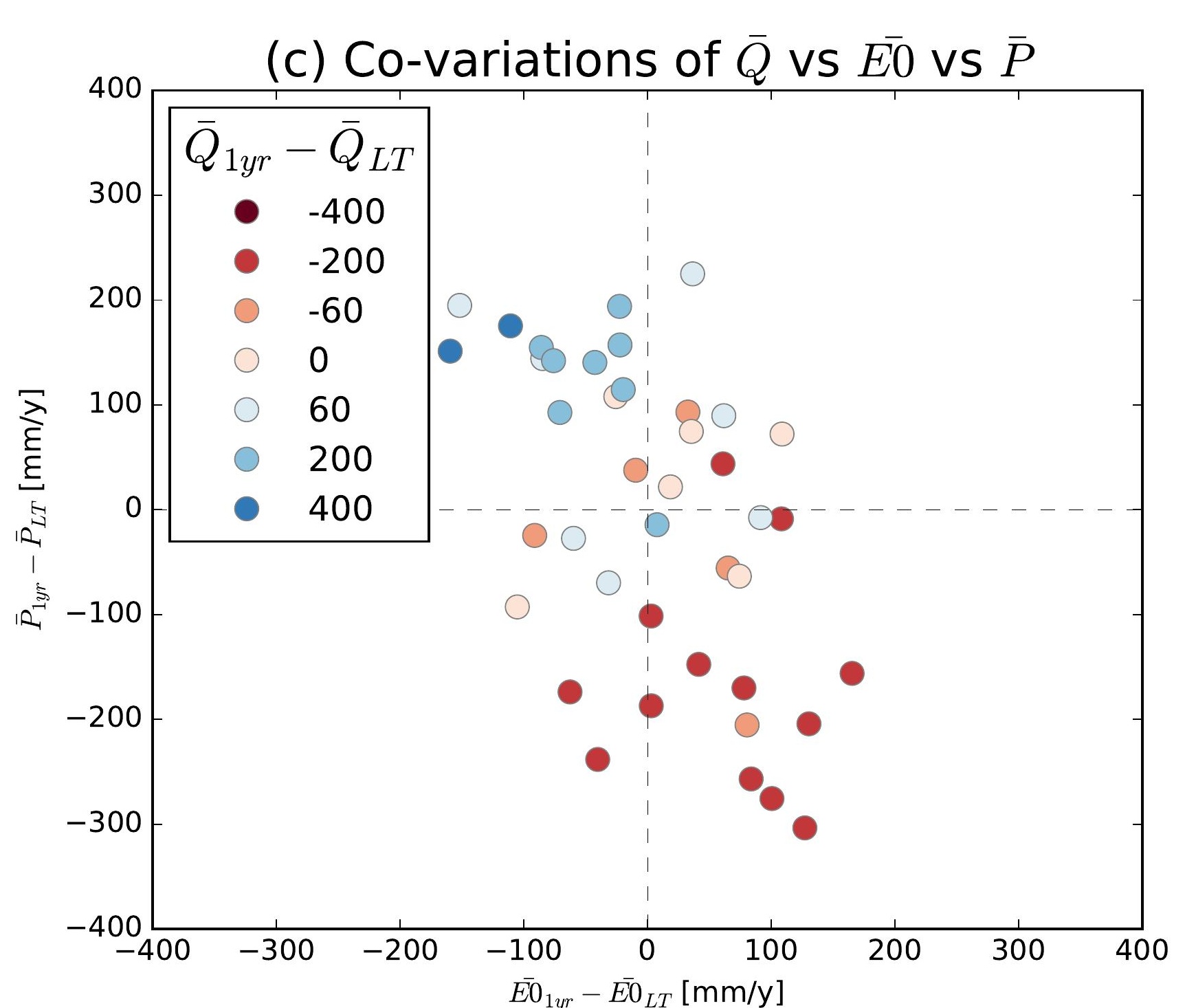
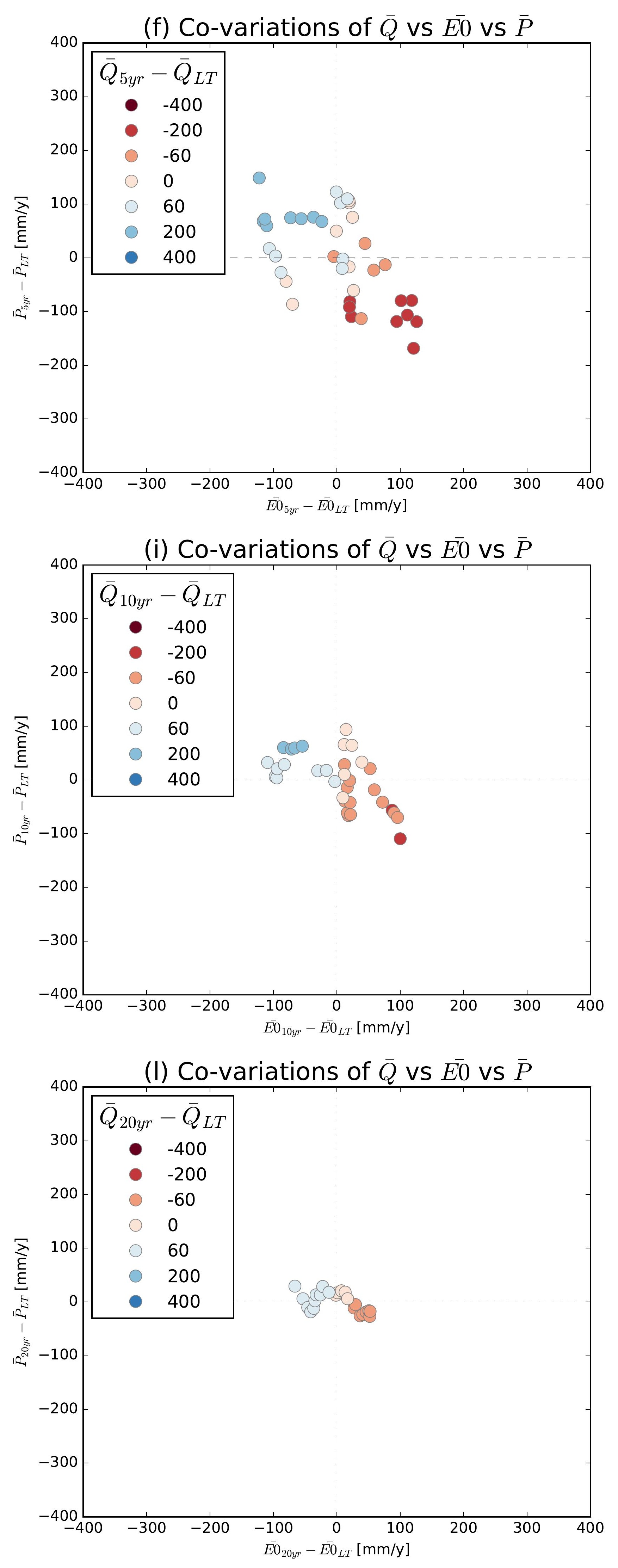

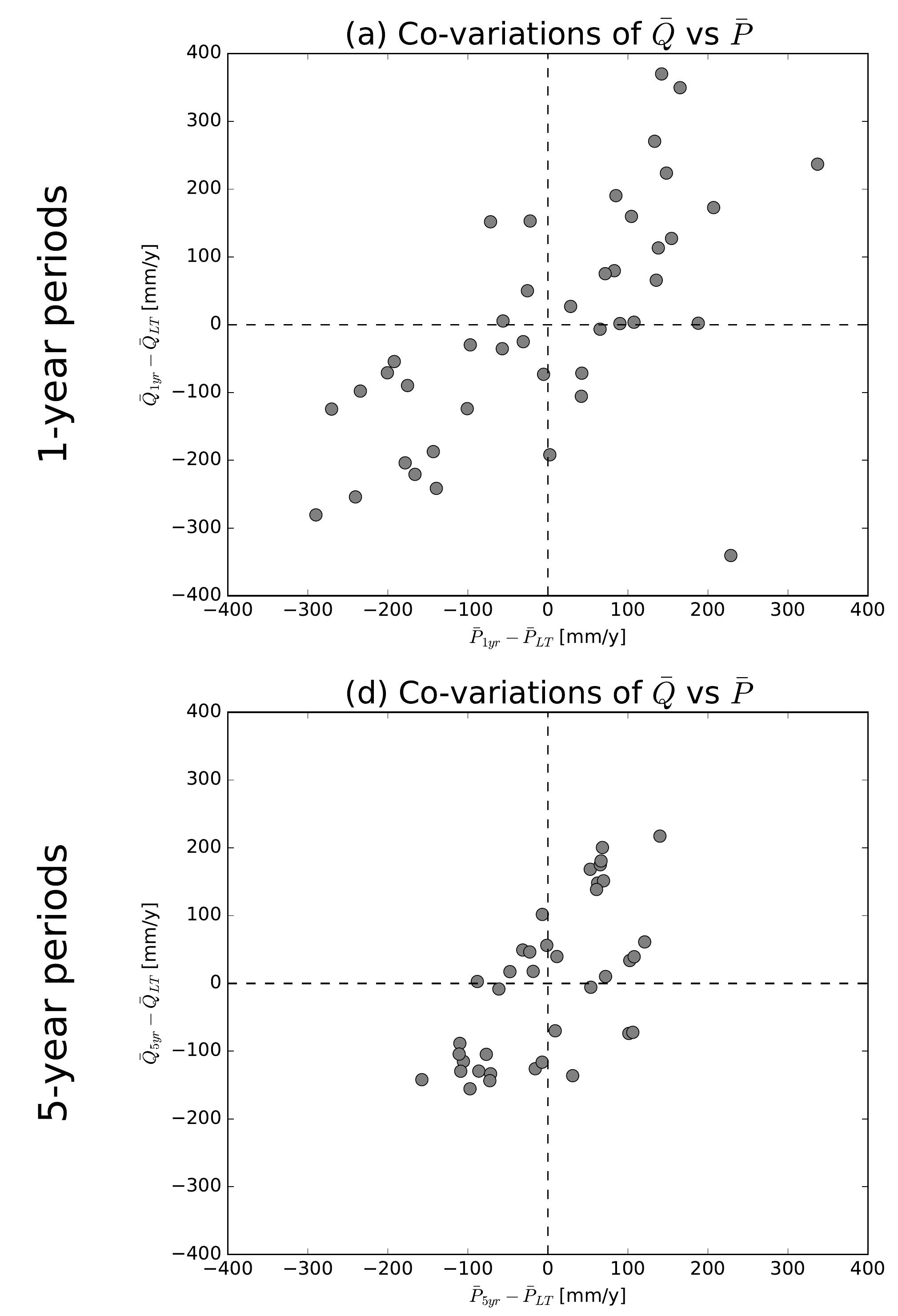

(g) Co-variations of $\bar{Q}$ vs $\bar{P}$
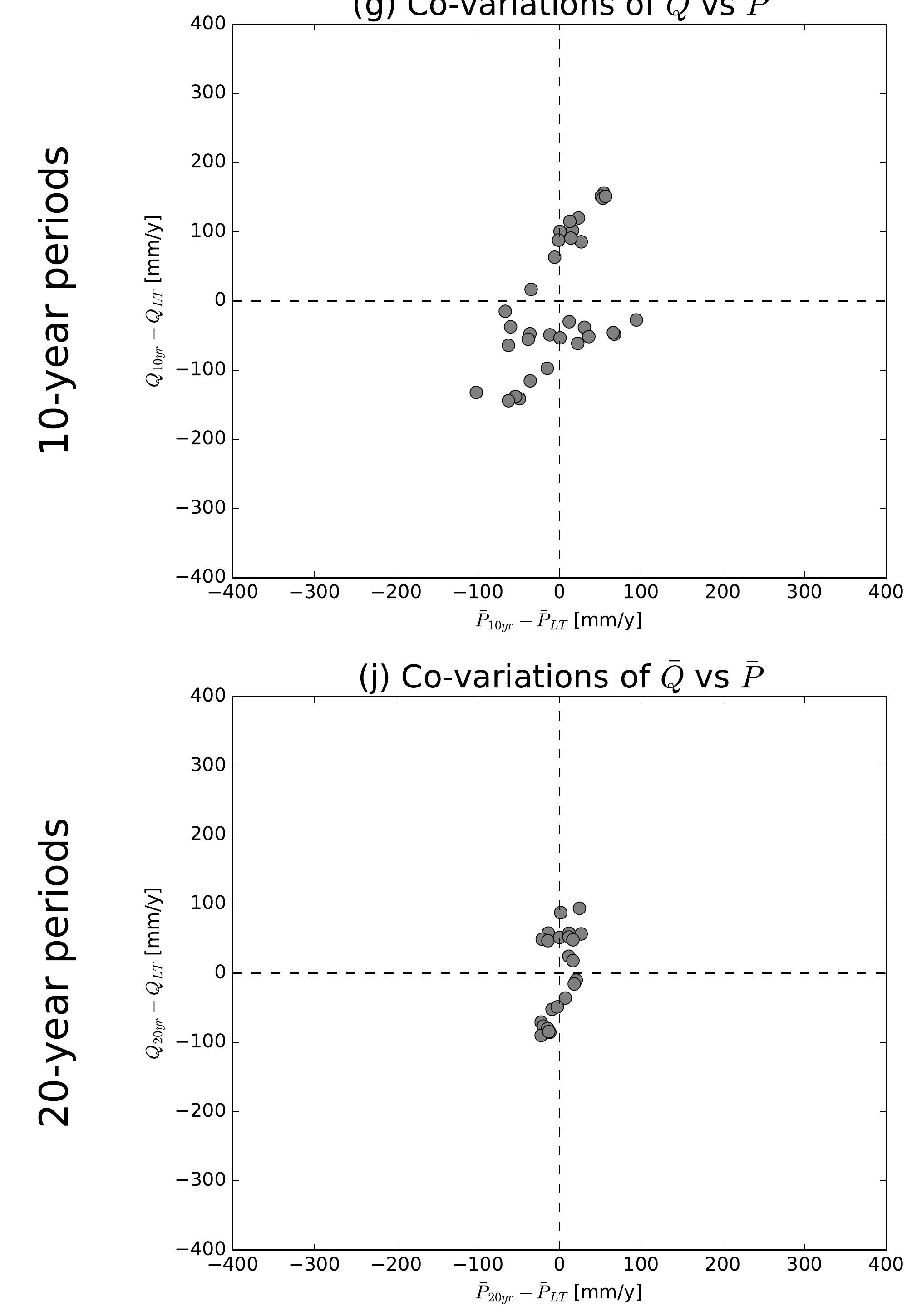

(b) Co-variations of $\bar{Q}$ vs $\overline{E 0}$

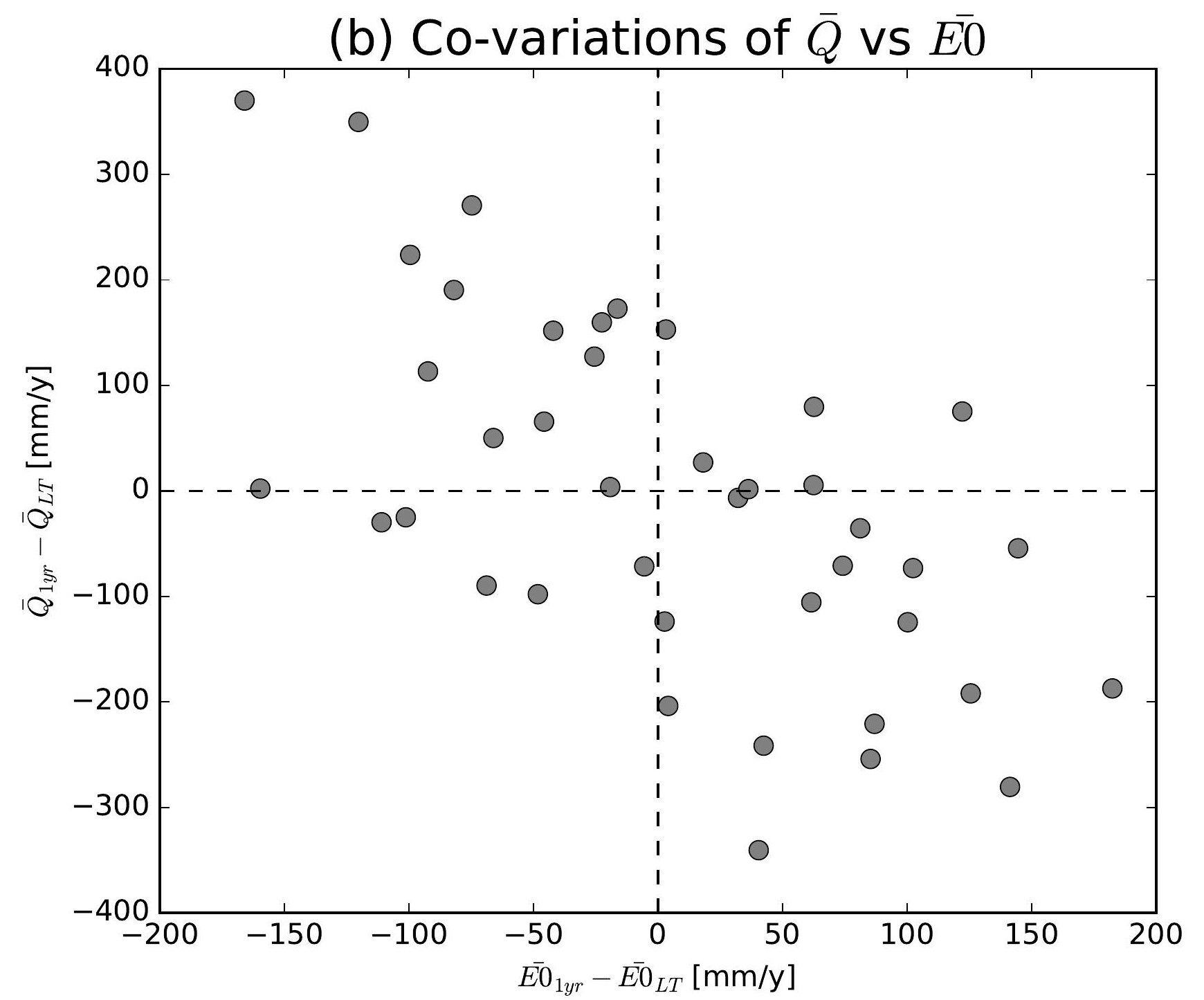

(e) Co-variations of $\bar{Q}$ vs $\overline{E 0}$

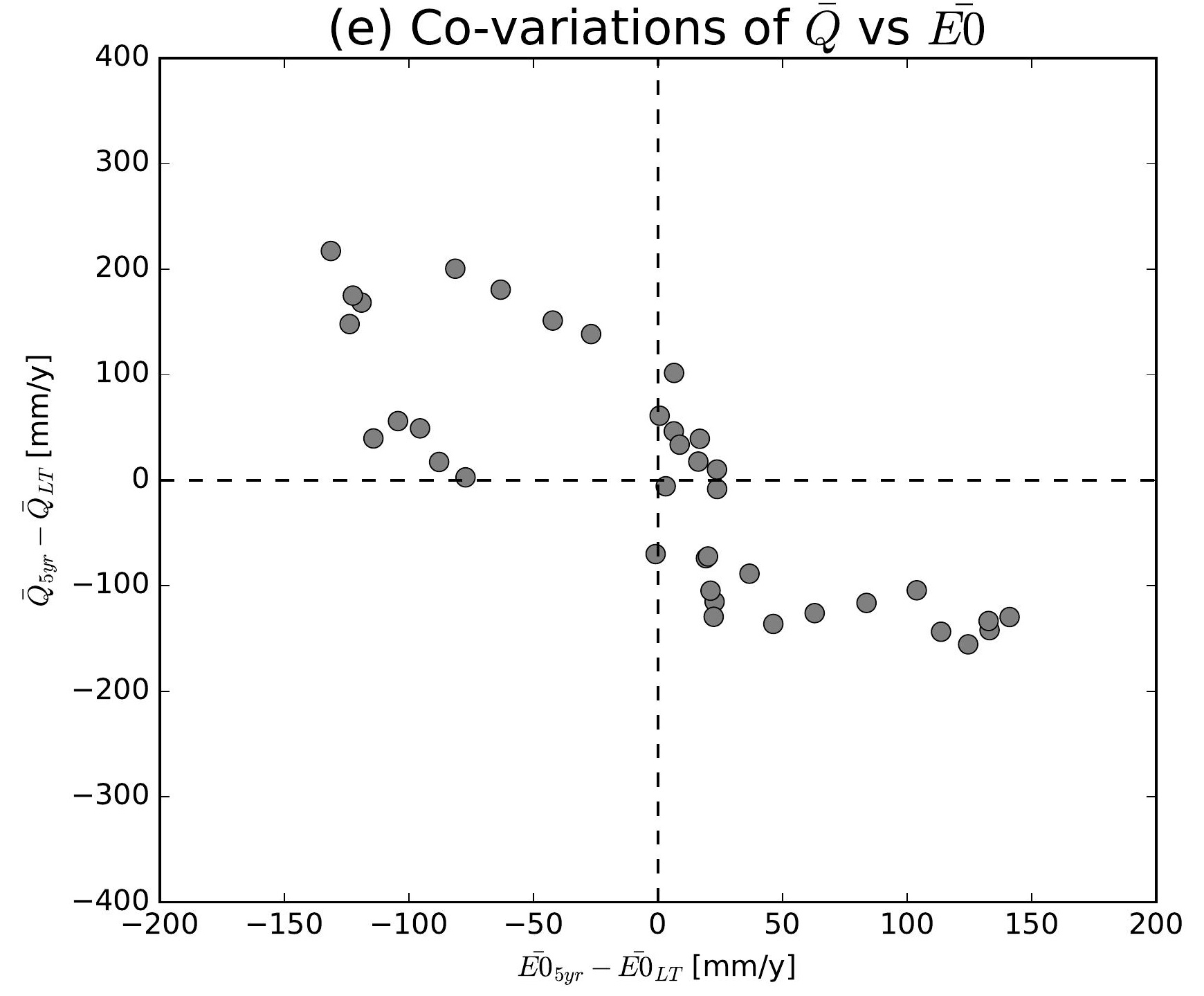

(h) Co-variations of $\bar{Q}$ vs $\overline{E 0}$

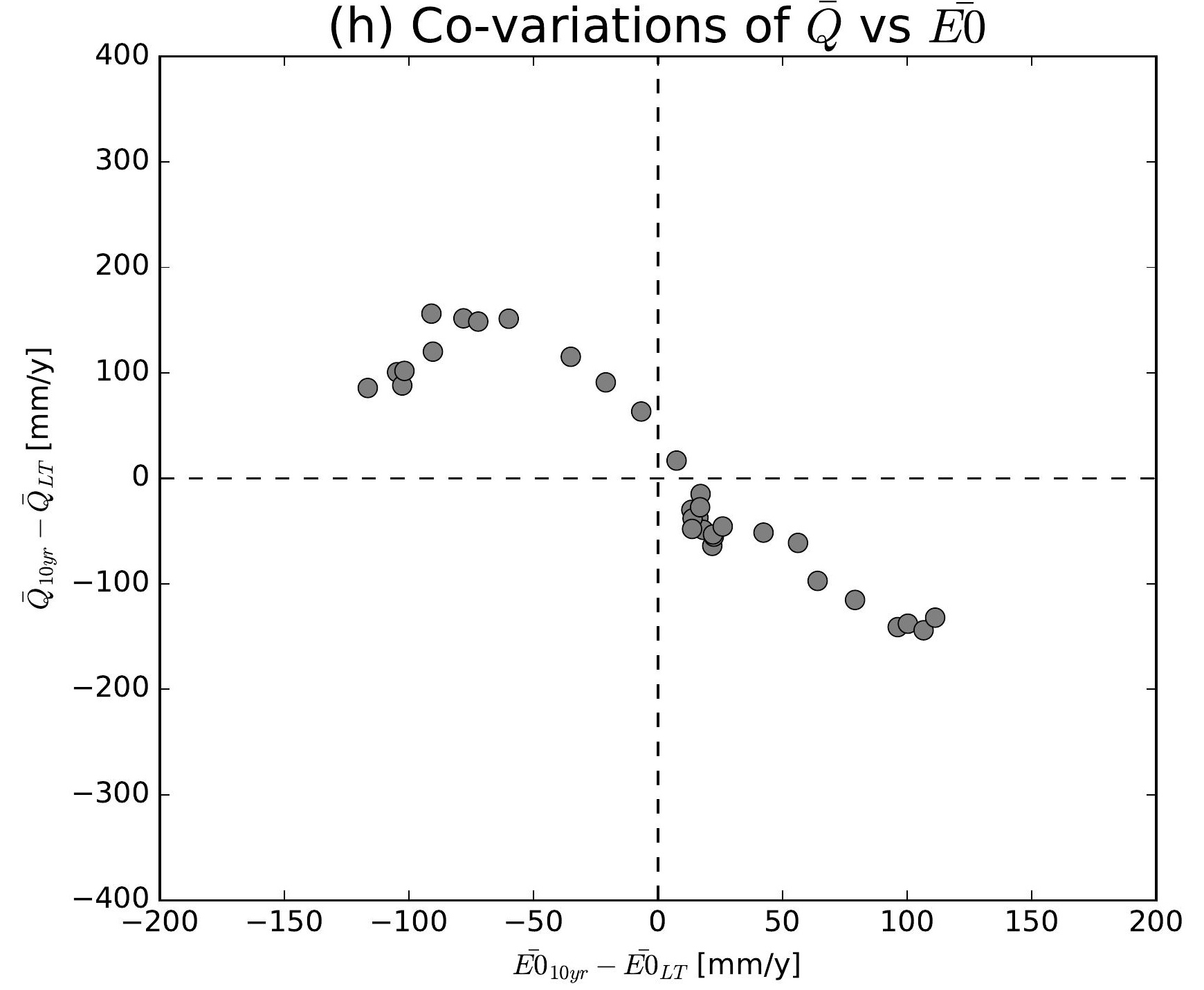

(k) Co-variations of $\bar{Q}$ vs $\overline{E 0}$

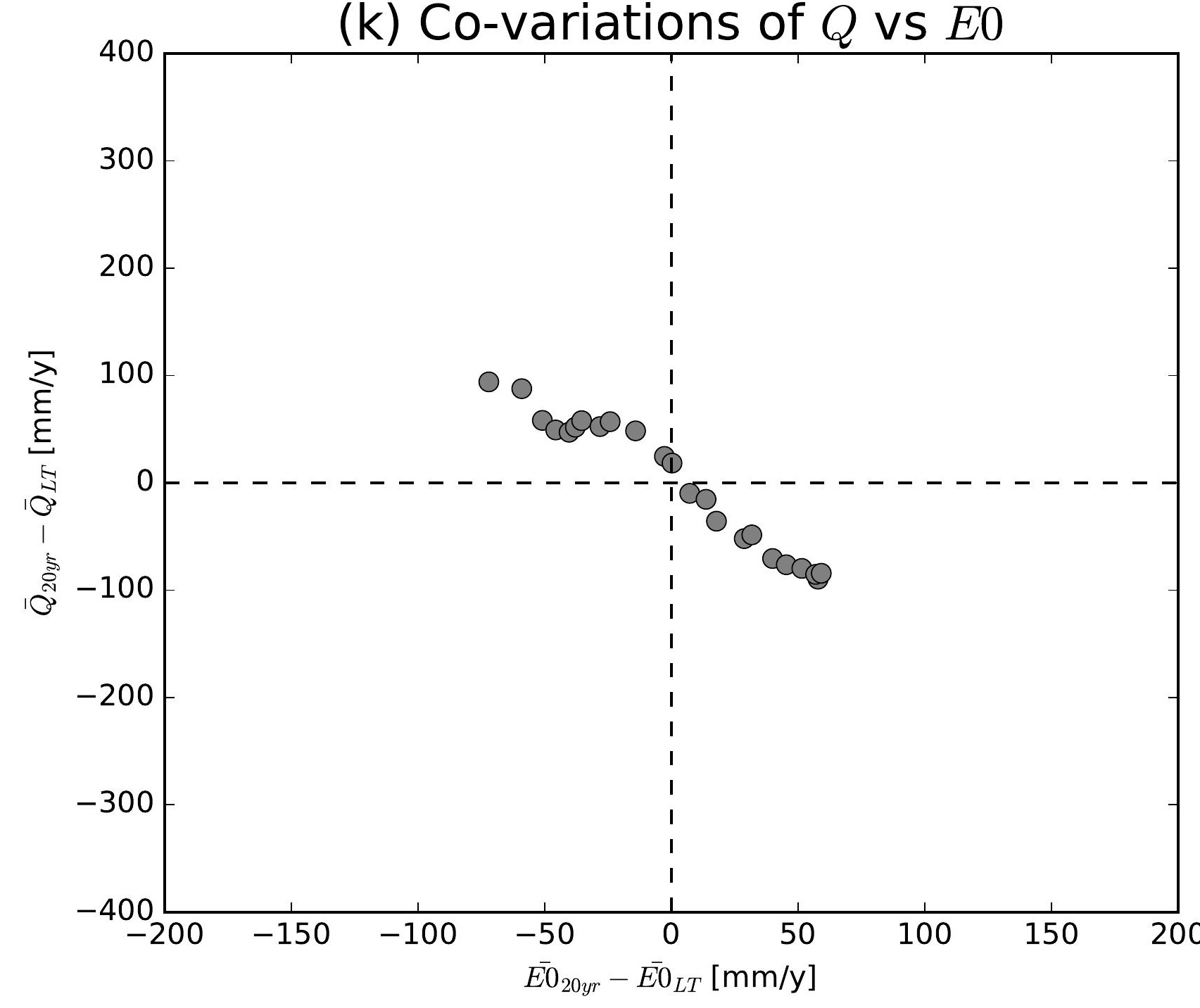

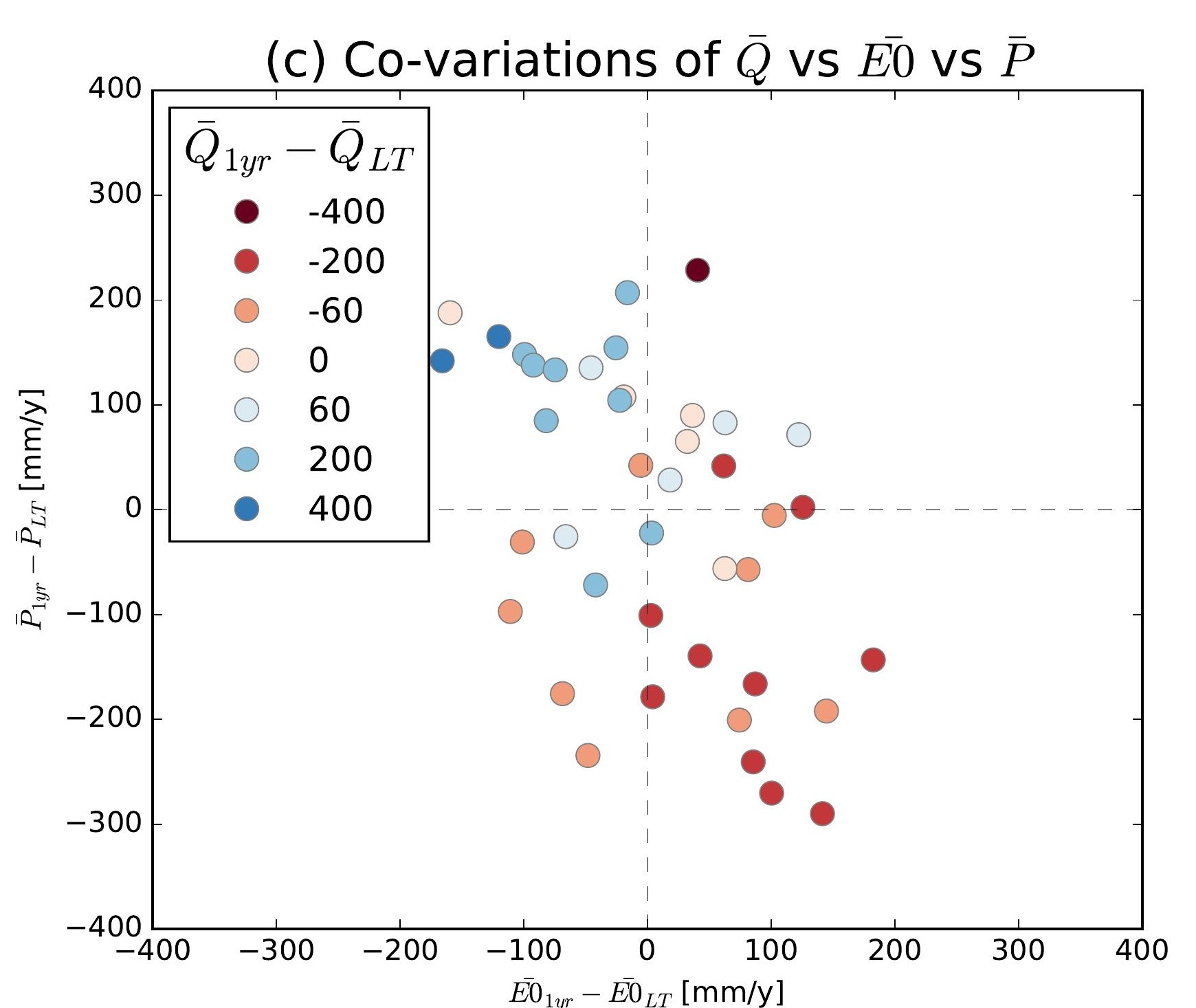
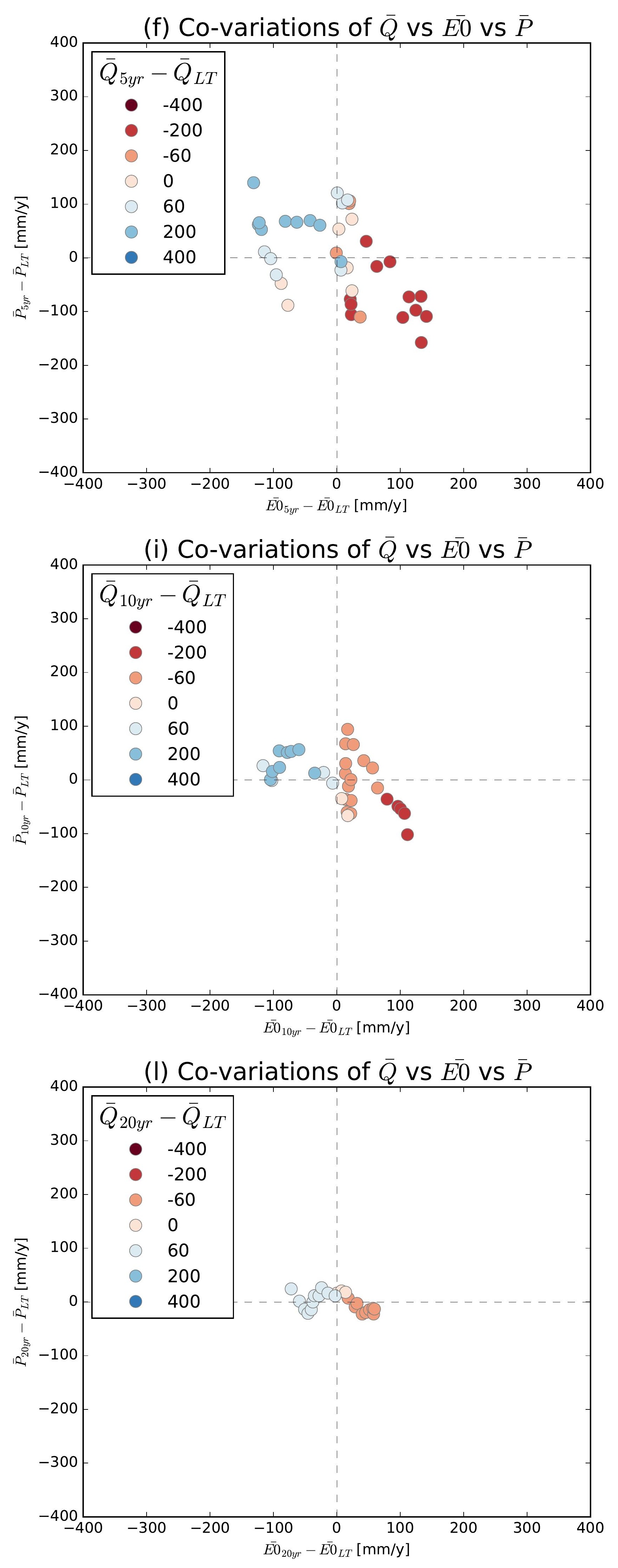

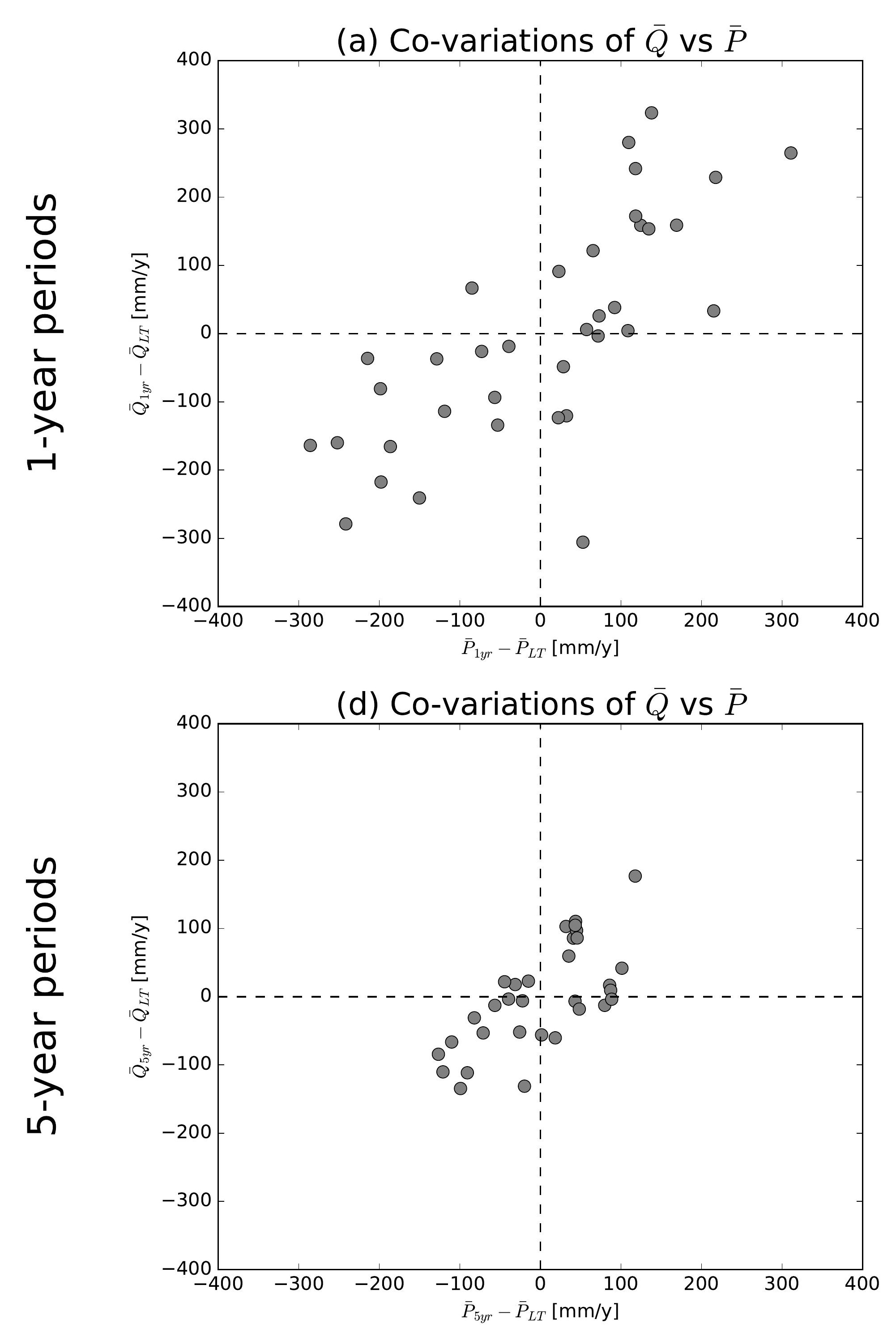

(g) Co-variations of $\bar{Q}$ vs $\bar{P}$

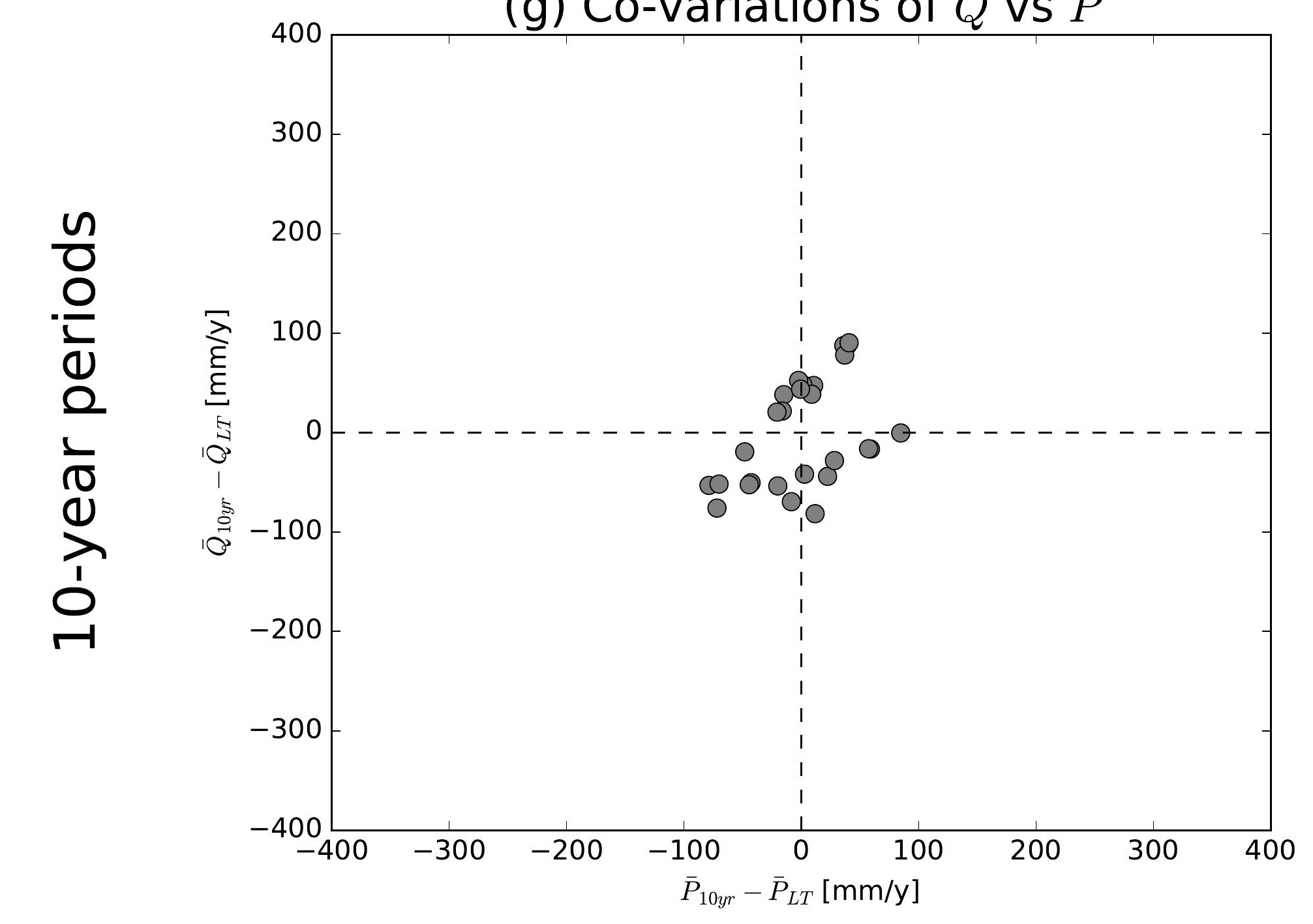

(j) Co-variations of $\bar{Q}$ vs $\bar{P}$

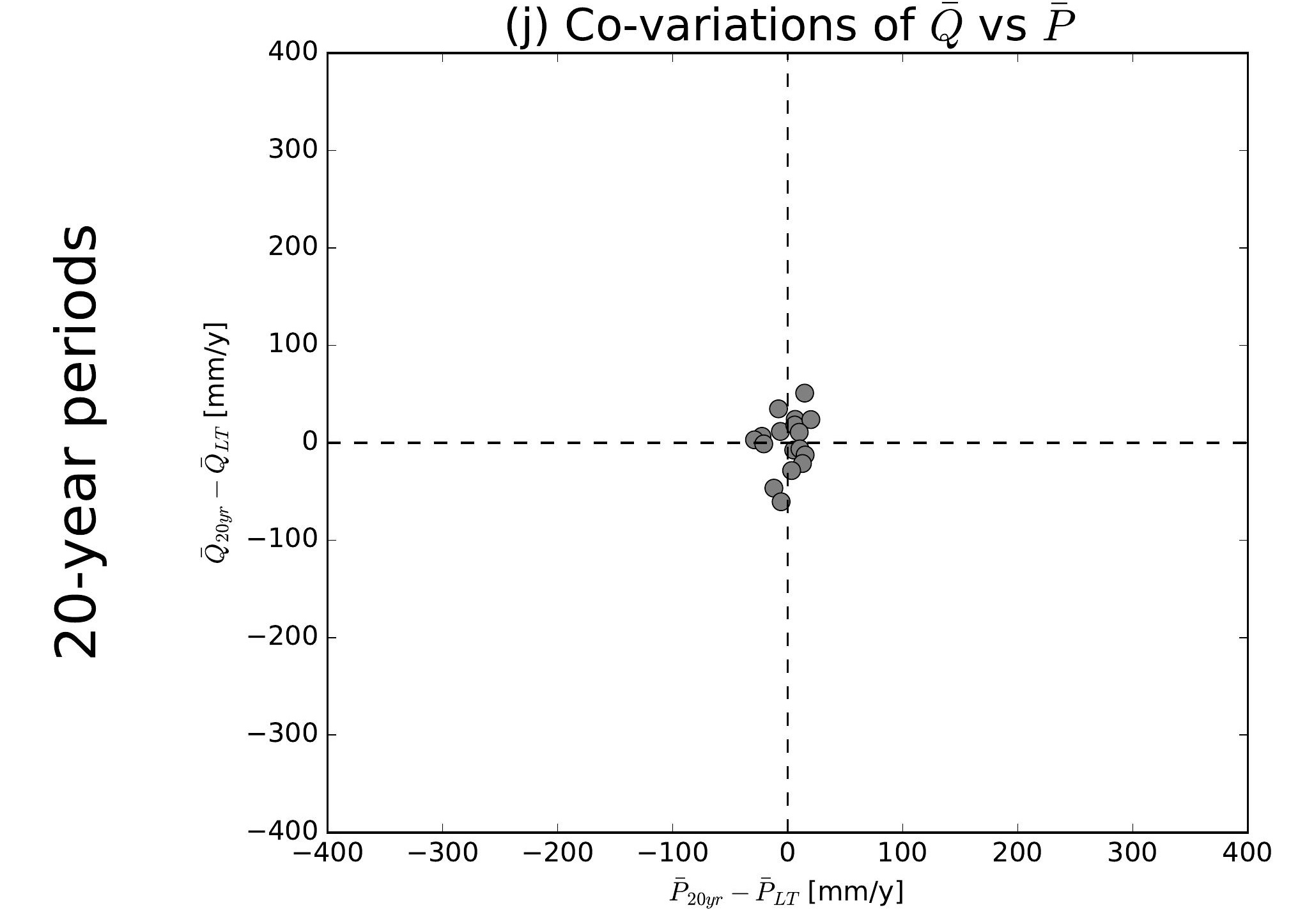

(b) Co-variations of $\bar{Q}$ vs $\overline{E 0}$

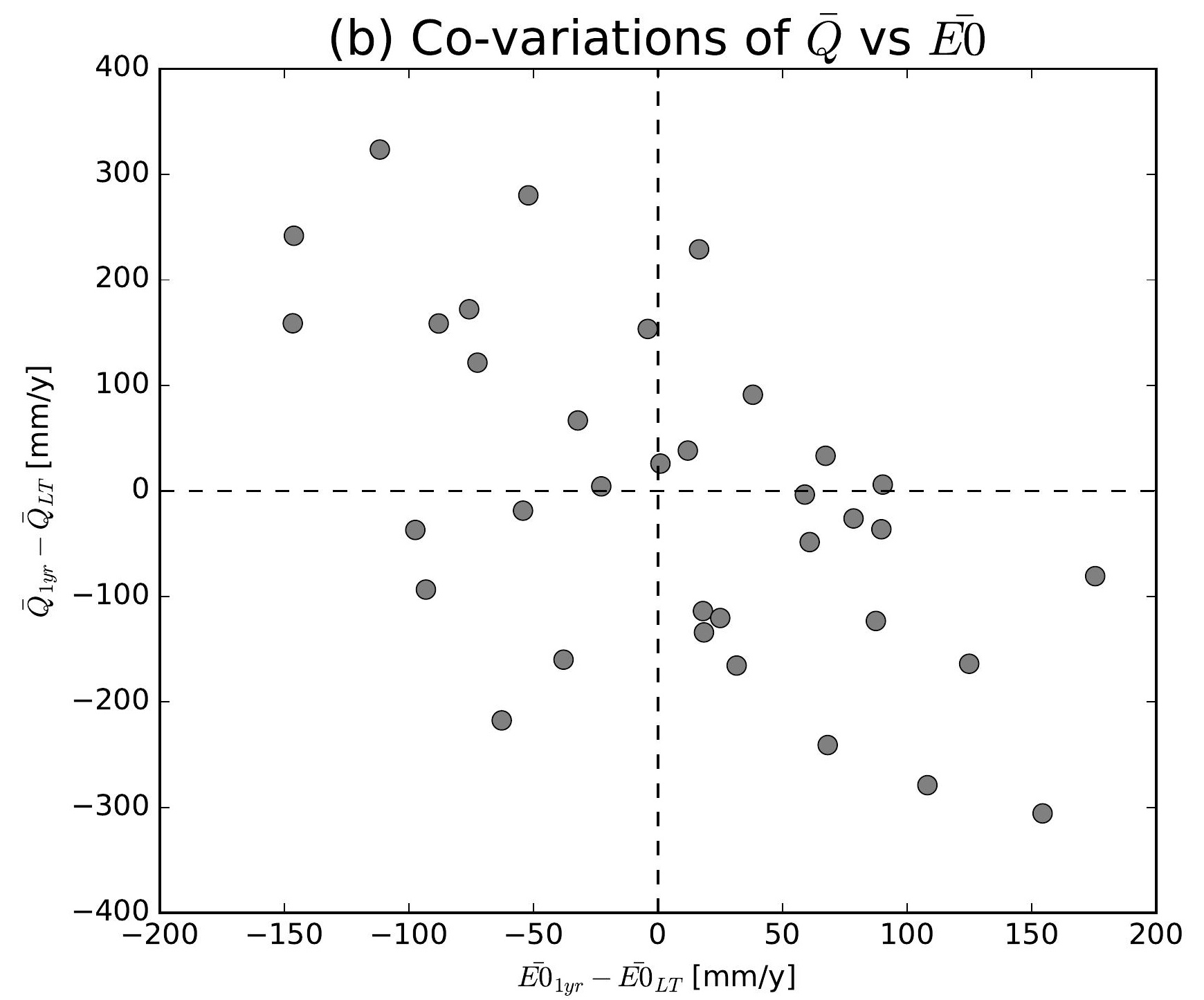

(e) Co-variations of $\bar{Q}$ vs $\overline{E 0}$

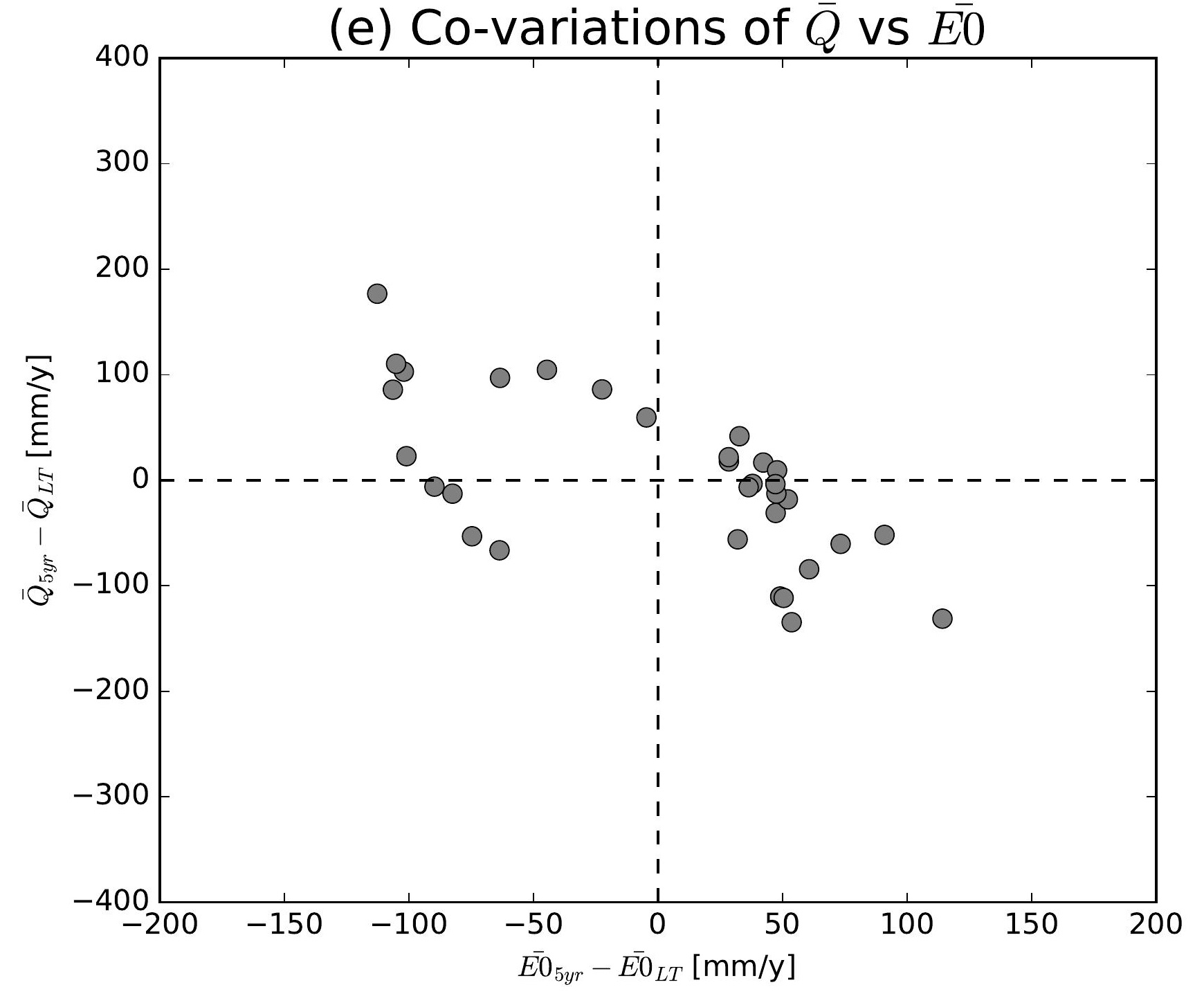

(h) Co-variations of $\bar{Q}$ vs $\overline{E 0}$

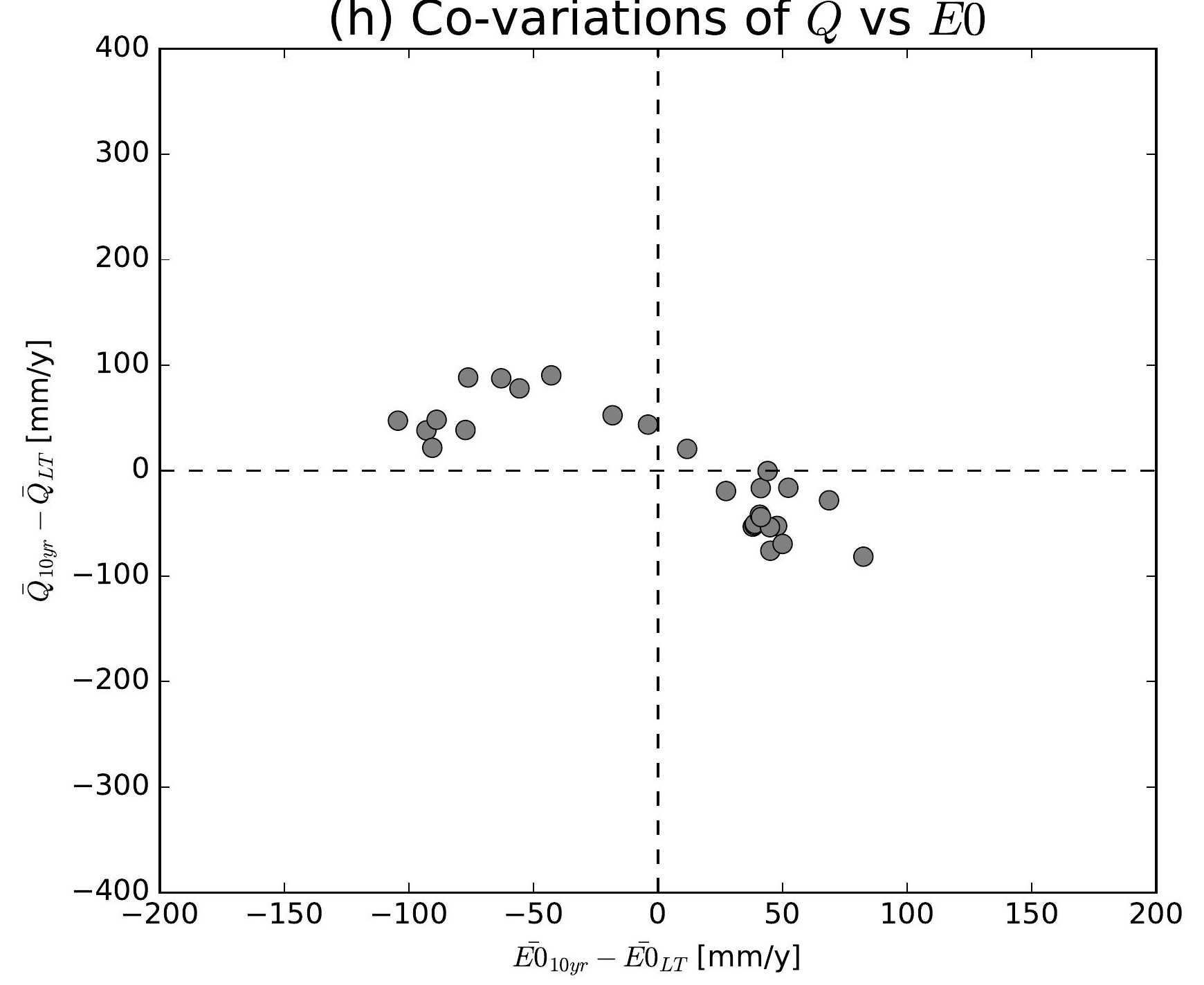

(k) Co-variations of $\bar{Q}$ vs $\overline{E 0}$

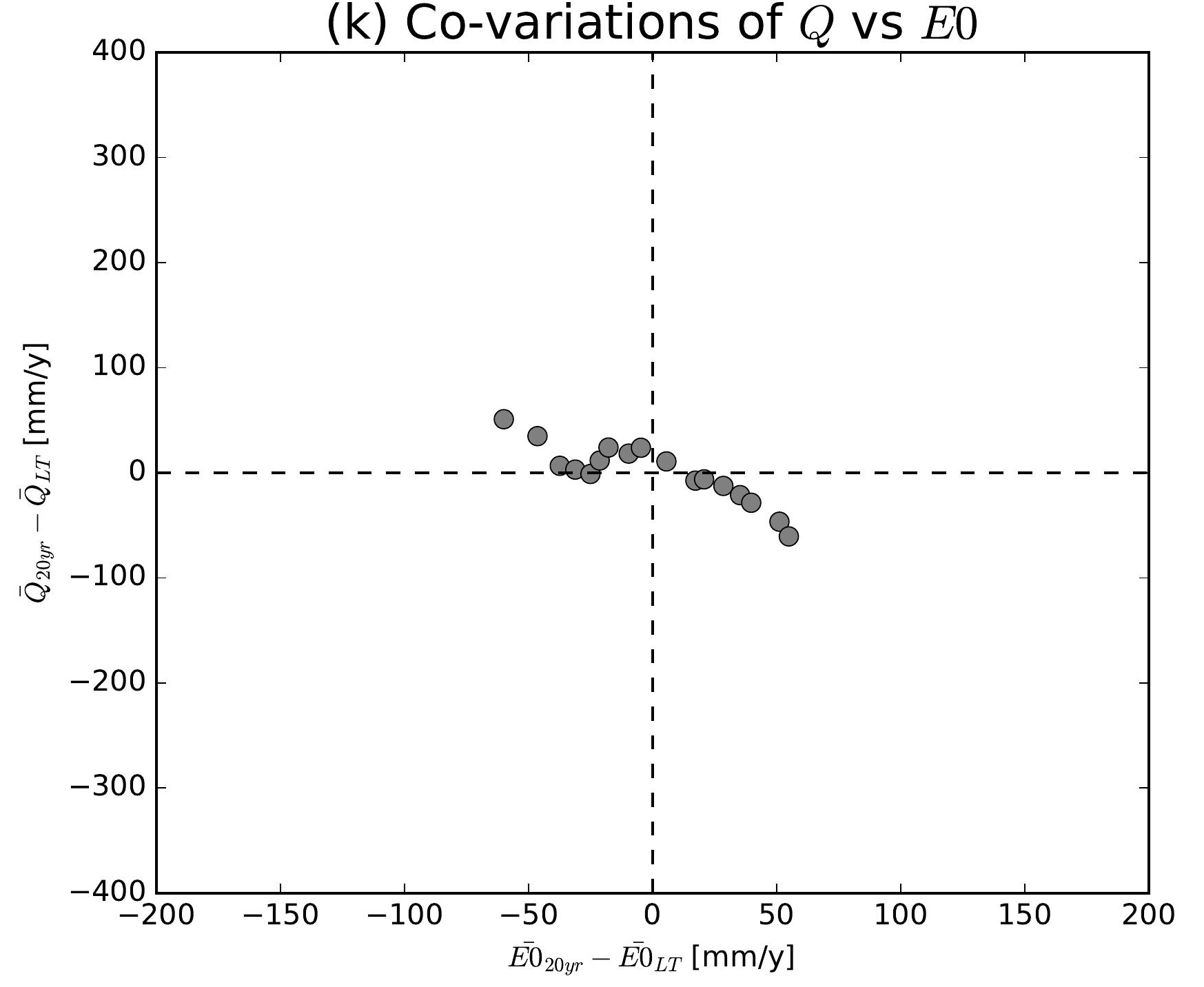

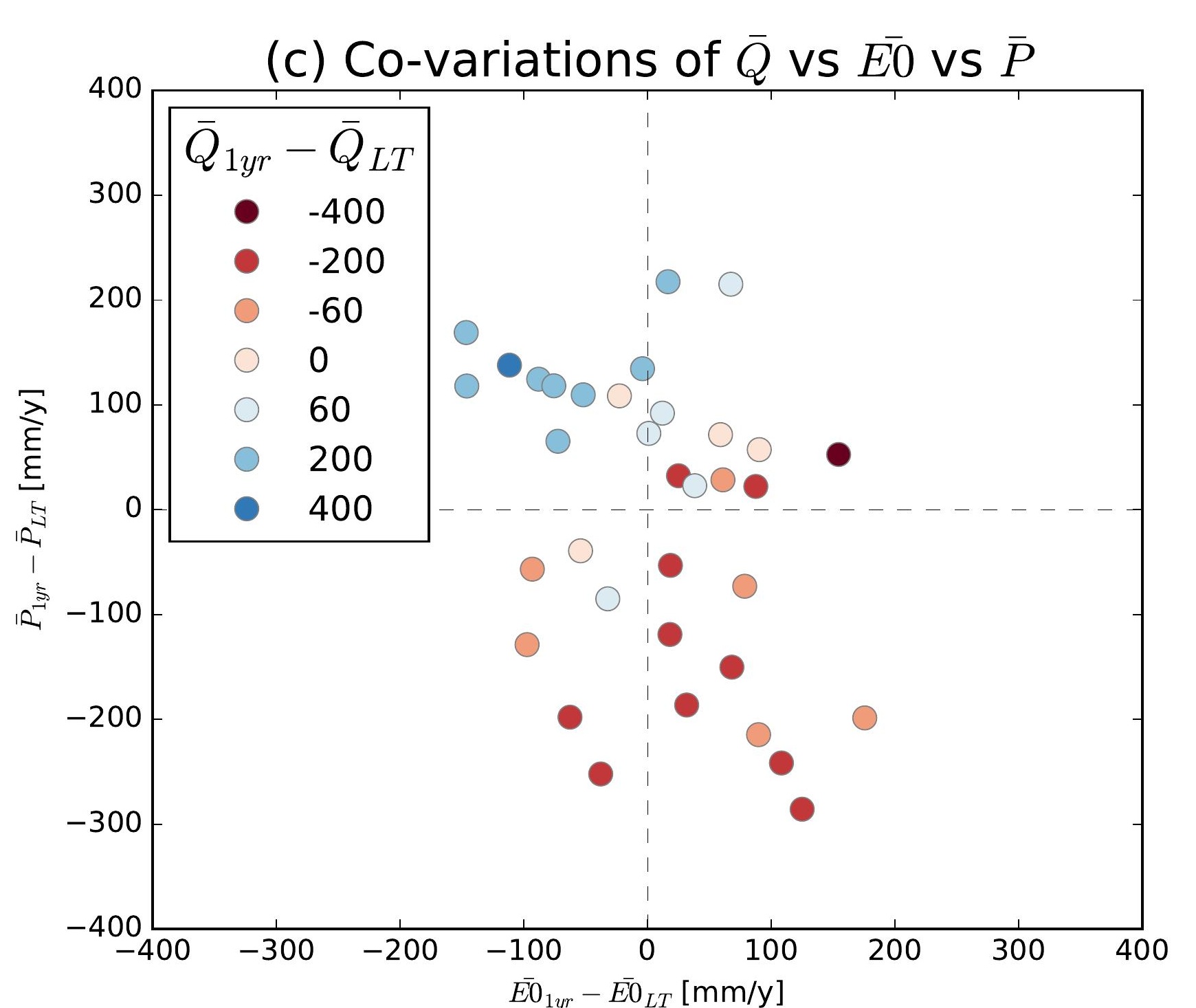
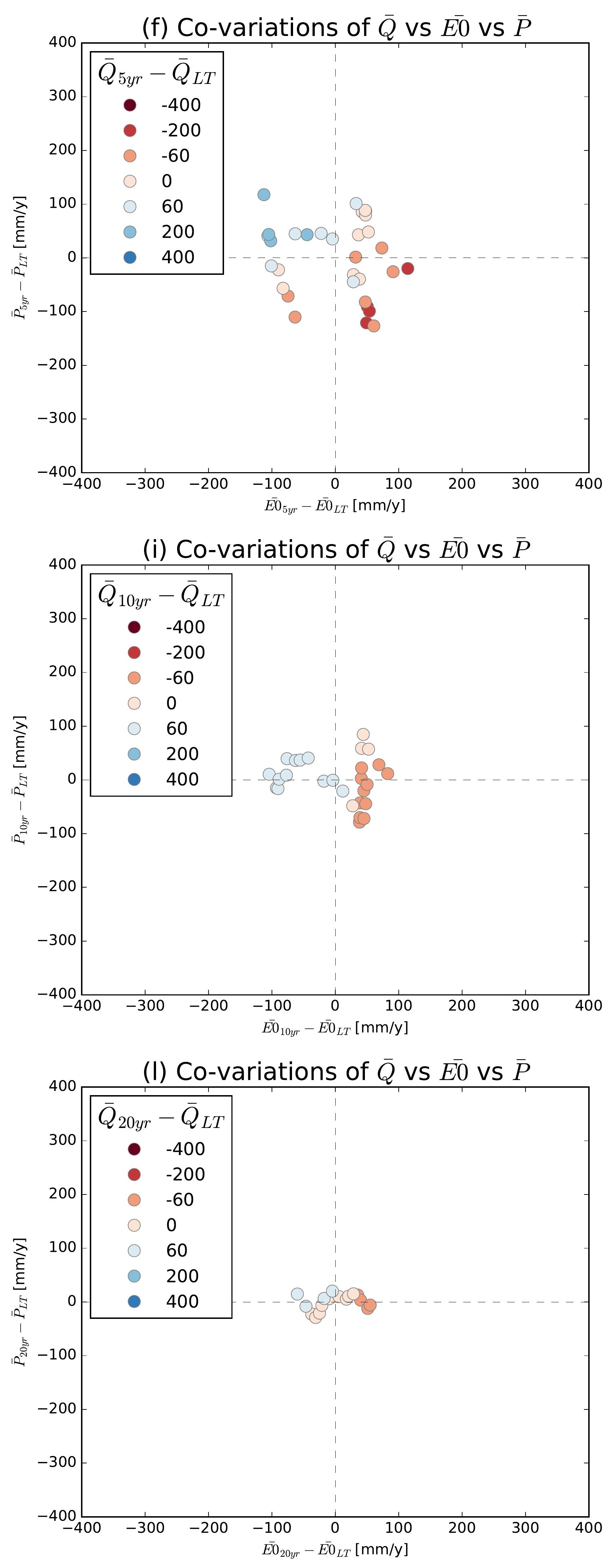

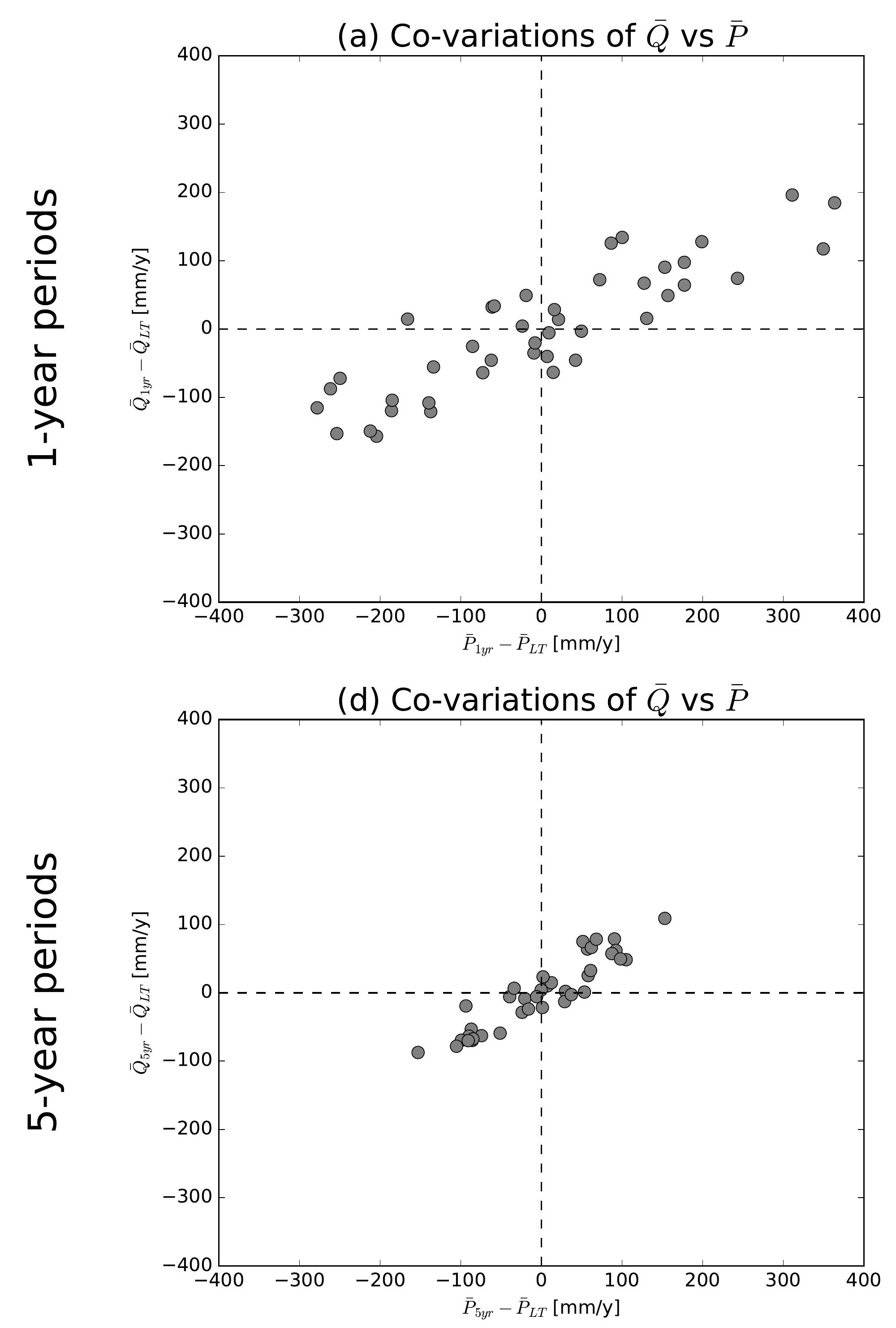

(g) Co-variations of $\bar{Q}$ vs $\bar{P}$
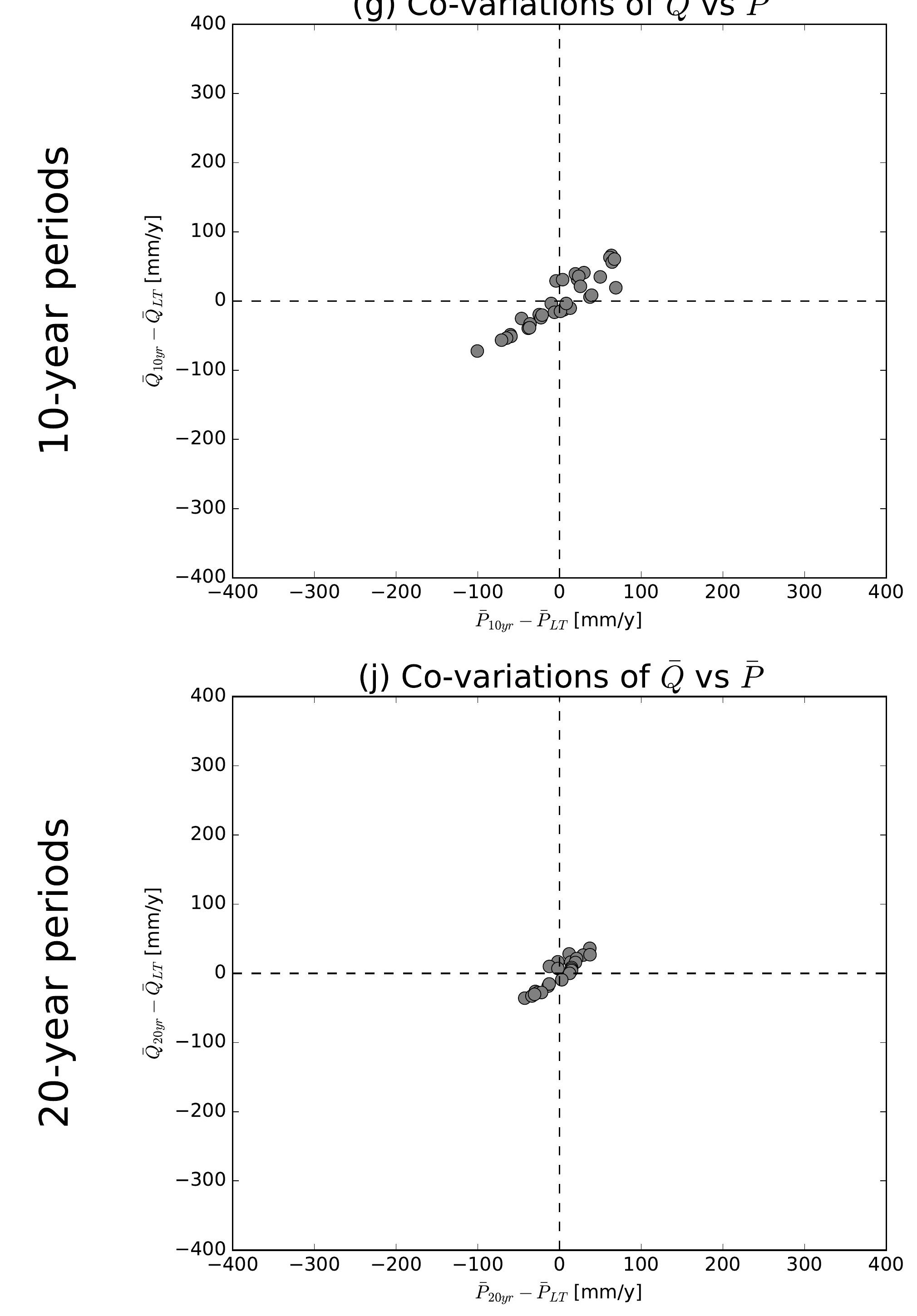

(b) Co-variations of $\bar{Q}$ vs $\overline{E 0}$

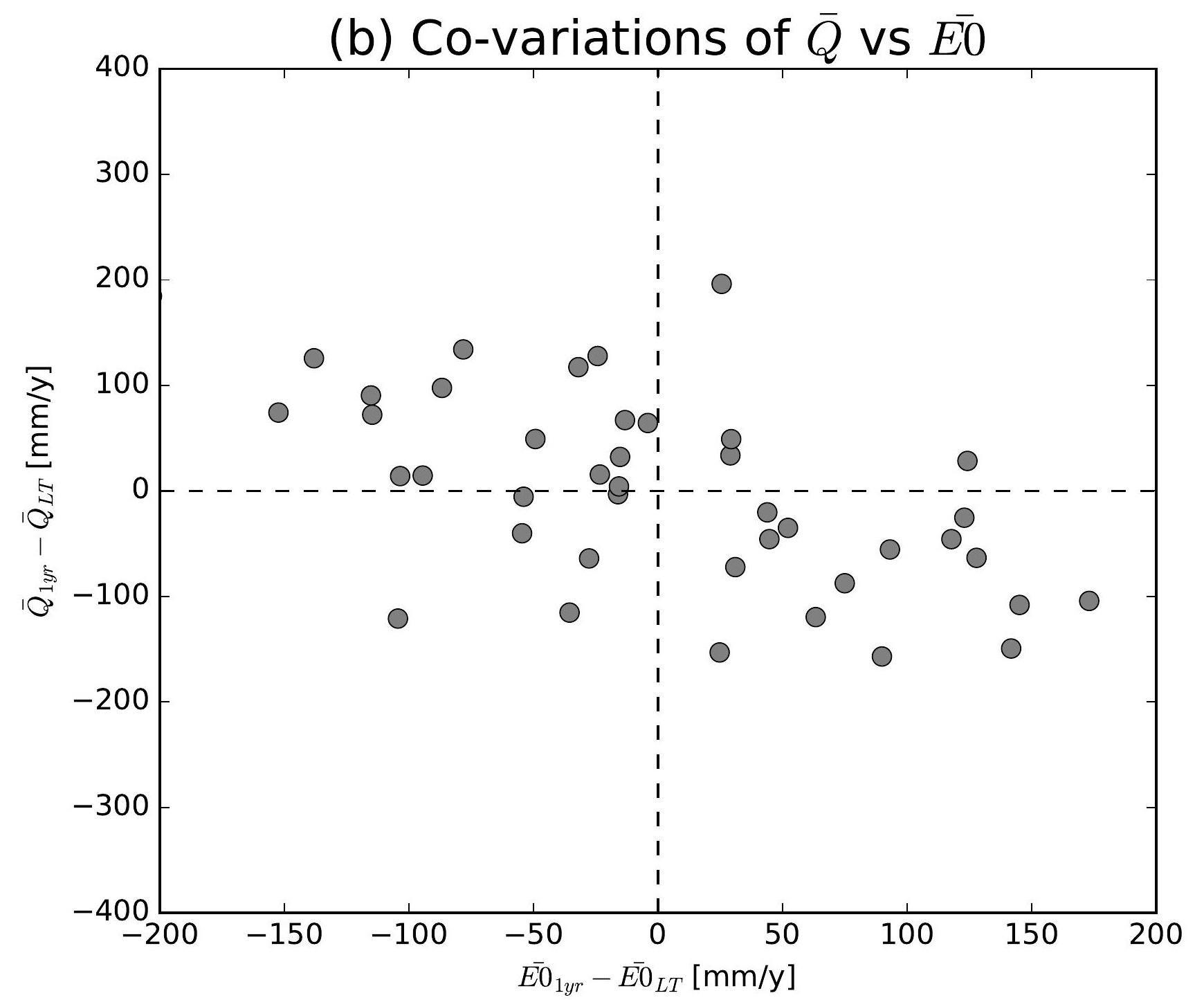

(e) Co-variations of $\bar{Q}$ vs $\overline{E 0}$

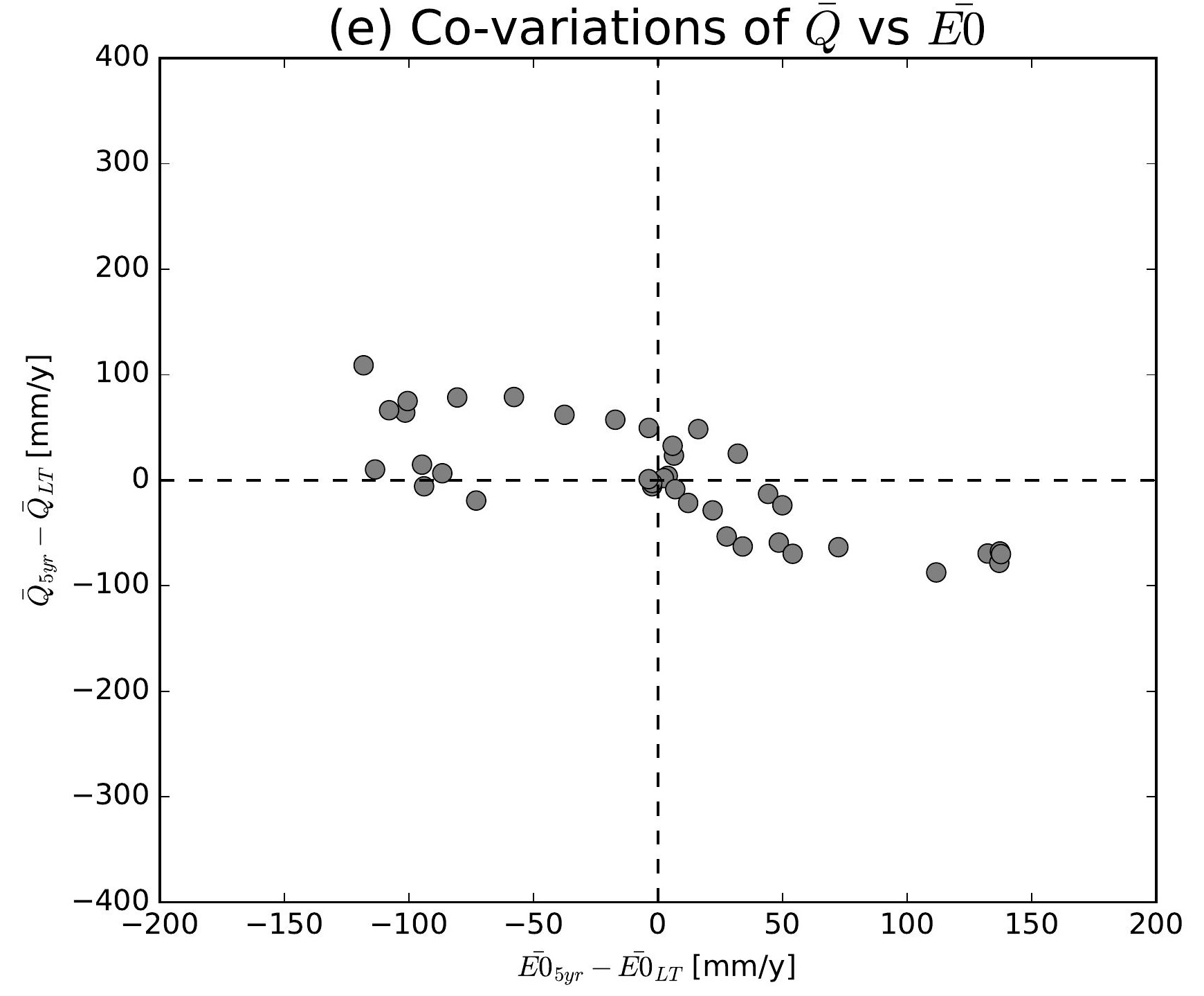

(h) Co-variations of $\bar{Q}$ vs $\overline{E 0}$

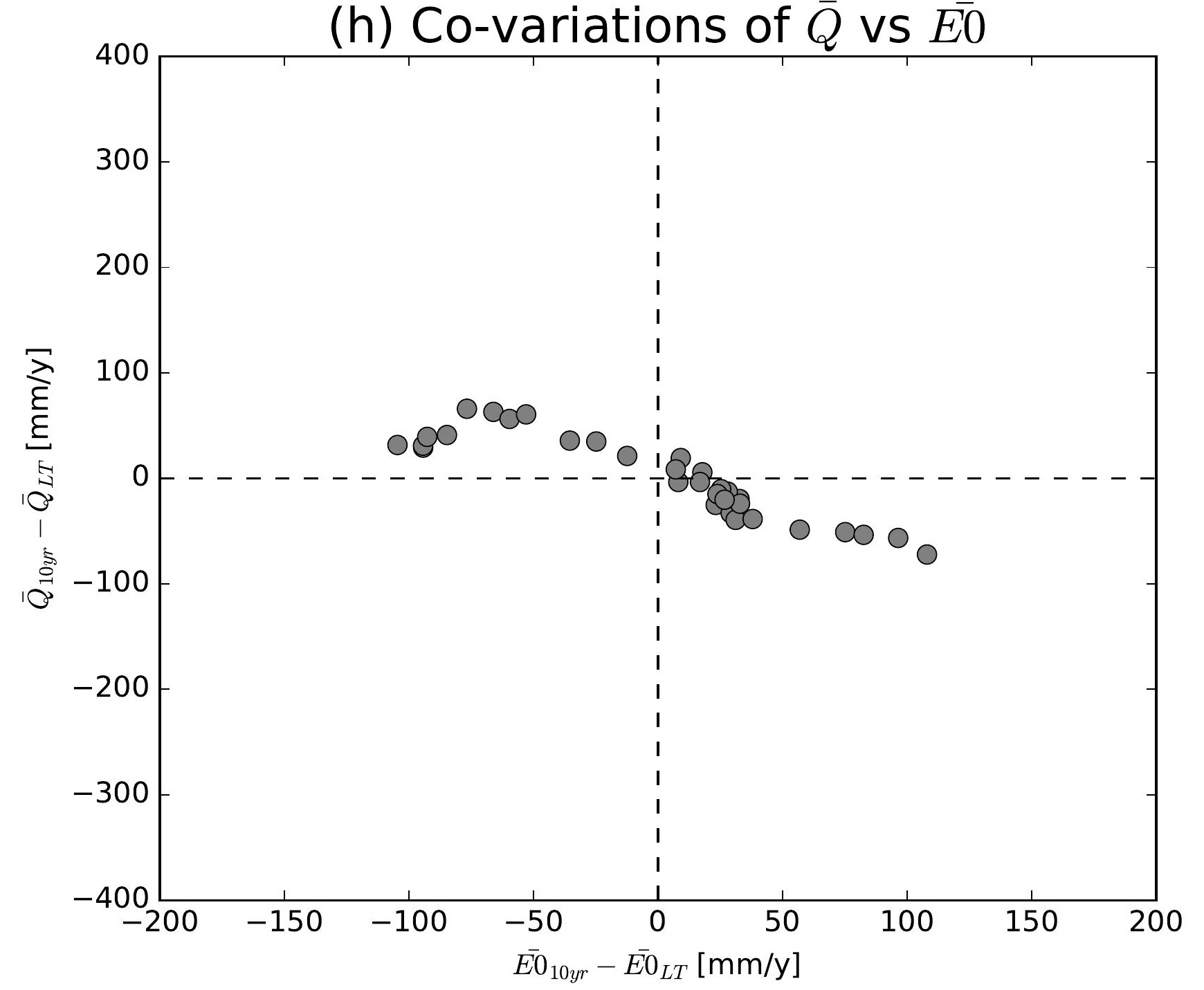

(k) Co-variations of $\bar{Q}$ vs $\overline{E 0}$

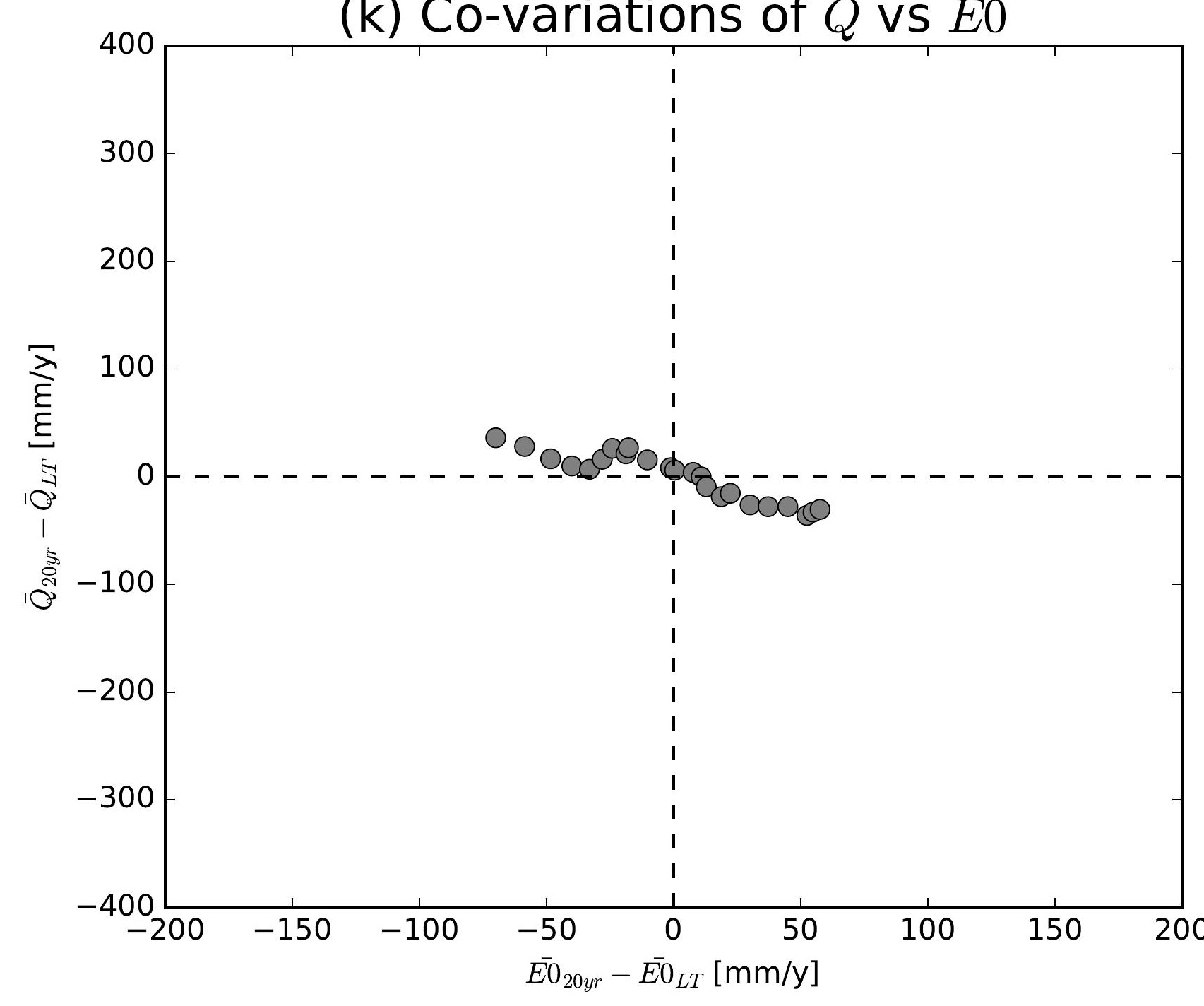

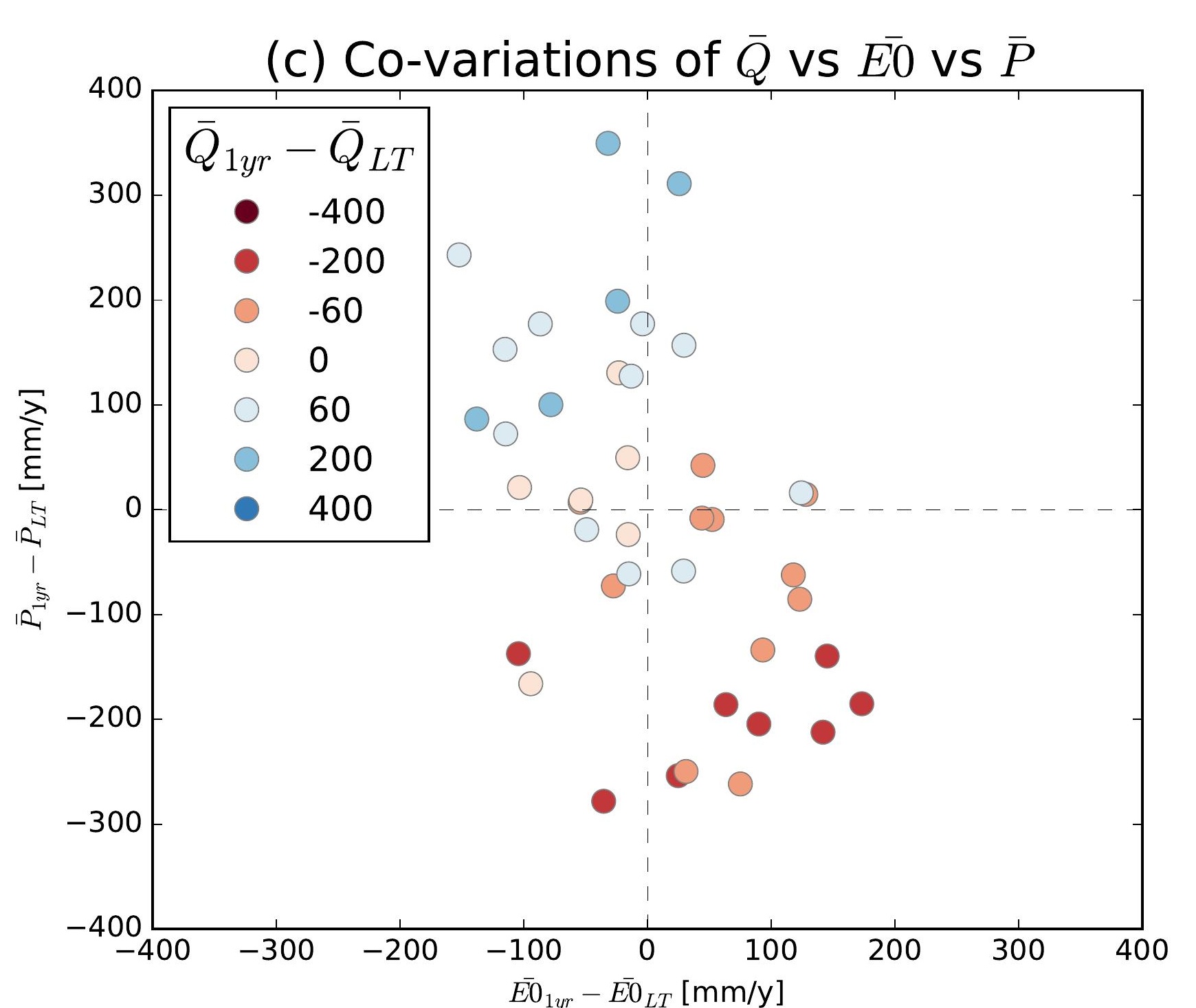
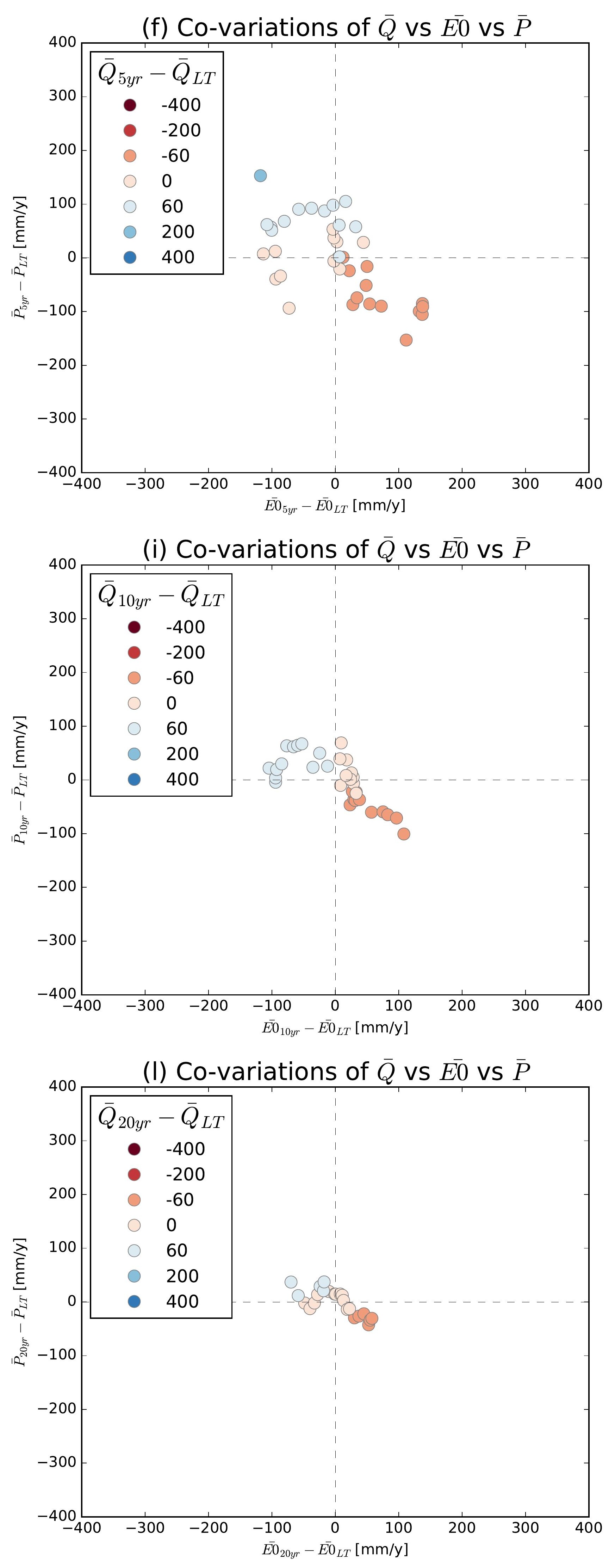

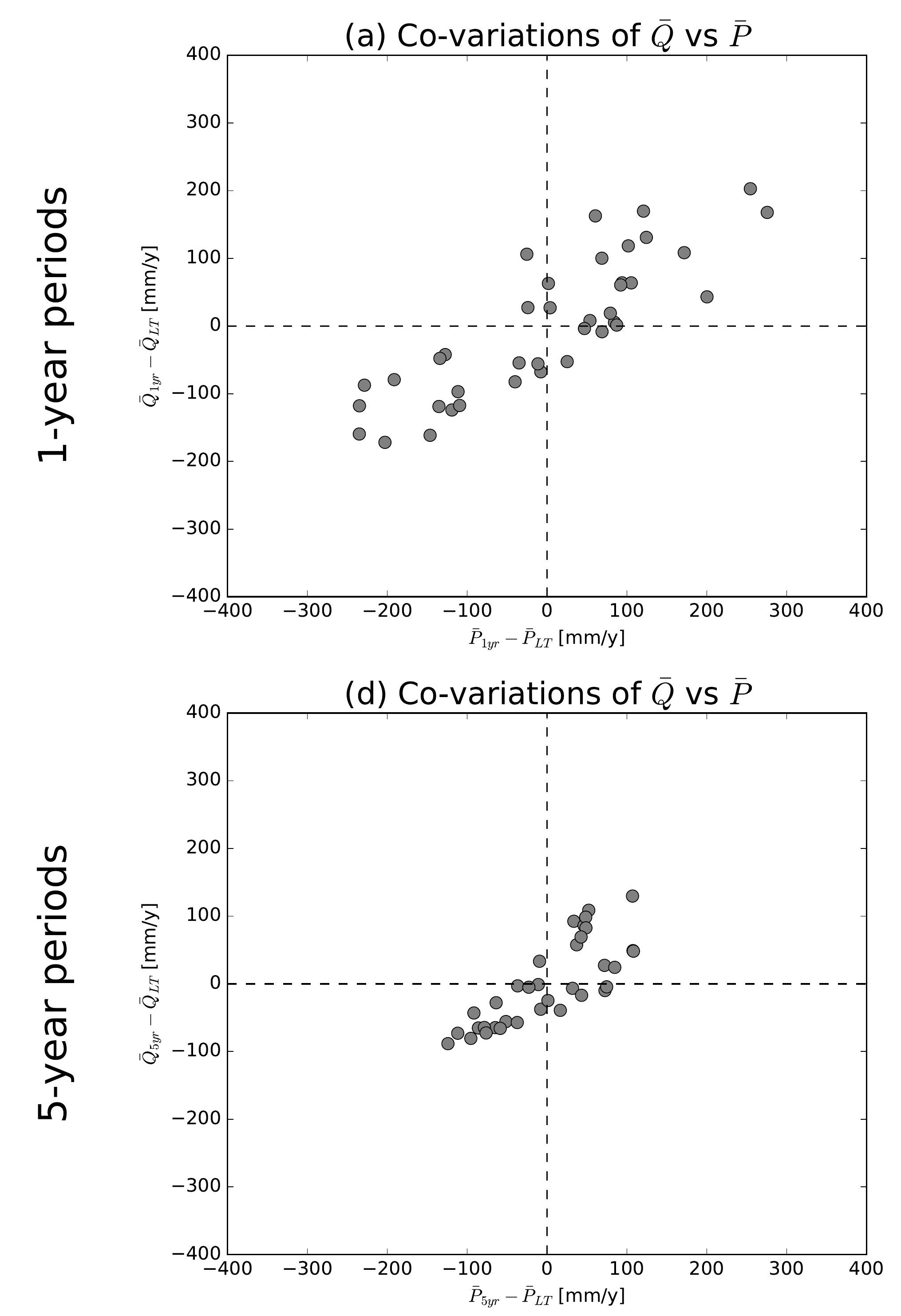

(g) Co-variations of $\bar{Q}$ vs $\bar{P}$
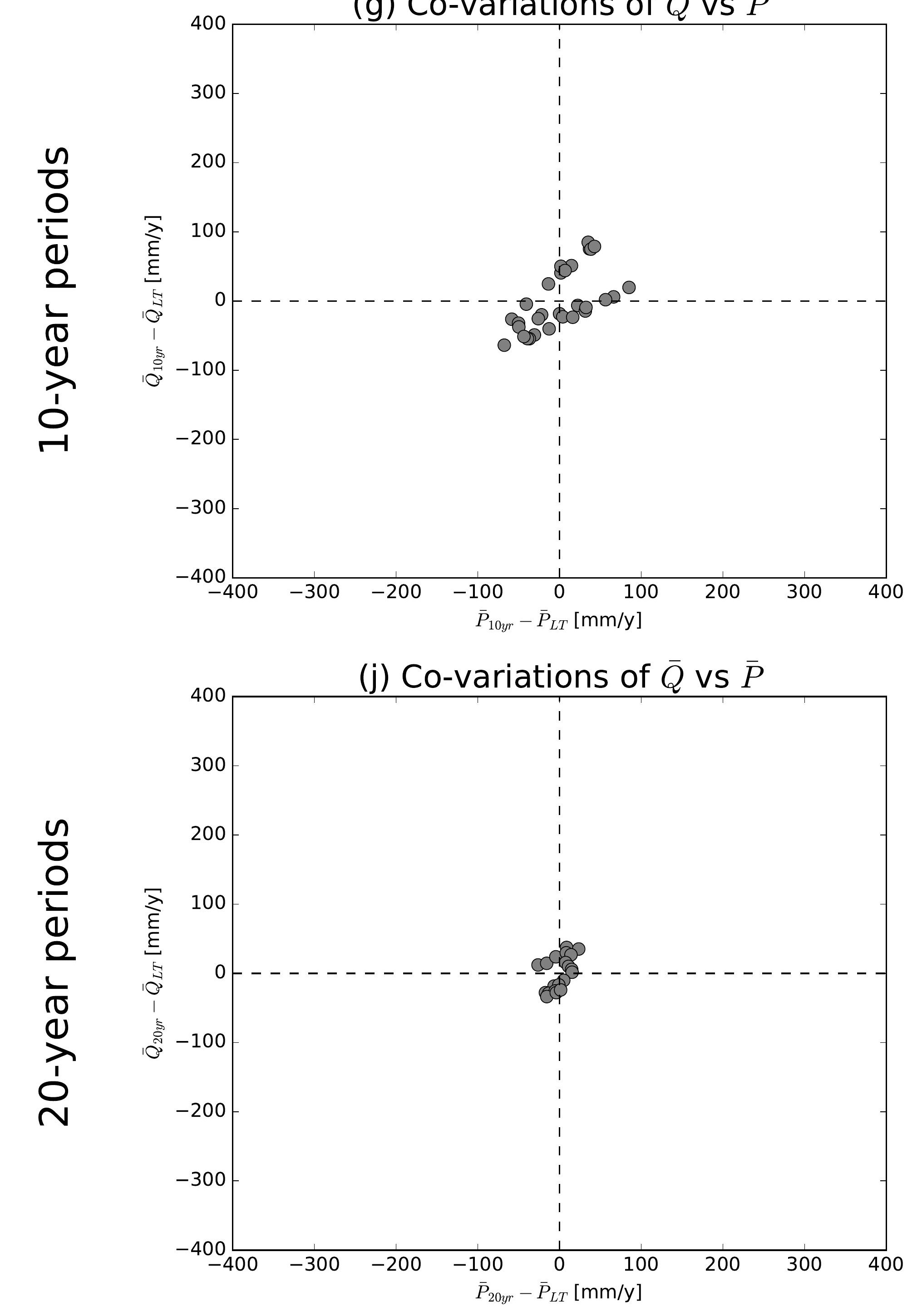

(b) Co-variations of $\bar{Q}$ vs $\overline{E 0}$

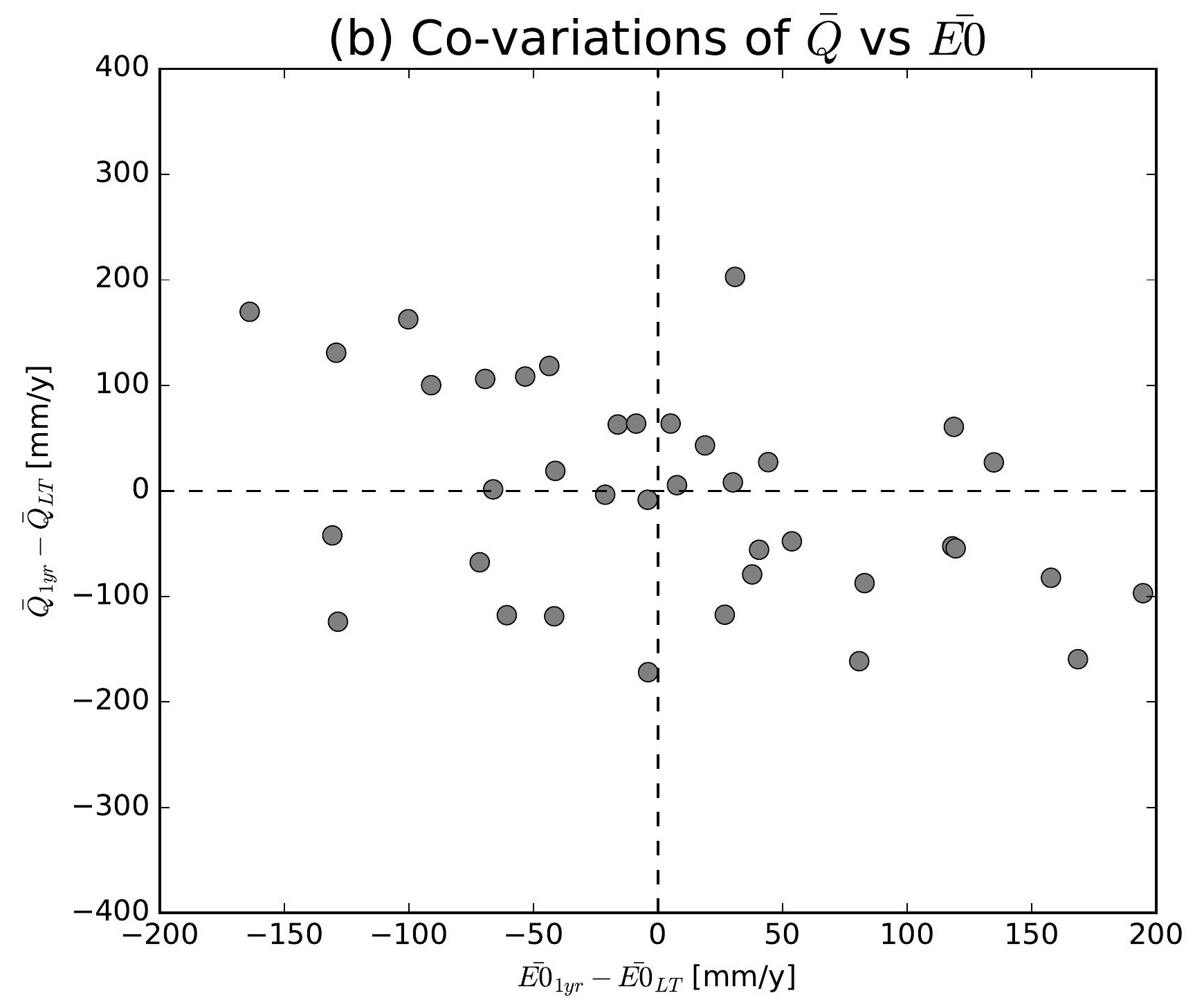

(e) Co-variations of $\bar{Q}$ vs $\overline{E 0}$

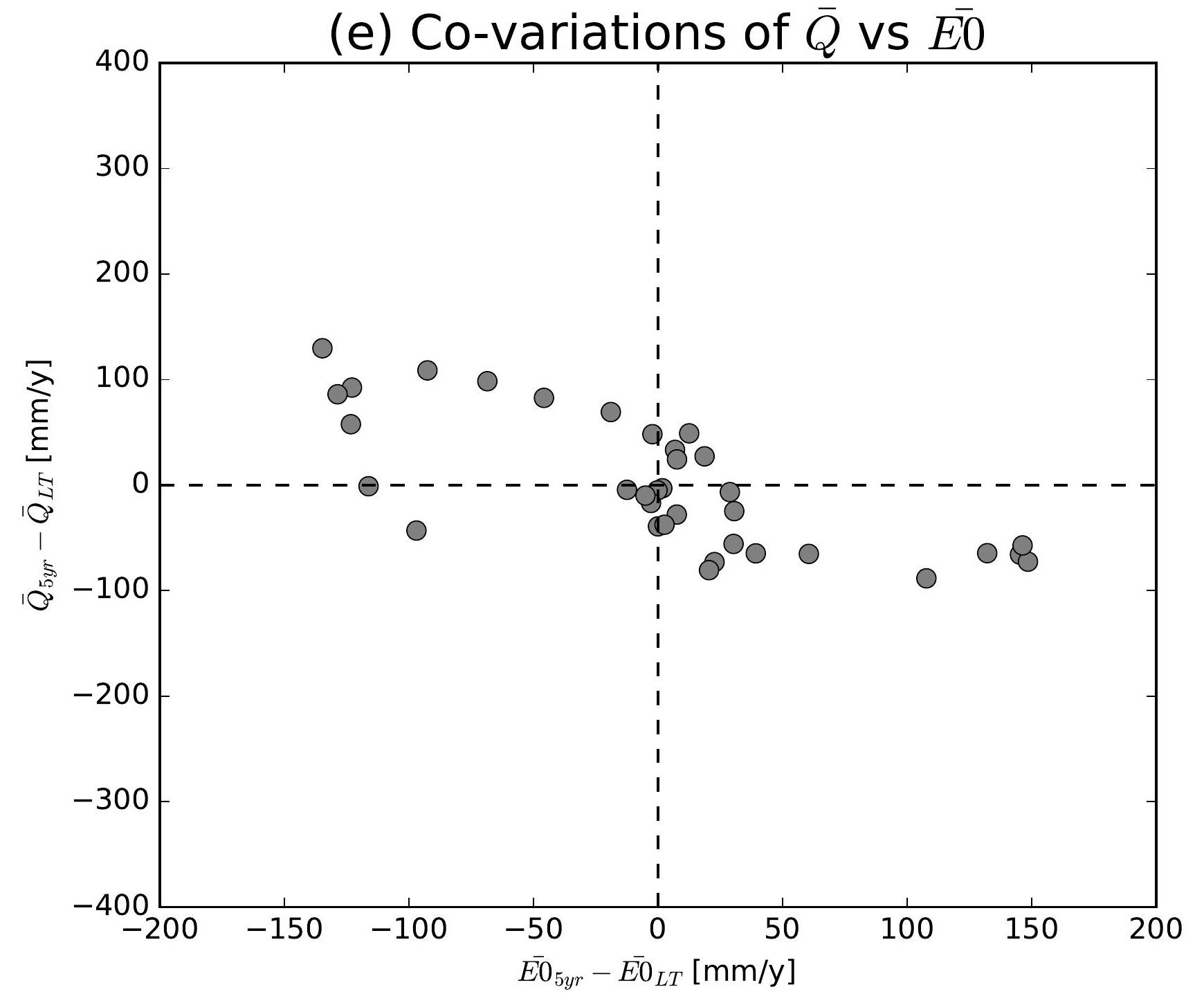

(h) Co-variations of $\bar{Q}$ vs $\overline{E 0}$

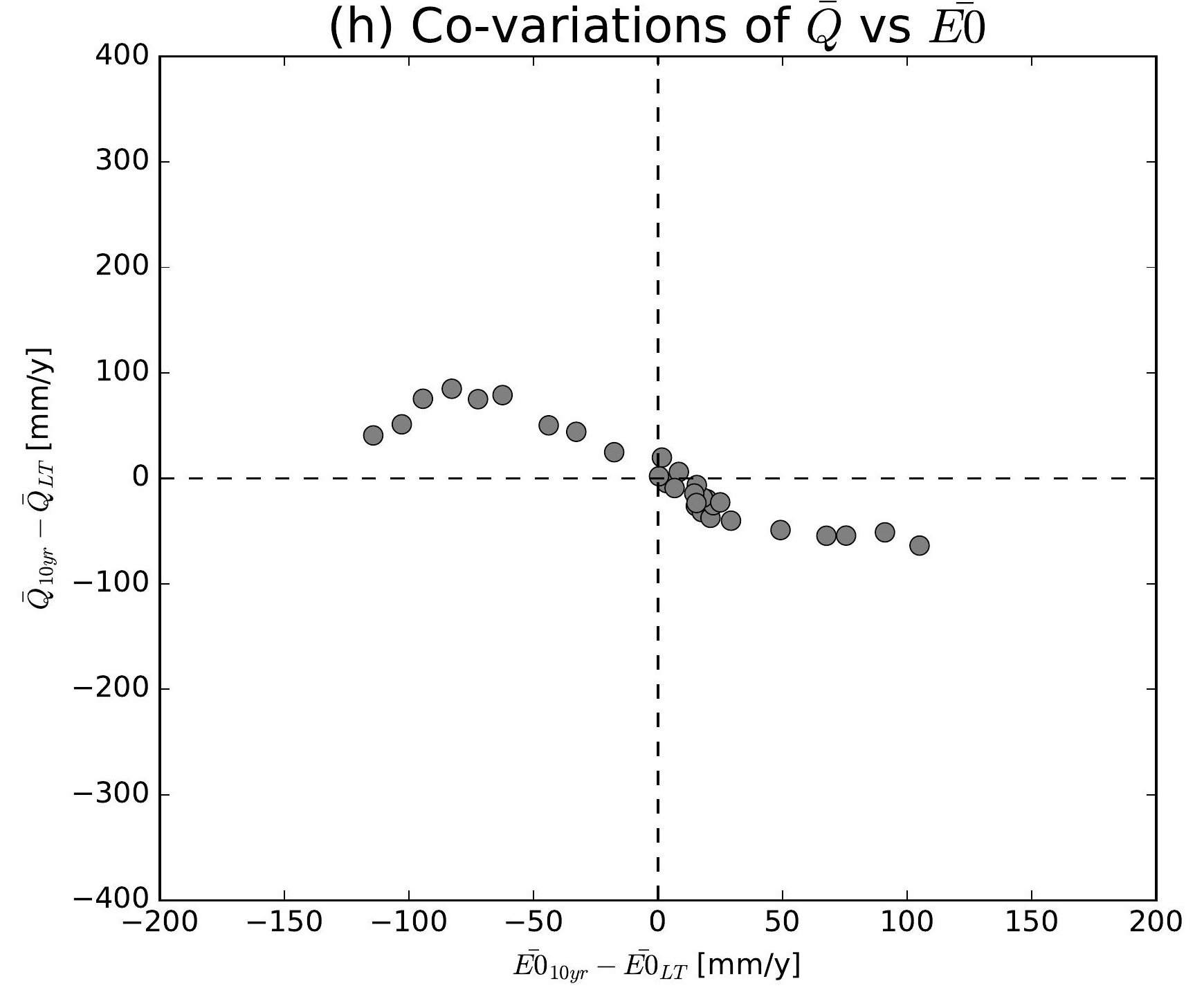

(k) Co-variations of $\bar{Q}$ vs $\overline{E 0}$

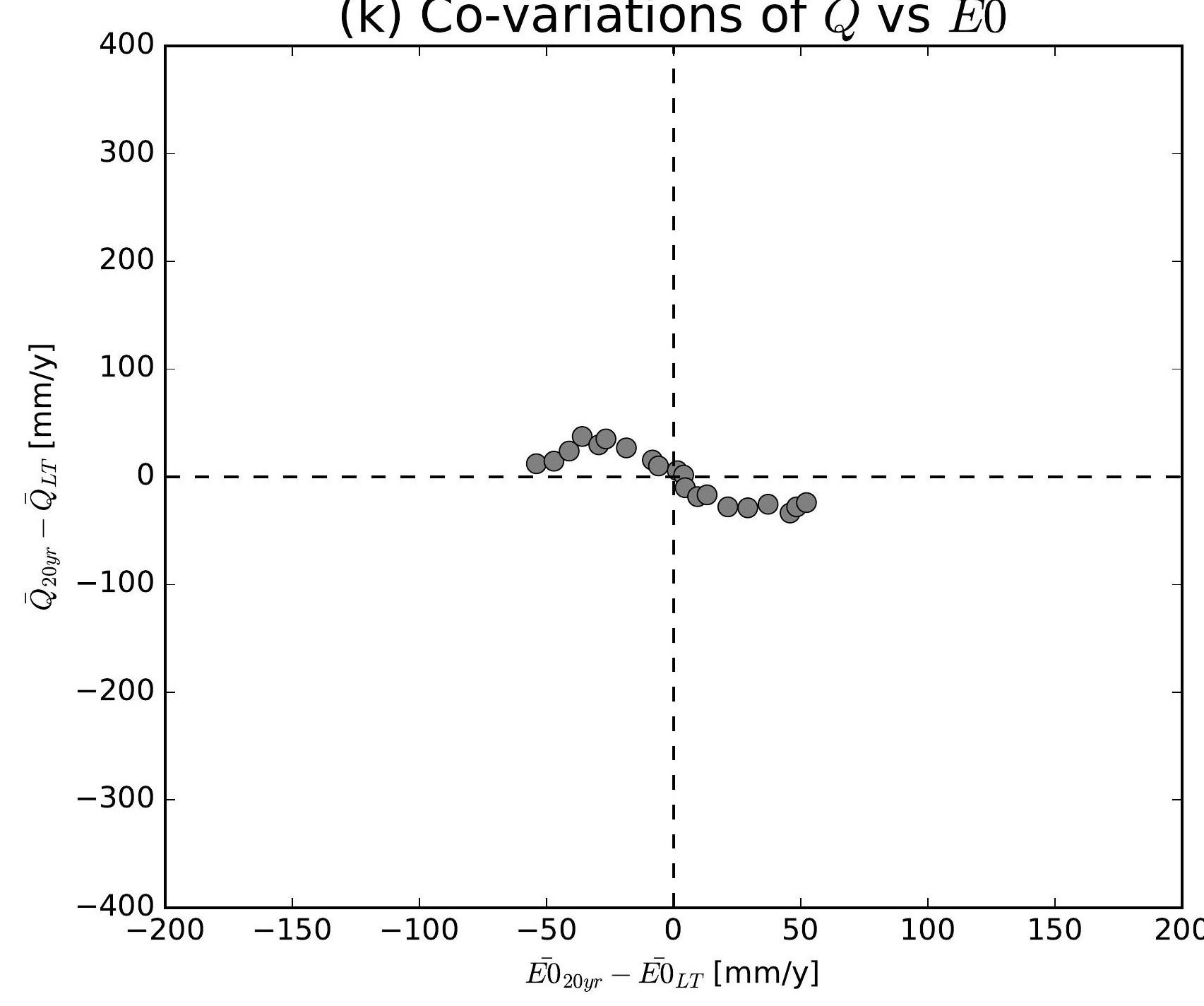

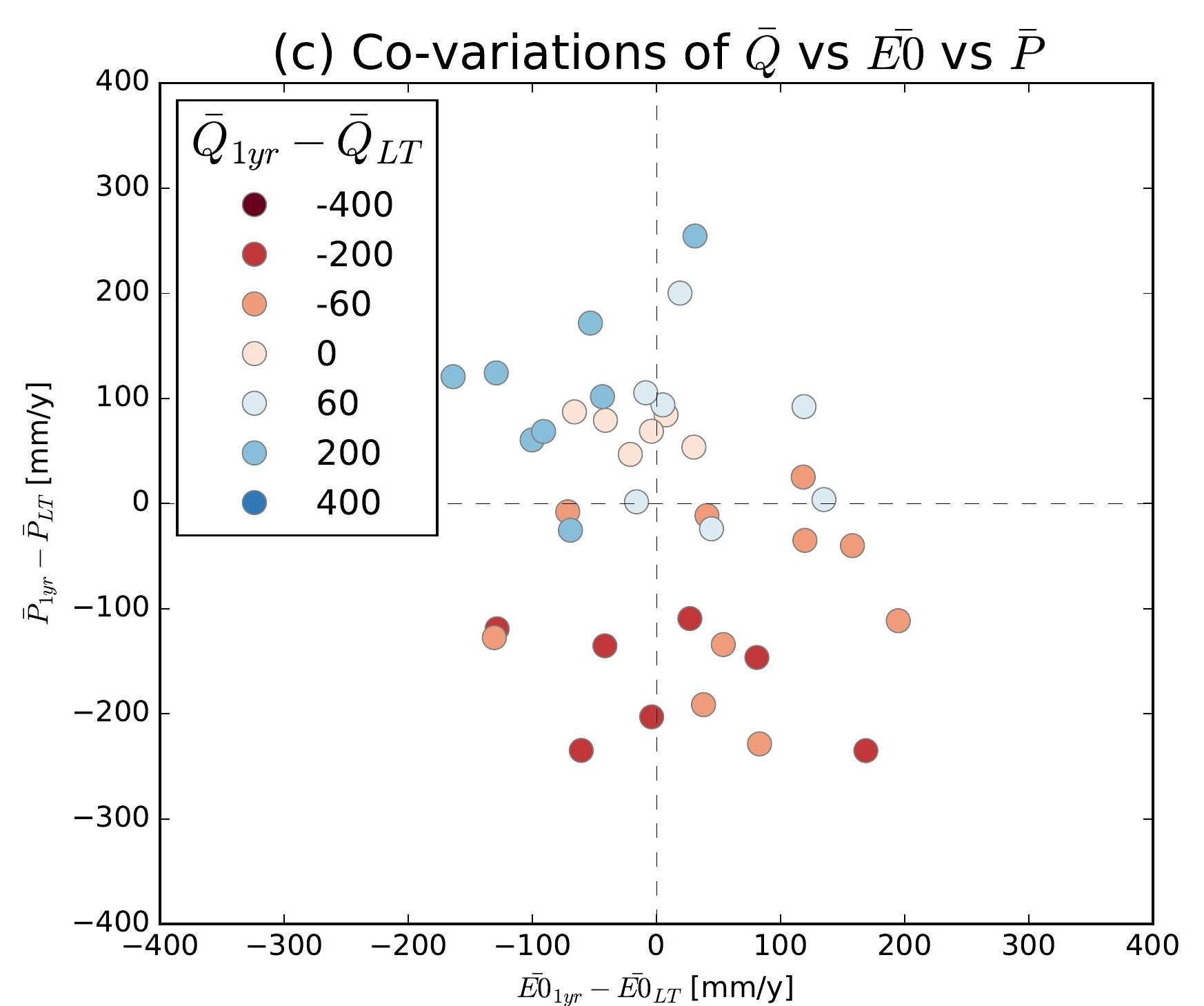
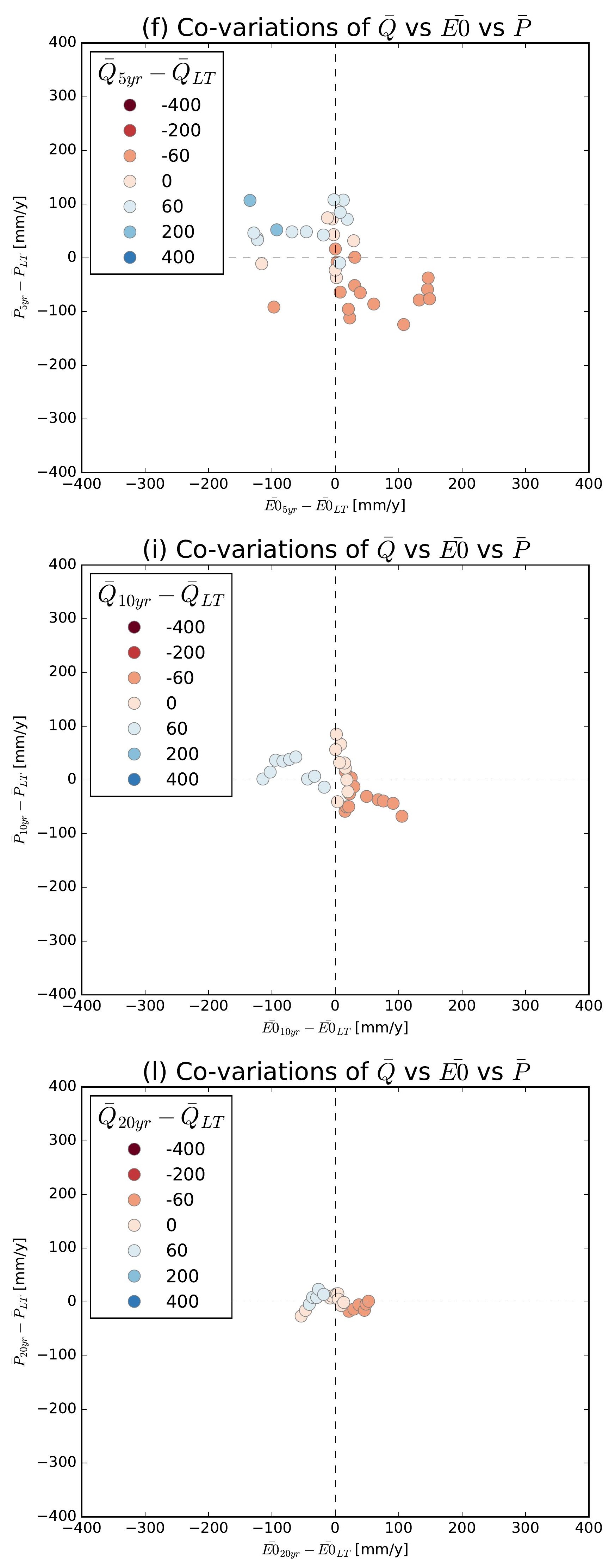

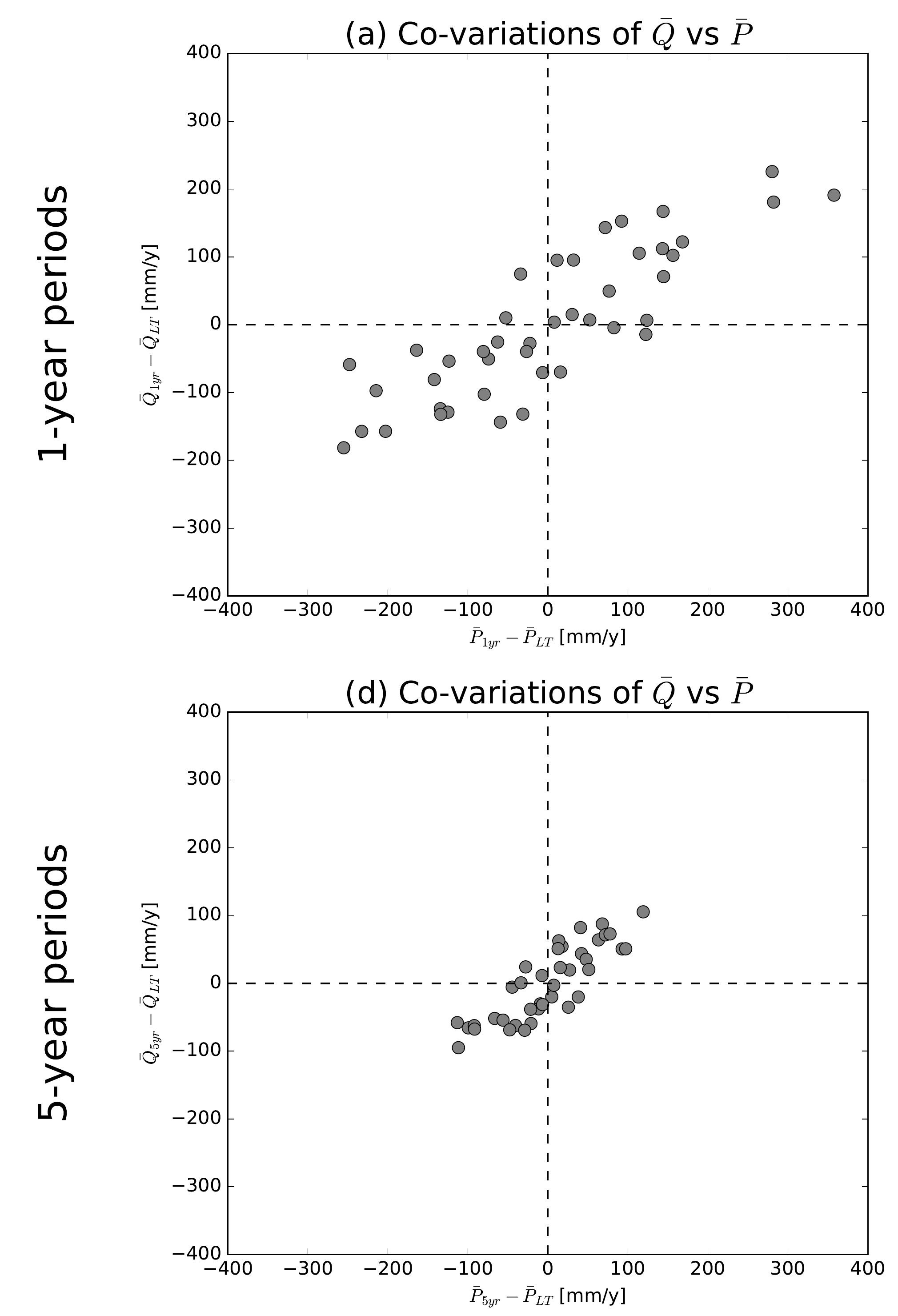

(g) Co-variations of $\bar{Q}$ vs $\bar{P}$
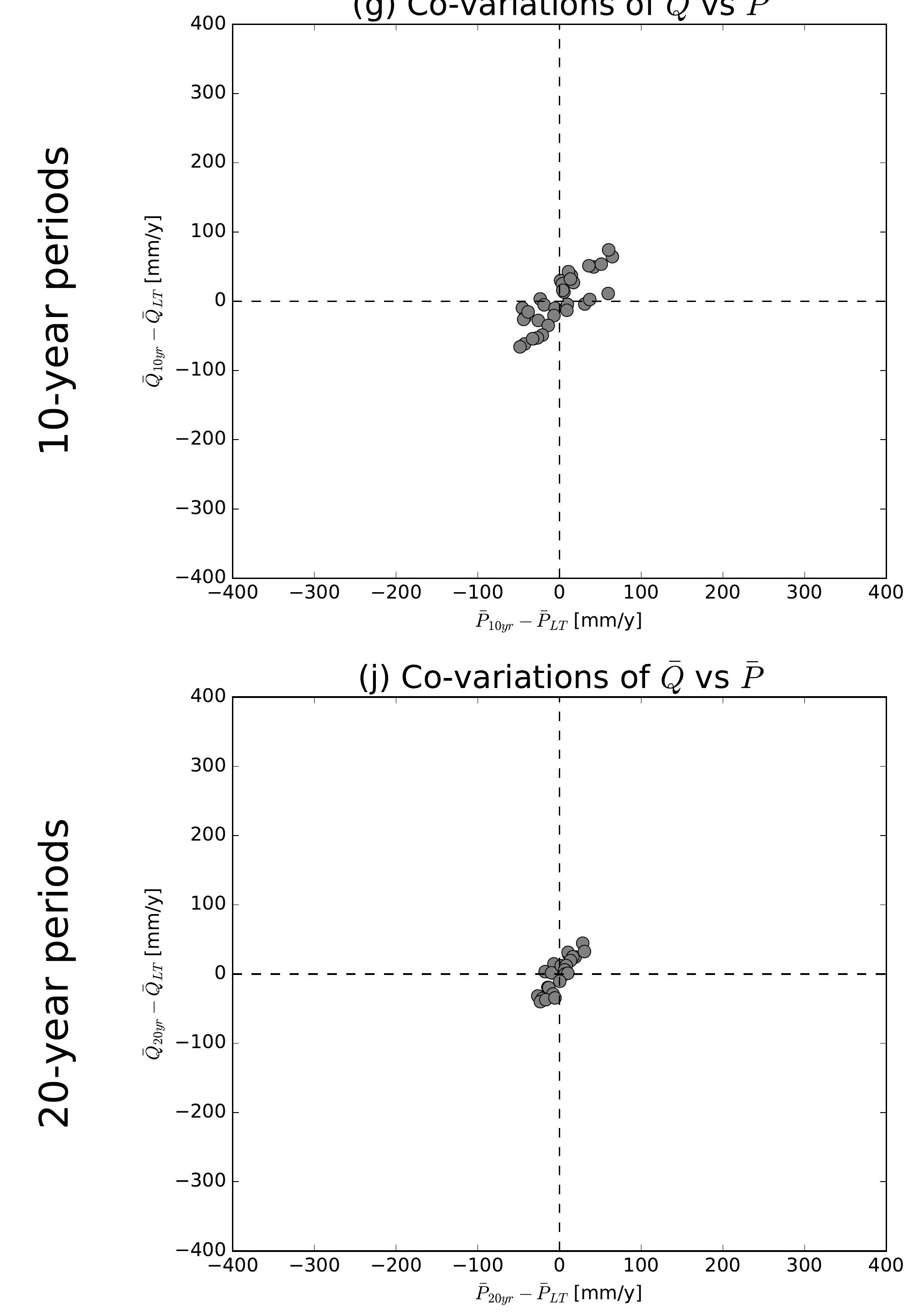

(b) Co-variations of $\bar{Q}$ vs $\overline{E 0}$

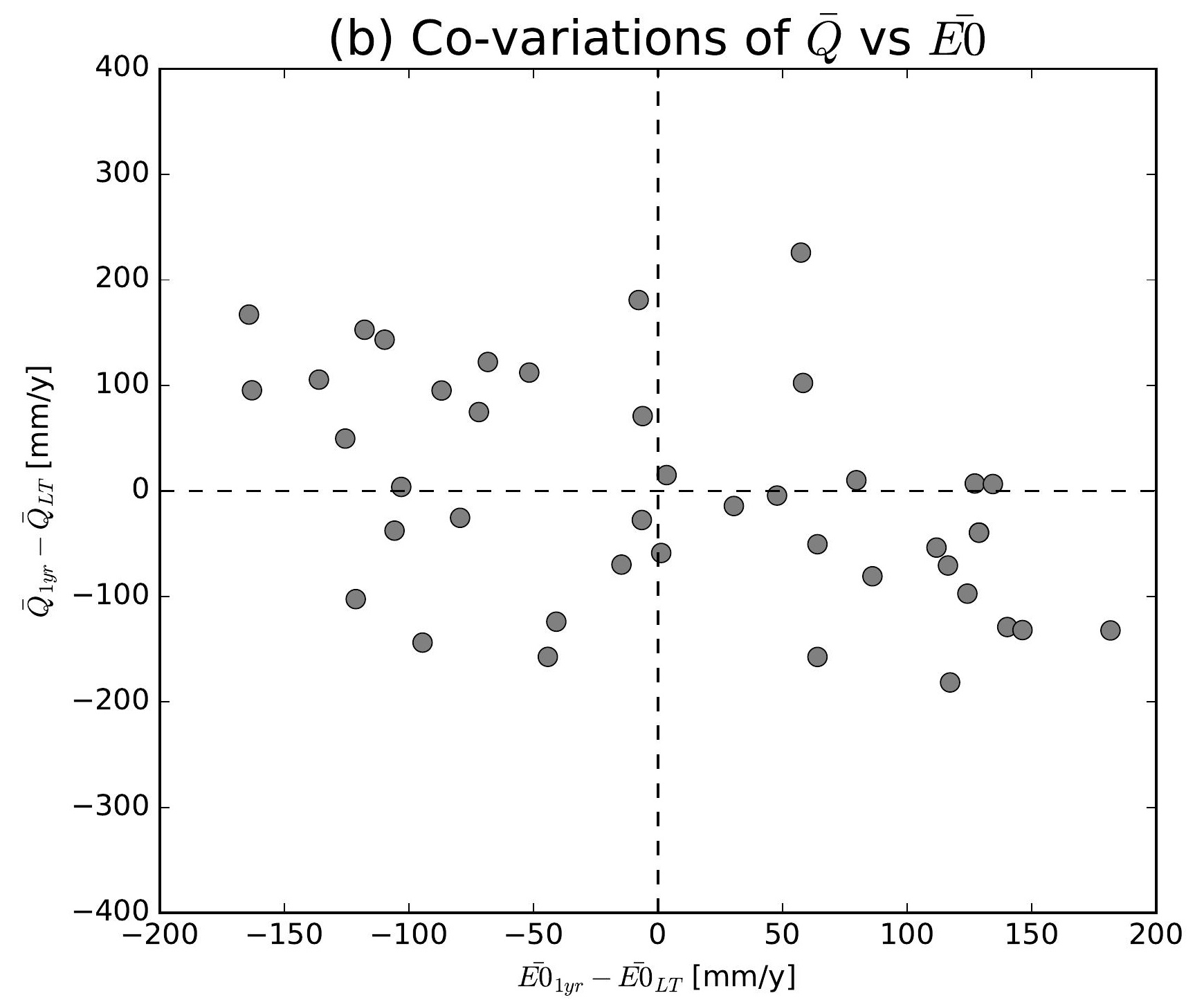

(e) Co-variations of $\bar{Q}$ vs $\overline{E 0}$

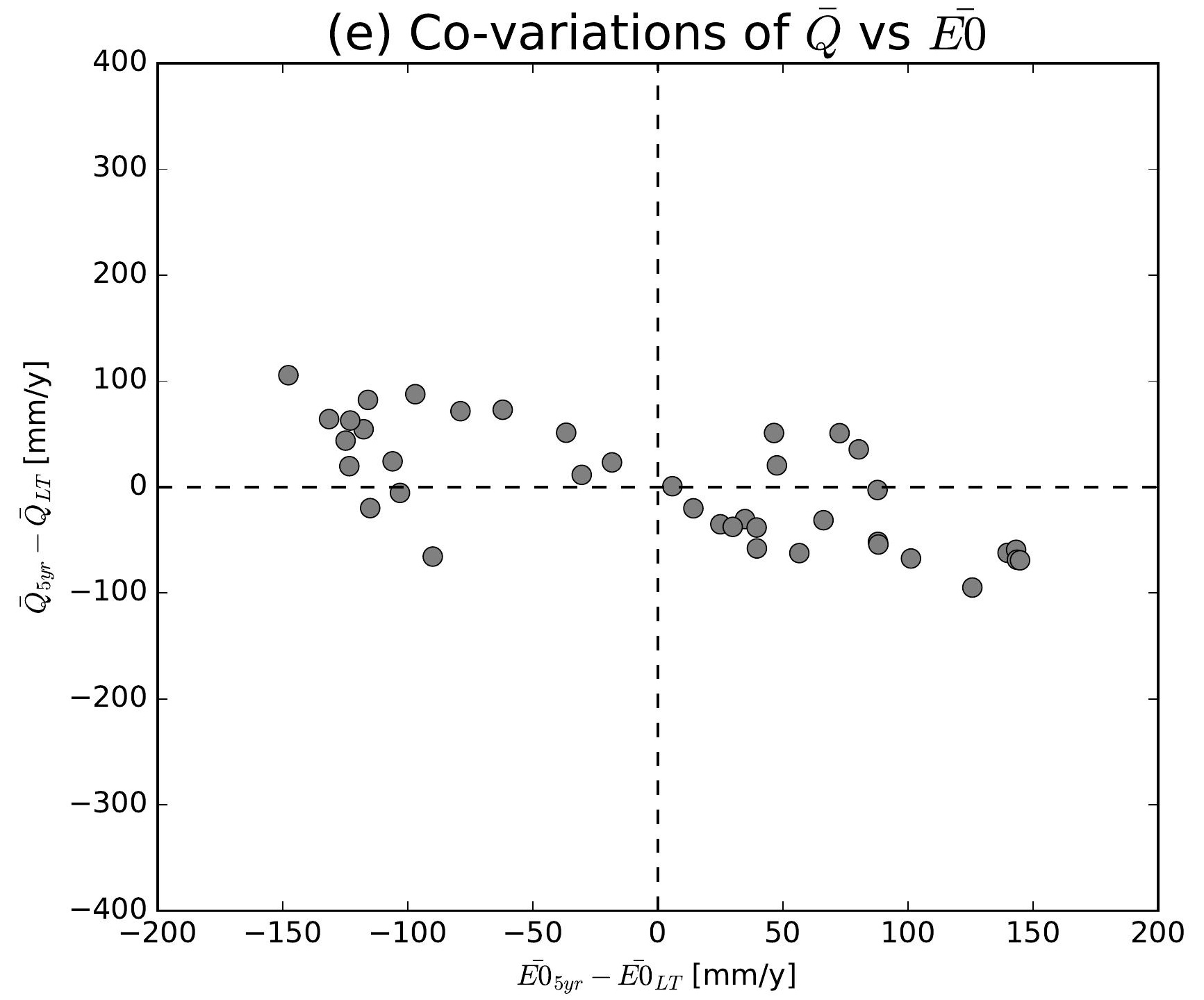

(h) Co-variations of $\bar{Q}$ vs $\overline{E 0}$

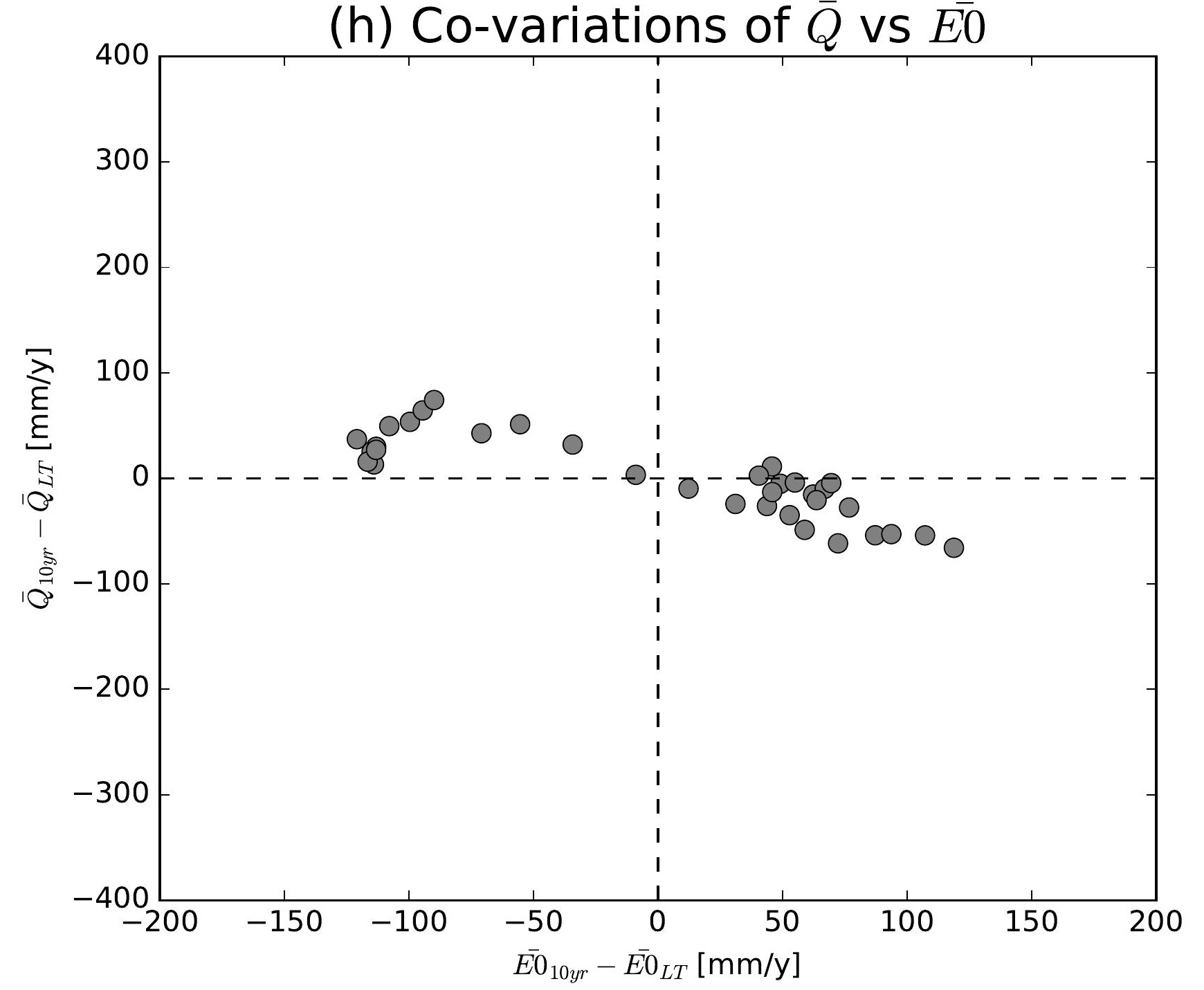

(k) Co-variations of $\bar{Q}$ vs $\overline{E 0}$

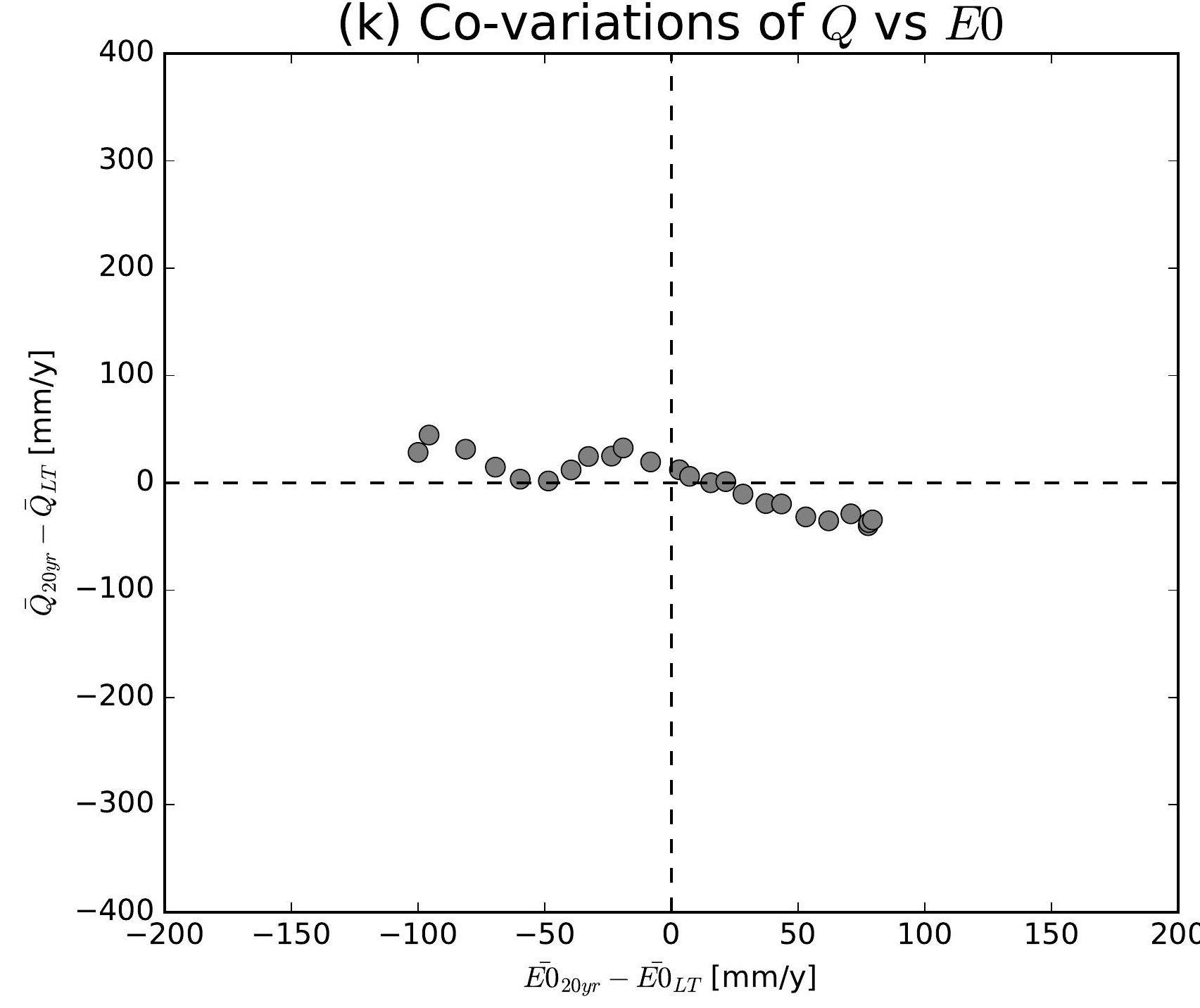

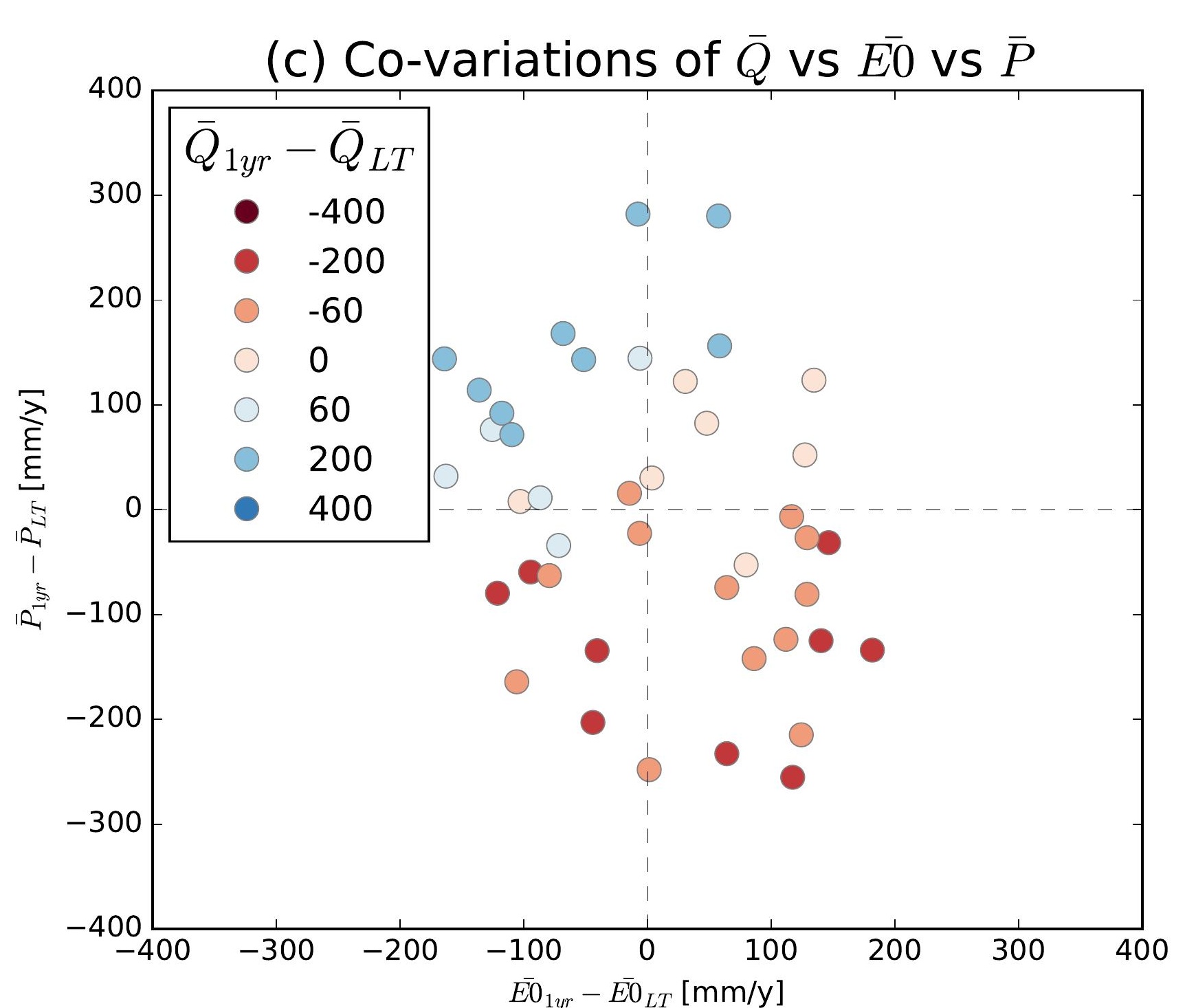
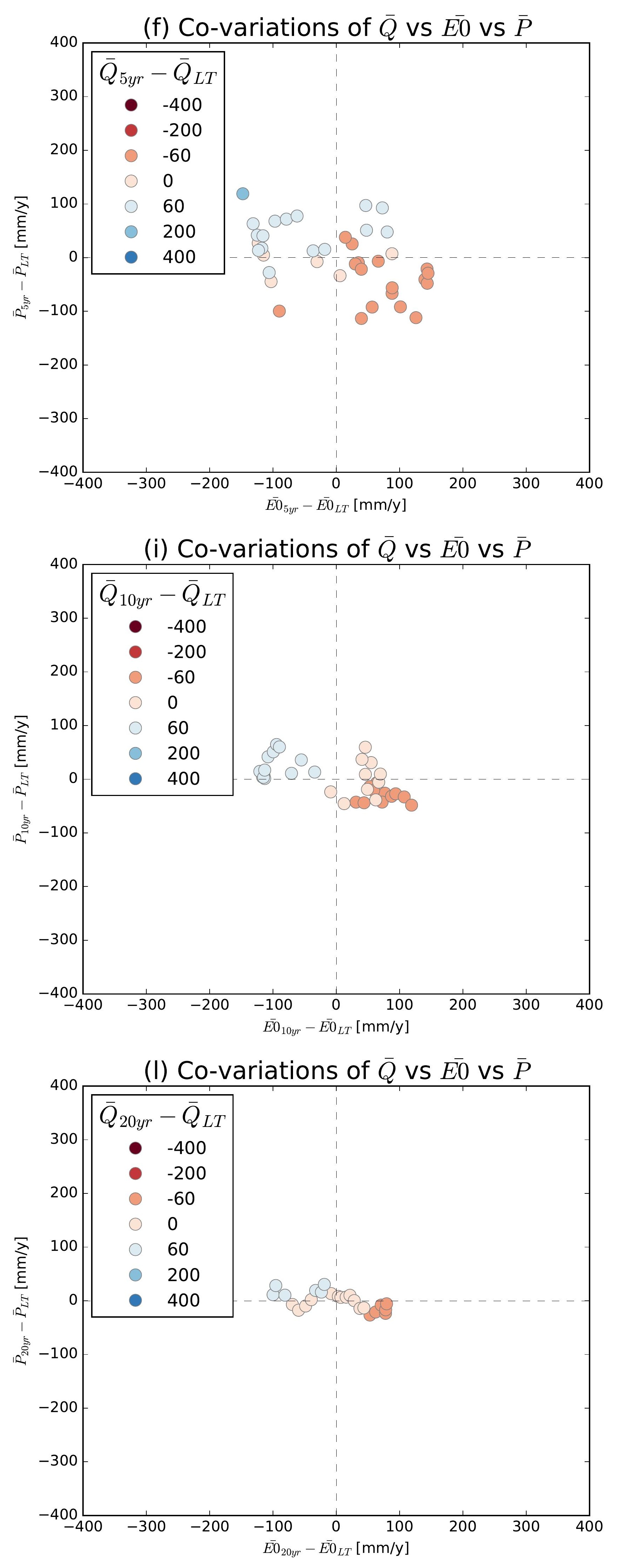

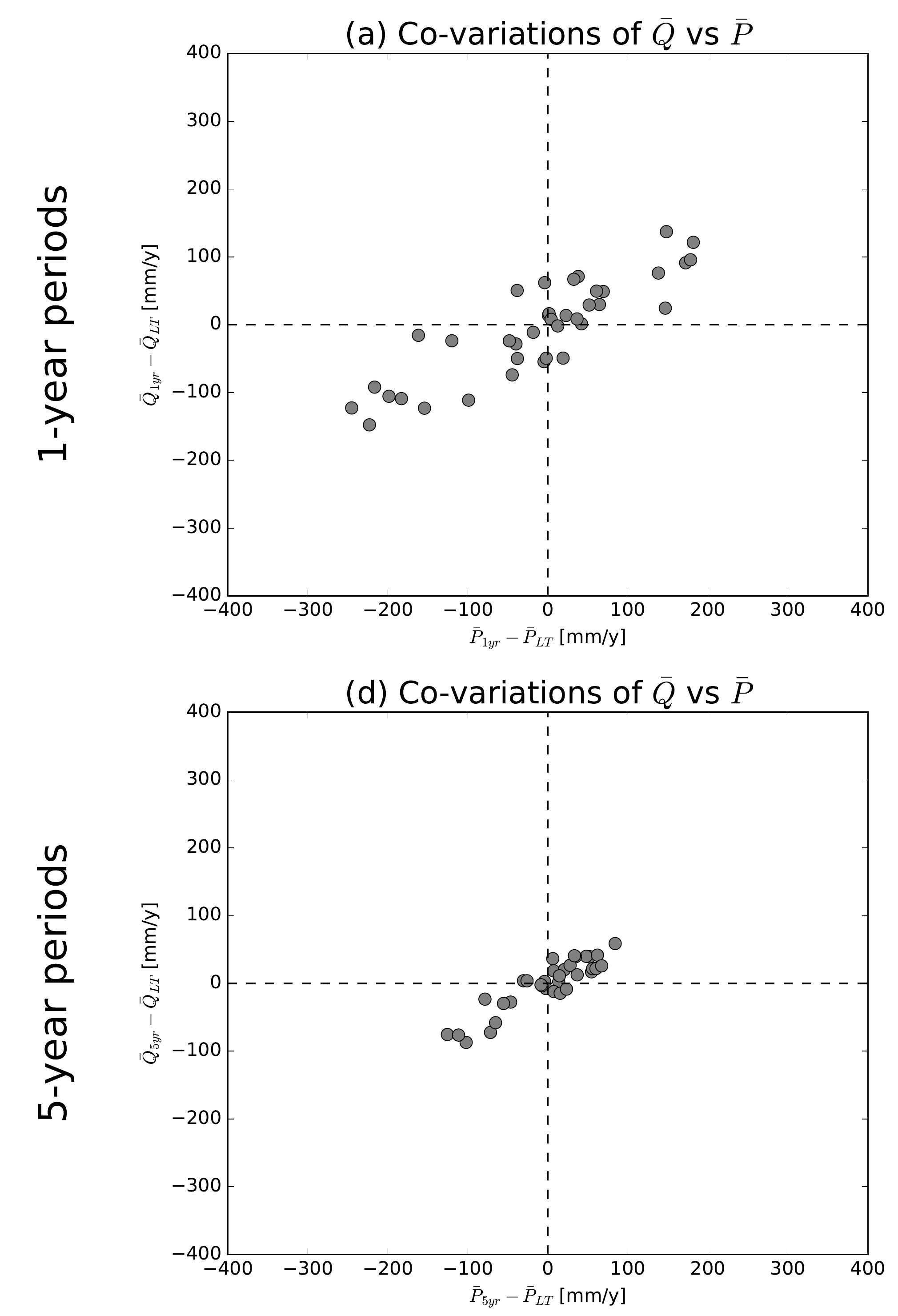

(g) Co-variations of $\bar{Q}$ vs $\bar{P}$
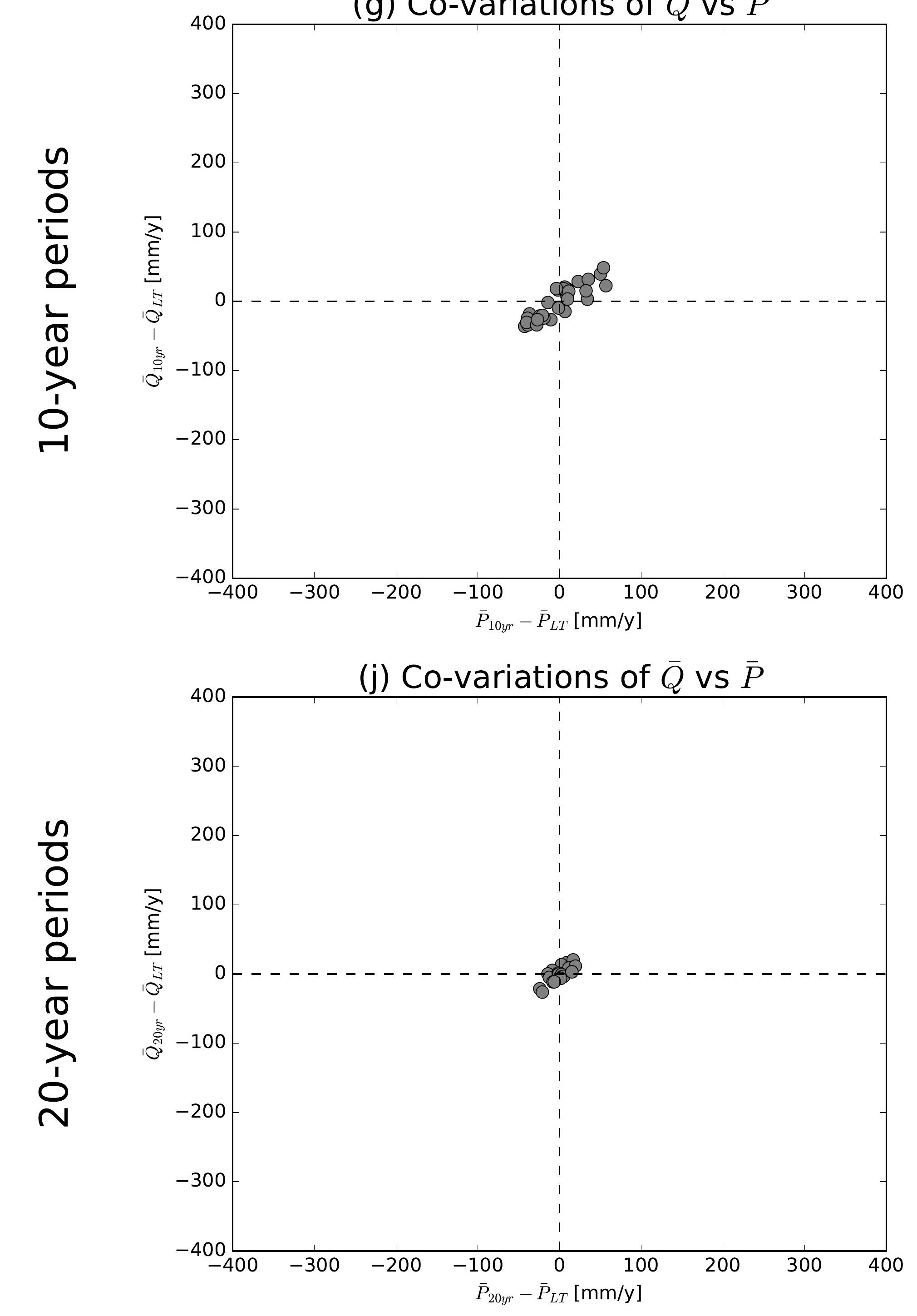

(b) Co-variations of $\bar{Q}$ vs $\overline{E 0}$

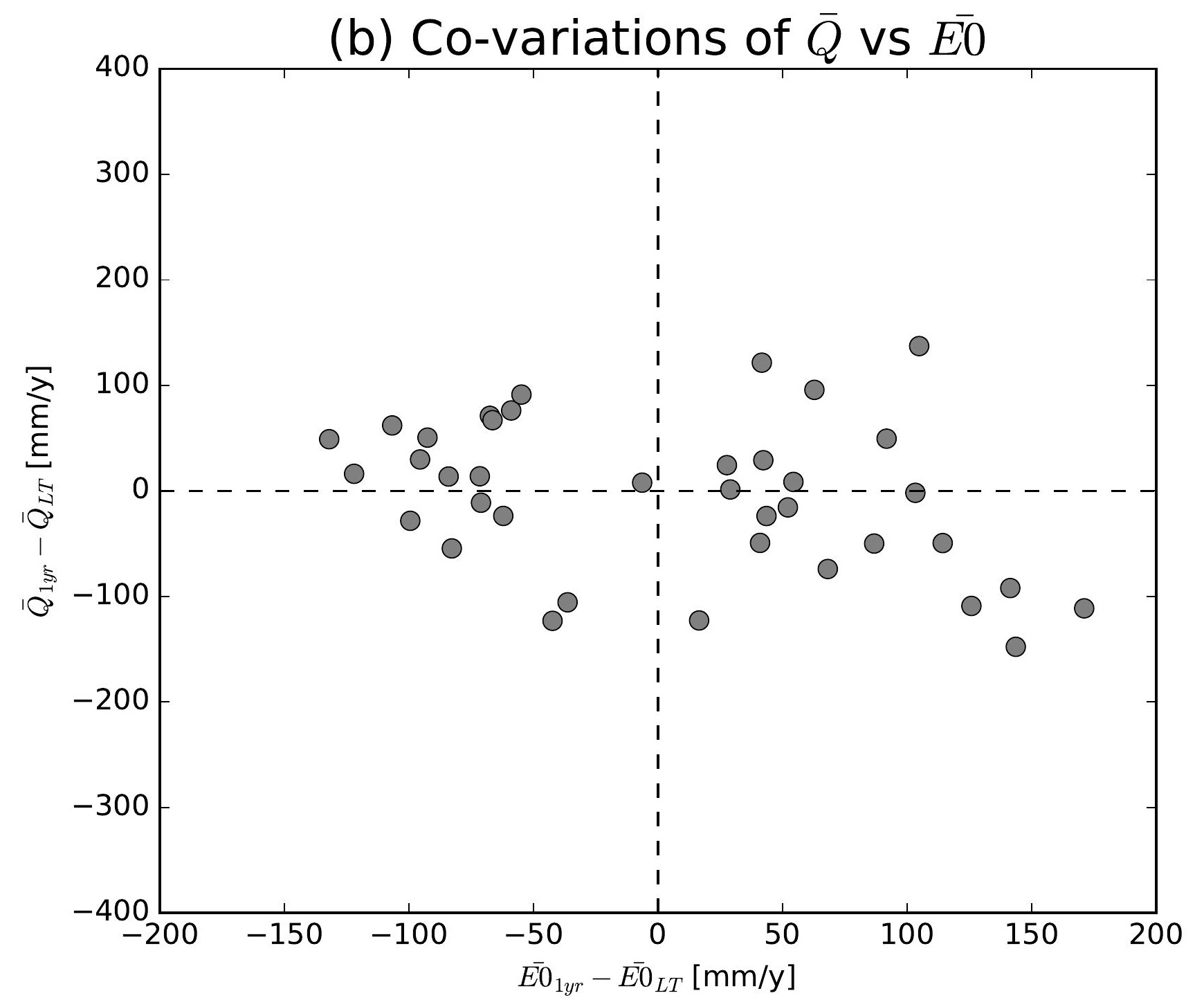

(e) Co-variations of $\bar{Q}$ vs $\overline{E 0}$

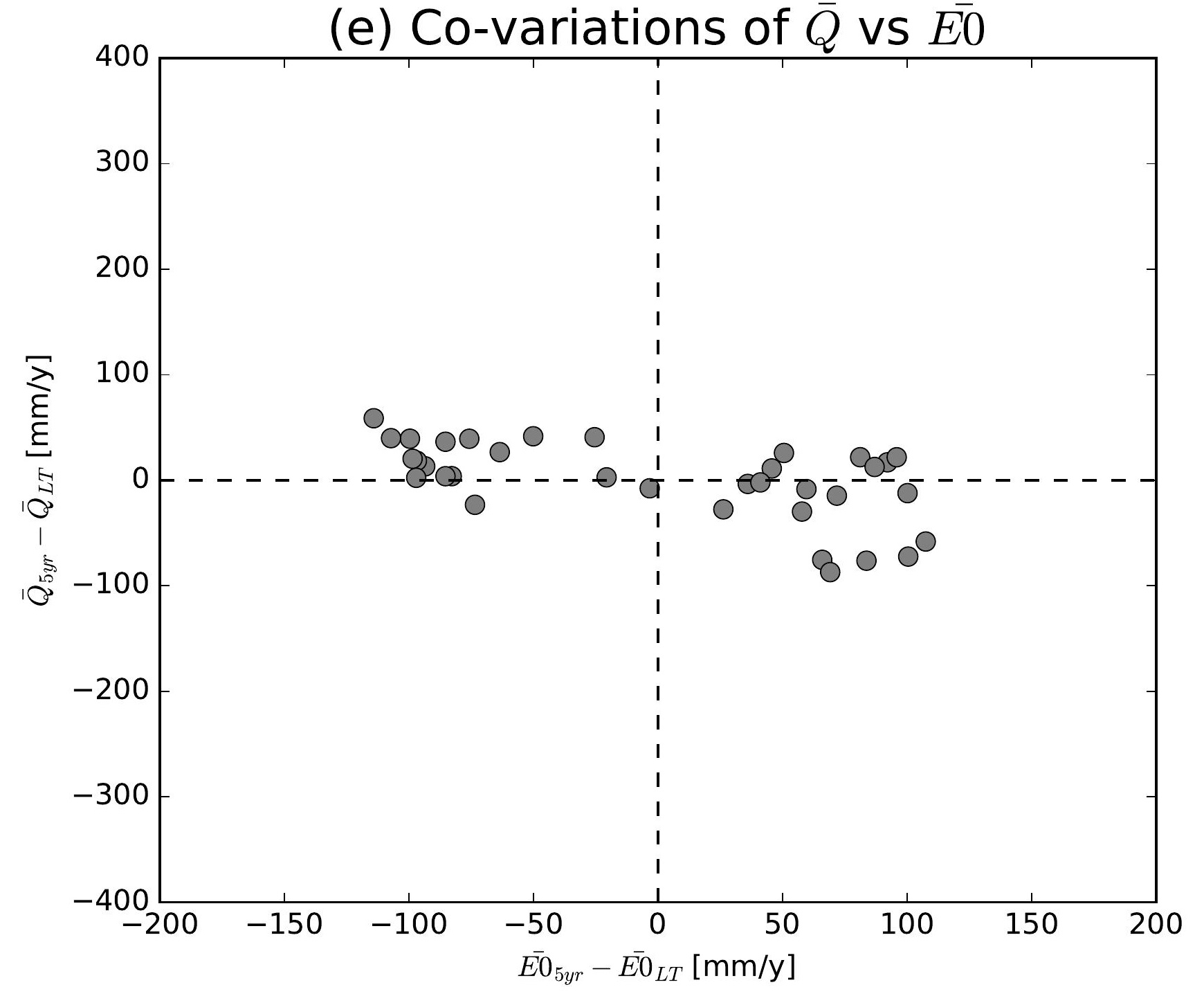

(h) Co-variations of $\bar{Q}$ vs $\overline{E 0}$

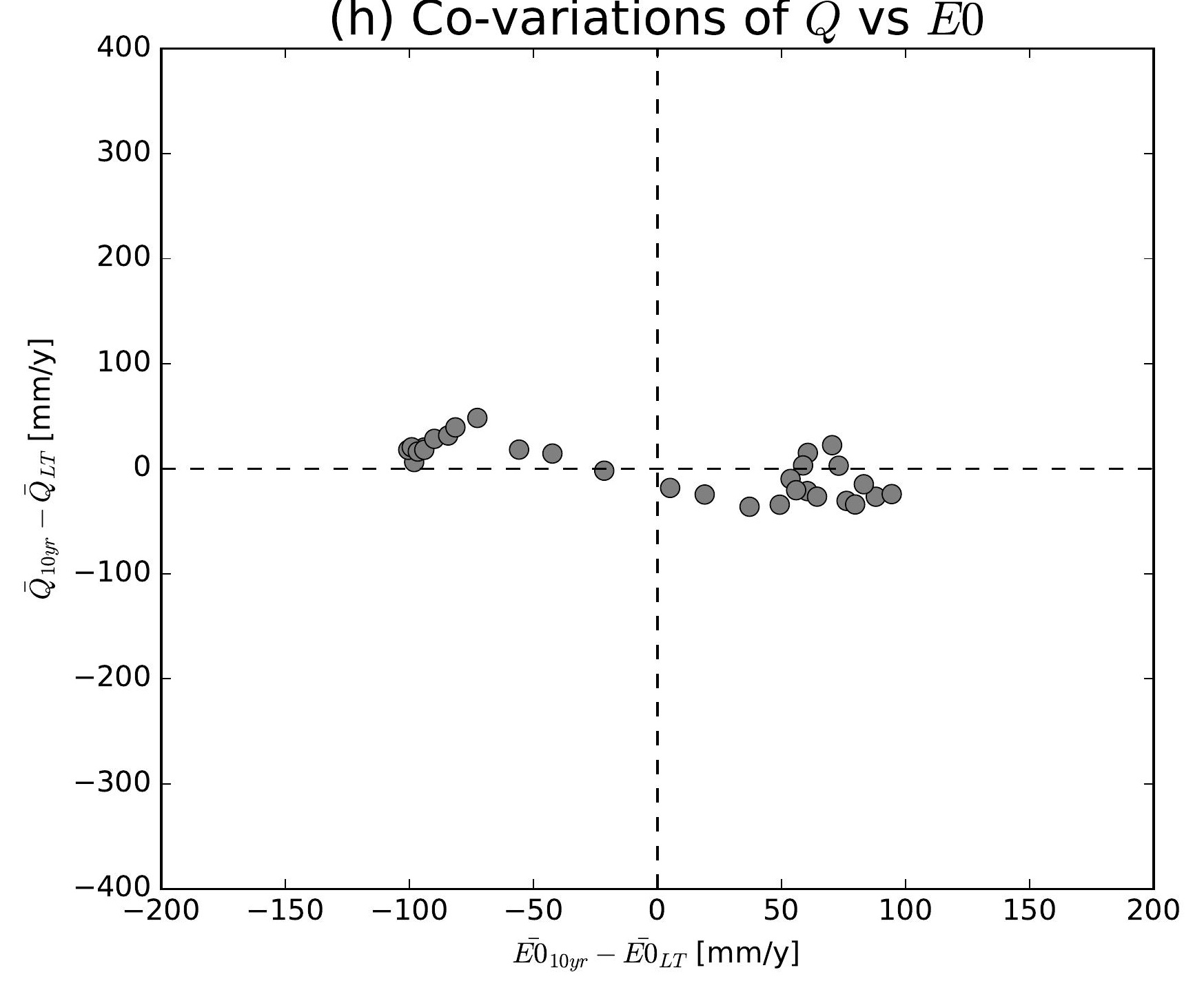

(k) Co-variations of $\bar{Q}$ vs $\overline{E 0}$

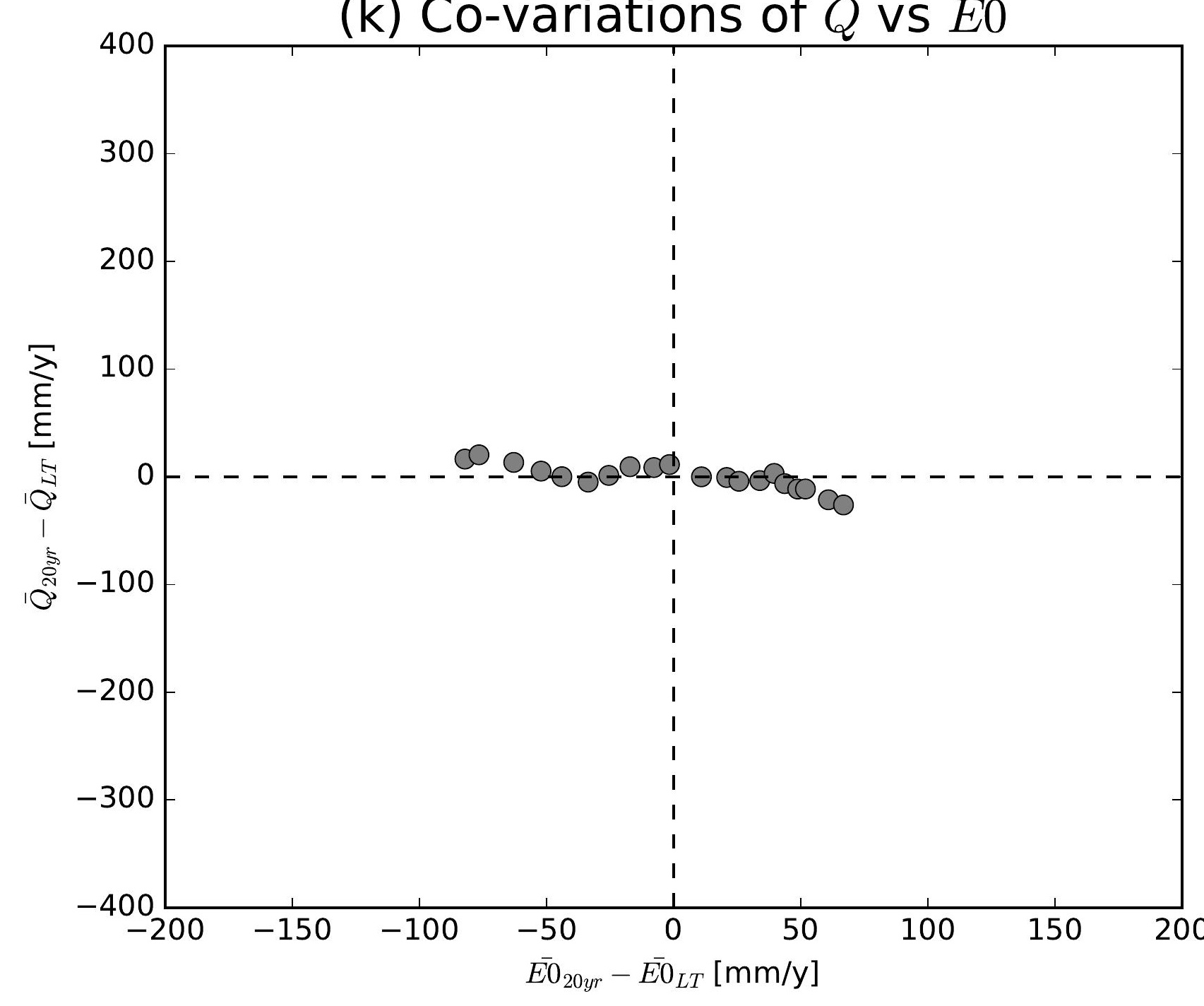

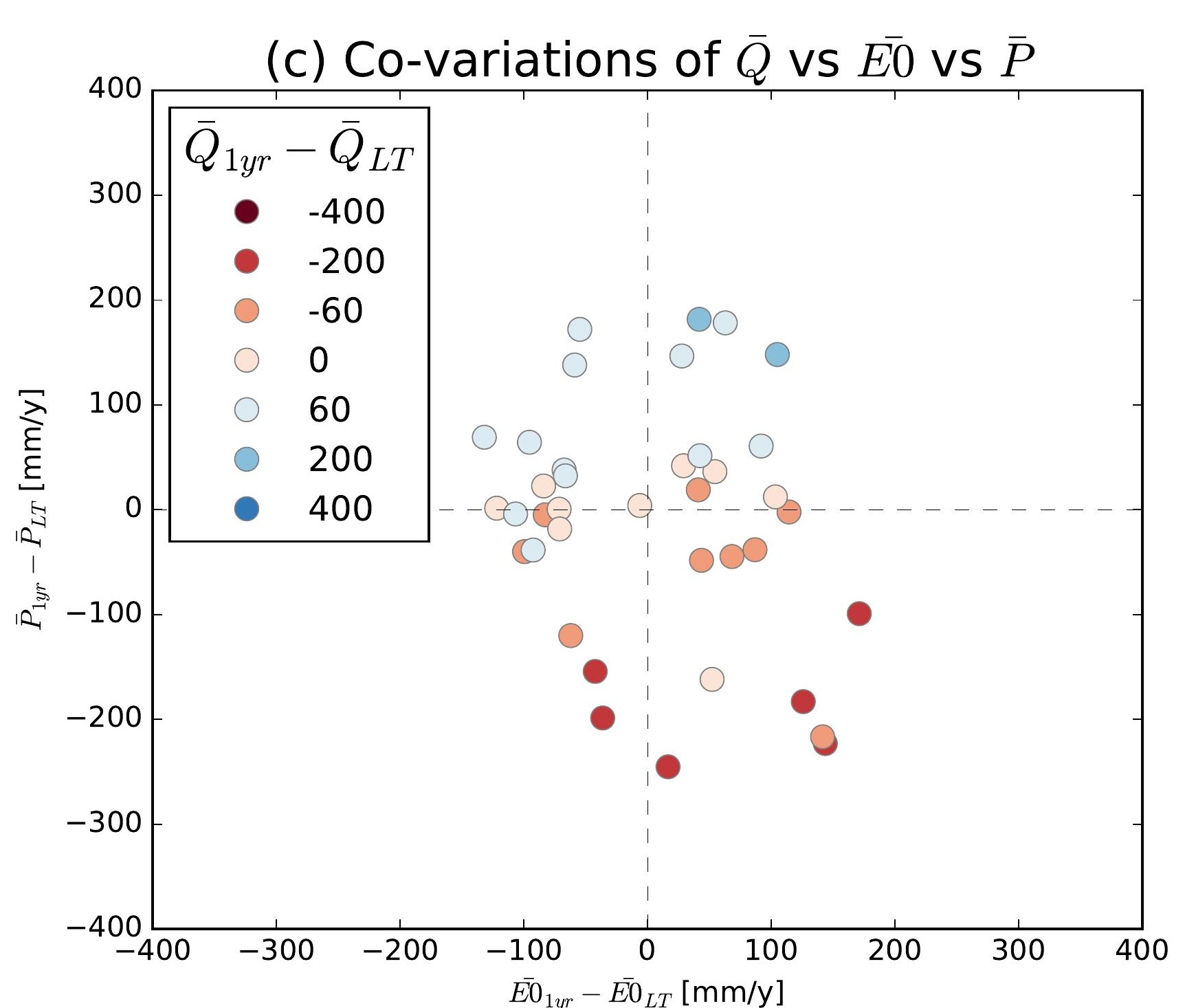
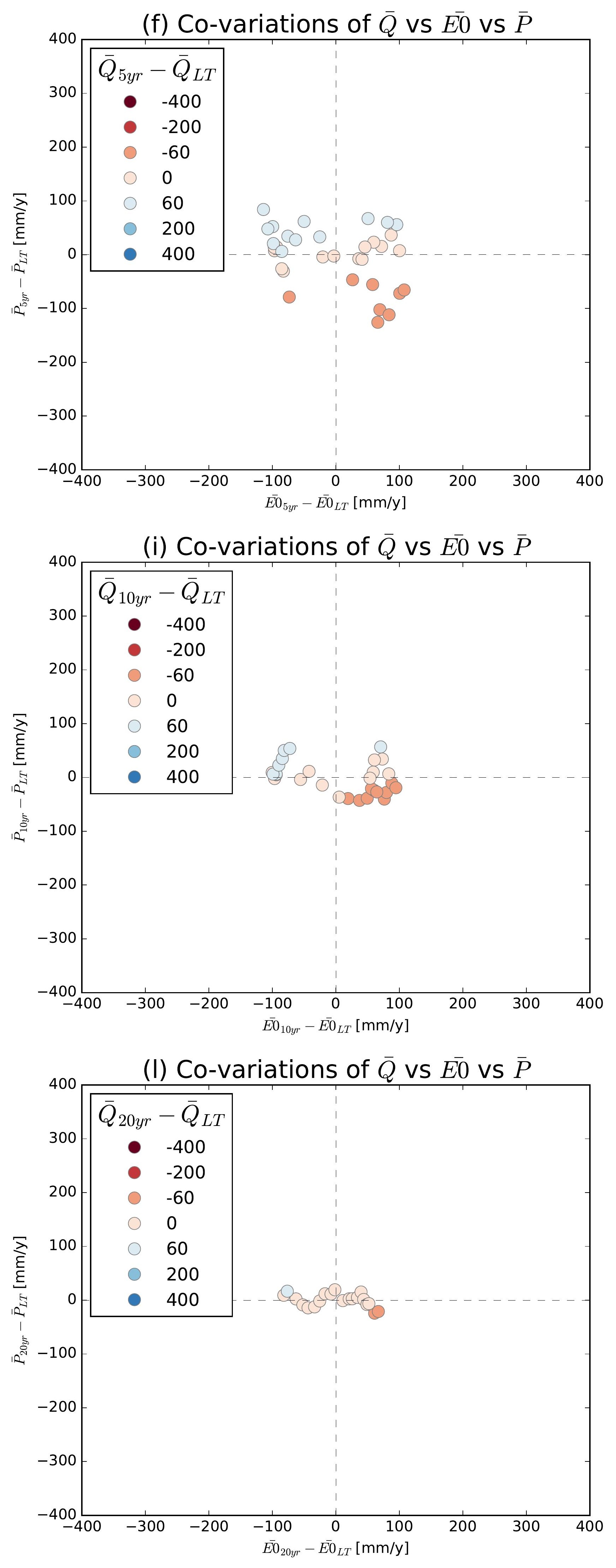

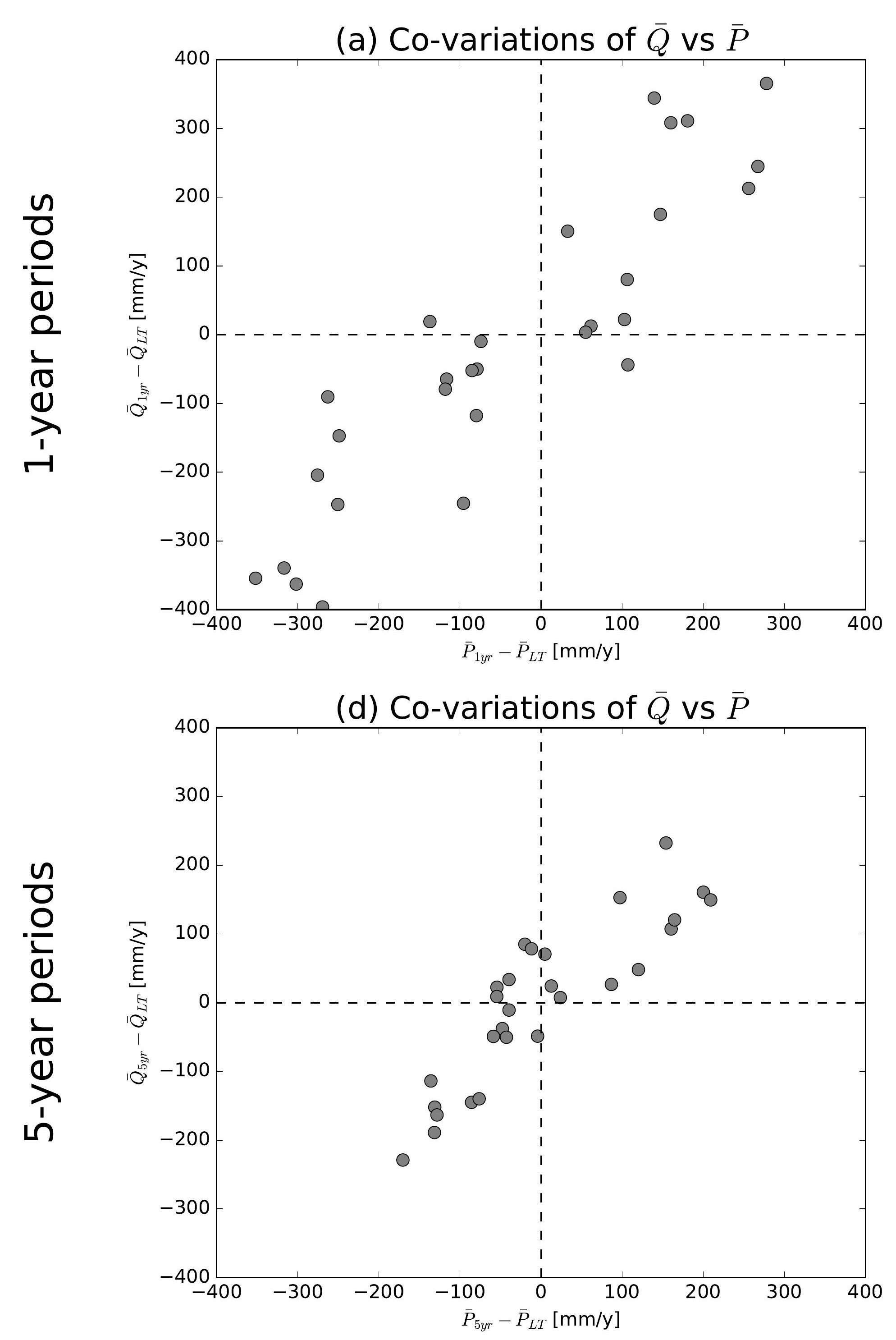

(g) Co-variations of $\bar{Q}$ vs $\bar{P}$

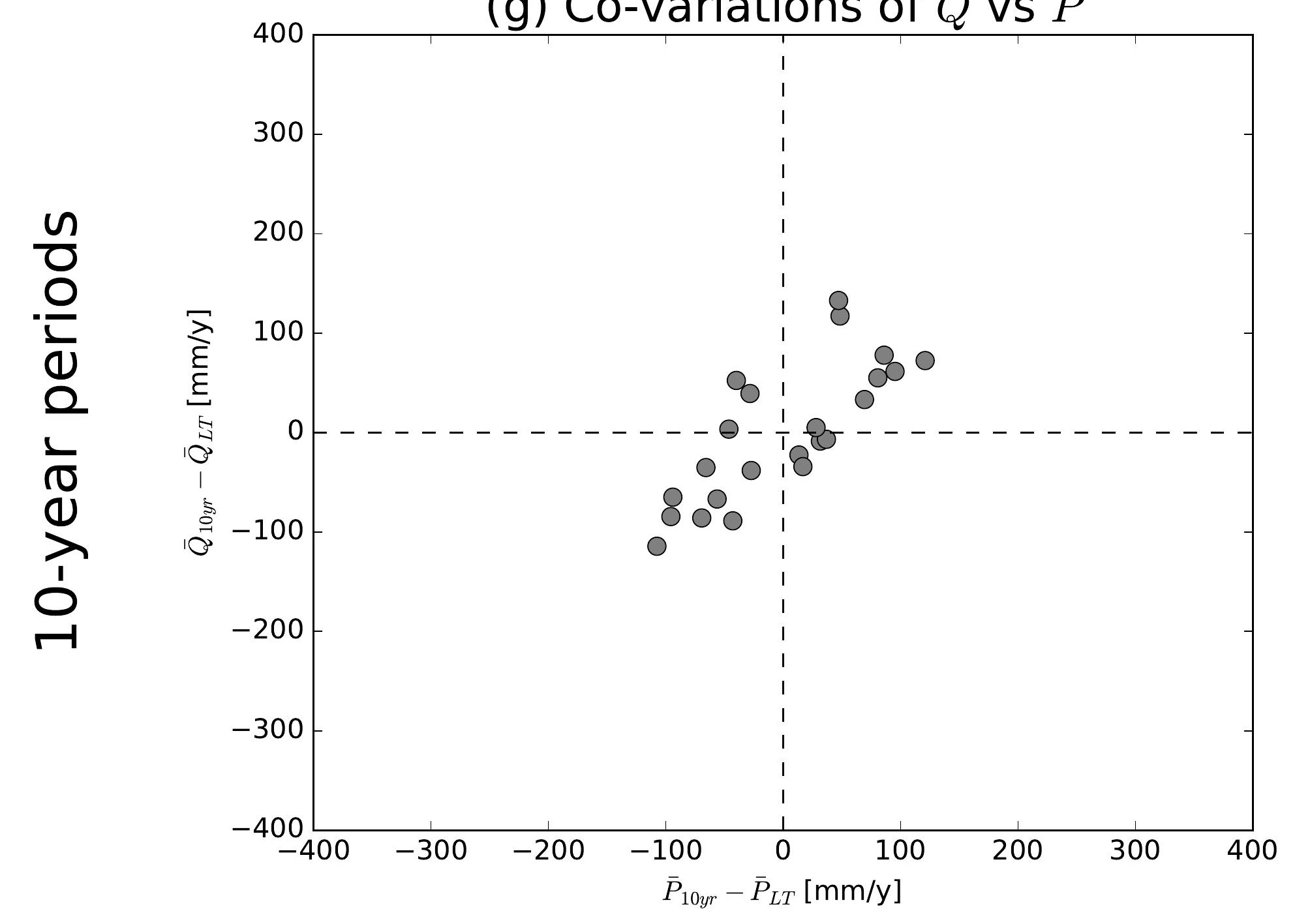

(j) Co-variations of $\bar{Q}$ vs $\bar{P}$

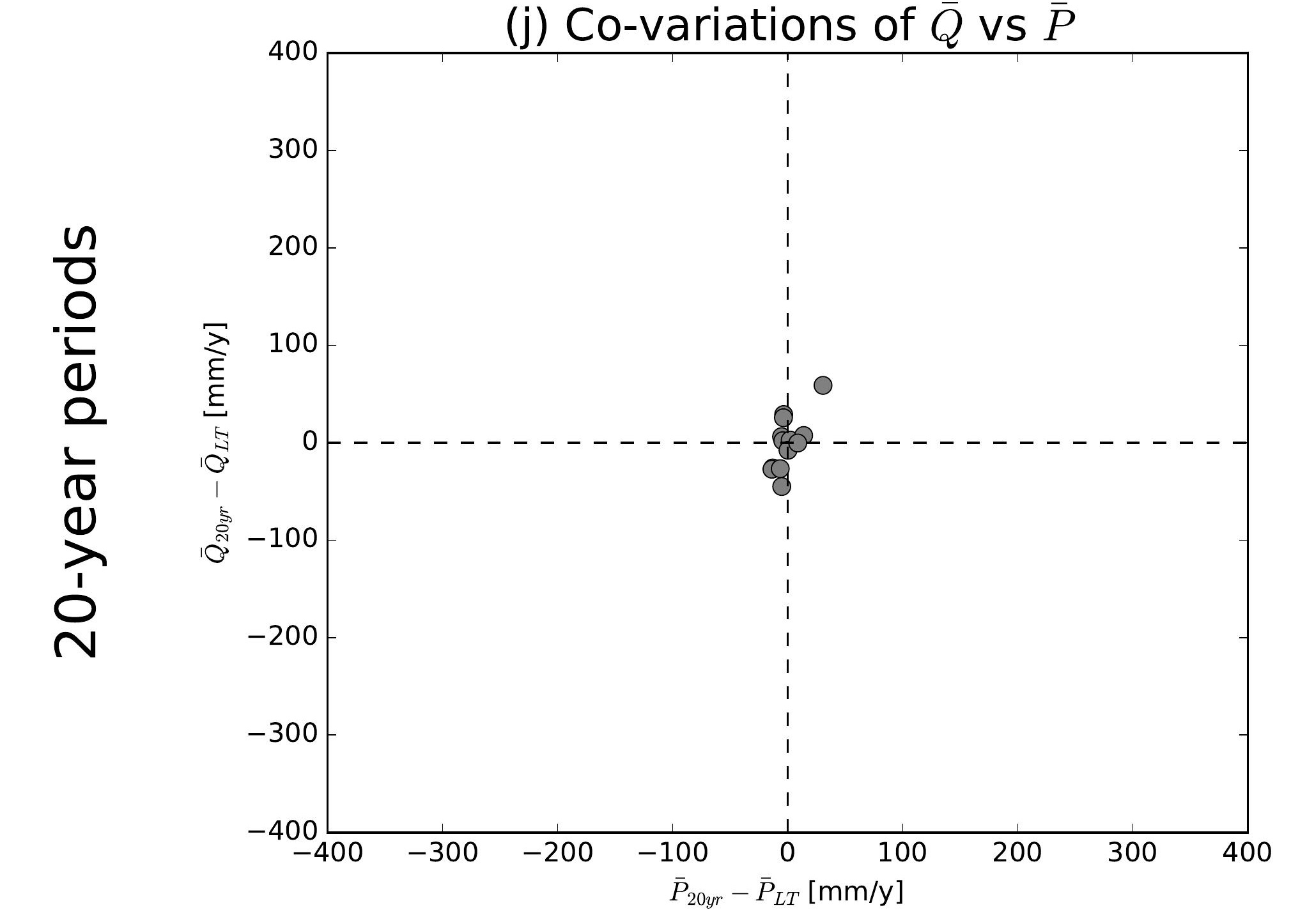

(b) Co-variations of $\bar{Q}$ vs $\overline{E 0}$

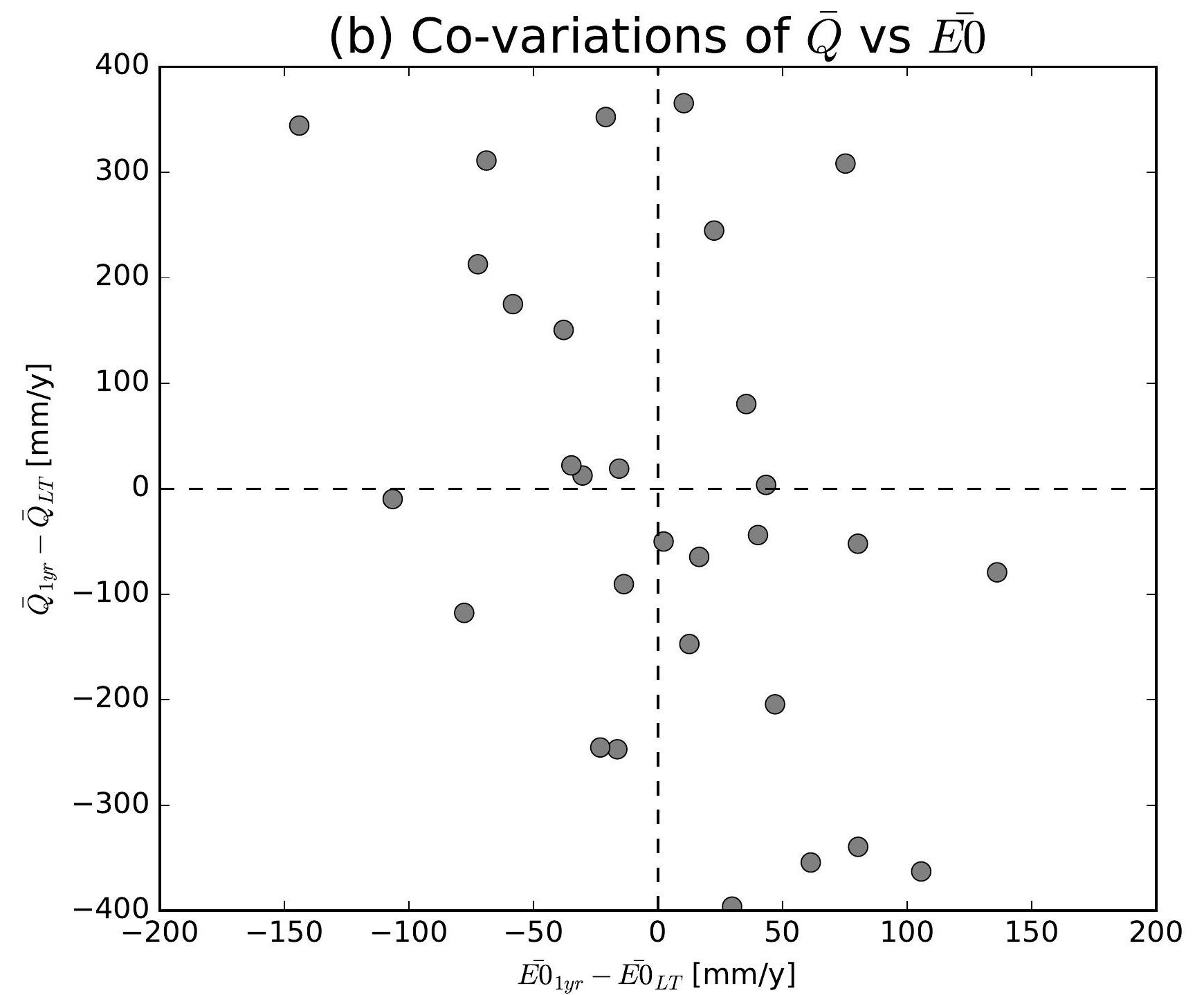

(e) Co-variations of $\bar{Q}$ vs $\overline{E 0}$

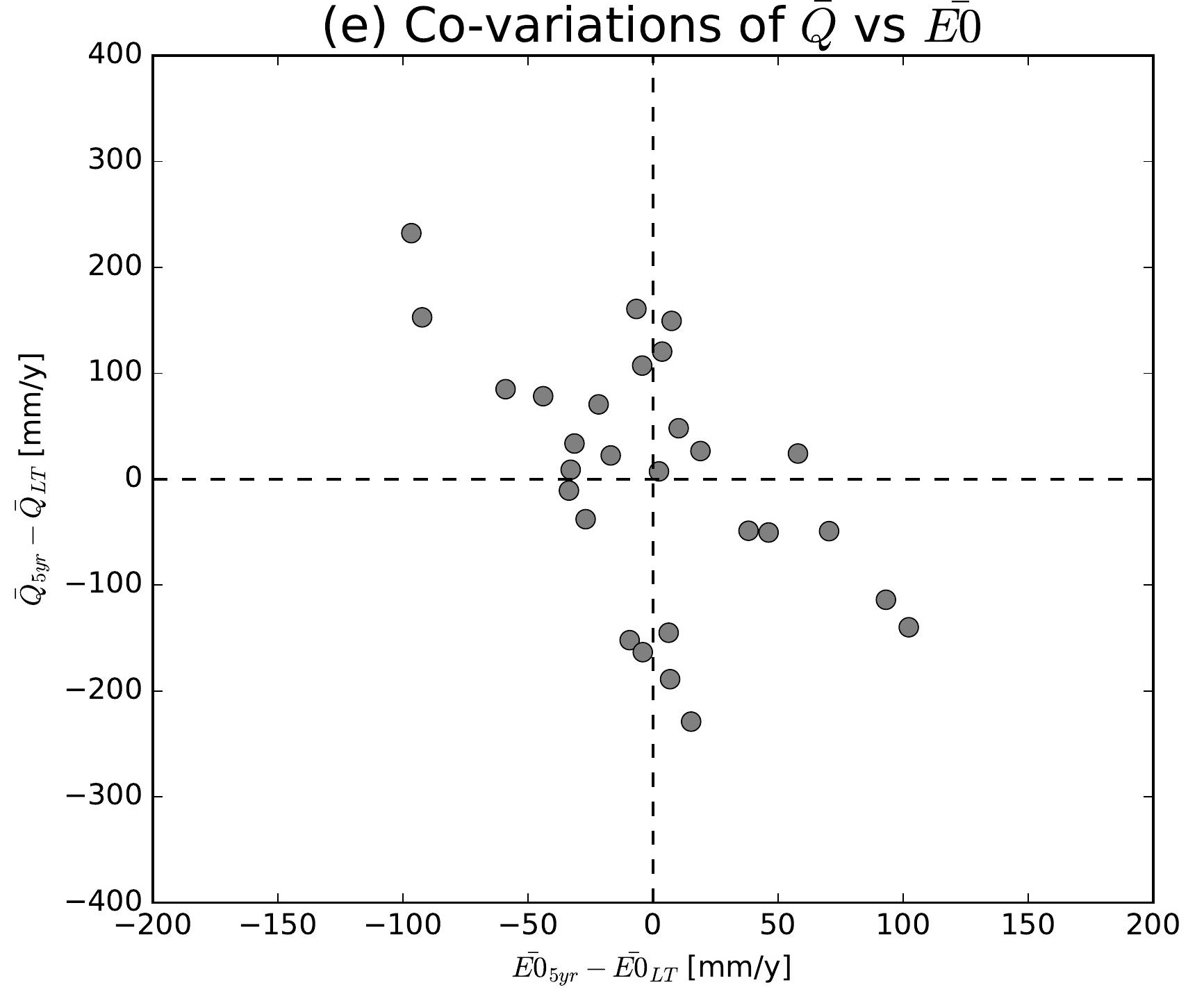

(h) Co-variations of $\bar{Q}$ vs $\overline{E 0}$

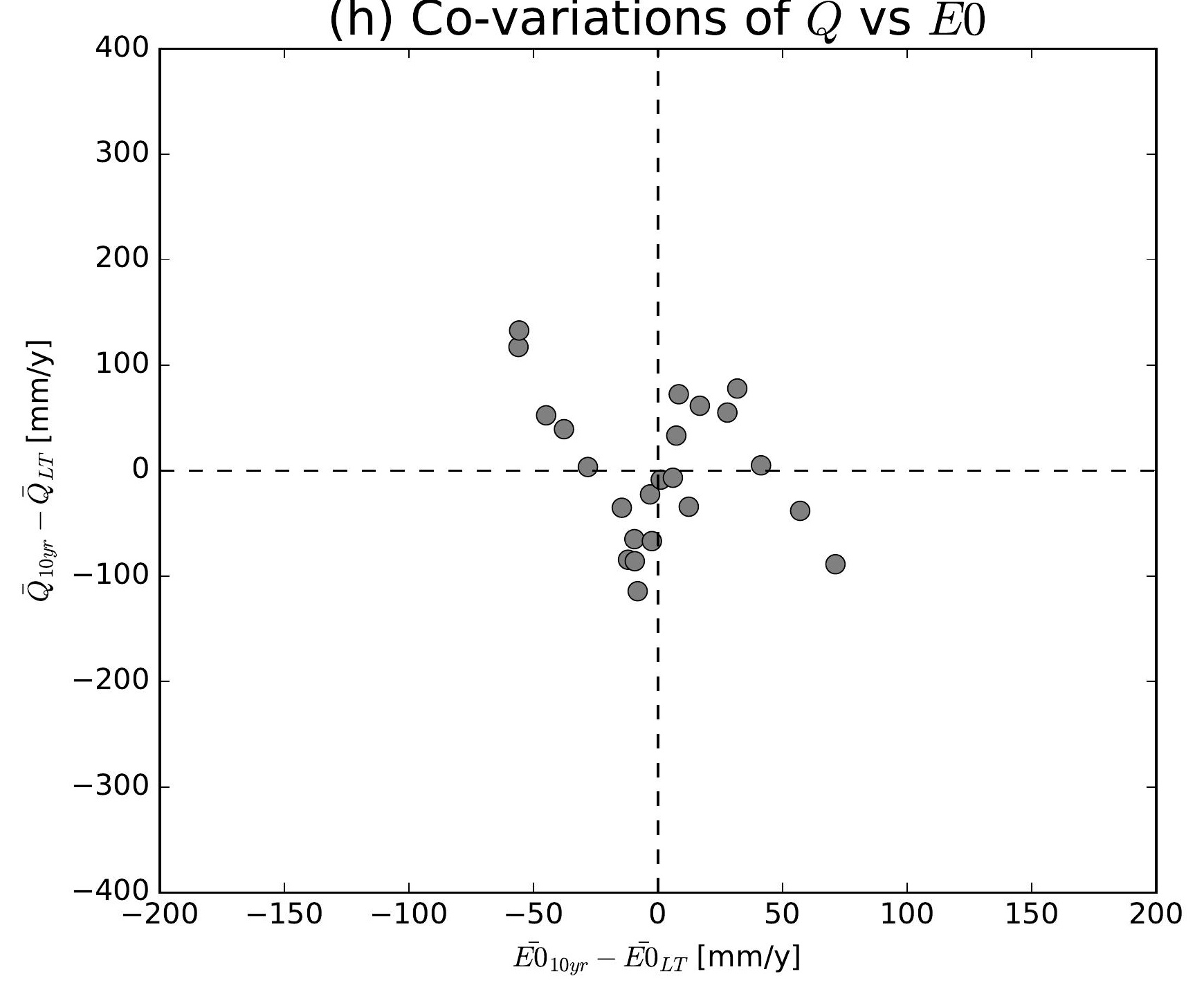

(k) Co-variations of $\bar{Q}$ vs $\overline{E 0}$

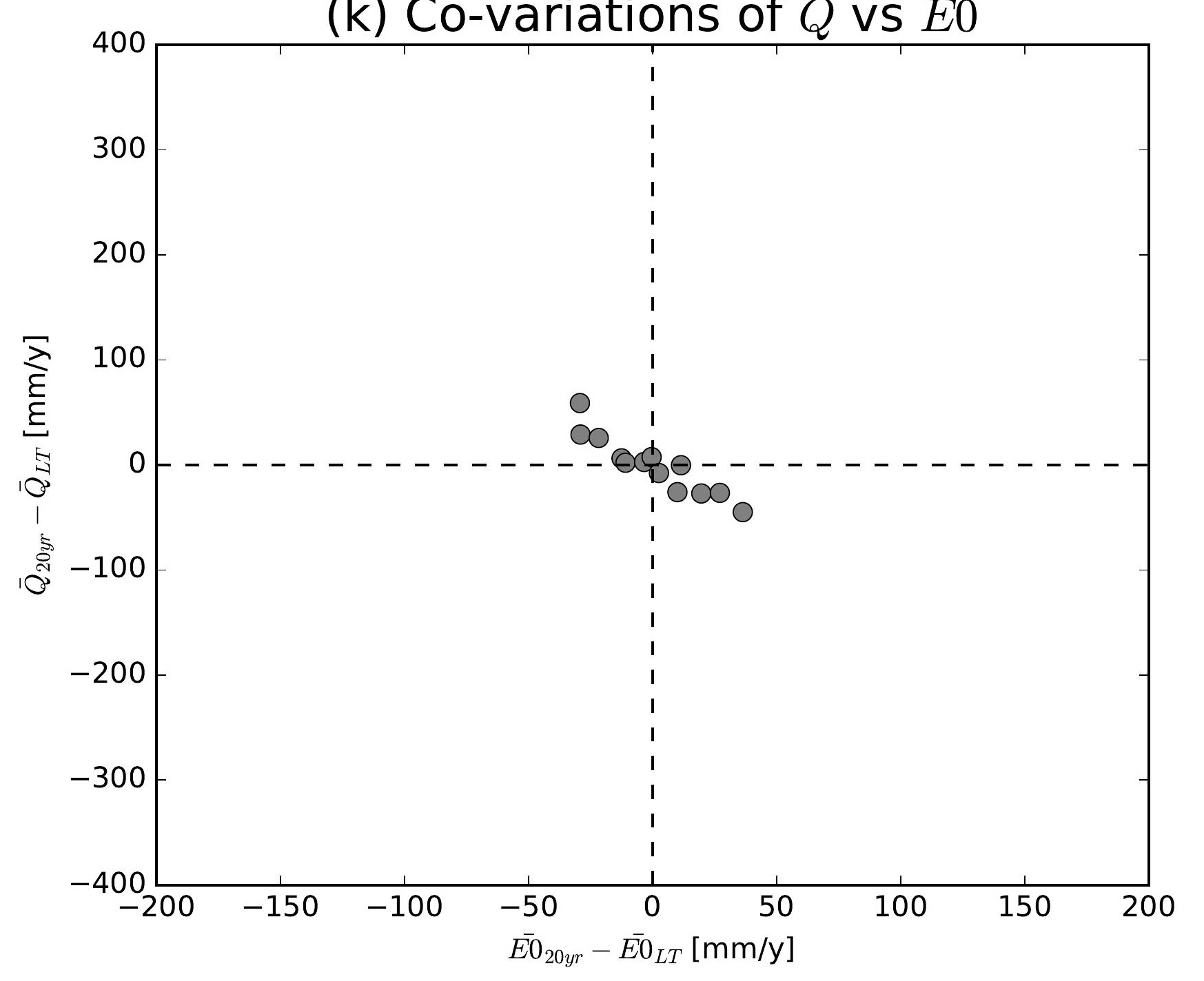

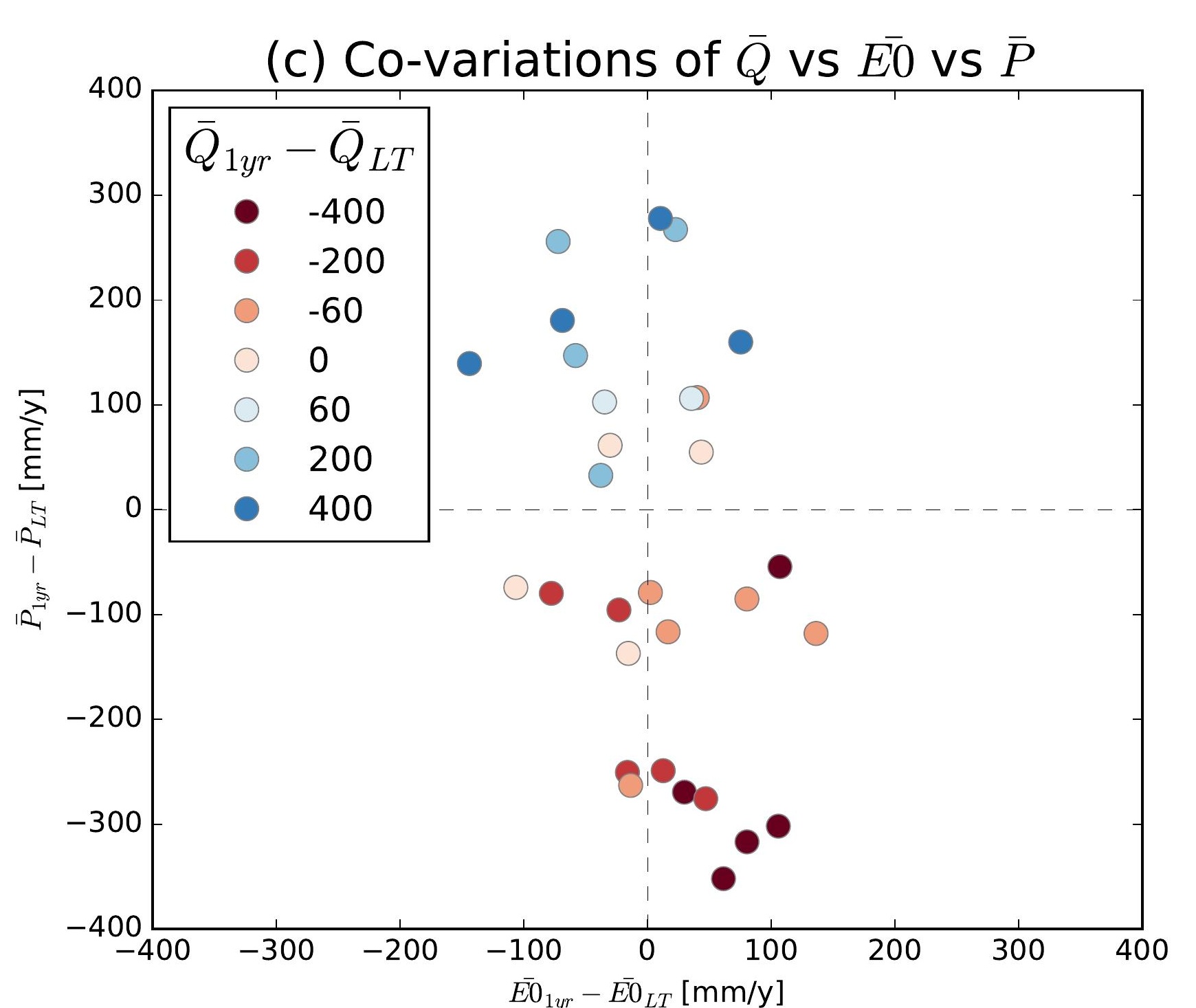
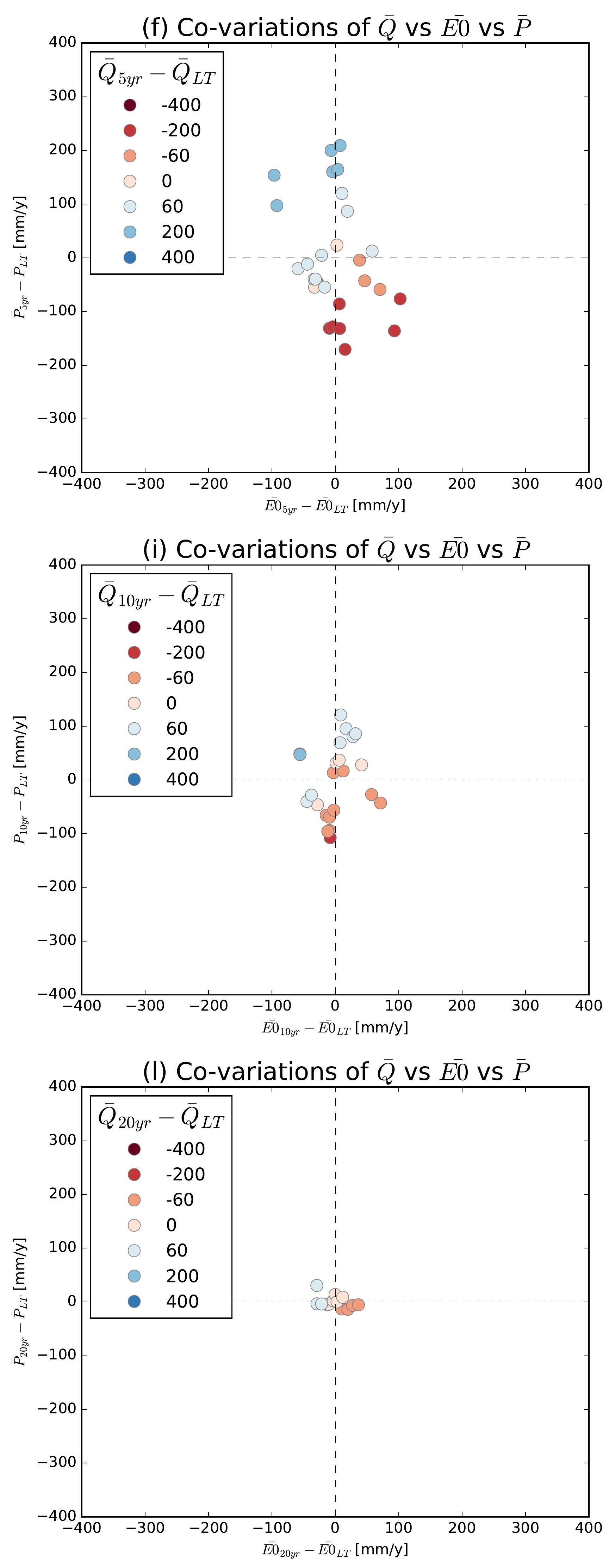

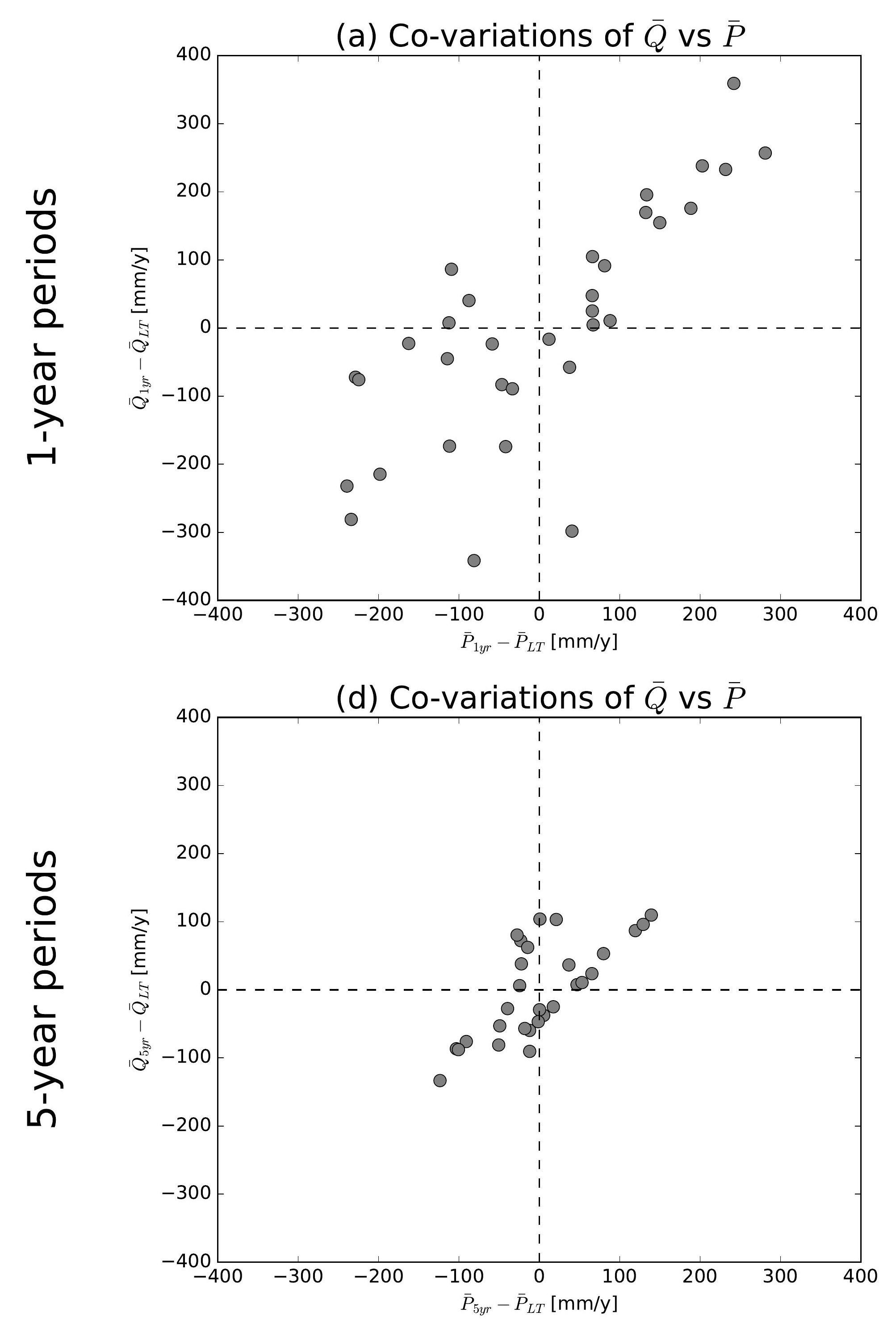

(g) Co-variations of $\bar{Q}$ vs $\bar{P}$

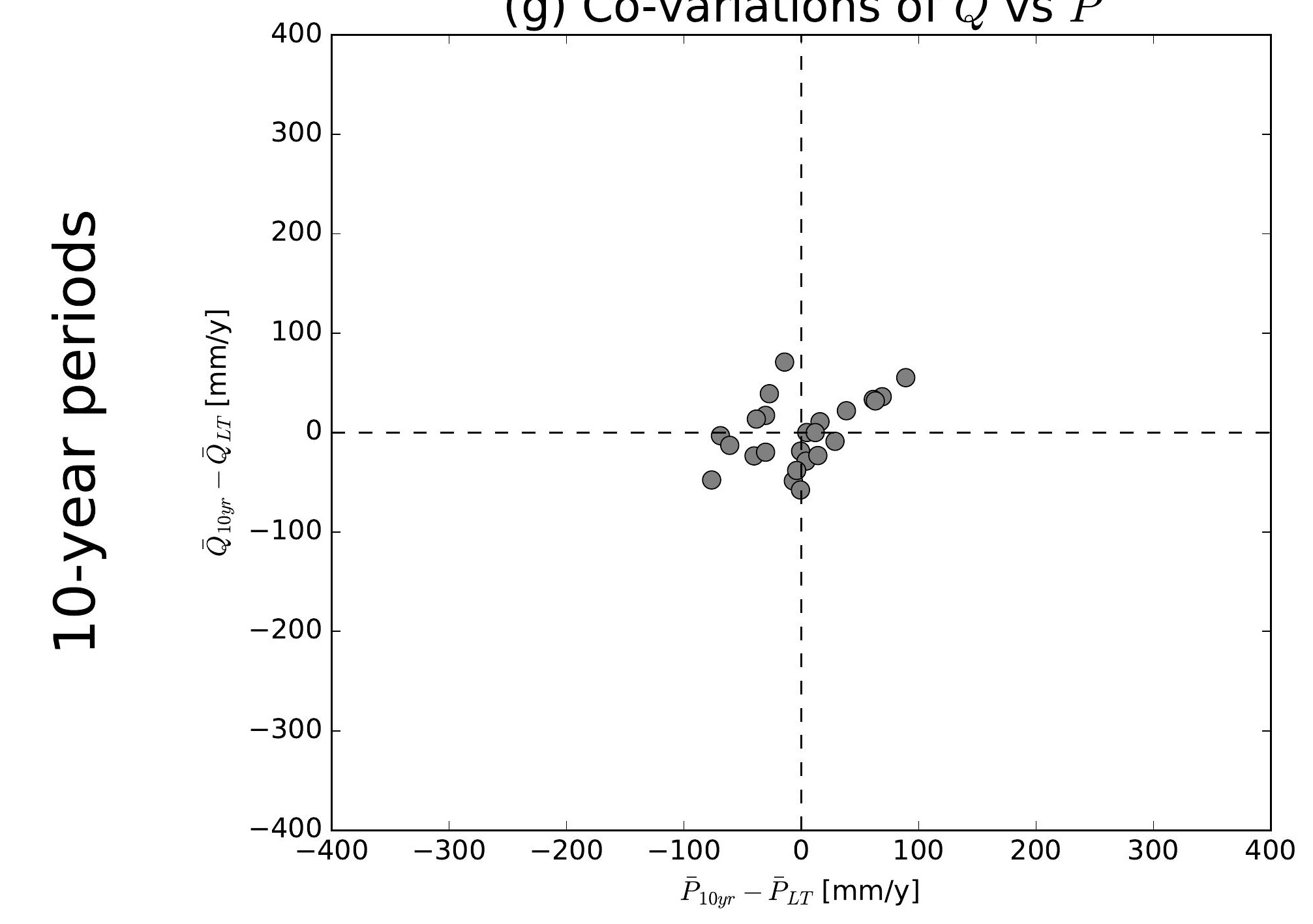

(j) Co-variations of $\bar{Q}$ vs $\bar{P}$

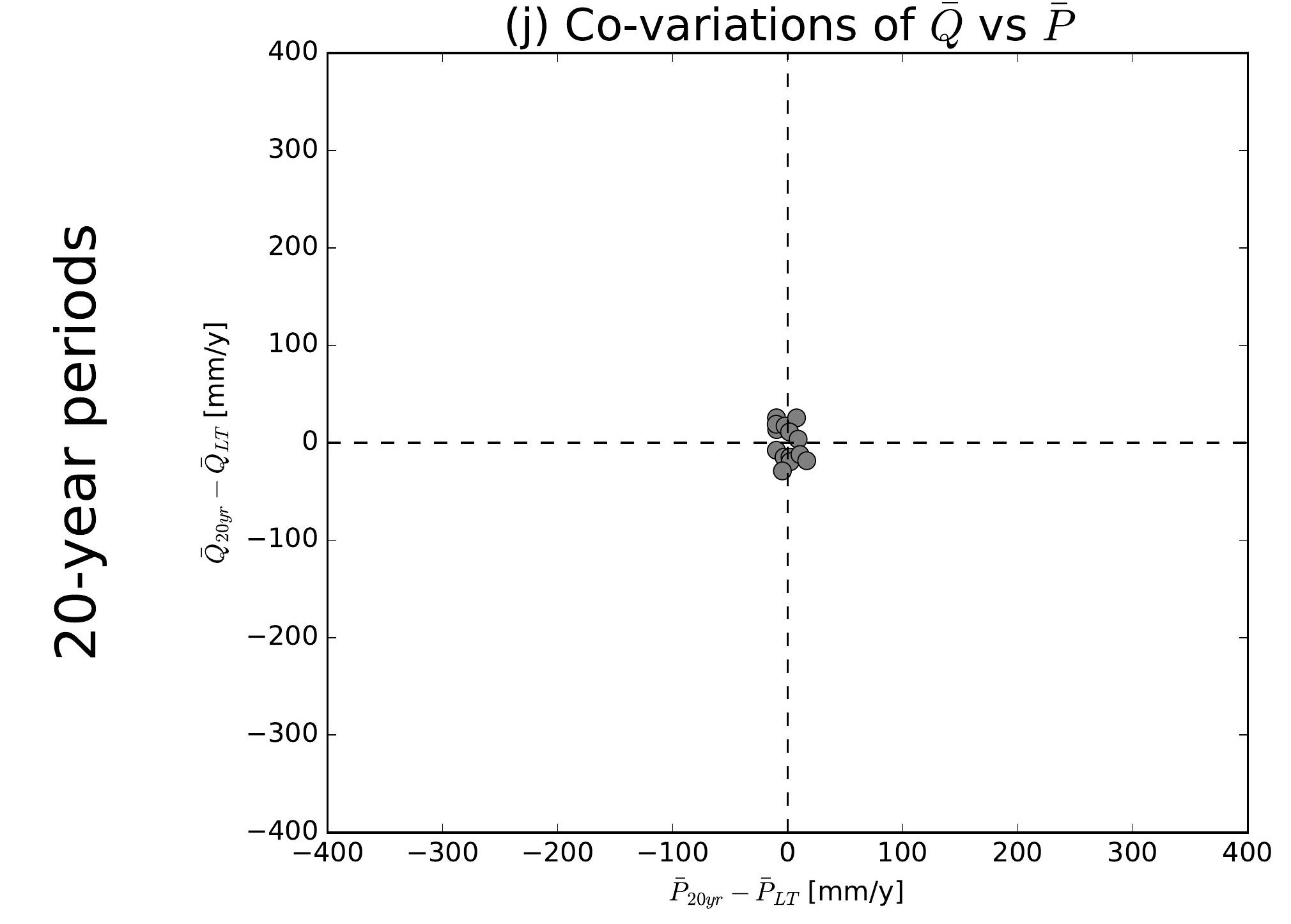

(b) Co-variations of $\bar{Q}$ vs $\overline{E 0}$

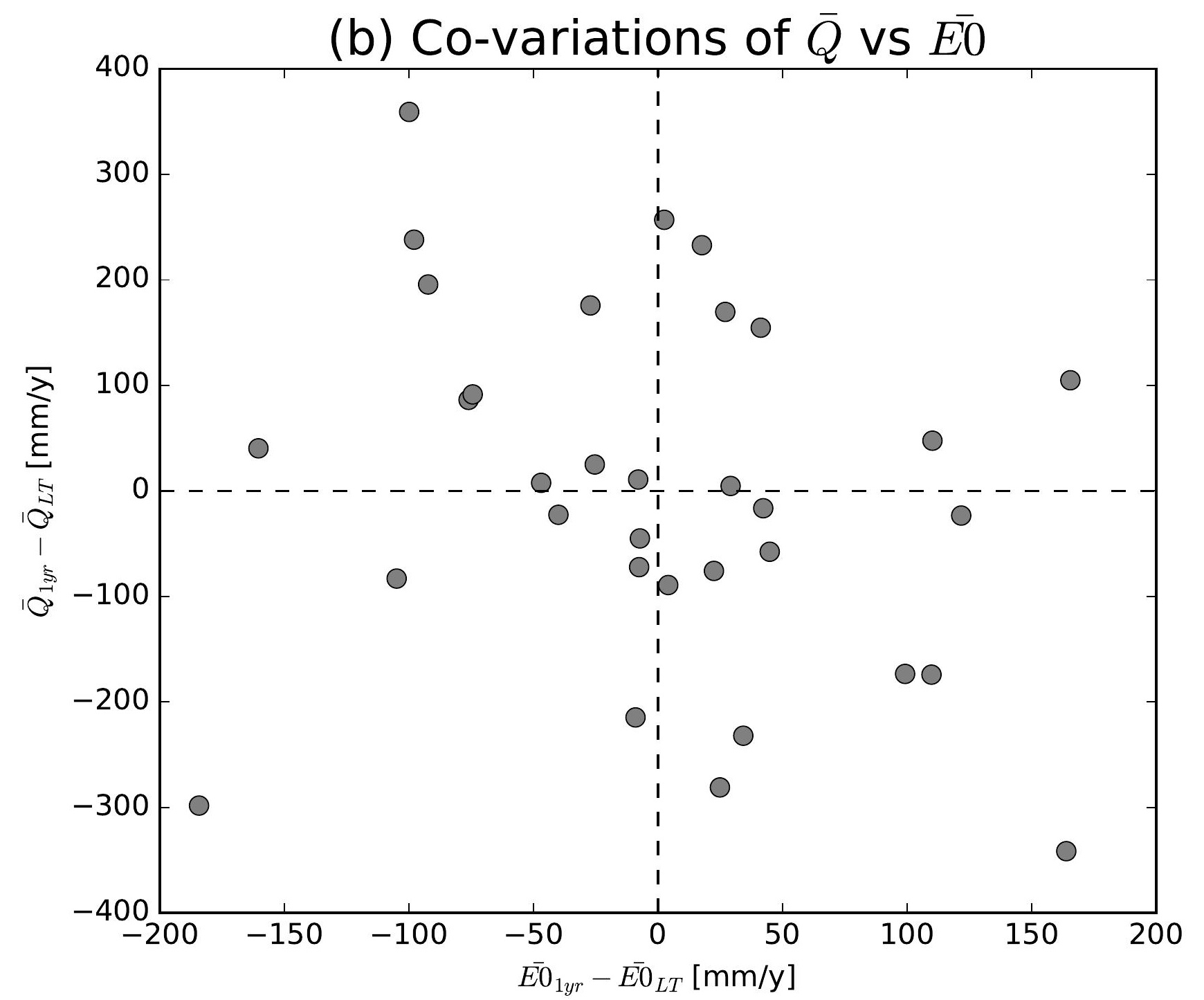

(e) Co-variations of $\bar{Q}$ vs $\overline{E 0}$

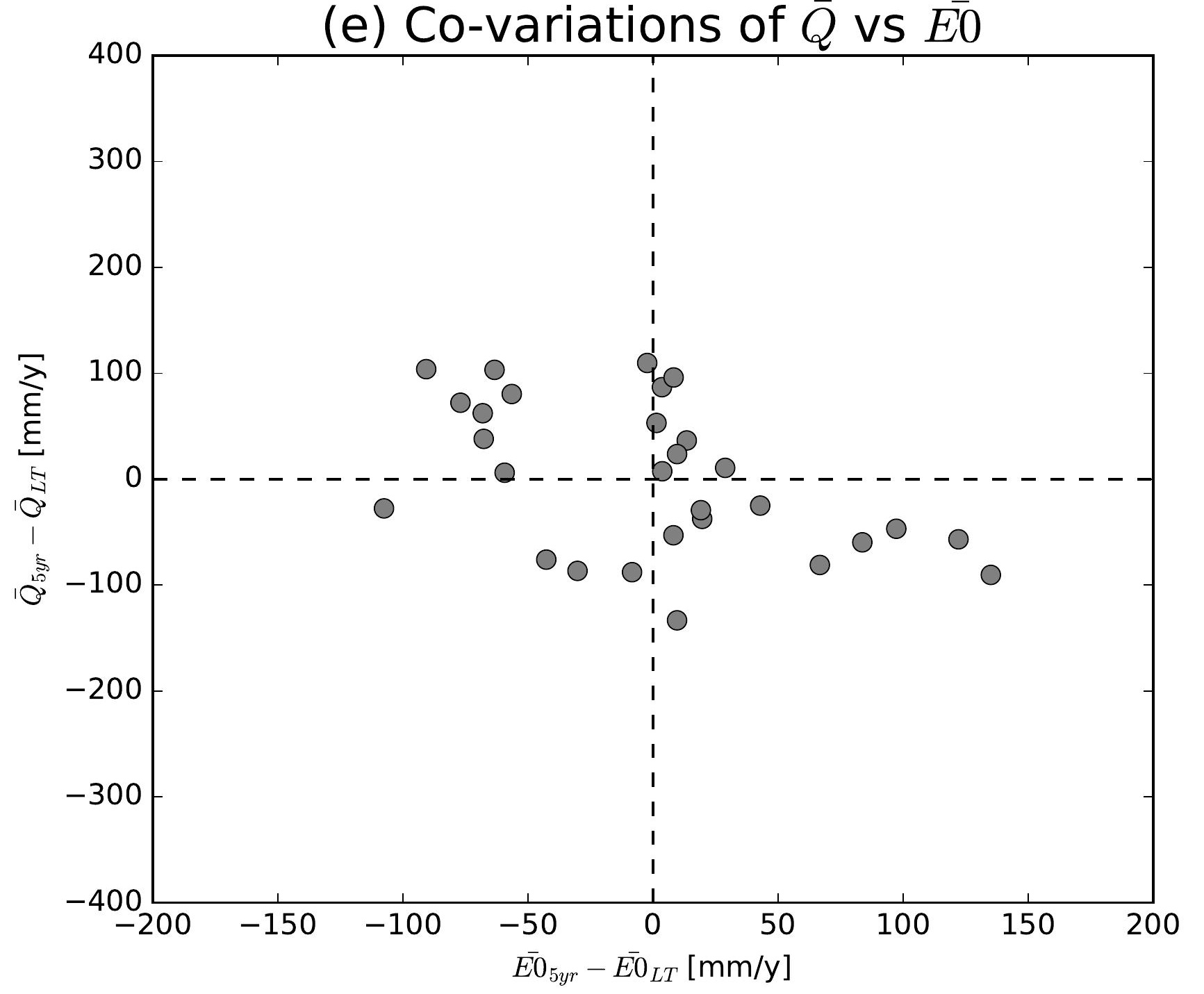

(h) Co-variations of $\bar{Q}$ vs $\overline{E 0}$

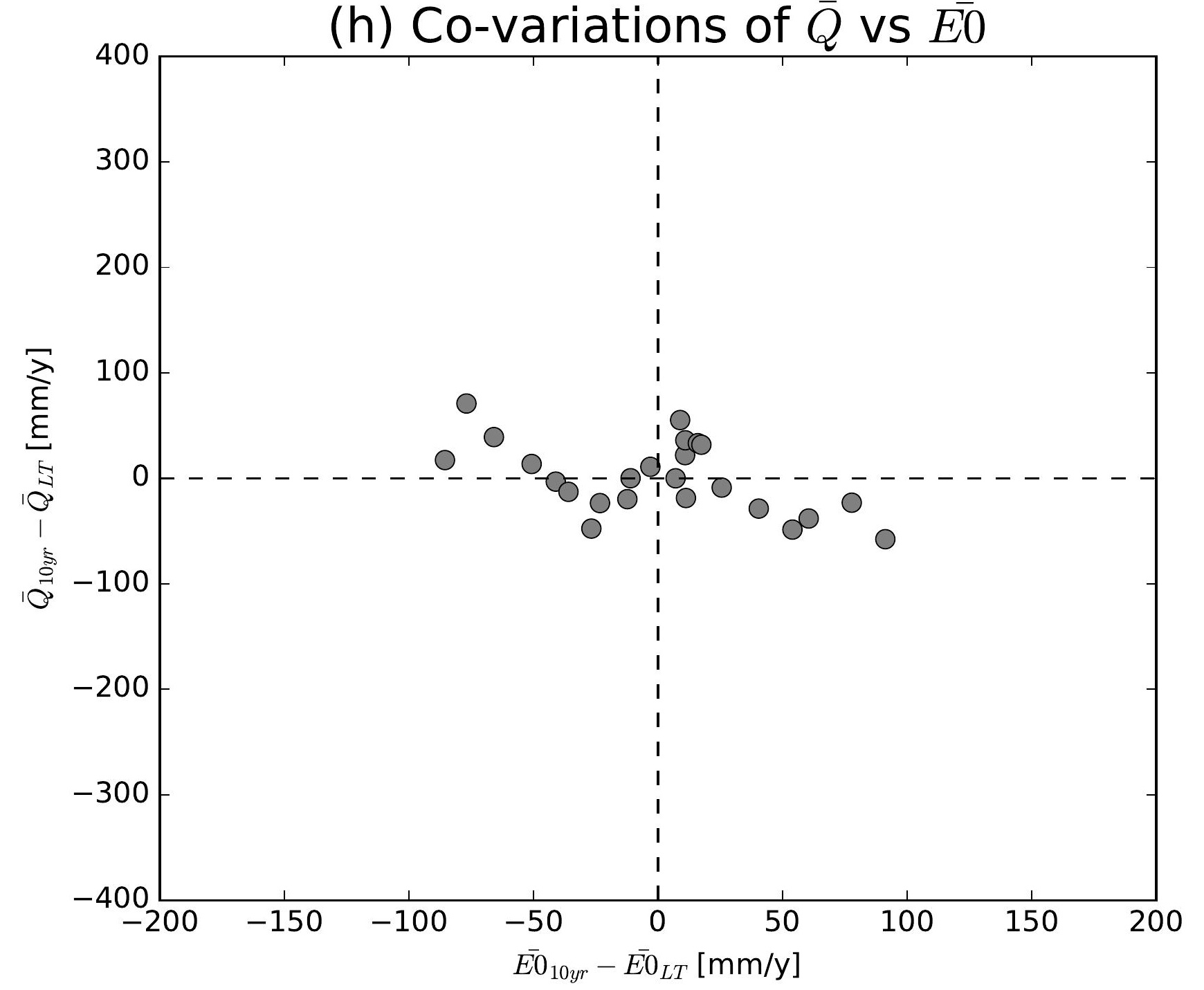

(k) Co-variations of $\bar{Q}$ vs $\overline{E 0}$

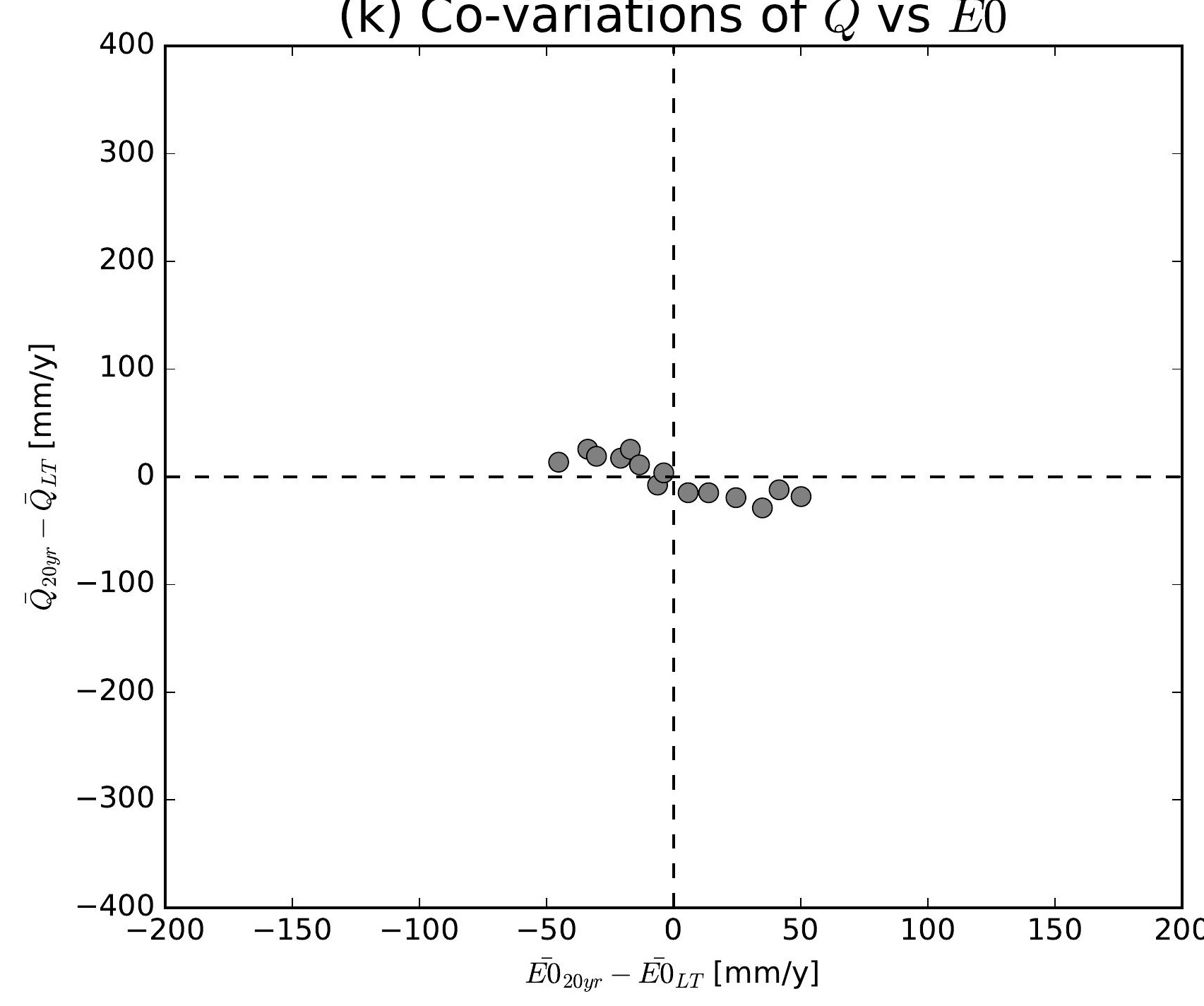

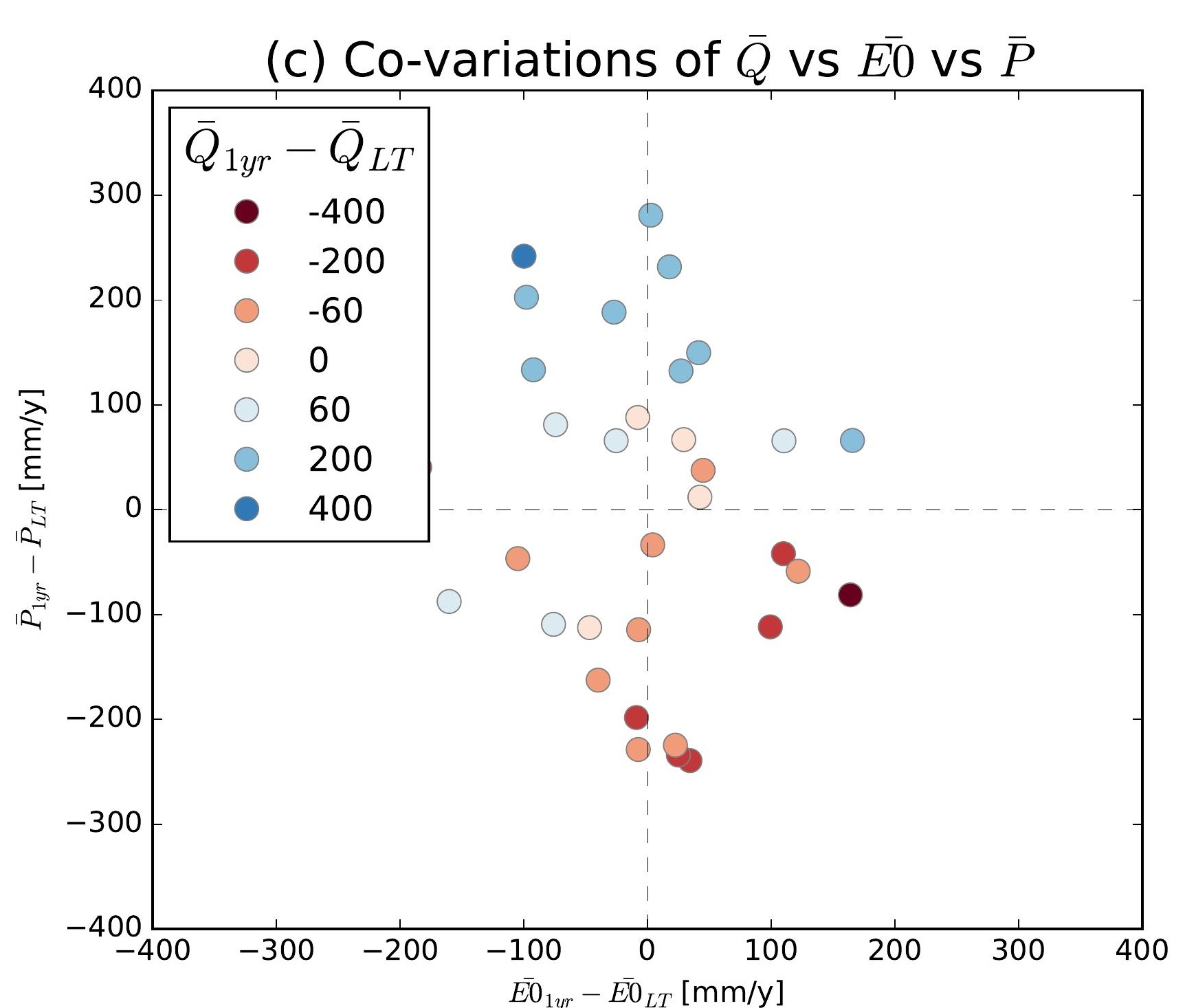
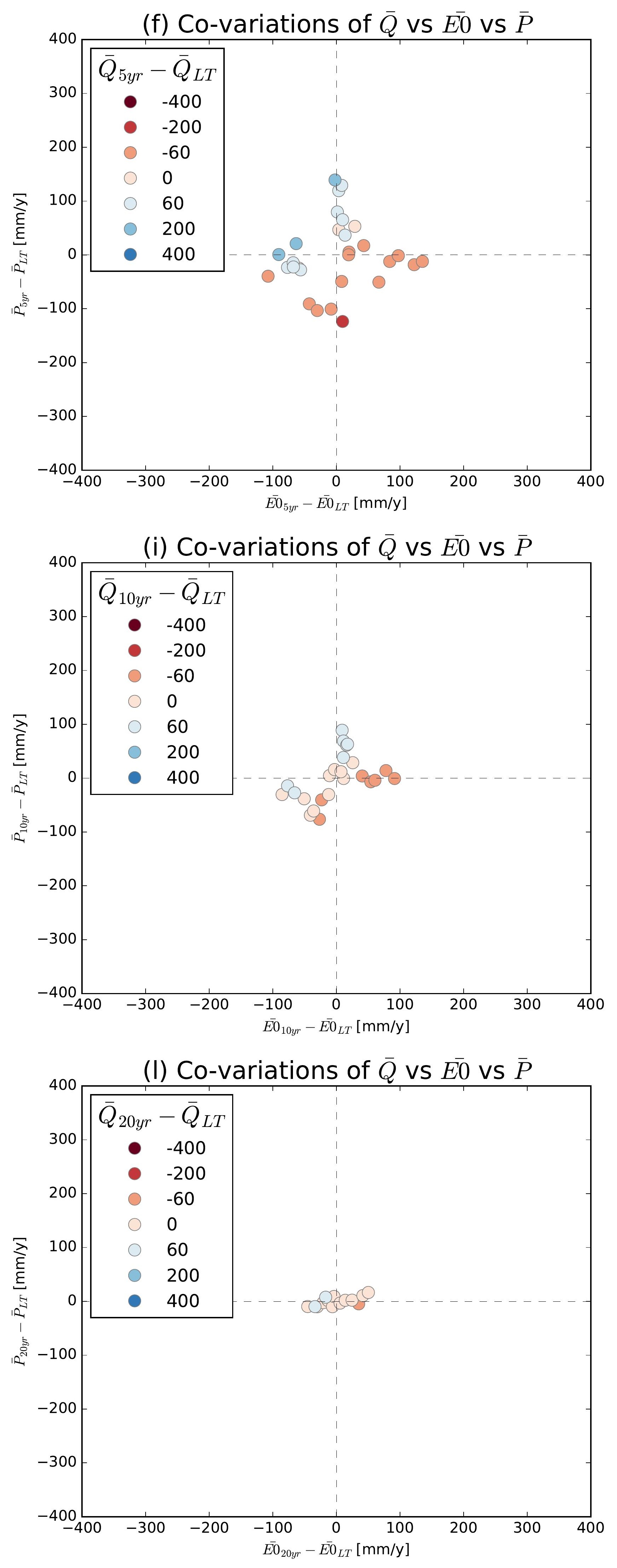

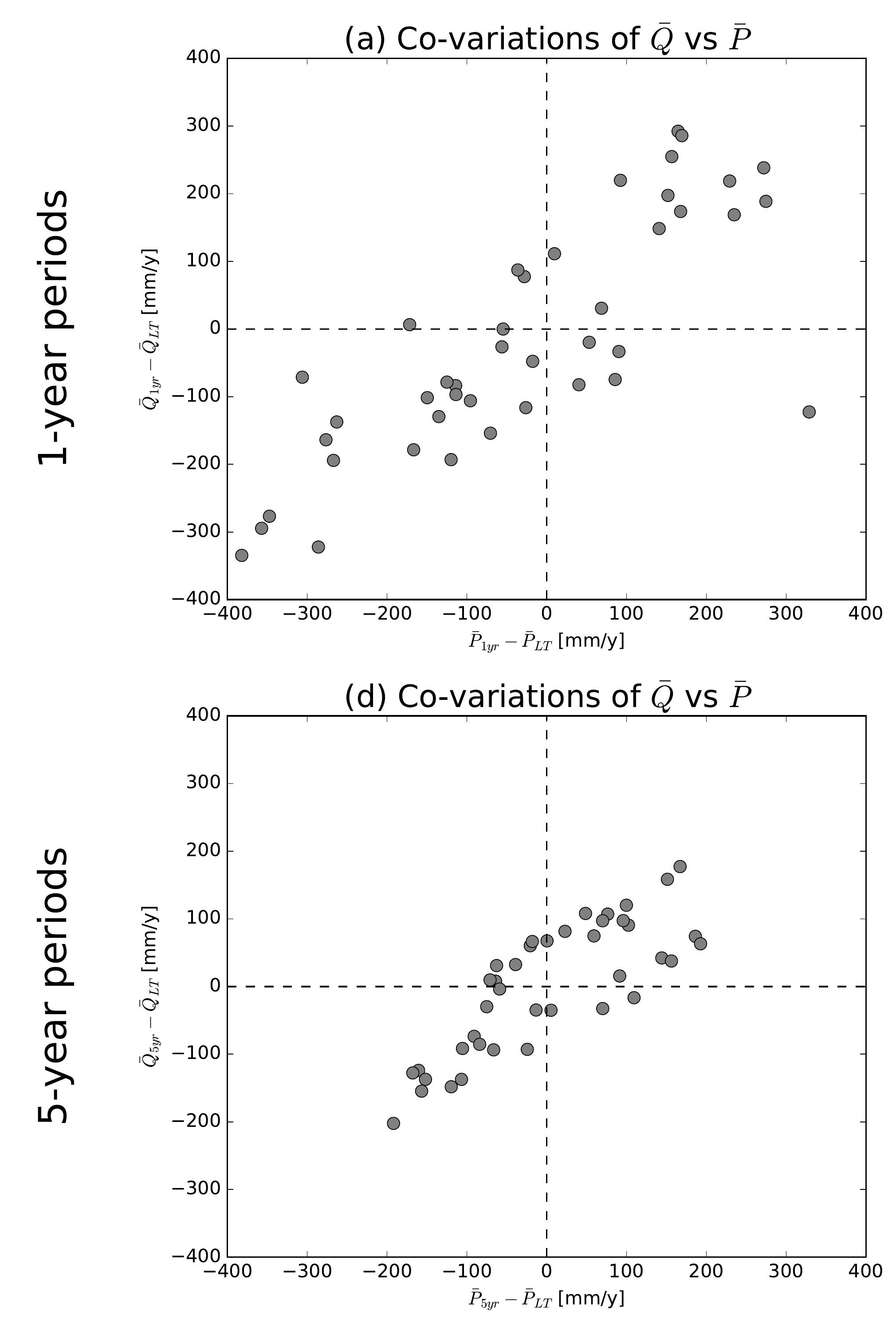

(g) Co-variations of $\bar{Q}$ vs $\bar{P}$

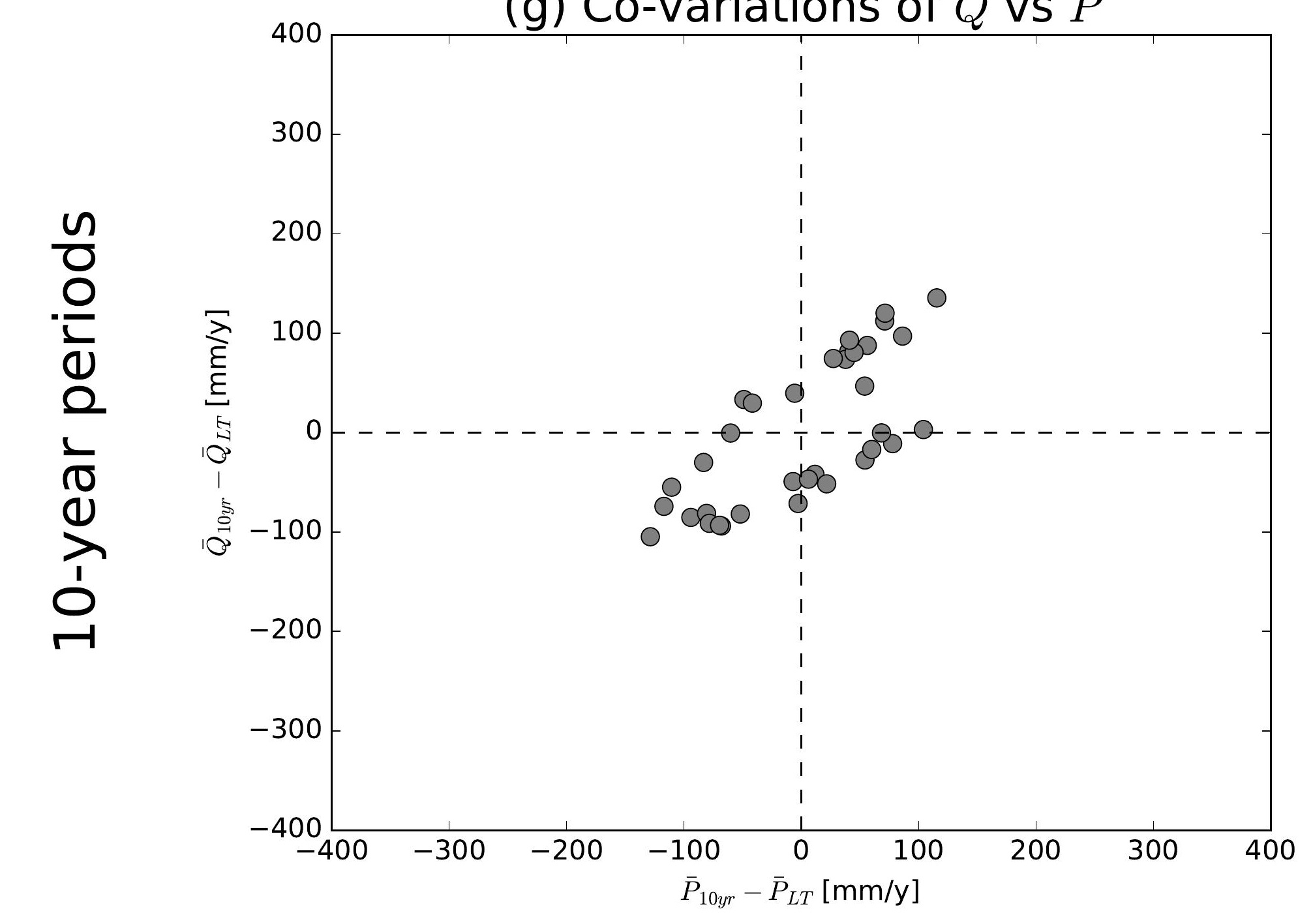

(j) Co-variations of $\bar{Q}$ vs $\bar{P}$

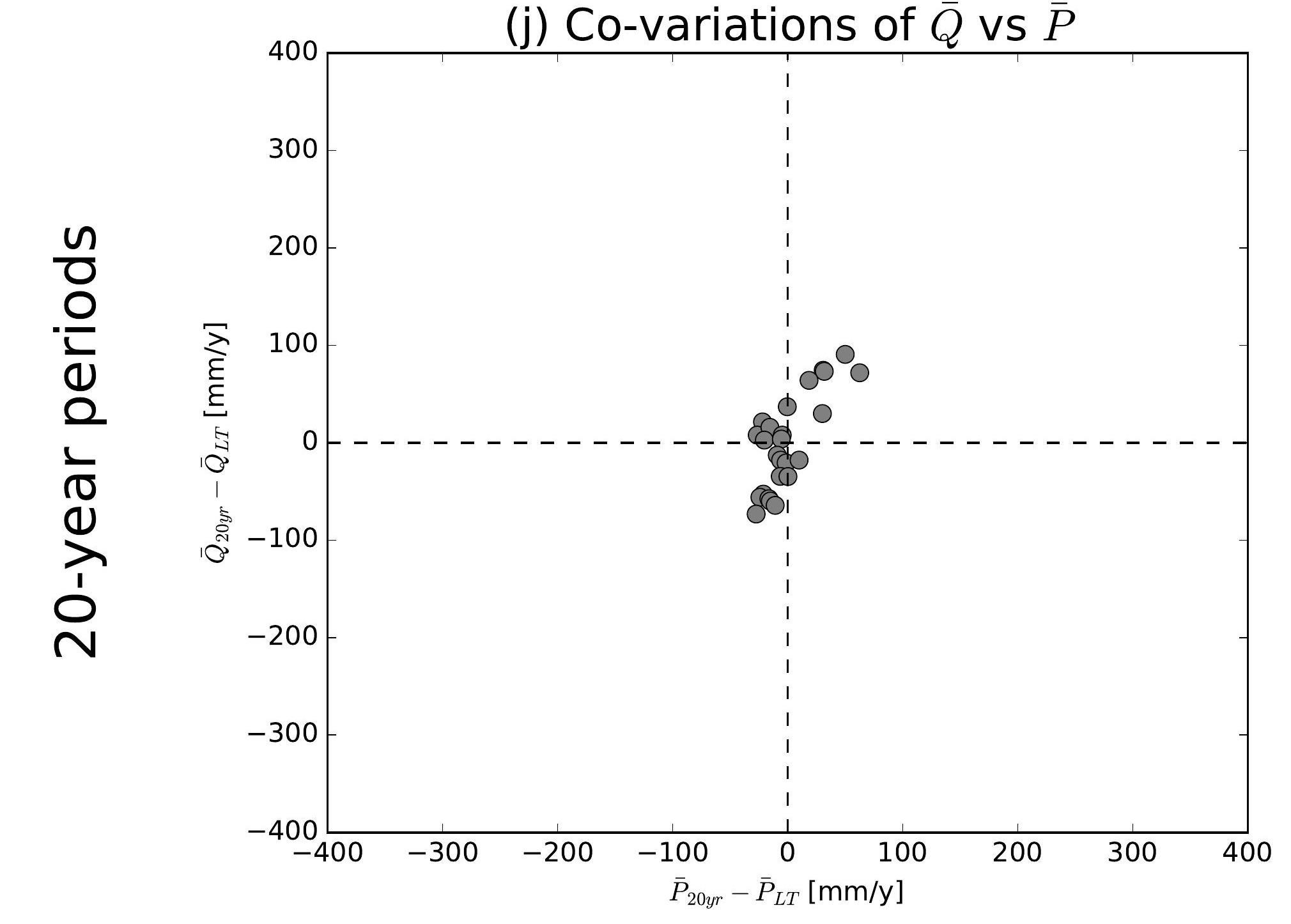

(b) Co-variations of $\bar{Q}$ vs $\overline{E 0}$

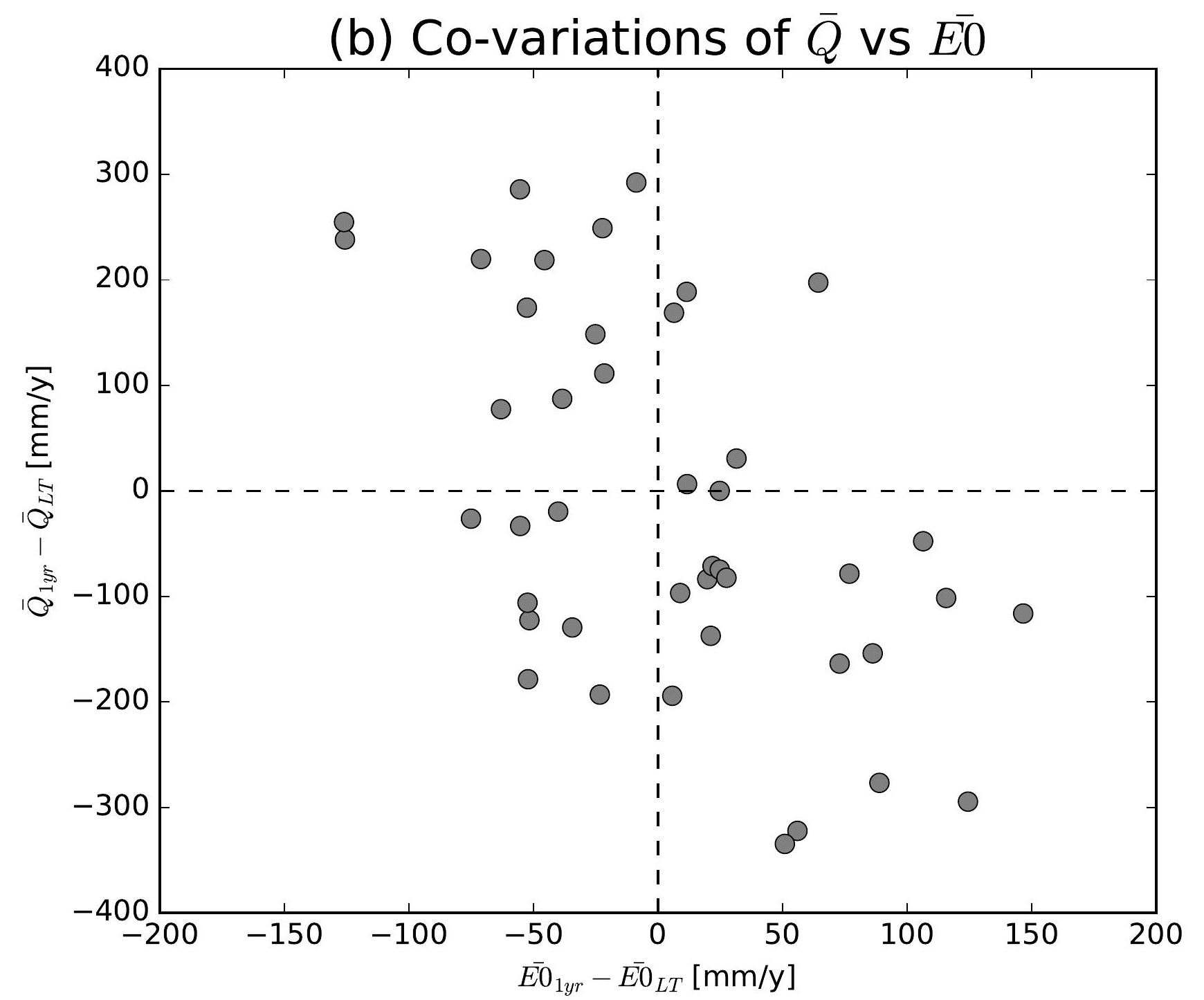

(e) Co-variations of $\bar{Q}$ vs $\overline{E 0}$

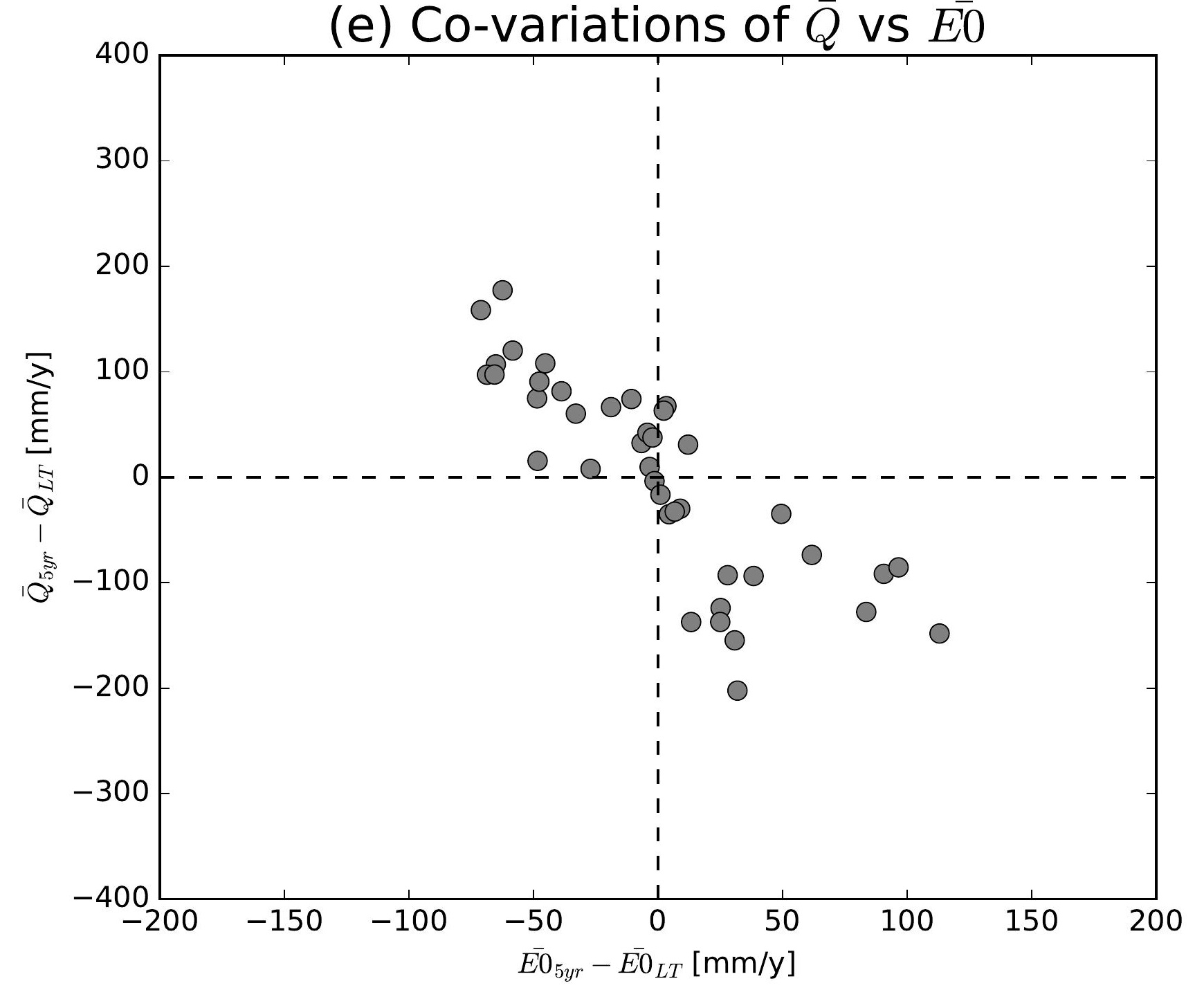

(h) Co-variations of $\bar{Q}$ vs $\overline{E 0}$

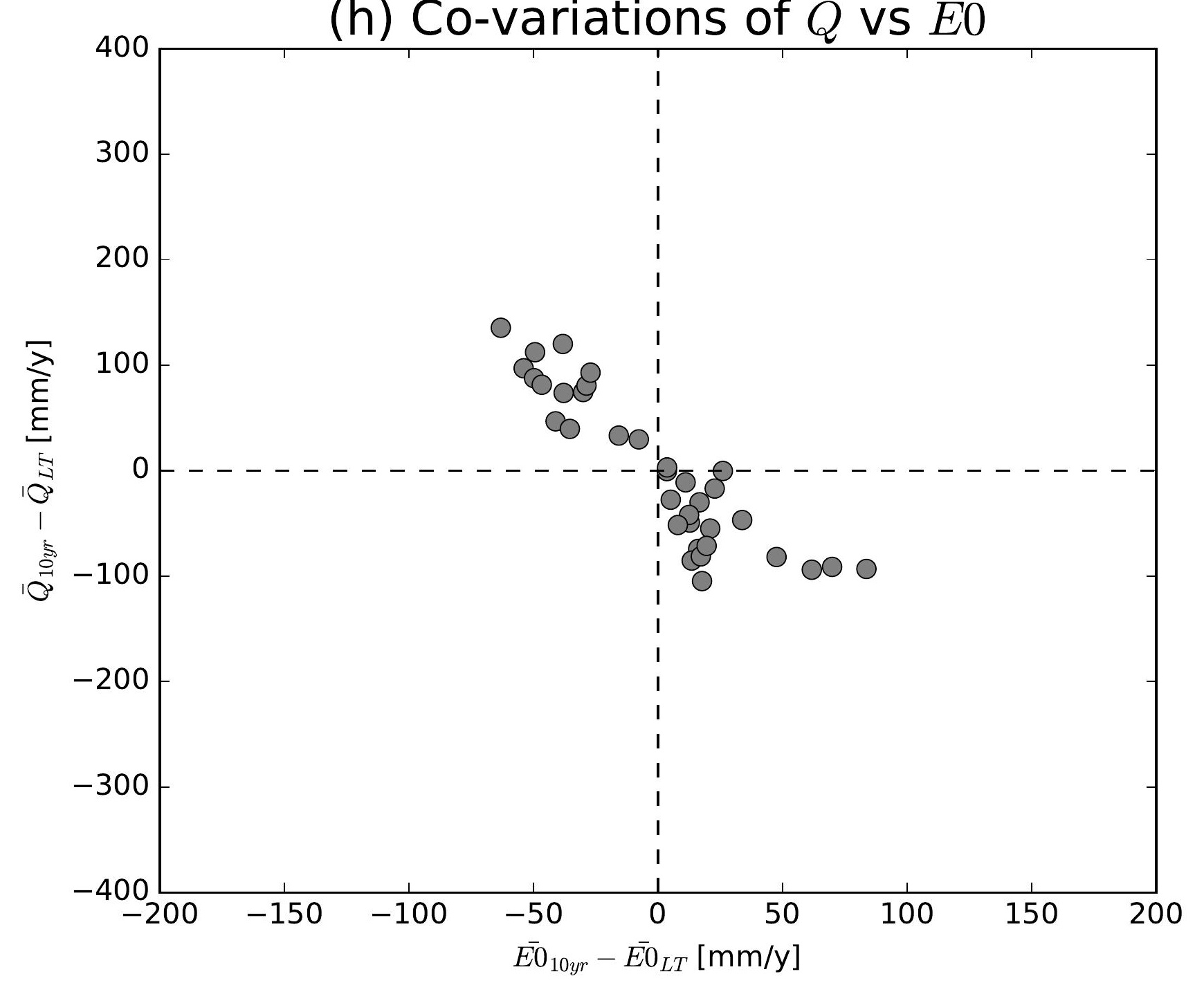

(k) Co-variations of $\bar{Q}$ vs $\overline{E 0}$

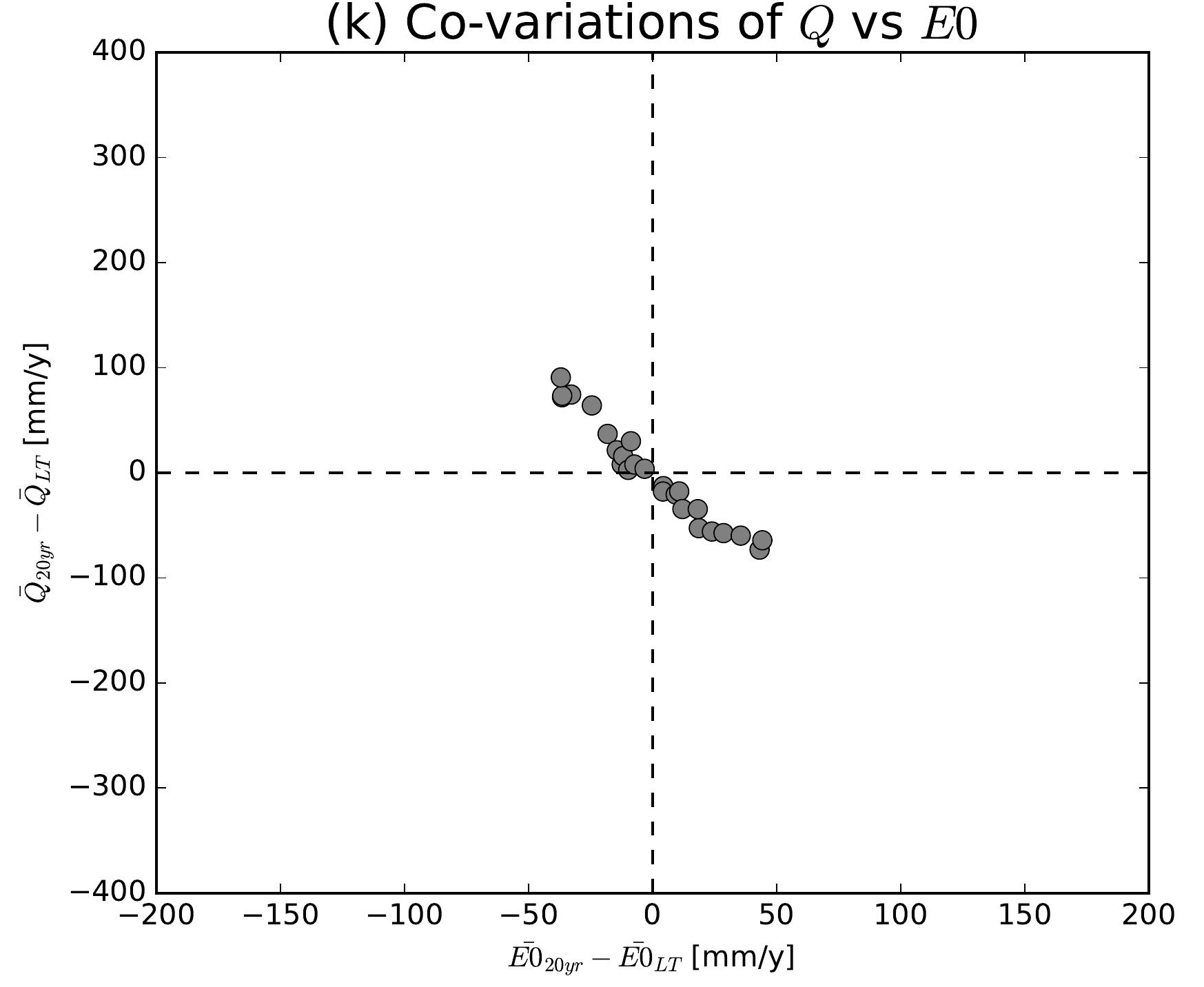

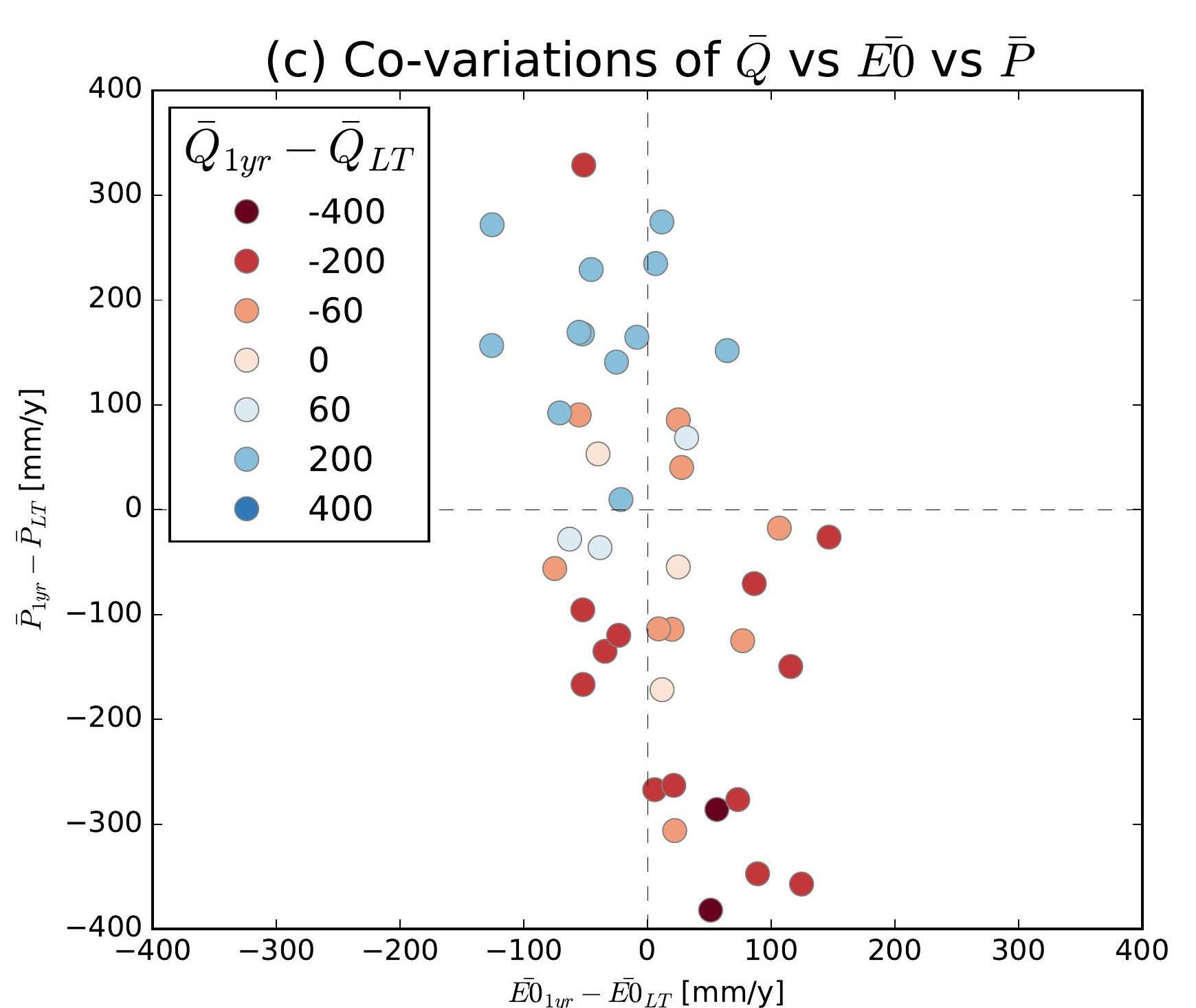
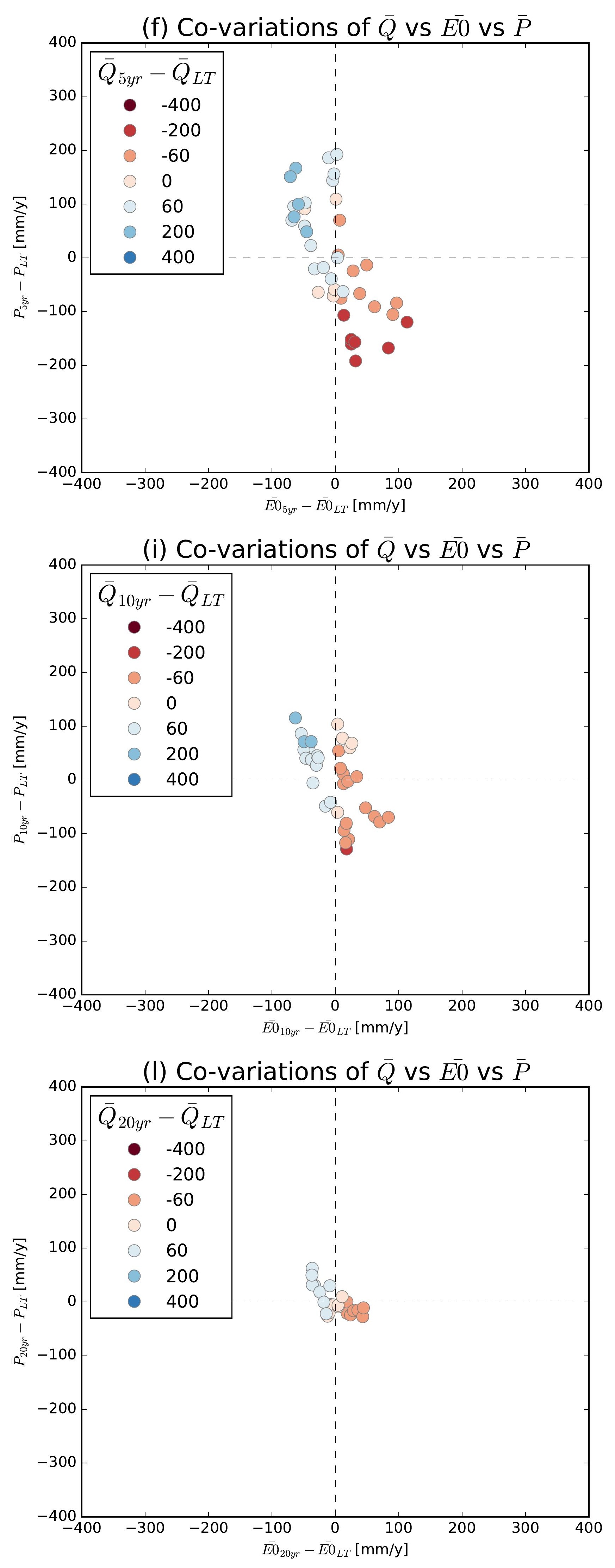

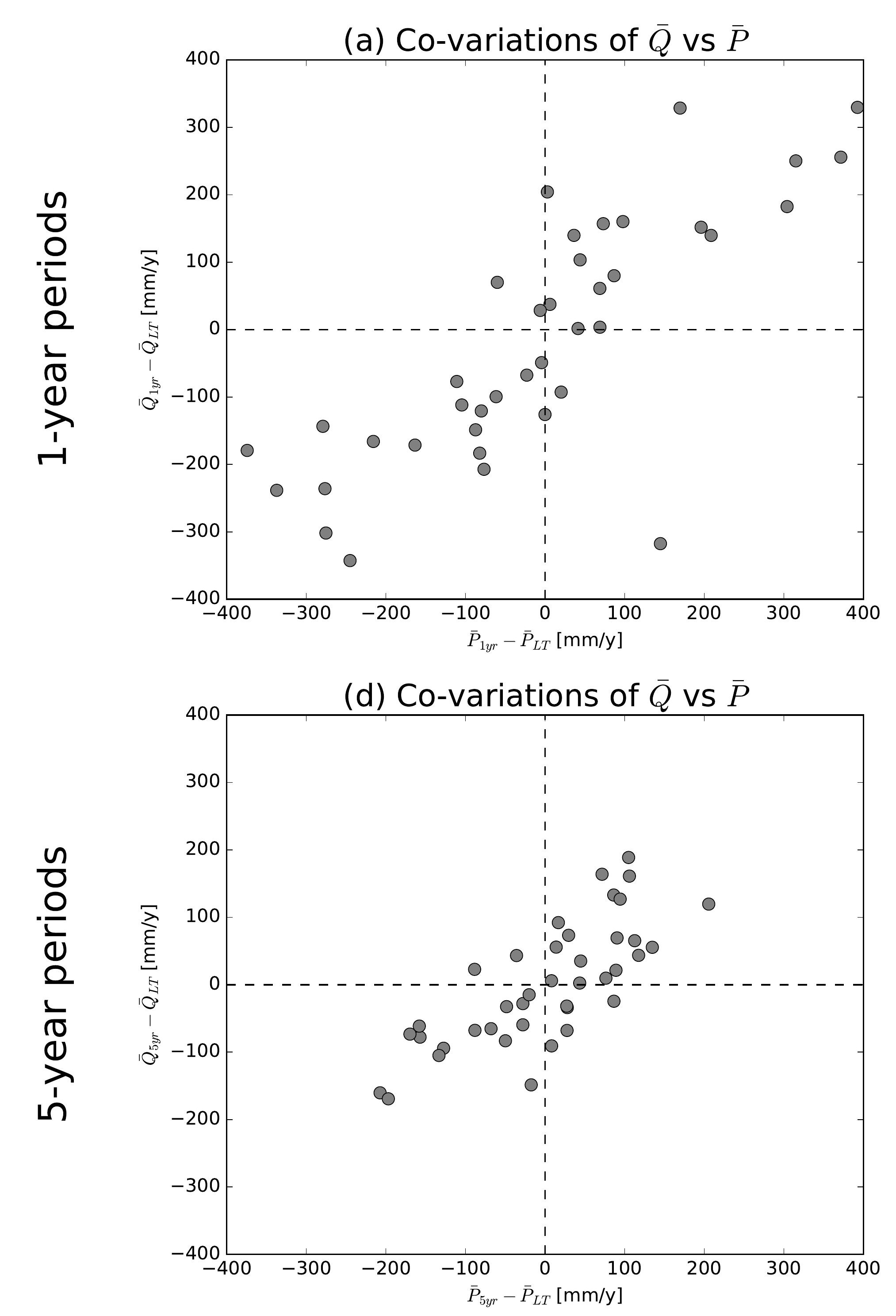

(g) Co-variations of $\bar{Q}$ vs $\bar{P}$

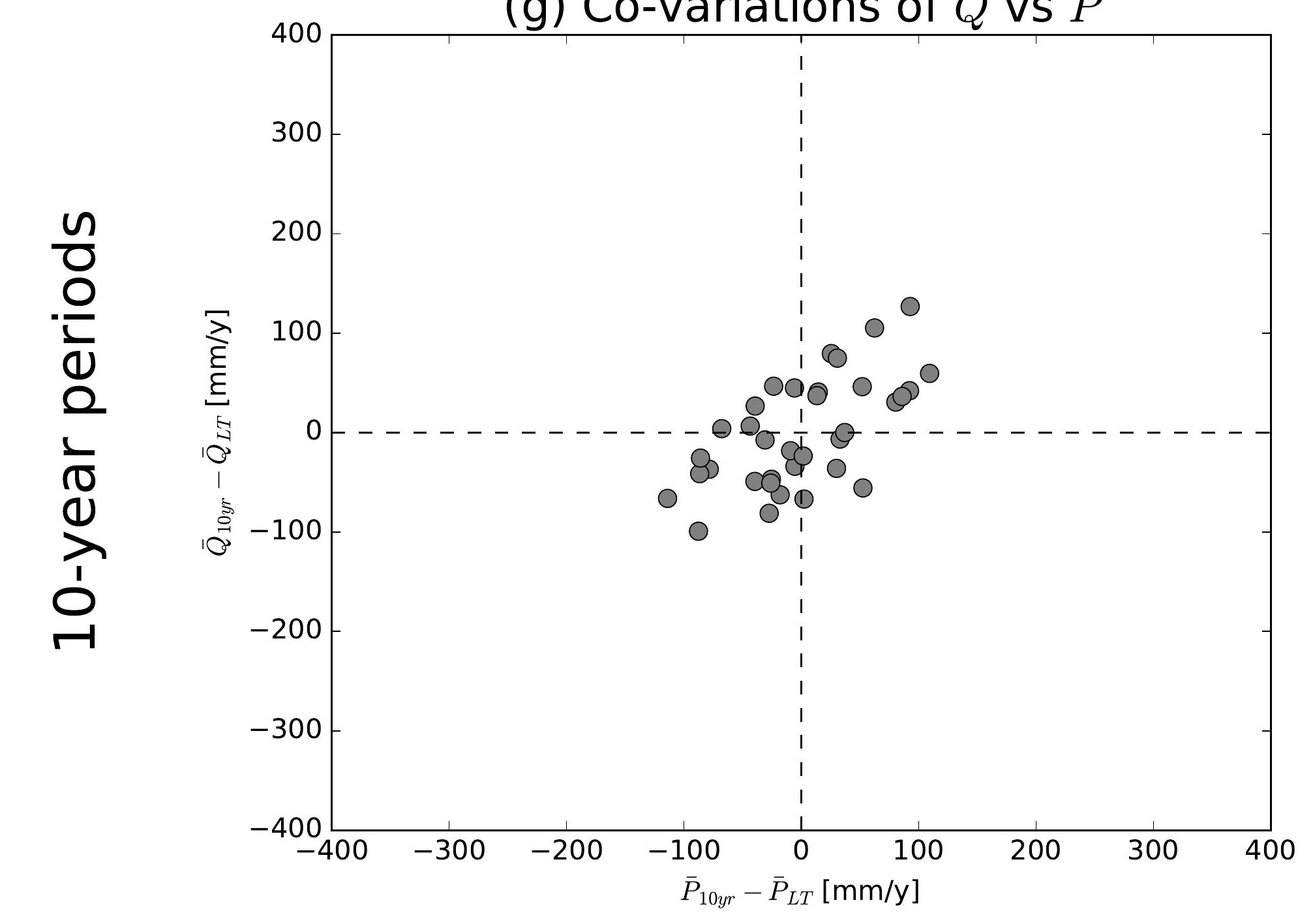

(j) Co-variations of $\bar{Q}$ vs $\bar{P}$

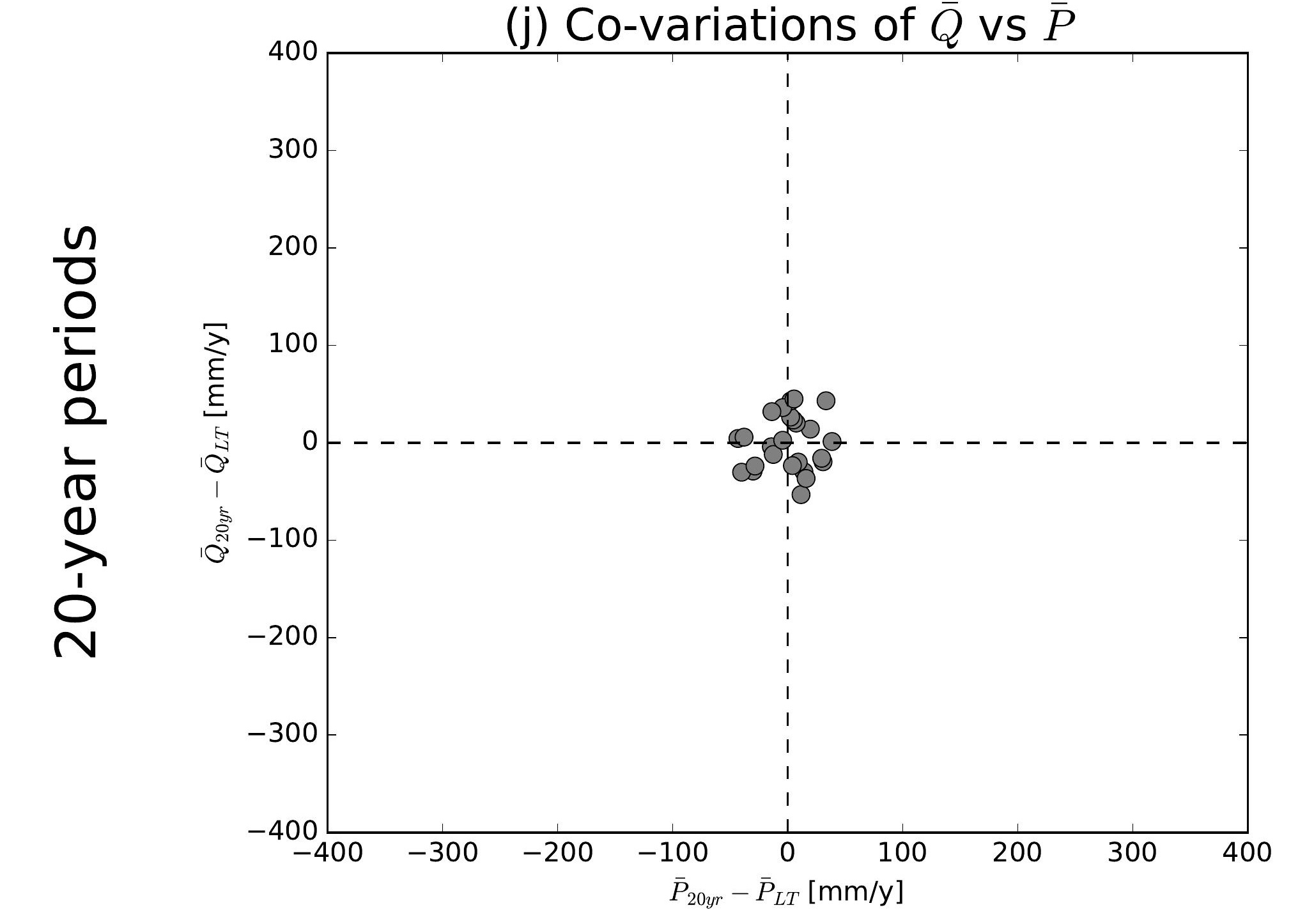

(b) Co-variations of $\bar{Q}$ vs $\overline{E 0}$

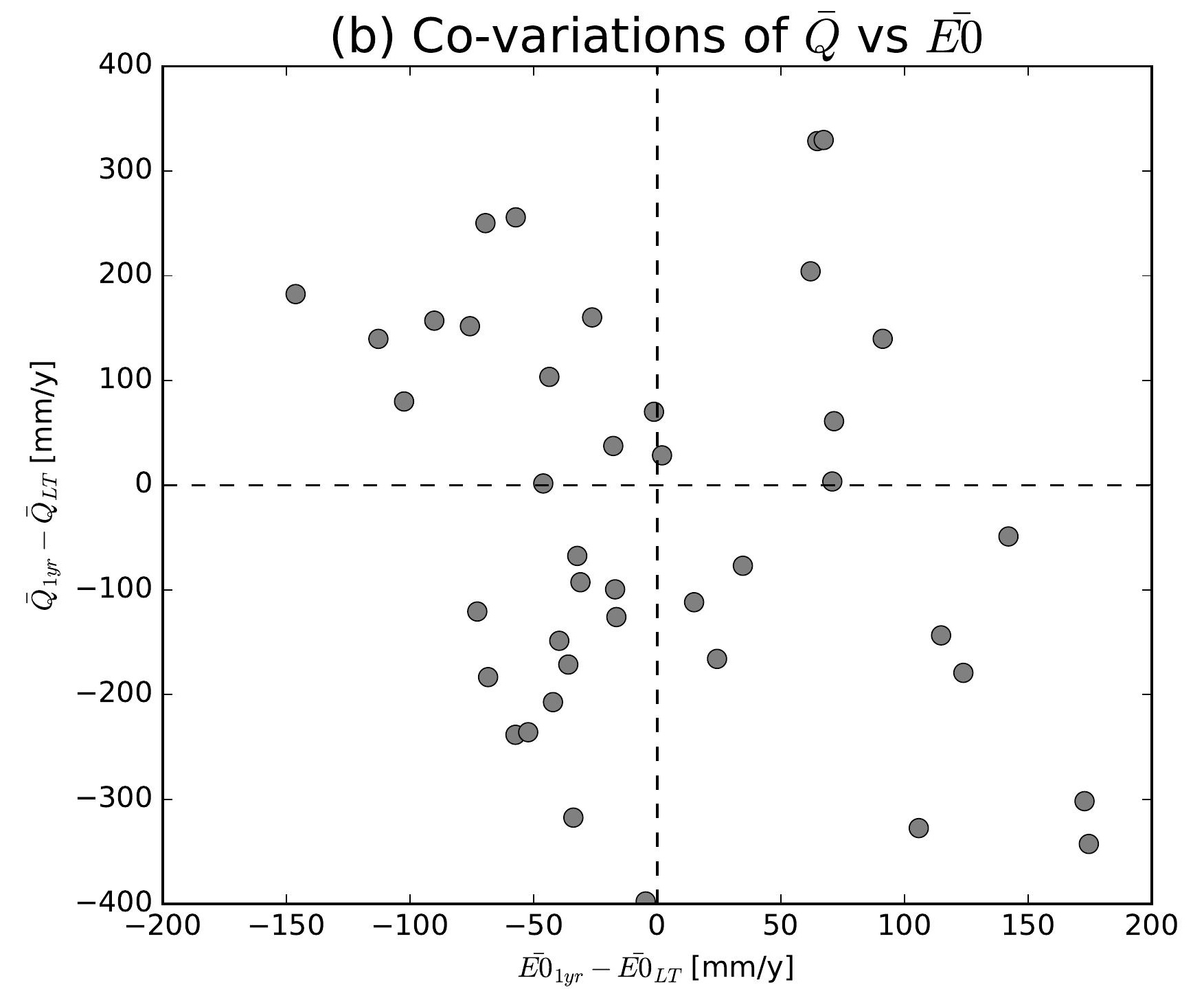

(e) Co-variations of $\bar{Q}$ vs $\overline{E 0}$

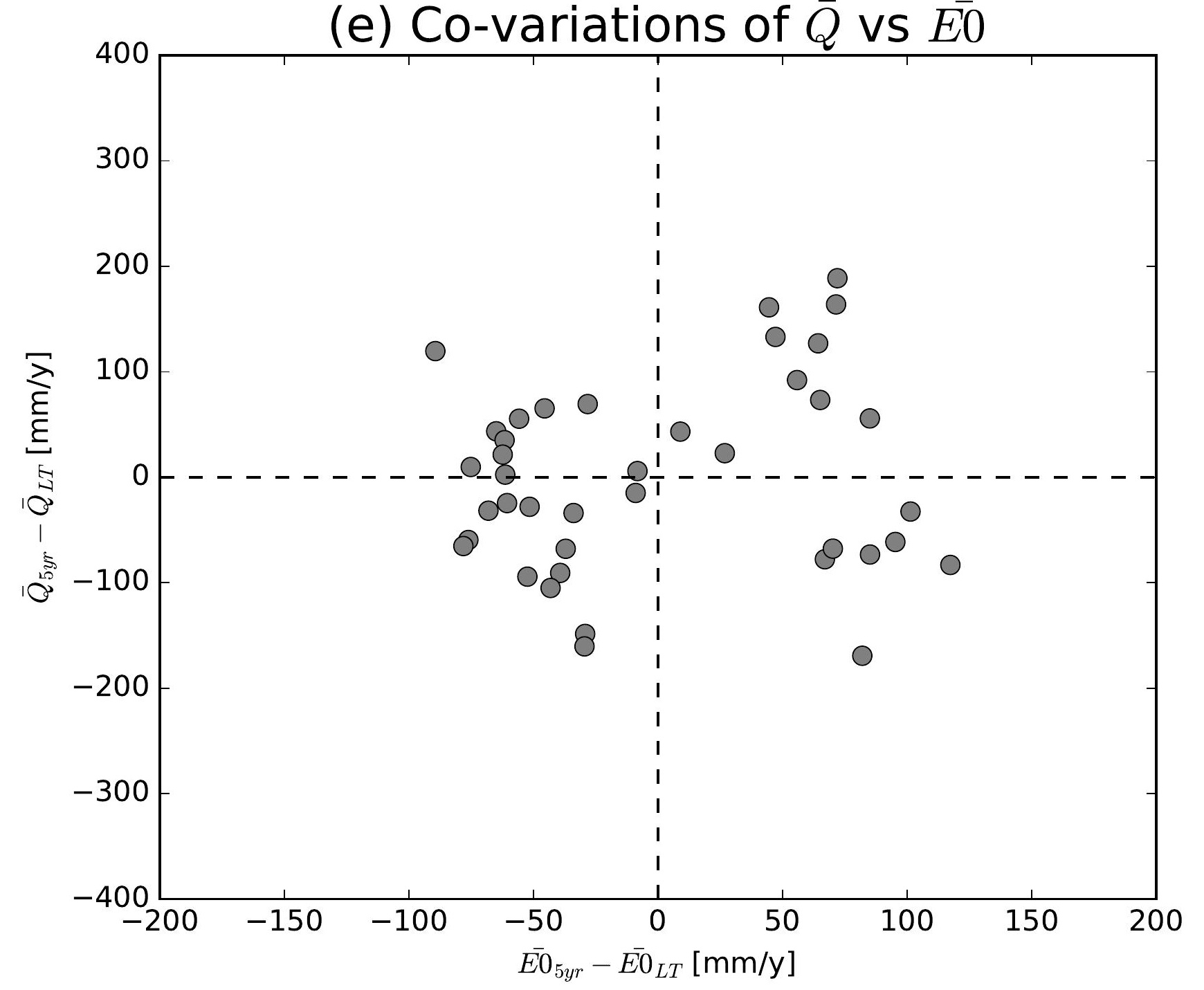

(h) Co-variations of $\bar{Q}$ vs $\overline{E 0}$

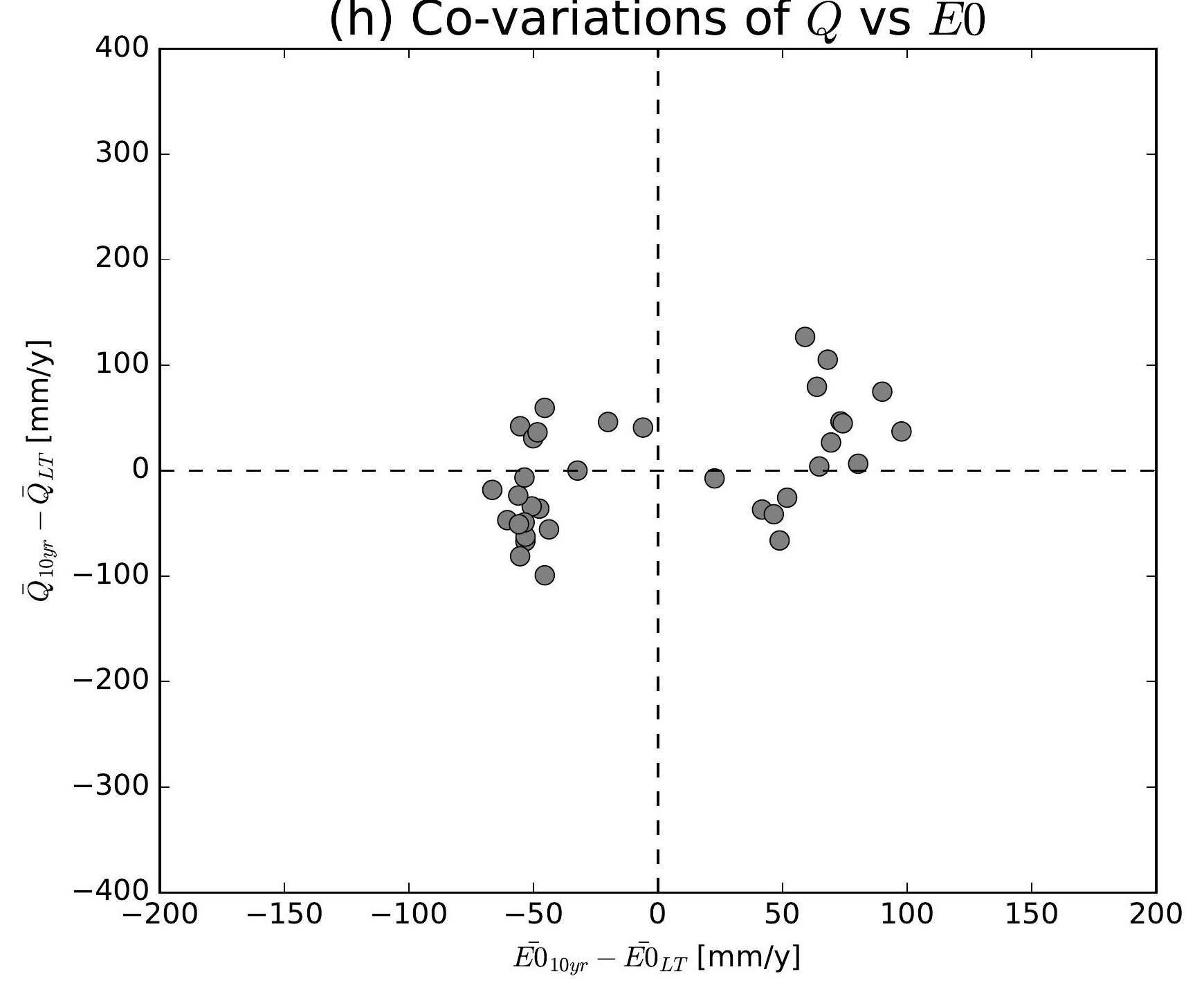

(k) Co-variations of $\bar{Q}$ vs $\overline{E 0}$

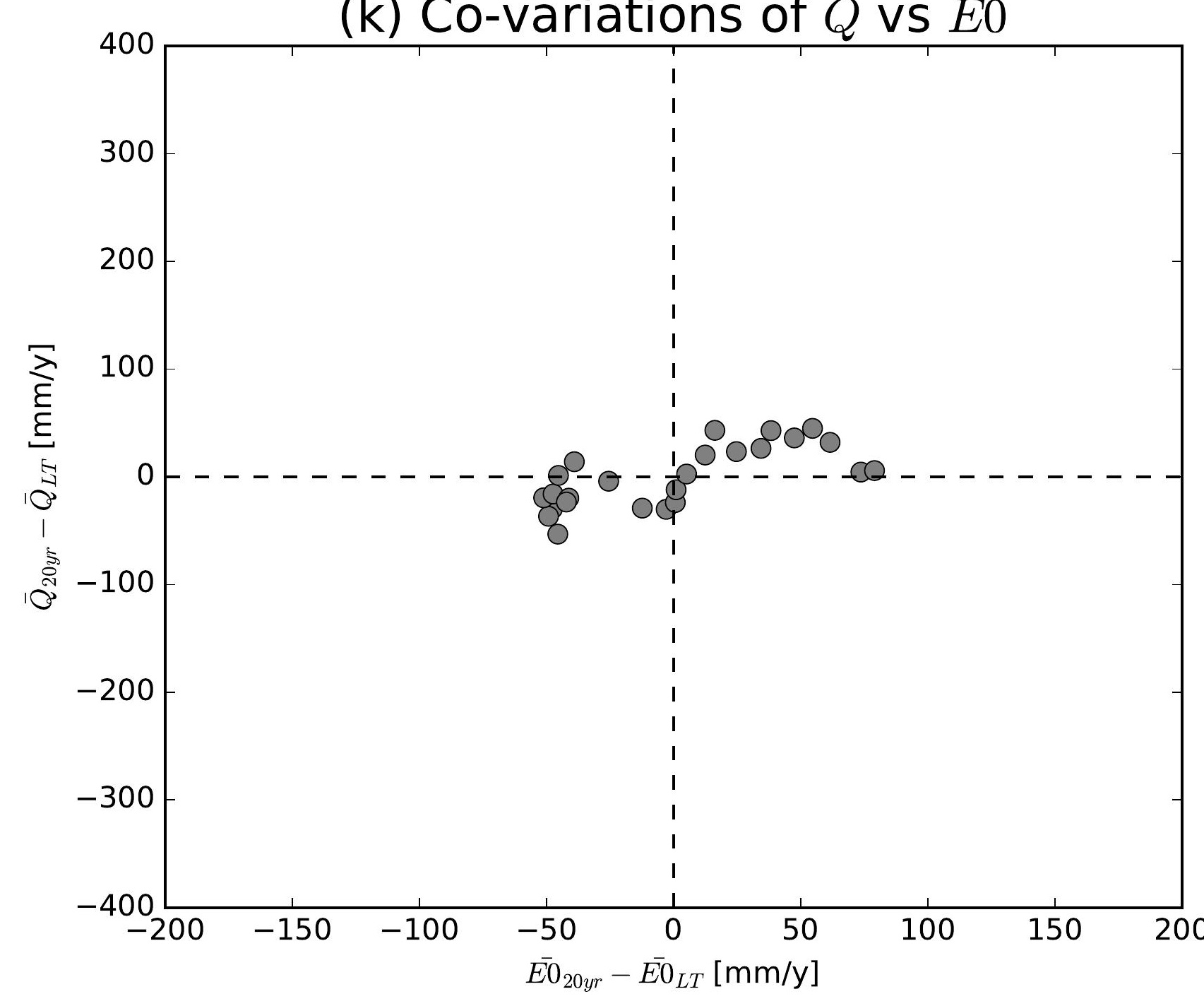

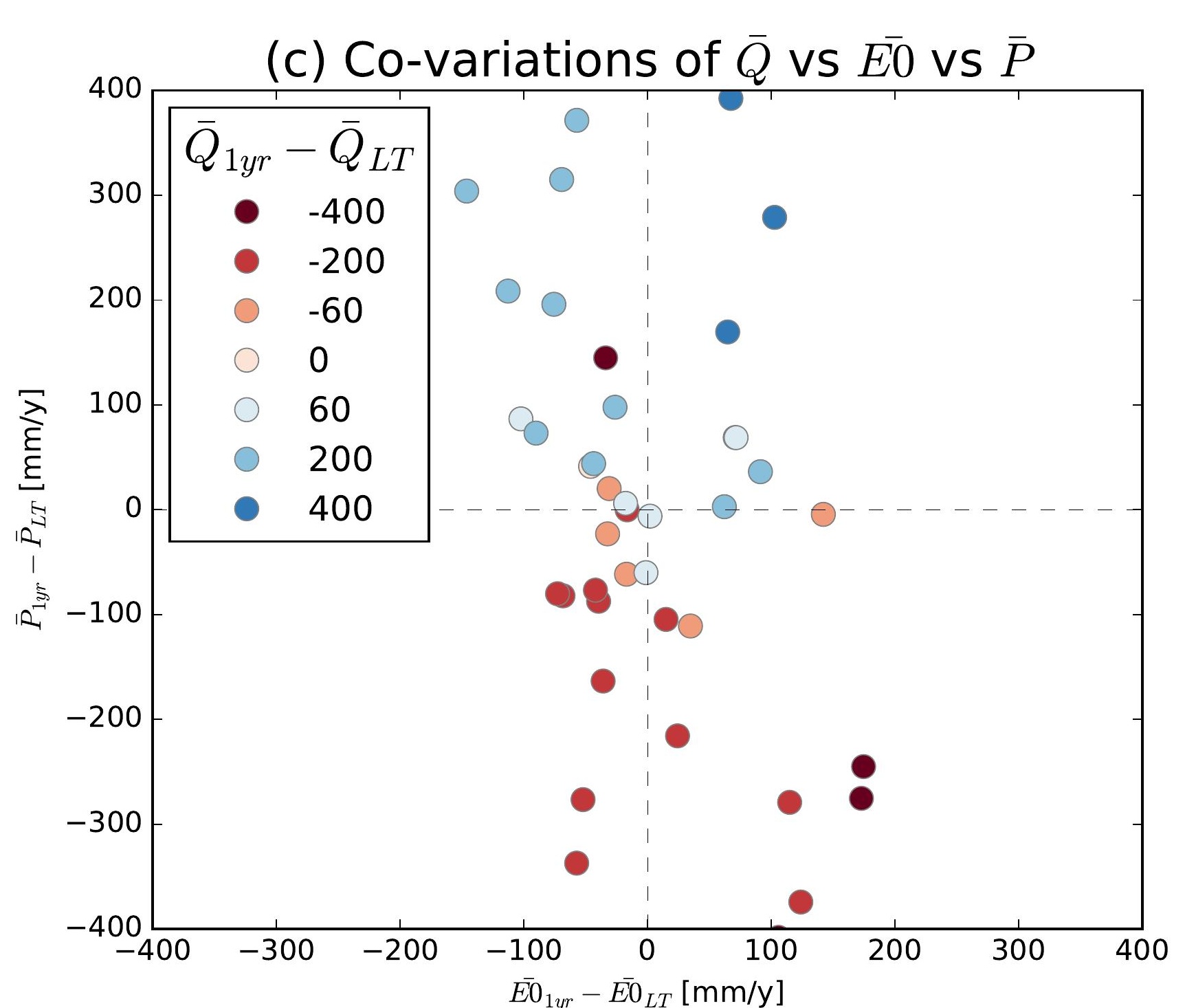
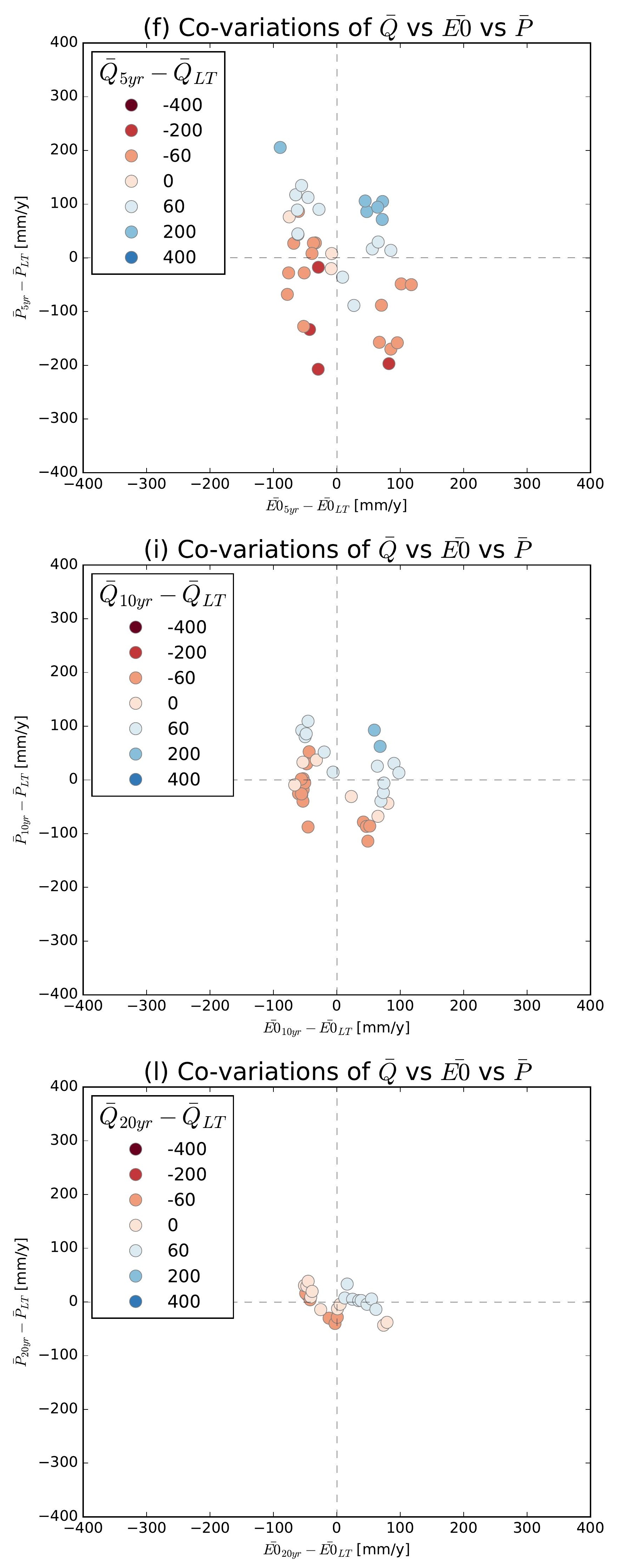

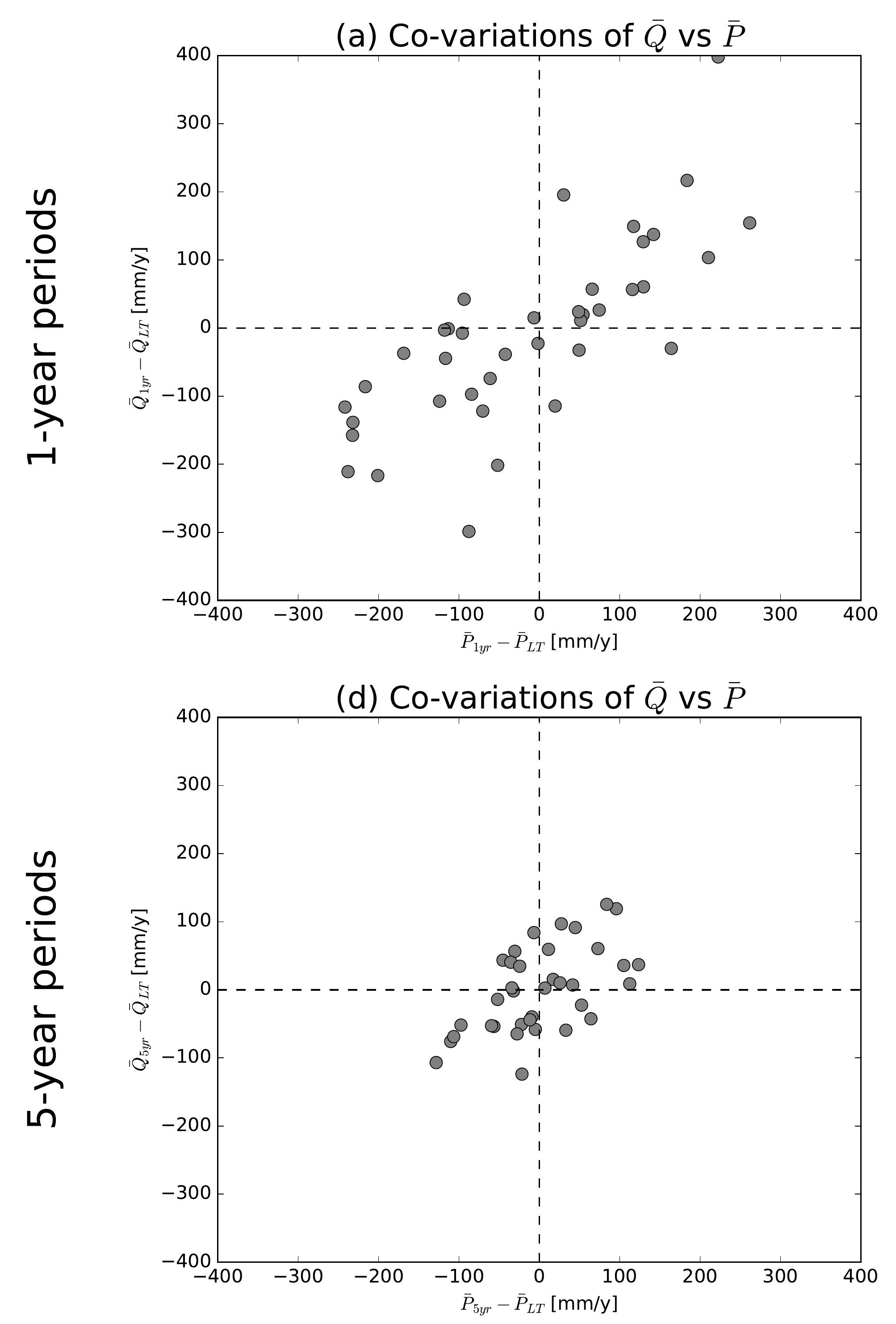

(g) Co-variations of $\bar{Q}$ vs $\bar{P}$

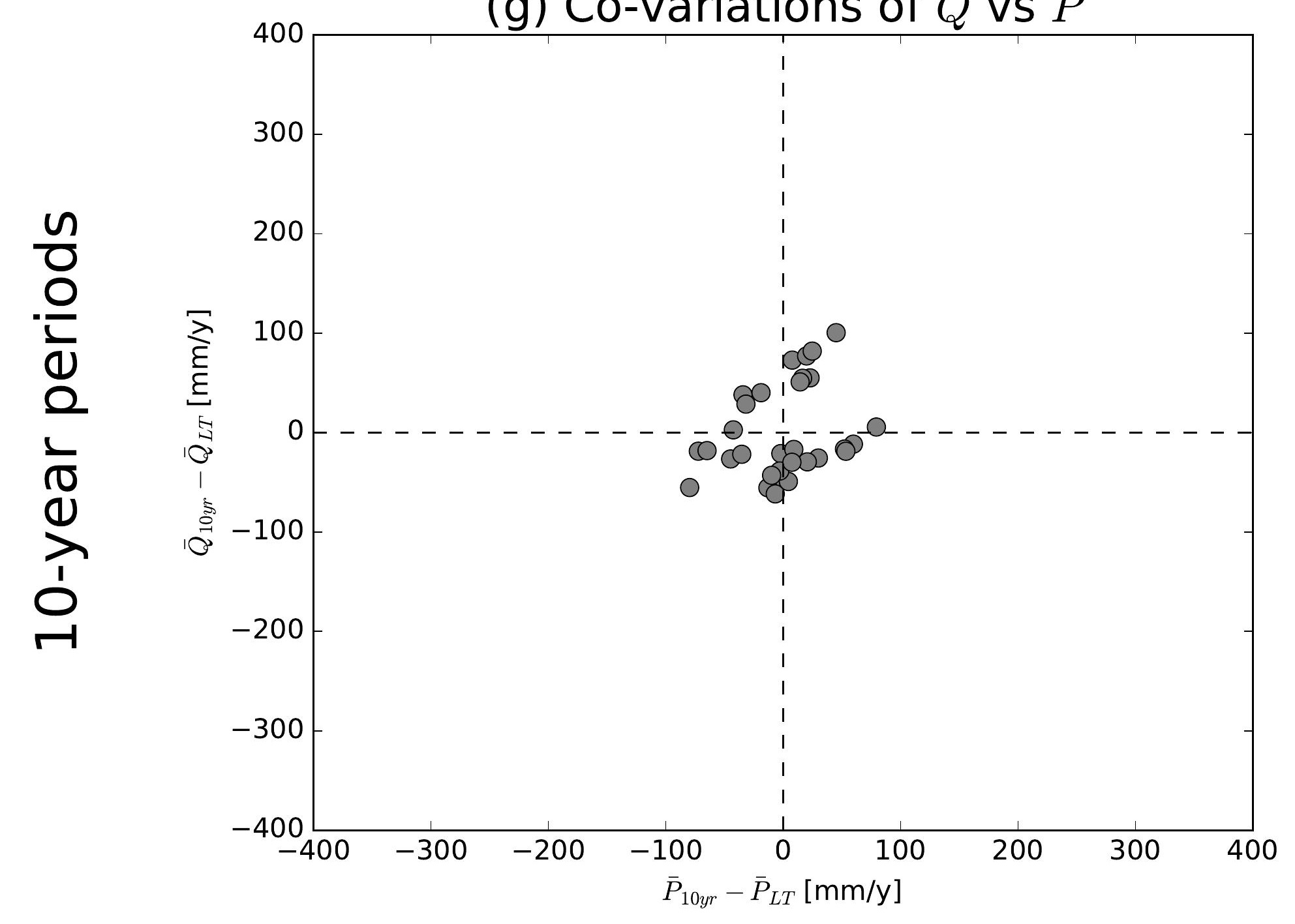

(j) Co-variations of $\bar{Q}$ vs $\bar{P}$

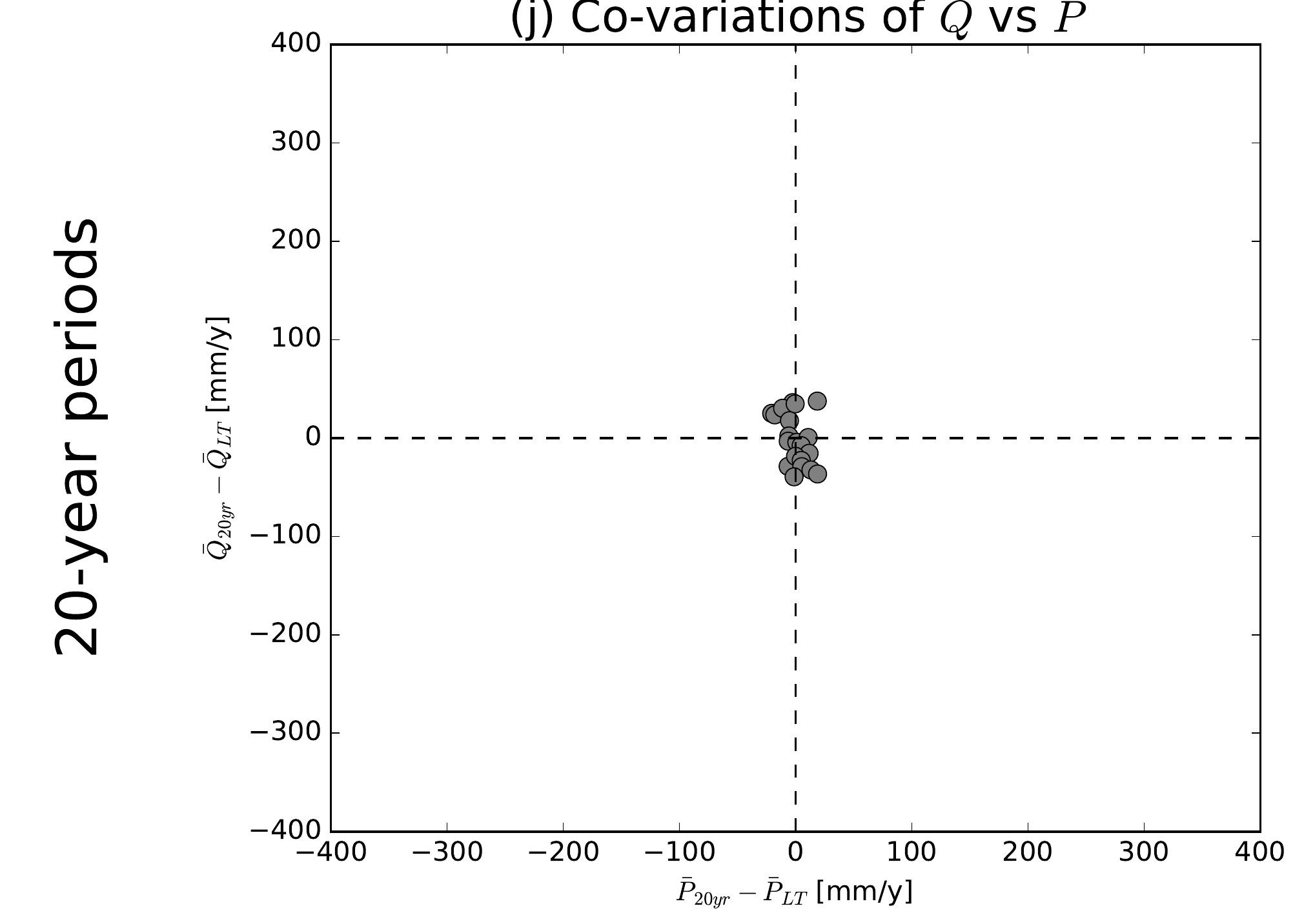

(b) Co-variations of $\bar{Q}$ vs $\overline{E 0}$

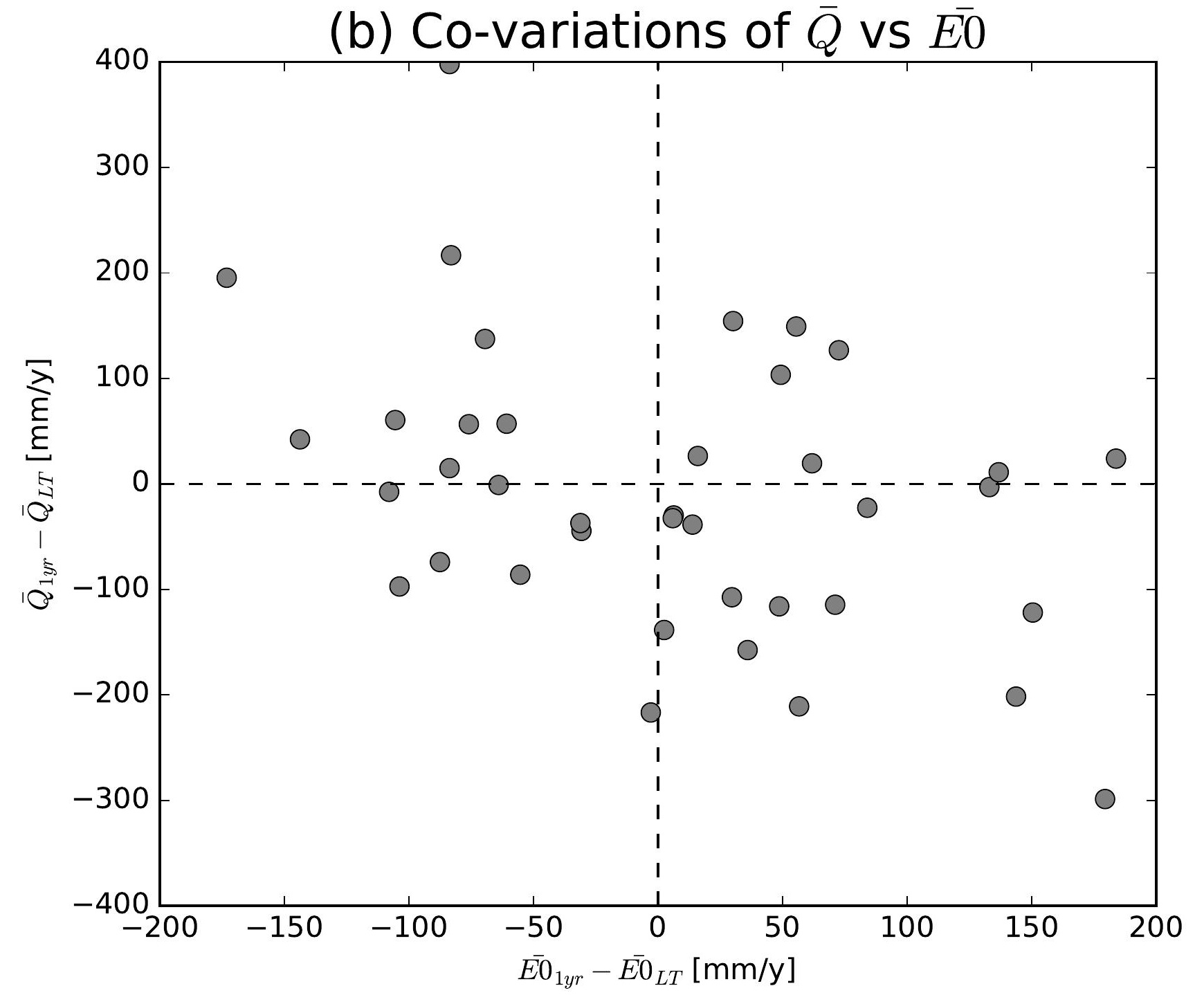

(e) Co-variations of $\bar{Q}$ vs $\overline{E 0}$

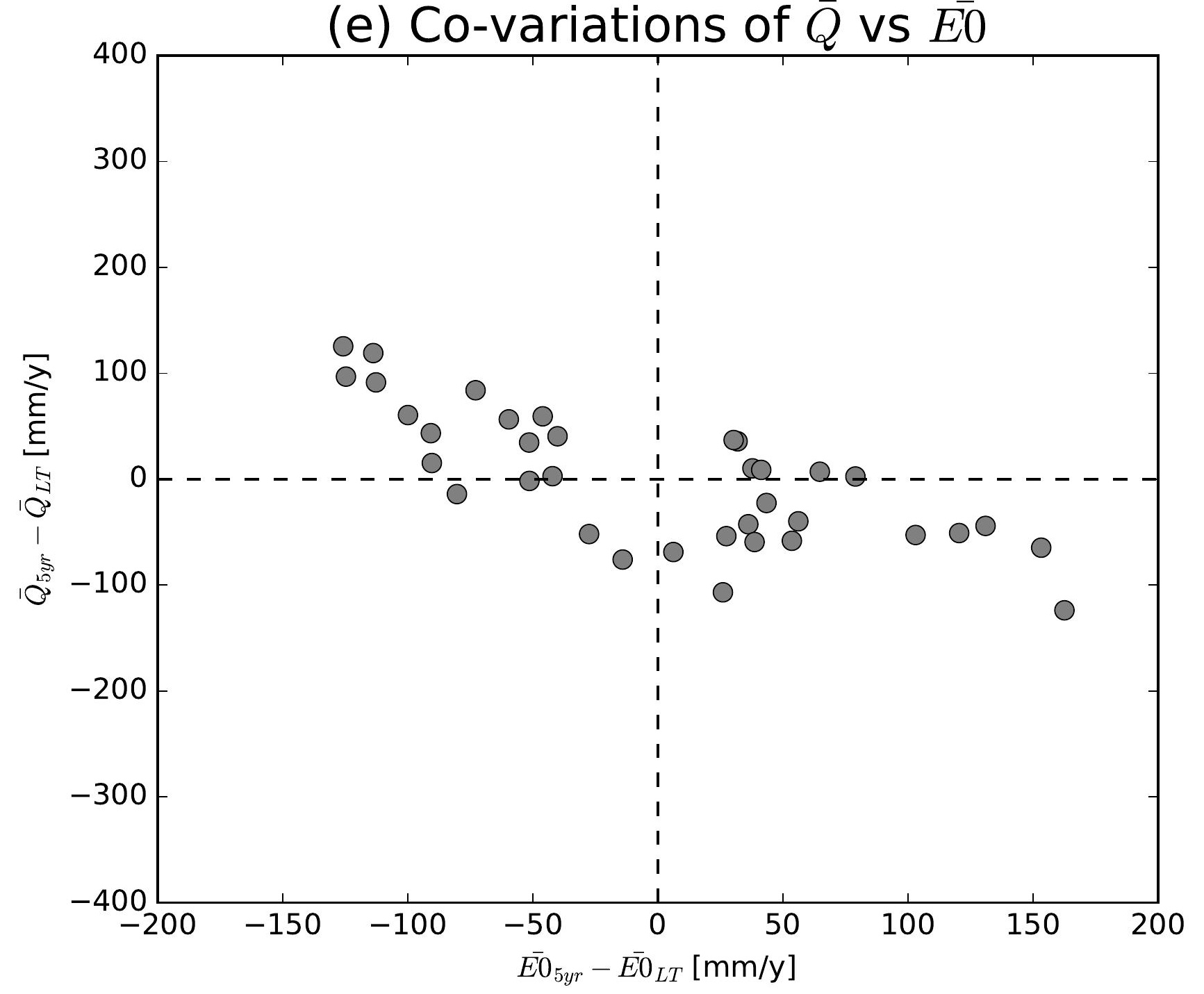

(h) Co-variations of $\bar{Q}$ vs $\overline{E 0}$

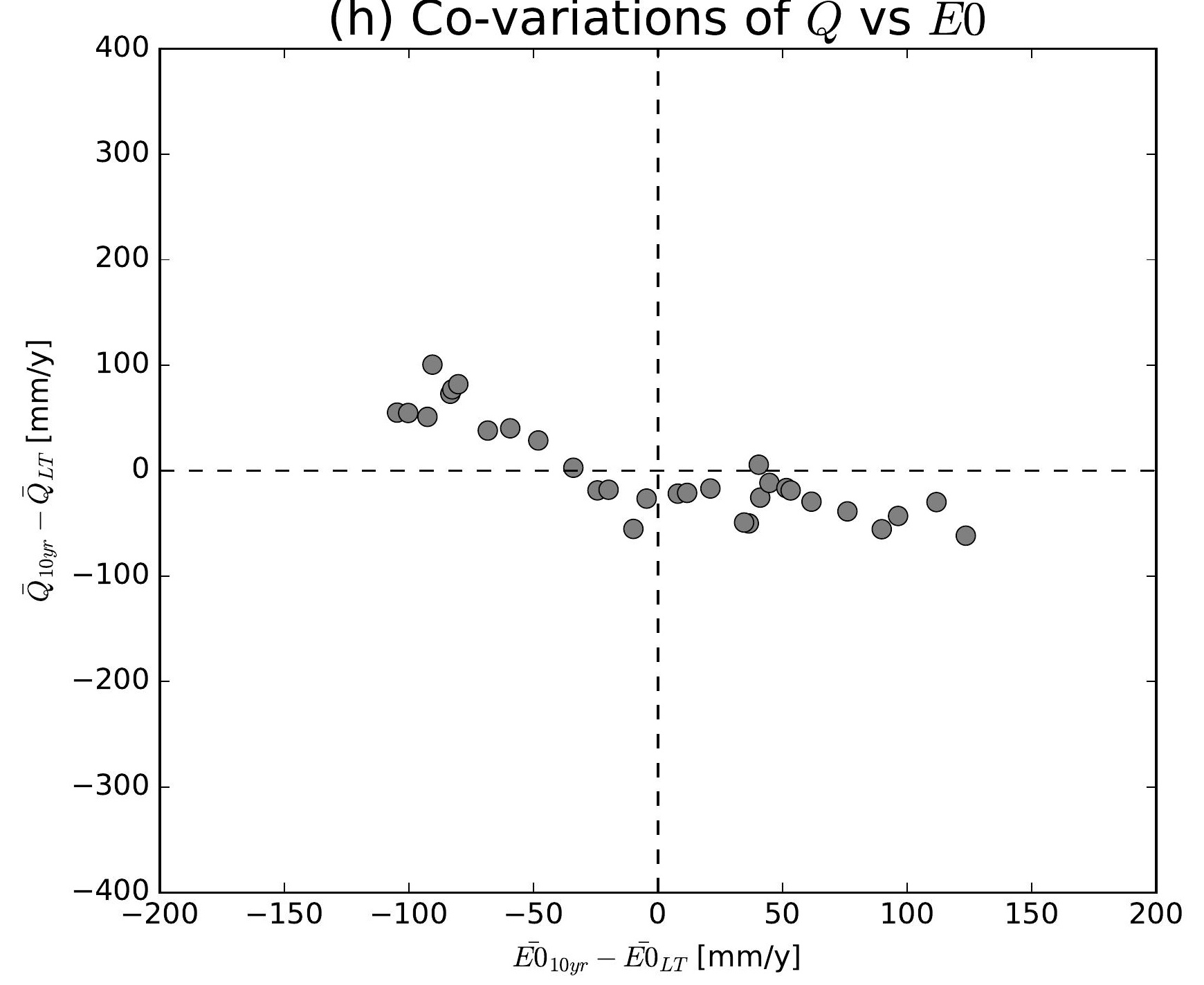

(k) Co-variations of $\bar{Q}$ vs $\overline{E 0}$

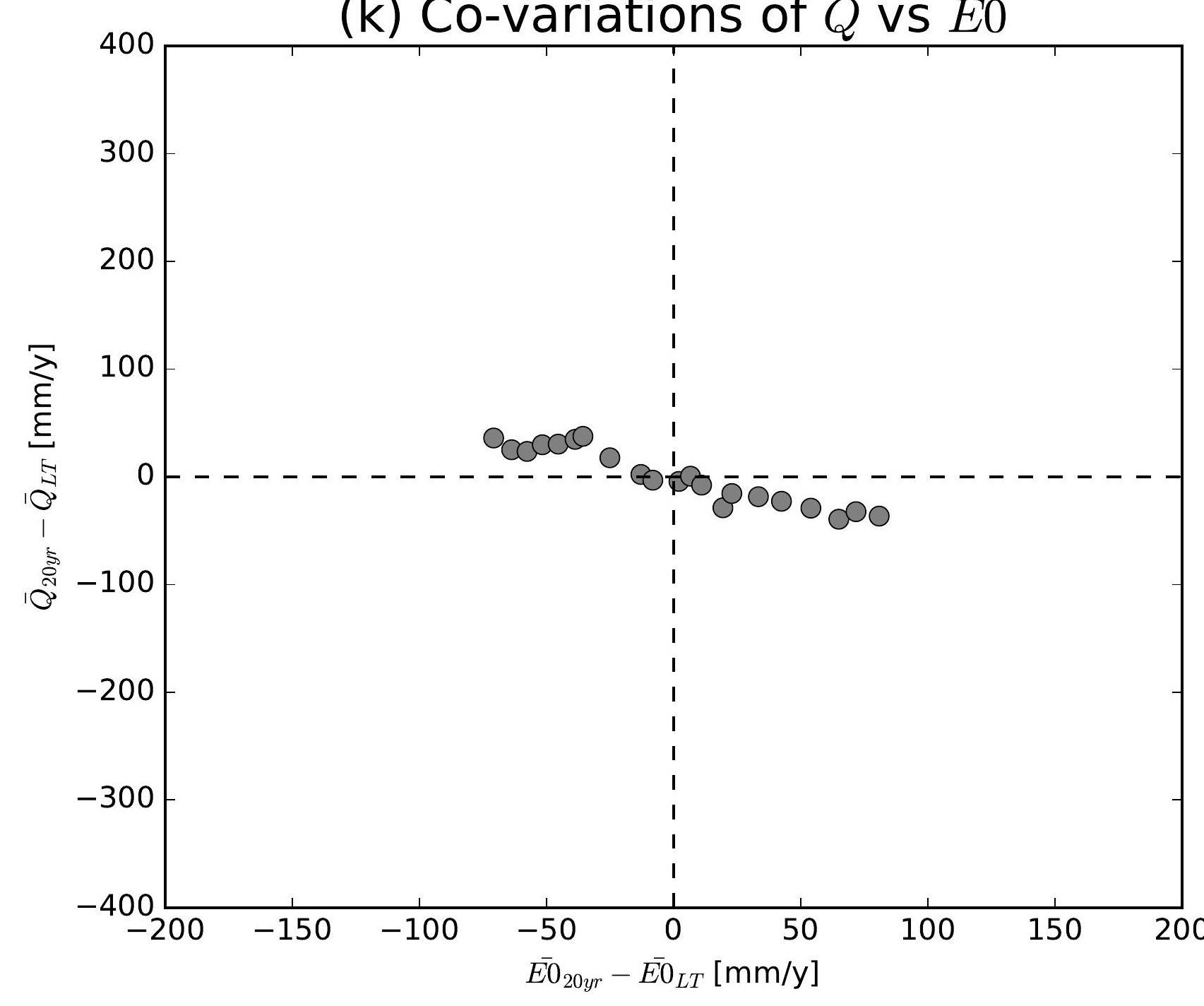

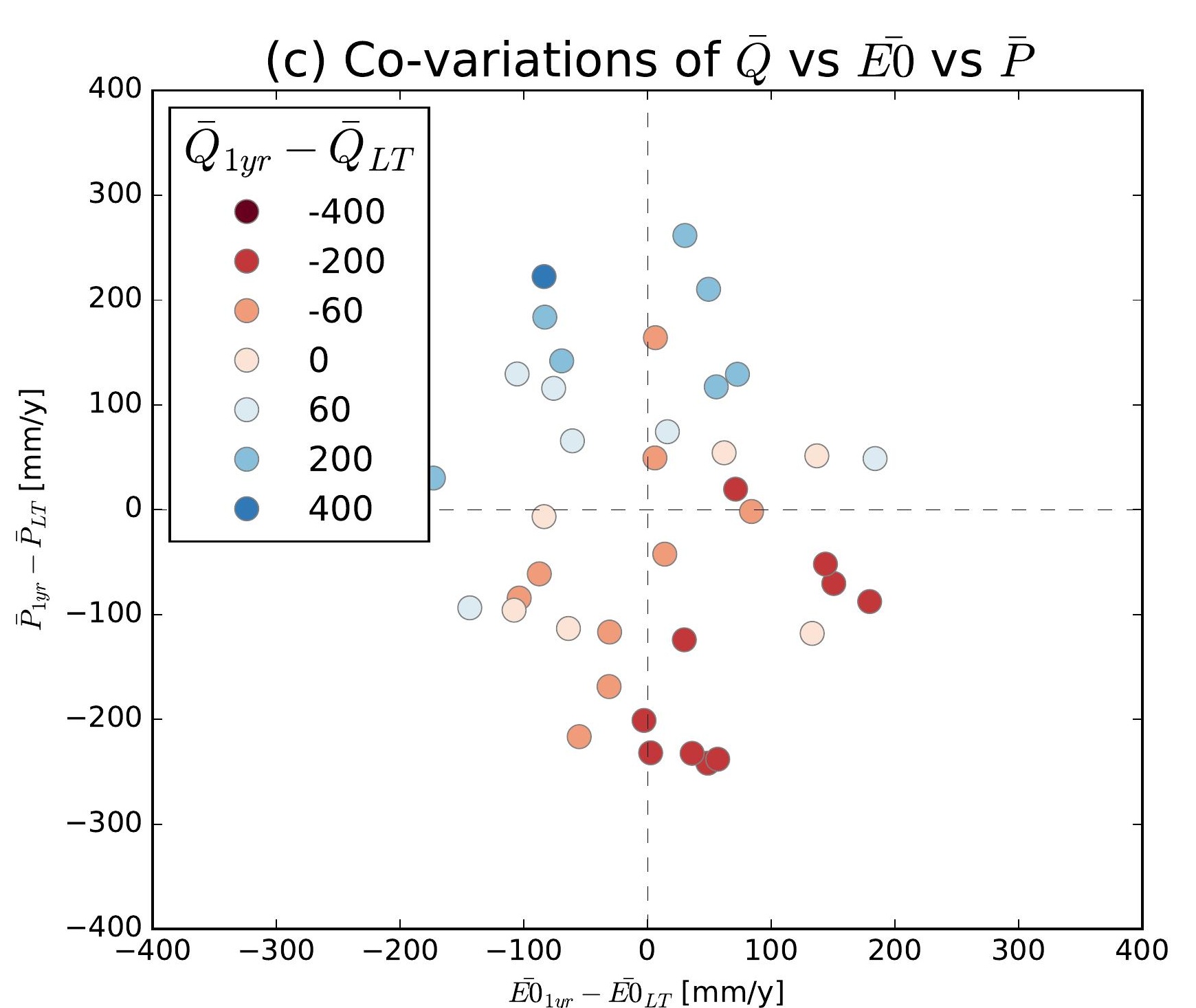
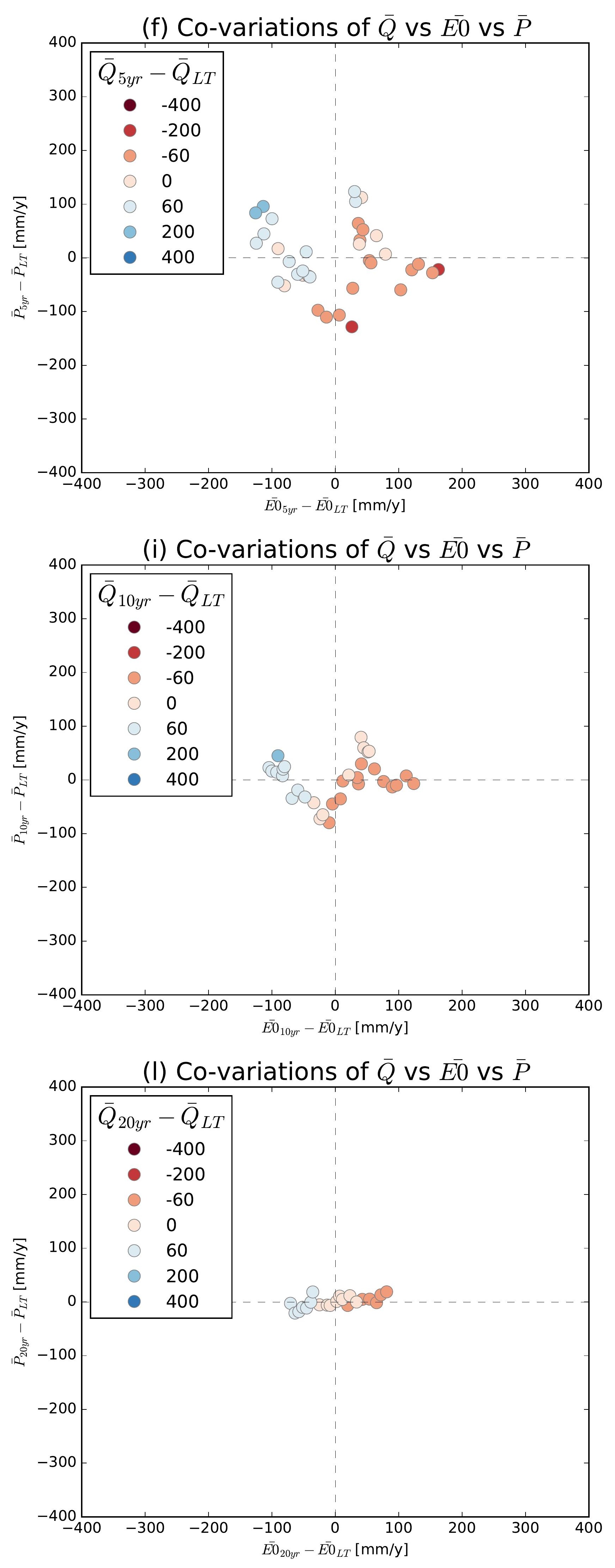

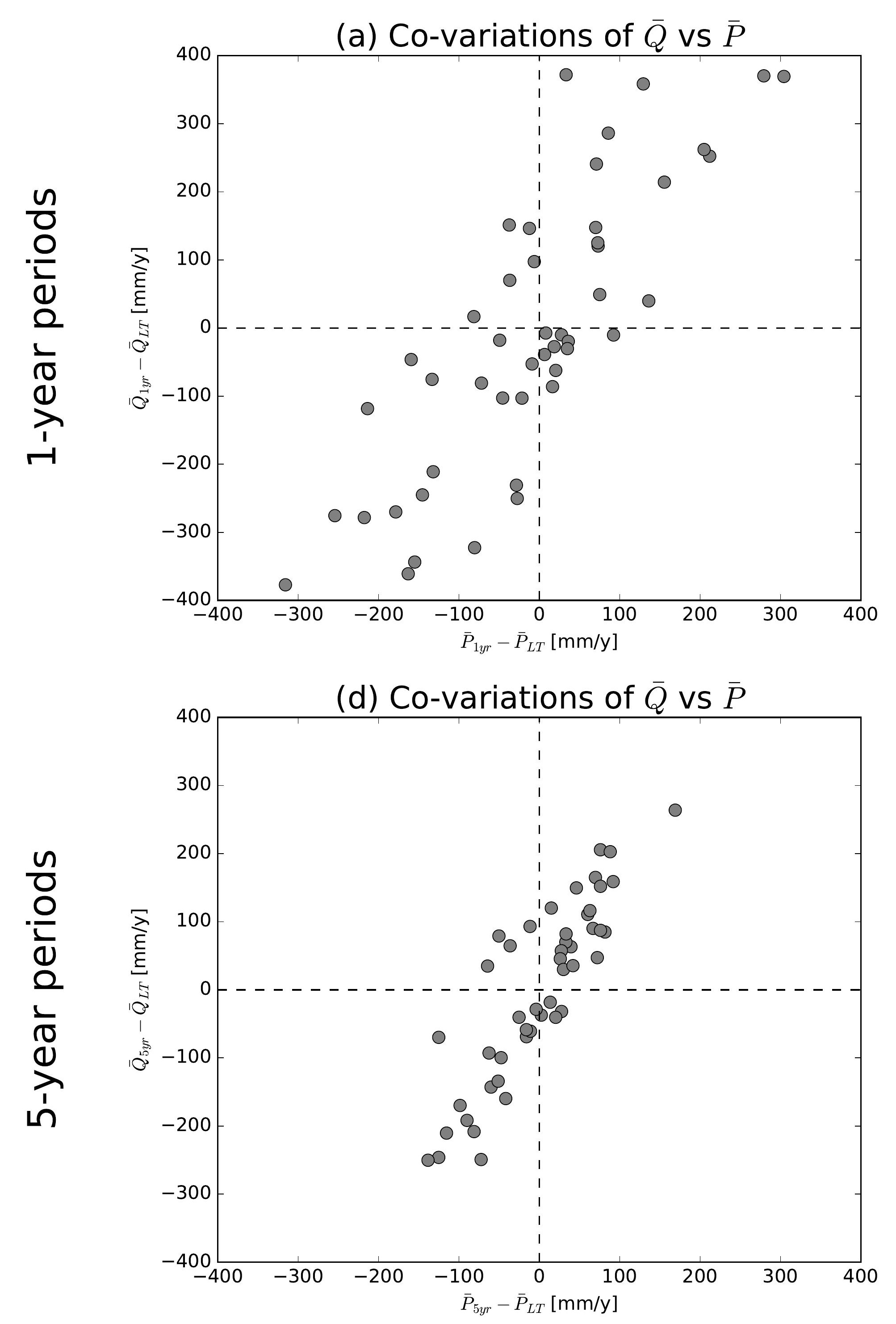

(g) Co-variations of $\bar{Q}$ vs $\bar{P}$
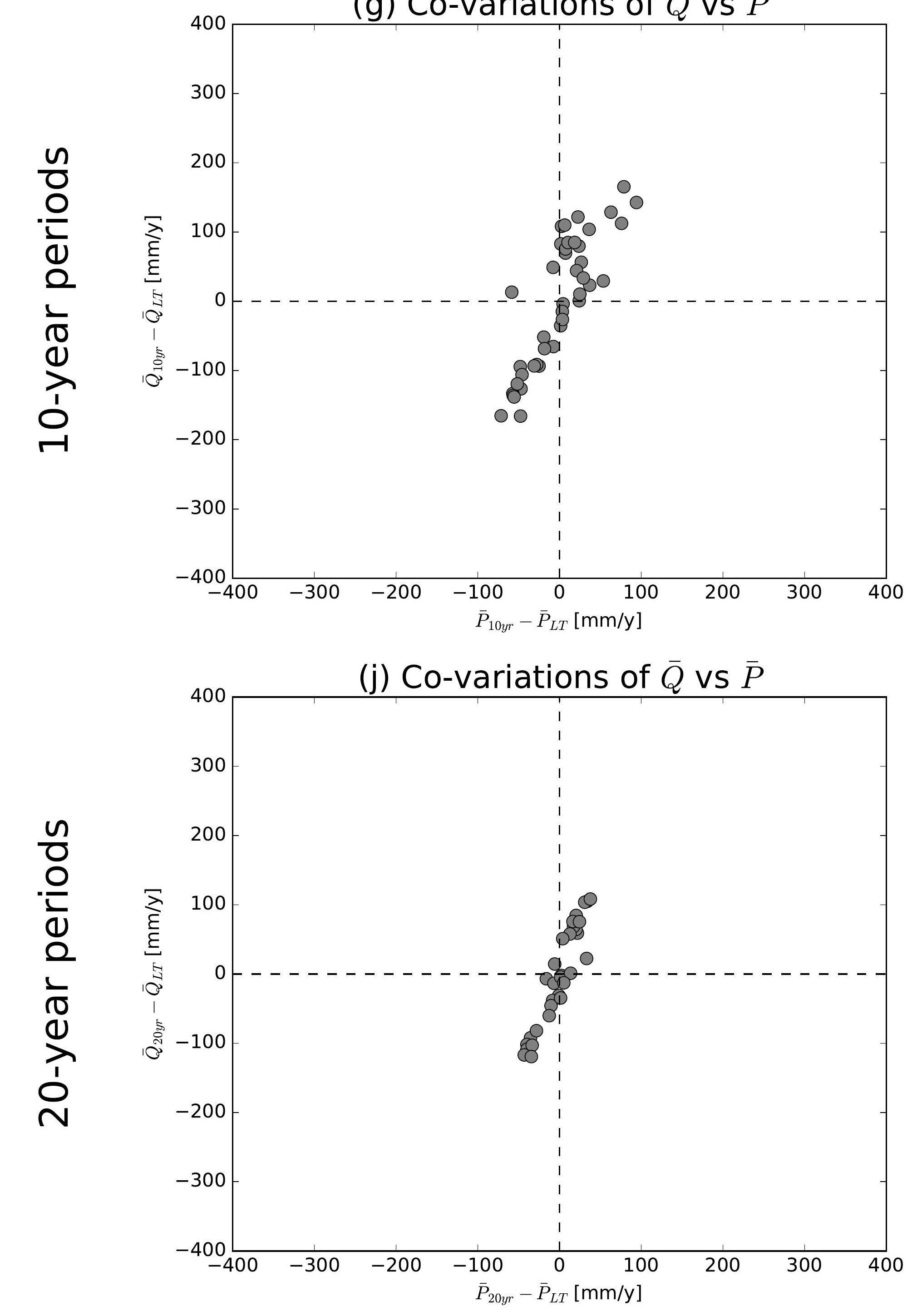

(b) Co-variations of $\bar{Q}$ vs $\overline{E 0}$

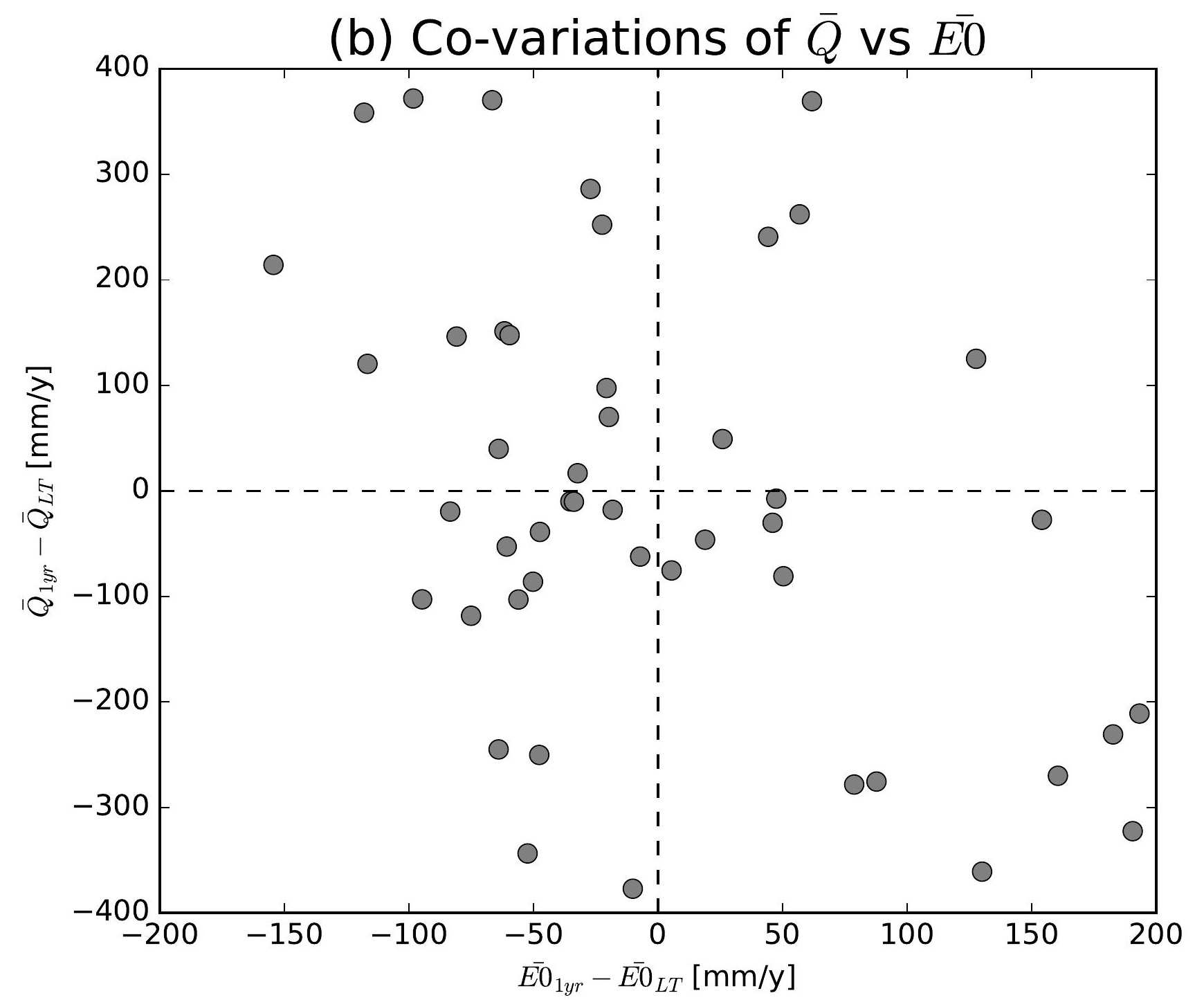

(e) Co-variations of $\bar{Q}$ vs $\overline{E 0}$

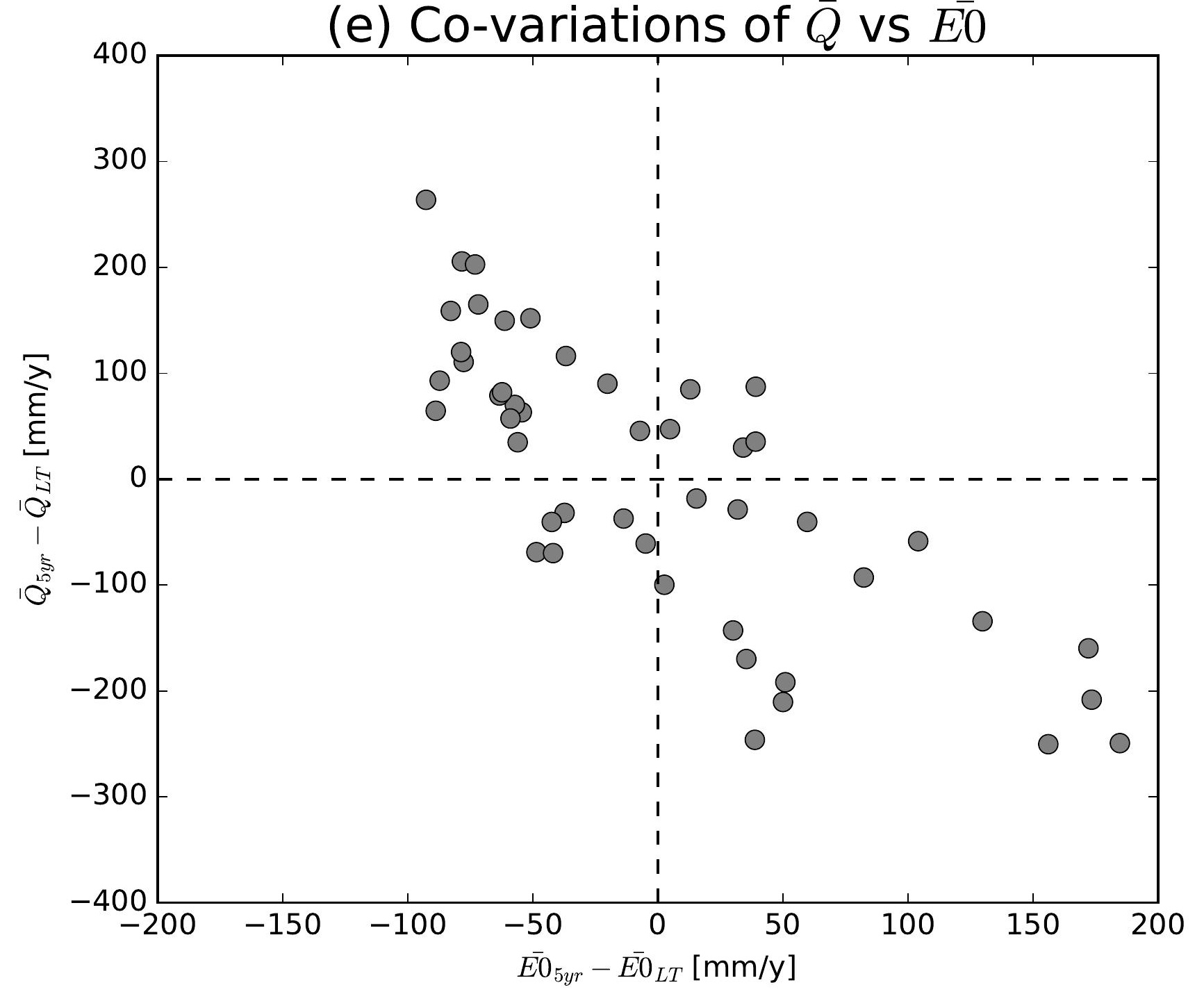

(h) Co-variations of $\bar{Q}$ vs $\overline{E 0}$

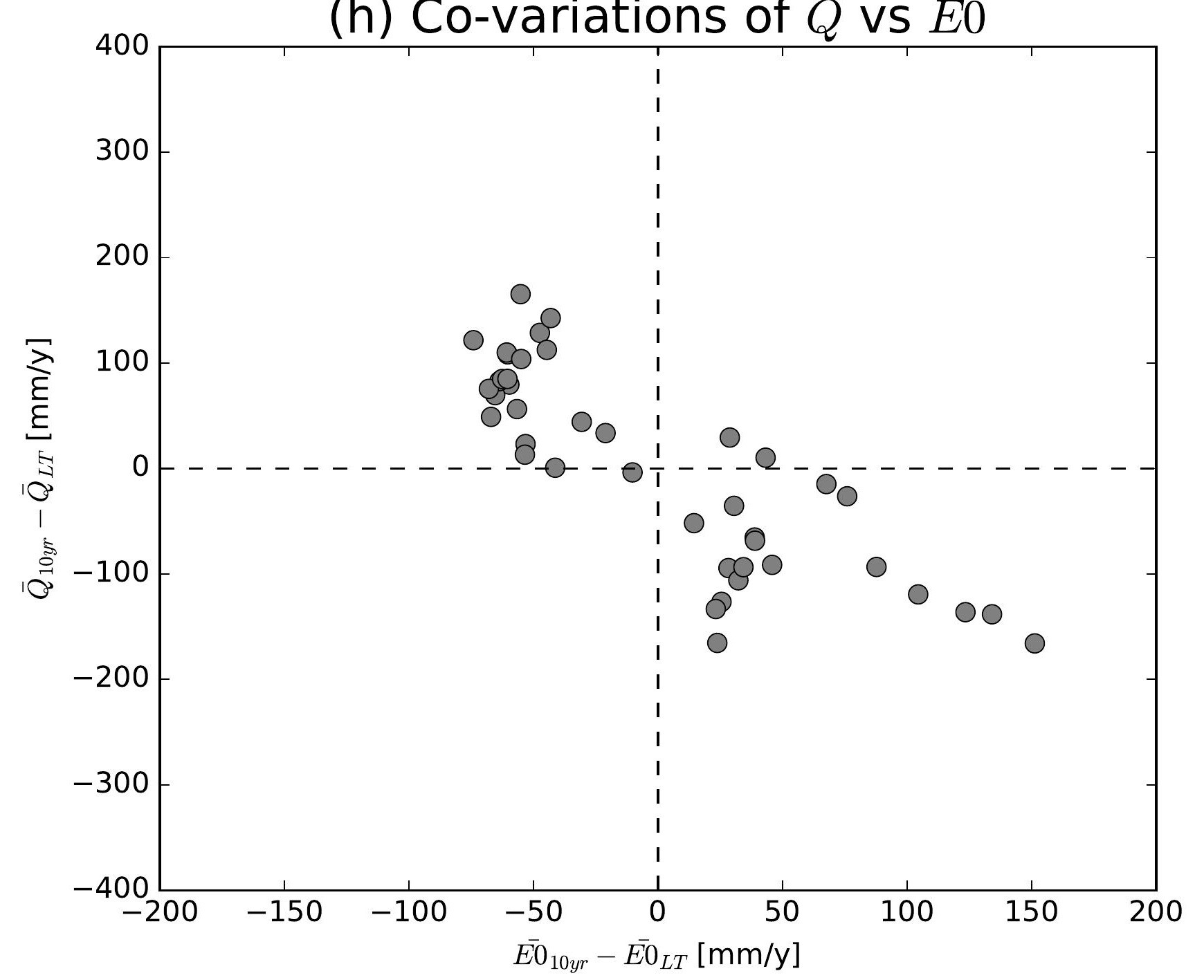

(k) Co-variations of $\bar{Q}$ vs $\overline{E 0}$

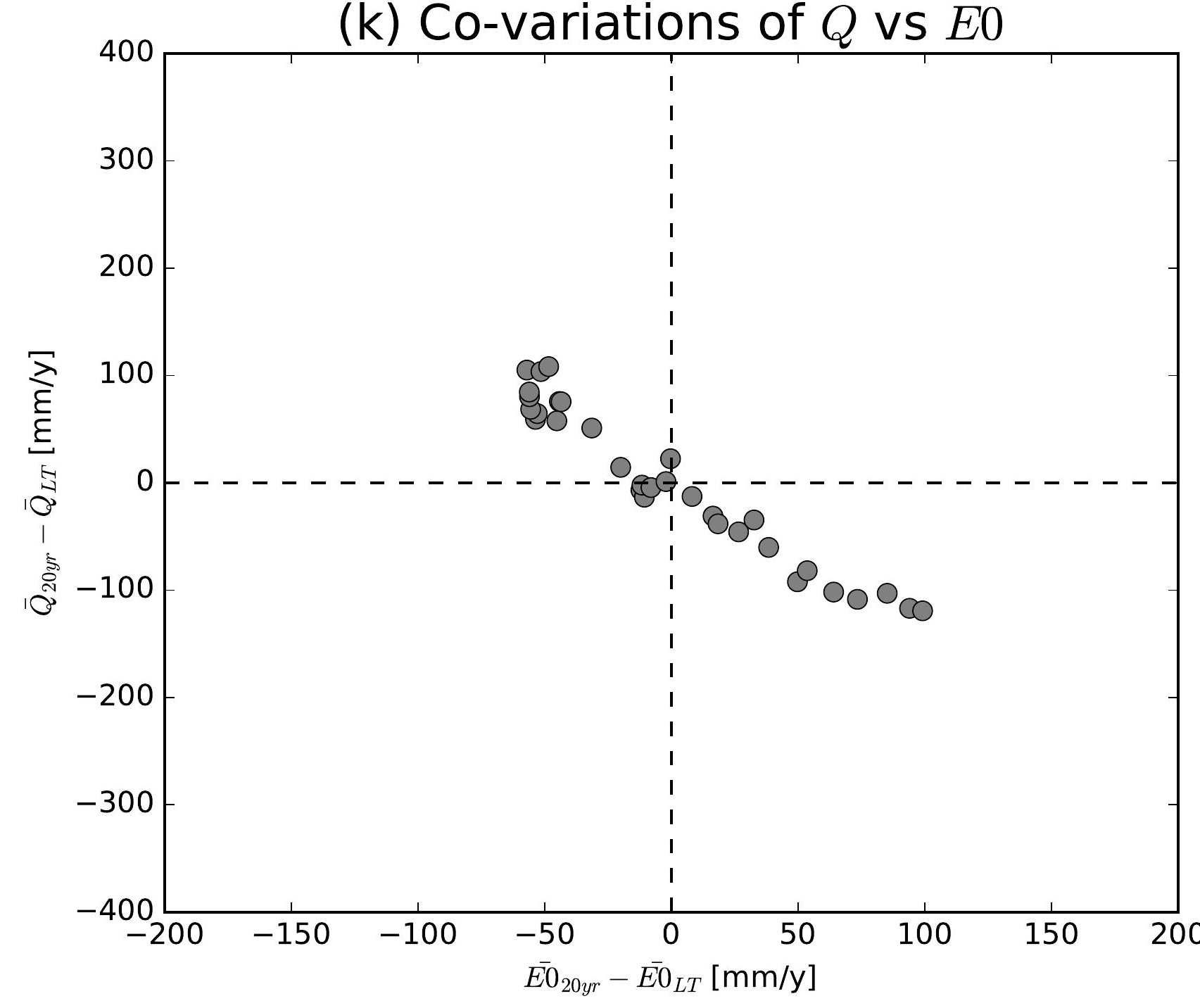

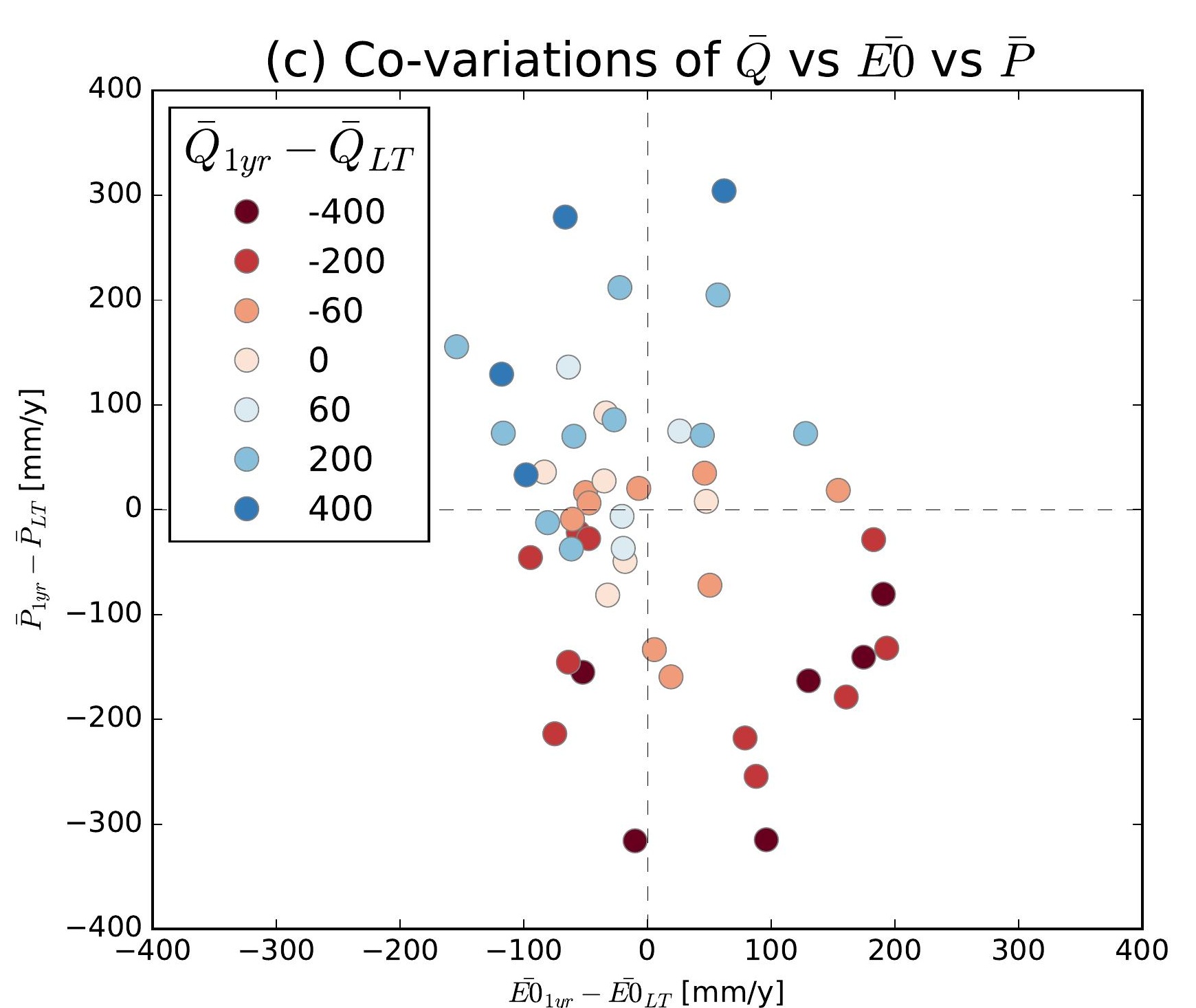
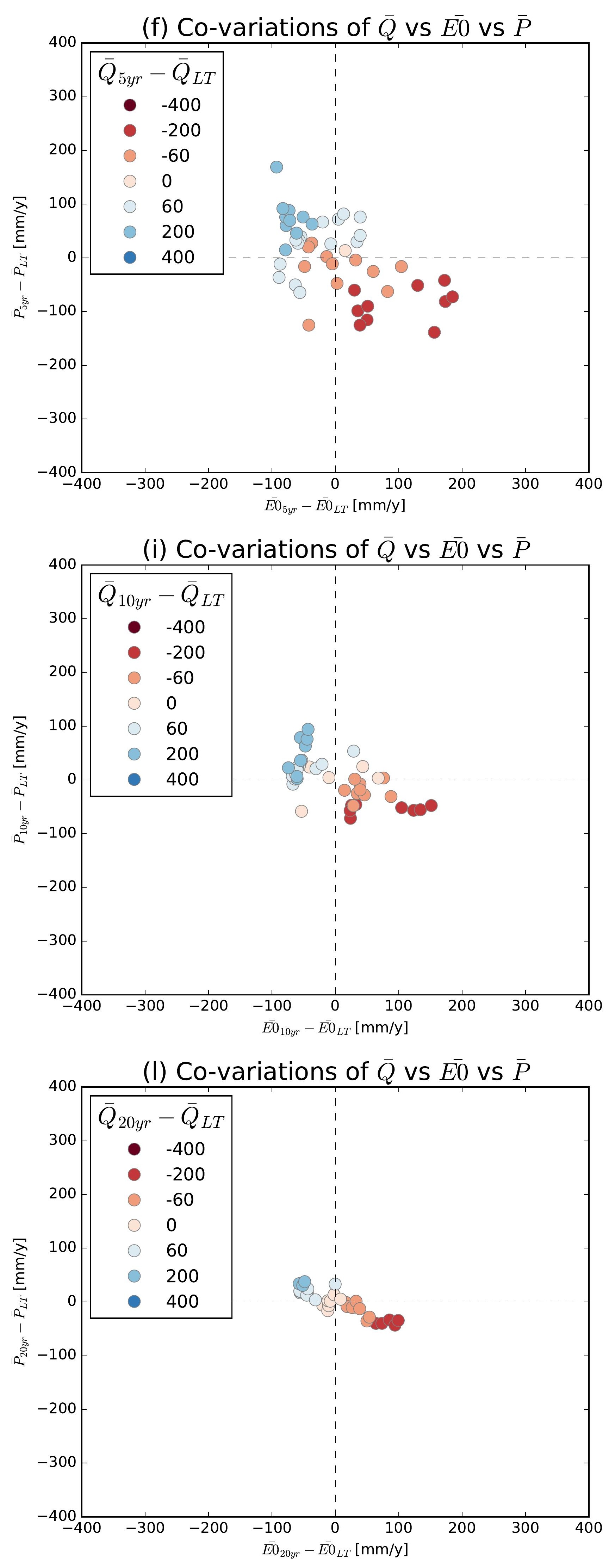

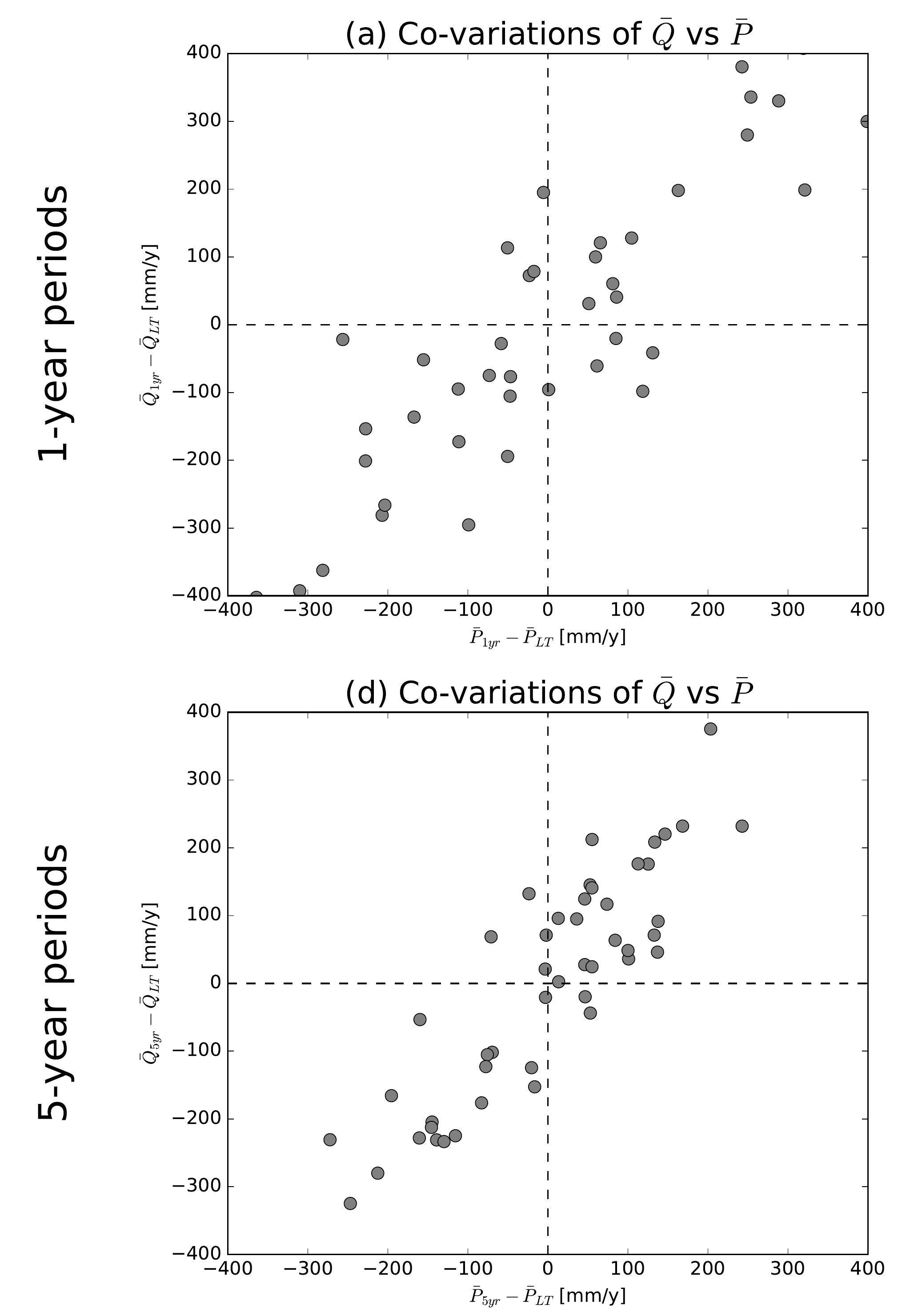

(g) Co-variations of $\bar{Q}$ vs $\bar{P}$
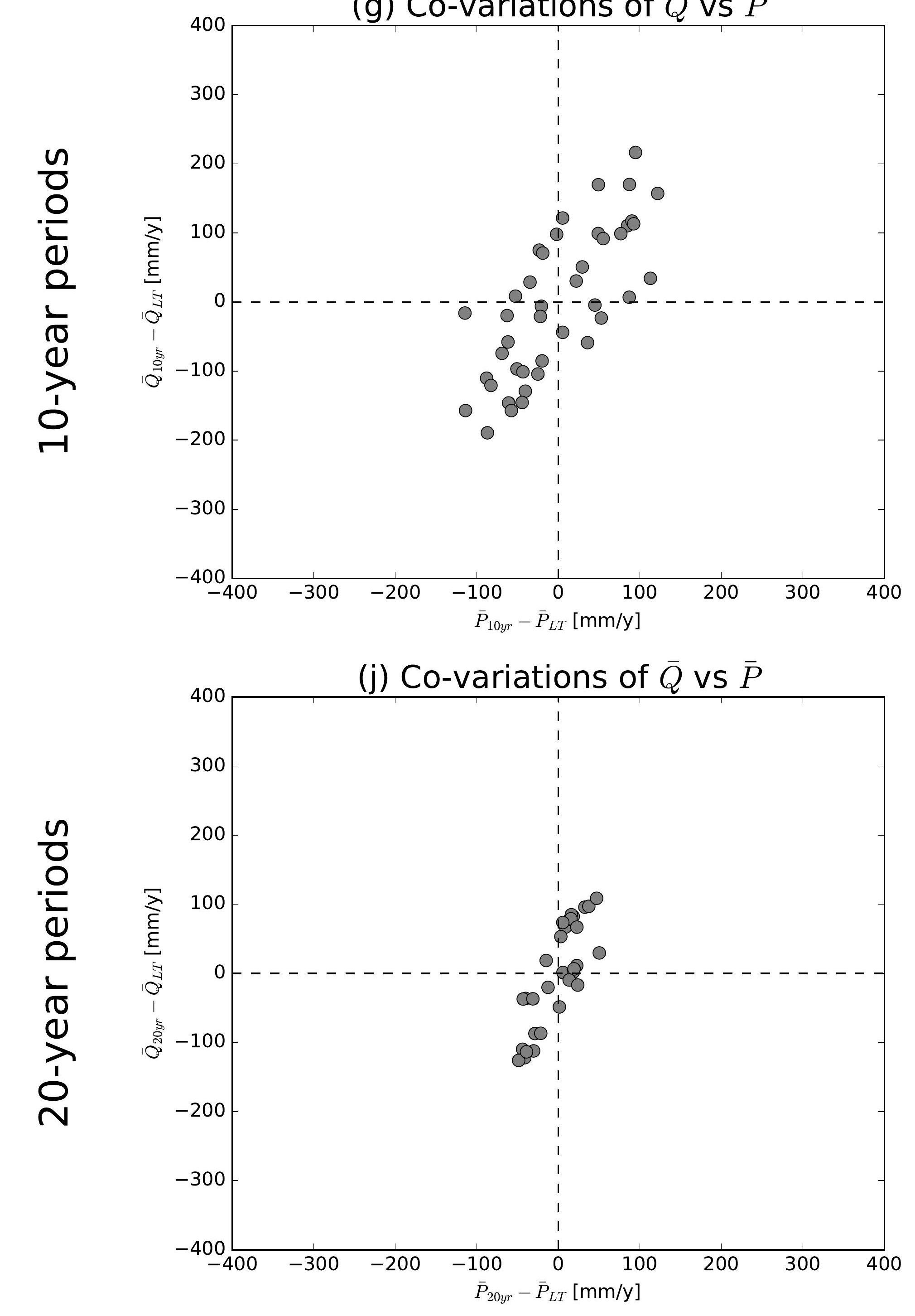

(b) Co-variations of $\bar{Q}$ vs $\overline{E 0}$

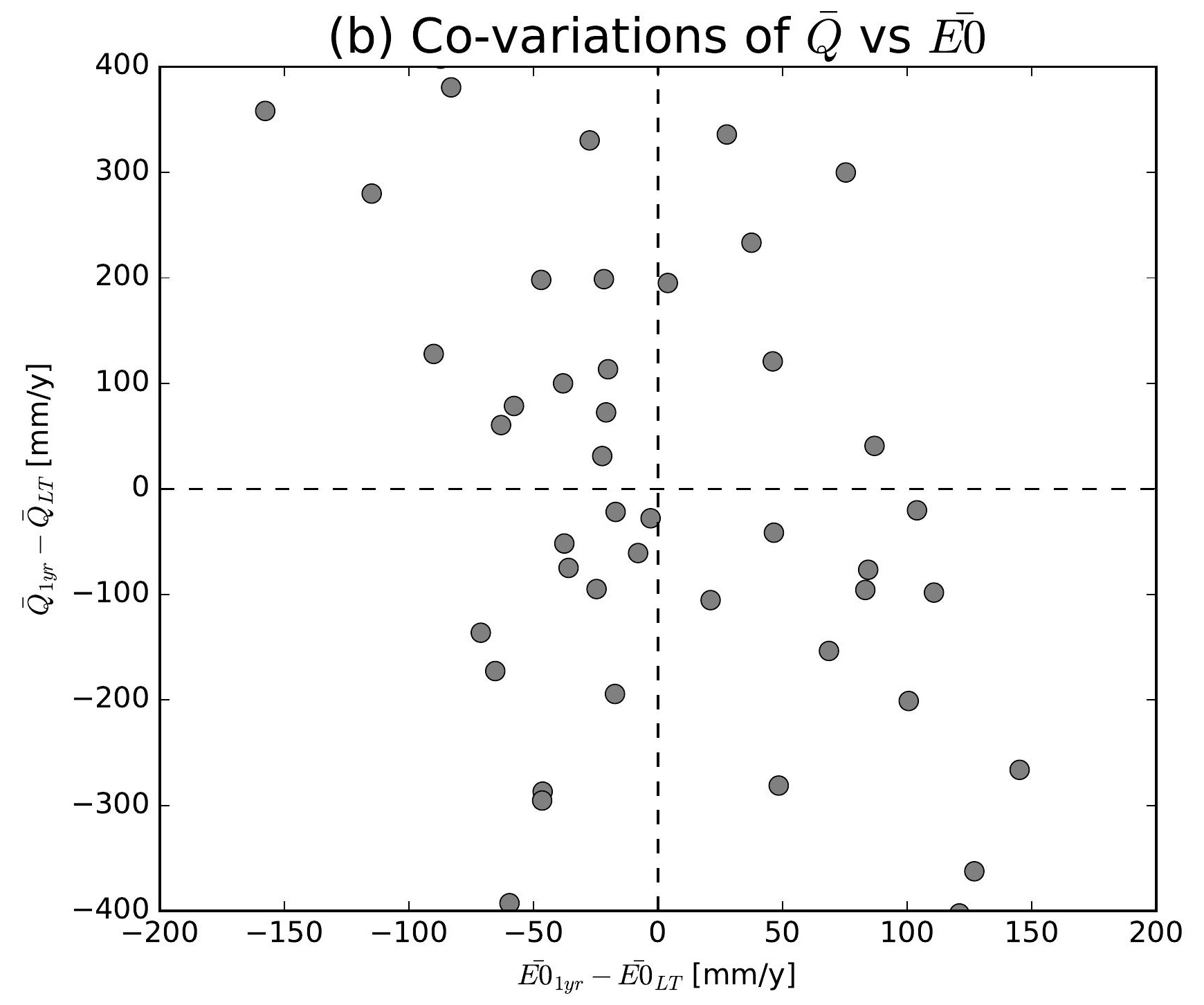

(e) Co-variations of $\bar{Q}$ vs $\overline{E 0}$

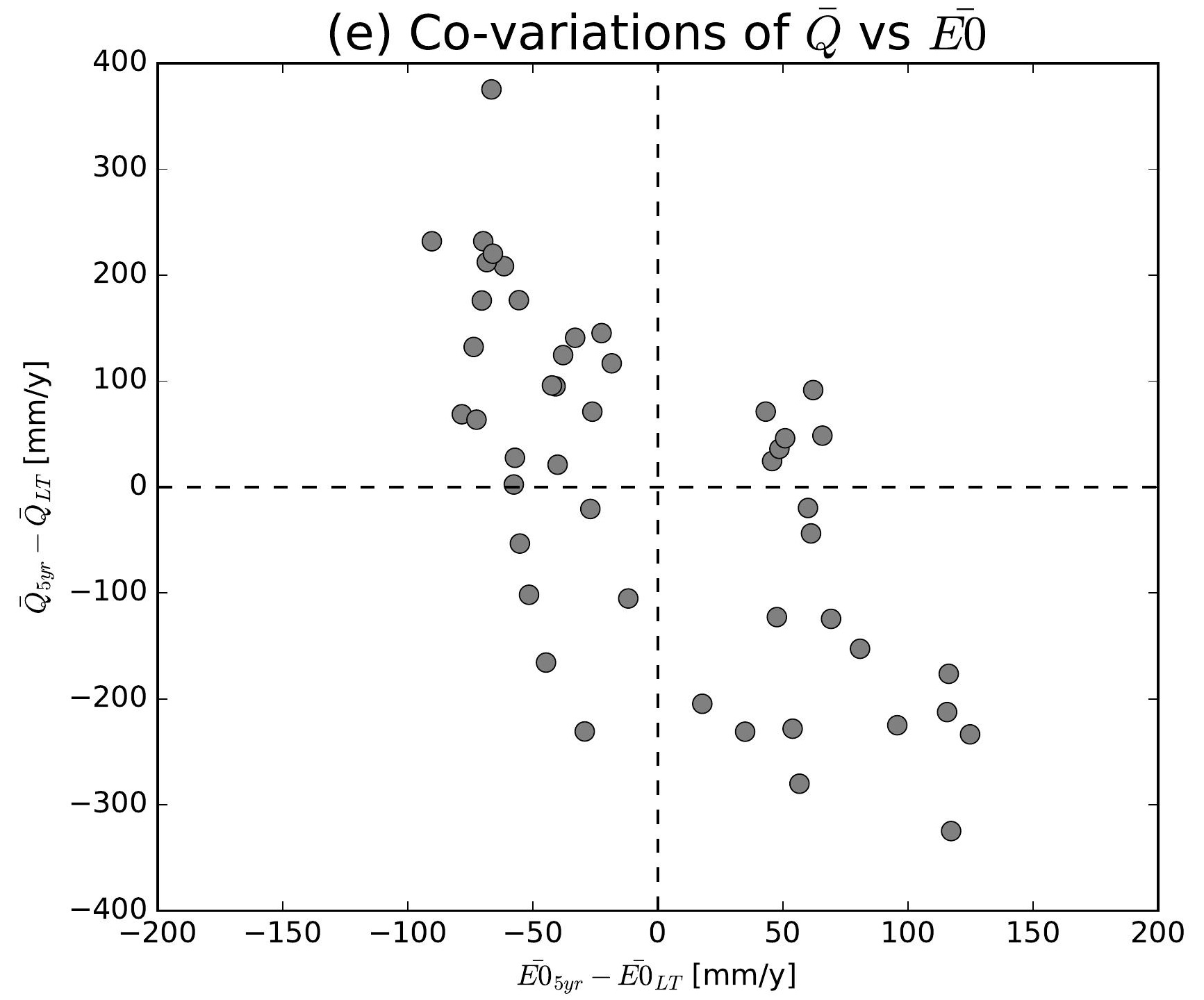

(h) Co-variations of $\bar{Q}$ vs $\overline{E 0}$

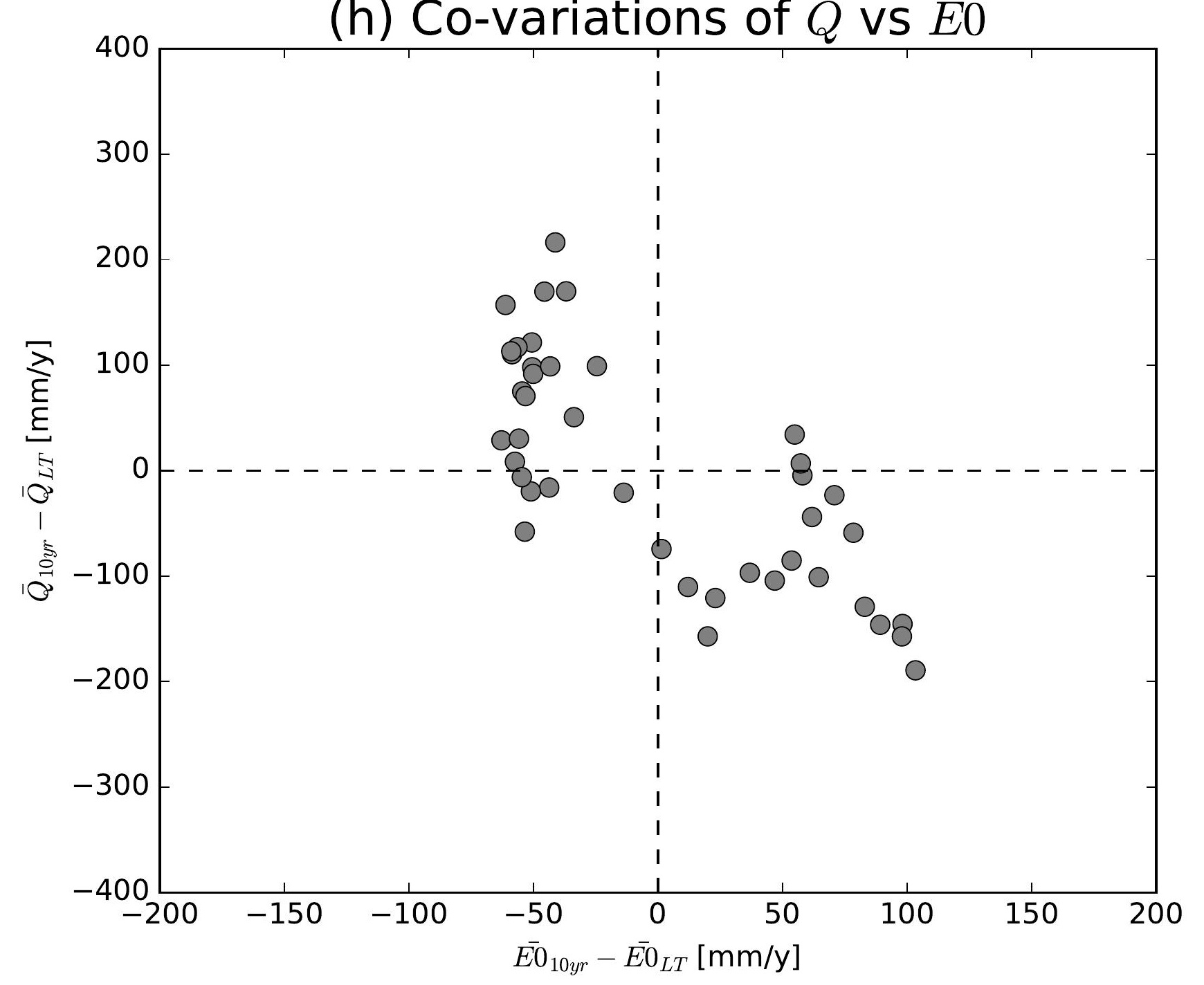

(k) Co-variations of $\bar{Q}$ vs $\overline{E 0}$

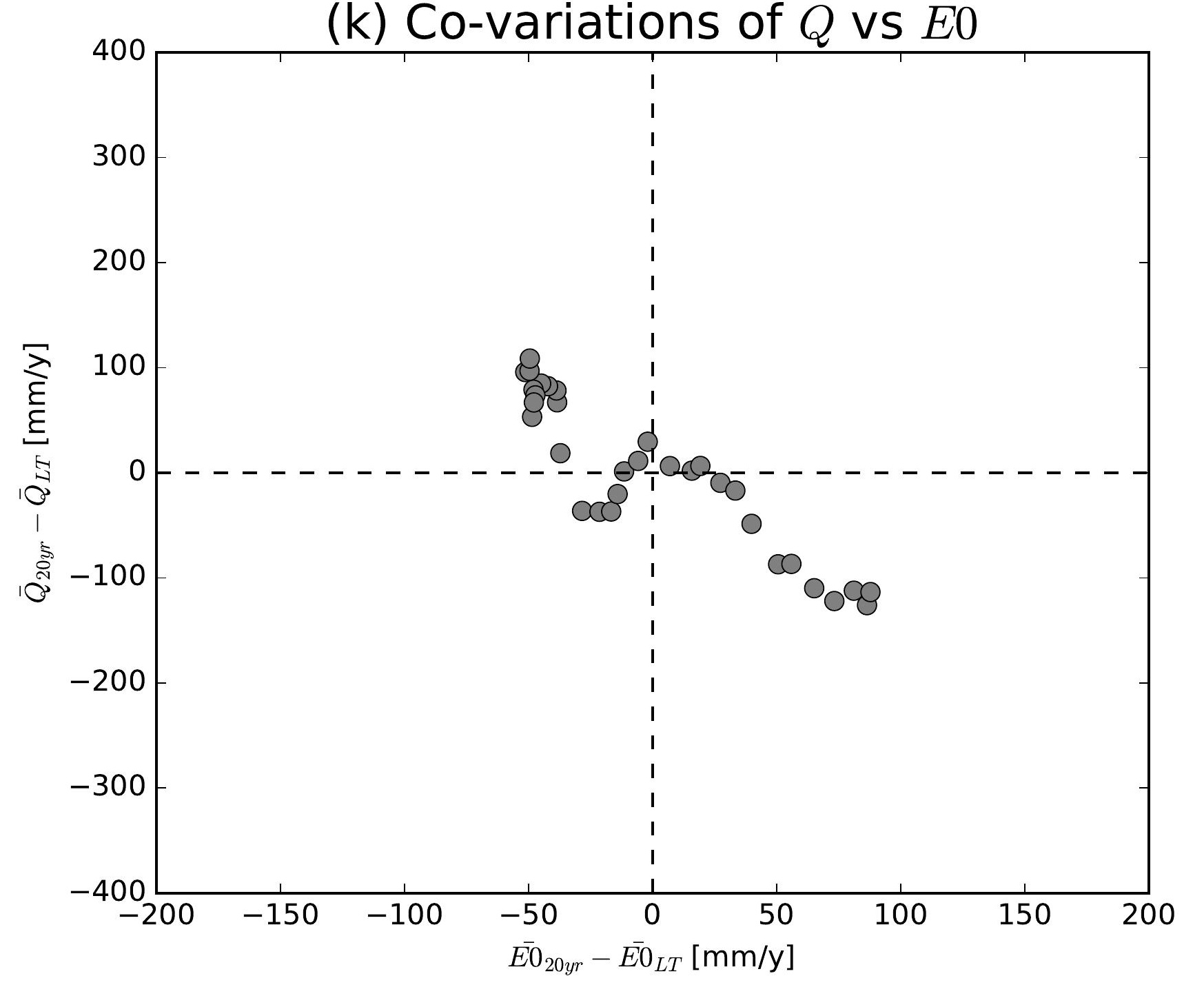

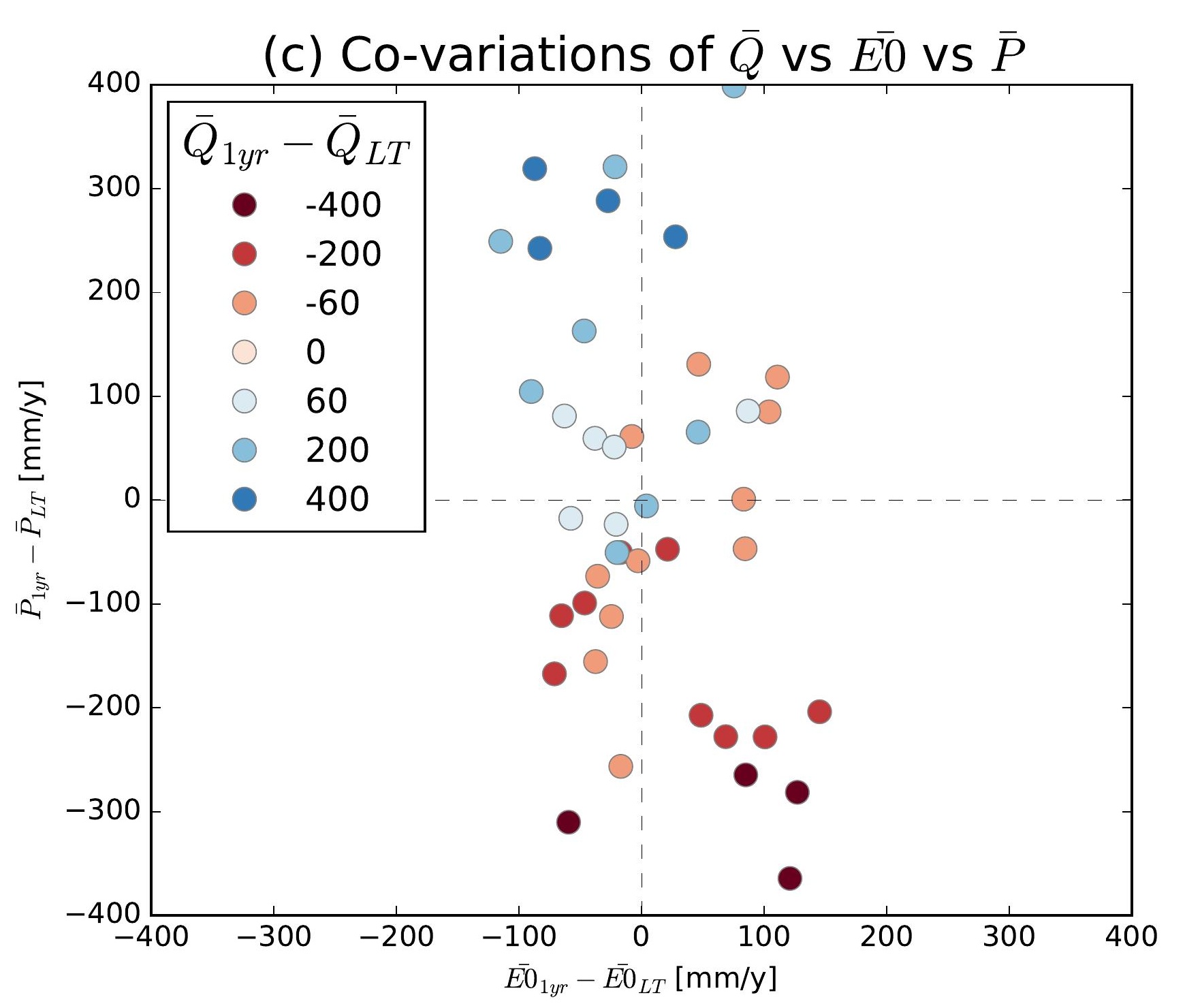
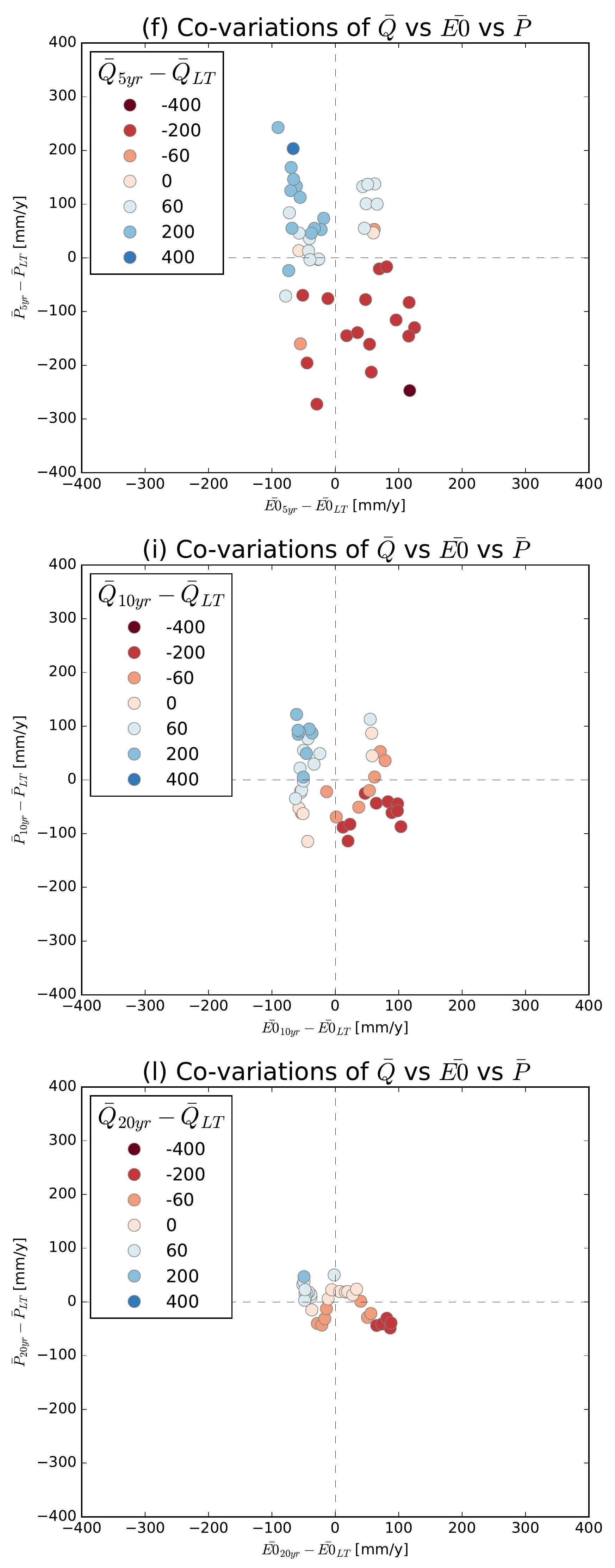

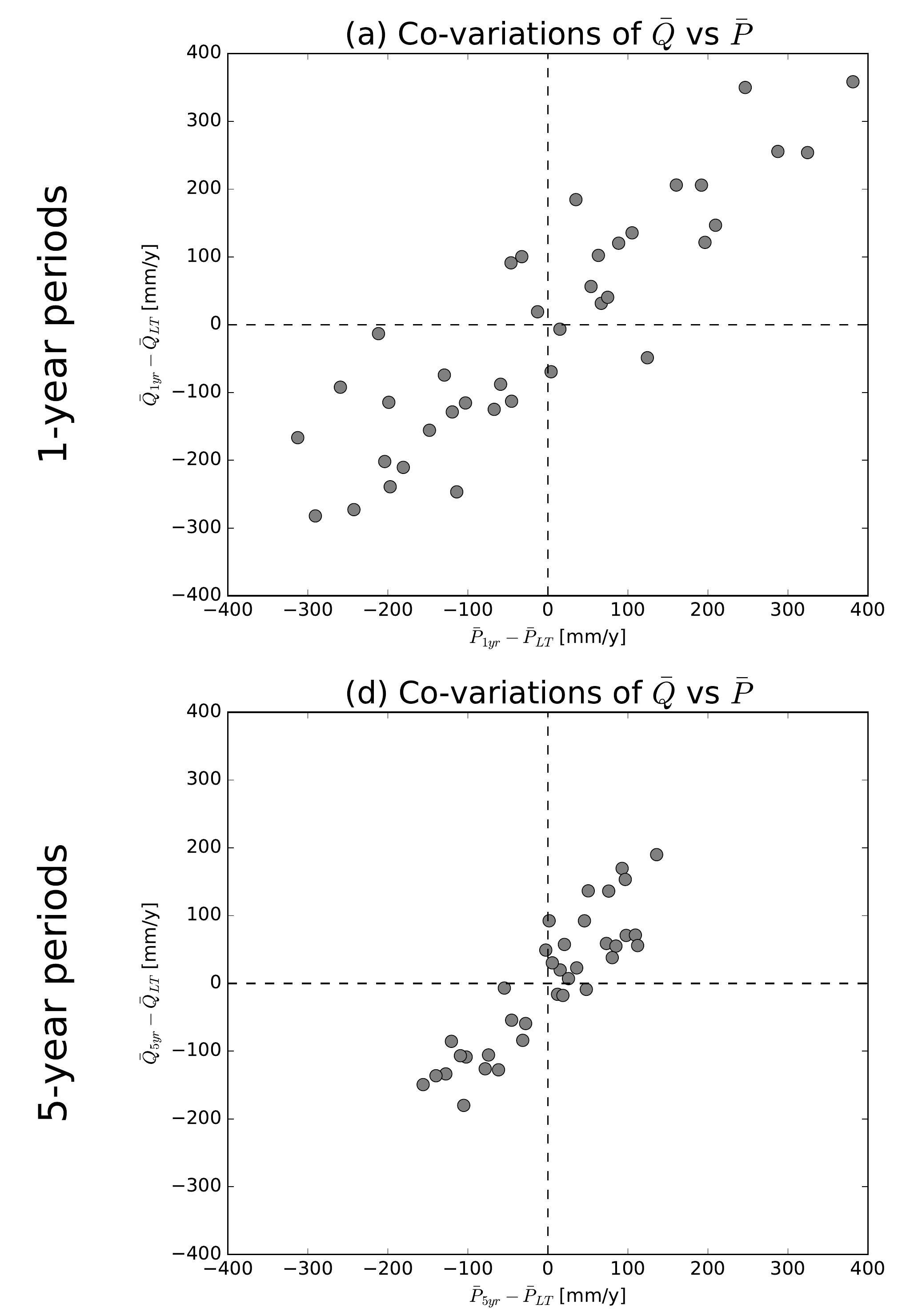

(g) Co-variations of $\bar{Q}$ vs $\bar{P}$
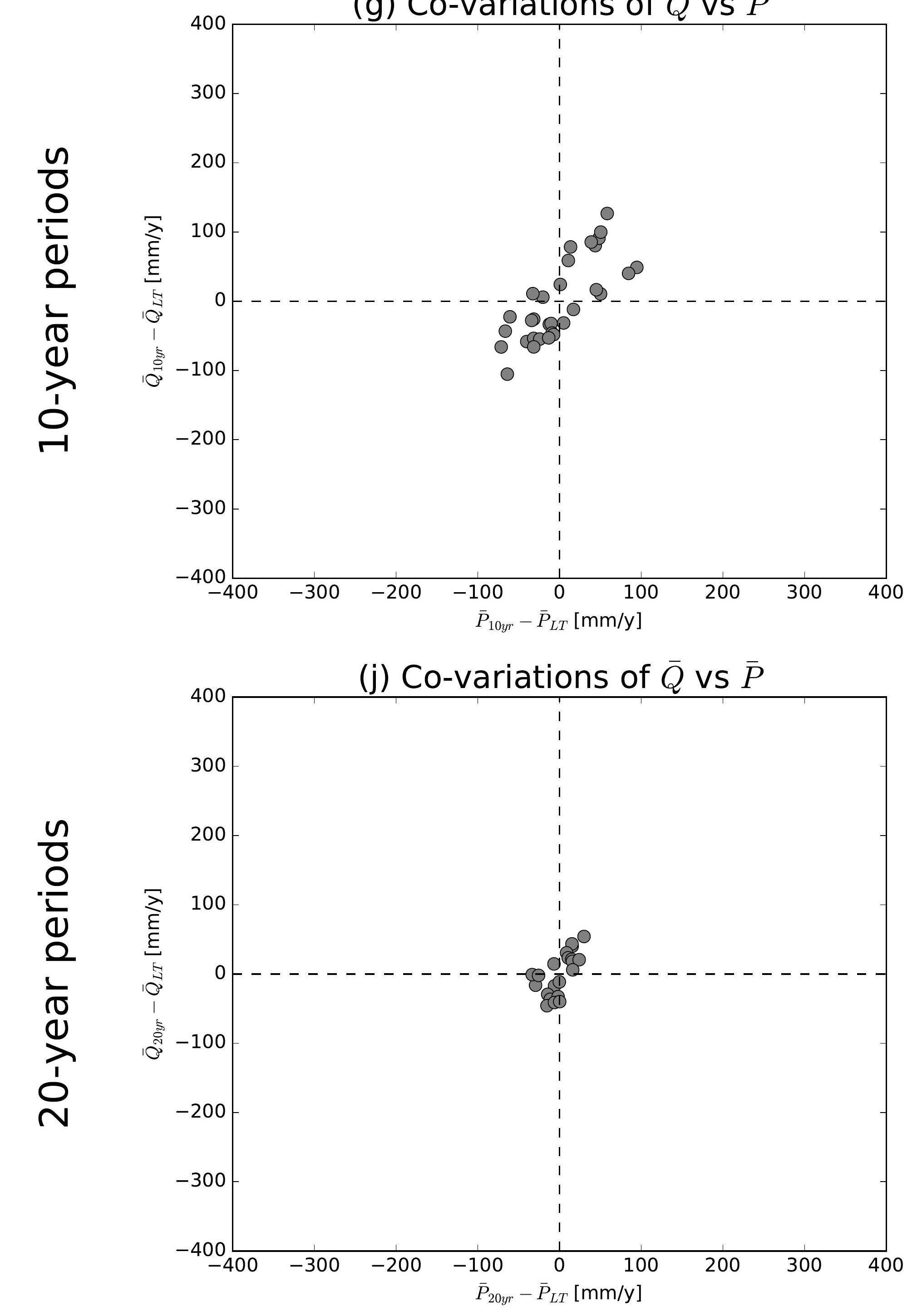

(b) Co-variations of $\bar{Q}$ vs $\overline{E 0}$

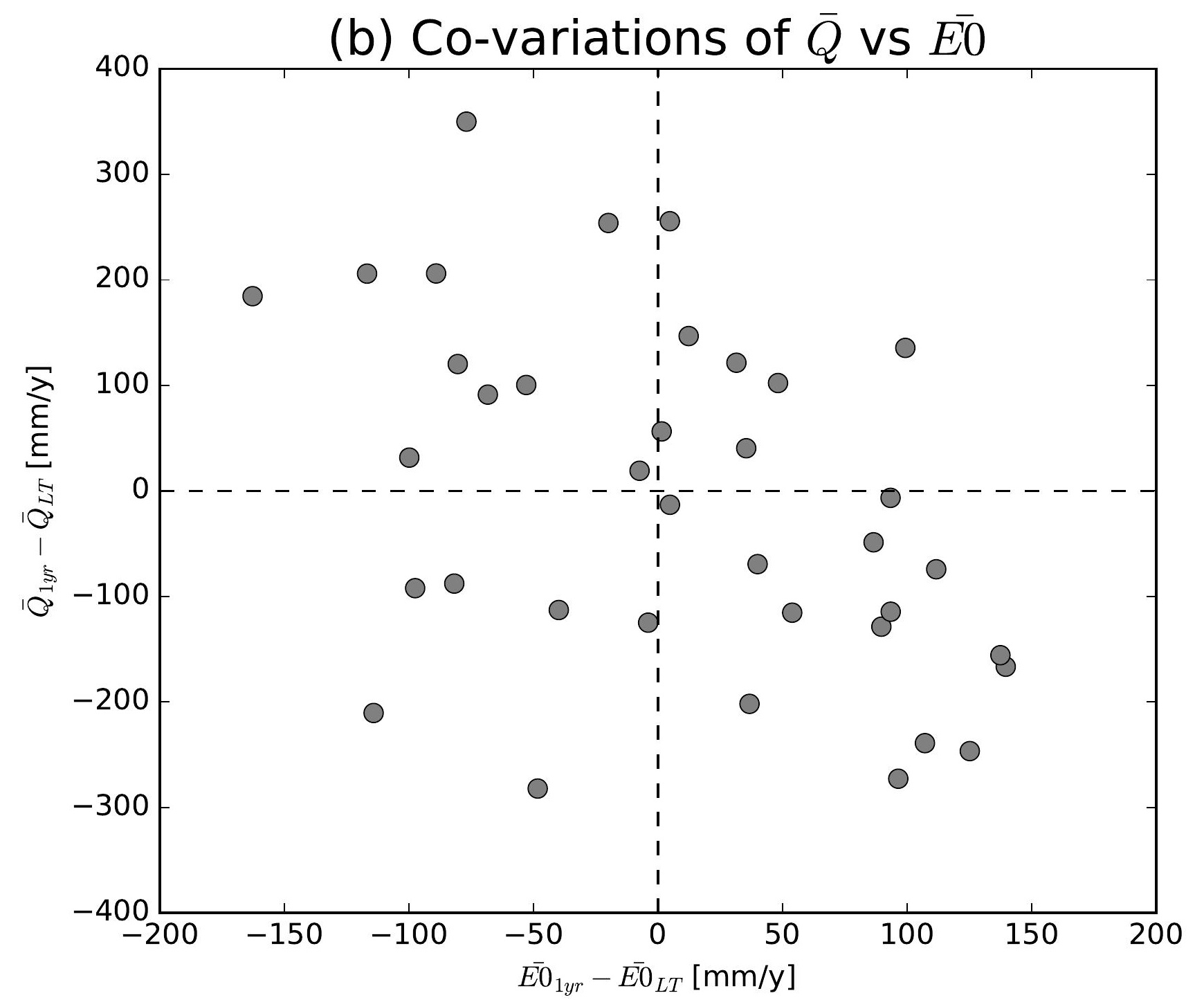

(e) Co-variations of $\bar{Q}$ vs $\overline{E 0}$

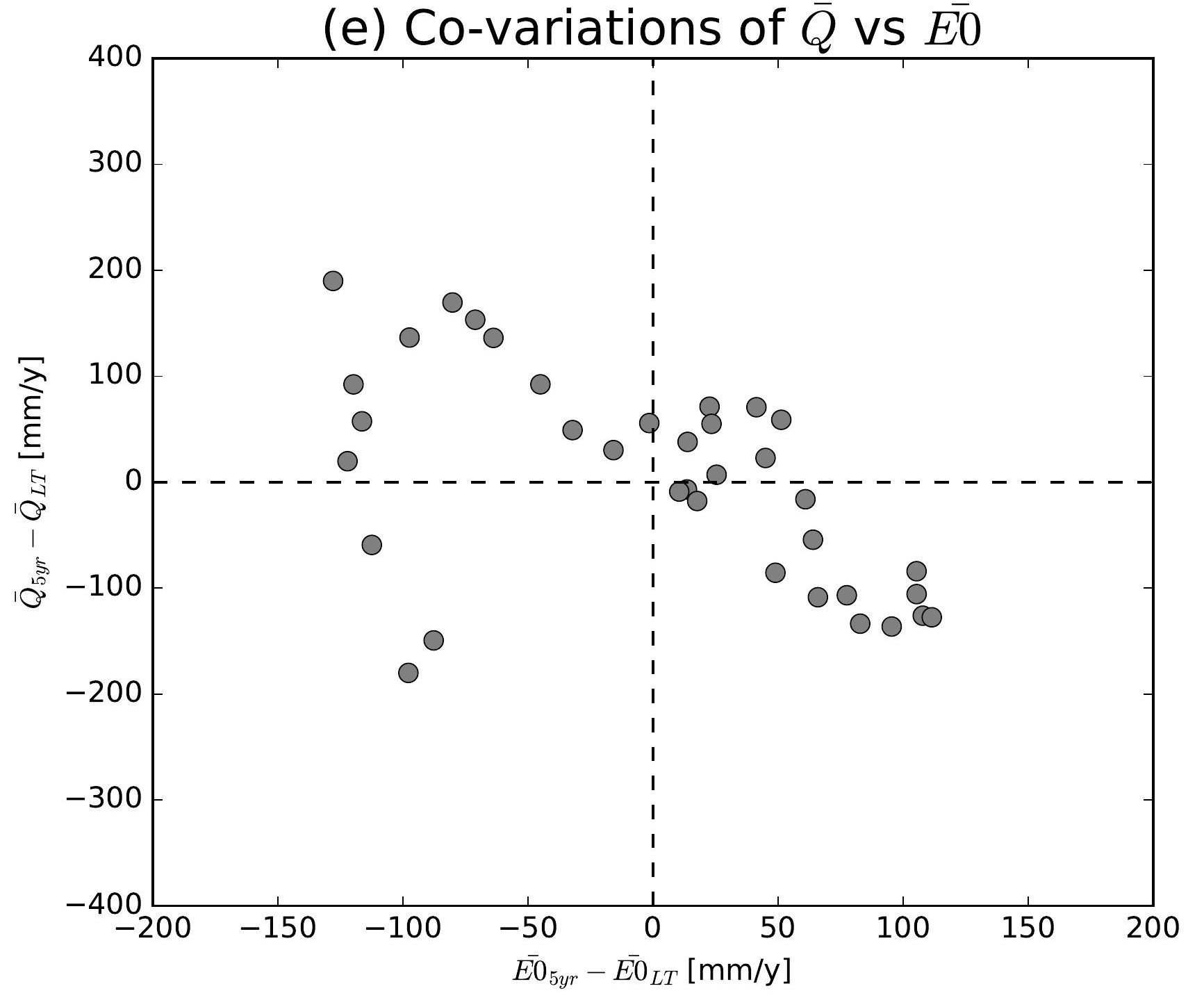

(h) Co-variations of $\bar{Q}$ vs $\overline{E 0}$

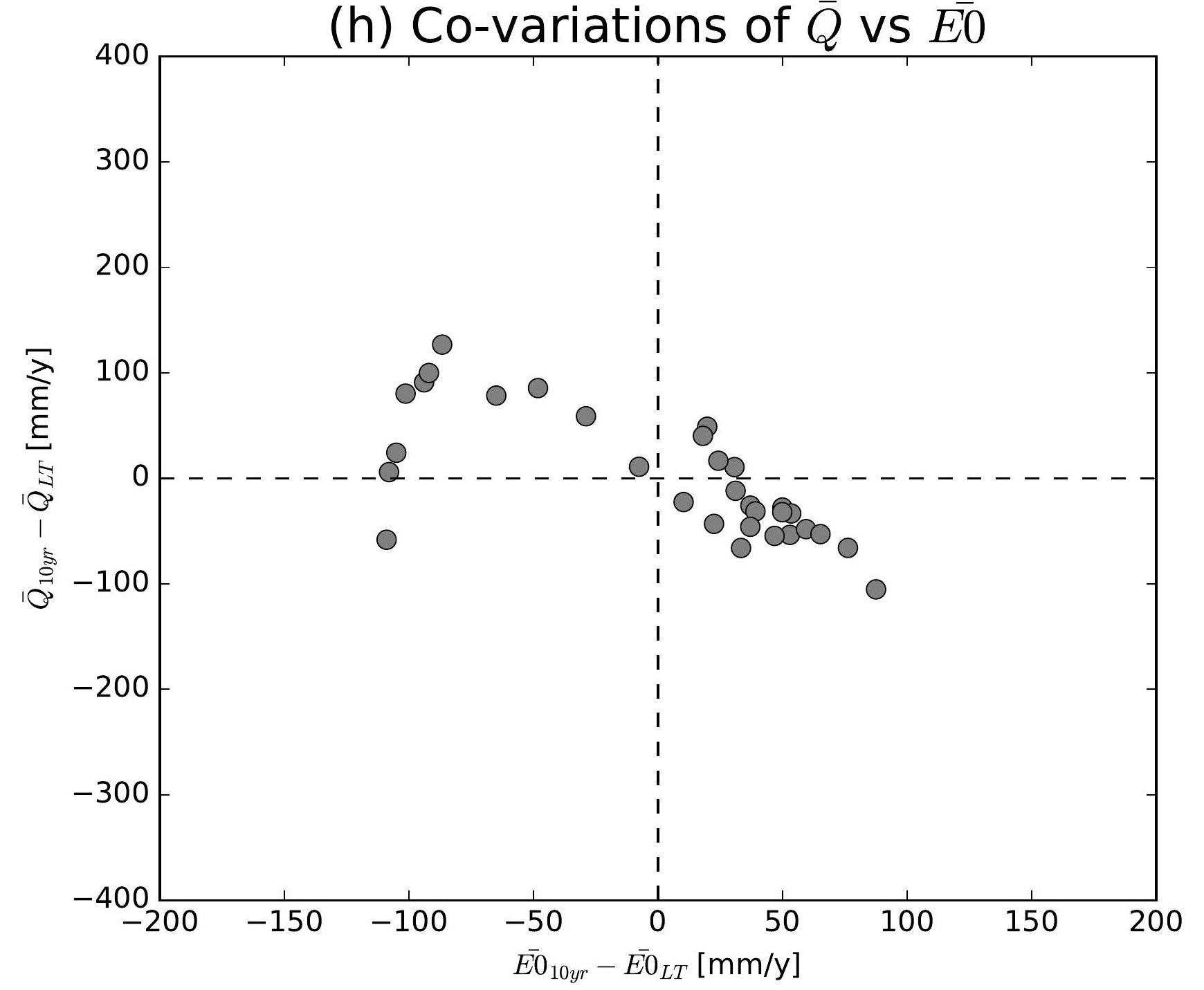

(k) Co-variations of $\bar{Q}$ vs $\overline{E 0}$

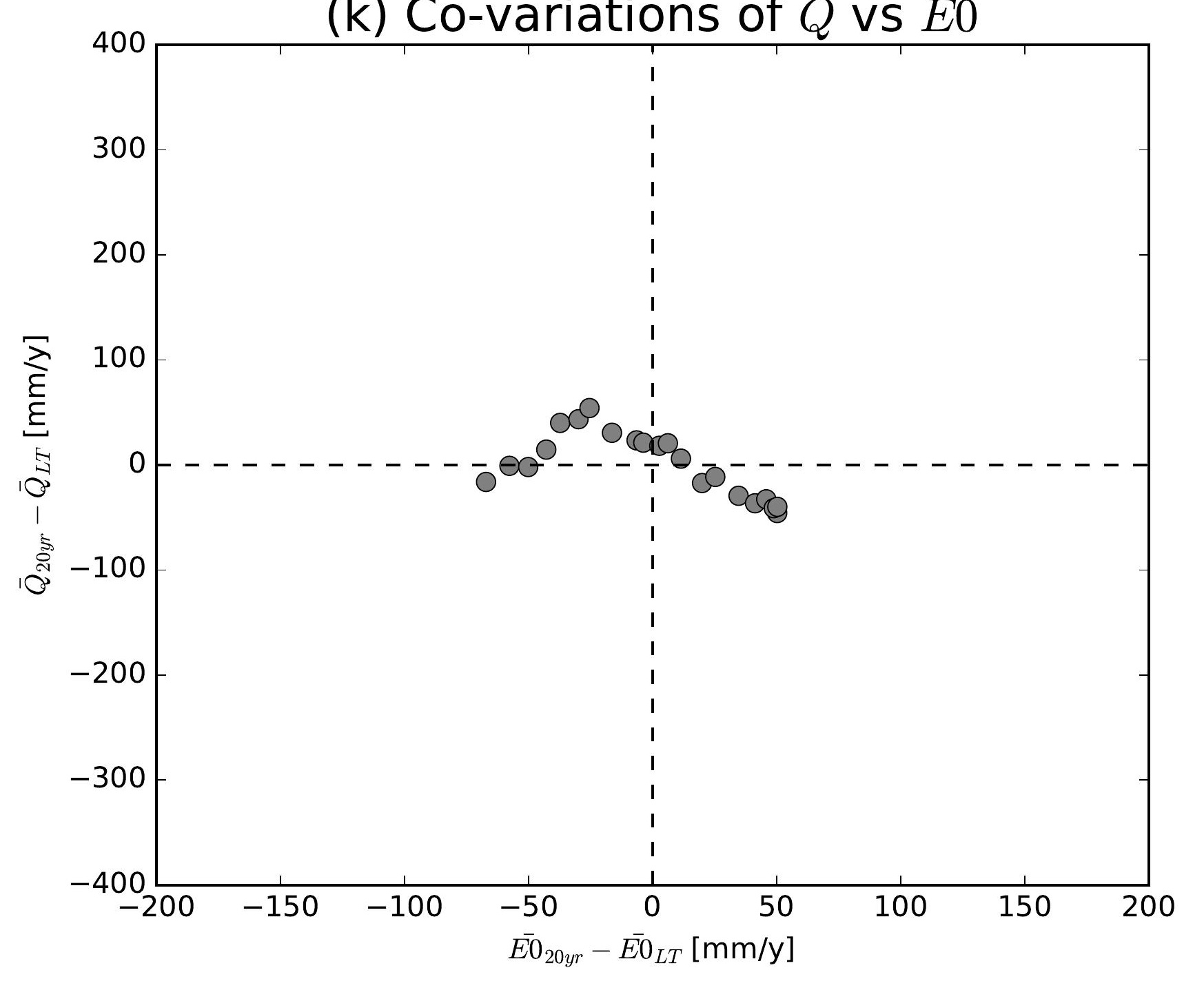

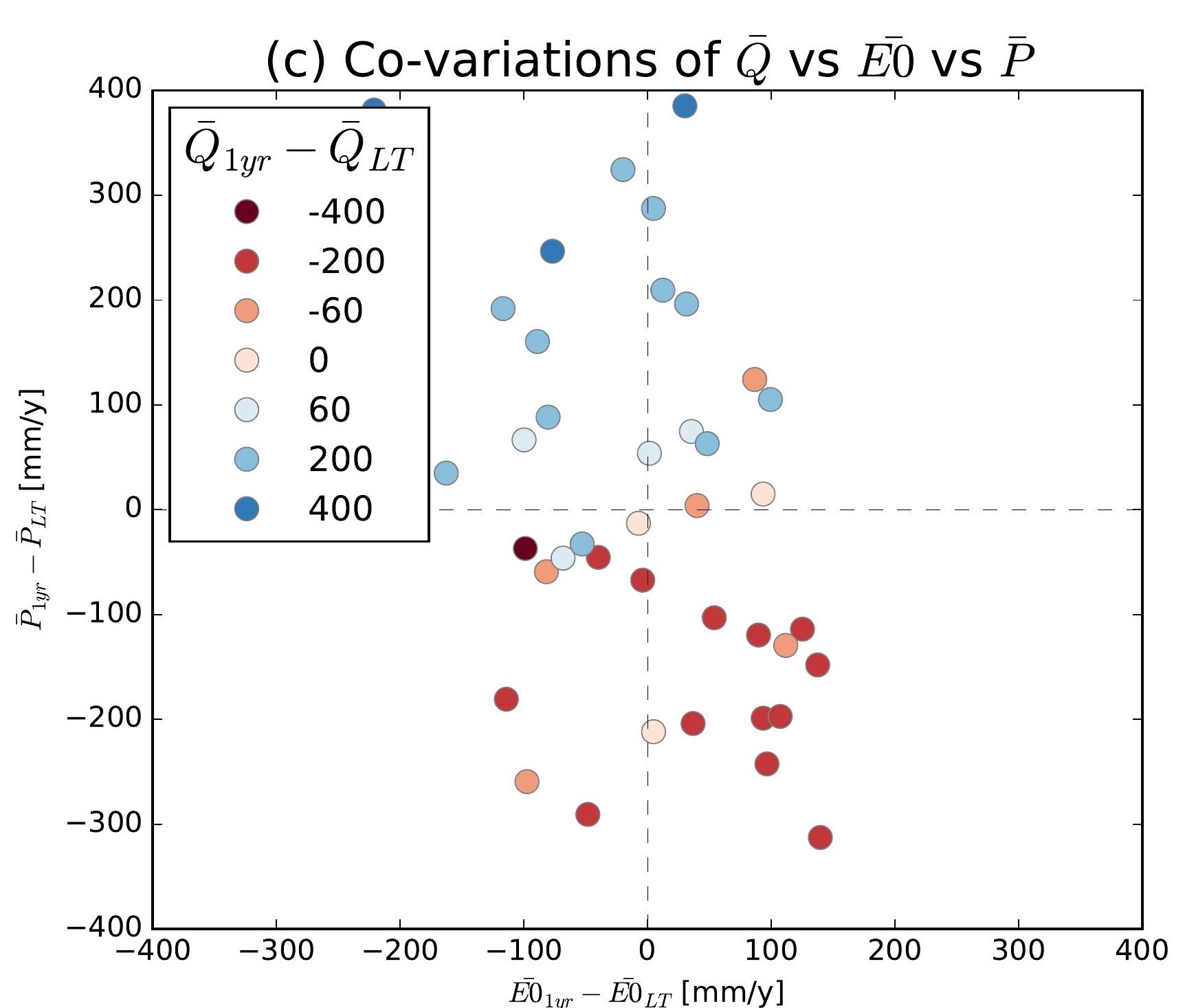
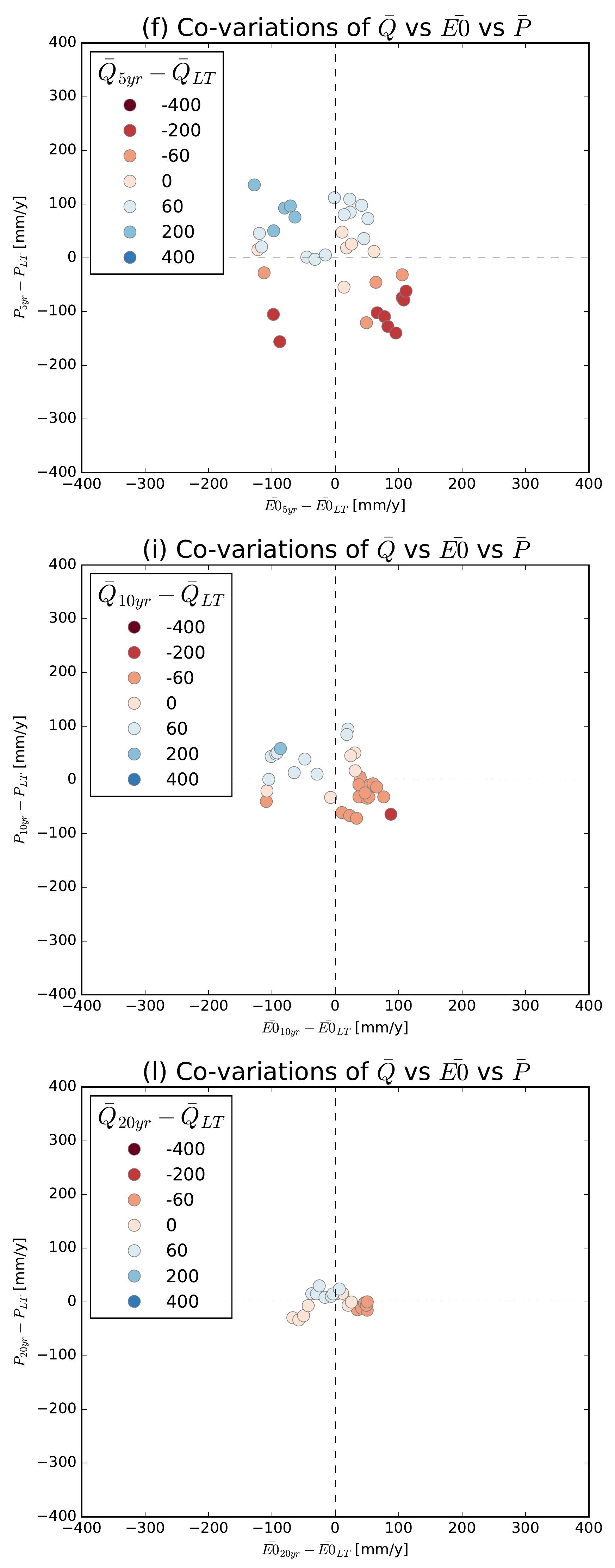

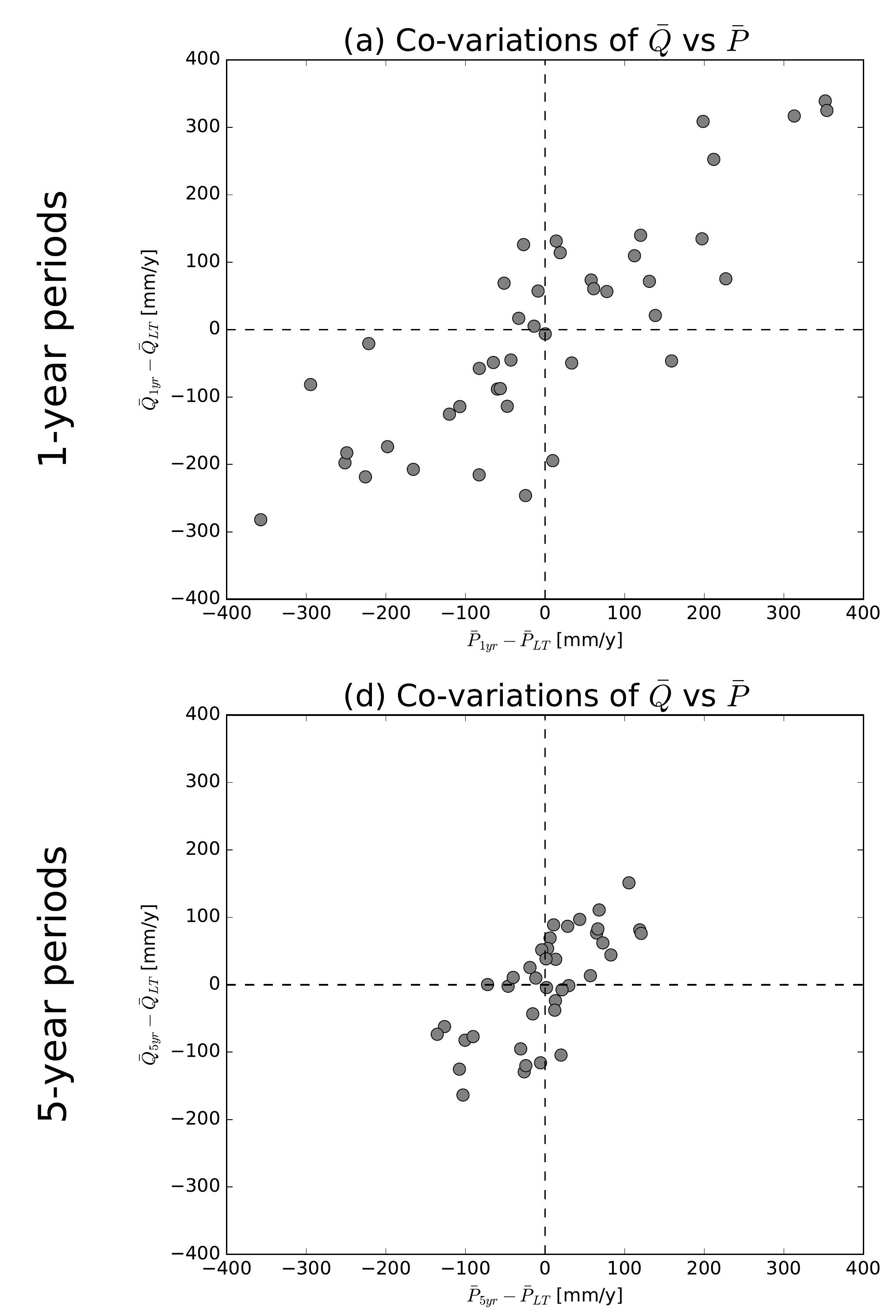

(g) Co-variations of $\bar{Q}$ vs $\bar{P}$
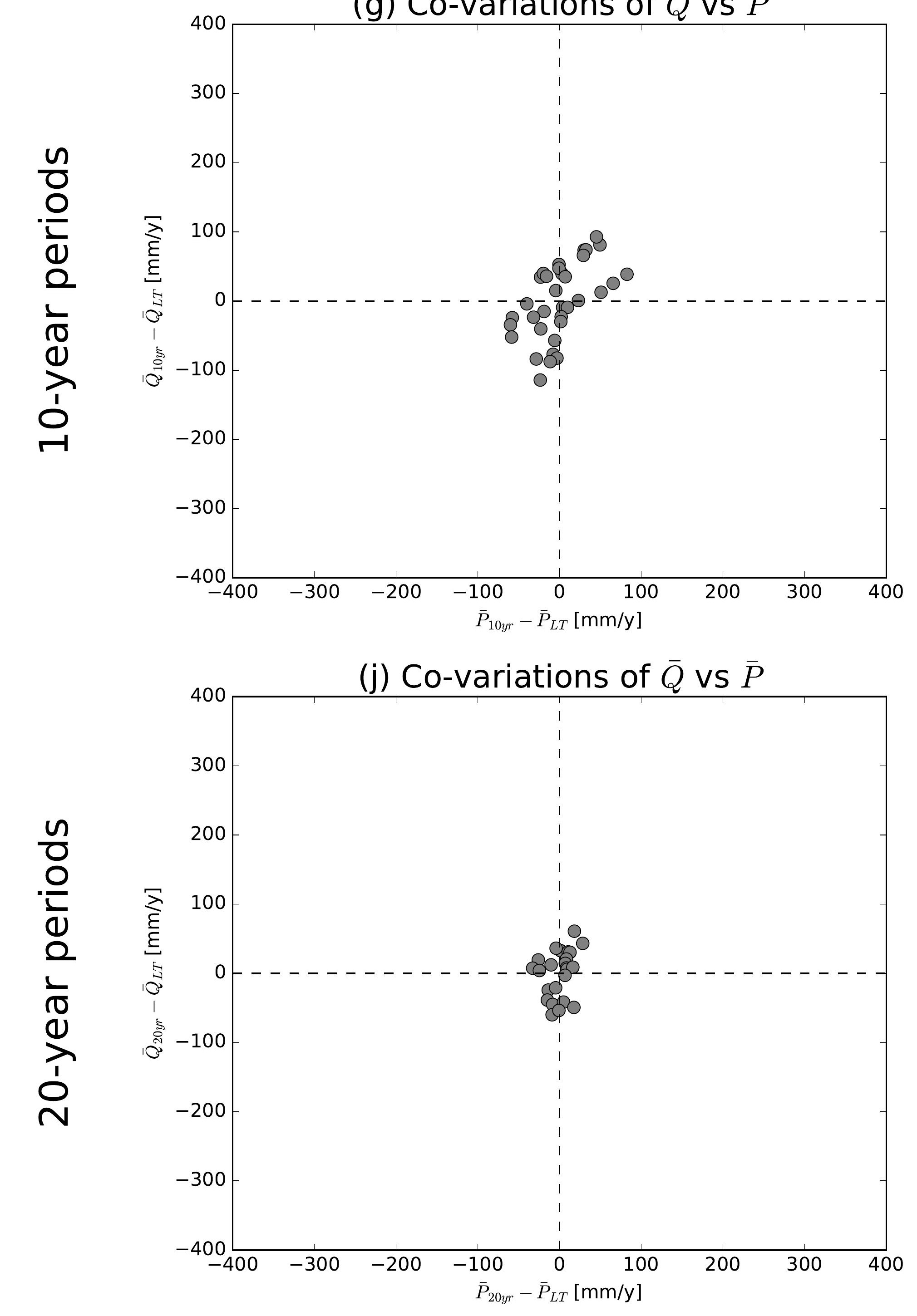

(b) Co-variations of $\bar{Q}$ vs $\overline{E 0}$

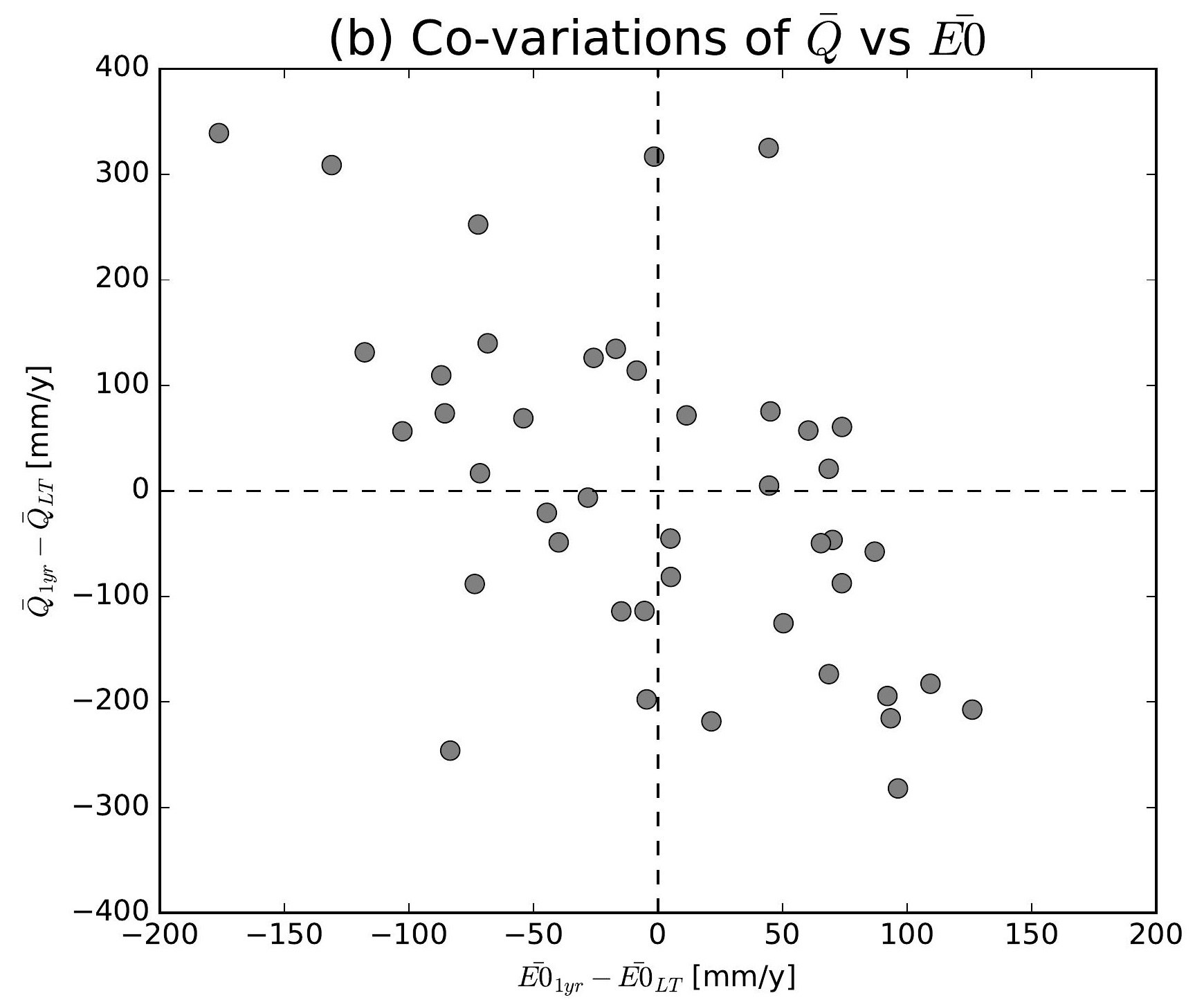

(e) Co-variations of $\bar{Q}$ vs $\overline{E 0}$

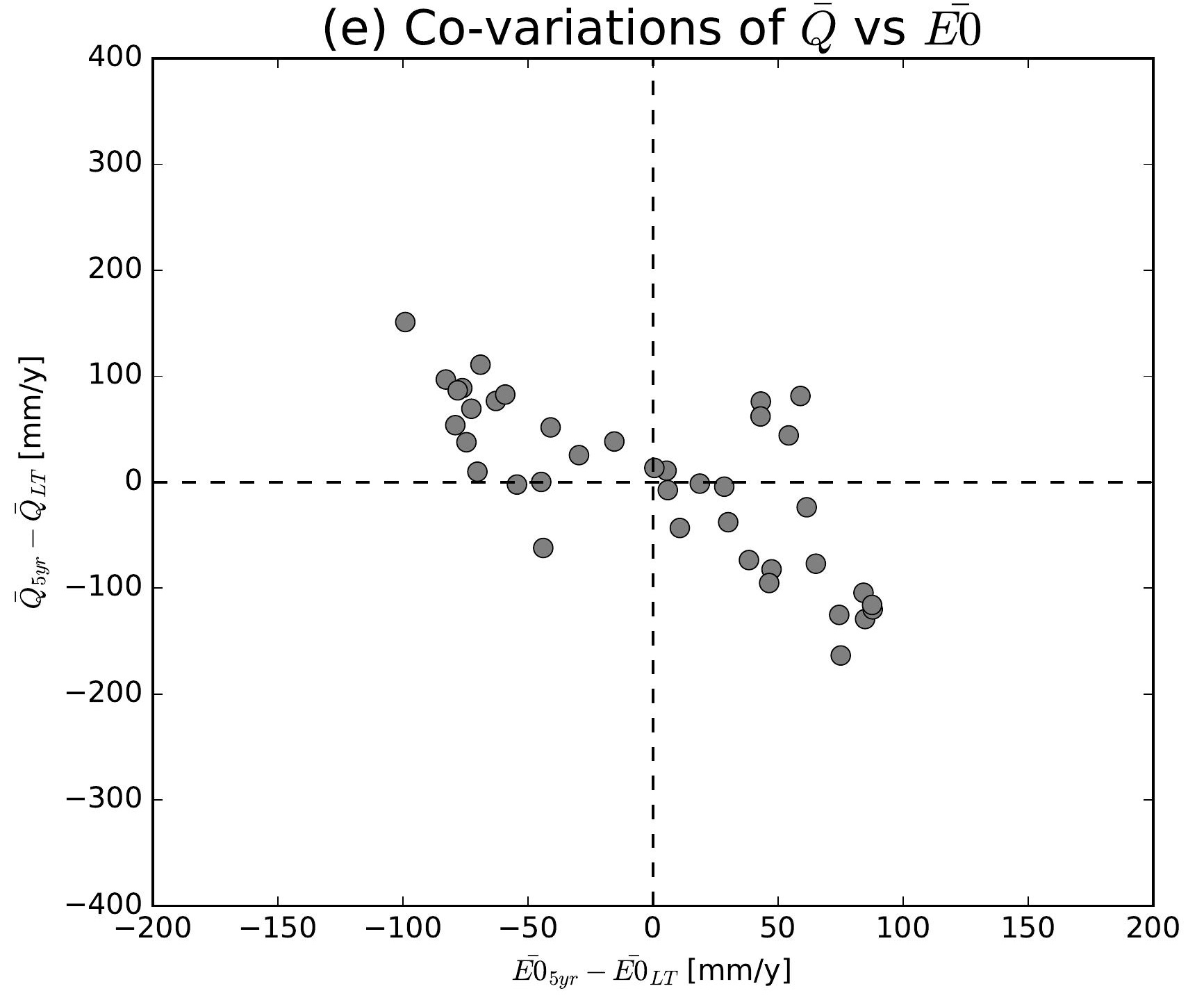

(h) Co-variations of $\bar{Q}$ vs $\overline{E 0}$

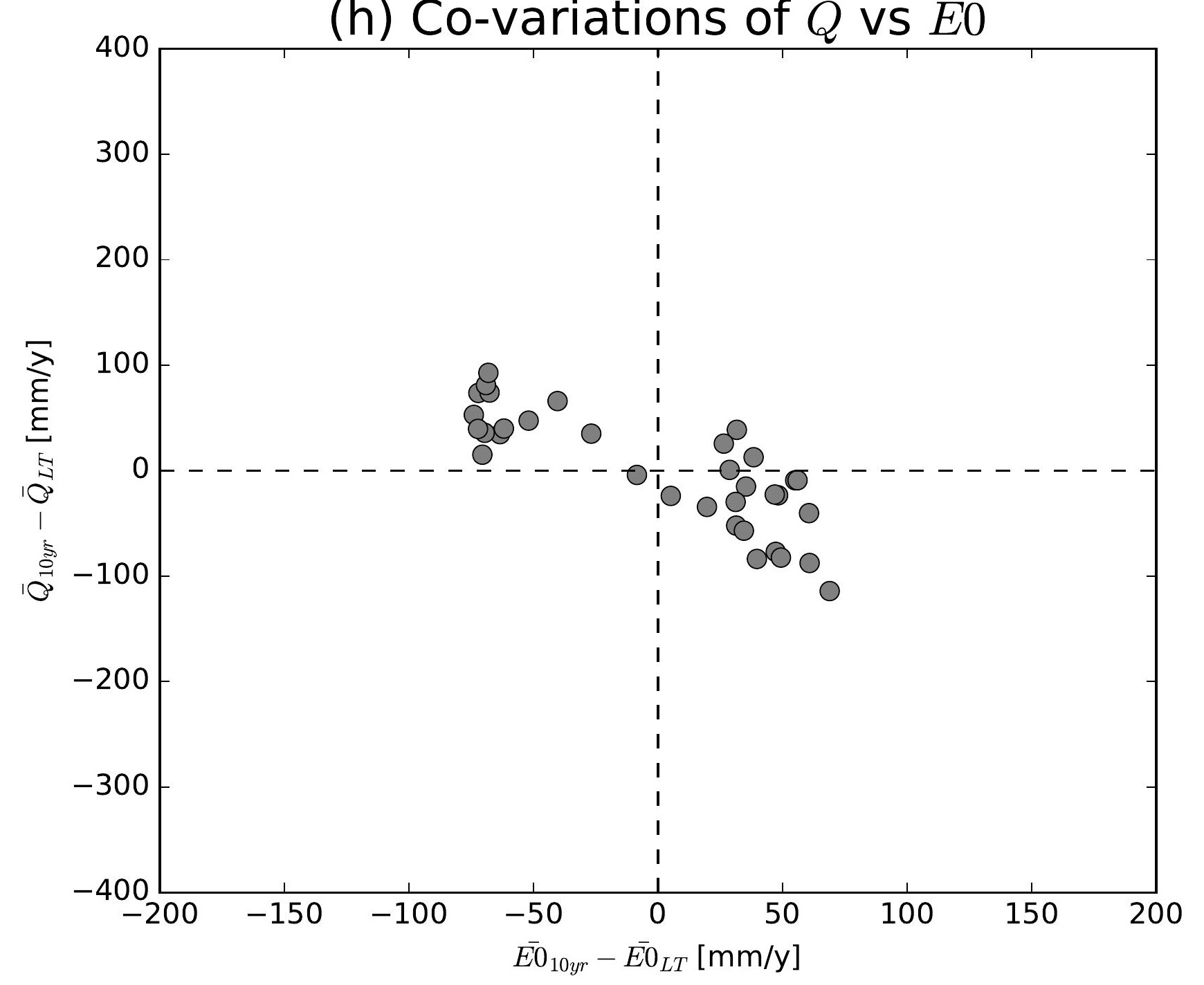

(k) Co-variations of $\bar{Q}$ vs $\overline{E 0}$

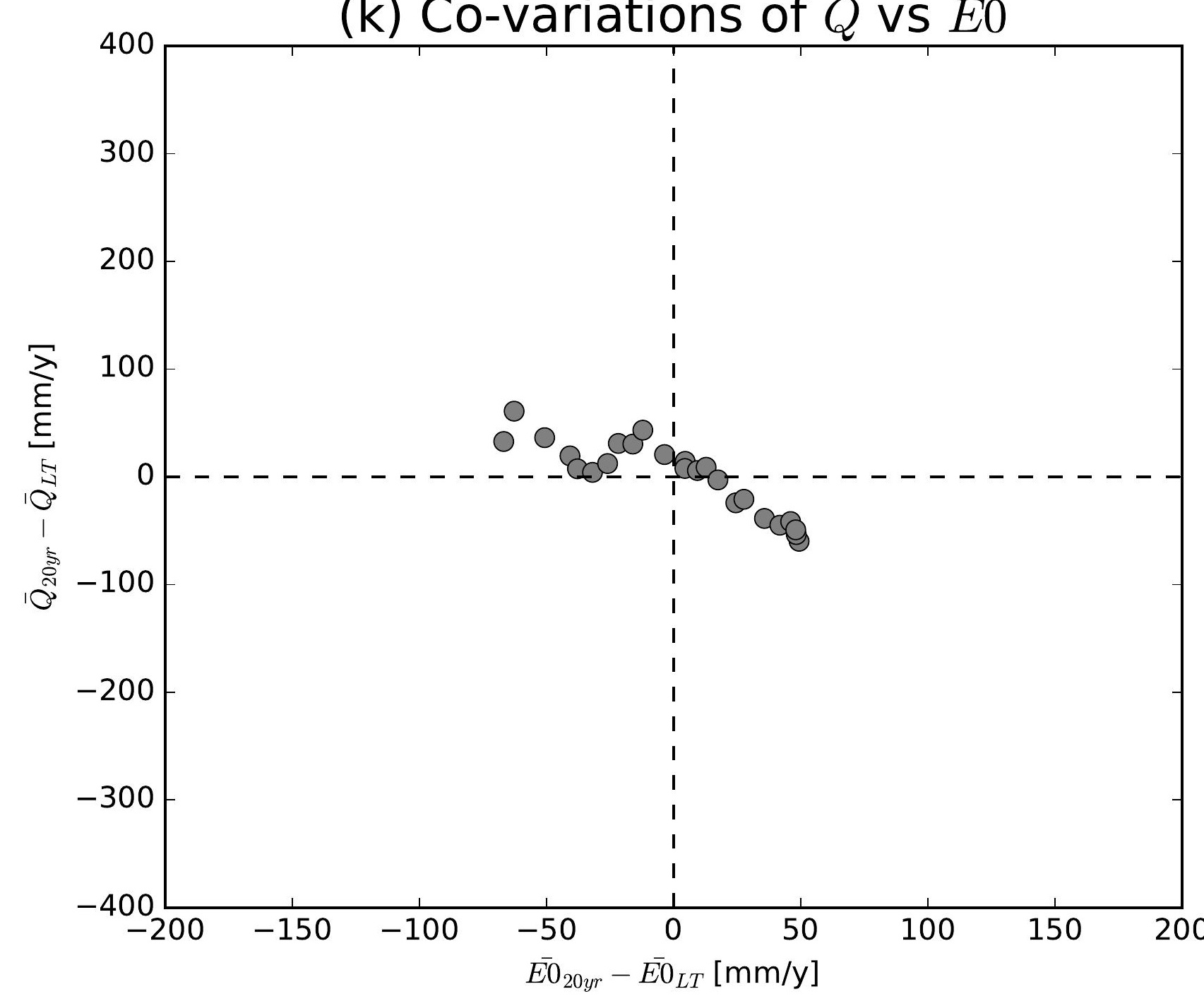

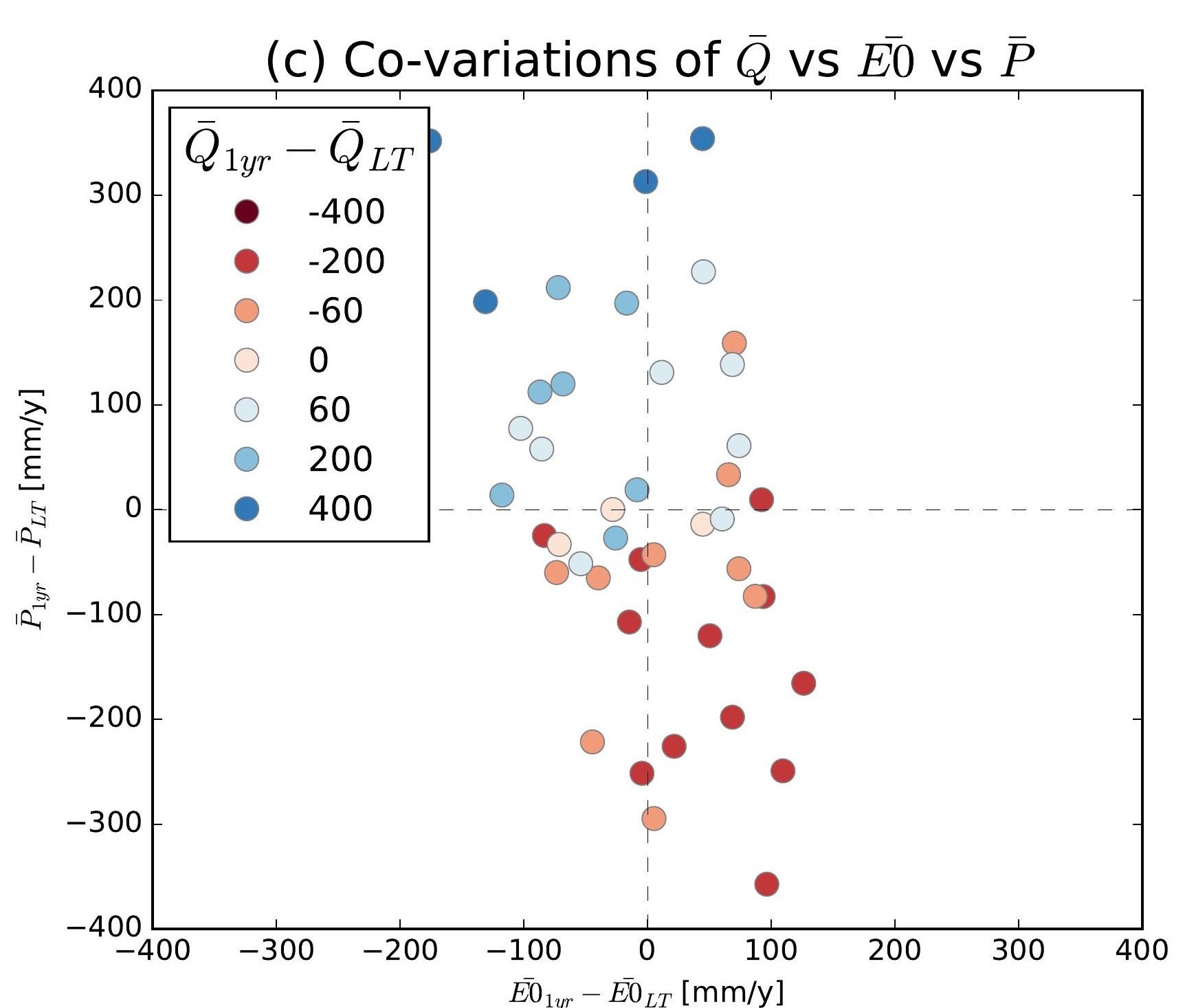
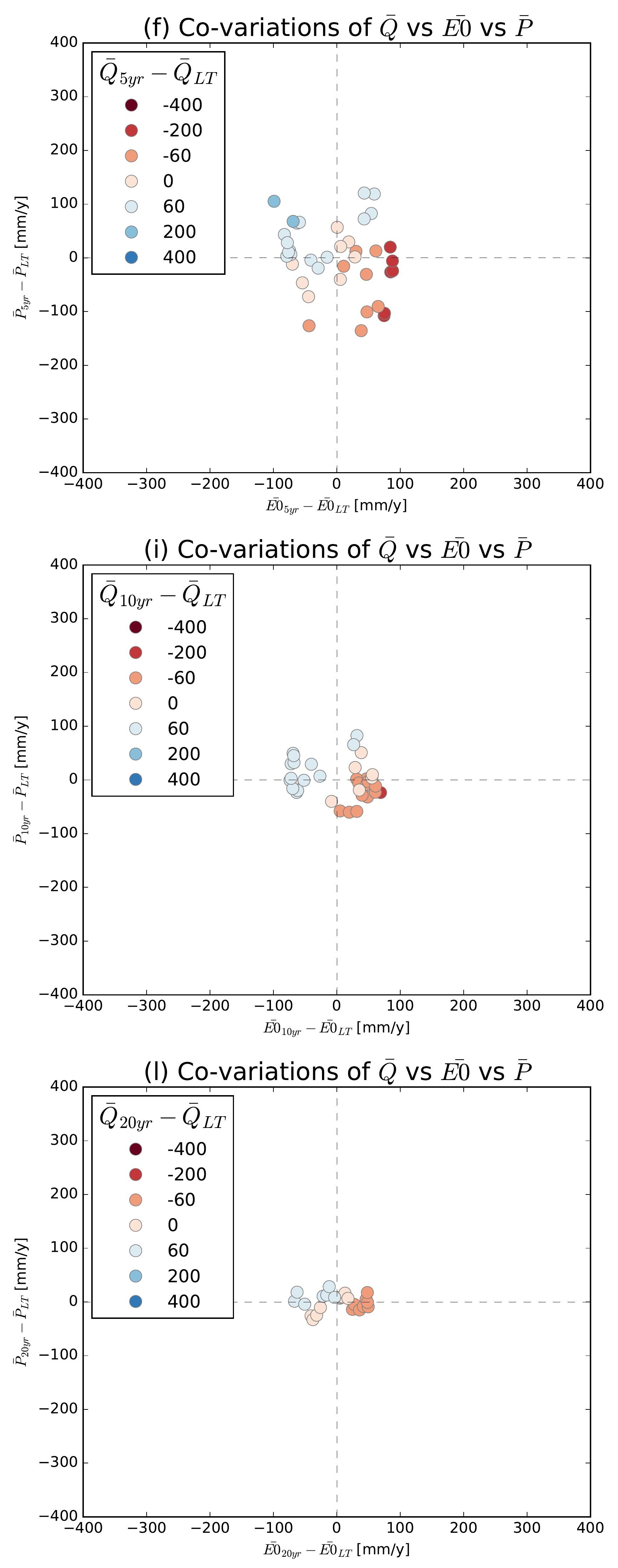

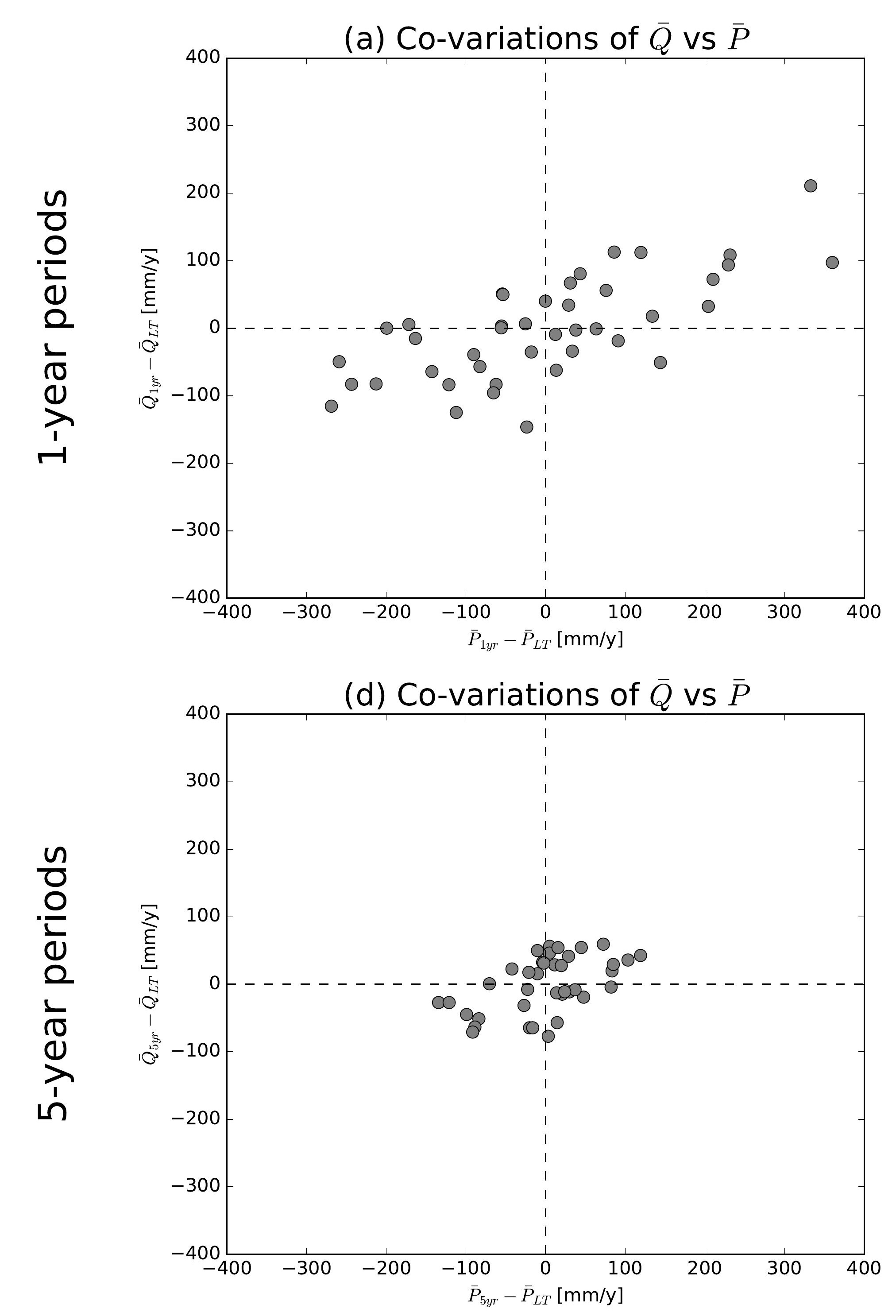

(g) Co-variations of $\bar{Q}$ vs $\bar{P}$
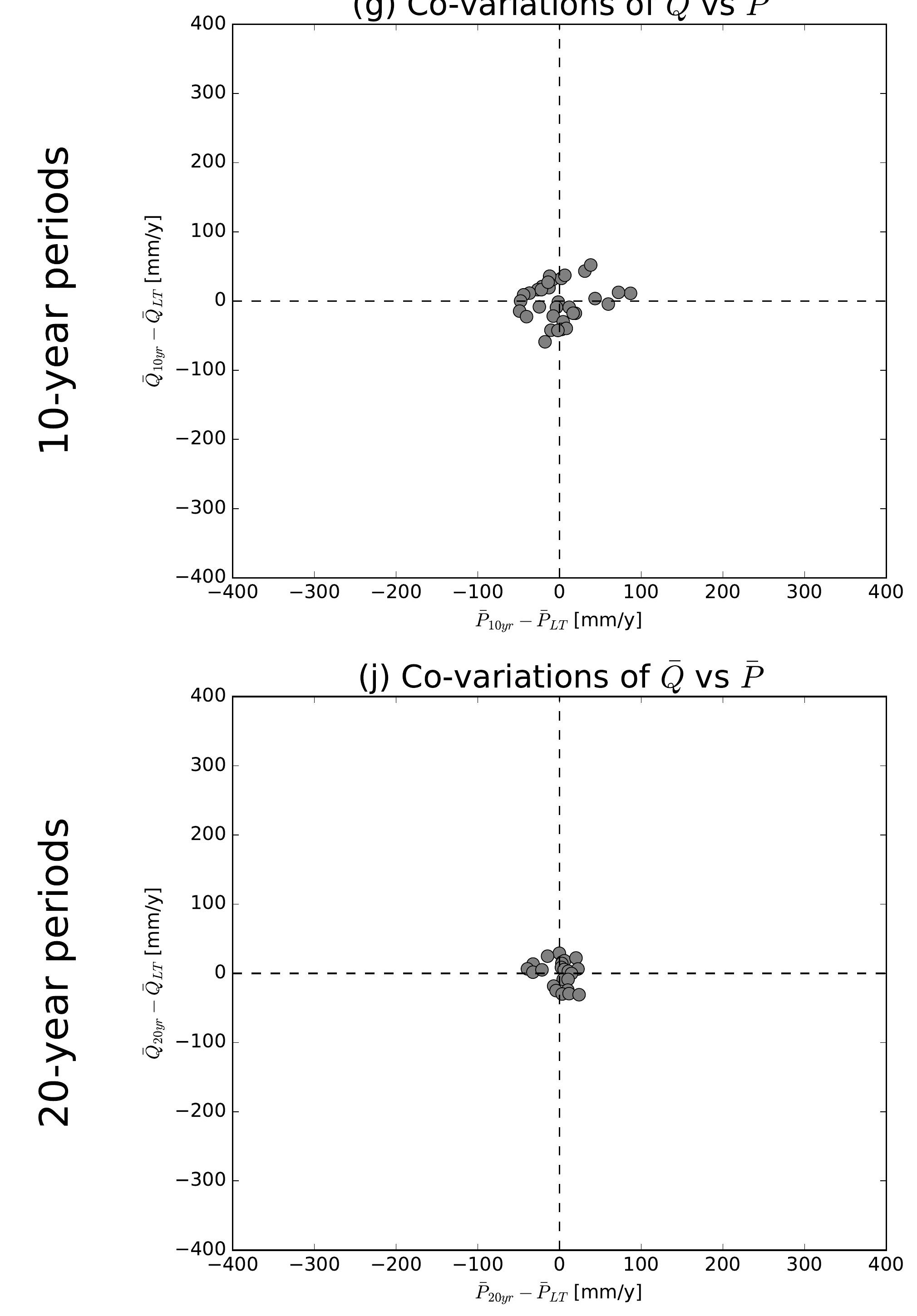

(b) Co-variations of $\bar{Q}$ vs $\overline{E 0}$

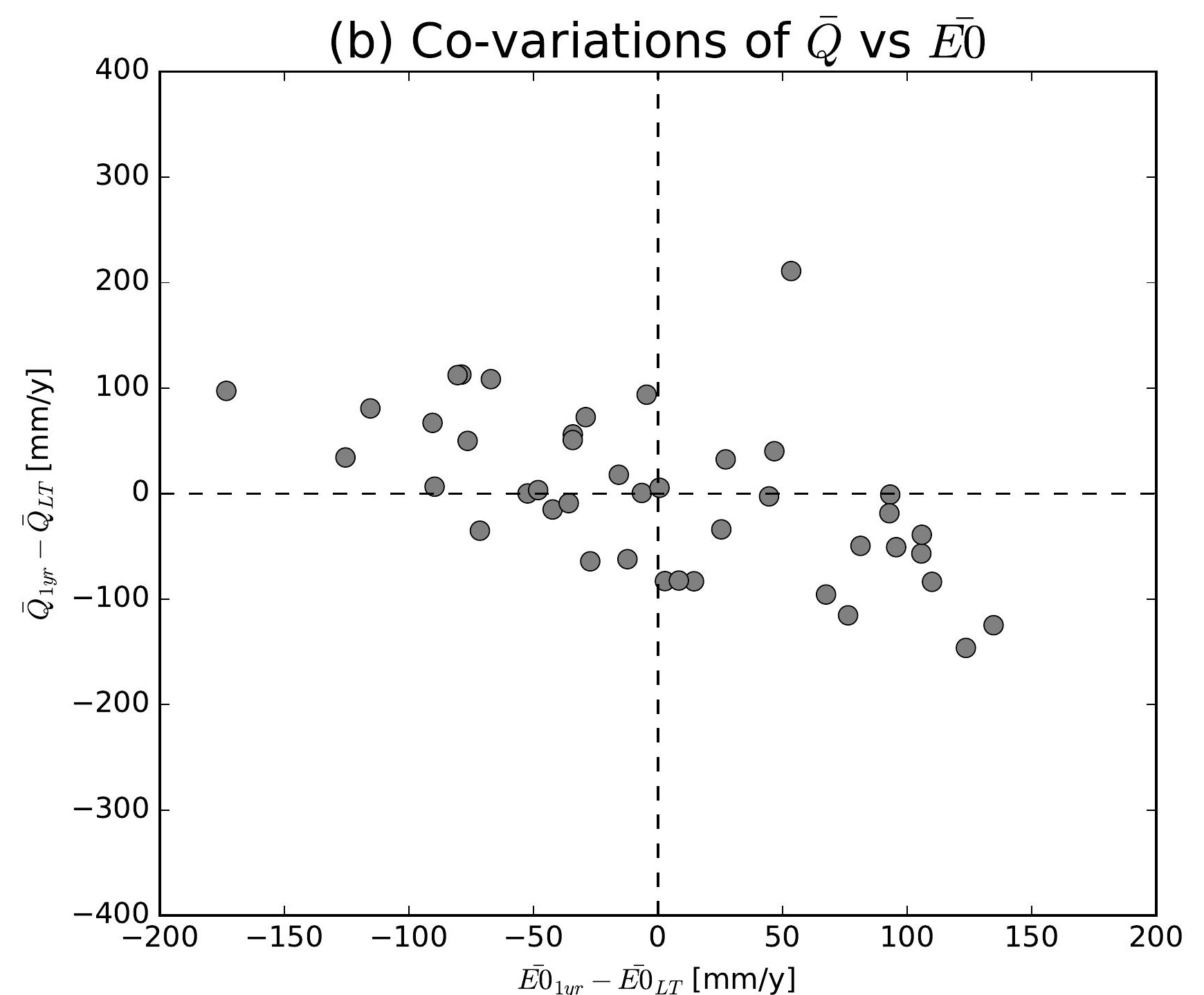

(e) Co-variations of $\bar{Q}$ vs $\overline{E 0}$

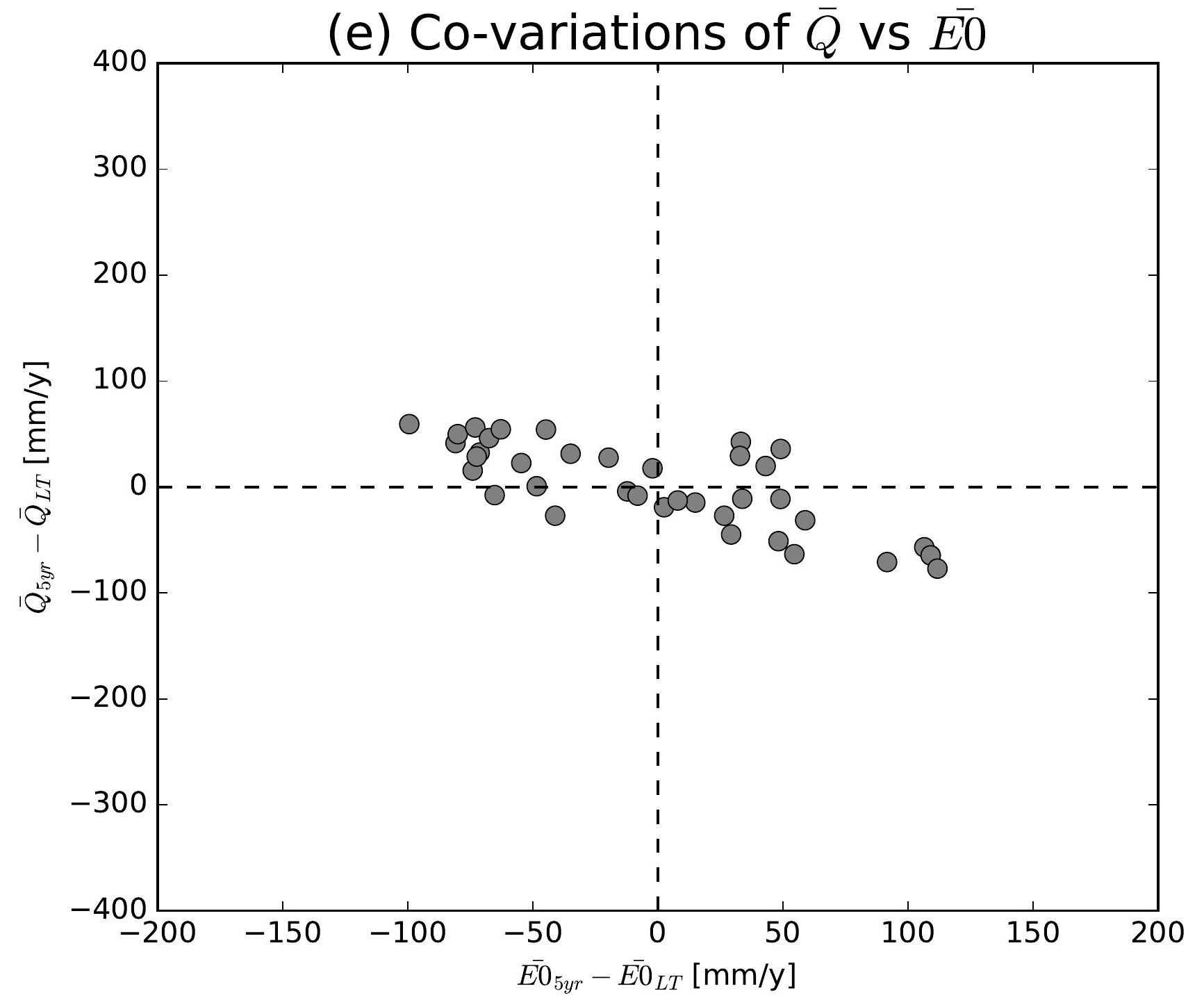

(h) Co-variations of $\bar{Q}$ vs $\overline{E 0}$

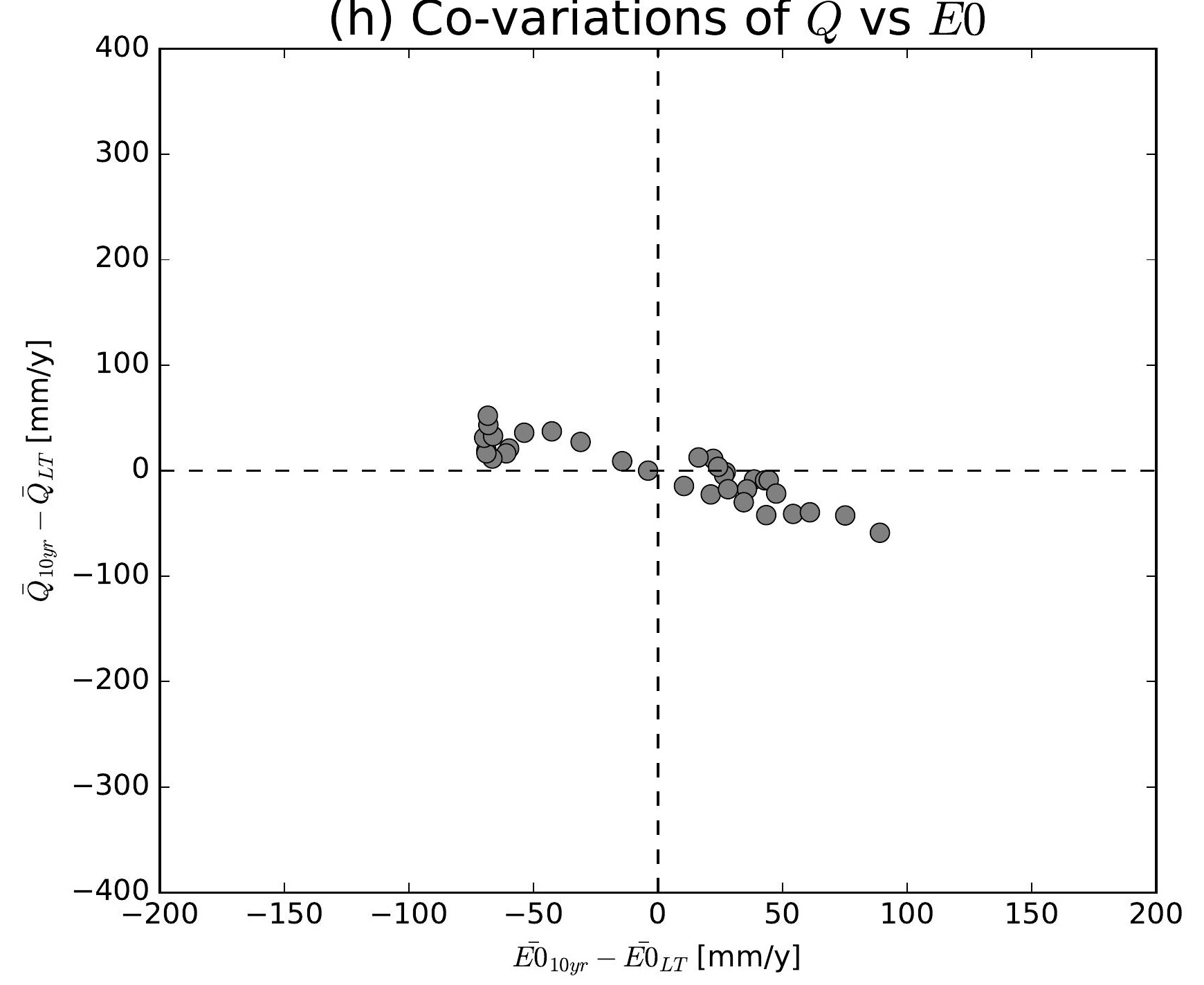

(k) Co-variations of $\bar{Q}$ vs $\overline{E 0}$

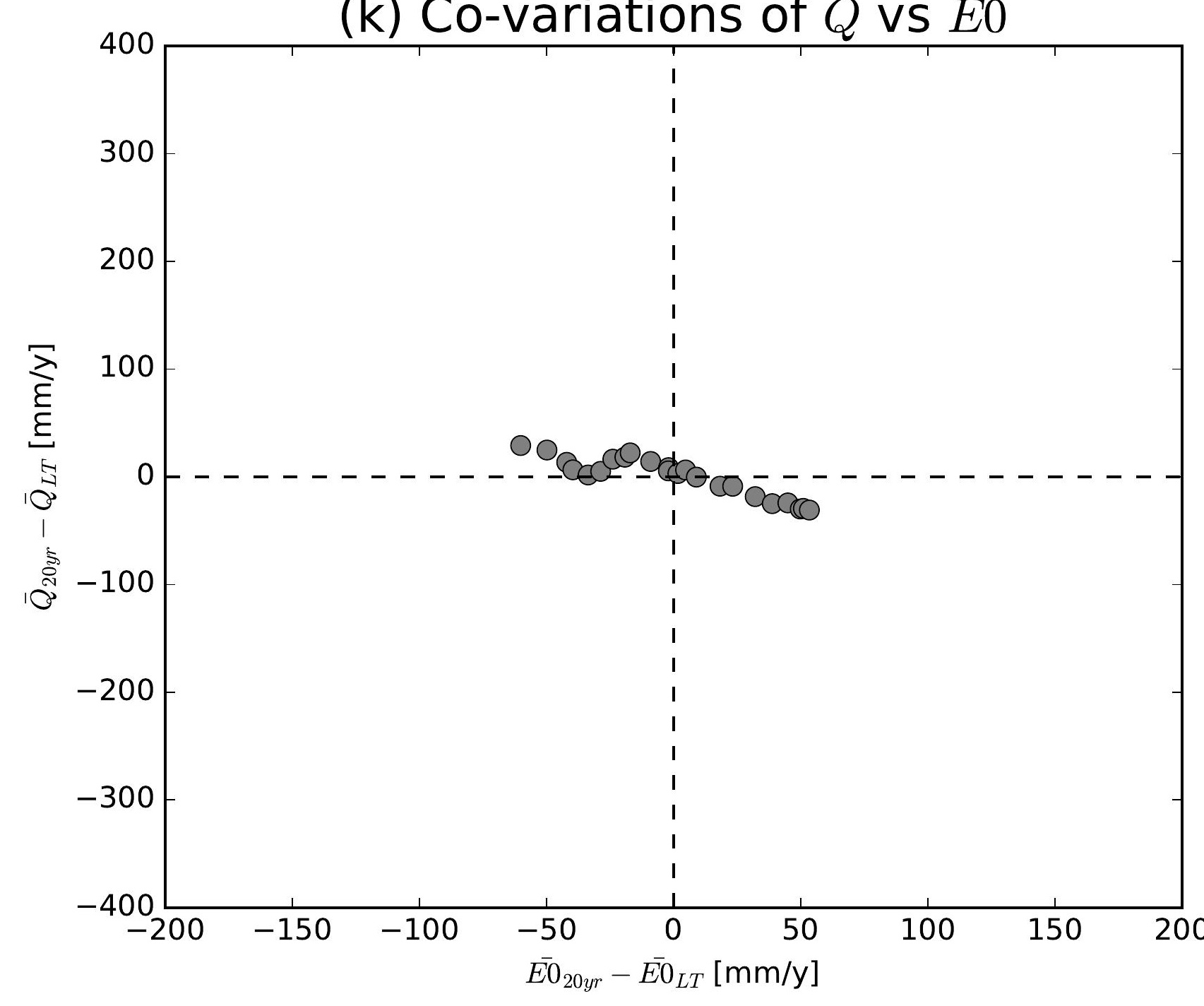

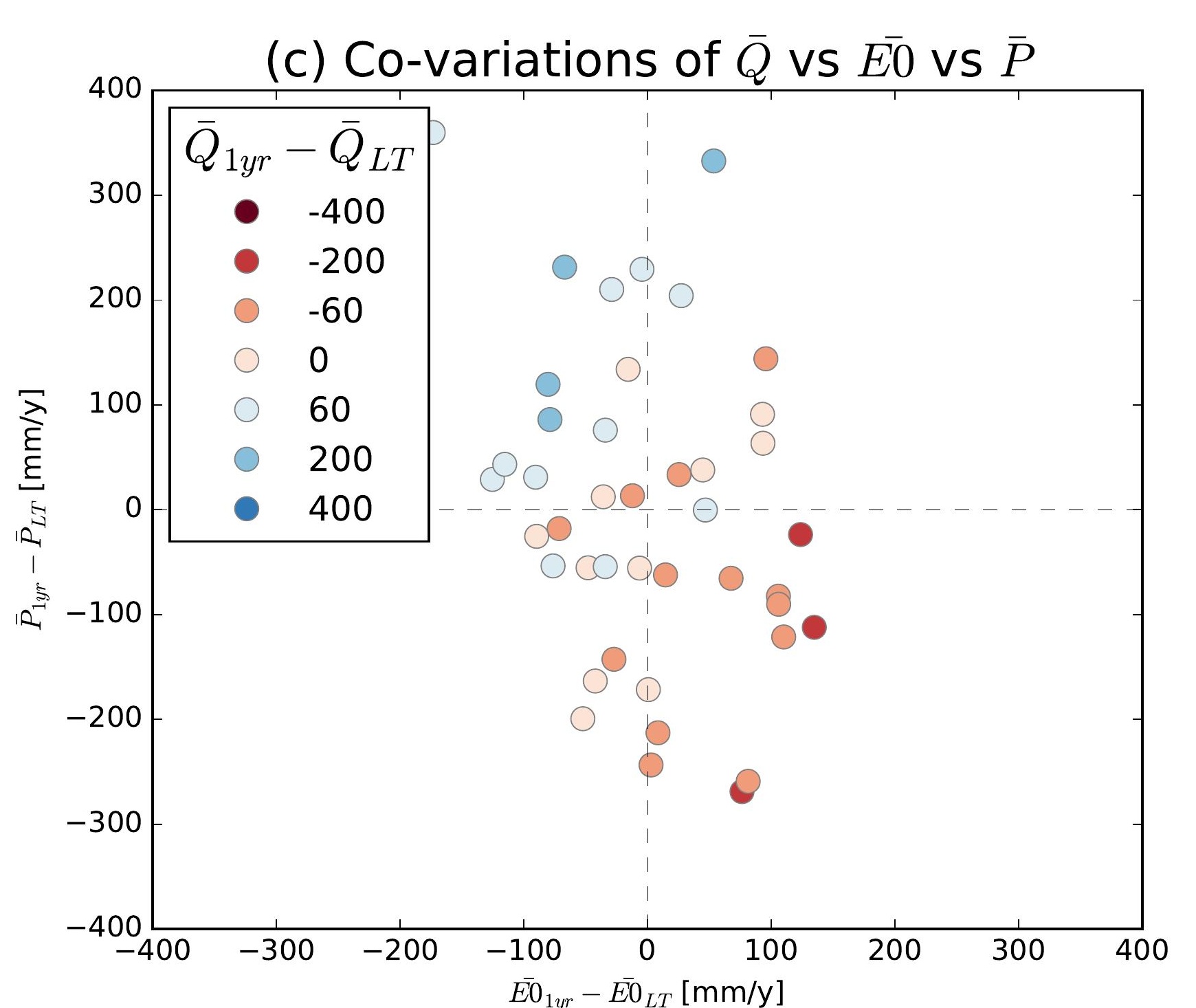
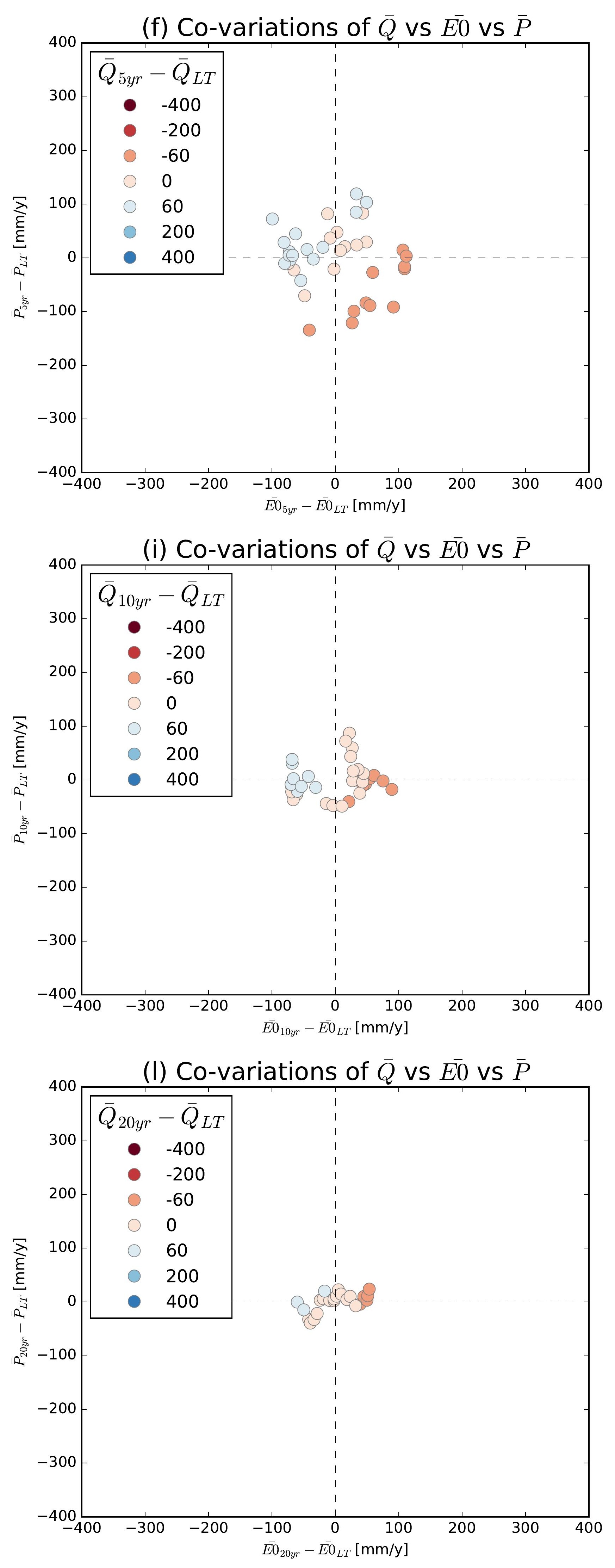

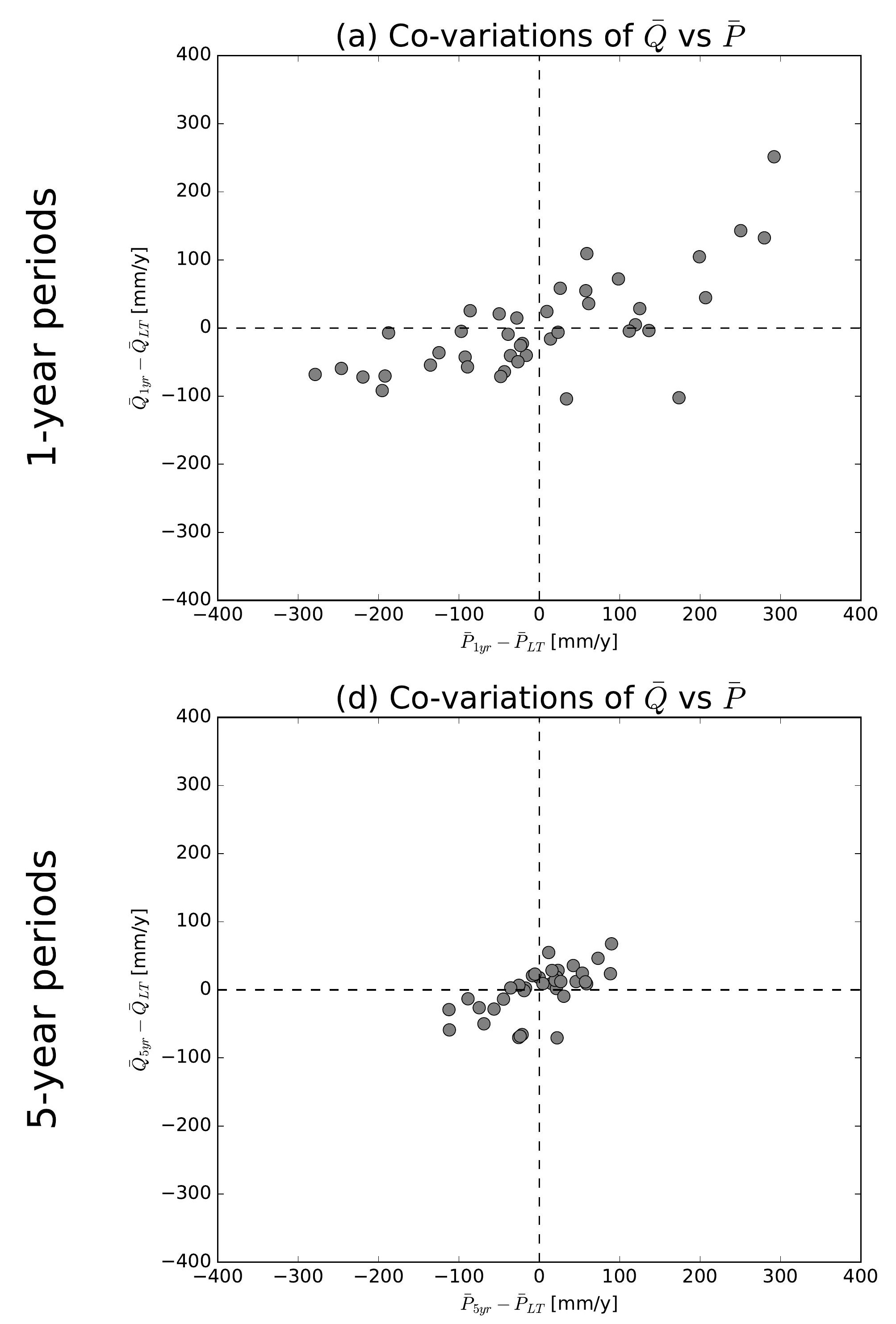

(g) Co-variations of $\bar{Q}$ vs $\bar{P}$

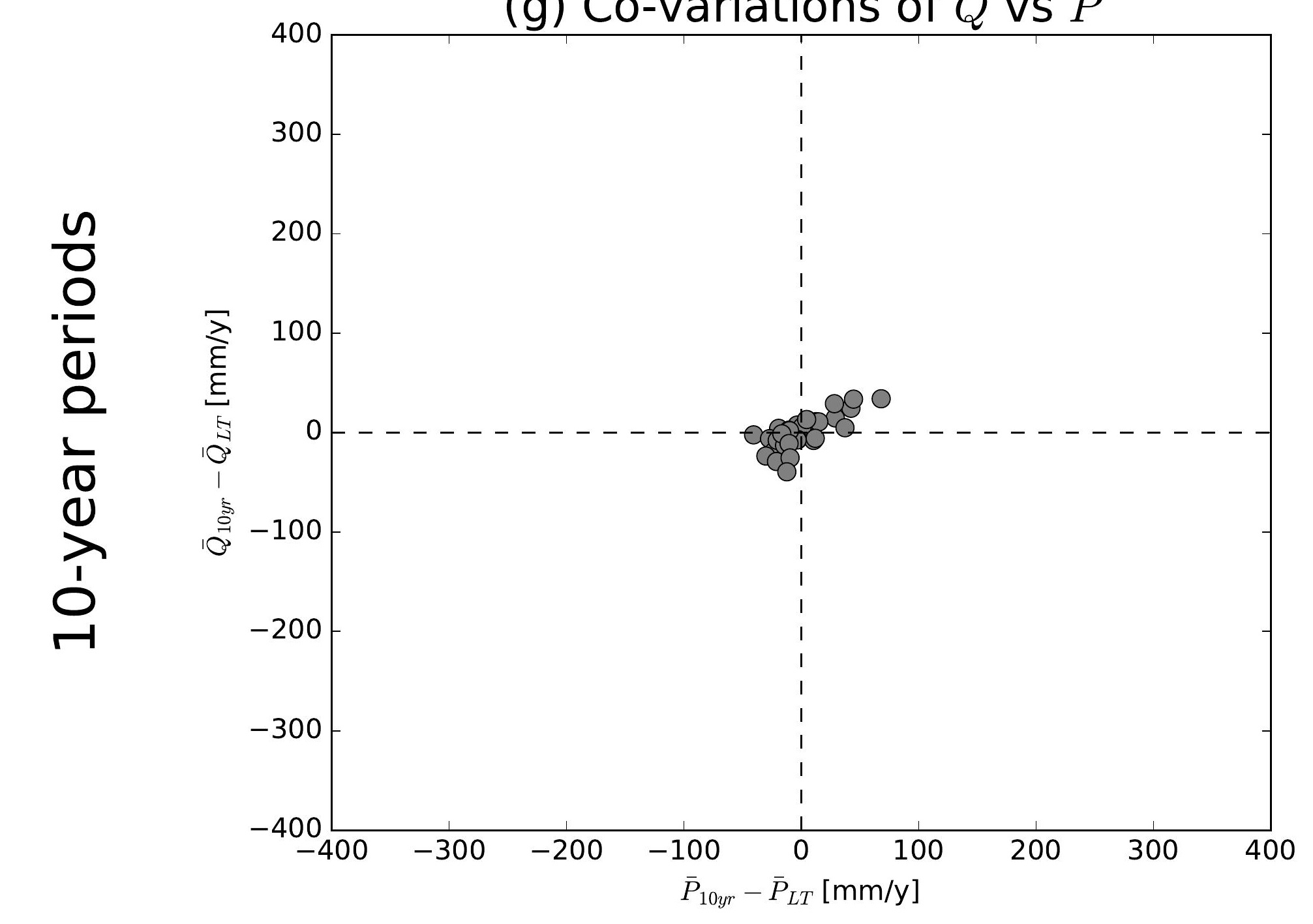

(j) Co-variations of $\bar{Q}$ vs $\bar{P}$

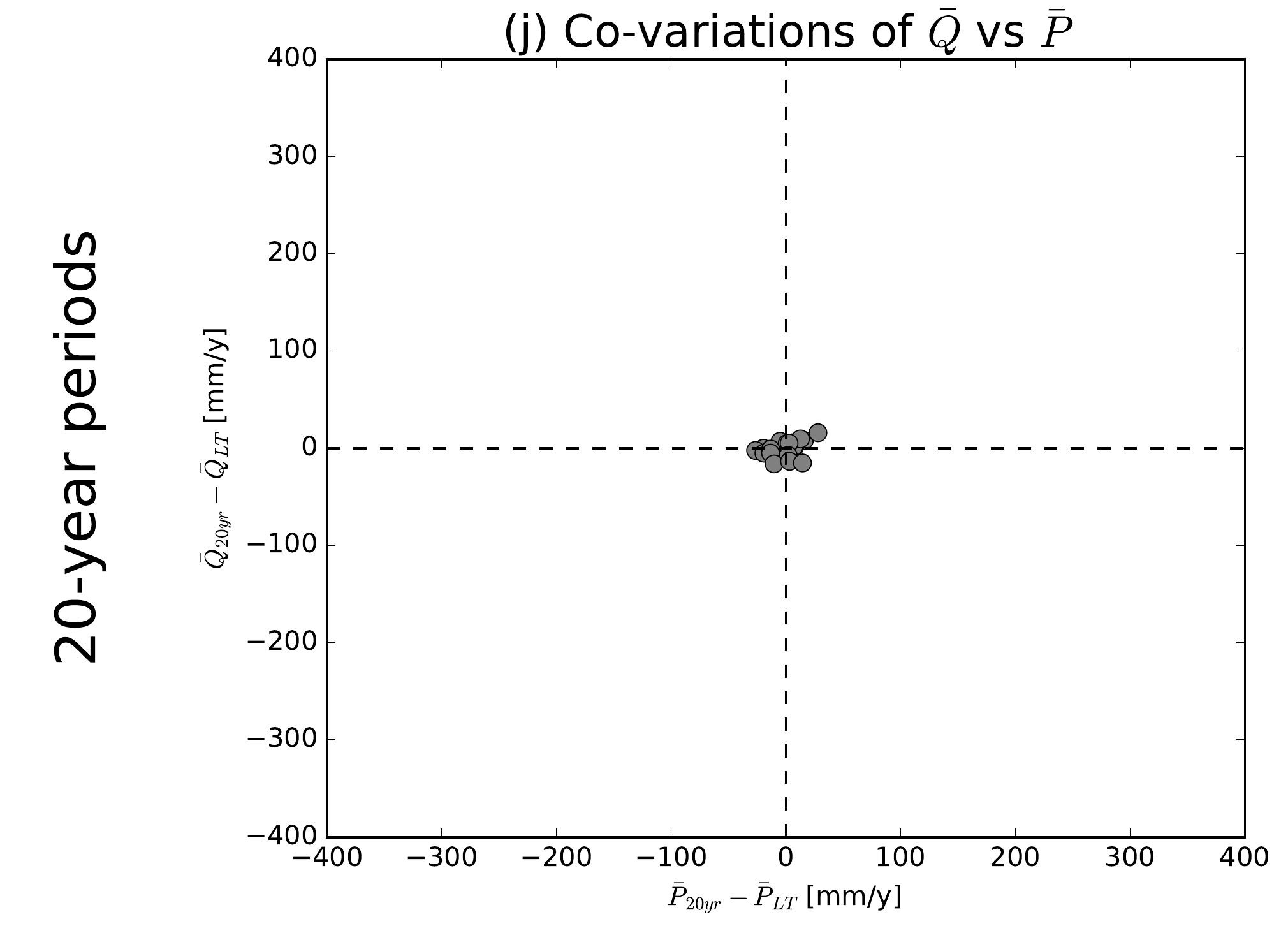

(b) Co-variations of $\bar{Q}$ vs $\overline{E 0}$

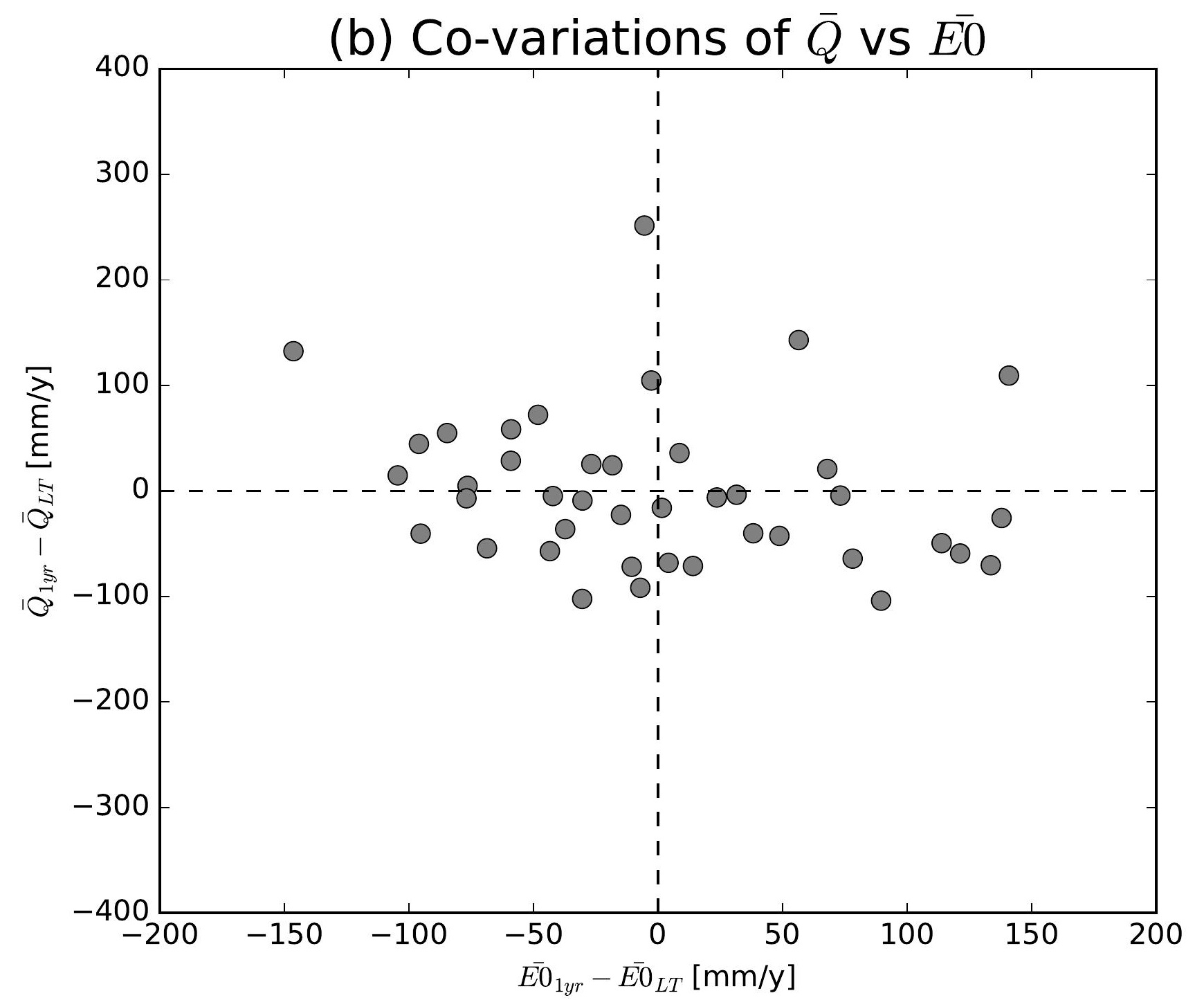

(e) Co-variations of $\bar{Q}$ vs $\overline{E 0}$

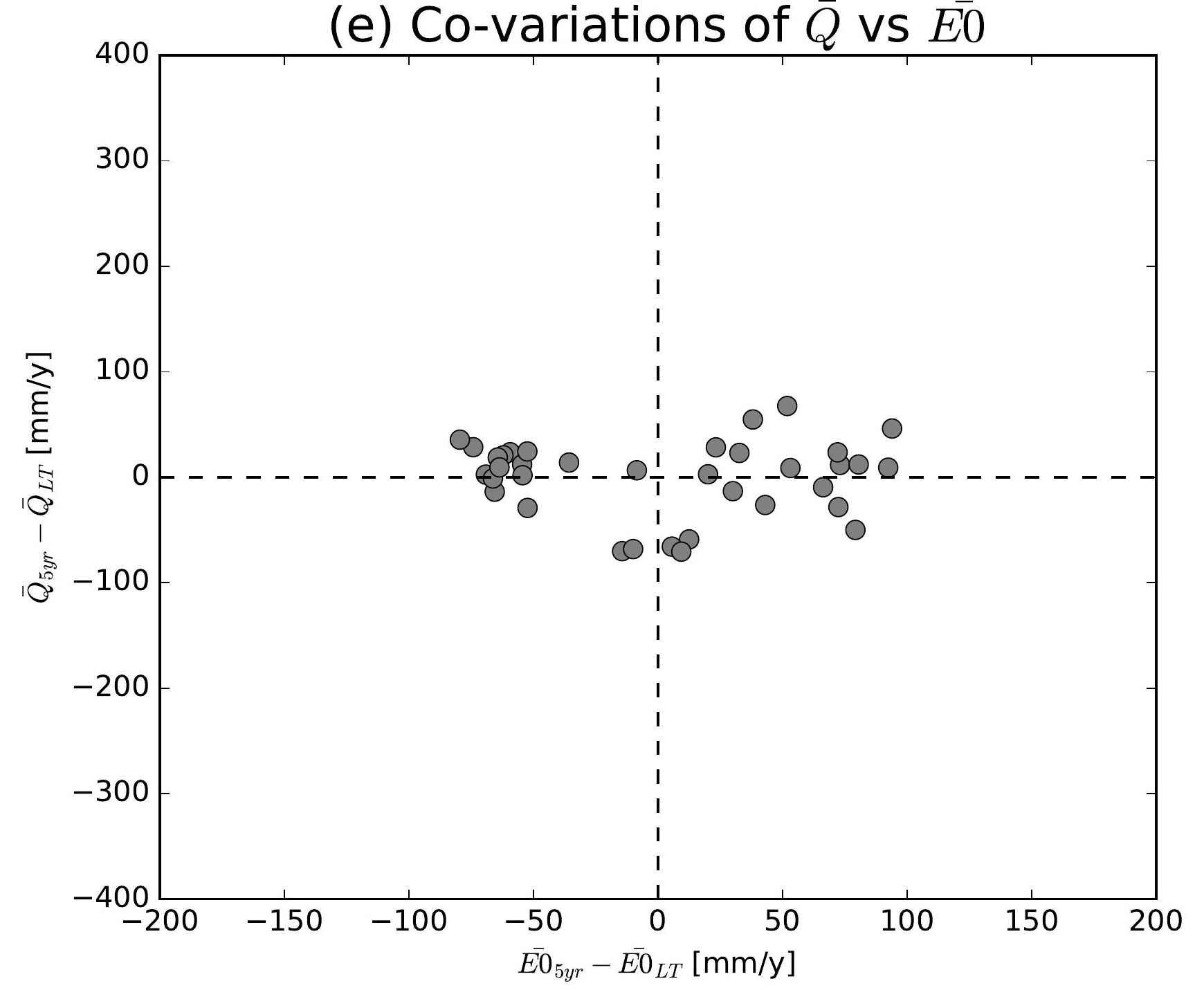

(h) Co-variations of $\bar{Q}$ vs $\overline{E 0}$

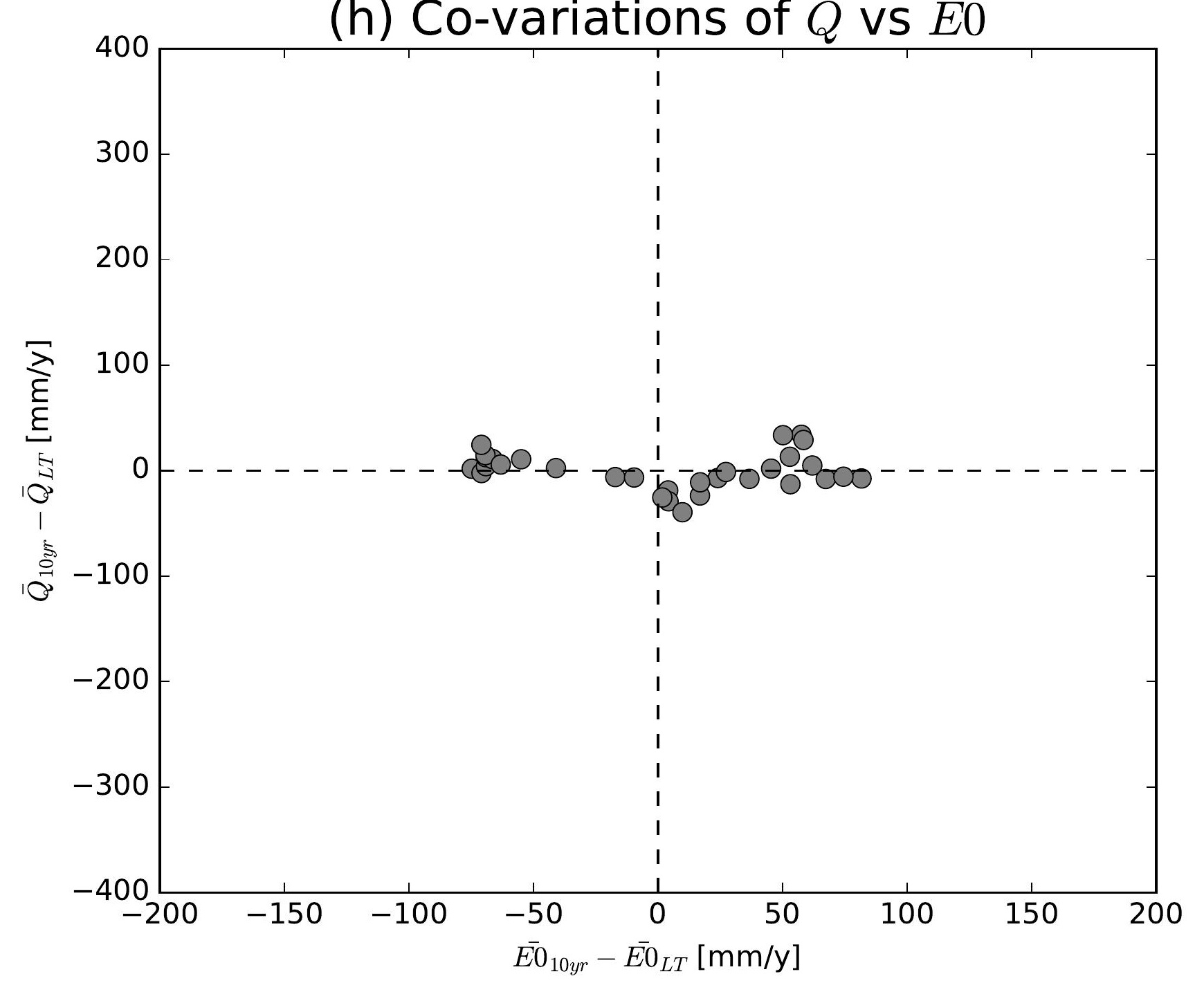

(k) Co-variations of $\bar{Q}$ vs $\overline{E 0}$

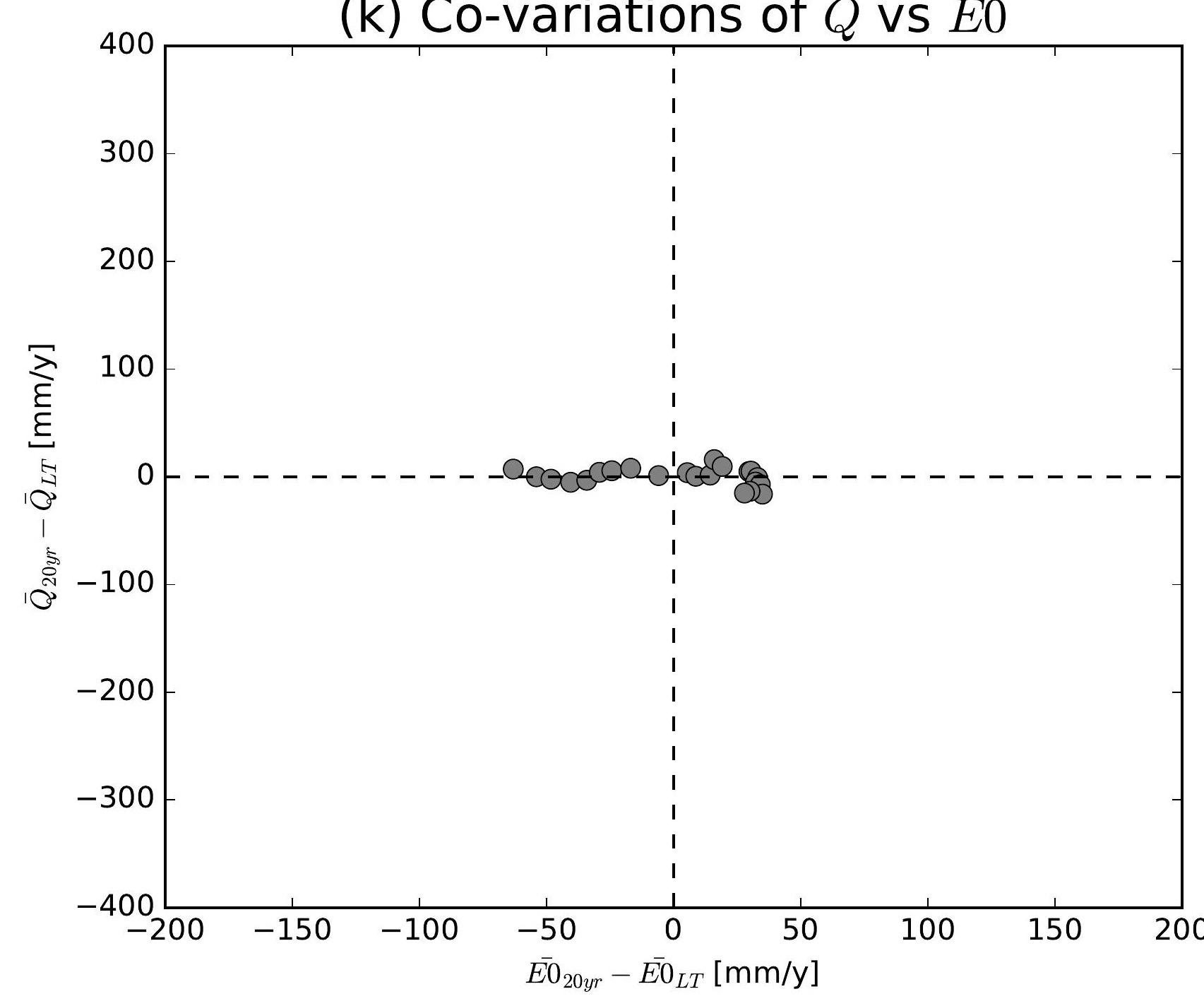

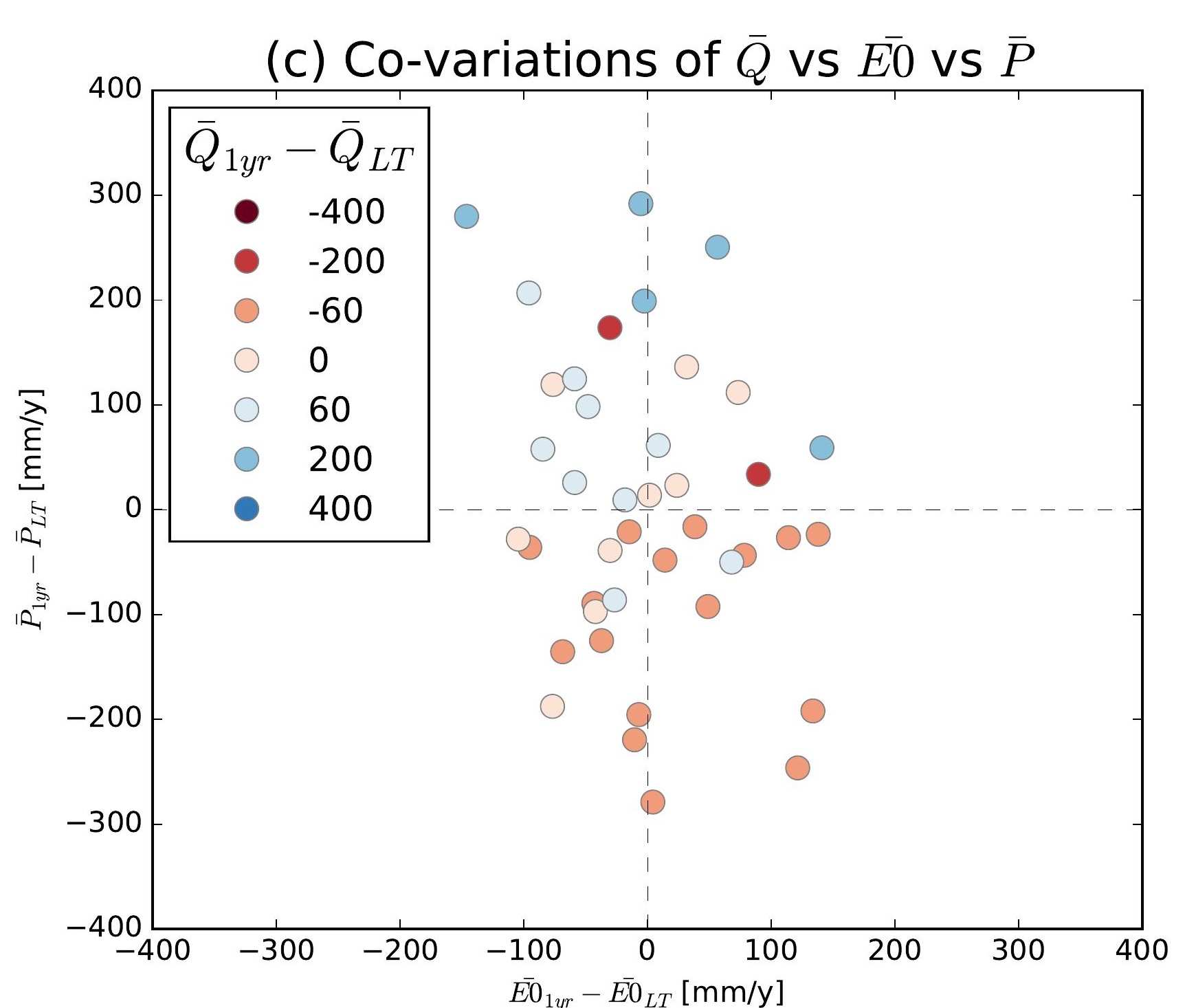
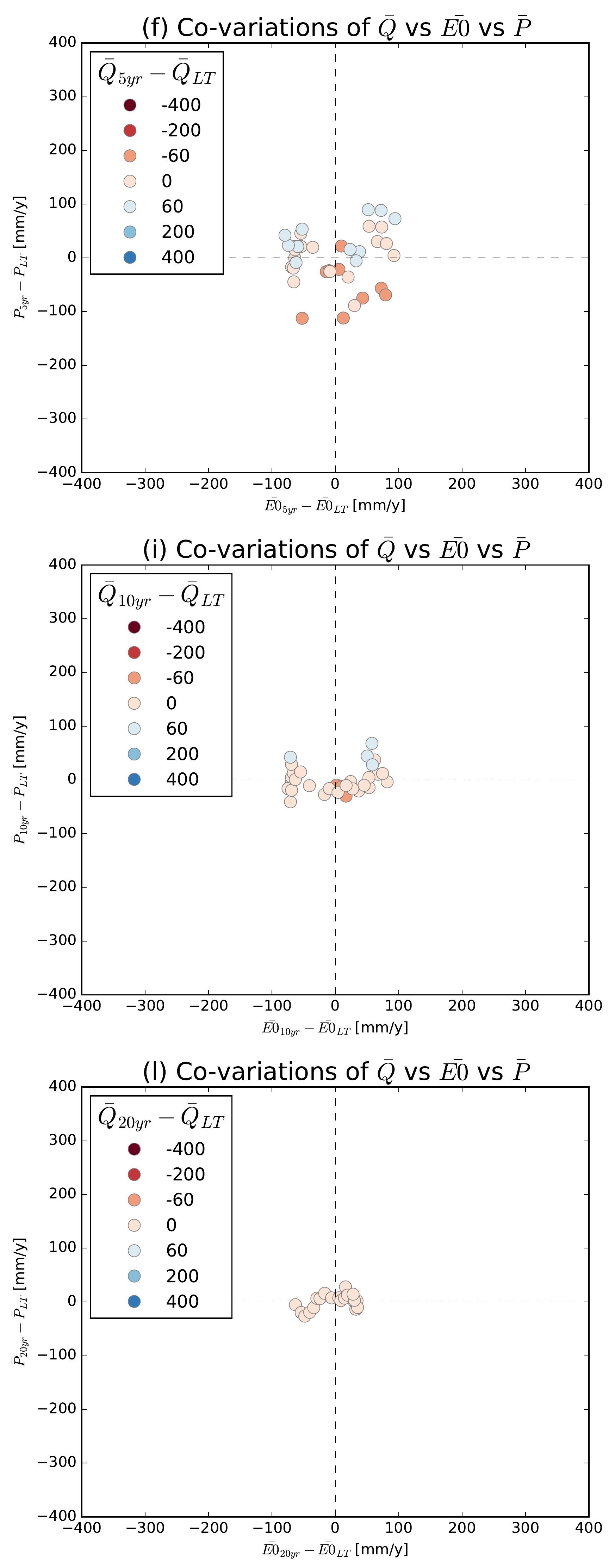

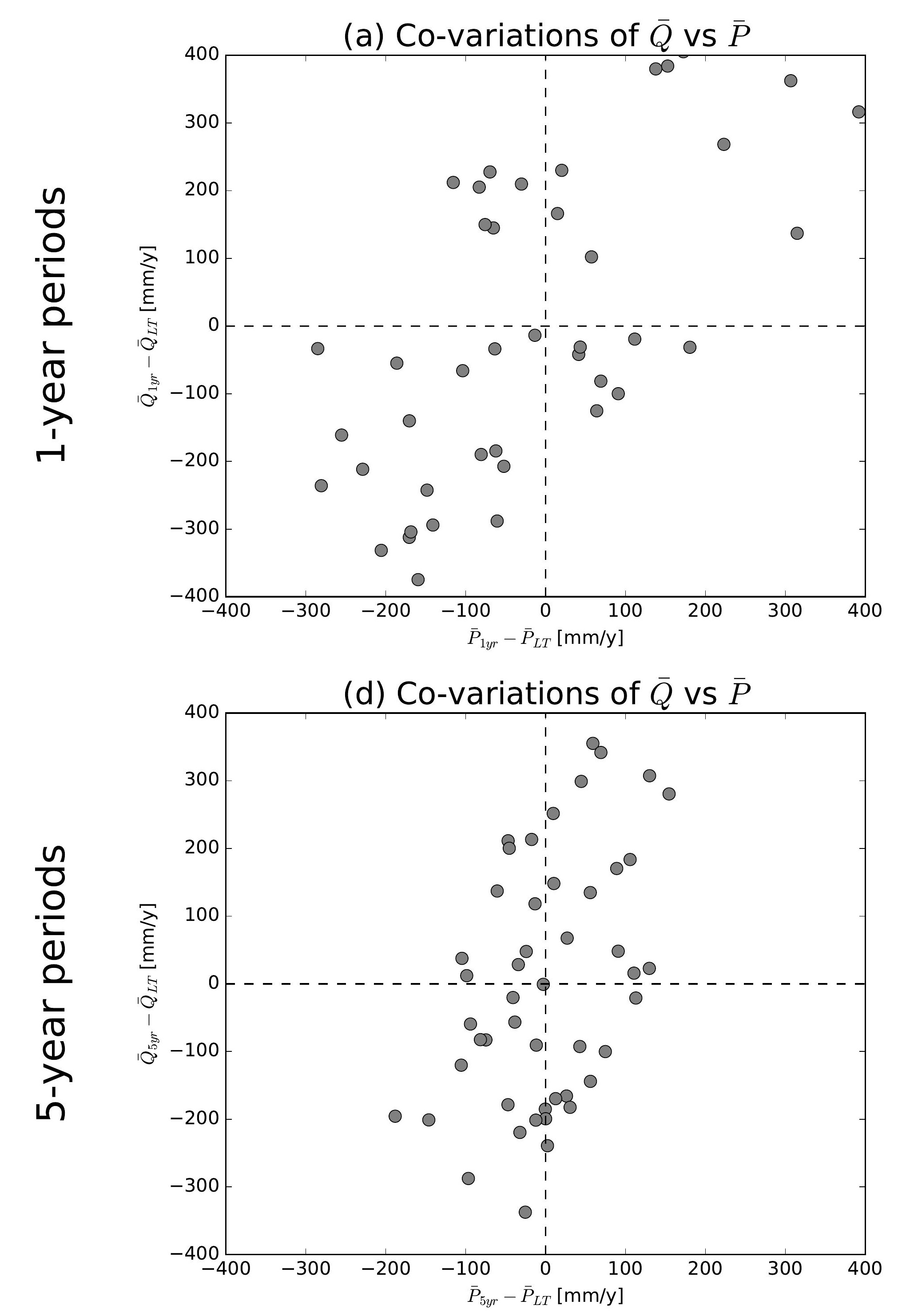

(g) Co-variations of $\bar{Q}$ vs $\bar{P}$

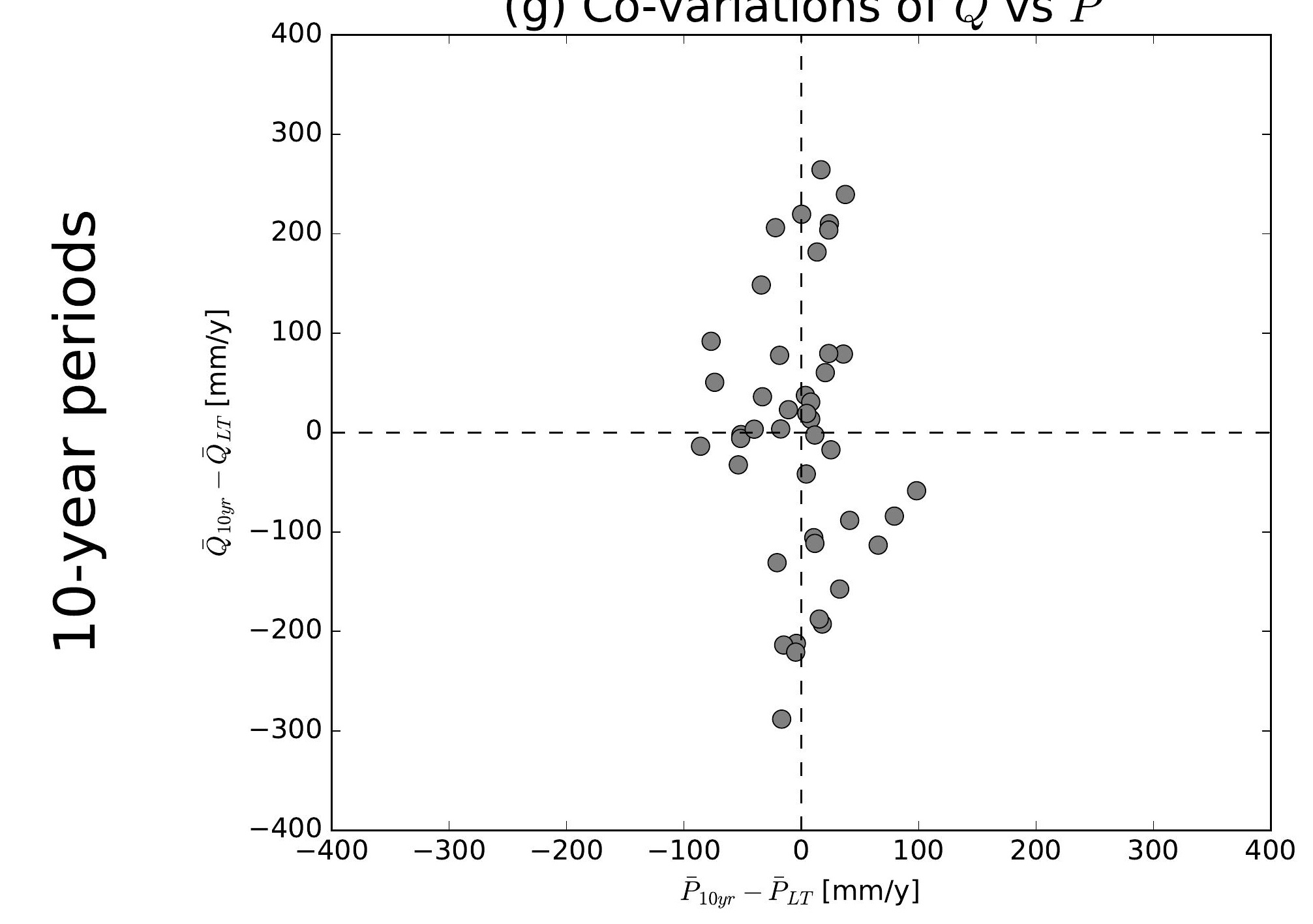

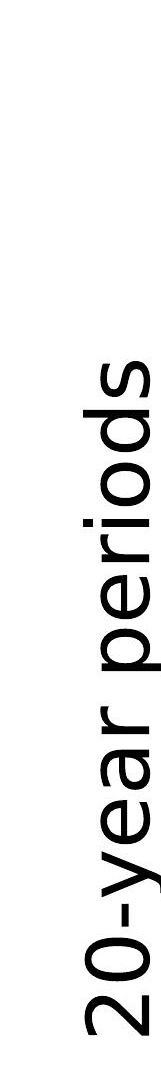

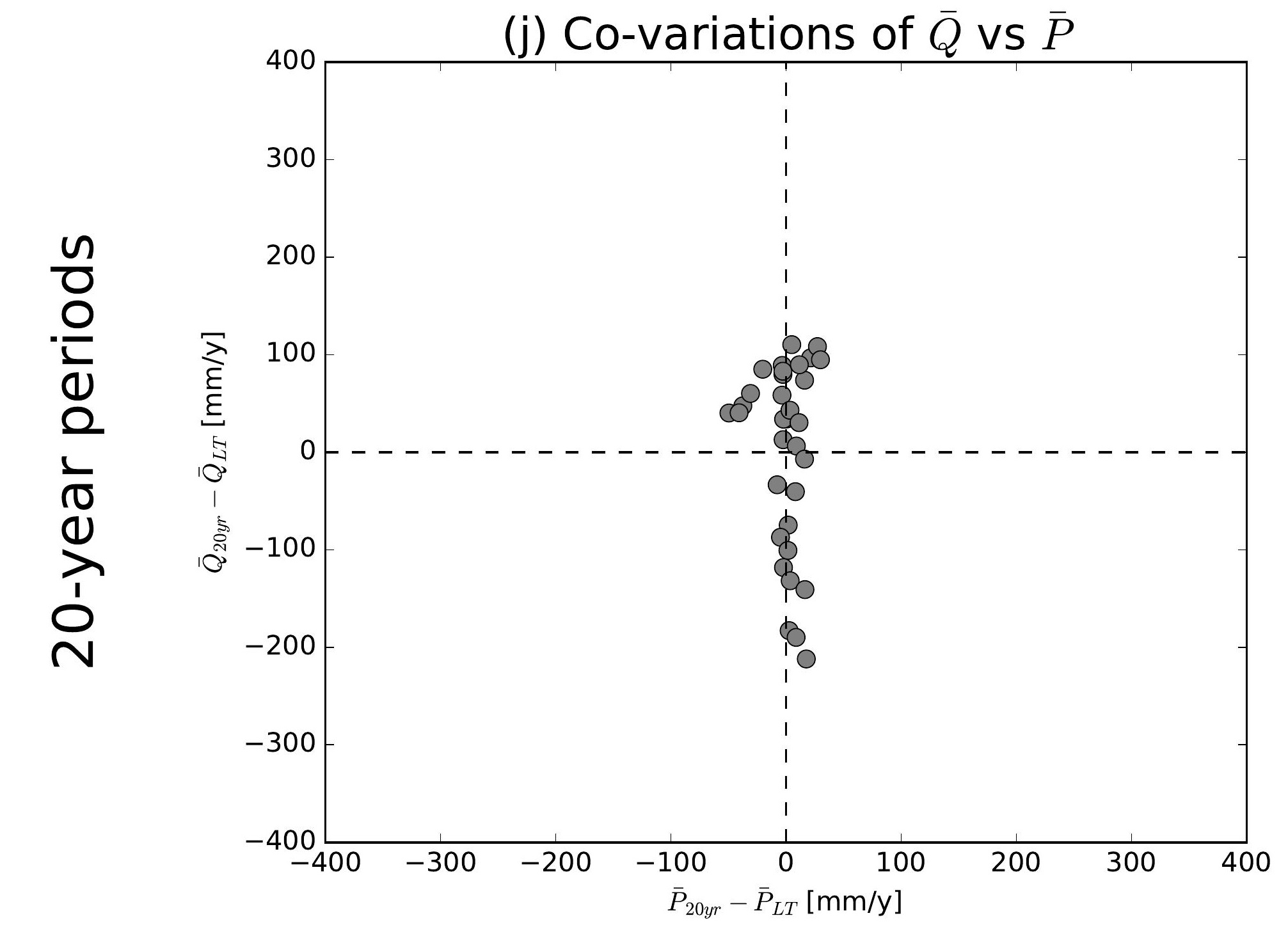

(b) Co-variations of $\bar{Q}$ vs $\overline{E 0}$

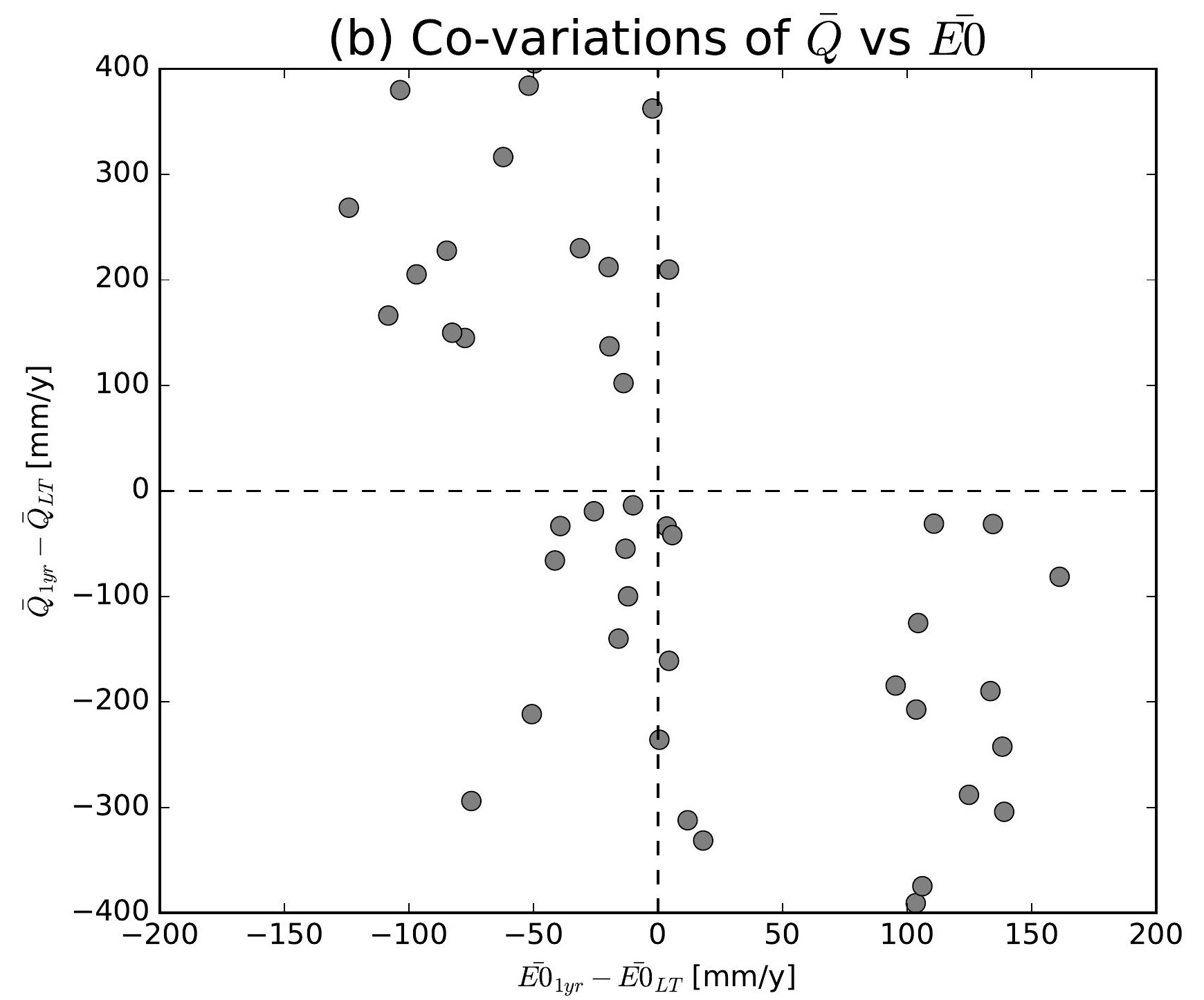

(e) Co-variations of $\bar{Q}$ vs $\overline{E 0}$

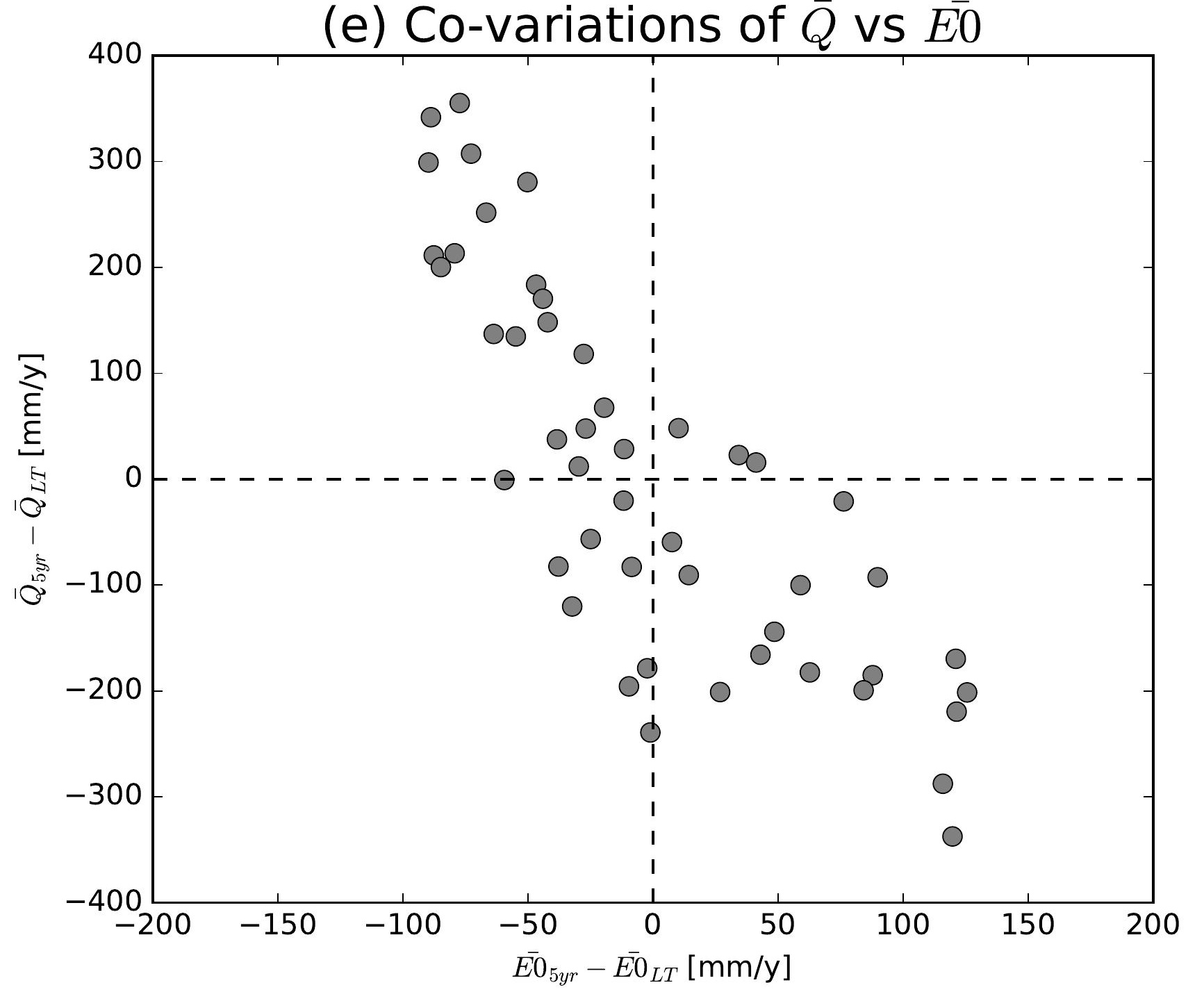

(h) Co-variations of $\bar{Q}$ vs $\overline{E 0}$

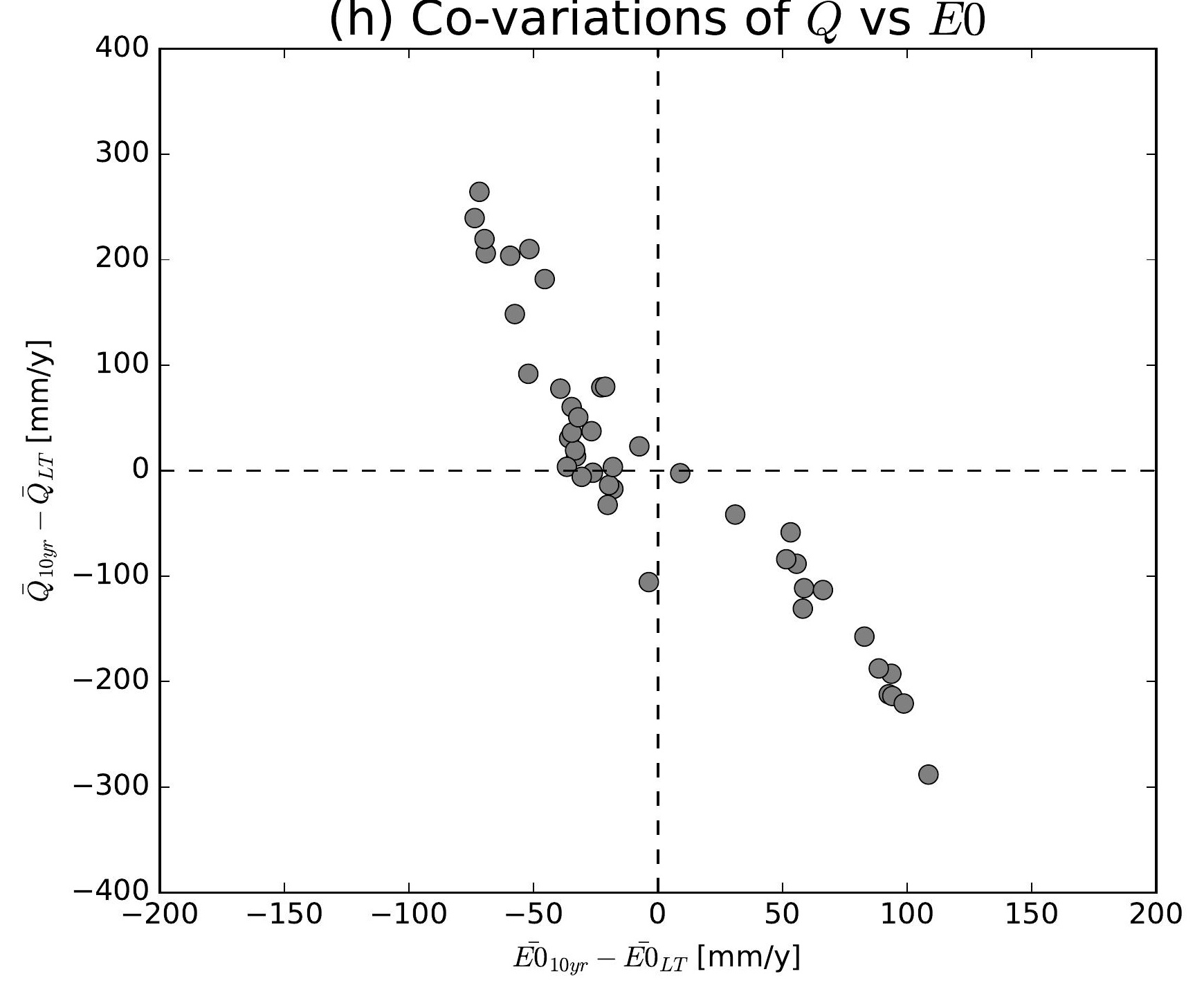

(k) Co-variations of $\bar{Q}$ vs $\overline{E 0}$

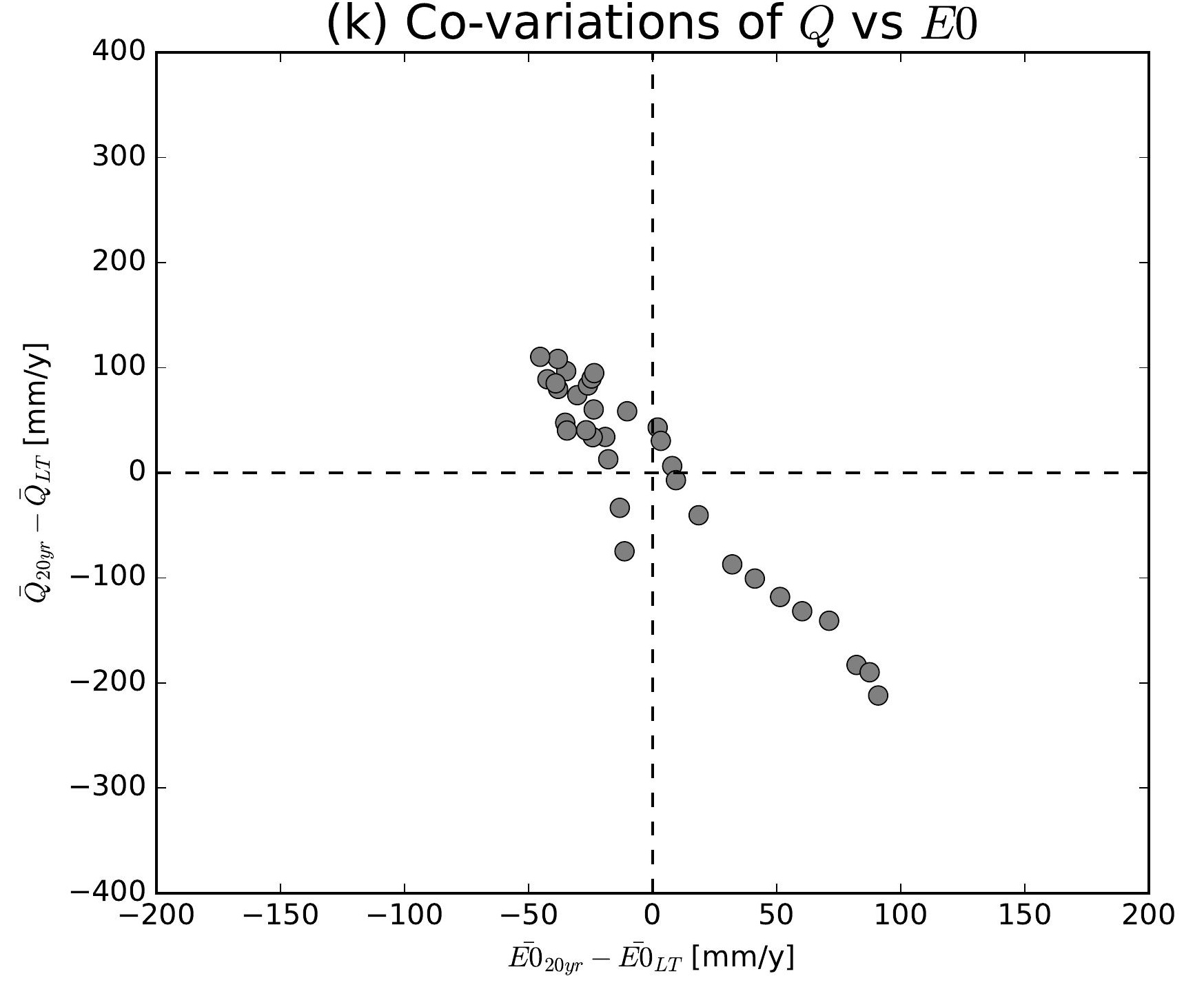

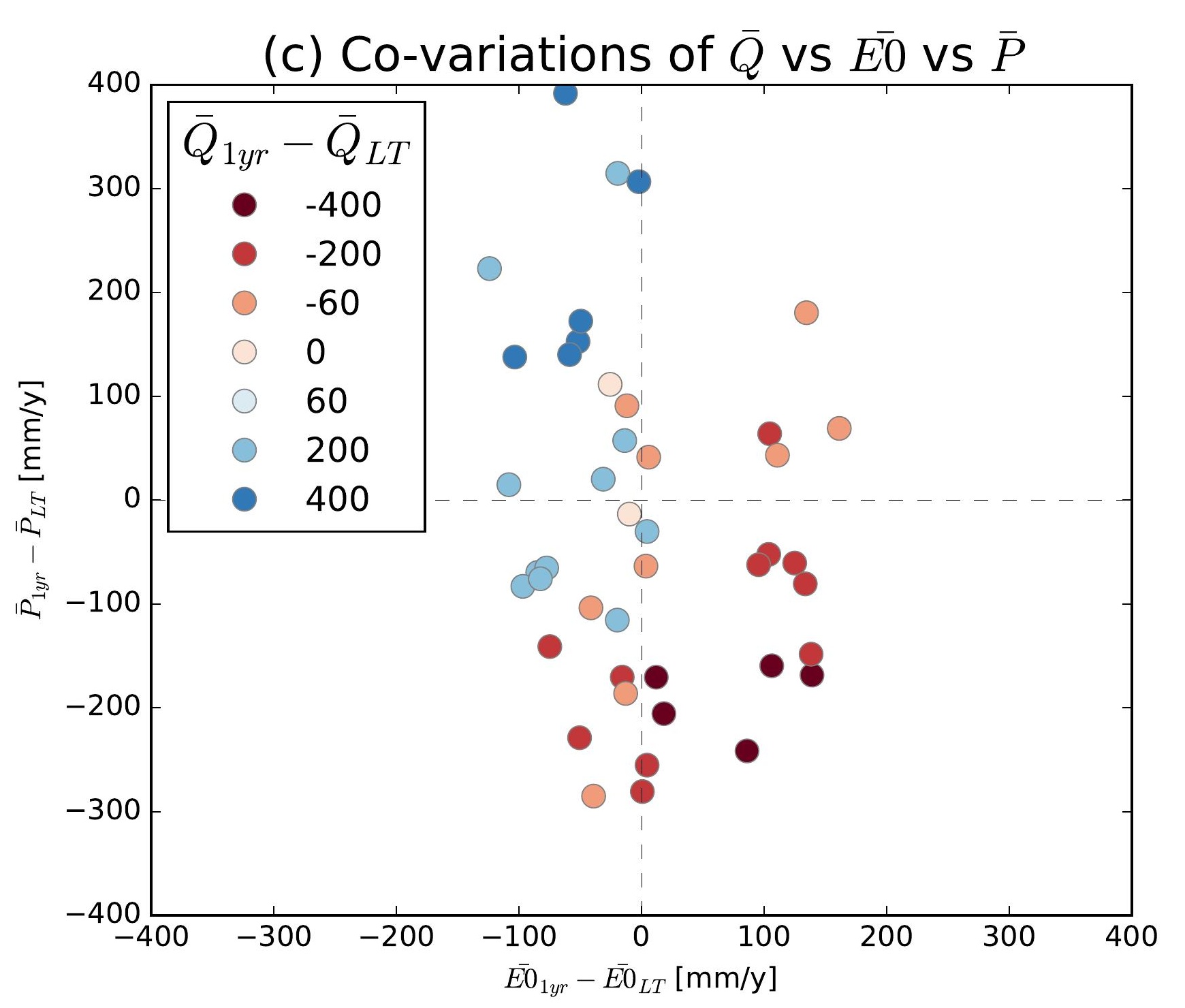
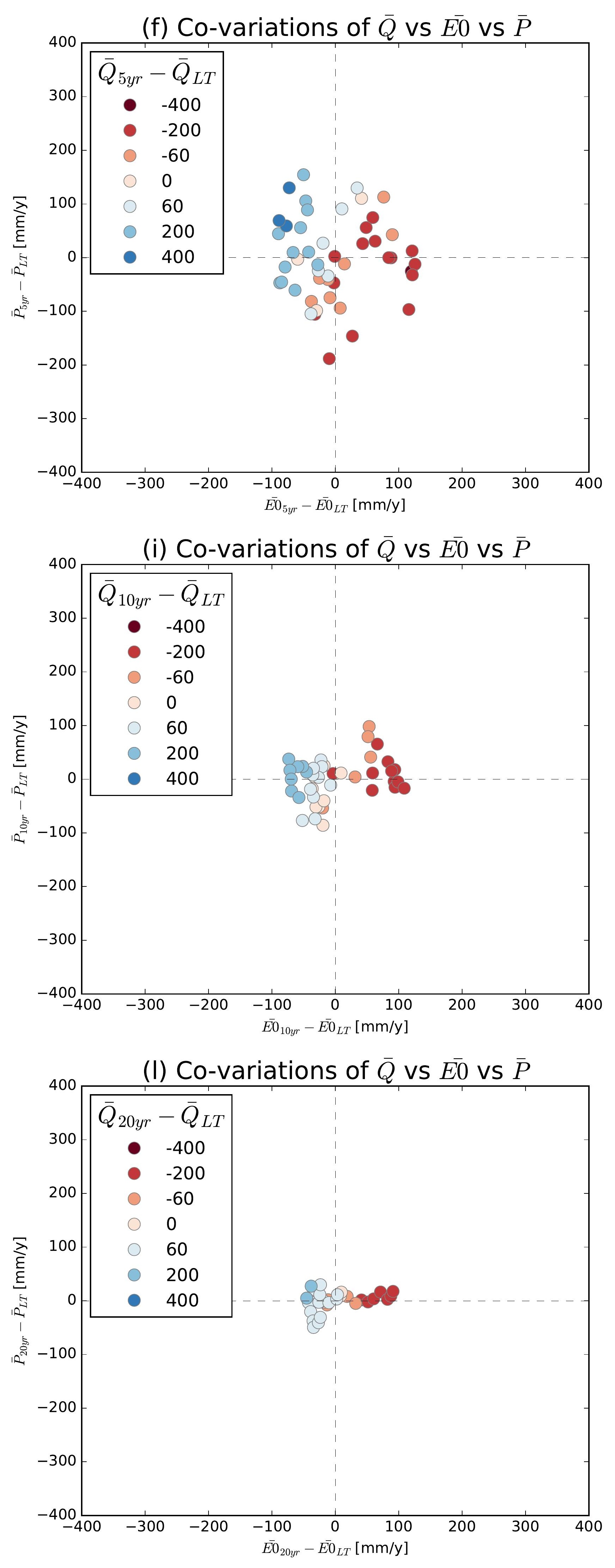

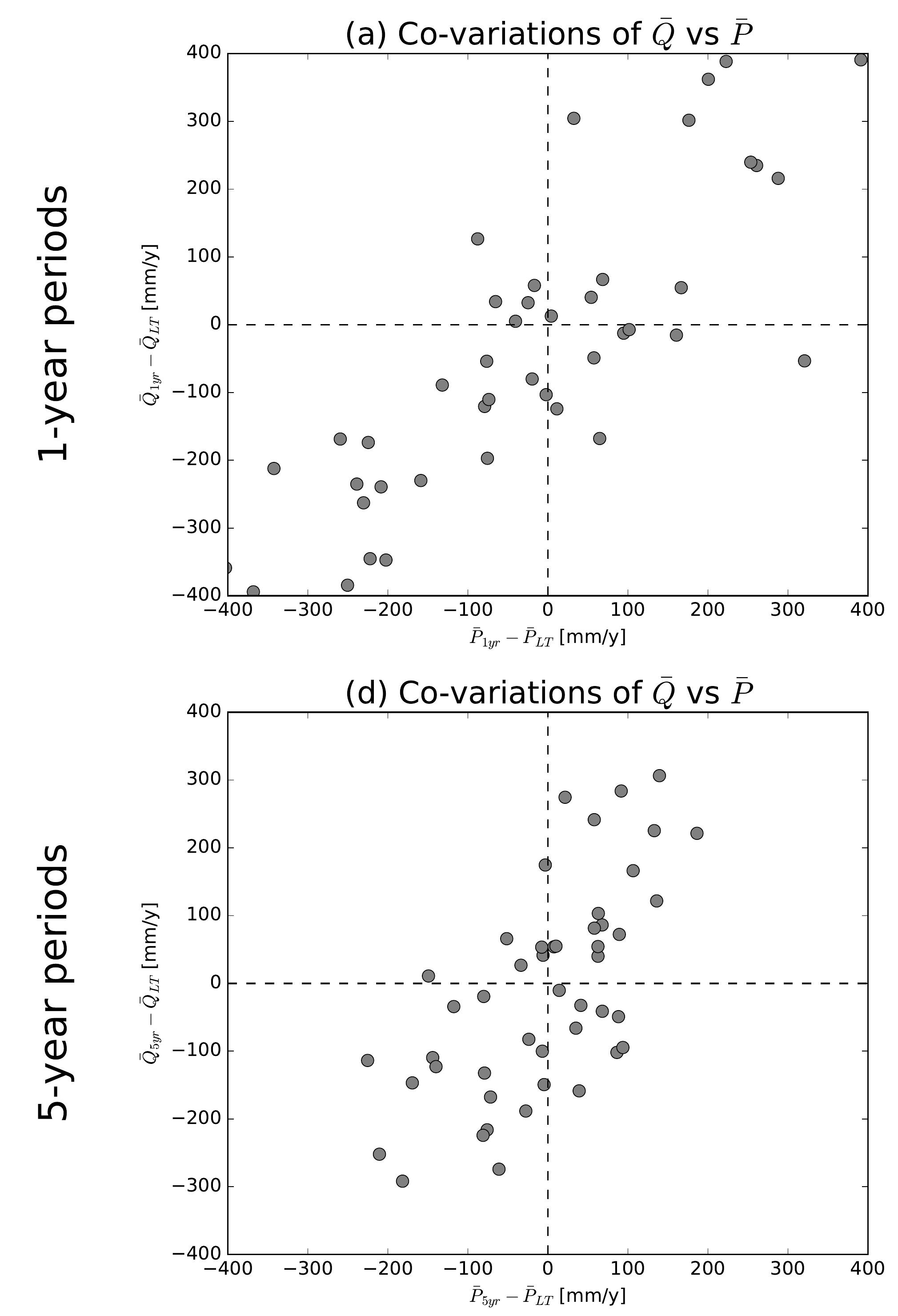

(g) Co-variations of $\bar{Q}$ vs $\bar{P}$
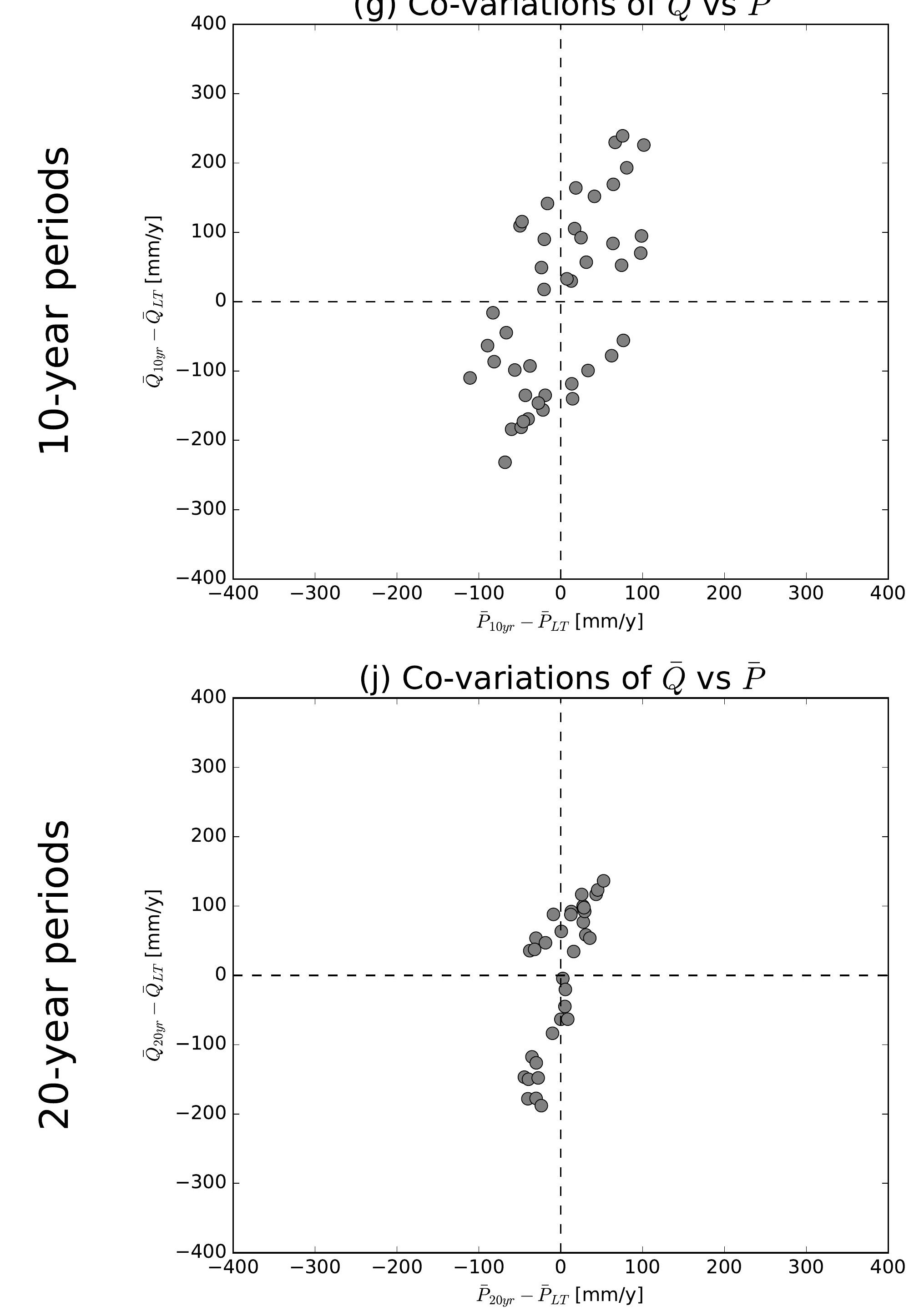

(b) Co-variations of $\bar{Q}$ vs $\overline{E 0}$

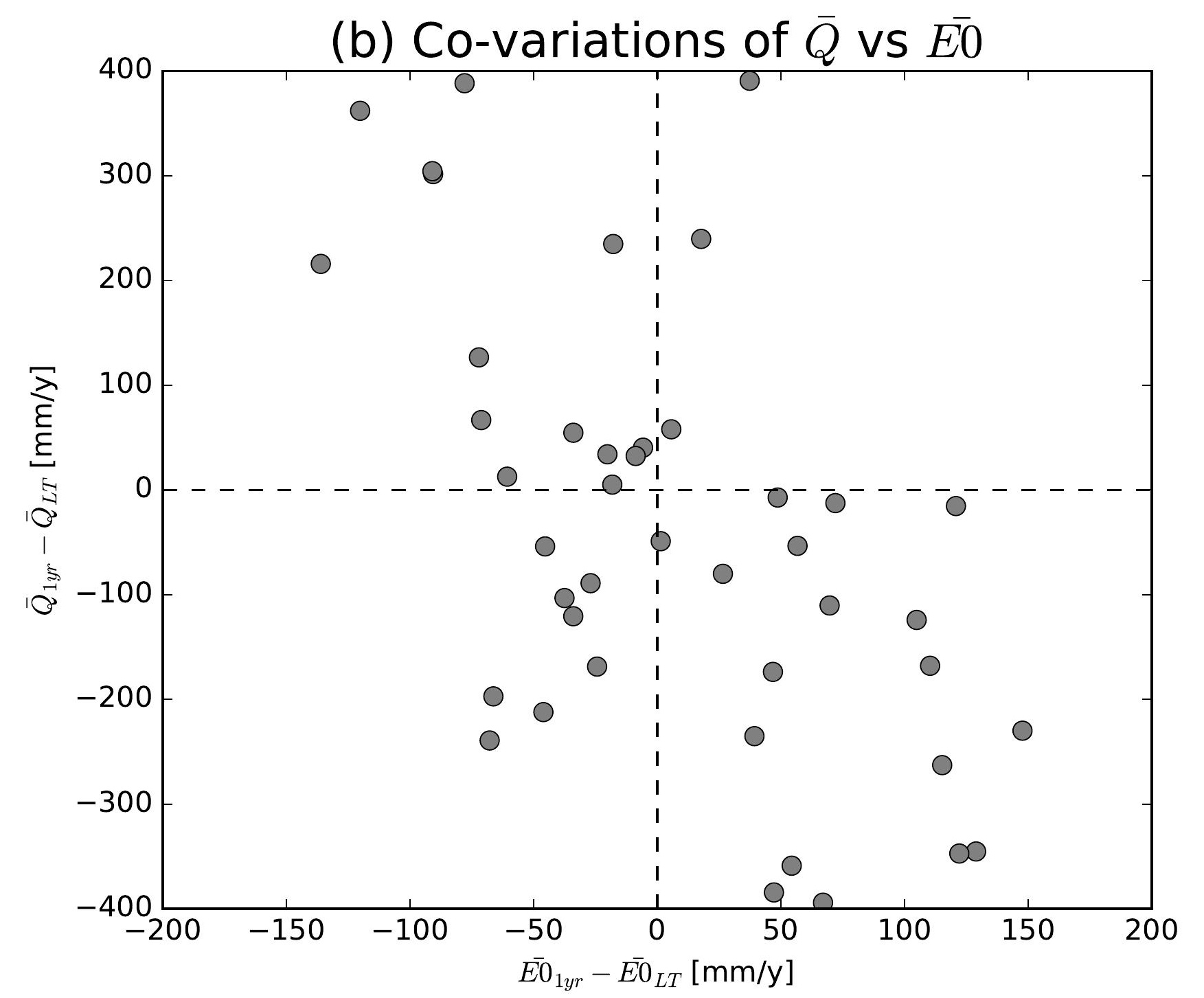

(e) Co-variations of $\bar{Q}$ vs $\overline{E 0}$

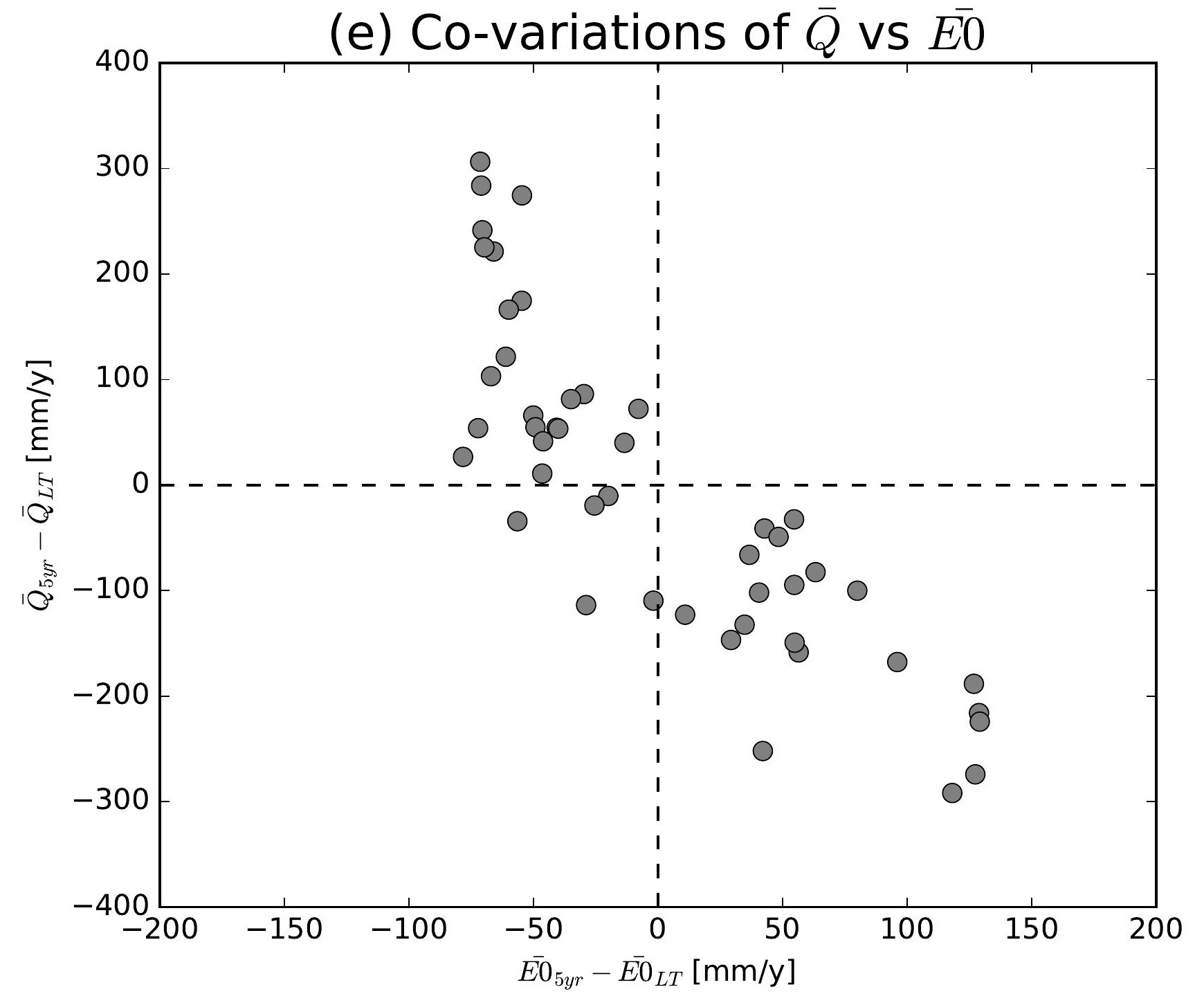

(h) Co-variations of $\bar{Q}$ vs $\overline{E 0}$

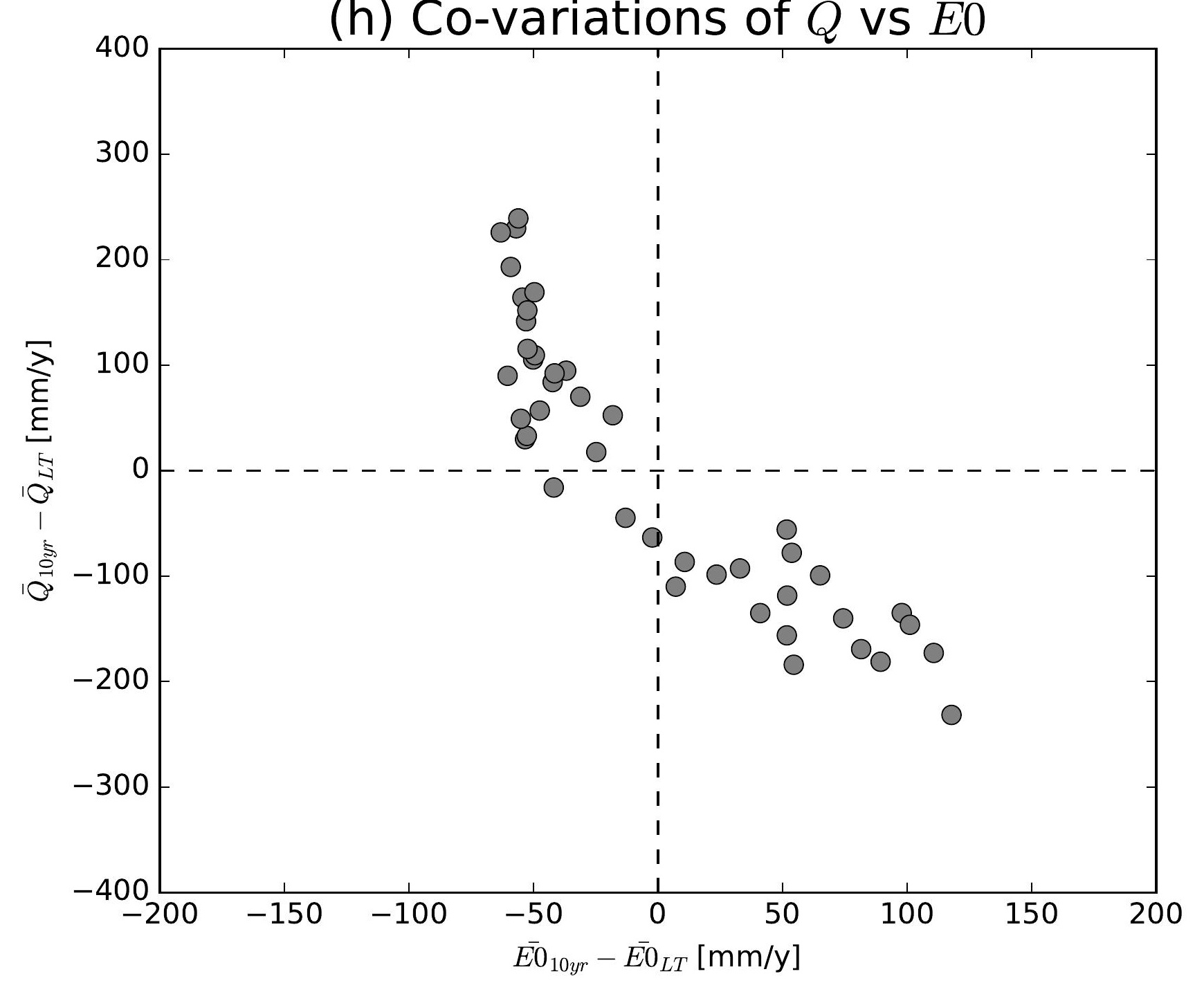

(k) Co-variations of $\bar{Q}$ vs $\overline{E 0}$

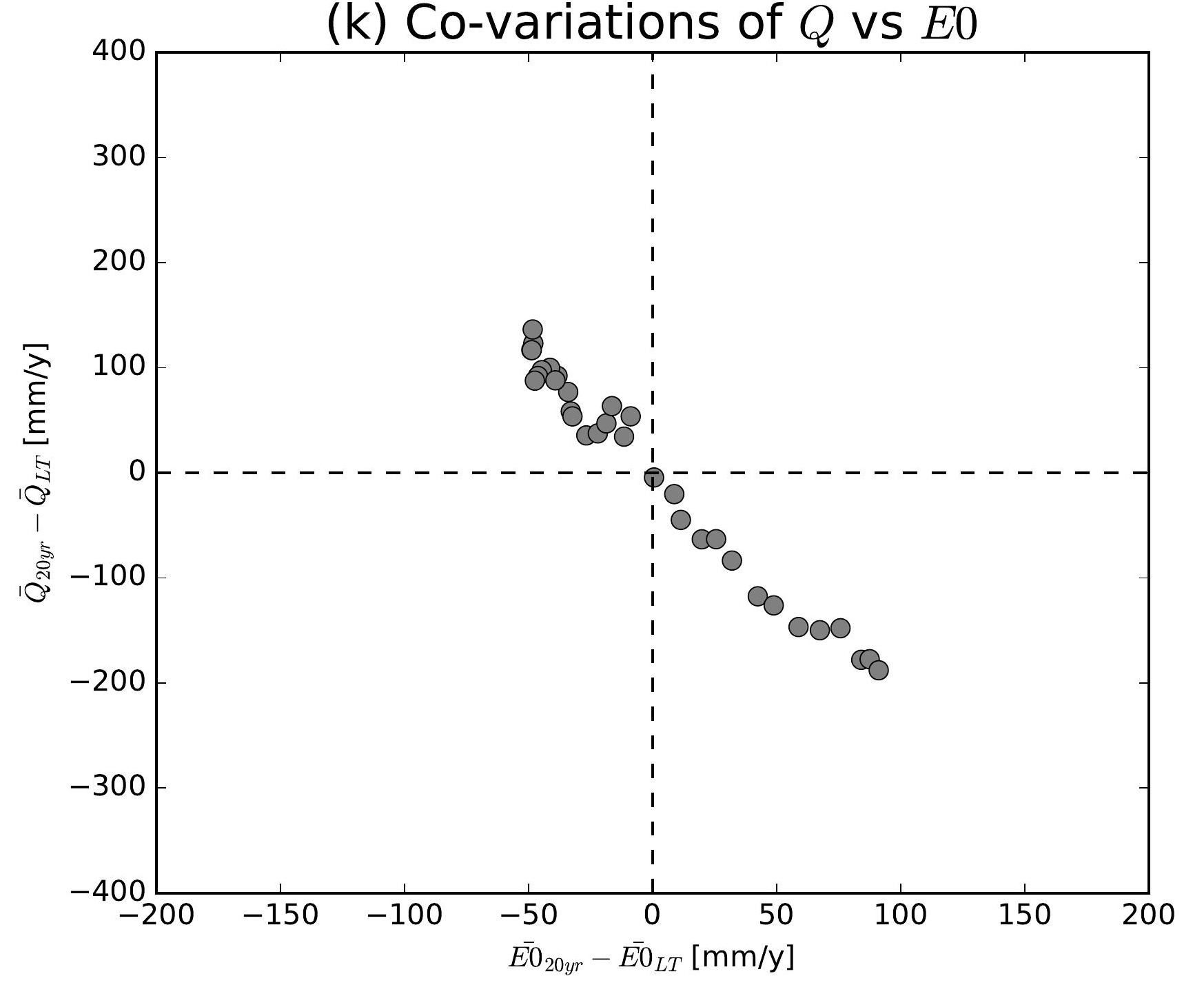

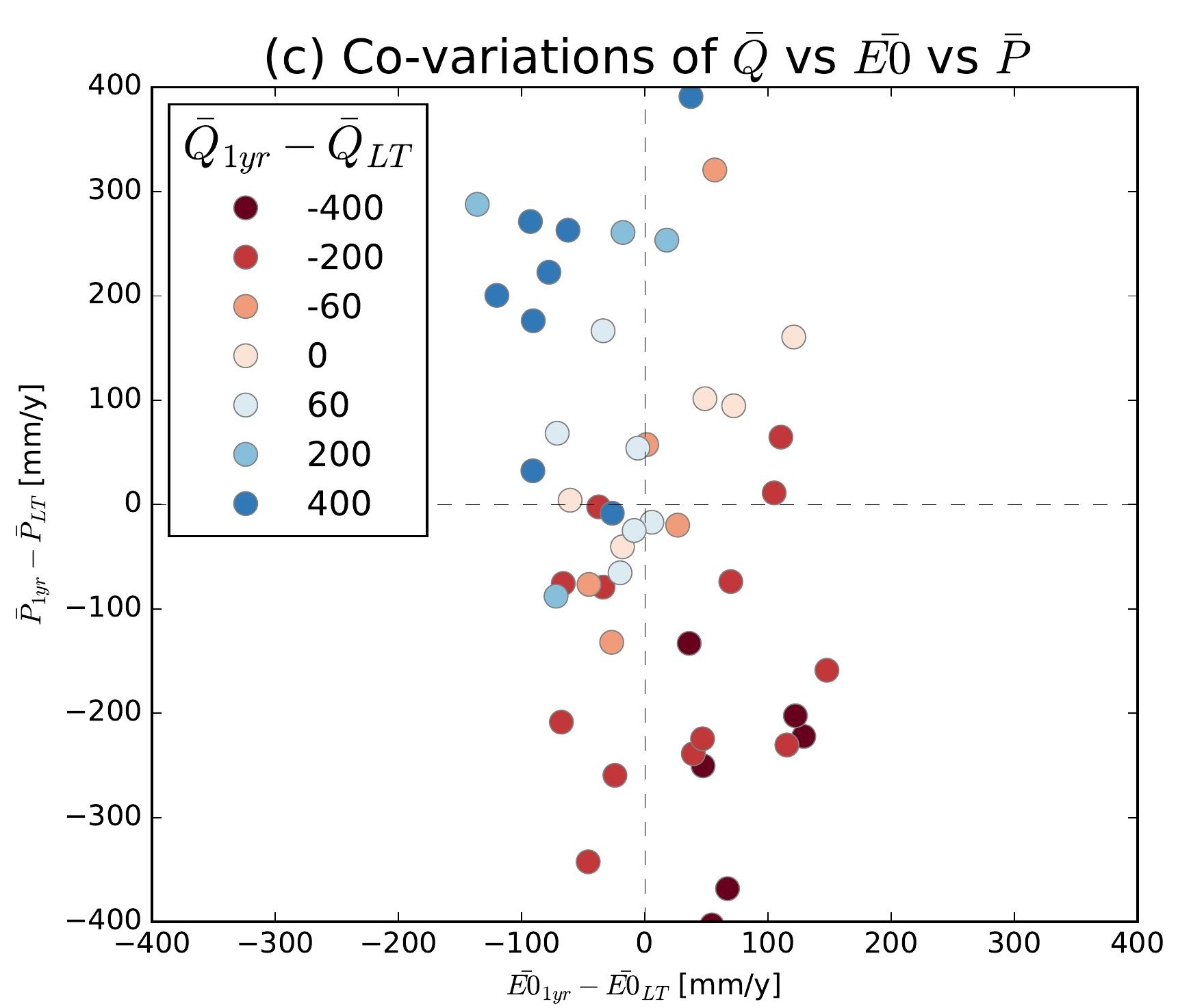
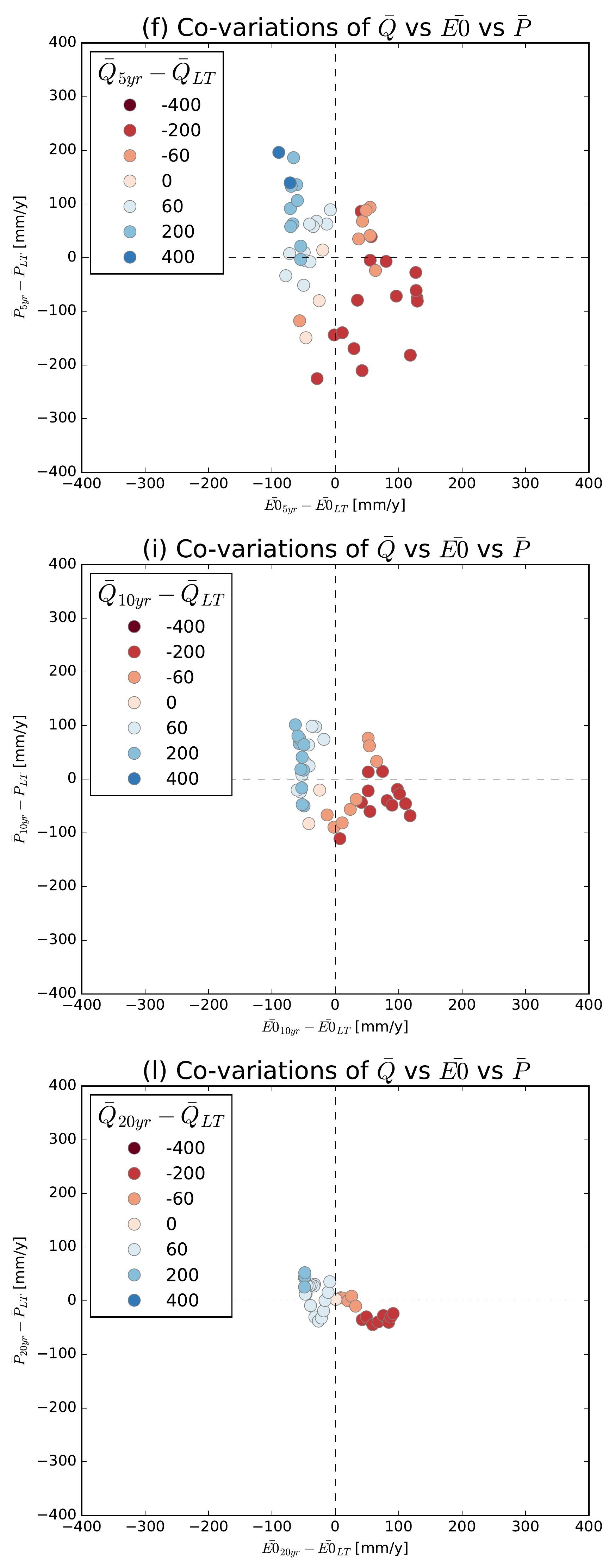

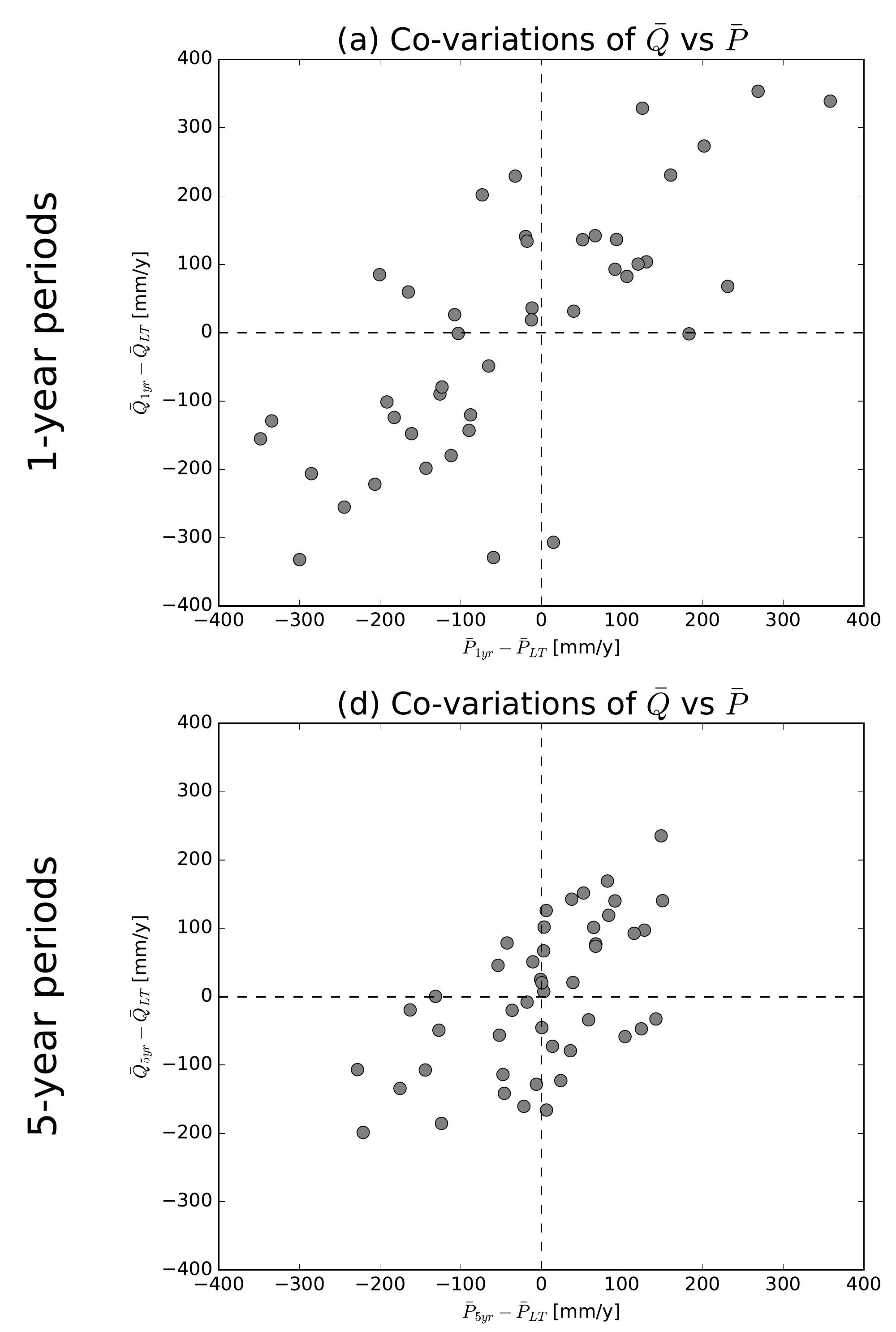

(g) Co-variations of $\bar{Q}$ vs $\bar{P}$

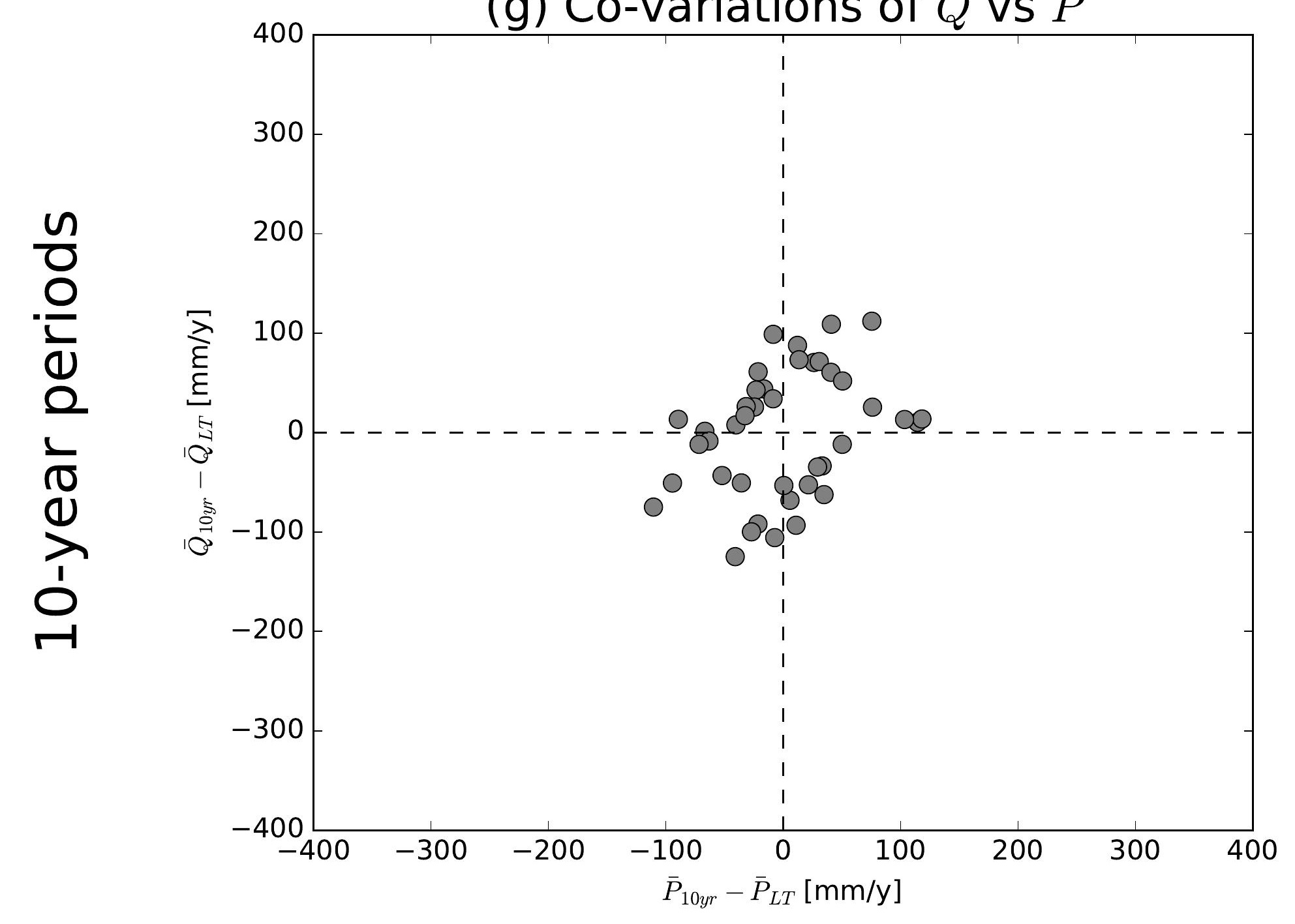

(j) Co-variations of $\bar{Q}$ vs $\bar{P}$

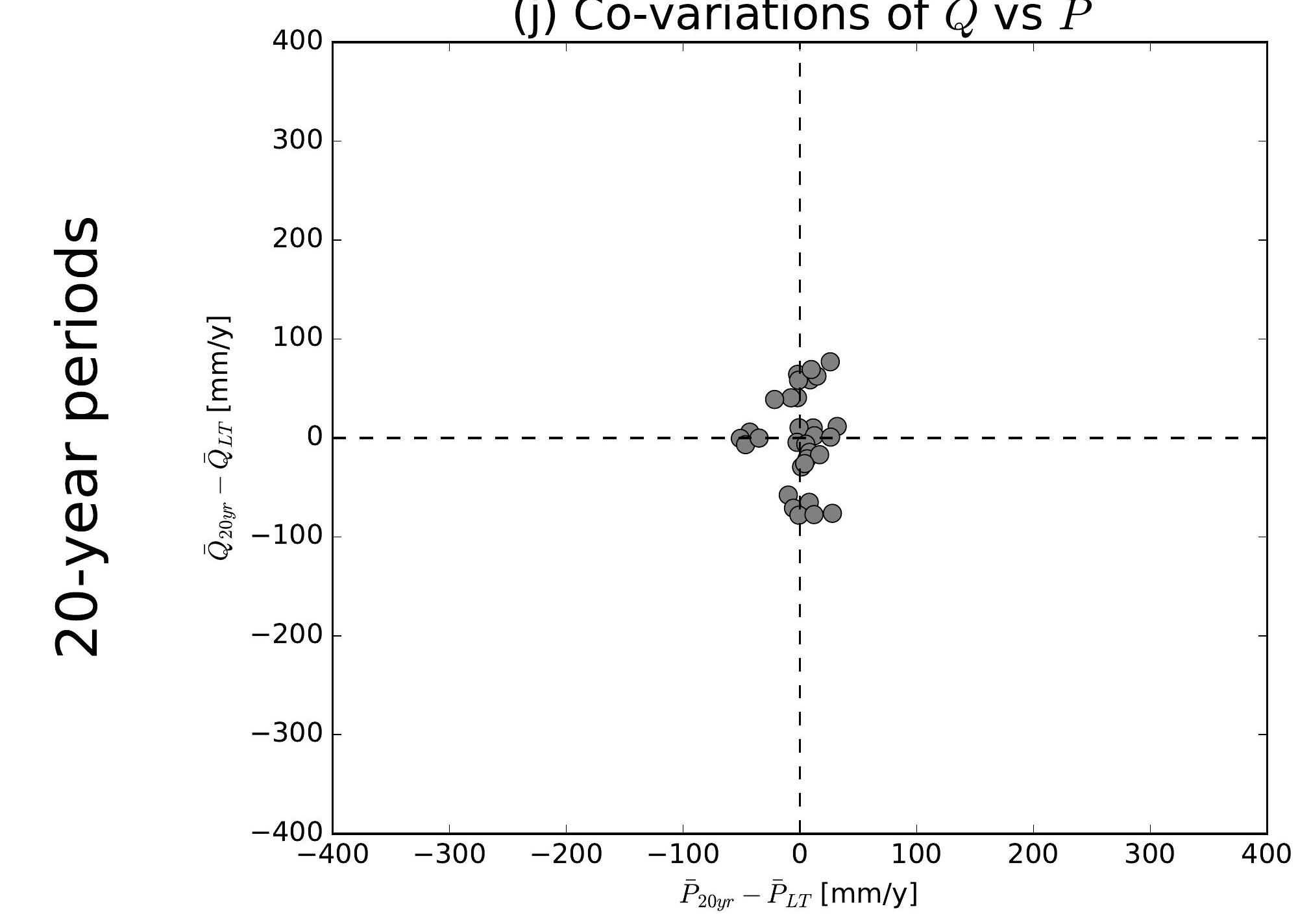

(b) Co-variations of $\bar{Q}$ vs $\overline{E 0}$

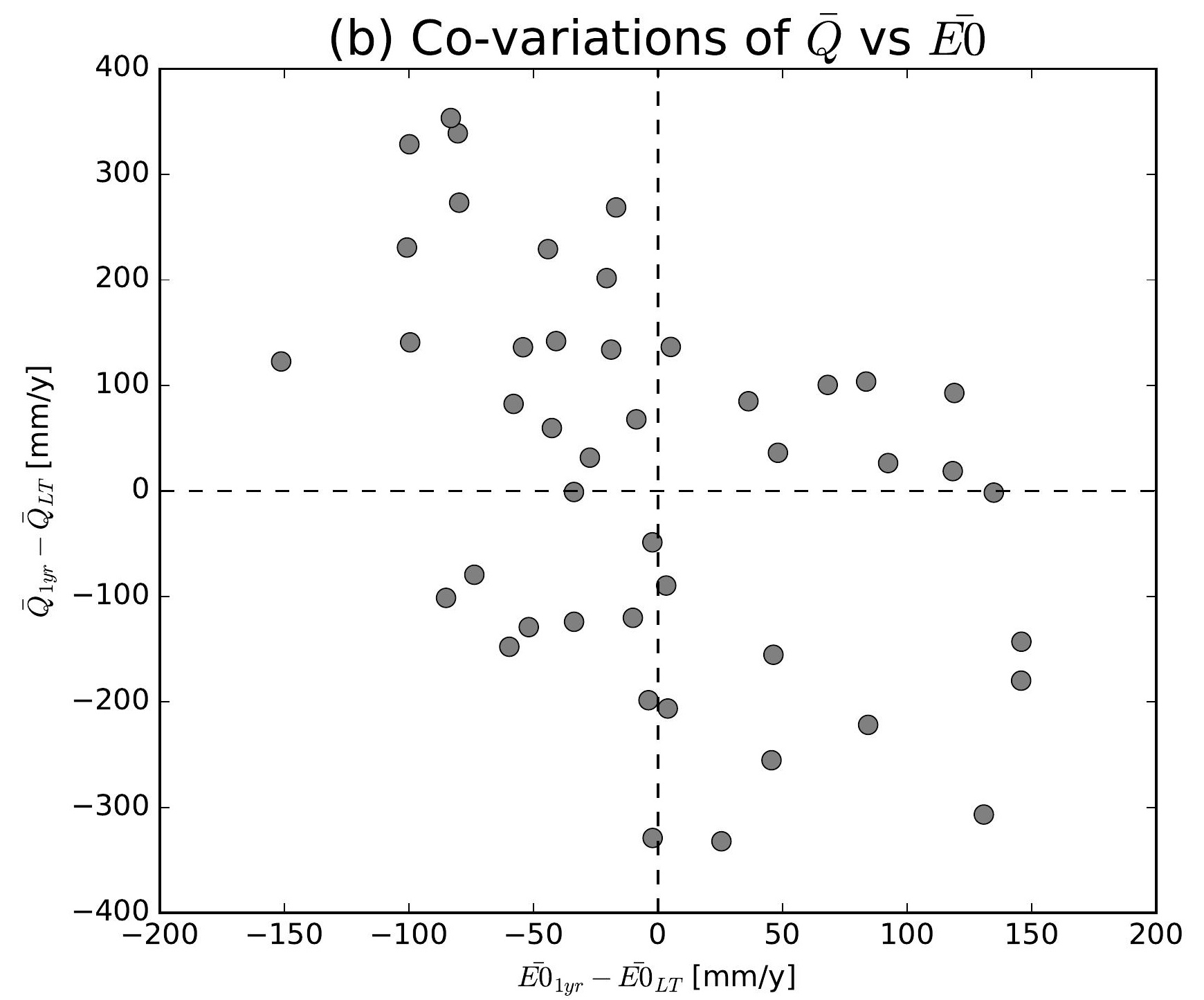

(e) Co-variations of $\bar{Q}$ vs $\overline{E 0}$

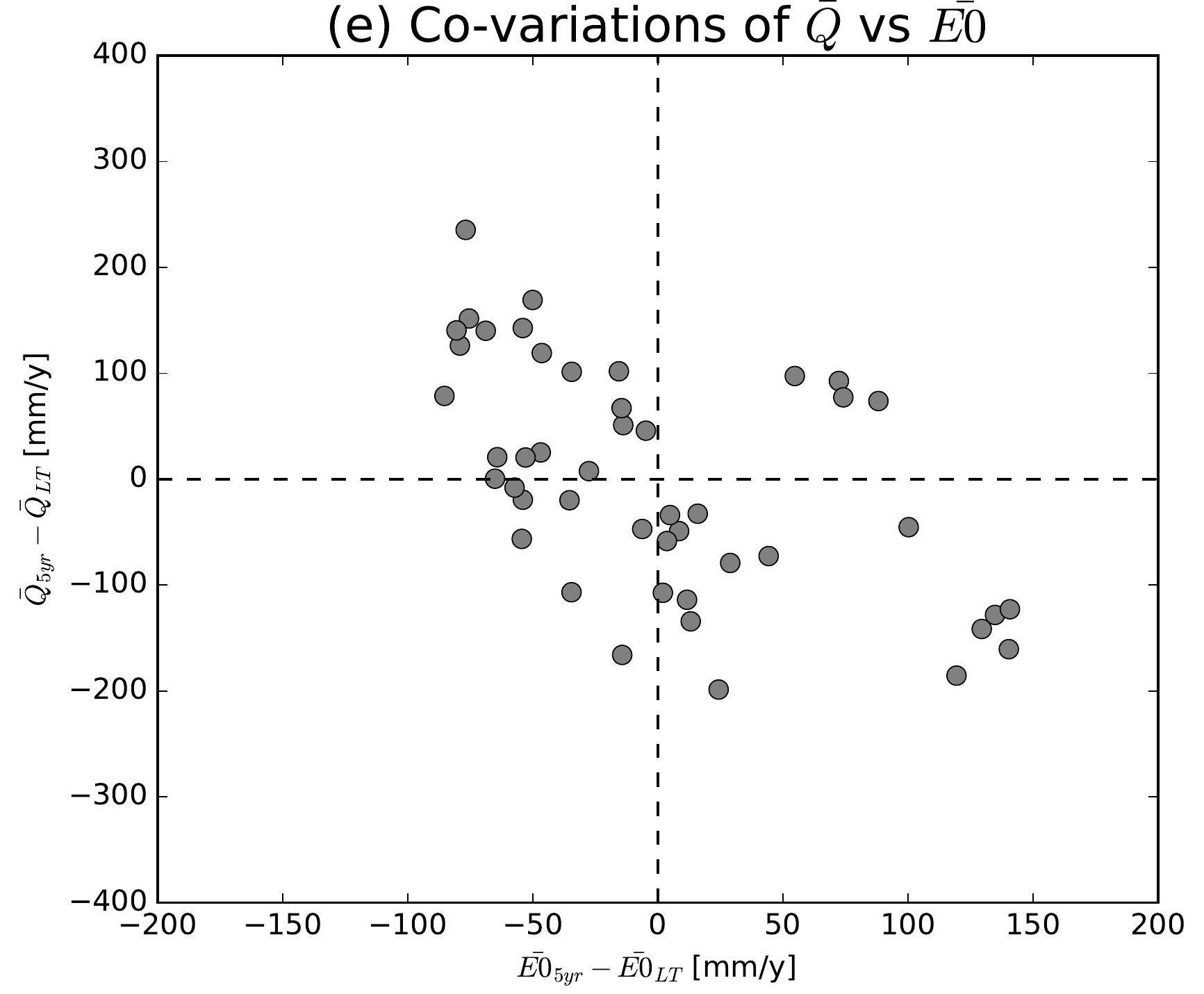

(h) Co-variations of $\bar{Q}$ vs $\overline{E 0}$

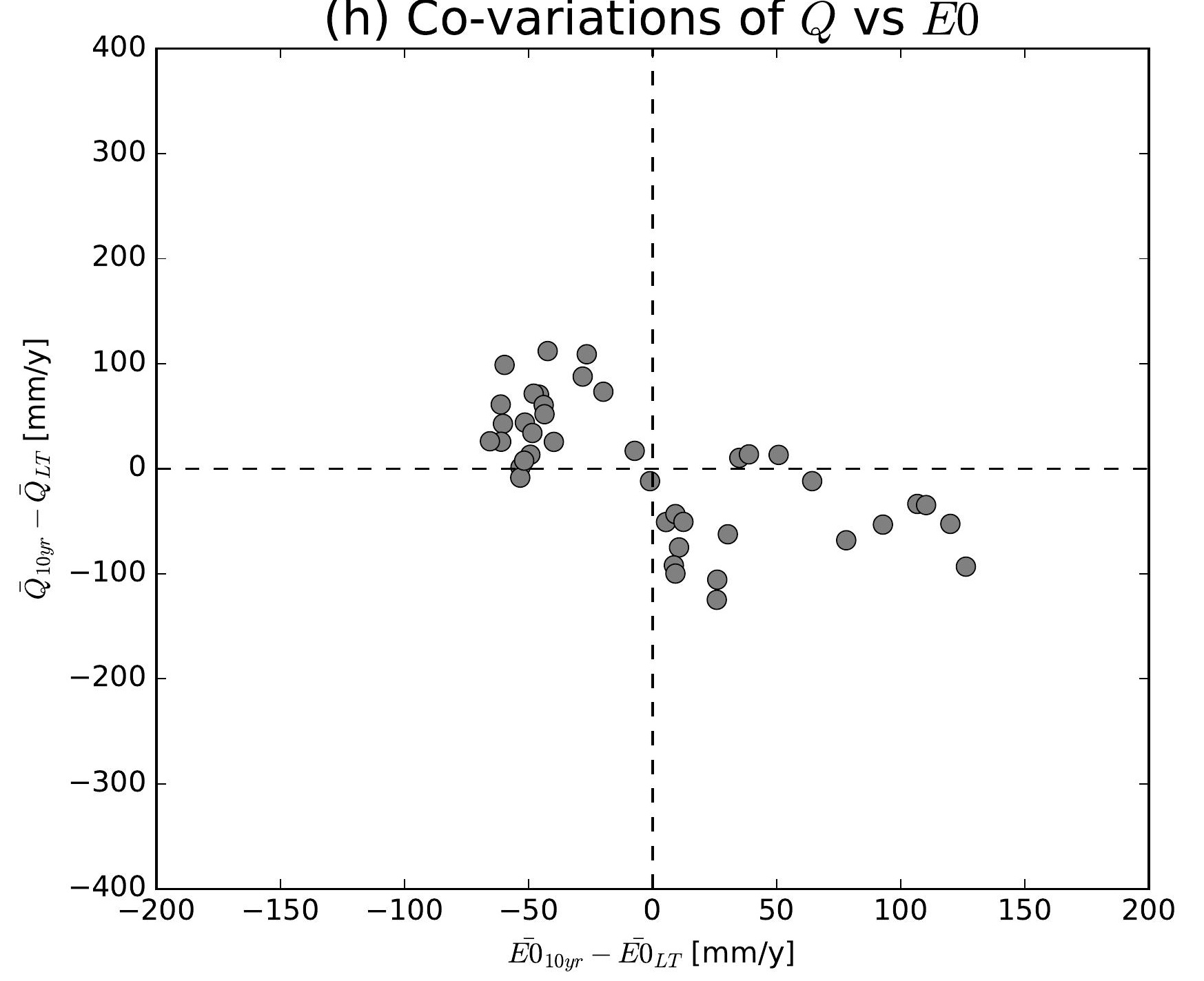

(k) Co-variations of $\bar{Q}$ vs $\overline{E 0}$

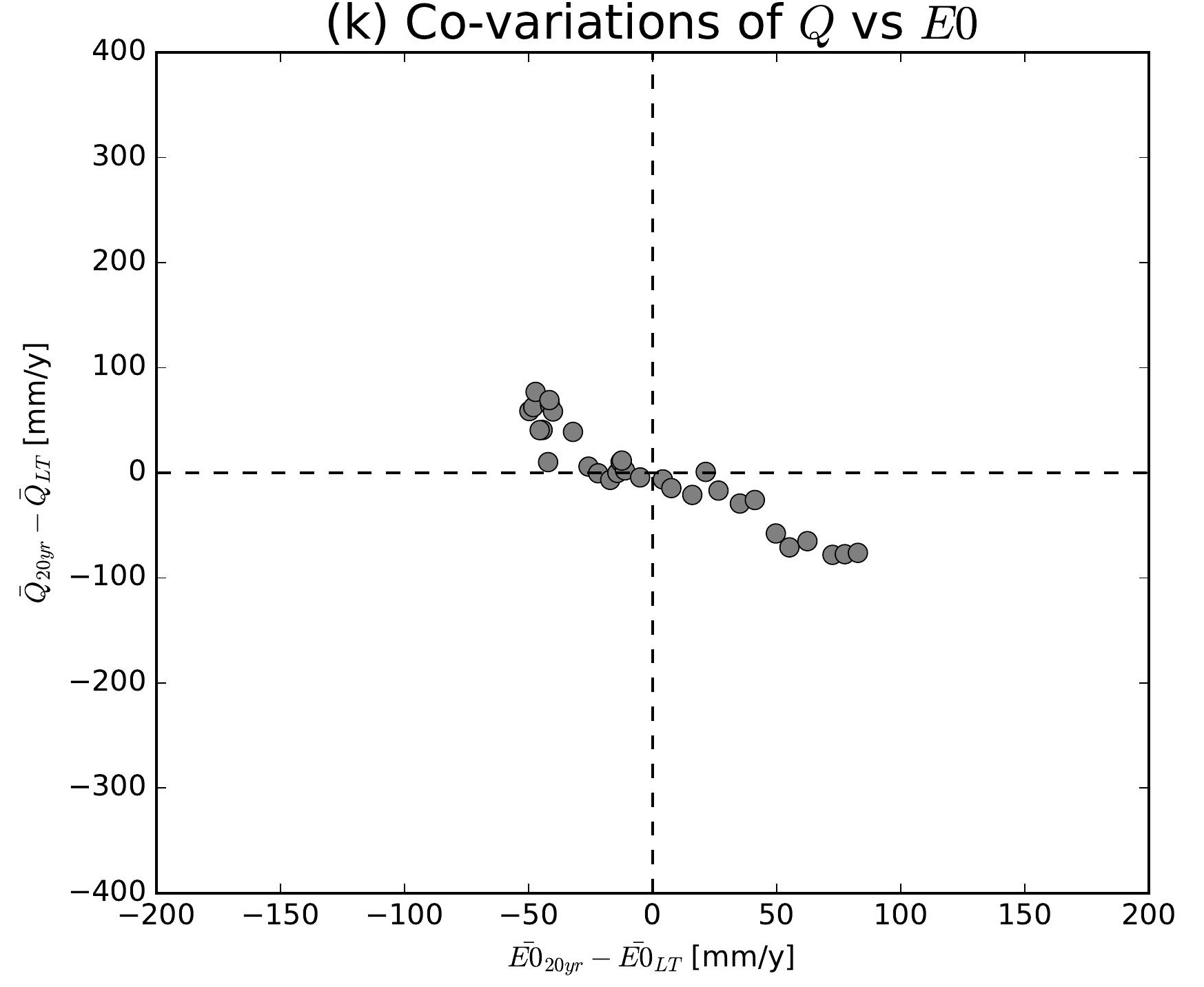

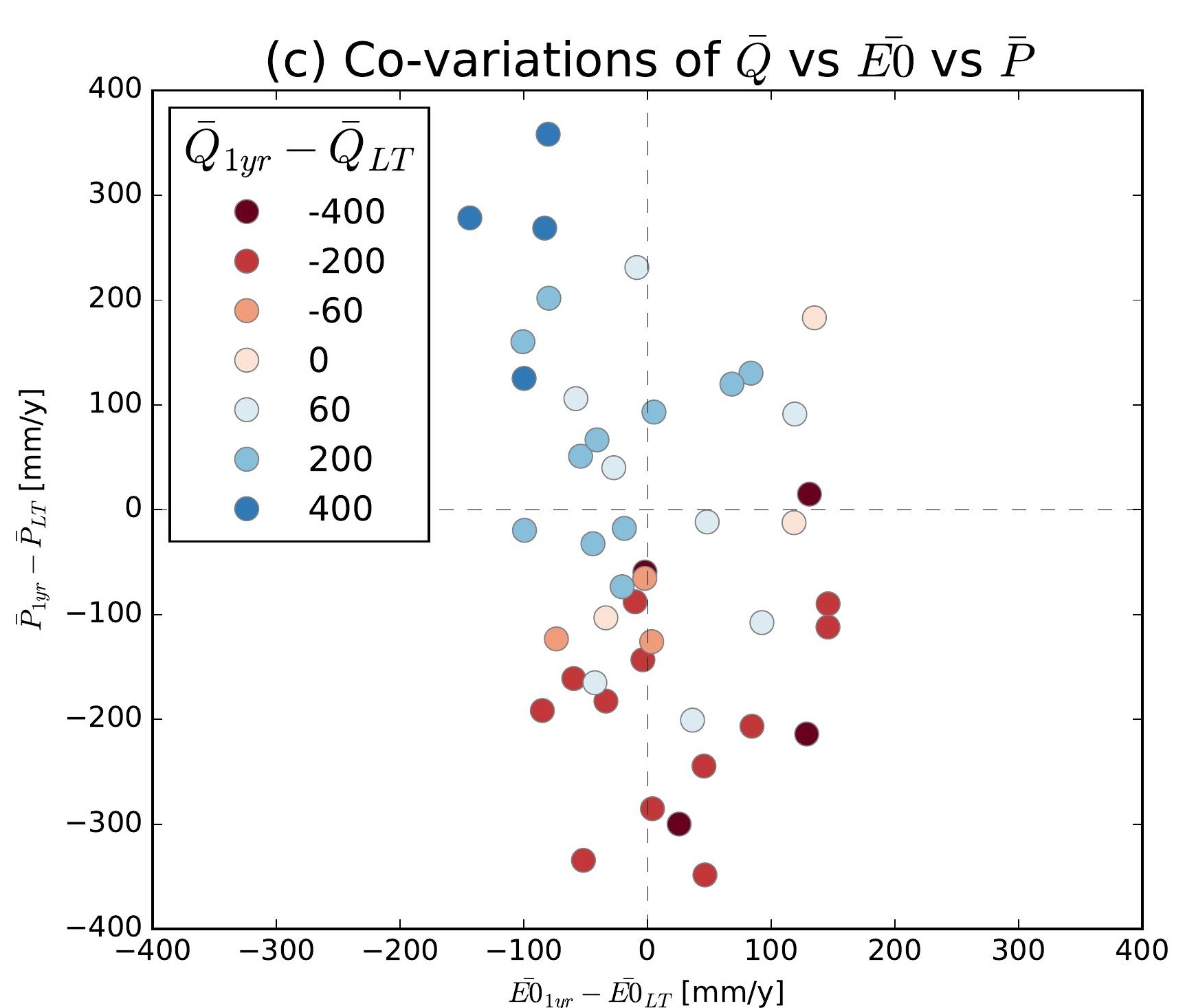
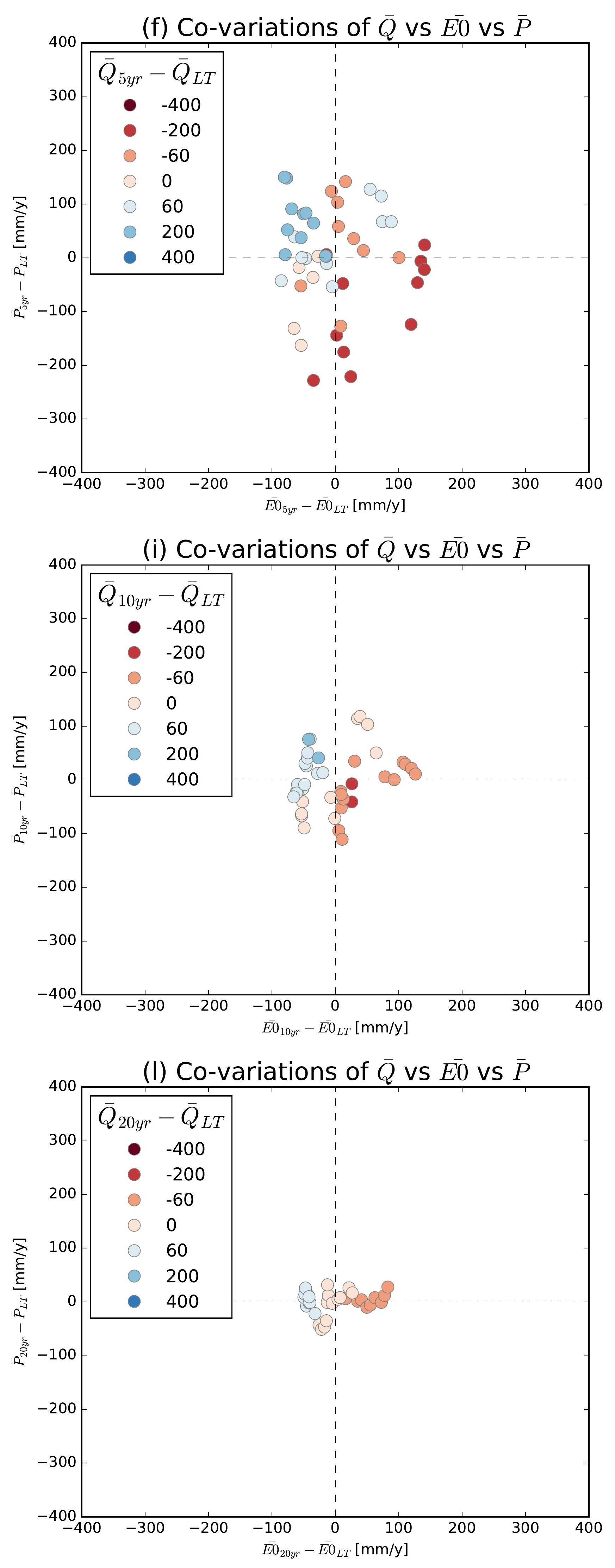

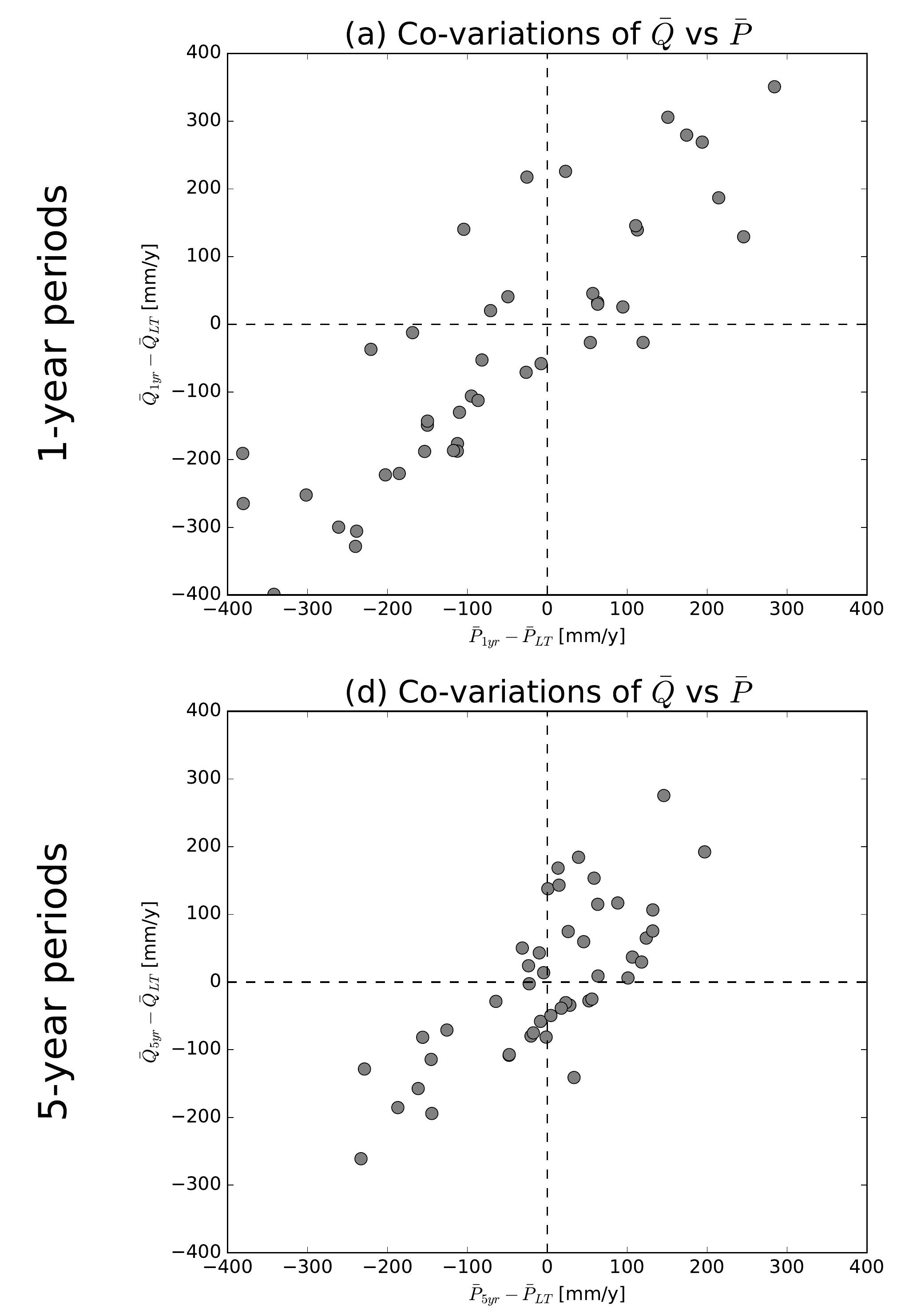

(g) Co-variations of $\bar{Q}$ vs $\bar{P}$
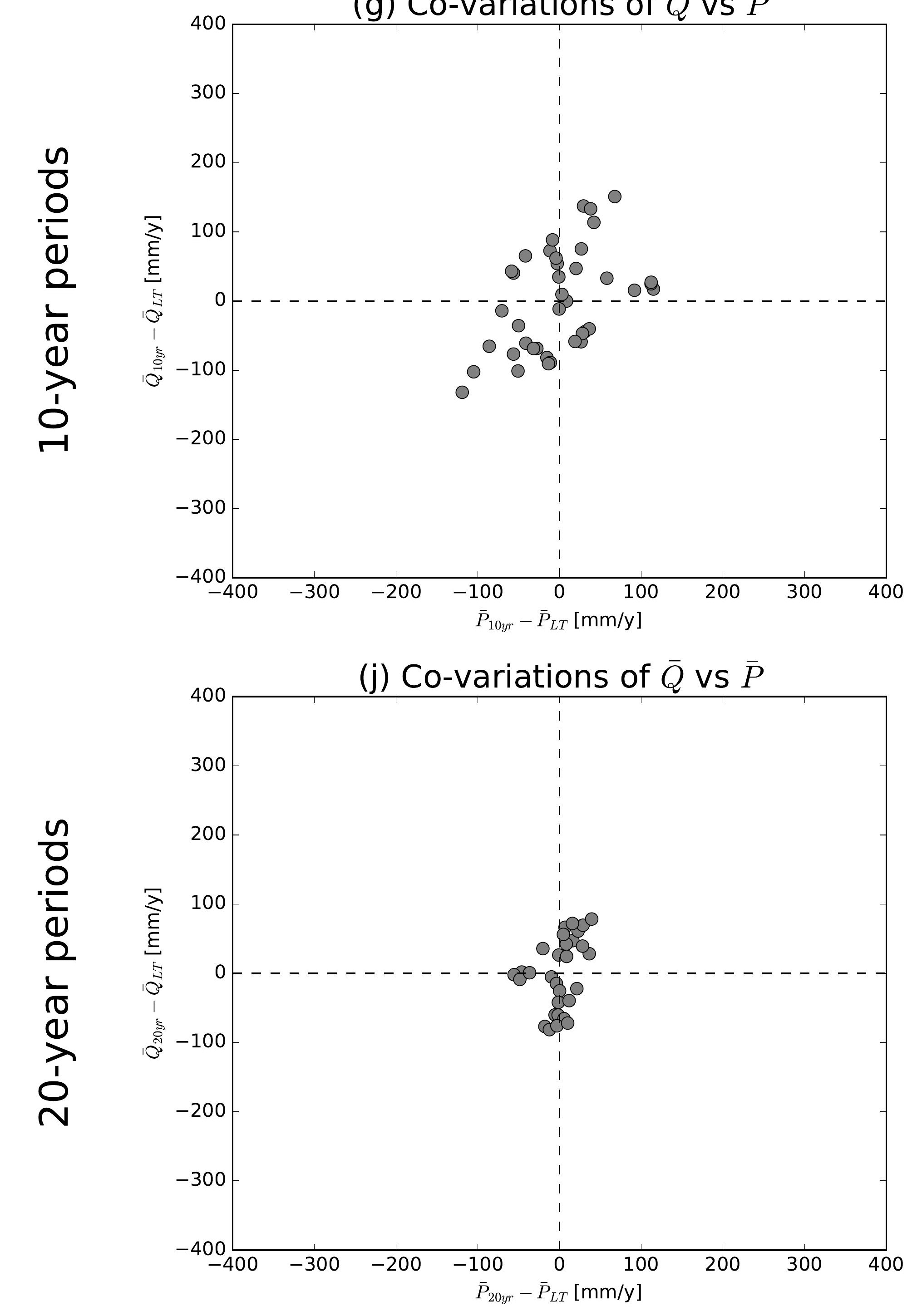

(b) Co-variations of $\bar{Q}$ vs $\overline{E 0}$

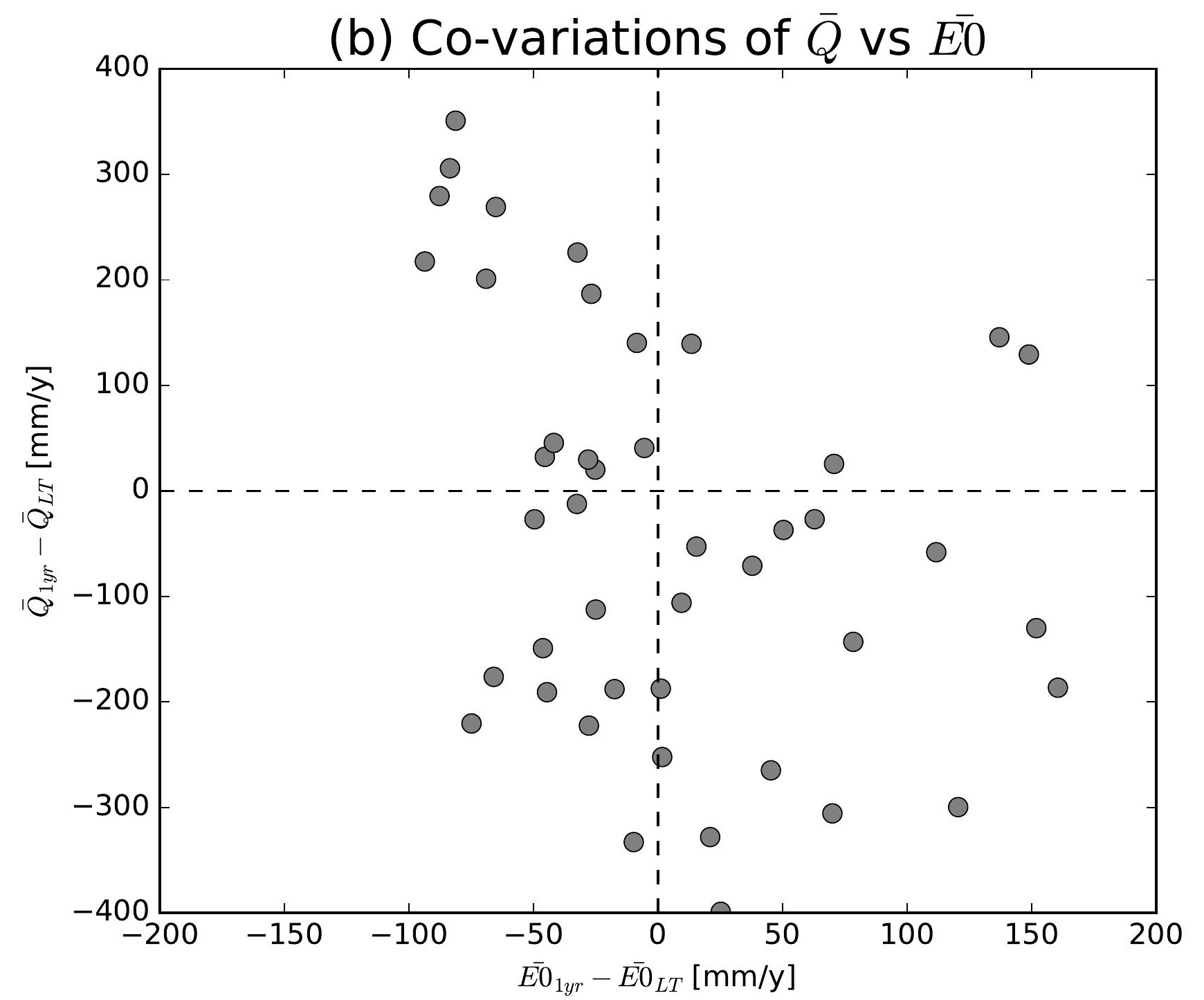

(e) Co-variations of $\bar{Q}$ vs $\overline{E 0}$

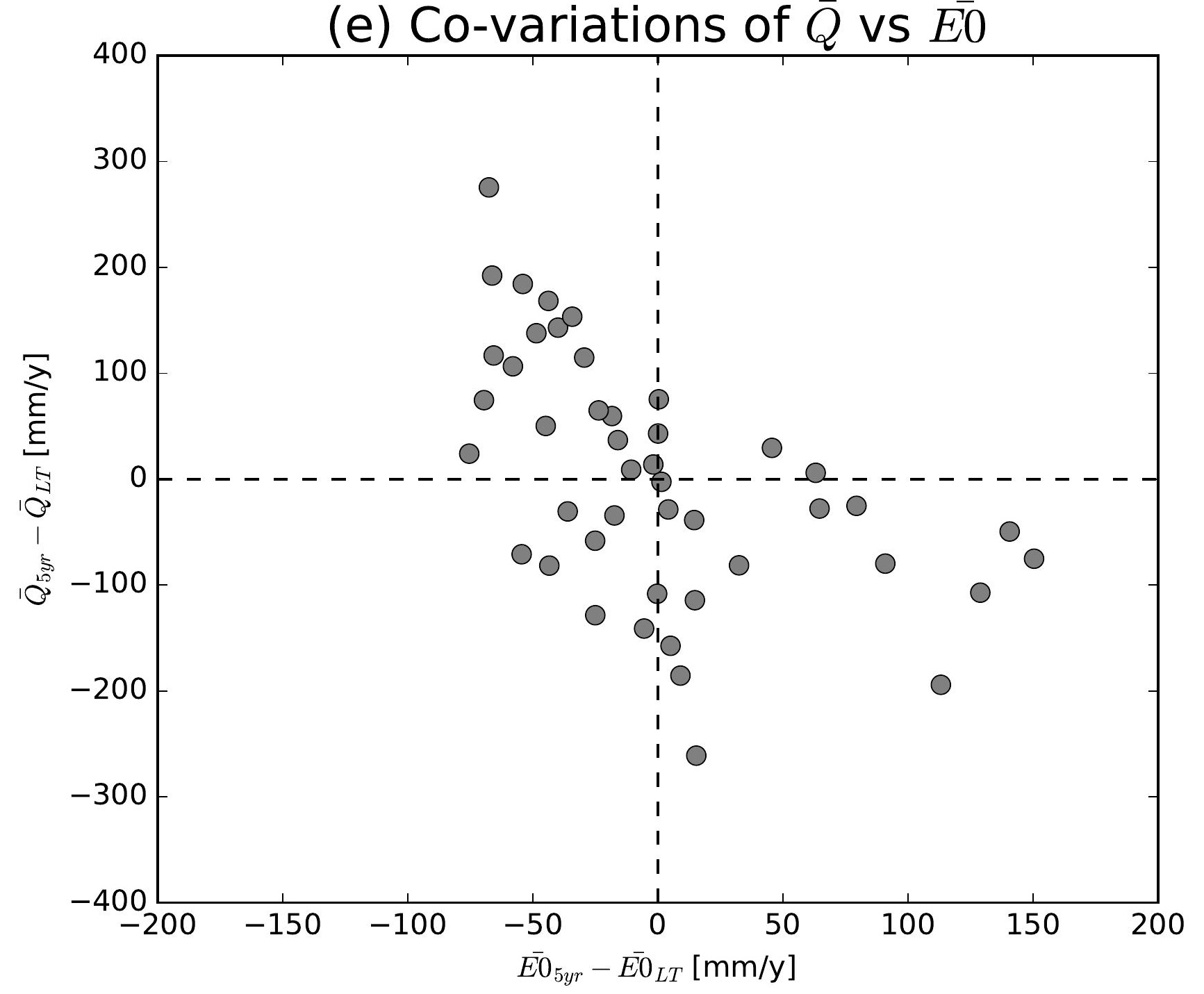

(h) Co-variations of $\bar{Q}$ vs $\overline{E 0}$

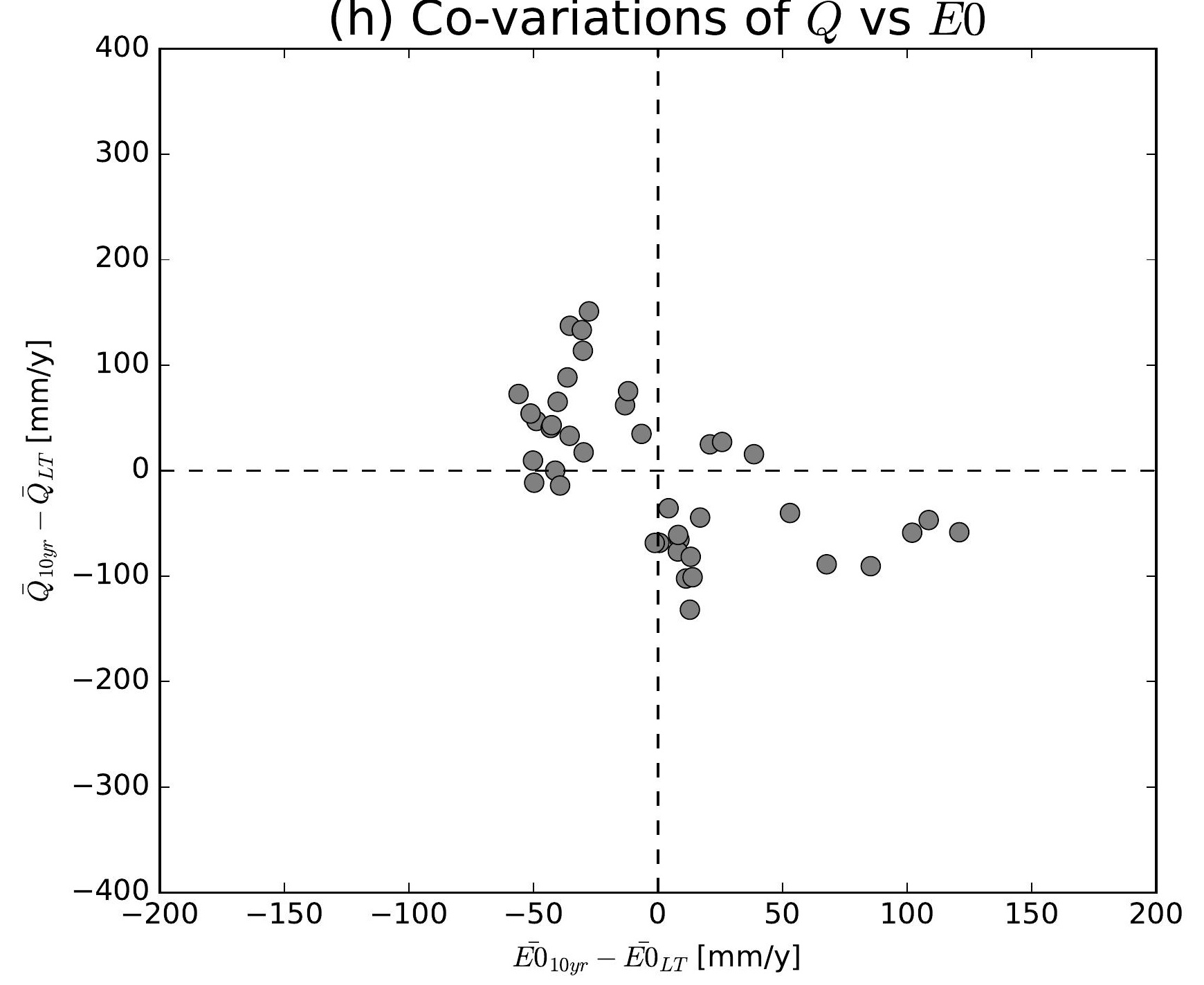

(k) Co-variations of $\bar{Q}$ vs $\overline{E 0}$

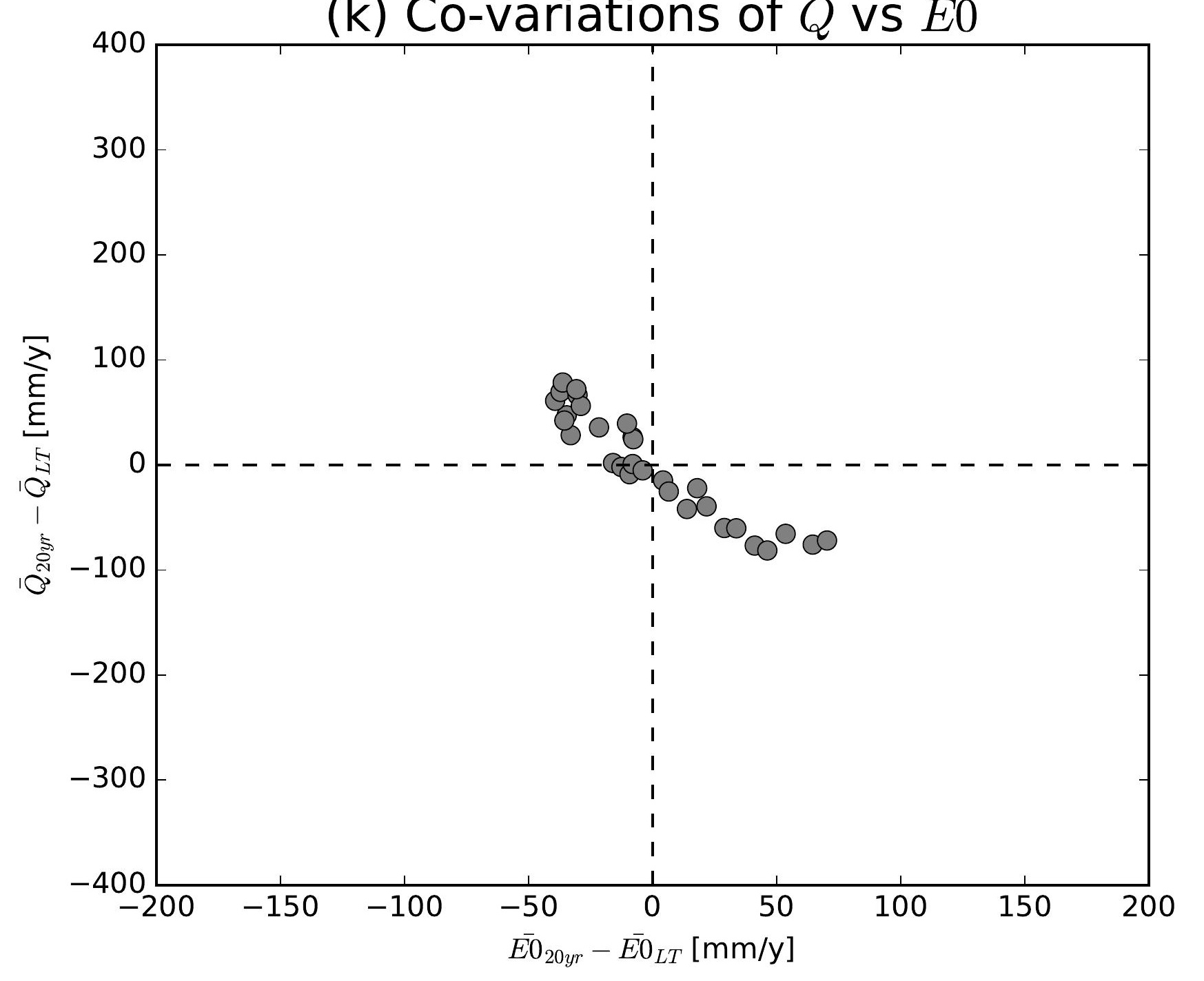

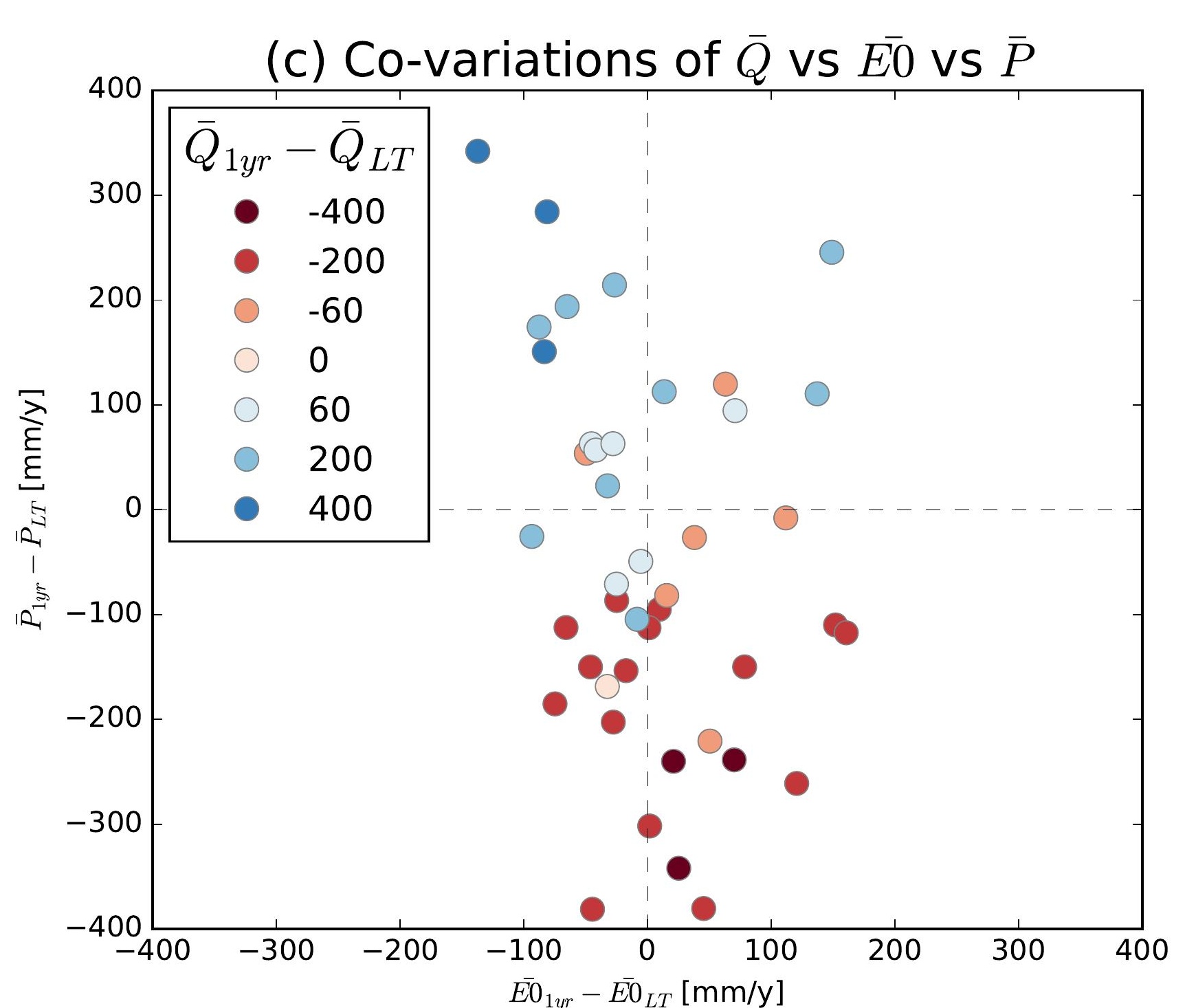
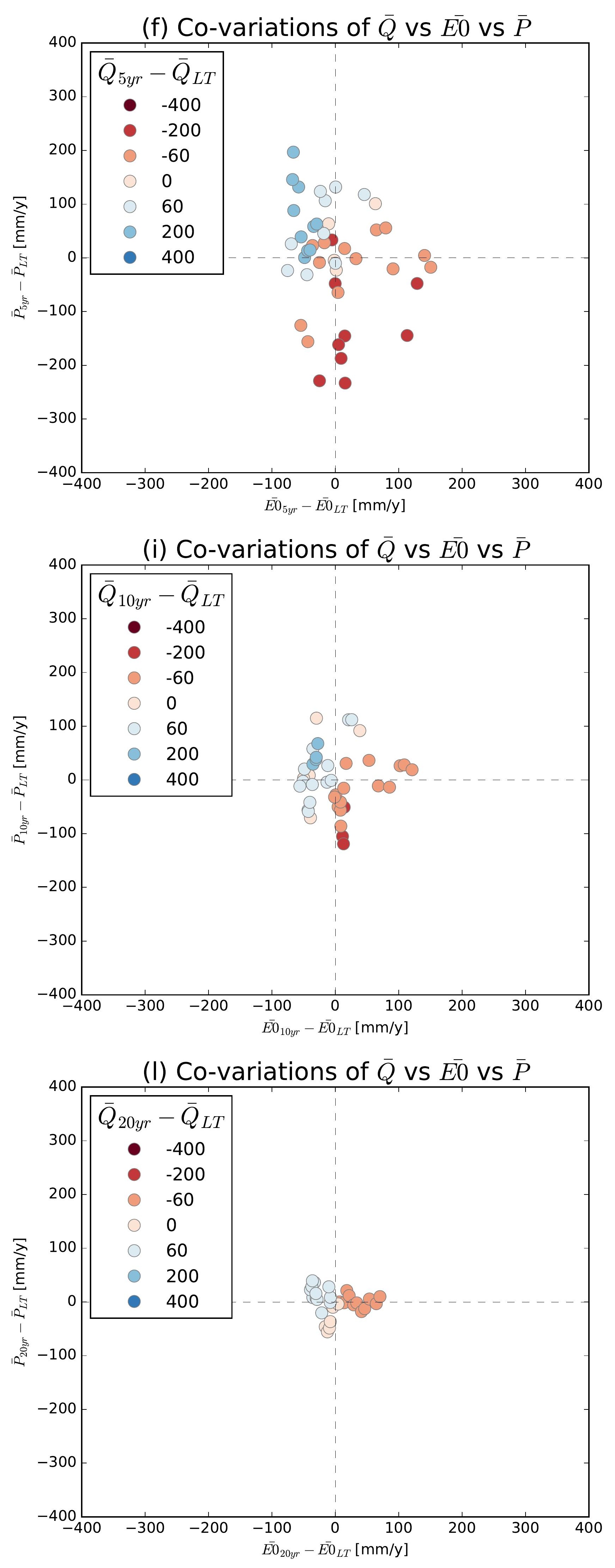

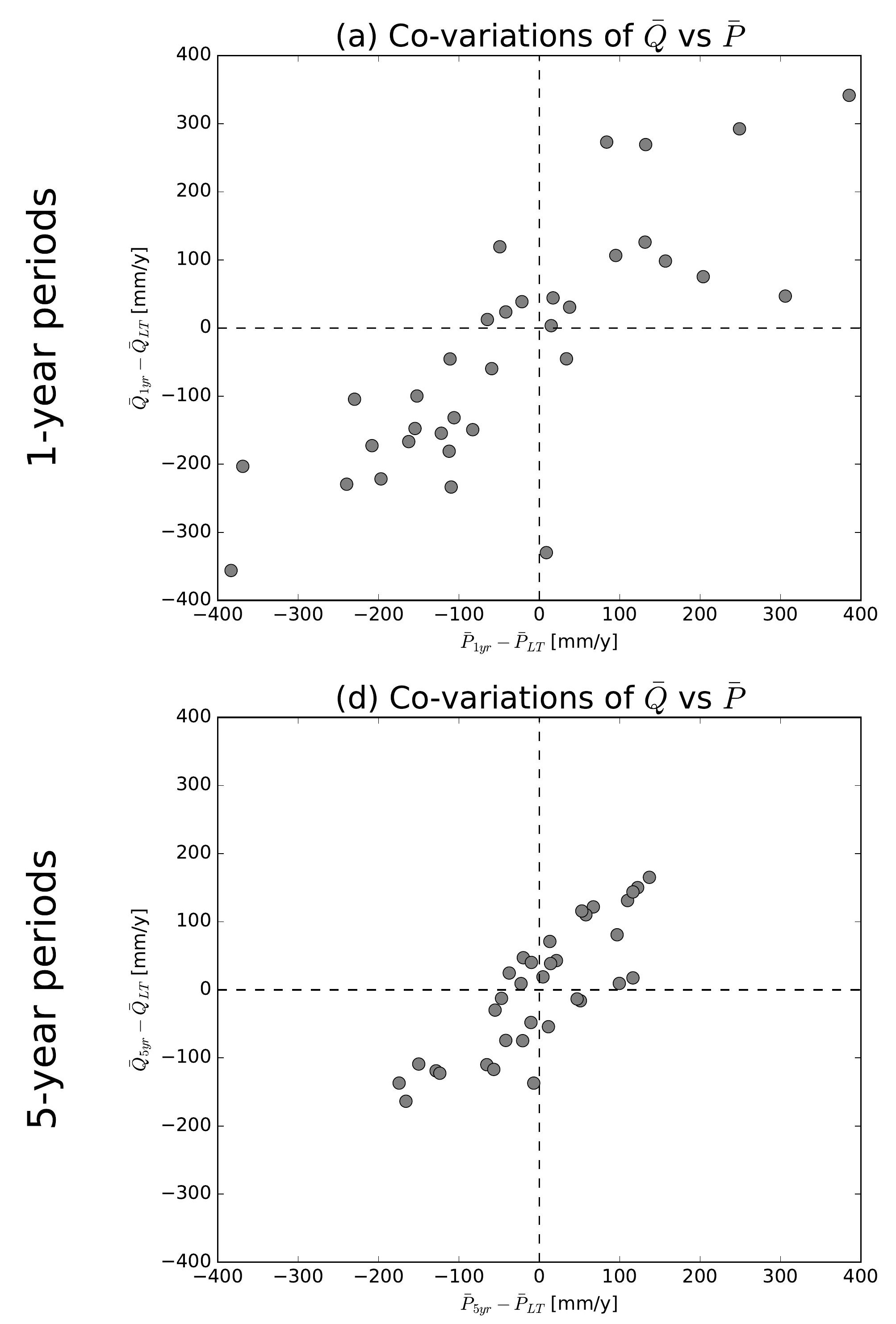

(g) Co-variations of $\bar{Q}$ vs $\bar{P}$

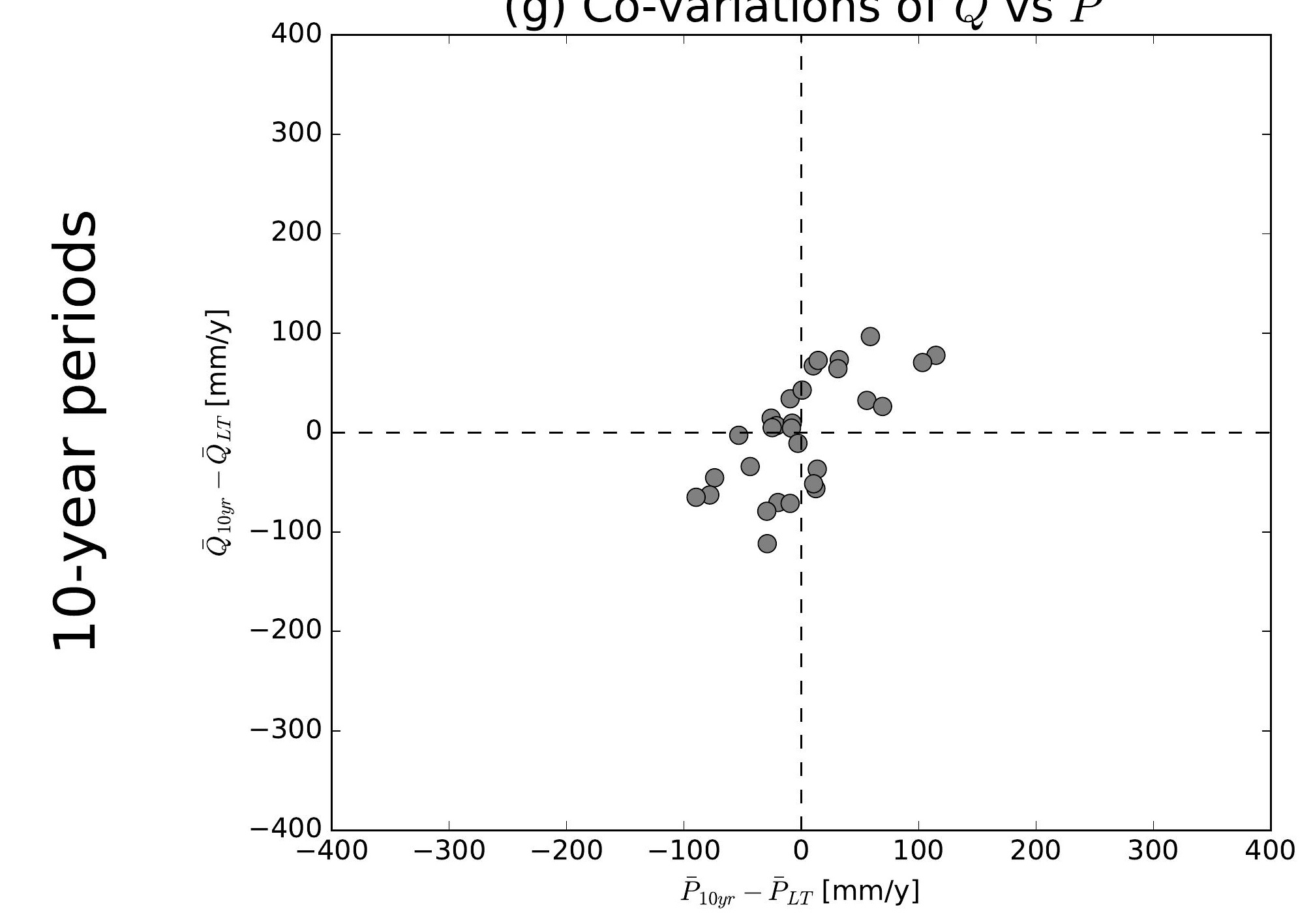

(j) Co-variations of $\bar{Q}$ vs $\bar{P}$

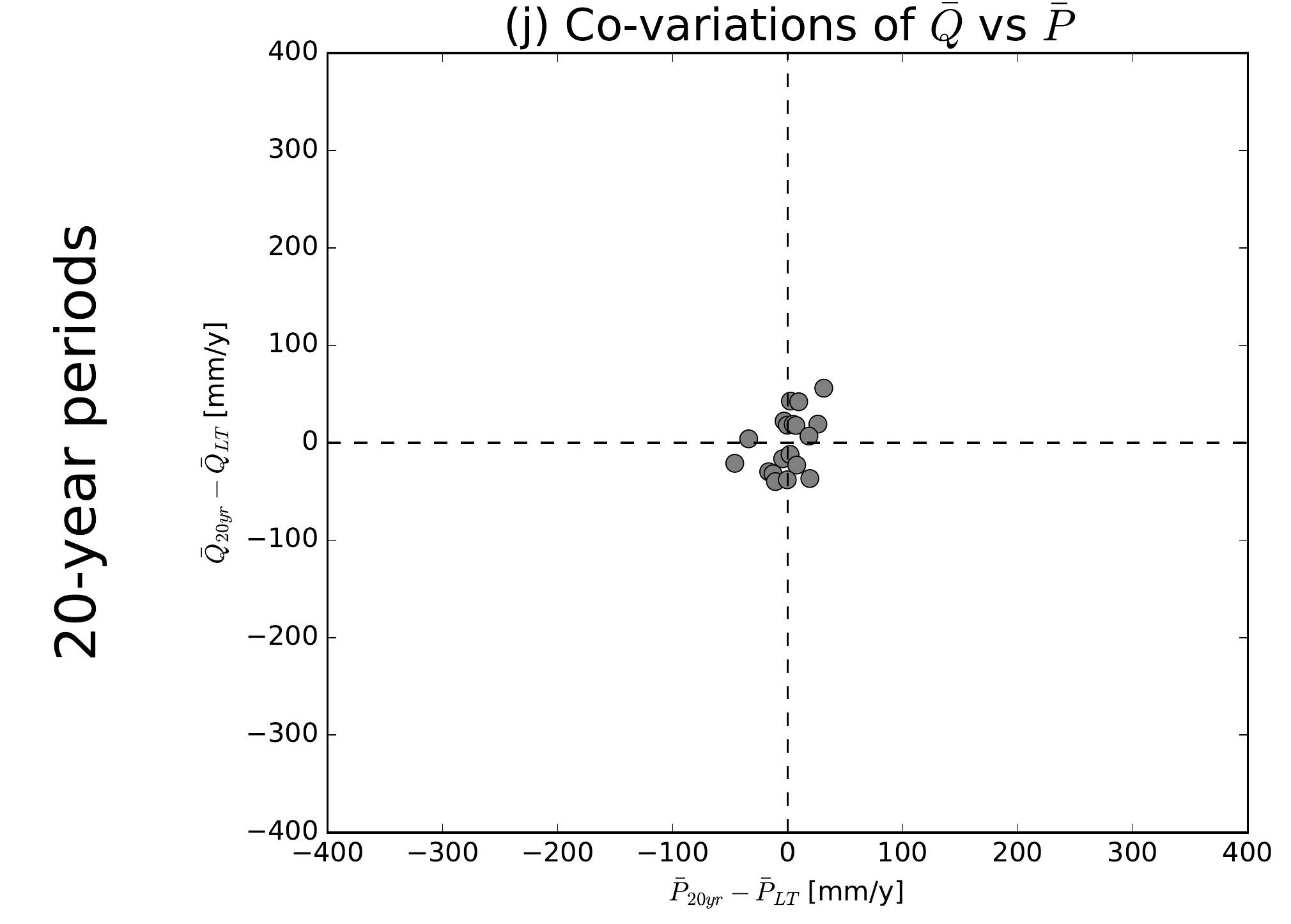

(b) Co-variations of $\bar{Q}$ vs $\overline{E 0}$

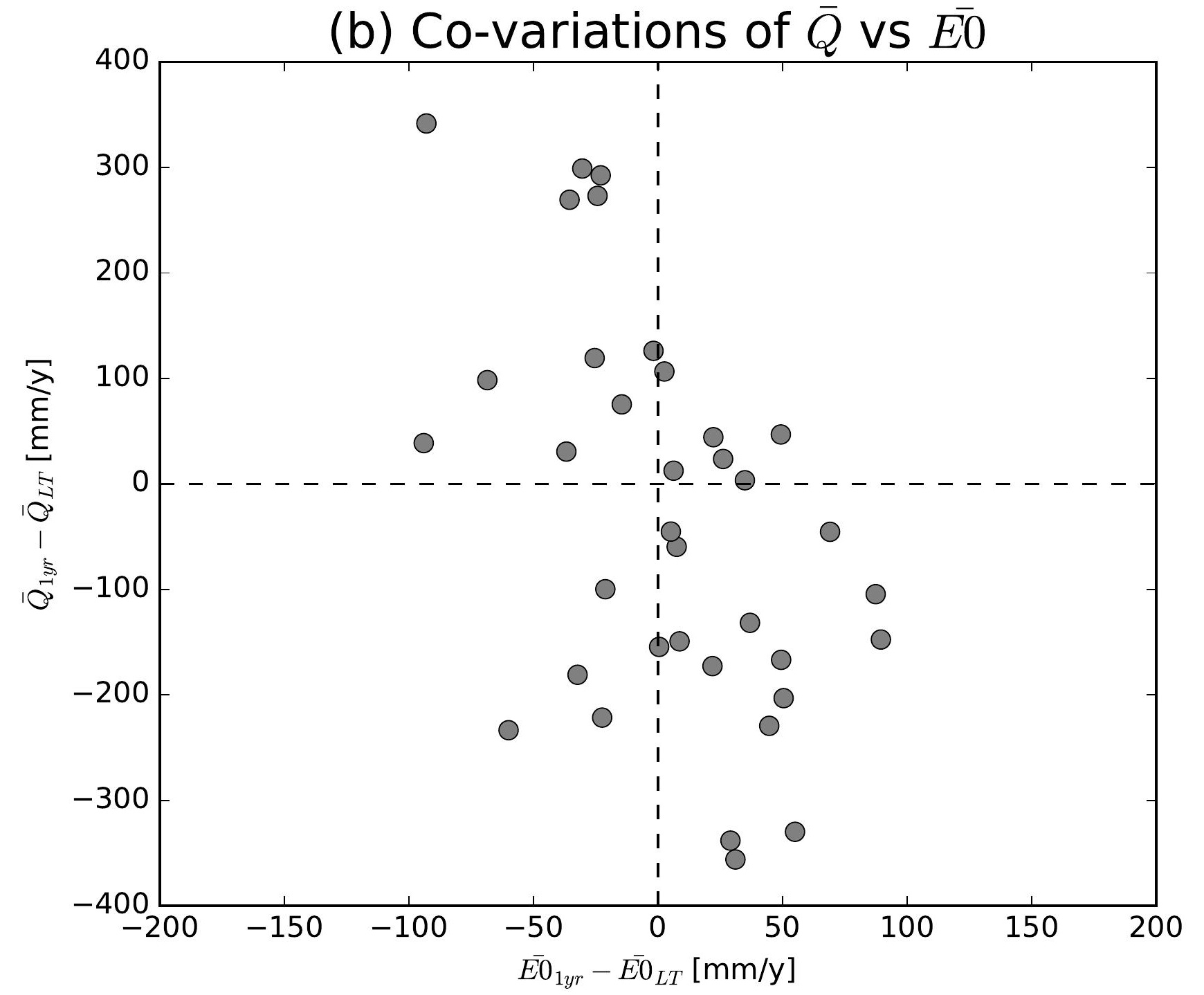

(e) Co-variations of $\bar{Q}$ vs $\overline{E 0}$

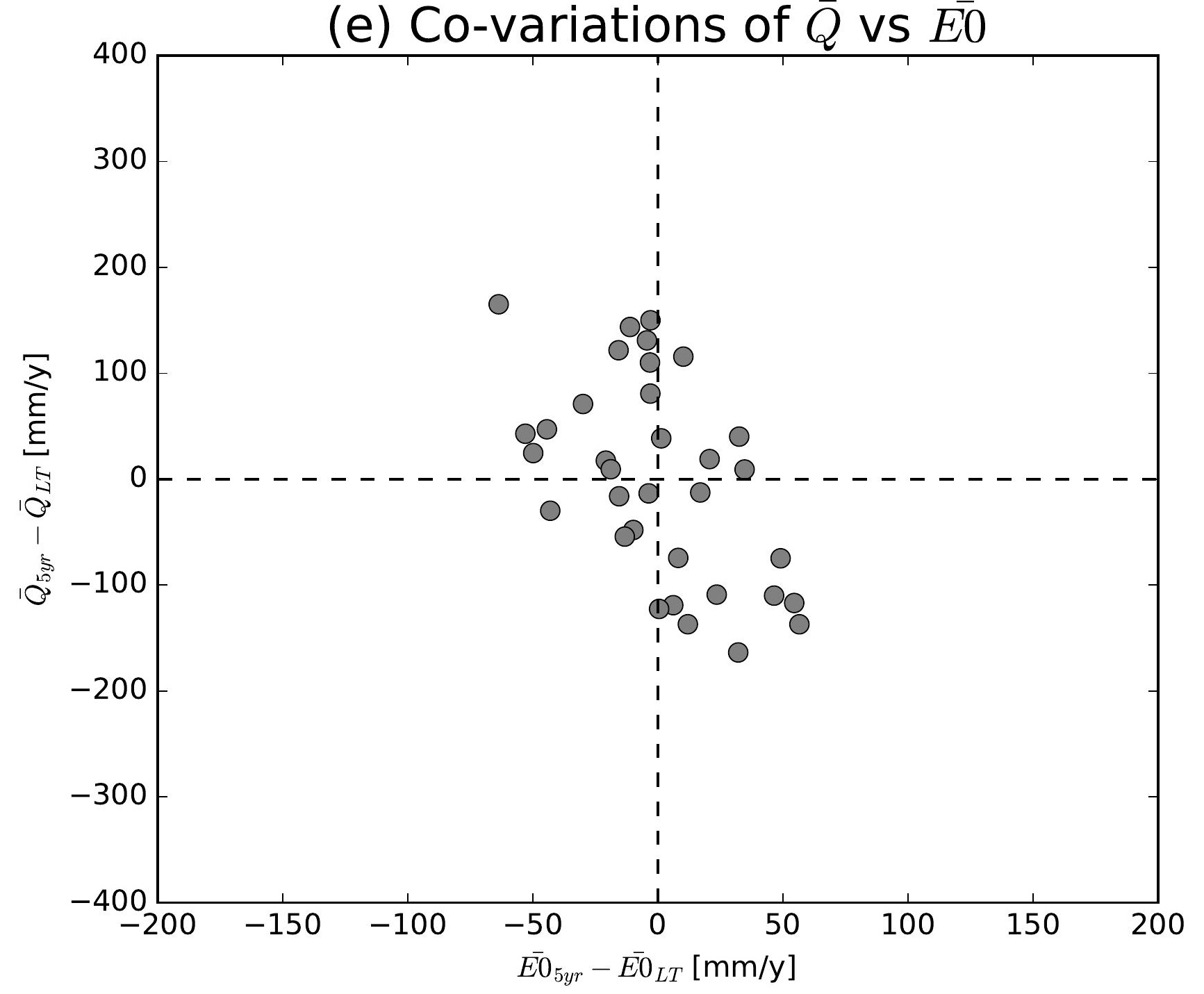

(h) Co-variations of $\bar{Q}$ vs $\overline{E 0}$

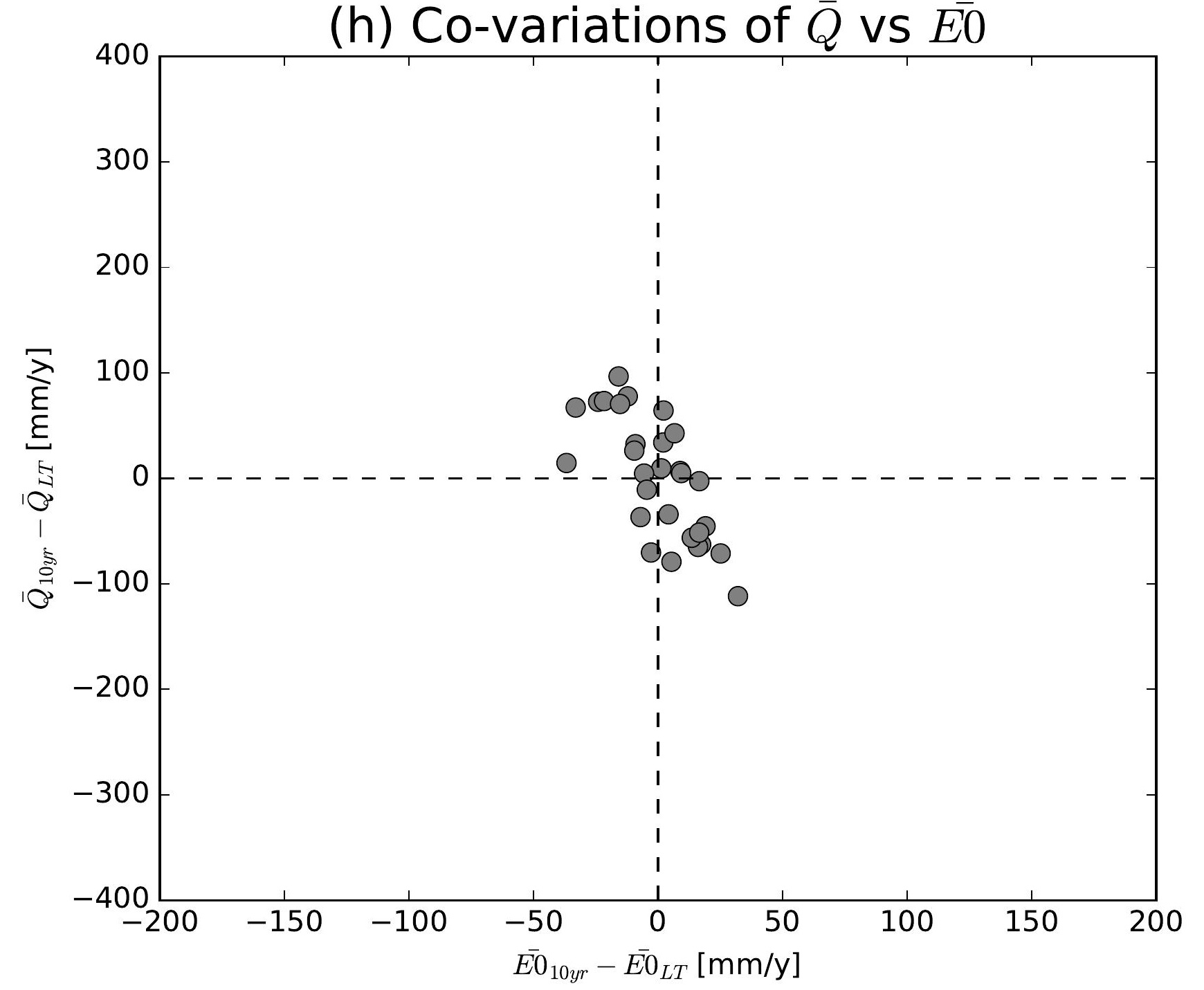

(k) Co-variations of $\bar{Q}$ vs $\overline{E 0}$

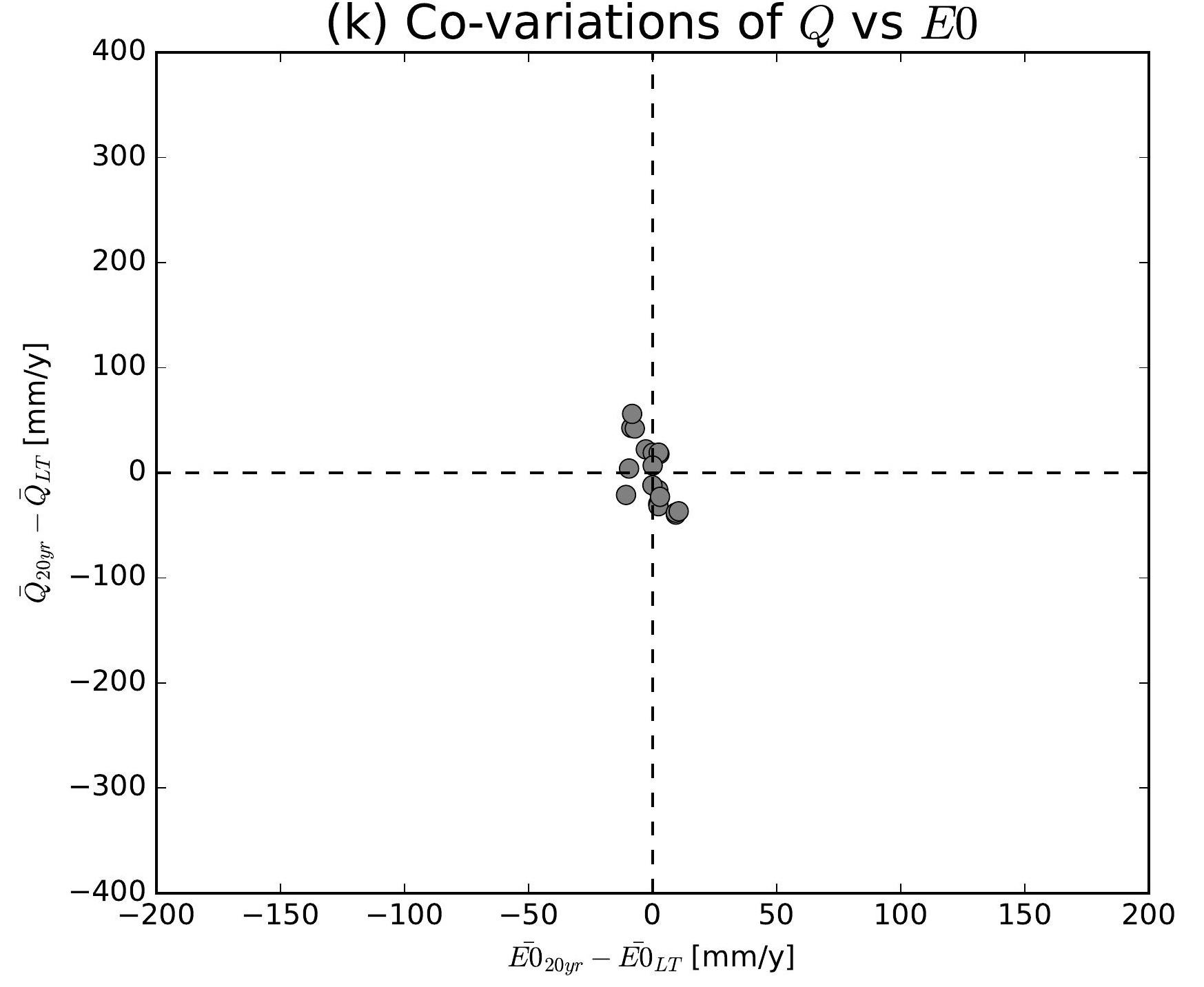

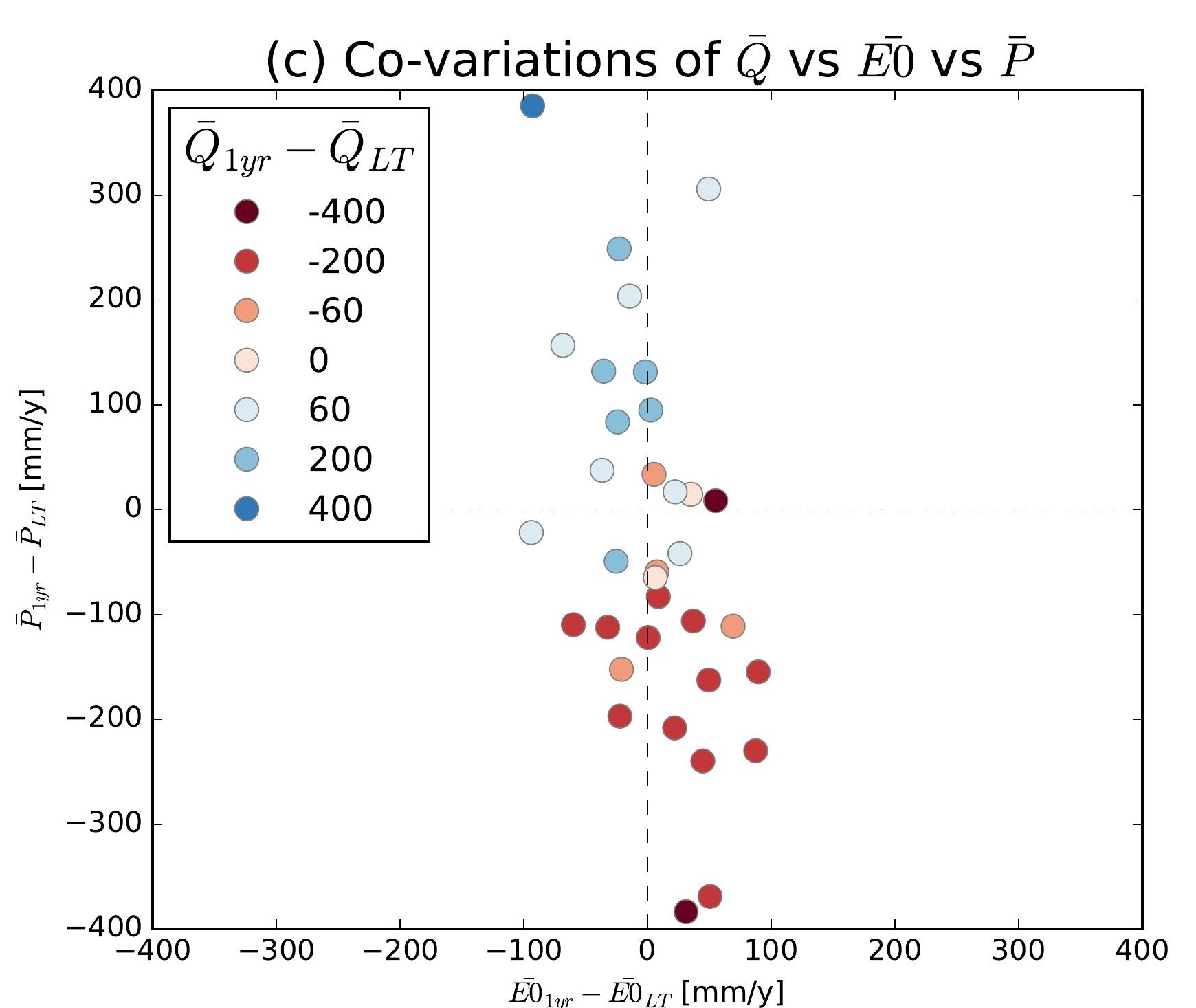
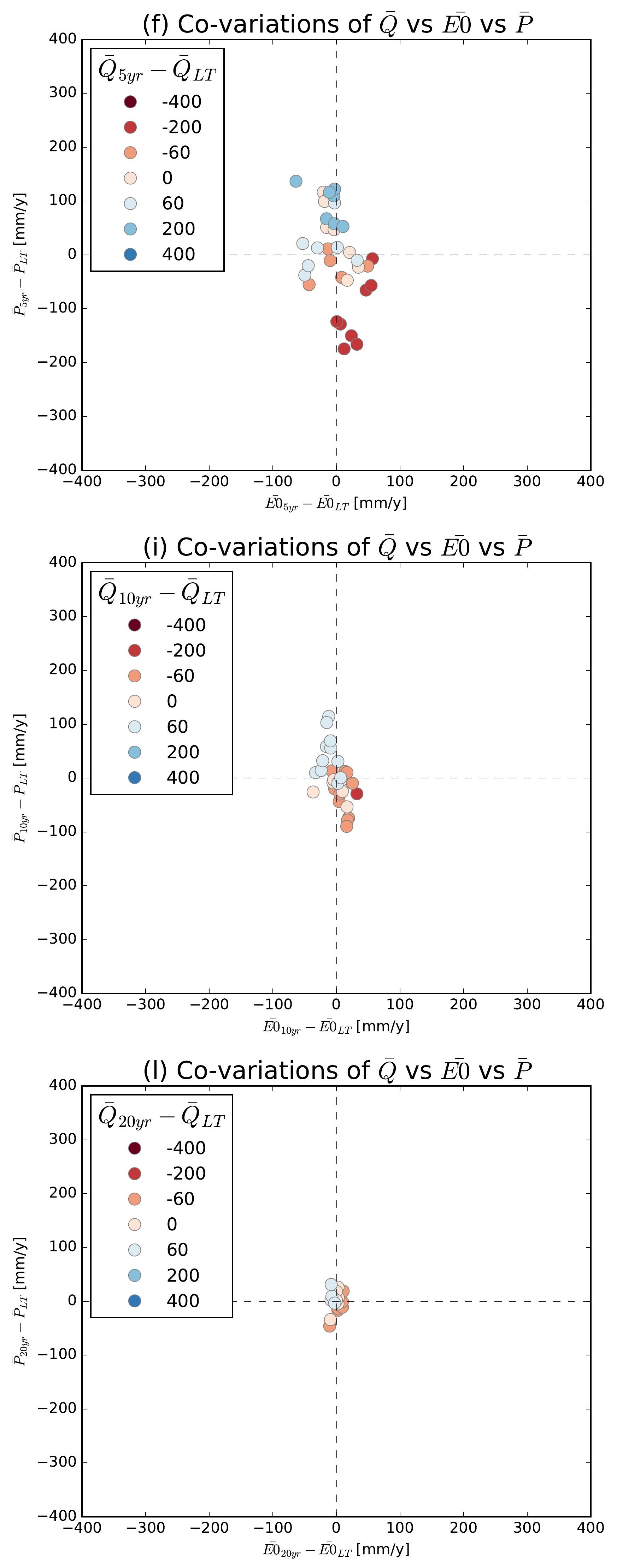

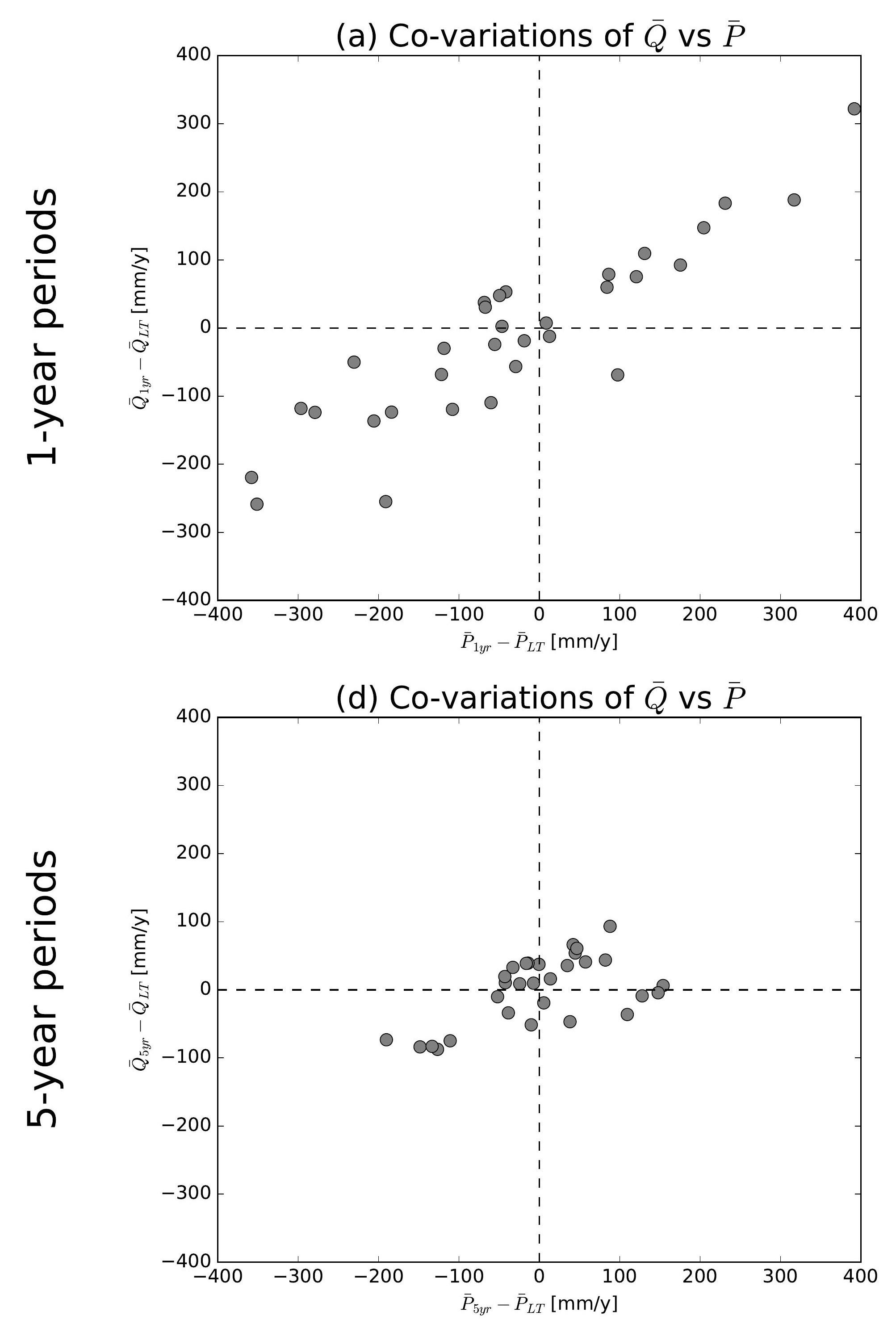

(g) Co-variations of $\bar{Q}$ vs $\bar{P}$

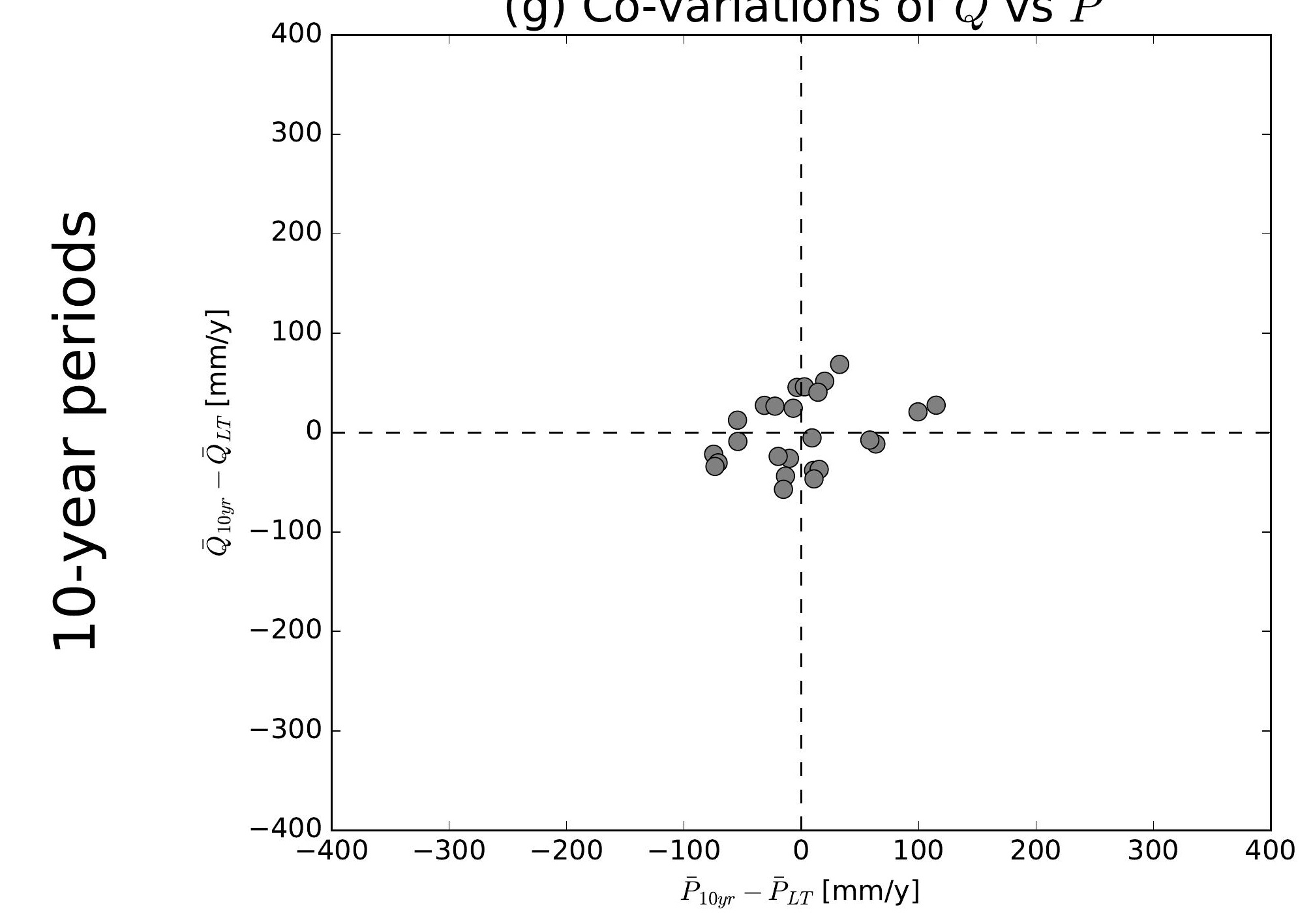

(j) Co-variations of $\bar{Q}$ vs $\bar{P}$

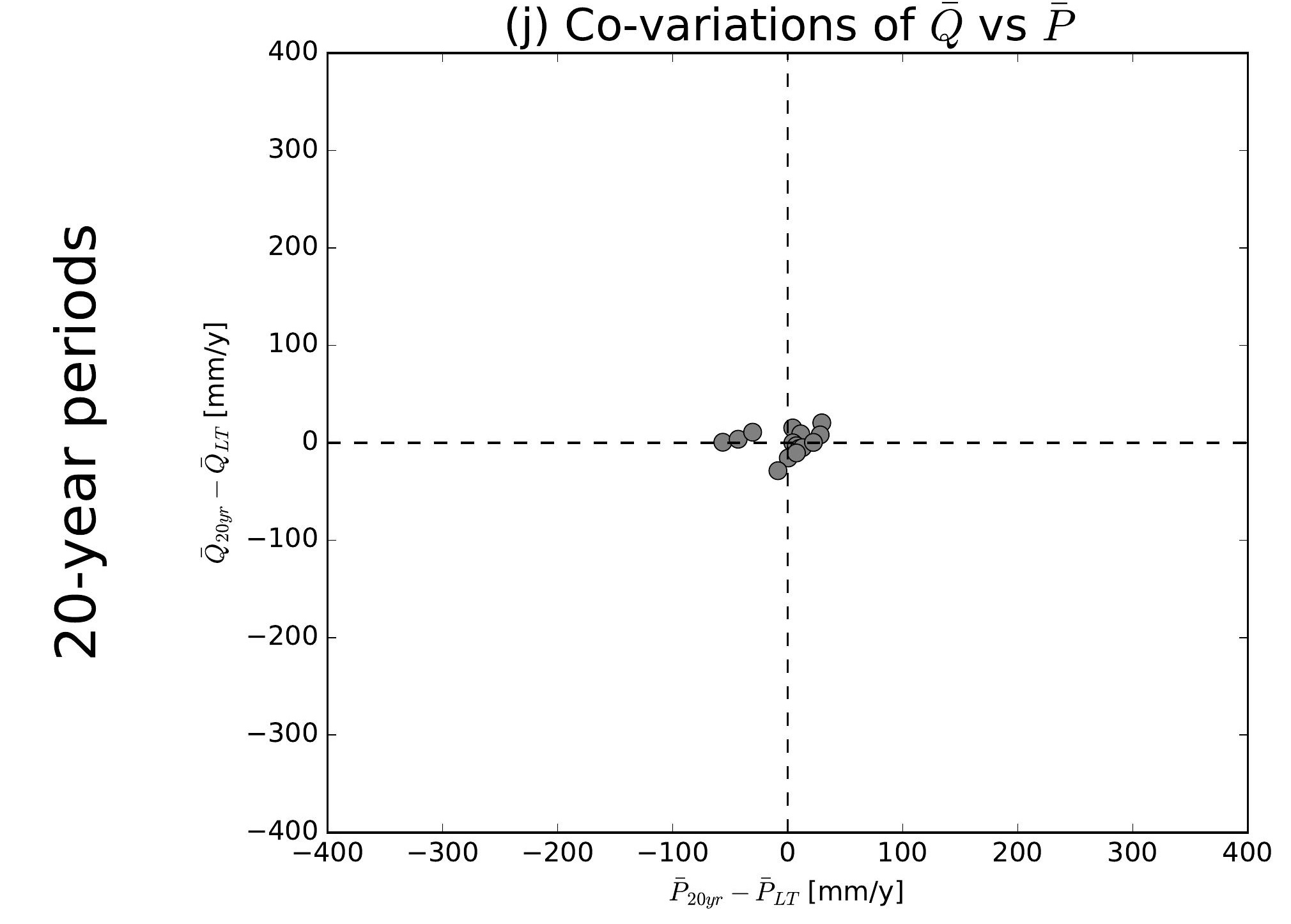

(b) Co-variations of $\bar{Q}$ vs $\overline{E 0}$

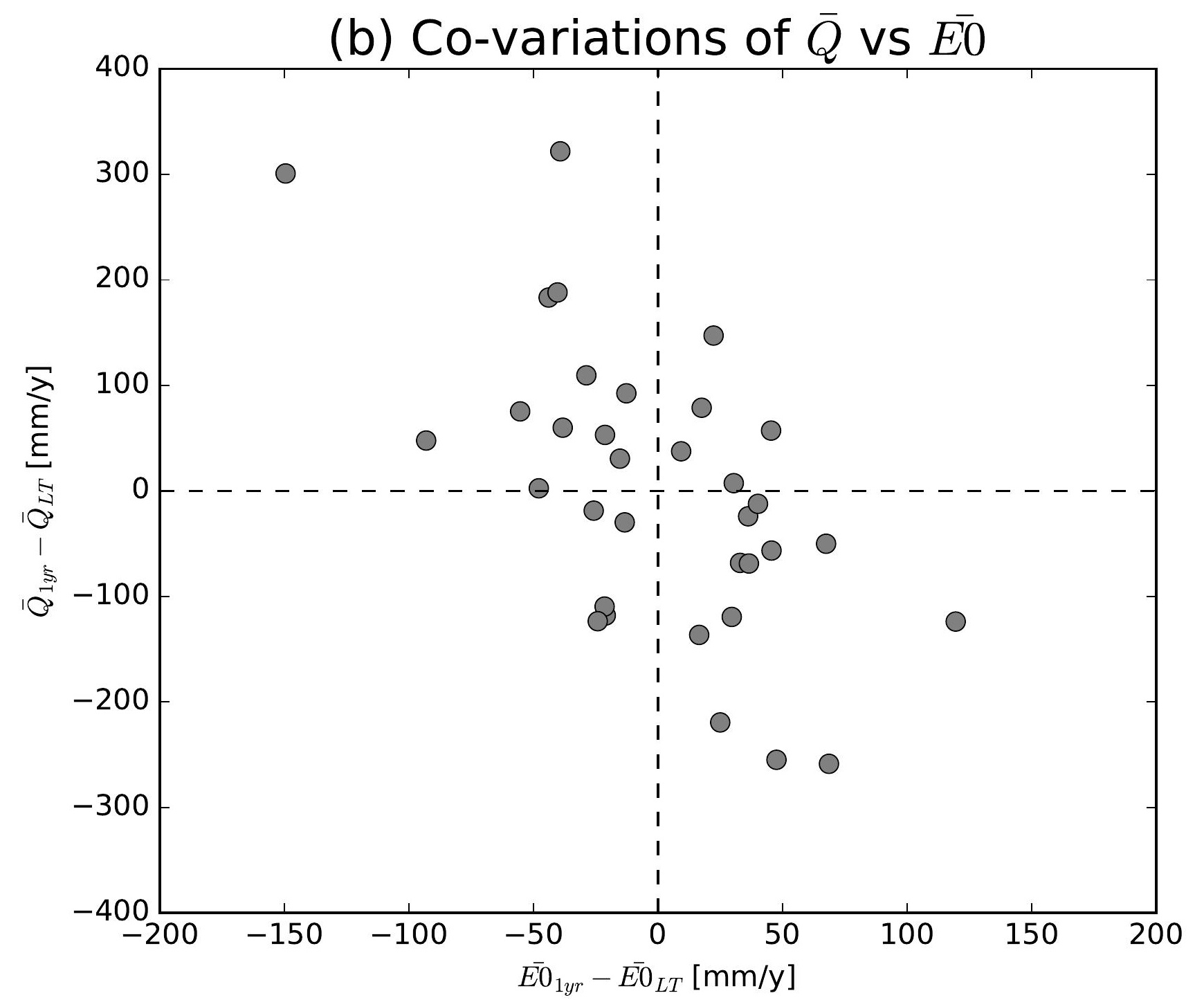

(e) Co-variations of $\bar{Q}$ vs $\overline{E 0}$

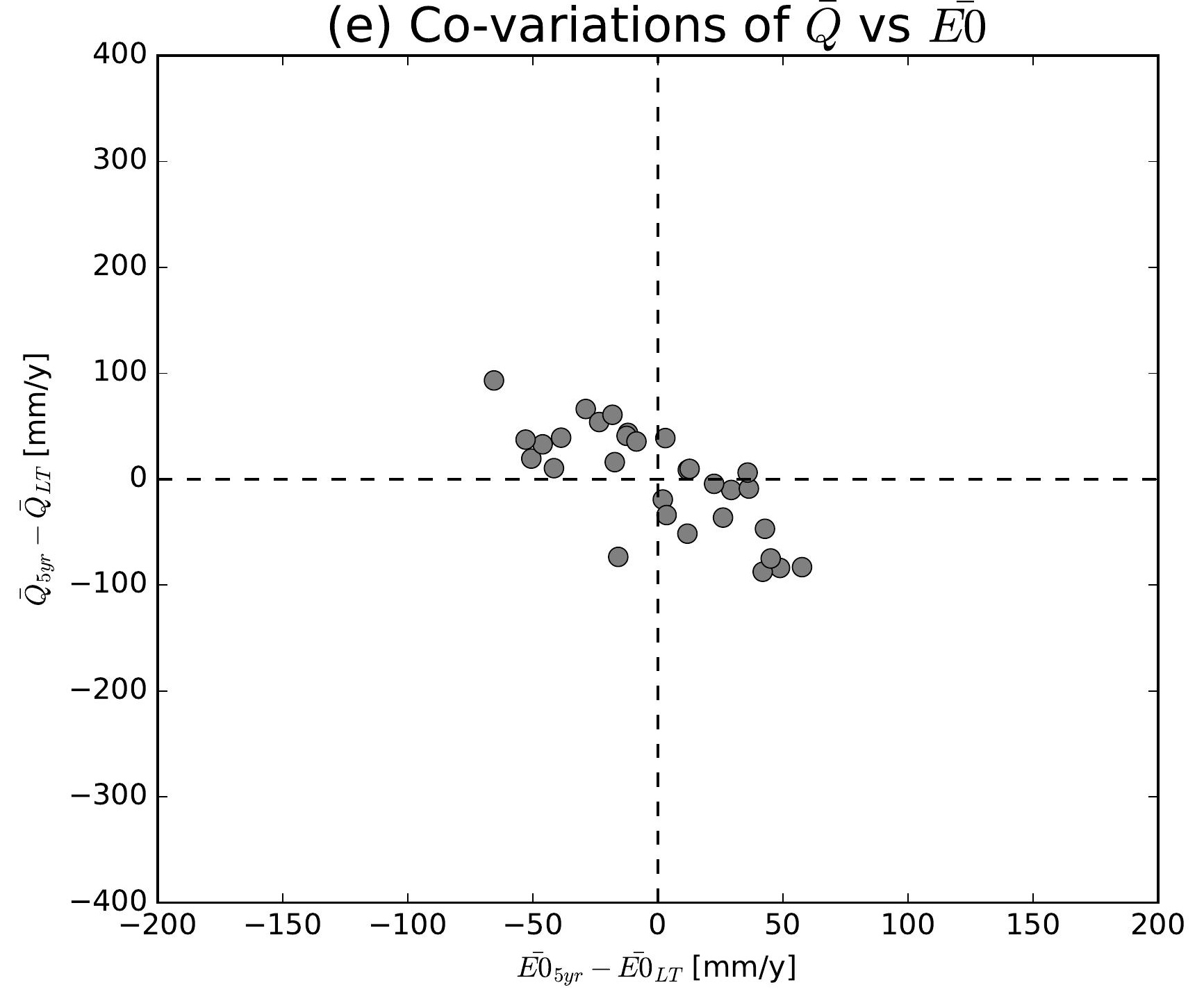

(h) Co-variations of $\bar{Q}$ vs $\overline{E 0}$

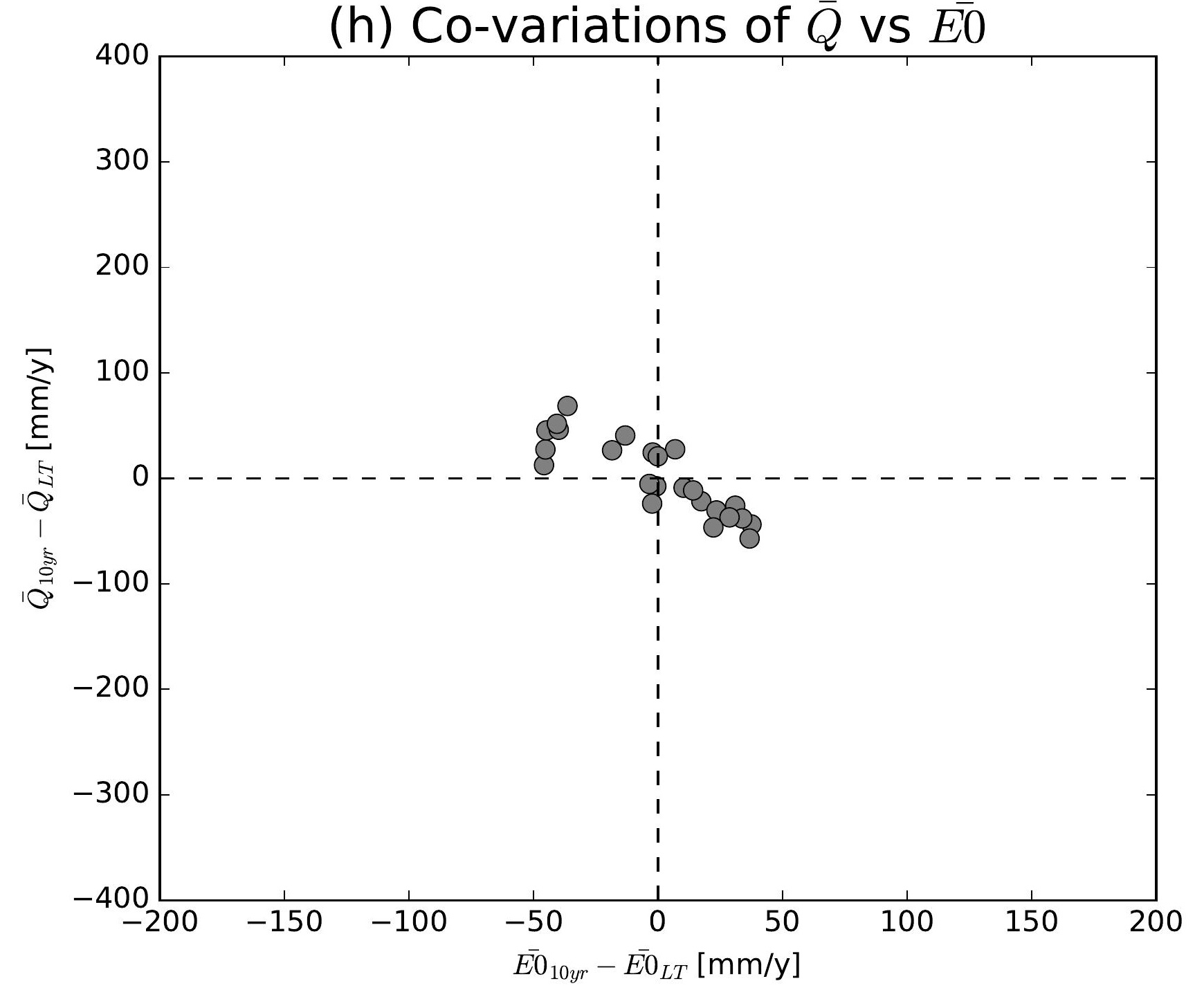

(k) Co-variations of $\bar{Q}$ vs $\overline{E 0}$

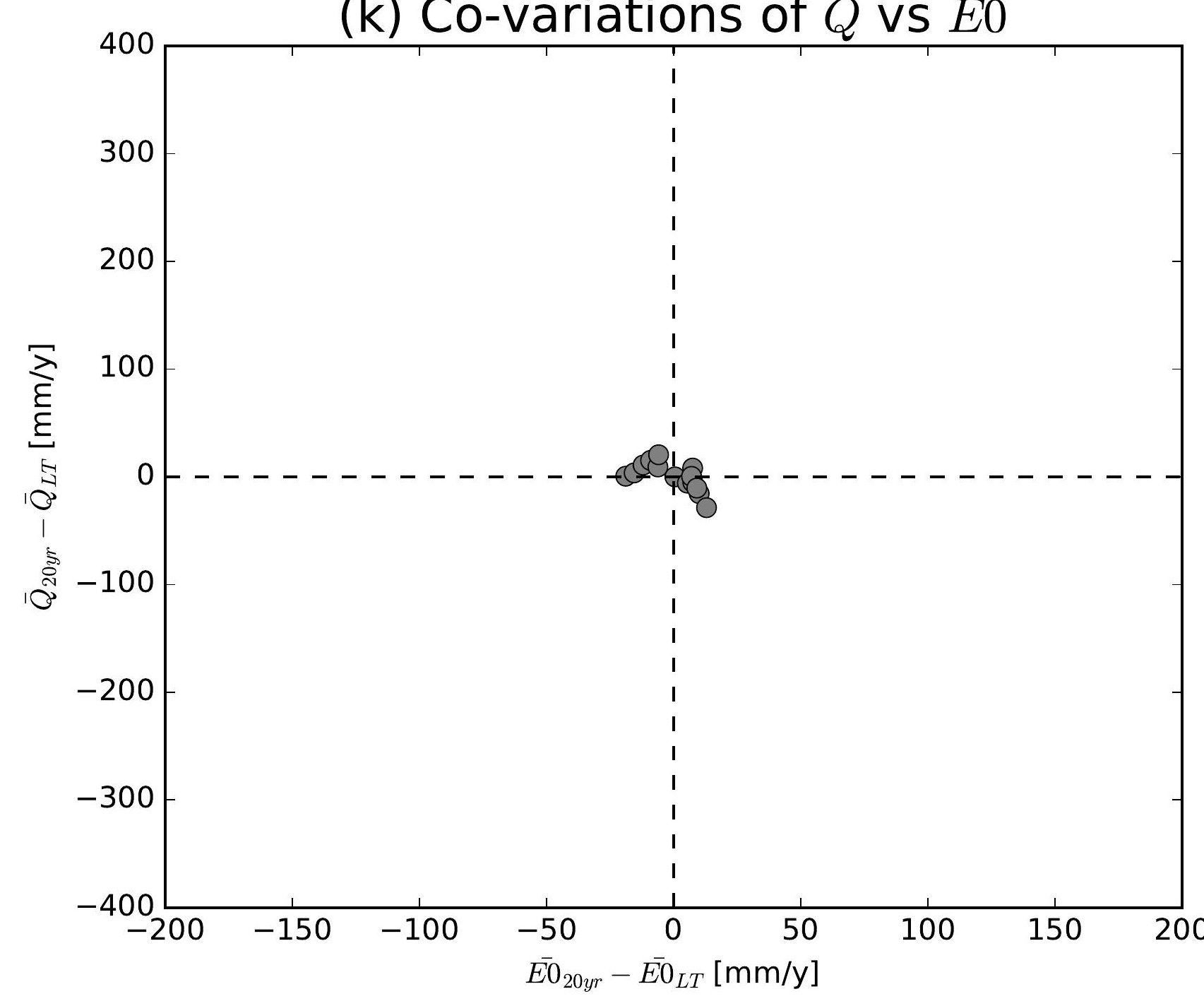

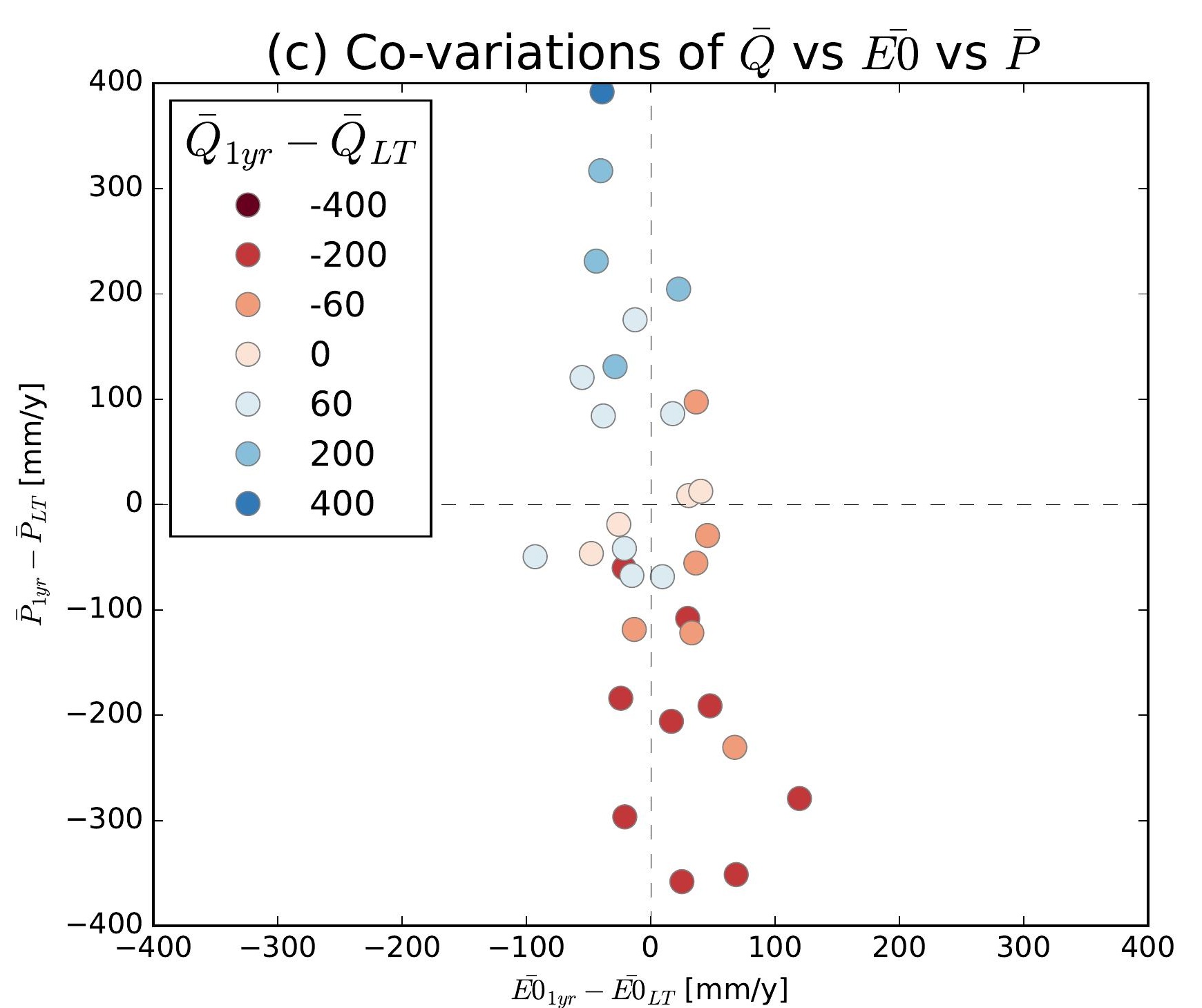
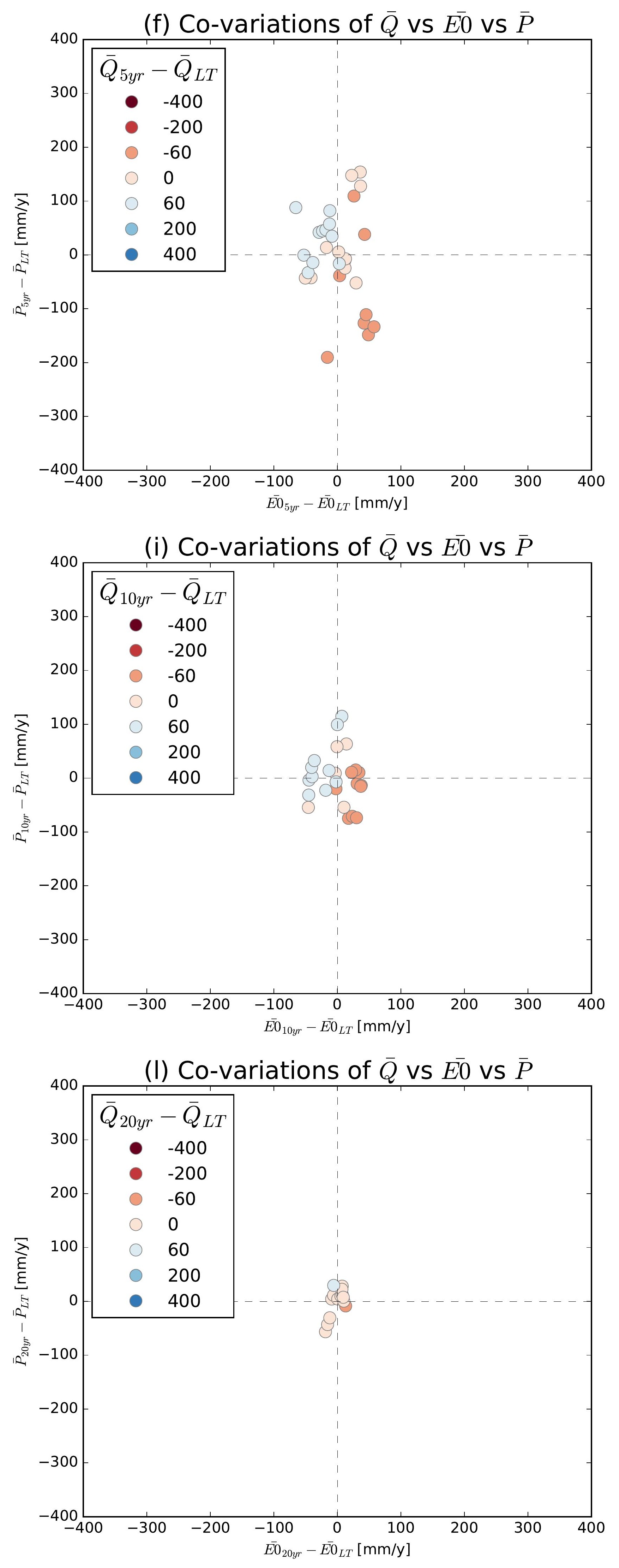

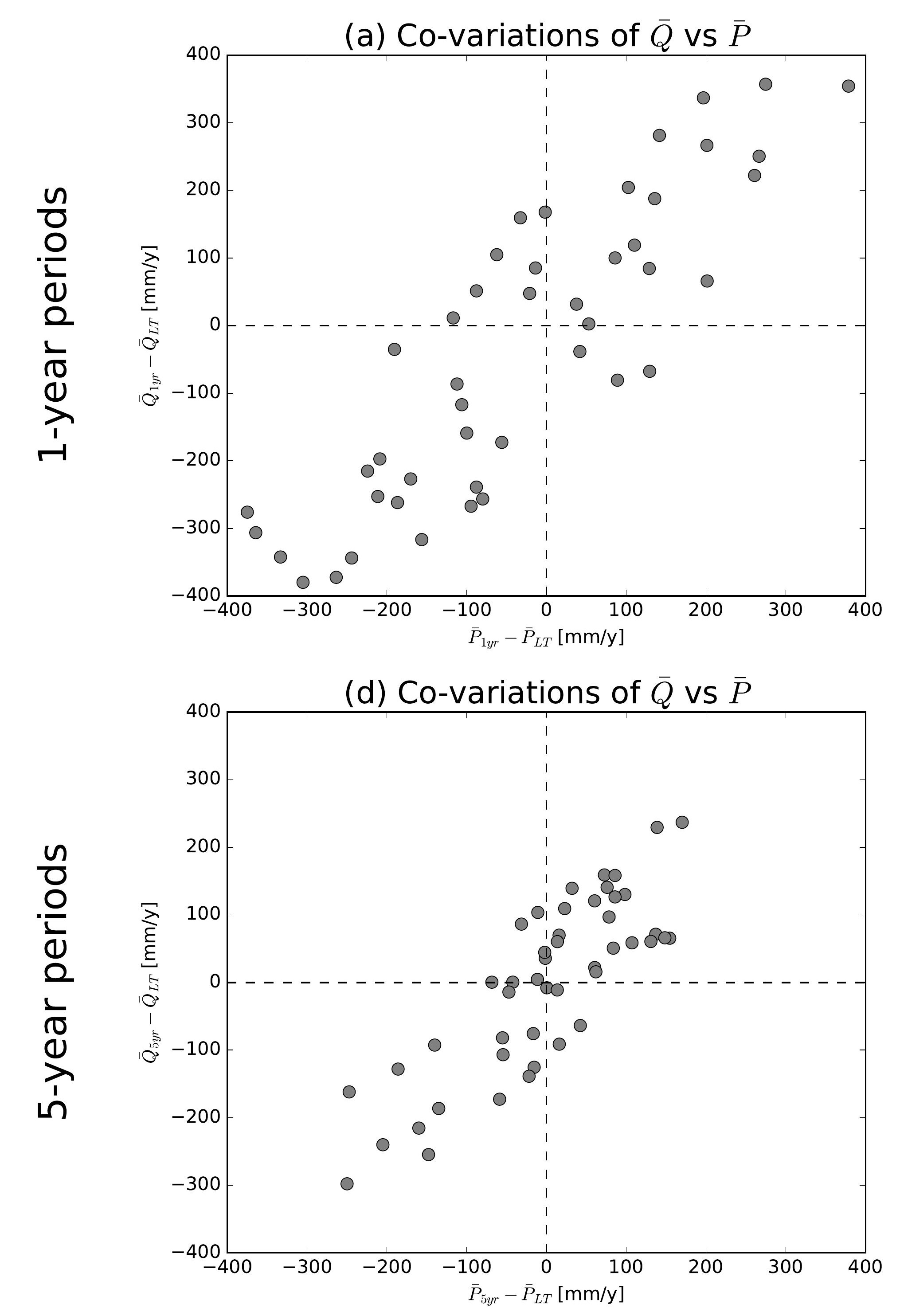

(g) Co-variations of $\bar{Q}$ vs $\bar{P}$

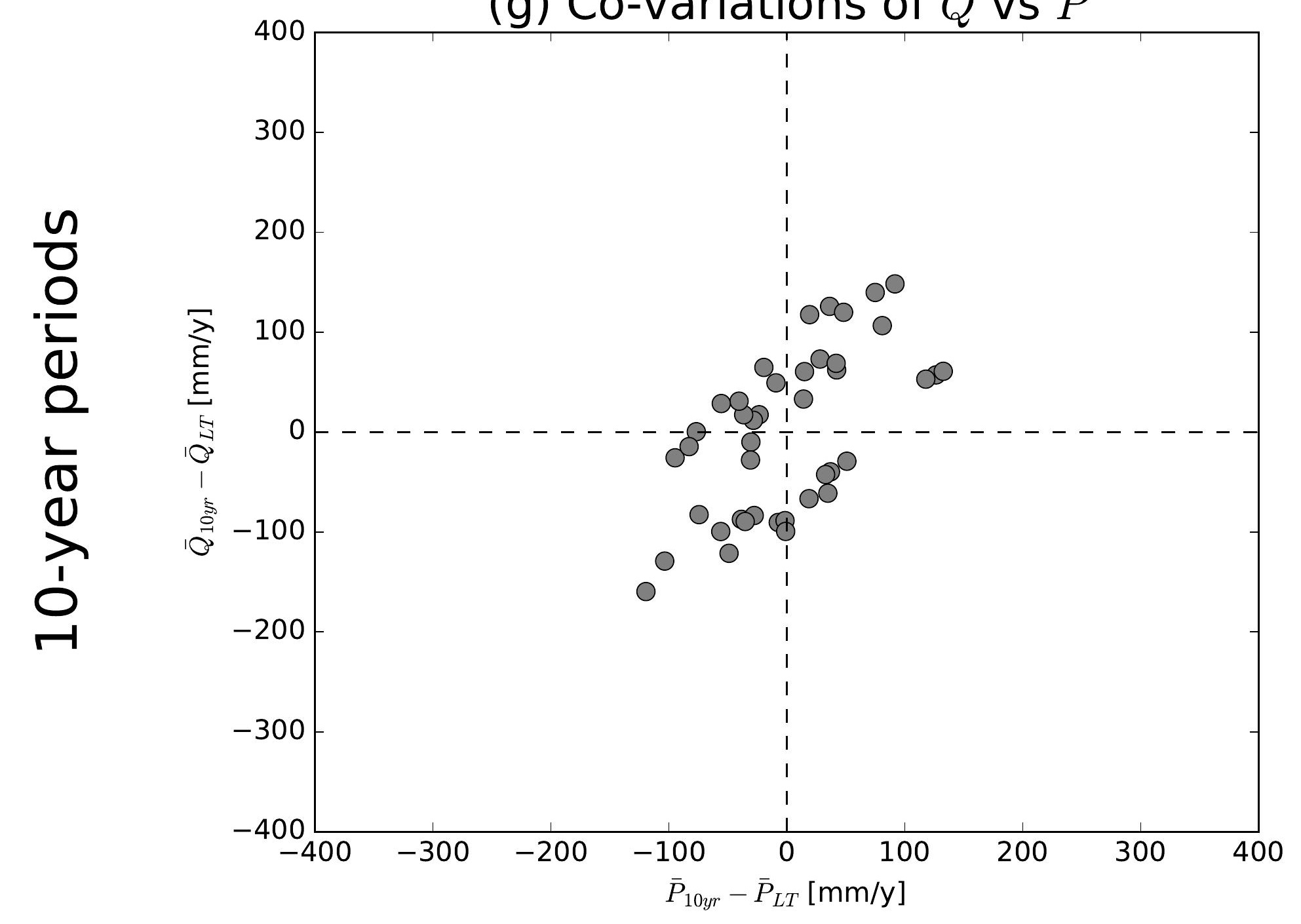

(j) Co-variations of $\bar{Q}$ vs $\bar{P}$

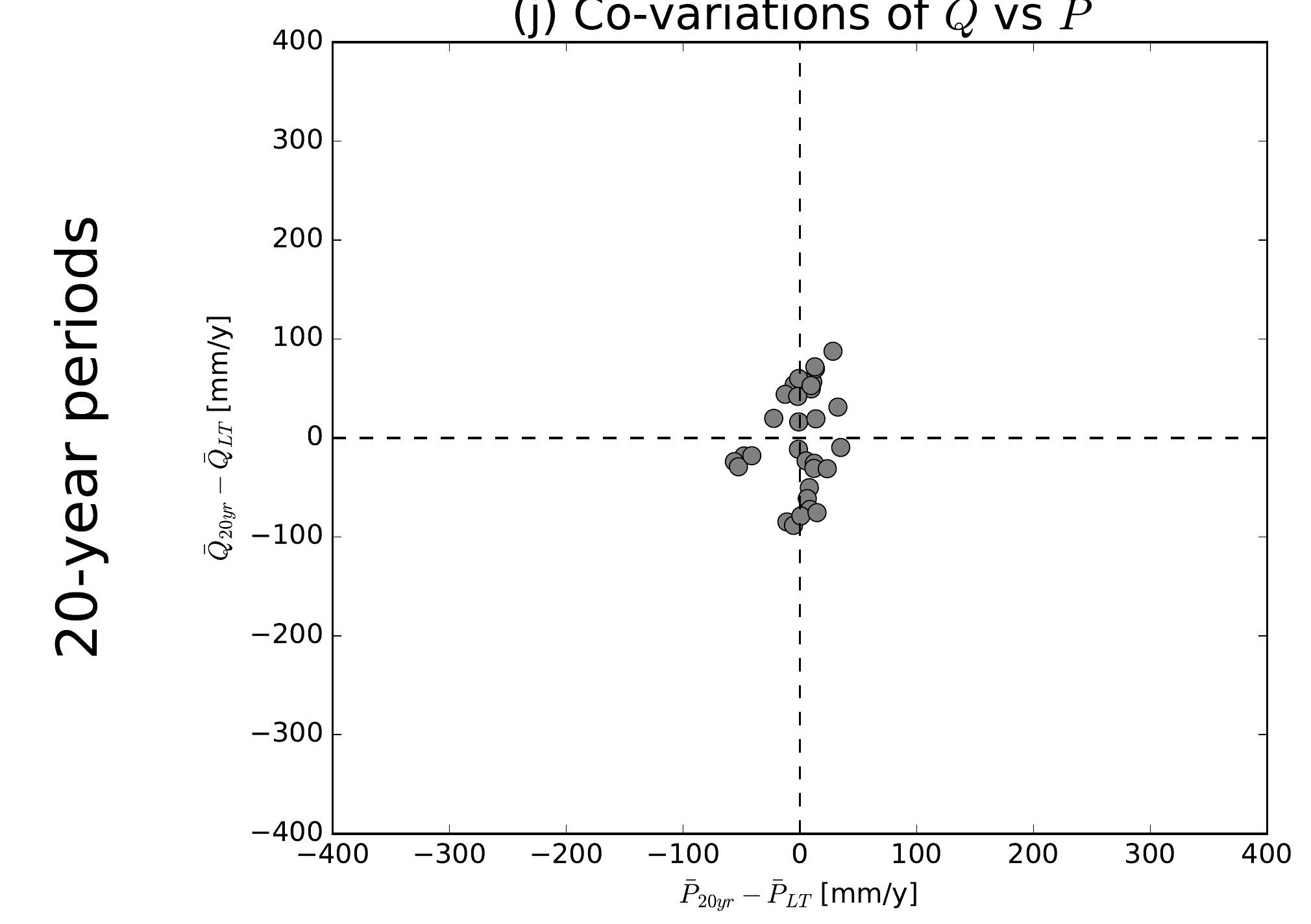

(b) Co-variations of $\bar{Q}$ vs $\overline{E 0}$

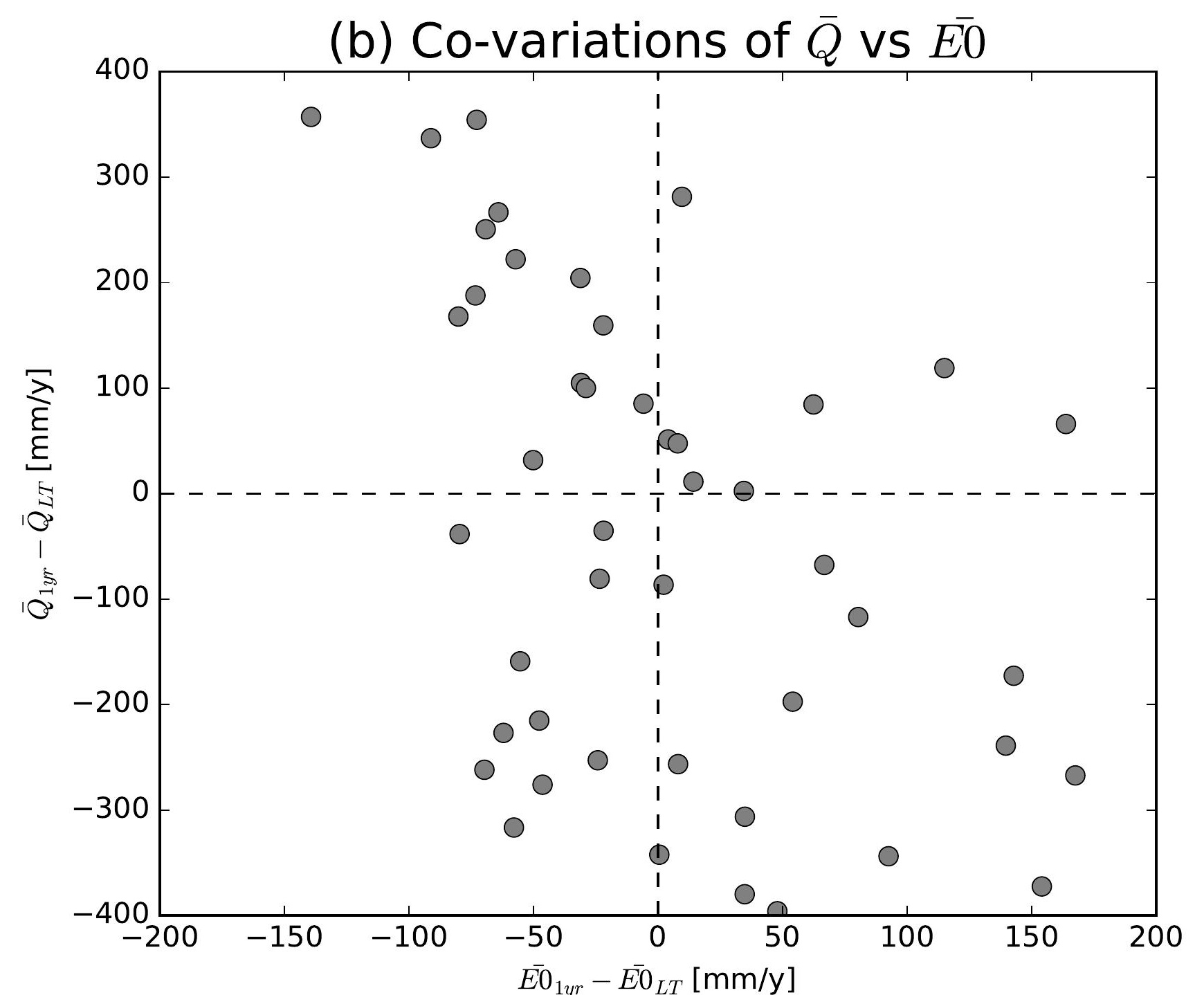

(e) Co-variations of $\bar{Q}$ vs $\overline{E 0}$

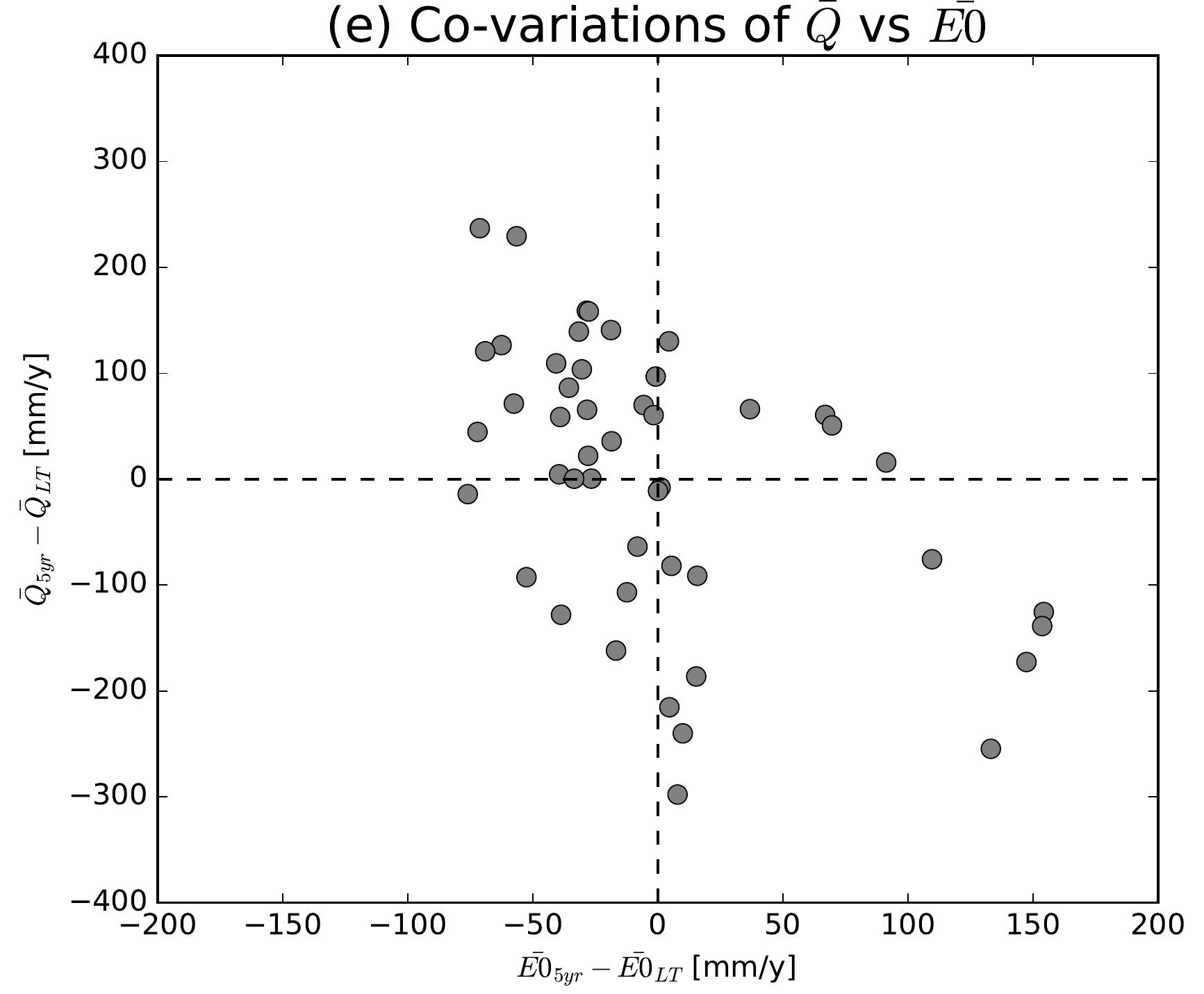

(h) Co-variations of $\bar{Q}$ vs $\overline{E 0}$

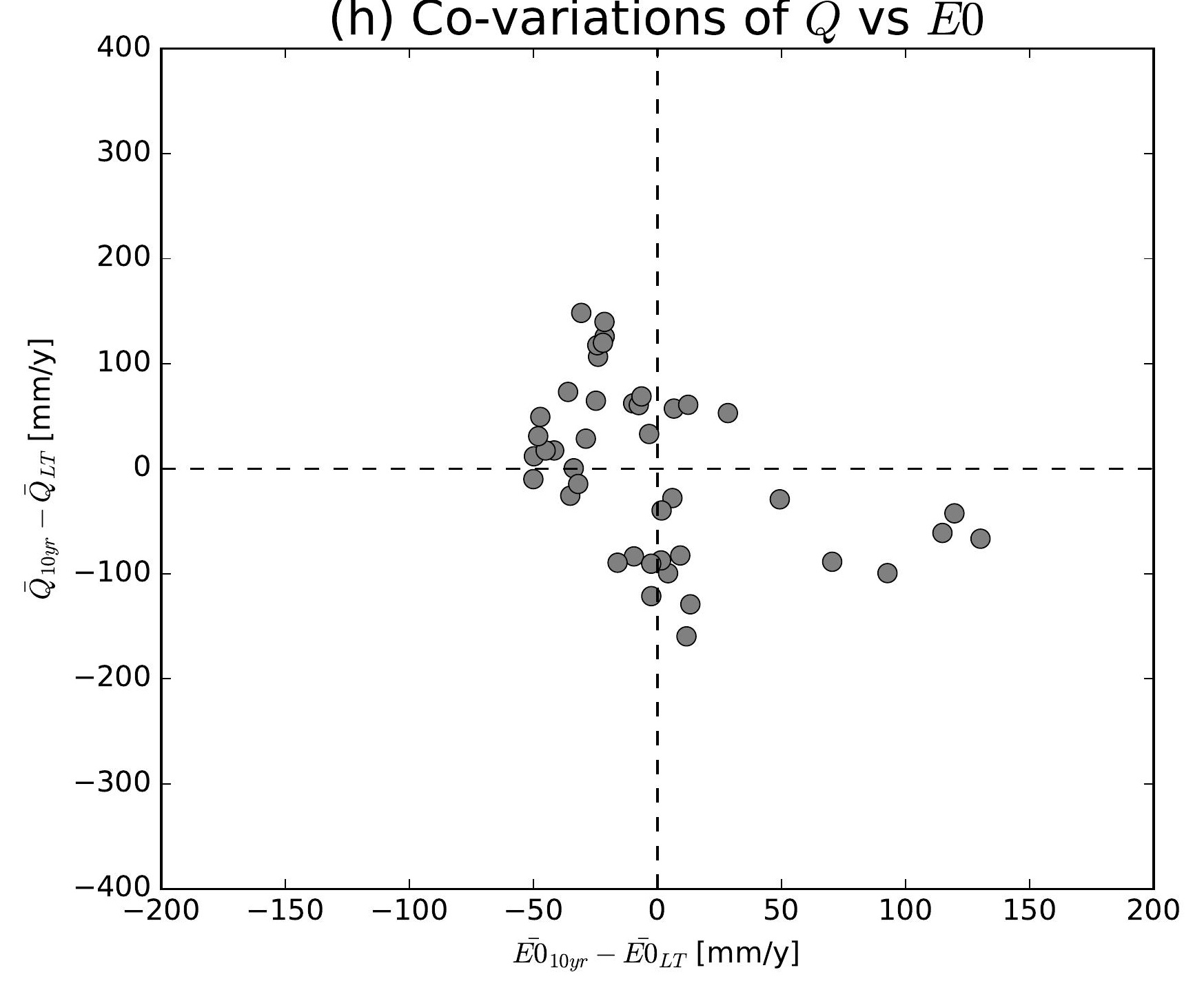

(k) Co-variations of $\bar{Q}$ vs $\overline{E 0}$

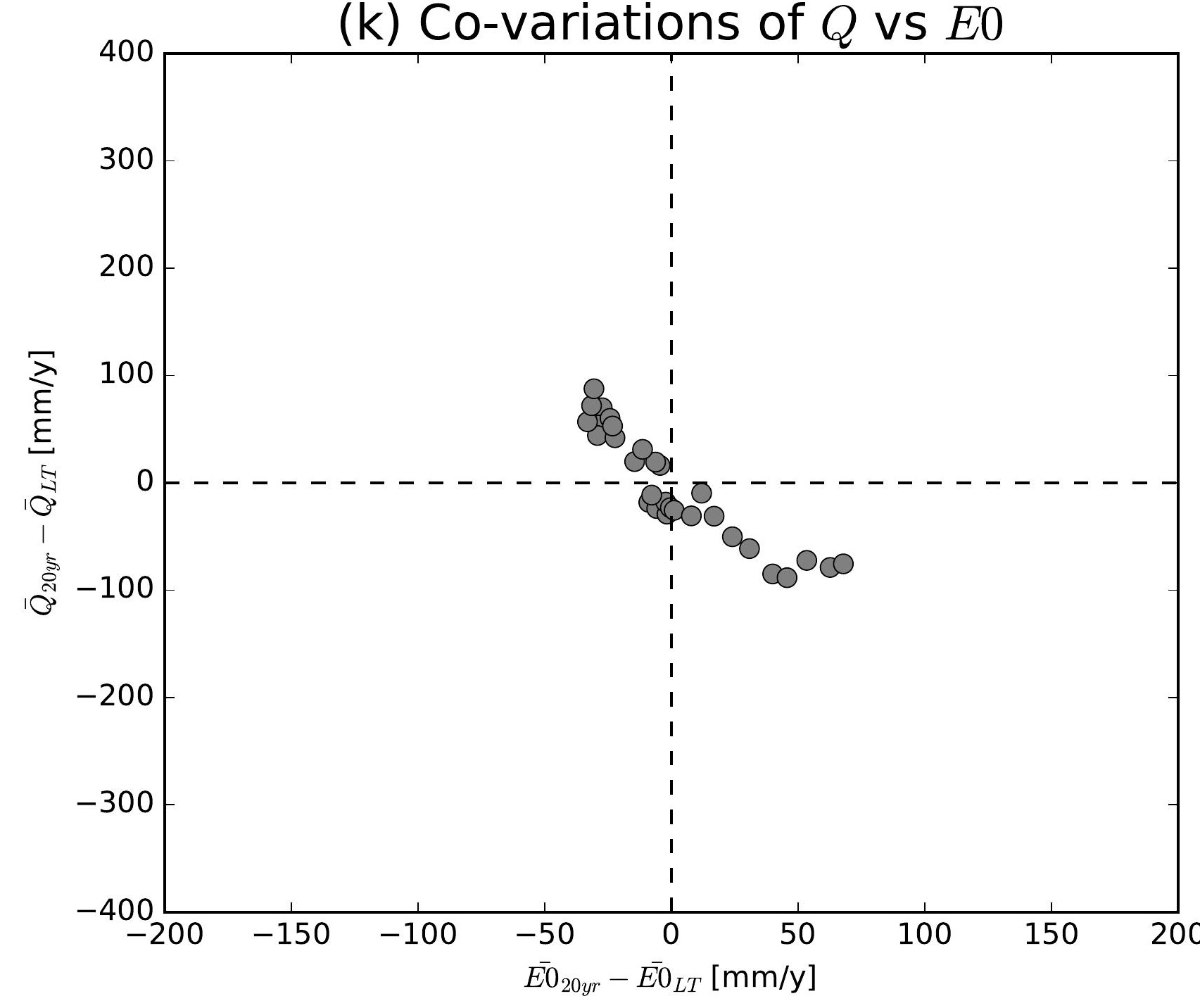

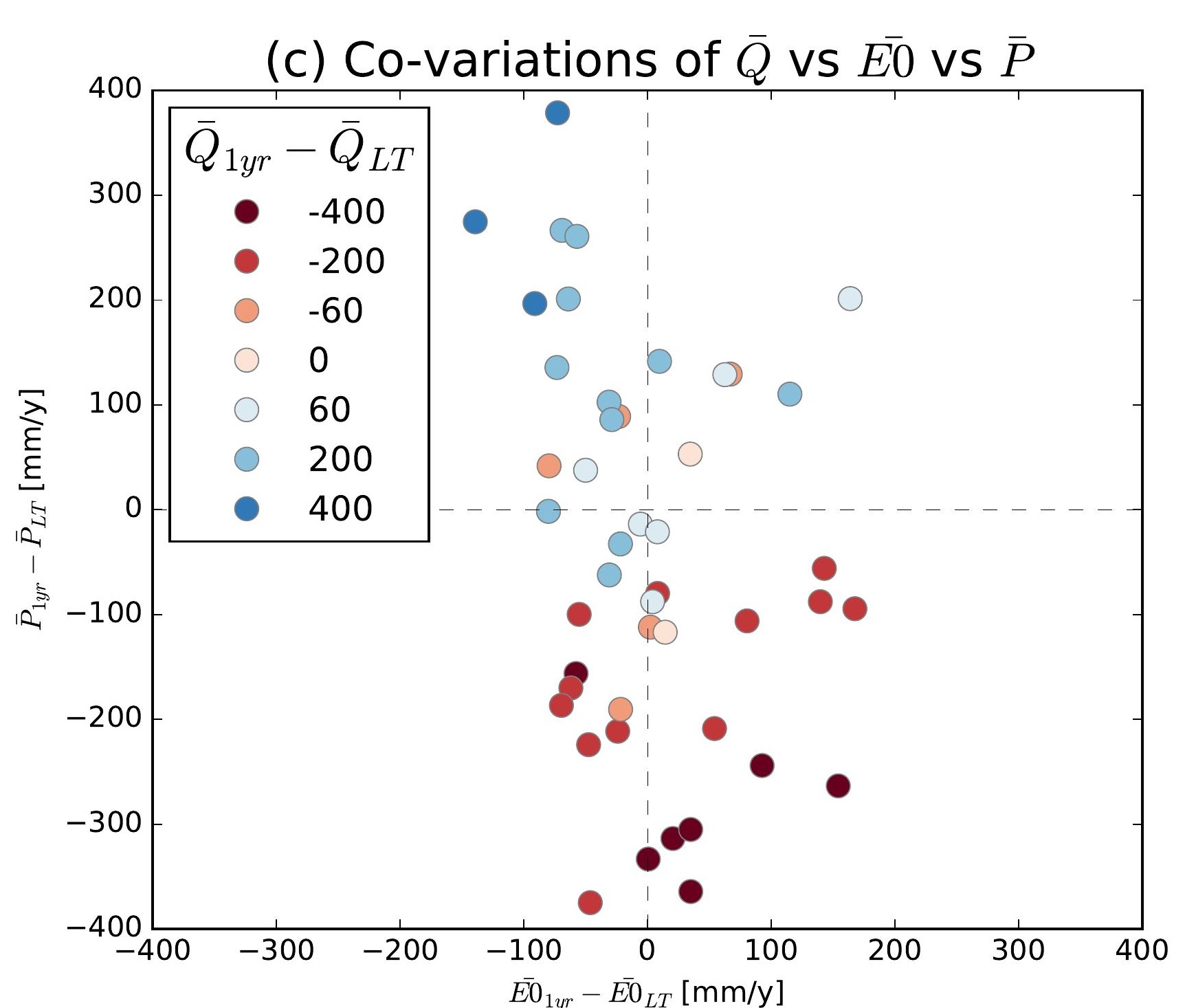
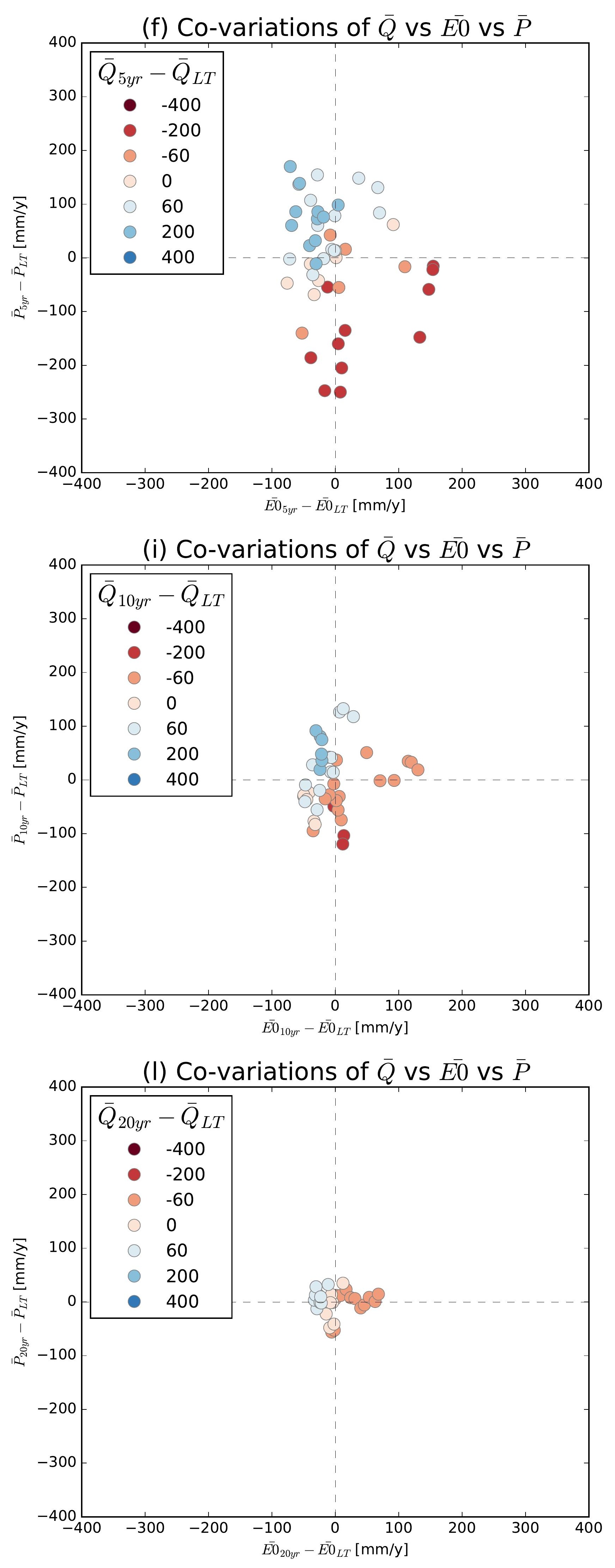

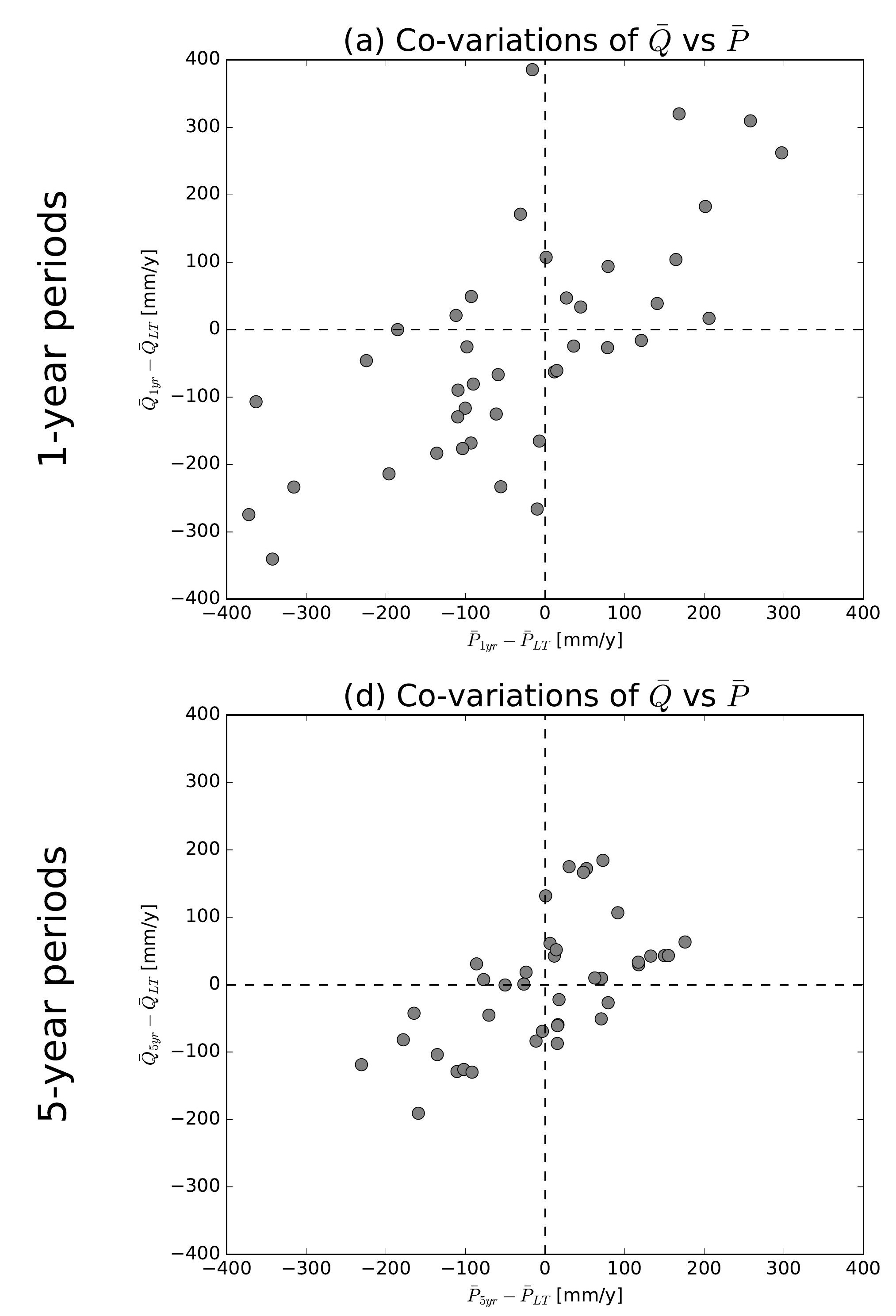

(g) Co-variations of $\bar{Q}$ vs $\bar{P}$

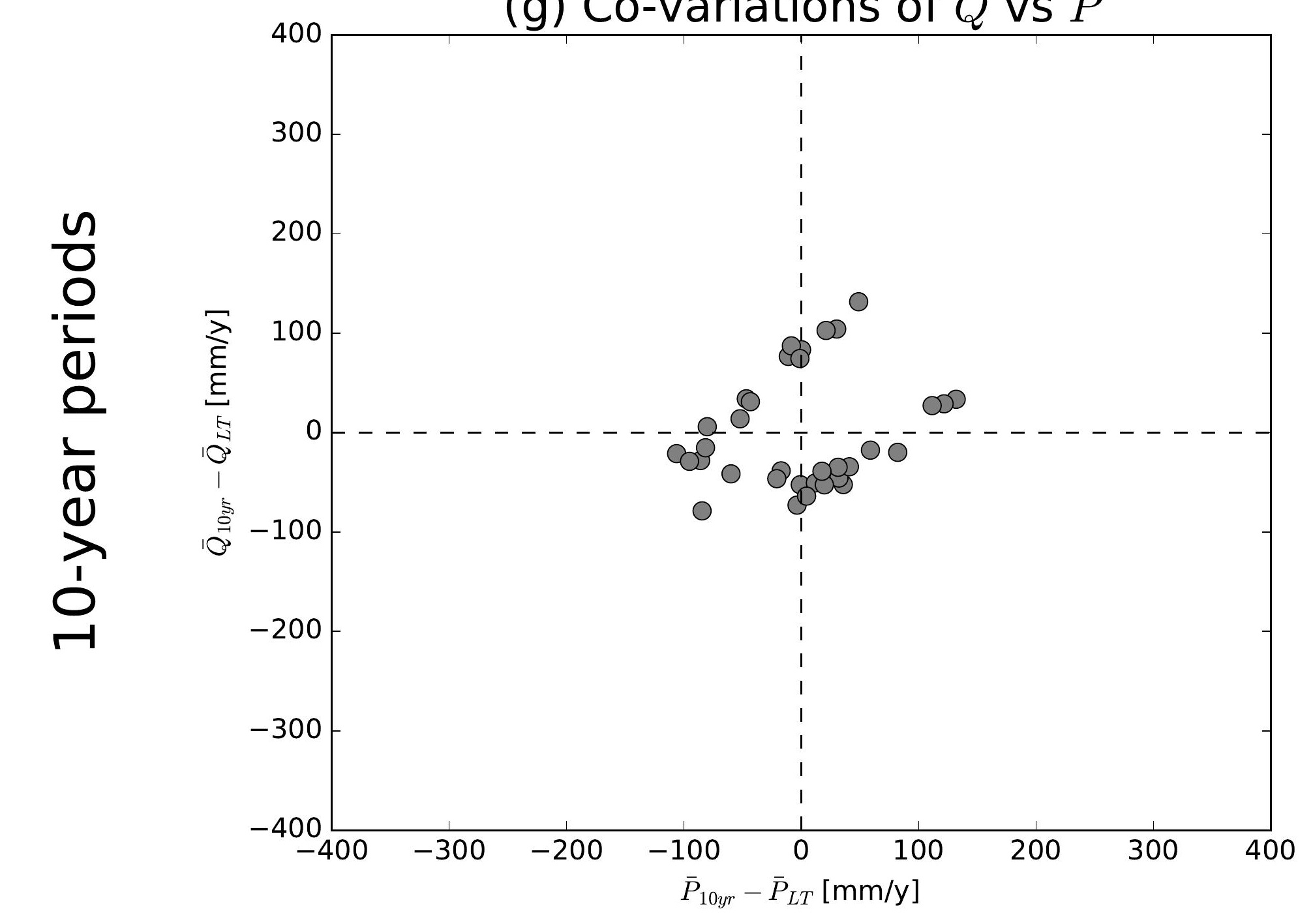

(j) Co-variations of $\bar{Q}$ vs $\bar{P}$

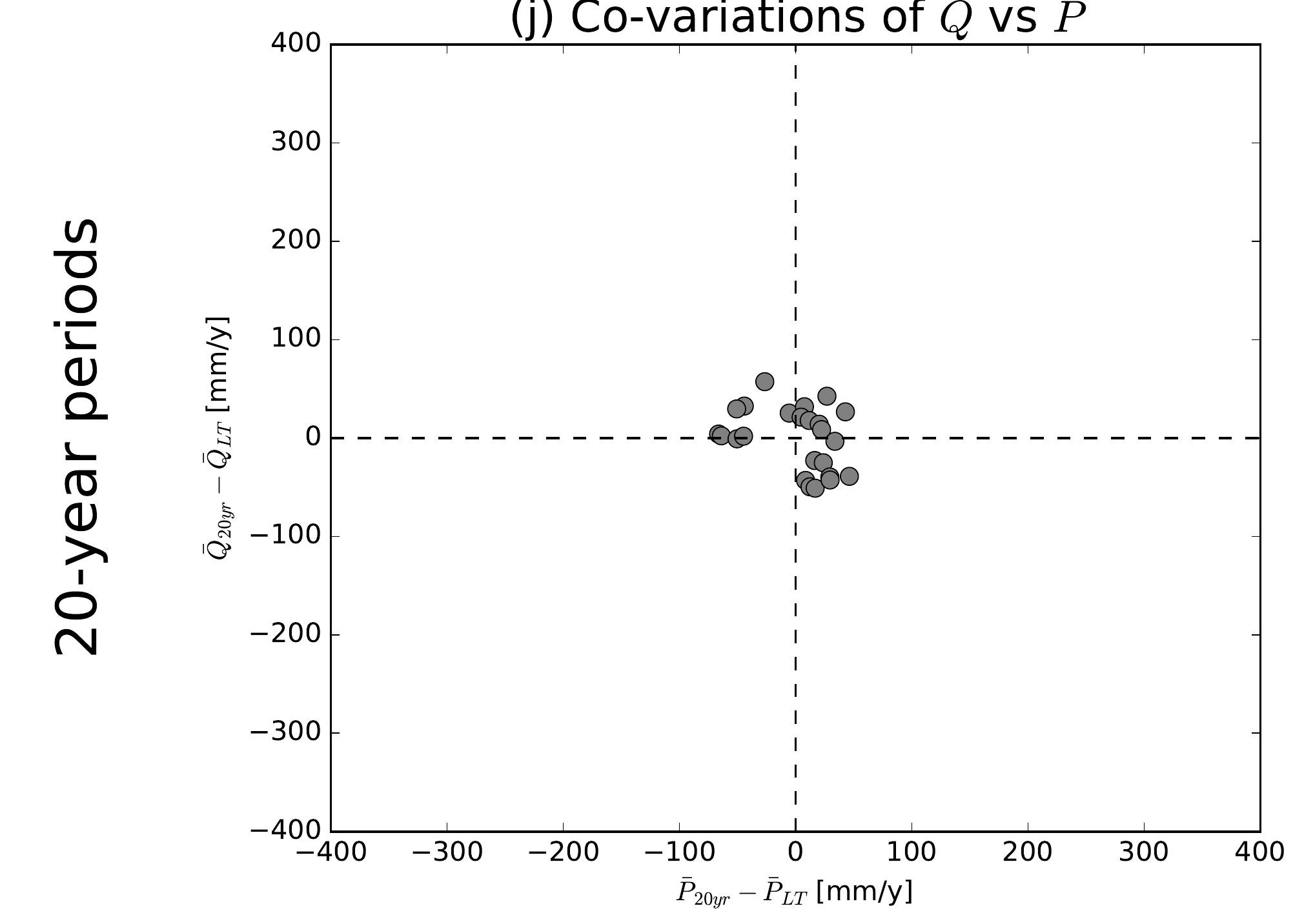

(b) Co-variations of $\bar{Q}$ vs $\overline{E 0}$

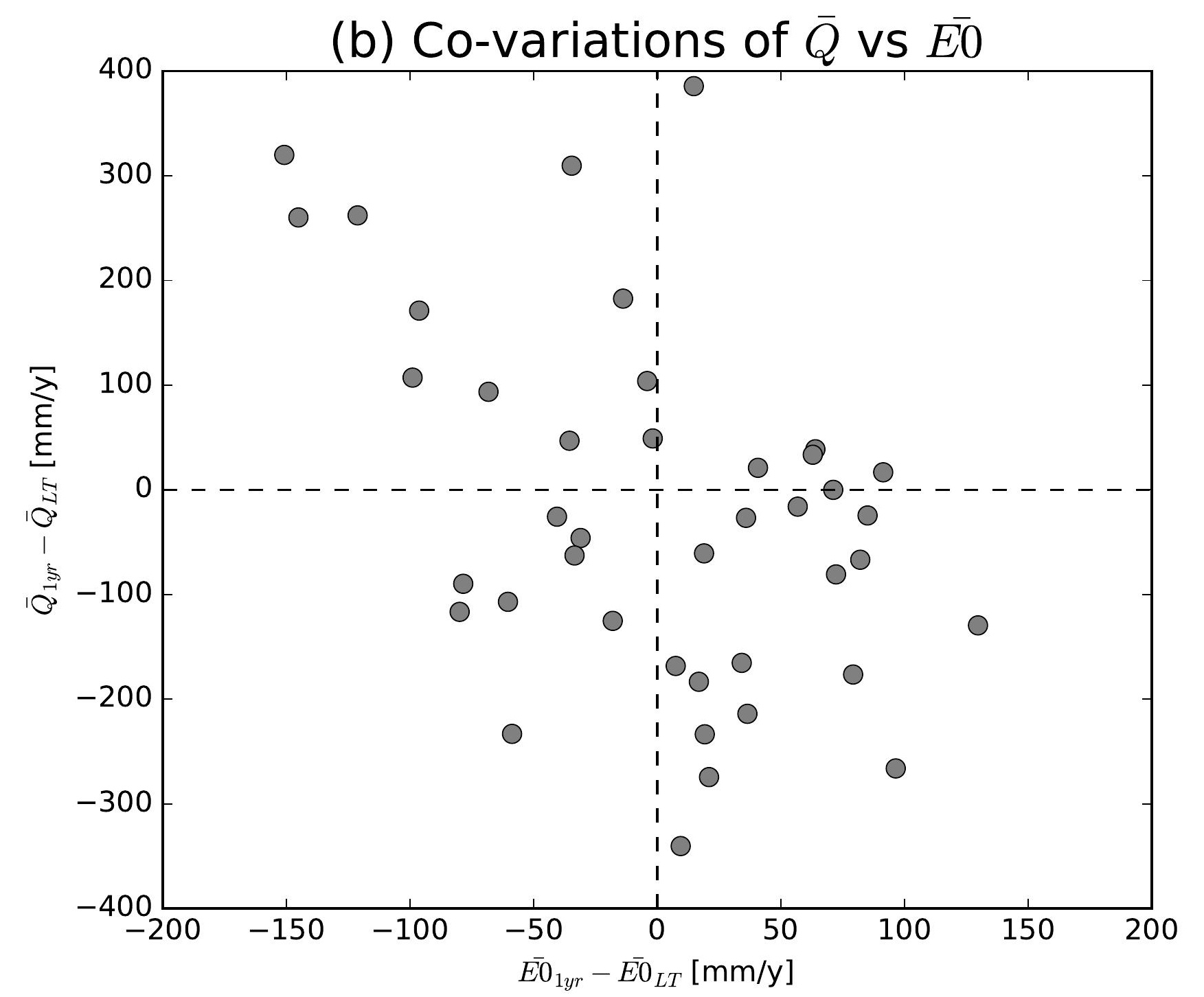

(e) Co-variations of $\bar{Q}$ vs $\overline{E 0}$

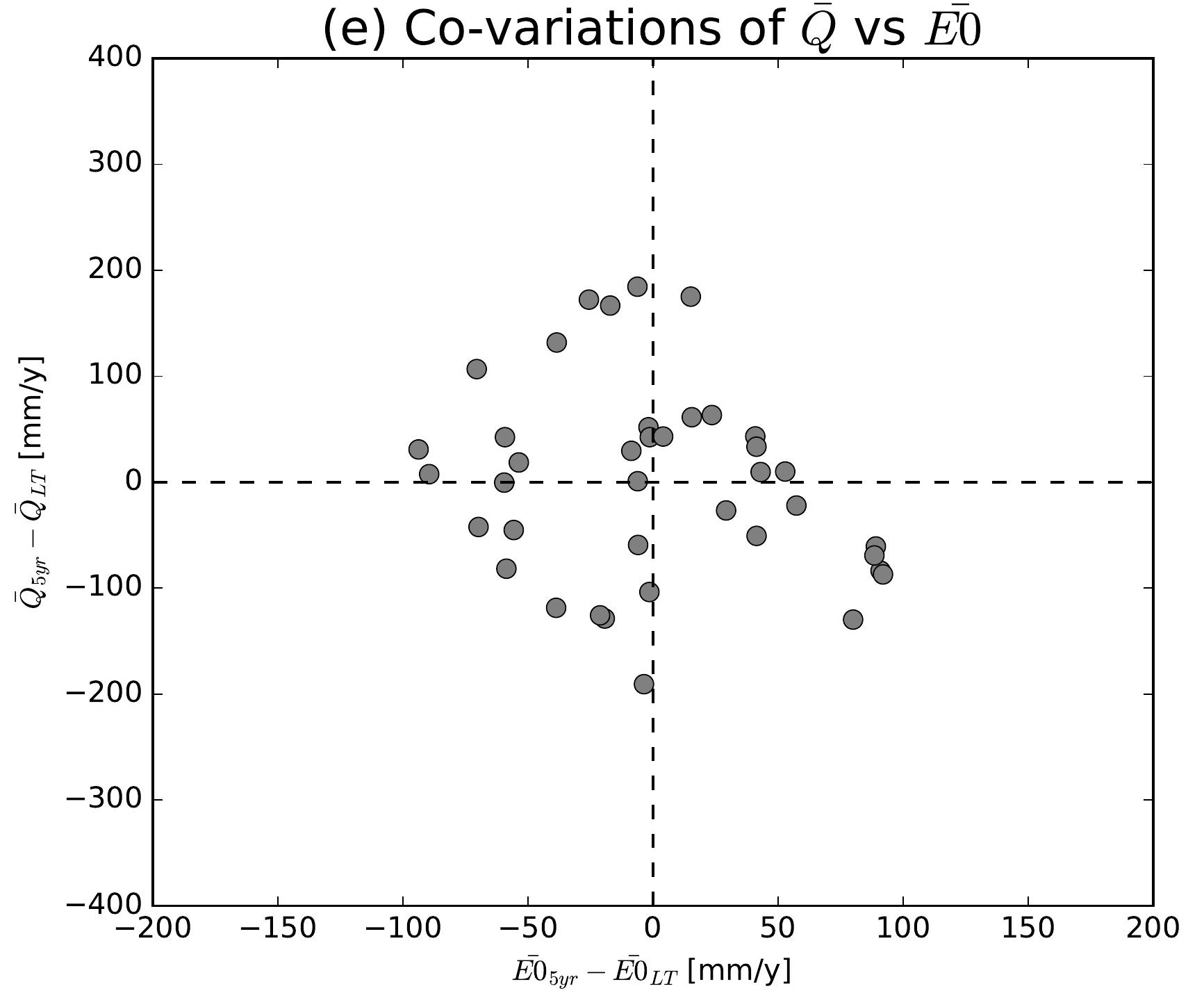

(h) Co-variations of $\bar{Q}$ vs $\overline{E 0}$

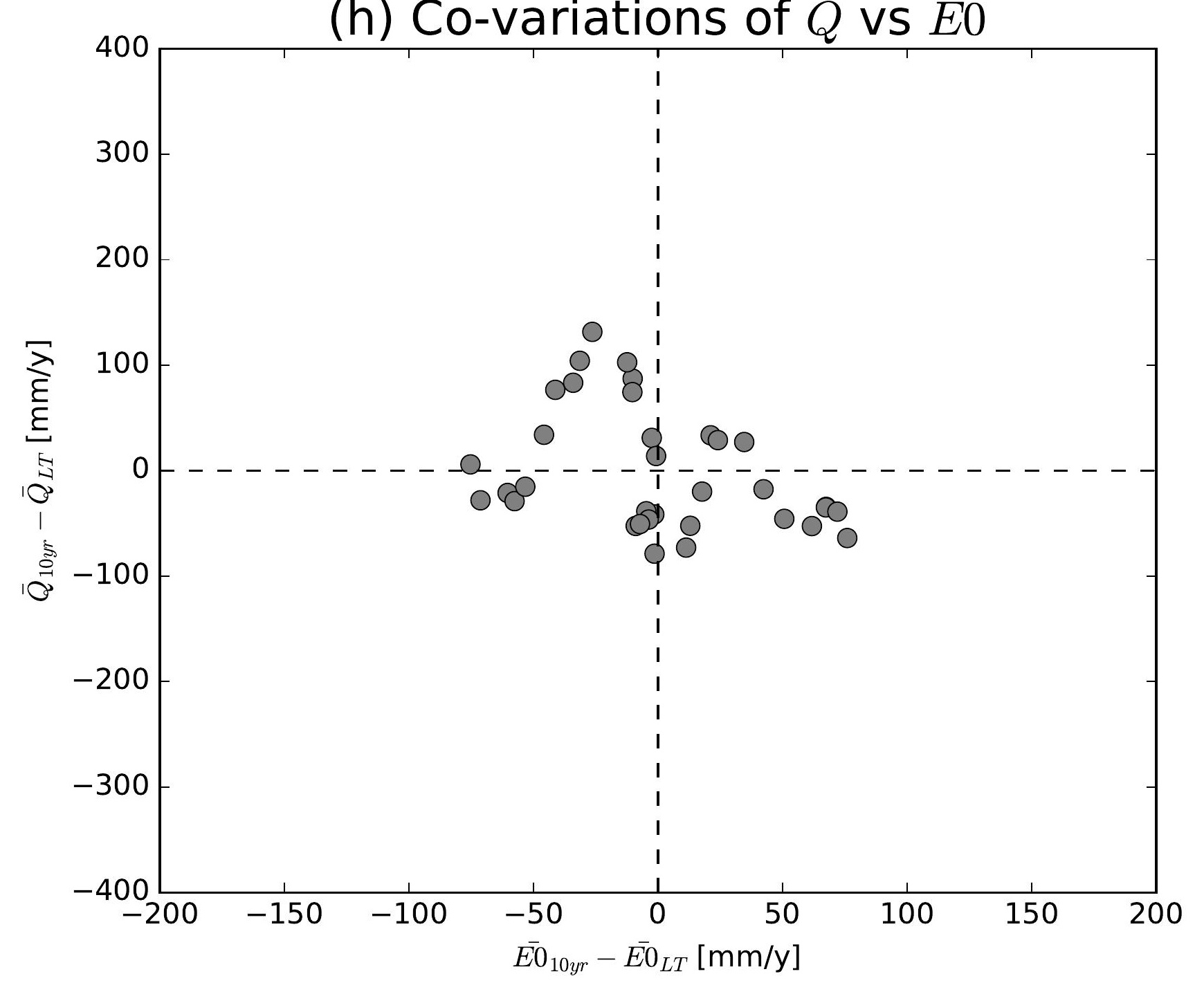

(k) Co-variations of $\bar{Q}$ vs $\overline{E 0}$

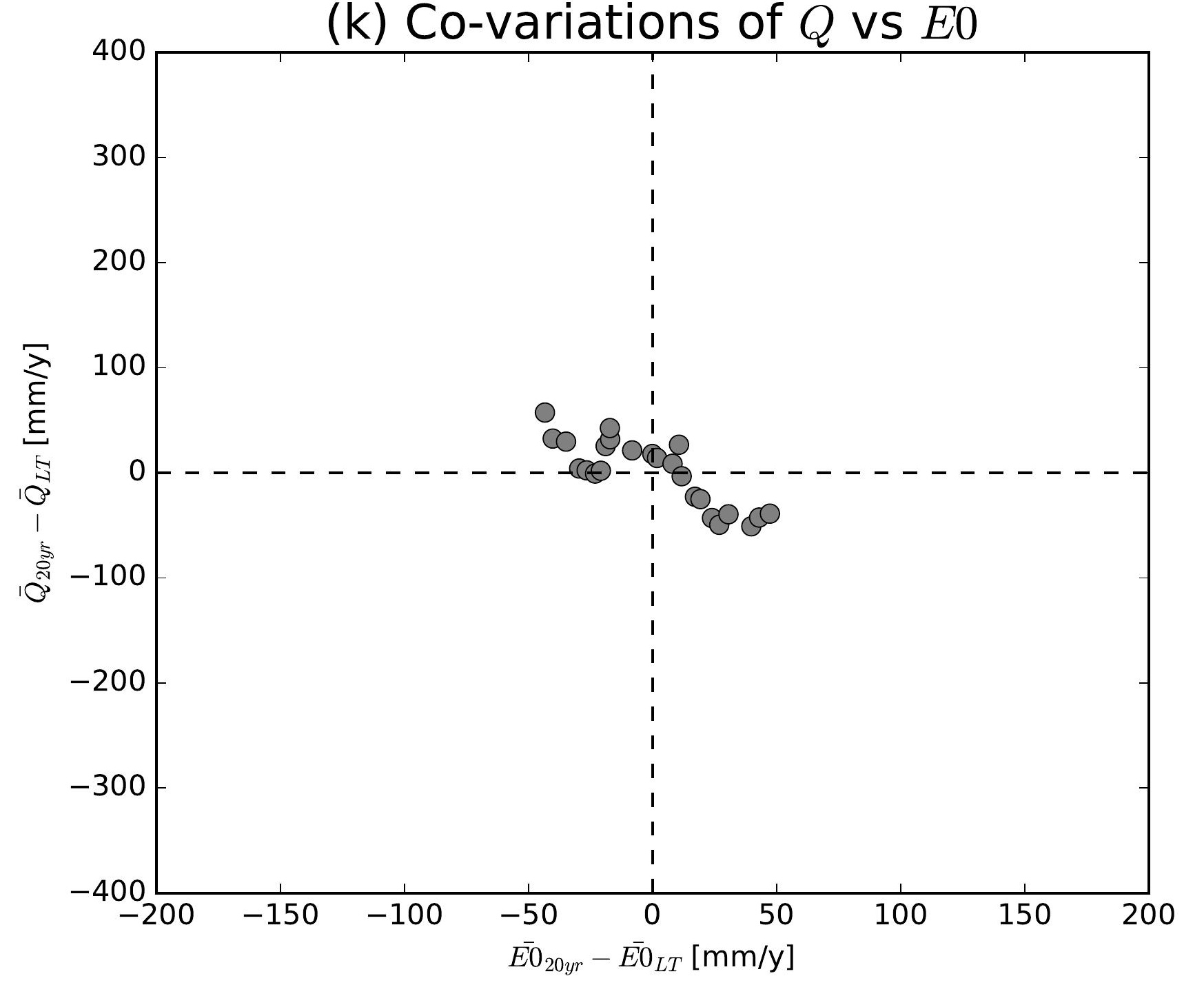

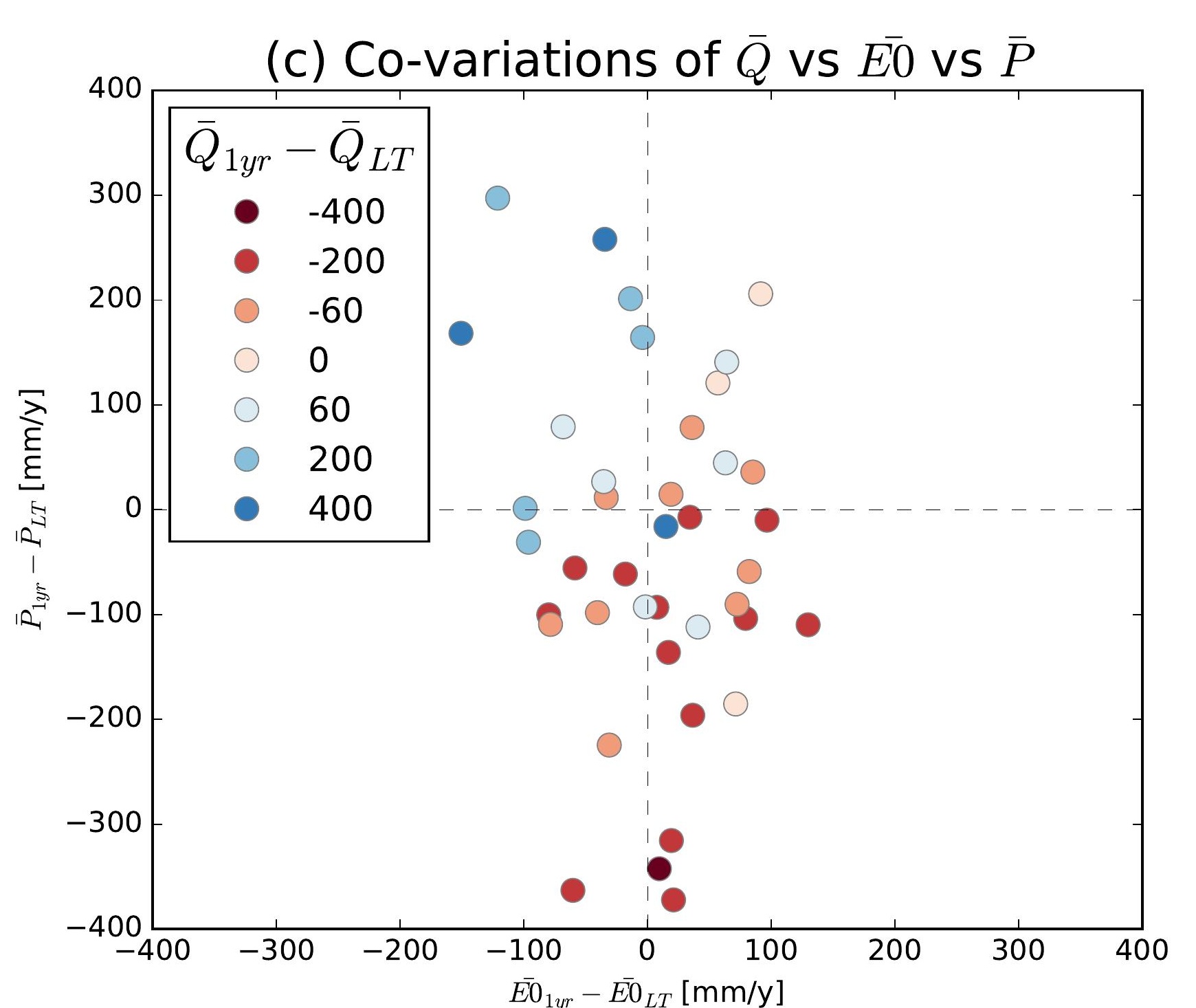
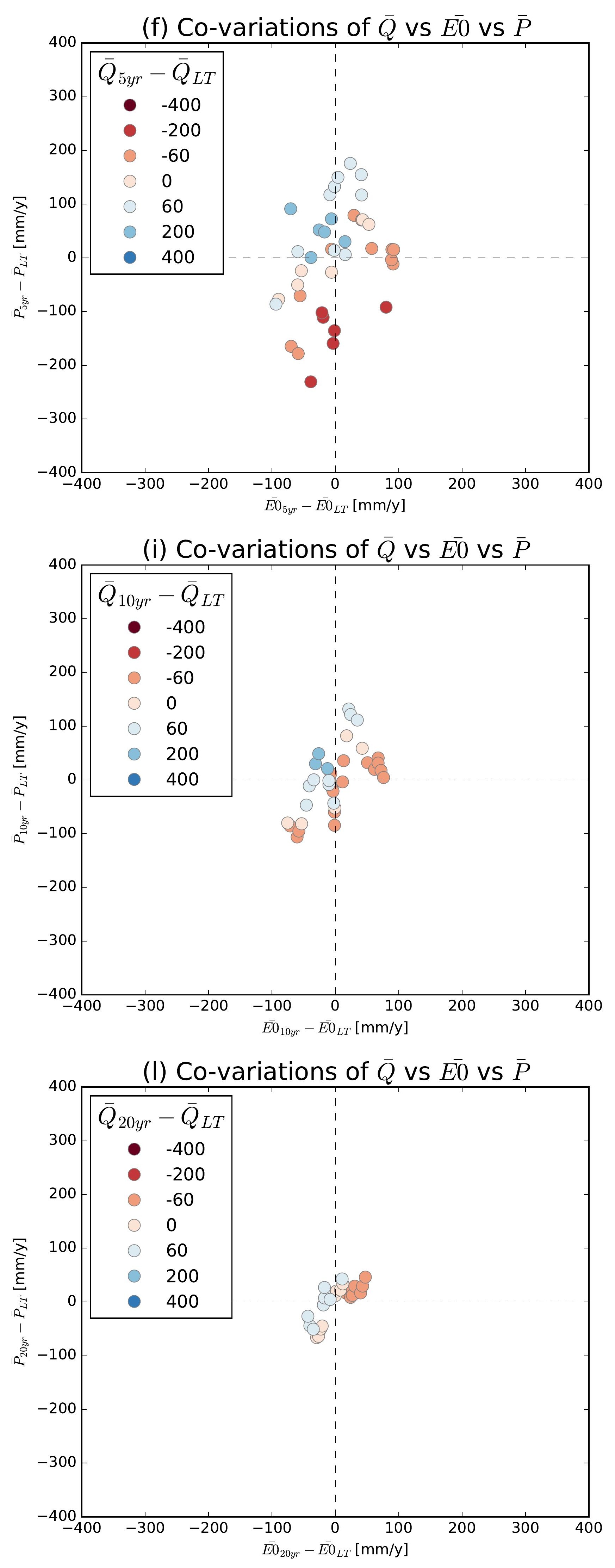

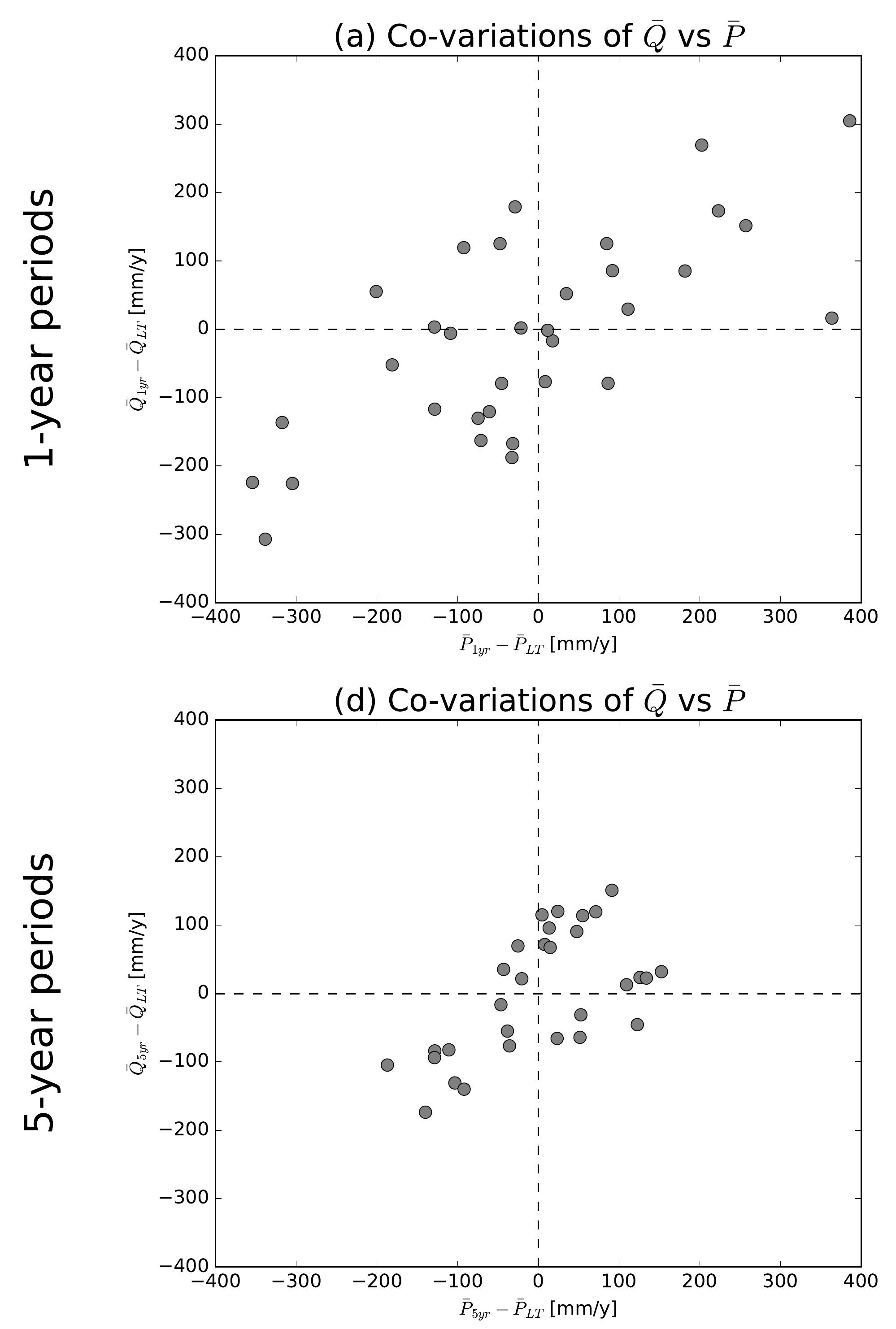

(g) Co-variations of $\bar{Q}$ vs $\bar{P}$

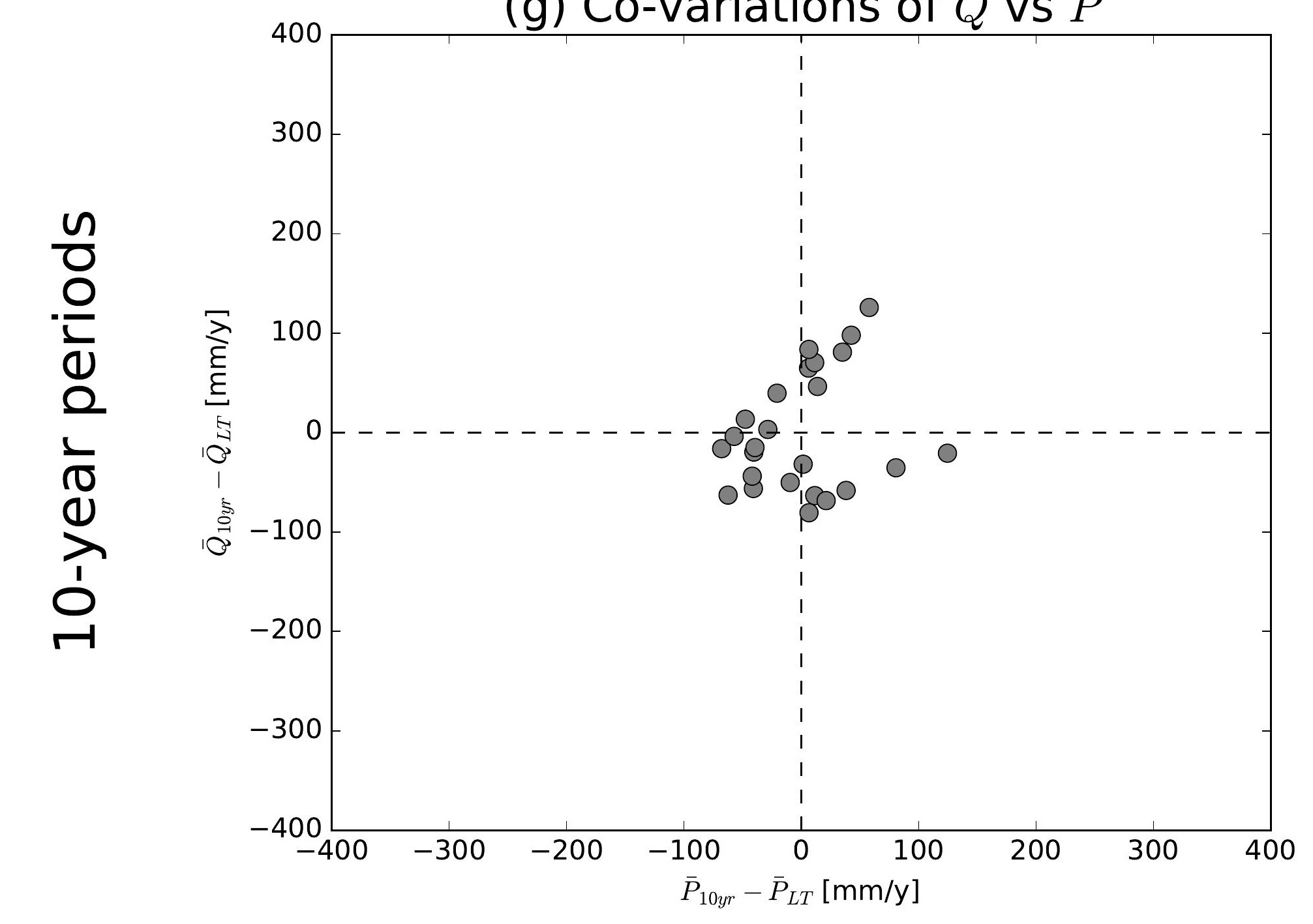

(j) Co-variations of $\bar{Q}$ vs $\bar{P}$

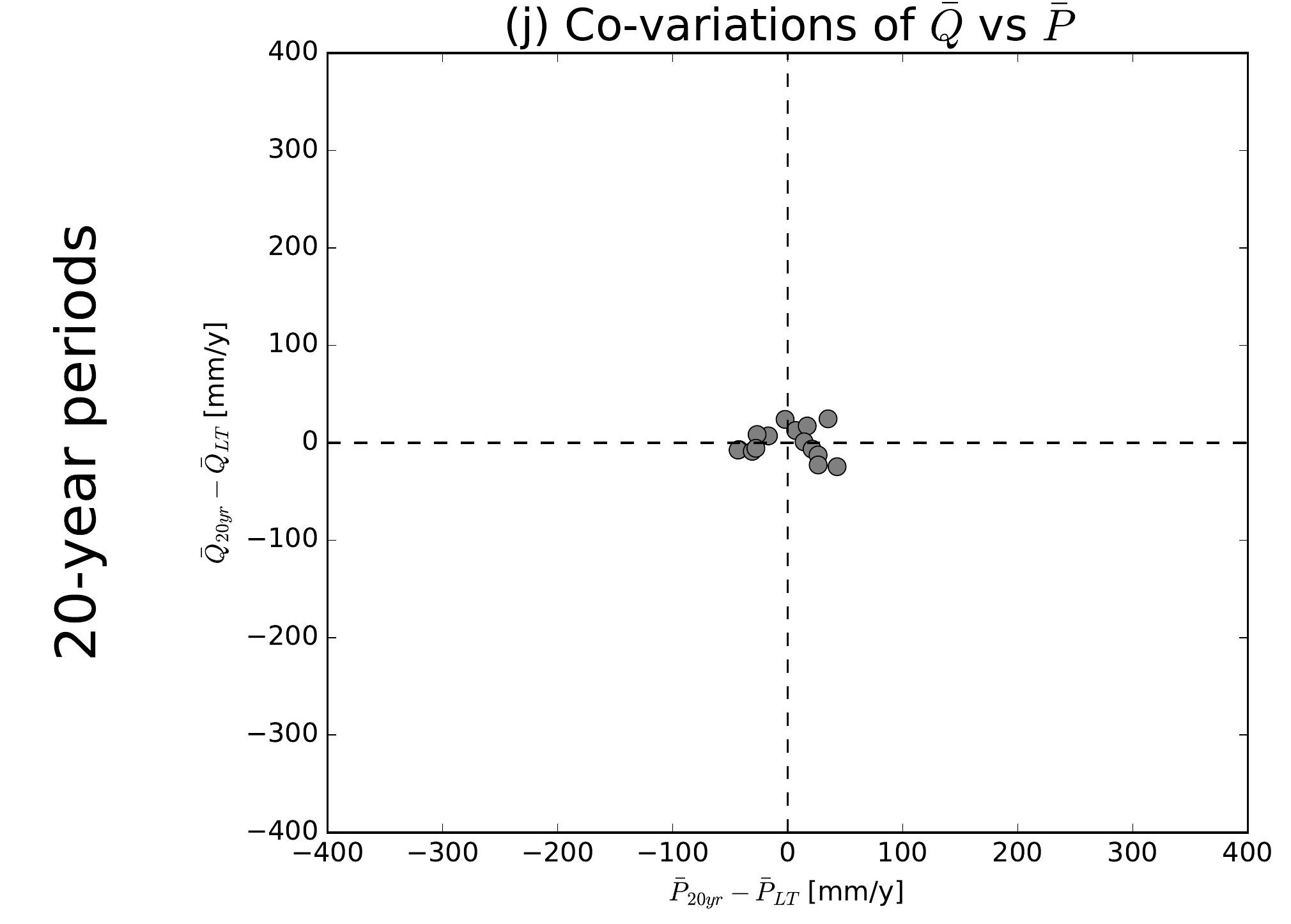

(b) Co-variations of $\bar{Q}$ vs $\overline{E 0}$

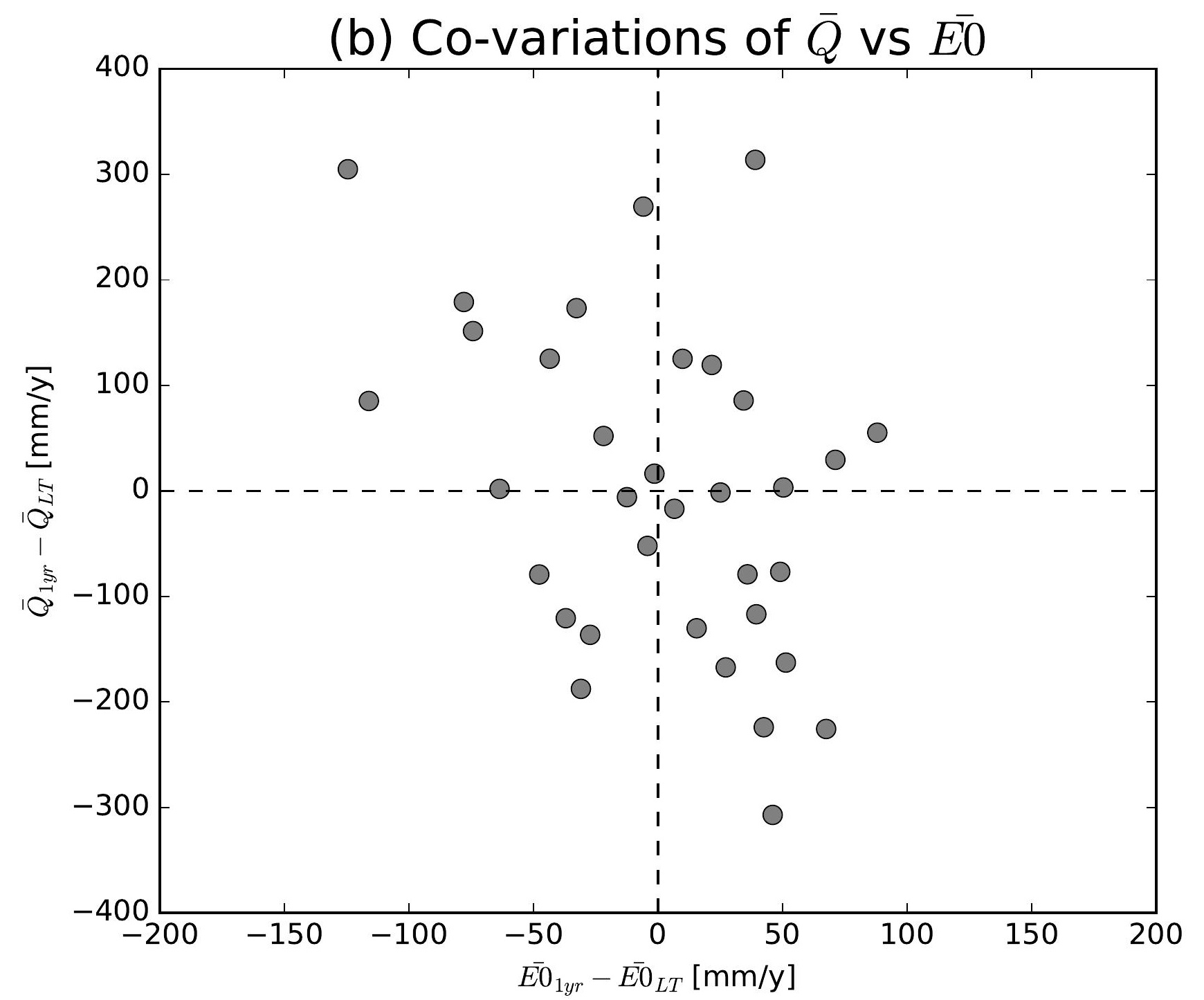

(e) Co-variations of $\bar{Q}$ vs $\overline{E 0}$

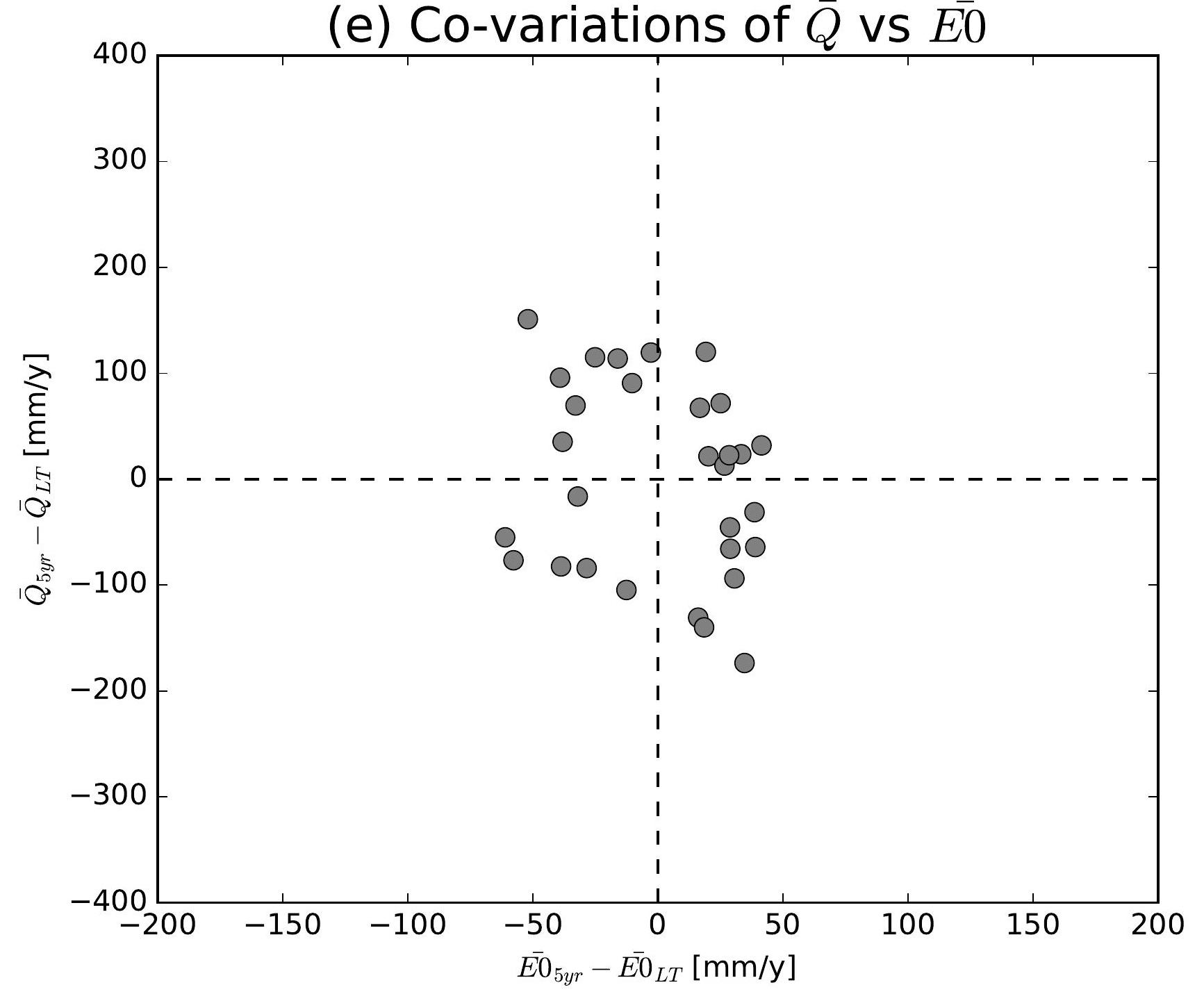

(h) Co-variations of $\bar{Q}$ vs $\overline{E 0}$

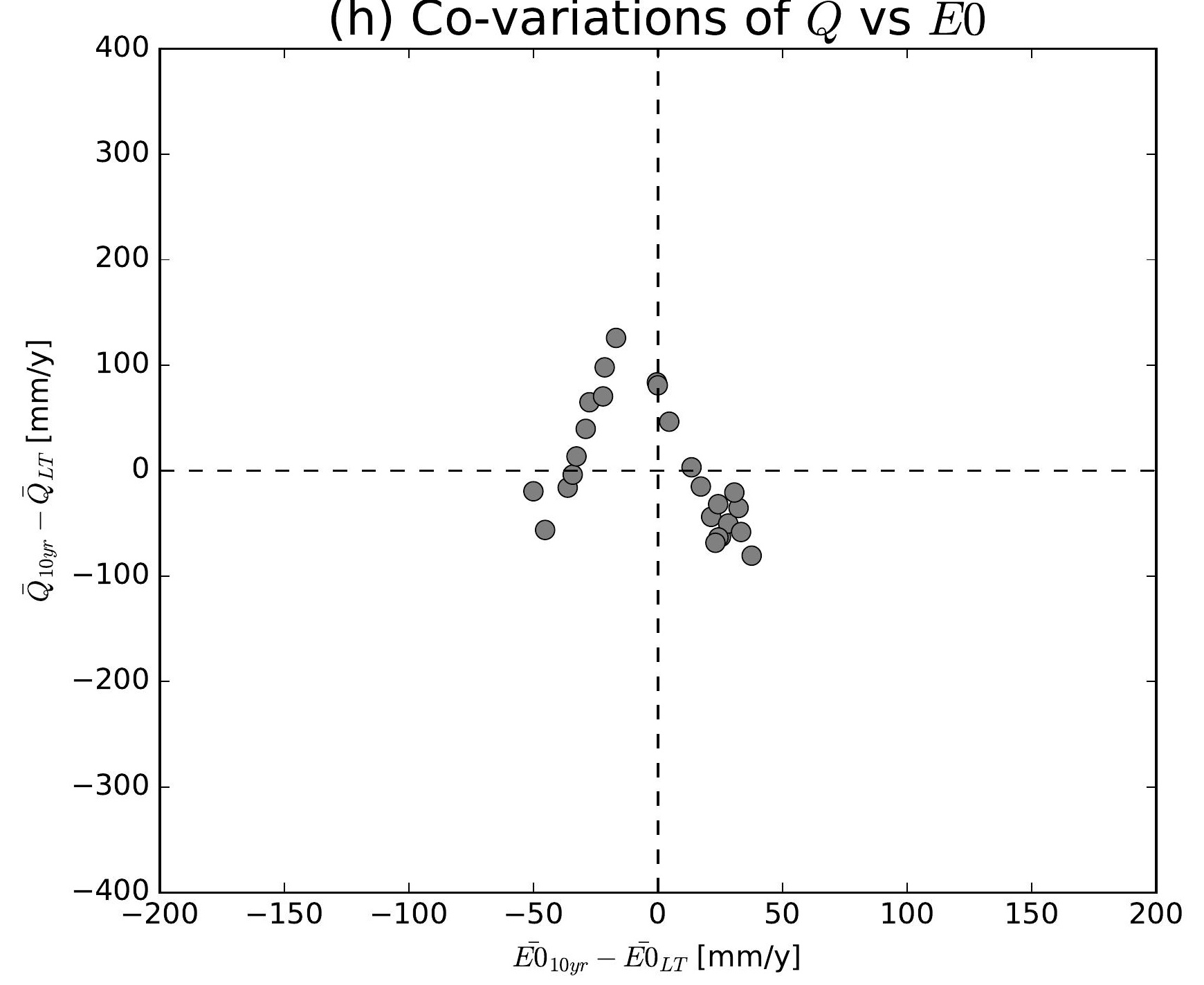

(k) Co-variations of $\bar{Q}$ vs $\overline{E 0}$

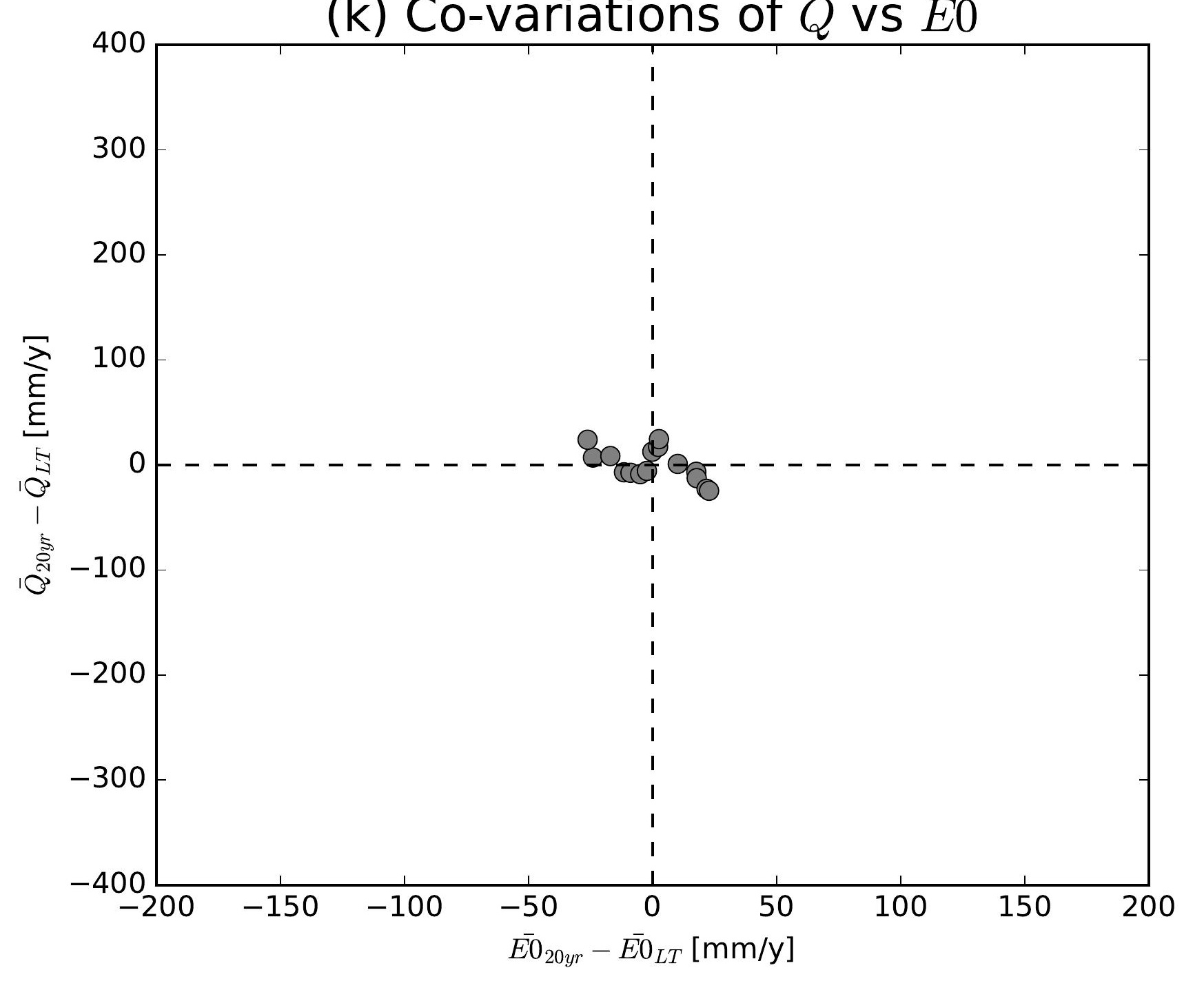

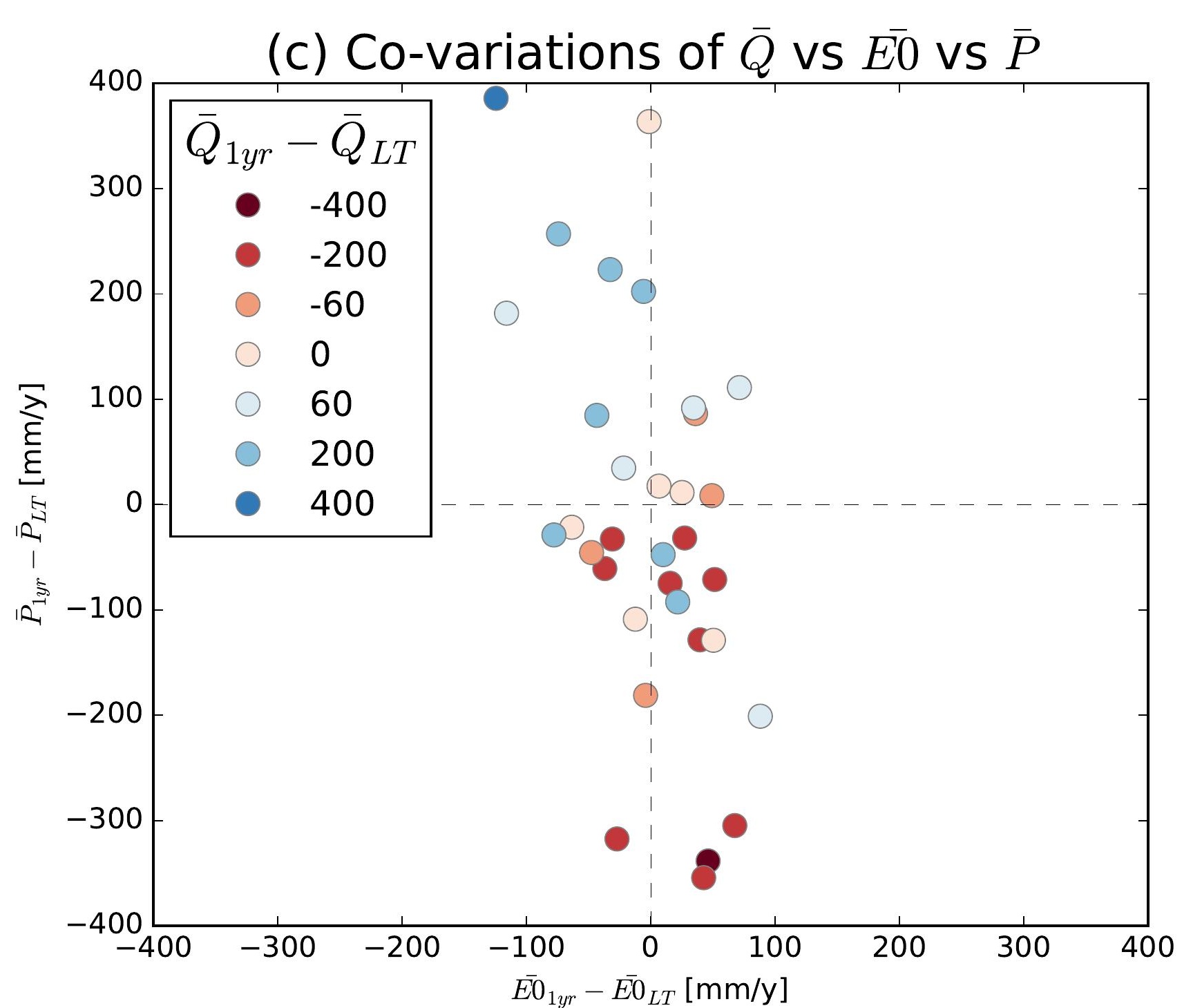
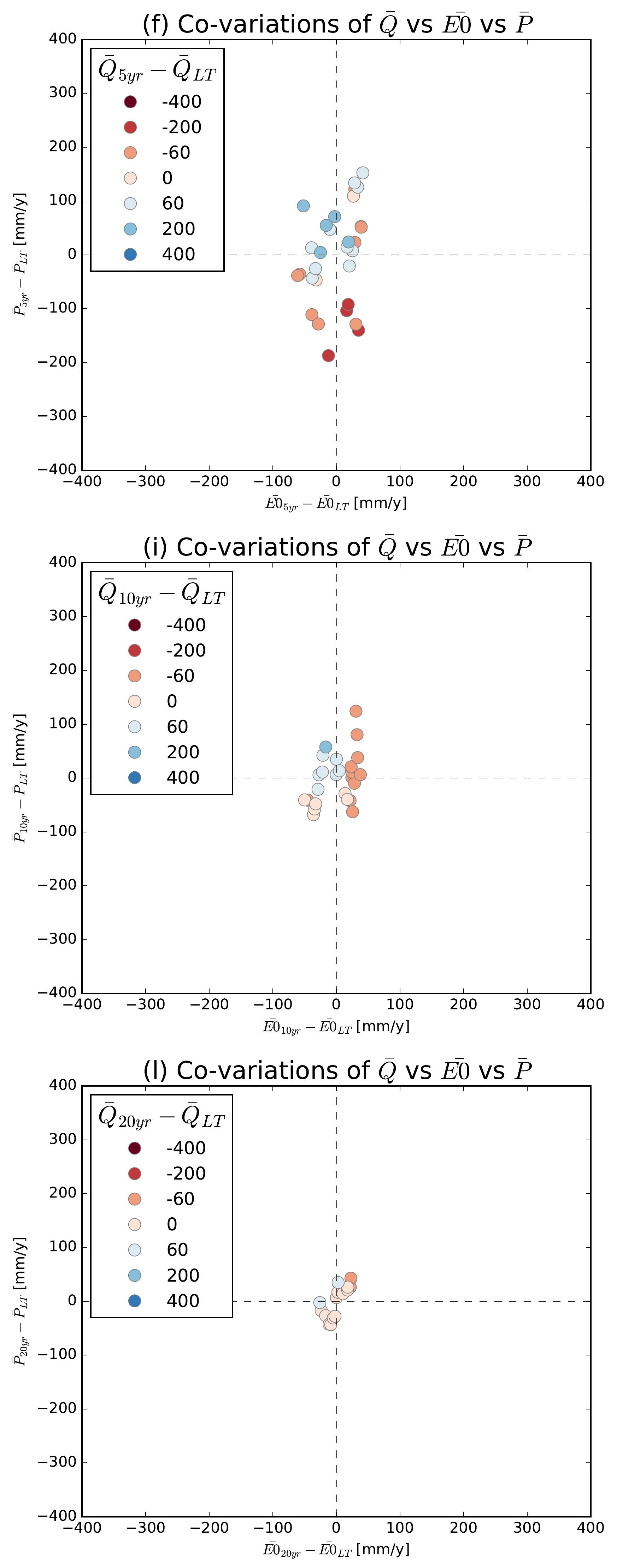

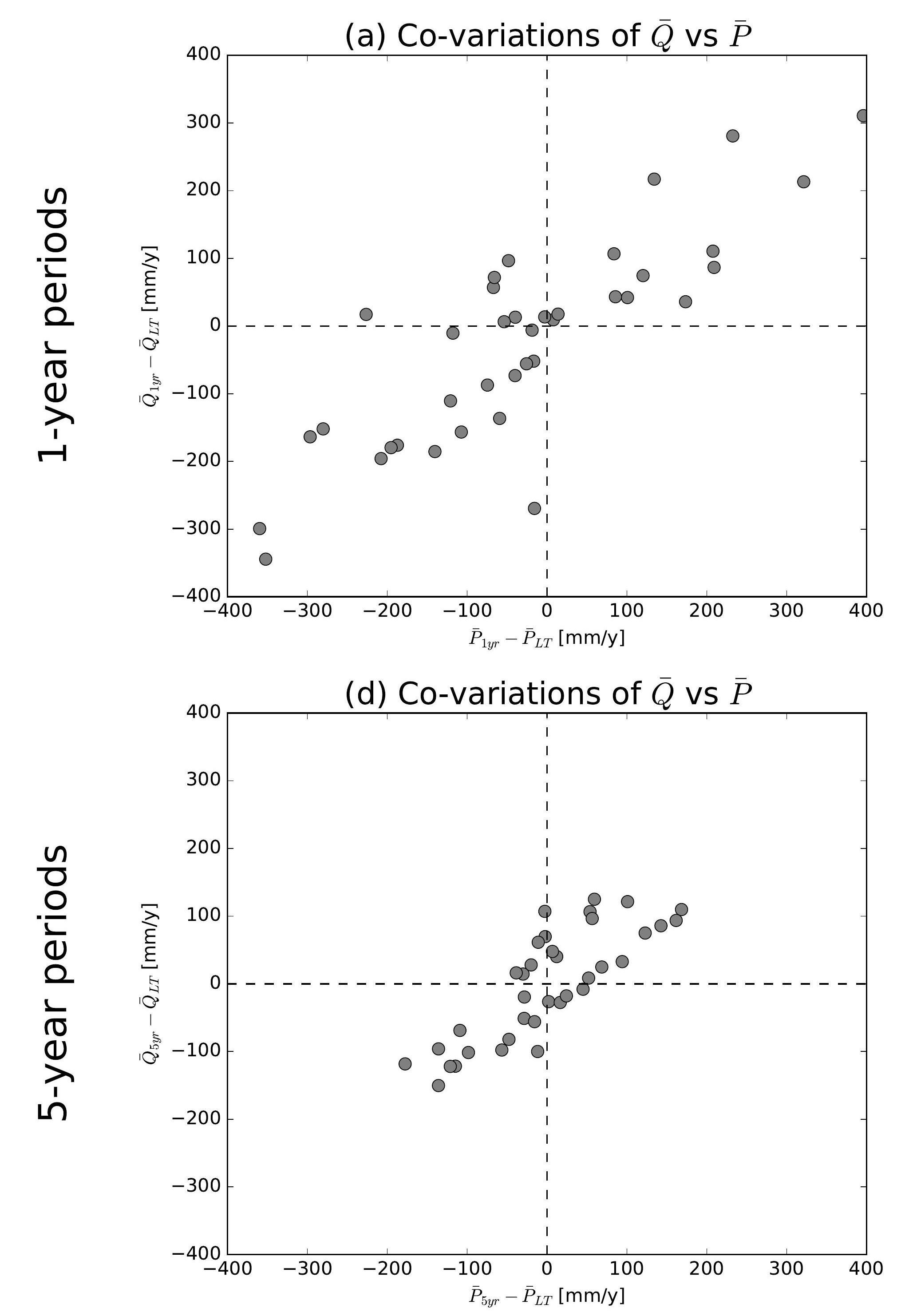

(g) Co-variations of $\bar{Q}$ vs $\bar{P}$

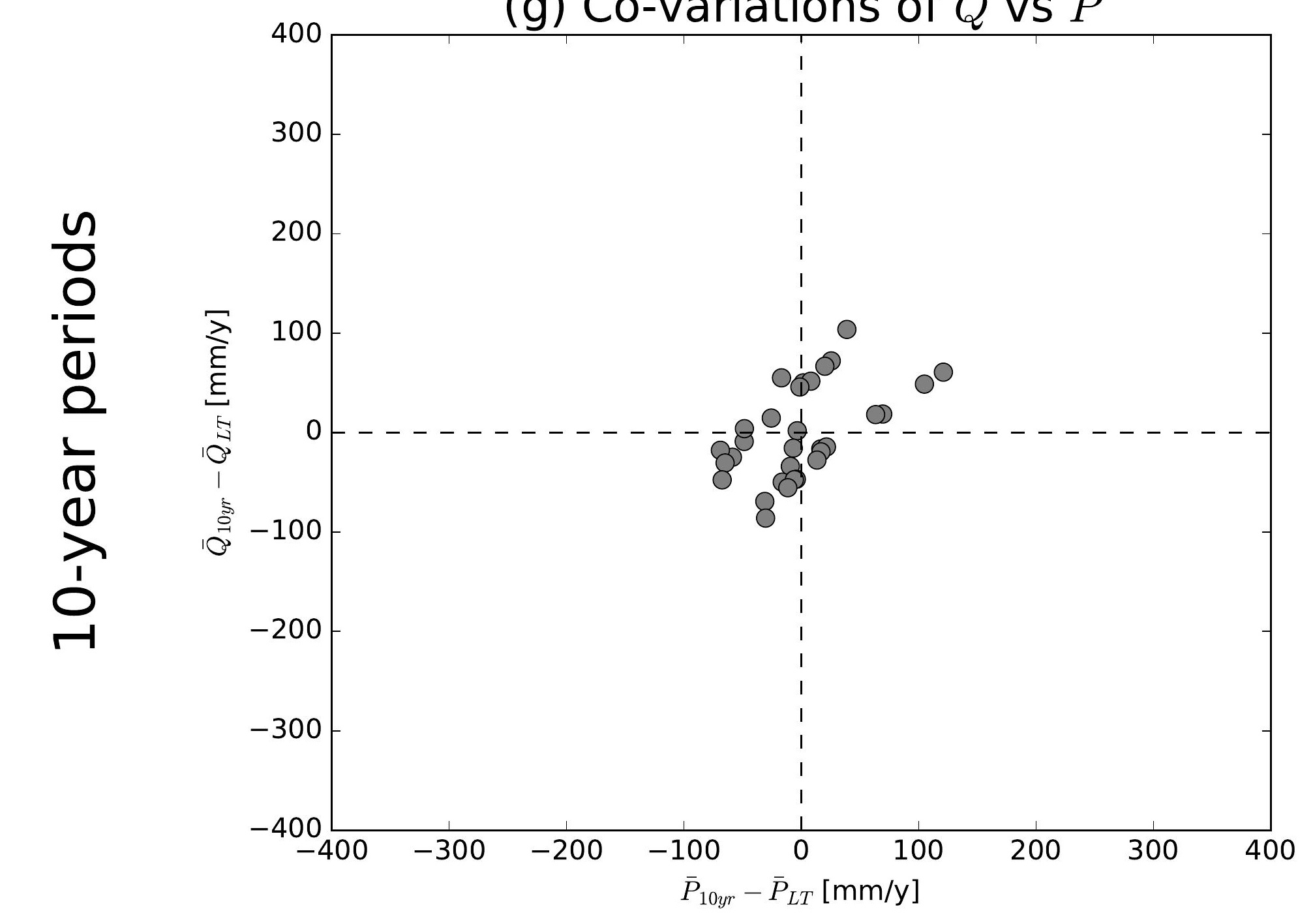

(j) Co-variations of $\bar{Q}$ vs $\bar{P}$

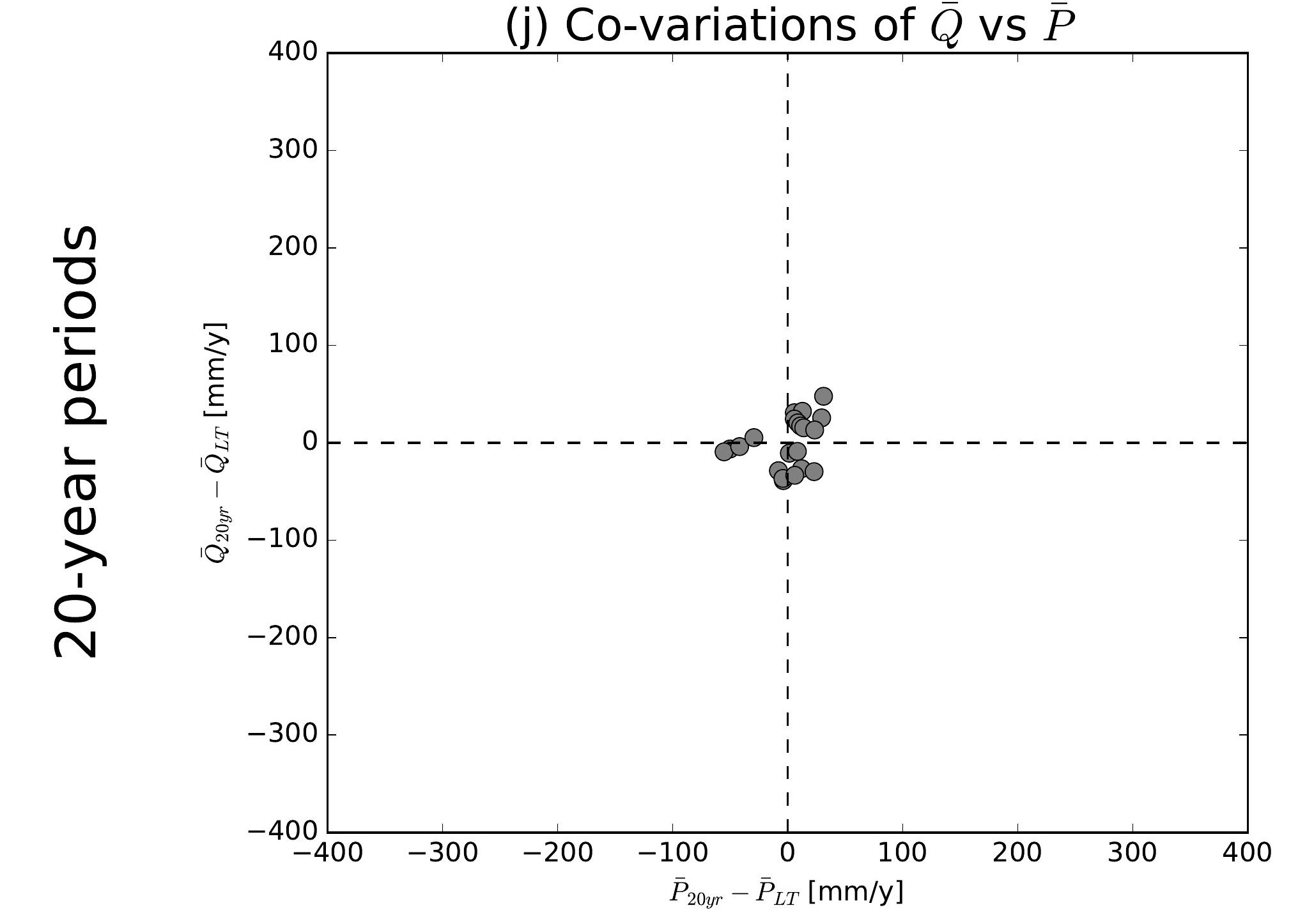

(b) Co-variations of $\bar{Q}$ vs $\overline{E 0}$

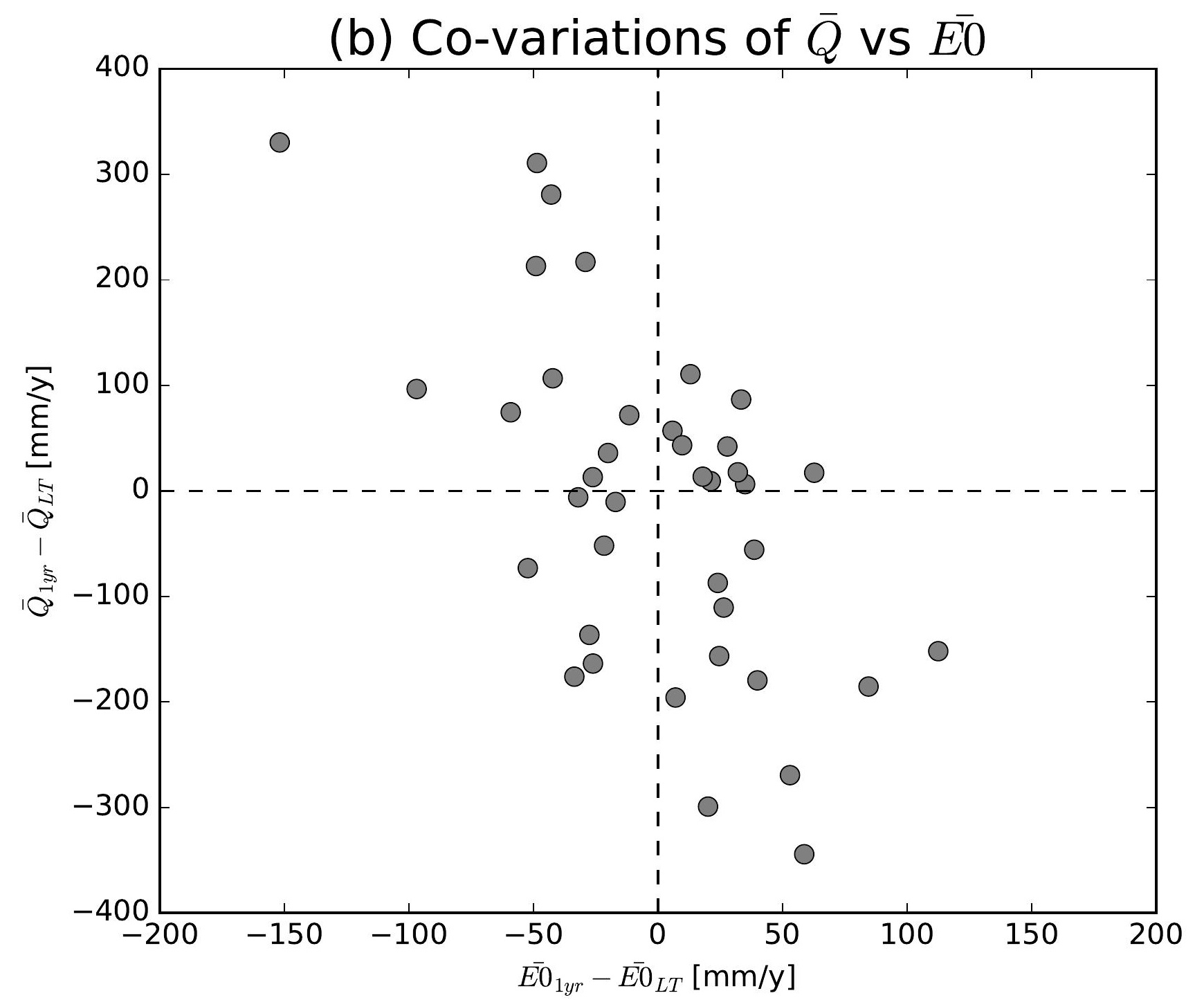

(e) Co-variations of $\bar{Q}$ vs $\overline{E 0}$

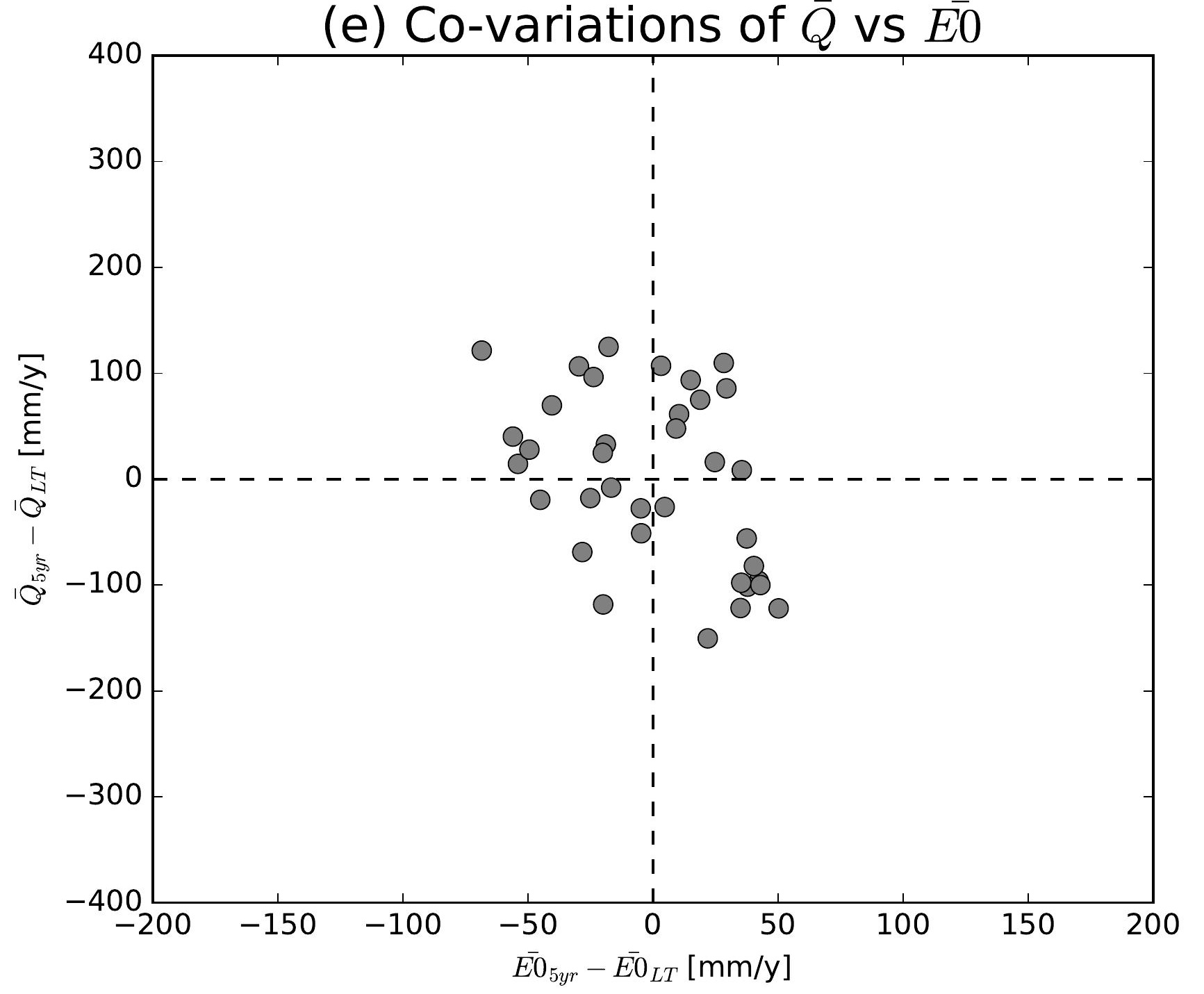

(h) Co-variations of $\bar{Q}$ vs $\overline{E 0}$

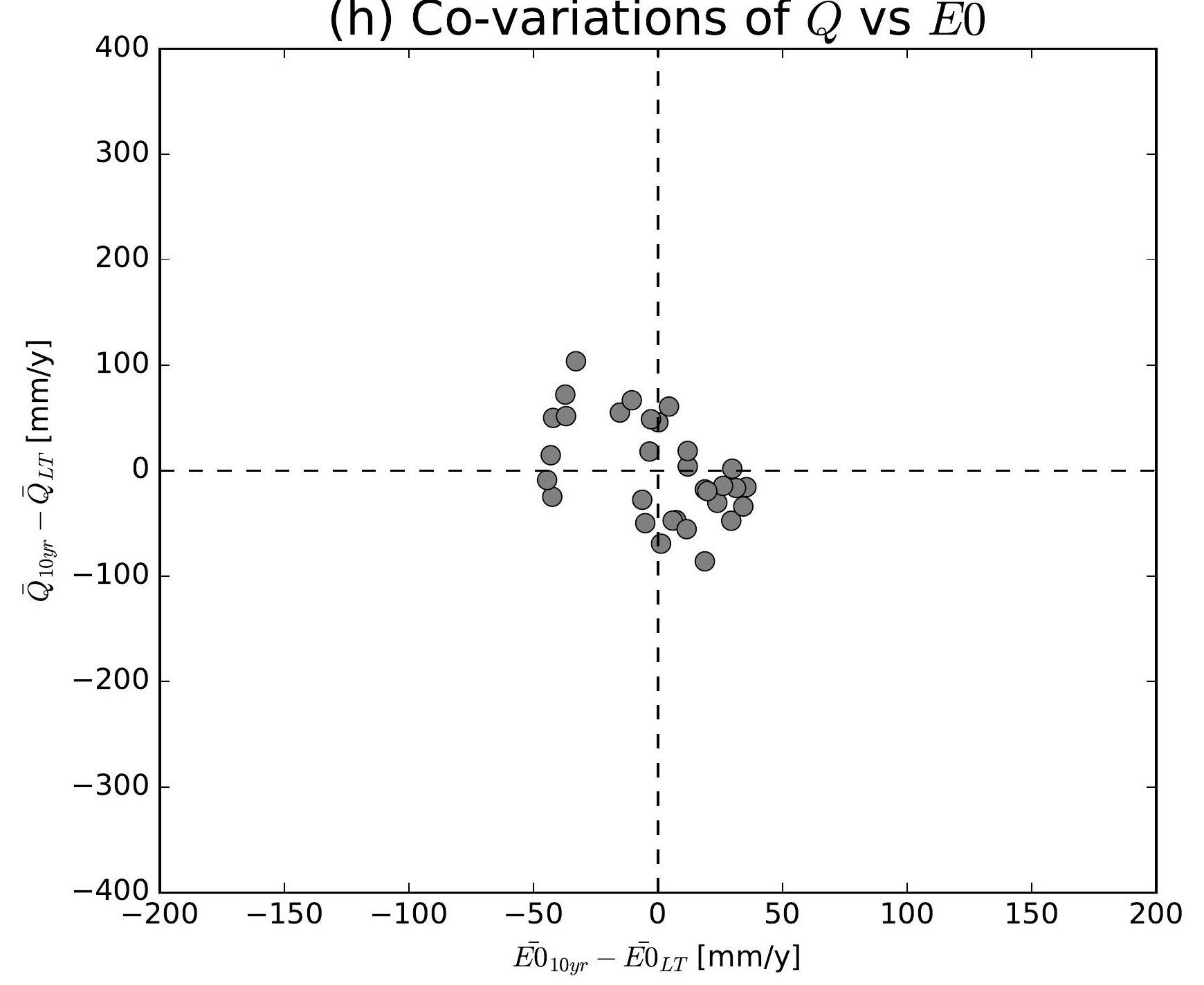

(k) Co-variations of $\bar{Q}$ vs $\overline{E 0}$

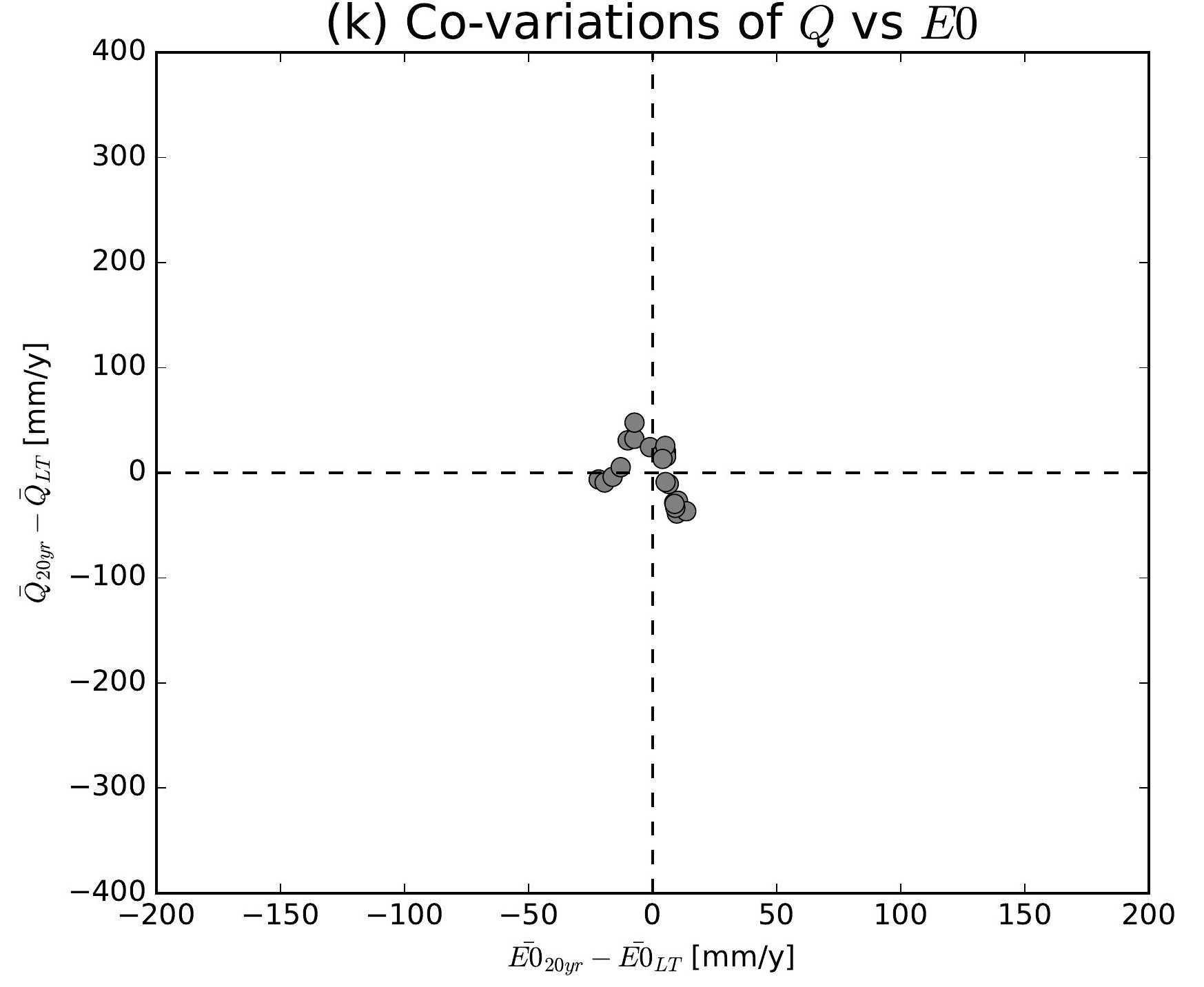

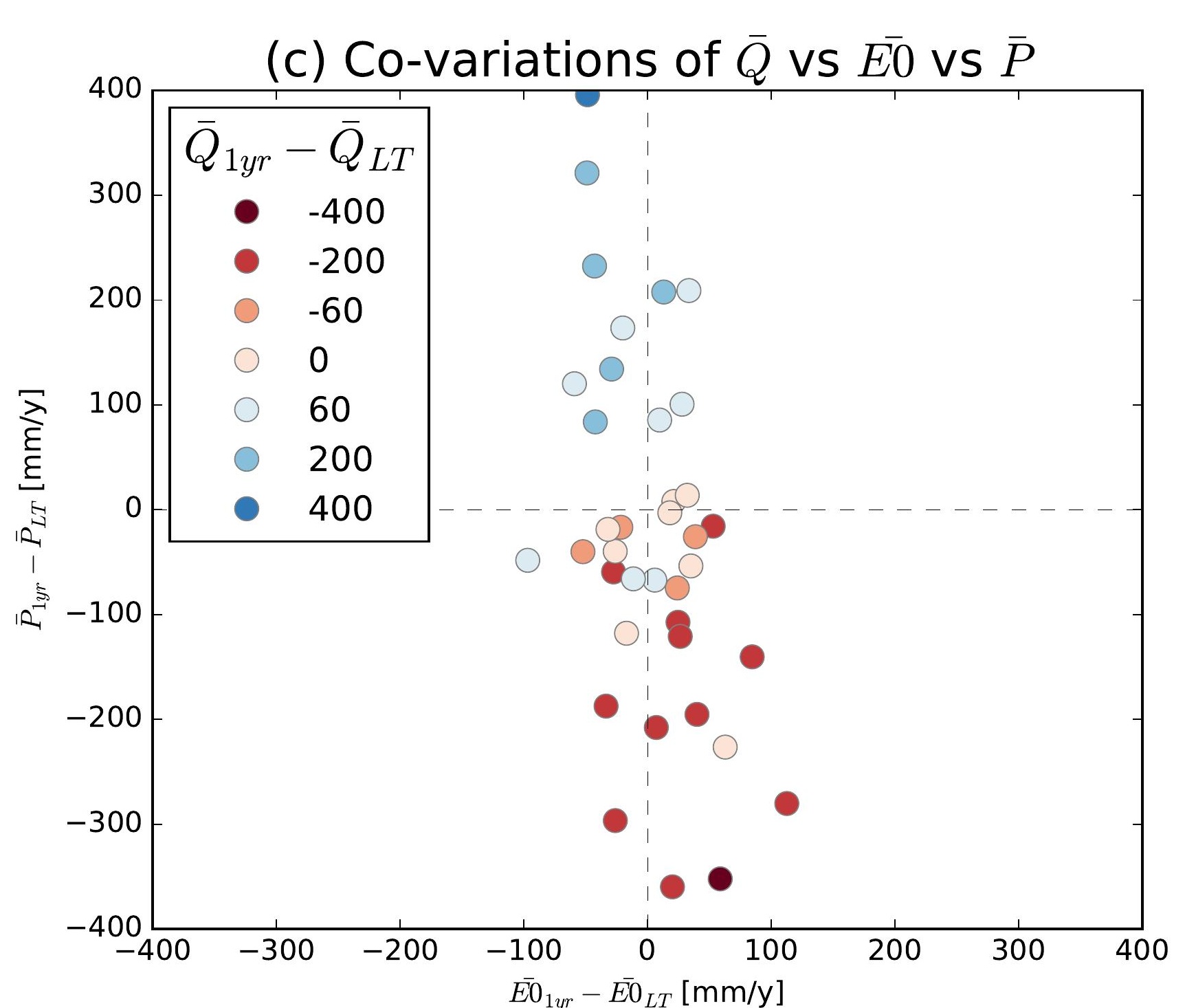
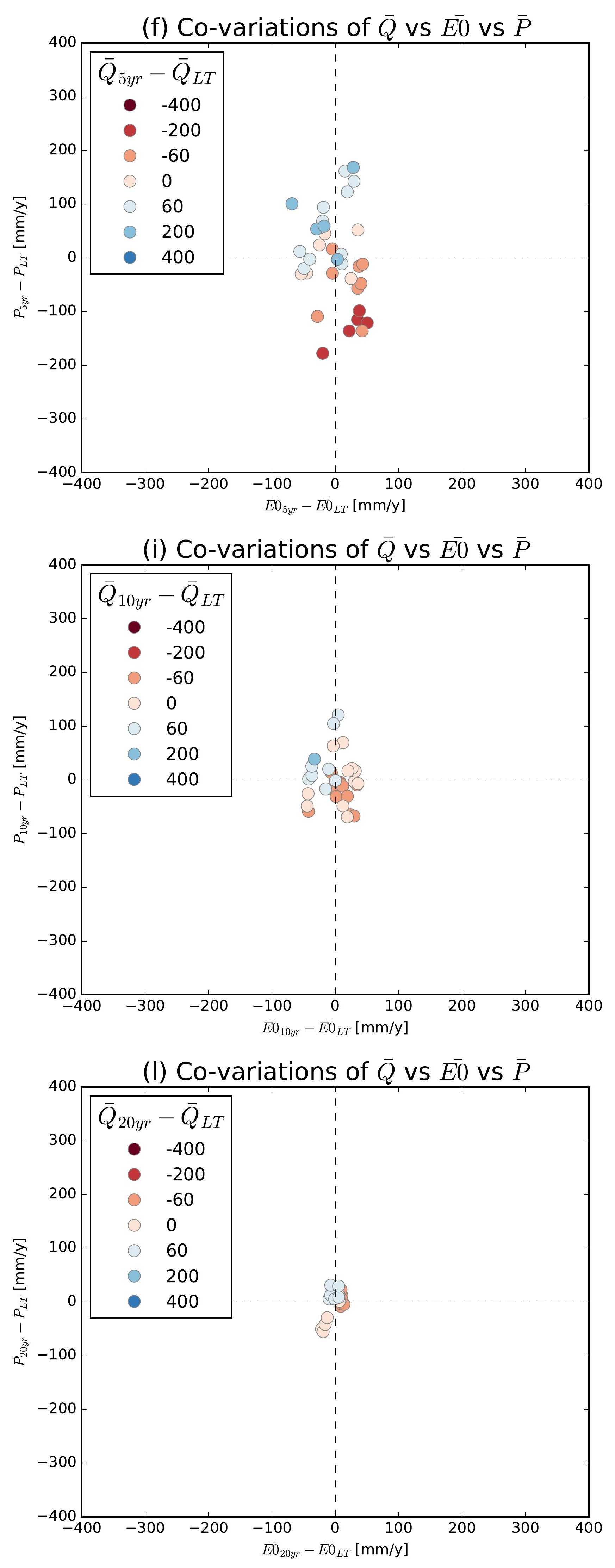

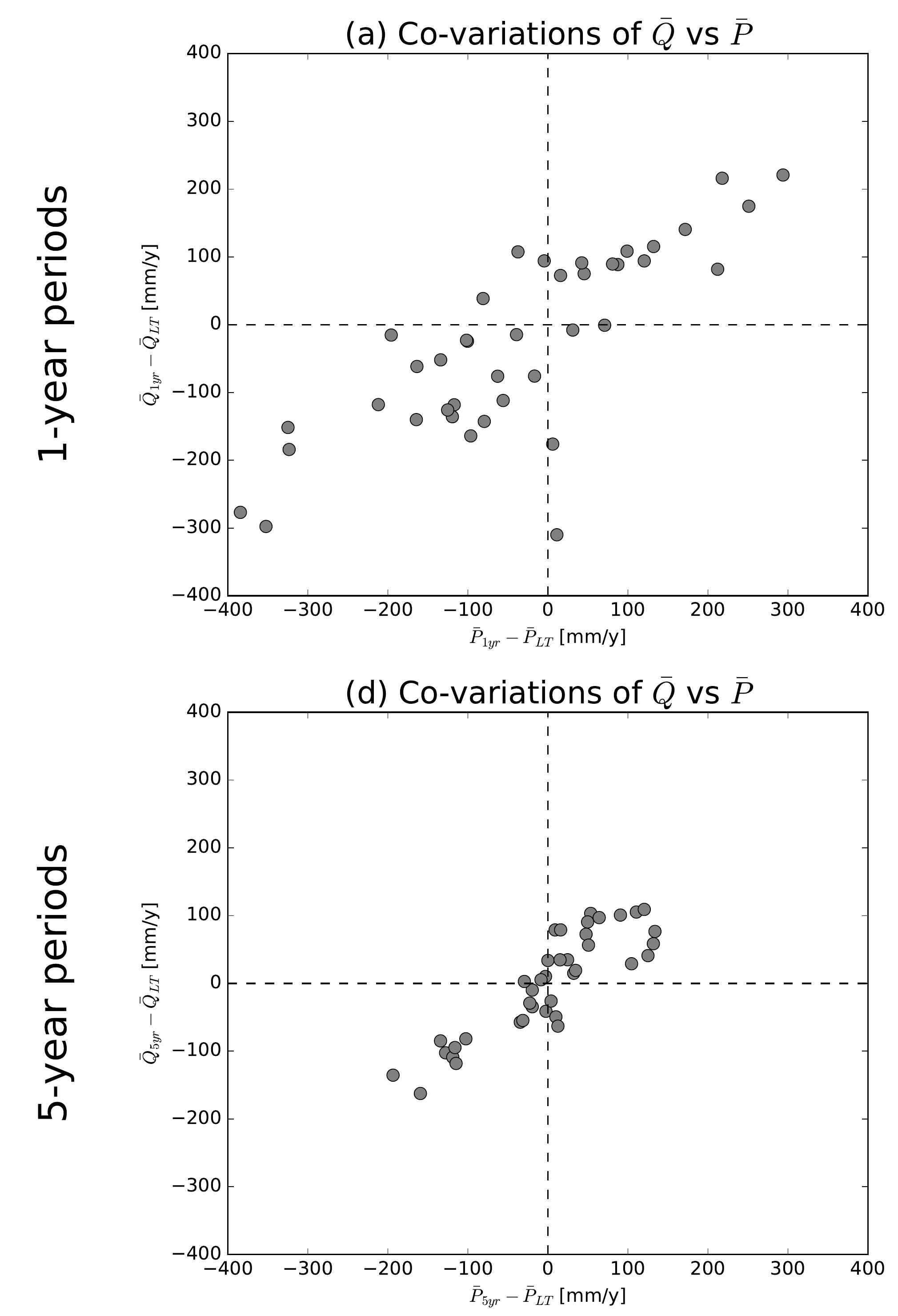

(g) Co-variations of $\bar{Q}$ vs $\bar{P}$
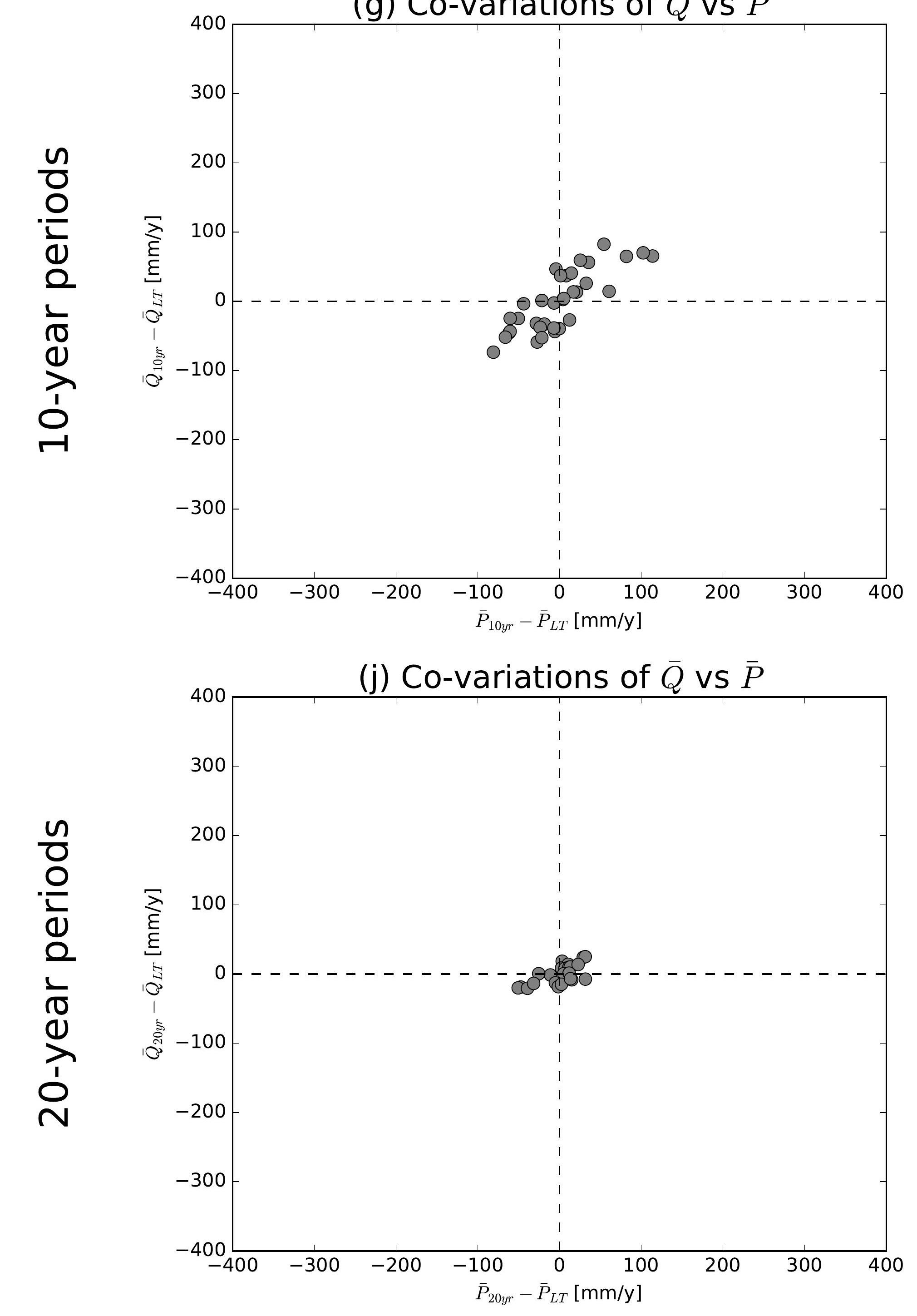

(b) Co-variations of $\bar{Q}$ vs $\overline{E 0}$

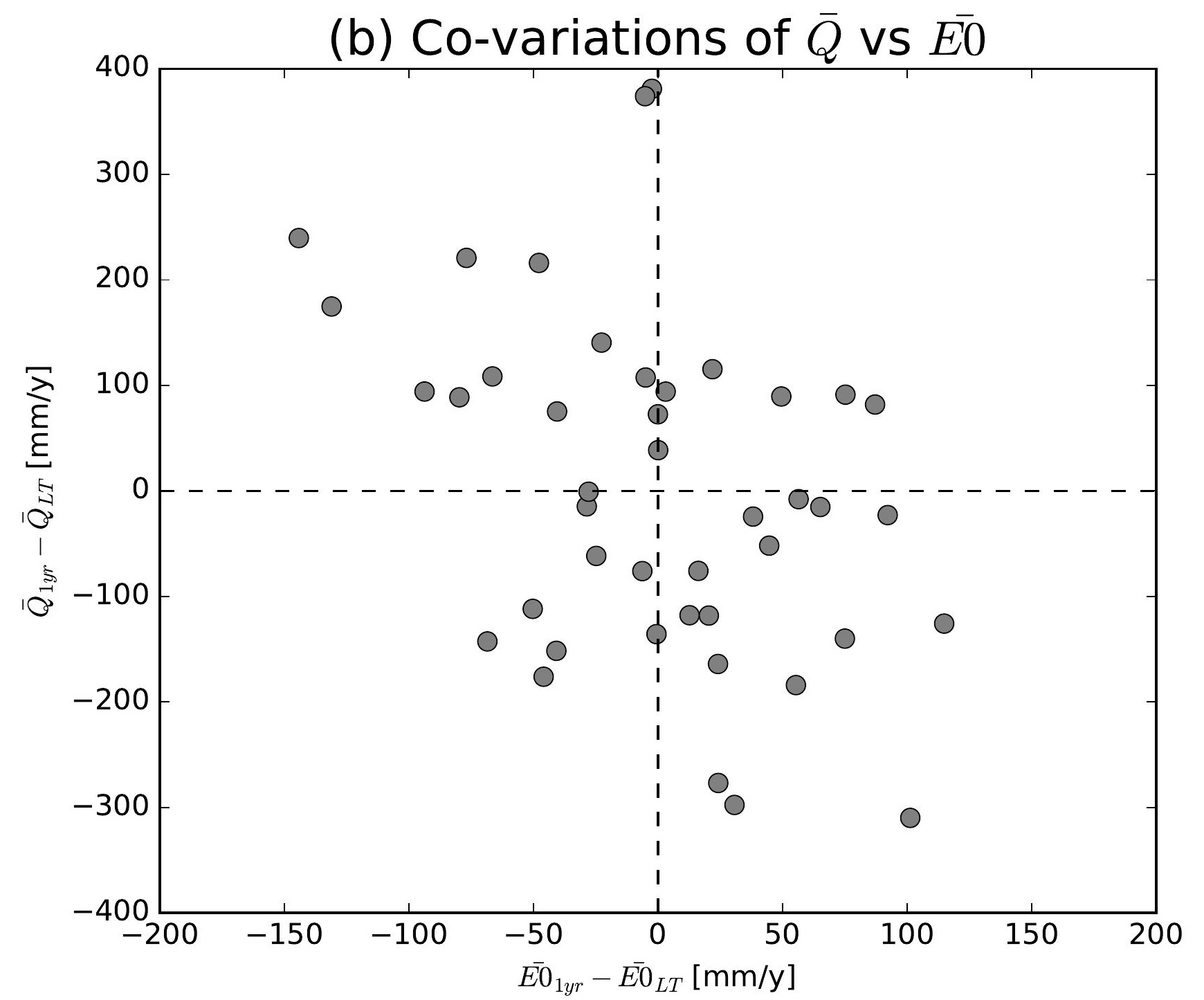

(e) Co-variations of $\bar{Q}$ vs $\overline{E 0}$

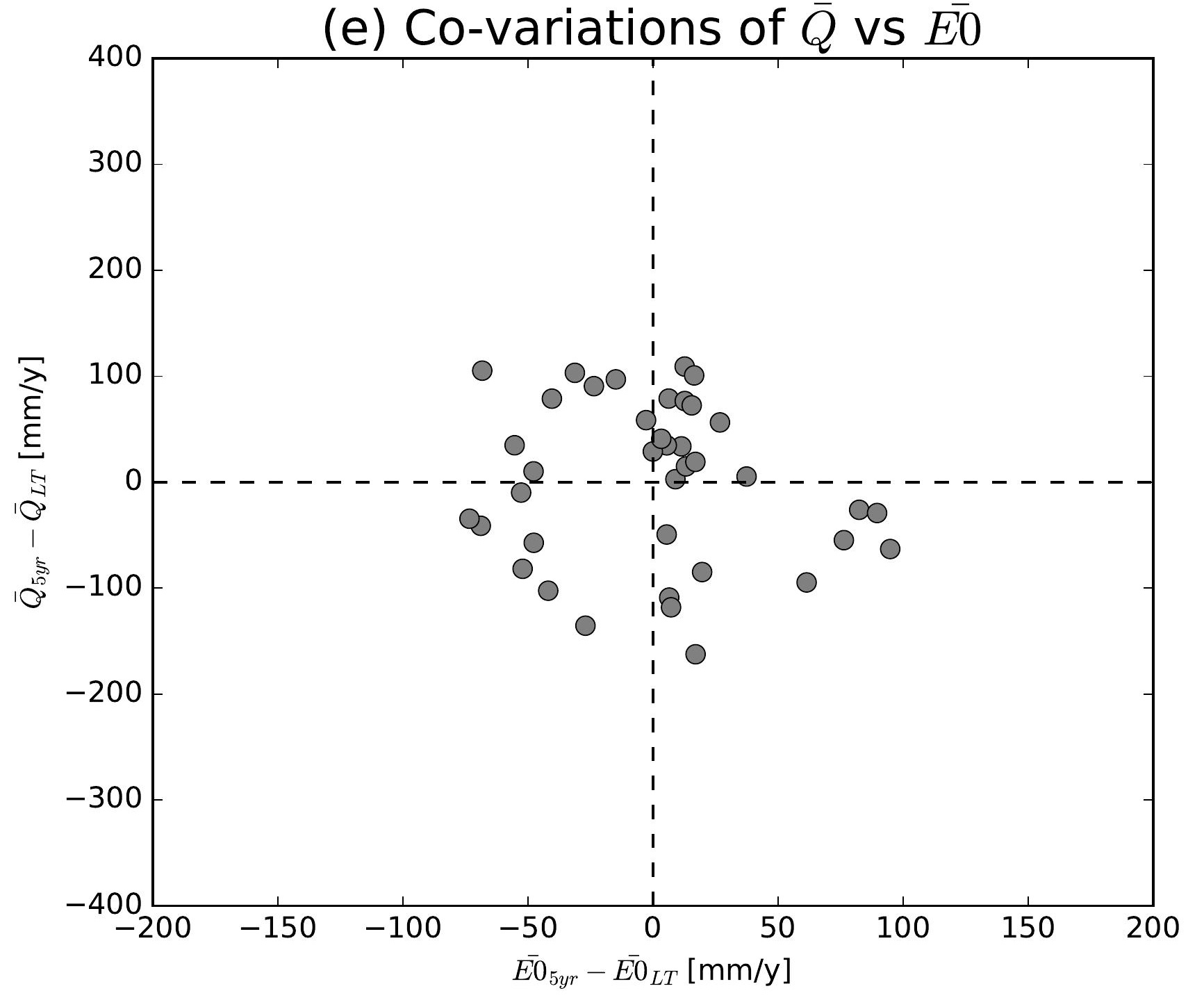

(h) Co-variations of $\bar{Q}$ vs $\overline{E 0}$

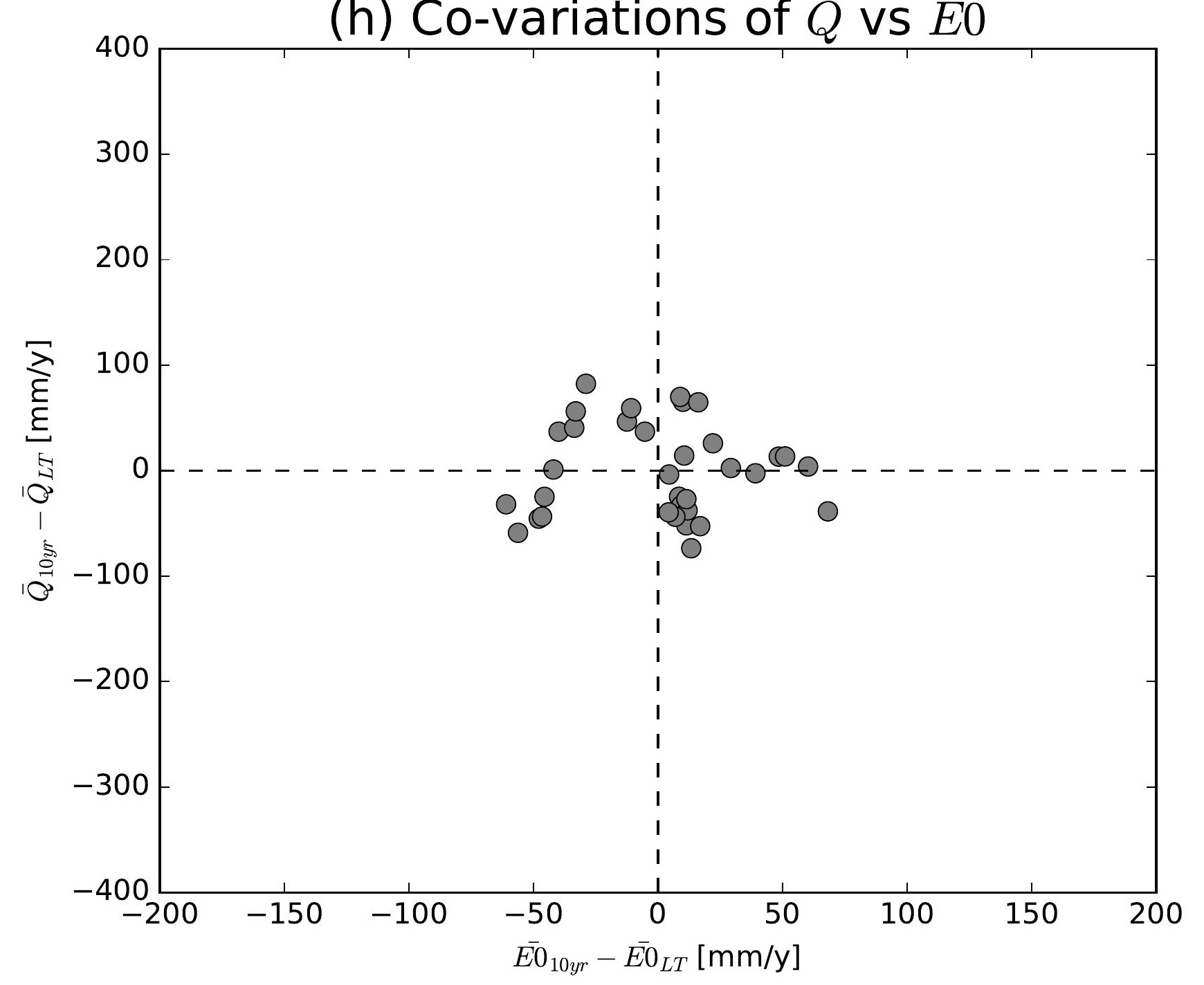

(k) Co-variations of $\bar{Q}$ vs $\overline{E 0}$

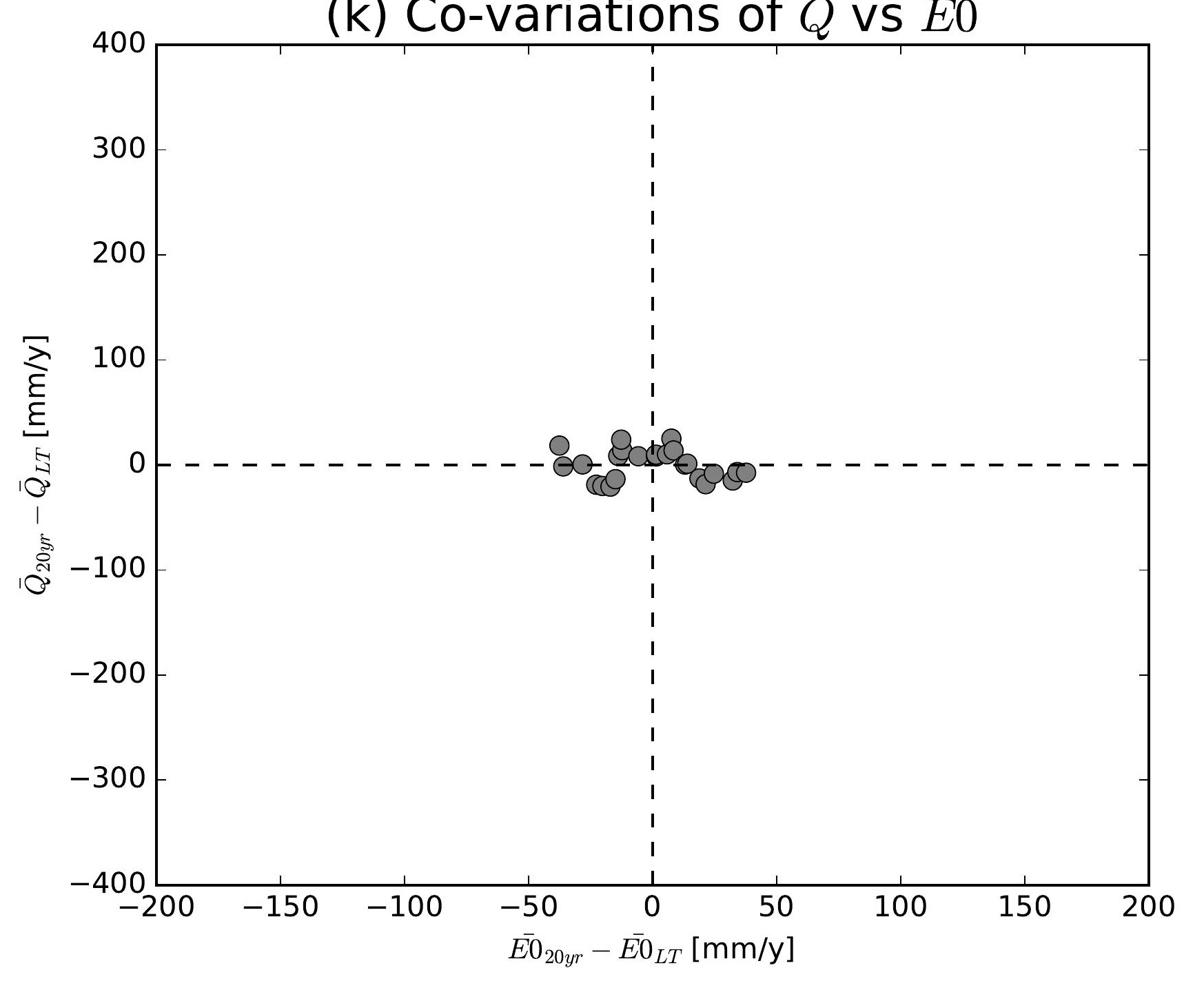

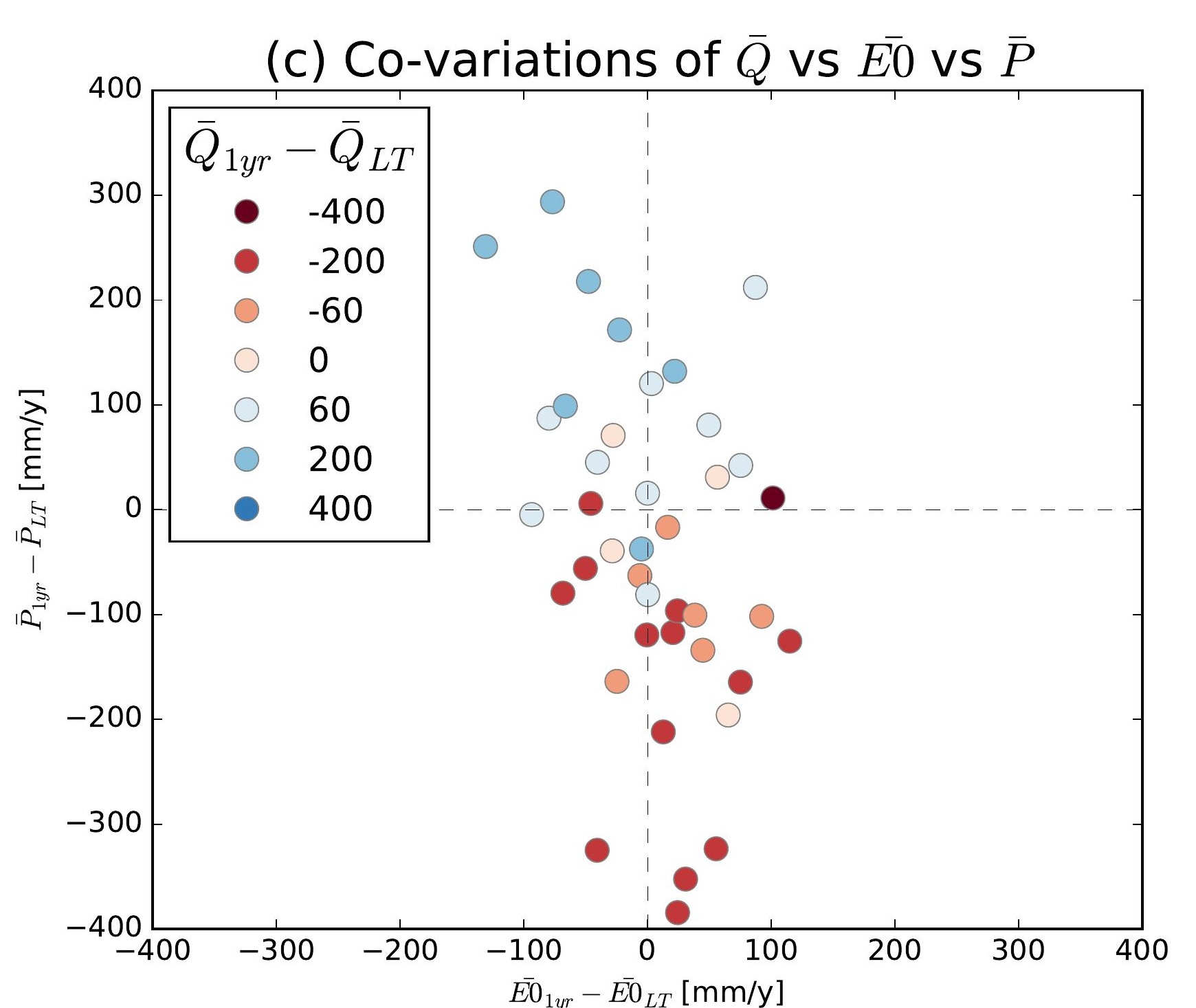
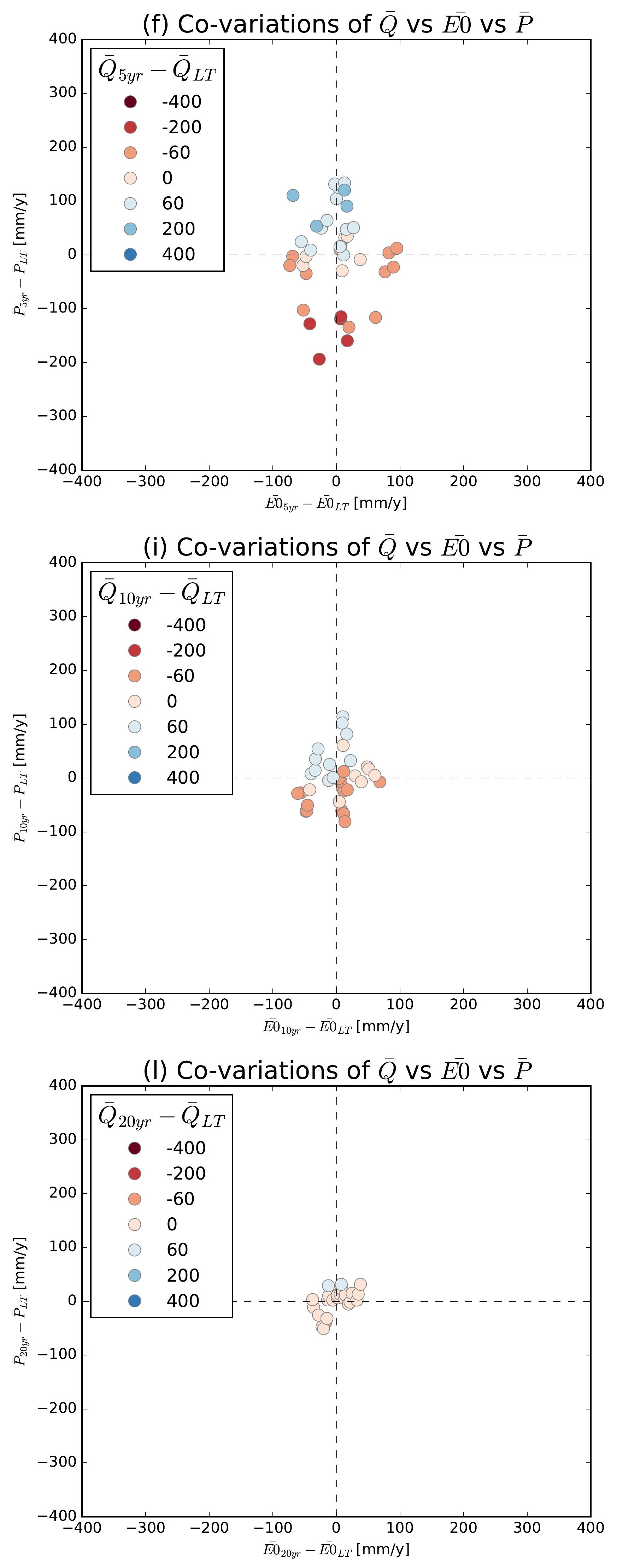

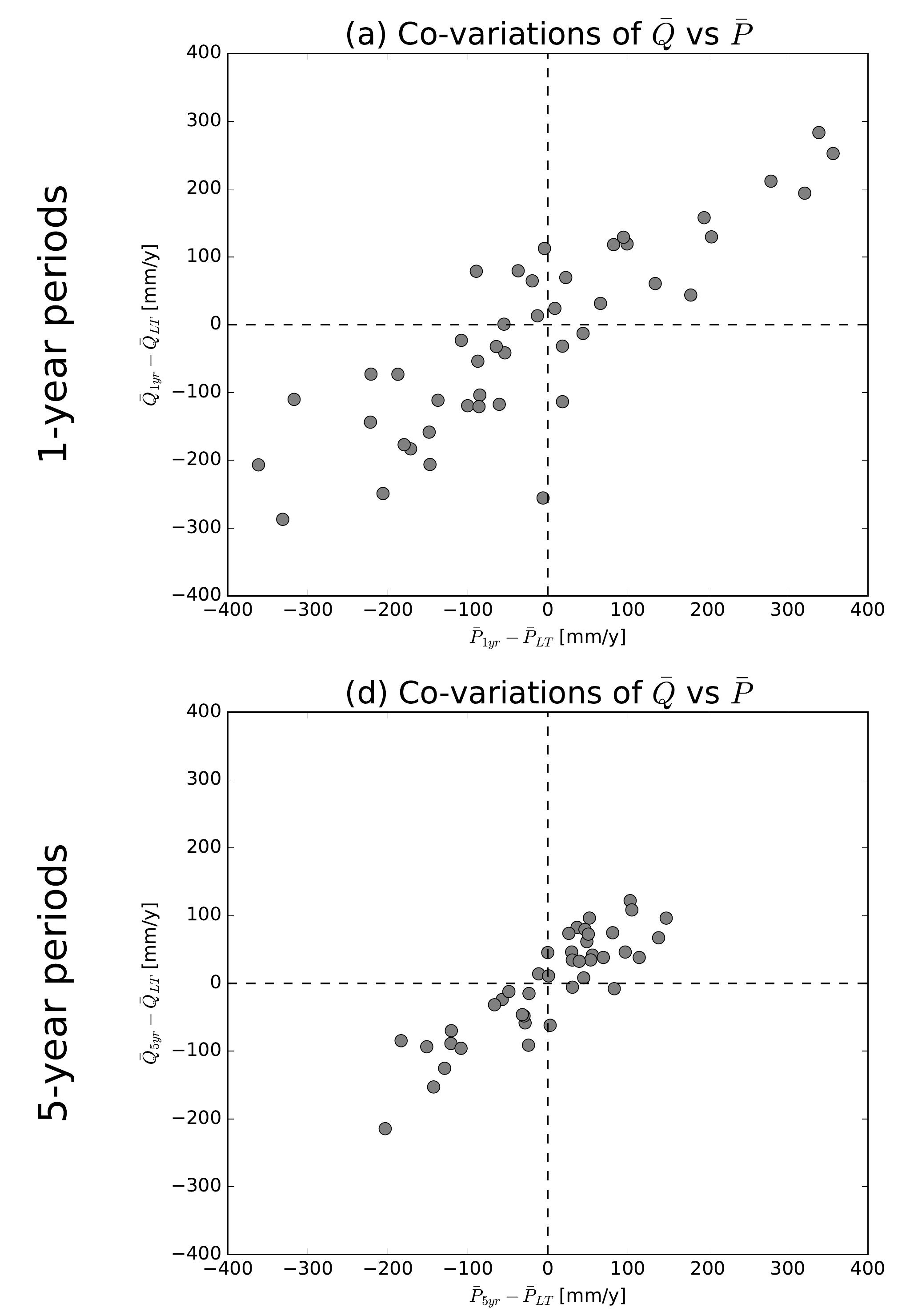

(g) Co-variations of $\bar{Q}$ vs $\bar{P}$
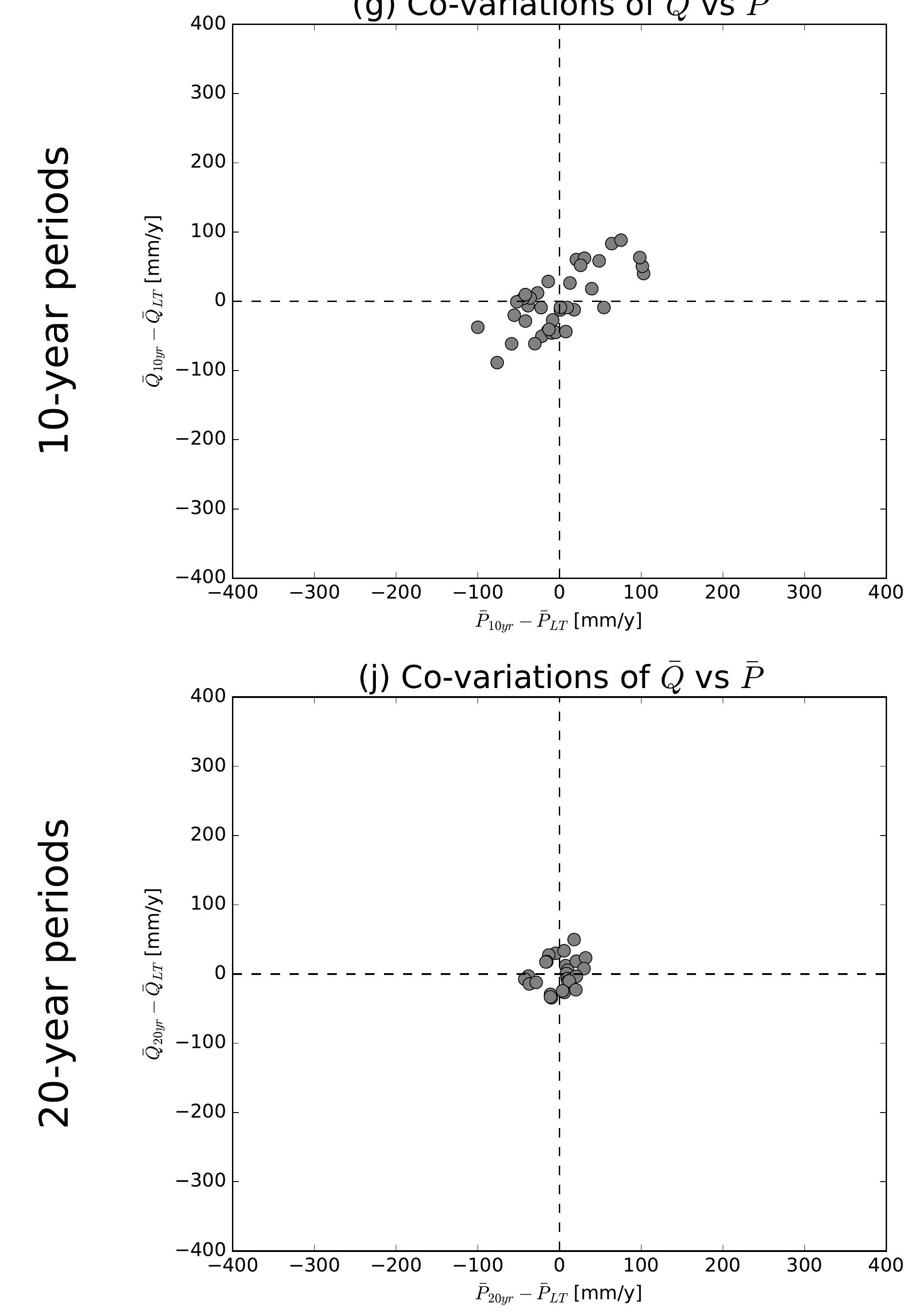

(b) Co-variations of $\bar{Q}$ vs $\overline{E 0}$

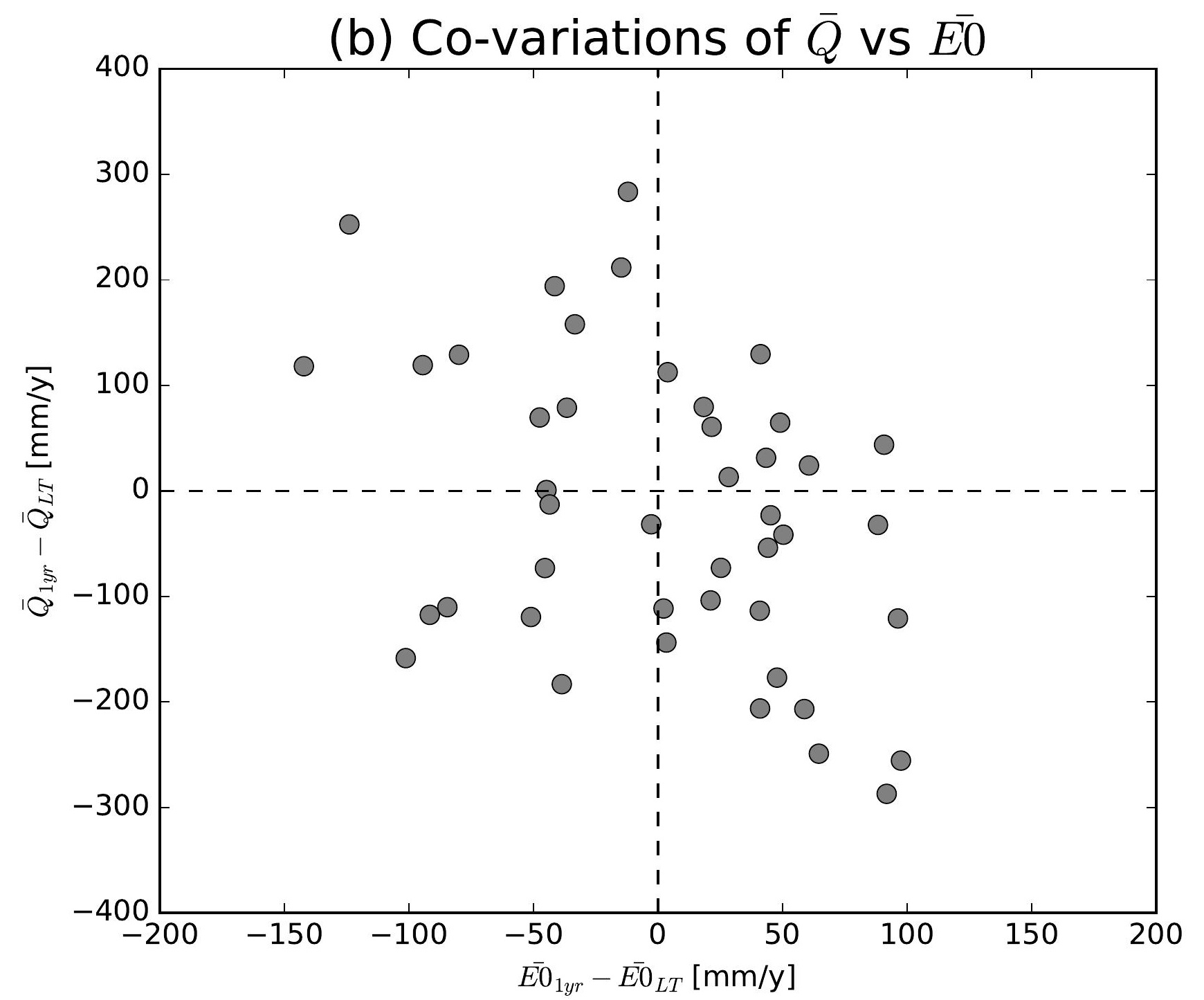

(e) Co-variations of $\bar{Q}$ vs $\overline{E 0}$

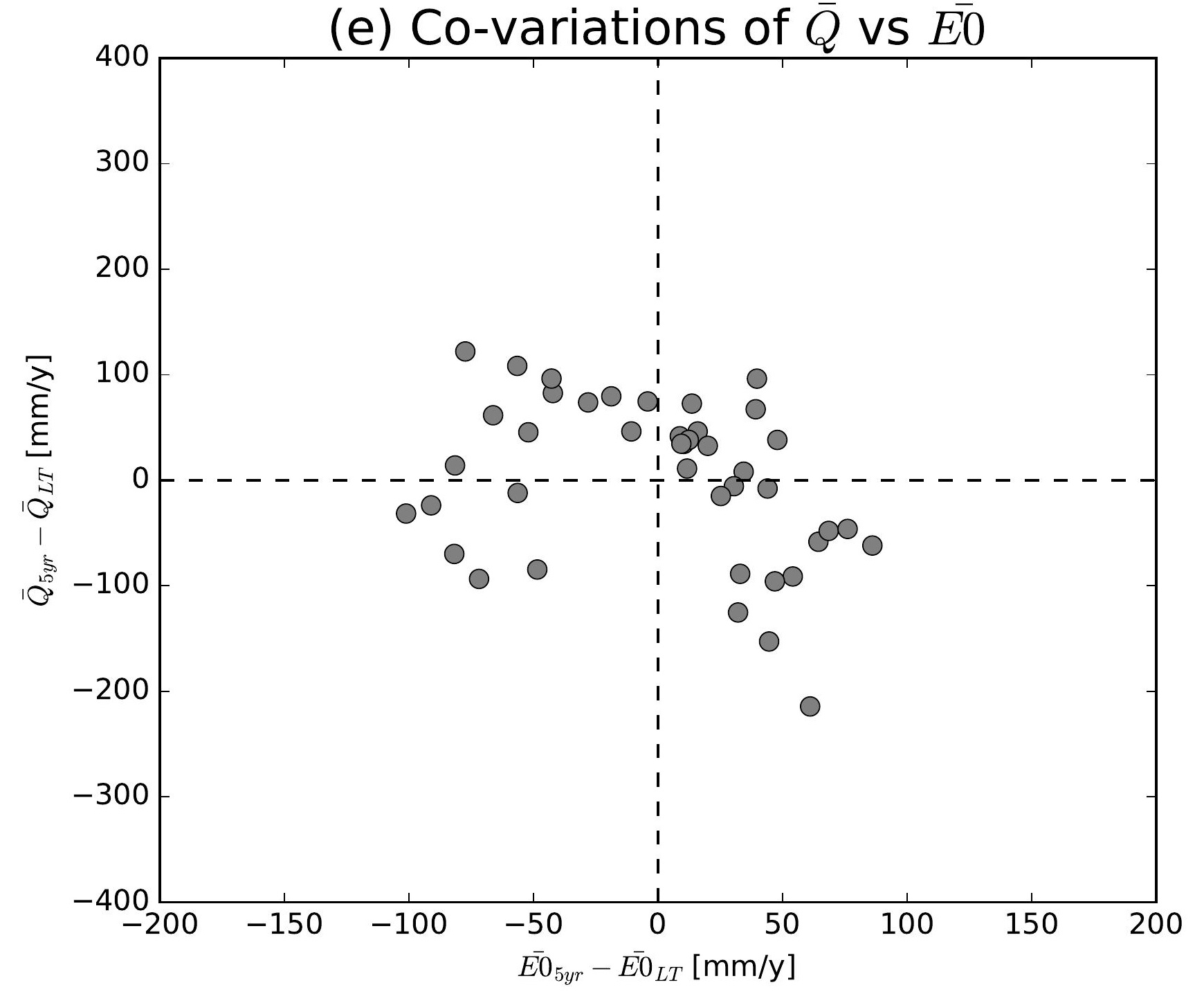

(h) Co-variations of $\bar{Q}$ vs $\overline{E 0}$

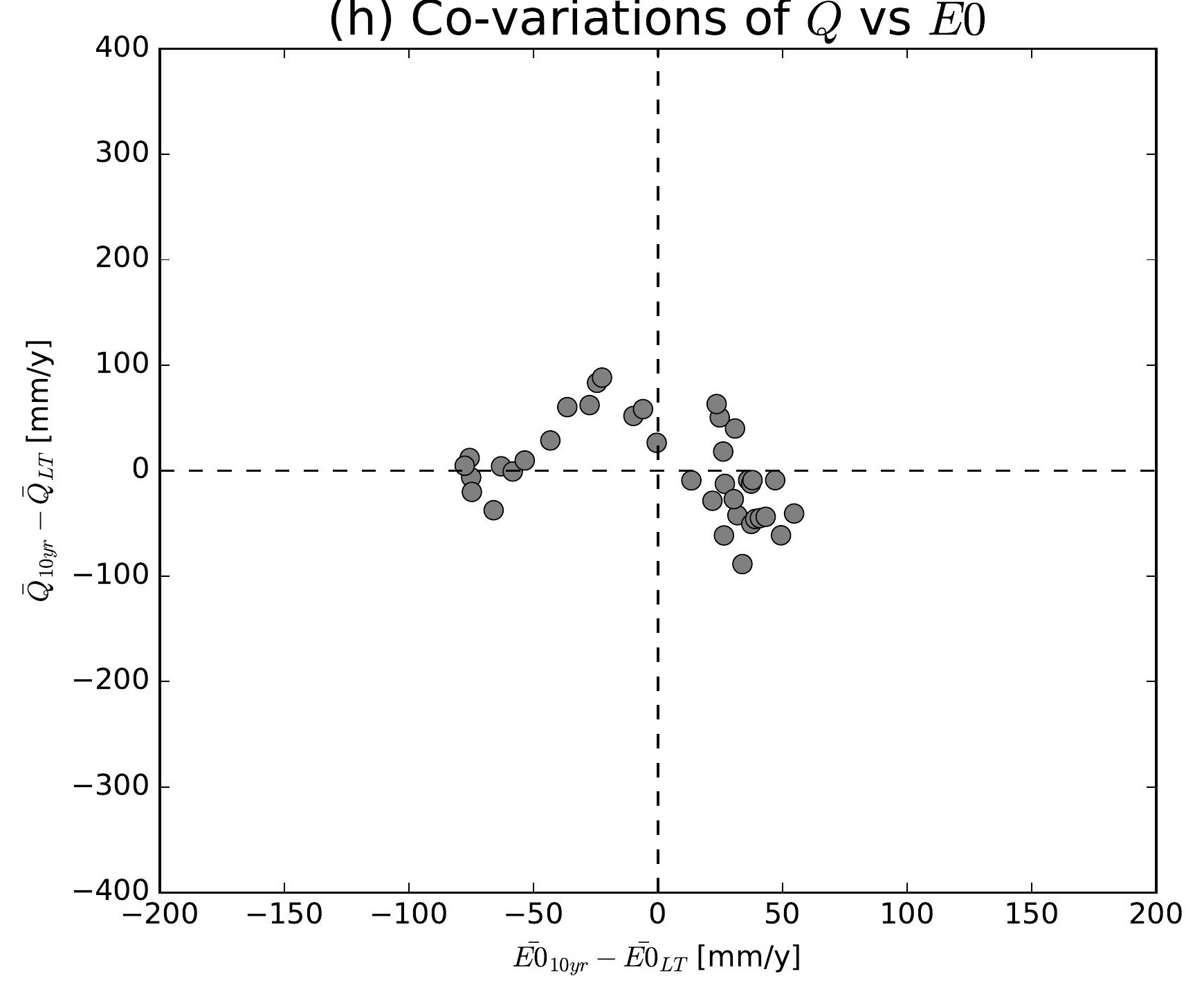

(k) Co-variations of $\bar{Q}$ vs $\overline{E 0}$

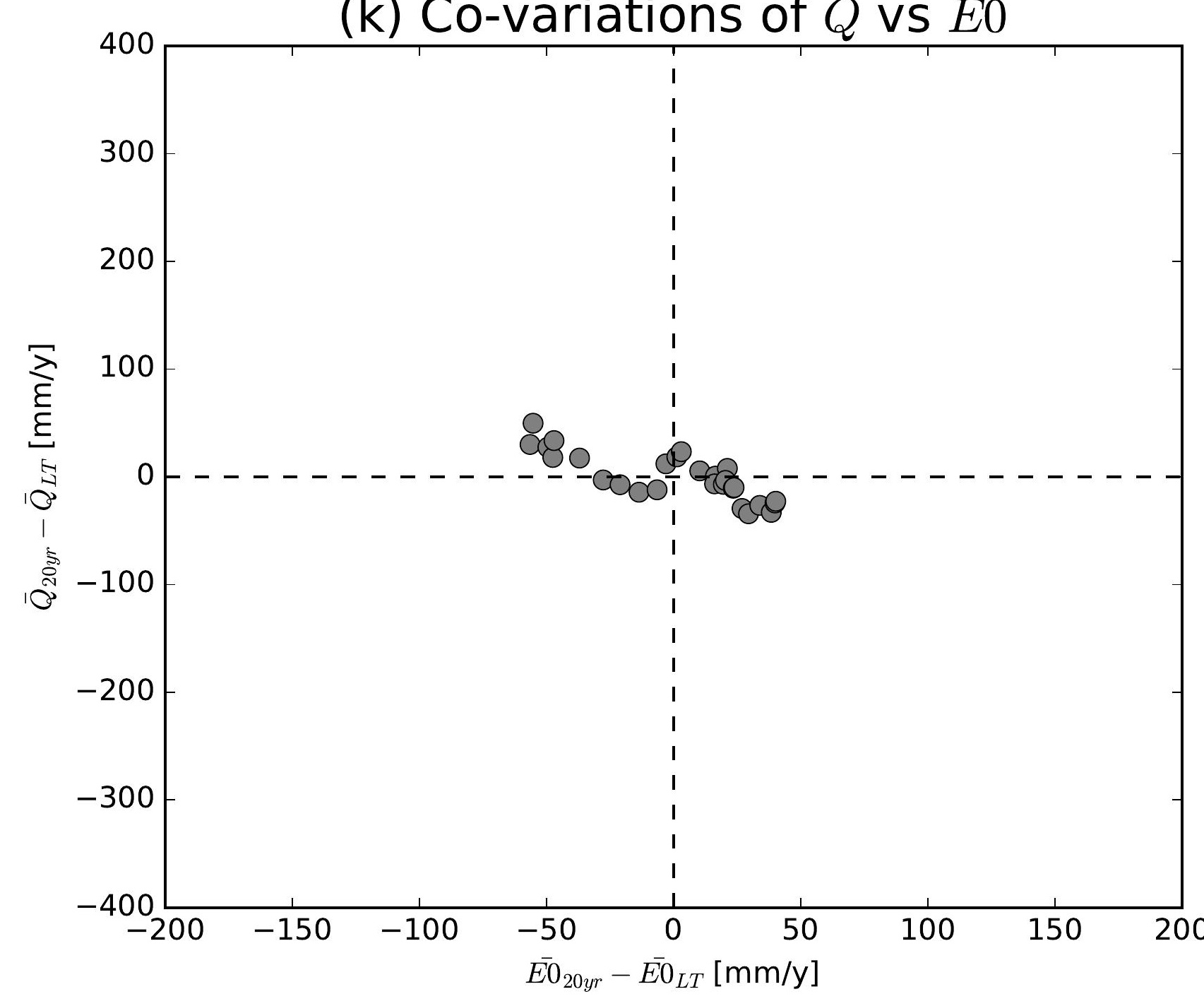

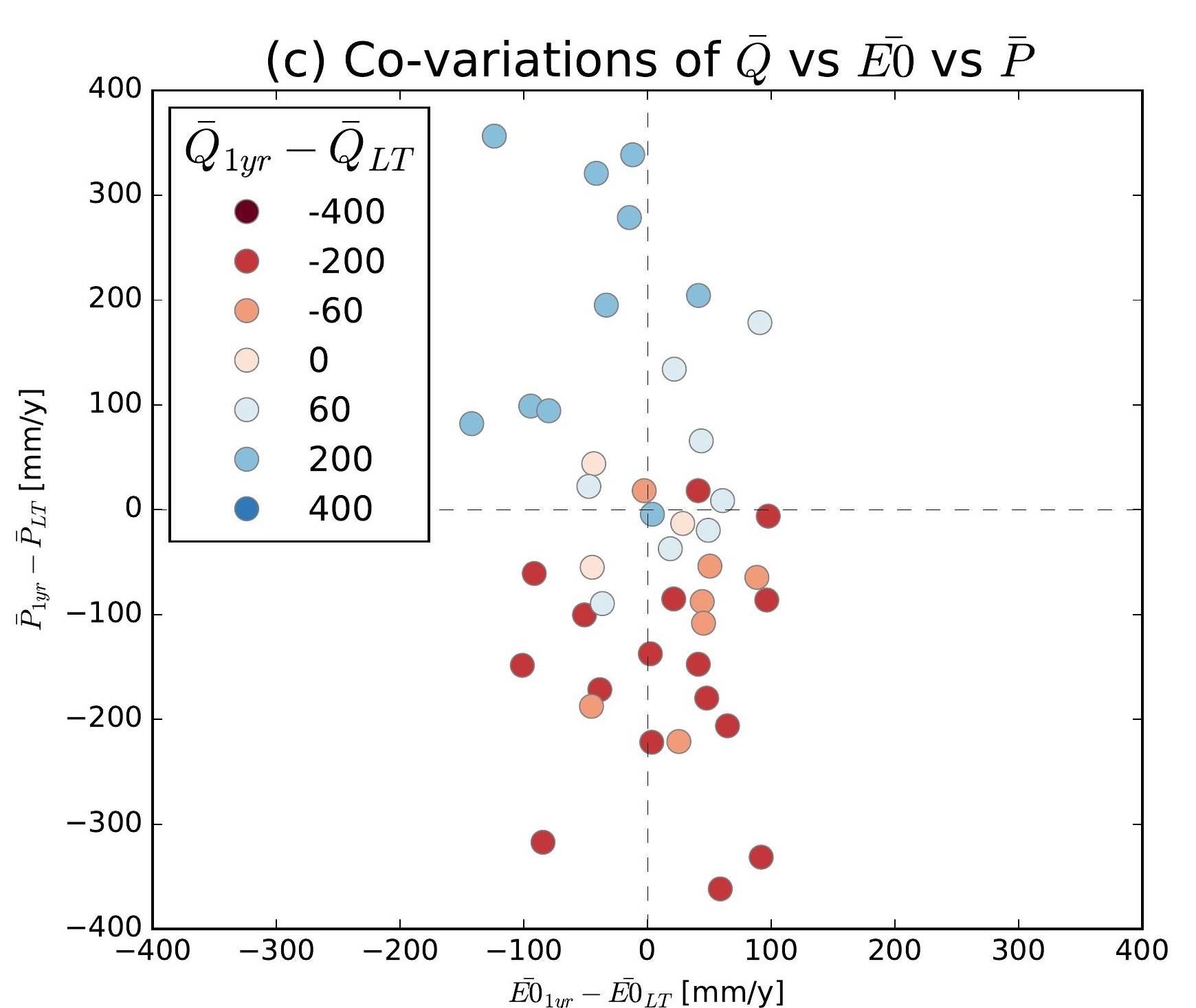
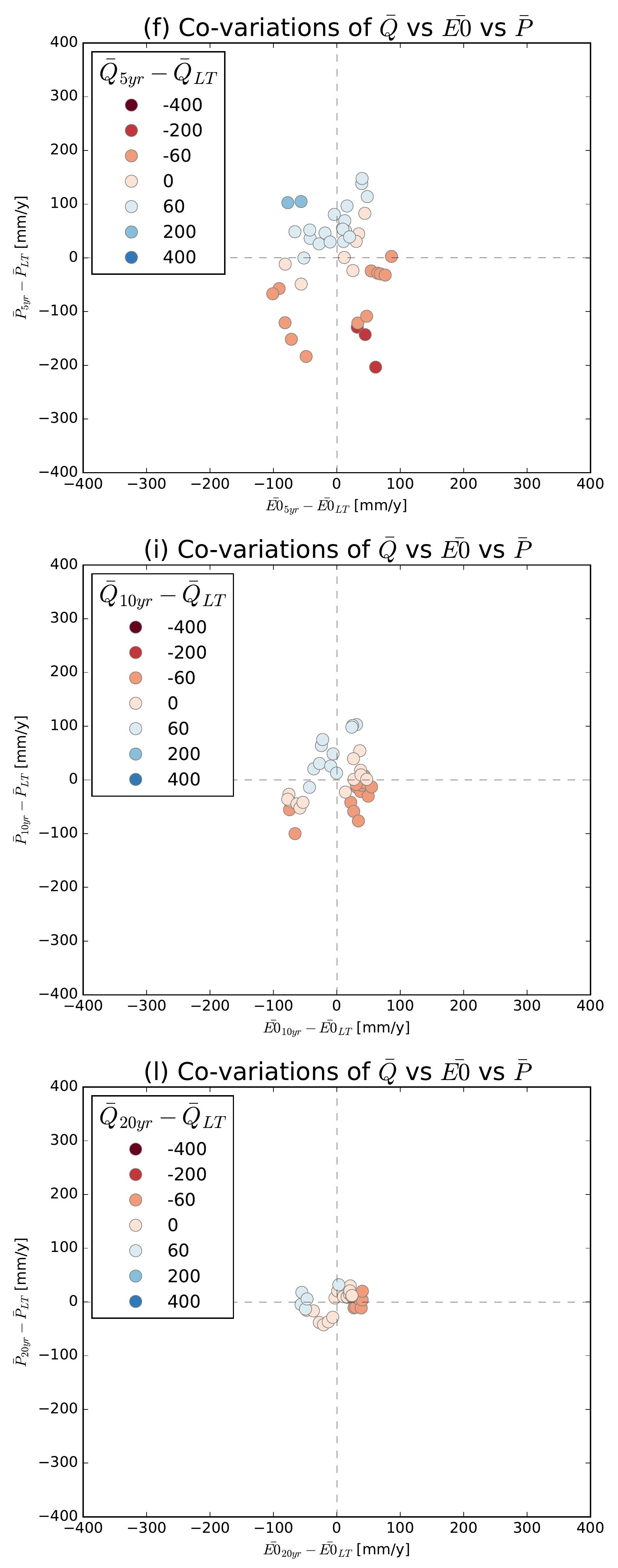

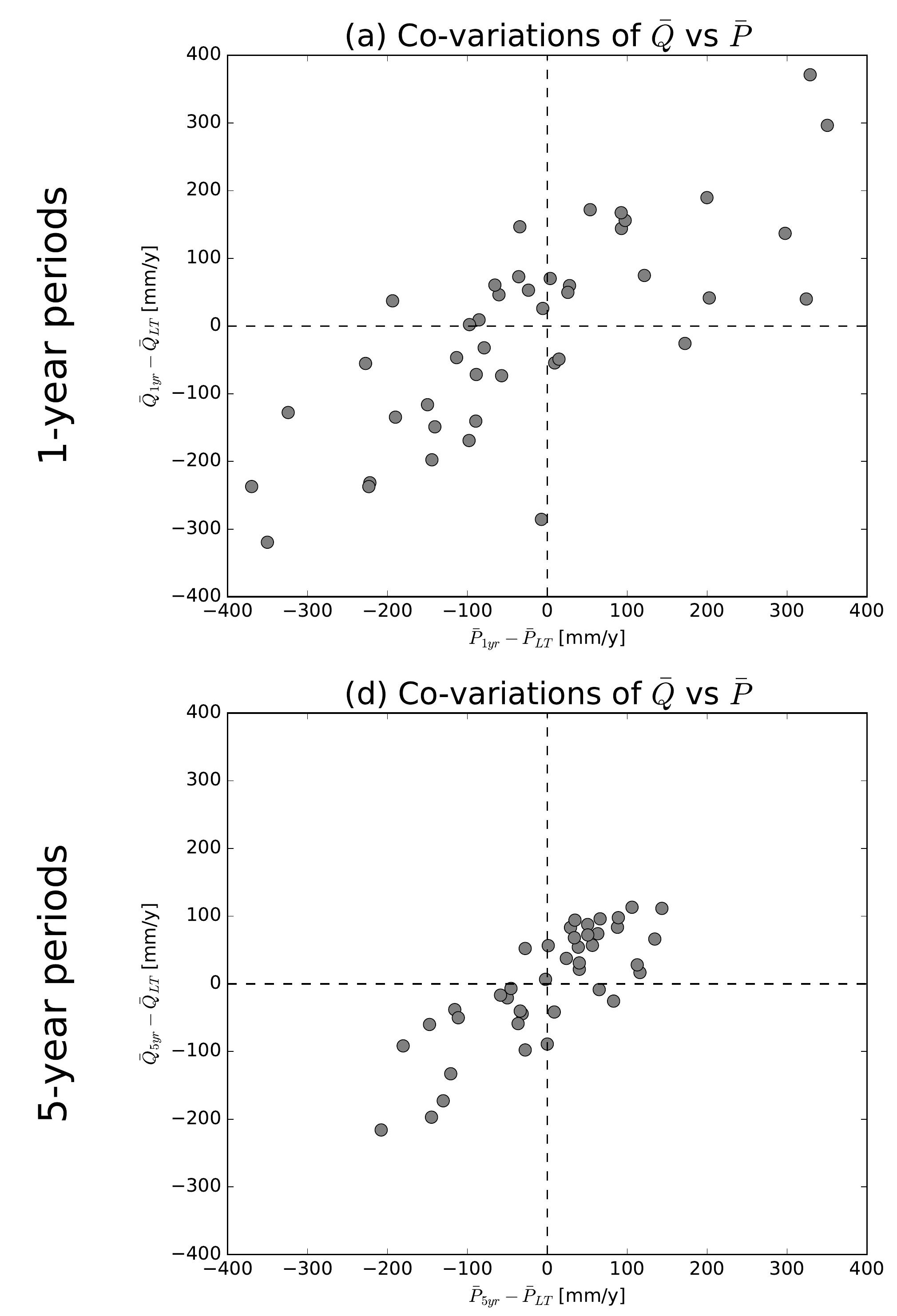

(g) Co-variations of $\bar{Q}$ vs $\bar{P}$

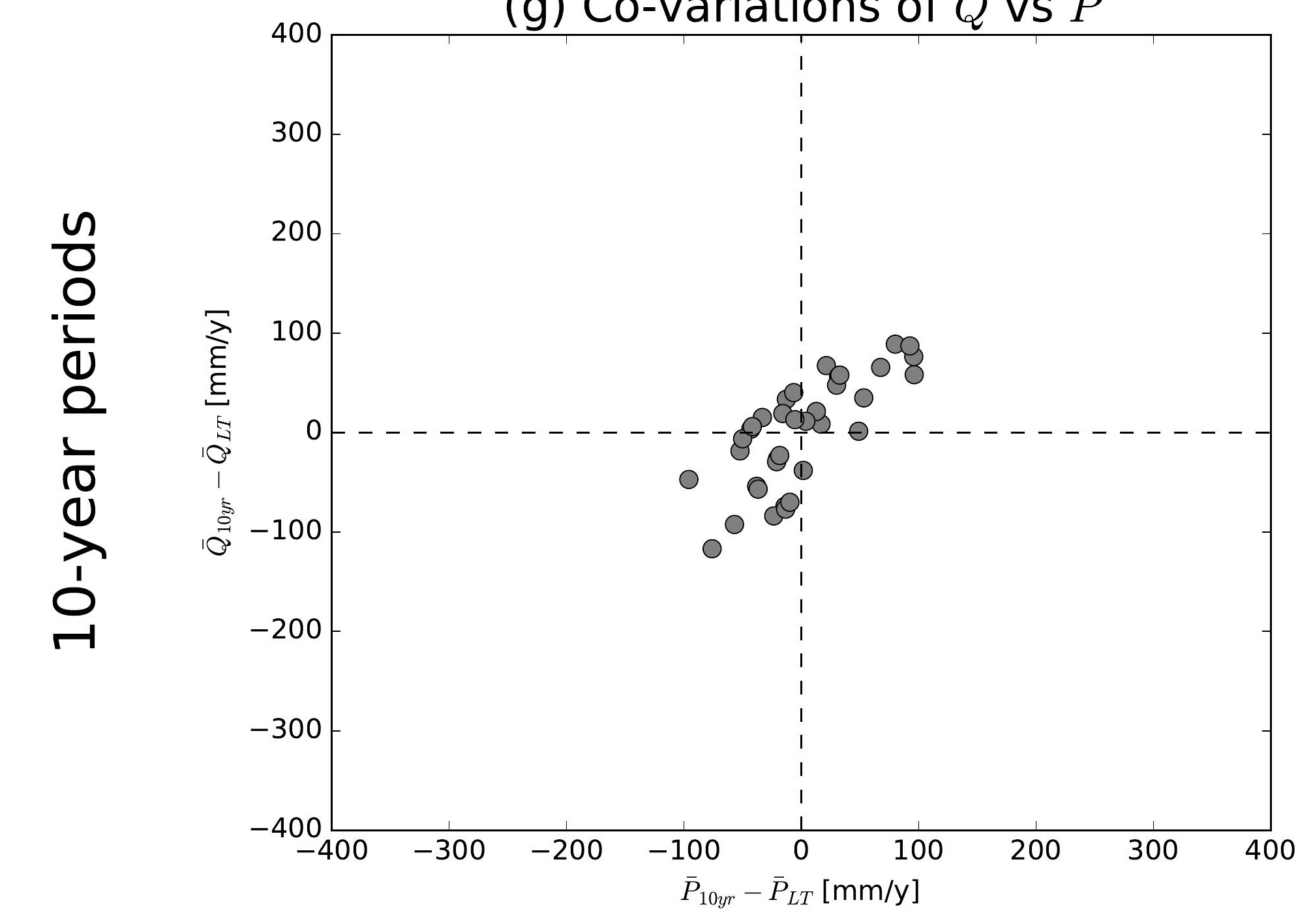

(j) Co-variations of $\bar{Q}$ vs $\bar{P}$

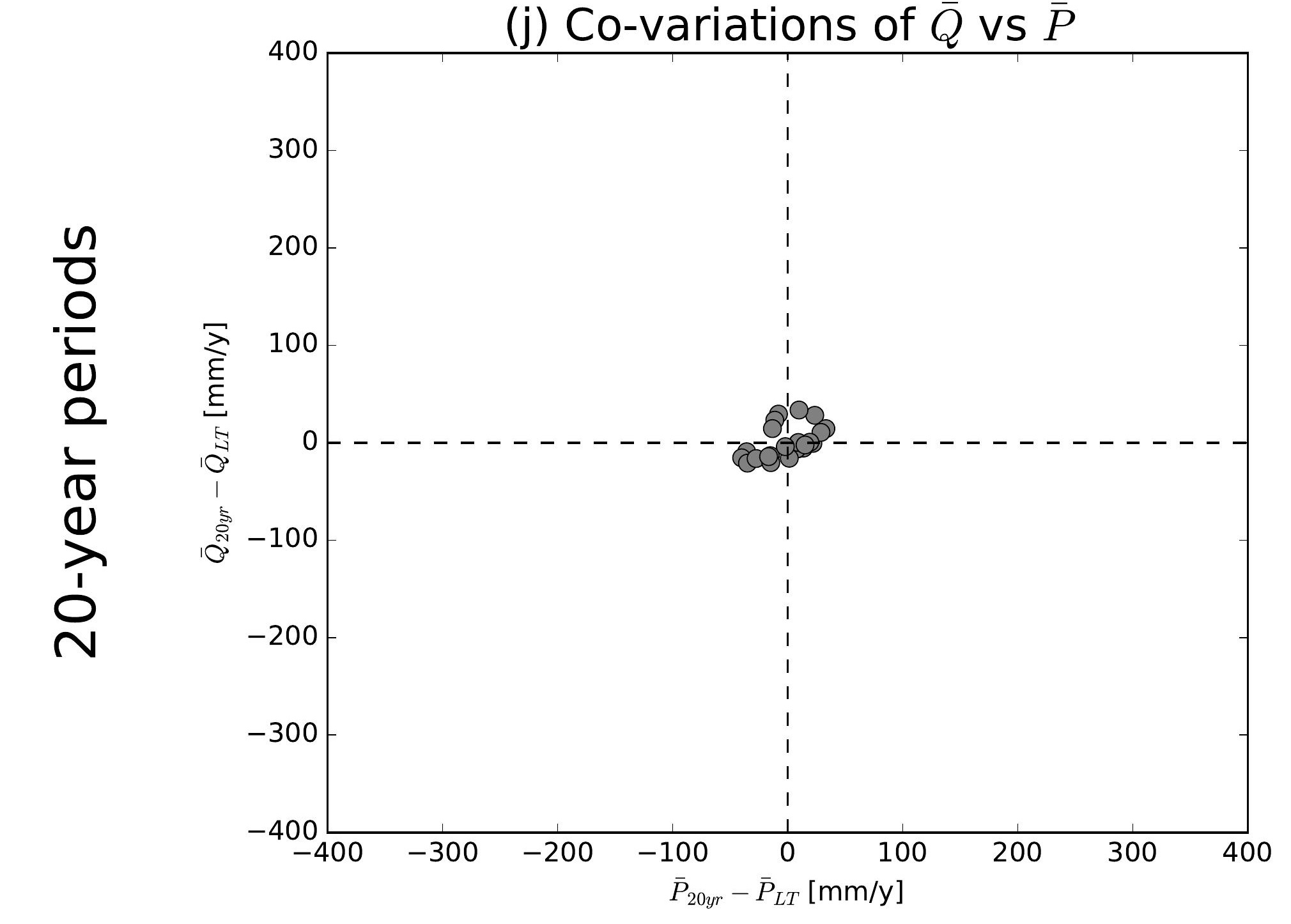

(b) Co-variations of $\bar{Q}$ vs $\overline{E 0}$

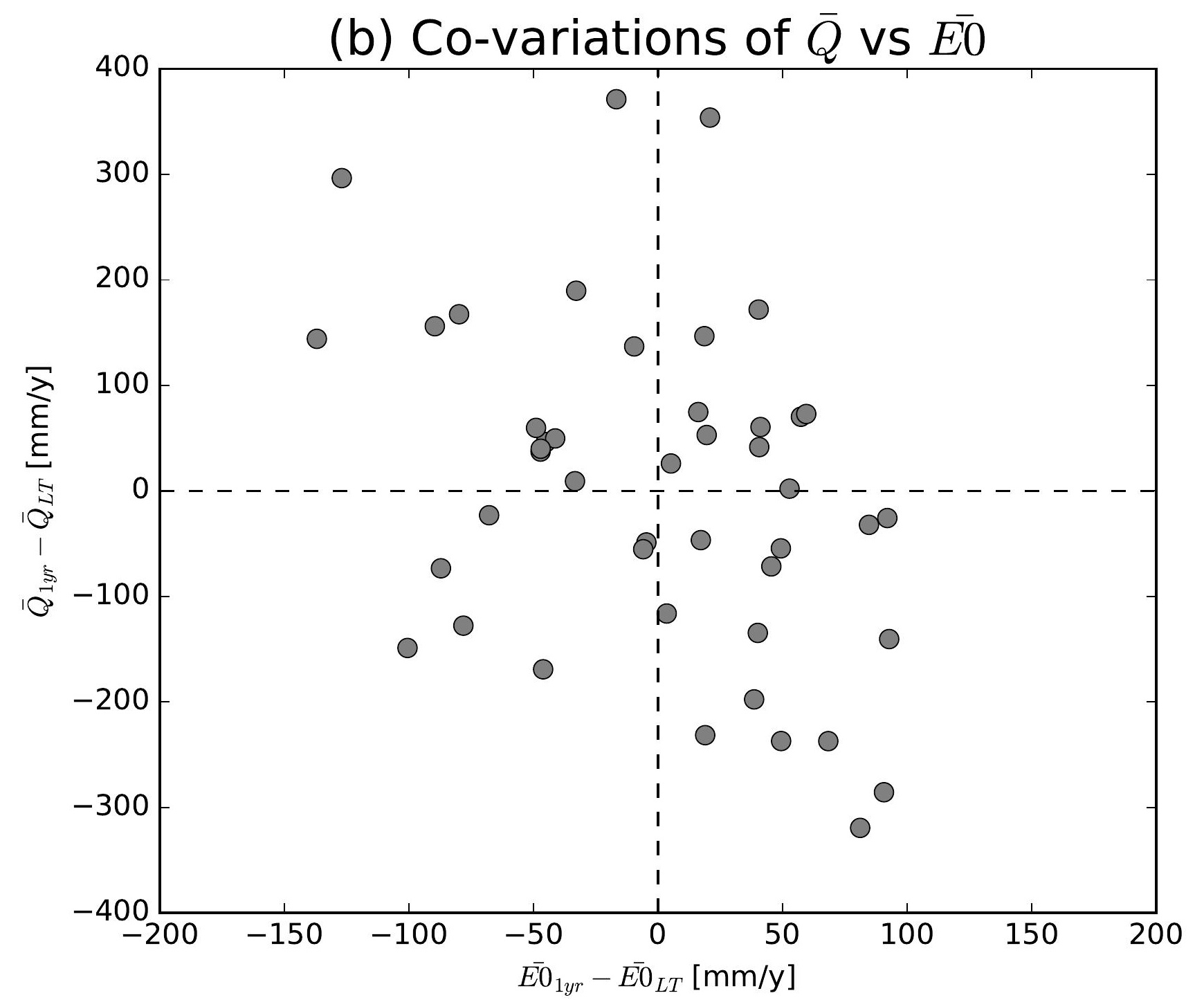

(e) Co-variations of $\bar{Q}$ vs $\overline{E 0}$

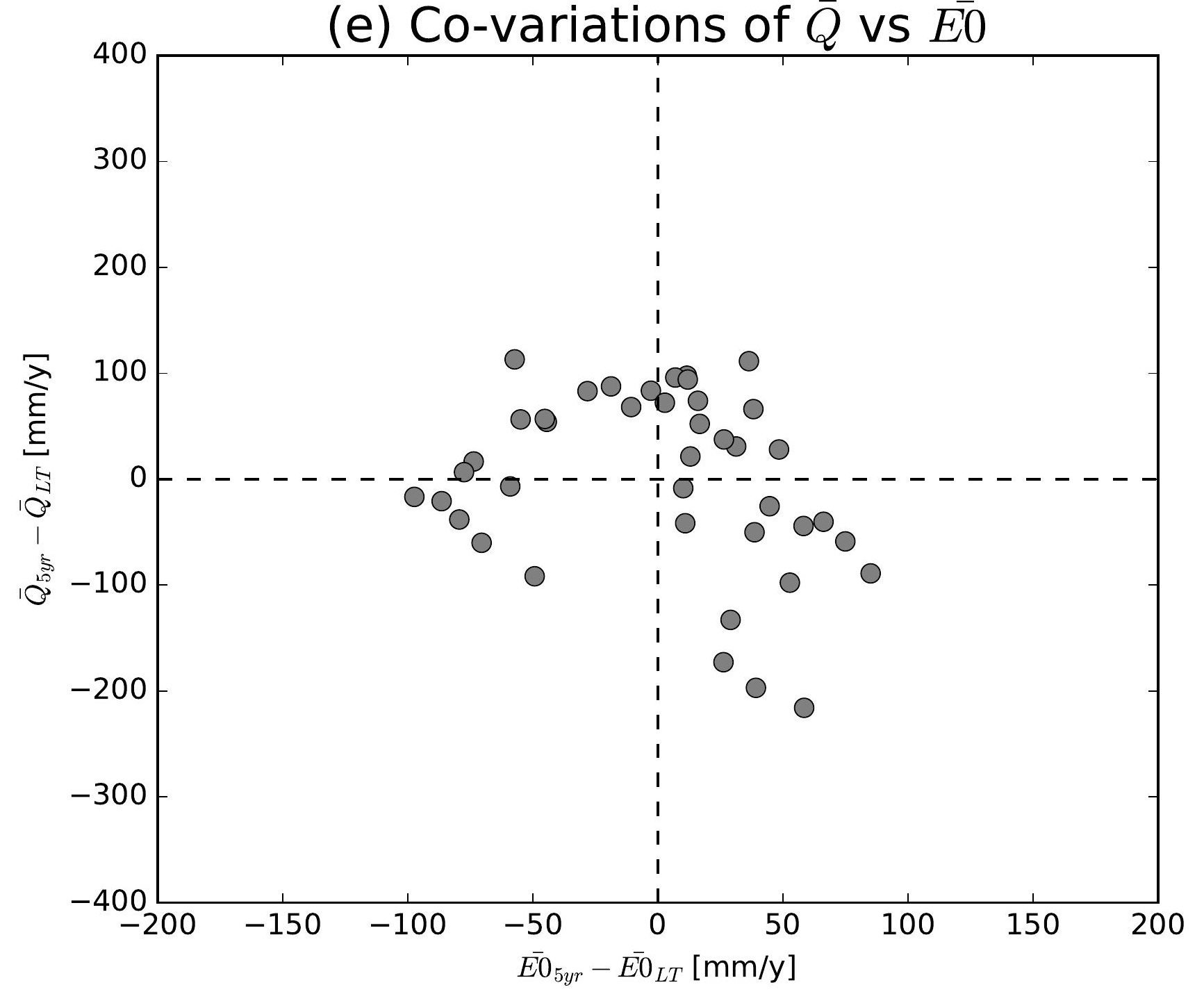

(h) Co-variations of $\bar{Q}$ vs $\overline{E 0}$

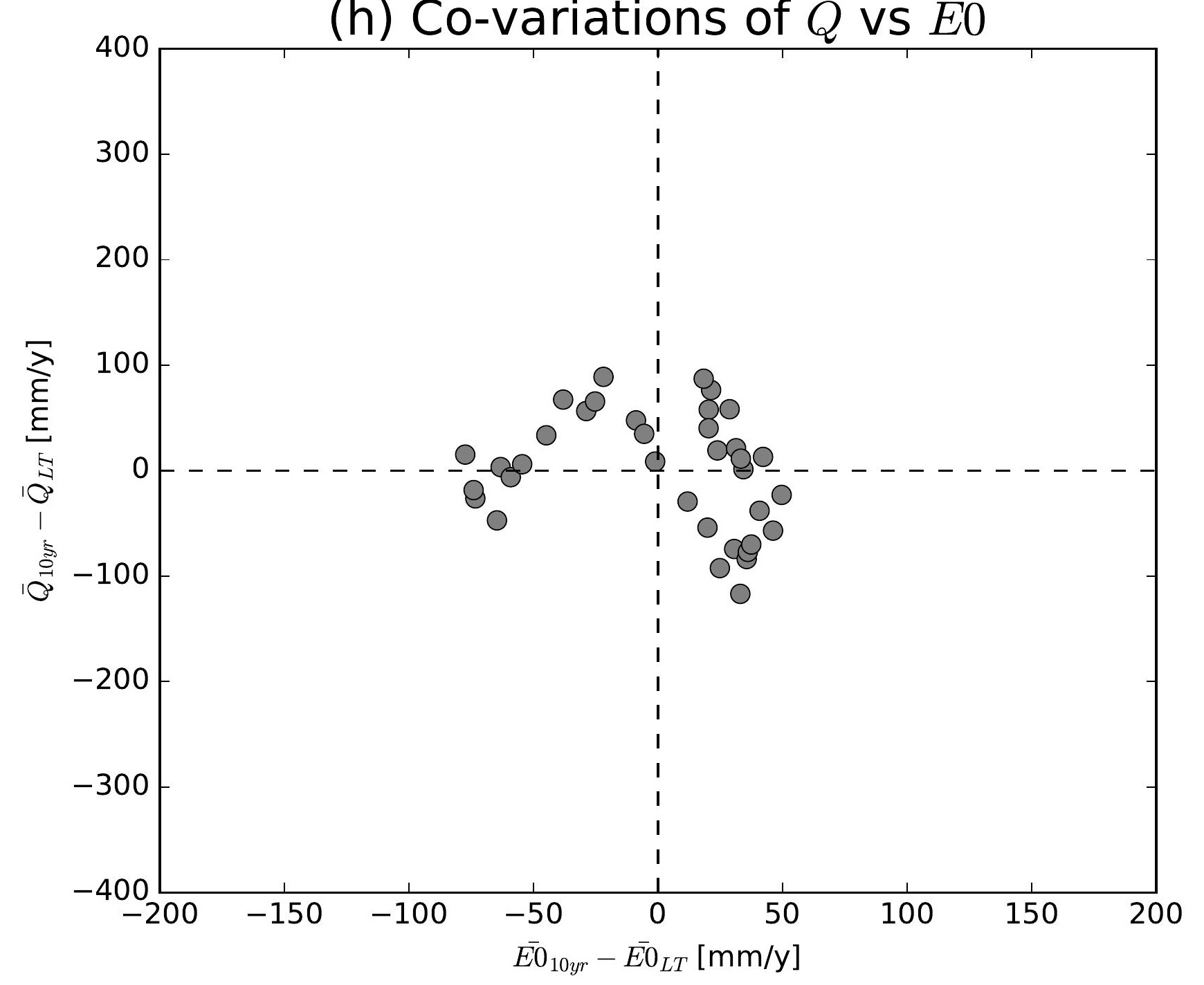

(k) Co-variations of $\bar{Q}$ vs $\overline{E 0}$

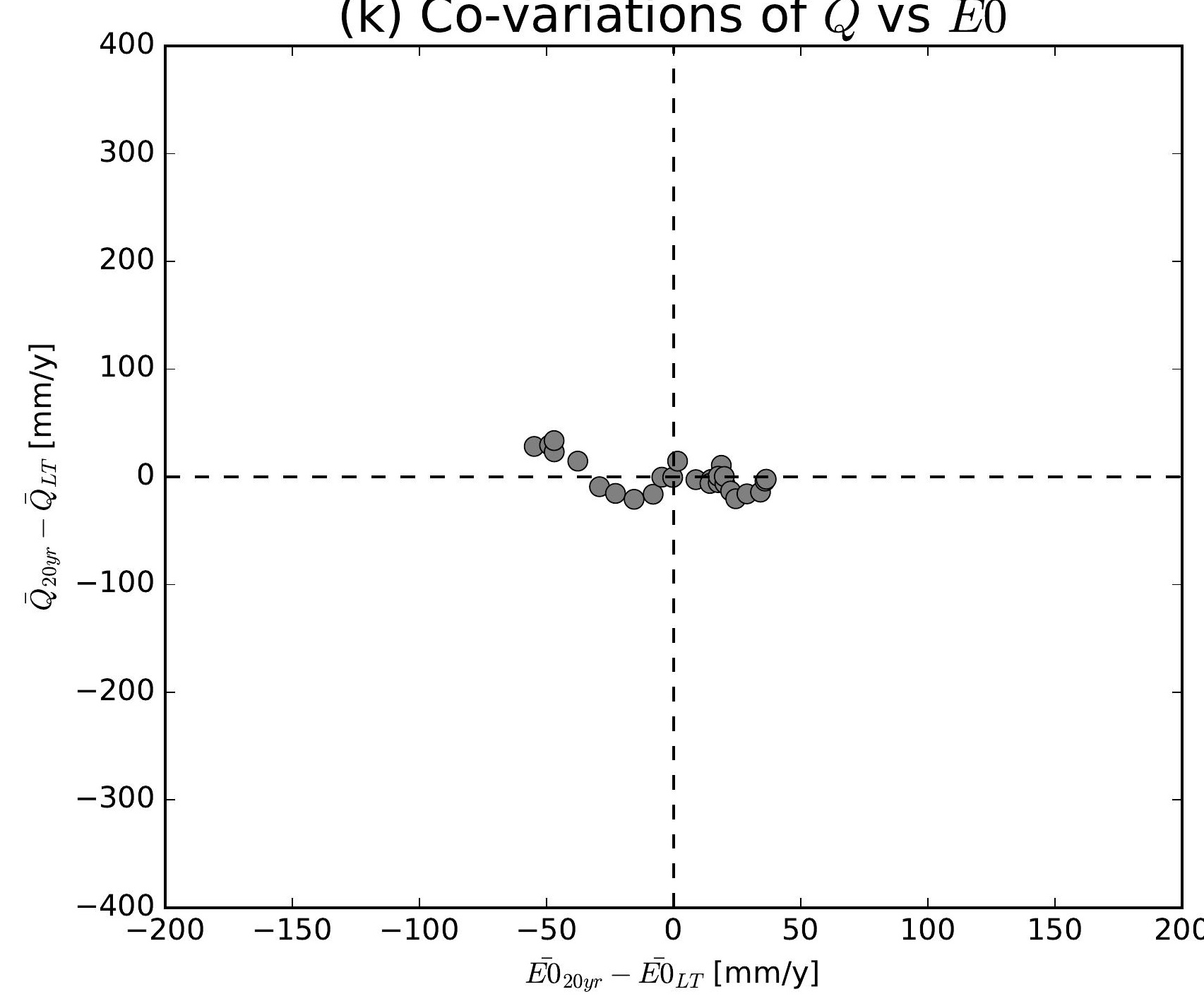

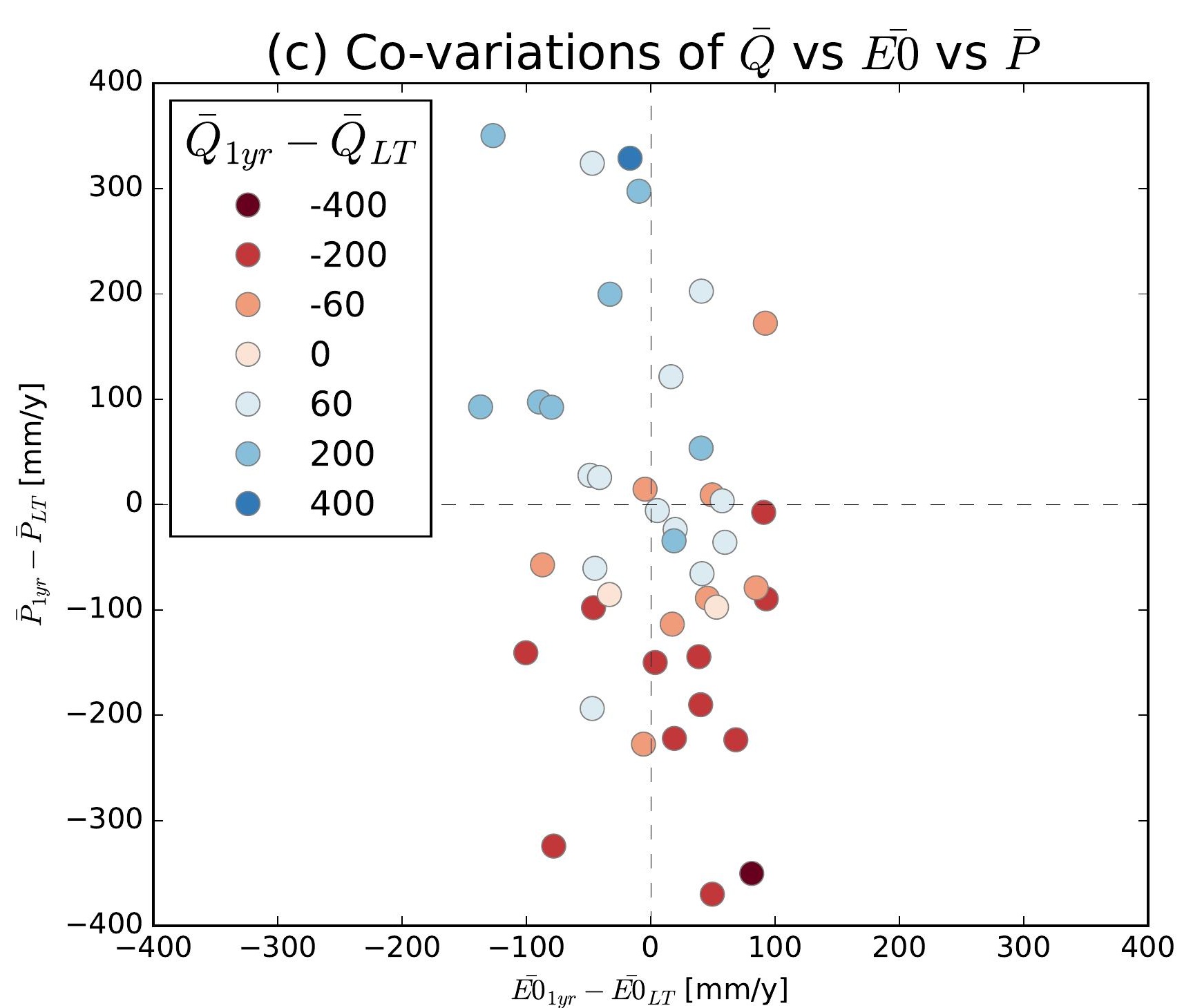
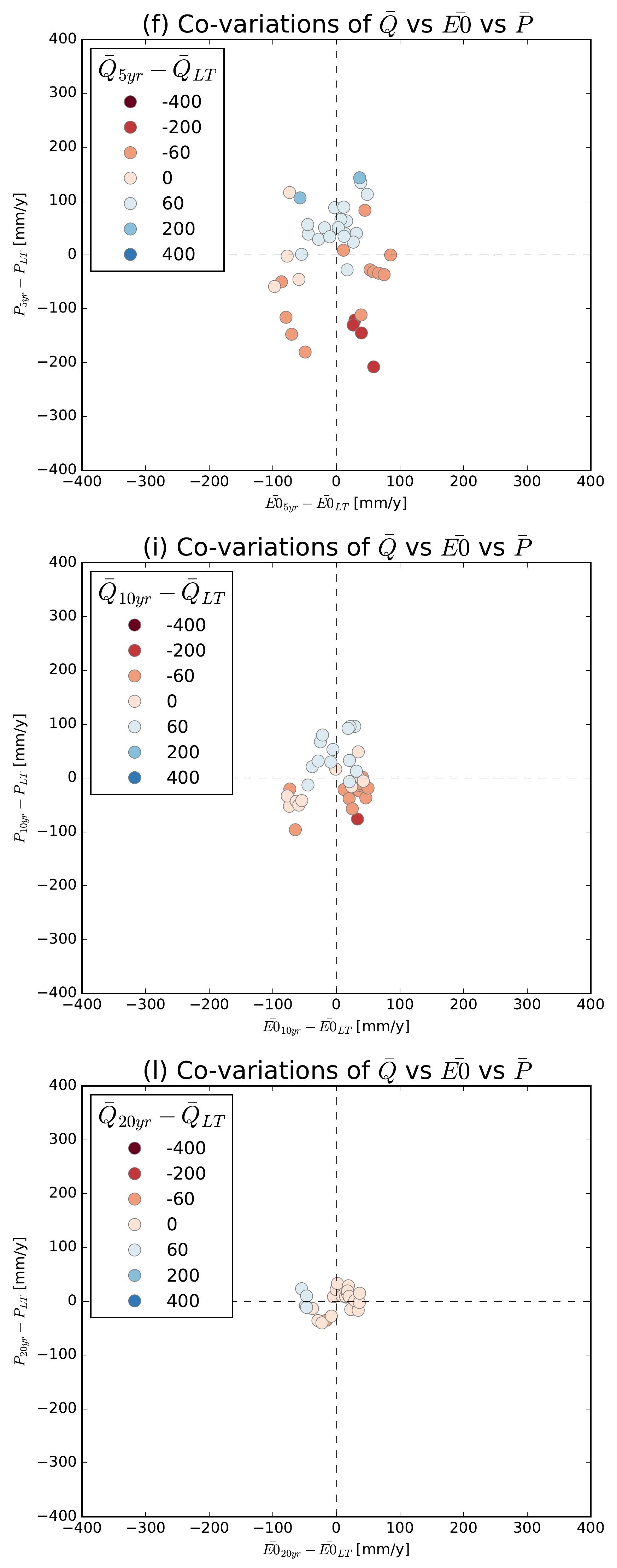

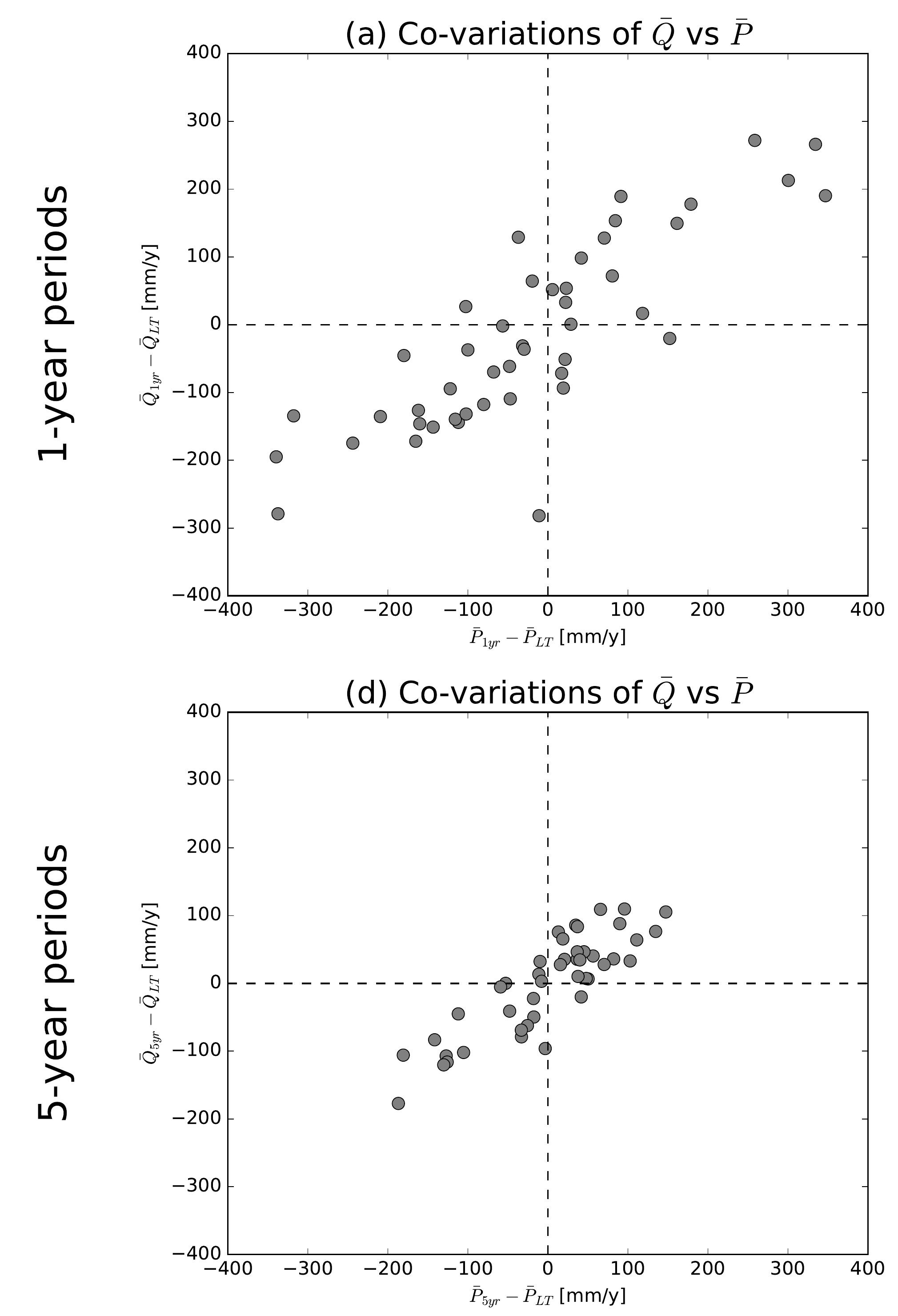

(g) Co-variations of $\bar{Q}$ vs $\bar{P}$
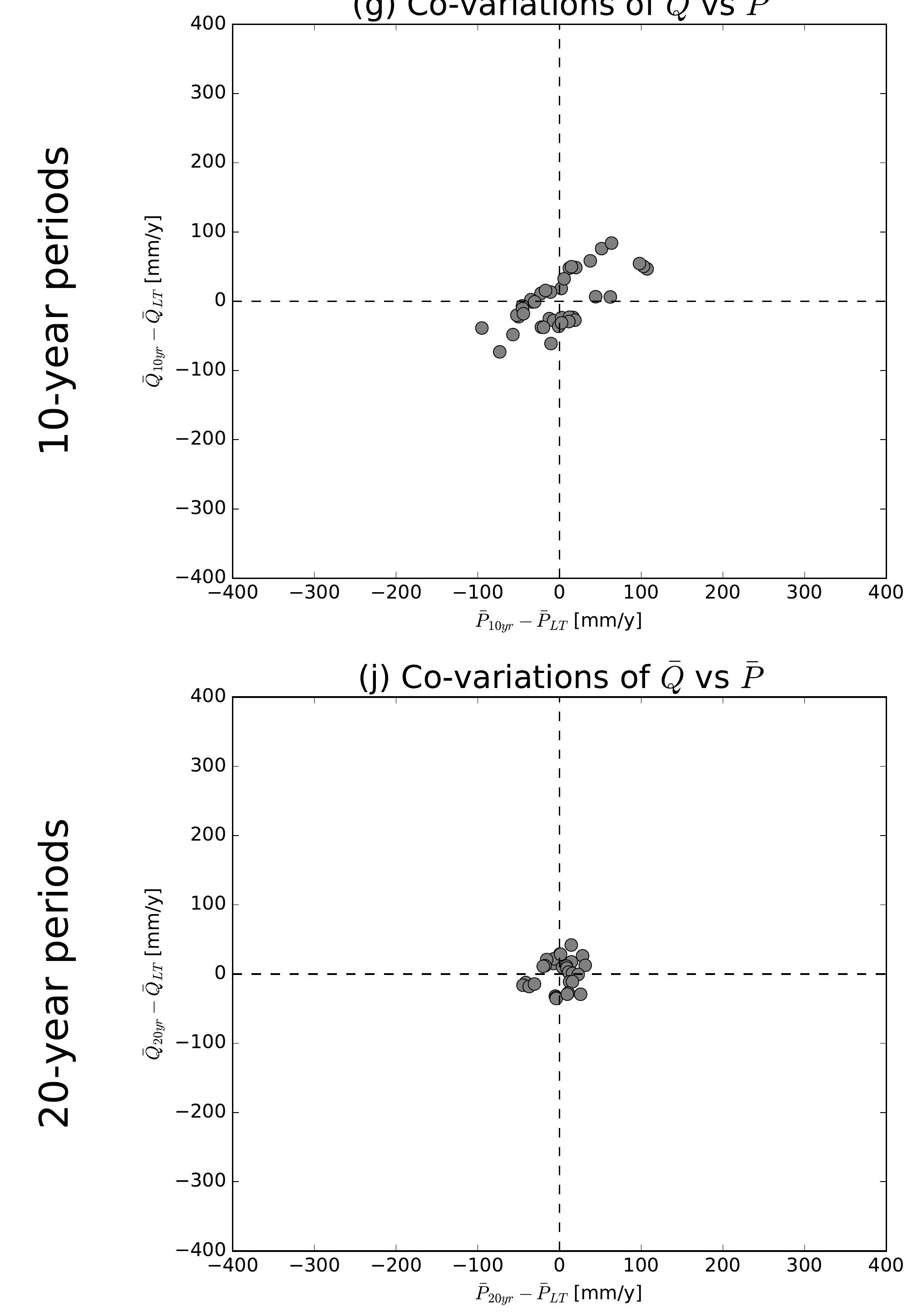

(b) Co-variations of $\bar{Q}$ vs $\overline{E 0}$

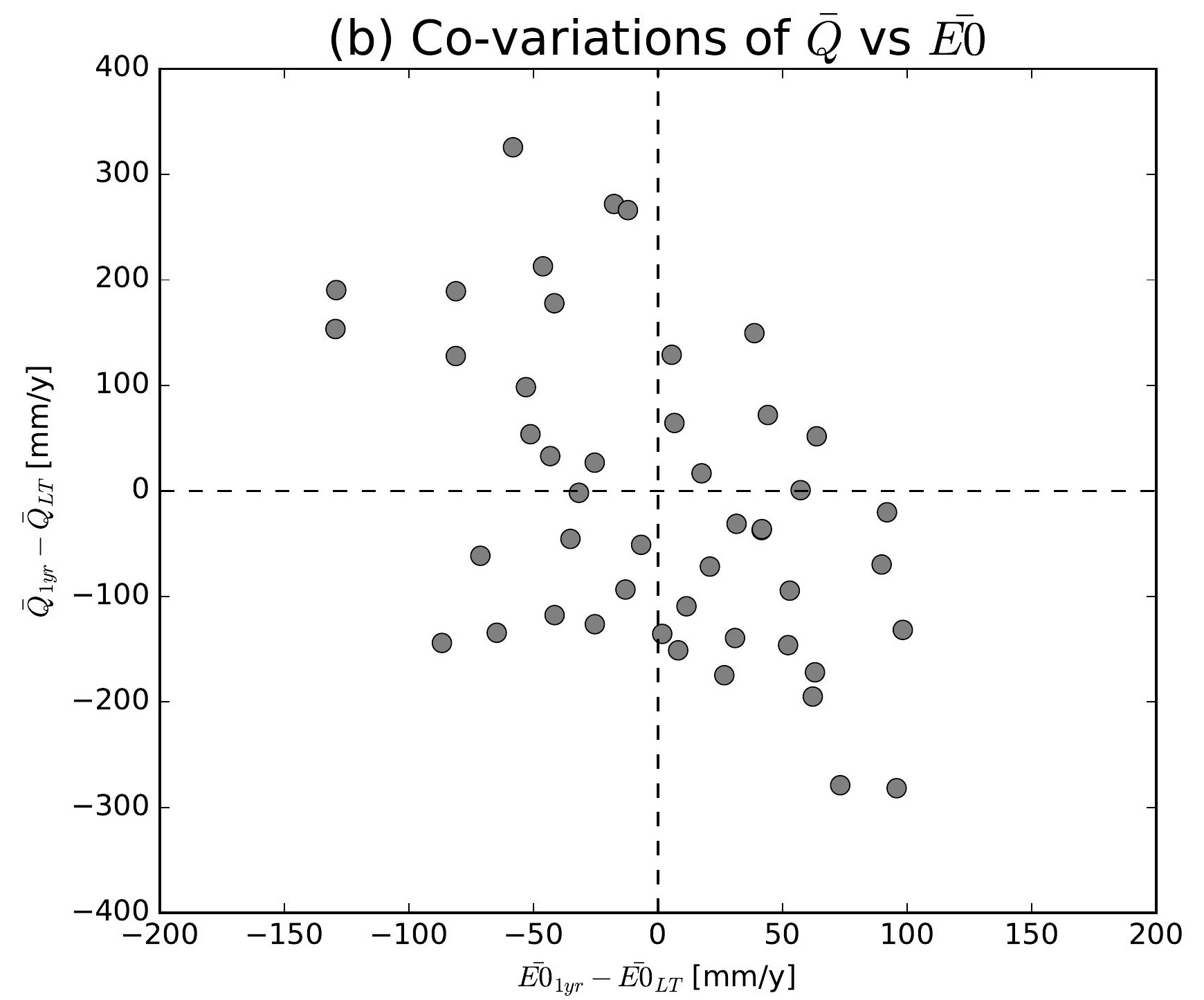

(e) Co-variations of $\bar{Q}$ vs $\overline{E 0}$

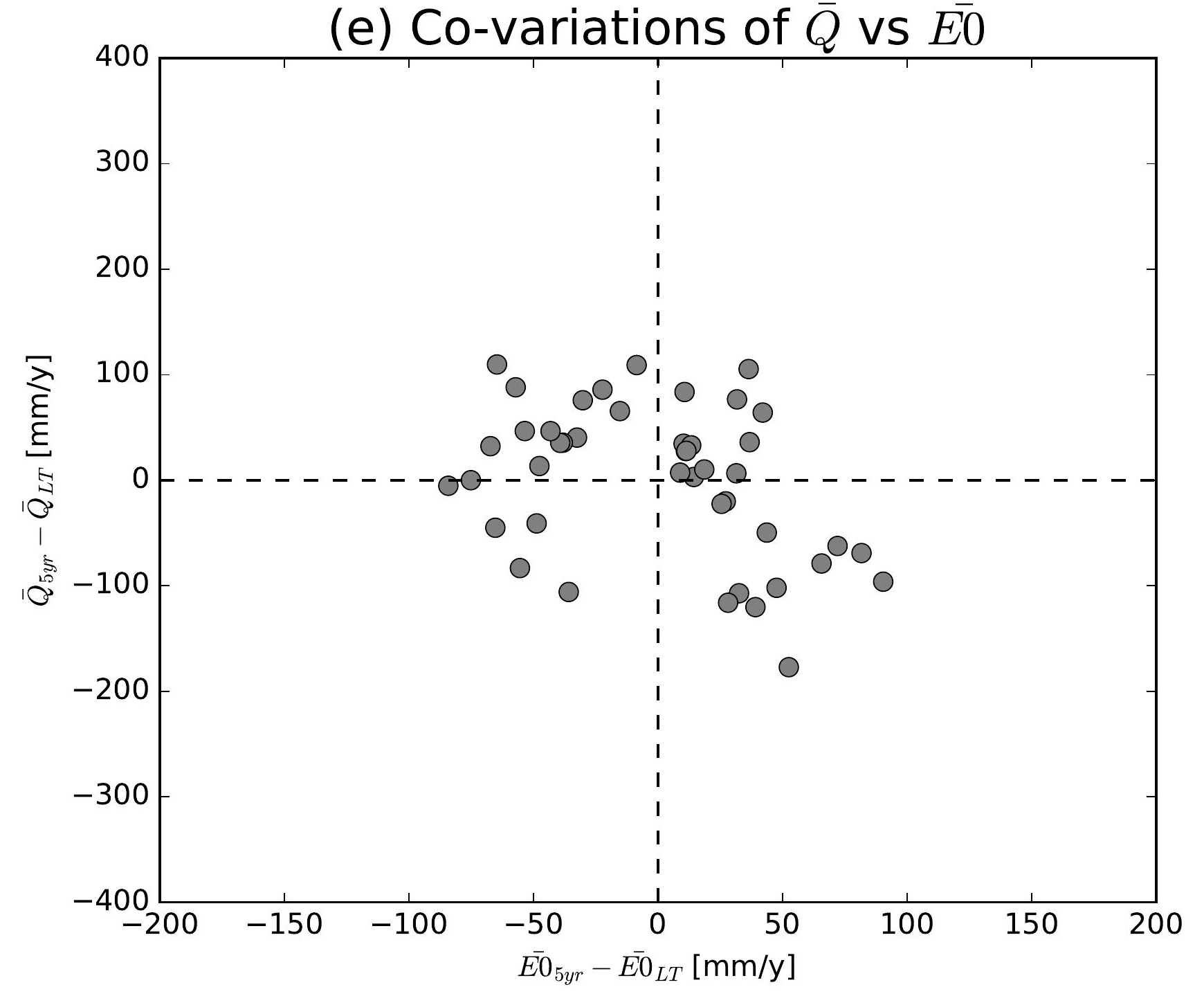

(h) Co-variations of $\bar{Q}$ vs $\overline{E 0}$

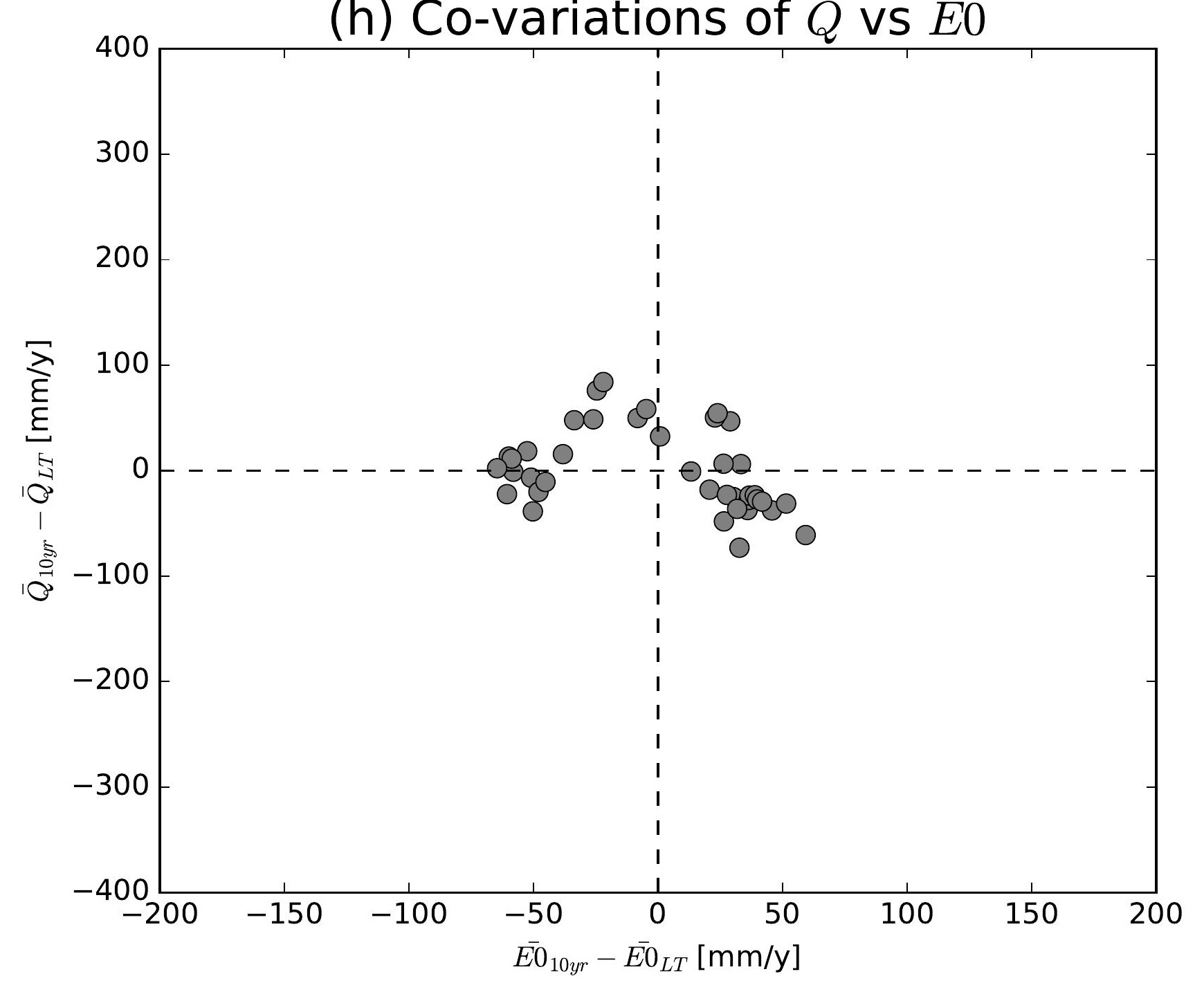

(k) Co-variations of $\bar{Q}$ vs $\overline{E 0}$

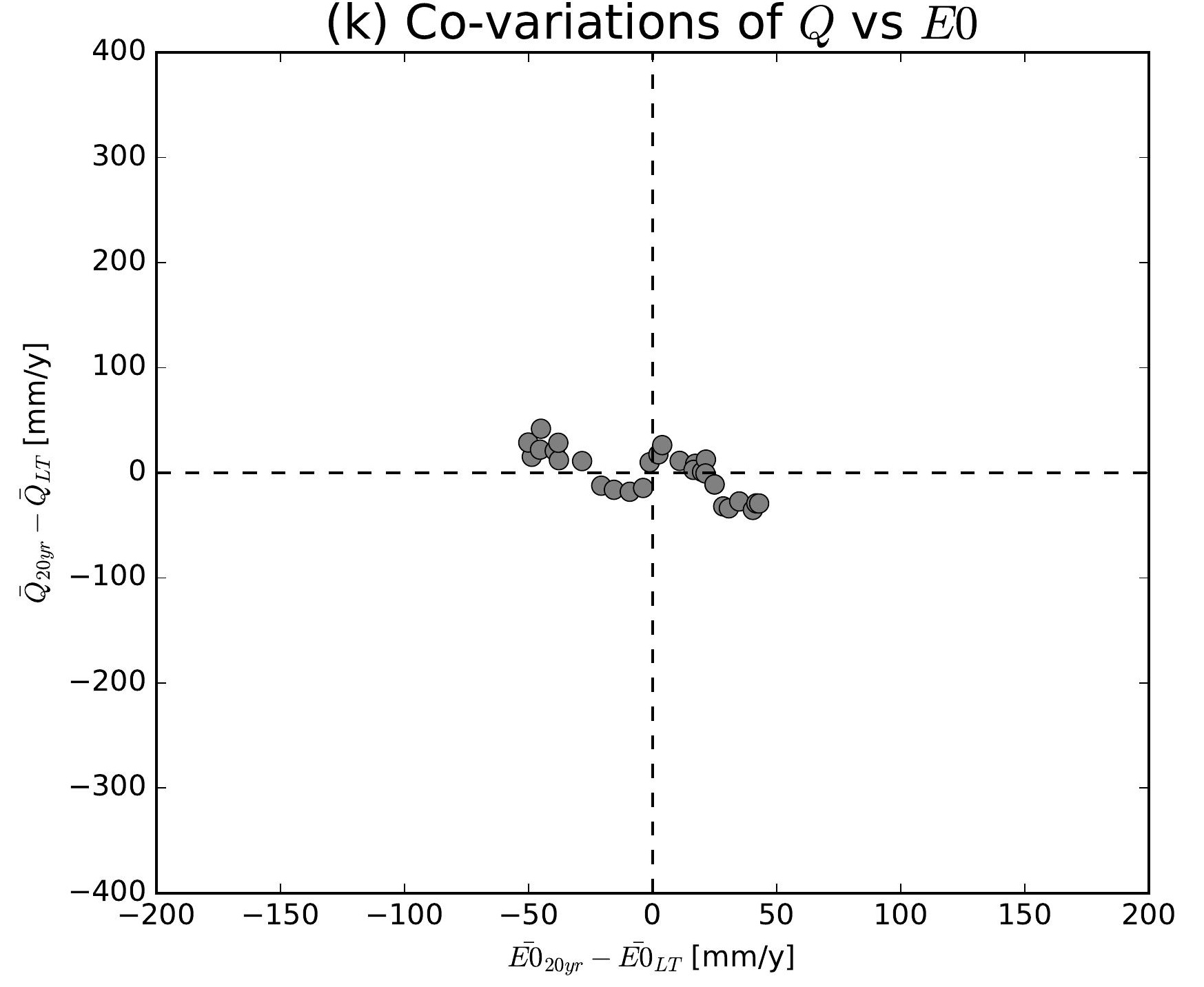

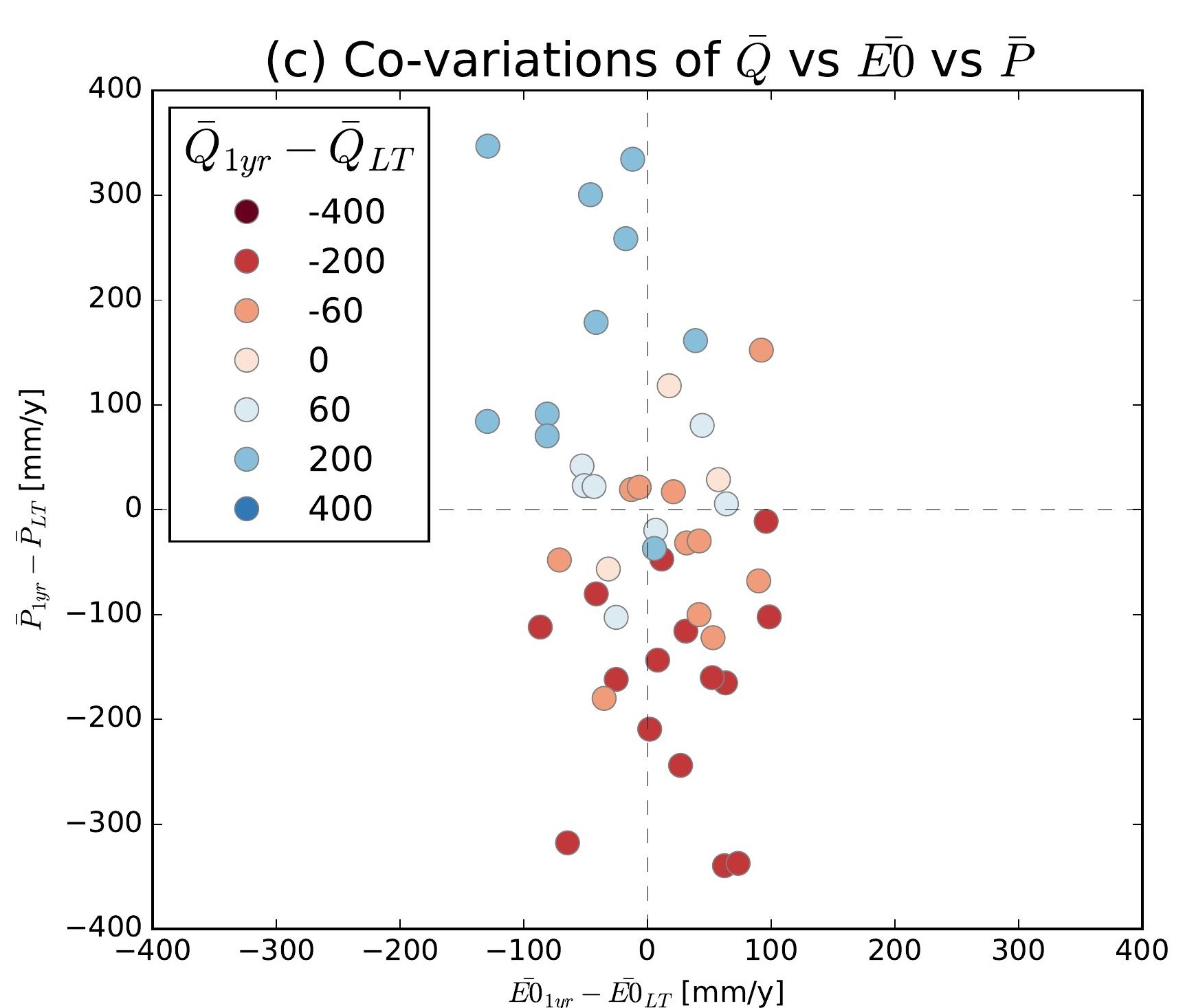
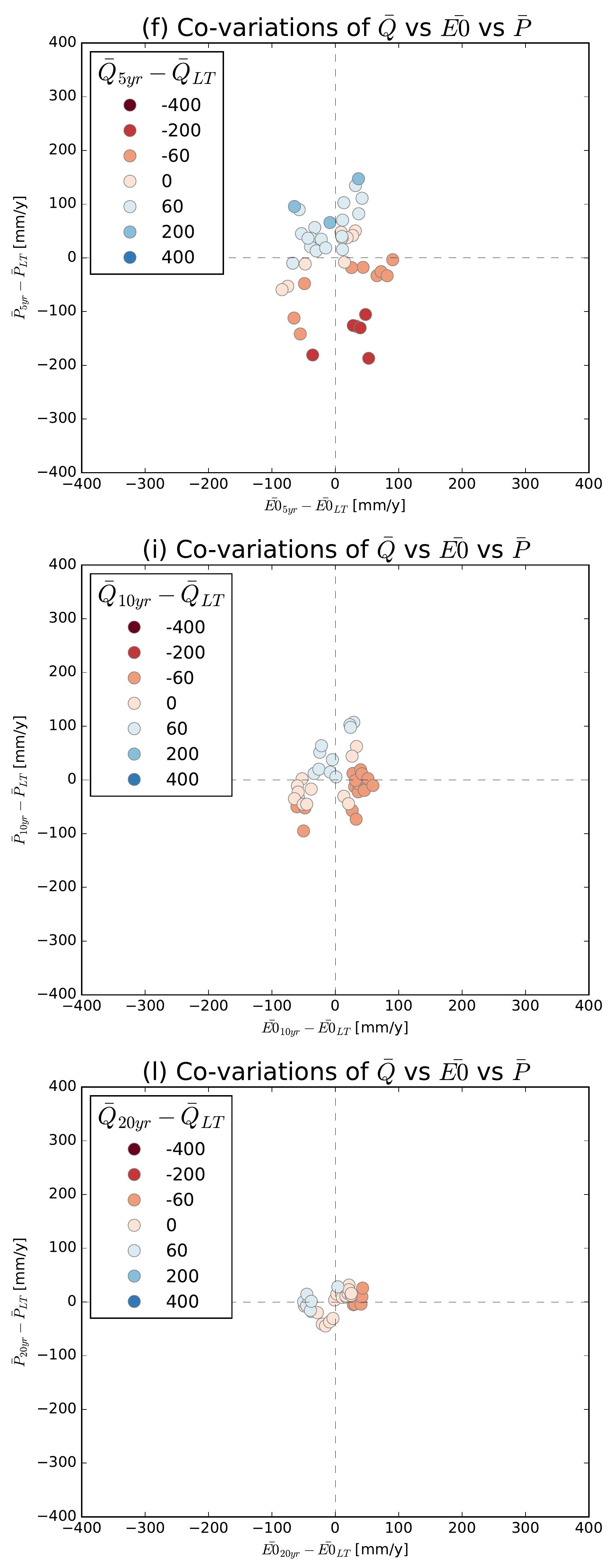

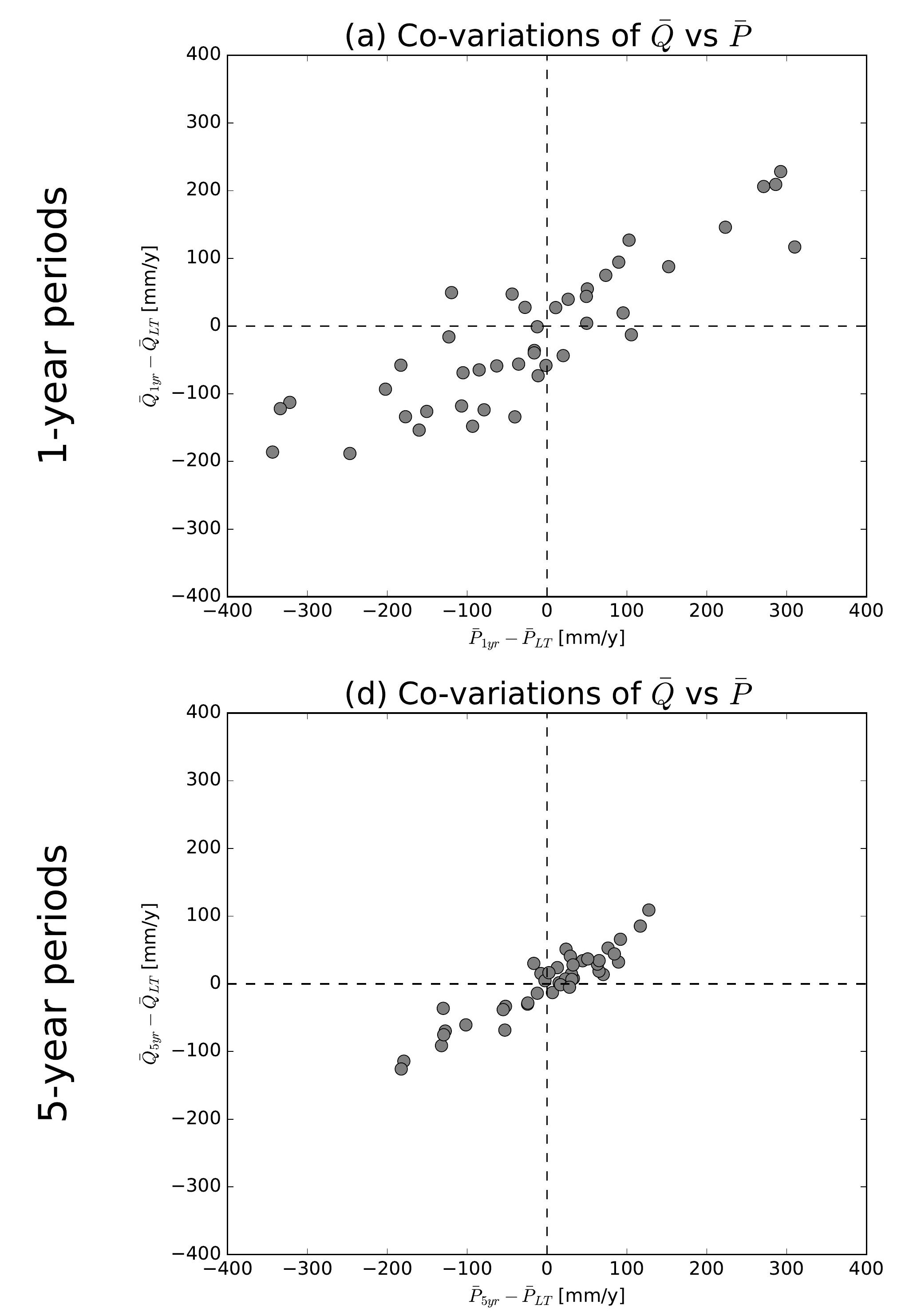

(g) Co-variations of $\bar{Q}$ vs $\bar{P}$

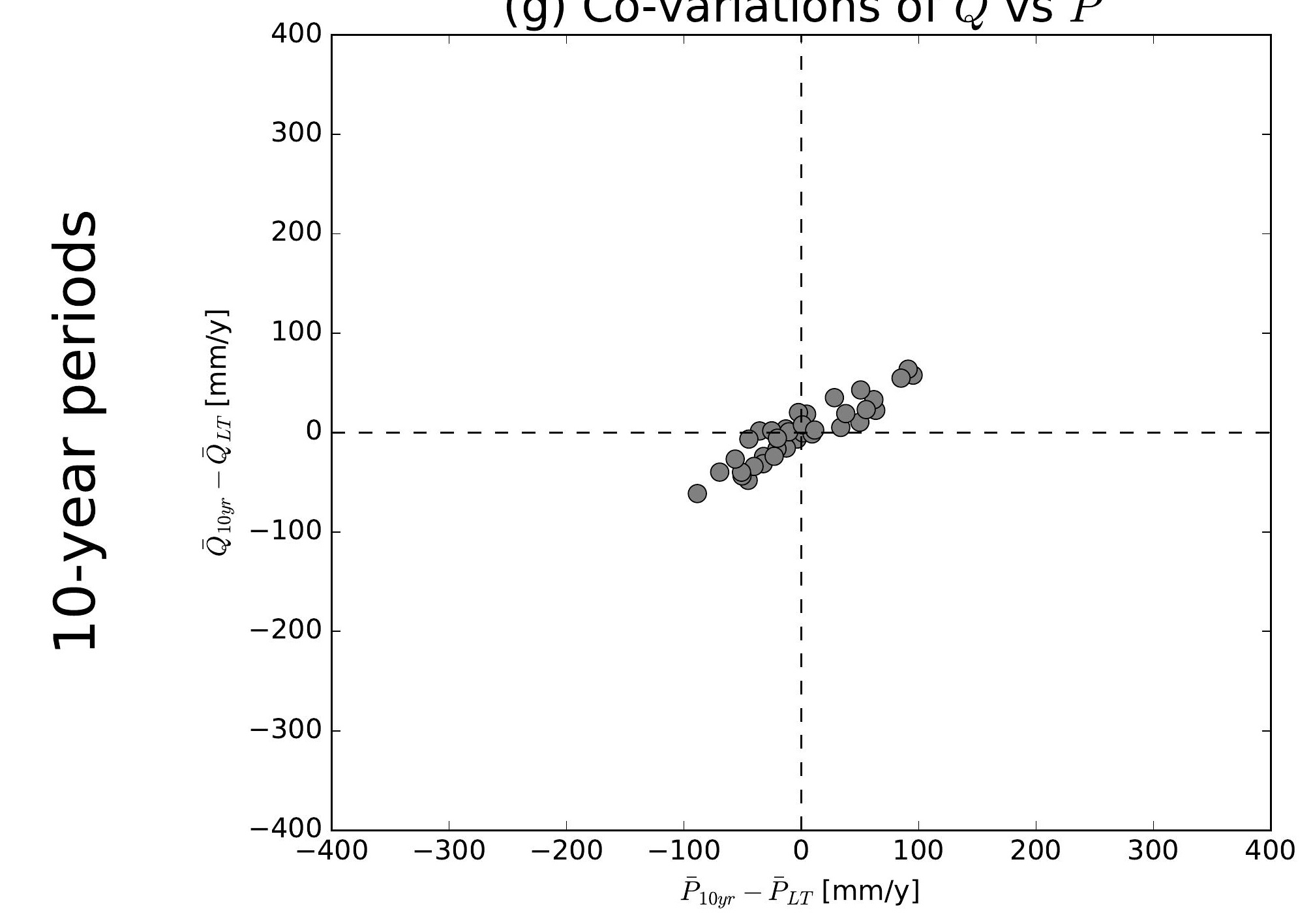

(j) Co-variations of $\bar{Q}$ vs $\bar{P}$

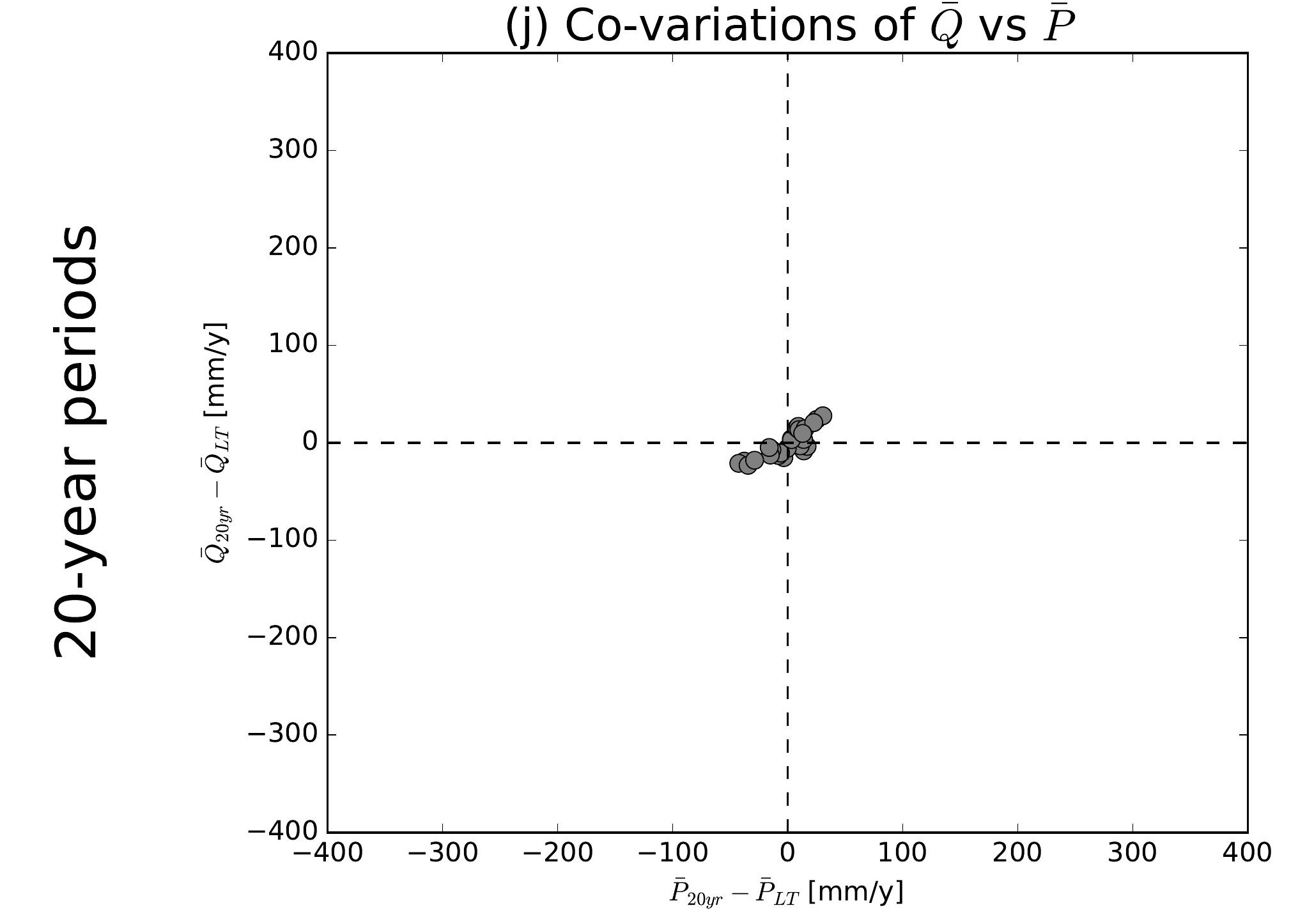

(b) Co-variations of $\bar{Q}$ vs $\overline{E 0}$

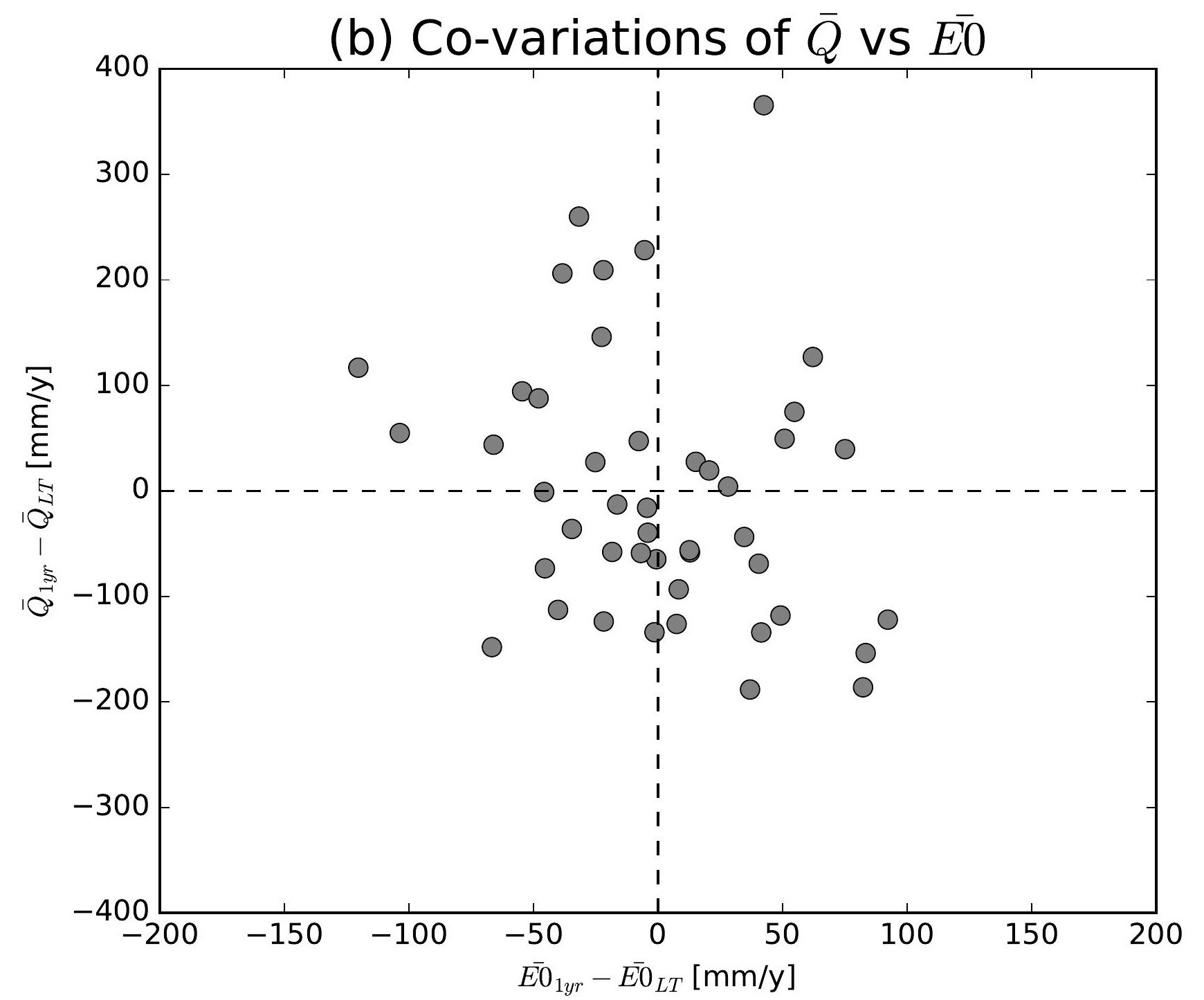

(e) Co-variations of $\bar{Q}$ vs $\overline{E 0}$

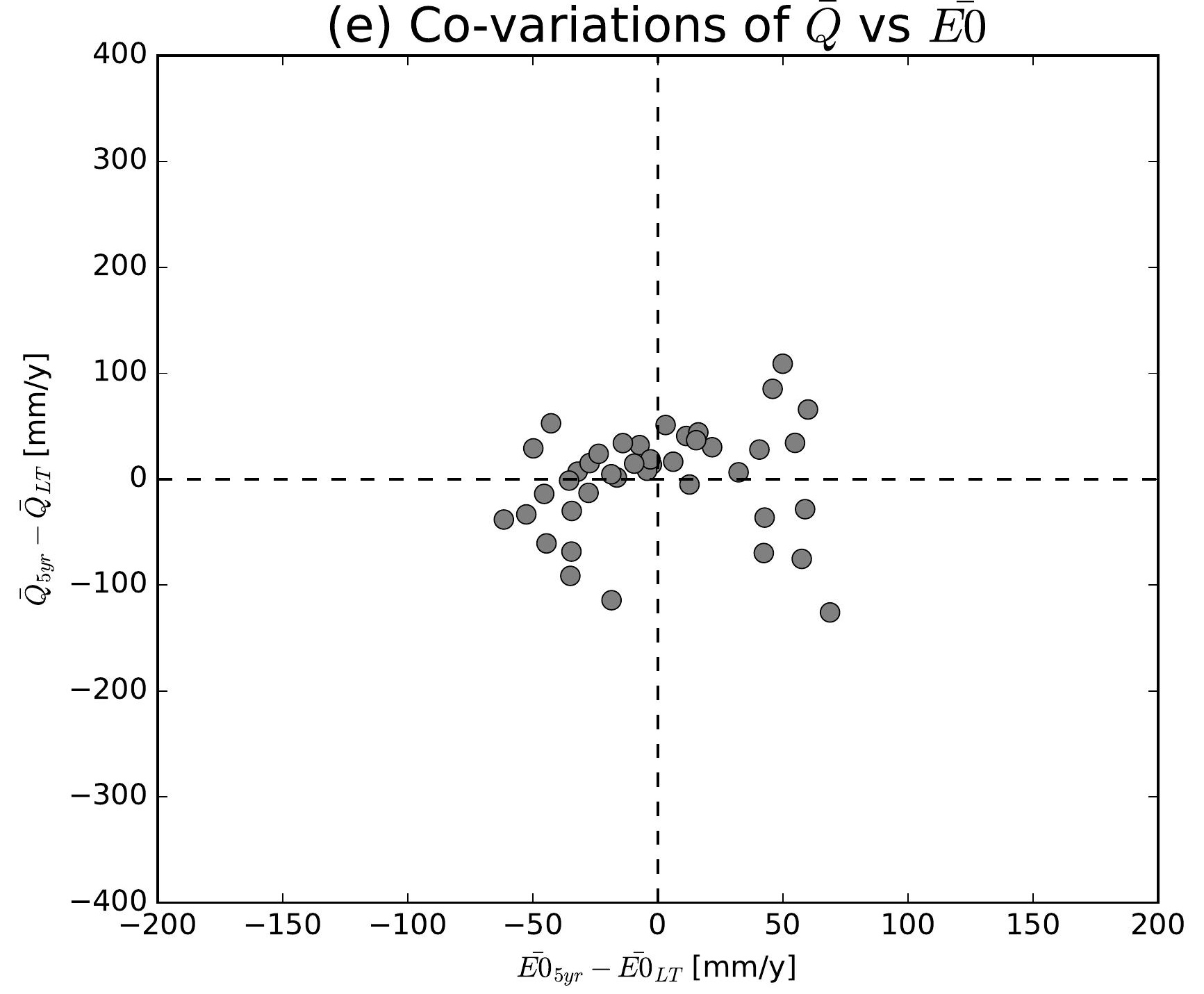

(h) Co-variations of $\bar{Q}$ vs $\overline{E 0}$

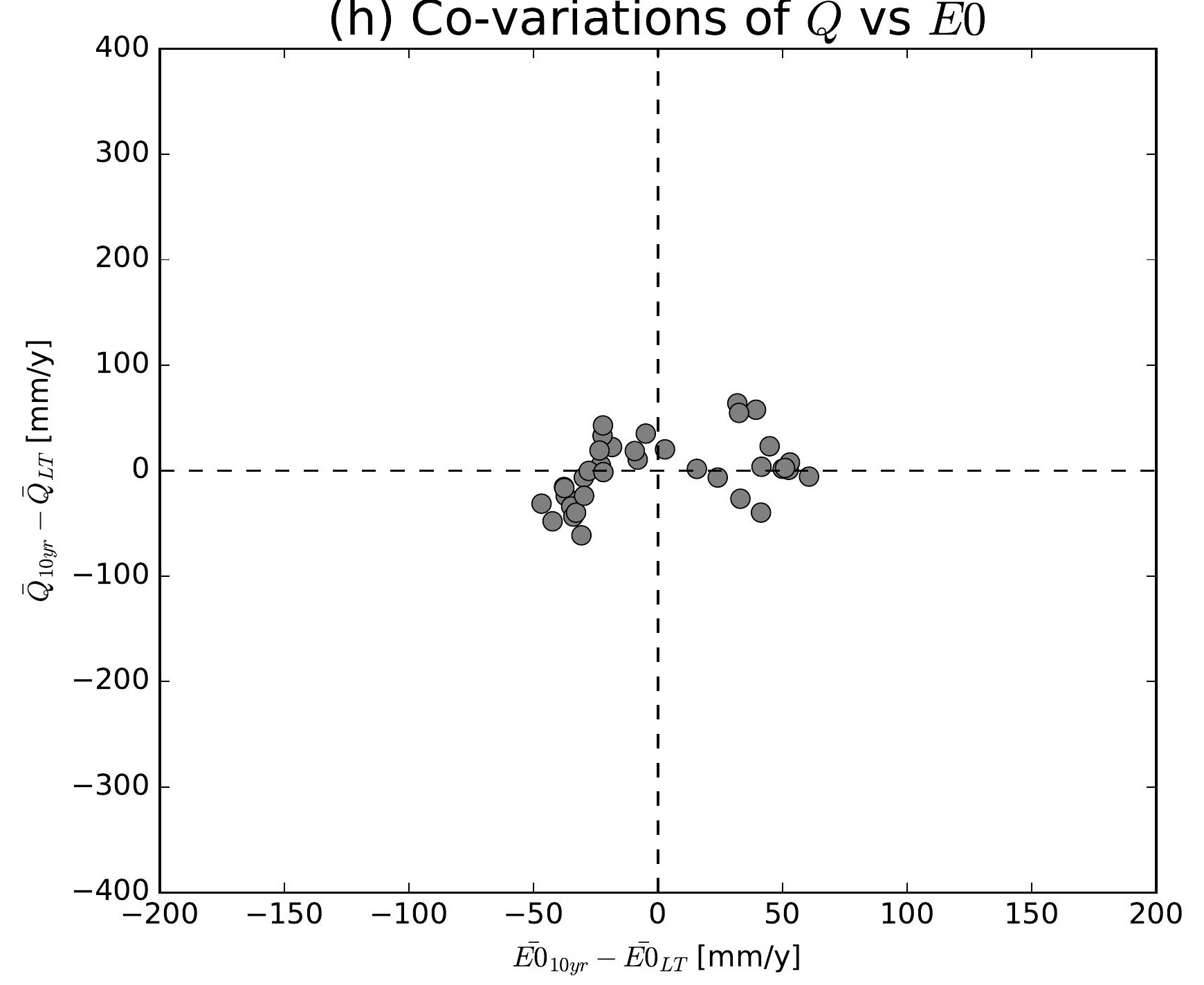

(k) Co-variations of $\bar{Q}$ vs $\overline{E 0}$

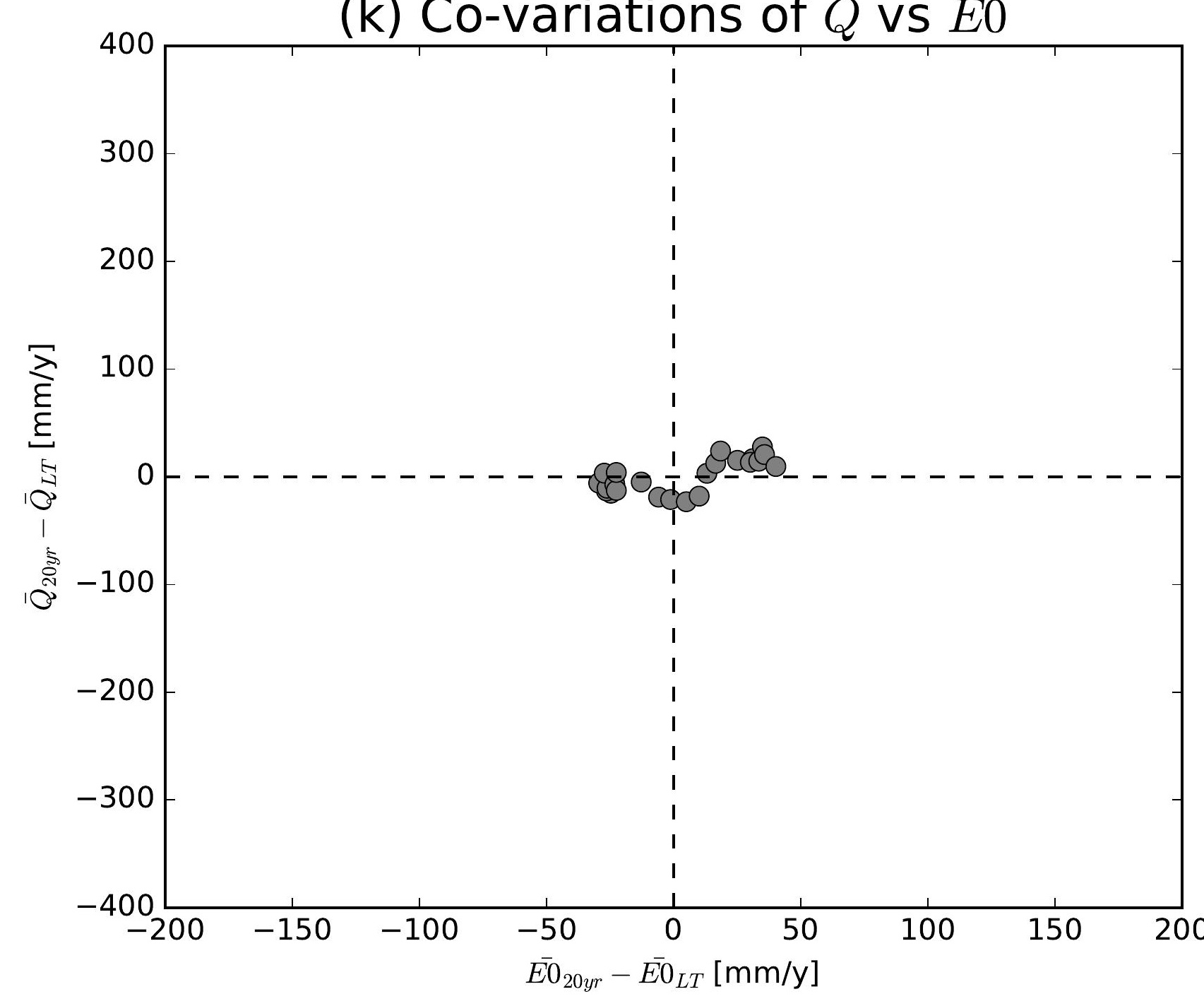

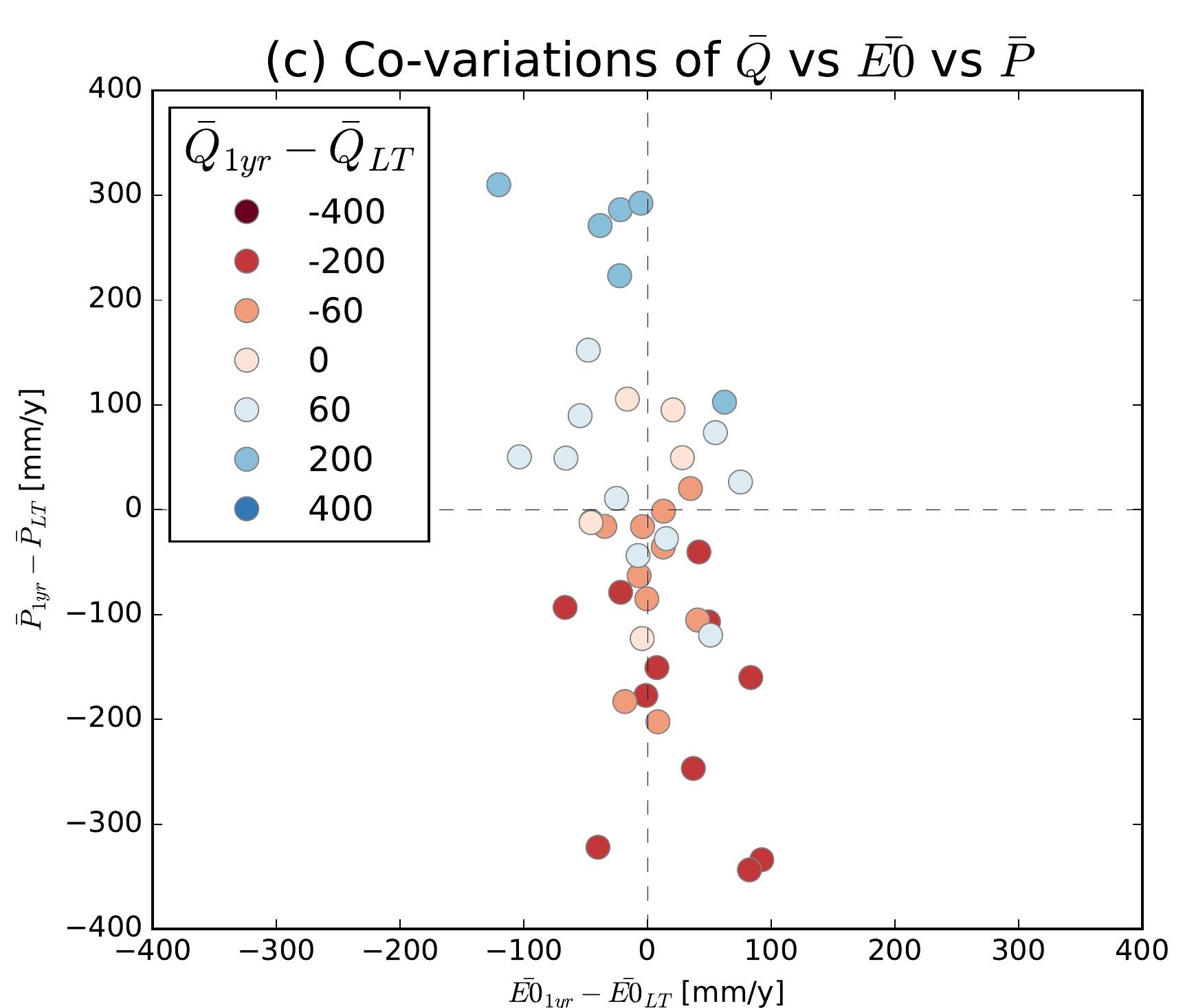
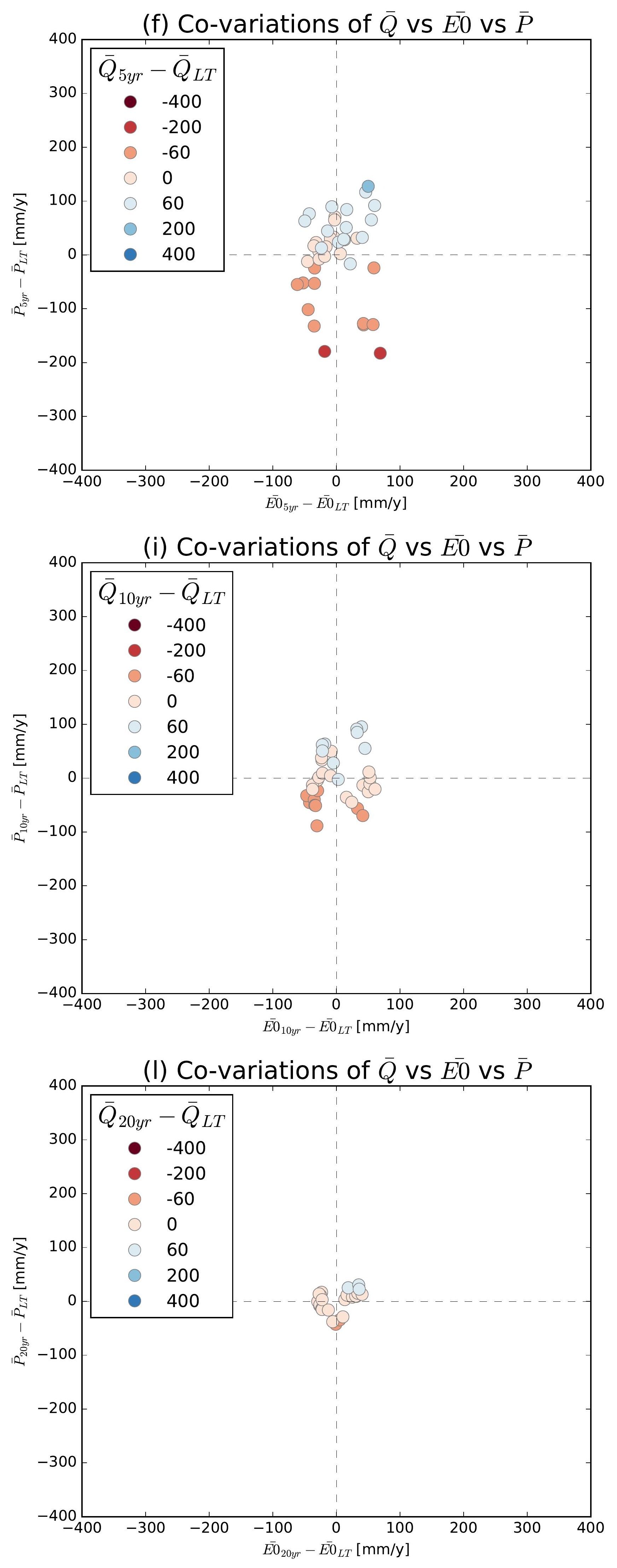

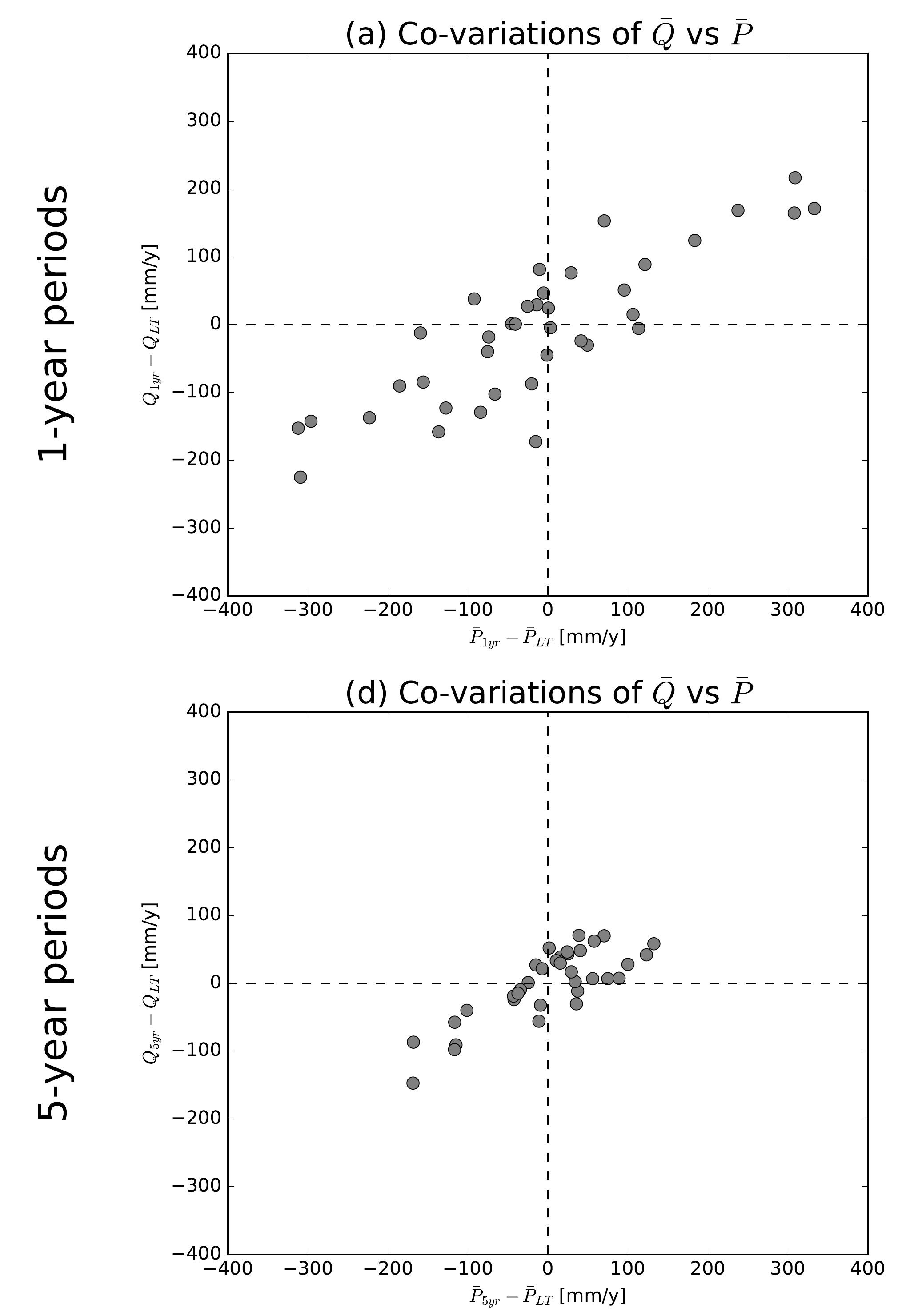

(g) Co-variations of $\bar{Q}$ vs $\bar{P}$
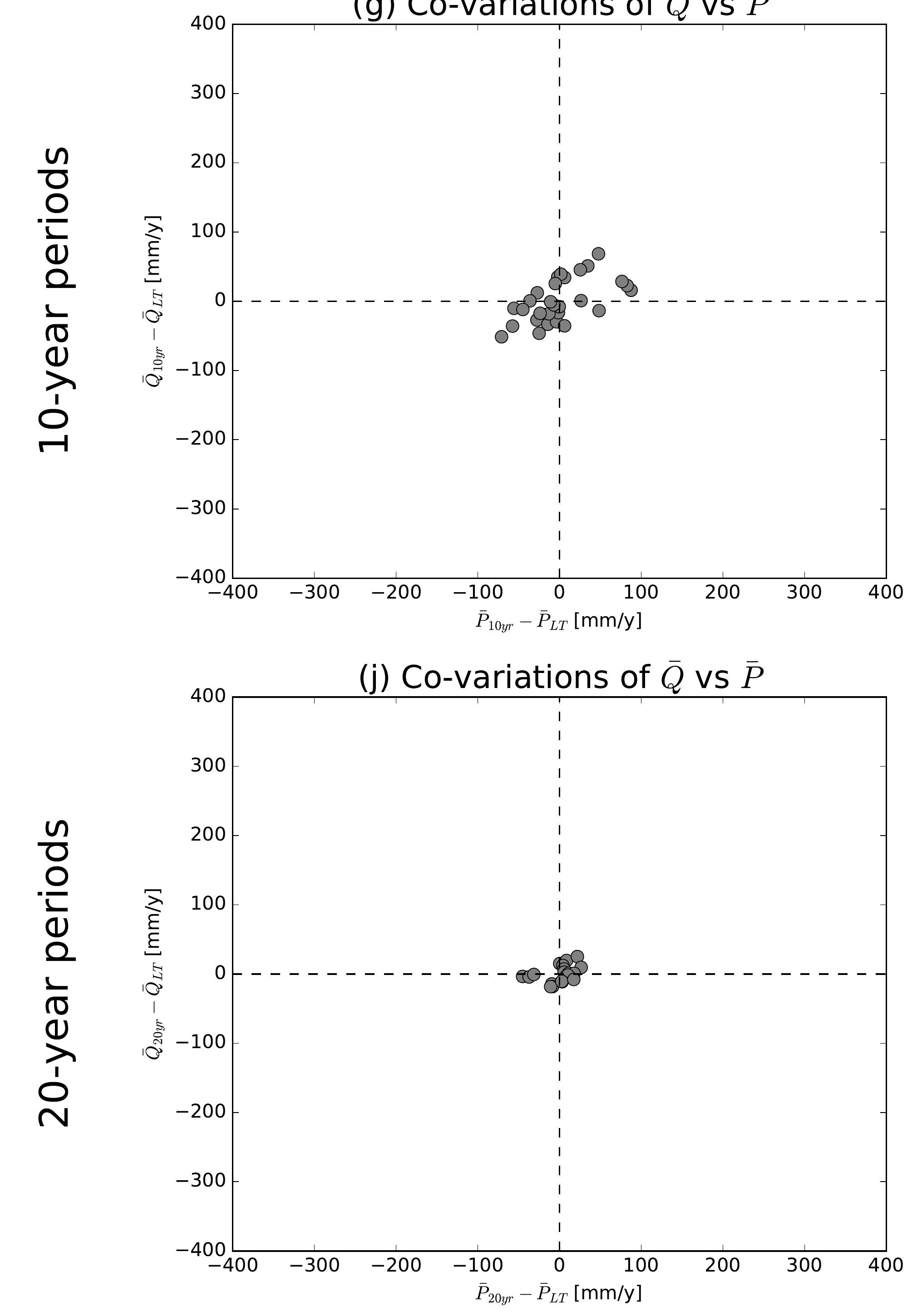

(b) Co-variations of $\bar{Q}$ vs $\overline{E 0}$

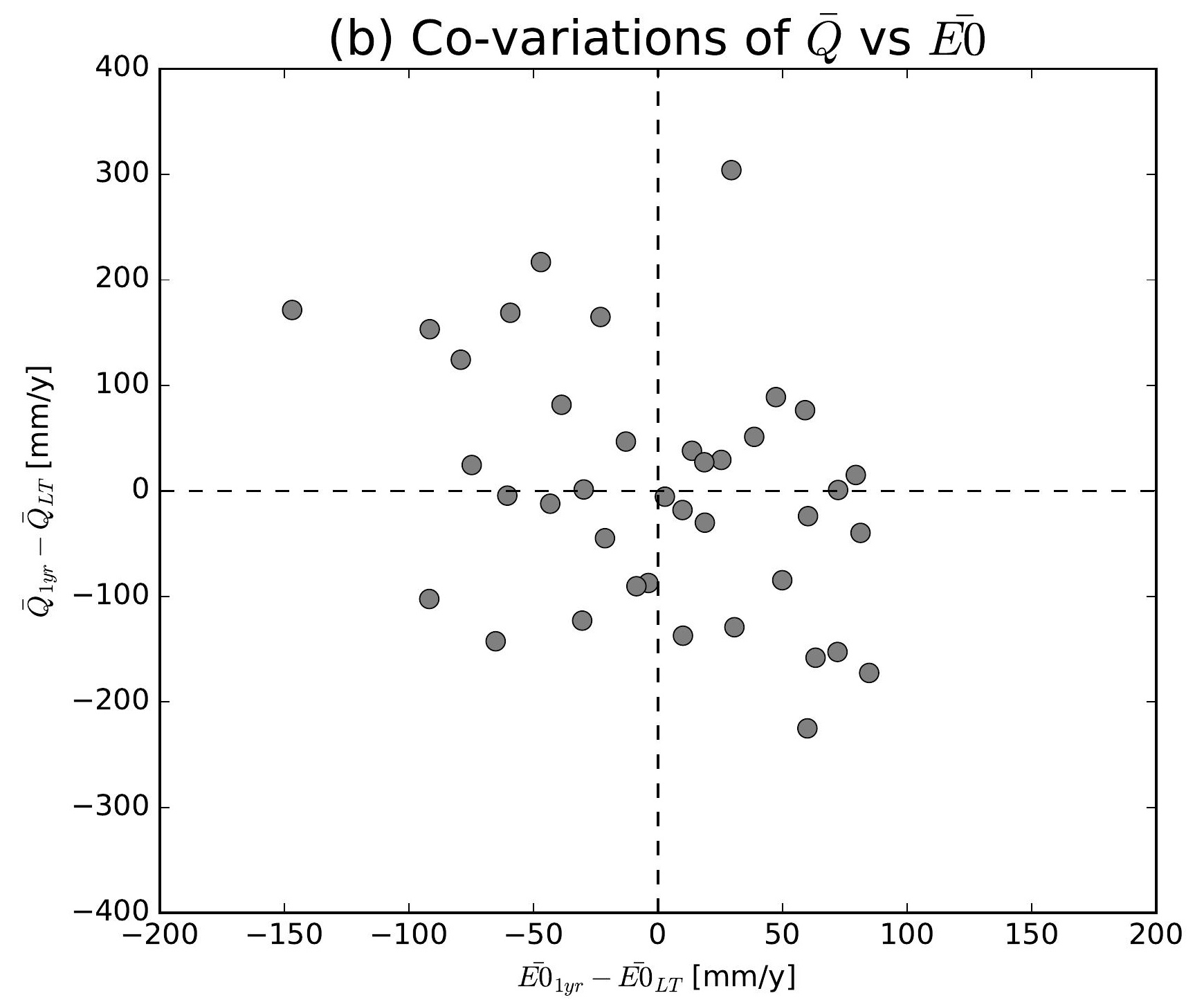

(e) Co-variations of $\bar{Q}$ vs $\overline{E 0}$

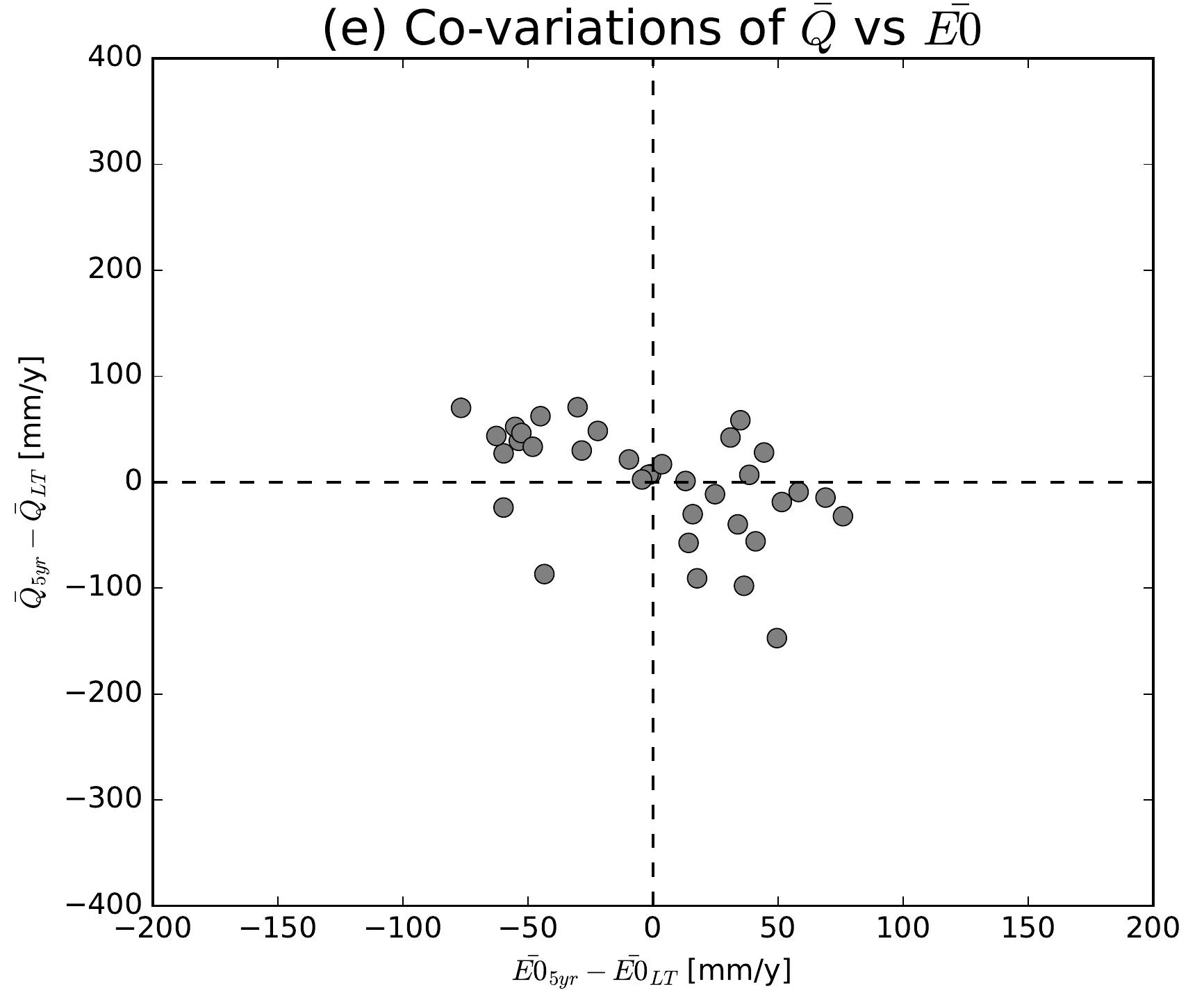

(h) Co-variations of $\bar{Q}$ vs $\overline{E 0}$

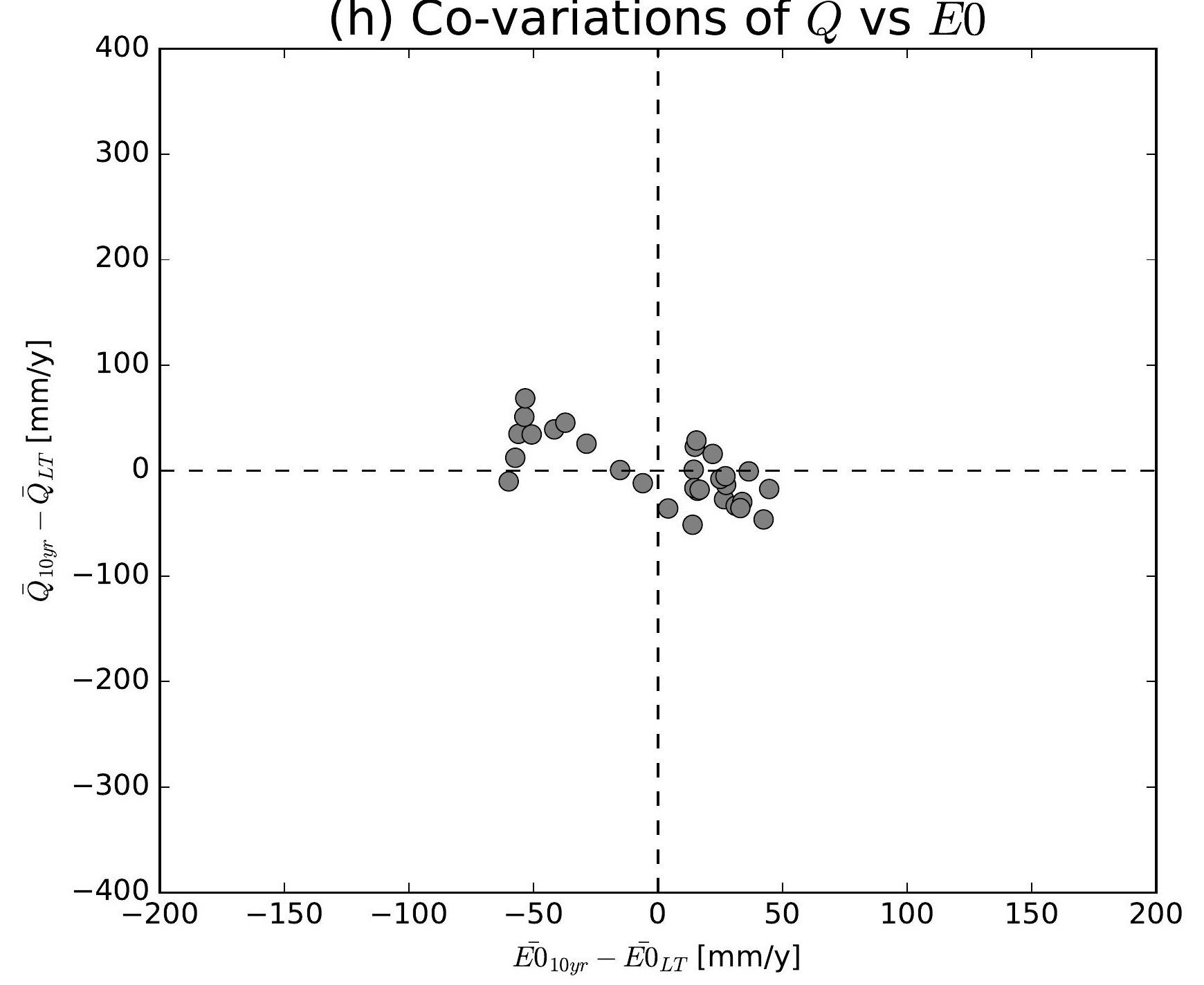

(k) Co-variations of $\bar{Q}$ vs $\overline{E 0}$

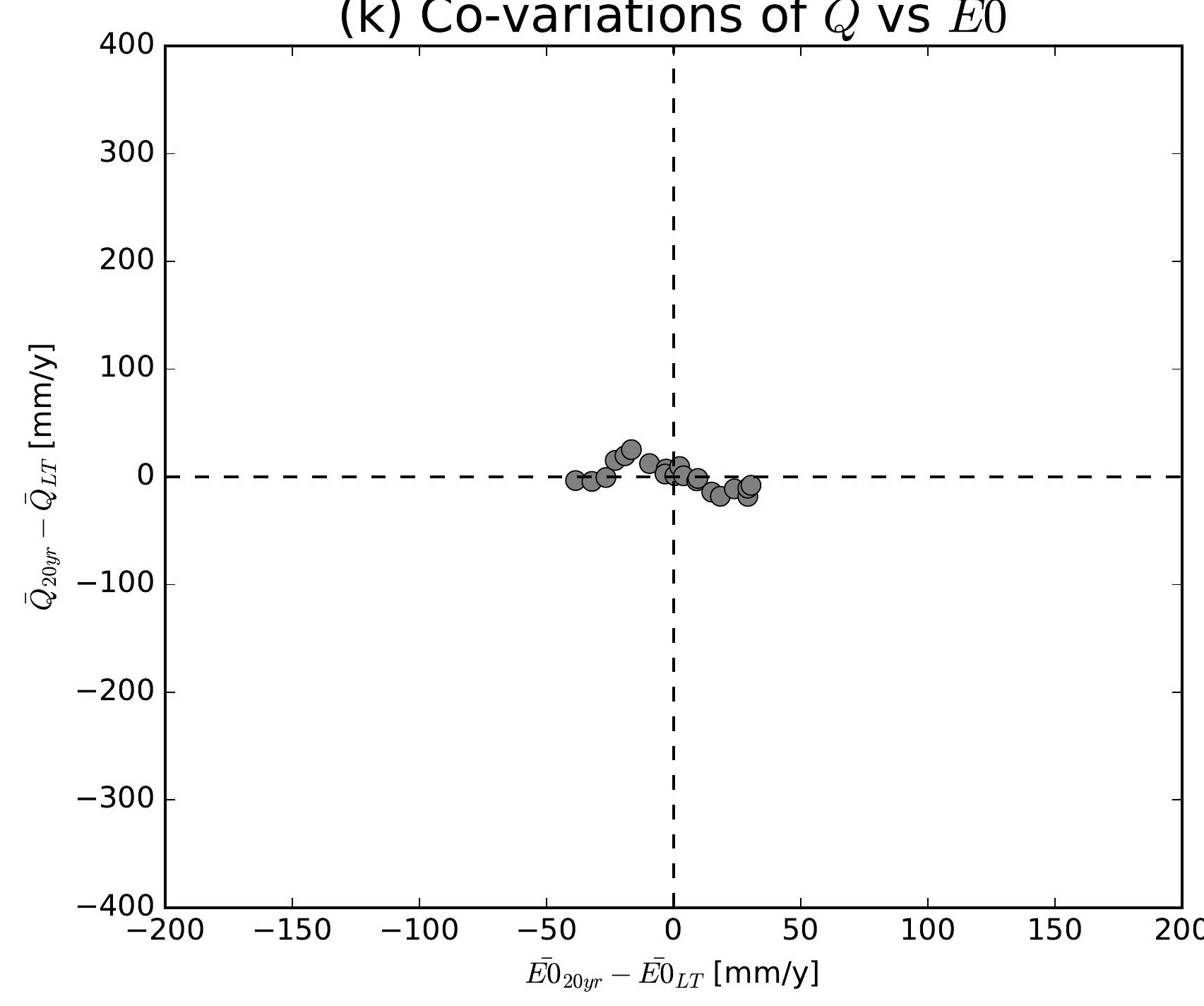

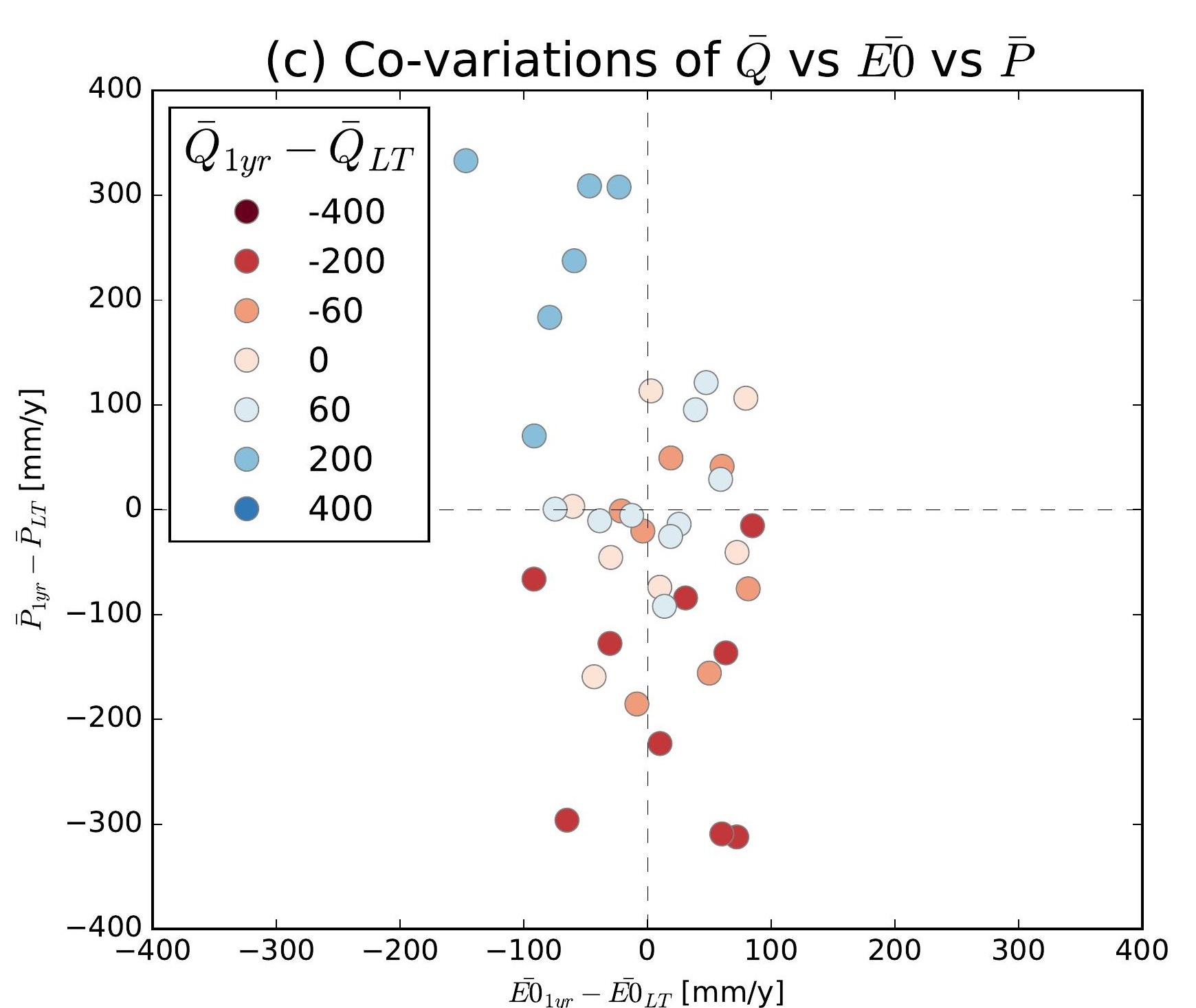
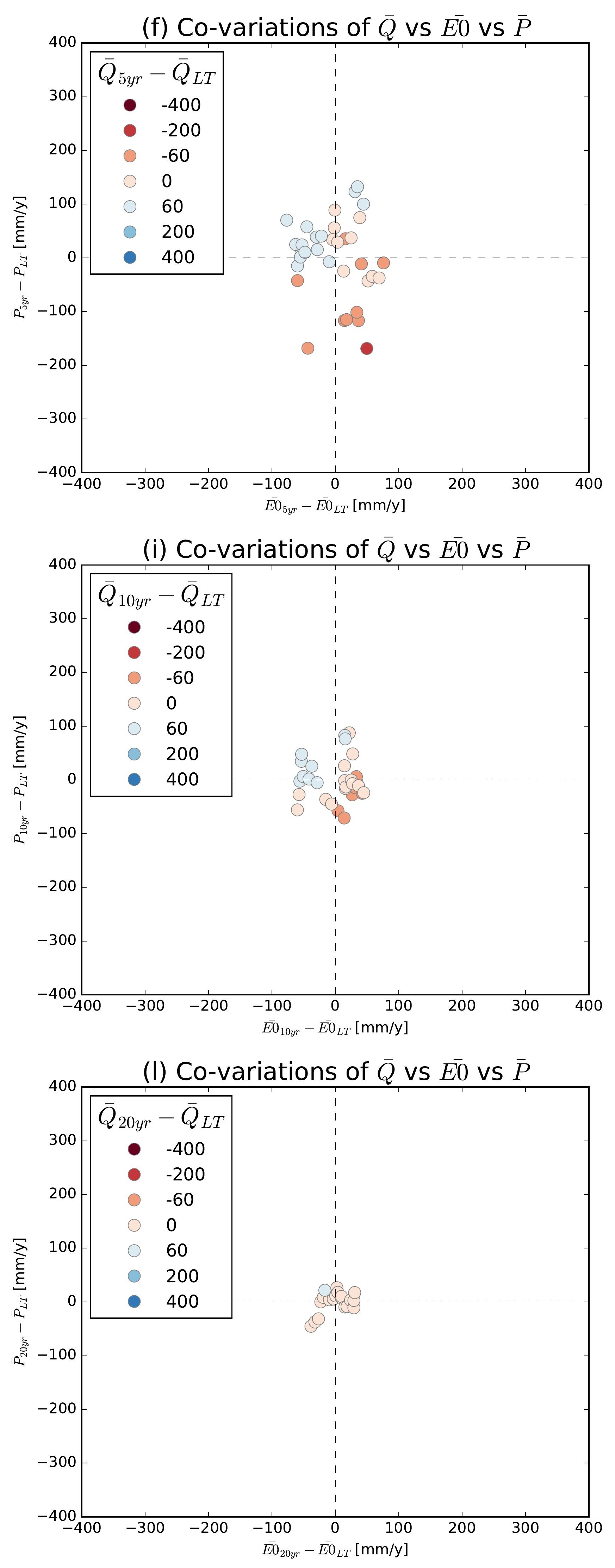

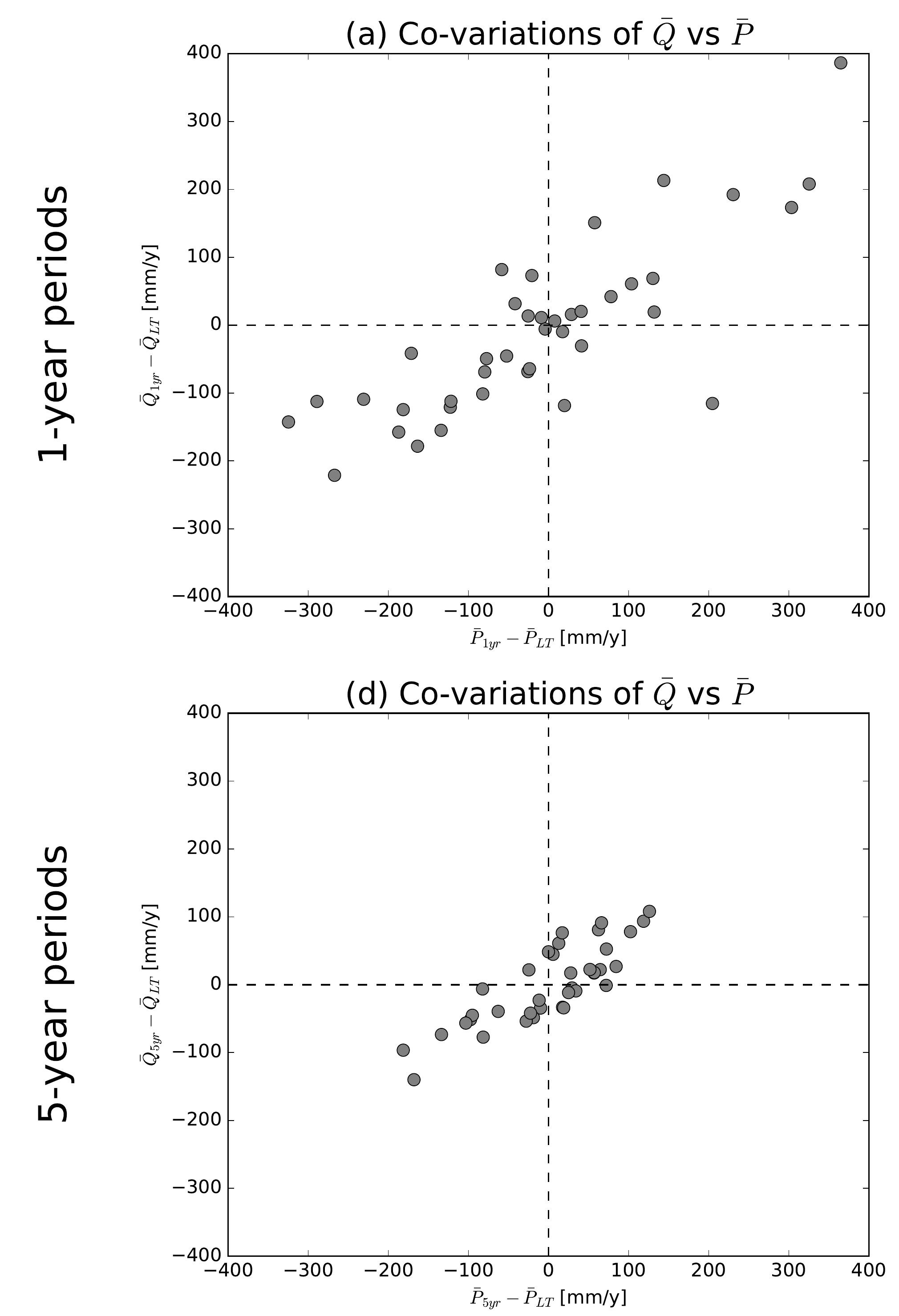

(g) Co-variations of $\bar{Q}$ vs $\bar{P}$
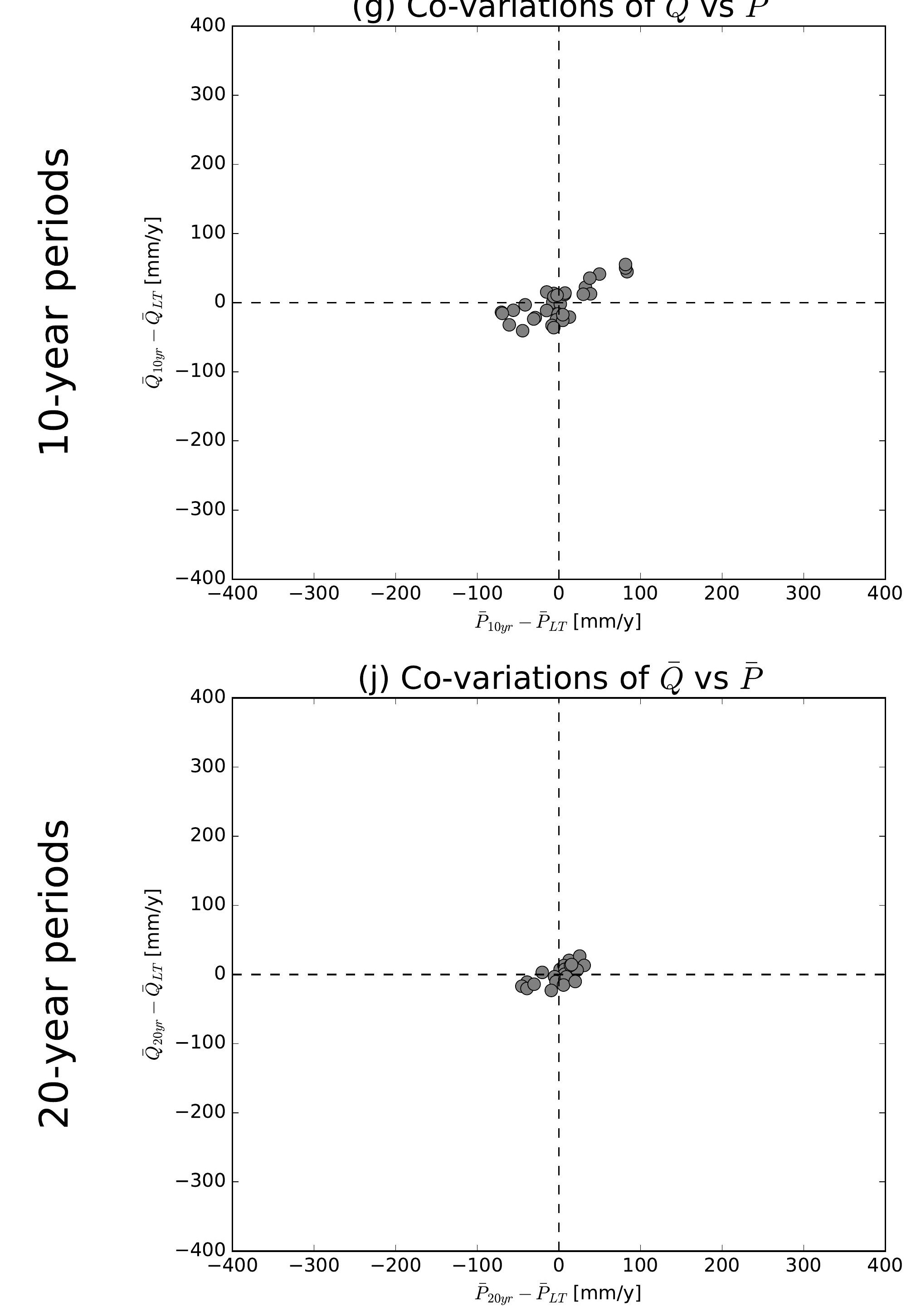

(b) Co-variations of $\bar{Q}$ vs $\overline{E 0}$

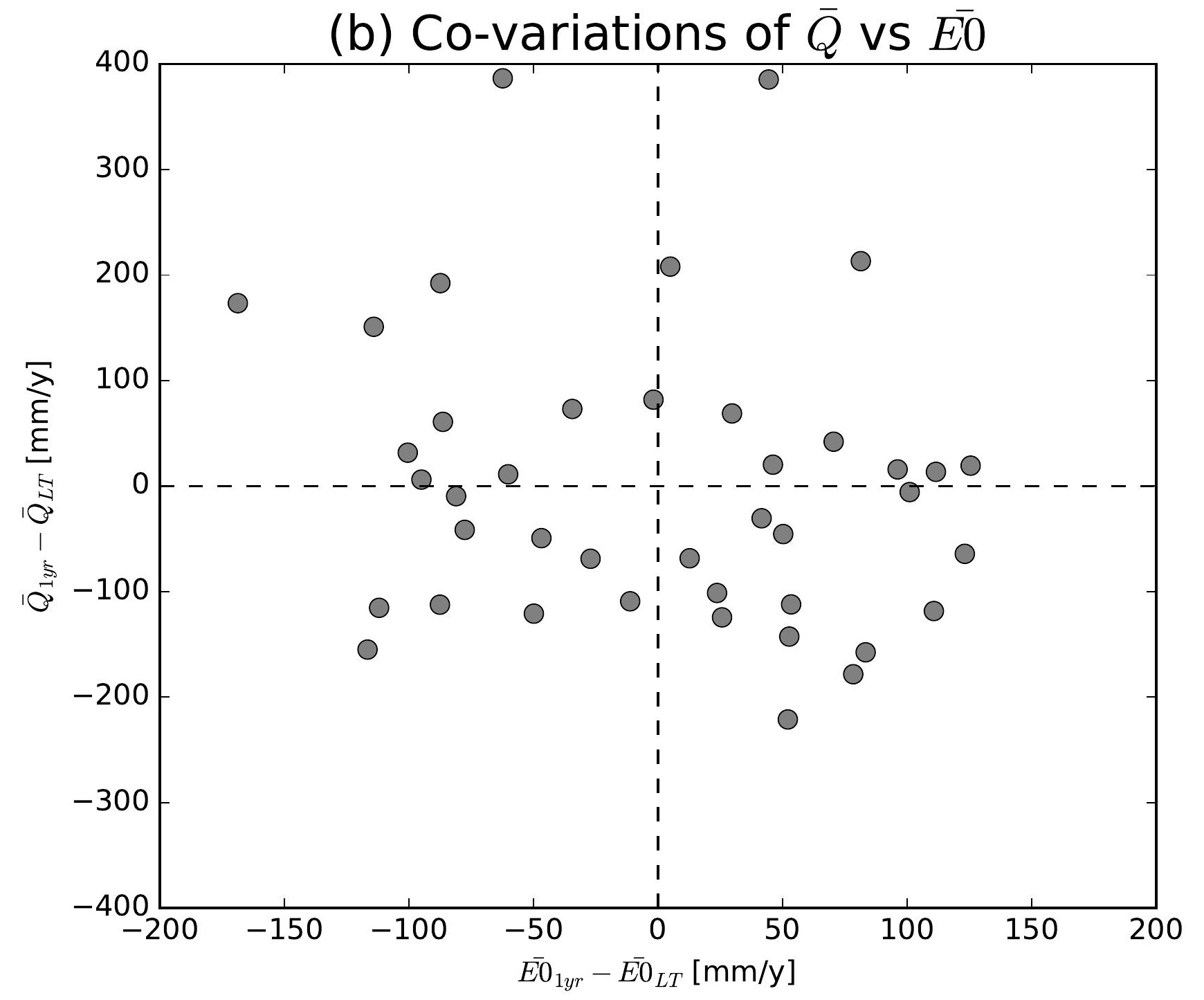

(e) Co-variations of $\bar{Q}$ vs $\overline{E 0}$

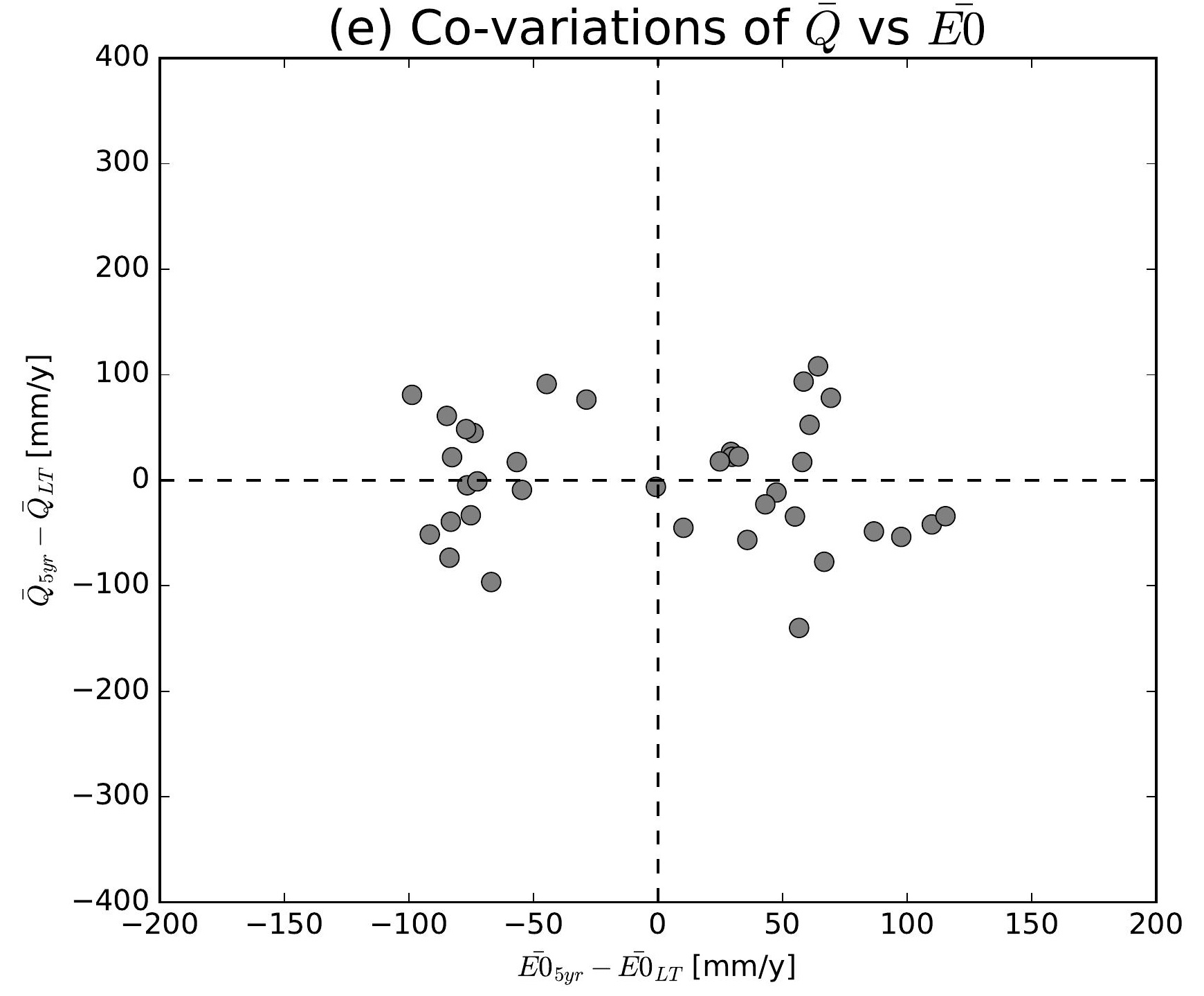

(h) Co-variations of $\bar{Q}$ vs $\overline{E 0}$

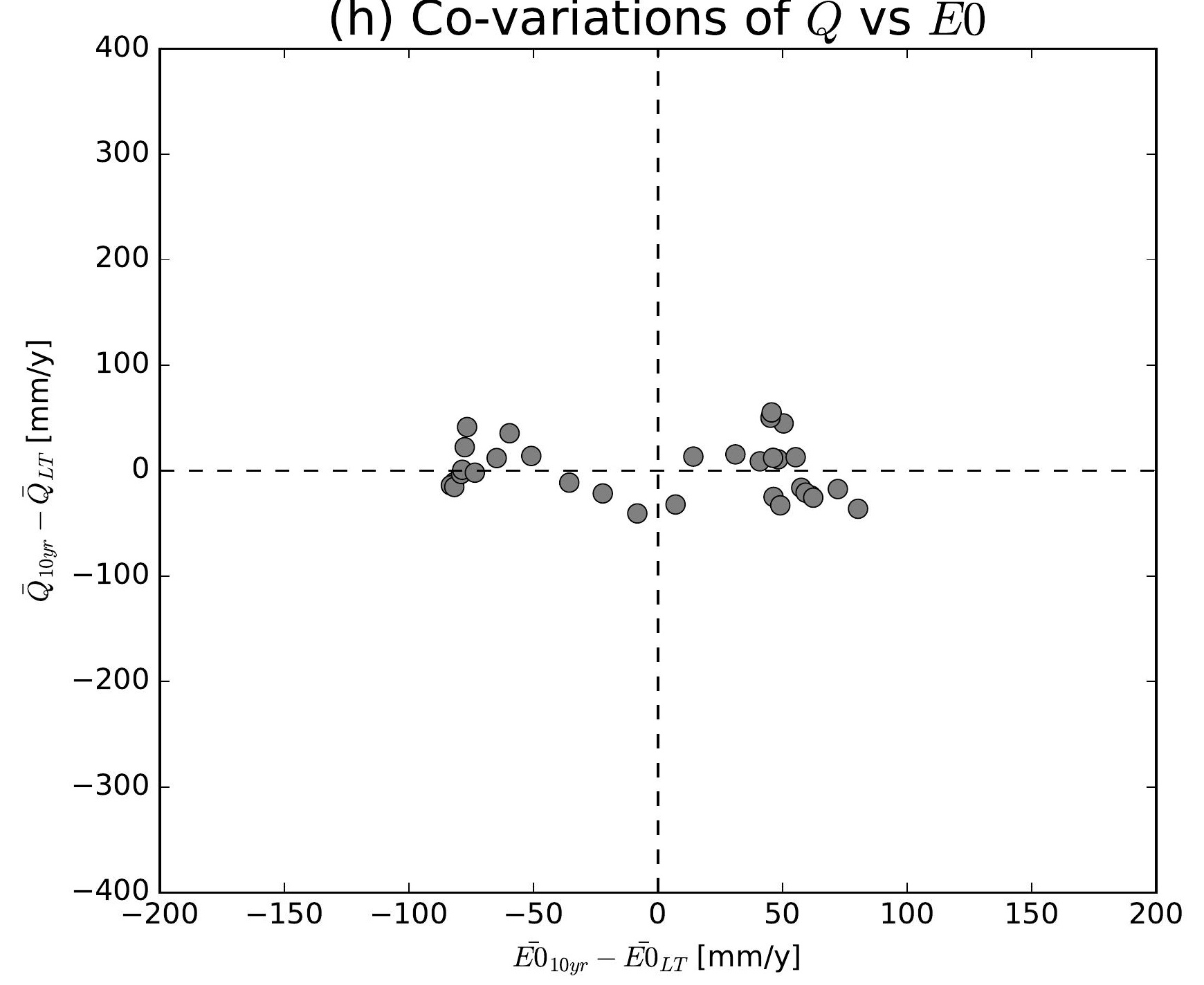

(k) Co-variations of $\bar{Q}$ vs $\overline{E 0}$

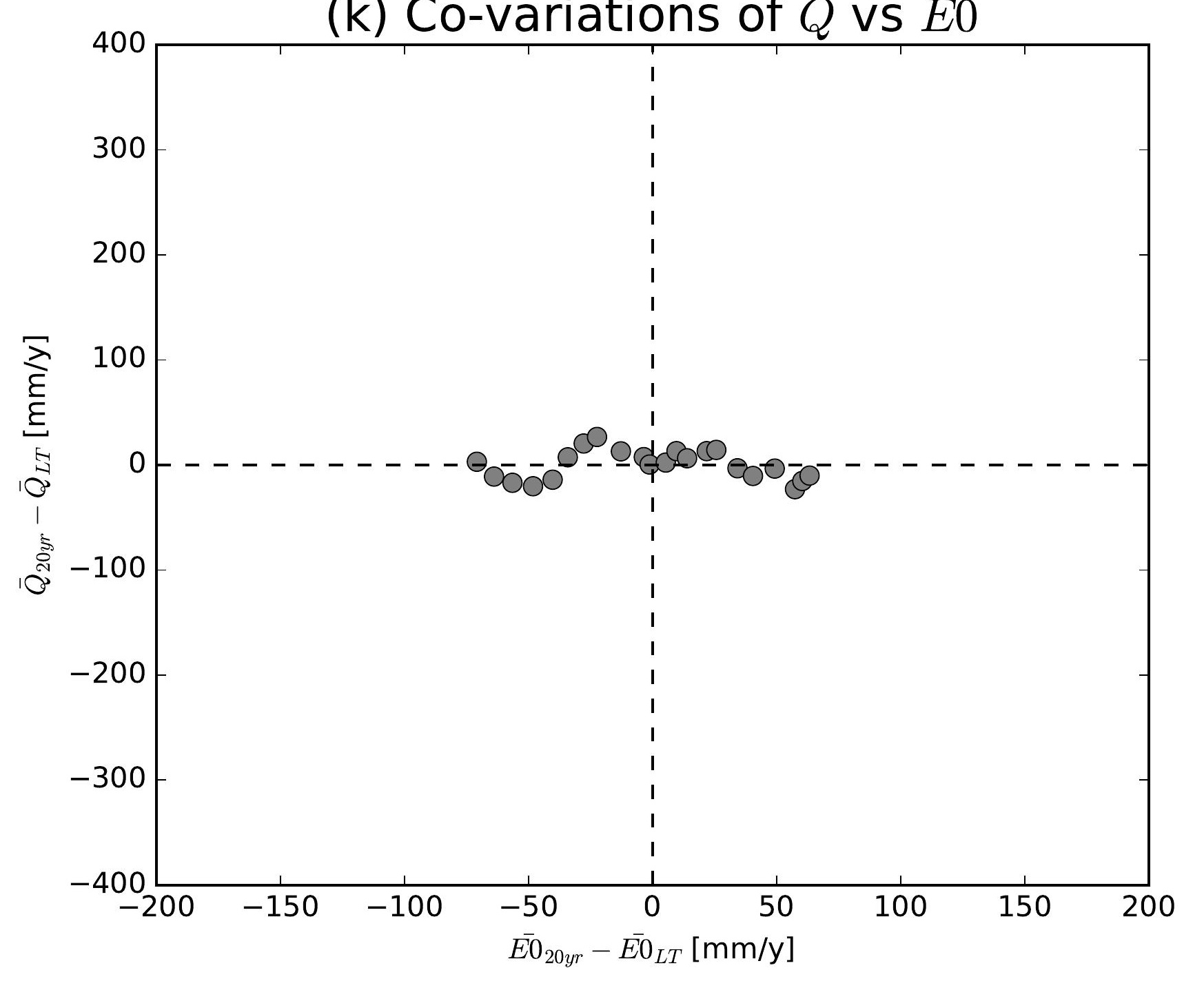

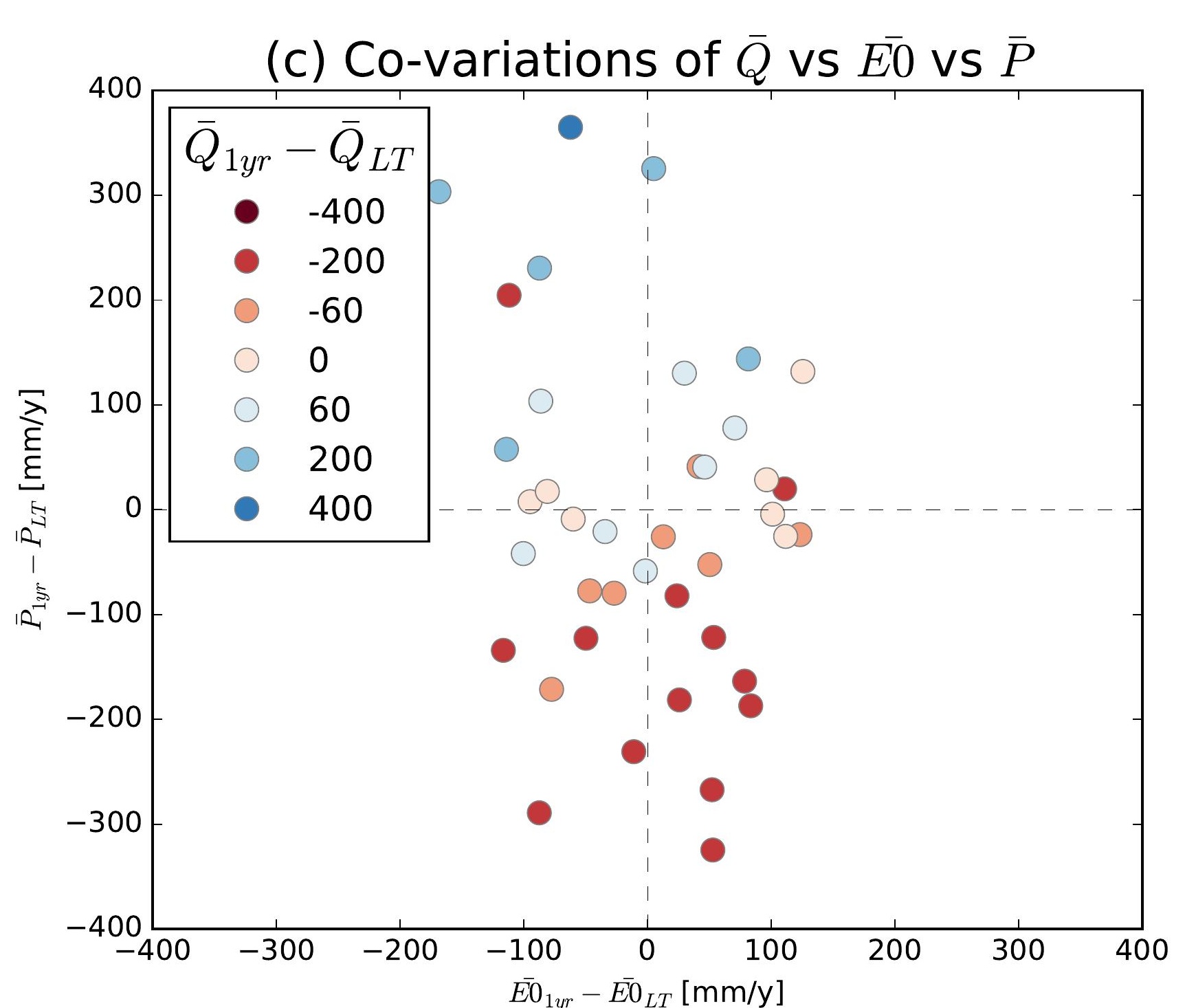
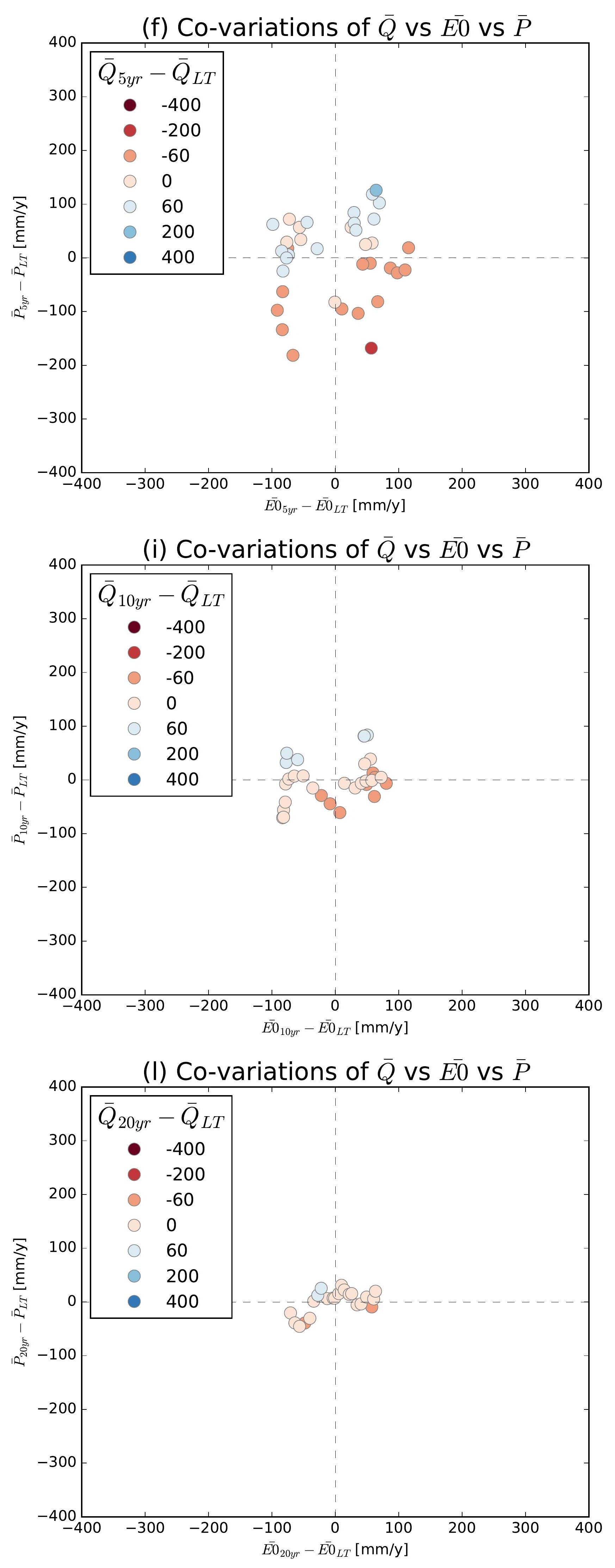

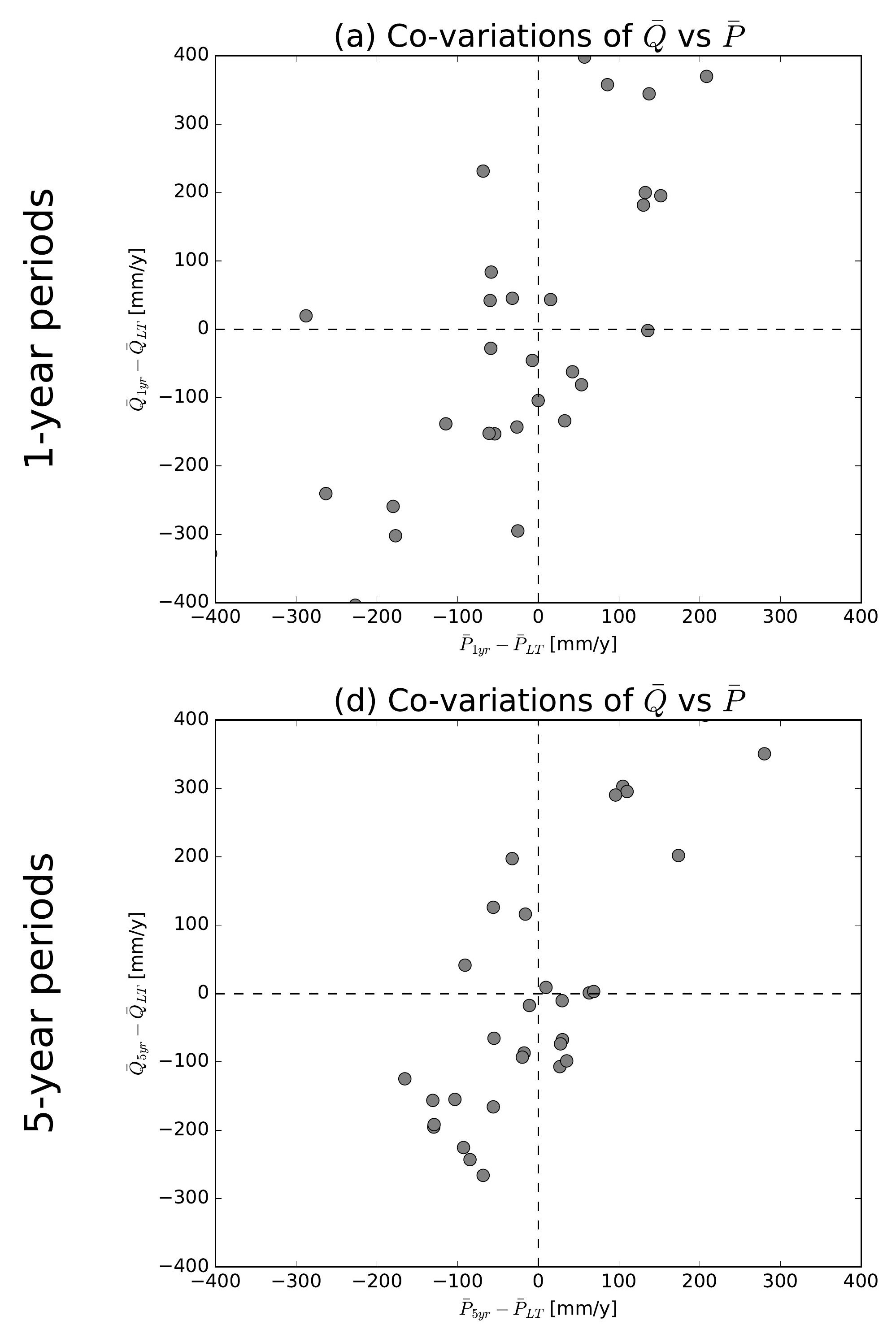

(g) Co-variations of $\bar{Q}$ vs $\bar{P}$

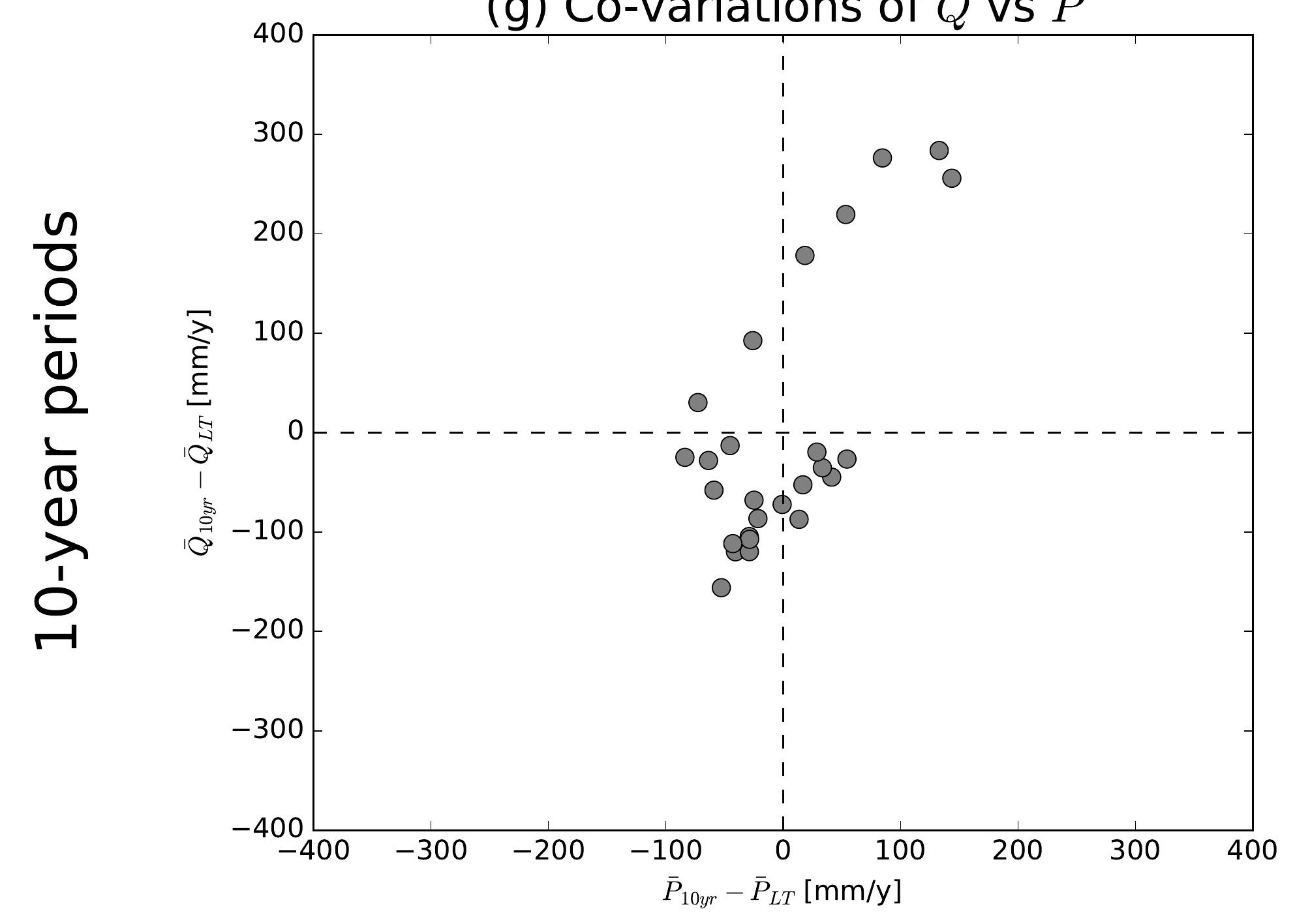

(j) Co-variations of $\bar{Q}$ vs $\bar{P}$

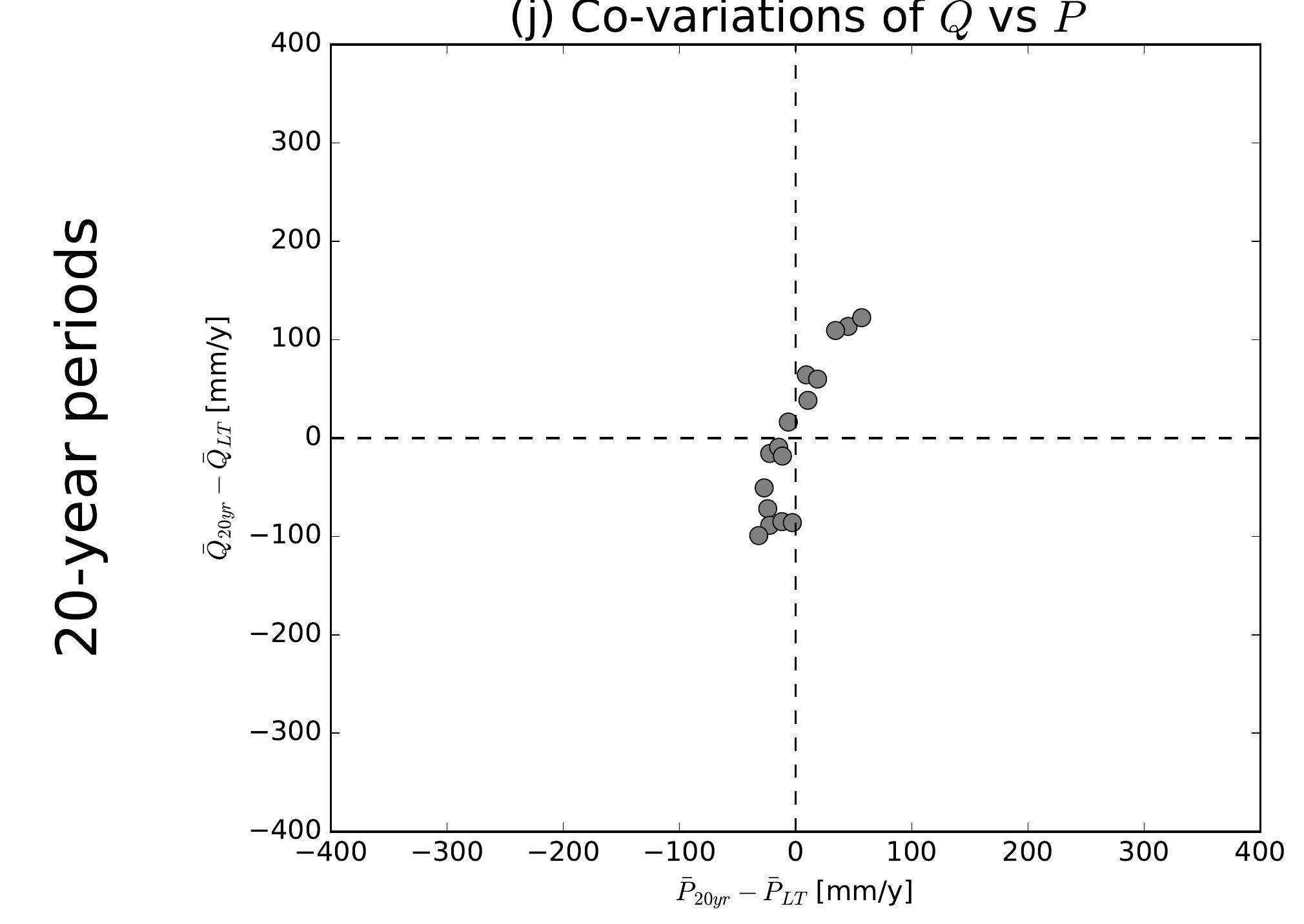

(b) Co-variations of $\bar{Q}$ vs $\overline{E 0}$

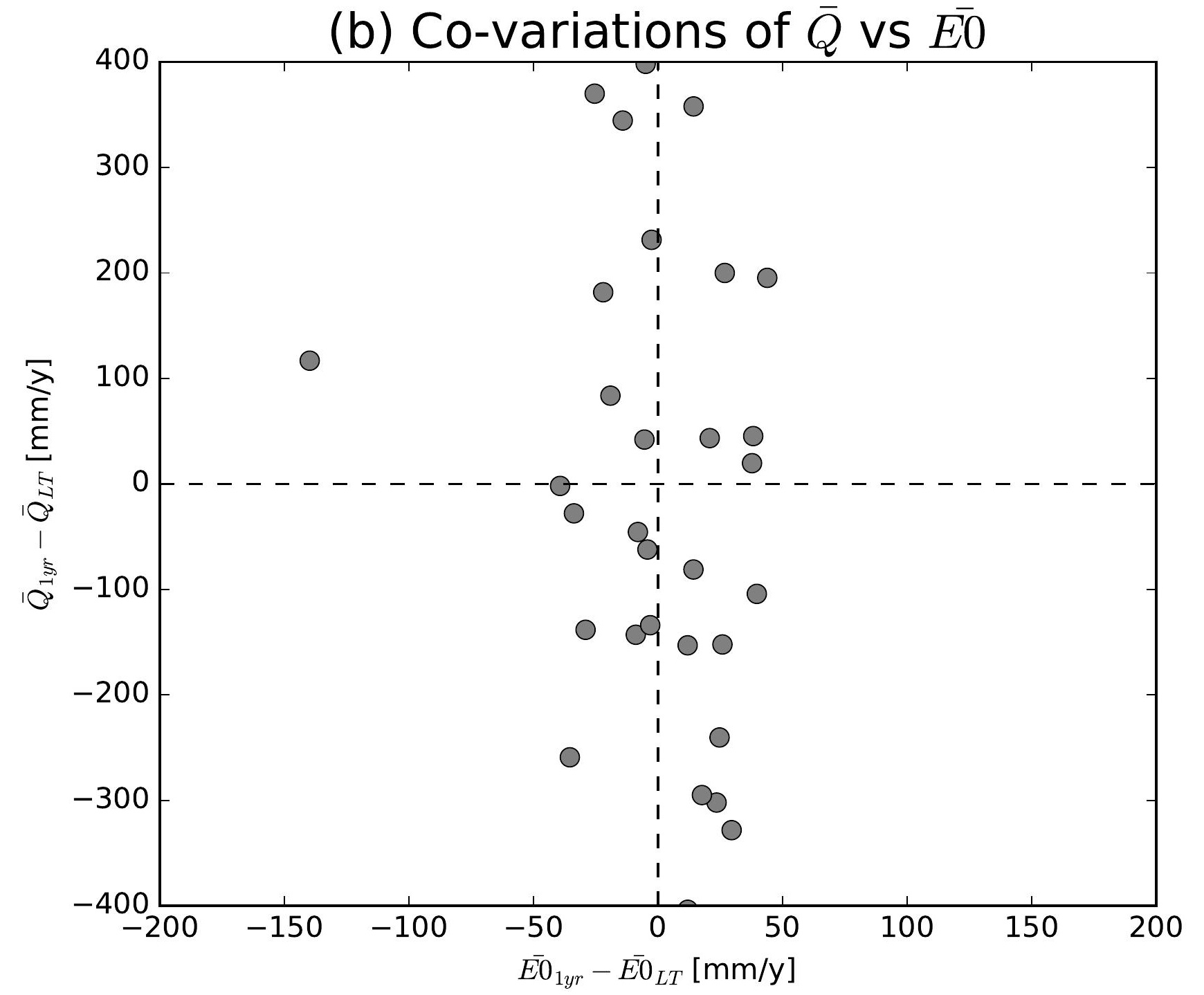

(e) Co-variations of $\bar{Q}$ vs $\overline{E 0}$

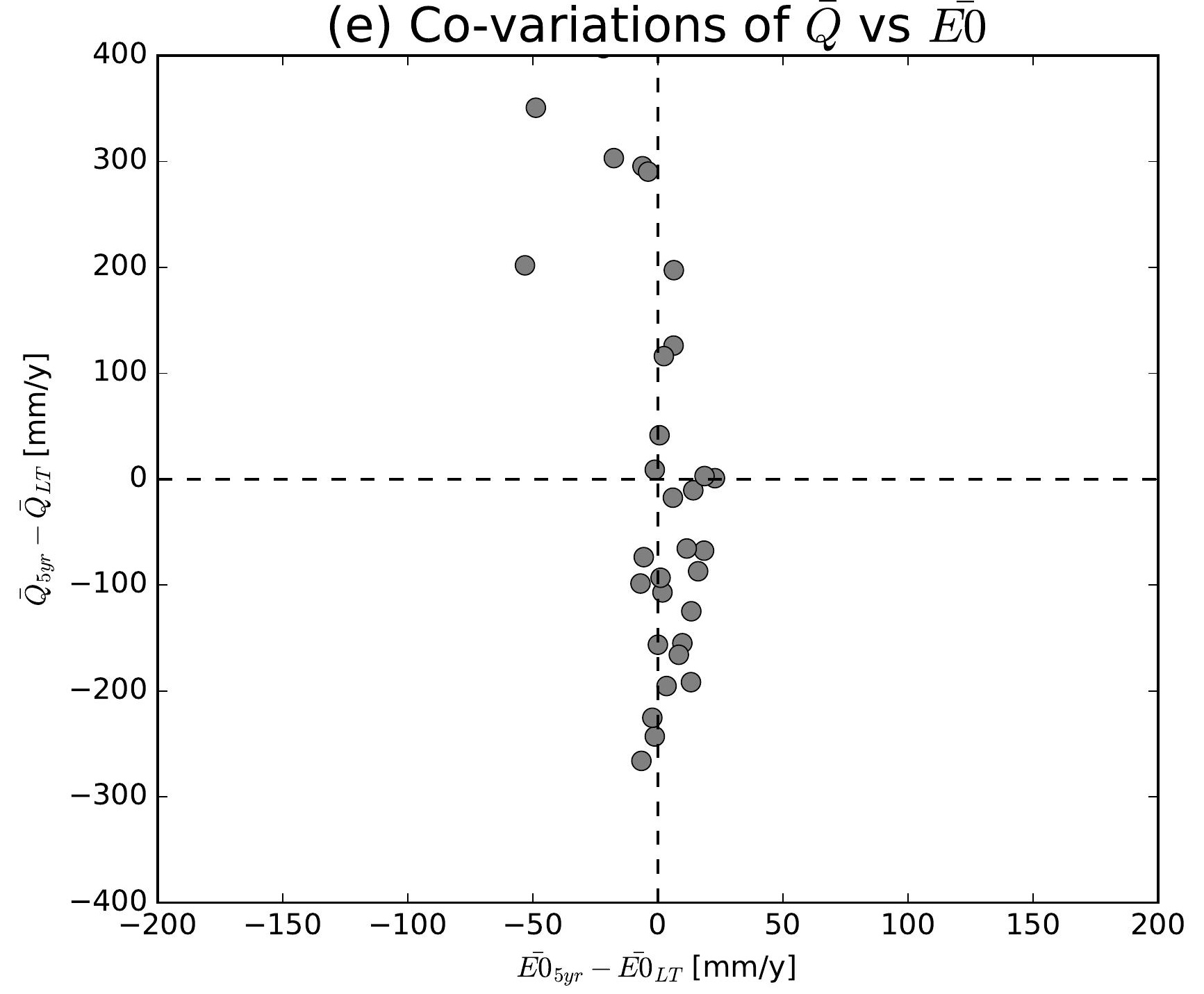

(h) Co-variations of $\bar{Q}$ vs $\overline{E 0}$

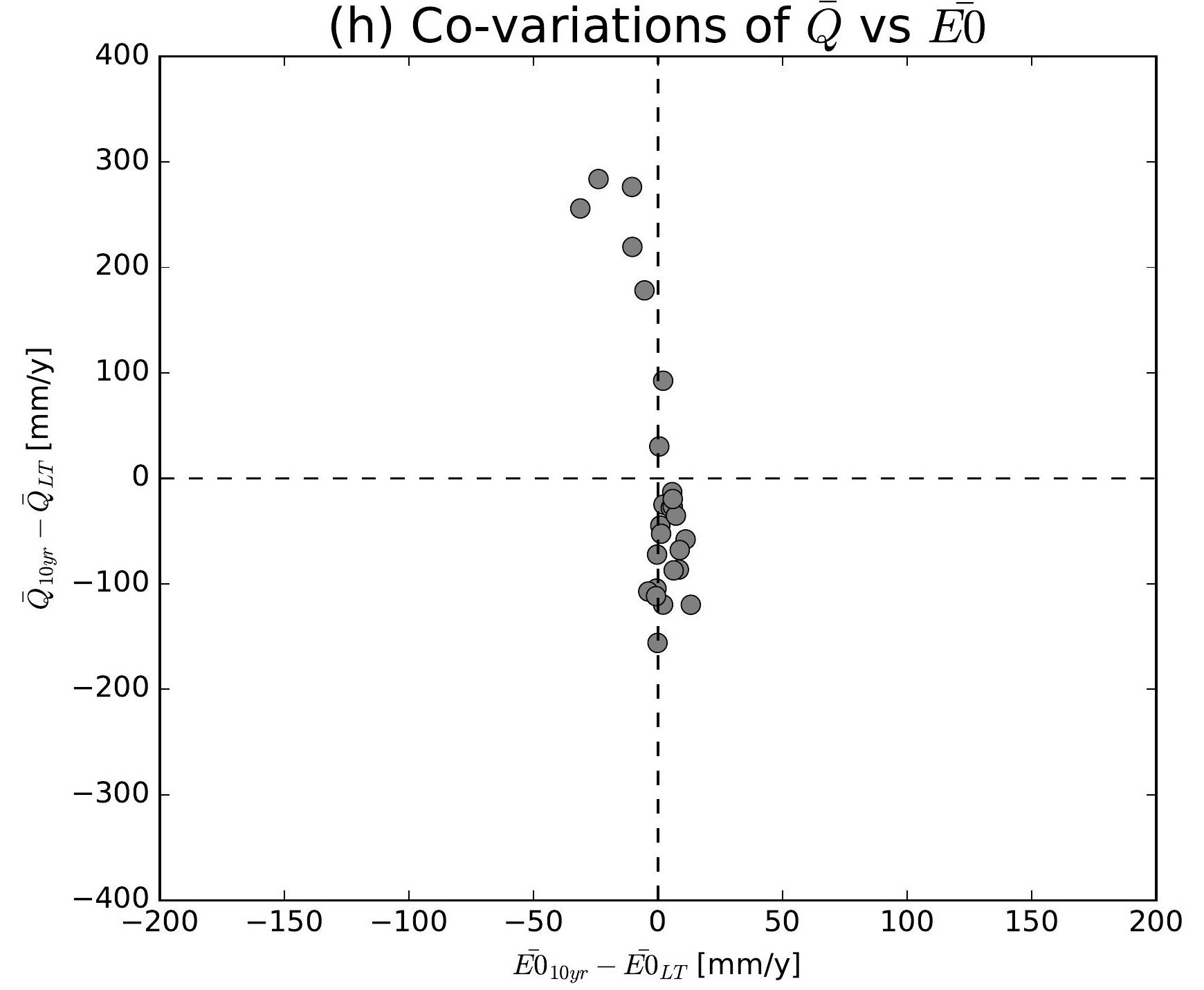

(k) Co-variations of $\bar{Q}$ vs $\overline{E 0}$

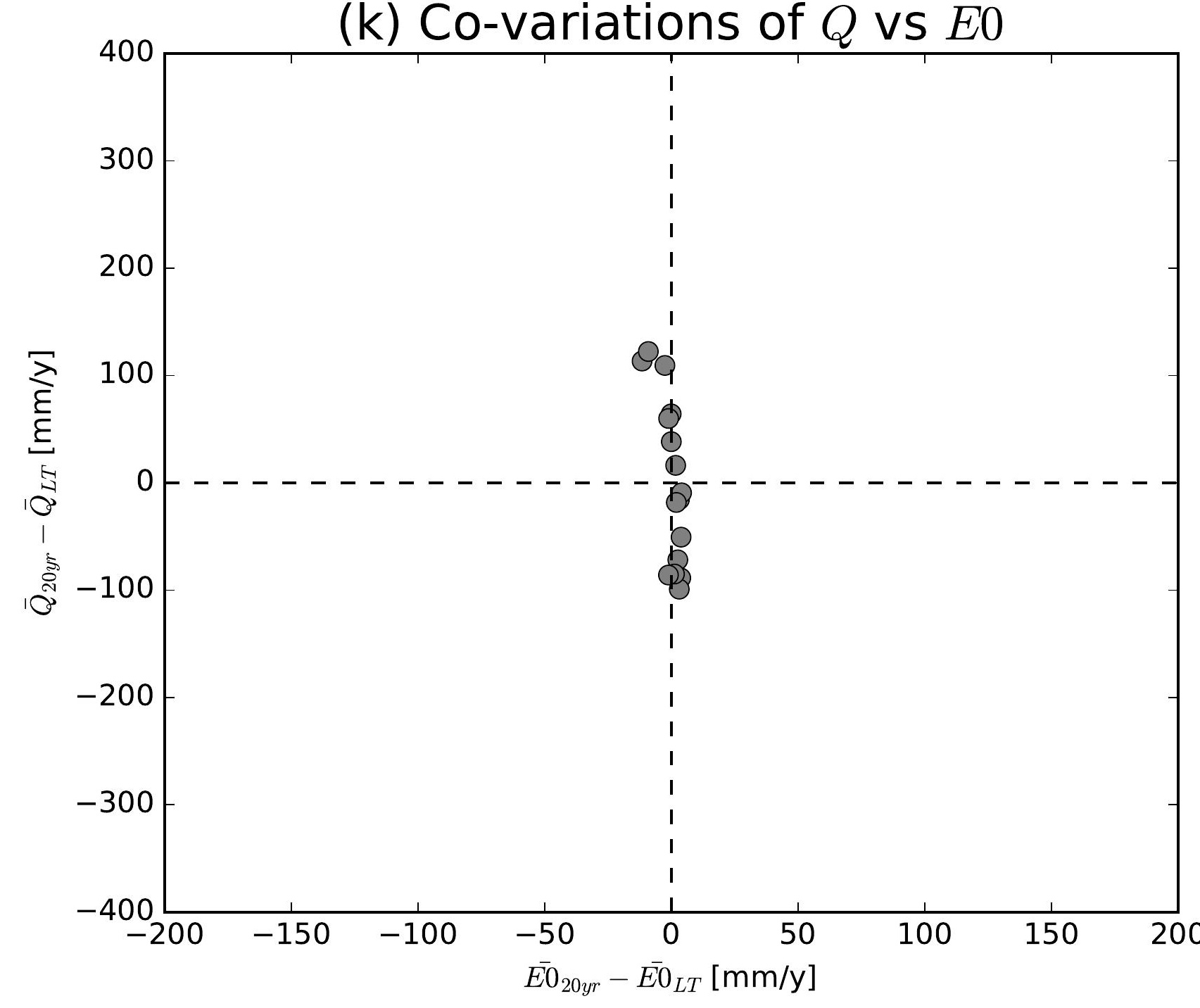

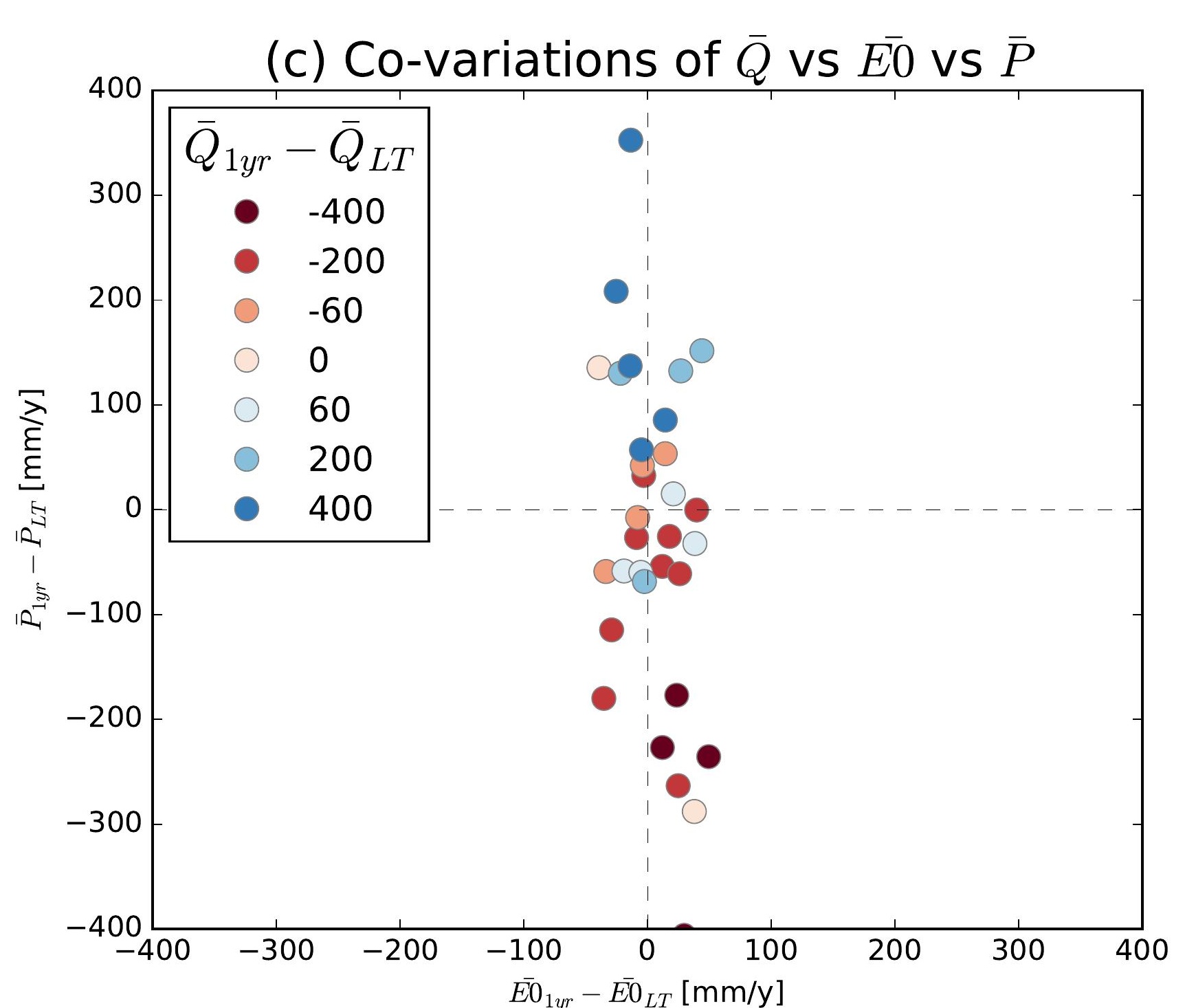
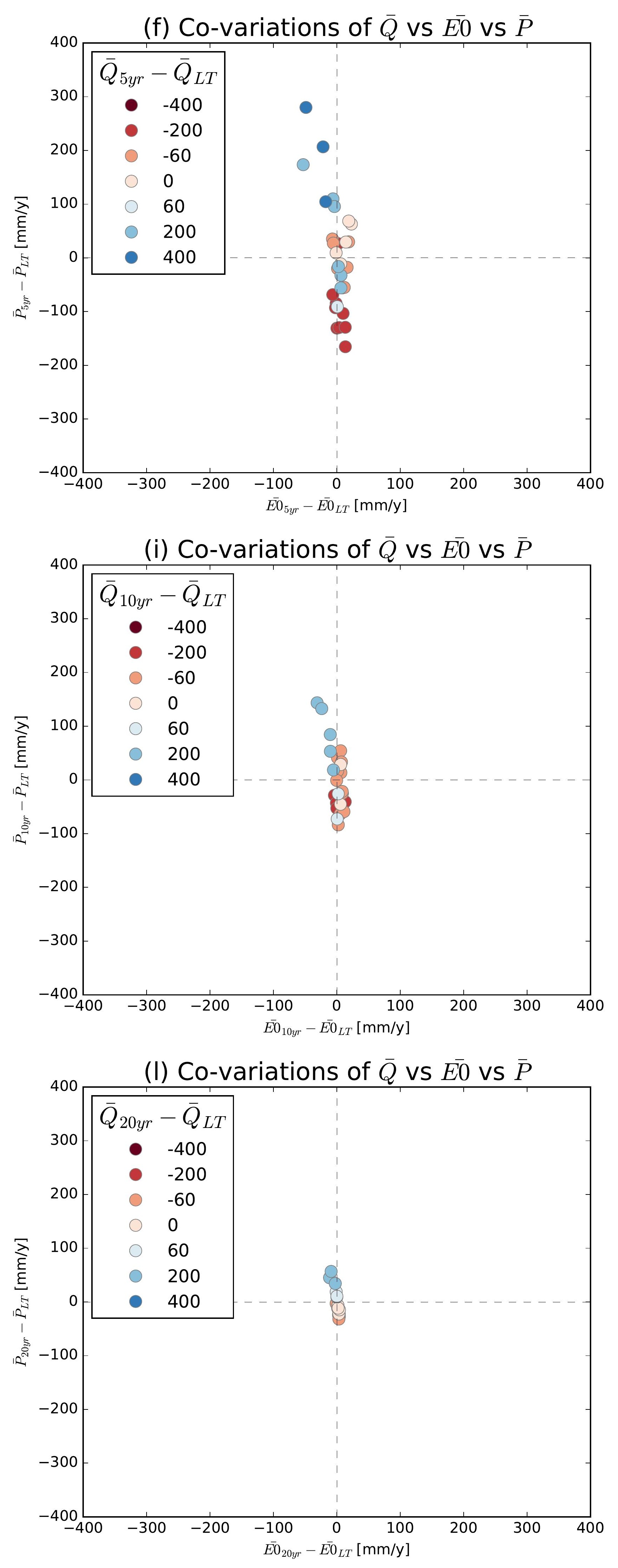

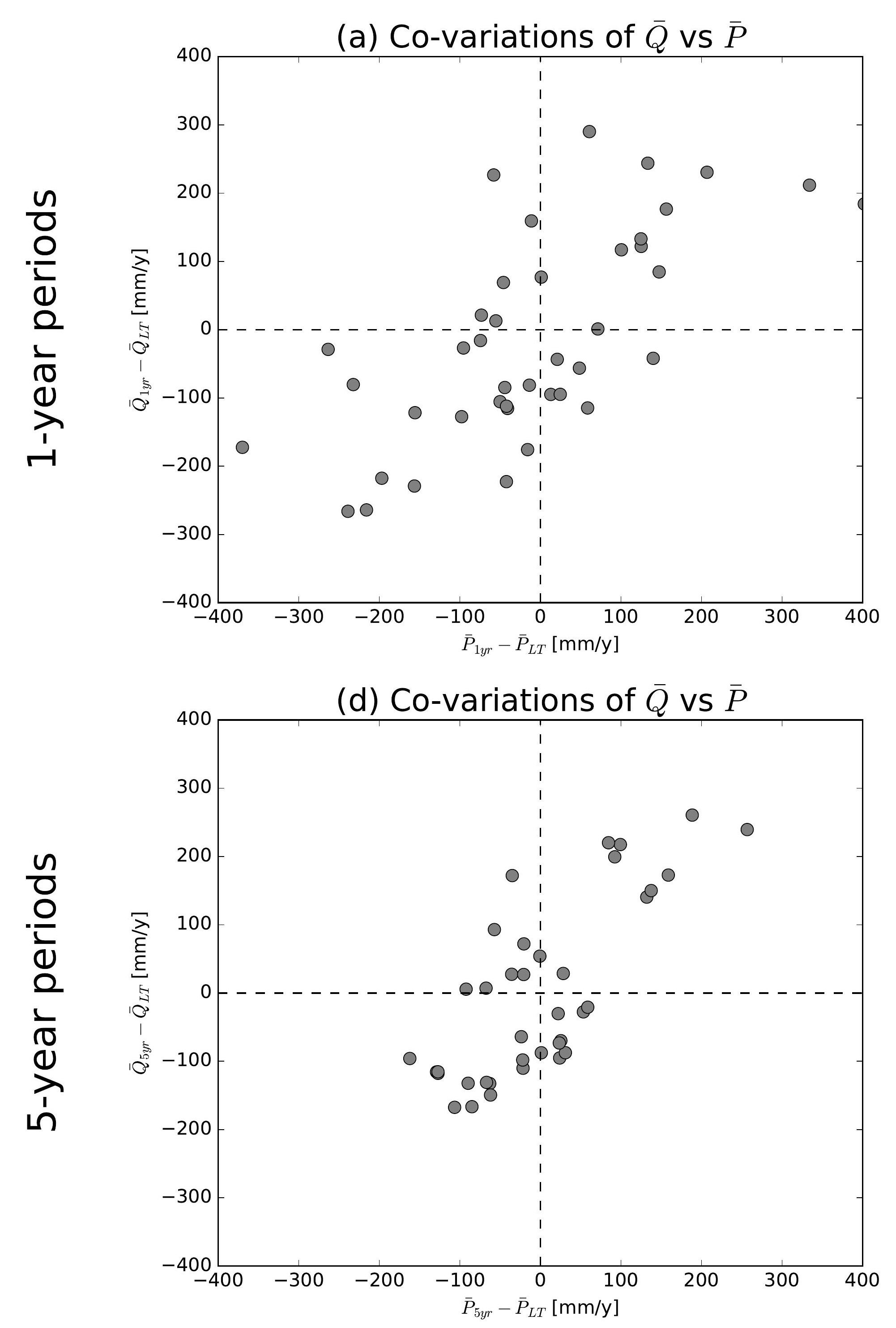

(g) Co-variations of $\bar{Q}$ vs $\bar{P}$

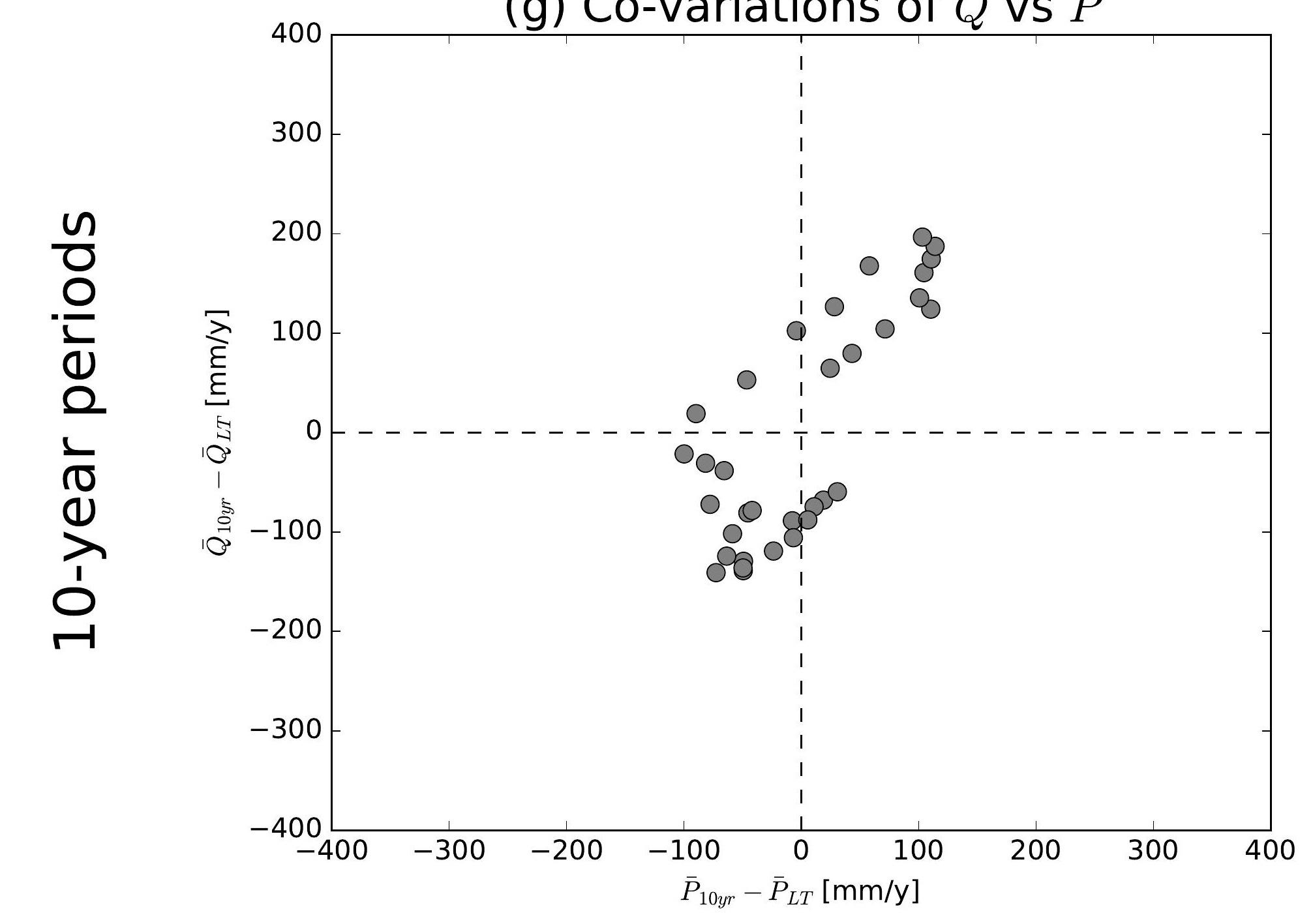

(j) Co-variations of $\bar{Q}$ vs $\bar{P}$

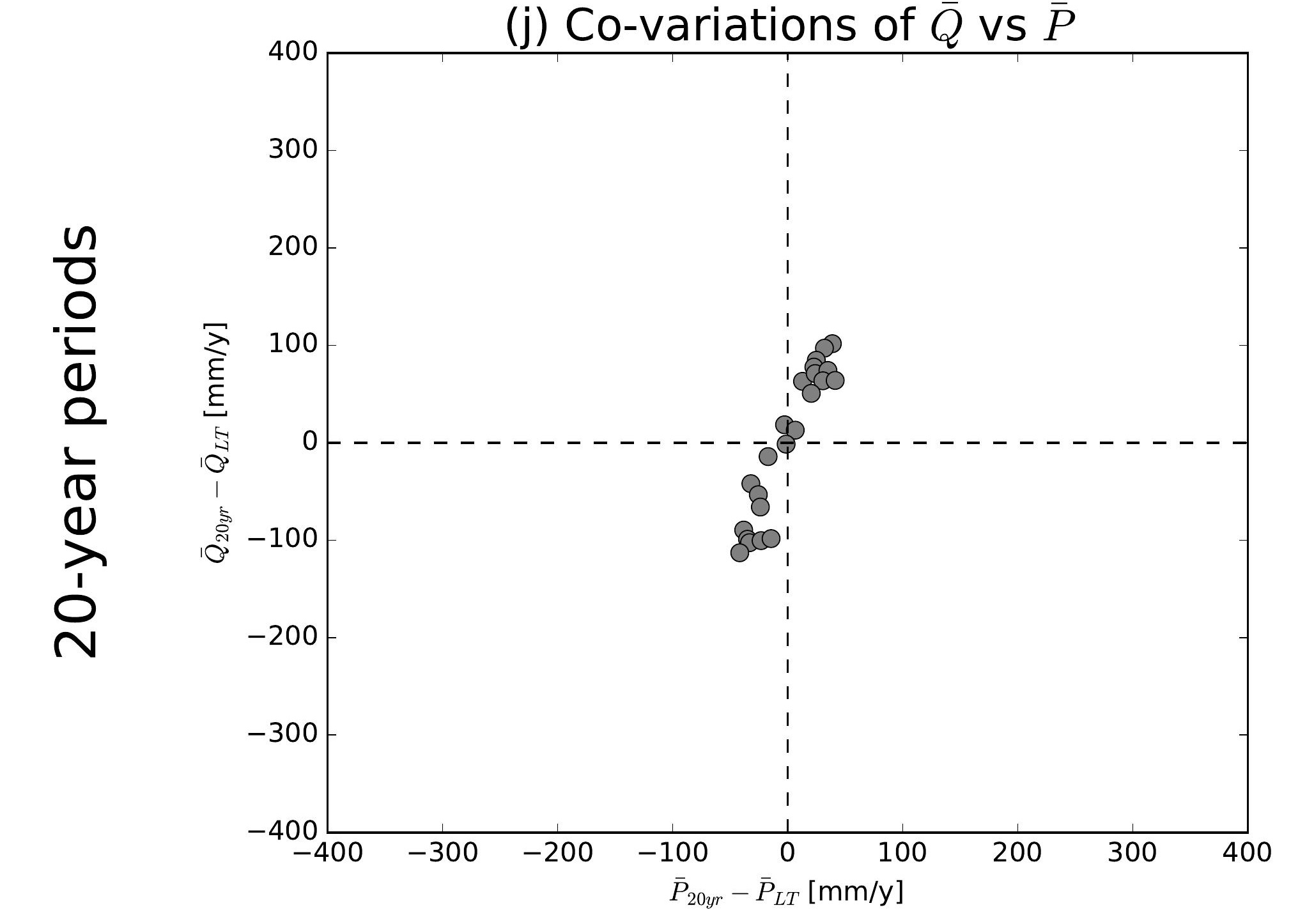

(b) Co-variations of $\bar{Q}$ vs $\overline{E 0}$

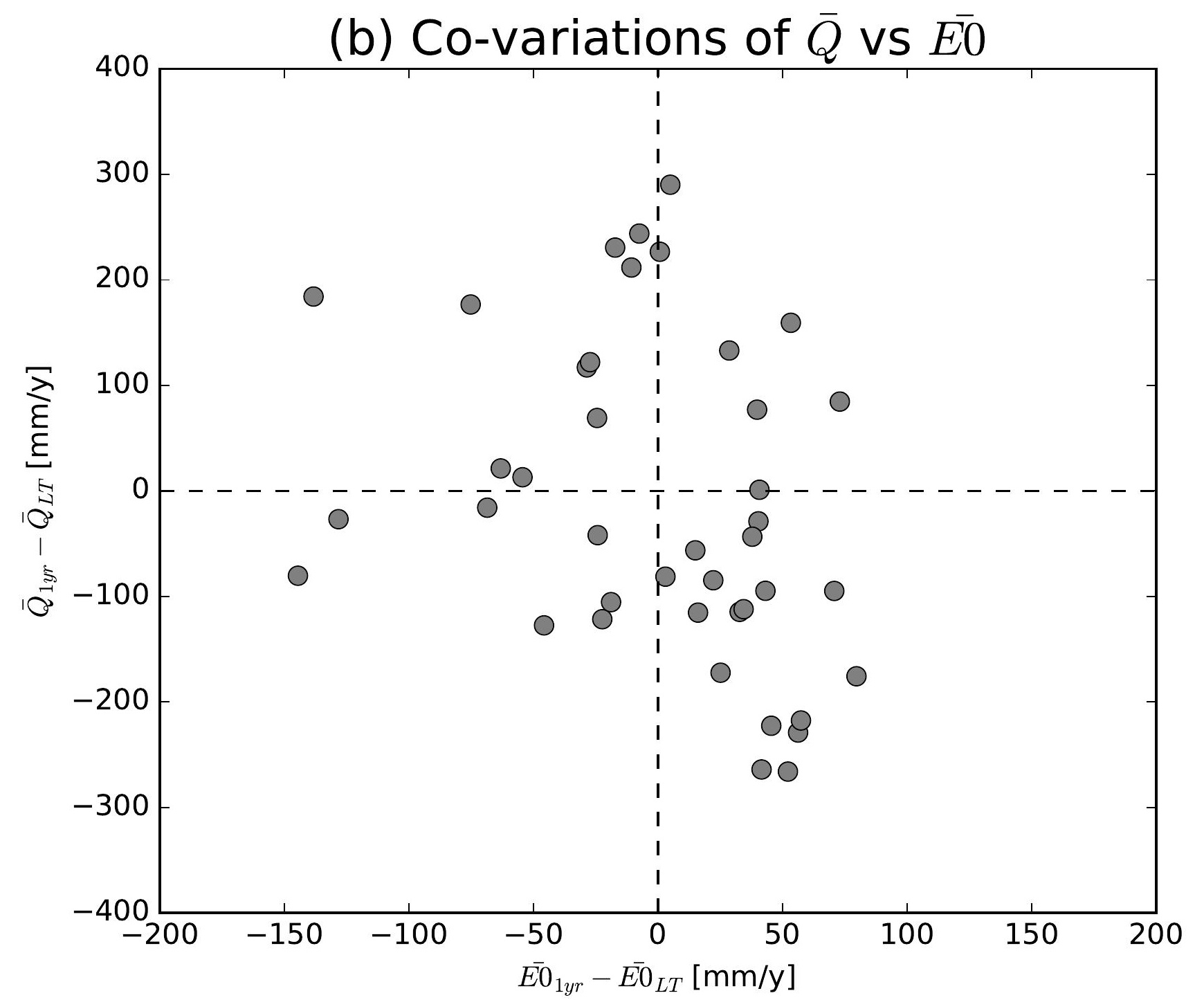

(e) Co-variations of $\bar{Q}$ vs $\overline{E 0}$

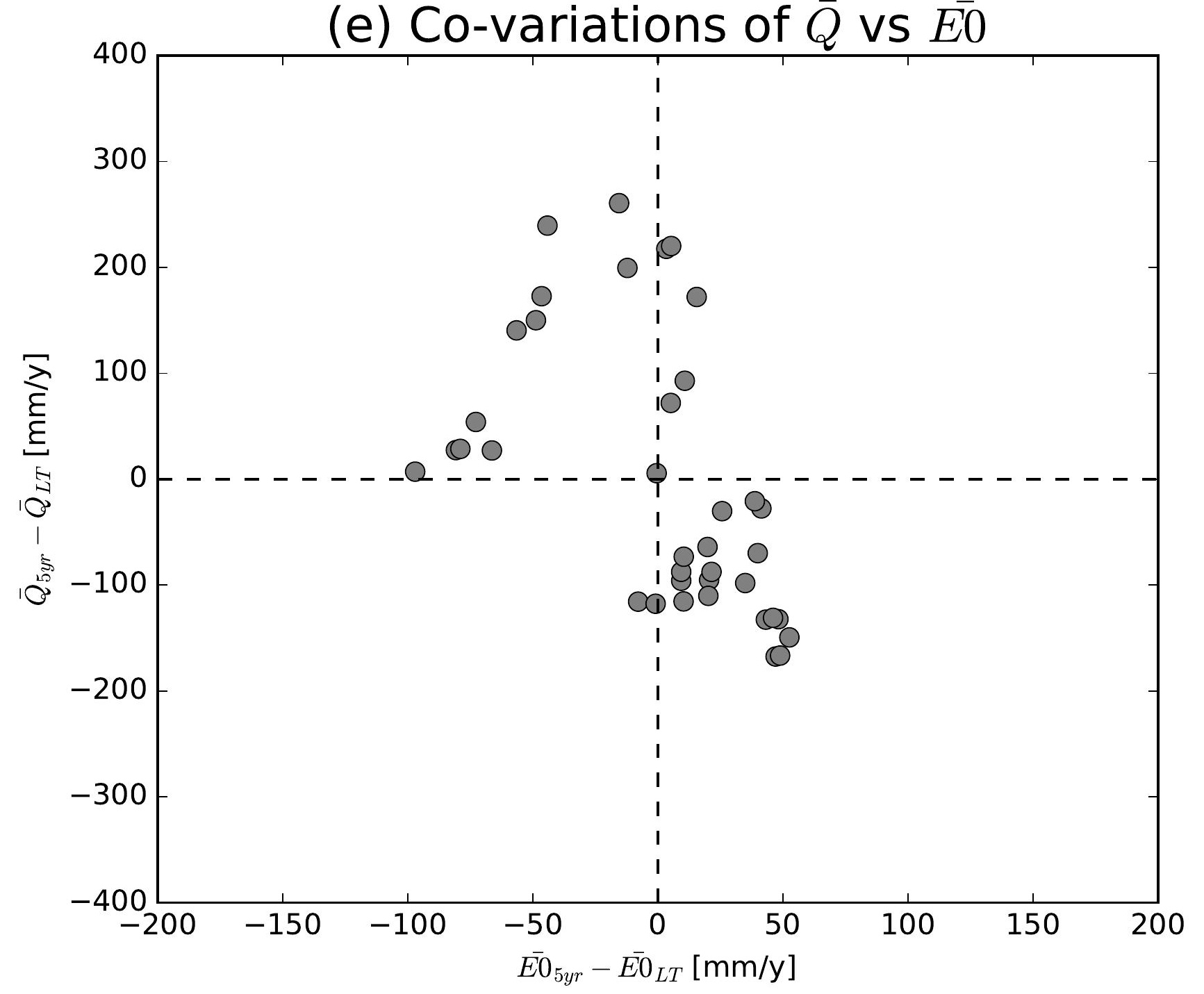

(h) Co-variations of $\bar{Q}$ vs $\overline{E 0}$

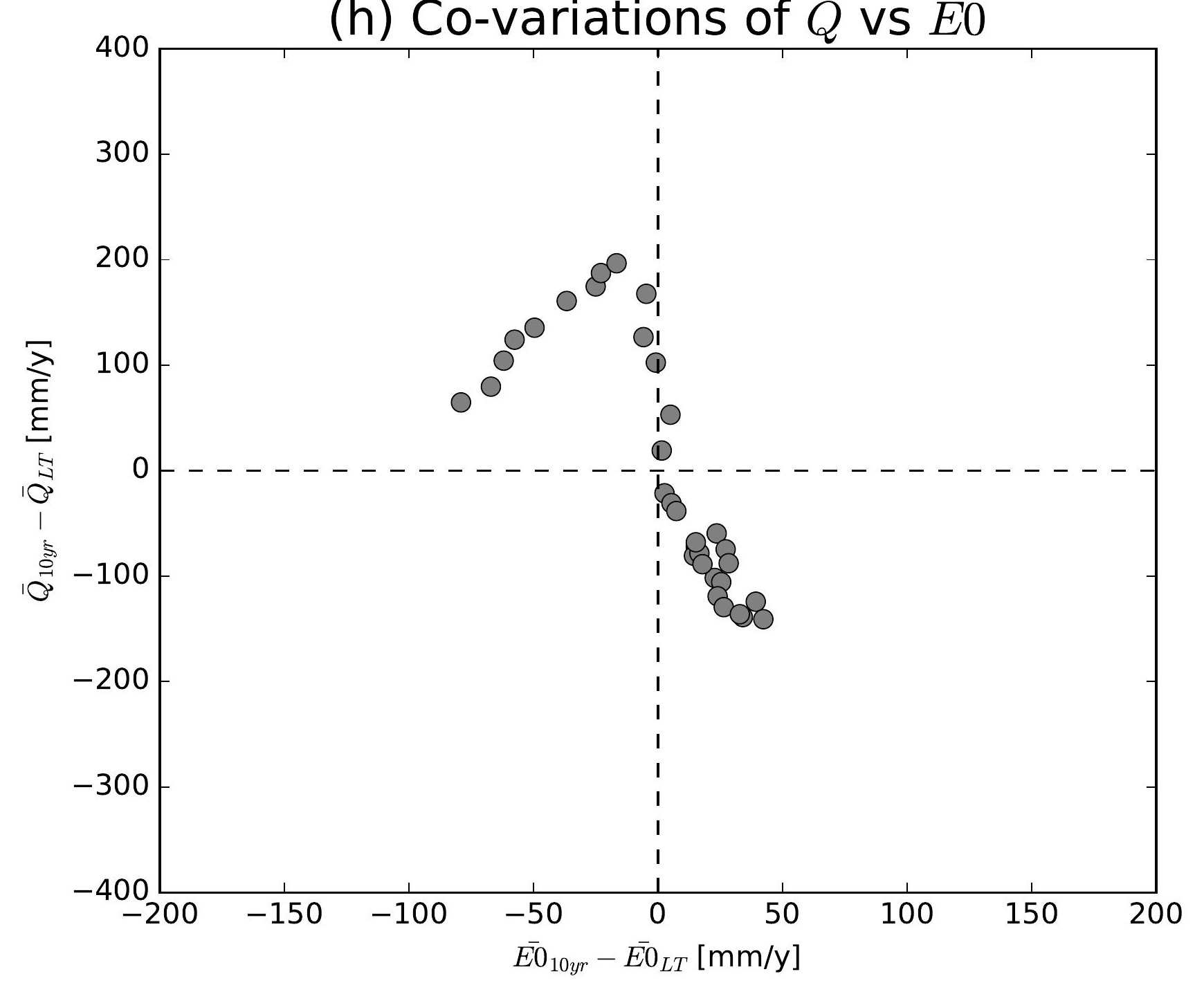

(k) Co-variations of $\bar{Q}$ vs $\overline{E 0}$

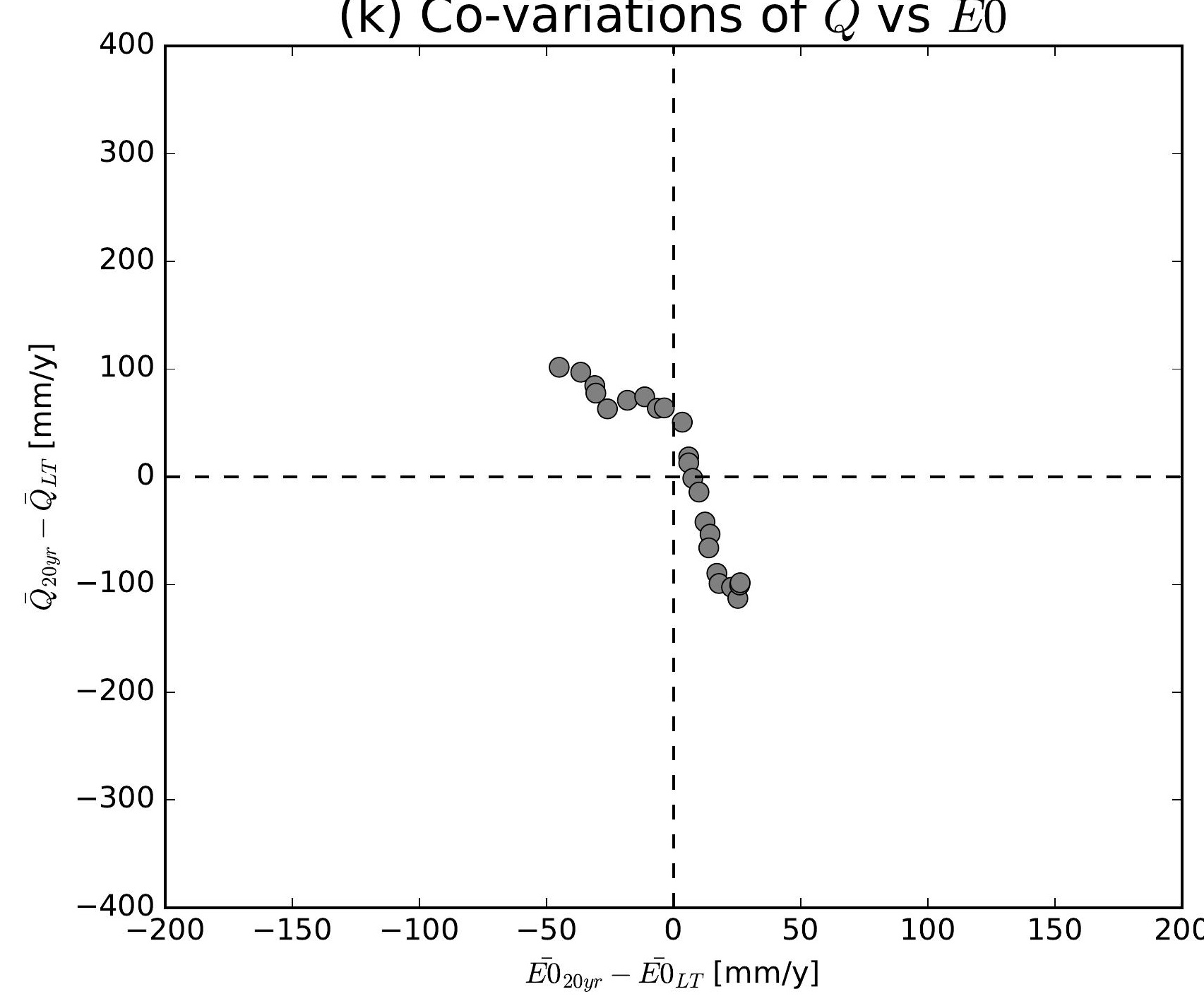

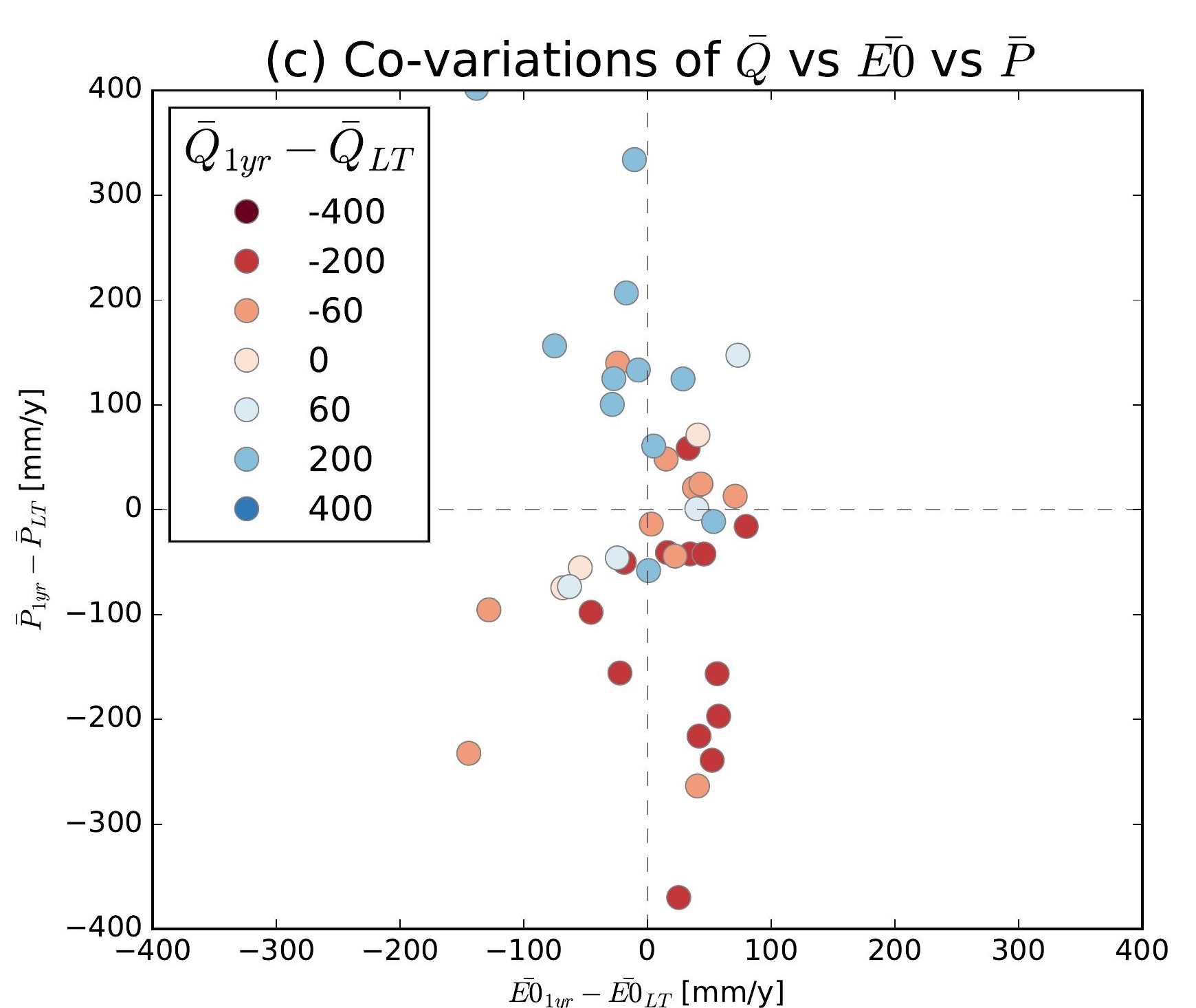
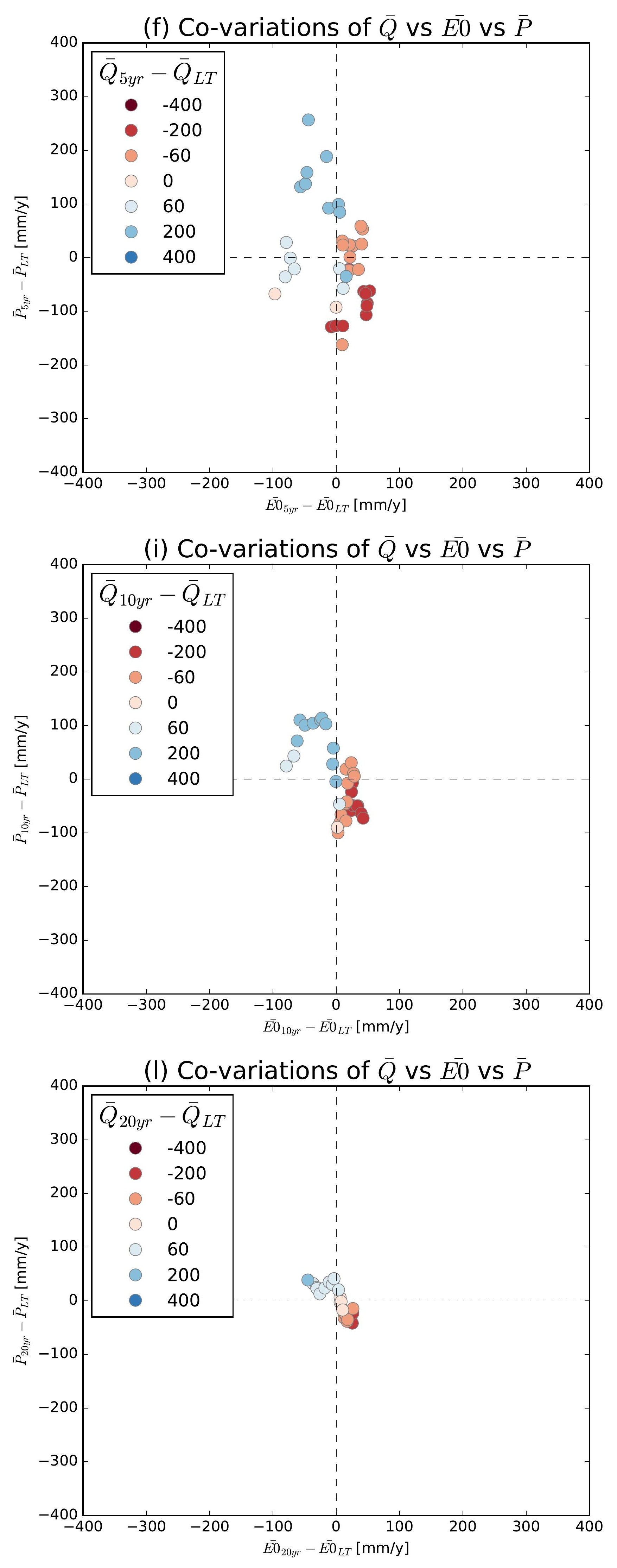

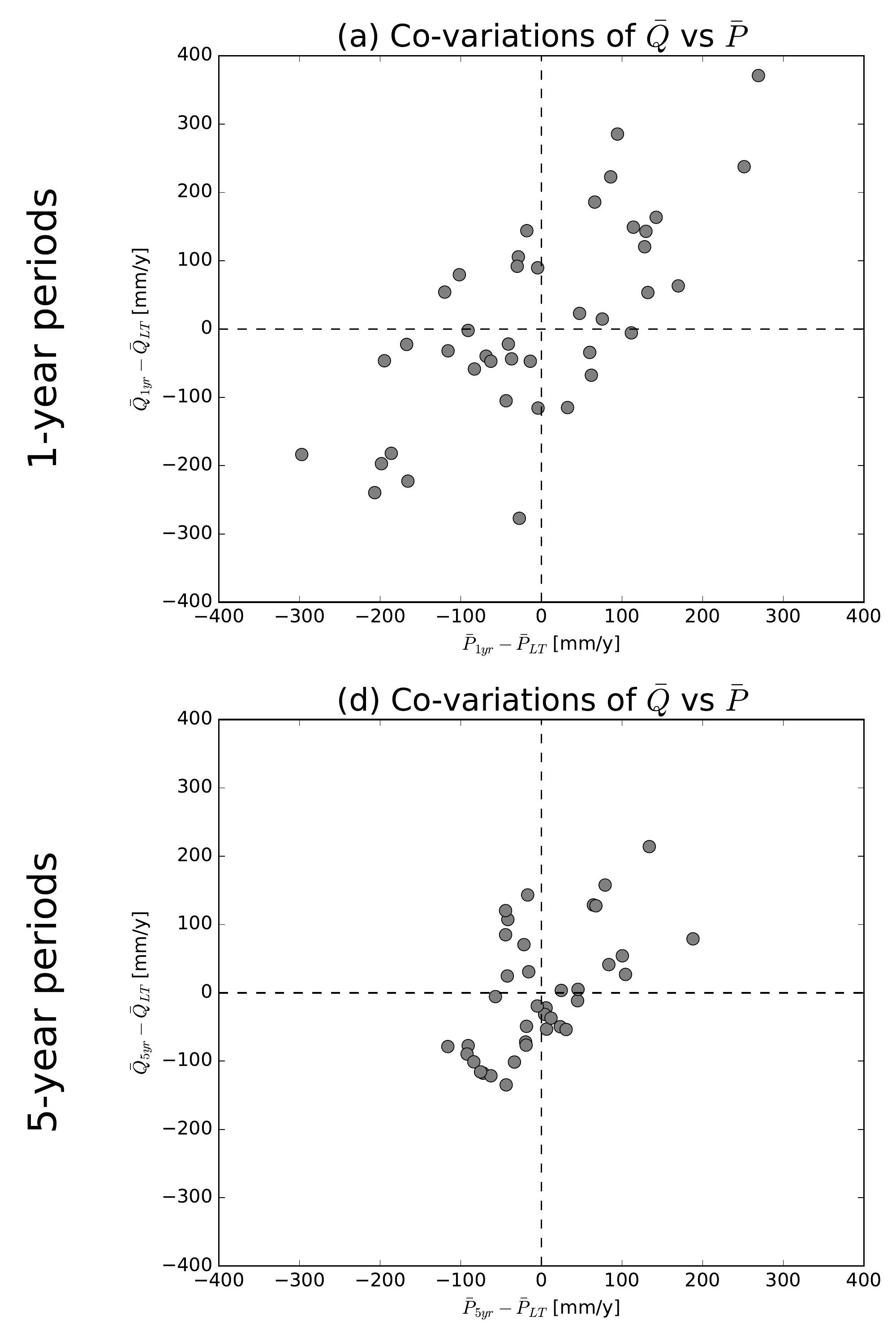

(g) Co-variations of $\bar{Q}$ vs $\bar{P}$

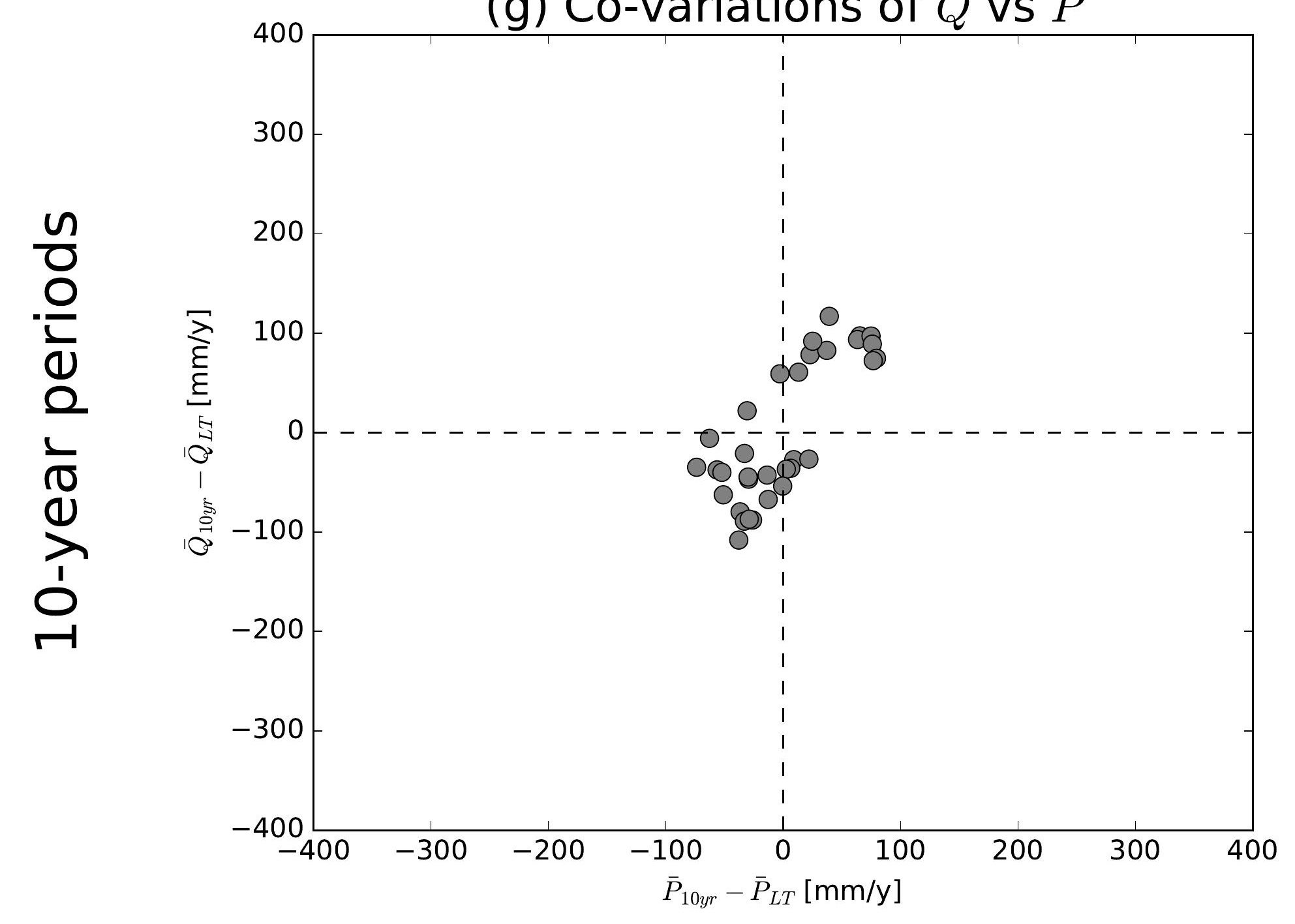

(j) Co-variations of $\bar{Q}$ vs $\bar{P}$

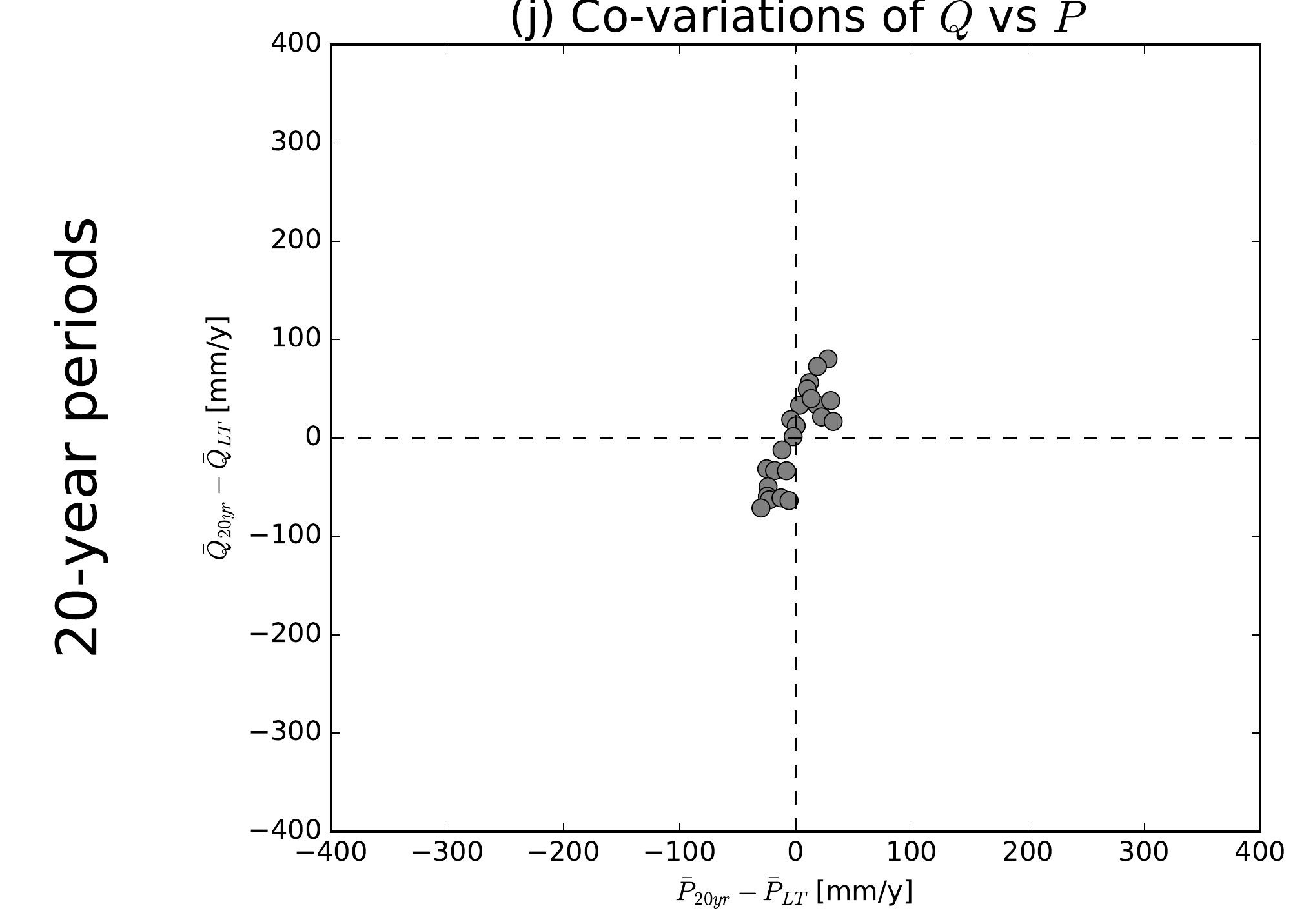

(b) Co-variations of $\bar{Q}$ vs $\overline{E 0}$

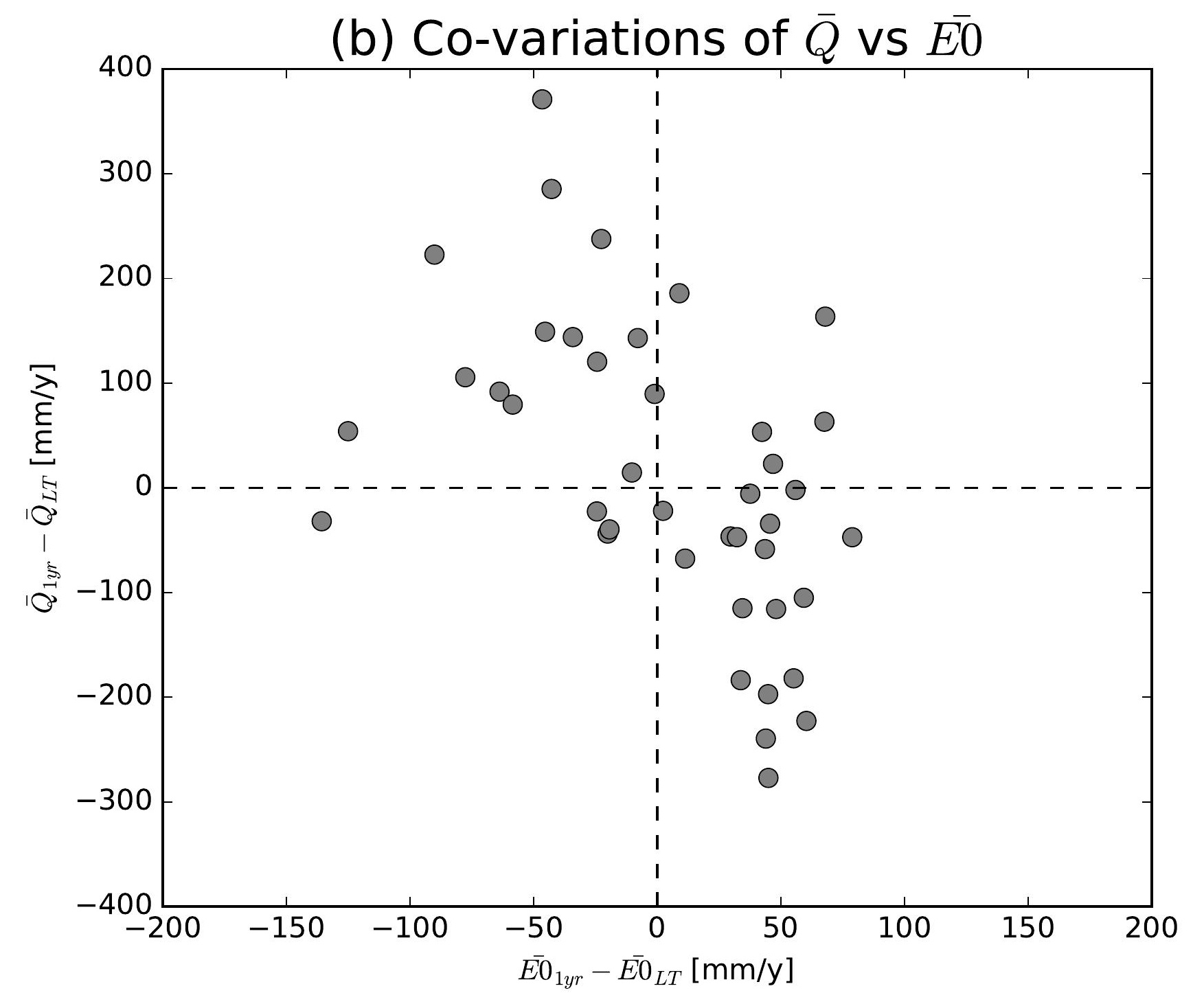

(e) Co-variations of $\bar{Q}$ vs $\overline{E 0}$

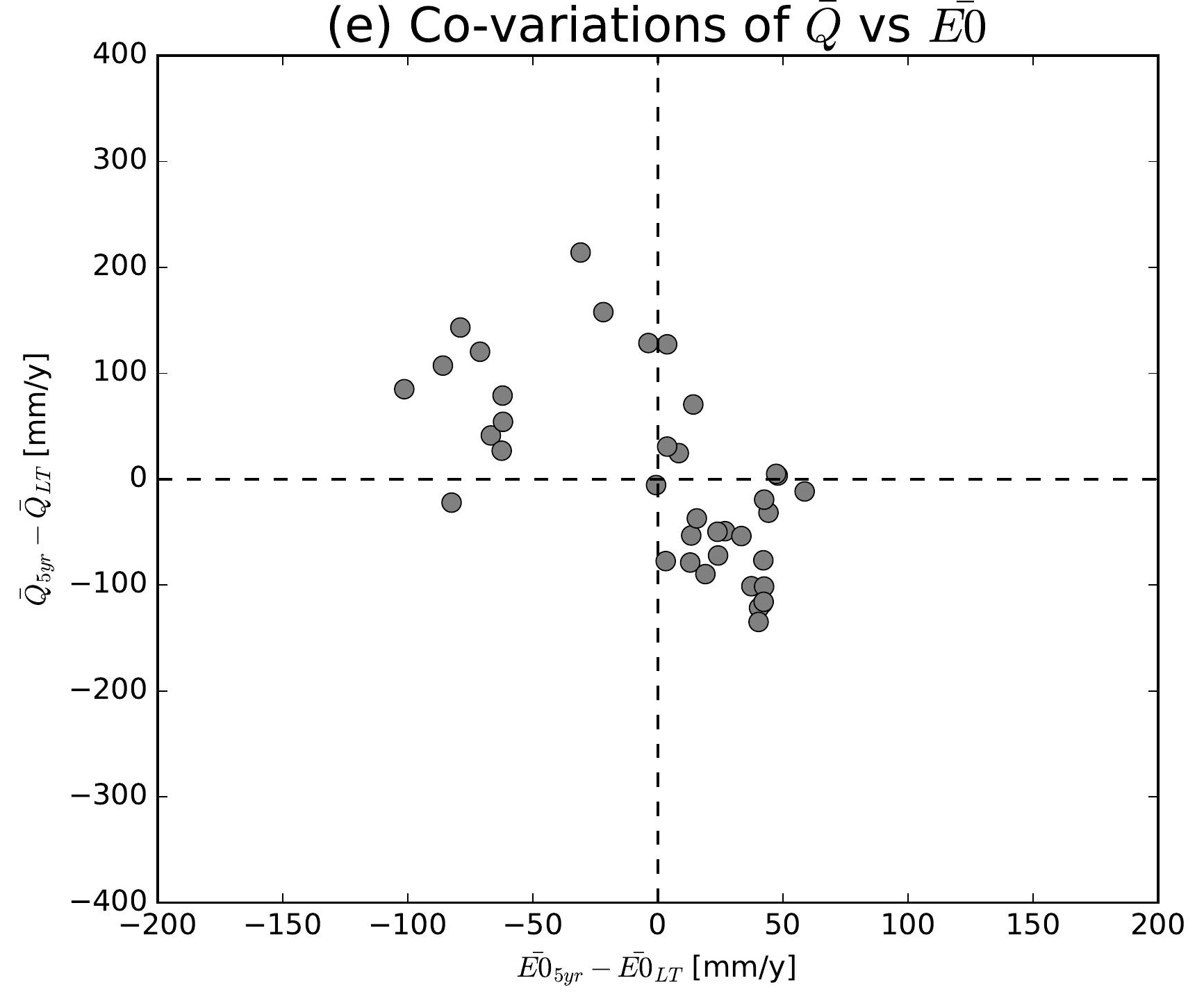

(h) Co-variations of $\bar{Q}$ vs $\overline{E 0}$

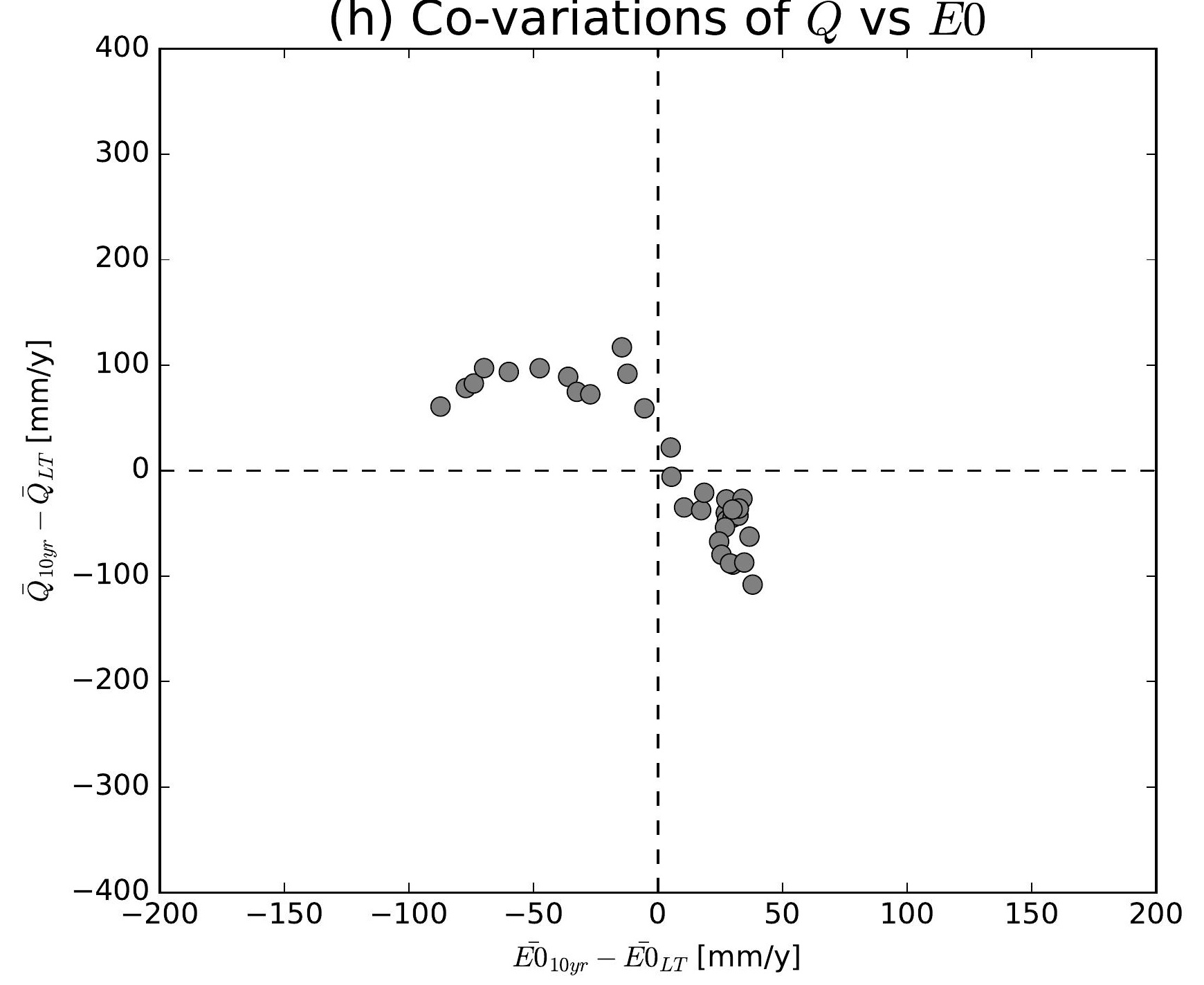

(k) Co-variations of $\bar{Q}$ vs $\overline{E 0}$

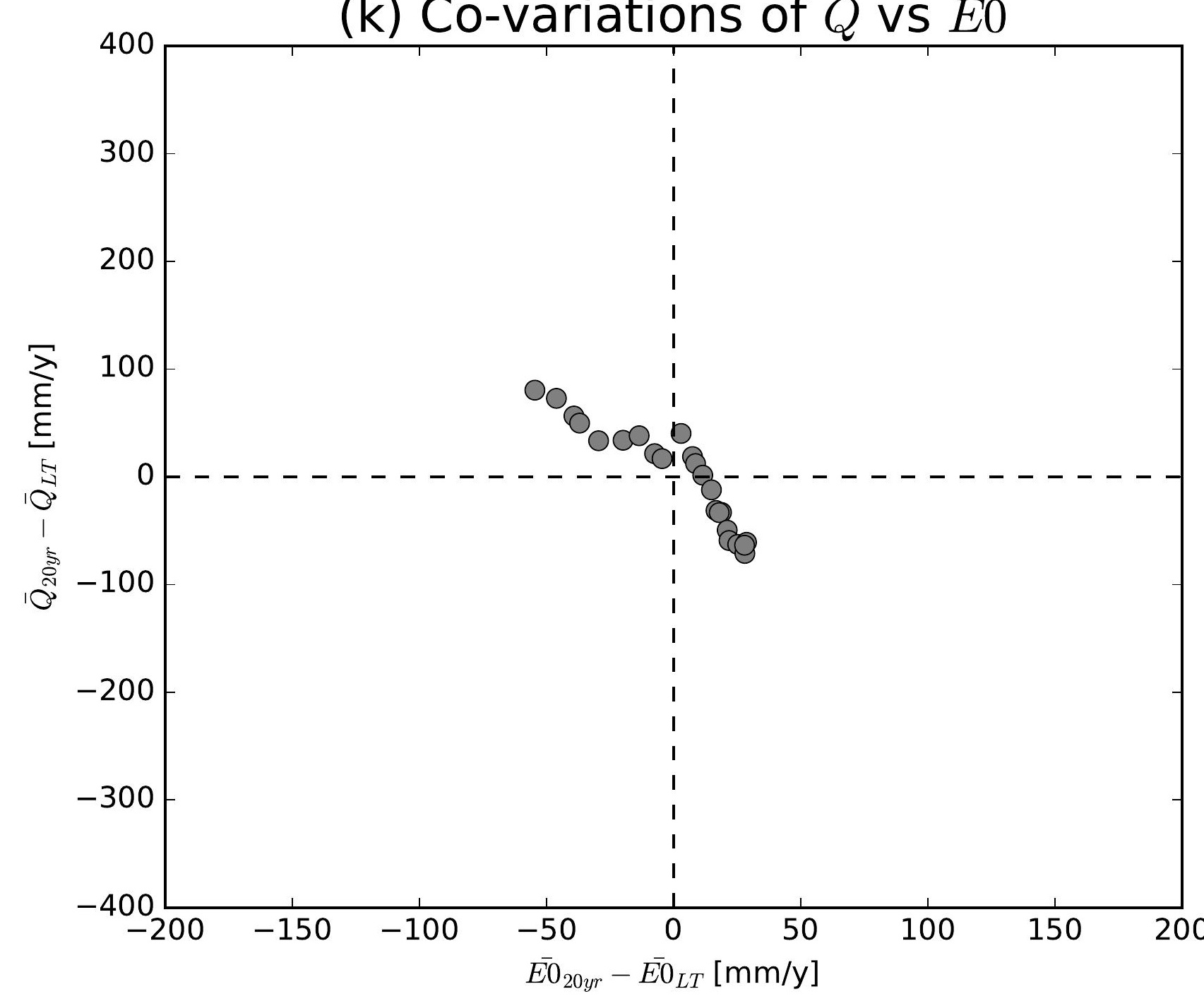

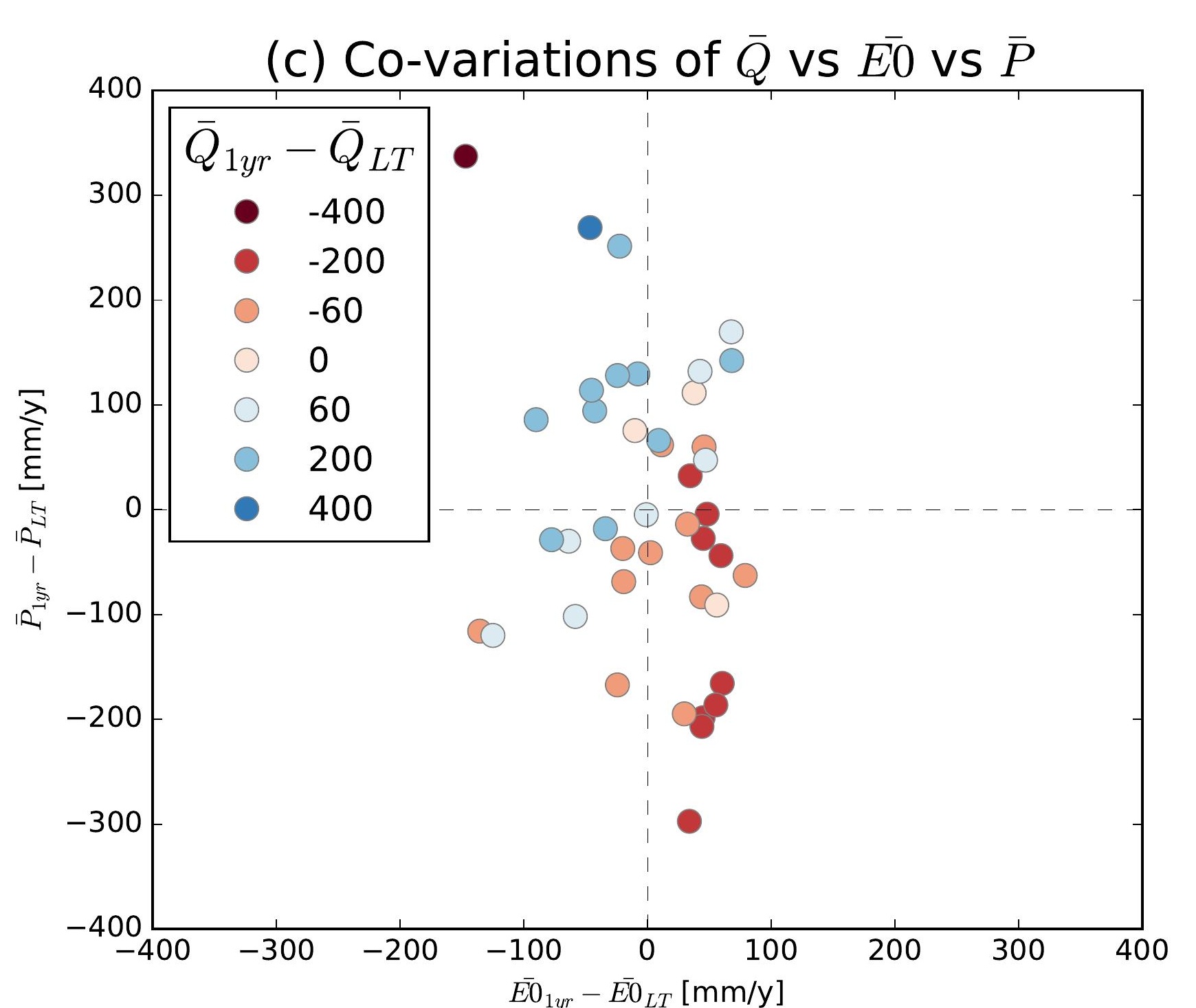
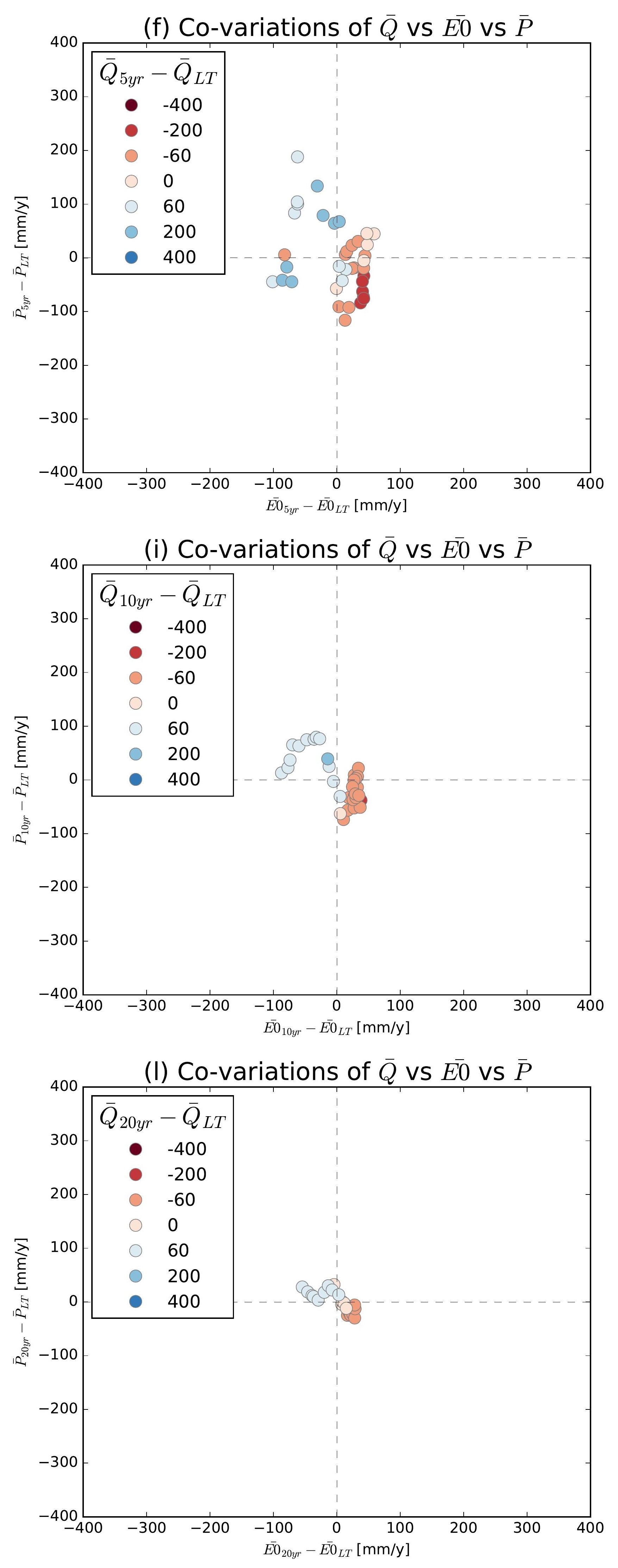

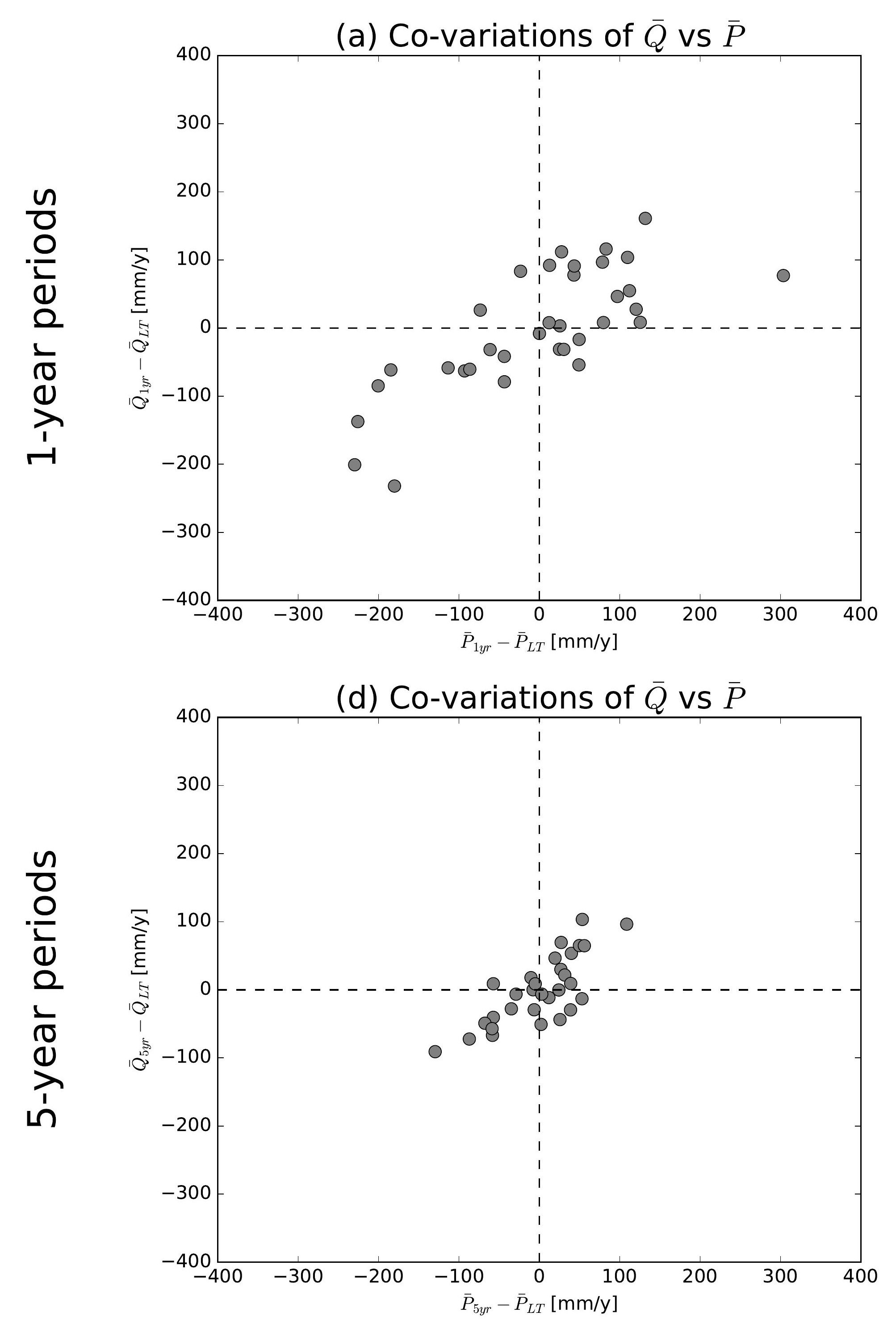

(g) Co-variations of $\bar{Q}$ vs $\bar{P}$
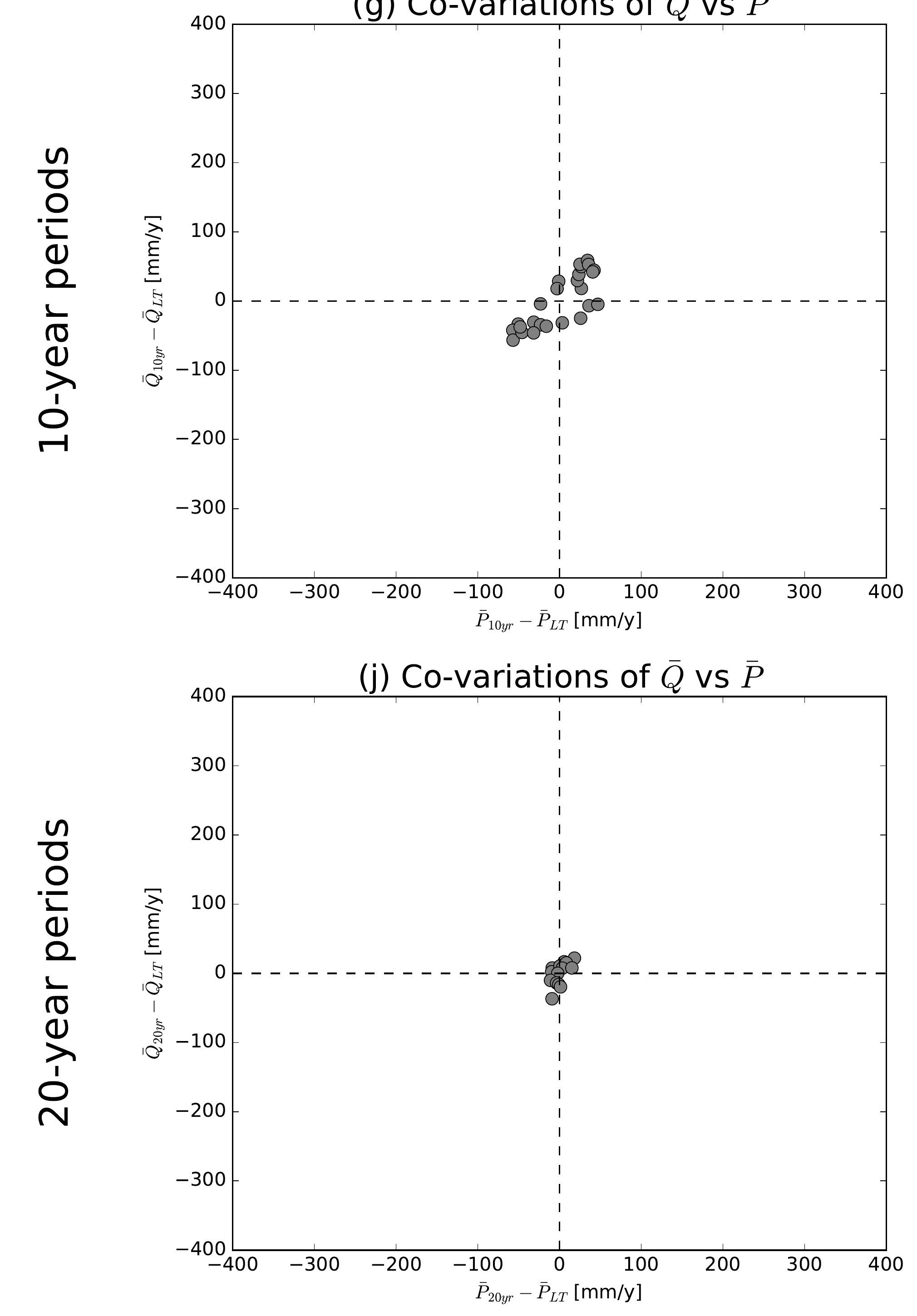

(b) Co-variations of $\bar{Q}$ vs $\overline{E 0}$

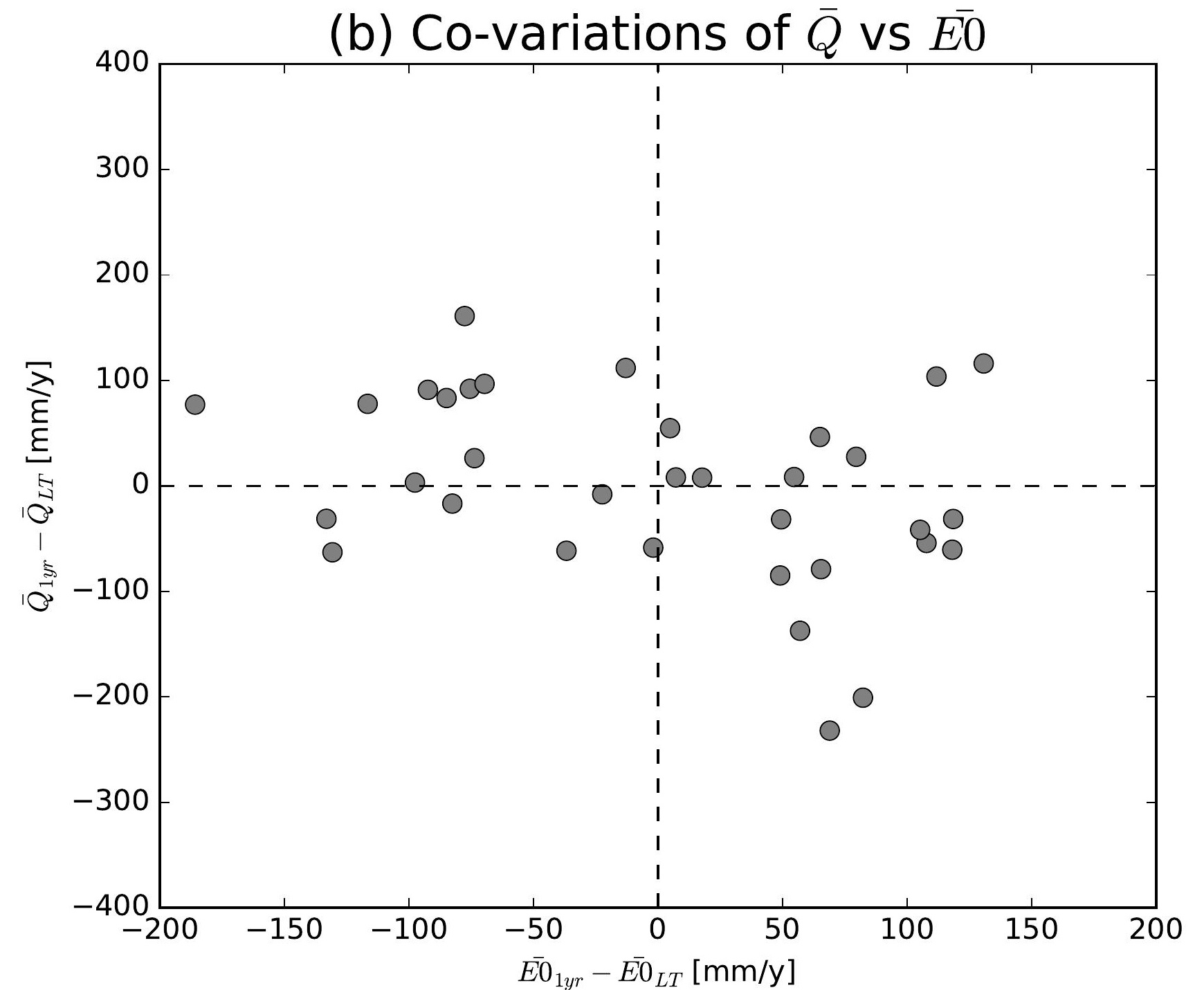

(e) Co-variations of $\bar{Q}$ vs $\overline{E 0}$

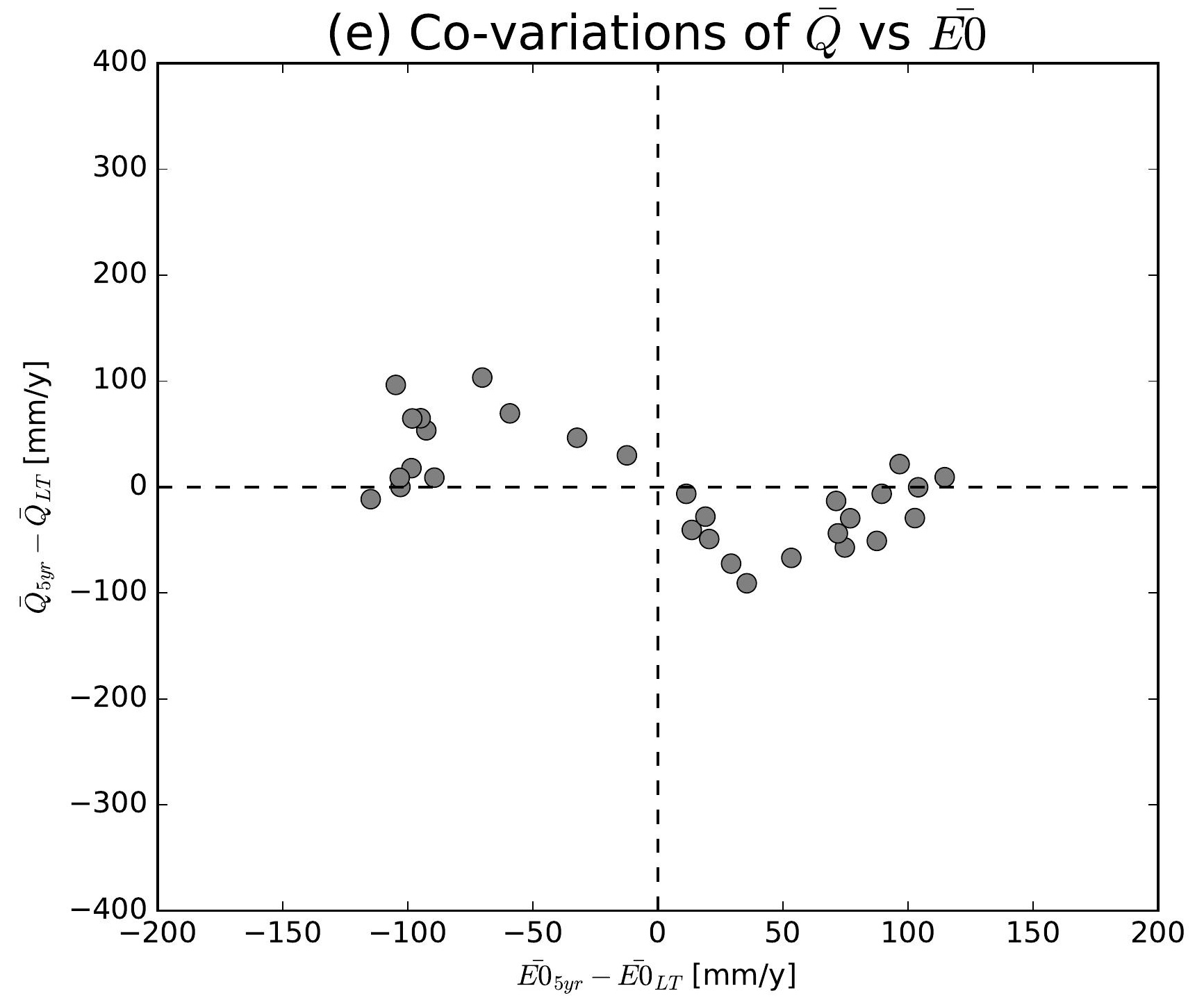

(h) Co-variations of $\bar{Q}$ vs $\overline{E 0}$

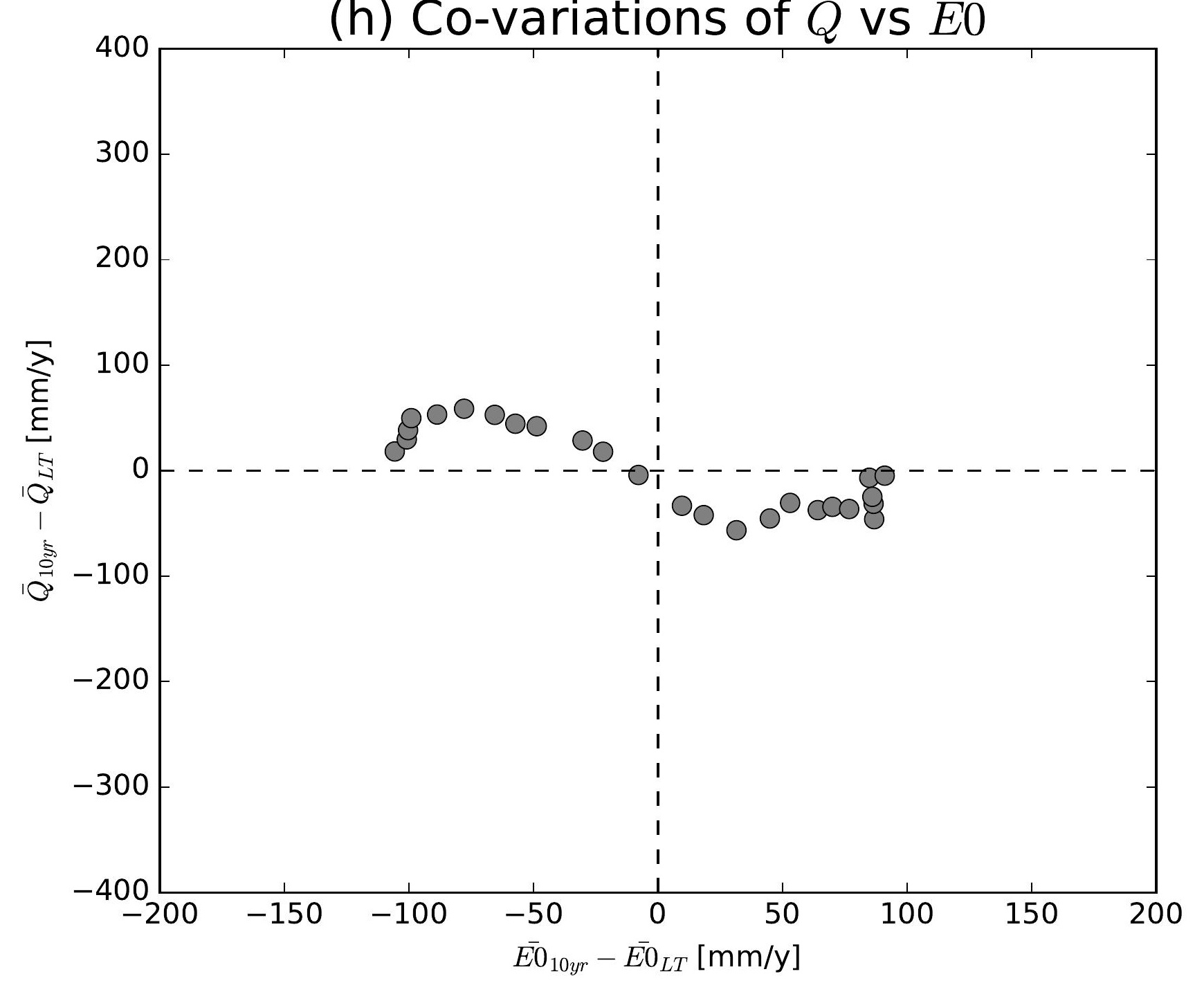

(k) Co-variations of $\bar{Q}$ vs $\overline{E 0}$

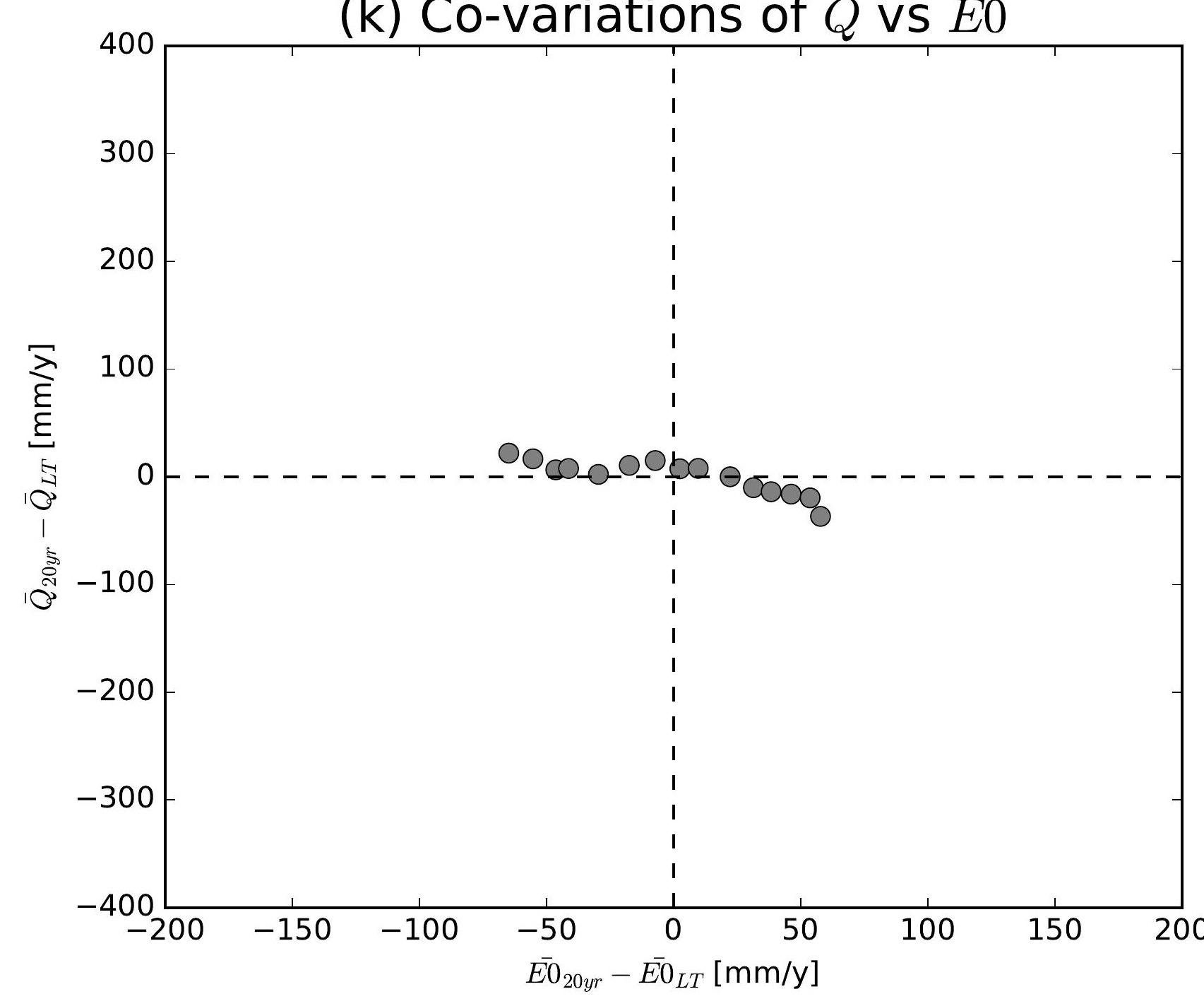

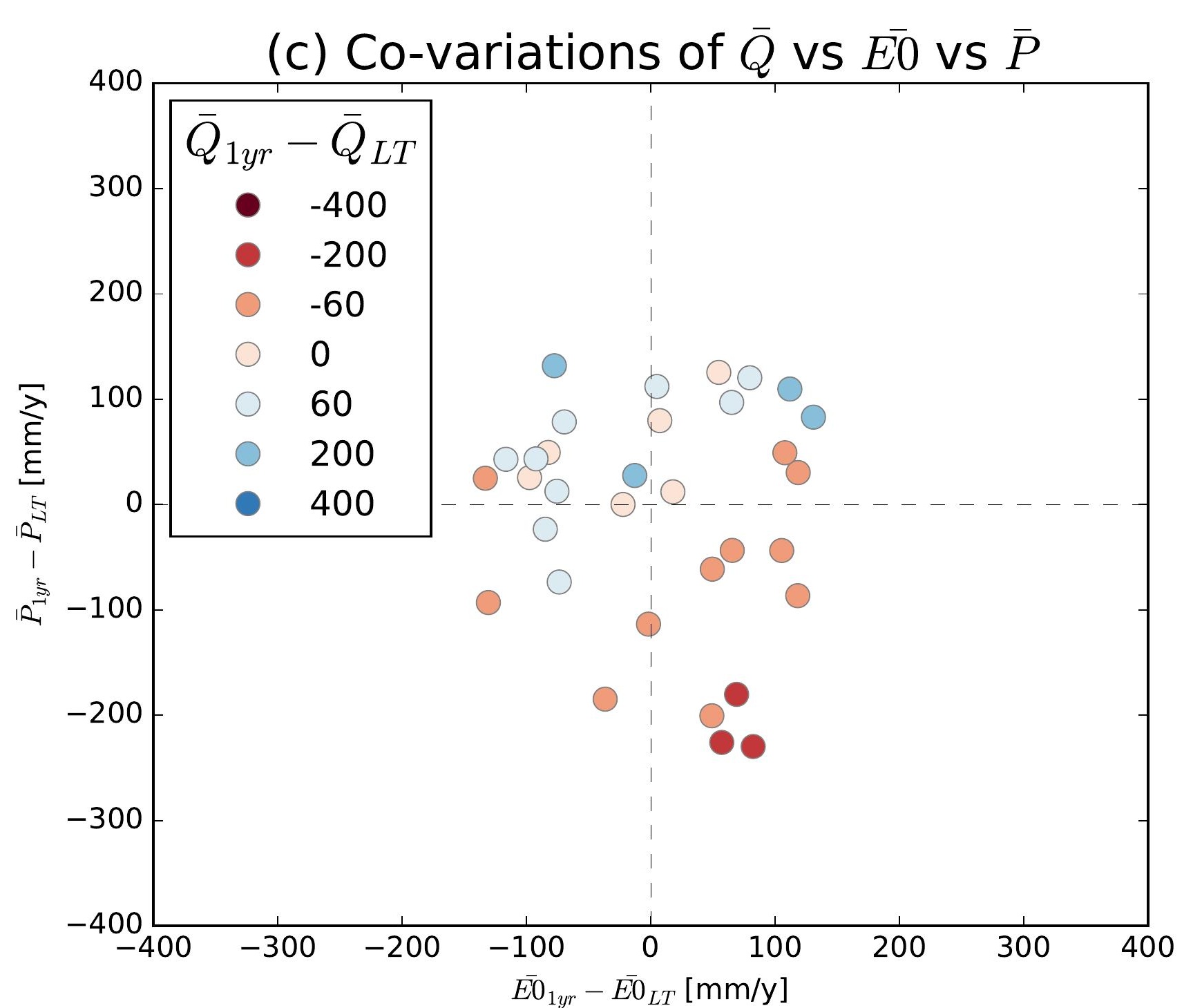
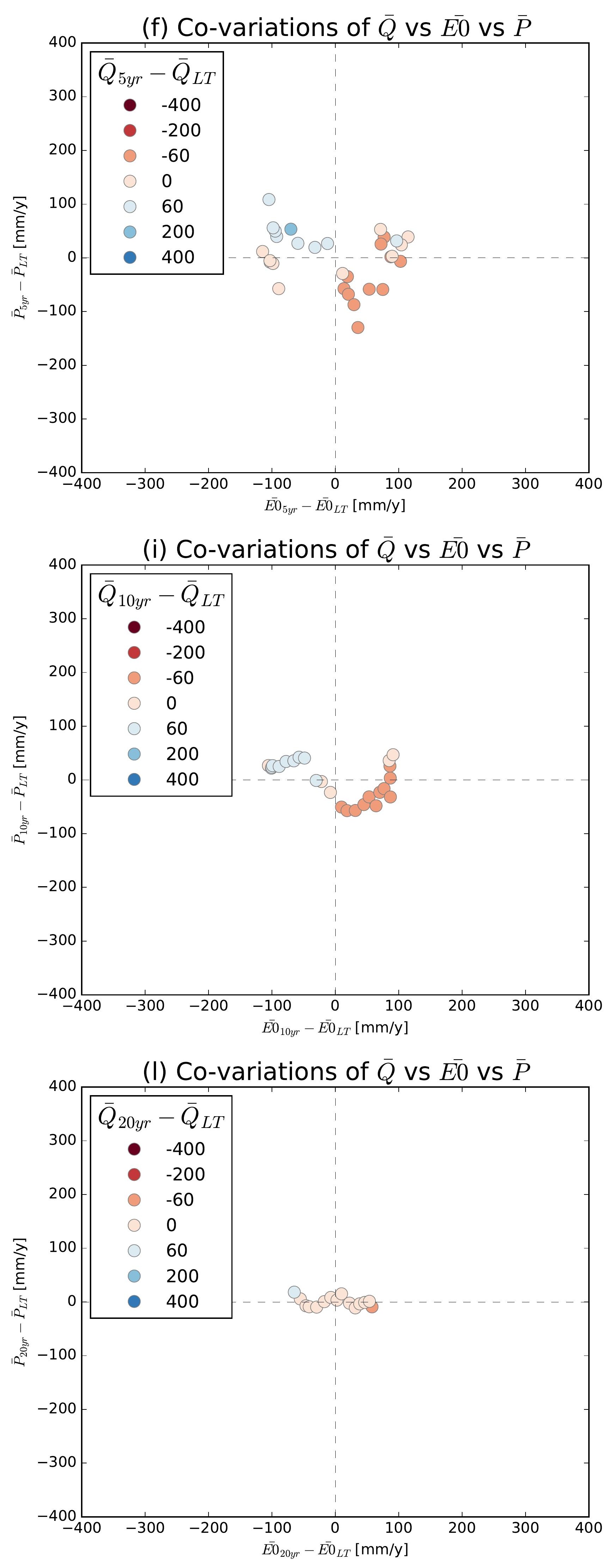

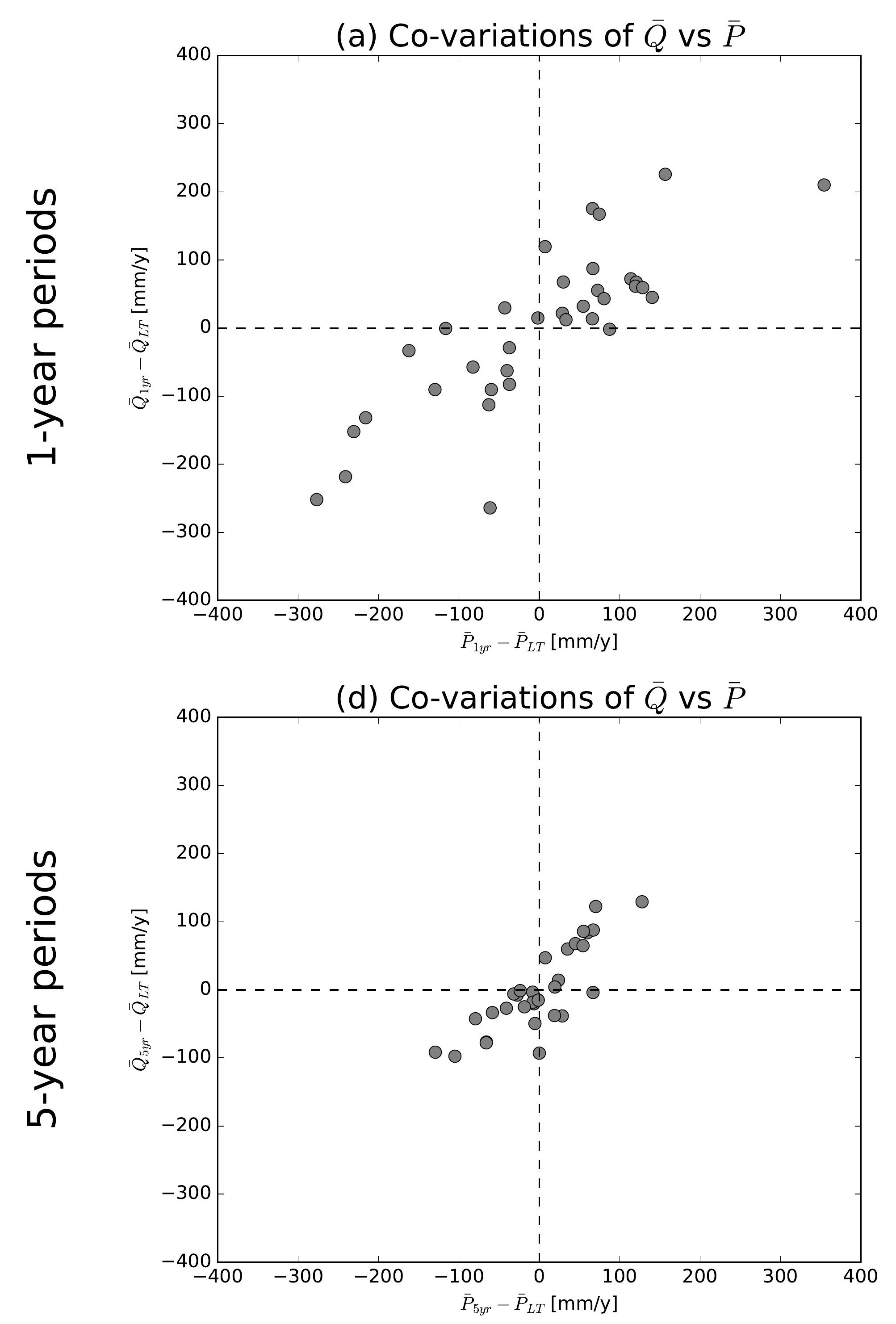

(g) Co-variations of $\bar{Q}$ vs $\bar{P}$
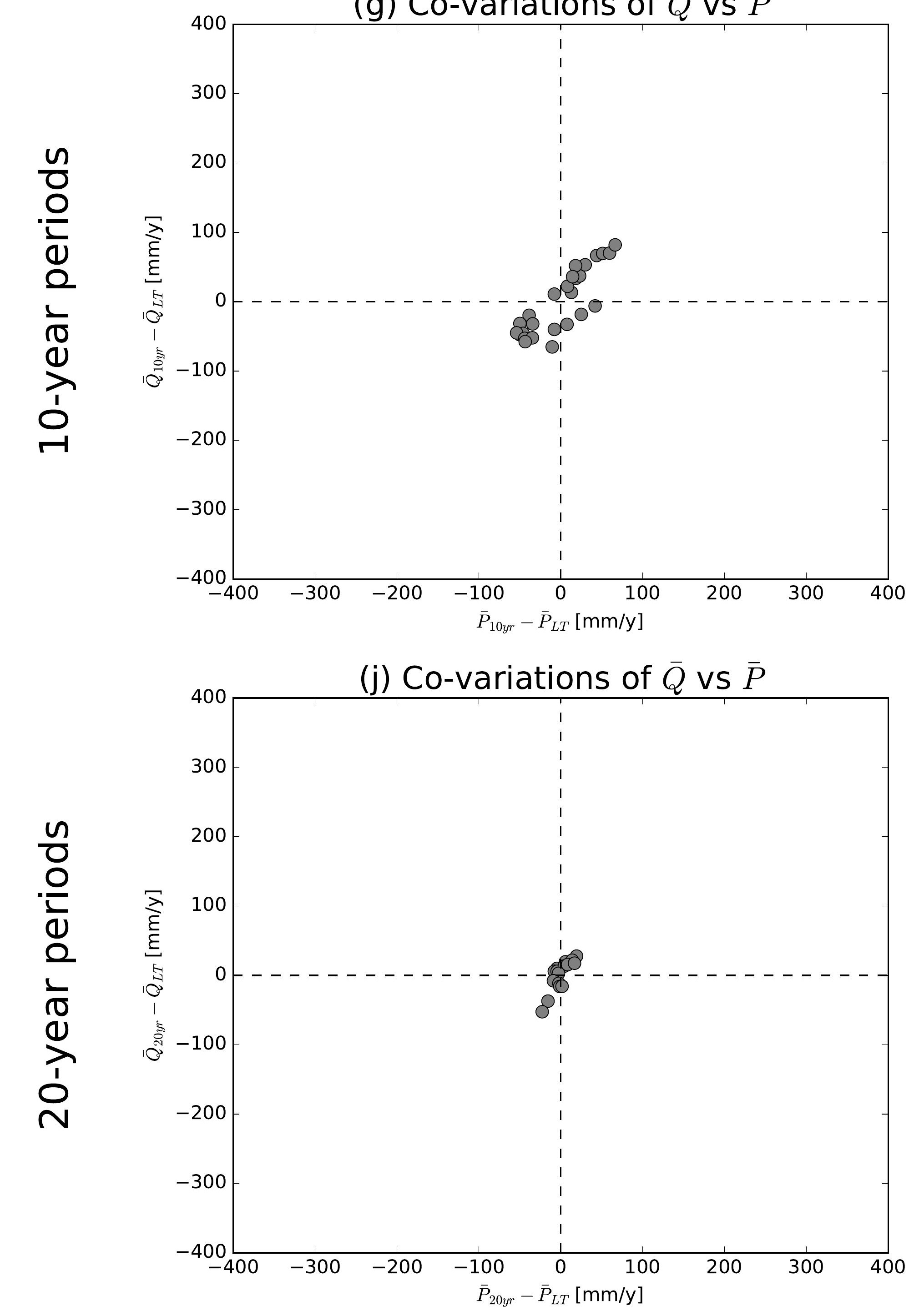

(b) Co-variations of $\bar{Q}$ vs $\overline{E 0}$

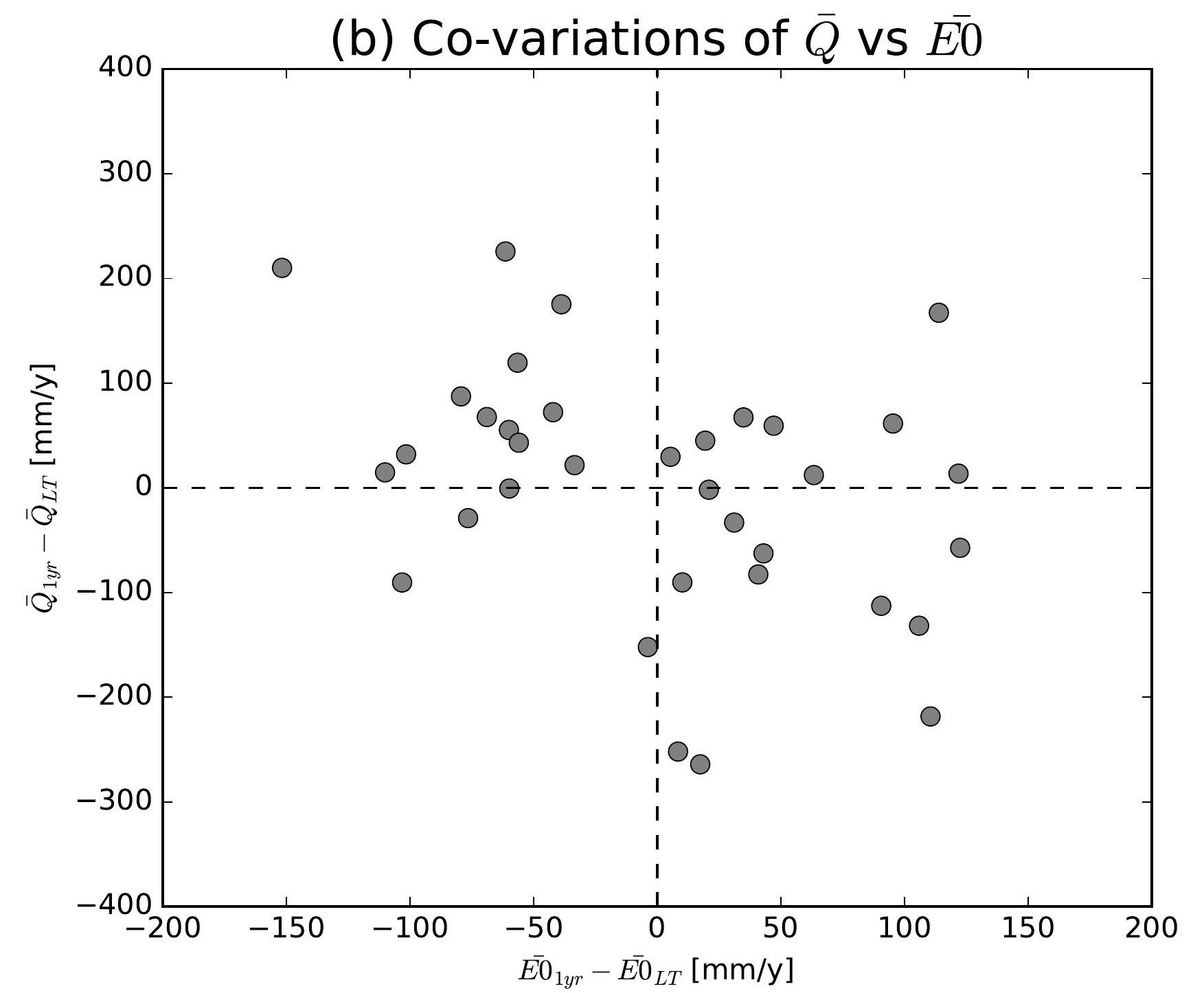

(e) Co-variations of $\bar{Q}$ vs $\overline{E 0}$

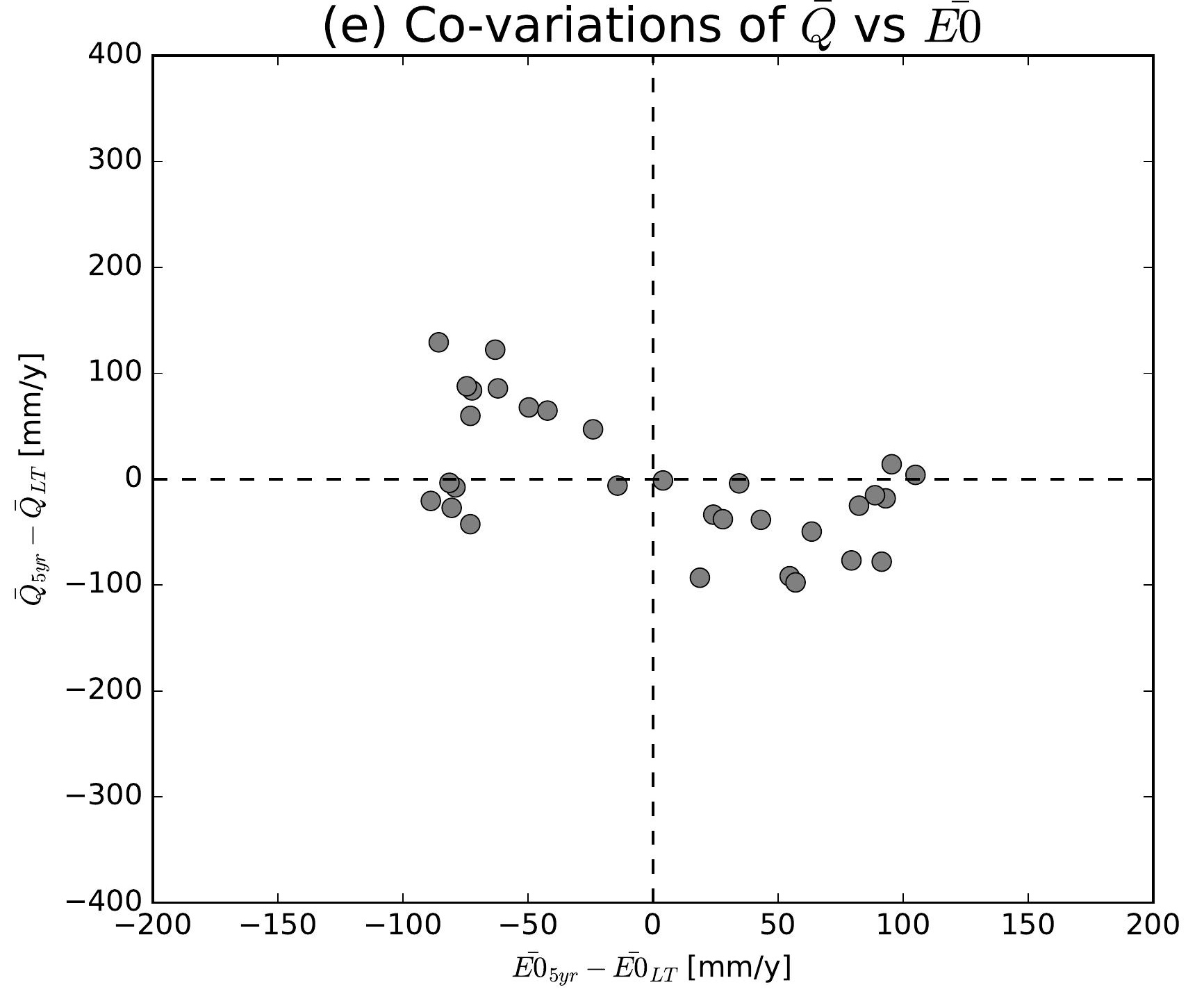

(h) Co-variations of $\bar{Q}$ vs $\overline{E 0}$

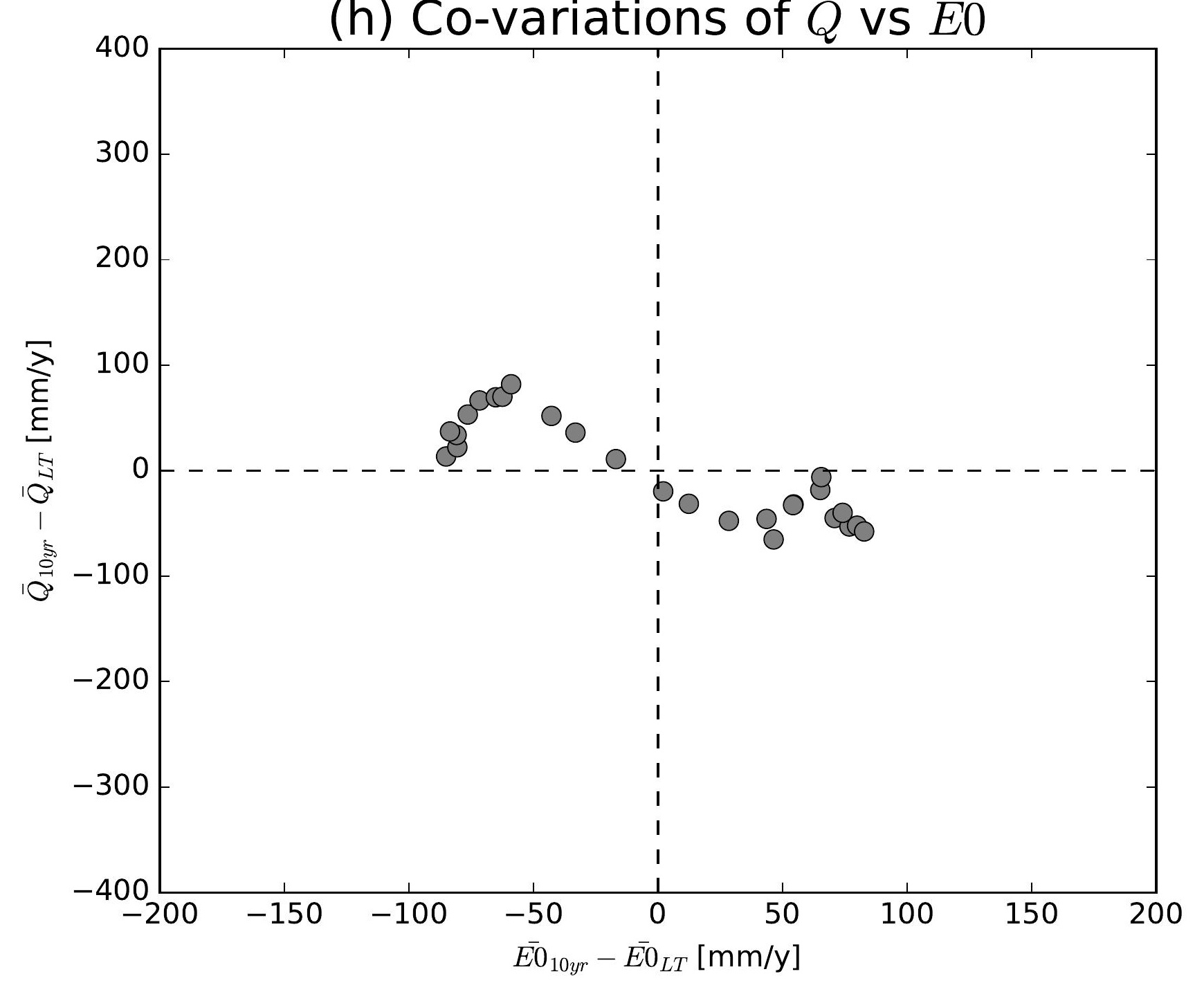

(k) Co-variations of $\bar{Q}$ vs $\overline{E 0}$

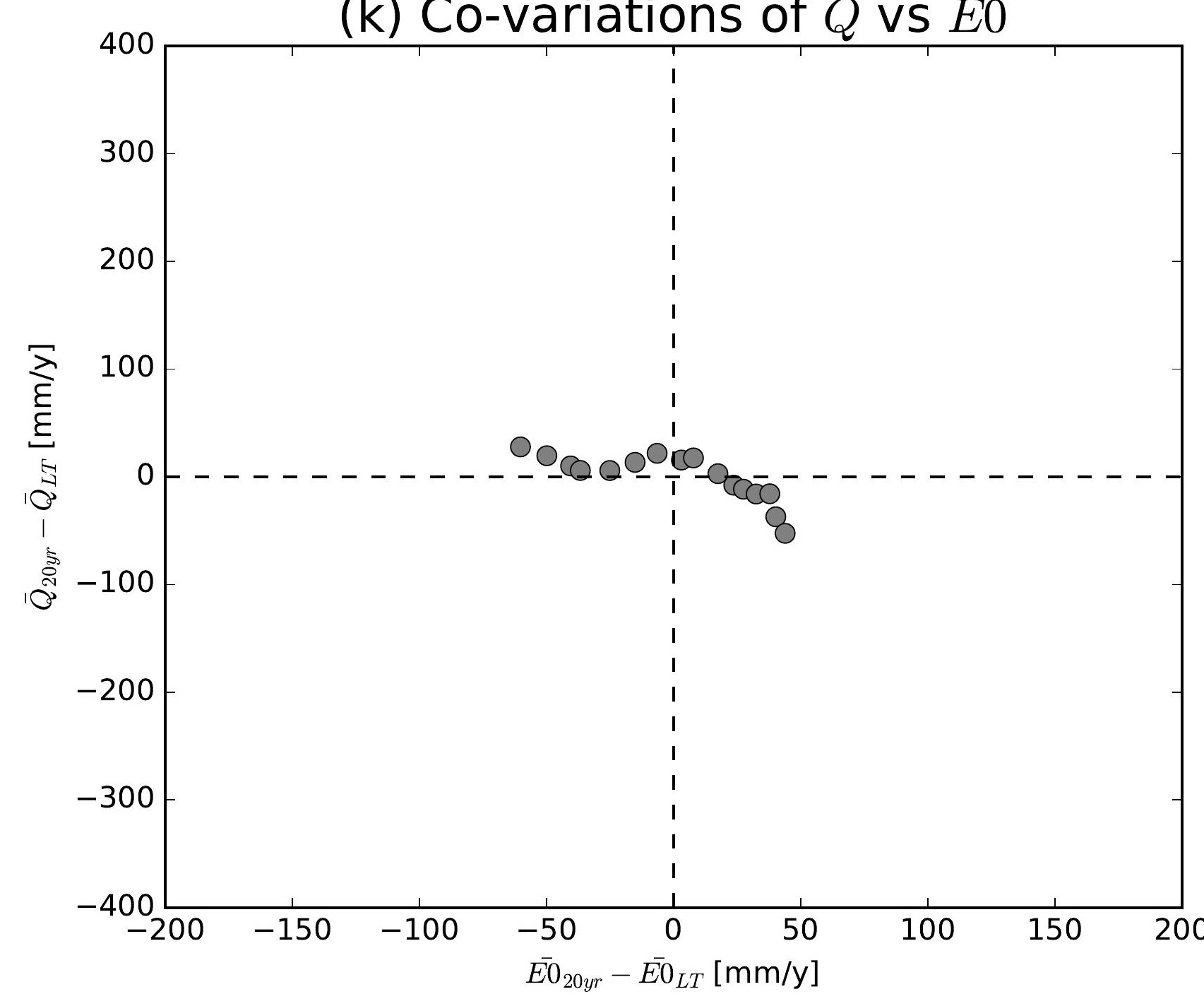

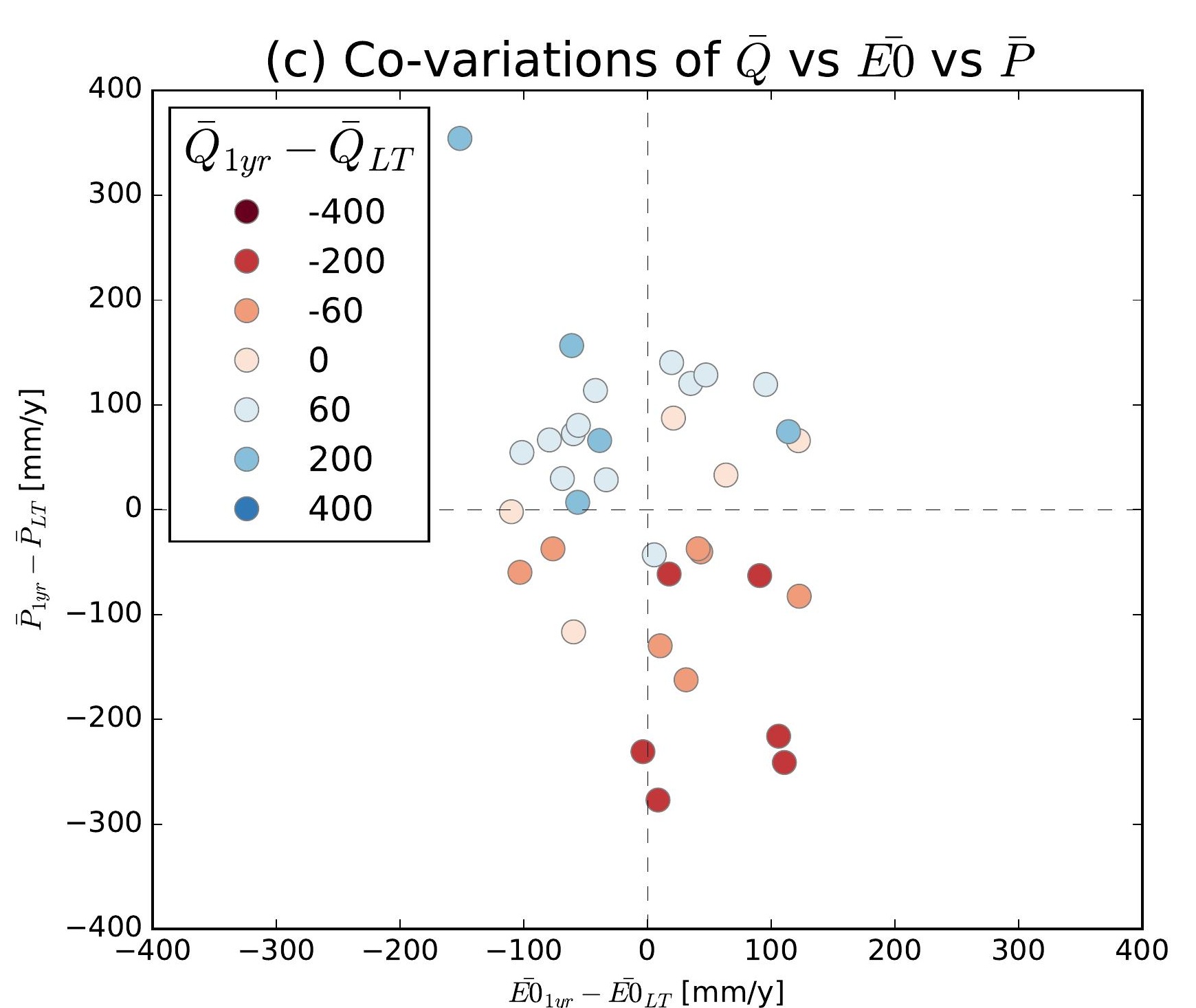
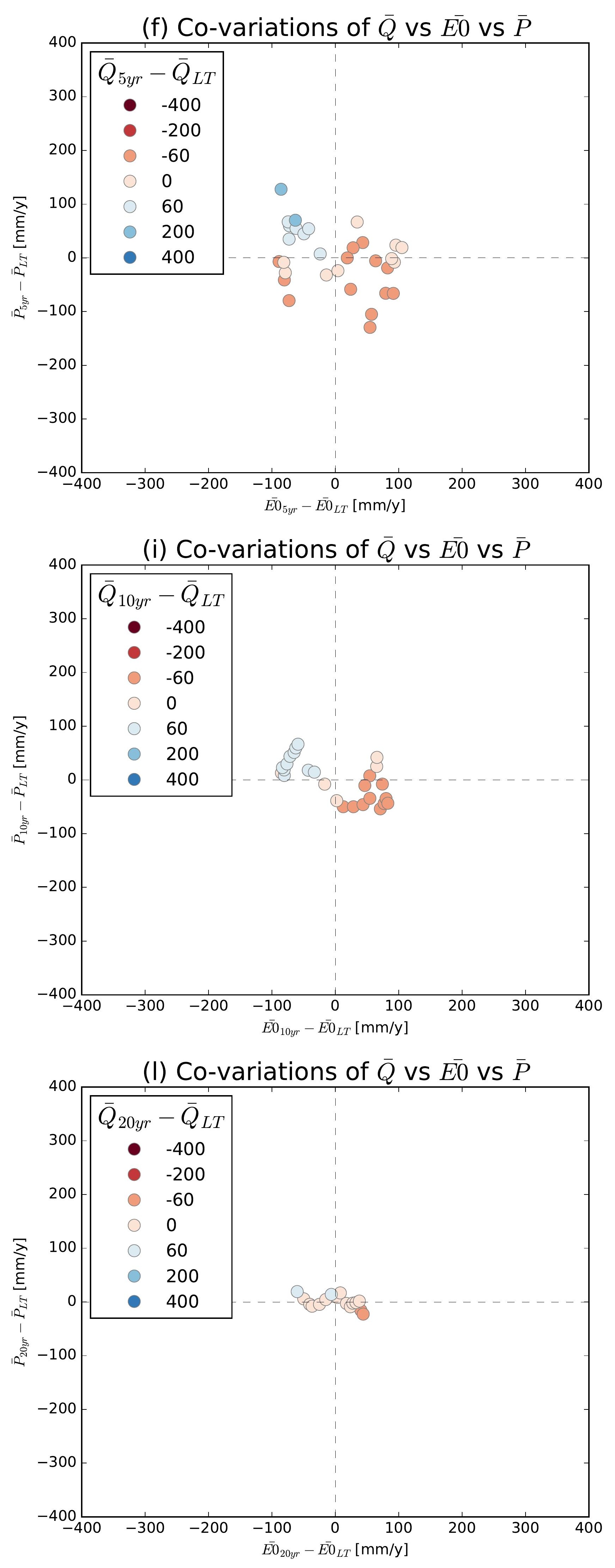

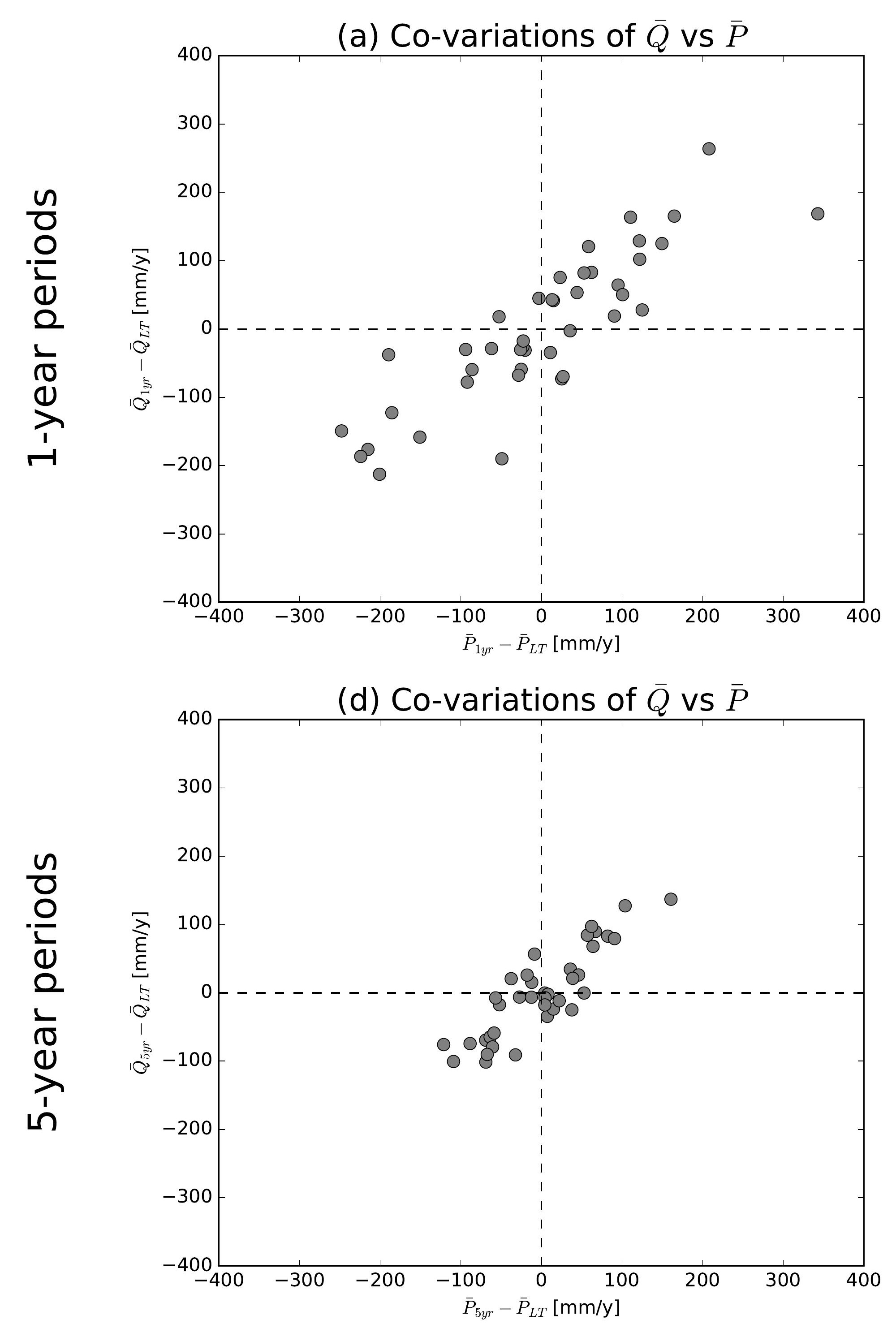

(g) Co-variations of $\bar{Q}$ vs $\bar{P}$

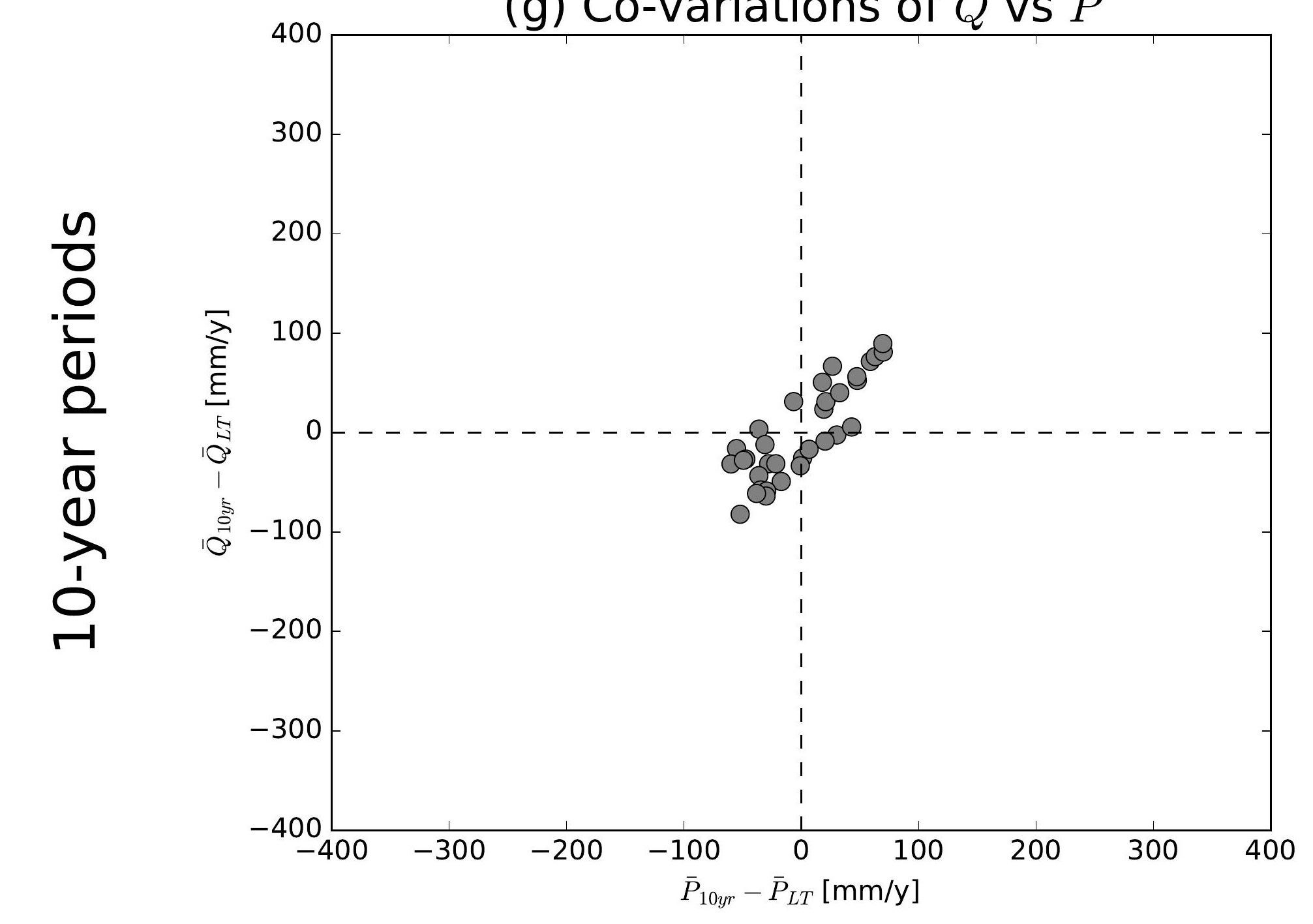

(j) Co-variations of $\bar{Q}$ vs $\bar{P}$

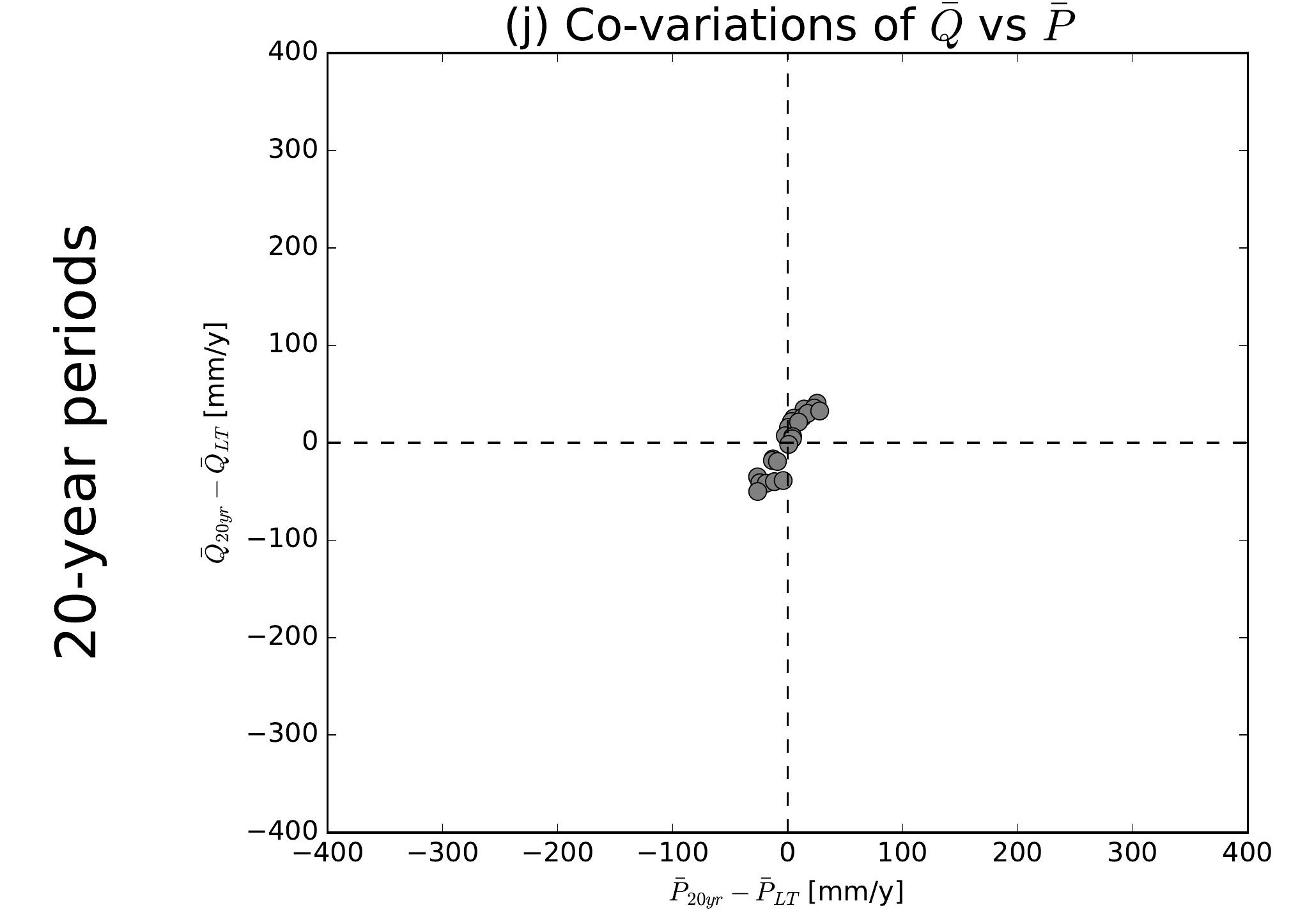

(b) Co-variations of $\bar{Q}$ vs $\overline{E 0}$

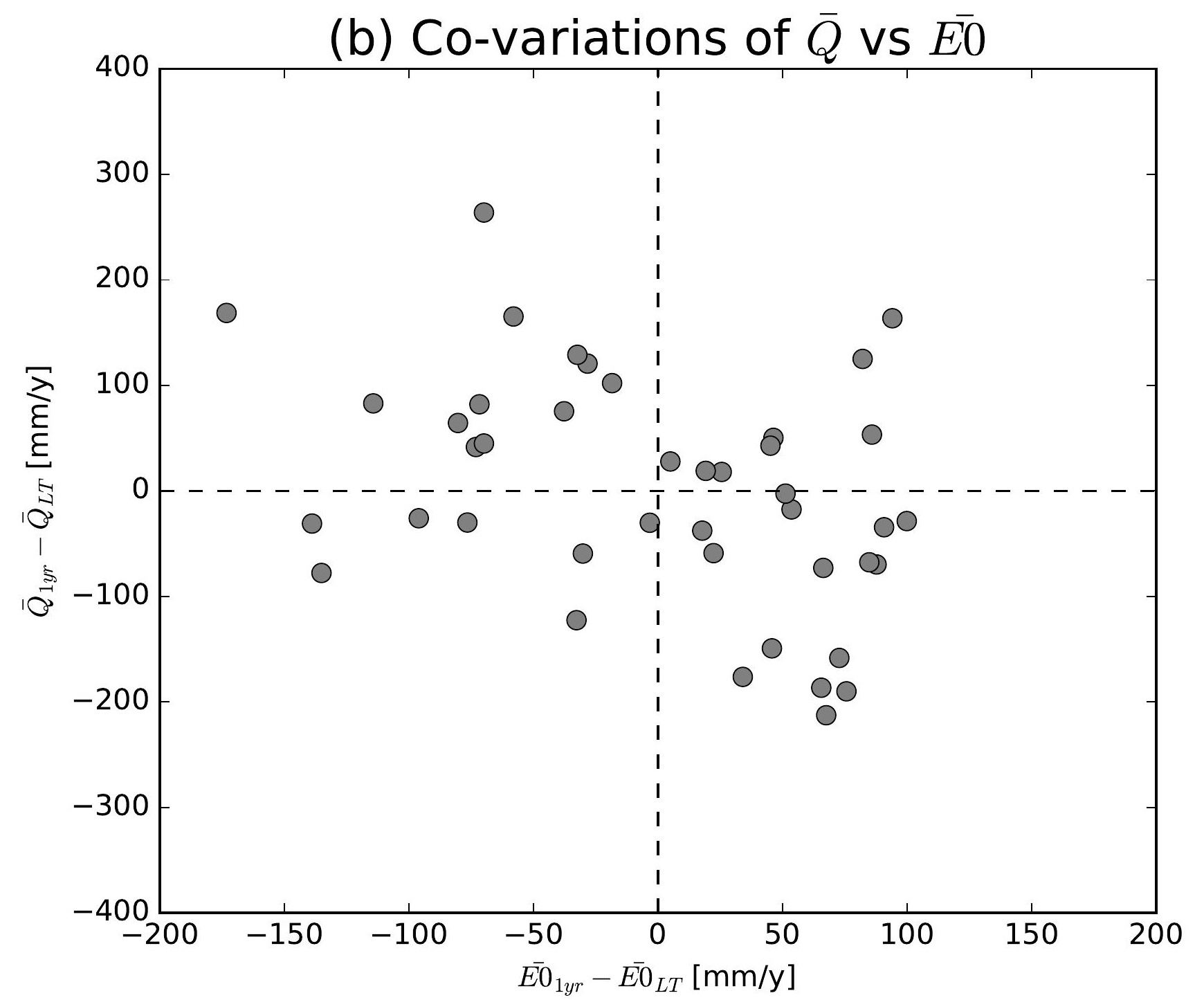

(e) Co-variations of $\bar{Q}$ vs $\overline{E 0}$

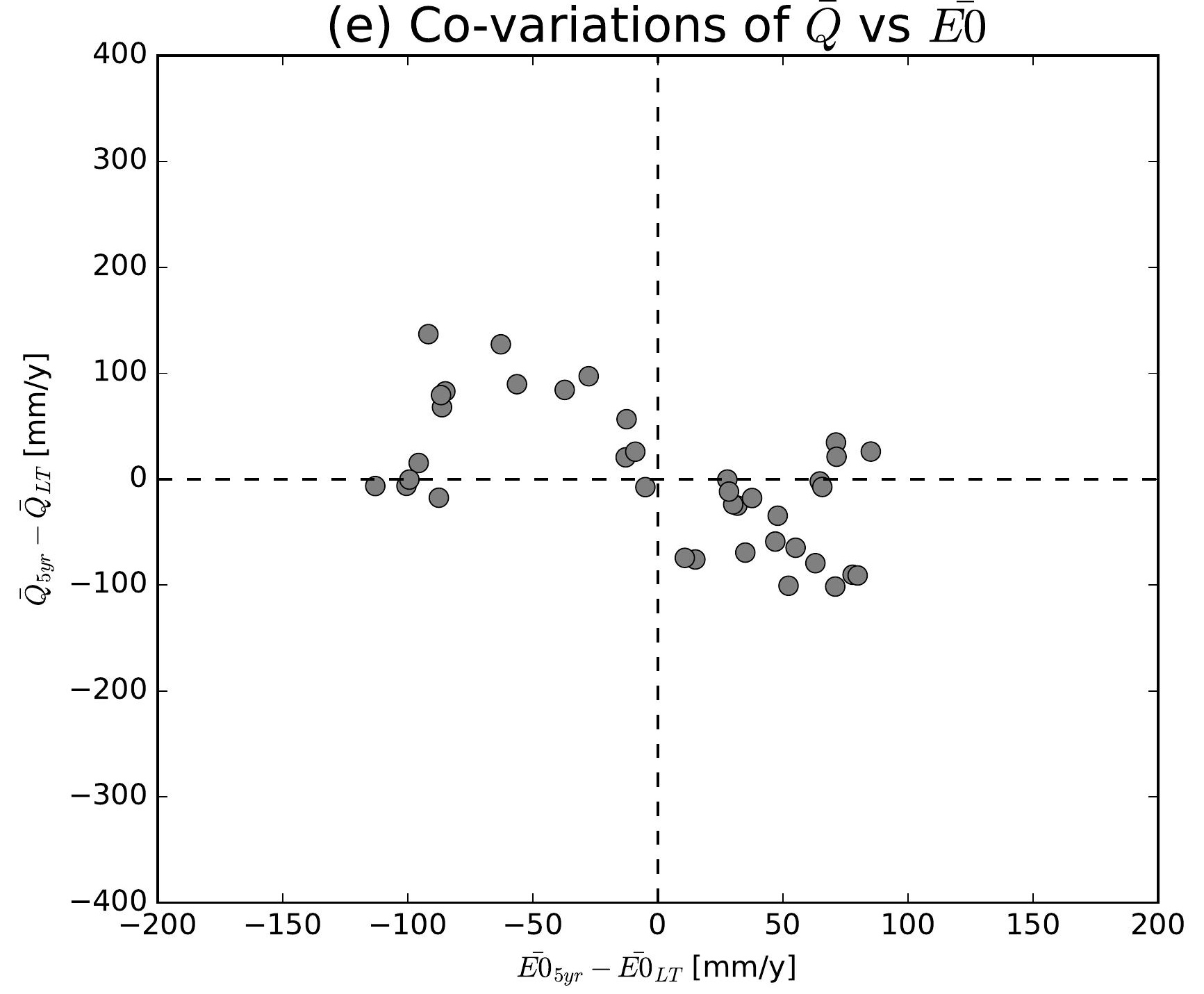

(h) Co-variations of $\bar{Q}$ vs $\overline{E 0}$

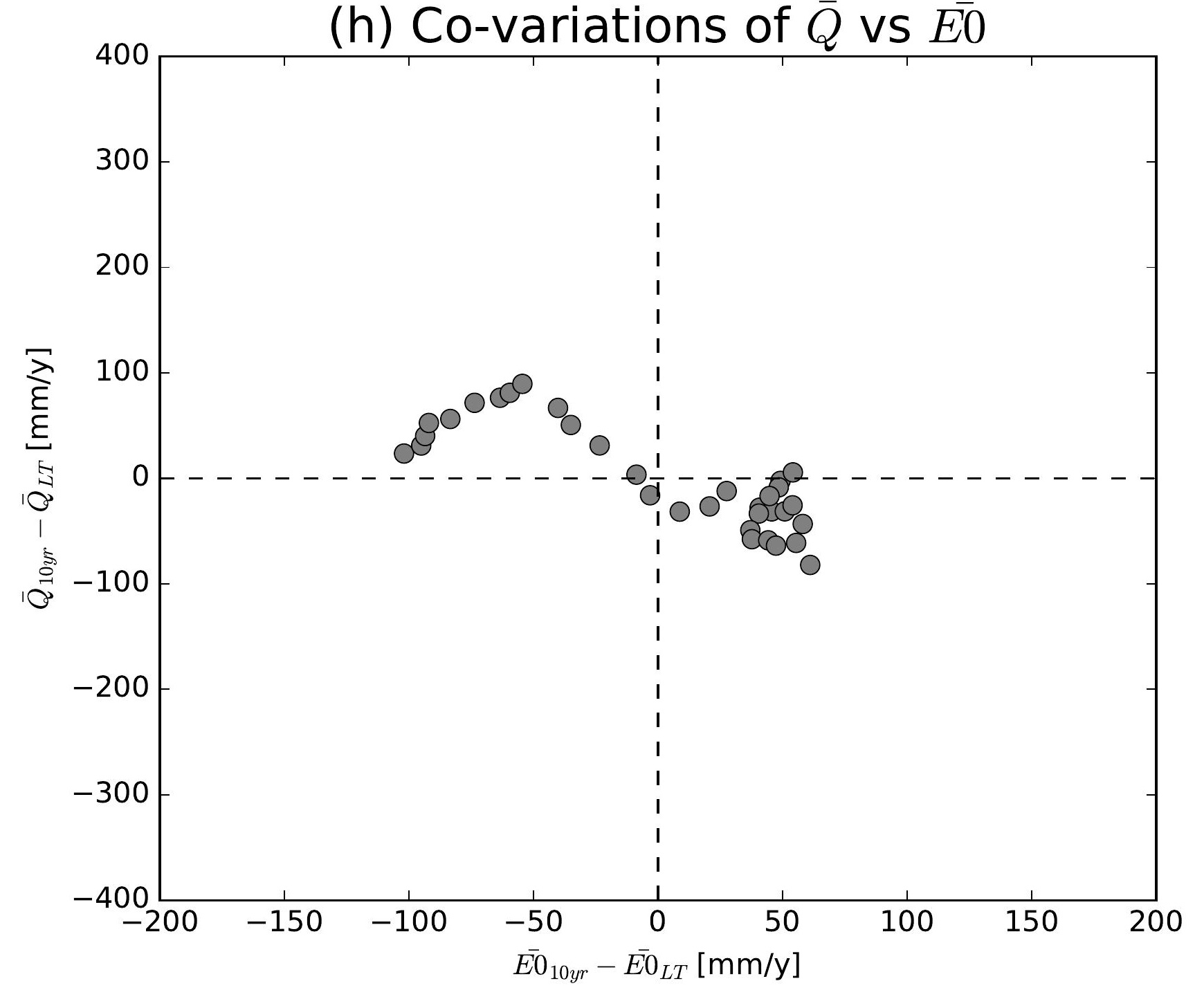

(k) Co-variations of $\bar{Q}$ vs $\overline{E 0}$

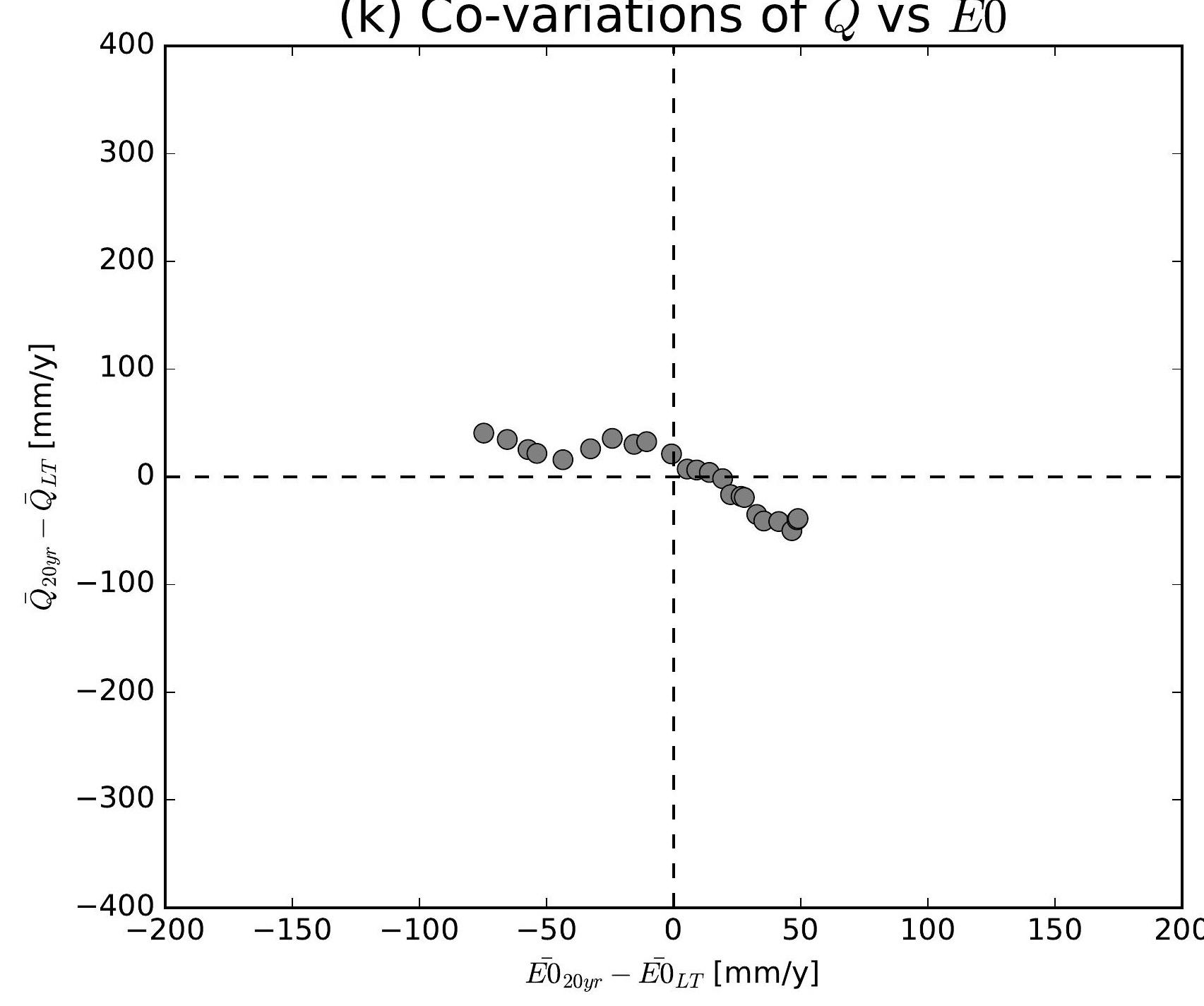

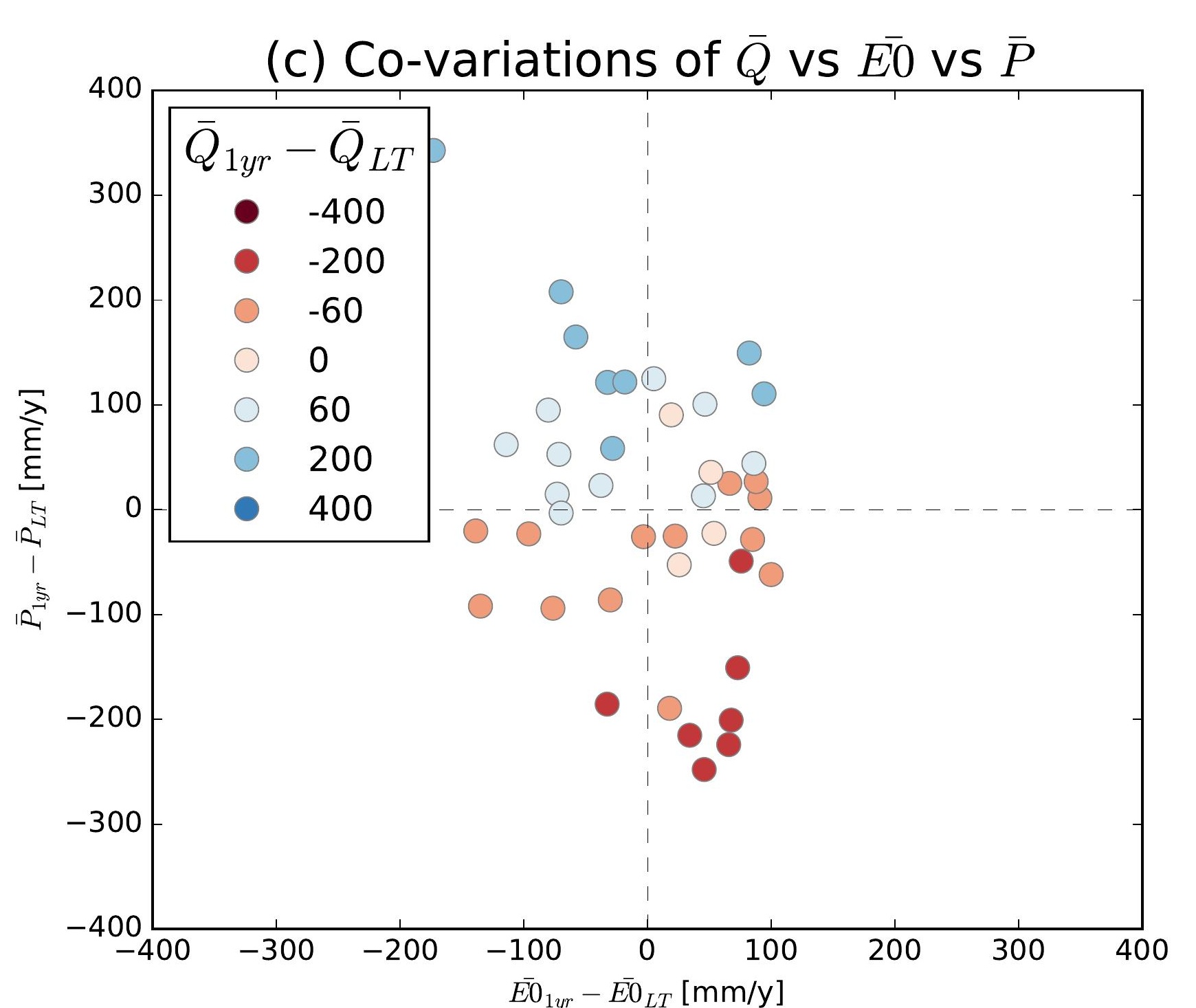
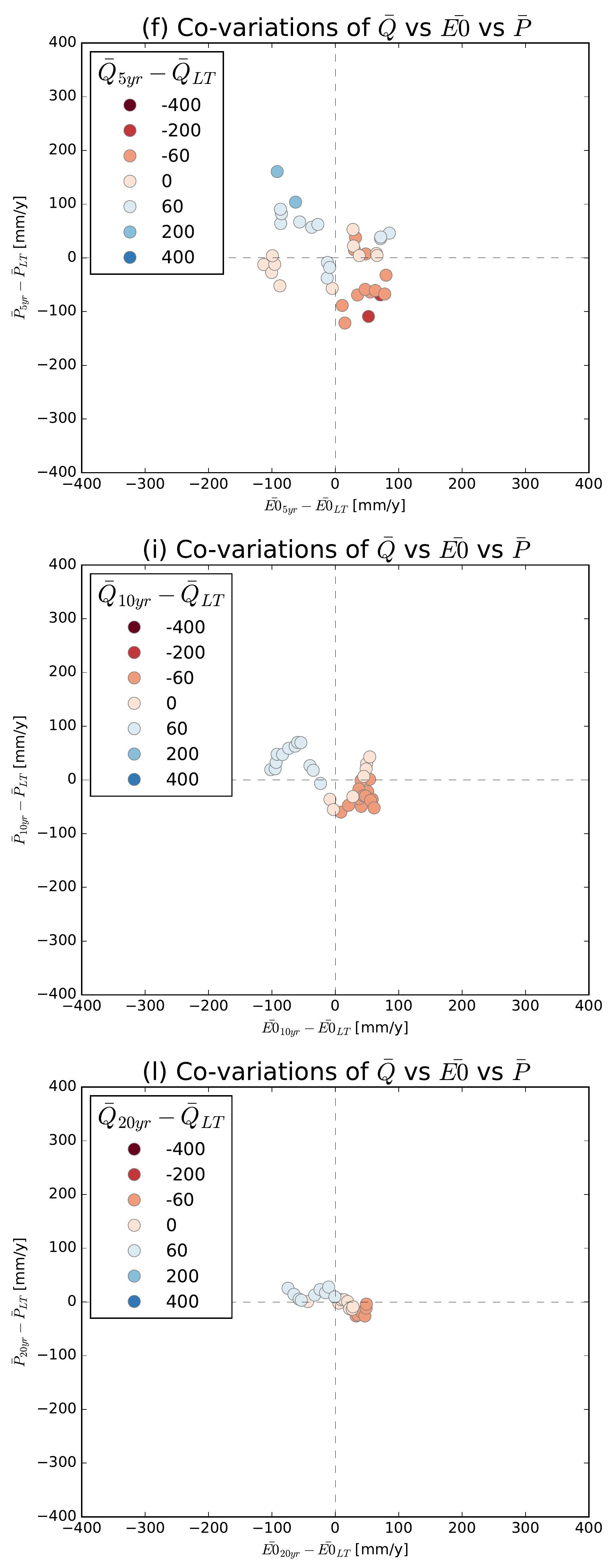

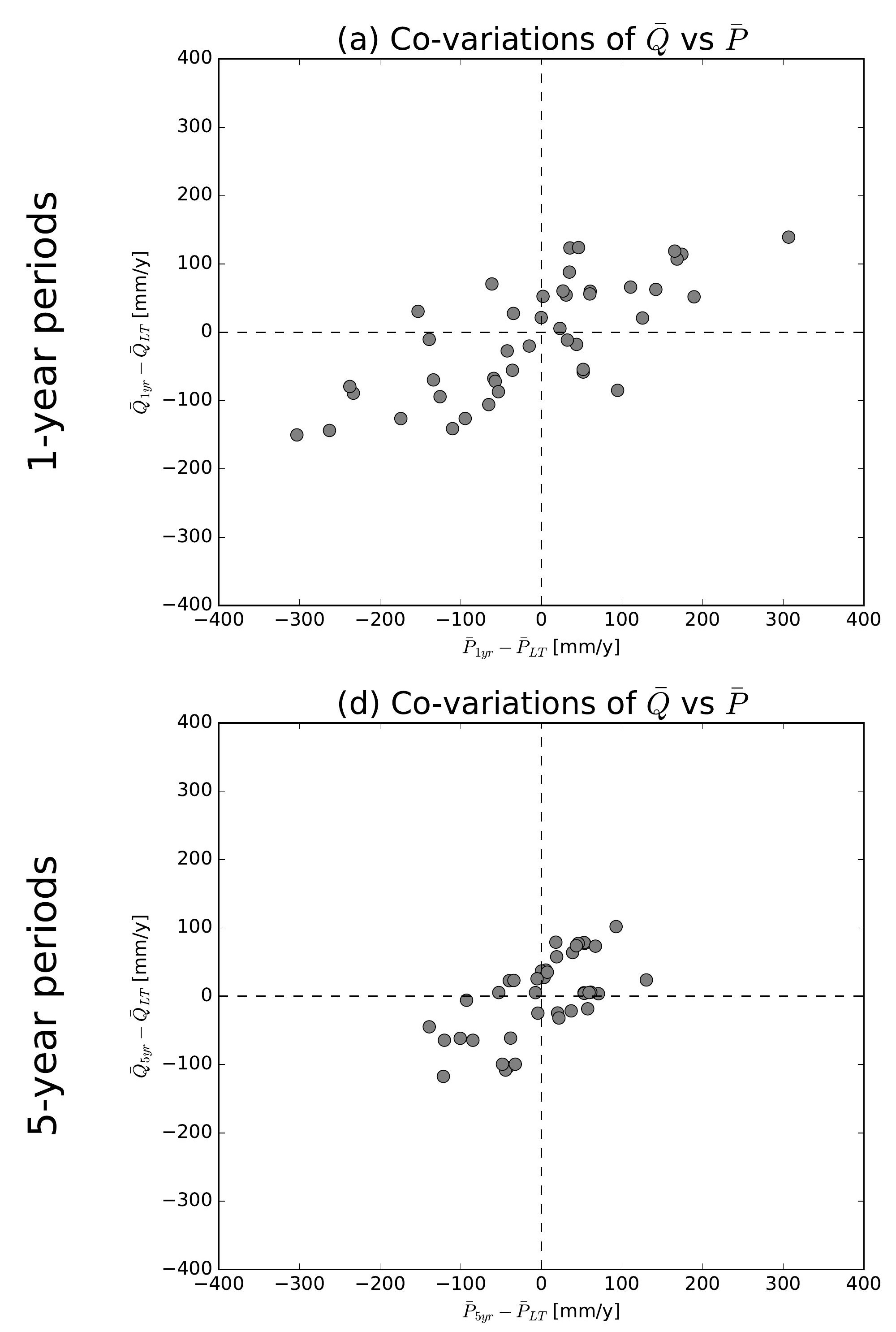

(g) Co-variations of $\bar{Q}$ vs $\bar{P}$
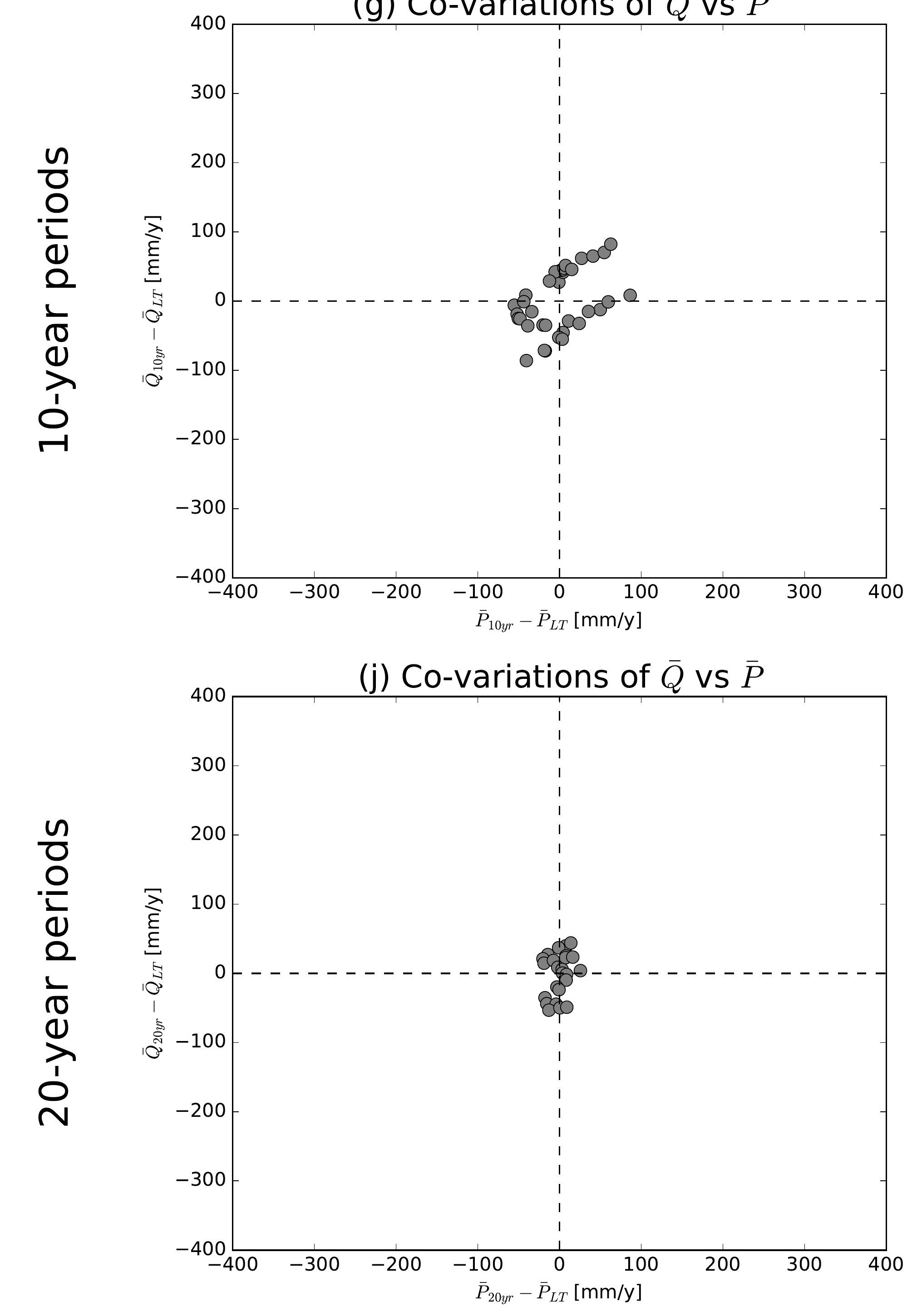

(b) Co-variations of $\bar{Q}$ vs $\overline{E 0}$

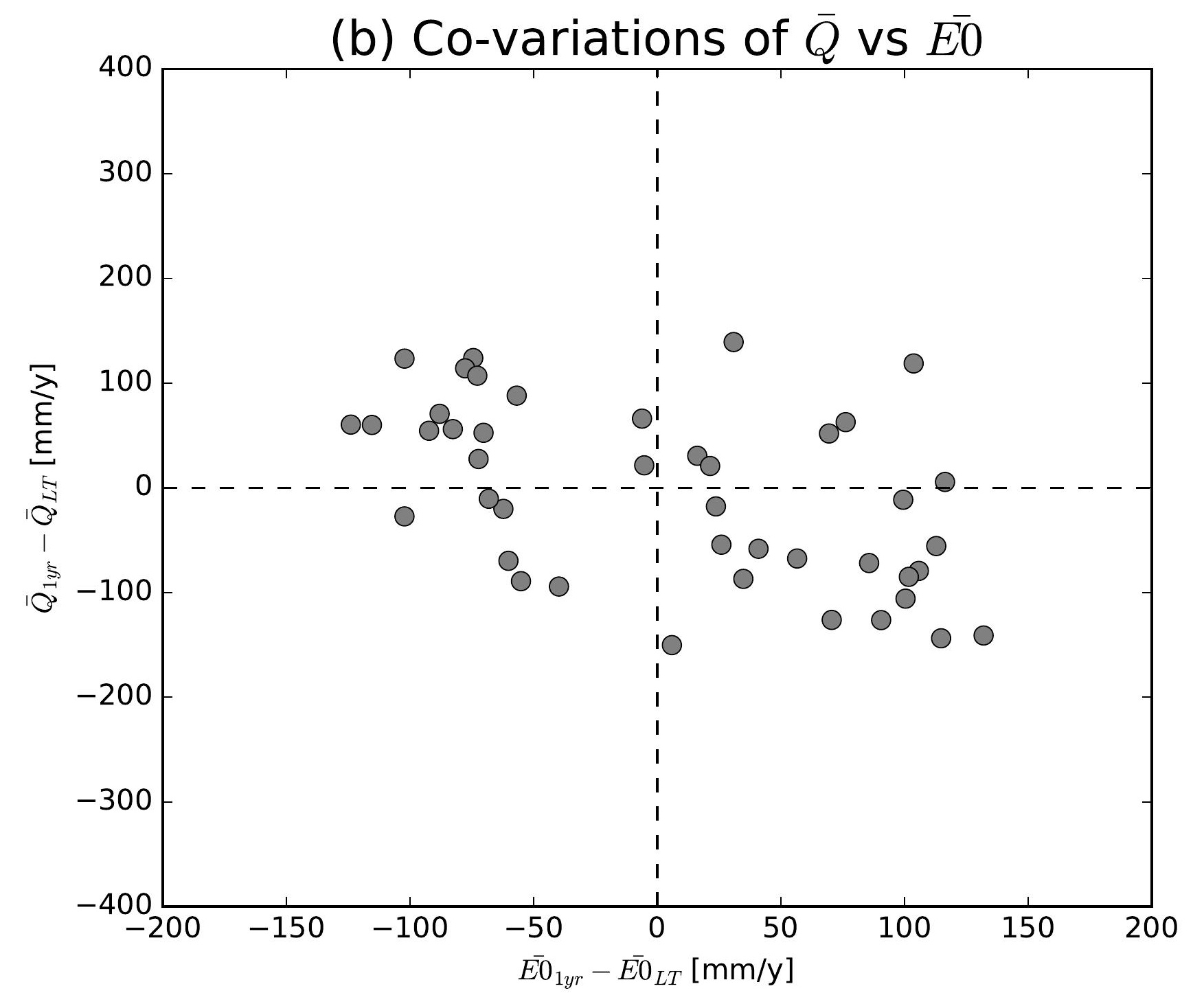

(e) Co-variations of $\bar{Q}$ vs $\overline{E 0}$

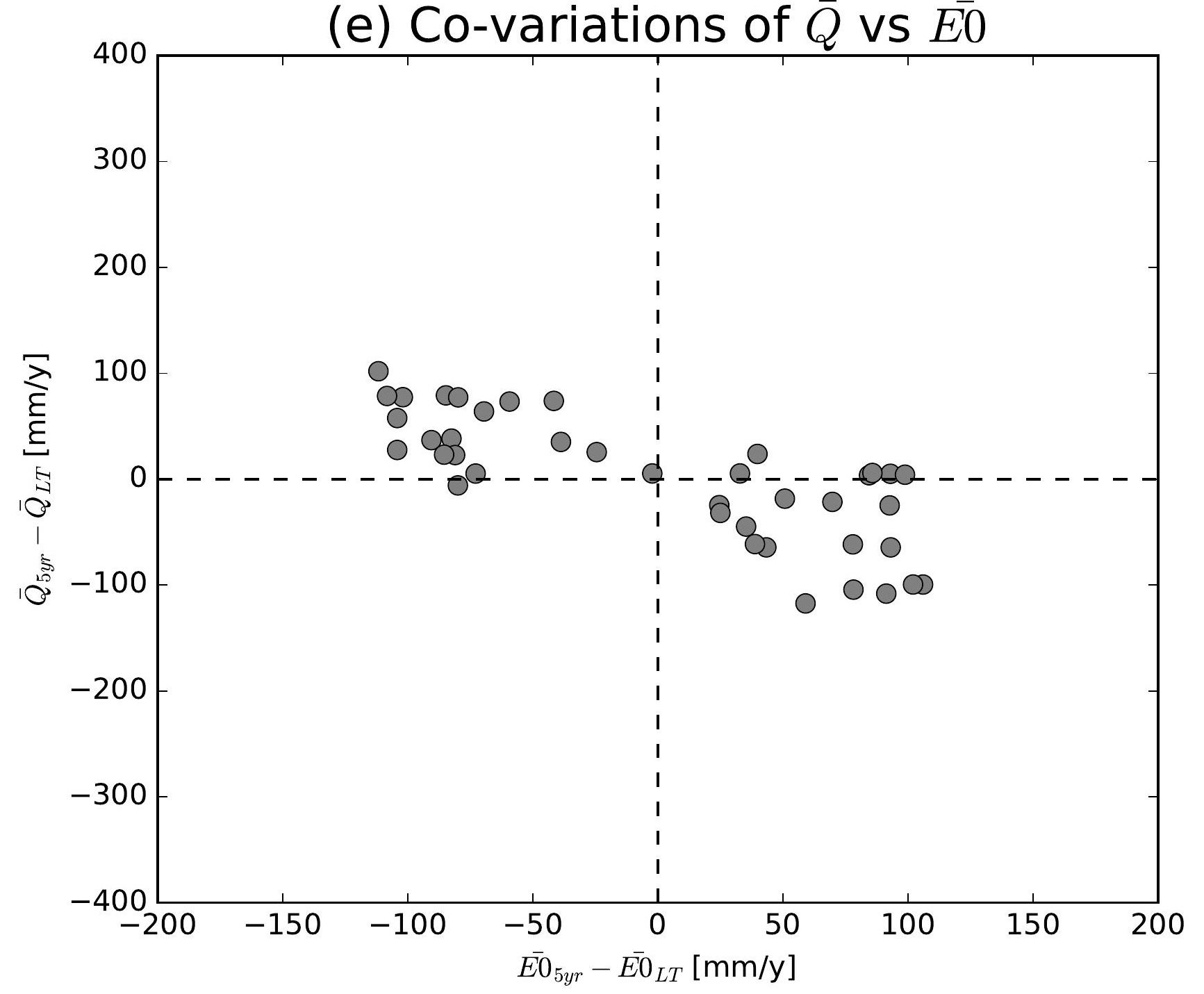

(h) Co-variations of $\bar{Q}$ vs $\overline{E 0}$

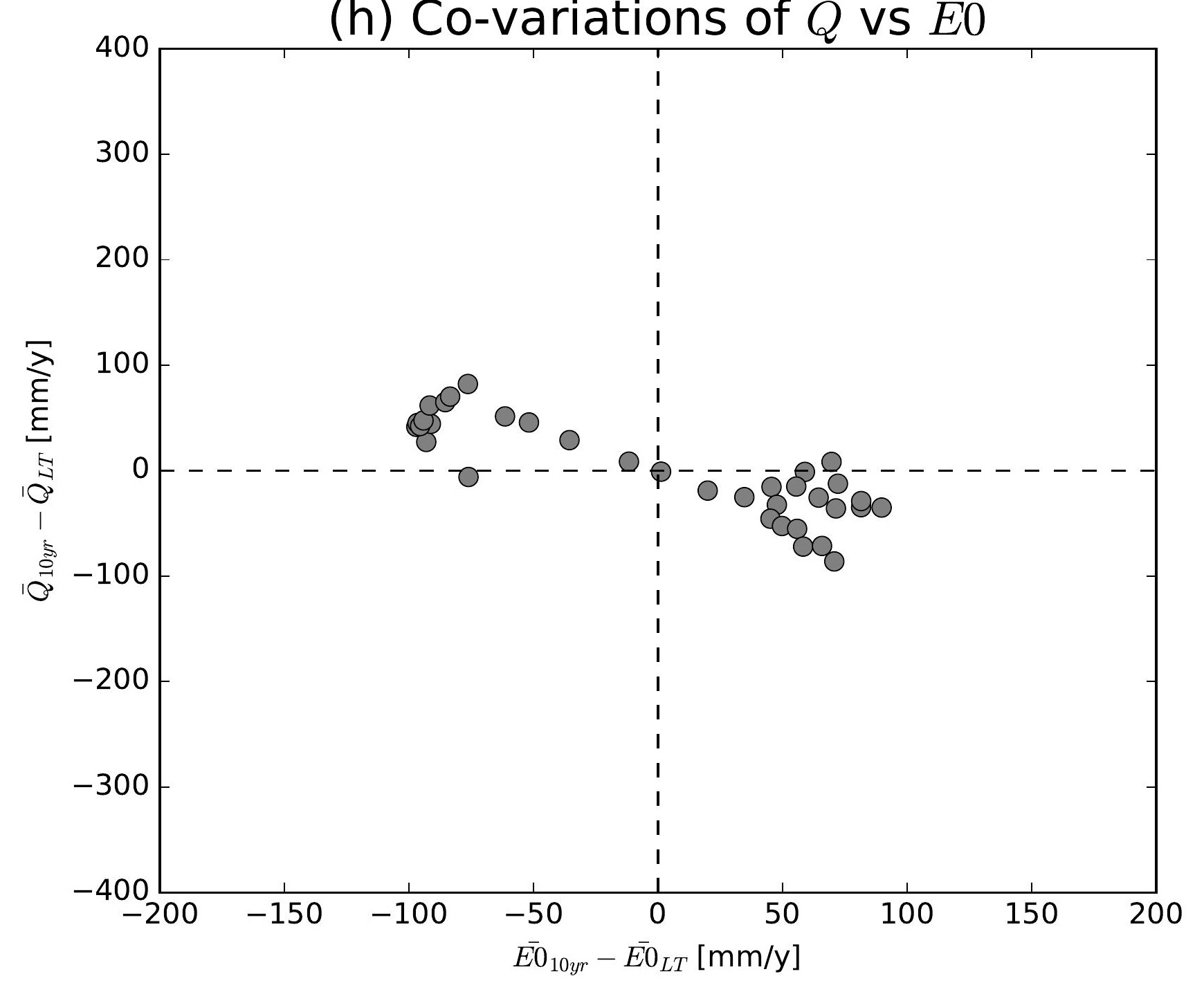

(k) Co-variations of $\bar{Q}$ vs $\overline{E 0}$

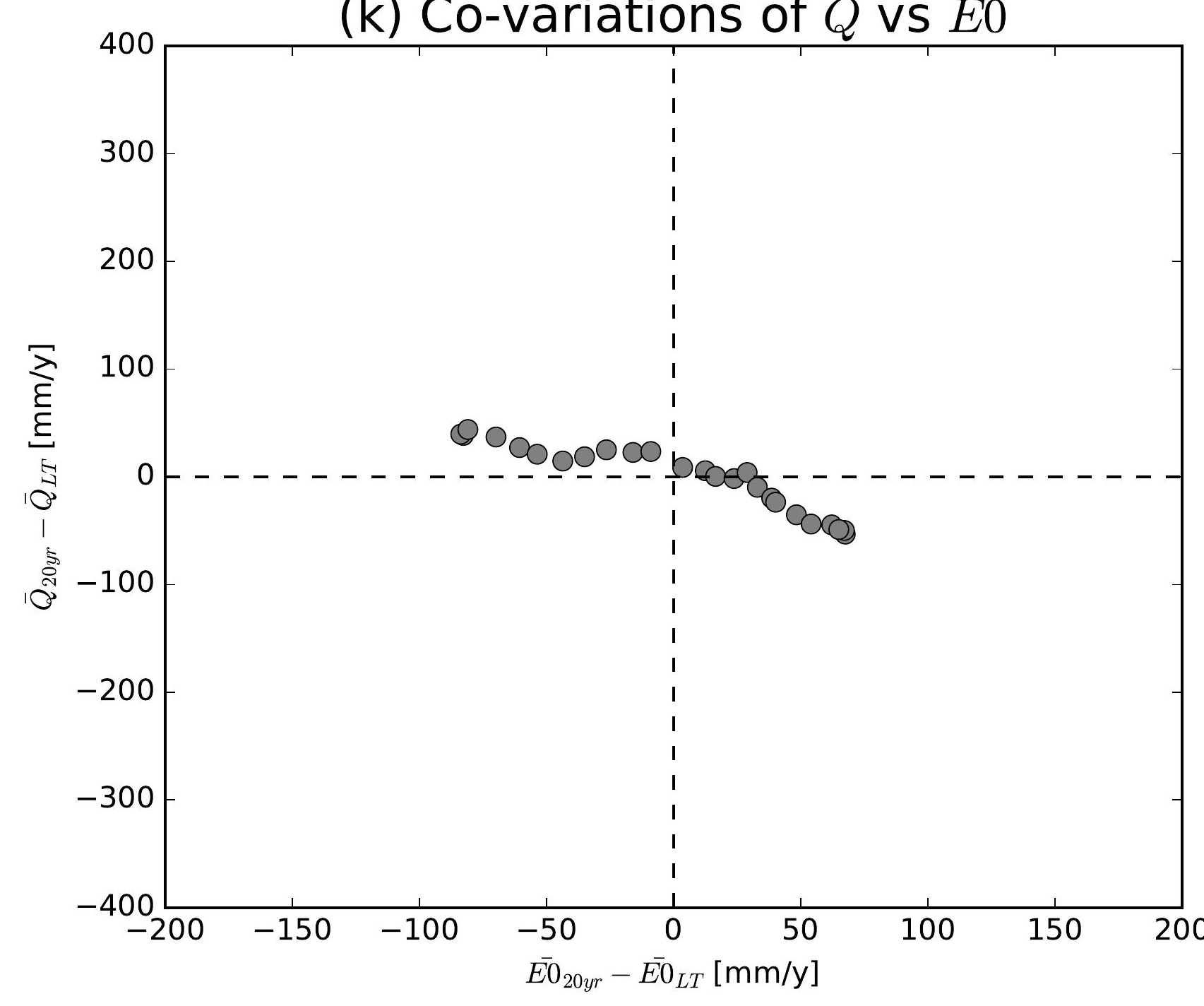

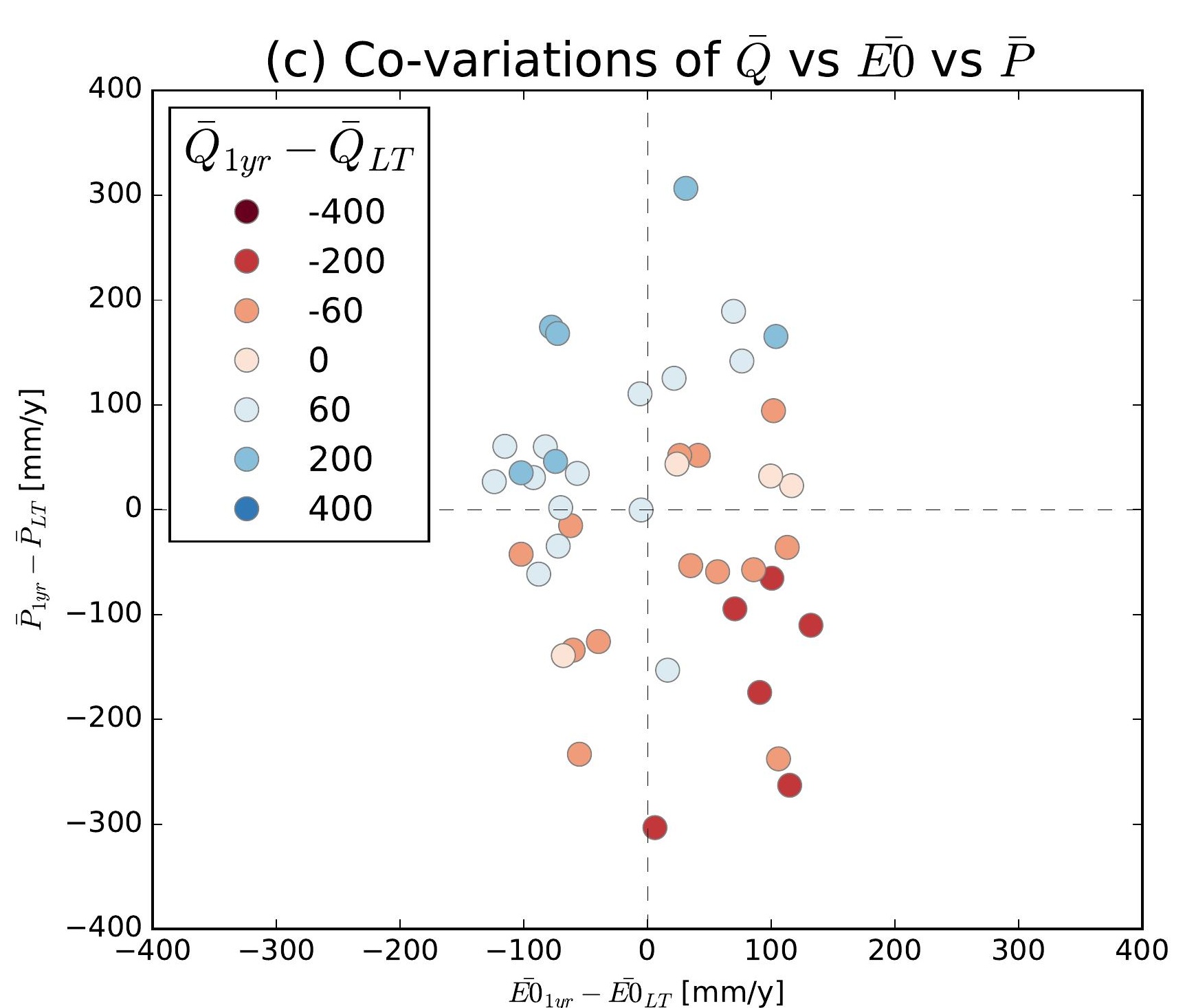
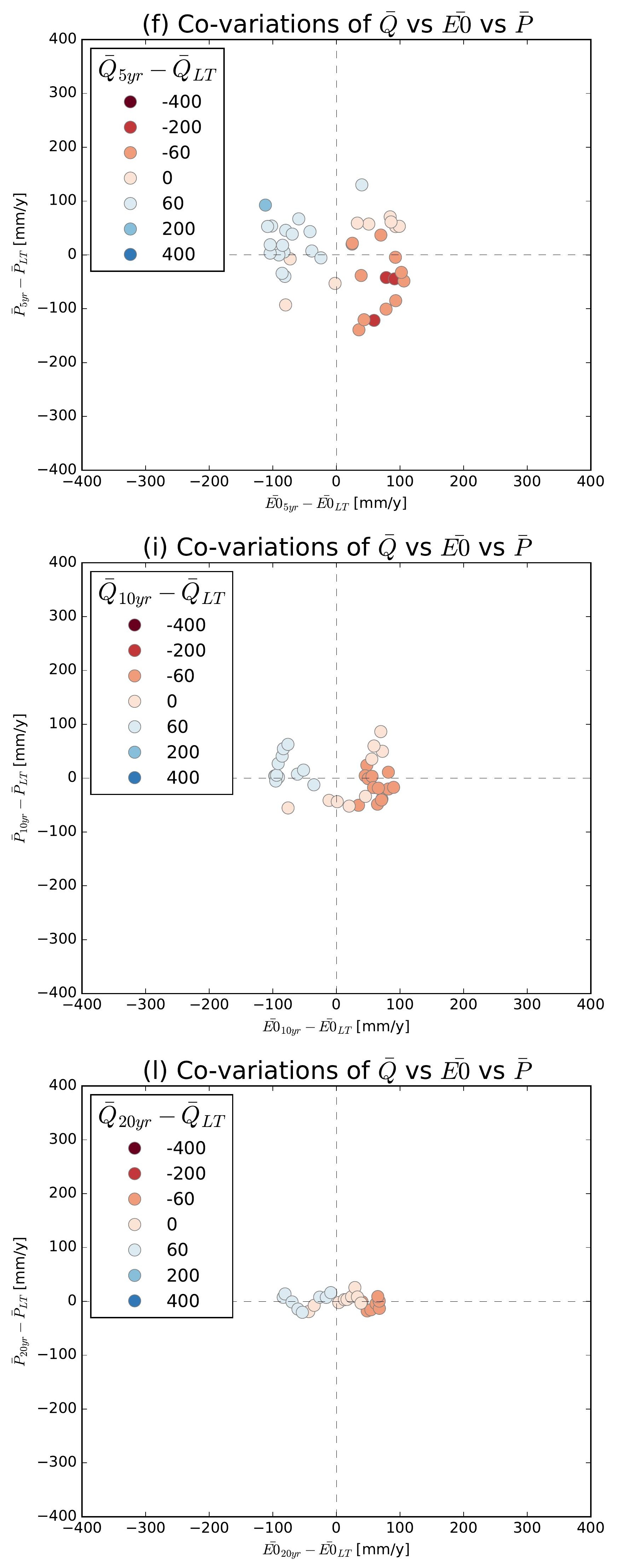

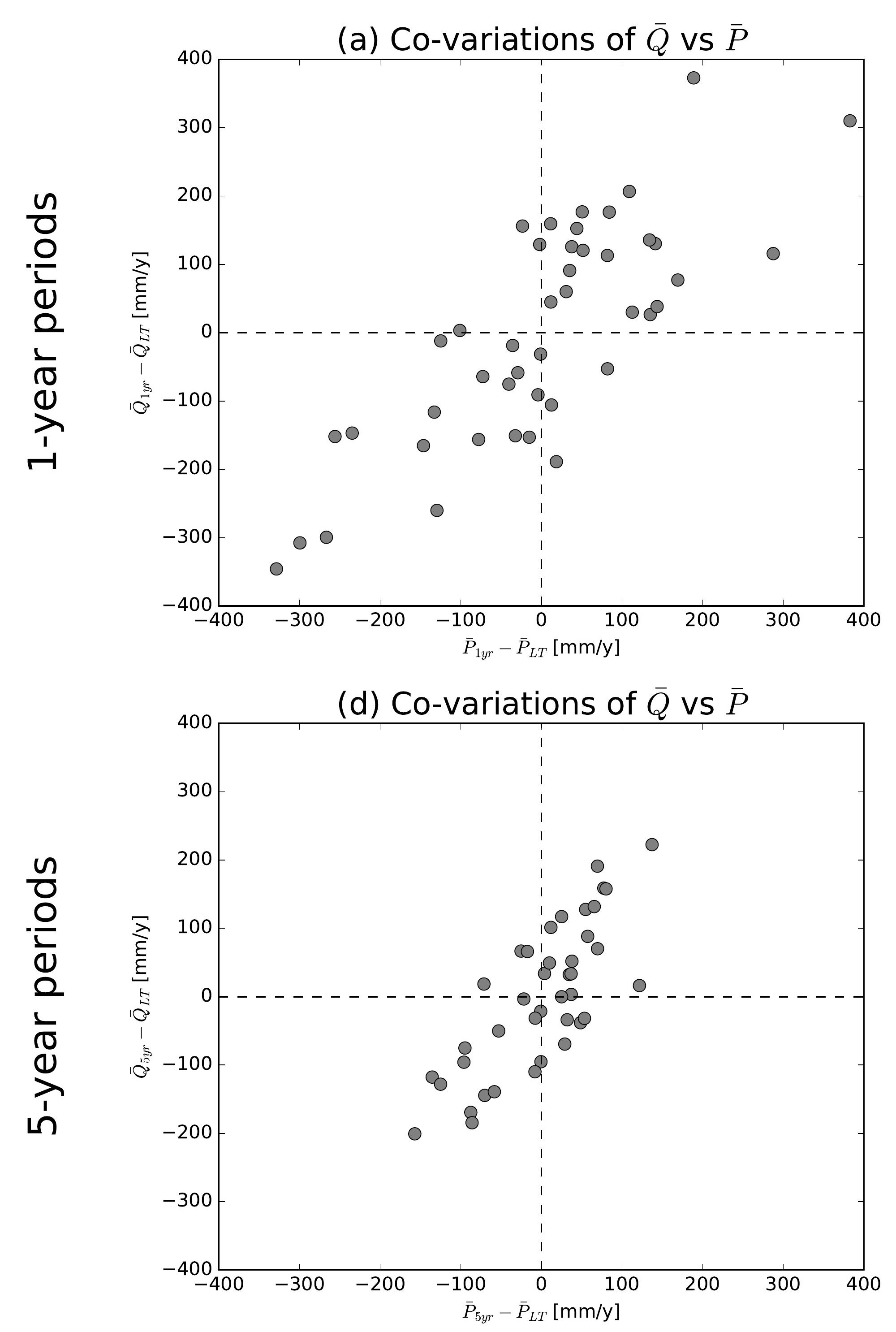

(g) Co-variations of $\bar{Q}$ vs $\bar{P}$

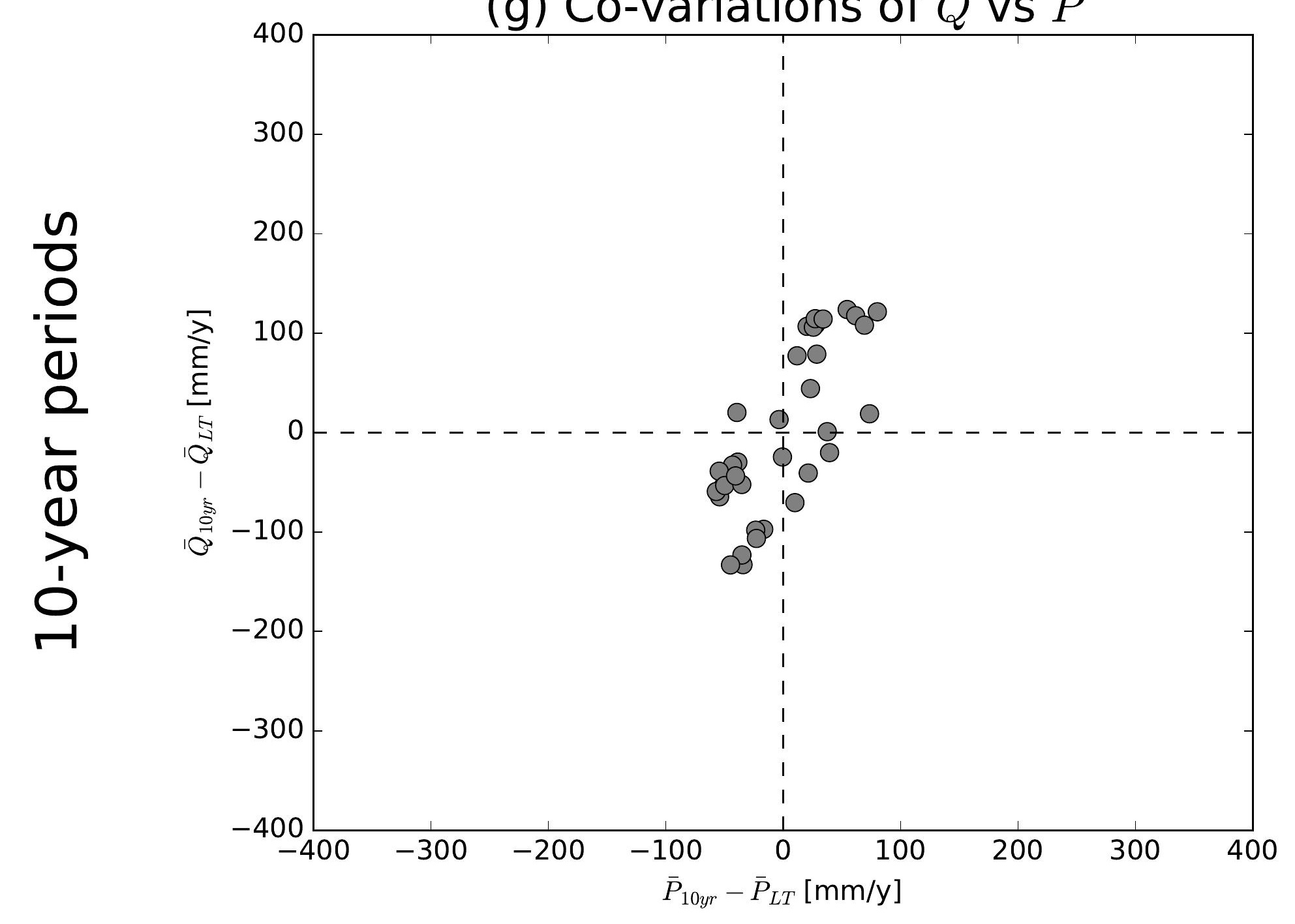

(j) Co-variations of $\bar{Q}$ vs $\bar{P}$

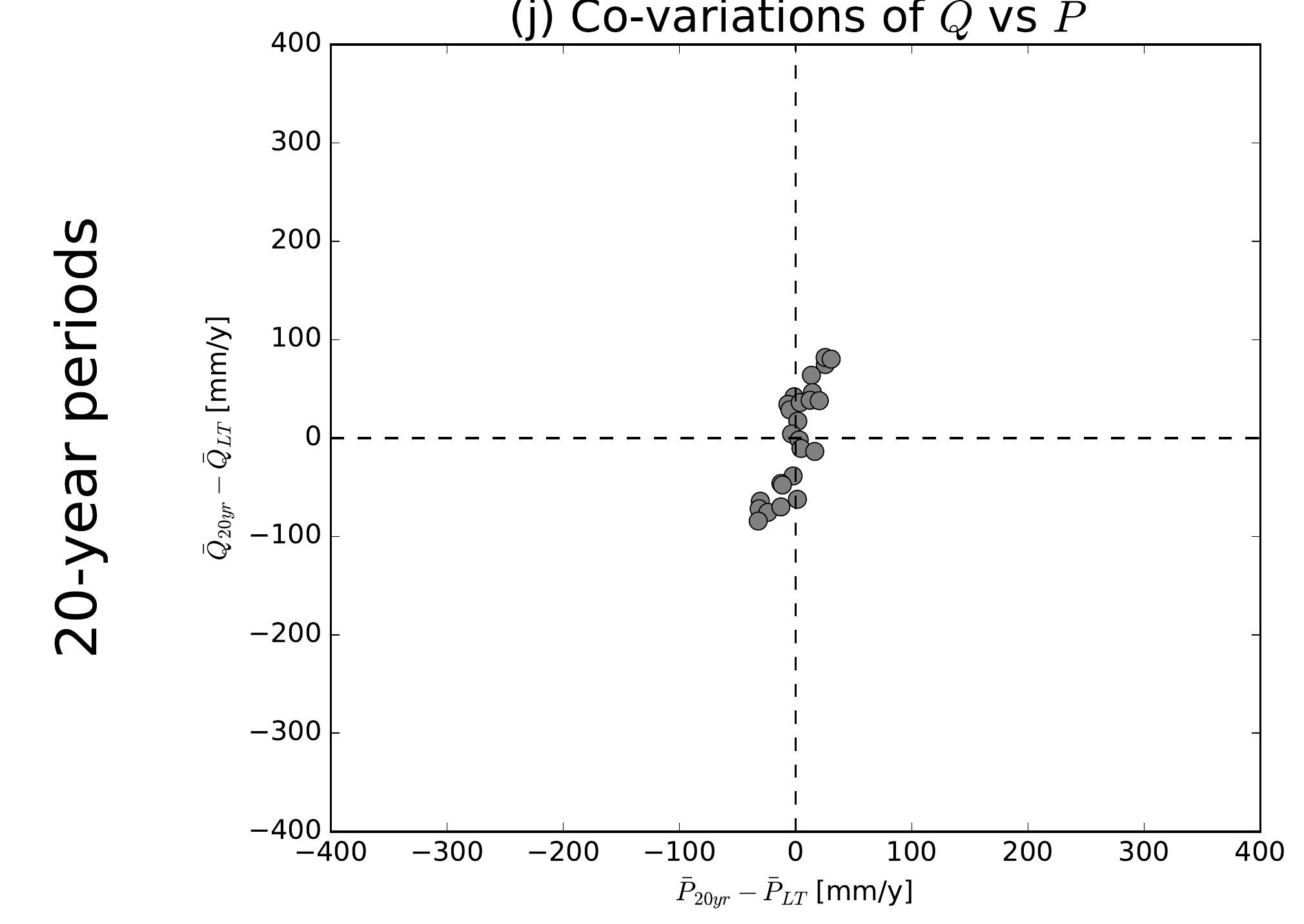

(b) Co-variations of $\bar{Q}$ vs $\overline{E 0}$

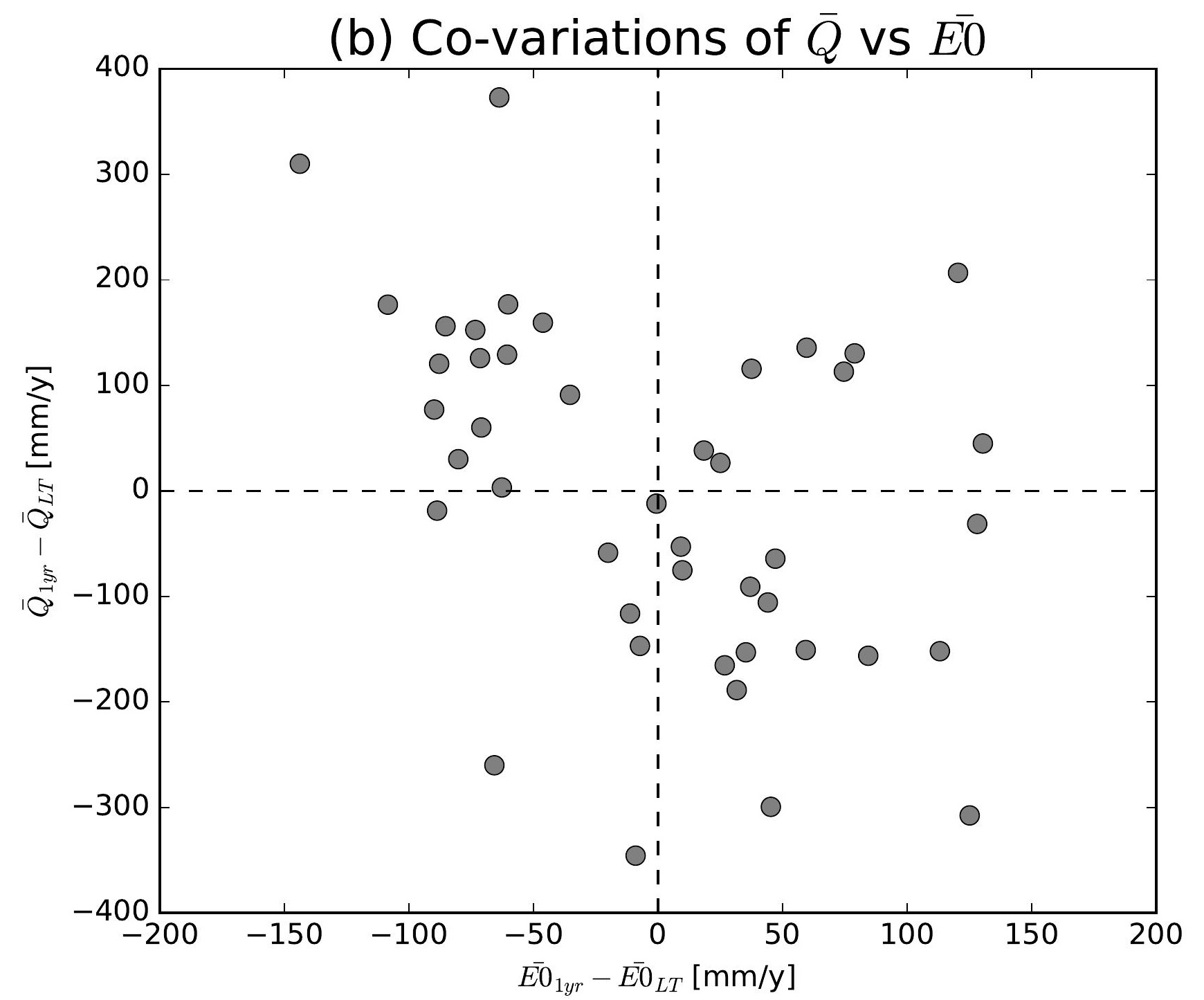

(e) Co-variations of $\bar{Q}$ vs $\overline{E 0}$

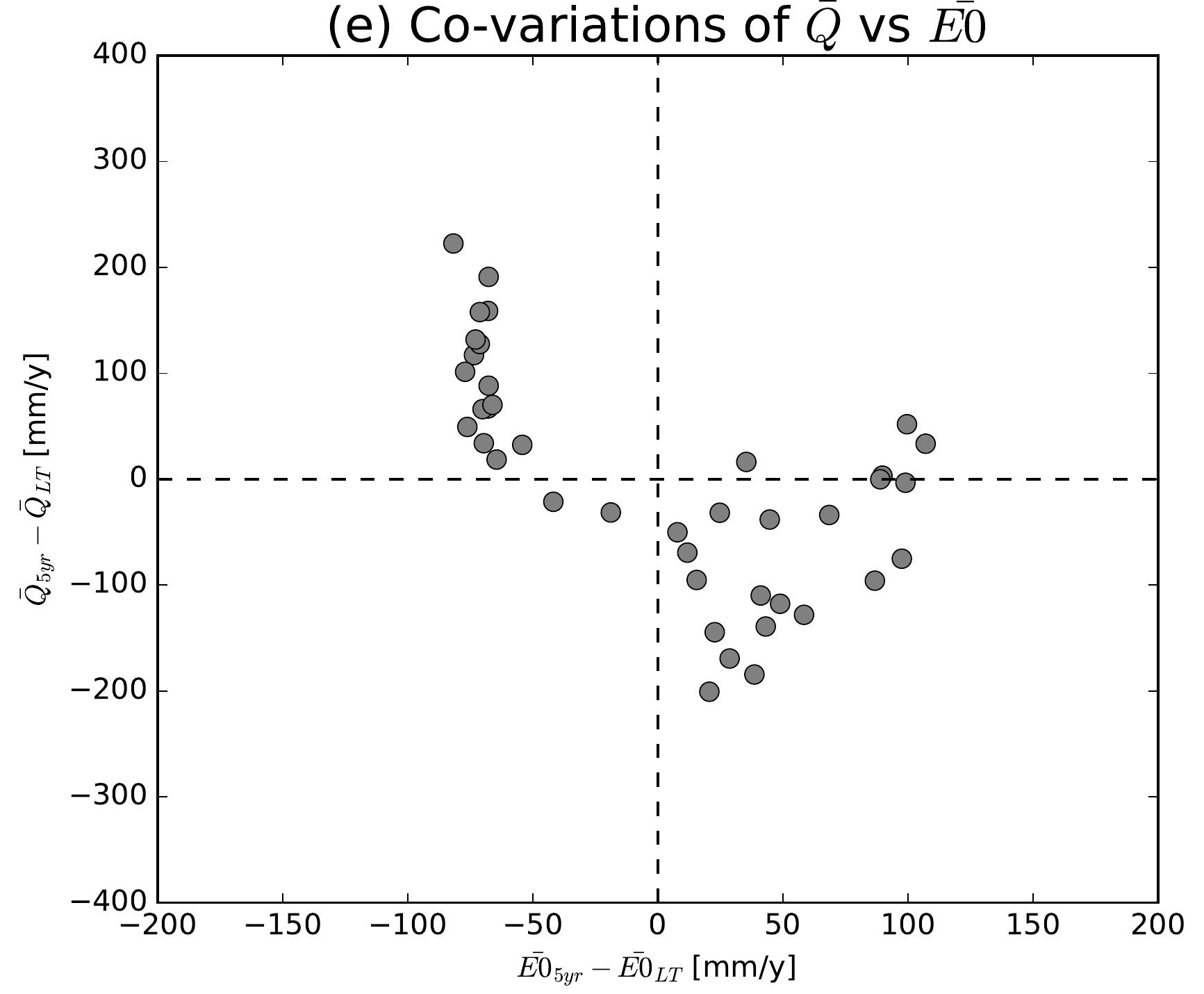

(h) Co-variations of $\bar{Q}$ vs $\overline{E 0}$

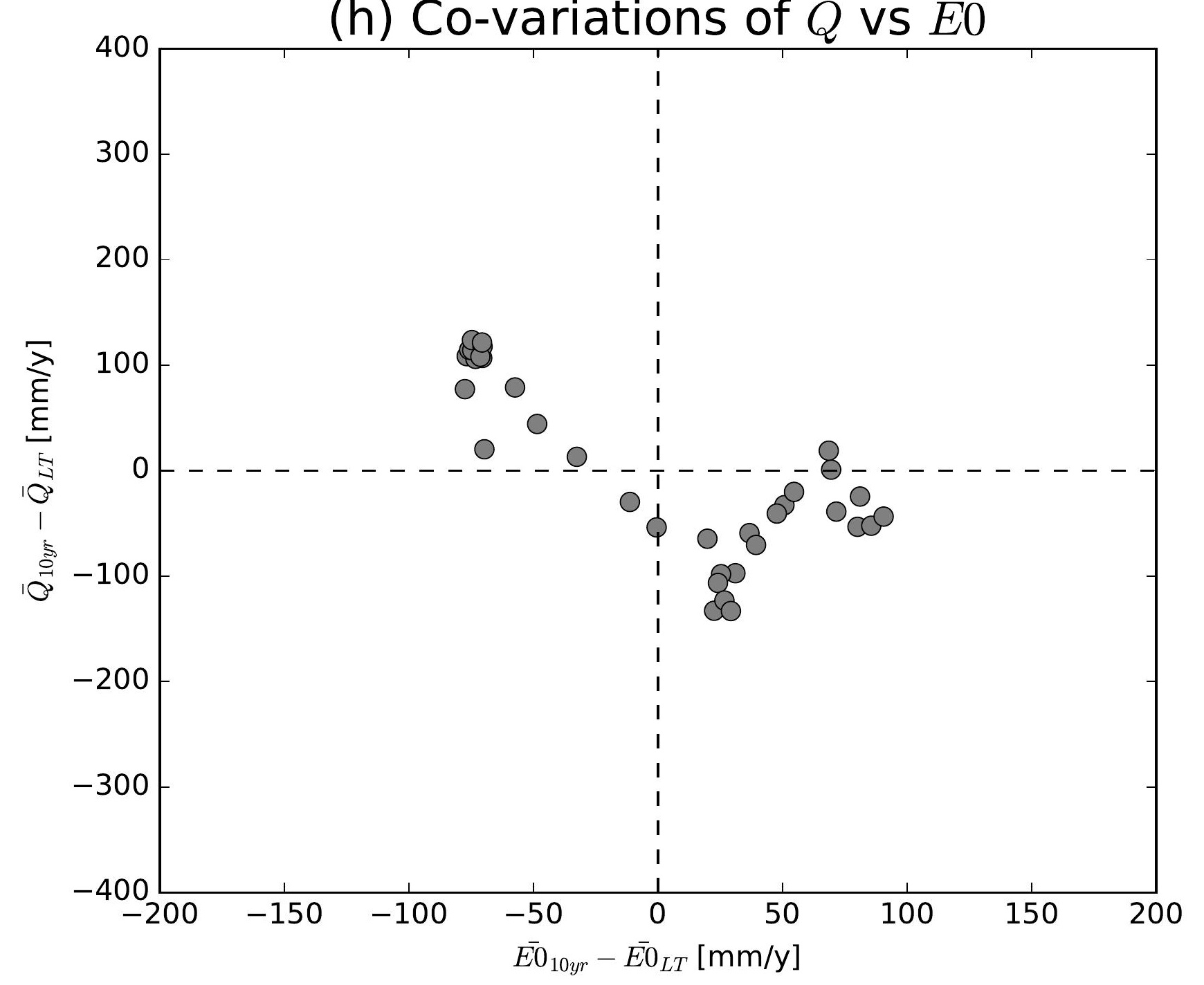

(k) Co-variations of $\bar{Q}$ vs $\overline{E 0}$

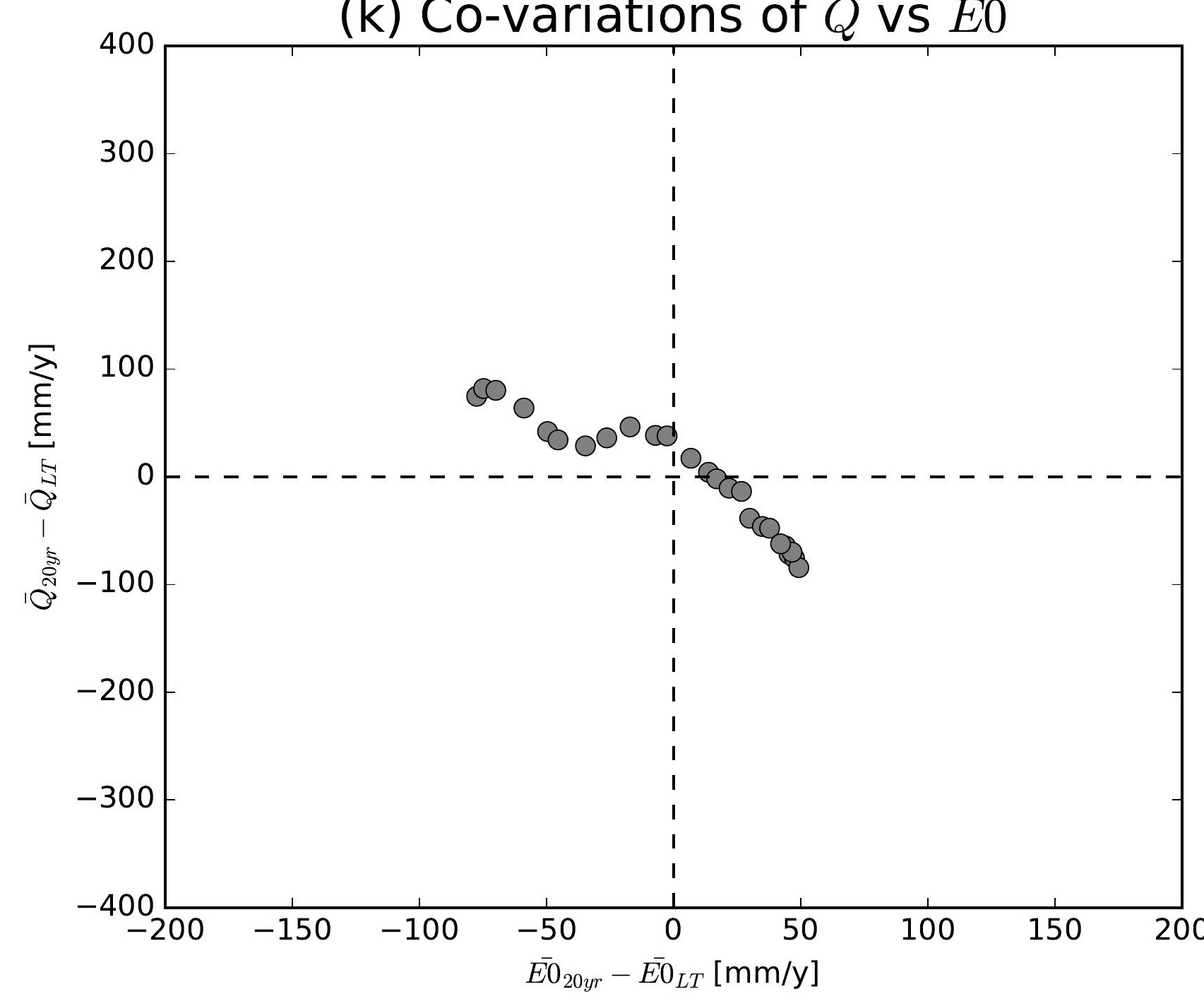

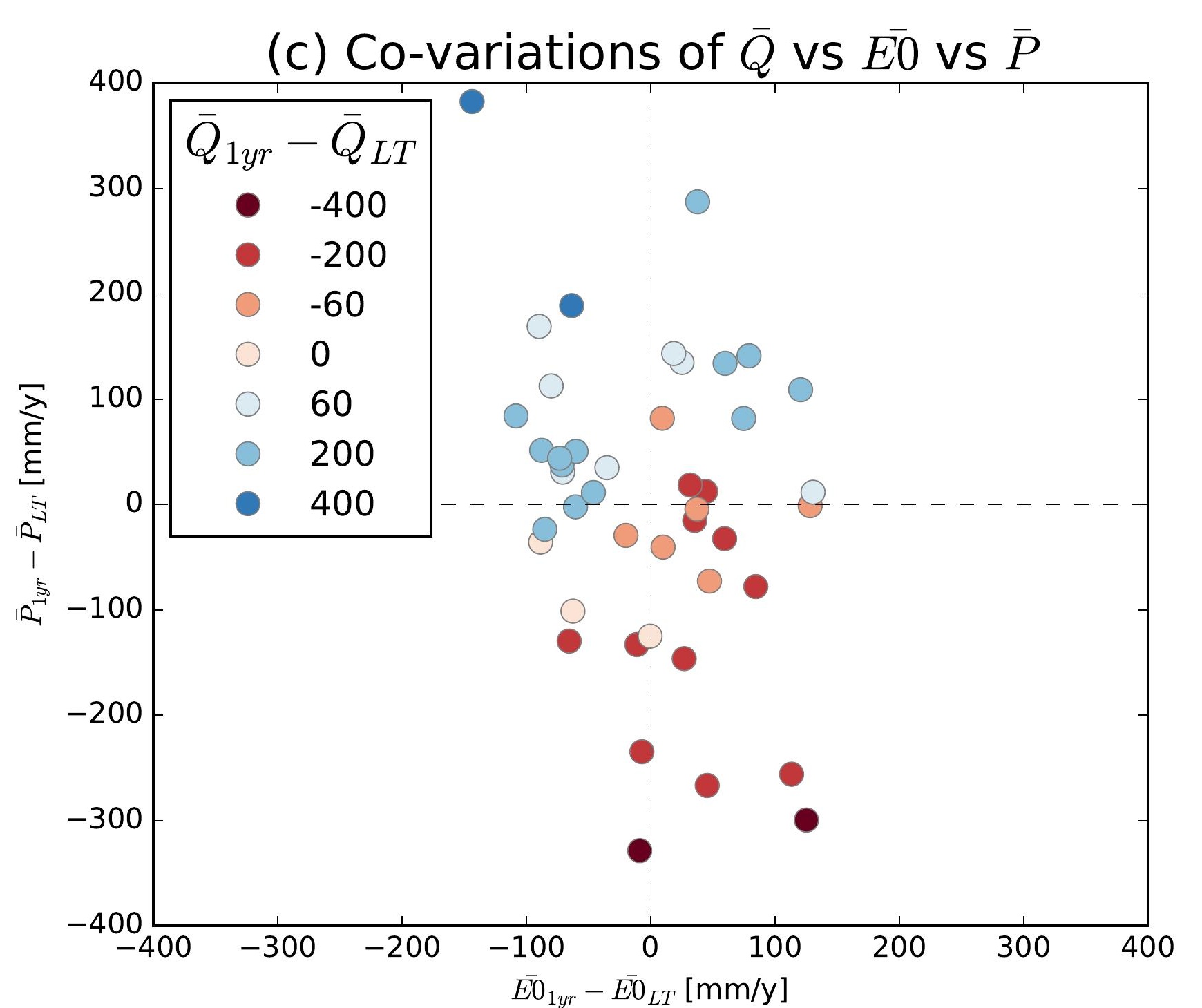
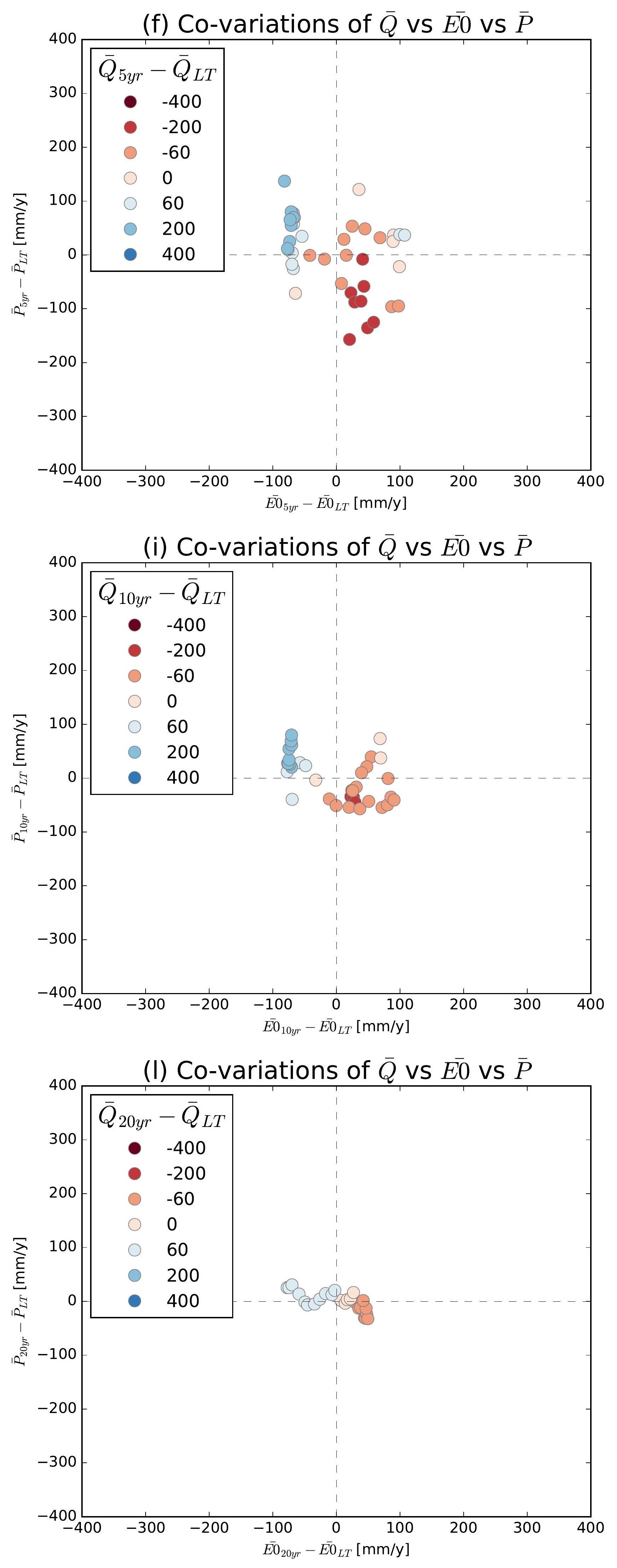


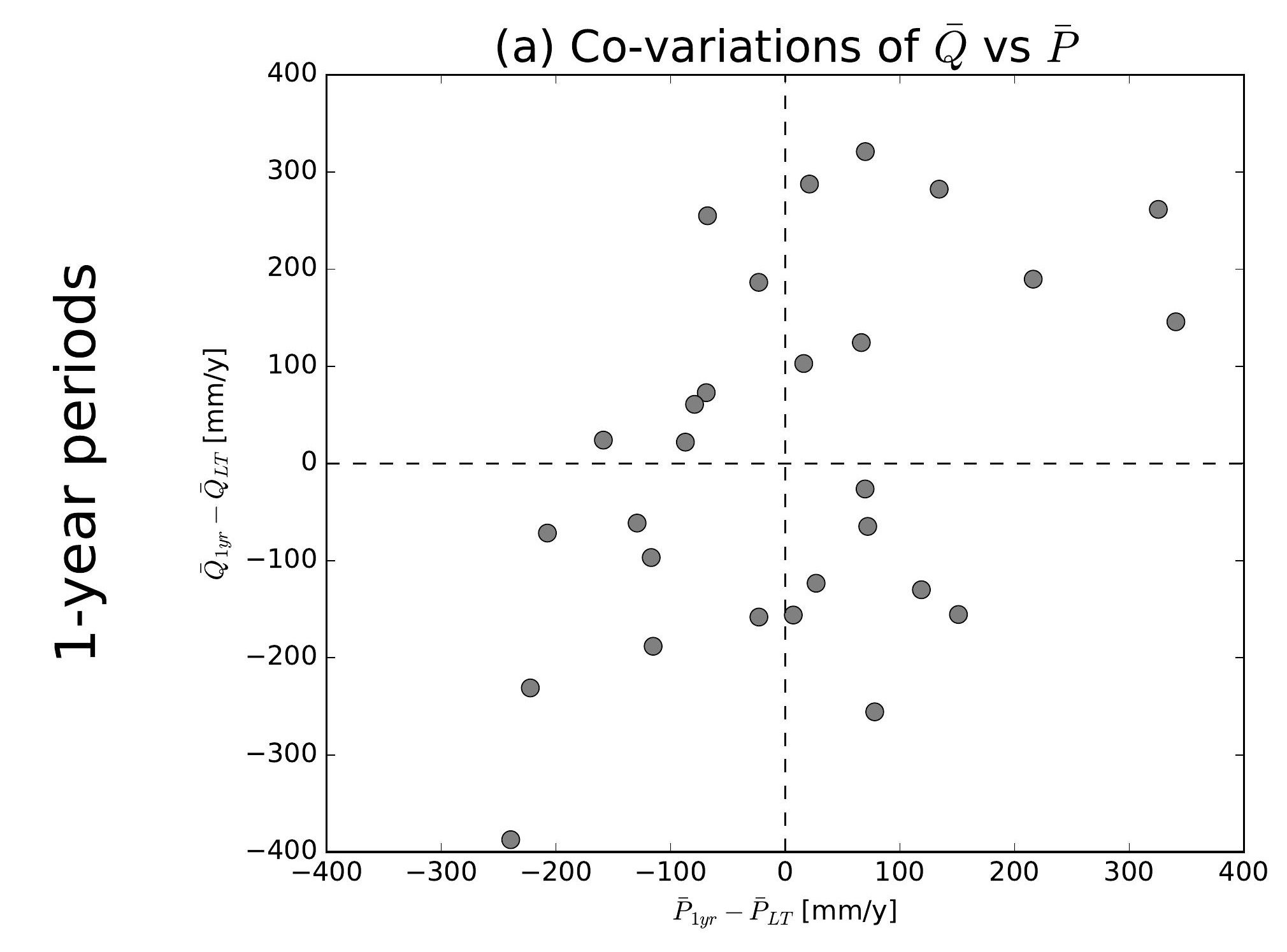

(d) Co-variations of $\bar{Q}$ vs $\bar{P}$

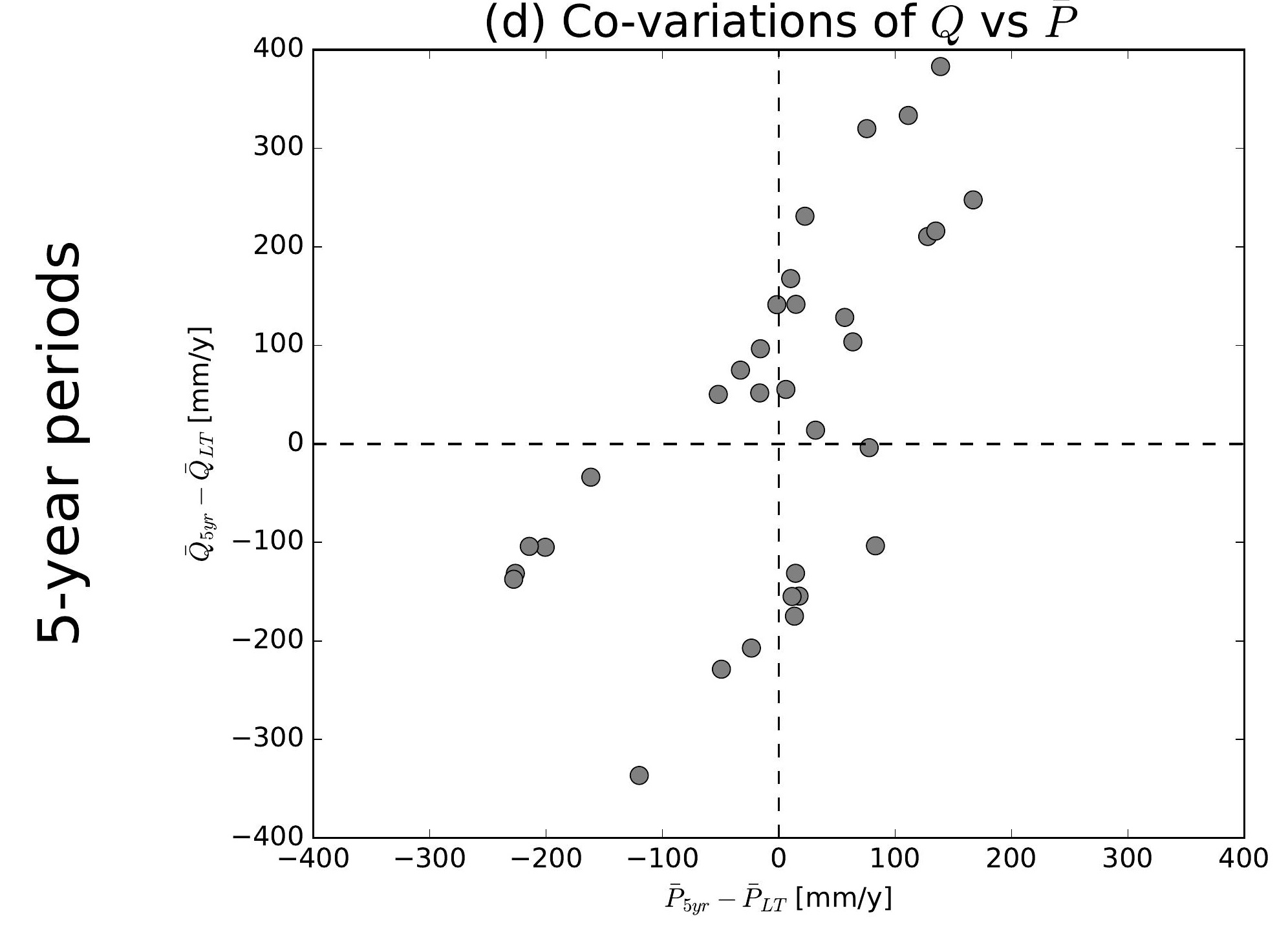

(g) Co-variations of $\bar{Q}$ vs $\bar{P}$

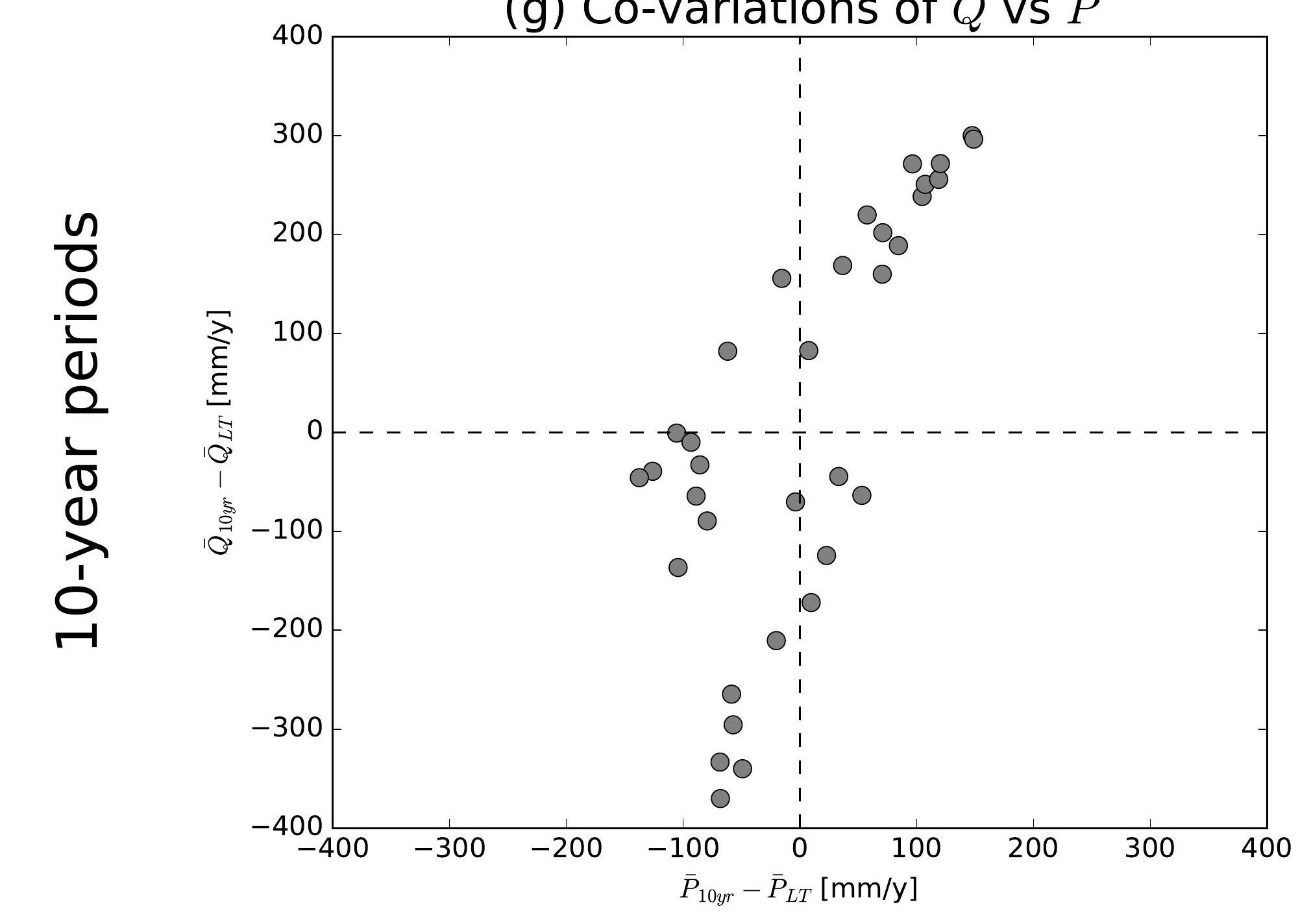

(j) Co-variations of $\bar{Q}$ vs $\bar{P}$

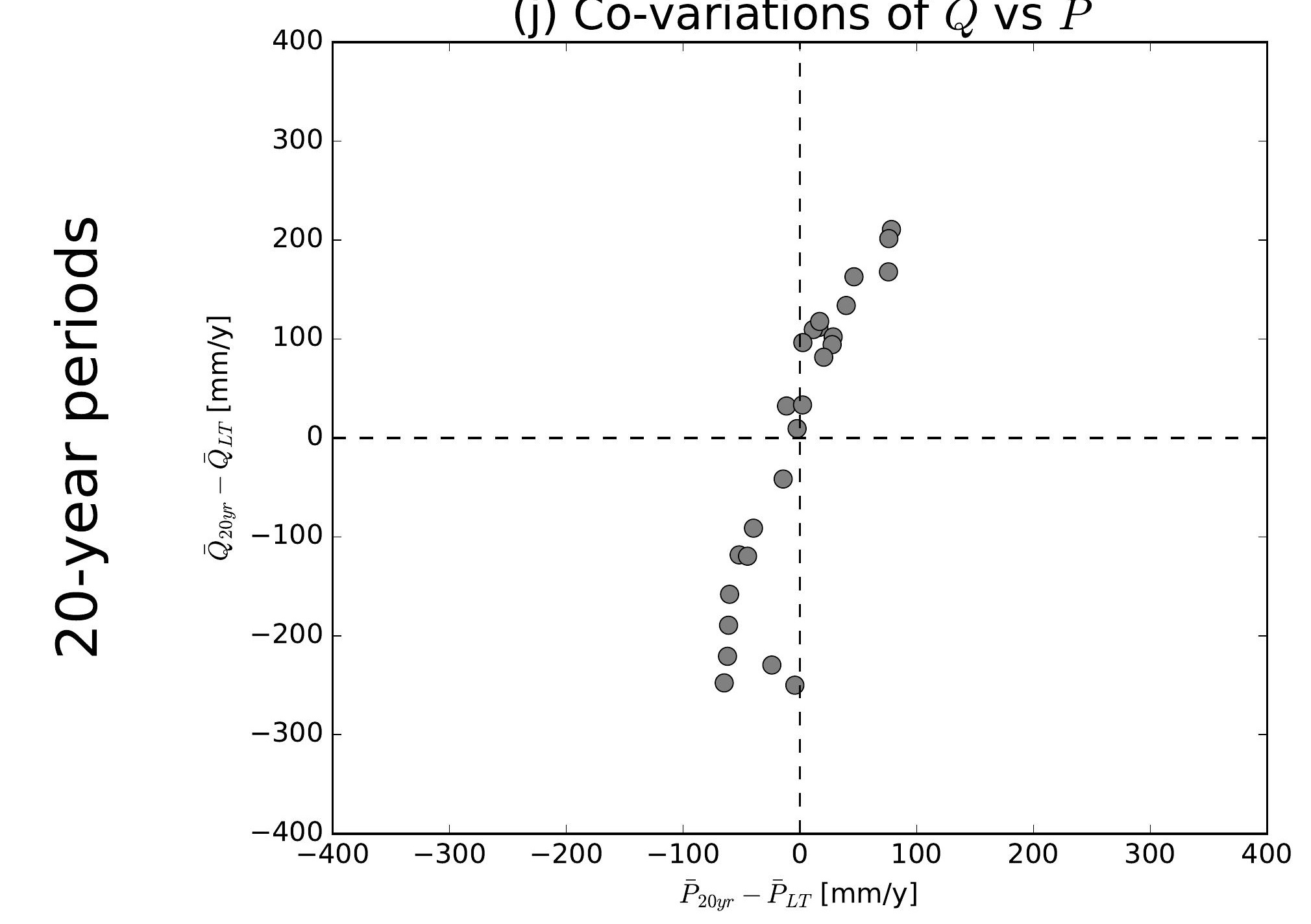

(b) Co-variations of $\bar{Q}$ vs $\overline{E 0}$

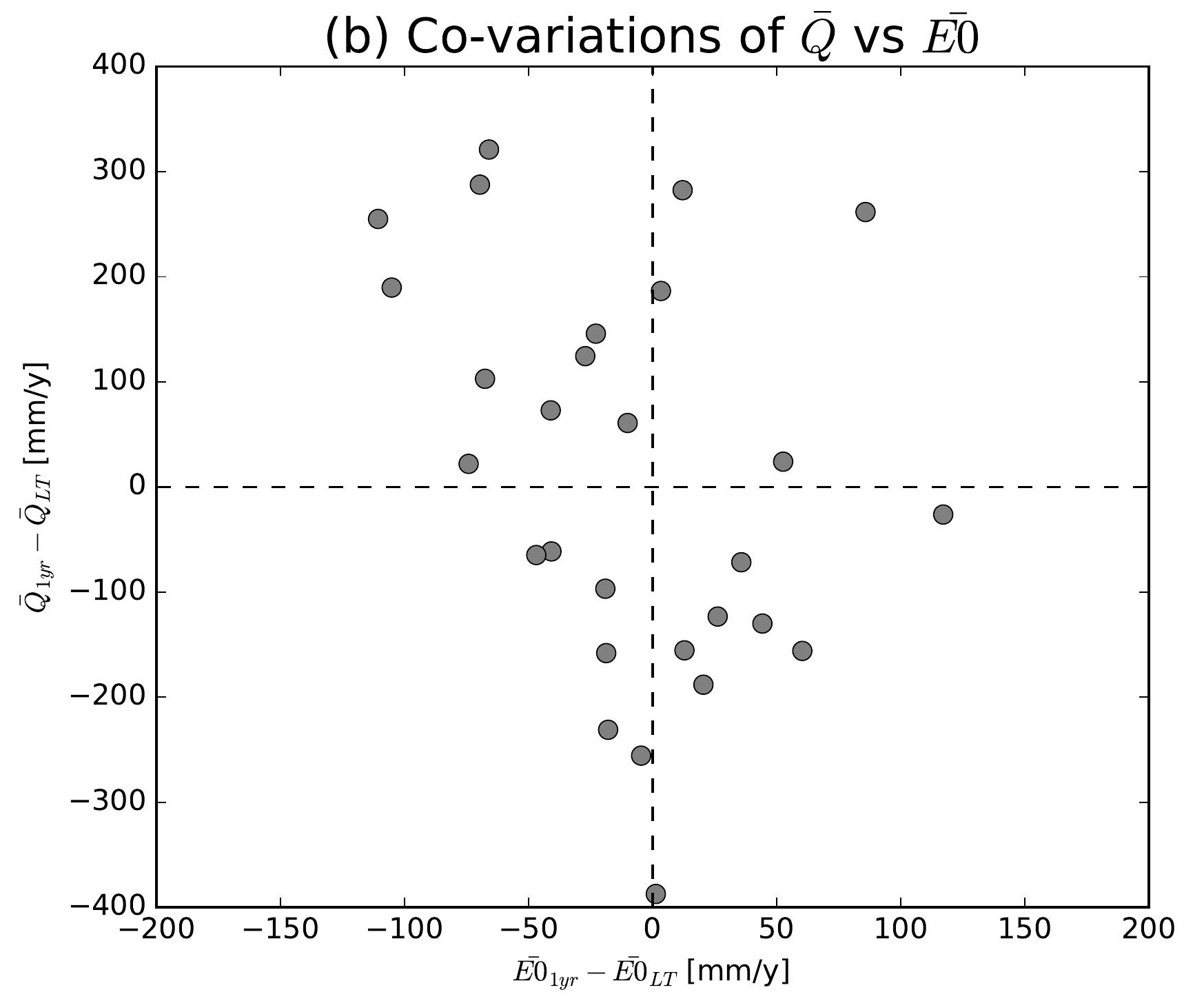

(e) Co-variations of $\bar{Q}$ vs $\overline{E 0}$

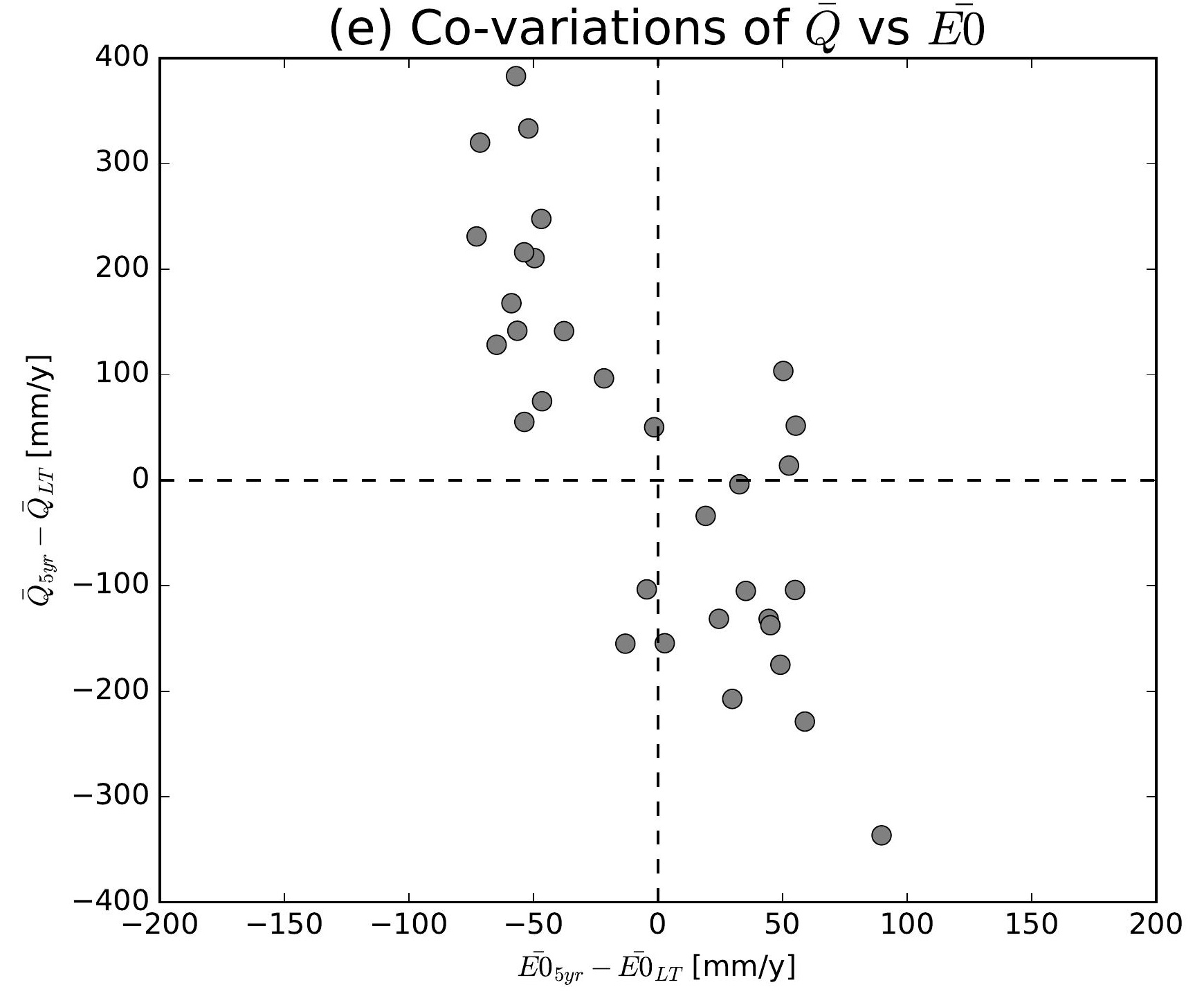

(h) Co-variations of $\bar{Q}$ vs $\overline{E 0}$

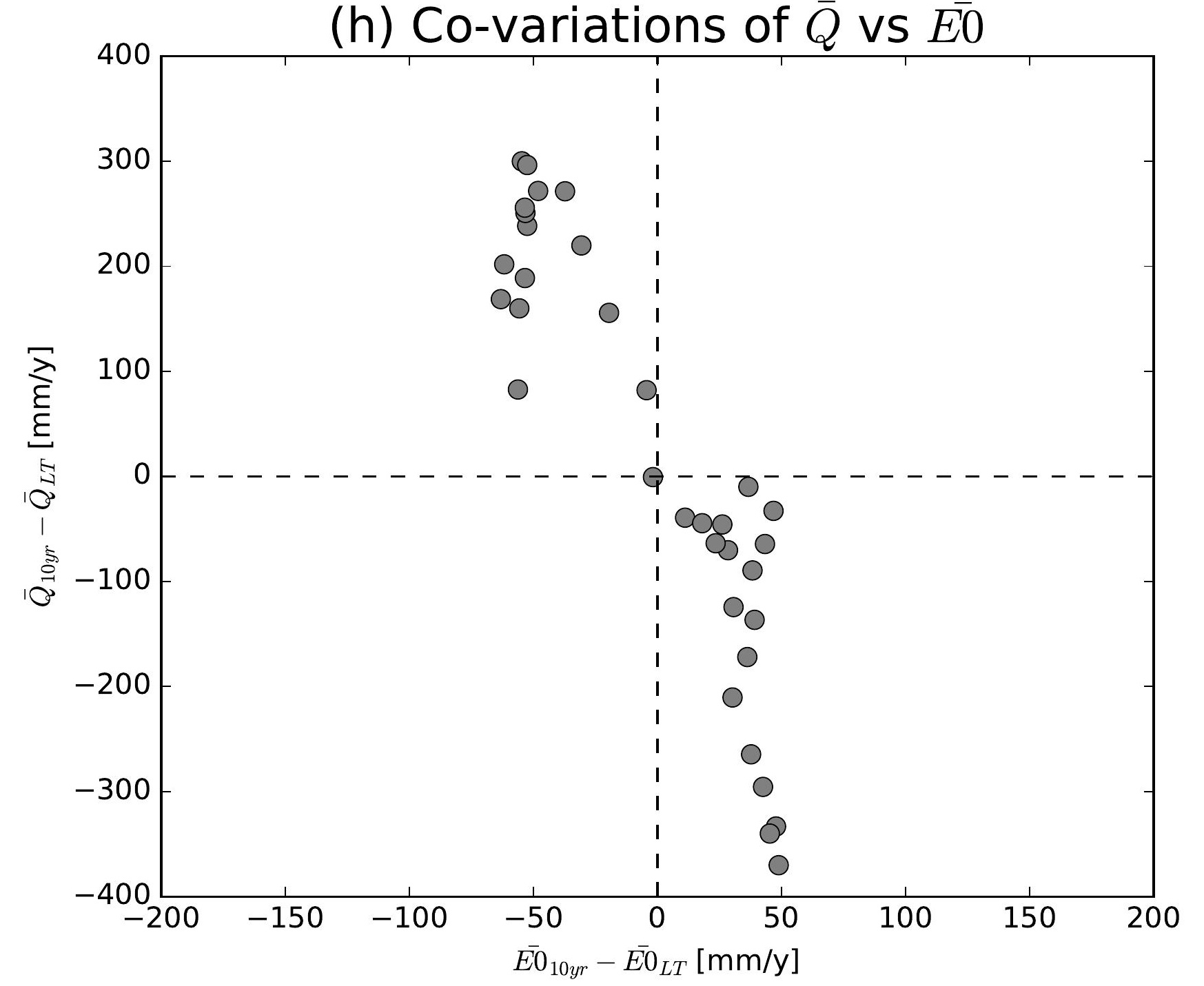

(k) Co-variations of $\bar{Q}$ vs $\overline{E 0}$

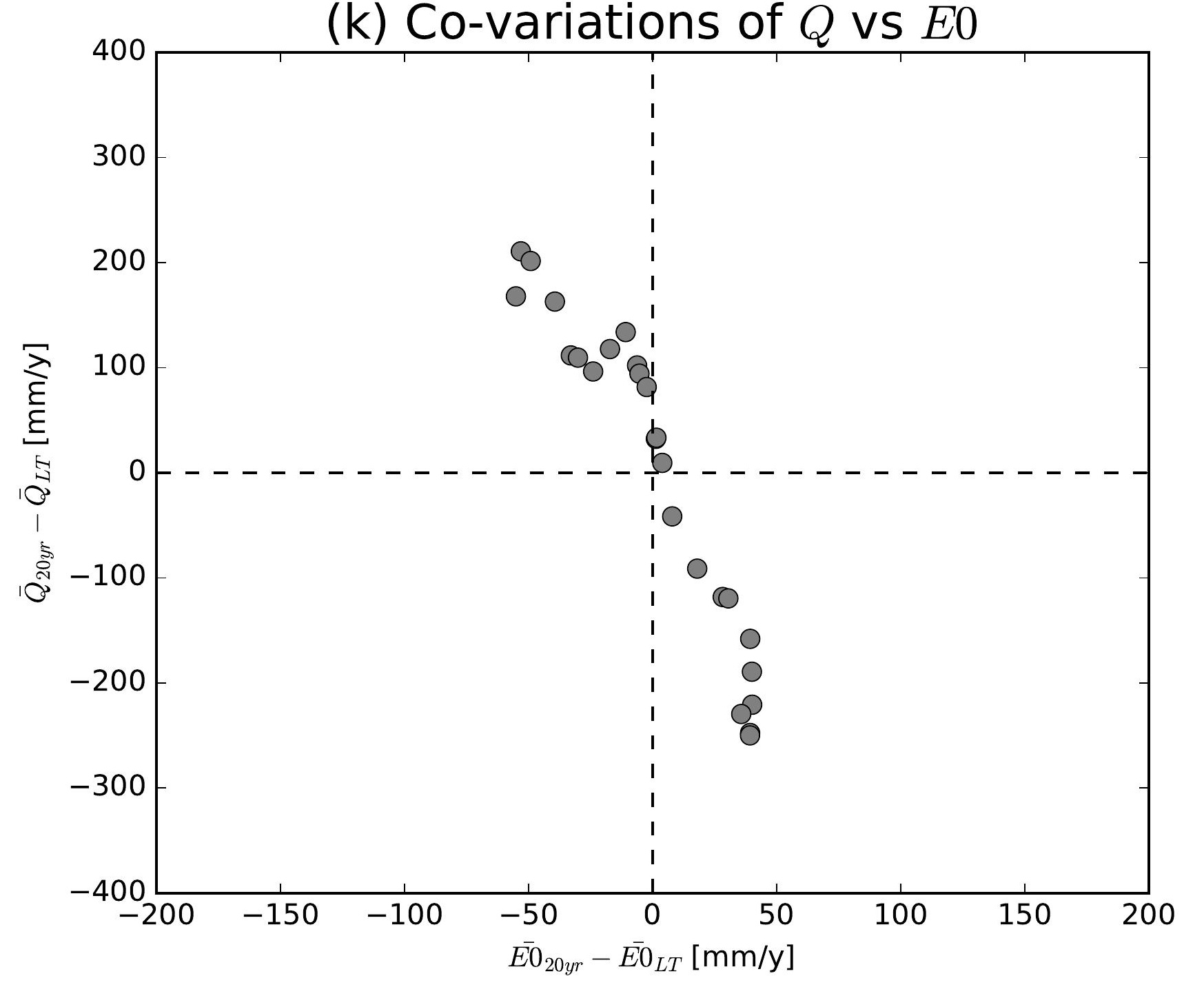

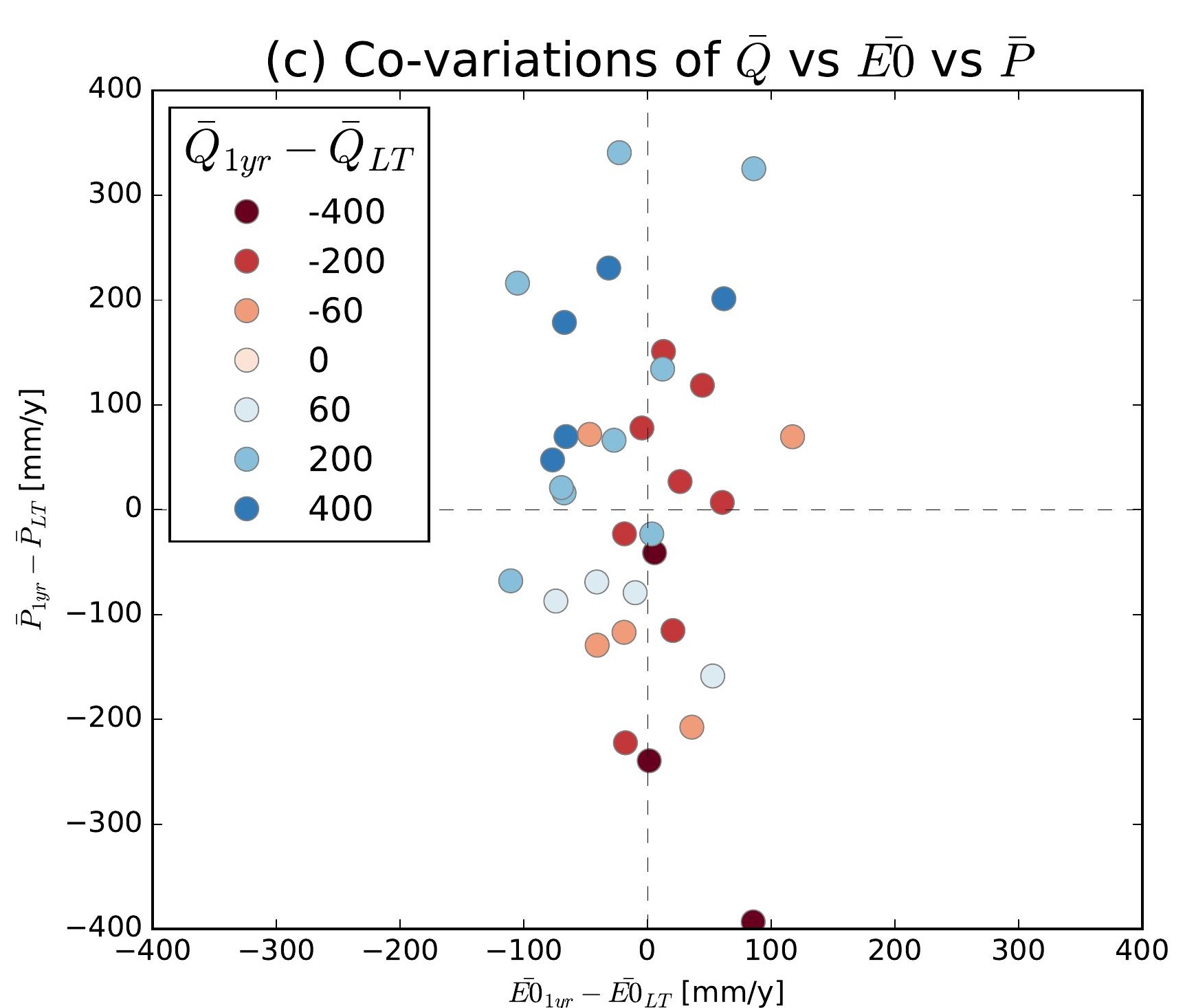
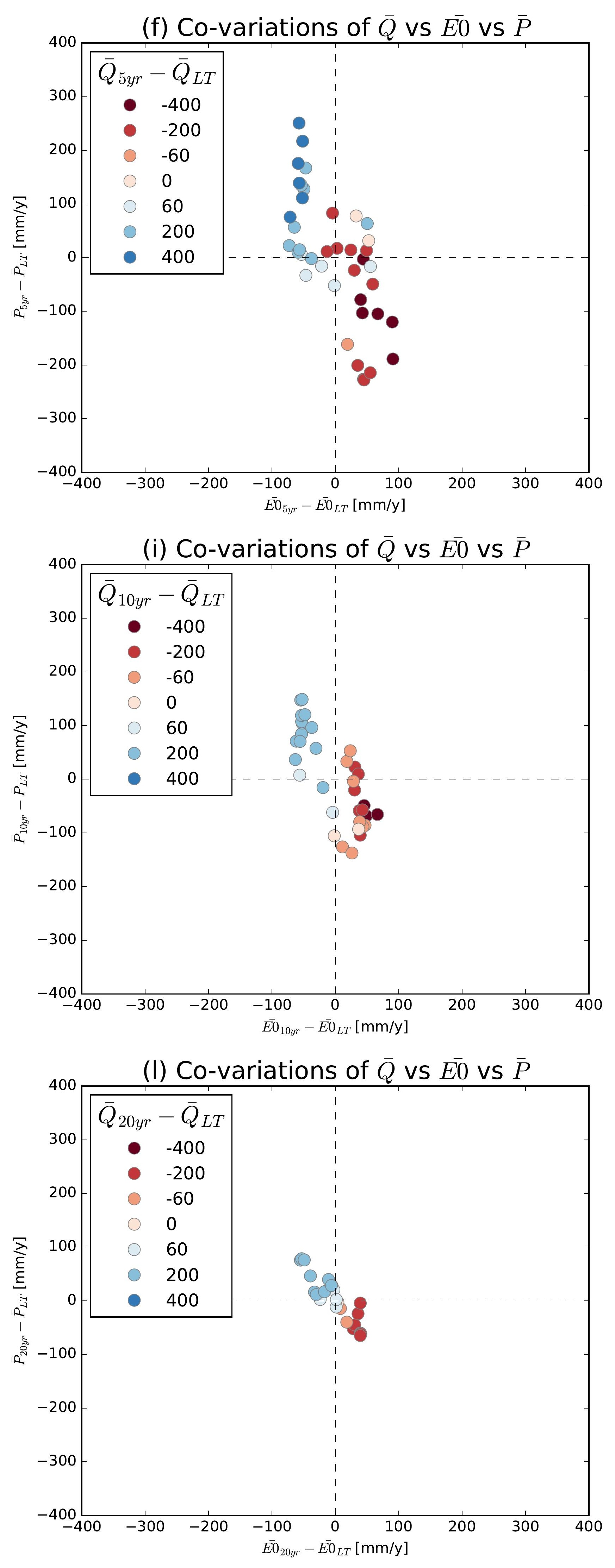

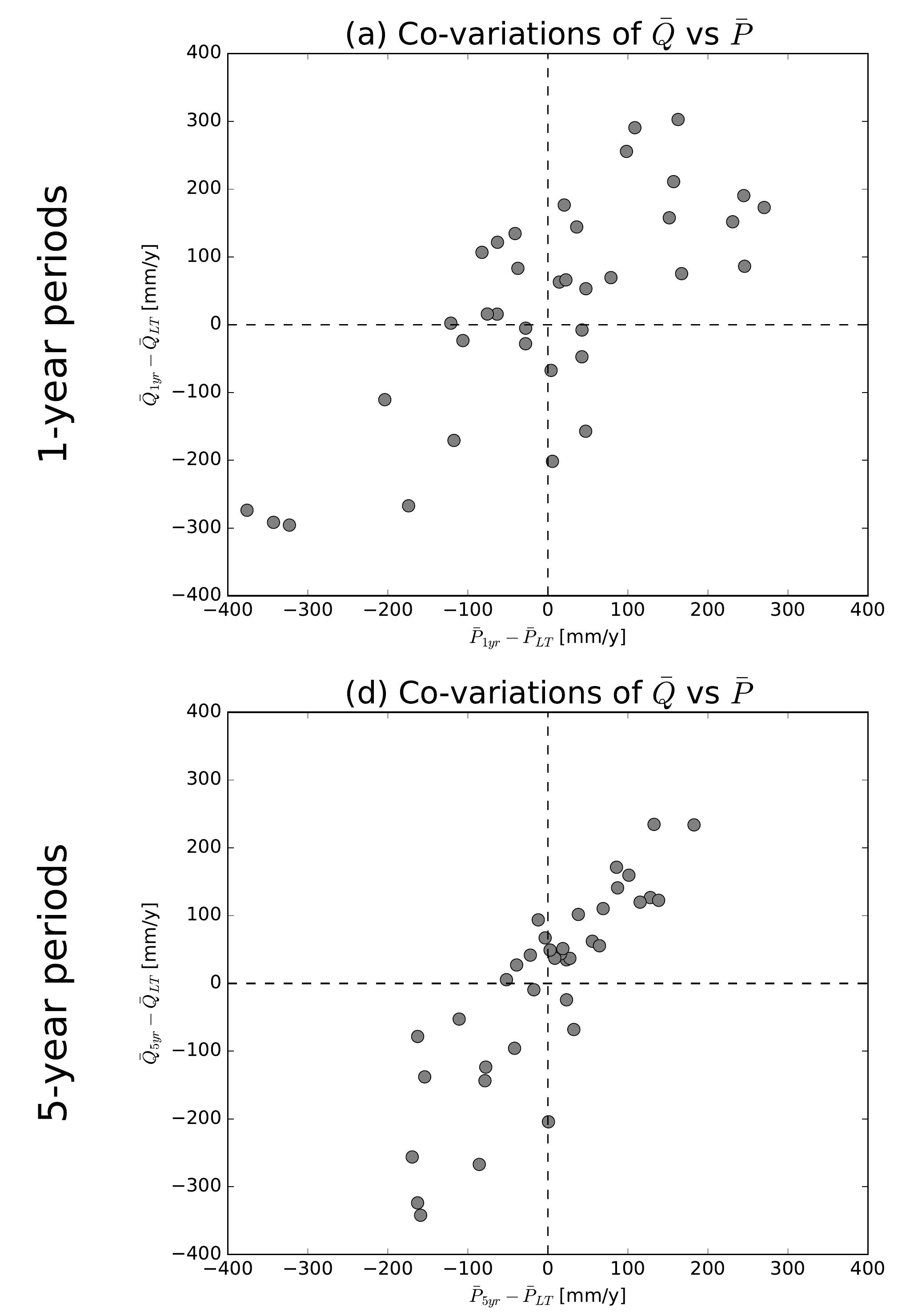

(g) Co-variations of $\bar{Q}$ vs $\bar{P}$
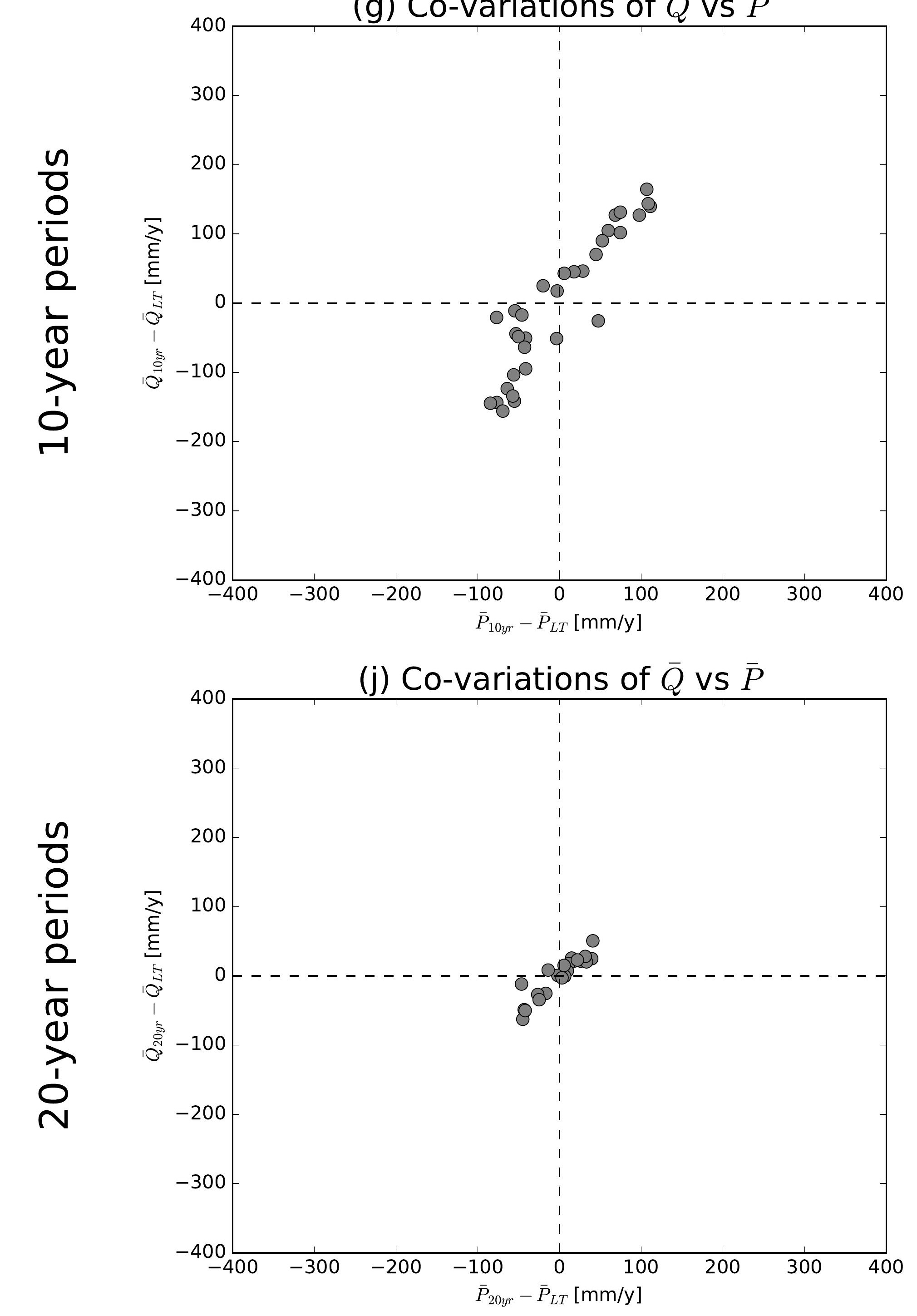

(b) Co-variations of $\bar{Q}$ vs $\overline{E 0}$

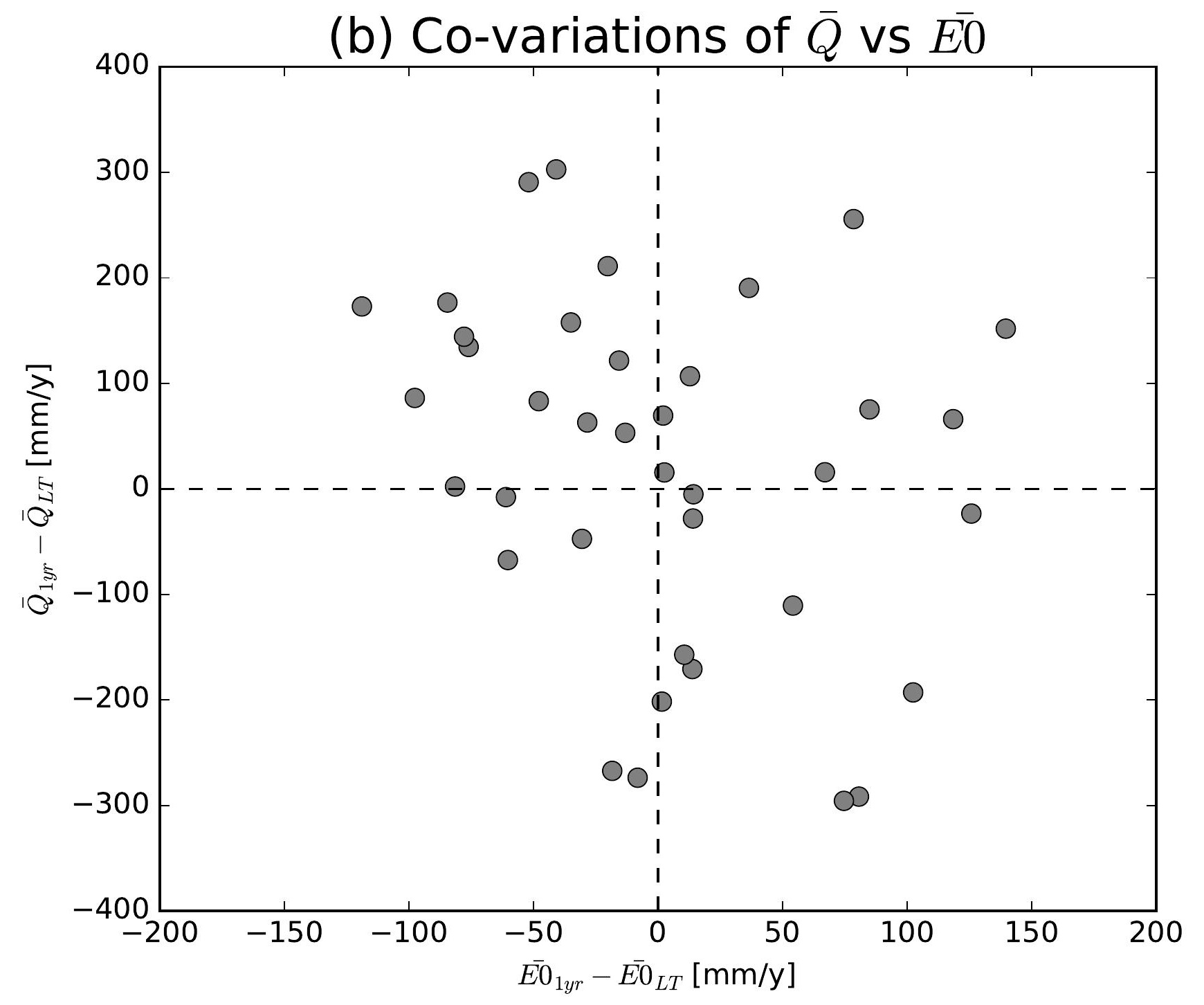

(e) Co-variations of $\bar{Q}$ vs $\overline{E 0}$

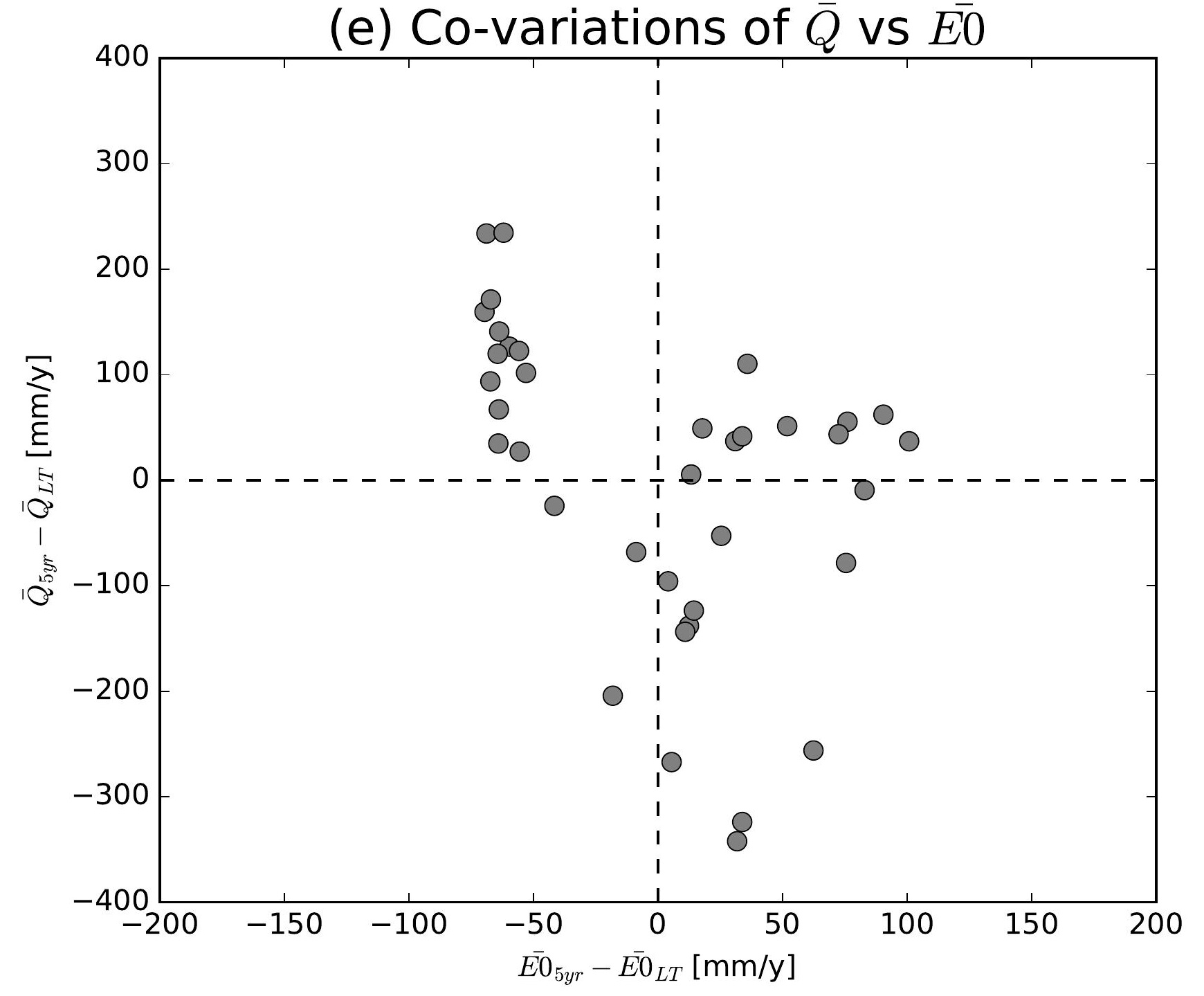

(h) Co-variations of $\bar{Q}$ vs $\overline{E 0}$

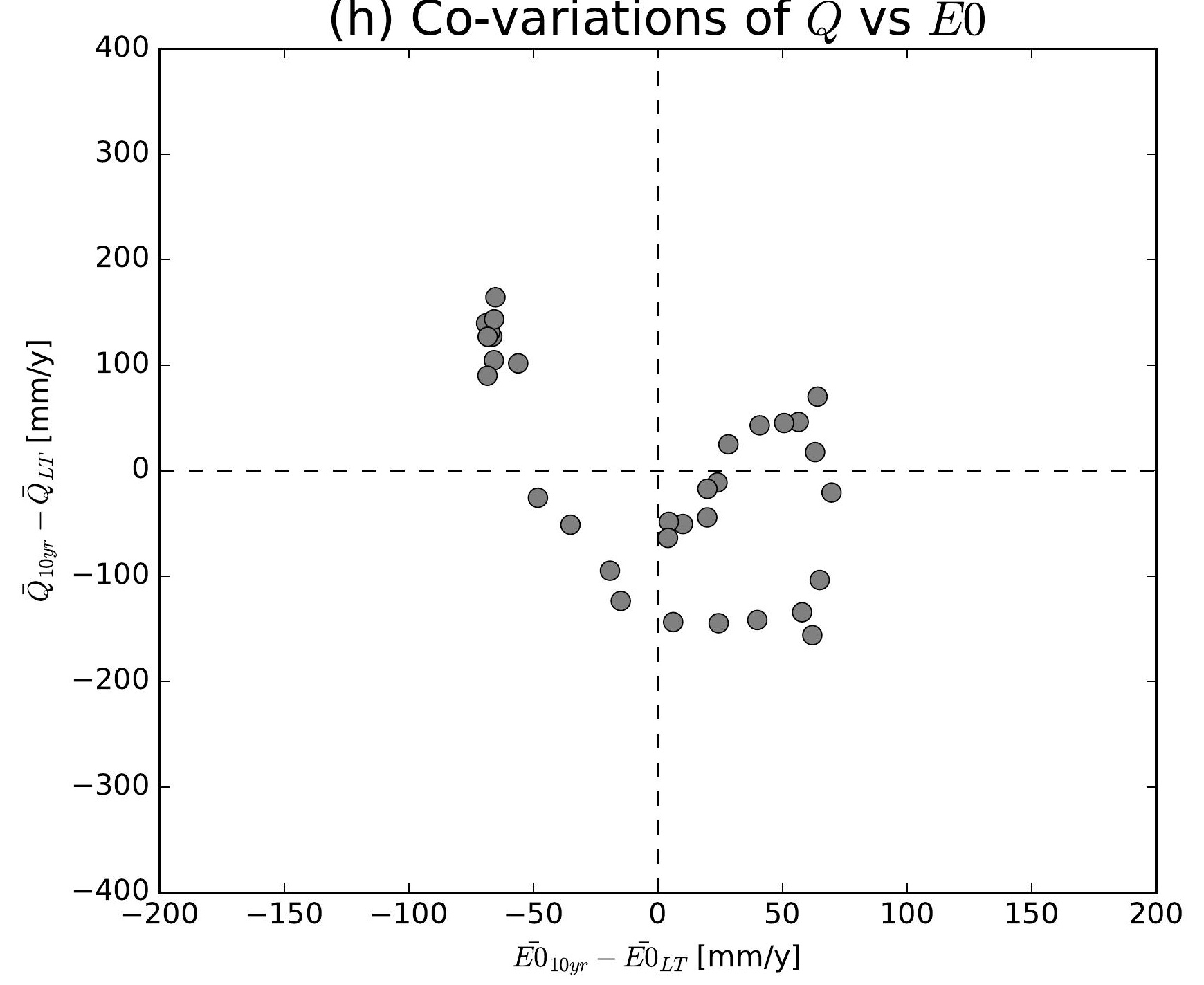

(k) Co-variations of $\bar{Q}$ vs $\overline{E 0}$

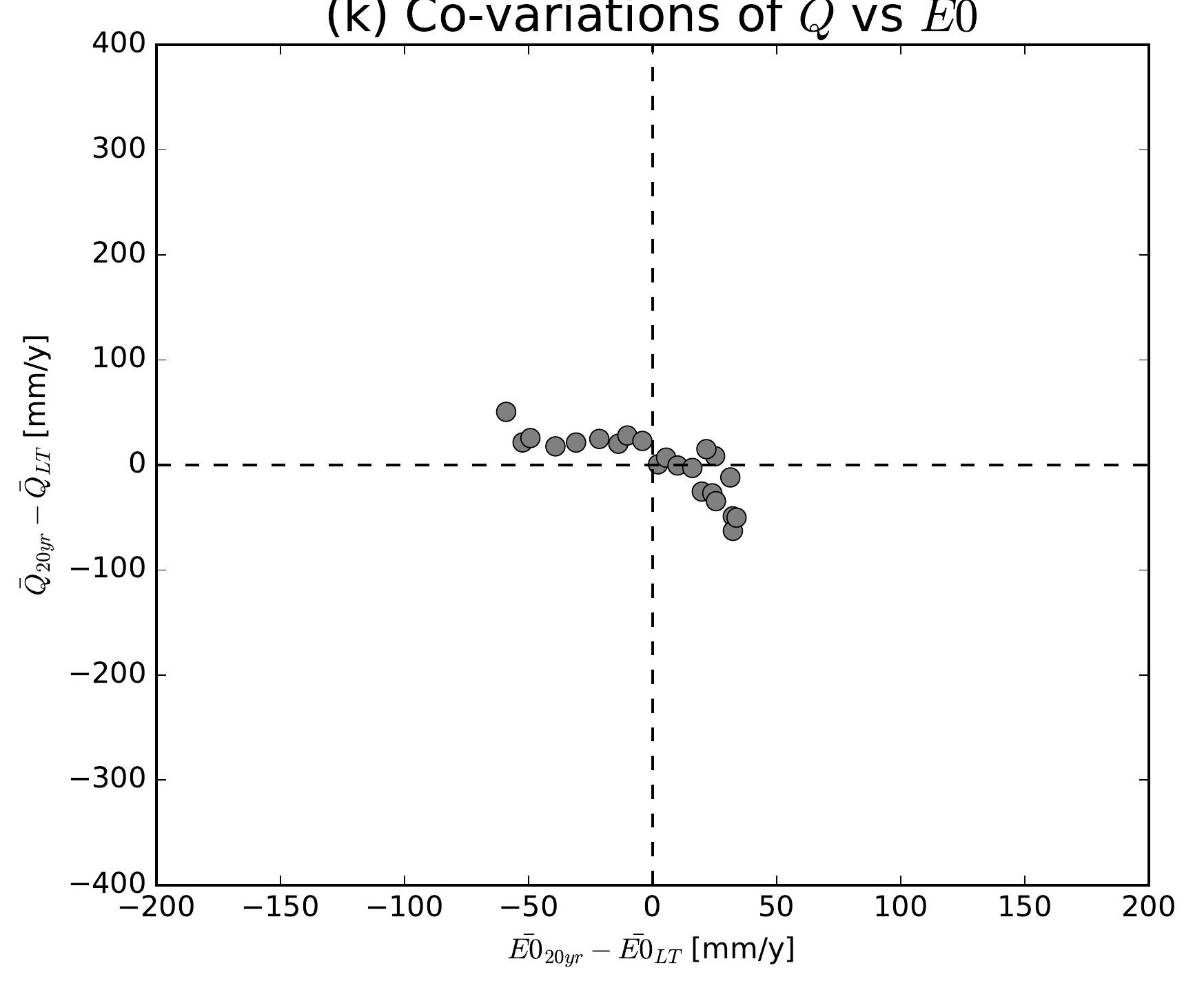

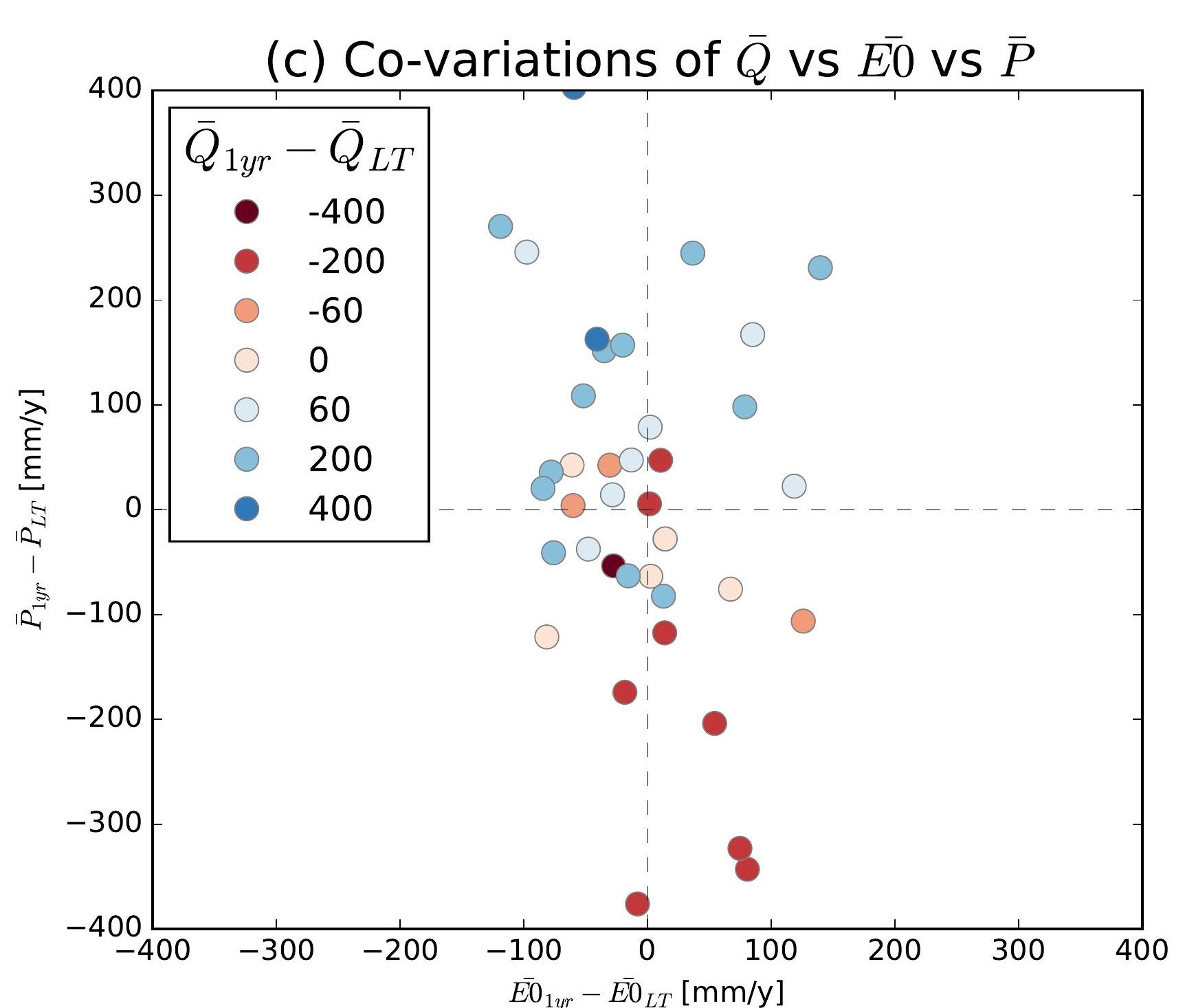
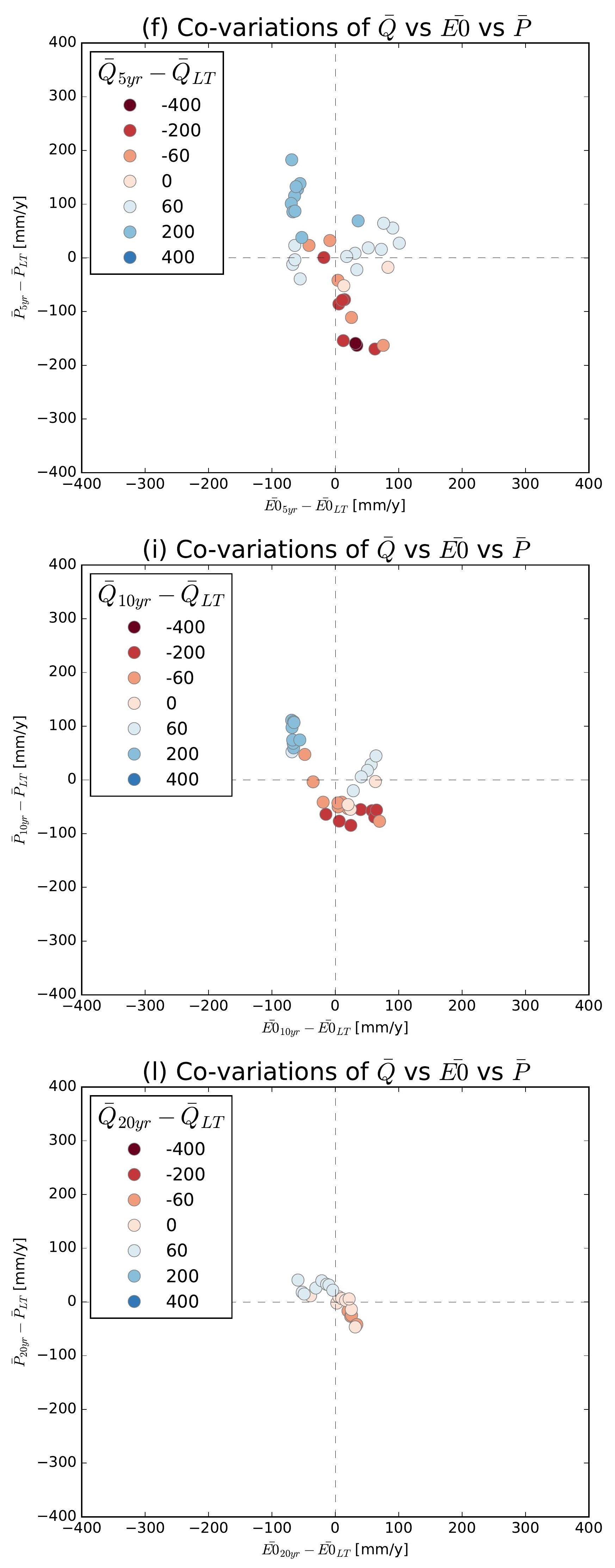

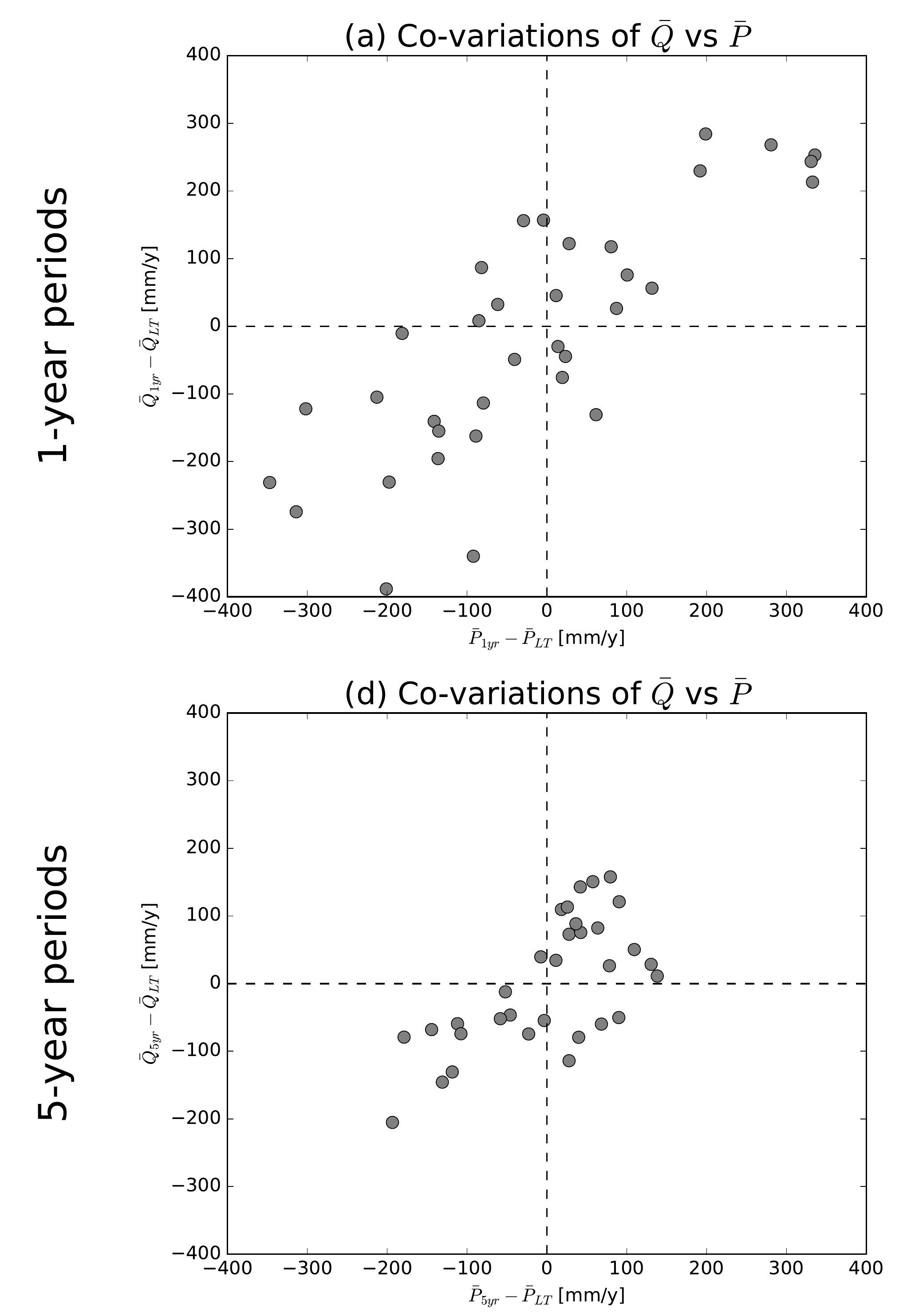

(g) Co-variations of $\bar{Q}$ vs $\bar{P}$

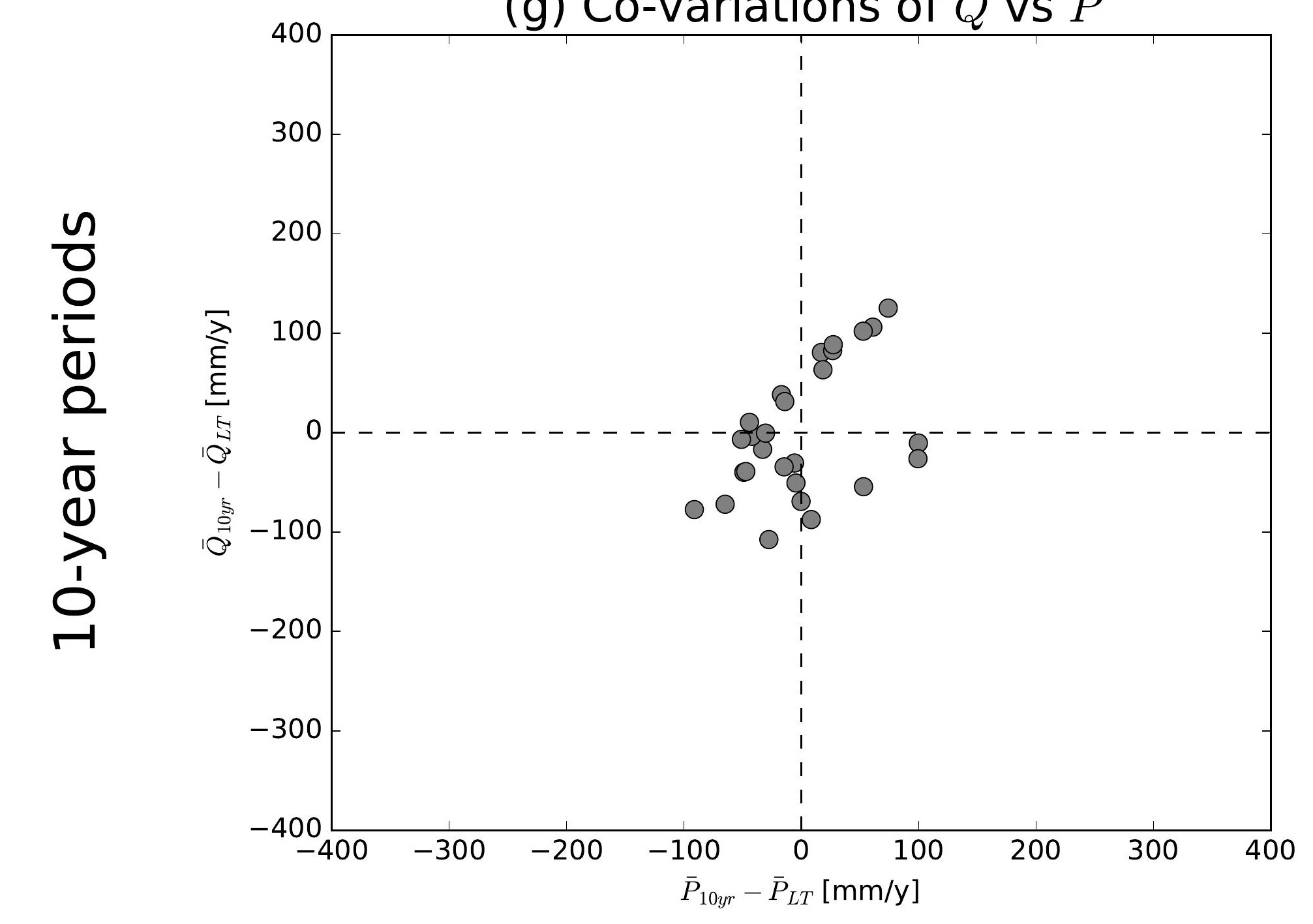

(j) Co-variations of $\bar{Q}$ vs $\bar{P}$

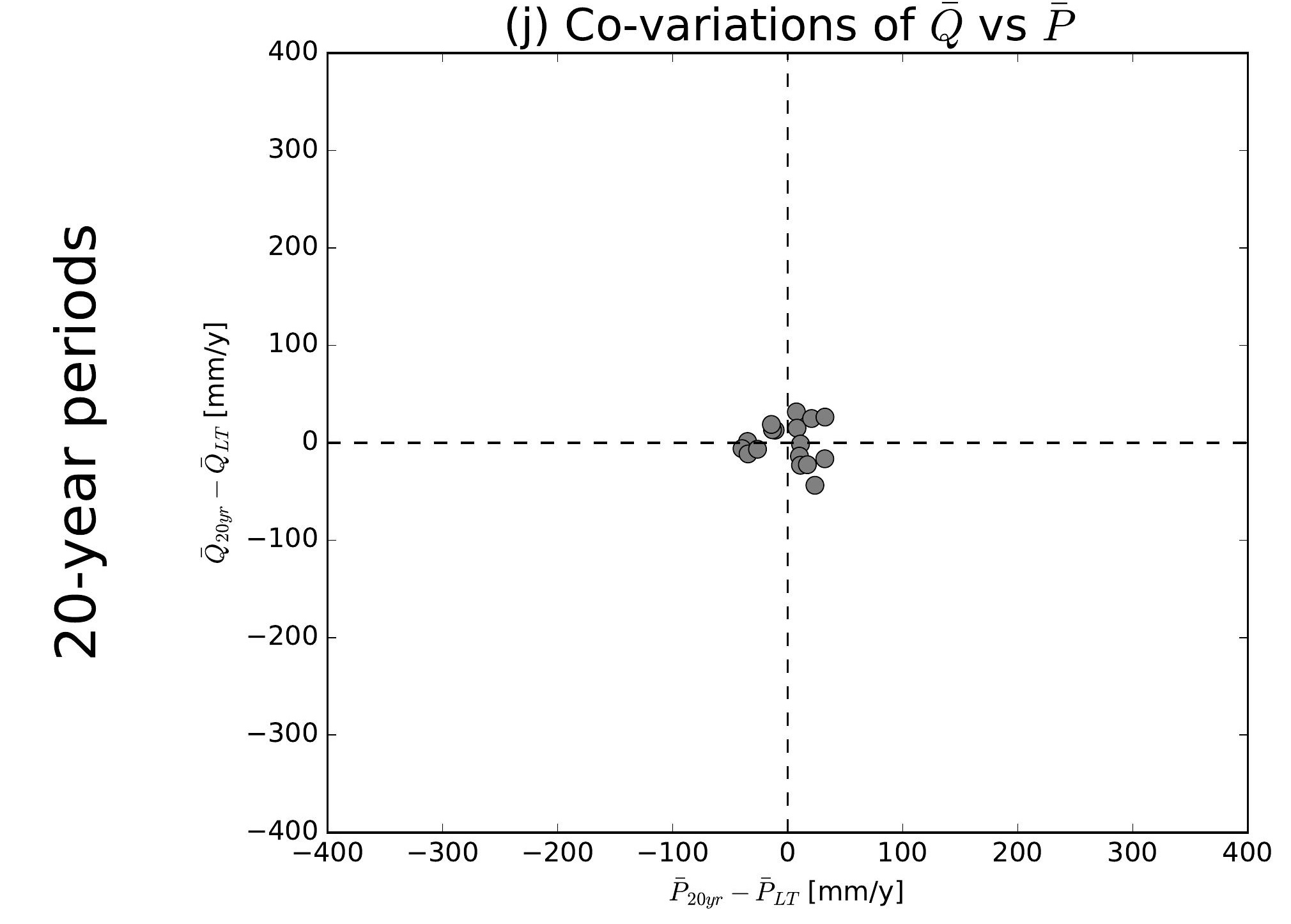

(b) Co-variations of $\bar{Q}$ vs $\overline{E 0}$

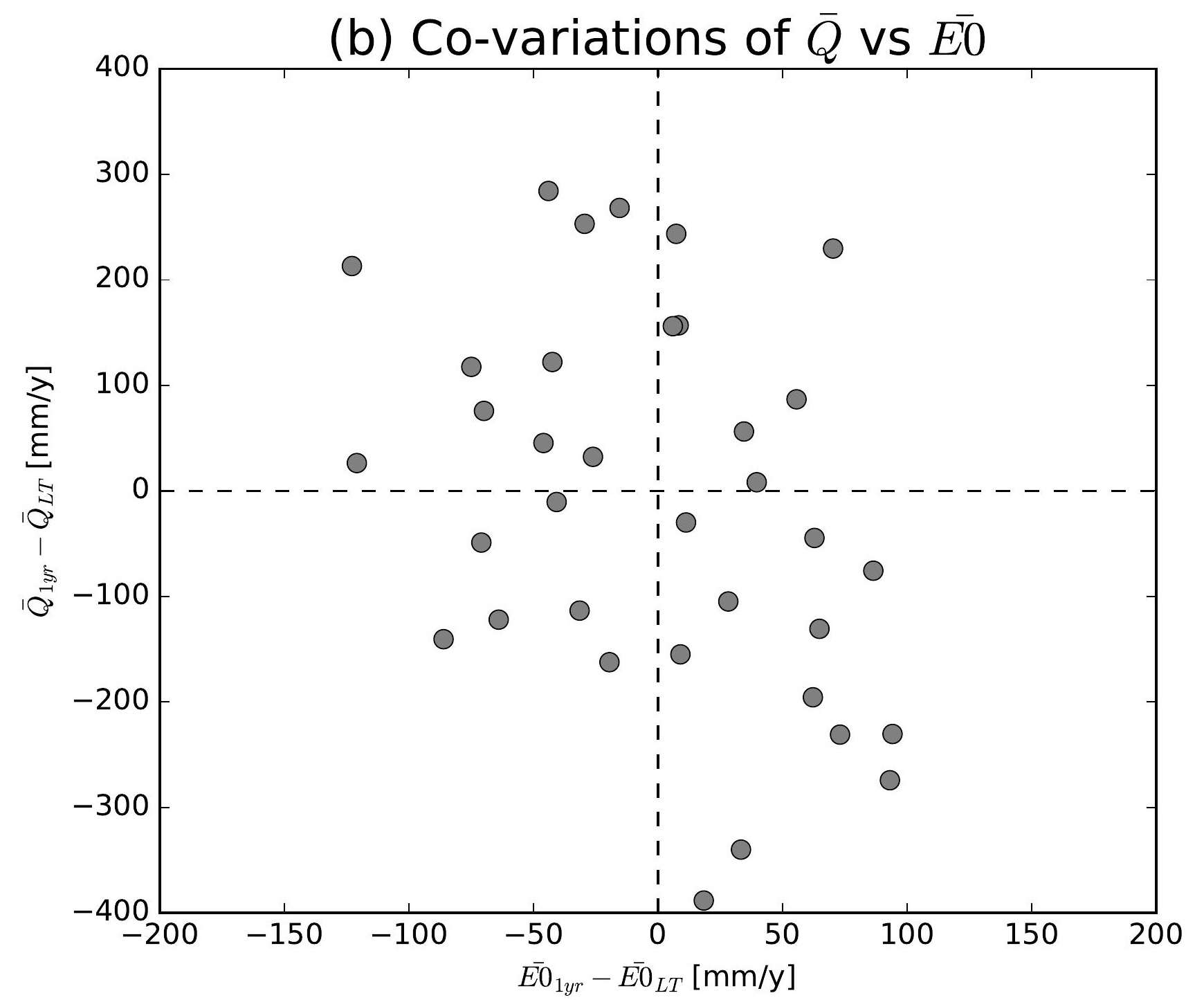

(e) Co-variations of $\bar{Q}$ vs $\overline{E 0}$

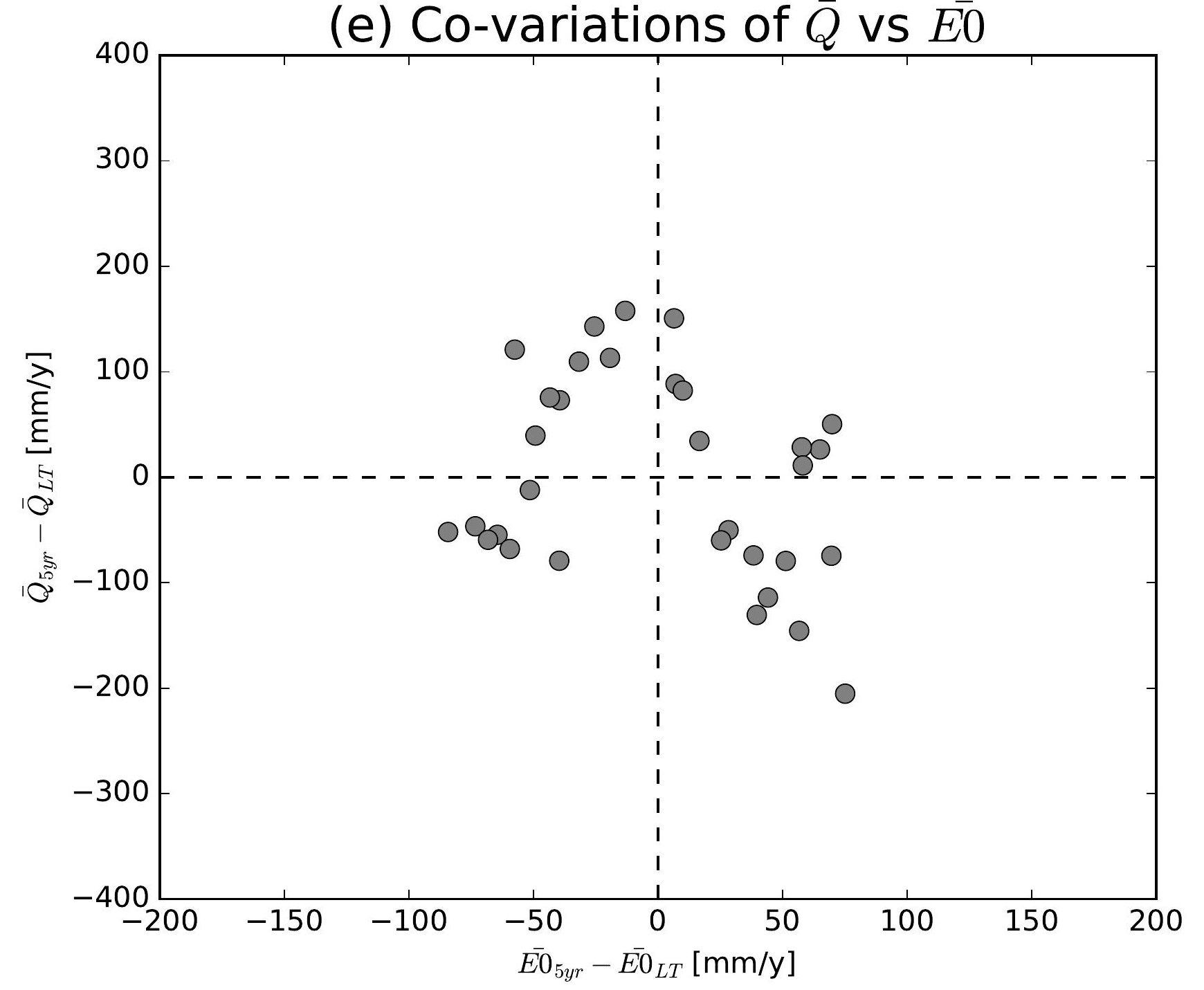

(h) Co-variations of $\bar{Q}$ vs $\overline{E 0}$

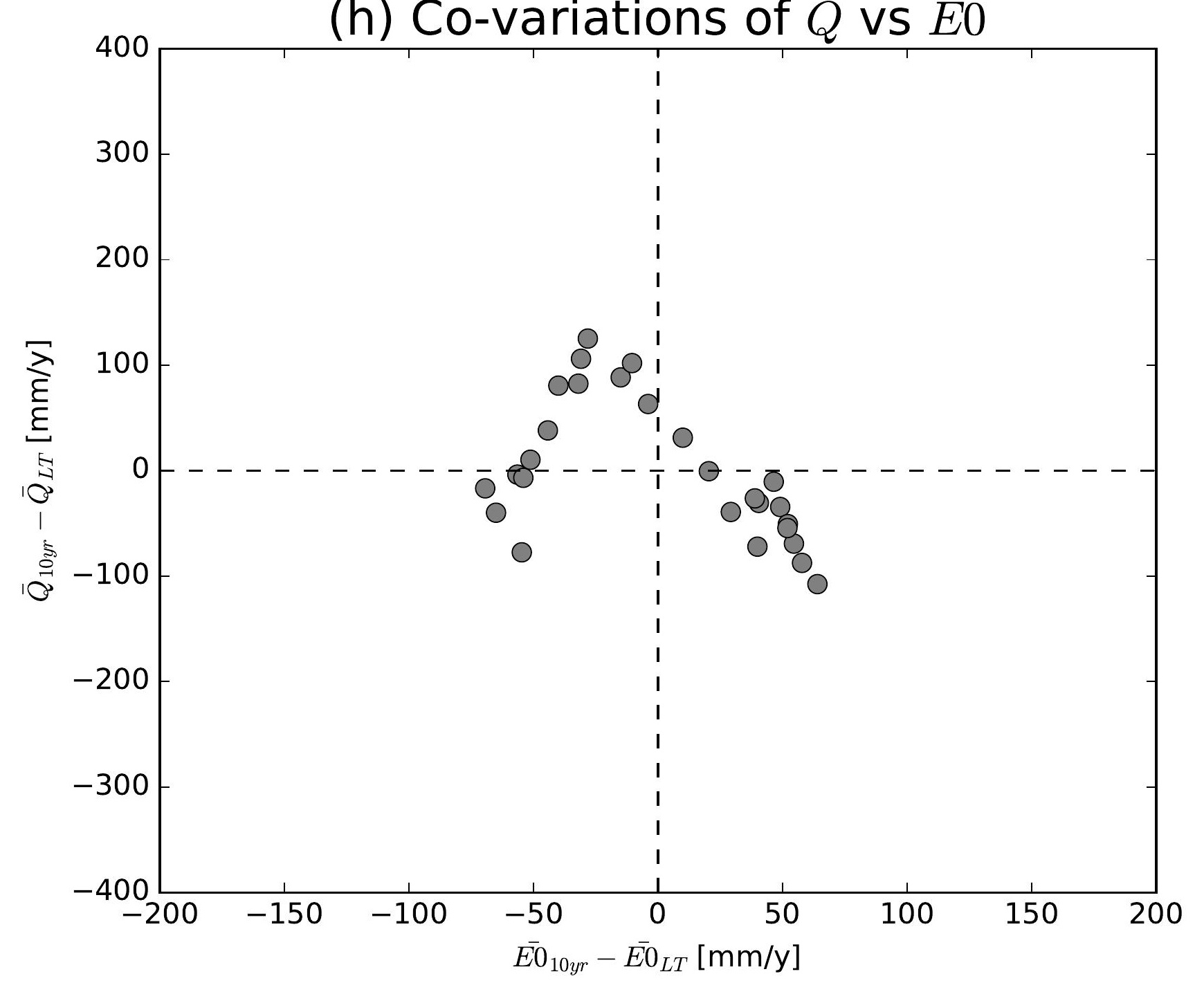

(k) Co-variations of $\bar{Q}$ vs $\overline{E 0}$

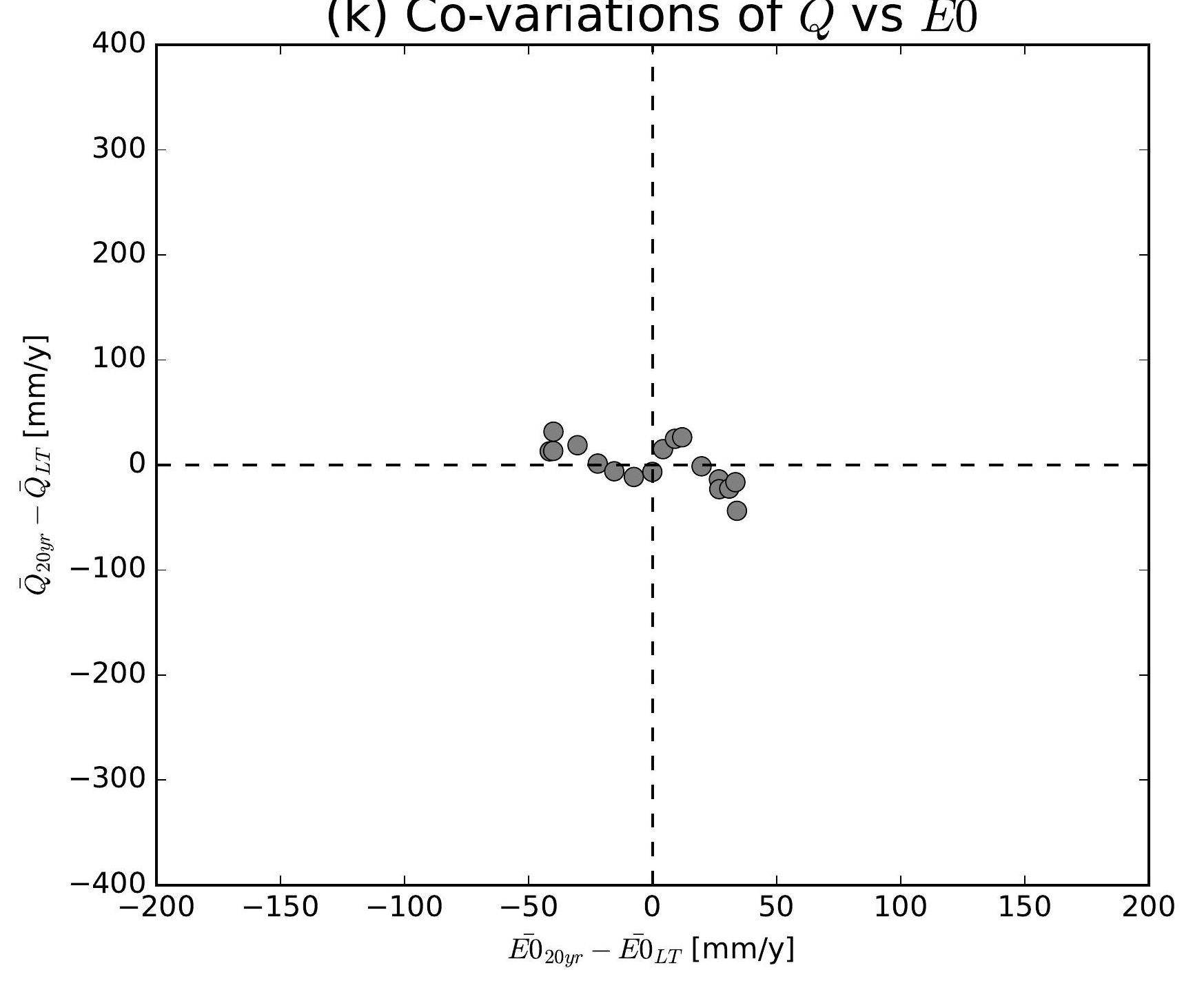

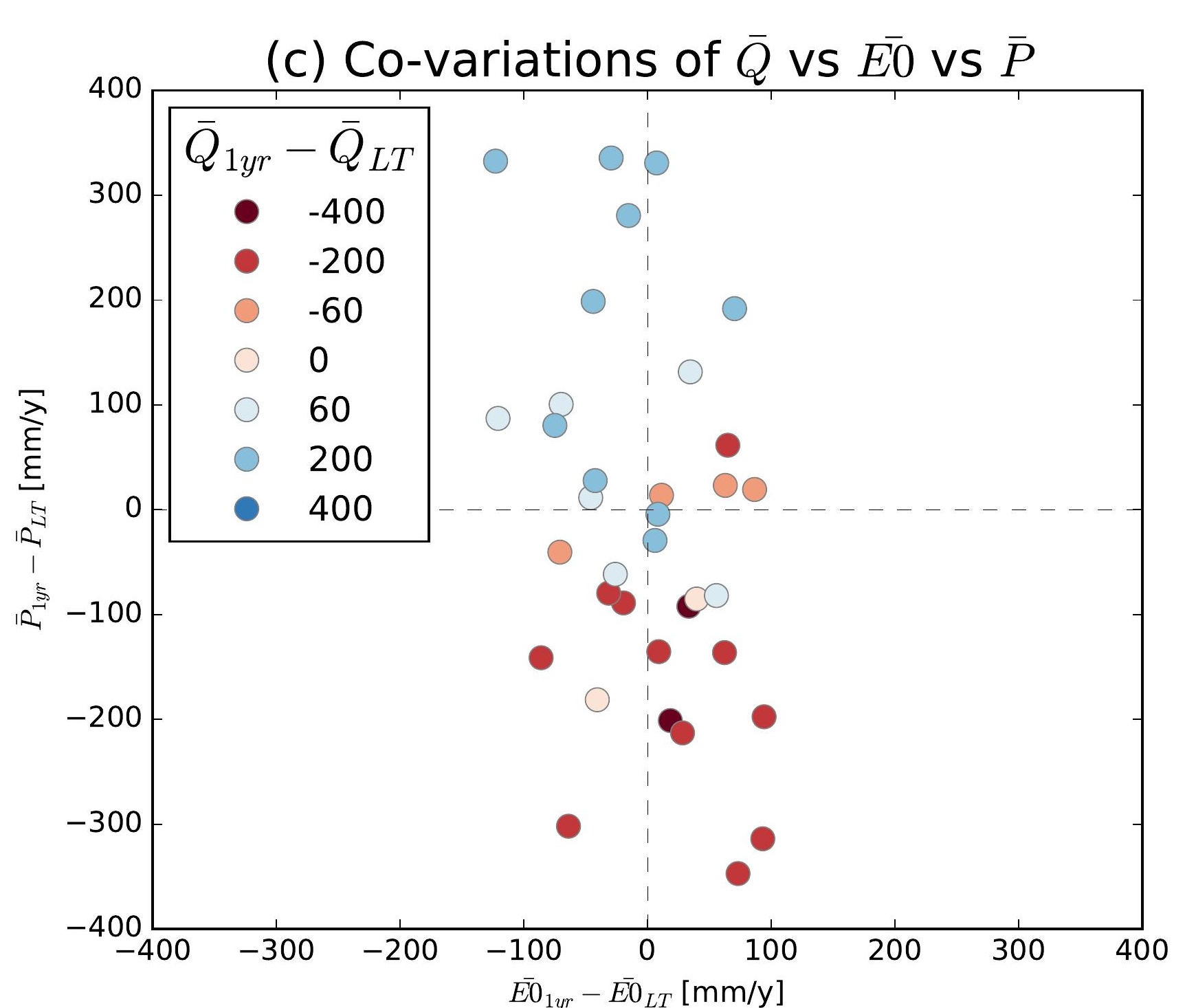
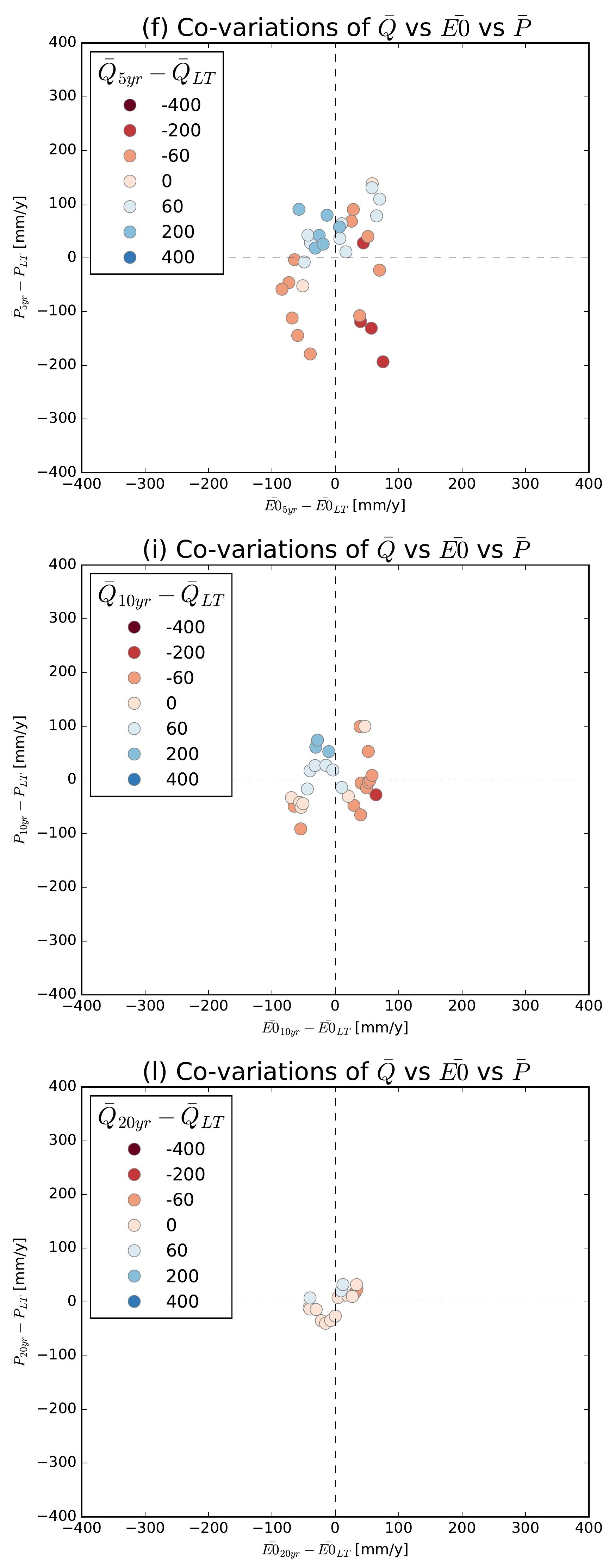

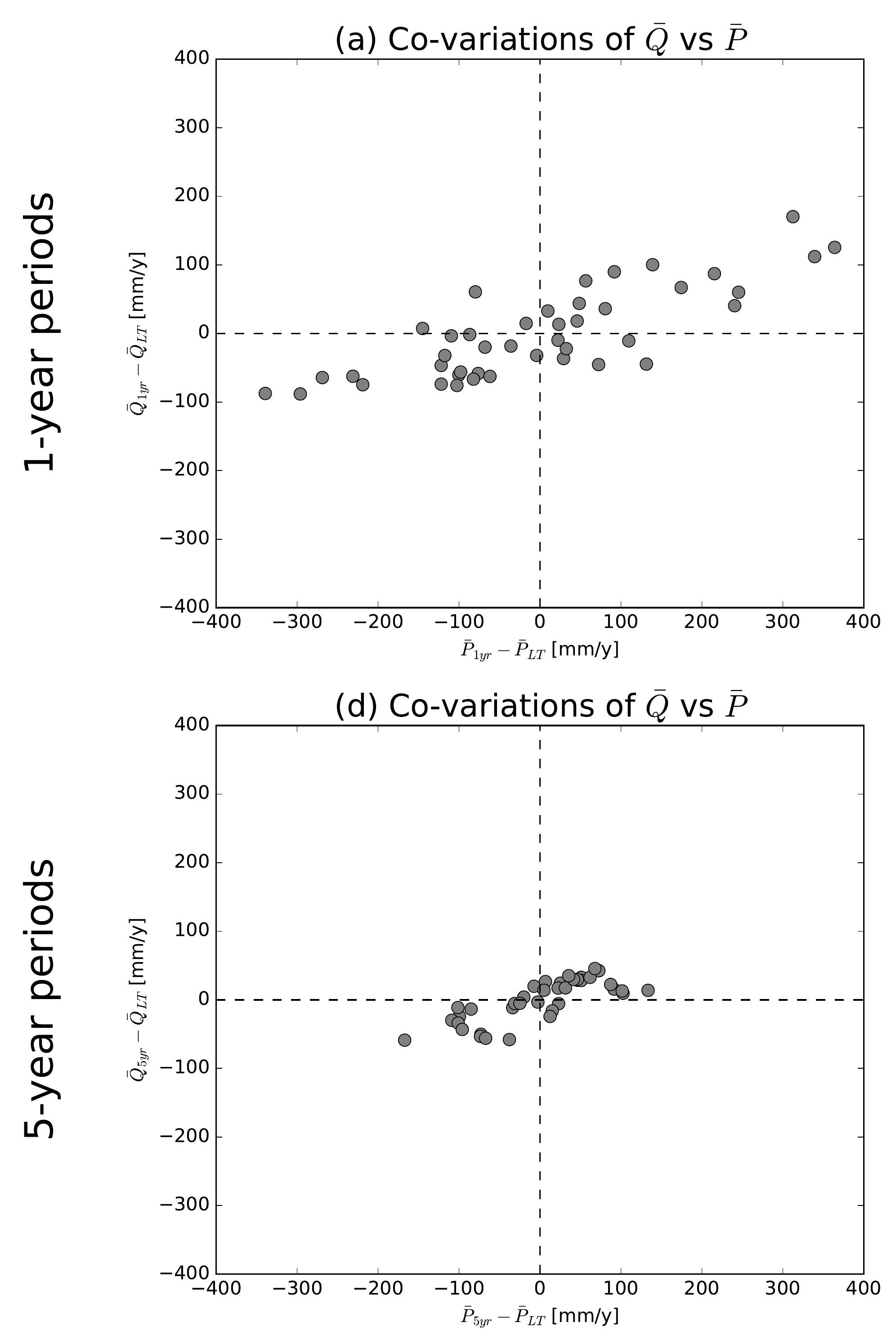

(g) Co-variations of $\bar{Q}$ vs $\bar{P}$
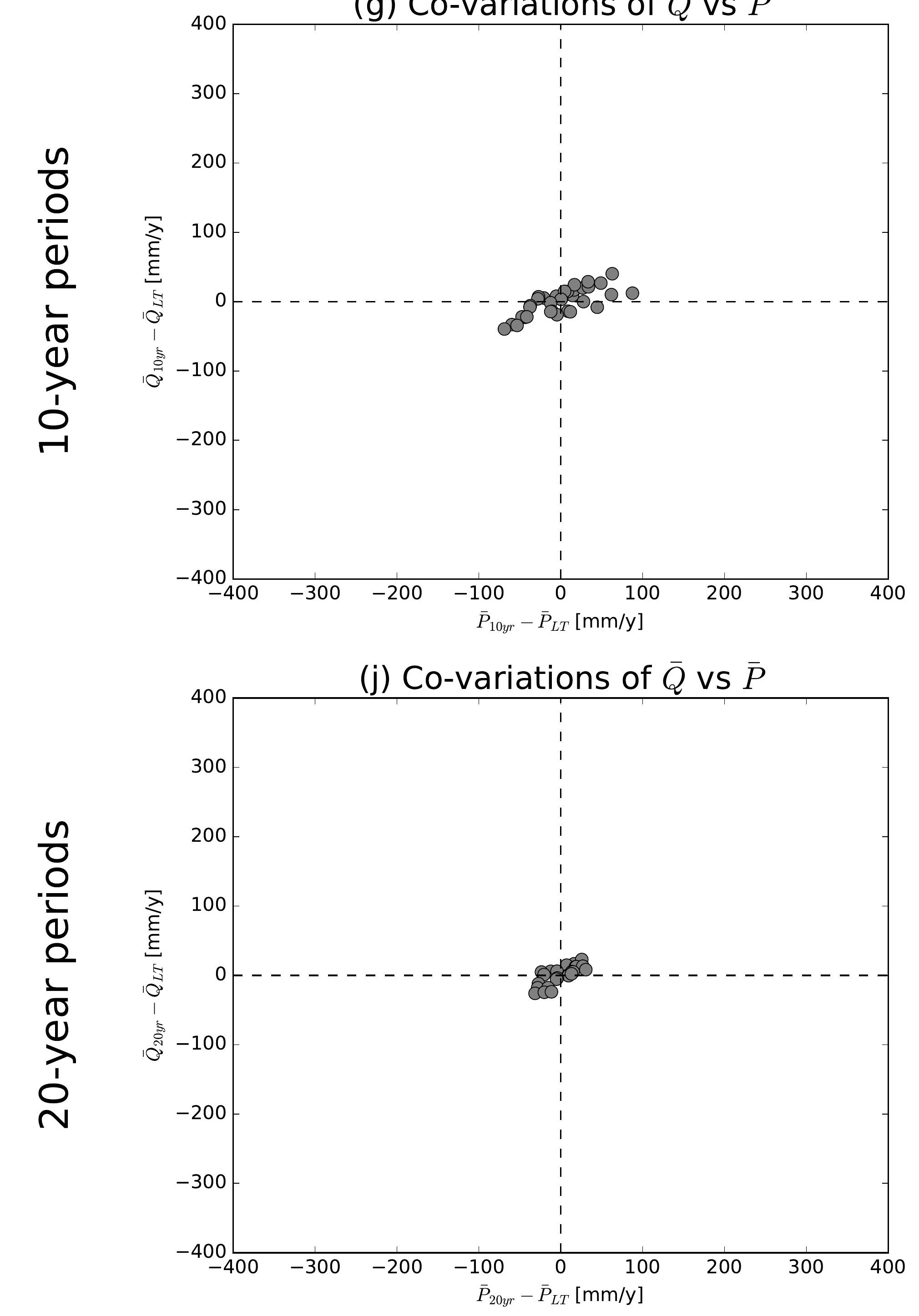

(b) Co-variations of $\bar{Q}$ vs $\overline{E 0}$

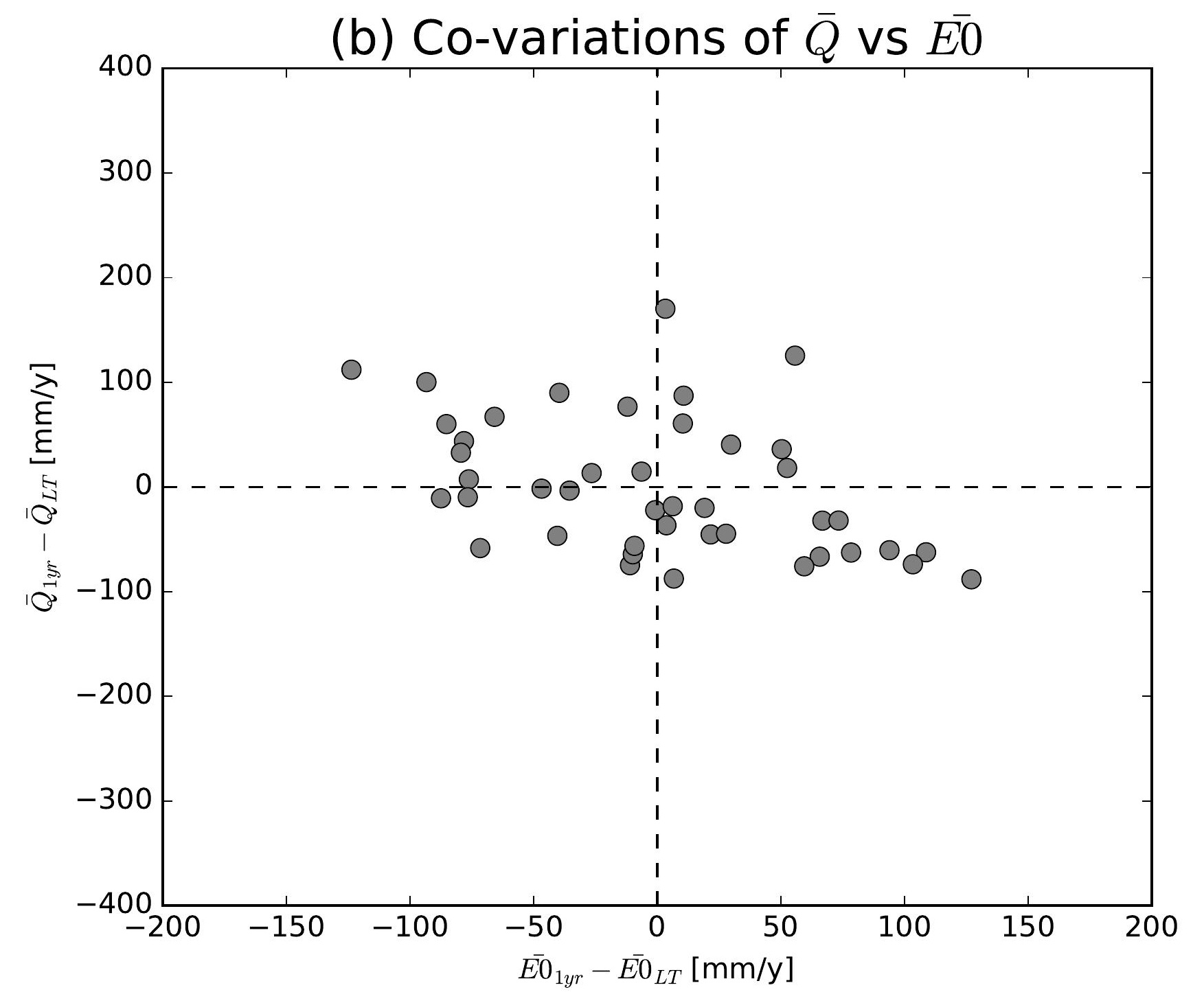

(e) Co-variations of $\bar{Q}$ vs $\overline{E 0}$

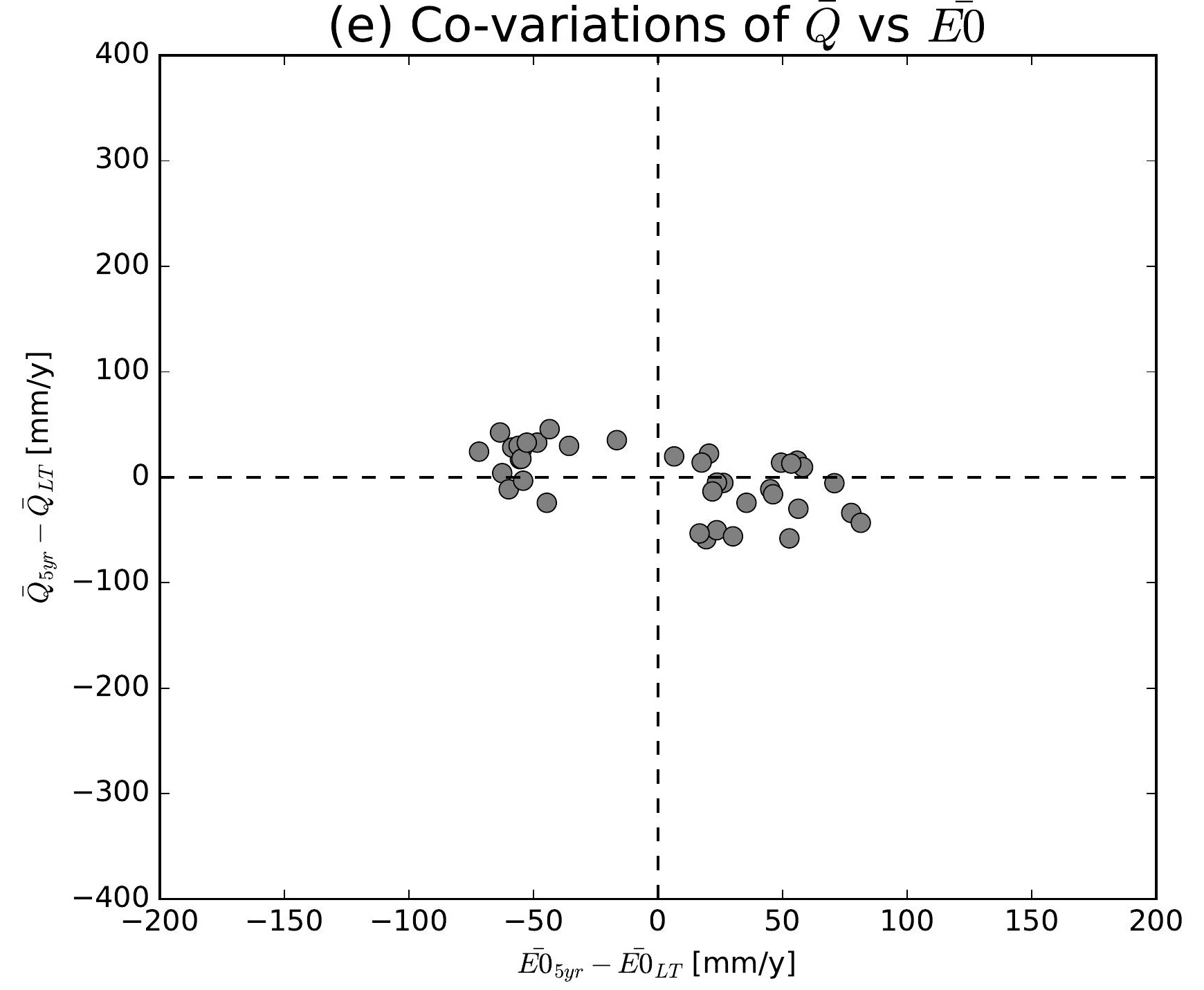

(h) Co-variations of $\bar{Q}$ vs $\overline{E 0}$

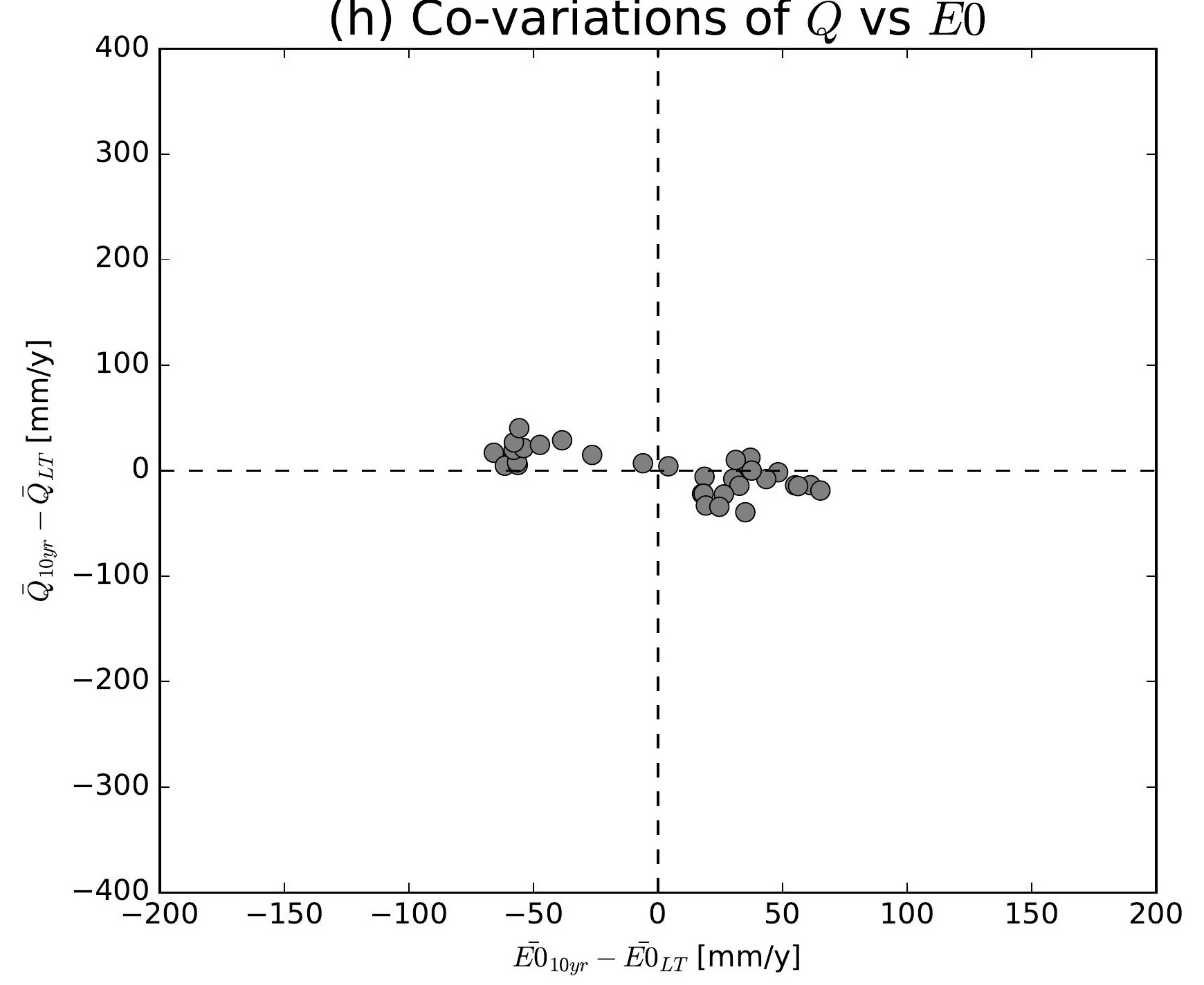

(k) Co-variations of $\bar{Q}$ vs $\overline{E 0}$

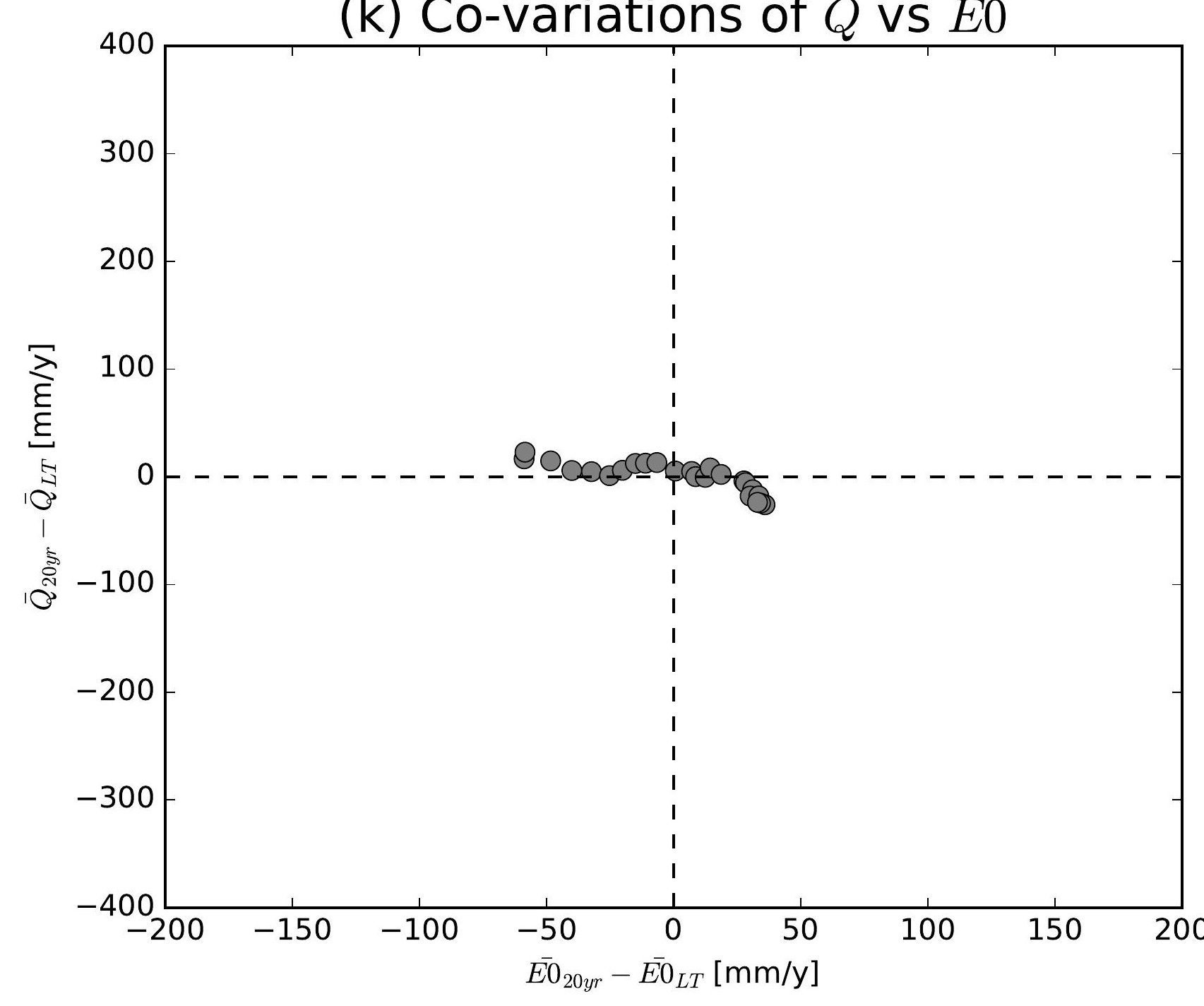

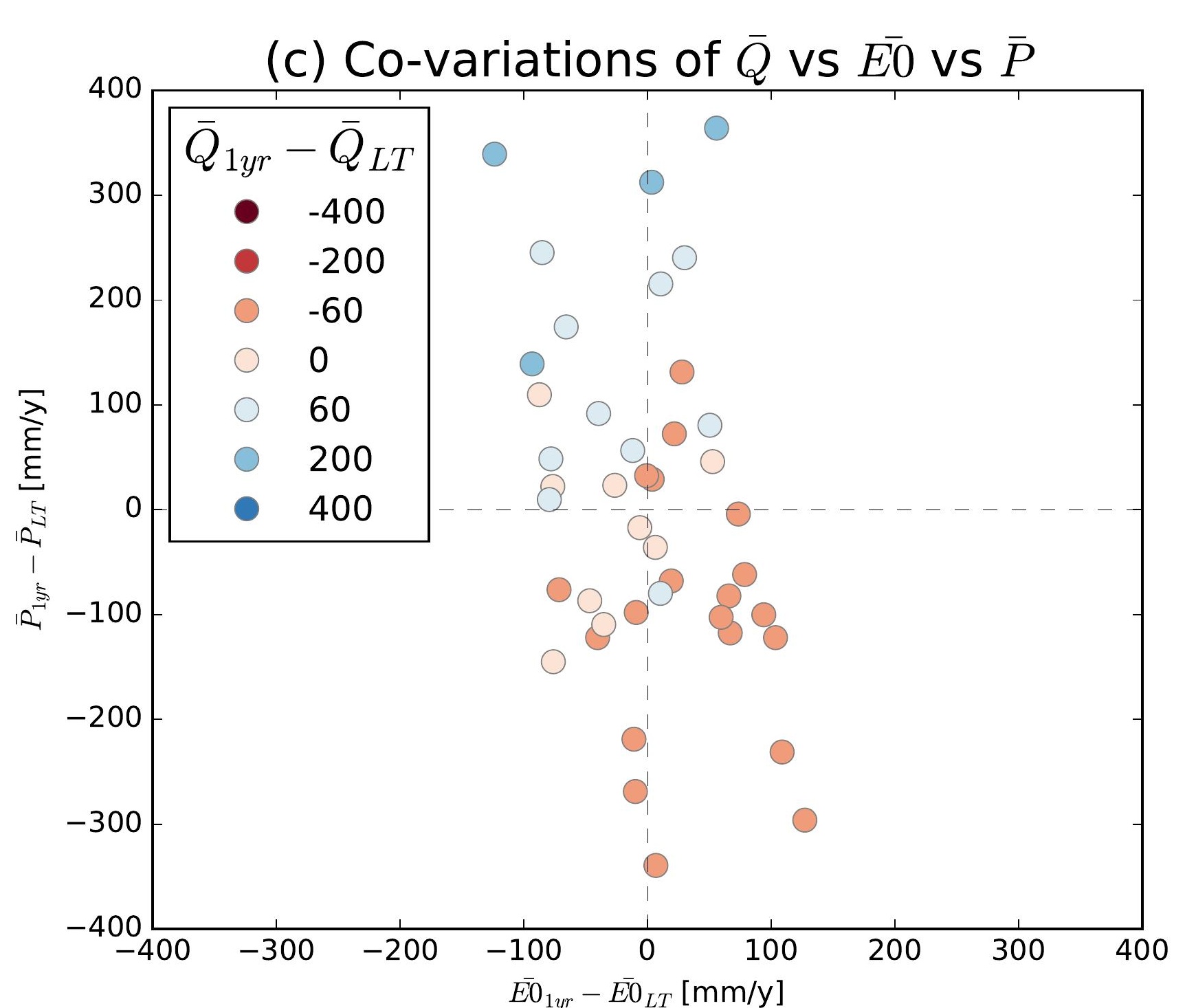
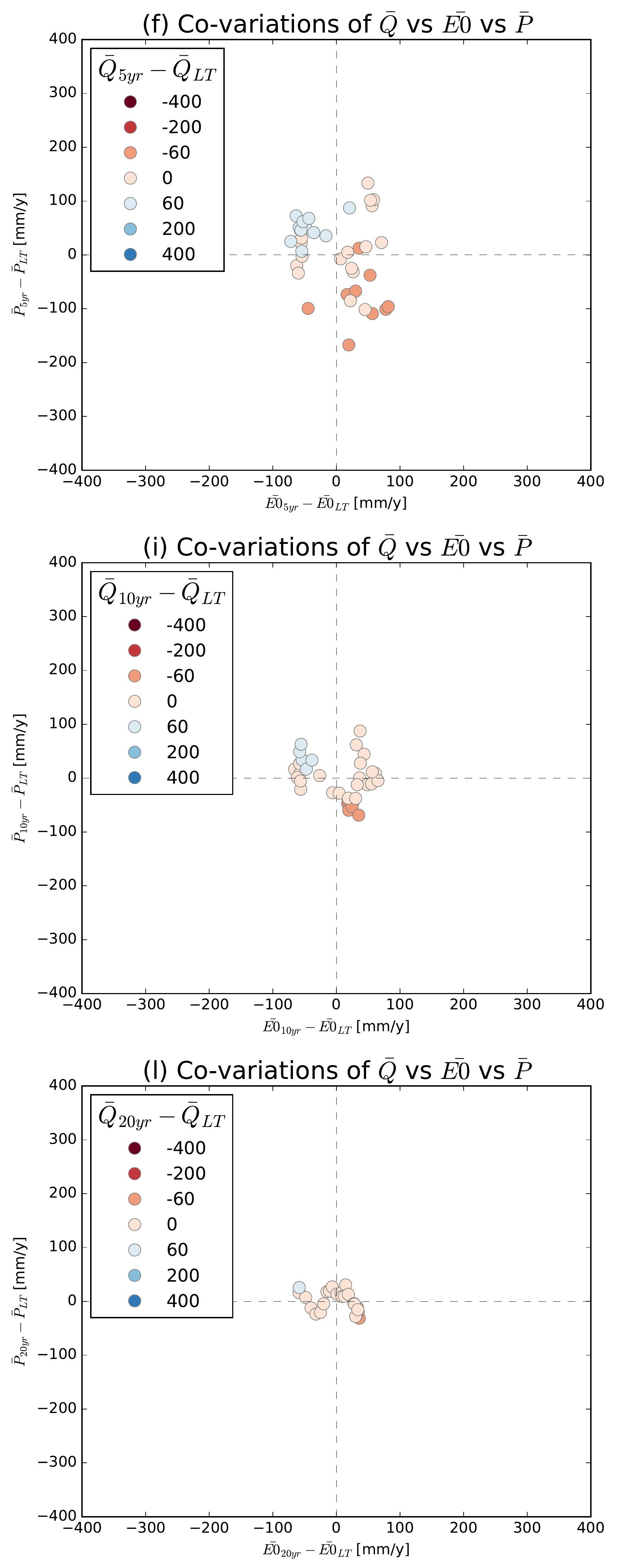

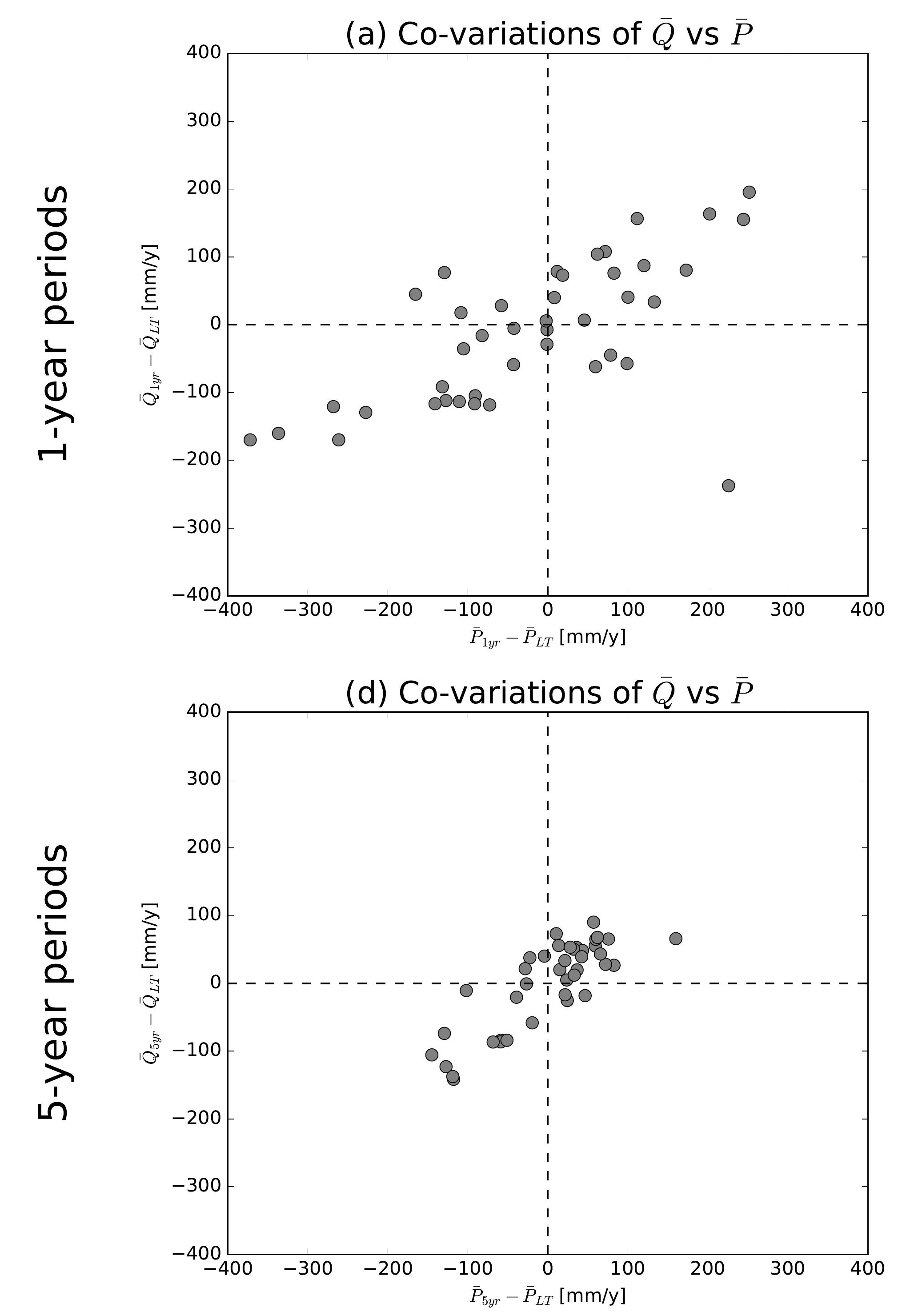

(g) Co-variations of $\bar{Q}$ vs $\bar{P}$
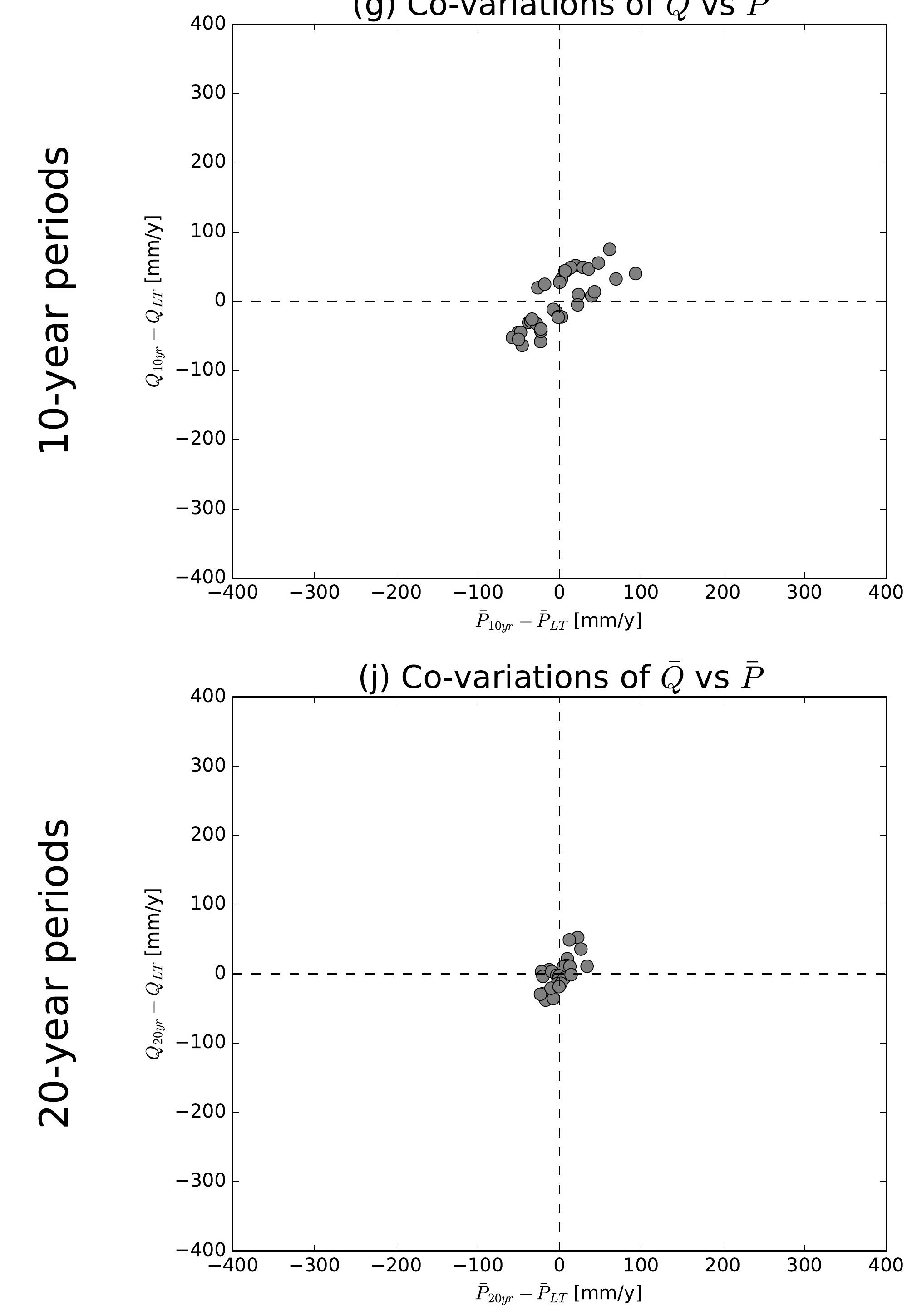

(b) Co-variations of $\bar{Q}$ vs $\overline{E 0}$

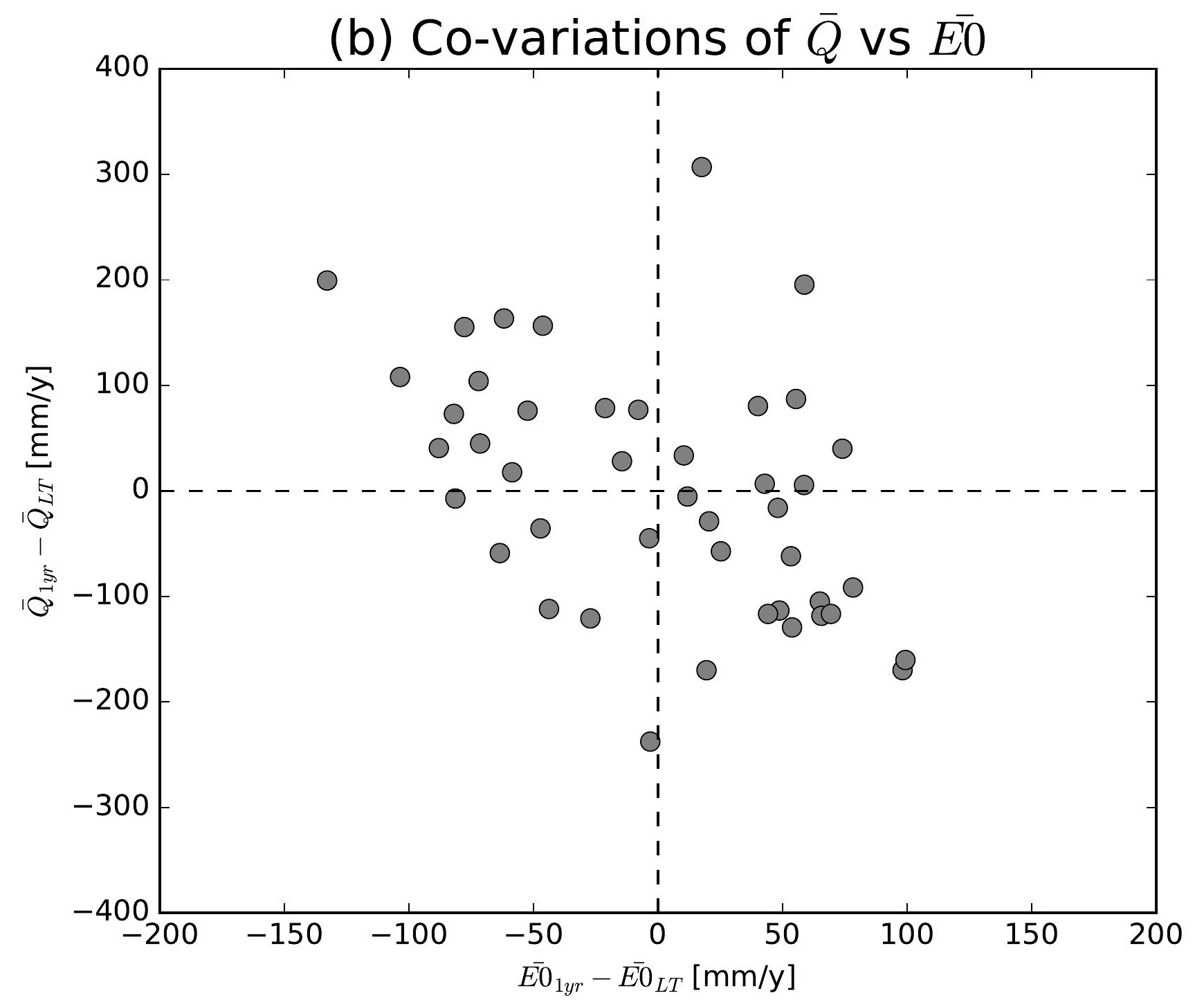

(e) Co-variations of $\bar{Q}$ vs $\overline{E 0}$

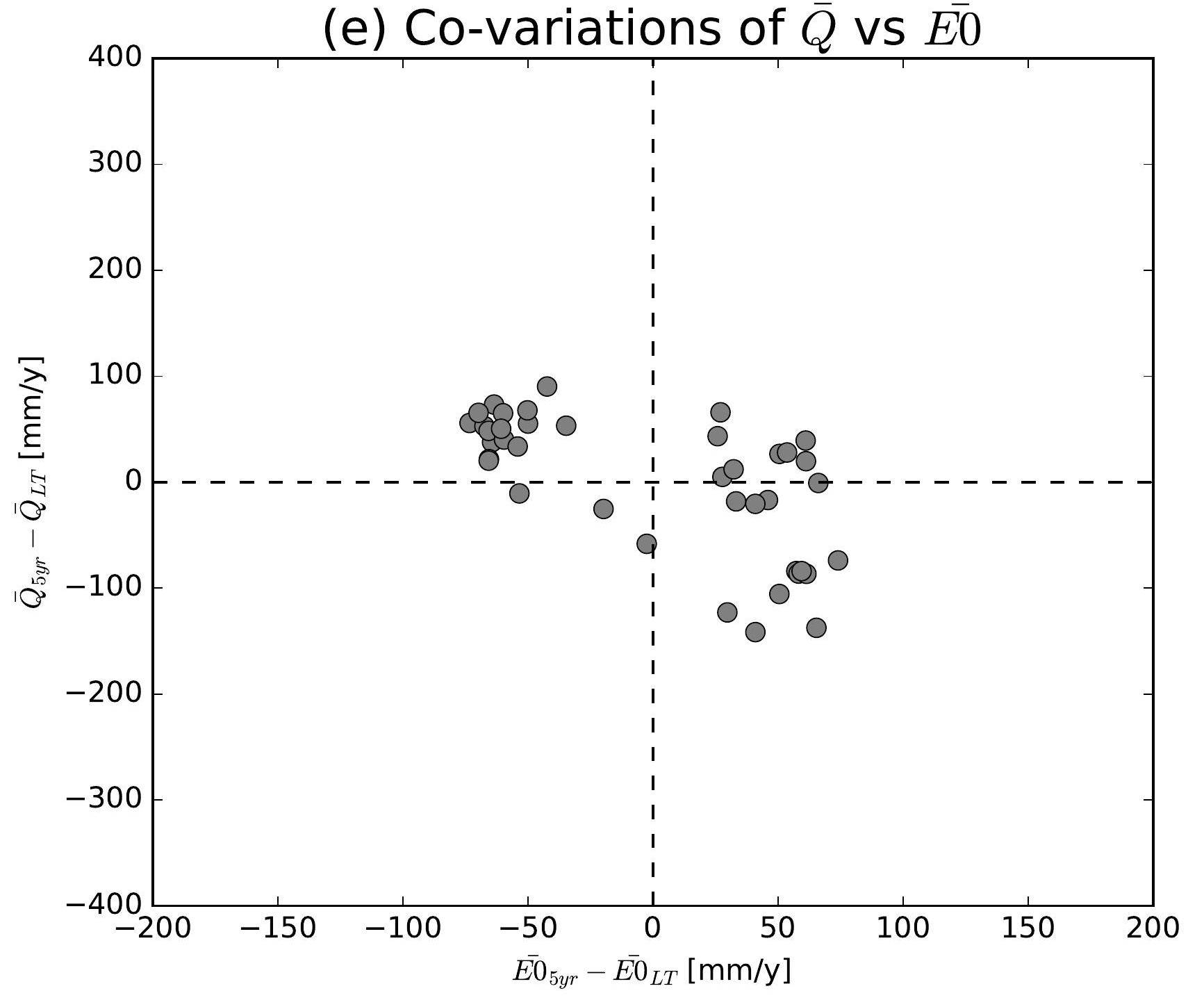

(h) Co-variations of $\bar{Q}$ vs $\overline{E 0}$

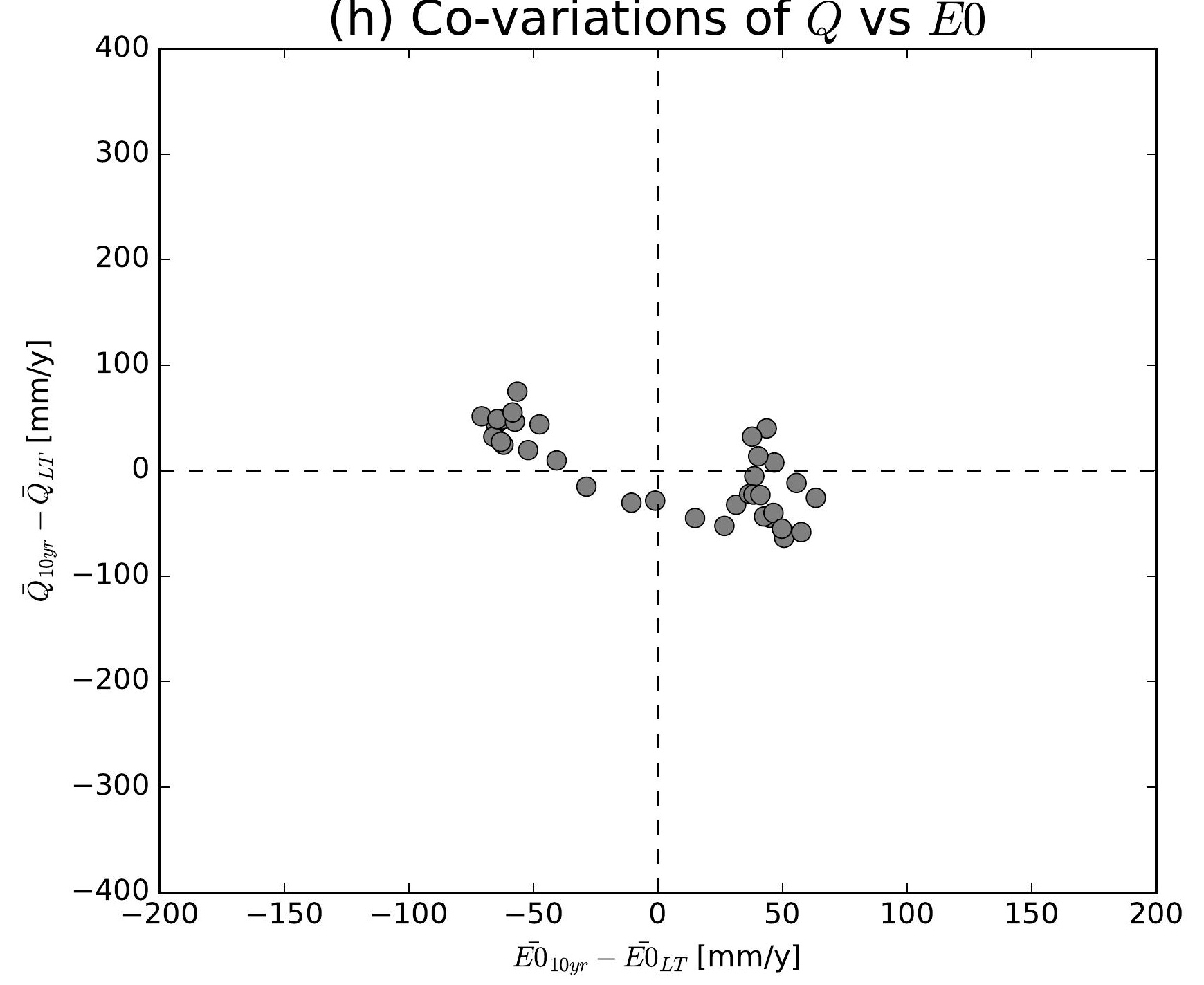

(k) Co-variations of $\bar{Q}$ vs $\overline{E 0}$

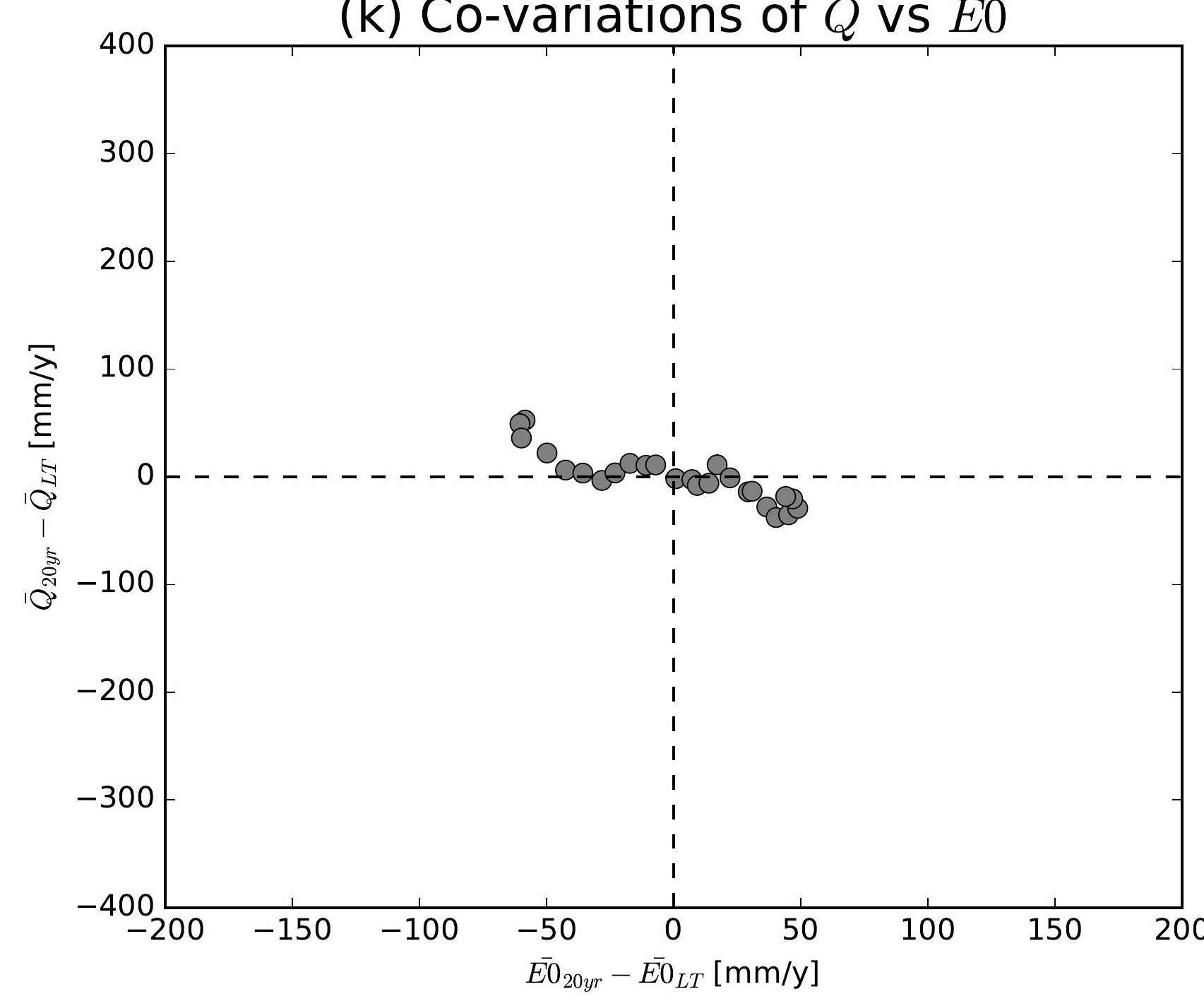

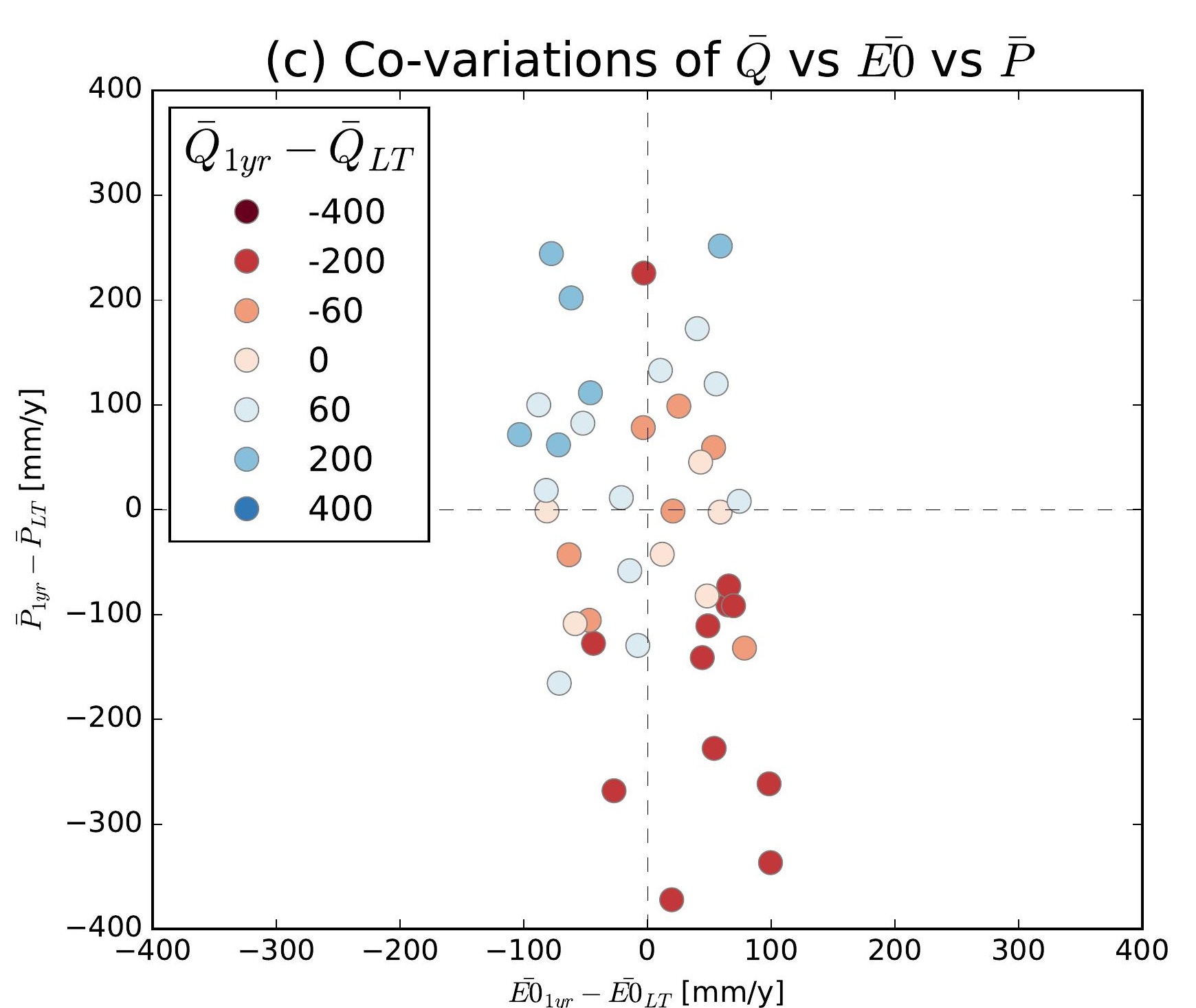
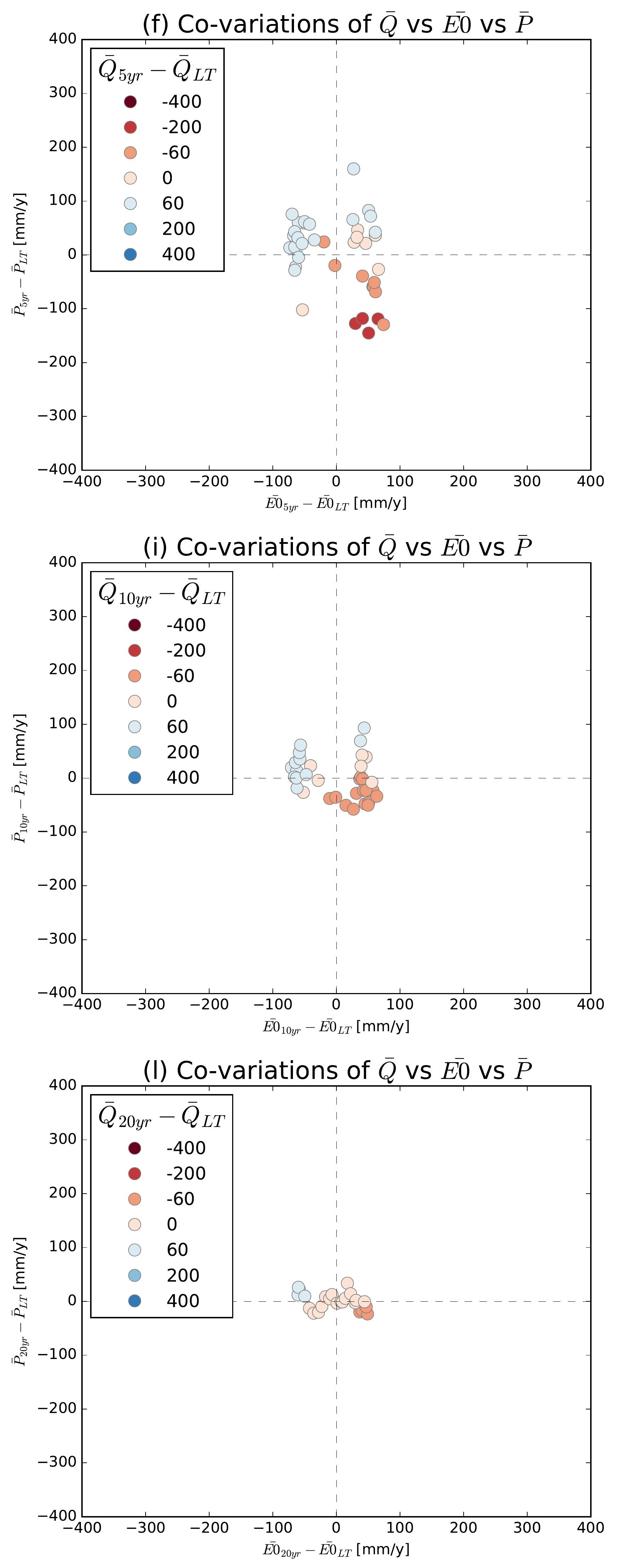

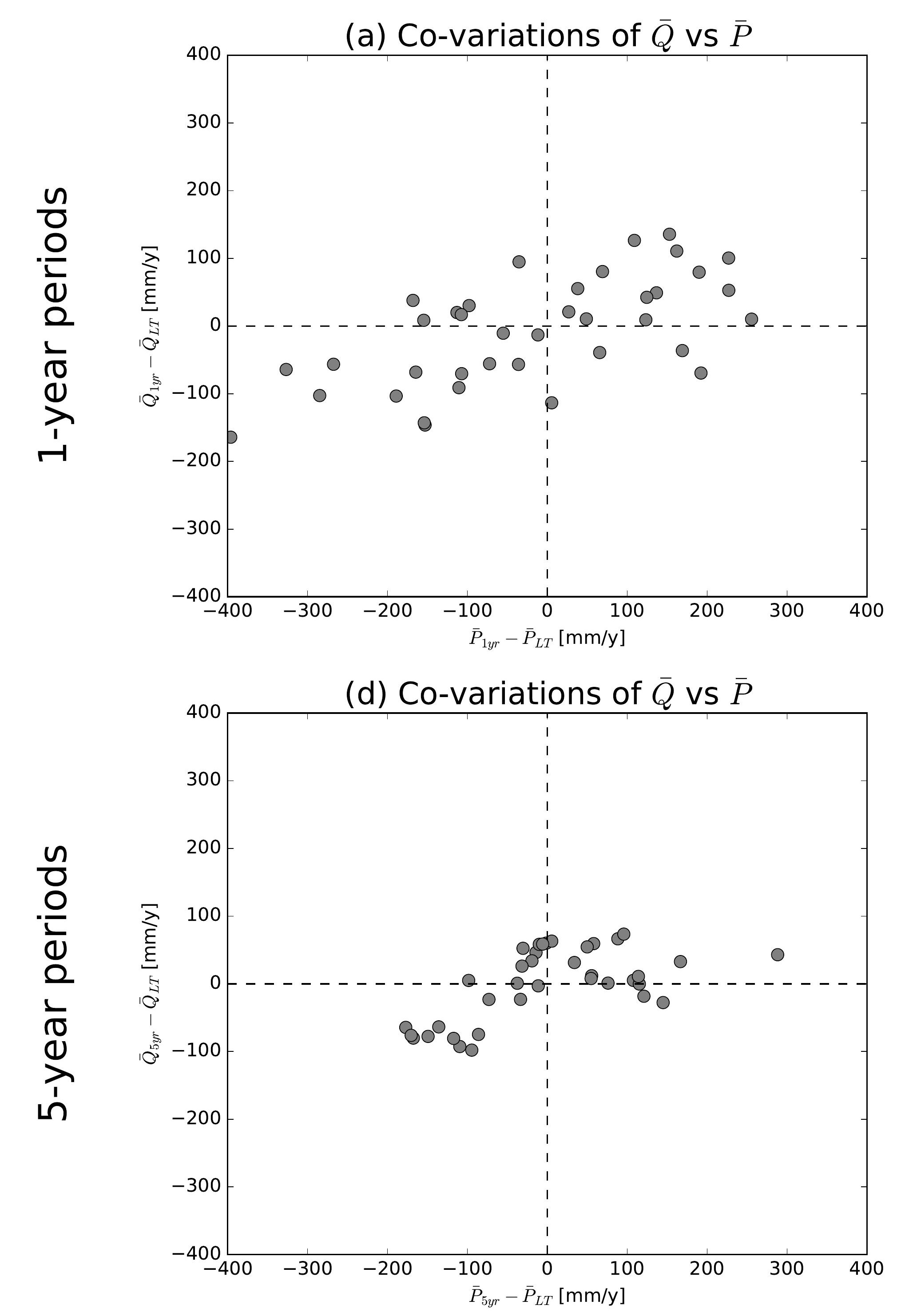

(g) Co-variations of $\bar{Q}$ vs $\bar{P}$

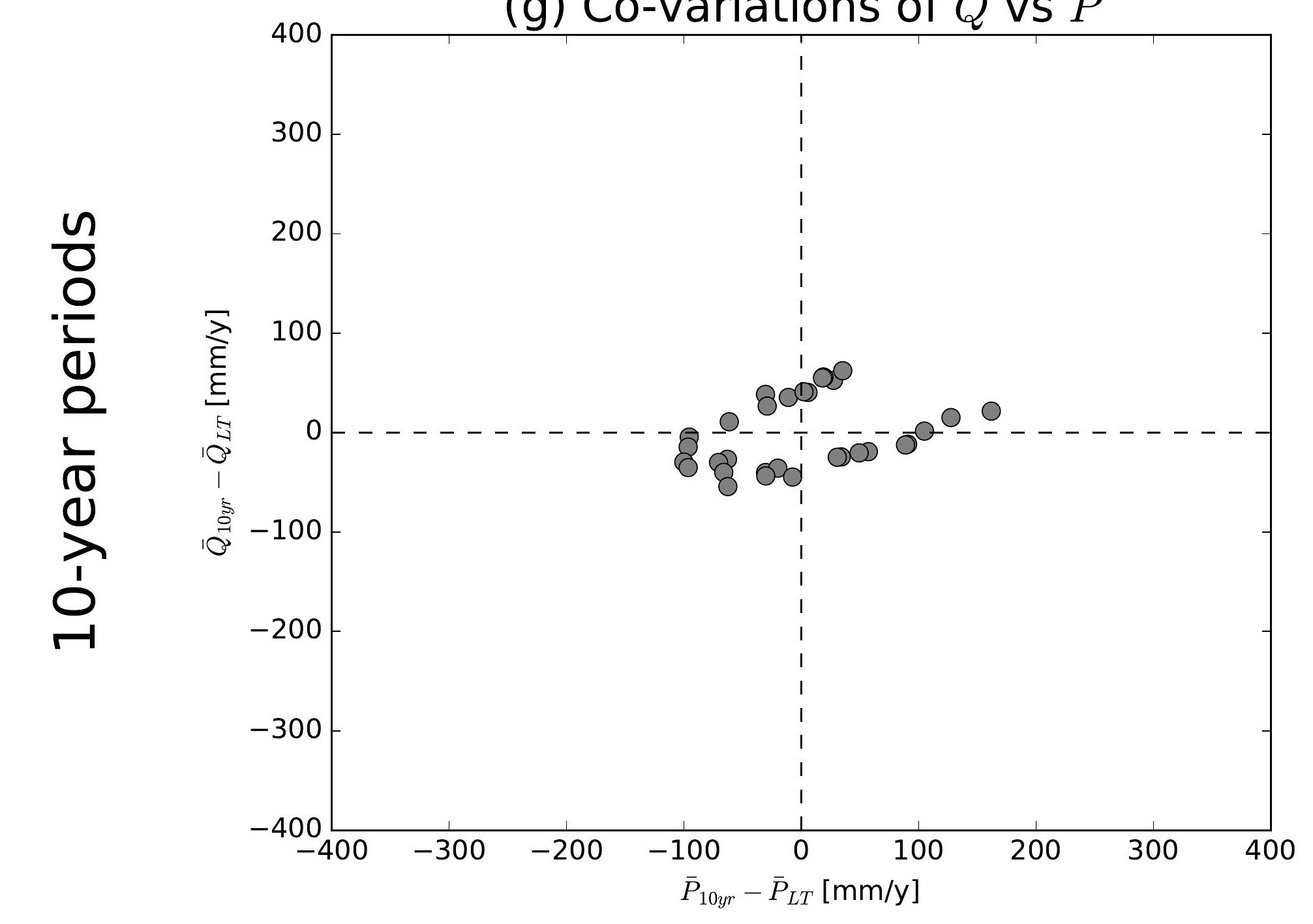

(j) Co-variations of $\bar{Q}$ vs $\bar{P}$

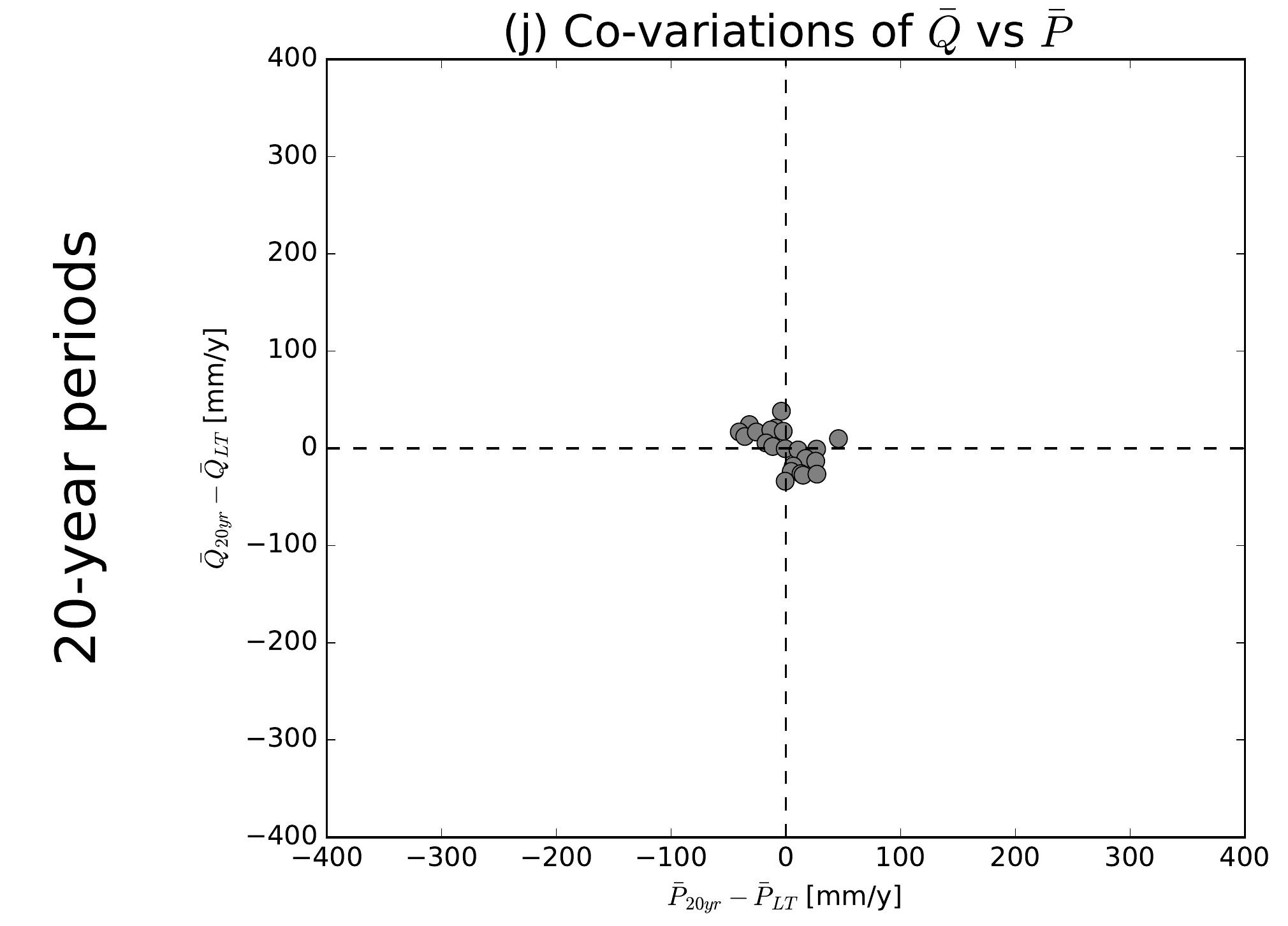

(b) Co-variations of $\bar{Q}$ vs $\overline{E 0}$

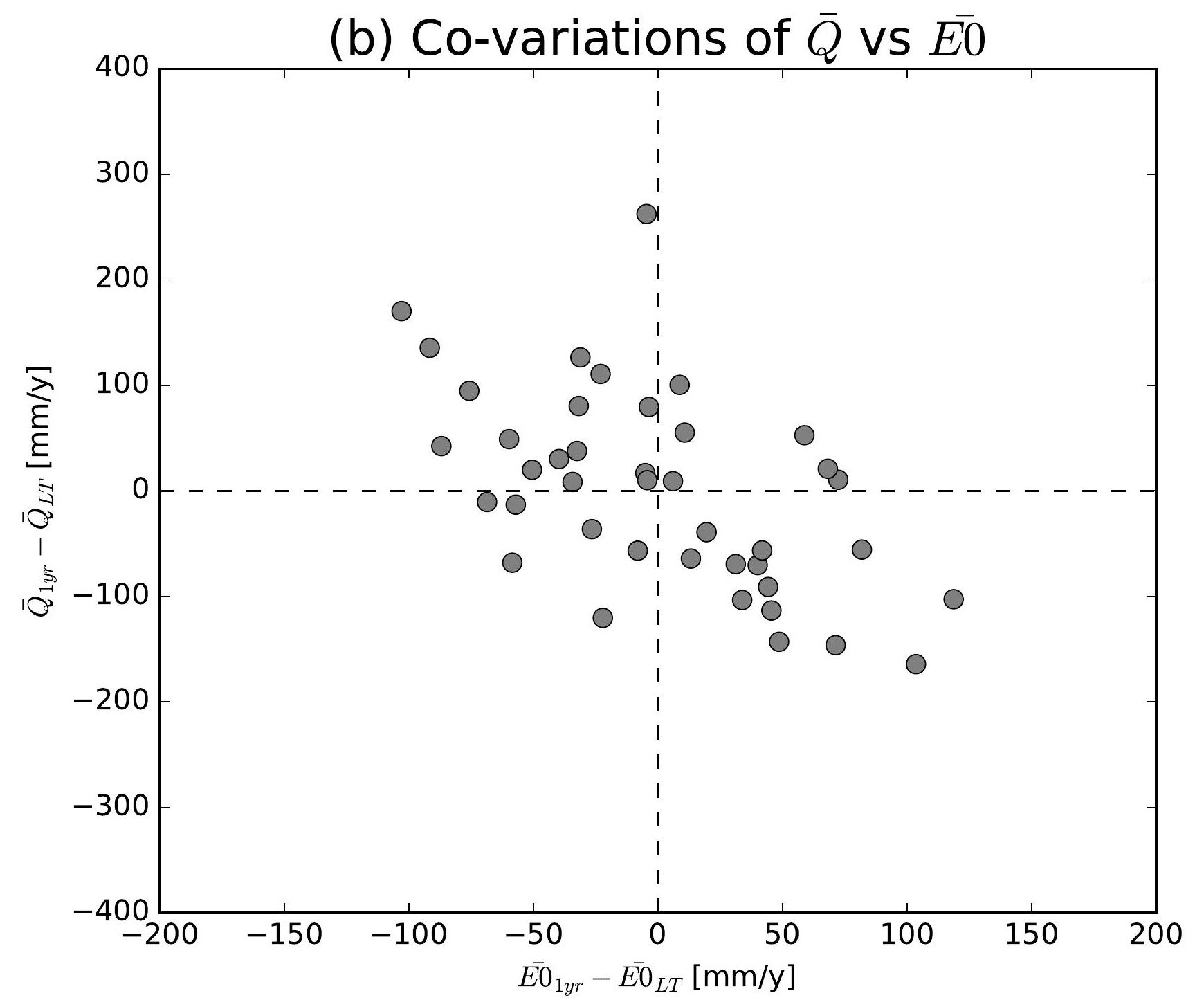

(e) Co-variations of $\bar{Q}$ vs $\overline{E 0}$

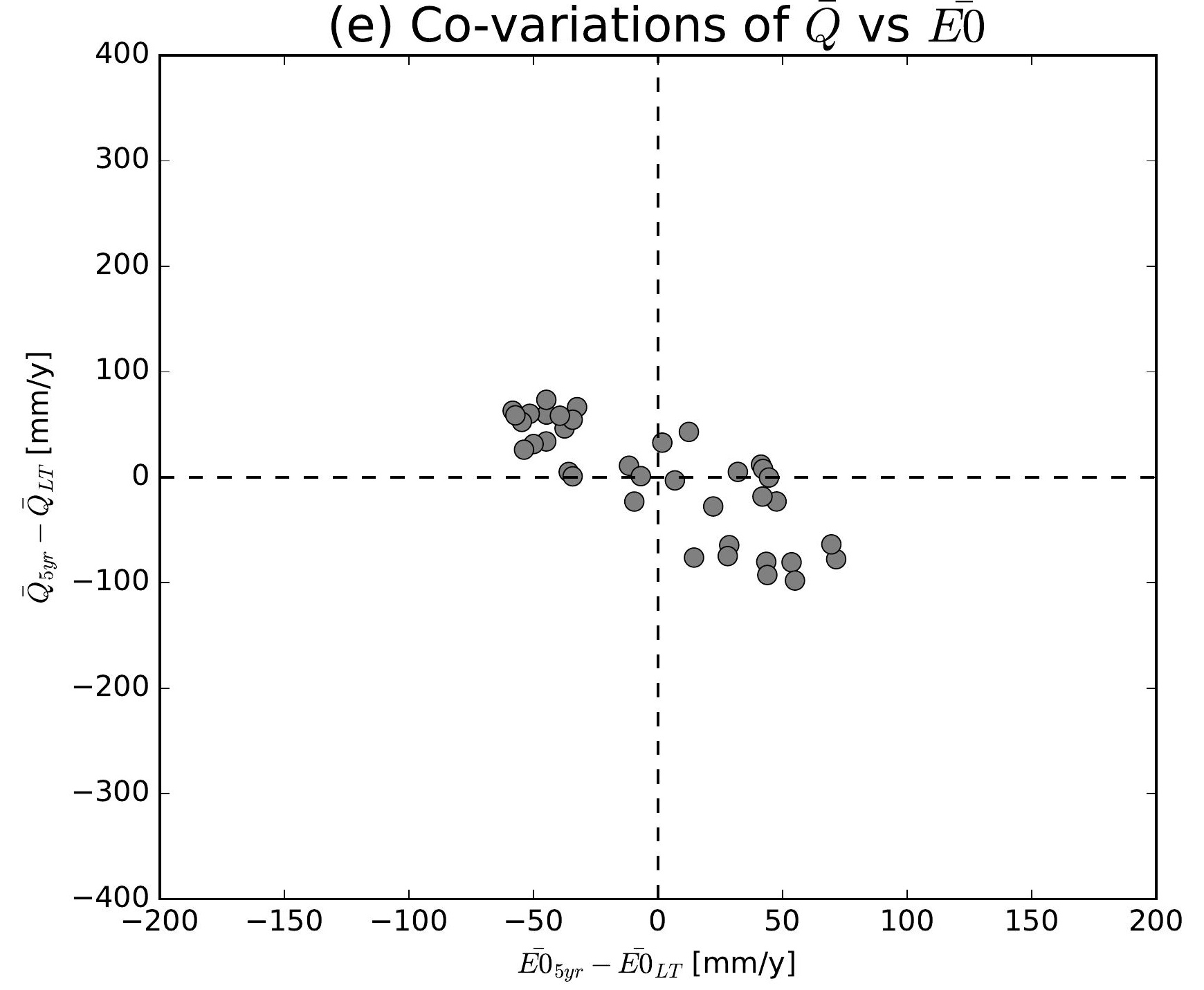

(h) Co-variations of $\bar{Q}$ vs $\overline{E 0}$

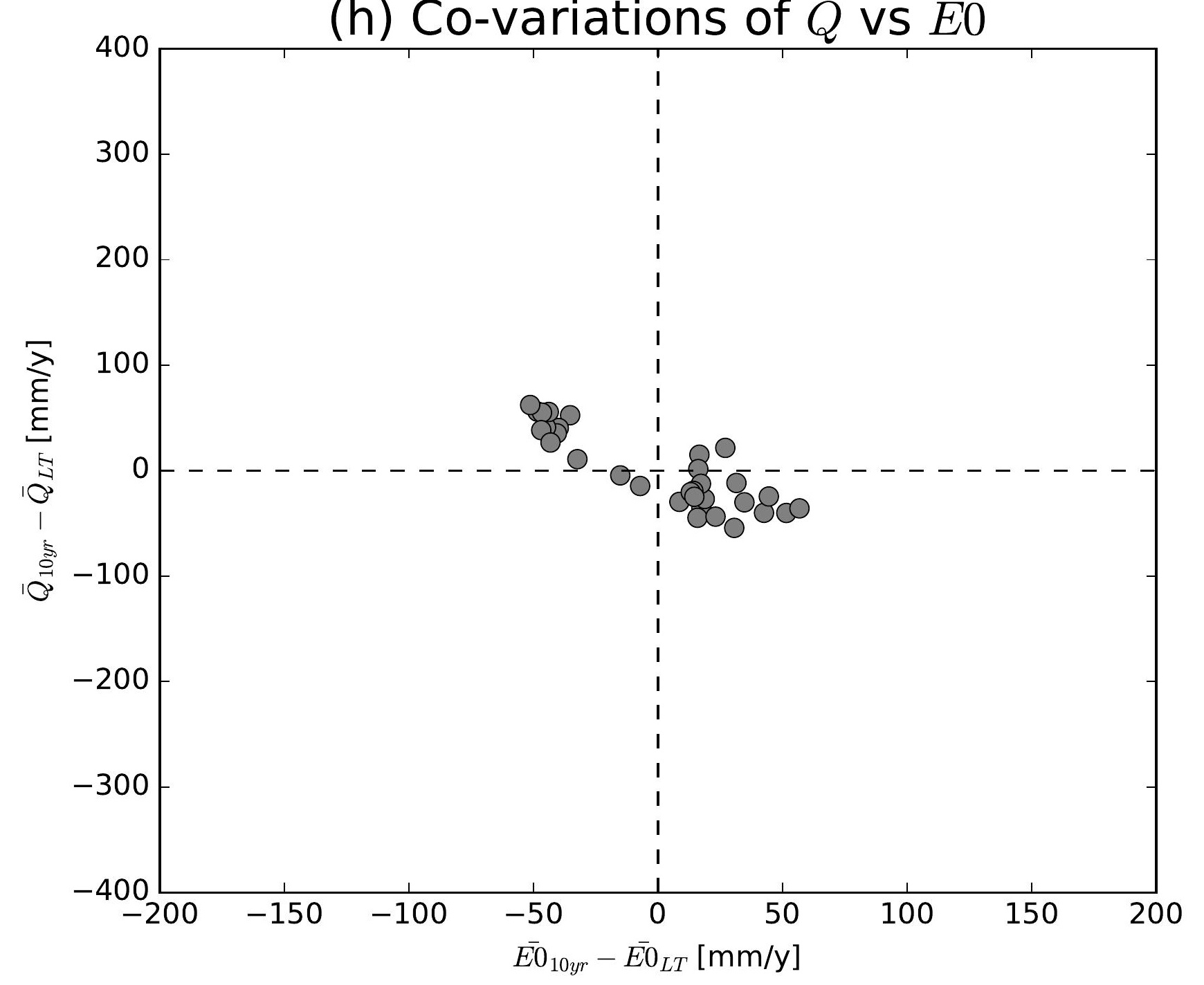

(k) Co-variations of $\bar{Q}$ vs $\overline{E 0}$

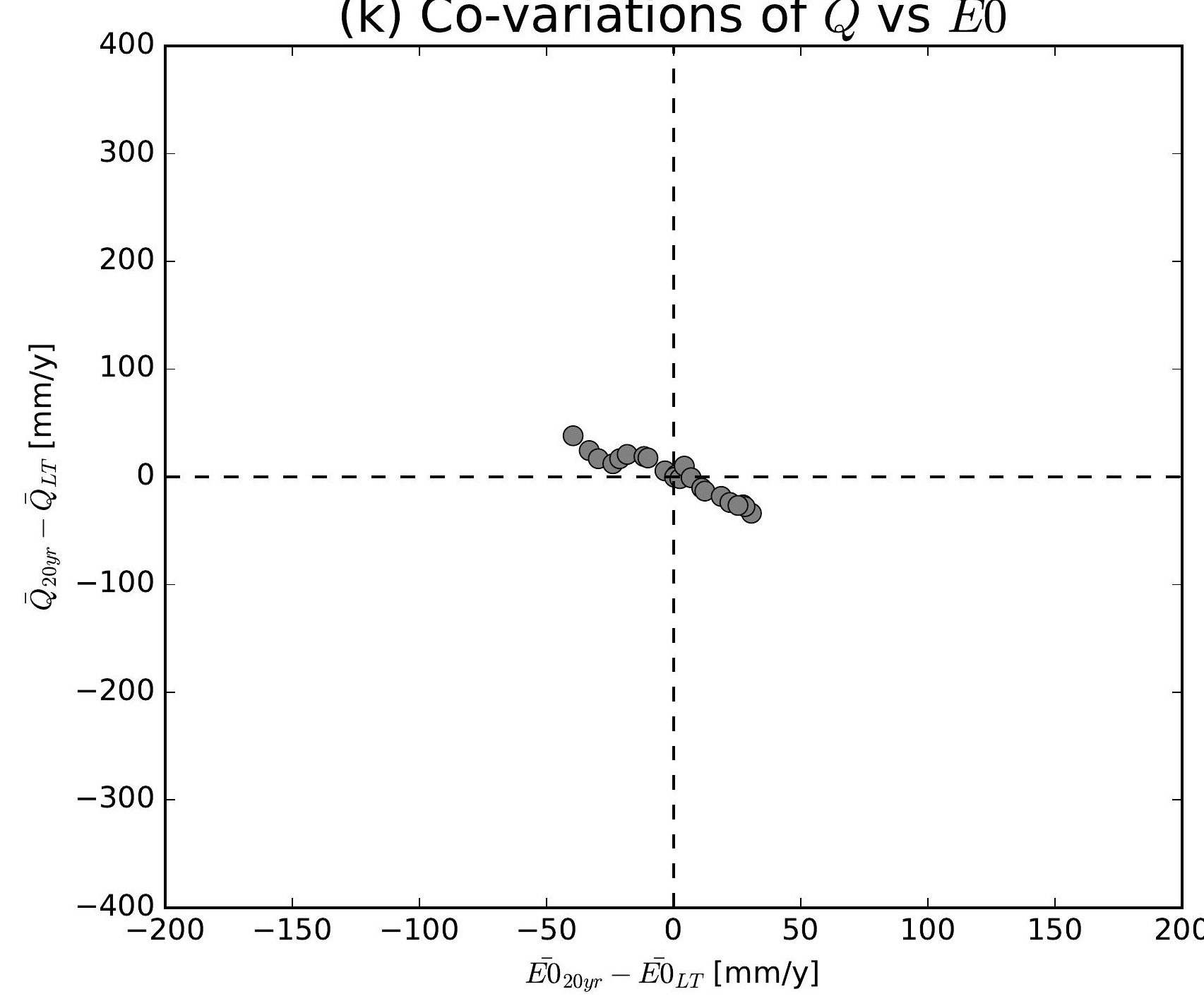

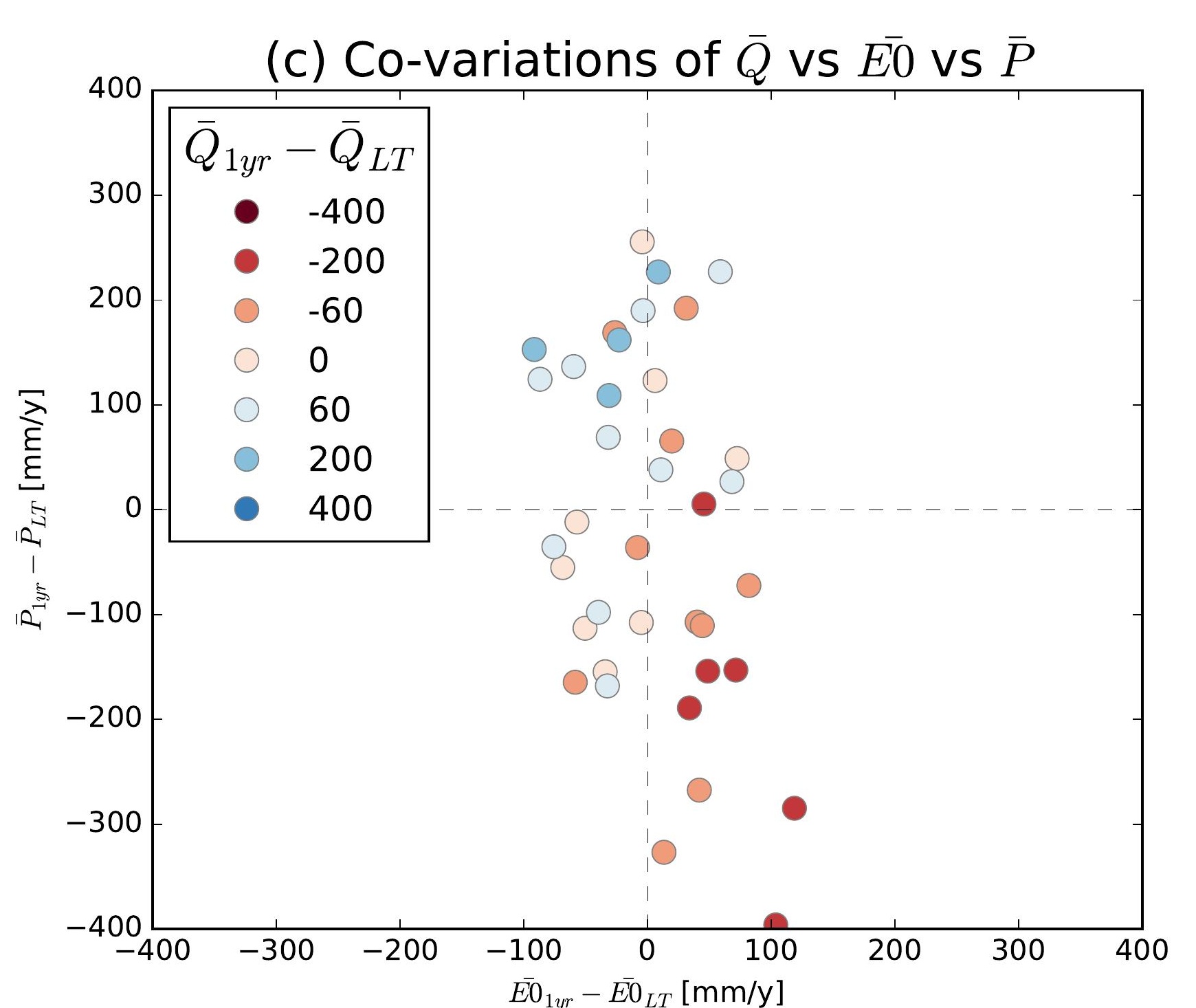
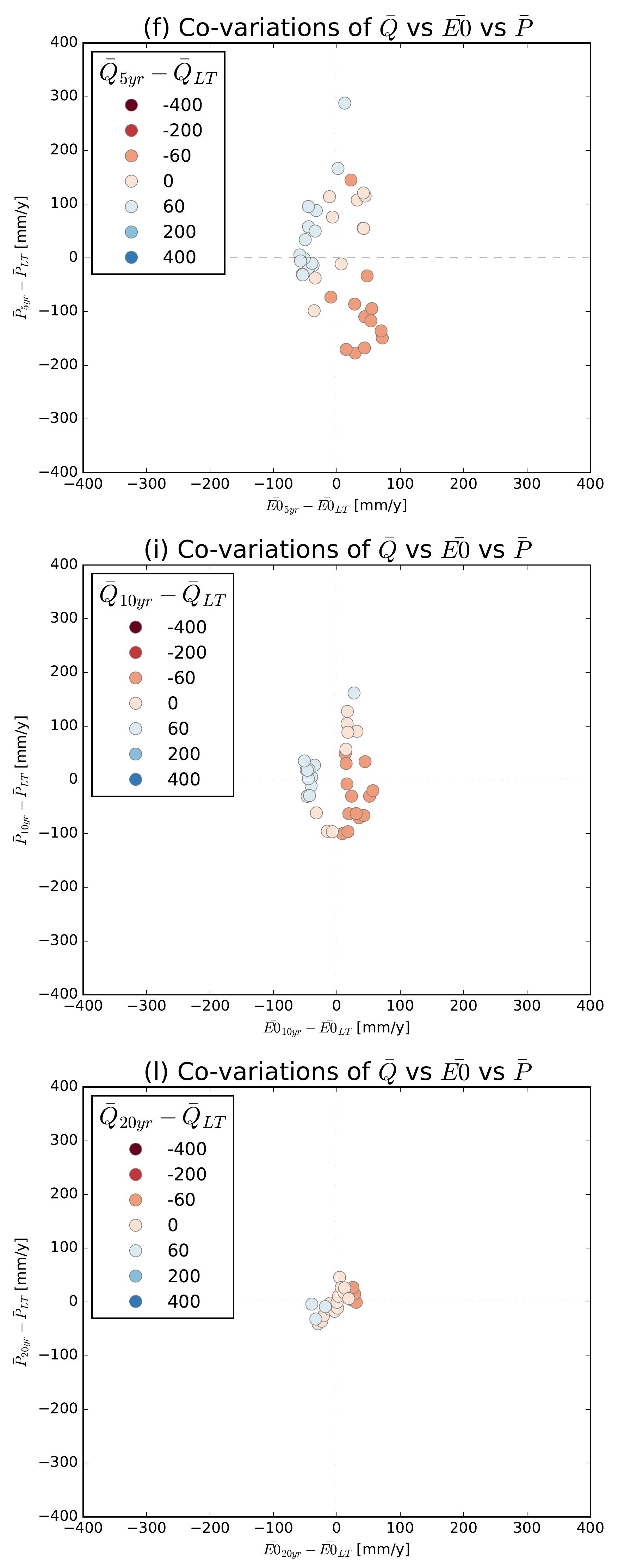

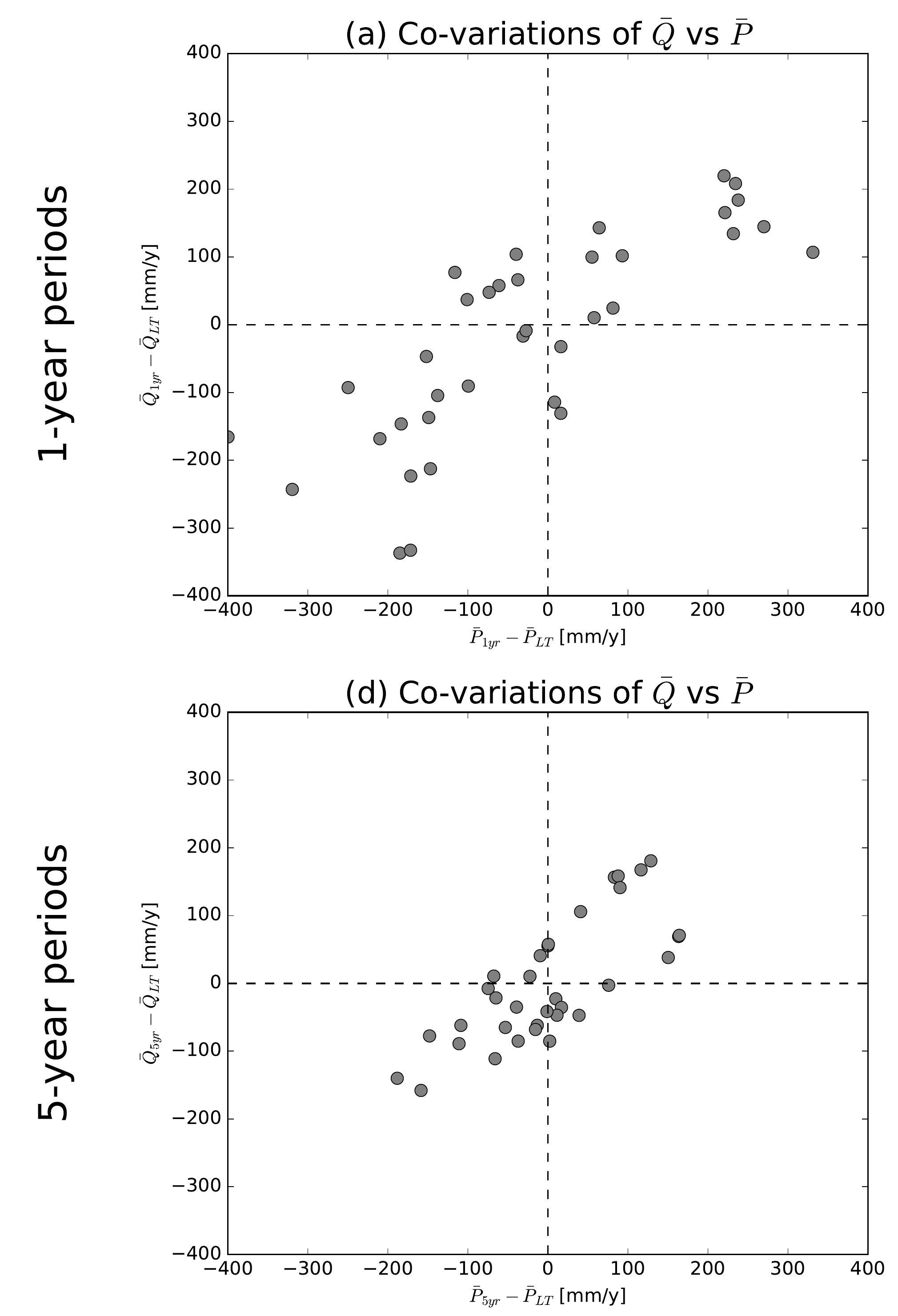

(g) Co-variations of $\bar{Q}$ vs $\bar{P}$
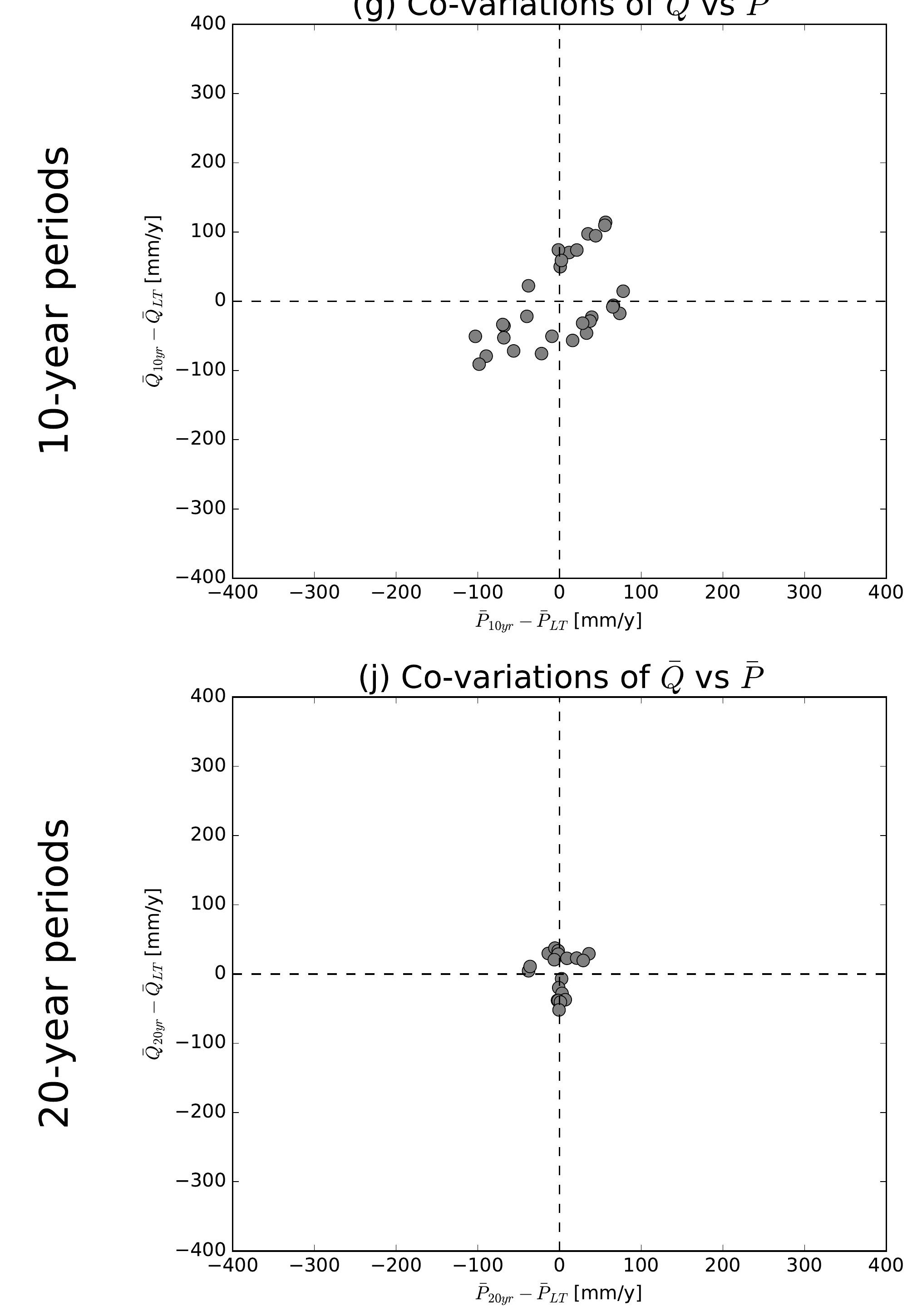

(b) Co-variations of $\bar{Q}$ vs $\overline{E 0}$

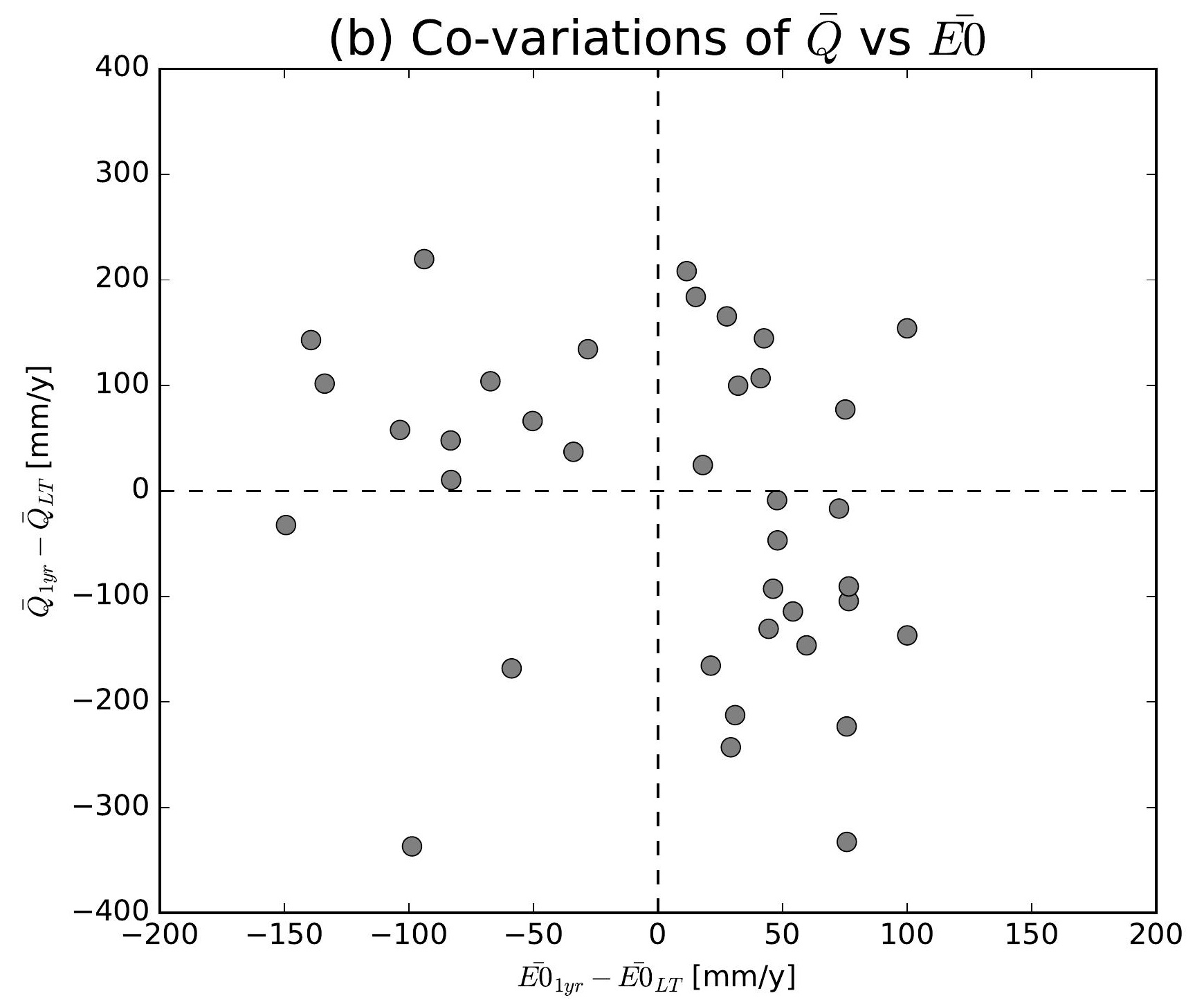

(e) Co-variations of $\bar{Q}$ vs $\overline{E 0}$

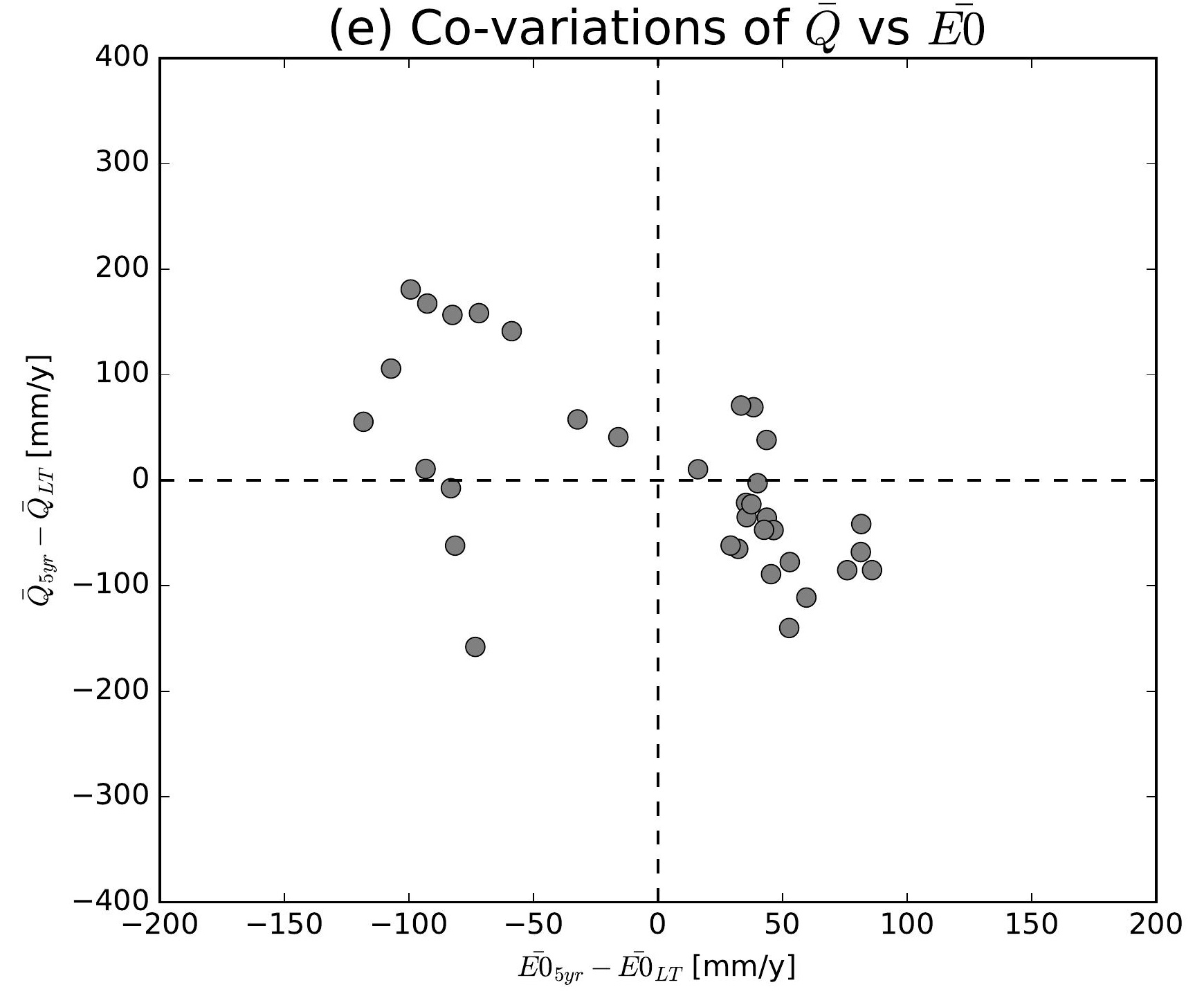

(h) Co-variations of $\bar{Q}$ vs $\overline{E 0}$

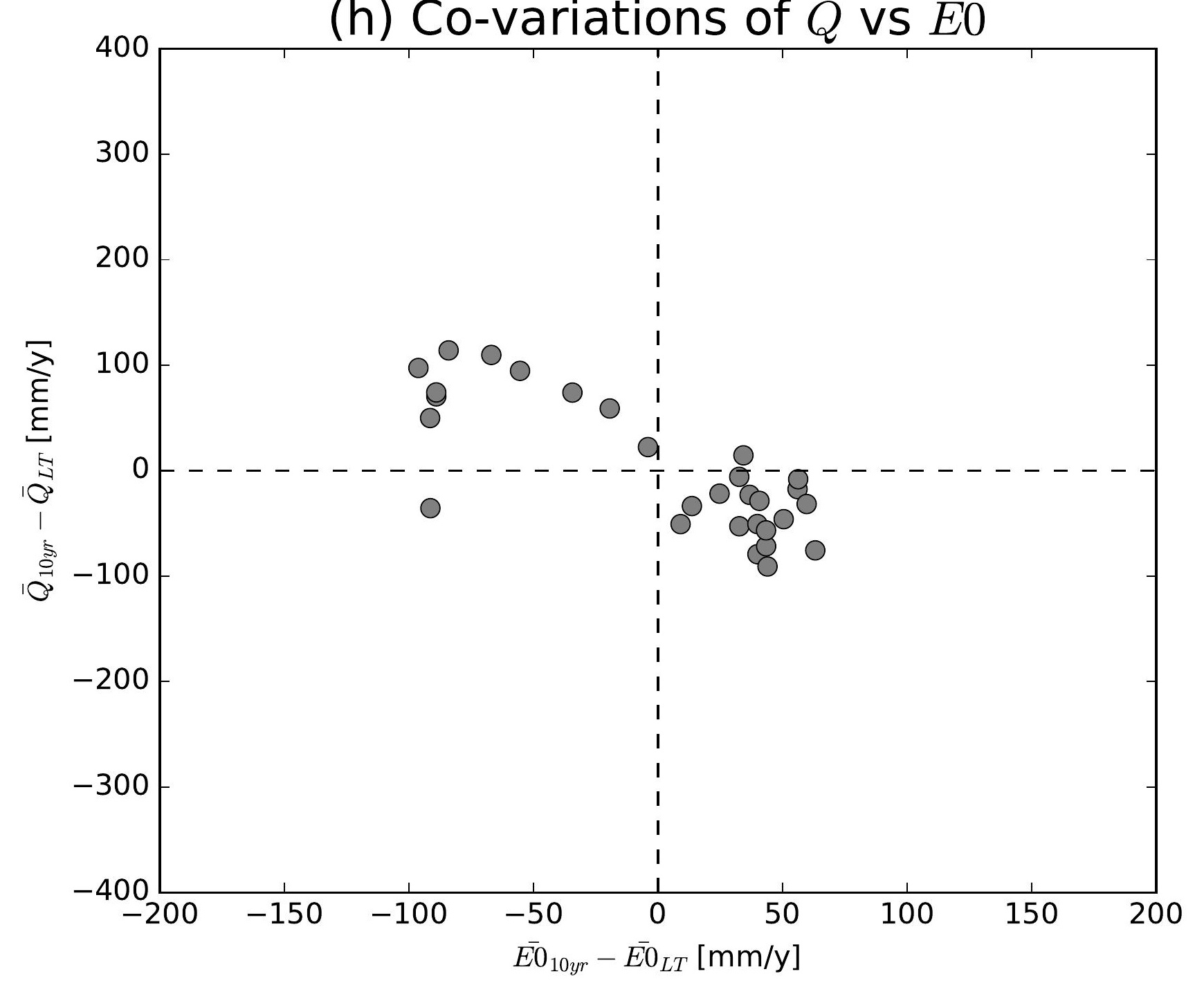

(k) Co-variations of $\bar{Q}$ vs $\overline{E 0}$

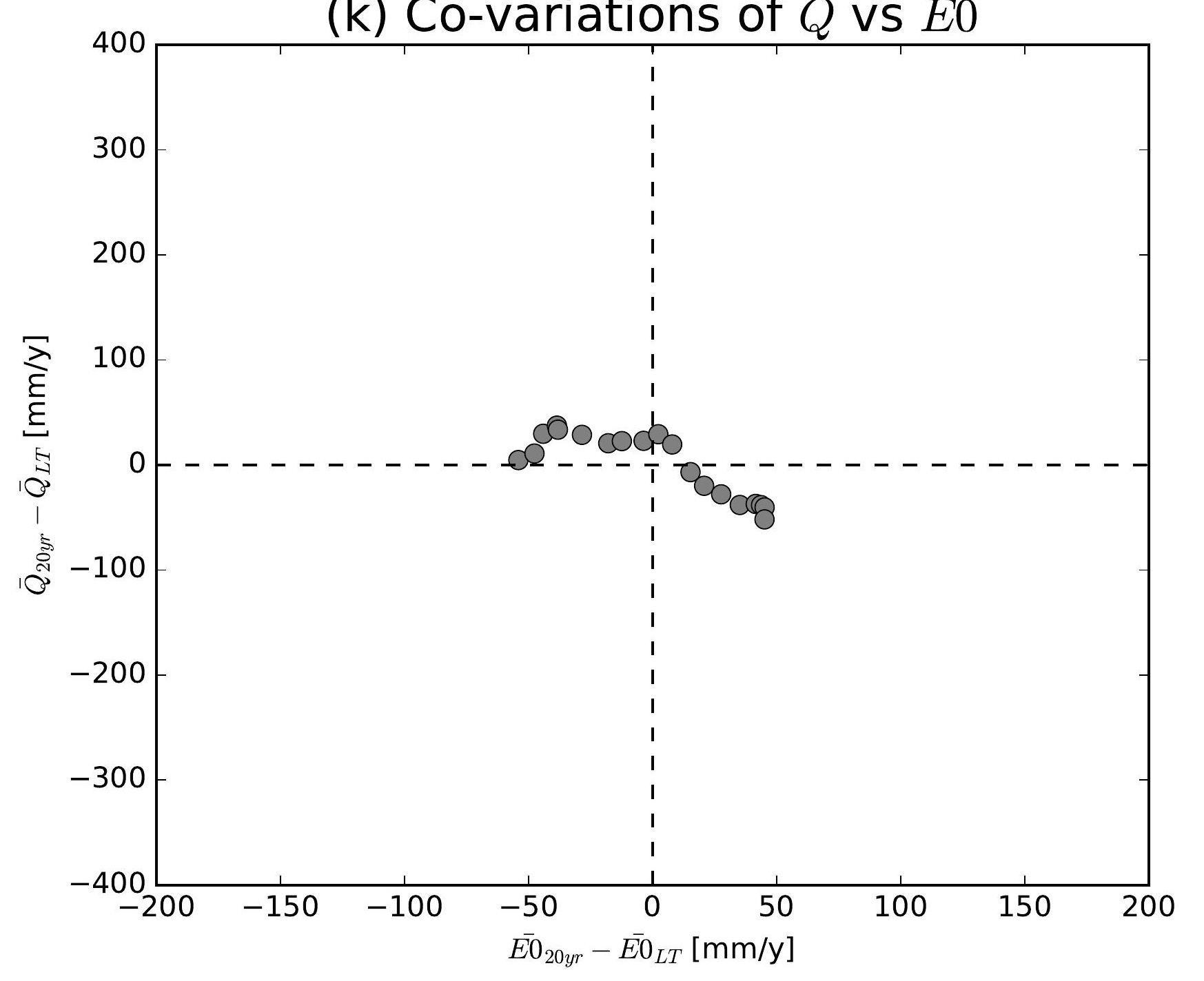

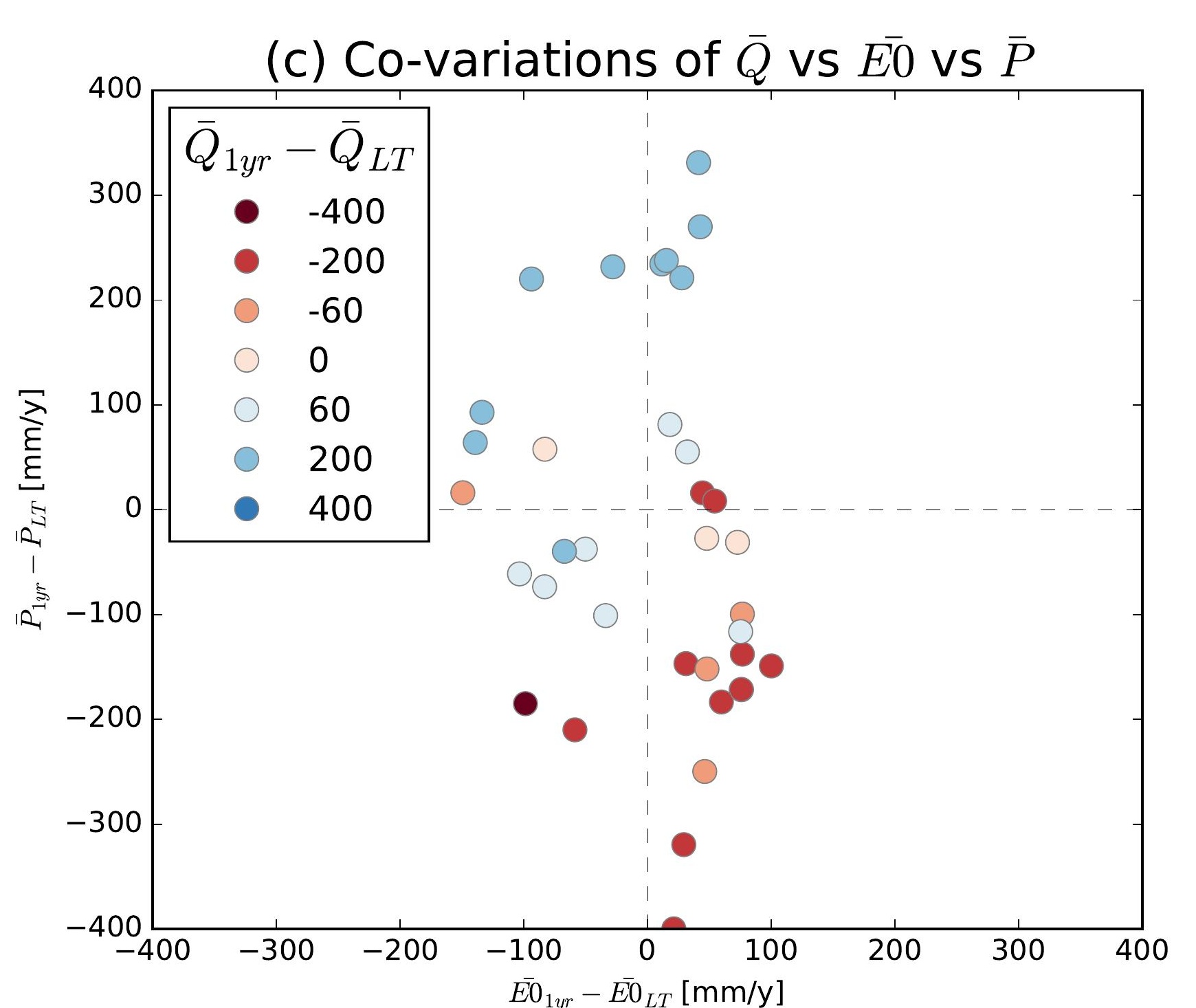
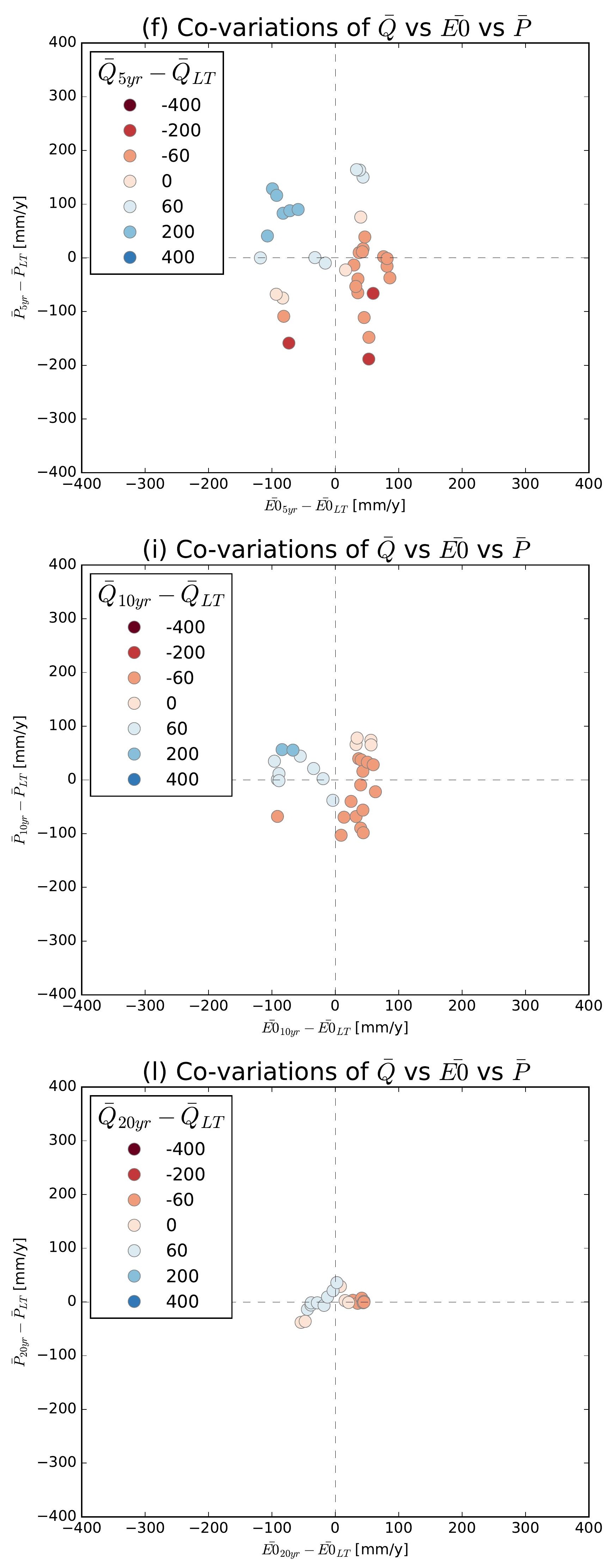

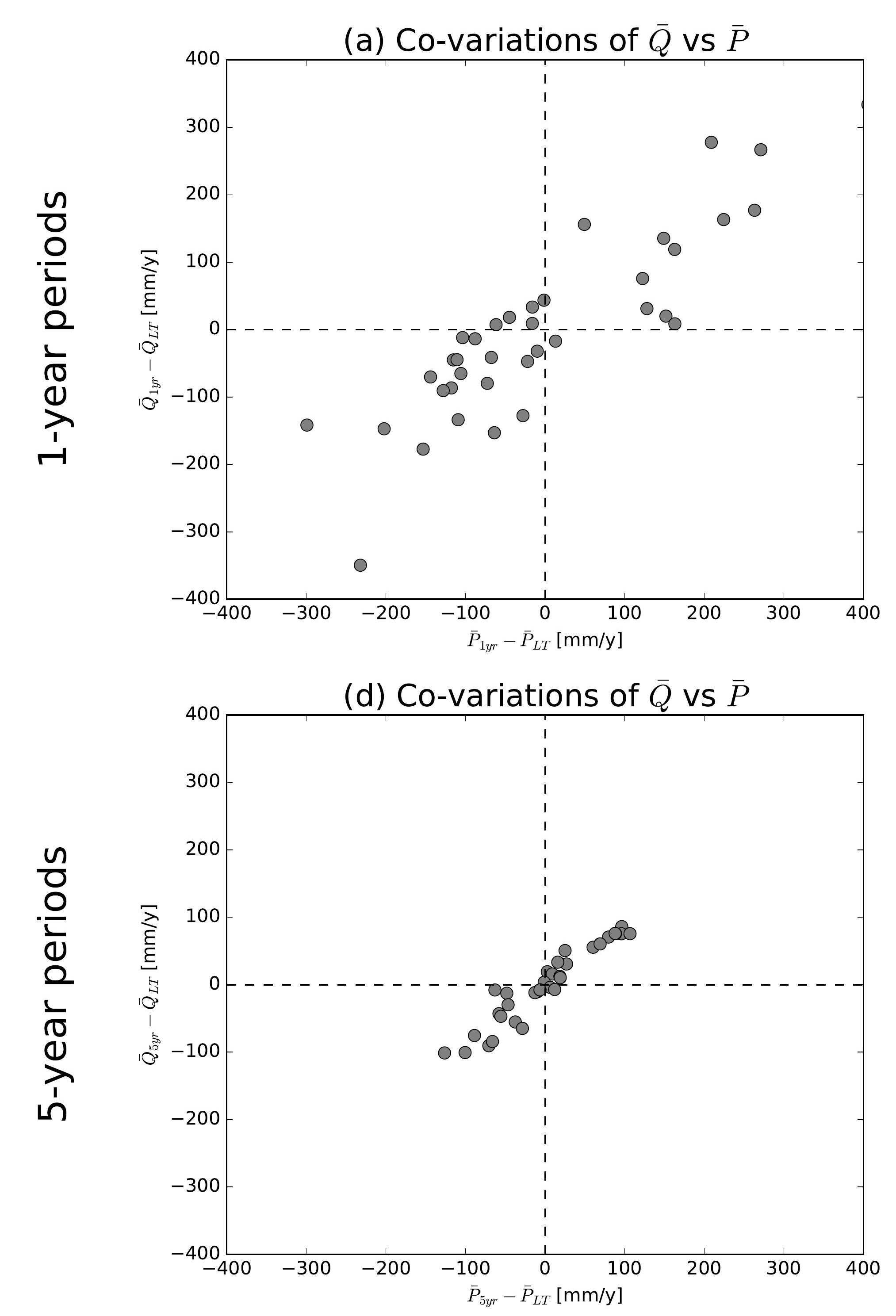

(g) Co-variations of $\bar{Q}$ vs $\bar{P}$
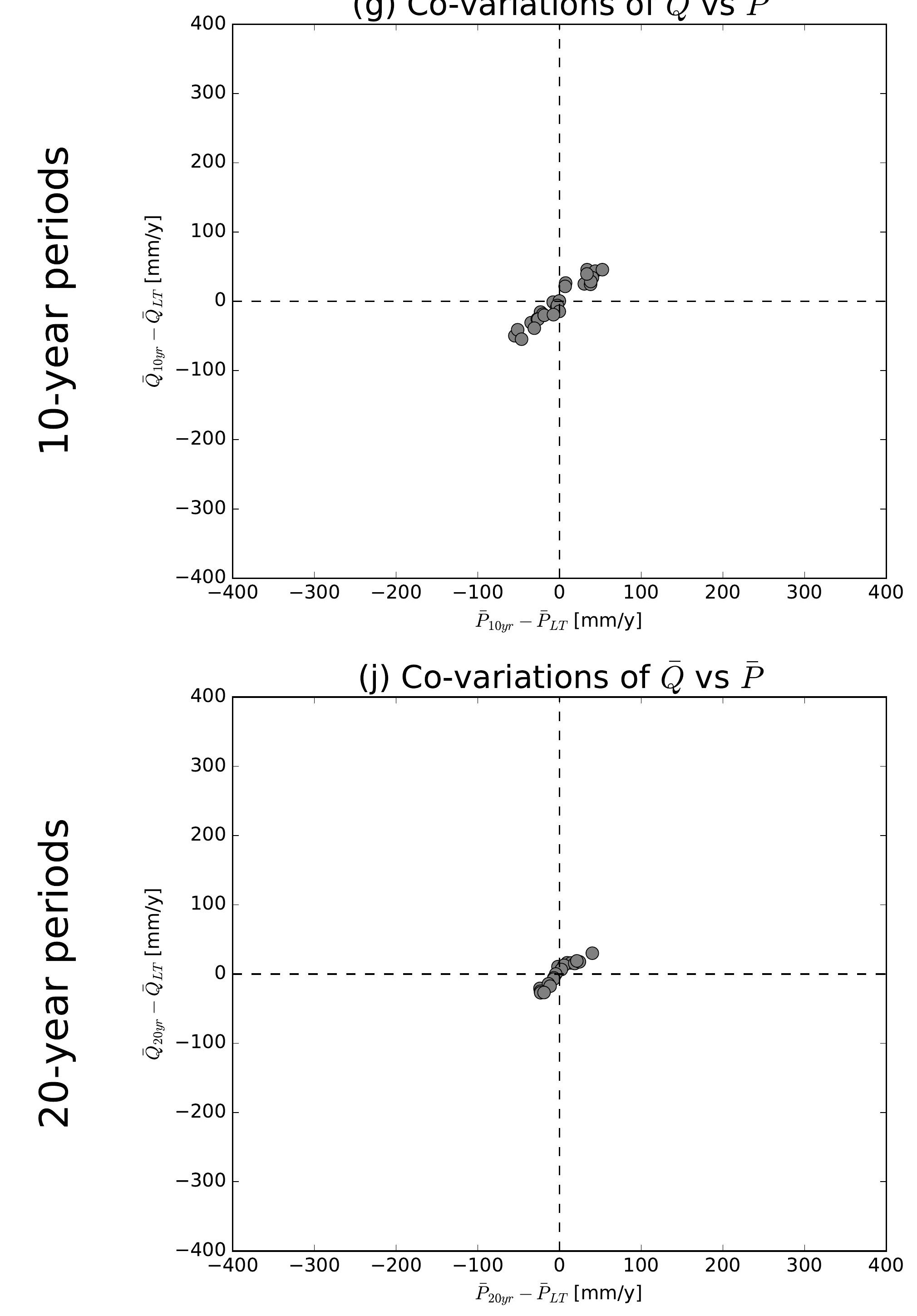

(b) Co-variations of $\bar{Q}$ vs $\overline{E 0}$

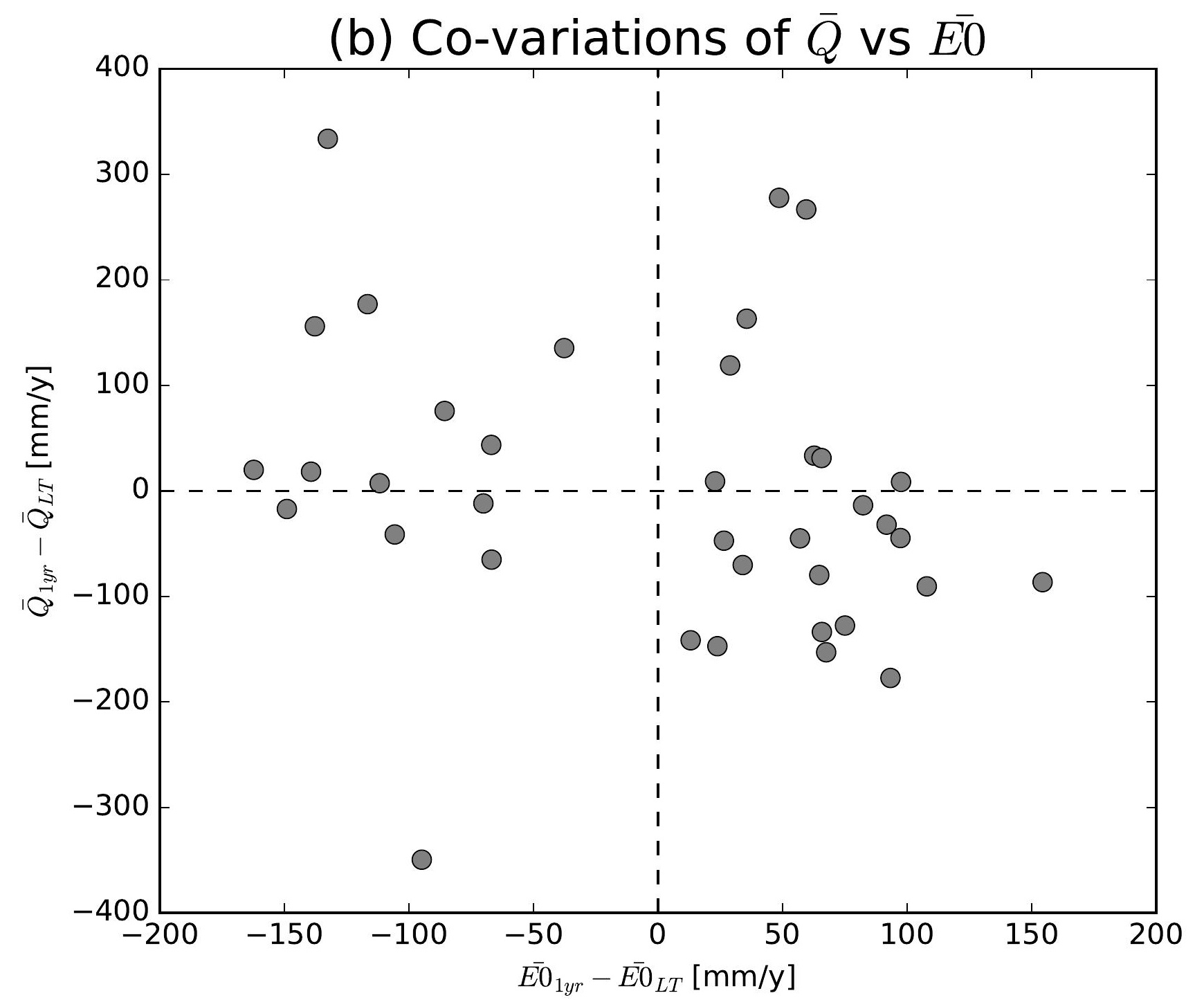

(e) Co-variations of $\bar{Q}$ vs $\overline{E 0}$

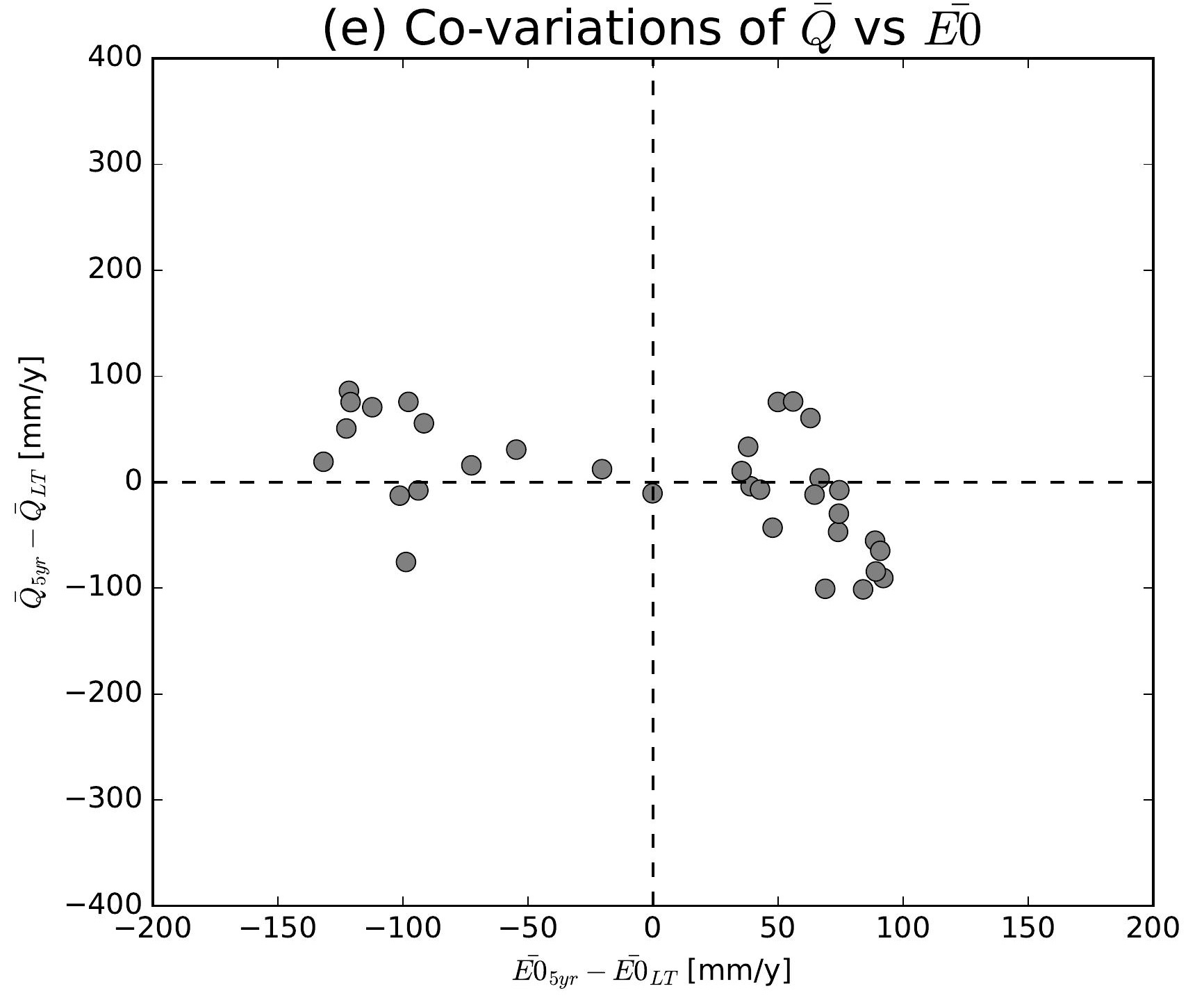

(h) Co-variations of $\bar{Q}$ vs $\overline{E 0}$

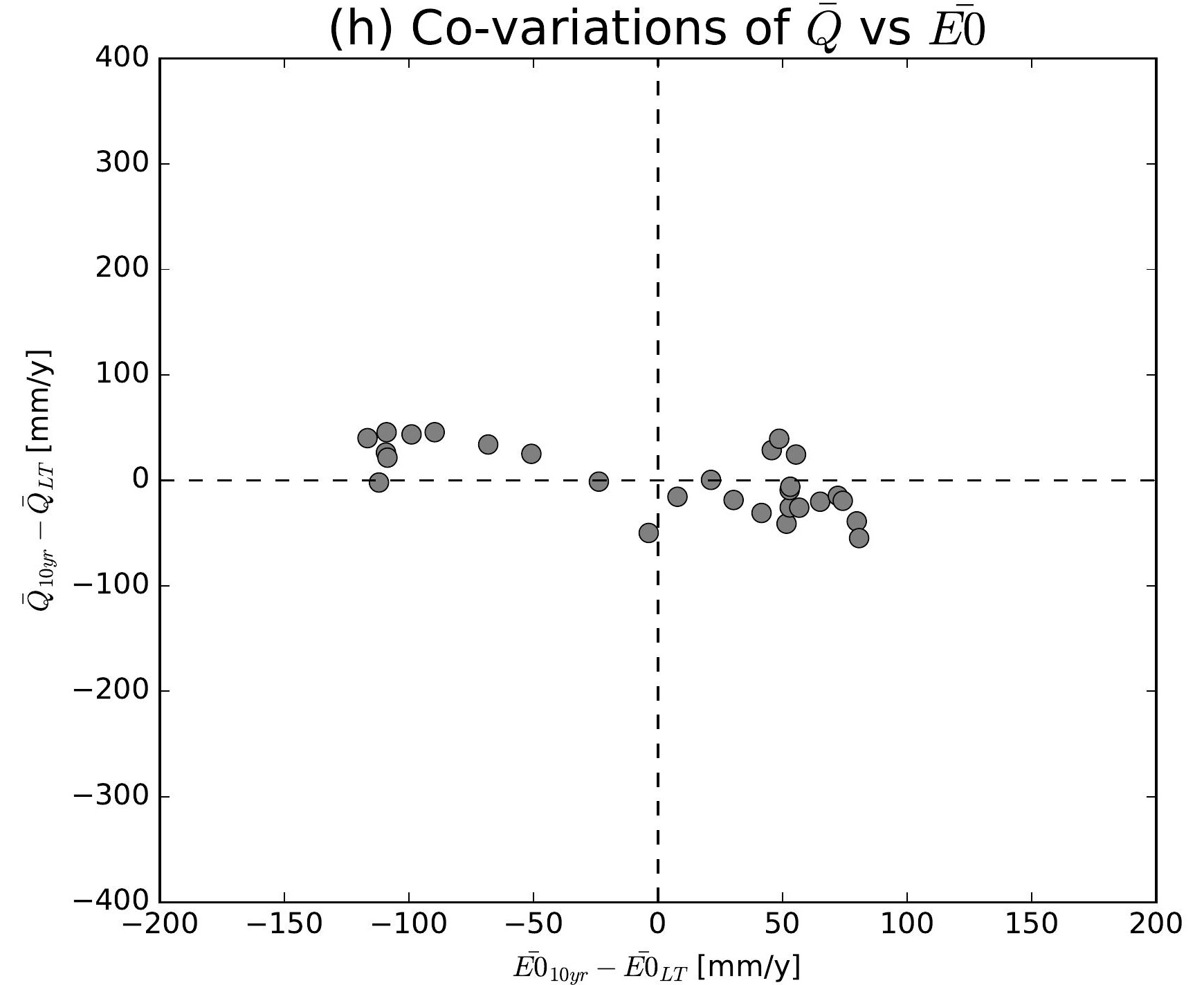

(k) Co-variations of $\bar{Q}$ vs $\overline{E 0}$

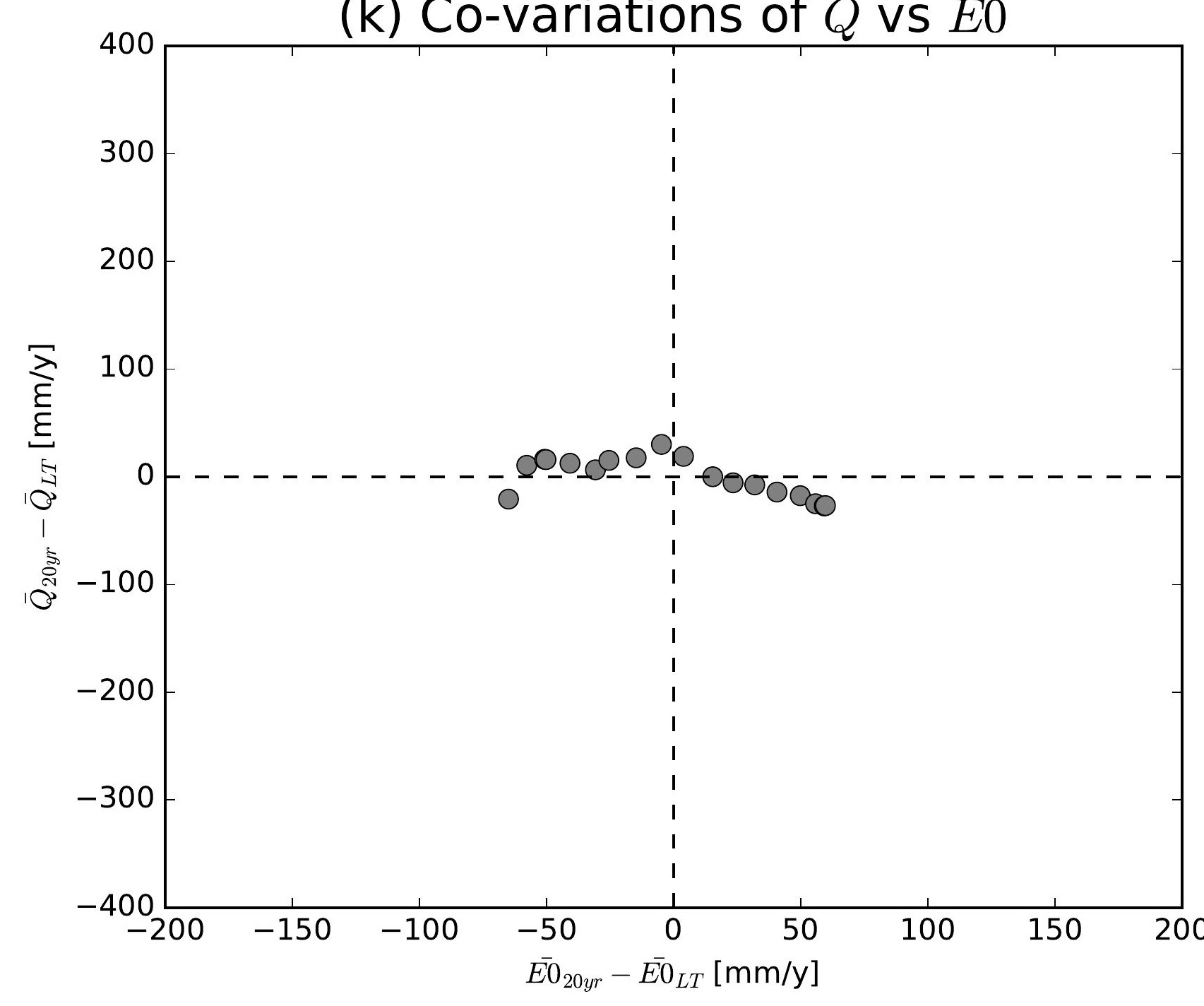

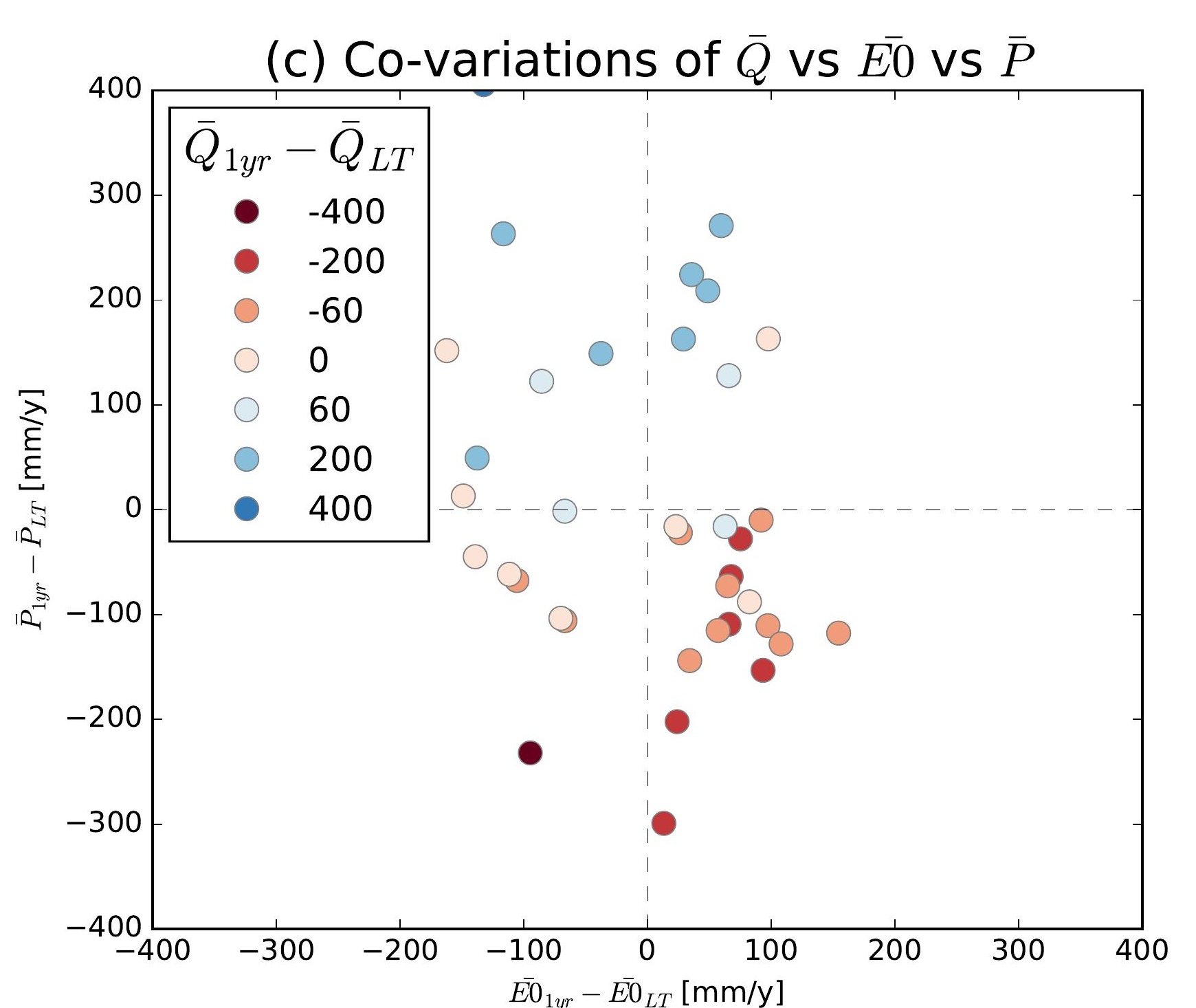
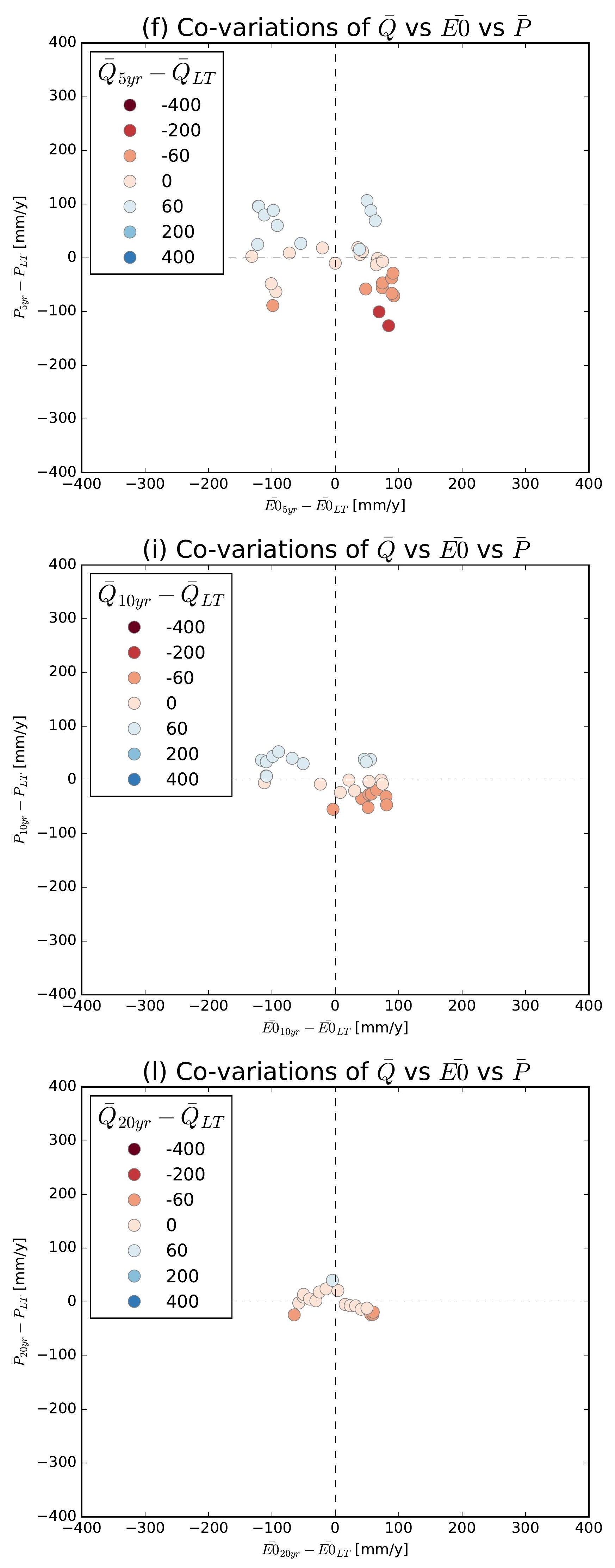

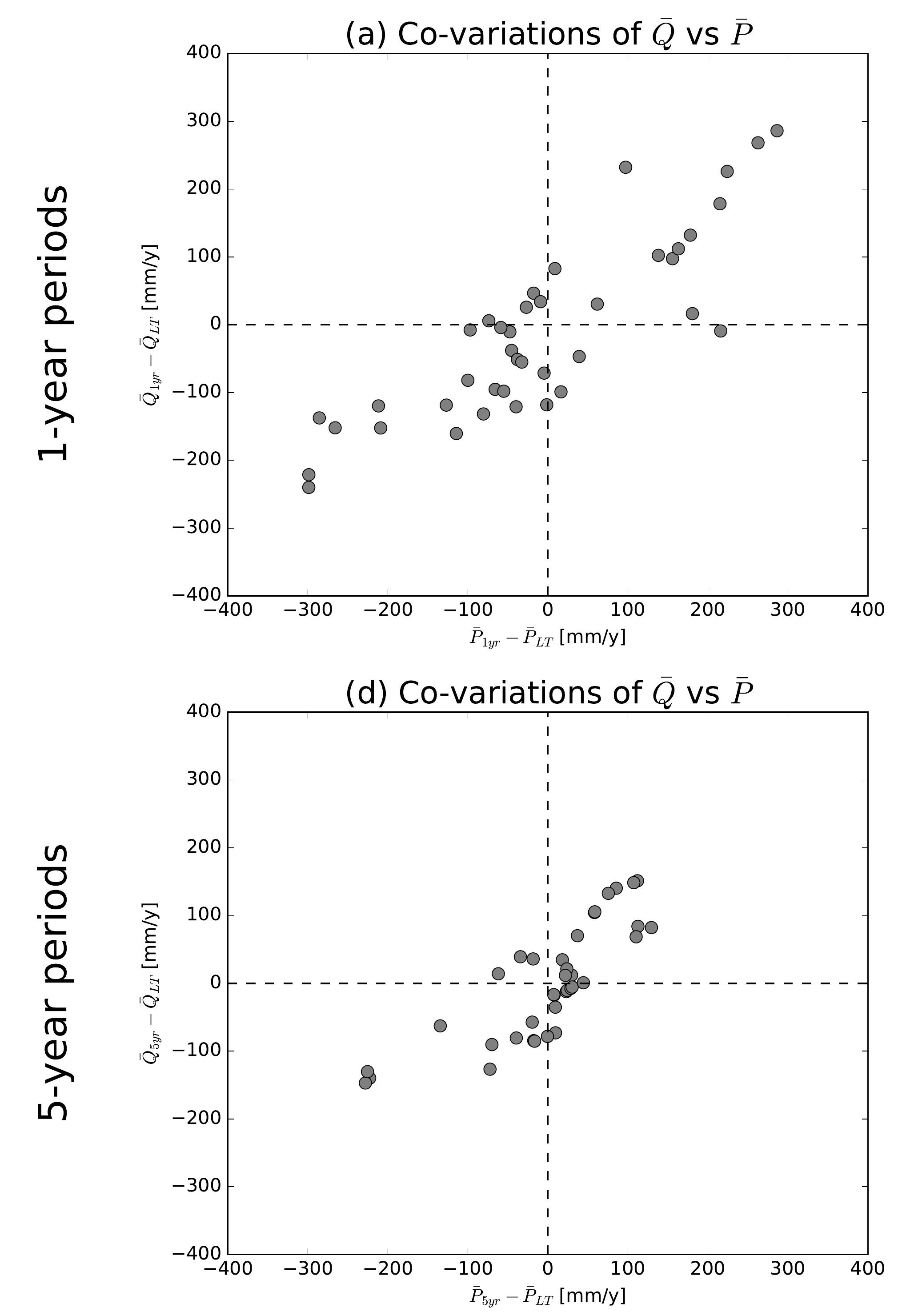

(g) Co-variations of $\bar{Q}$ vs $\bar{P}$
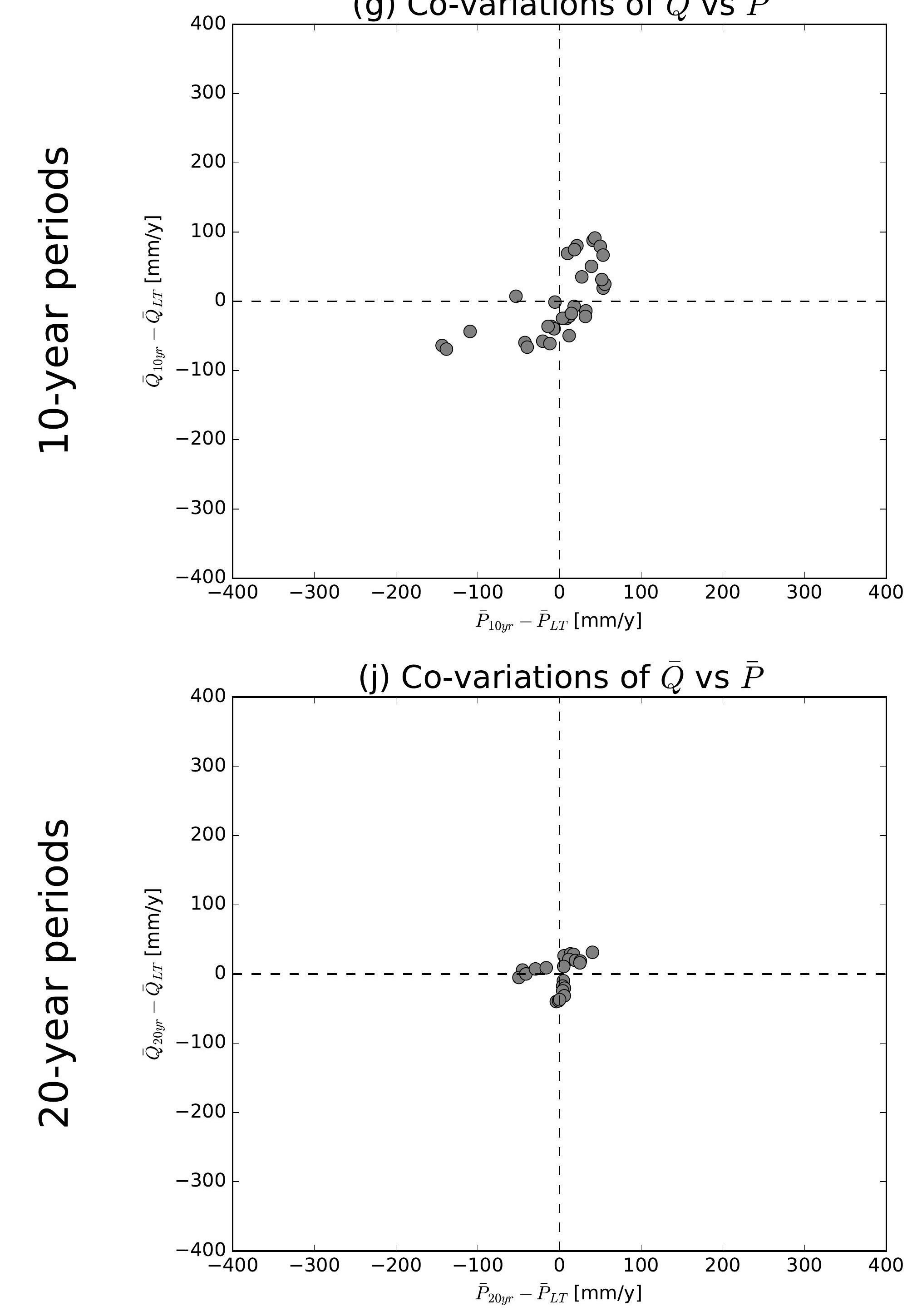

(b) Co-variations of $\bar{Q}$ vs $\overline{E 0}$

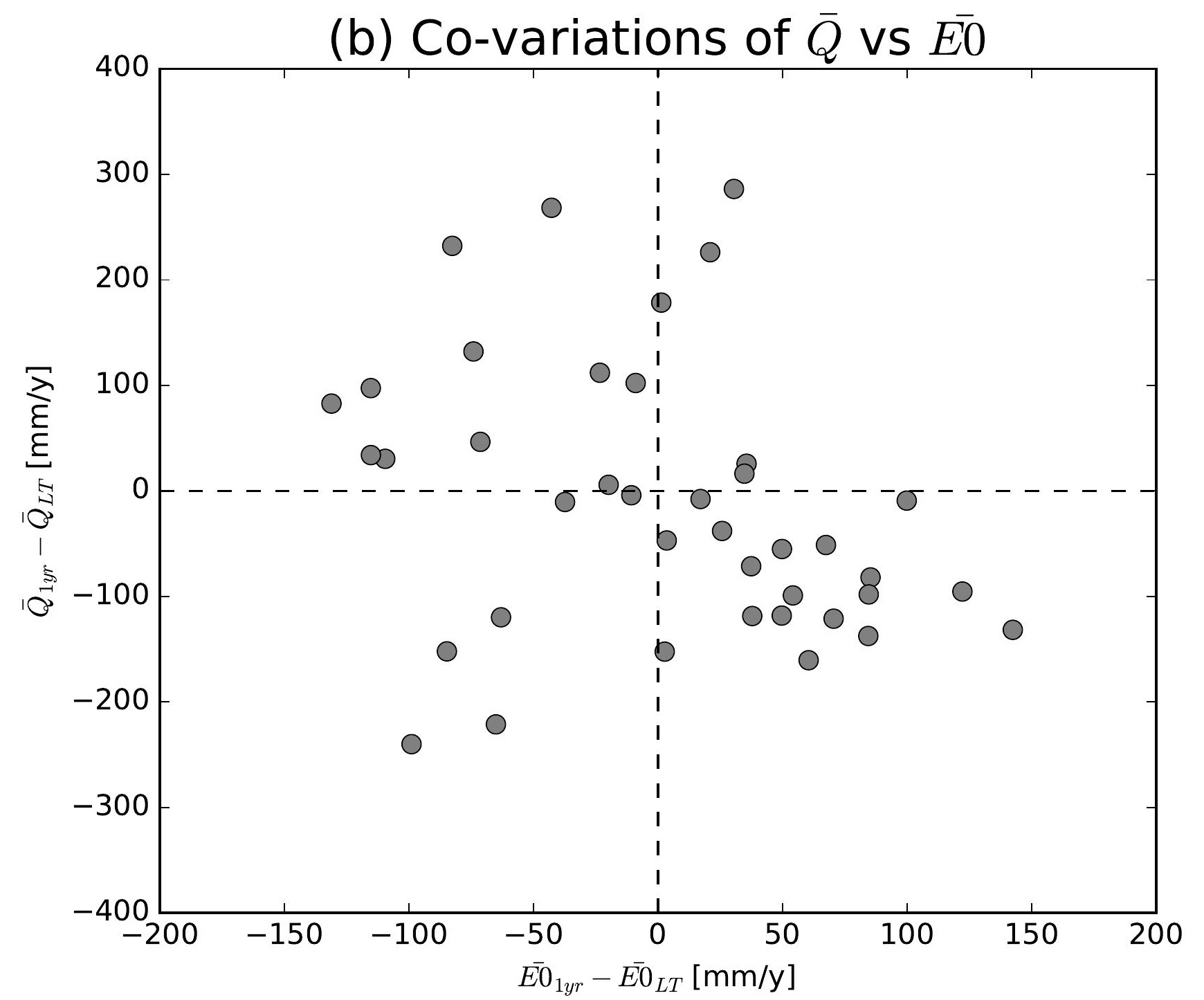

(e) Co-variations of $\bar{Q}$ vs $\overline{E 0}$

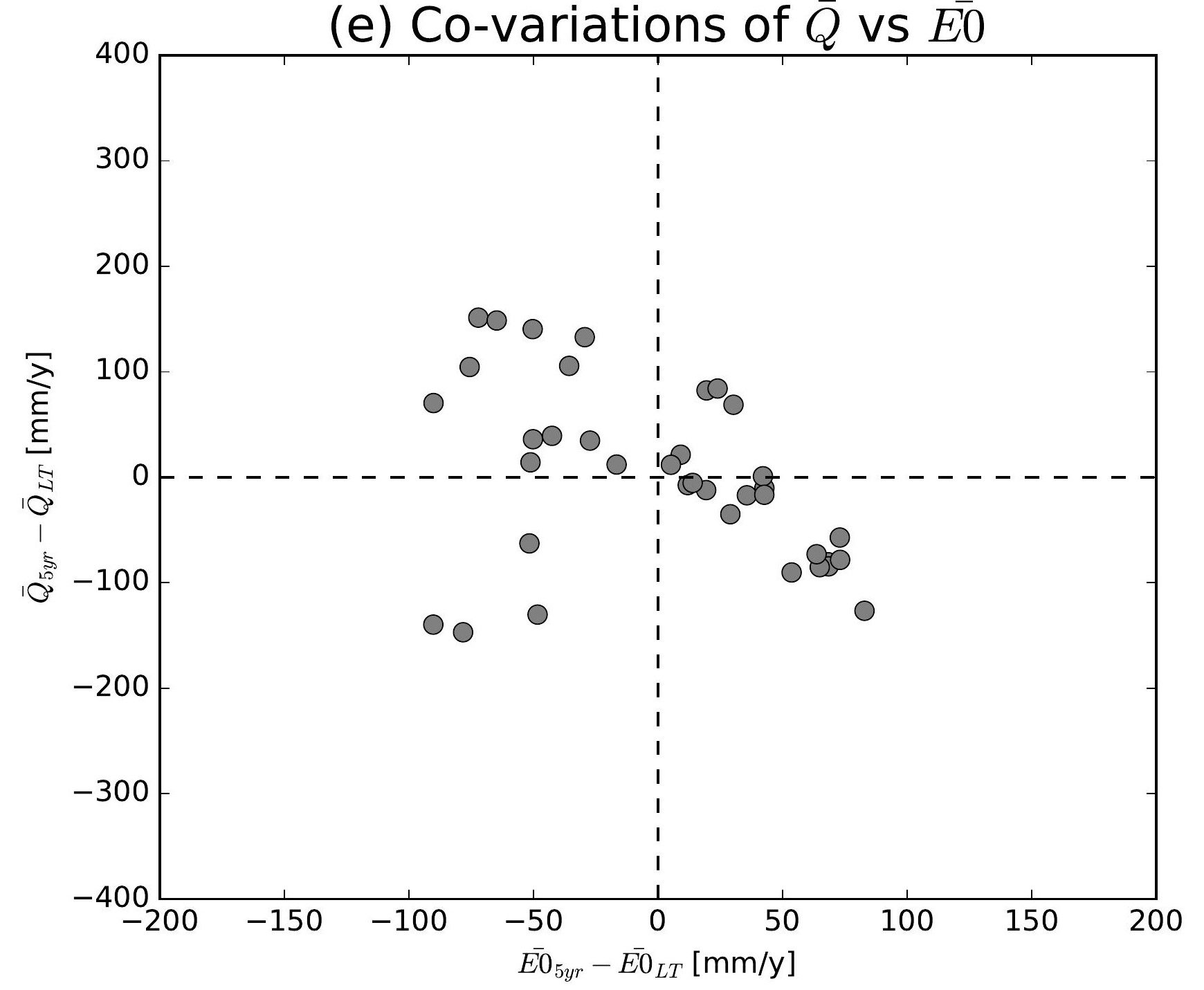

(h) Co-variations of $\bar{Q}$ vs $\overline{E 0}$

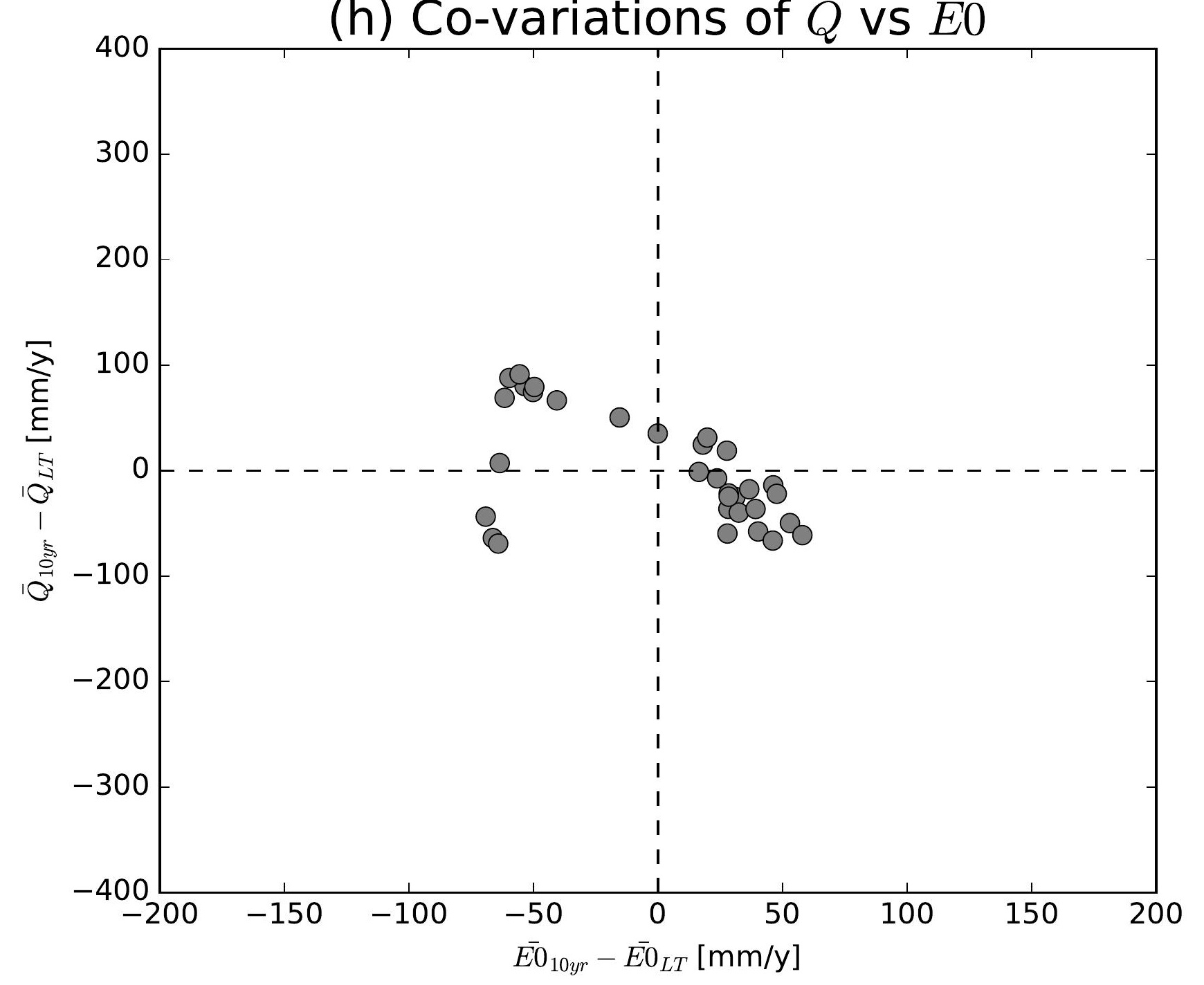

(k) Co-variations of $\bar{Q}$ vs $\overline{E 0}$

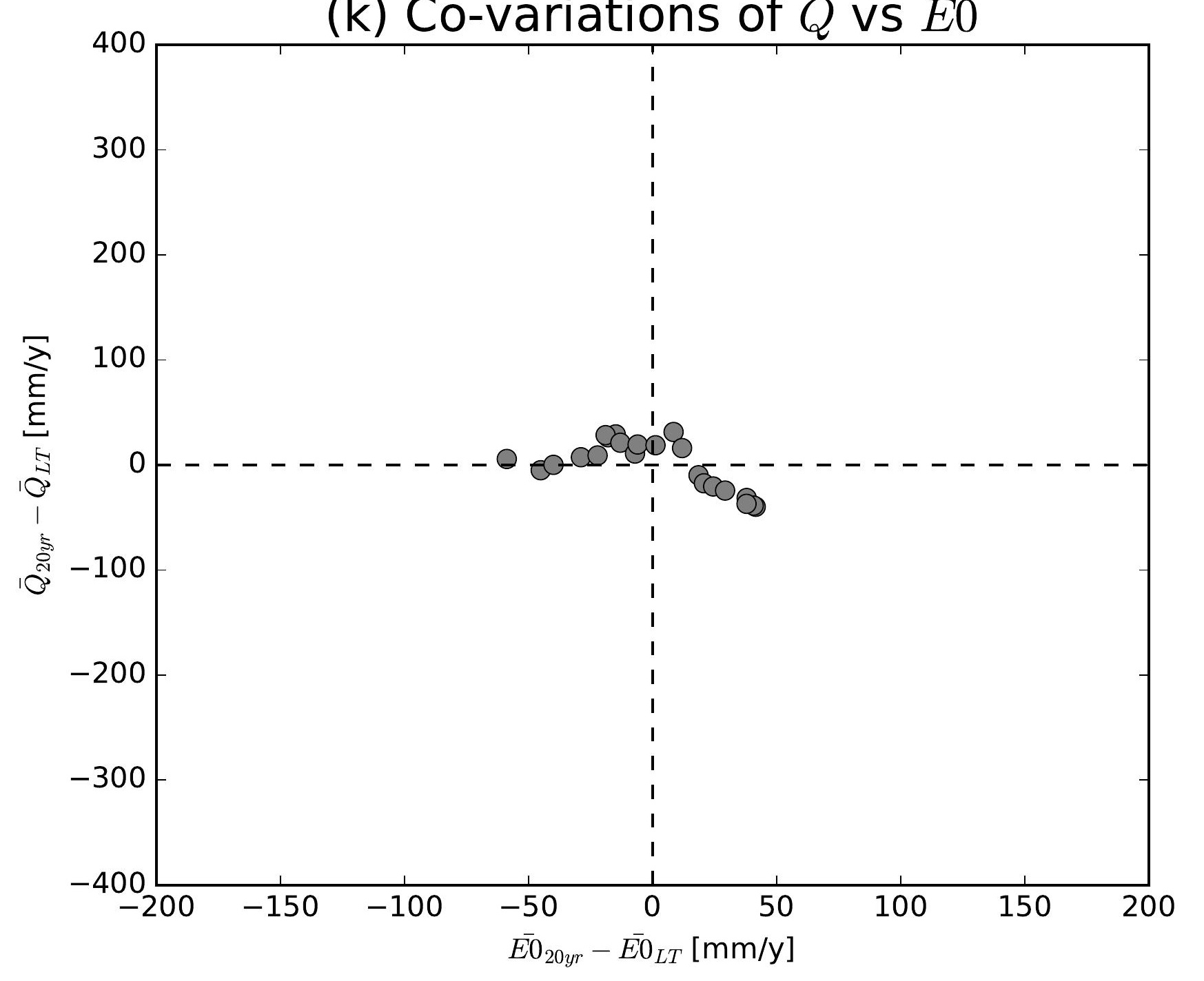

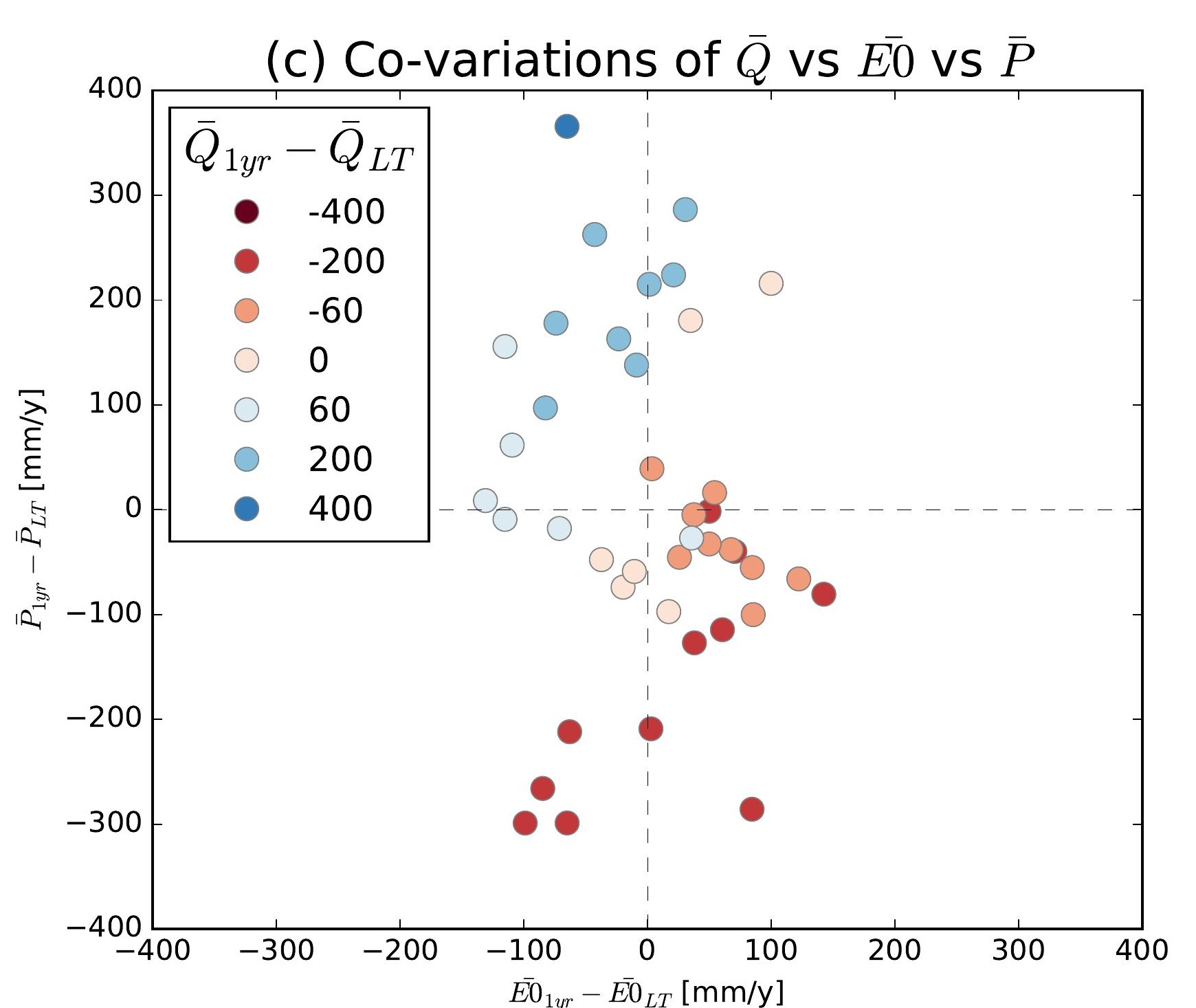
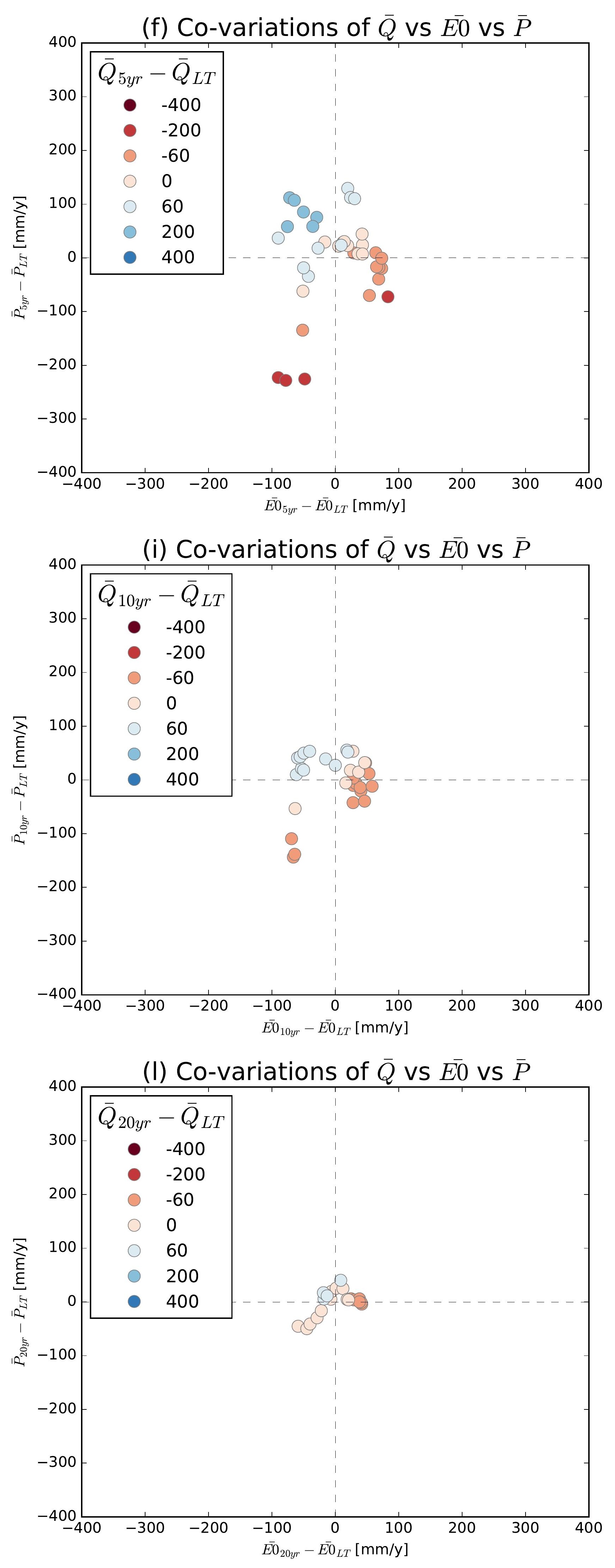

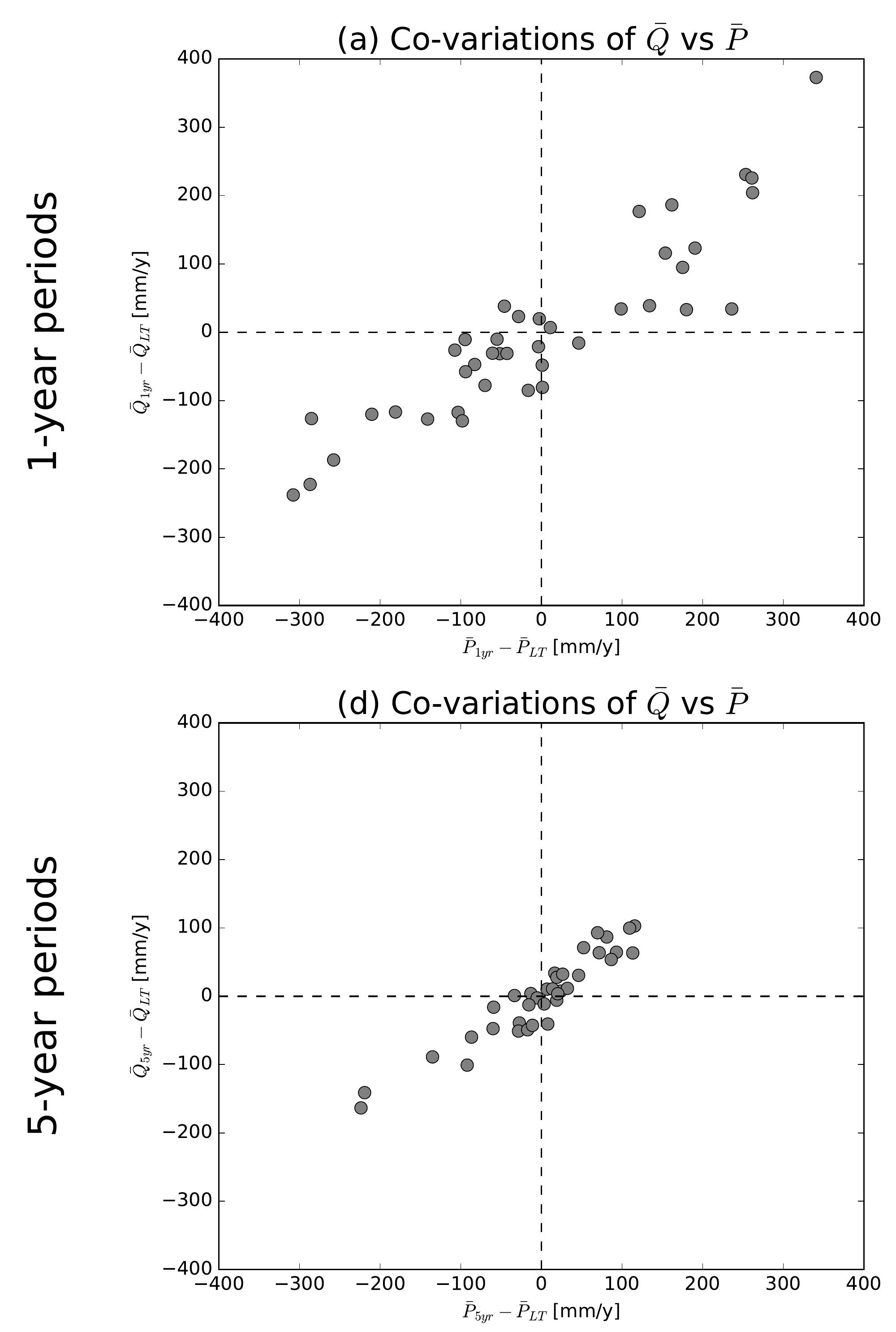

(g) Co-variations of $\bar{Q}$ vs $\bar{P}$

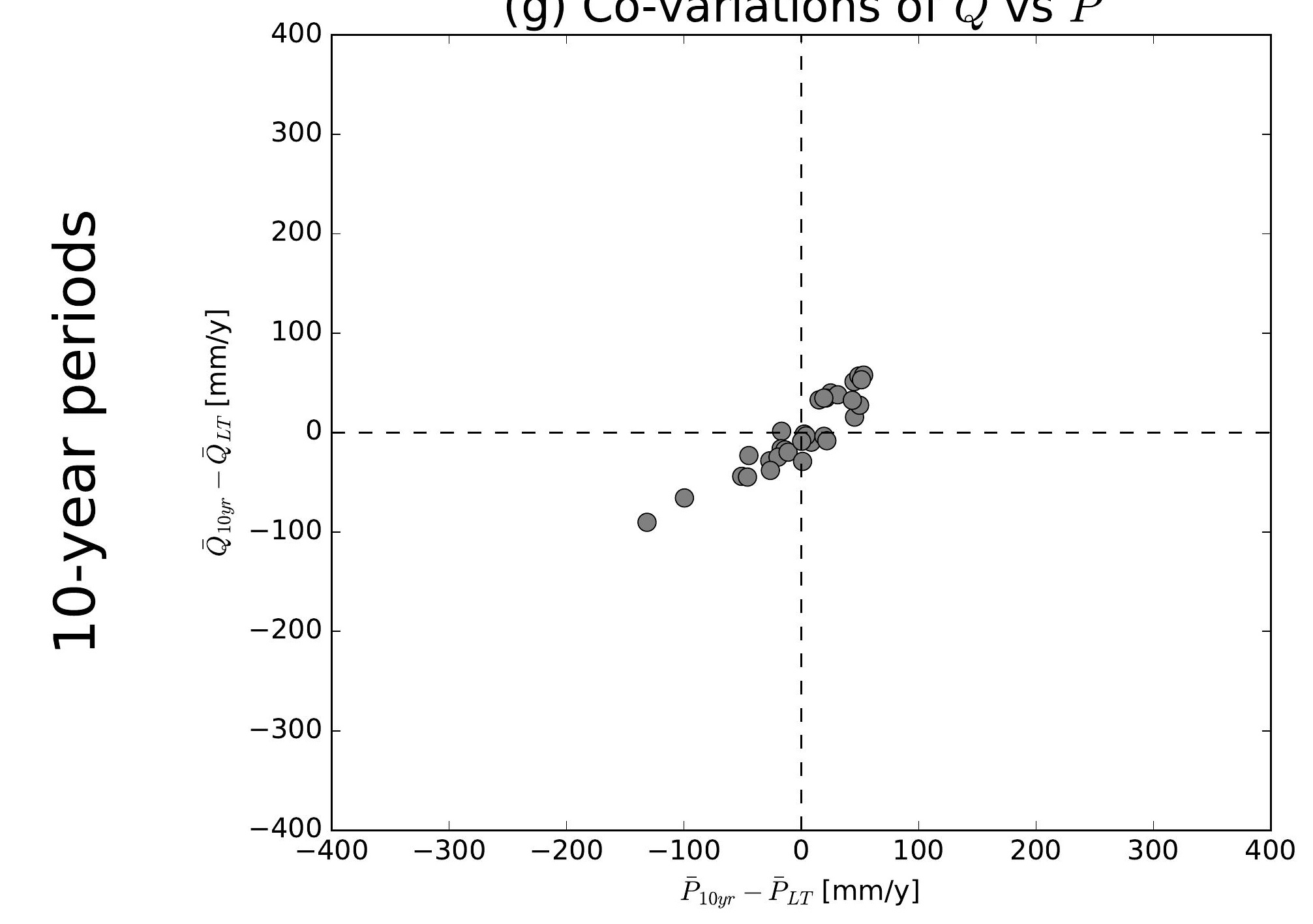

(j) Co-variations of $\bar{Q}$ vs $\bar{P}$

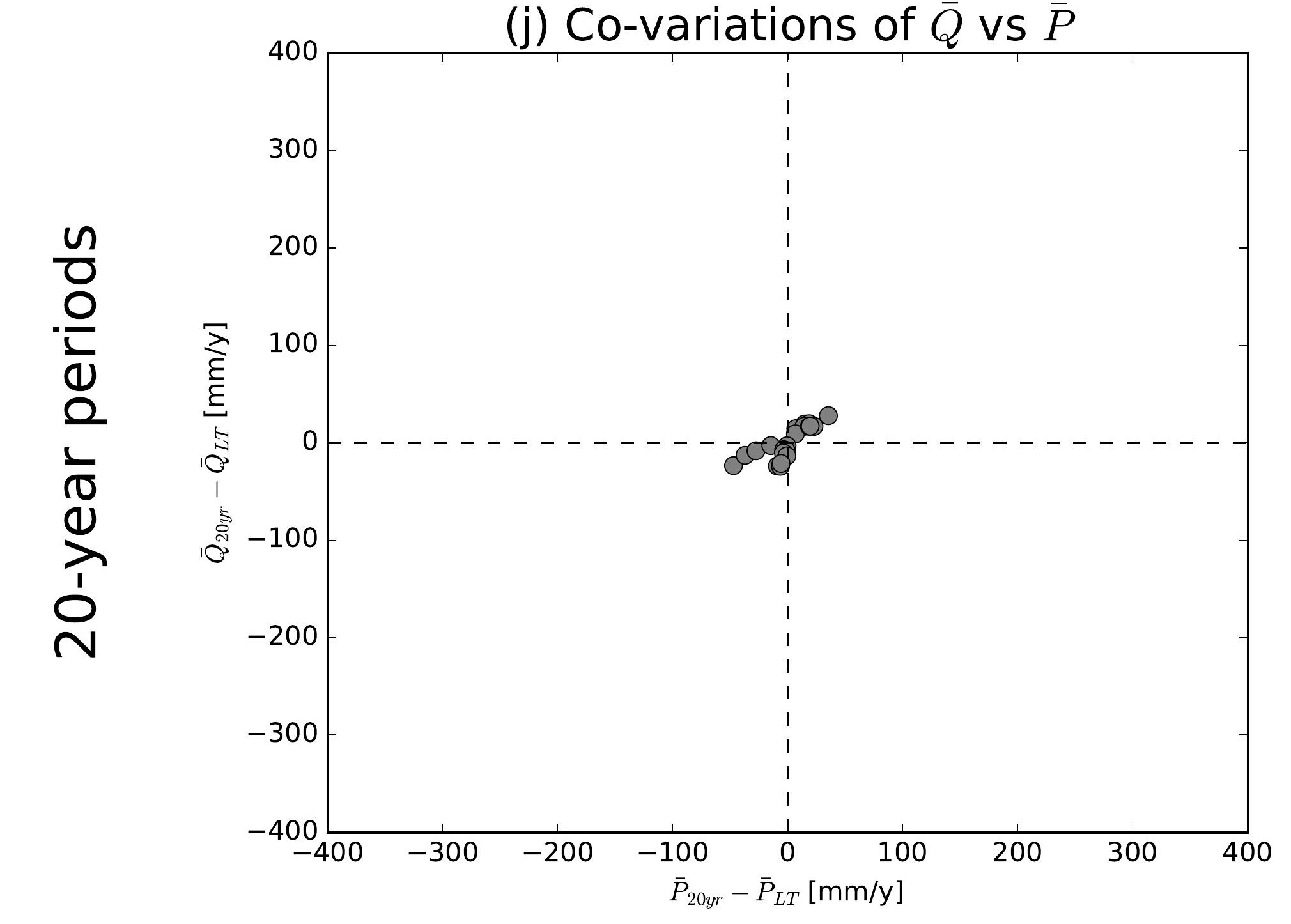

(b) Co-variations of $\bar{Q}$ vs $\overline{E 0}$

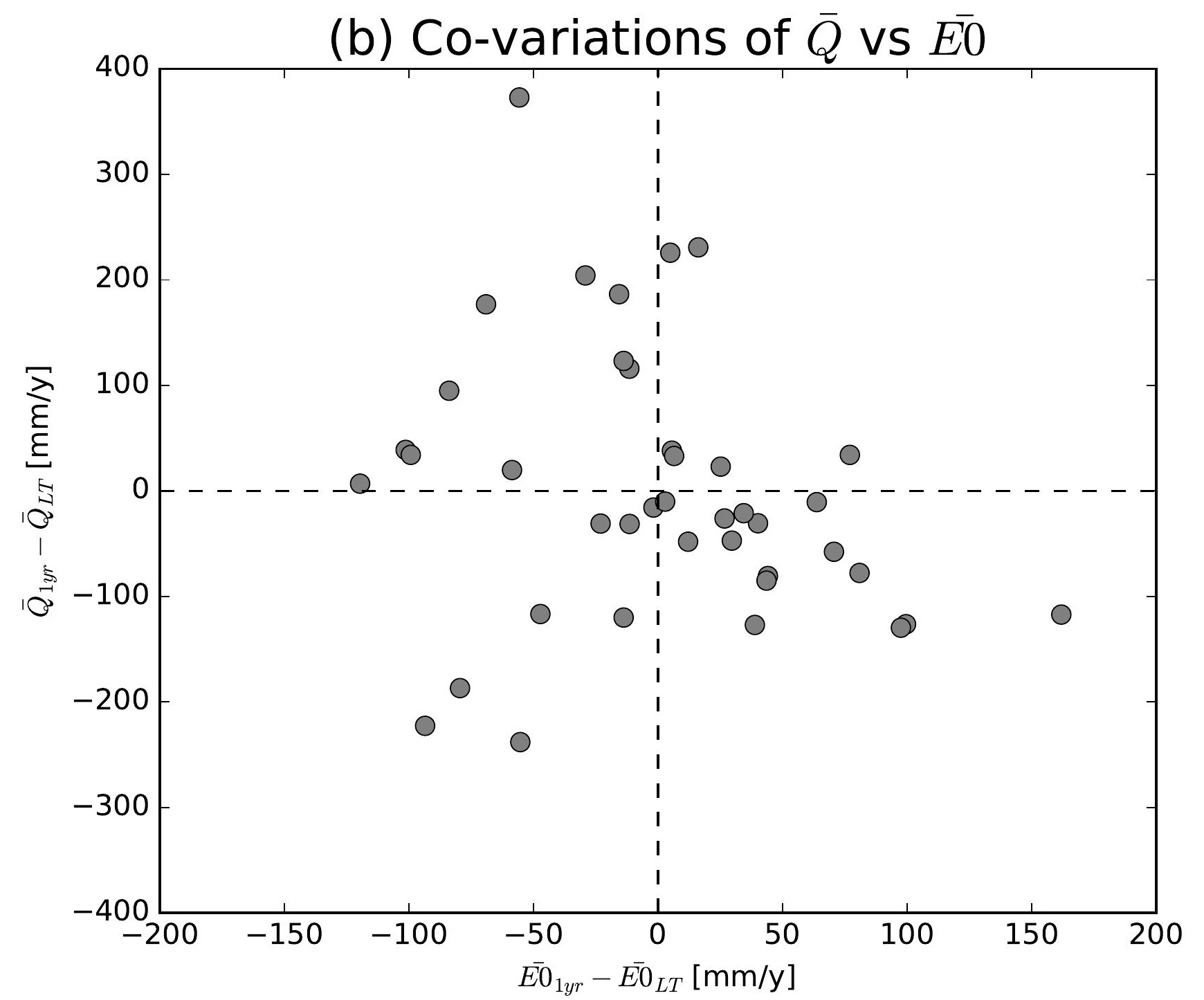

(e) Co-variations of $\bar{Q}$ vs $\overline{E 0}$

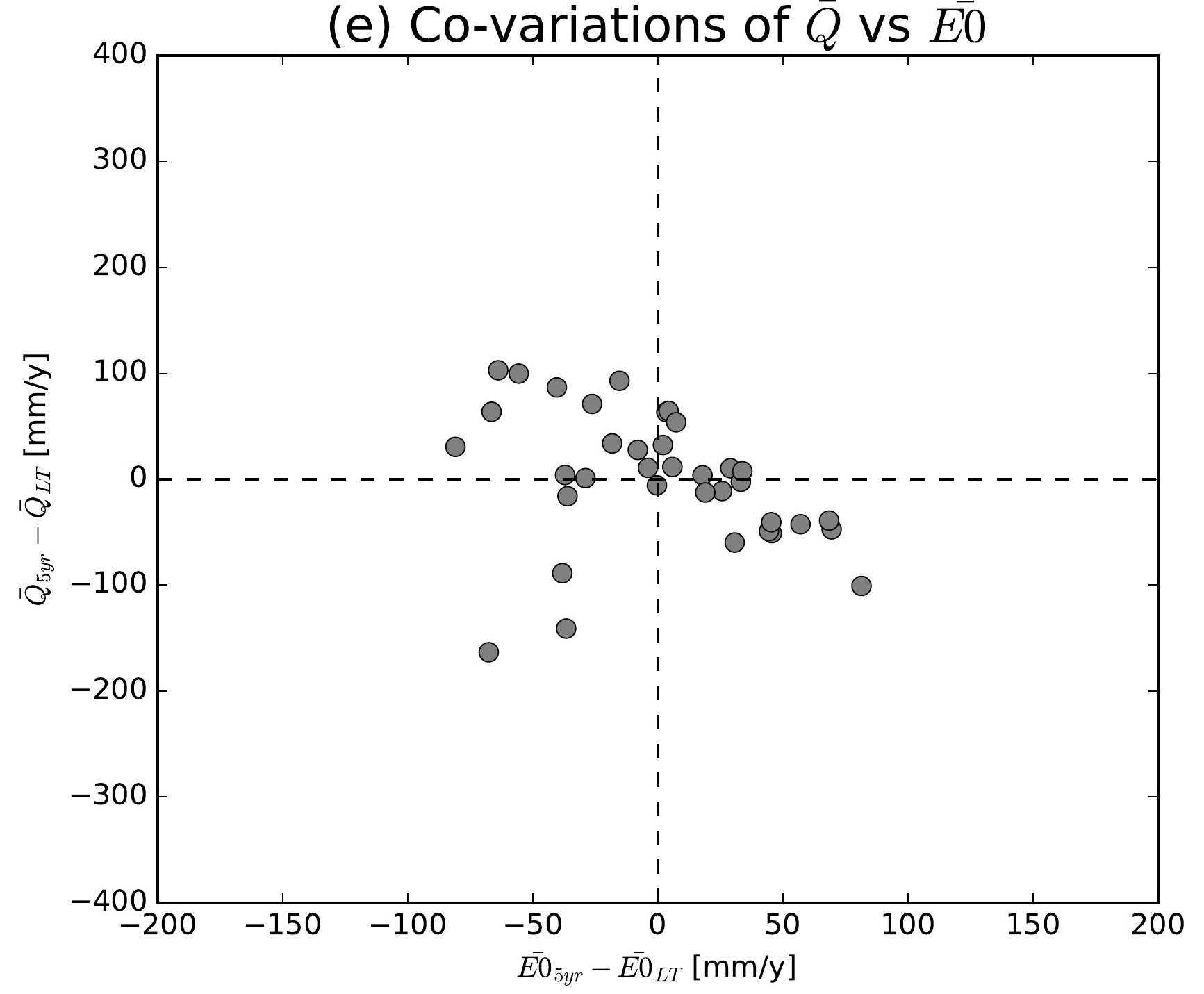

(h) Co-variations of $\bar{Q}$ vs $\overline{E 0}$

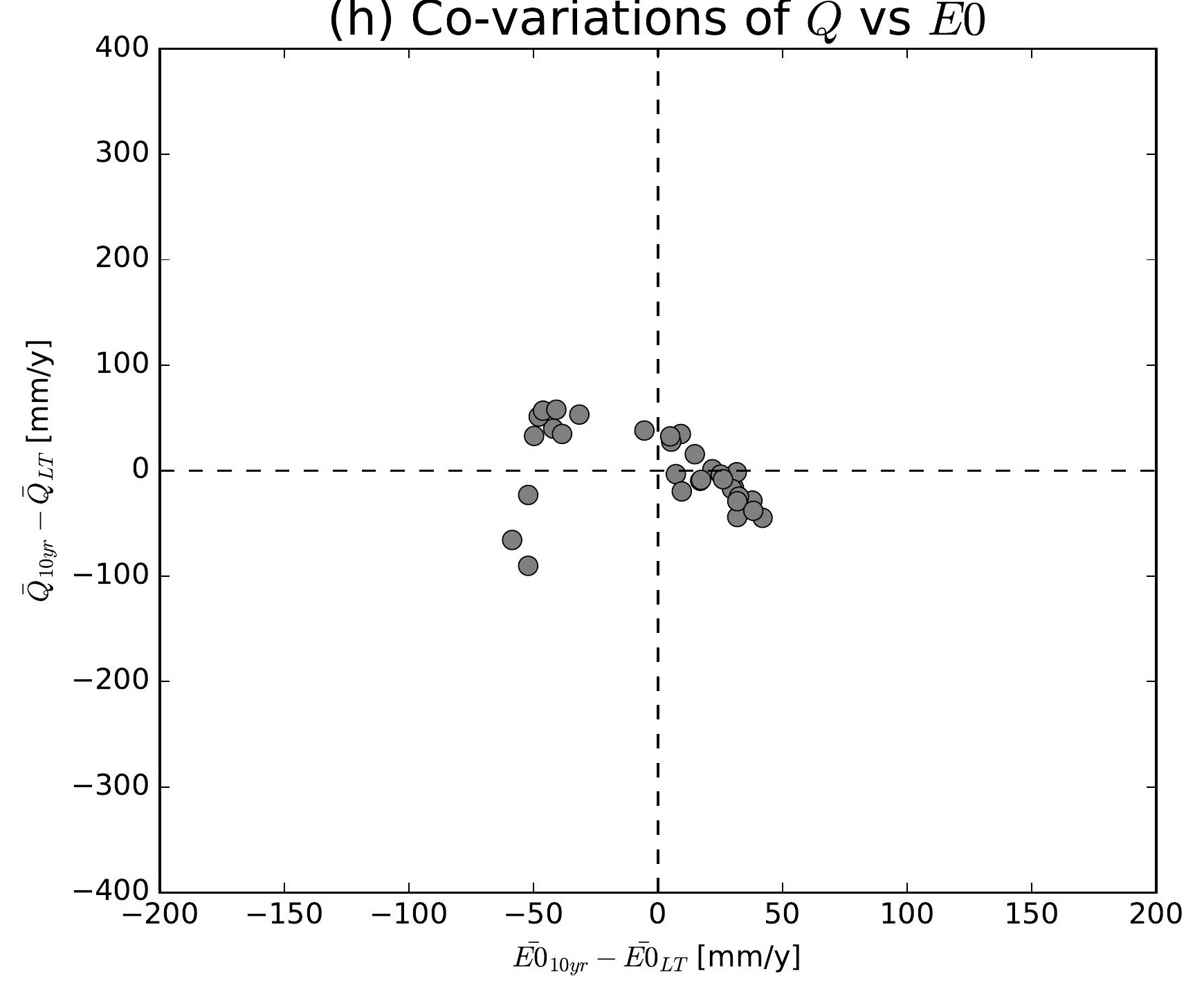

(k) Co-variations of $\bar{Q}$ vs $\overline{E 0}$

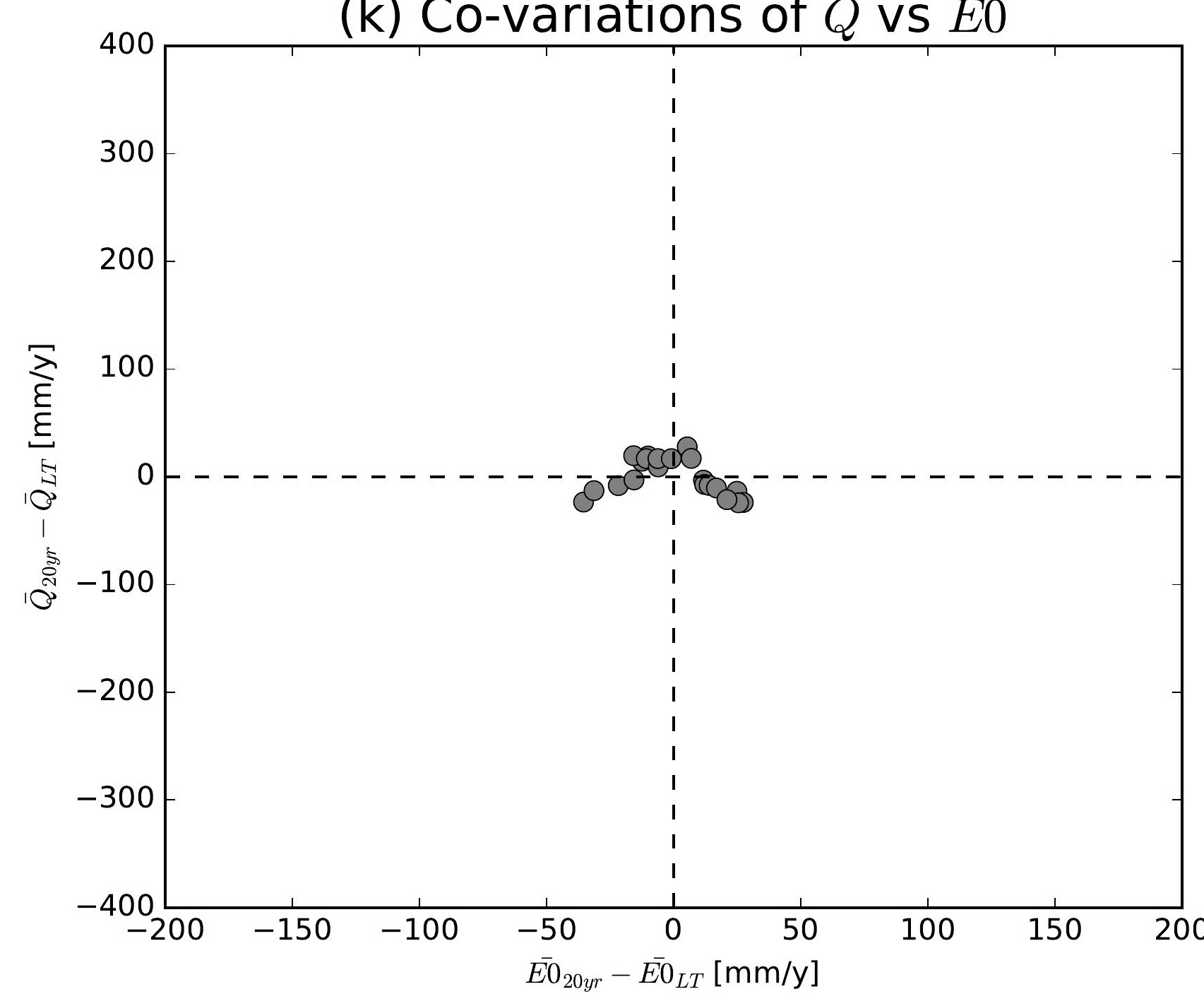

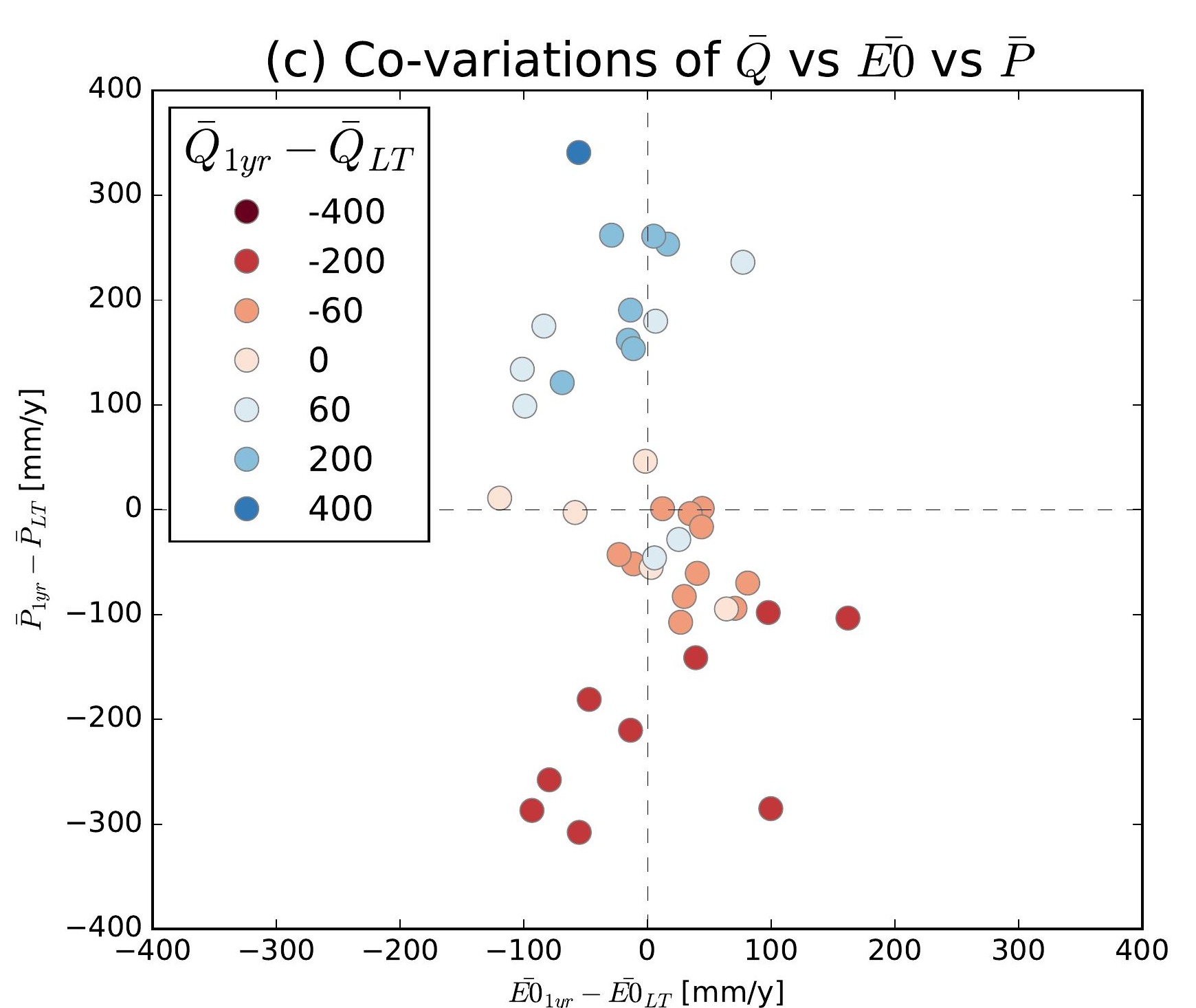
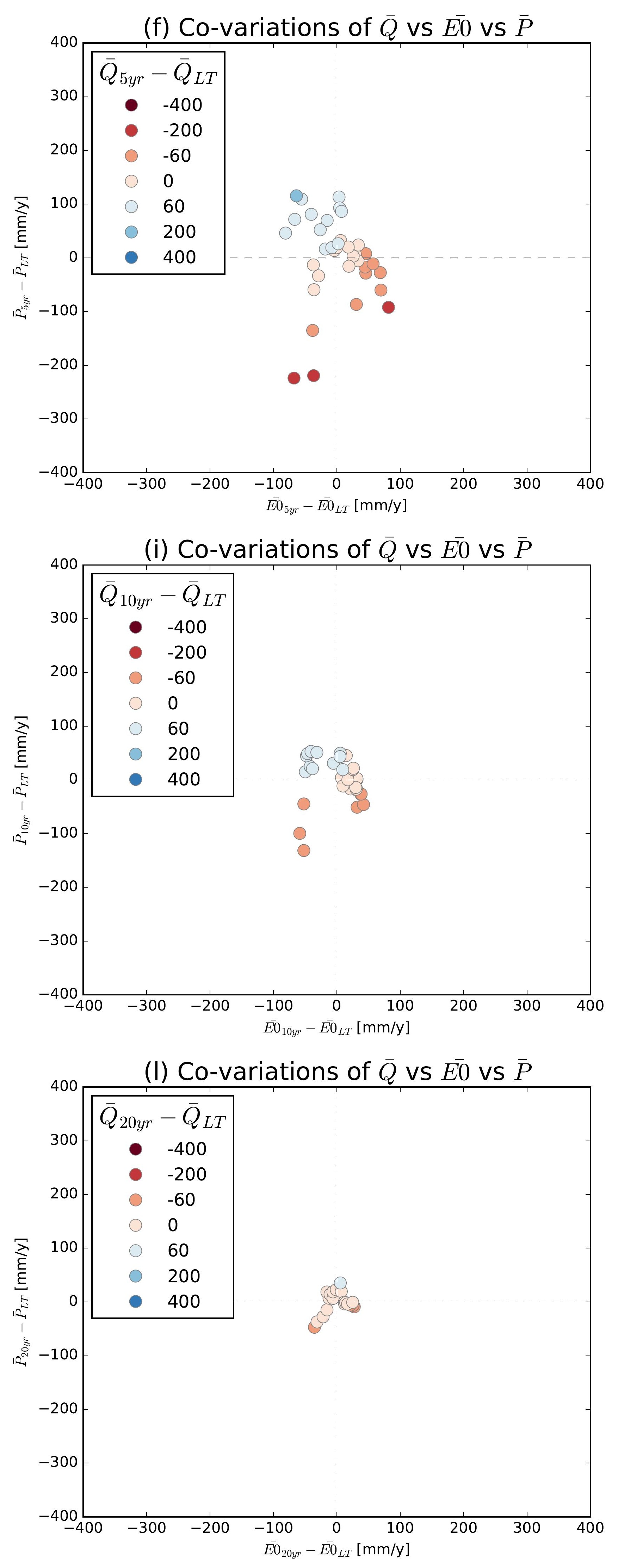

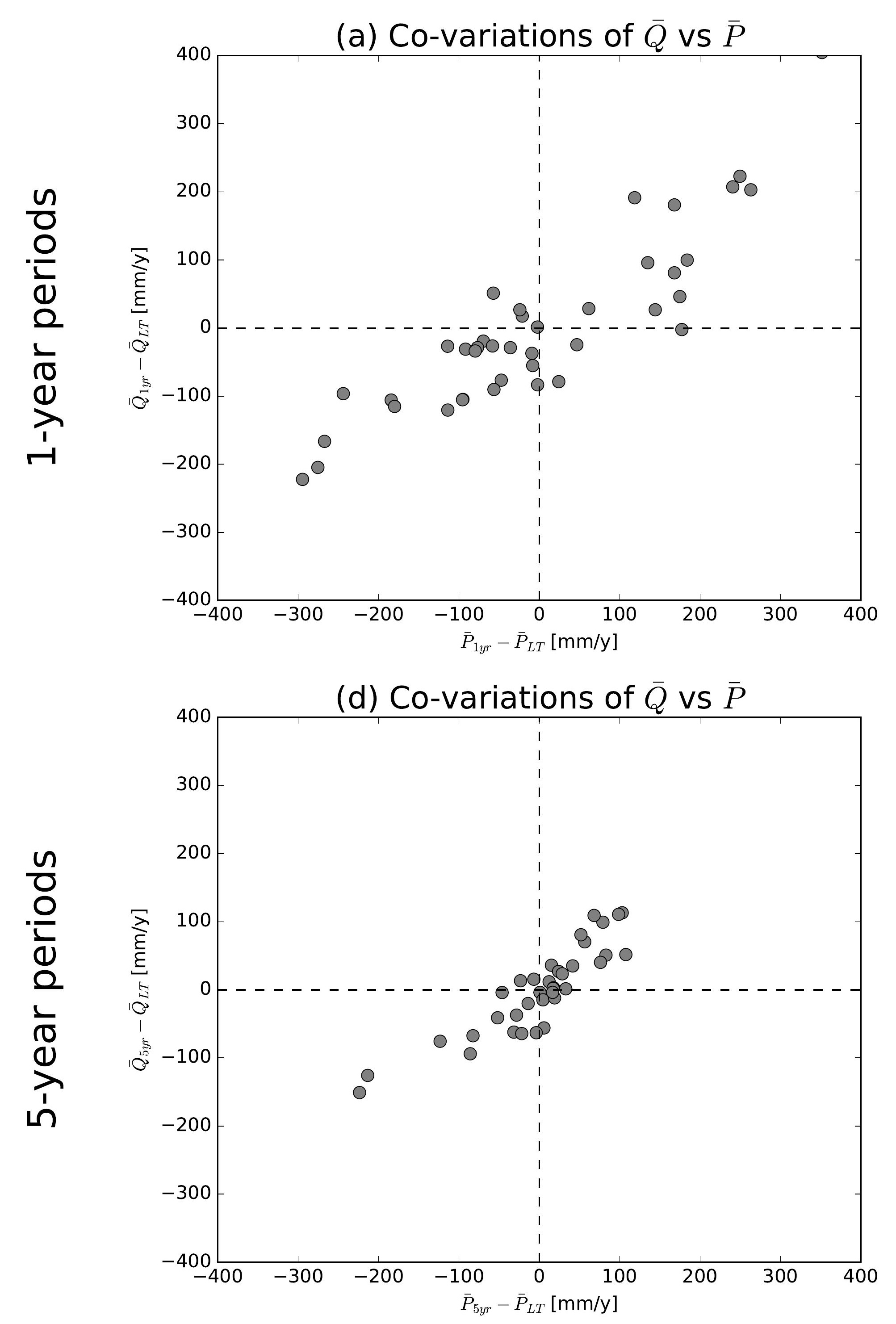

(g) Co-variations of $\bar{Q}$ vs $\bar{P}$

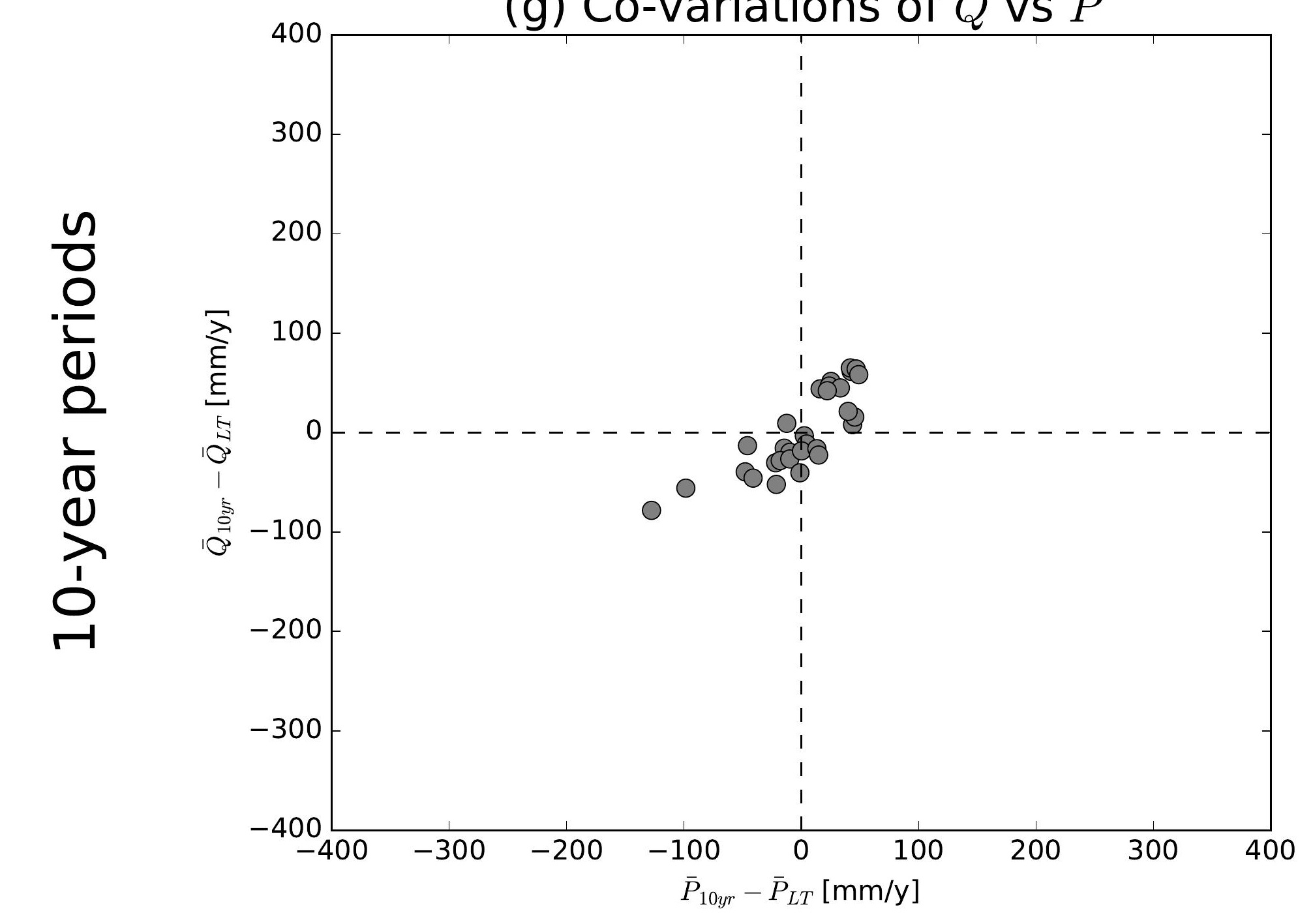

(j) Co-variations of $\bar{Q}$ vs $\bar{P}$

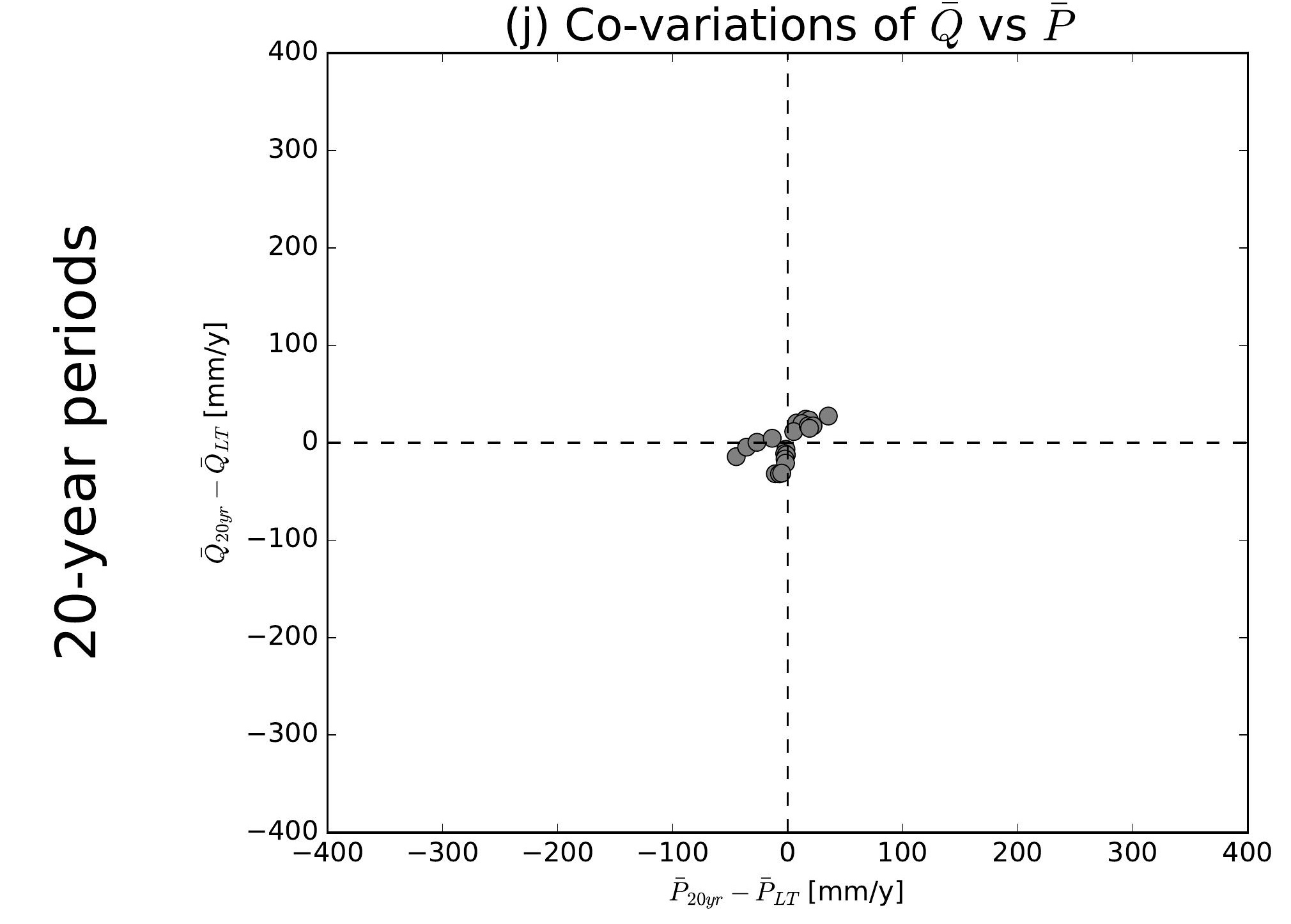

(b) Co-variations of $\bar{Q}$ vs $\overline{E 0}$

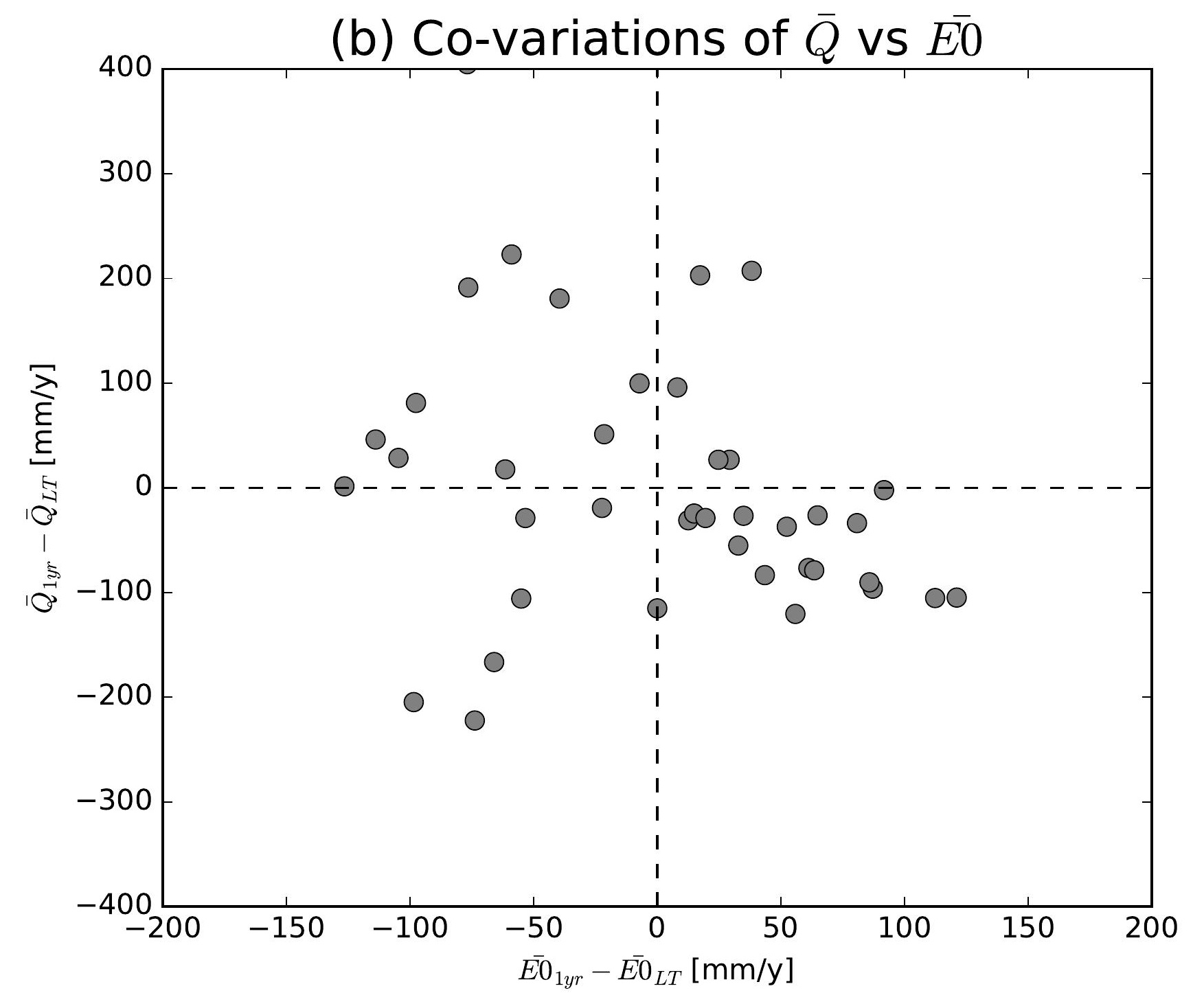

(e) Co-variations of $\bar{Q}$ vs $\overline{E 0}$

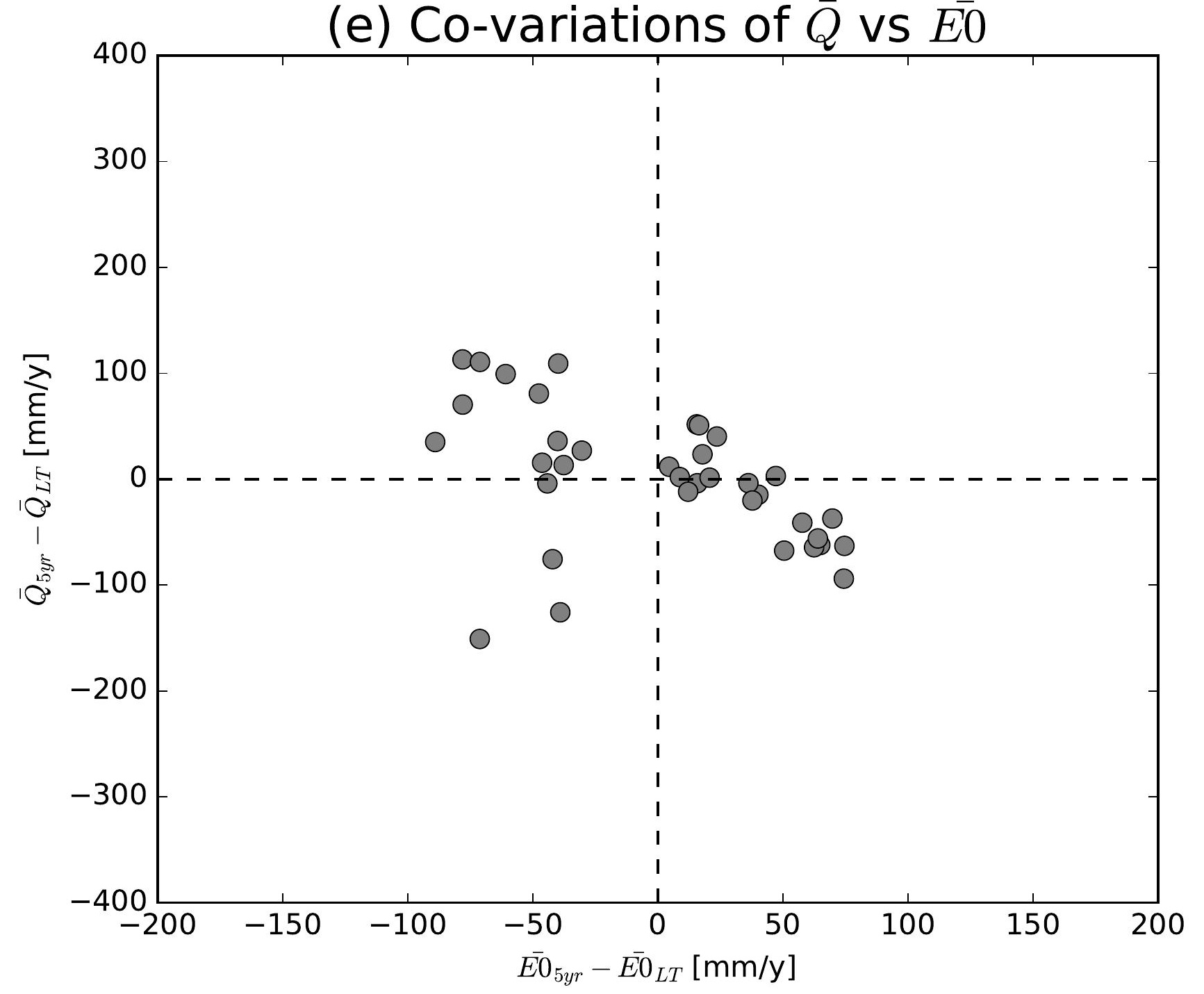

(h) Co-variations of $\bar{Q}$ vs $\overline{E 0}$

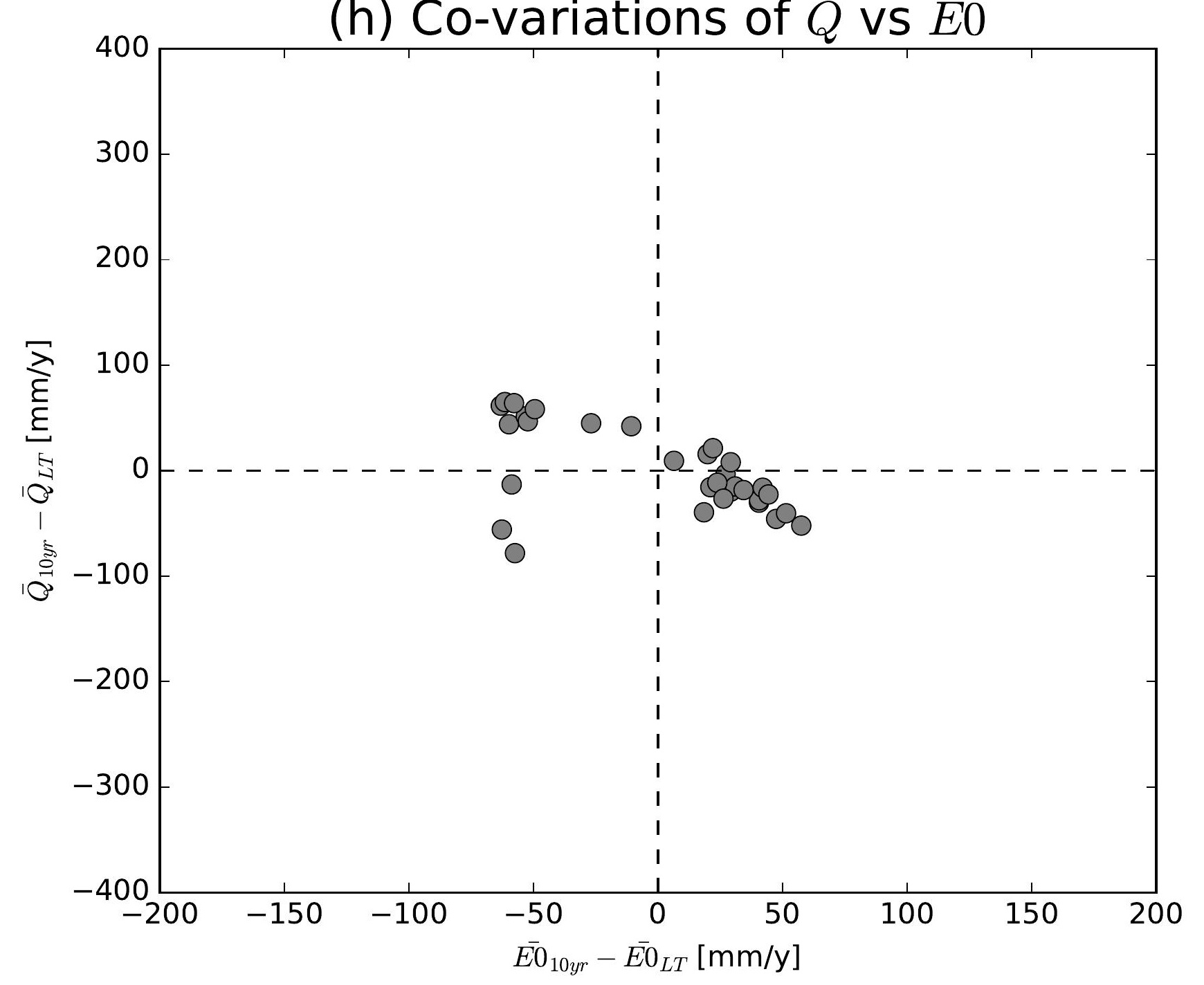

(k) Co-variations of $\bar{Q}$ vs $\overline{E 0}$

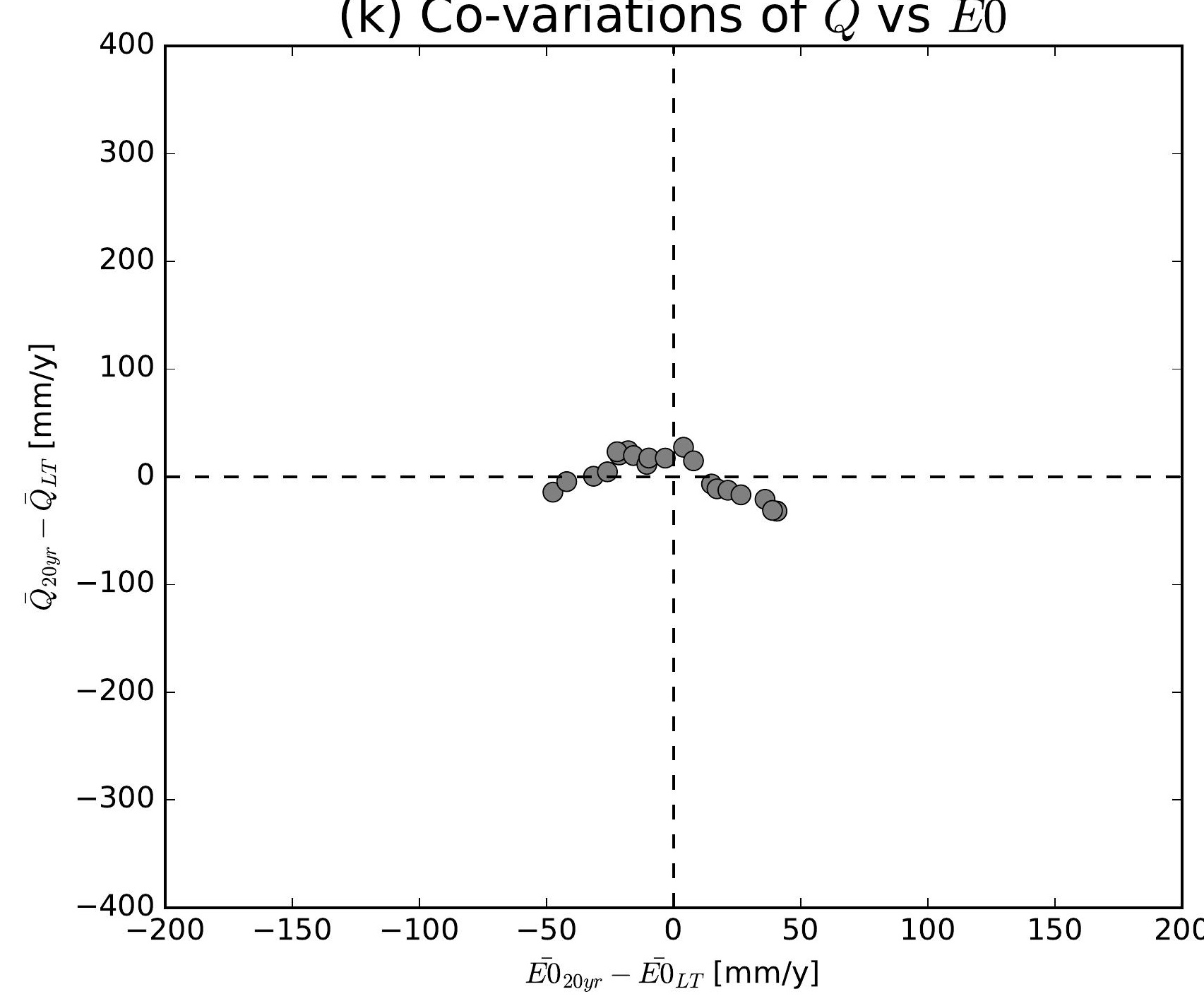

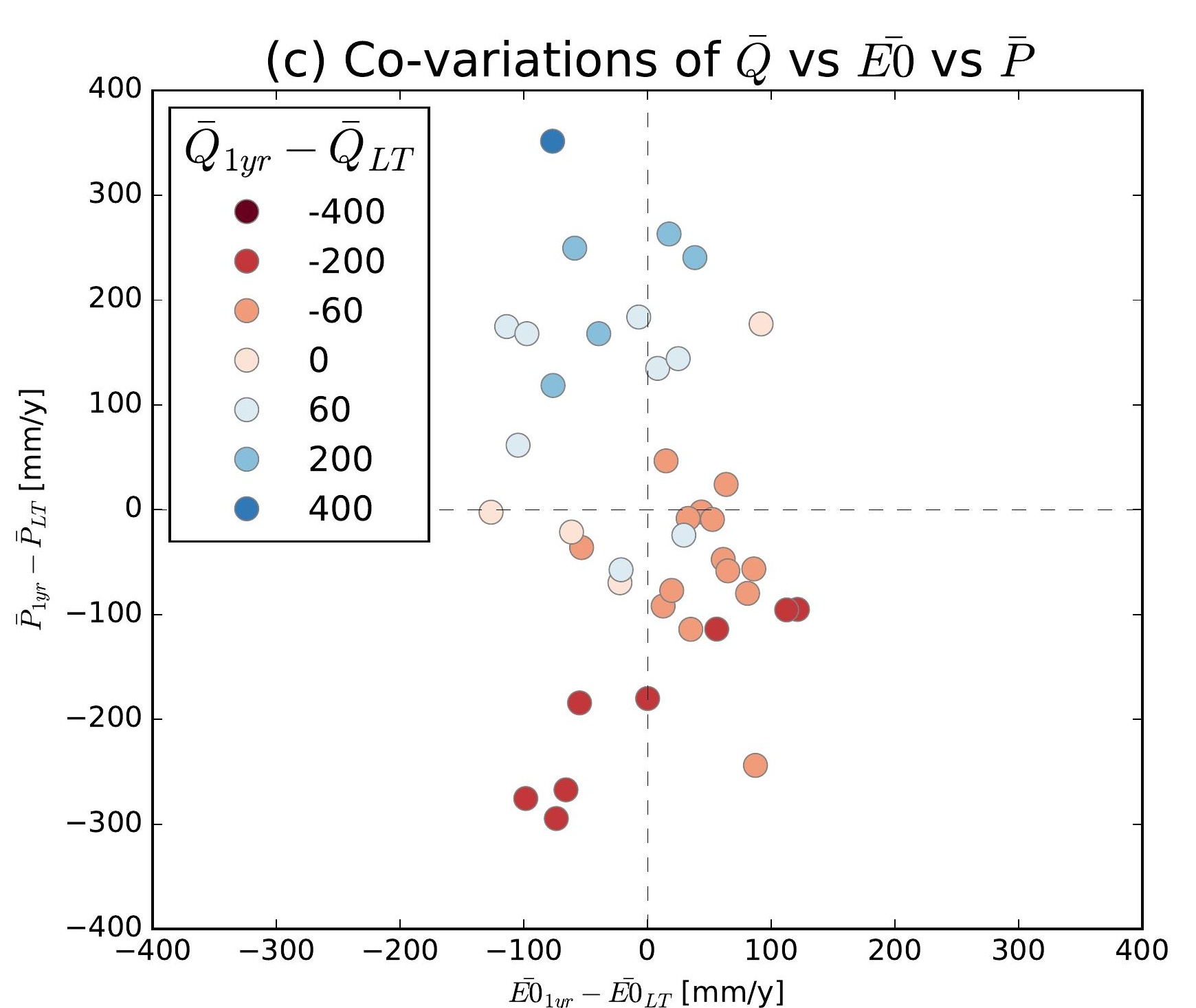
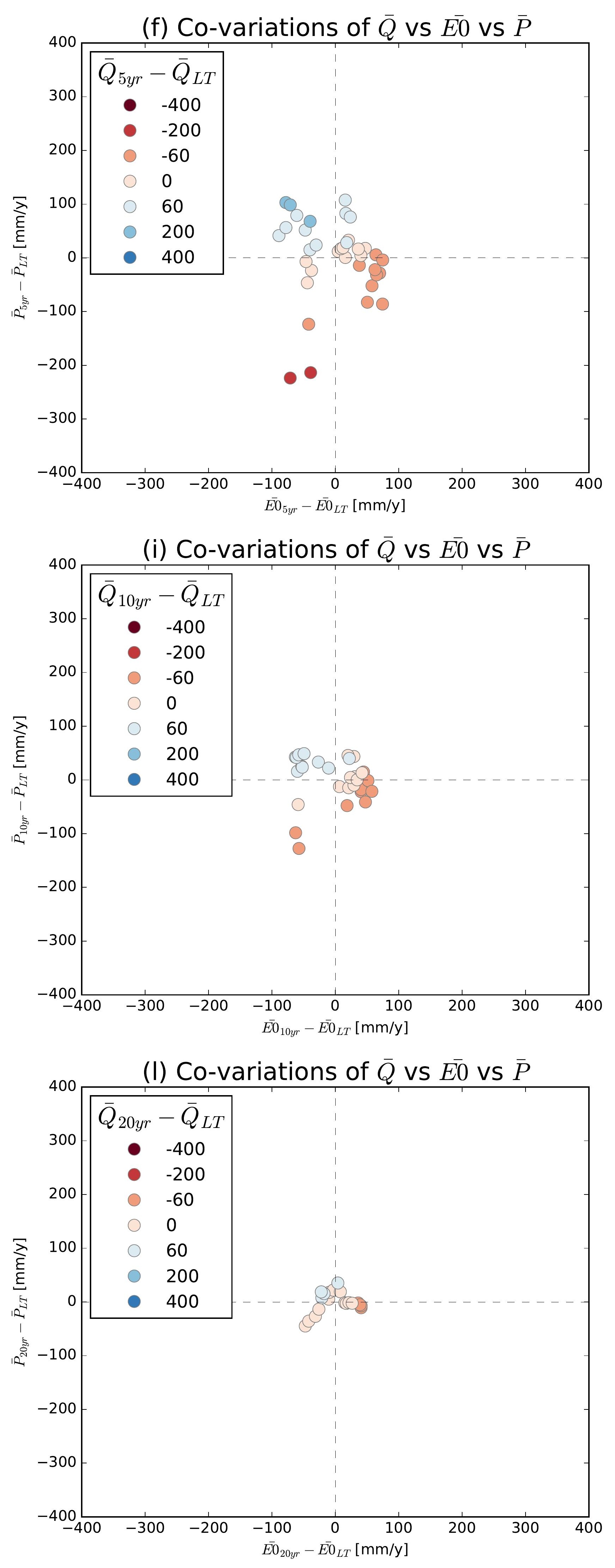

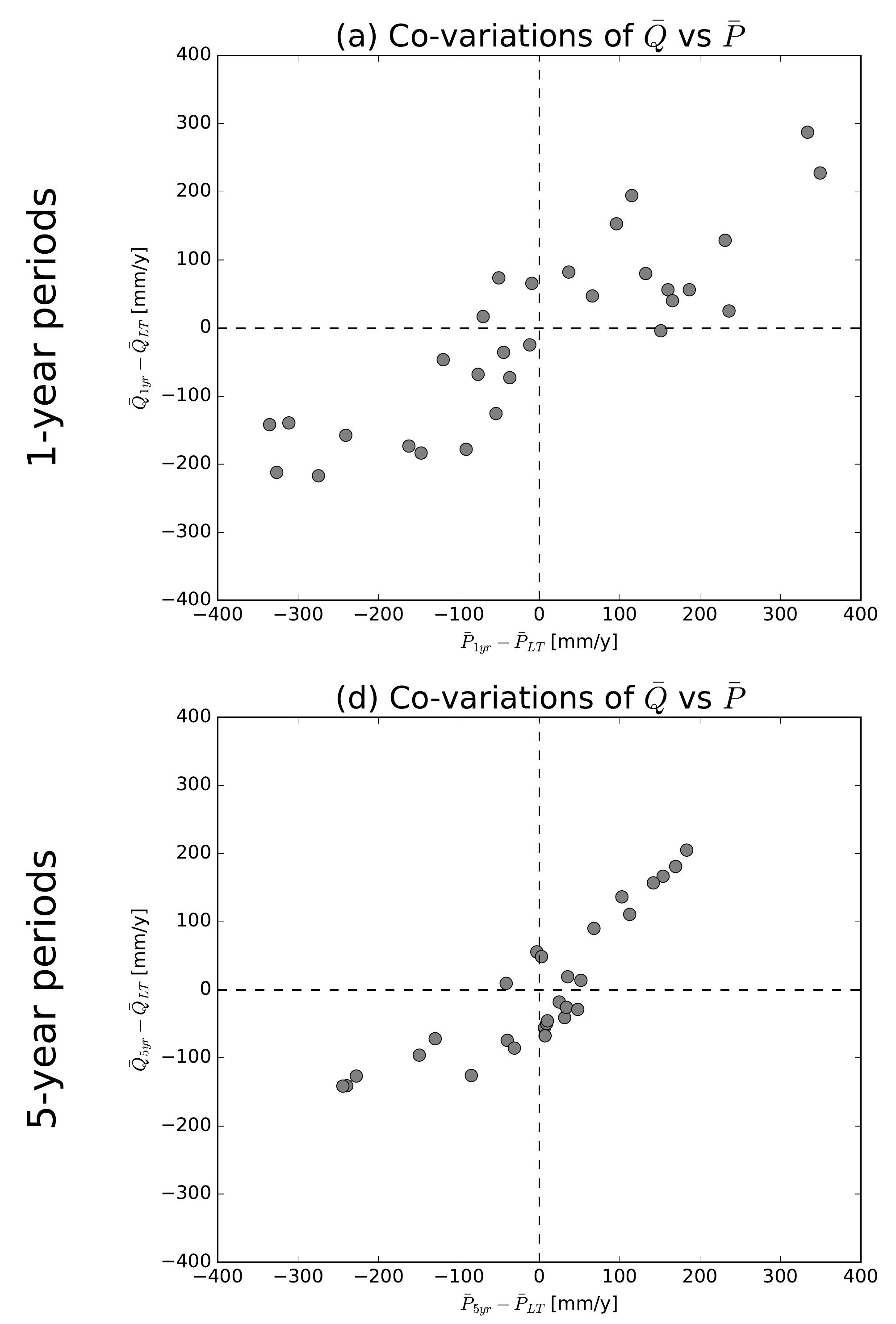

(g) Co-variations of $\bar{Q}$ vs $\bar{P}$

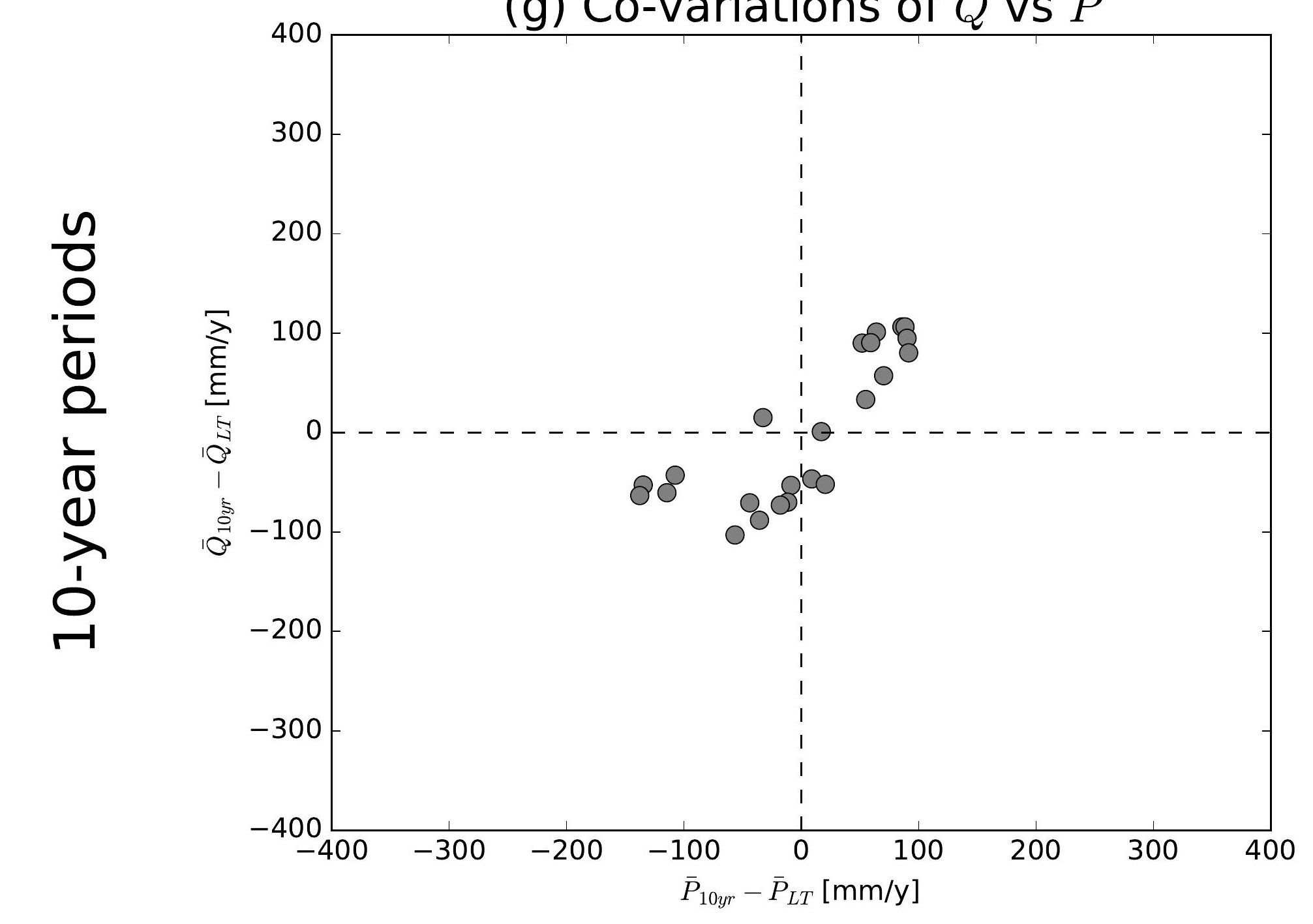

(j) Co-variations of $\bar{Q}$ vs $\bar{P}$

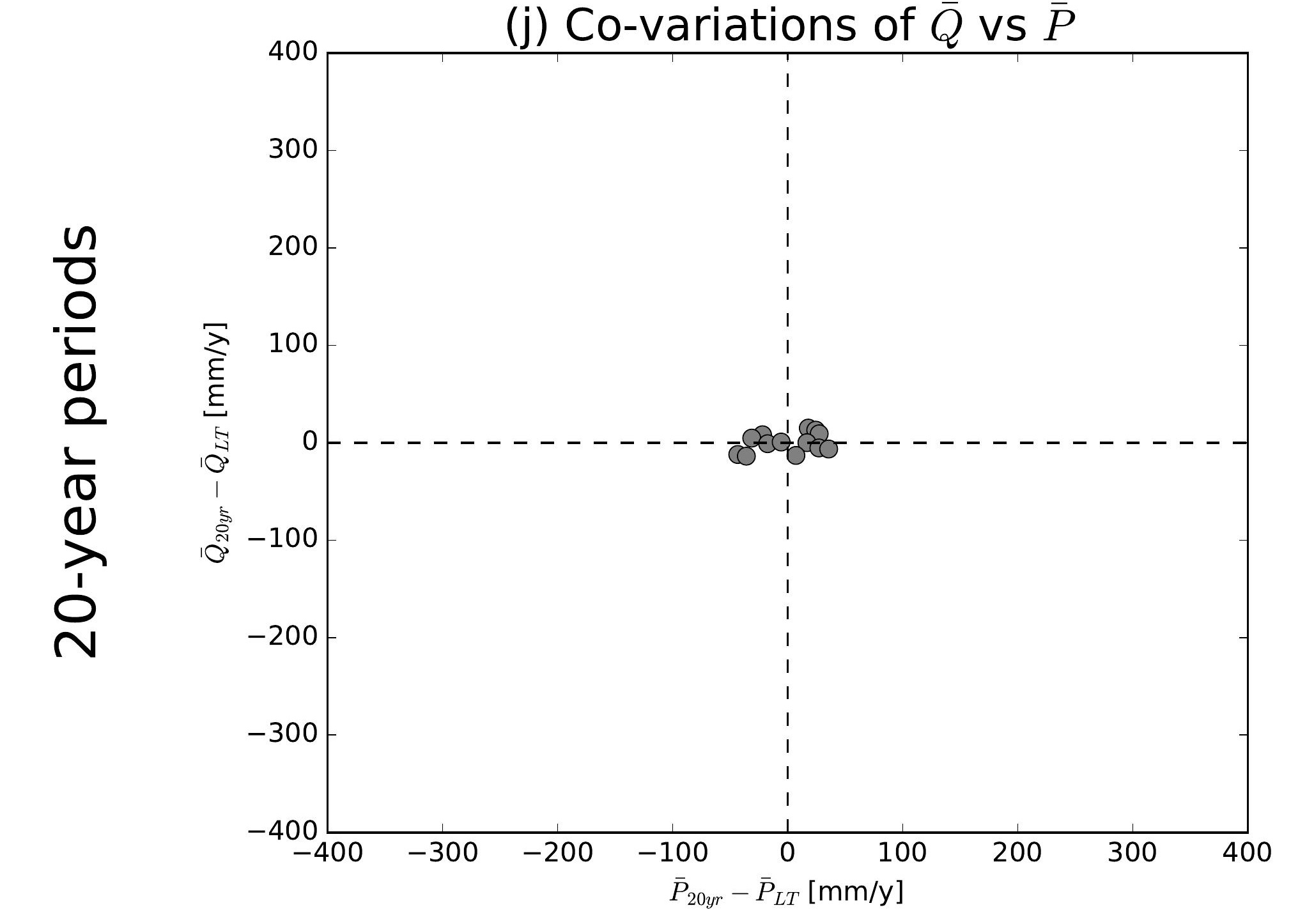

(b) Co-variations of $\bar{Q}$ vs $\overline{E 0}$

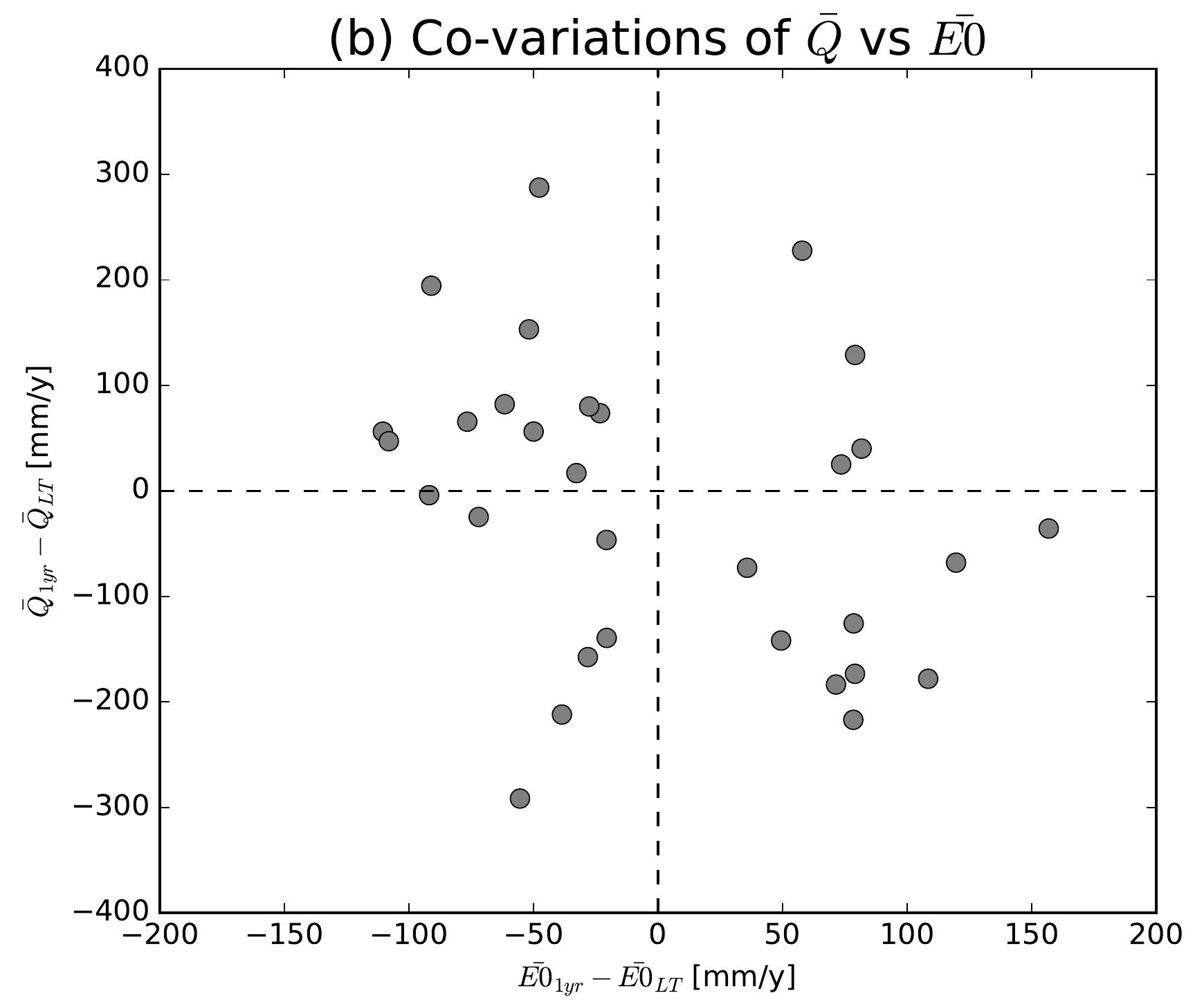

(e) Co-variations of $\bar{Q}$ vs $\overline{E 0}$

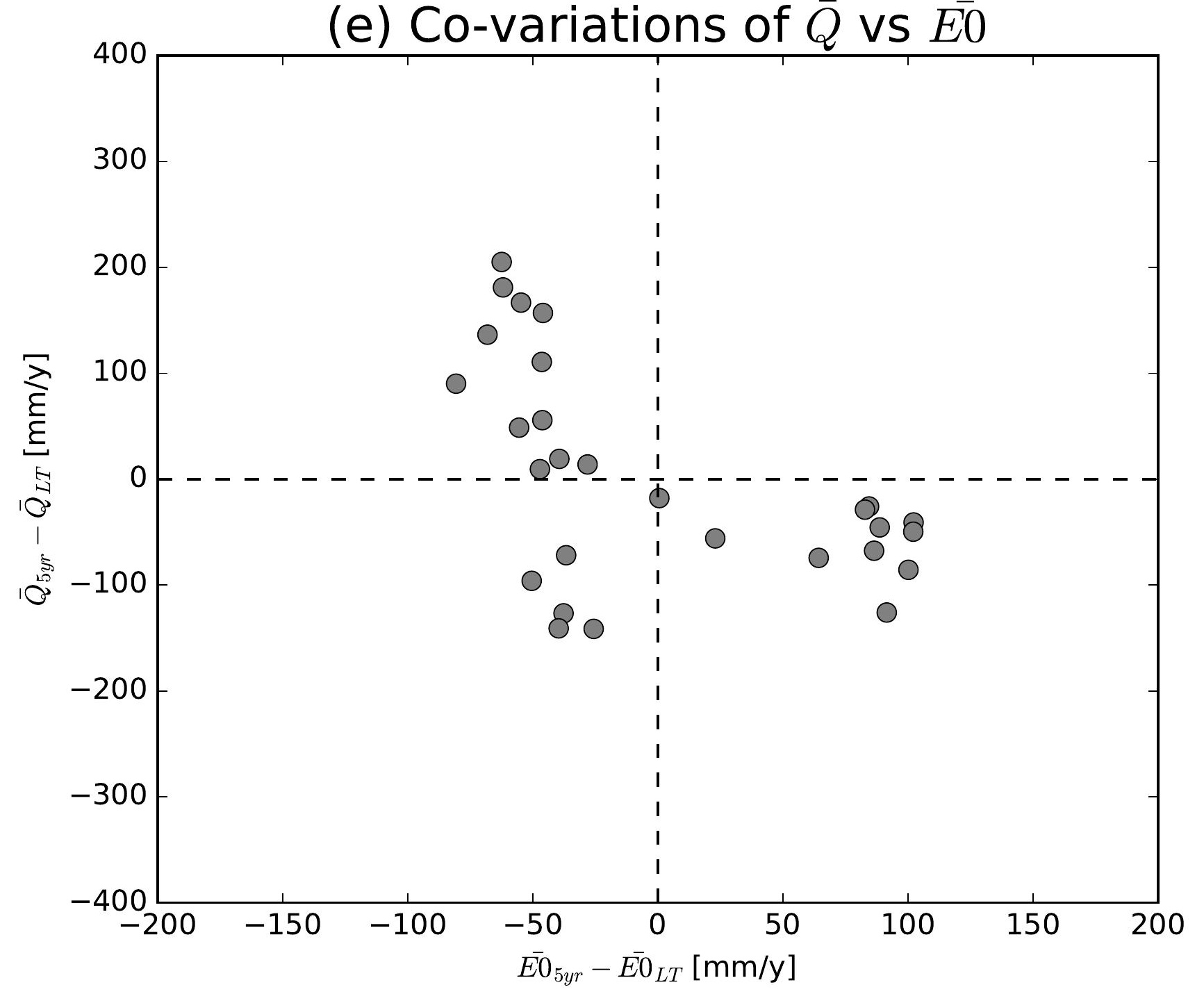

(h) Co-variations of $\bar{Q}$ vs $\overline{E 0}$

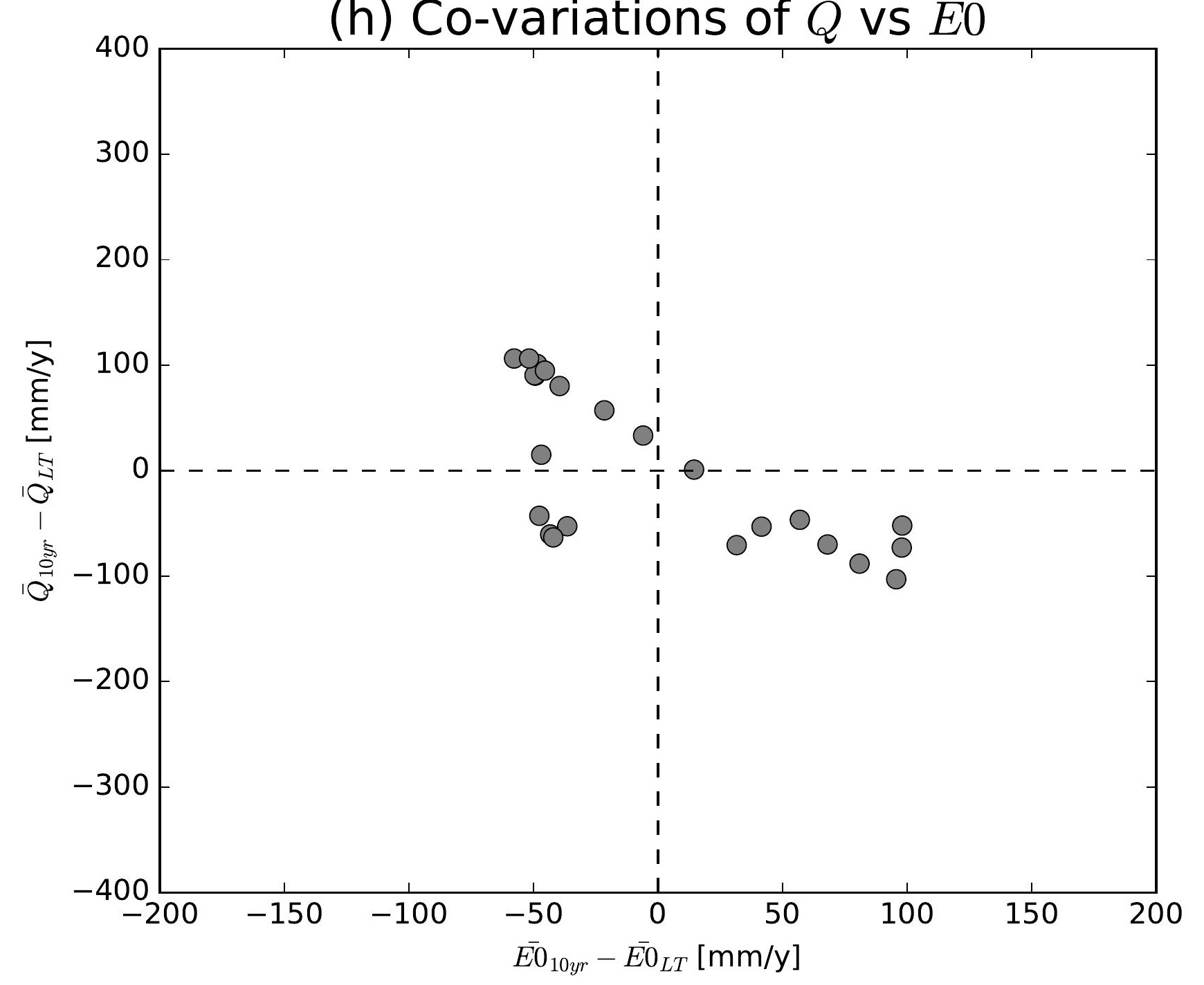

(k) Co-variations of $\bar{Q}$ vs $\overline{E 0}$

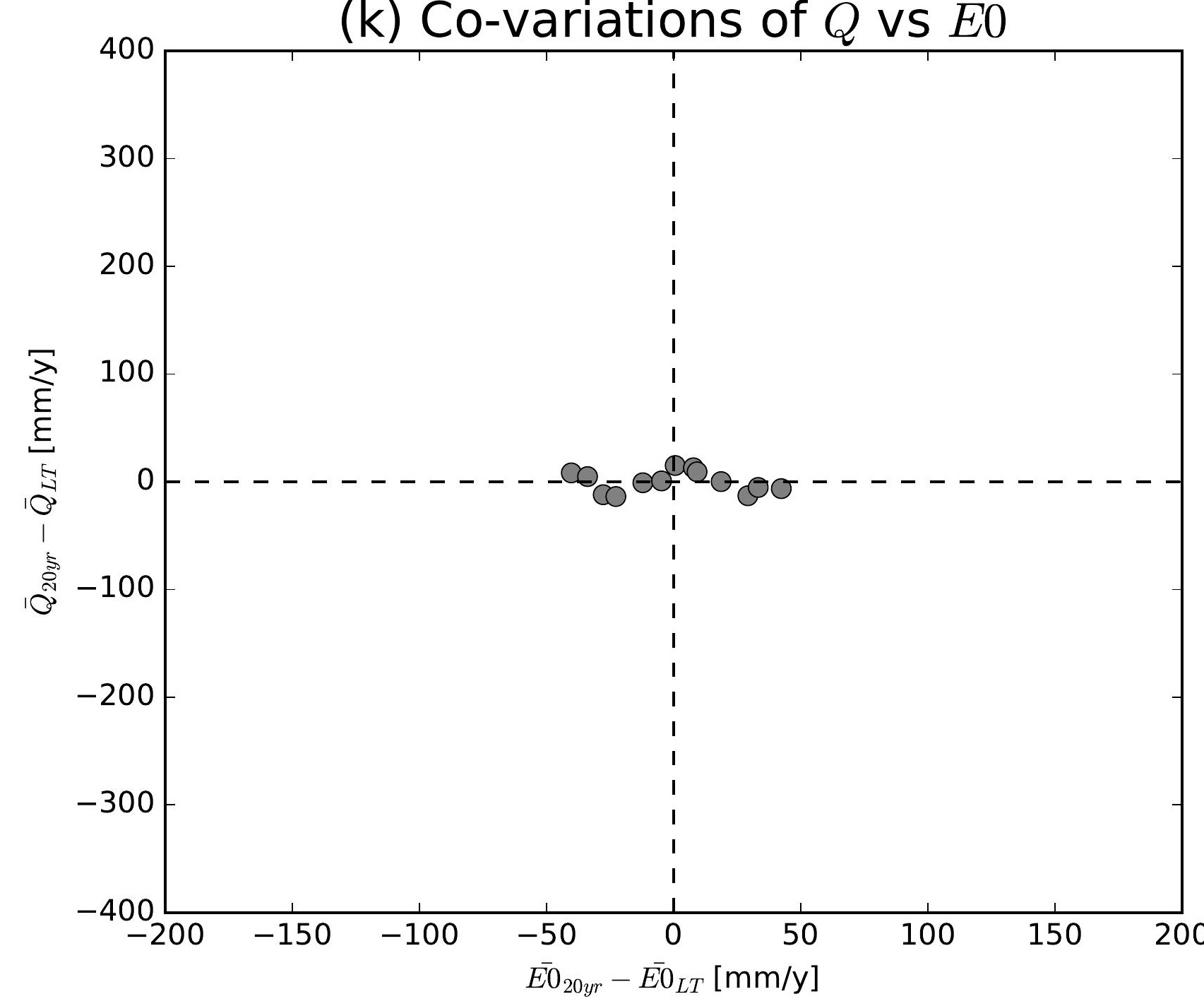

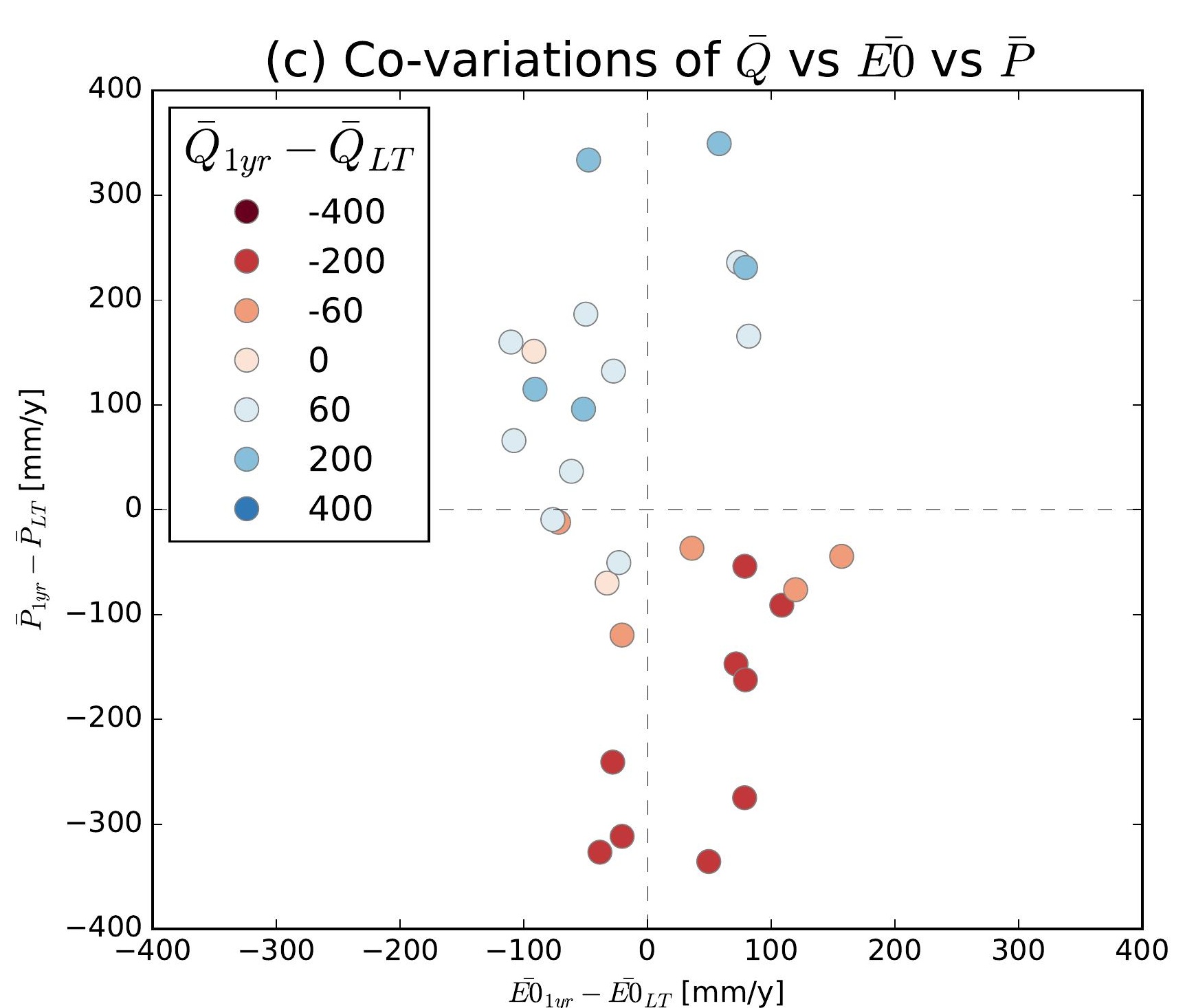
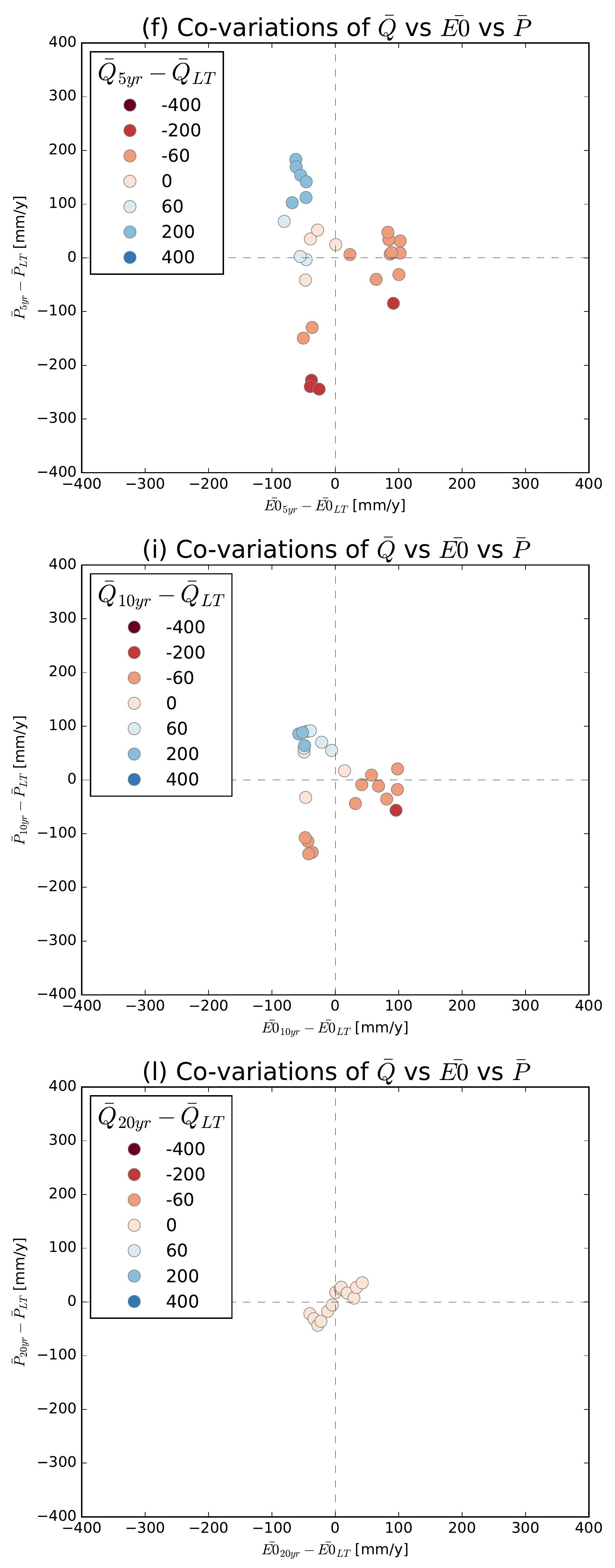

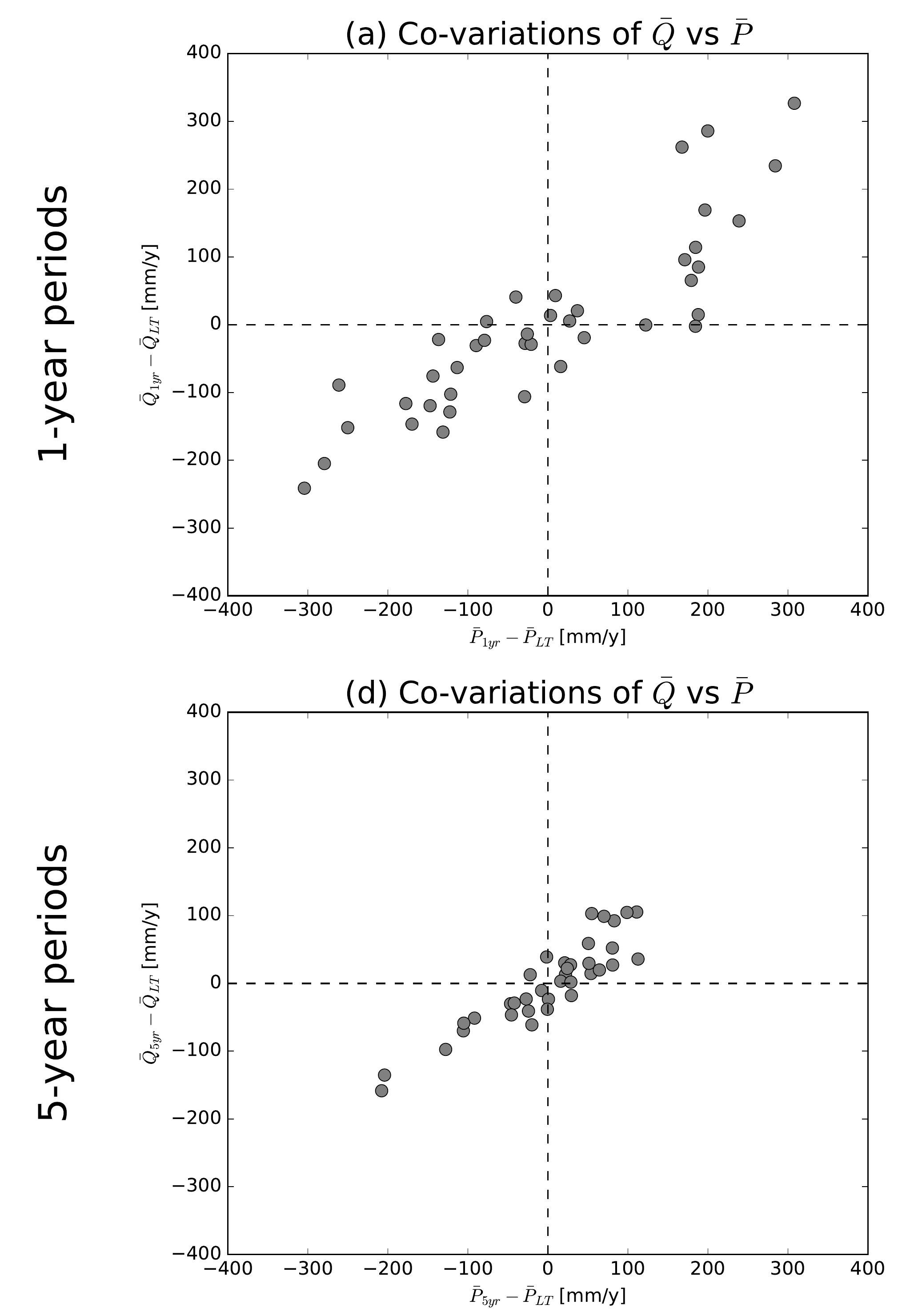

(g) Co-variations of $\bar{Q}$ vs $\bar{P}$

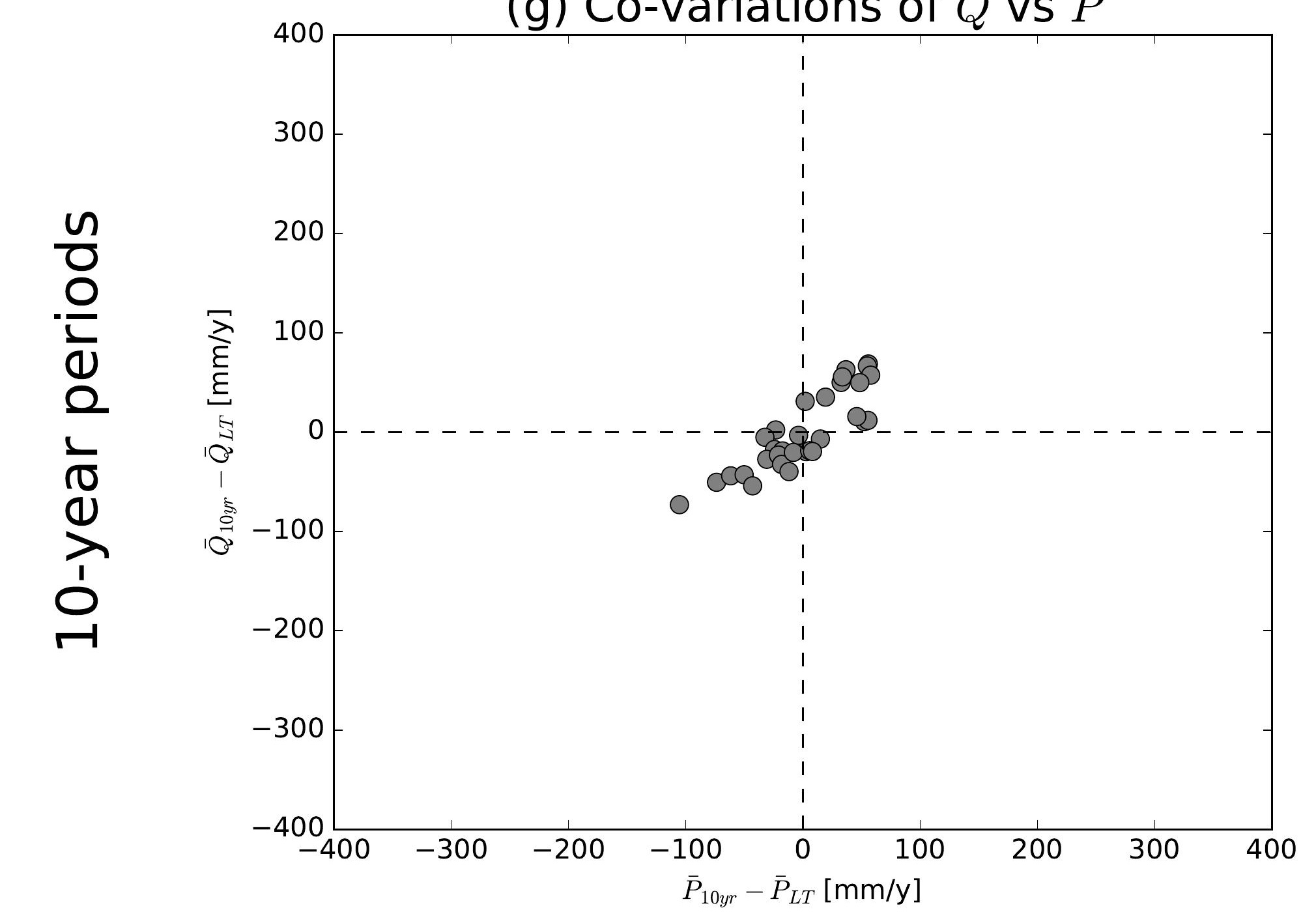

(j) Co-variations of $\bar{Q}$ vs $\bar{P}$

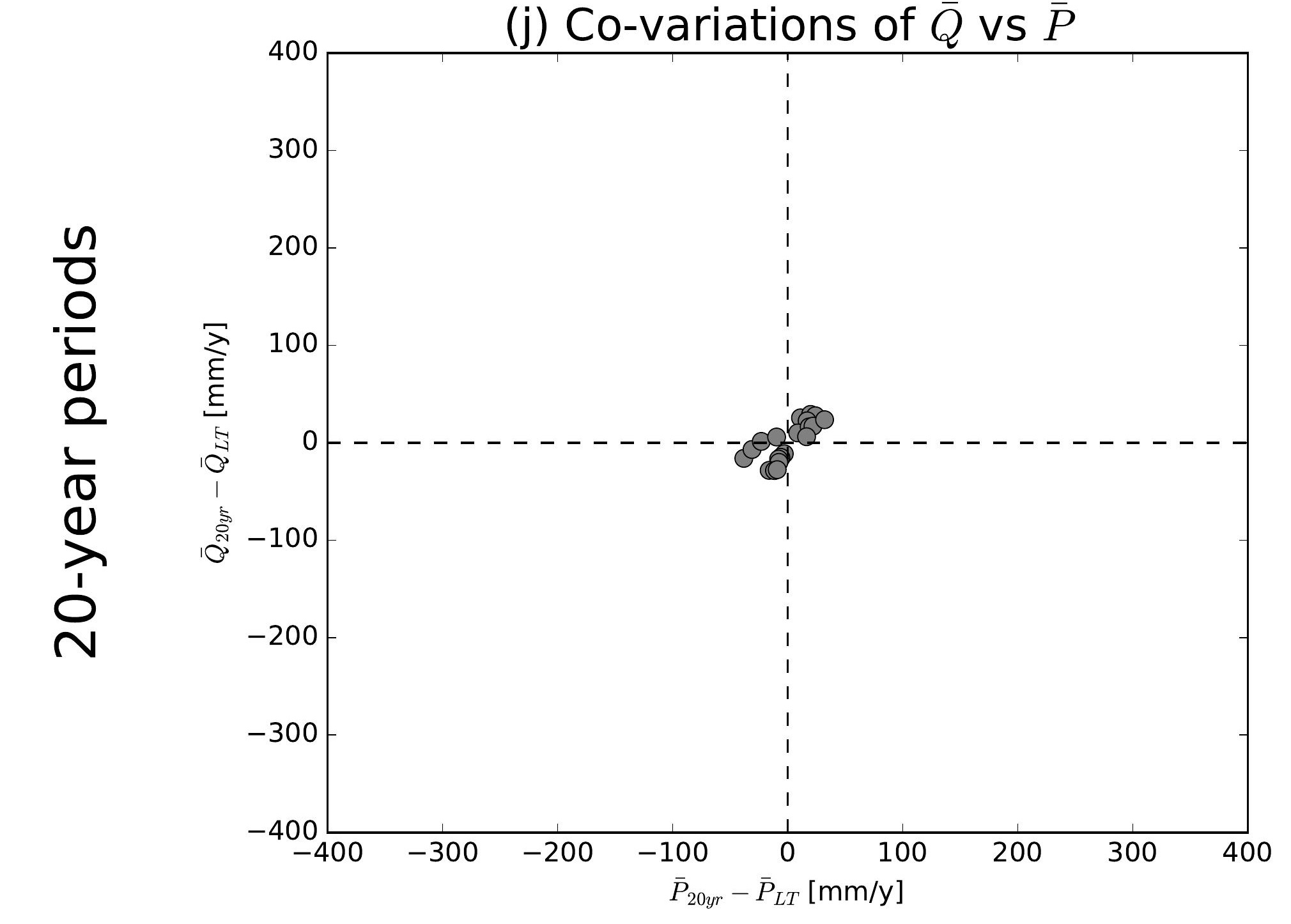

(b) Co-variations of $\bar{Q}$ vs $\overline{E 0}$

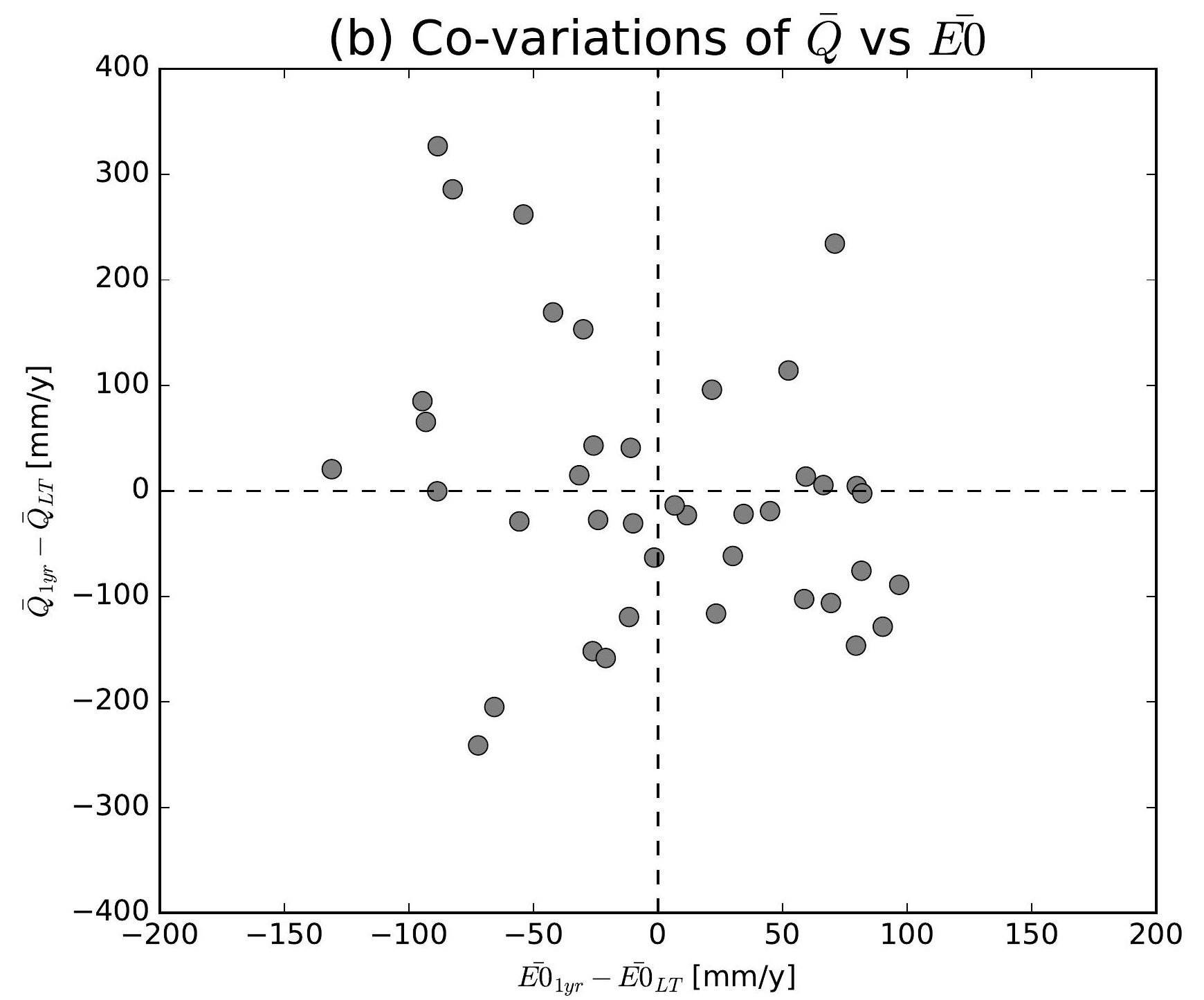

(e) Co-variations of $\bar{Q}$ vs $\overline{E 0}$

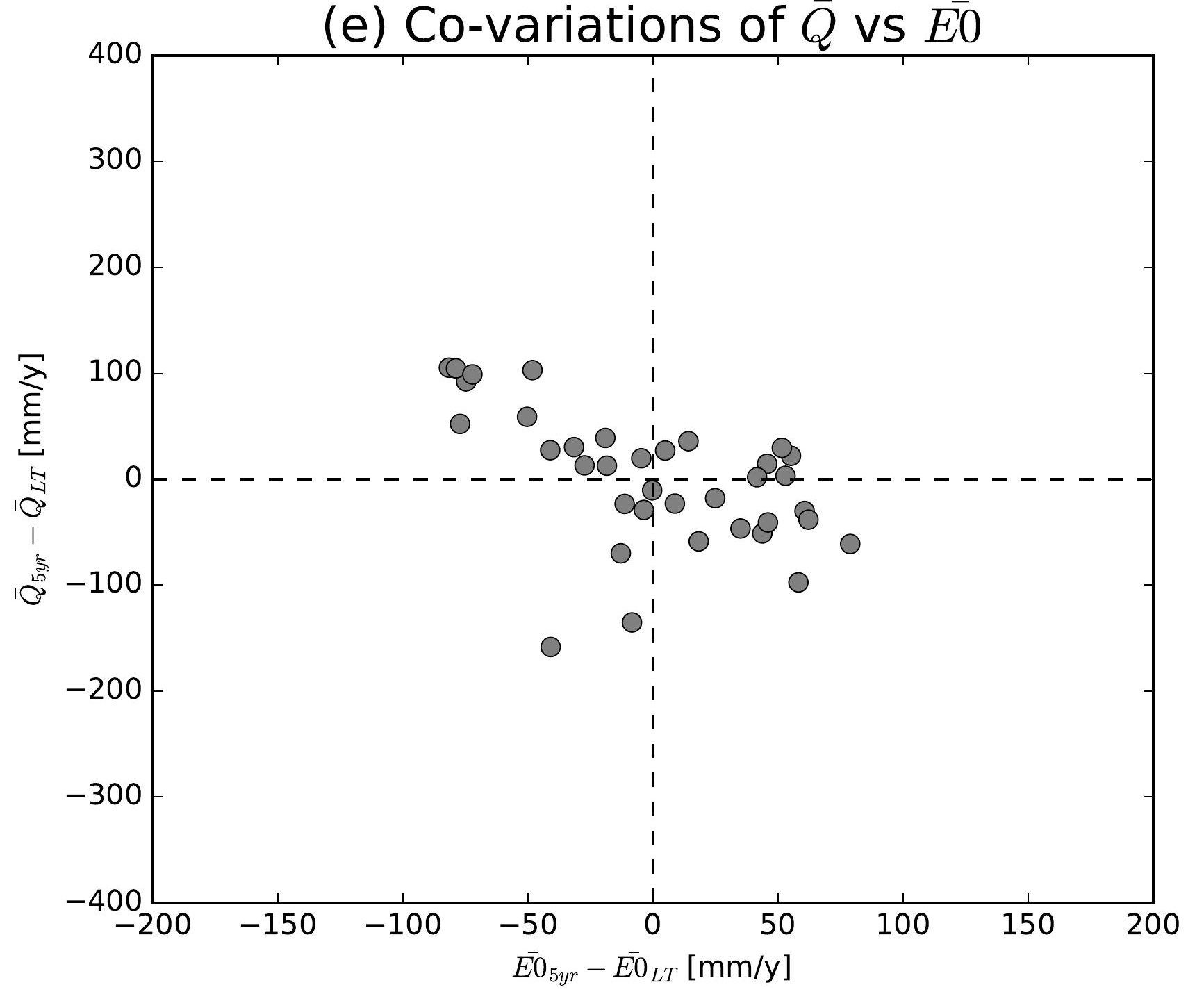

(h) Co-variations of $\bar{Q}$ vs $\overline{E 0}$

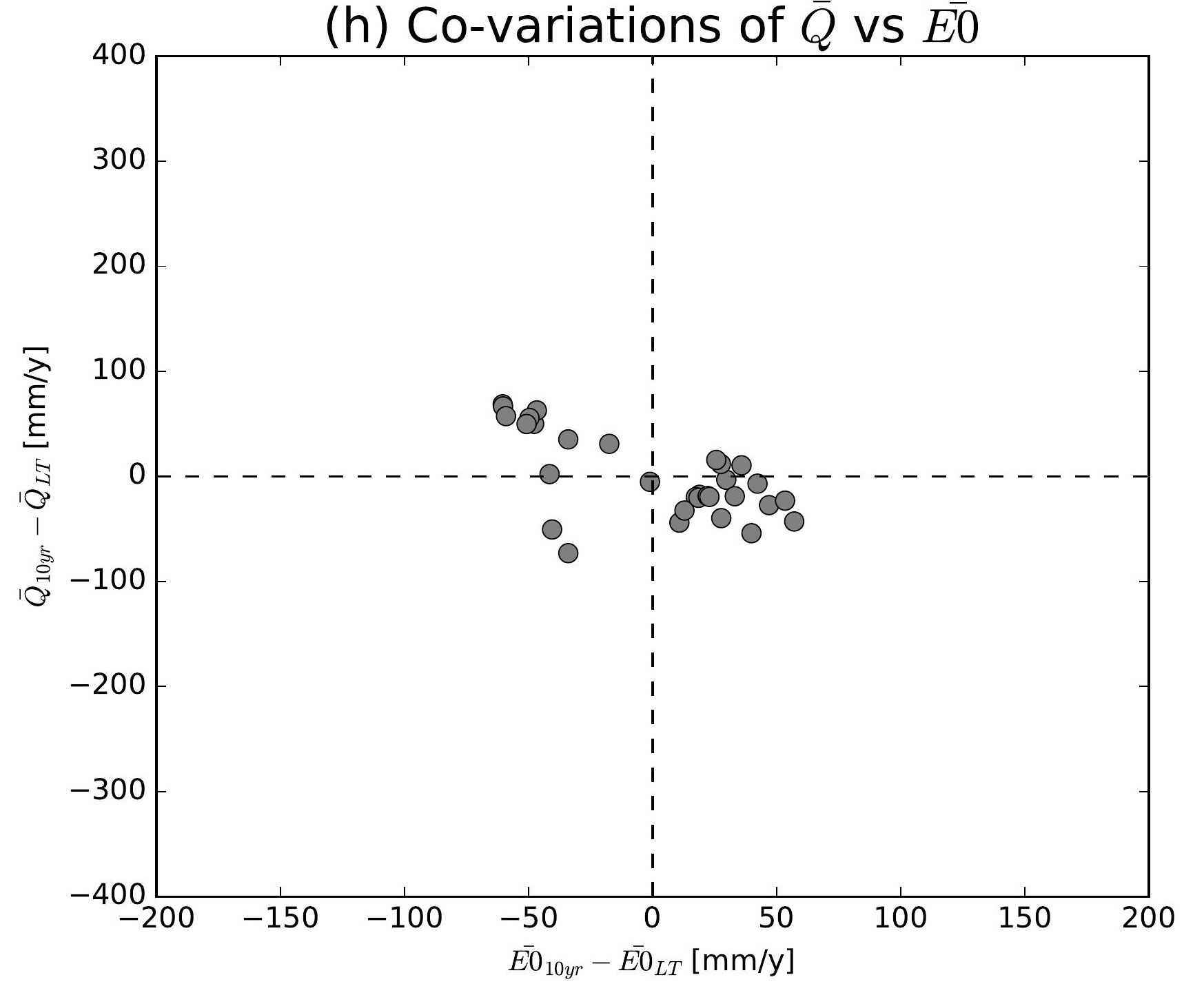

(k) Co-variations of $\bar{Q}$ vs $\overline{E 0}$

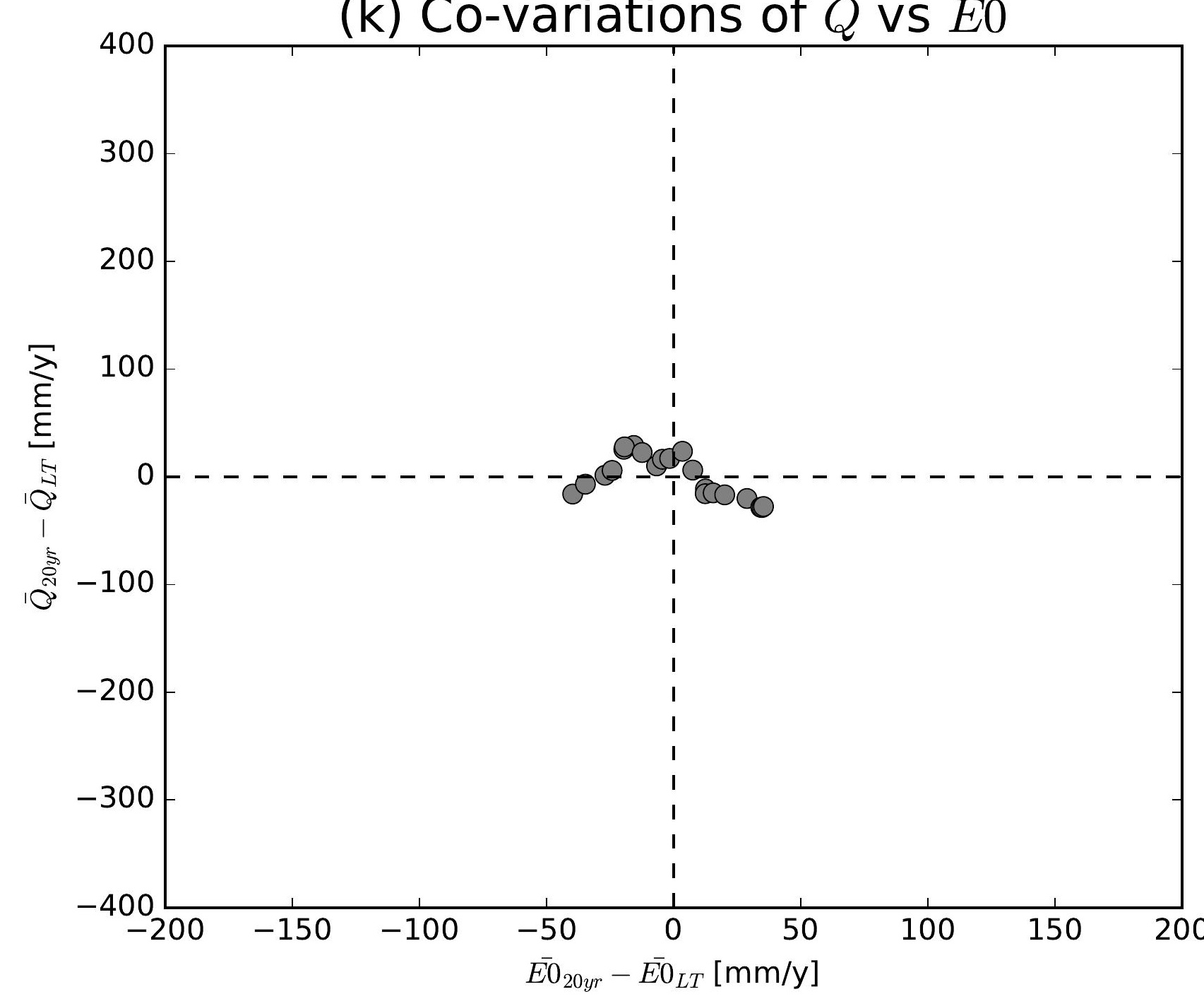

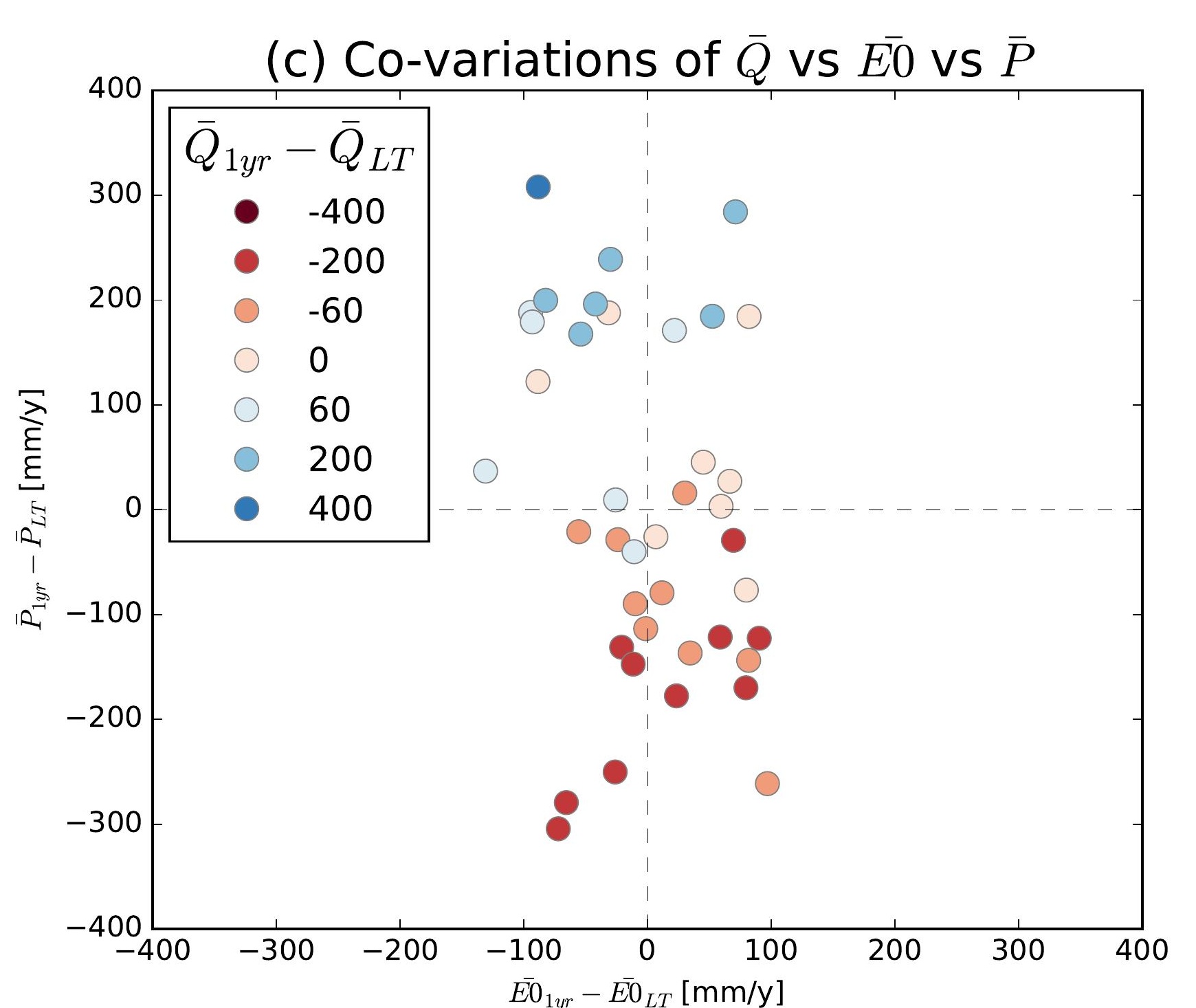
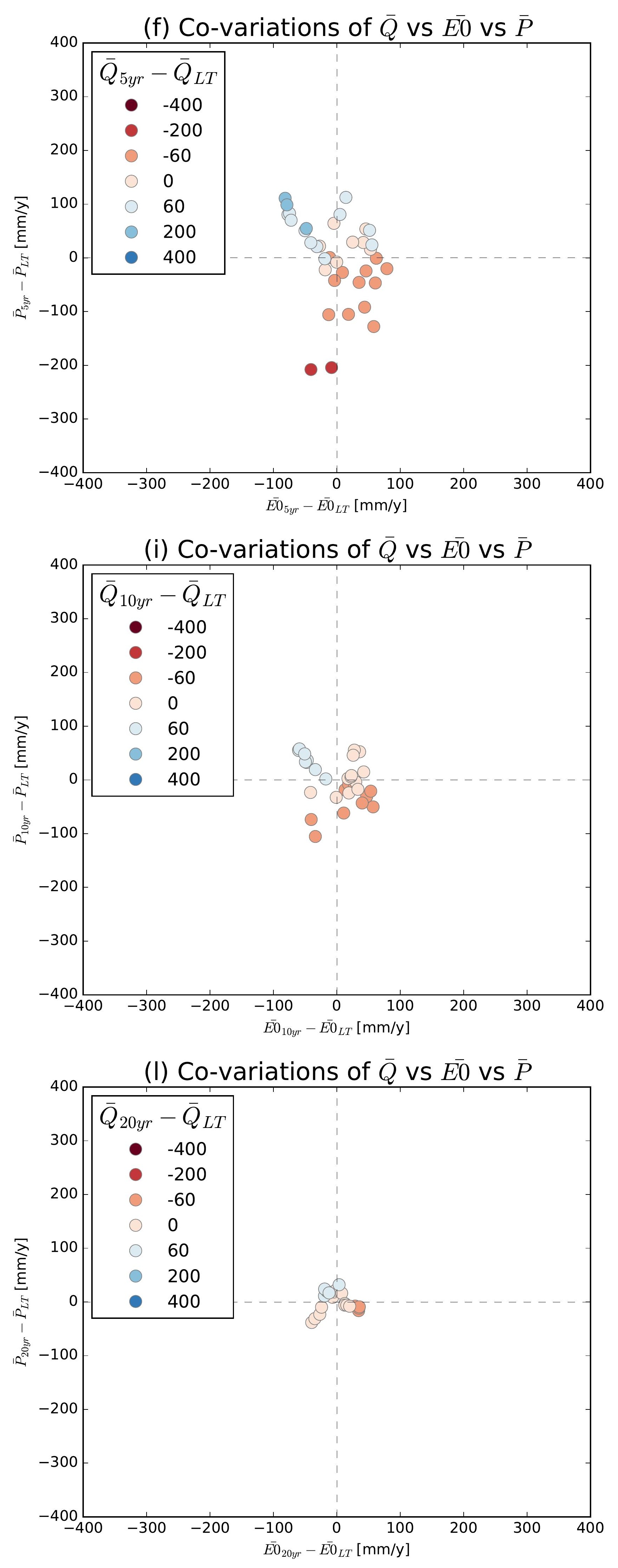

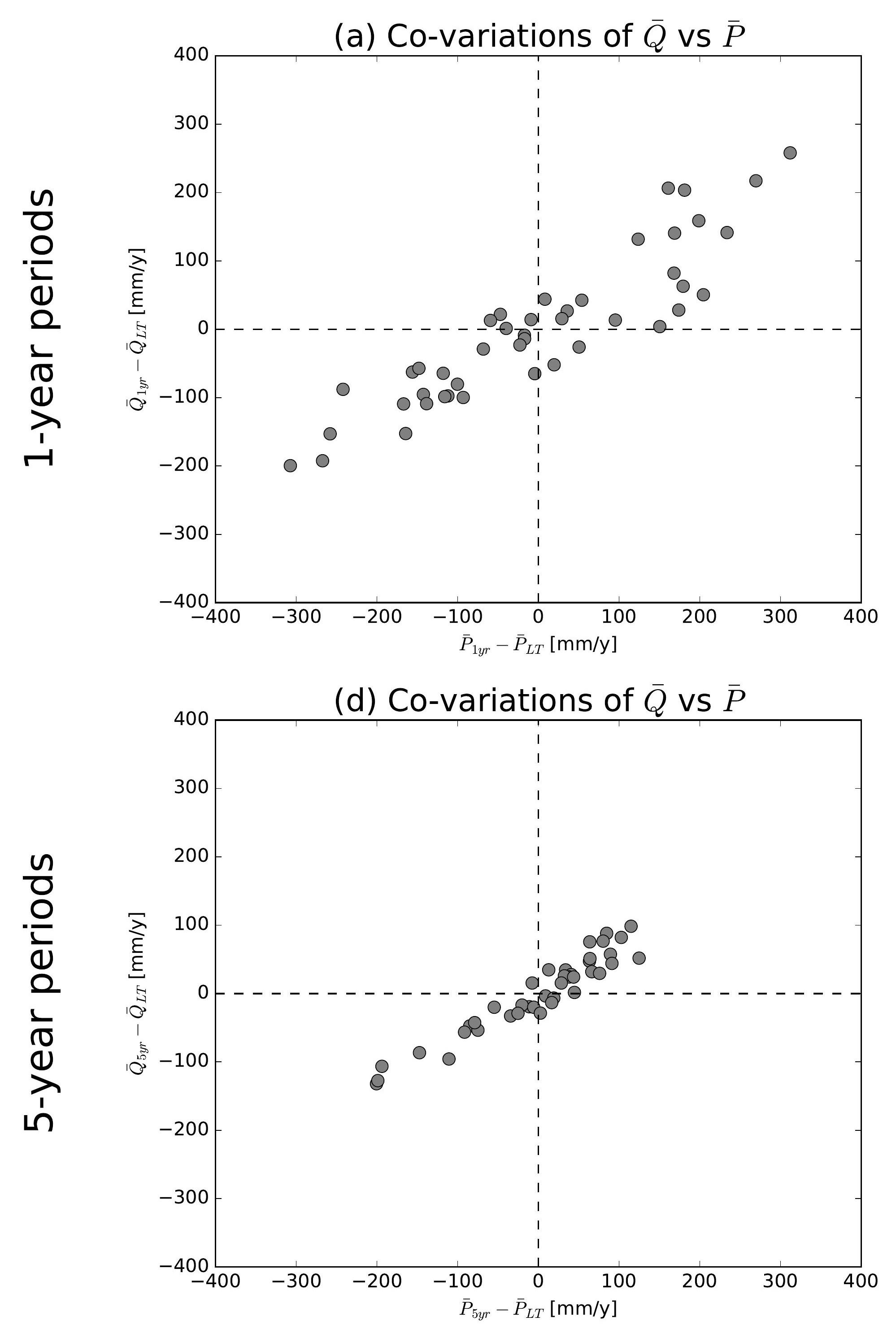

(g) Co-variations of $\bar{Q}$ vs $\bar{P}$

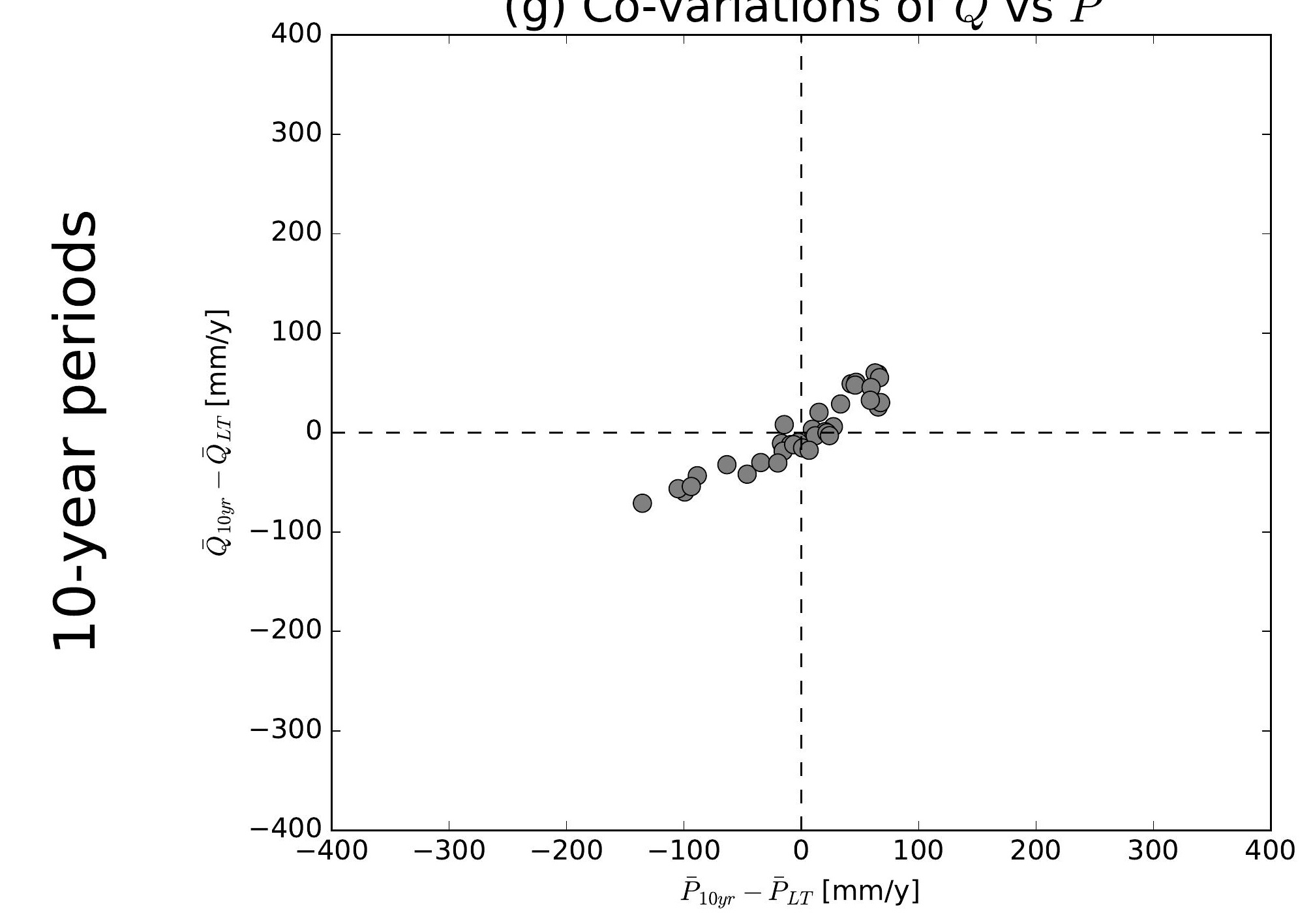

(j) Co-variations of $\bar{Q}$ vs $\bar{P}$

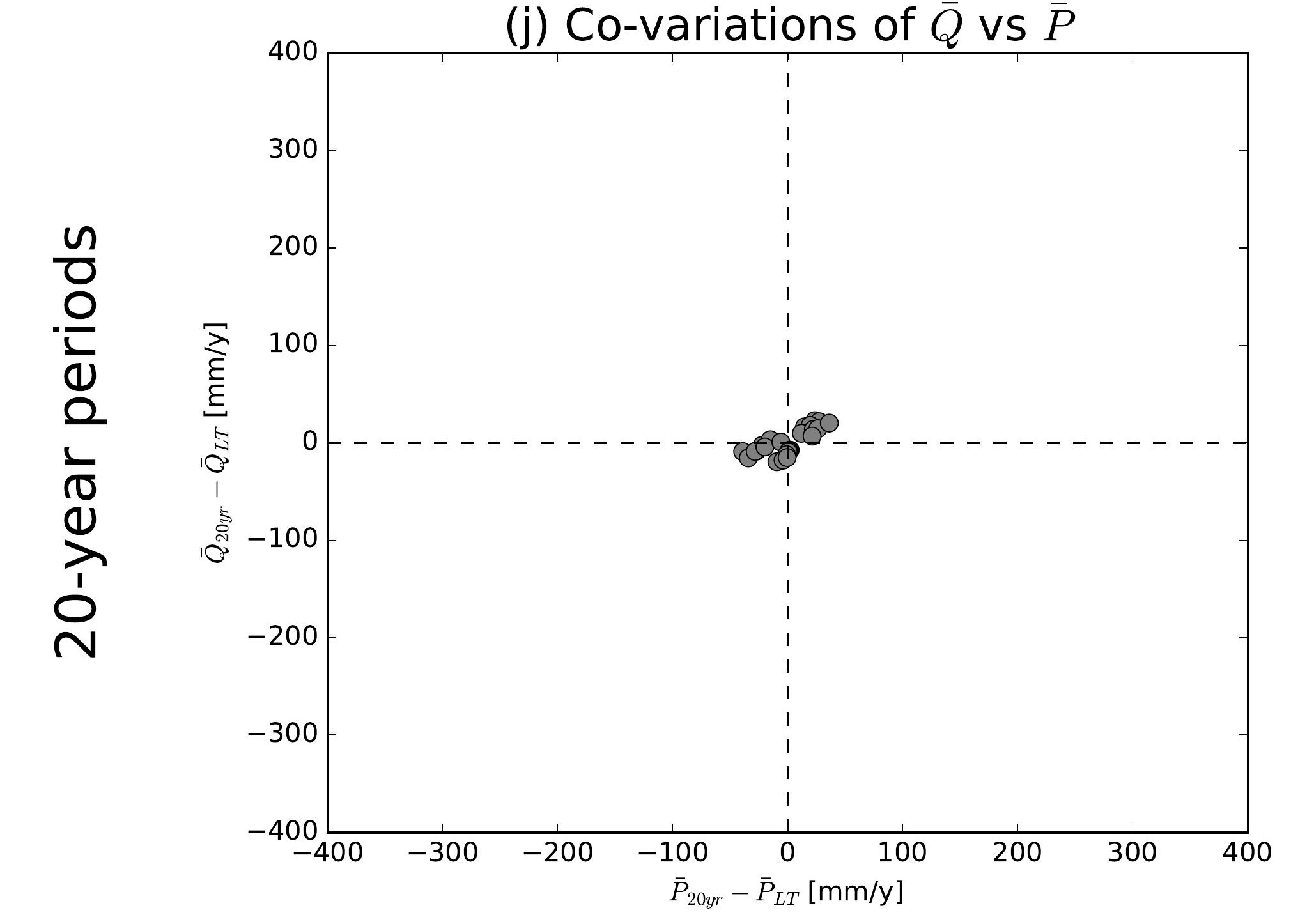

(b) Co-variations of $\bar{Q}$ vs $\overline{E 0}$

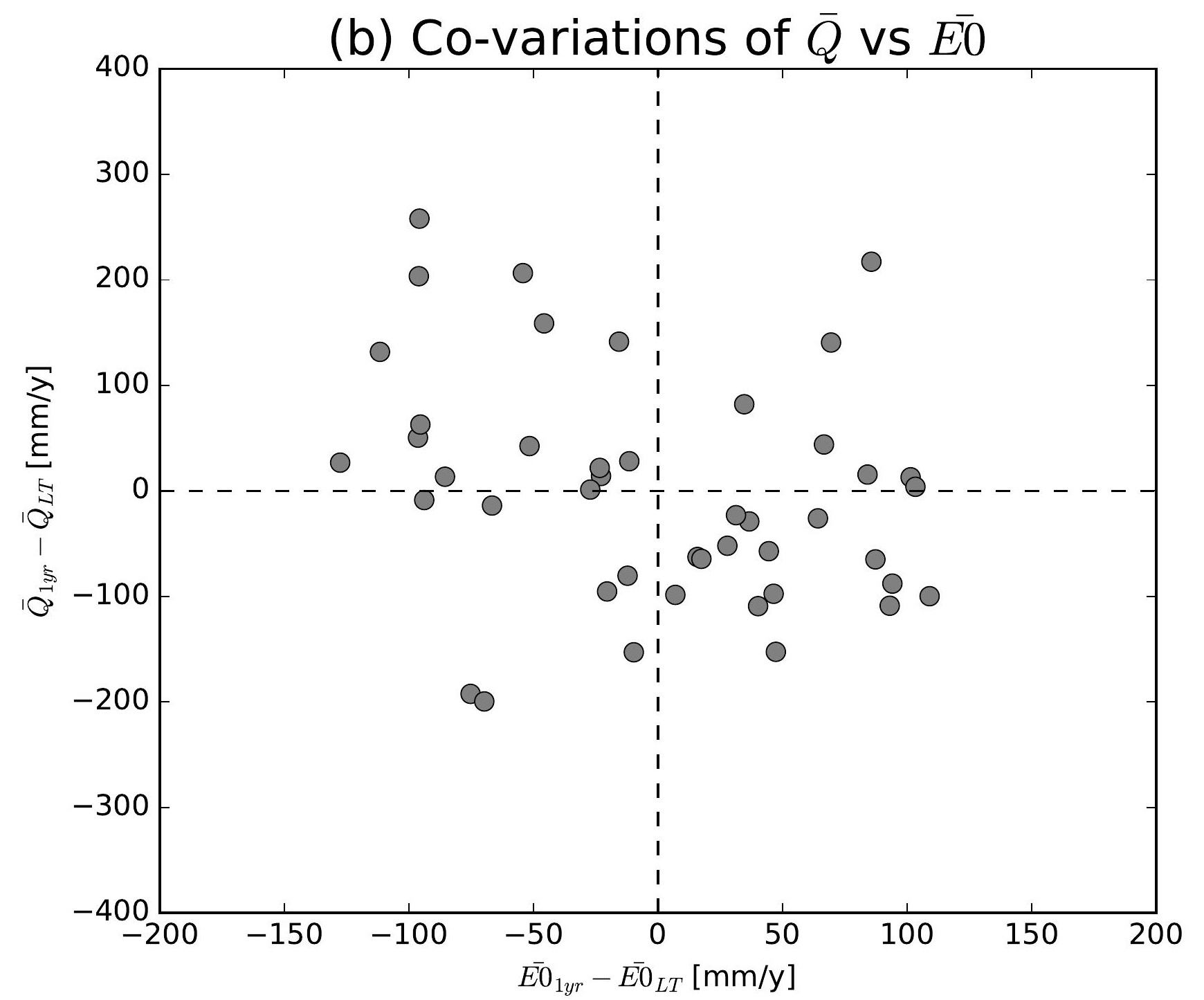

(e) Co-variations of $\bar{Q}$ vs $\overline{E 0}$

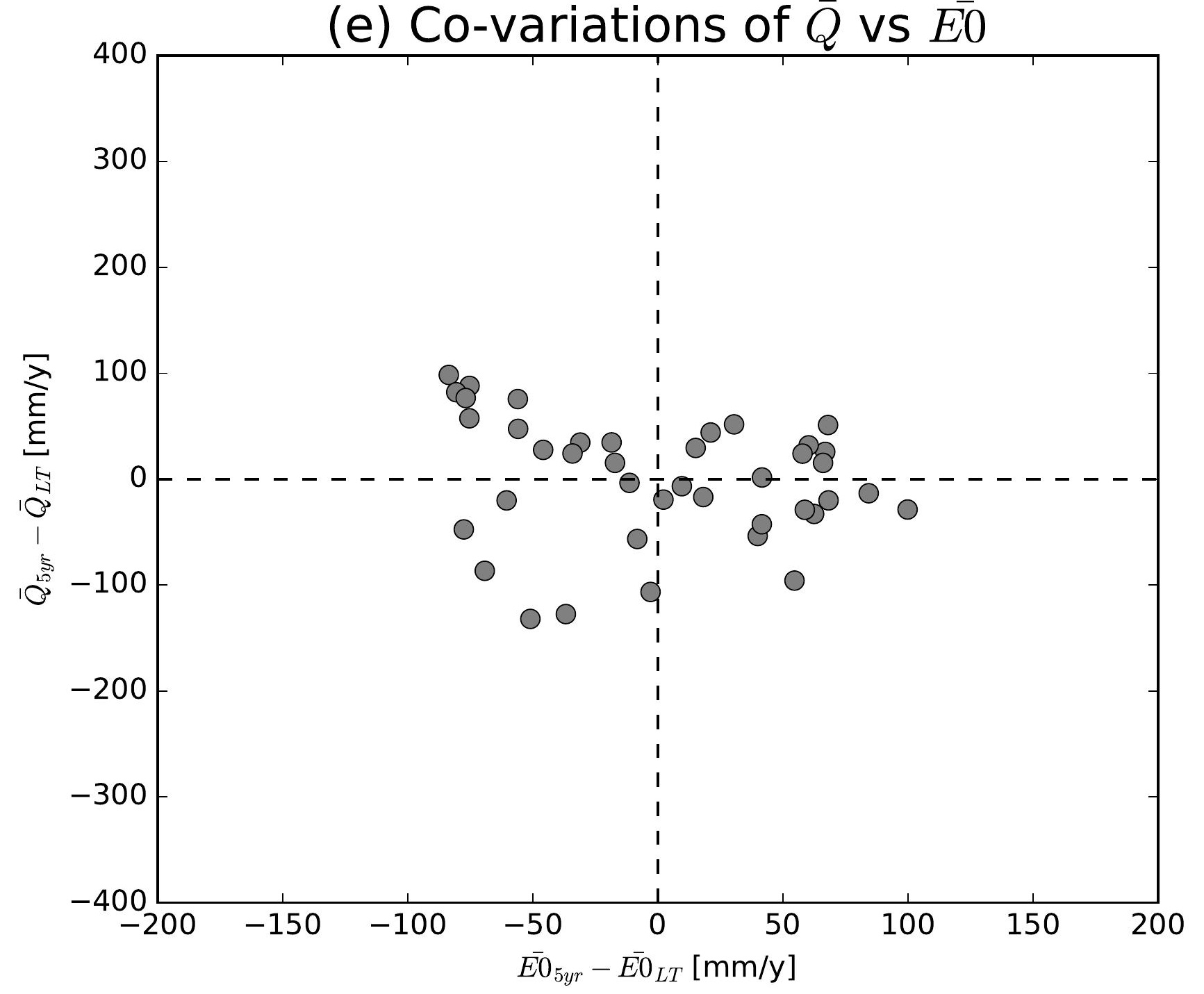

(h) Co-variations of $\bar{Q}$ vs $\overline{E 0}$

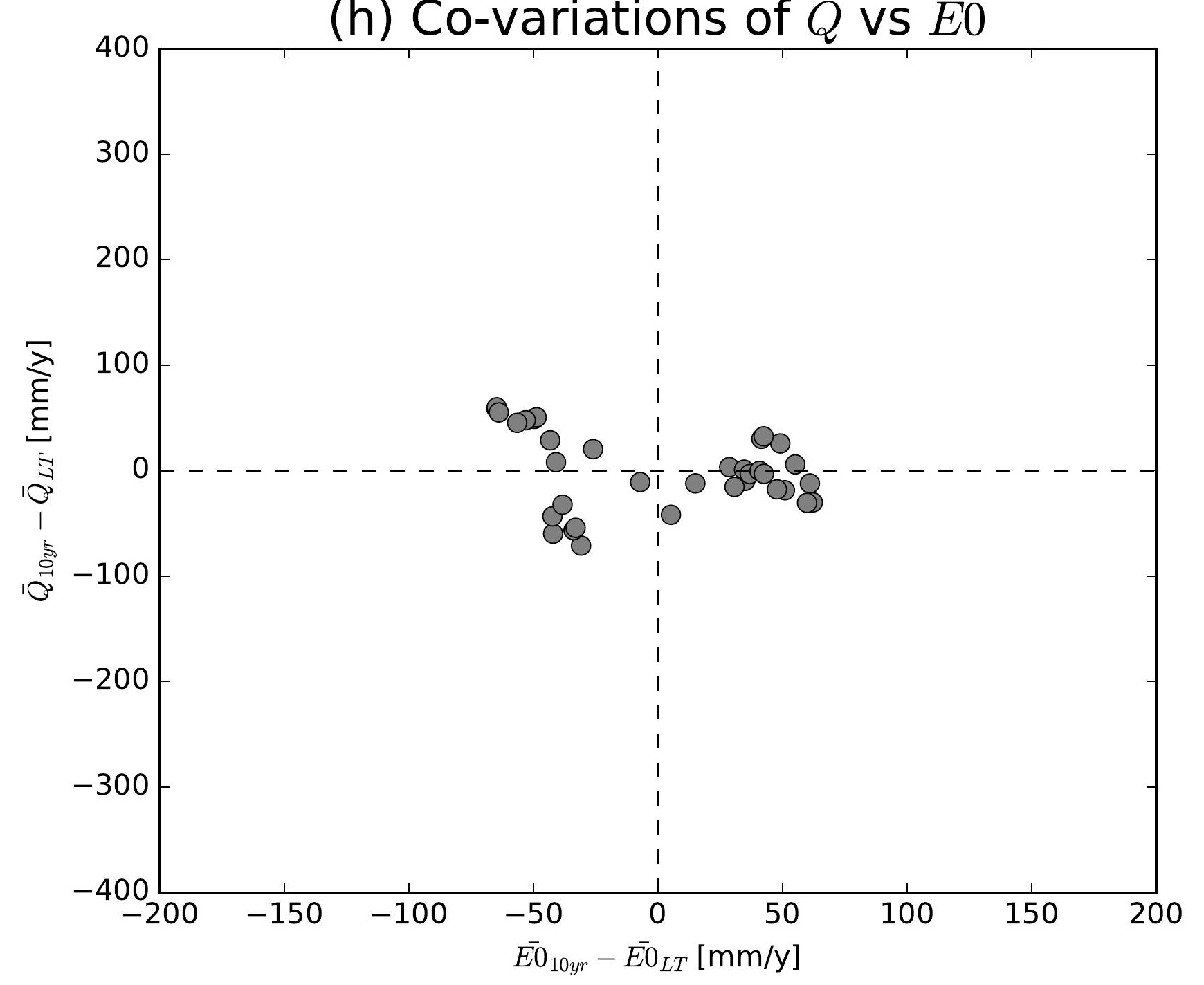

(k) Co-variations of $\bar{Q}$ vs $\overline{E 0}$

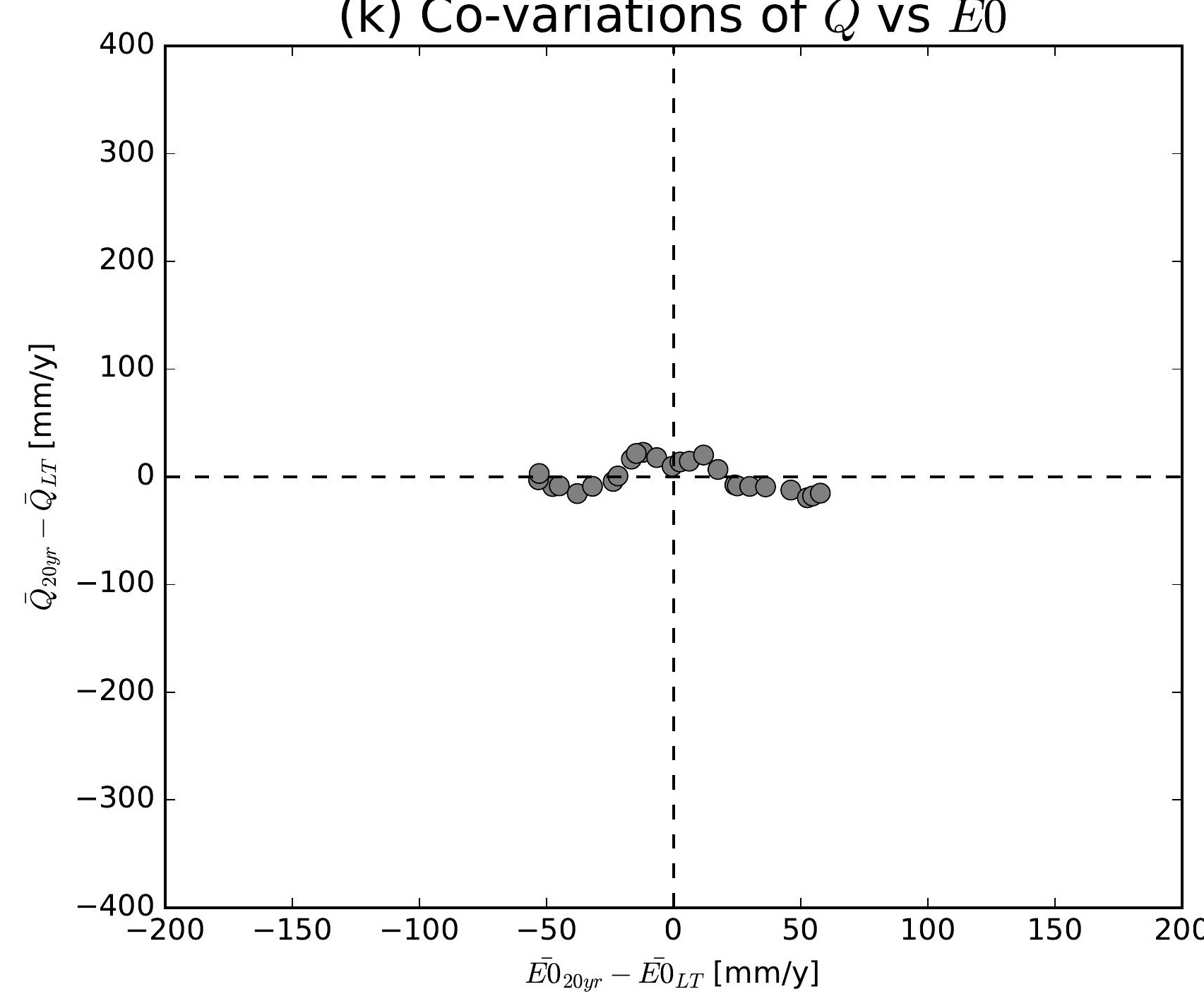

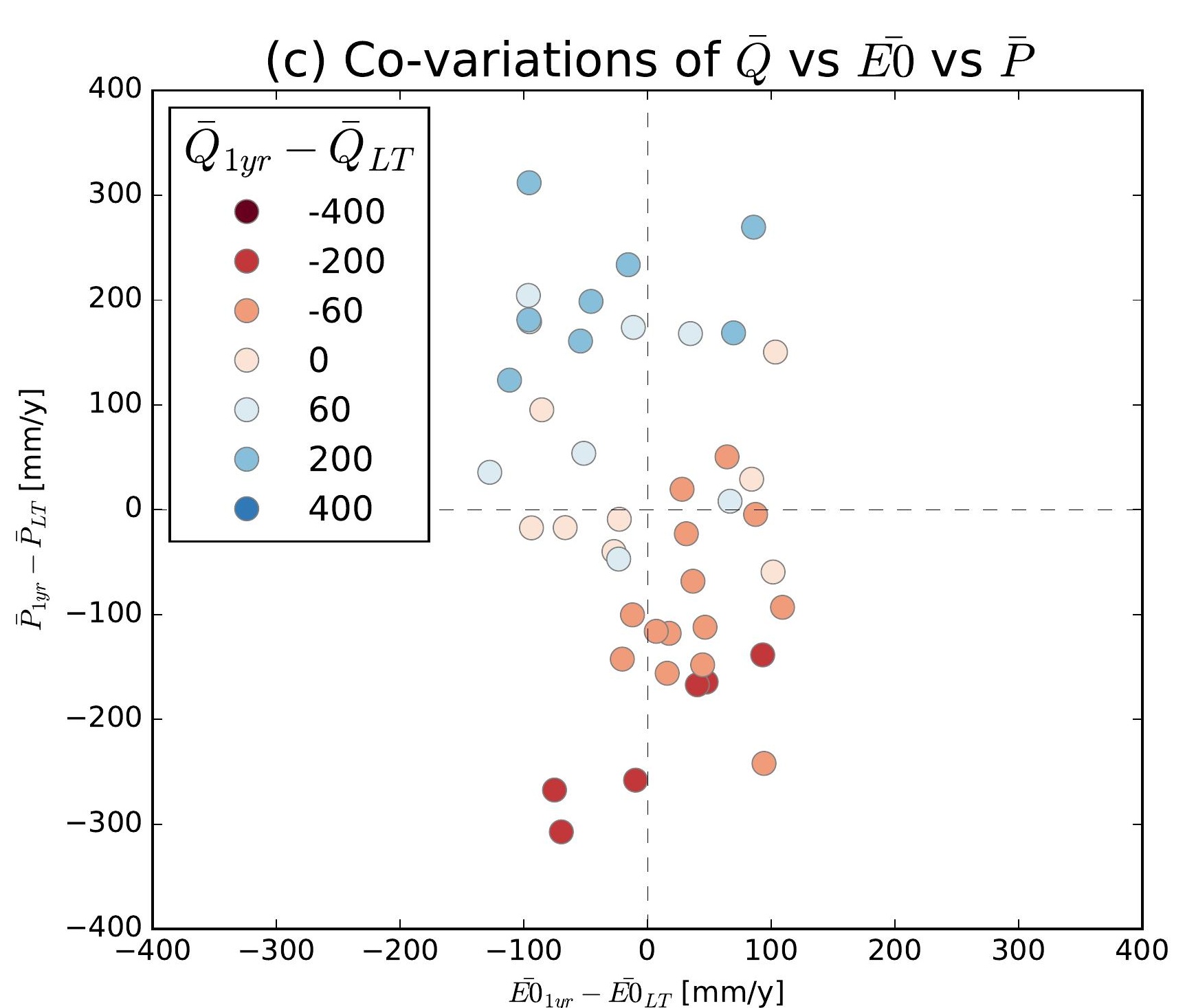
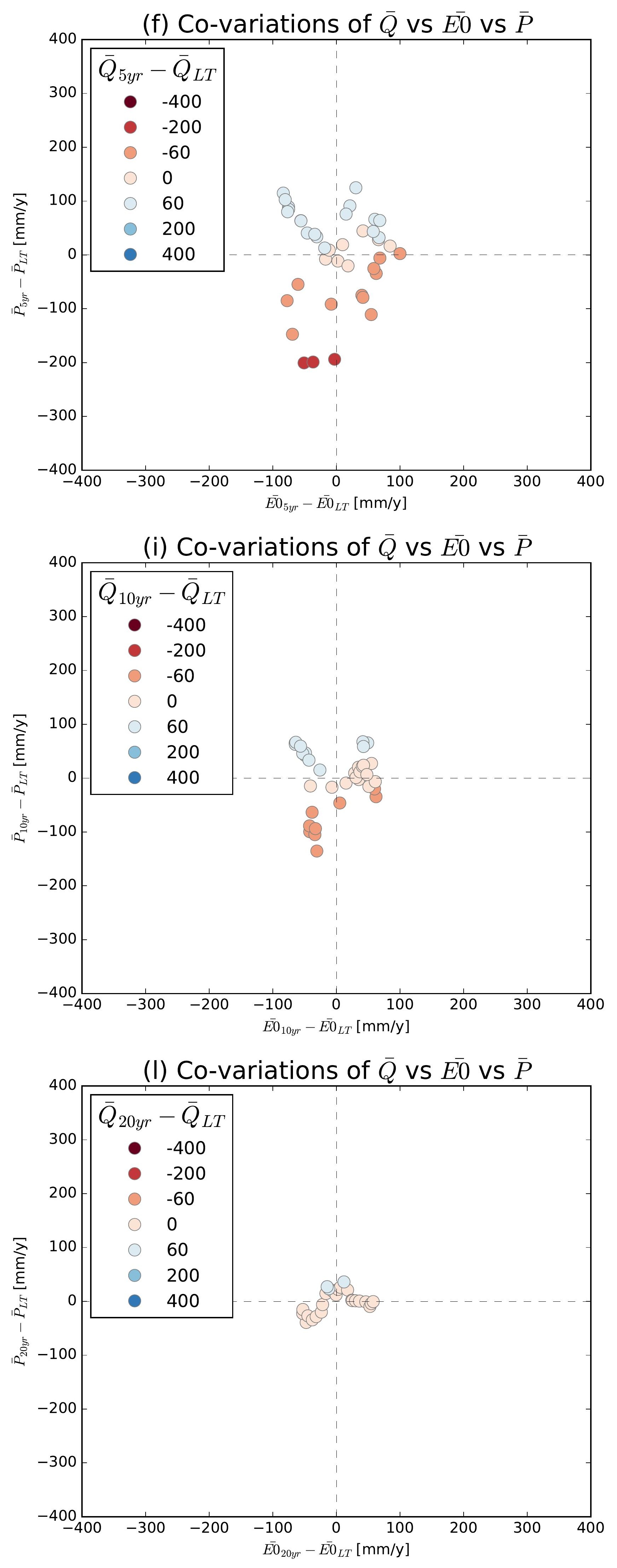

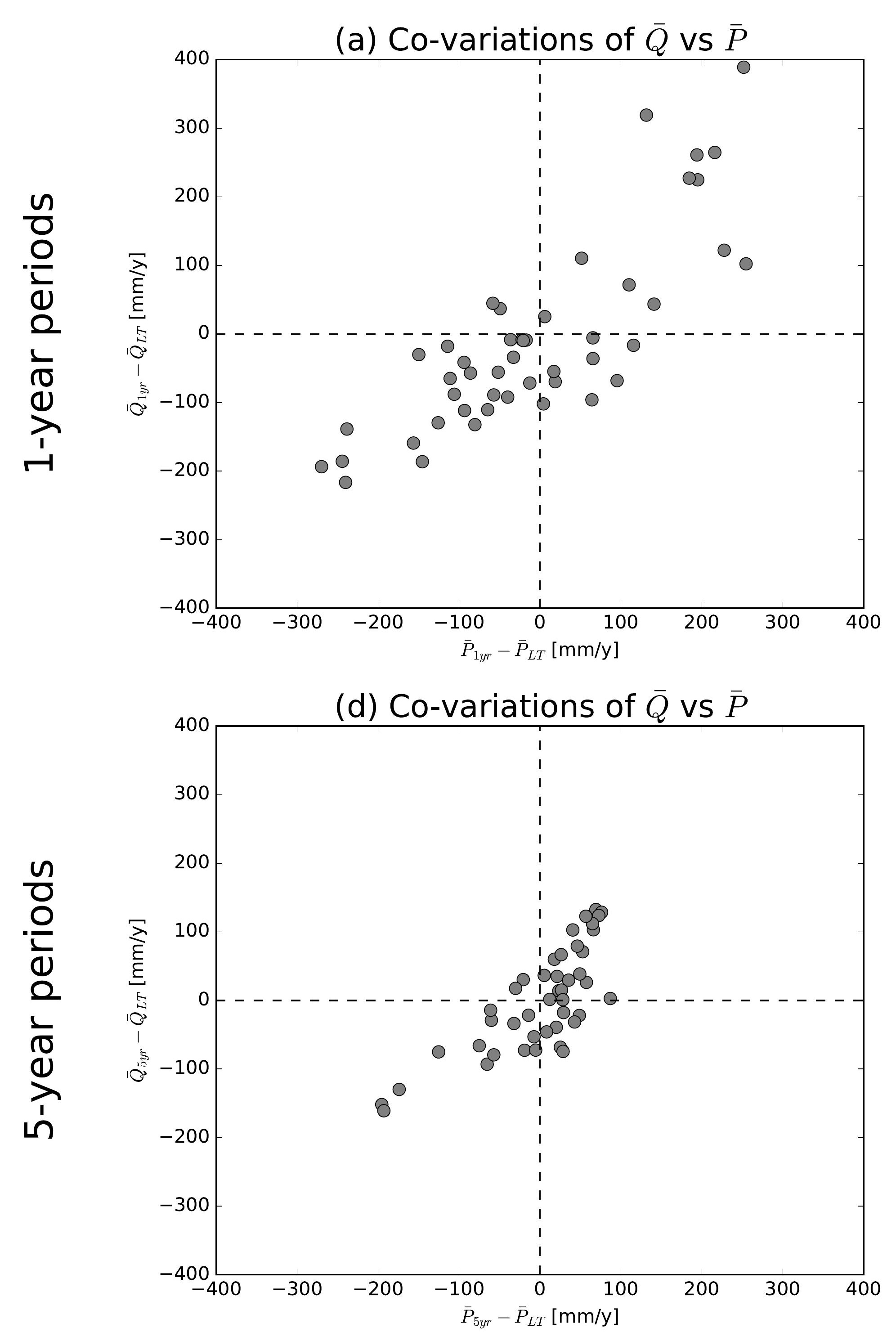

(g) Co-variations of $\bar{Q}$ vs $\bar{P}$

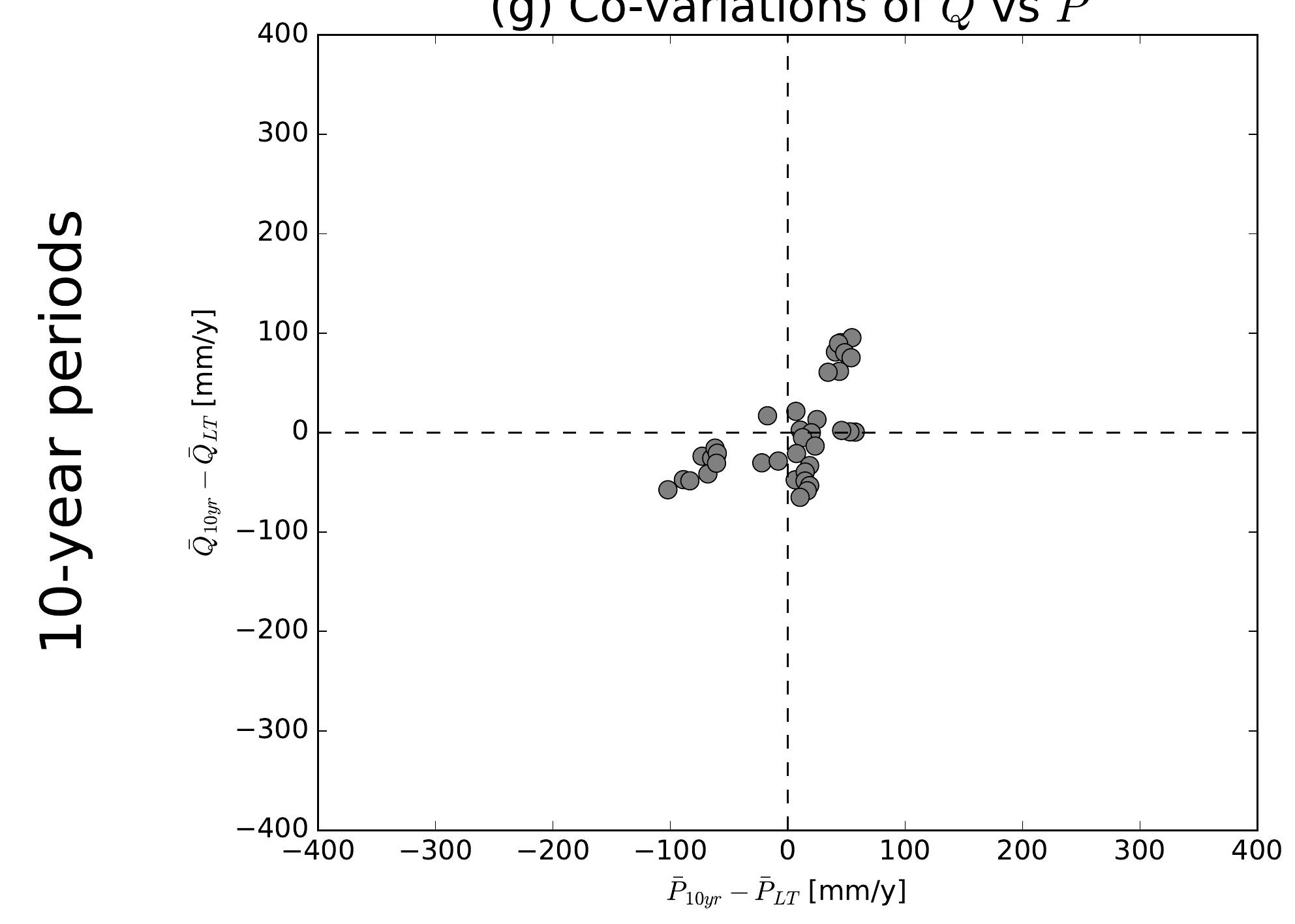

(j) Co-variations of $\bar{Q}$ vs $\bar{P}$

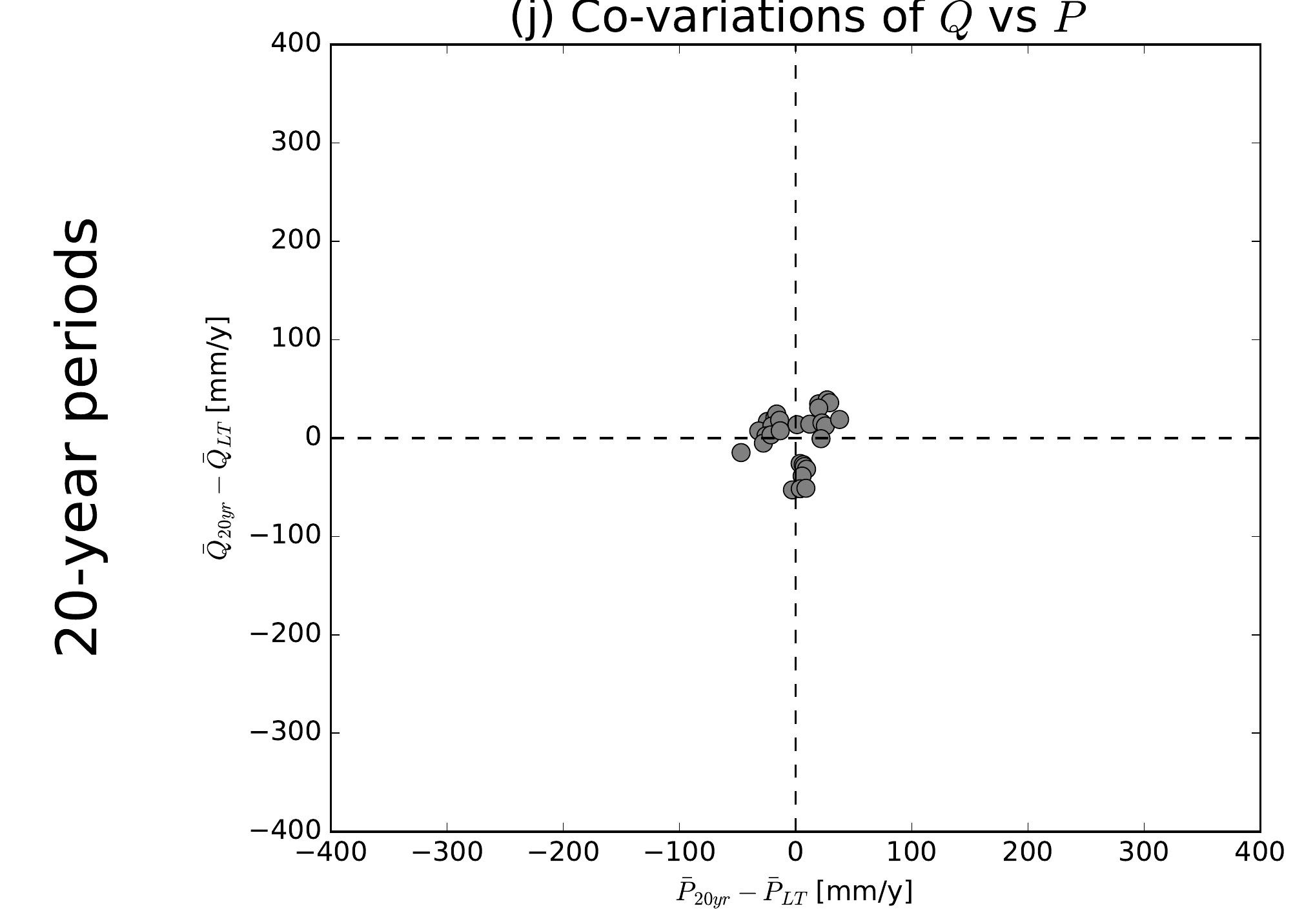

(b) Co-variations of $\bar{Q}$ vs $\overline{E 0}$

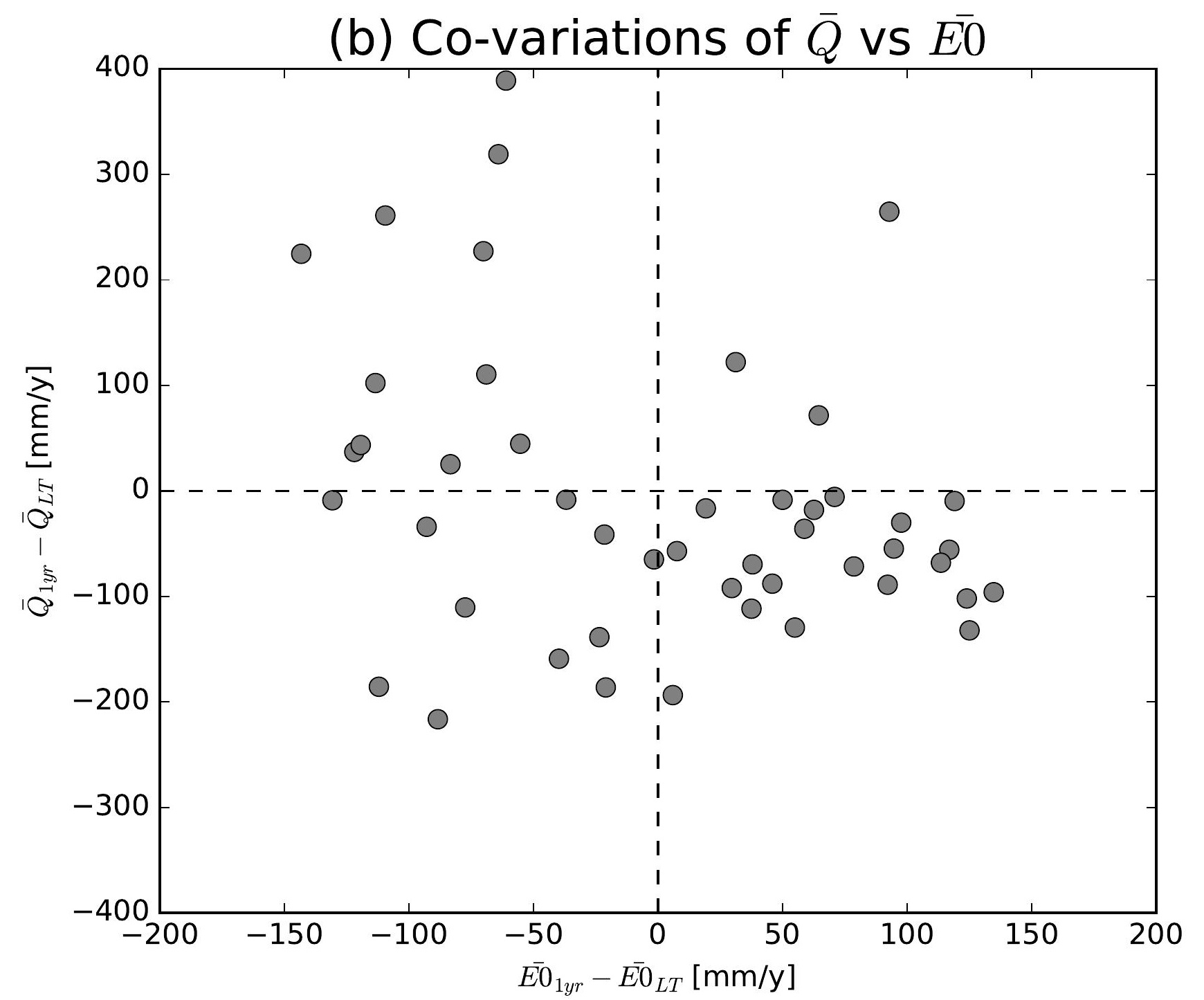

(e) Co-variations of $\bar{Q}$ vs $\overline{E 0}$

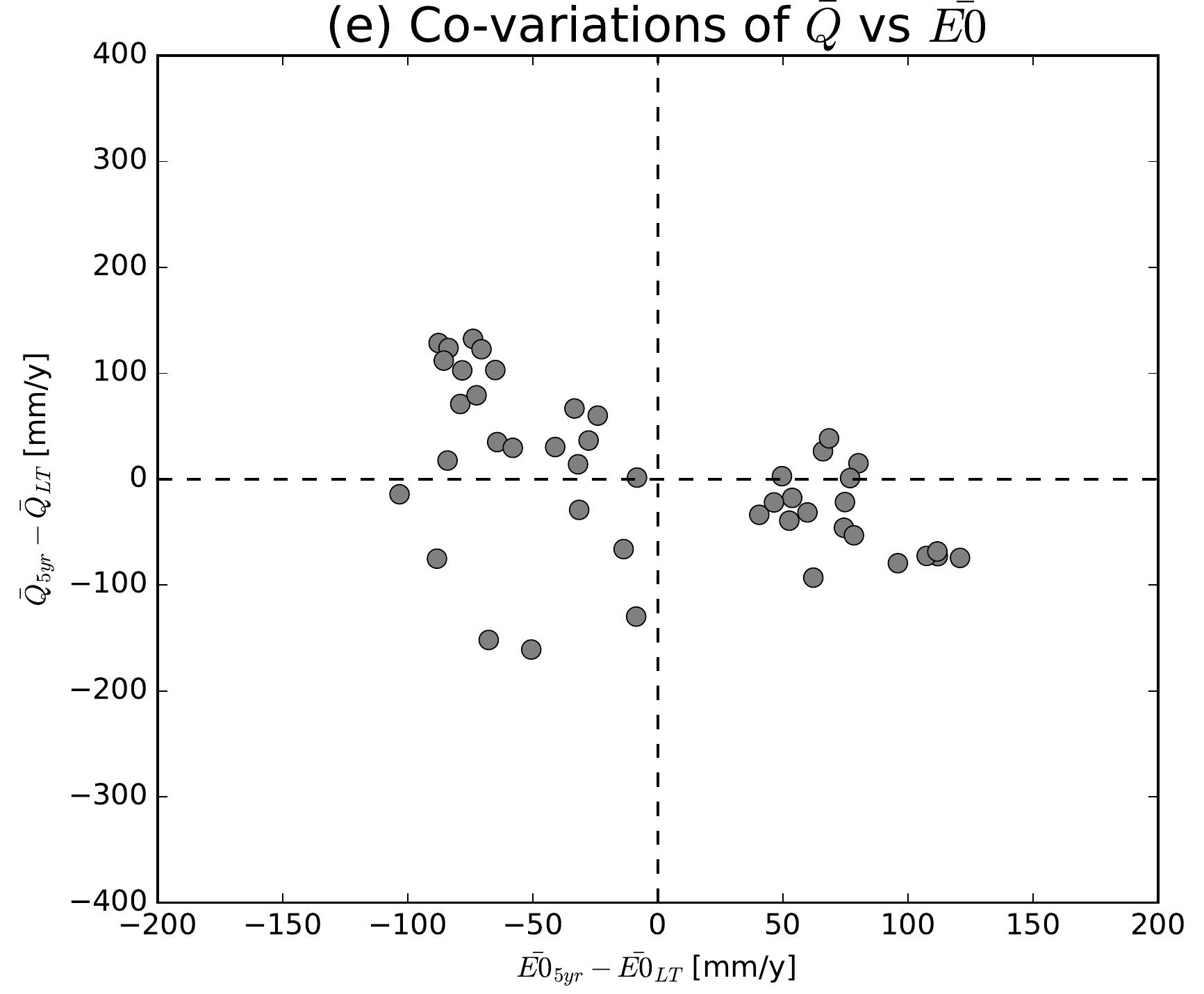

(h) Co-variations of $\bar{Q}$ vs $\overline{E 0}$

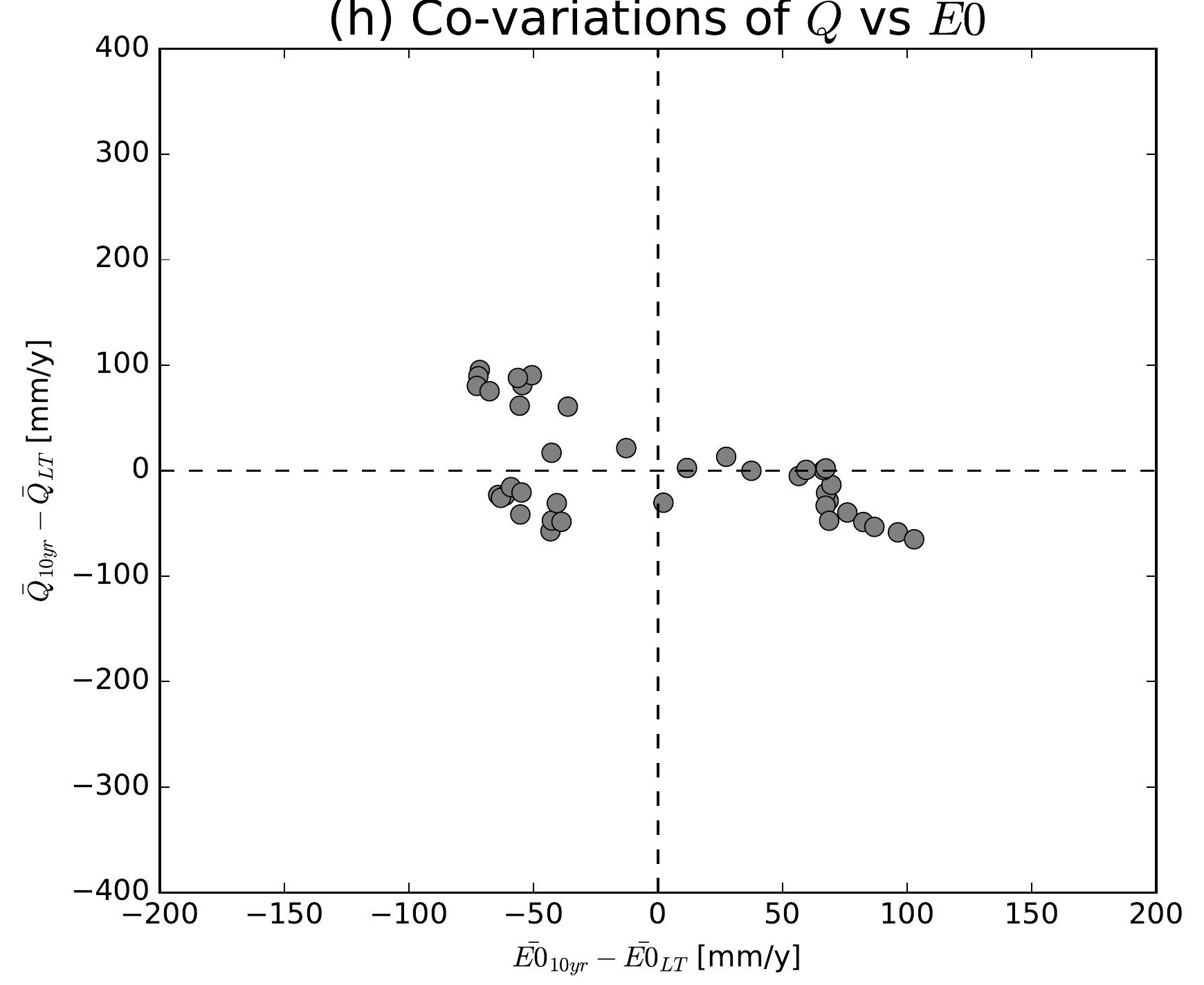

(k) Co-variations of $\bar{Q}$ vs $\overline{E 0}$

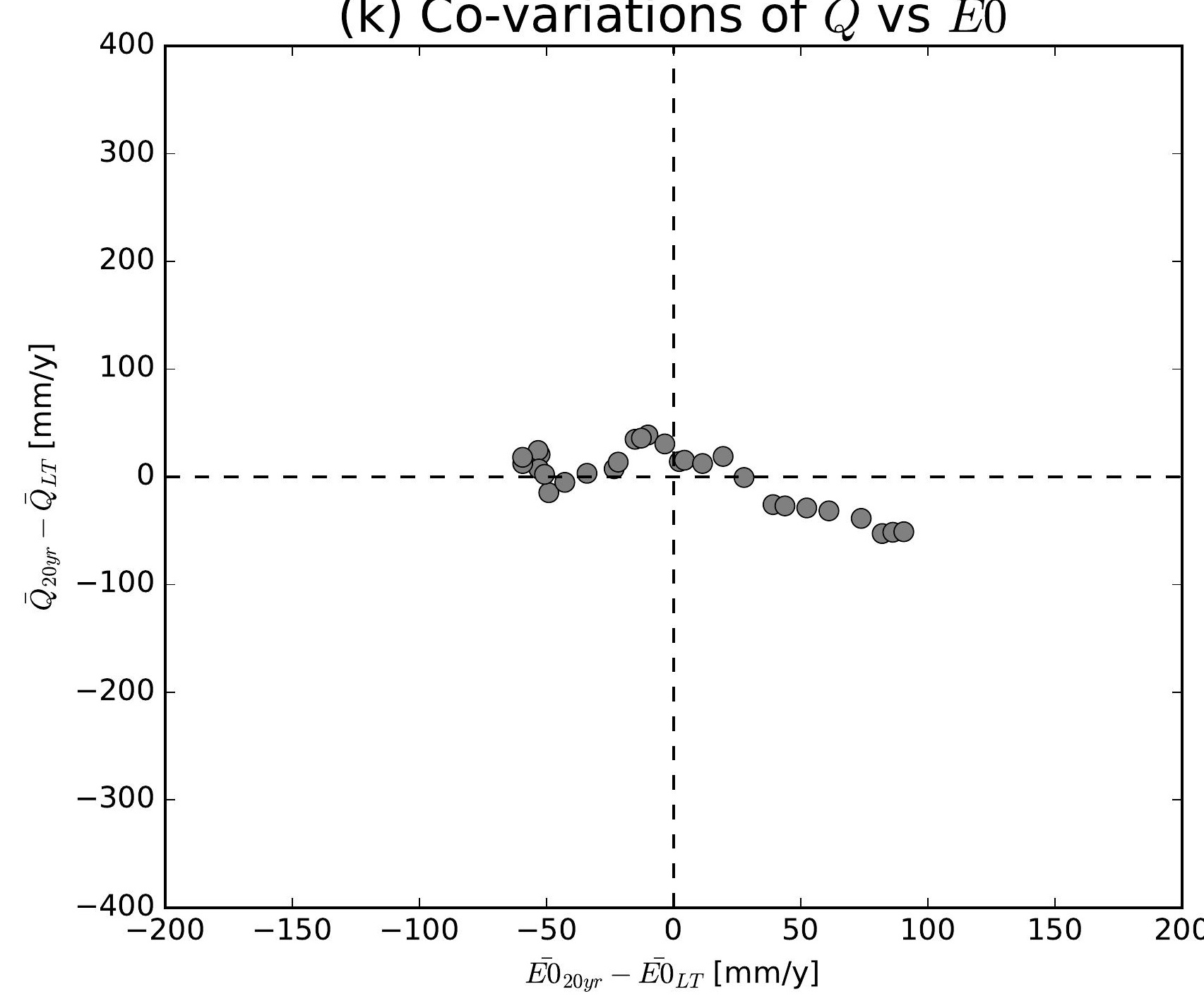

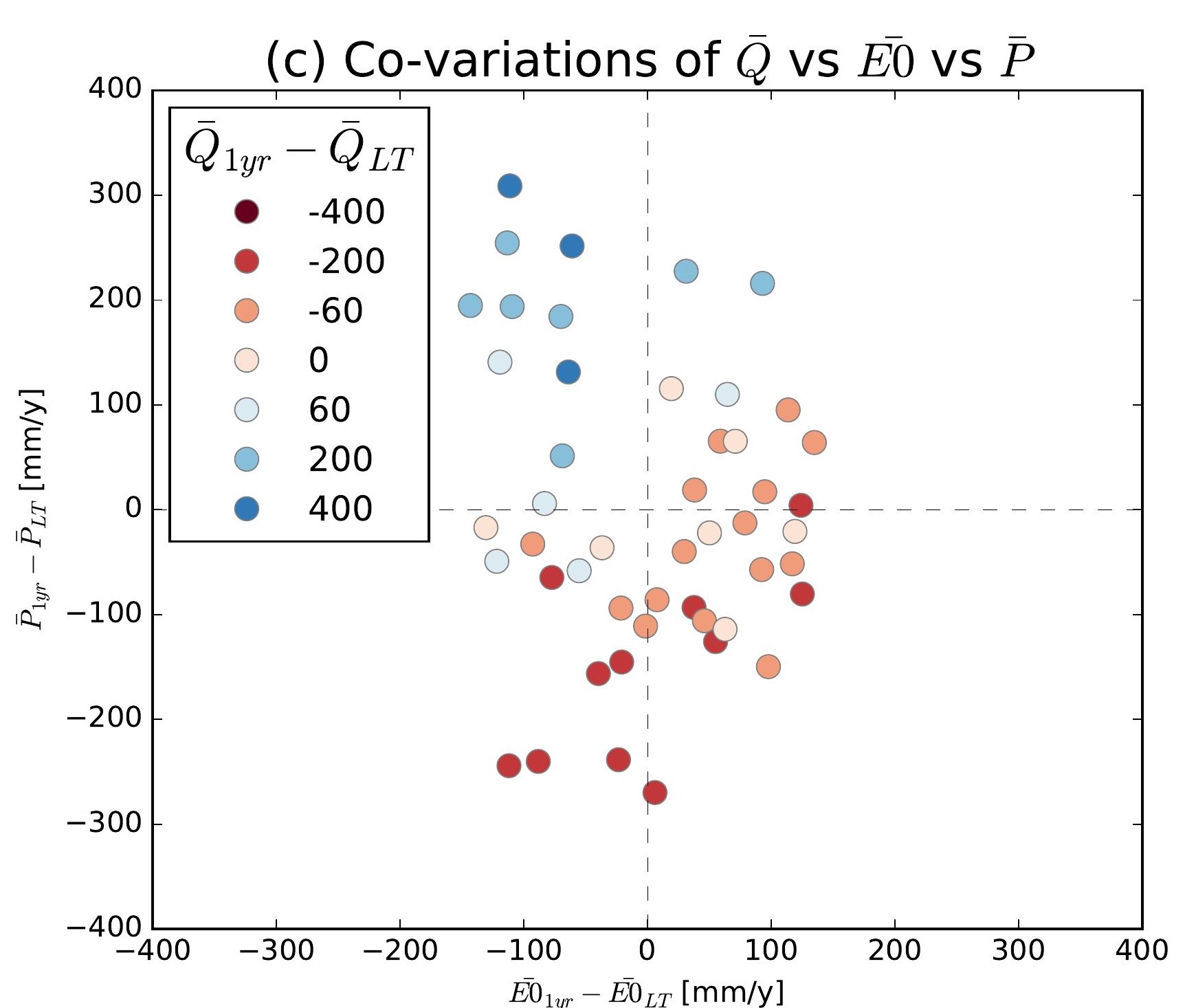
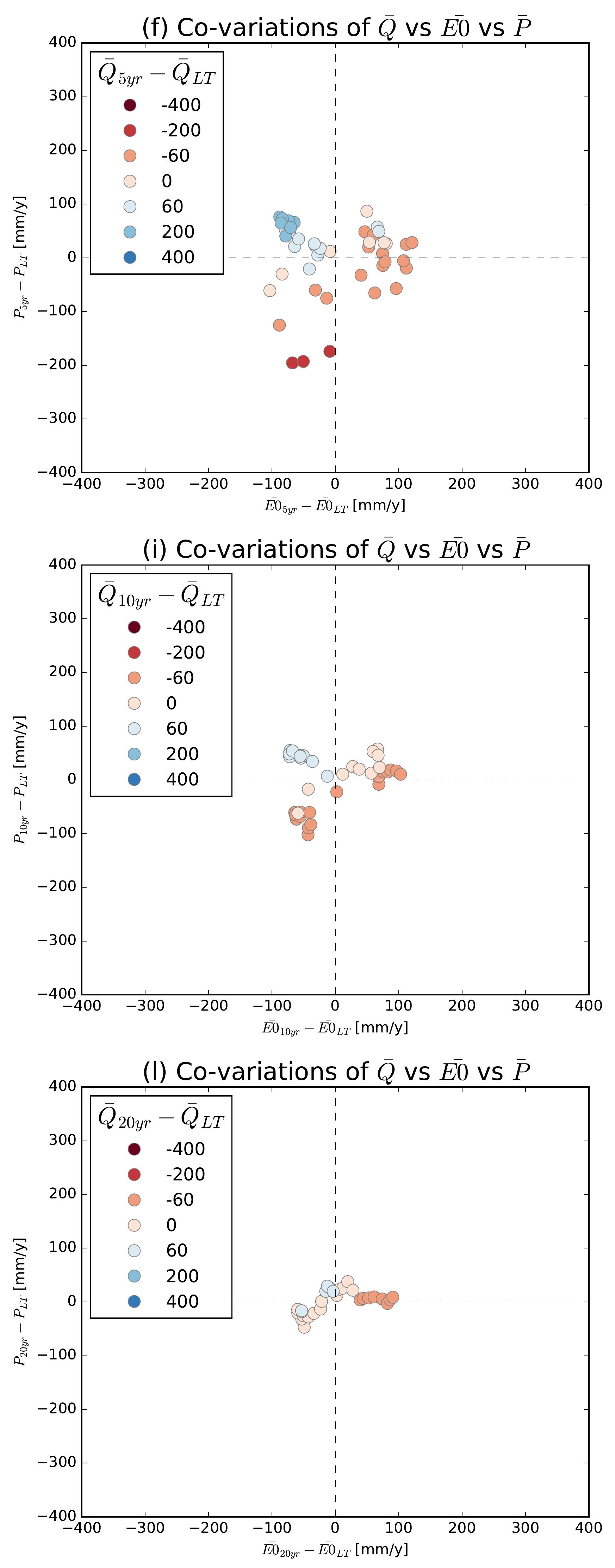

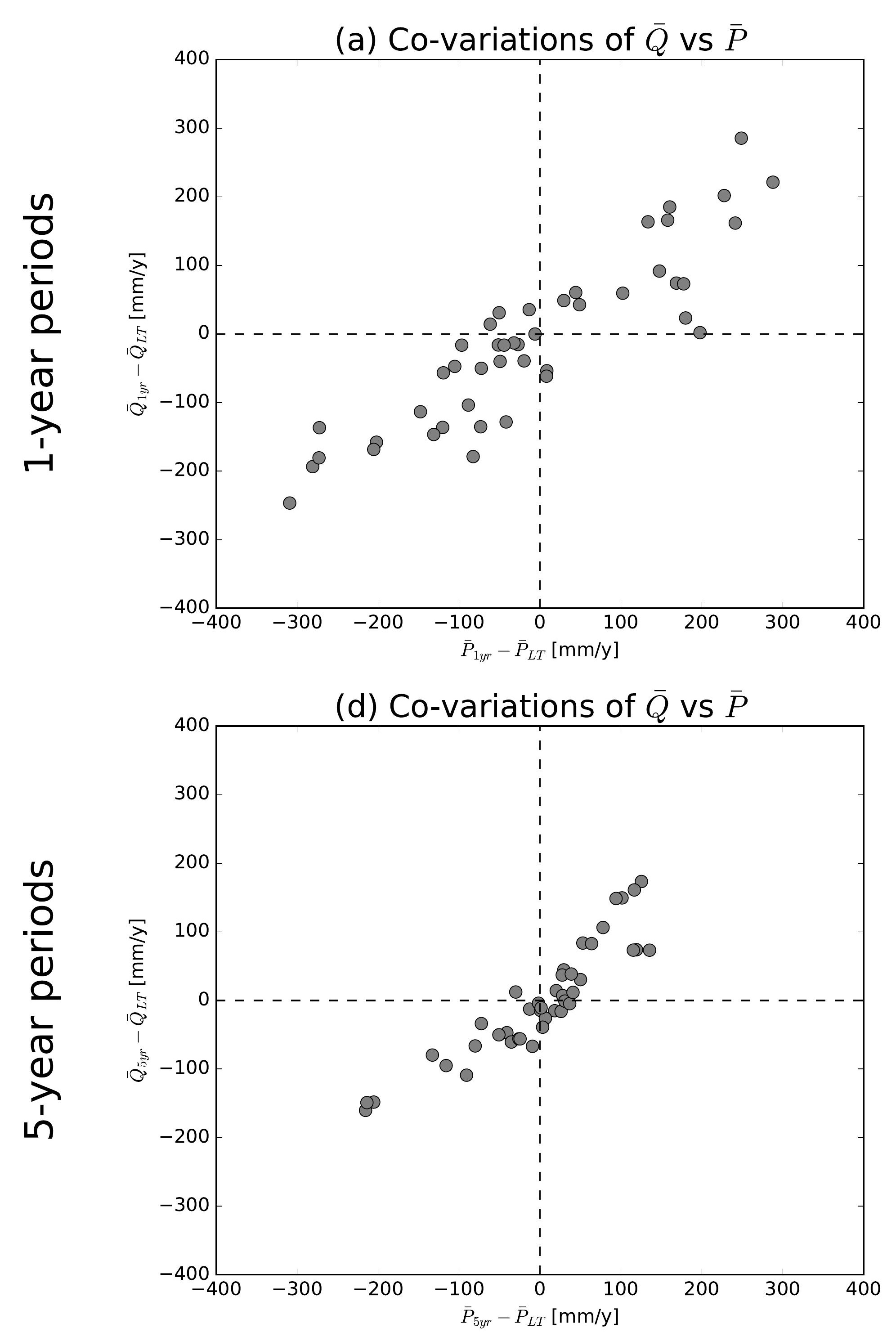

(g) Co-variations of $\bar{Q}$ vs $\bar{P}$

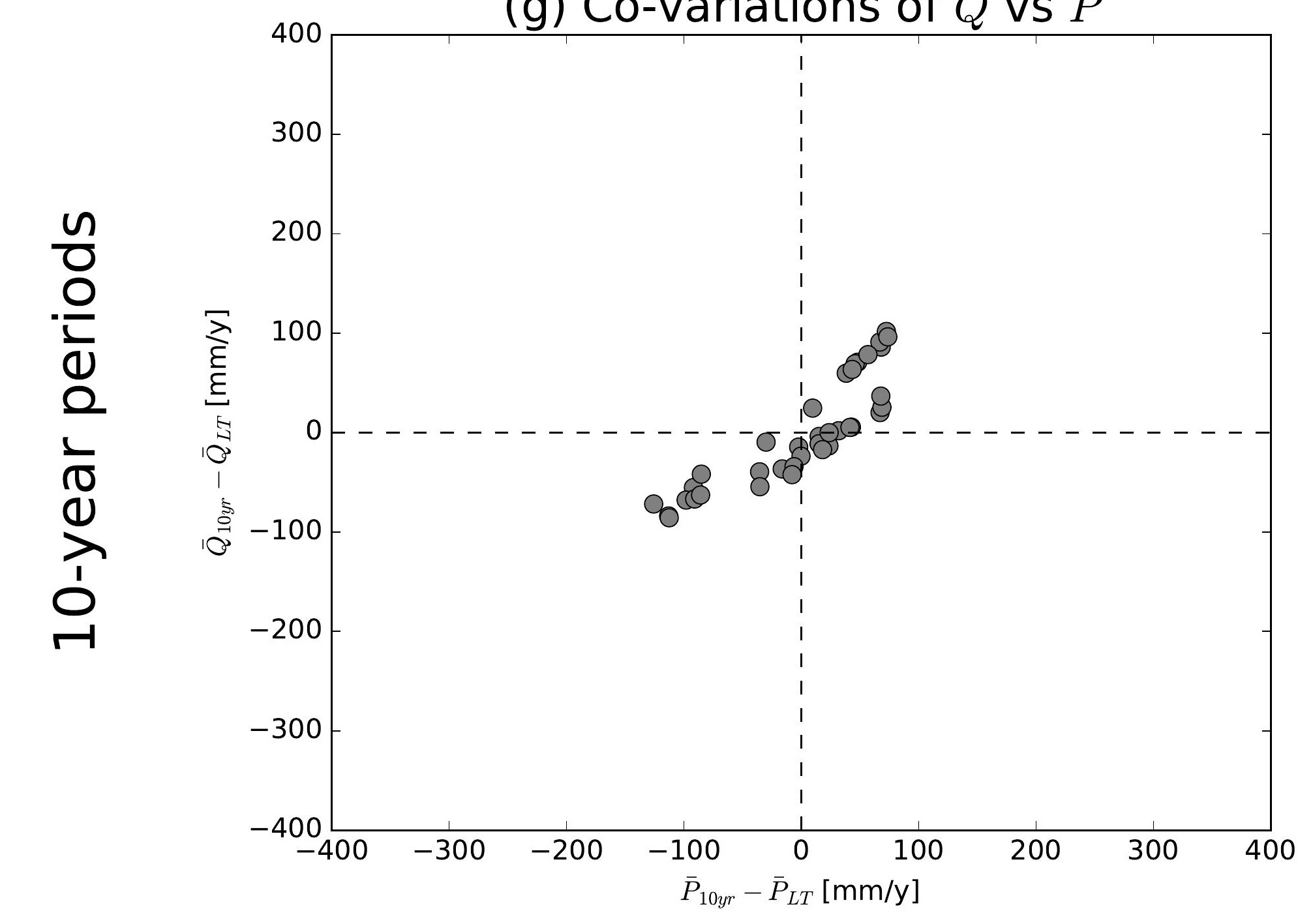

(j) Co-variations of $\bar{Q}$ vs $\bar{P}$

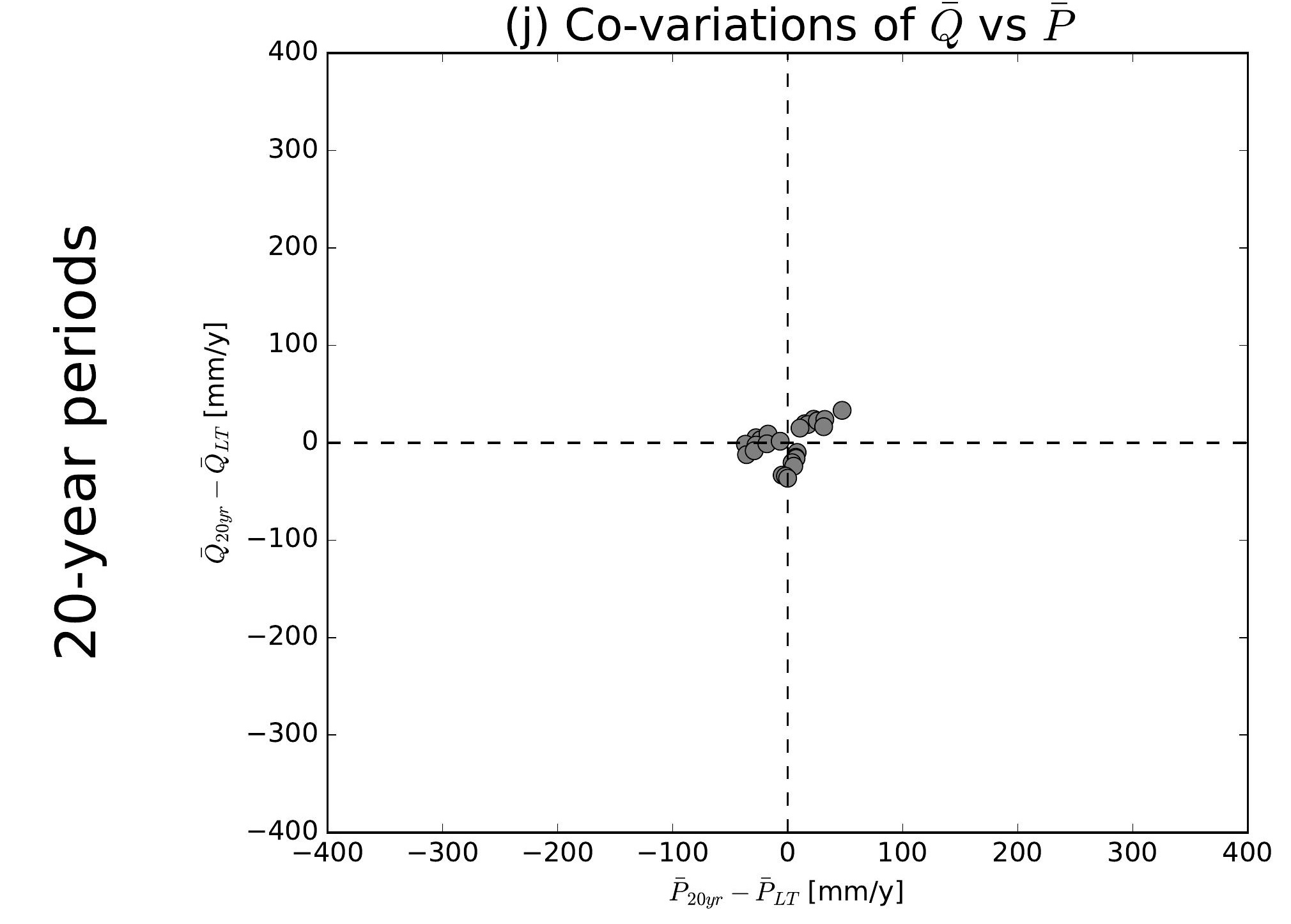

(b) Co-variations of $\bar{Q}$ vs $\overline{E 0}$

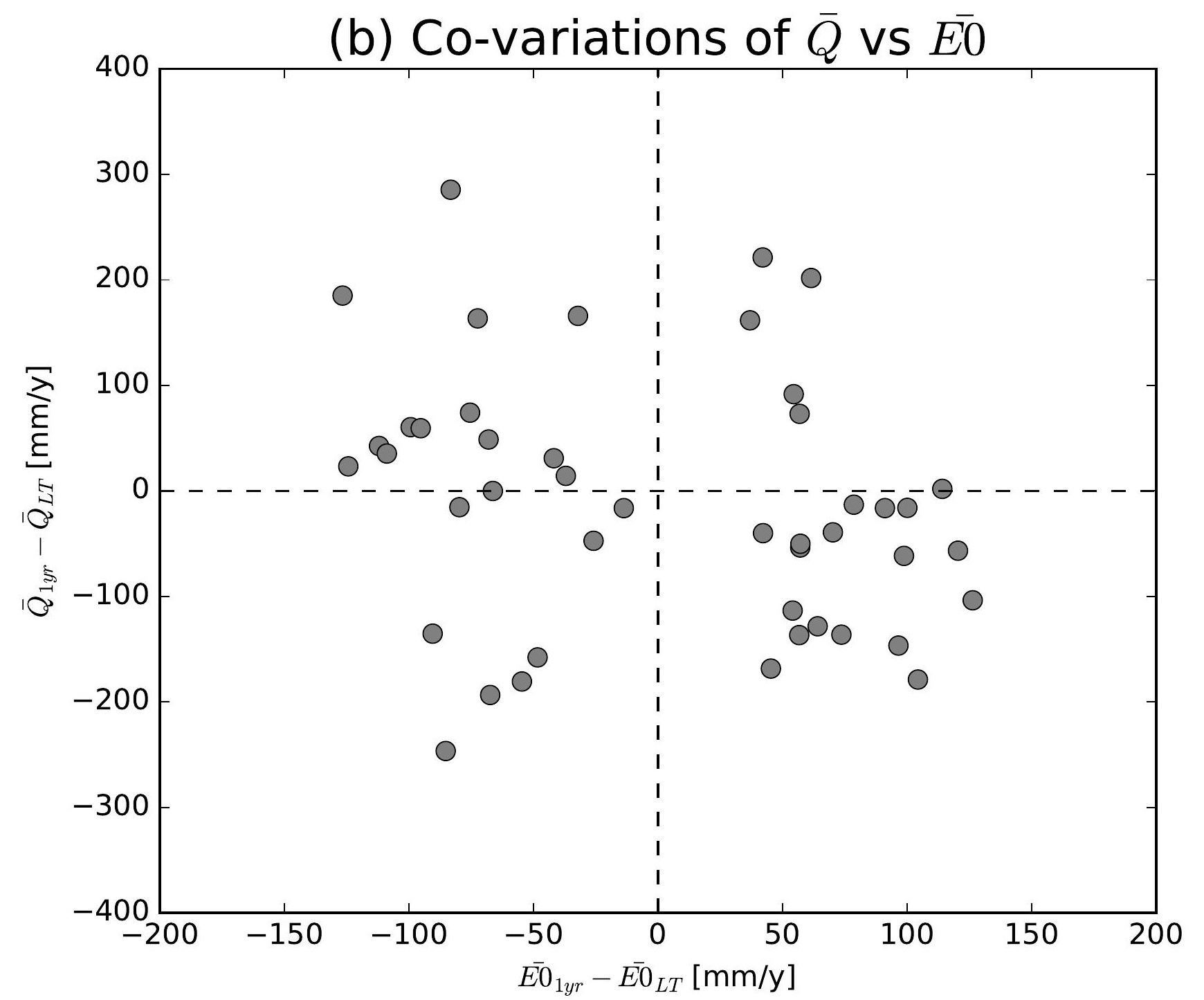

(e) Co-variations of $\bar{Q}$ vs $\overline{E 0}$

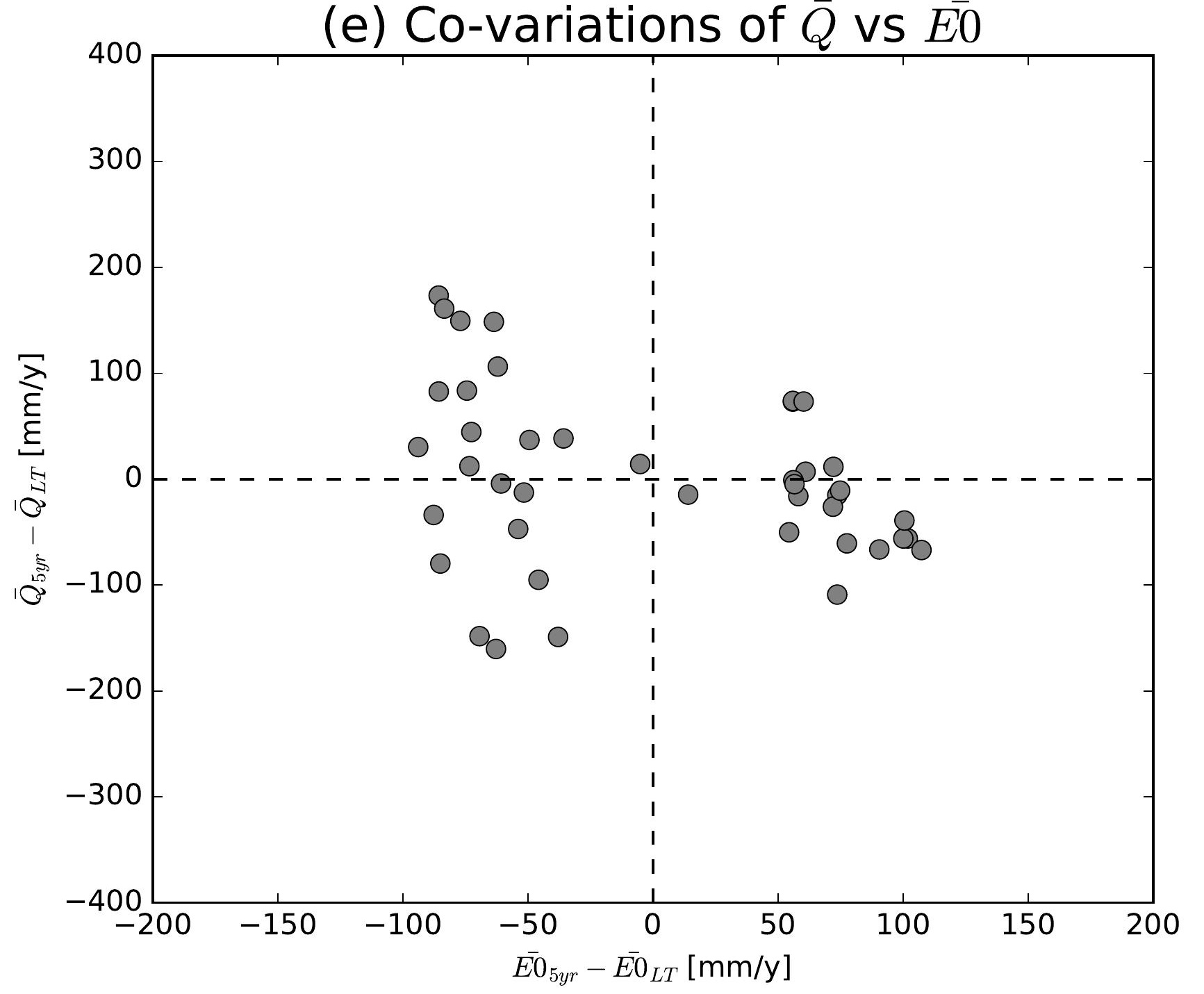

(h) Co-variations of $\bar{Q}$ vs $\overline{E 0}$

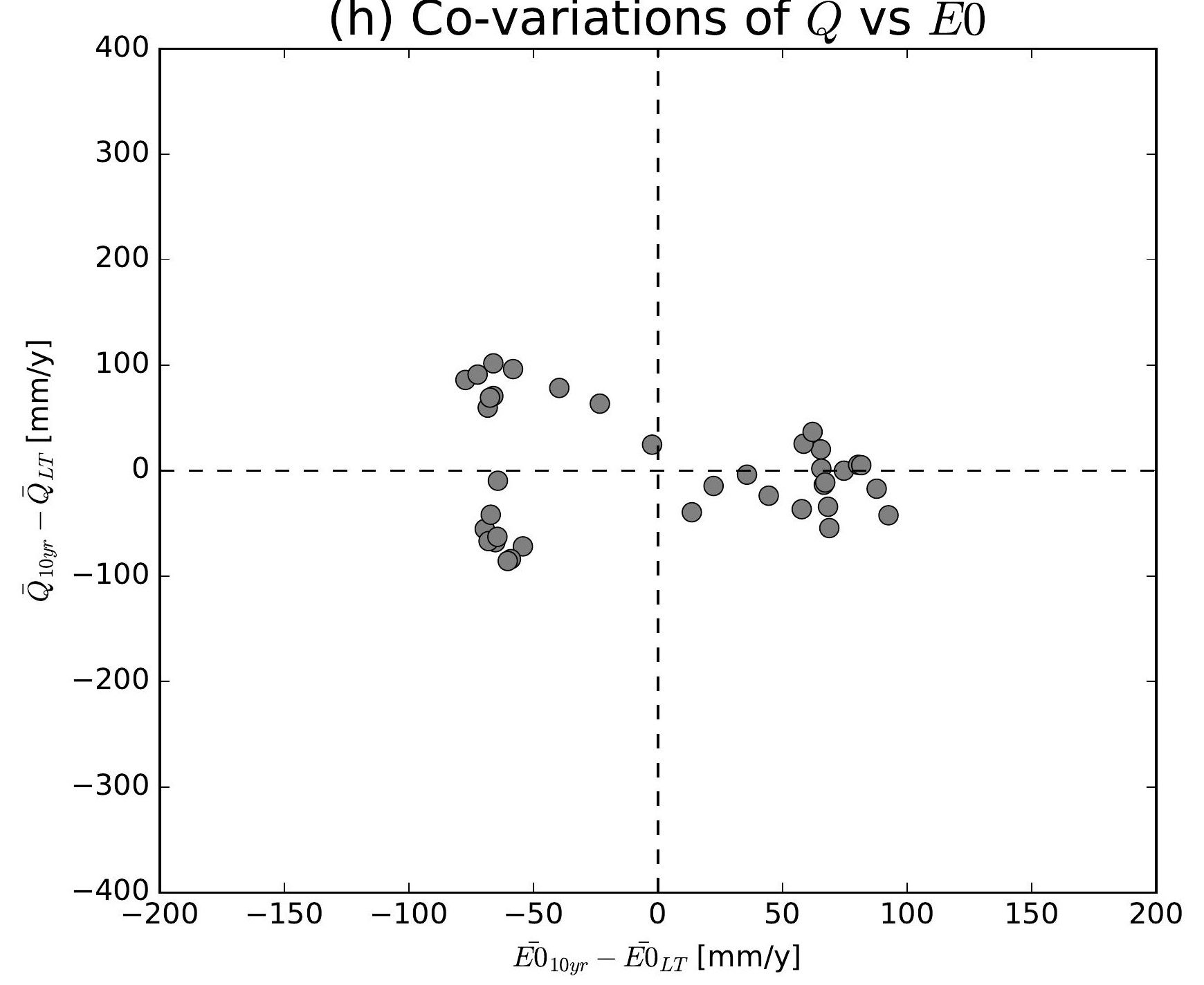

(k) Co-variations of $\bar{Q}$ vs $\overline{E 0}$

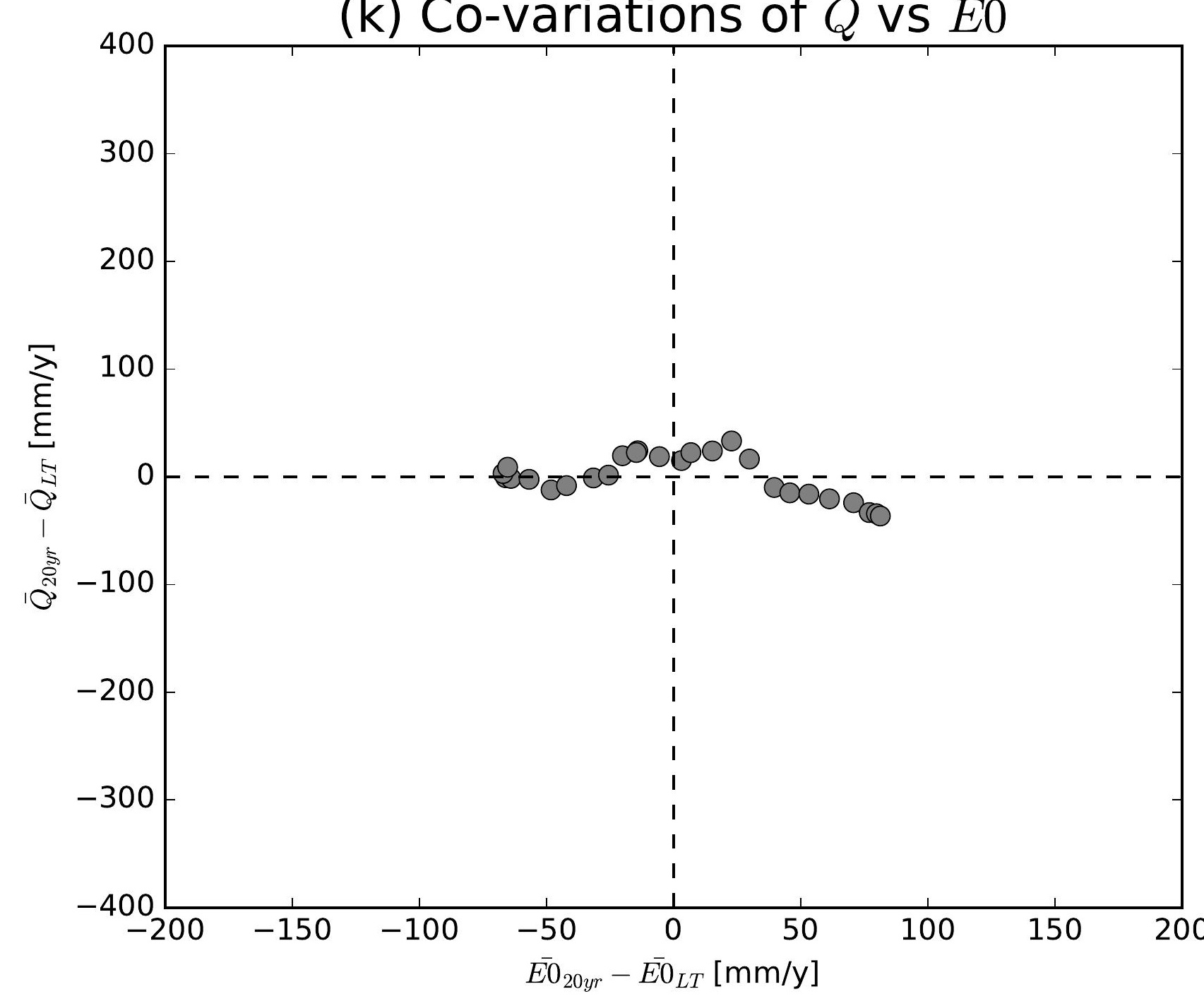

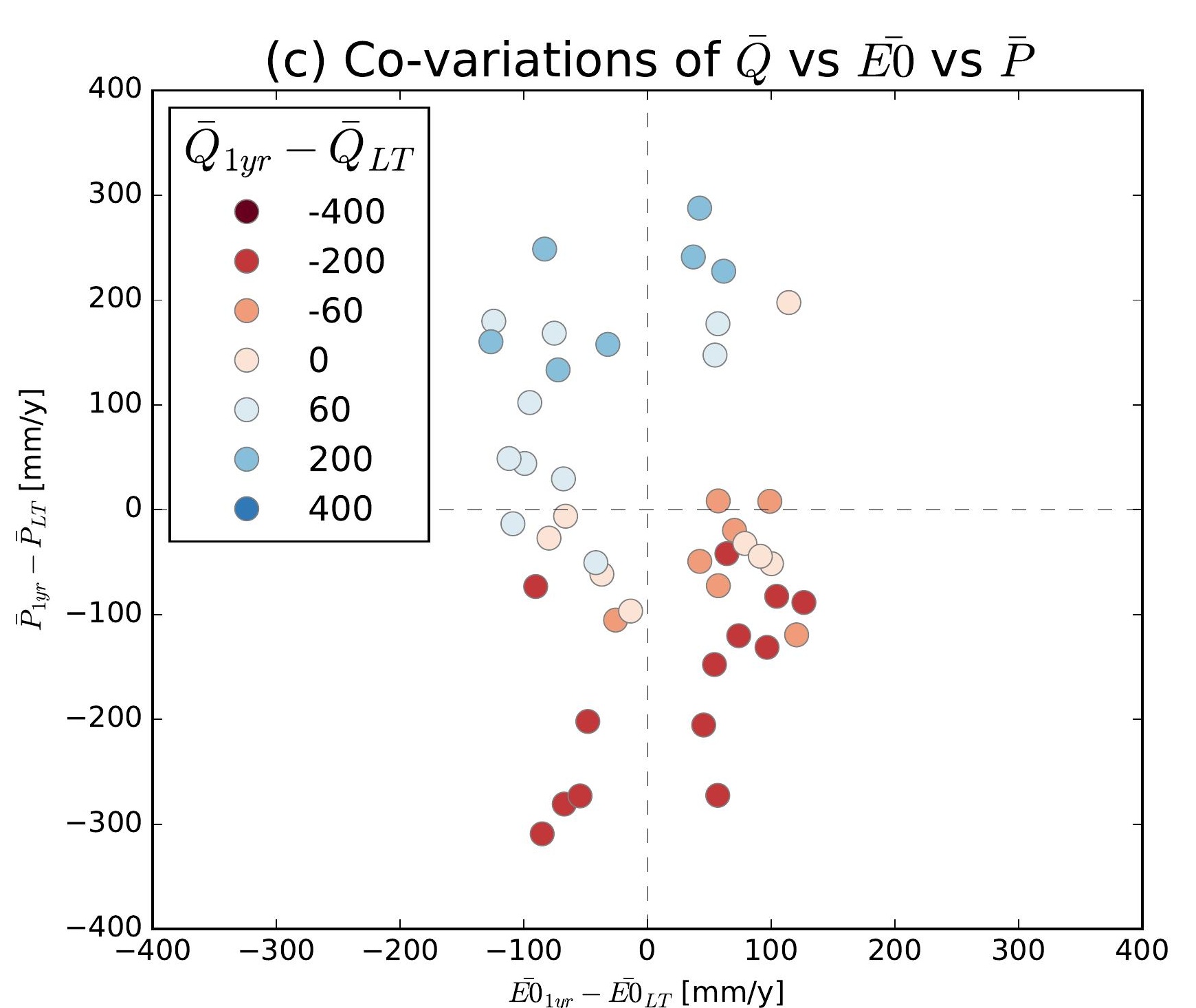
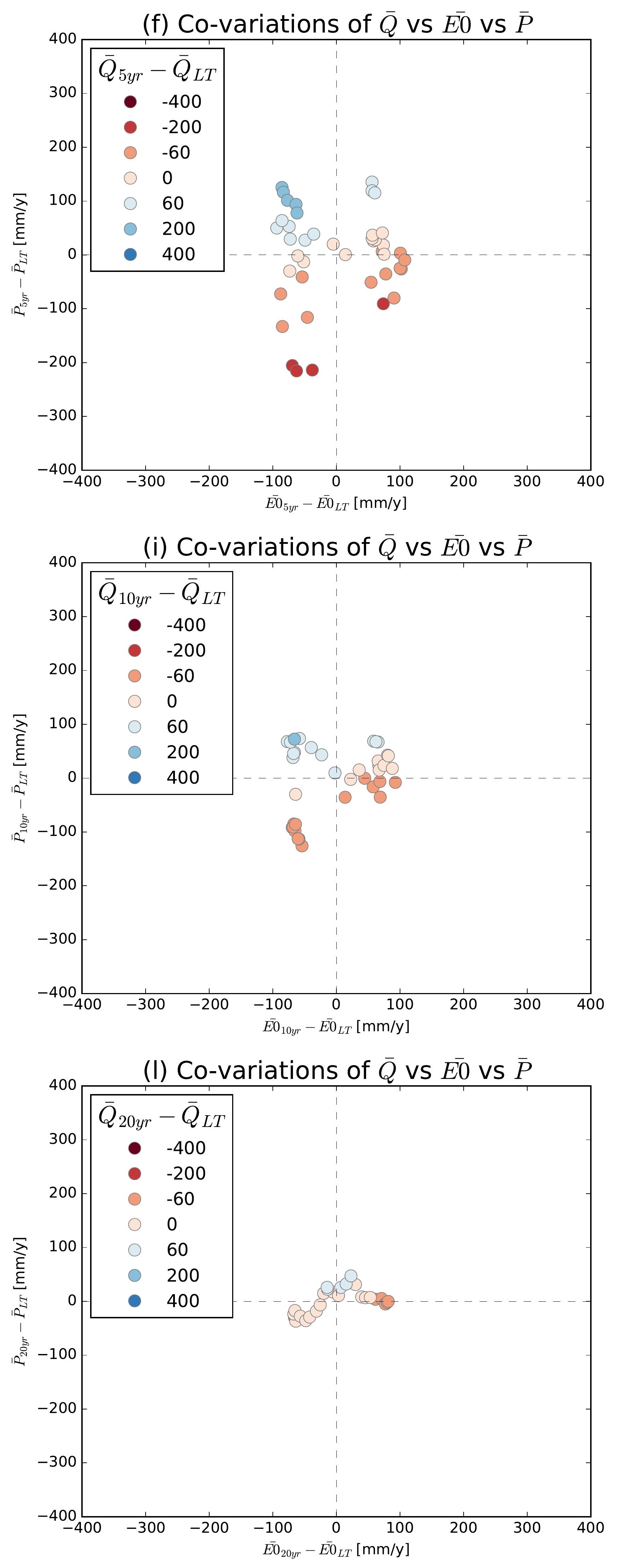

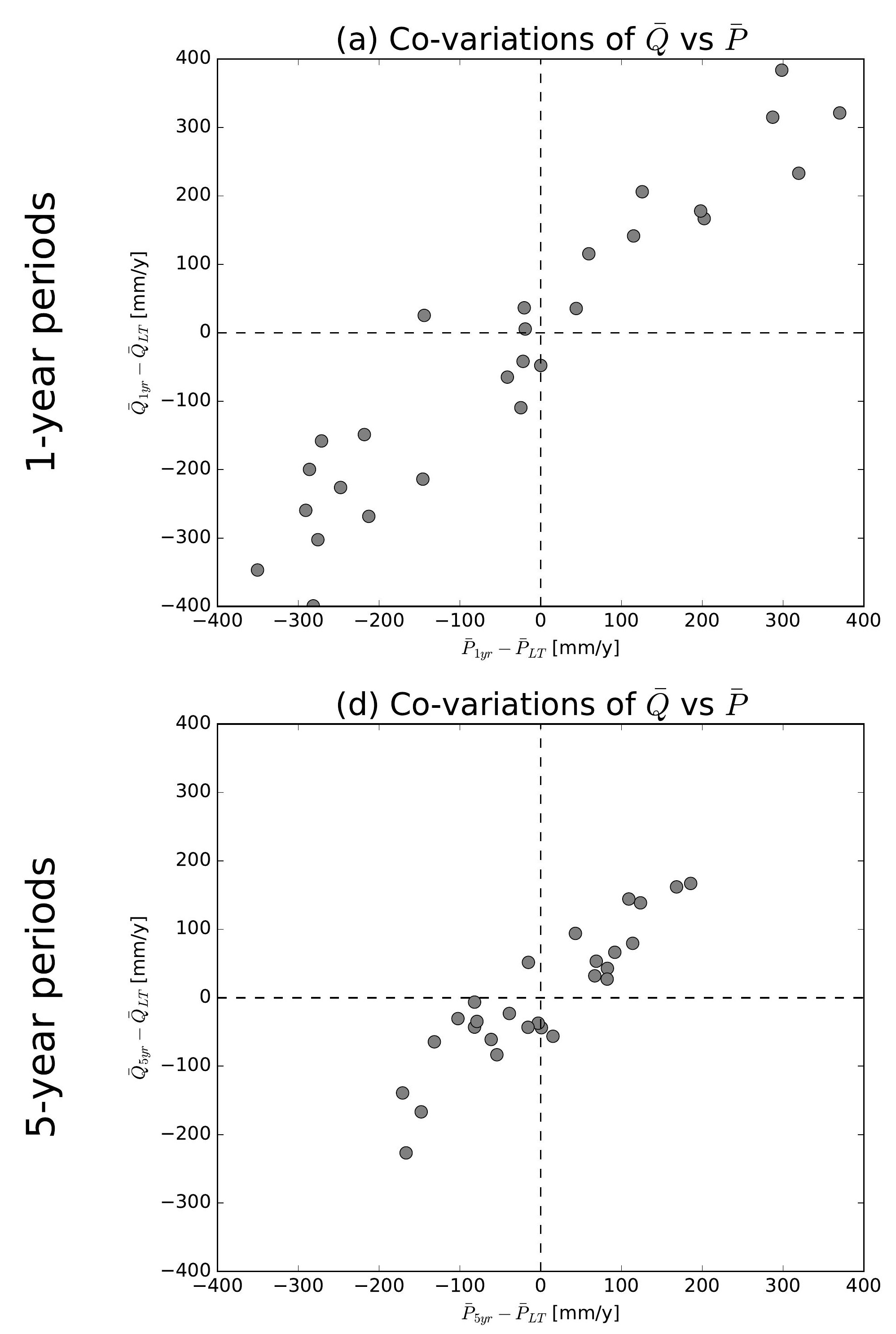

(g) Co-variations of $\bar{Q}$ vs $\bar{P}$

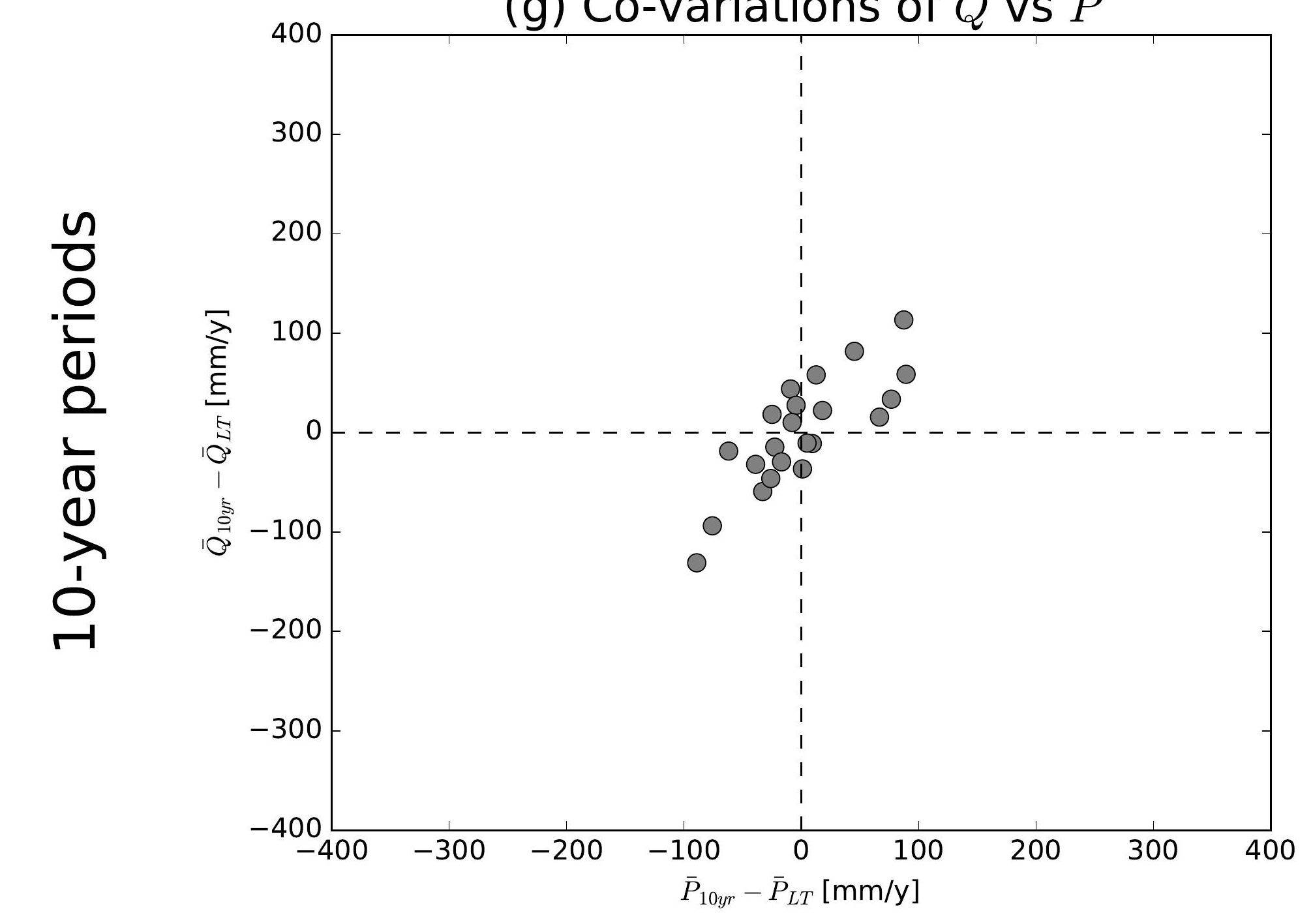

(j) Co-variations of $\bar{Q}$ vs $\bar{P}$

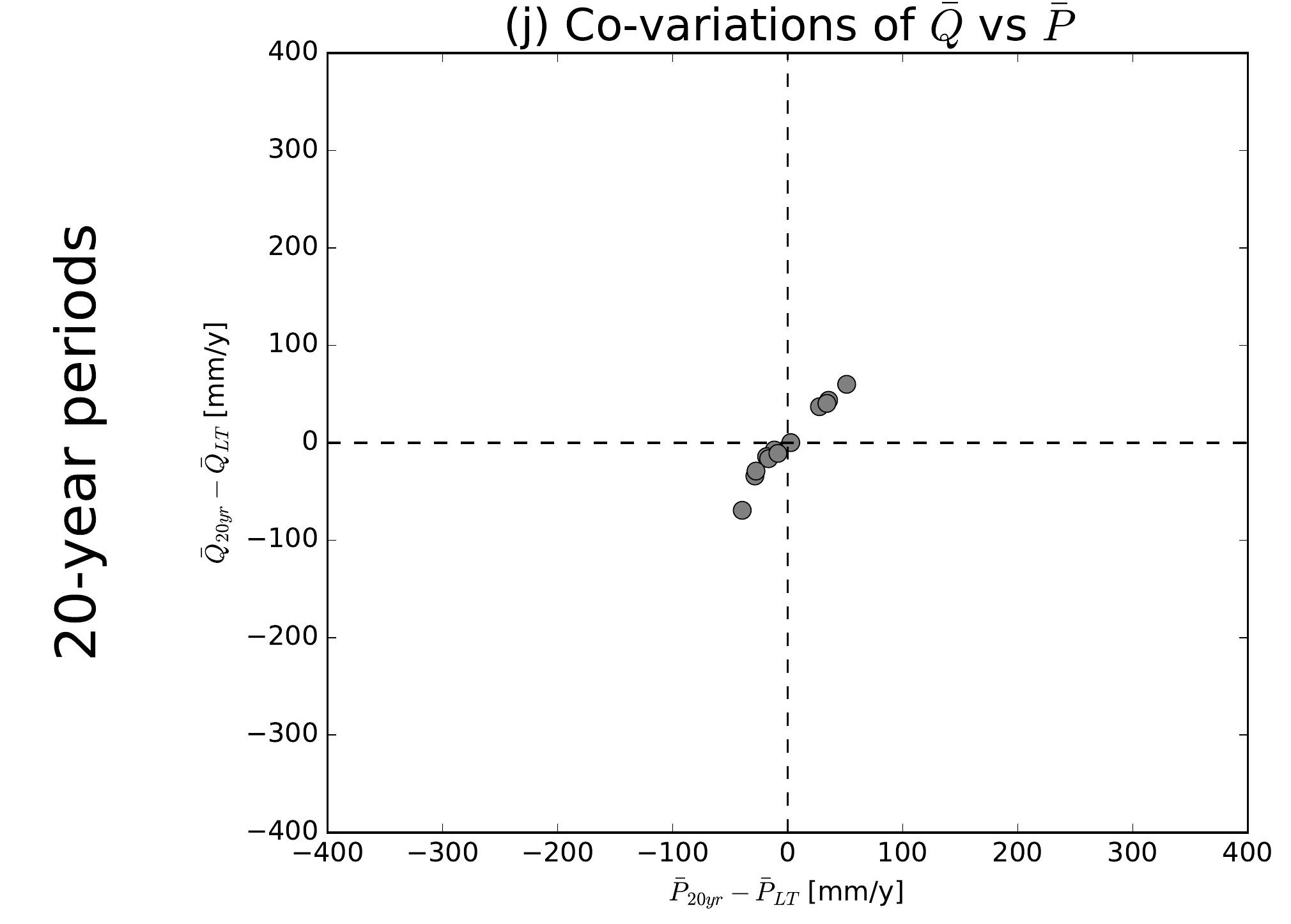

(b) Co-variations of $\bar{Q}$ vs $\overline{E 0}$

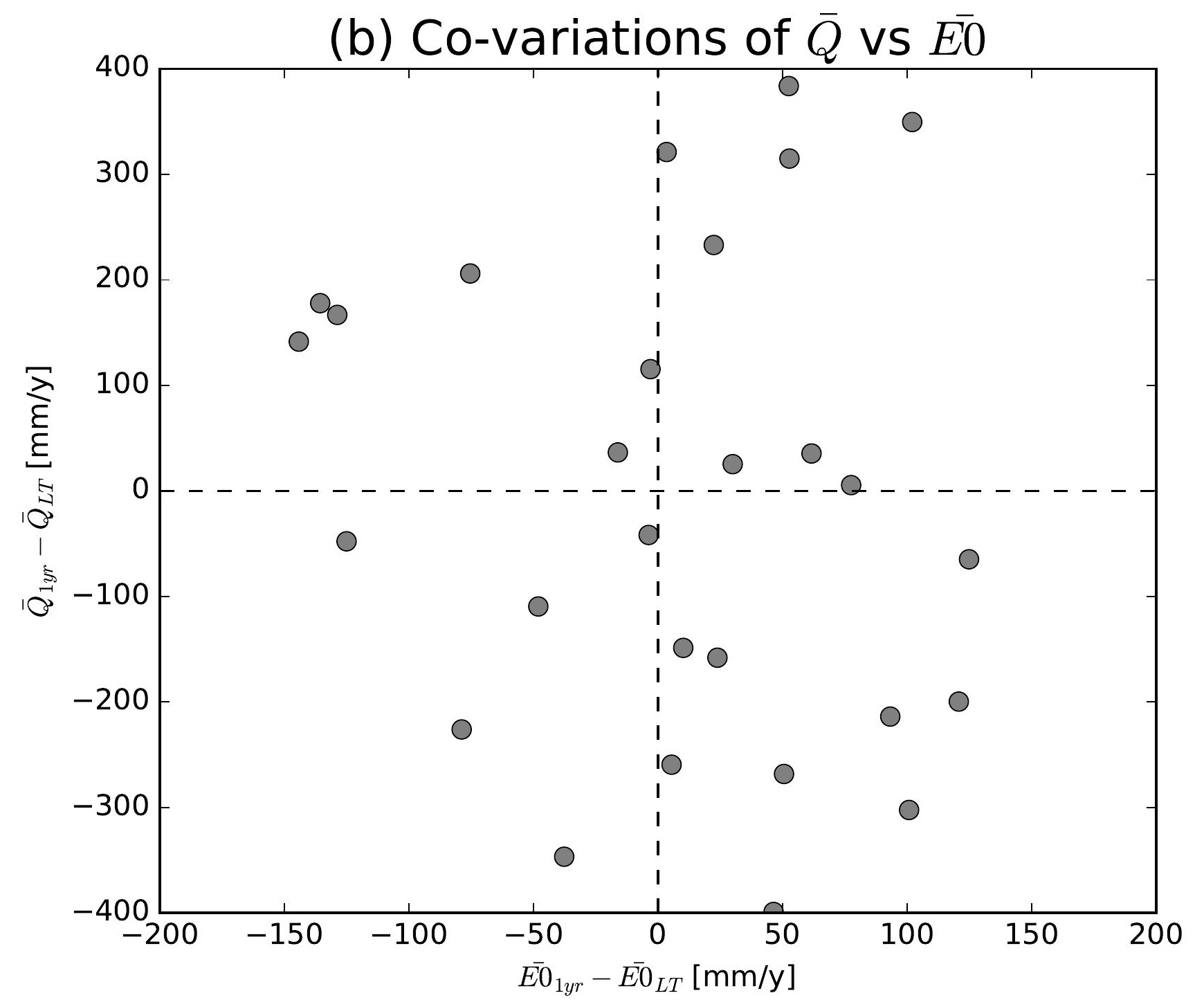

(e) Co-variations of $\bar{Q}$ vs $\overline{E 0}$

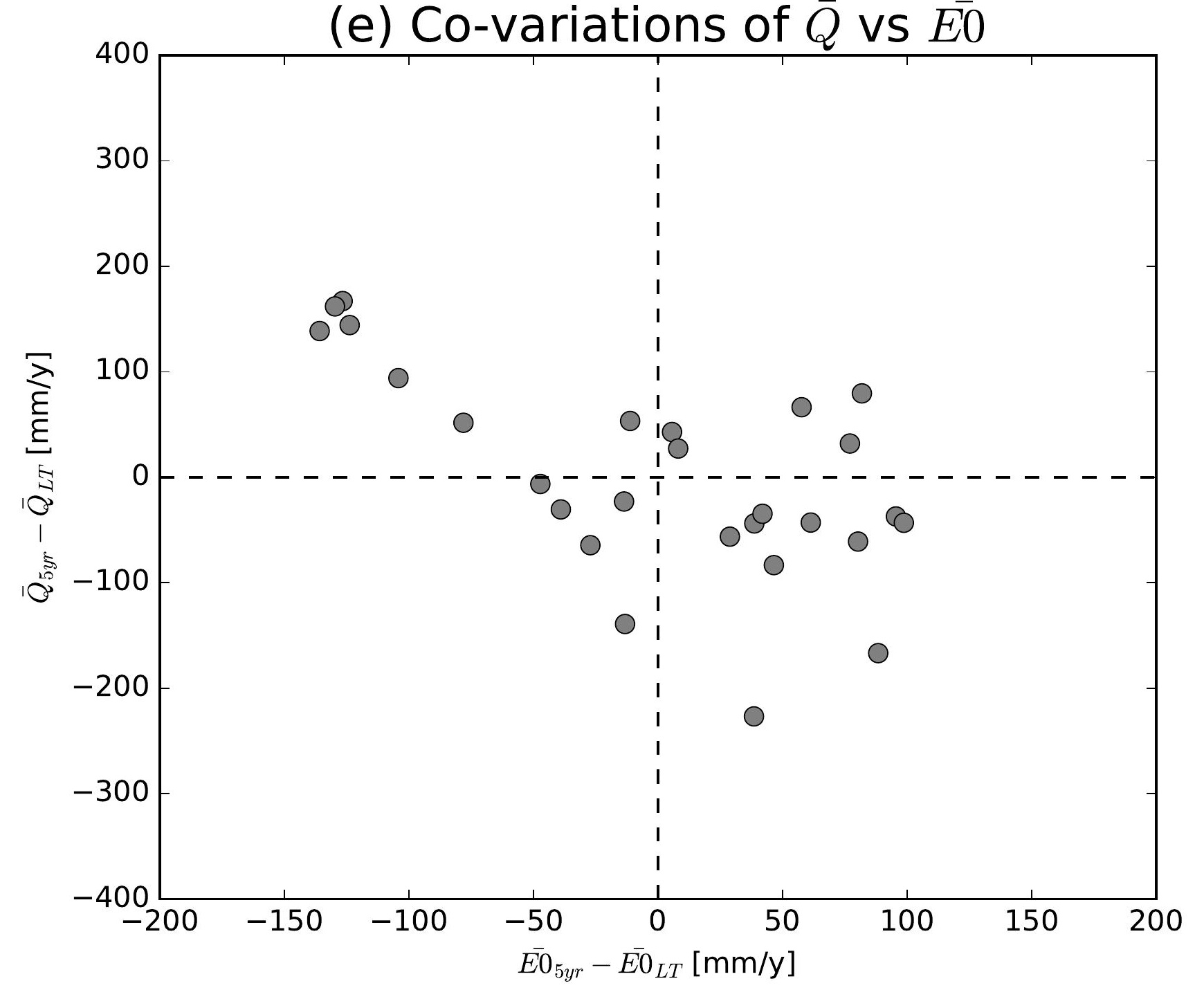

(h) Co-variations of $\bar{Q}$ vs $\overline{E 0}$

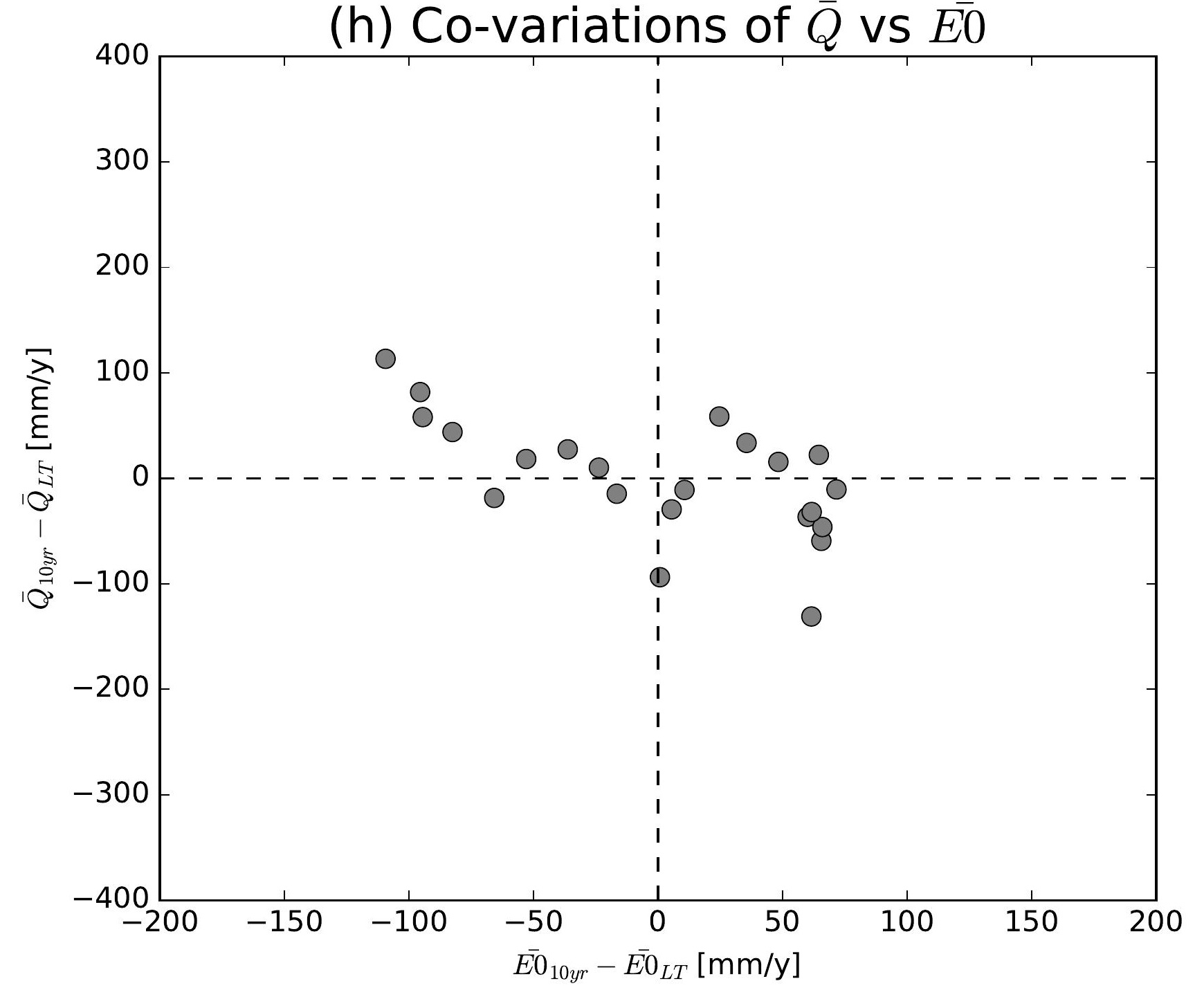

(k) Co-variations of $\bar{Q}$ vs $\overline{E 0}$

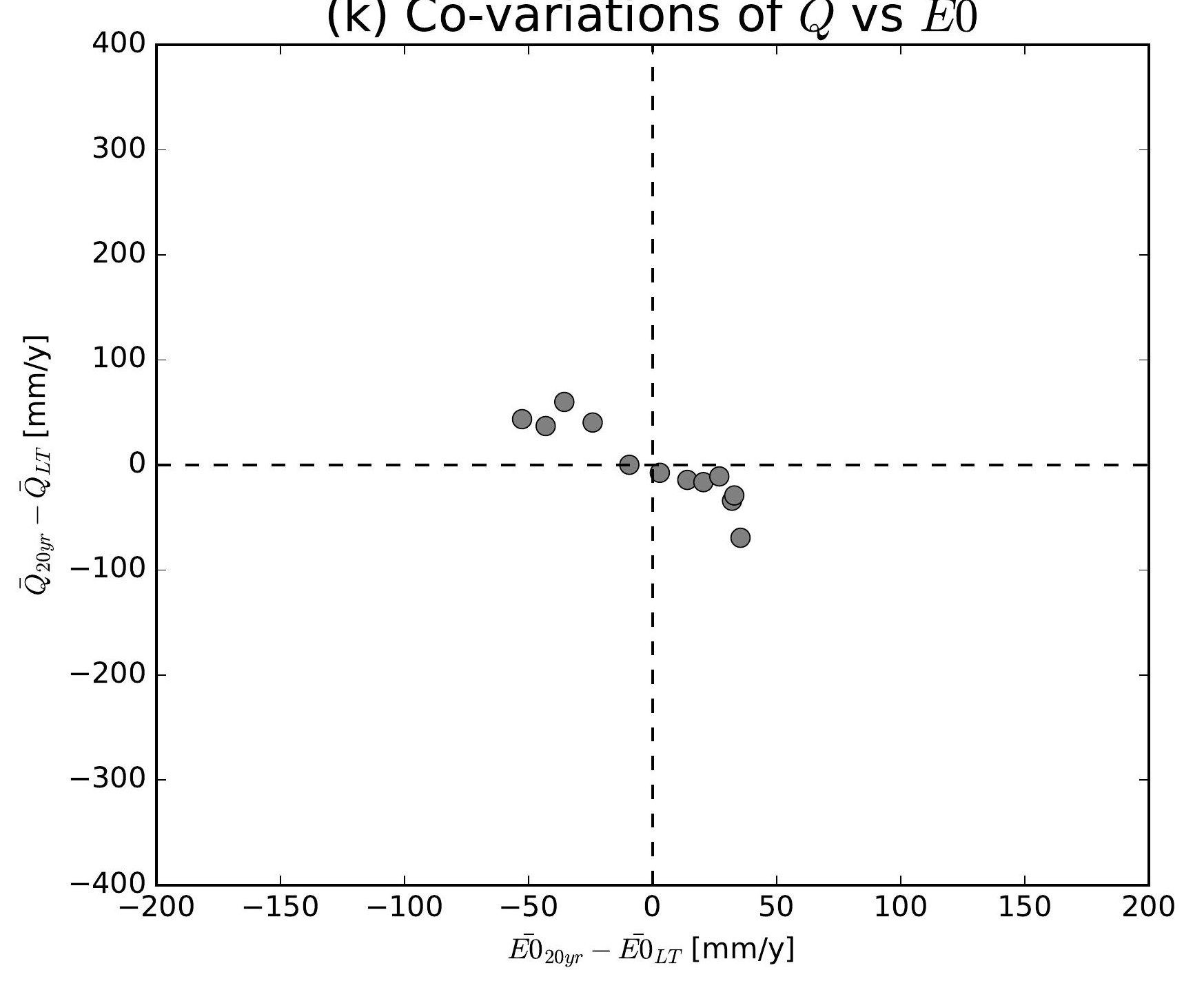

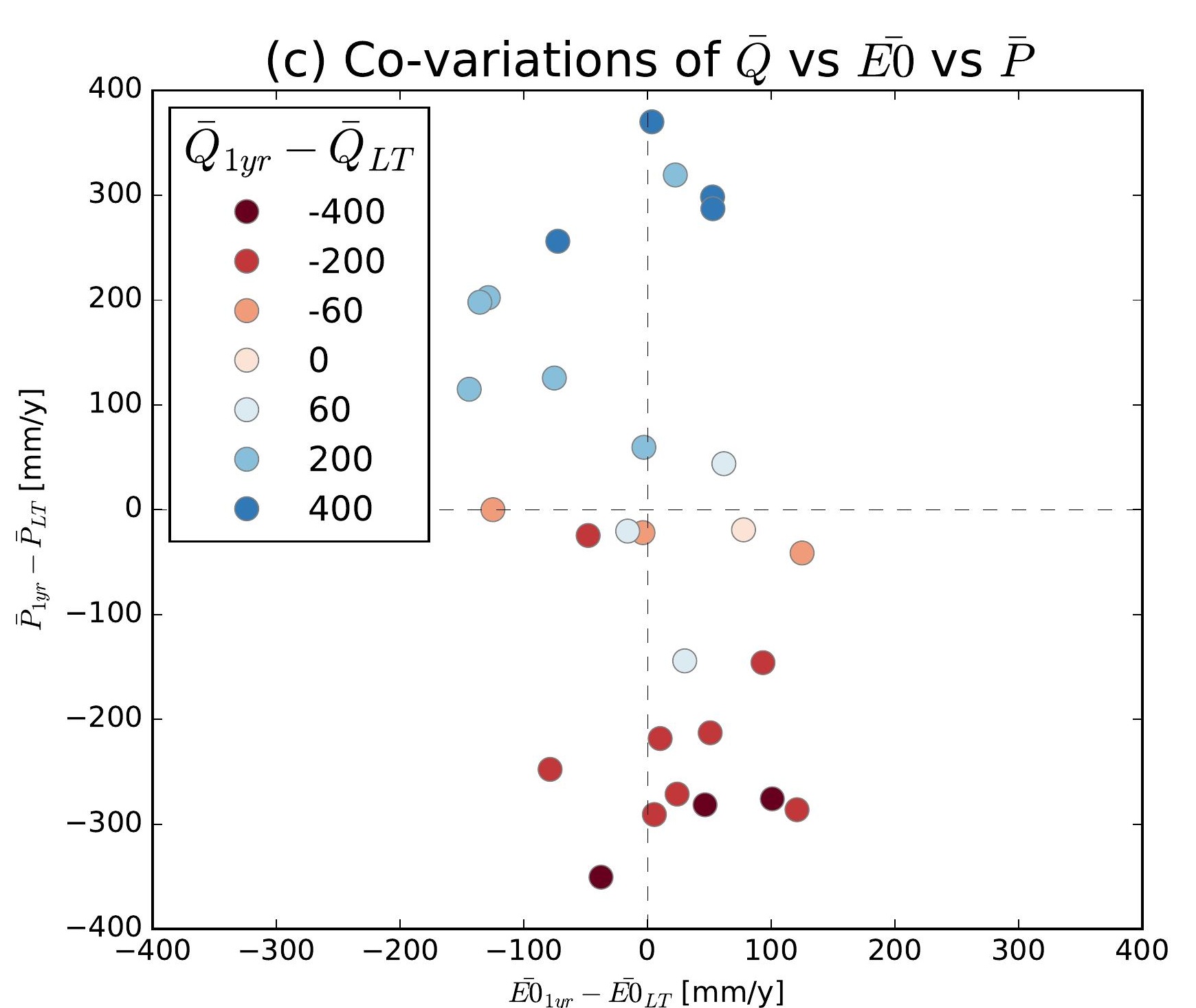
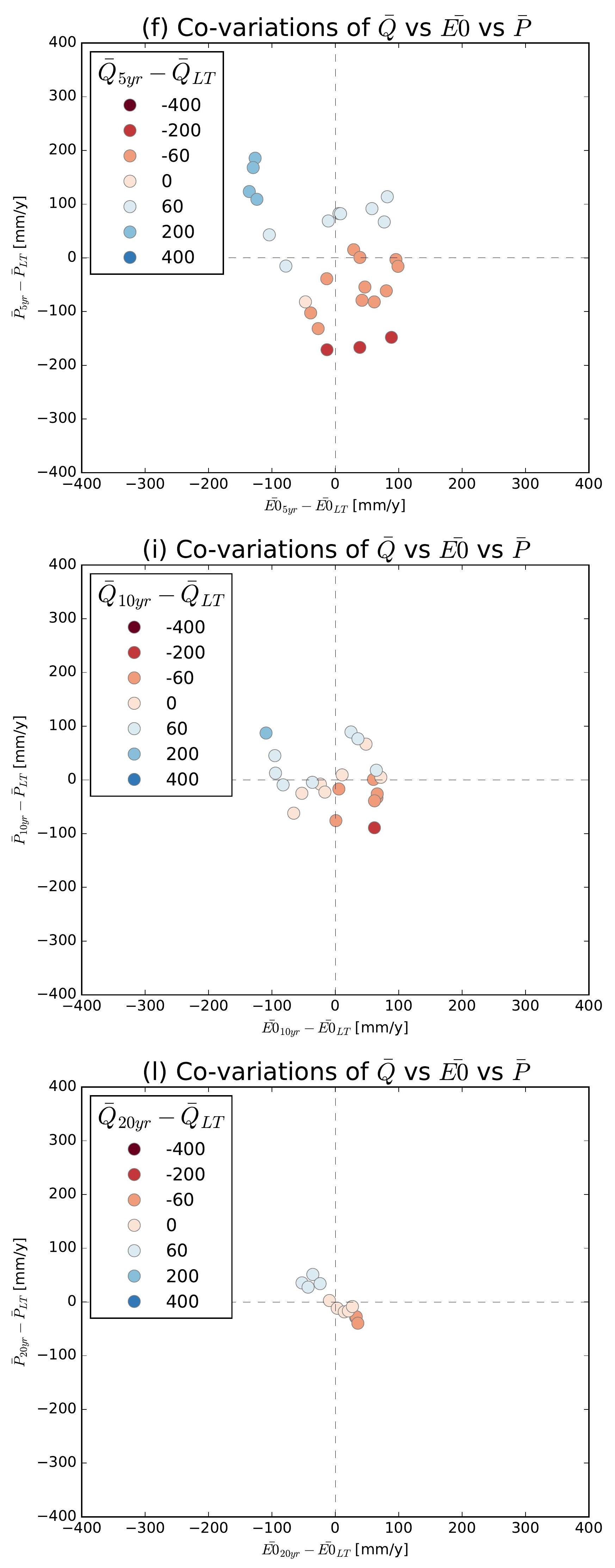

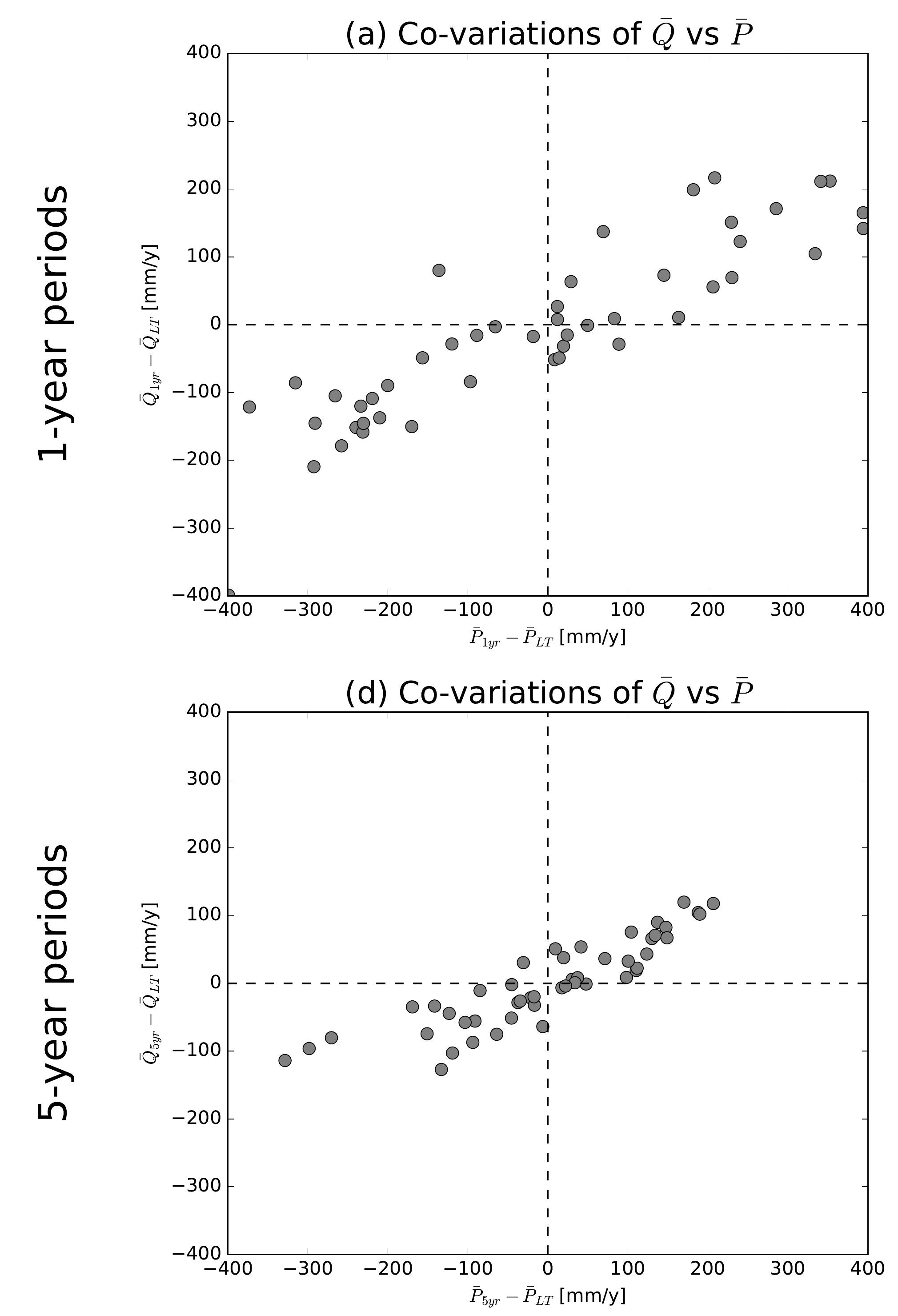

(g) Co-variations of $\bar{Q}$ vs $\bar{P}$

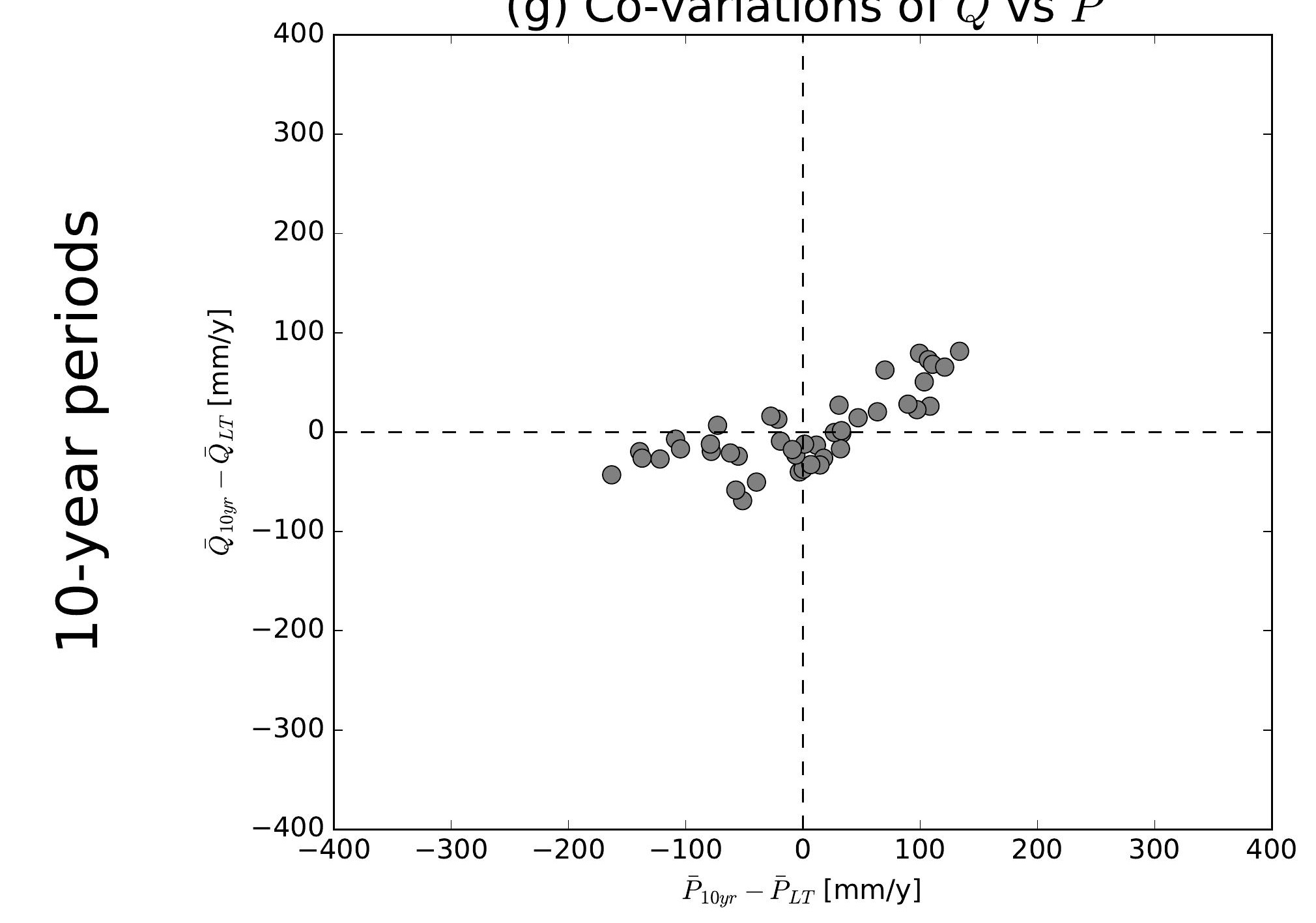

$\frac{4}{2}$

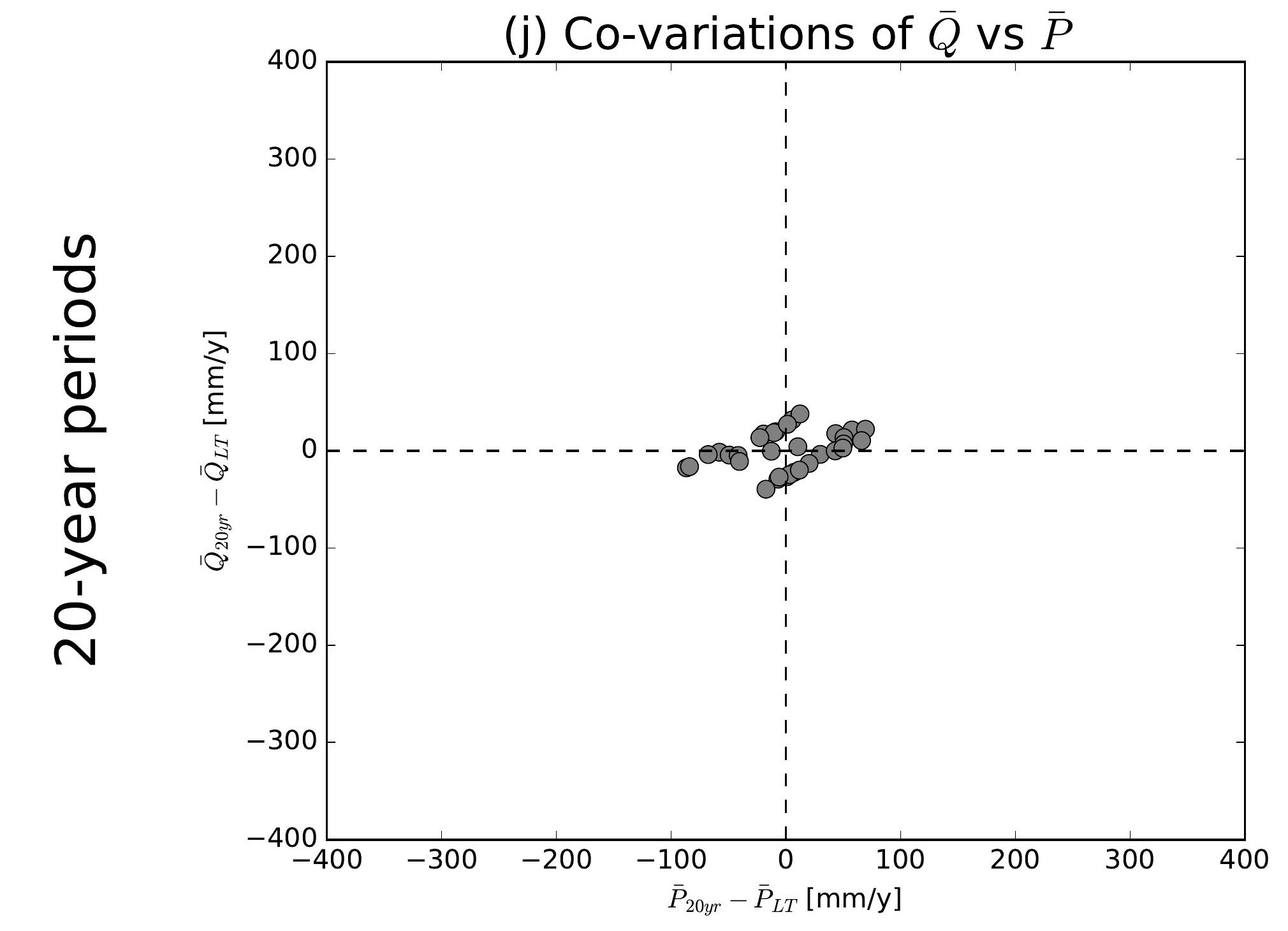

(b) Co-variations of $\bar{Q}$ vs $\overline{E 0}$

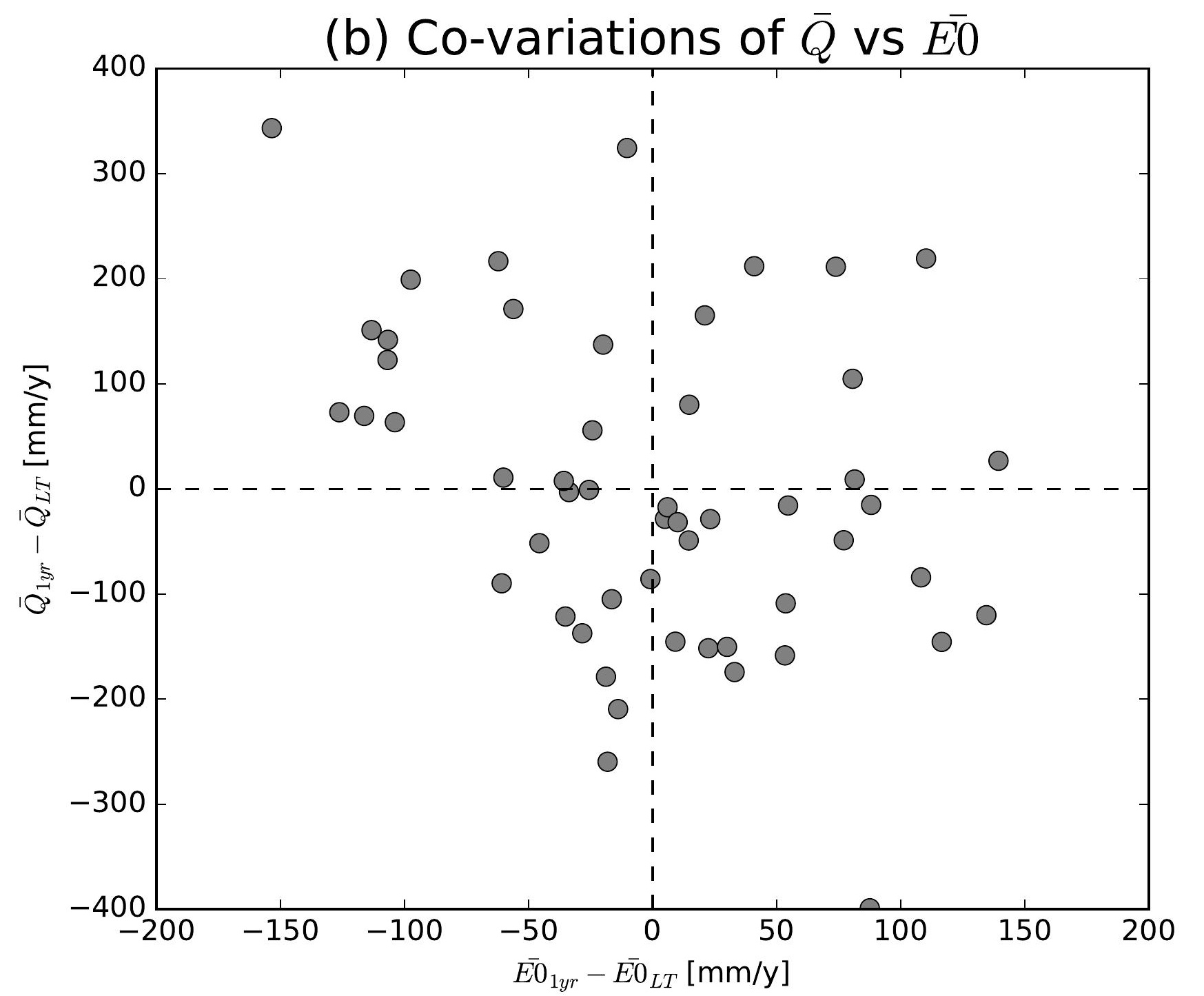

(e) Co-variations of $\bar{Q}$ vs $\overline{E 0}$

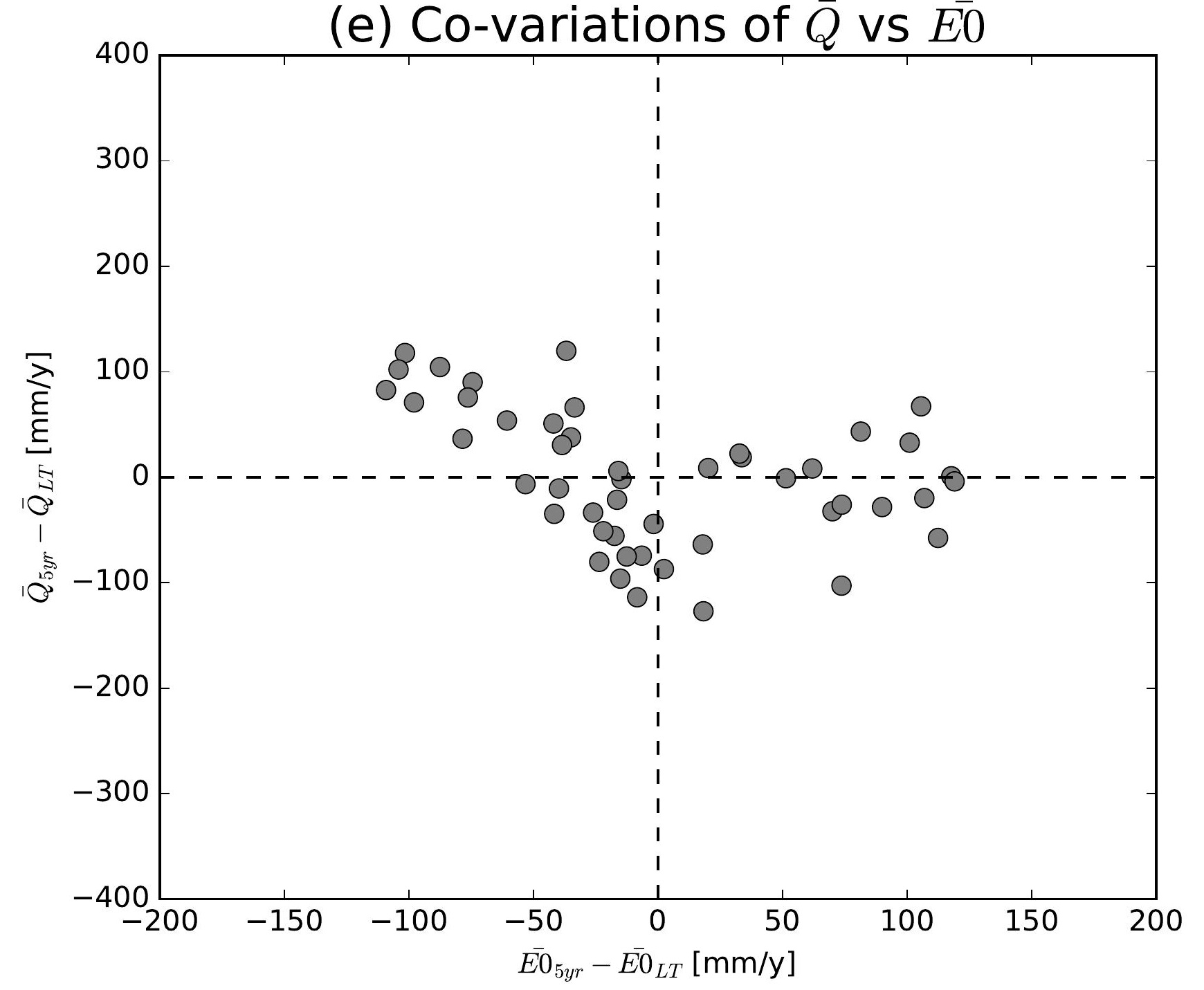

(h) Co-variations of $\bar{Q}$ vs $\overline{E 0}$

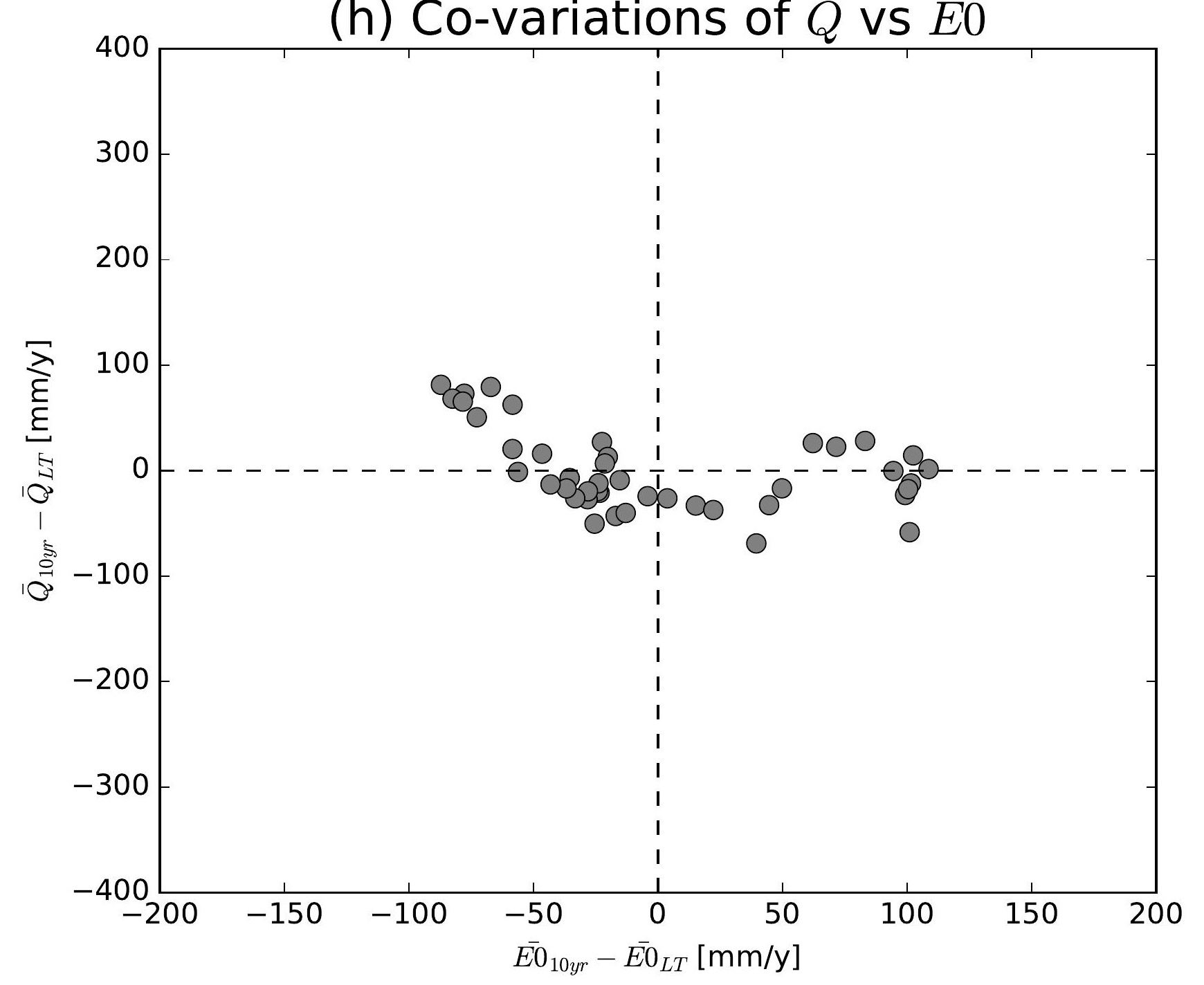

(k) Co-variations of $\bar{Q}$ vs $\overline{E 0}$

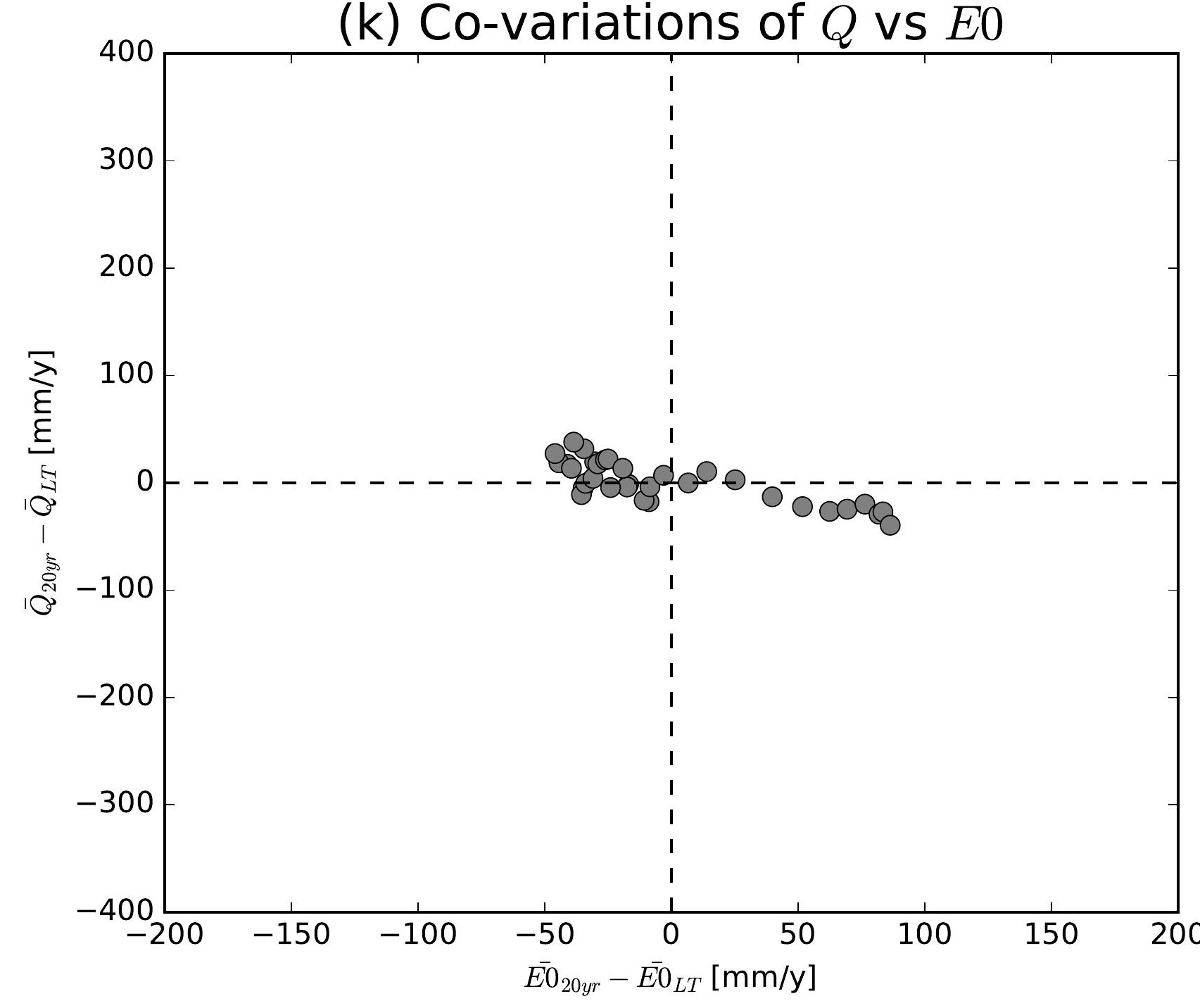

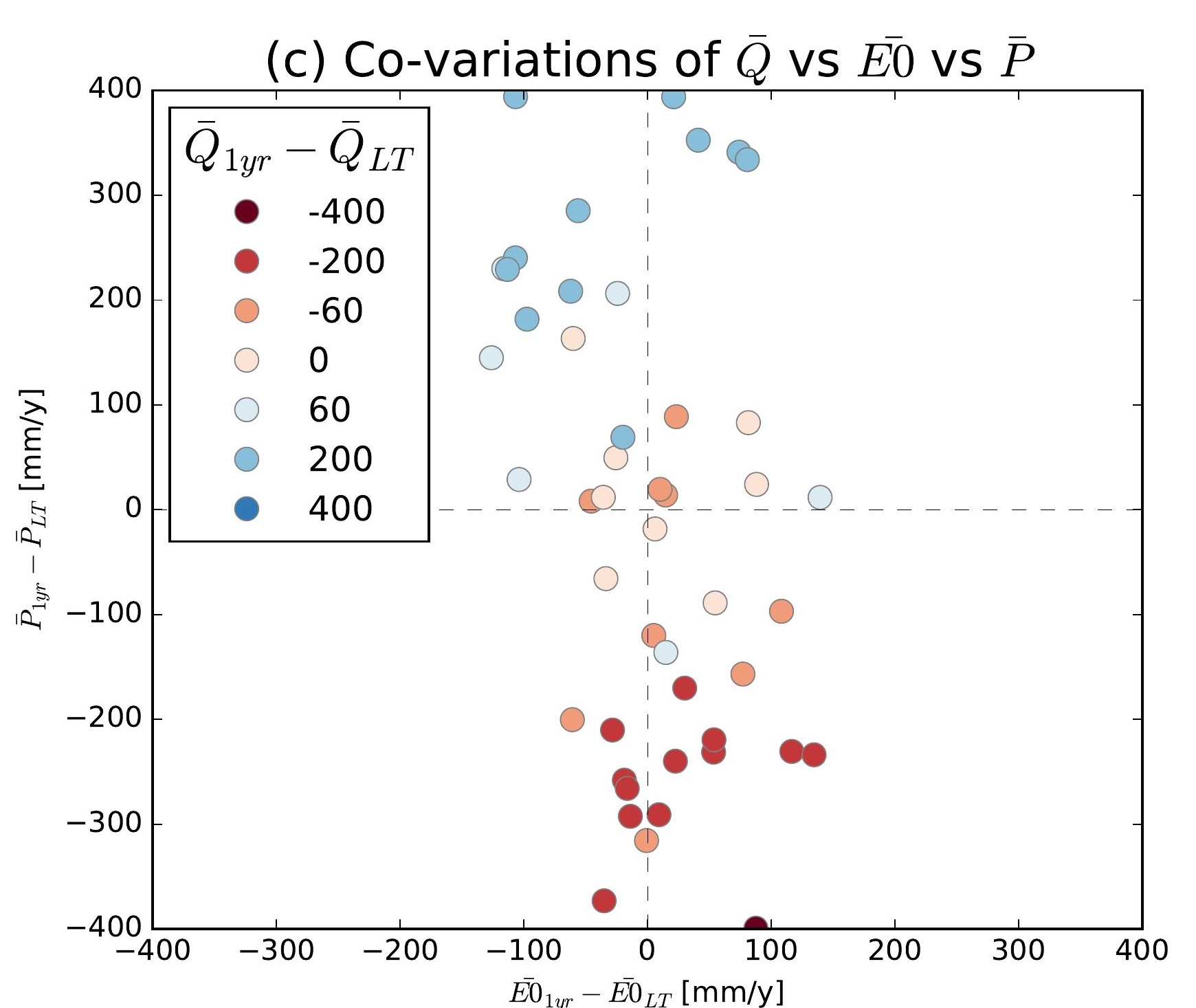
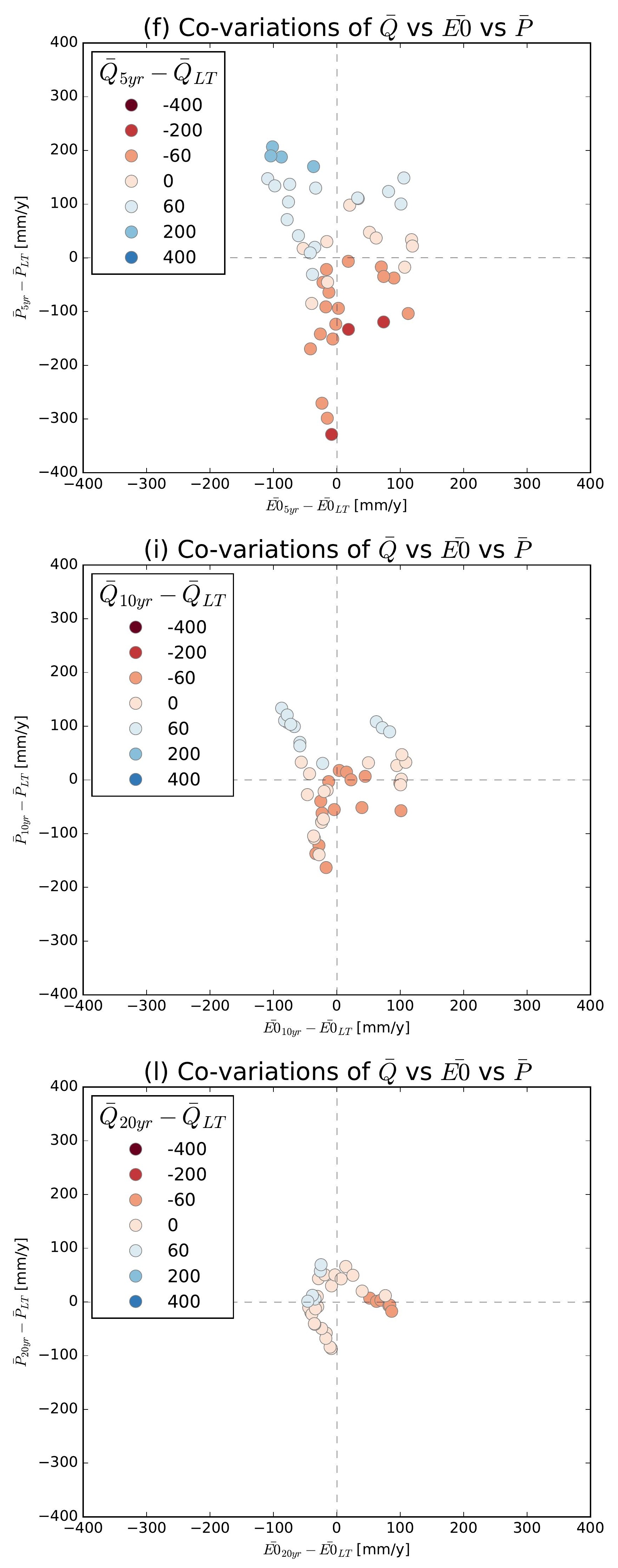

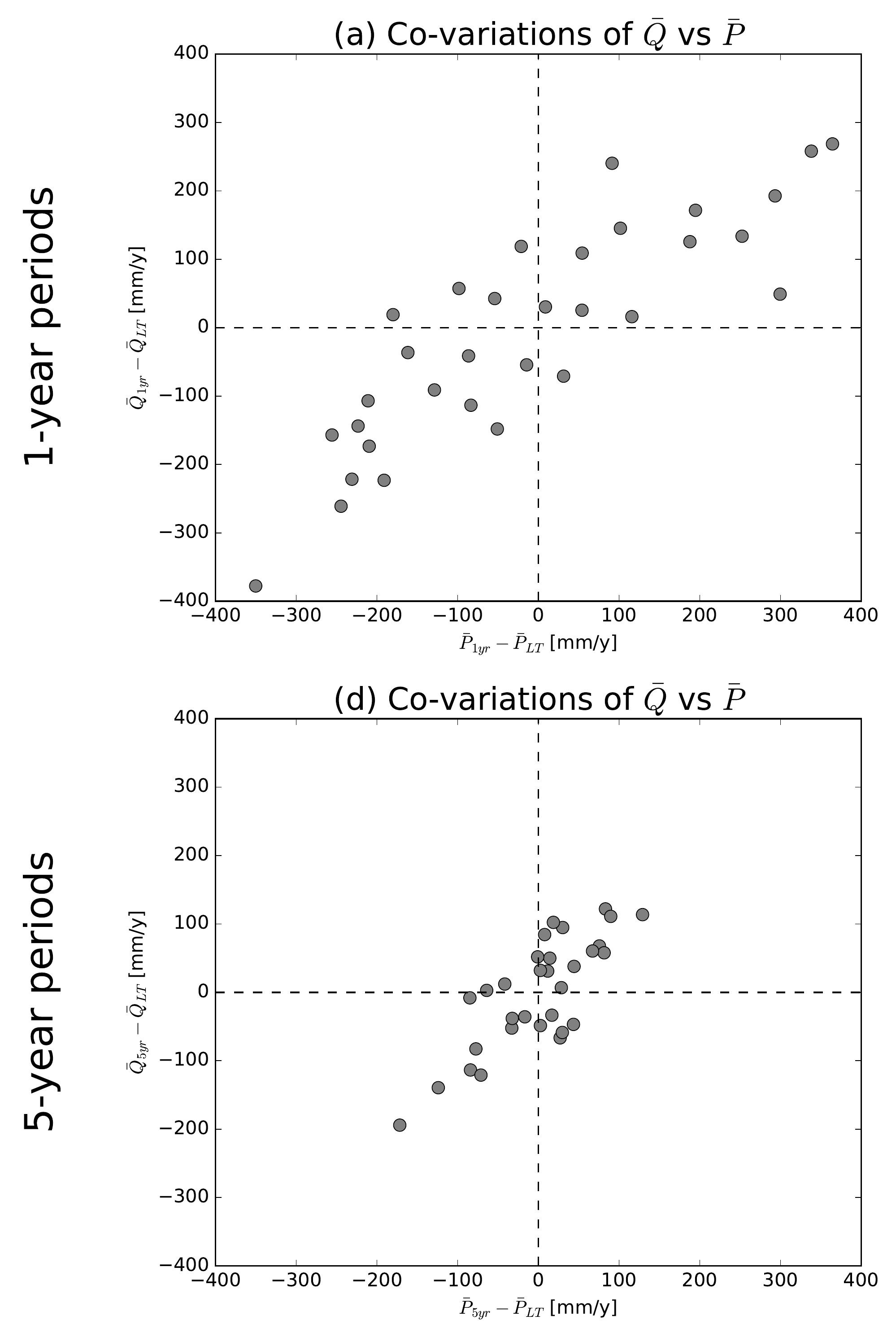

(g) Co-variations of $\bar{Q}$ vs $\bar{P}$
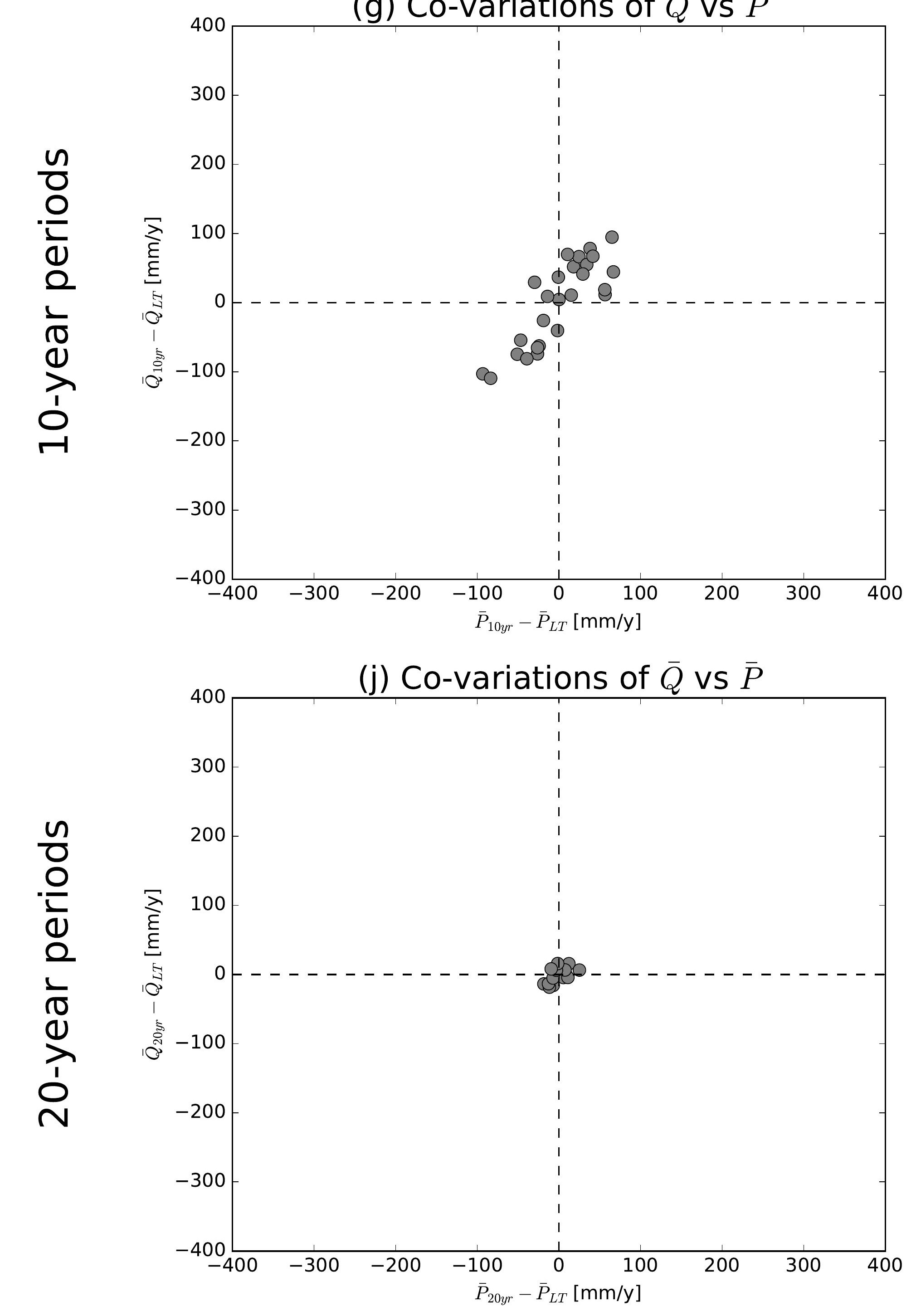

(b) Co-variations of $\bar{Q}$ vs $\overline{E 0}$

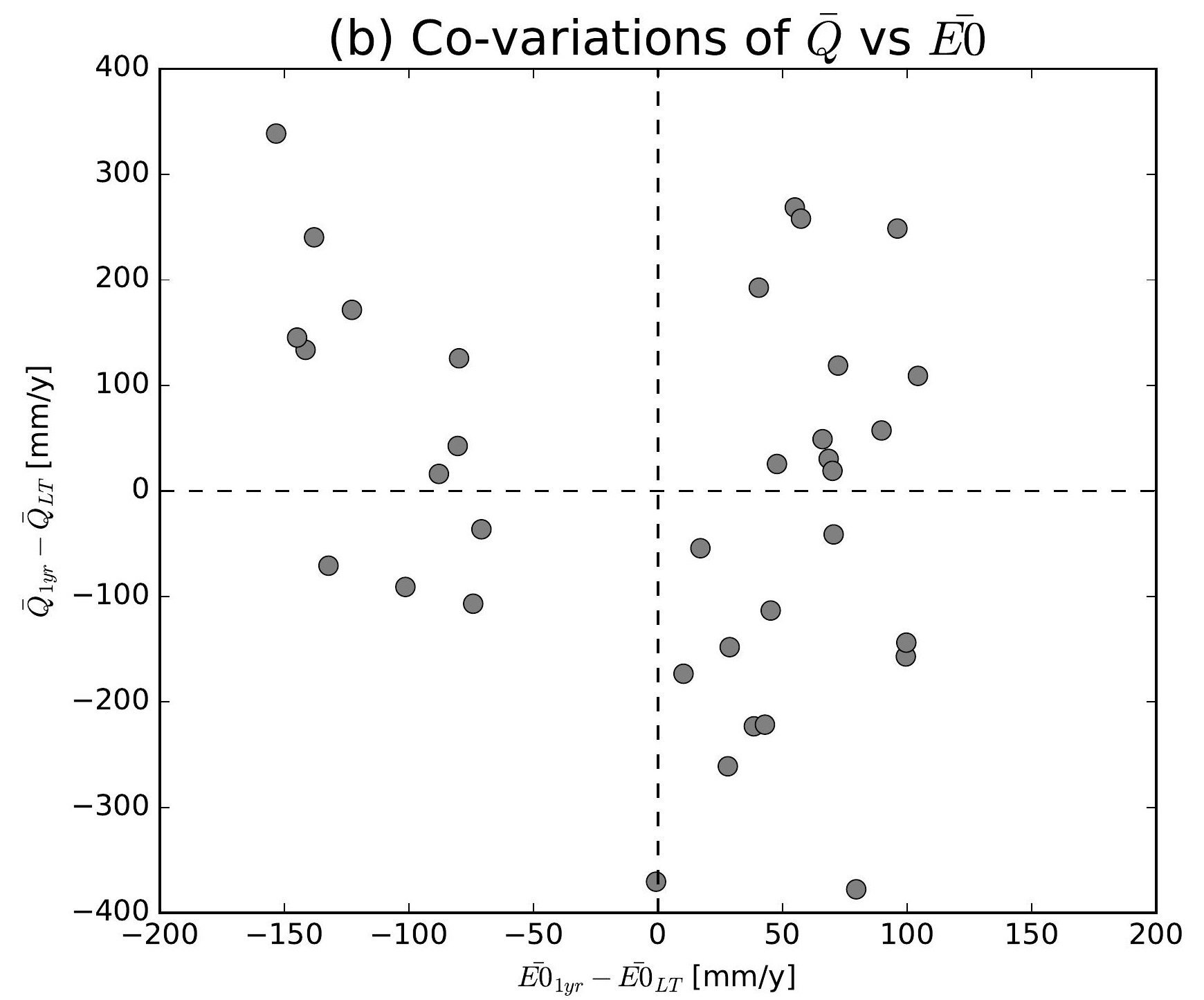

(e) Co-variations of $\bar{Q}$ vs $\overline{E 0}$

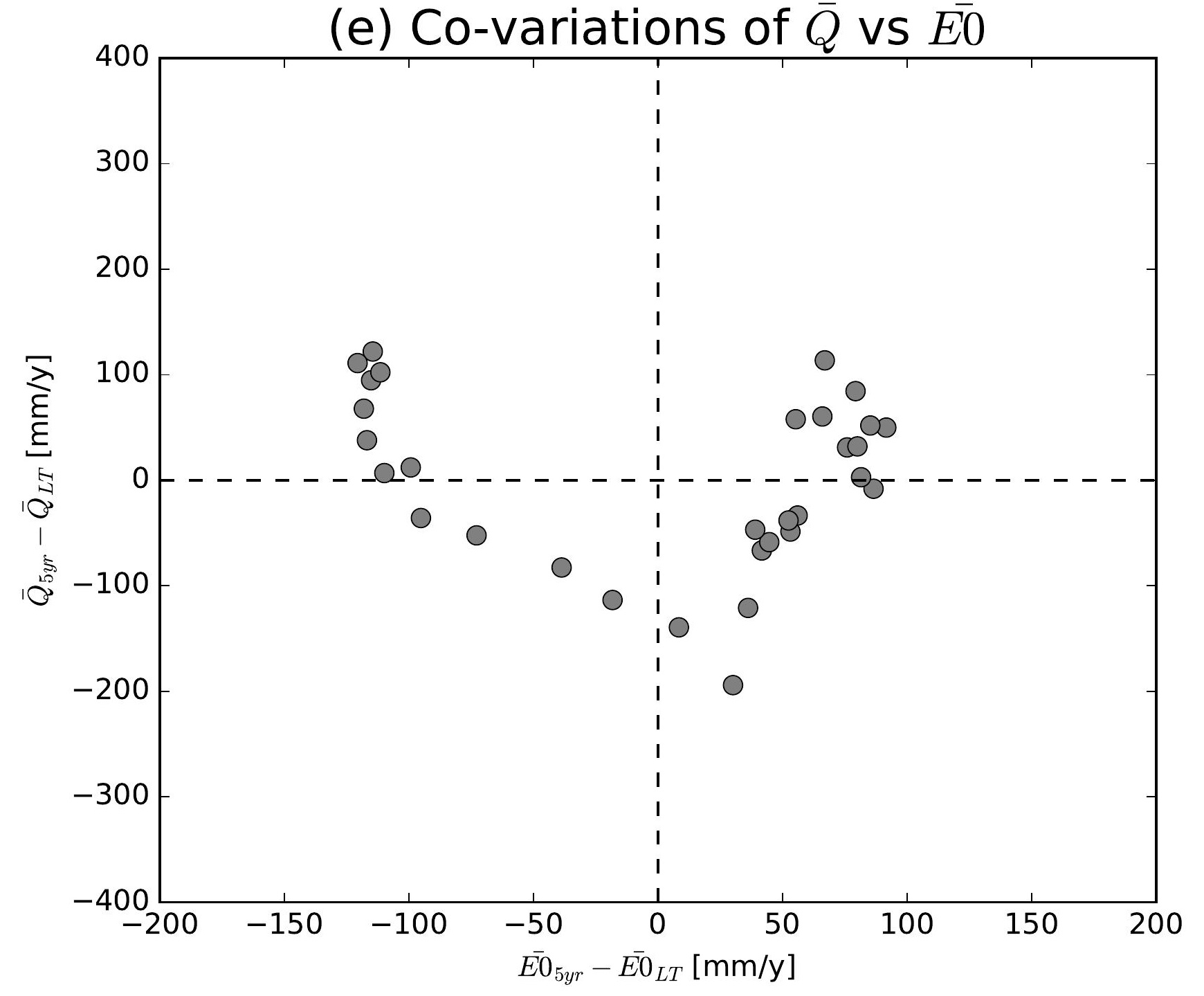

(h) Co-variations of $\bar{Q}$ vs $\overline{E 0}$

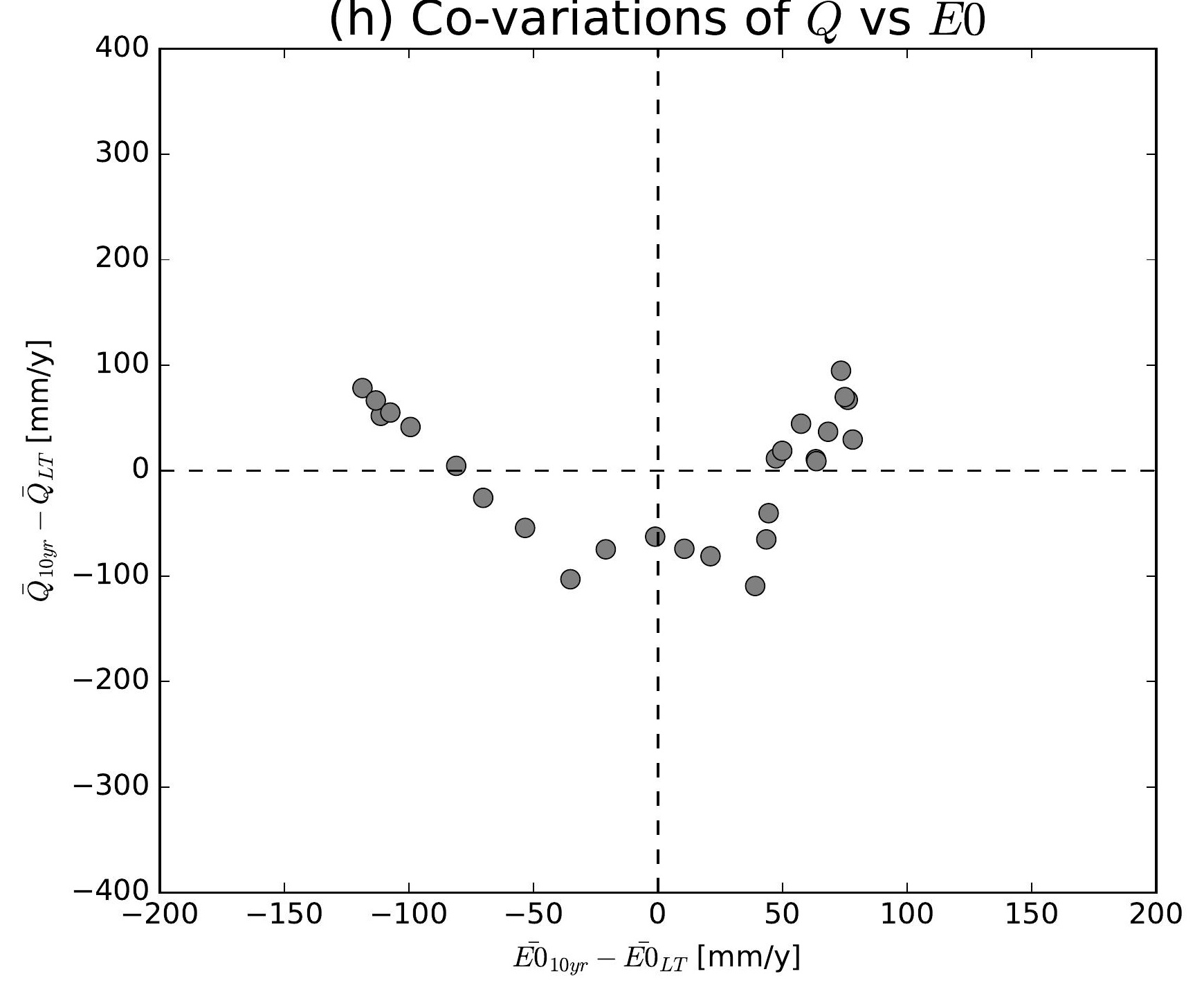

(k) Co-variations of $\bar{Q}$ vs $\overline{E 0}$

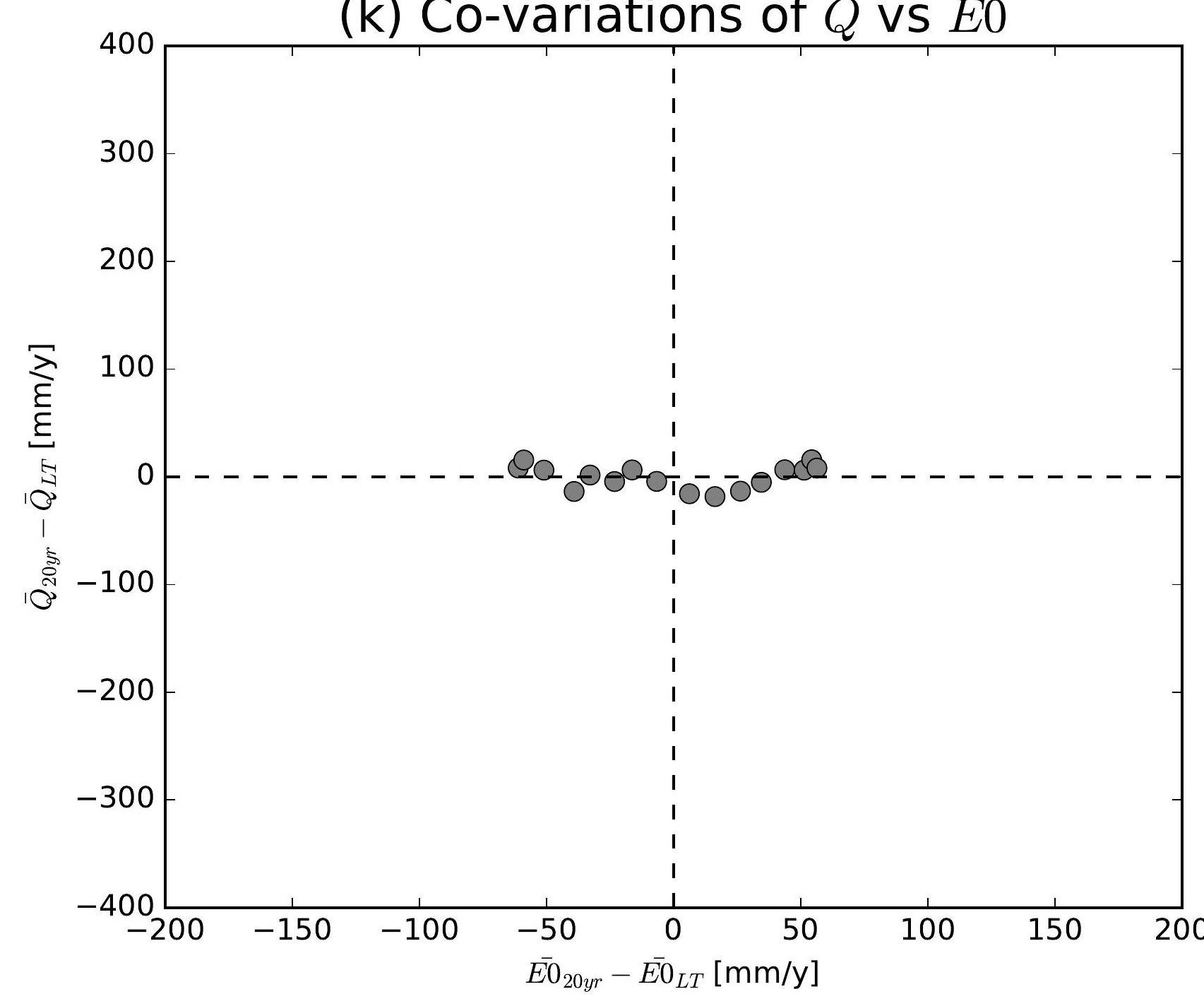

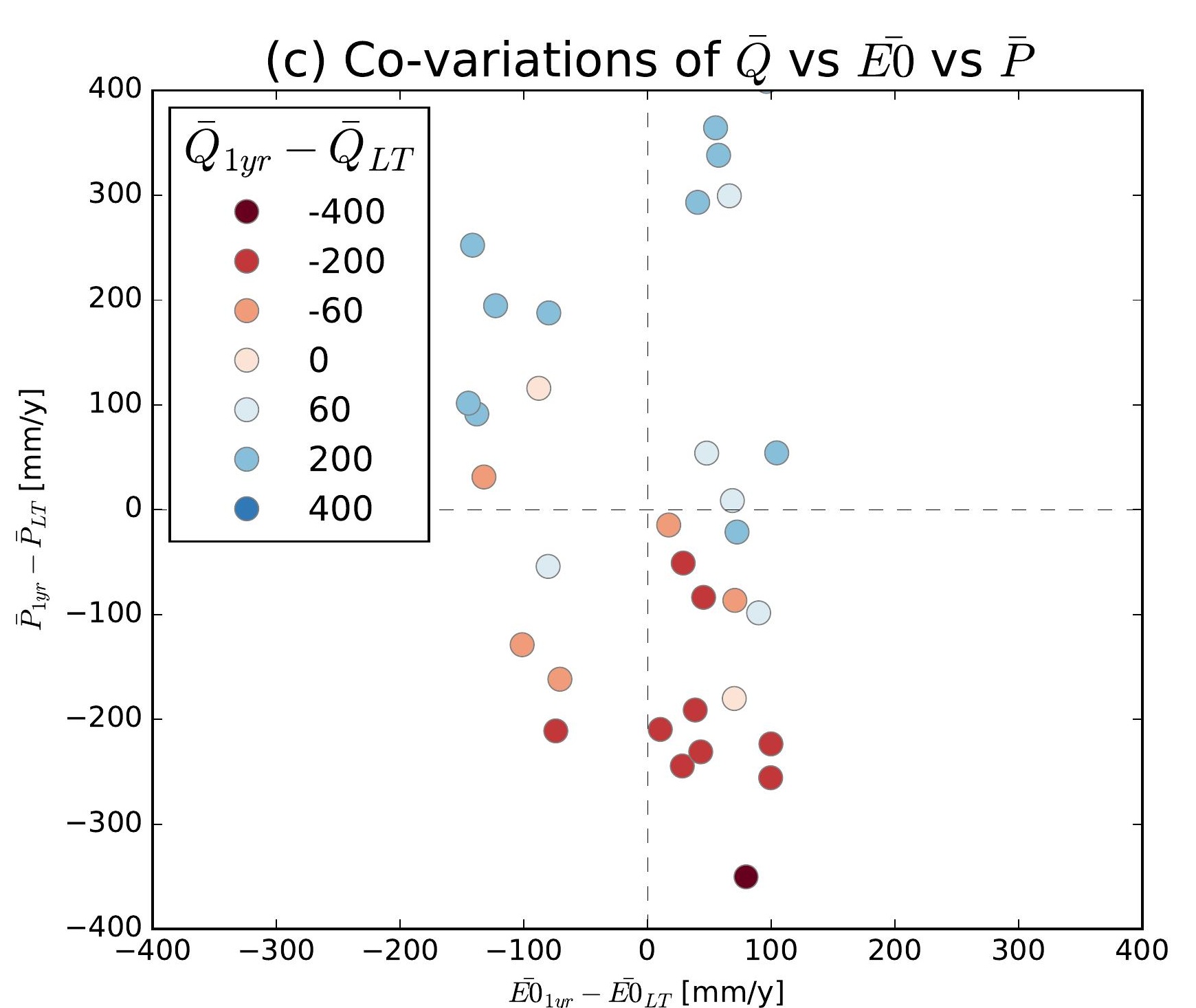
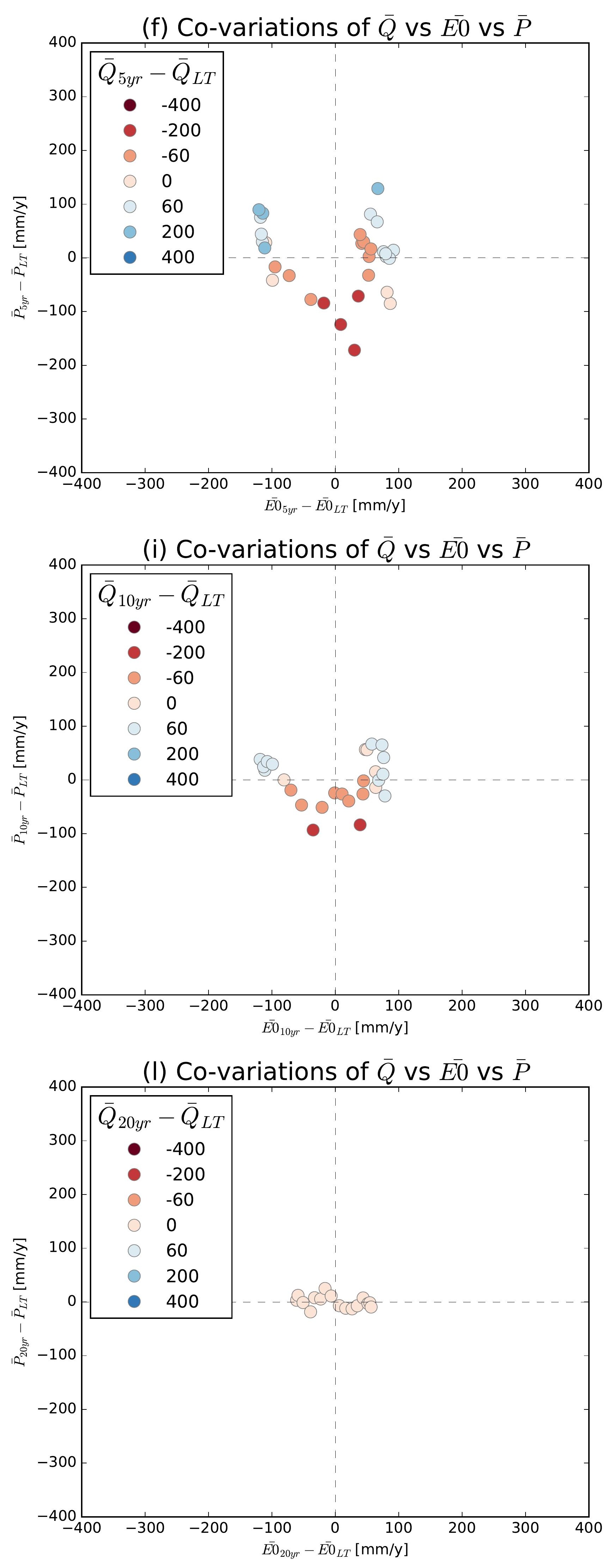

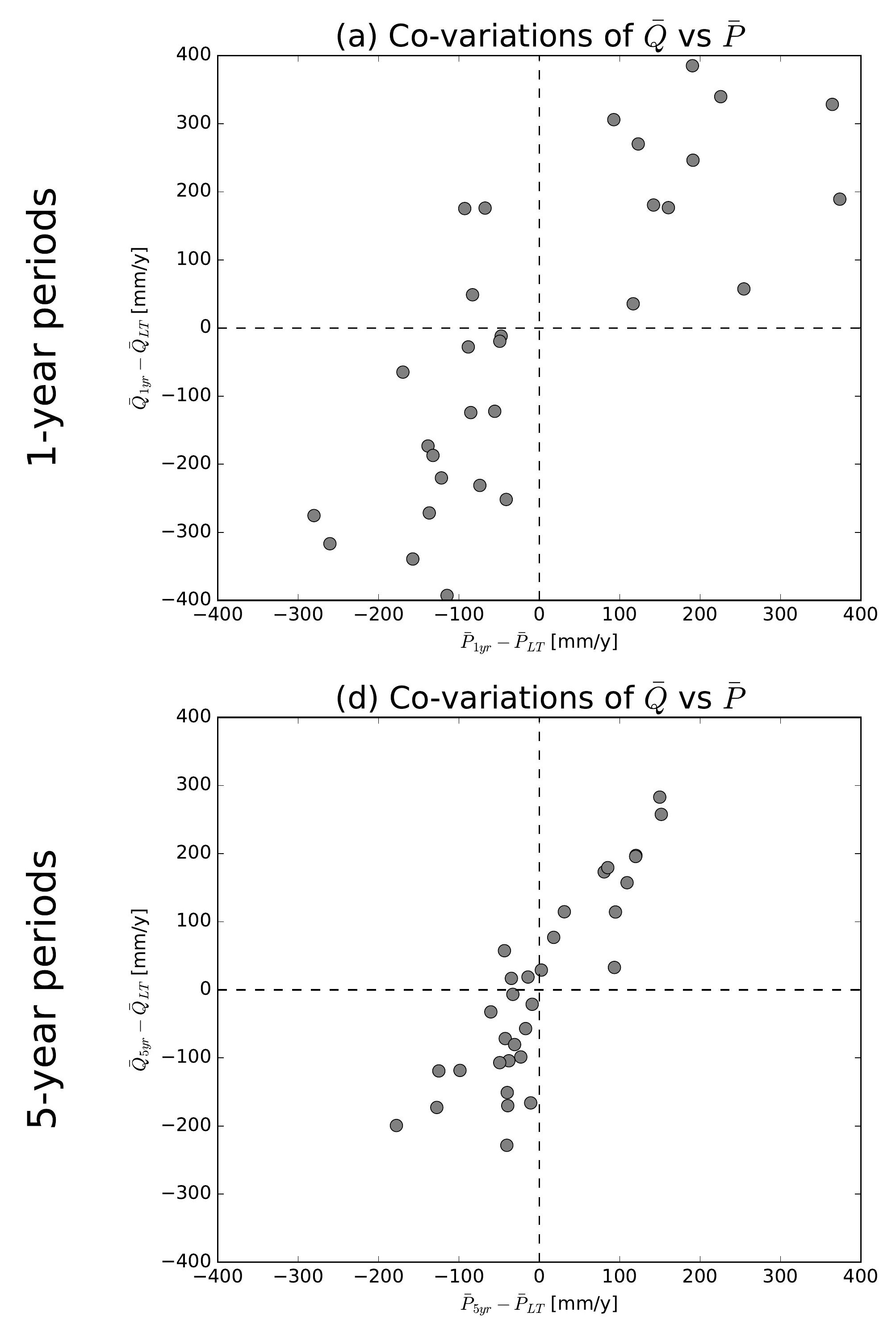

(g) Co-variations of $\bar{Q}$ vs $\bar{P}$

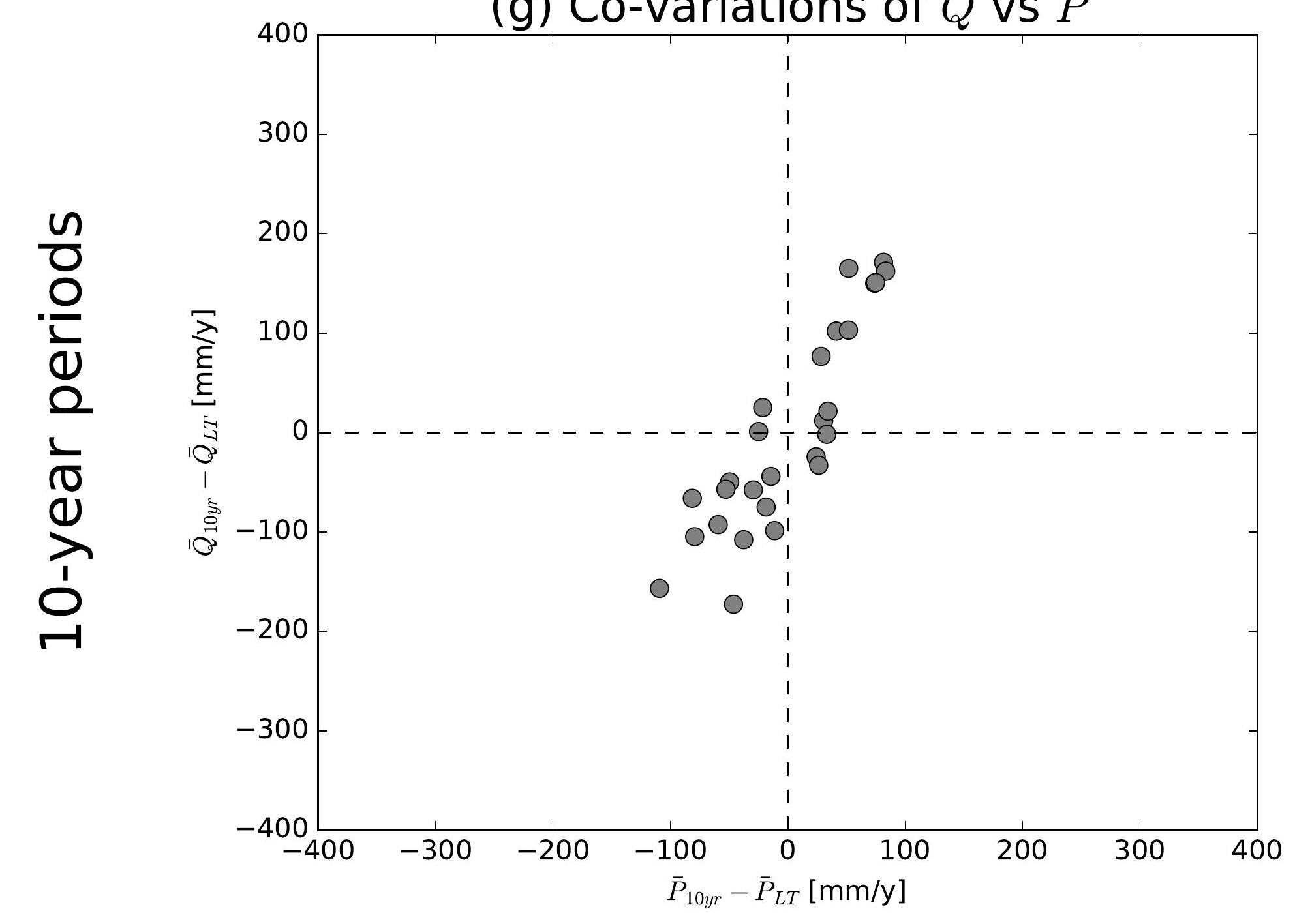

(j) Co-variations of $\bar{Q}$ vs $\bar{P}$

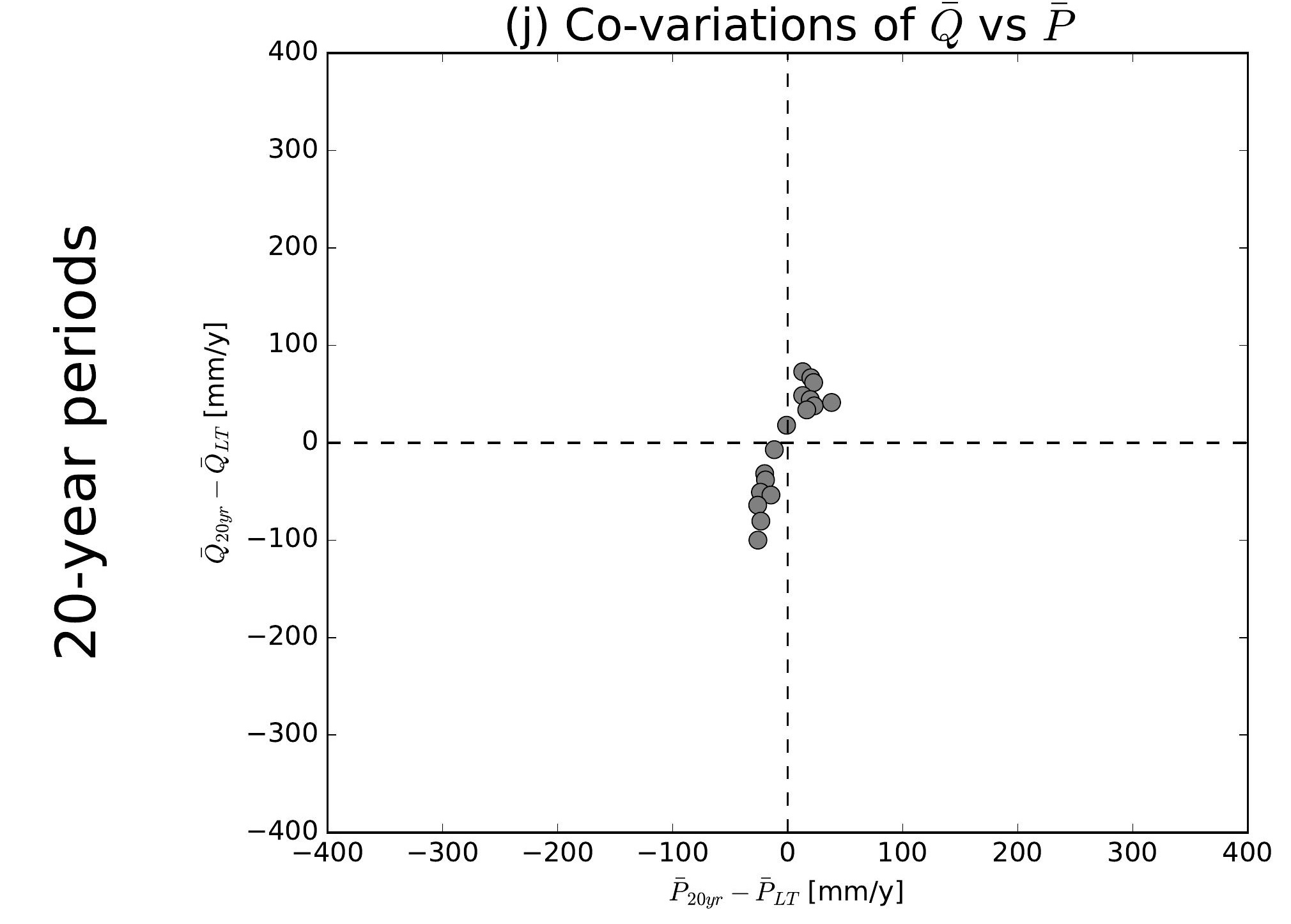

(b) Co-variations of $\bar{Q}$ vs $\overline{E 0}$

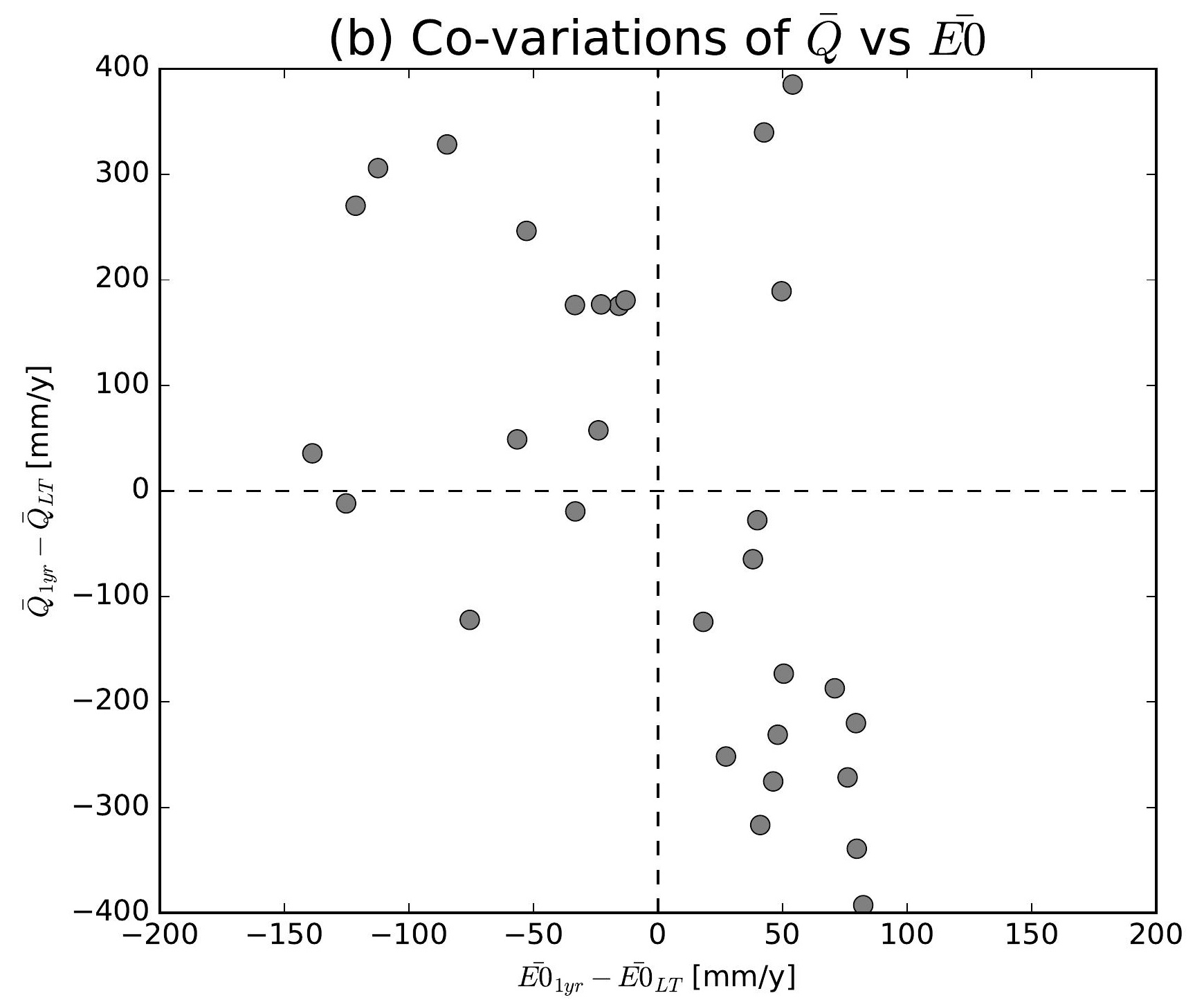

(e) Co-variations of $\bar{Q}$ vs $\overline{E 0}$

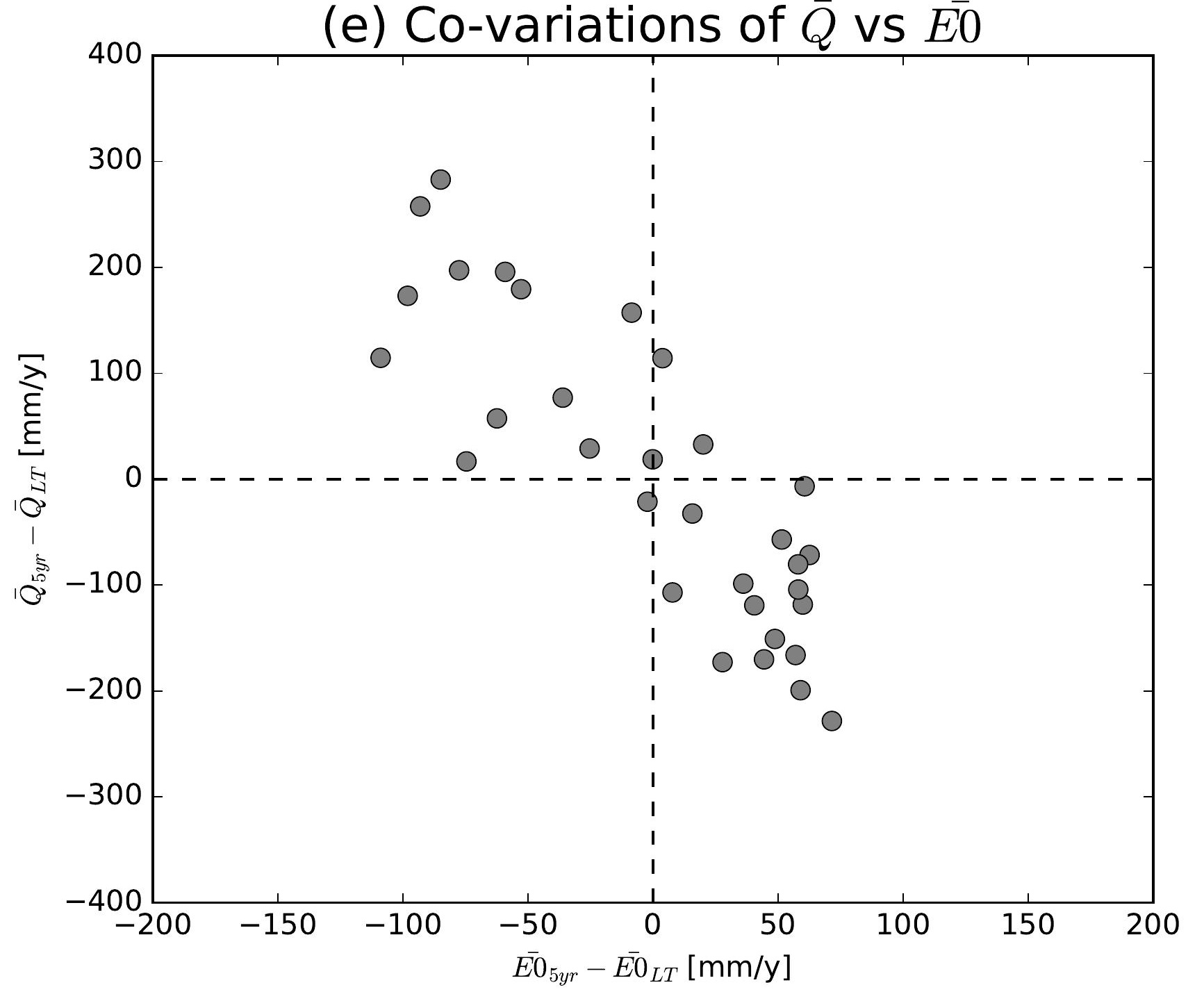

(h) Co-variations of $\bar{Q}$ vs $\overline{E 0}$

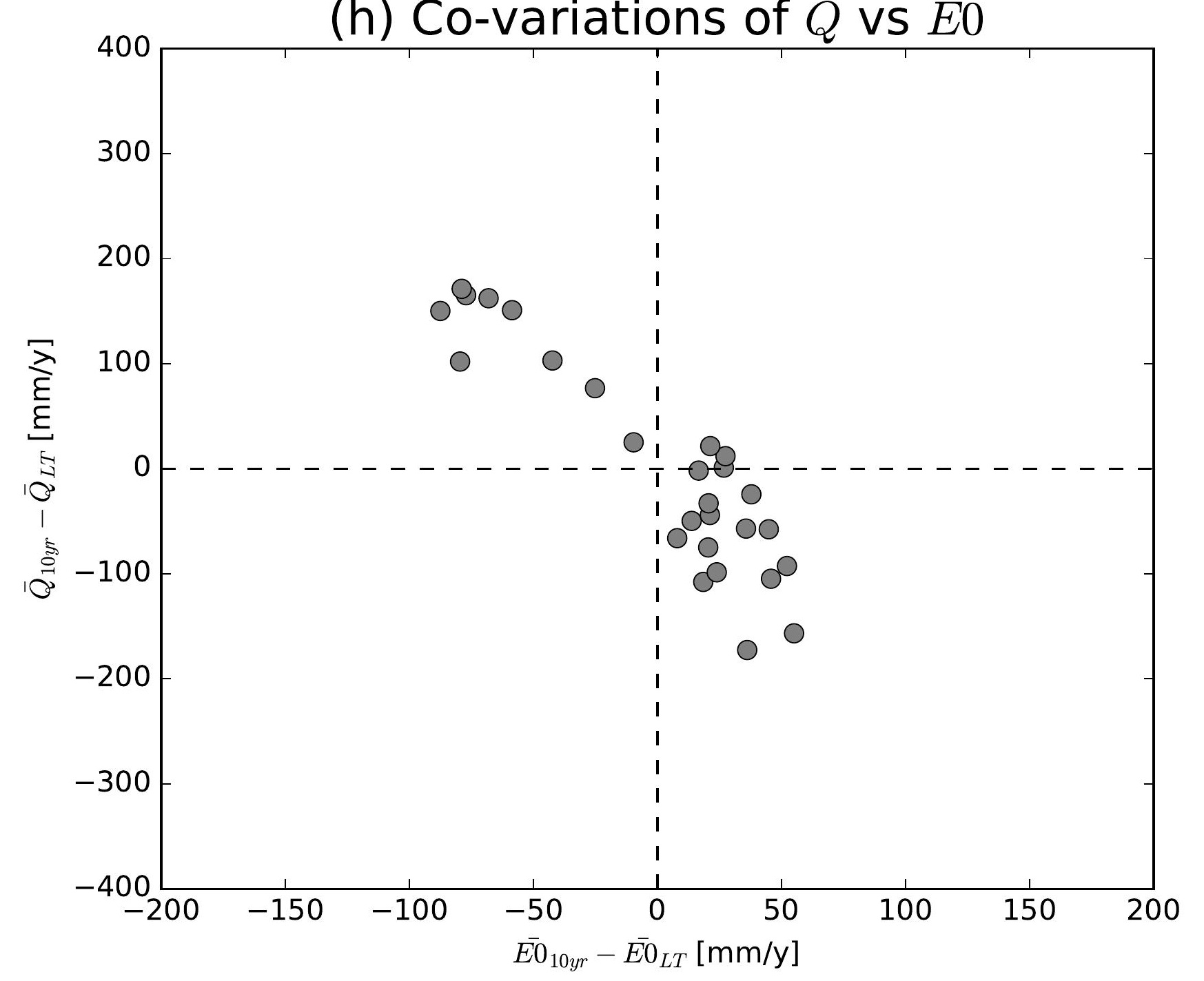

(k) Co-variations of $\bar{Q}$ vs $\overline{E 0}$

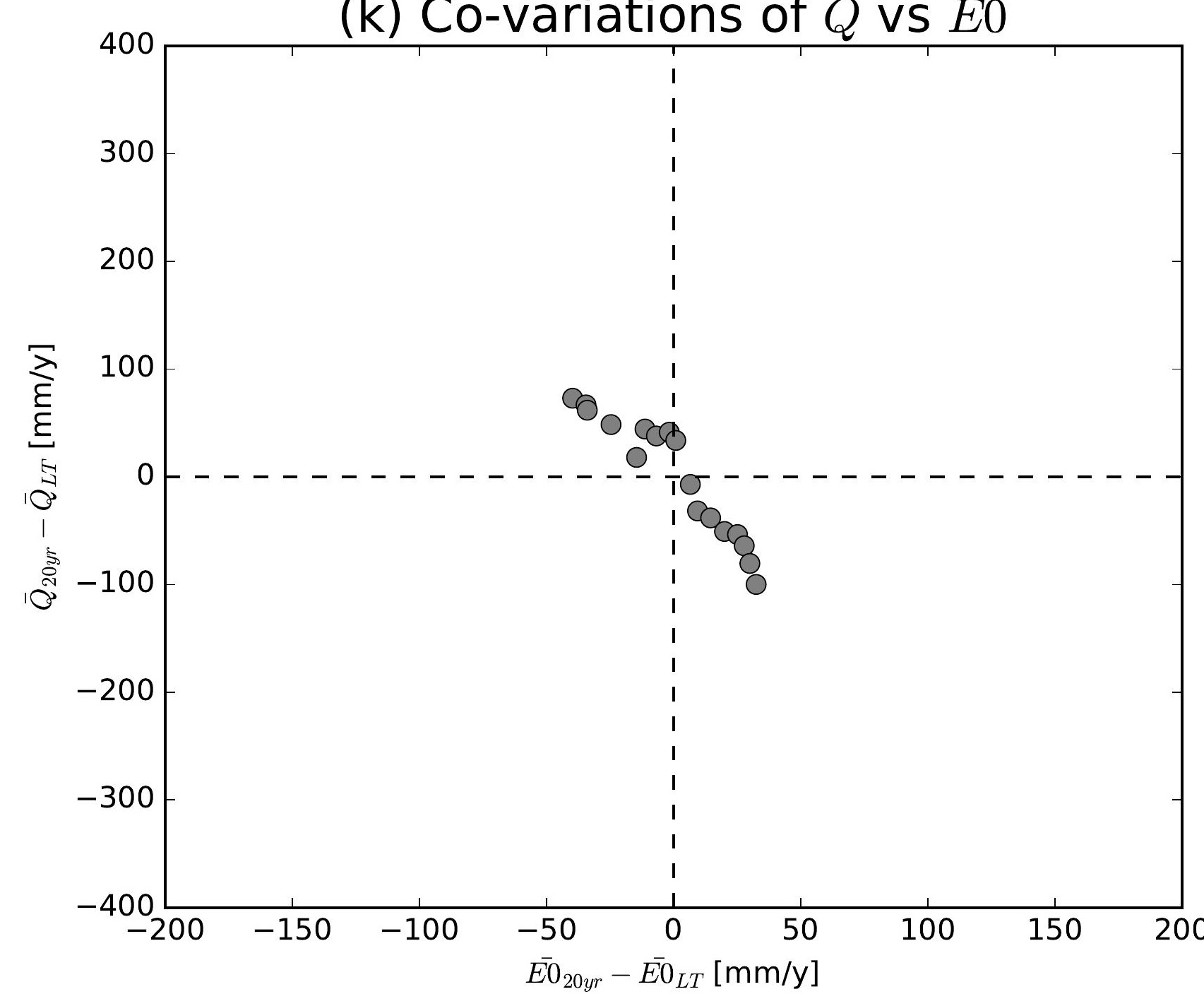

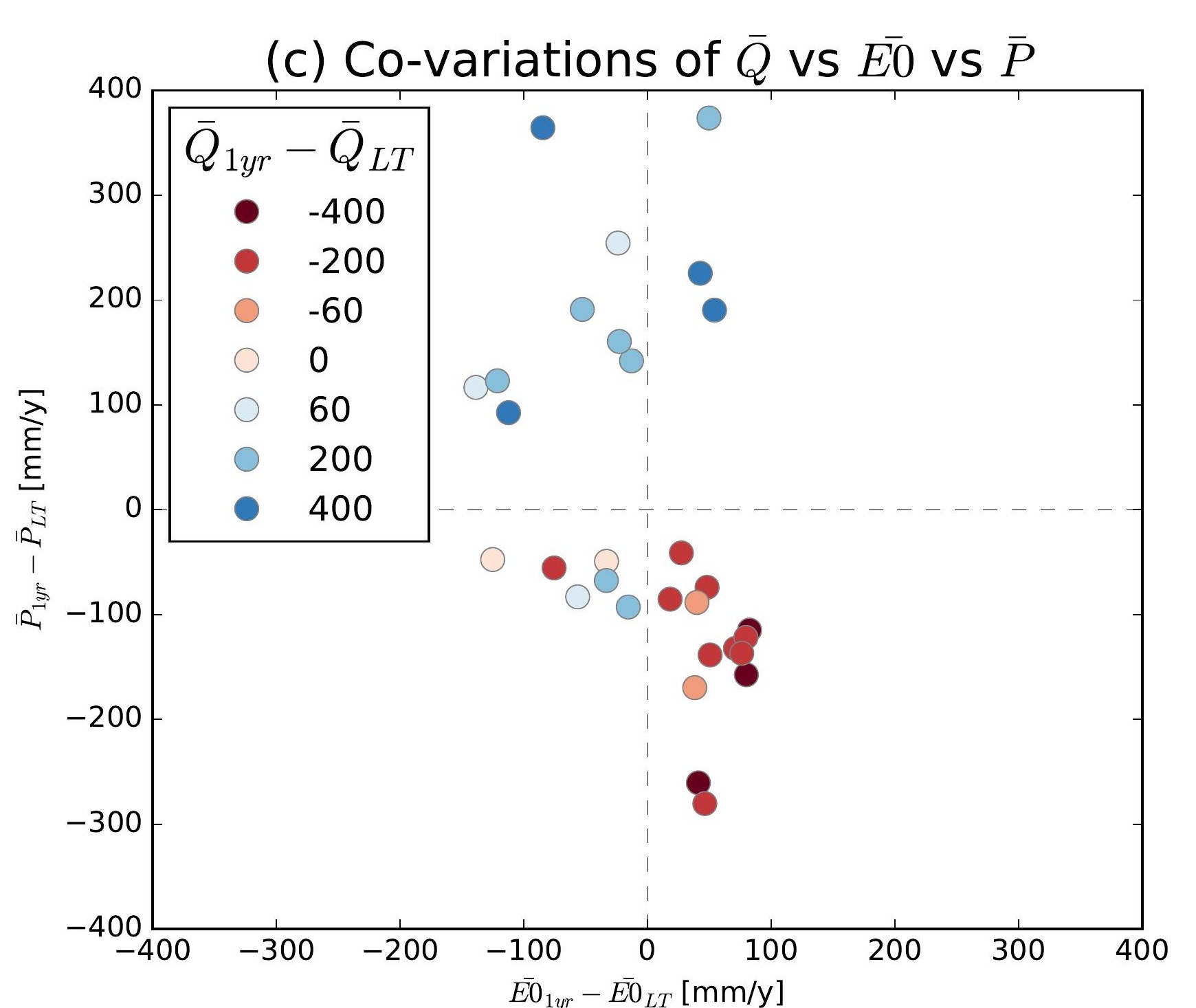
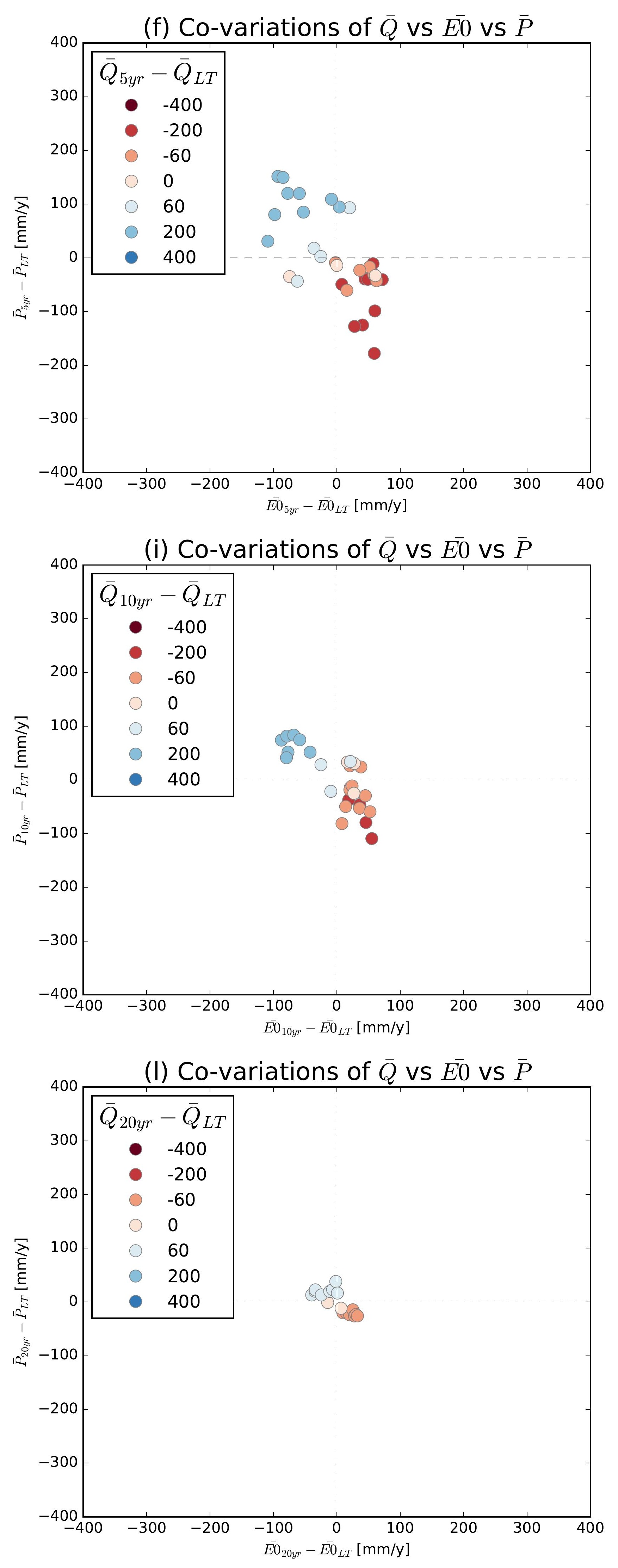

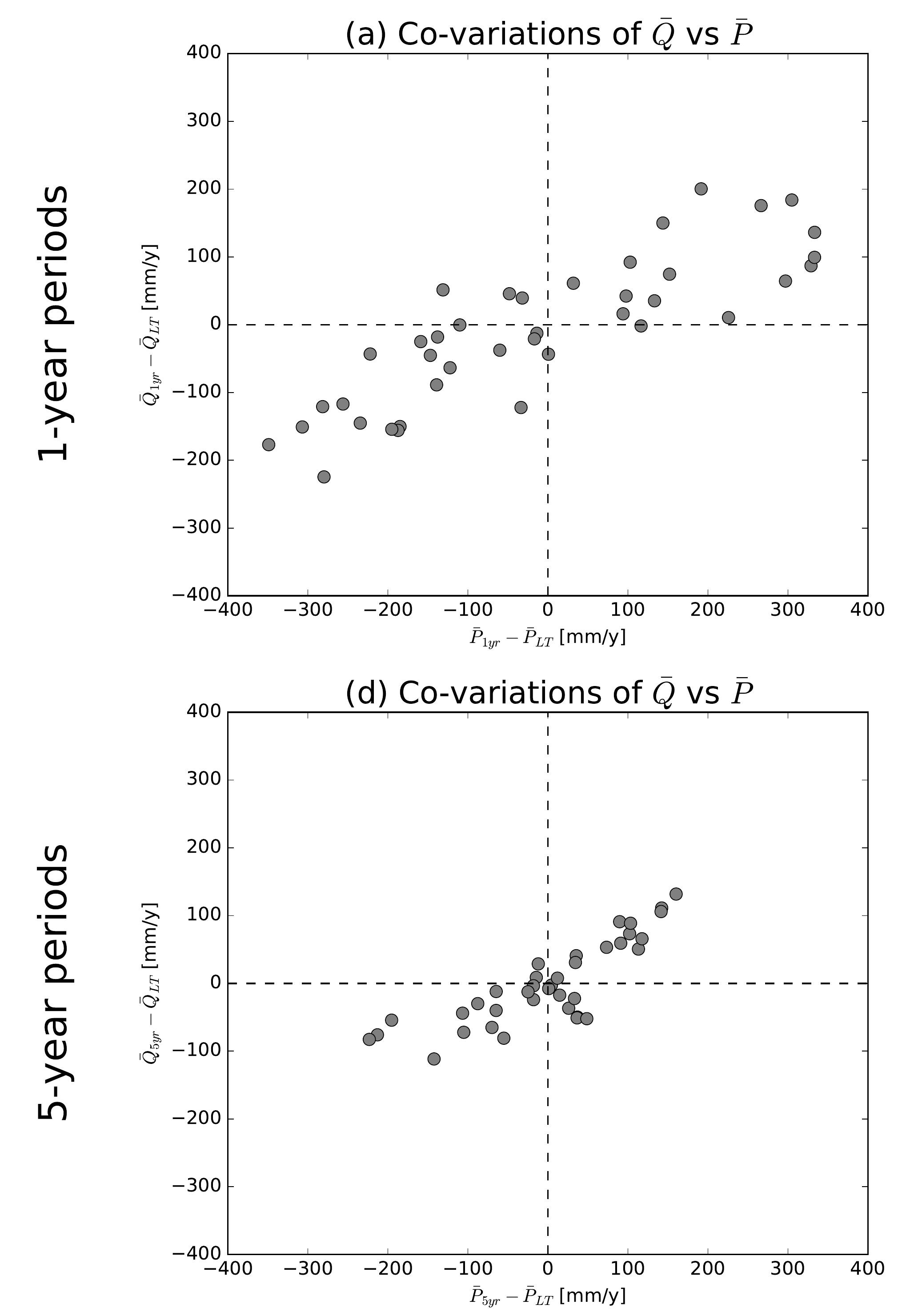

(g) Co-variations of $\bar{Q}$ vs $\bar{P}$
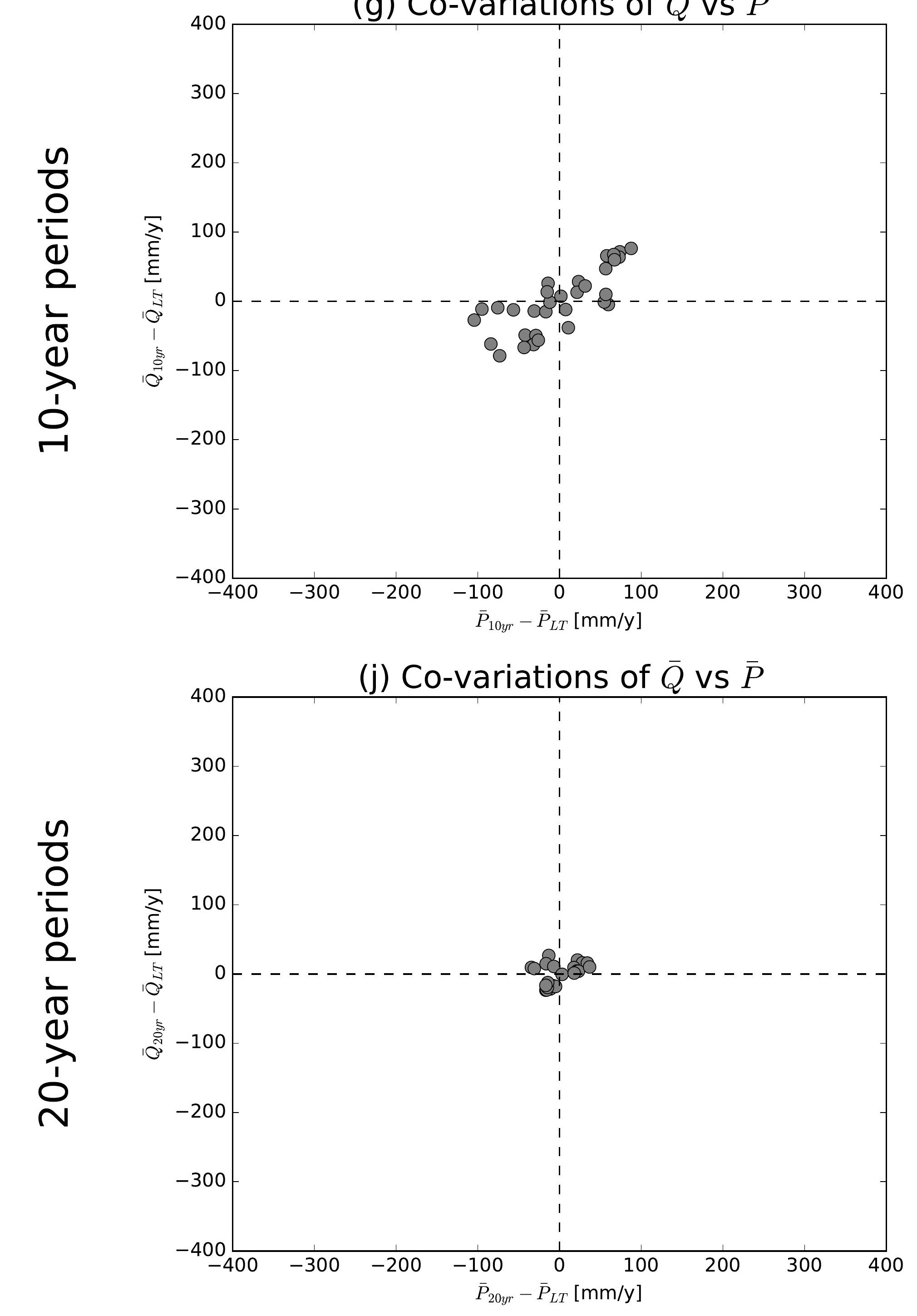

(b) Co-variations of $\bar{Q}$ vs $\overline{E 0}$

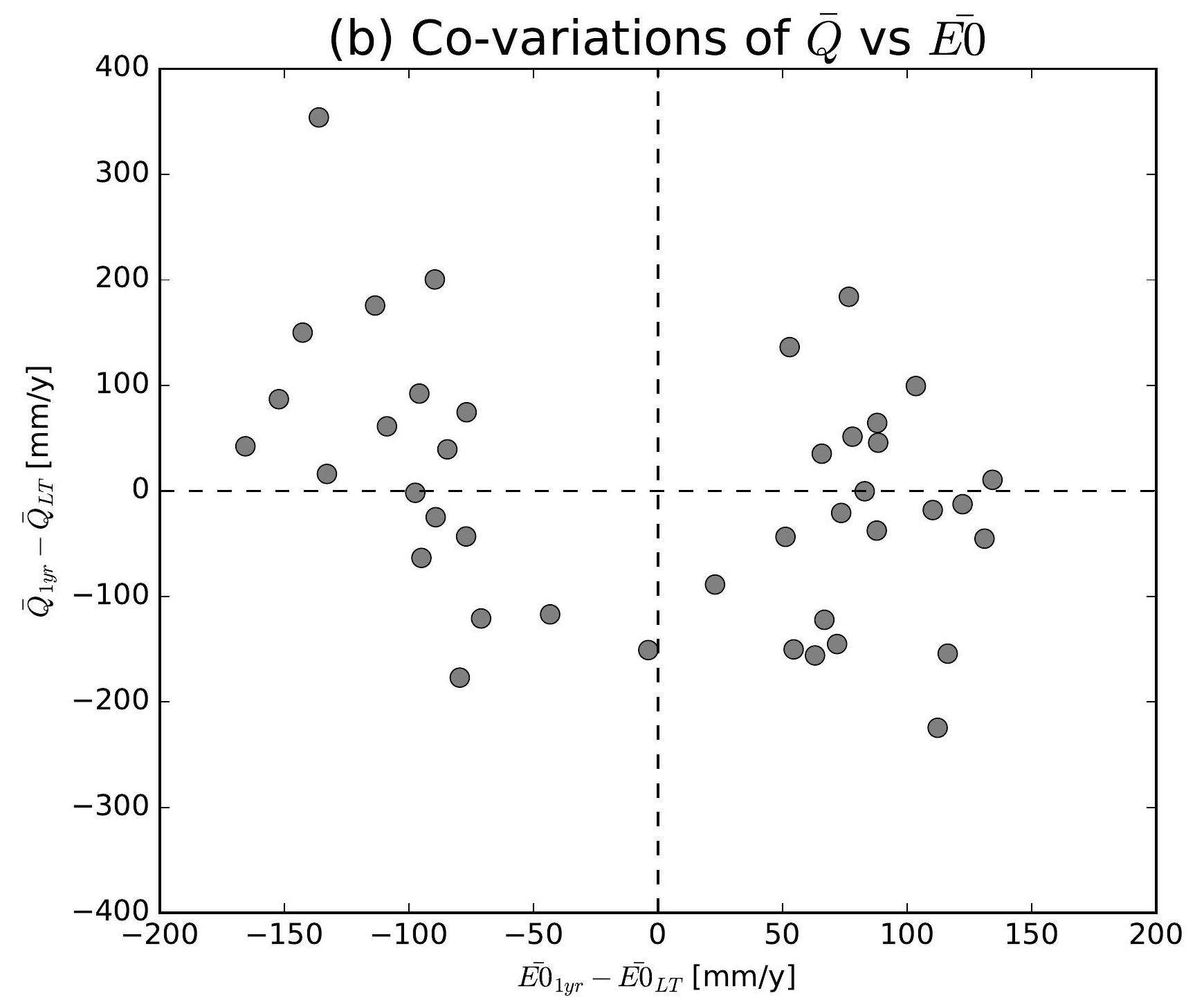

(e) Co-variations of $\bar{Q}$ vs $\overline{E 0}$

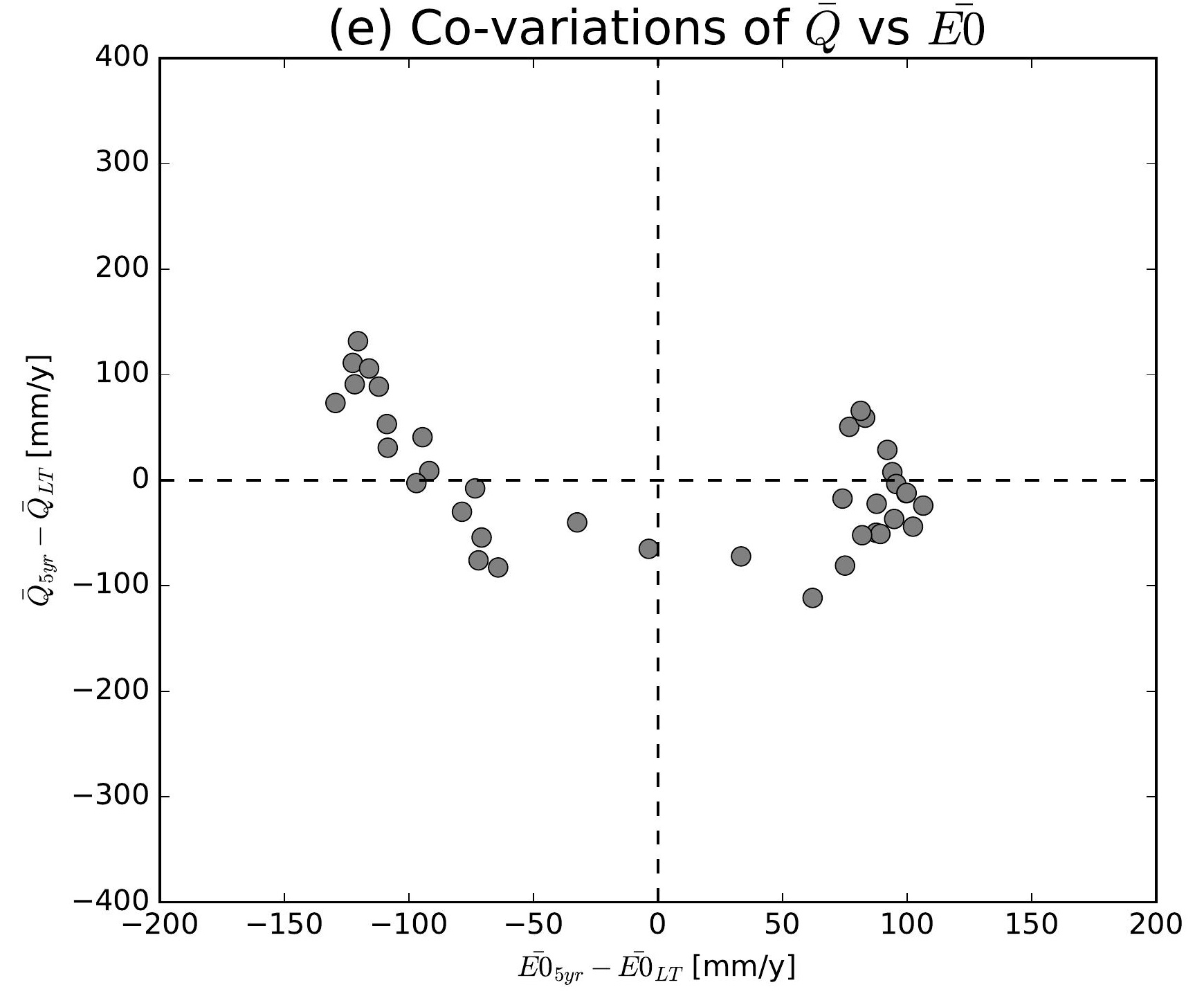

(h) Co-variations of $\bar{Q}$ vs $\overline{E 0}$

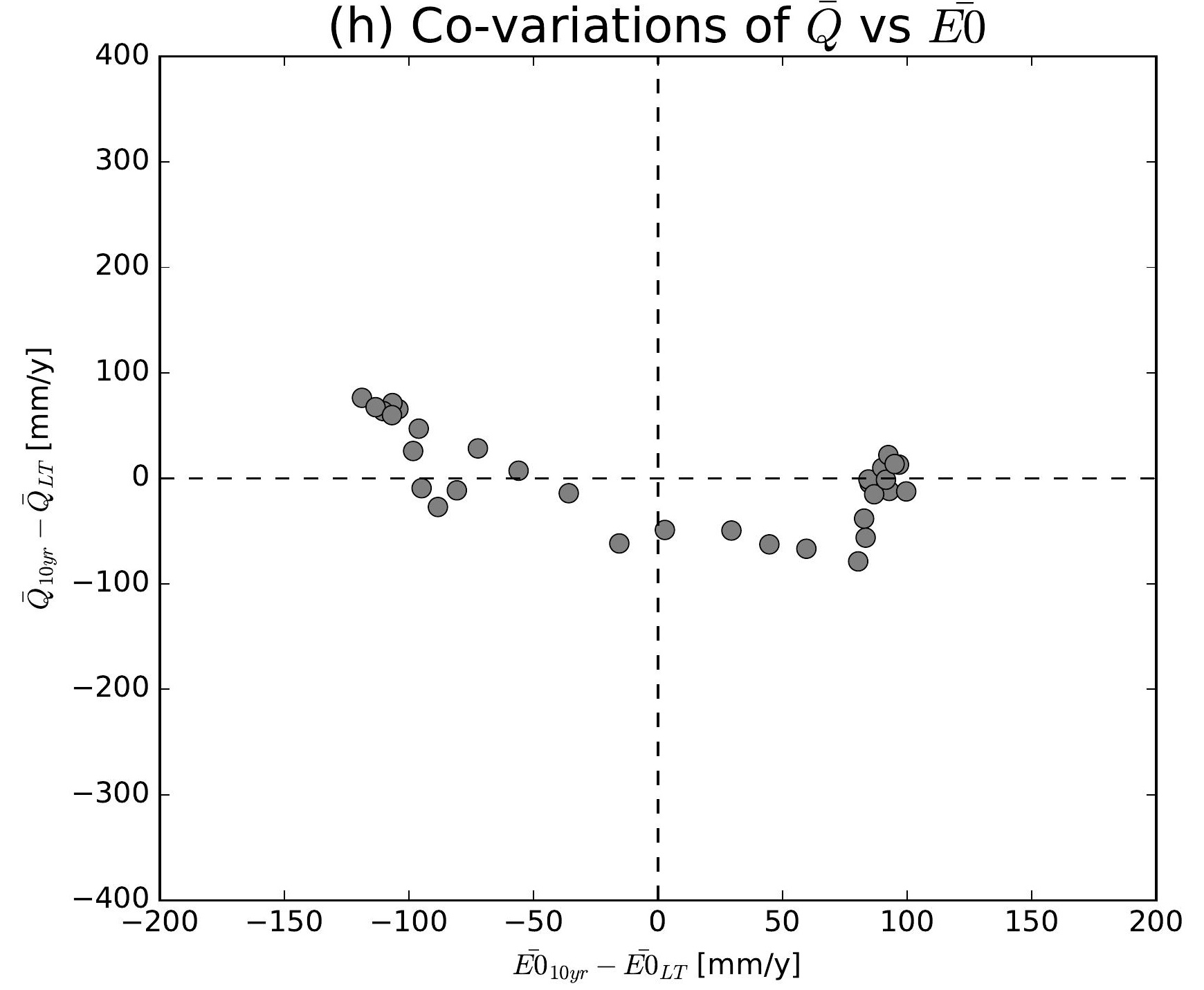

(k) Co-variations of $\bar{Q}$ vs $\overline{E 0}$

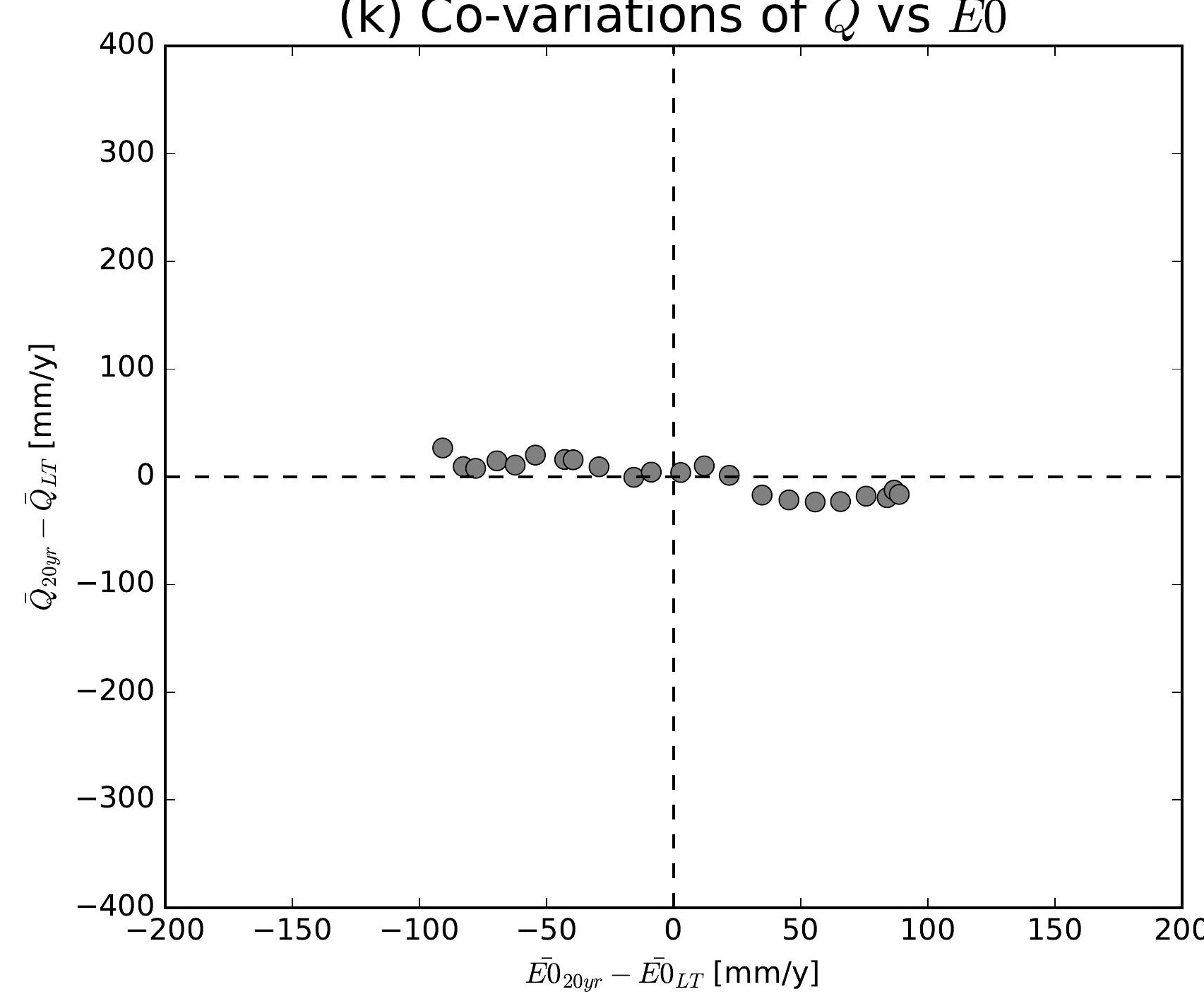

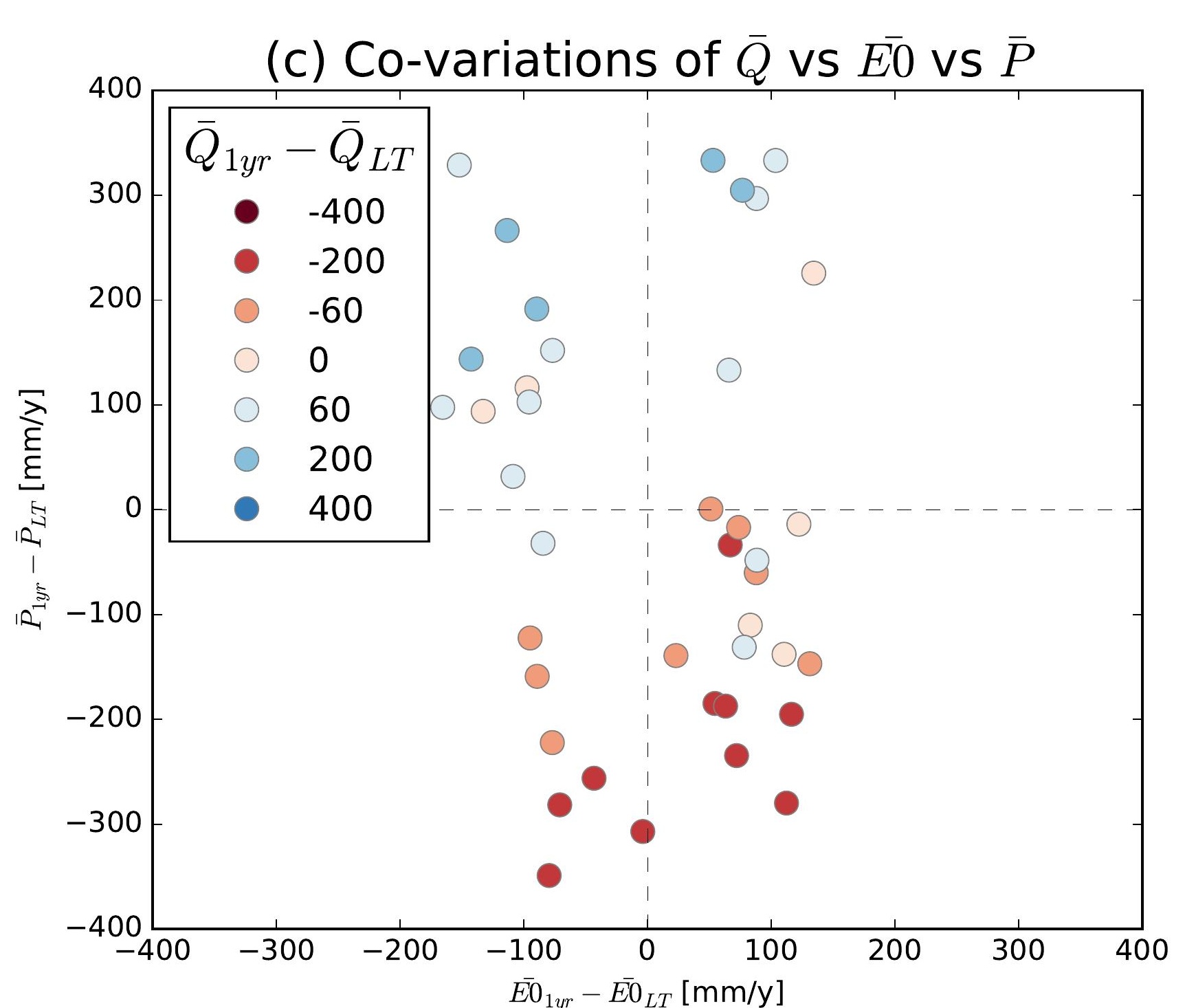
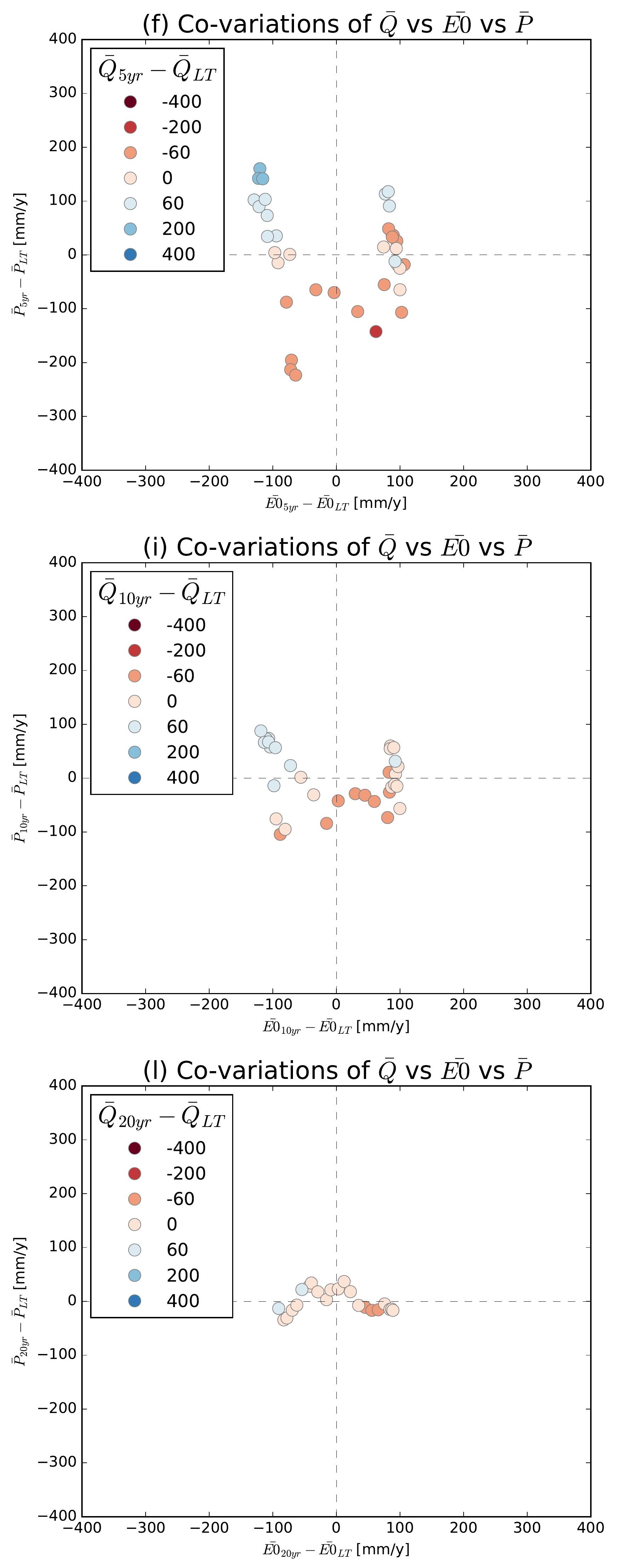

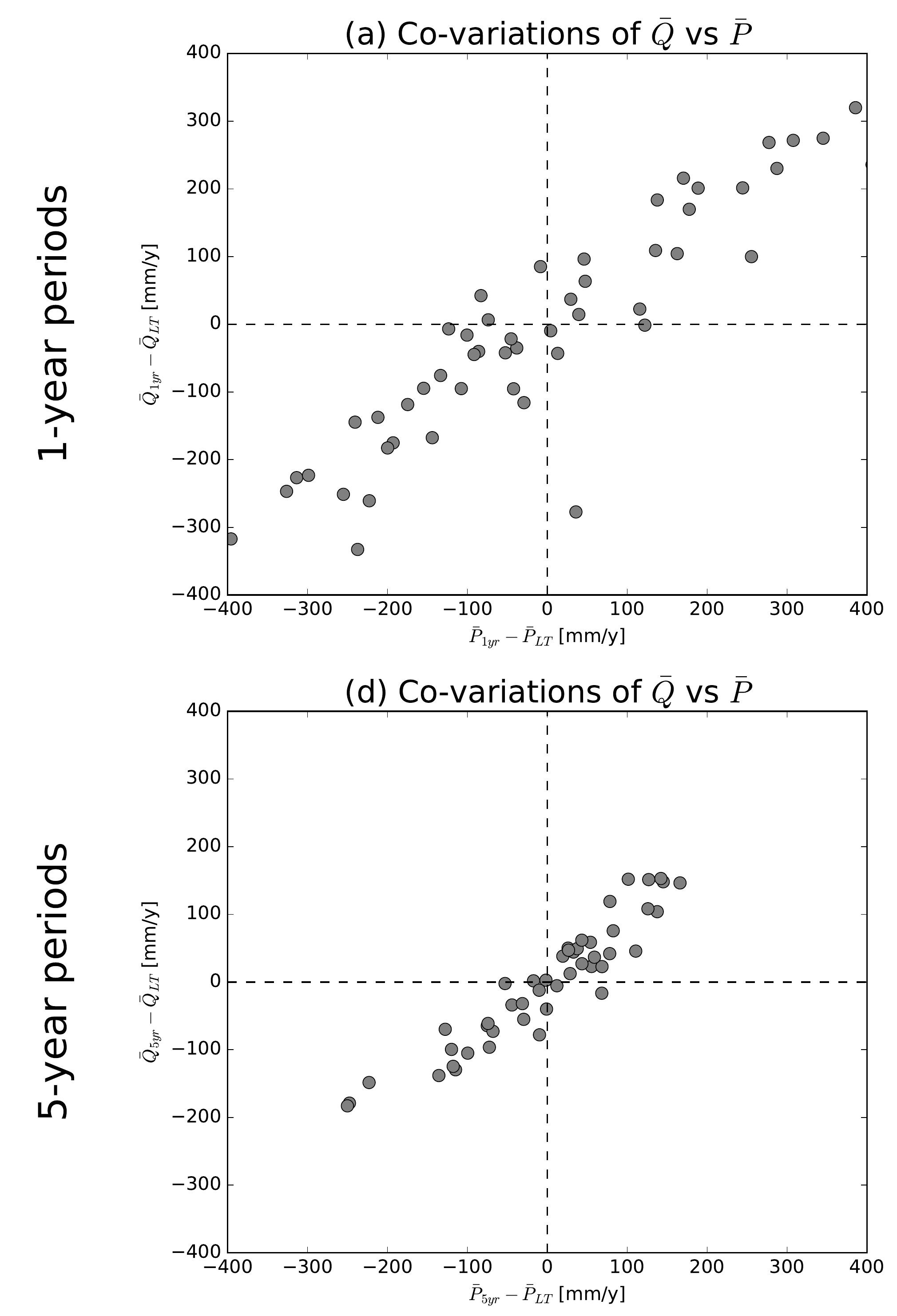

(g) Co-variations of $\bar{Q}$ vs $\bar{P}$
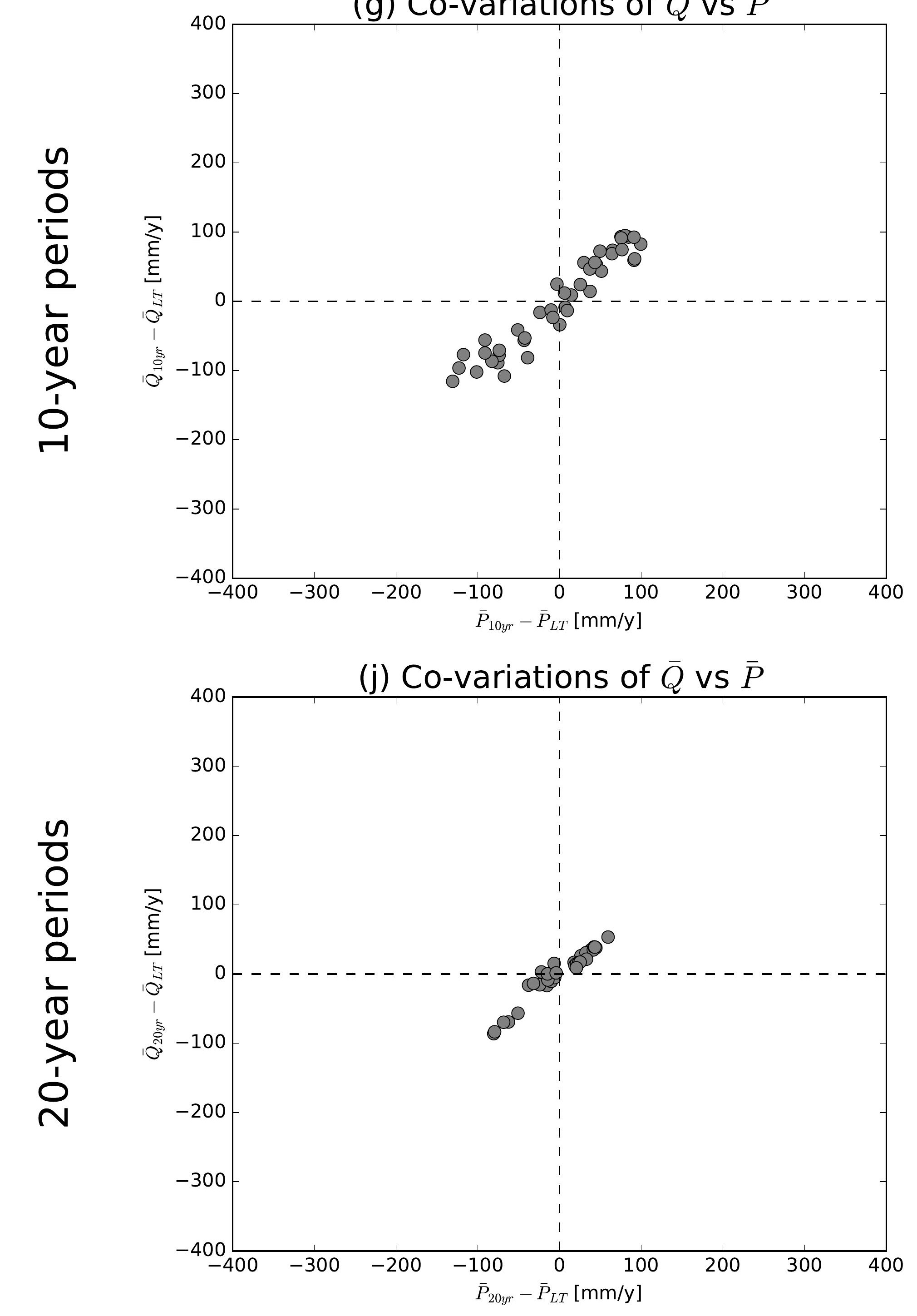

(b) Co-variations of $\bar{Q}$ vs $\overline{E 0}$

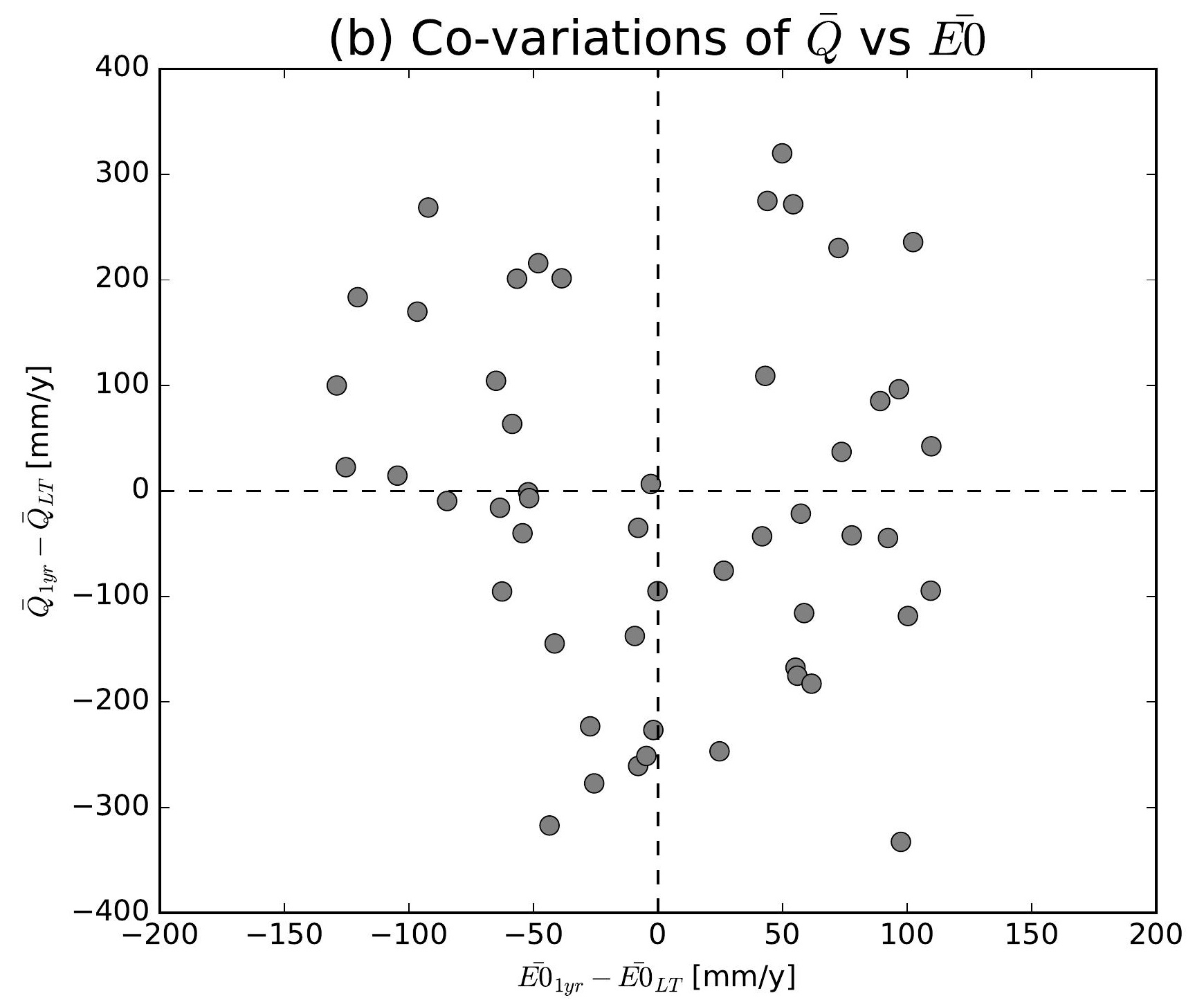

(e) Co-variations of $\bar{Q}$ vs $\overline{E 0}$

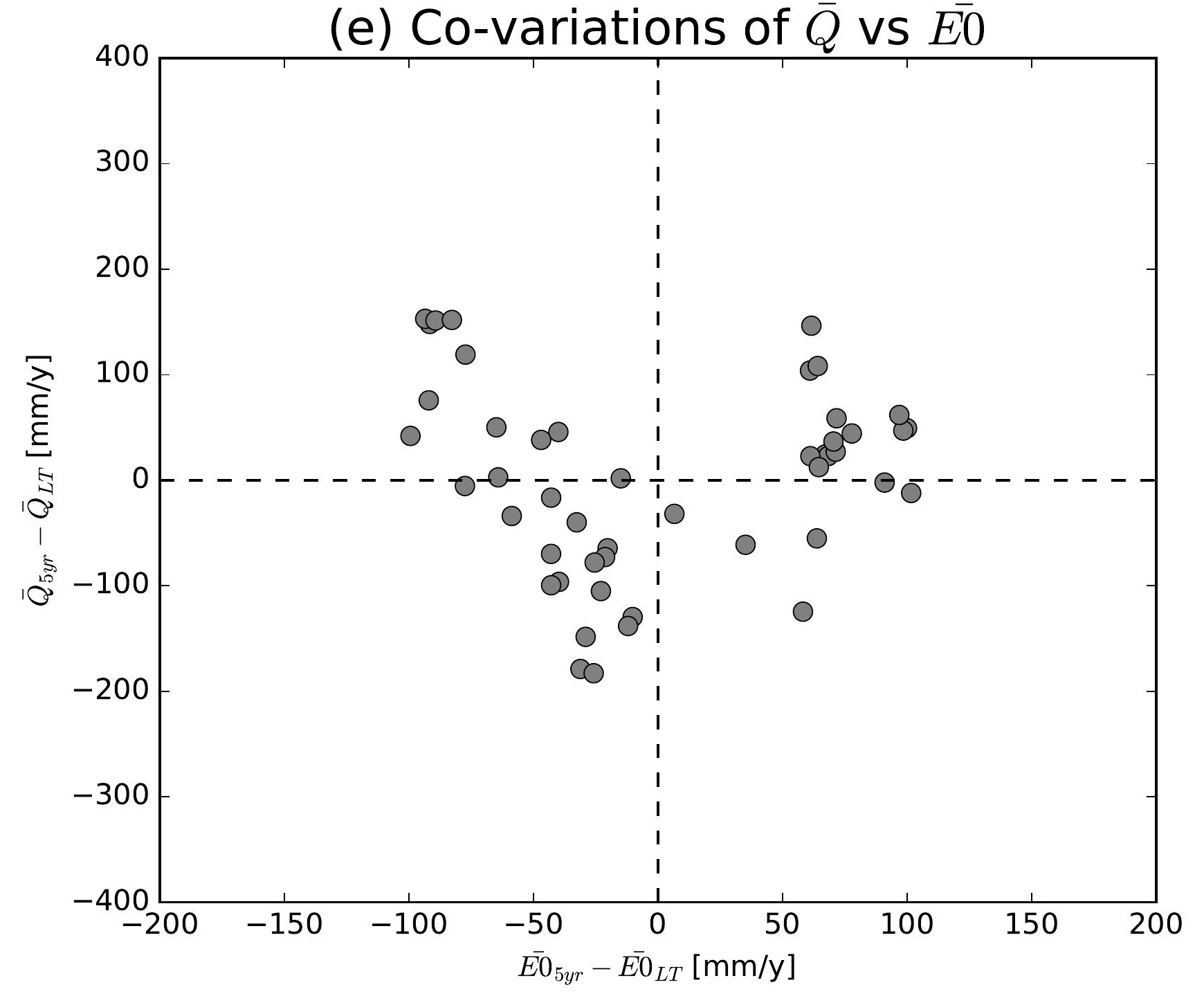

(h) Co-variations of $\bar{Q}$ vs $\overline{E 0}$

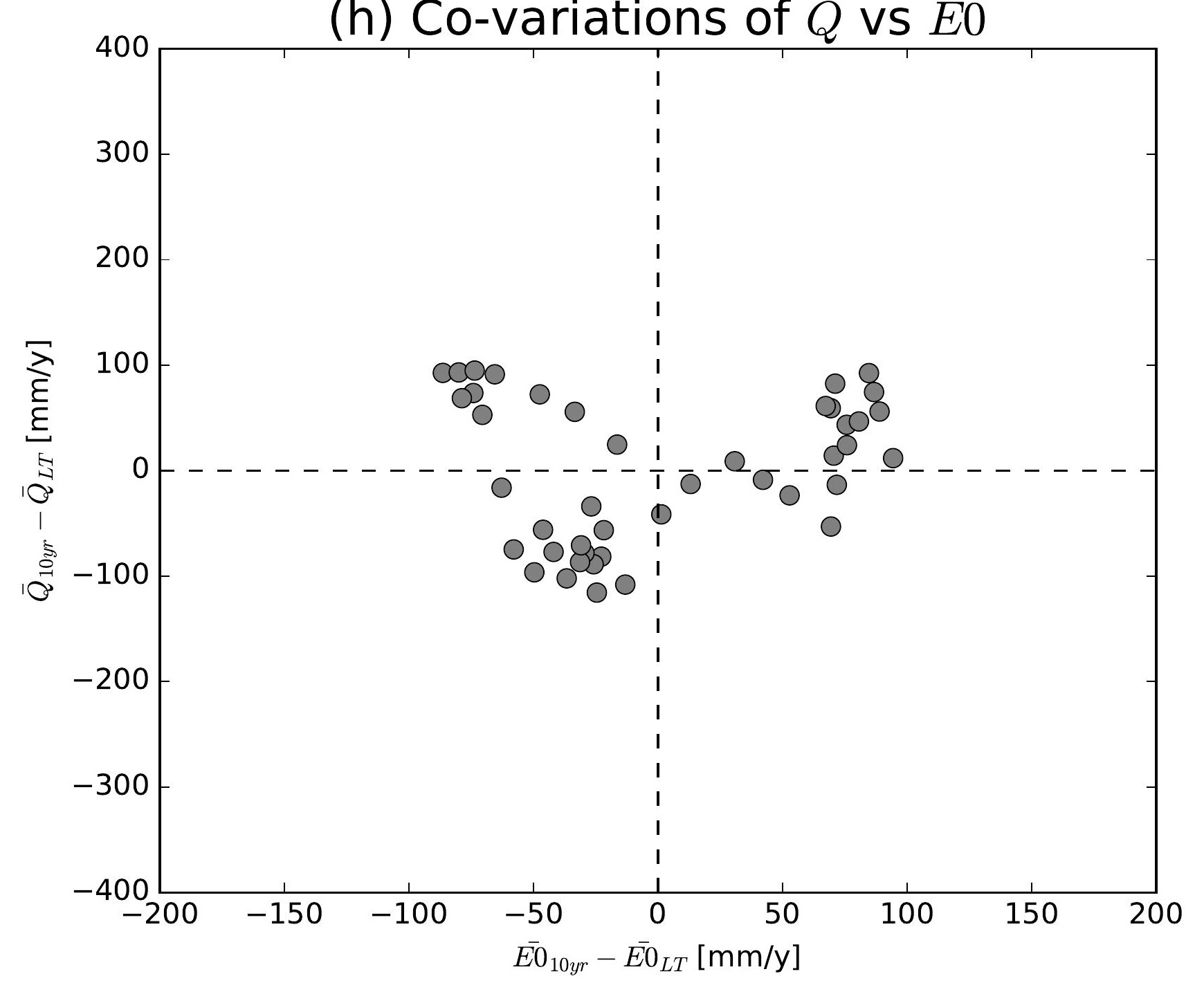

(k) Co-variations of $\bar{Q}$ vs $\overline{E 0}$

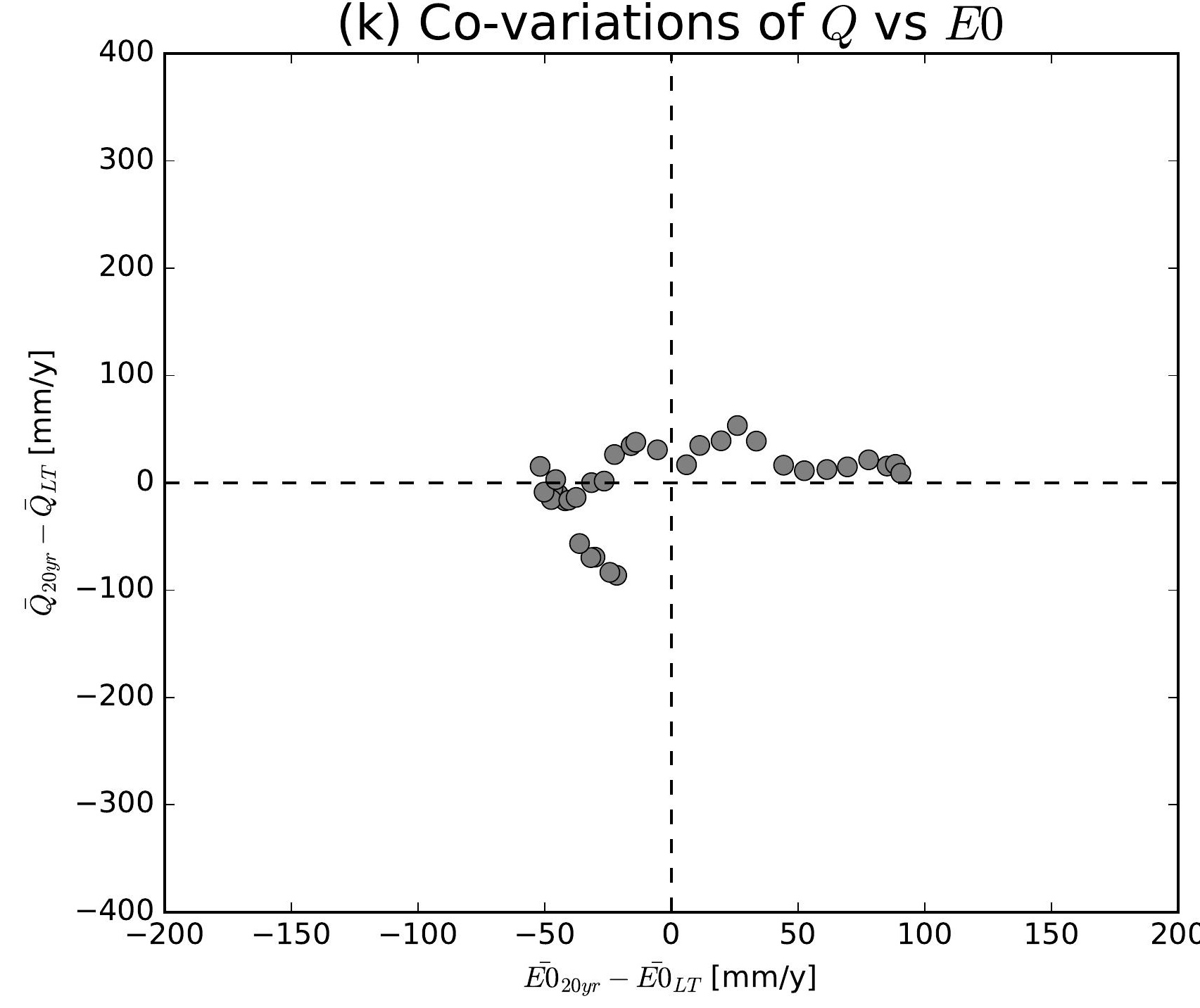

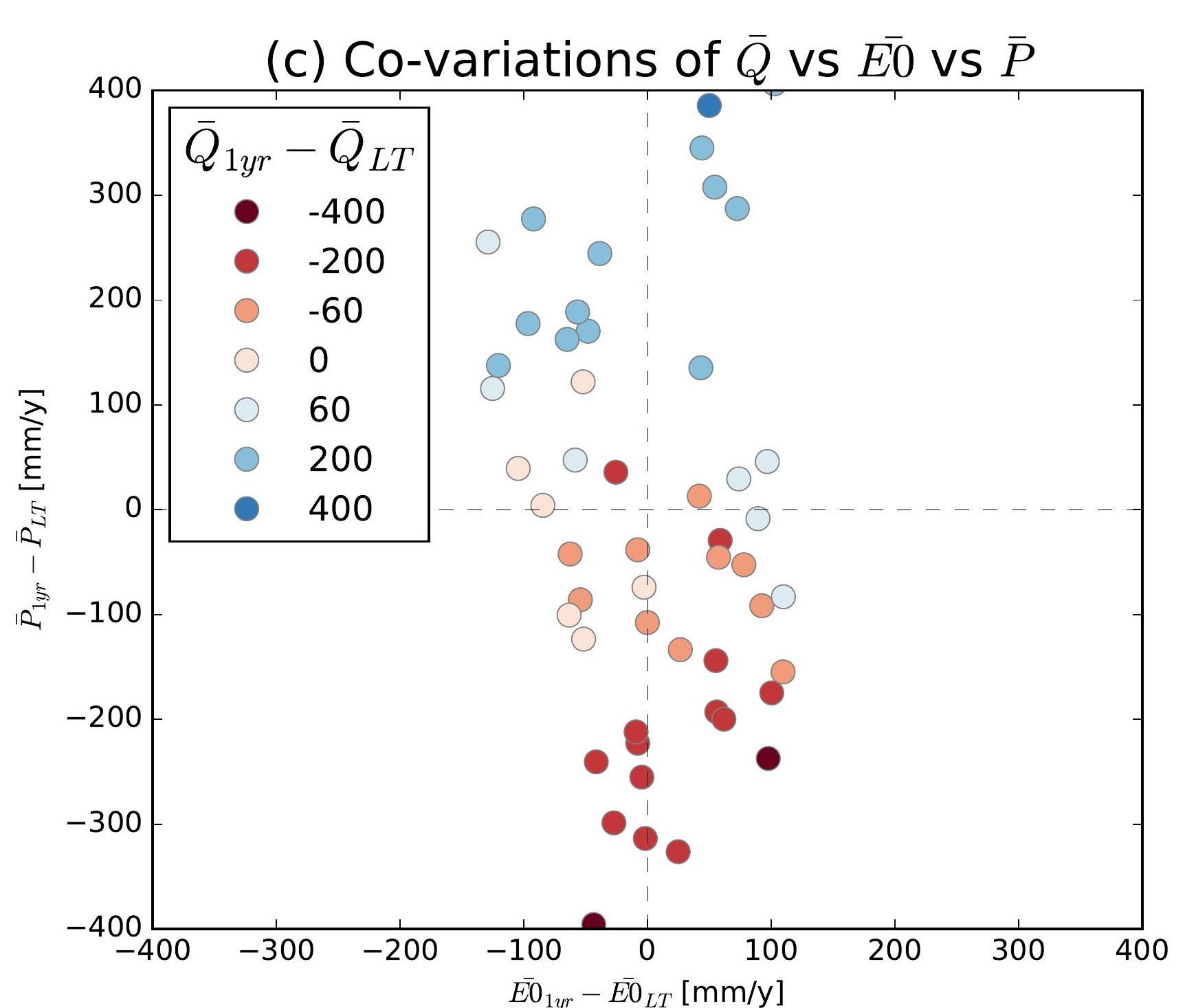
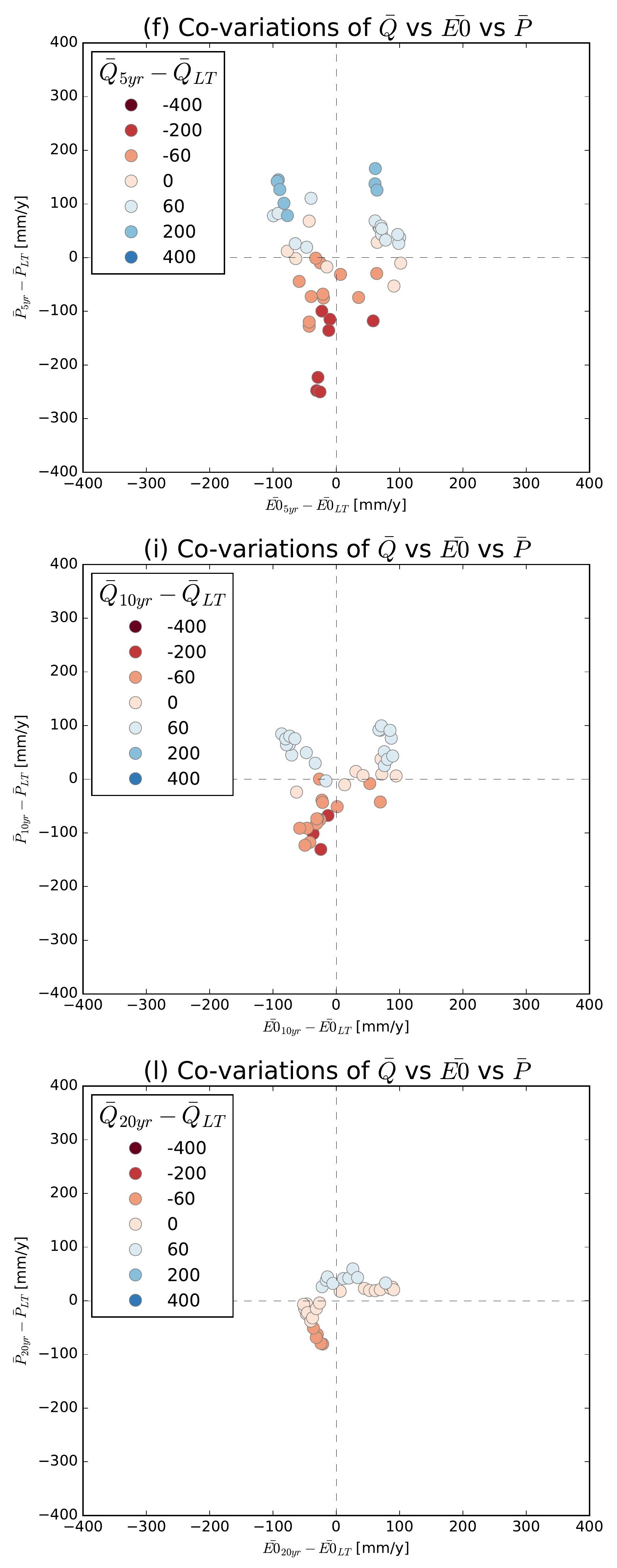

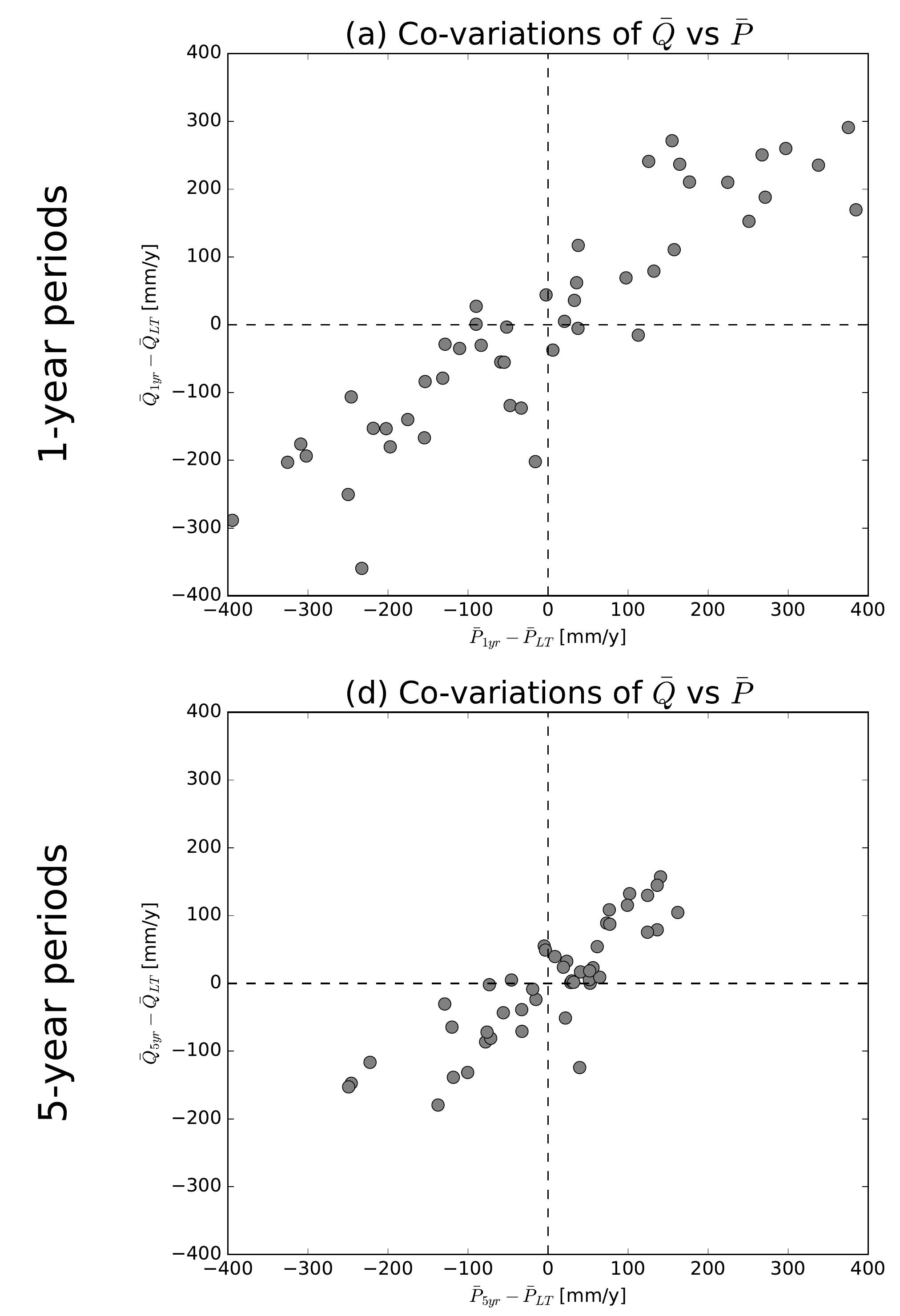

(g) Co-variations of $\bar{Q}$ vs $\bar{P}$

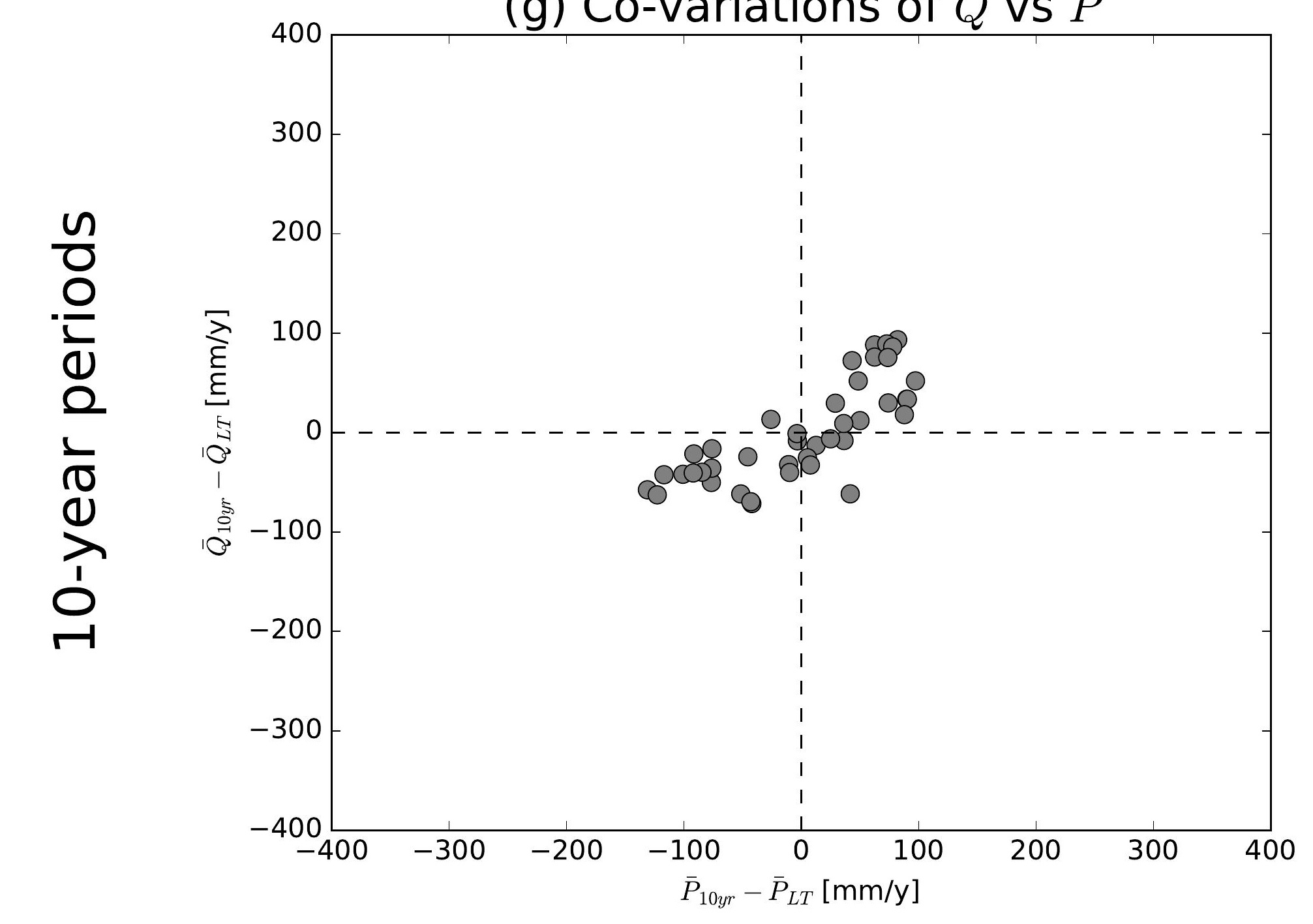

(j) Co-variations of $\bar{Q}$ vs $\bar{P}$

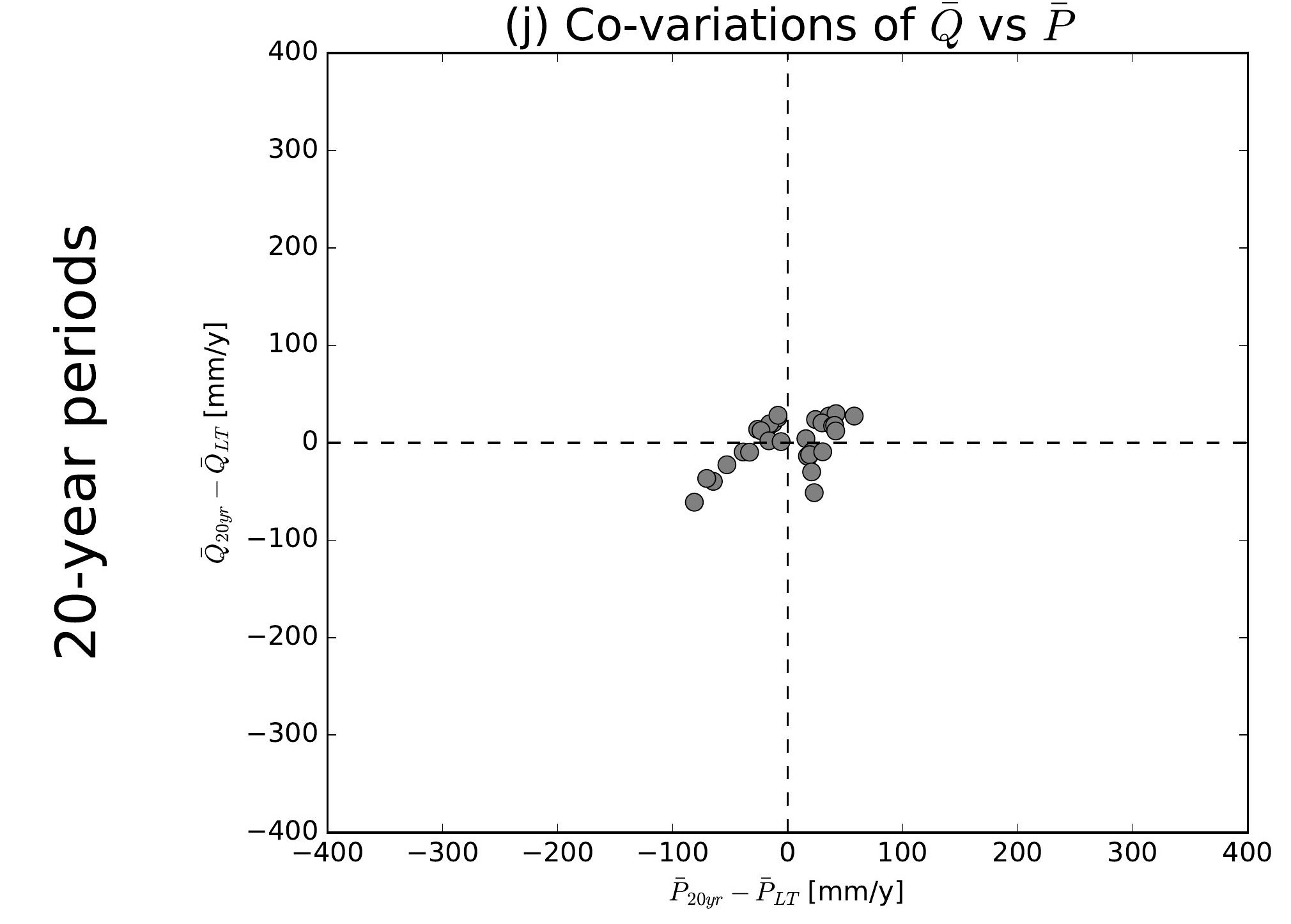

(b) Co-variations of $\bar{Q}$ vs $\overline{E 0}$

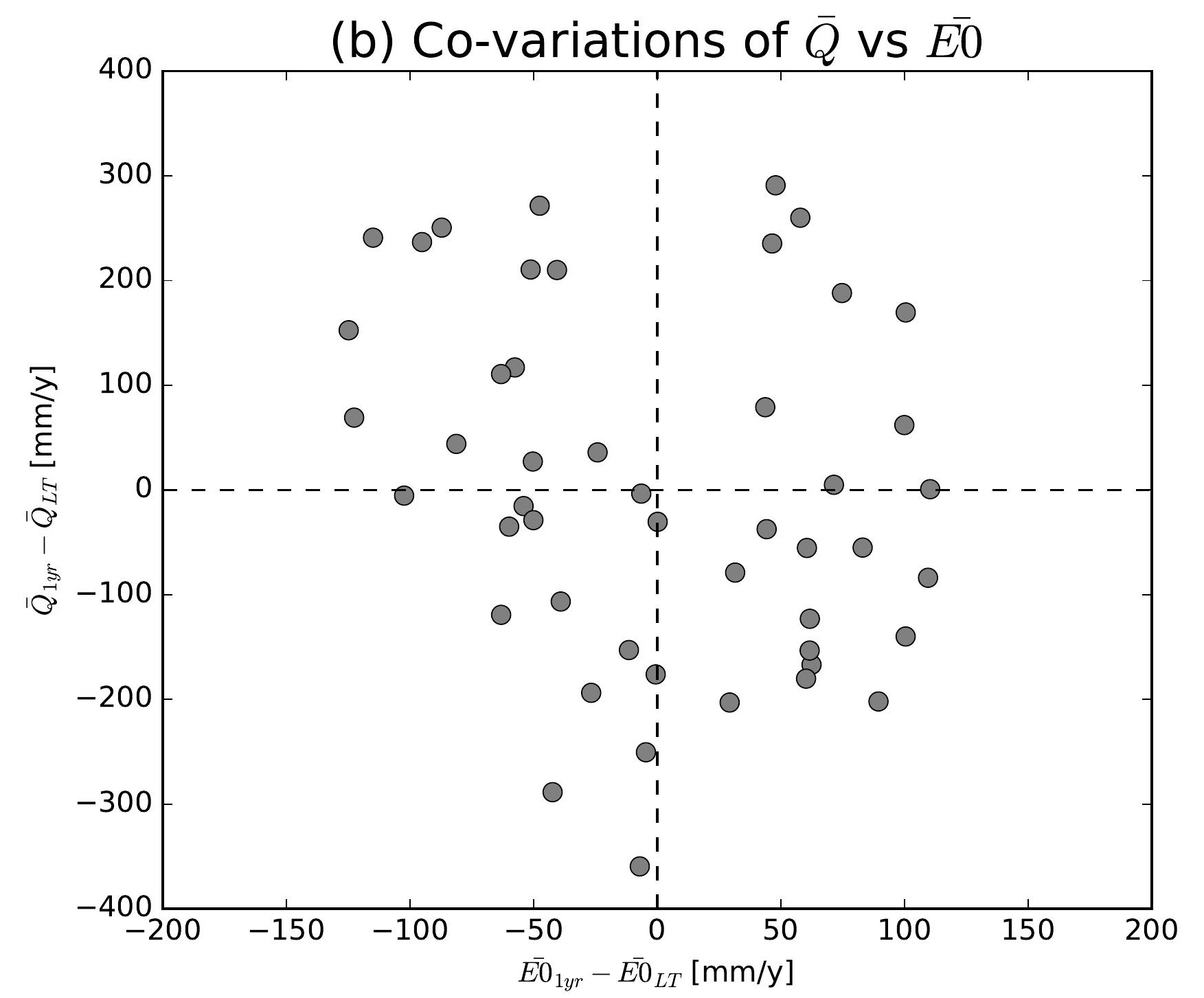

(e) Co-variations of $\bar{Q}$ vs $\overline{E 0}$

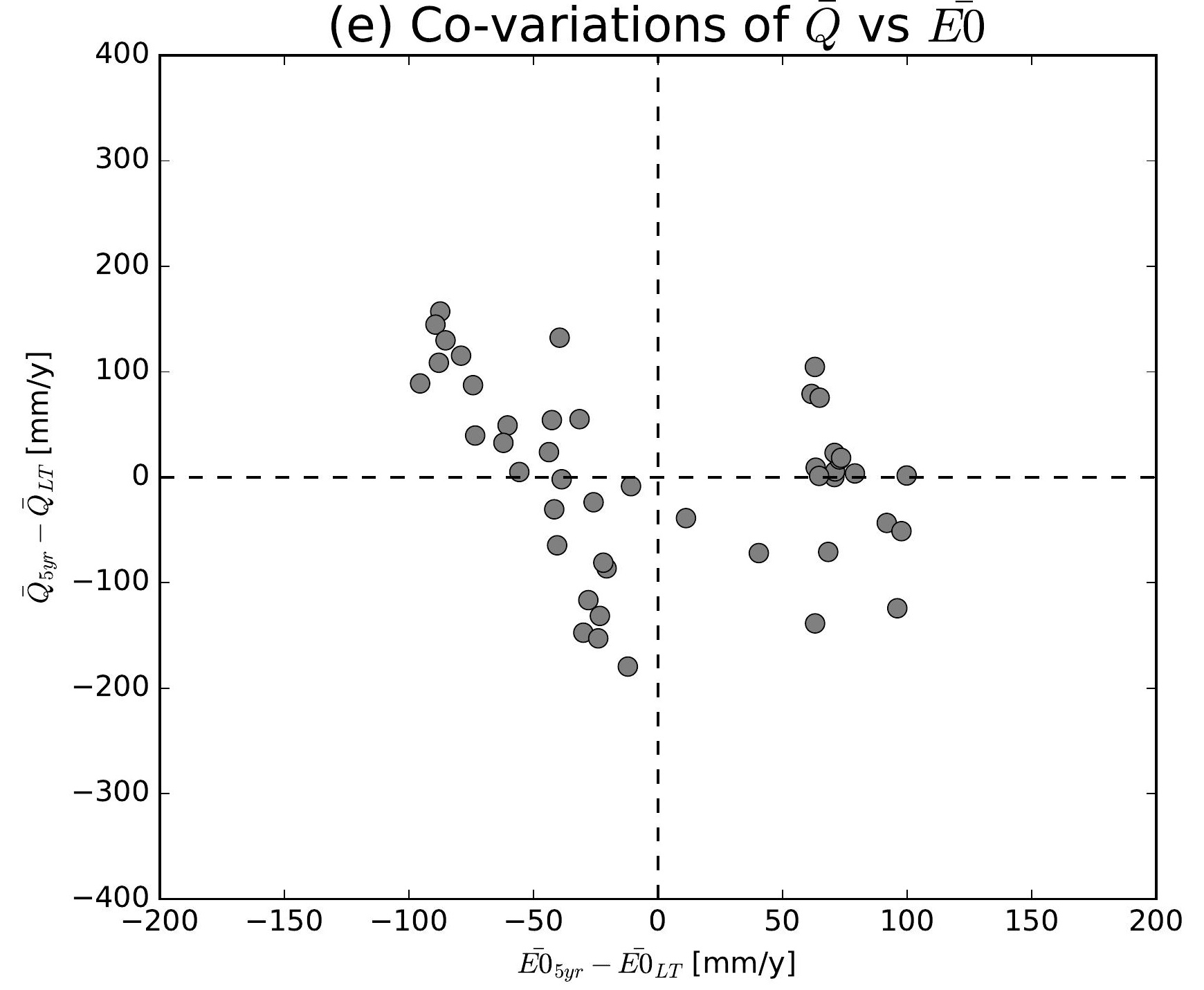

(h) Co-variations of $\bar{Q}$ vs $\overline{E 0}$

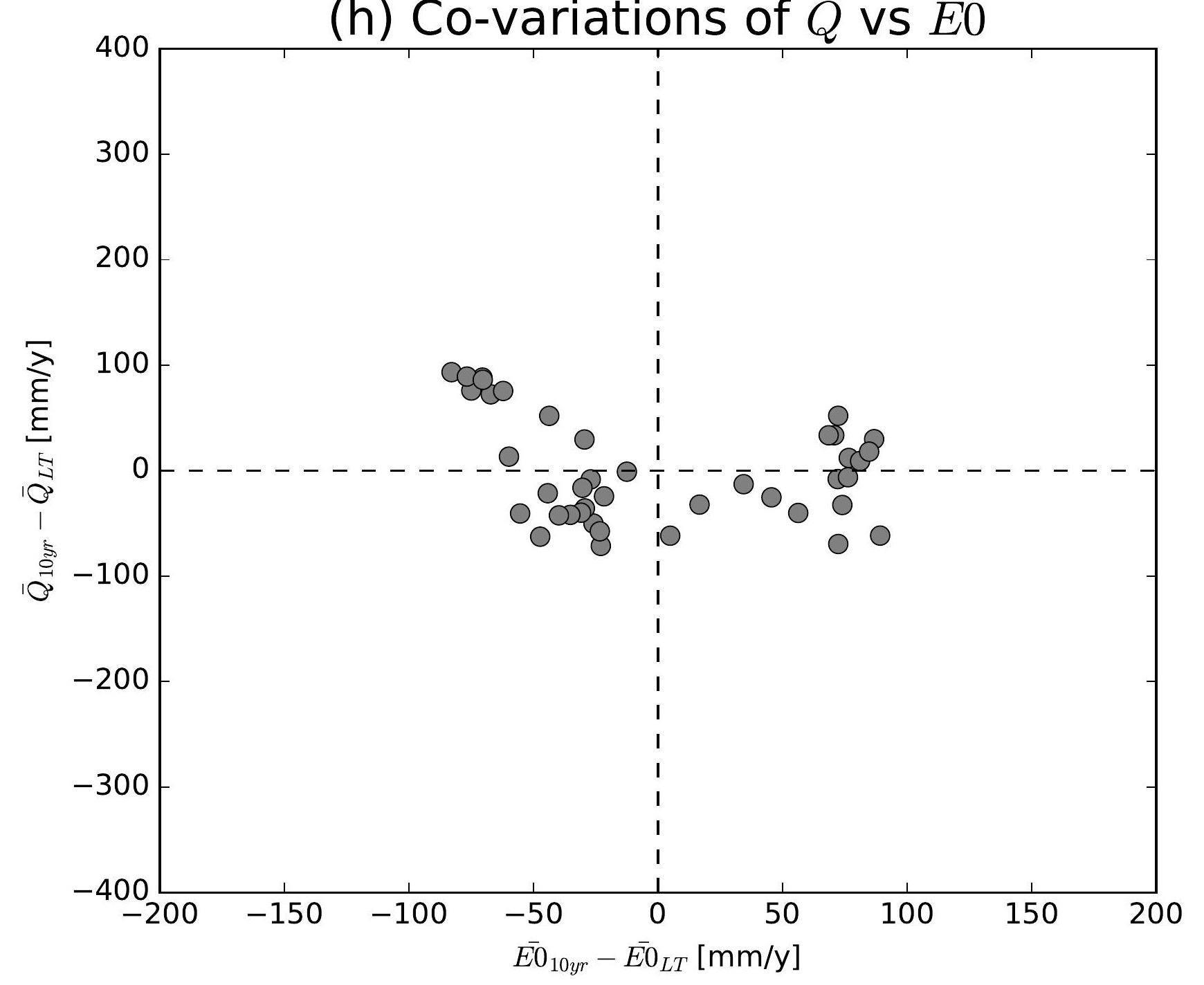

(k) Co-variations of $\bar{Q}$ vs $\overline{E 0}$

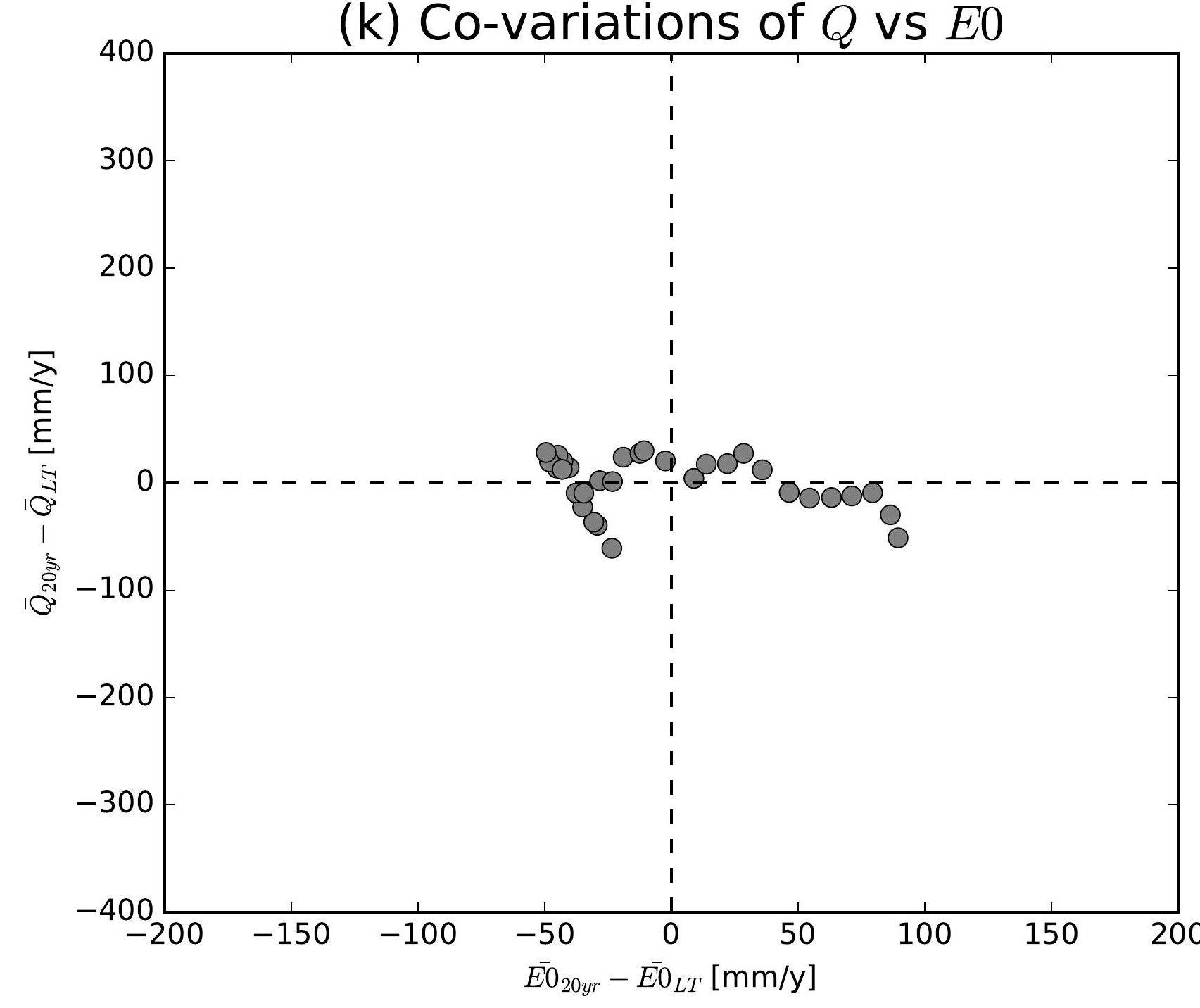

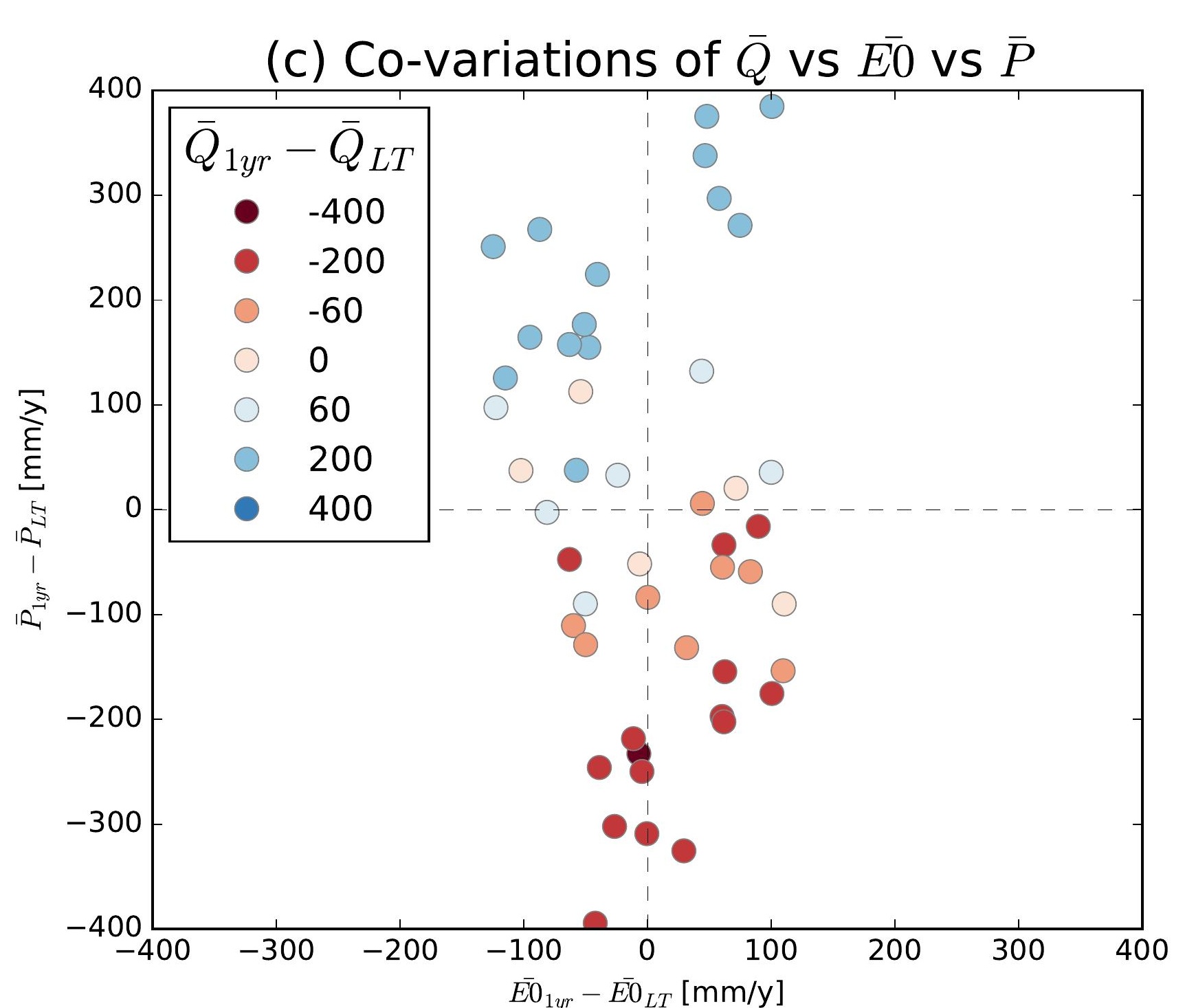
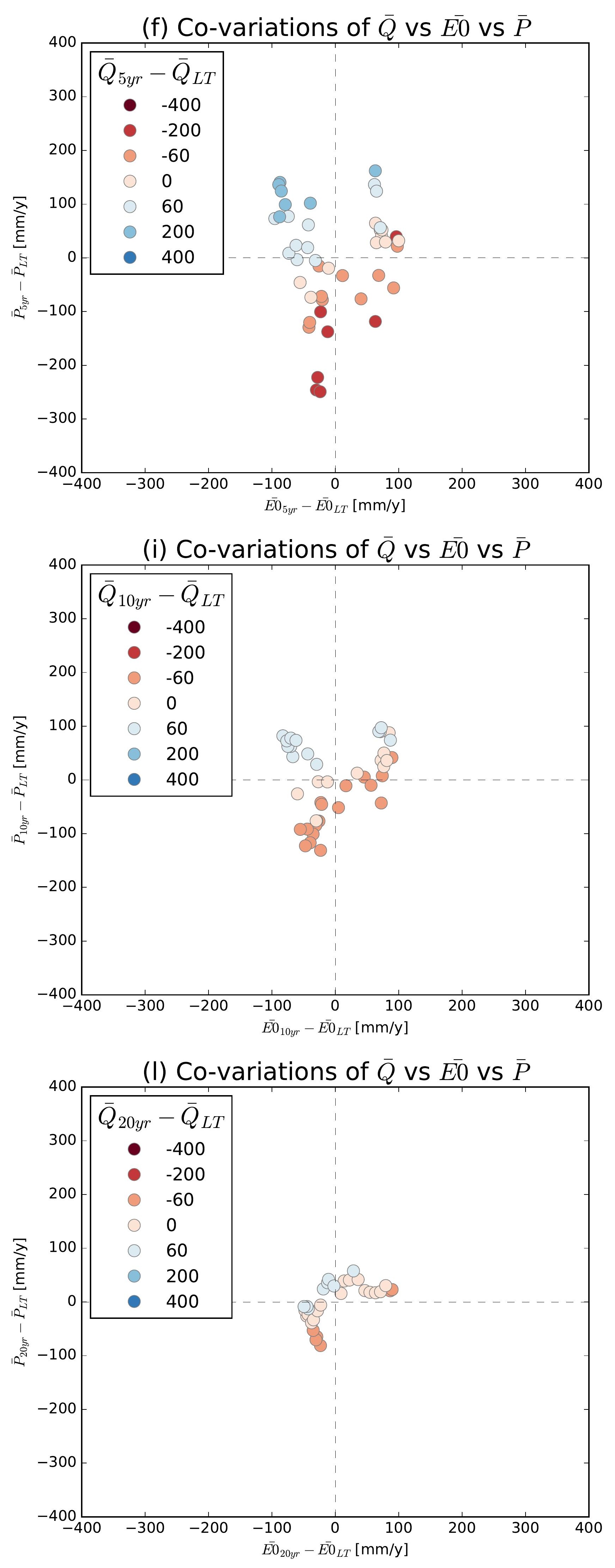

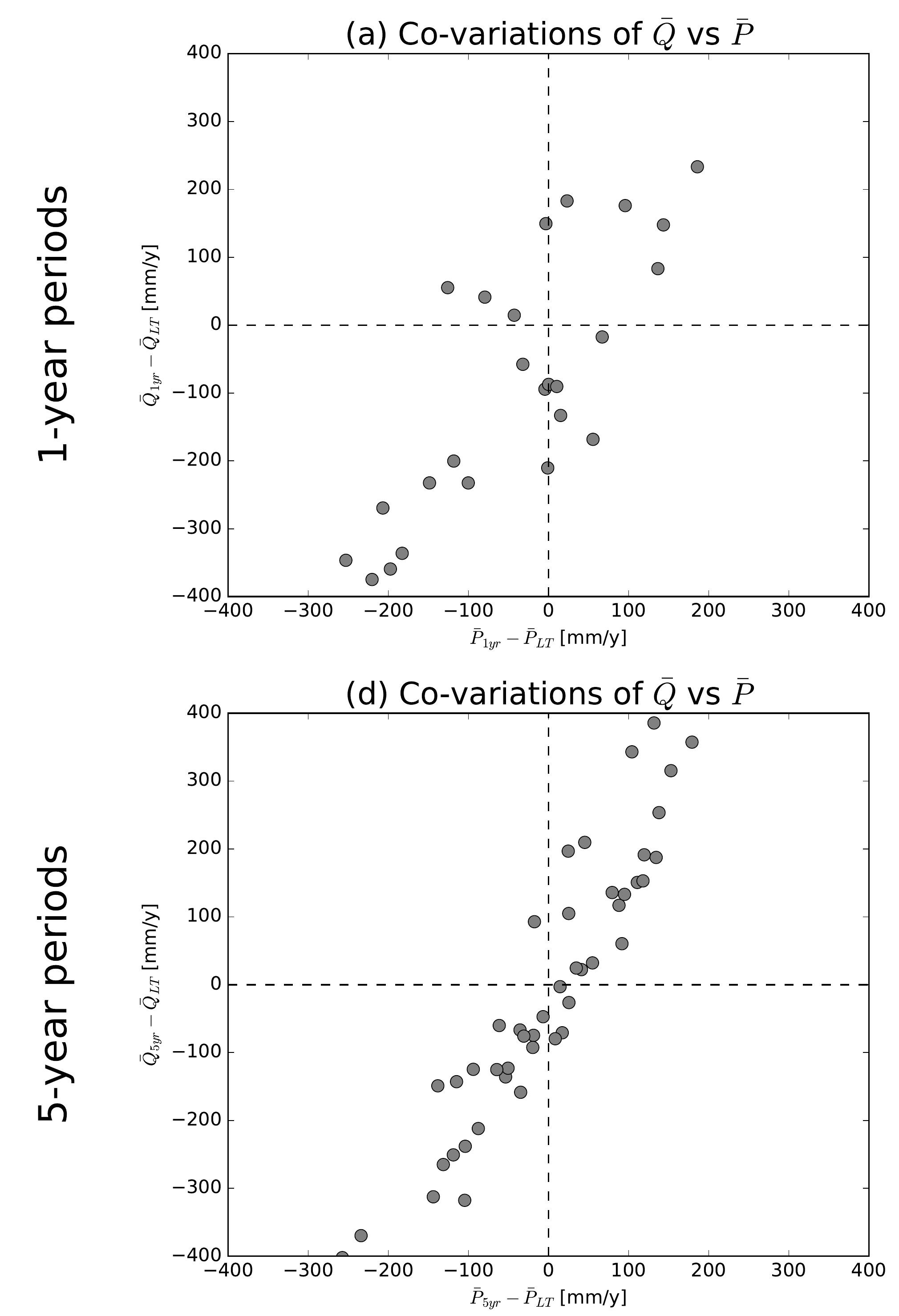

(g) Co-variations of $\bar{Q}$ vs $\bar{P}$
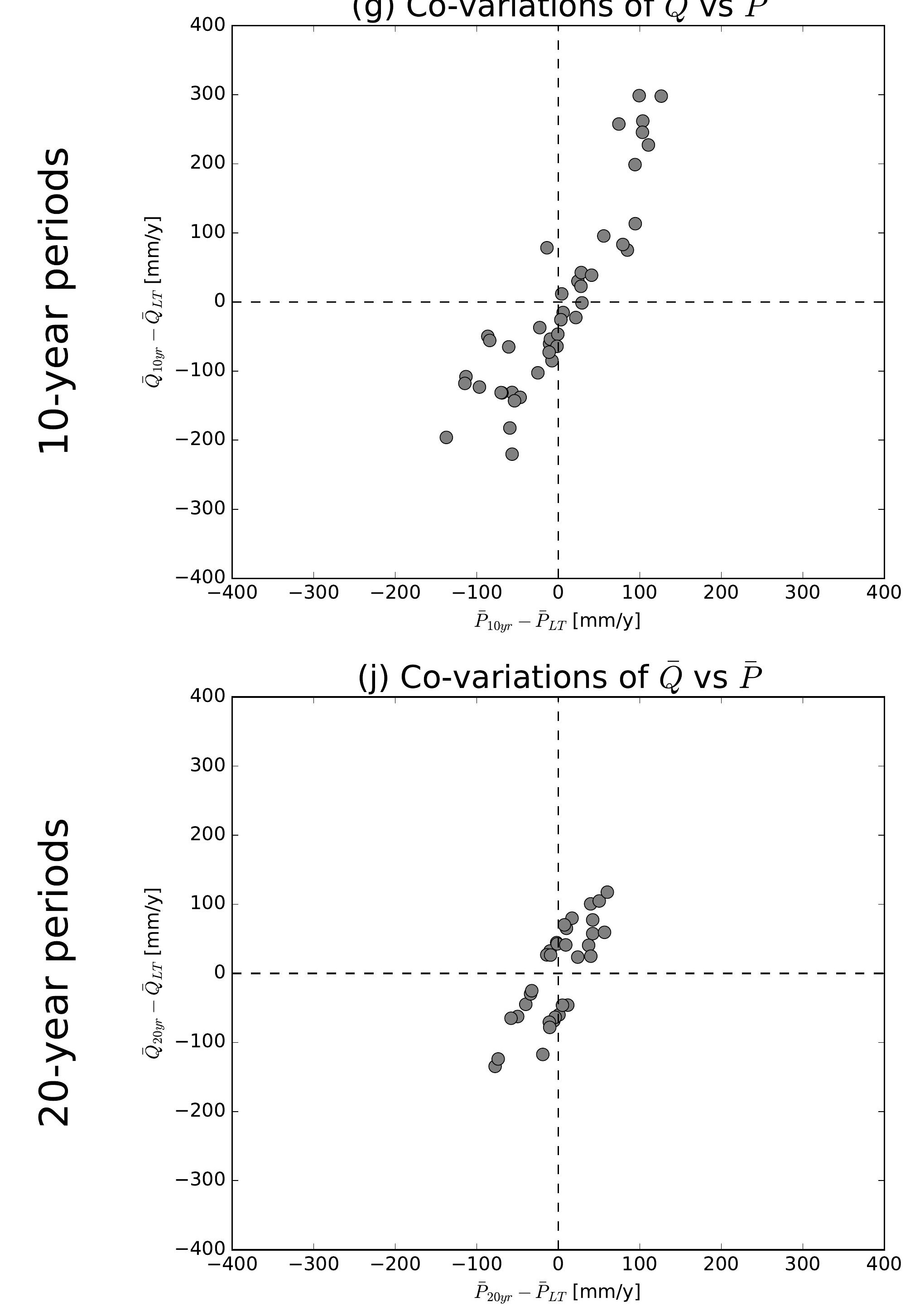

(b) Co-variations of $\bar{Q}$ vs $\overline{E 0}$

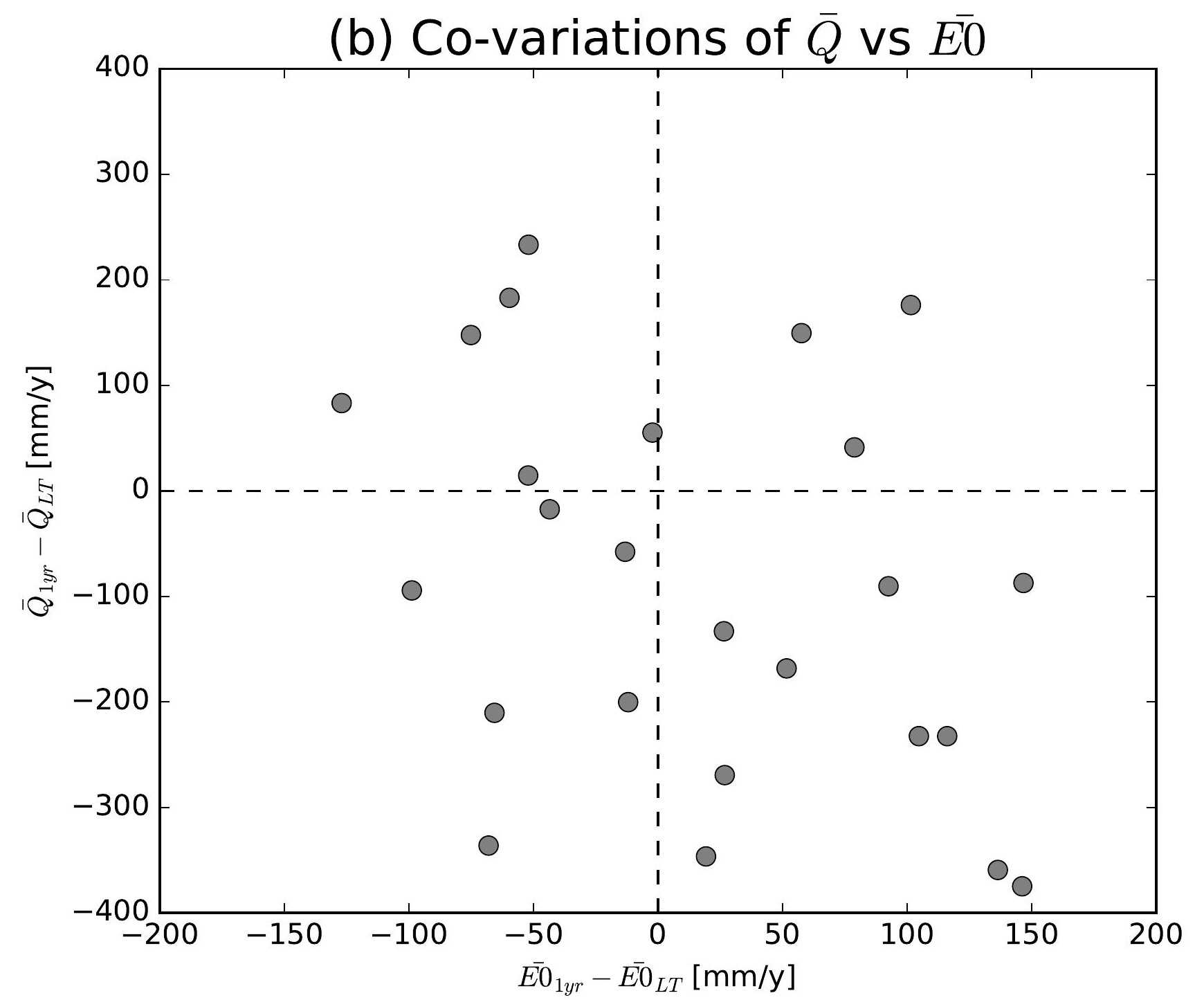

(e) Co-variations of $\bar{Q}$ vs $\overline{E 0}$

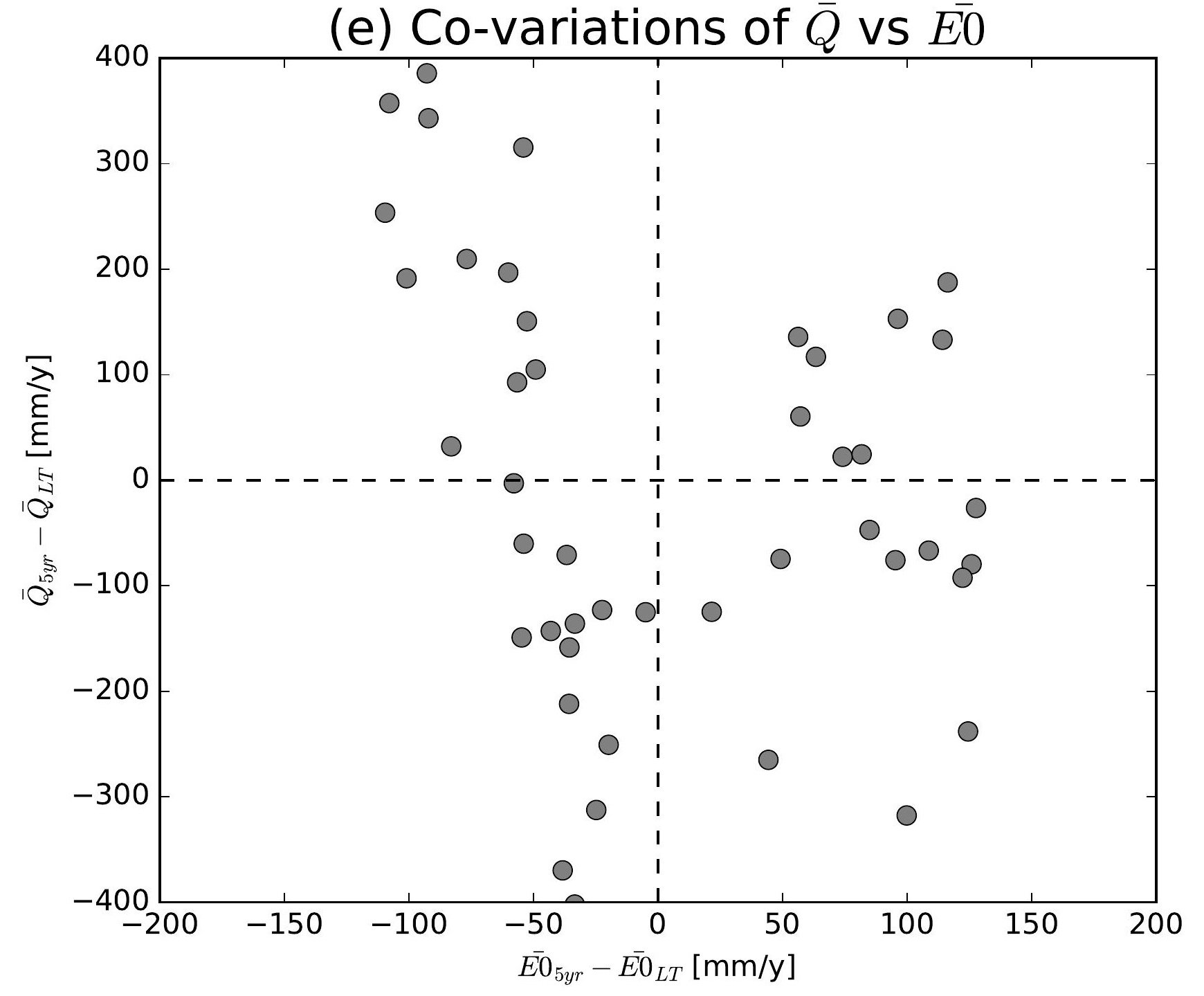

(h) Co-variations of $\bar{Q}$ vs $\overline{E 0}$

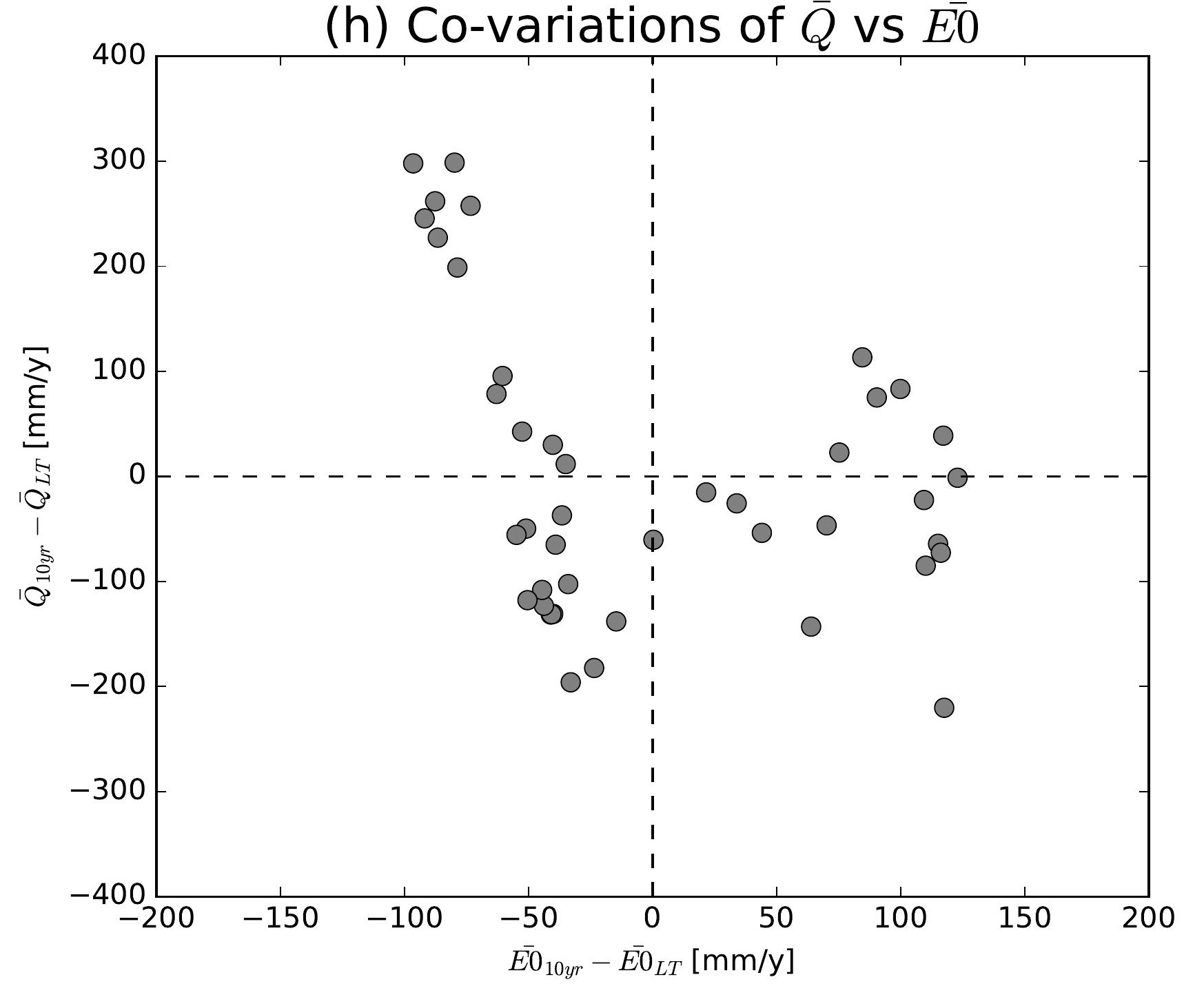

(k) Co-variations of $\bar{Q}$ vs $\overline{E 0}$

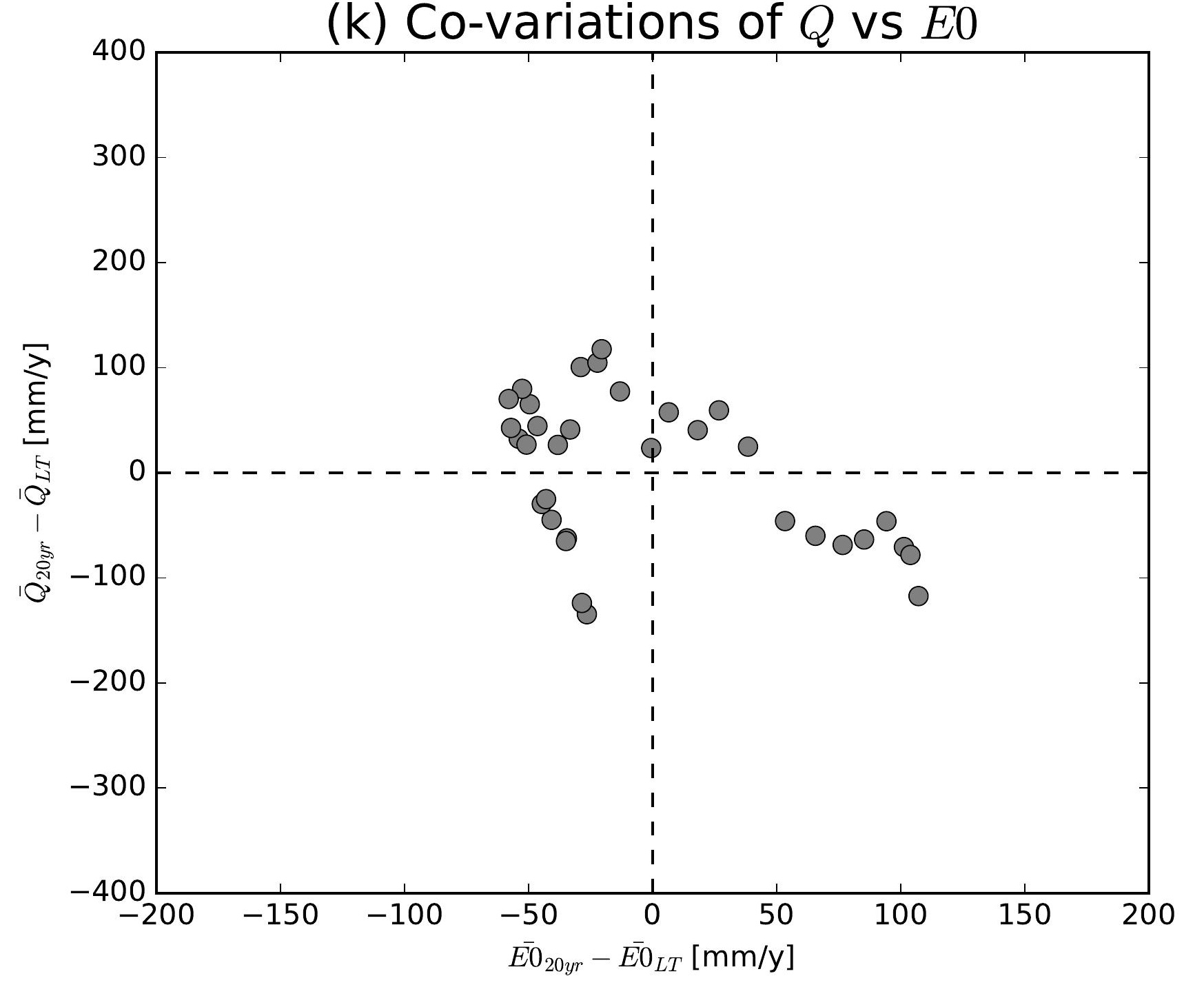

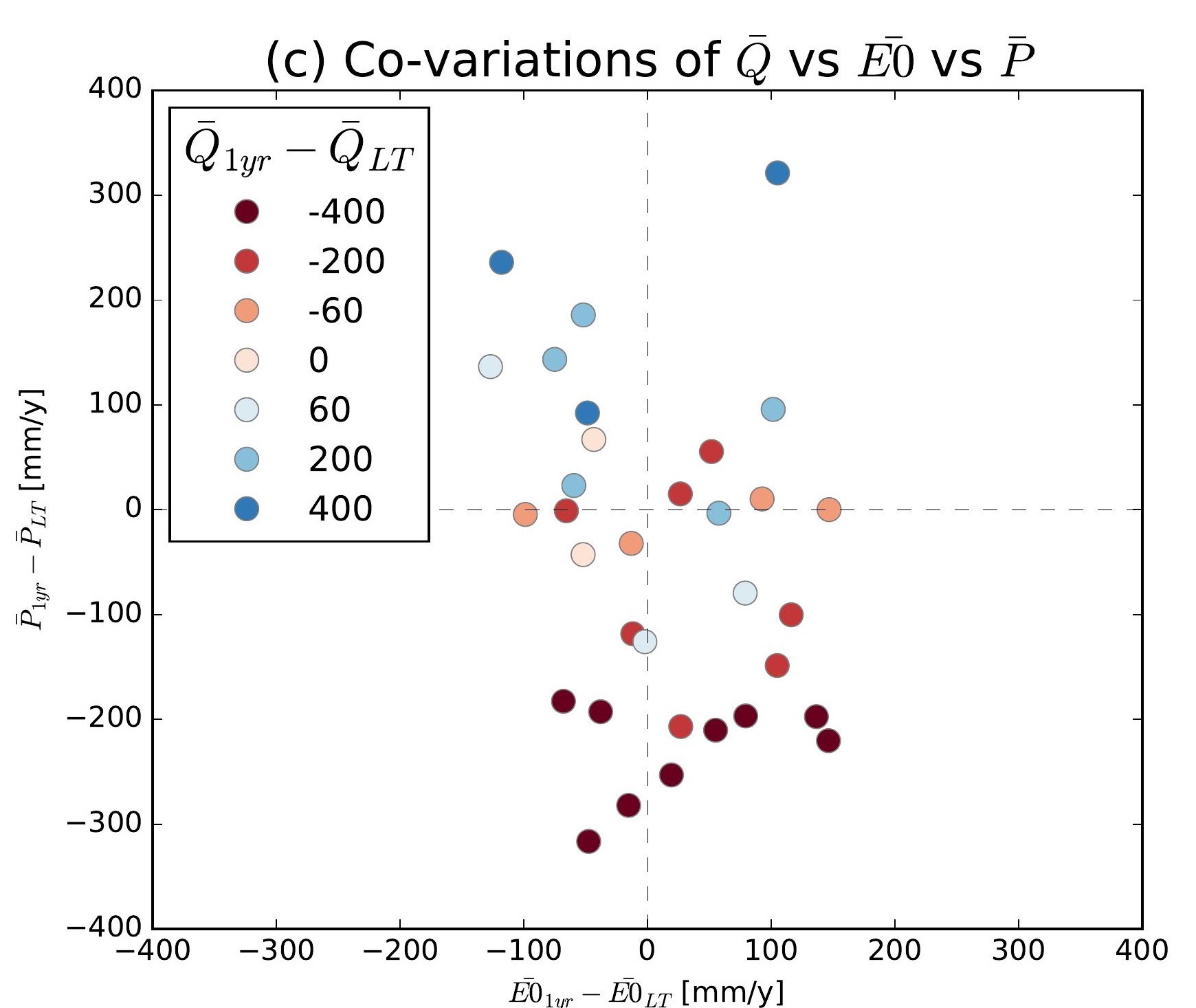
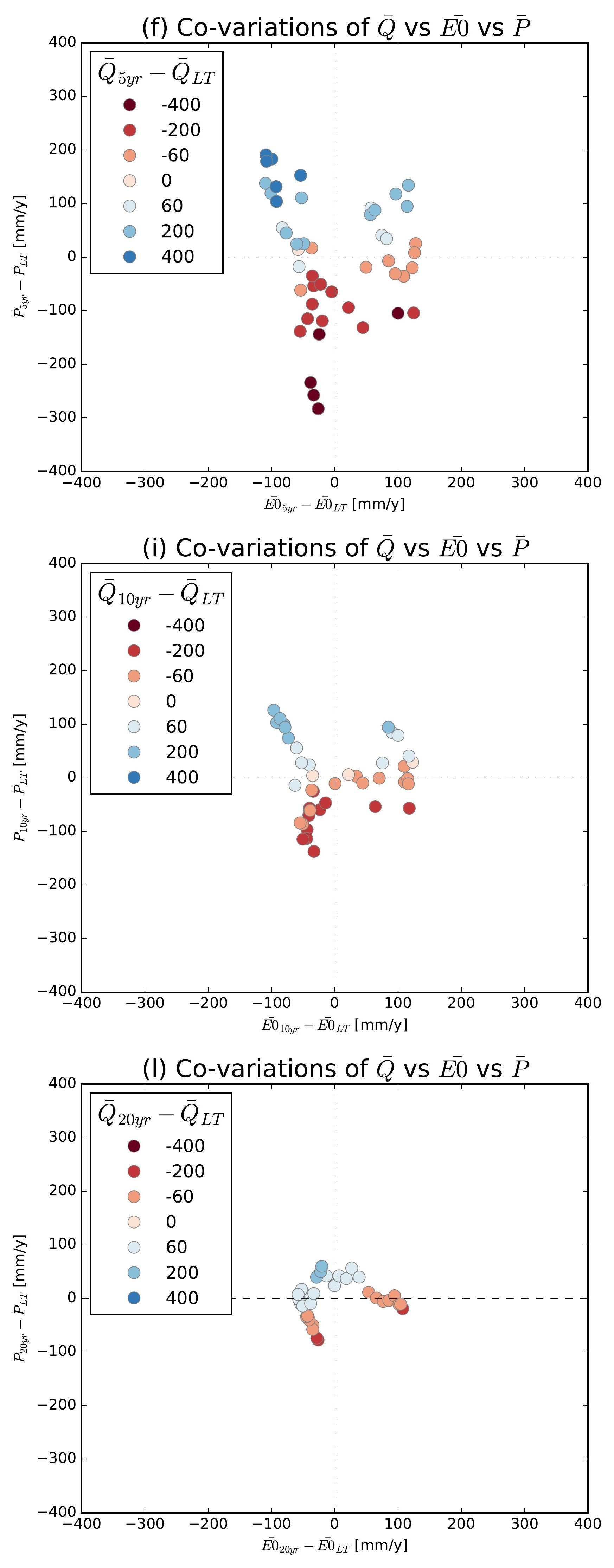

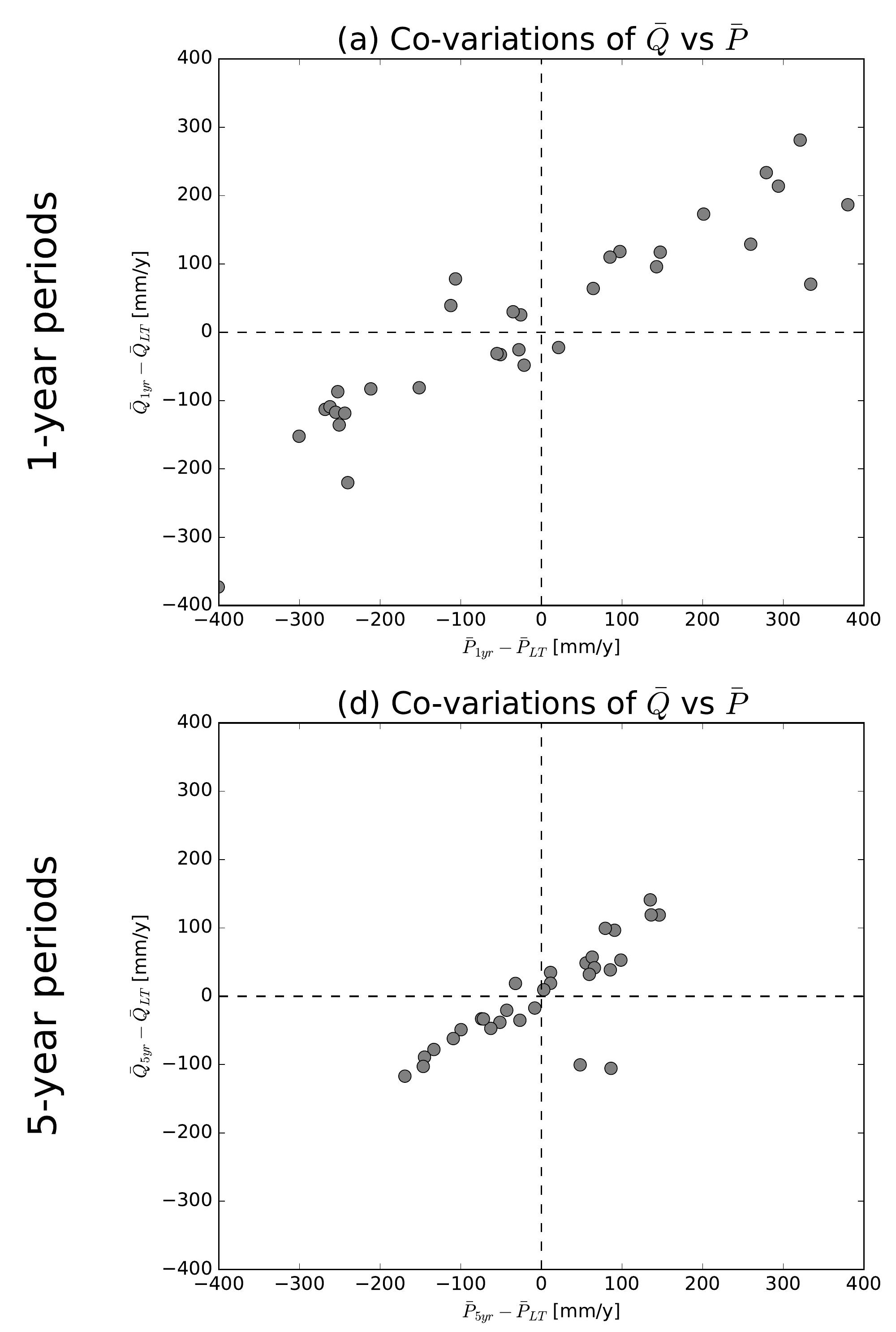

(g) Co-variations of $\bar{Q}$ vs $\bar{P}$

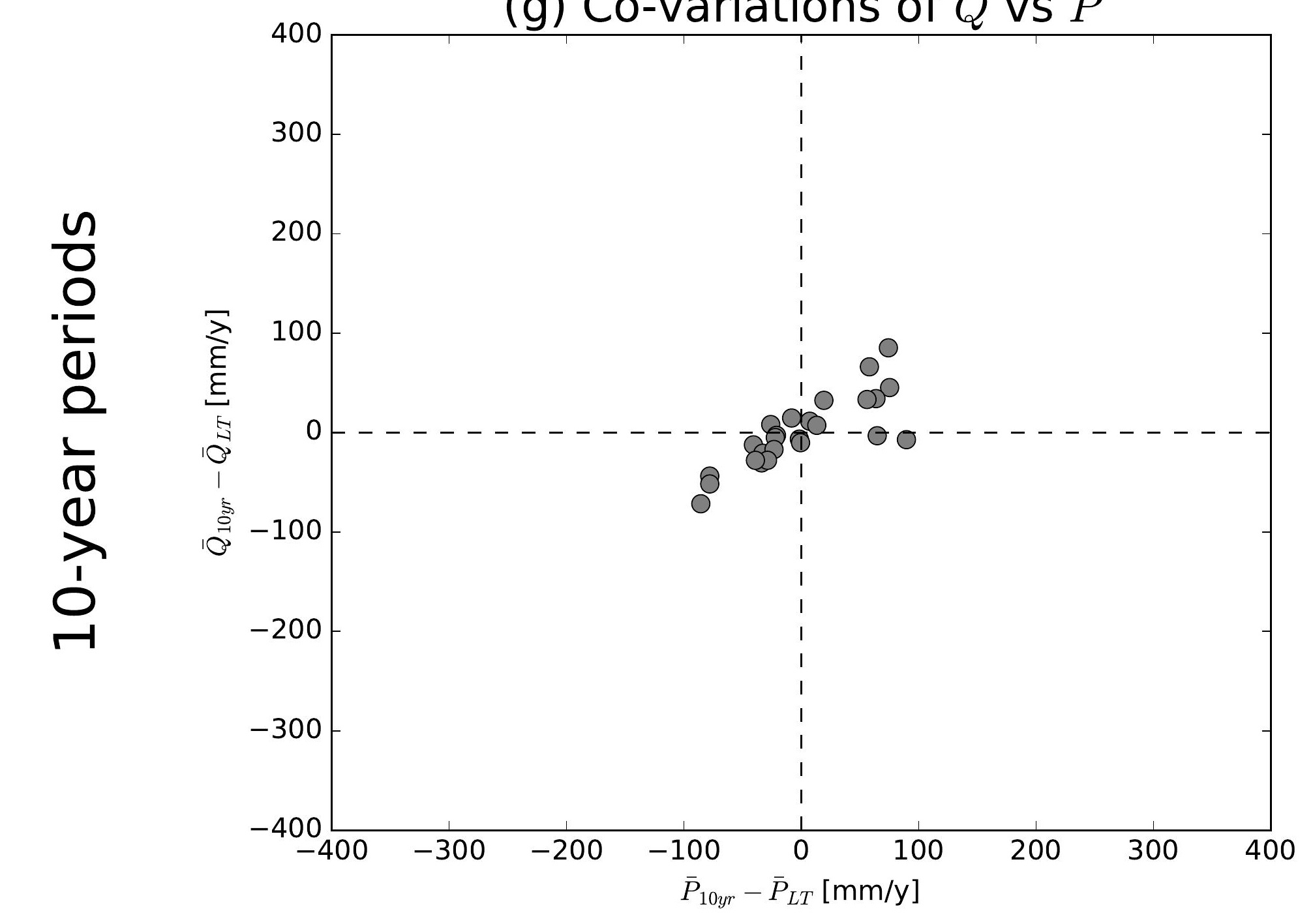

(j) Co-variations of $\bar{Q}$ vs $\bar{P}$

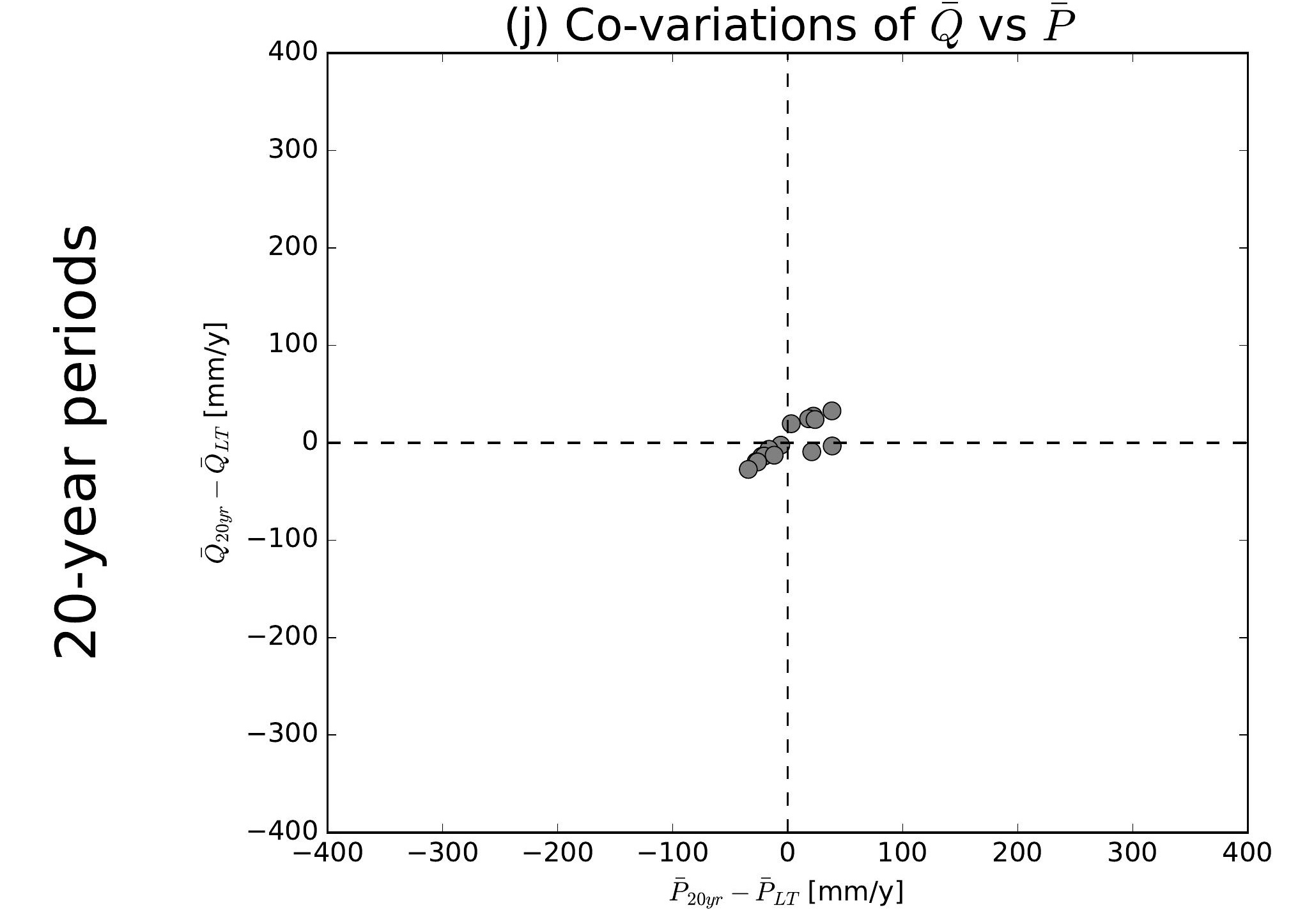

(b) Co-variations of $\bar{Q}$ vs $\overline{E 0}$

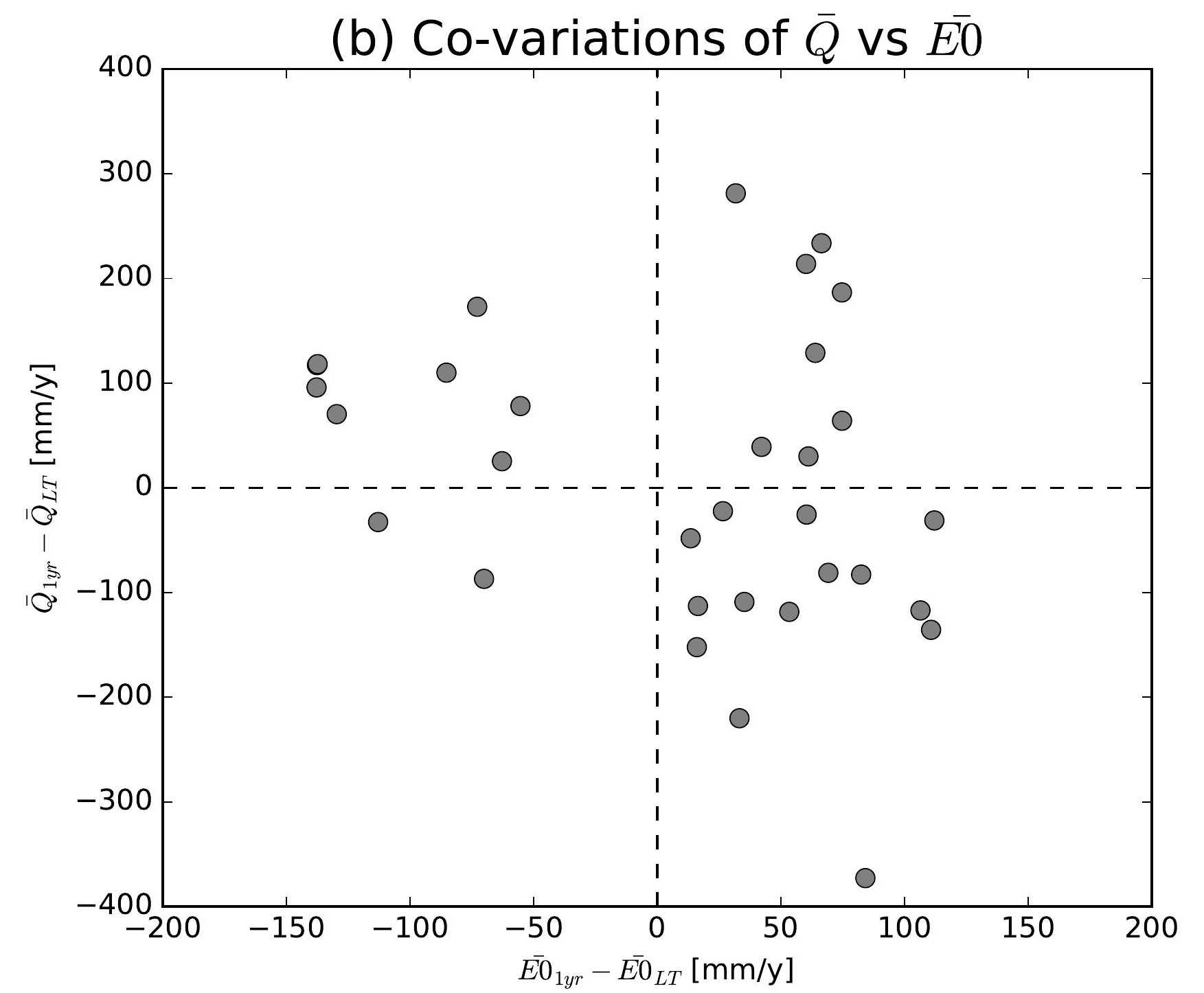

(e) Co-variations of $\bar{Q}$ vs $\overline{E 0}$

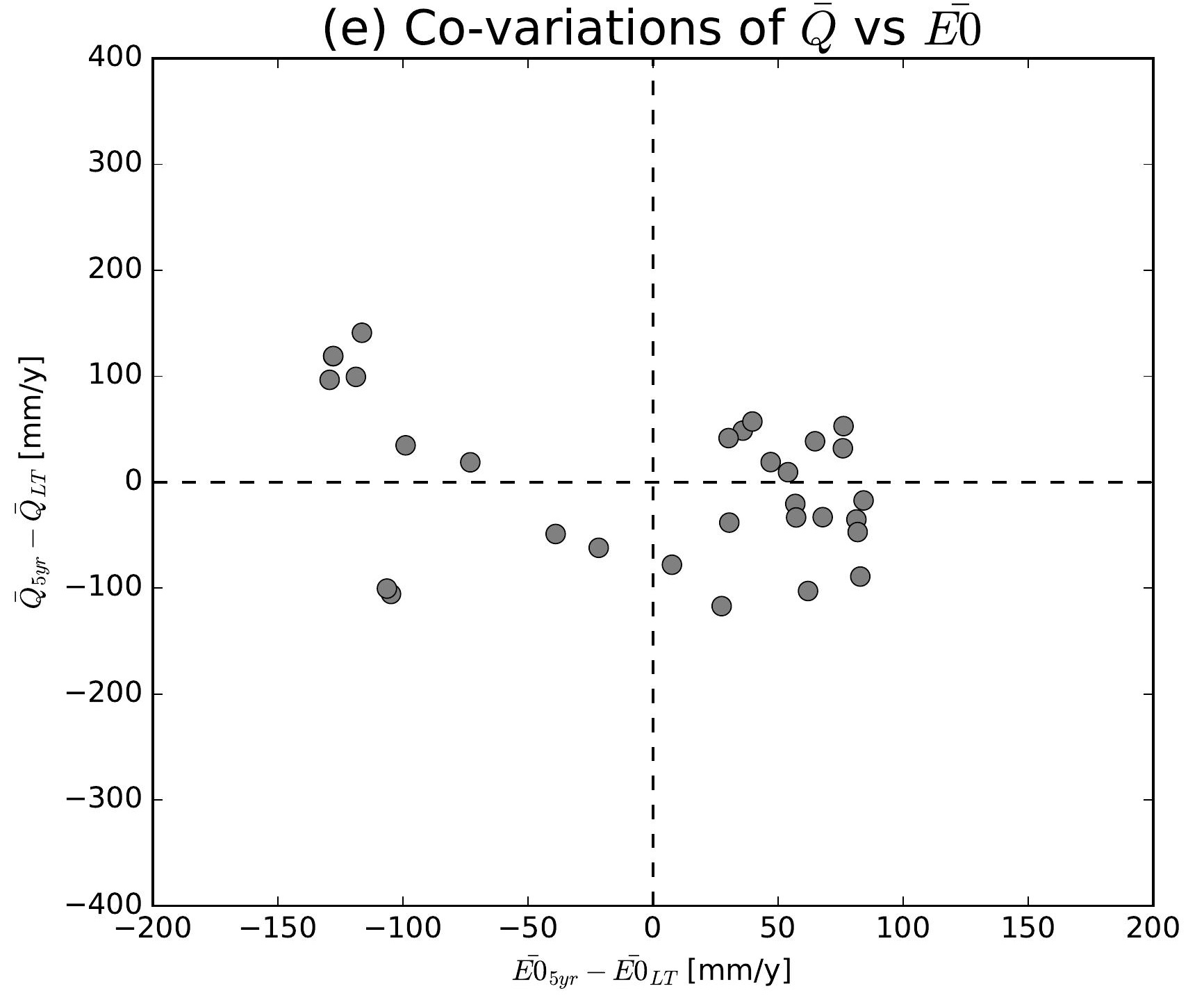

(h) Co-variations of $\bar{Q}$ vs $\overline{E 0}$

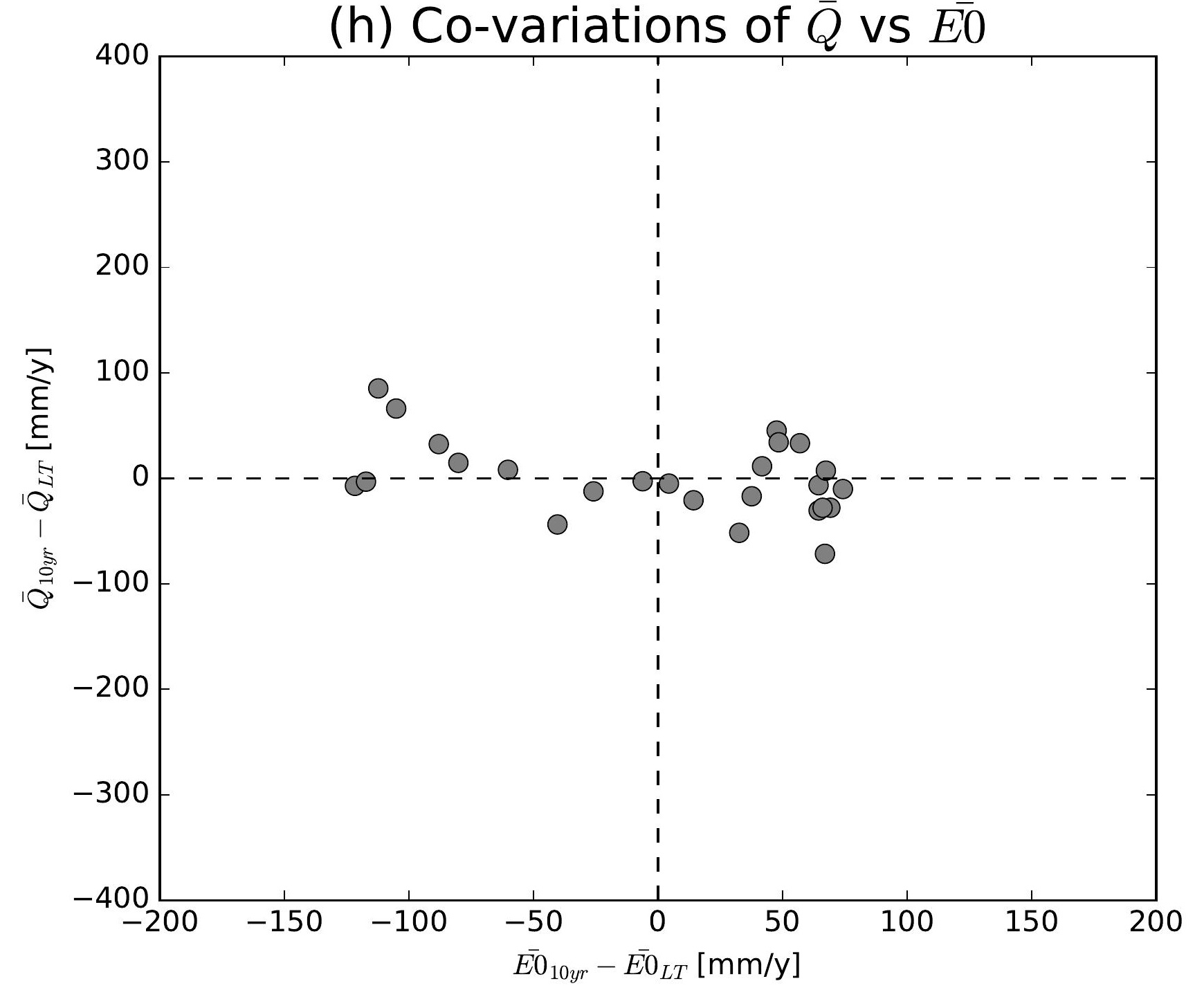

(k) Co-variations of $\bar{Q}$ vs $\overline{E 0}$

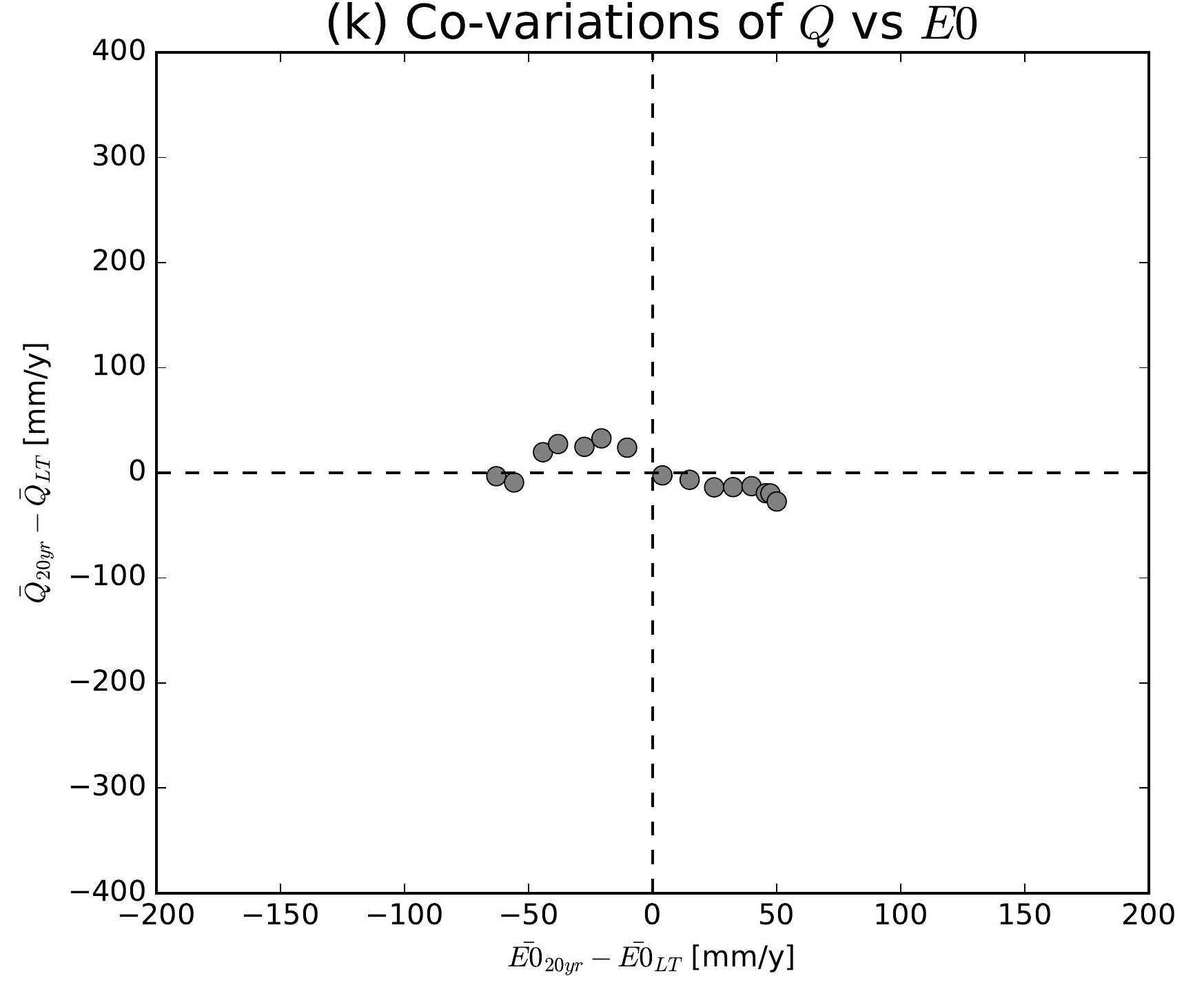

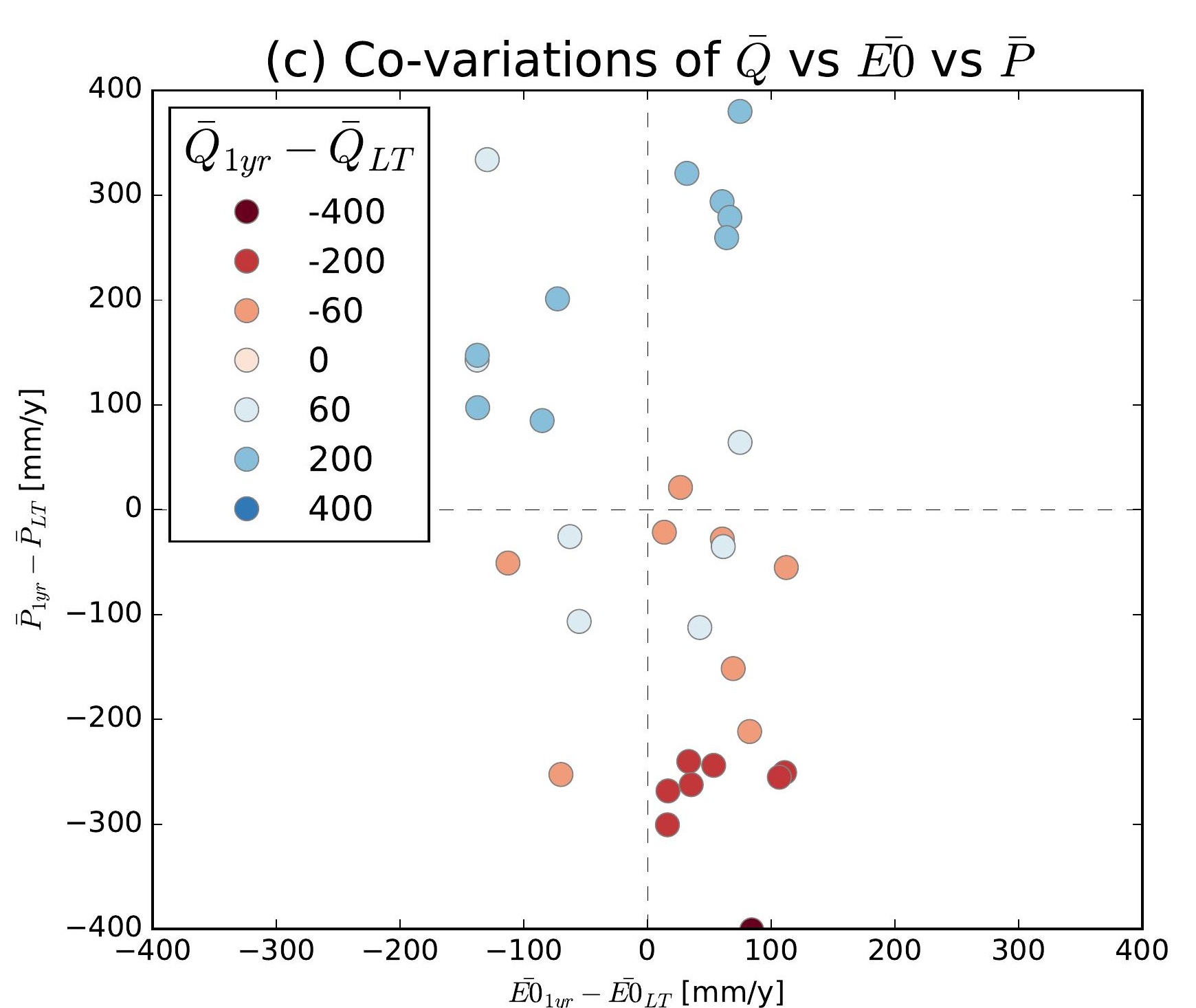
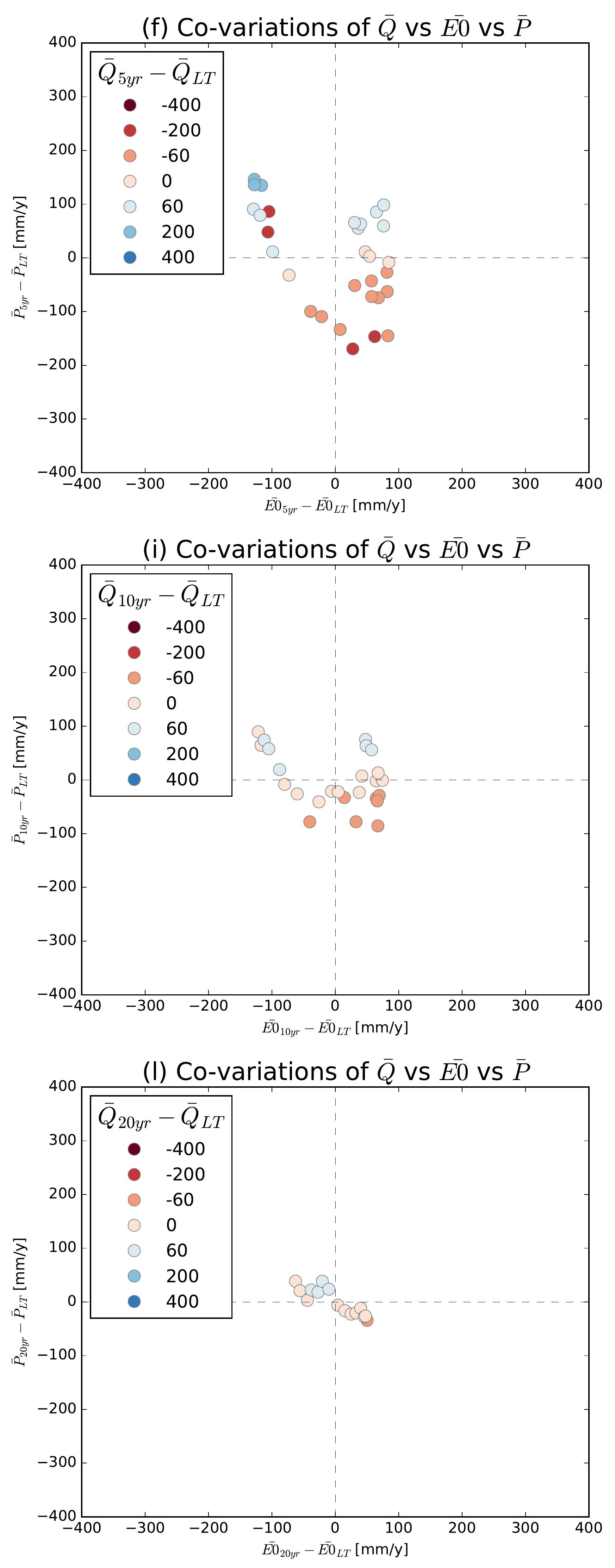

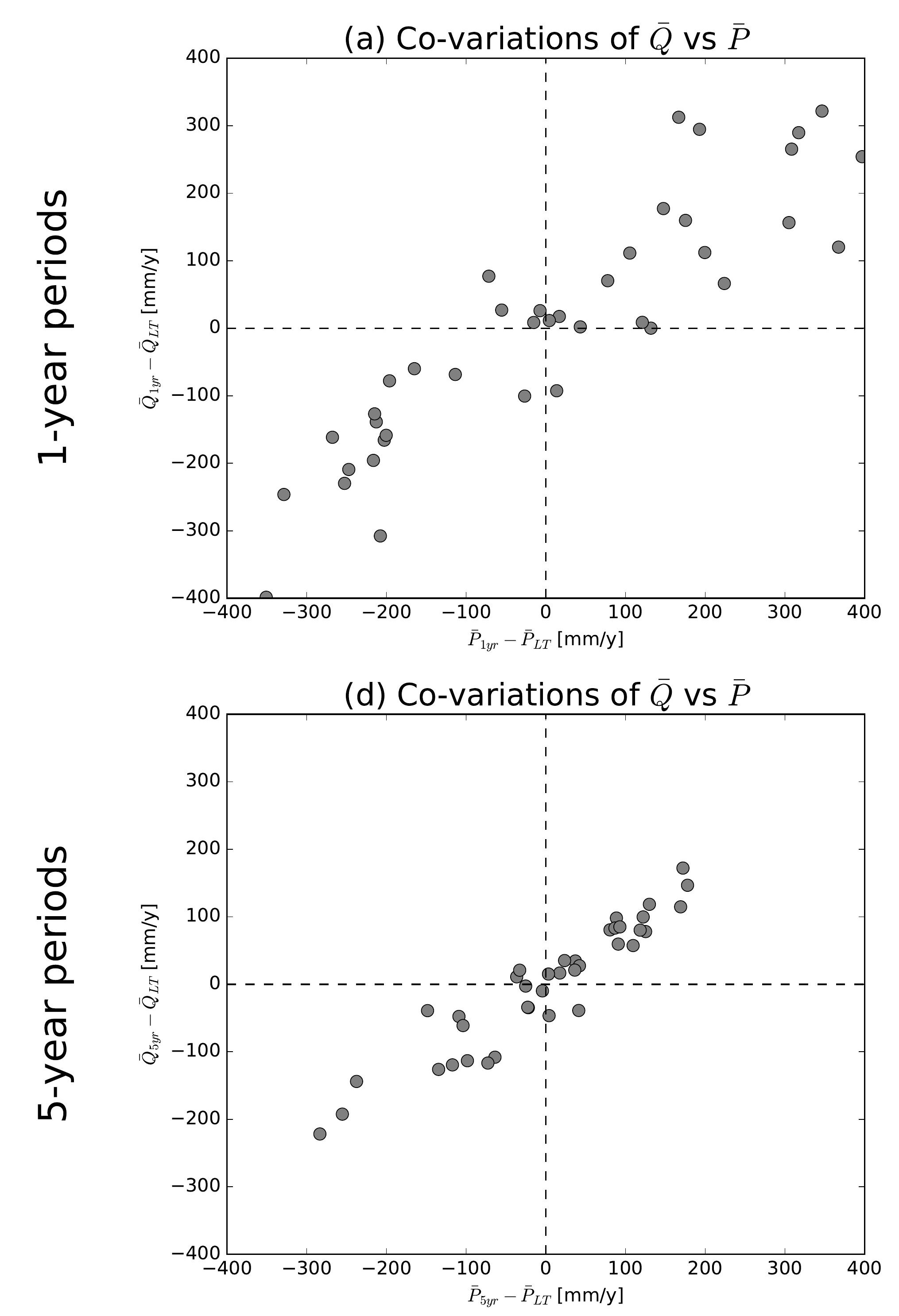

(g) Co-variations of $\bar{Q}$ vs $\bar{P}$

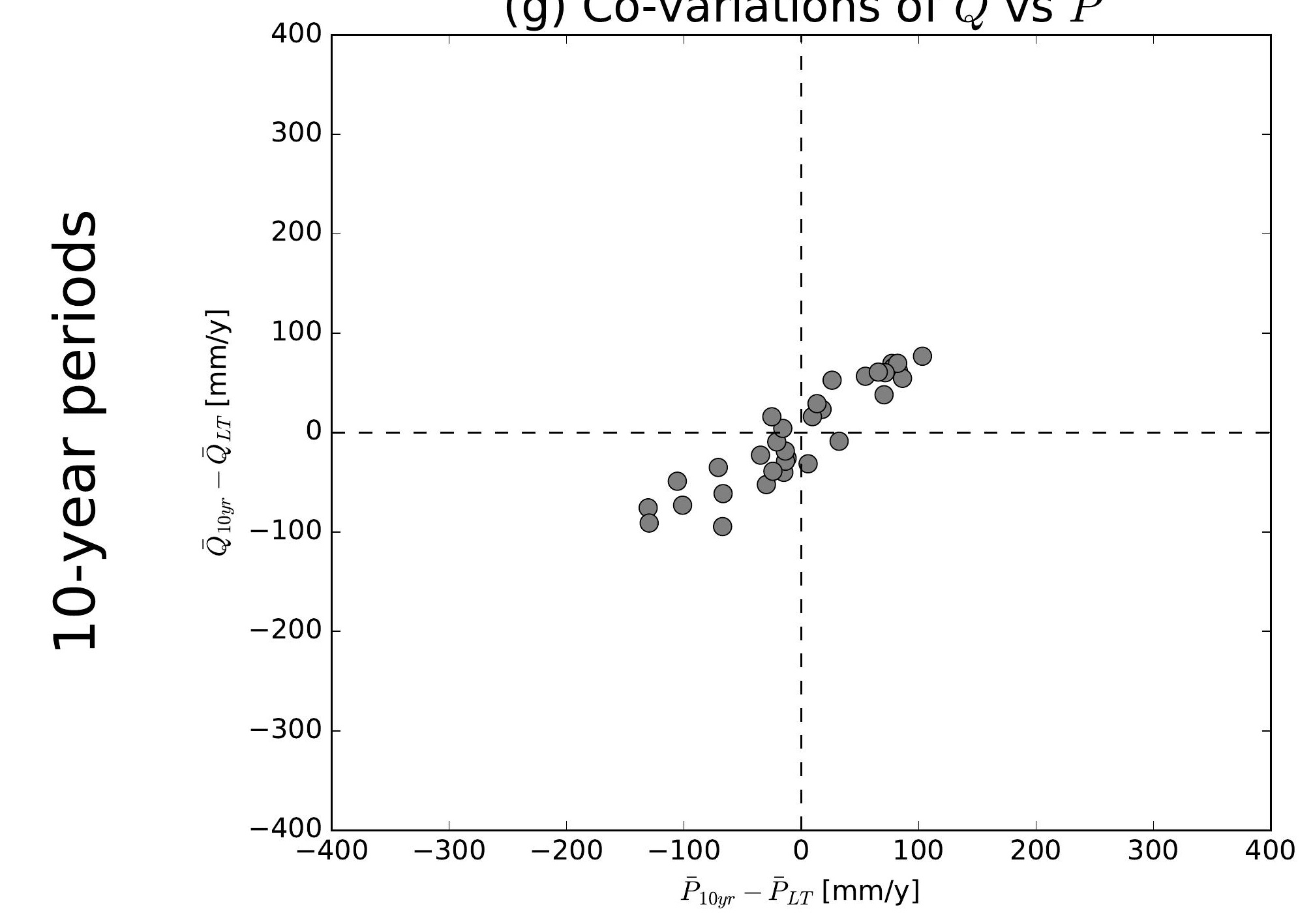

(j) Co-variations of $\bar{Q}$ vs $\bar{P}$

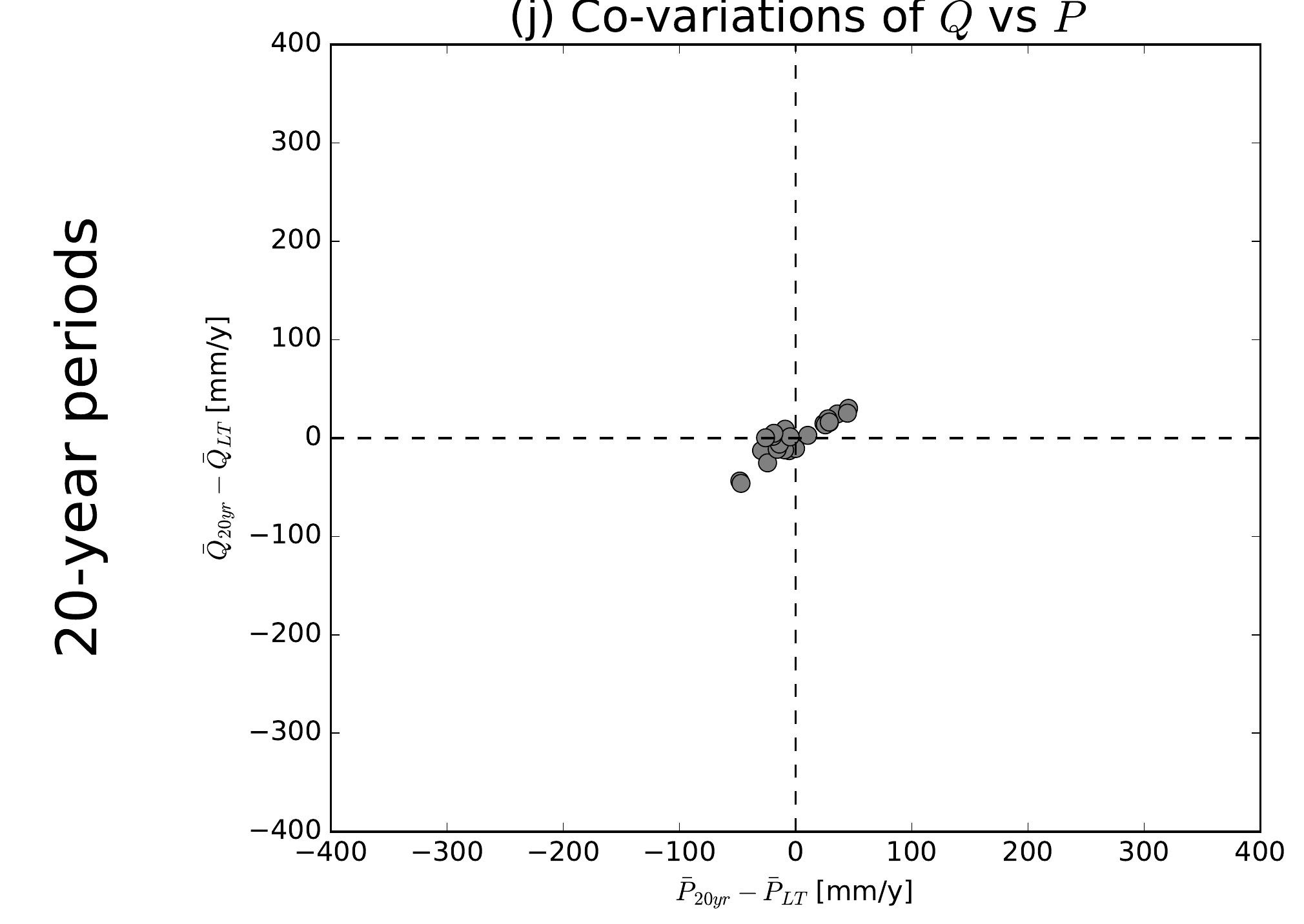

(b) Co-variations of $\bar{Q}$ vs $\overline{E 0}$

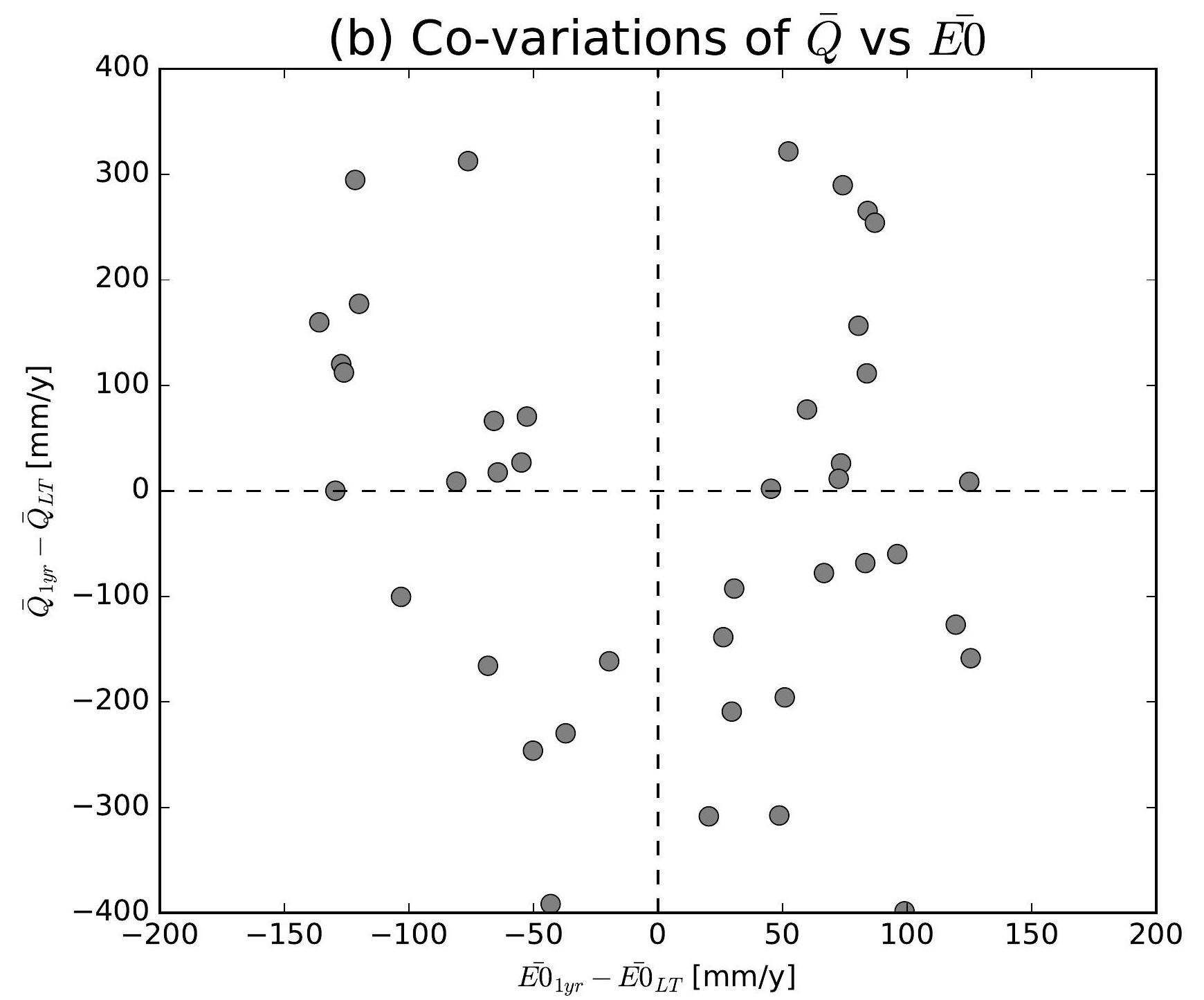

(e) Co-variations of $\bar{Q}$ vs $\overline{E 0}$

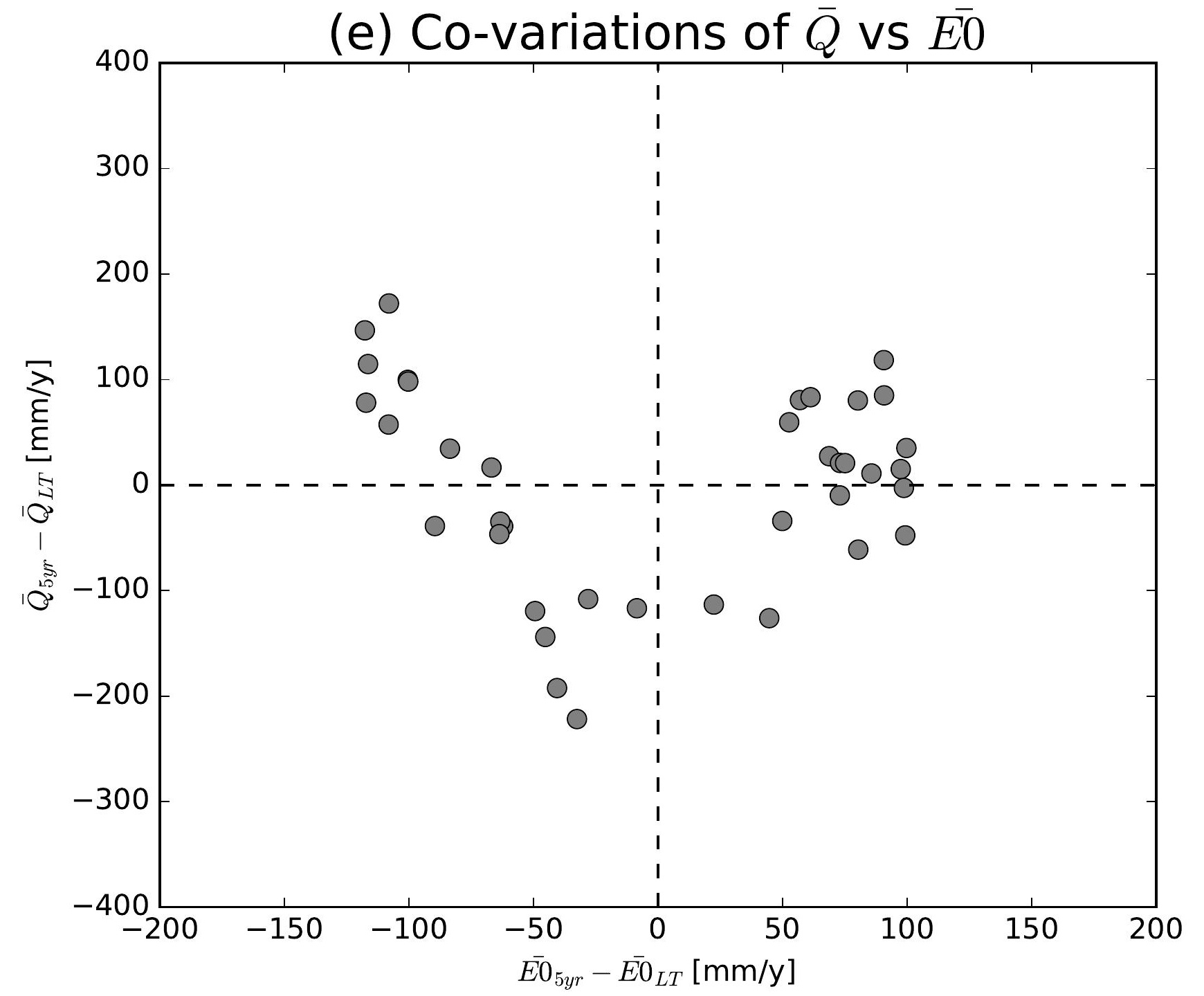

(h) Co-variations of $\bar{Q}$ vs $\overline{E 0}$

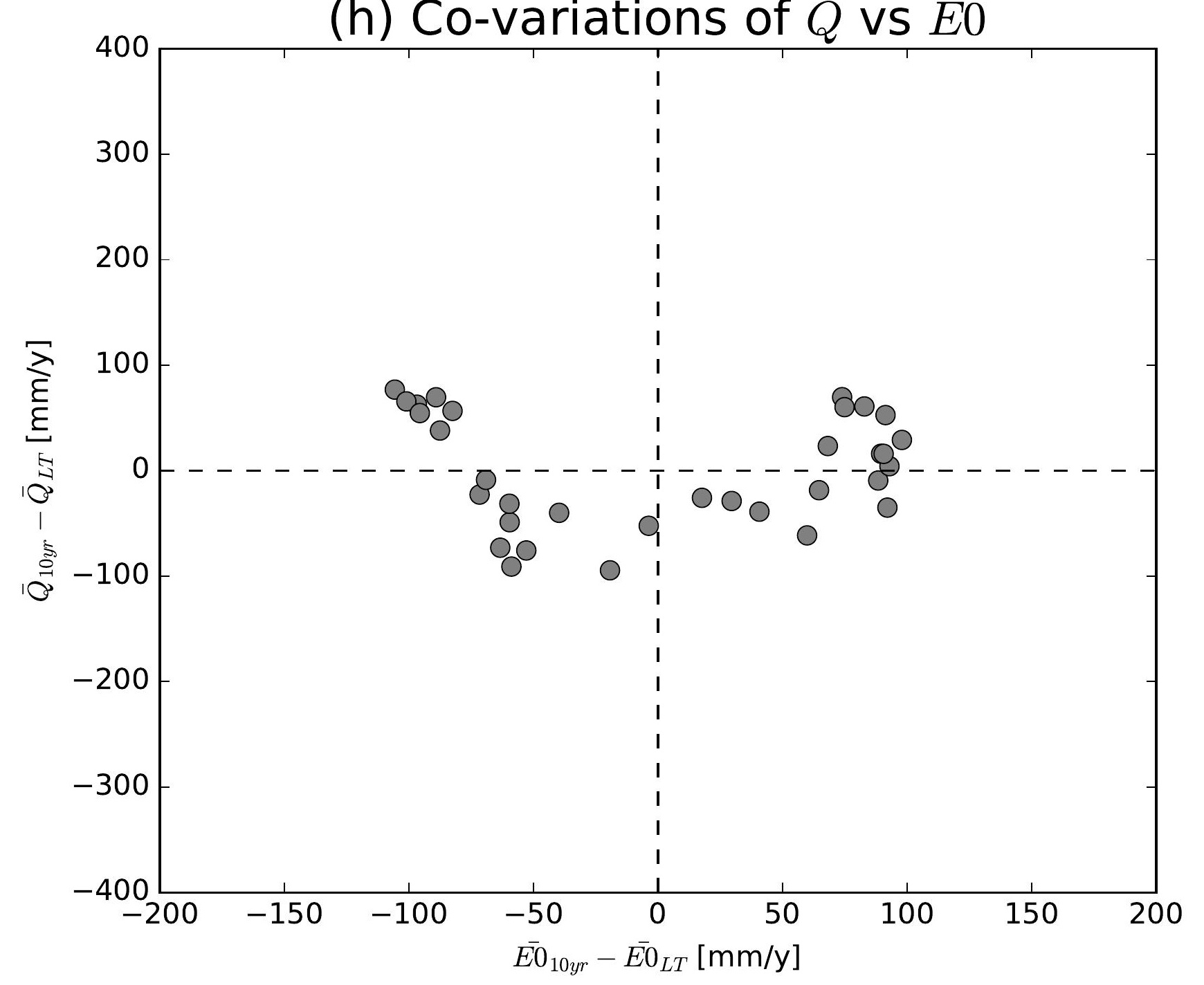

(k) Co-variations of $\bar{Q}$ vs $\overline{E 0}$

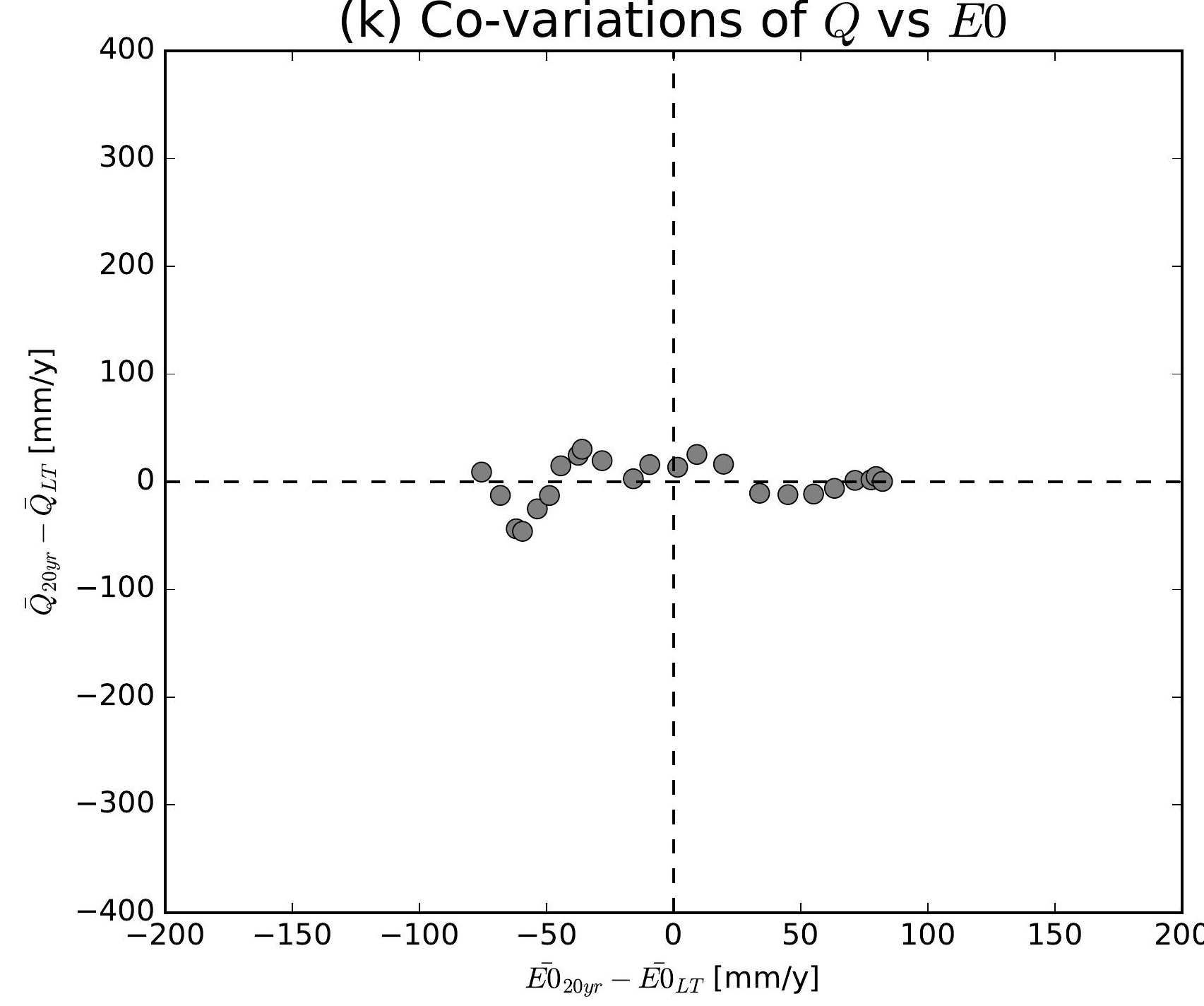

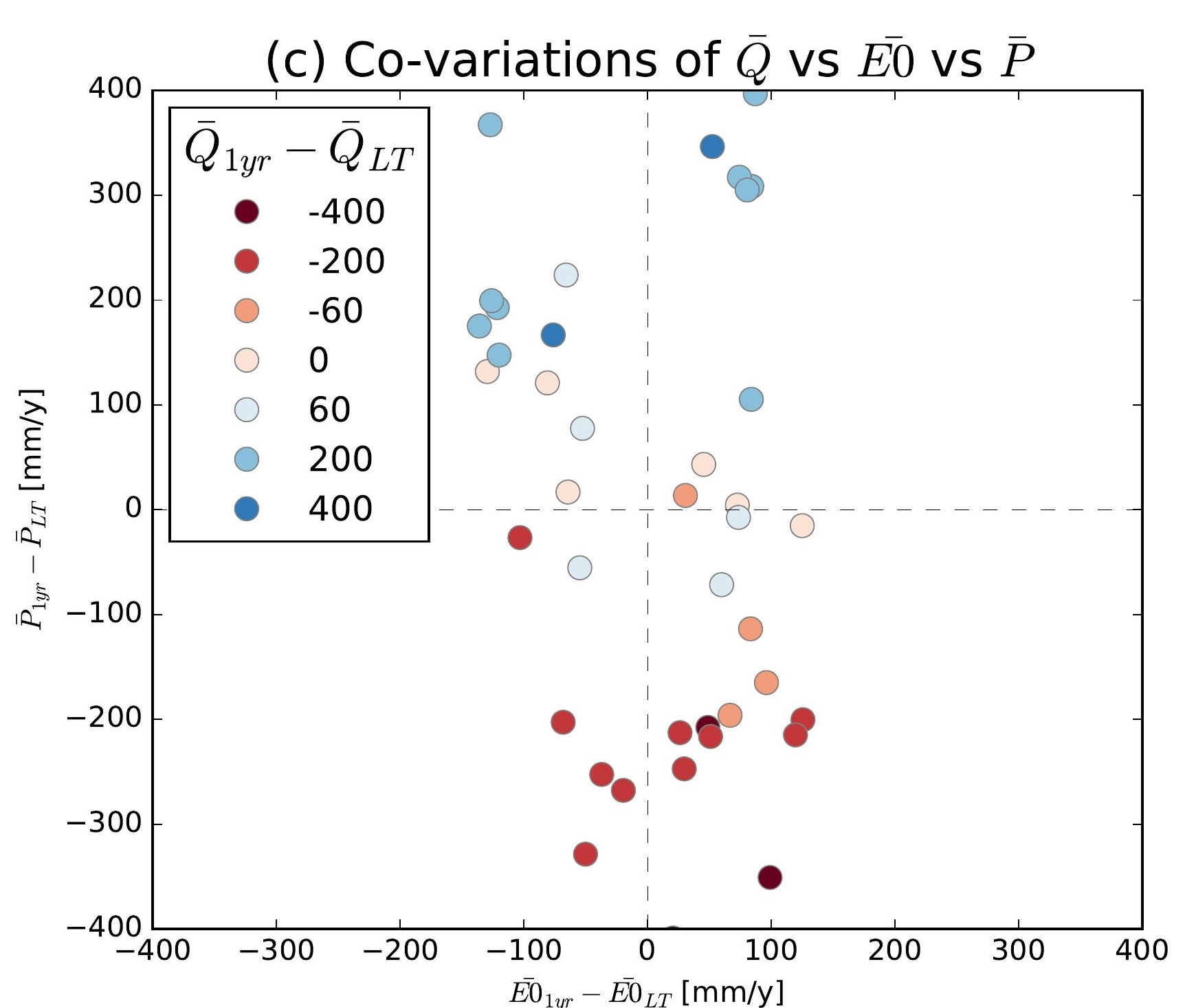
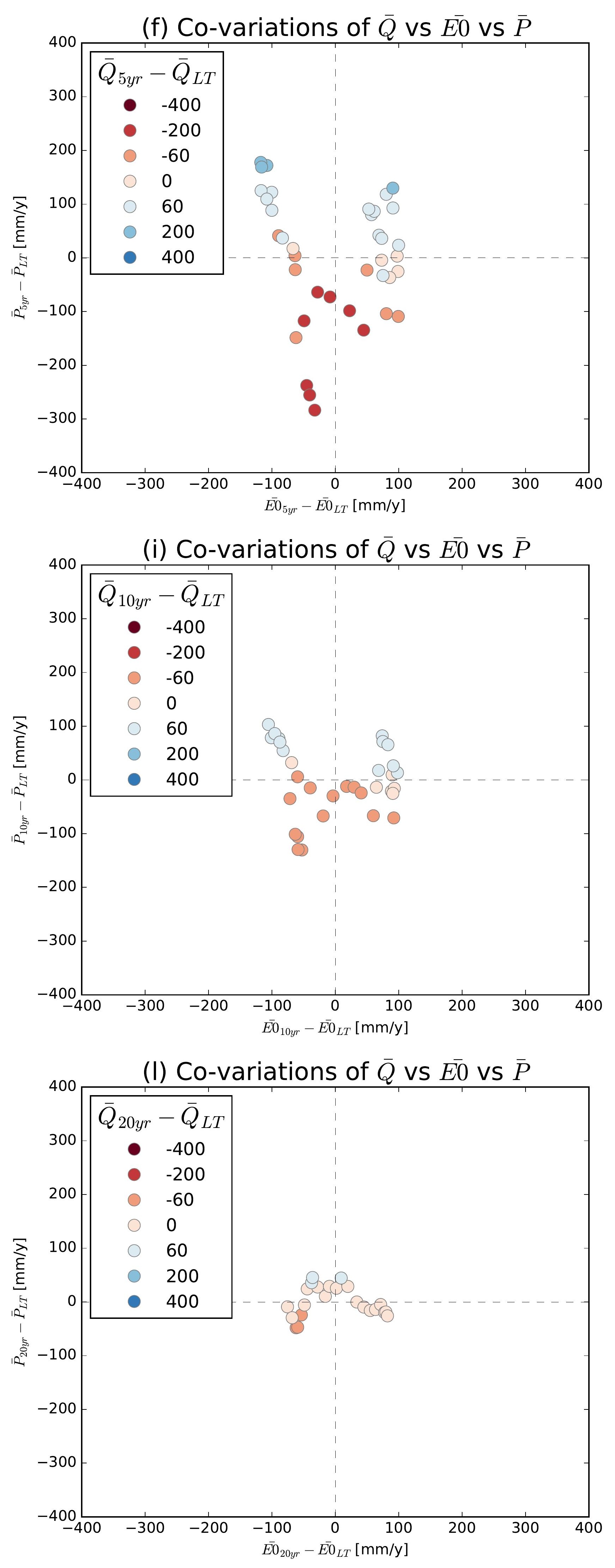

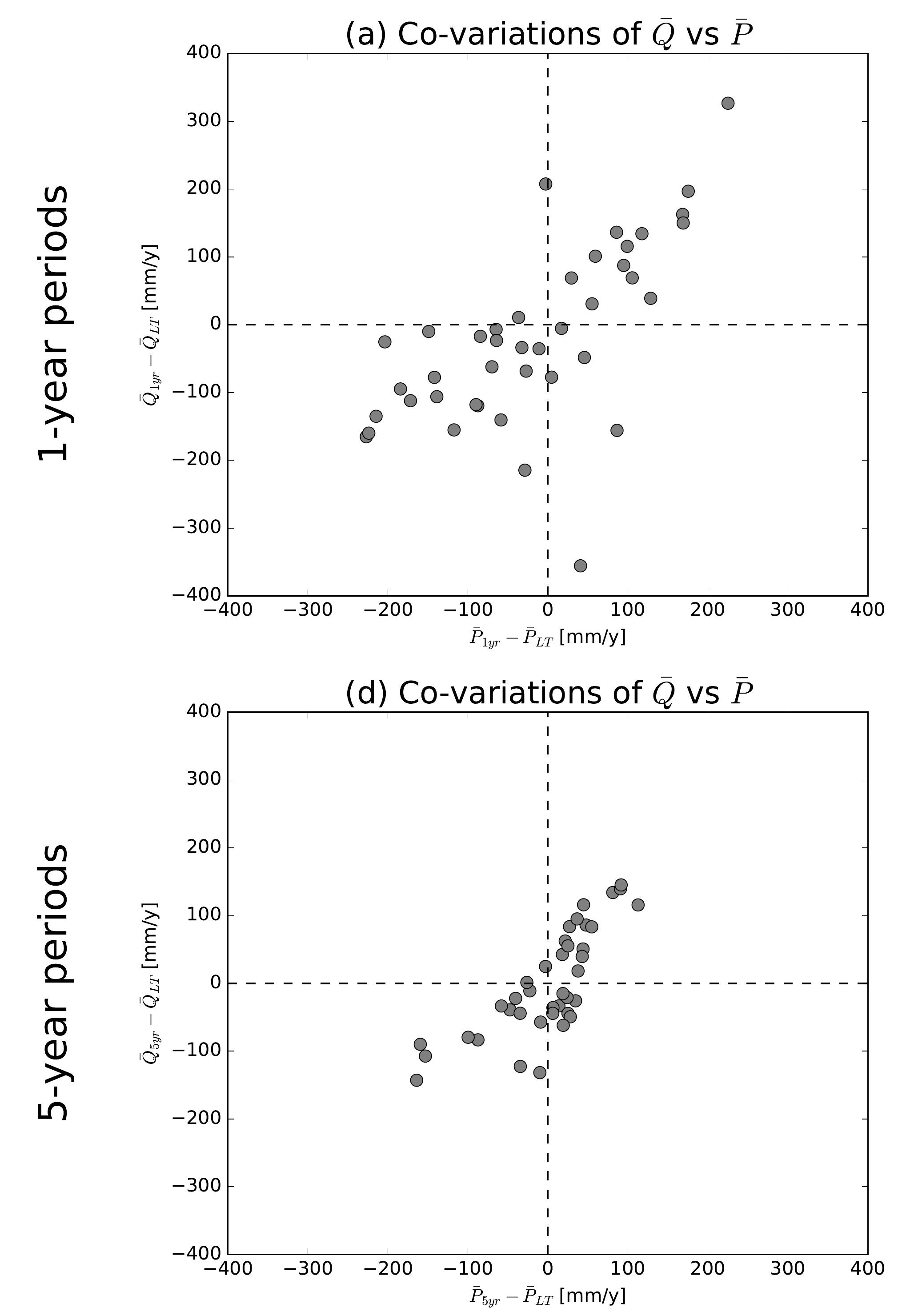

(g) Co-variations of $\bar{Q}$ vs $\bar{P}$
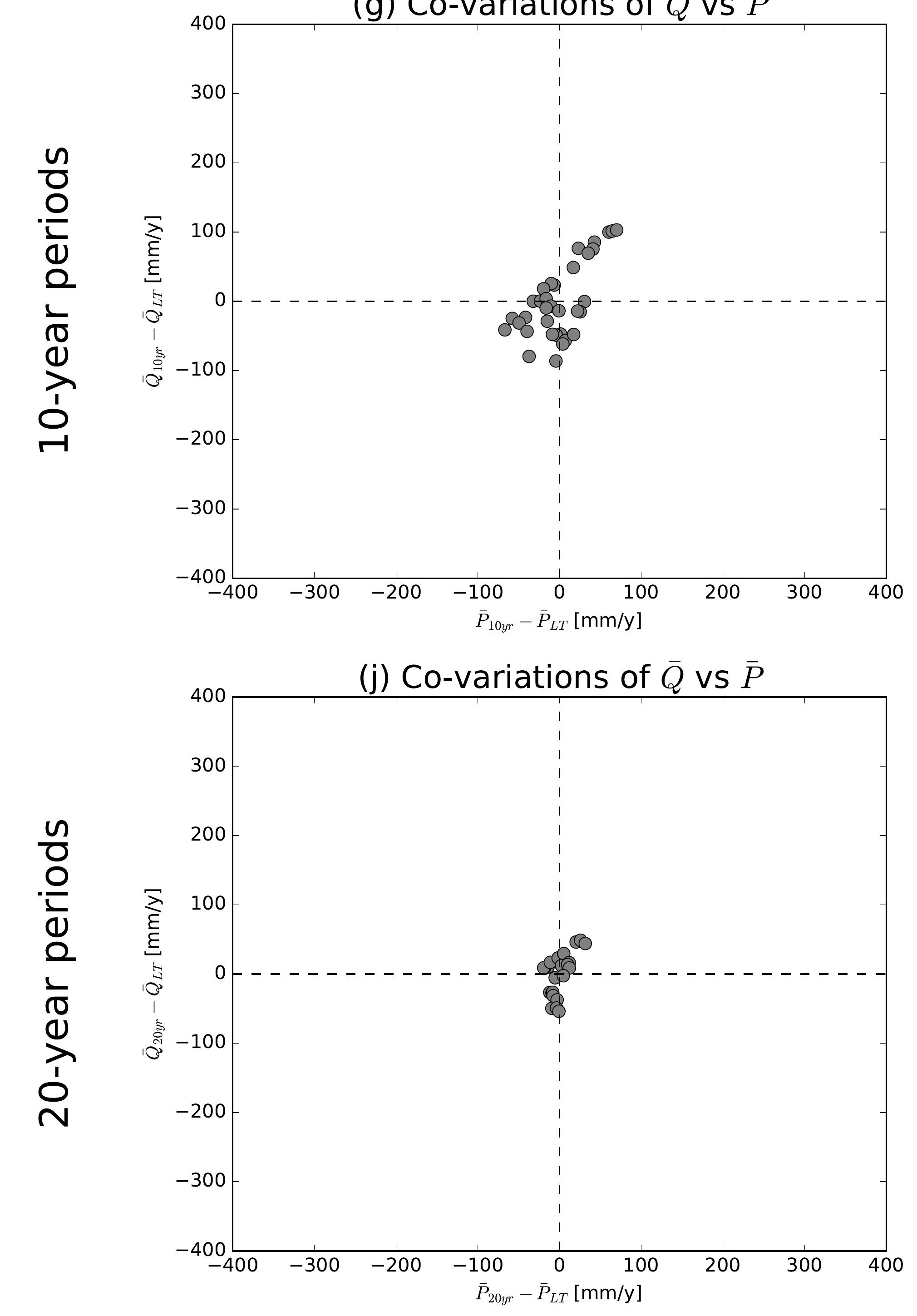

(b) Co-variations of $\bar{Q}$ vs $\overline{E 0}$

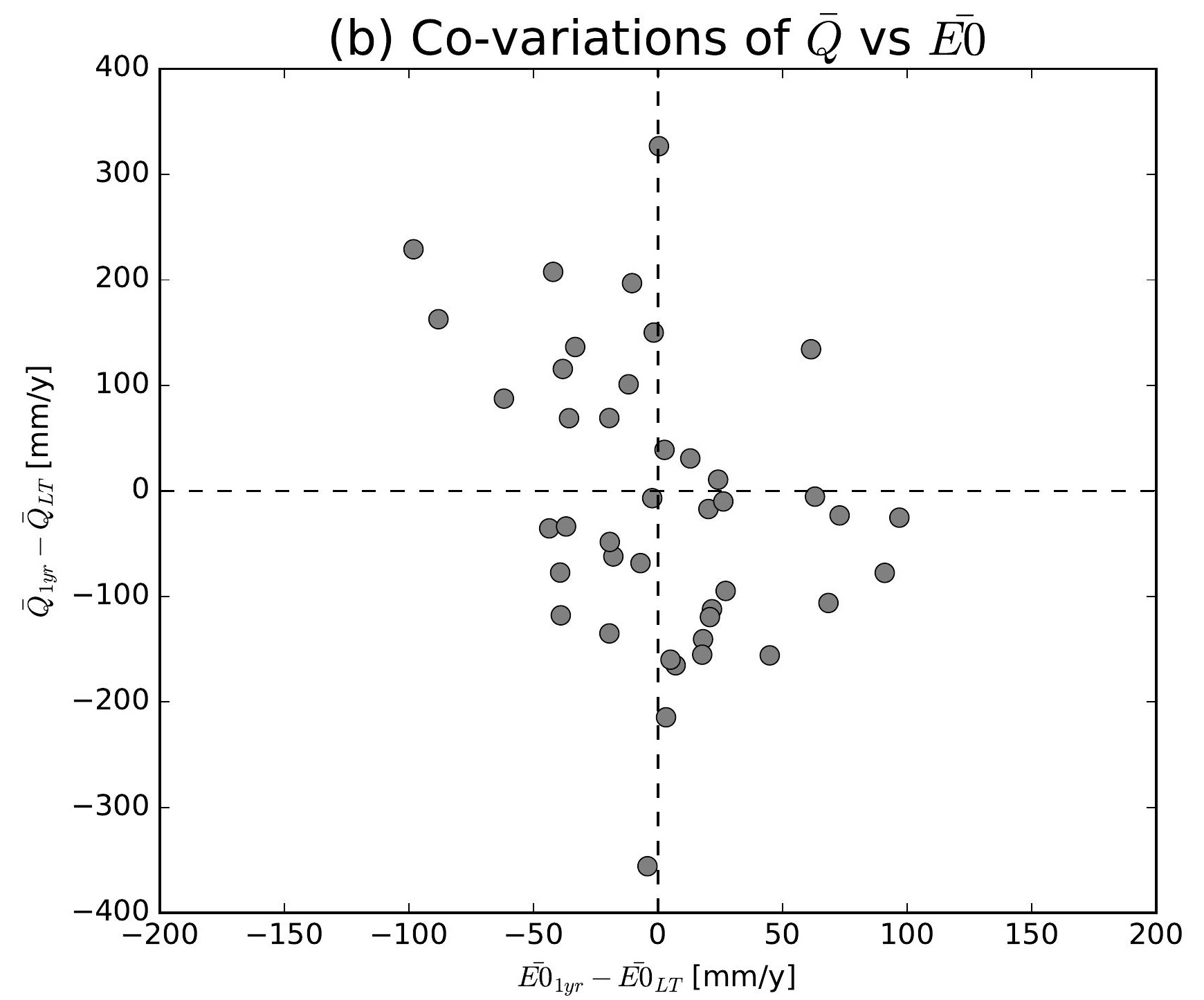

(e) Co-variations of $\bar{Q}$ vs $\overline{E 0}$

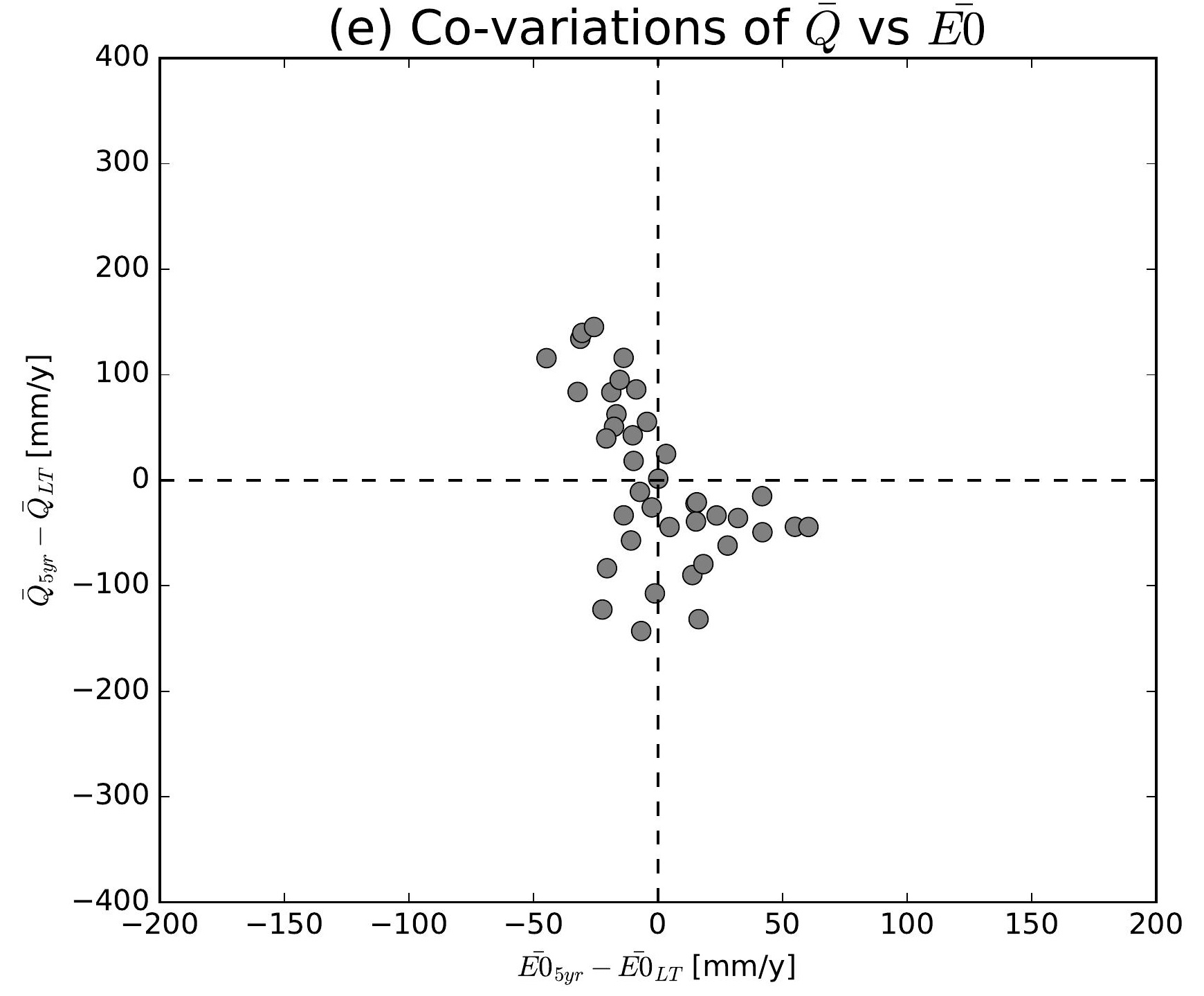

(h) Co-variations of $\bar{Q}$ vs $\overline{E 0}$

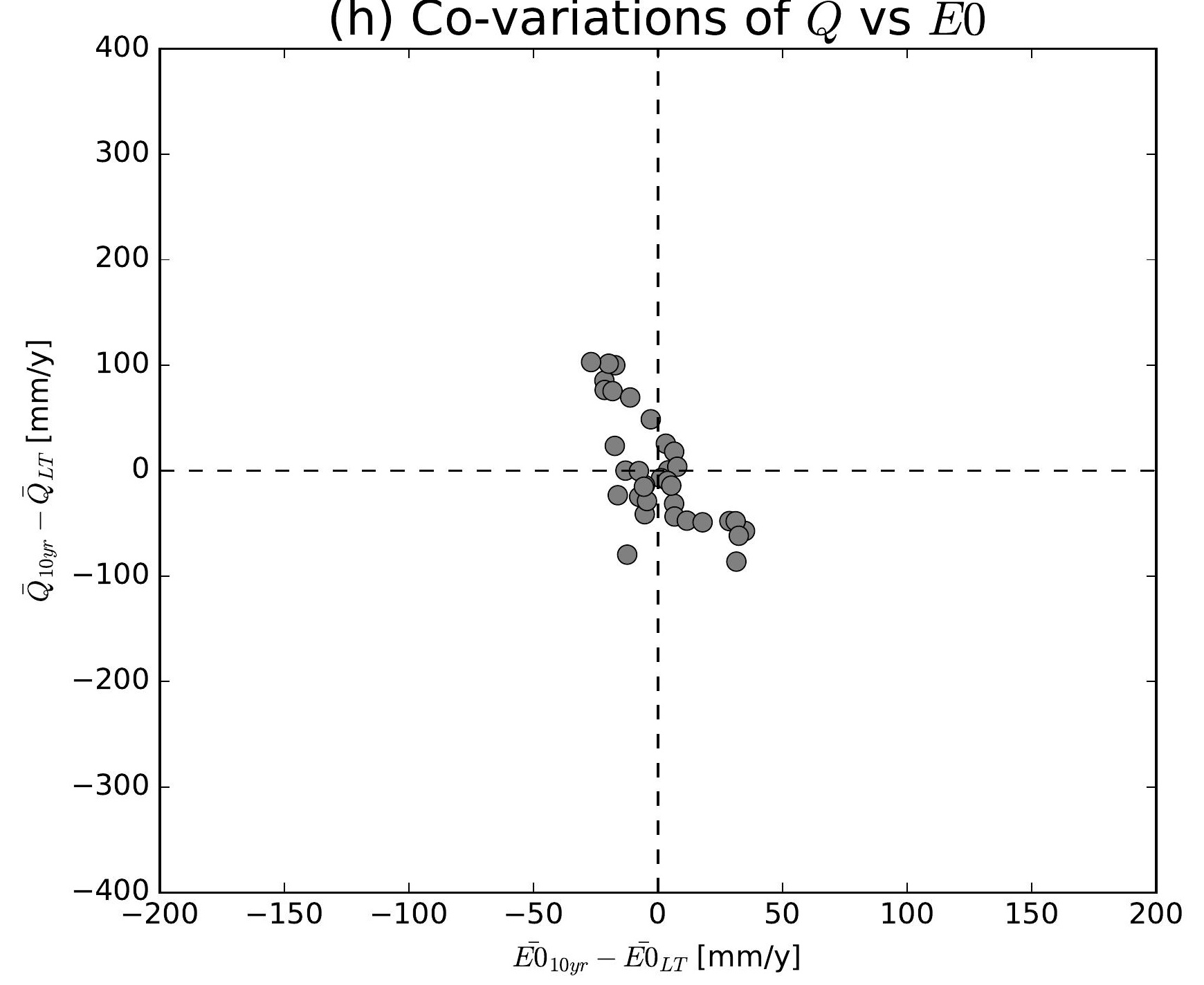

(k) Co-variations of $\bar{Q}$ vs $\overline{E 0}$

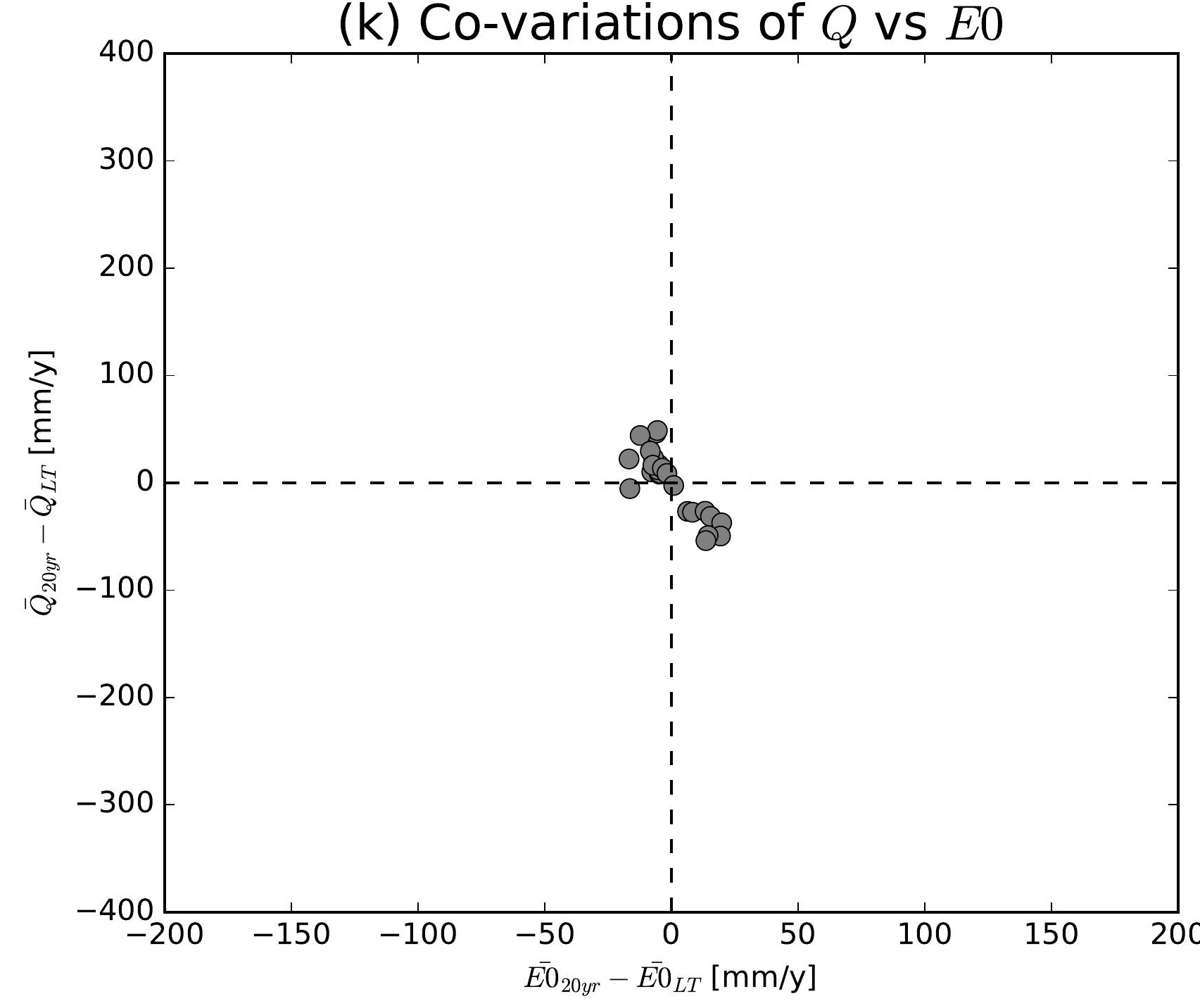

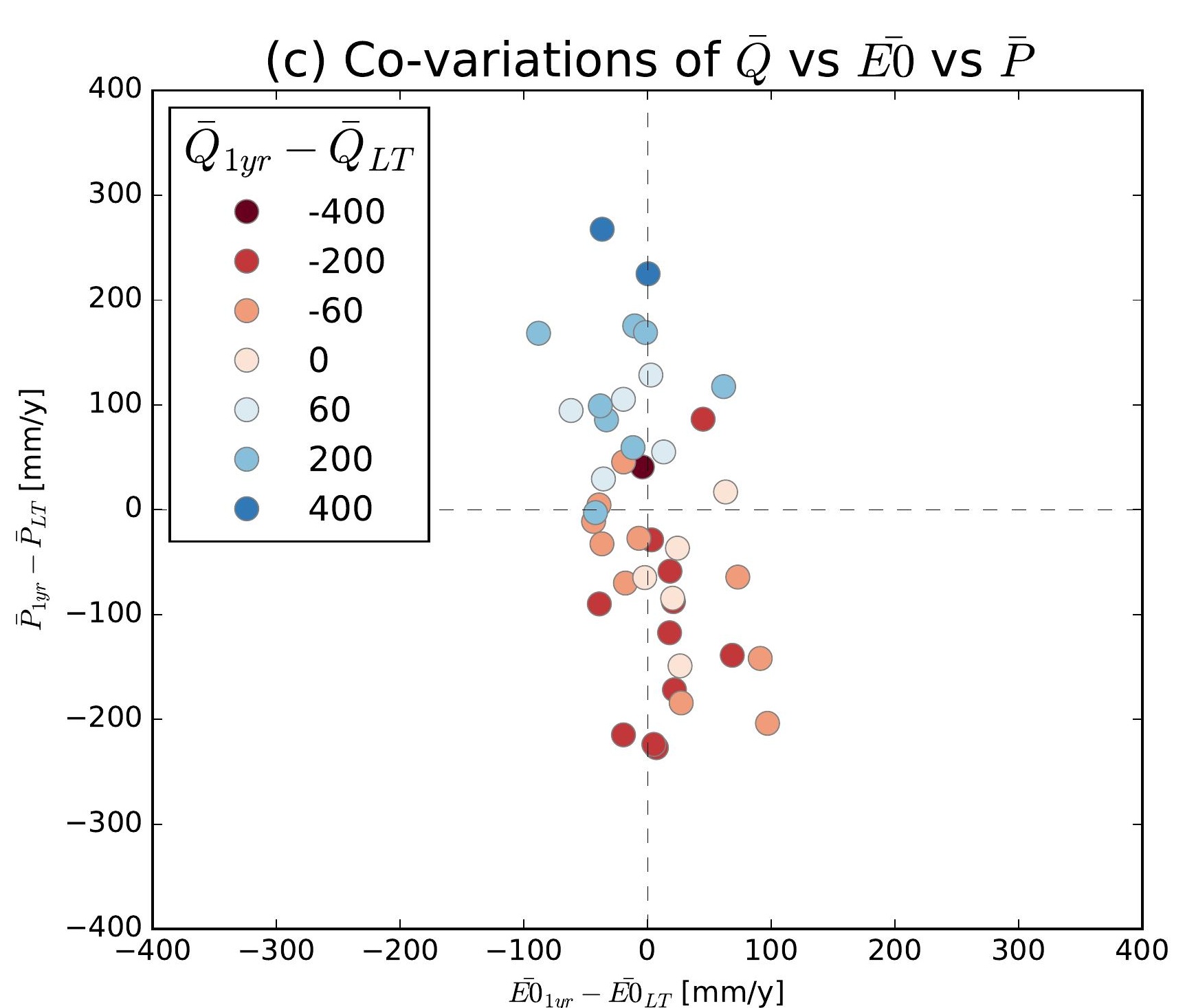
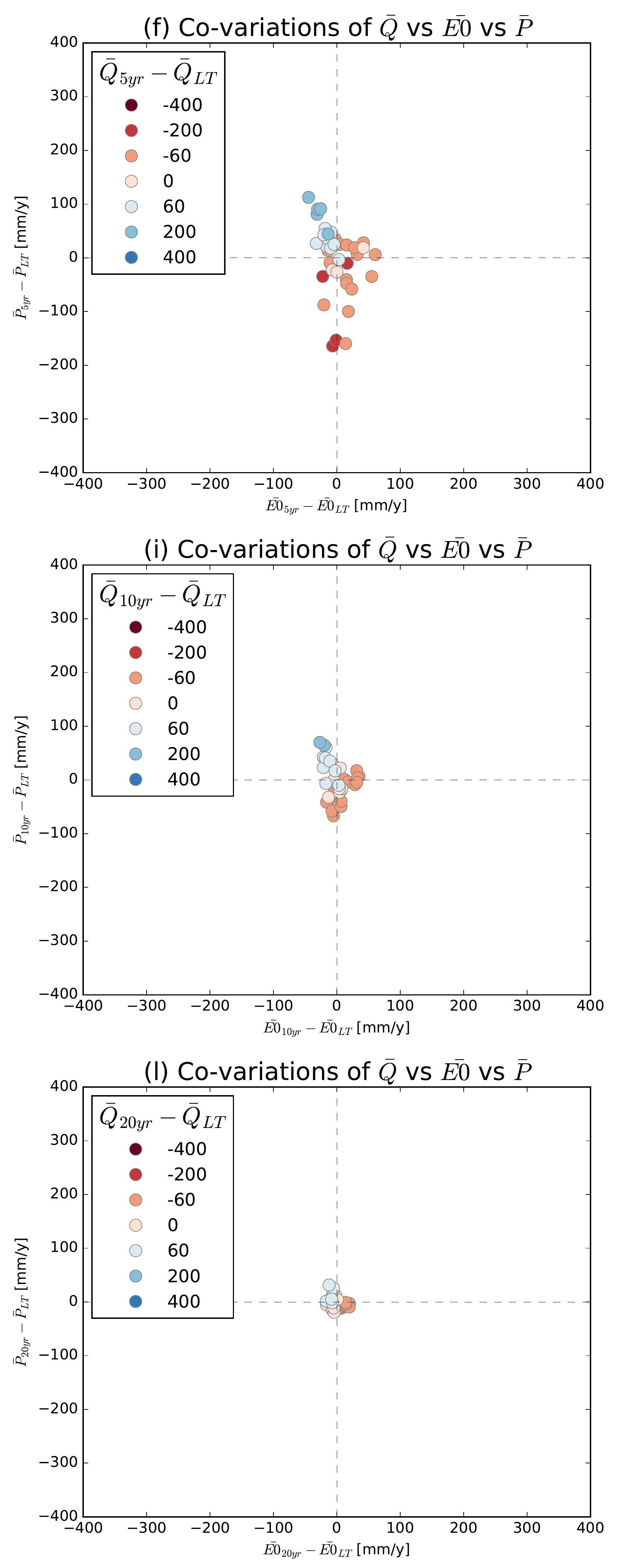

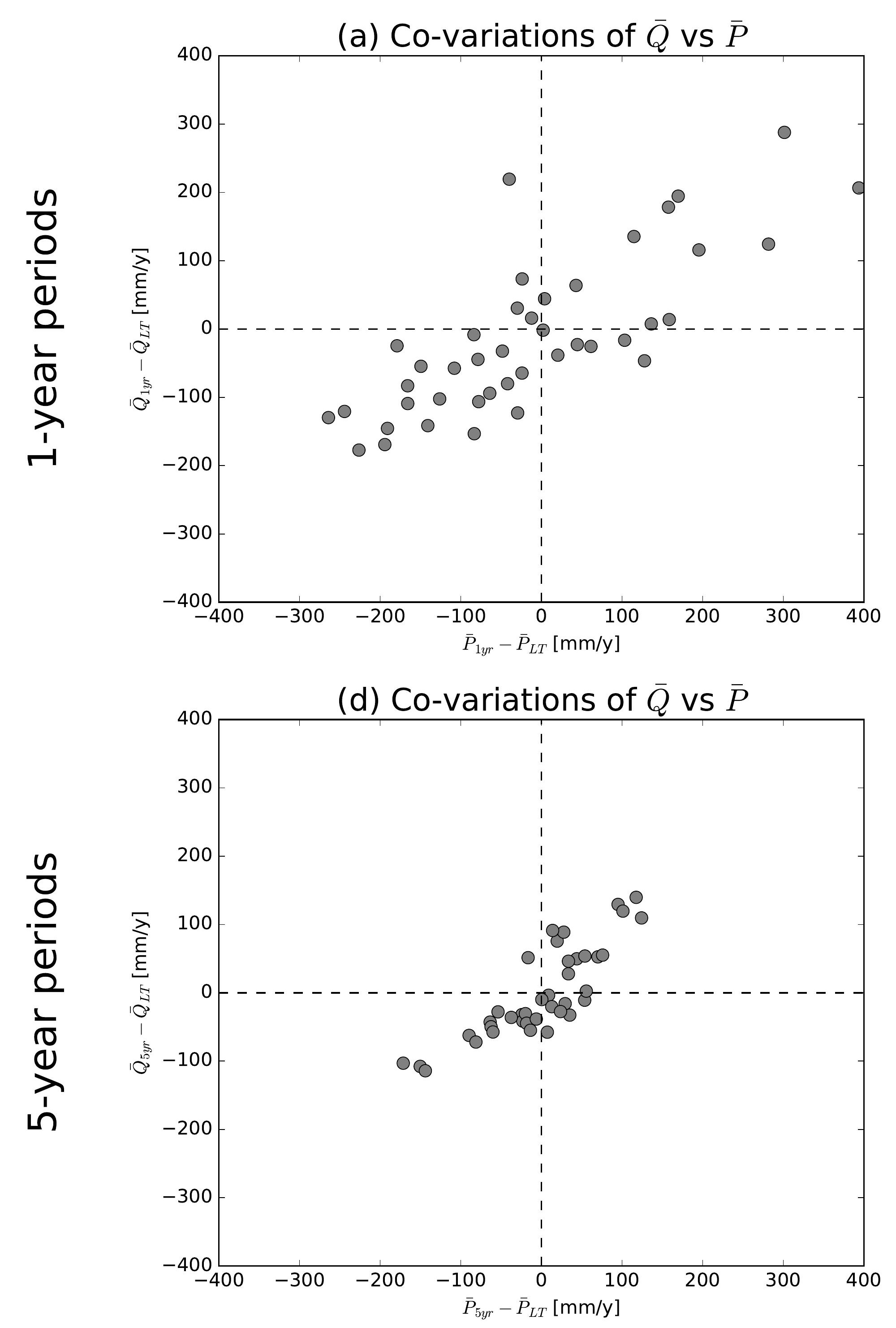

(g) Co-variations of $\bar{Q}$ vs $\bar{P}$

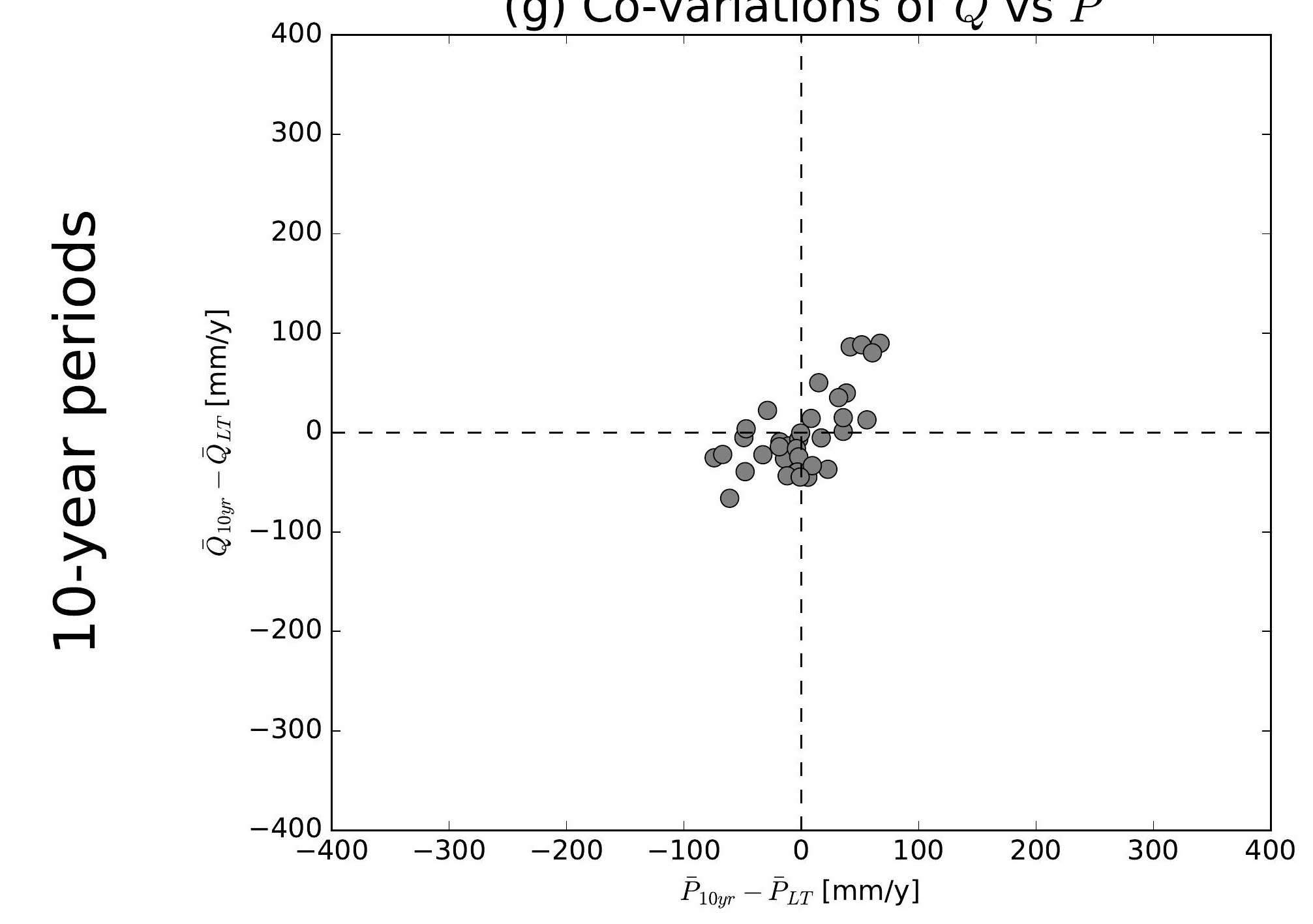

(j) Co-variations of $\bar{Q}$ vs $\bar{P}$

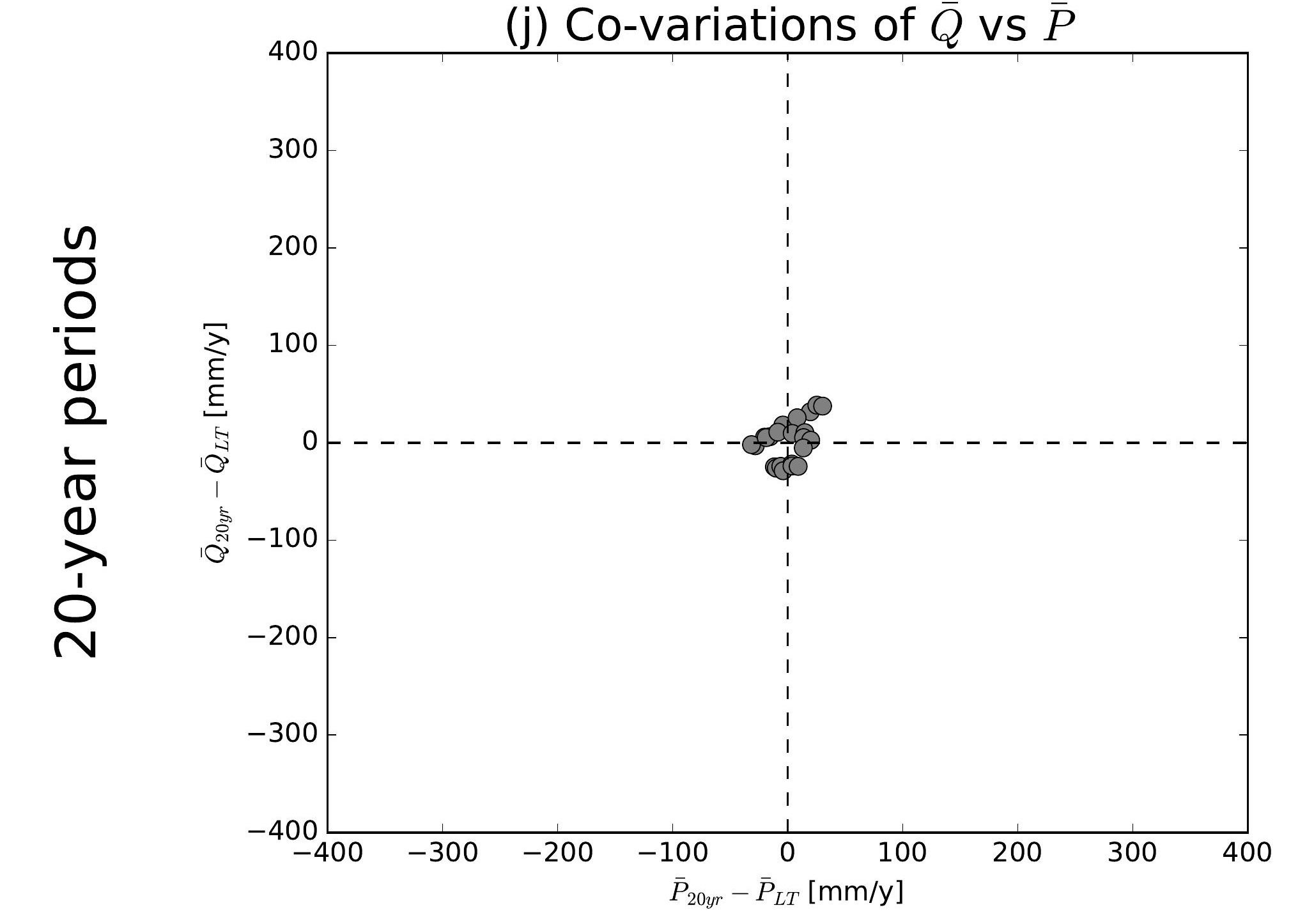

(b) Co-variations of $\bar{Q}$ vs $\overline{E 0}$

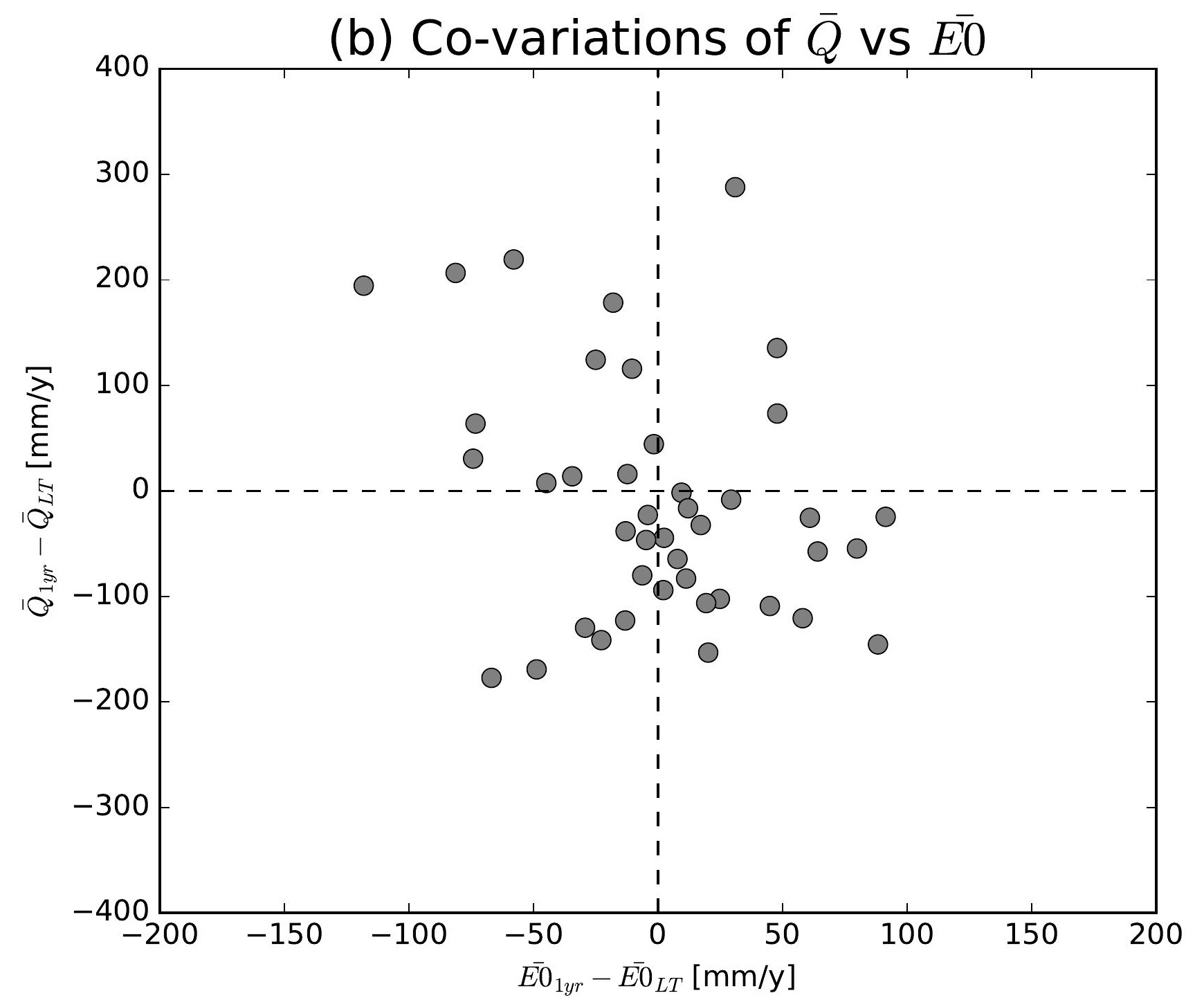

(e) Co-variations of $\bar{Q}$ vs $\overline{E 0}$

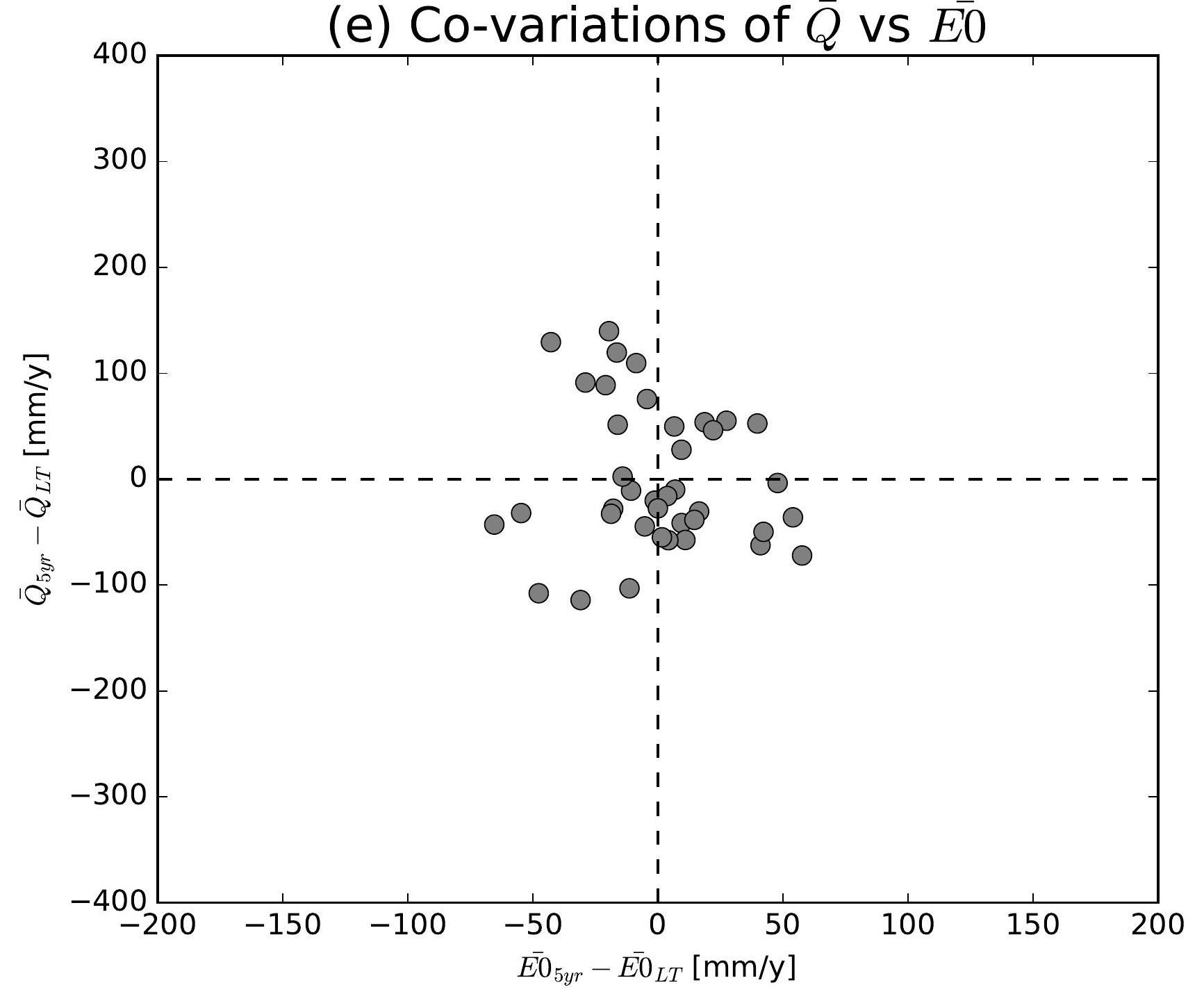

(h) Co-variations of $\bar{Q}$ vs $\overline{E 0}$

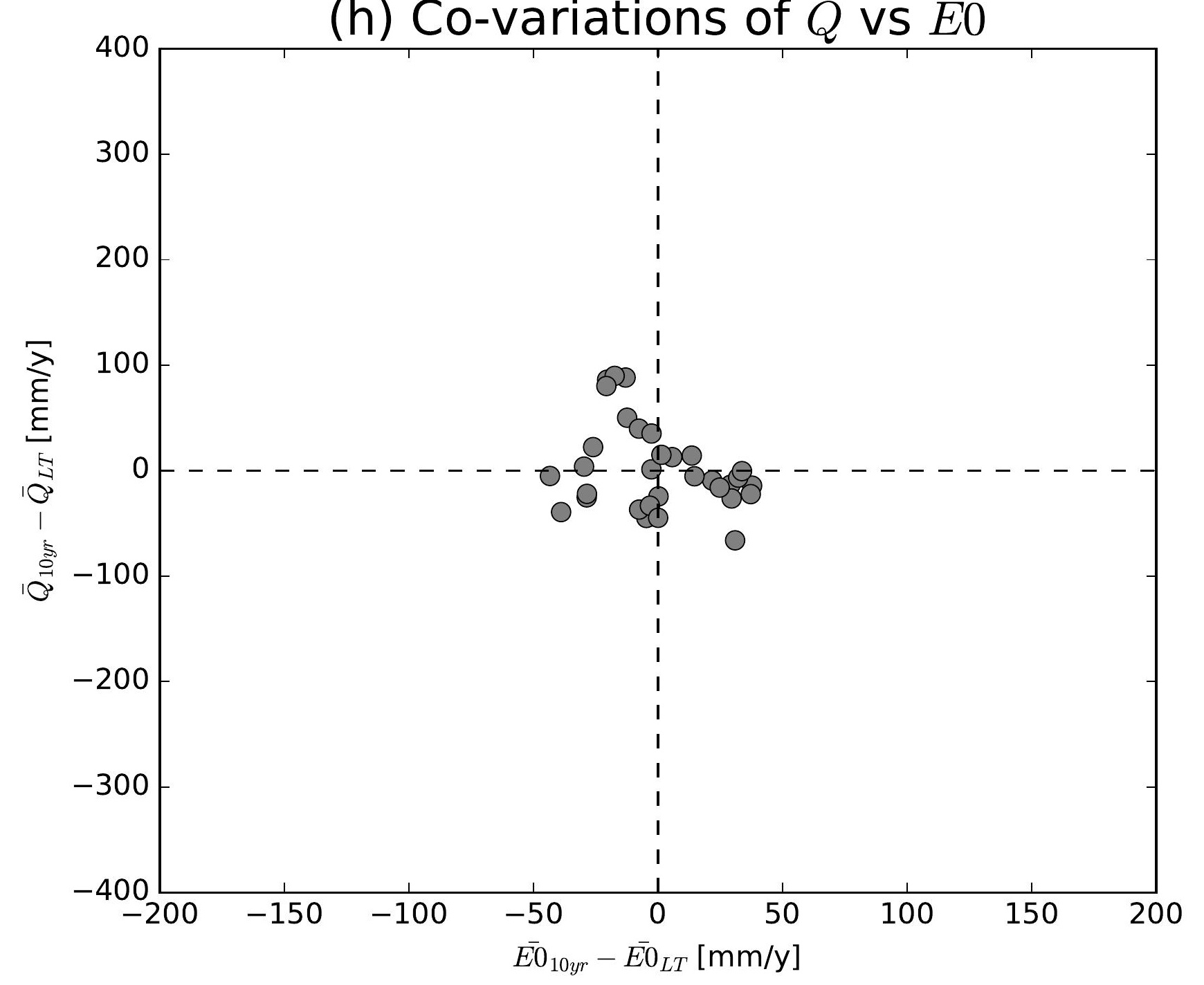

(k) Co-variations of $\bar{Q}$ vs $\overline{E 0}$

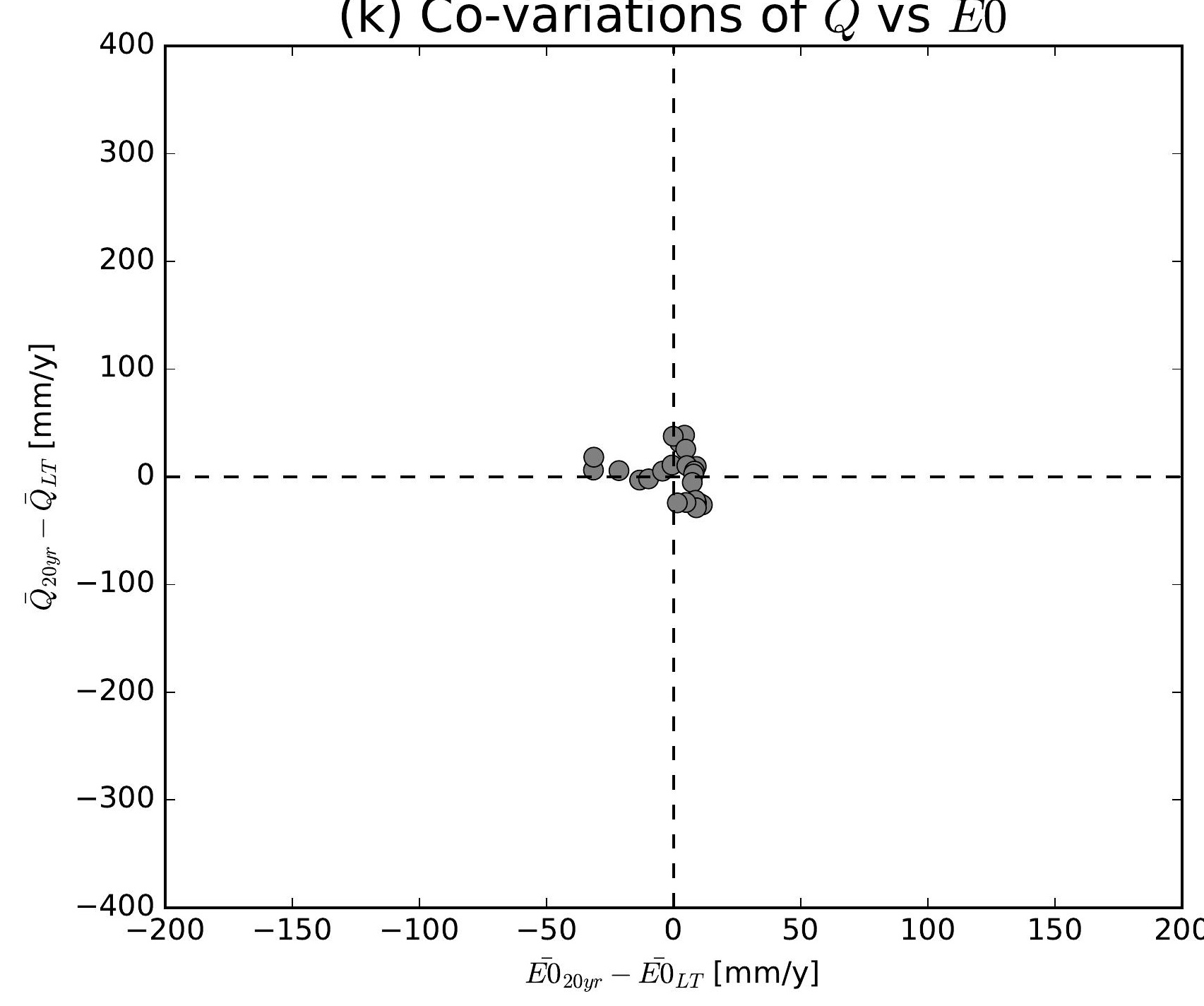

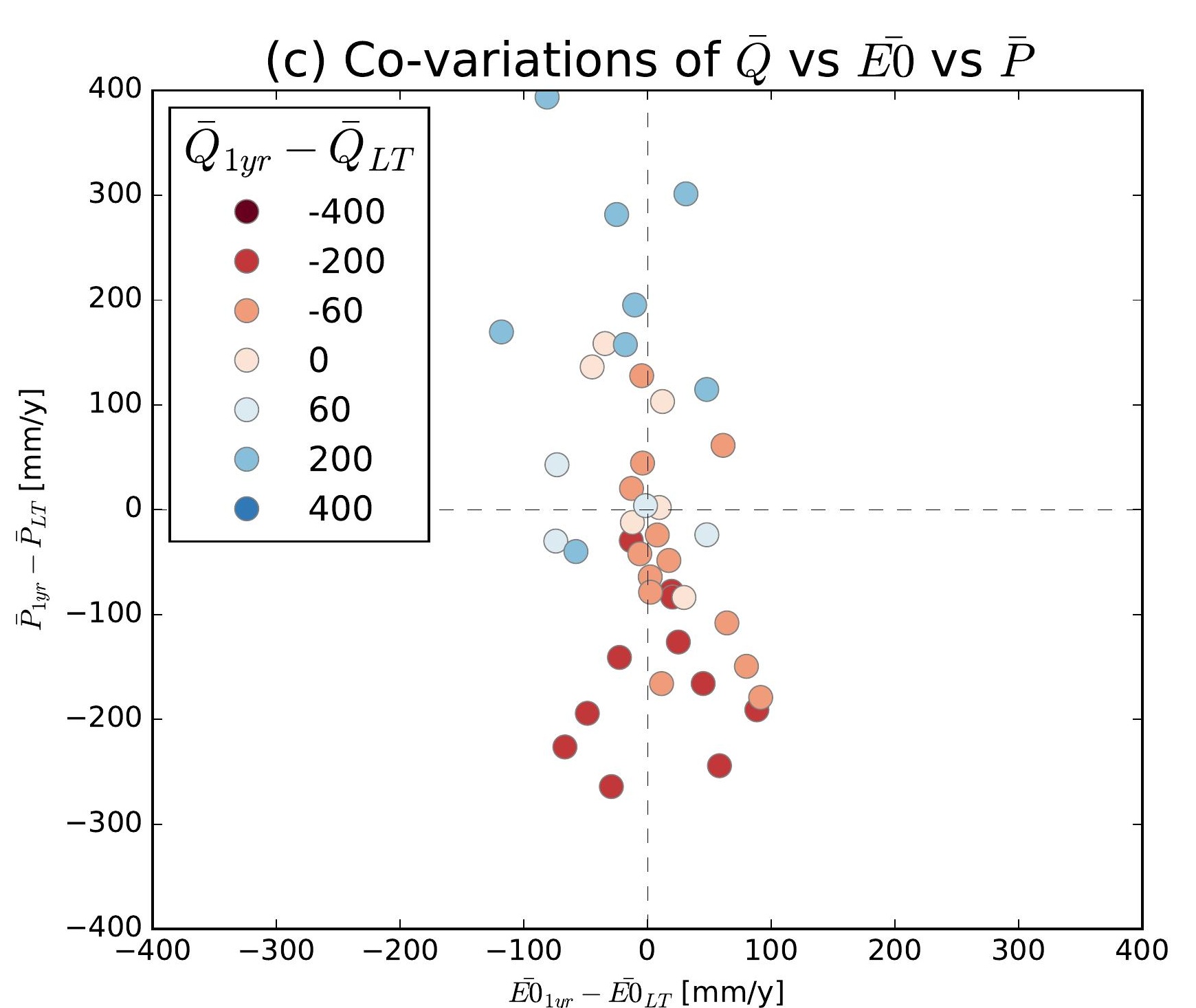
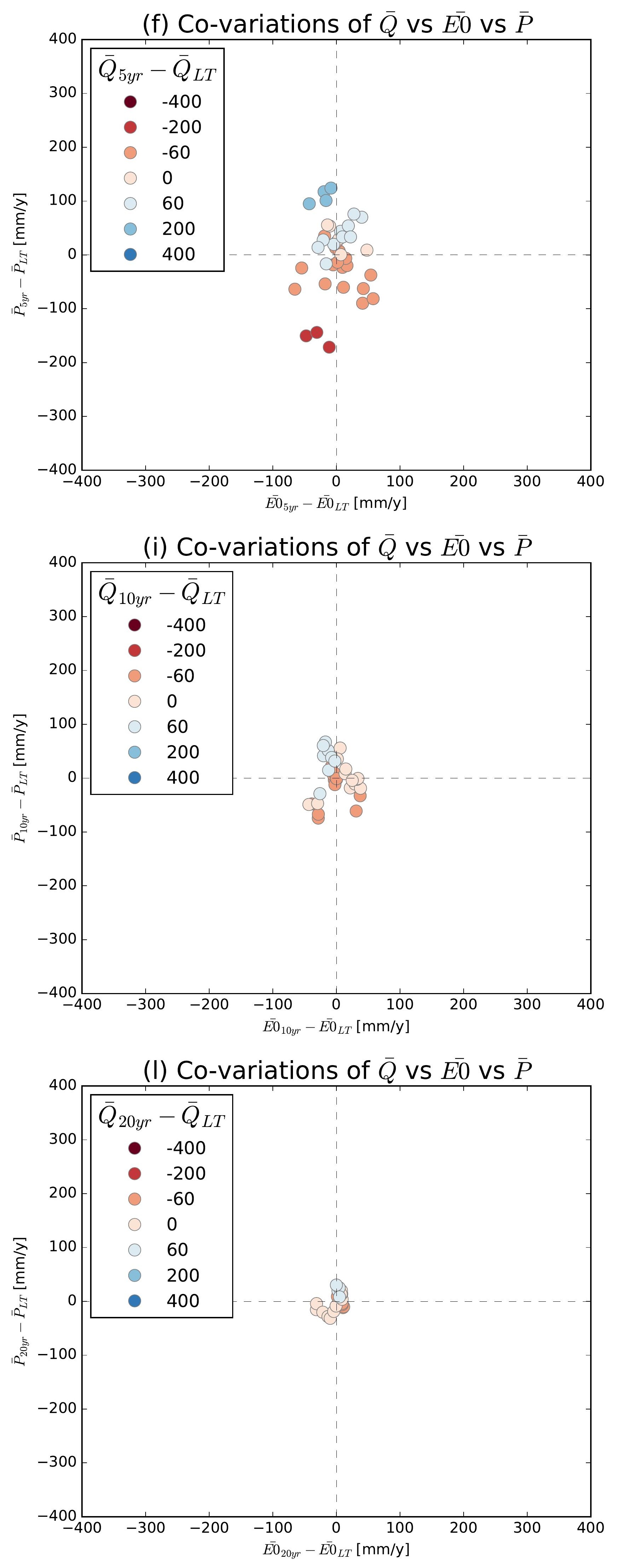

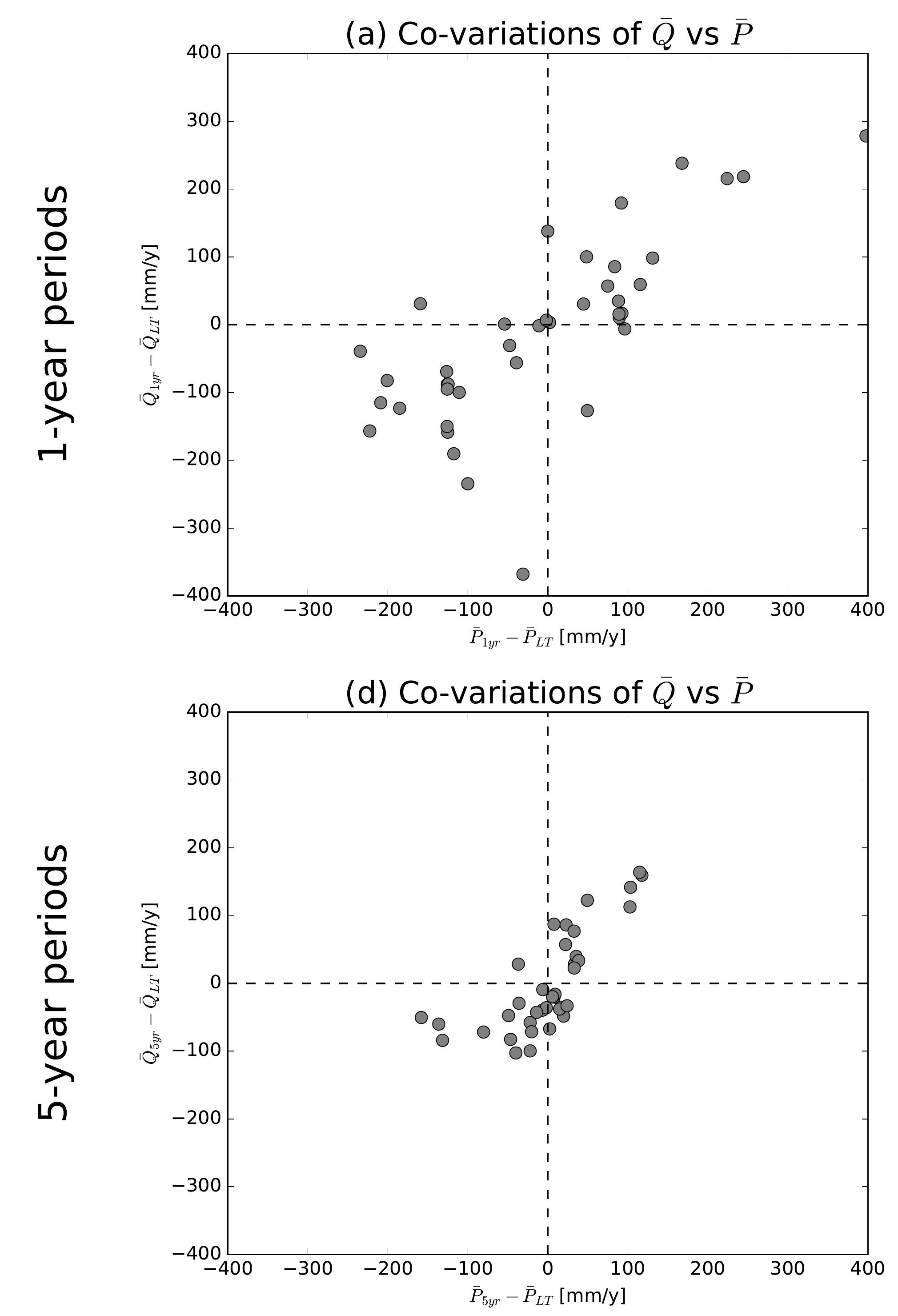

(g) Co-variations of $\bar{Q}$ vs $\bar{P}$
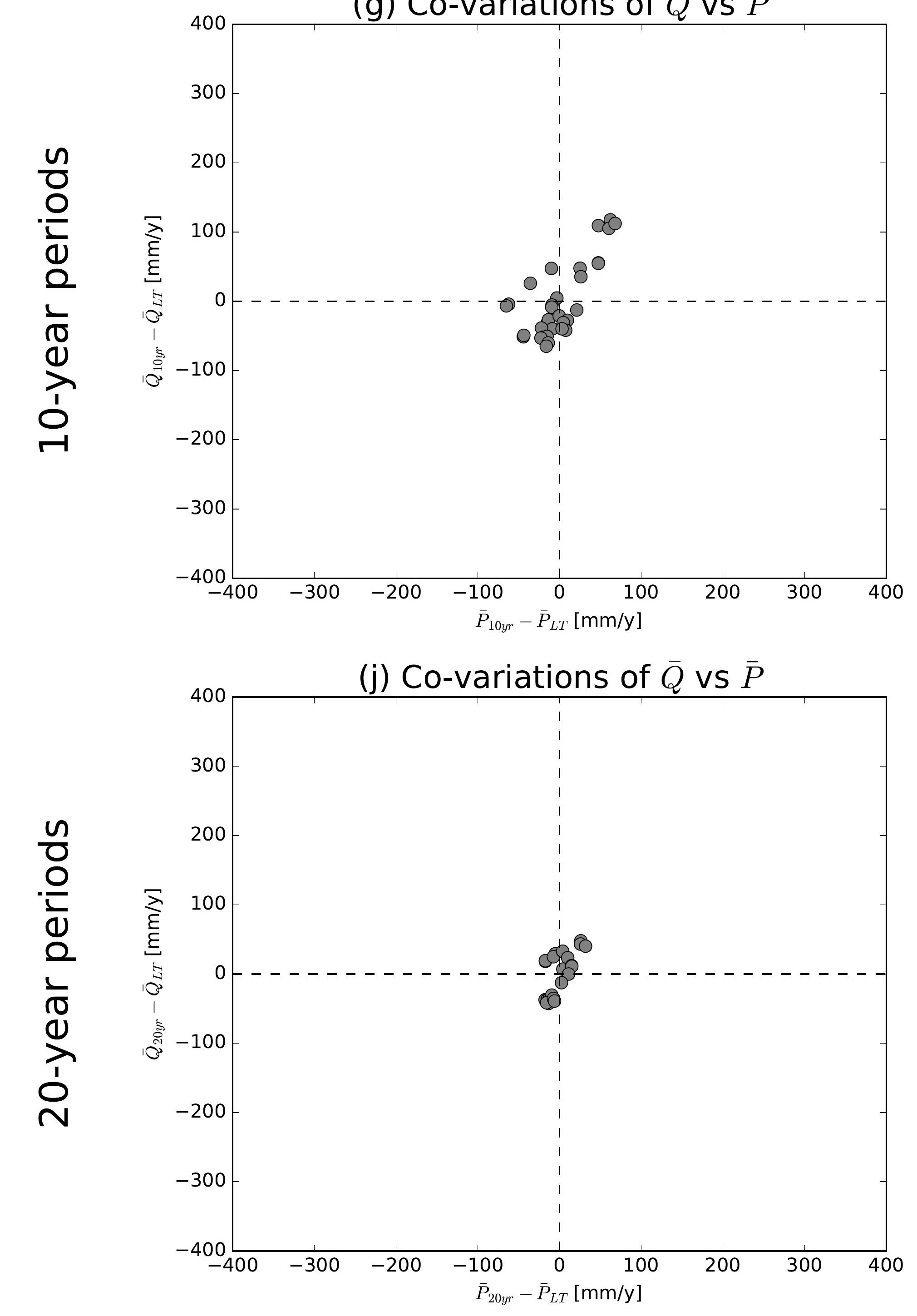

(b) Co-variations of $\bar{Q}$ vs $\overline{E 0}$

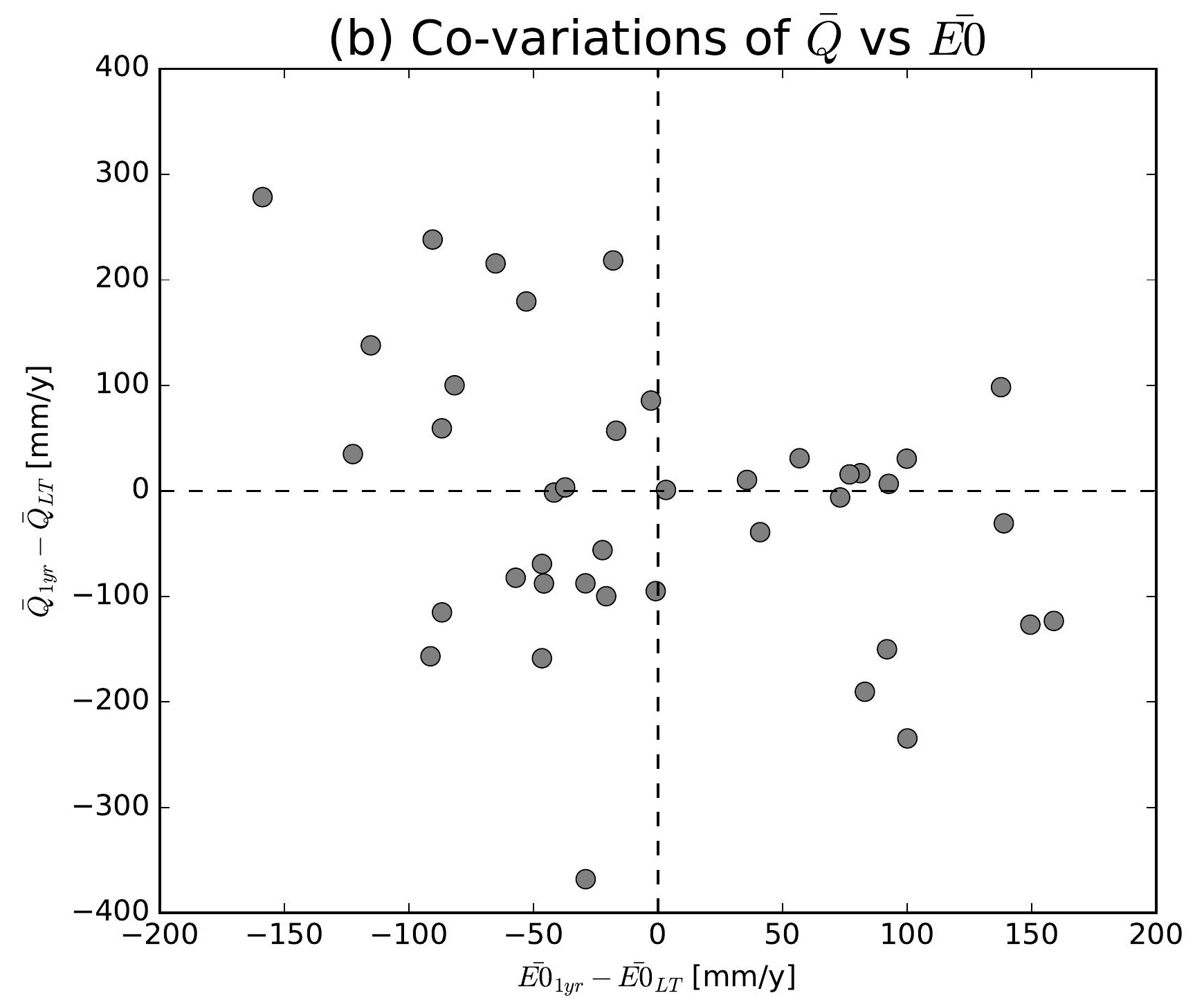

(e) Co-variations of $\bar{Q}$ vs $\overline{E 0}$

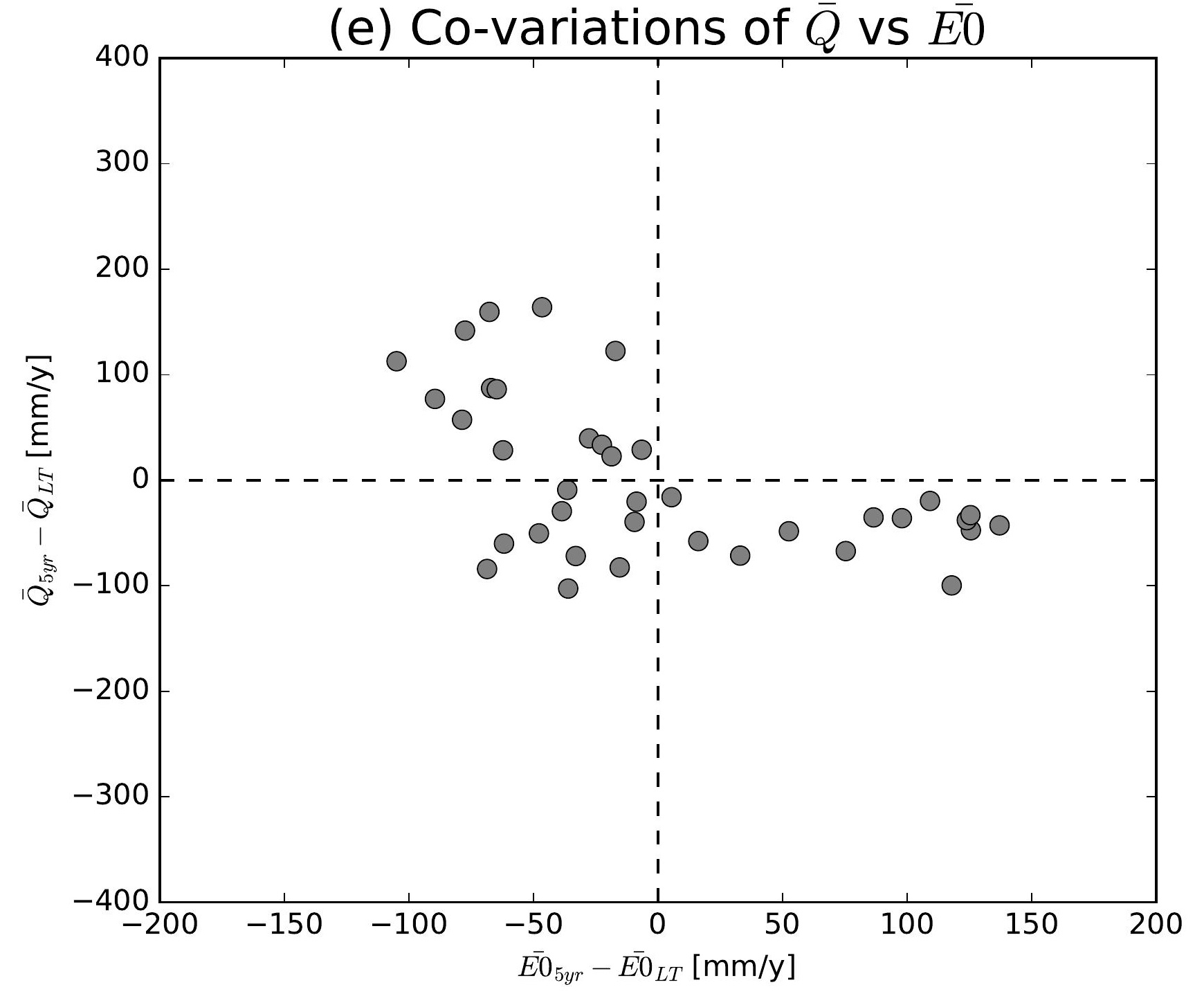

(h) Co-variations of $\bar{Q}$ vs $\overline{E 0}$

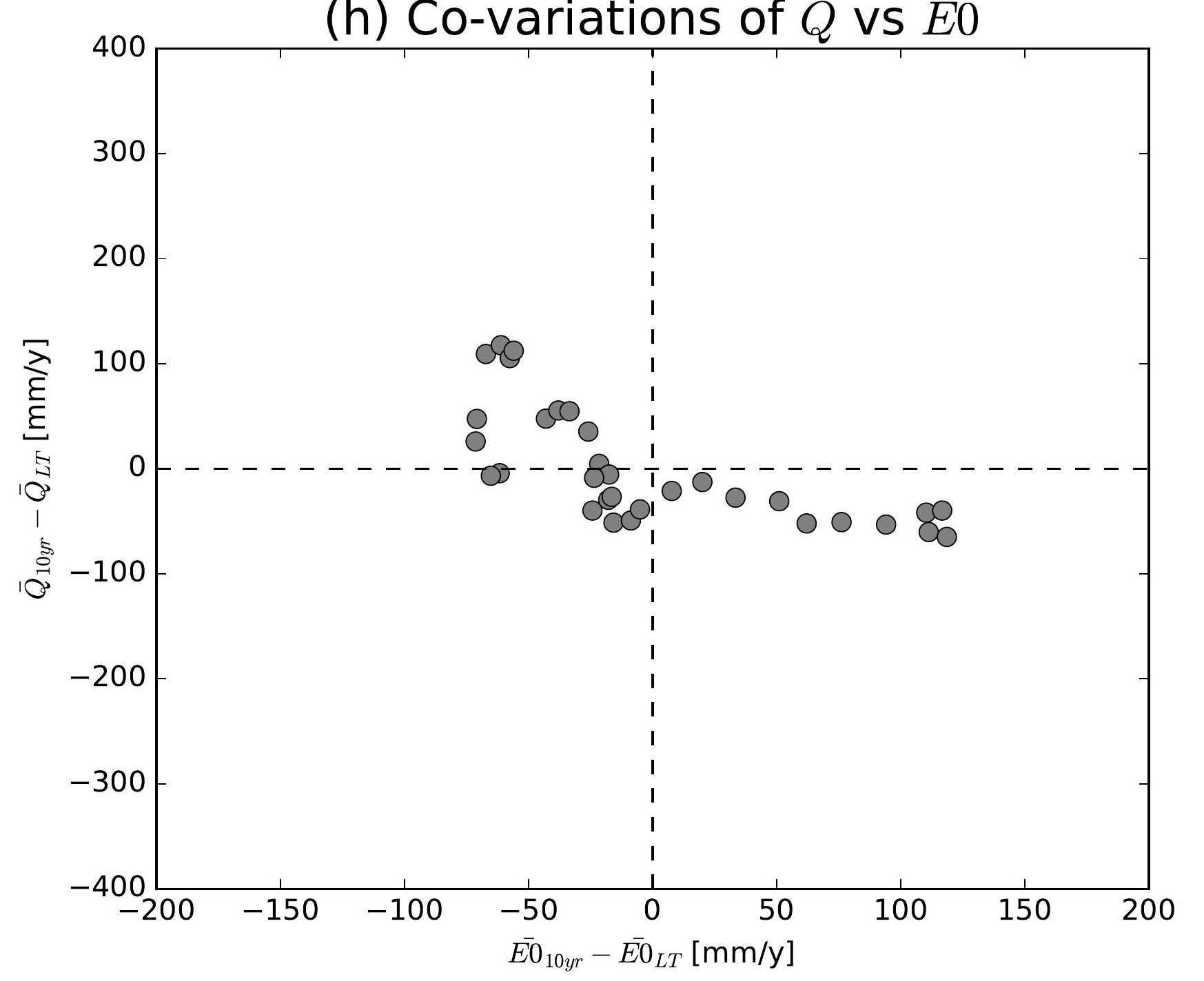

(k) Co-variations of $\bar{Q}$ vs $\overline{E 0}$

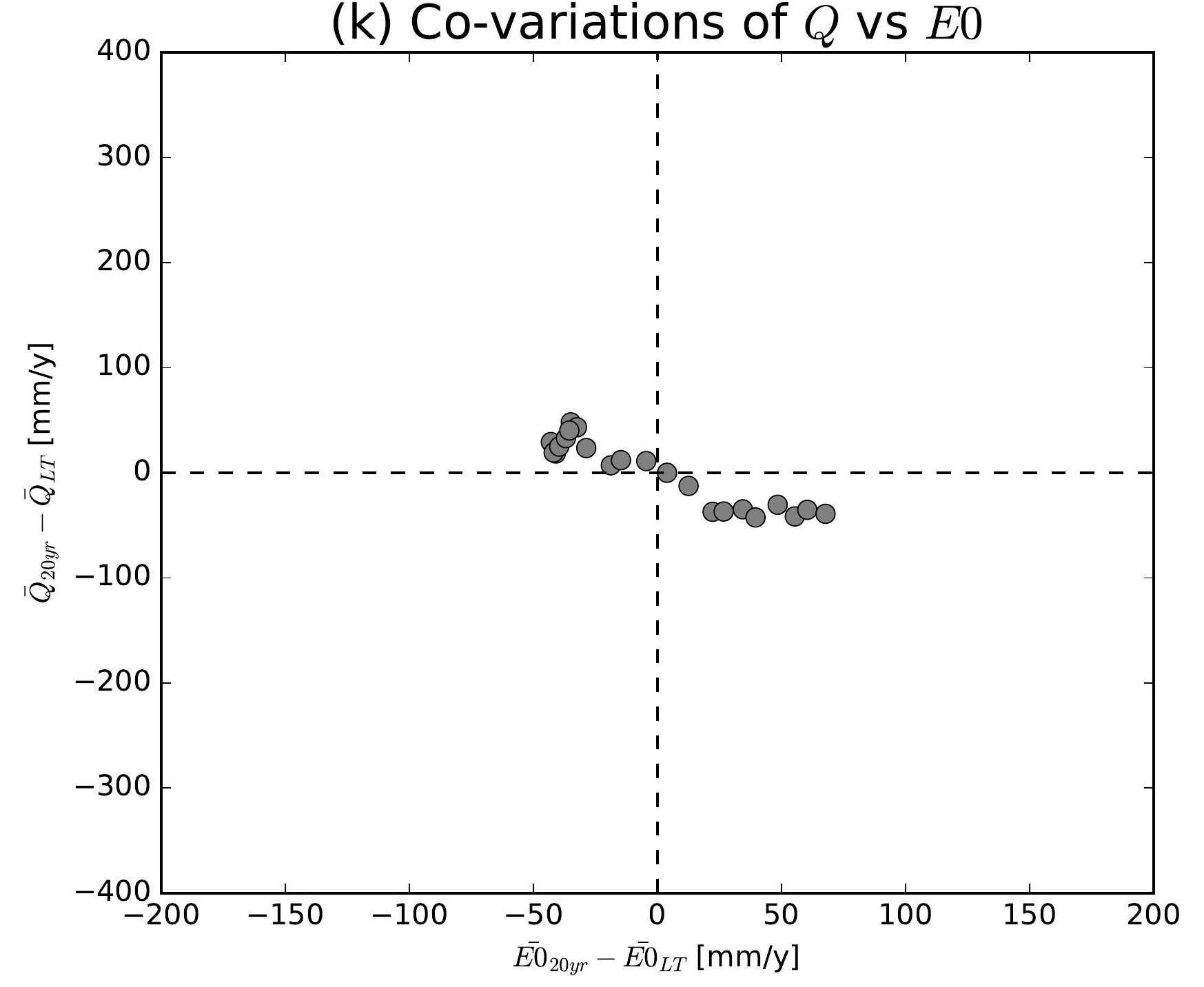

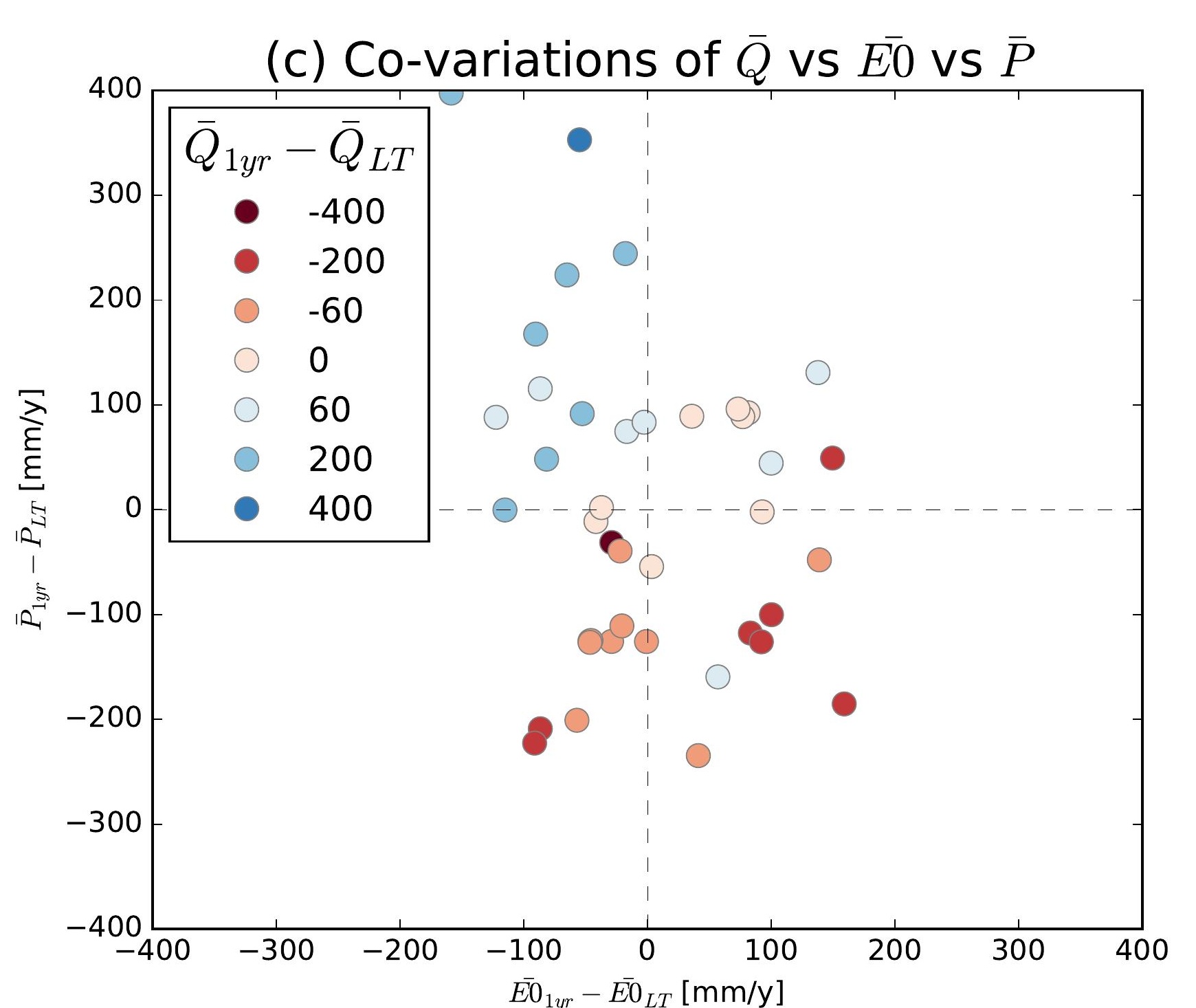
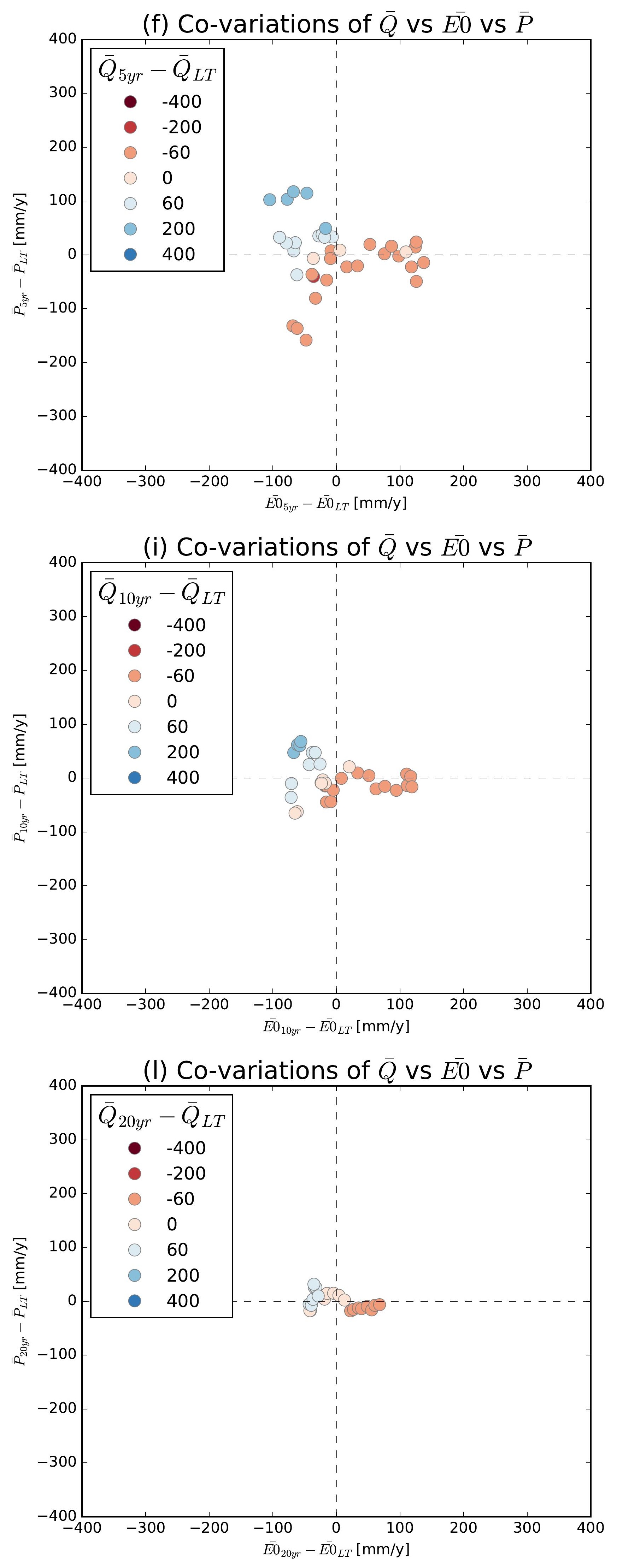

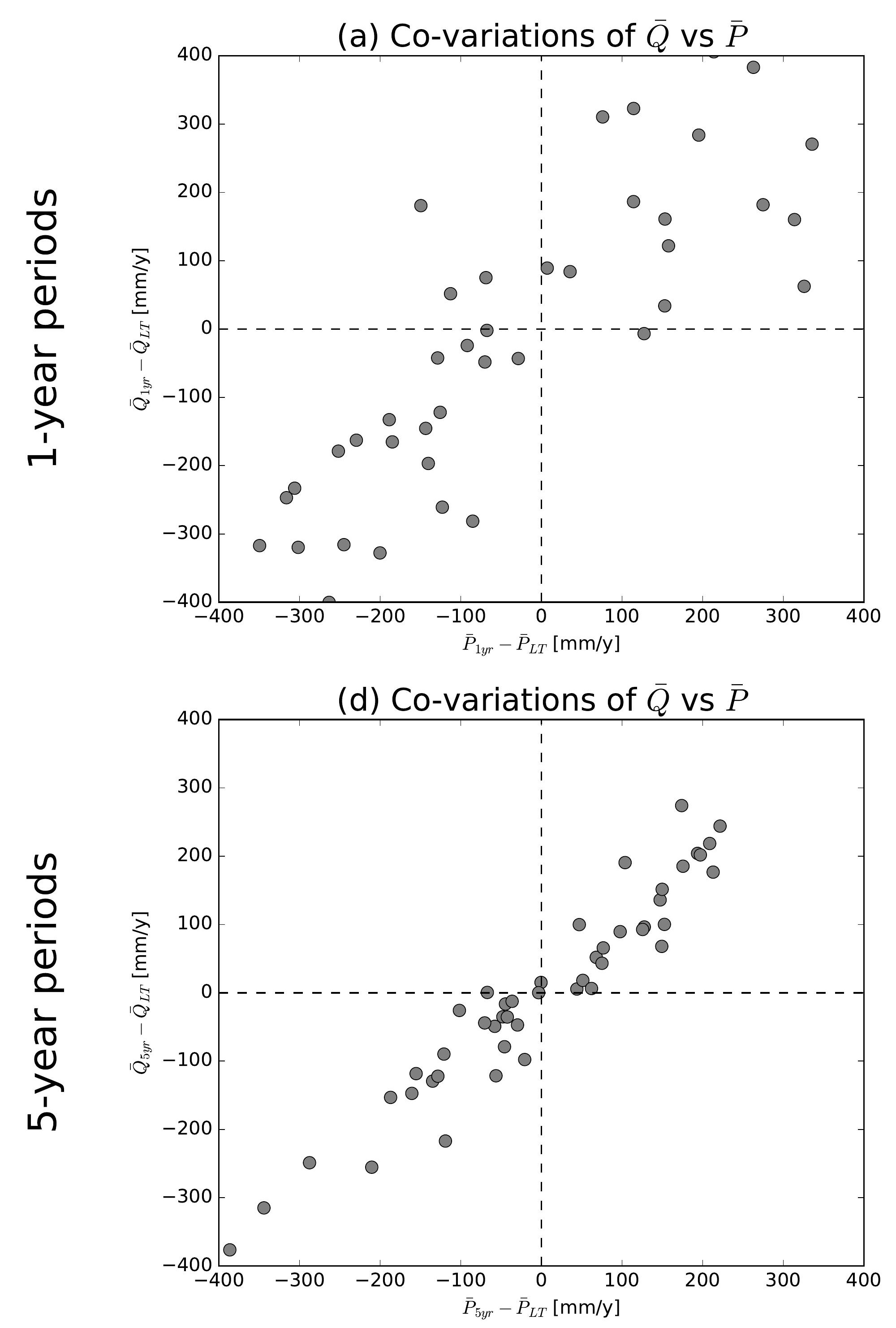

(g) Co-variations of $\bar{Q}$ vs $\bar{P}$

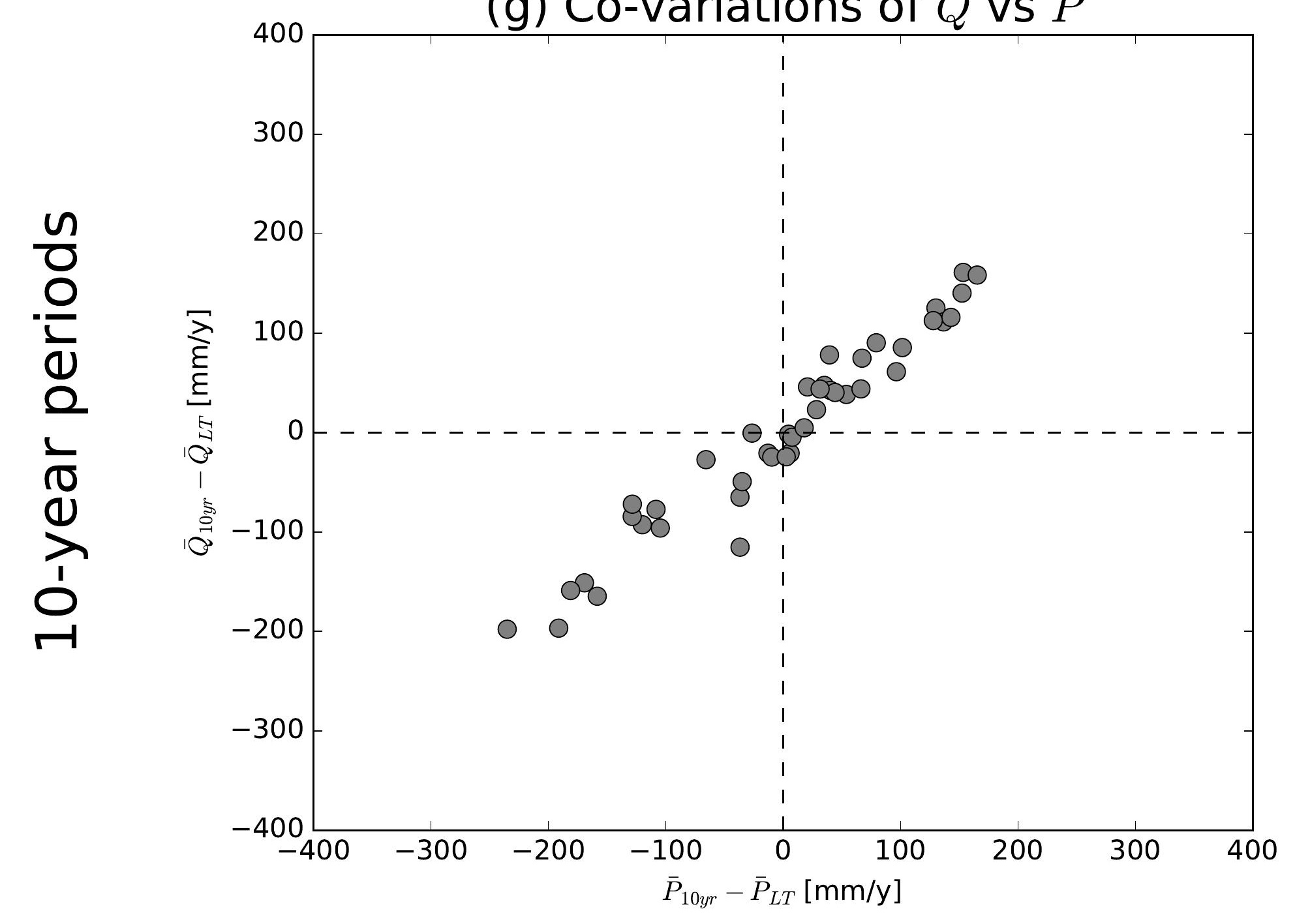

(j) Co-variations of $\bar{Q}$ vs $\bar{P}$

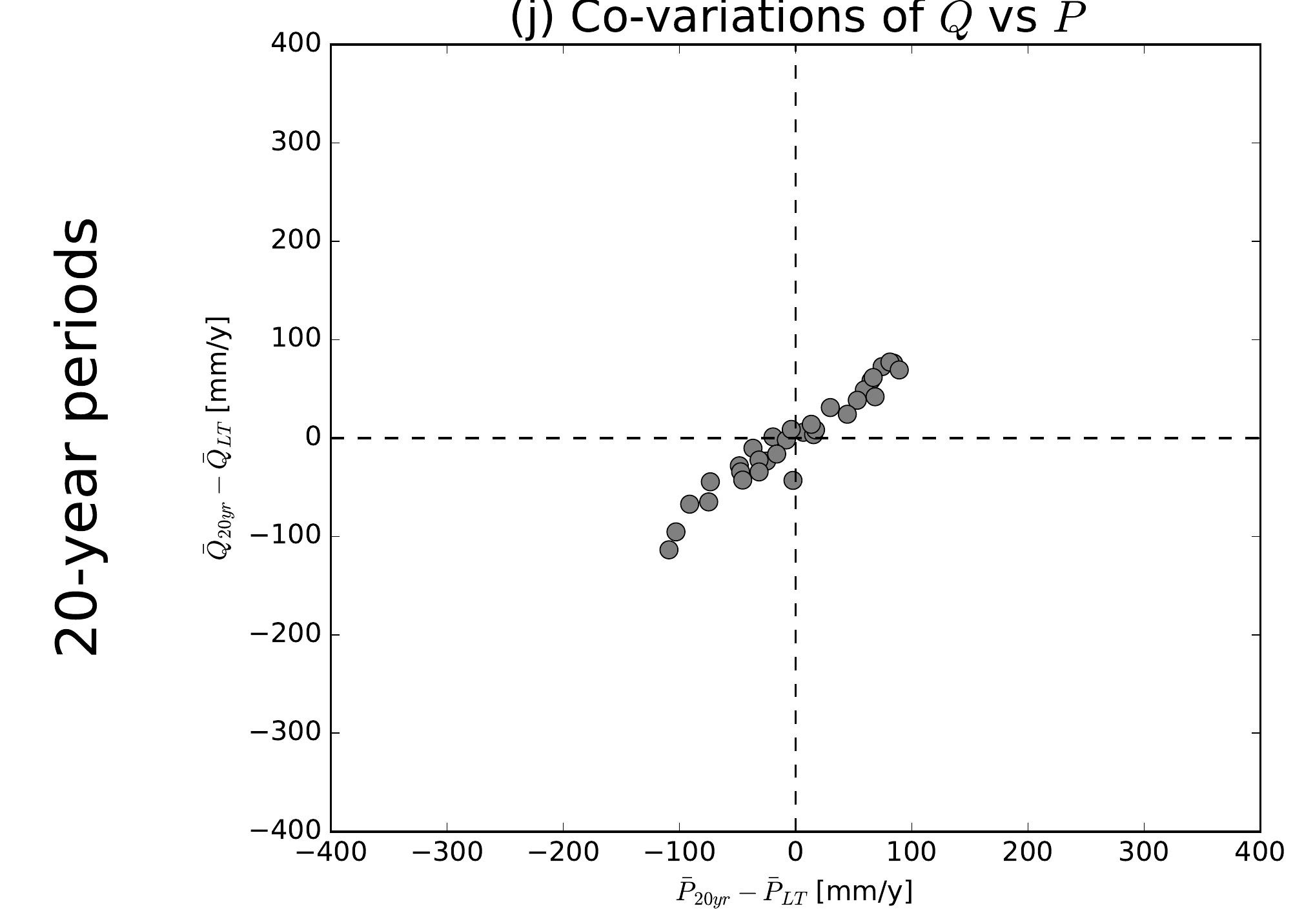

(b) Co-variations of $\bar{Q}$ vs $\overline{E 0}$

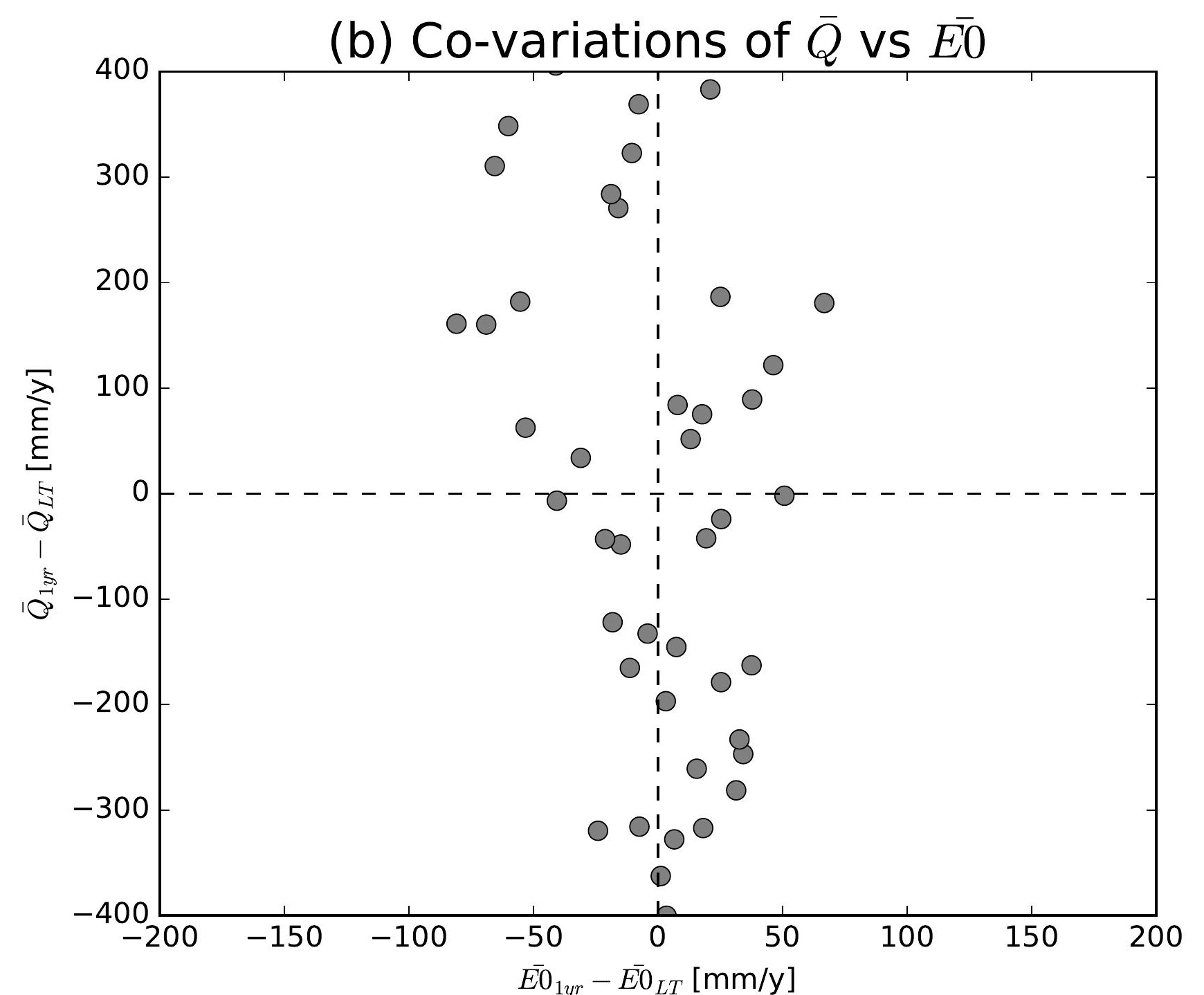

(e) Co-variations of $\bar{Q}$ vs $\overline{E 0}$

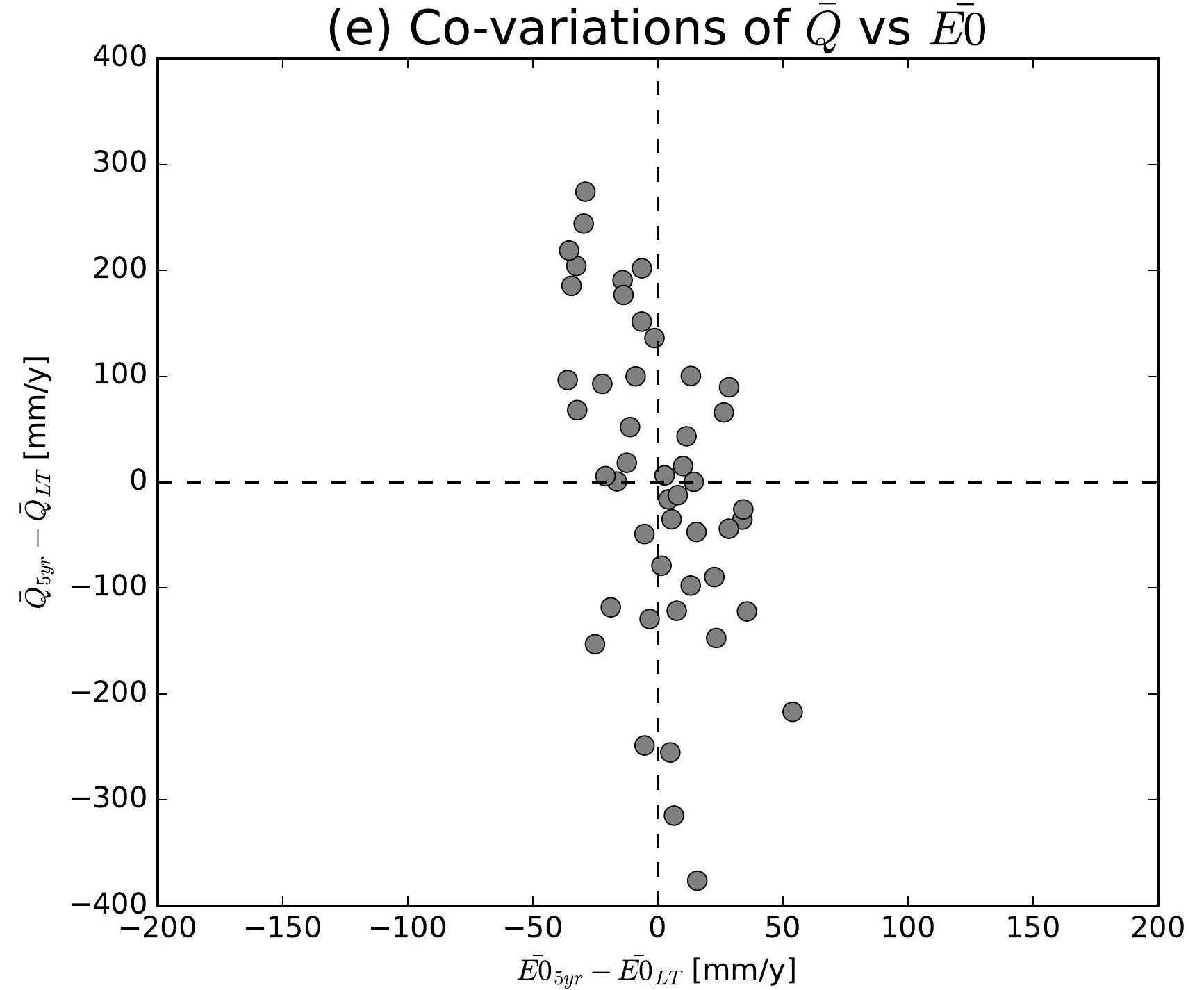

(h) Co-variations of $\bar{Q}$ vs $\overline{E 0}$

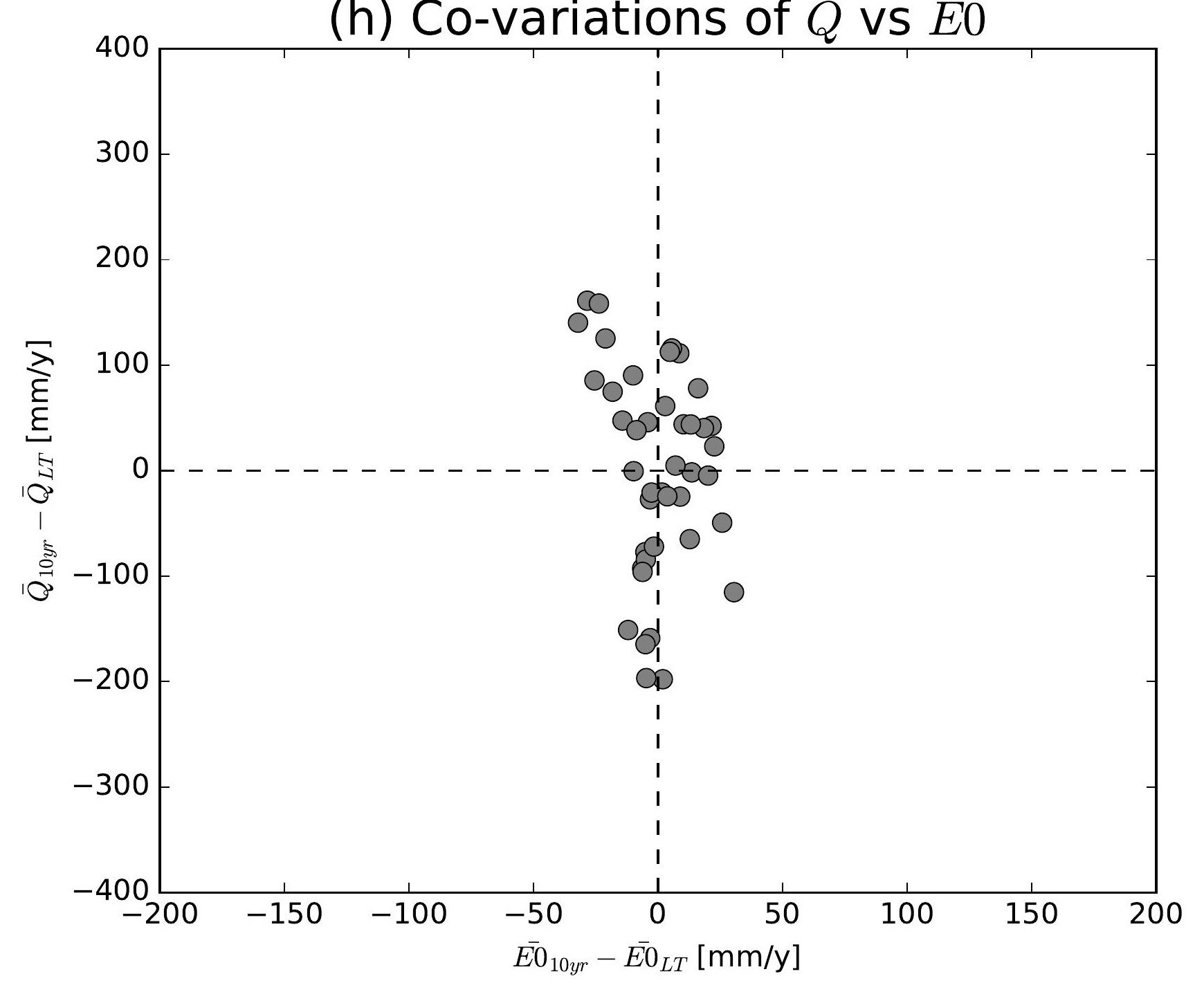

(k) Co-variations of $\bar{Q}$ vs $\overline{E 0}$

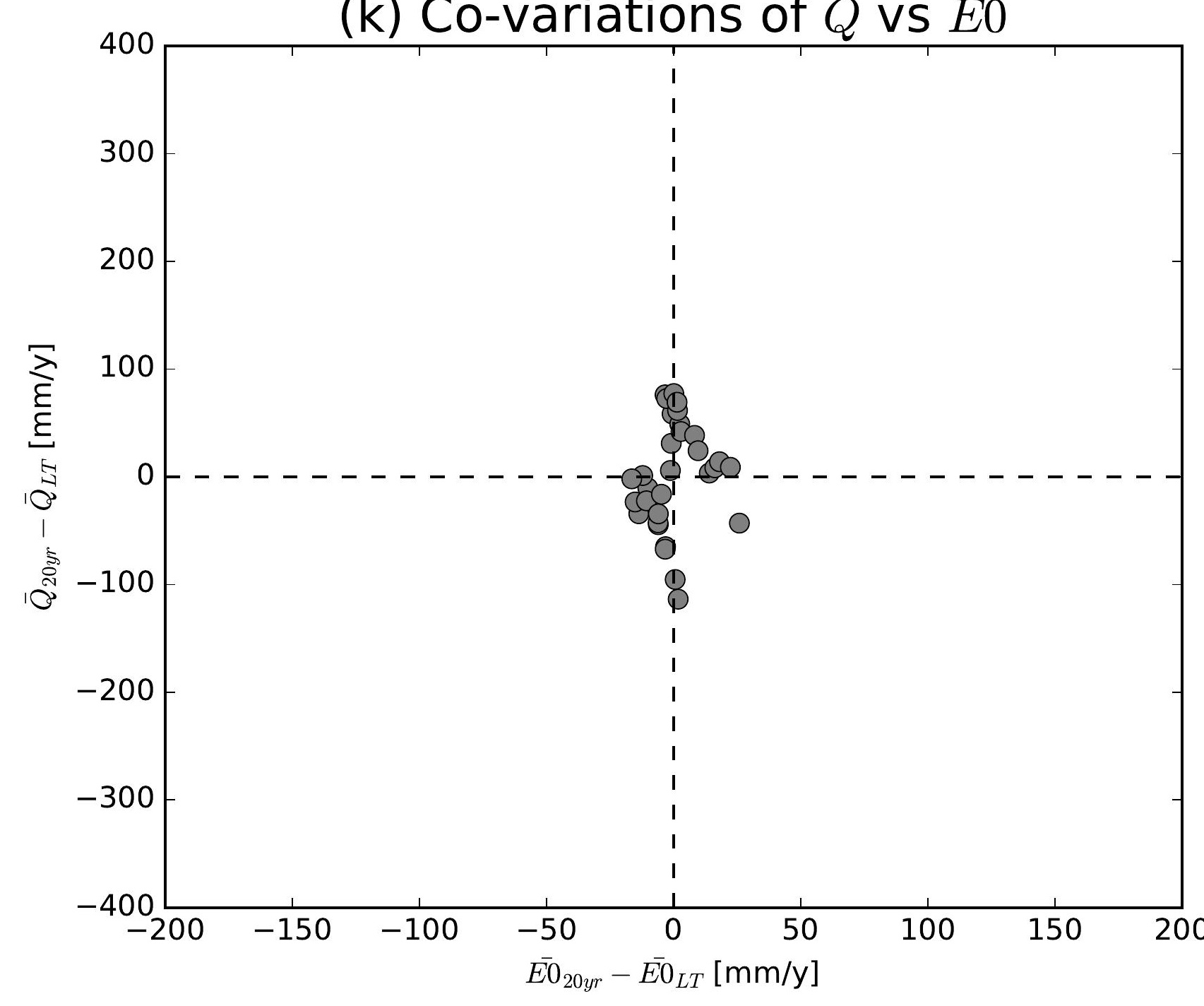

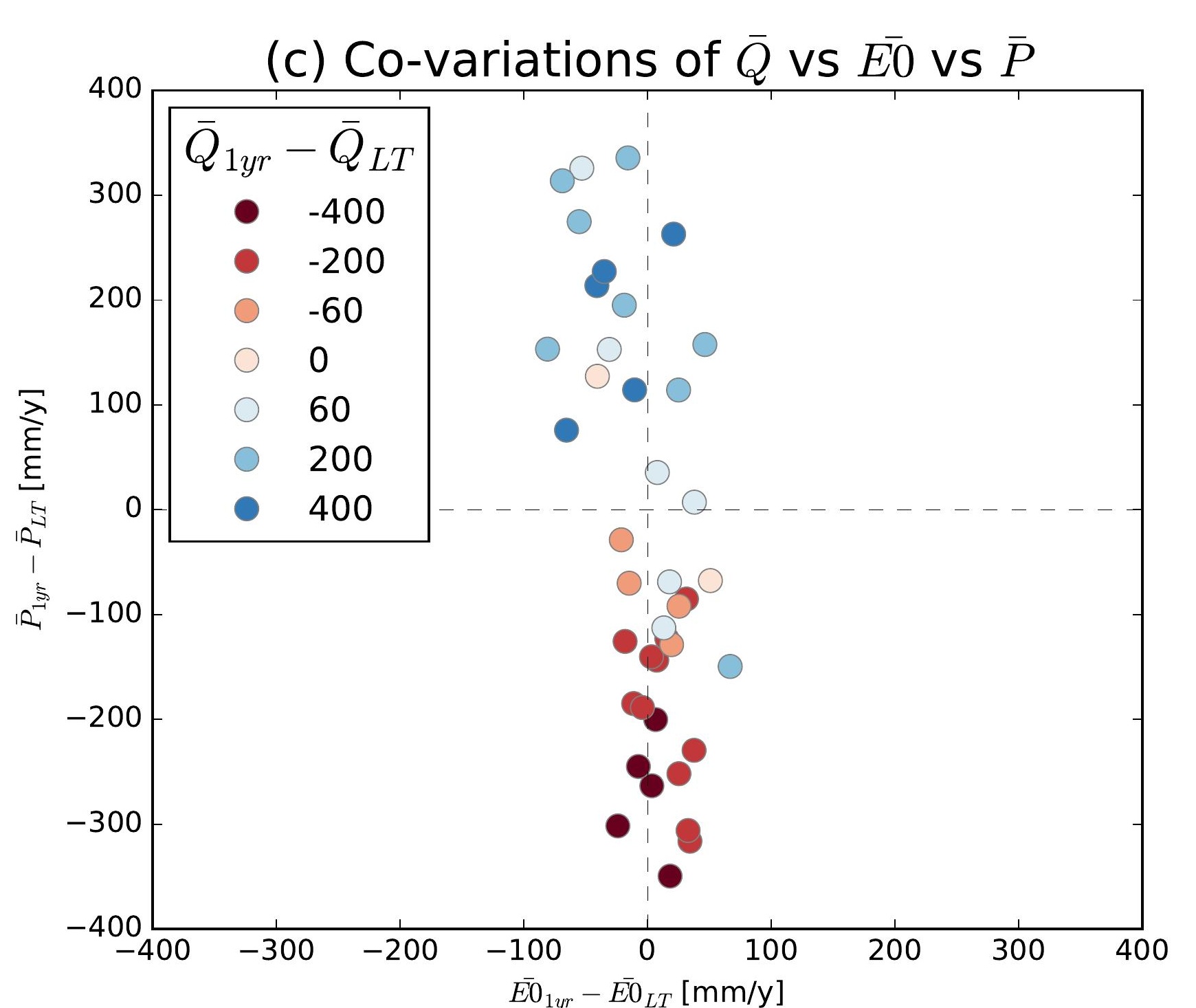
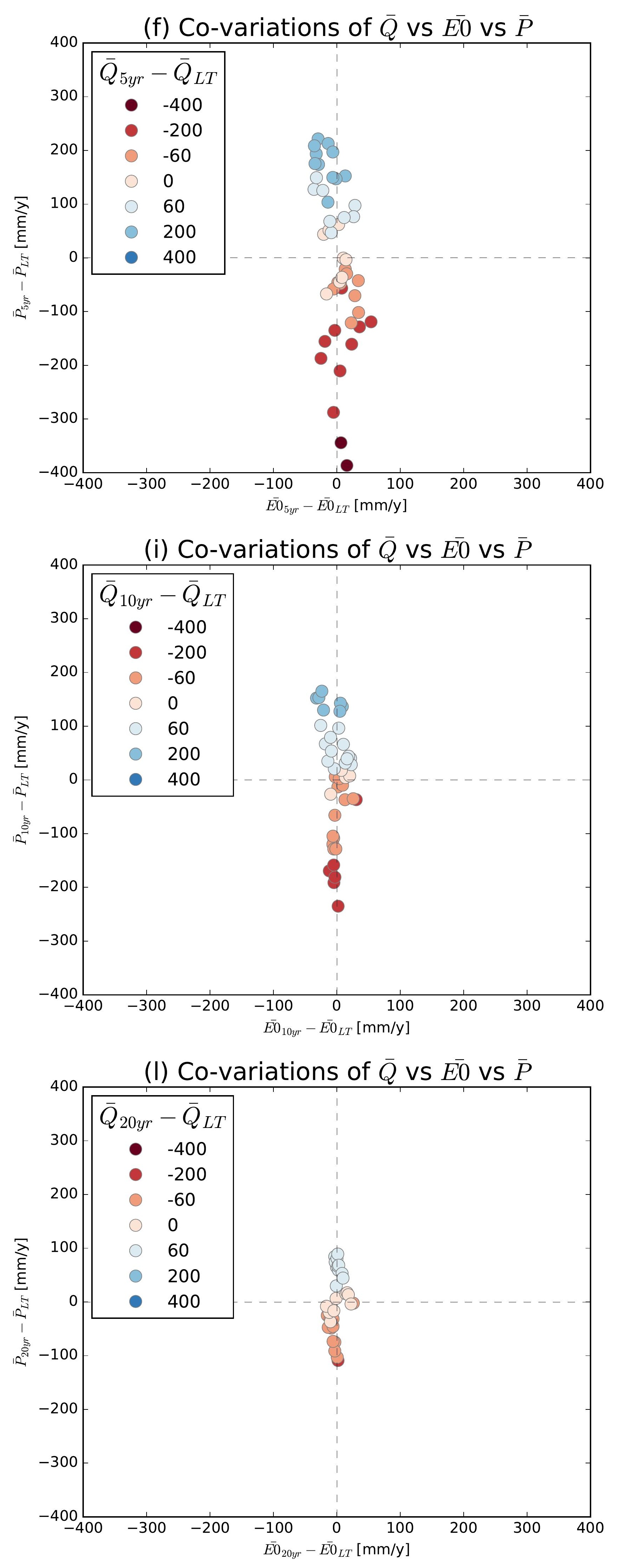

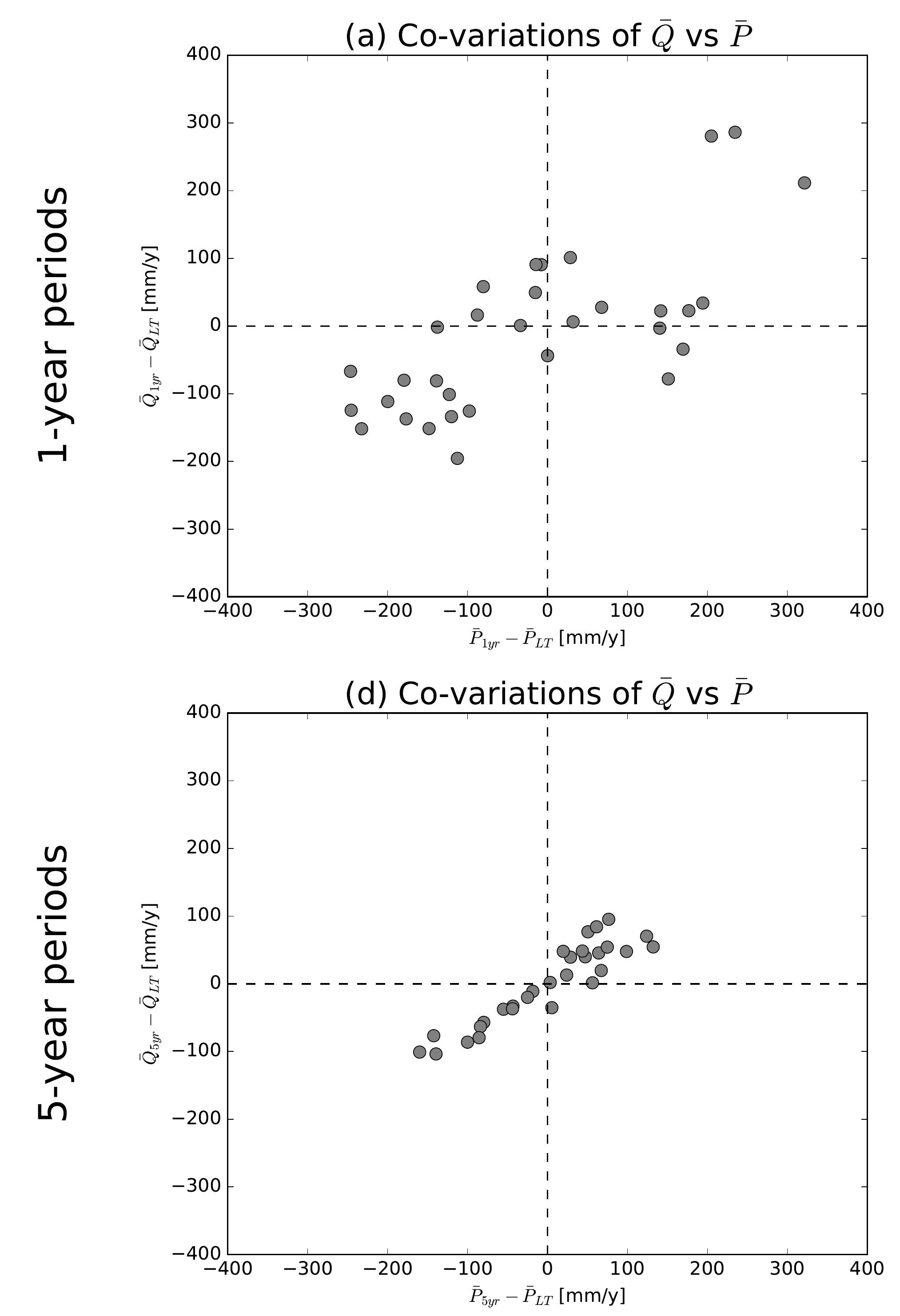

(g) Co-variations of $\bar{Q}$ vs $\bar{P}$
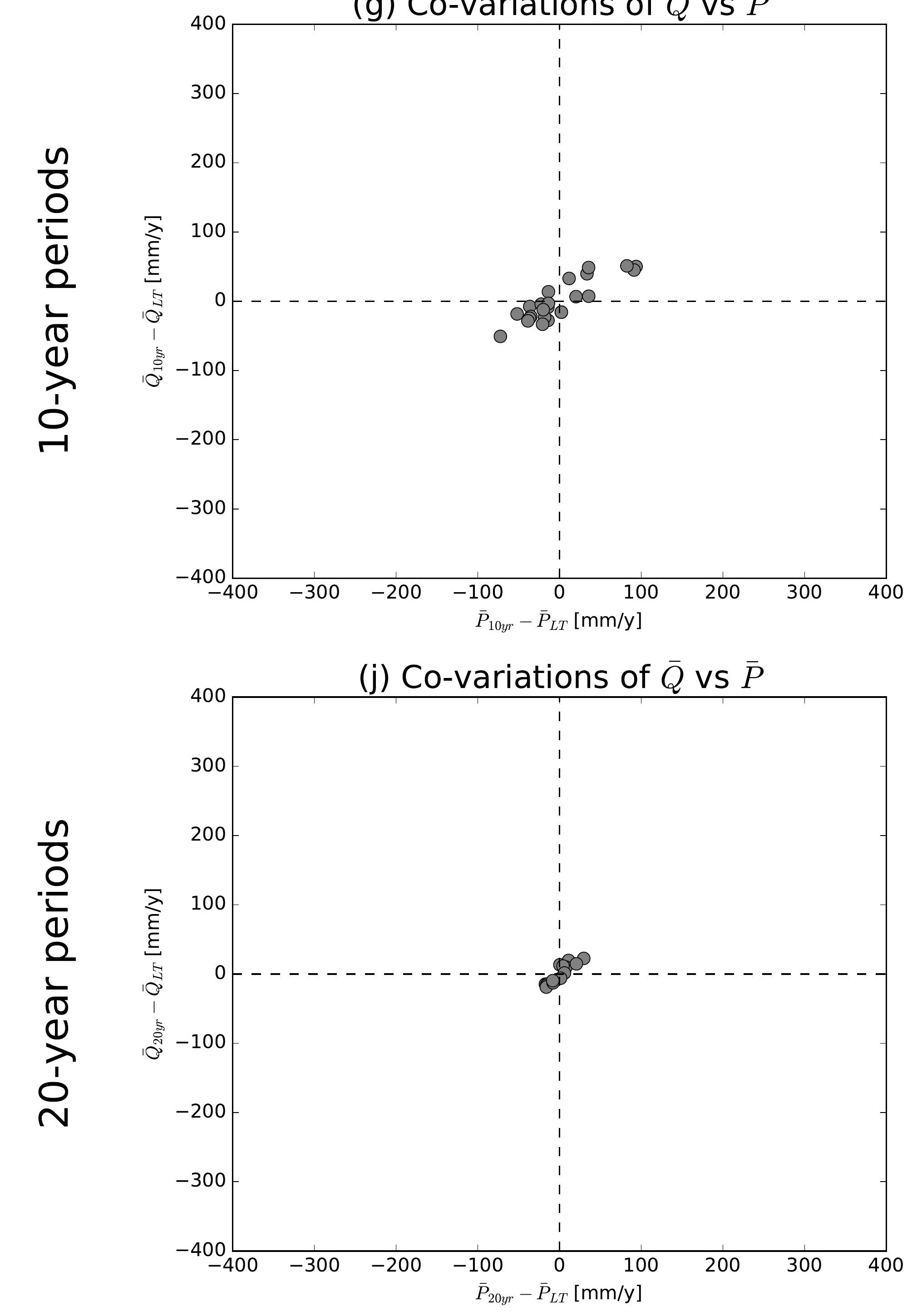

(b) Co-variations of $\bar{Q}$ vs $\overline{E 0}$

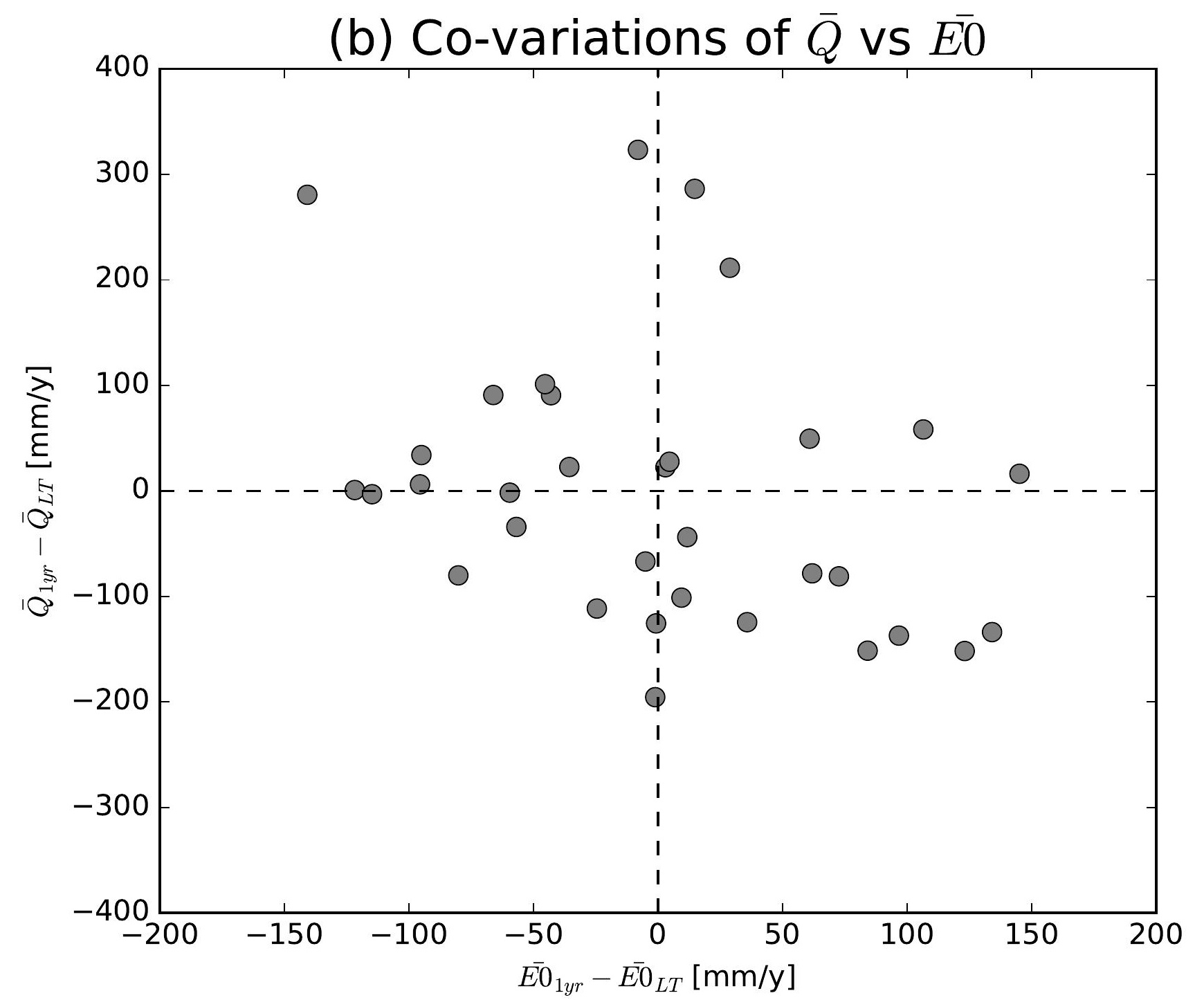

(e) Co-variations of $\bar{Q}$ vs $\overline{E 0}$

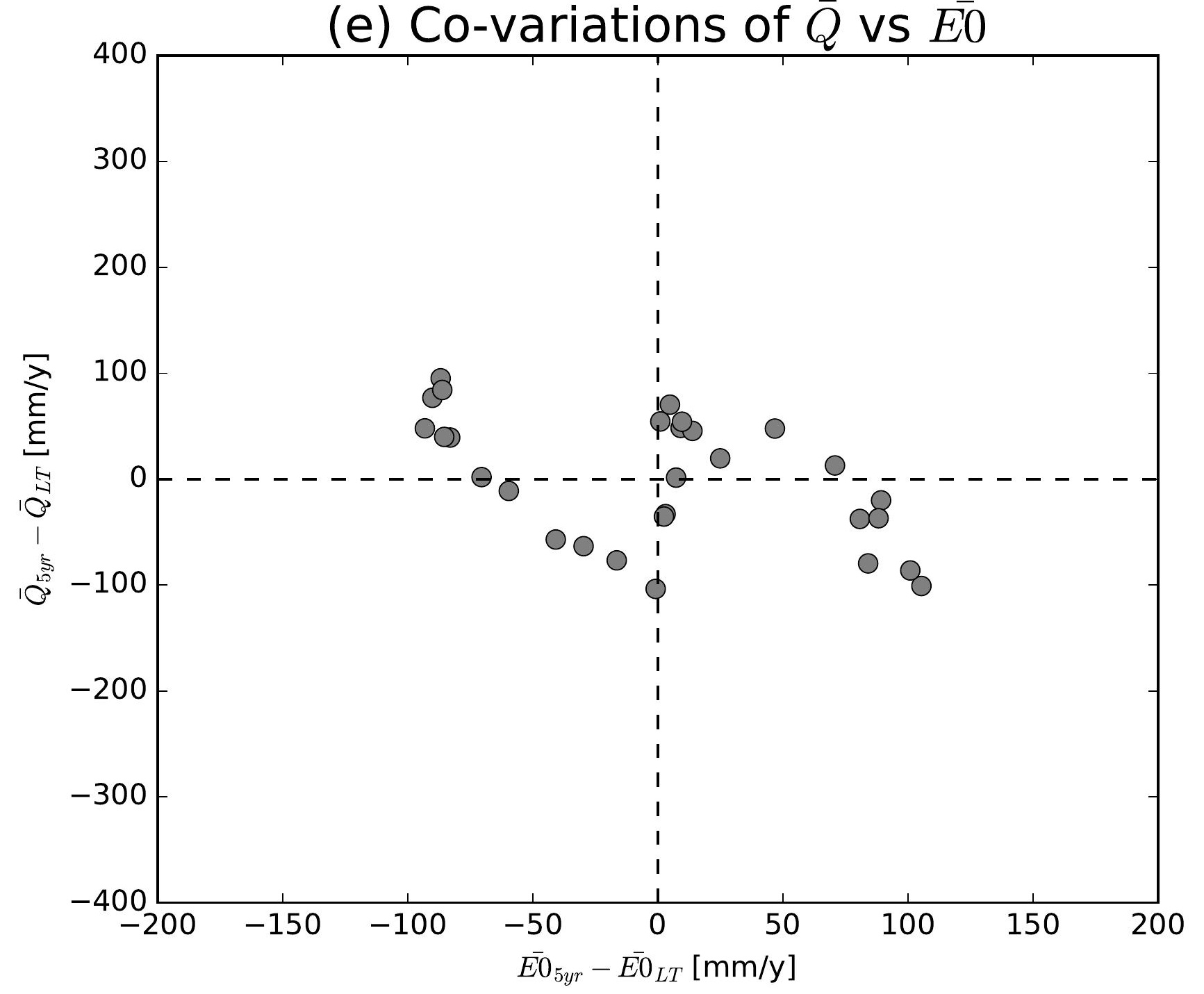

(h) Co-variations of $\bar{Q}$ vs $\overline{E 0}$

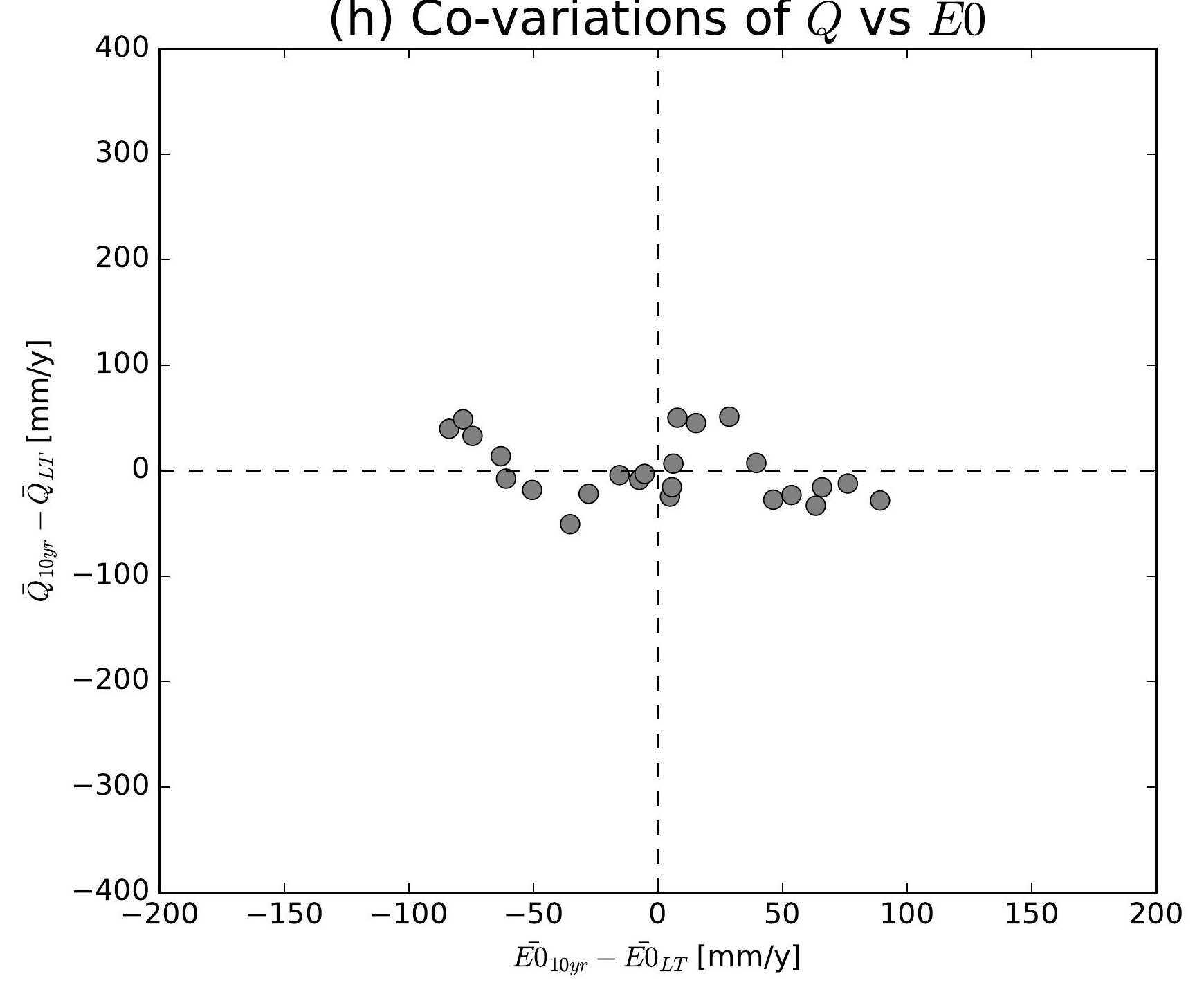

(k) Co-variations of $\bar{Q}$ vs $\overline{E 0}$

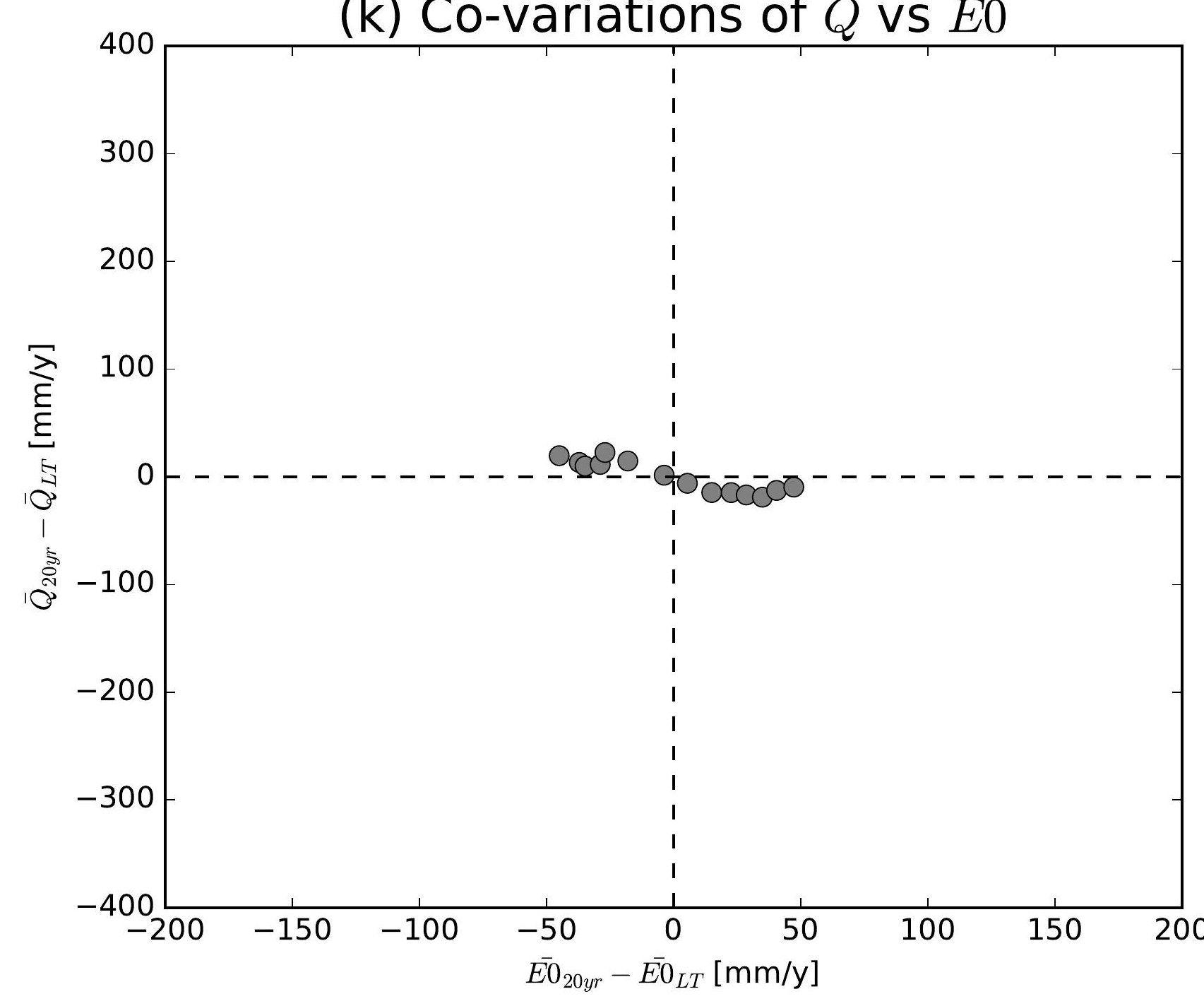

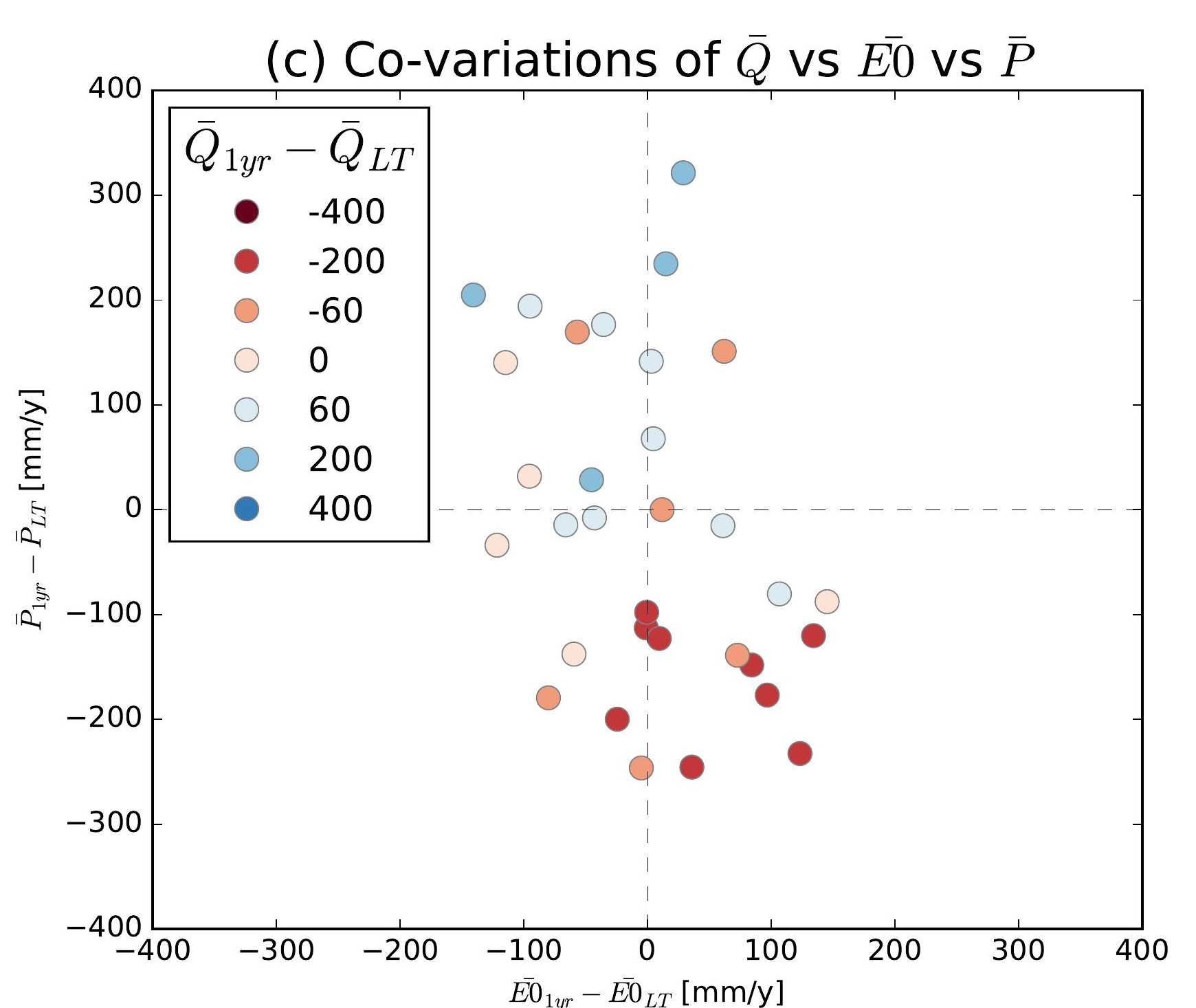
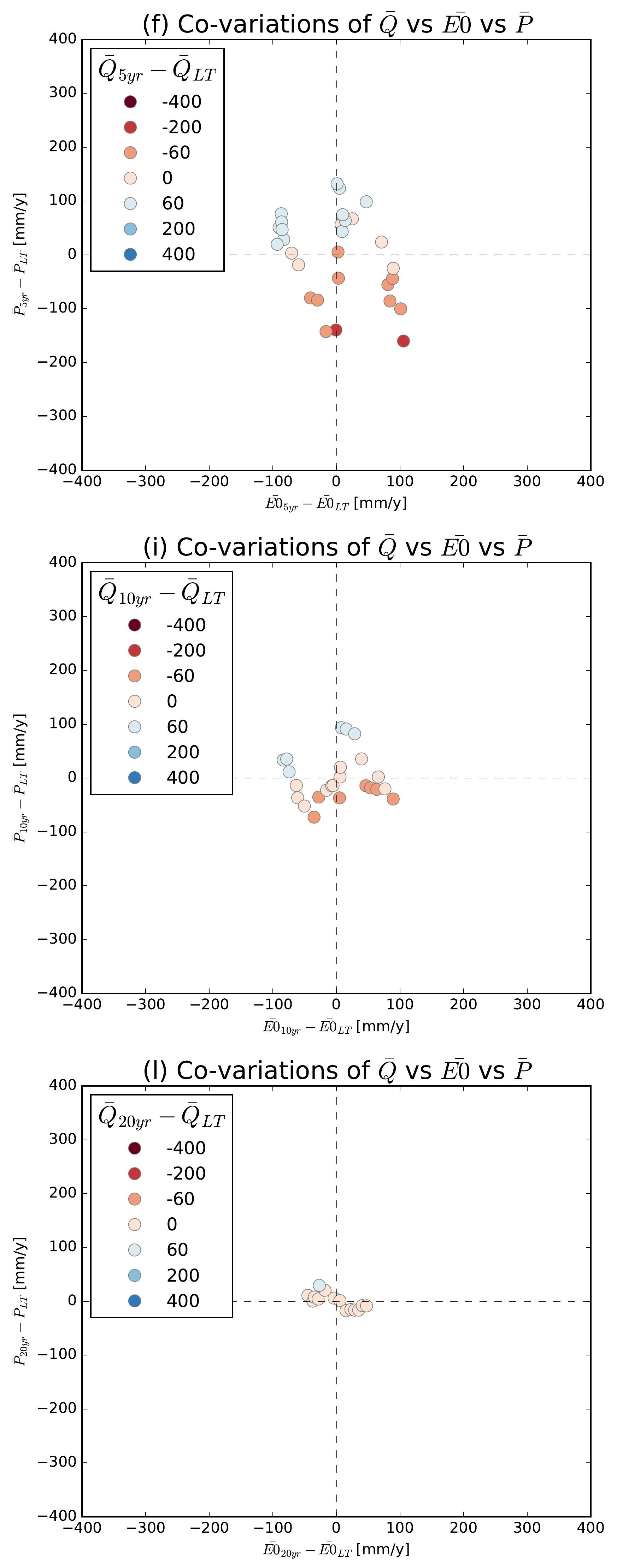

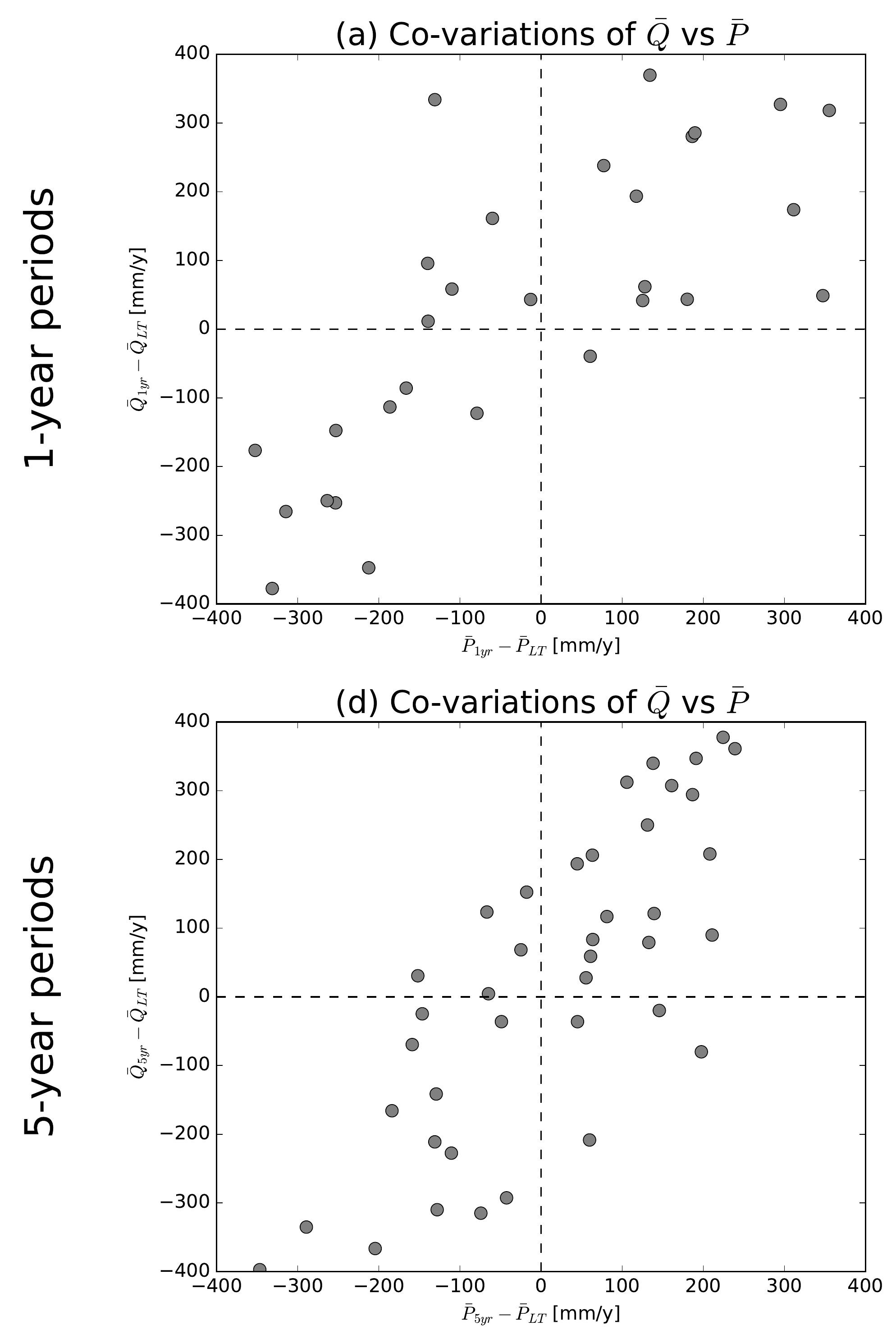

(g) Co-variations of $\bar{Q}$ vs $\bar{P}$

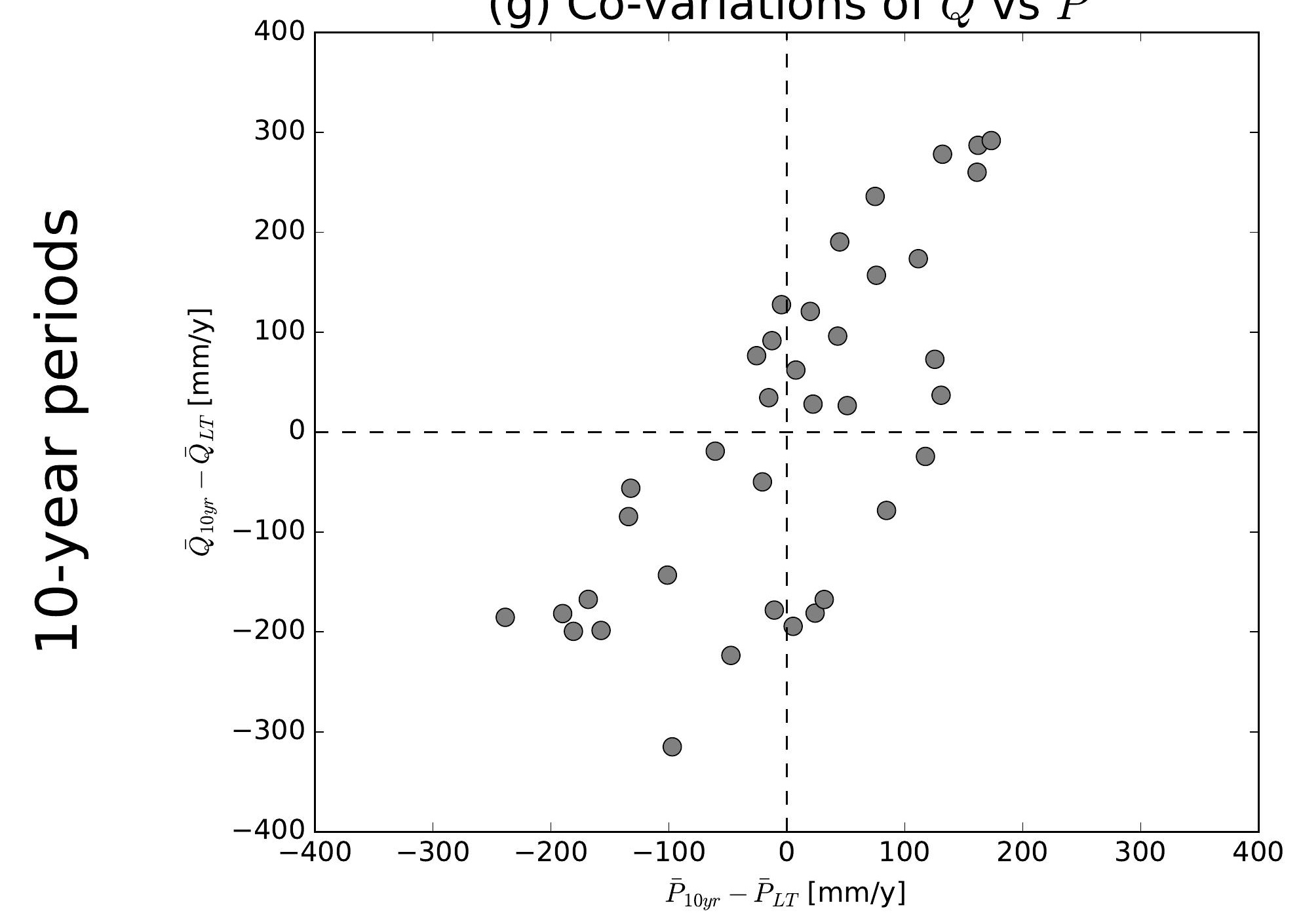

(j) Co-variations of $\bar{Q}$ vs $\bar{P}$

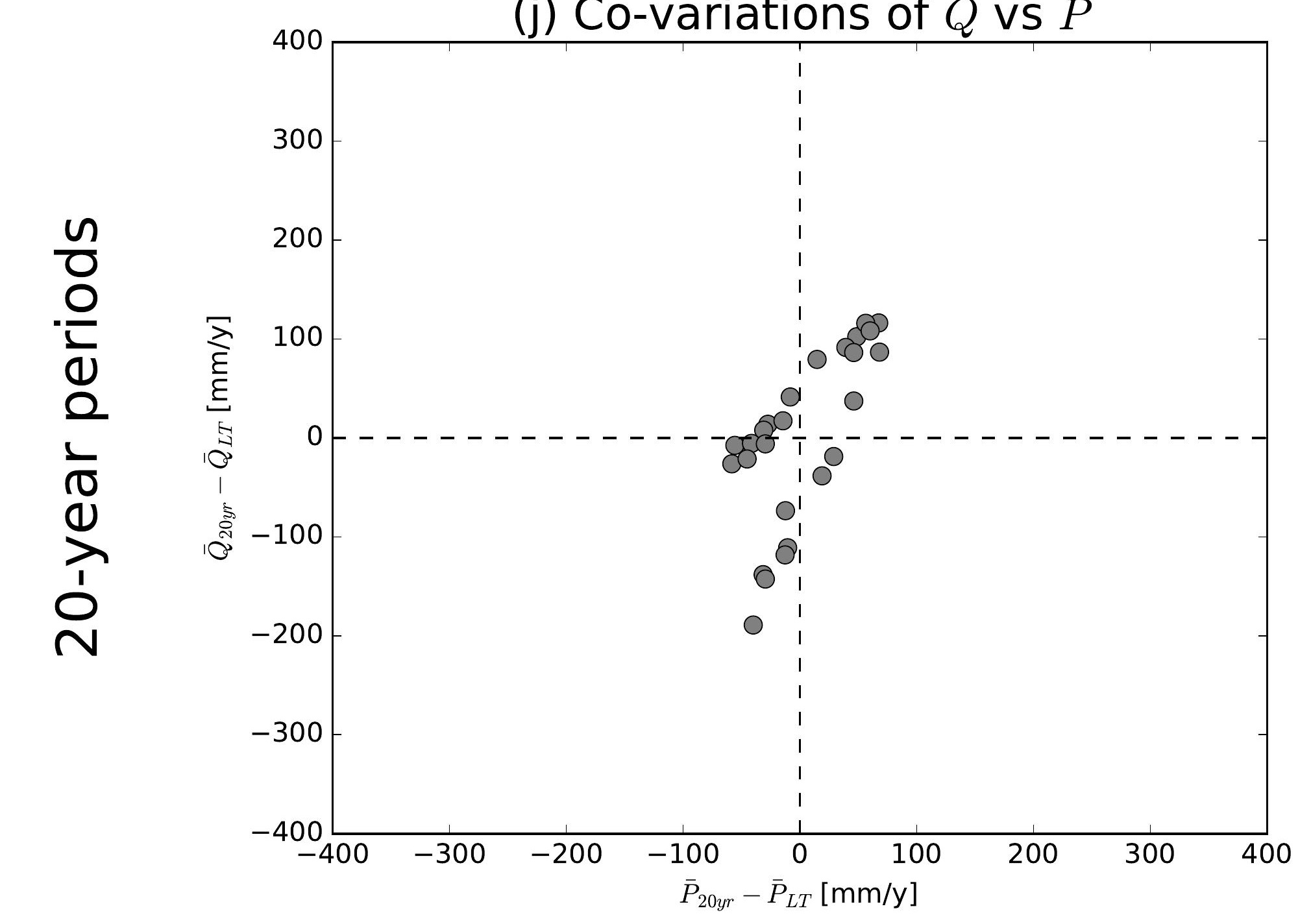

(b) Co-variations of $\bar{Q}$ vs $\overline{E 0}$

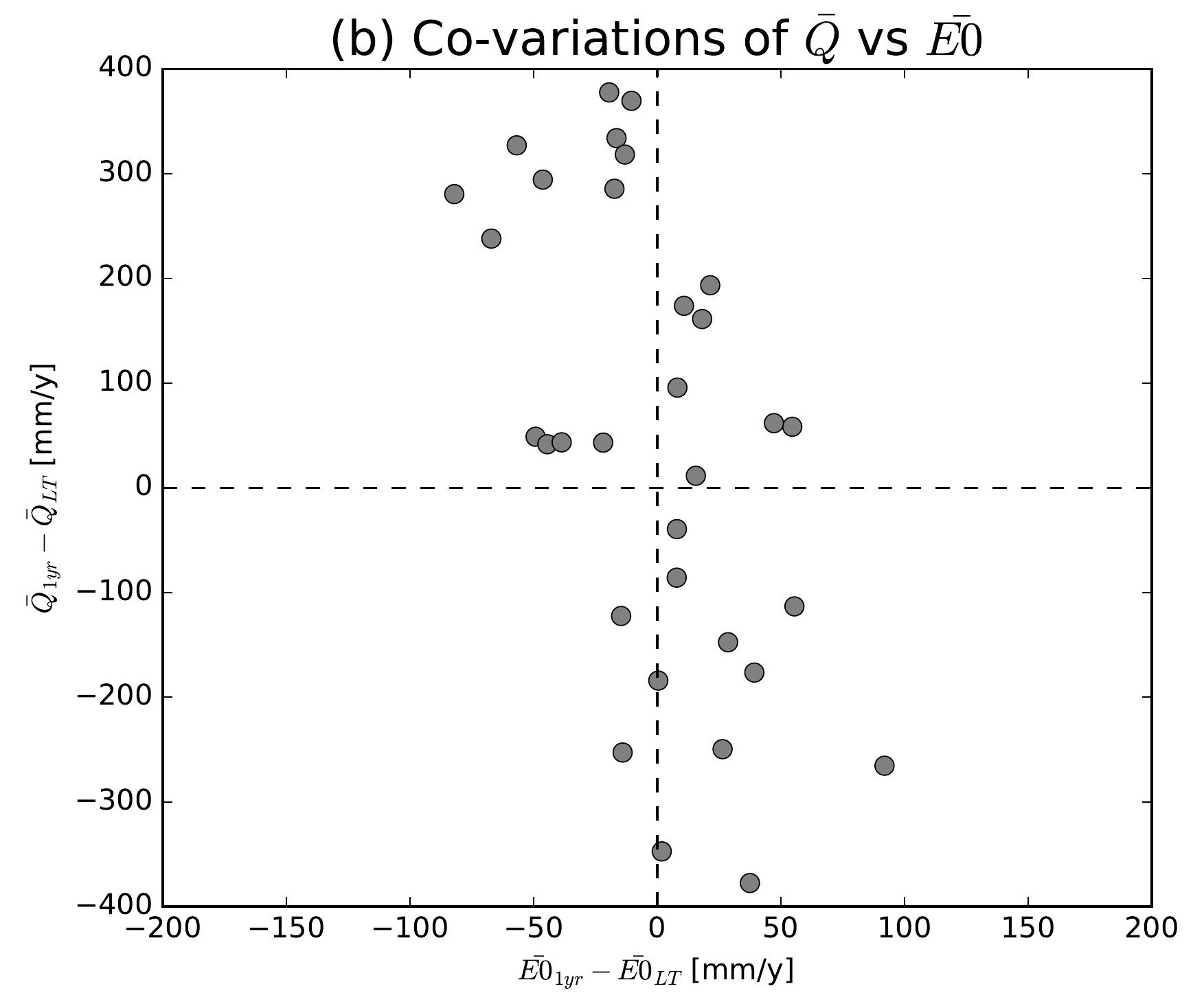

(e) Co-variations of $\bar{Q}$ vs $\overline{E 0}$

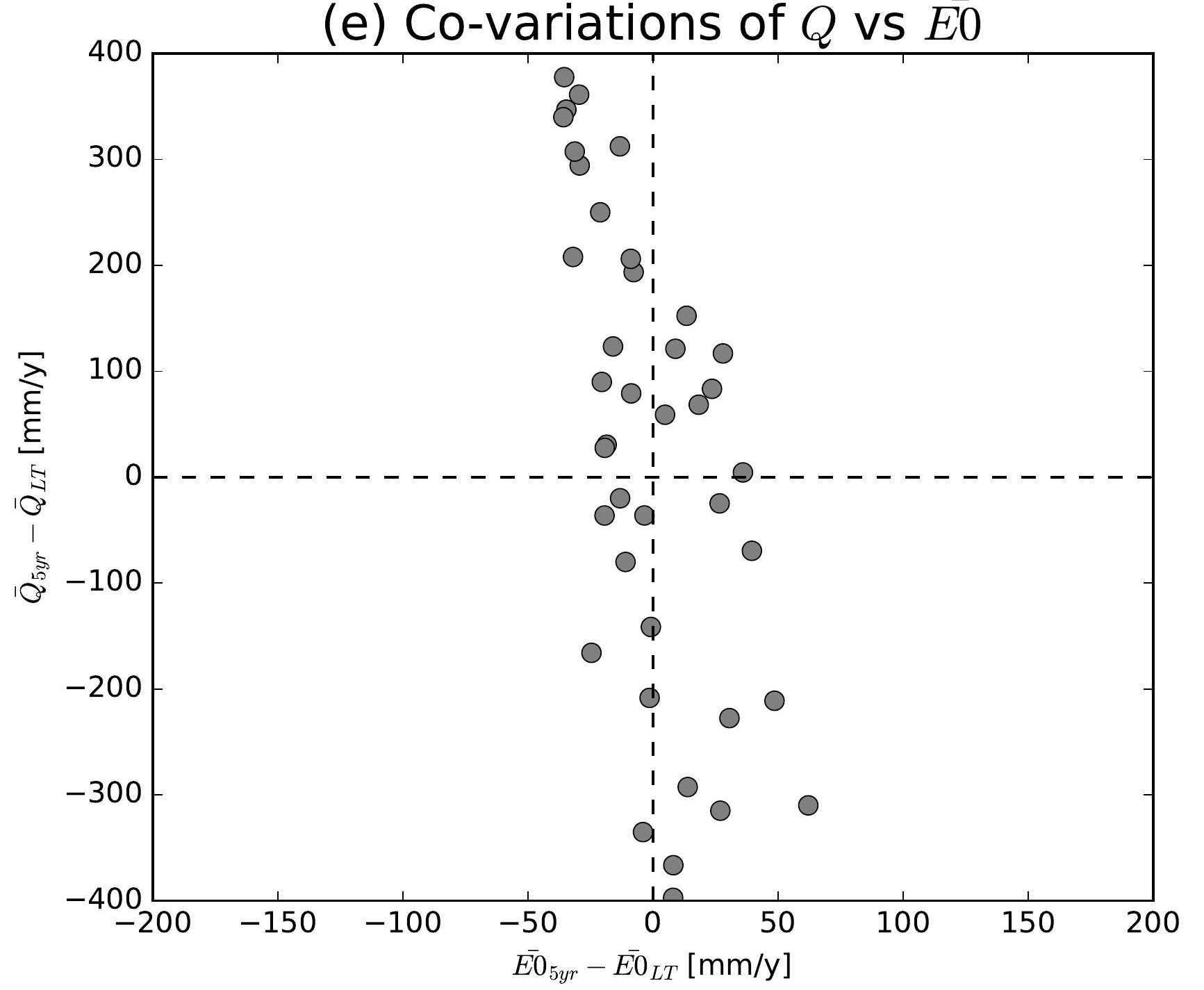

(h) Co-variations of $\bar{Q}$ vs $\overline{E 0}$

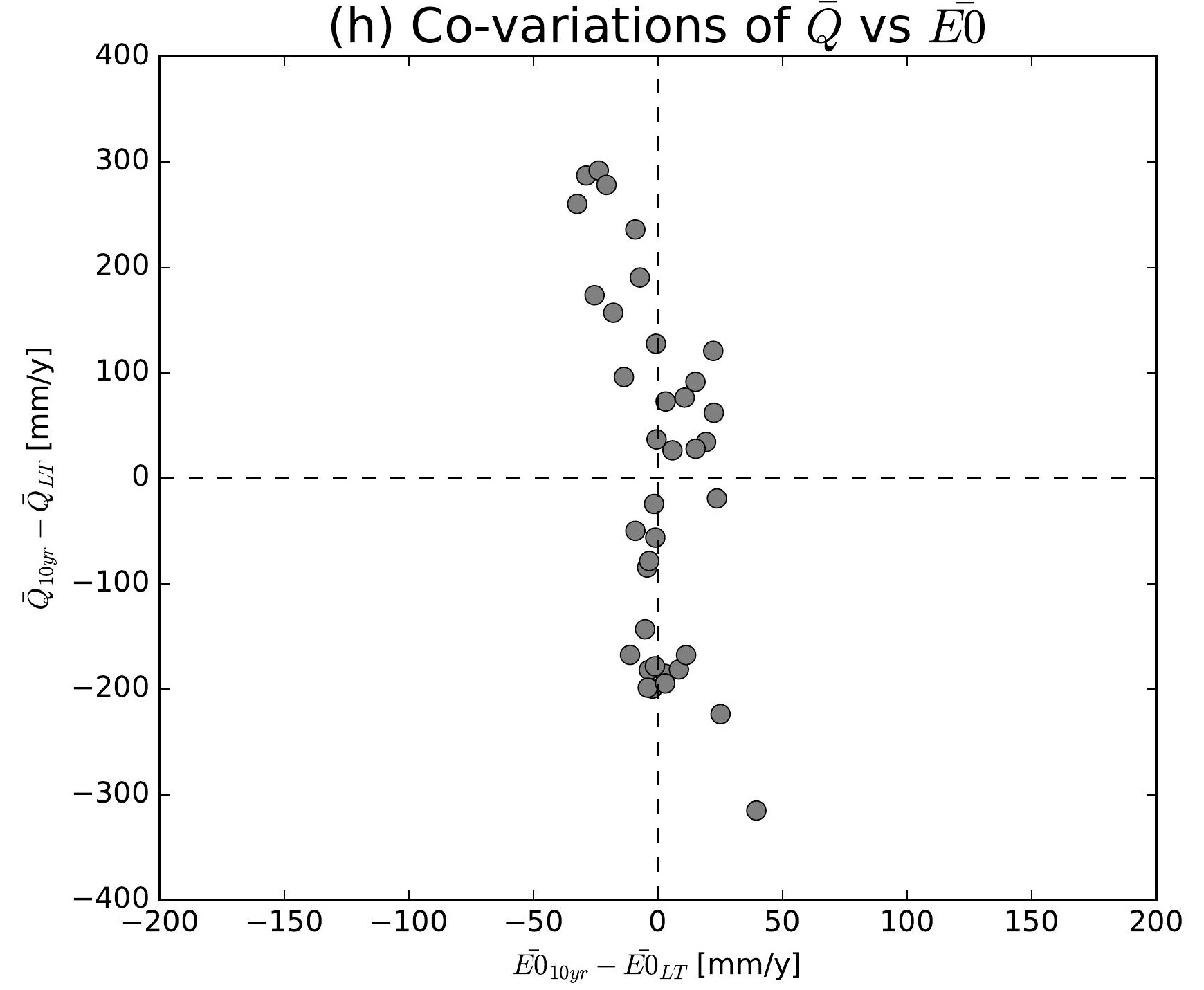

(k) Co-variations of $\bar{Q}$ vs $\overline{E 0}$

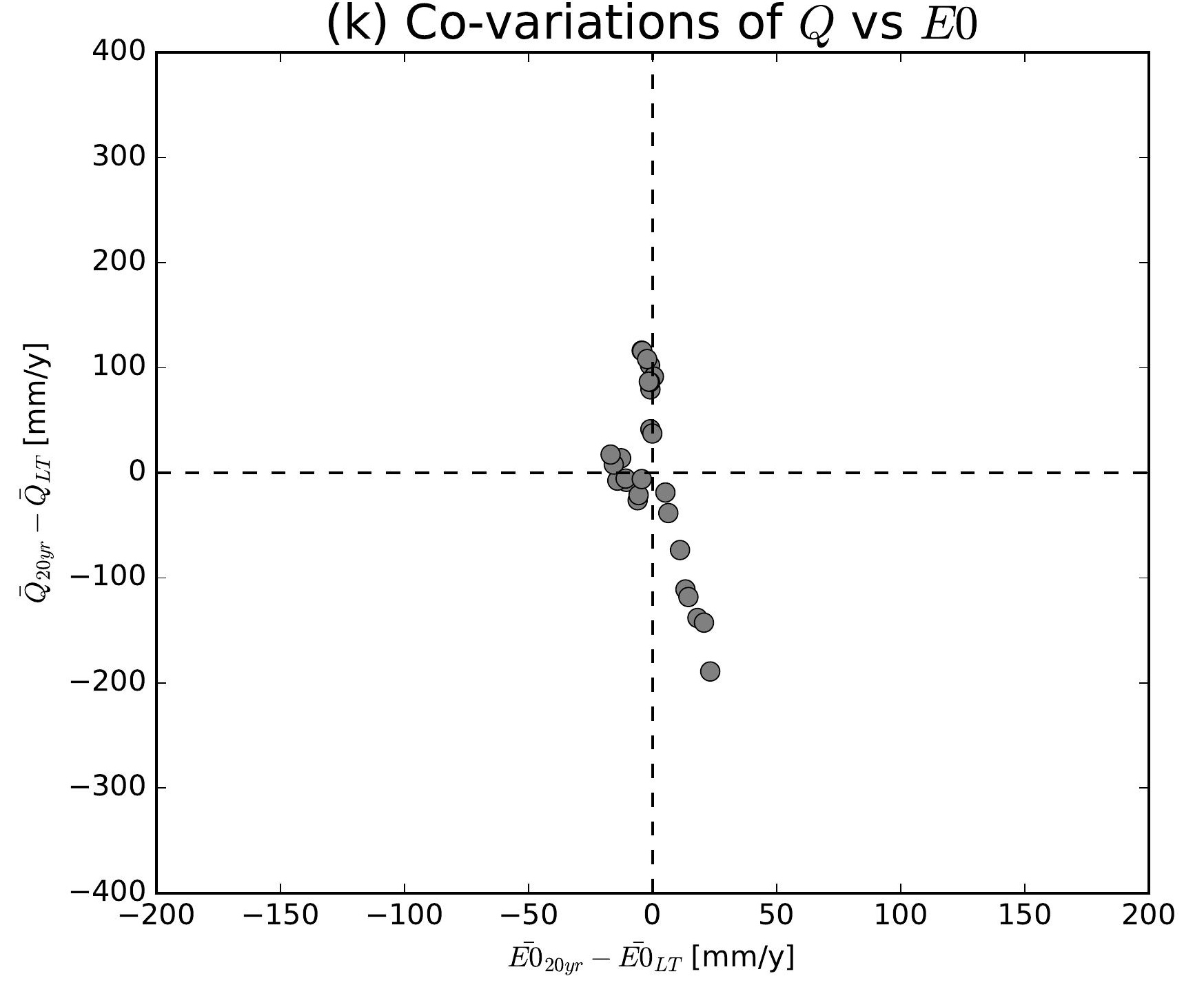

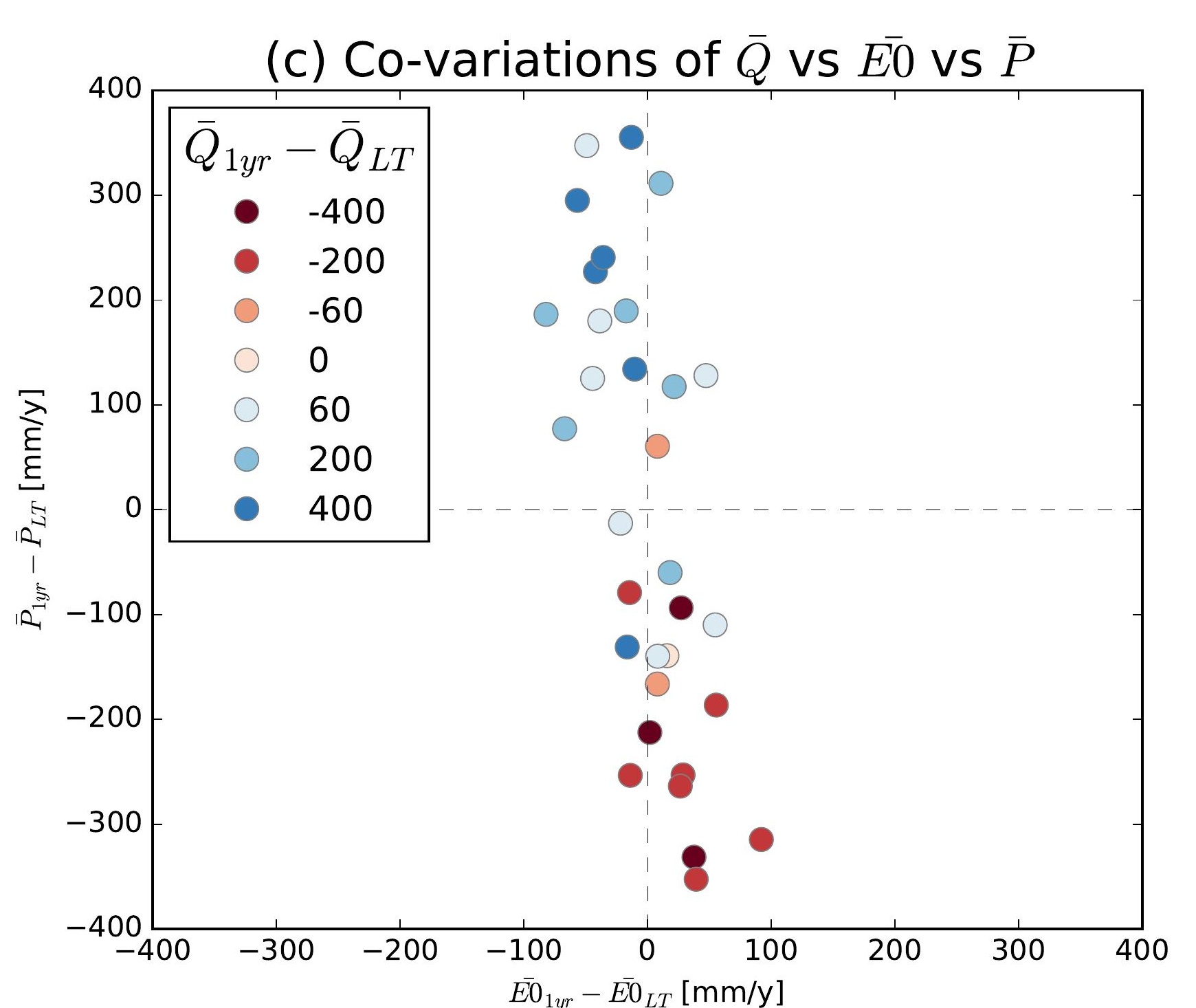
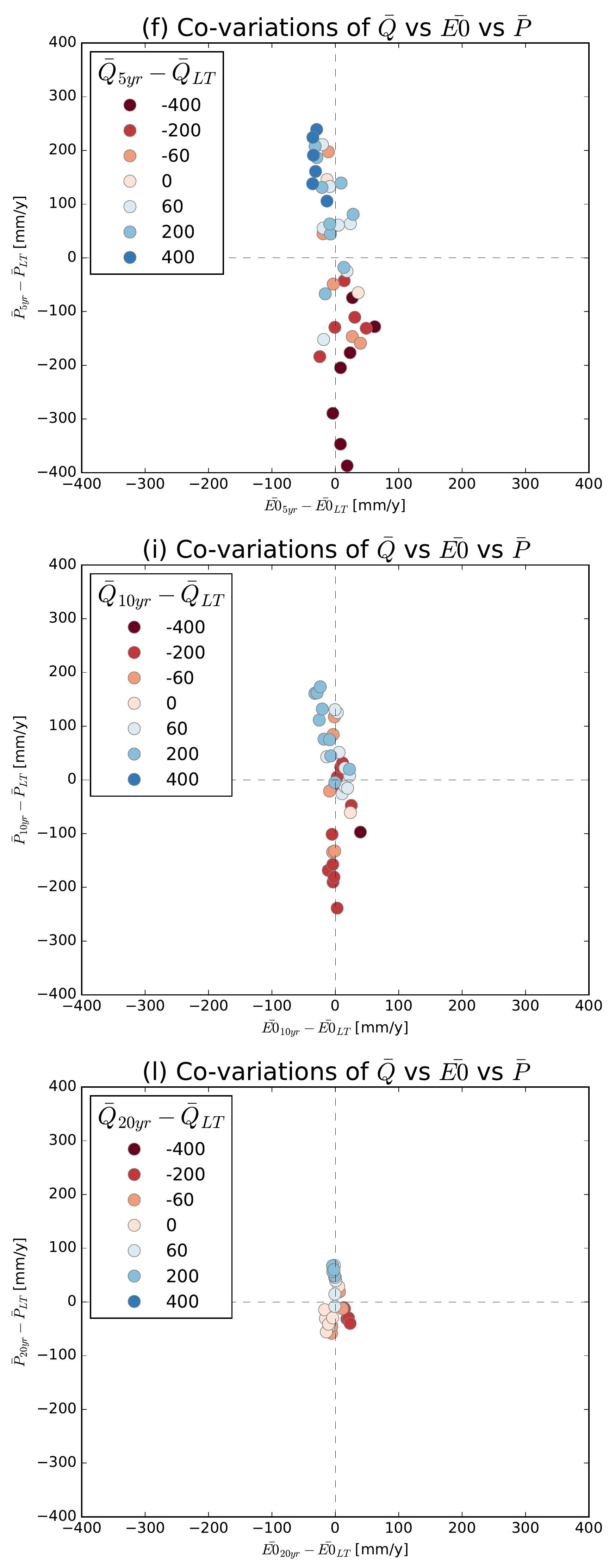

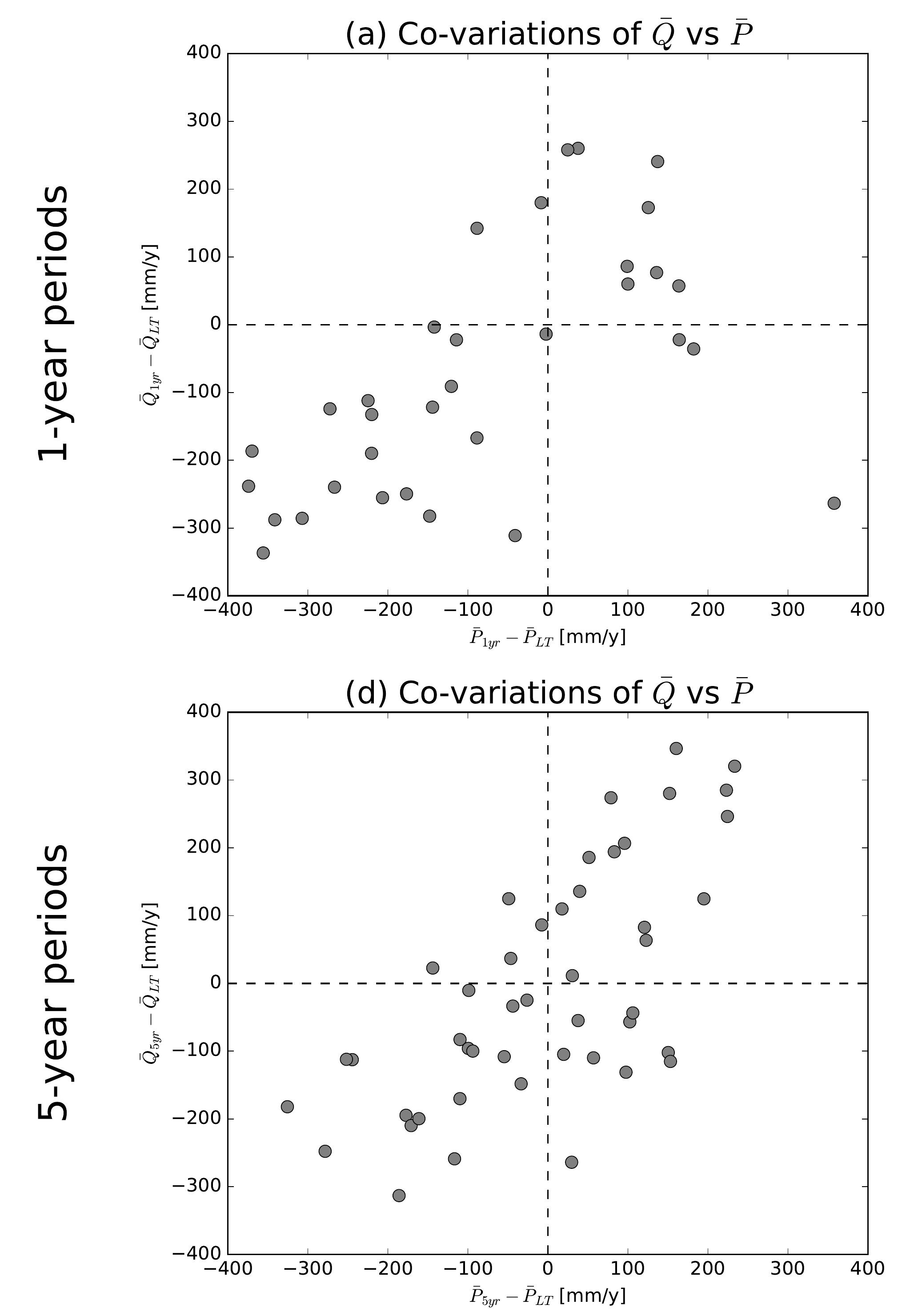

(g) Co-variations of $\bar{Q}$ vs $\bar{P}$
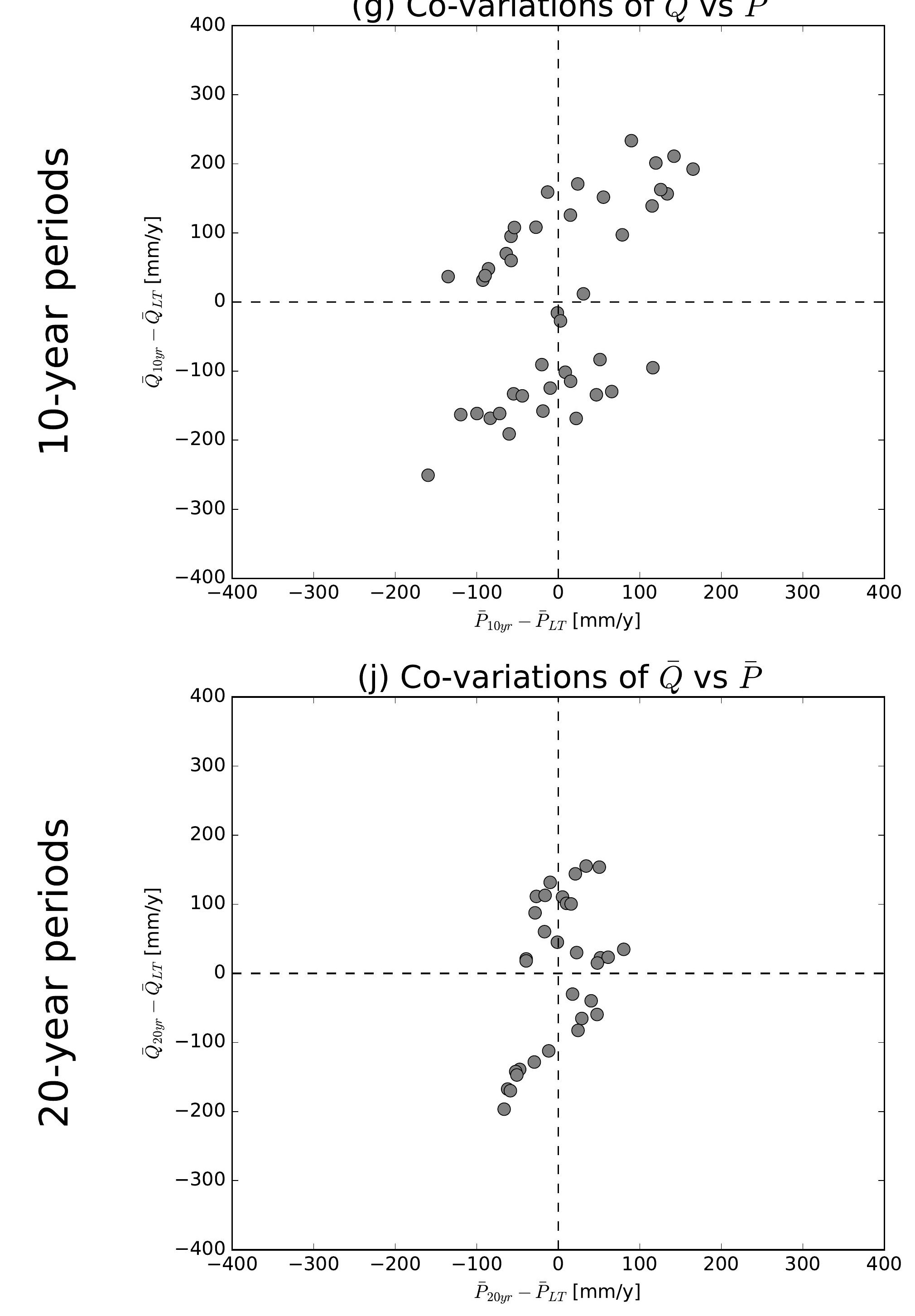

(b) Co-variations of $\bar{Q}$ vs $\overline{E 0}$

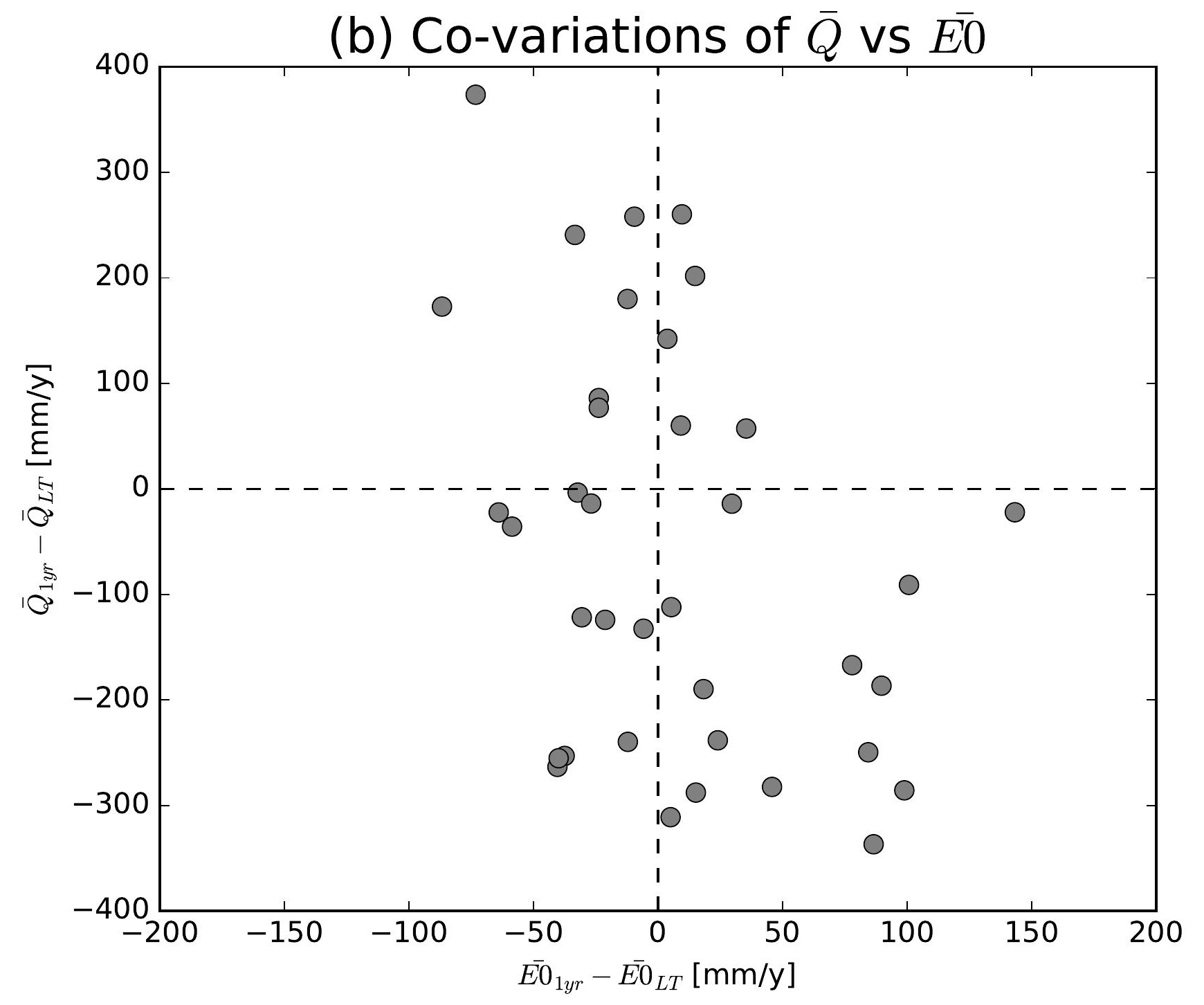

(e) Co-variations of $\bar{Q}$ vs $\overline{E 0}$

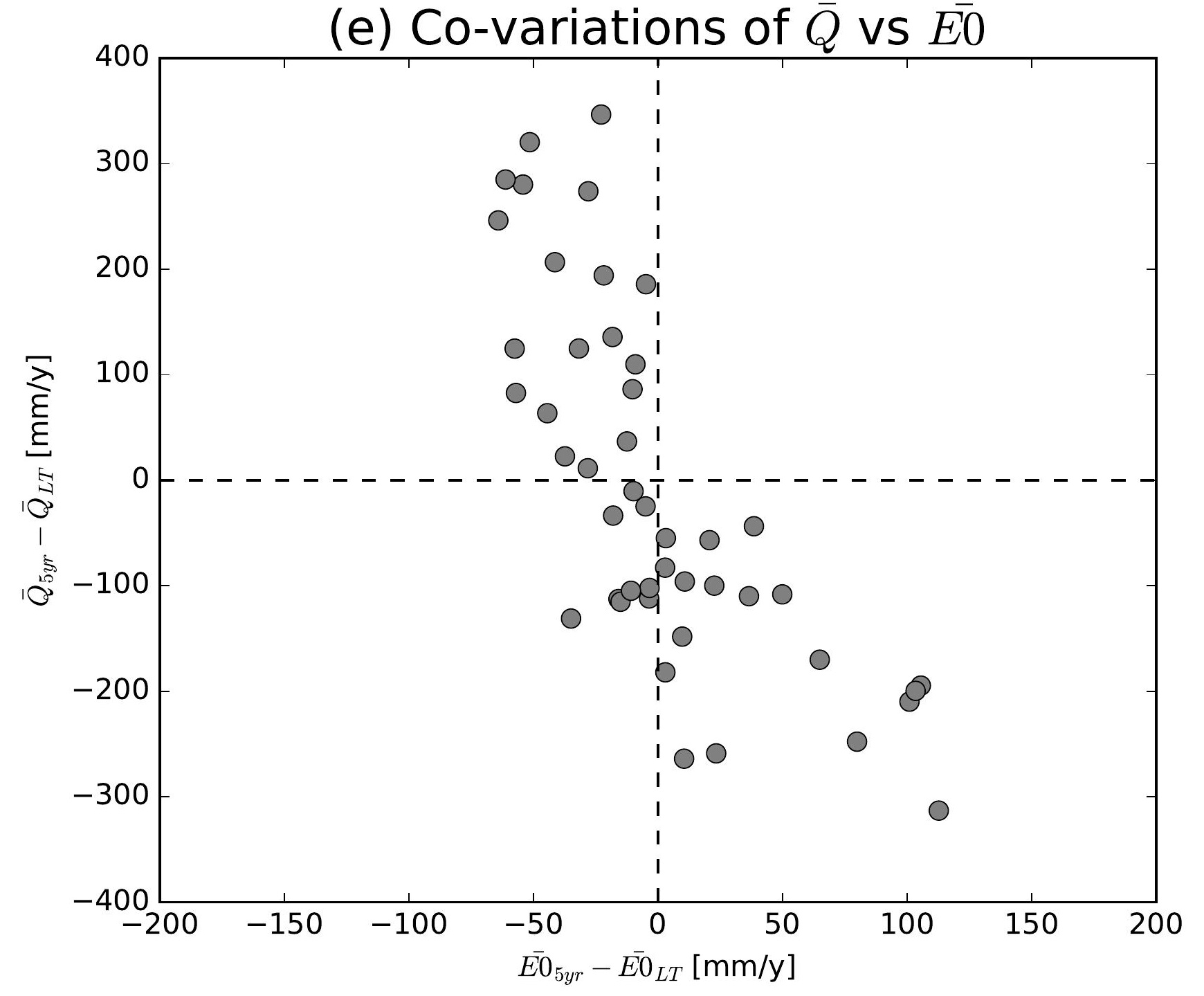

(h) Co-variations of $\bar{Q}$ vs $\overline{E 0}$

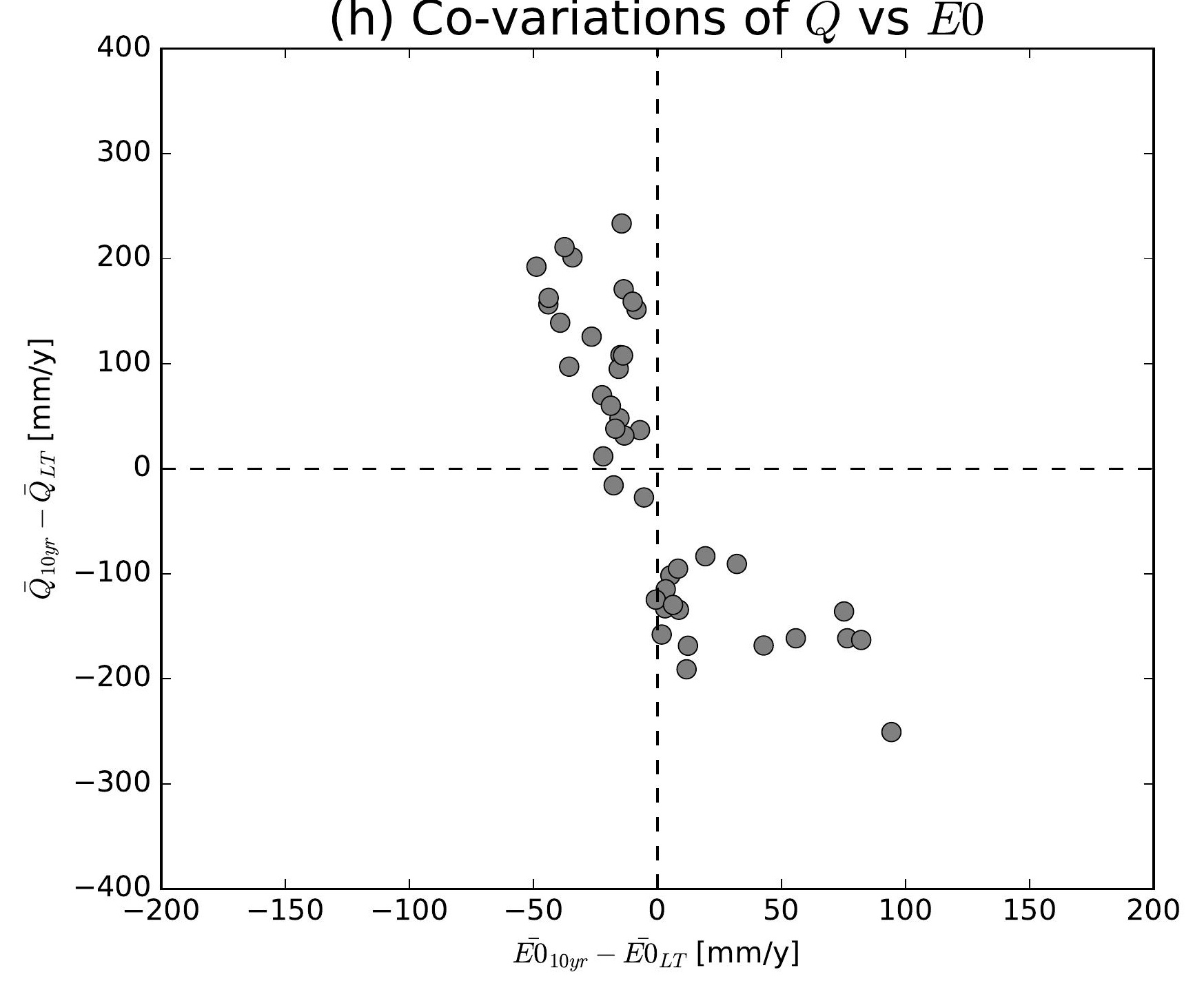

(k) Co-variations of $\bar{Q}$ vs $\overline{E 0}$

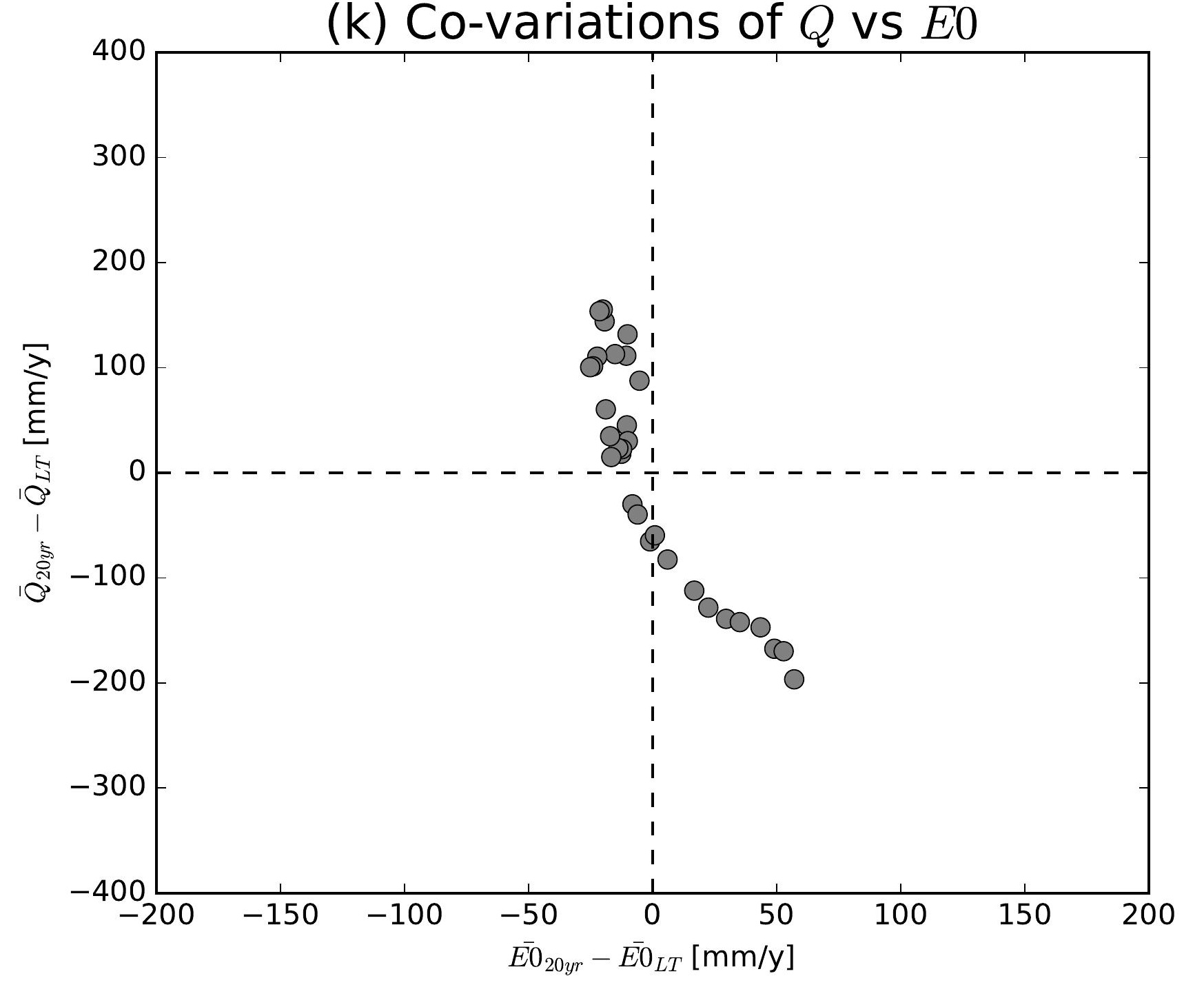

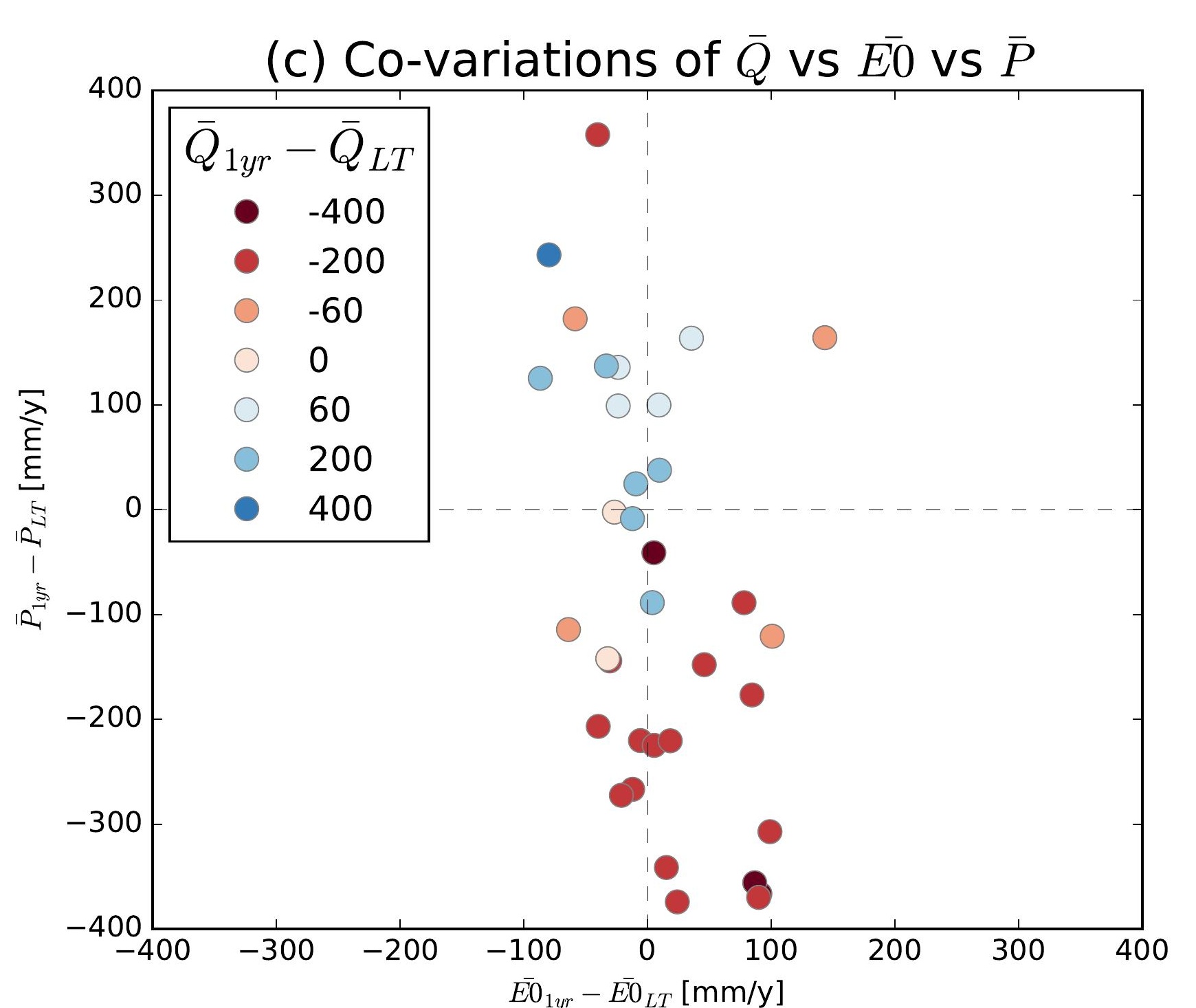
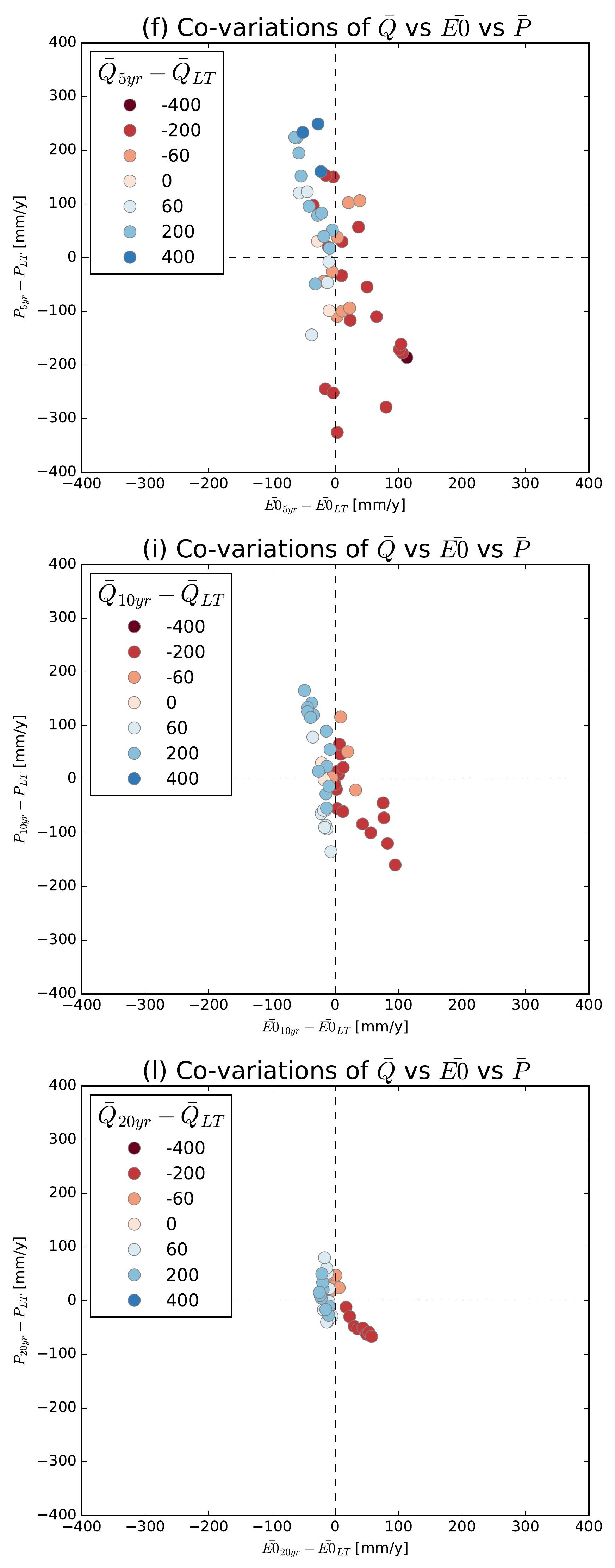

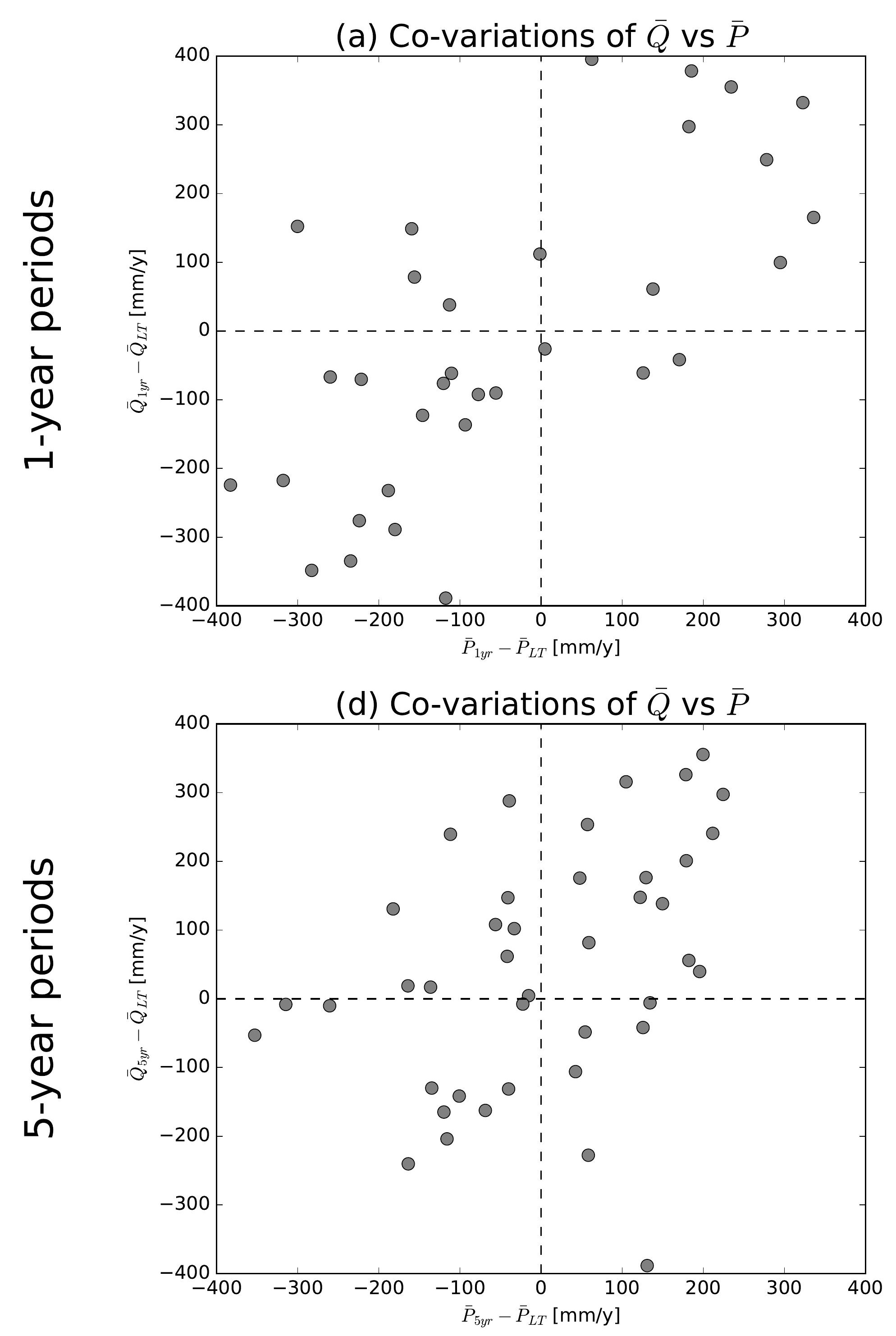

(g) Co-variations of $\bar{Q}$ vs $\bar{P}$

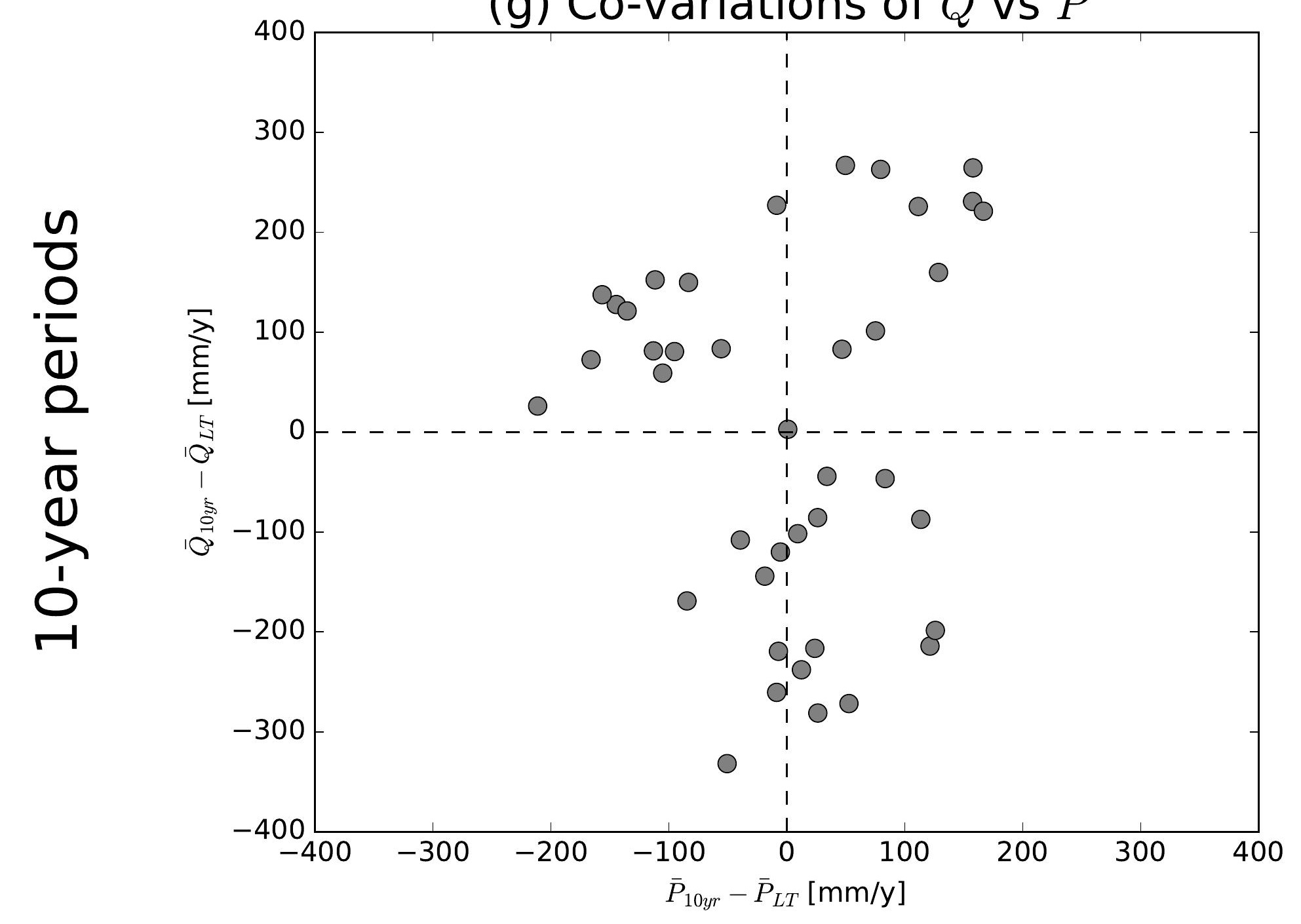

(j) Co-variations of $\bar{Q}$ vs $\bar{P}$

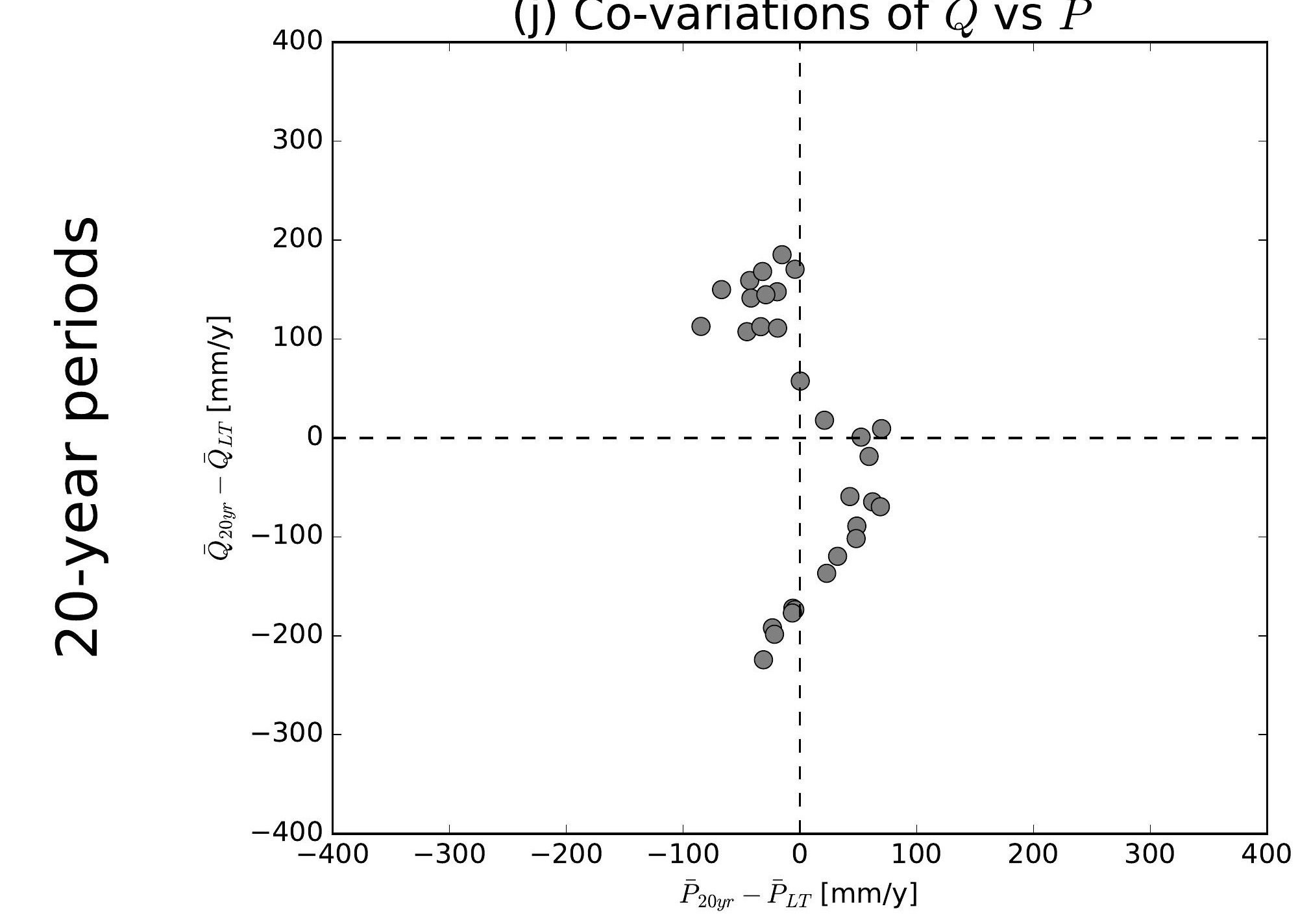

(b) Co-variations of $\bar{Q}$ vs $\overline{E 0}$

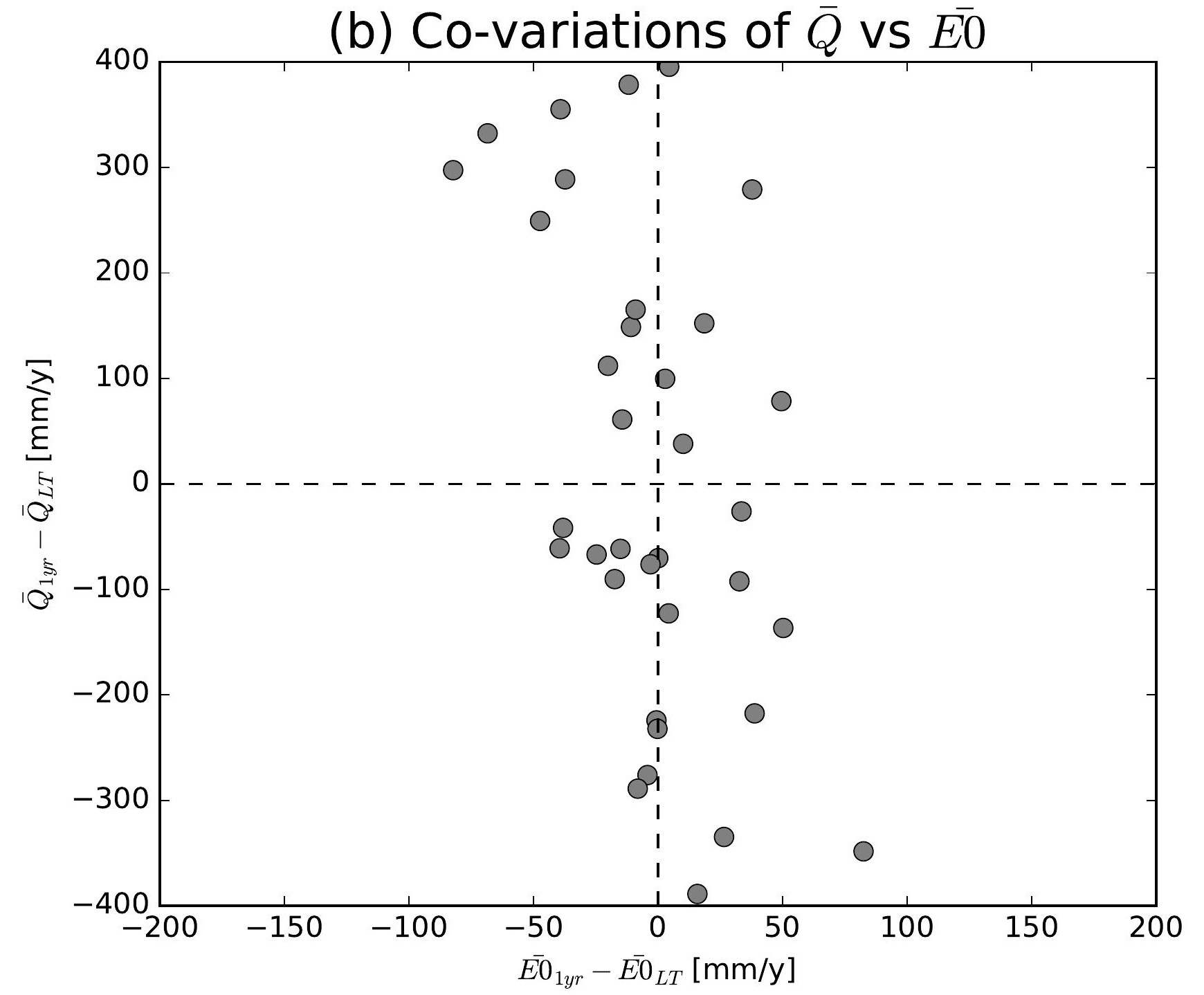

(e) Co-variations of $\bar{Q}$ vs $\overline{E 0}$

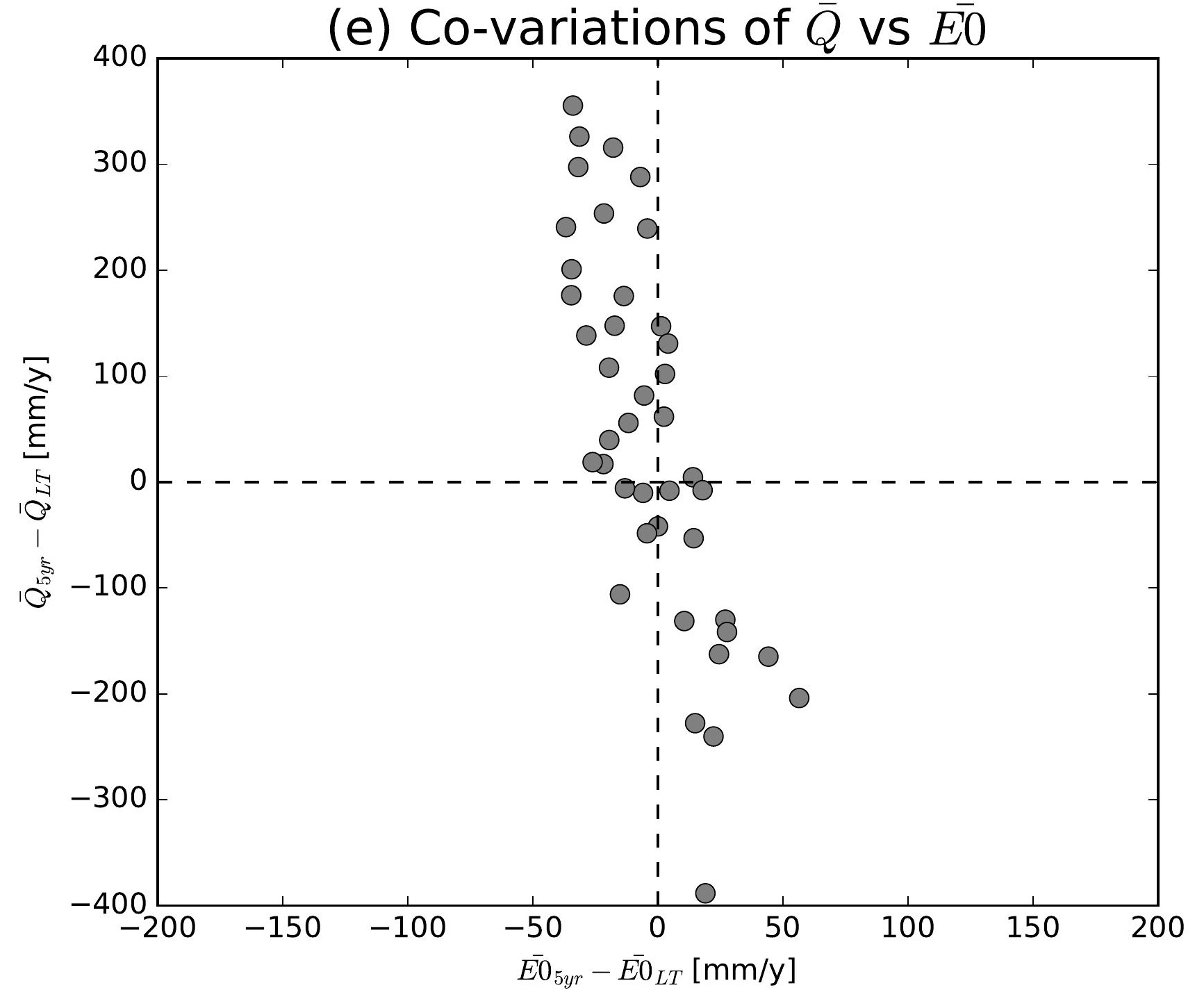

(h) Co-variations of $\bar{Q}$ vs $\overline{E 0}$

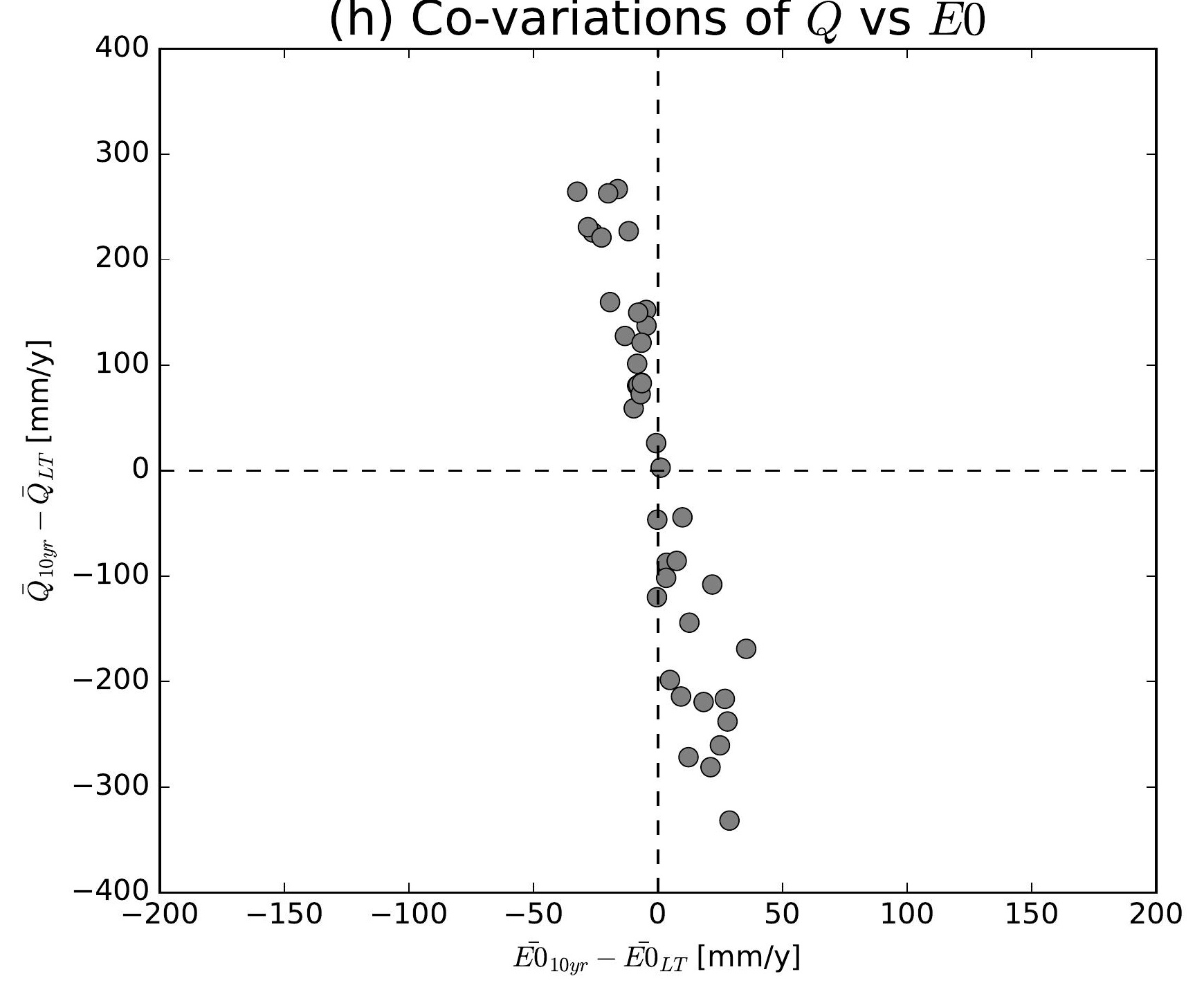

(k) Co-variations of $\bar{Q}$ vs $\overline{E 0}$

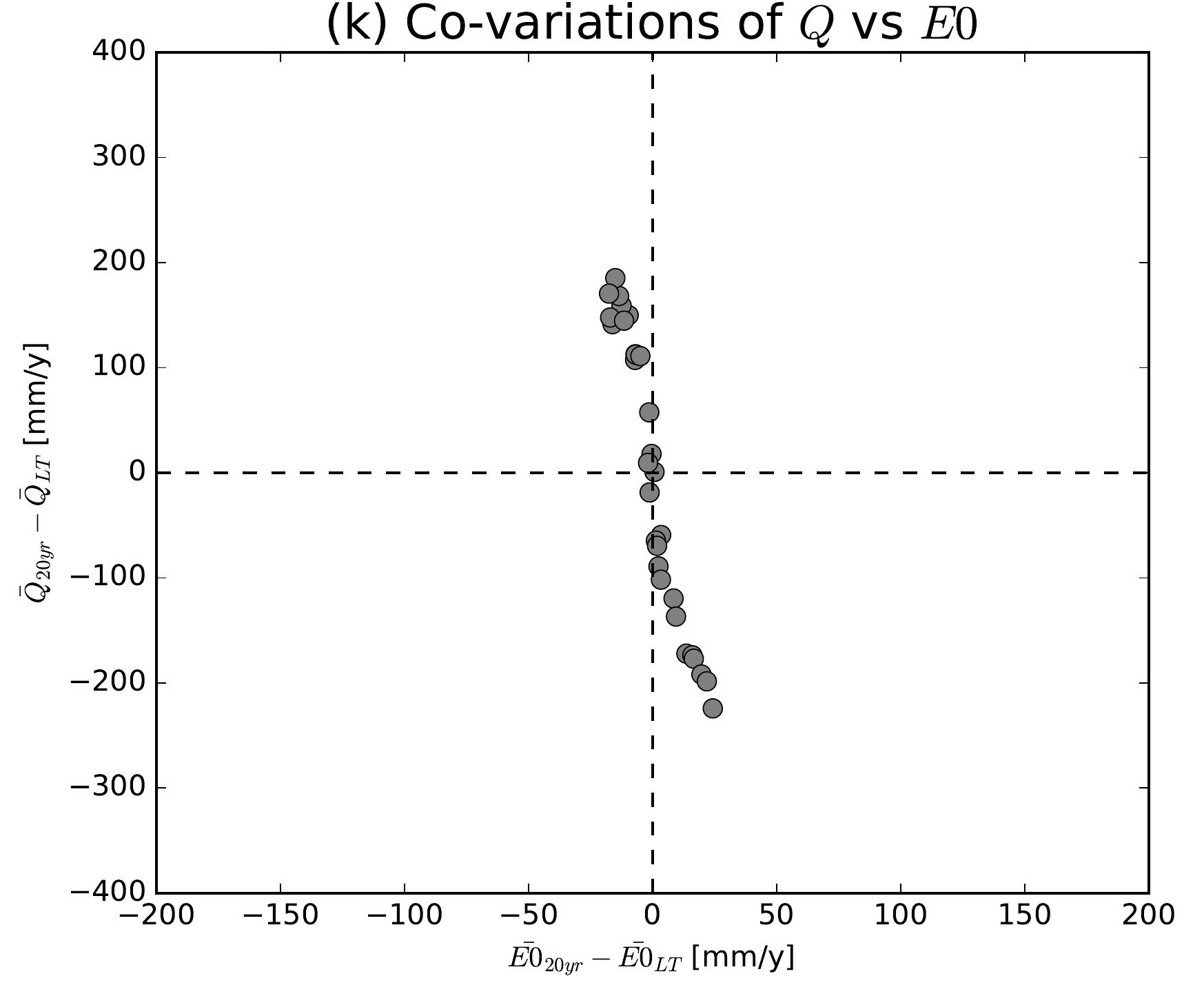

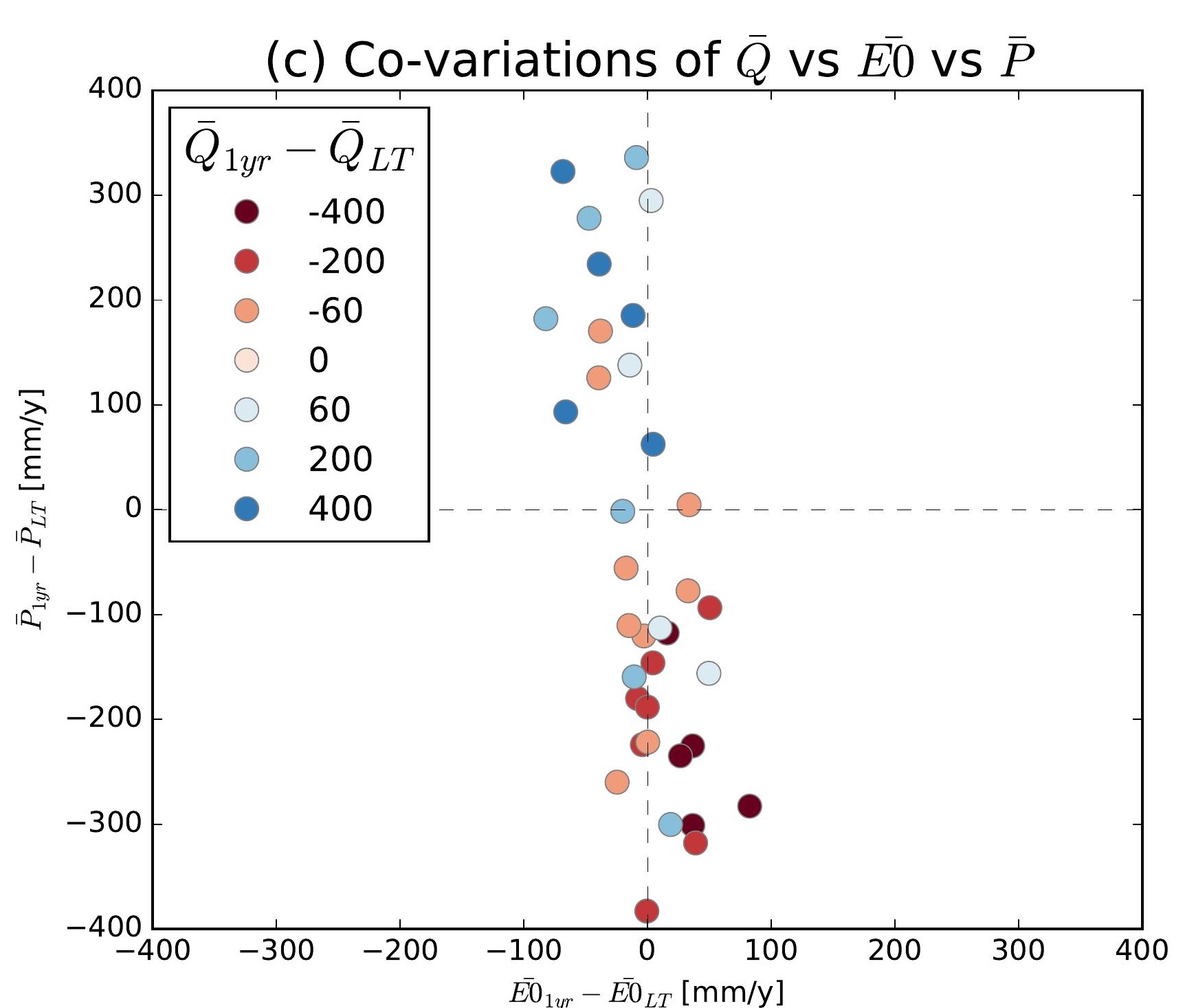
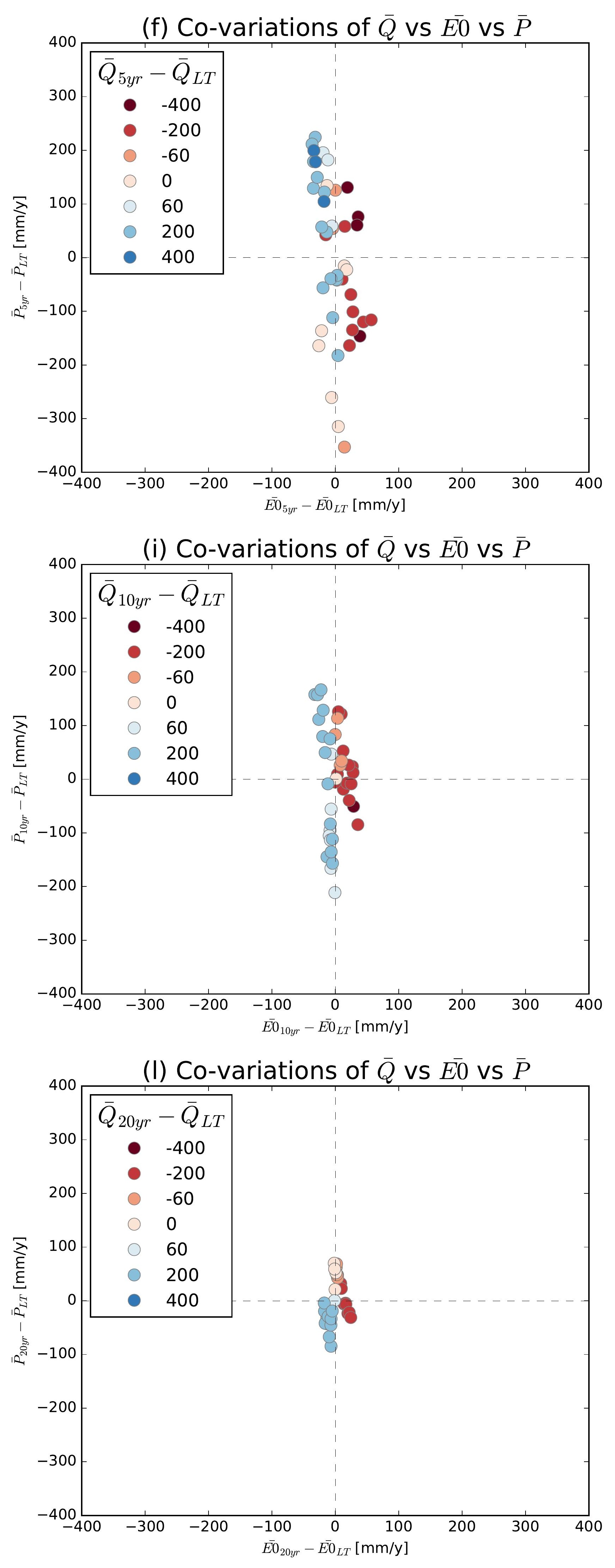

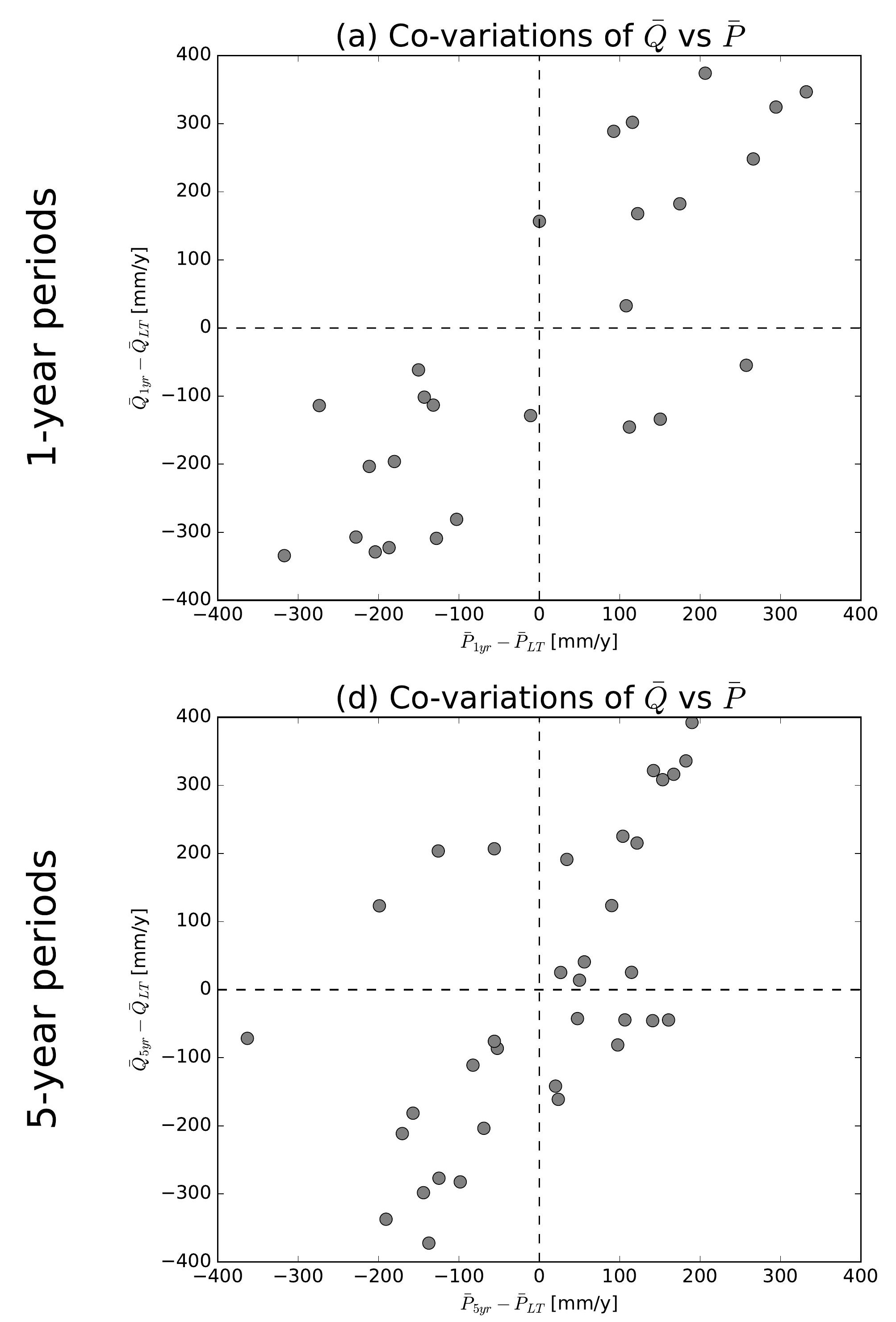

(g) Co-variations of $\bar{Q}$ vs $\bar{P}$

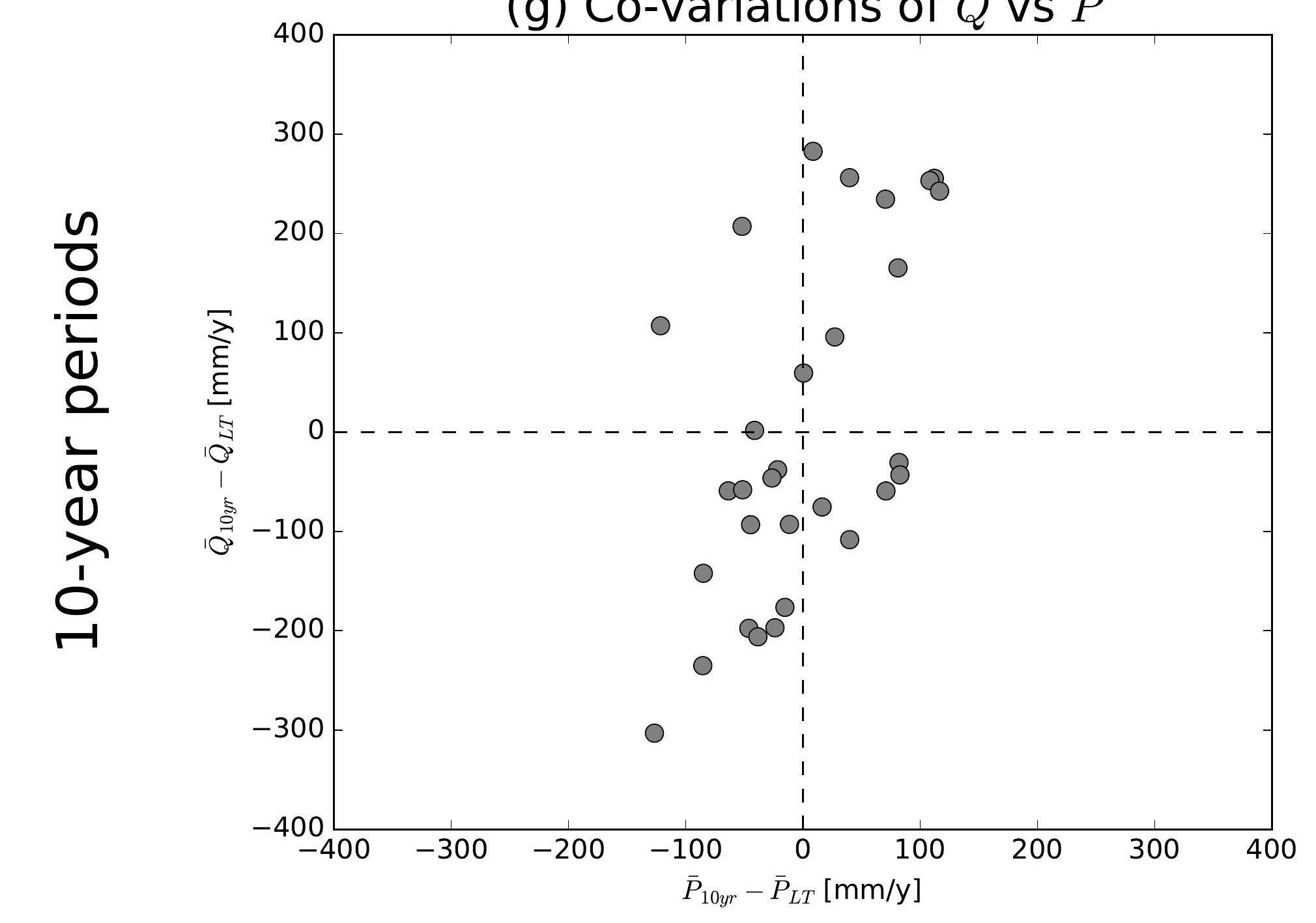

(j) Co-variations of $\bar{Q}$ vs $\bar{P}$

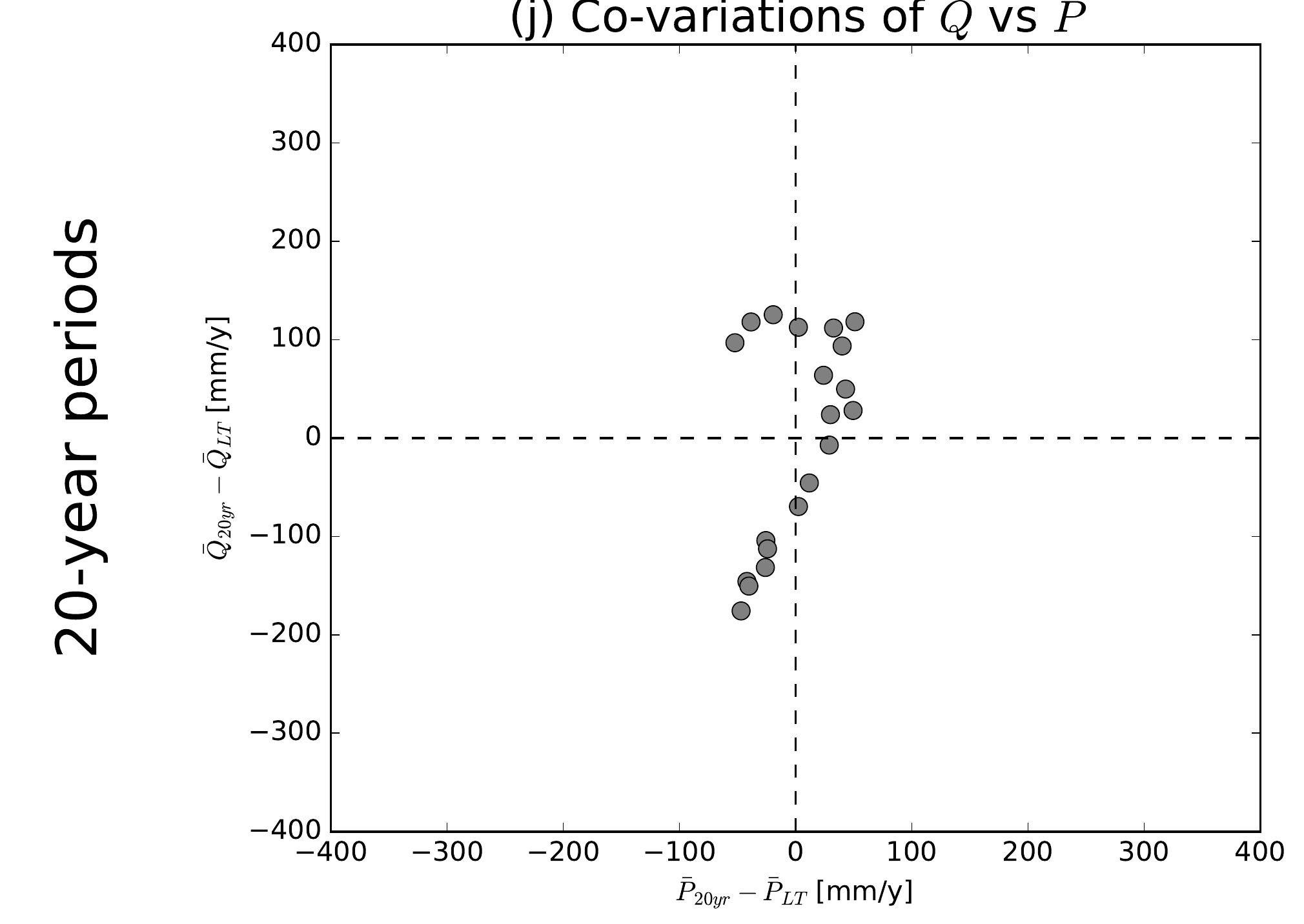

(b) Co-variations of $\bar{Q}$ vs $\overline{E 0}$

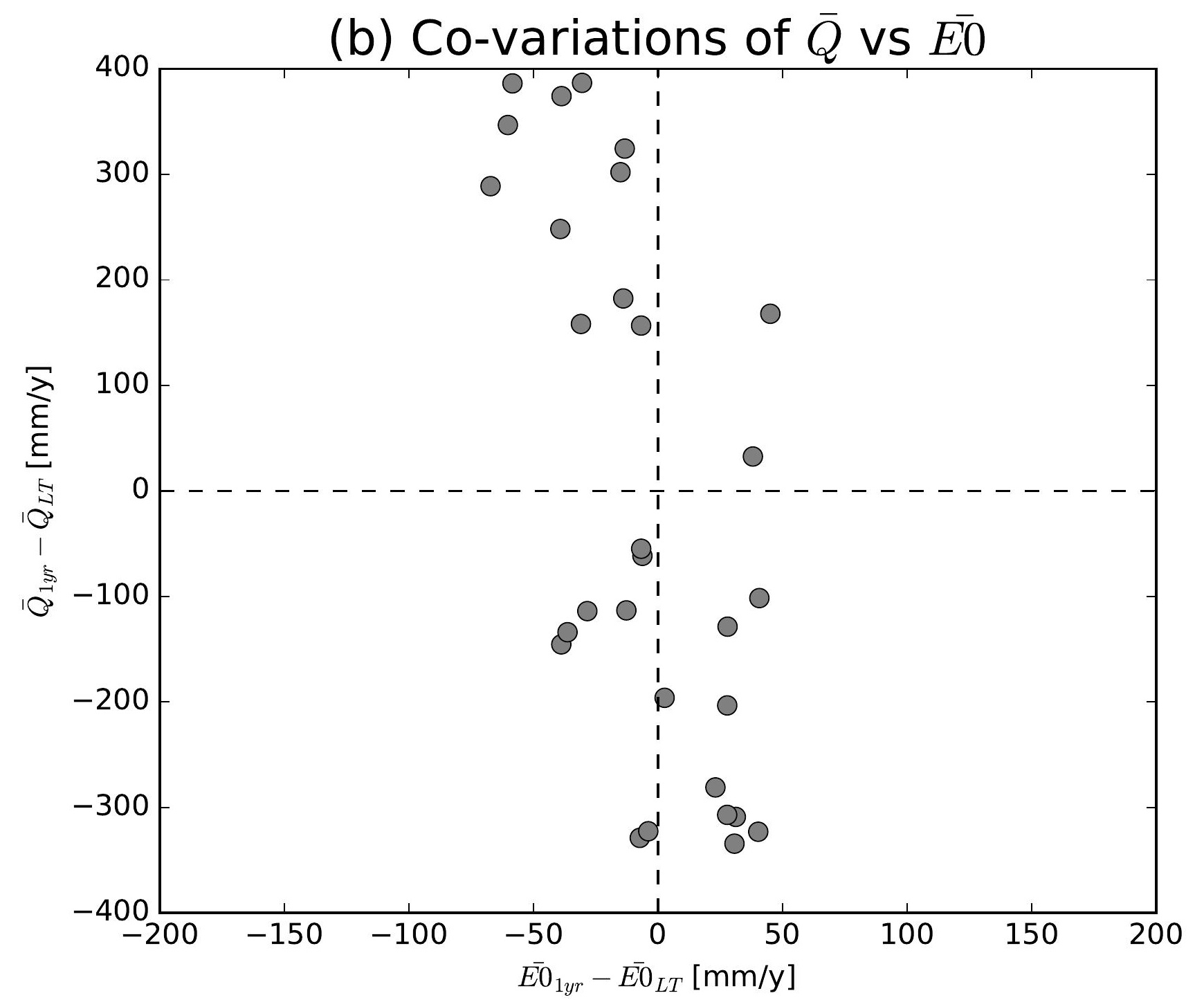

(e) Co-variations of $\bar{Q}$ vs $\overline{E 0}$

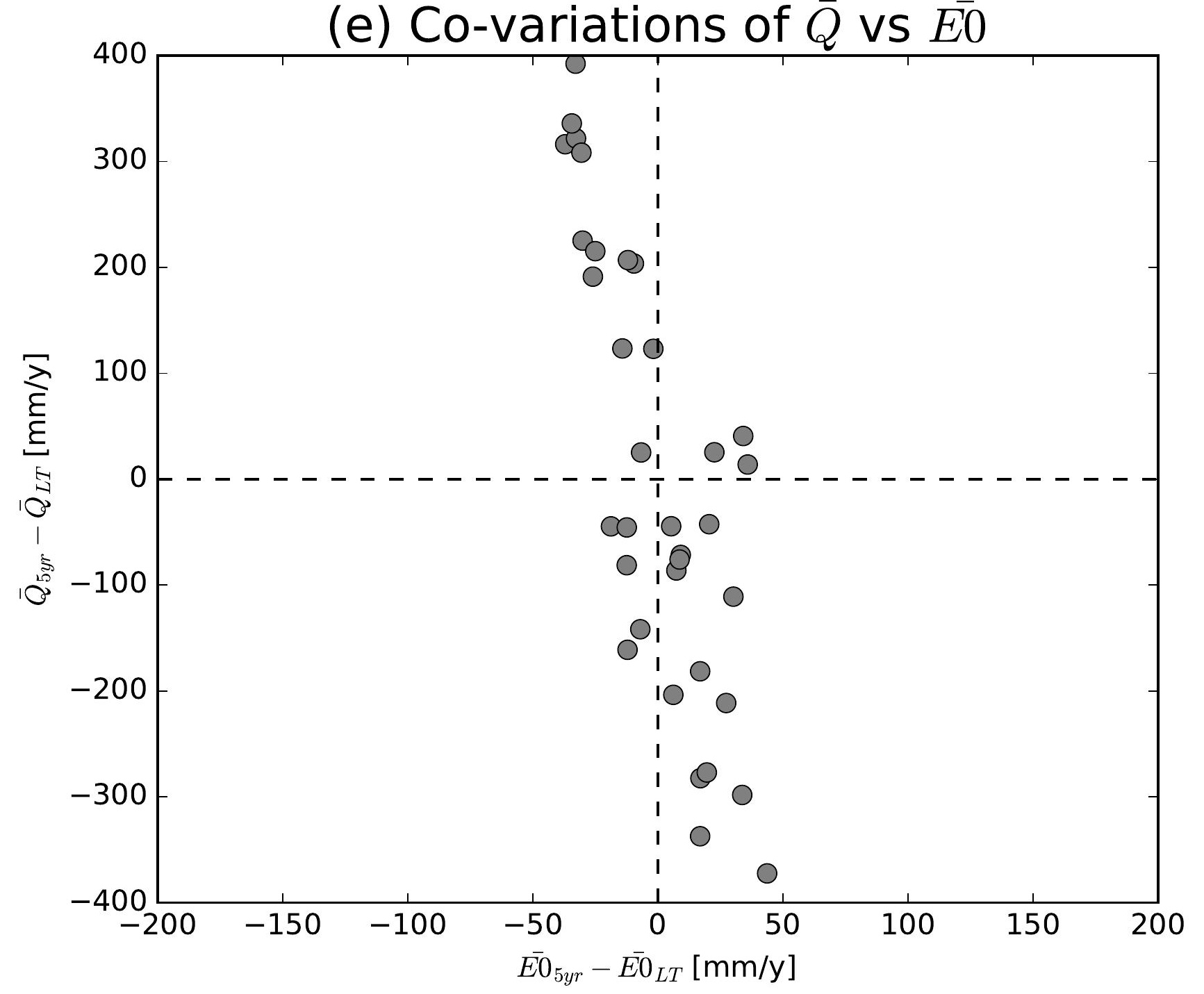

(h) Co-variations of $\bar{Q}$ vs $\overline{E 0}$

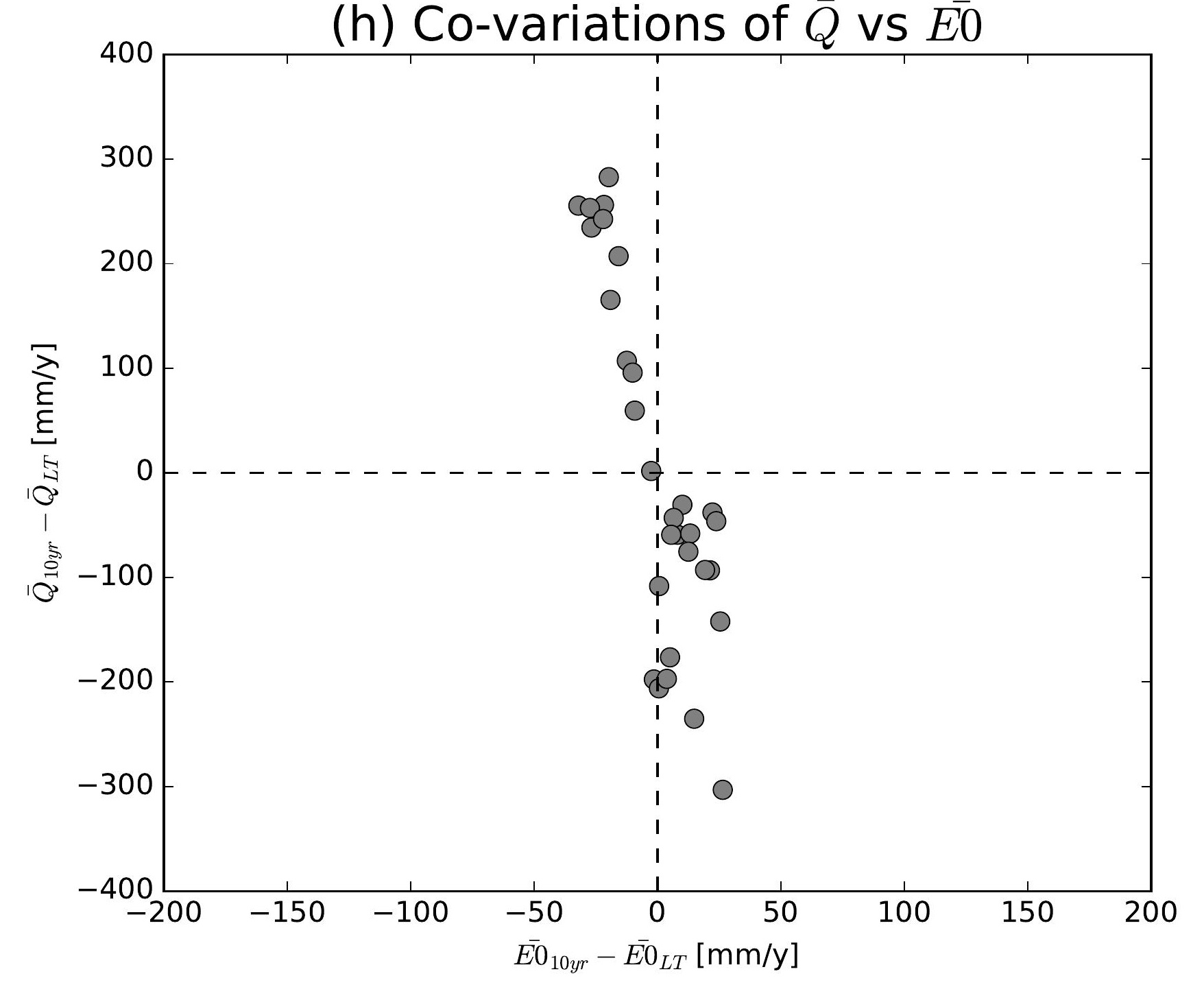

(k) Co-variations of $\bar{Q}$ vs $\overline{E 0}$

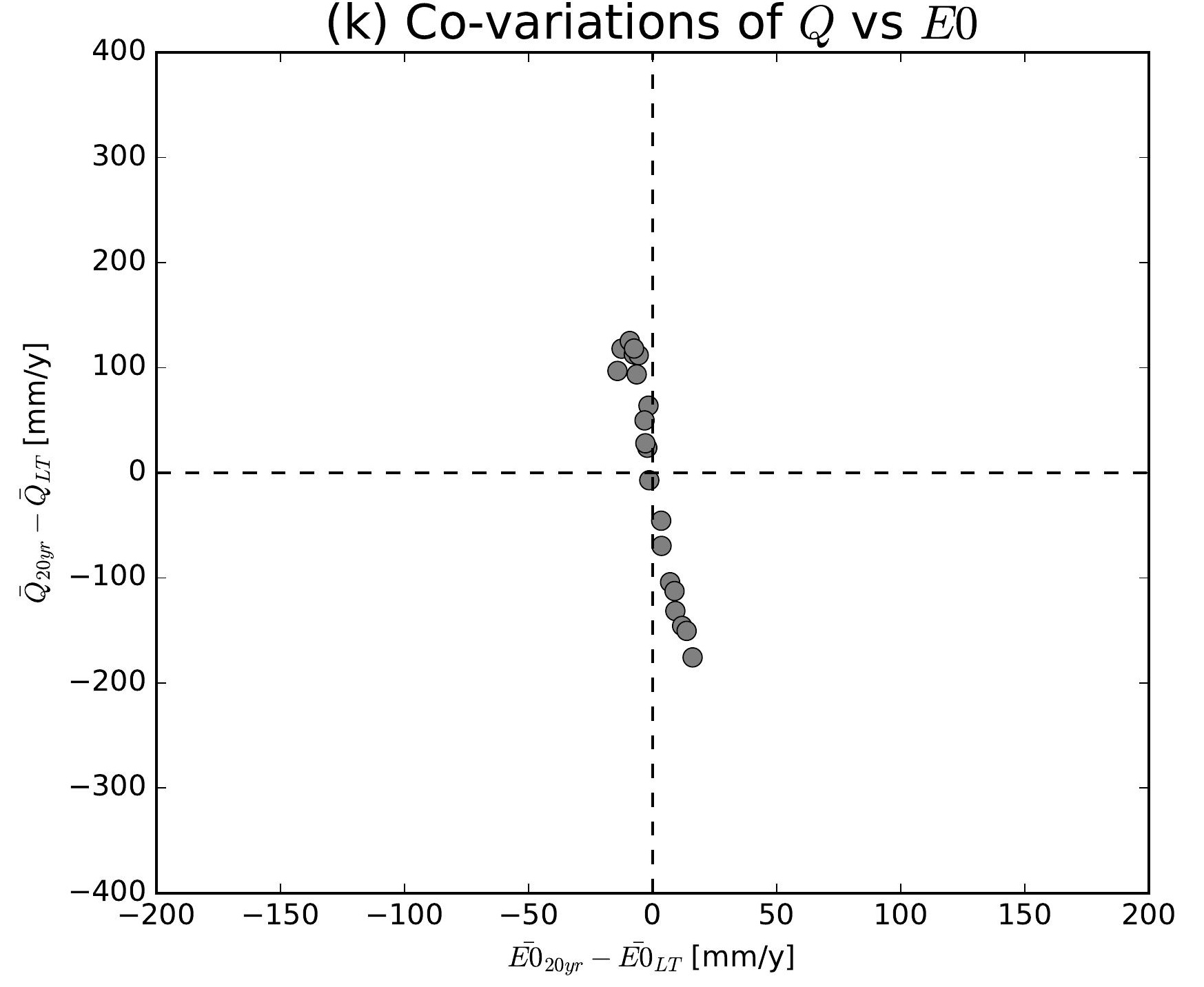

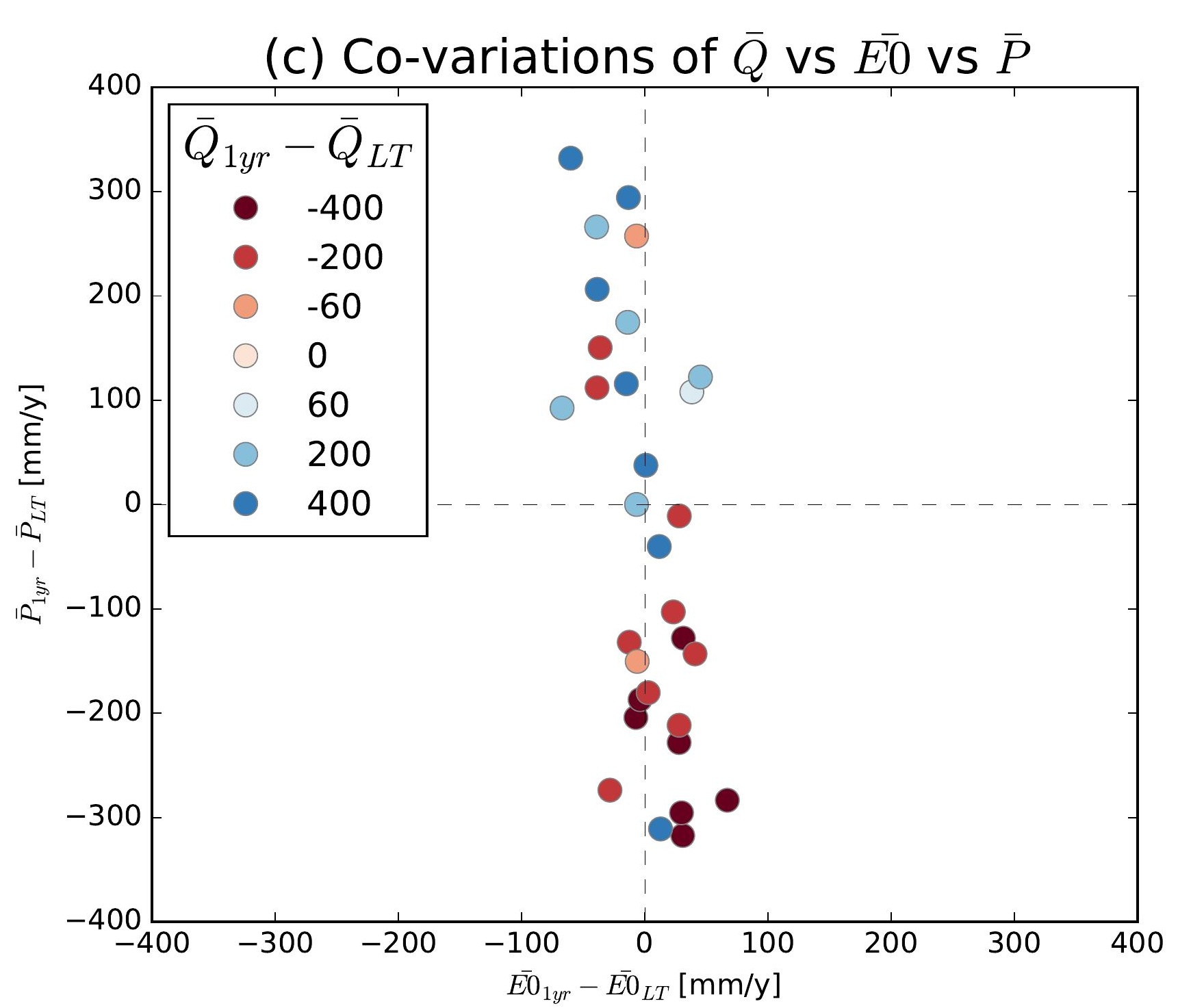
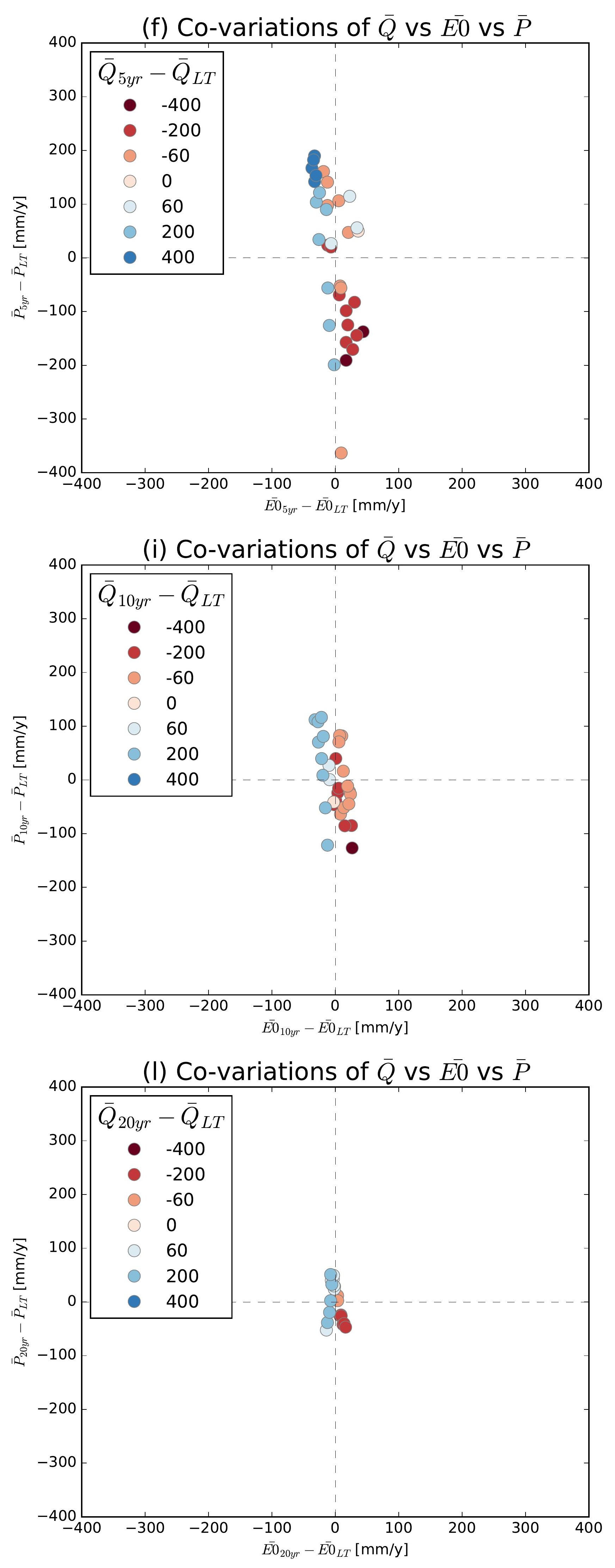

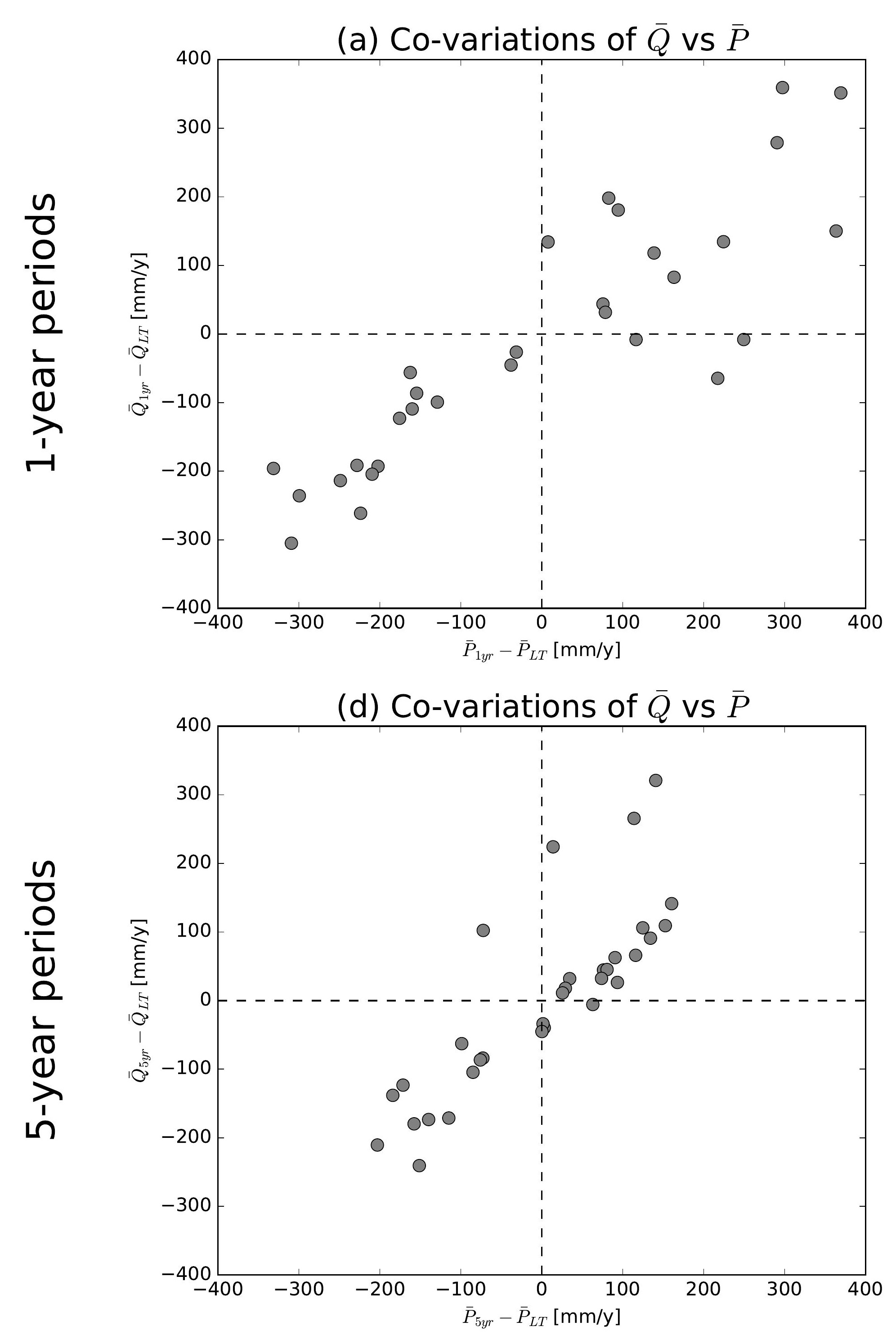

(g) Co-variations of $\bar{Q}$ vs $\bar{P}$

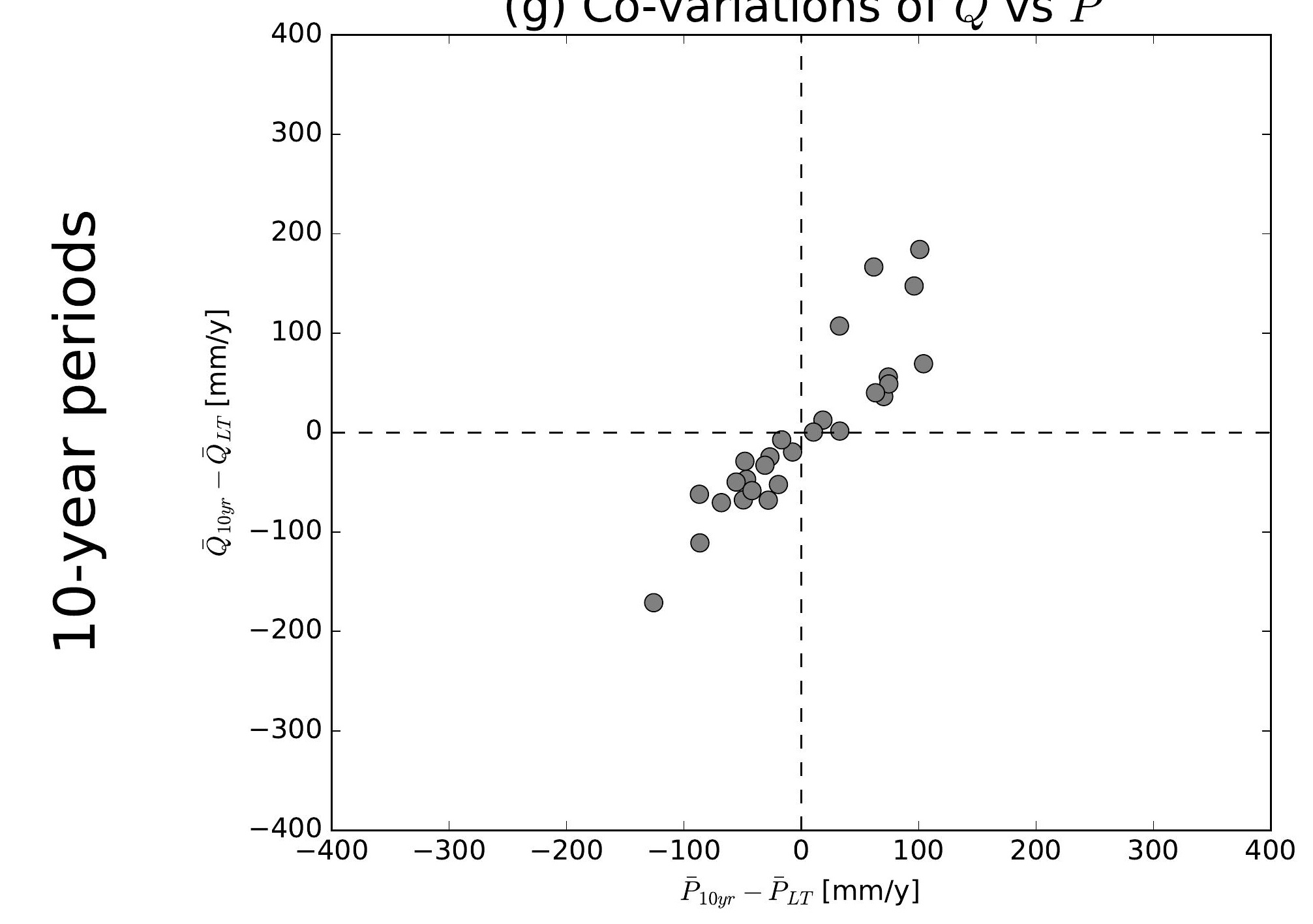

(j) Co-variations of $\bar{Q}$ vs $\bar{P}$

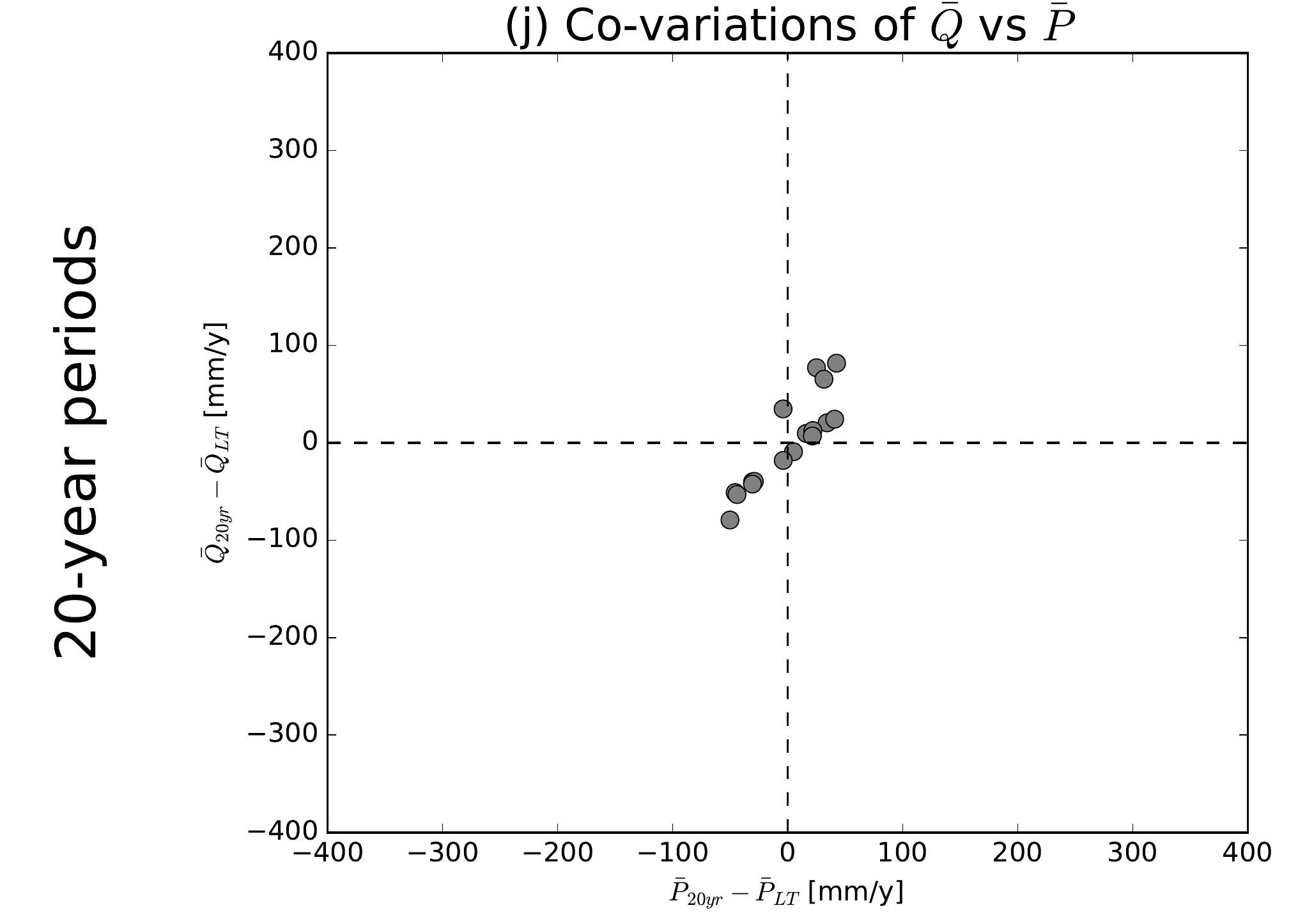

(b) Co-variations of $\bar{Q}$ vs $\overline{E 0}$

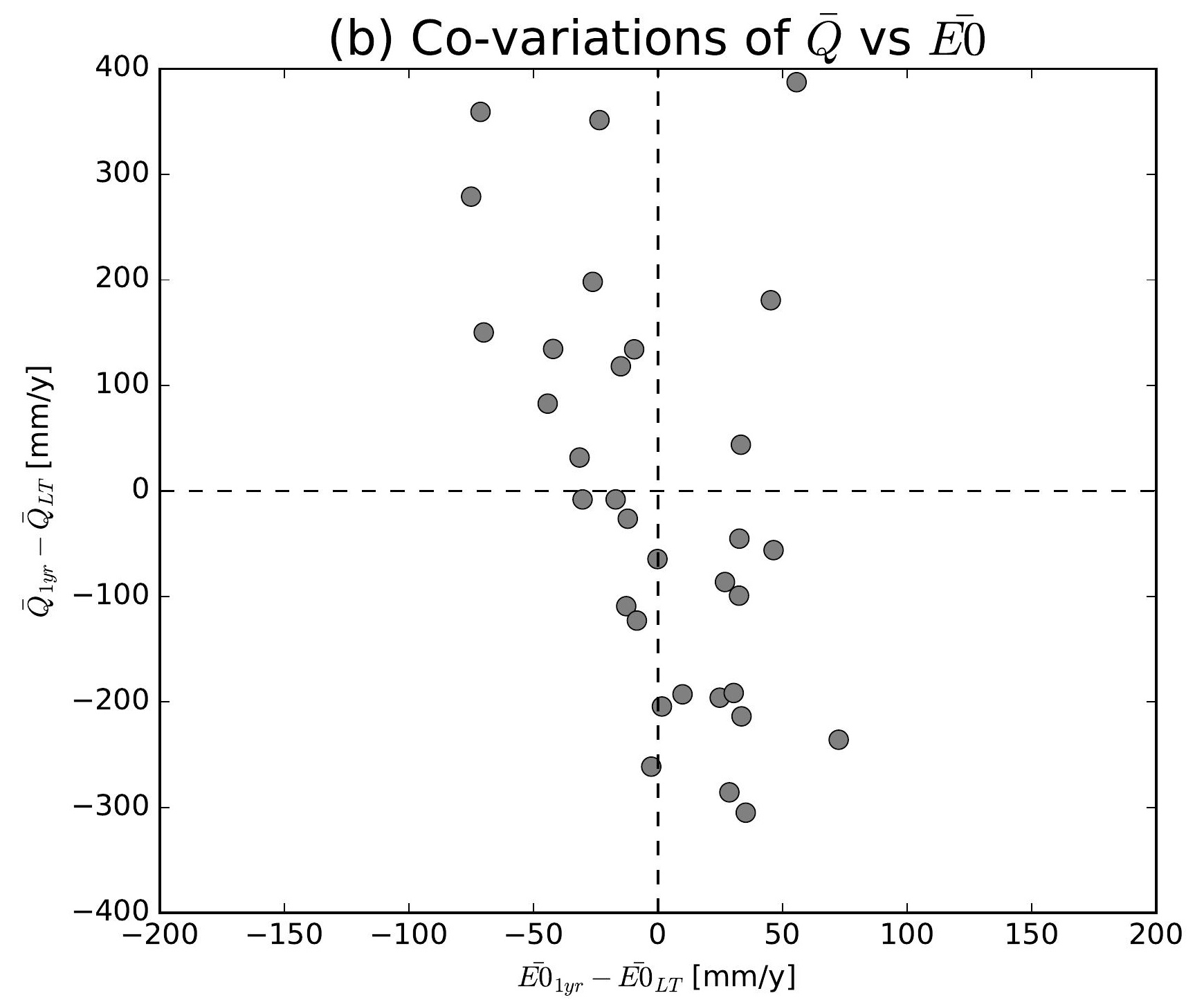

(e) Co-variations of $\bar{Q}$ vs $\overline{E 0}$

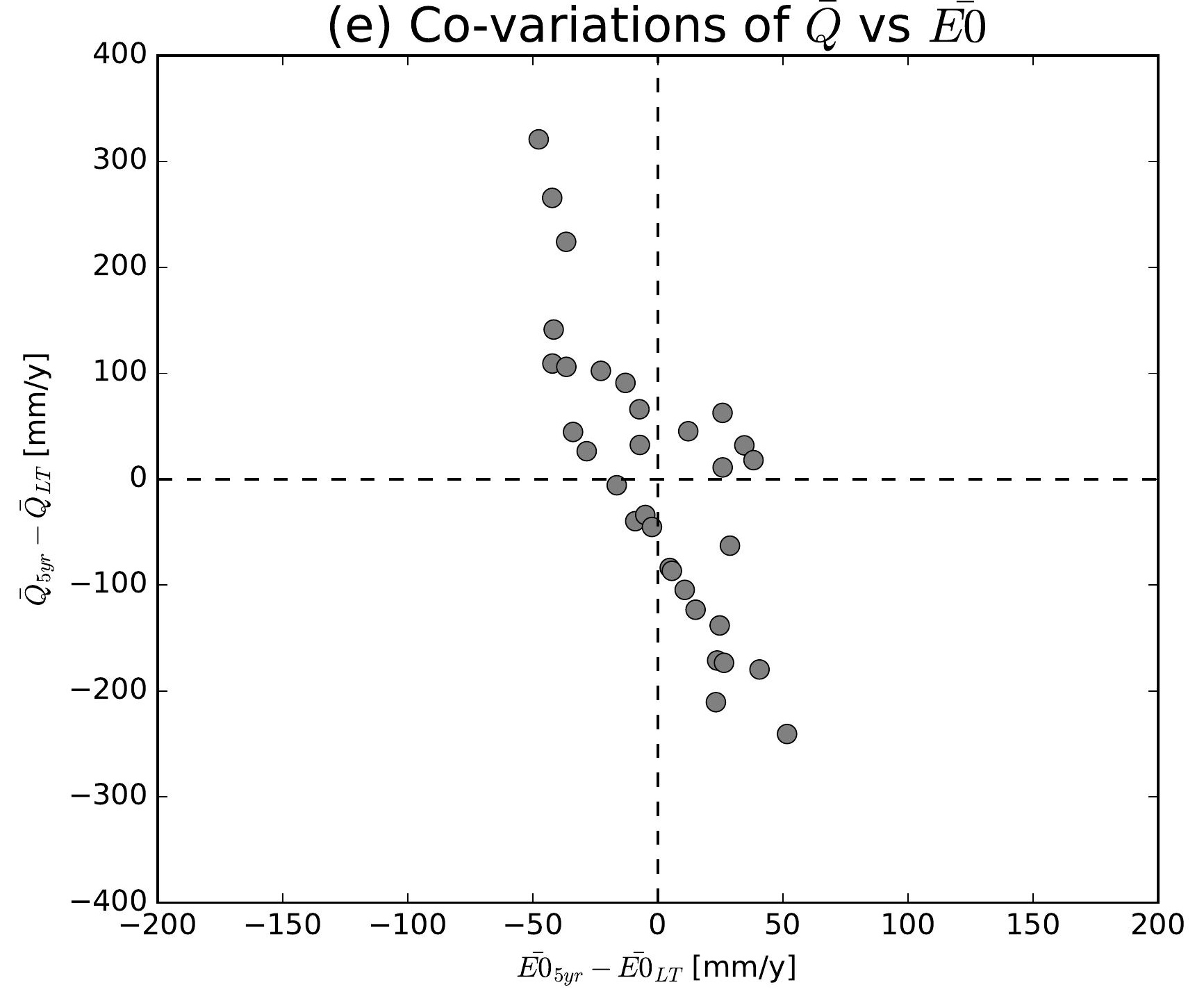

(h) Co-variations of $\bar{Q}$ vs $\overline{E 0}$

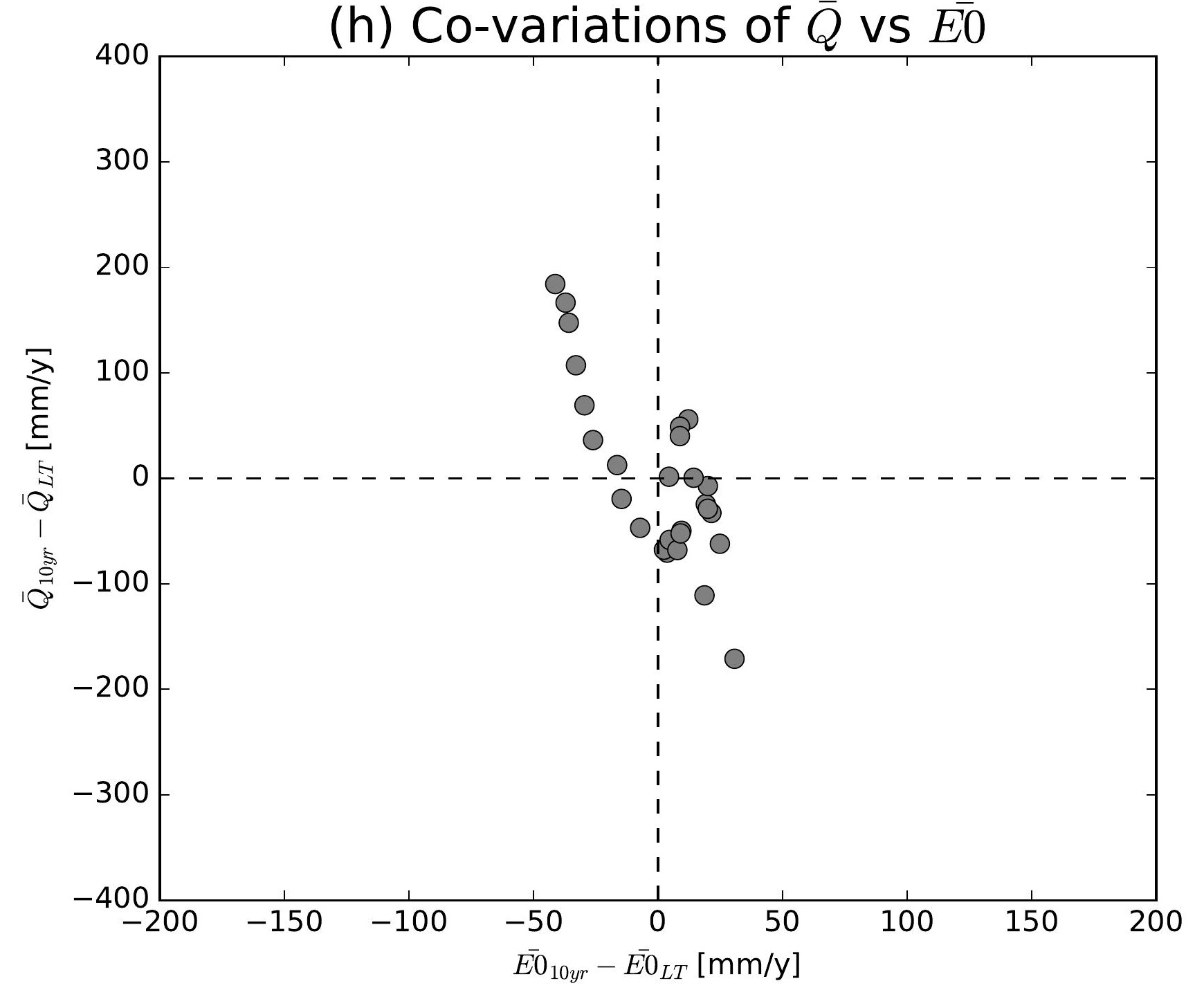

(k) Co-variations of $\bar{Q}$ vs $\overline{E 0}$

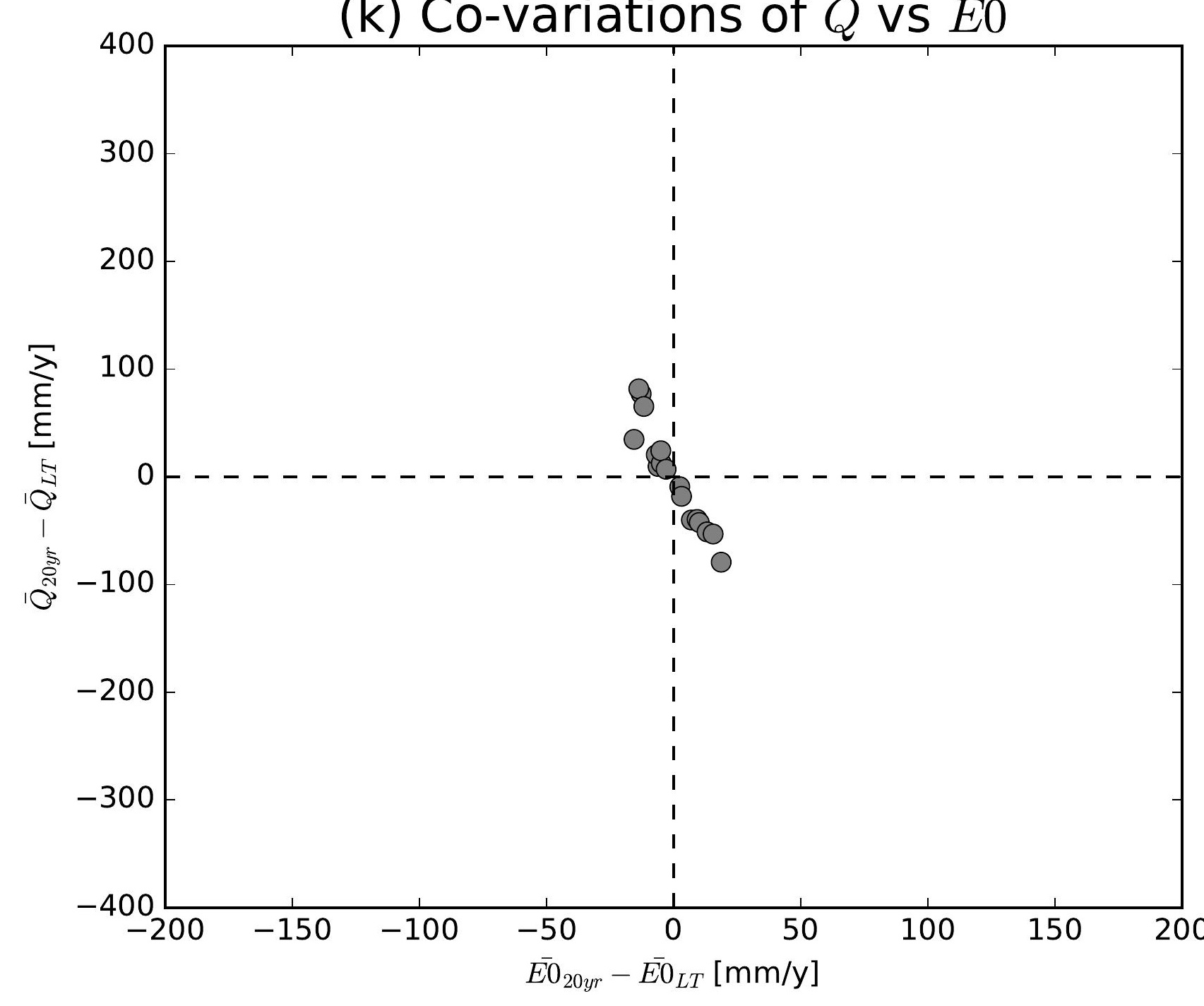

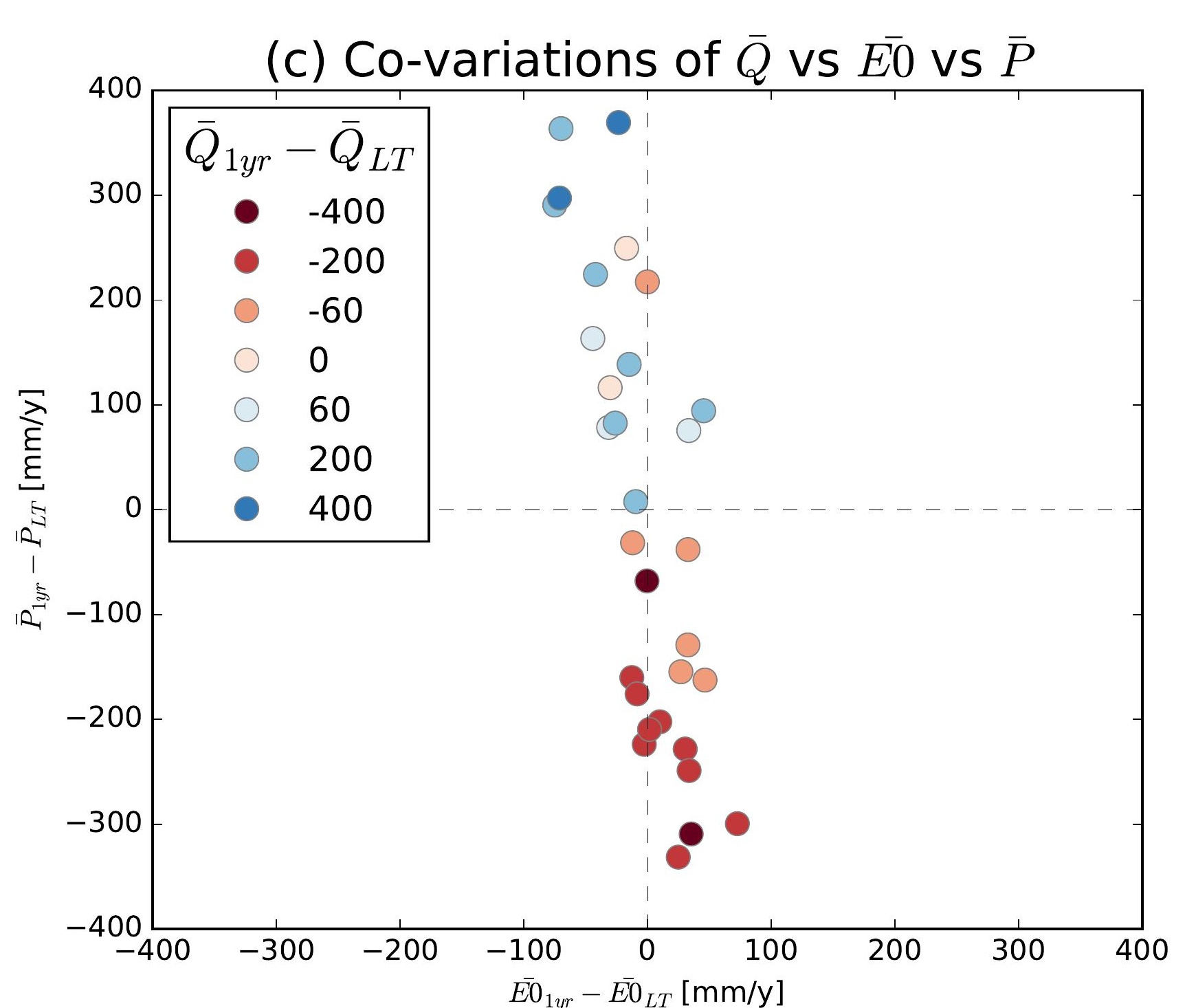
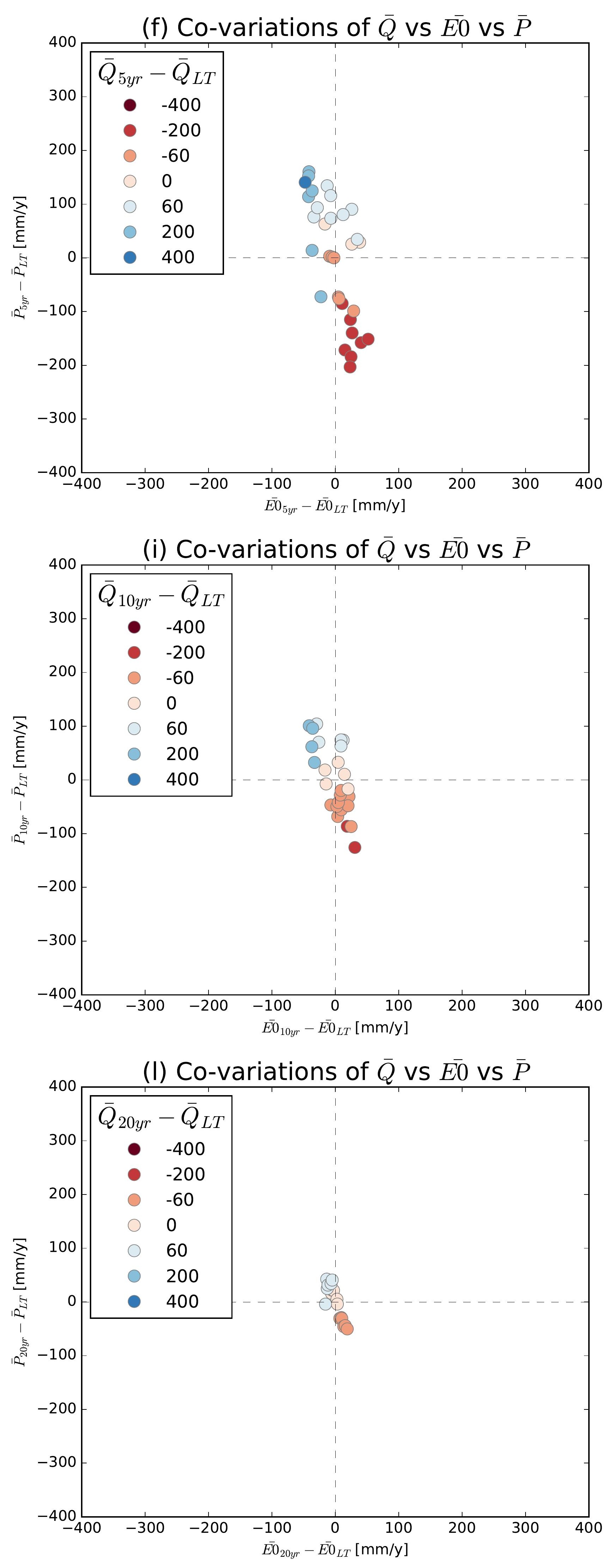

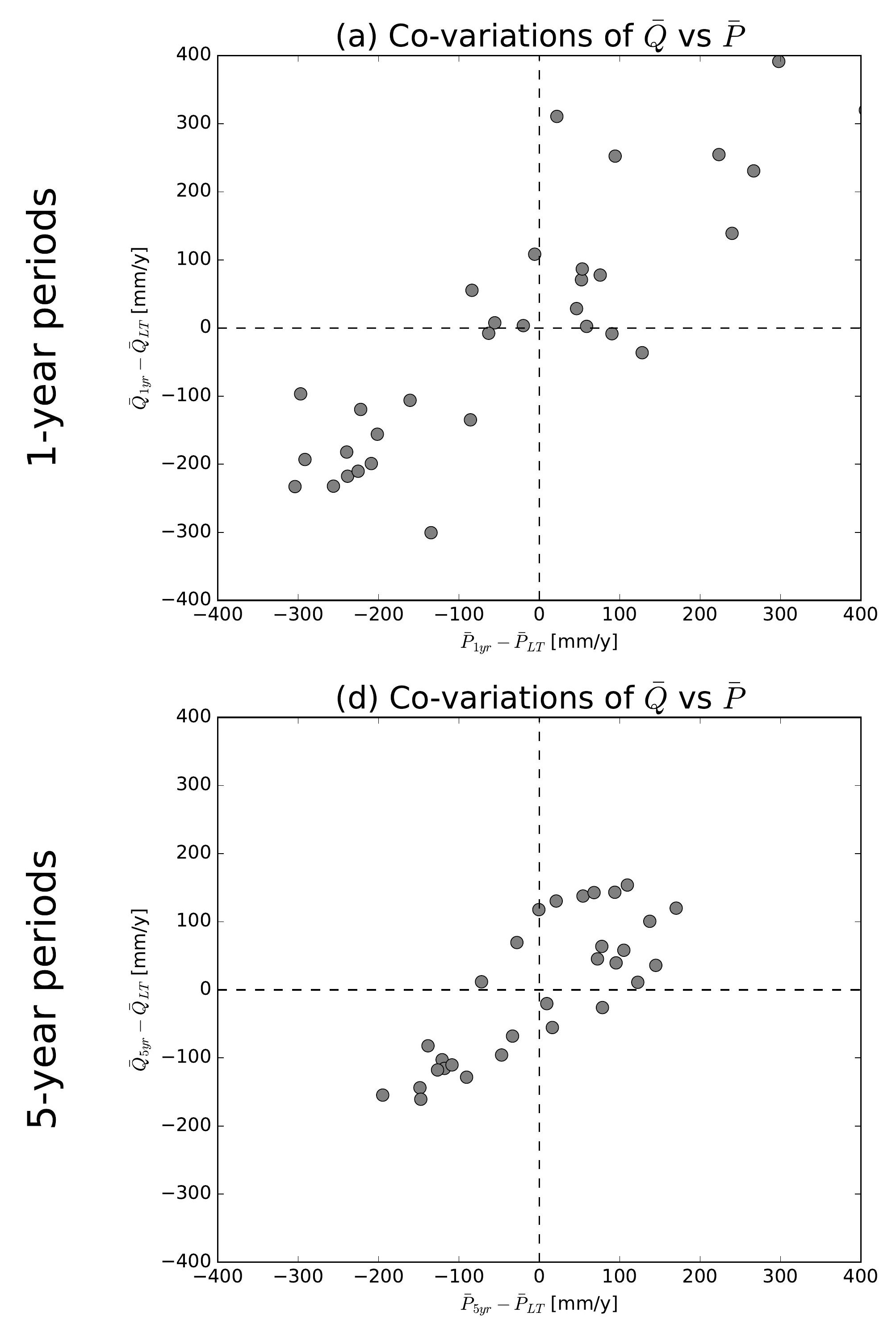

(g) Co-variations of $\bar{Q}$ vs $\bar{P}$

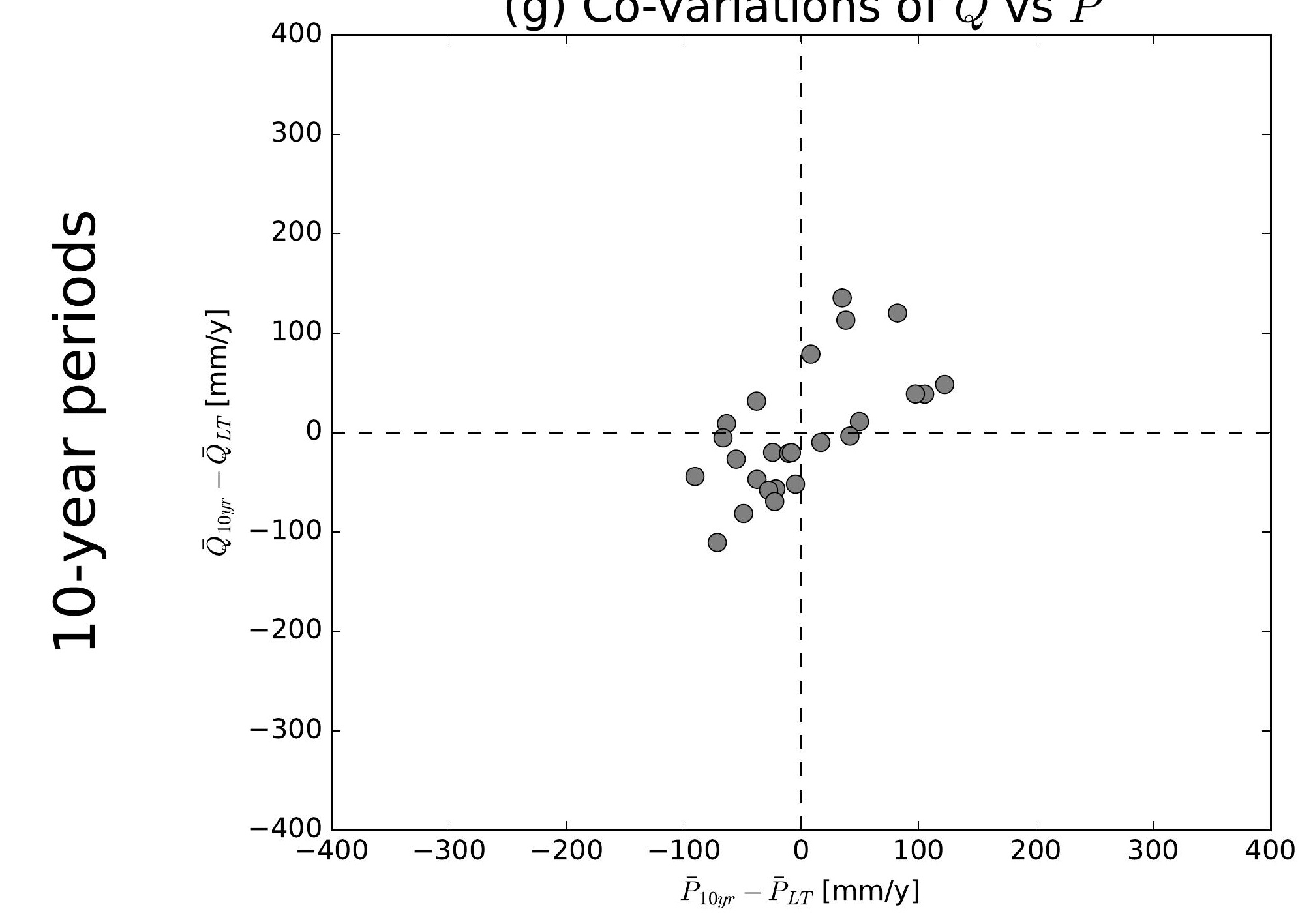

(j) Co-variations of $\bar{Q}$ vs $\bar{P}$

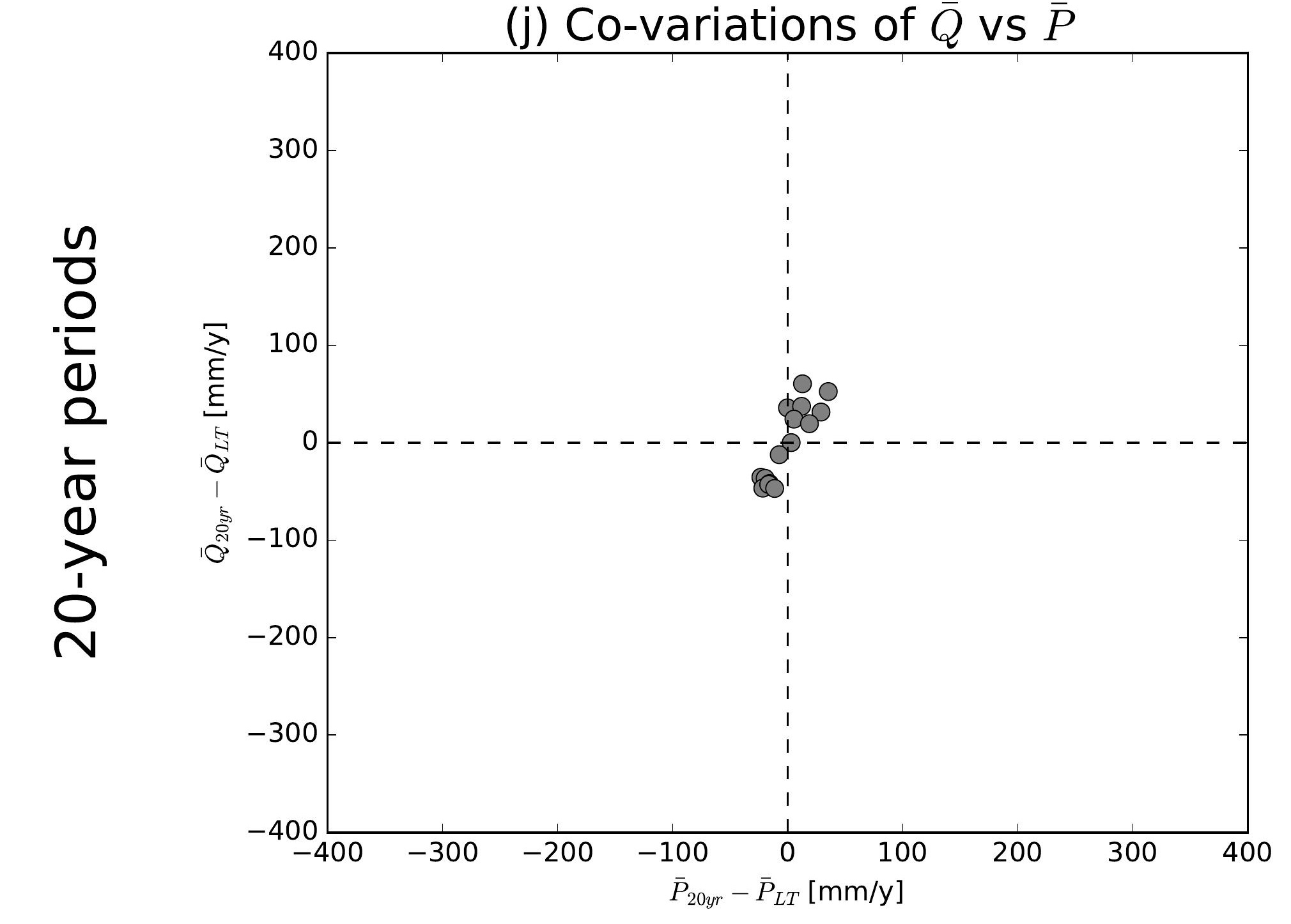

(b) Co-variations of $\bar{Q}$ vs $\overline{E 0}$

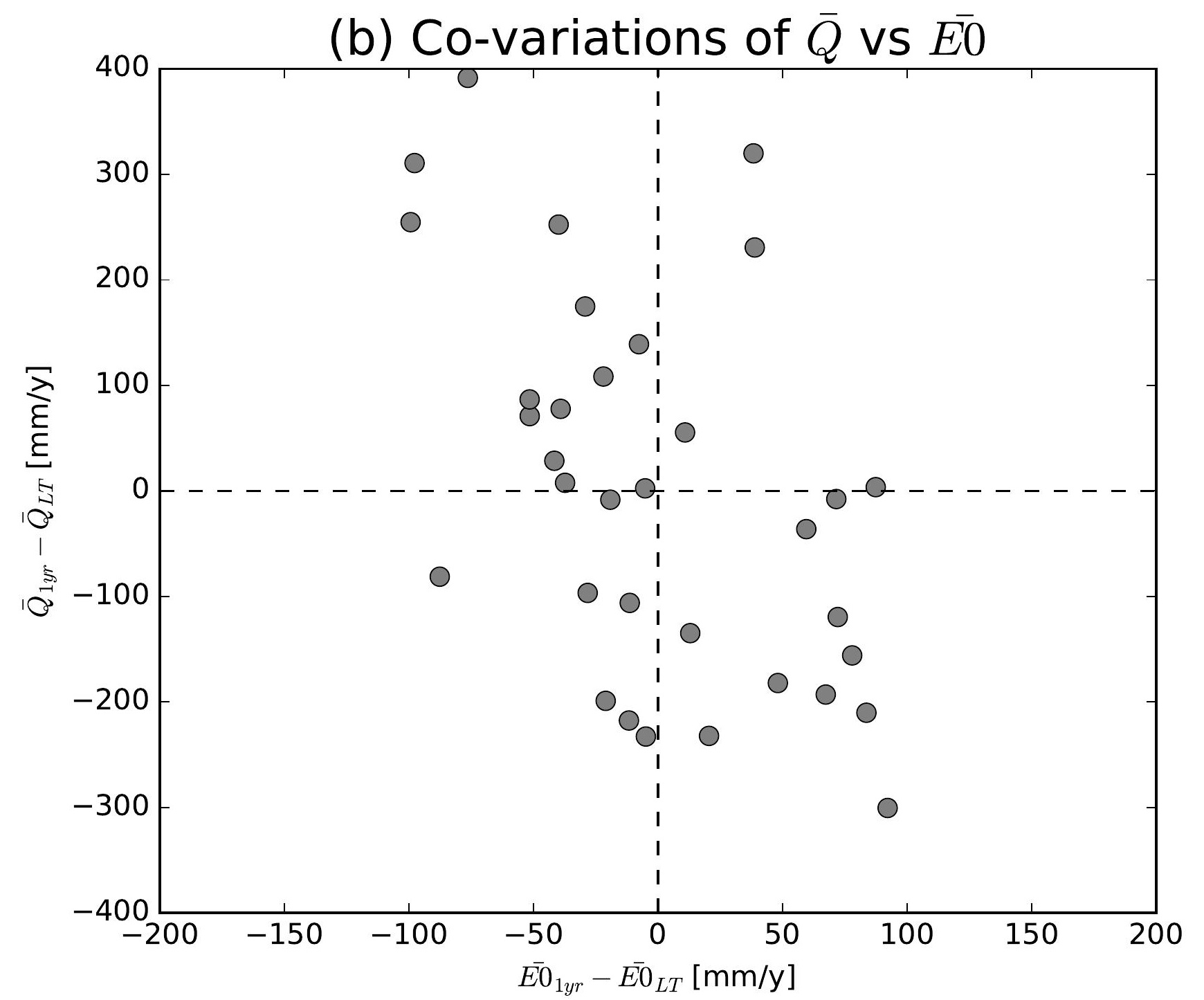

(e) Co-variations of $\bar{Q}$ vs $\overline{E 0}$

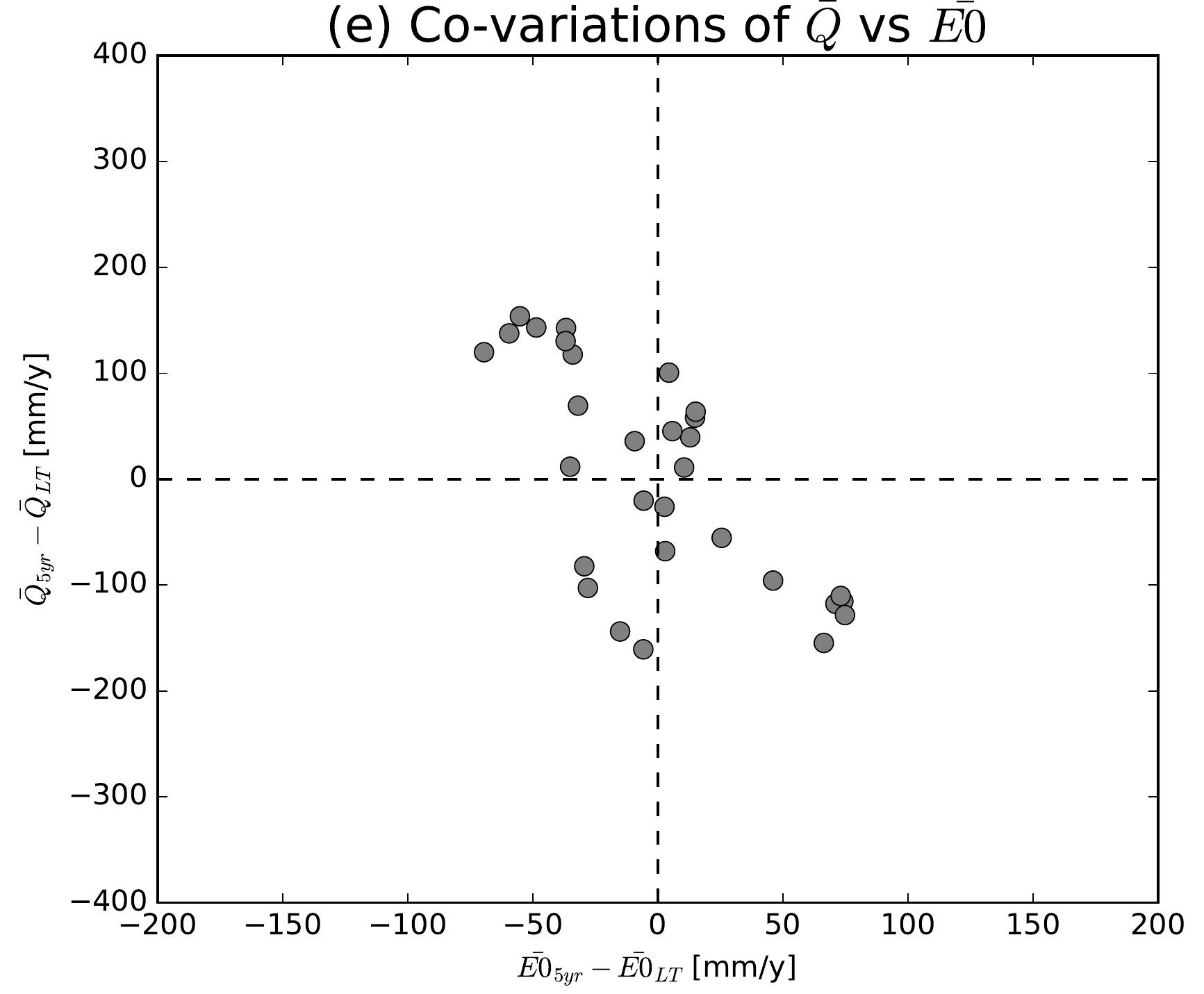

(h) Co-variations of $\bar{Q}$ vs $\overline{E 0}$

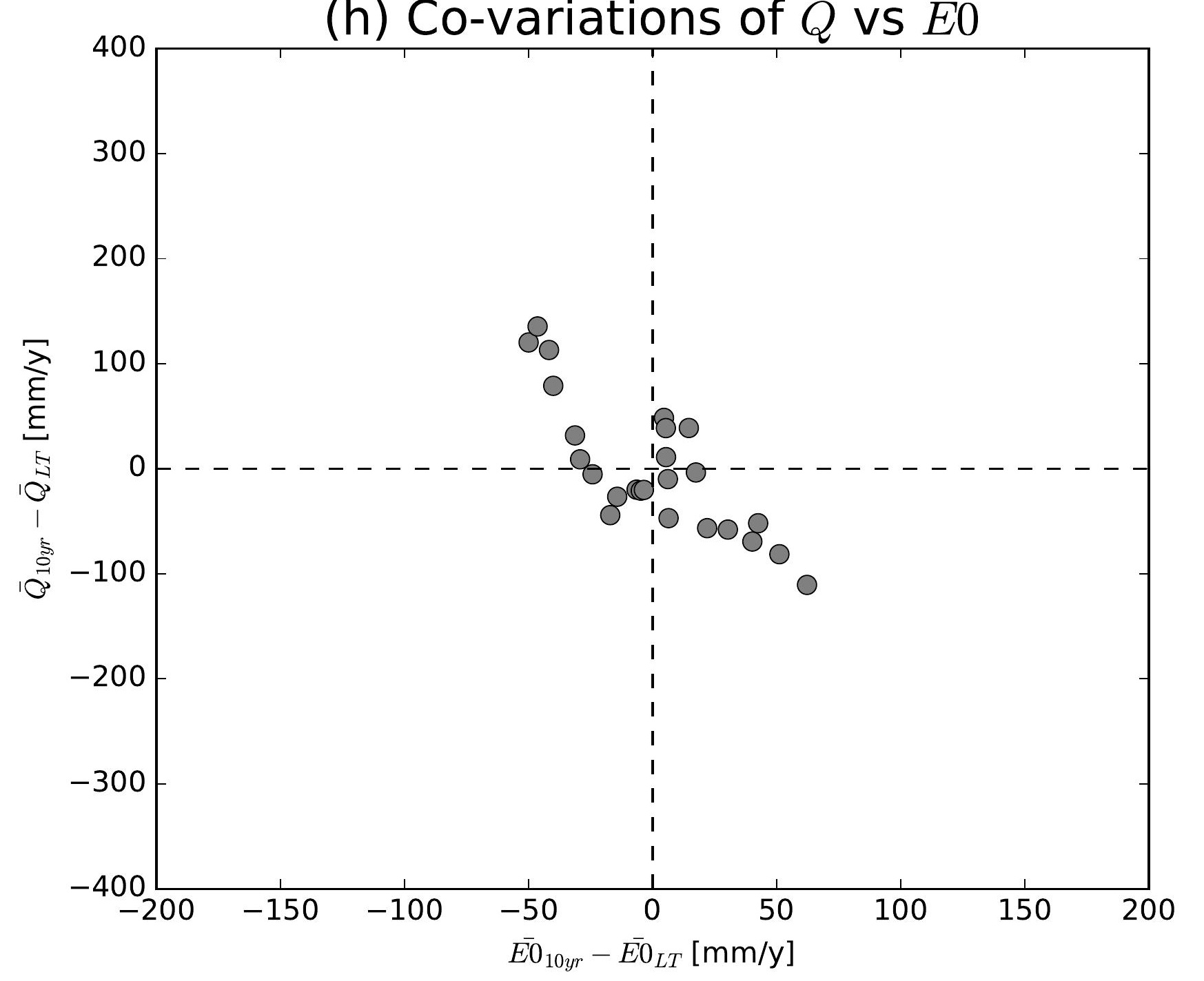

(k) Co-variations of $\bar{Q}$ vs $\overline{E 0}$

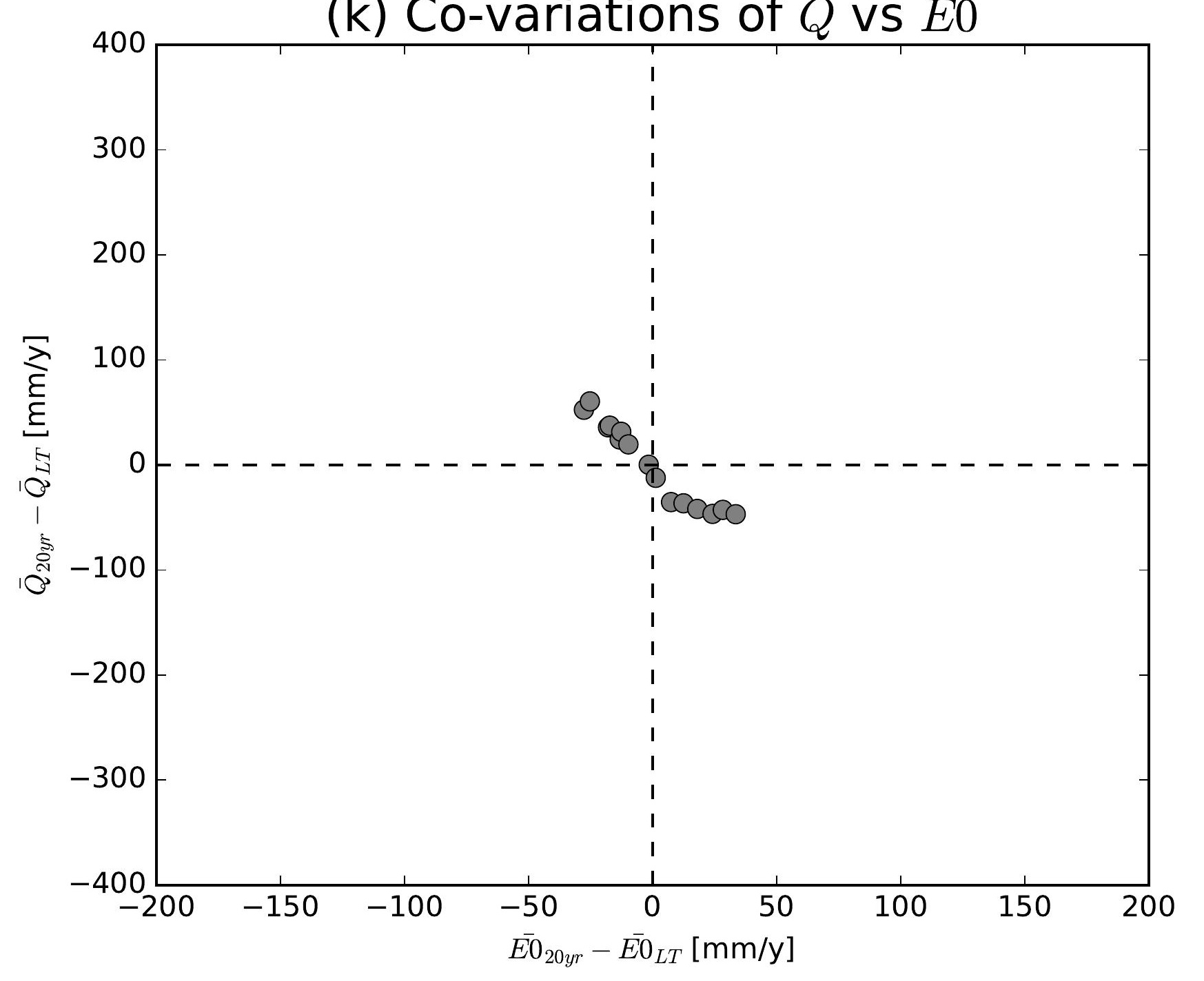

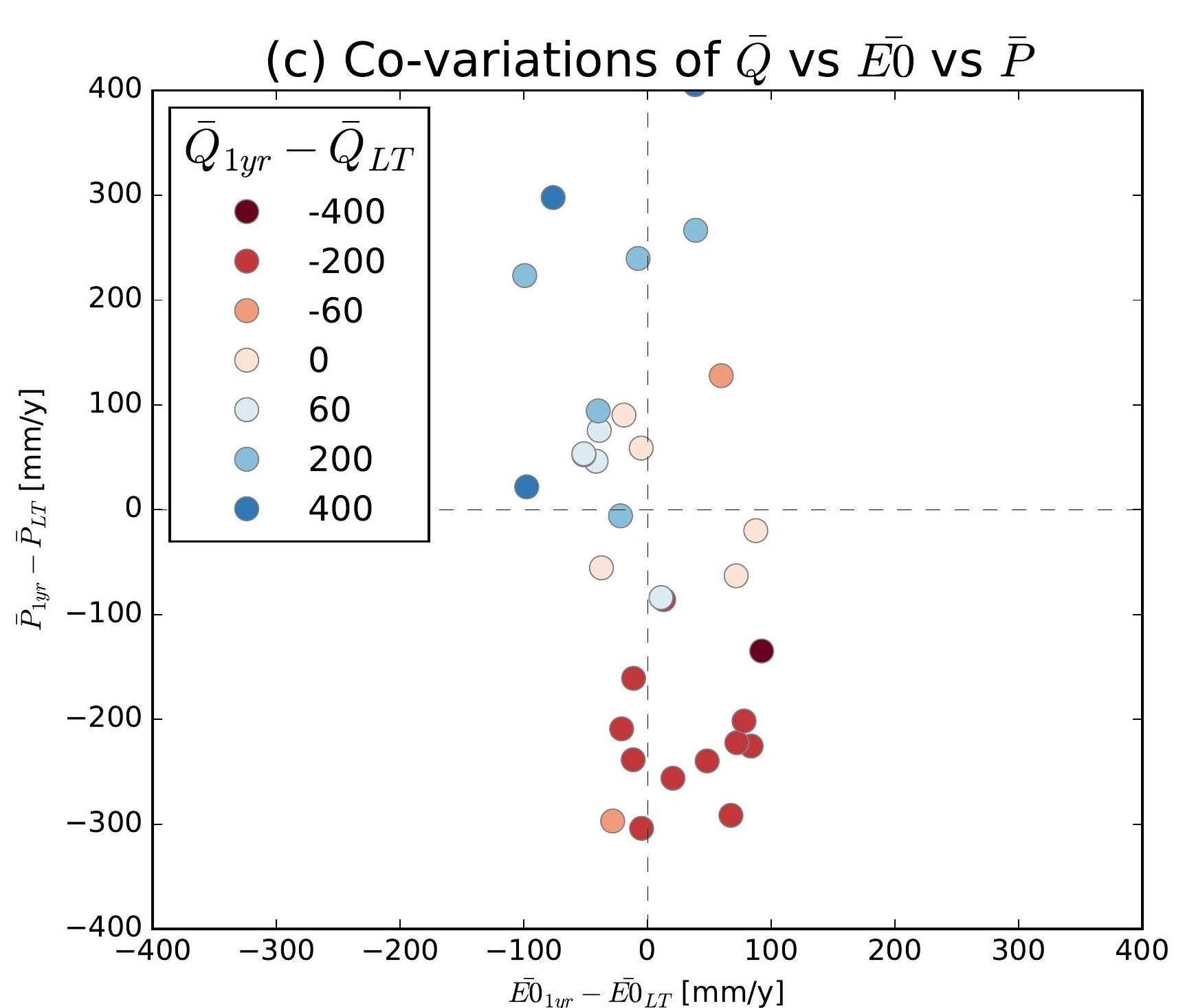
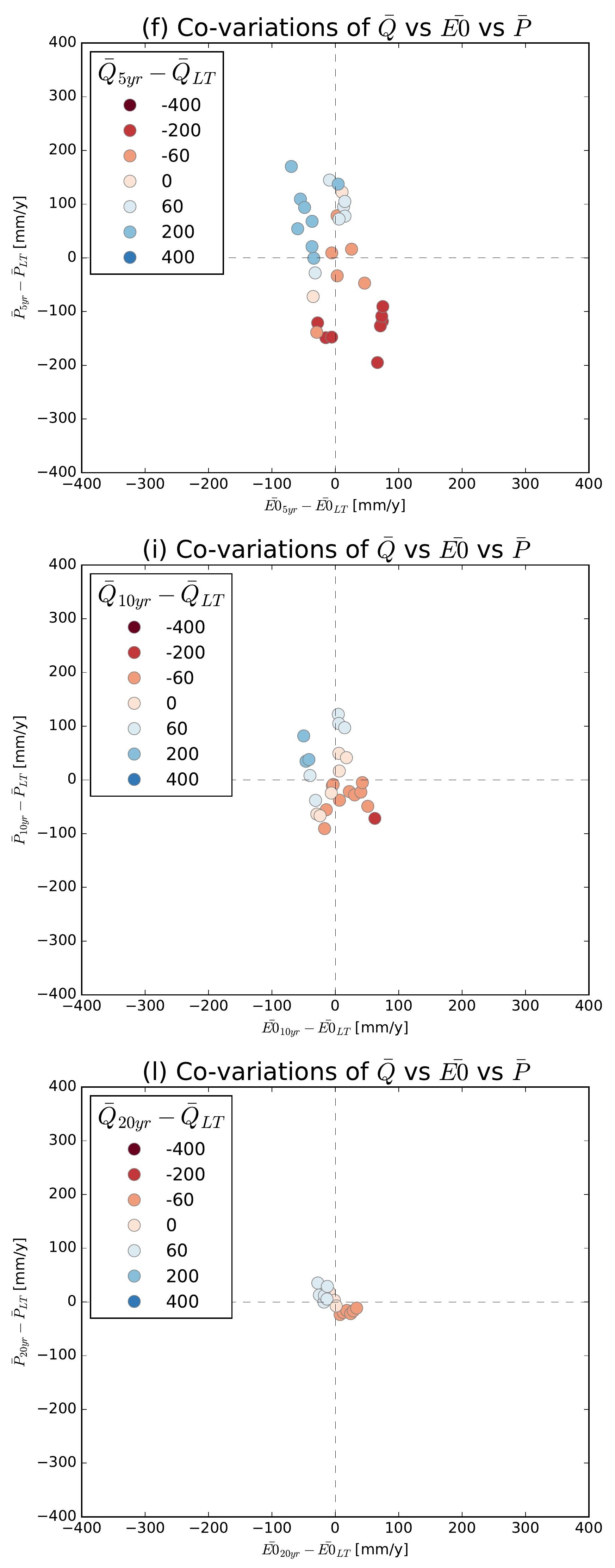

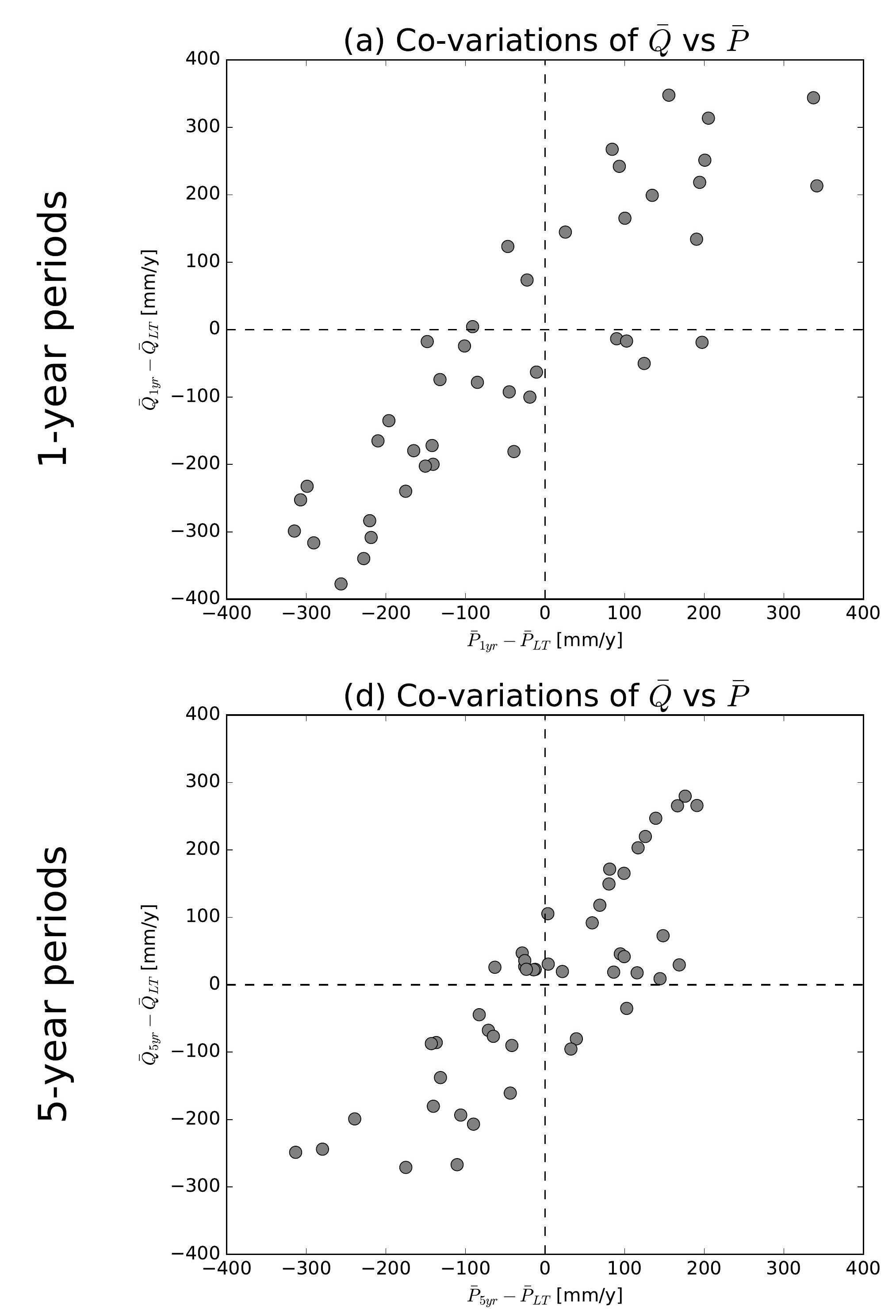

(g) Co-variations of $\bar{Q}$ vs $\bar{P}$

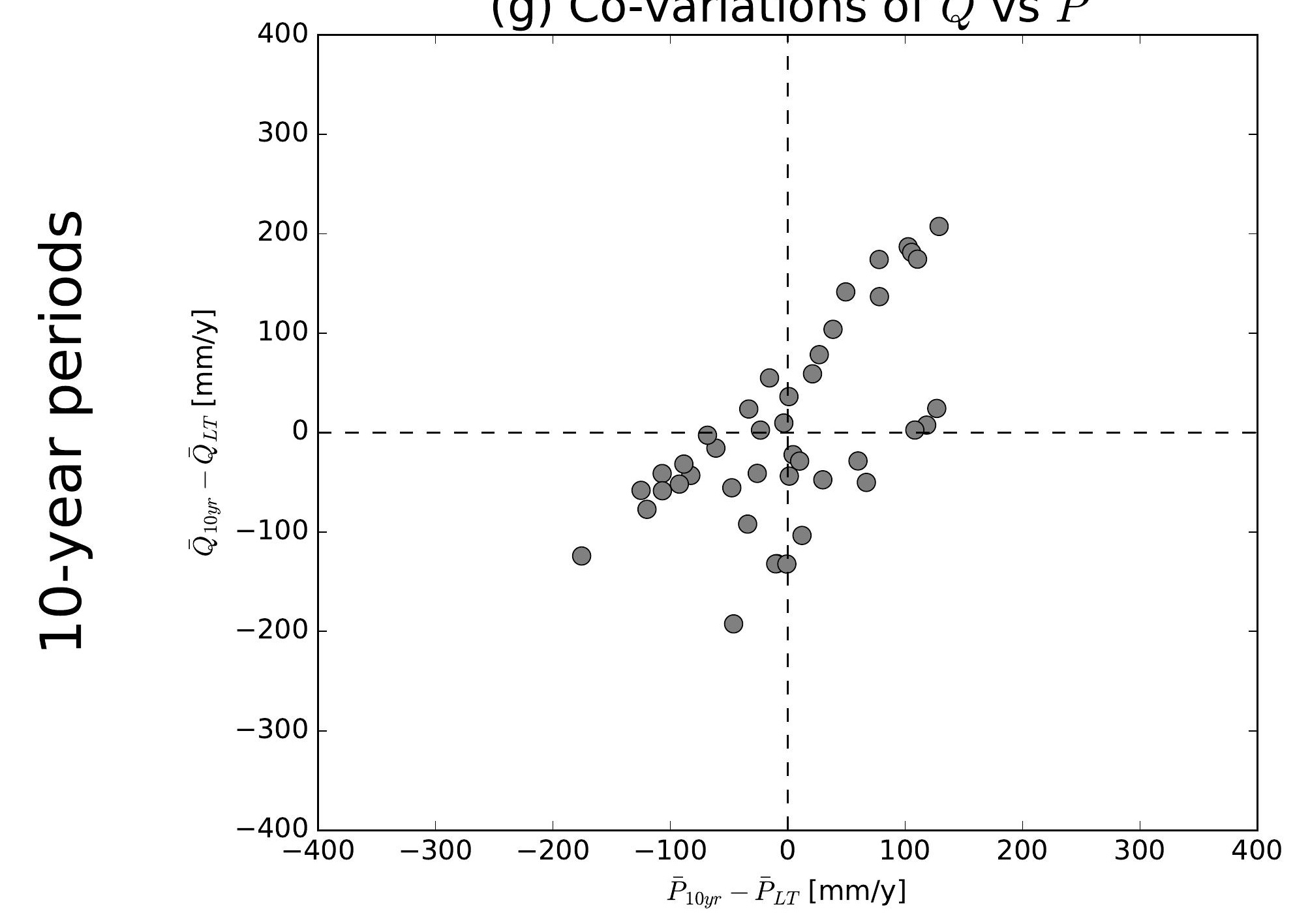

(j) Co-variations of $\bar{Q}$ vs $\bar{P}$

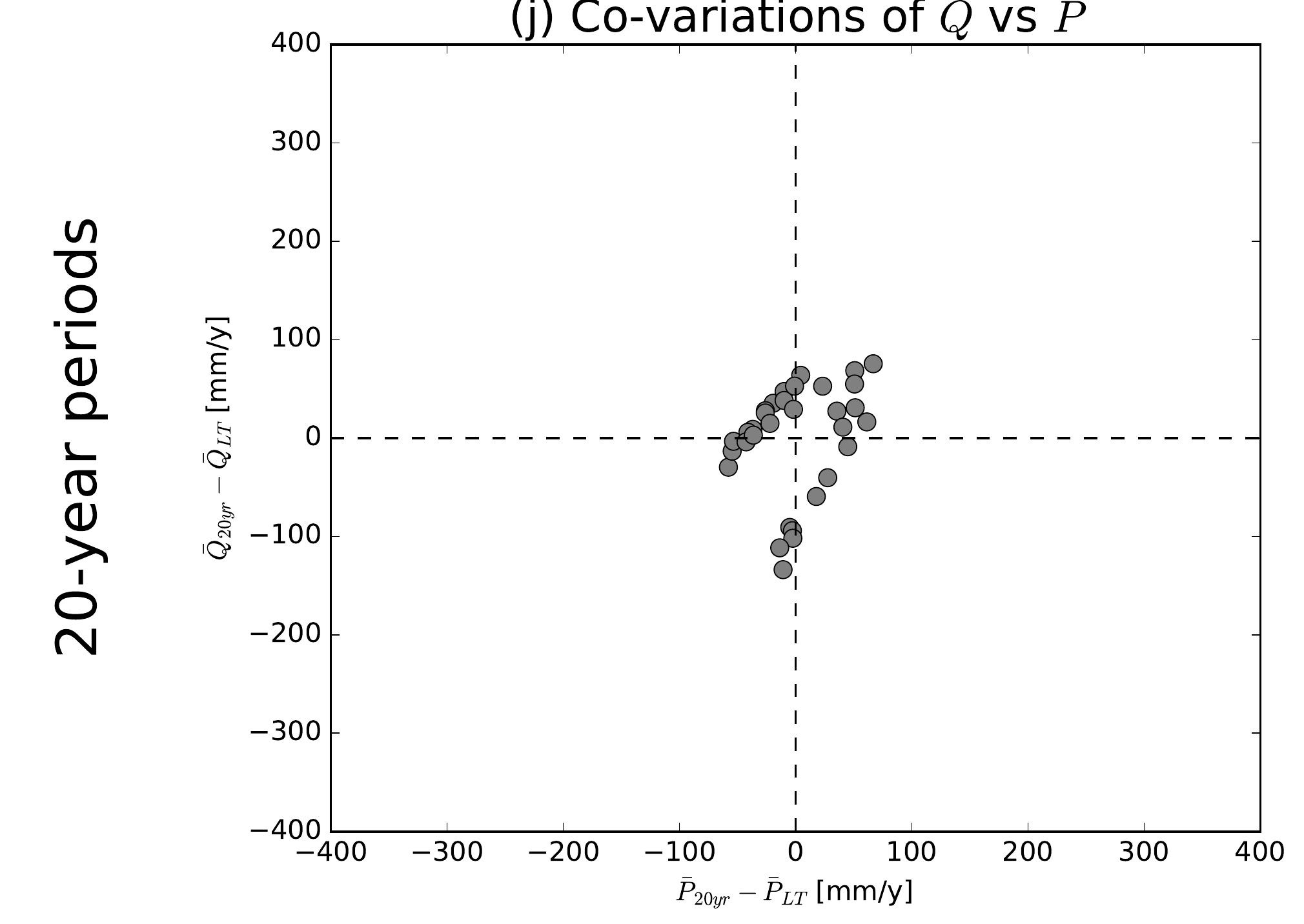

(b) Co-variations of $\bar{Q}$ vs $\overline{E 0}$

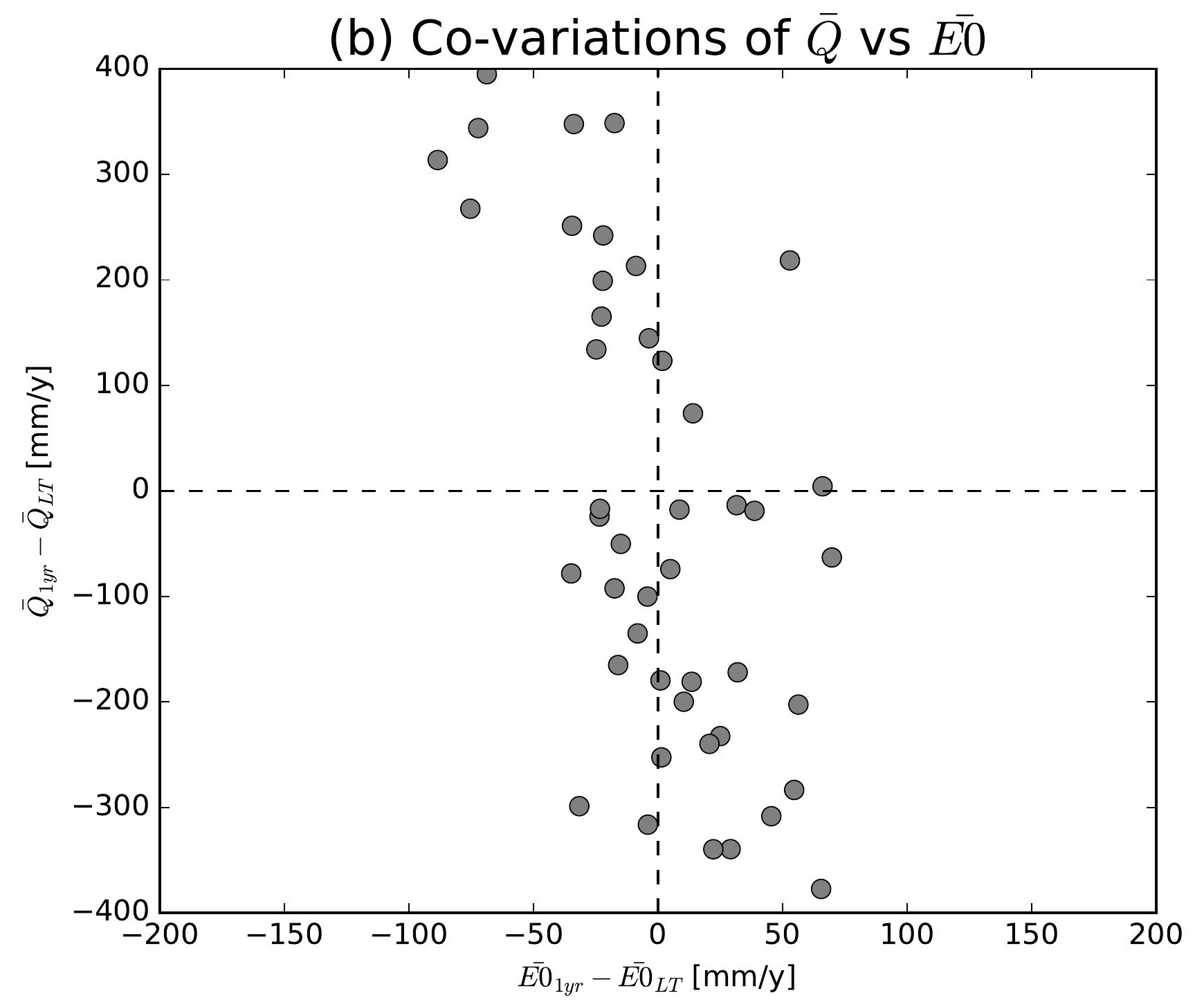

(e) Co-variations of $\bar{Q}$ vs $\overline{E 0}$

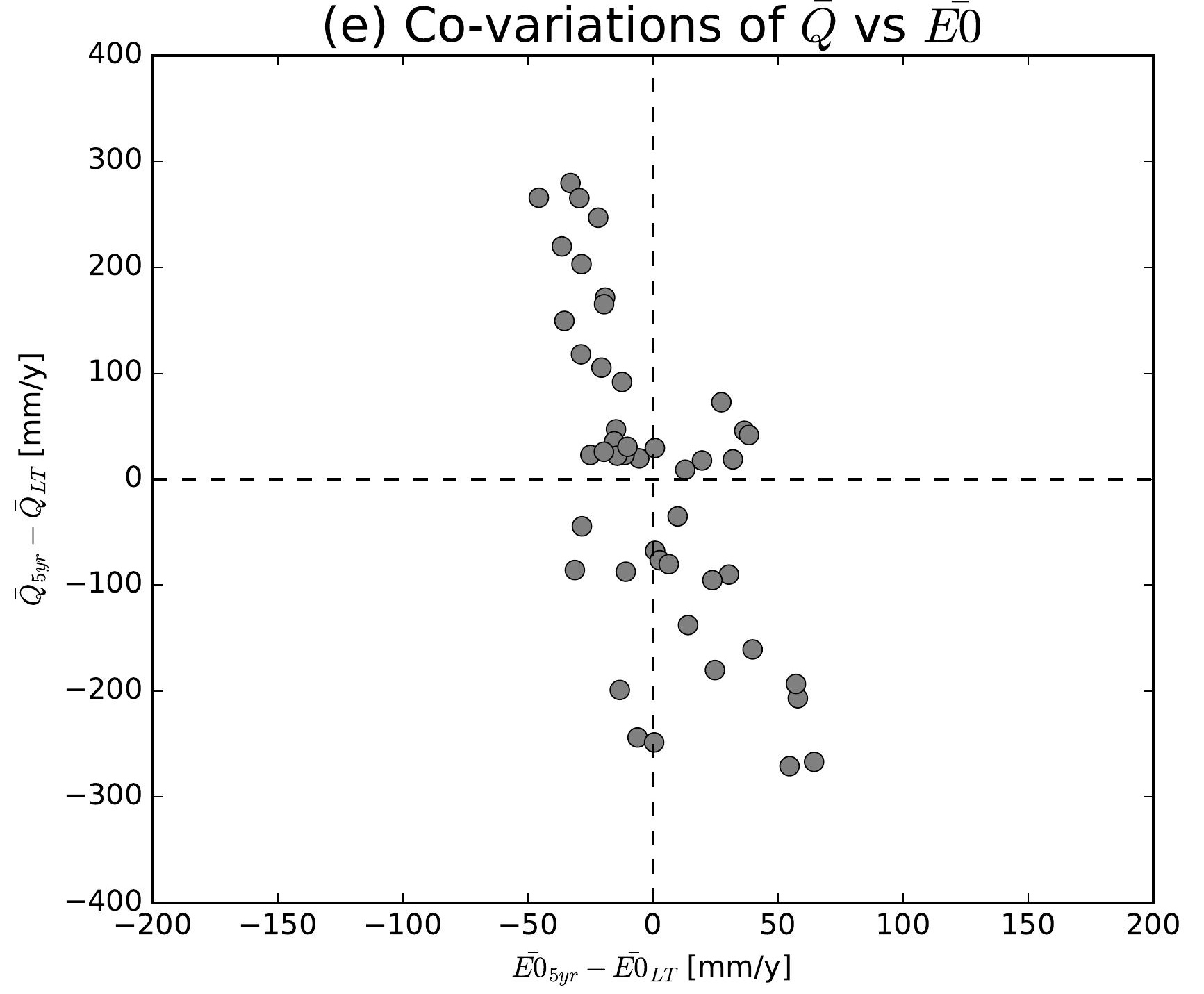

(h) Co-variations of $\bar{Q}$ vs $\overline{E 0}$

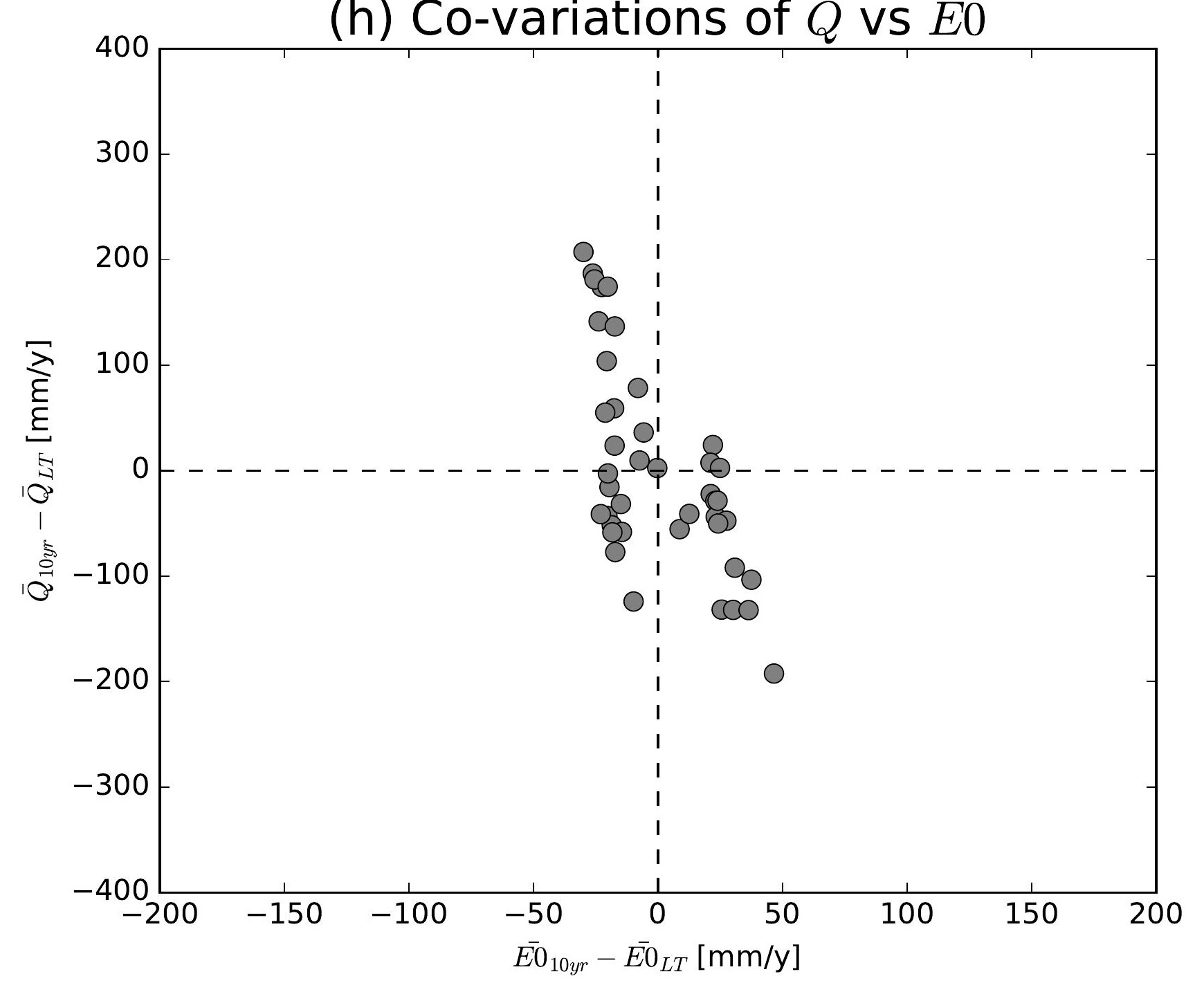

(k) Co-variations of $\bar{Q}$ vs $\overline{E 0}$

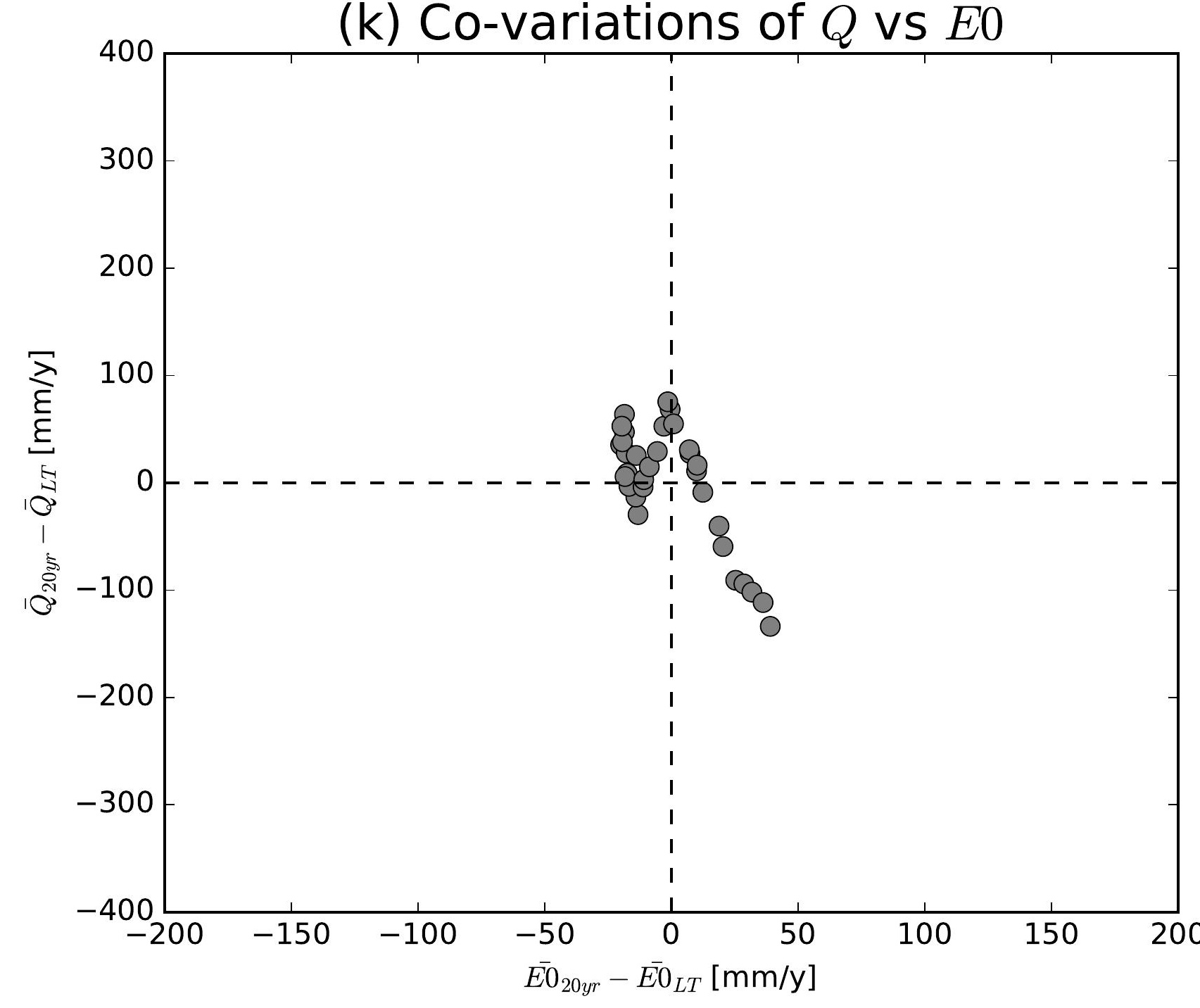

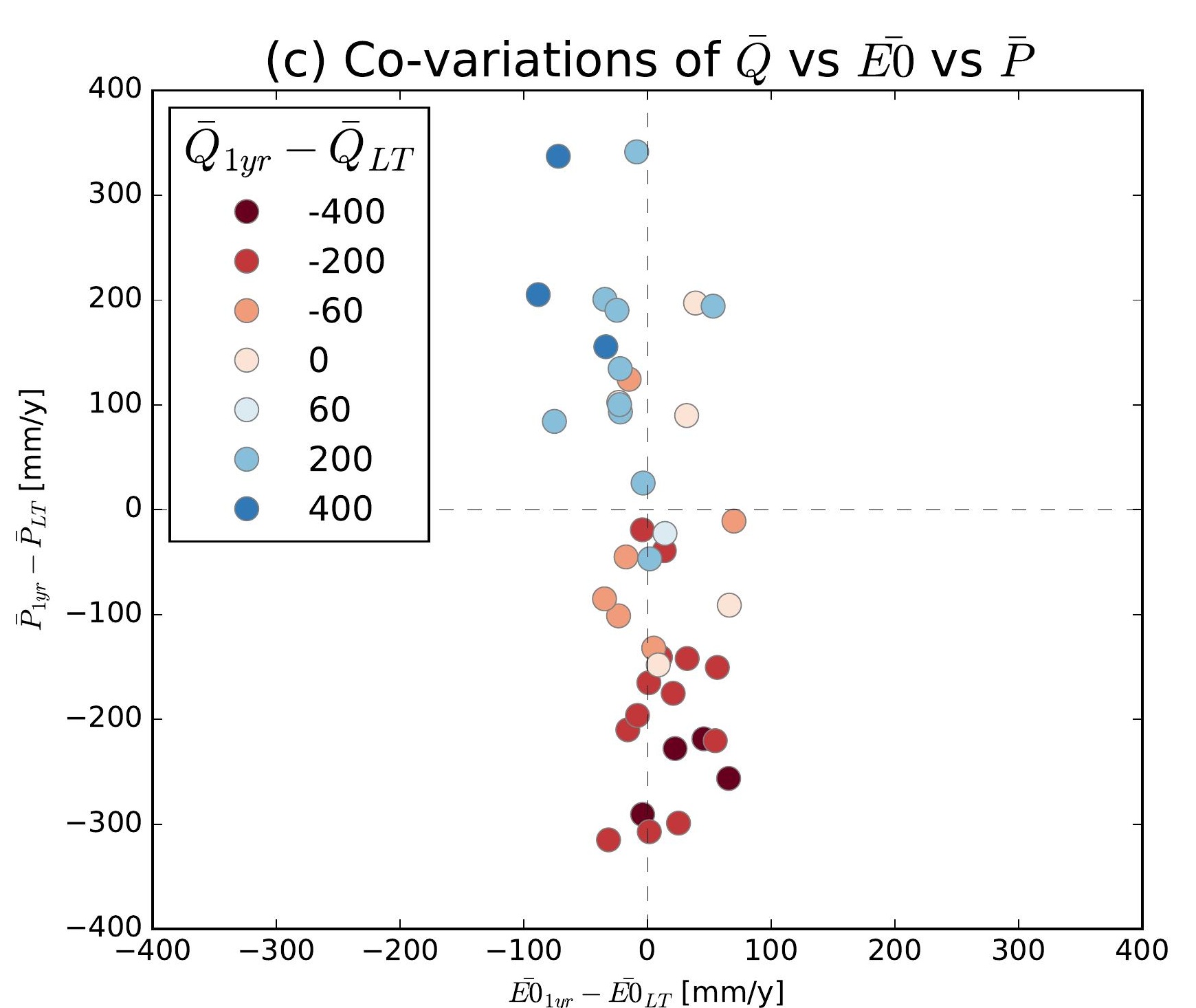
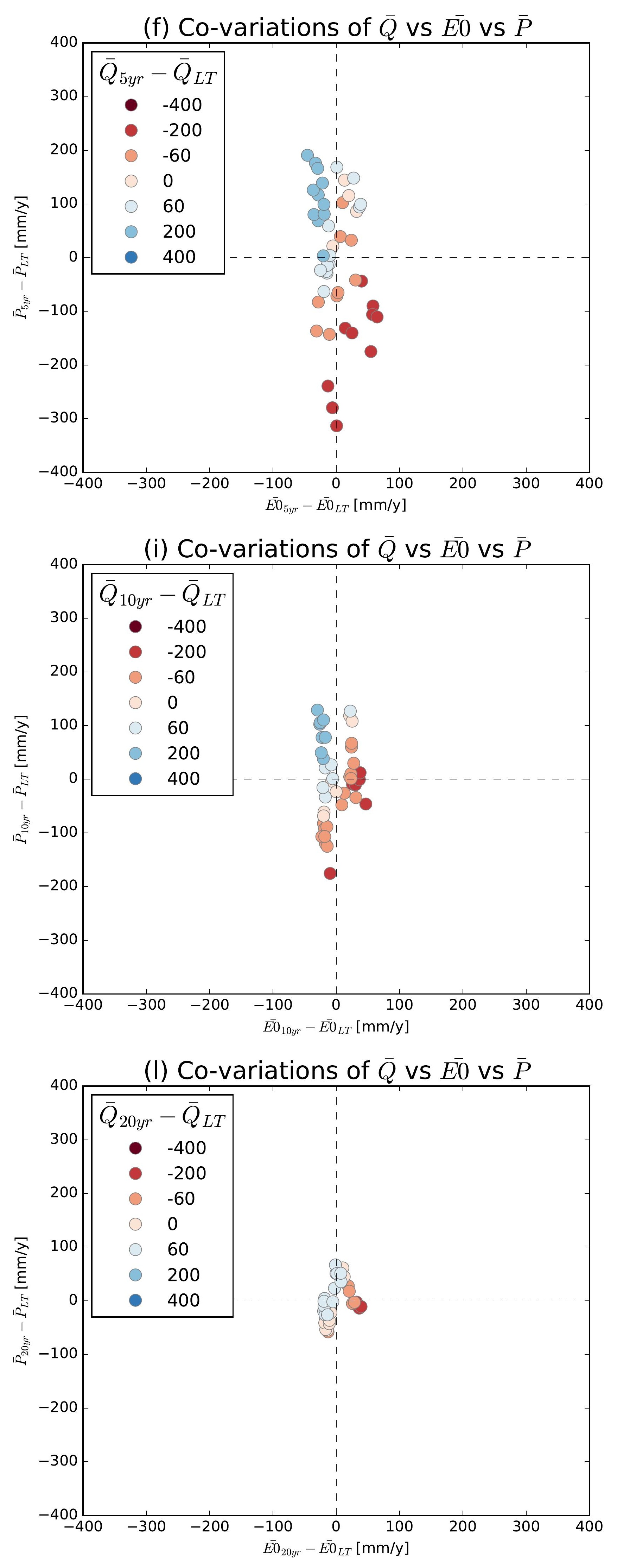

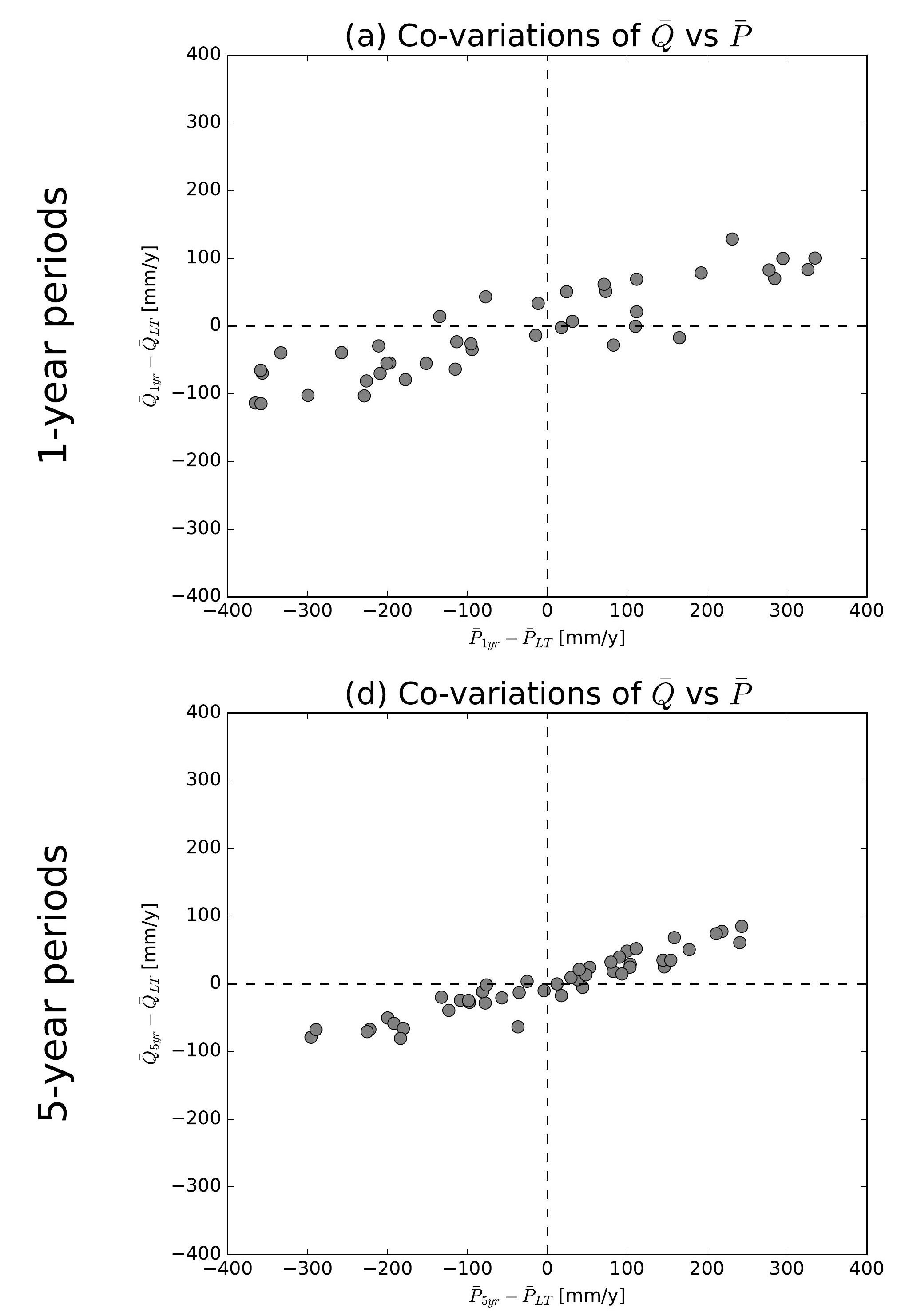

(g) Co-variations of $\bar{Q}$ vs $\bar{P}$

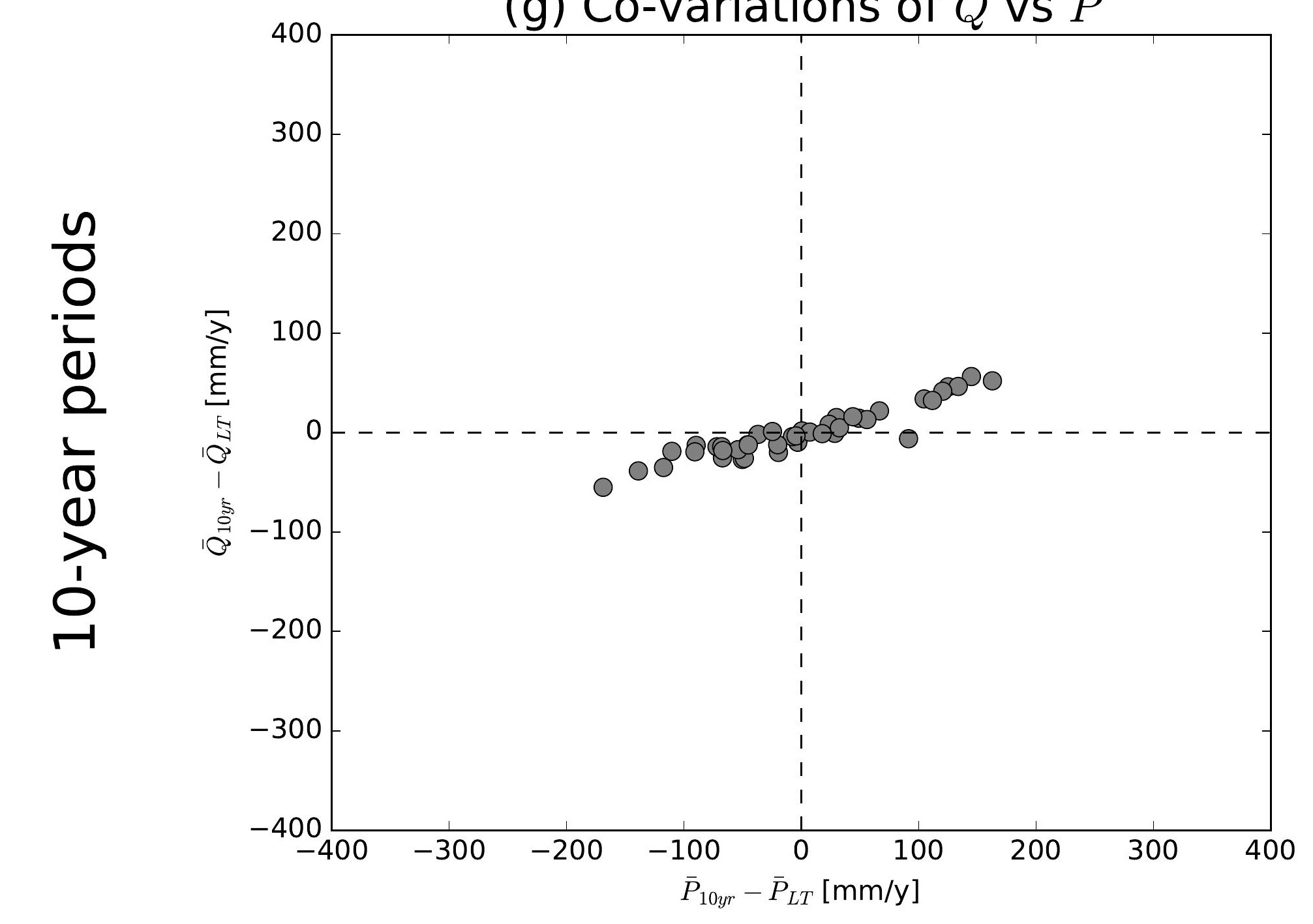

(j) Co-variations of $\bar{Q}$ vs $\bar{P}$

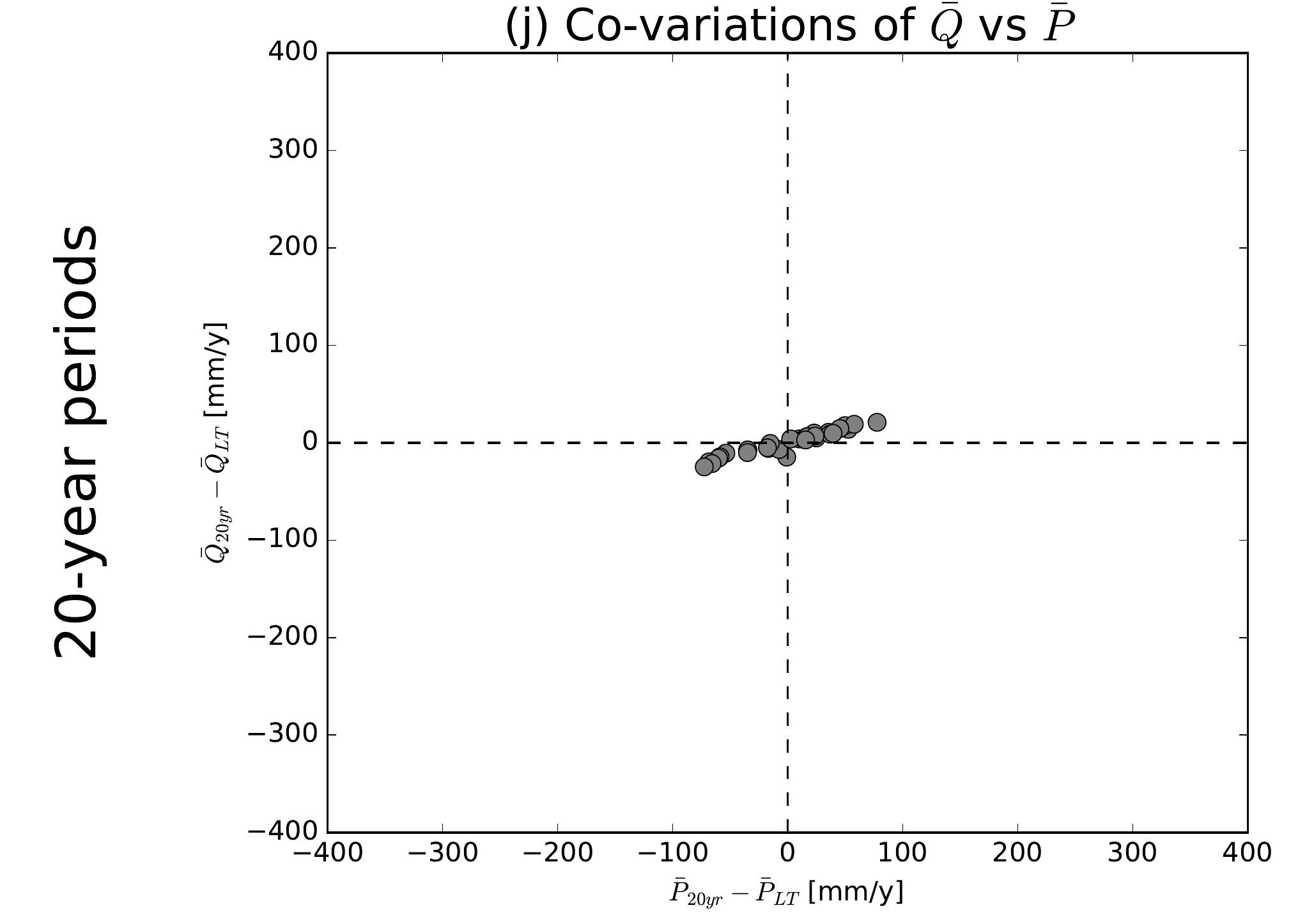

(b) Co-variations of $\bar{Q}$ vs $\overline{E 0}$

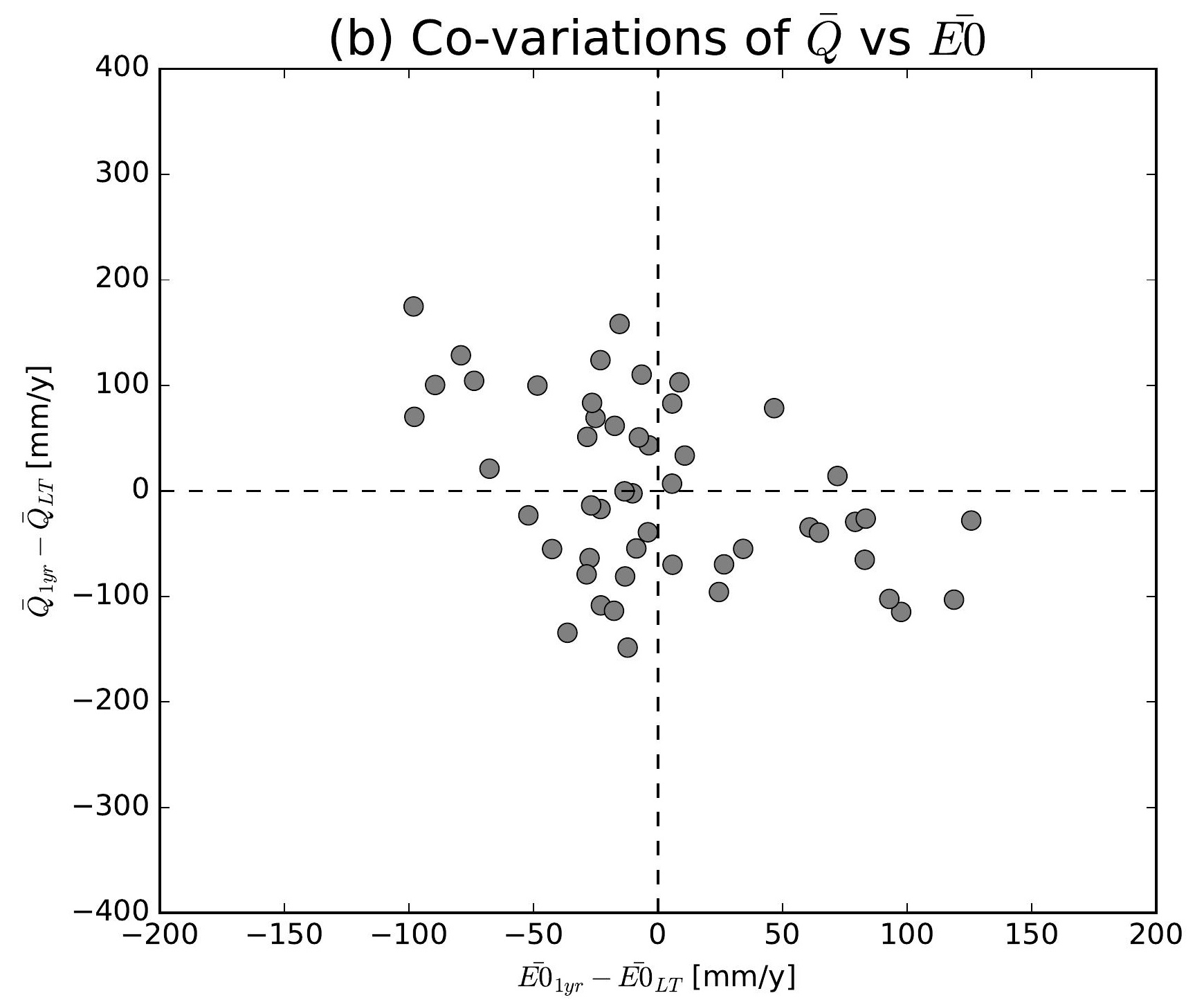

(e) Co-variations of $\bar{Q}$ vs $\overline{E 0}$

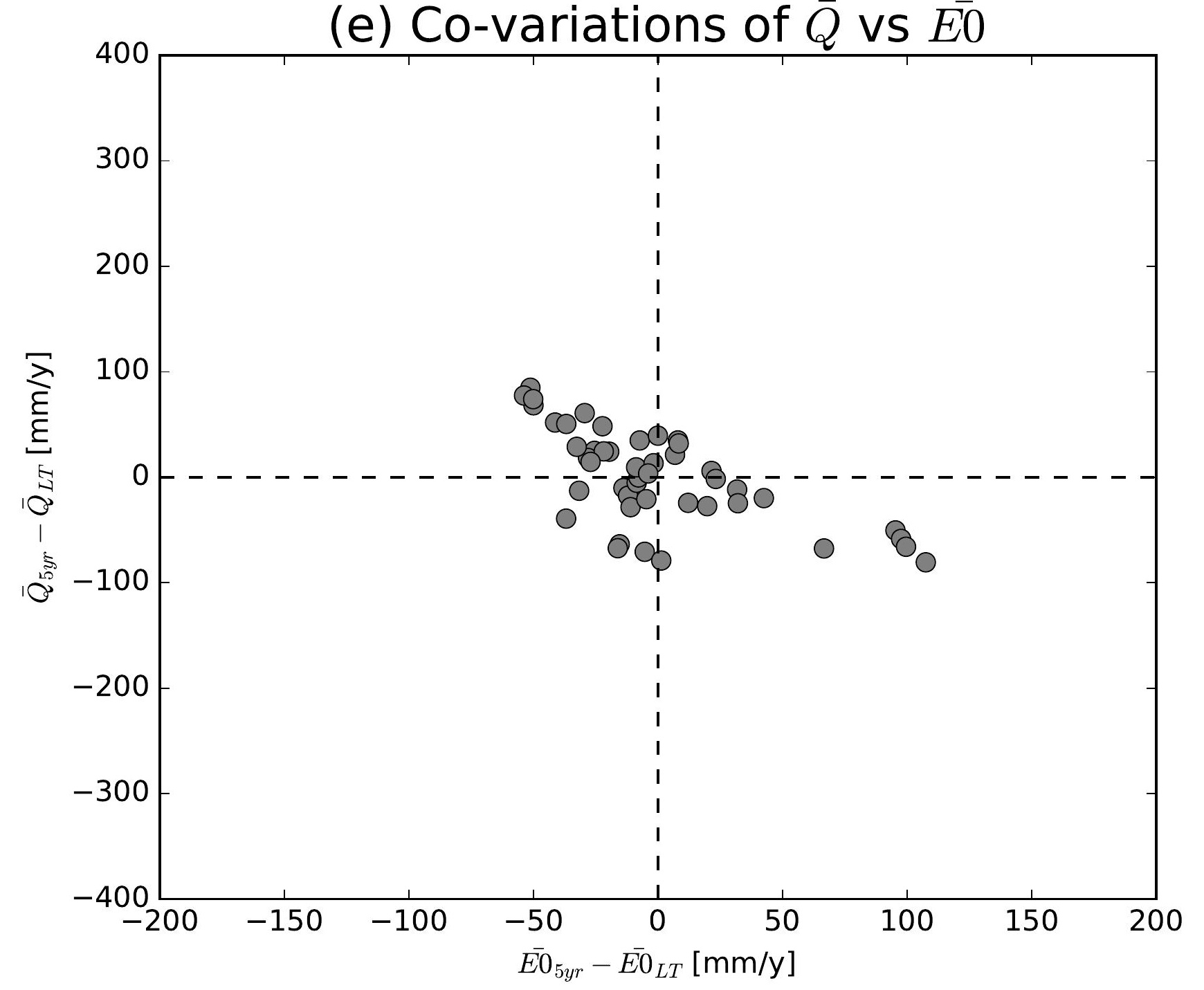

(h) Co-variations of $\bar{Q}$ vs $\overline{E 0}$

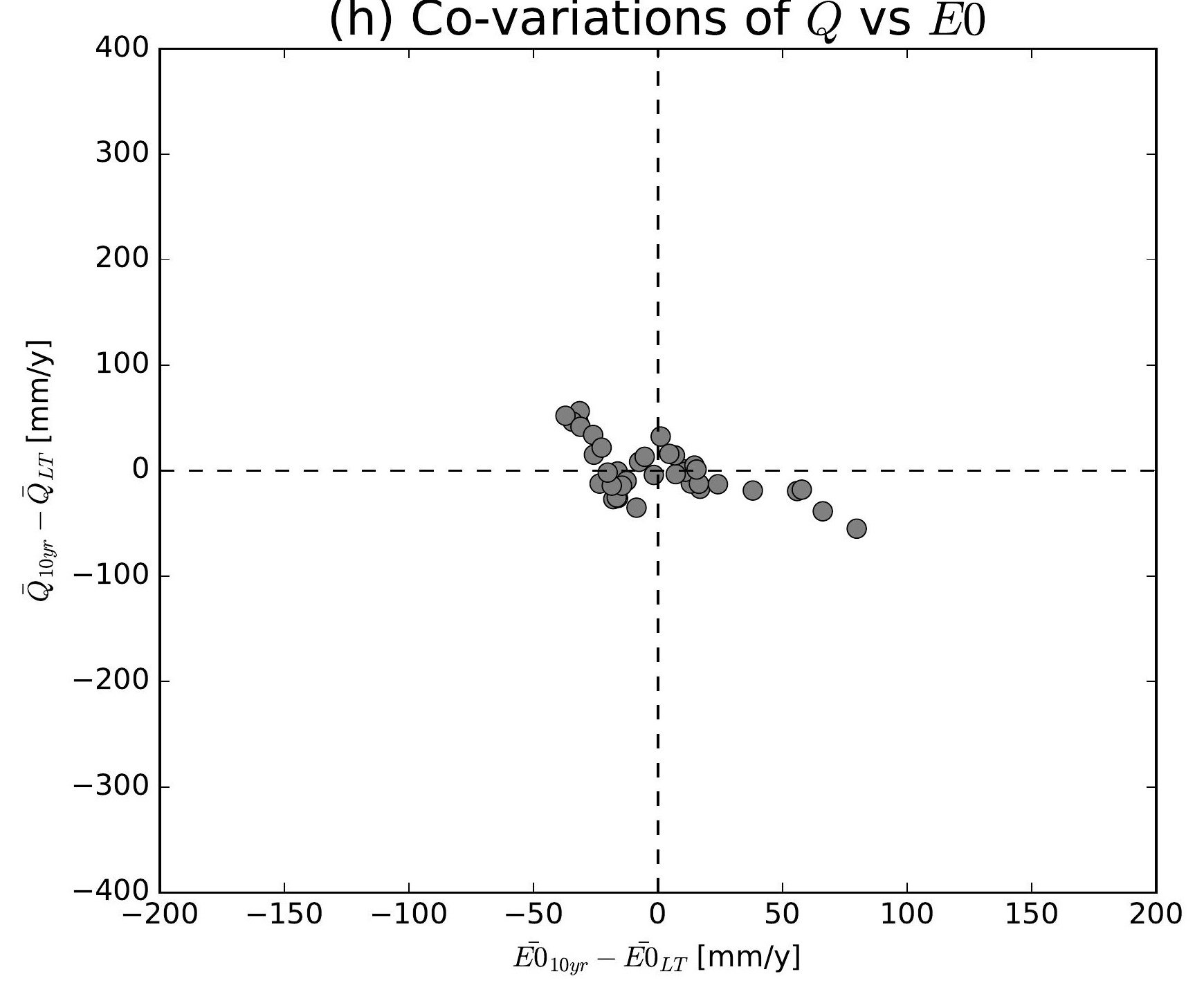

(k) Co-variations of $\bar{Q}$ vs $\overline{E 0}$

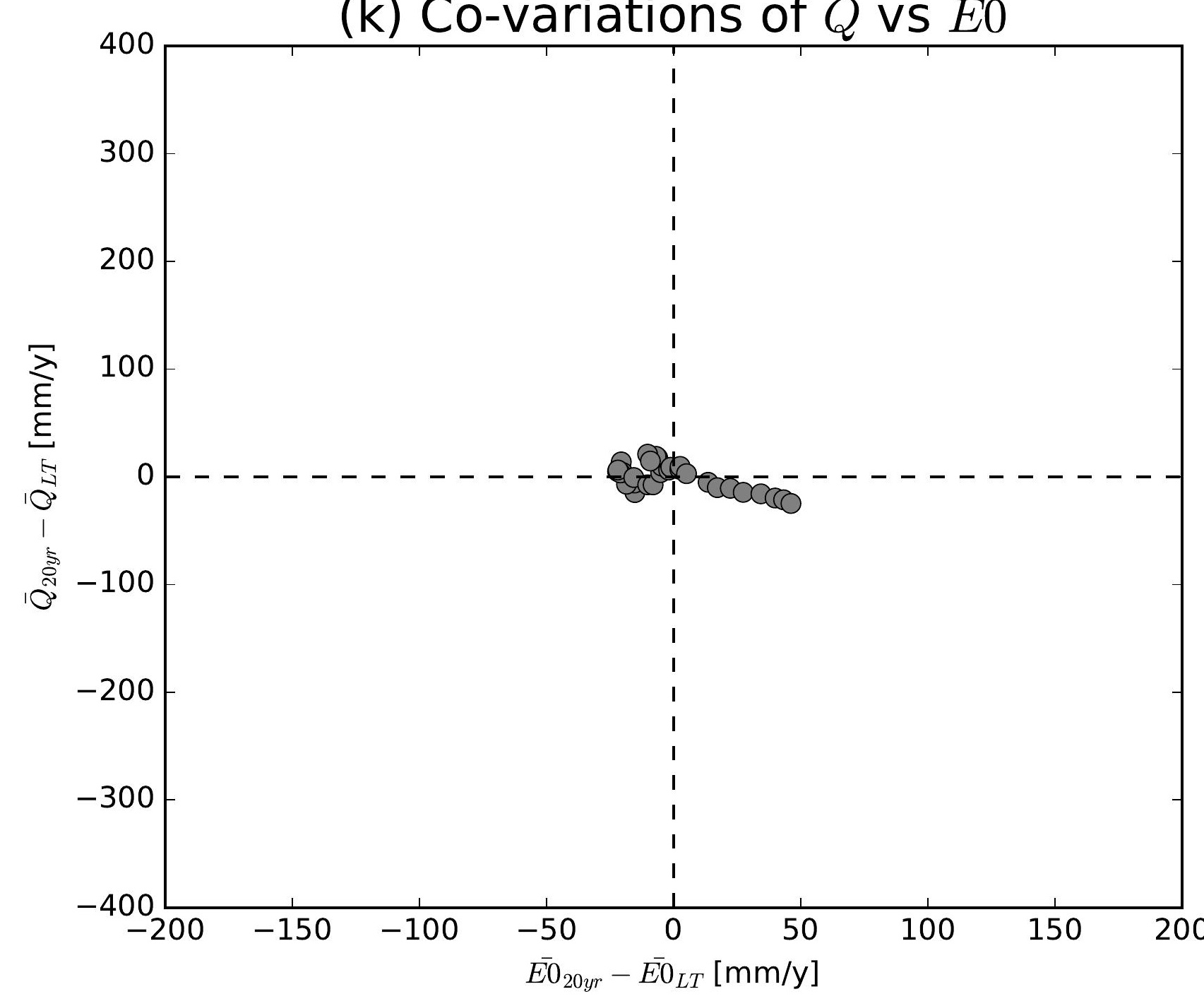

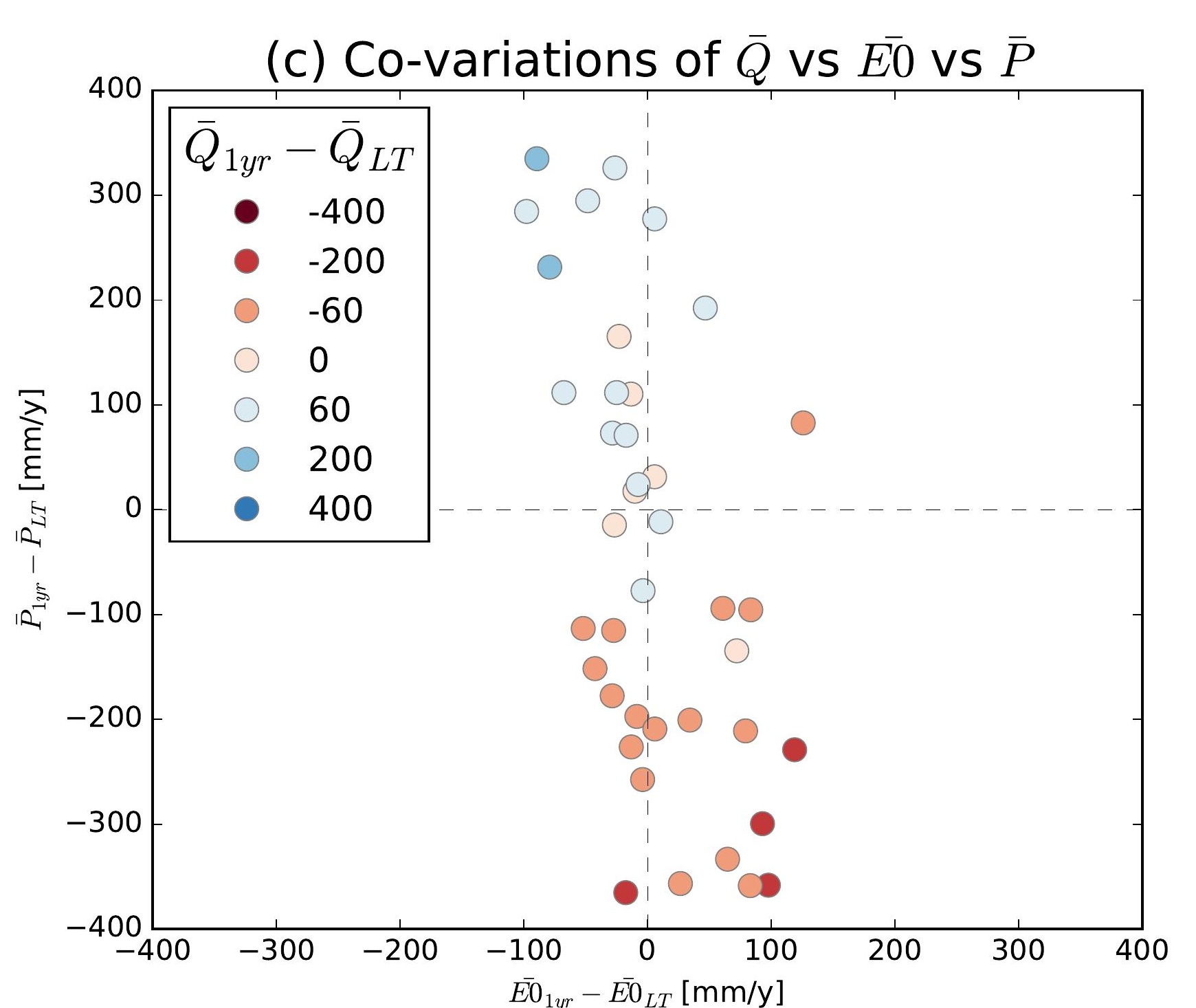
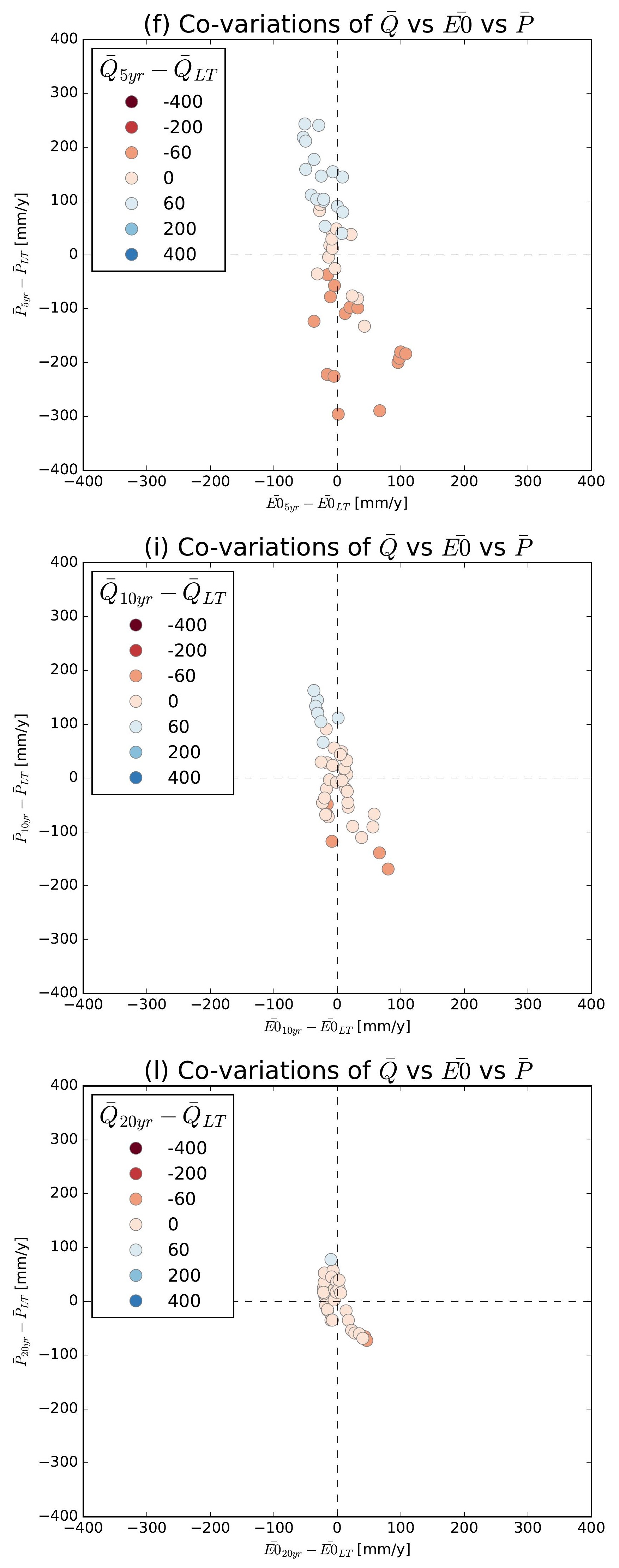

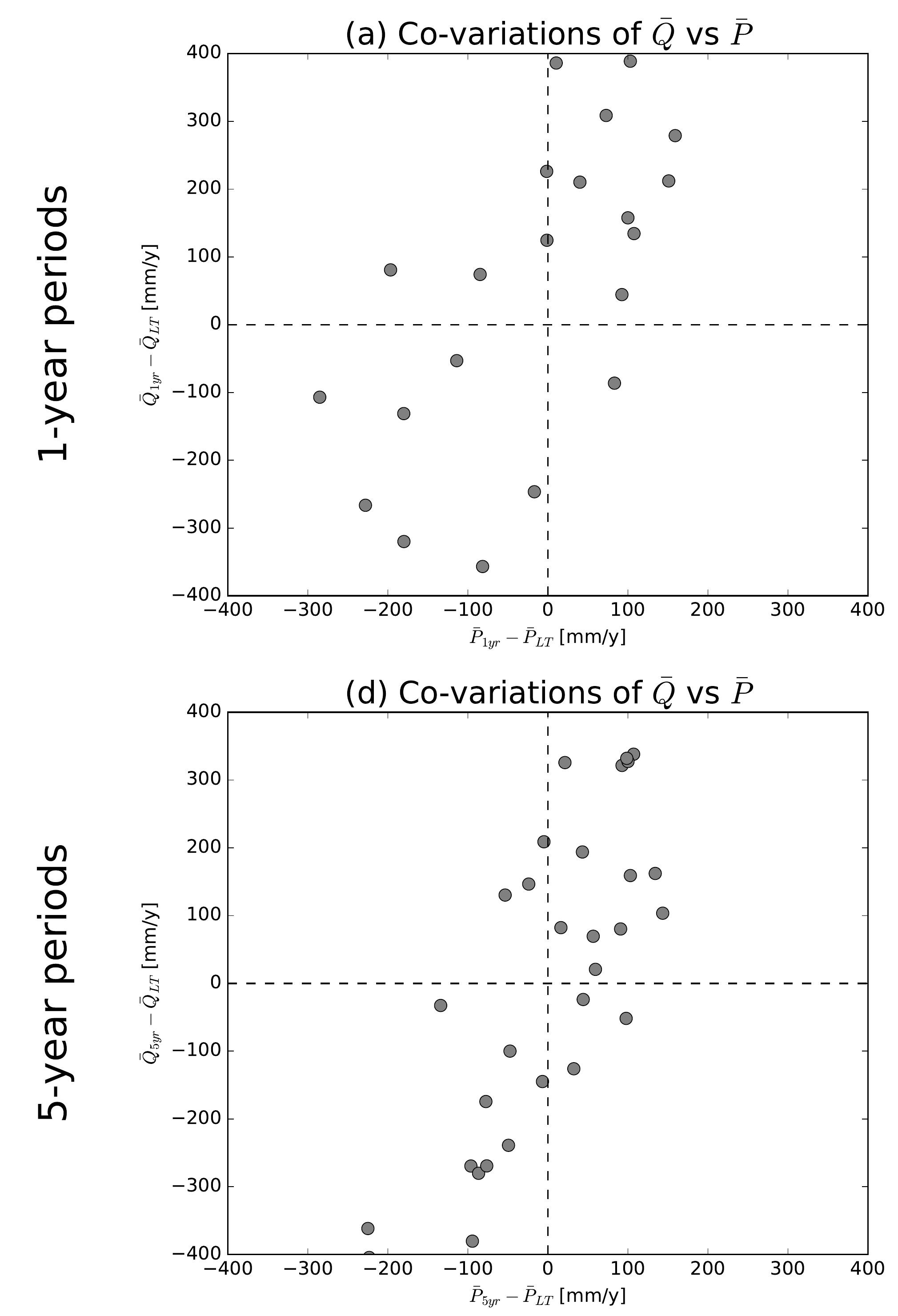

(g) Co-variations of $\bar{Q}$ vs $\bar{P}$

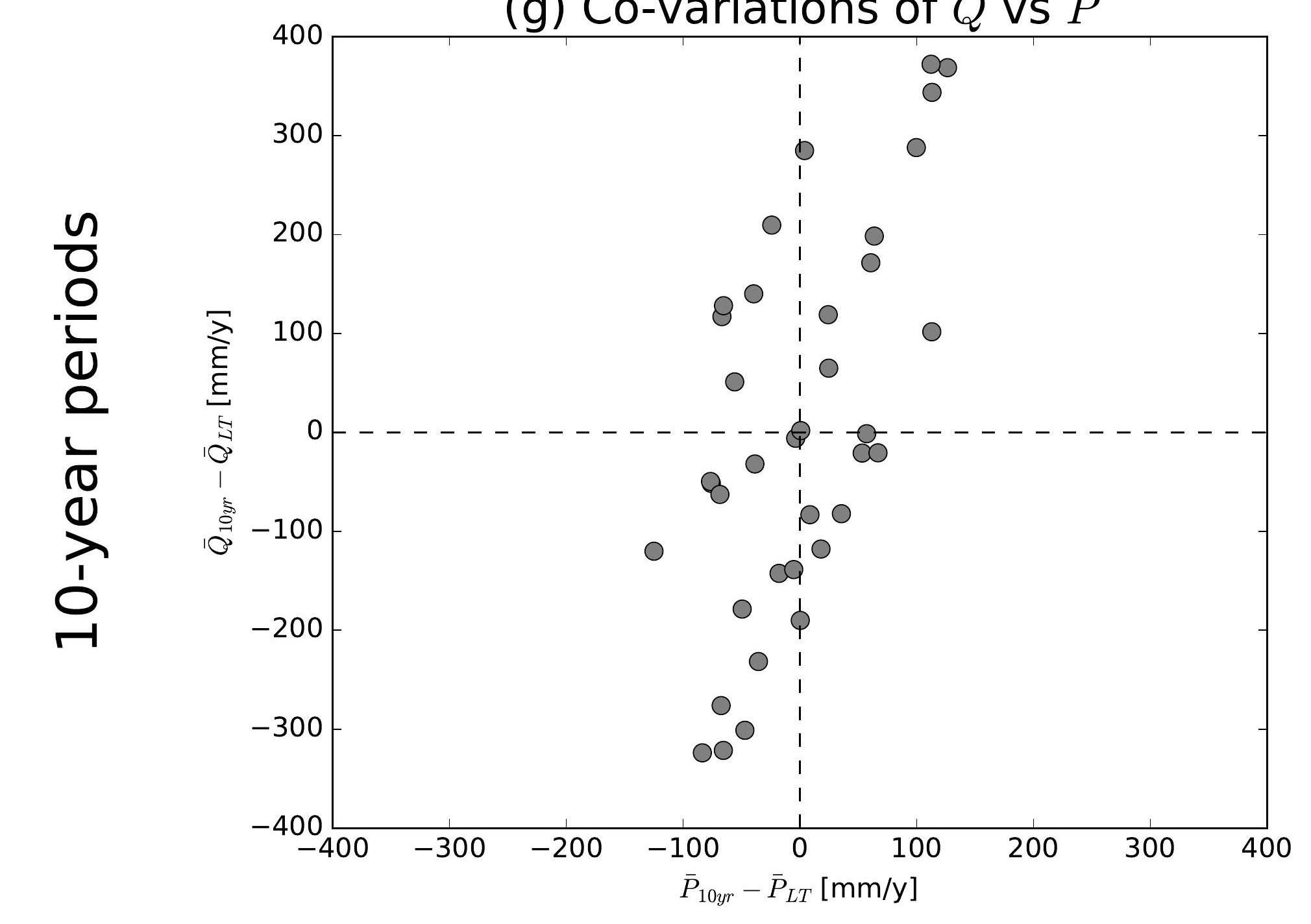

(j) Co-variations of $\bar{Q}$ vs $\bar{P}$

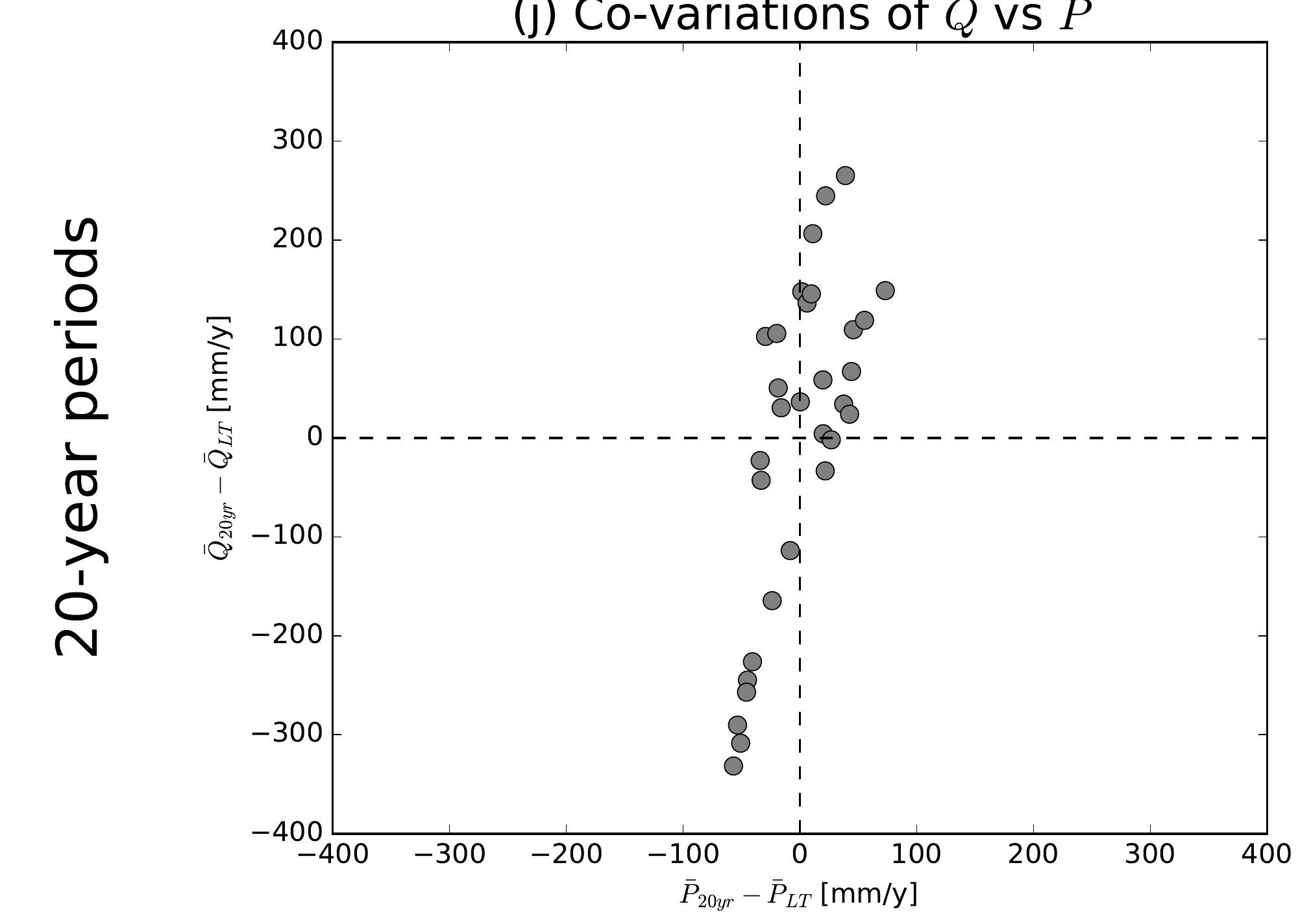

(b) Co-variations of $\bar{Q}$ vs $\overline{E 0}$

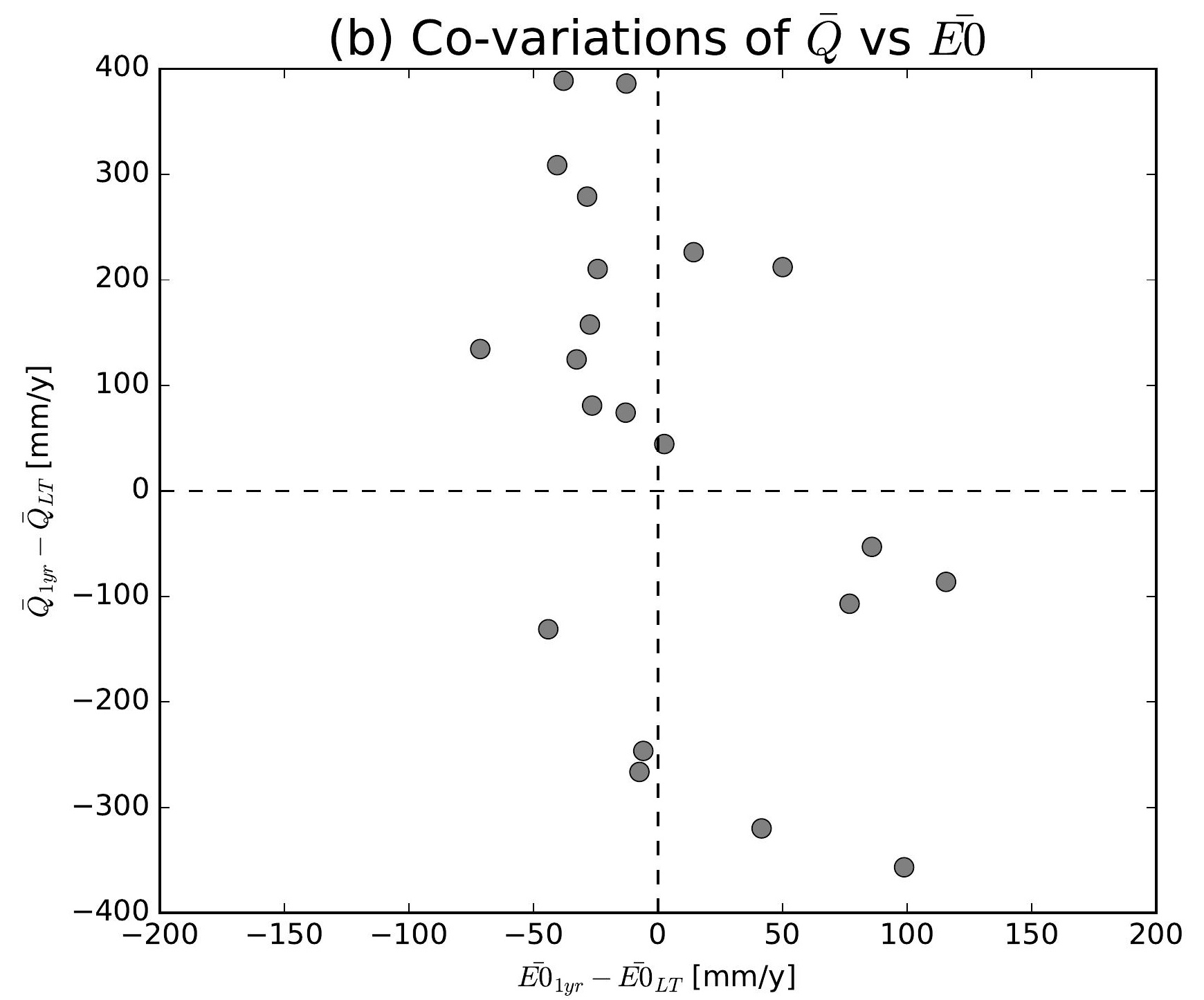

(e) Co-variations of $\bar{Q}$ vs $\overline{E 0}$

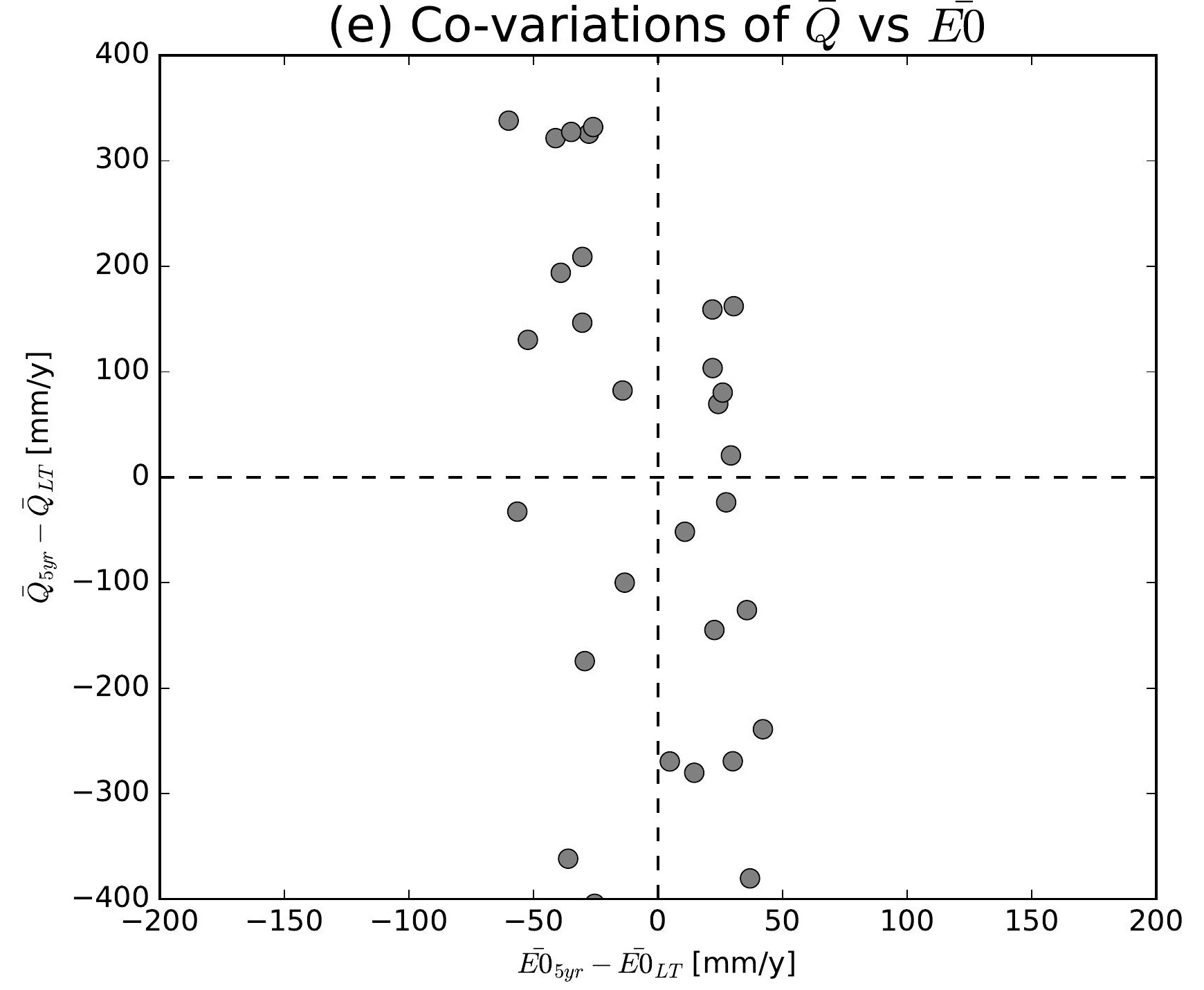

(h) Co-variations of $\bar{Q}$ vs $\overline{E 0}$

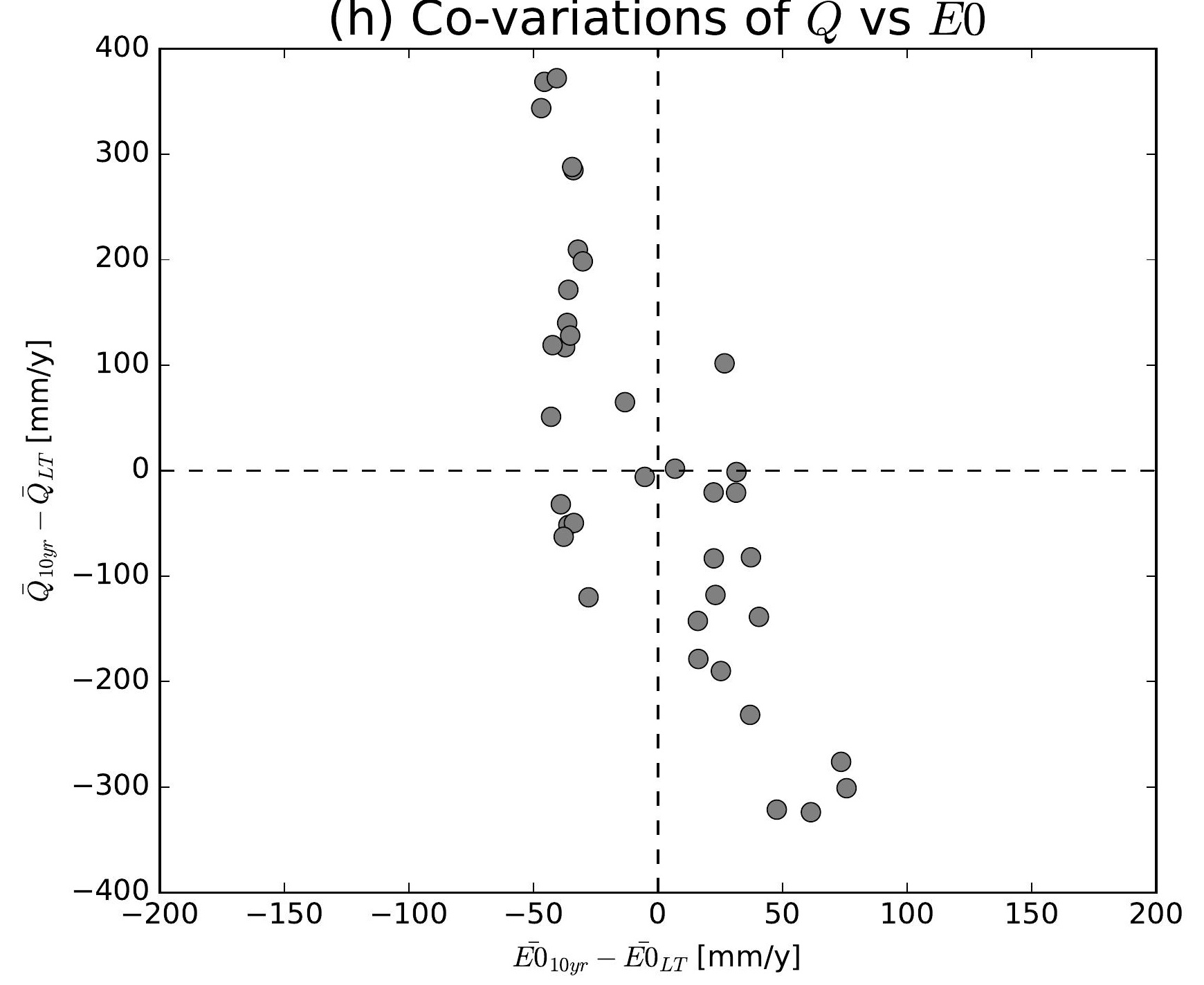

(k) Co-variations of $\bar{Q}$ vs $\overline{E 0}$

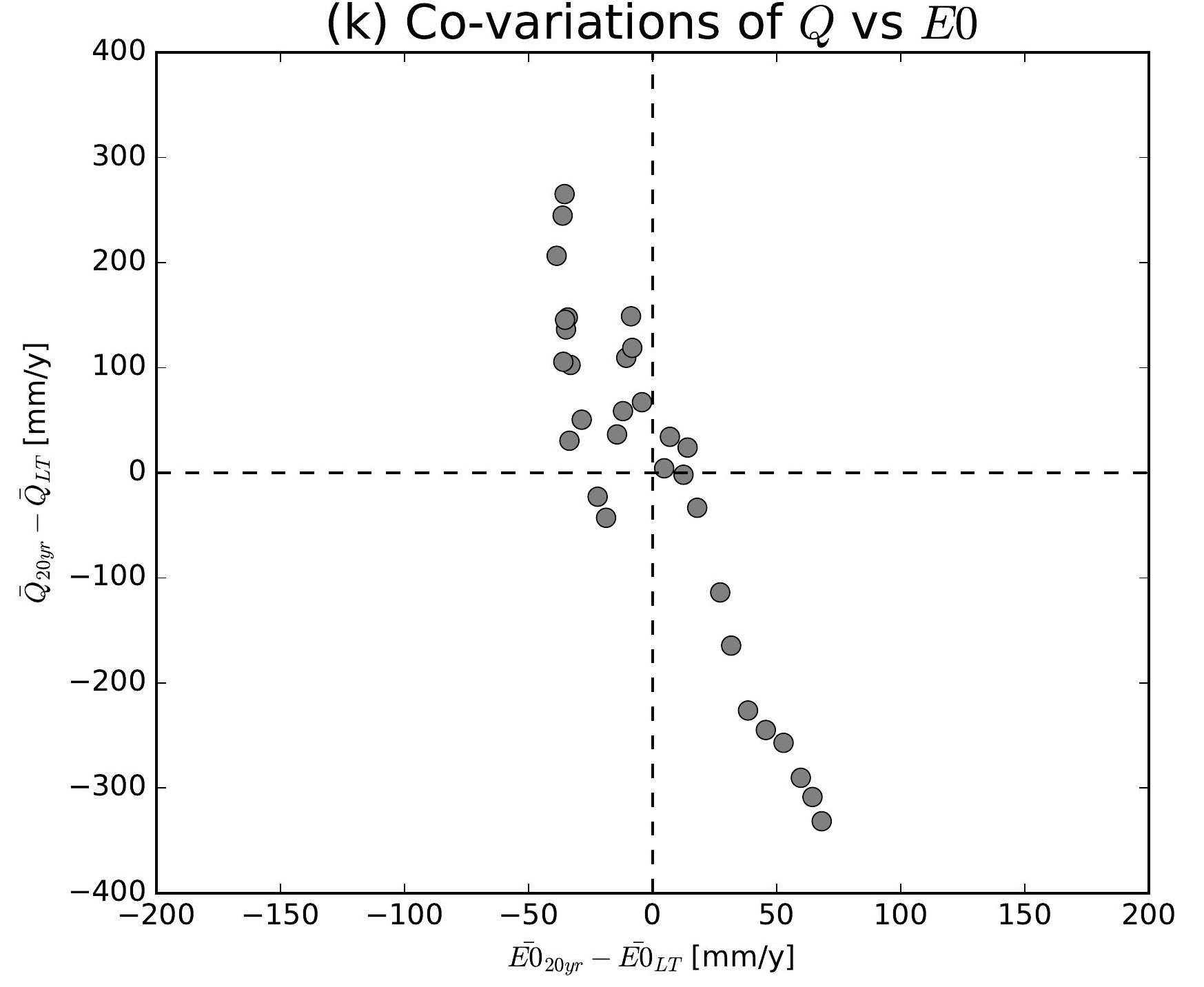

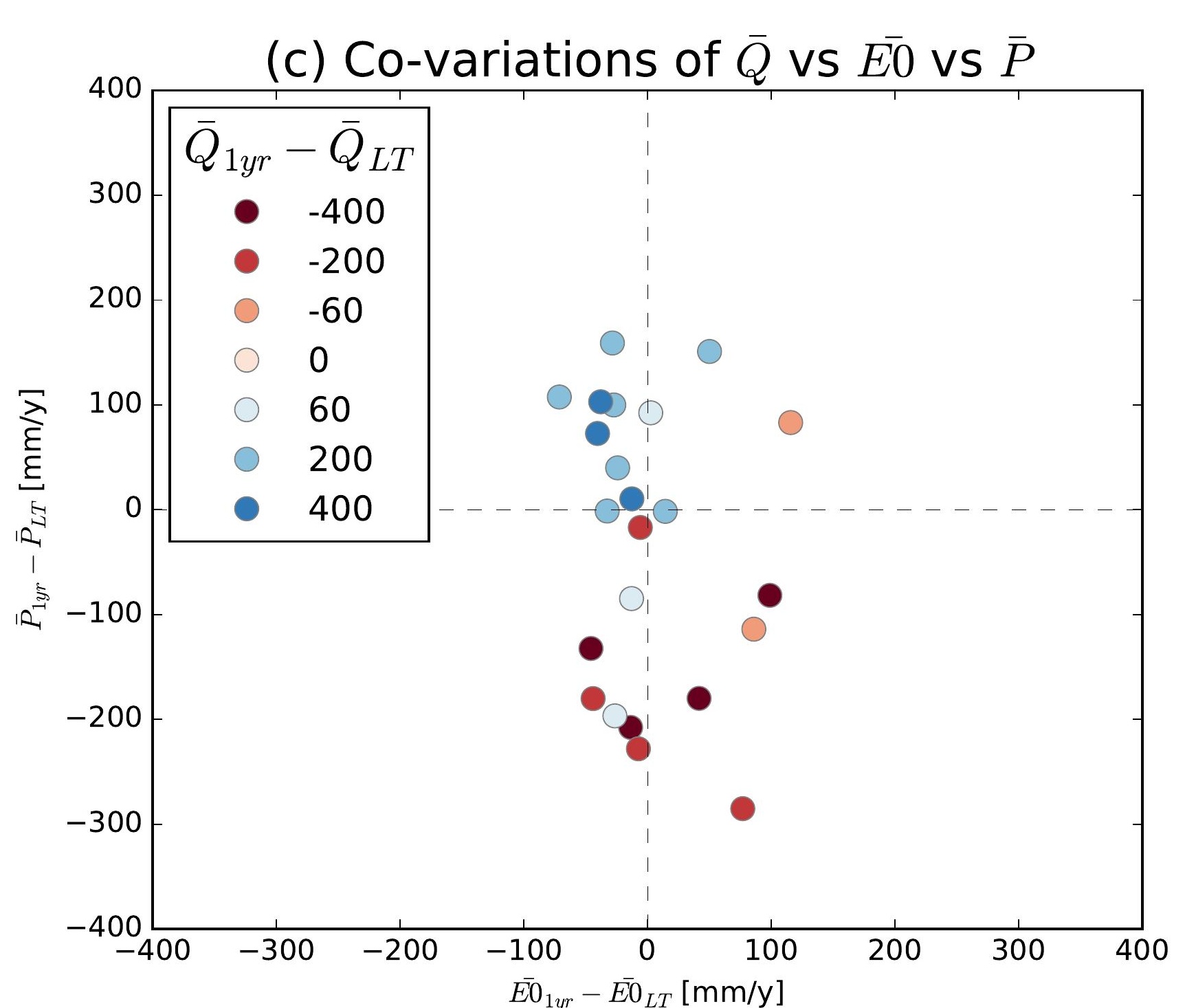
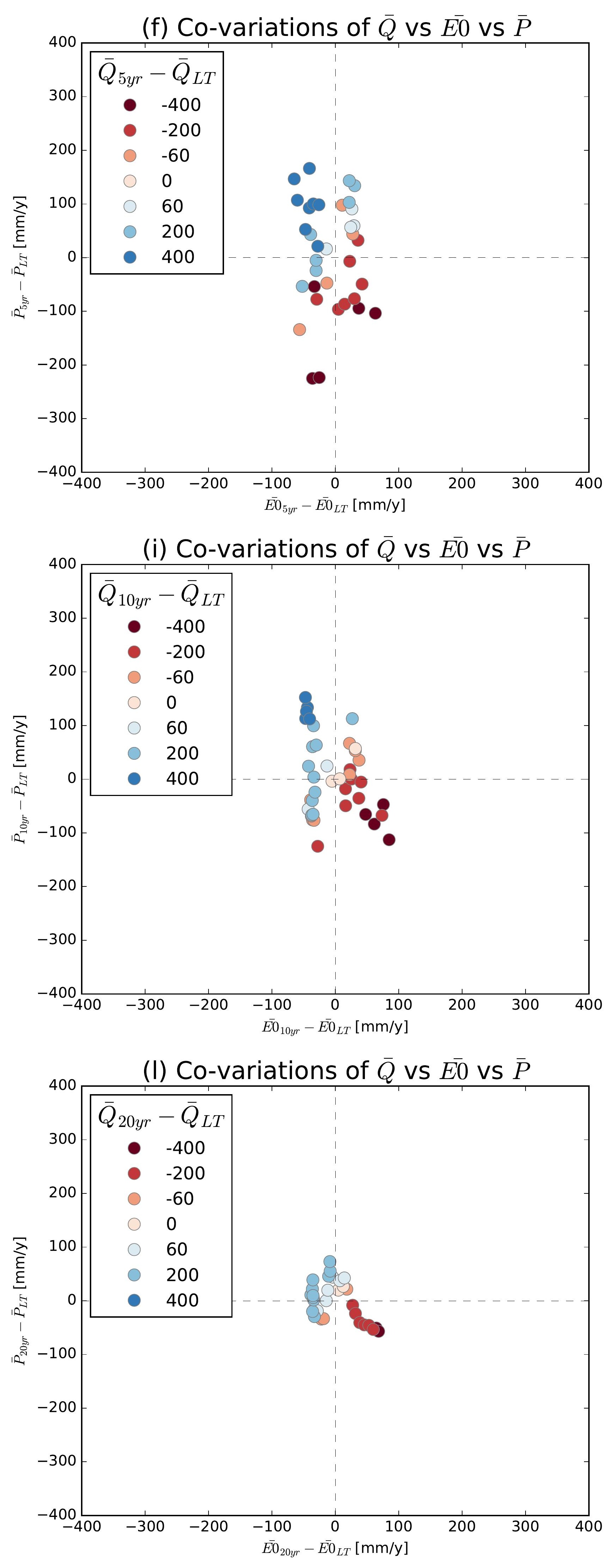

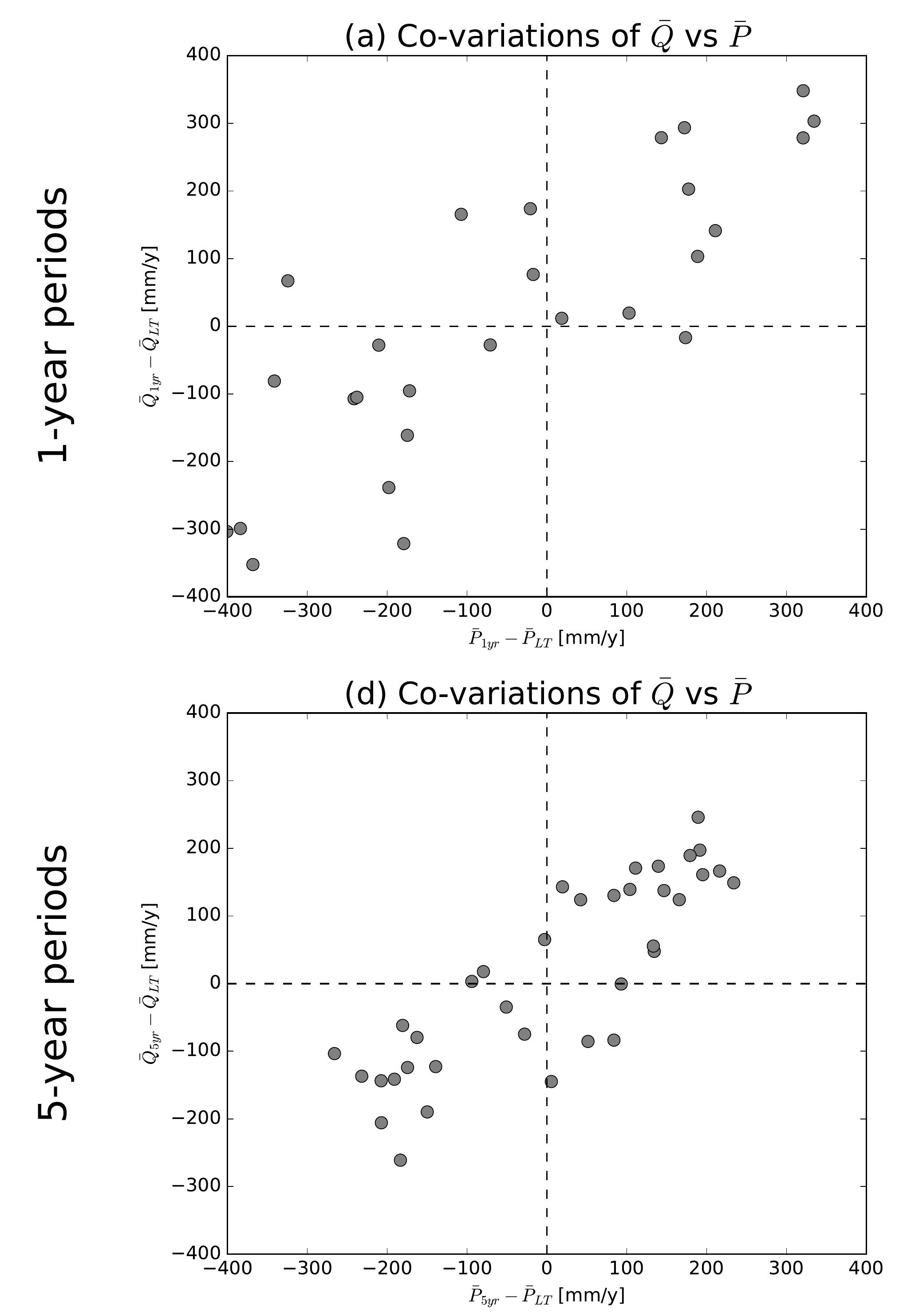

(g) Co-variations of $\bar{Q}$ vs $\bar{P}$

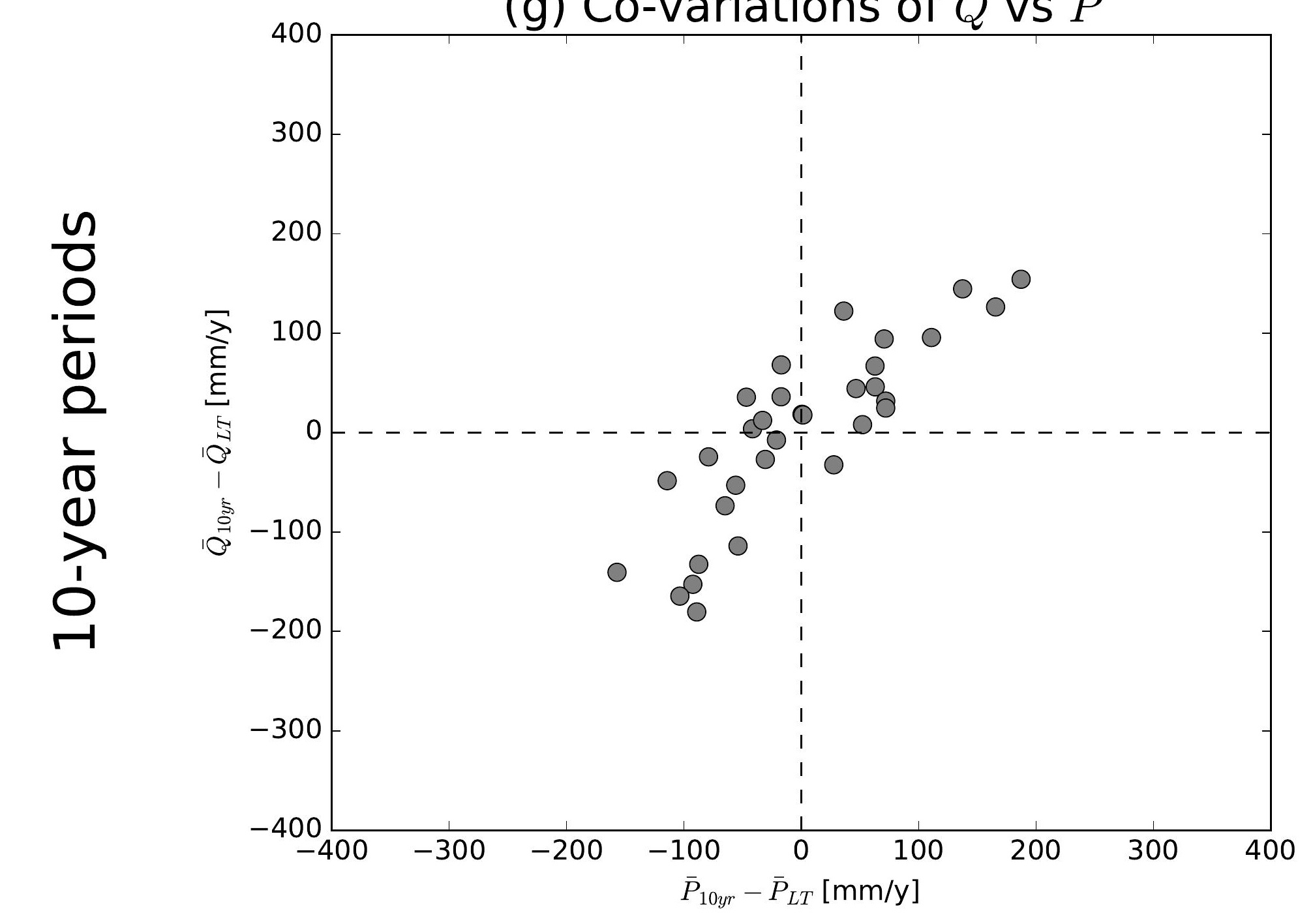

(j) Co-variations of $\bar{Q}$ vs $\bar{P}$

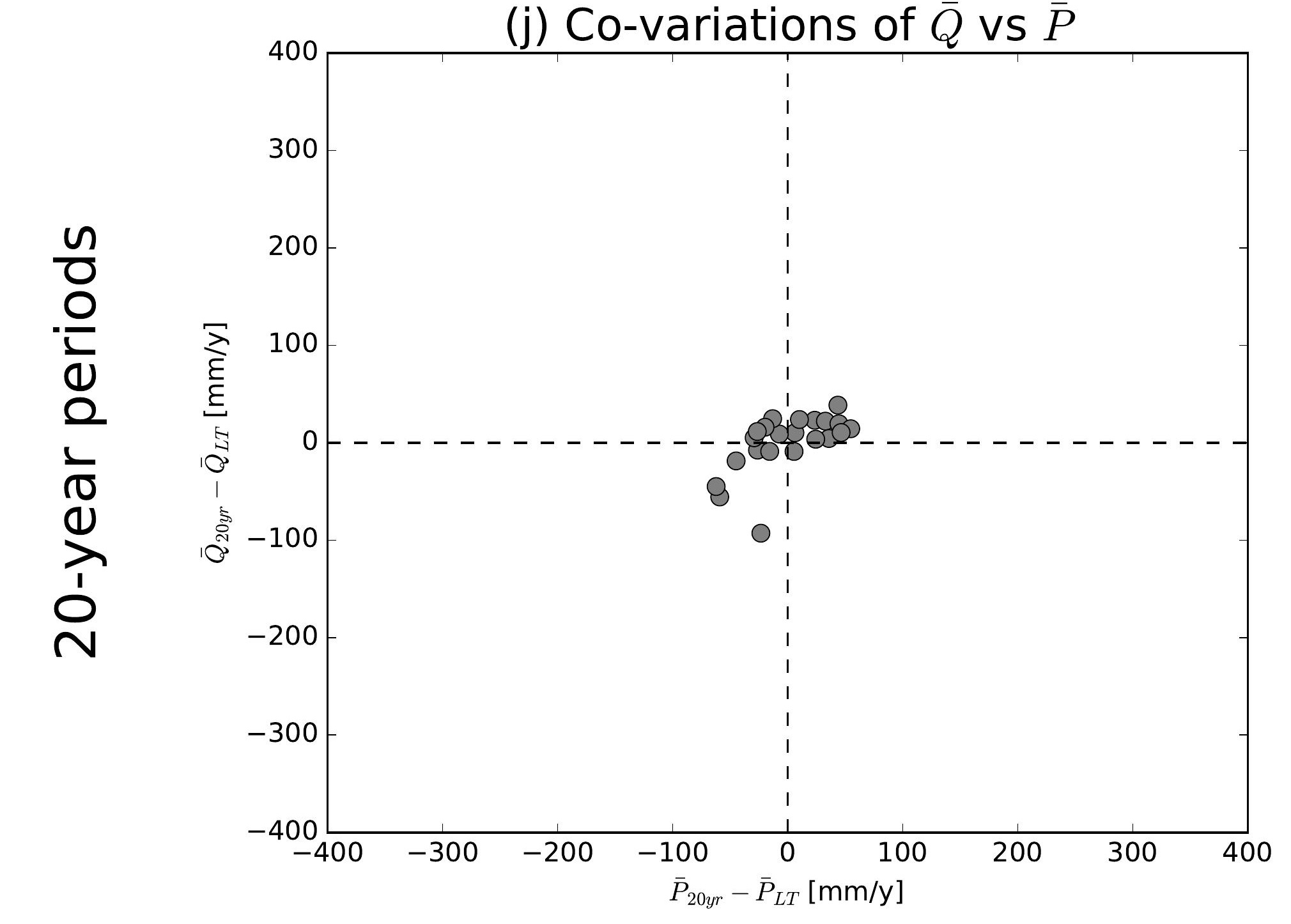

(b) Co-variations of $\bar{Q}$ vs $\overline{E 0}$

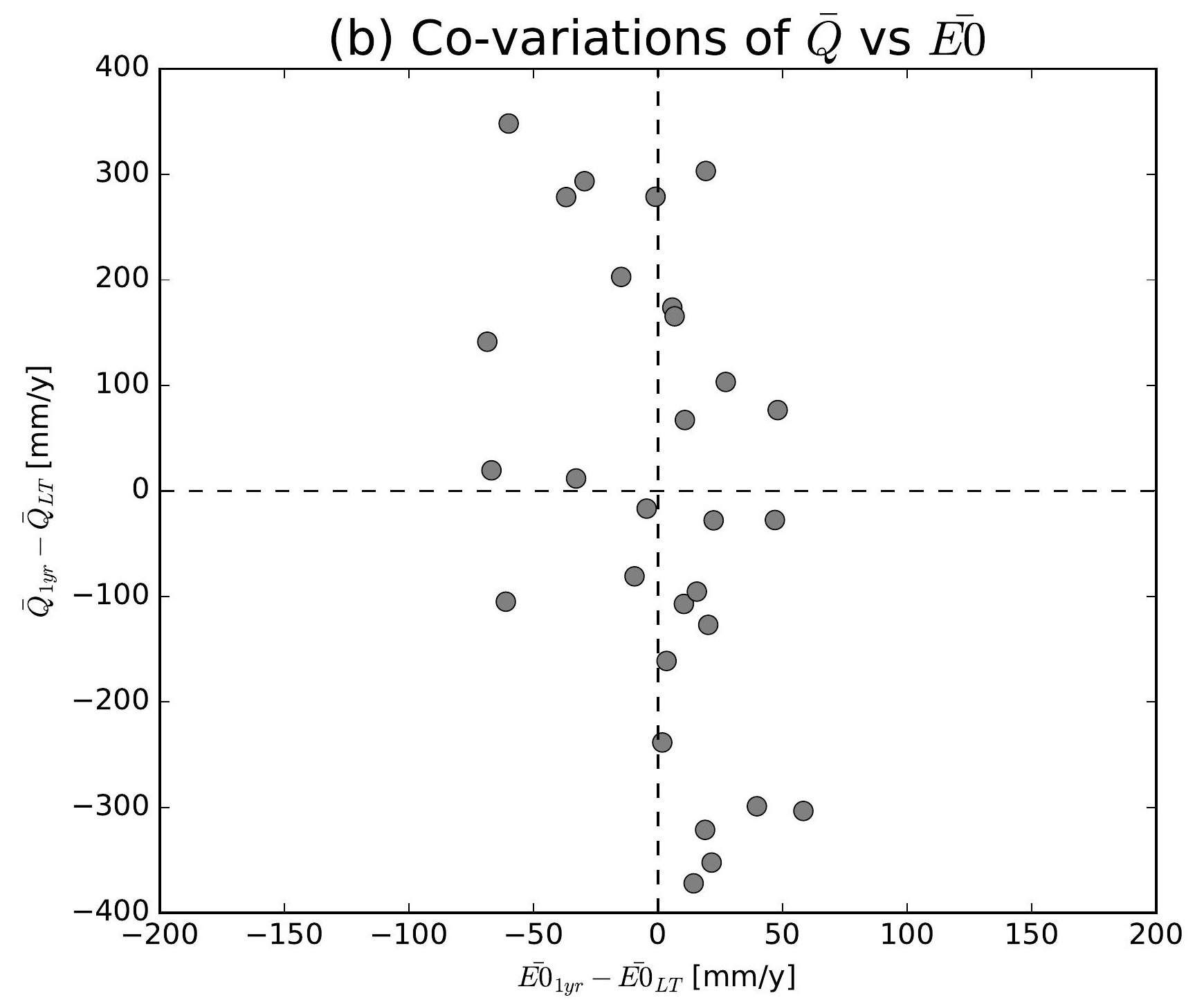

(e) Co-variations of $\bar{Q}$ vs $\overline{E 0}$

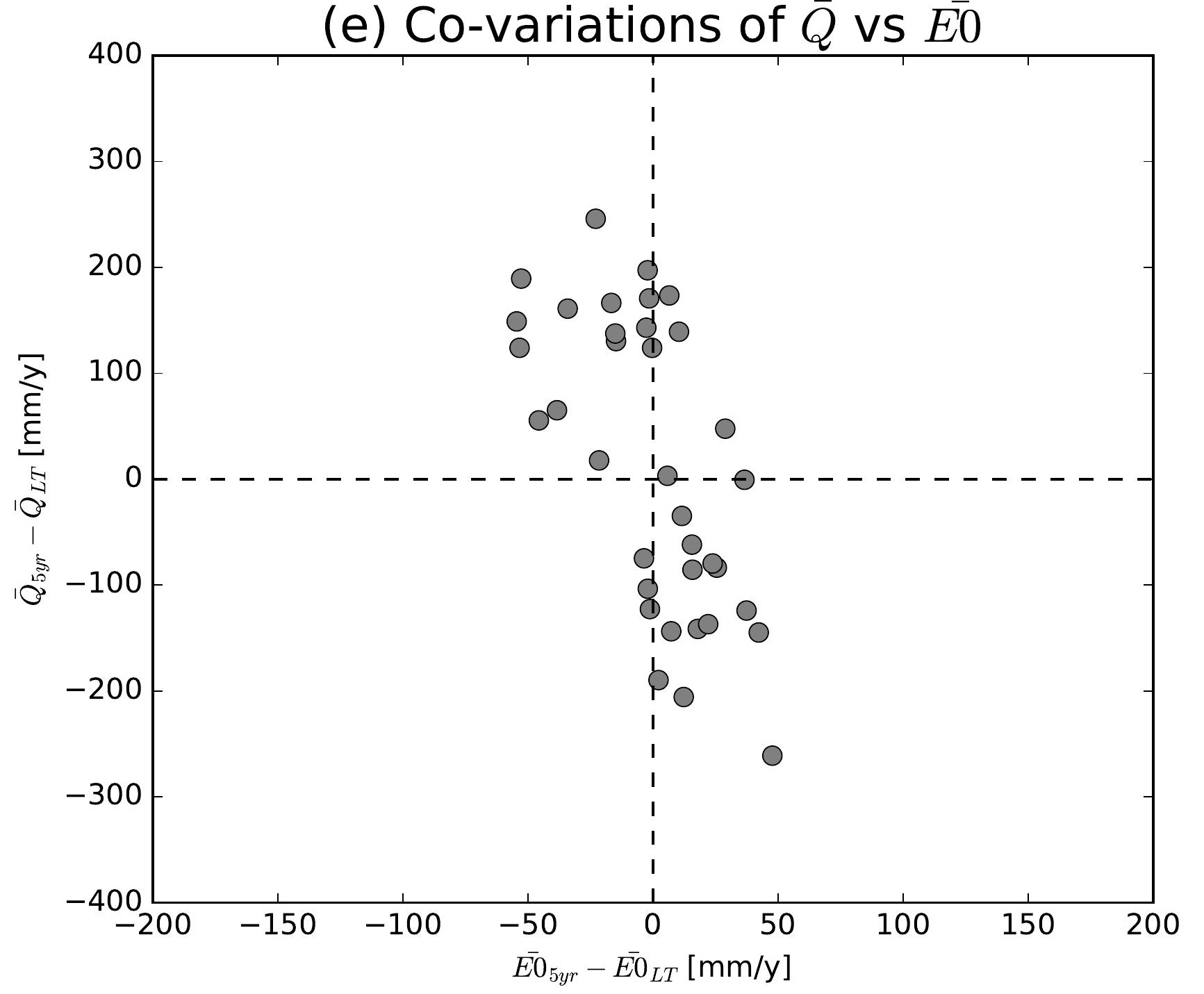

(h) Co-variations of $\bar{Q}$ vs $\overline{E 0}$

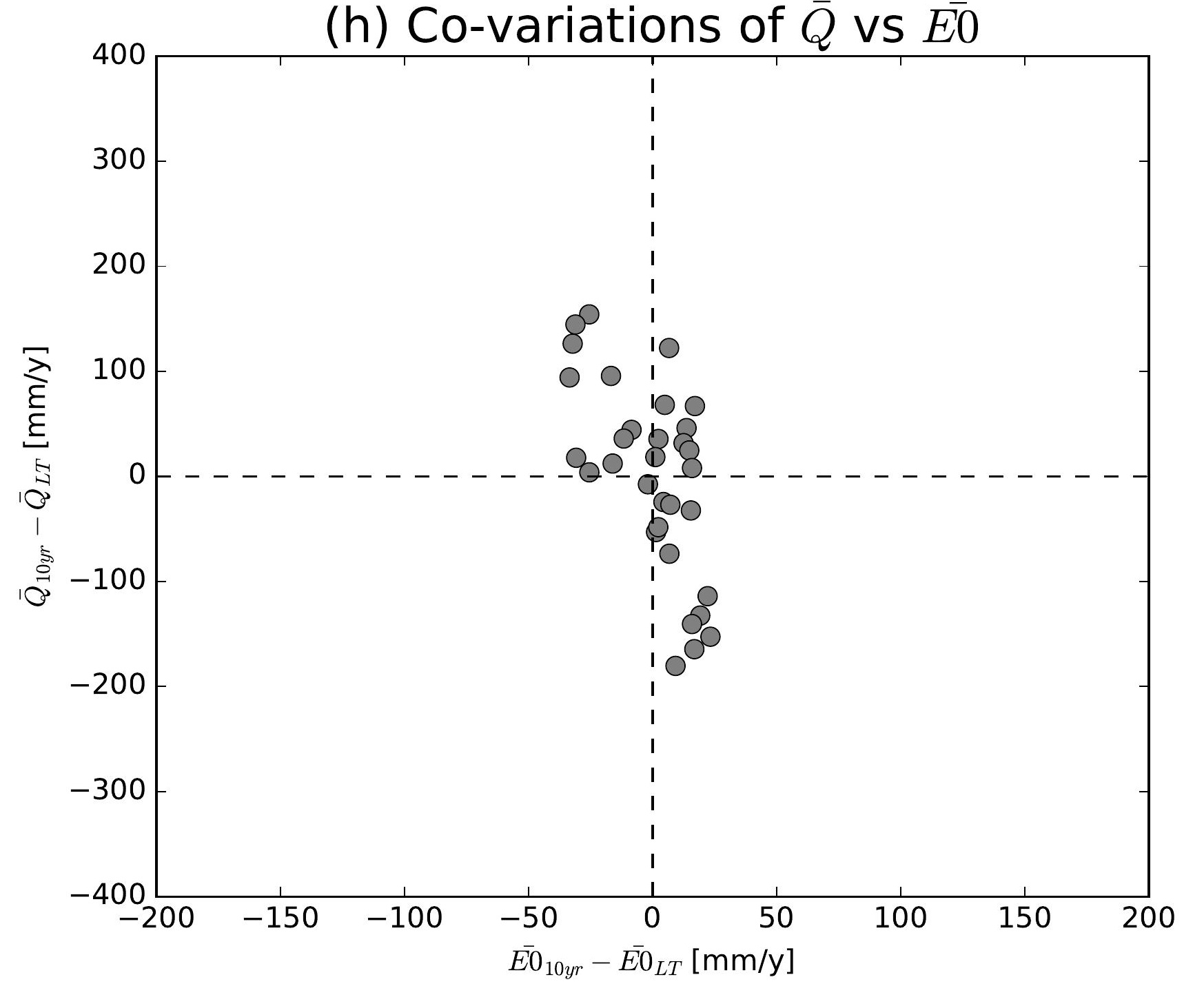

(k) Co-variations of $\bar{Q}$ vs $\overline{E 0}$

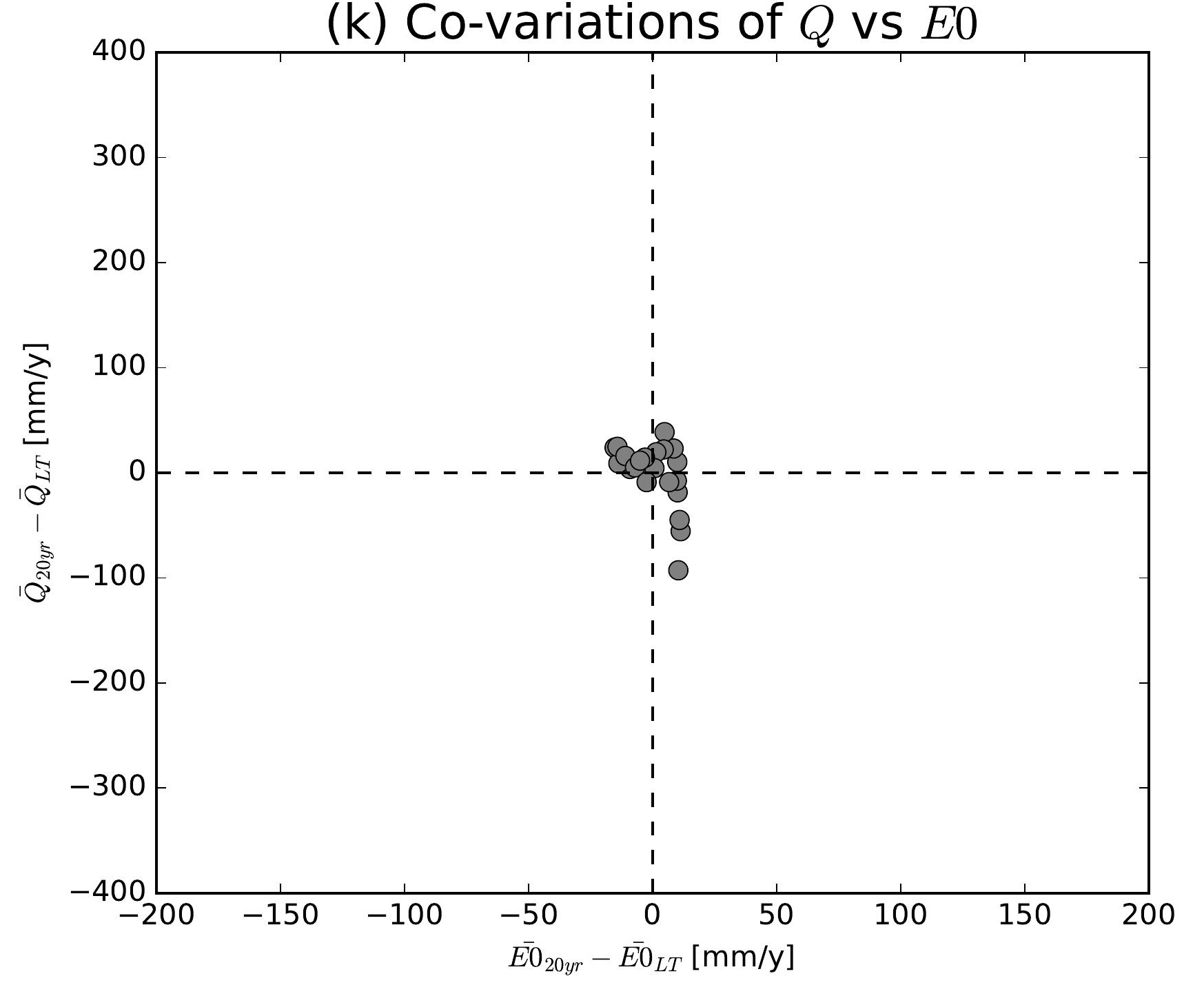

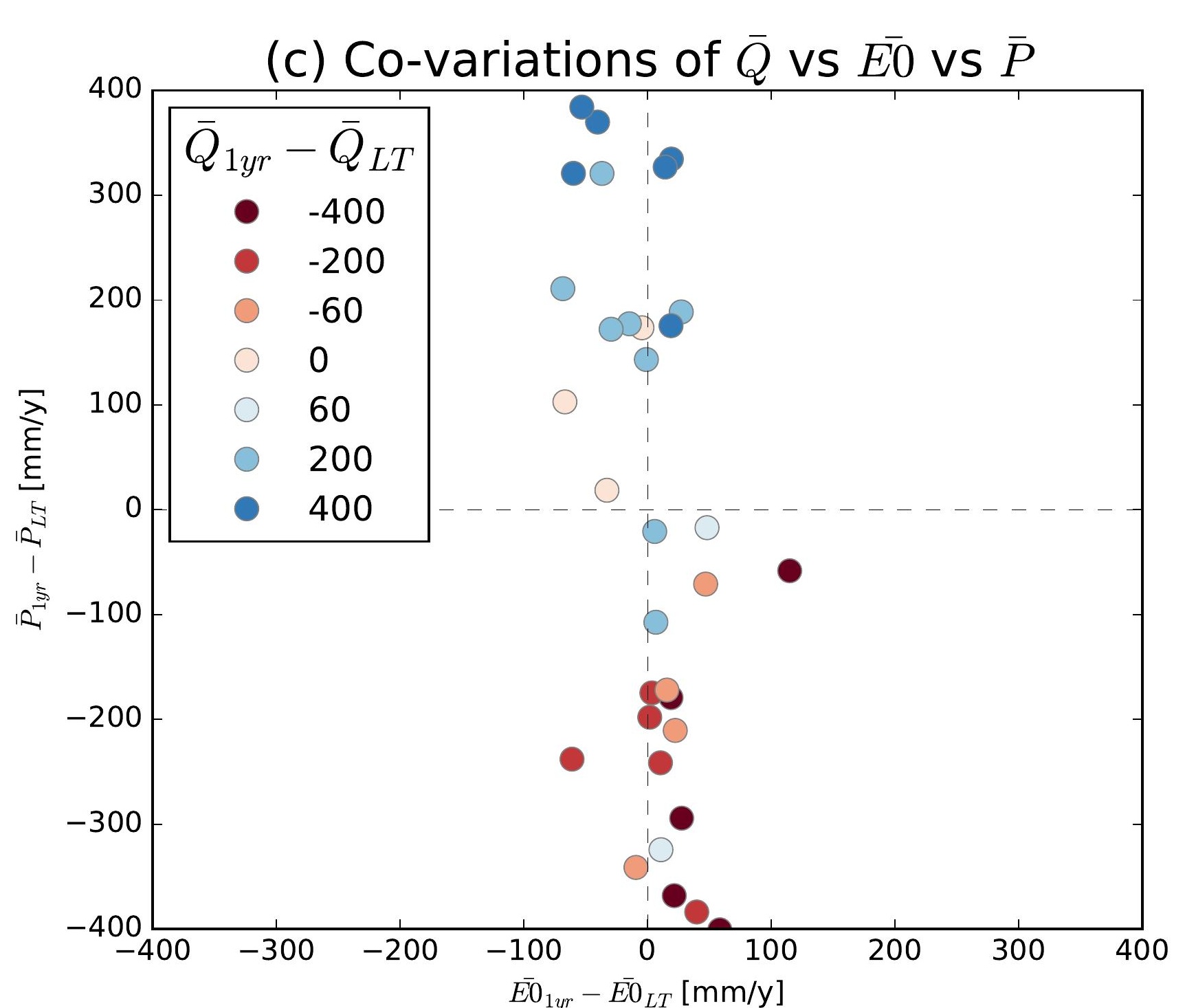
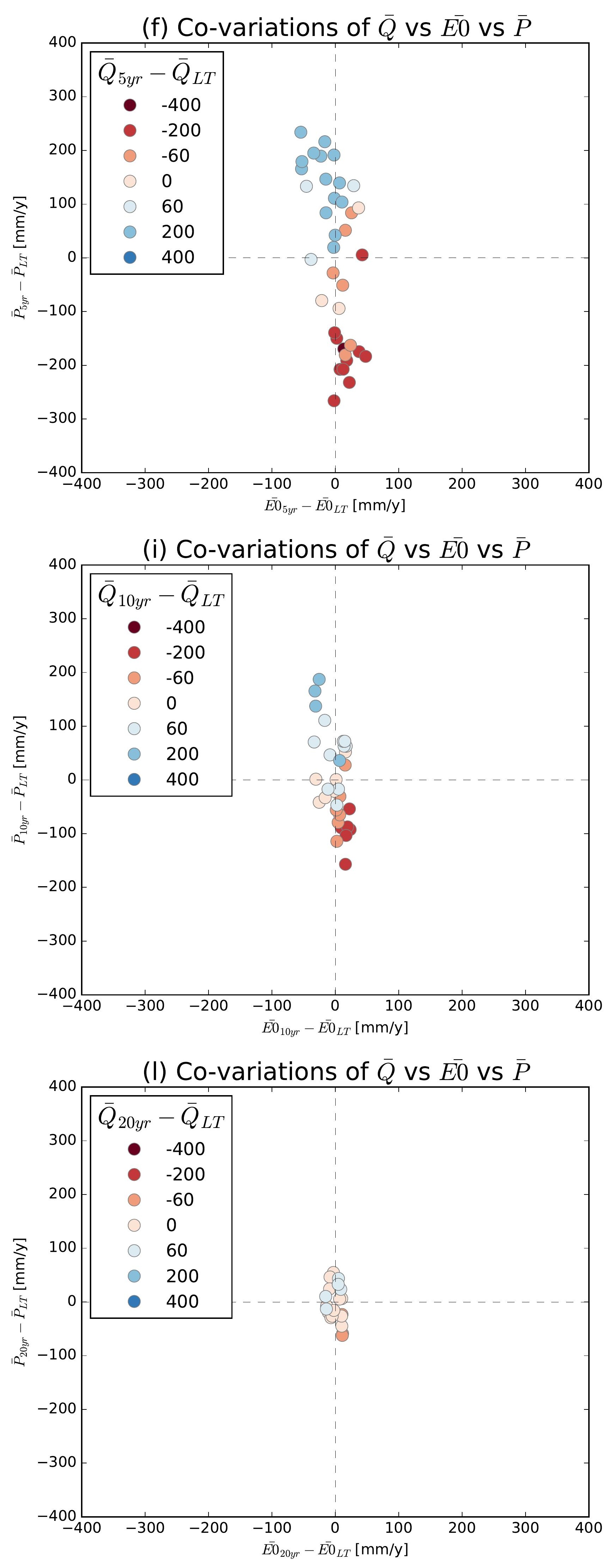

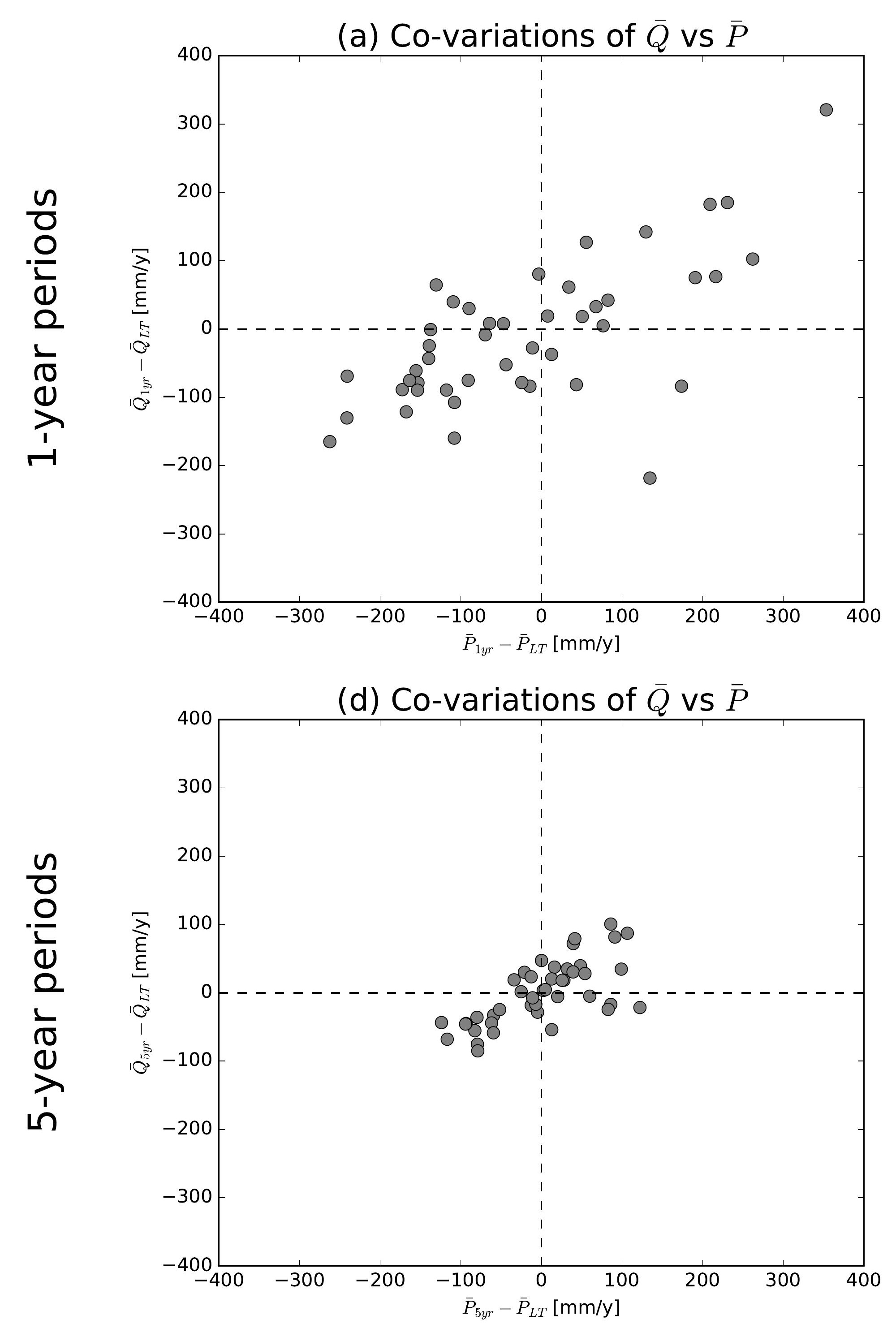

(g) Co-variations of $\bar{Q}$ vs $\bar{P}$

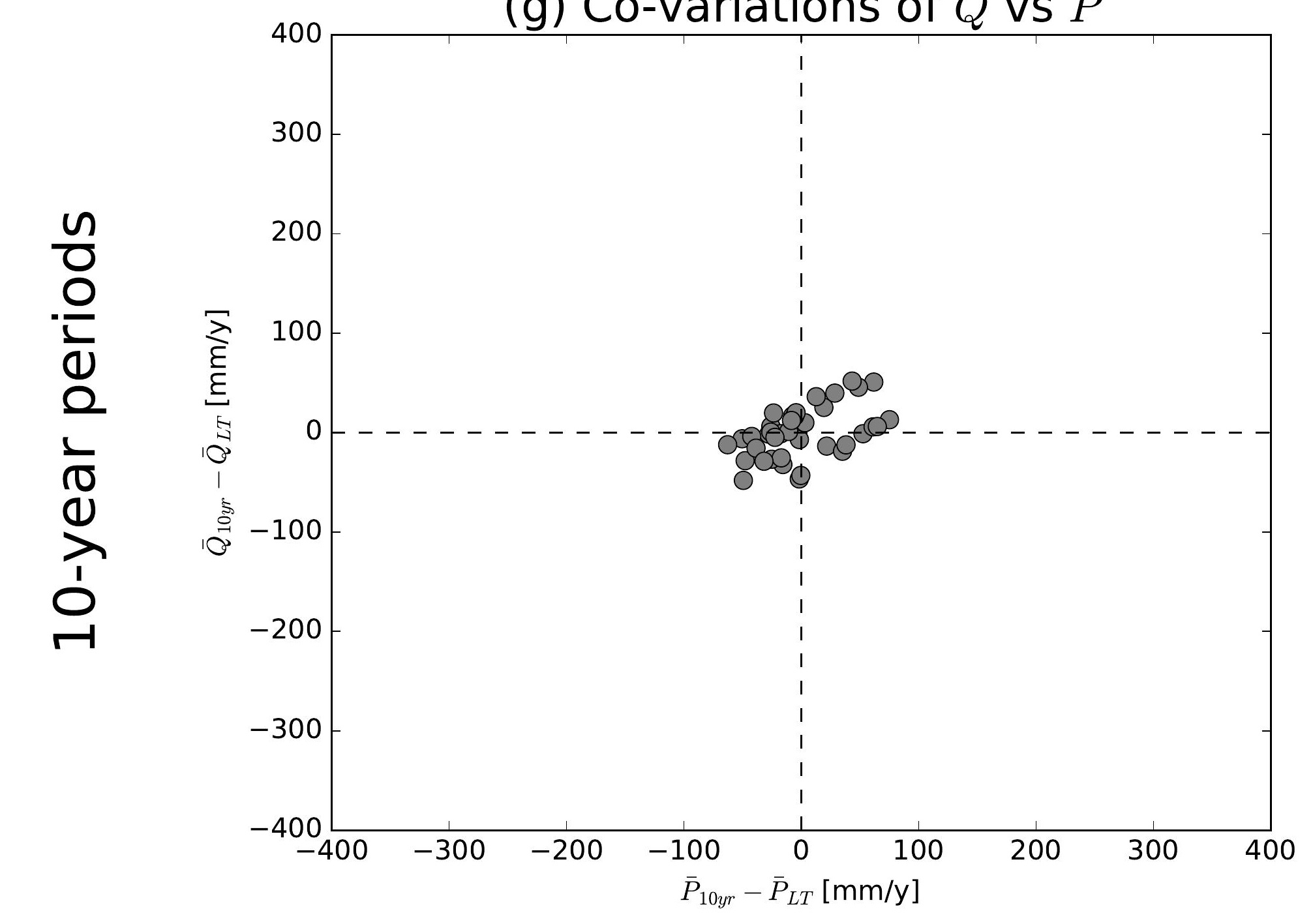

(j) Co-variations of $\bar{Q}$ vs $\bar{P}$

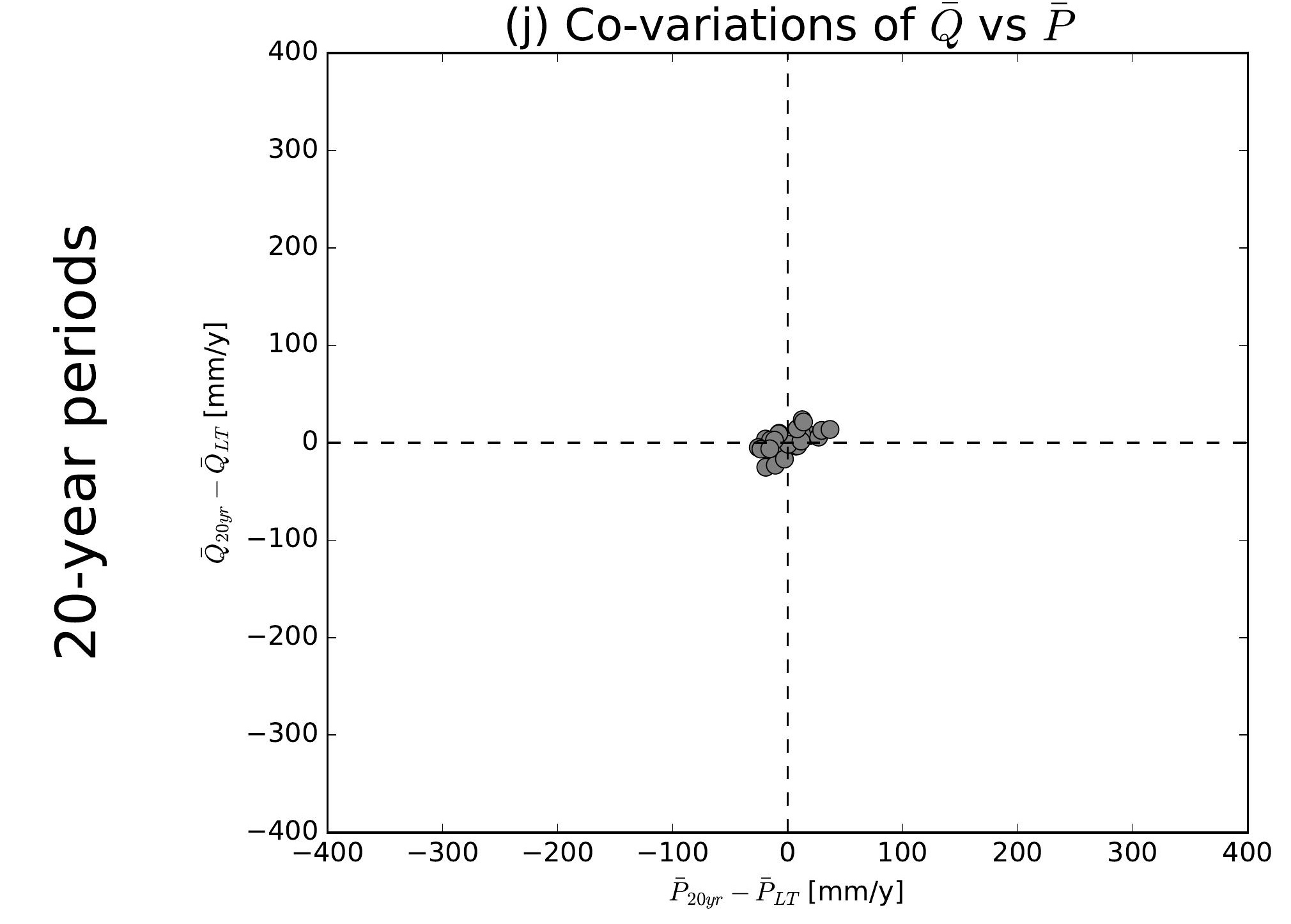

(b) Co-variations of $\bar{Q}$ vs $\overline{E 0}$

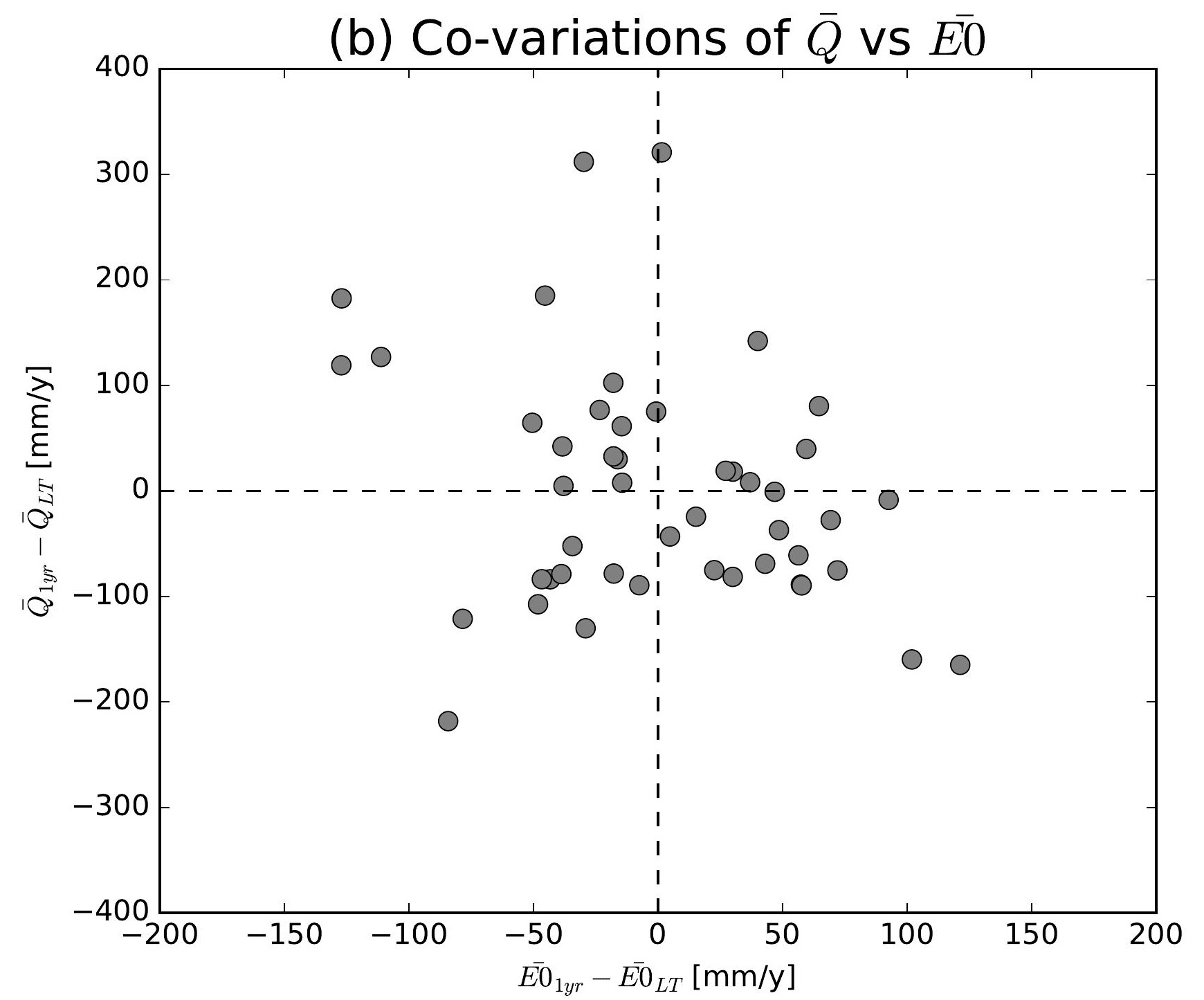

(e) Co-variations of $\bar{Q}$ vs $\overline{E 0}$

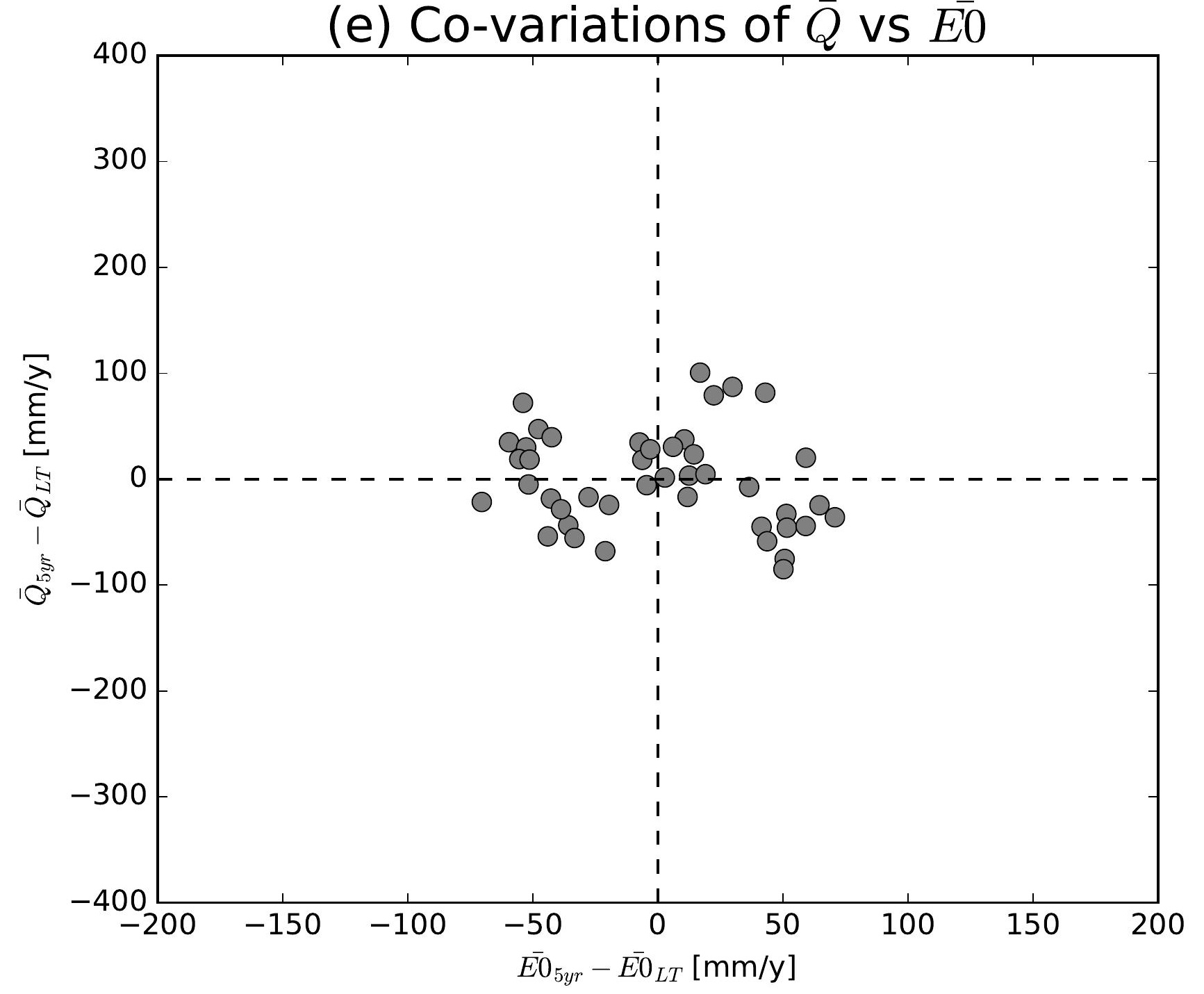

(h) Co-variations of $\bar{Q}$ vs $\overline{E 0}$

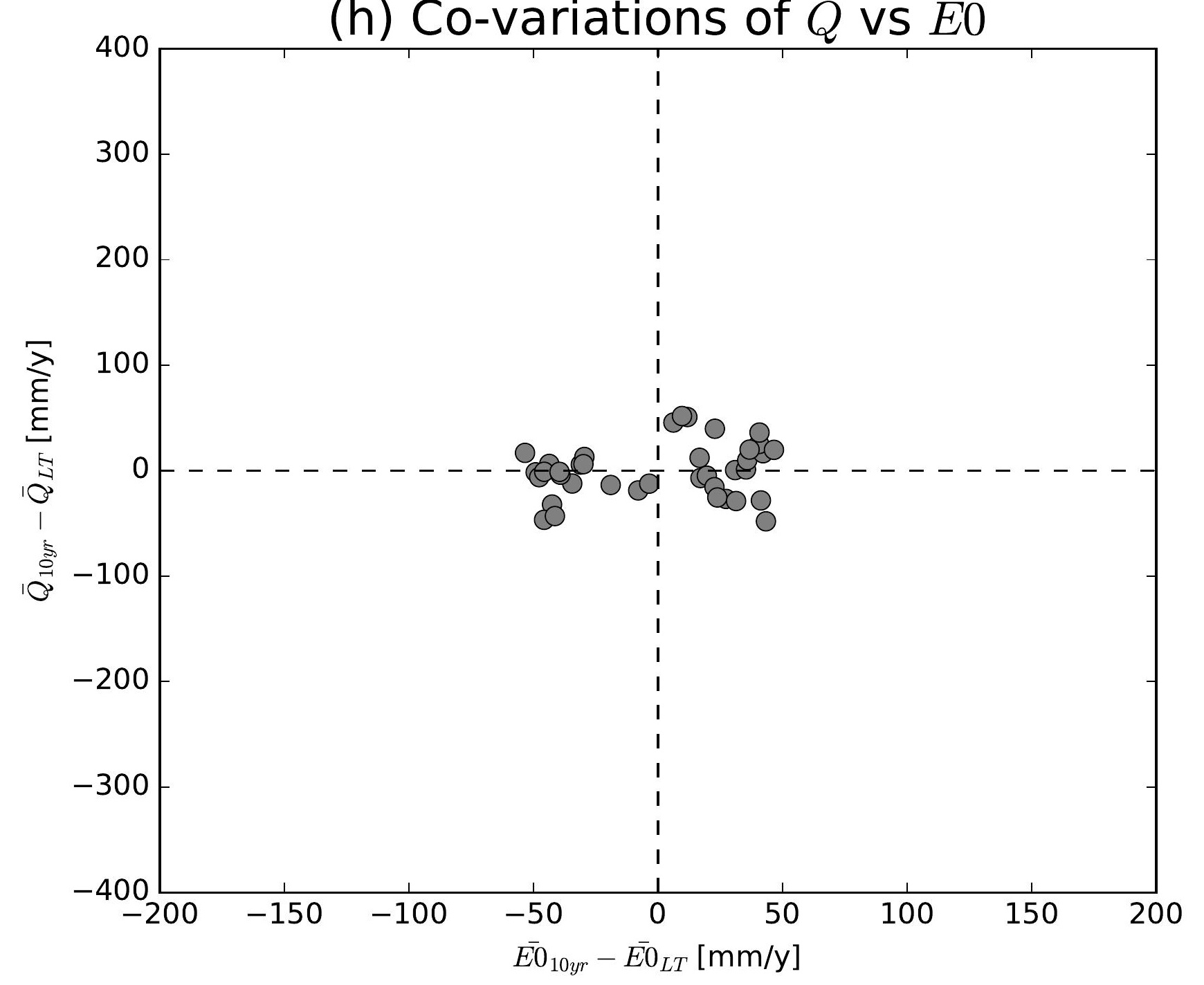

(k) Co-variations of $\bar{Q}$ vs $\overline{E 0}$

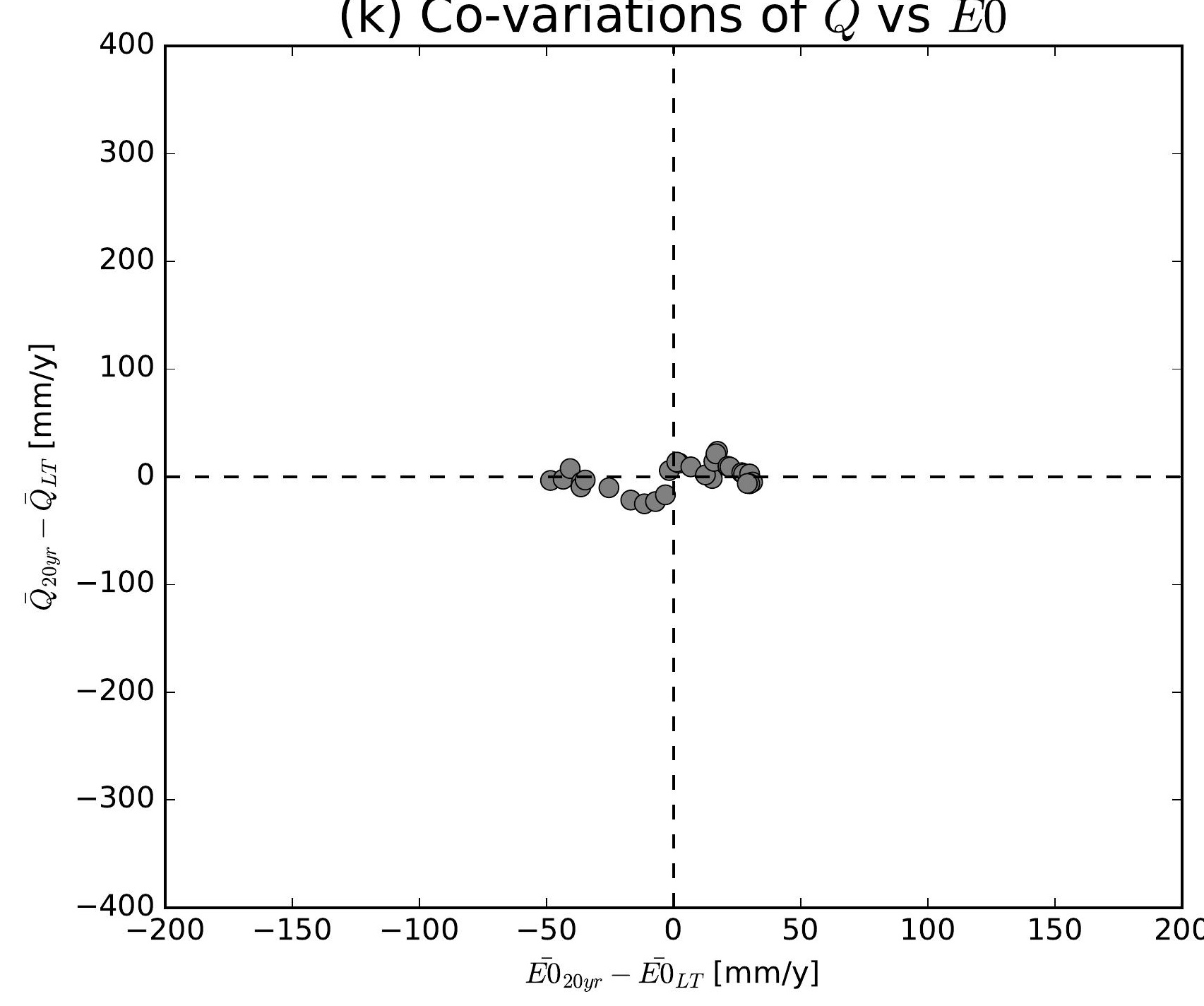

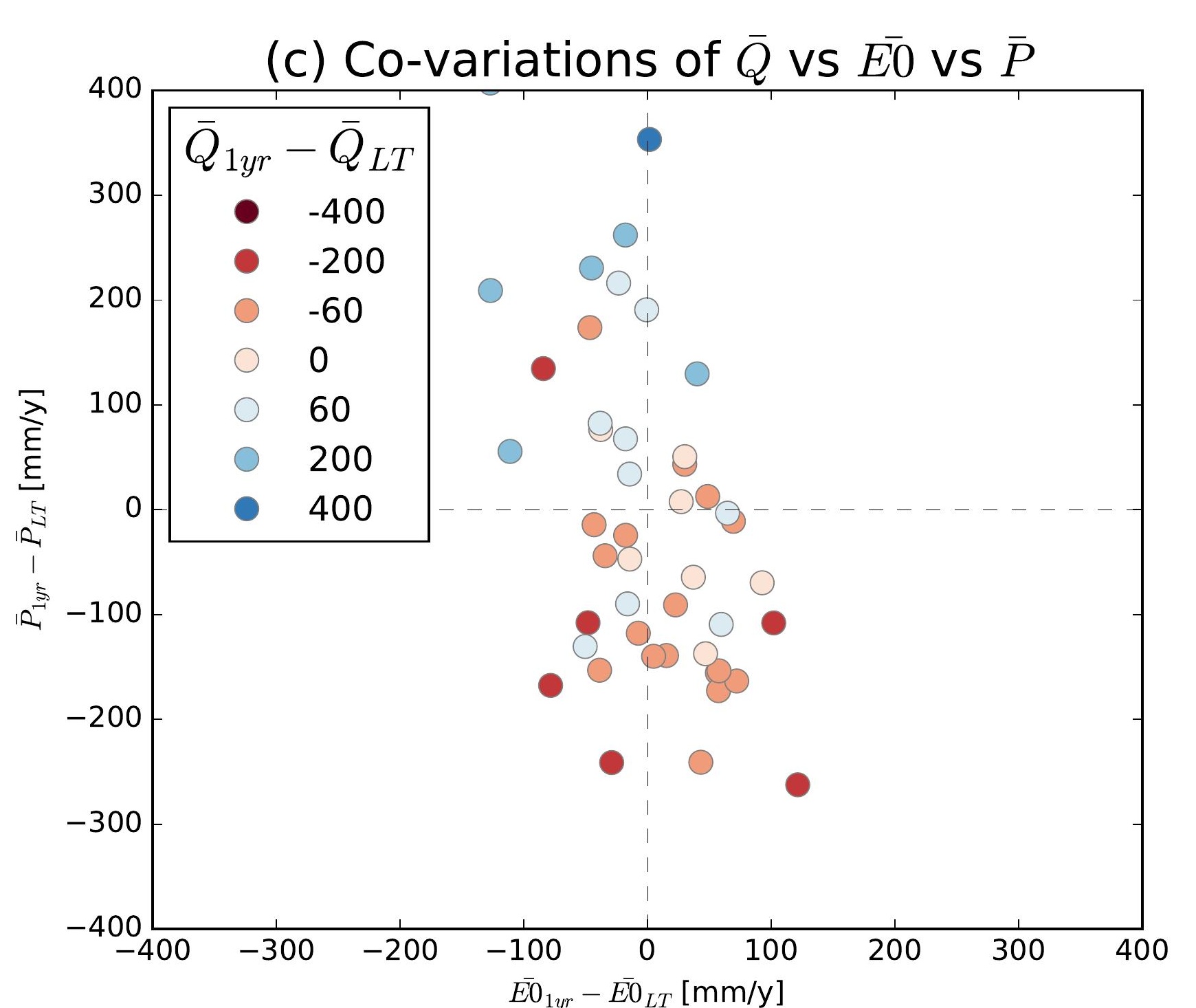
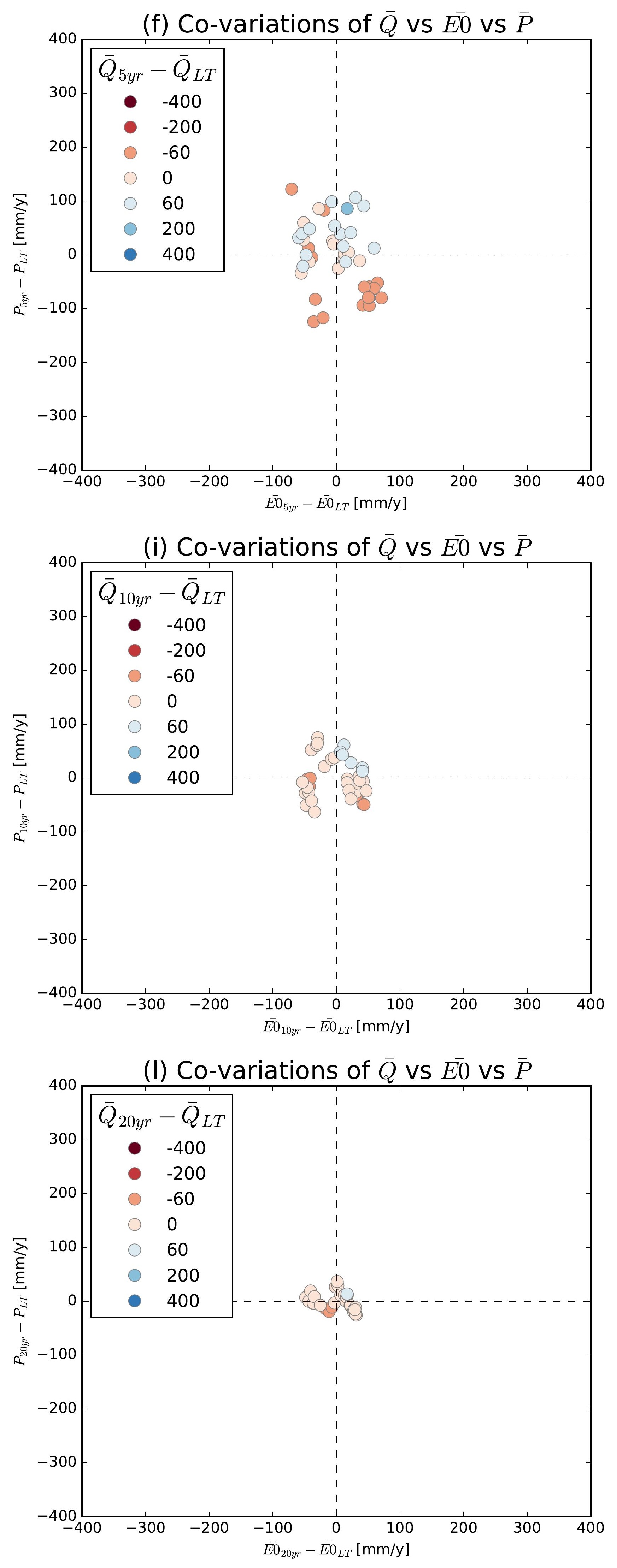

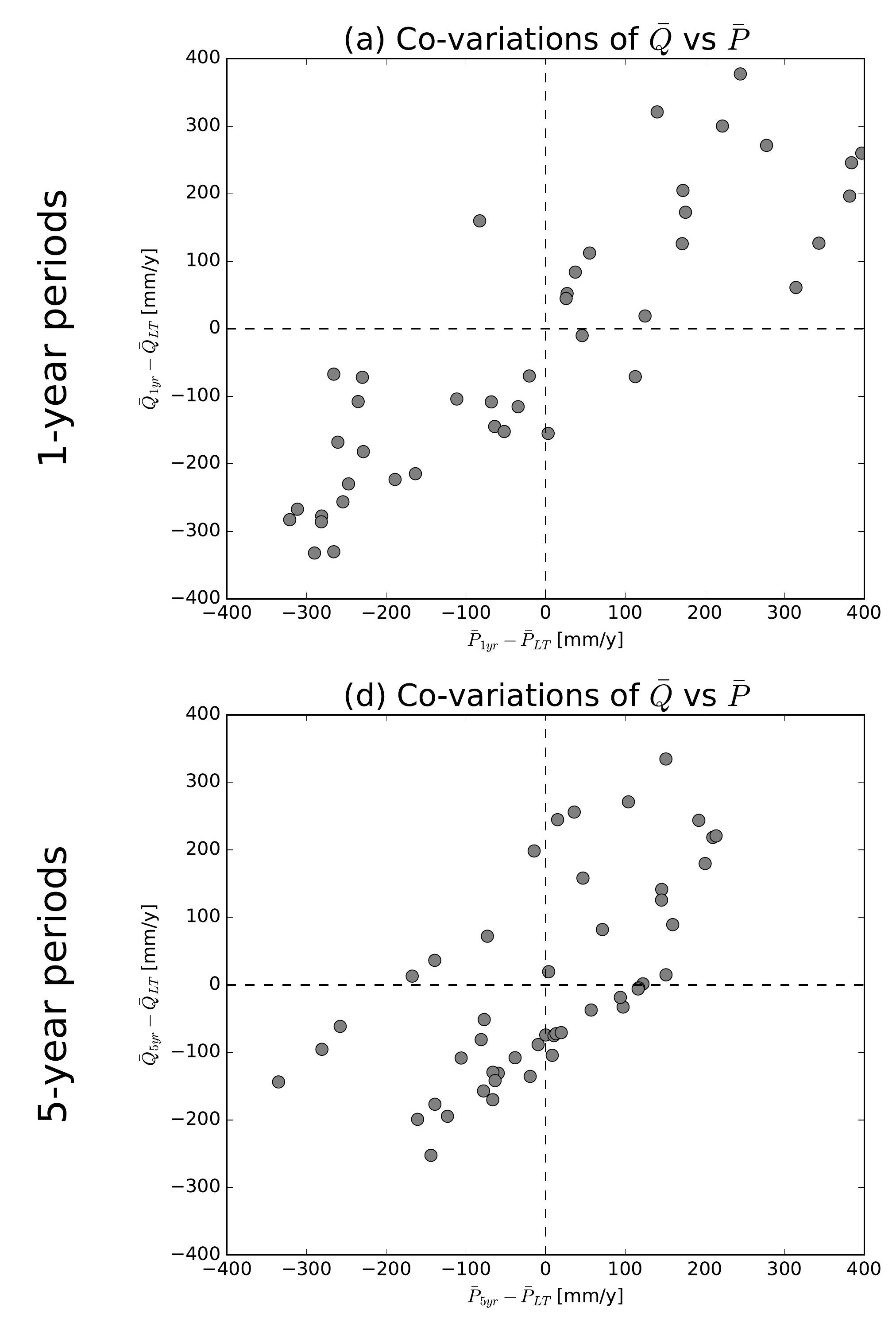

(g) Co-variations of $\bar{Q}$ vs $\bar{P}$

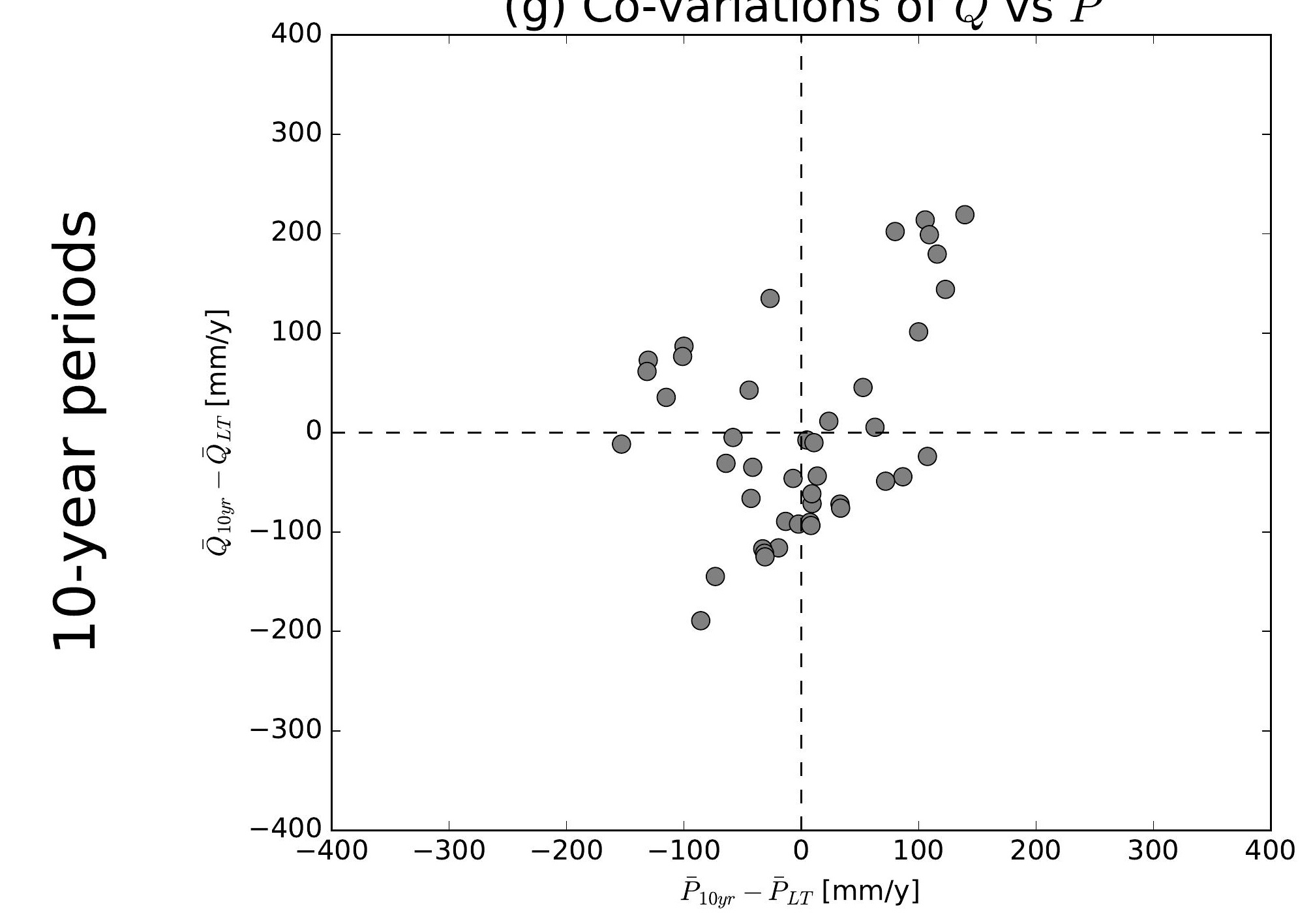

(j) Co-variations of $\bar{Q}$ vs $\bar{P}$

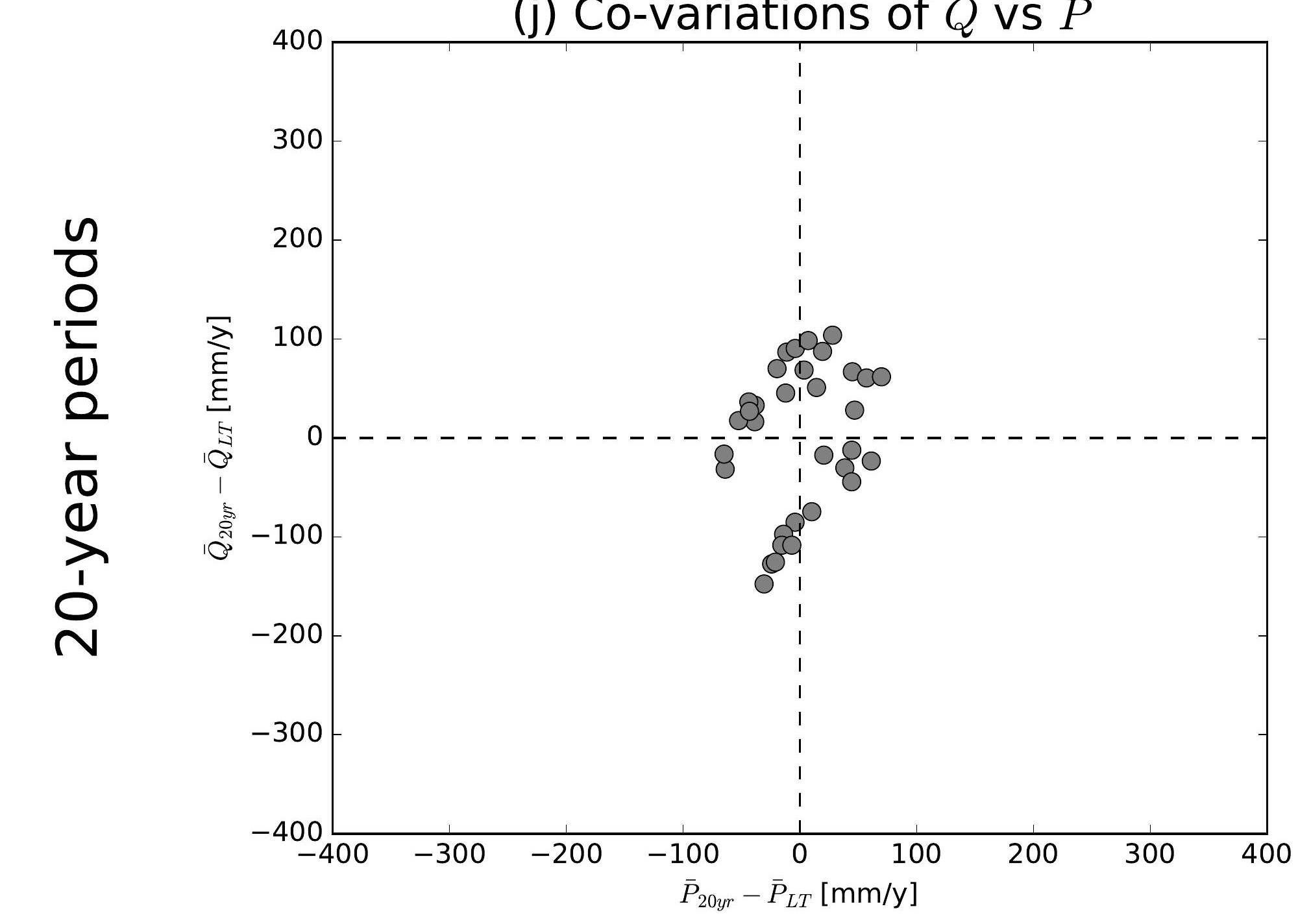

(b) Co-variations of $\bar{Q}$ vs $\overline{E 0}$

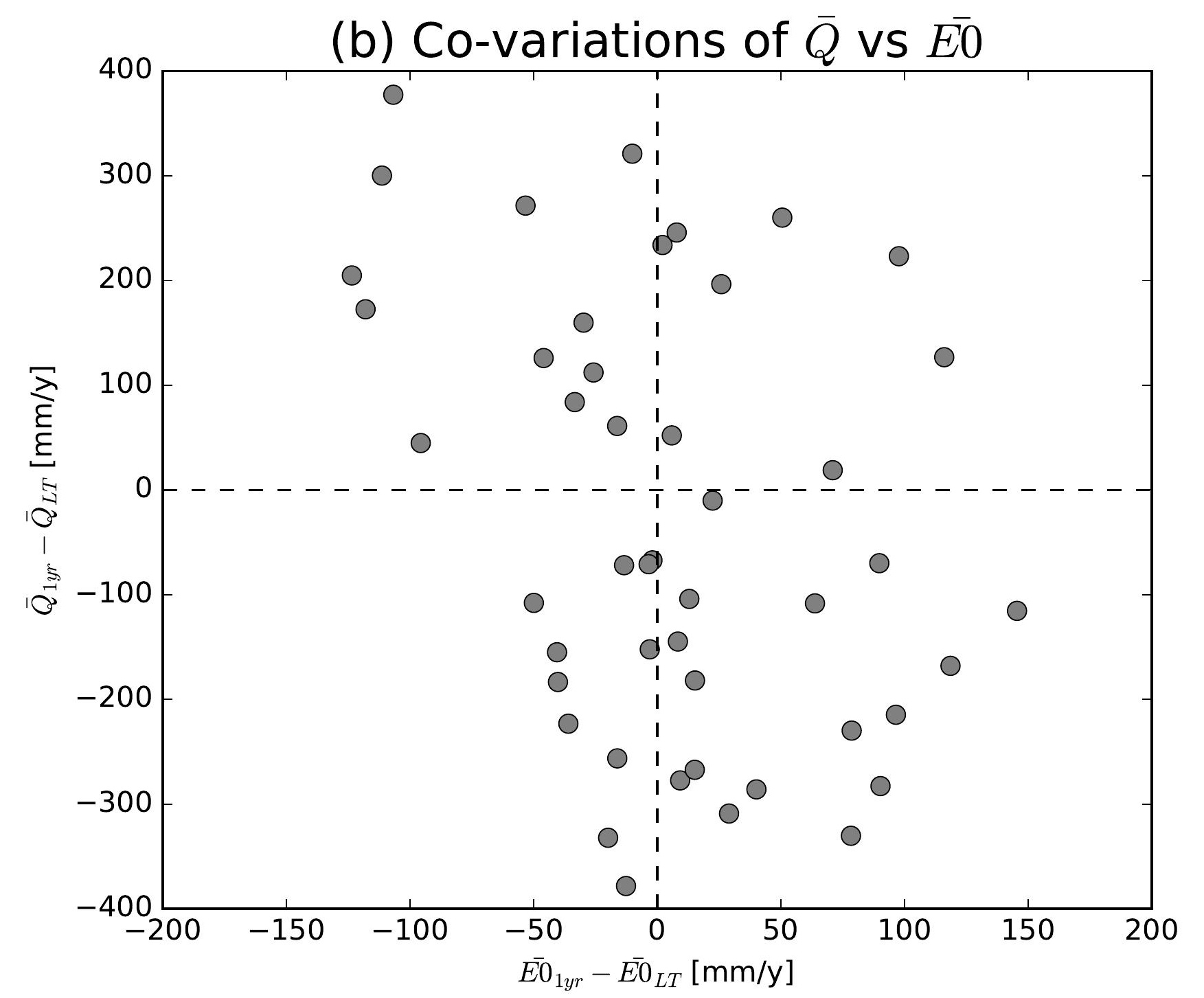

(e) Co-variations of $\bar{Q}$ vs $\overline{E 0}$

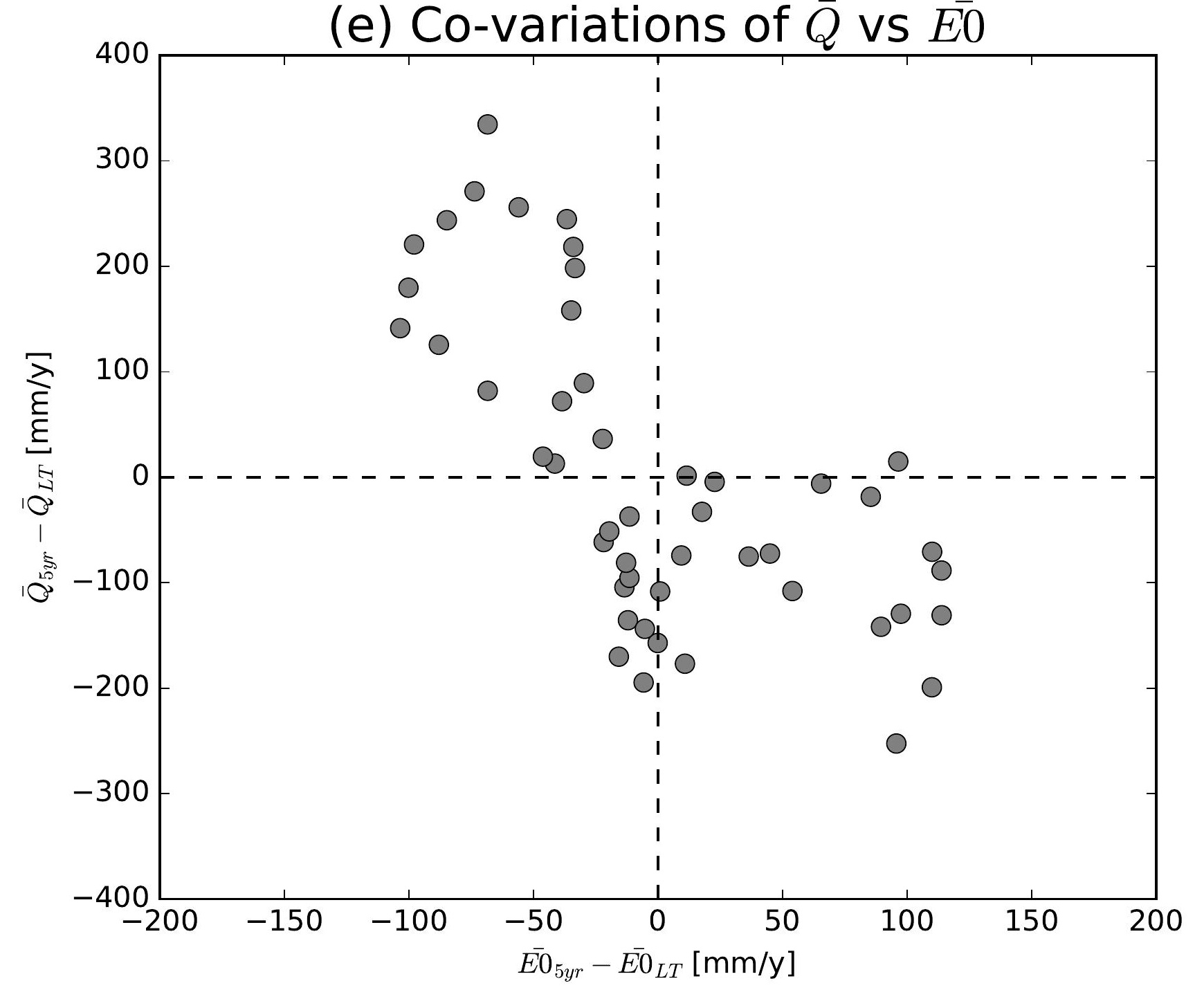

(h) Co-variations of $\bar{Q}$ vs $\overline{E 0}$

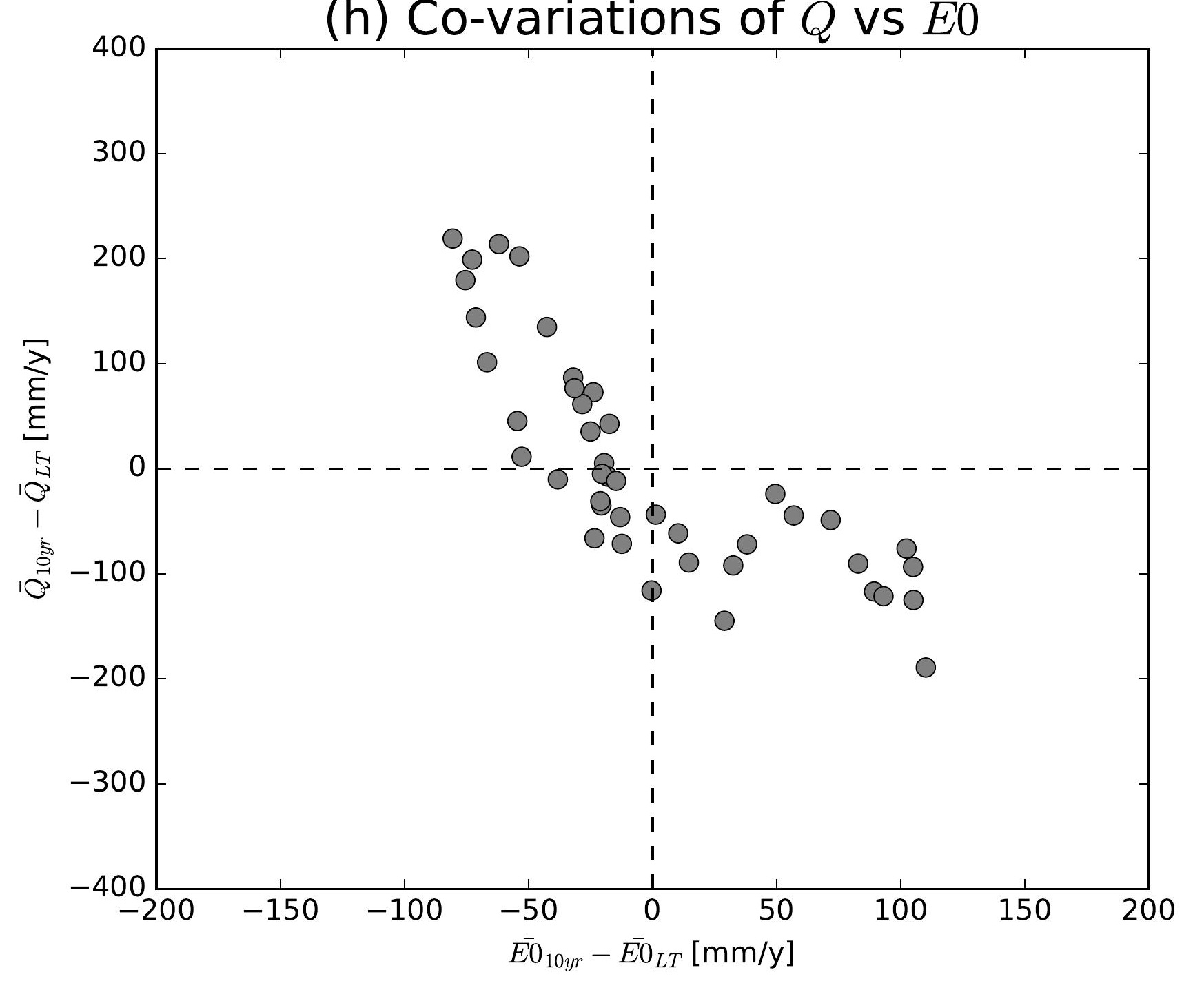

(k) Co-variations of $\bar{Q}$ vs $\overline{E 0}$

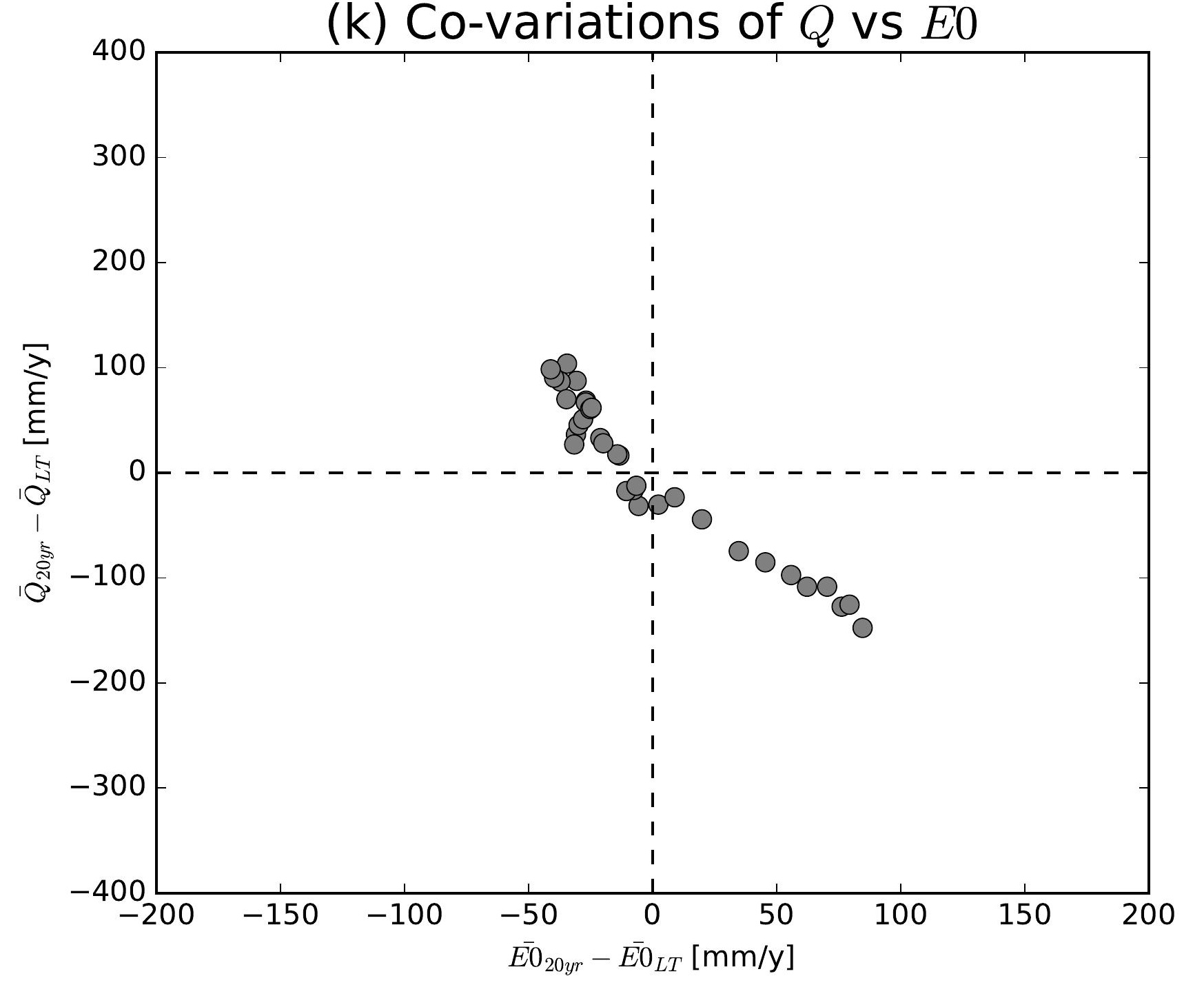

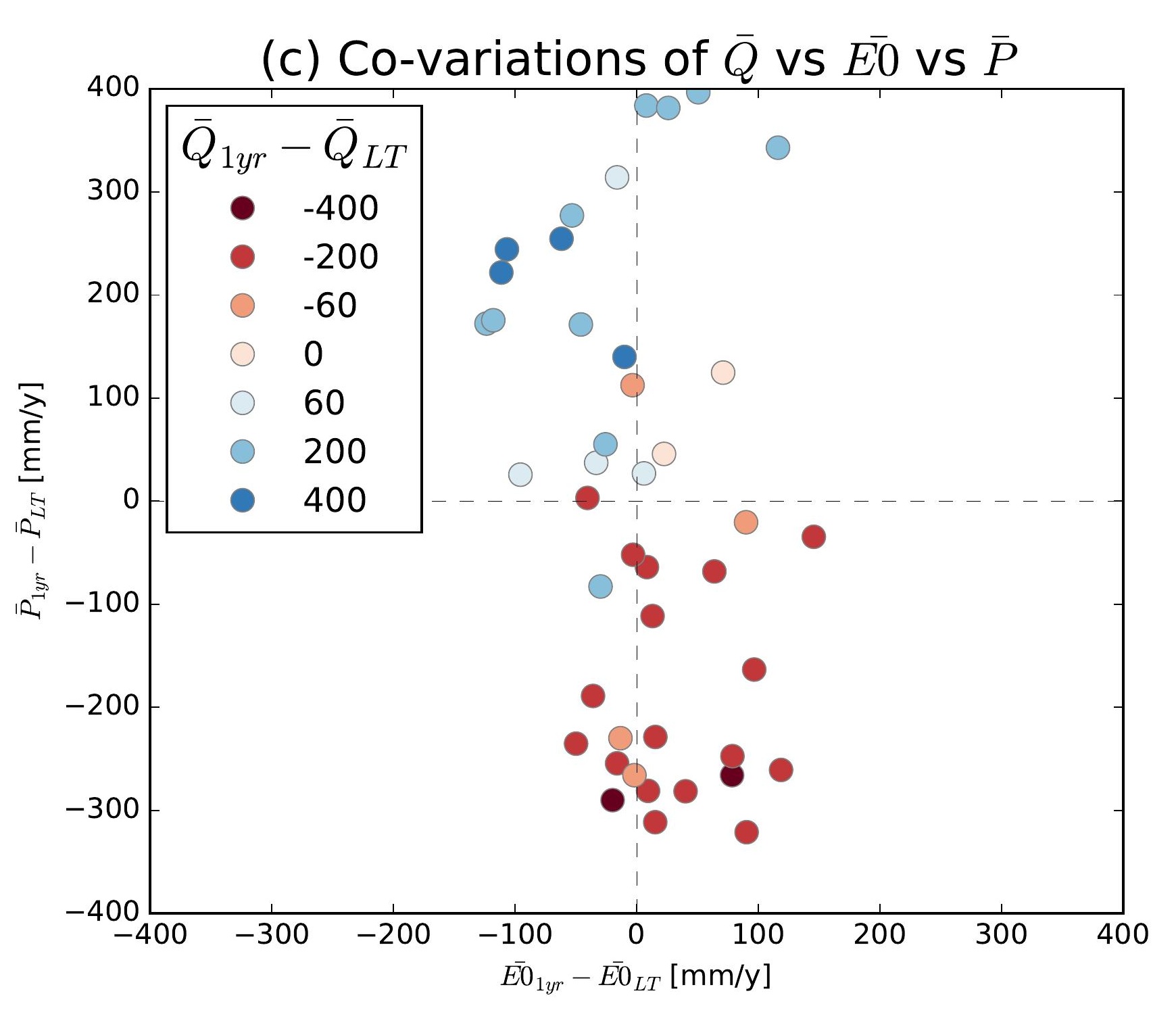
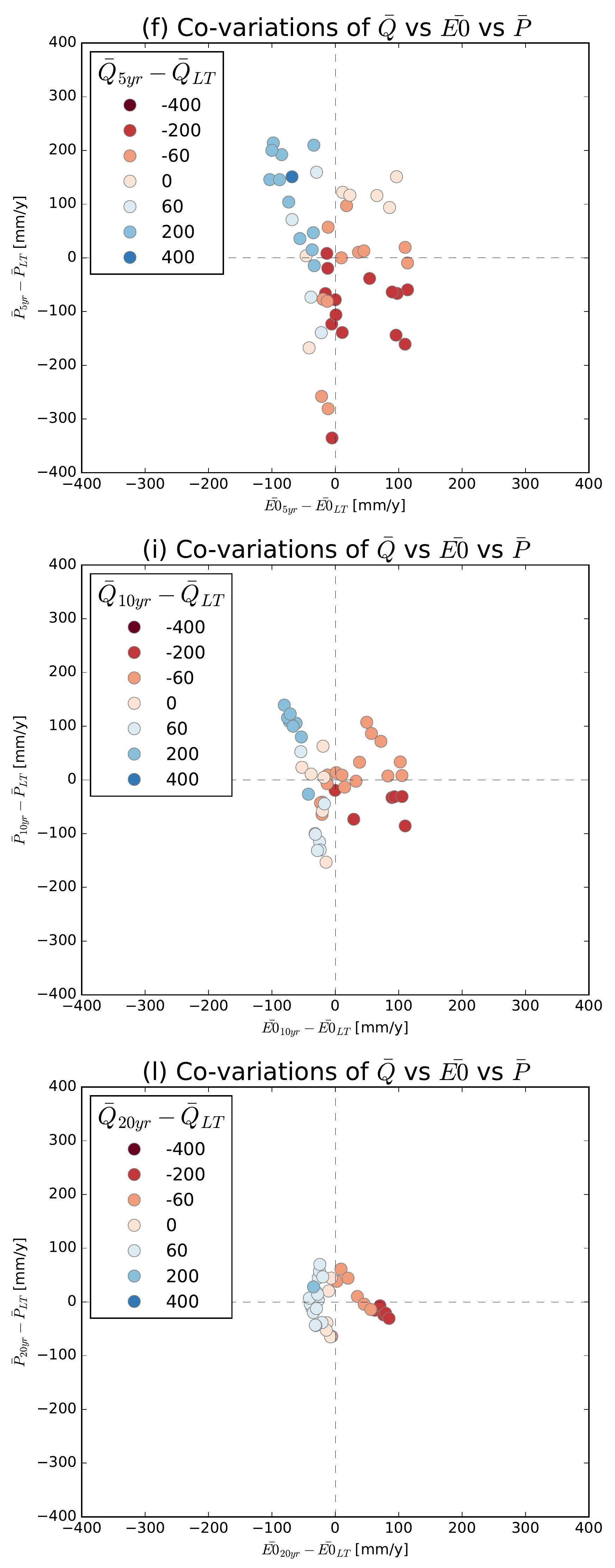

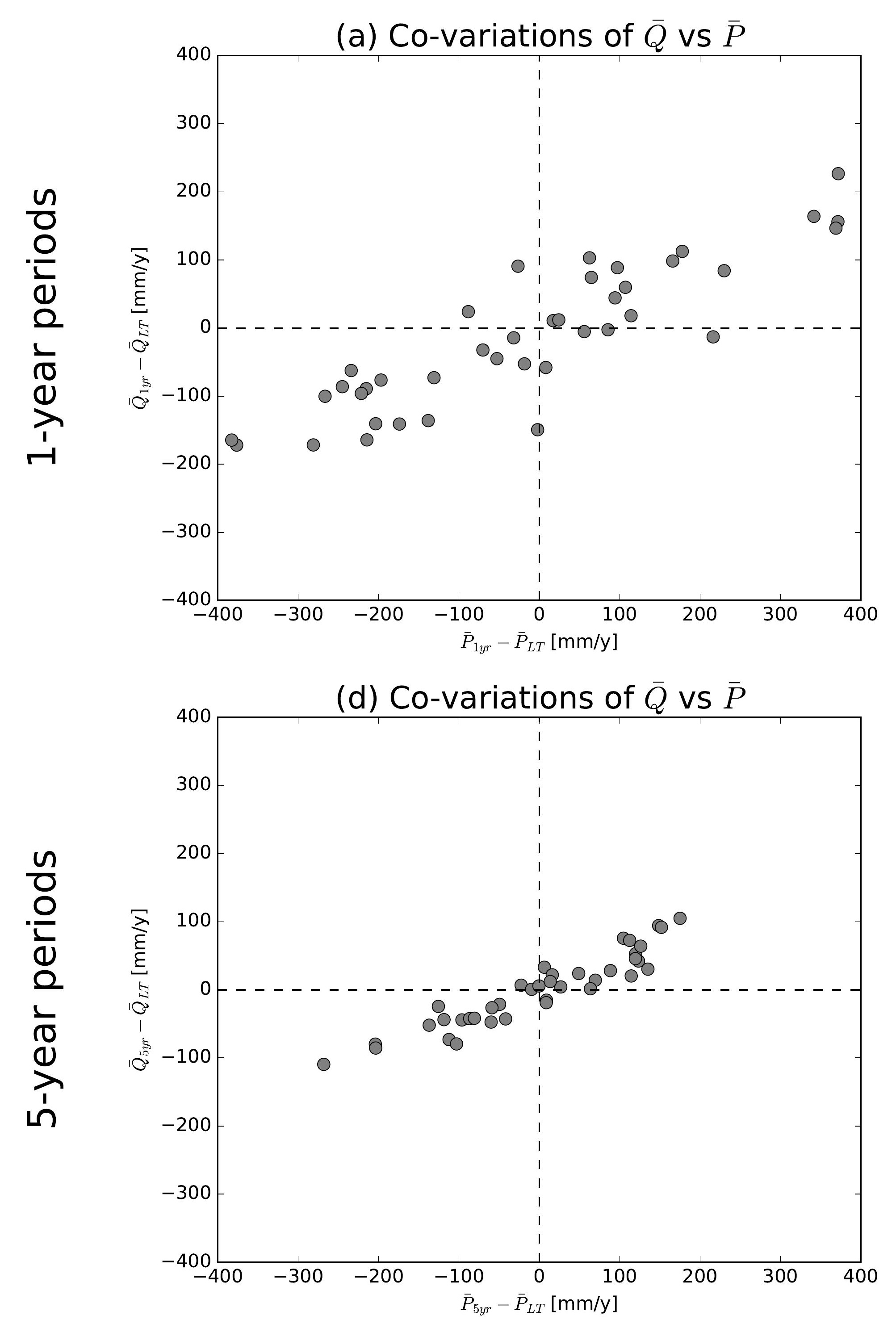

(g) Co-variations of $\bar{Q}$ vs $\bar{P}$

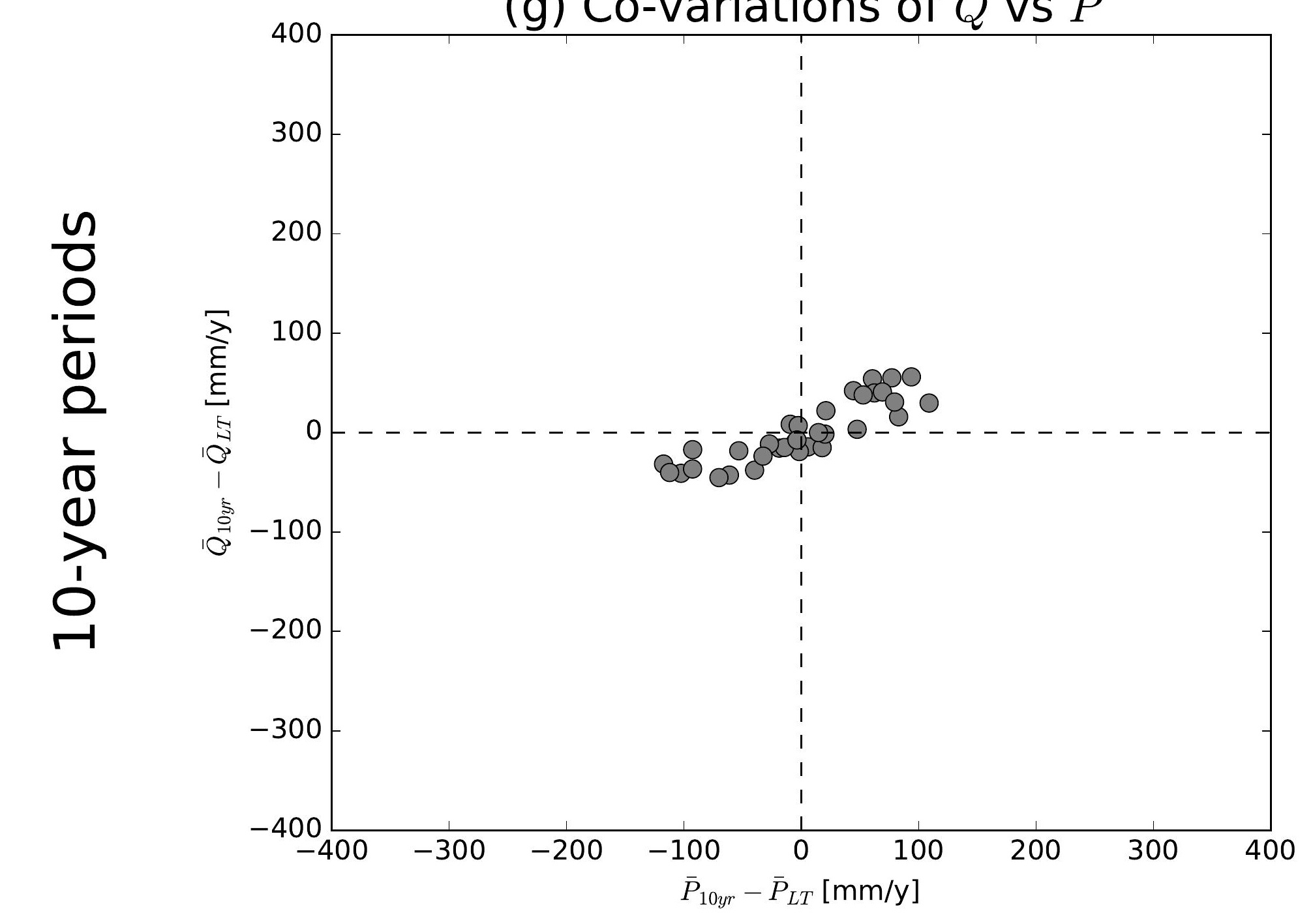

(j) Co-variations of $\bar{Q}$ vs $\bar{P}$

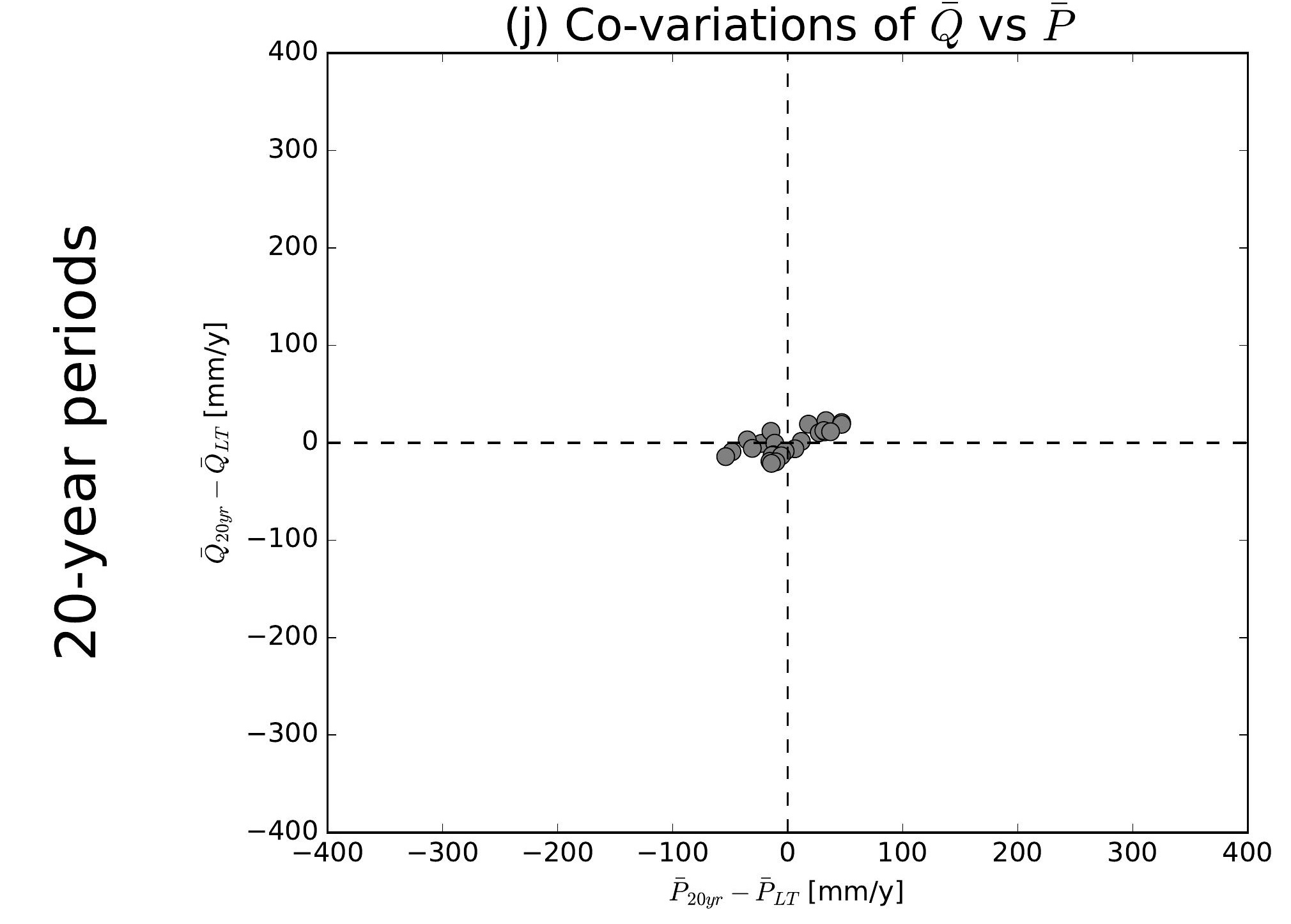

(b) Co-variations of $\bar{Q}$ vs $\overline{E 0}$

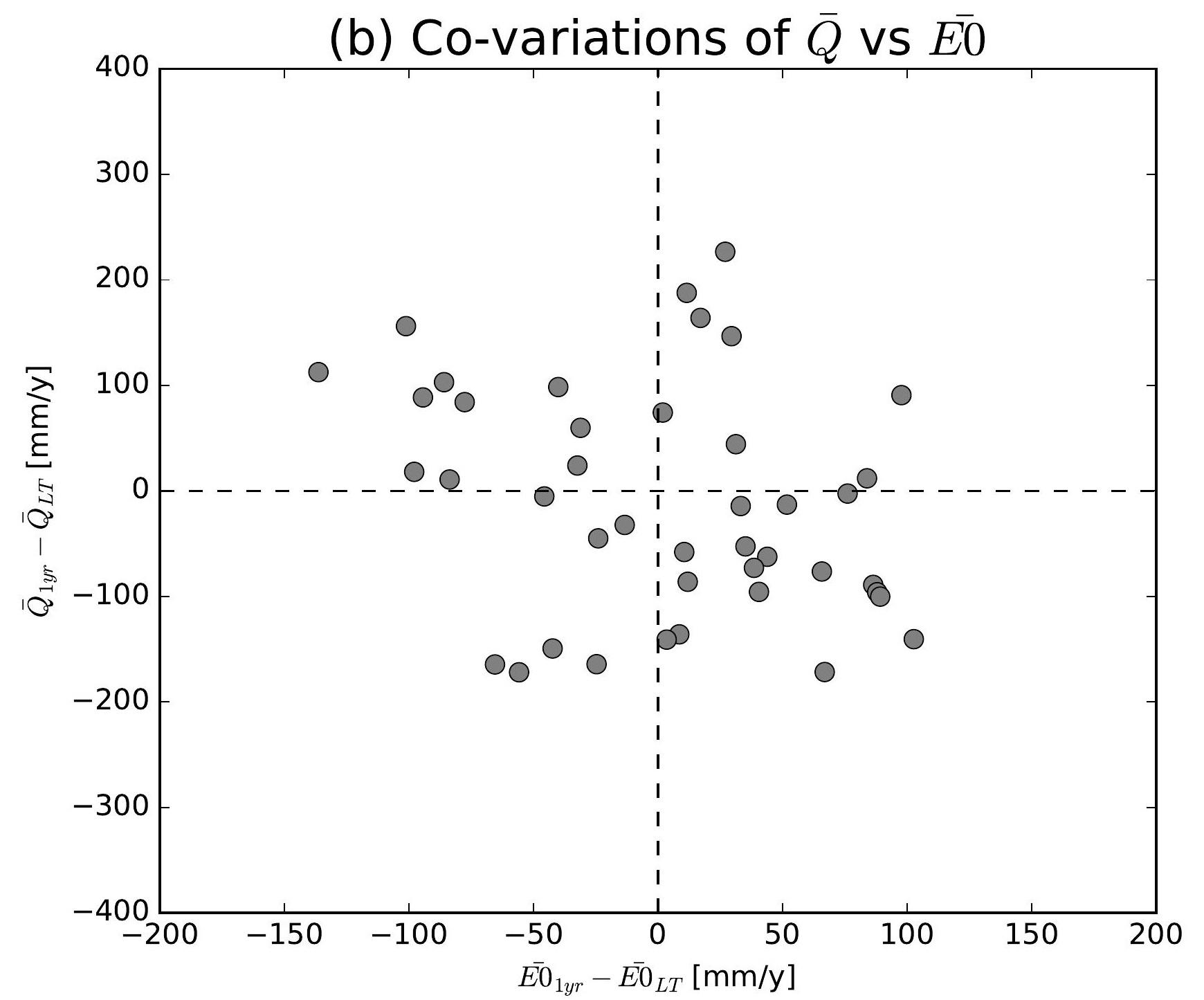

(e) Co-variations of $\bar{Q}$ vs $\overline{E 0}$

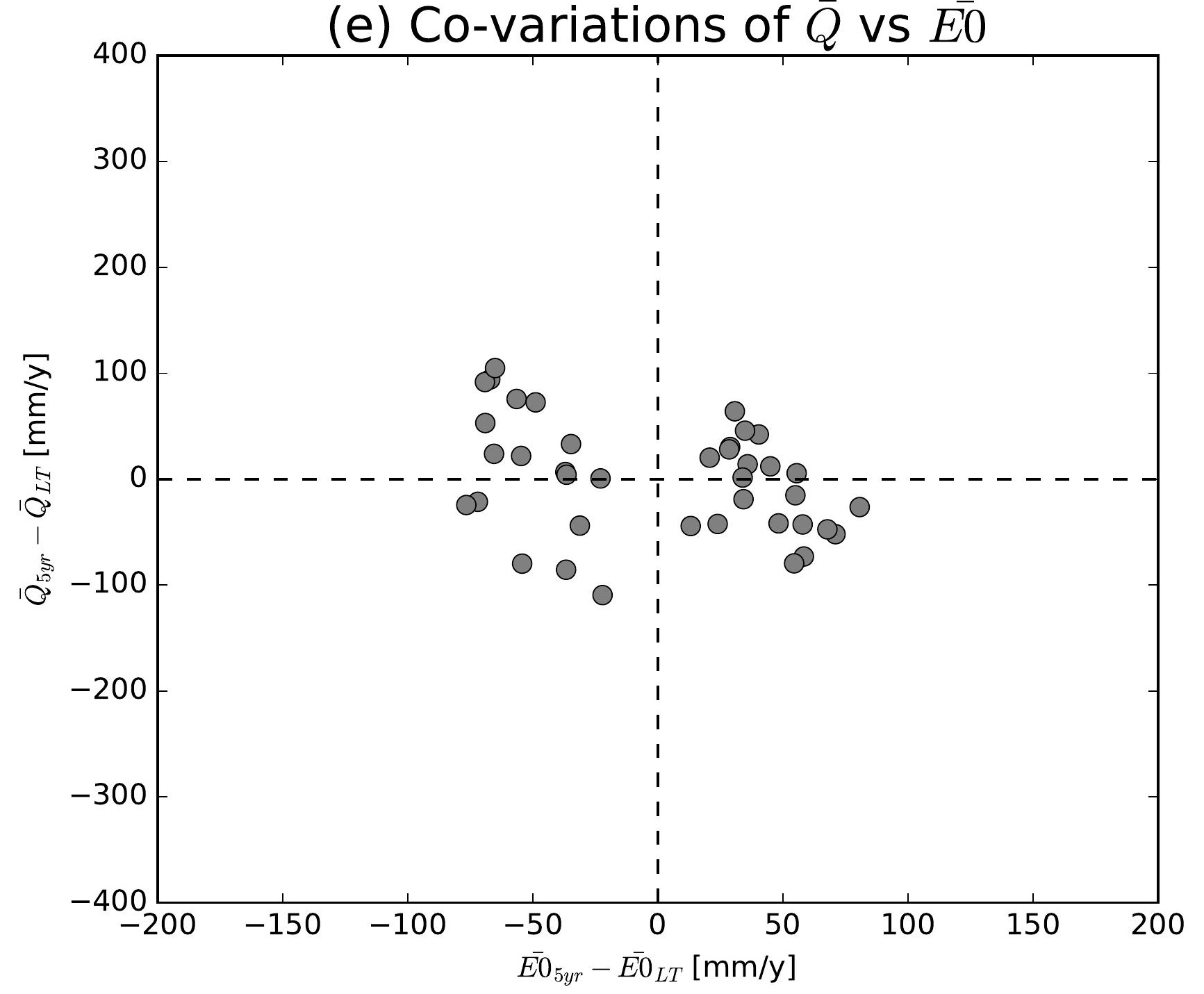

(h) Co-variations of $\bar{Q}$ vs $\overline{E 0}$

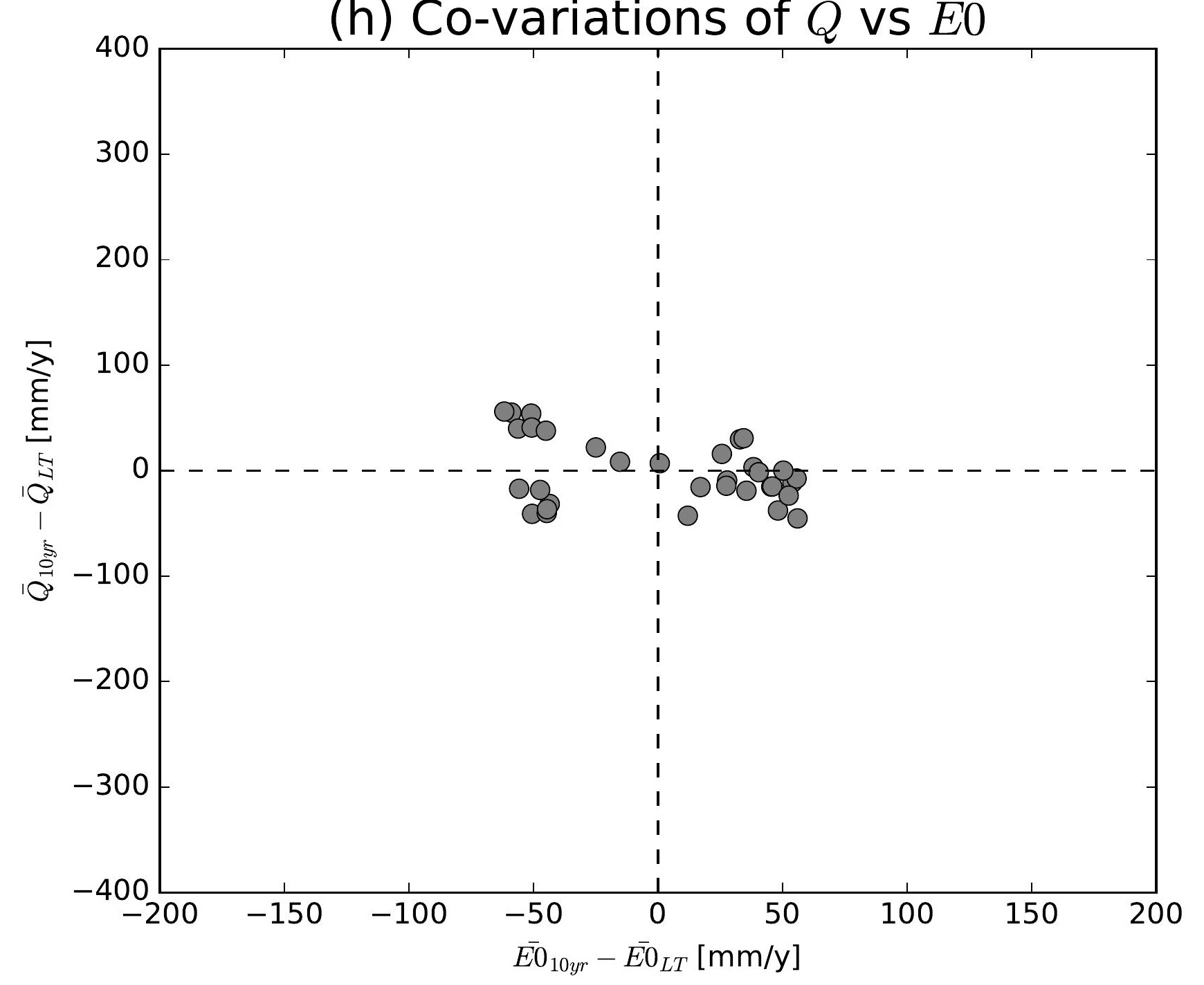

(k) Co-variations of $\bar{Q}$ vs $\overline{E 0}$

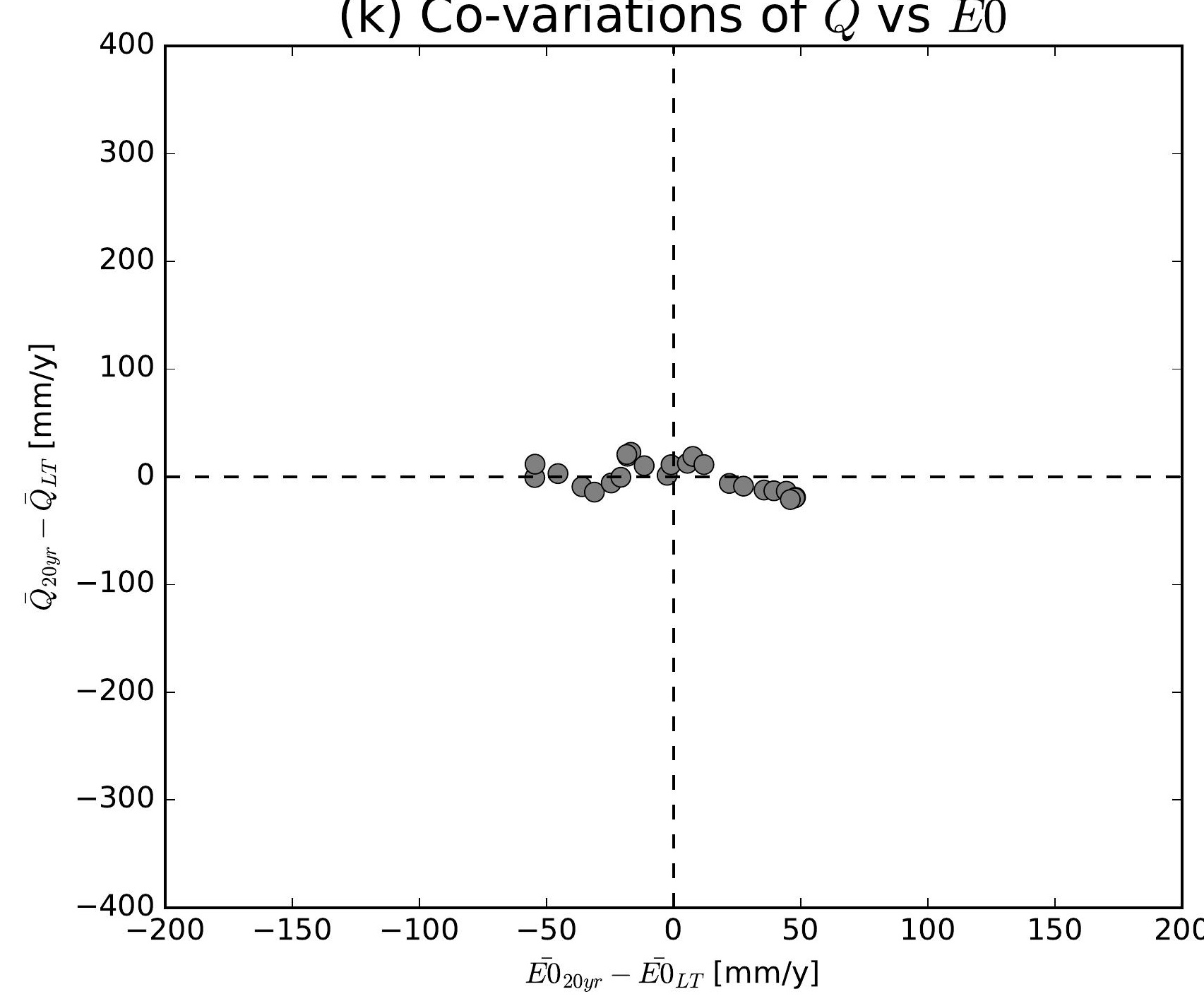

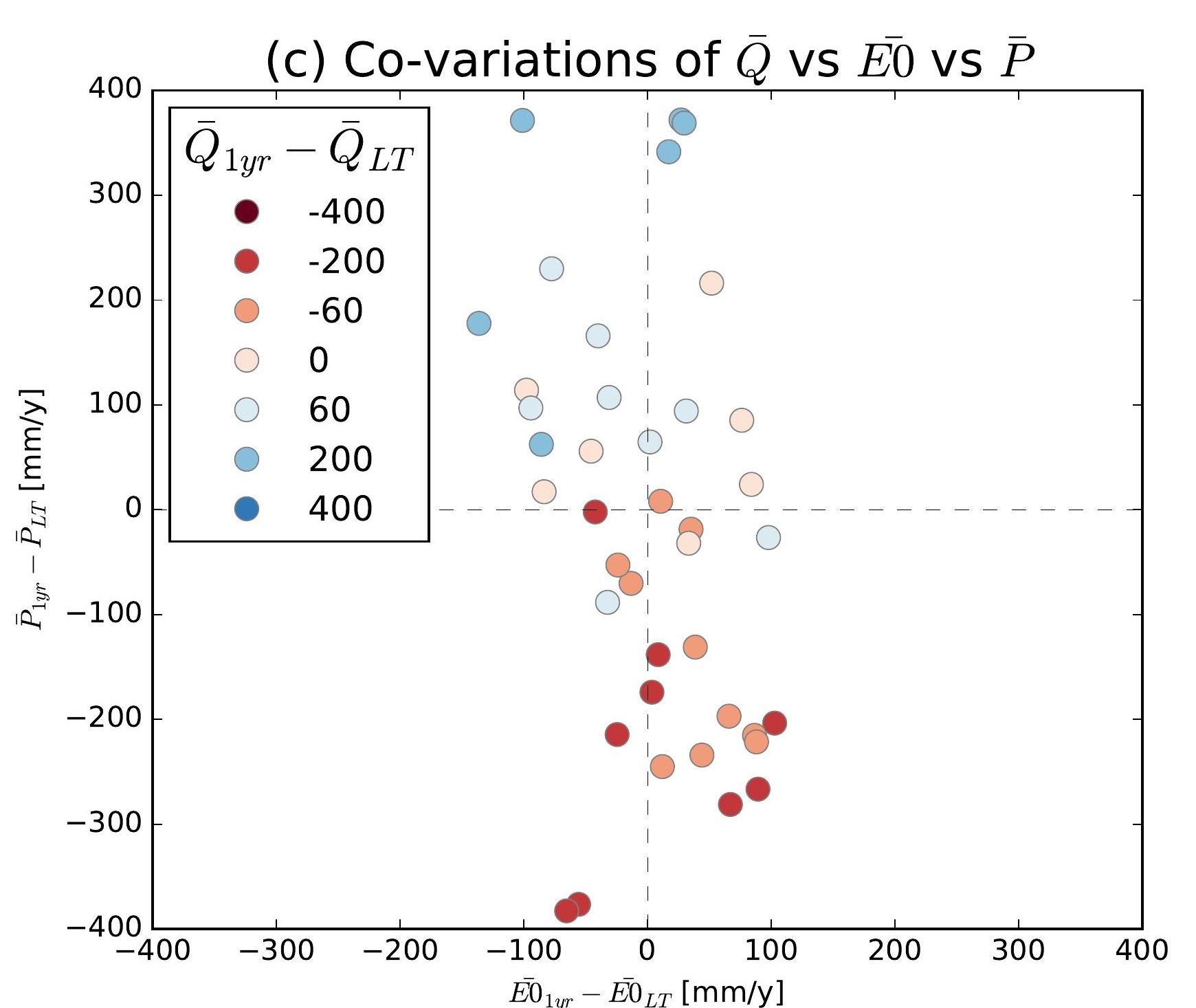
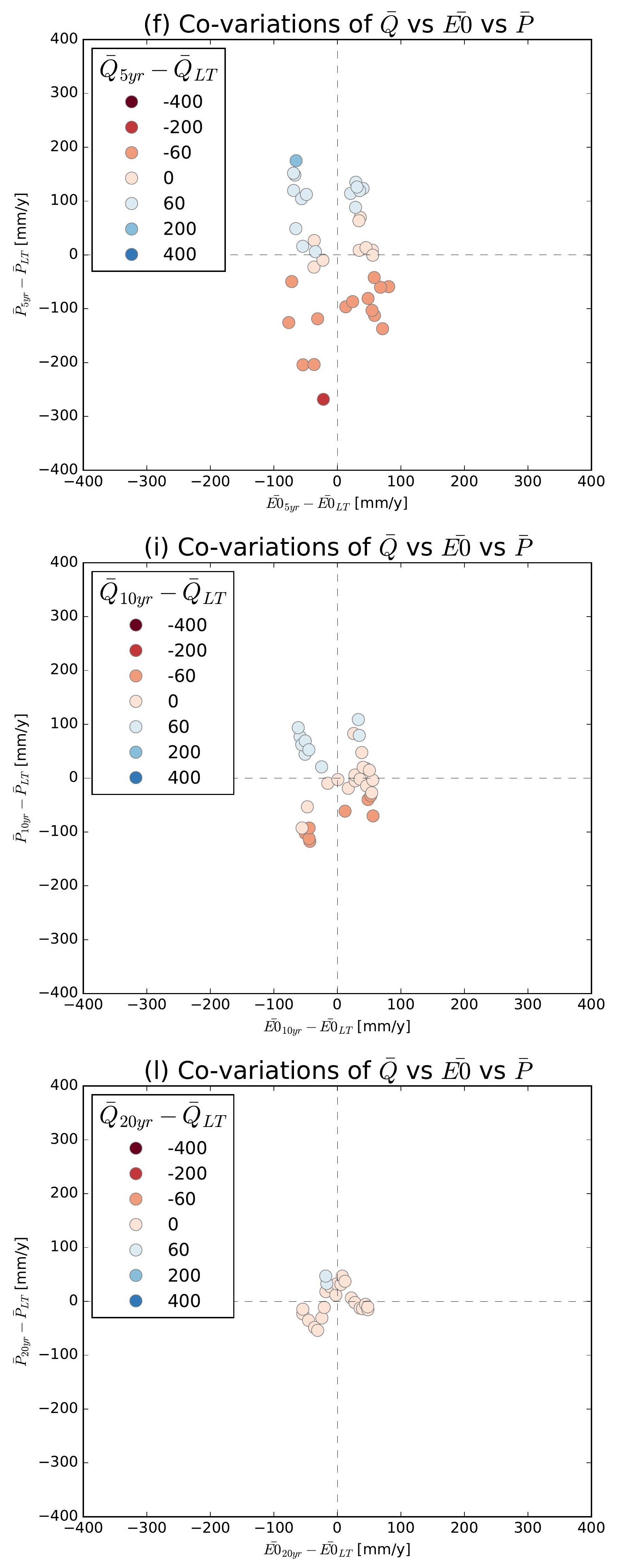

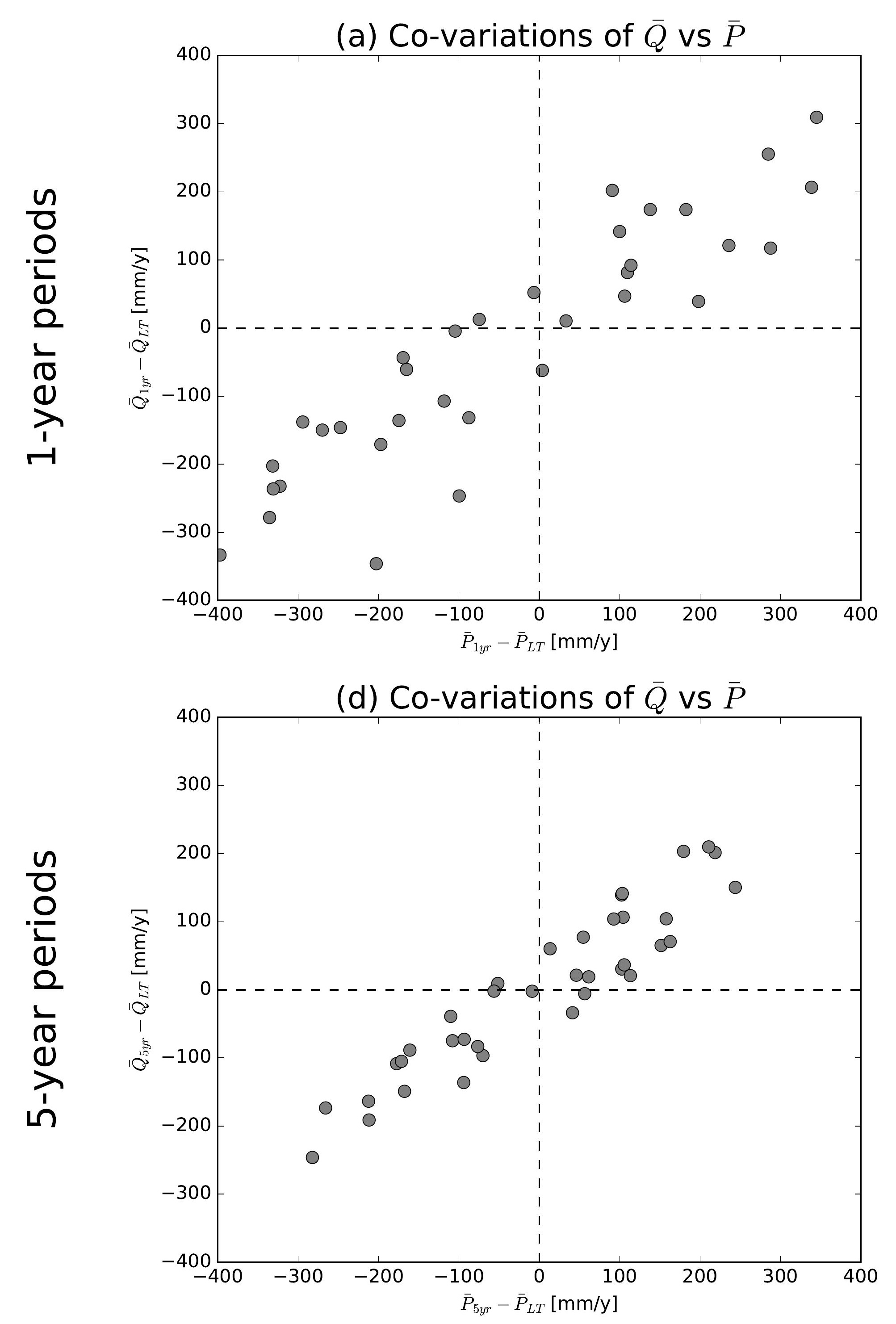

(g) Co-variations of $\bar{Q}$ vs $\bar{P}$

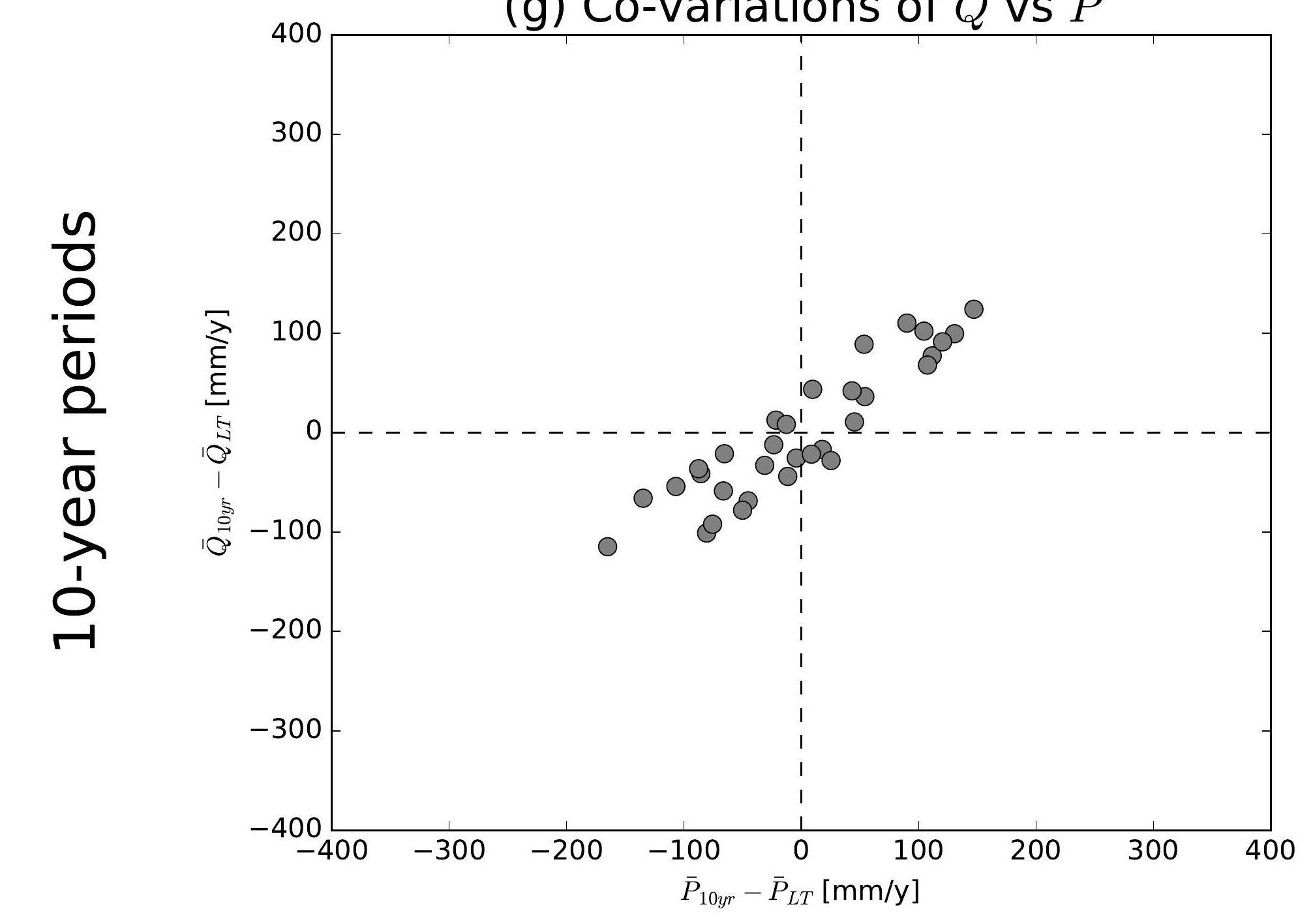

(j) Co-variations of $\bar{Q}$ vs $\bar{P}$

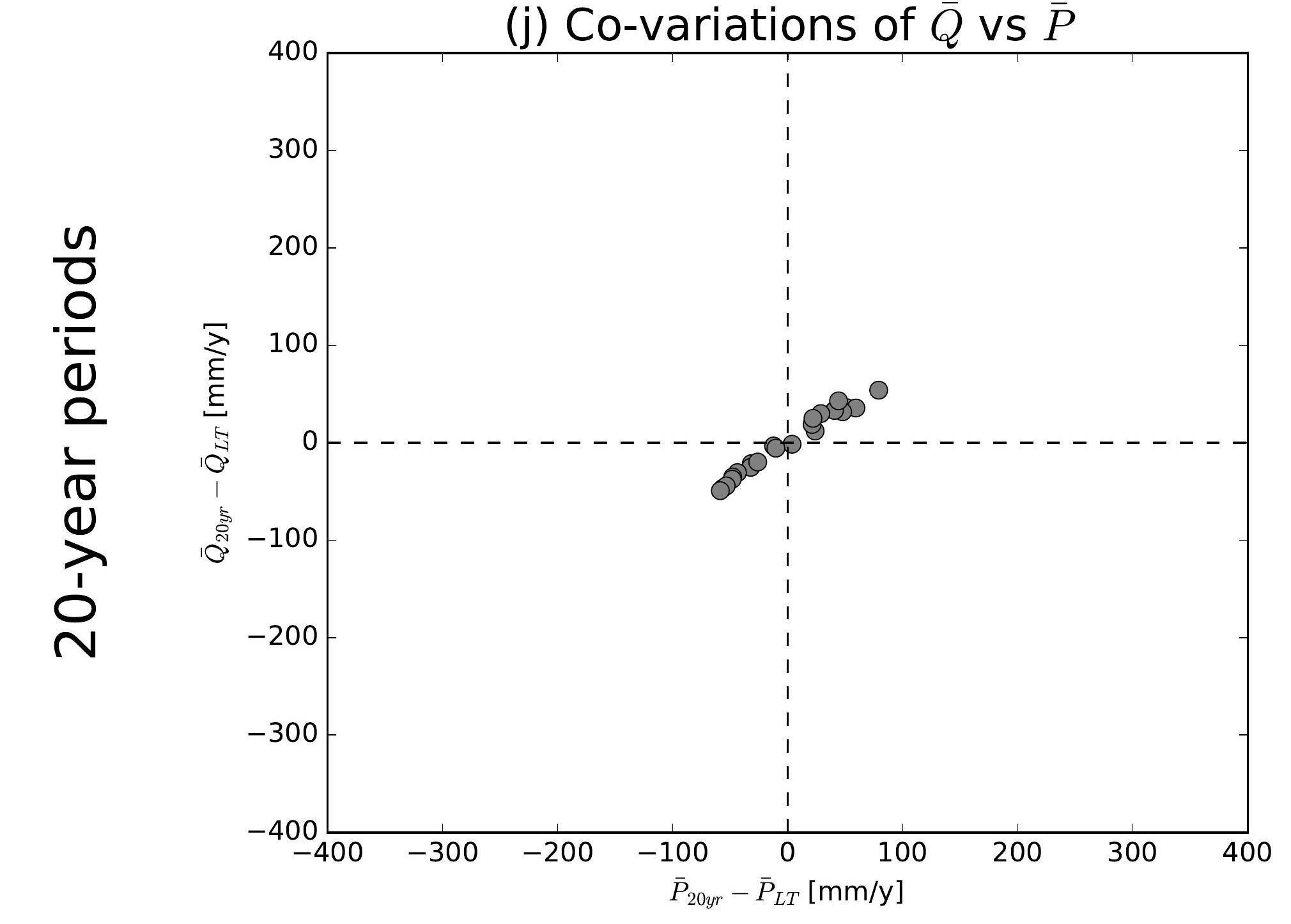

(b) Co-variations of $\bar{Q}$ vs $\overline{E 0}$

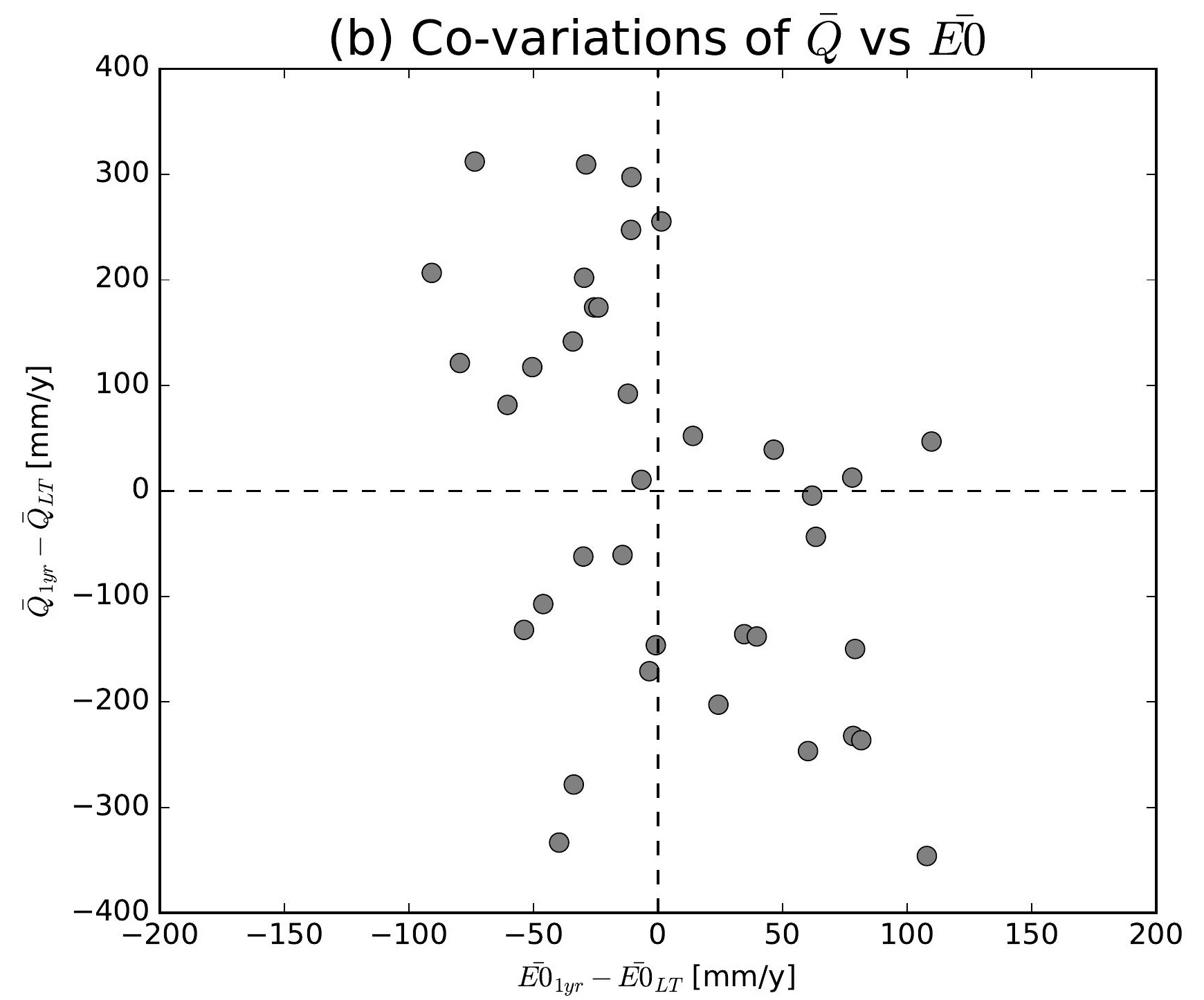

(e) Co-variations of $\bar{Q}$ vs $\overline{E 0}$

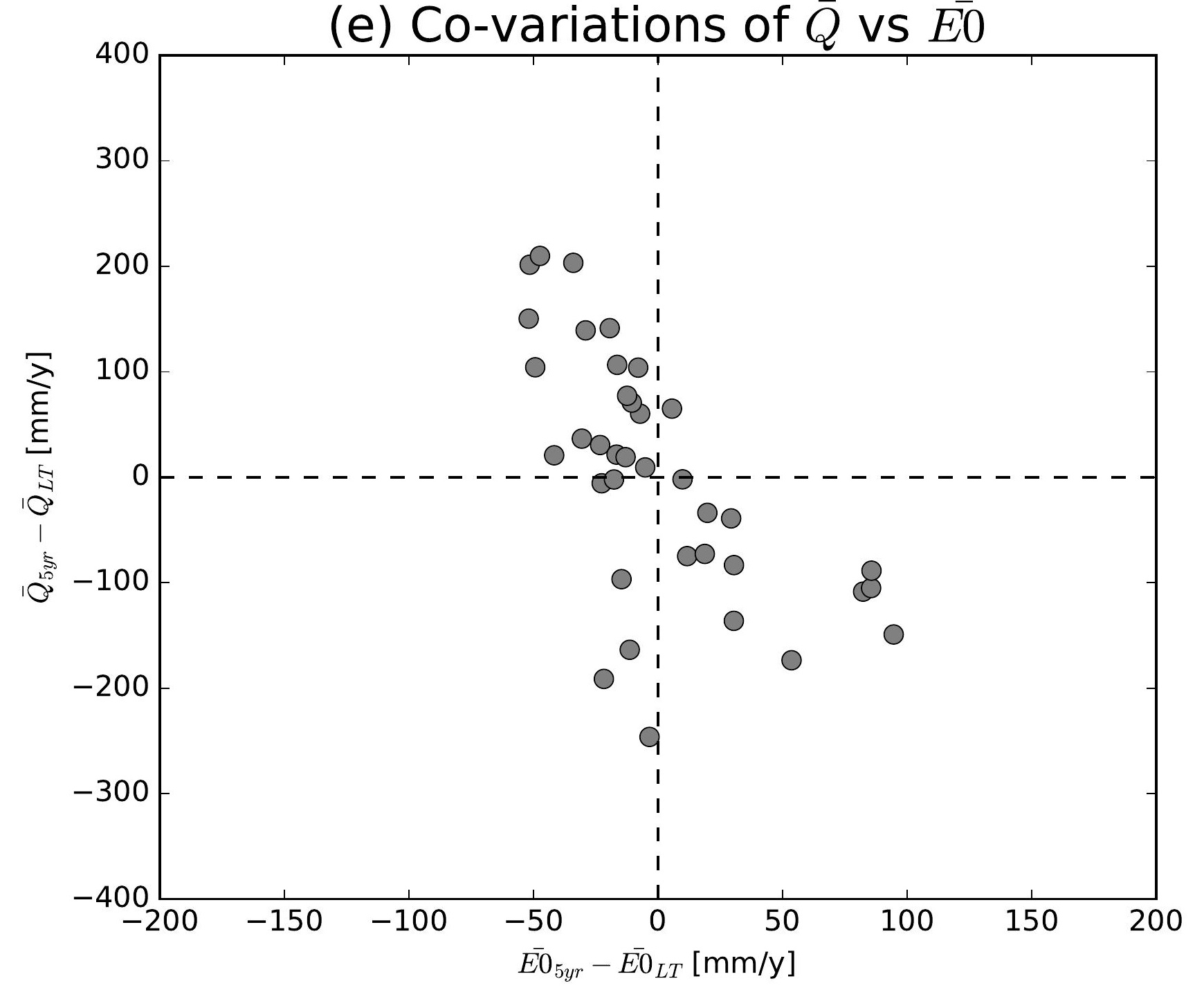

(h) Co-variations of $\bar{Q}$ vs $\overline{E 0}$

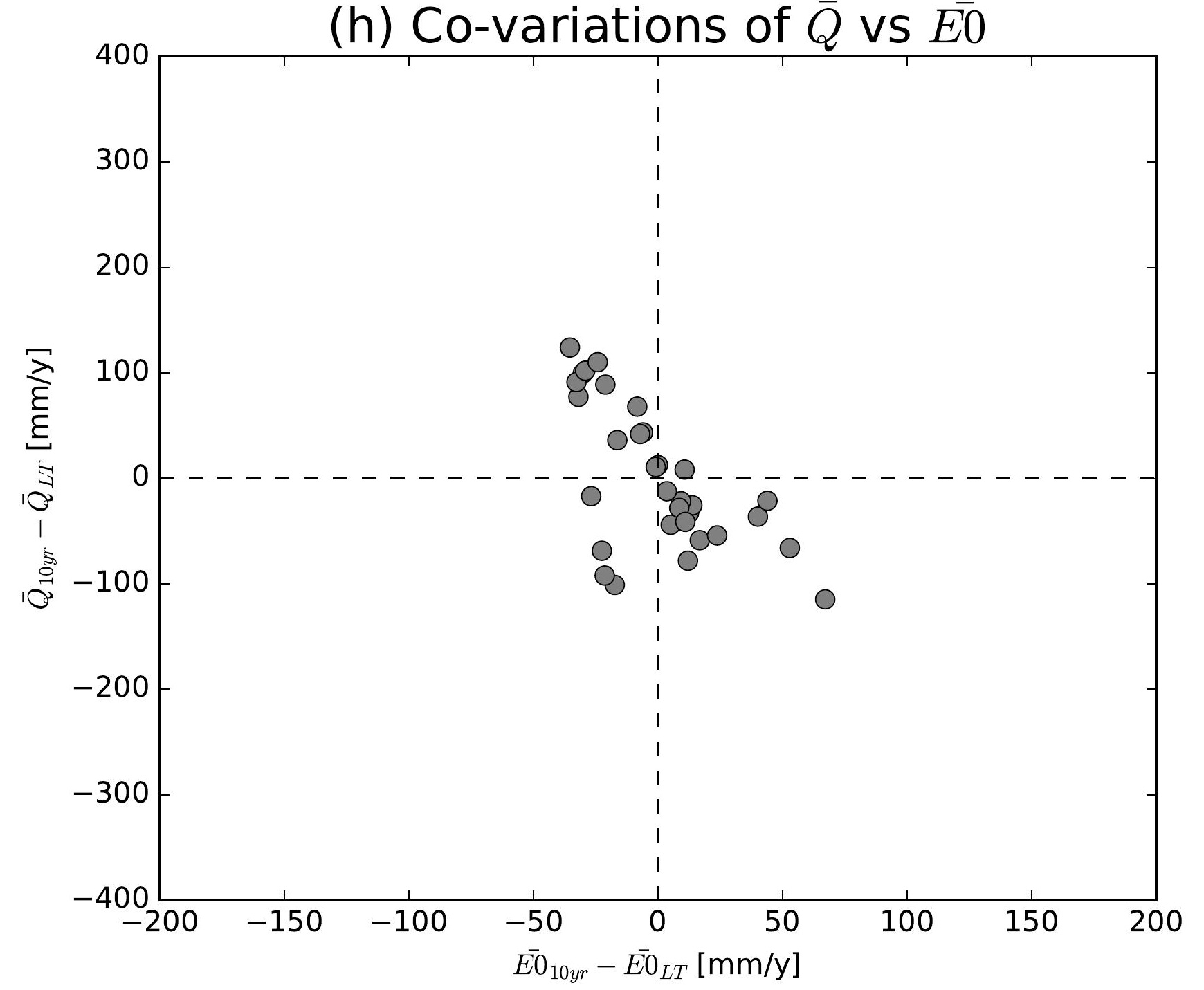

(k) Co-variations of $\bar{Q}$ vs $\overline{E 0}$

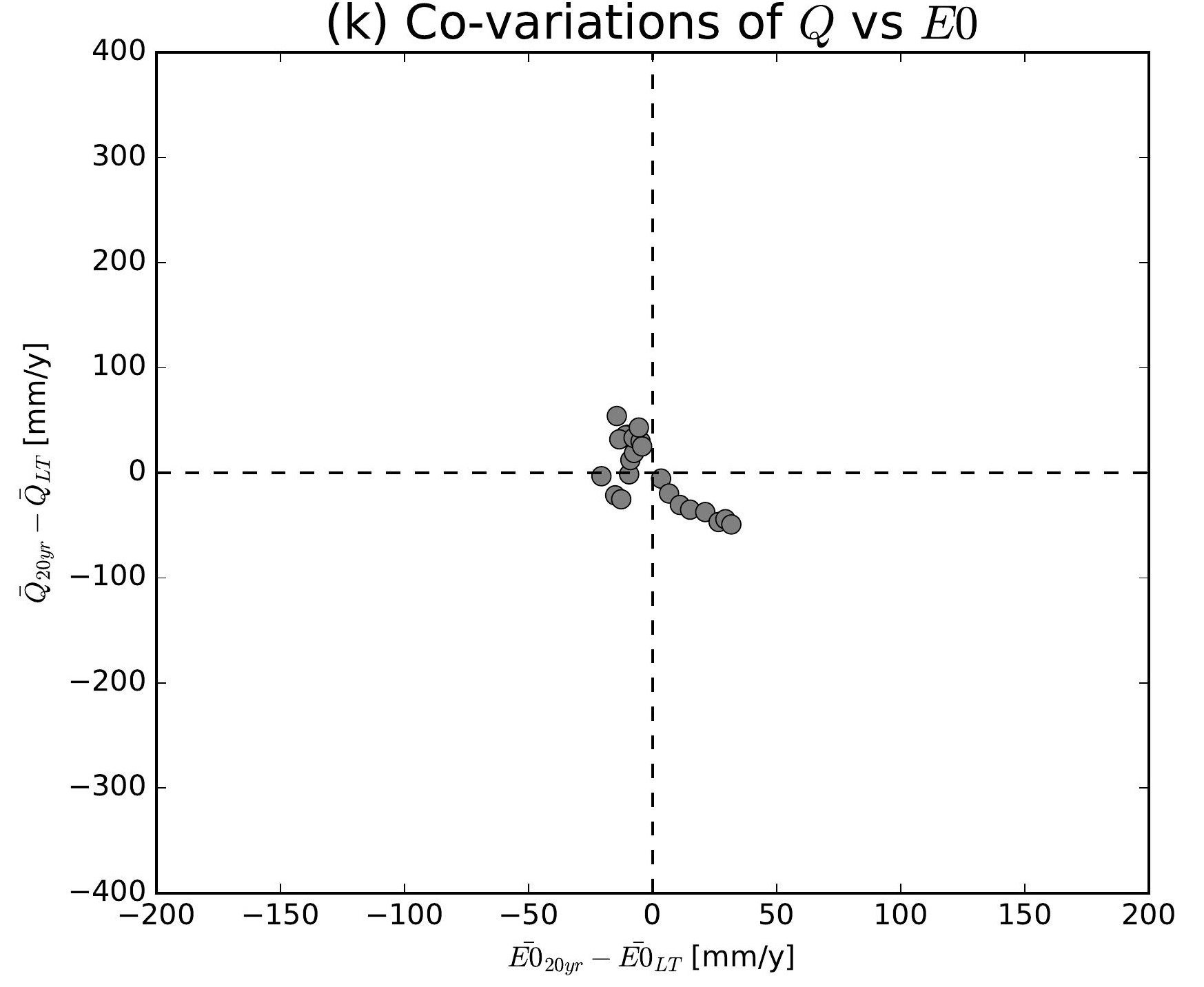

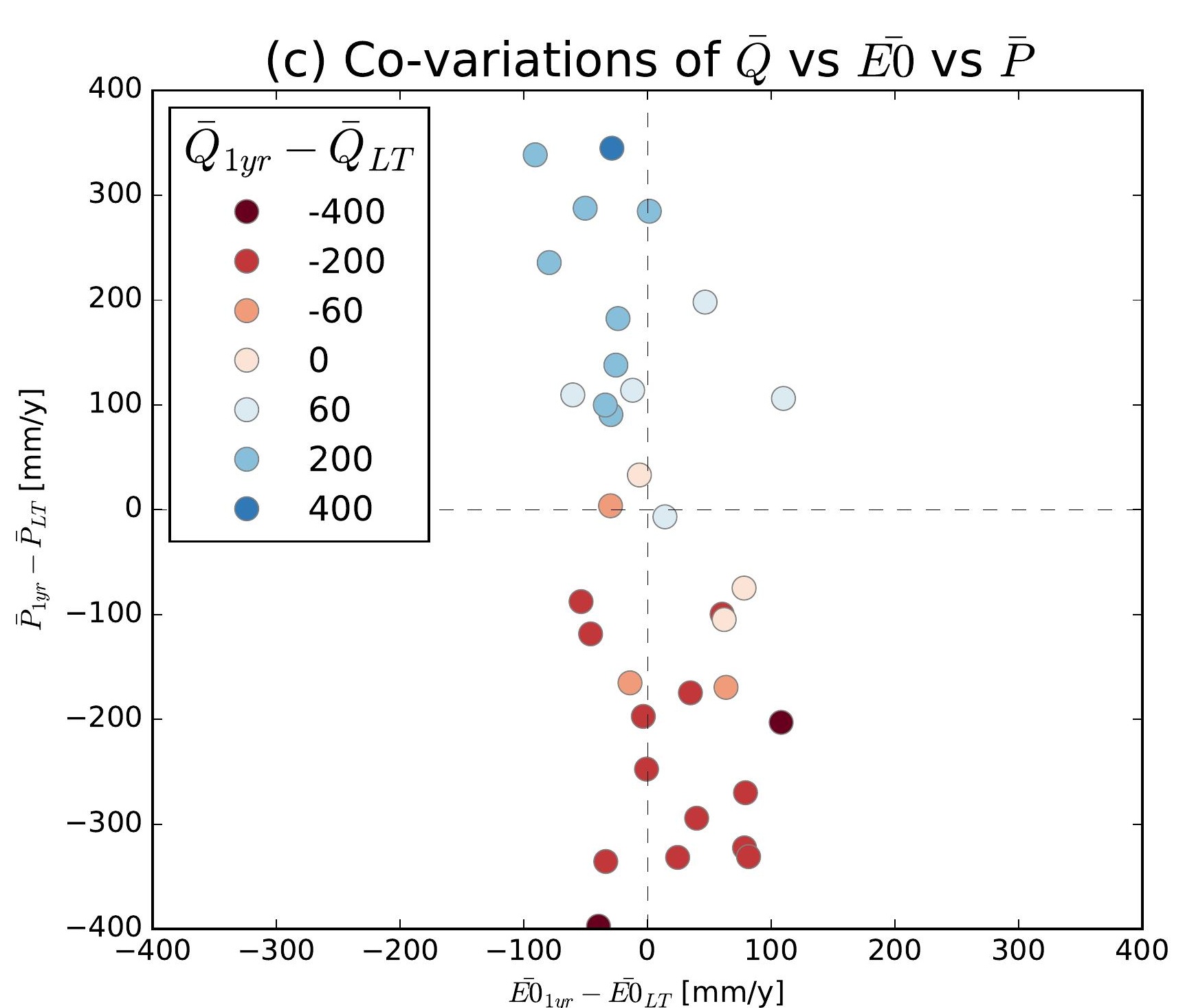
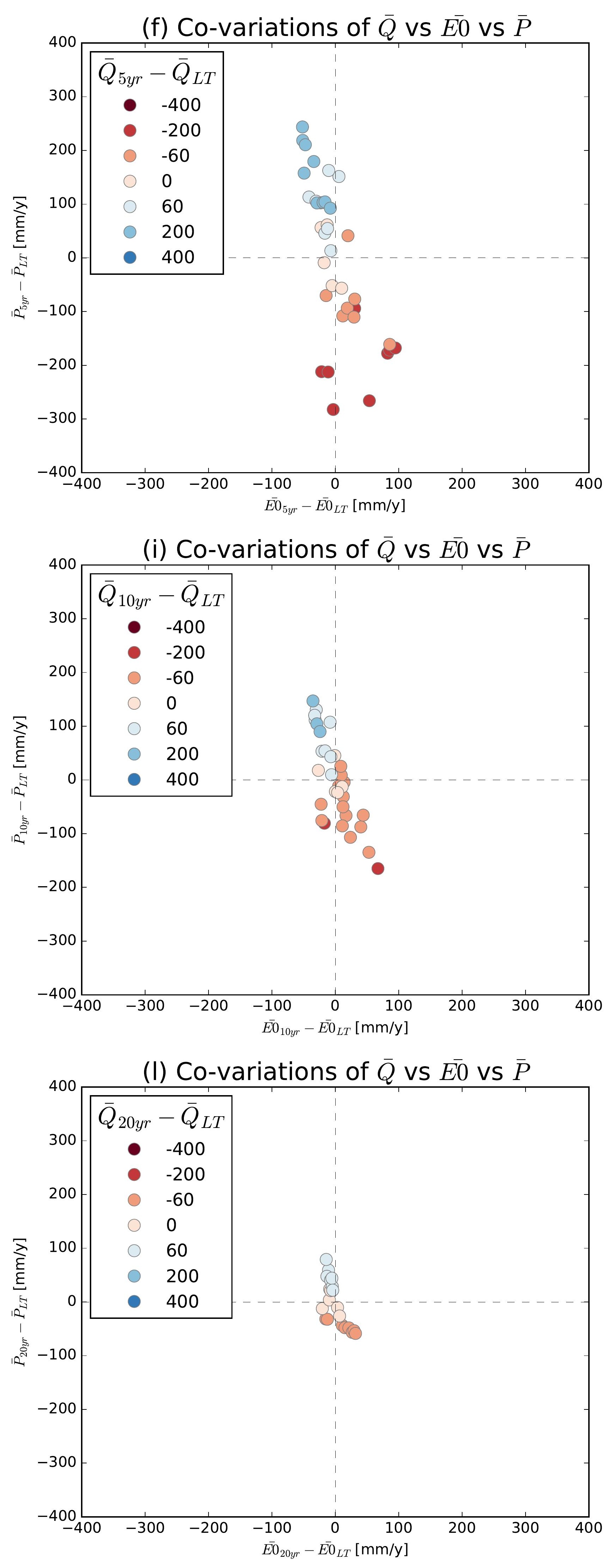

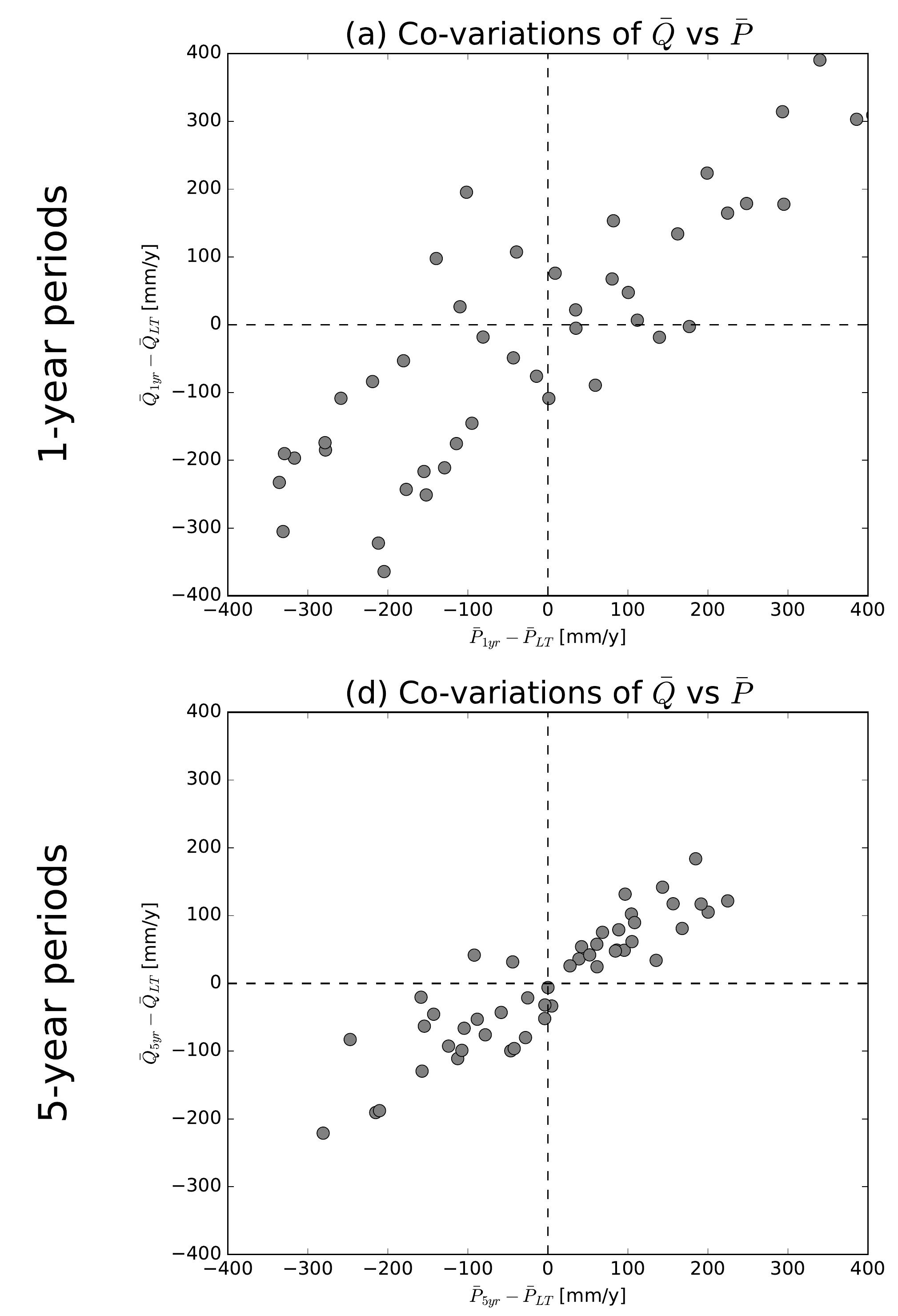

(g) Co-variations of $\bar{Q}$ vs $\bar{P}$

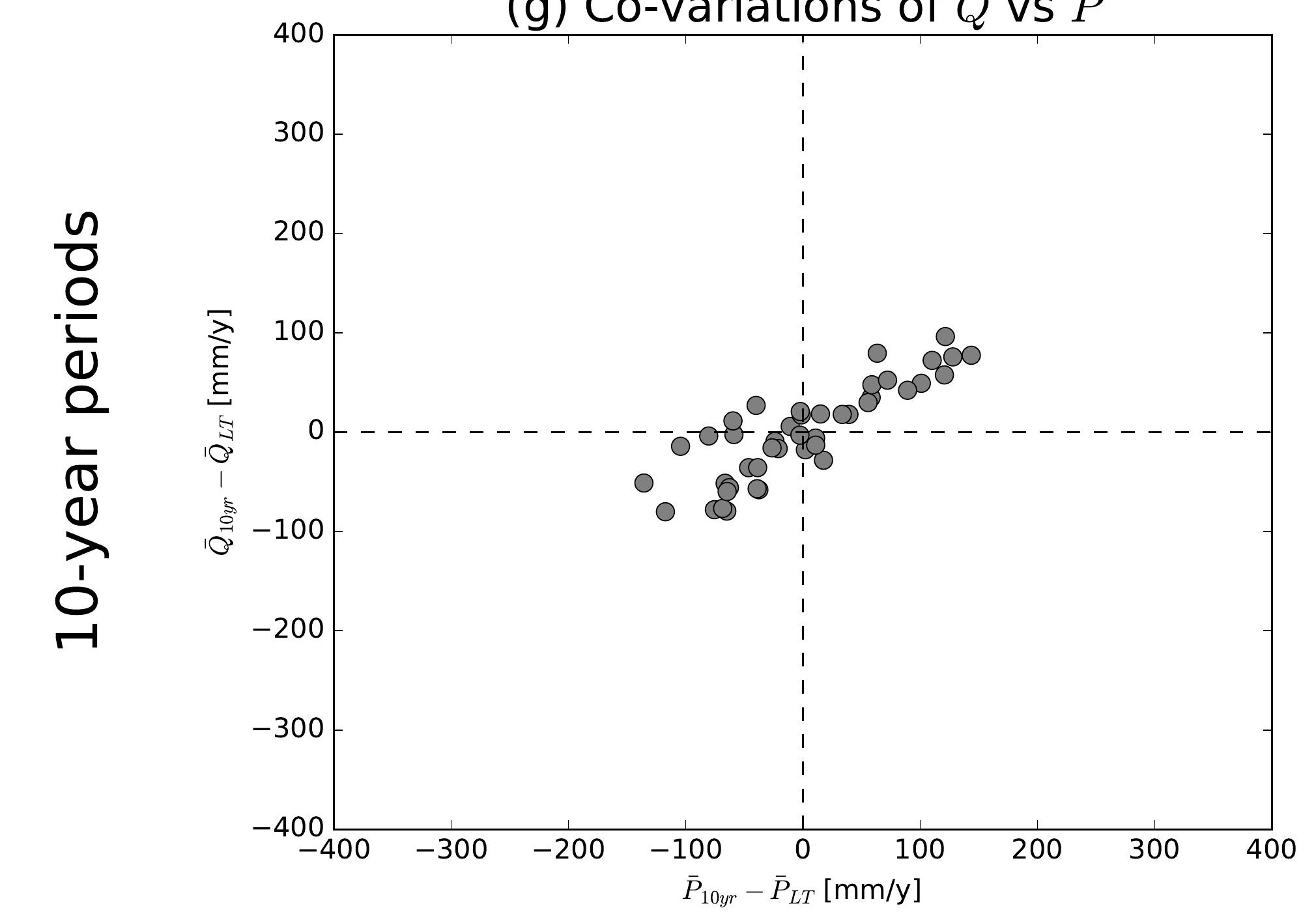

(j) Co-variations of $\bar{Q}$ vs $\bar{P}$

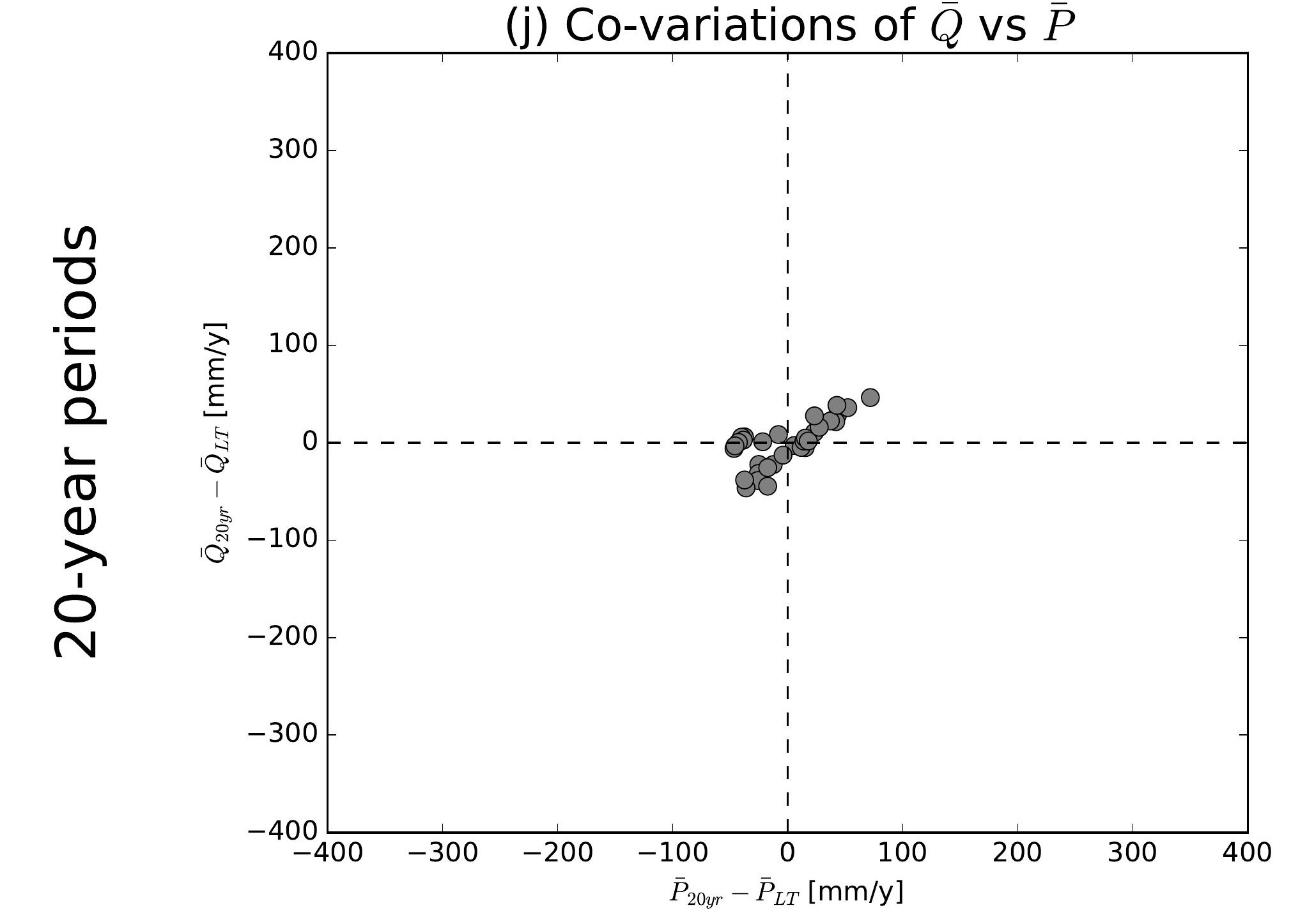

(b) Co-variations of $\bar{Q}$ vs $\overline{E 0}$

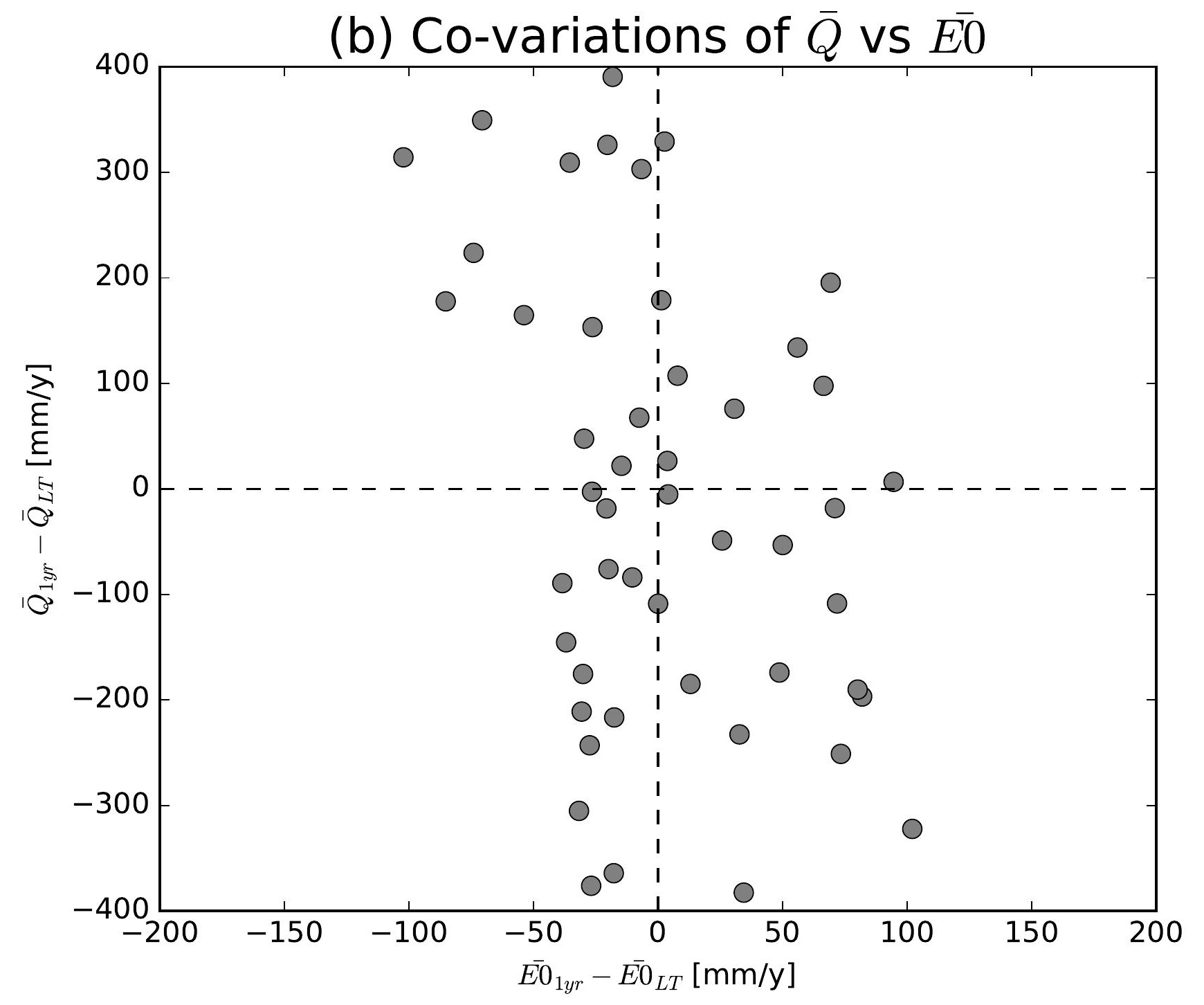

(e) Co-variations of $\bar{Q}$ vs $\overline{E 0}$

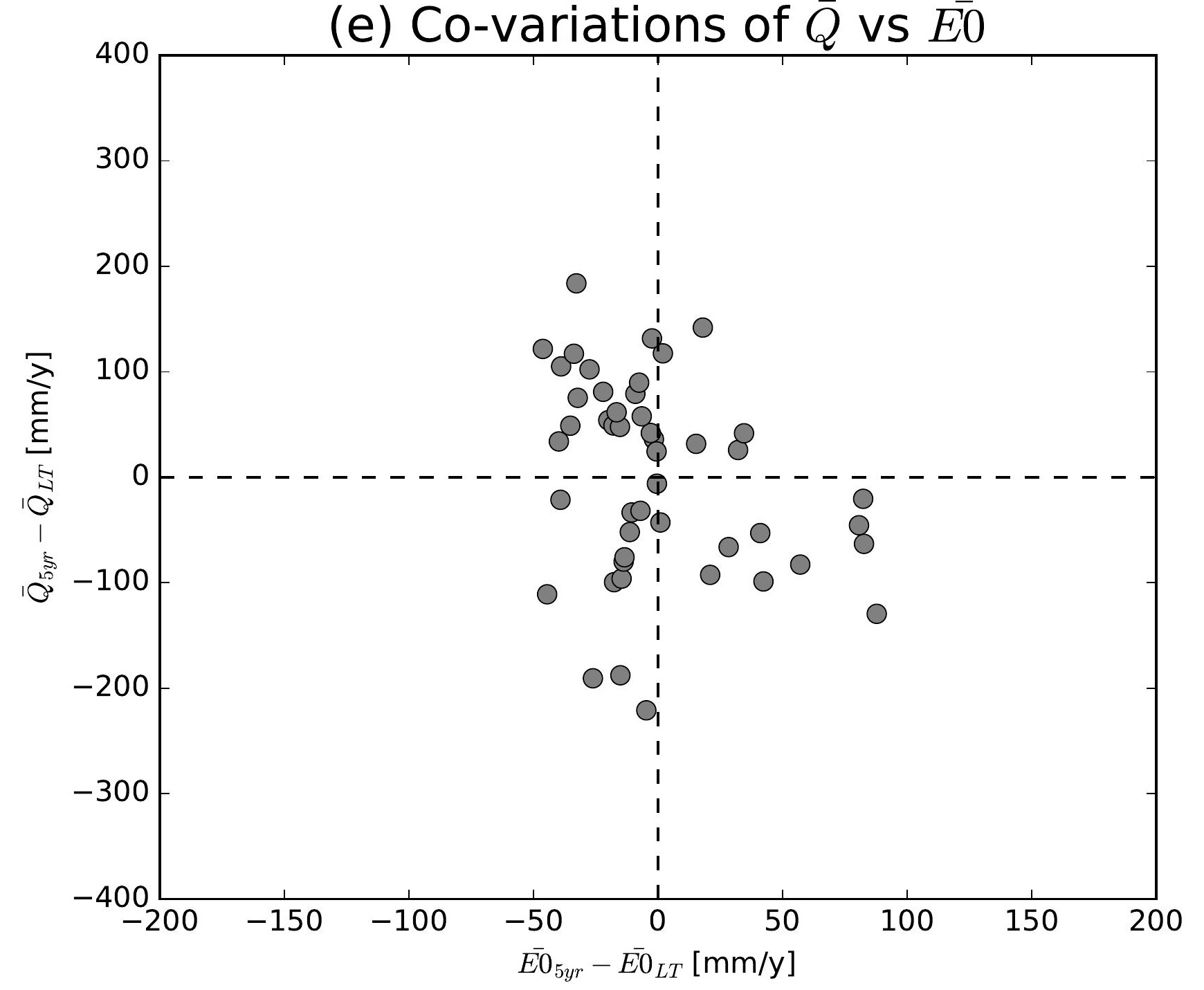

(h) Co-variations of $\bar{Q}$ vs $\overline{E 0}$

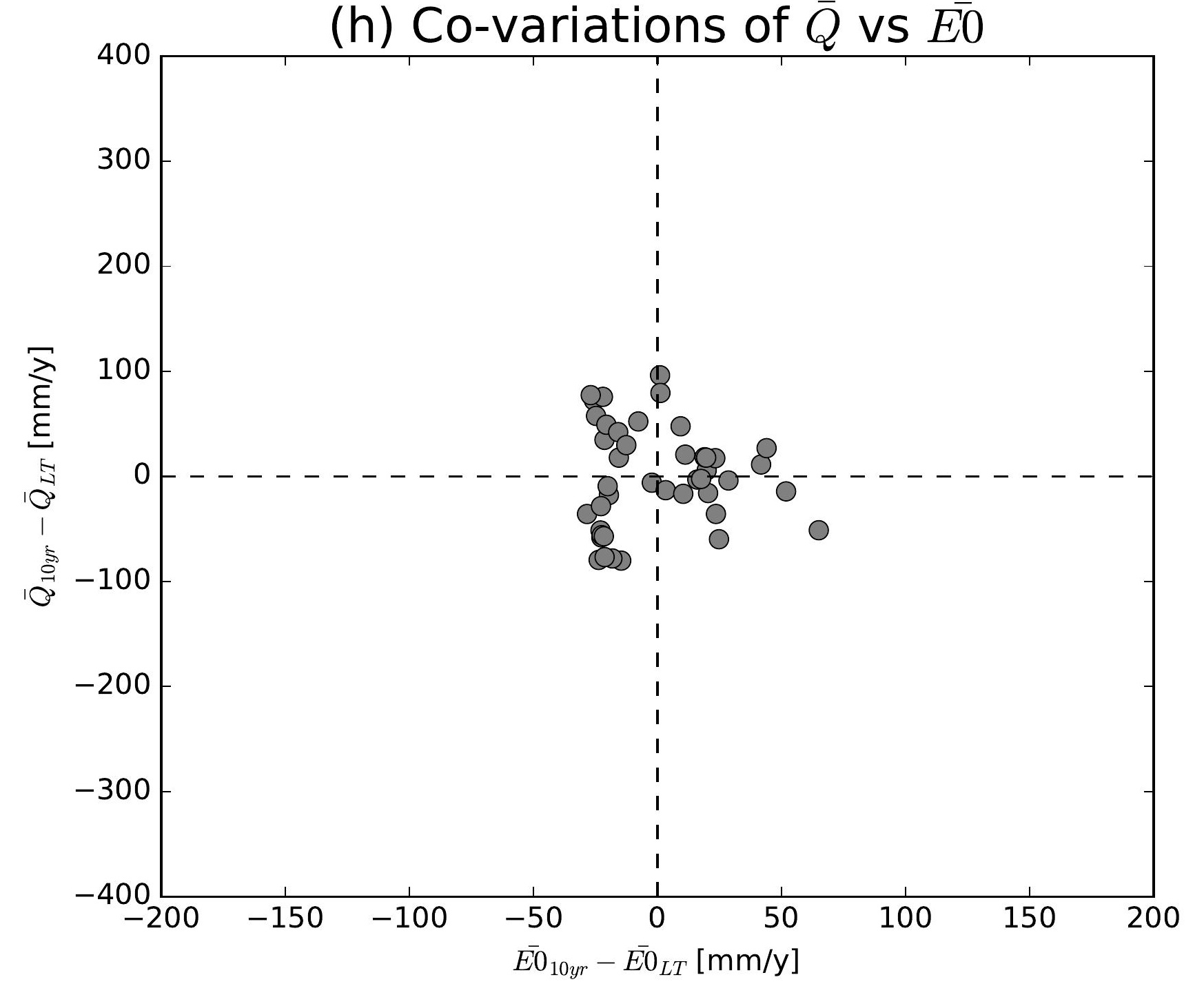

(k) Co-variations of $\bar{Q}$ vs $\overline{E 0}$

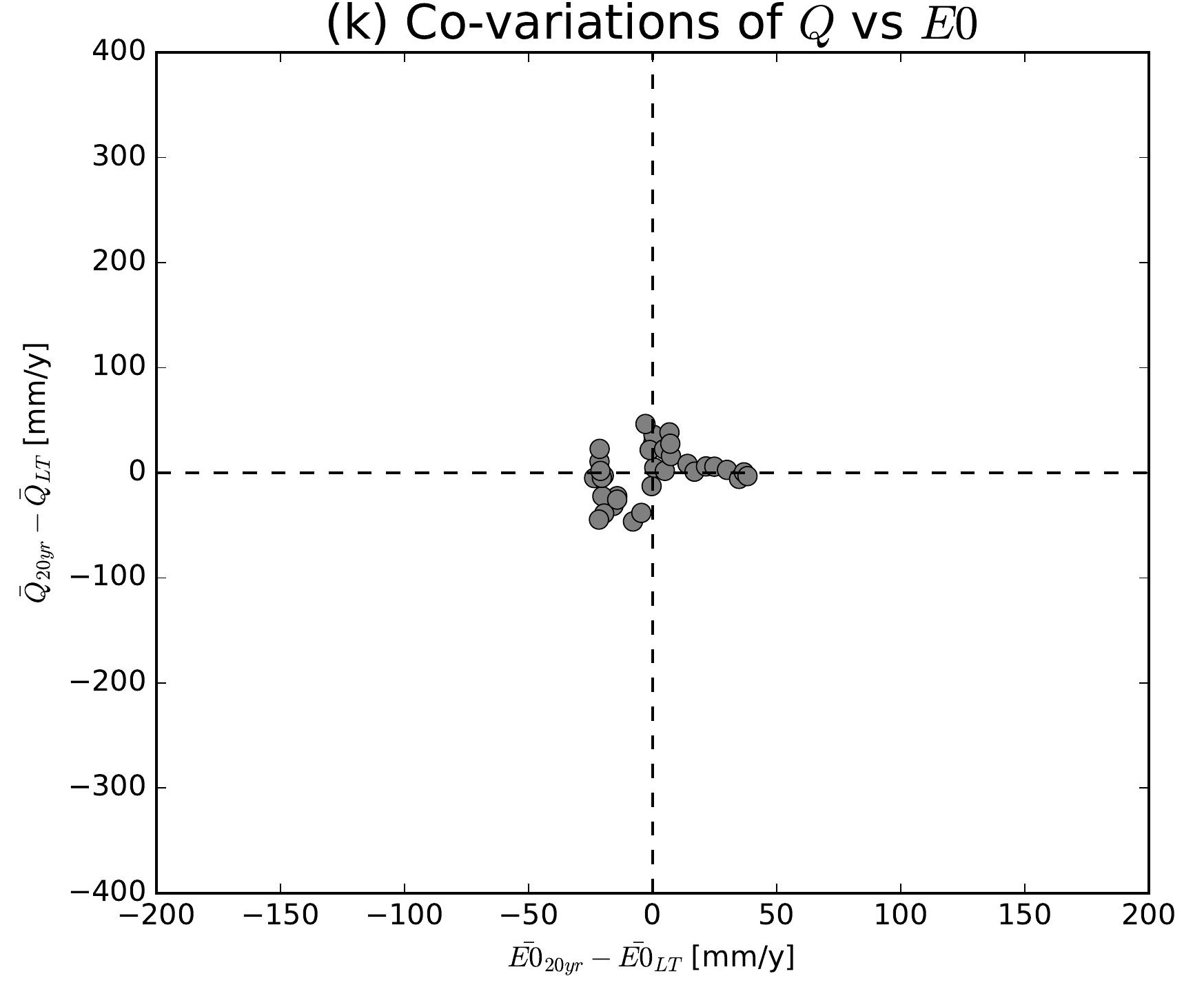

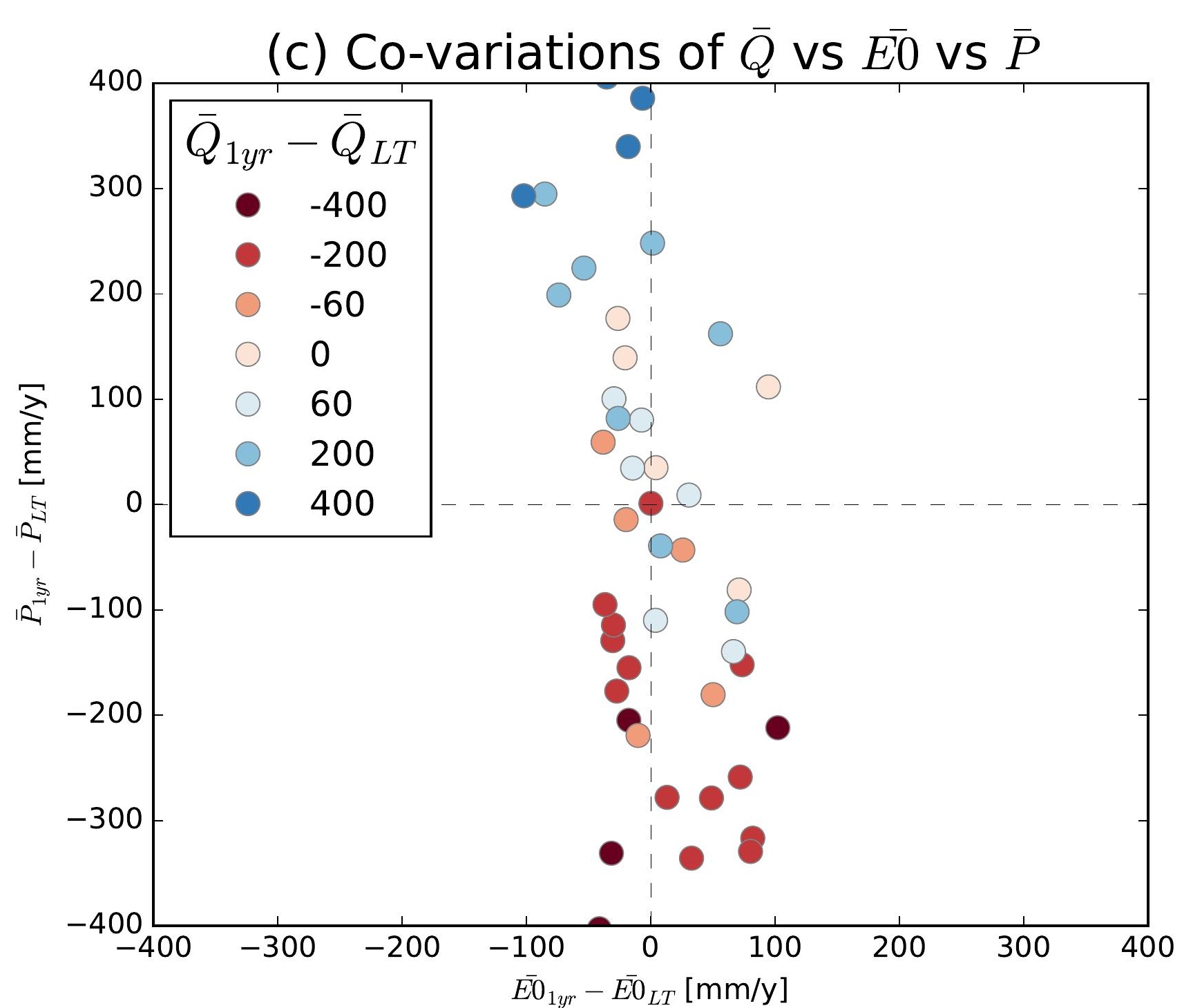
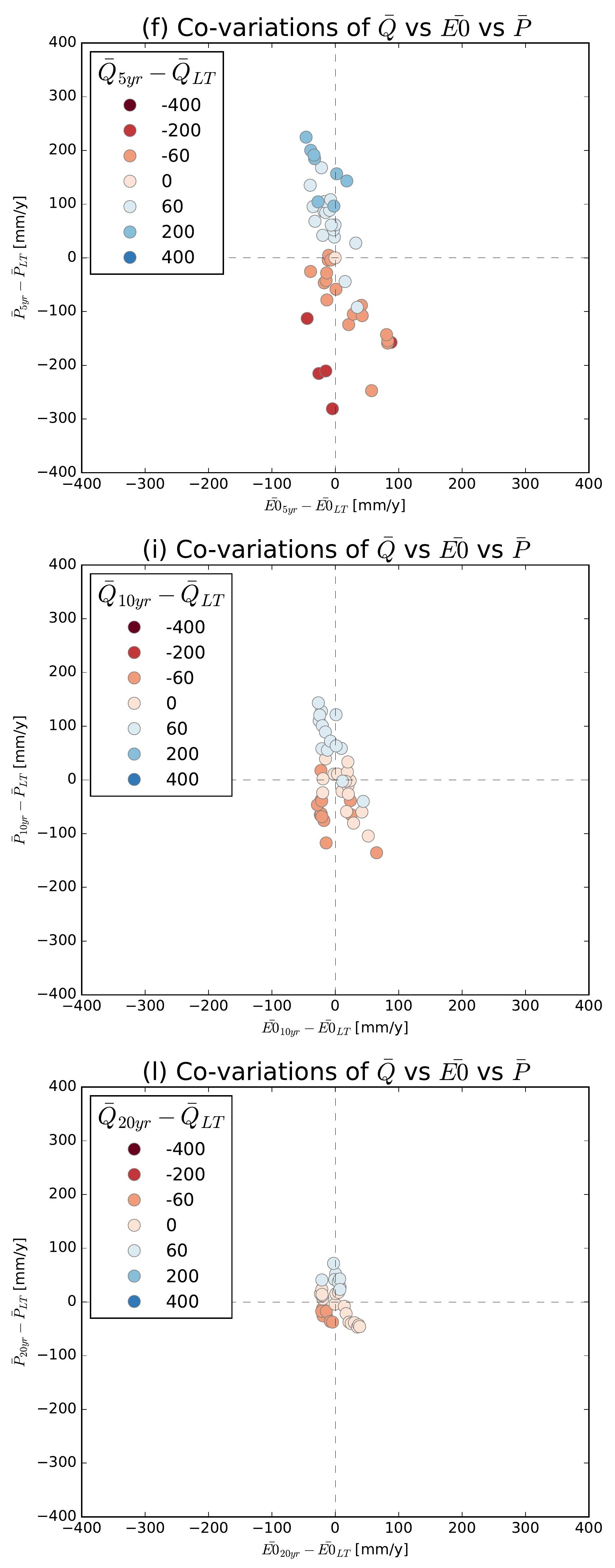

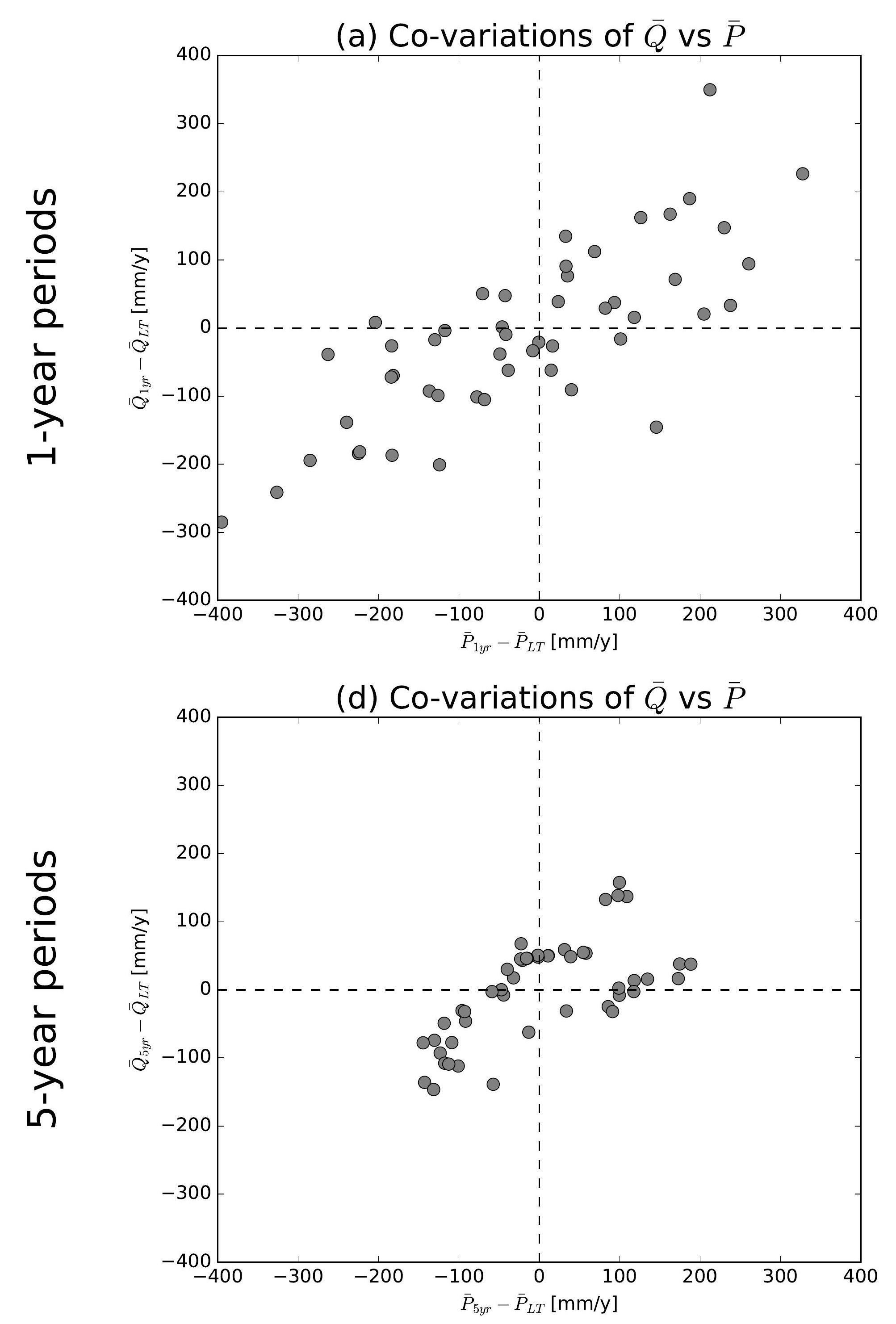

(g) Co-variations of $\bar{Q}$ vs $\bar{P}$

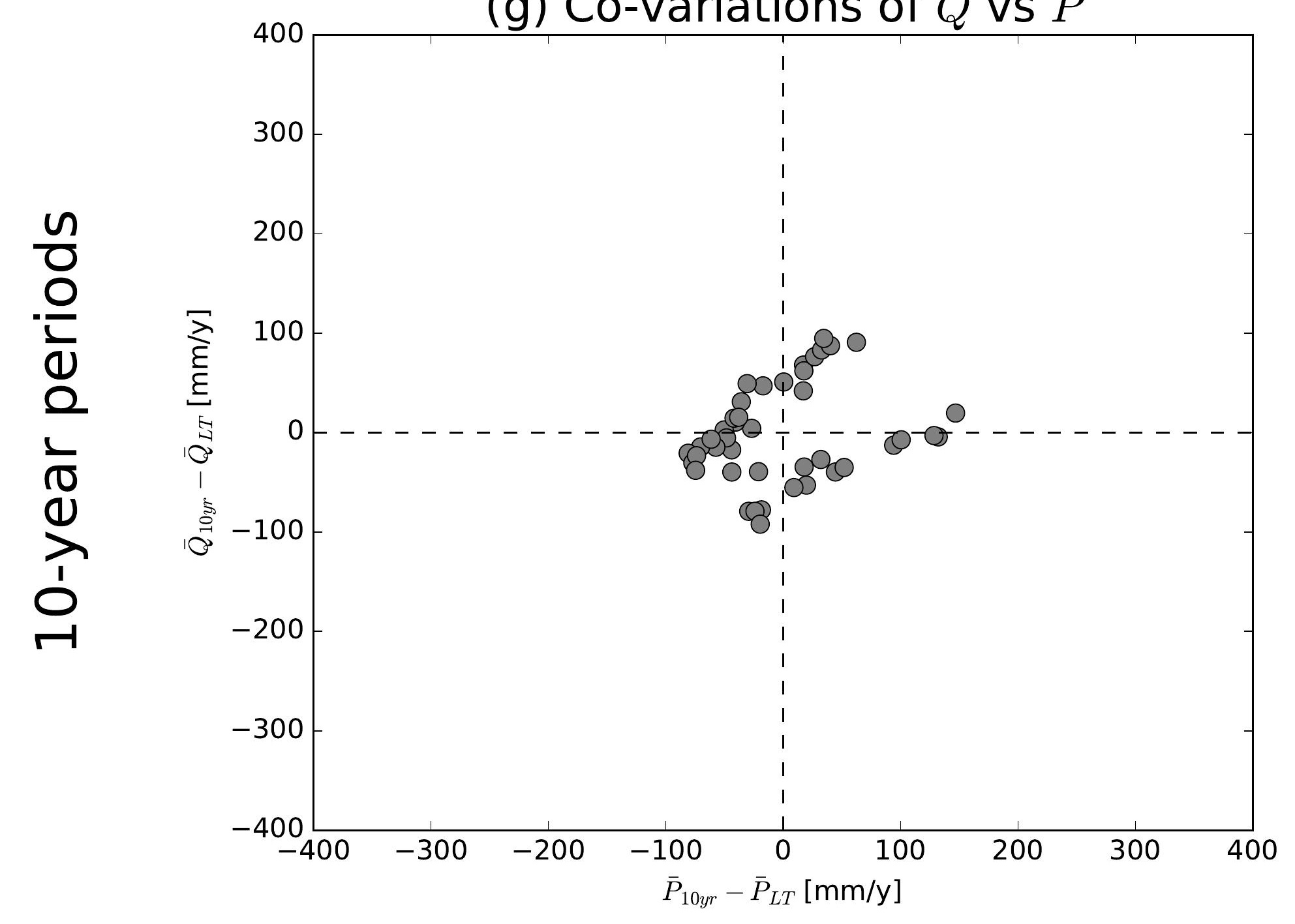

(j) Co-variations of $\bar{Q}$ vs $\bar{P}$

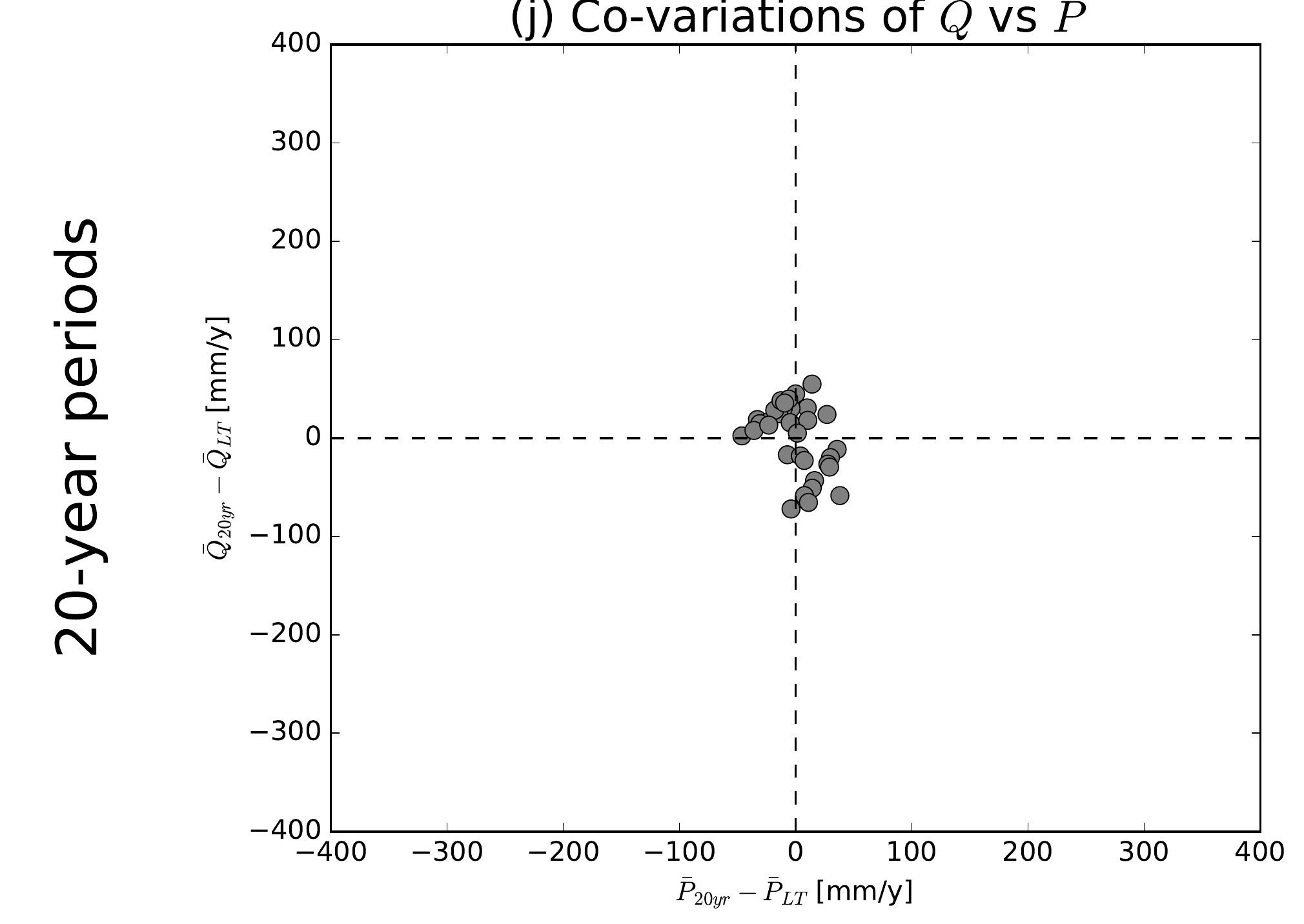

(b) Co-variations of $\bar{Q}$ vs $\overline{E 0}$

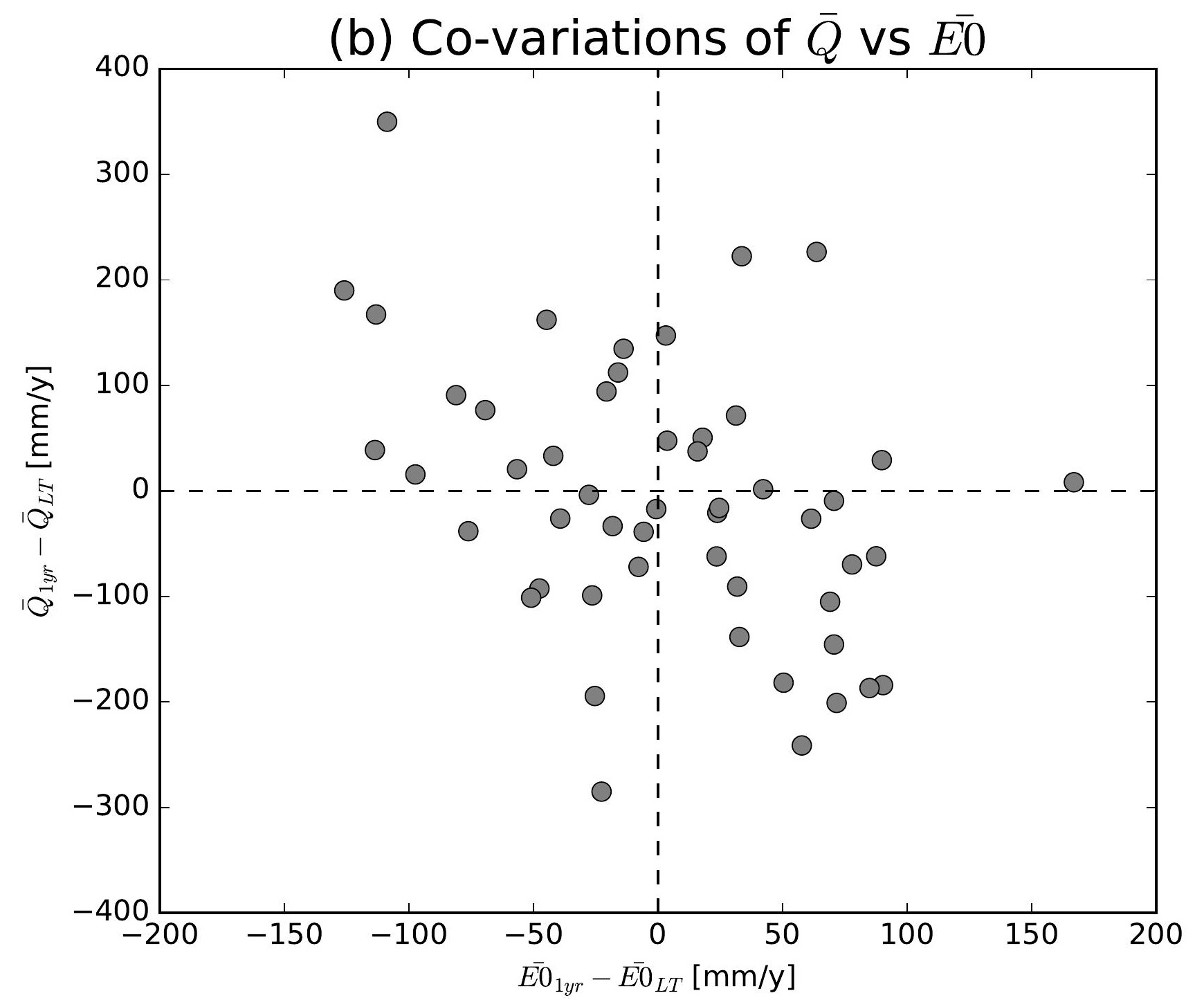

(e) Co-variations of $\bar{Q}$ vs $\overline{E 0}$

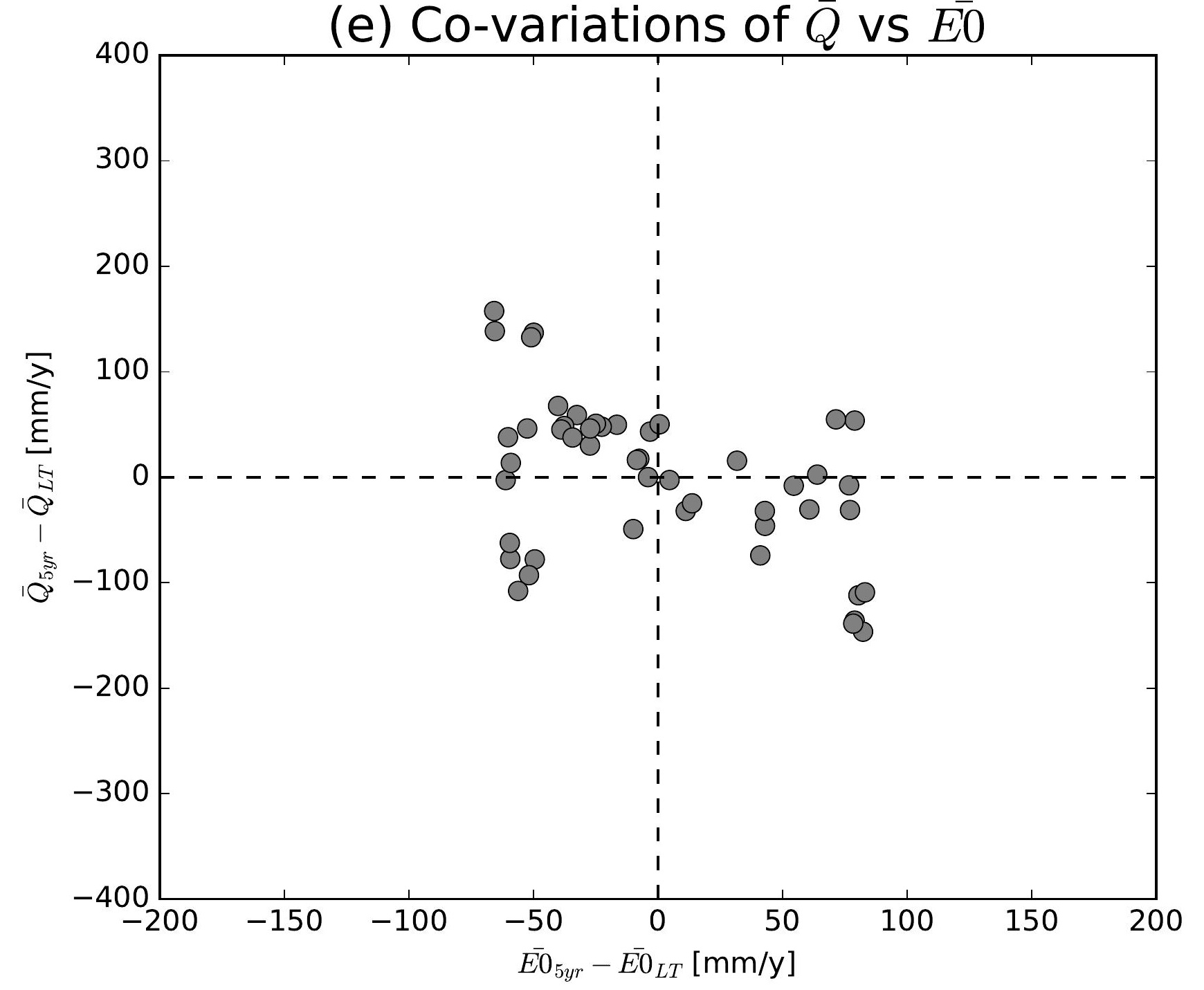

(h) Co-variations of $\bar{Q}$ vs $\overline{E 0}$

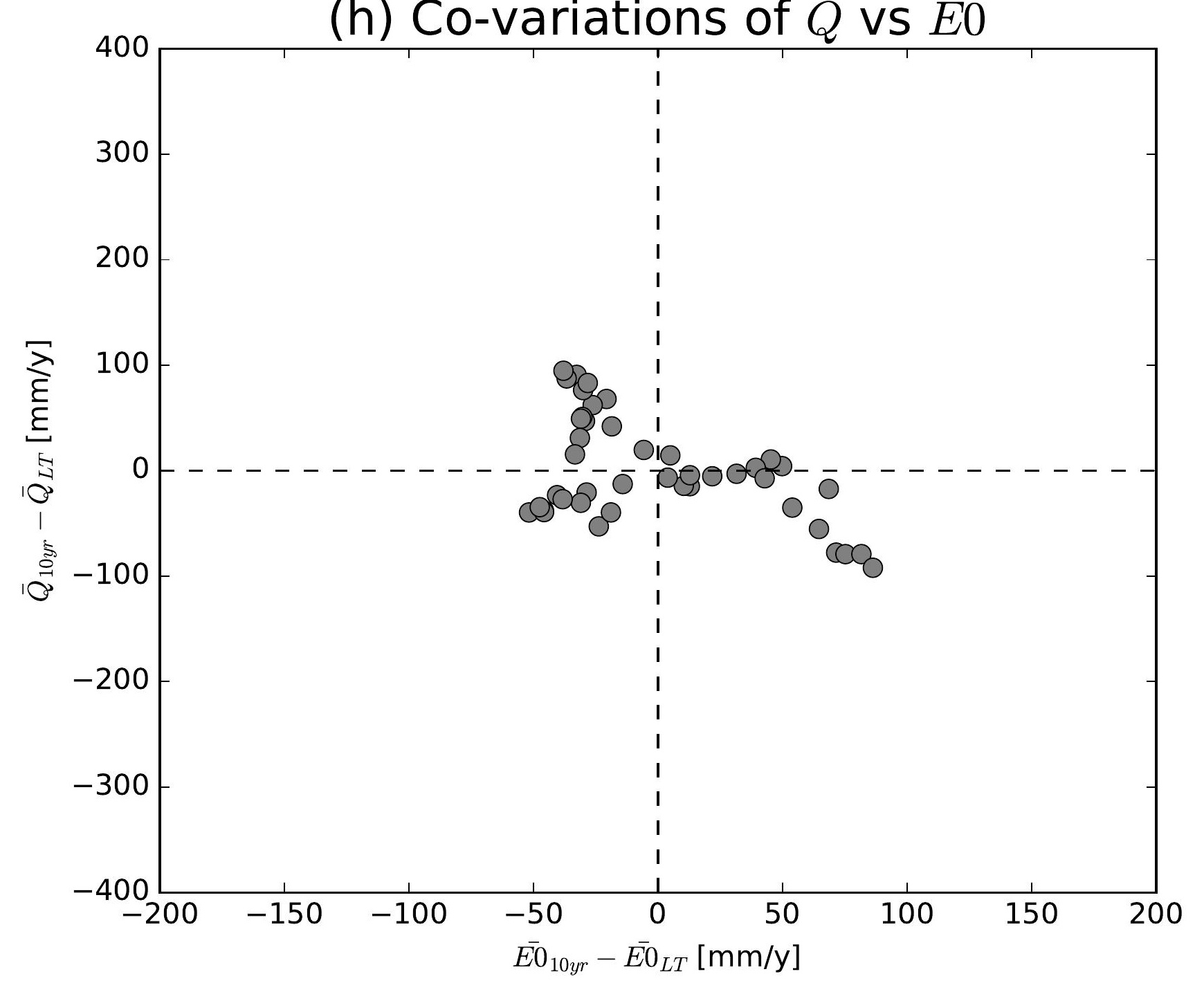

(k) Co-variations of $\bar{Q}$ vs $\overline{E 0}$

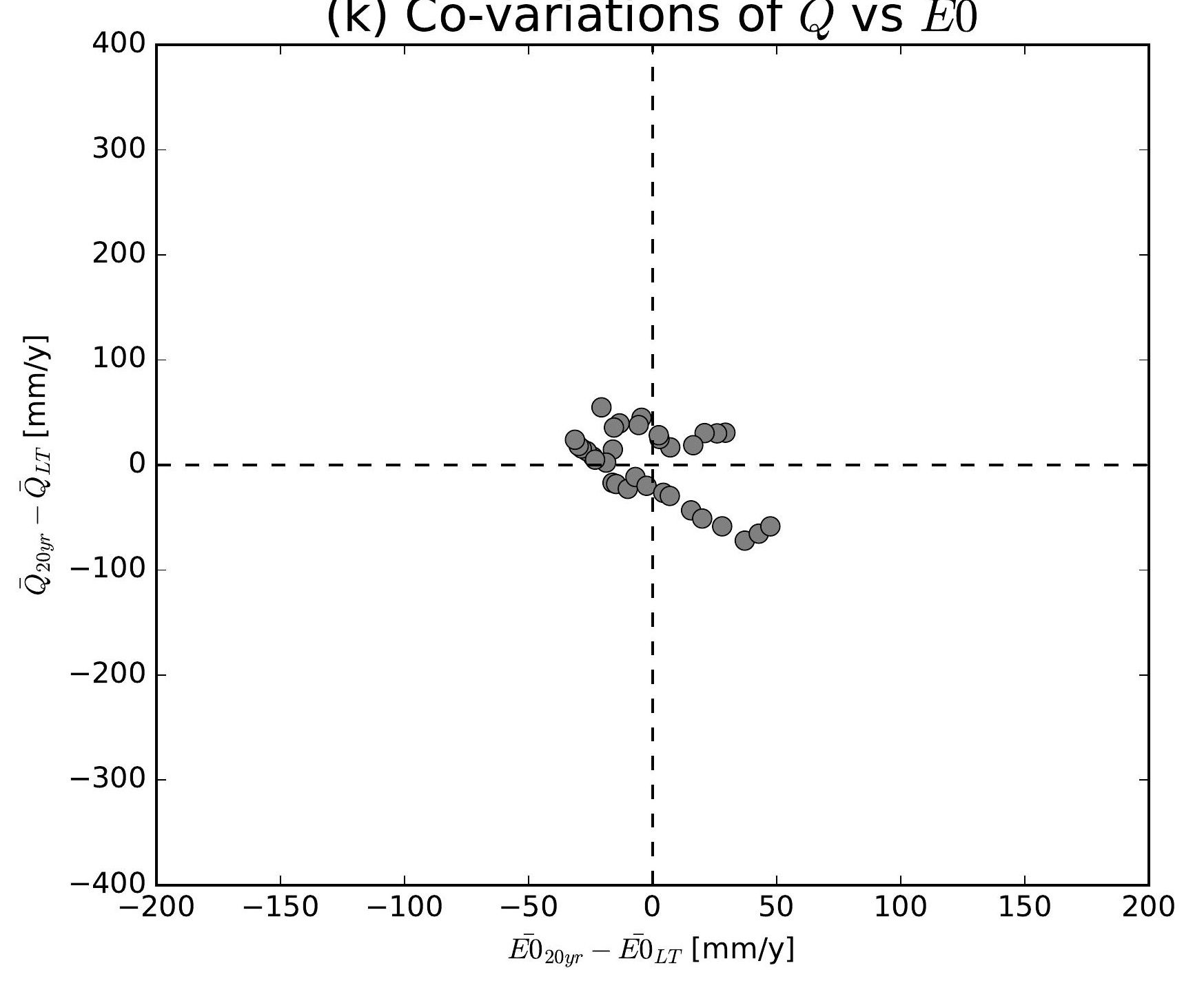

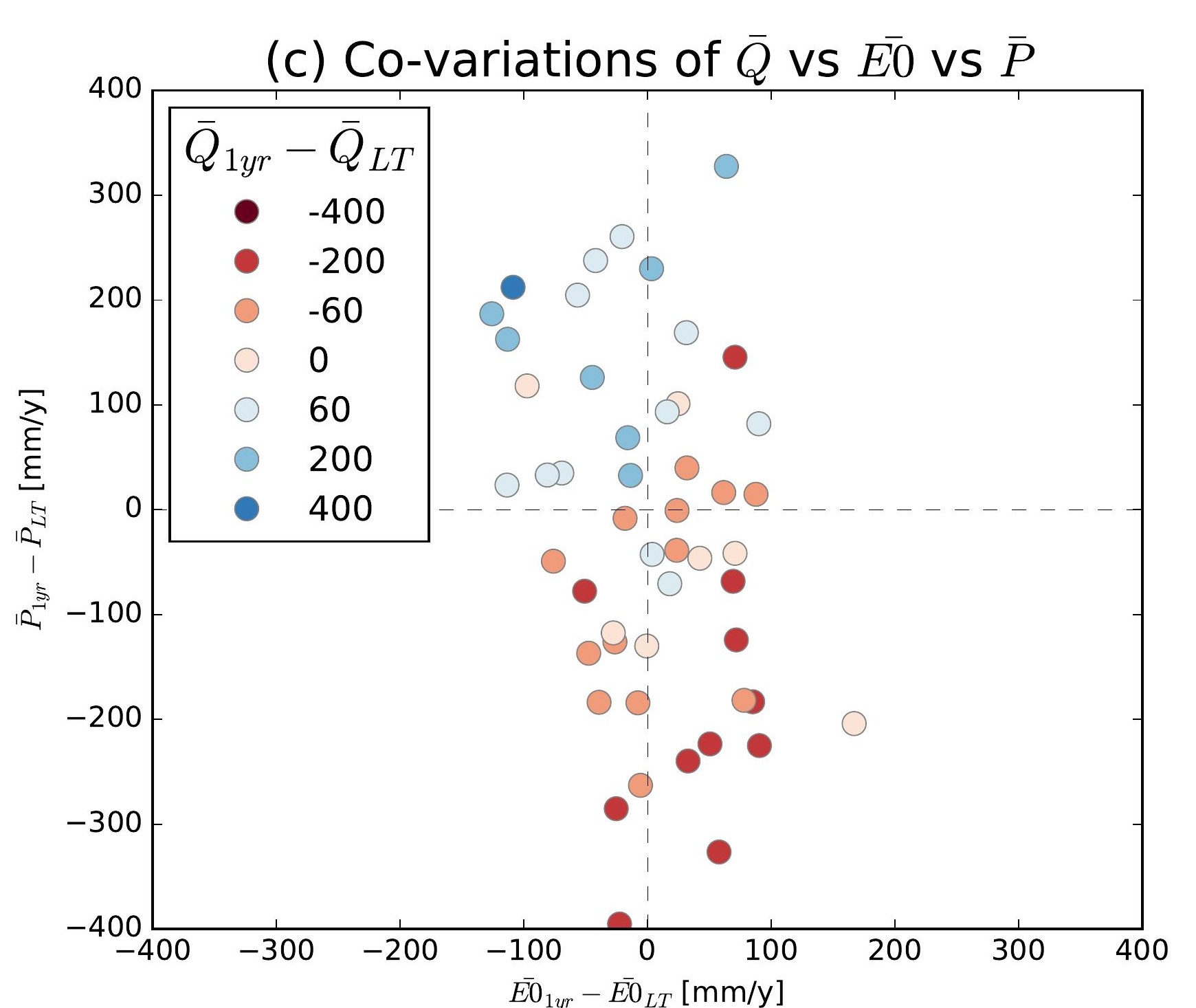
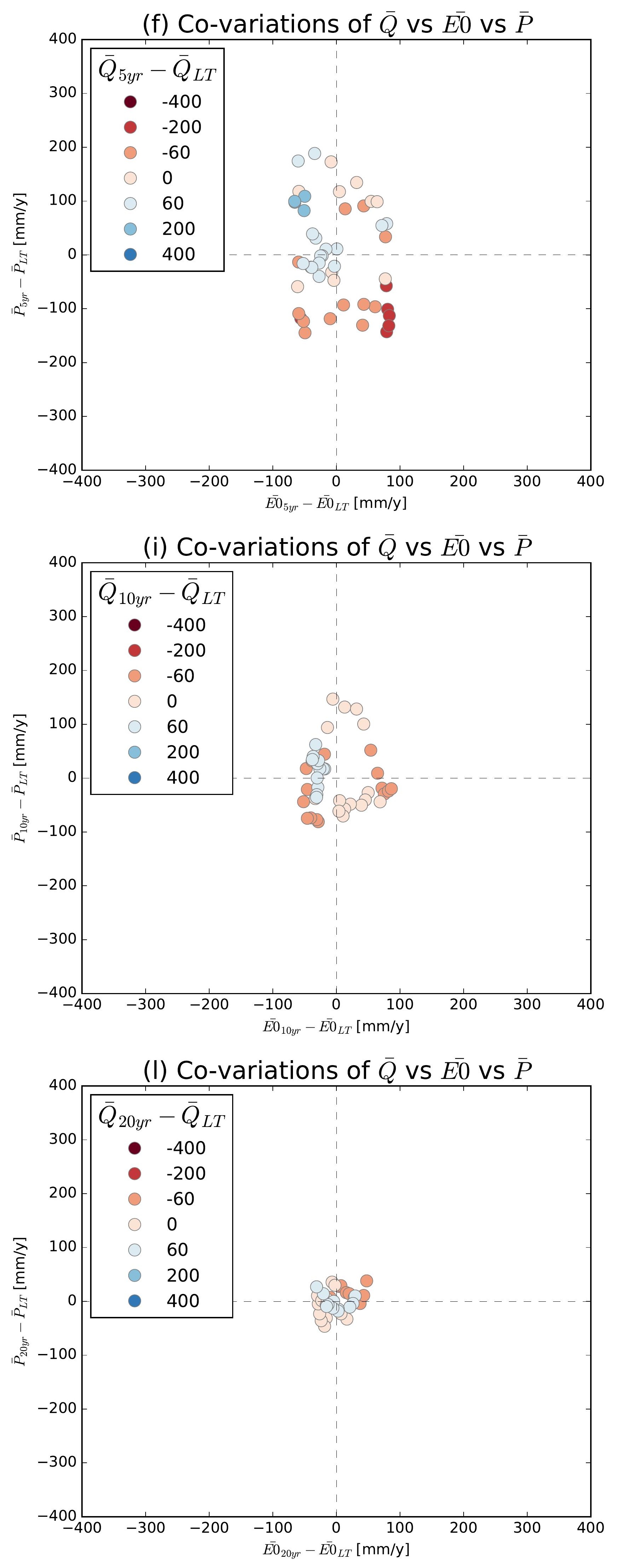

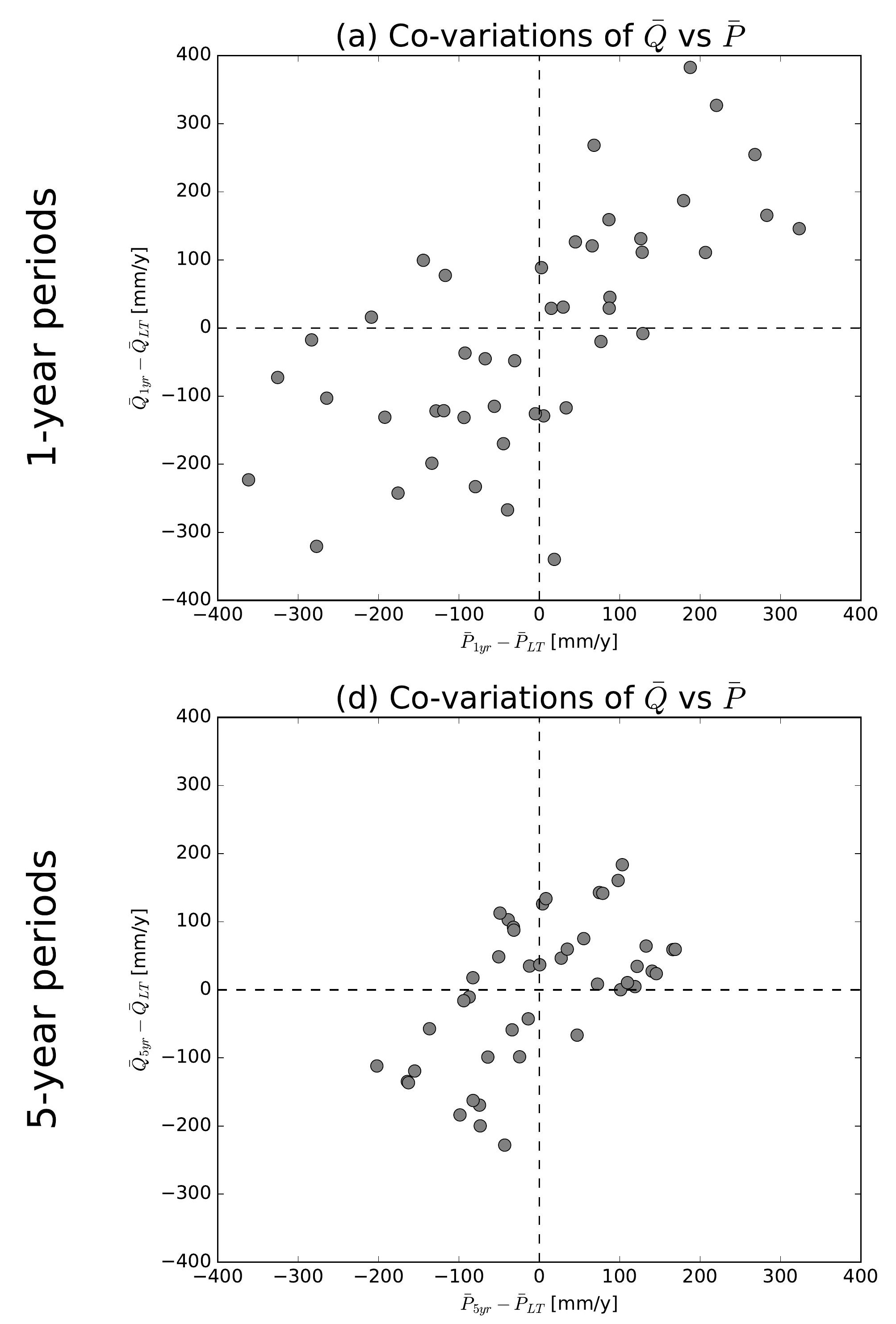

(g) Co-variations of $\bar{Q}$ vs $\bar{P}$

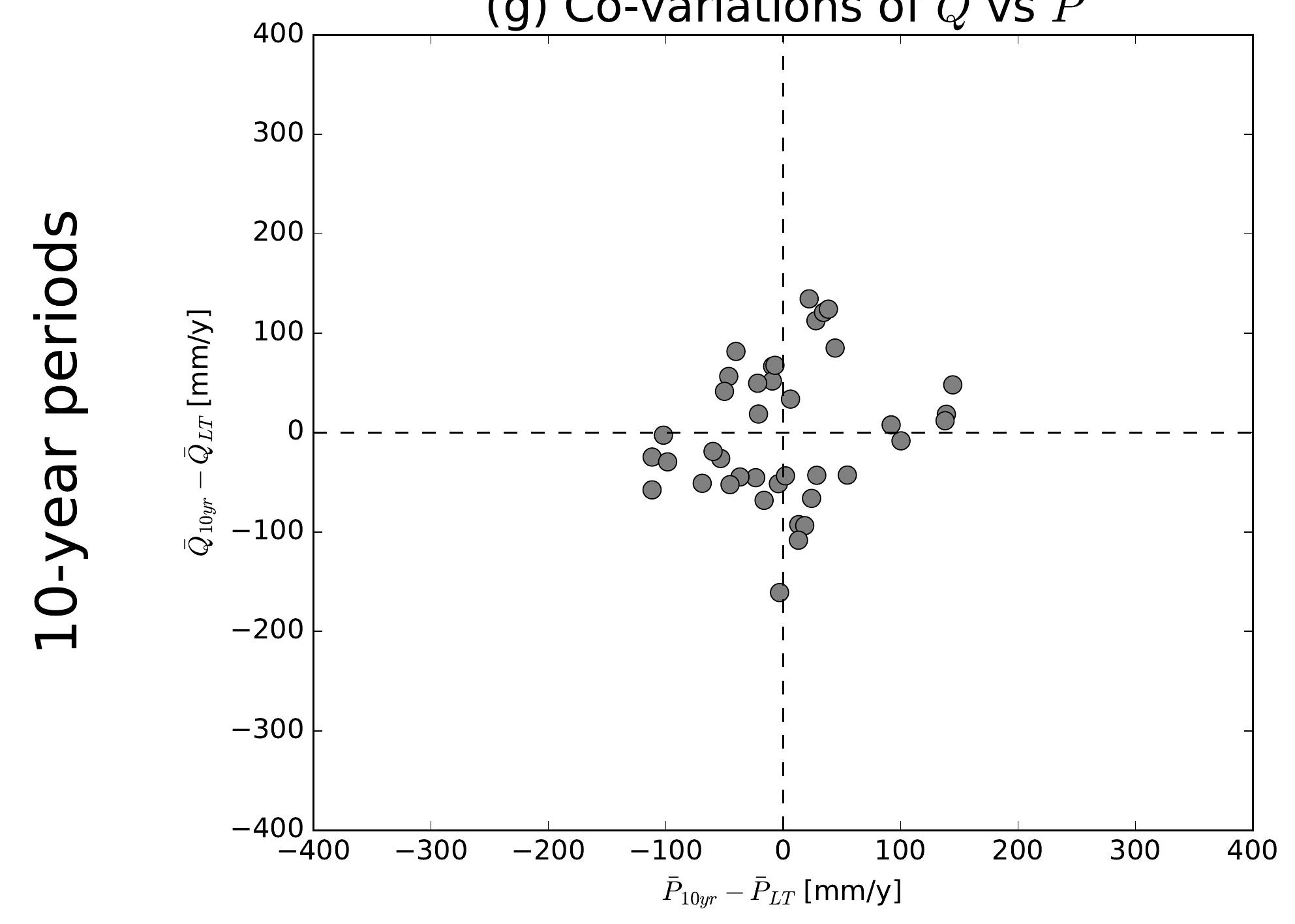

(j) Co-variations of $\bar{Q}$ vs $\bar{P}$

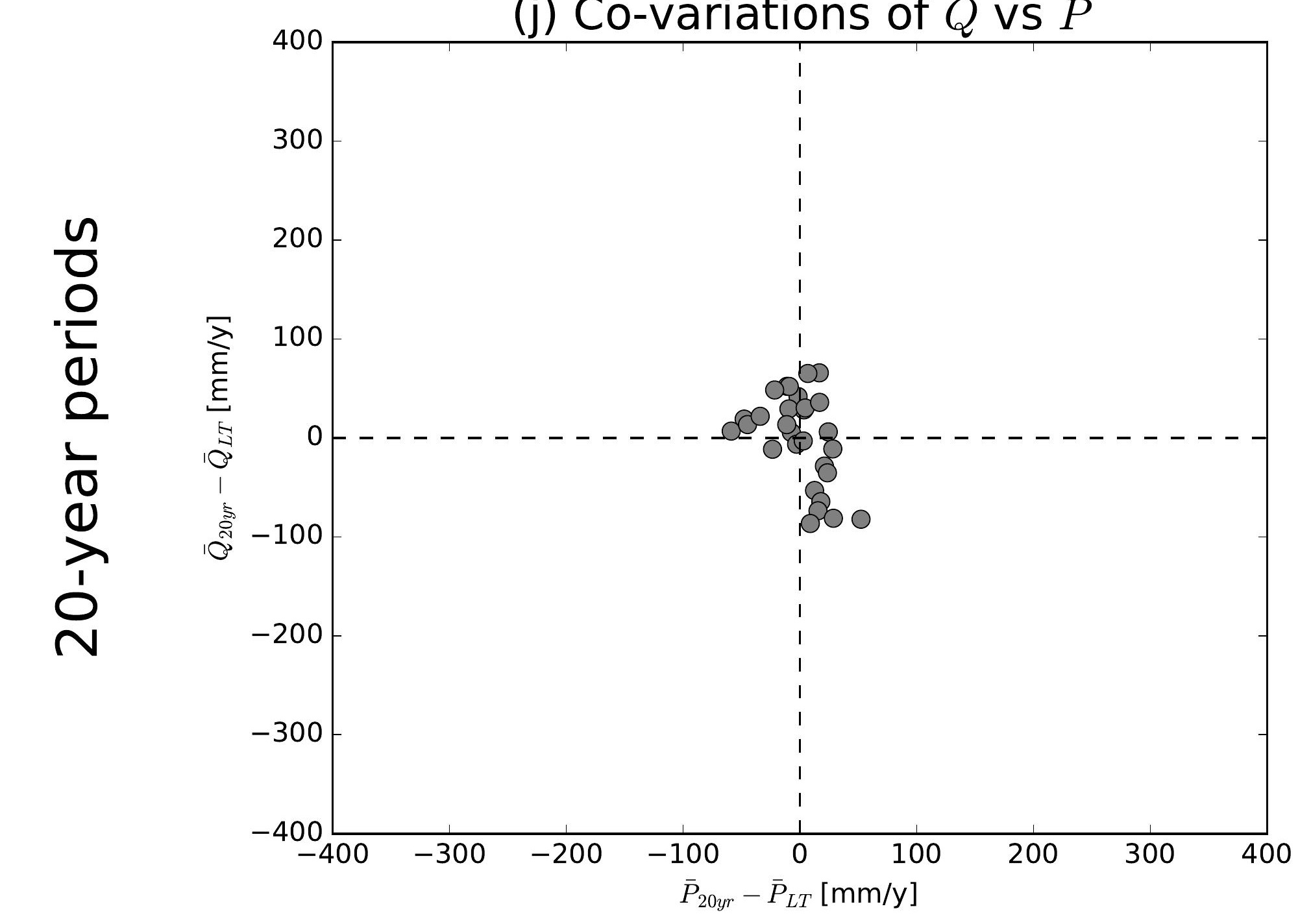

(b) Co-variations of $\bar{Q}$ vs $\overline{E 0}$

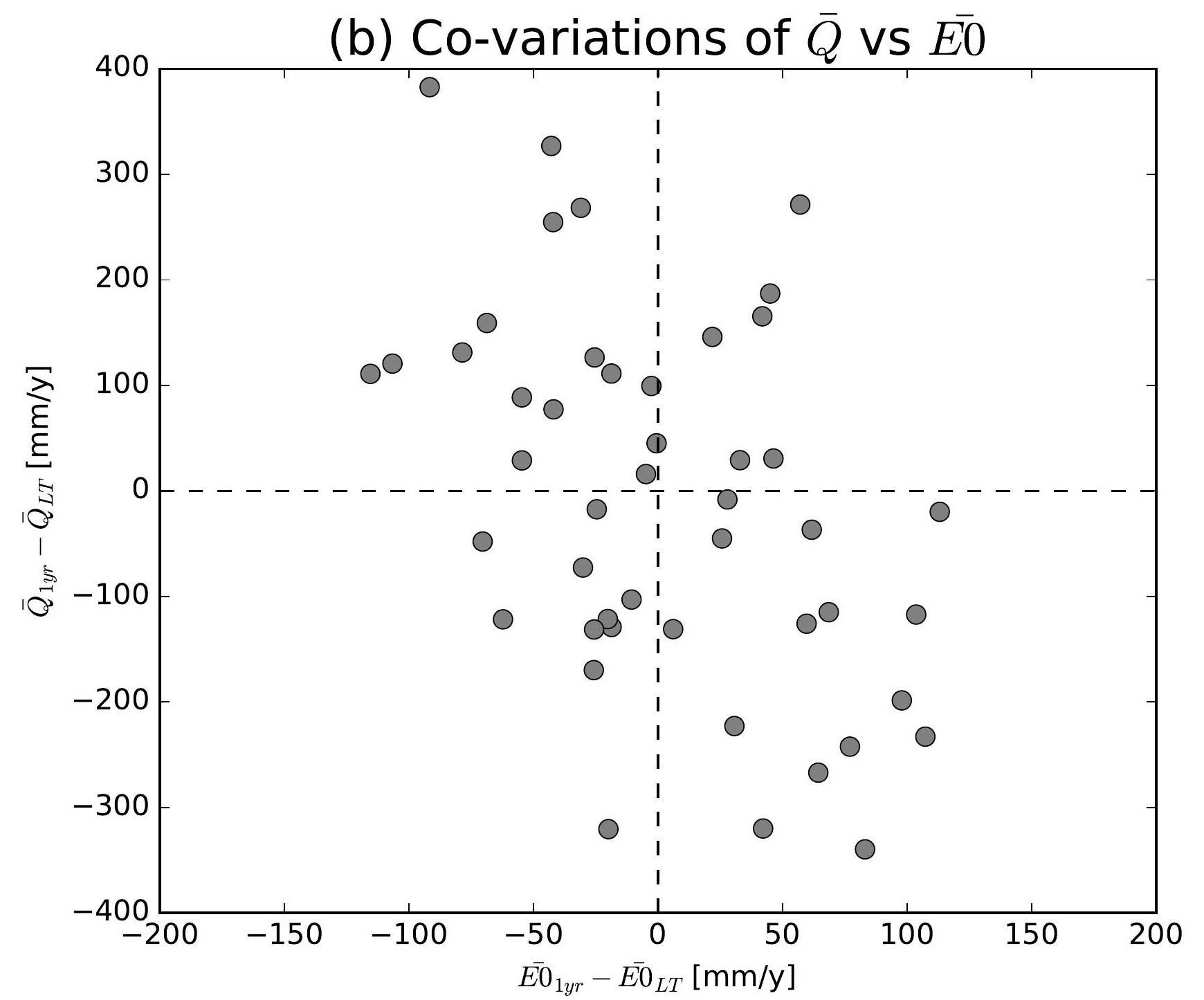

(e) Co-variations of $\bar{Q}$ vs $\overline{E 0}$

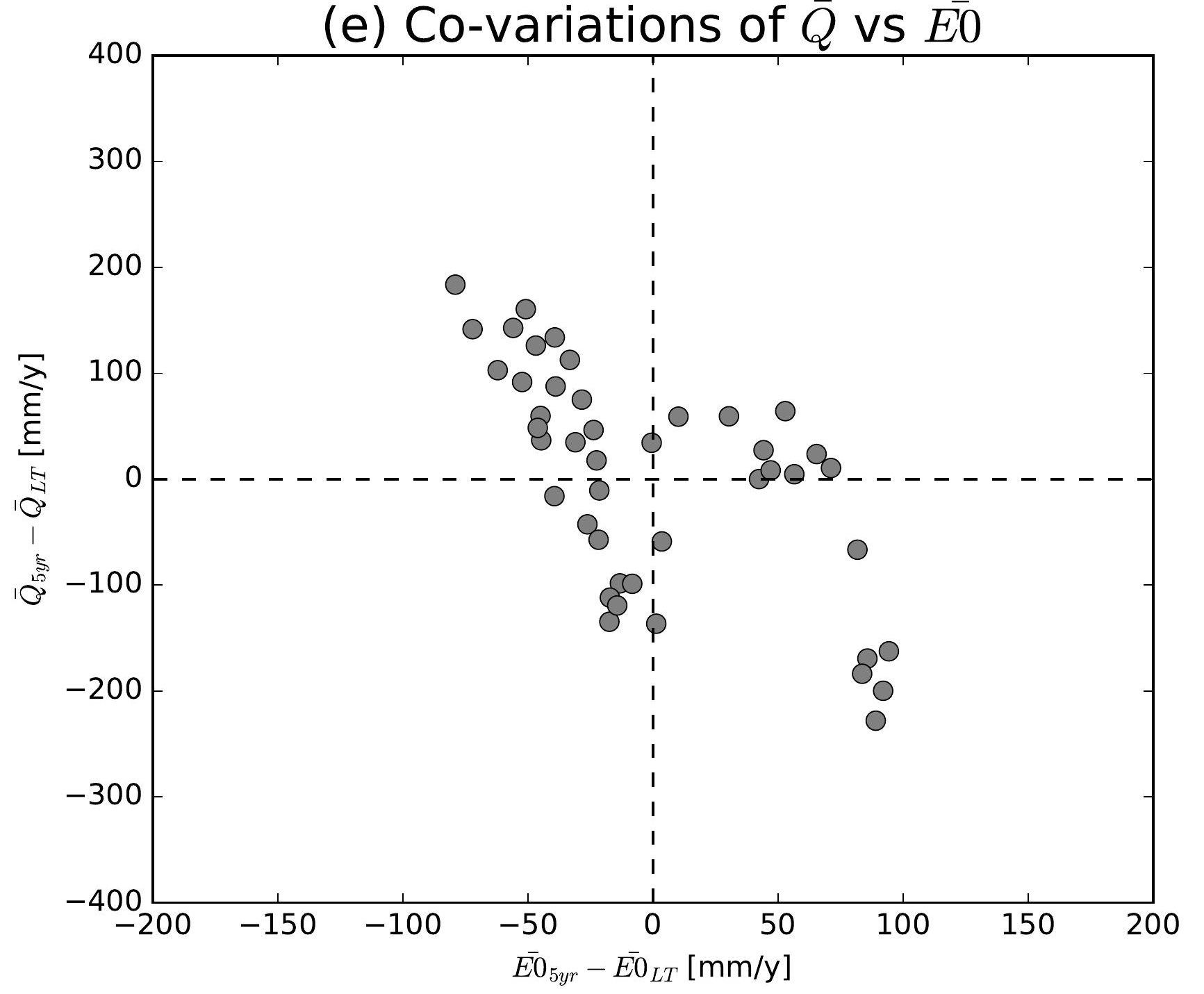

(h) Co-variations of $\bar{Q}$ vs $\overline{E 0}$

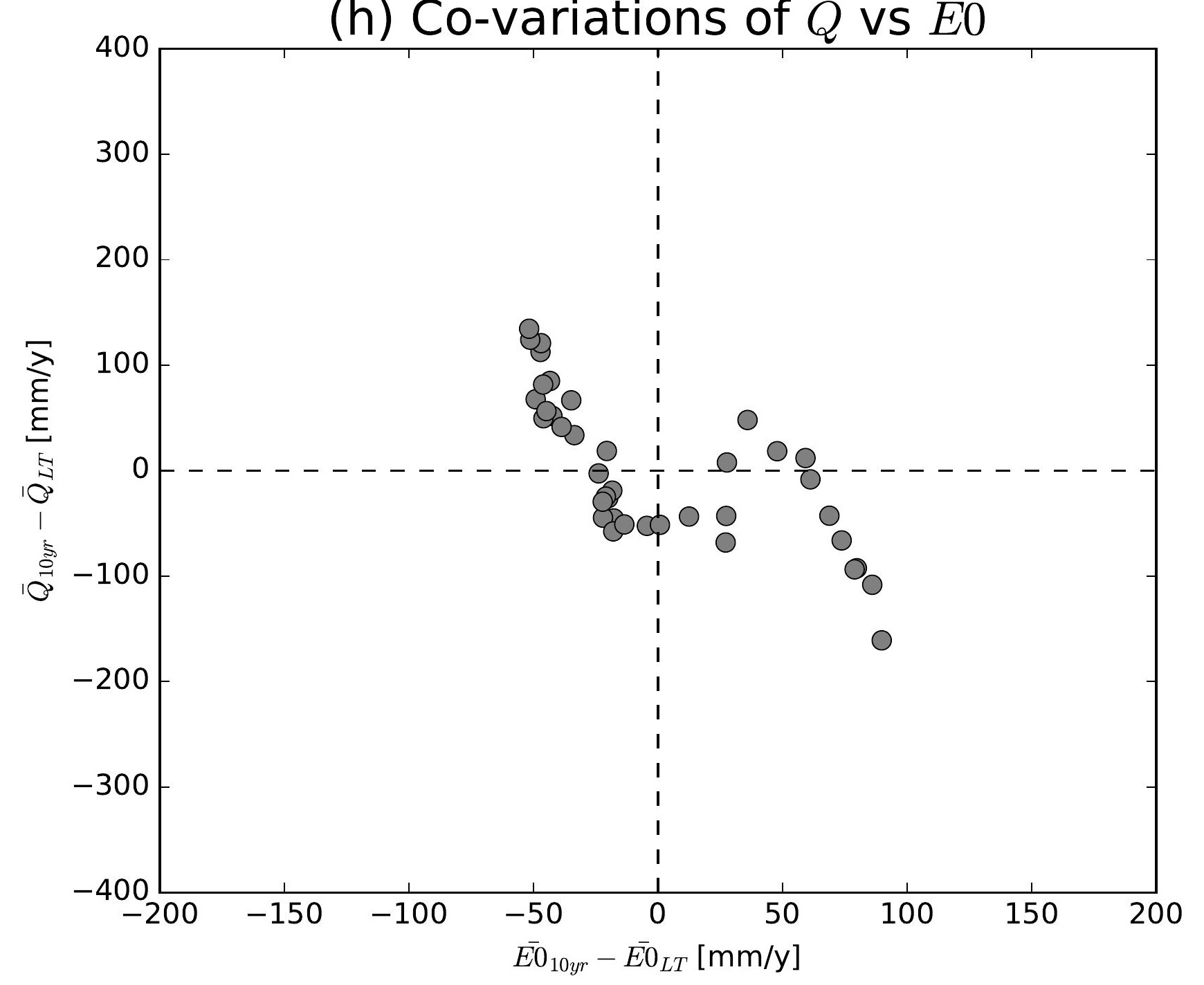

(k) Co-variations of $\bar{Q}$ vs $\overline{E 0}$

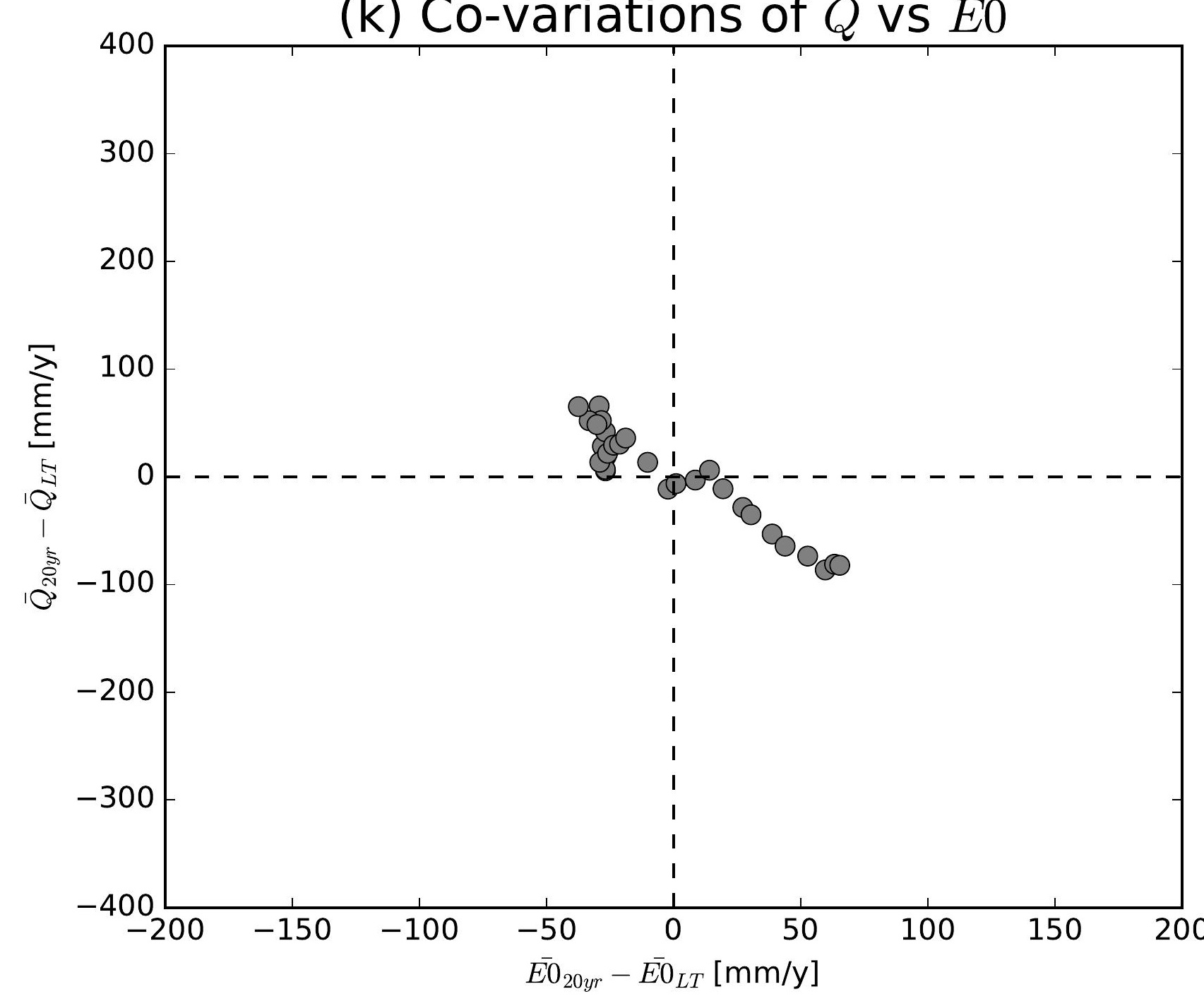

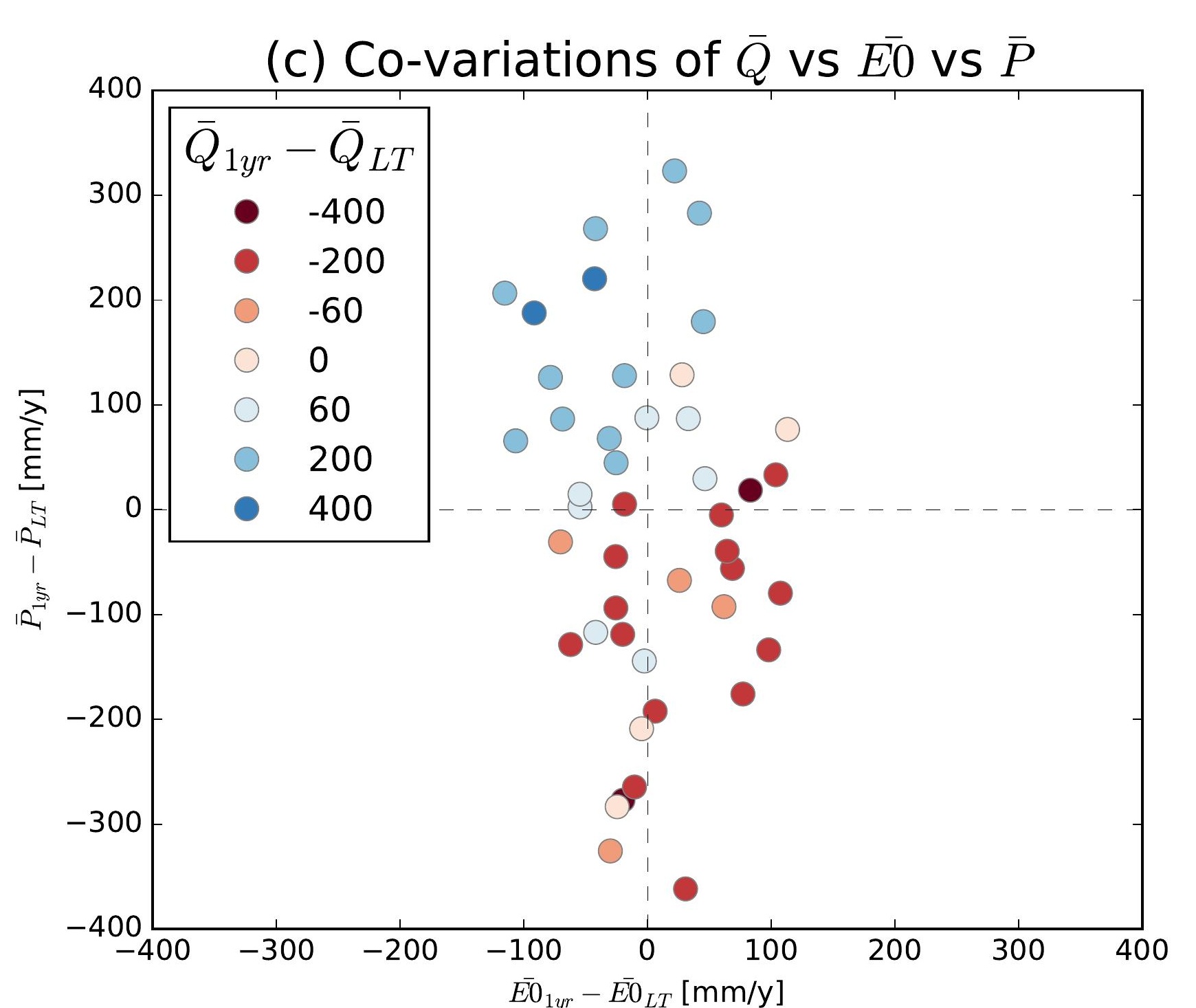
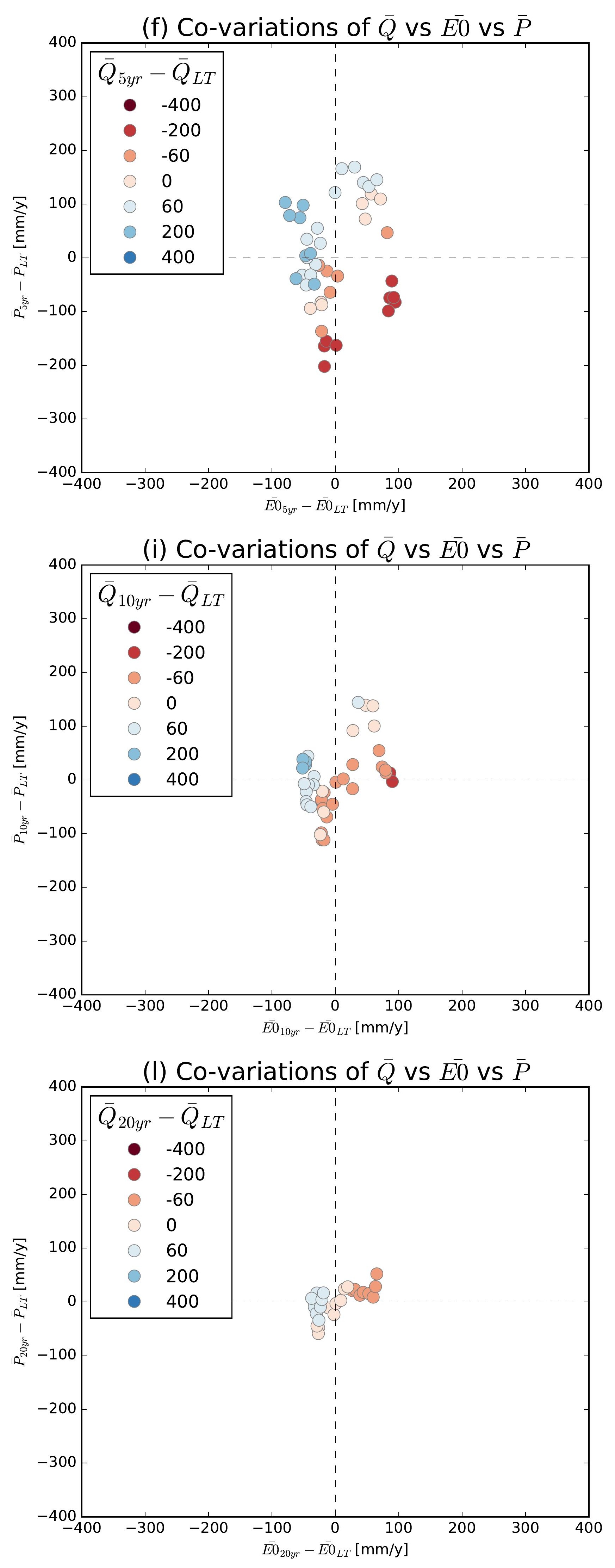

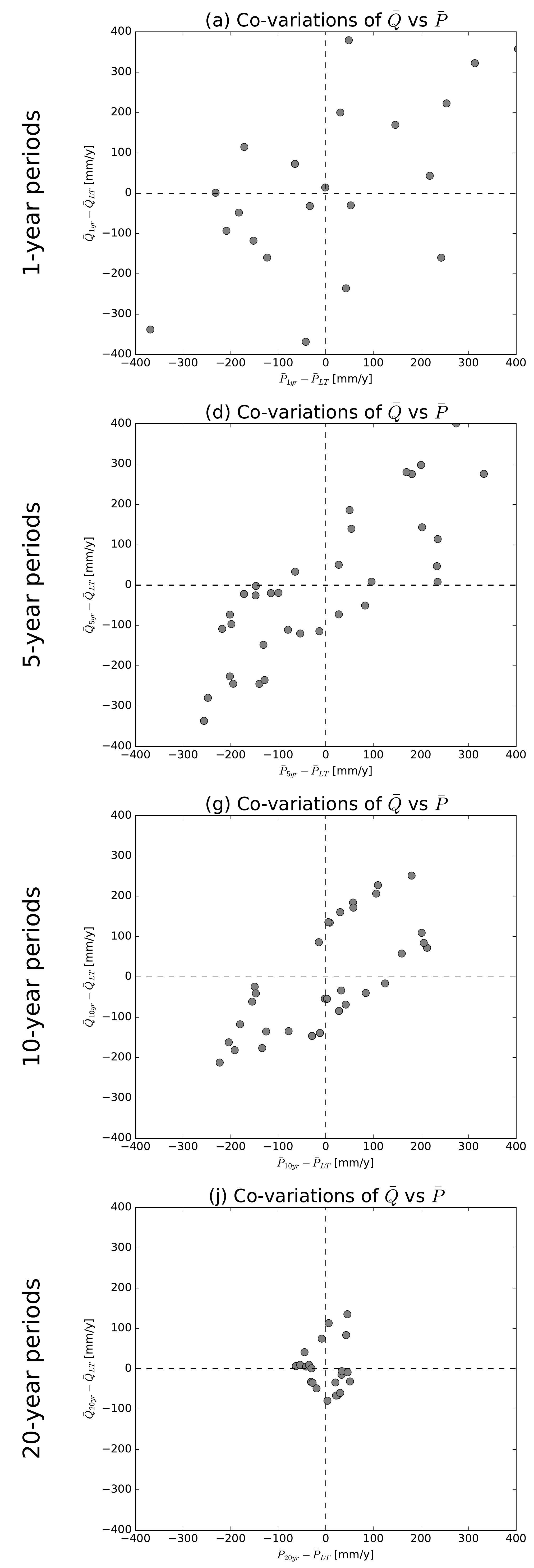

(b) Co-variations of $\bar{Q}$ vs $\overline{E 0}$

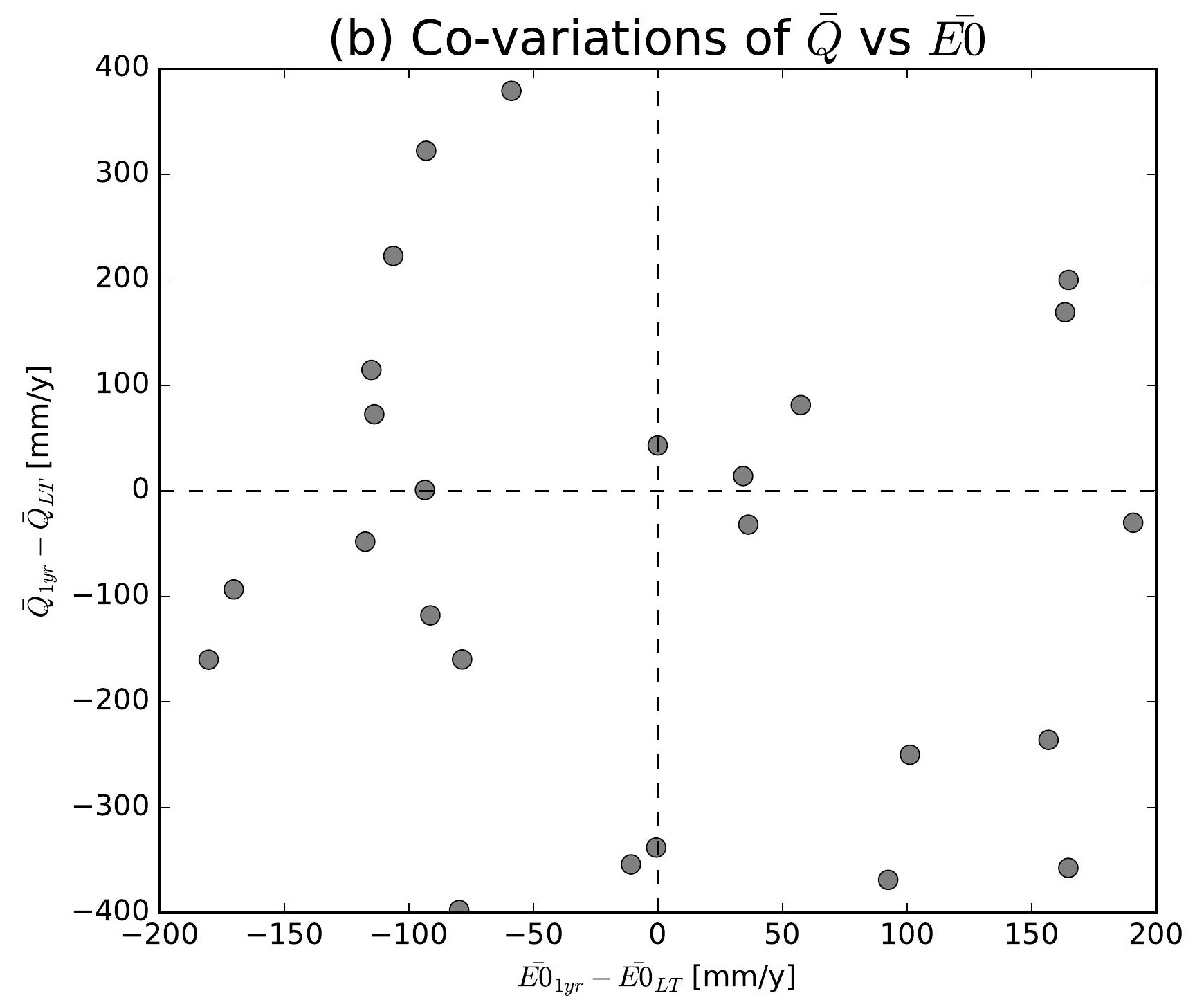

(e) Co-variations of $\bar{Q}$ vs $\overline{E 0}$

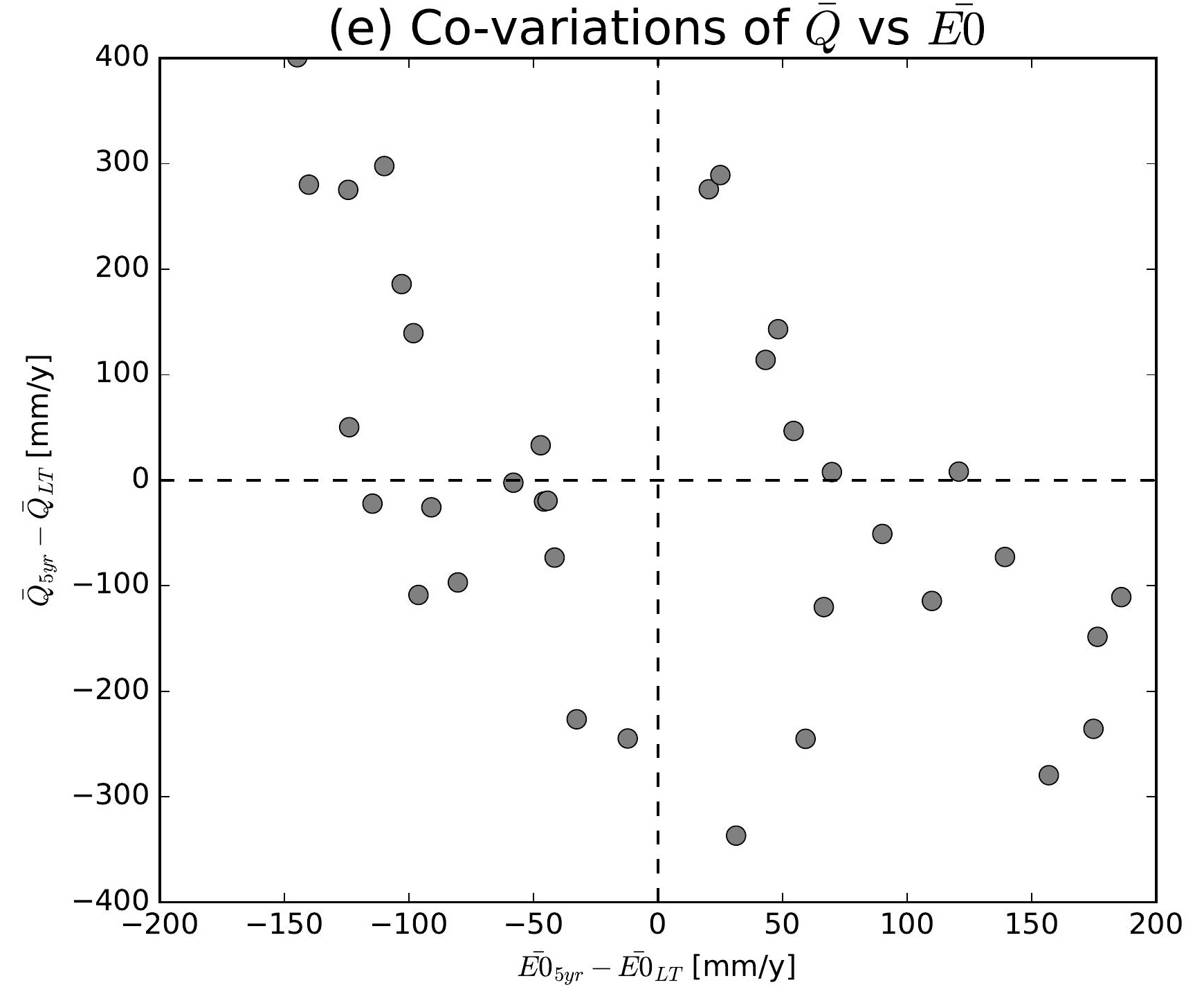

(h) Co-variations of $\bar{Q}$ vs $\overline{E 0}$

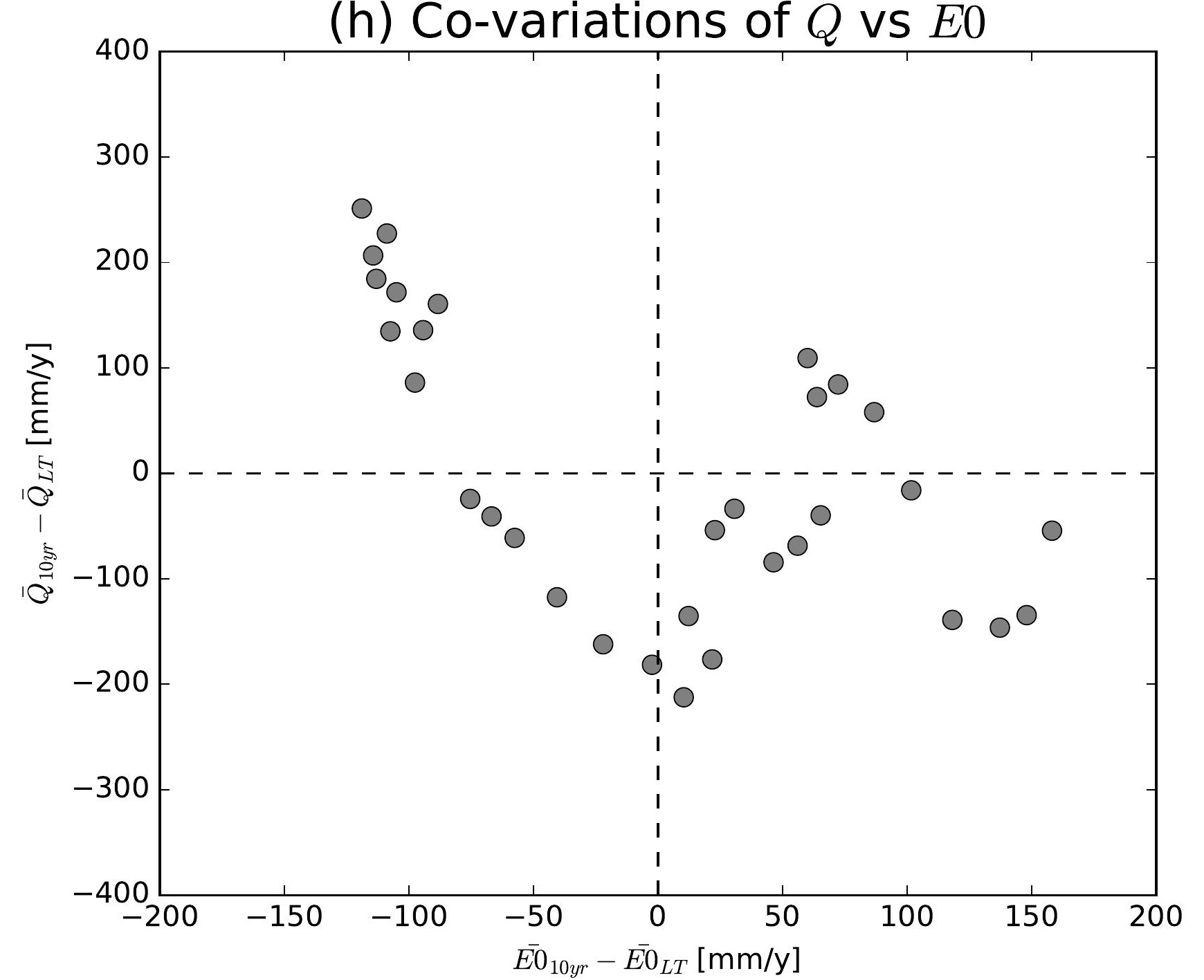

(k) Co-variations of $\bar{Q}$ vs $\overline{E 0}$

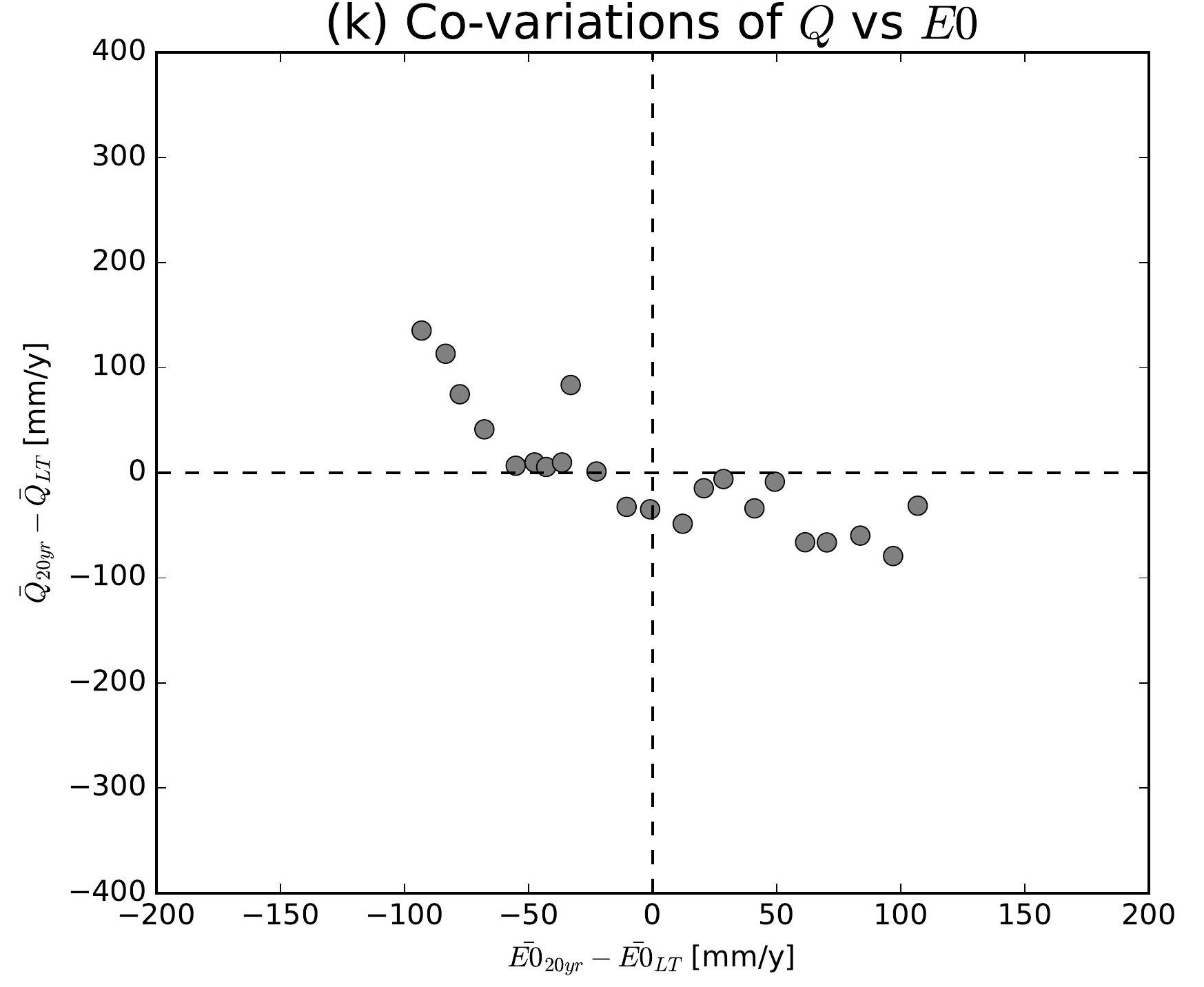

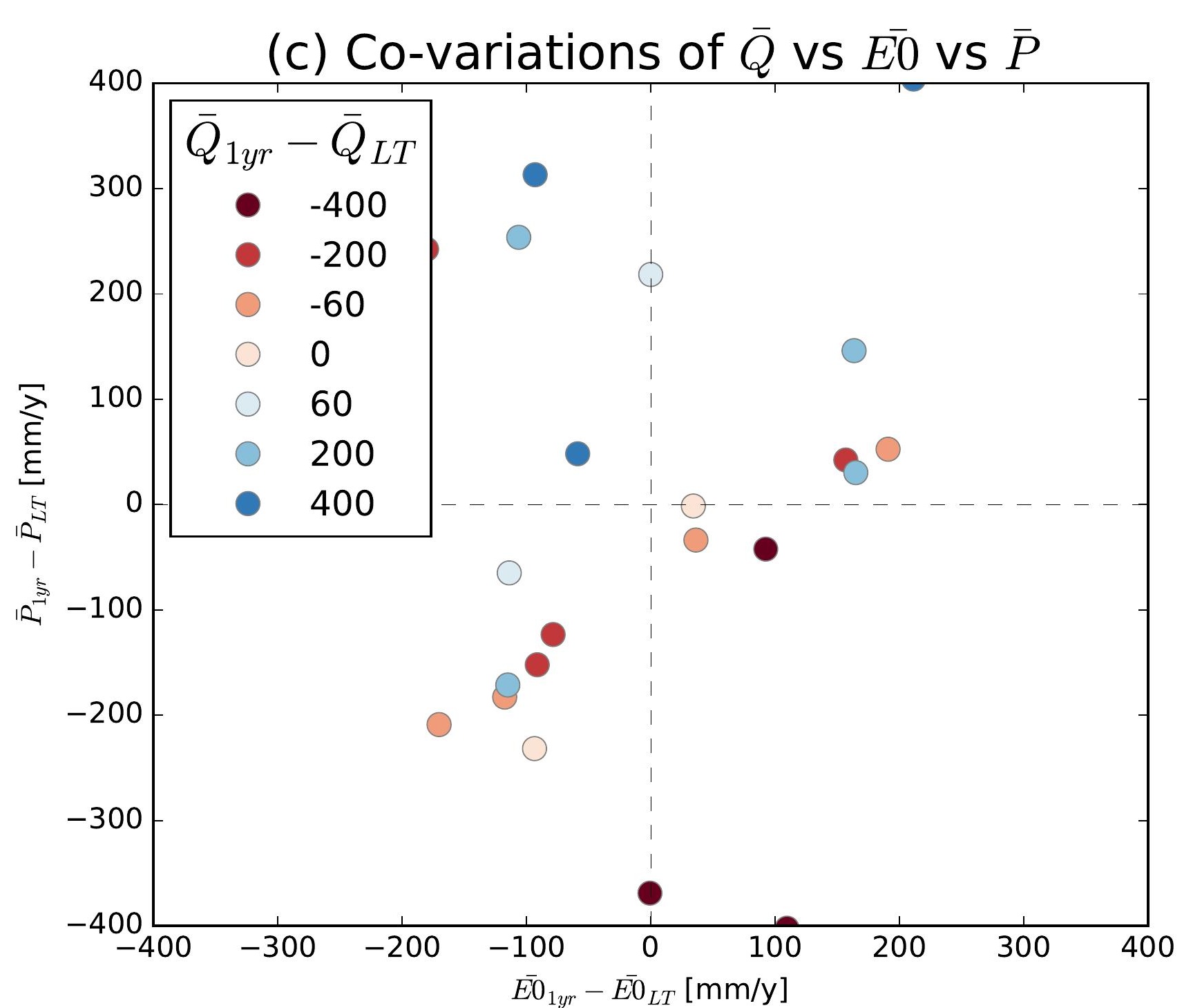
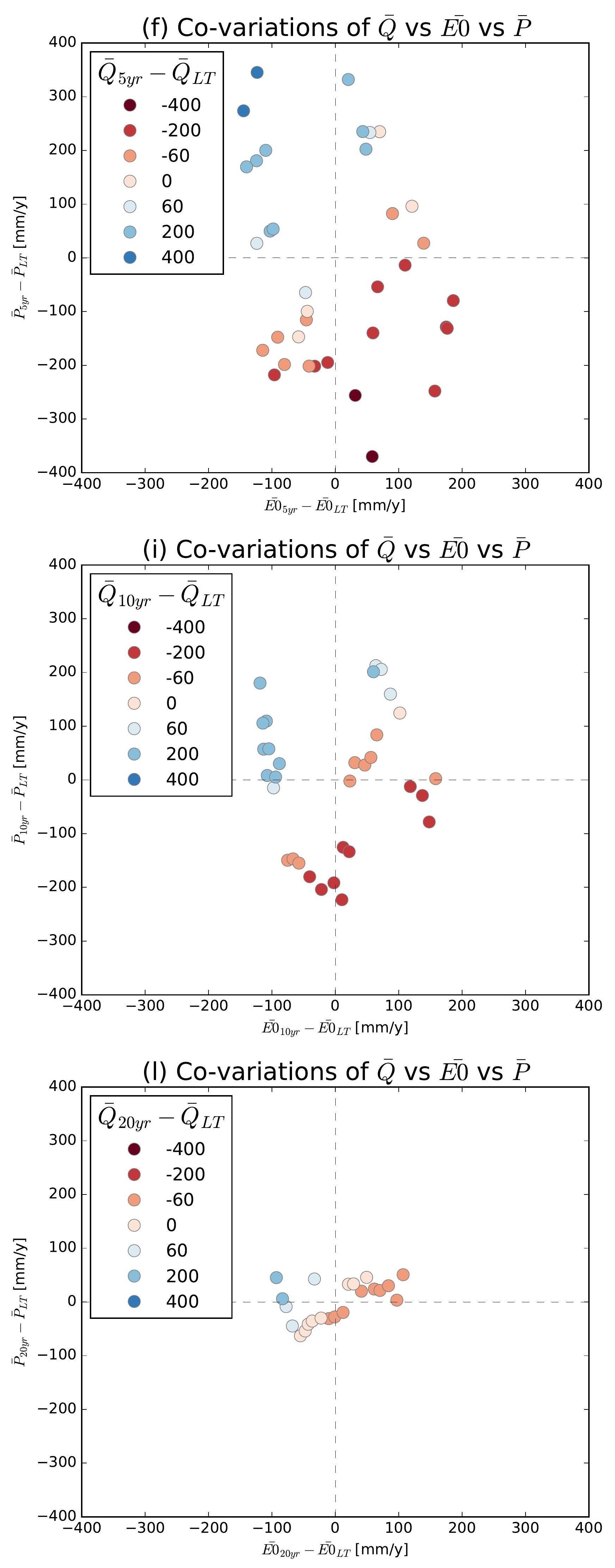

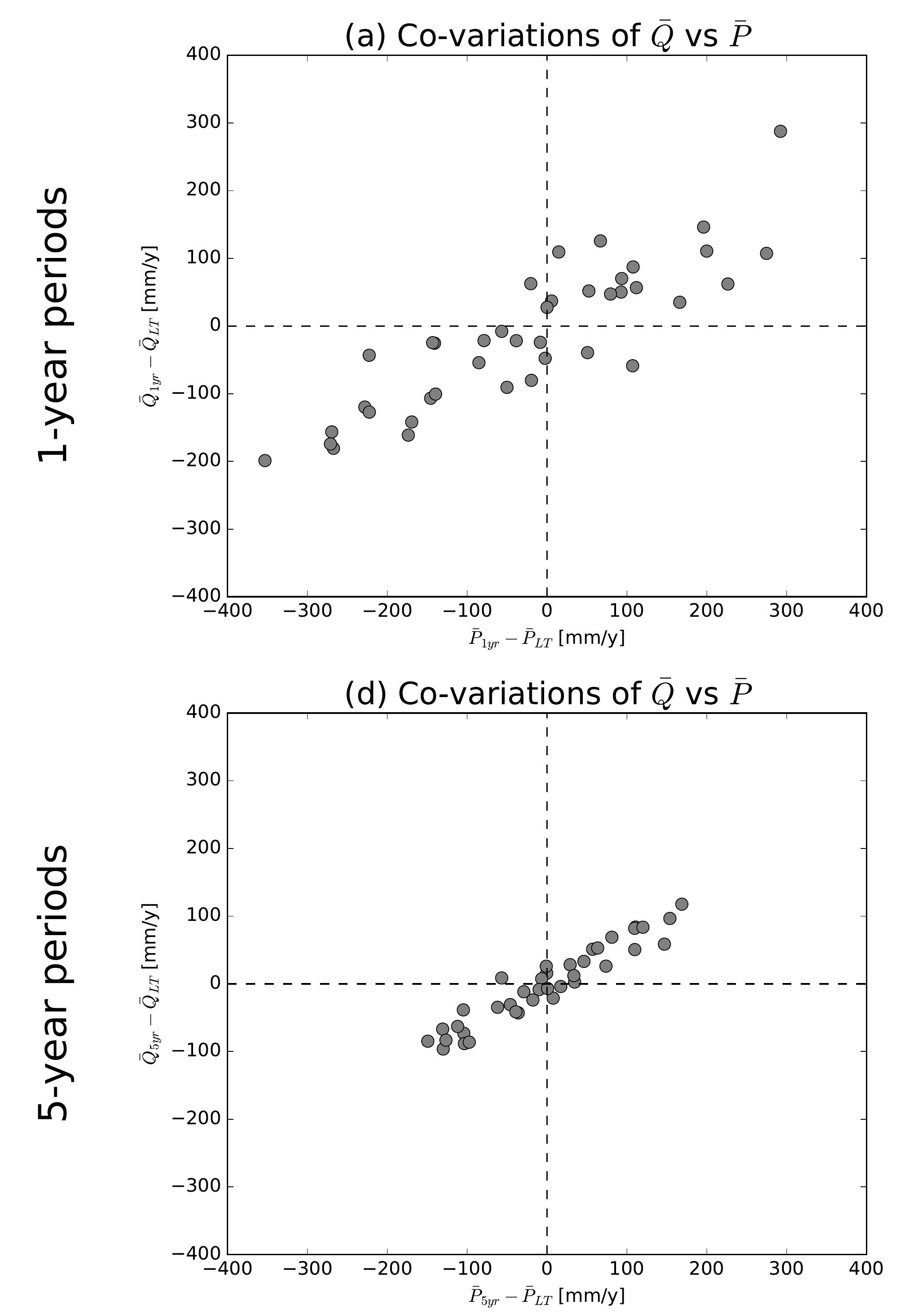

(g) Co-variations of $\bar{Q}$ vs $\bar{P}$

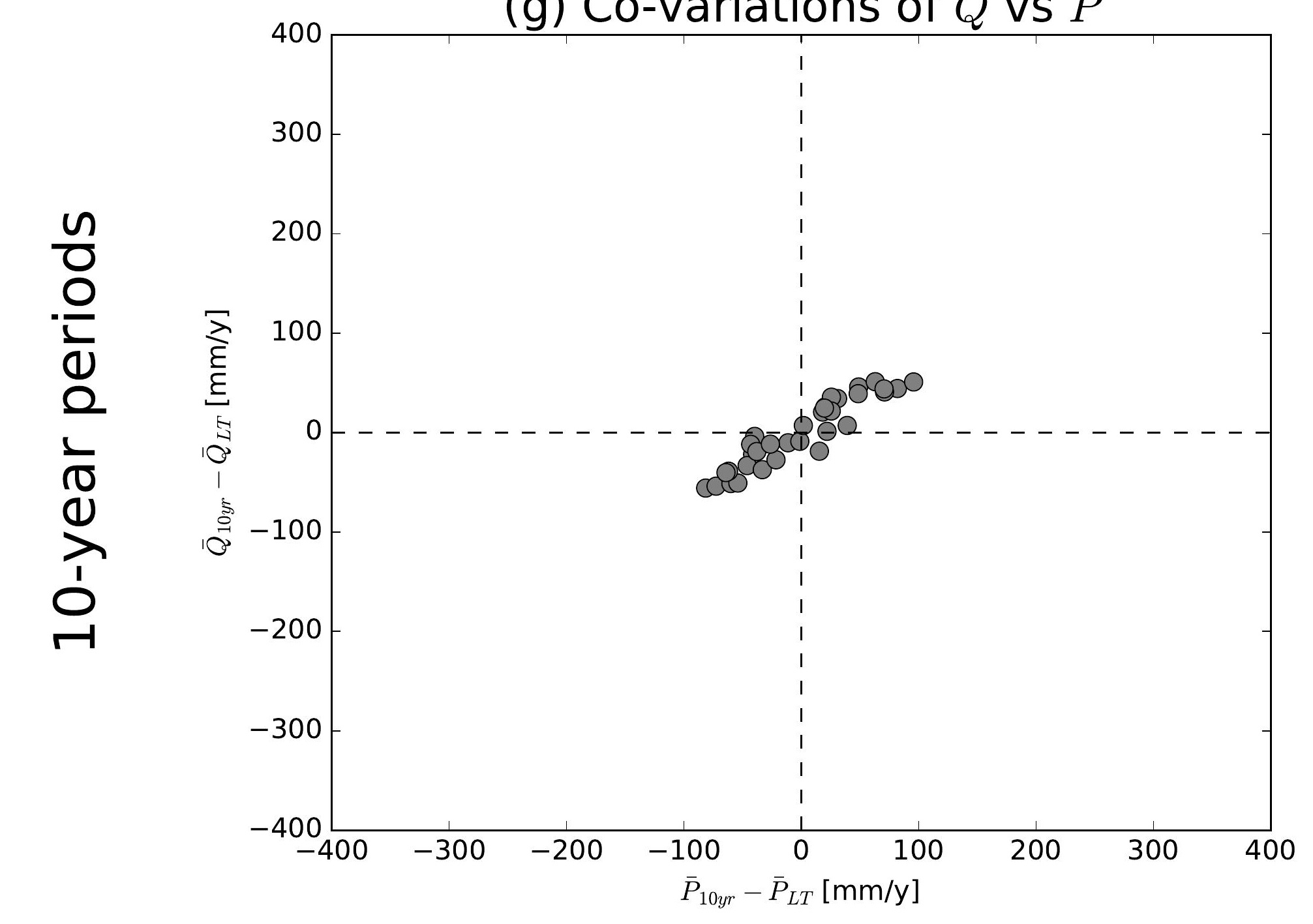

(j) Co-variations of $\bar{Q}$ vs $\bar{P}$

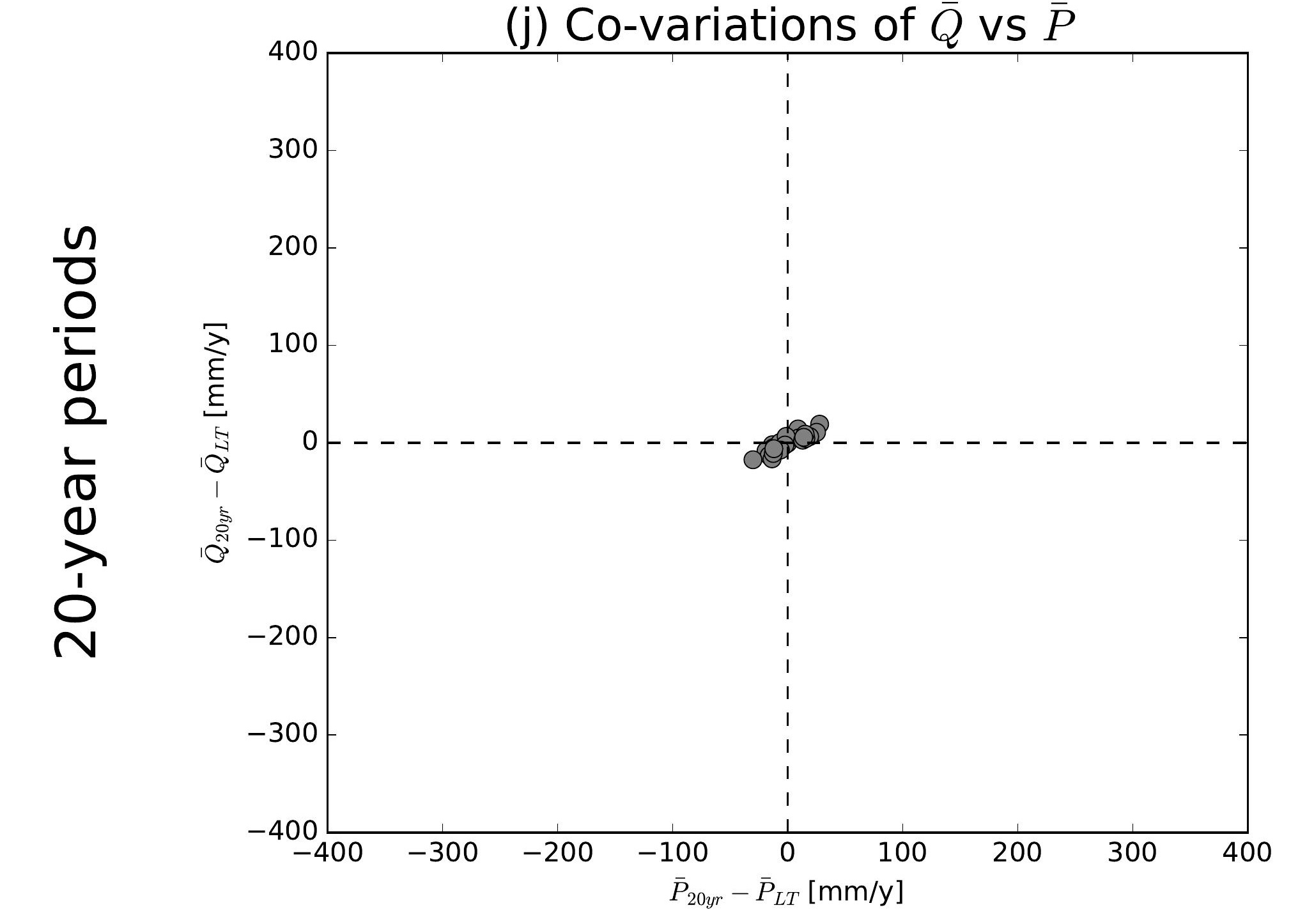

(b) Co-variations of $\bar{Q}$ vs $\overline{E 0}$

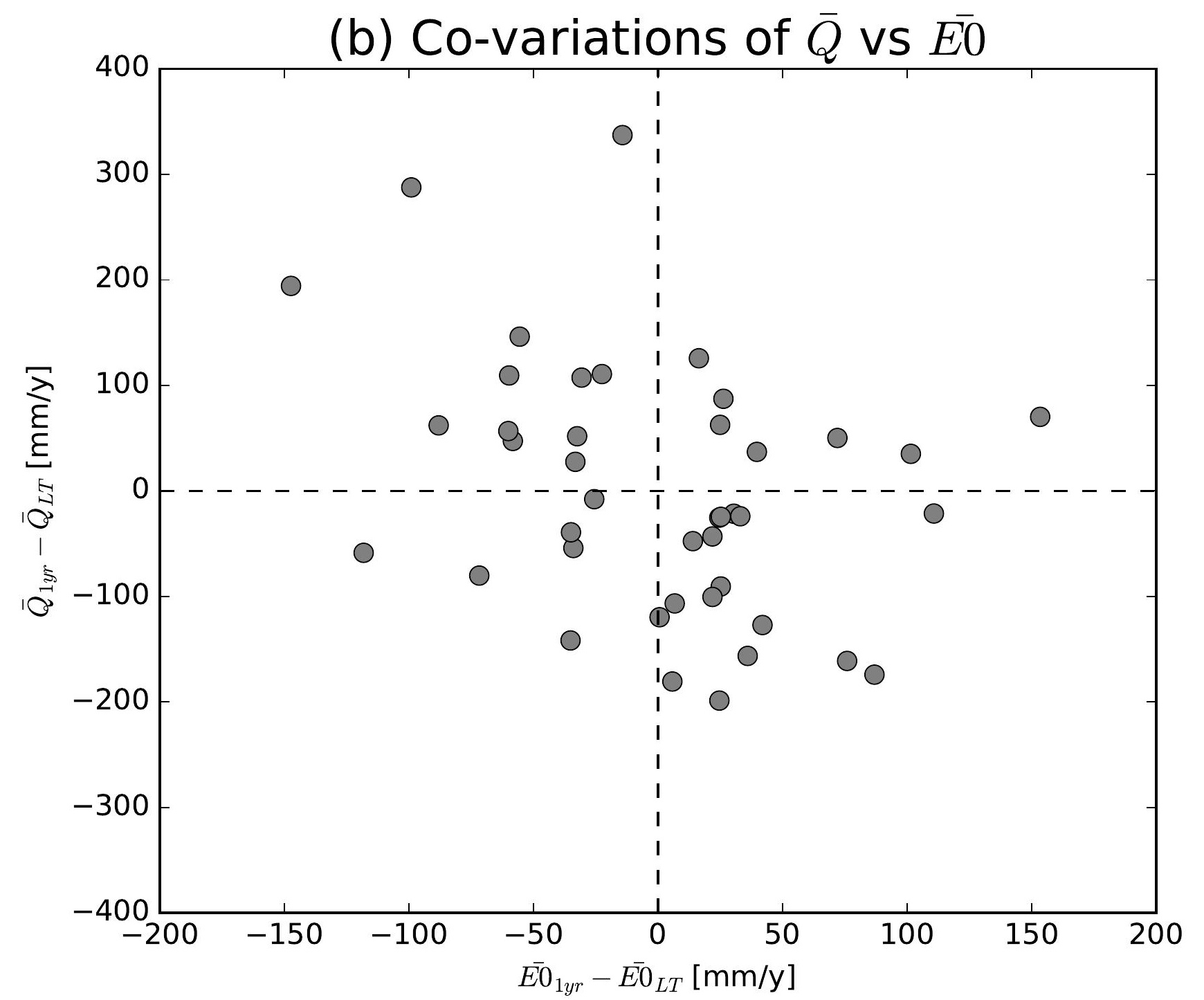

(e) Co-variations of $\bar{Q}$ vs $\overline{E 0}$

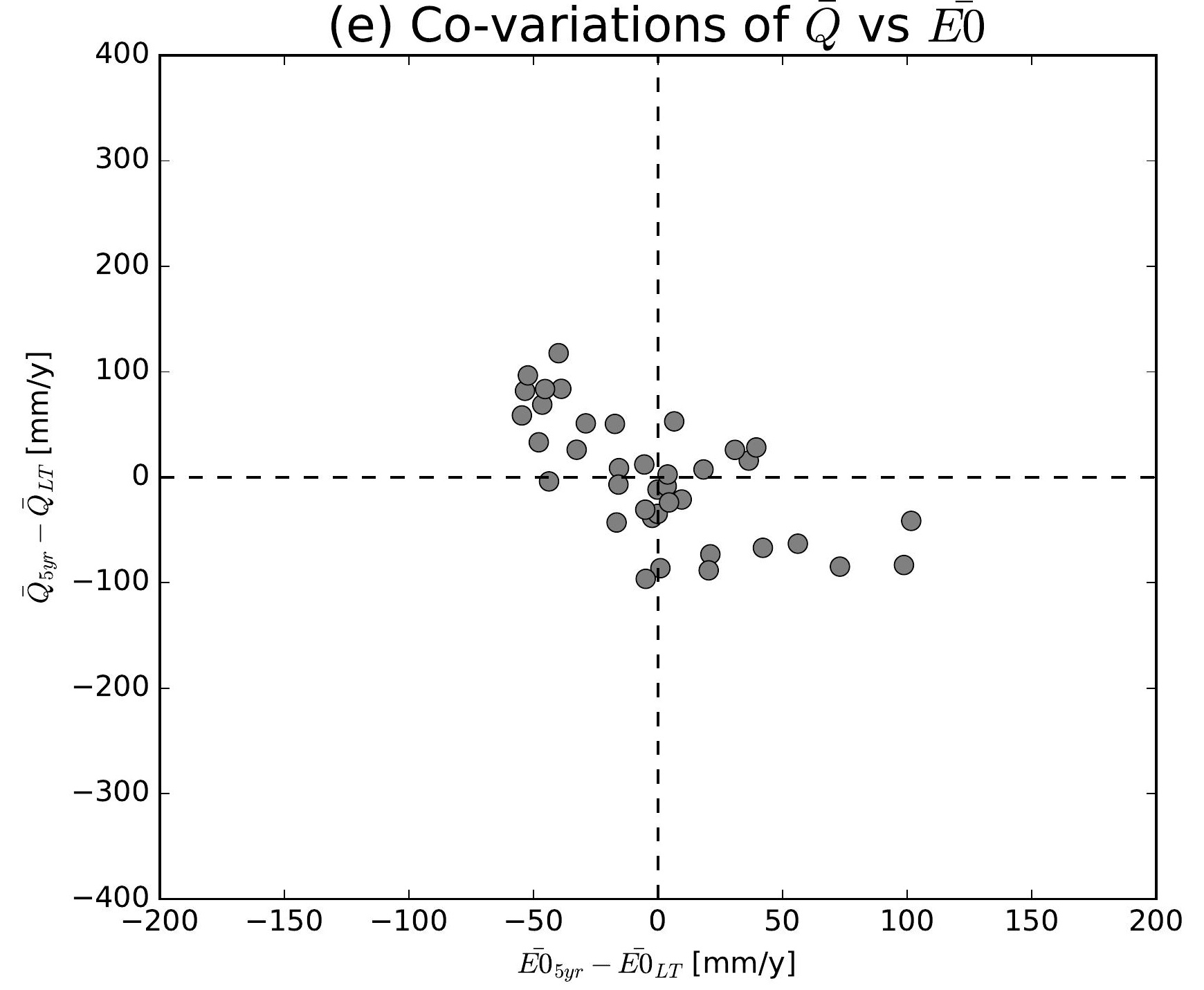

(h) Co-variations of $\bar{Q}$ vs $\overline{E 0}$

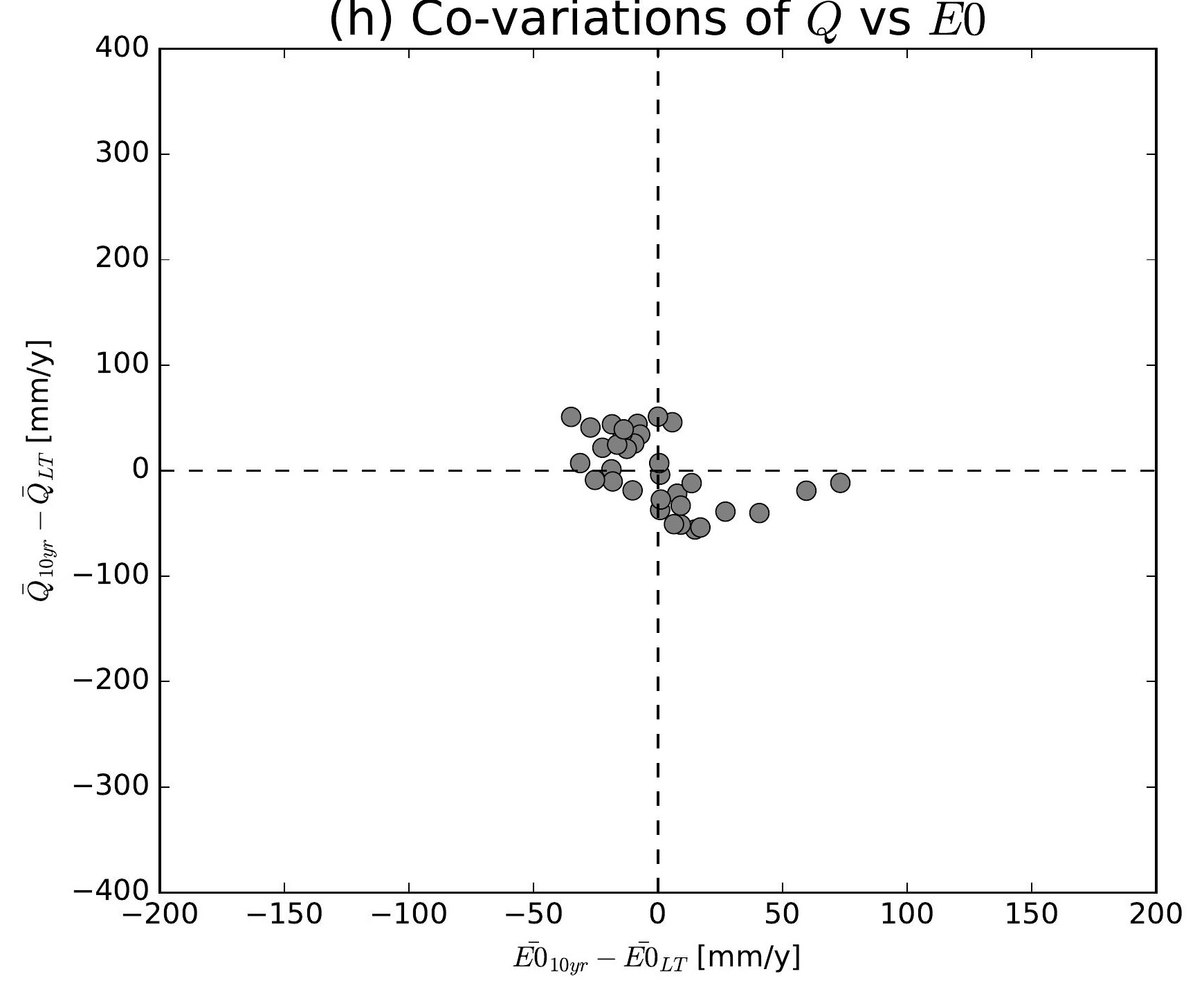

(k) Co-variations of $\bar{Q}$ vs $\overline{E 0}$

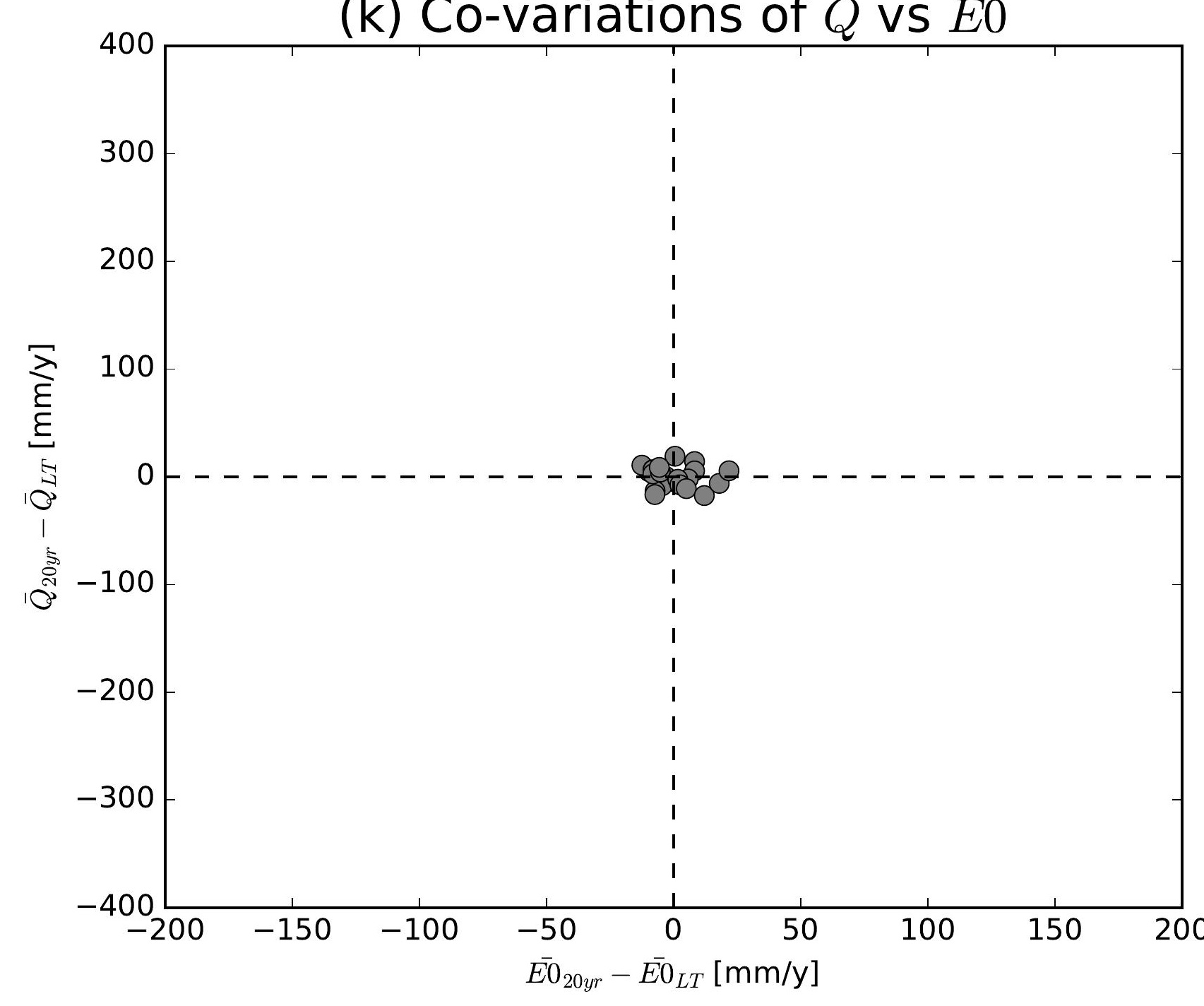

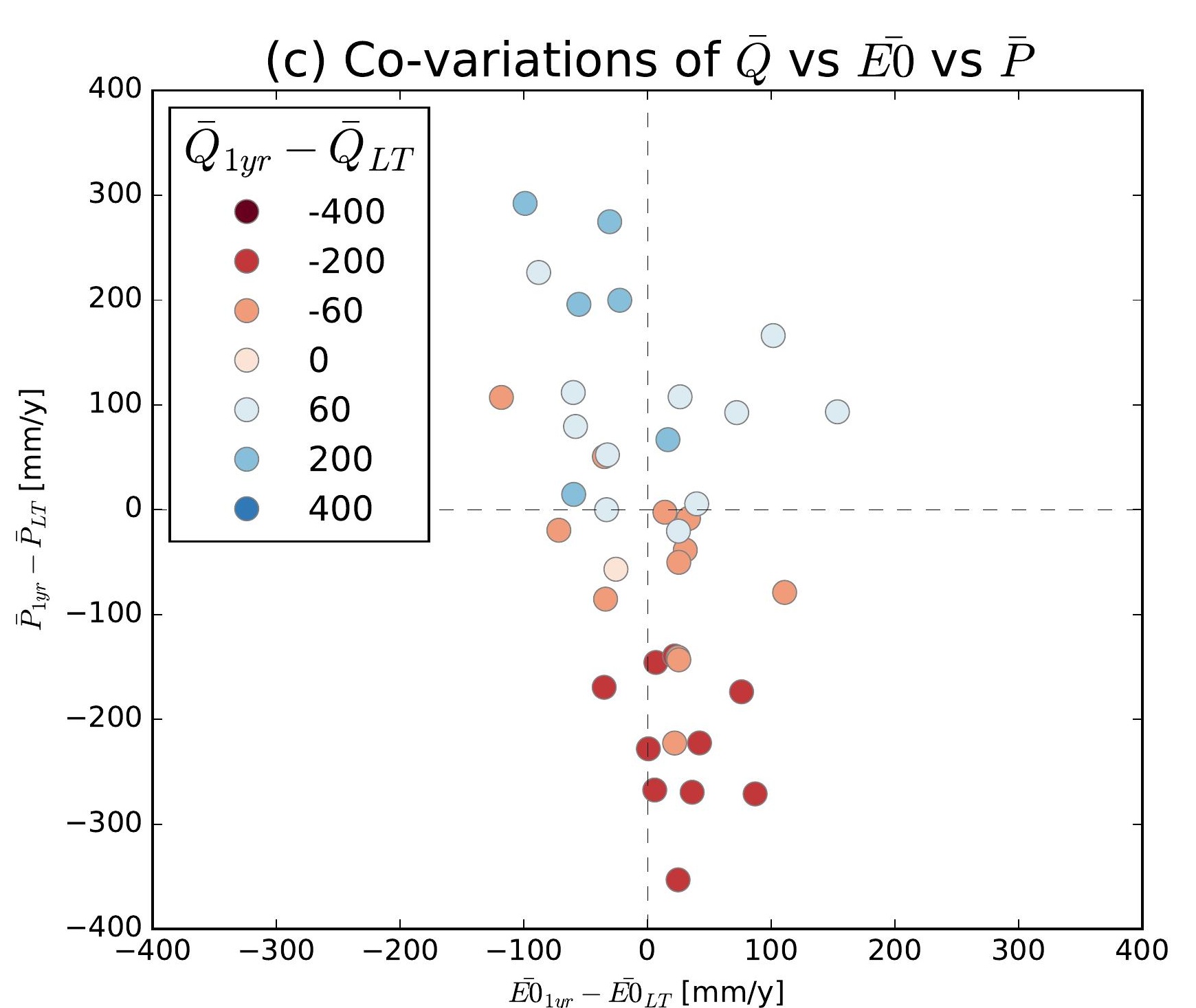
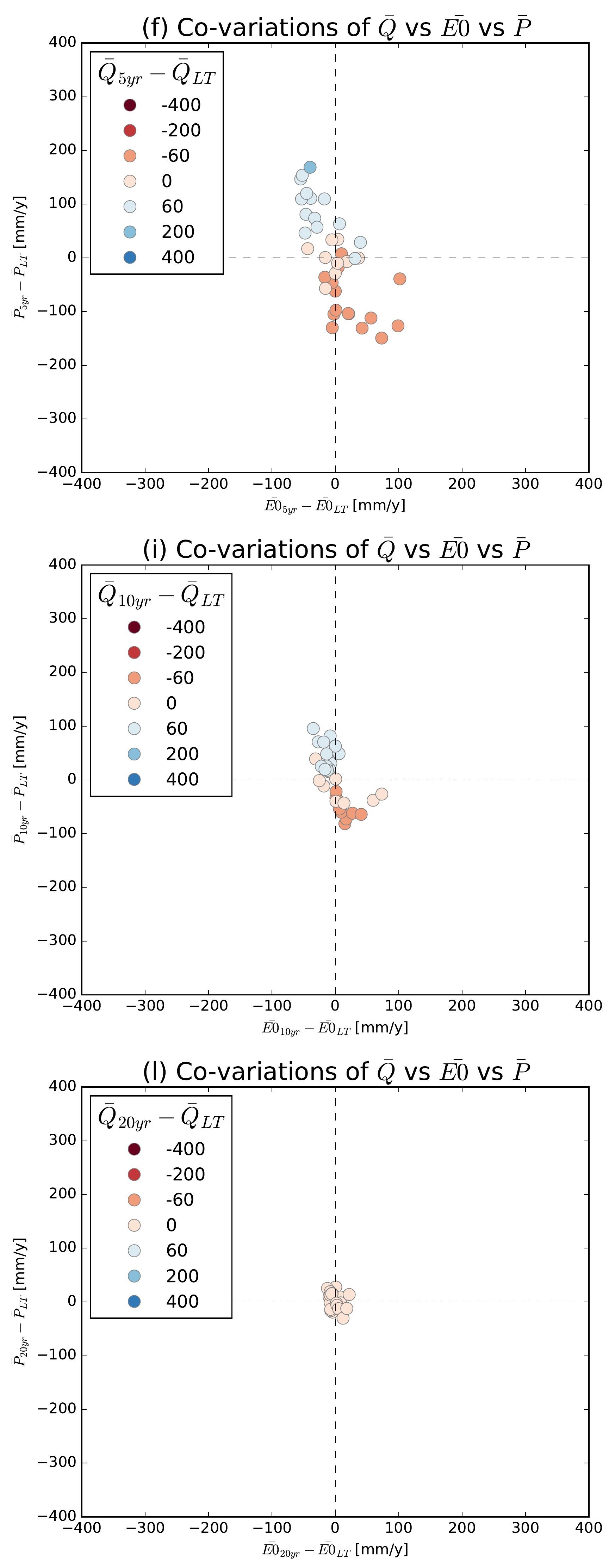

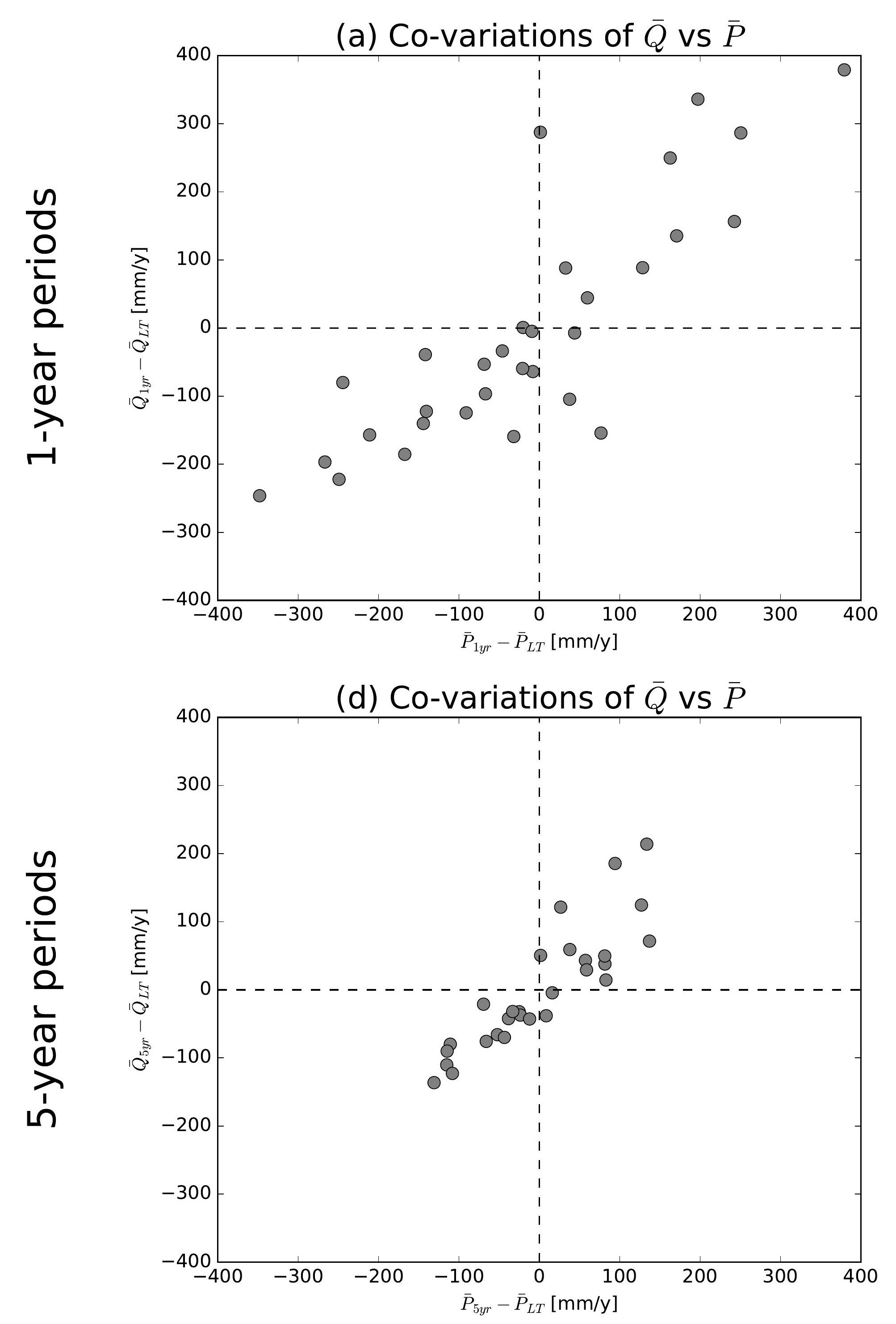

(g) Co-variations of $\bar{Q}$ vs $\bar{P}$

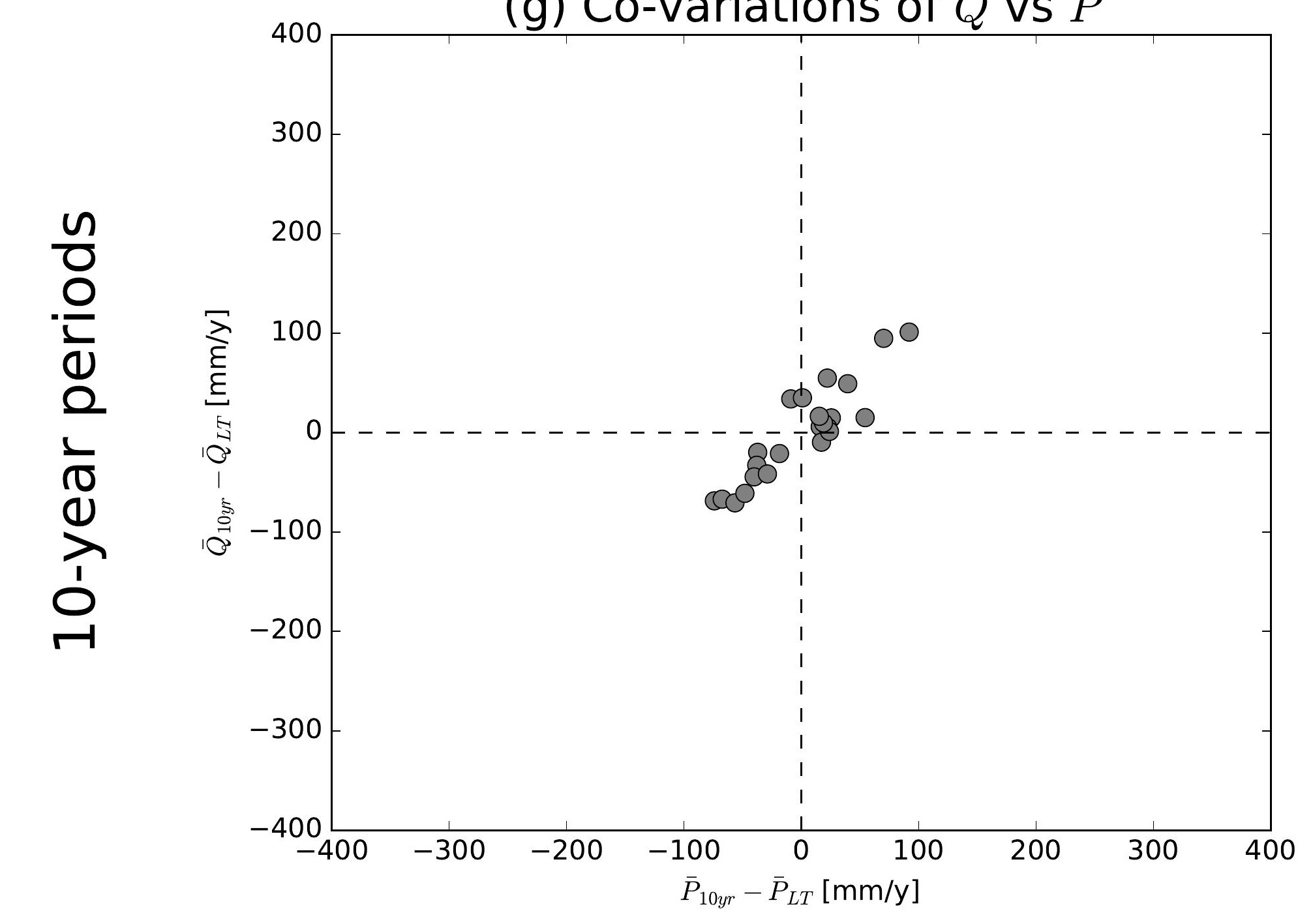

(j) Co-variations of $\bar{Q}$ vs $\bar{P}$

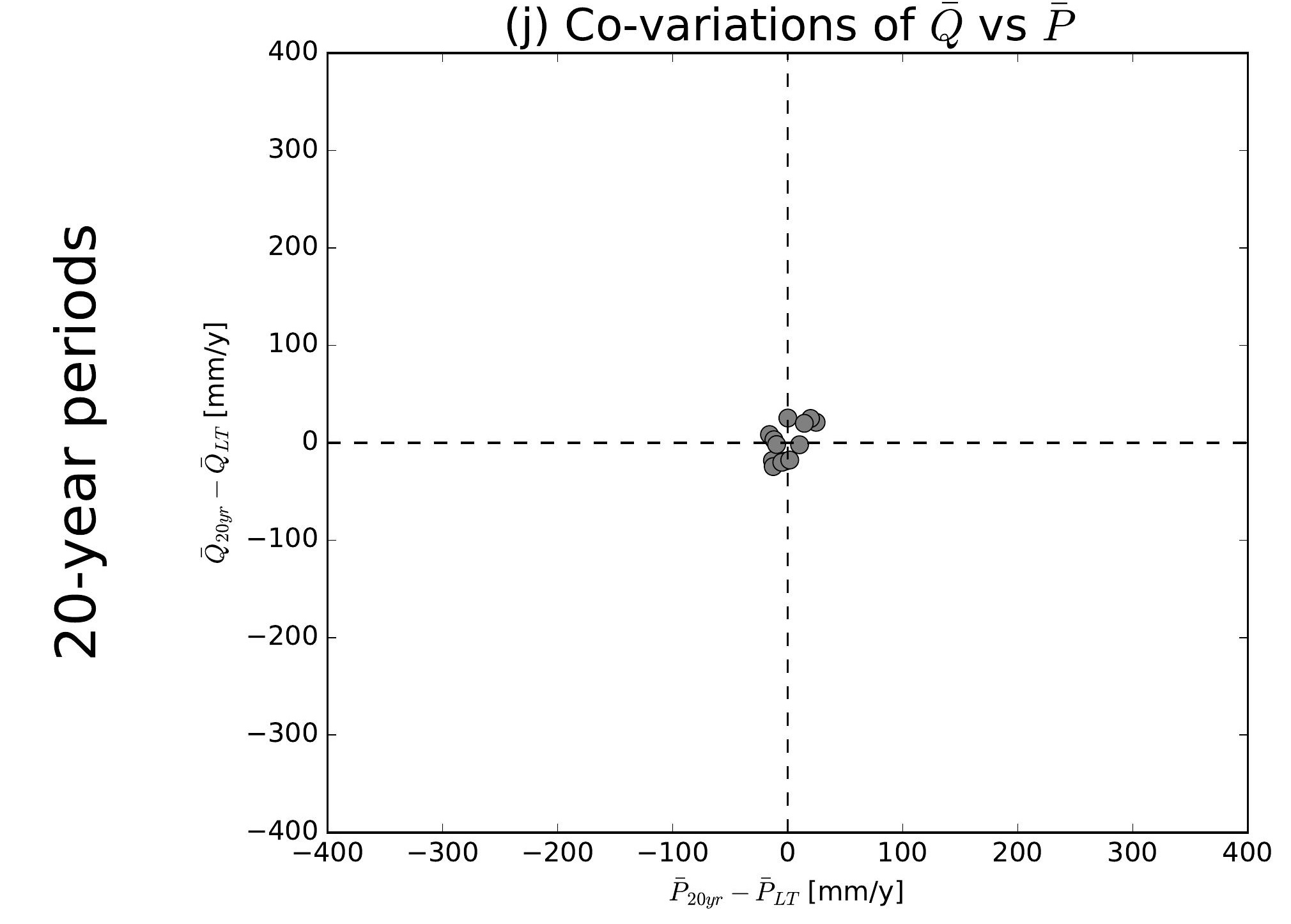

(b) Co-variations of $\bar{Q}$ vs $\overline{E 0}$

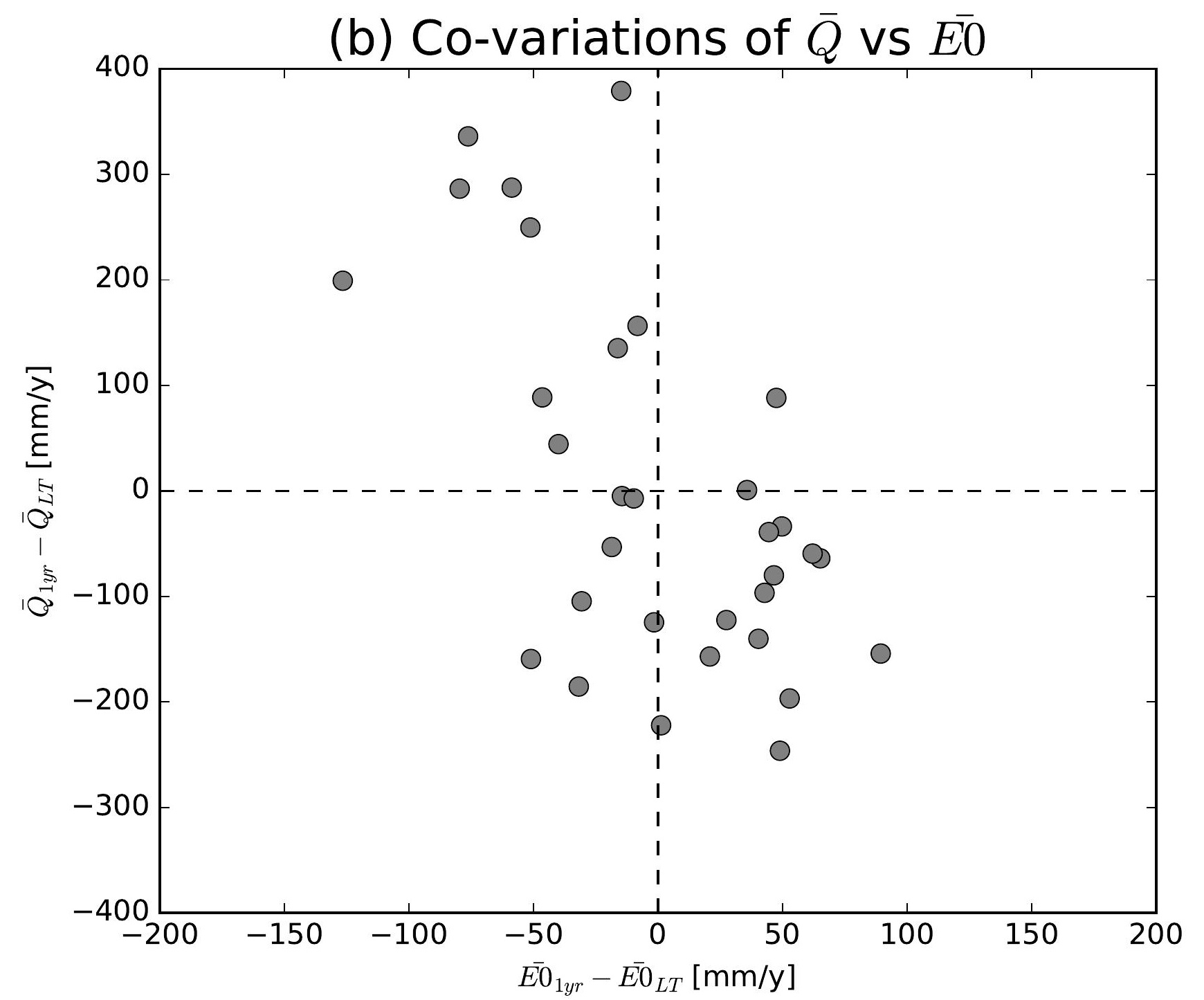

(e) Co-variations of $\bar{Q}$ vs $\overline{E 0}$

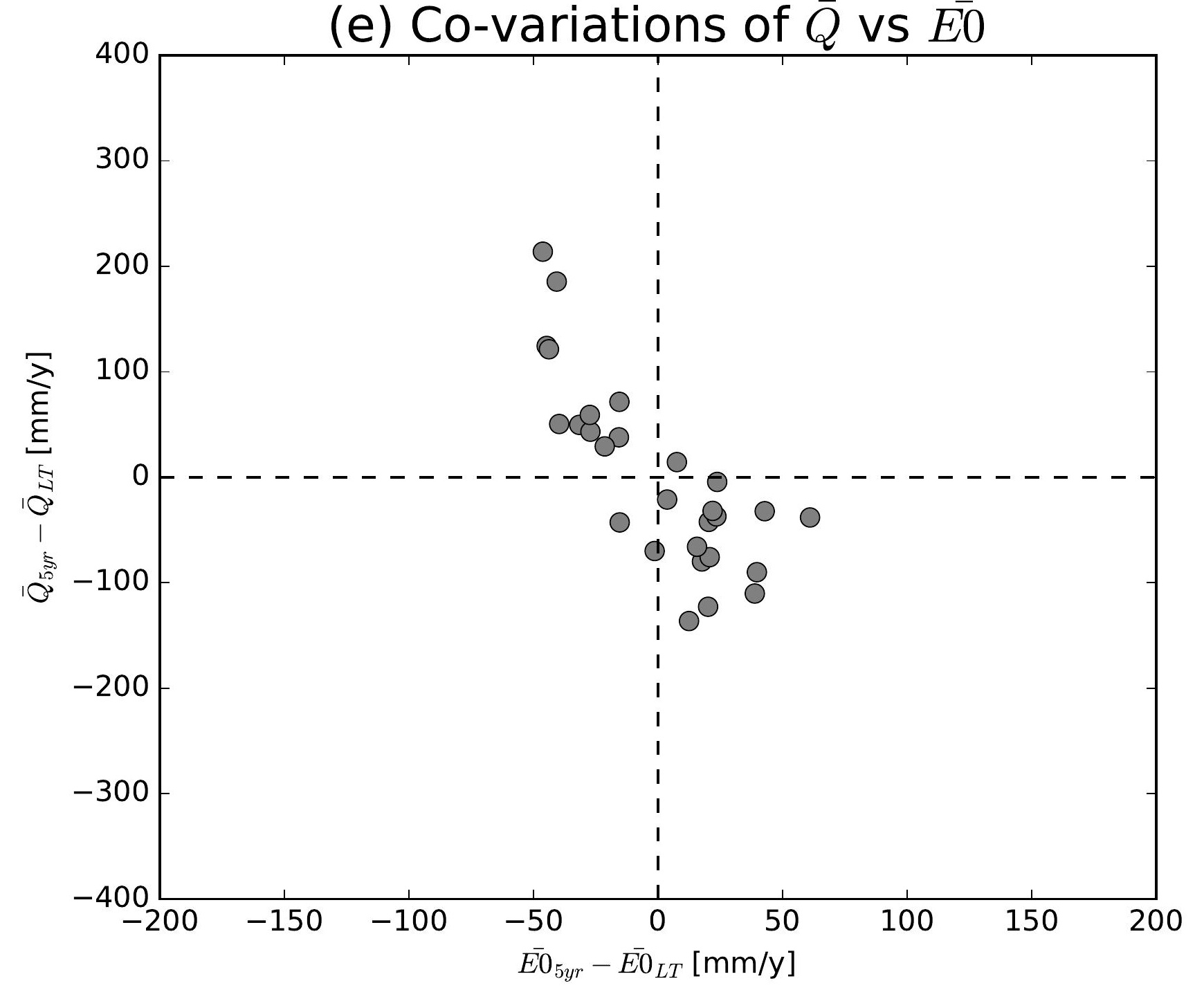

(h) Co-variations of $\bar{Q}$ vs $\overline{E 0}$

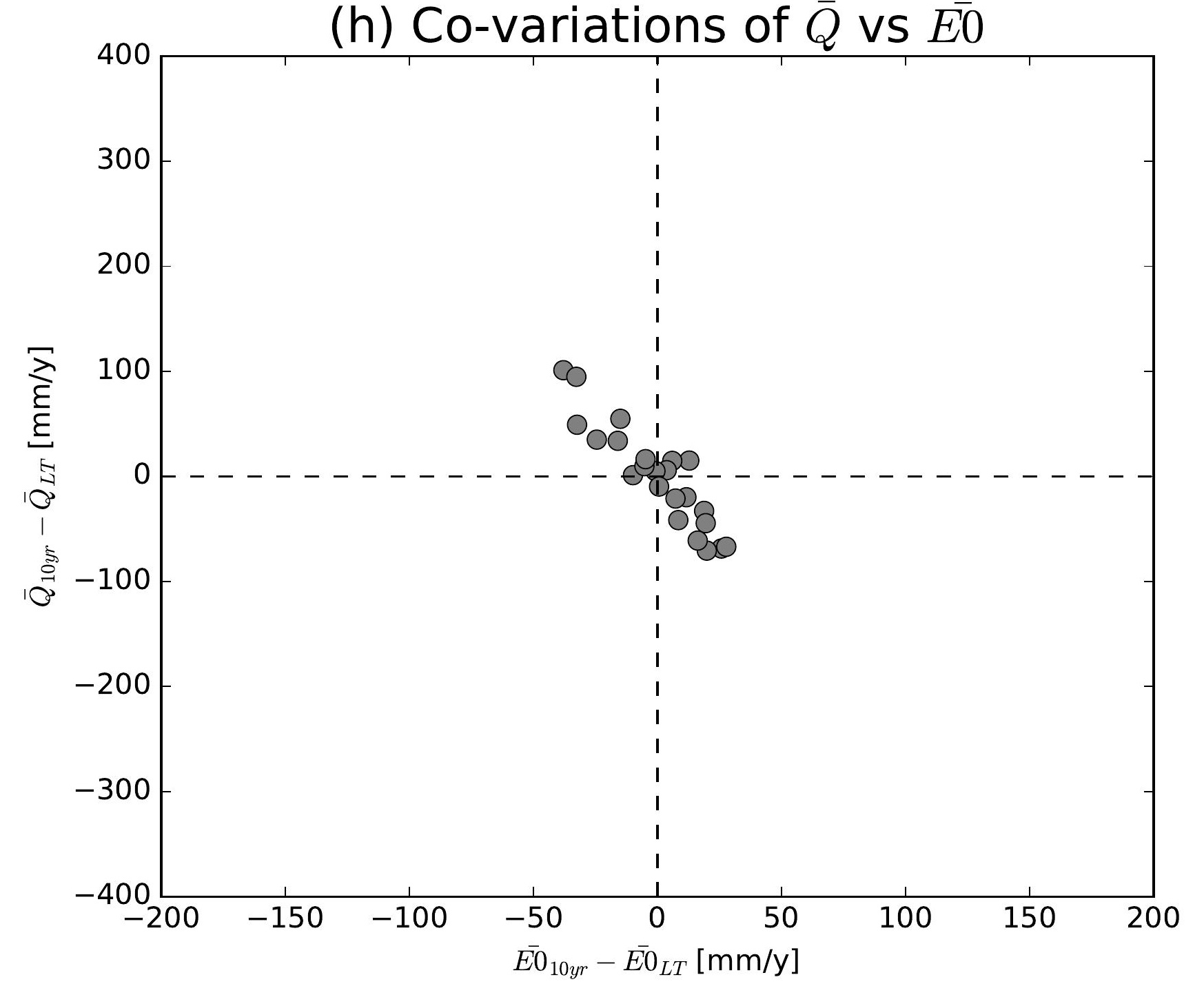

(k) Co-variations of $\bar{Q}$ vs $\overline{E 0}$

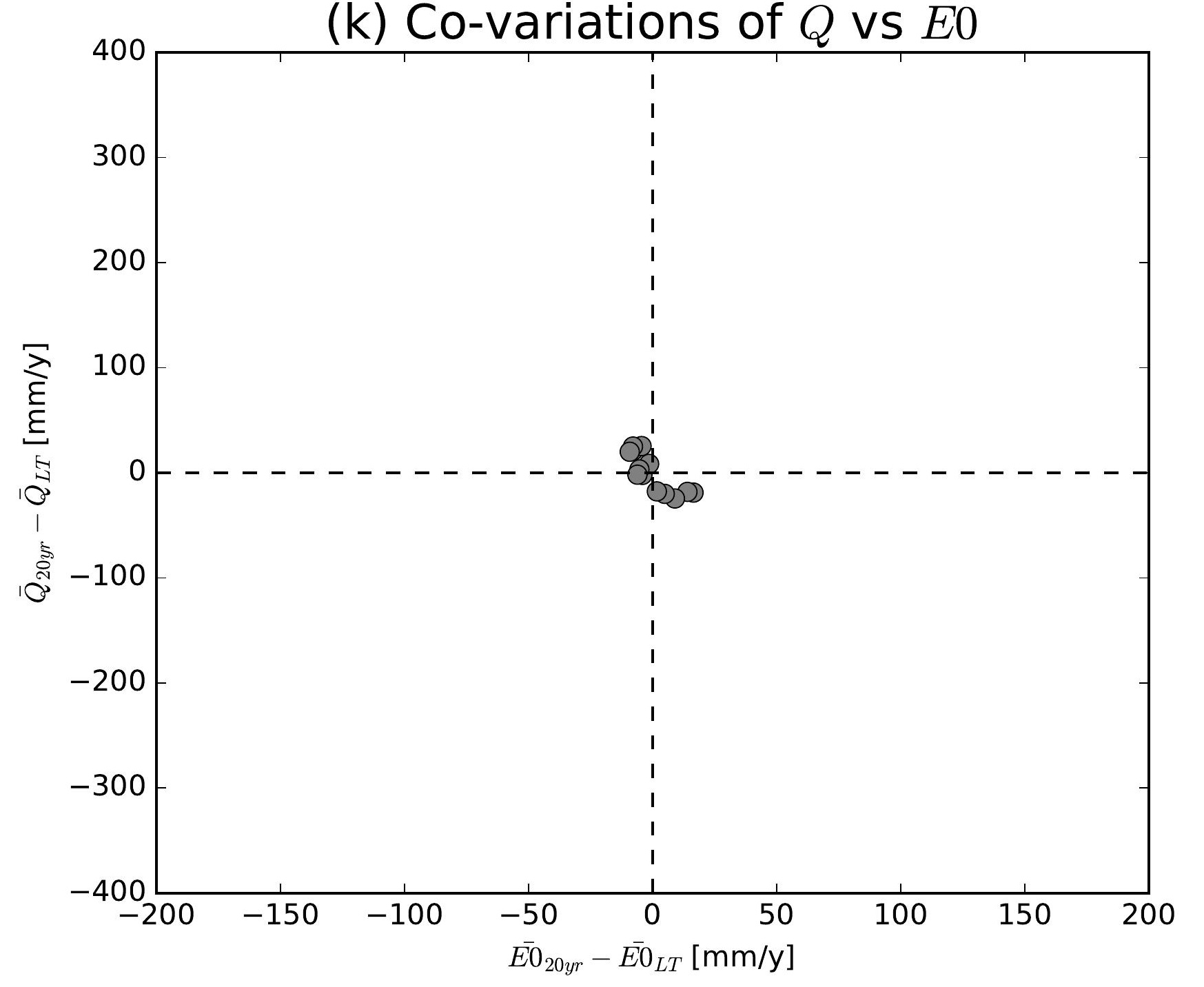

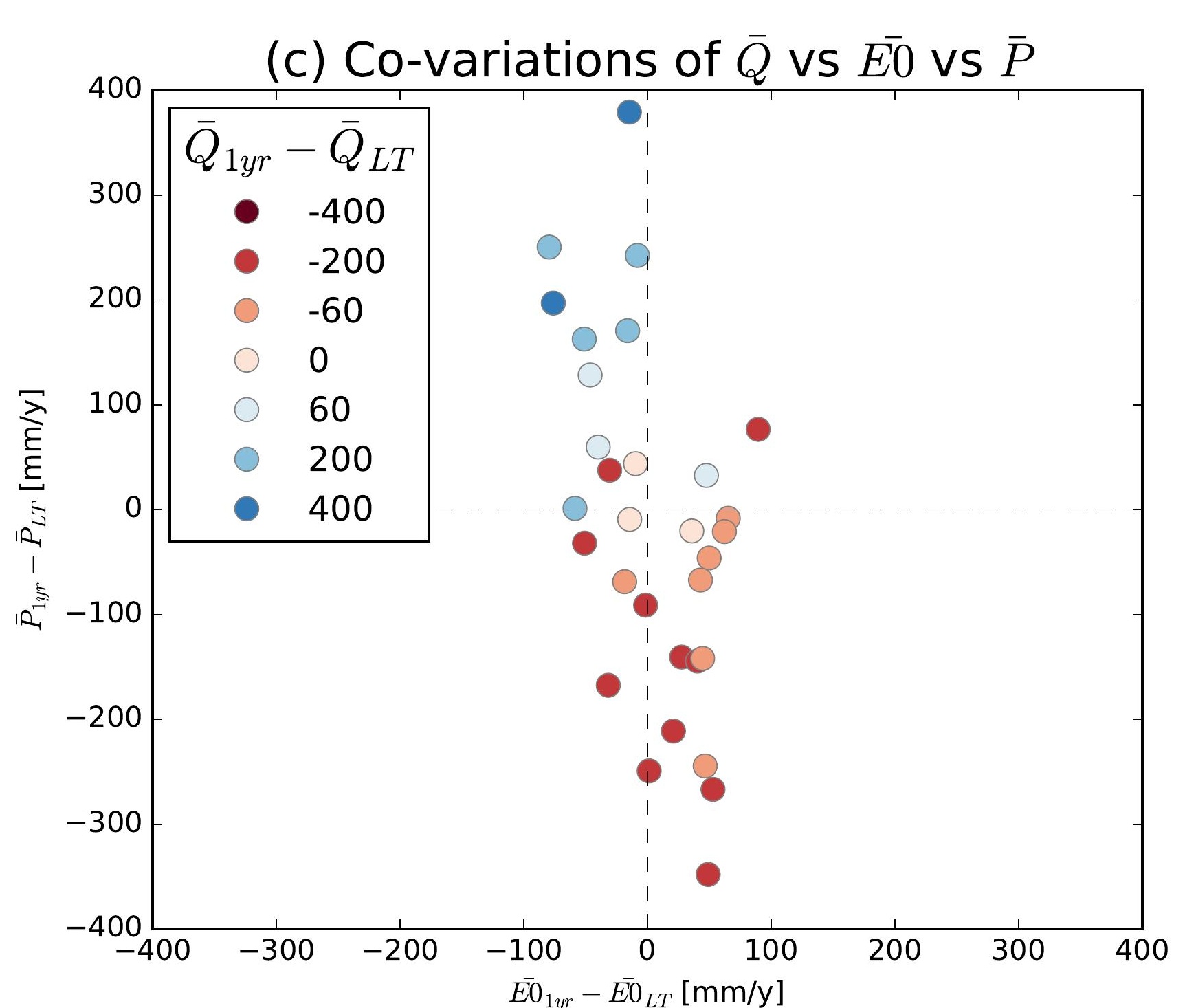
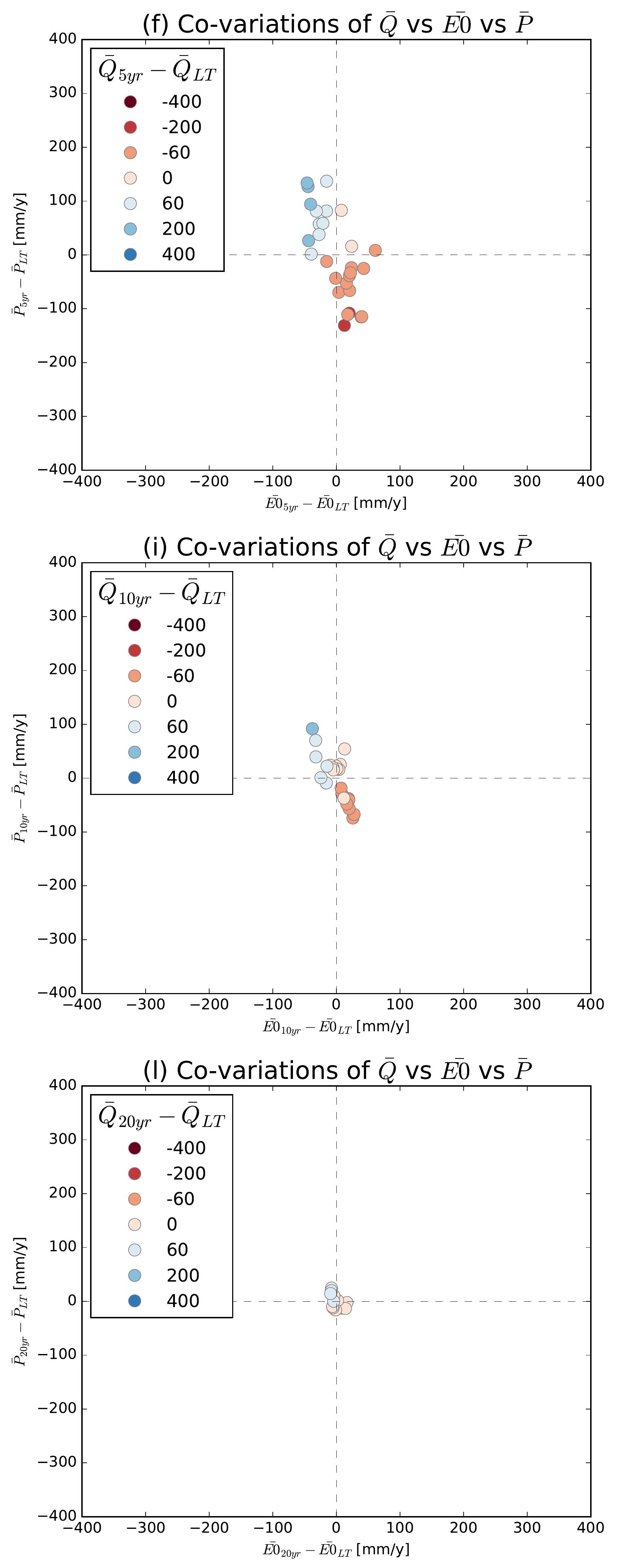
Catchment V7124010

(a) Co-variations of $\bar{Q}$ vs $\bar{P}$

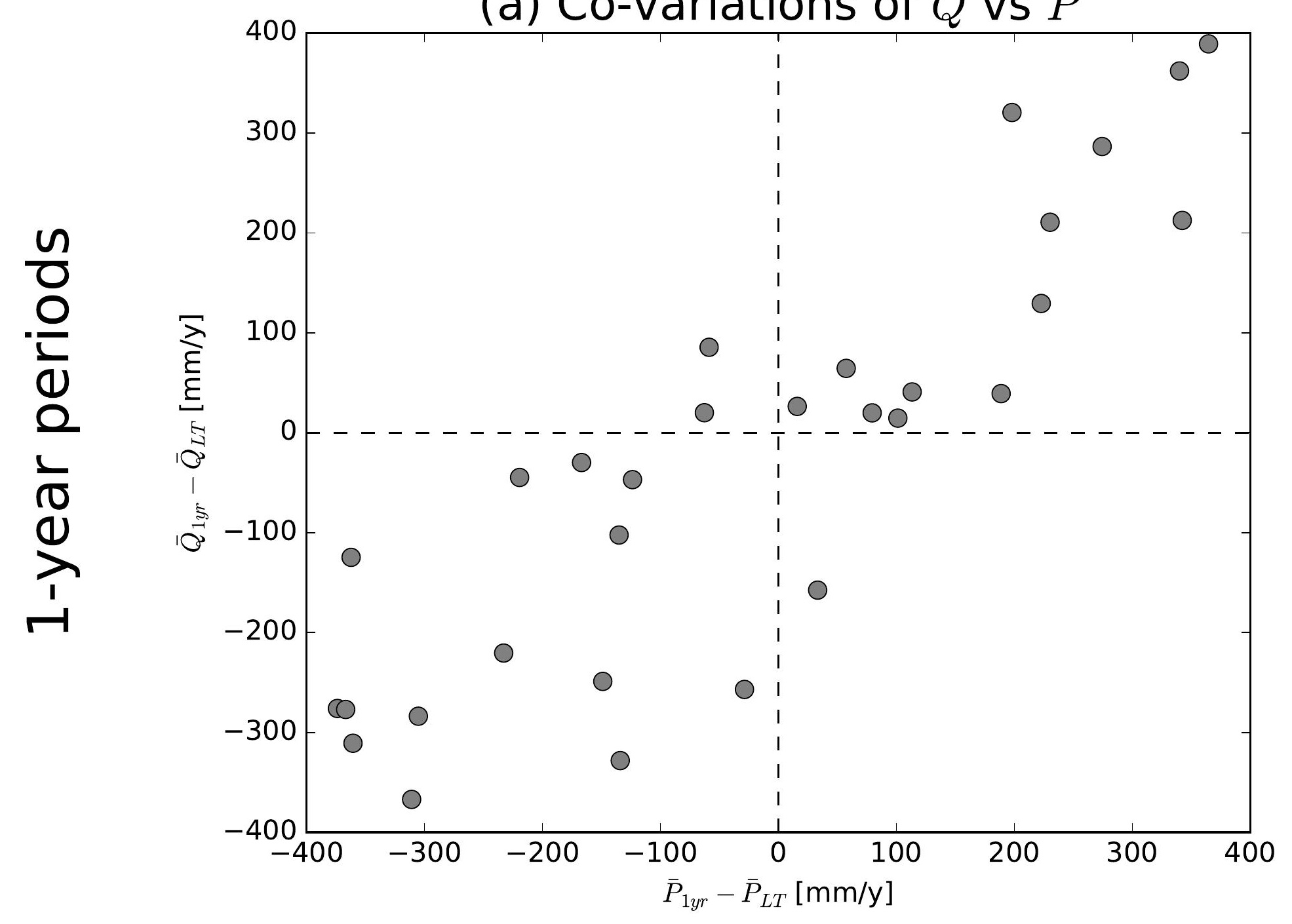

(d) Co-variations of $\bar{Q}$ vs $\bar{P}$

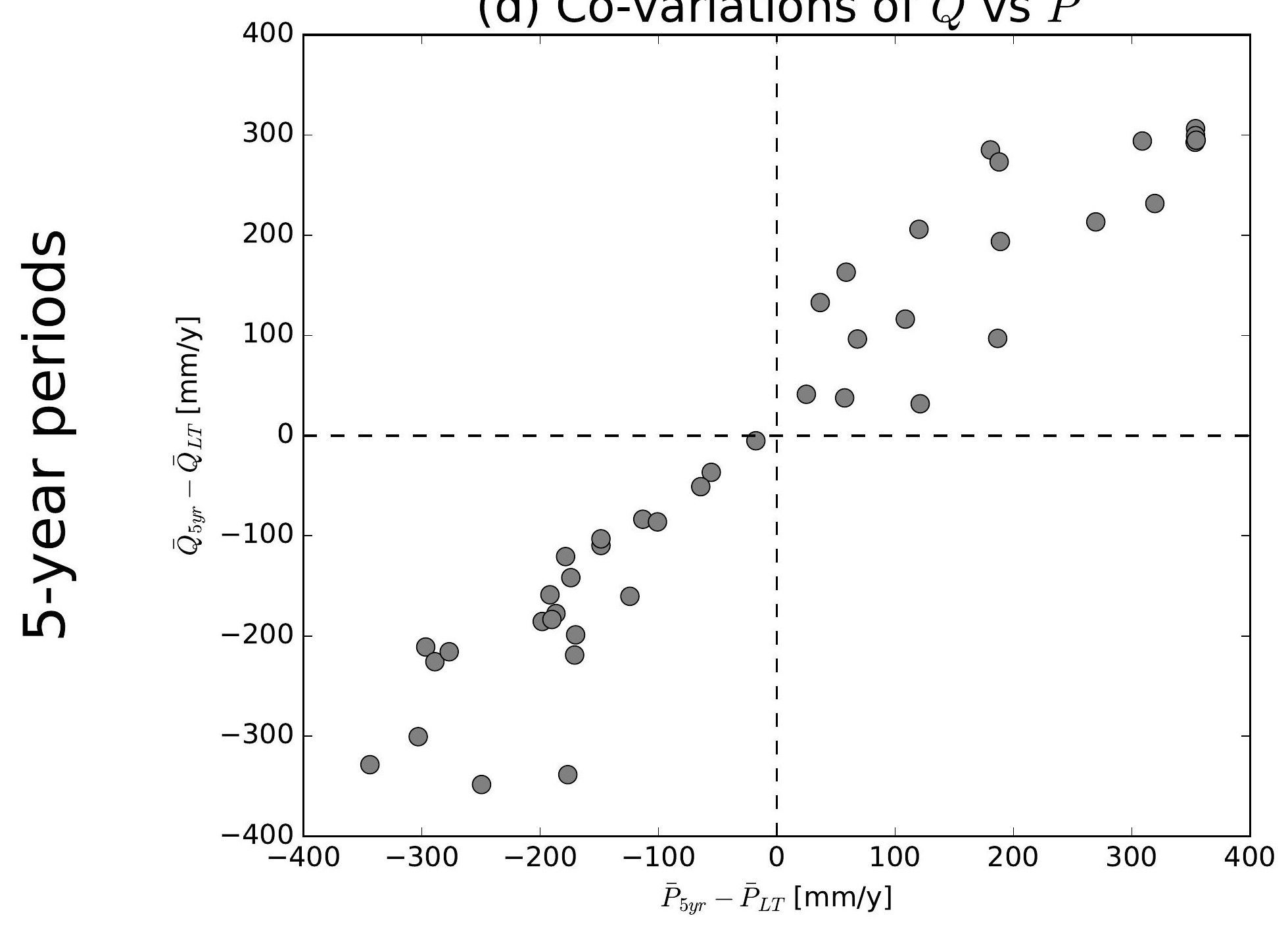

(g) Co-variations of $\bar{Q}$ vs $\bar{P}$

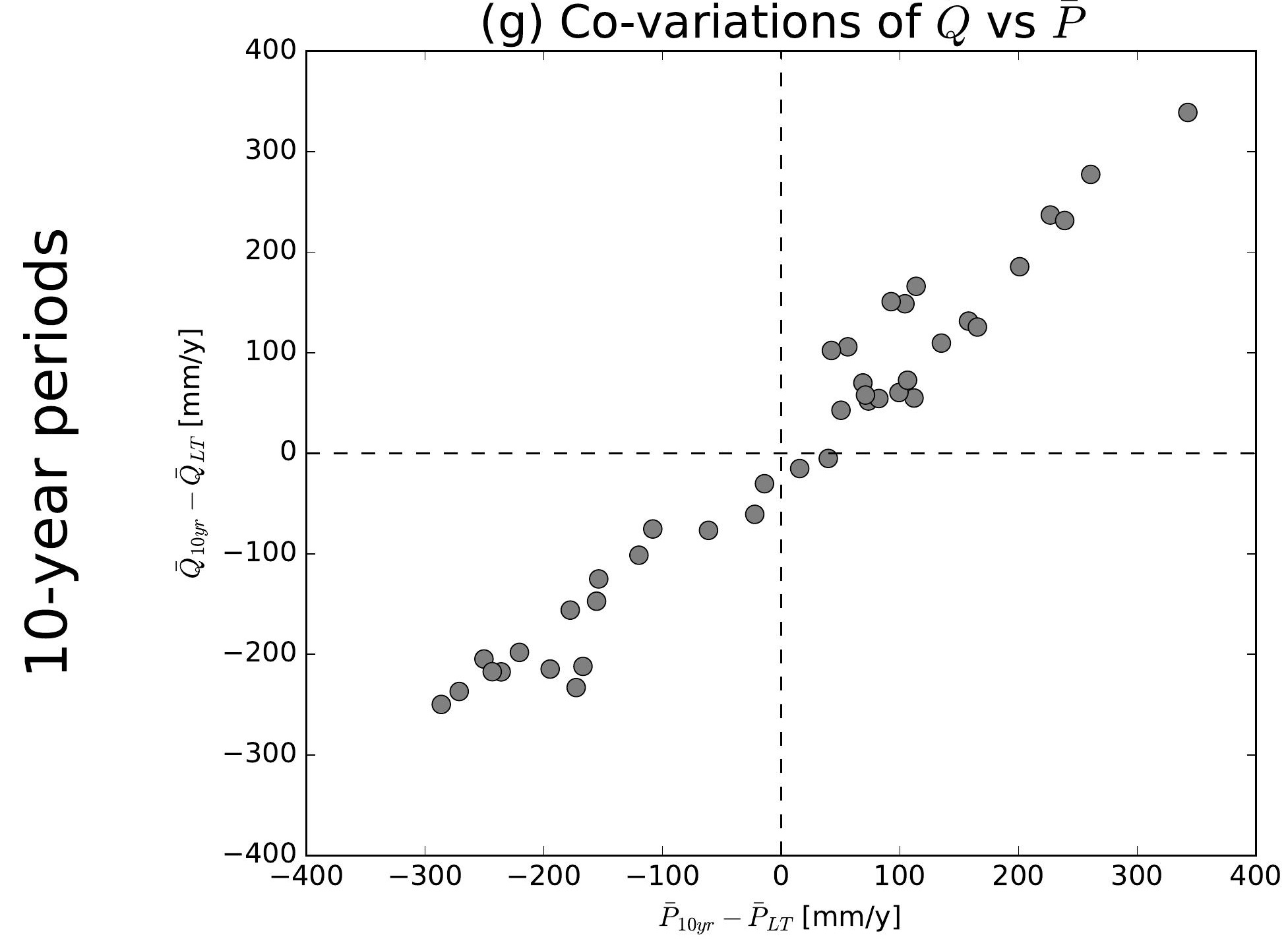

(j) Co-variations of $\bar{Q}$ vs $\bar{P}$

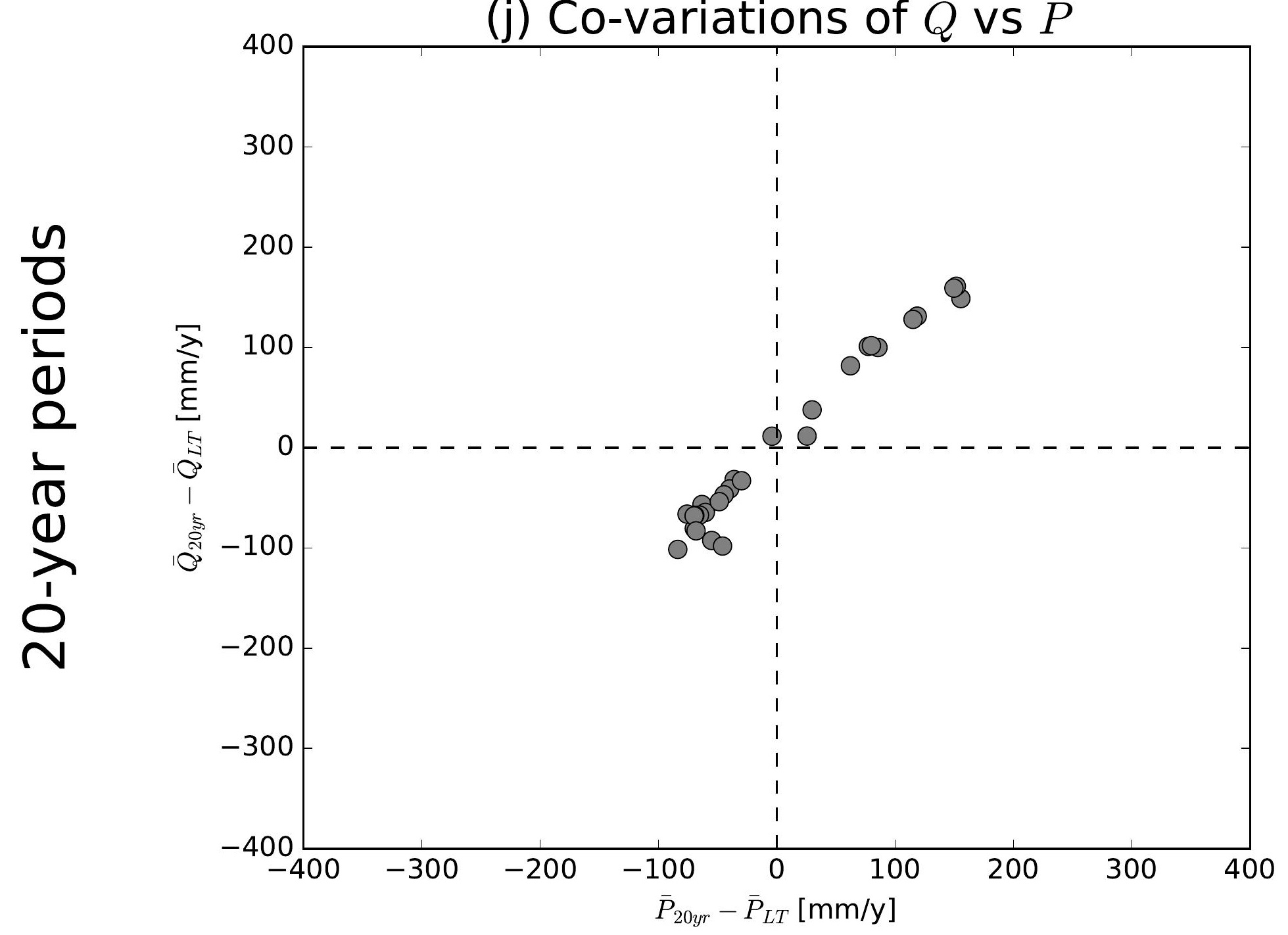

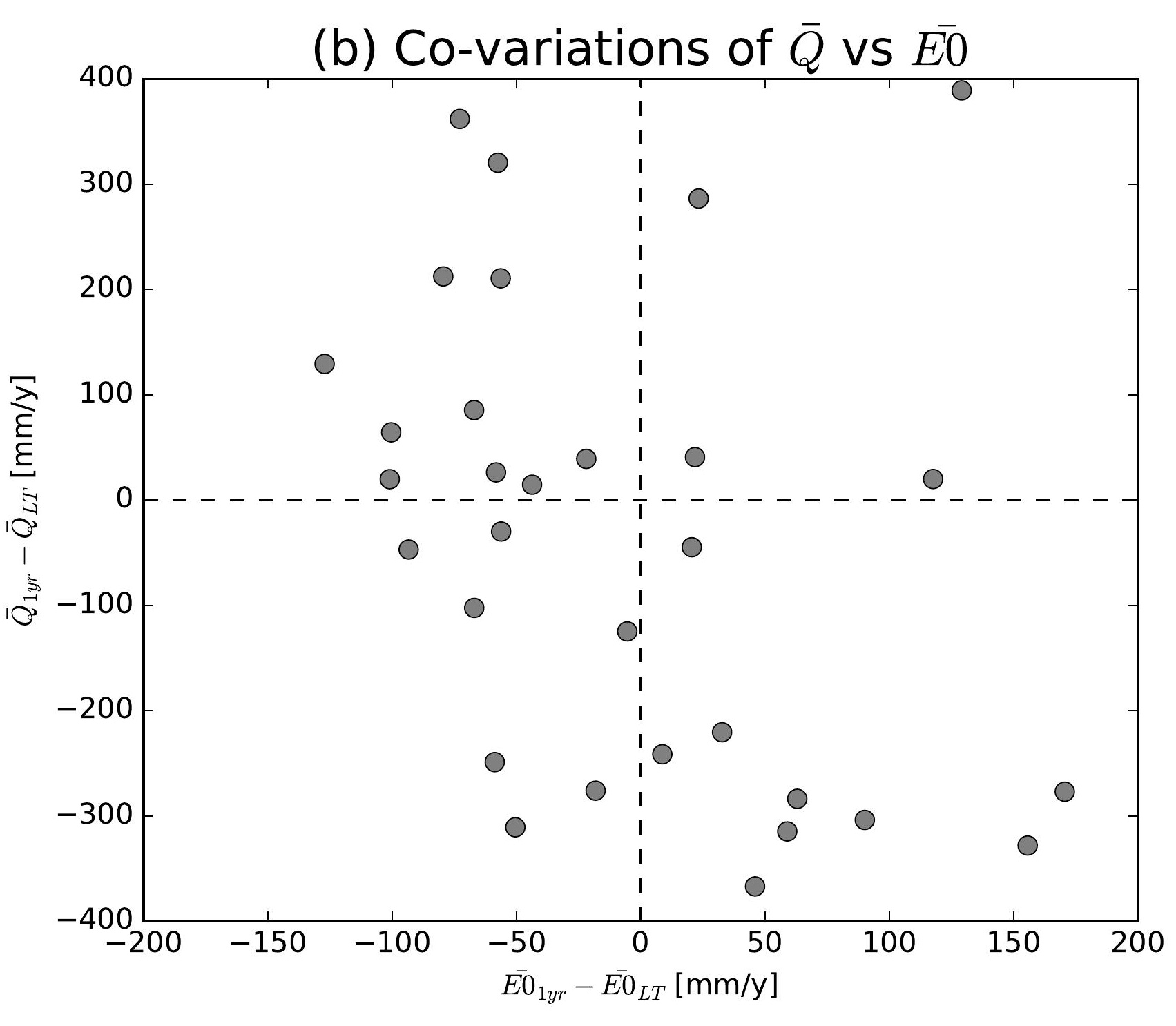
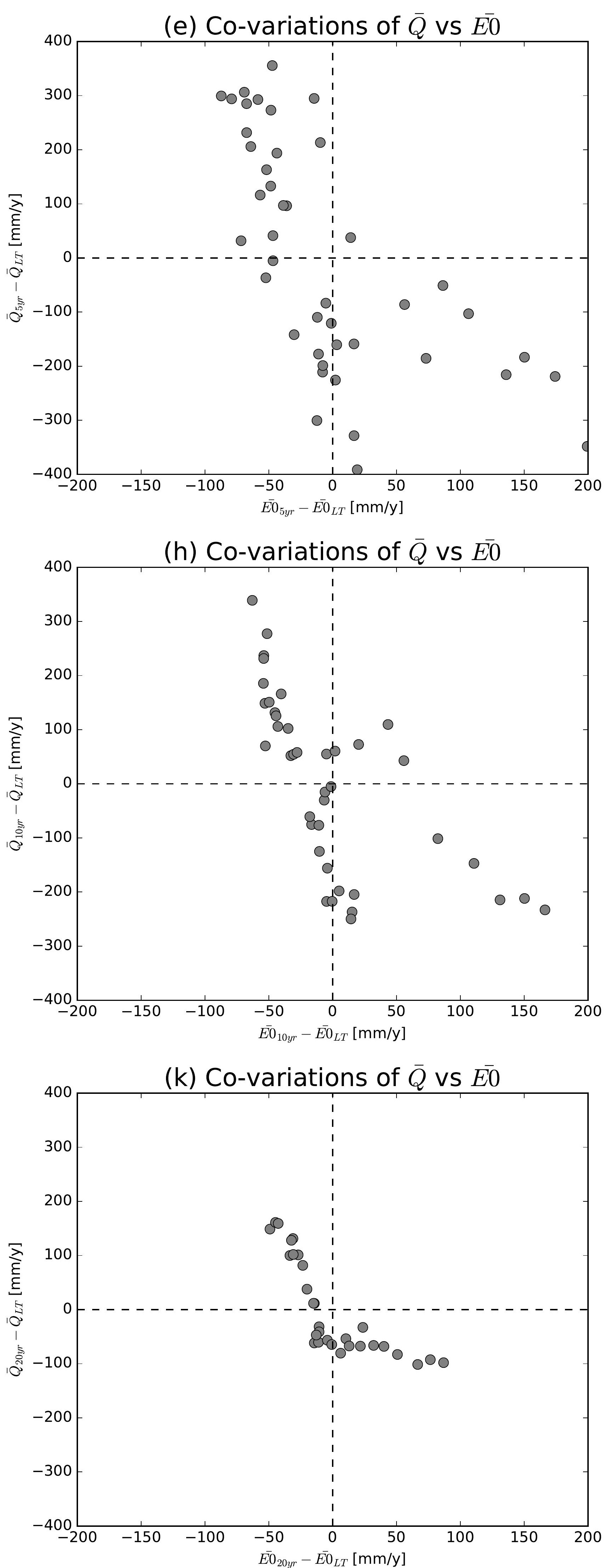
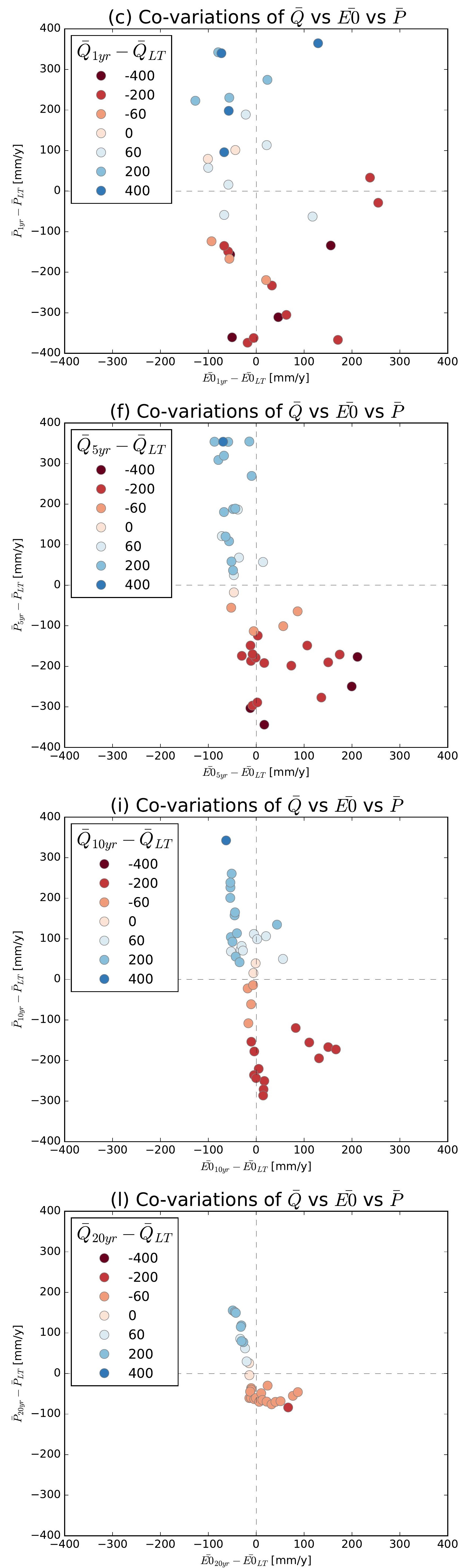

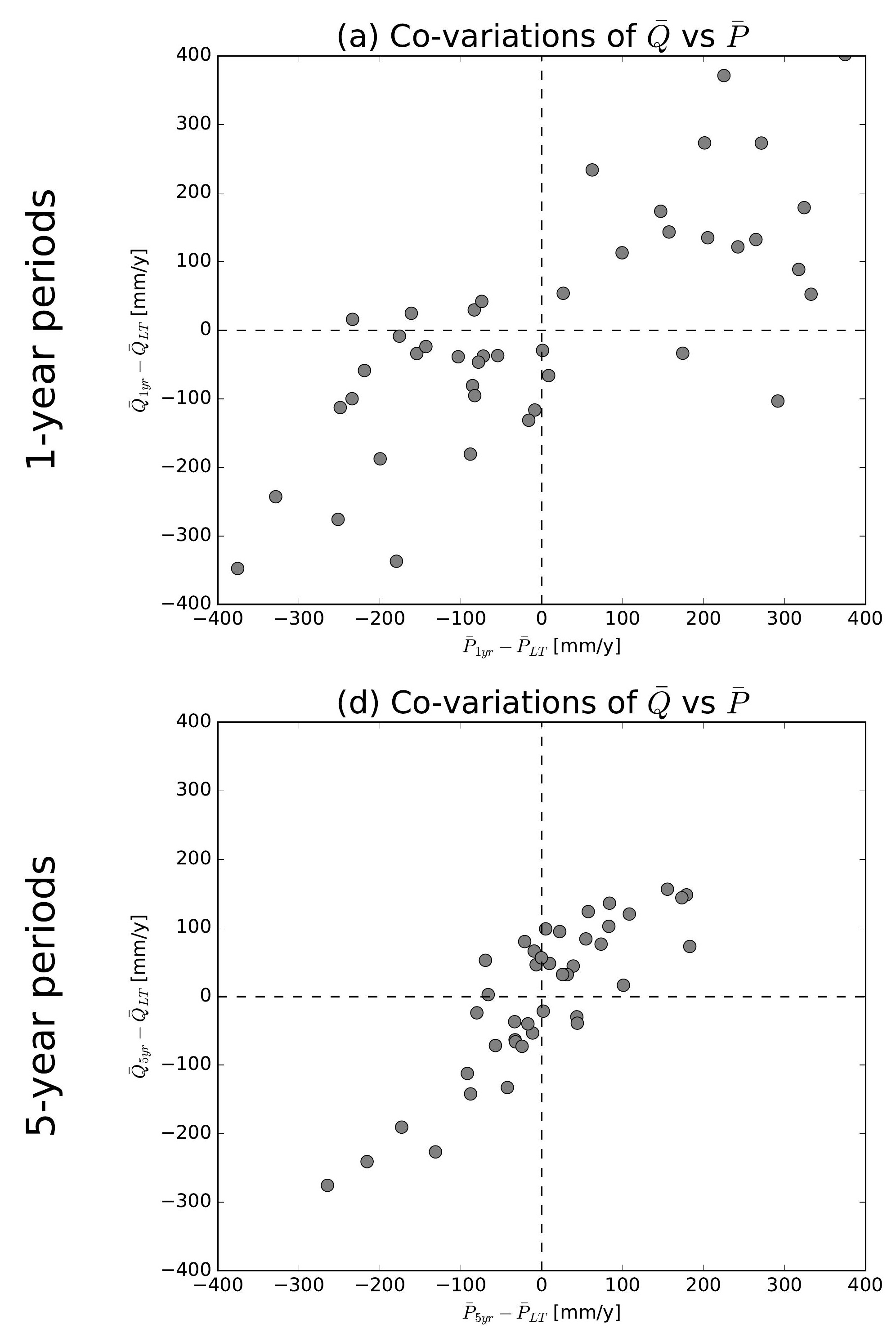

(g) Co-variations of $\bar{Q}$ vs $\bar{P}$

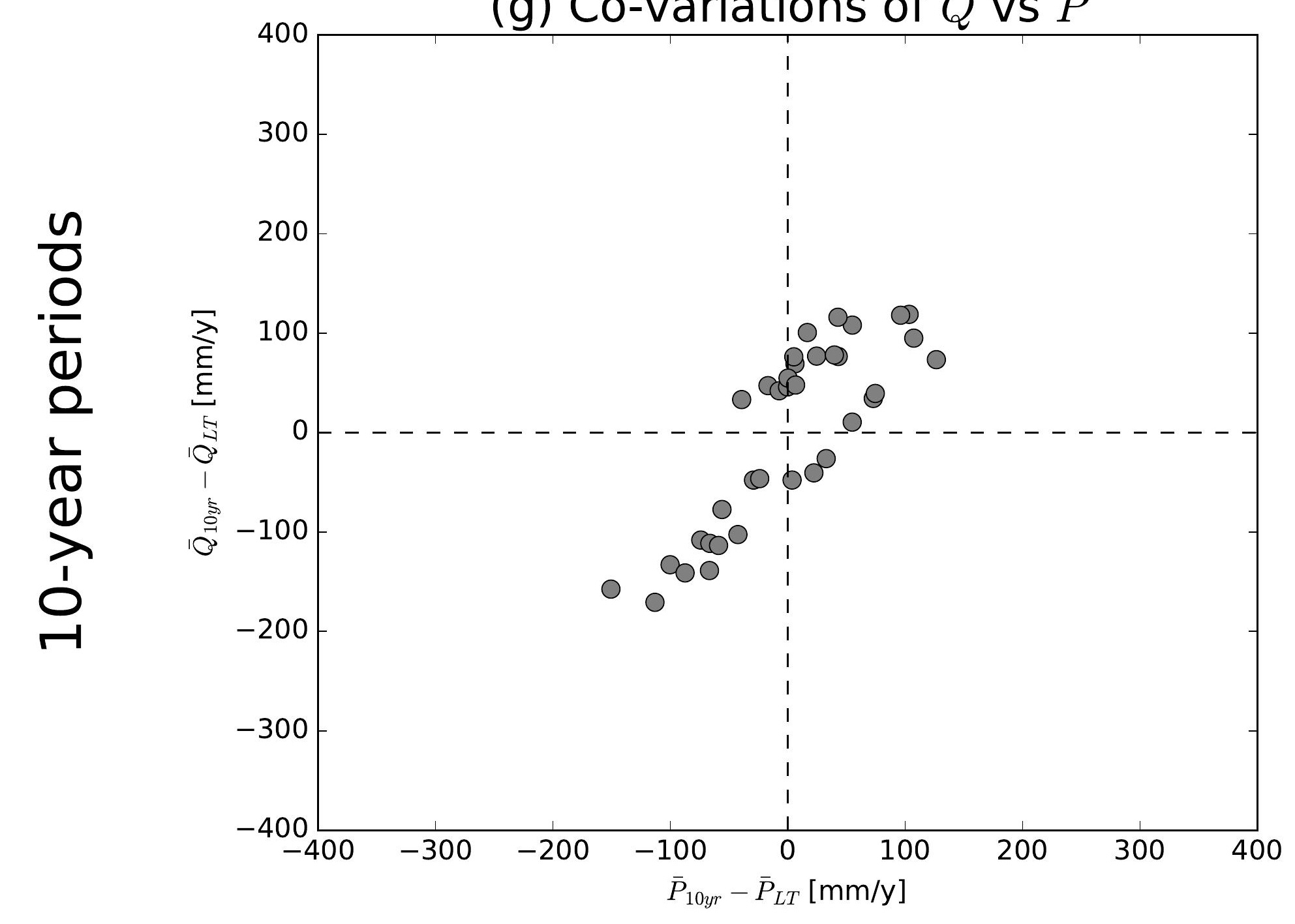

(j) Co-variations of $\bar{Q}$ vs $\bar{P}$

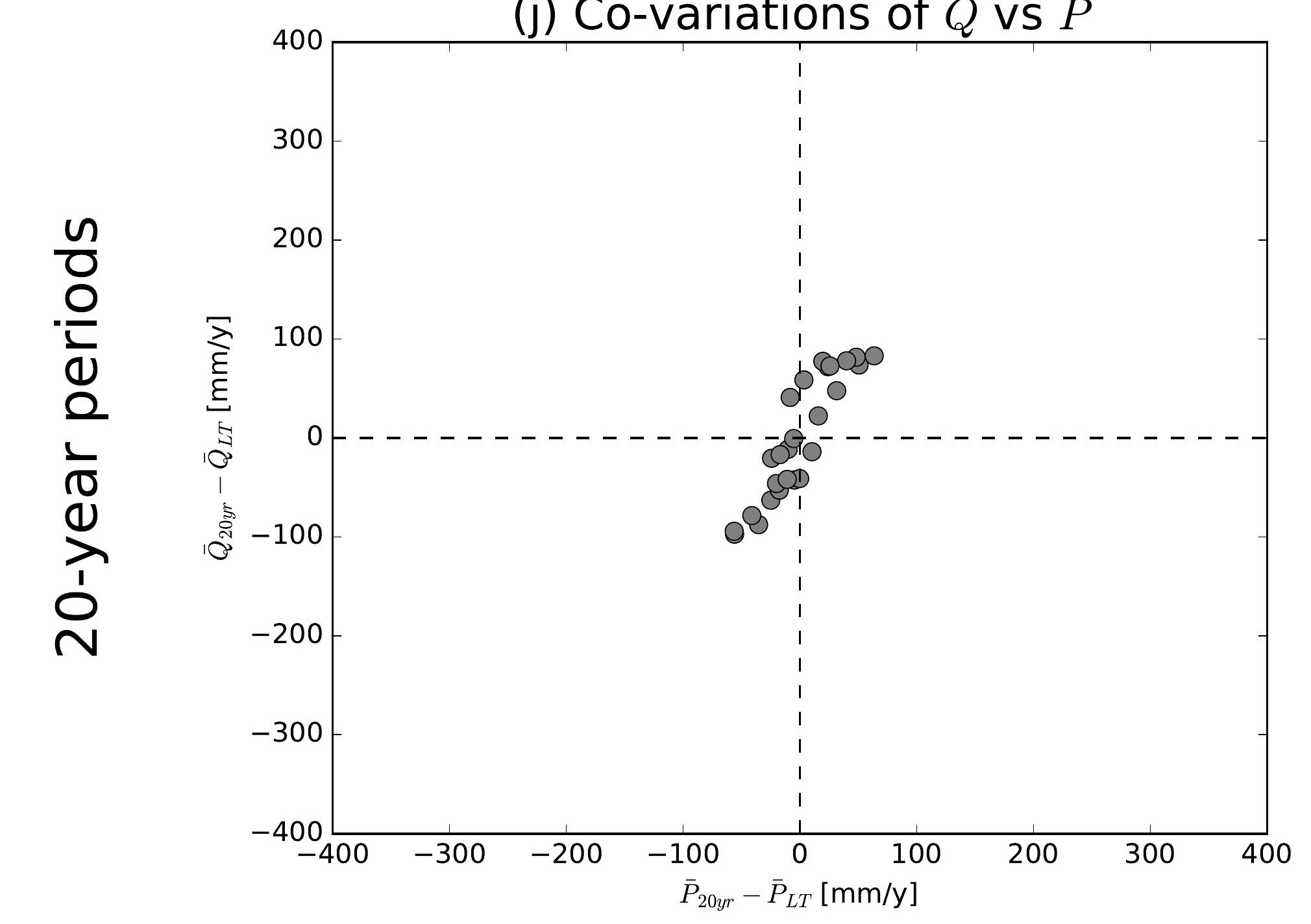

(b) Co-variations of $\bar{Q}$ vs $\overline{E 0}$

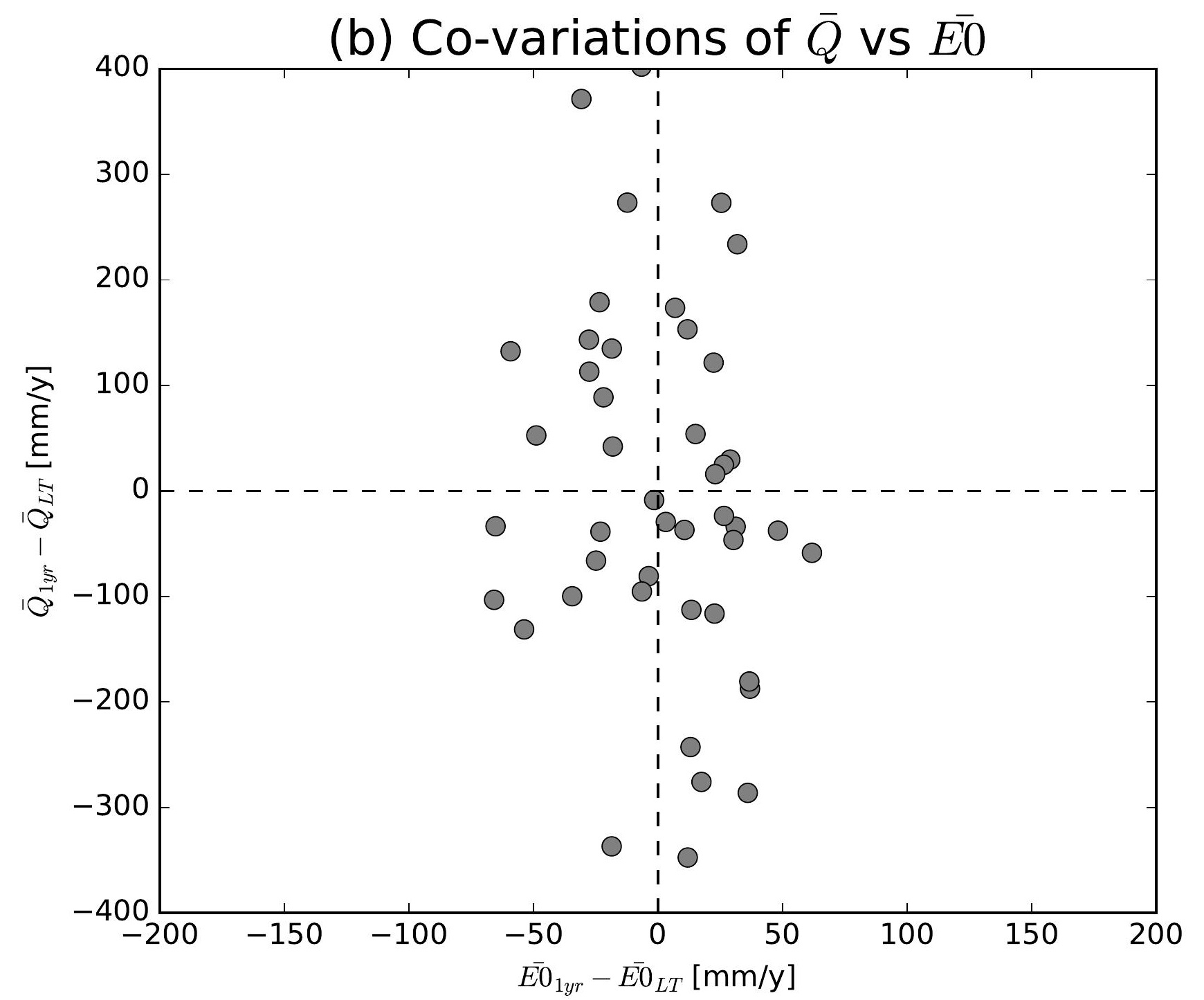

(e) Co-variations of $\bar{Q}$ vs $\overline{E 0}$

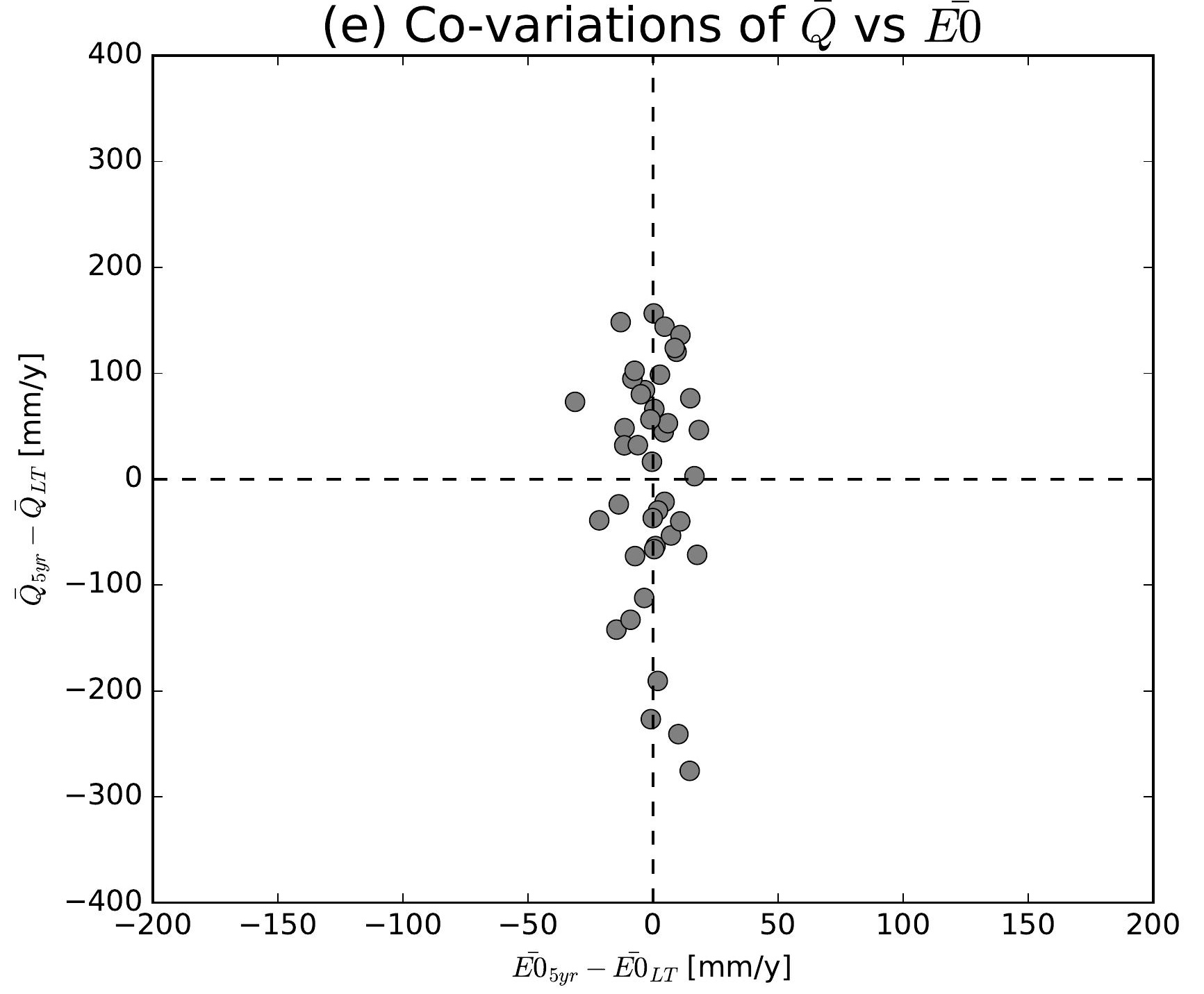

(h) Co-variations of $\bar{Q}$ vs $\overline{E 0}$

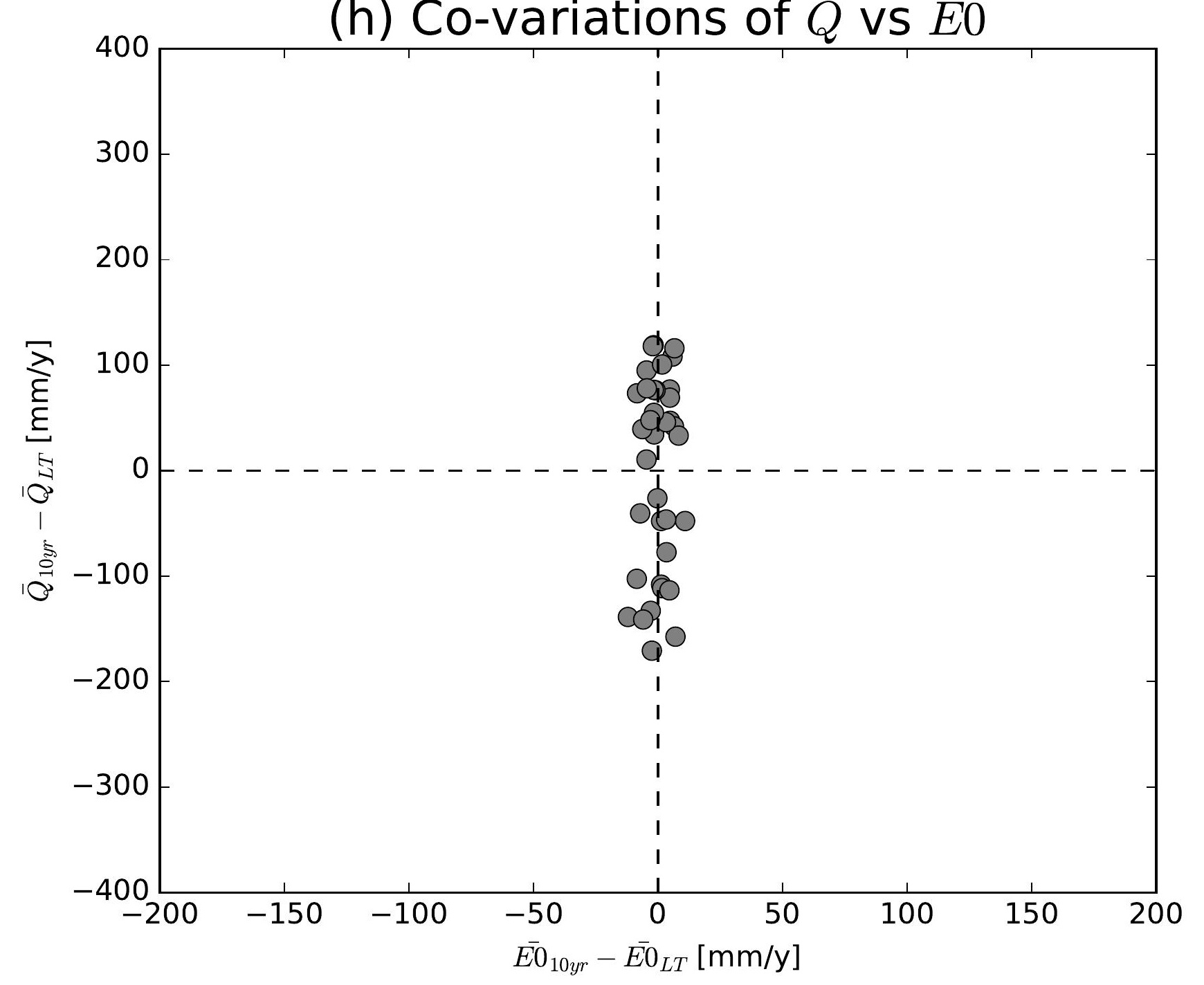

(k) Co-variations of $\bar{Q}$ vs $\overline{E 0}$

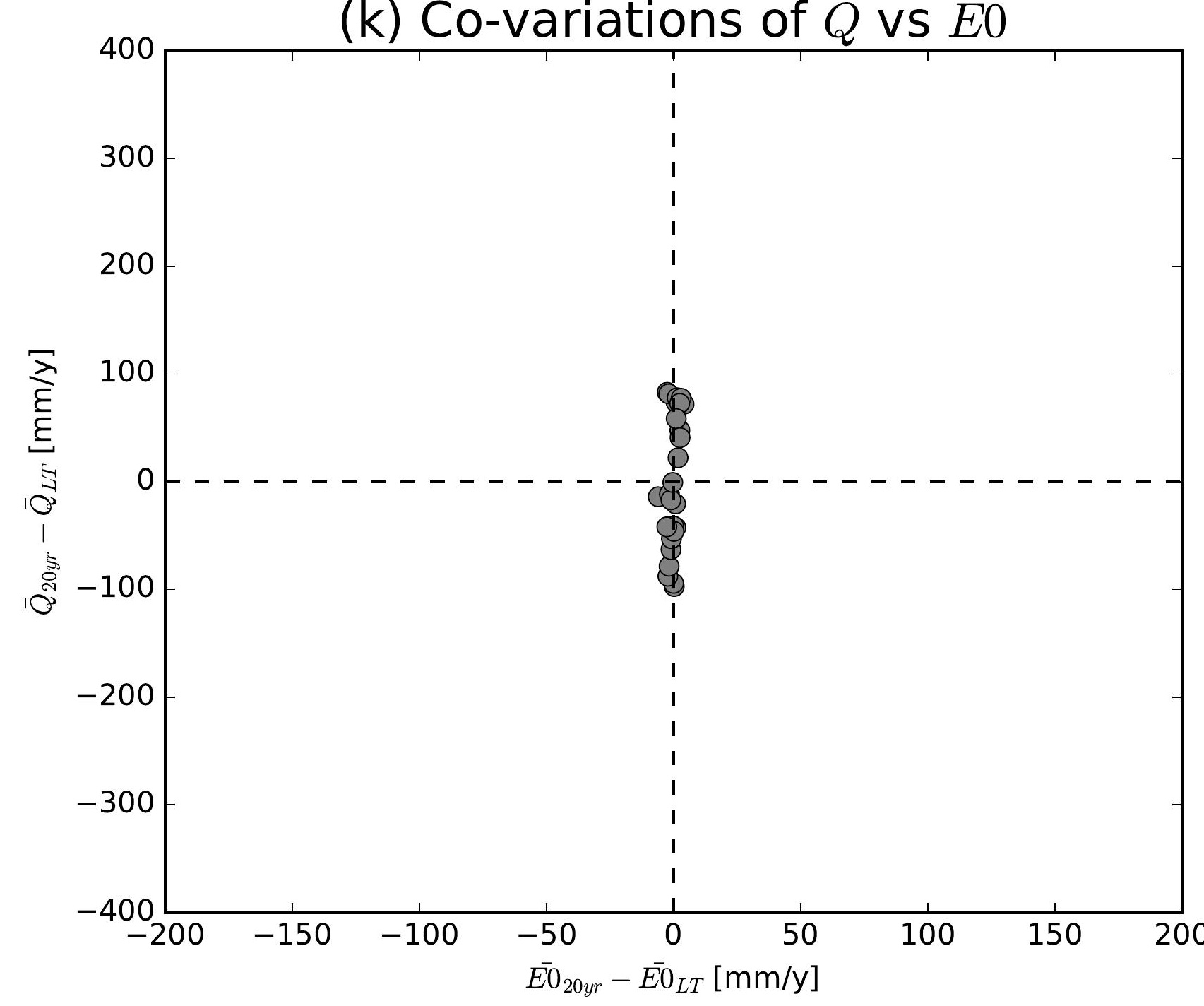

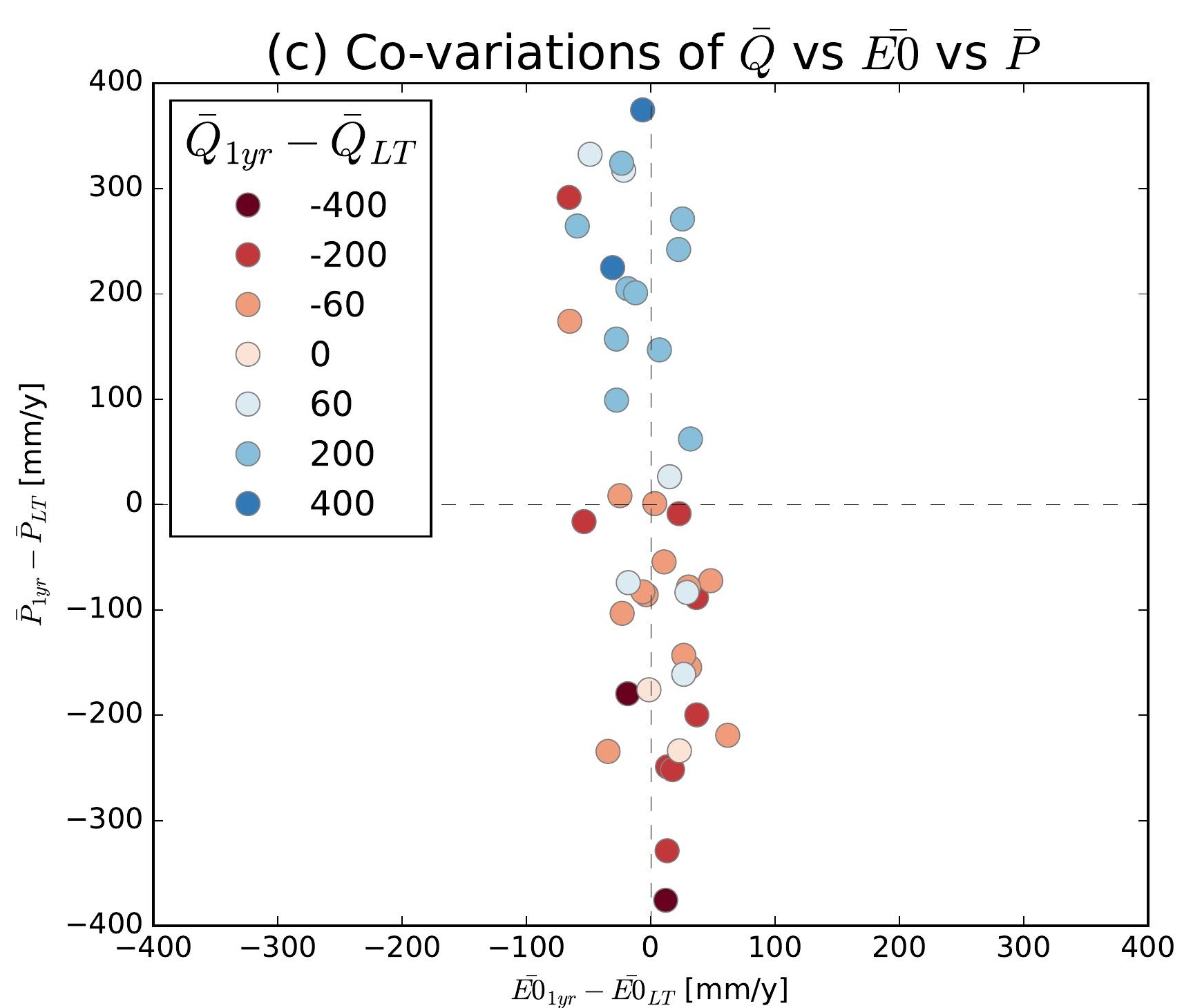
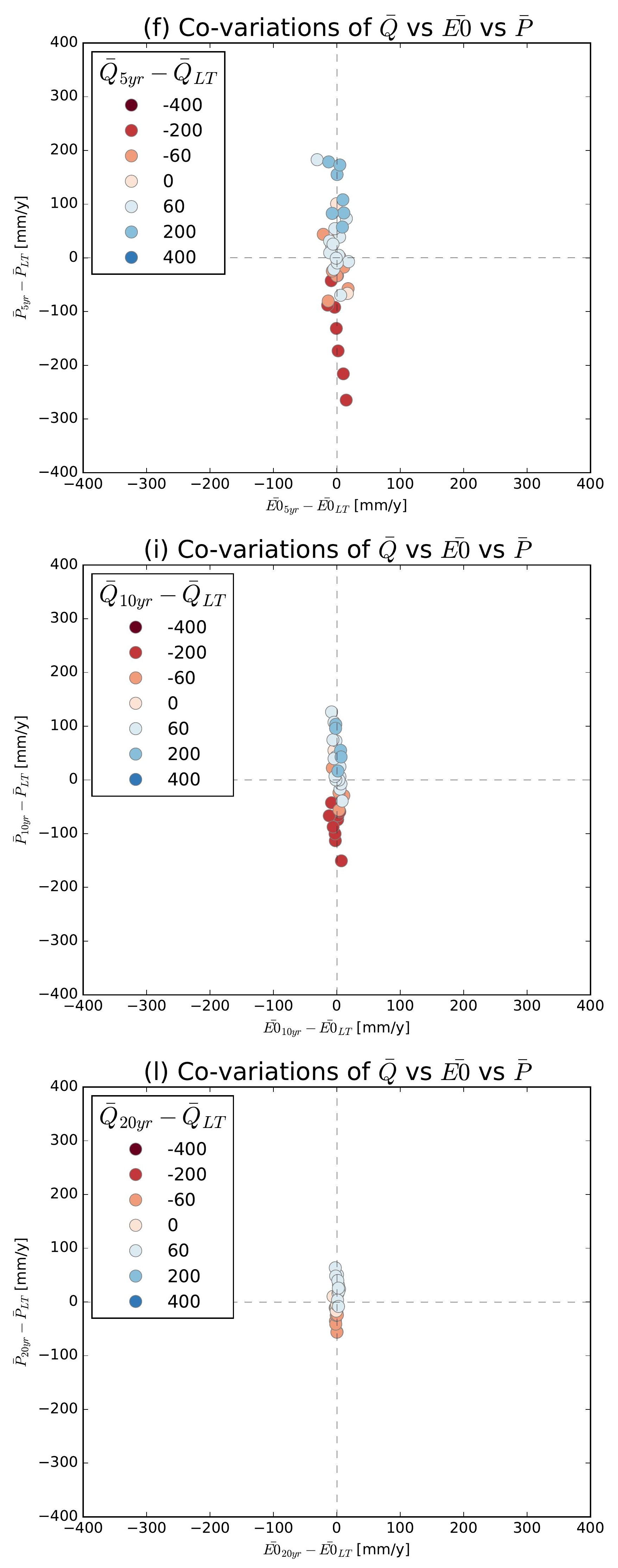

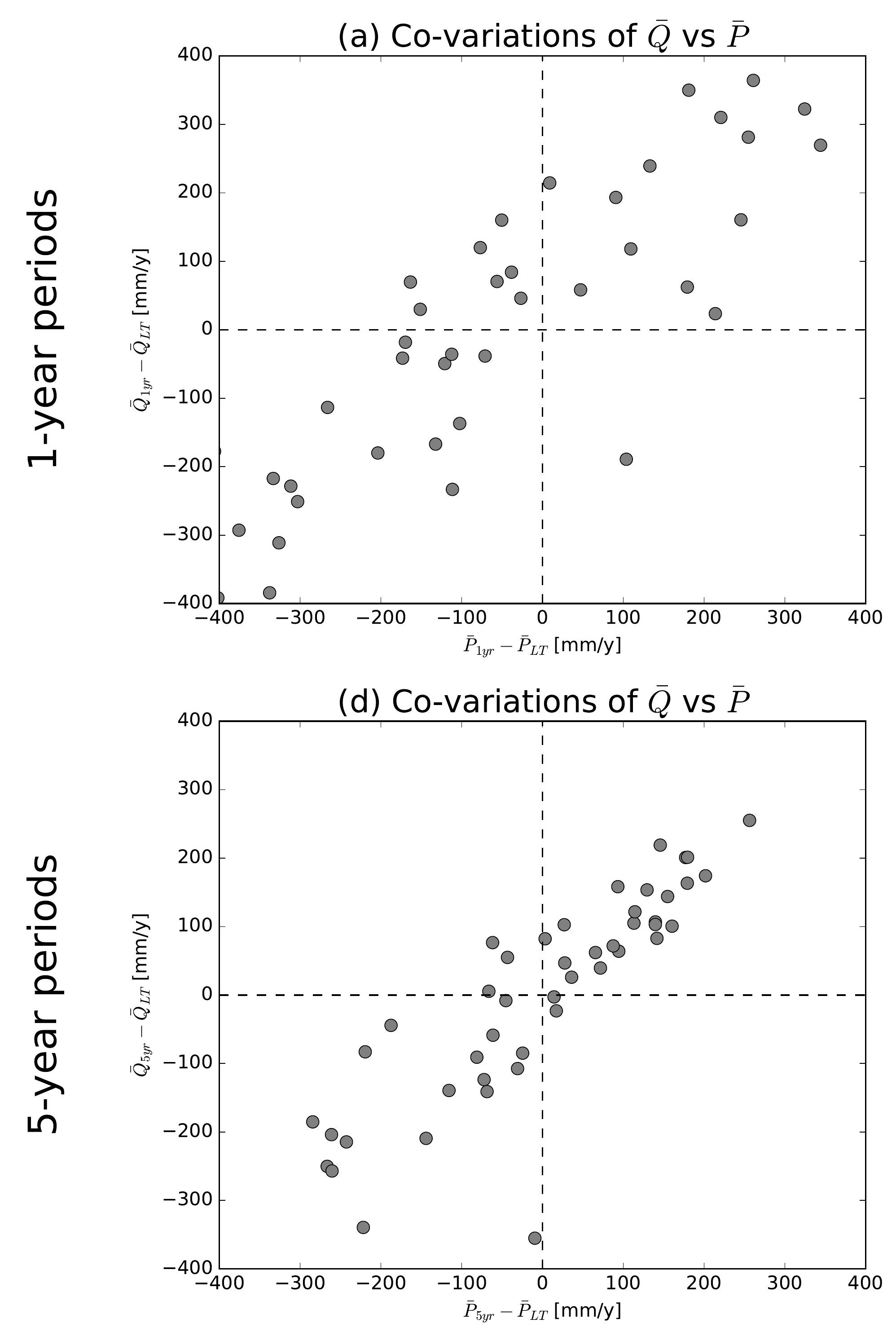

(g) Co-variations of $\bar{Q}$ vs $\bar{P}$

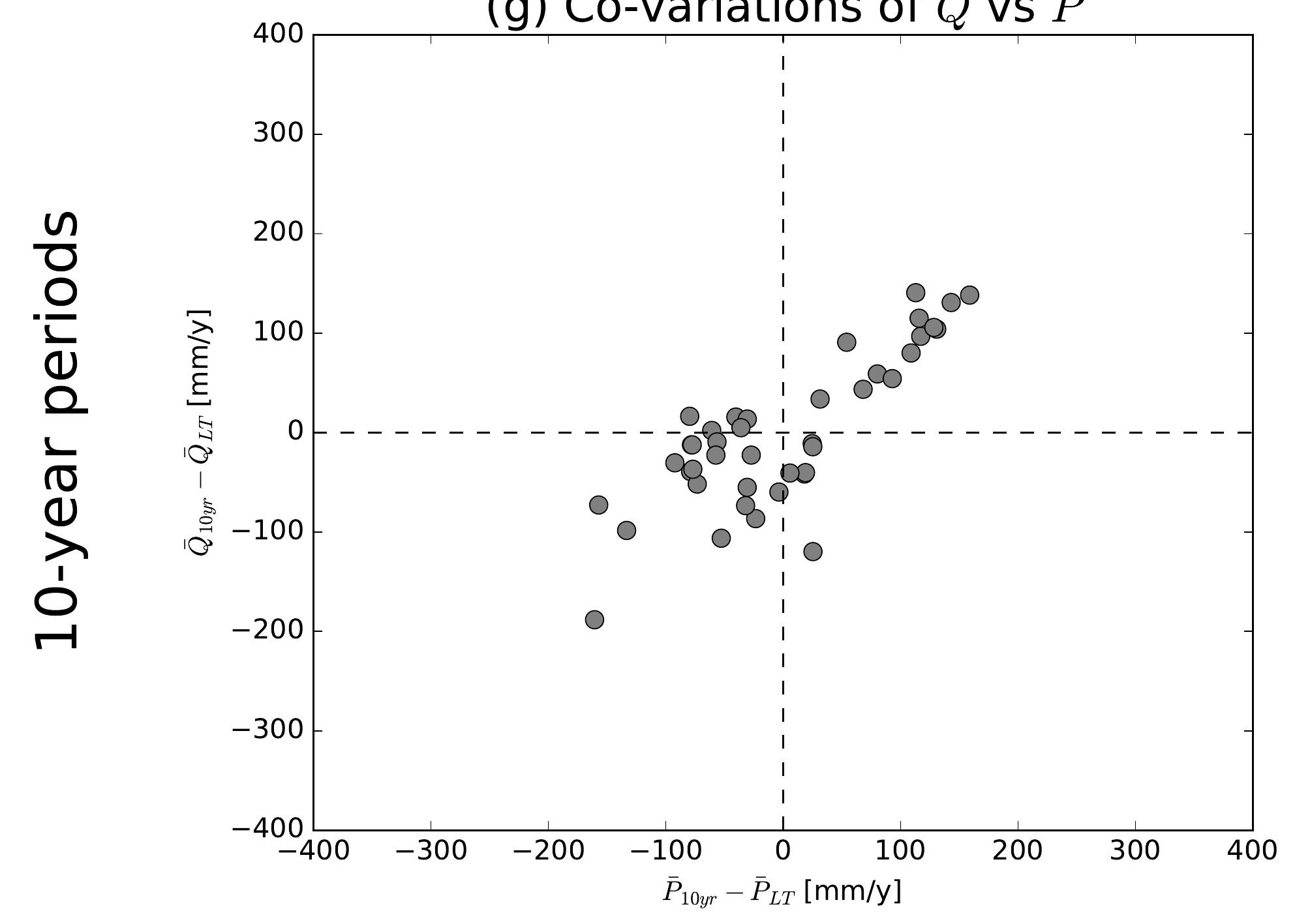

(j) Co-variations of $\bar{Q}$ vs $\bar{P}$

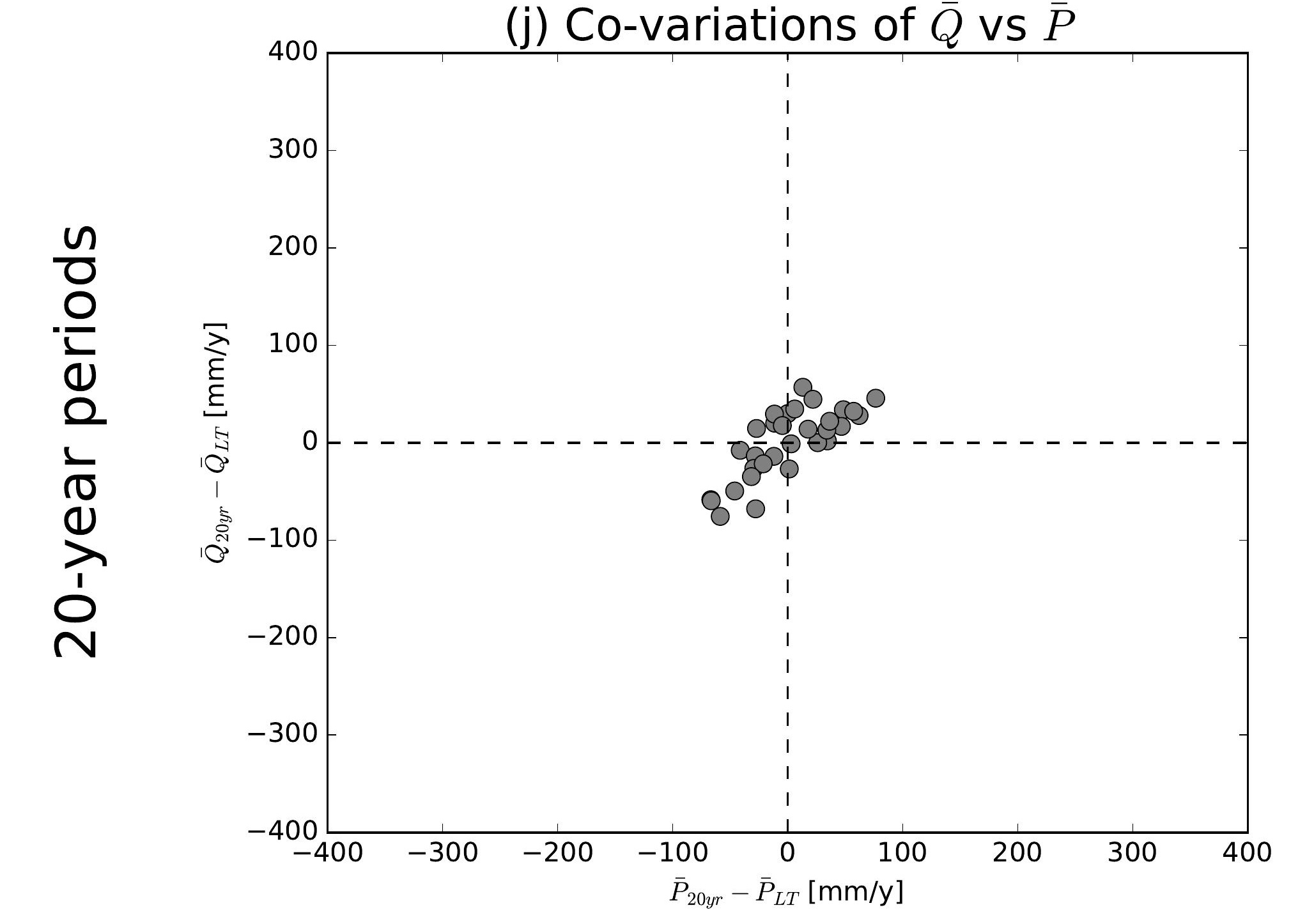

(b) Co-variations of $\bar{Q}$ vs $\overline{E 0}$

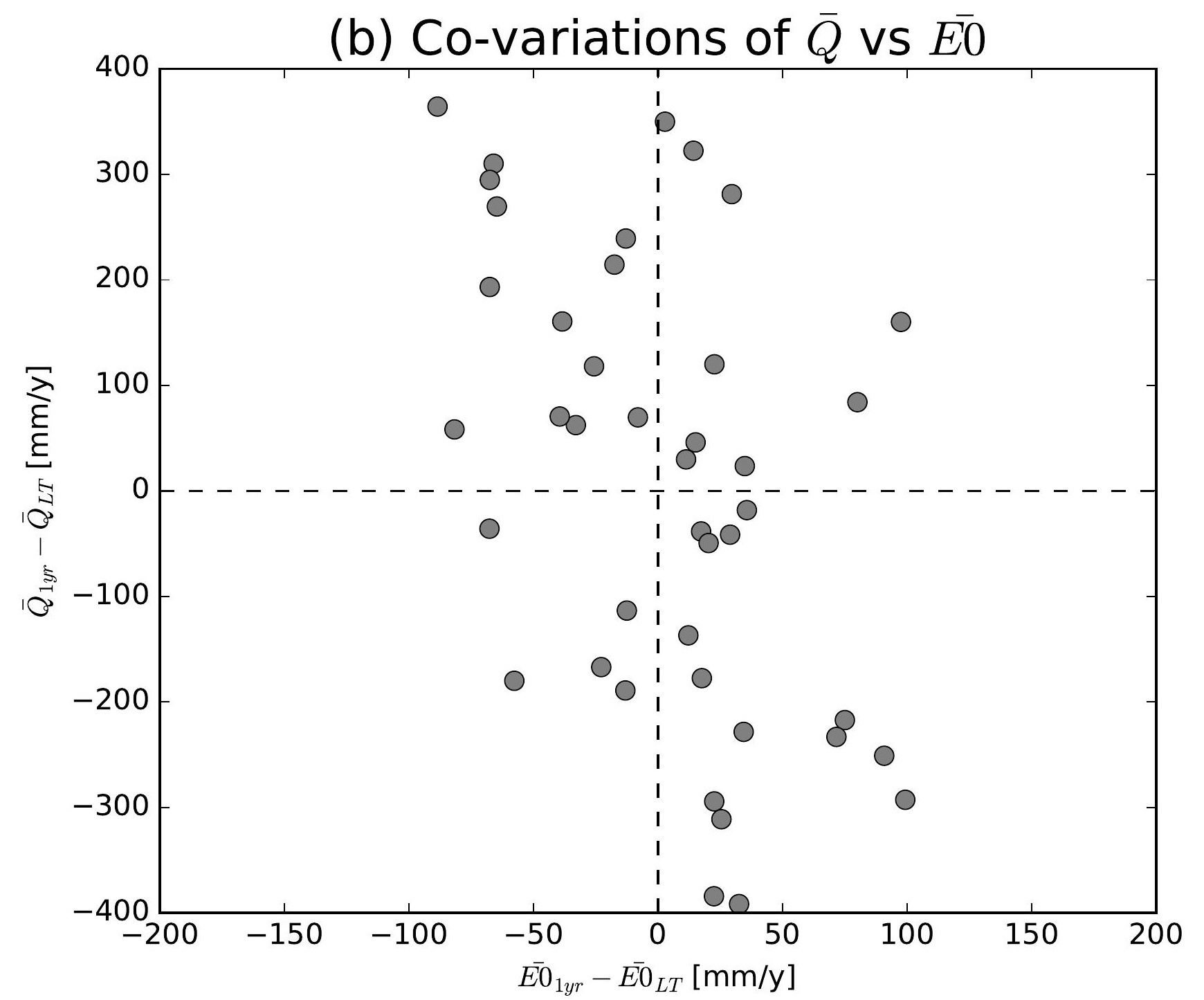

(e) Co-variations of $\bar{Q}$ vs $\overline{E 0}$

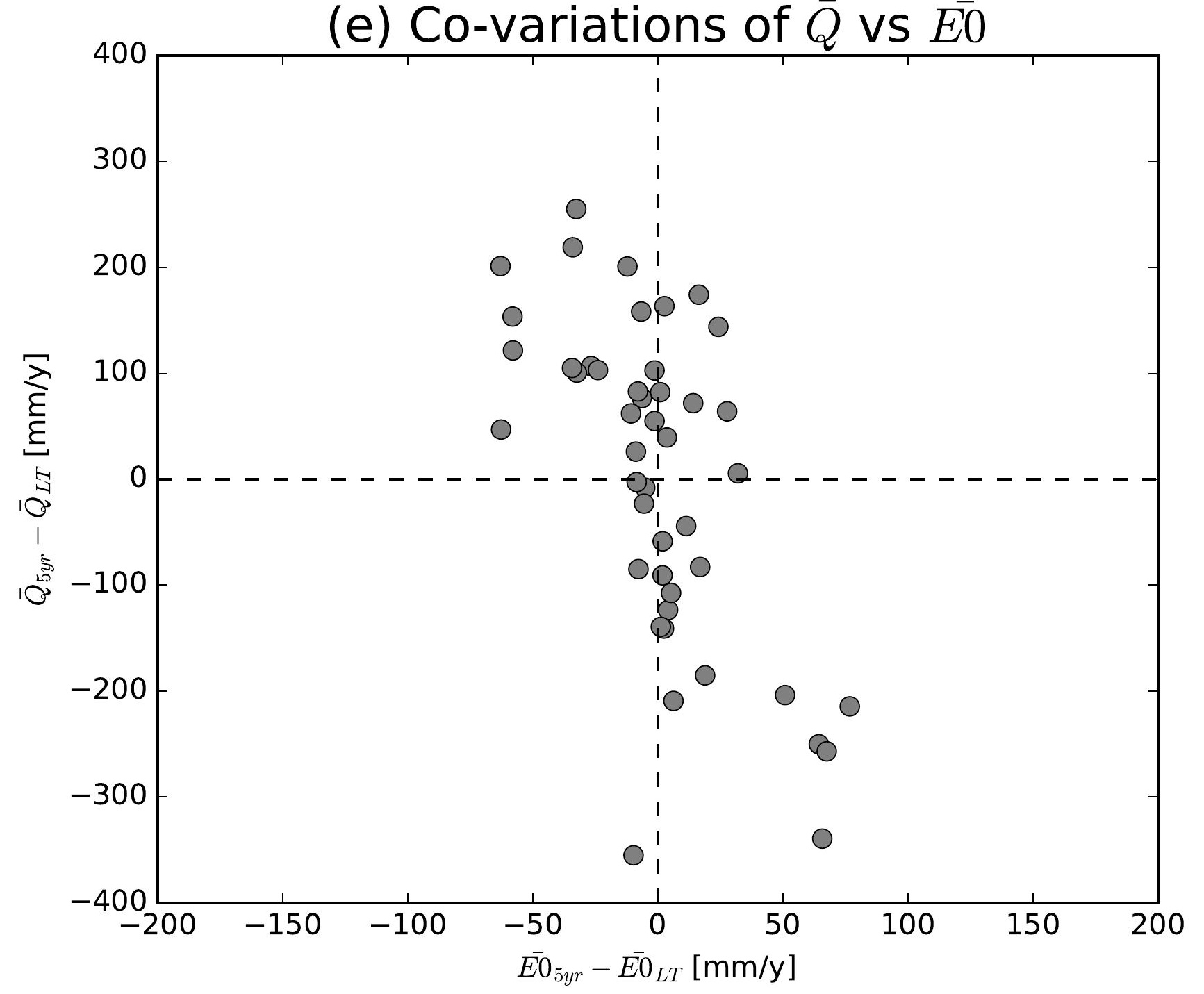

(h) Co-variations of $\bar{Q}$ vs $\overline{E 0}$

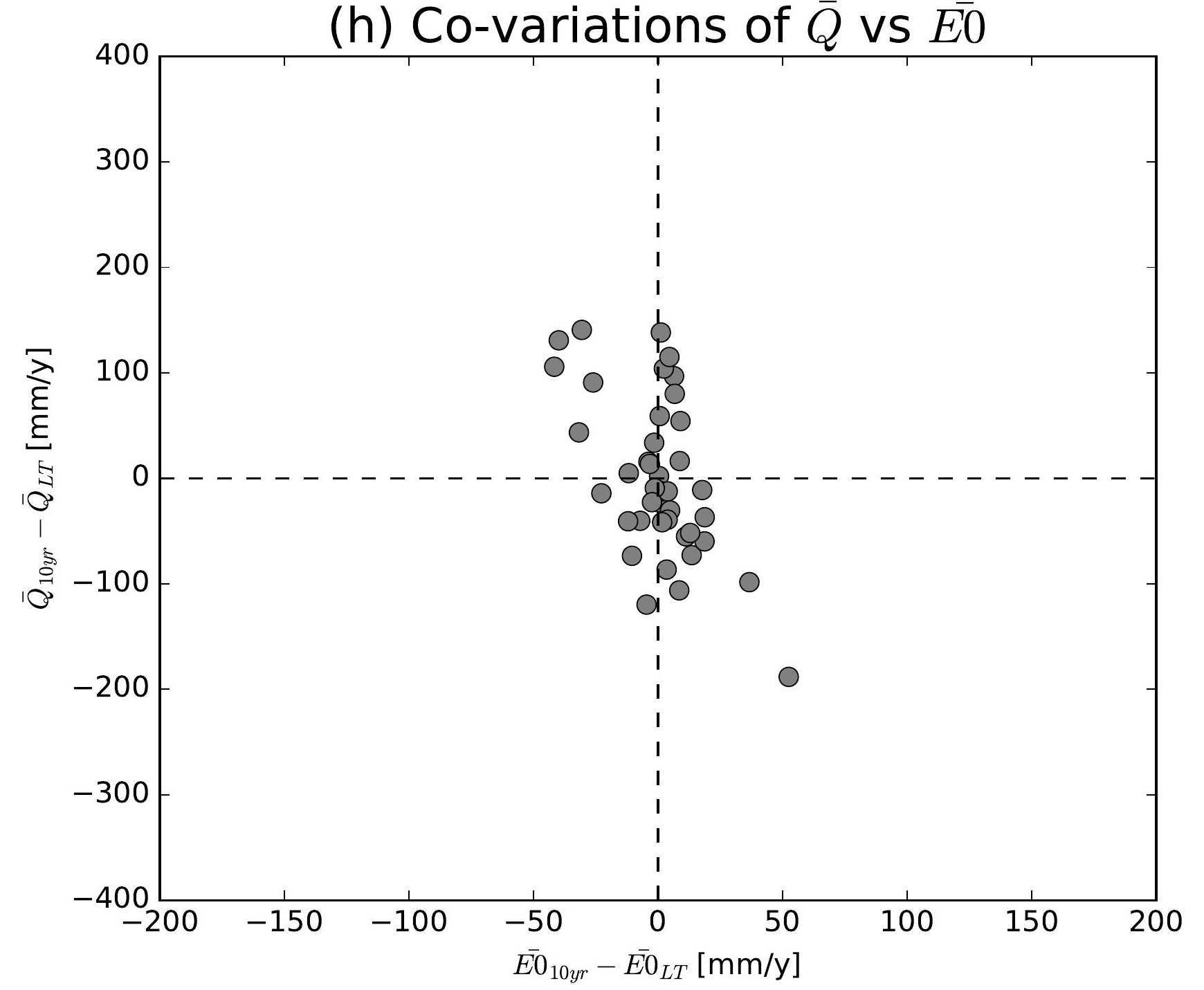

(k) Co-variations of $\bar{Q}$ vs $\overline{E 0}$

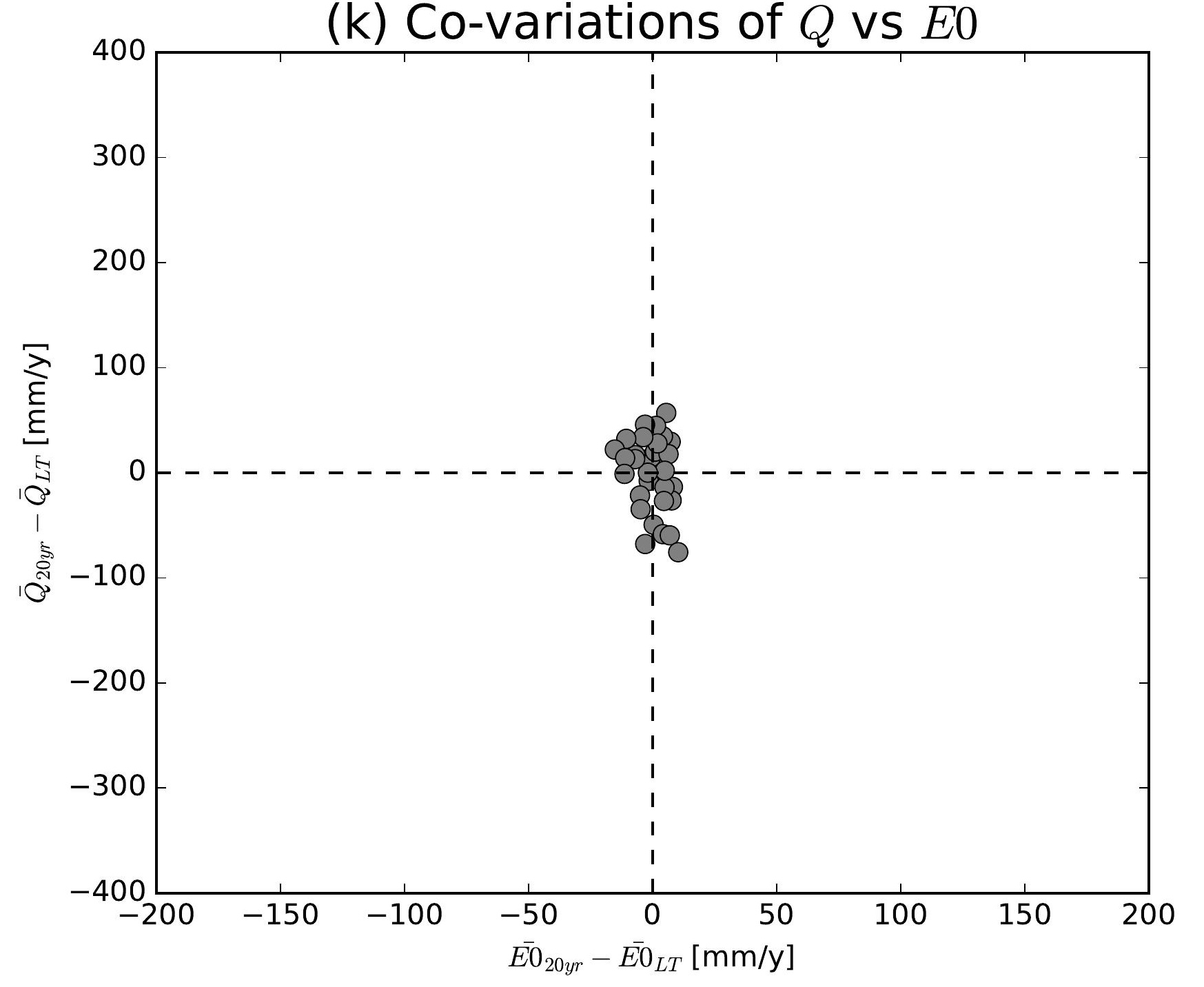

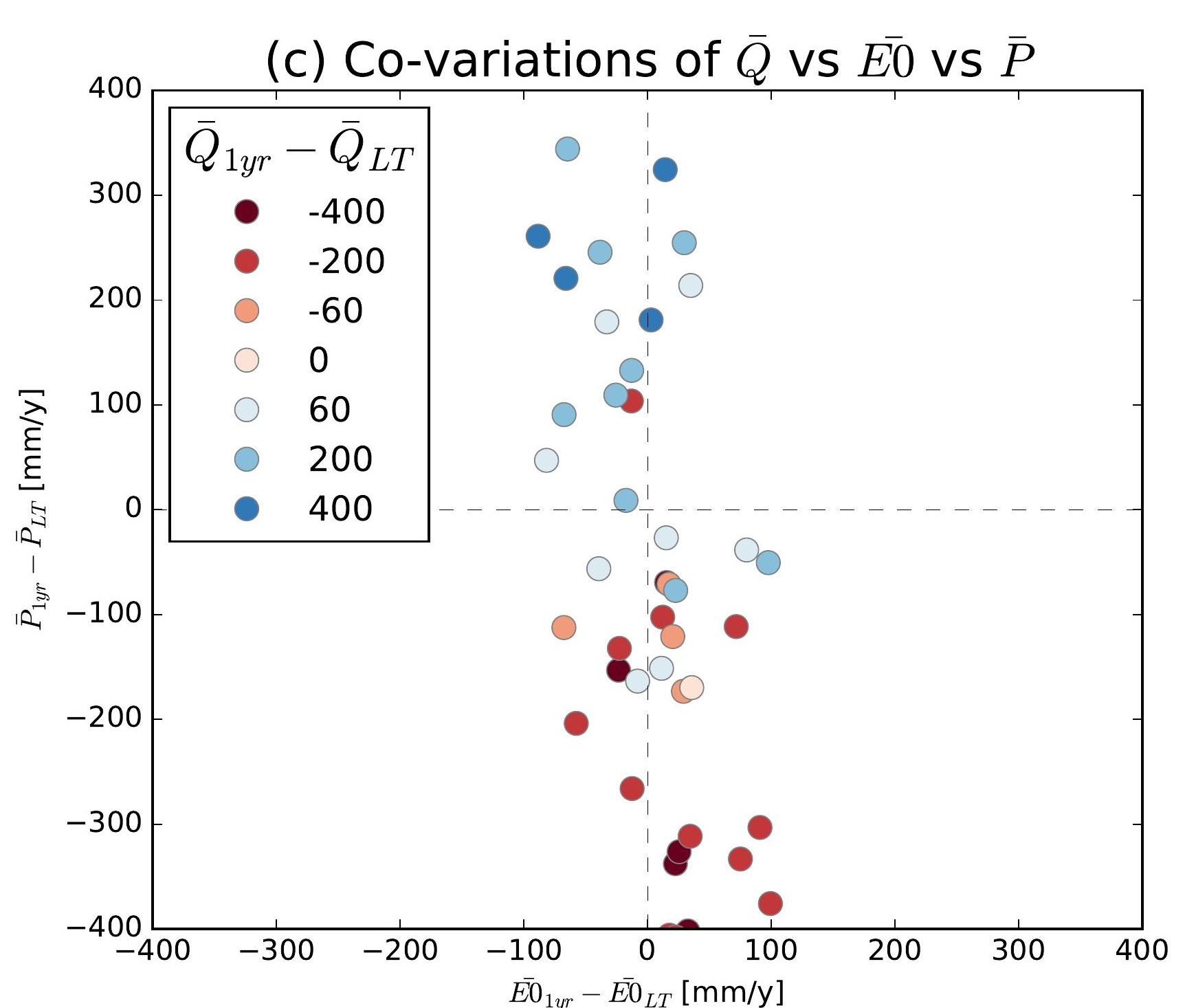
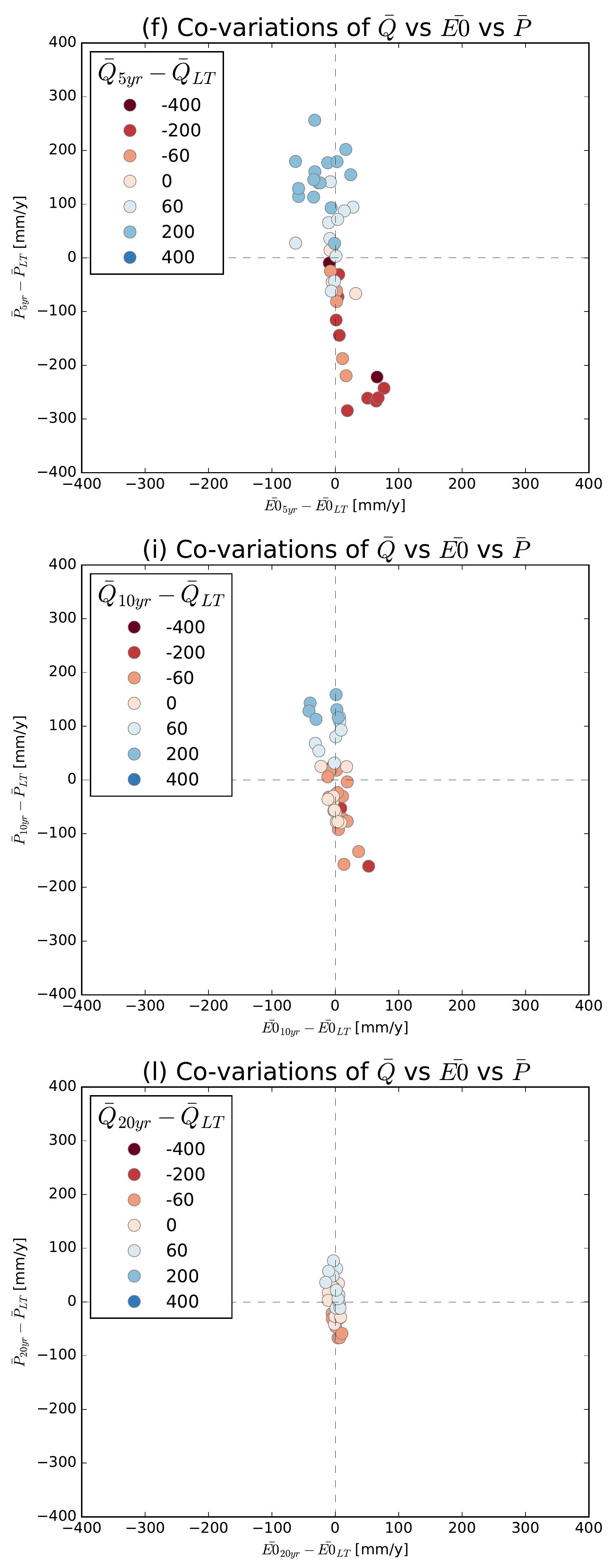

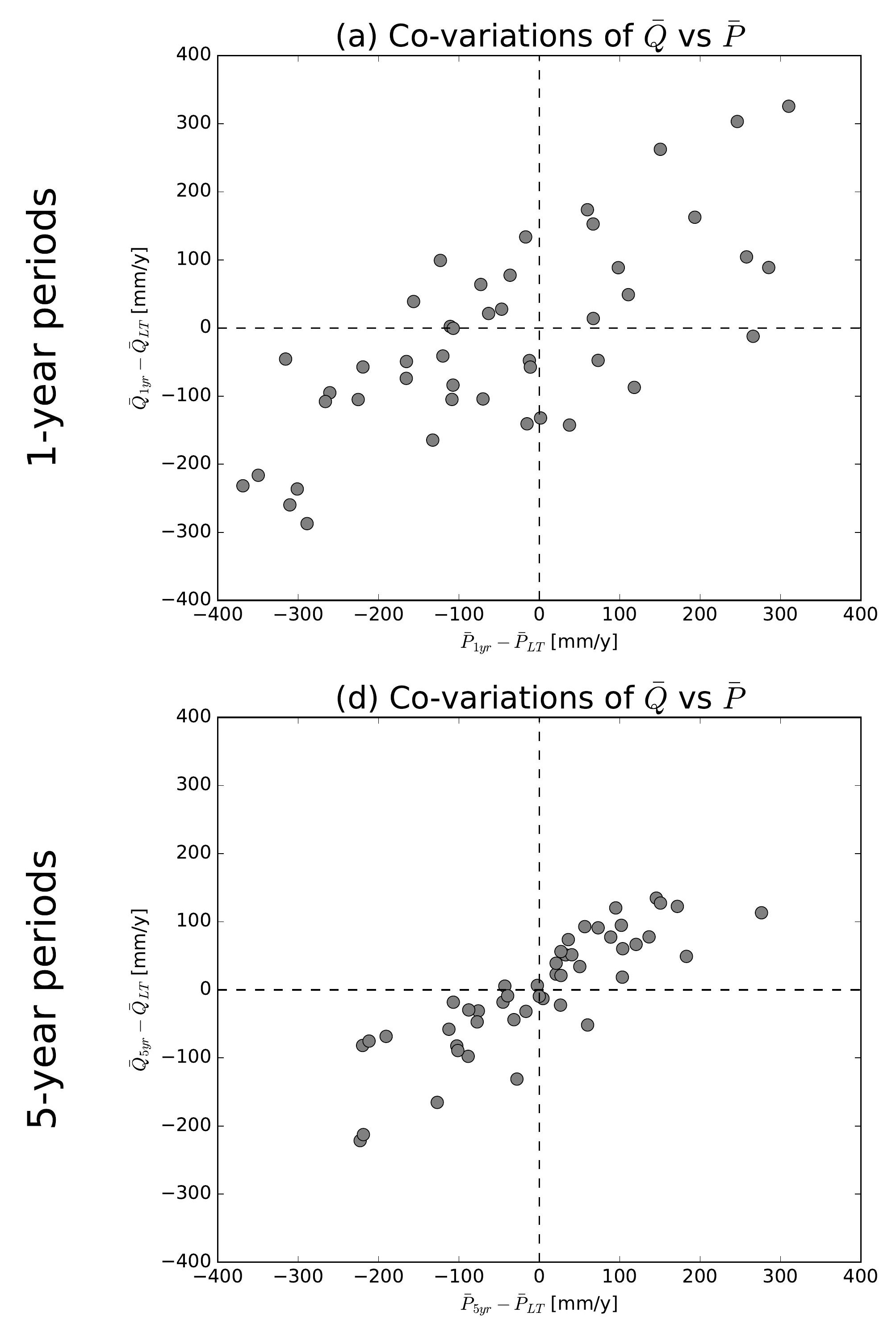

(g) Co-variations of $\bar{Q}$ vs $\bar{P}$

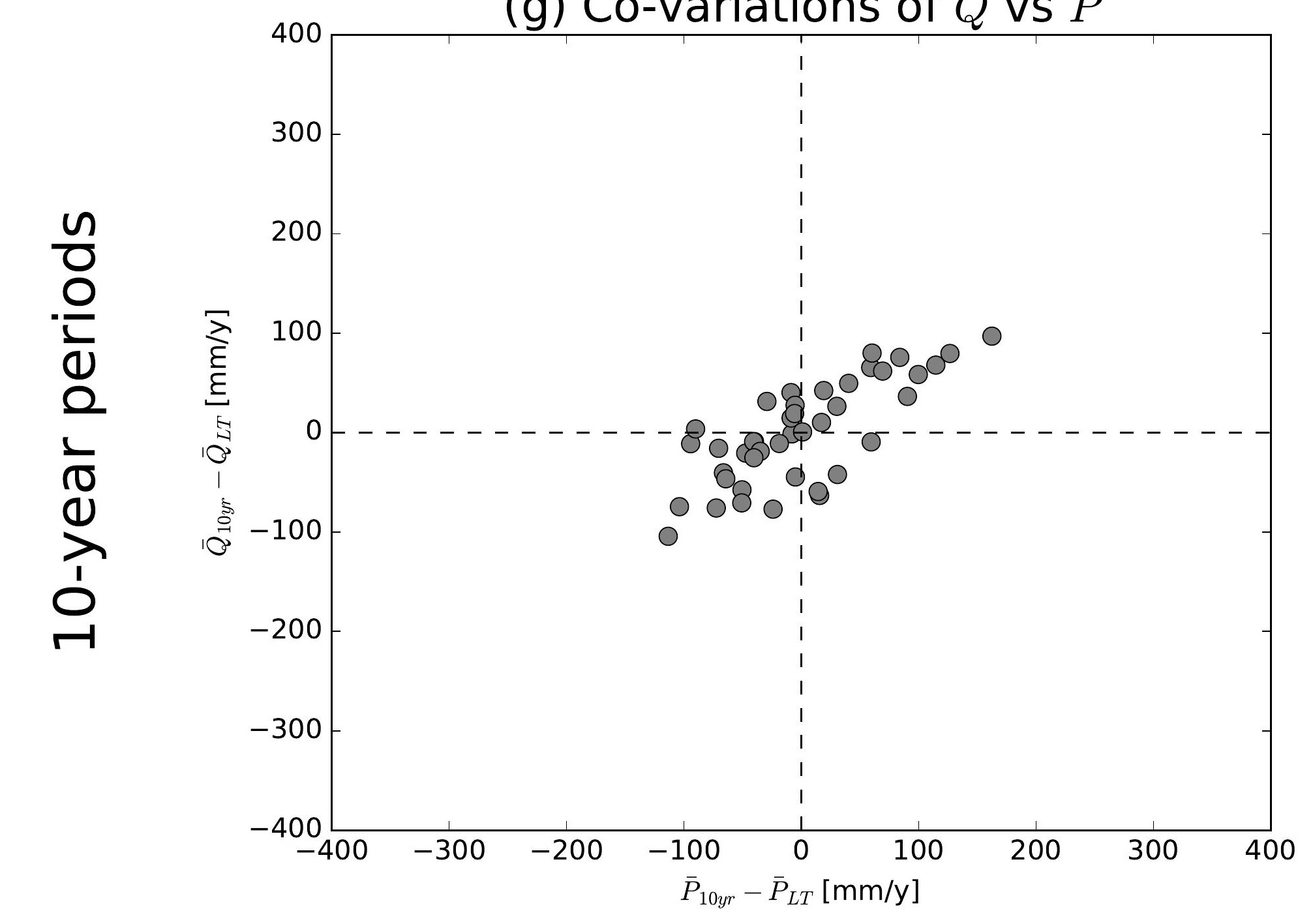

(j) Co-variations of $\bar{Q}$ vs $\bar{P}$

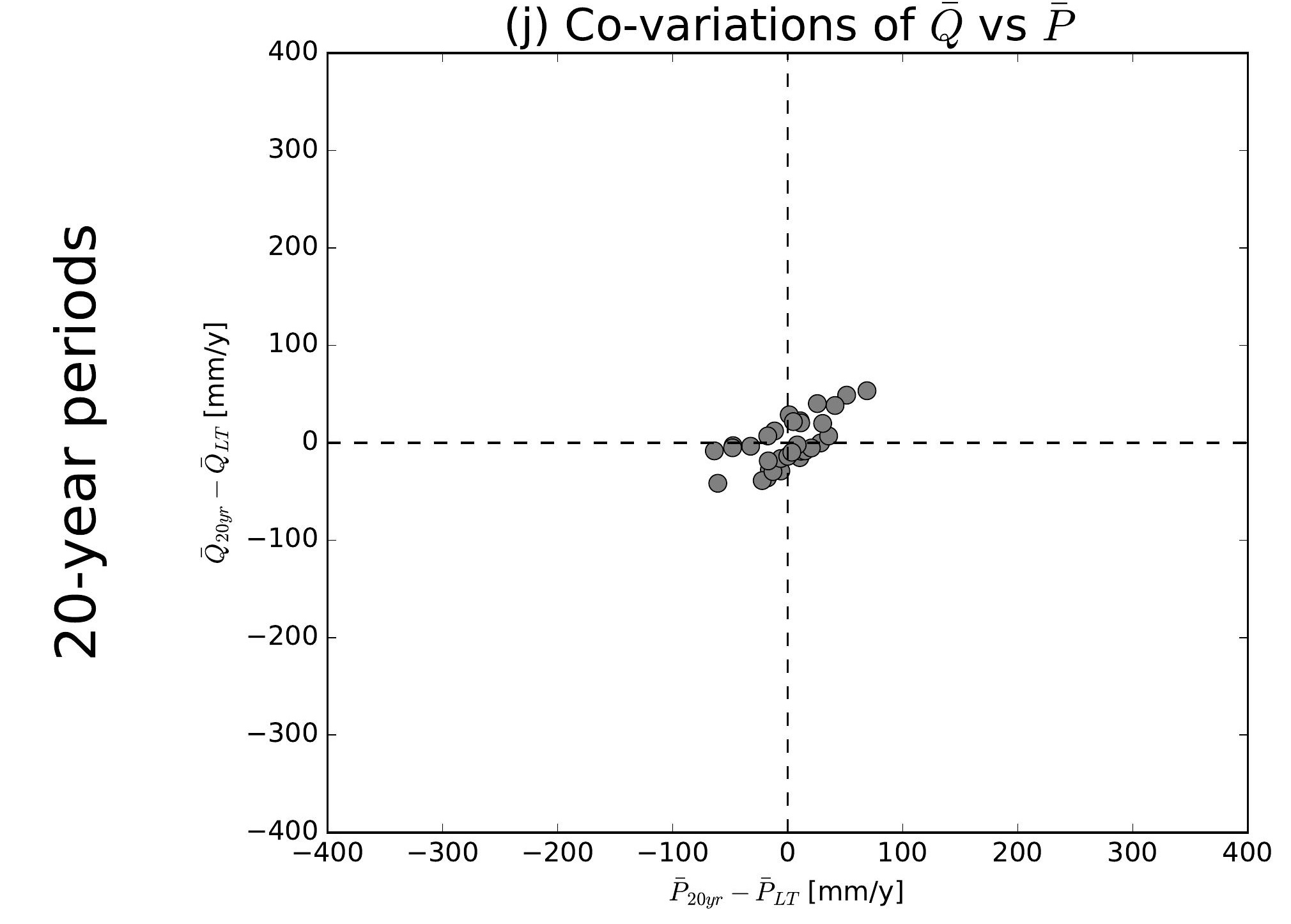

(b) Co-variations of $\bar{Q}$ vs $\overline{E 0}$

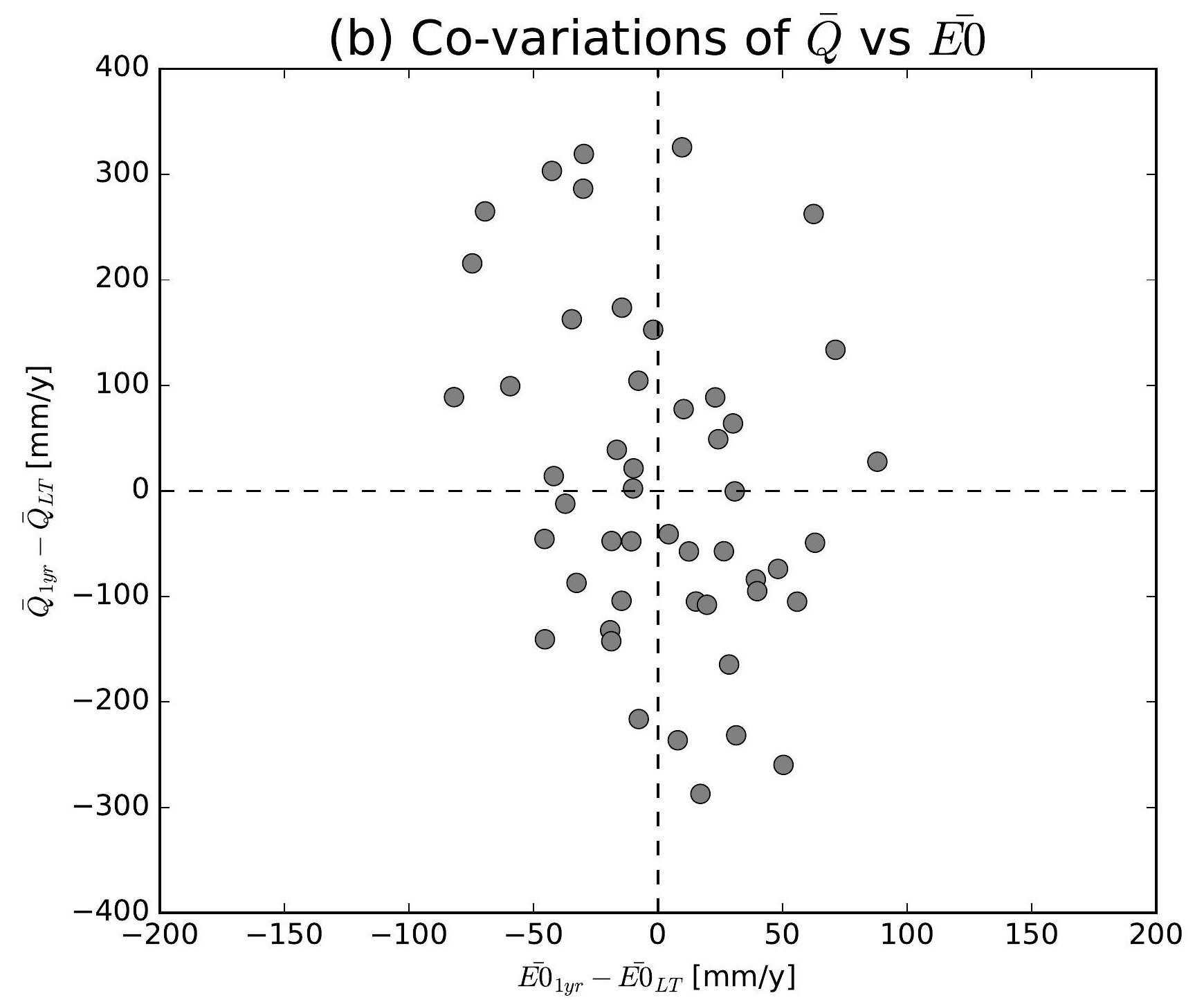

(e) Co-variations of $\bar{Q}$ vs $\overline{E 0}$

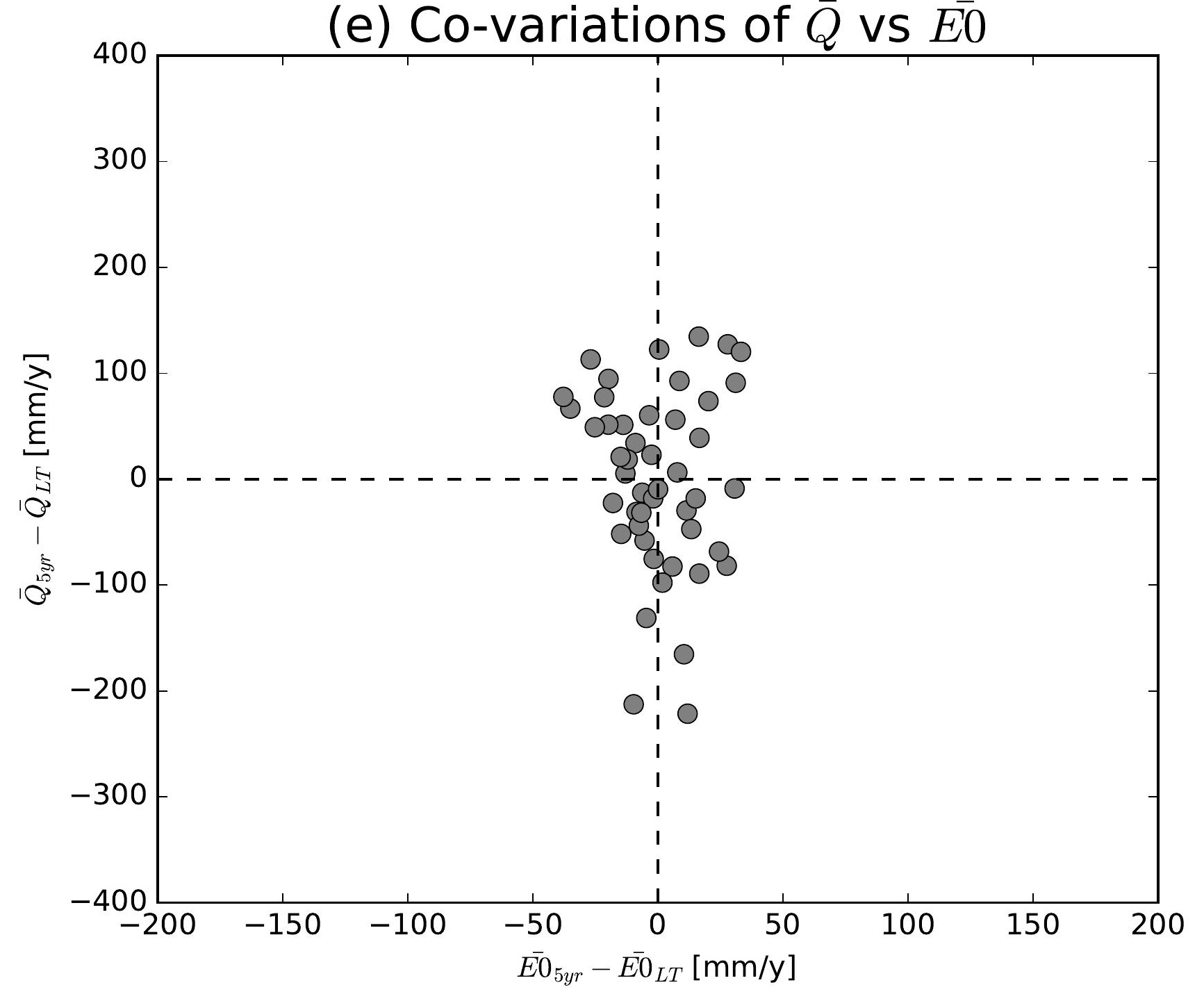

(h) Co-variations of $\bar{Q}$ vs $\overline{E 0}$

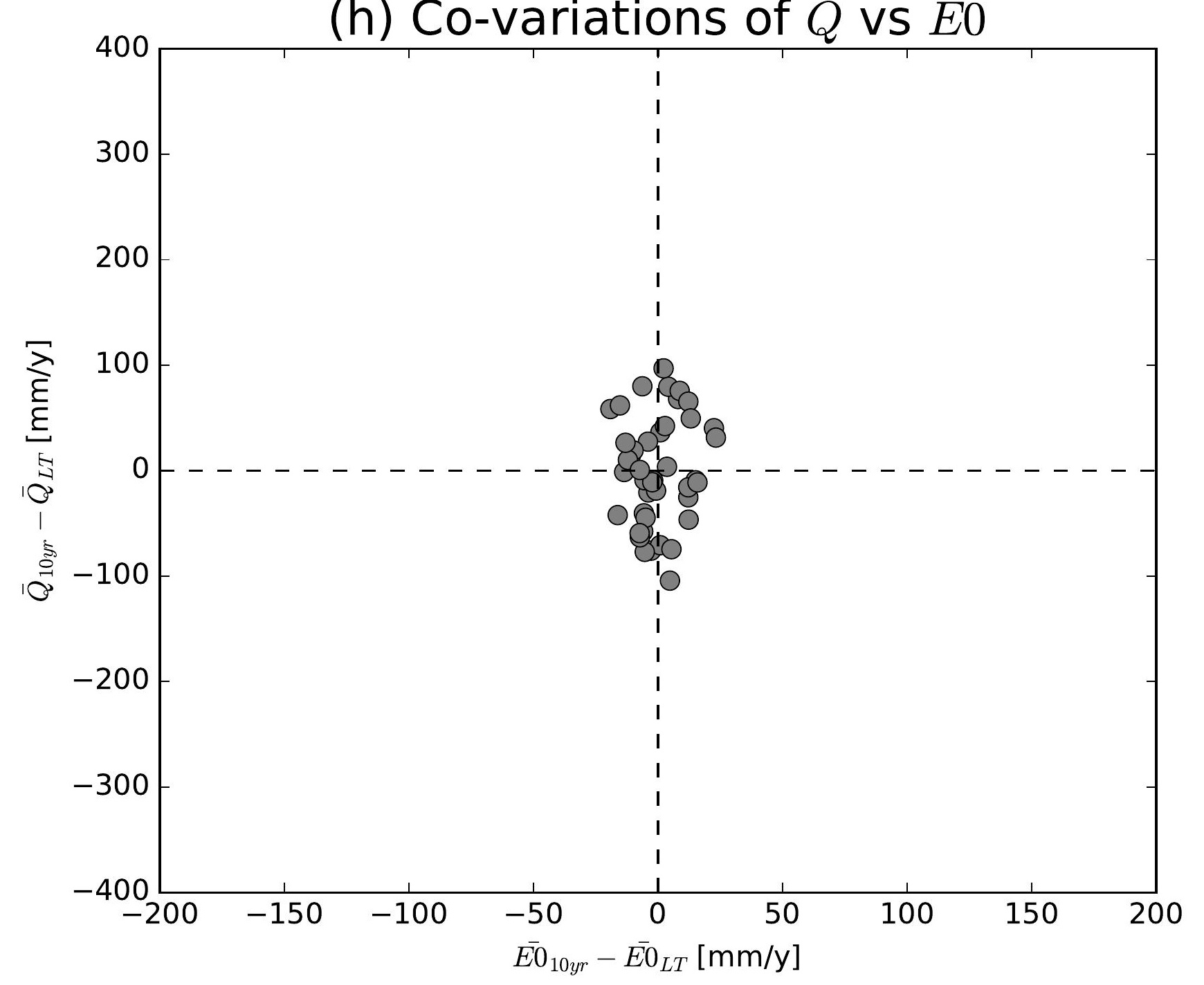

(k) Co-variations of $\bar{Q}$ vs $\overline{E 0}$

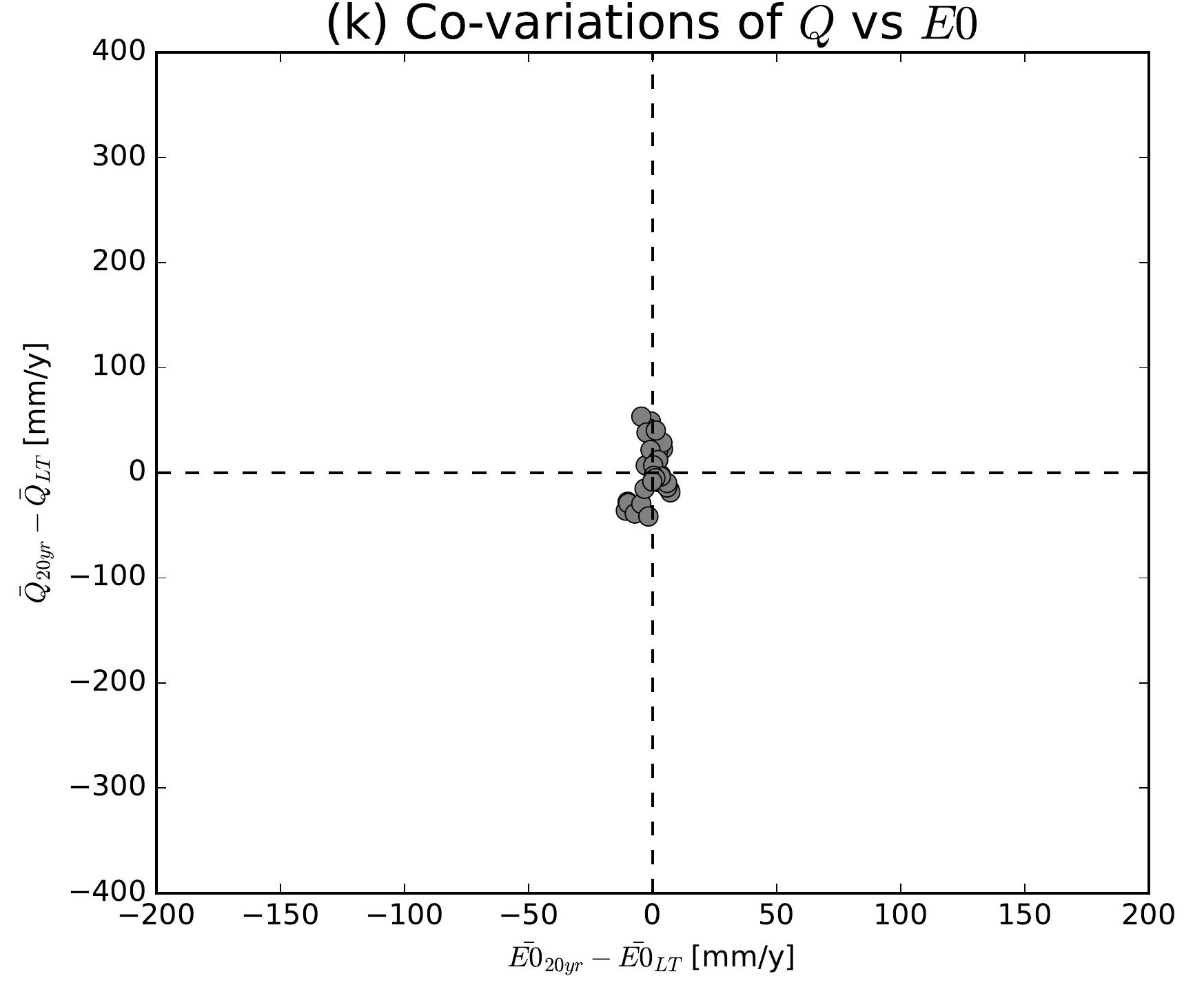

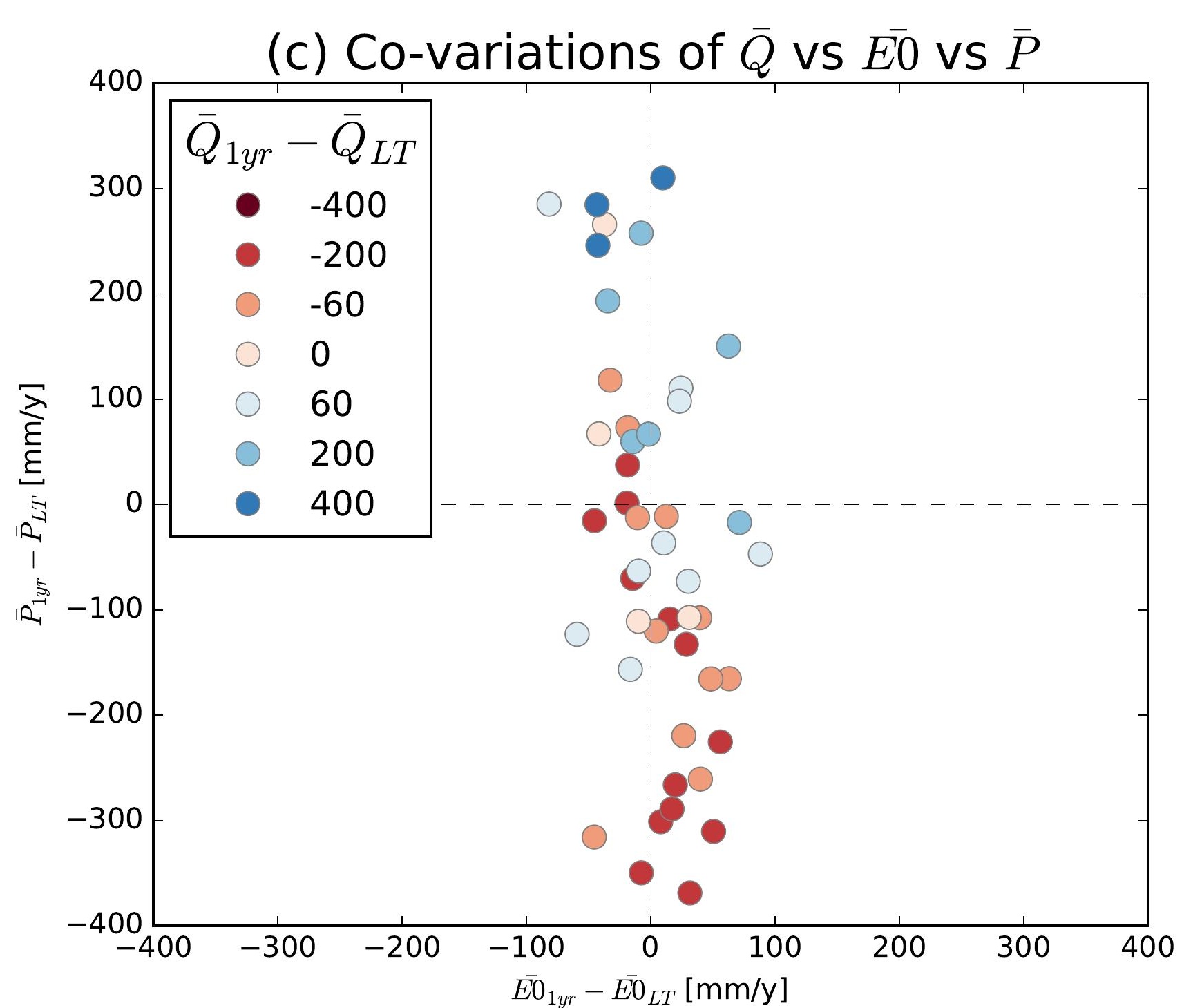
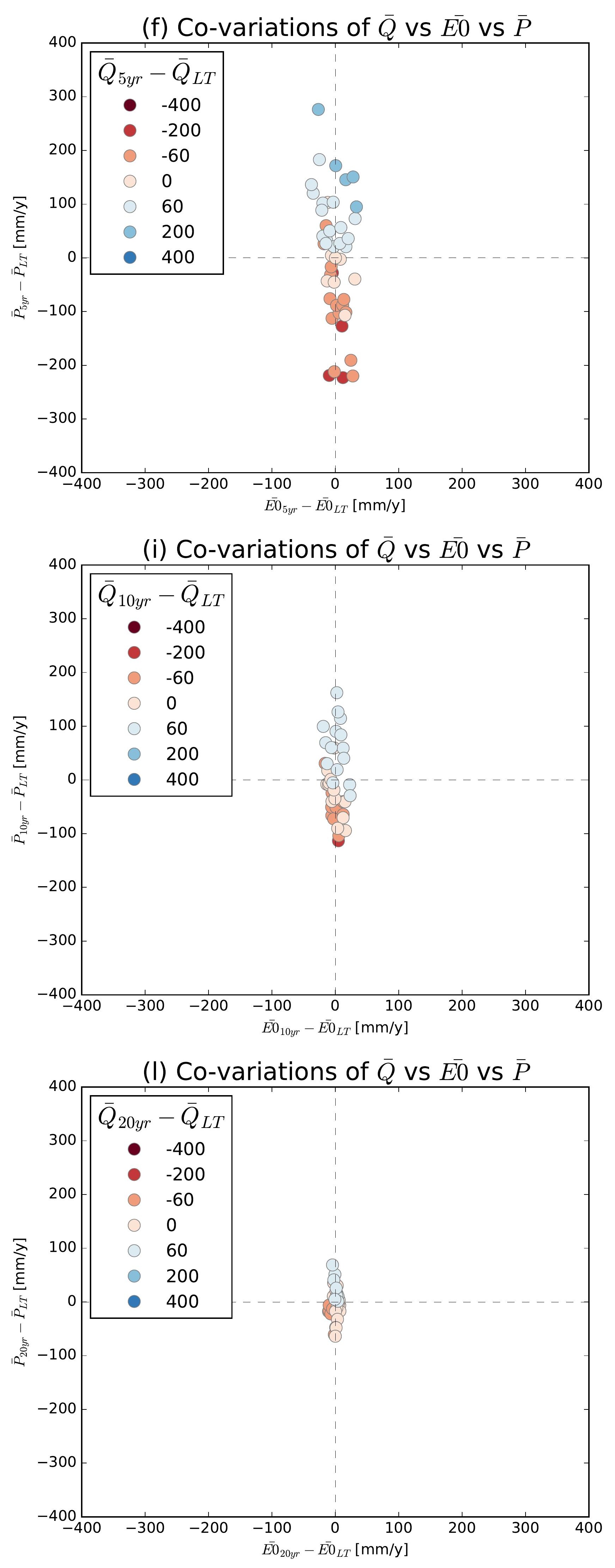

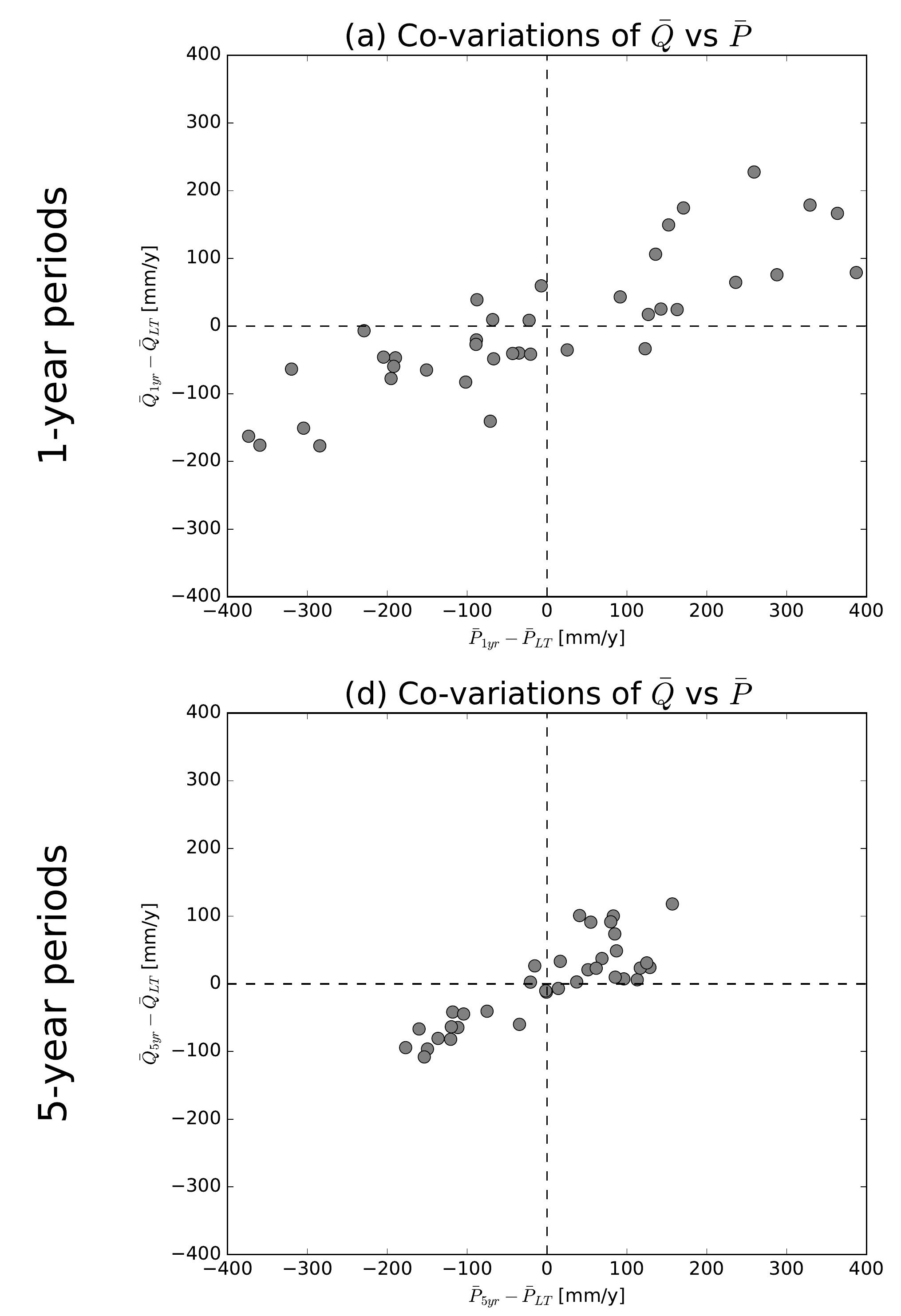

(g) Co-variations of $\bar{Q}$ vs $\bar{P}$

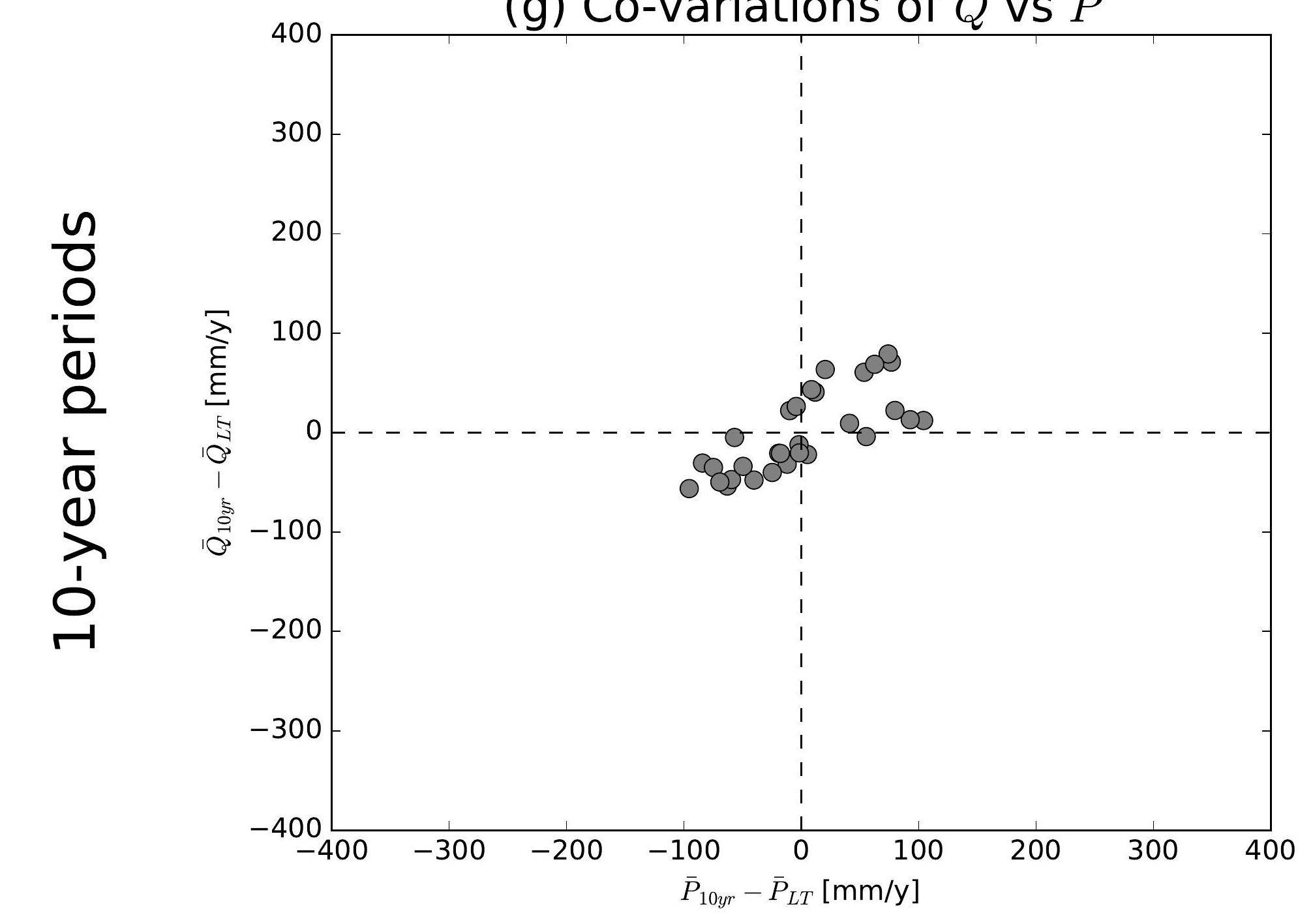

(j) Co-variations of $\bar{Q}$ vs $\bar{P}$

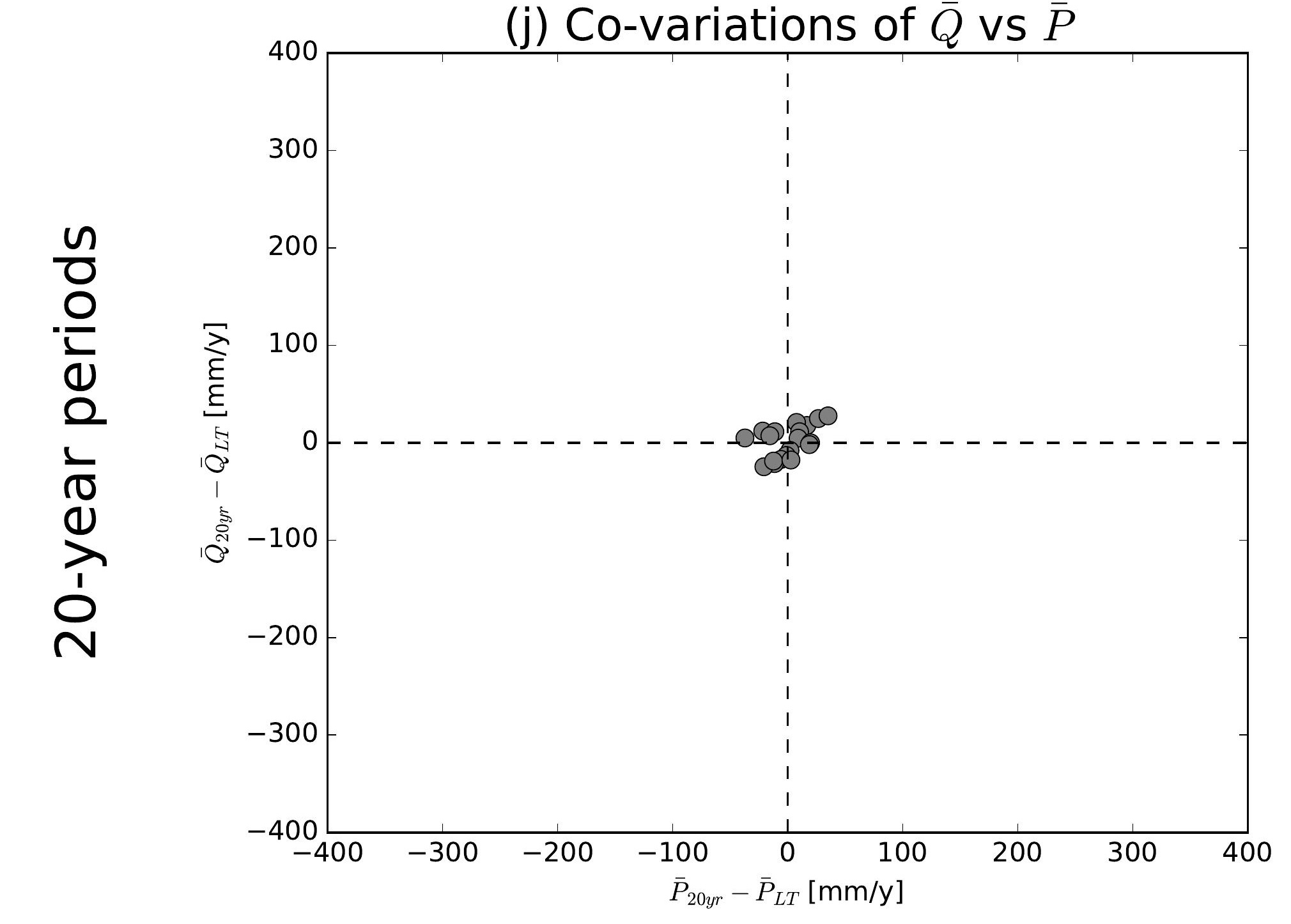

(b) Co-variations of $\bar{Q}$ vs $\overline{E 0}$

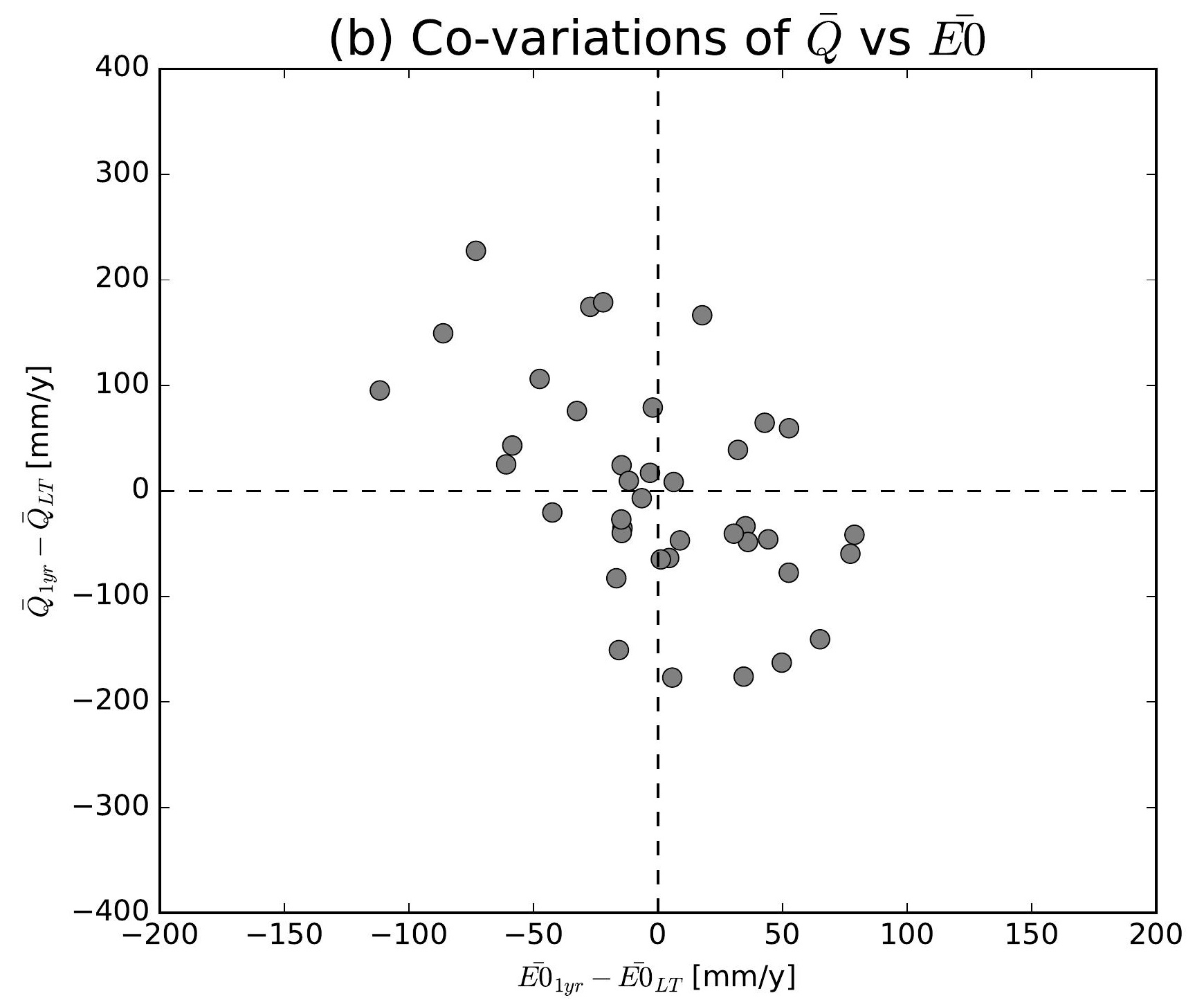

(e) Co-variations of $\bar{Q}$ vs $\overline{E 0}$

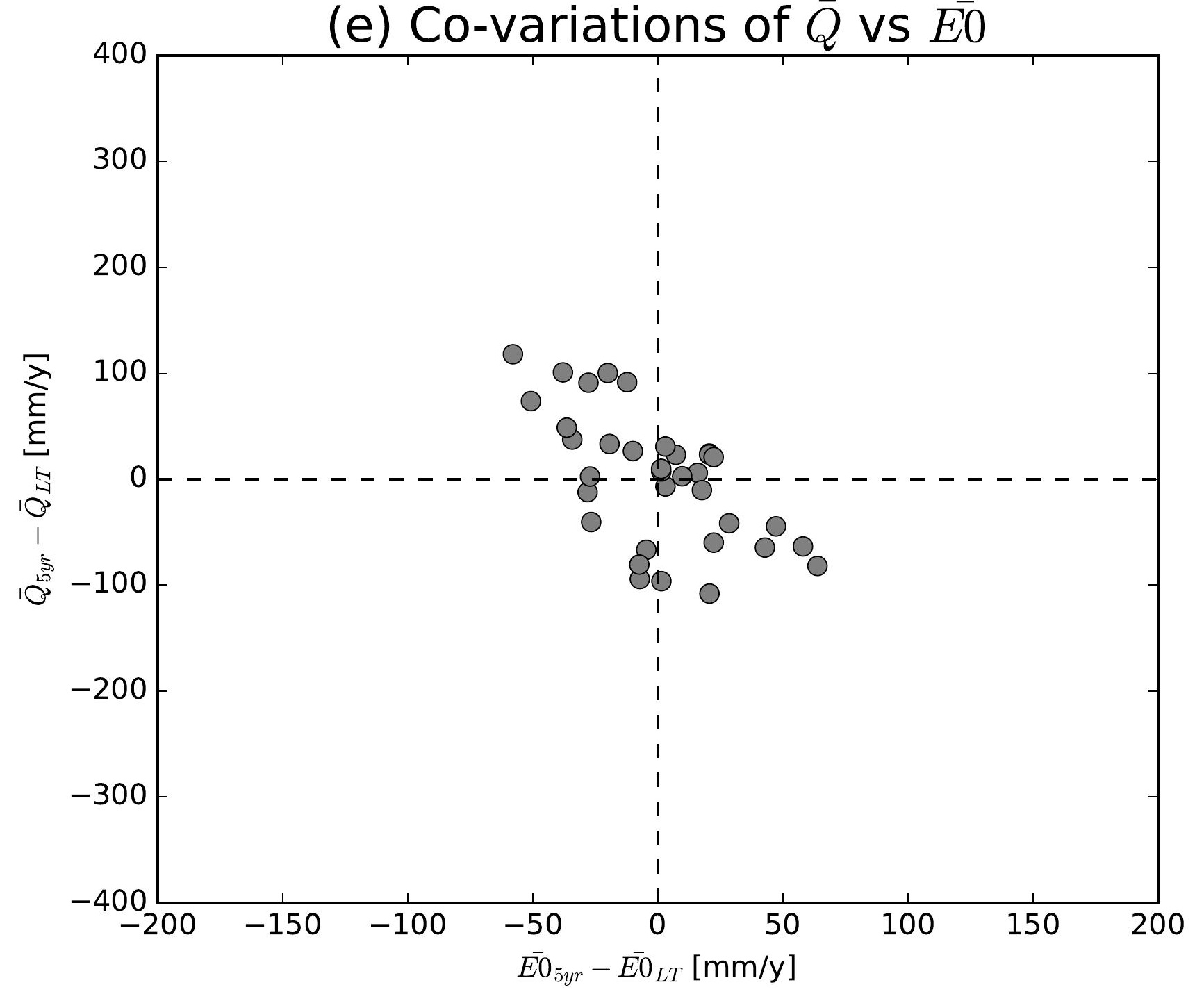

(h) Co-variations of $\bar{Q}$ vs $\overline{E 0}$

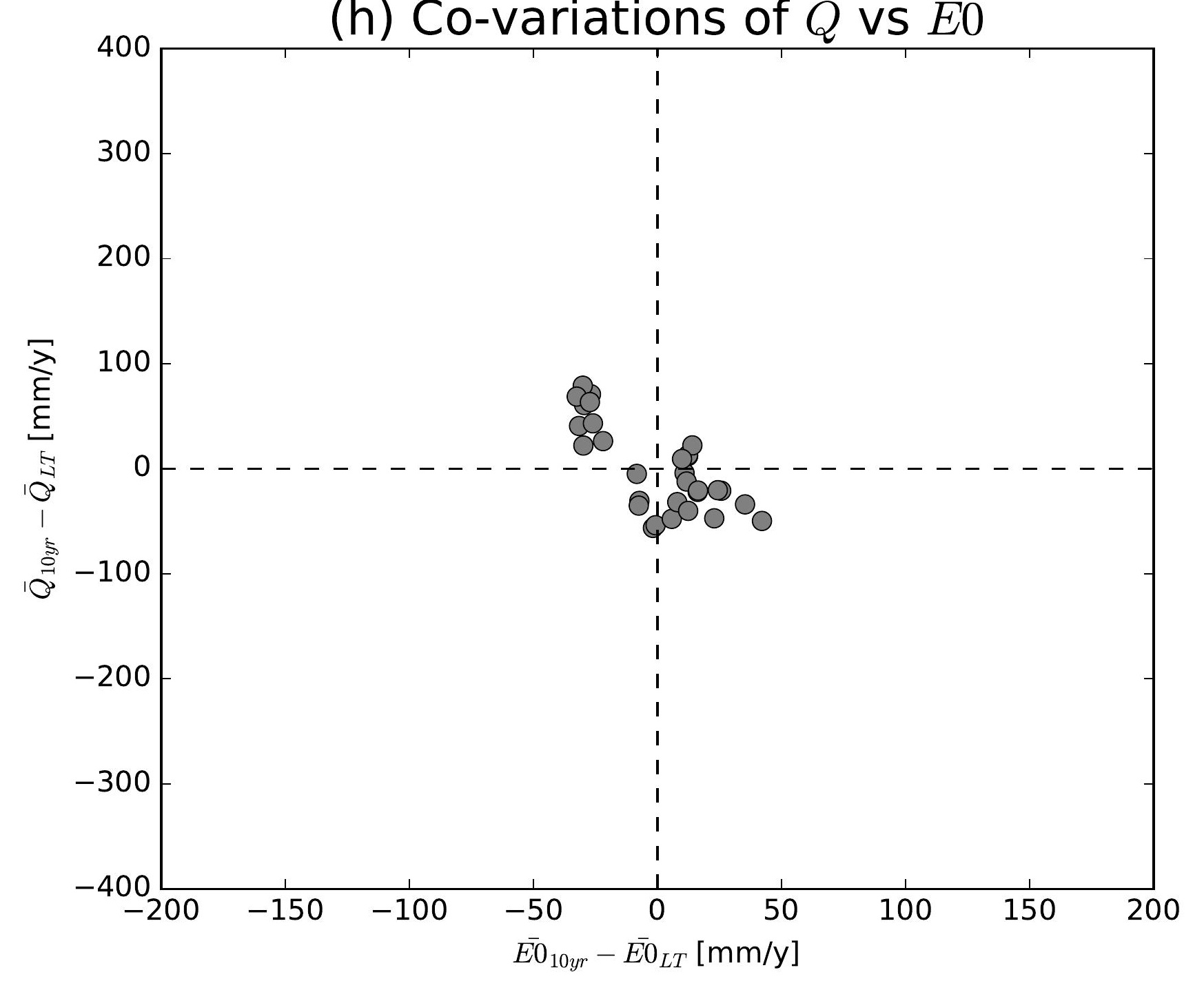

(k) Co-variations of $\bar{Q}$ vs $\overline{E 0}$

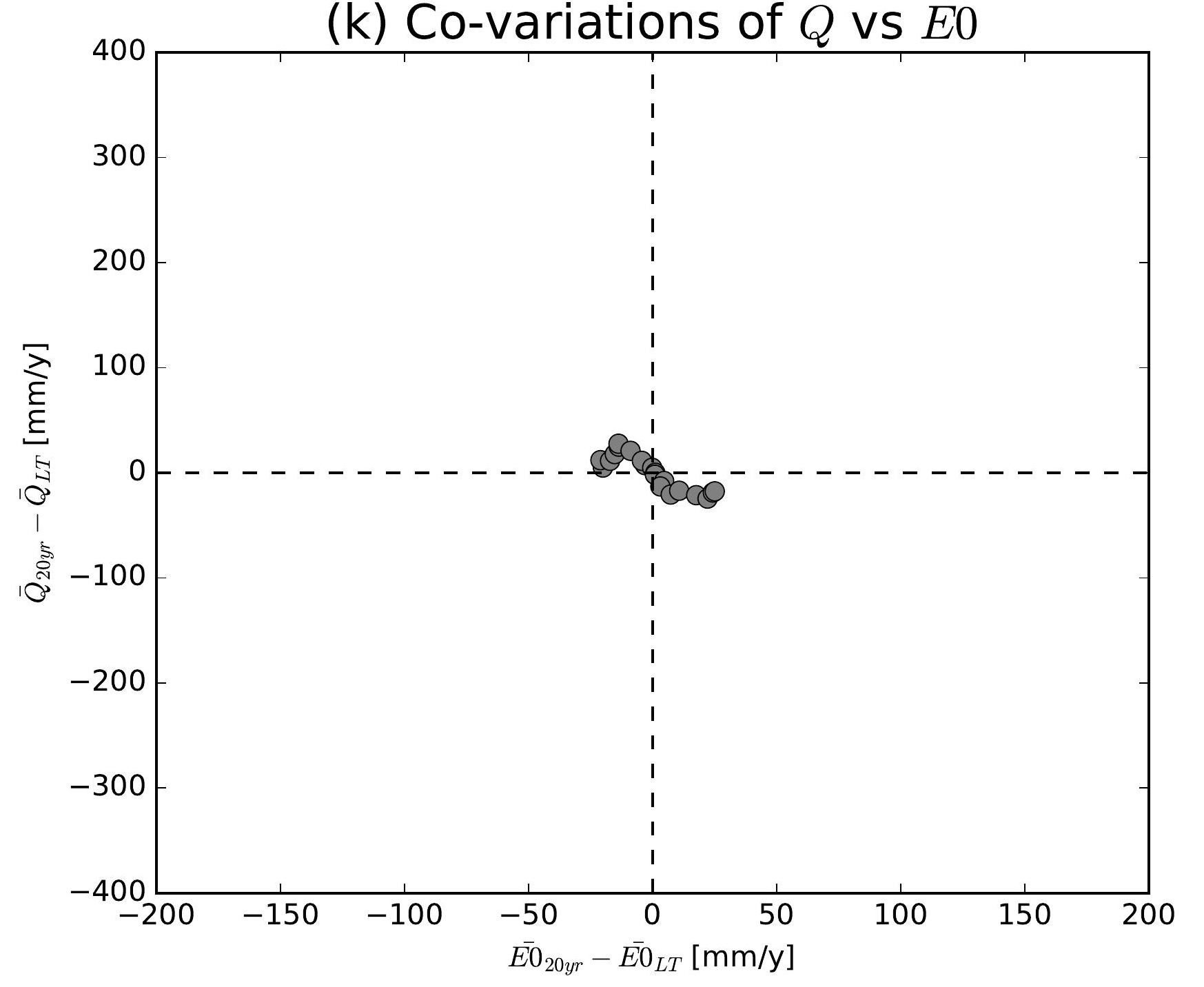

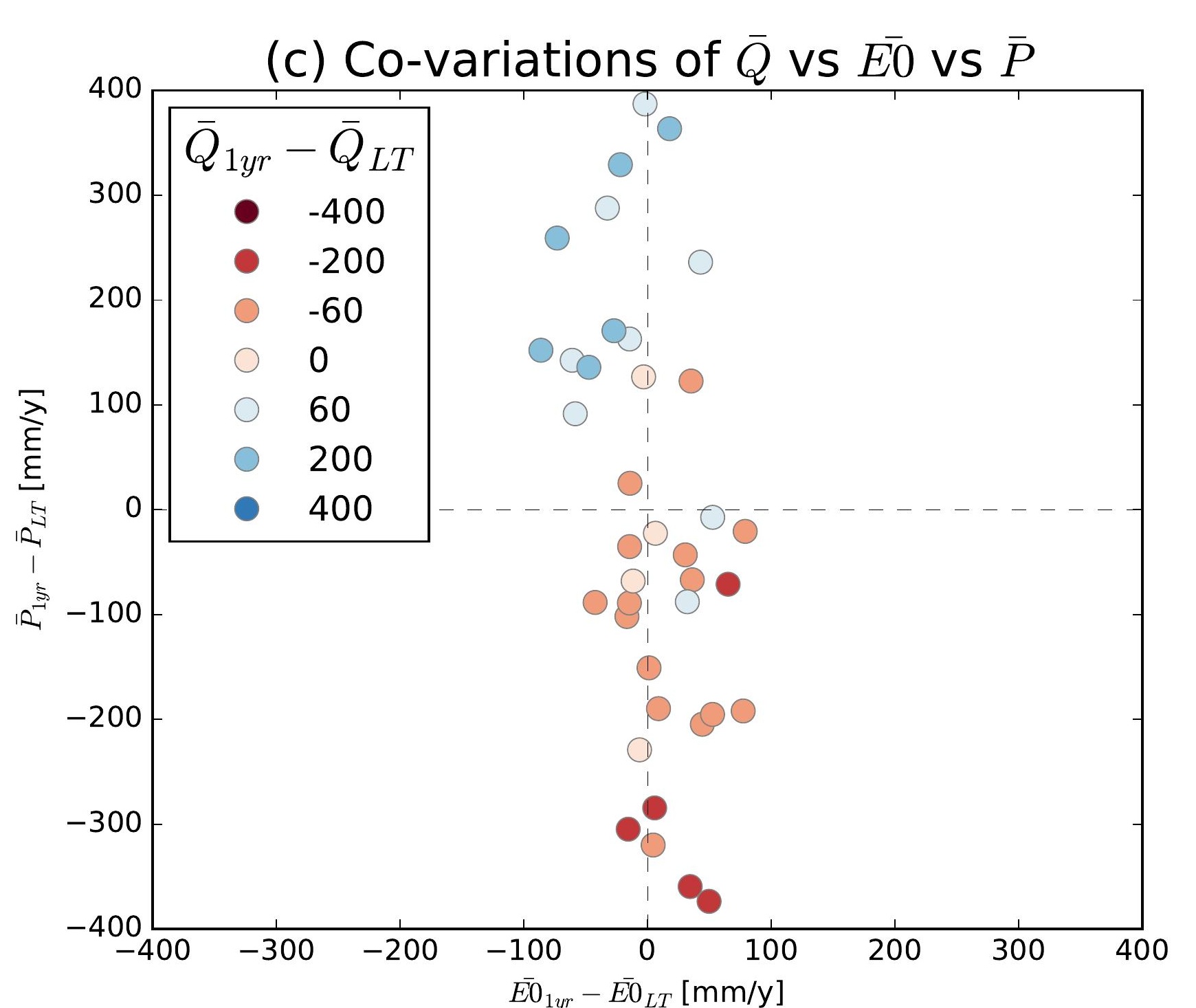
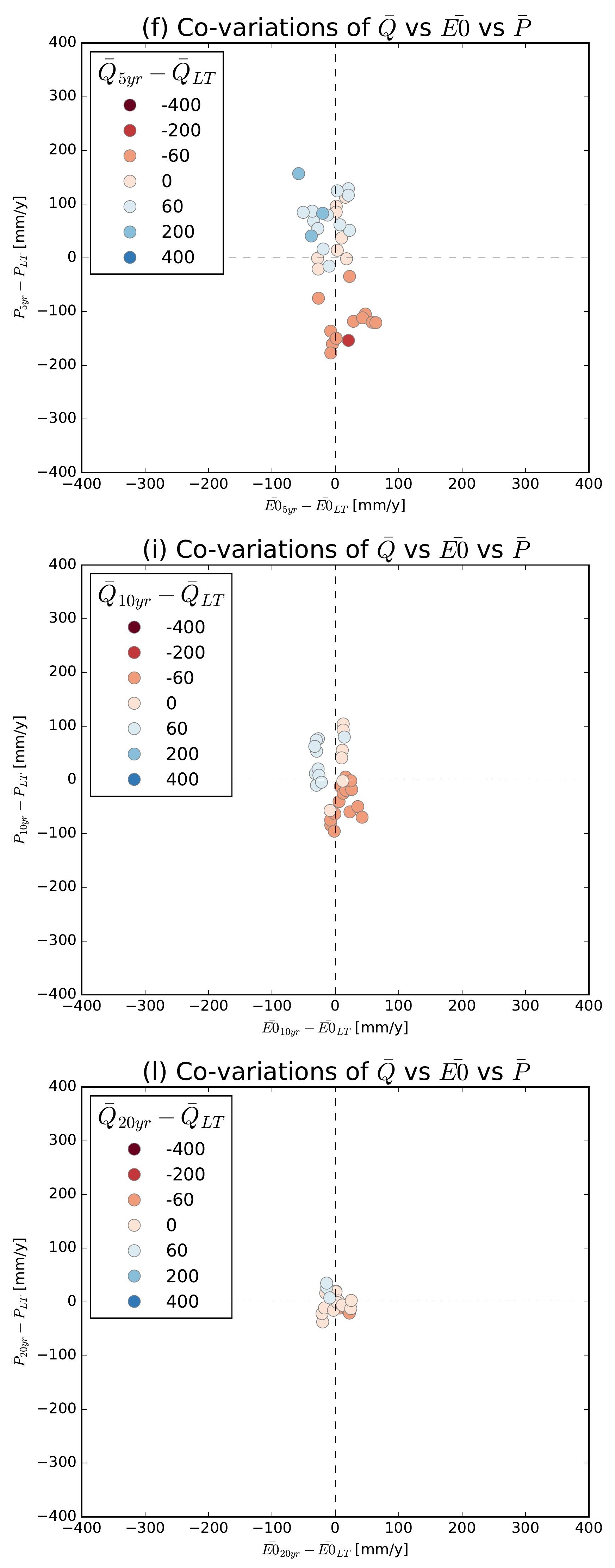

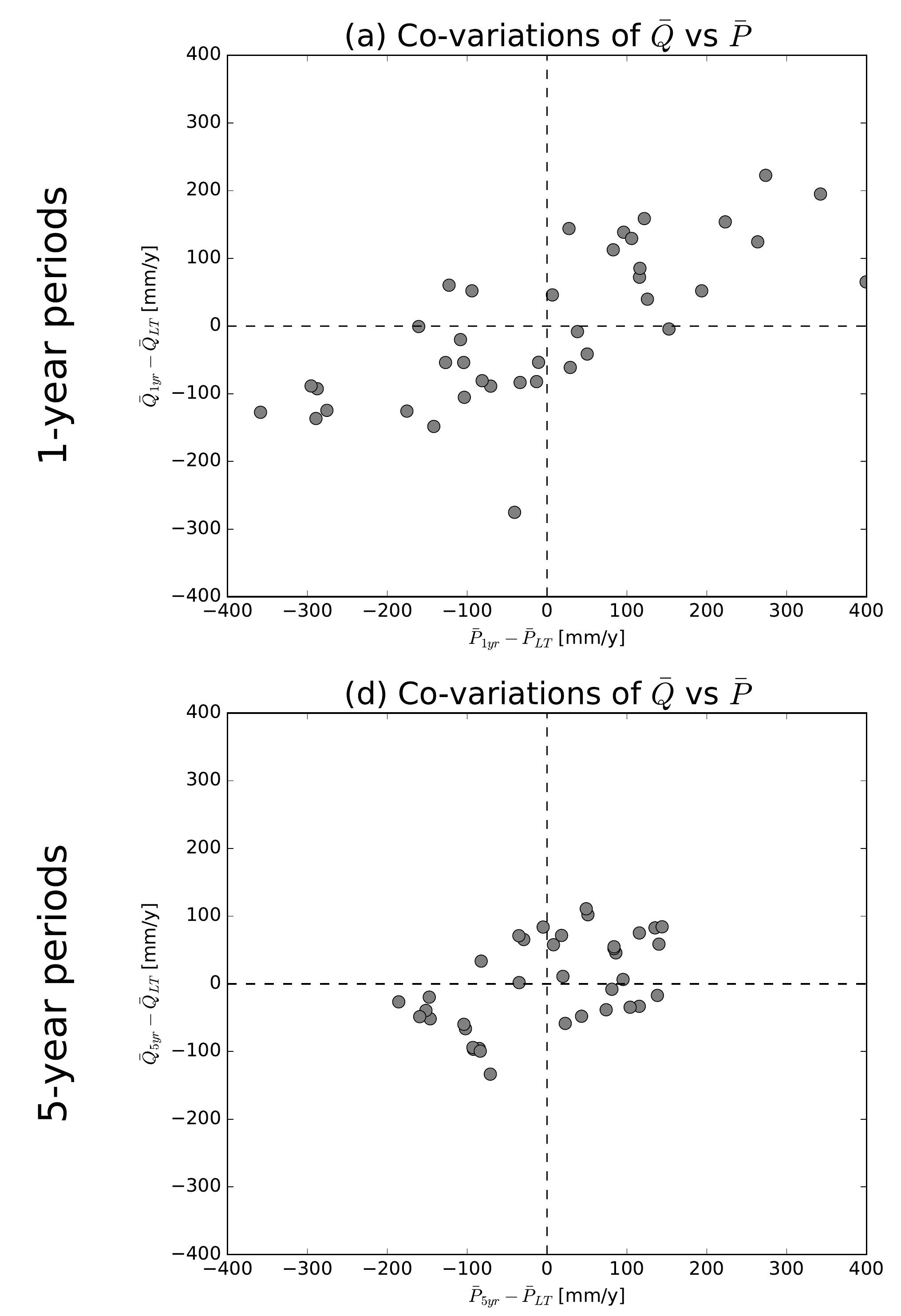

(g) Co-variations of $\bar{Q}$ vs $\bar{P}$

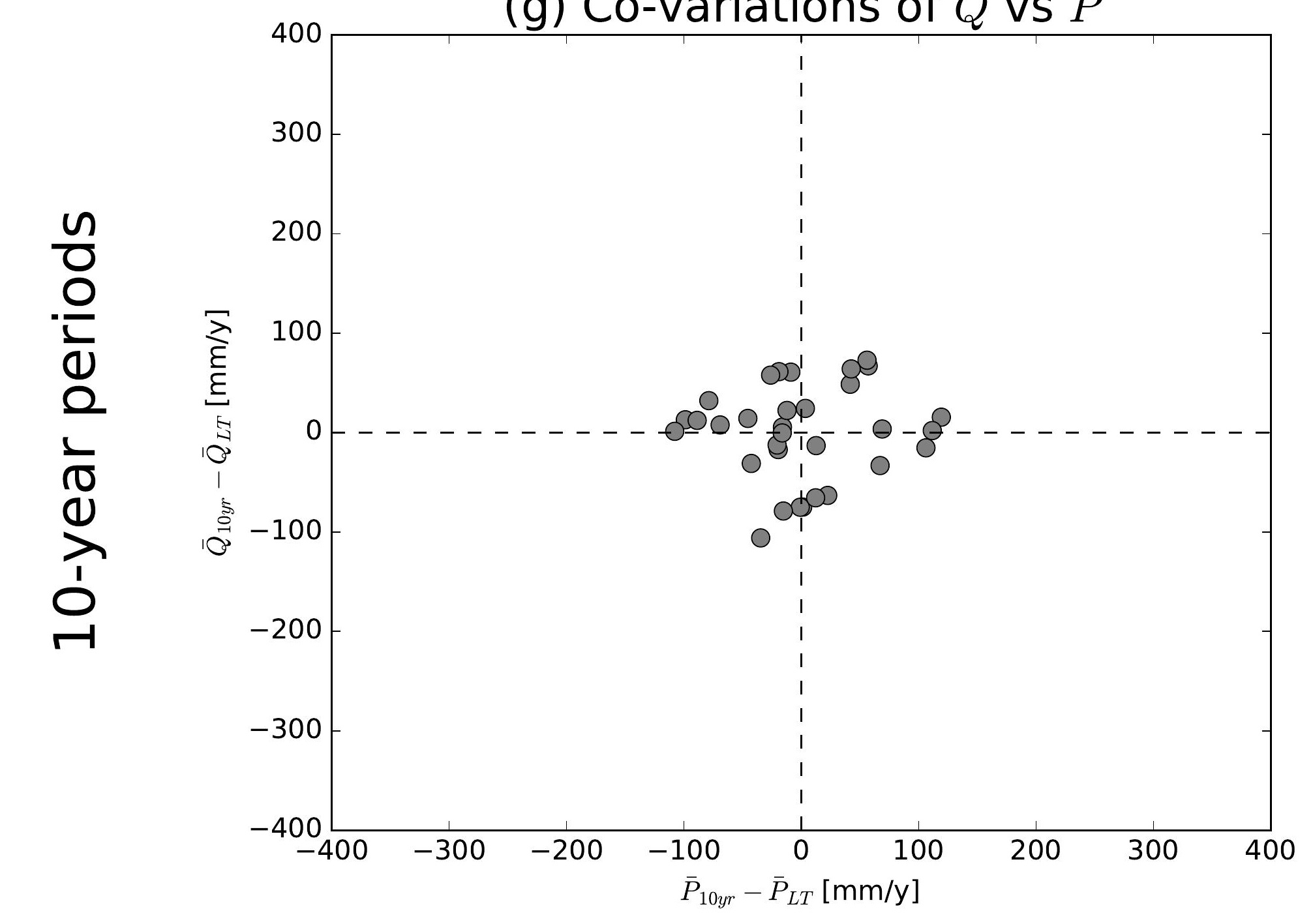

(j) Co-variations of $\bar{Q}$ vs $\bar{P}$

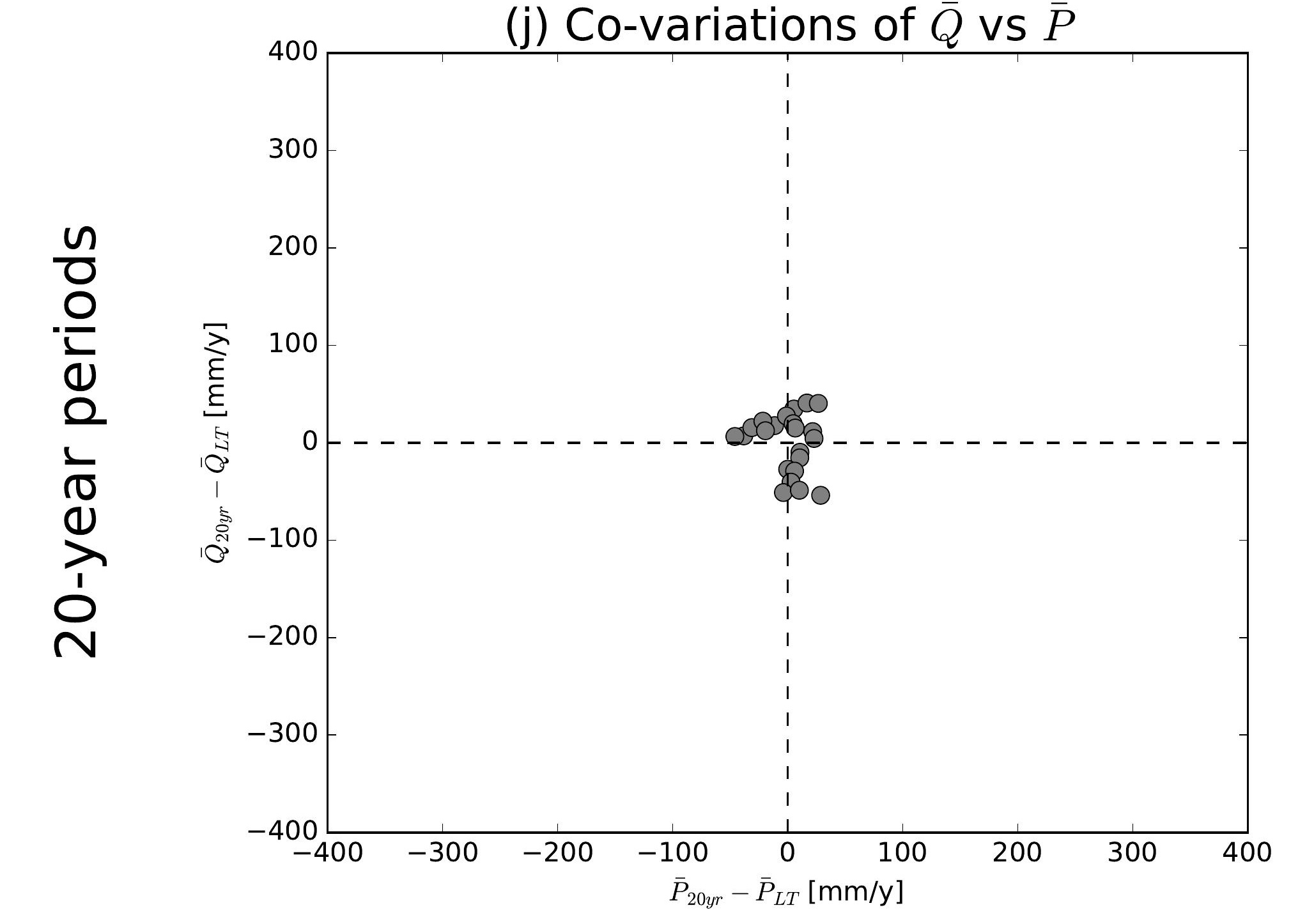

(b) Co-variations of $\bar{Q}$ vs $\overline{E 0}$

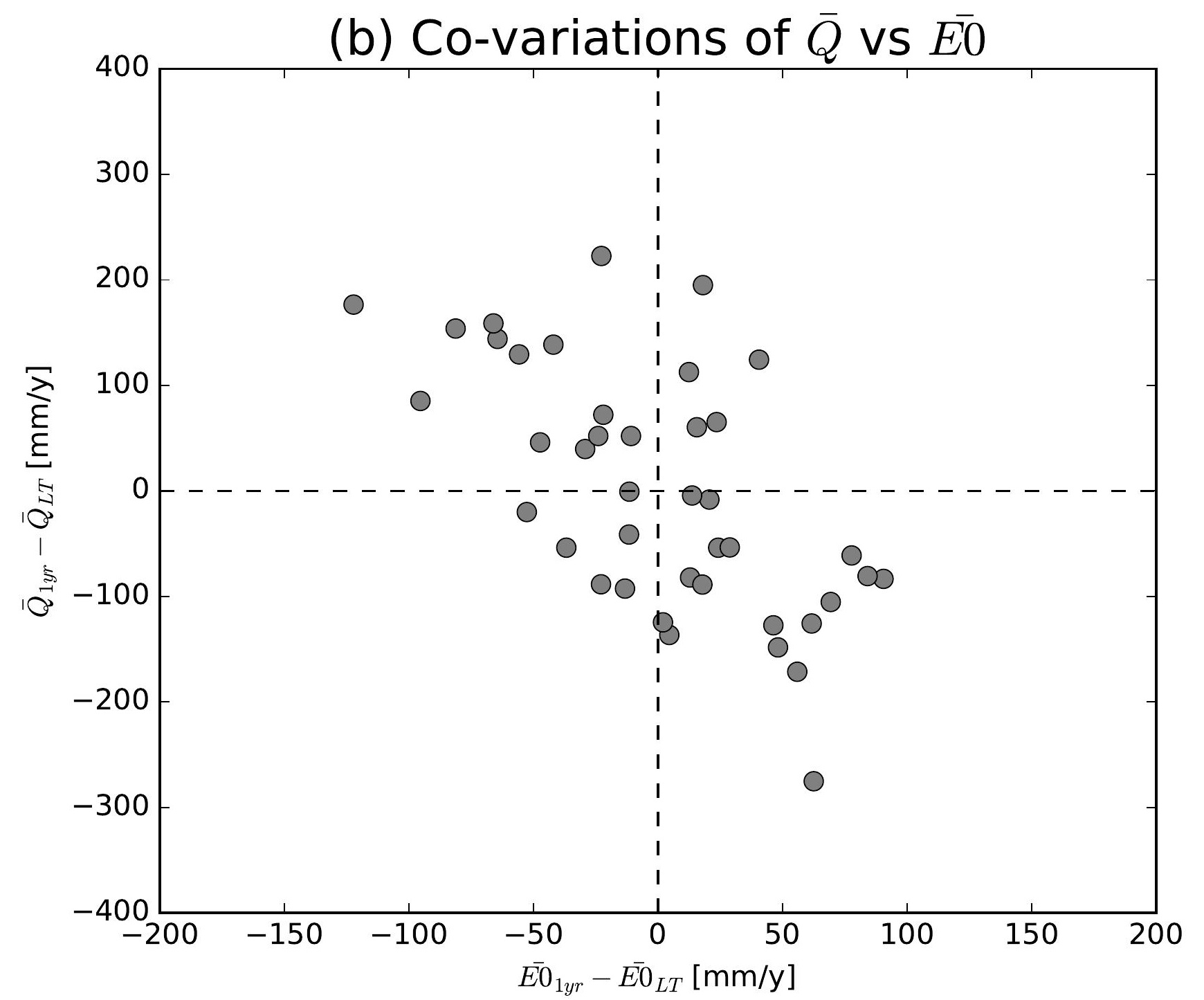

(e) Co-variations of $\bar{Q}$ vs $\overline{E 0}$

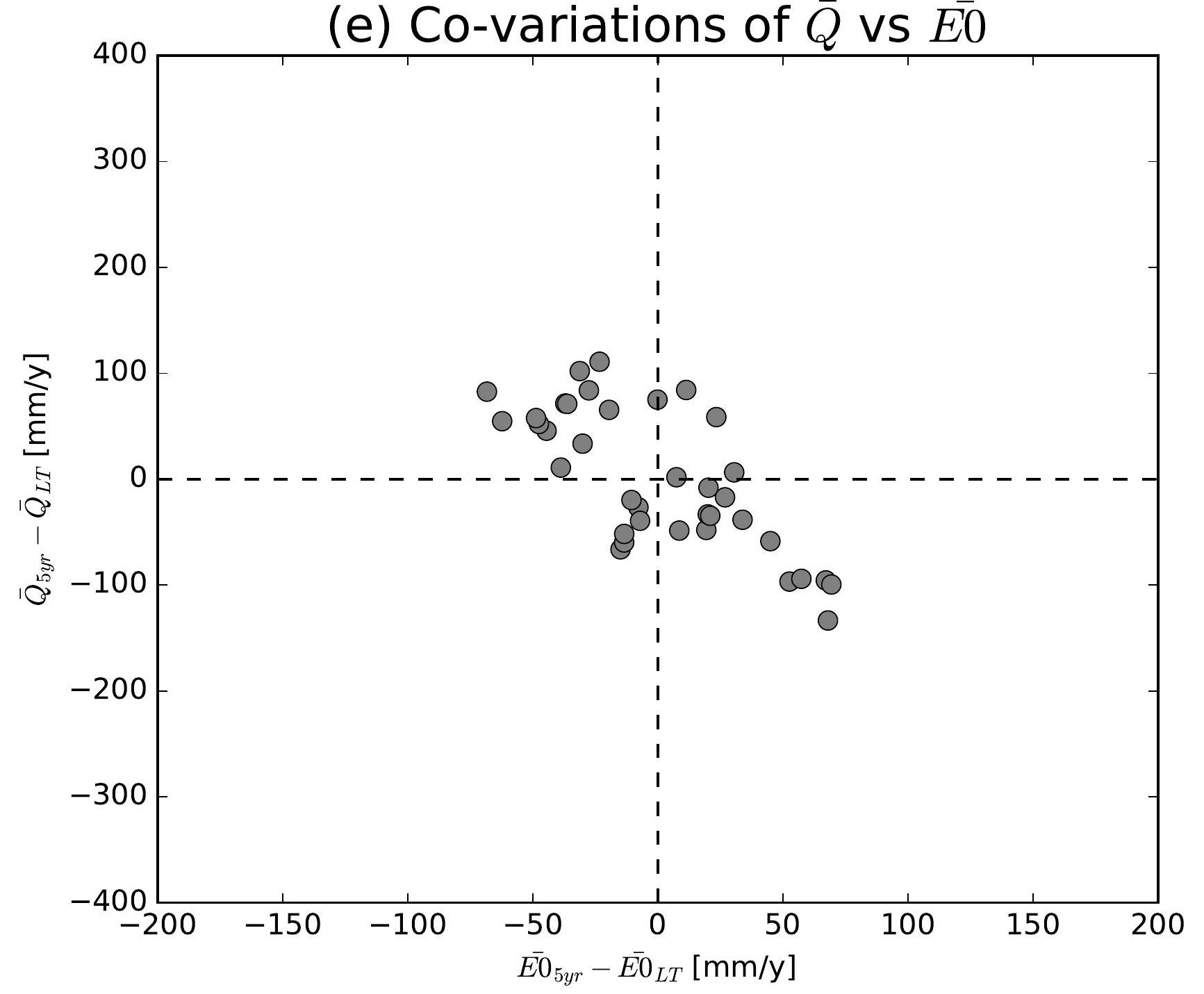

(h) Co-variations of $\bar{Q}$ vs $\overline{E 0}$

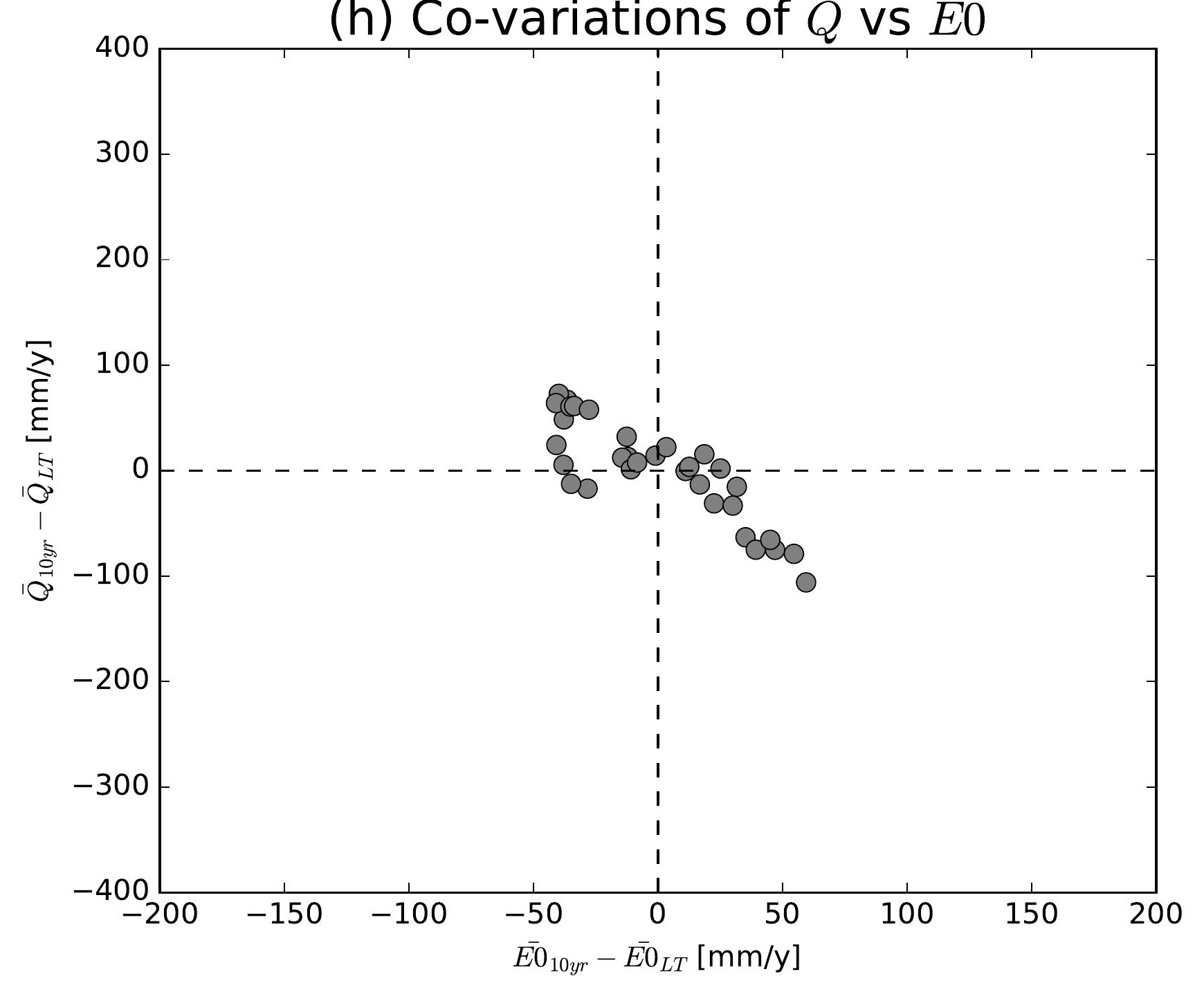

(k) Co-variations of $\bar{Q}$ vs $\overline{E 0}$

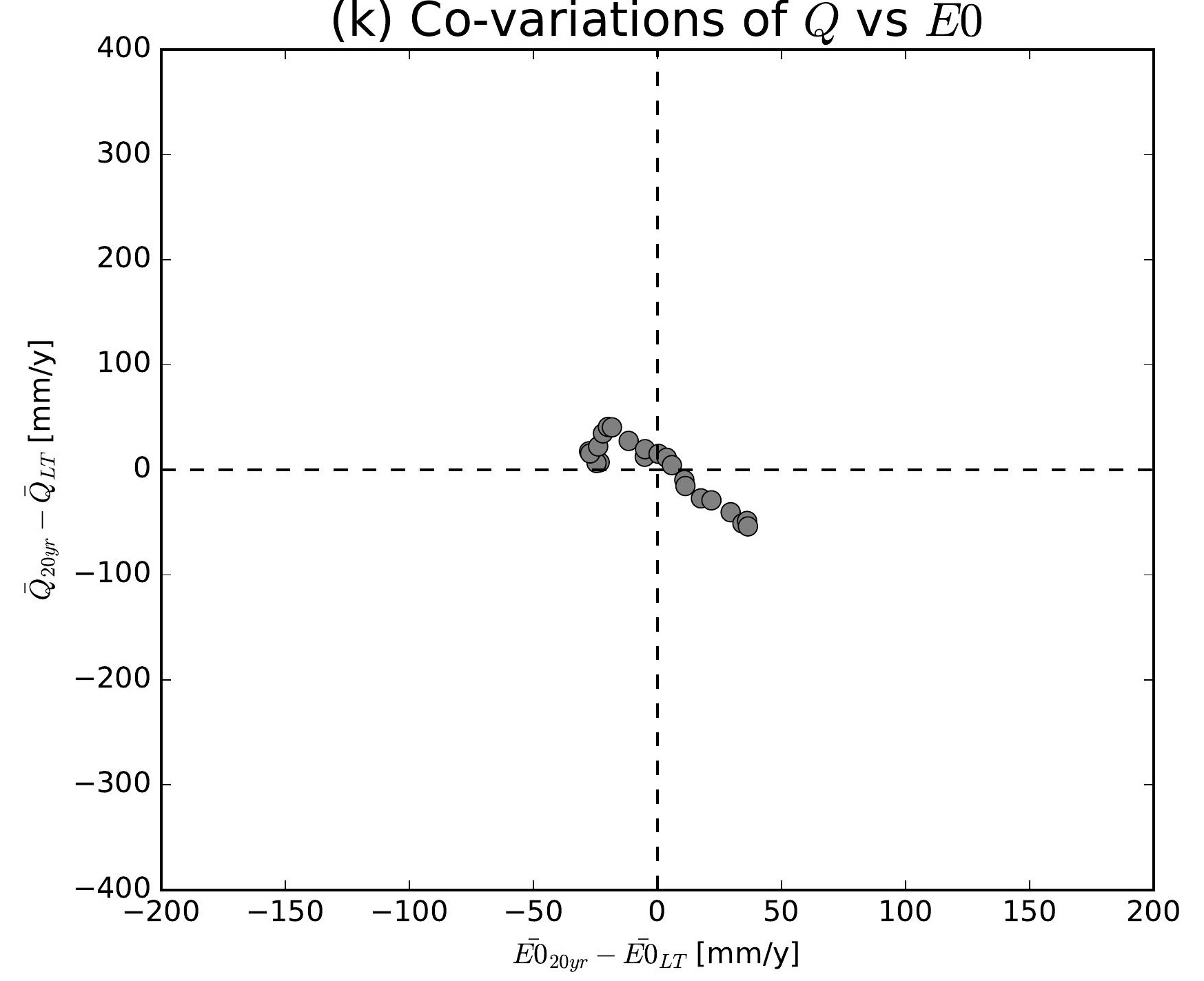

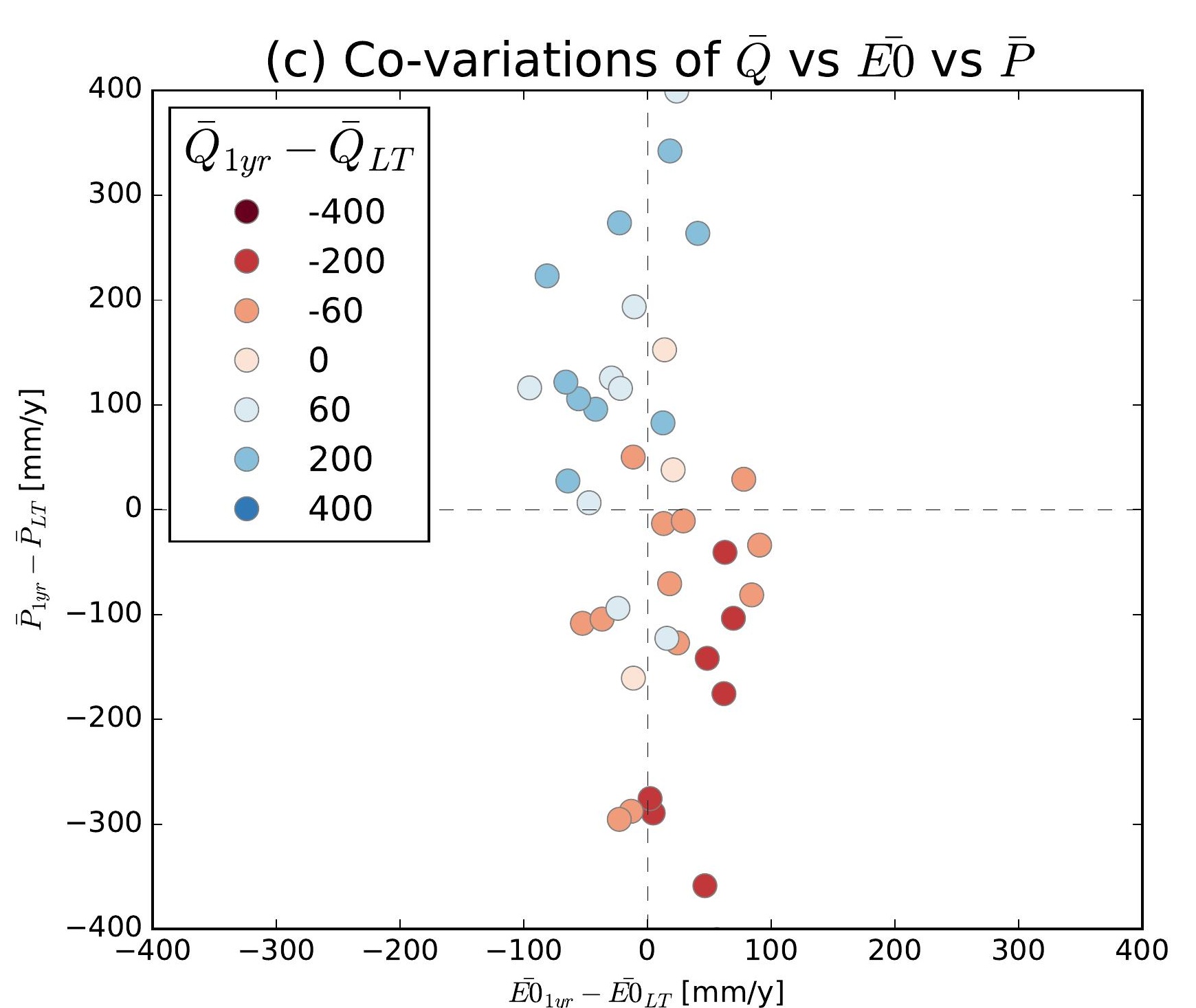
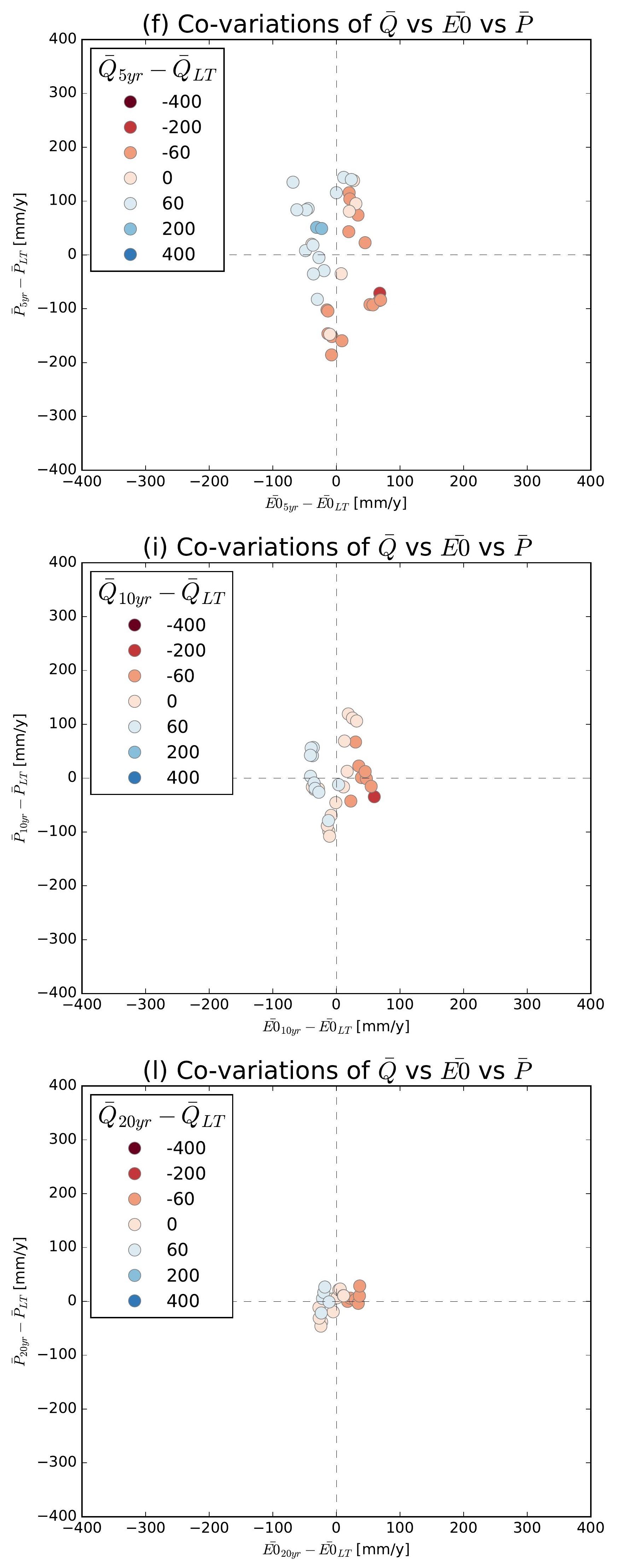

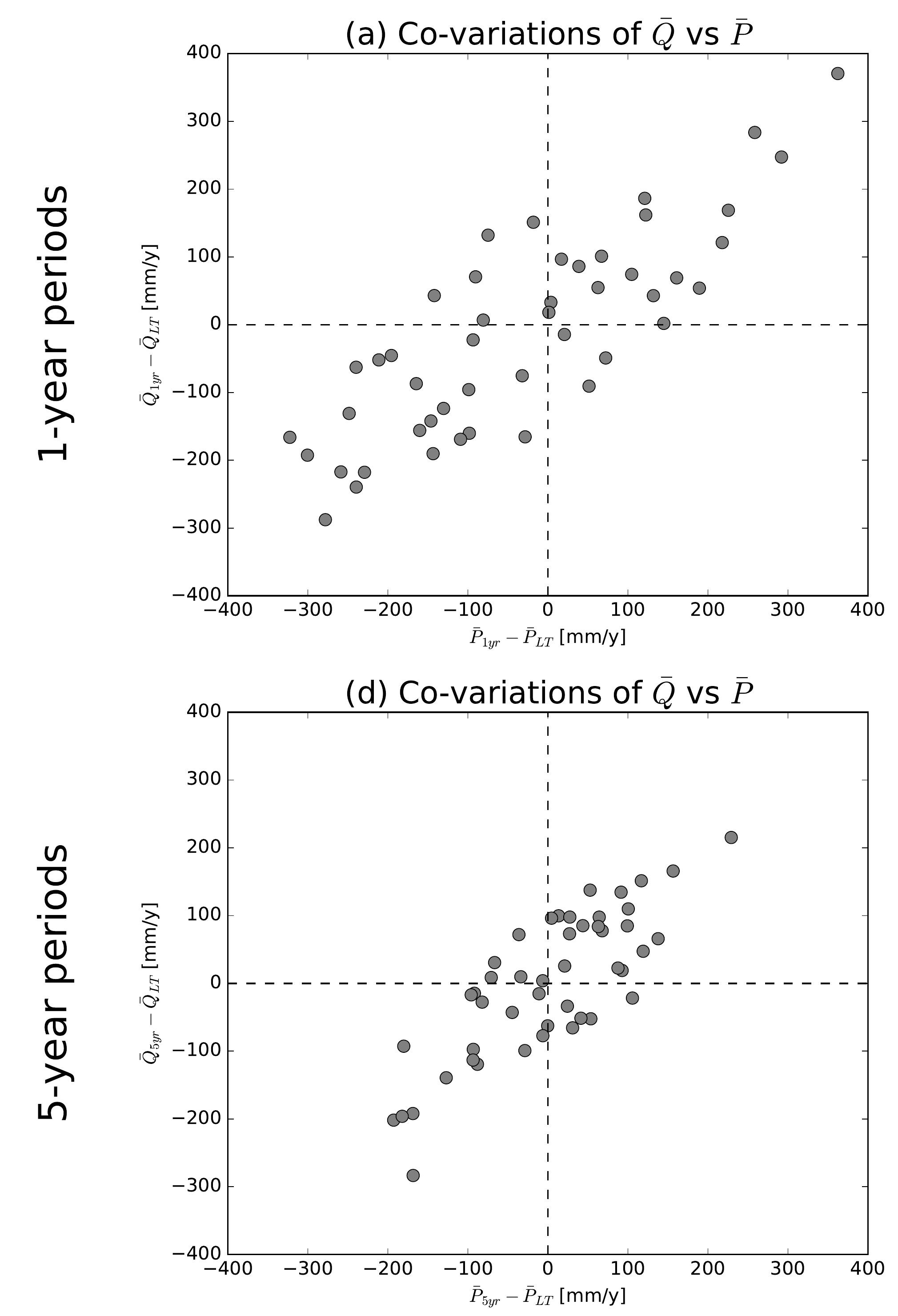

(g) Co-variations of $\bar{Q}$ vs $\bar{P}$

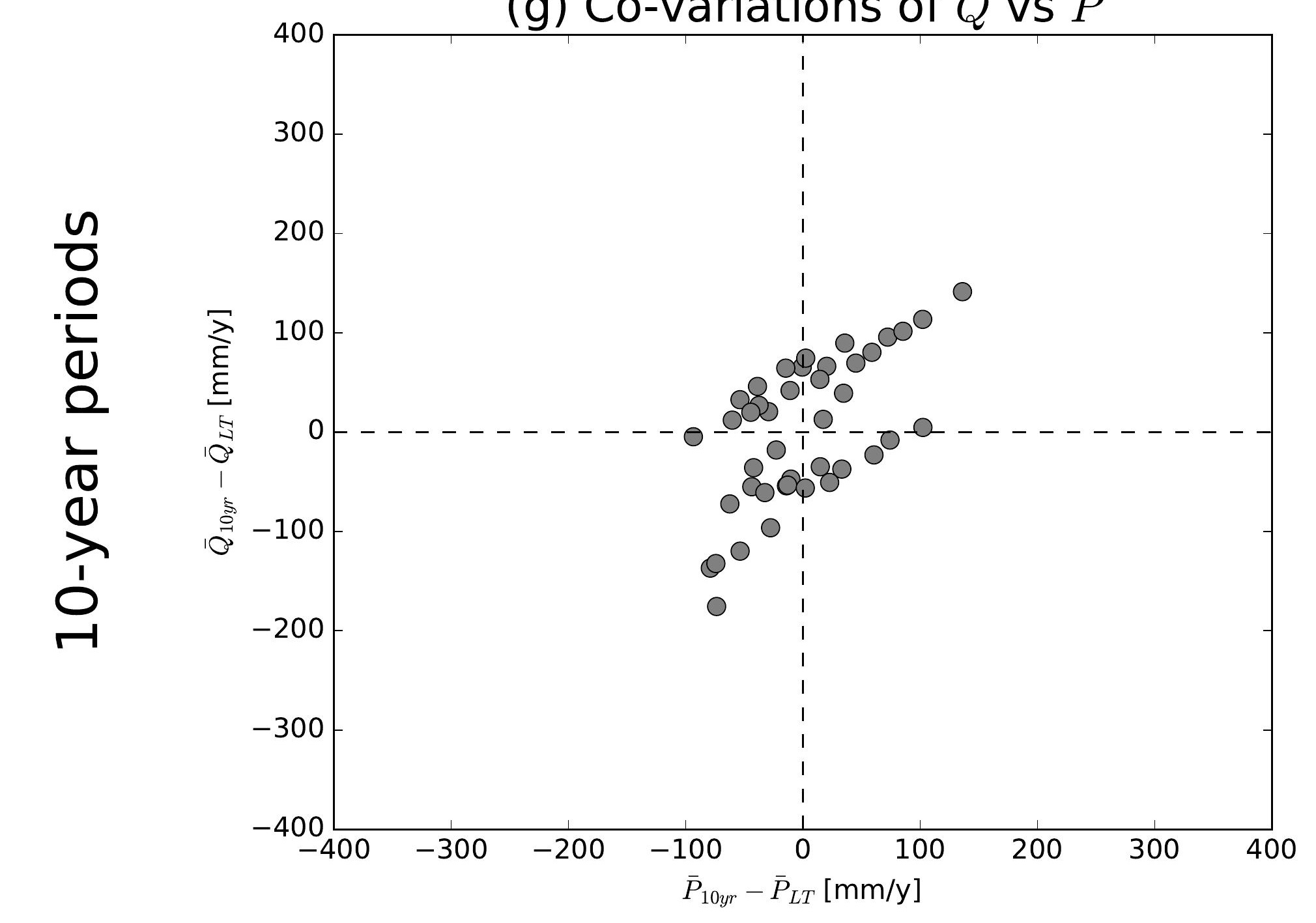

(j) Co-variations of $\bar{Q}$ vs $\bar{P}$

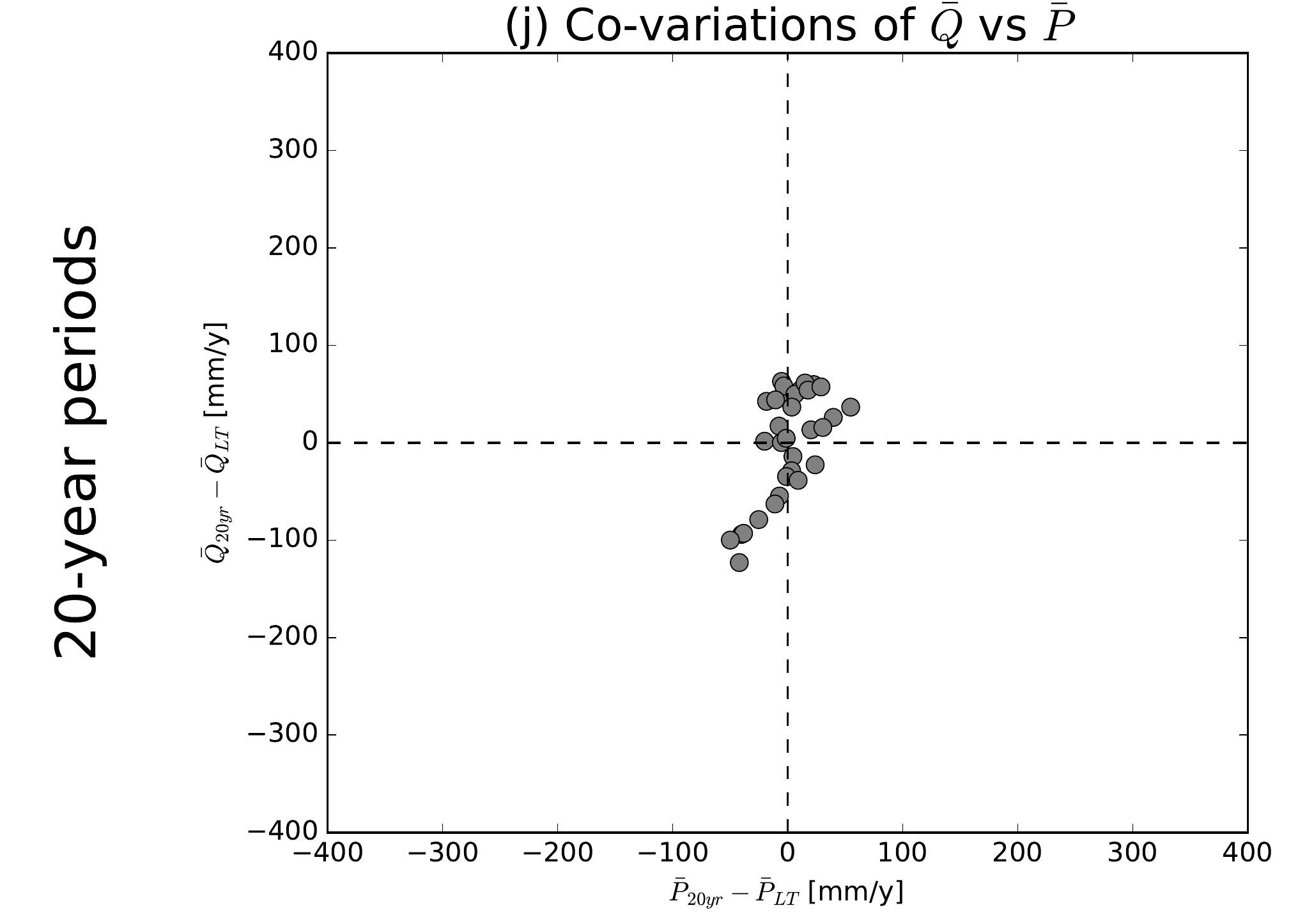

(b) Co-variations of $\bar{Q}$ vs $\overline{E 0}$

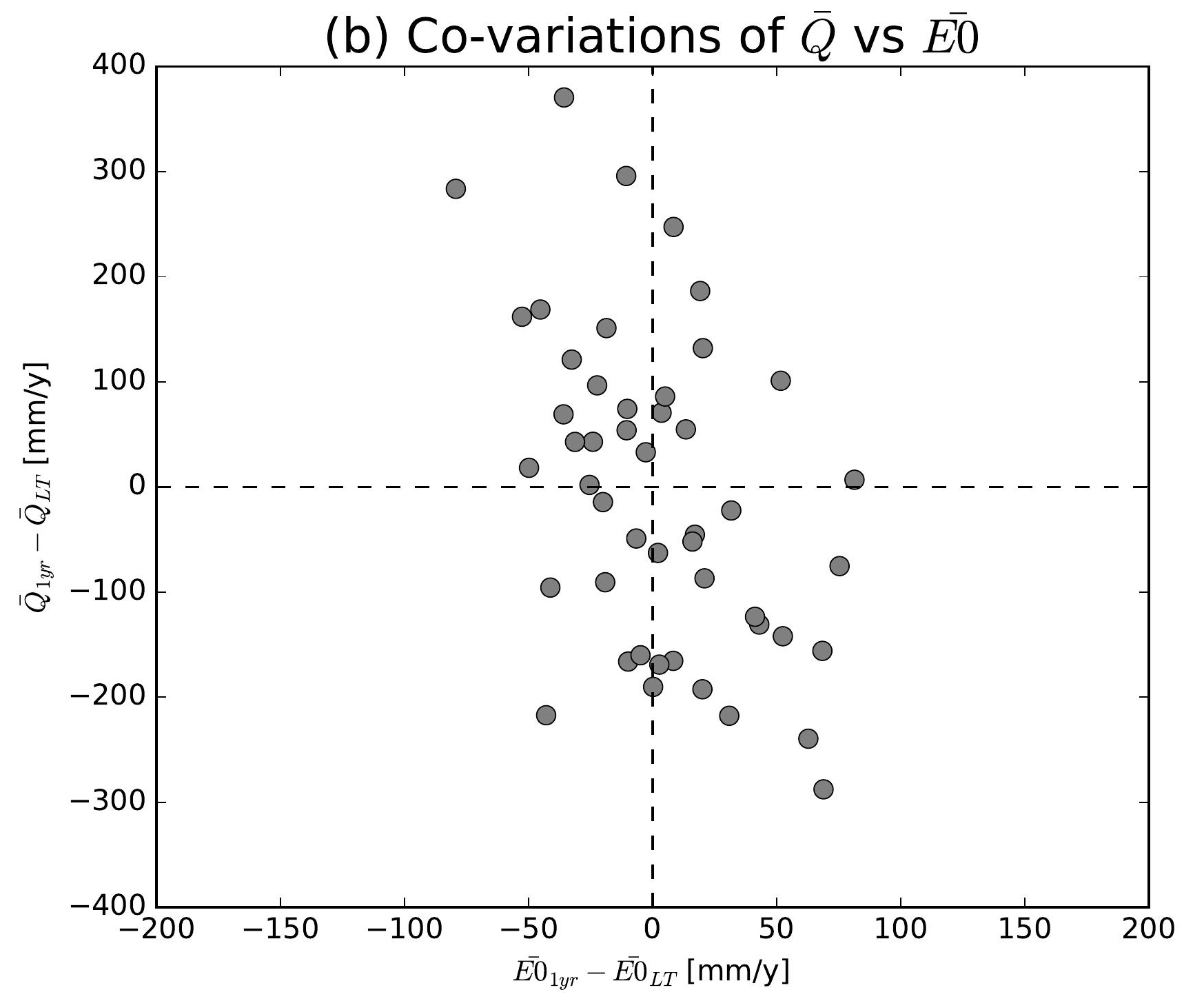

(e) Co-variations of $\bar{Q}$ vs $\overline{E 0}$

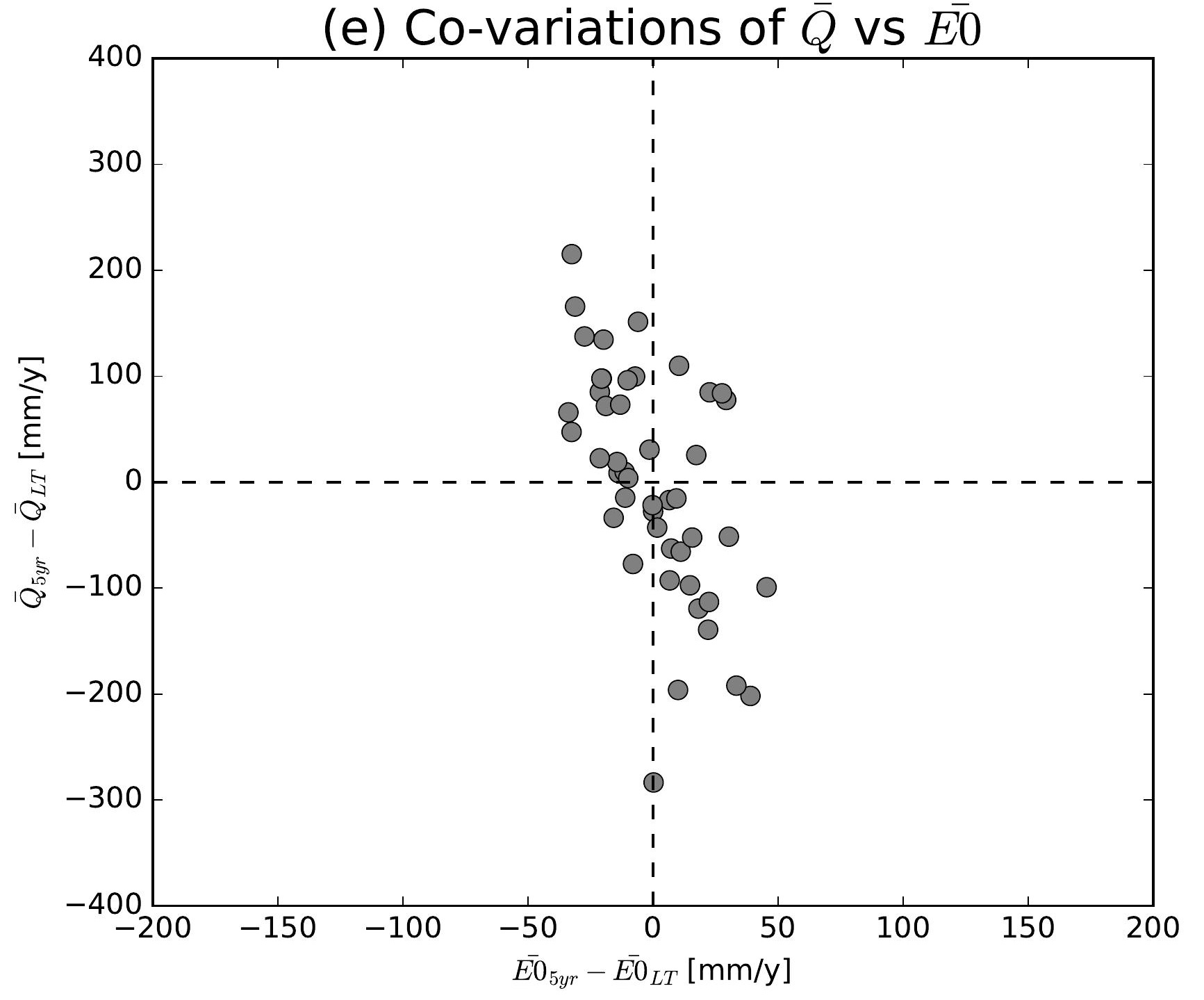

(h) Co-variations of $\bar{Q}$ vs $\overline{E 0}$

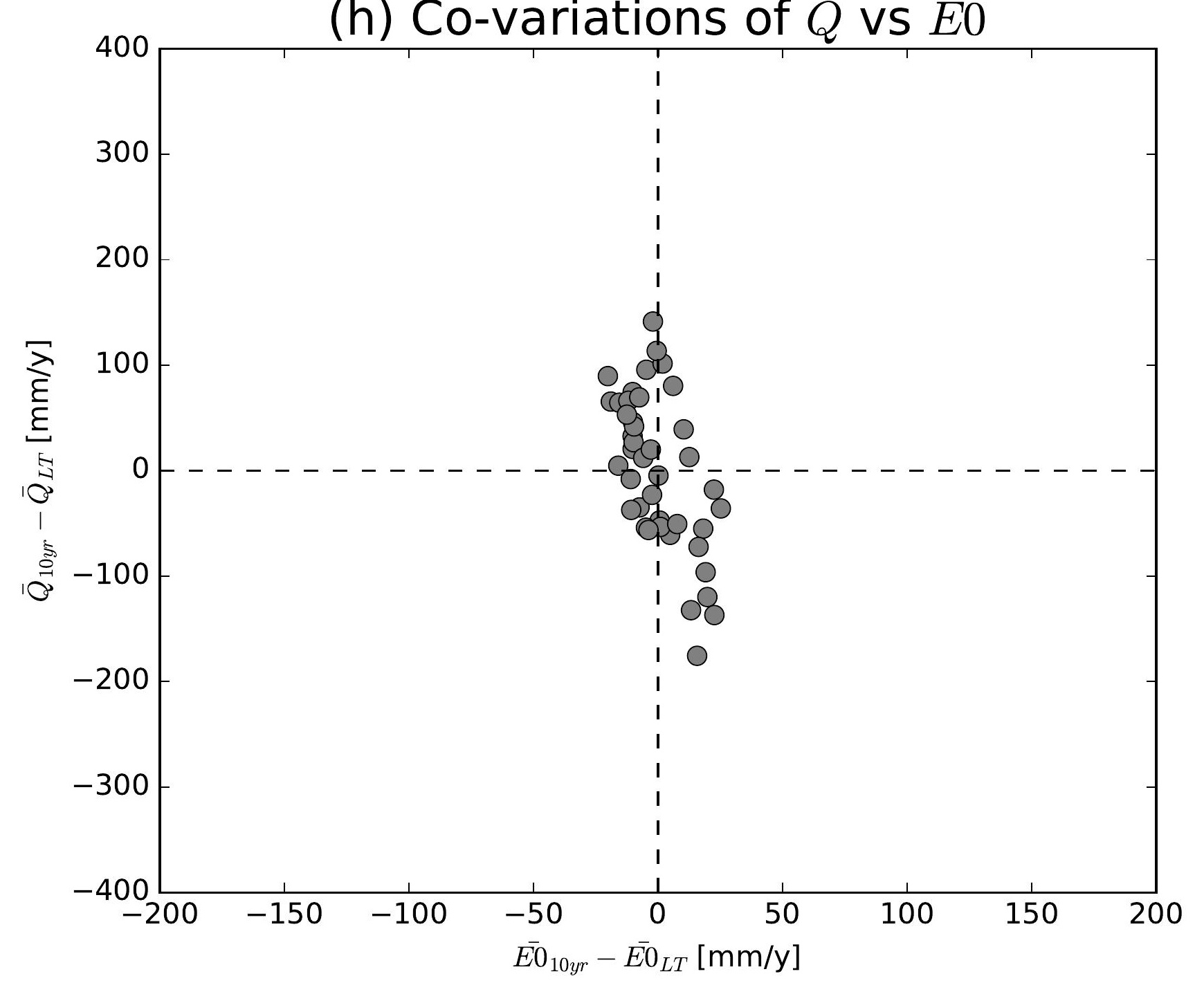

(k) Co-variations of $\bar{Q}$ vs $\overline{E 0}$

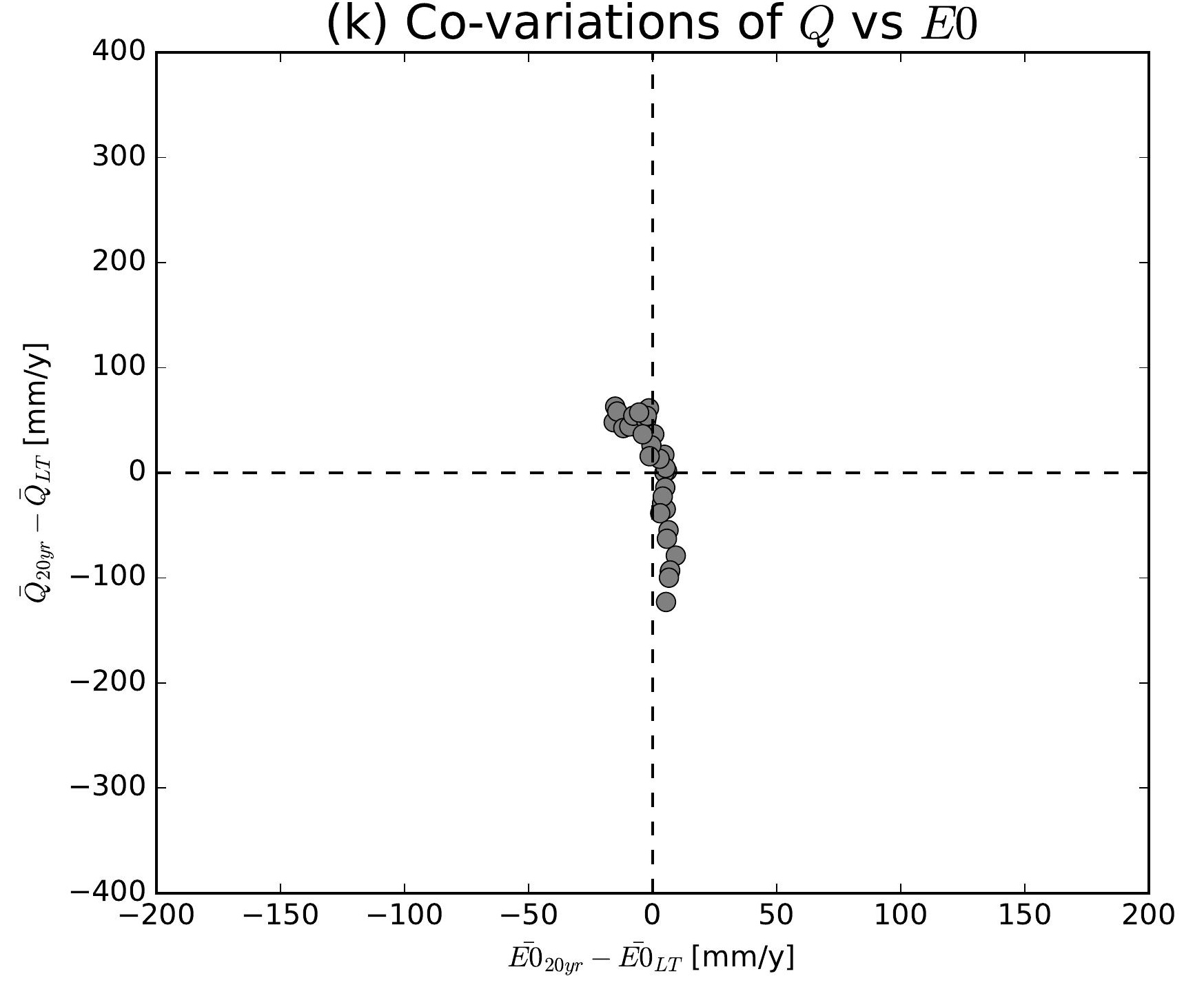

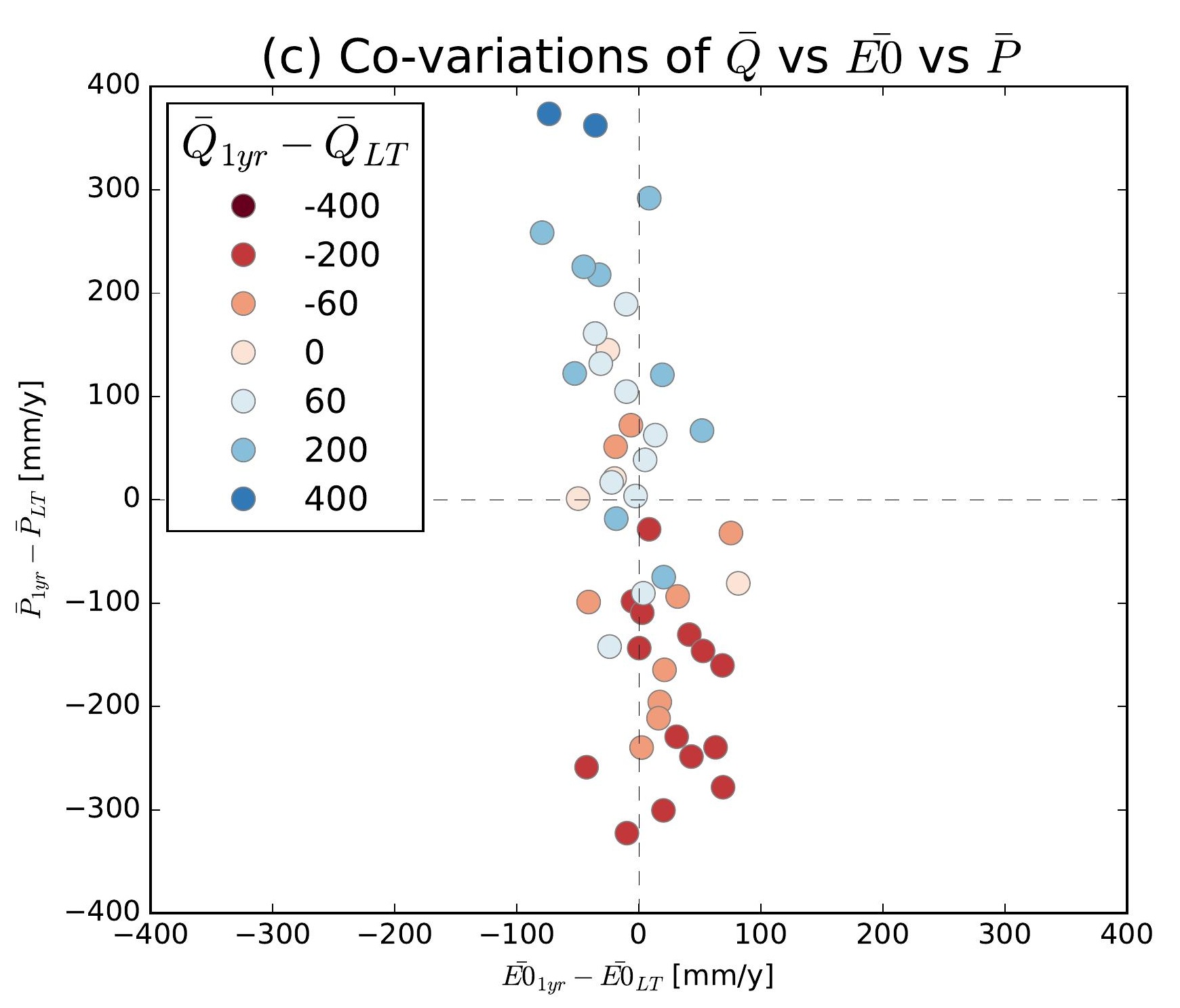
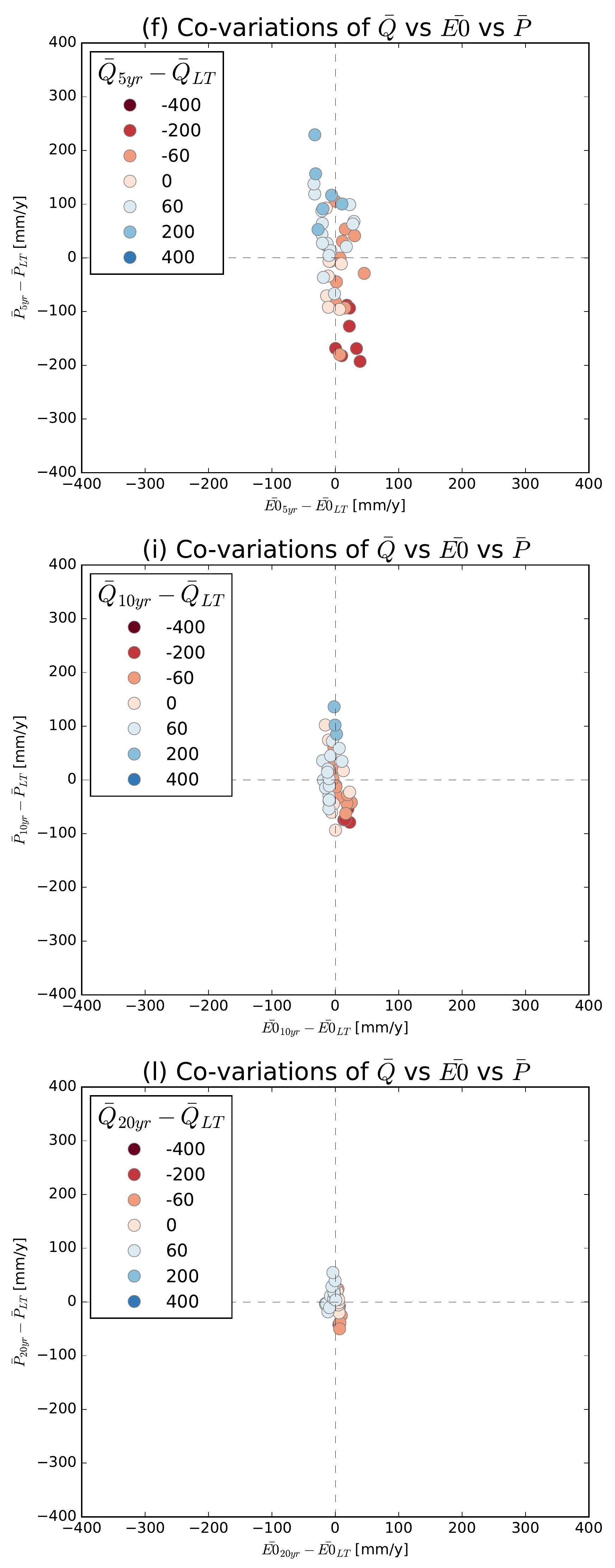

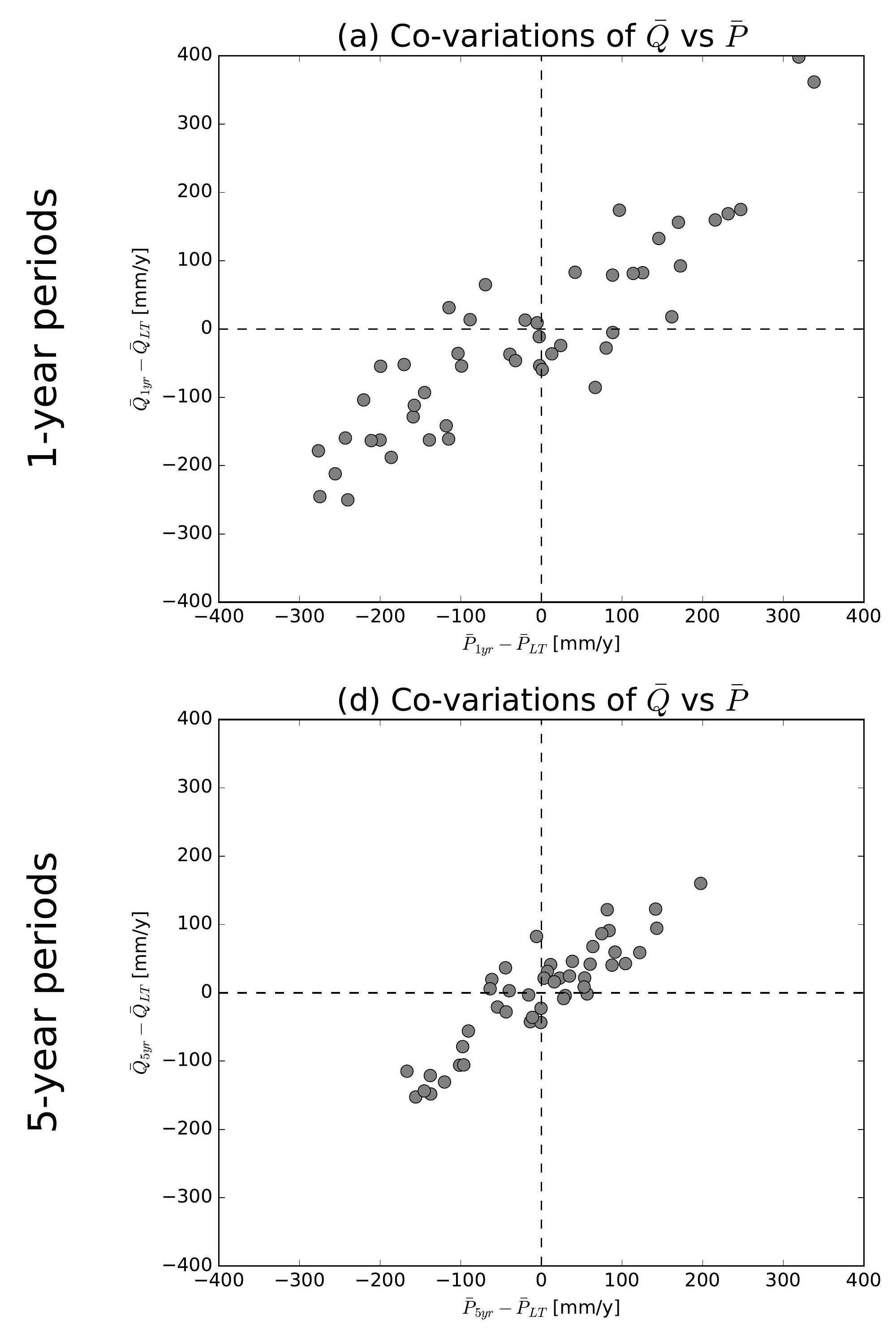

(g) Co-variations of $\bar{Q}$ vs $\bar{P}$

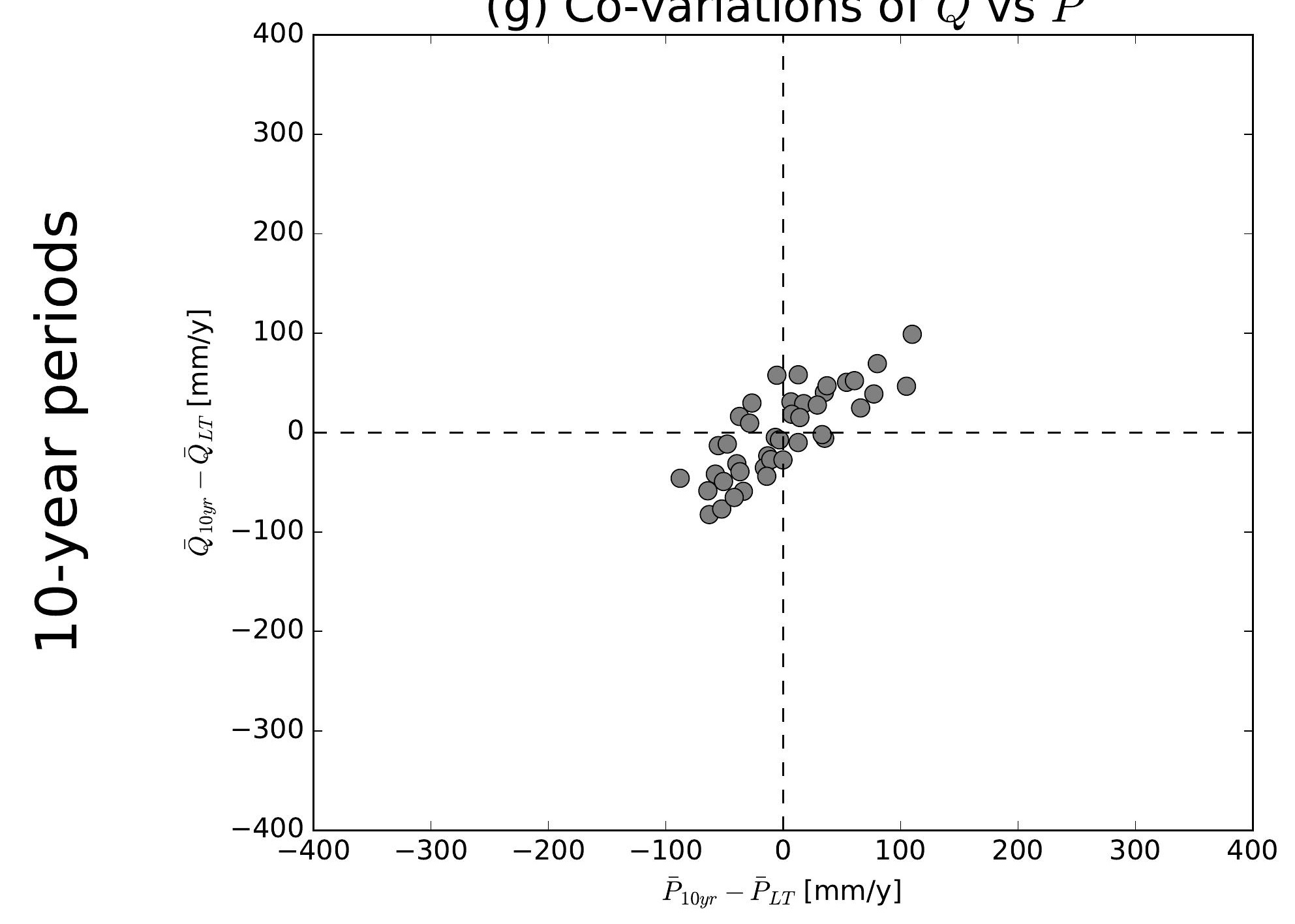

(j) Co-variations of $\bar{Q}$ vs $\bar{P}$

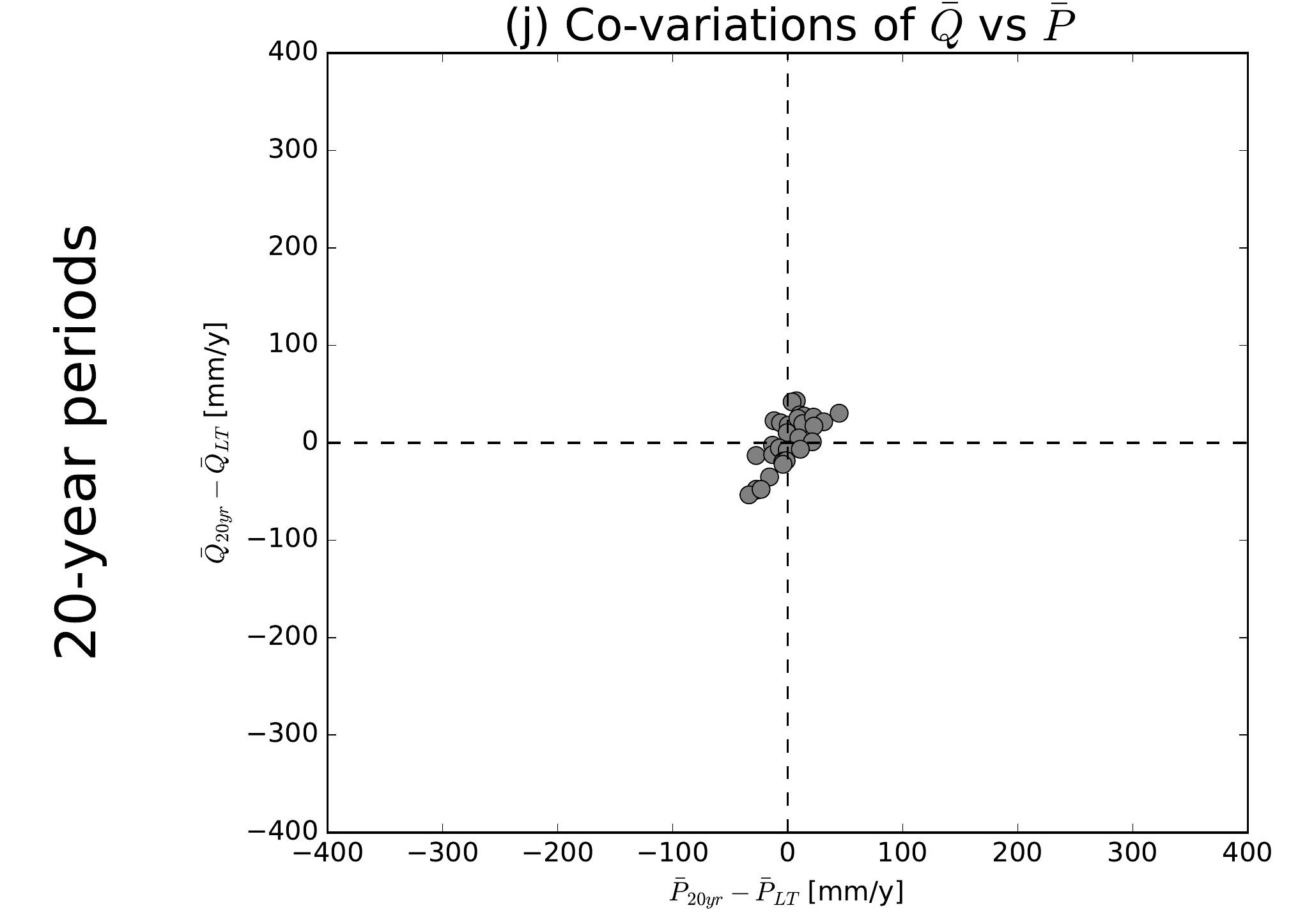

(b) Co-variations of $\bar{Q}$ vs $\overline{E 0}$

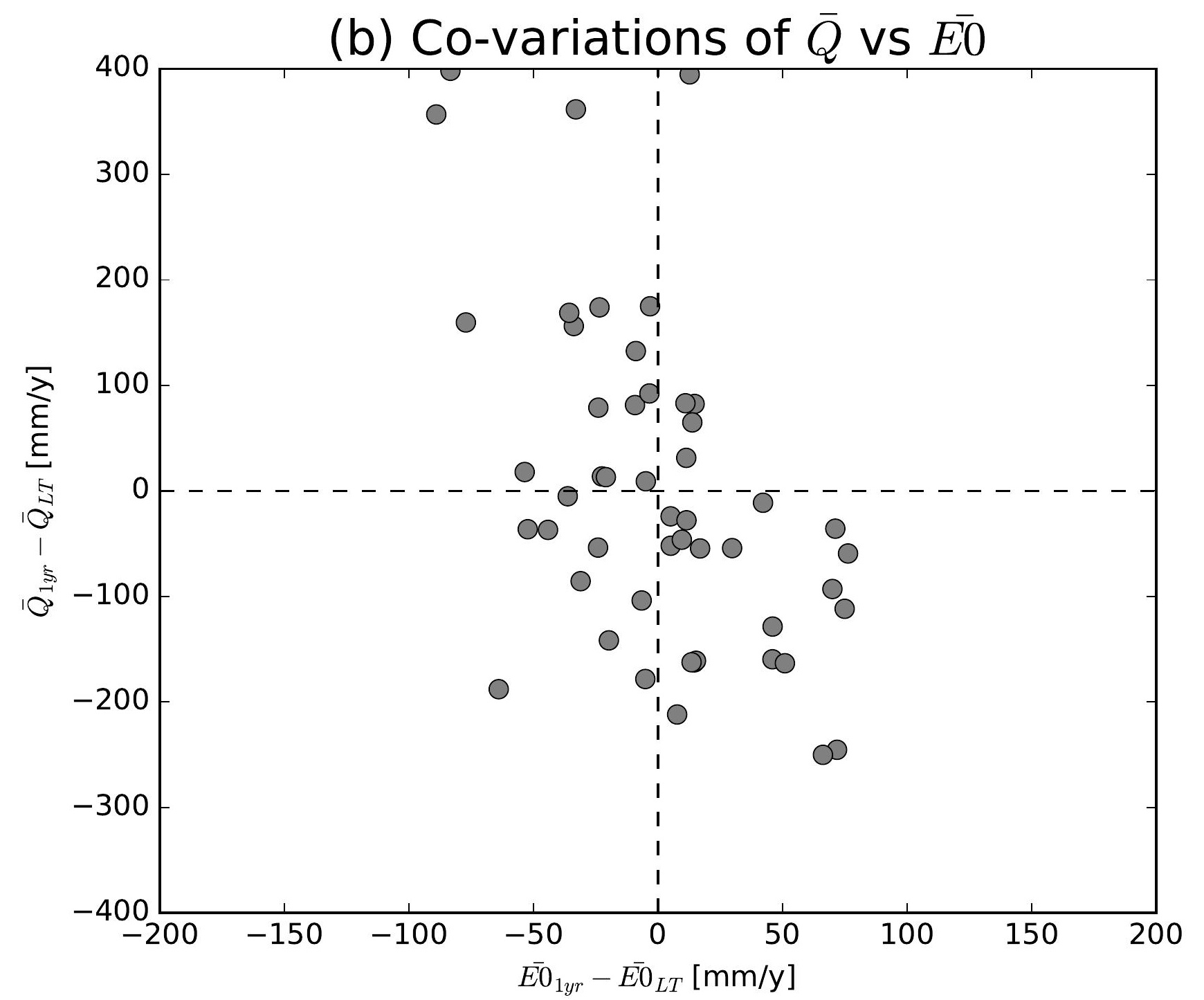

(e) Co-variations of $\bar{Q}$ vs $\overline{E 0}$

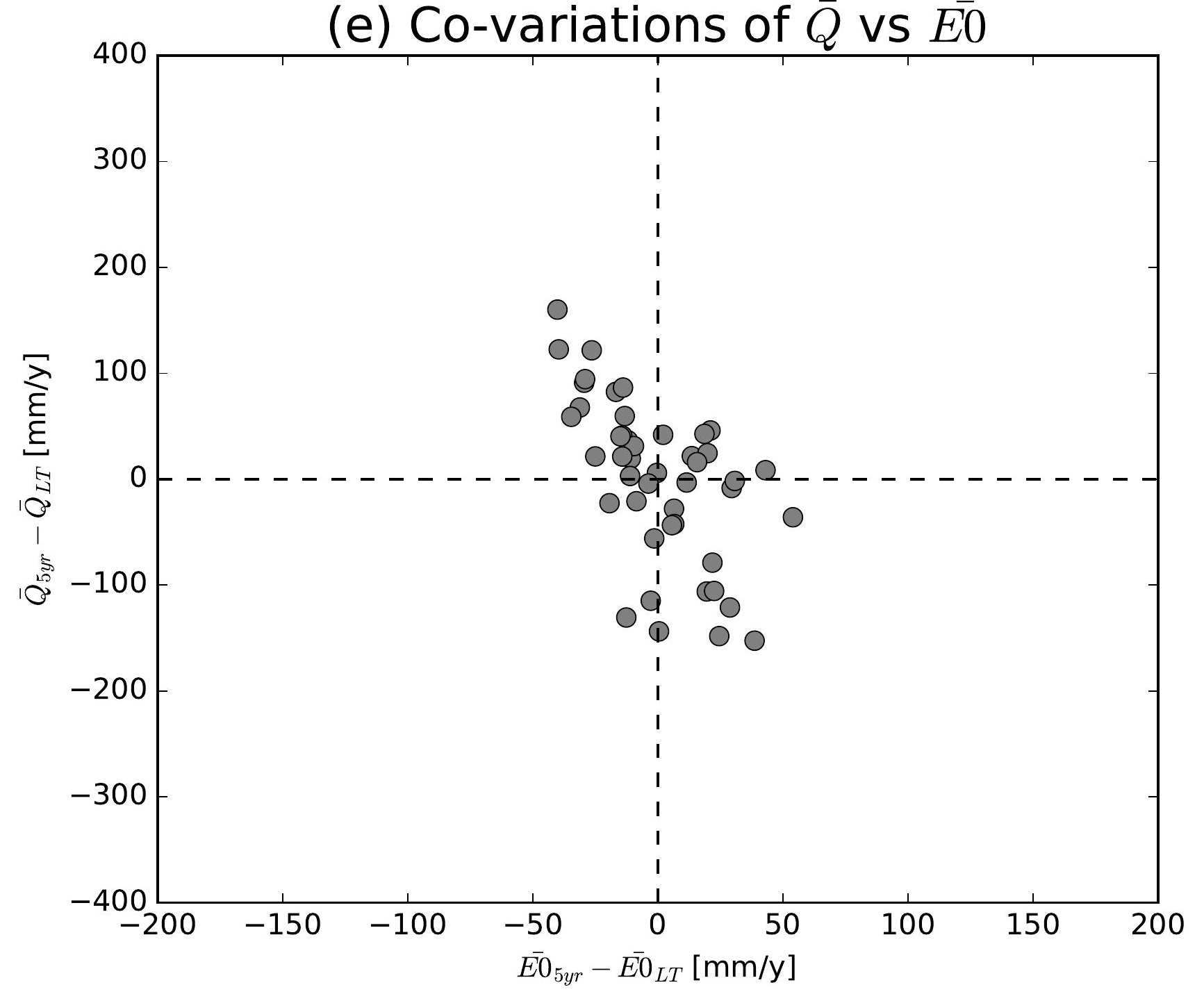

(h) Co-variations of $\bar{Q}$ vs $\overline{E 0}$

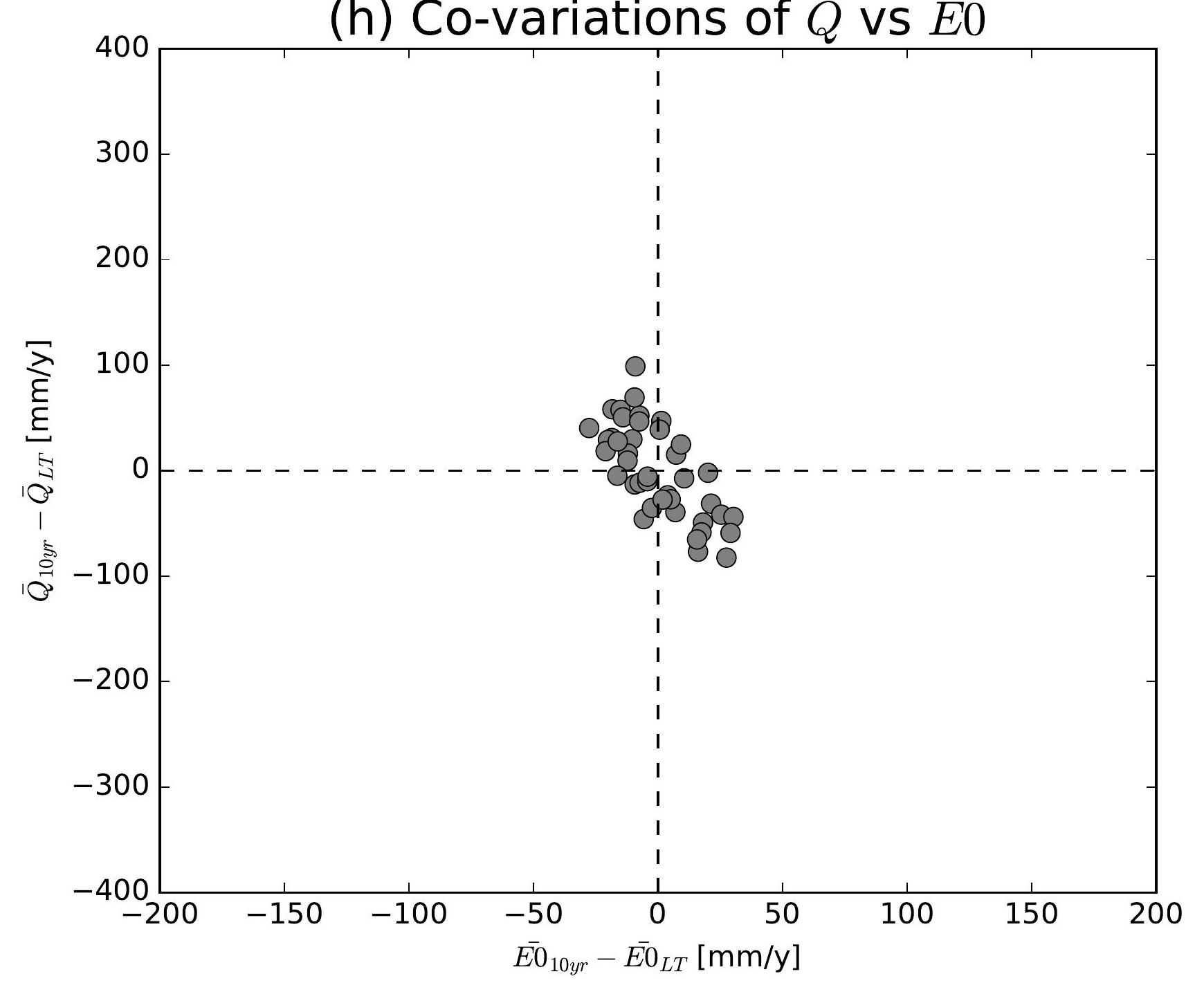

(k) Co-variations of $\bar{Q}$ vs $\overline{E 0}$

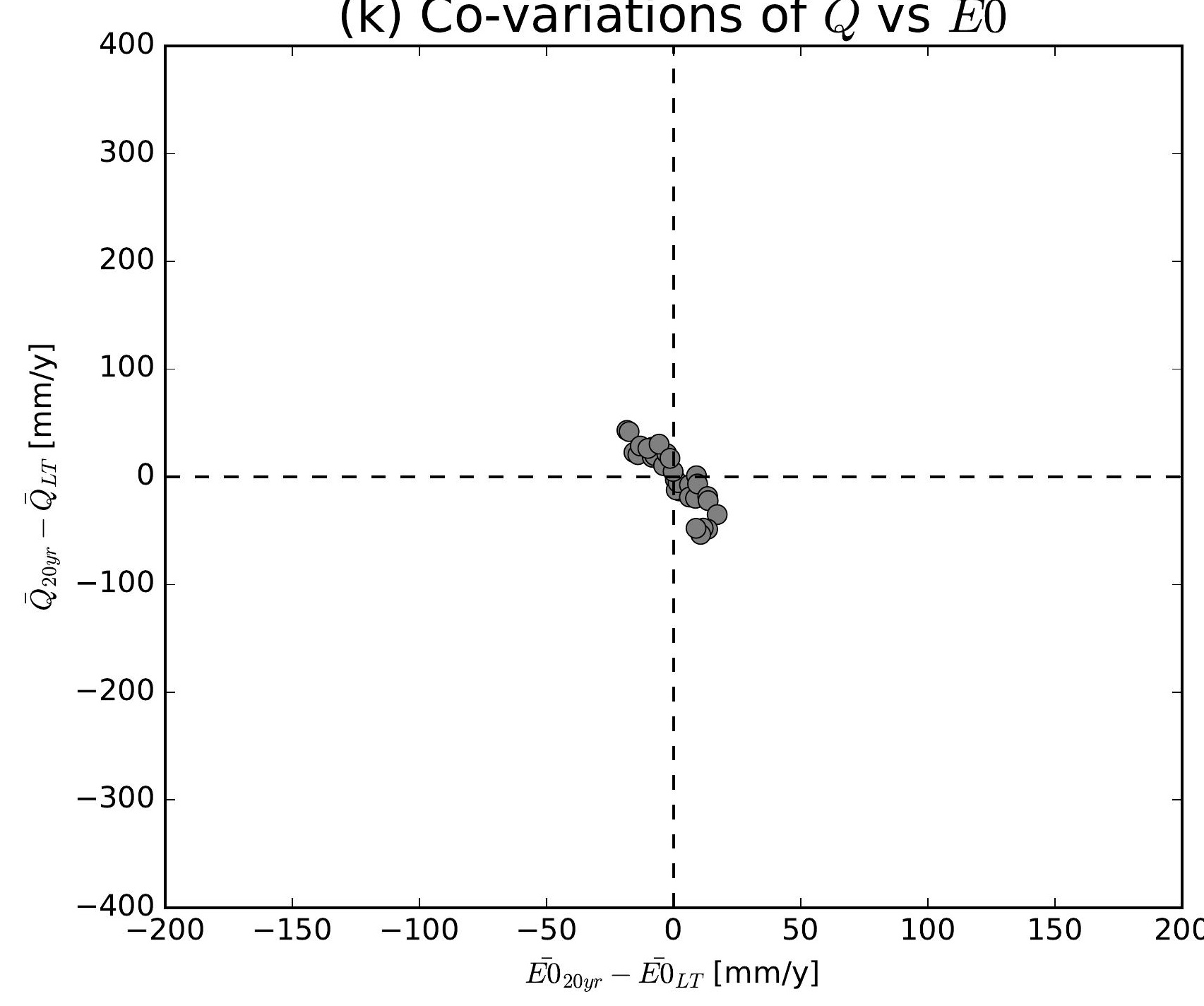

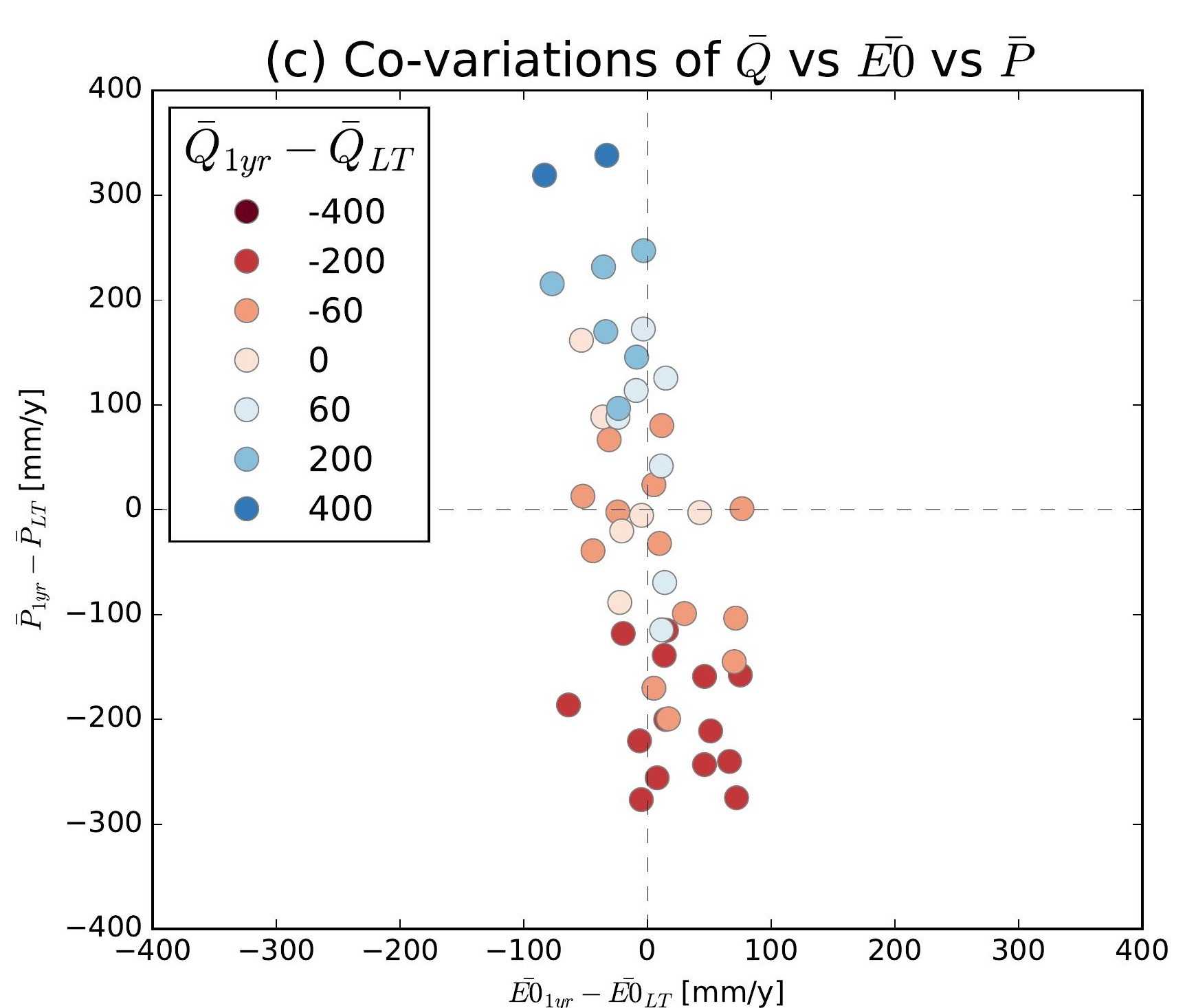
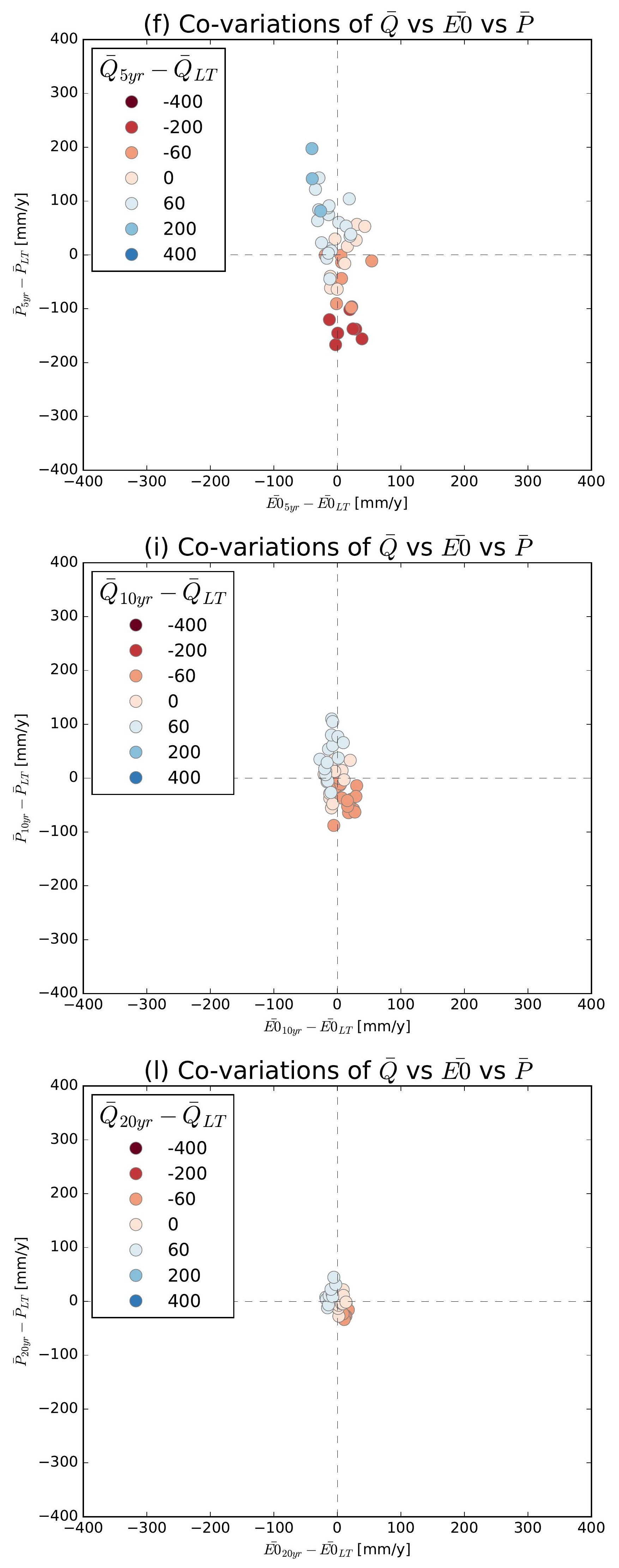

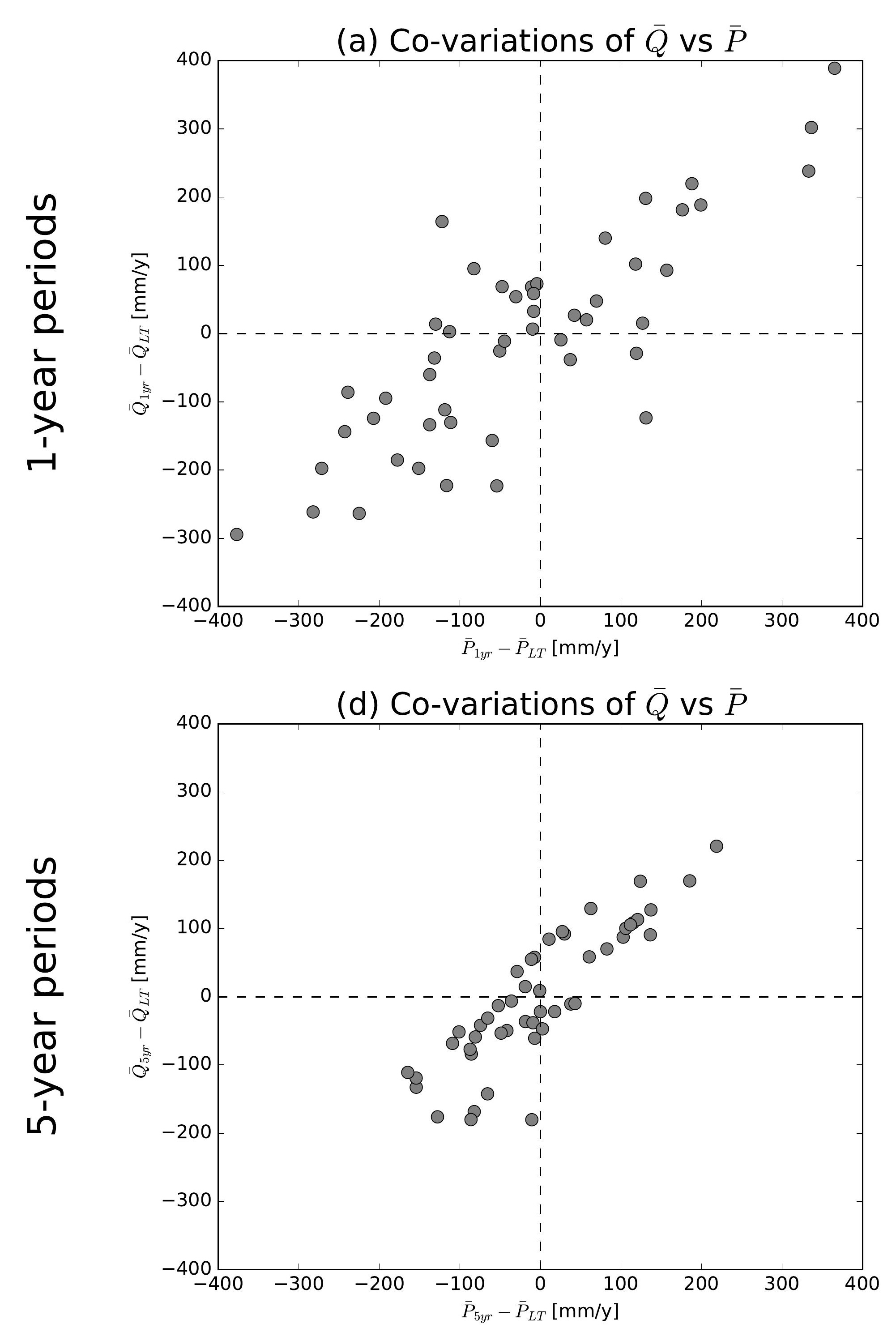

(g) Co-variations of $\bar{Q}$ vs $\bar{P}$

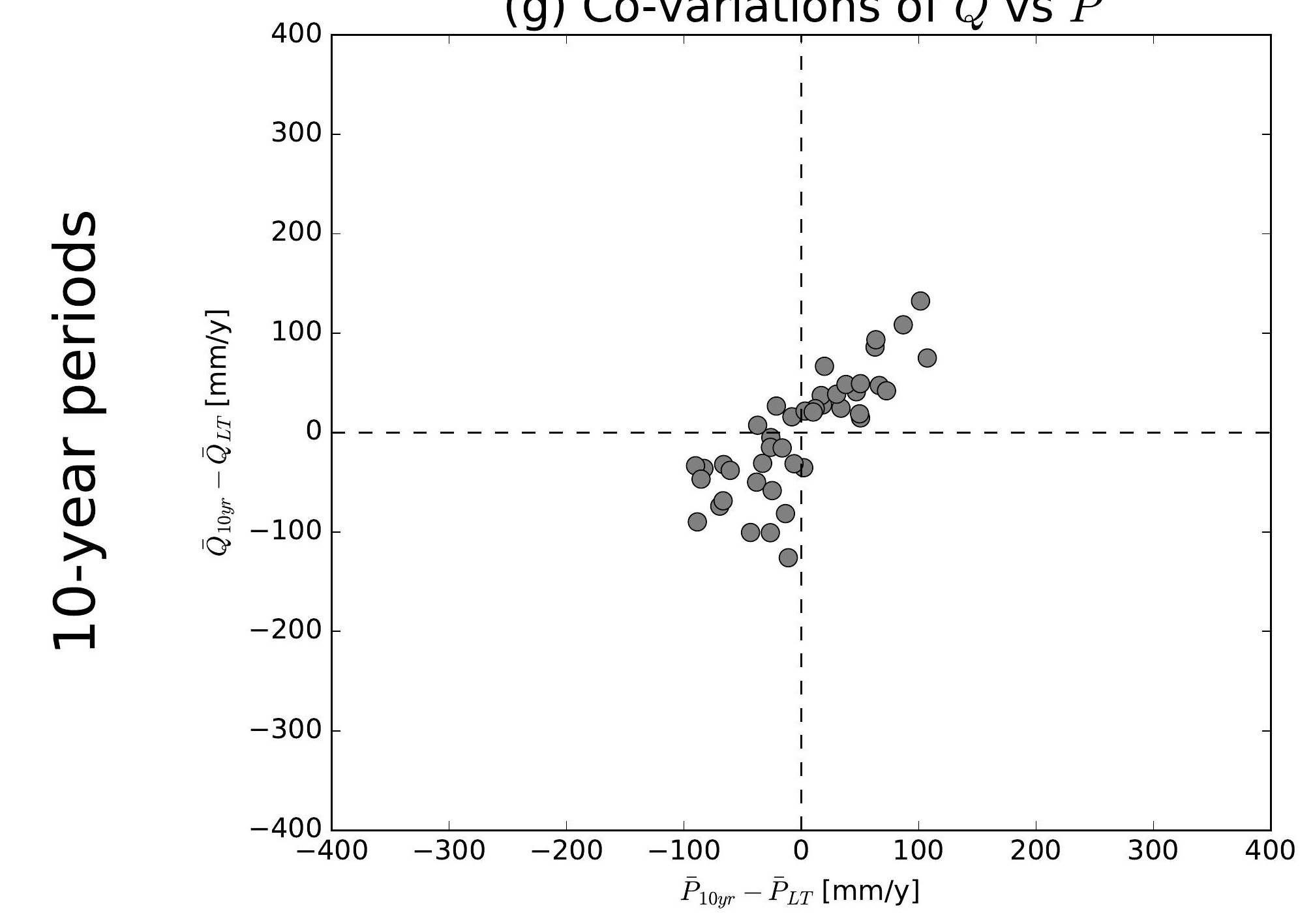

(j) Co-variations of $\bar{Q}$ vs $\bar{P}$

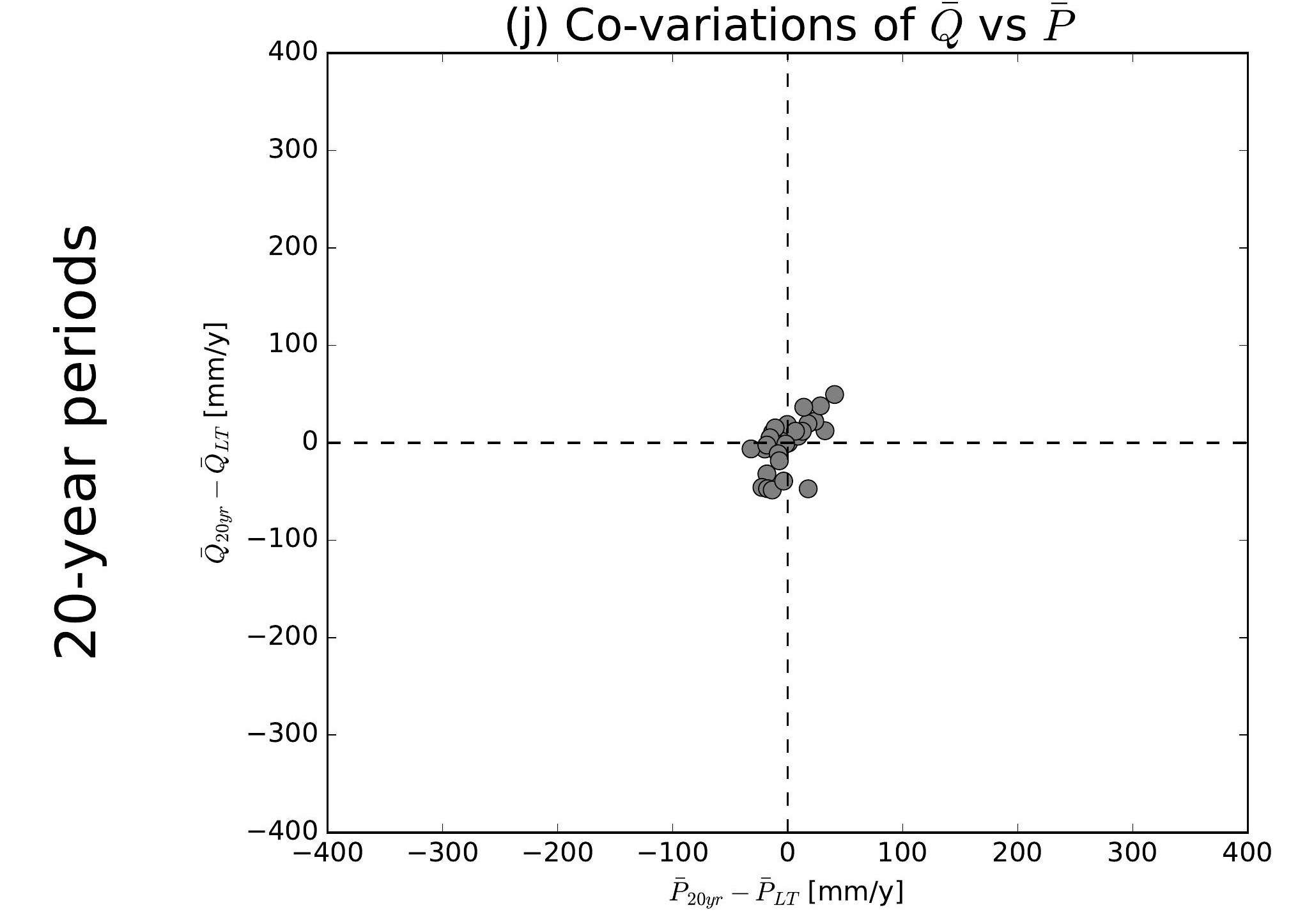

(b) Co-variations of $\bar{Q}$ vs $\overline{E 0}$

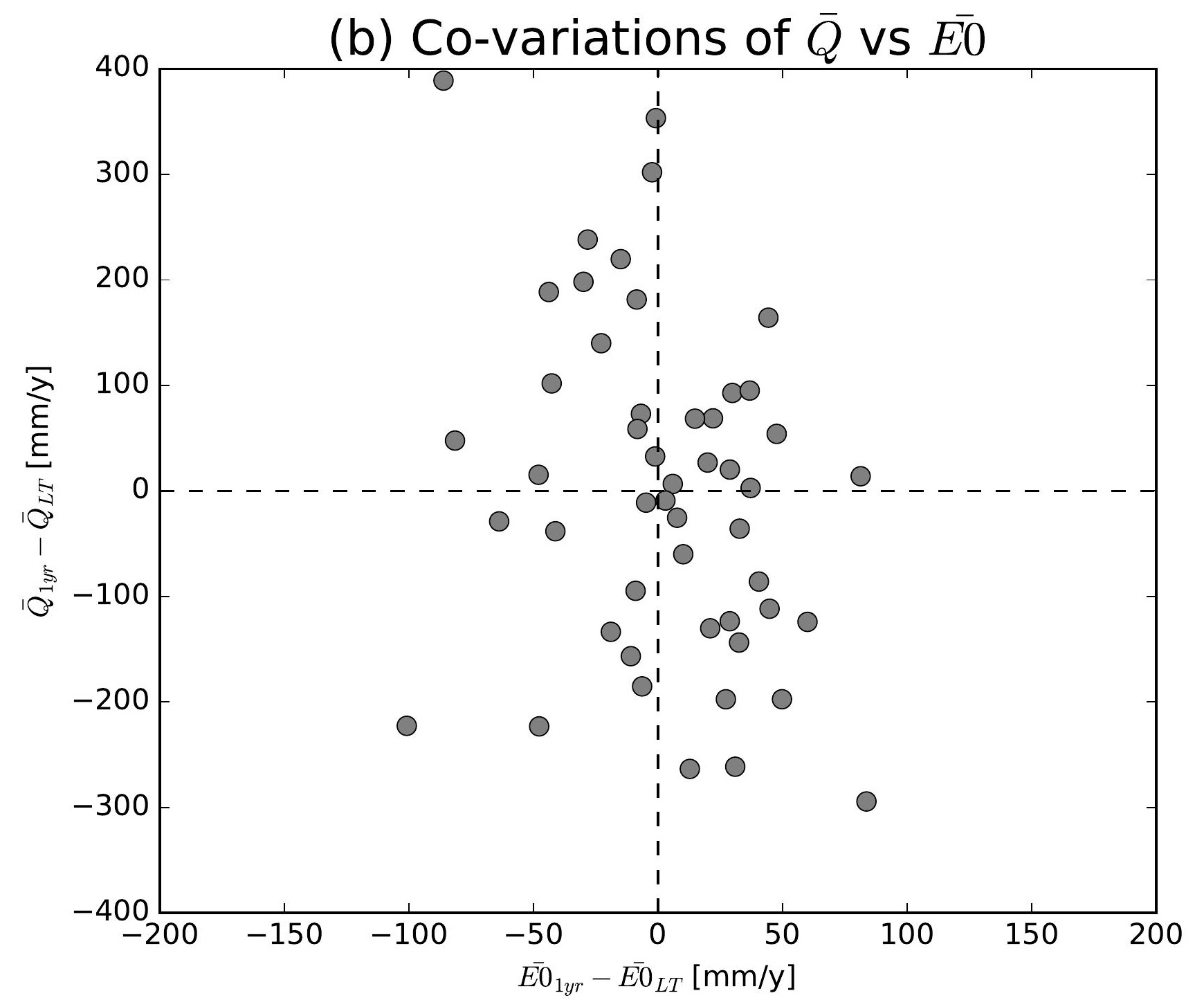

(e) Co-variations of $\bar{Q}$ vs $\overline{E 0}$

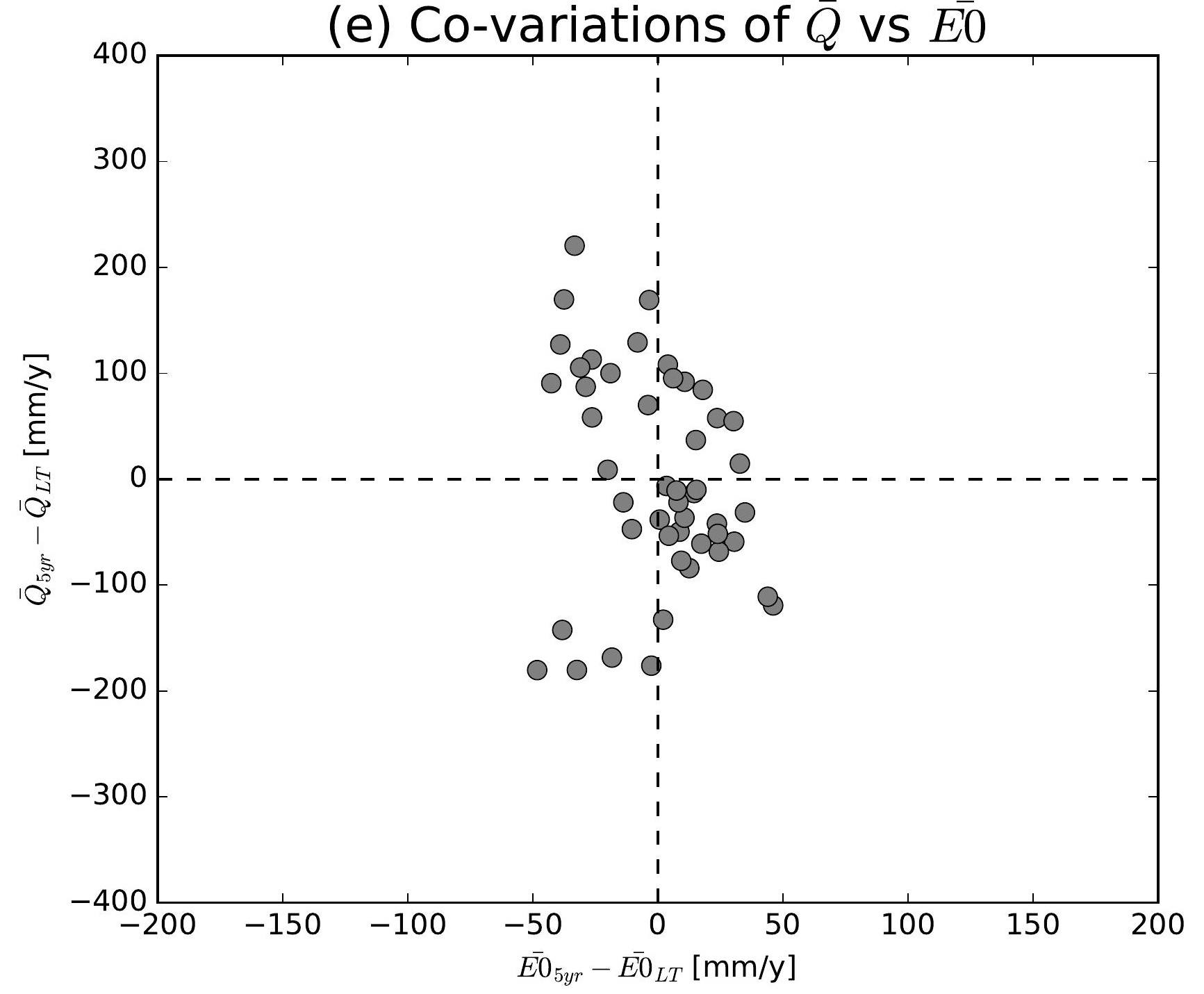

(h) Co-variations of $\bar{Q}$ vs $\overline{E 0}$

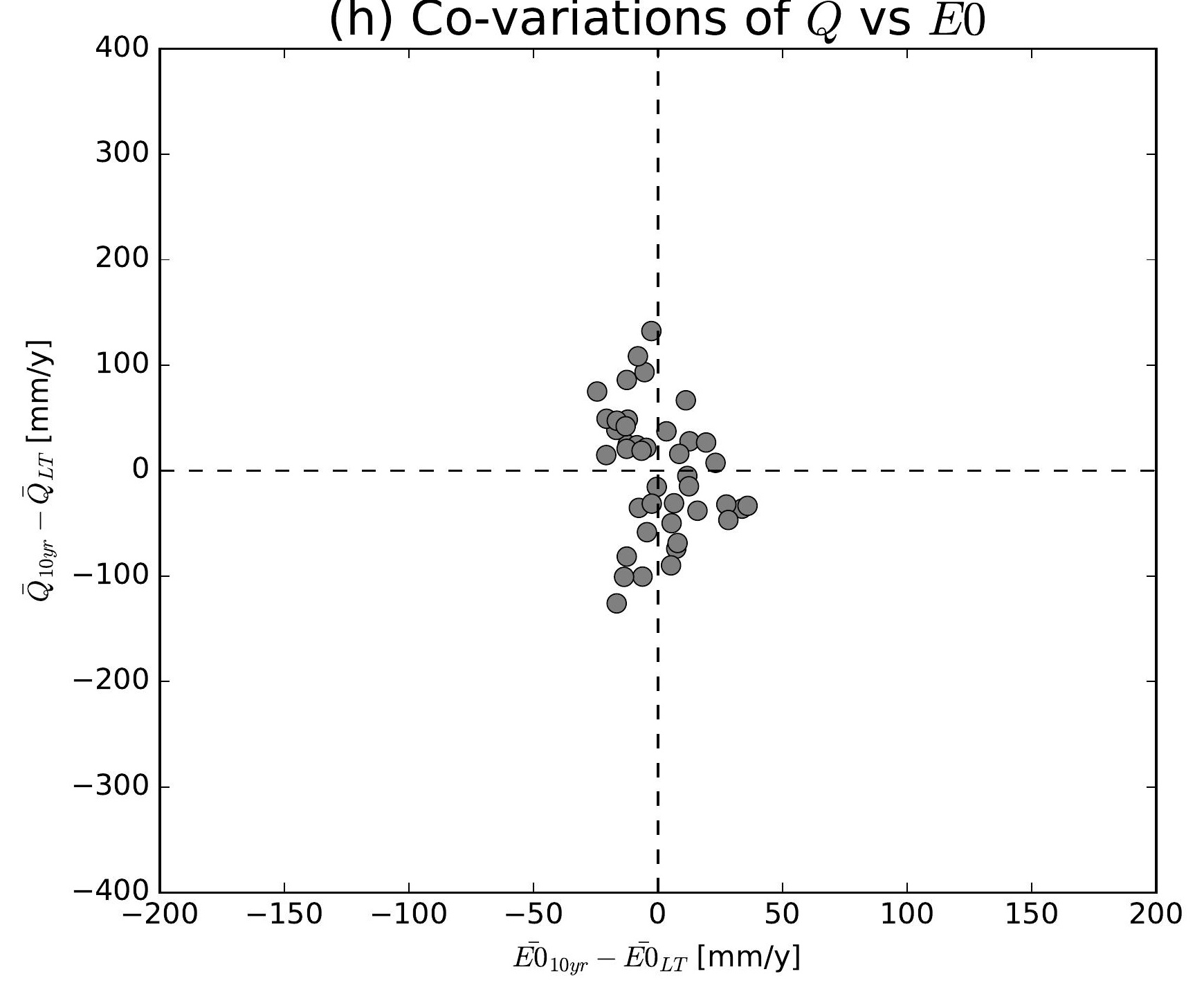

(k) Co-variations of $\bar{Q}$ vs $\overline{E 0}$

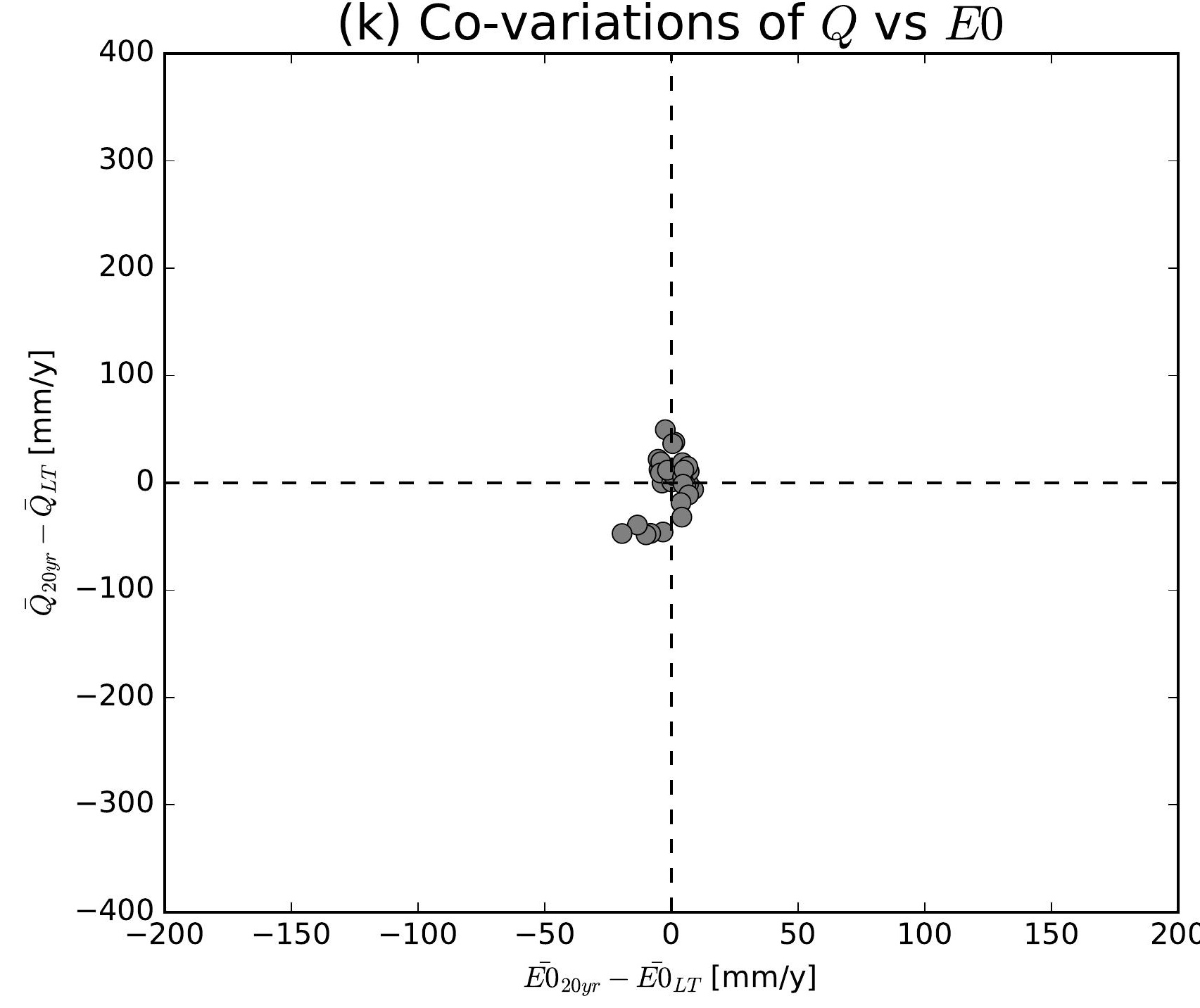

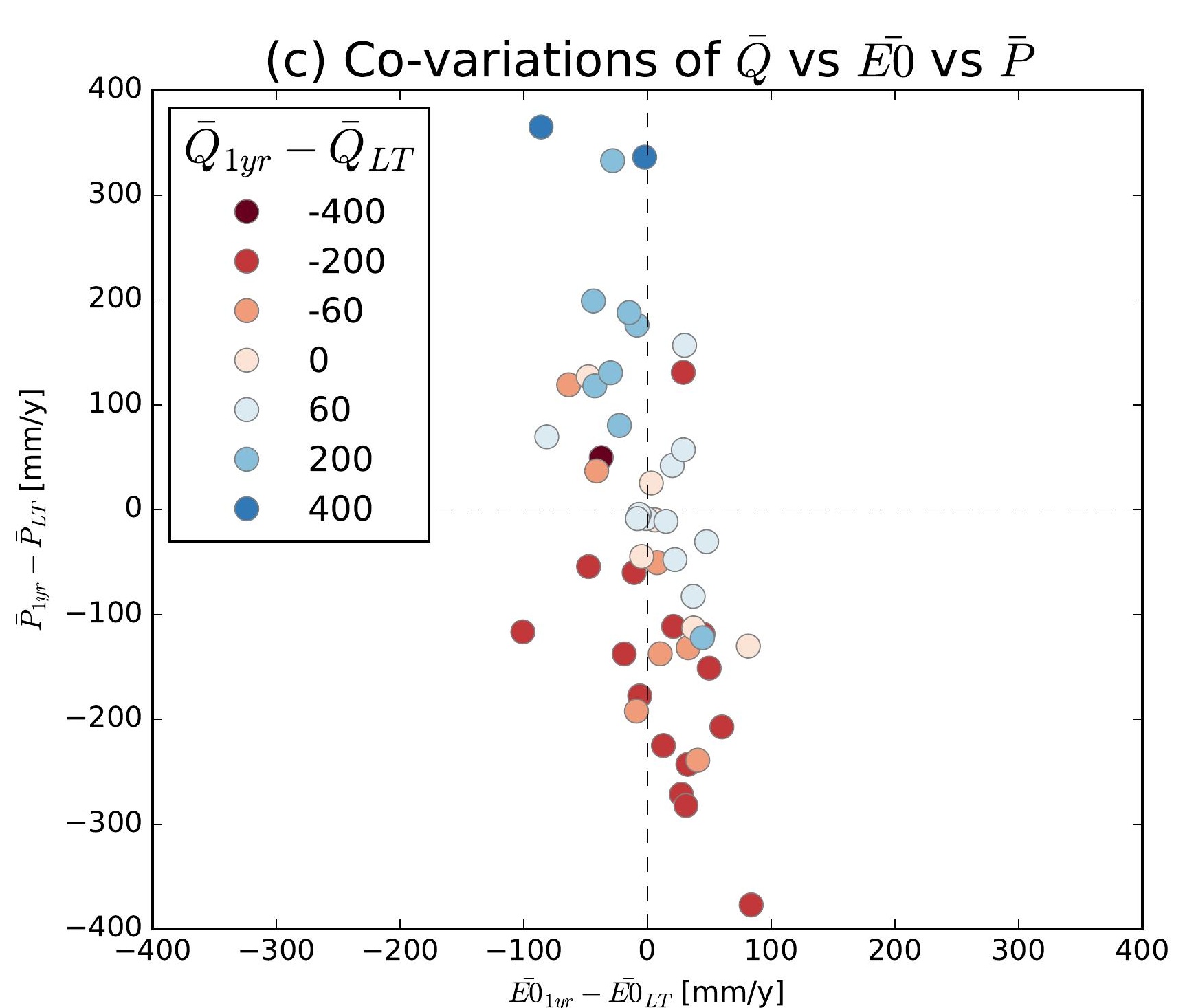
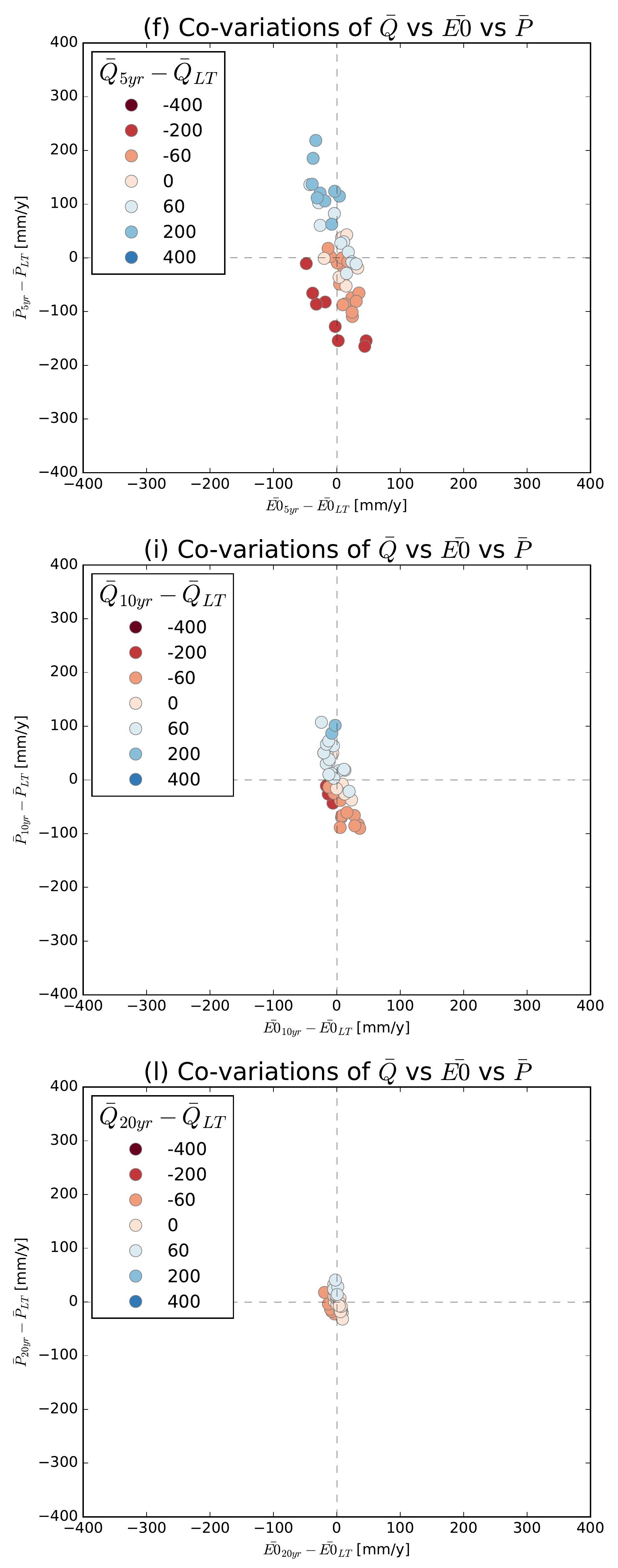

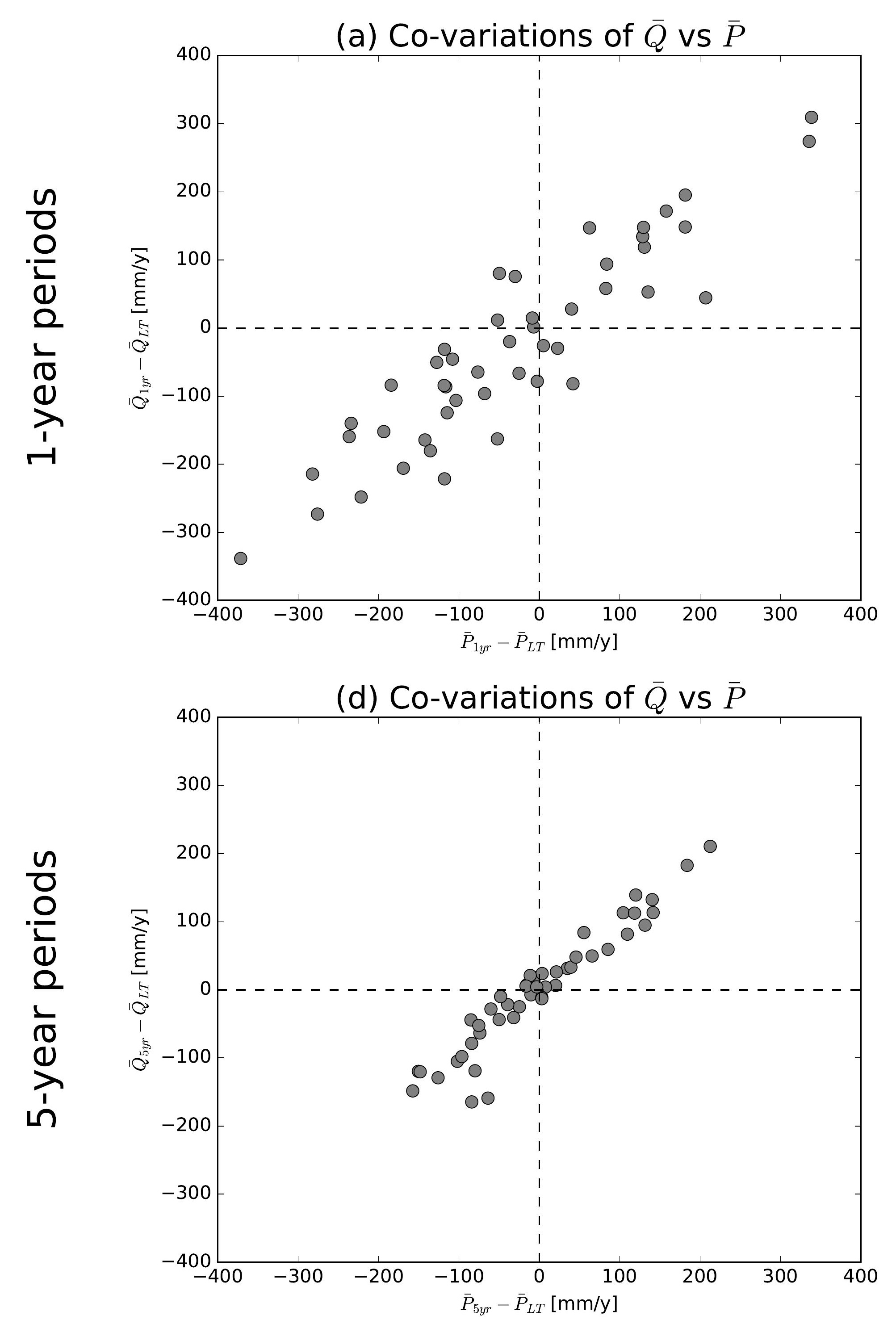

(g) Co-variations of $\bar{Q}$ vs $\bar{P}$

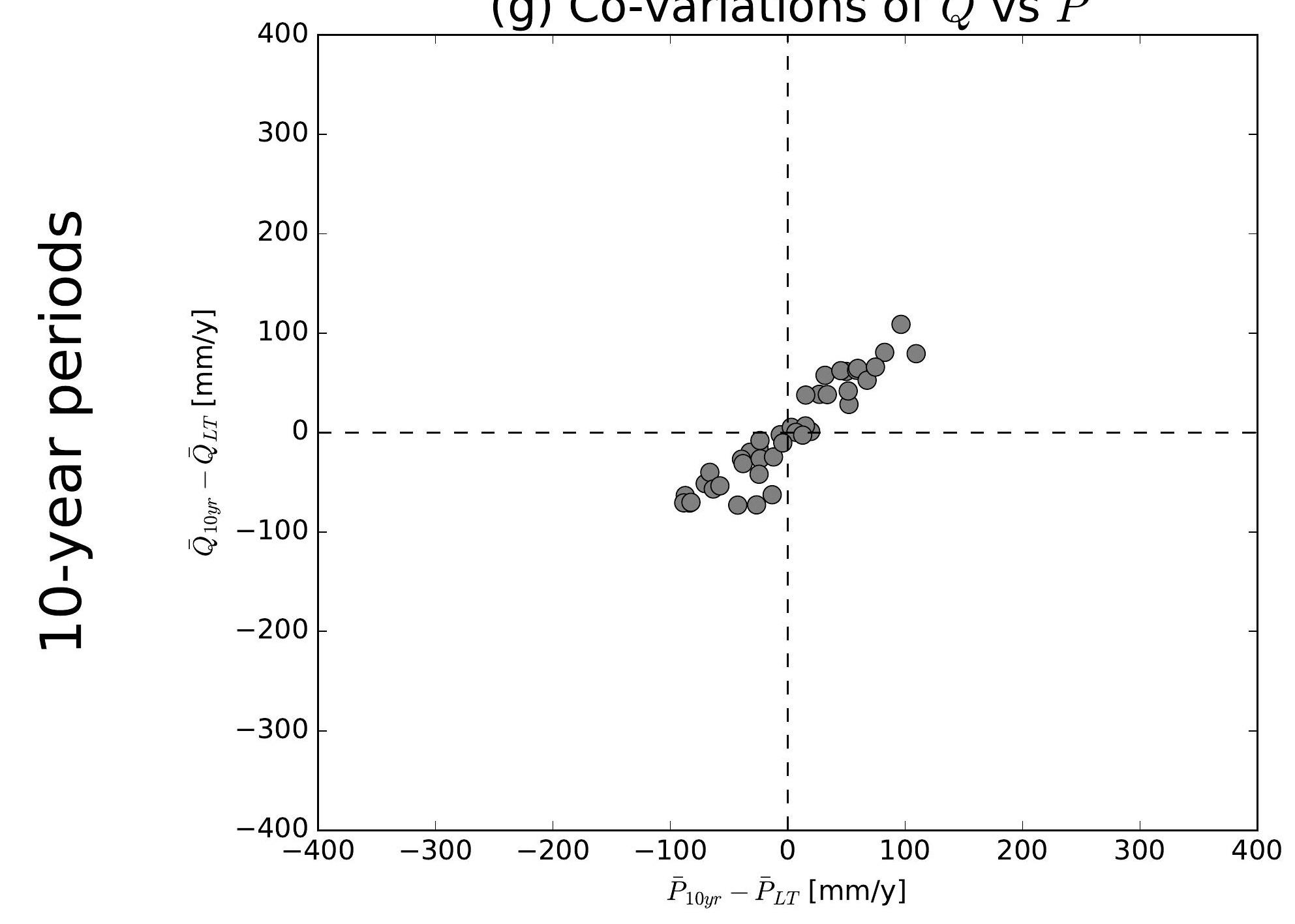

(j) Co-variations of $\bar{Q}$ vs $\bar{P}$

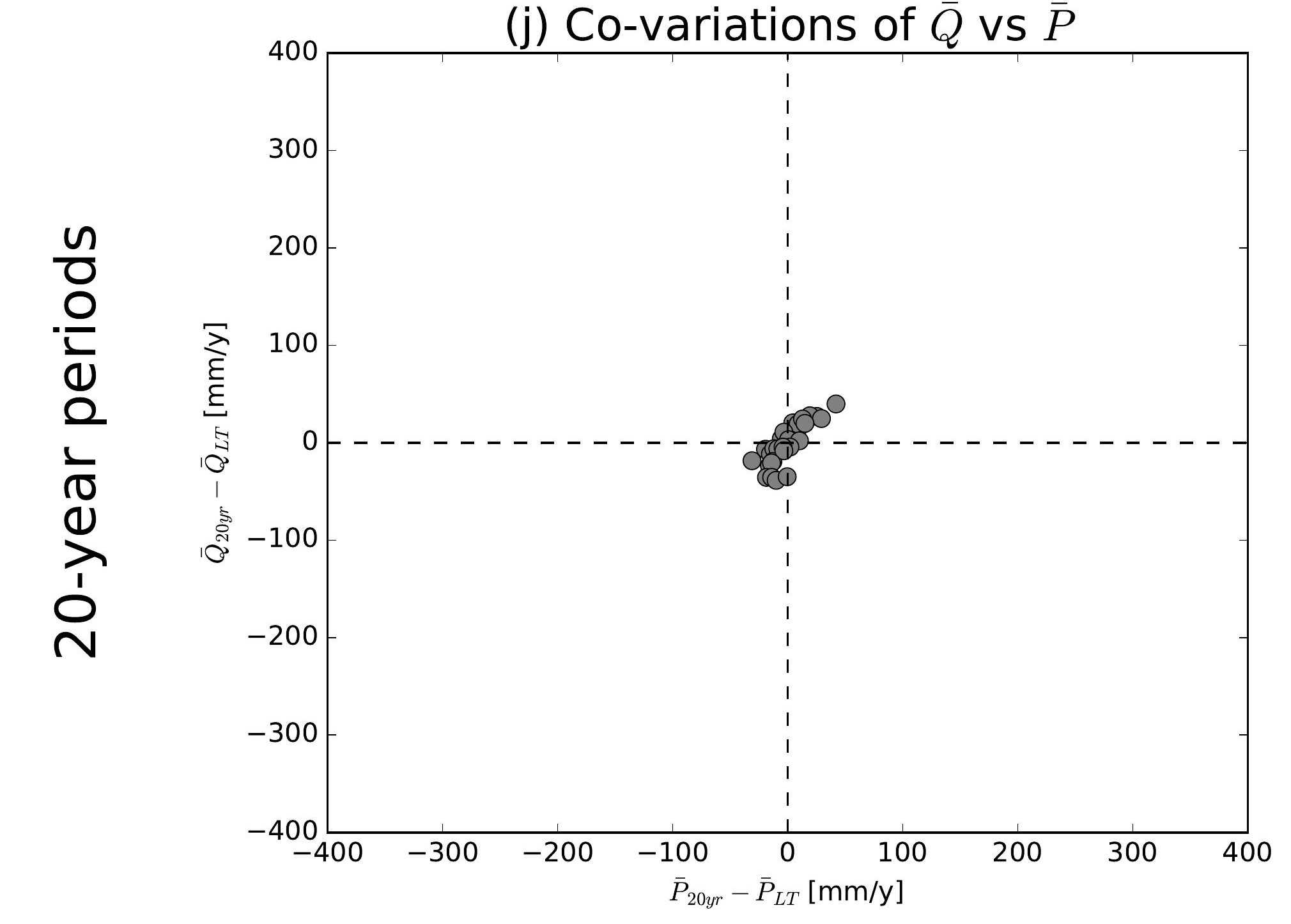

(b) Co-variations of $\bar{Q}$ vs $\overline{E 0}$

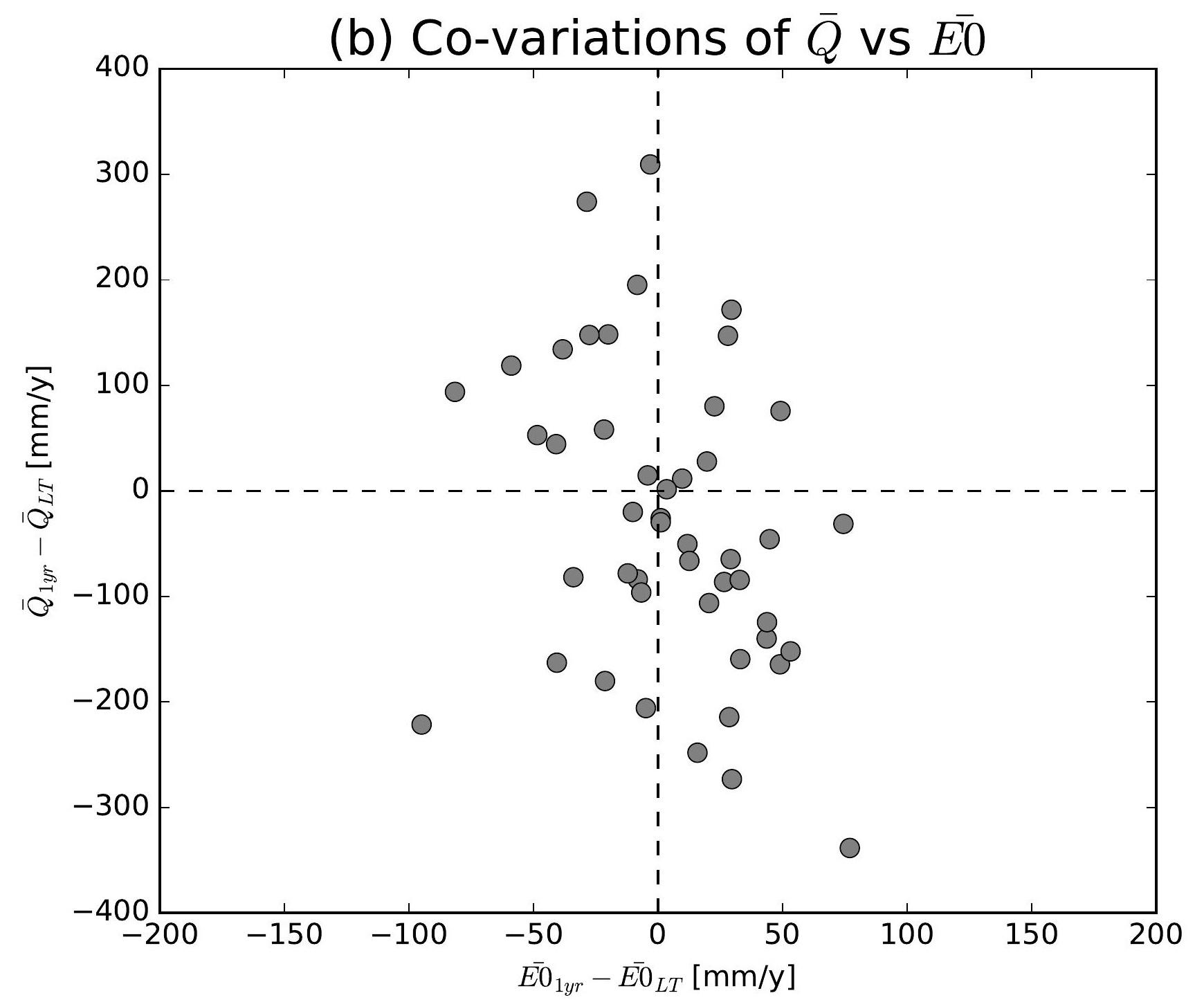

(e) Co-variations of $\bar{Q}$ vs $\overline{E 0}$

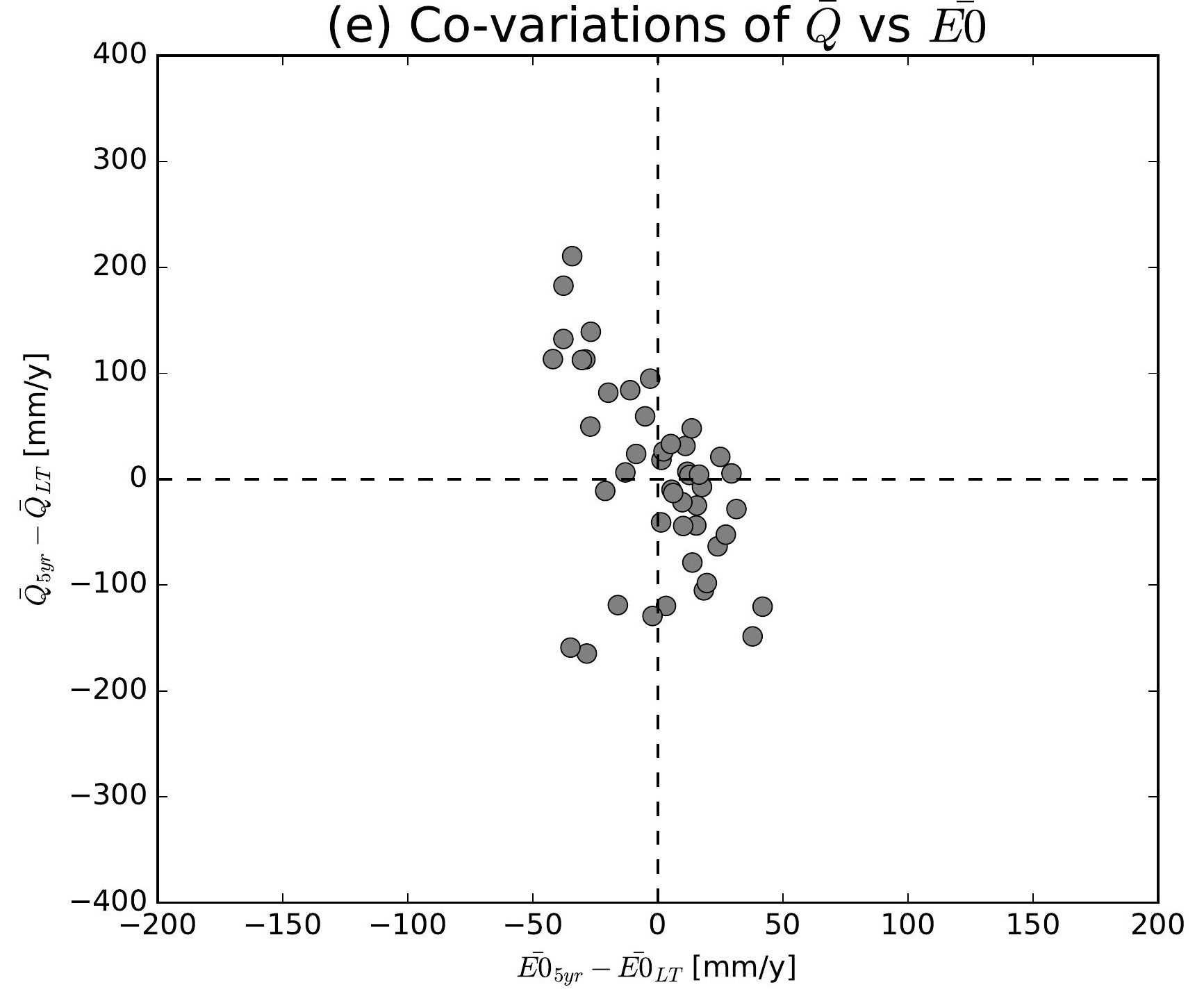

(h) Co-variations of $\bar{Q}$ vs $\overline{E 0}$

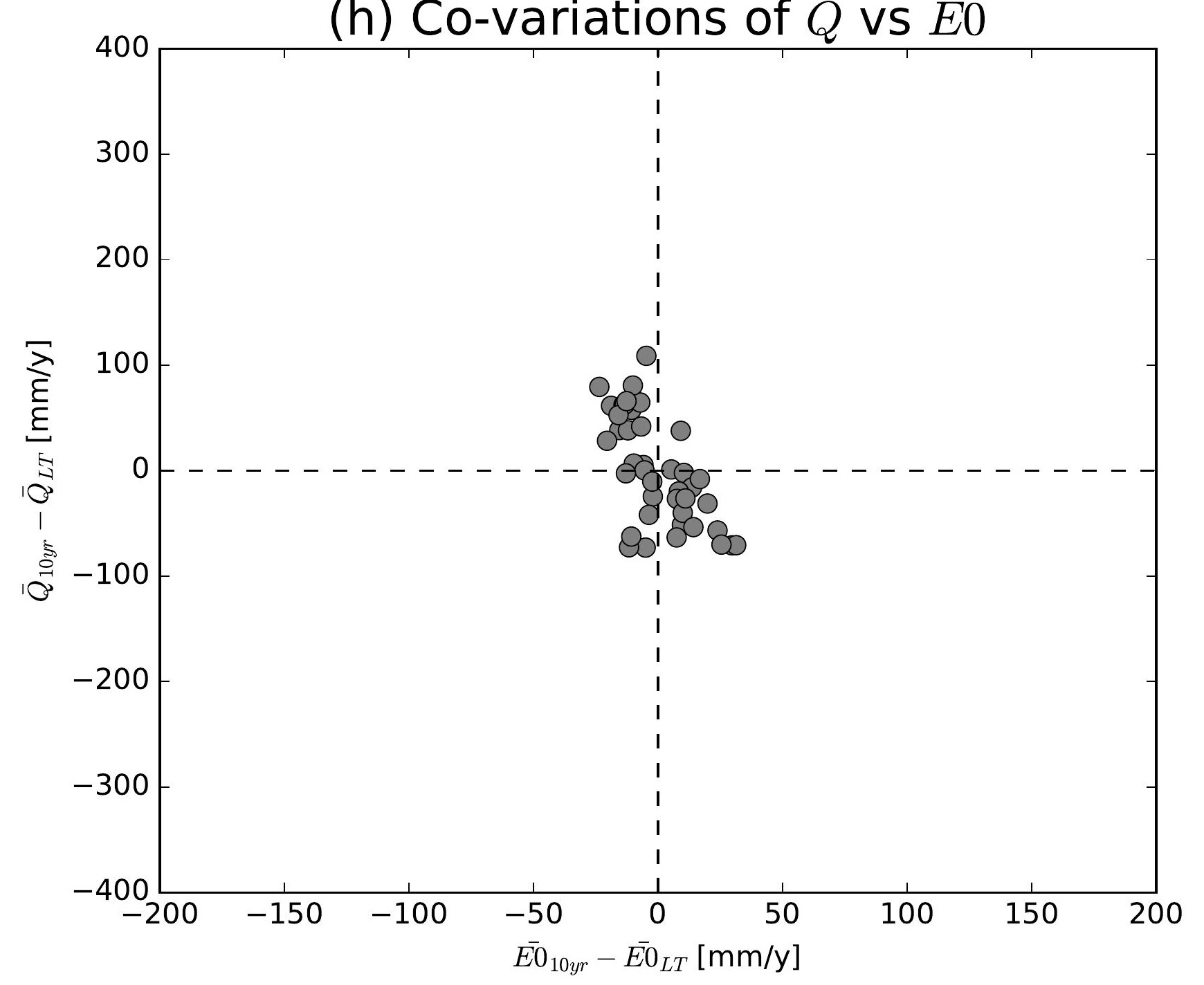

(k) Co-variations of $\bar{Q}$ vs $\overline{E 0}$

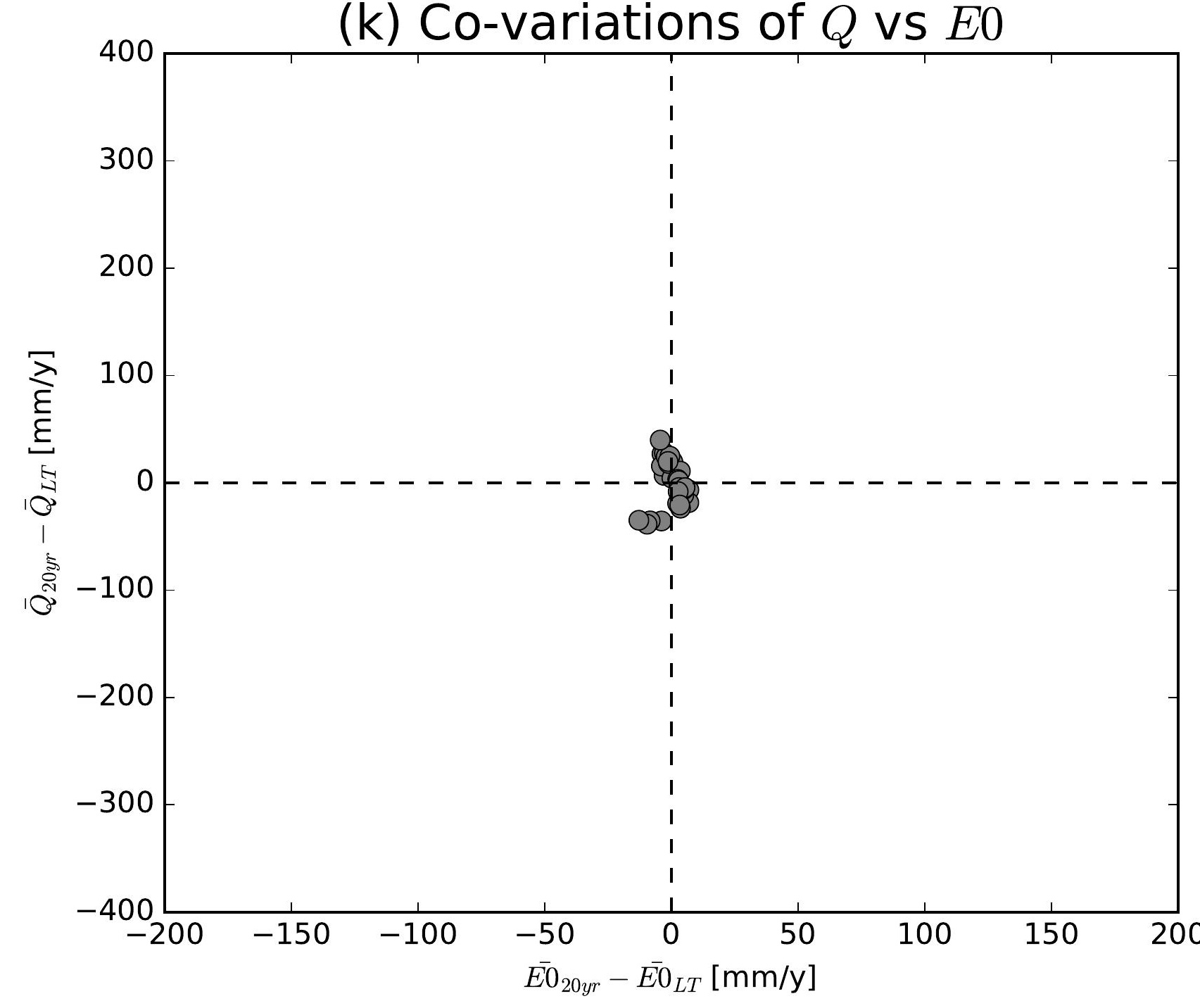

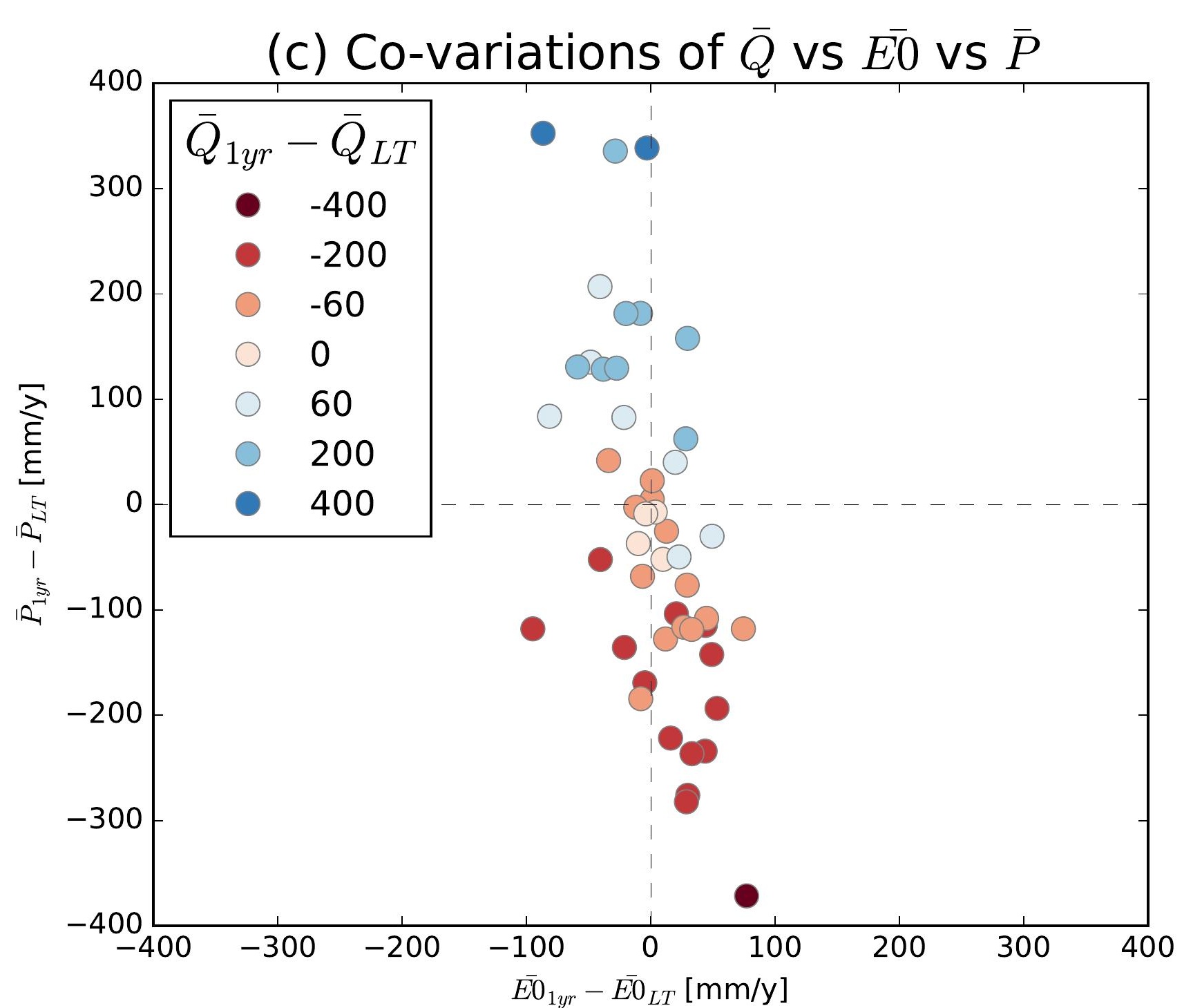
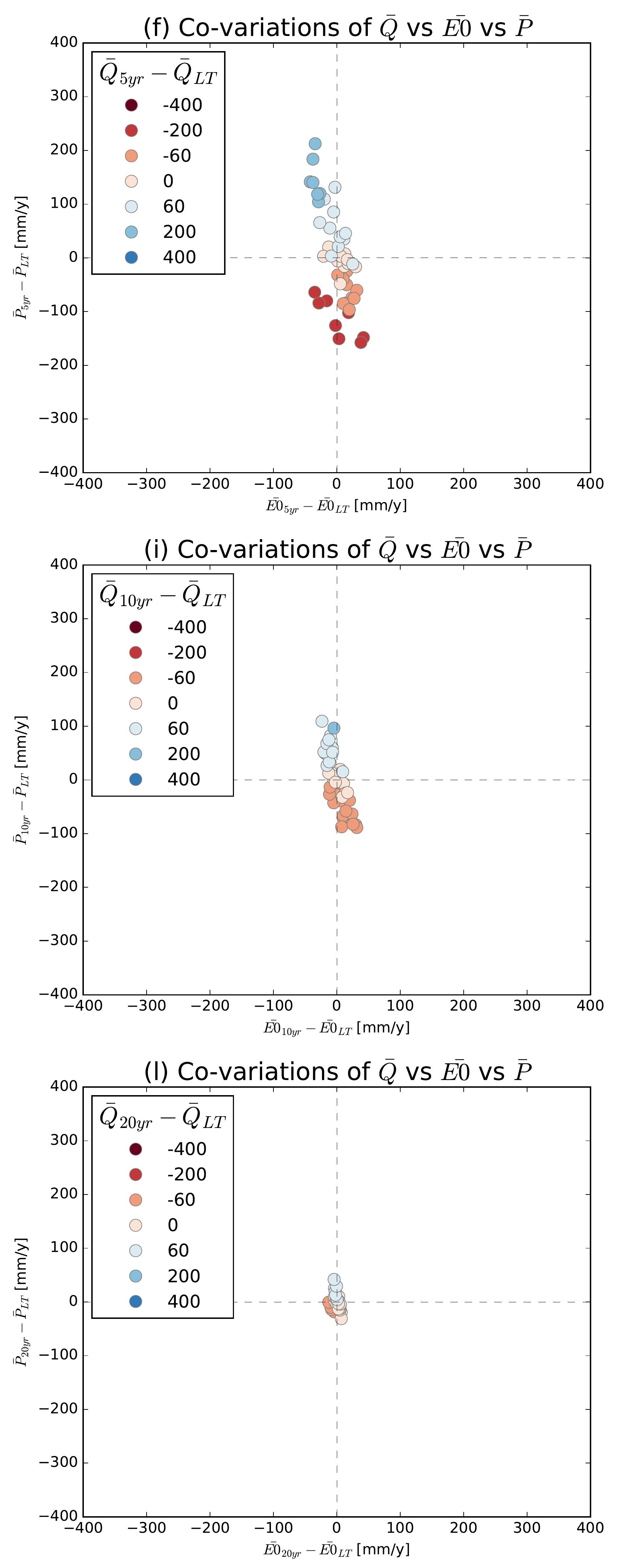

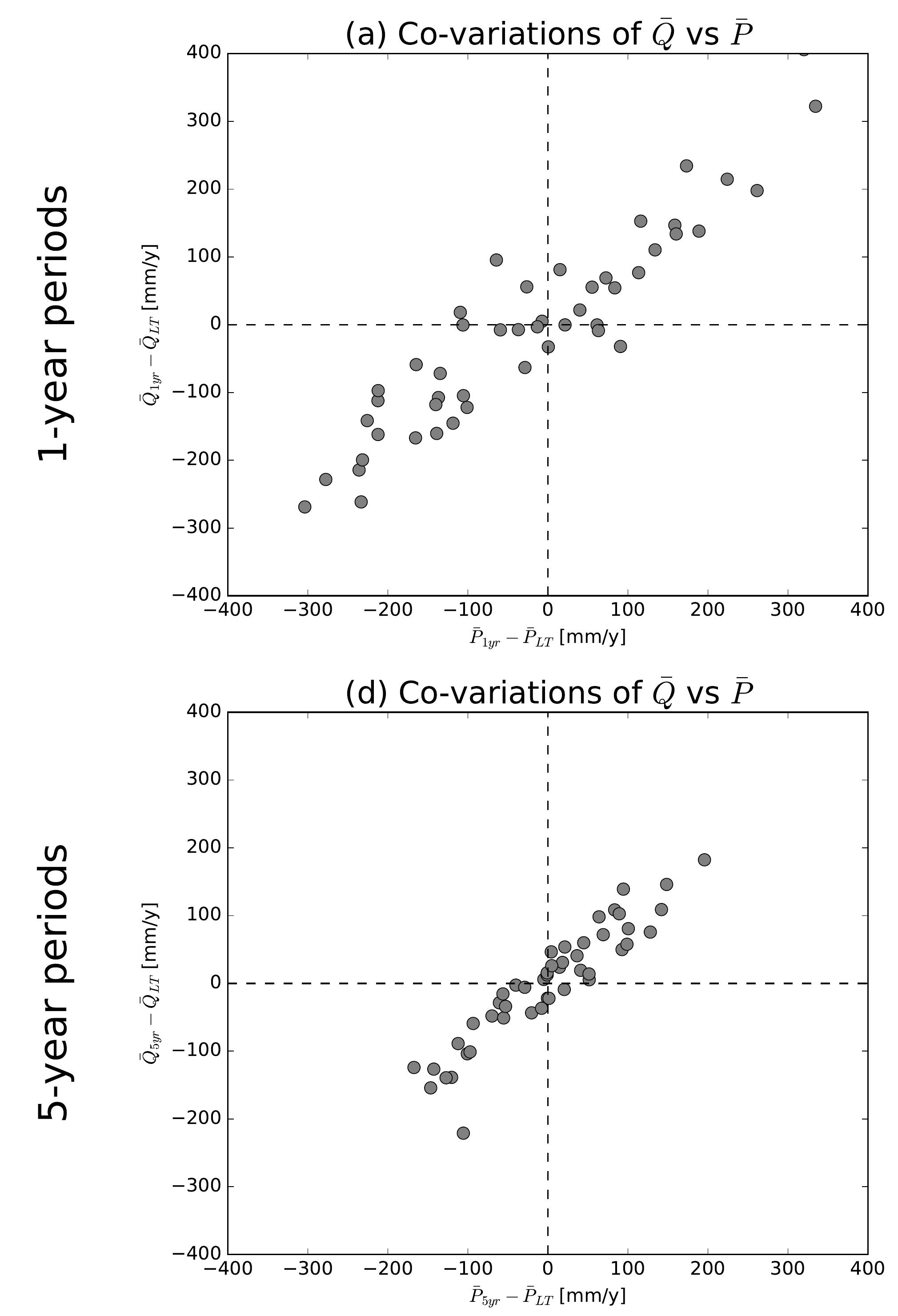

(g) Co-variations of $\bar{Q}$ vs $\bar{P}$

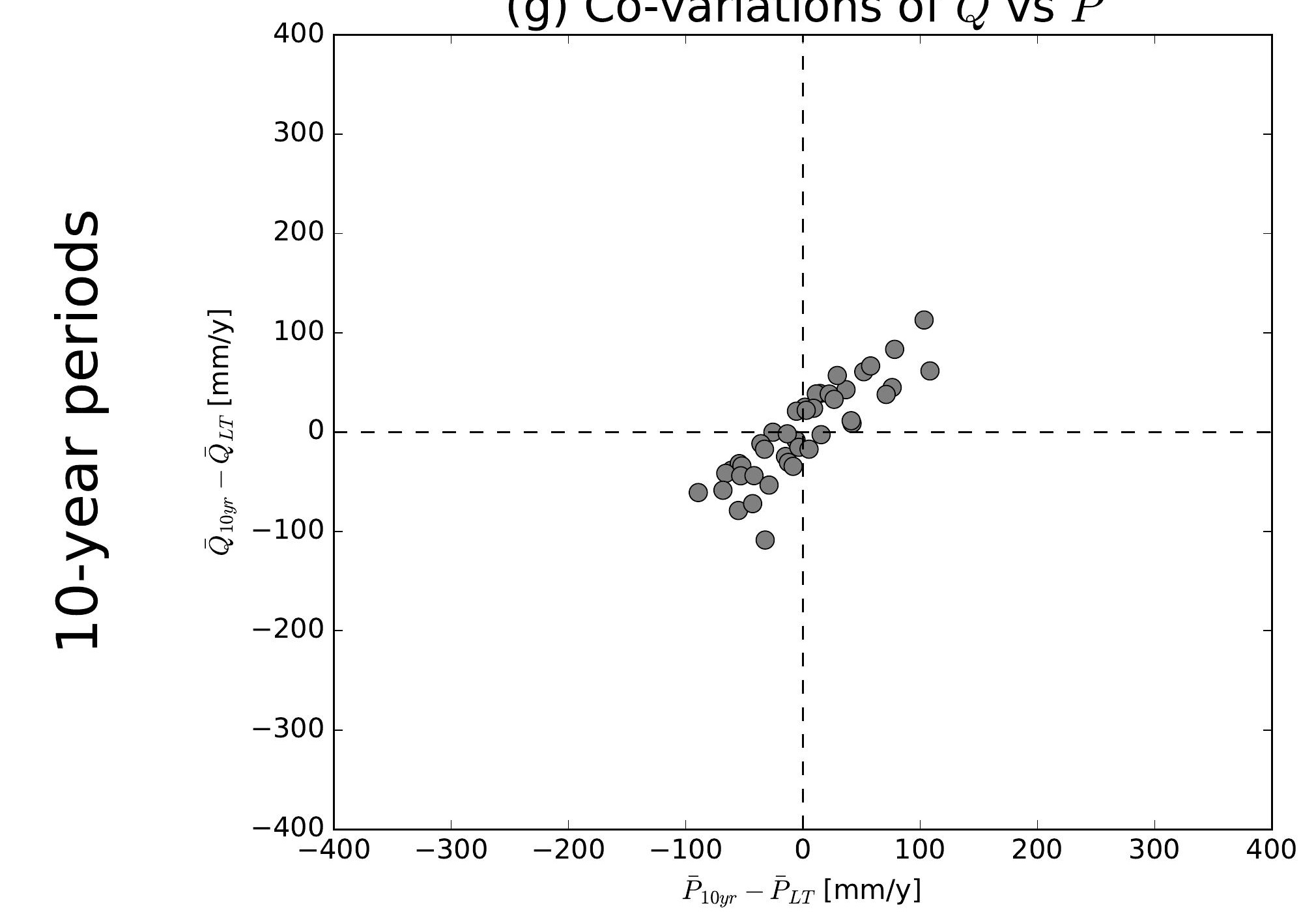

(j) Co-variations of $\bar{Q}$ vs $\bar{P}$

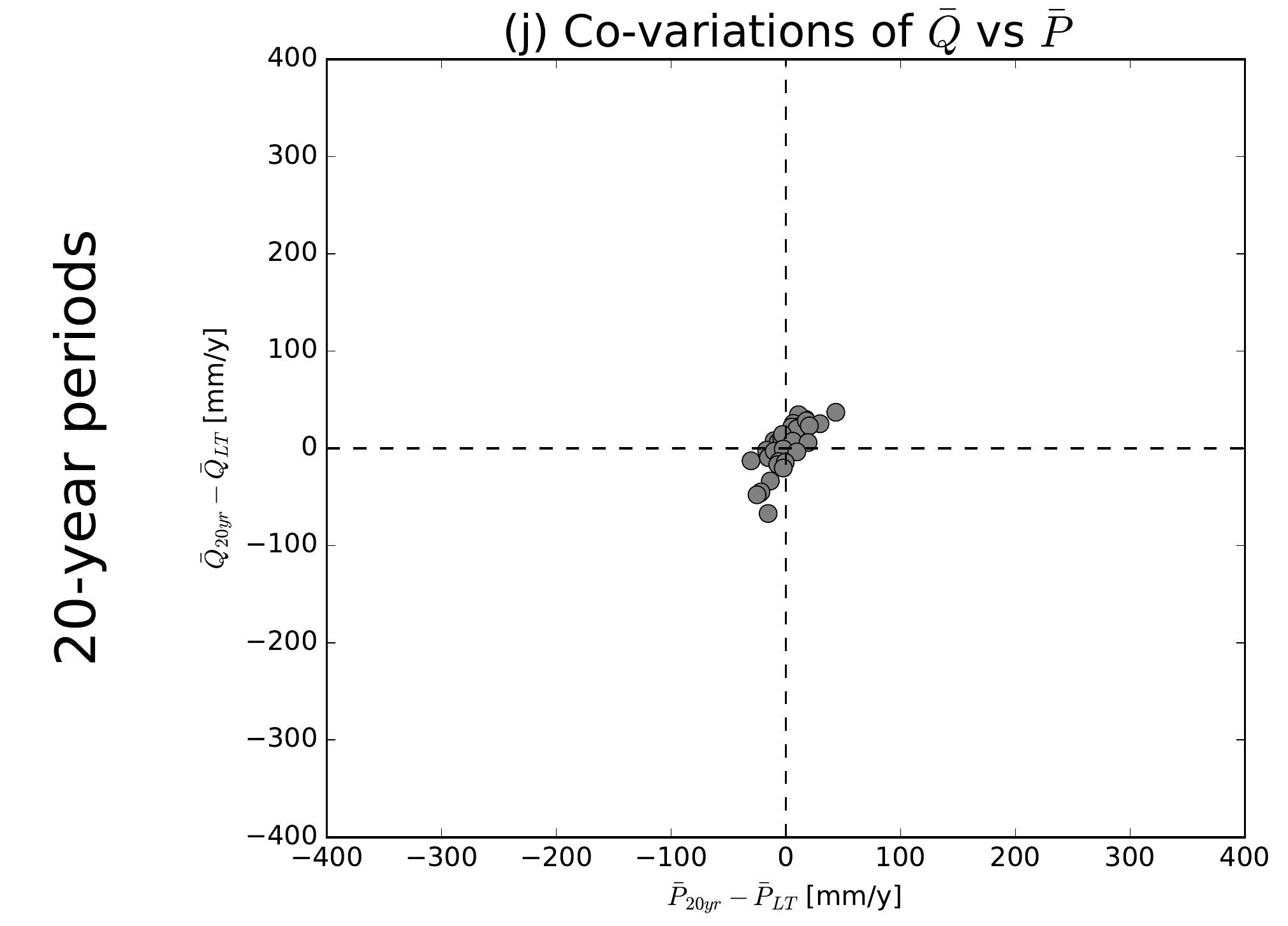

(b) Co-variations of $\bar{Q}$ vs $\overline{E 0}$

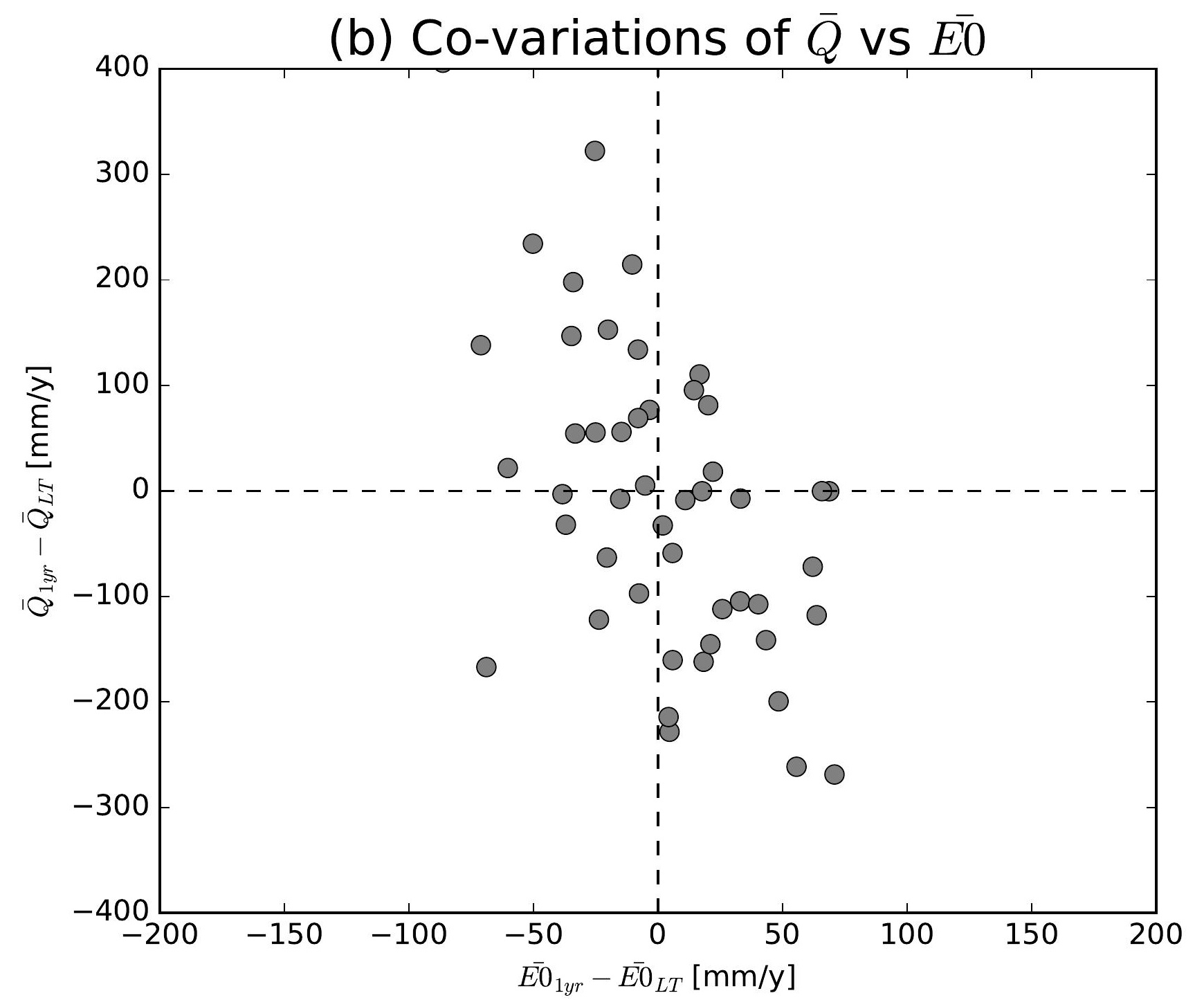

(e) Co-variations of $\bar{Q}$ vs $\overline{E 0}$

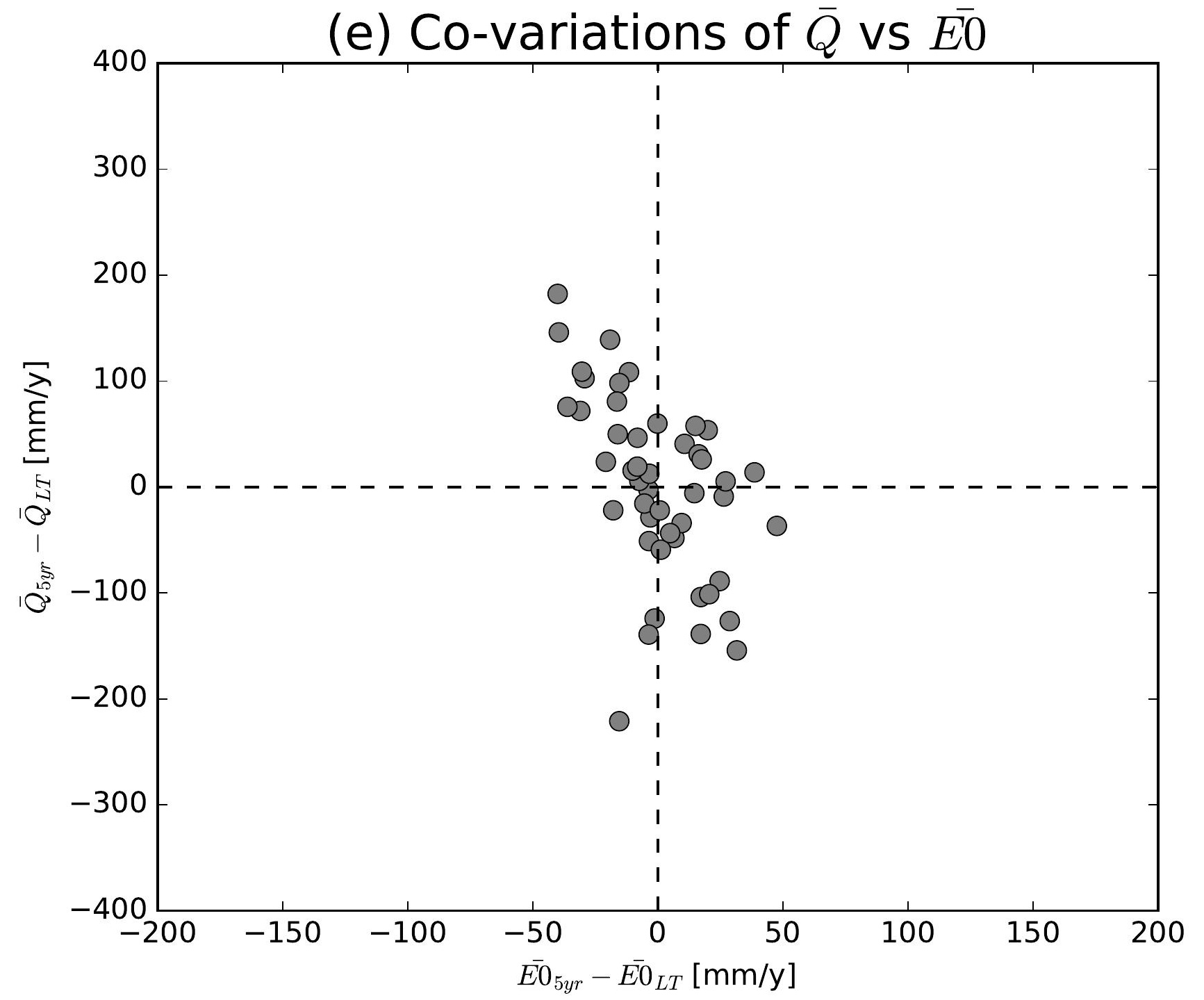

(h) Co-variations of $\bar{Q}$ vs $\overline{E 0}$

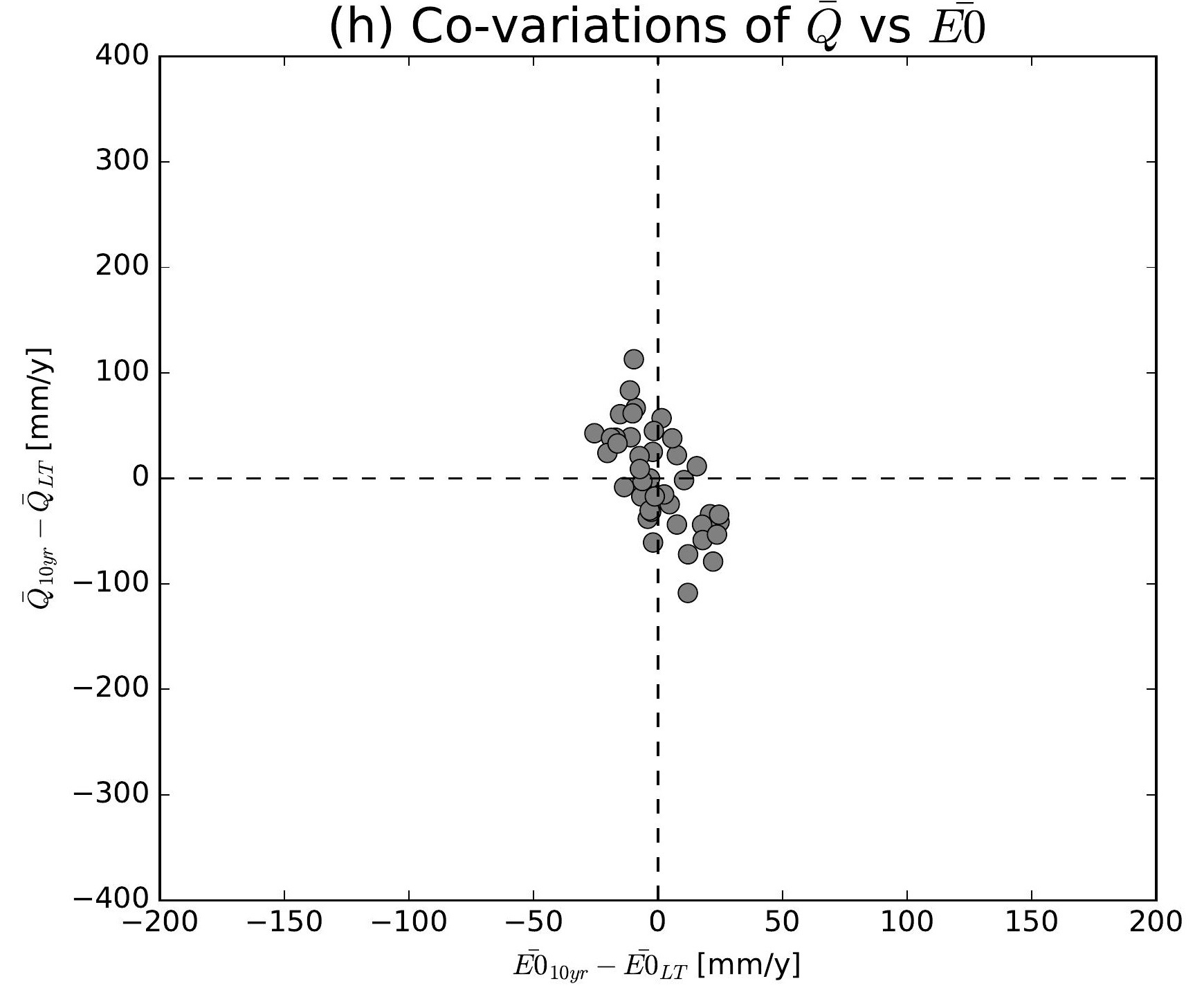

(k) Co-variations of $\bar{Q}$ vs $\overline{E 0}$

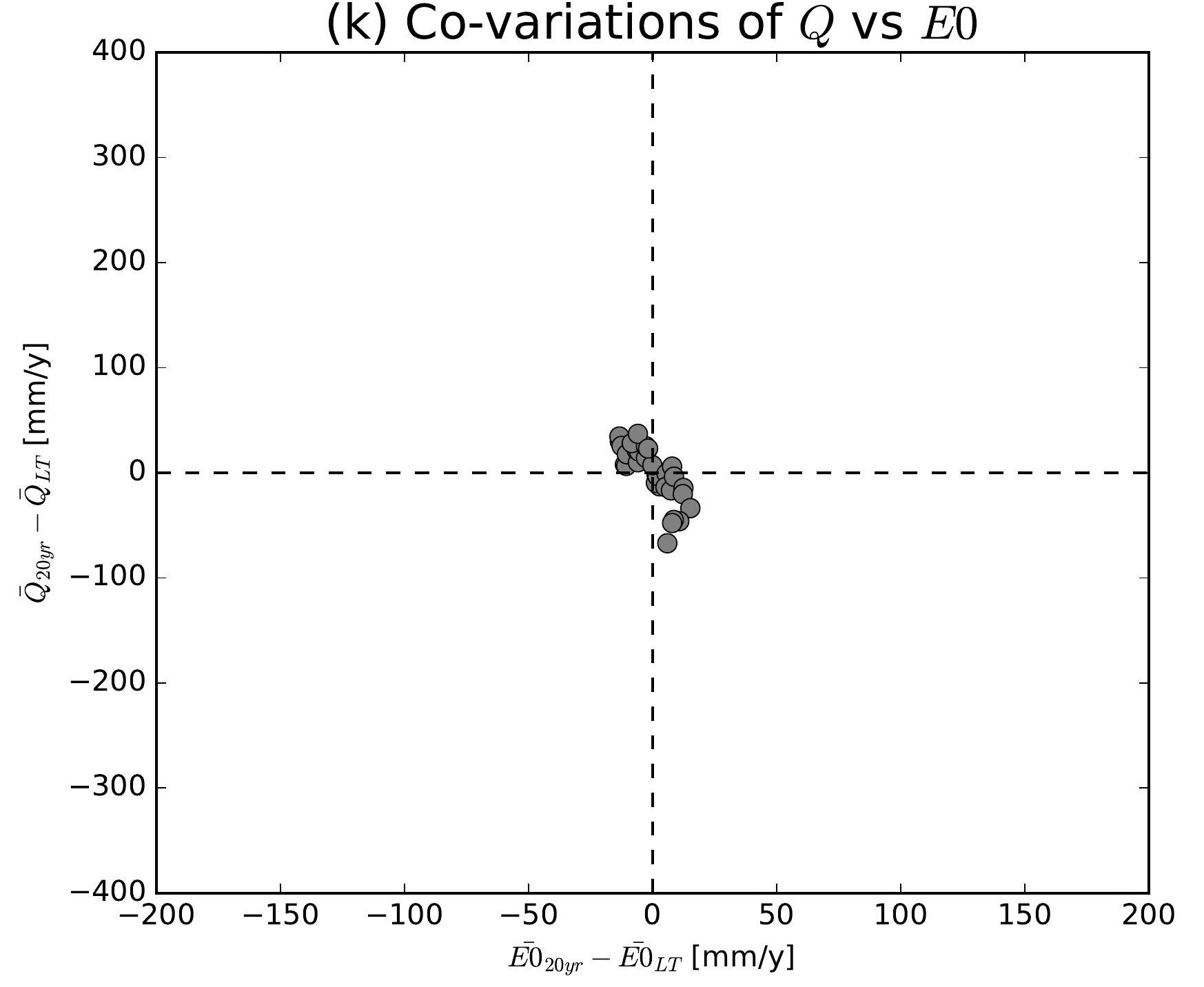

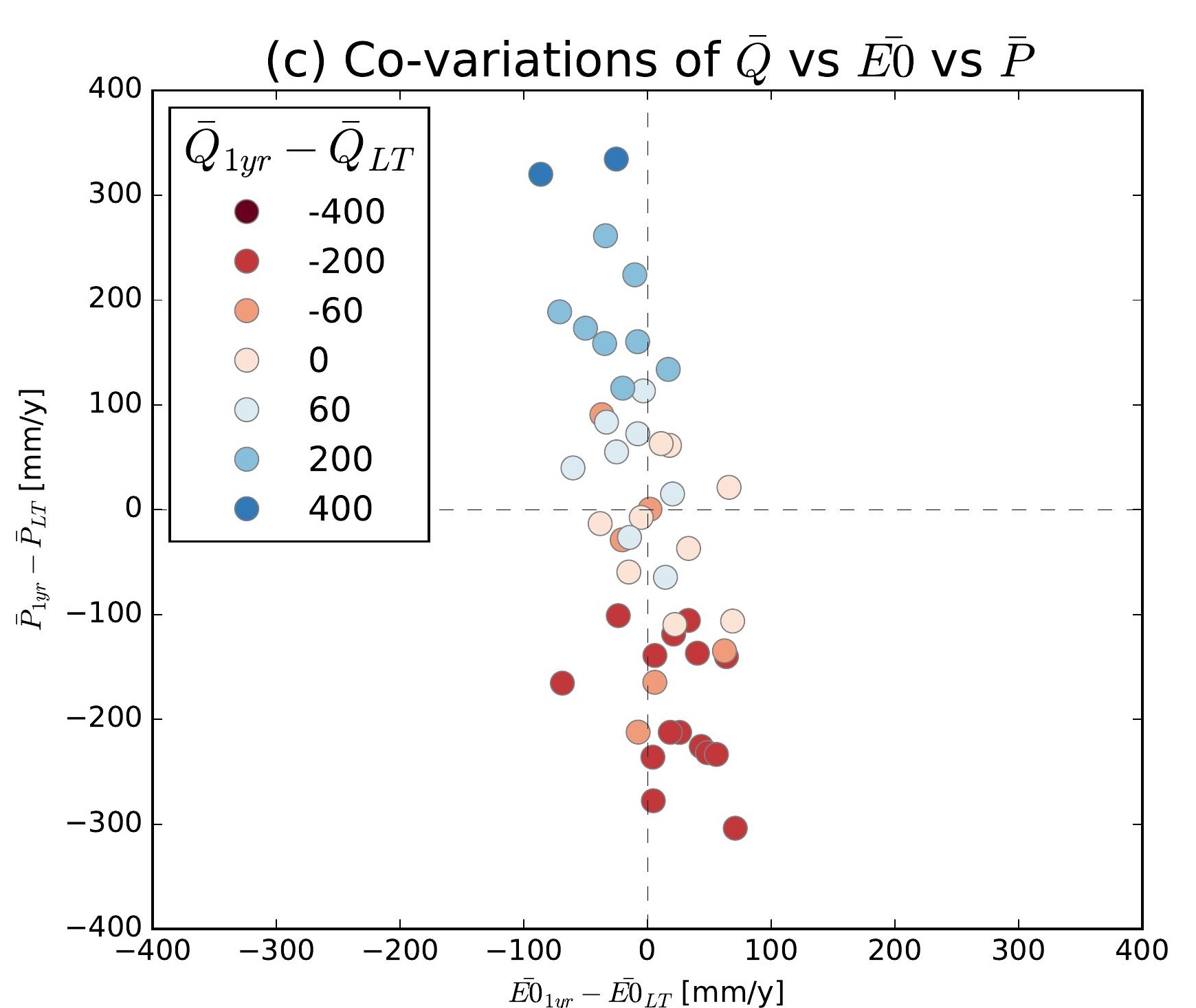
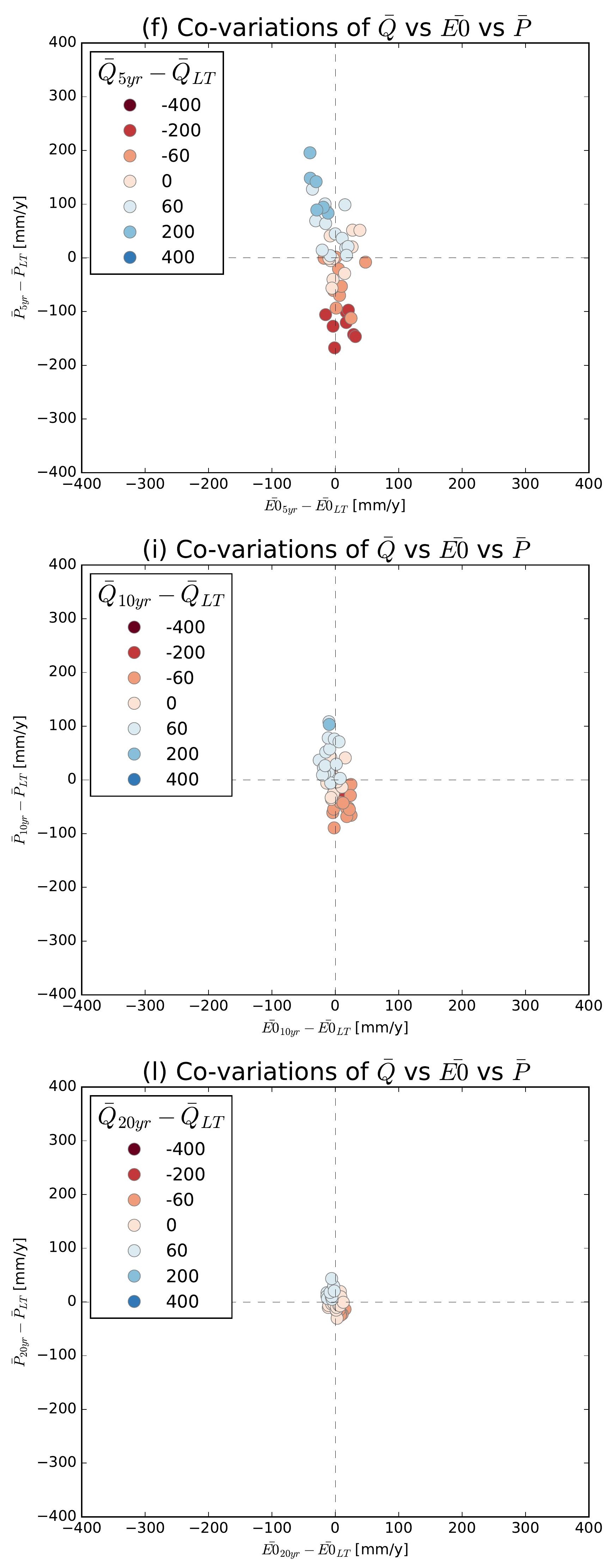

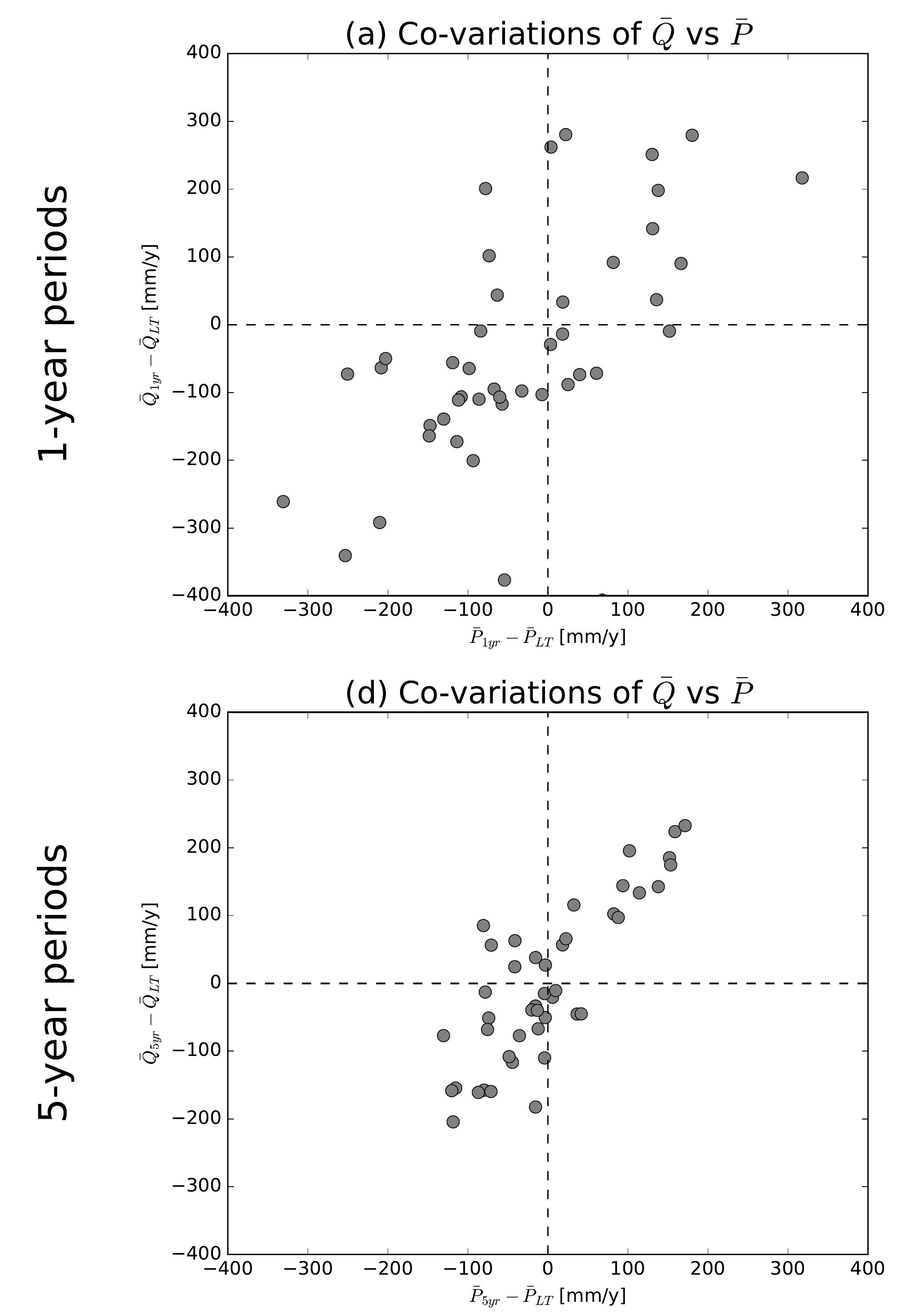

(g) Co-variations of $\bar{Q}$ vs $\bar{P}$
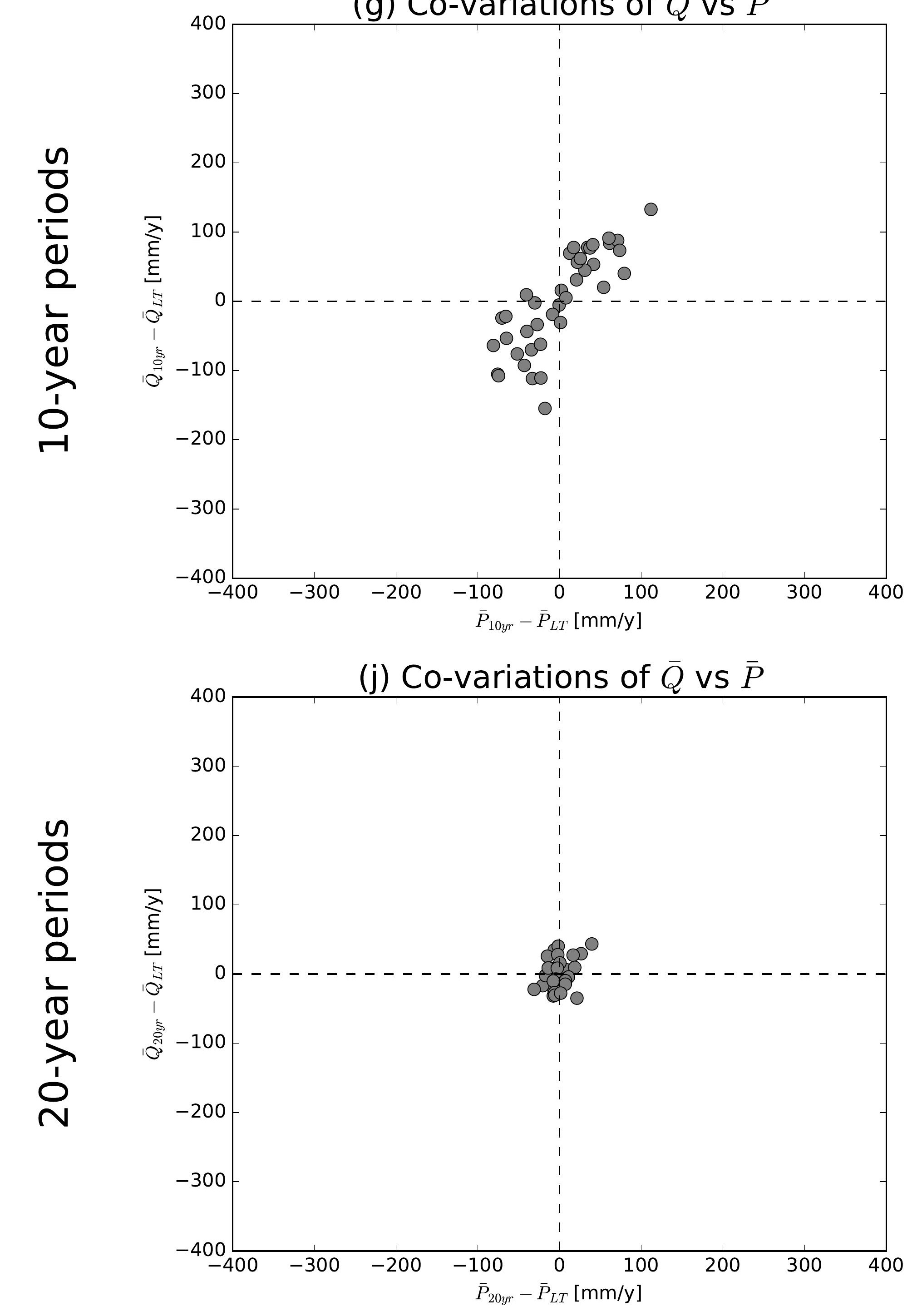

(b) Co-variations of $\bar{Q}$ vs $\overline{E 0}$

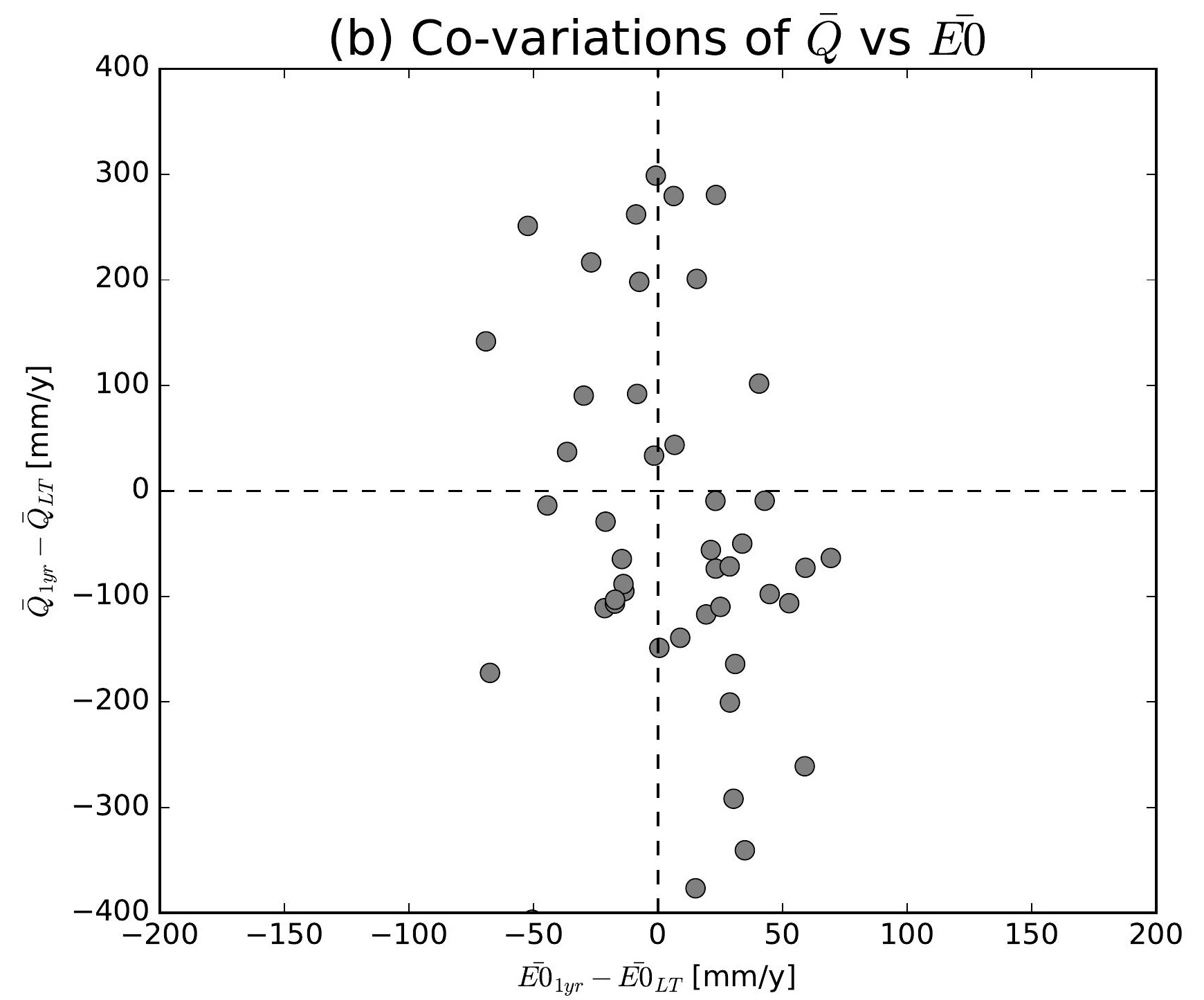

(e) Co-variations of $\bar{Q}$ vs $\overline{E 0}$

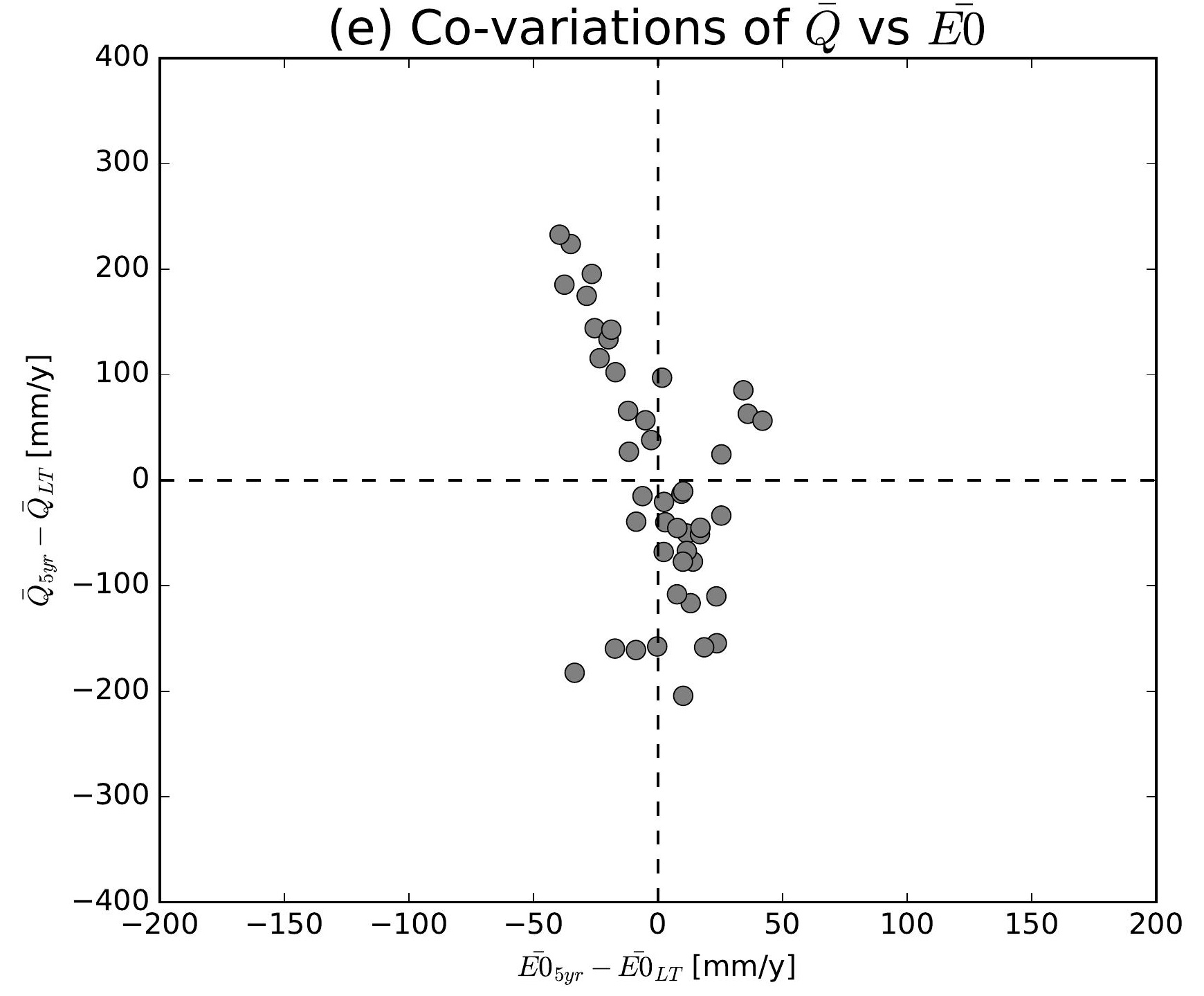

(h) Co-variations of $\bar{Q}$ vs $\overline{E 0}$

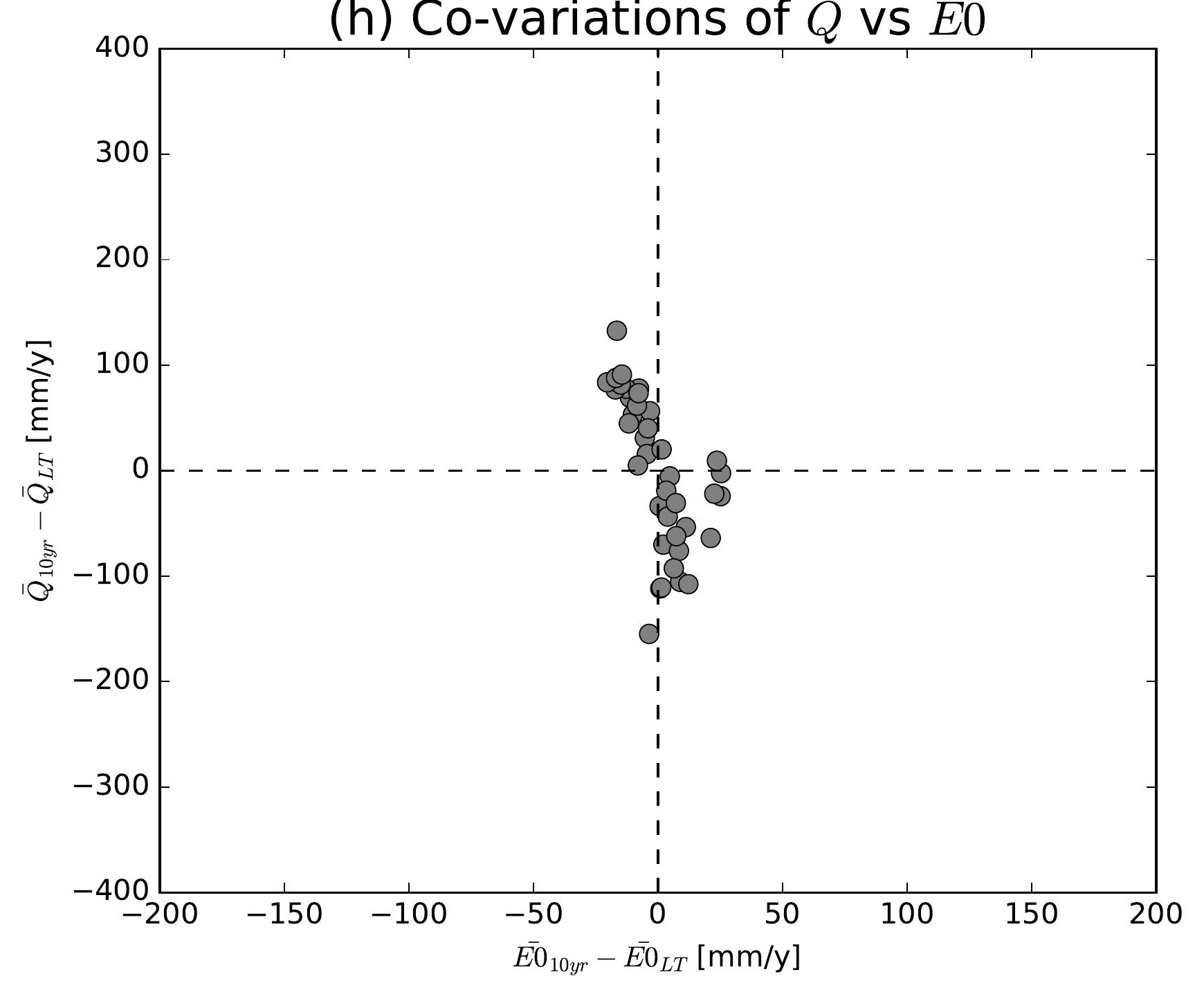

(k) Co-variations of $\bar{Q}$ vs $\overline{E 0}$

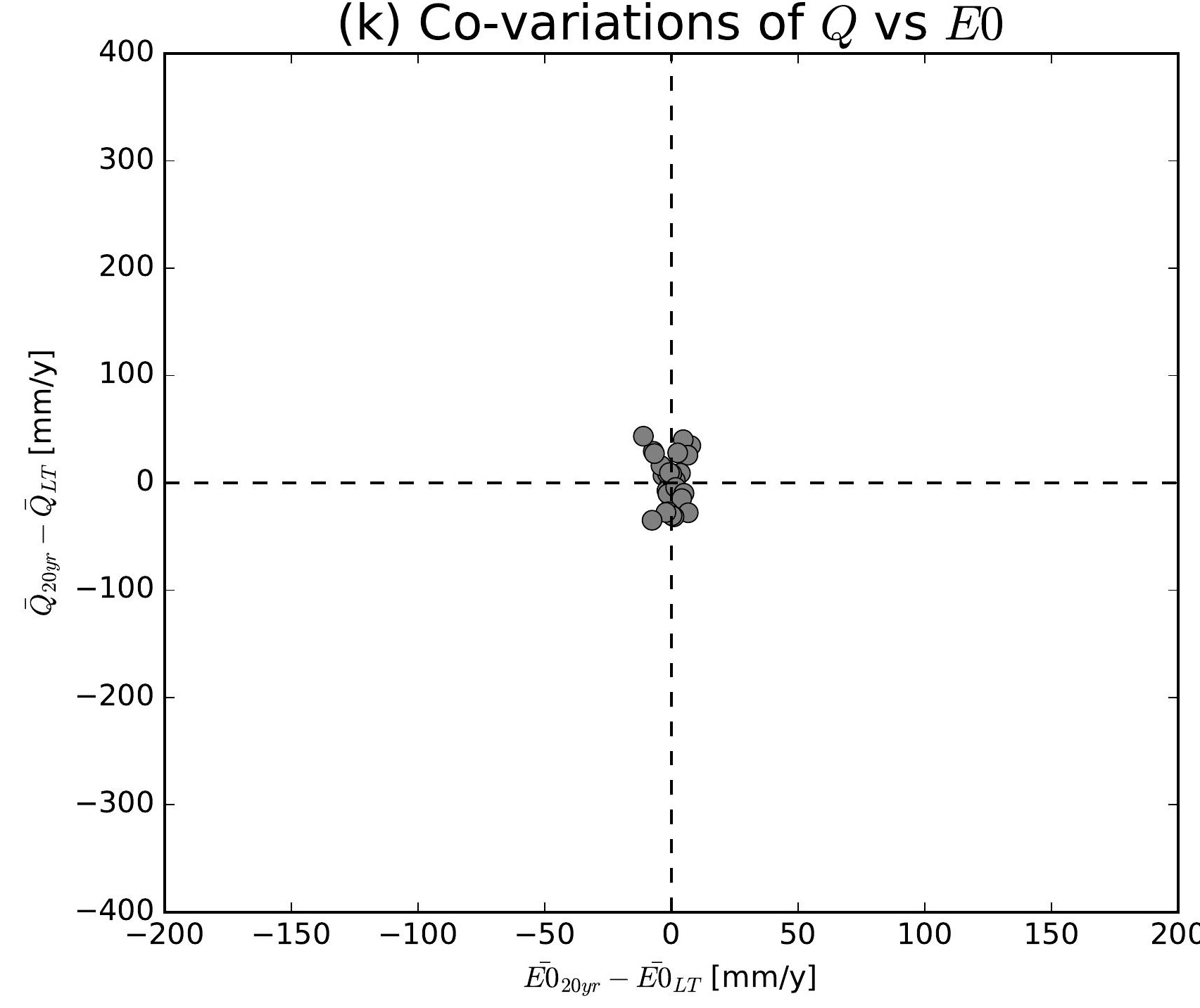

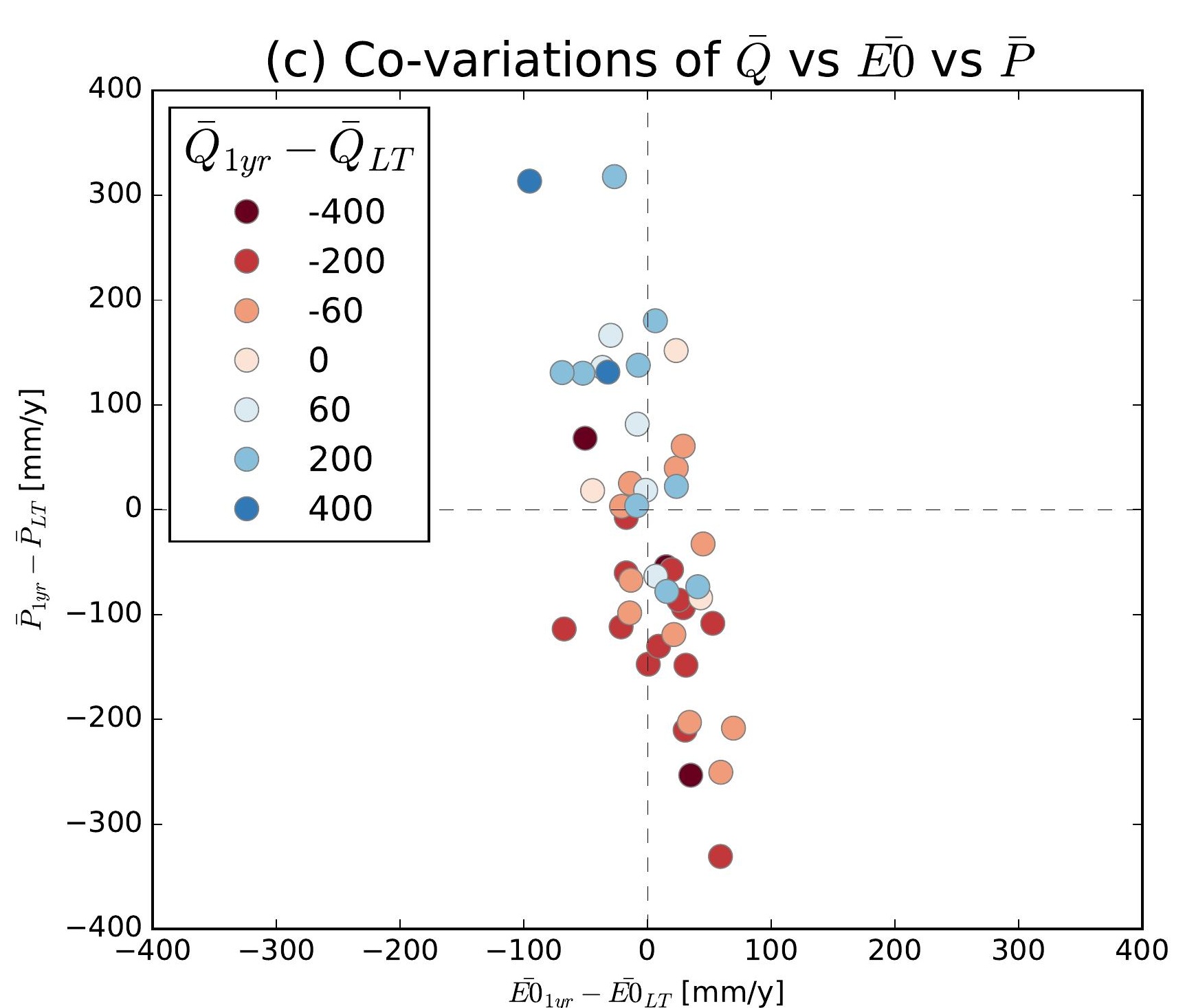
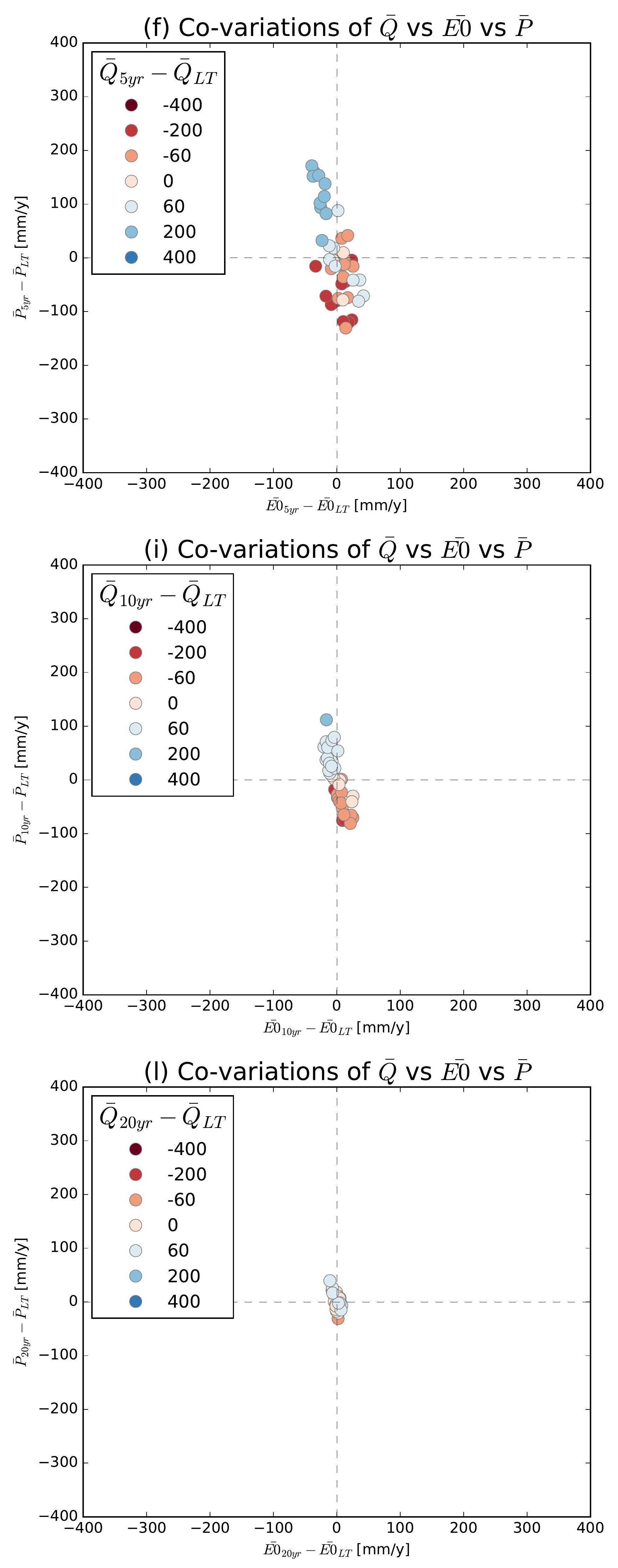

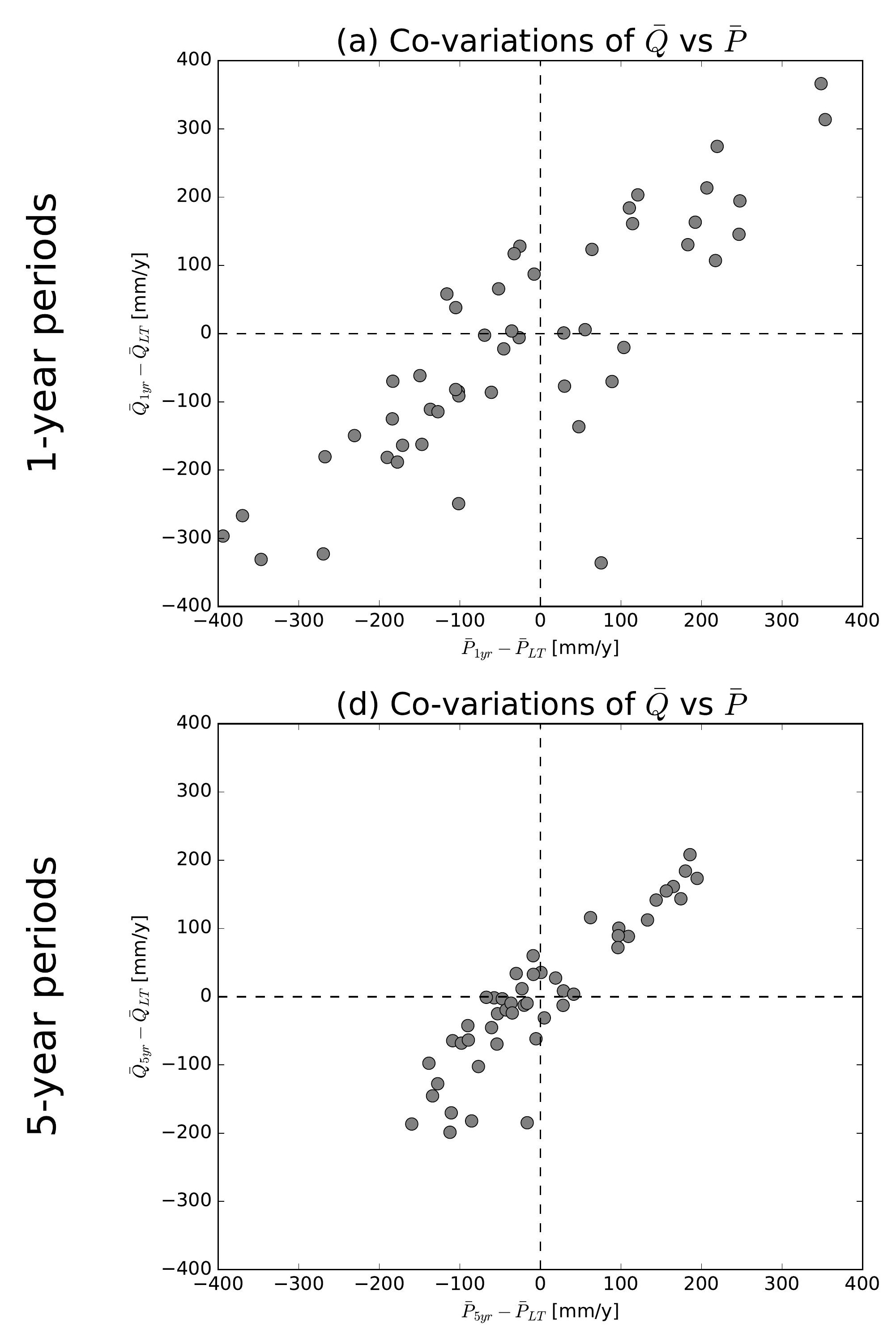

(g) Co-variations of $\bar{Q}$ vs $\bar{P}$

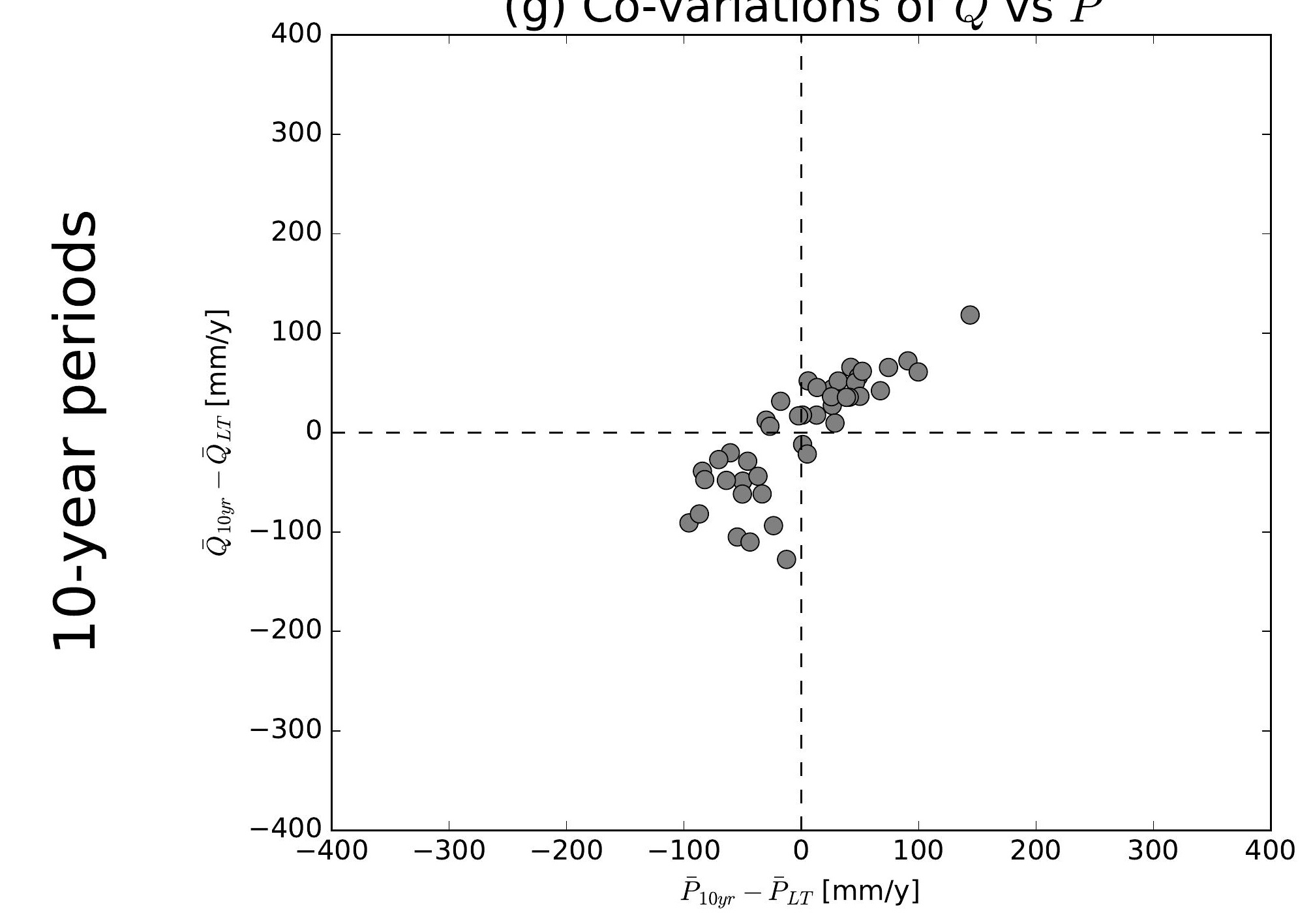

(j) Co-variations of $\bar{Q}$ vs $\bar{P}$

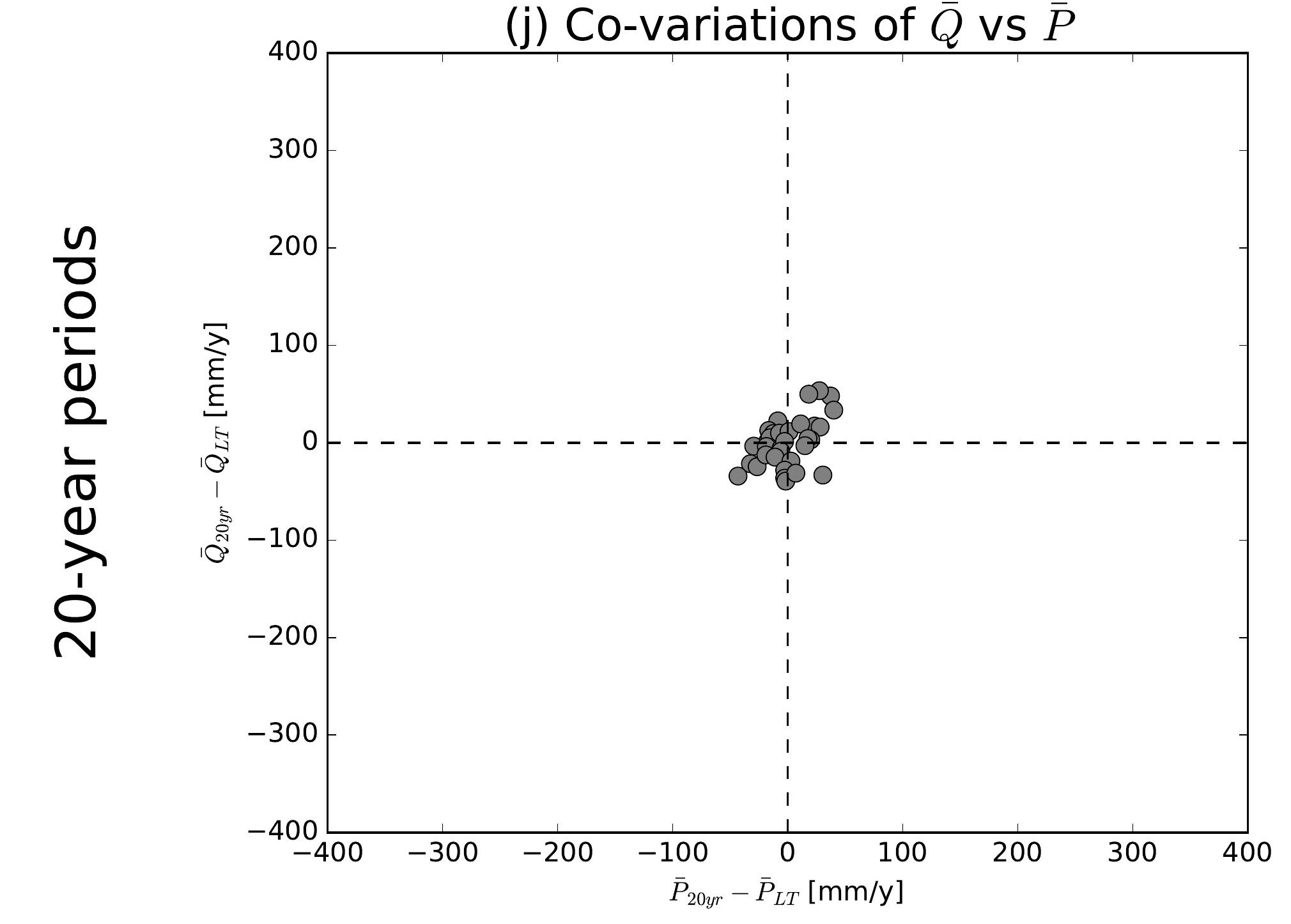

(b) Co-variations of $\bar{Q}$ vs $\overline{E 0}$

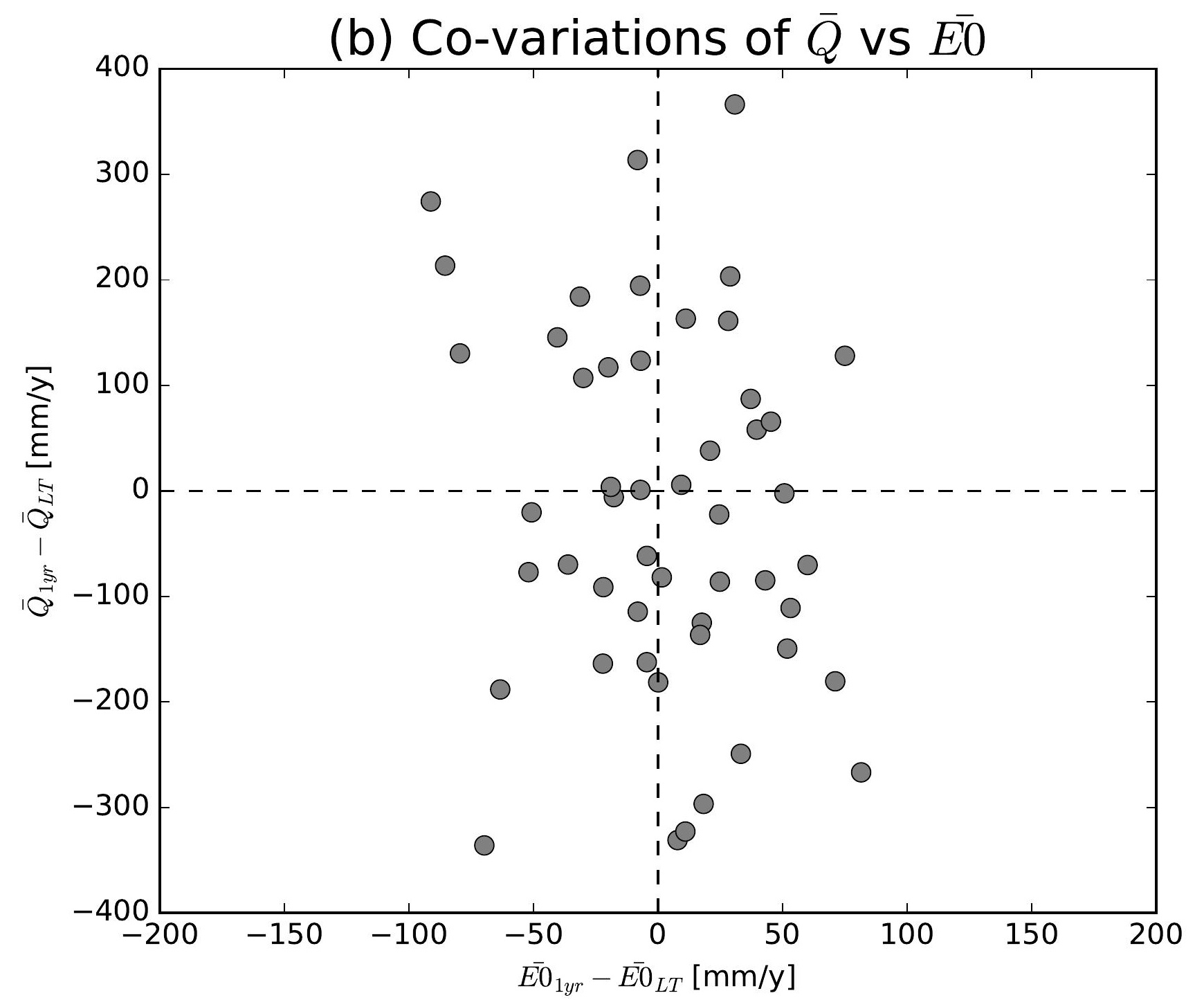

(e) Co-variations of $\bar{Q}$ vs $\overline{E 0}$

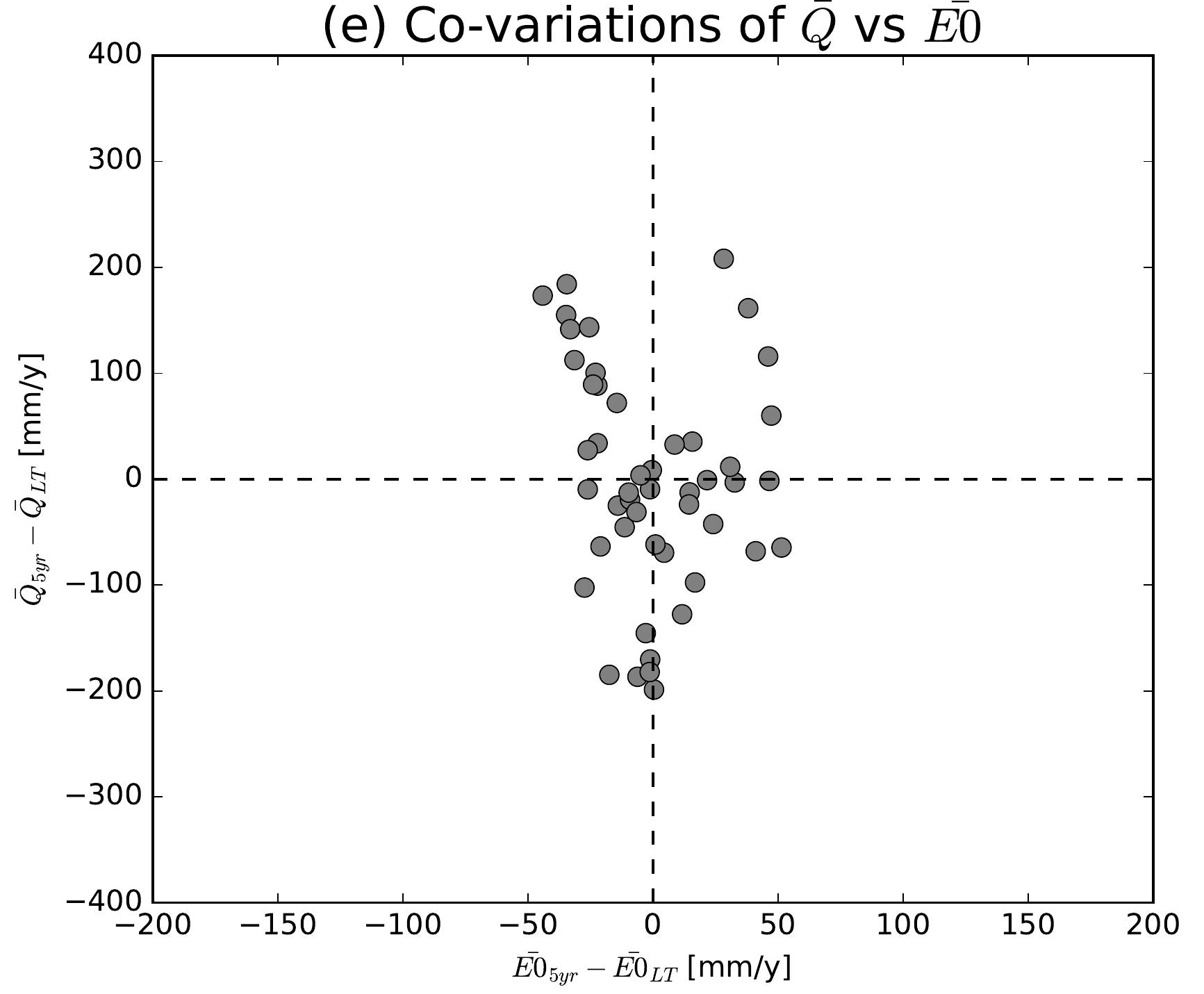

(h) Co-variations of $\bar{Q}$ vs $\overline{E 0}$

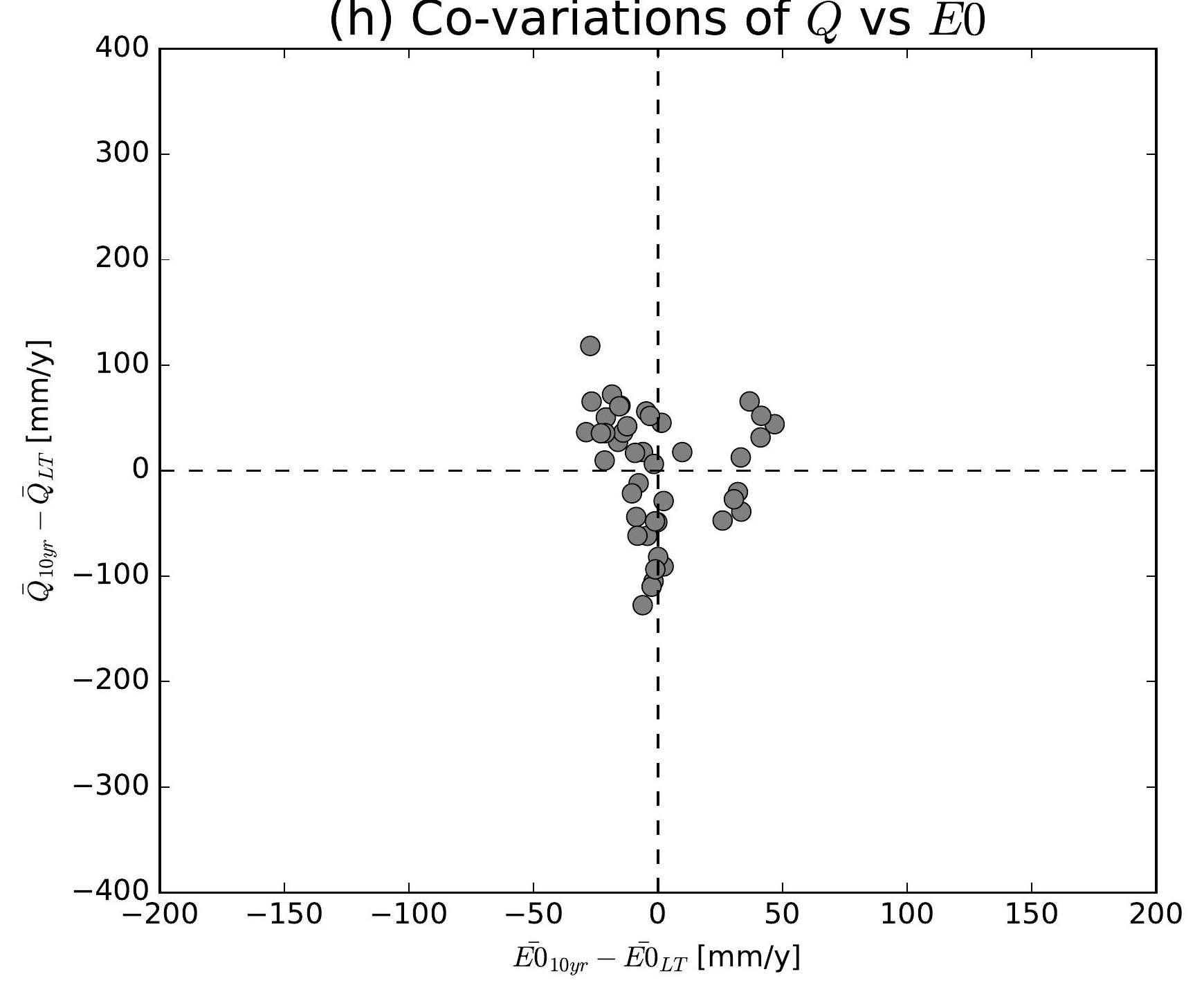

(k) Co-variations of $\bar{Q}$ vs $\overline{E 0}$

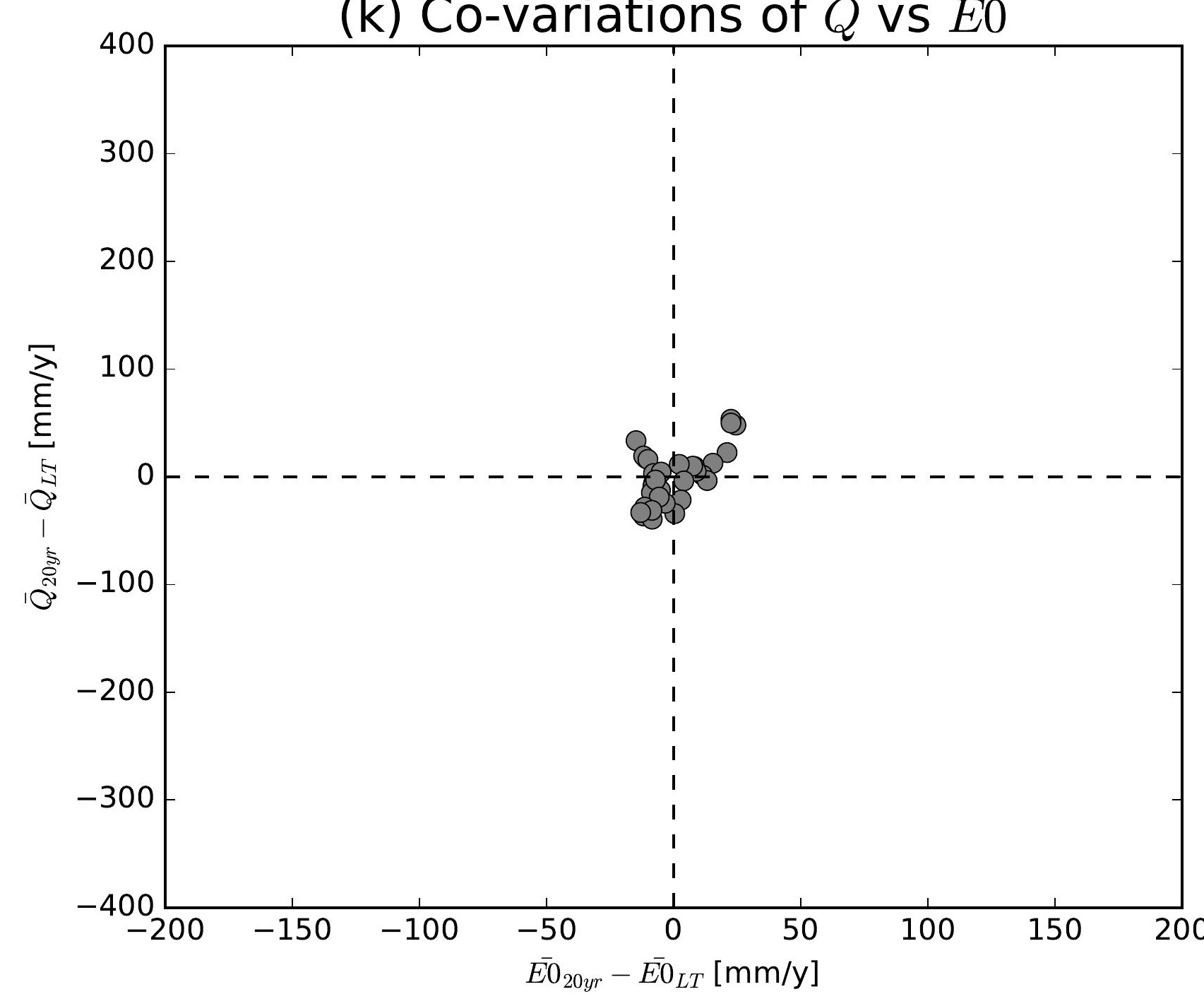

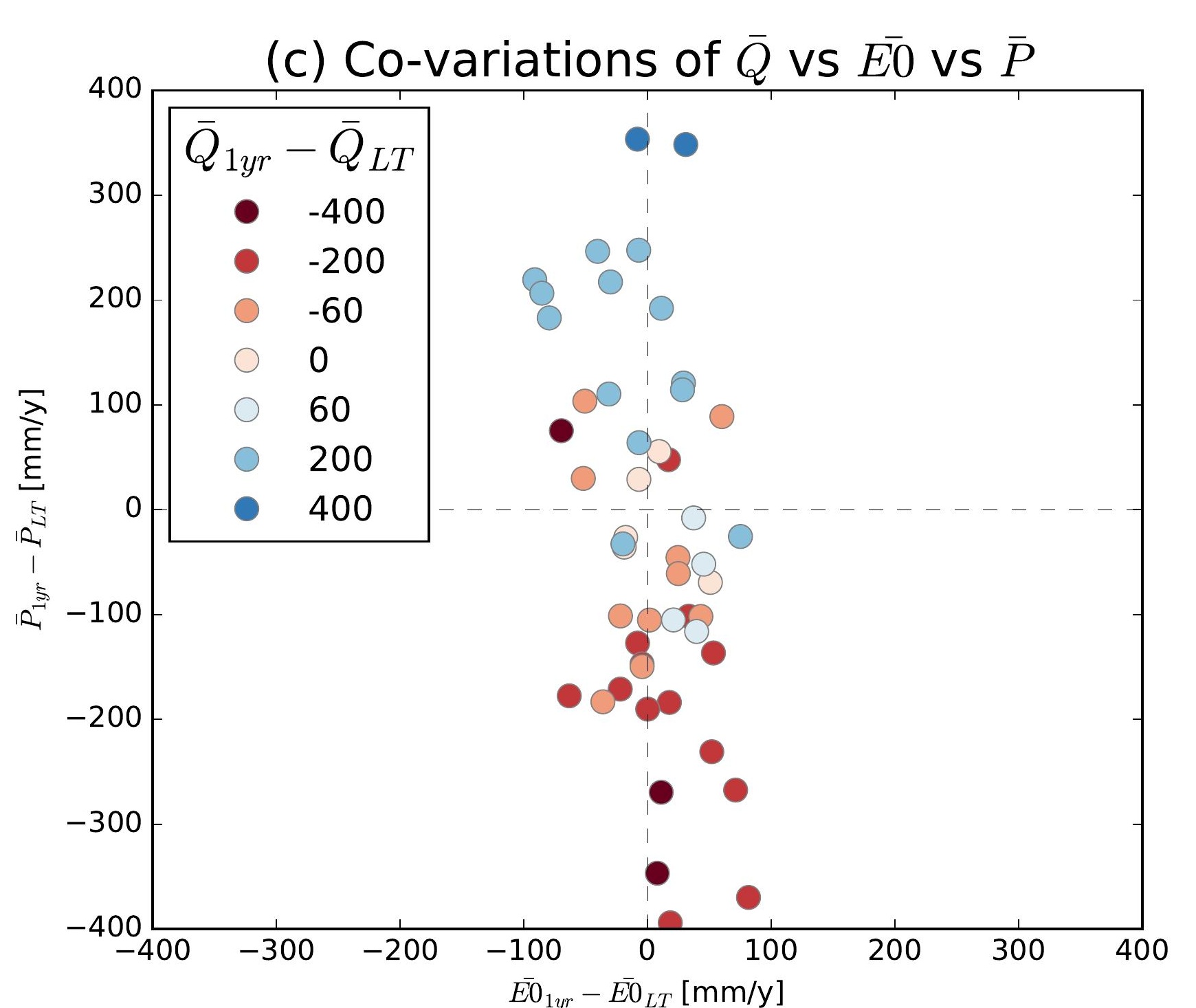
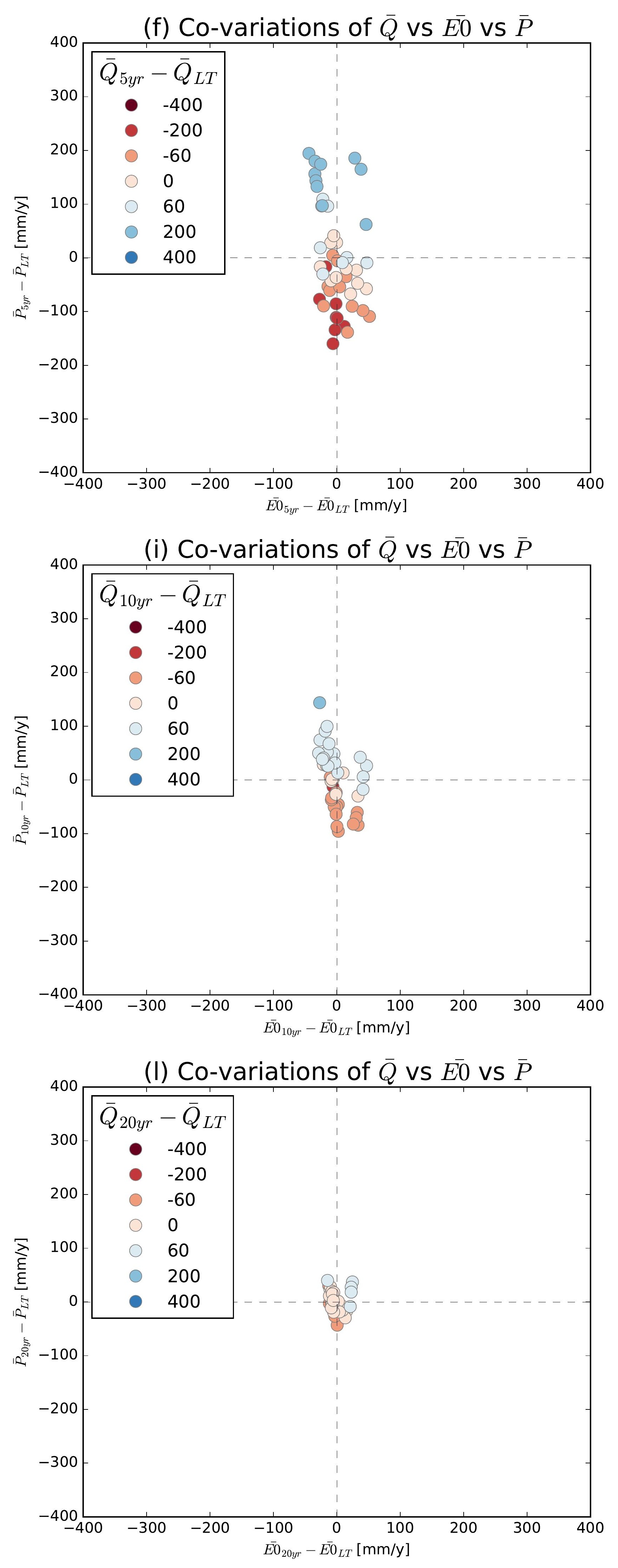

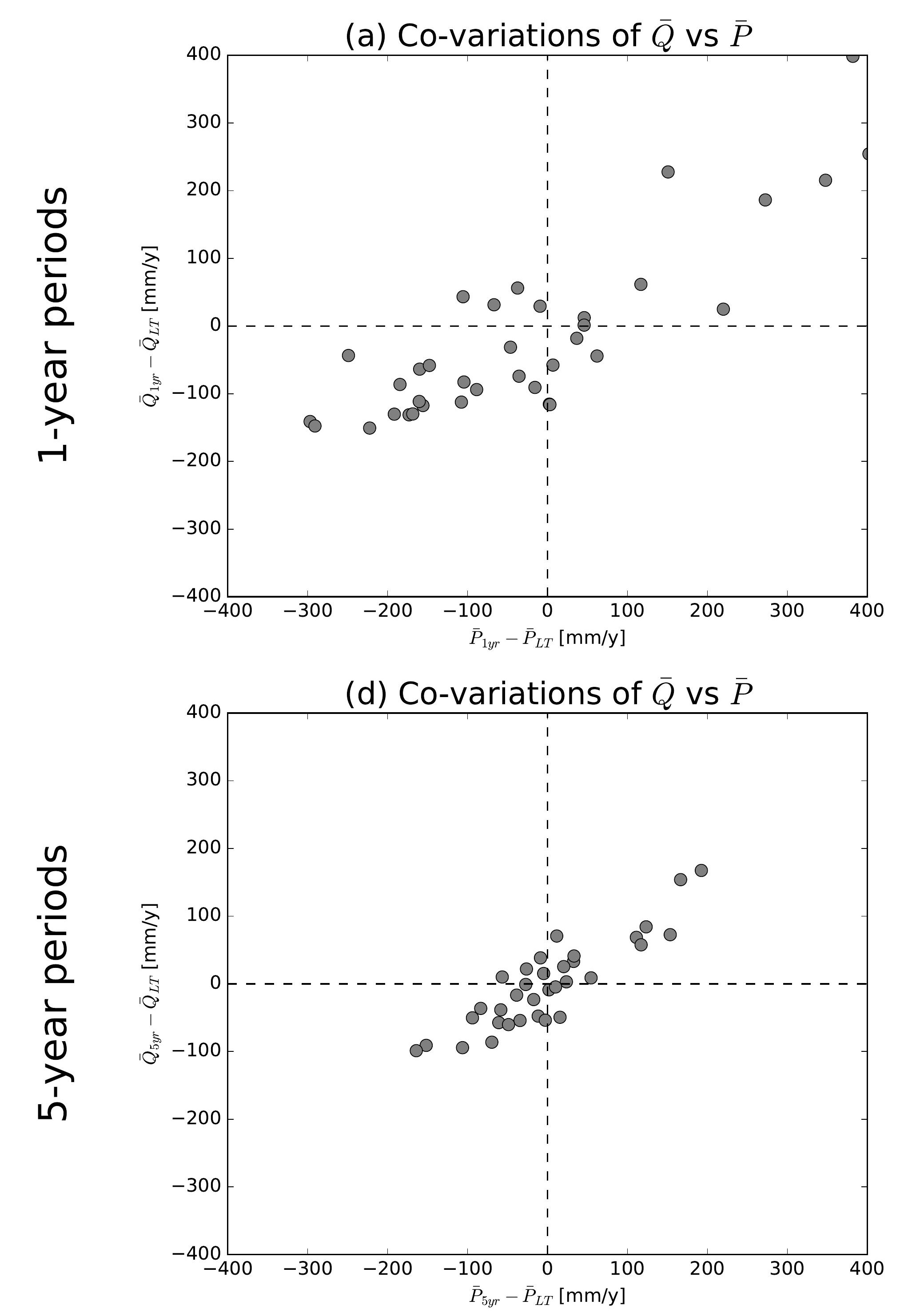

(g) Co-variations of $\bar{Q}$ vs $\bar{P}$
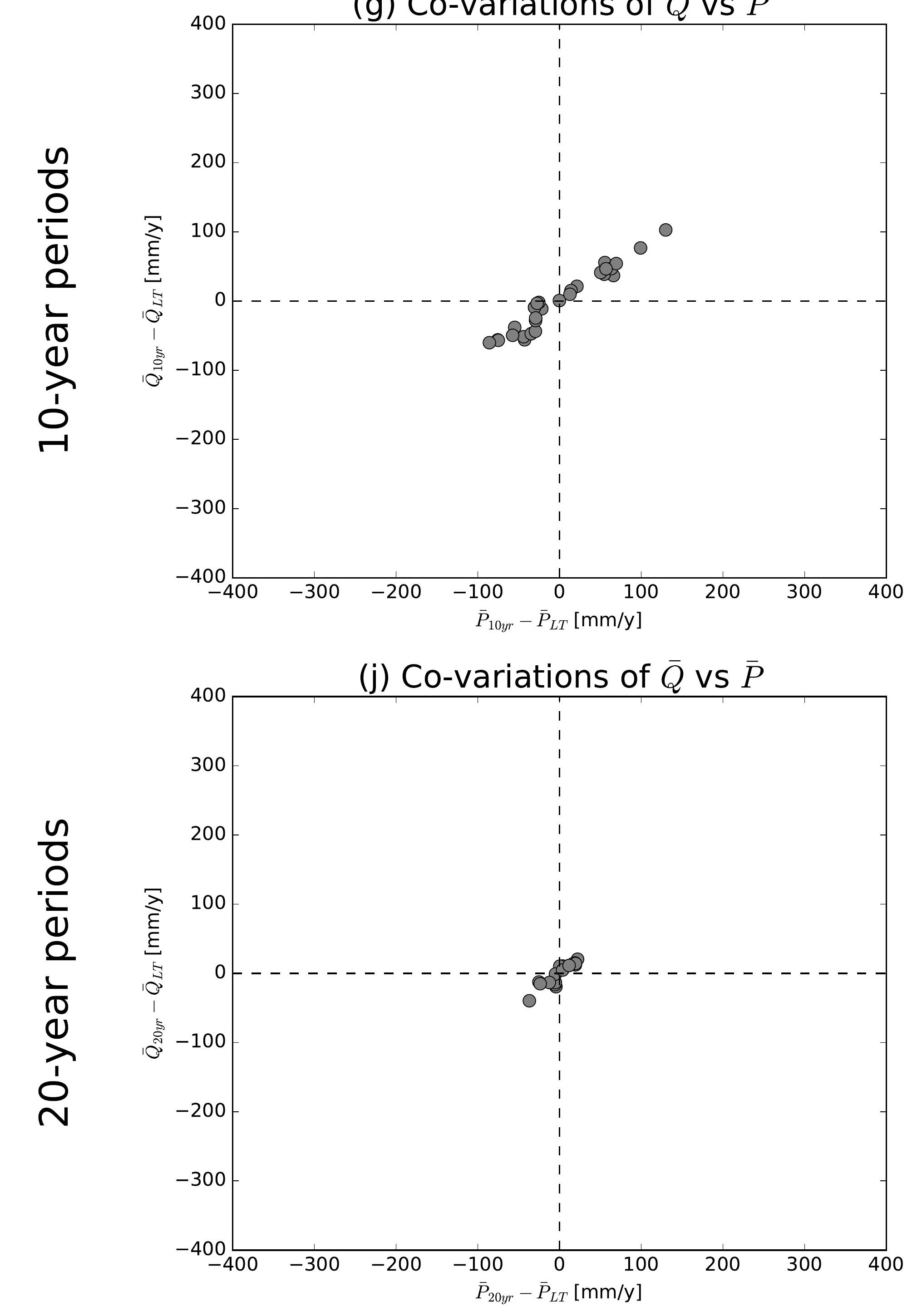

(b) Co-variations of $\bar{Q}$ vs $\overline{E 0}$

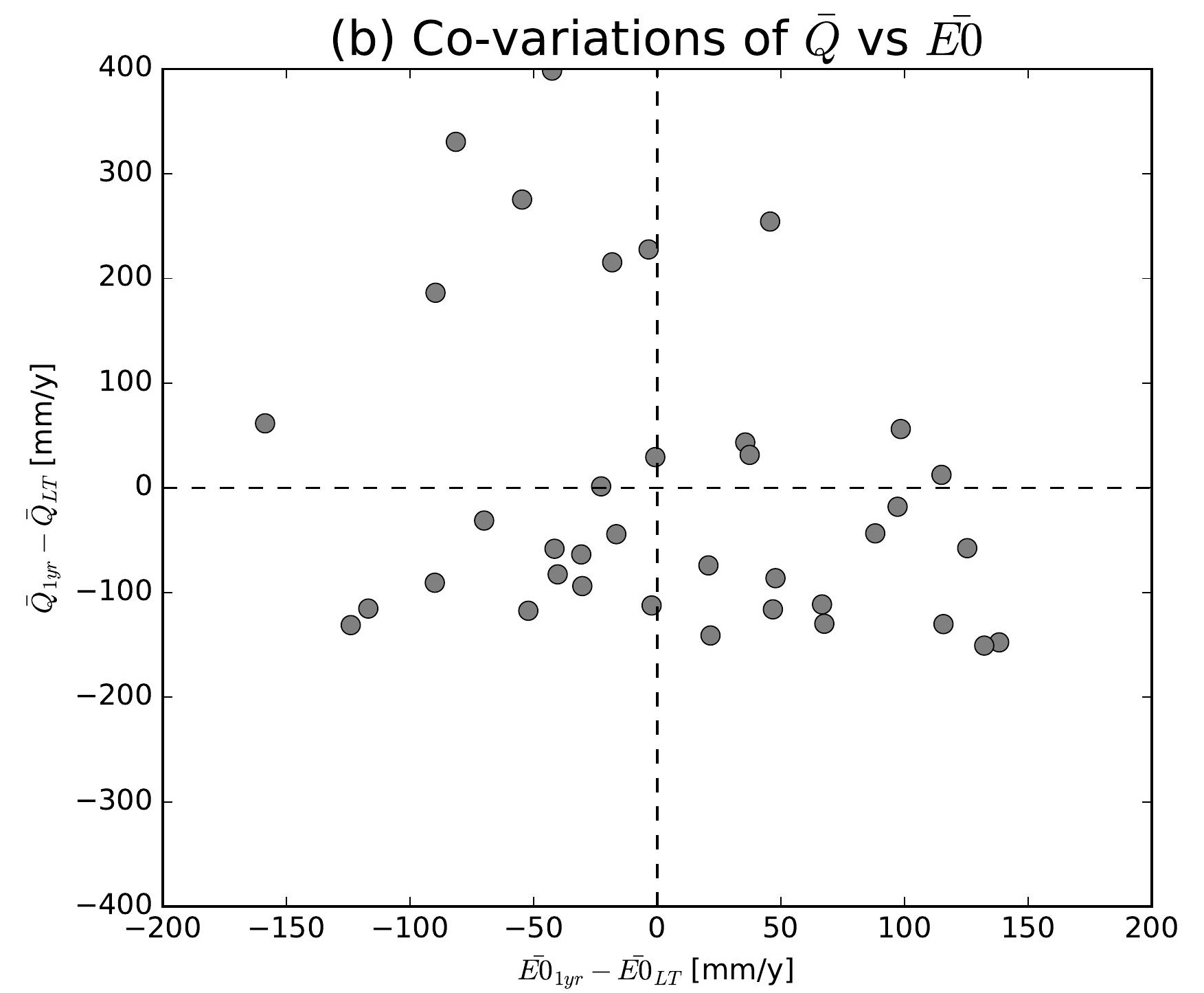

(e) Co-variations of $\bar{Q}$ vs $\overline{E 0}$

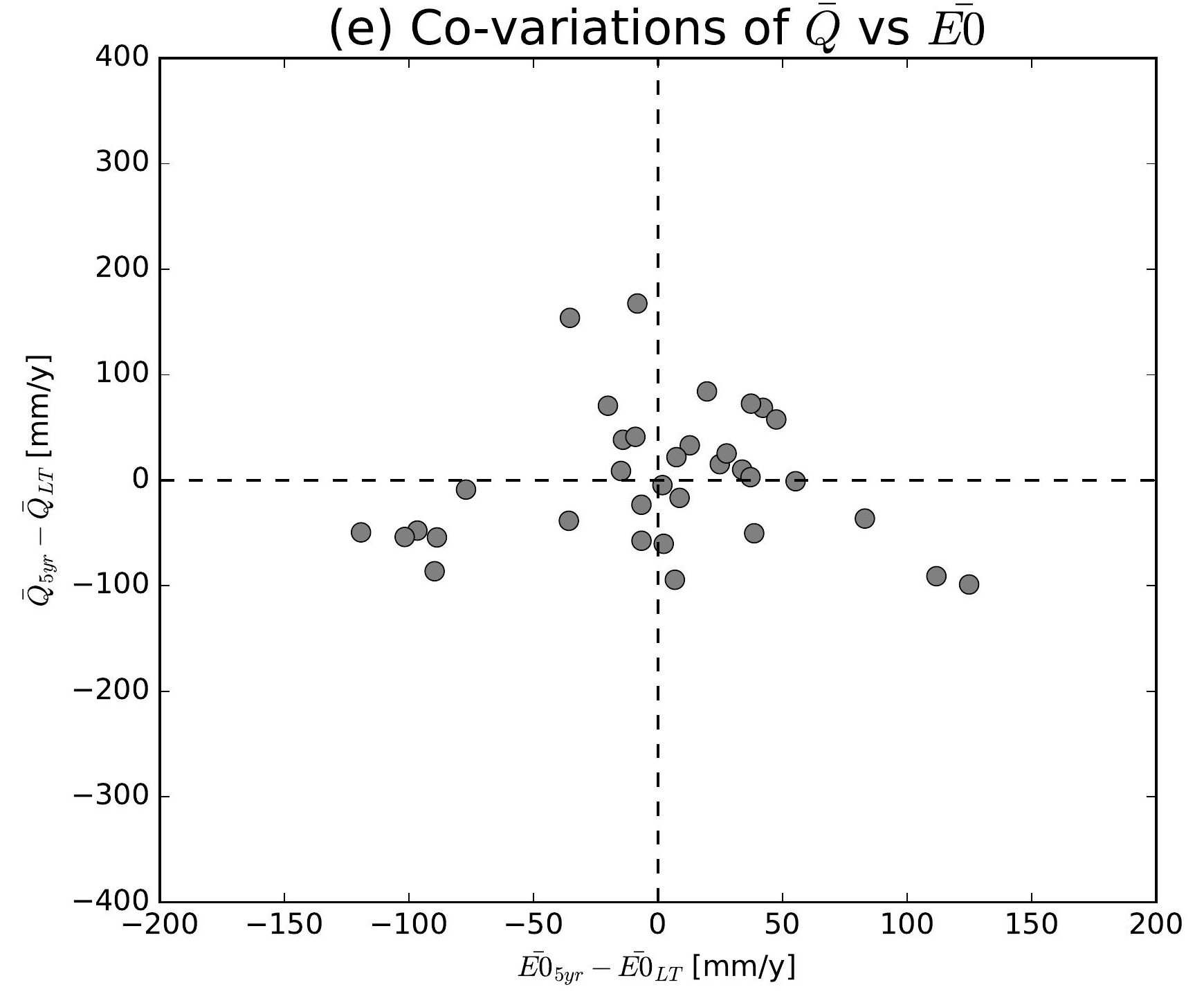

(h) Co-variations of $\bar{Q}$ vs $\overline{E 0}$

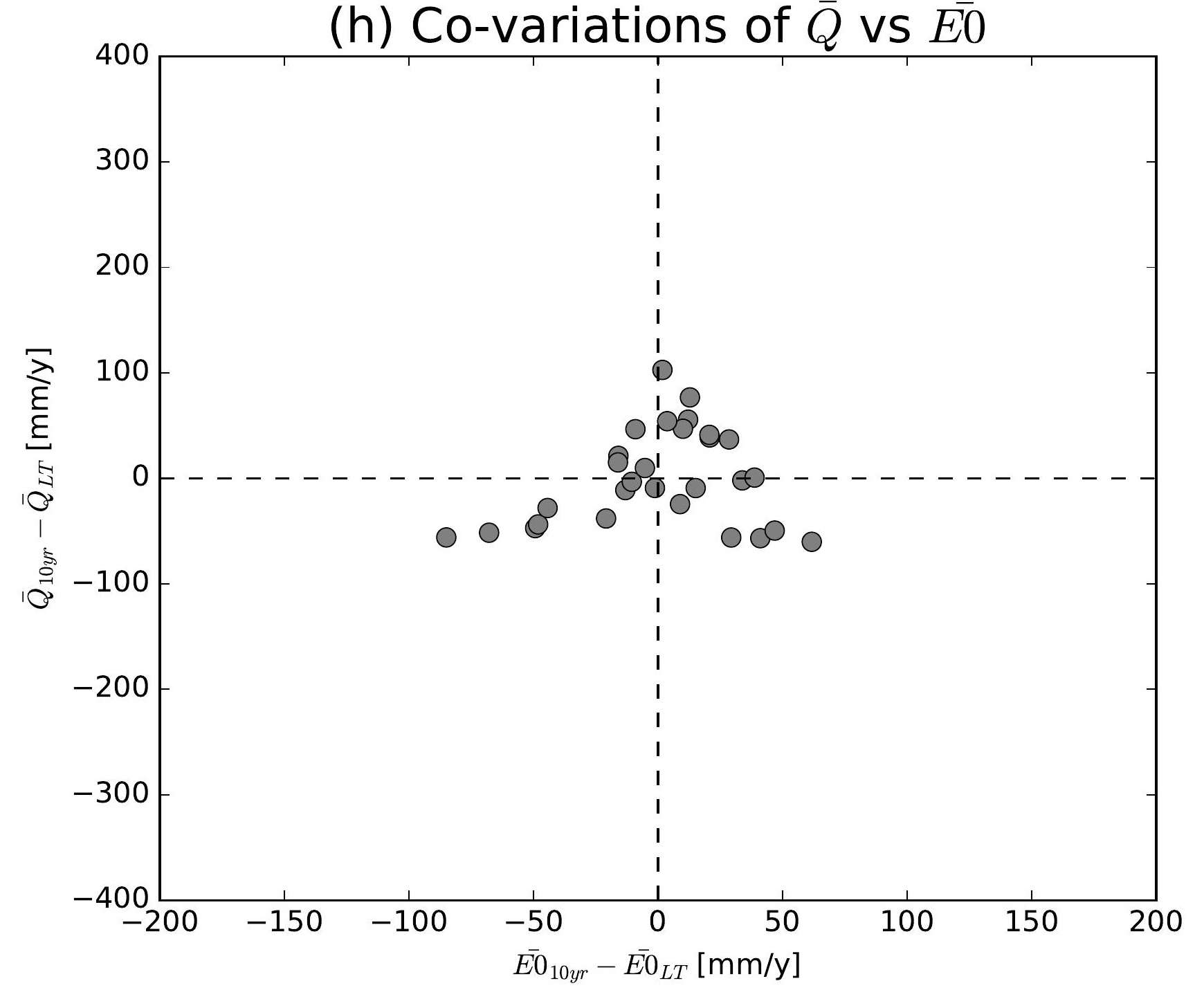

(k) Co-variations of $\bar{Q}$ vs $\overline{E 0}$

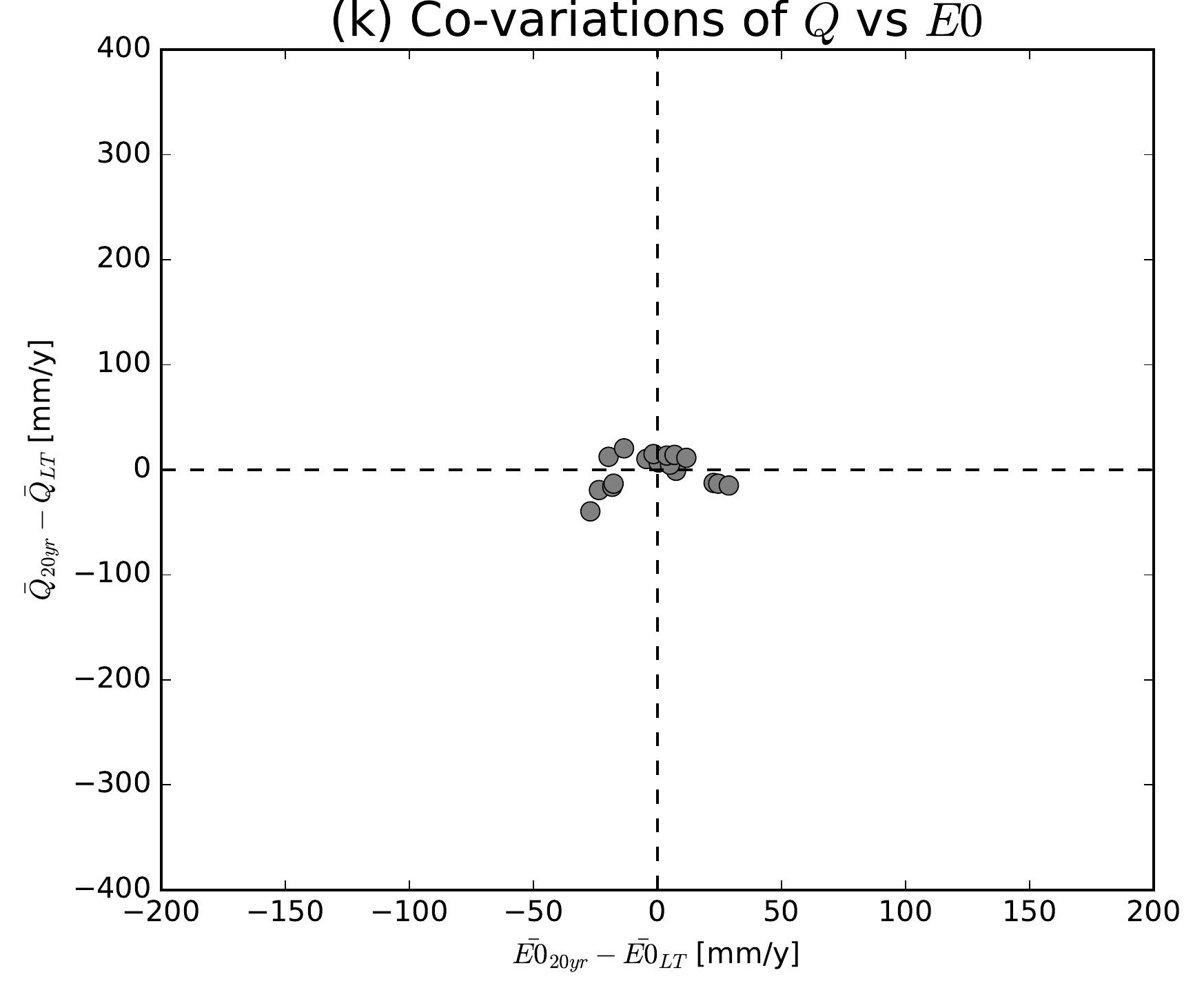

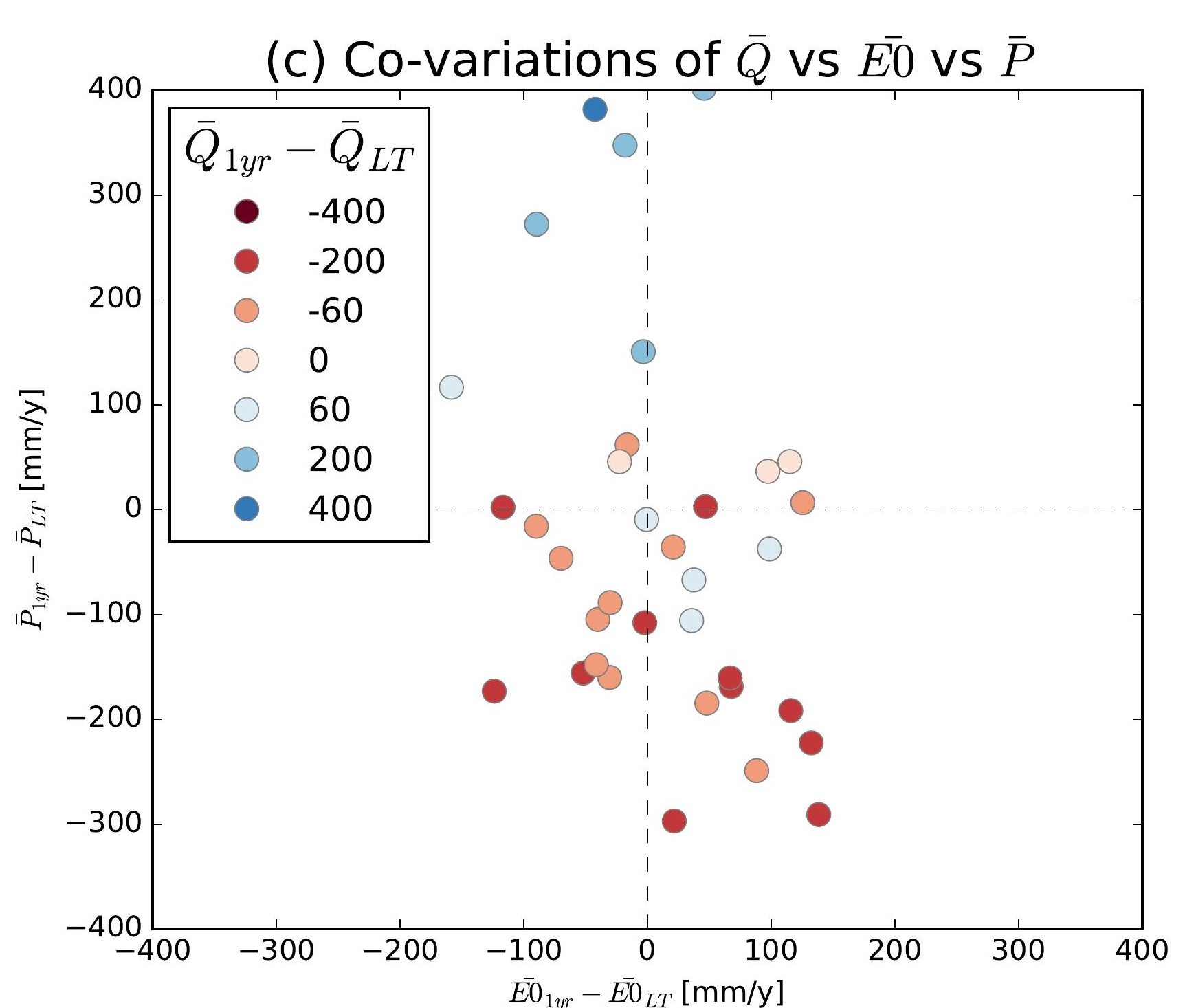
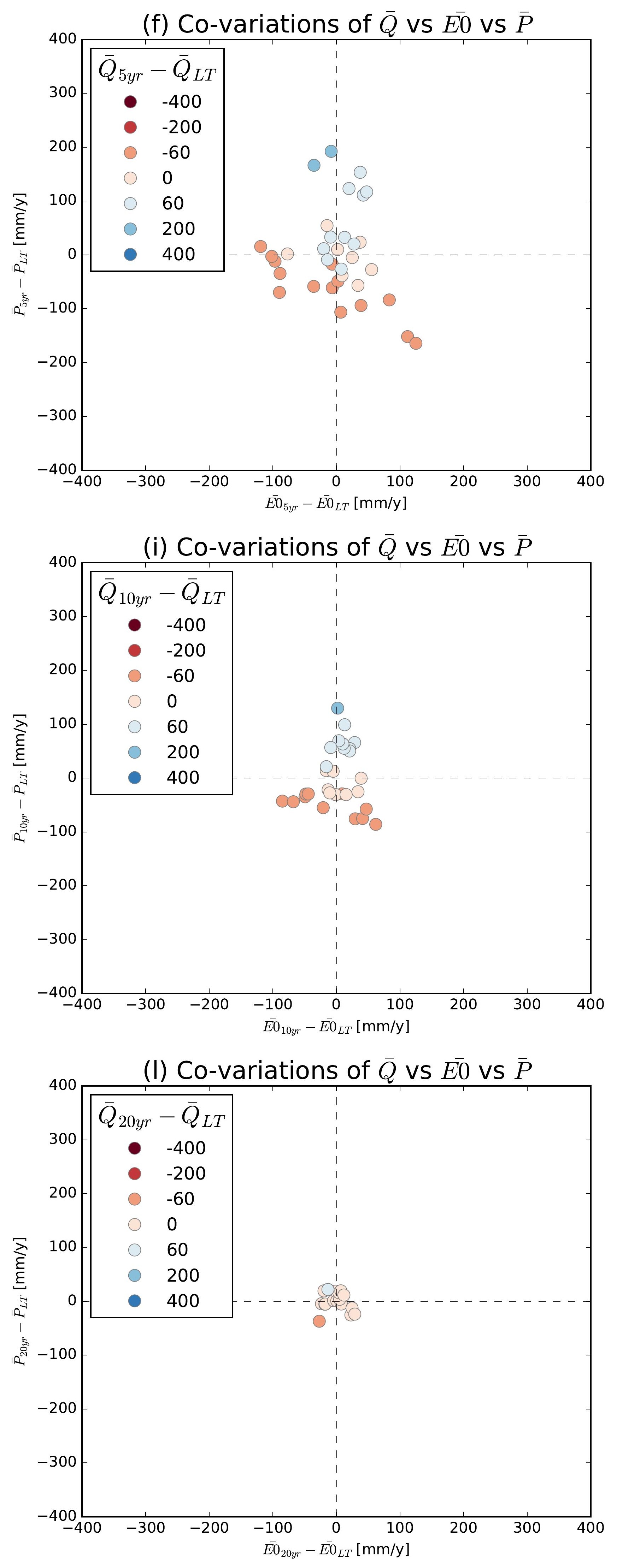

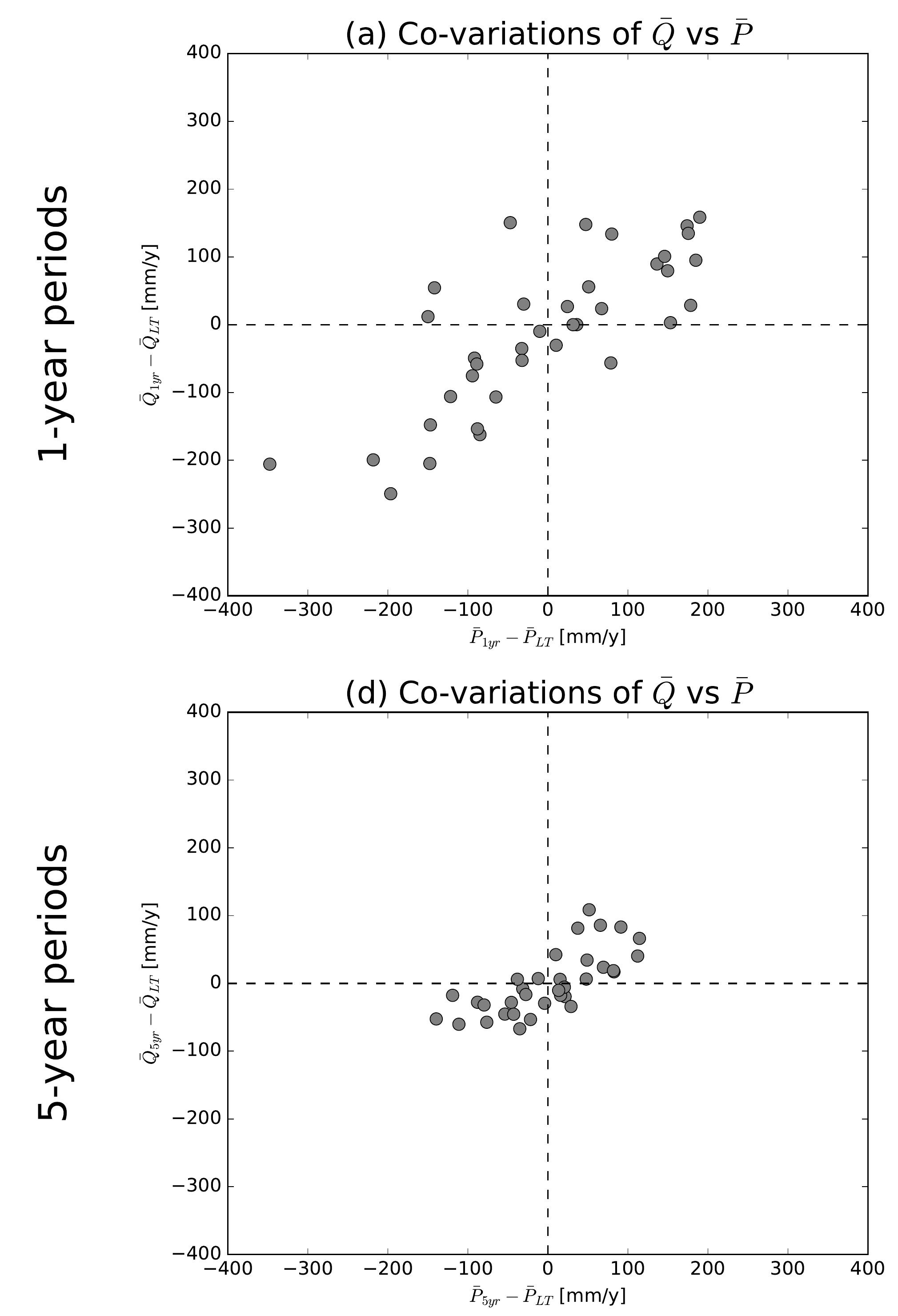

(g) Co-variations of $\bar{Q}$ vs $\bar{P}$
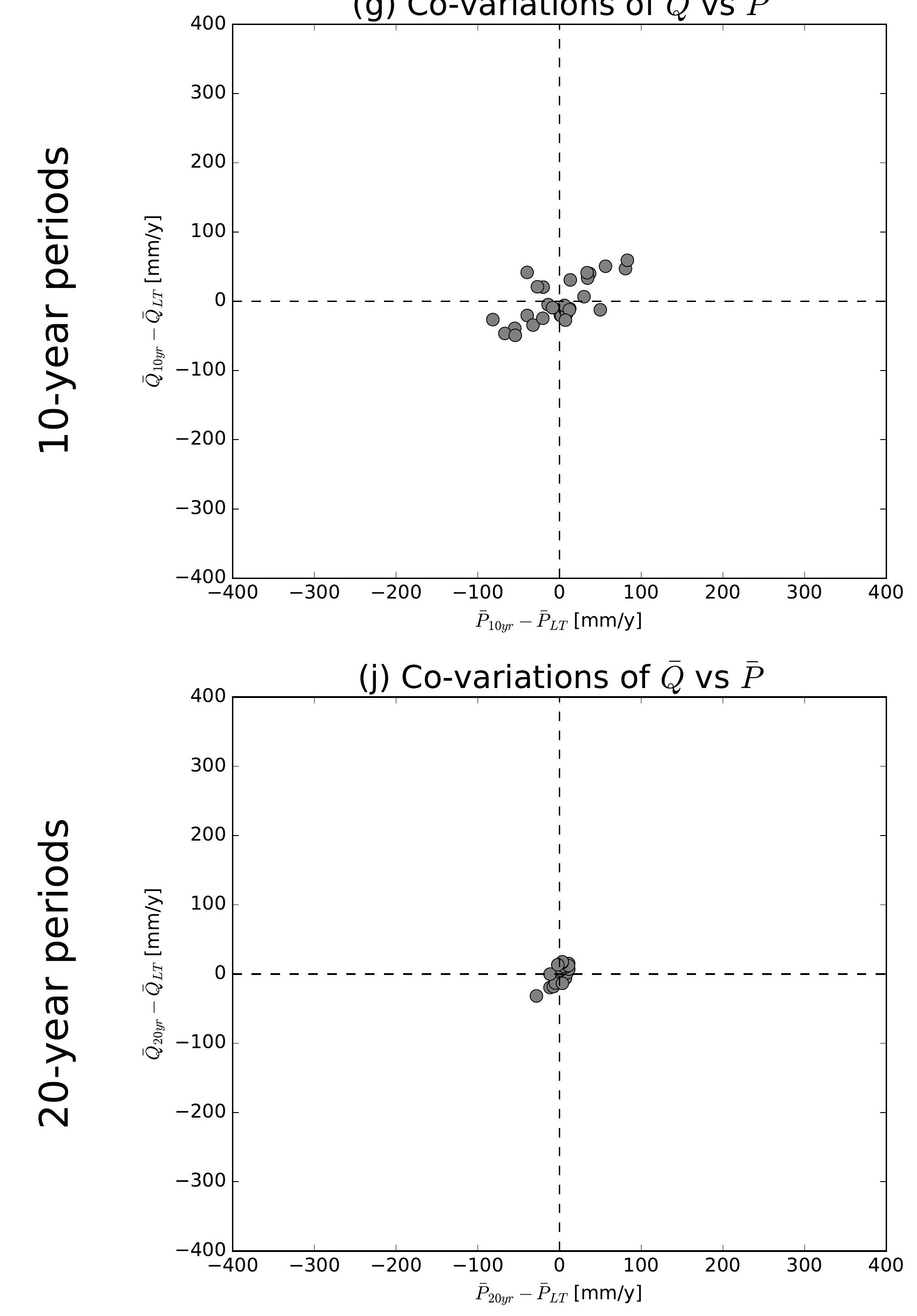

(b) Co-variations of $\bar{Q}$ vs $\overline{E 0}$

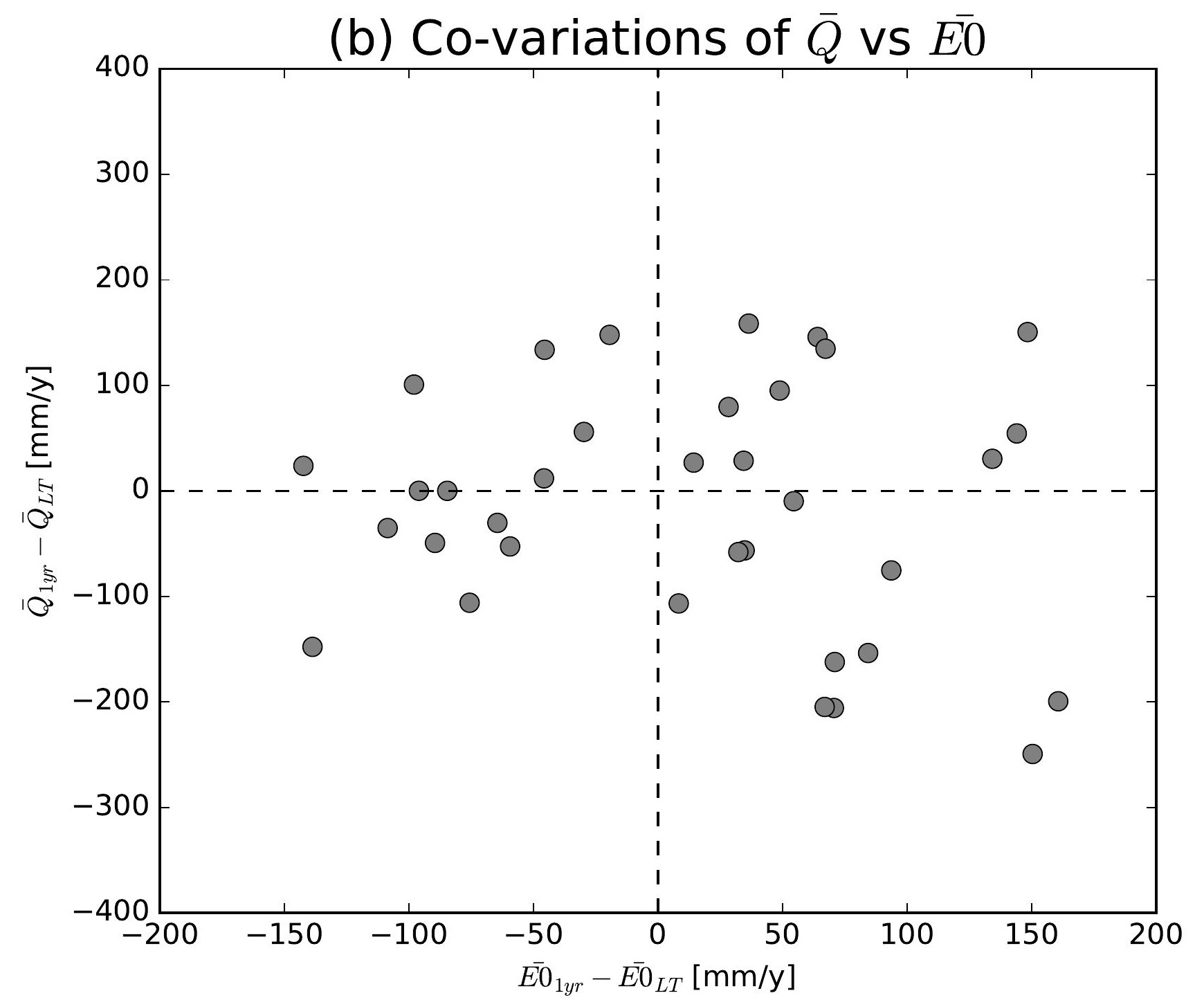

(e) Co-variations of $\bar{Q}$ vs $\overline{E 0}$

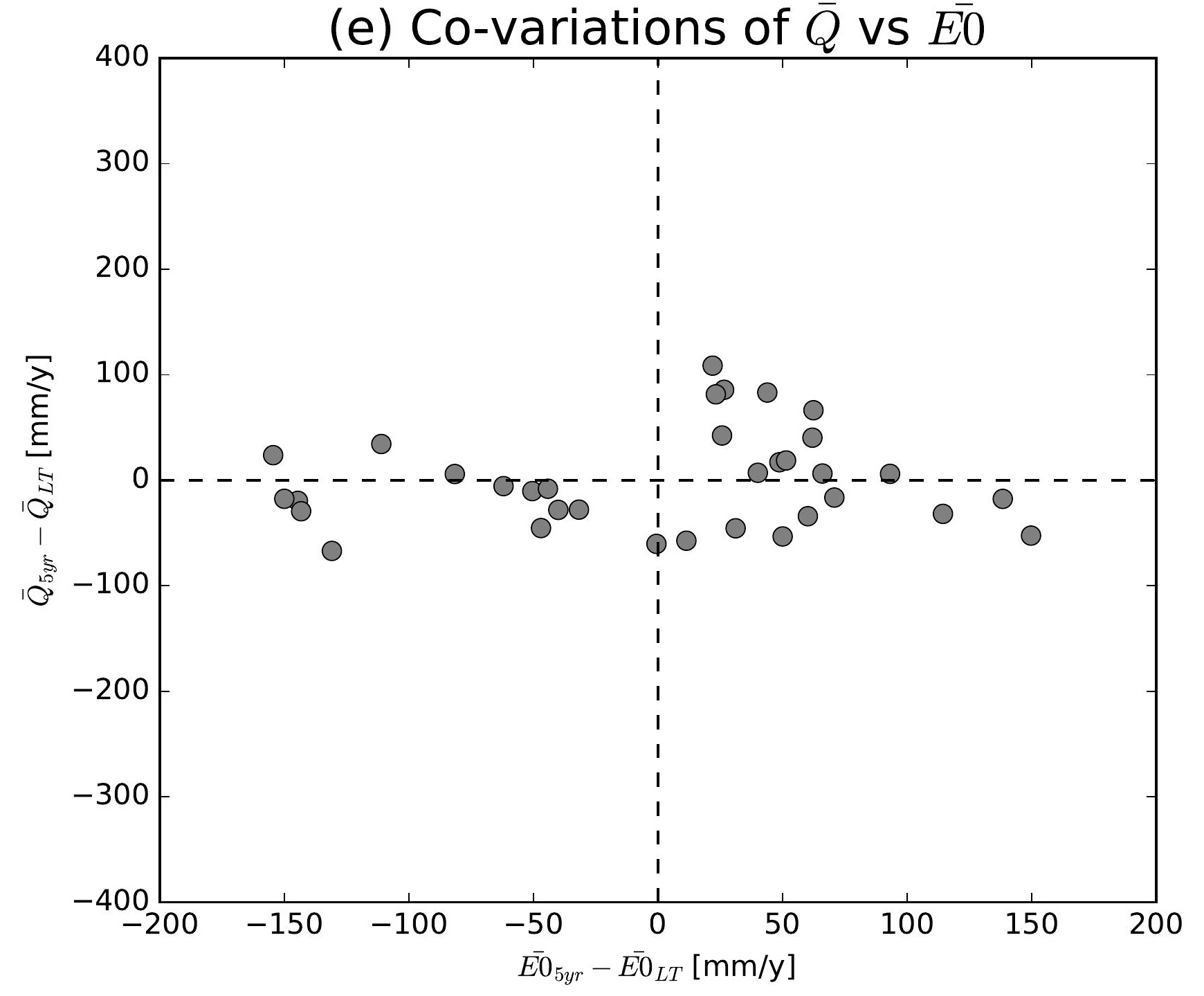

(h) Co-variations of $\bar{Q}$ vs $\overline{E 0}$

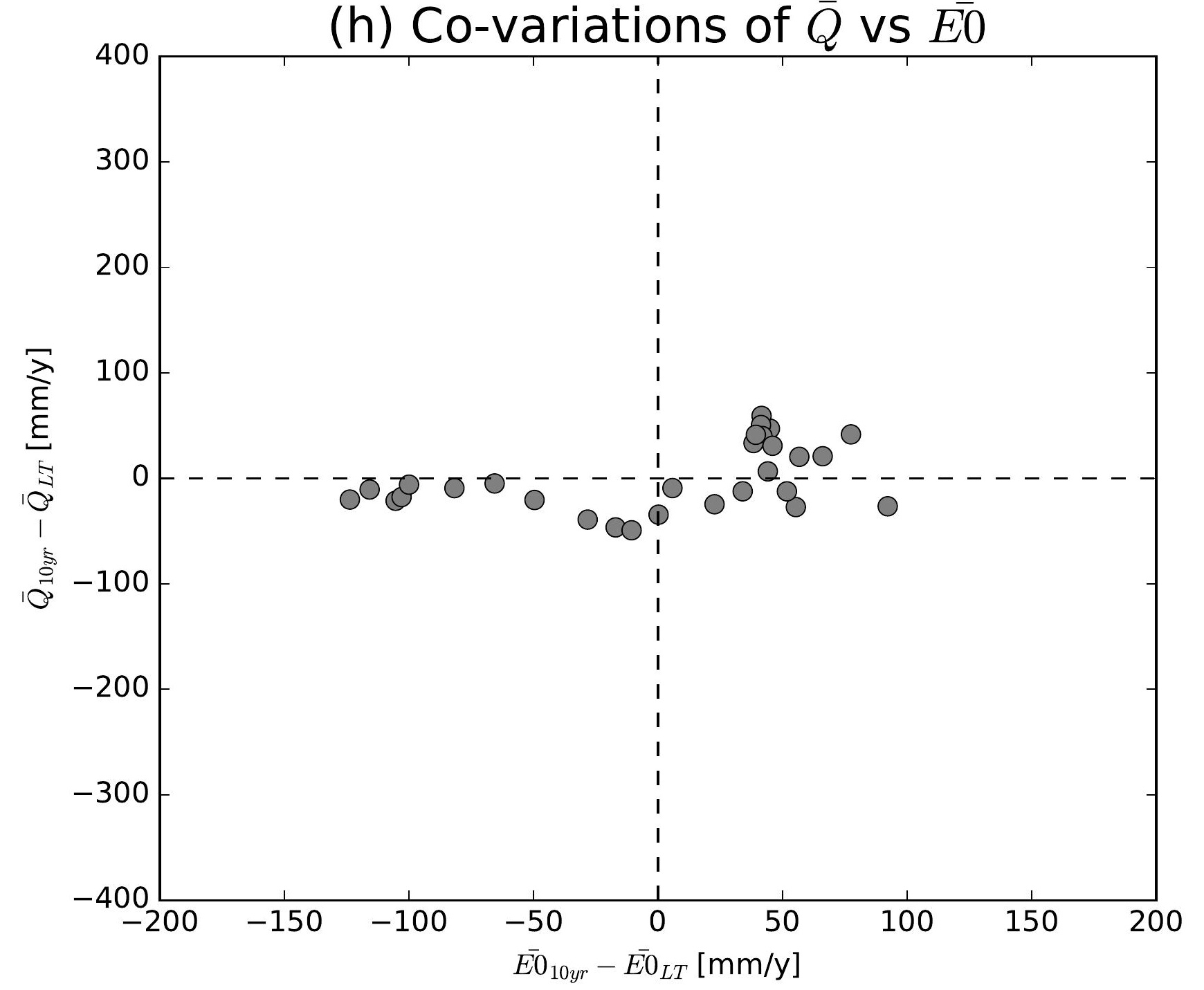

(k) Co-variations of $\bar{Q}$ vs $\overline{E 0}$

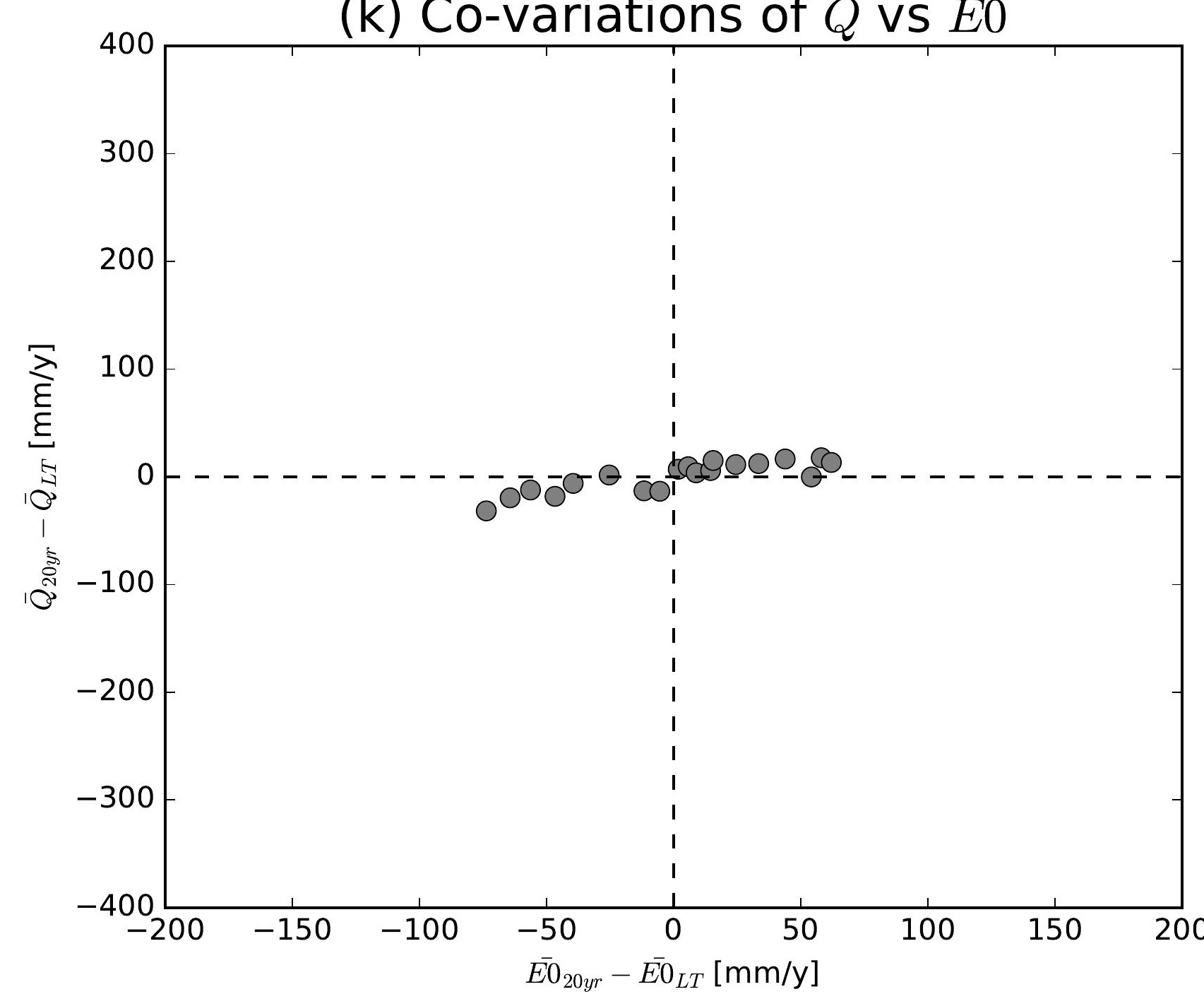

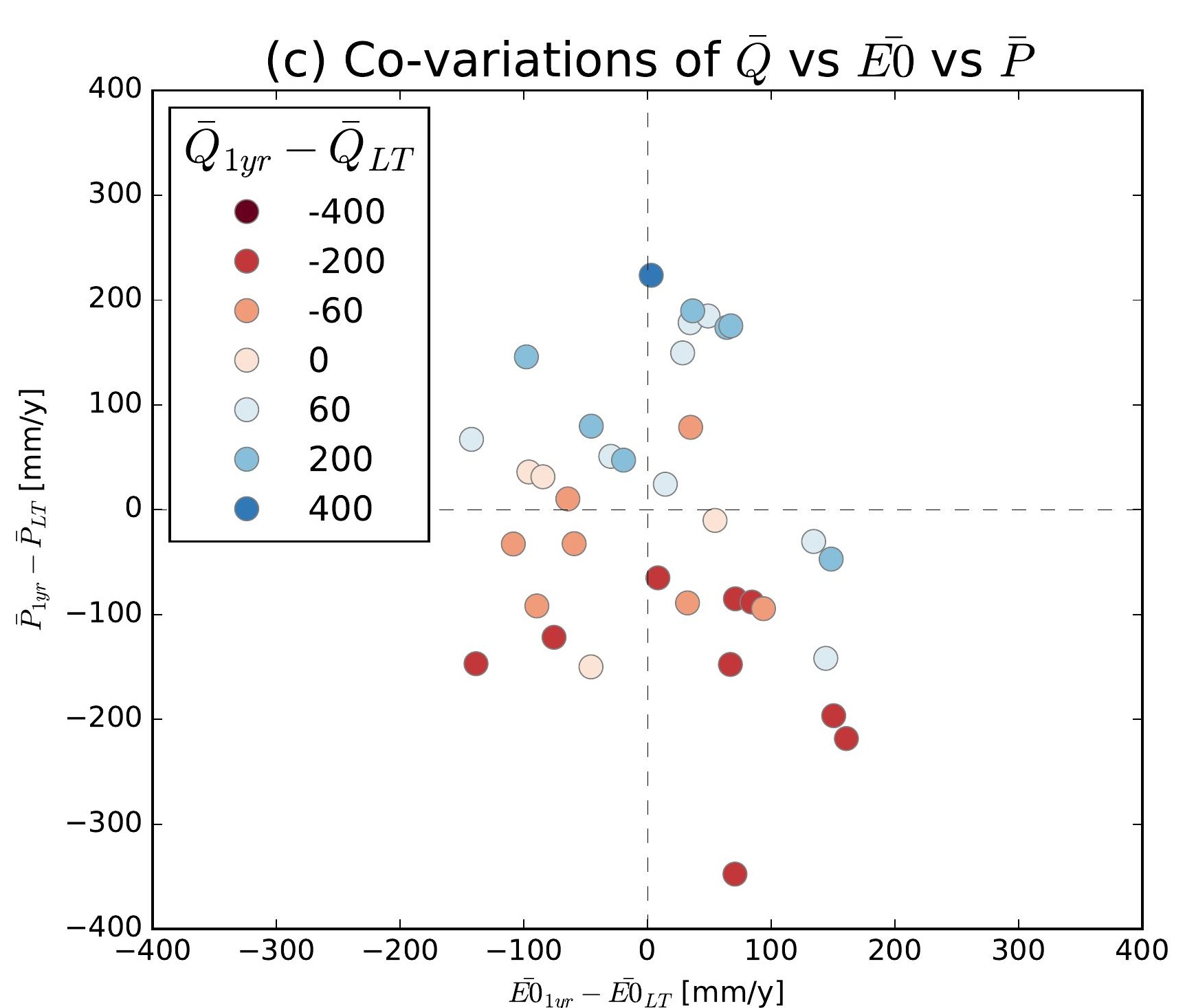
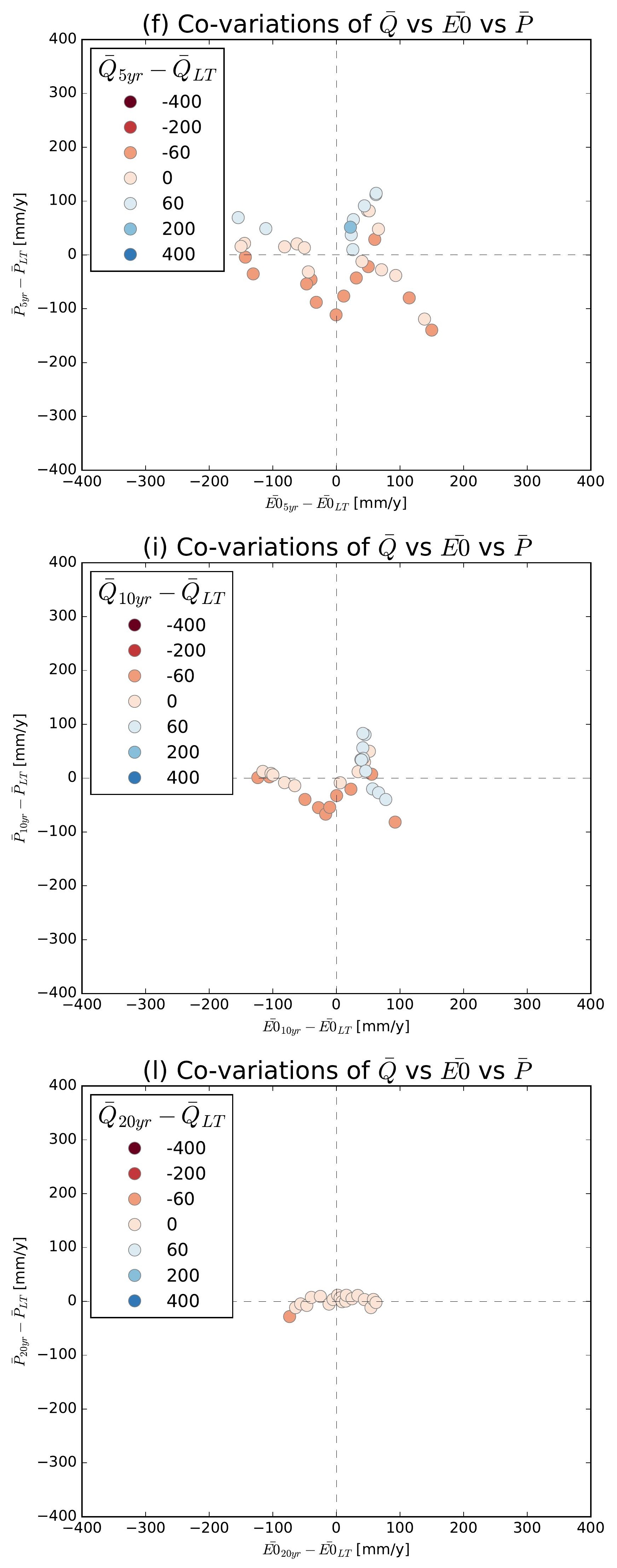

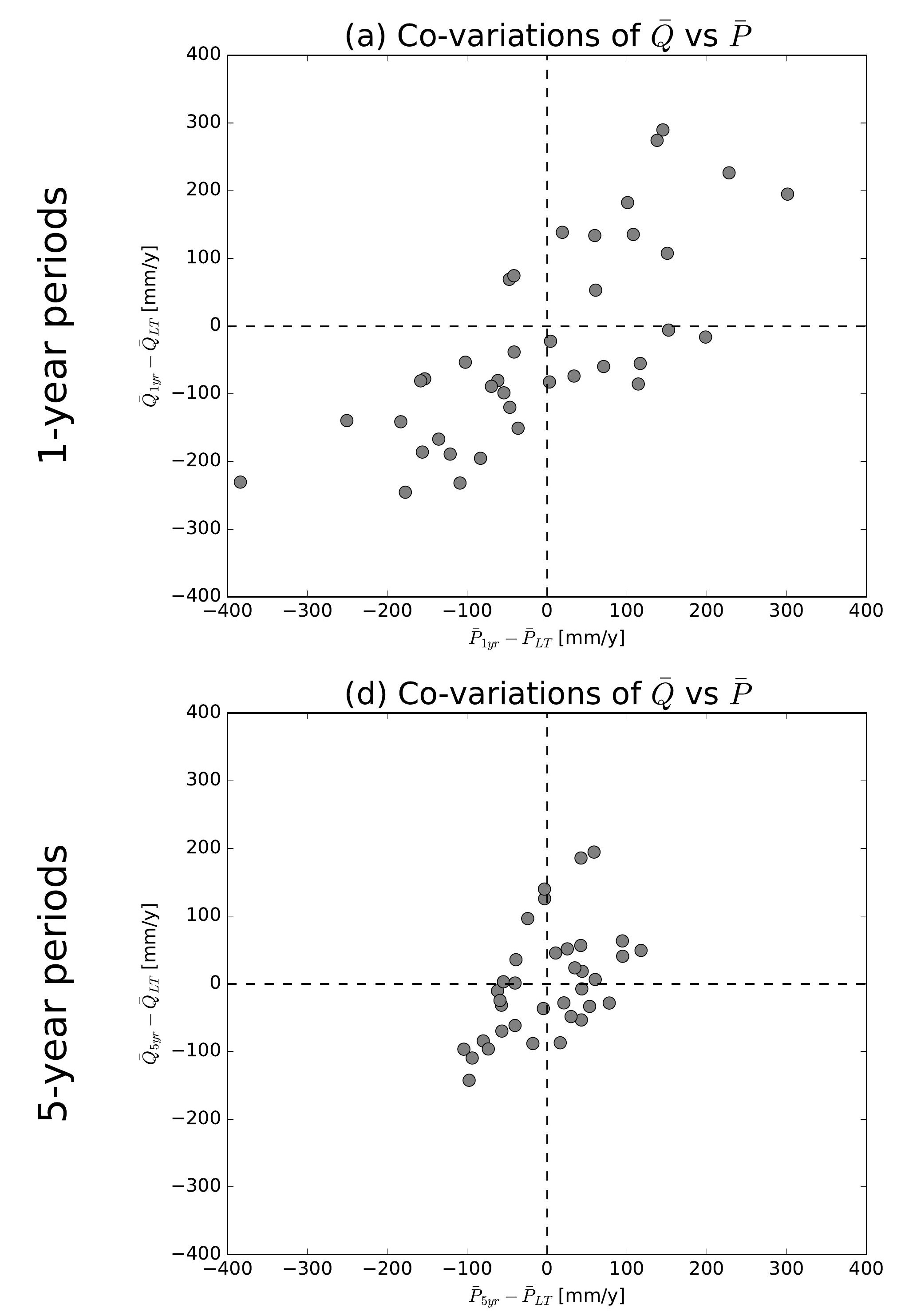

(g) Co-variations of $\bar{Q}$ vs $\bar{P}$
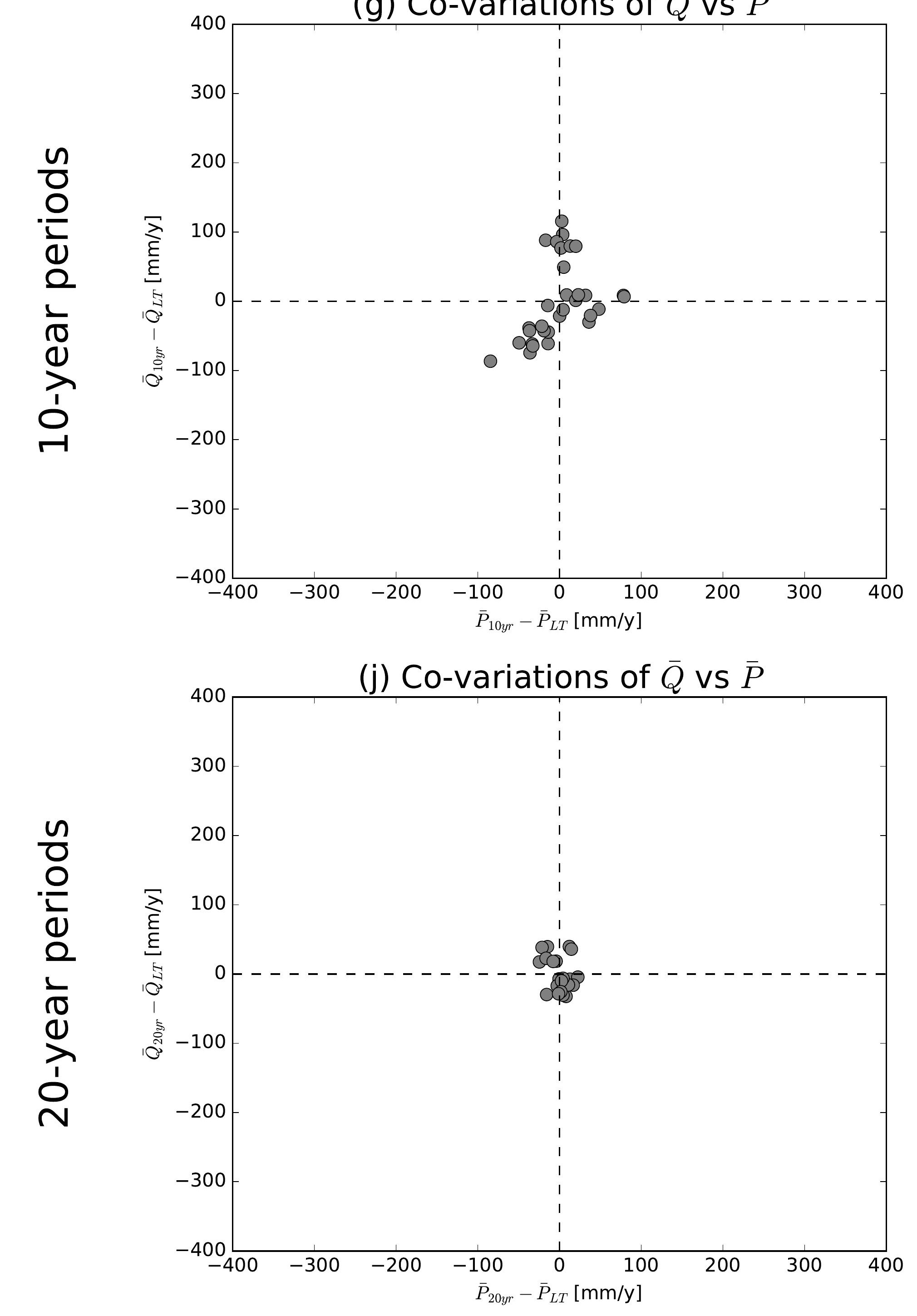

(b) Co-variations of $\bar{Q}$ vs $\overline{E 0}$

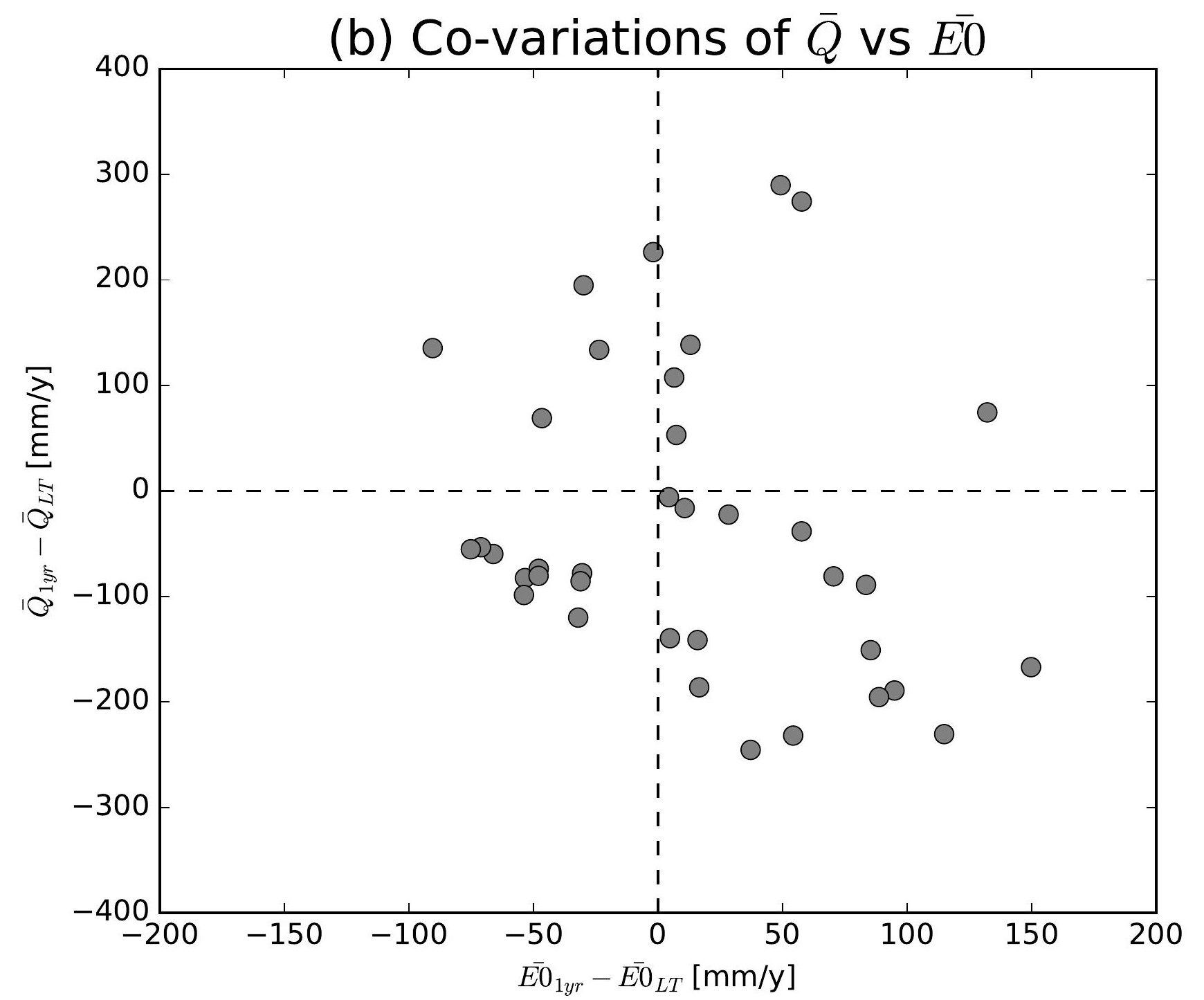

(e) Co-variations of $\bar{Q}$ vs $\overline{E 0}$

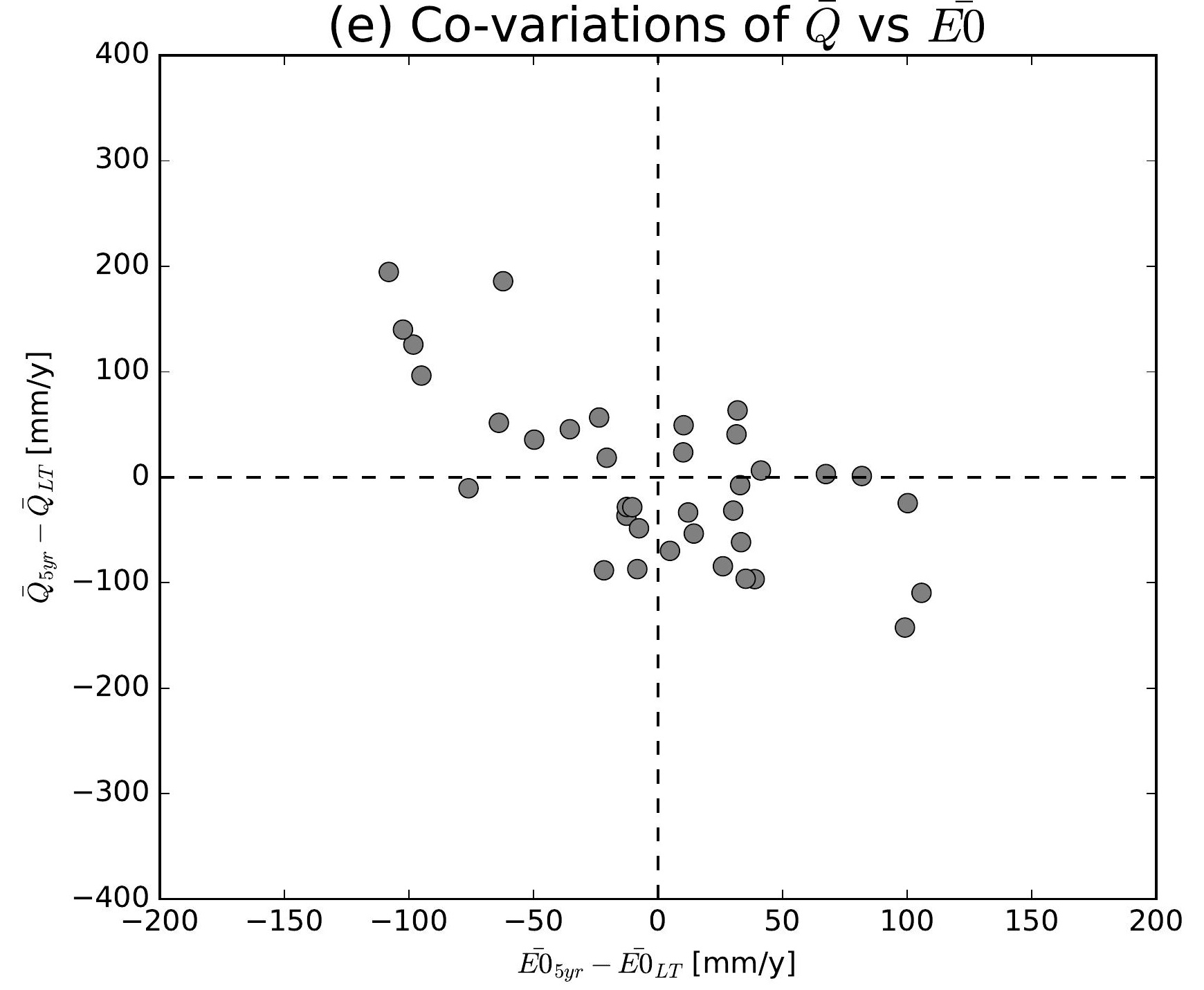

(h) Co-variations of $\bar{Q}$ vs $\overline{E 0}$

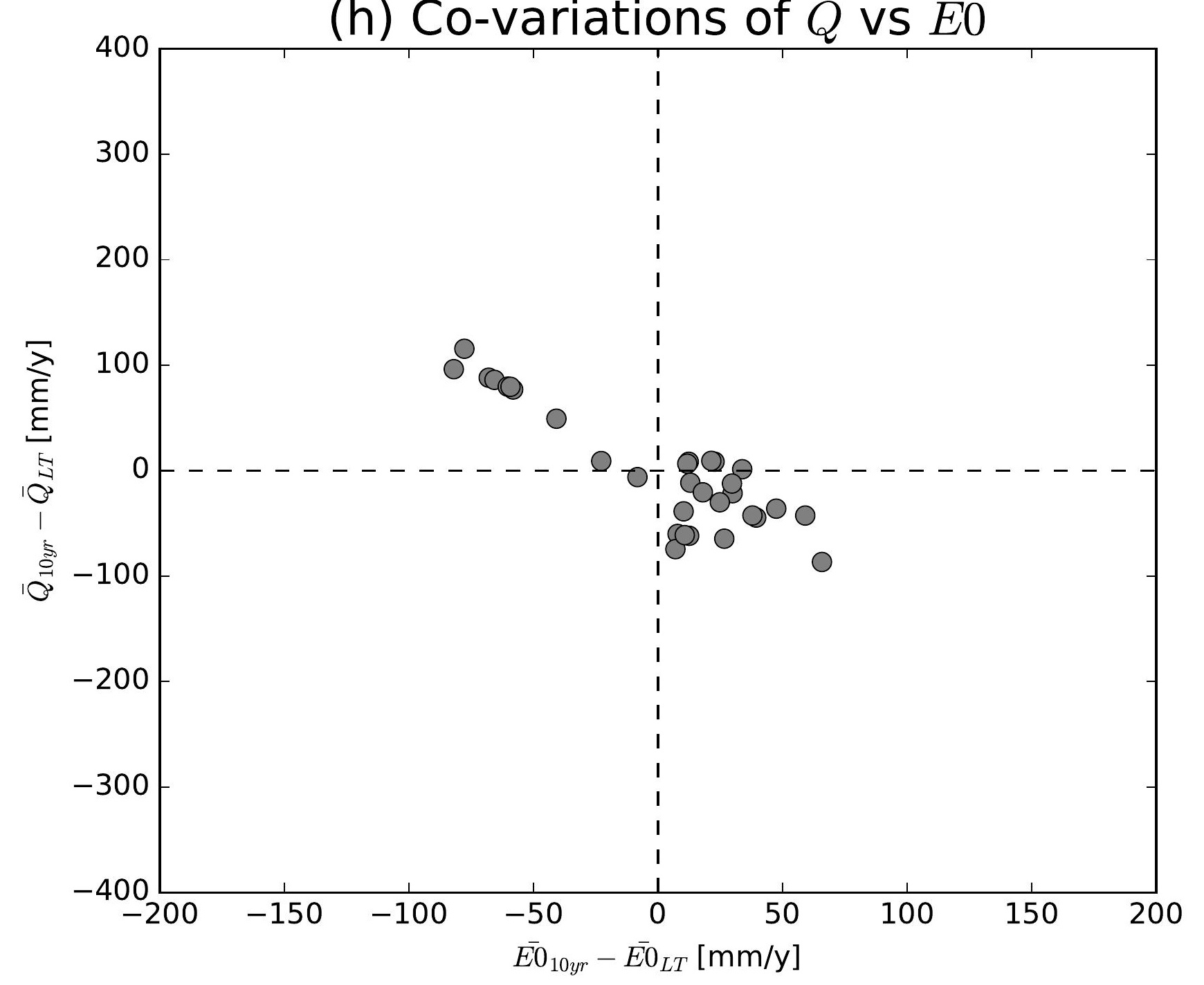

(k) Co-variations of $\bar{Q}$ vs $\overline{E 0}$

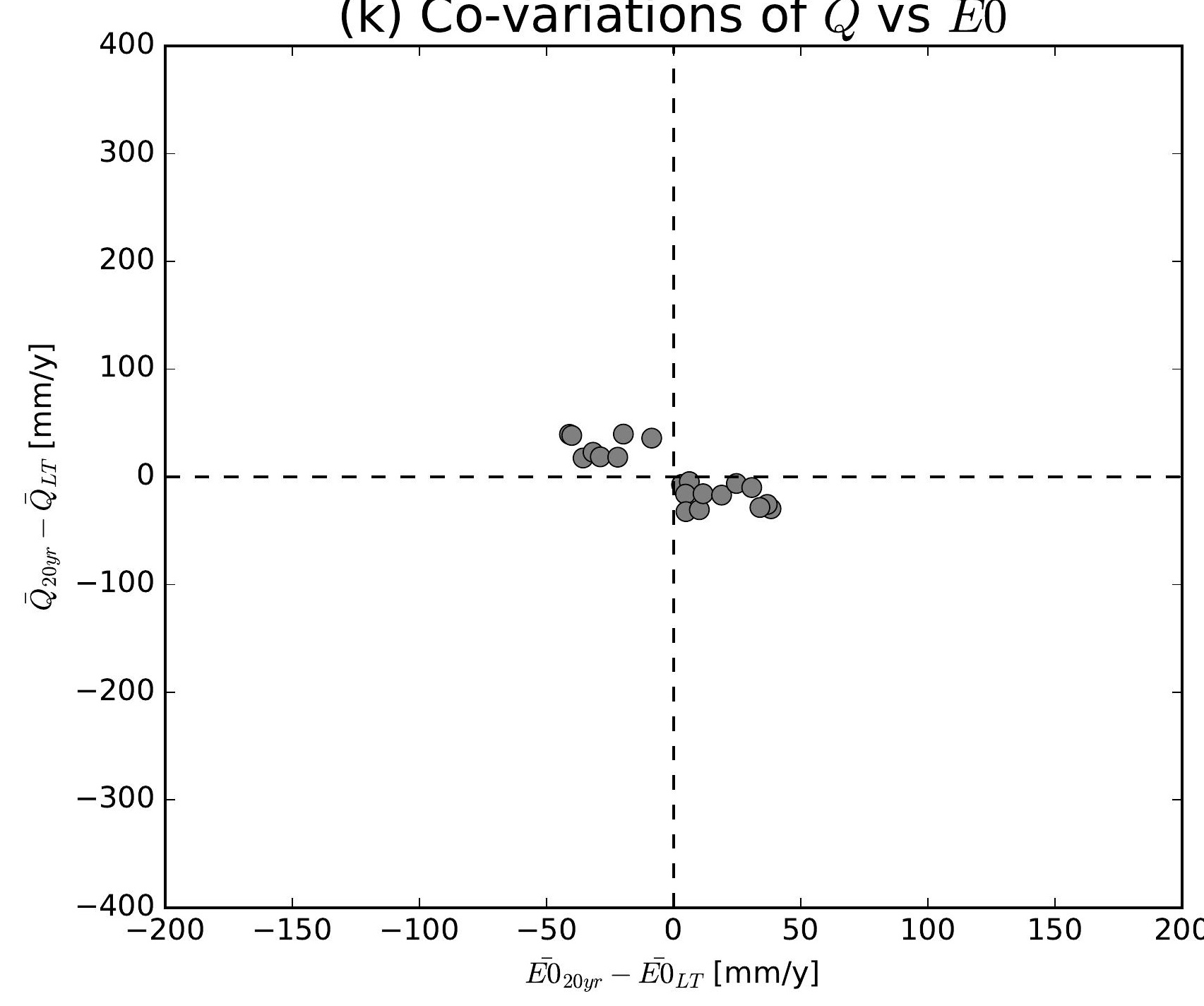

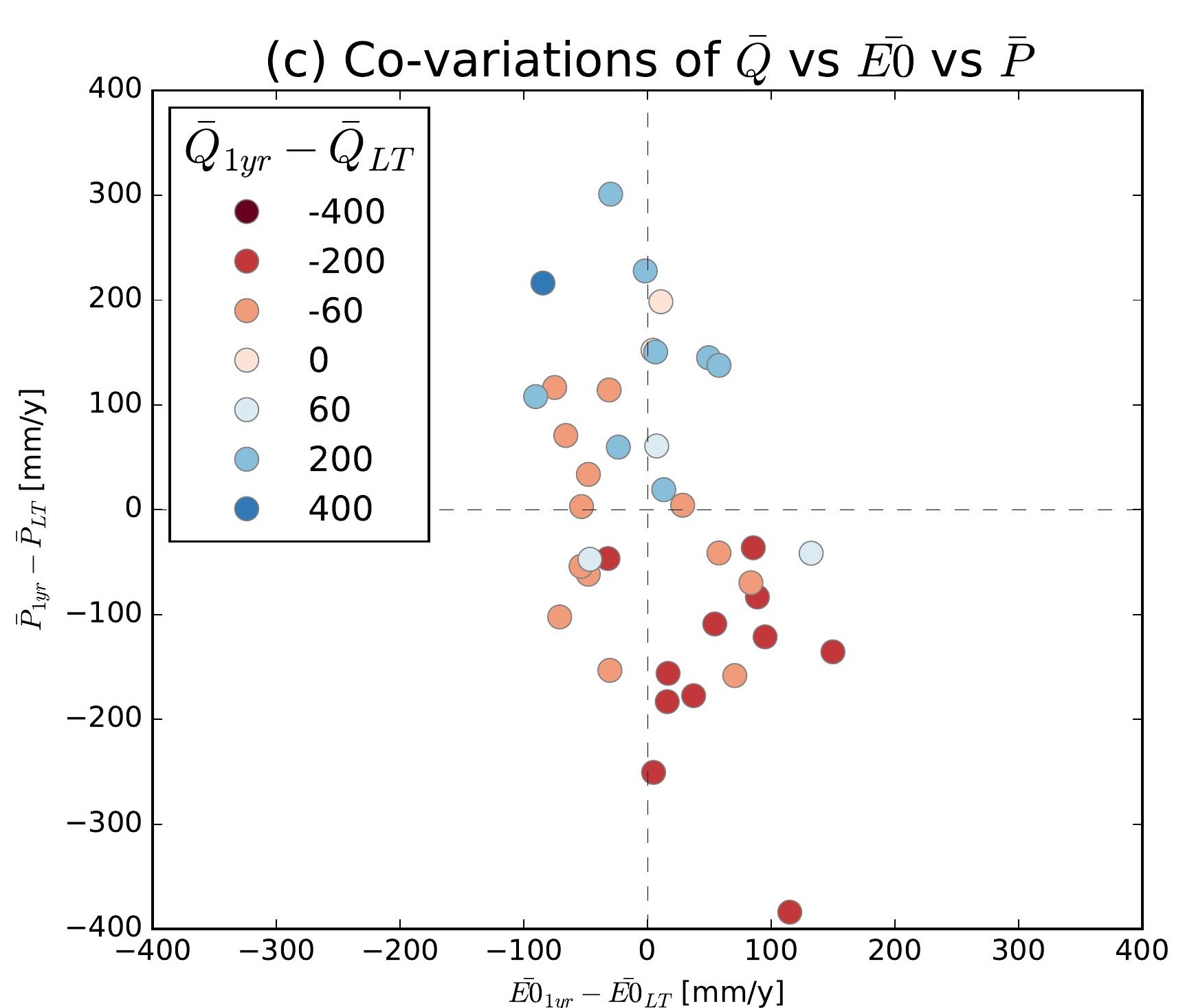
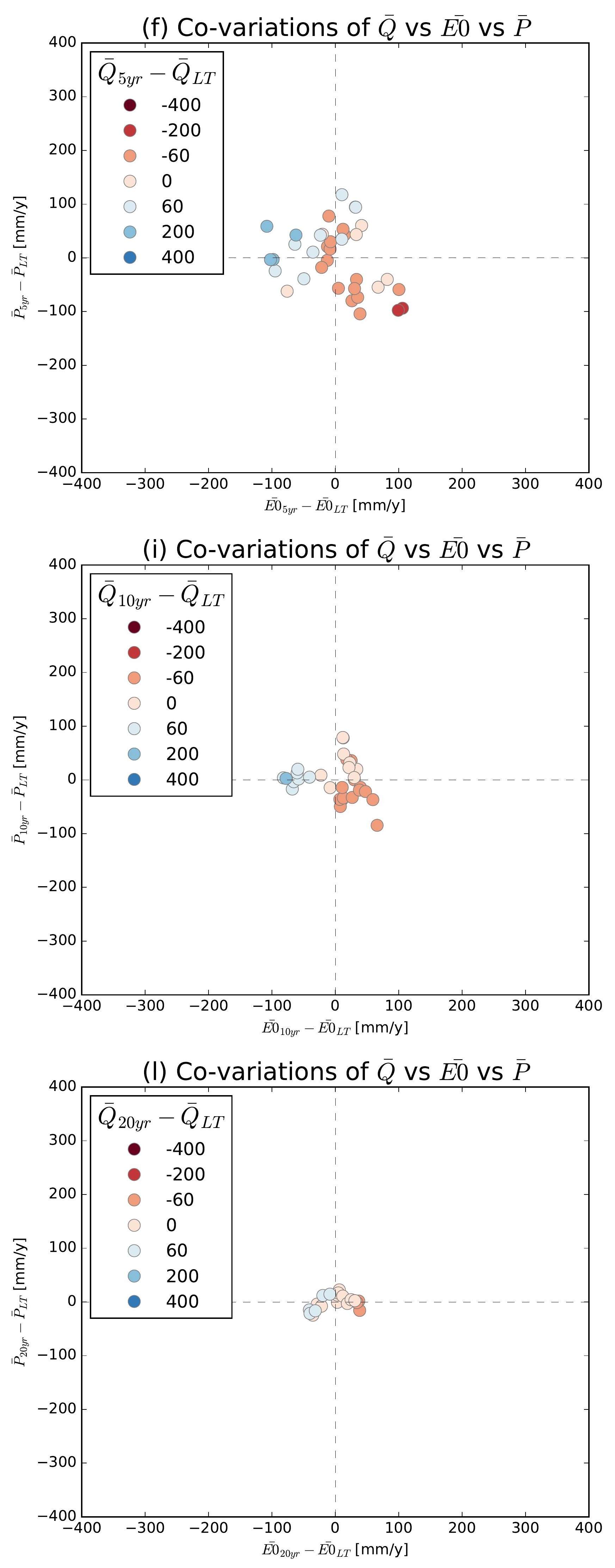
Catchment Y2015010

(a) Co-variations of $\bar{Q}$ vs $\bar{P}$

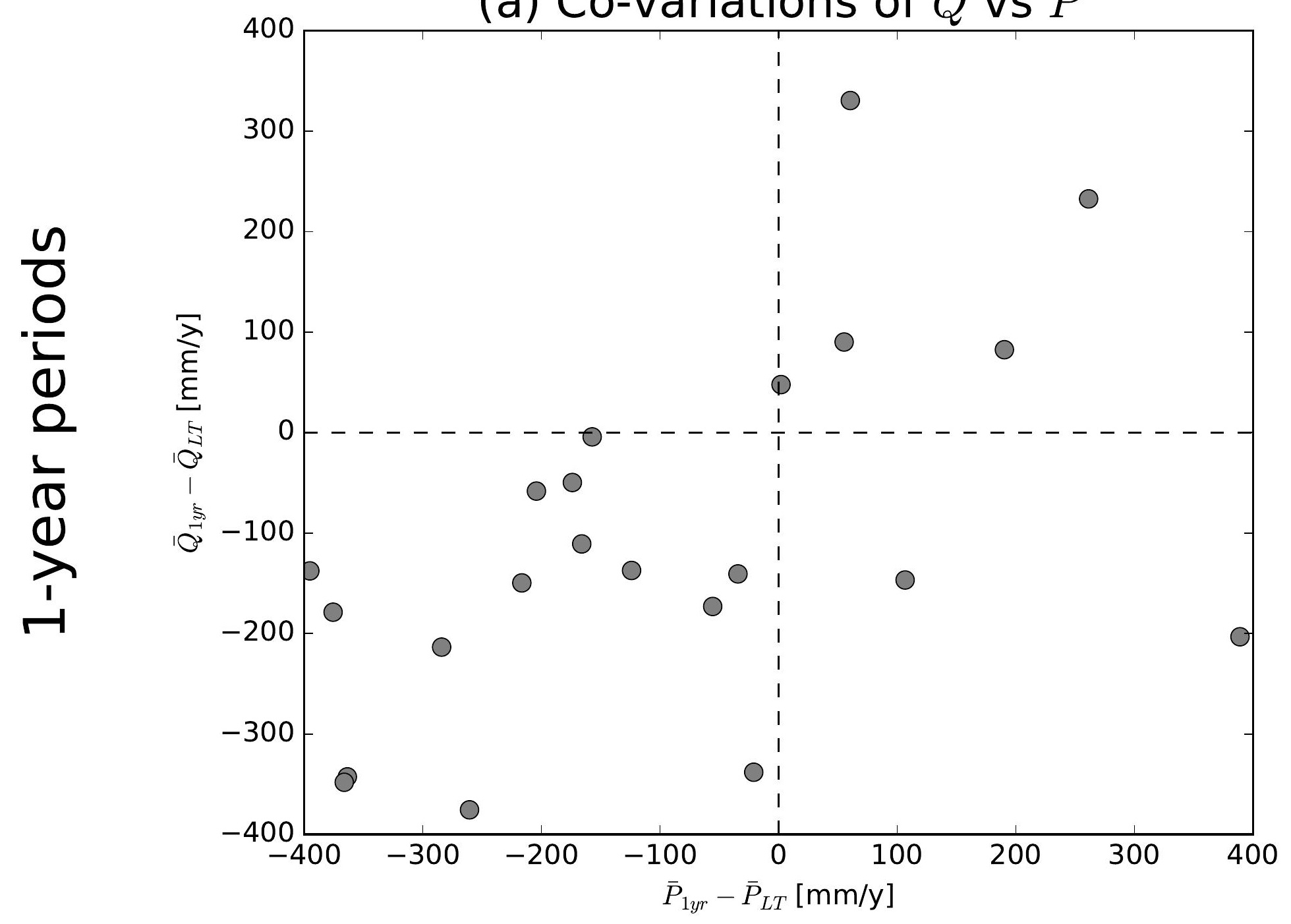

(d) Co-variations of $\bar{Q}$ vs $\bar{P}$

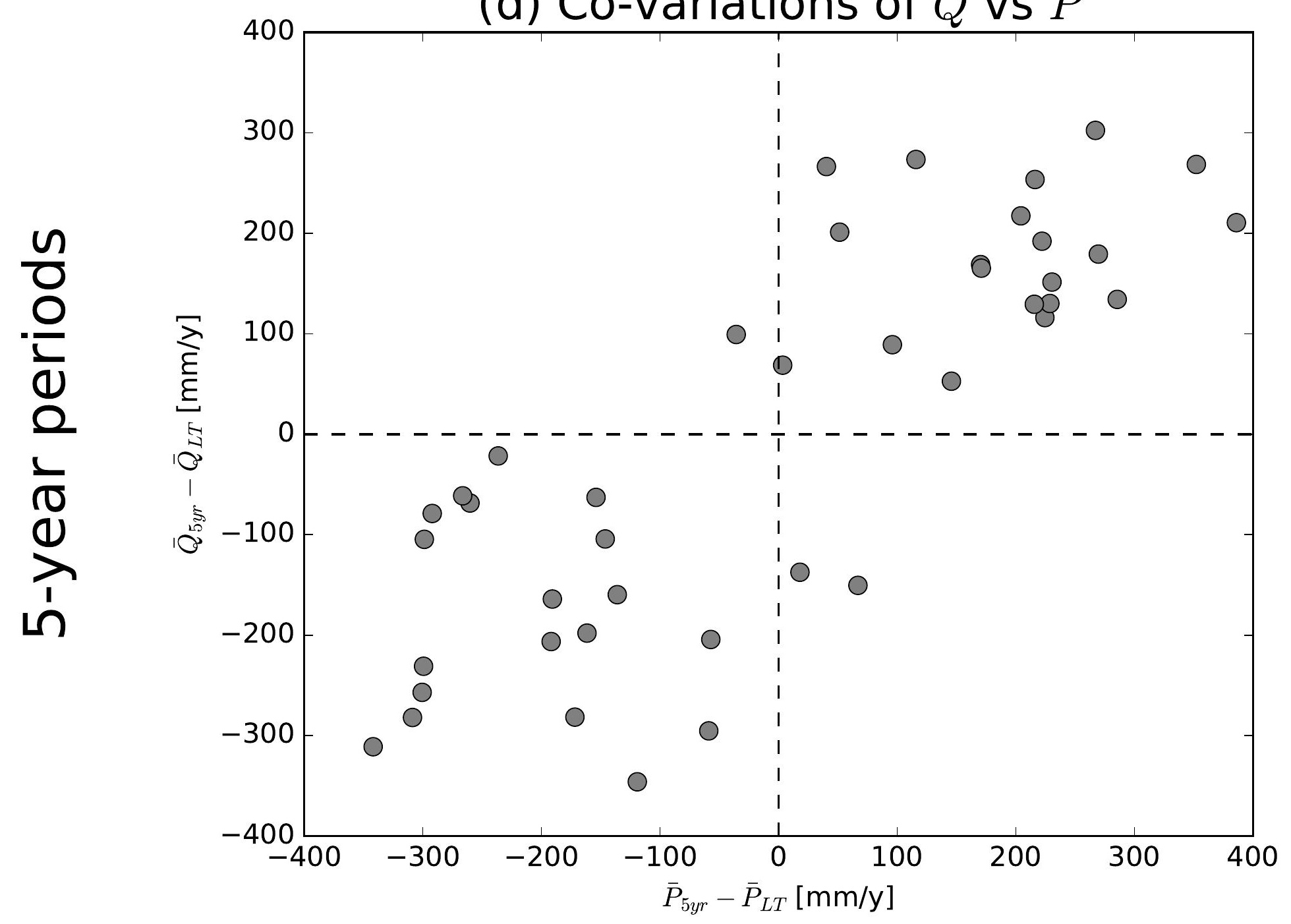

(g) Co-variations of $\bar{Q}$ vs $\bar{P}$

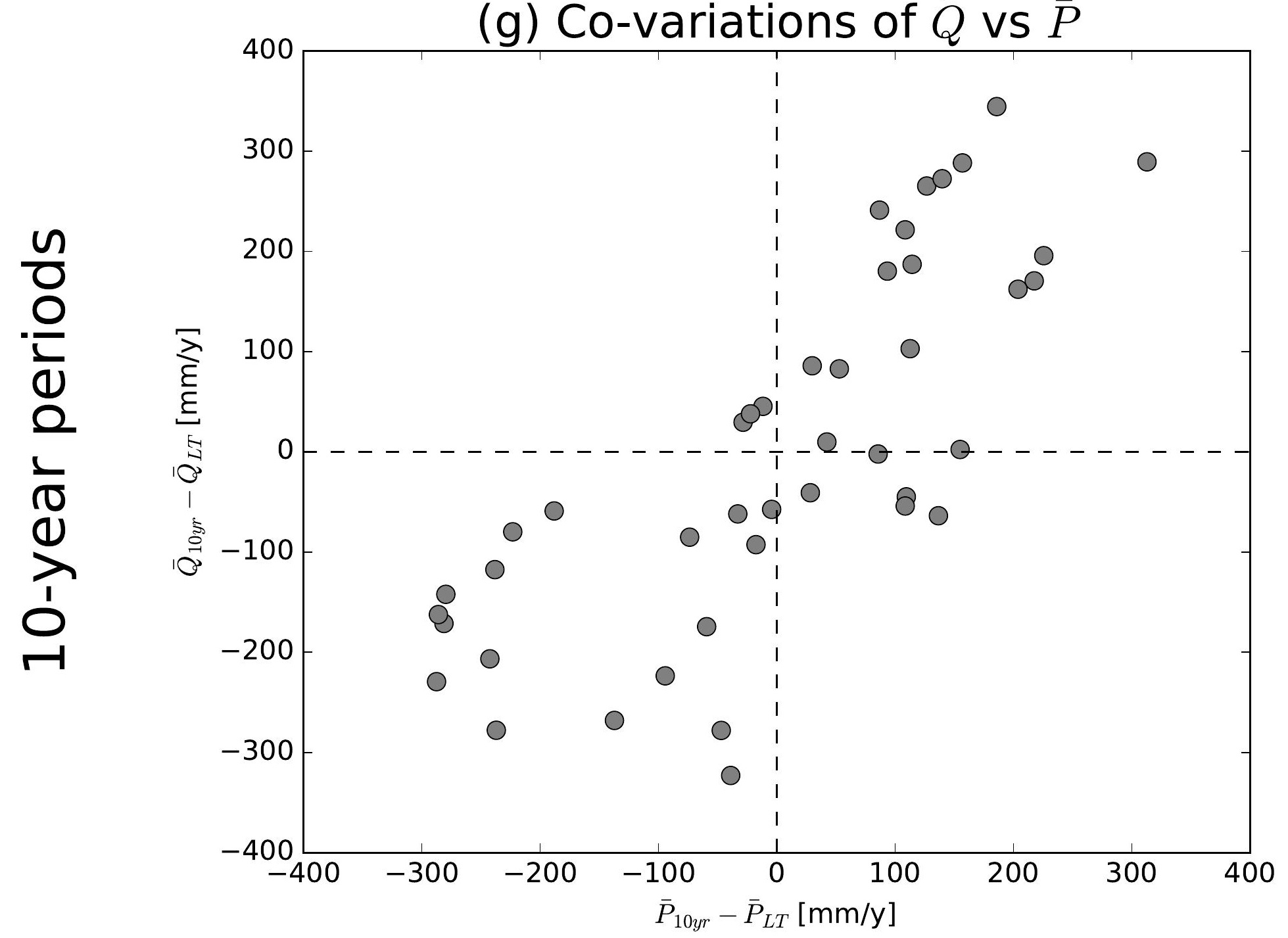

(j) Co-variations of $\bar{Q}$ vs $\bar{P}$

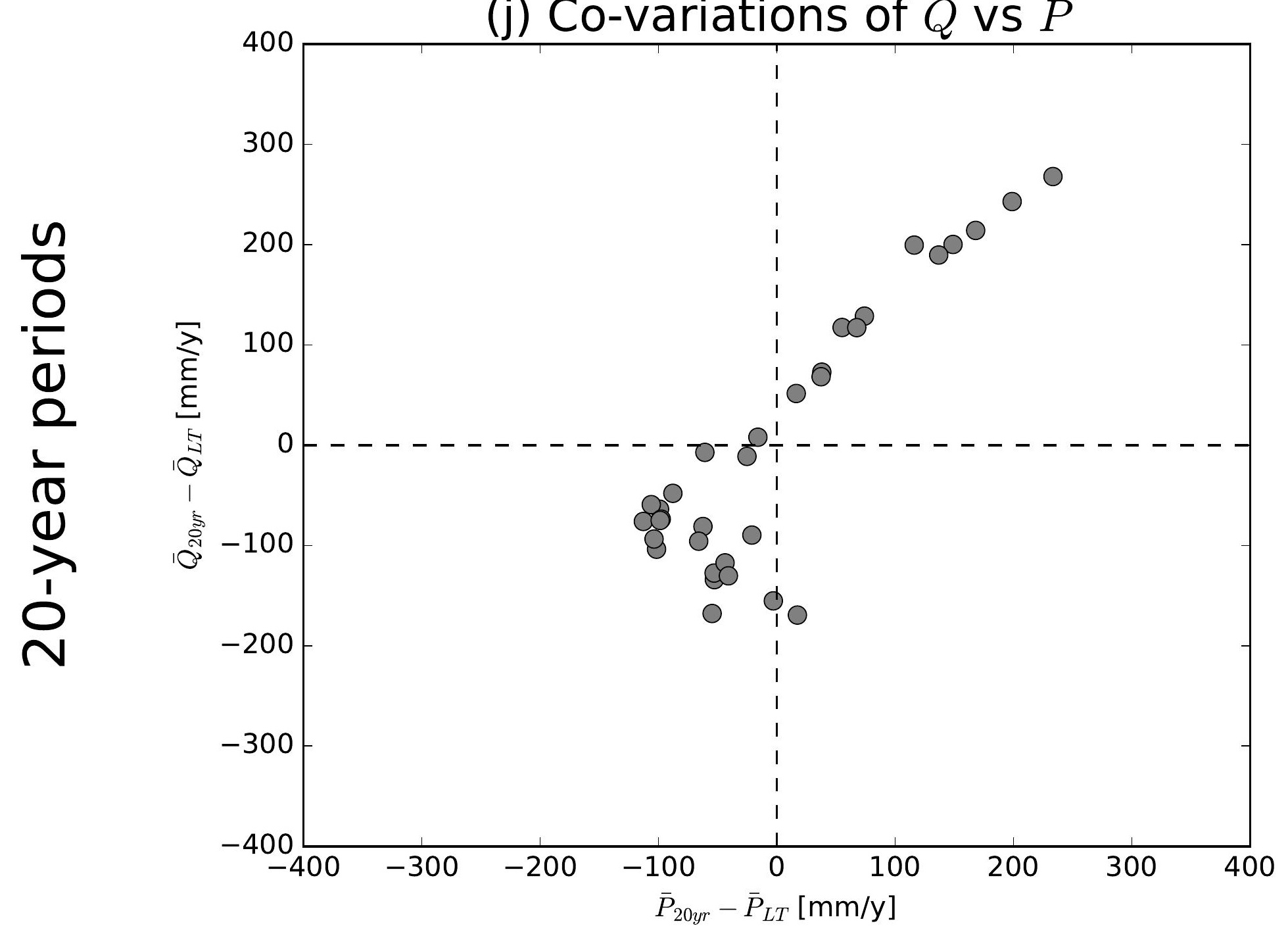

(b) Co-variations of $\bar{Q}$ vs $\overline{E 0}$

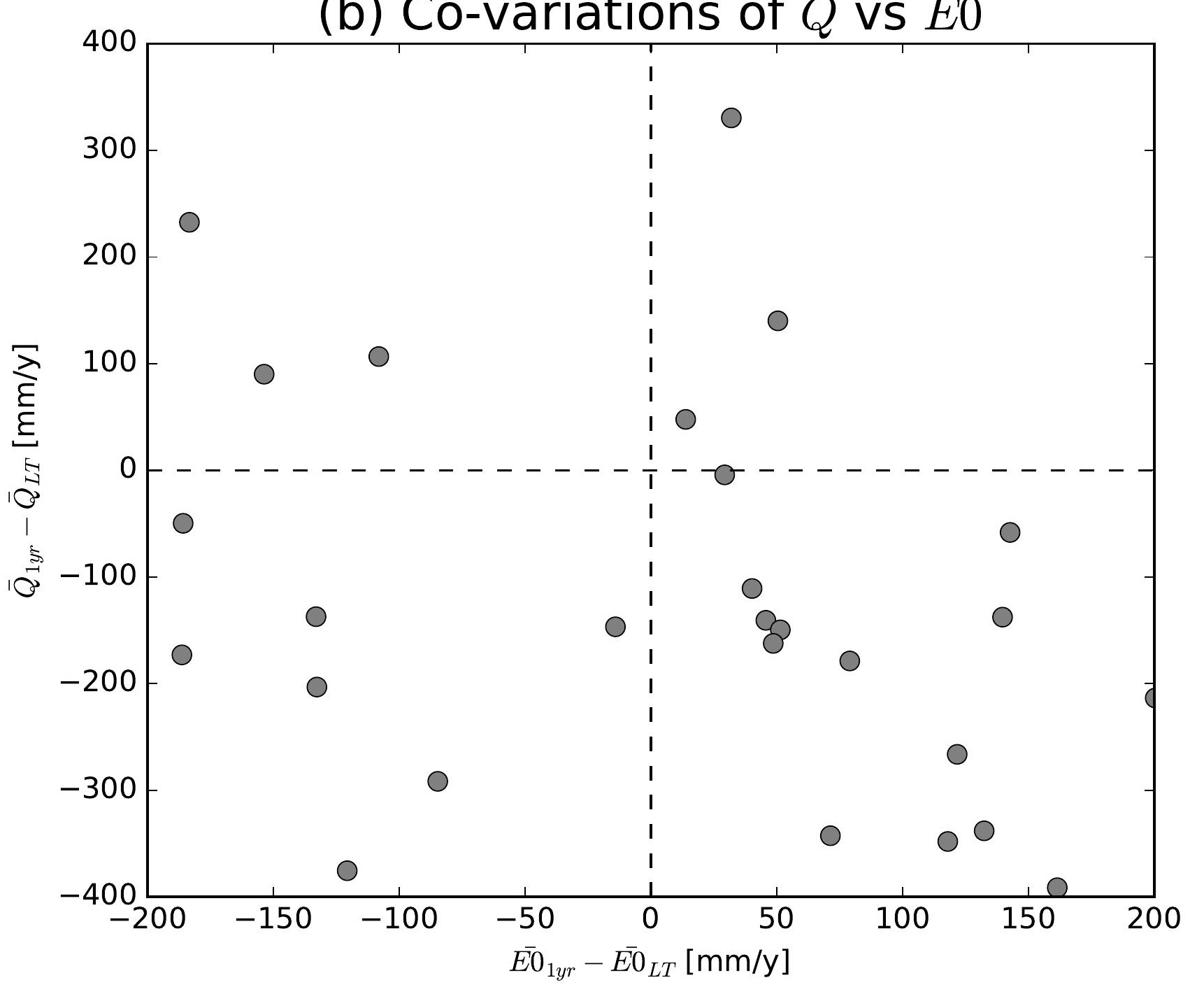

(e) Co-variations of $\bar{Q}$ vs $\overline{E 0}$

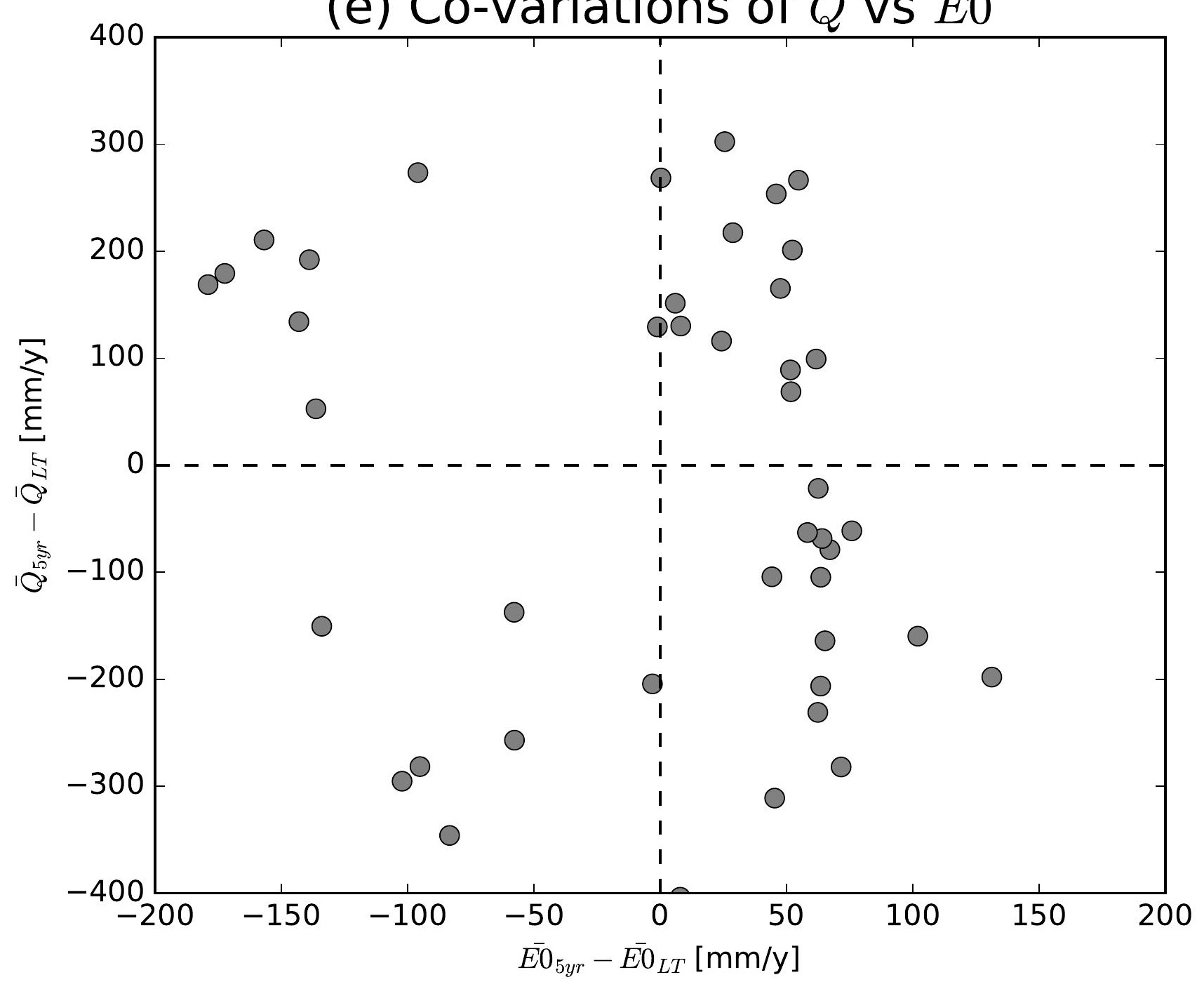

(h) Co-variations of $\bar{Q}$ vs $\overline{E 0}$

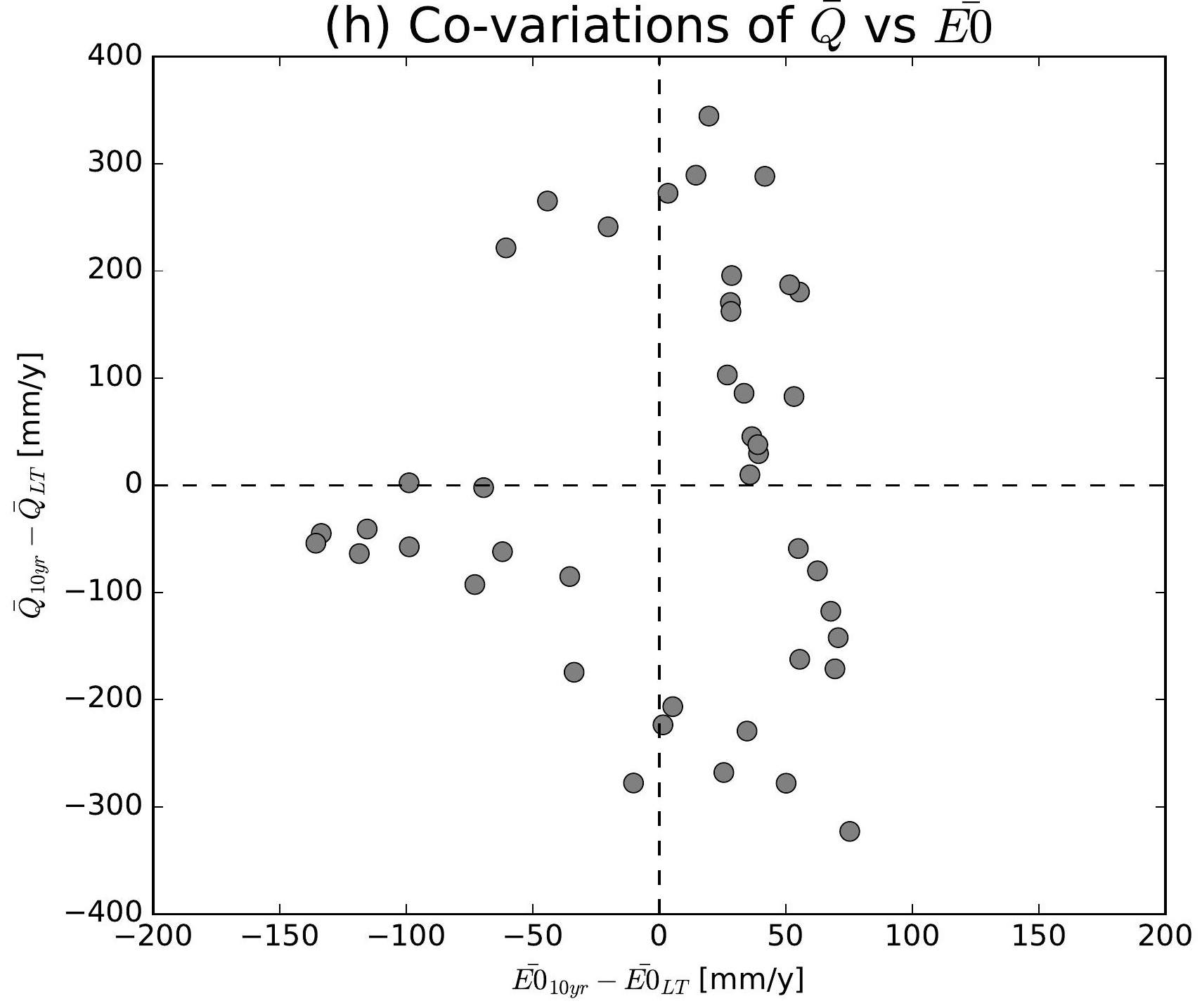

(k) Co-variations of $\bar{Q}$ vs $\overline{E 0}$

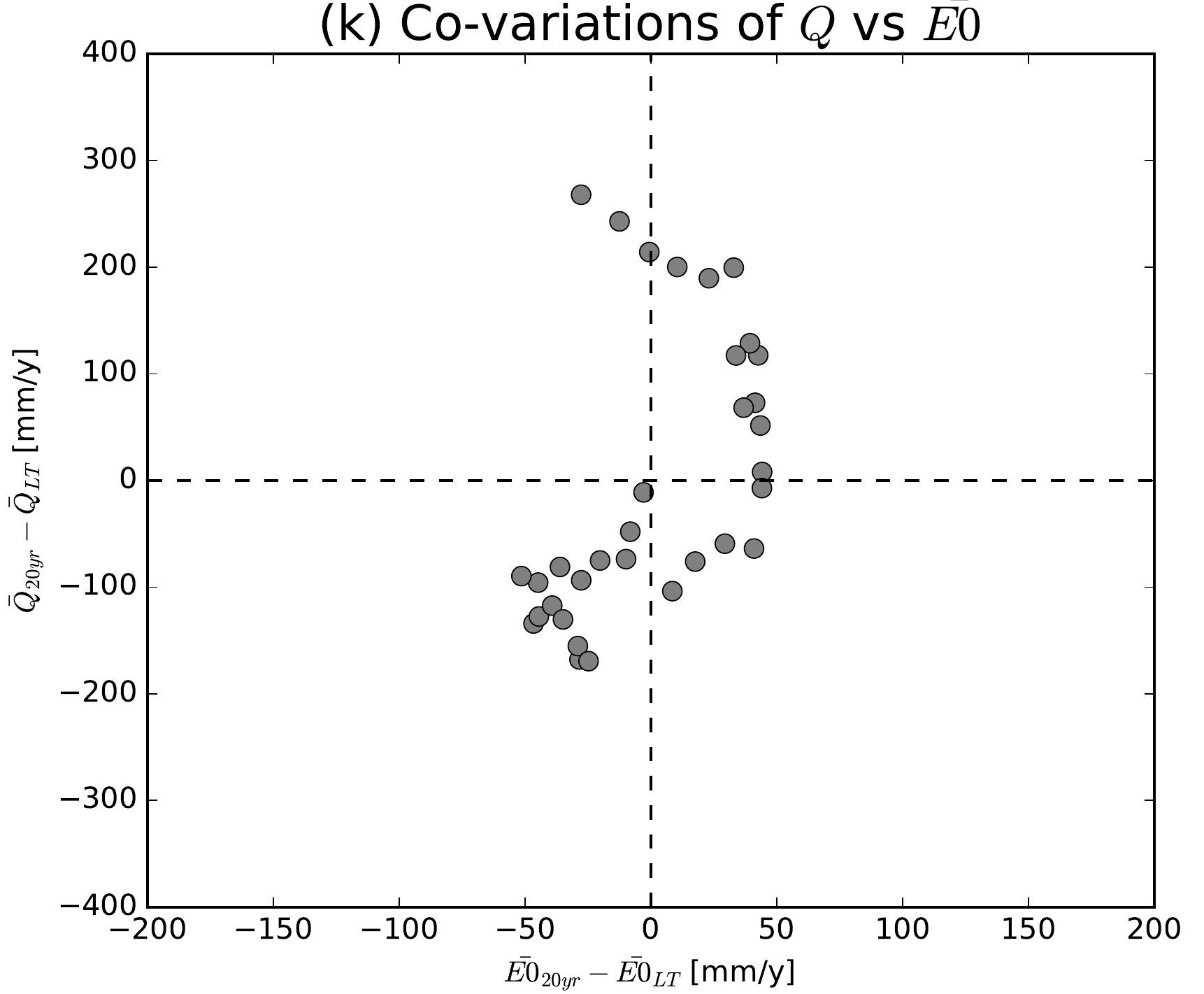

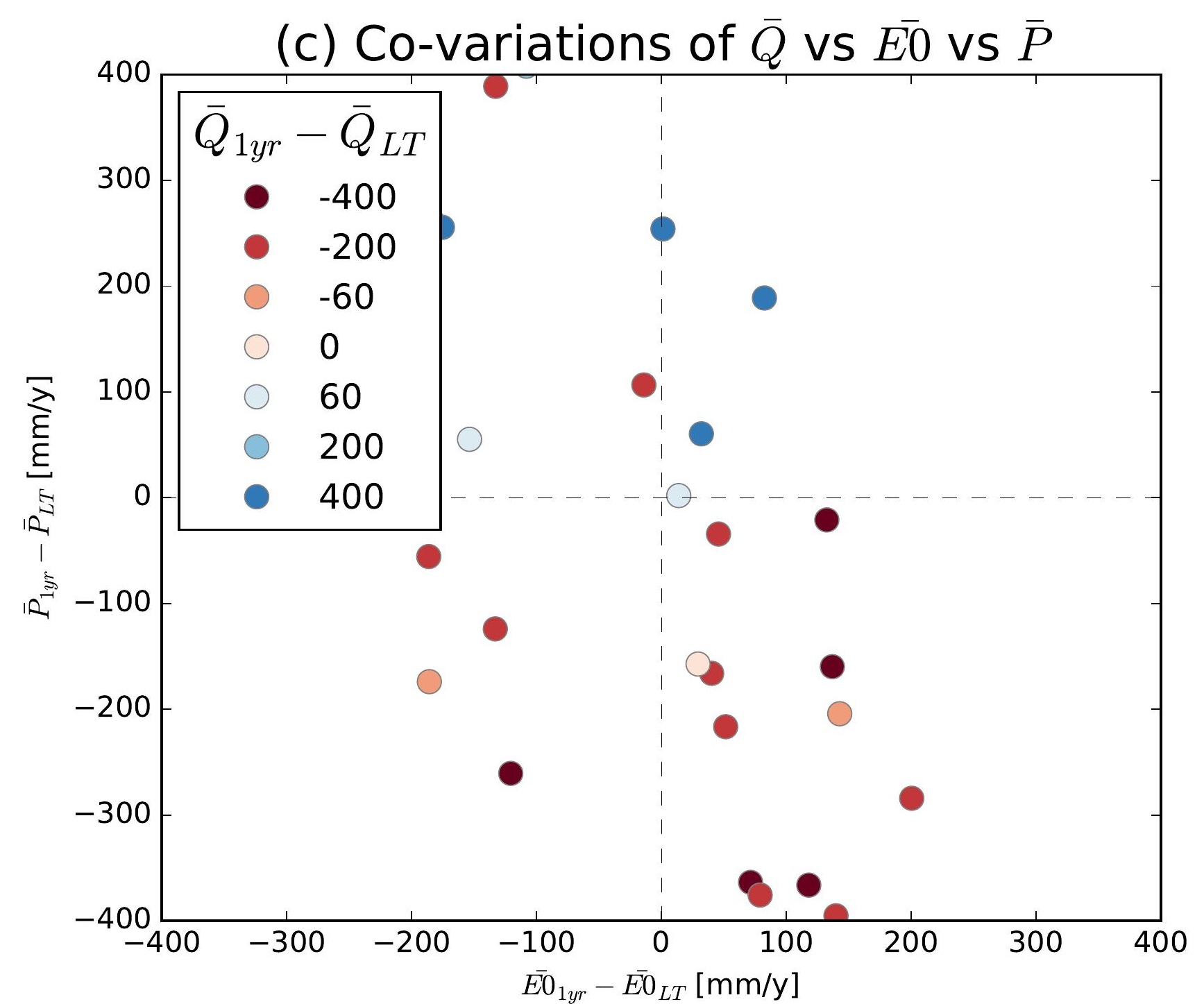
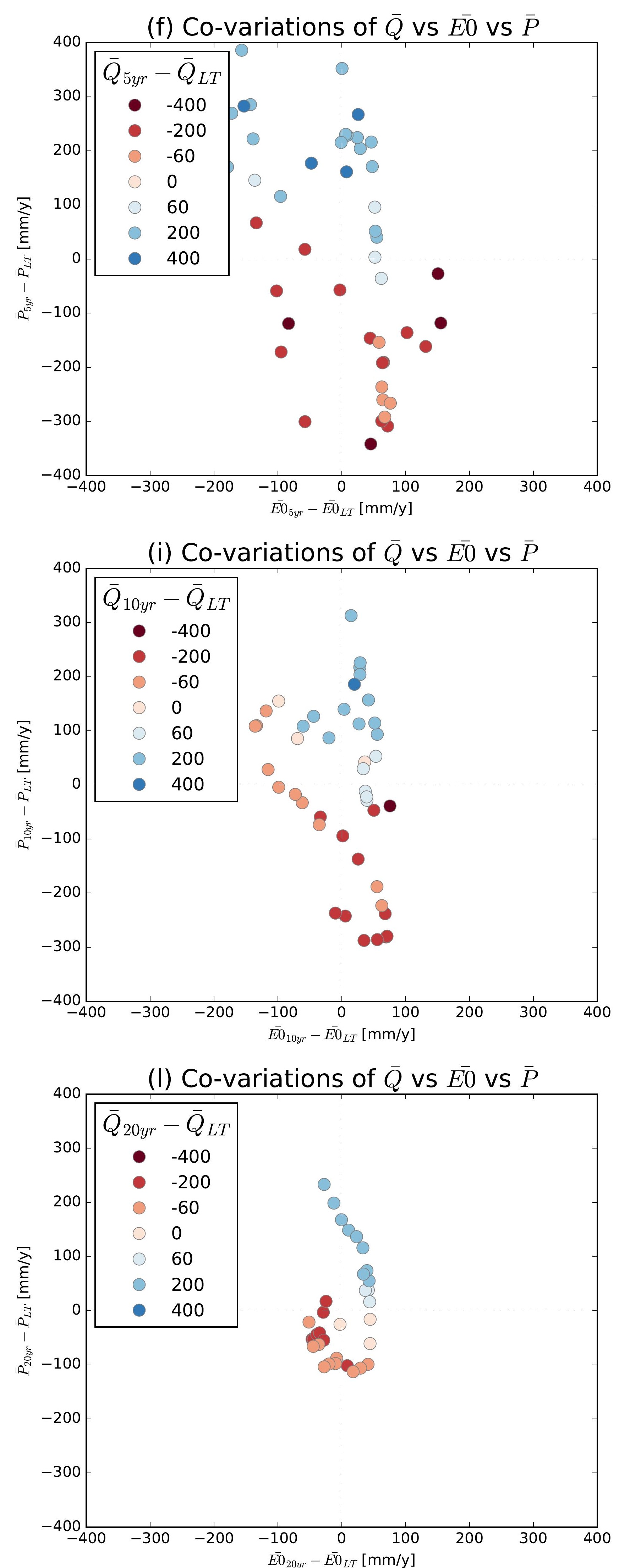

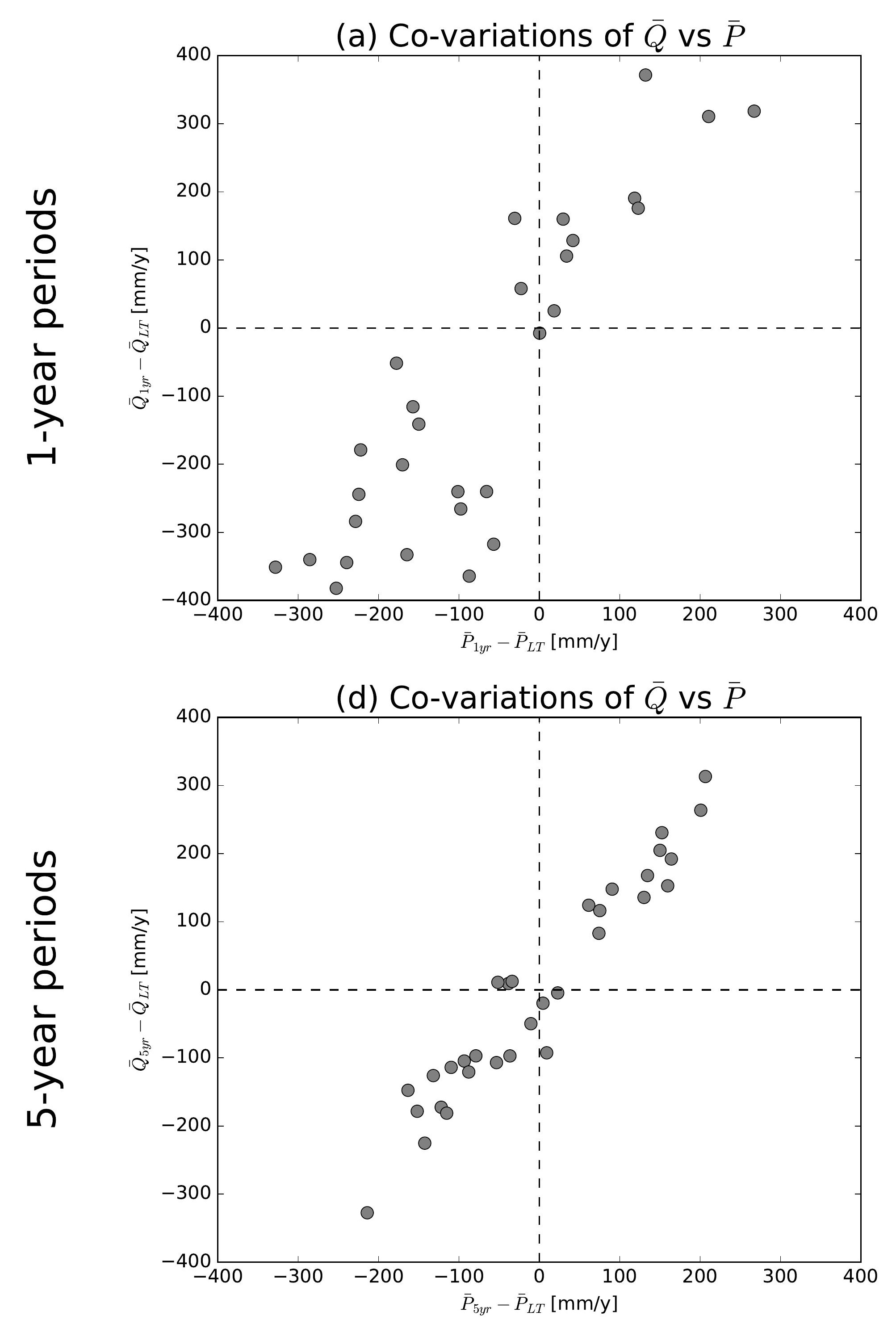

(g) Co-variations of $\bar{Q}$ vs $\bar{P}$

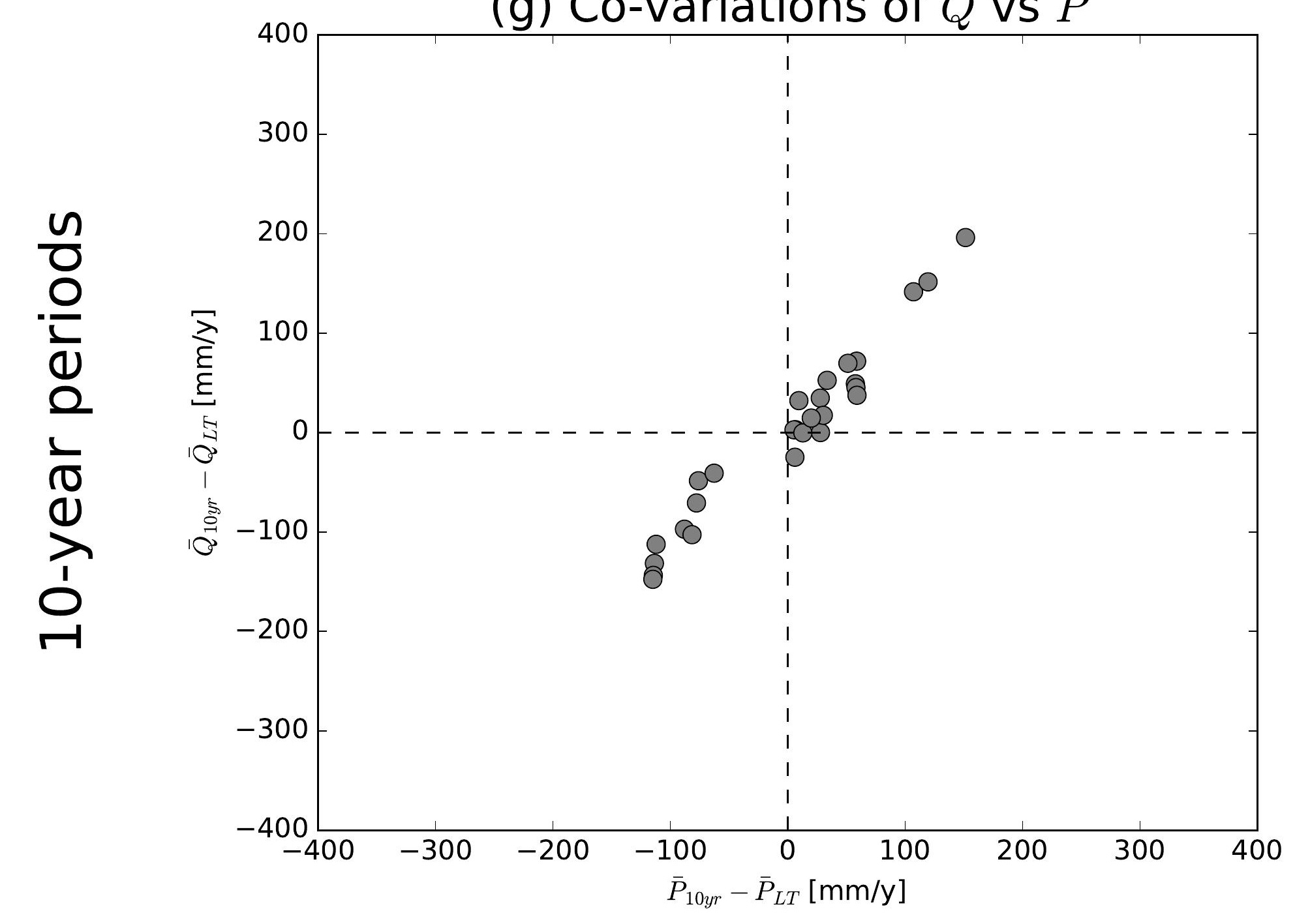

(j) Co-variations of $\bar{Q}$ vs $\bar{P}$

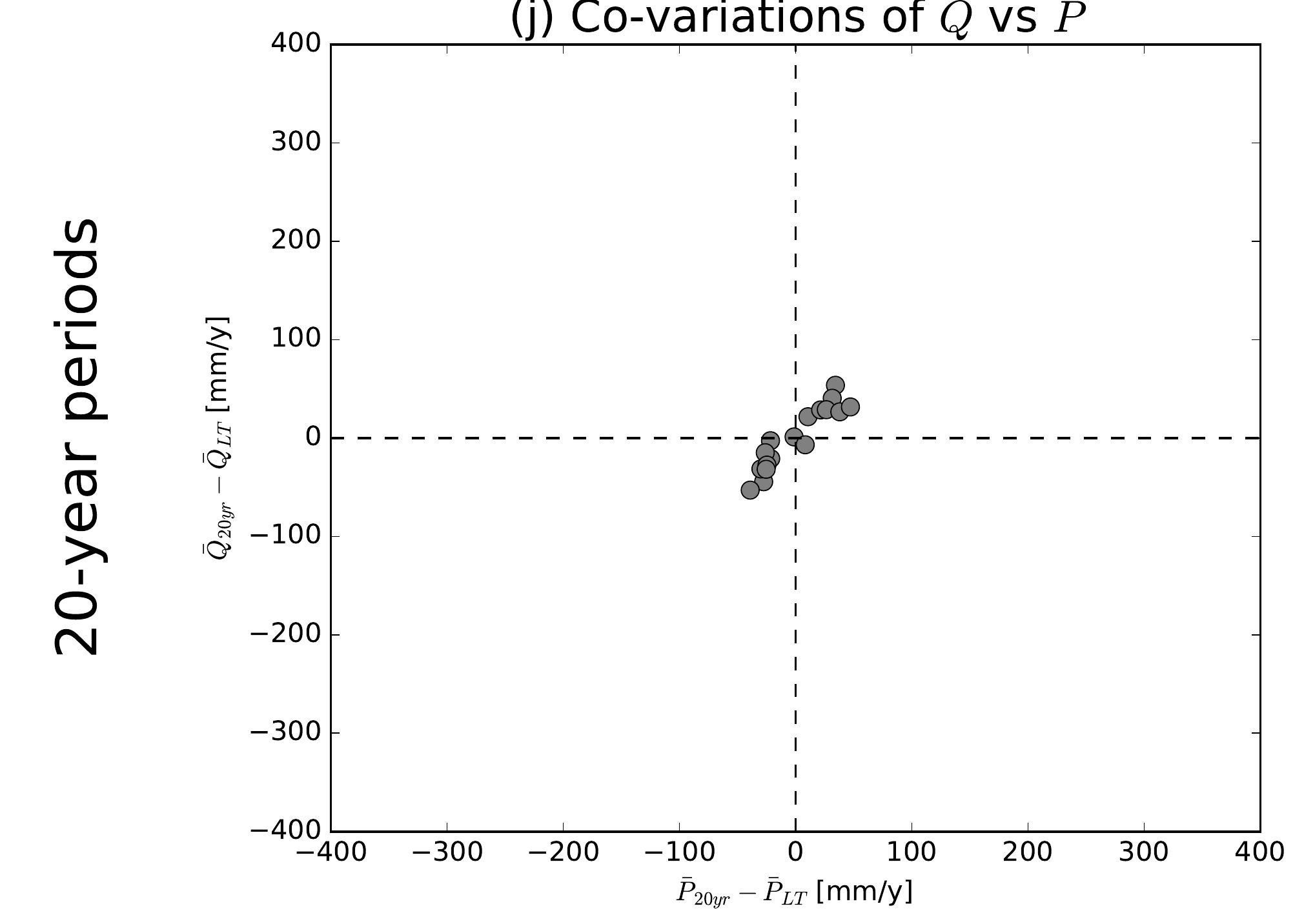

(b) Co-variations of $\bar{Q}$ vs $\overline{E 0}$

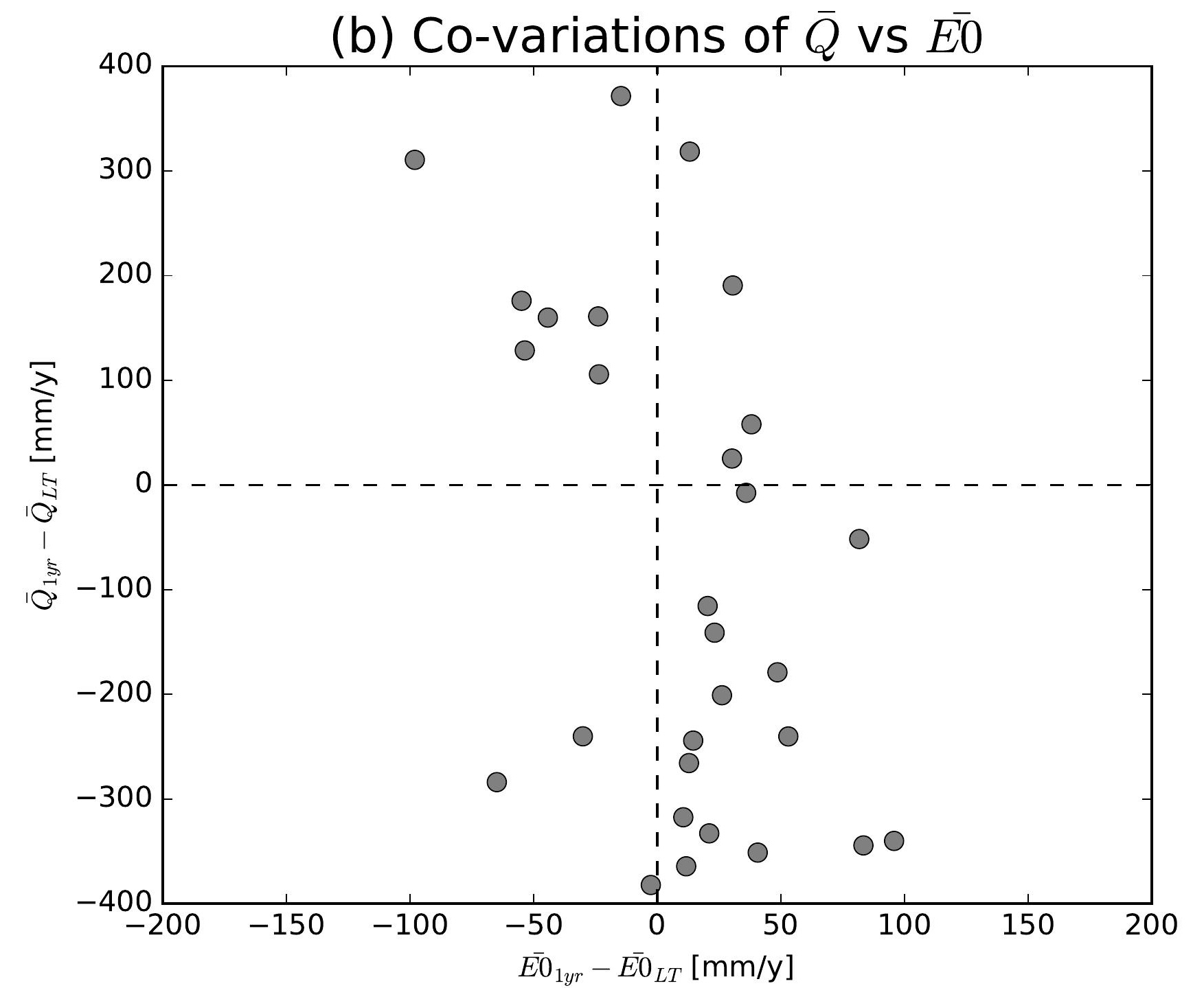

(e) Co-variations of $\bar{Q}$ vs $\overline{E 0}$

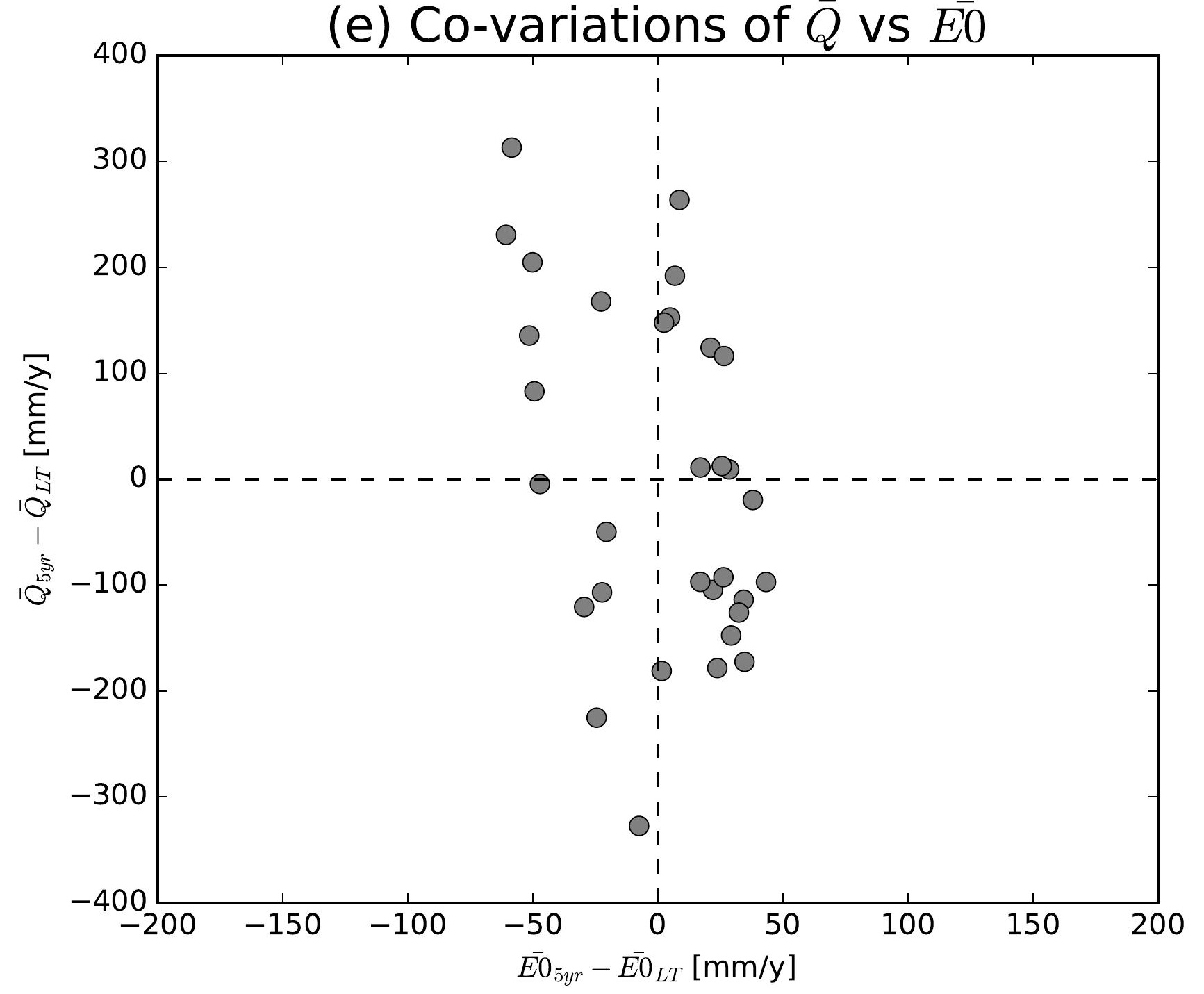

(h) Co-variations of $\bar{Q}$ vs $\overline{E 0}$

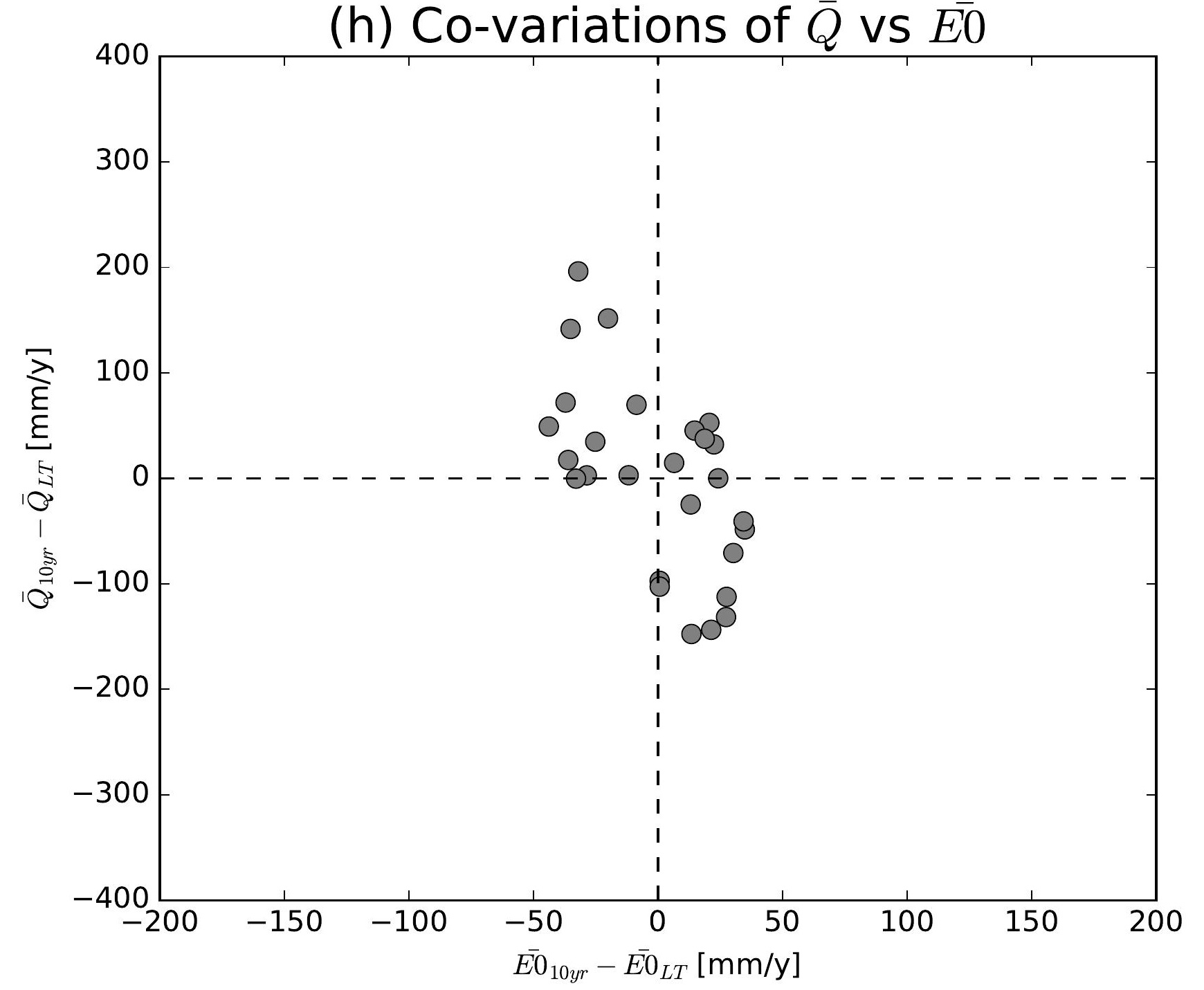

(k) Co-variations of $\bar{Q}$ vs $\overline{E 0}$

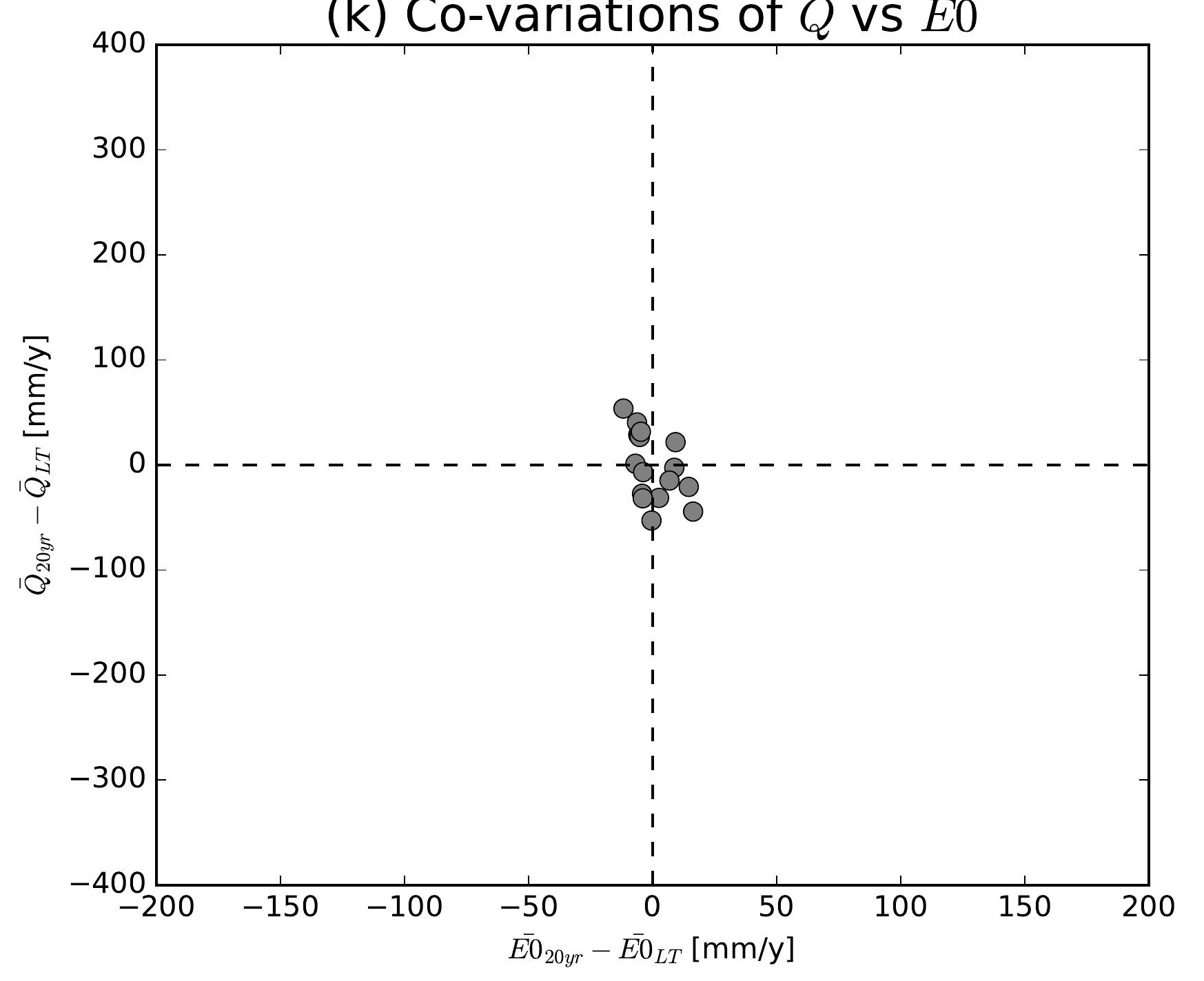

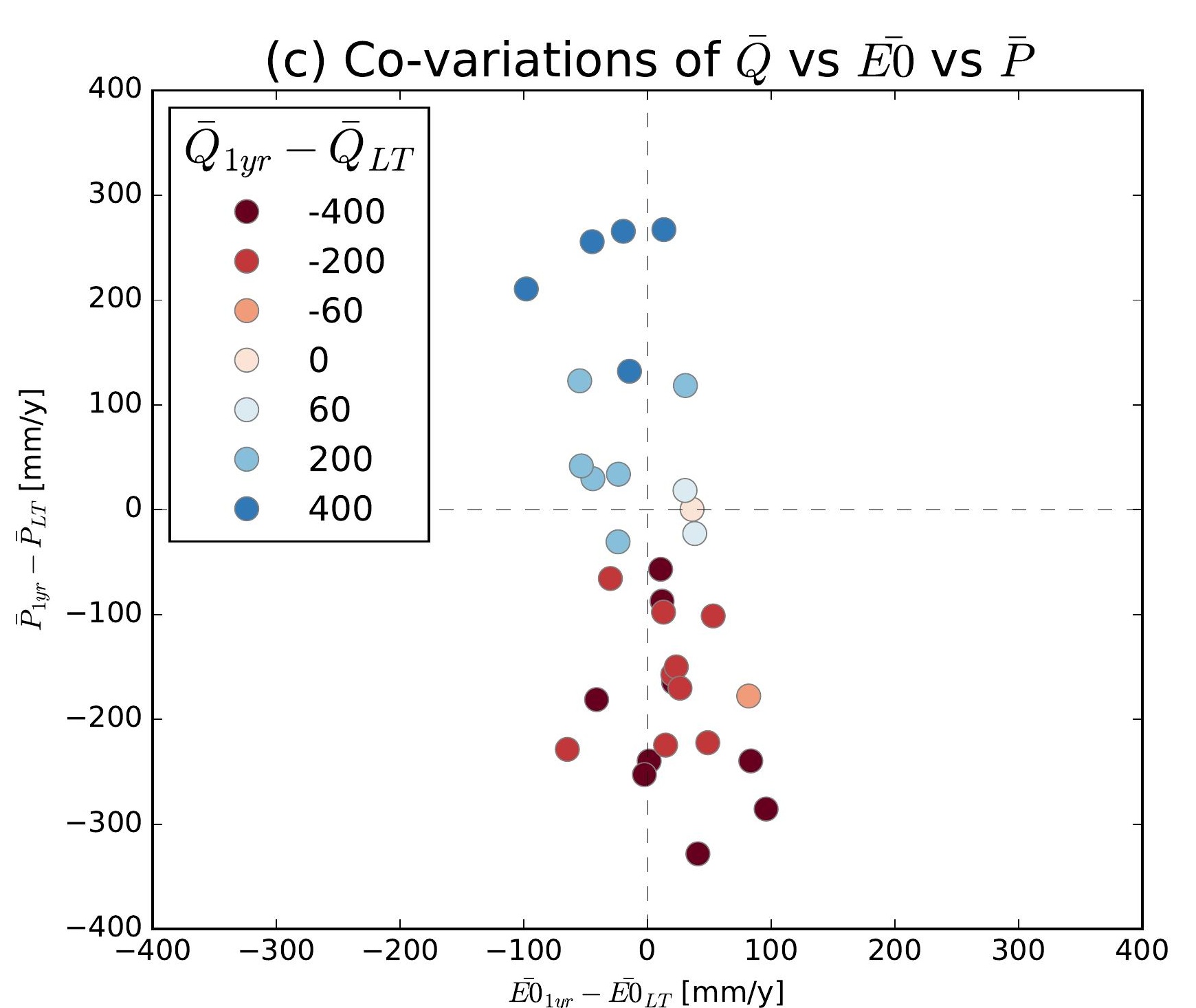
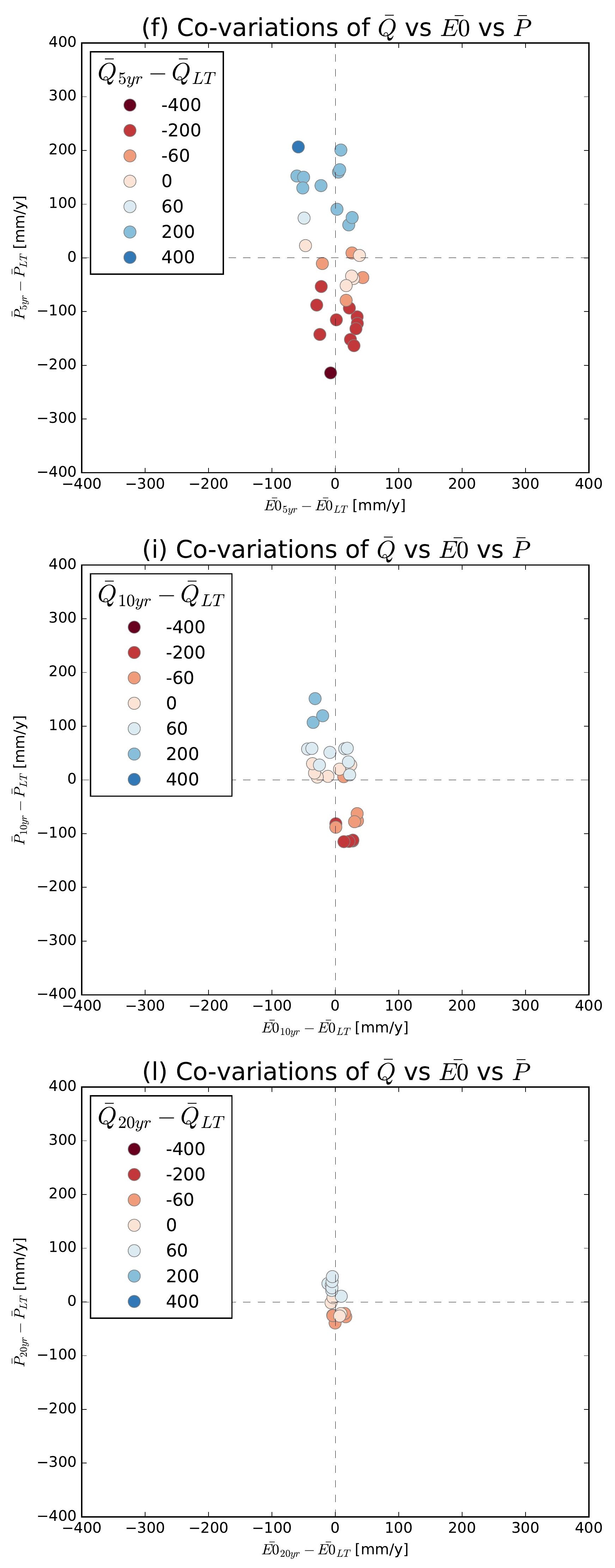

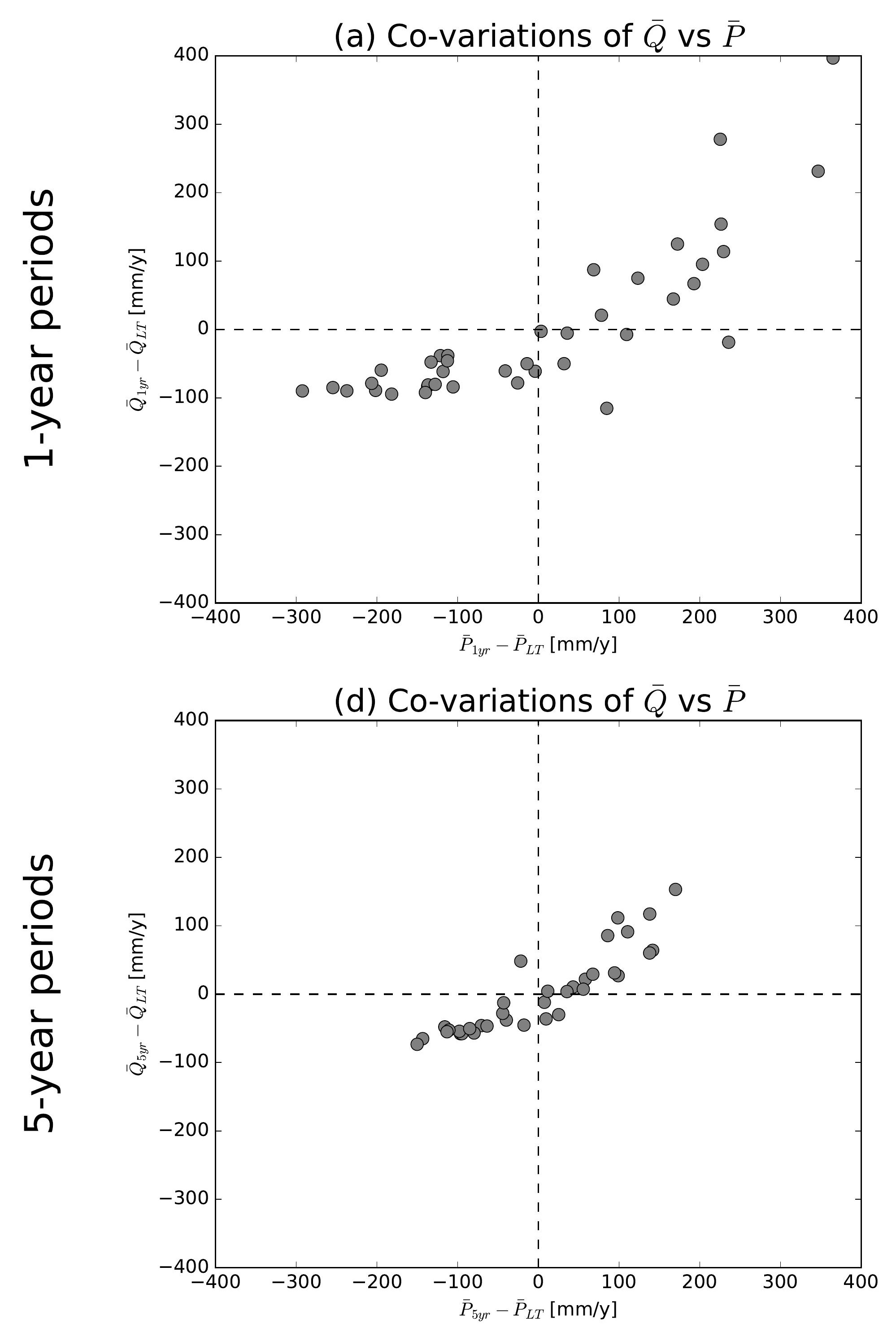

(g) Co-variations of $\bar{Q}$ vs $\bar{P}$
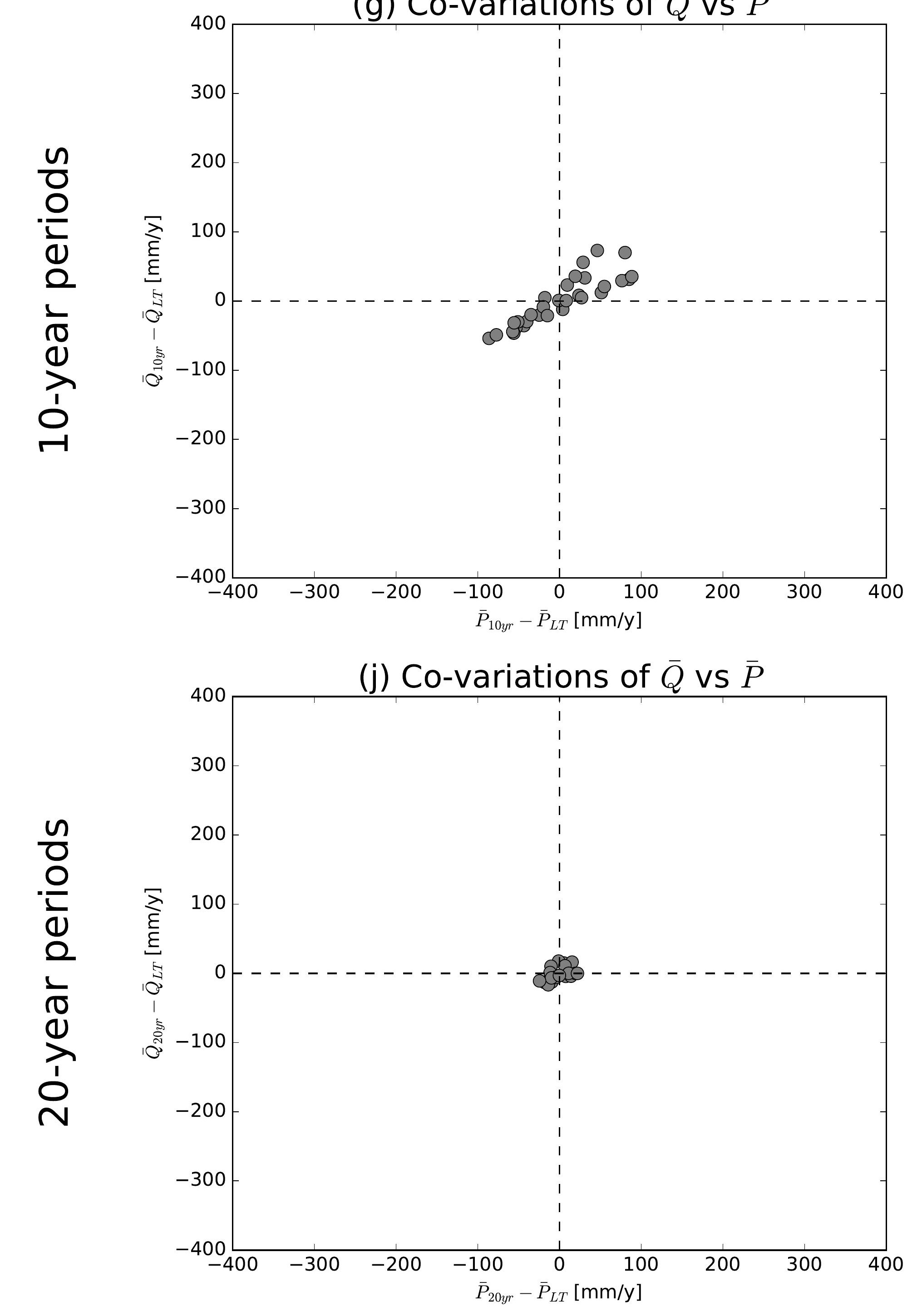

(b) Co-variations of $\bar{Q}$ vs $\overline{E 0}$

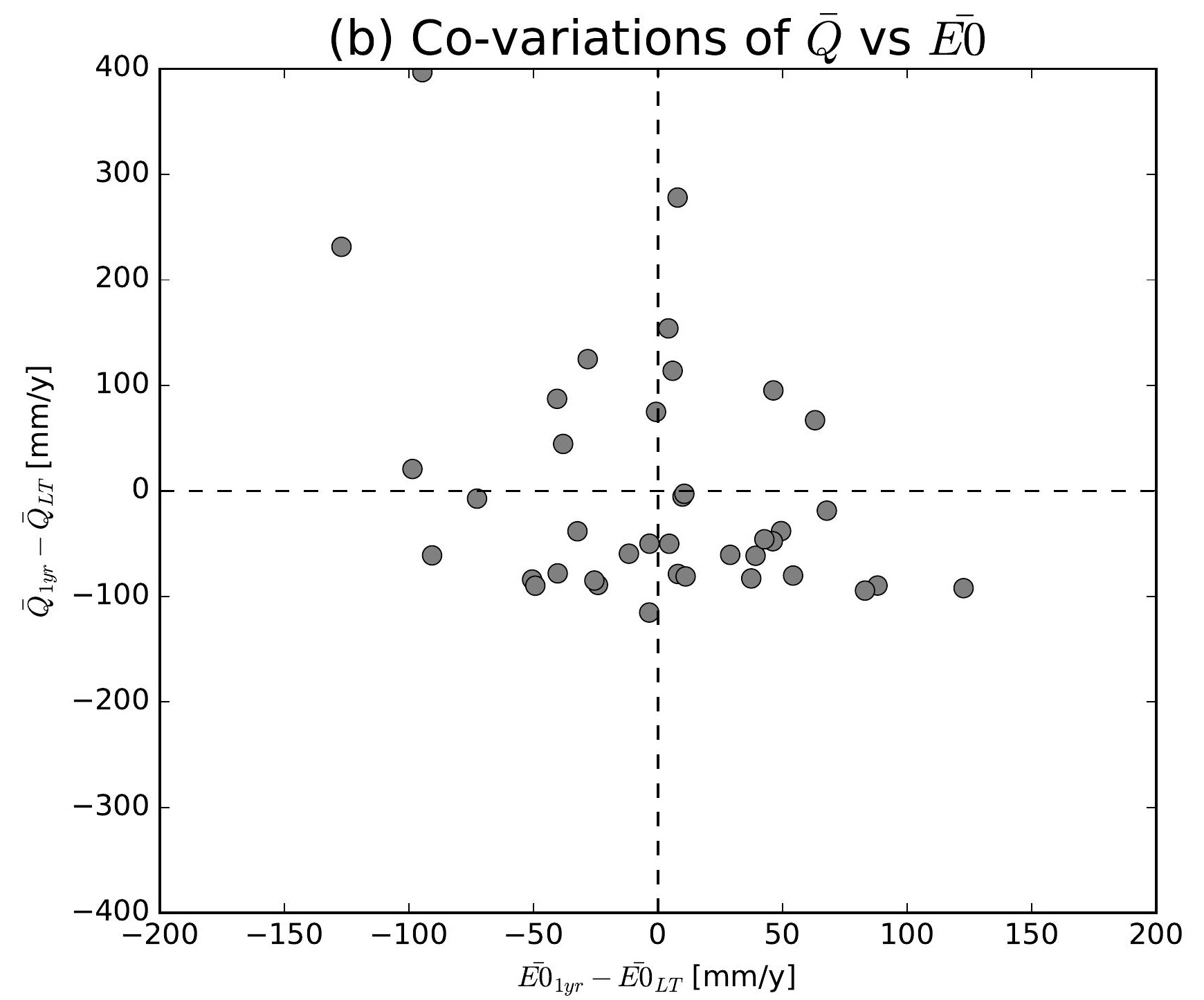

(e) Co-variations of $\bar{Q}$ vs $\overline{E 0}$

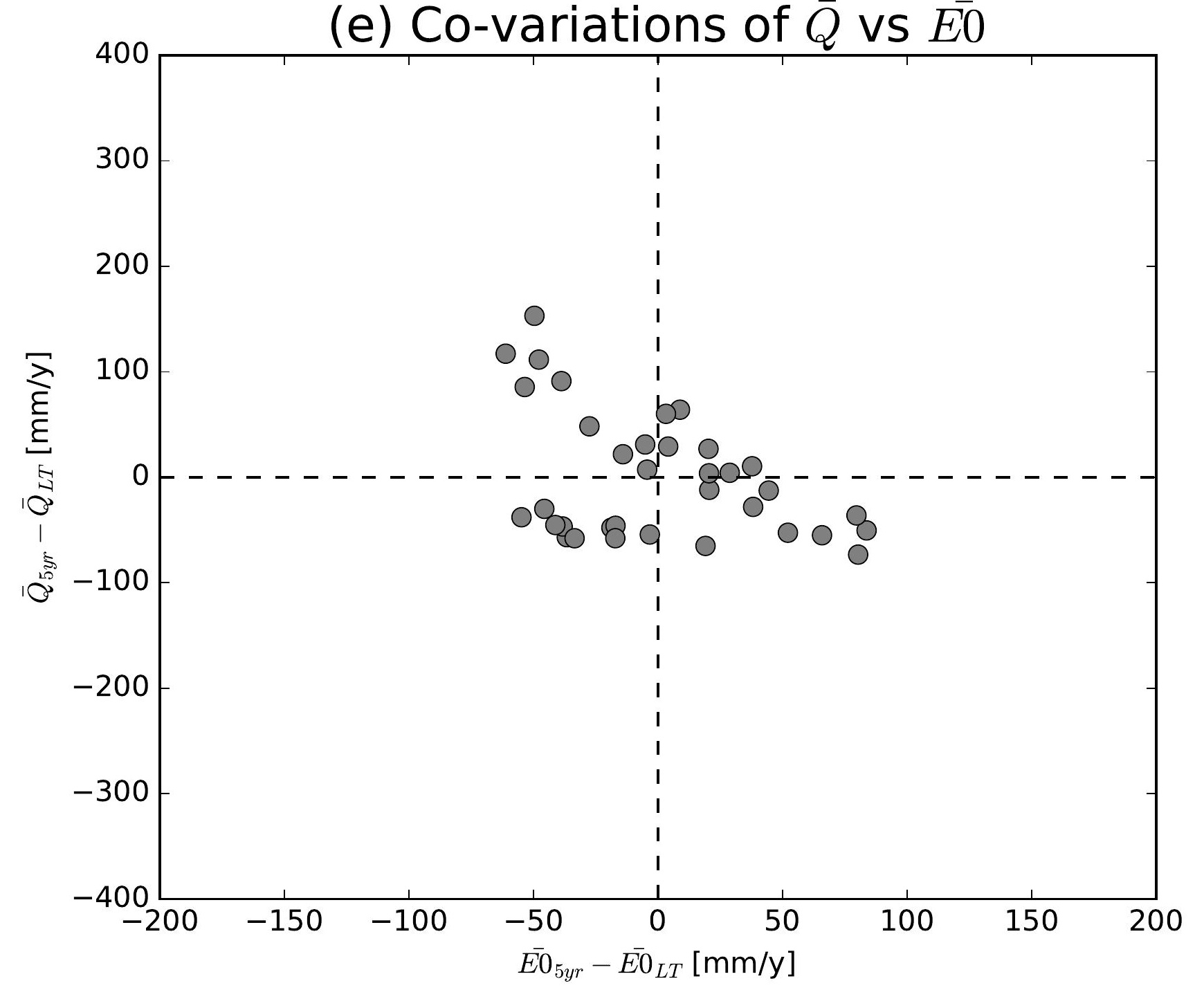

(h) Co-variations of $\bar{Q}$ vs $\overline{E 0}$

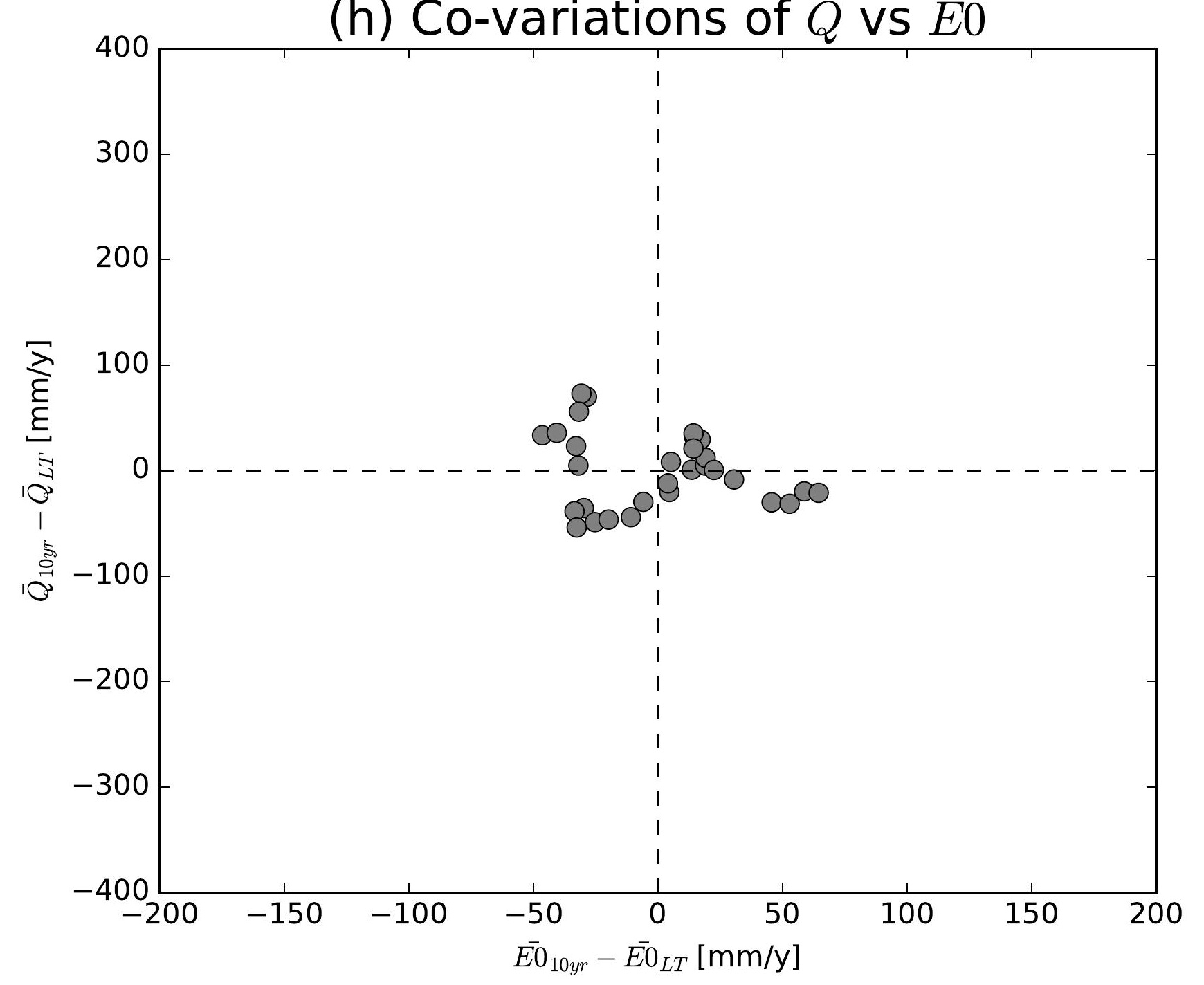

(k) Co-variations of $\bar{Q}$ vs $\overline{E 0}$

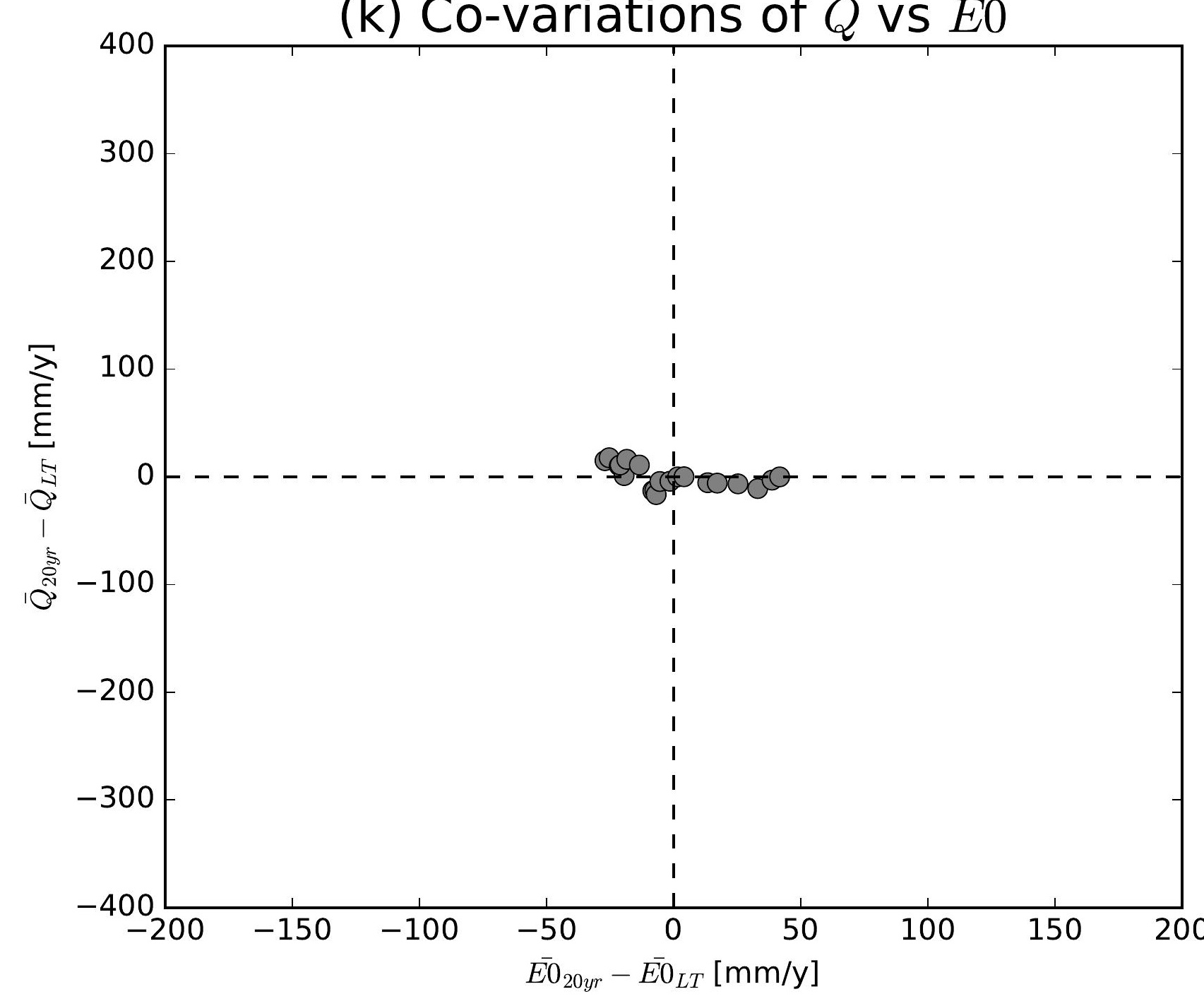

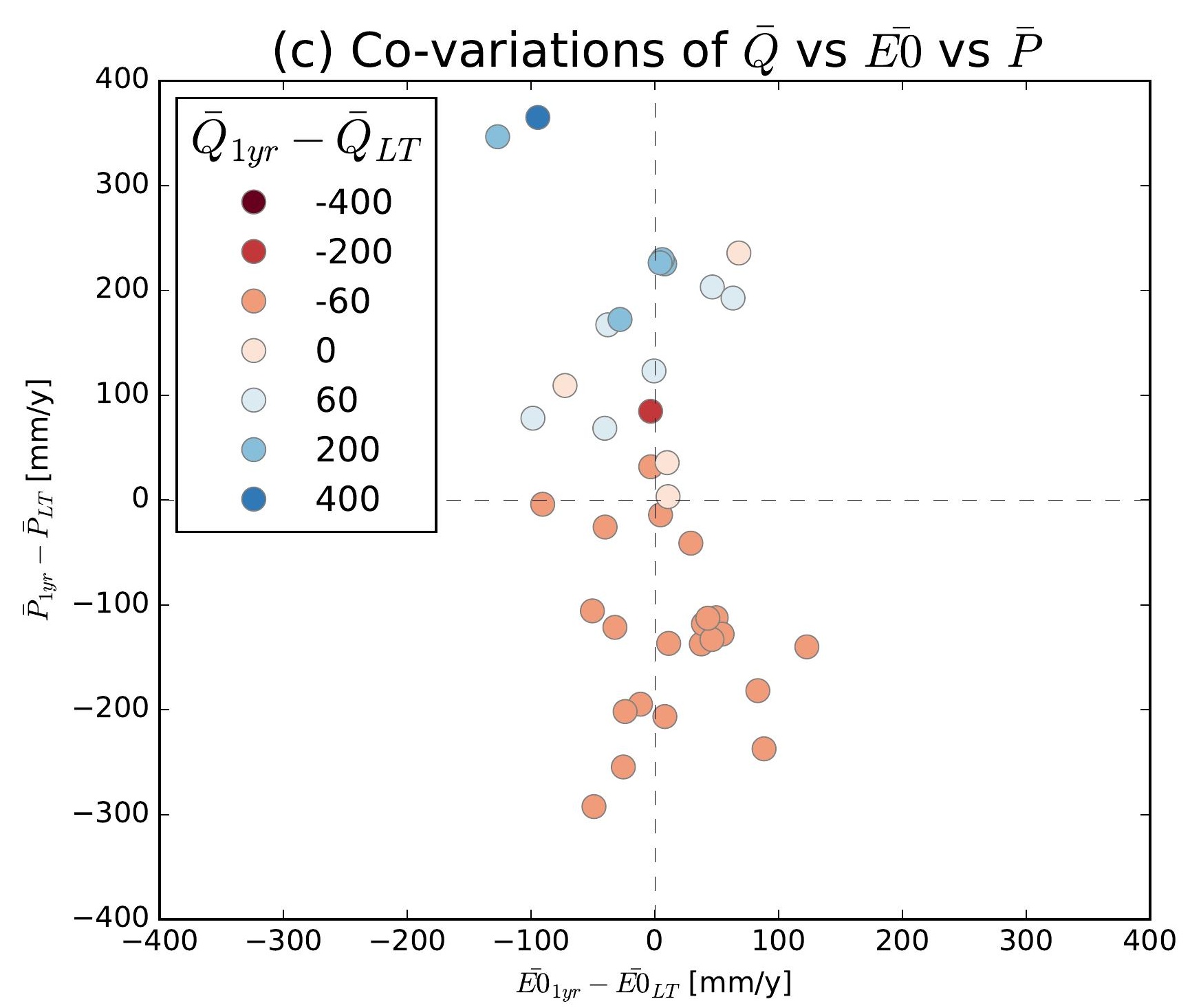
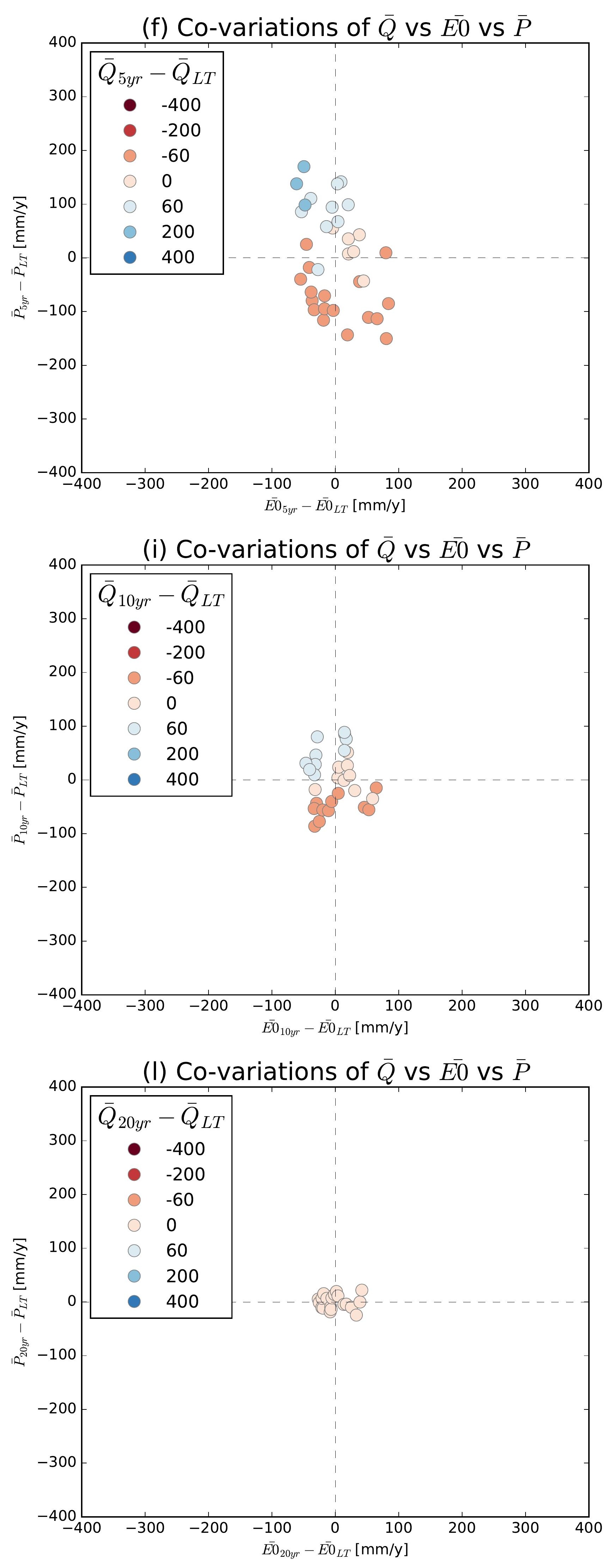

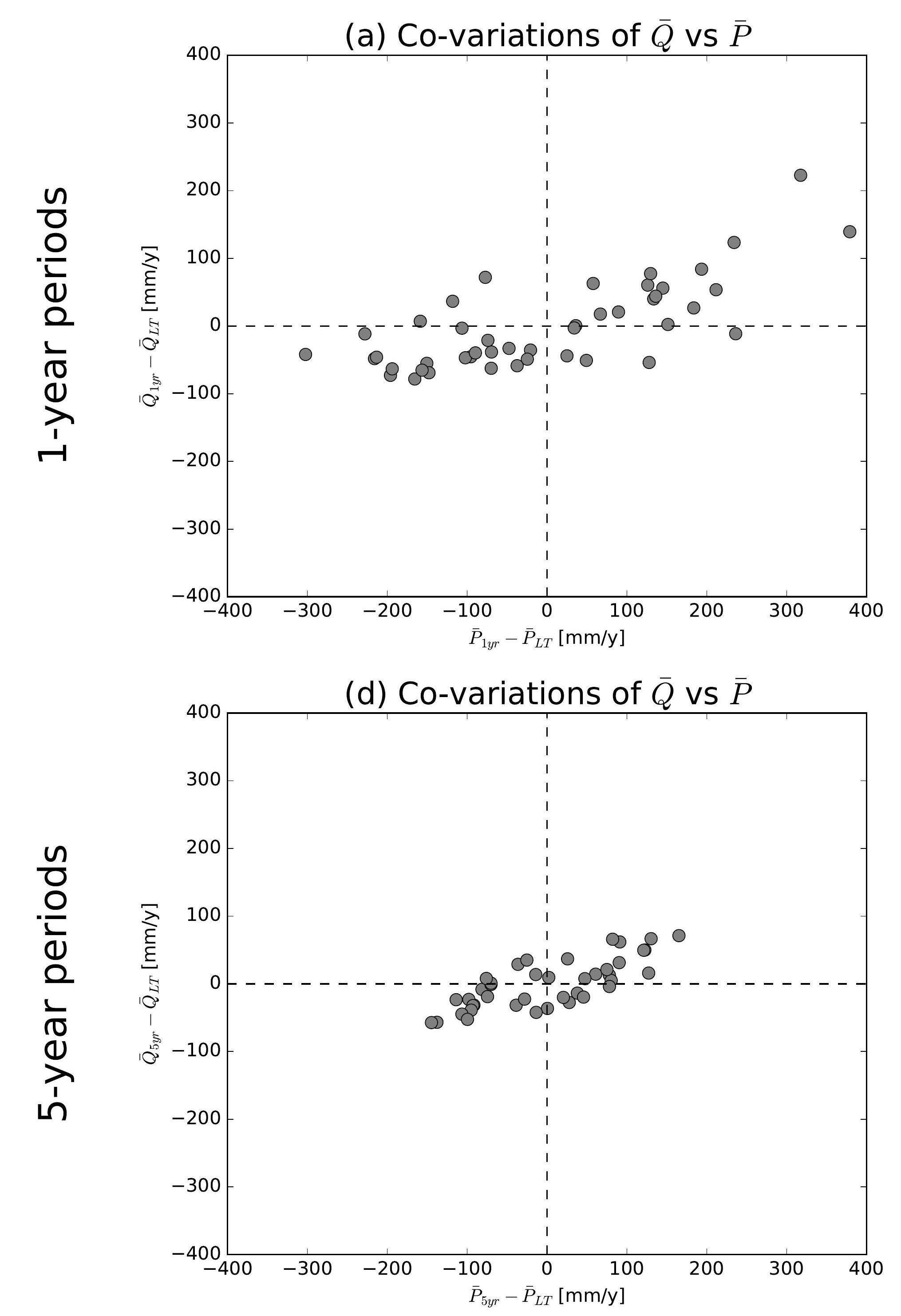

(g) Co-variations of $\bar{Q}$ vs $\bar{P}$

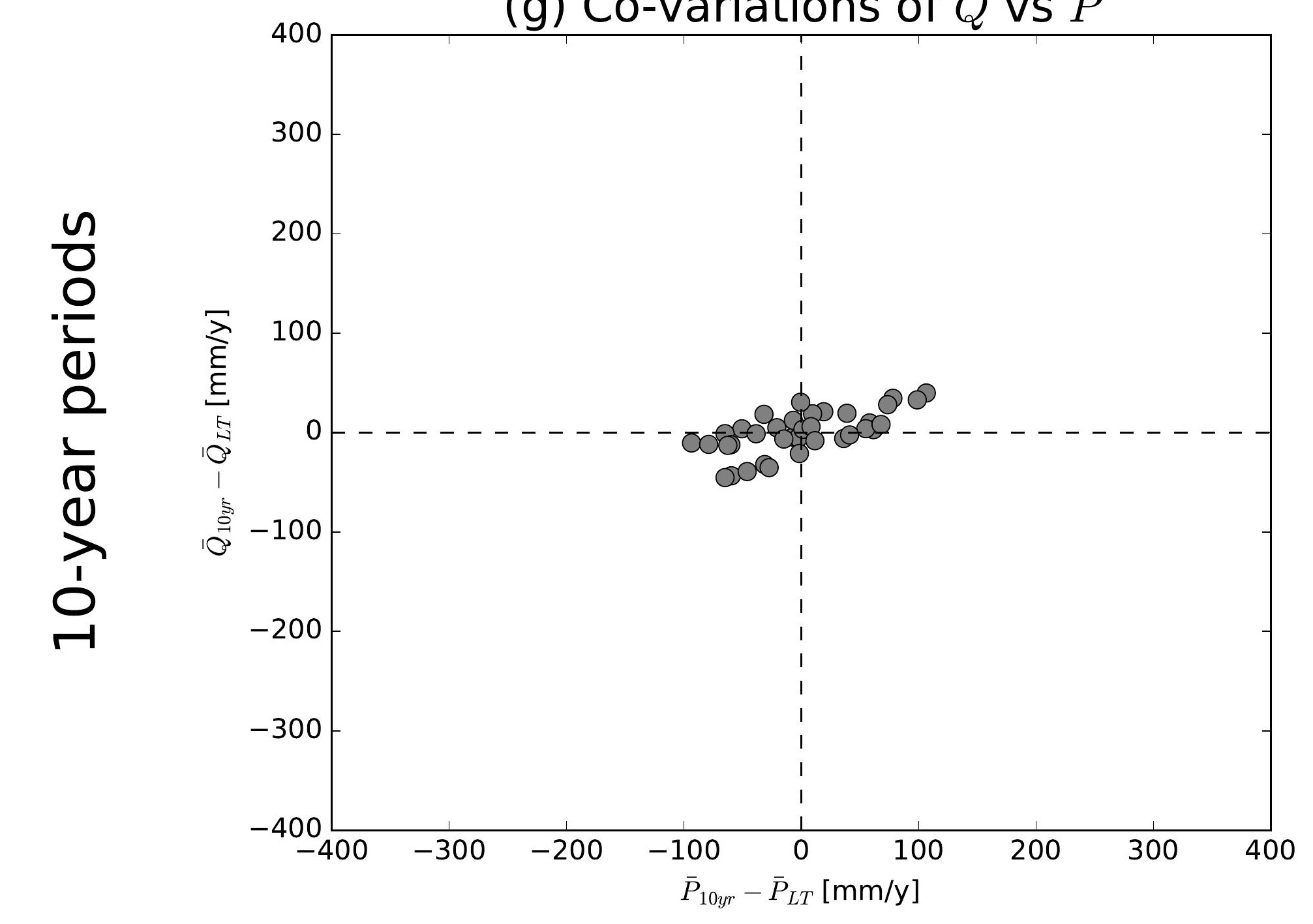

(j) Co-variations of $\bar{Q}$ vs $\bar{P}$

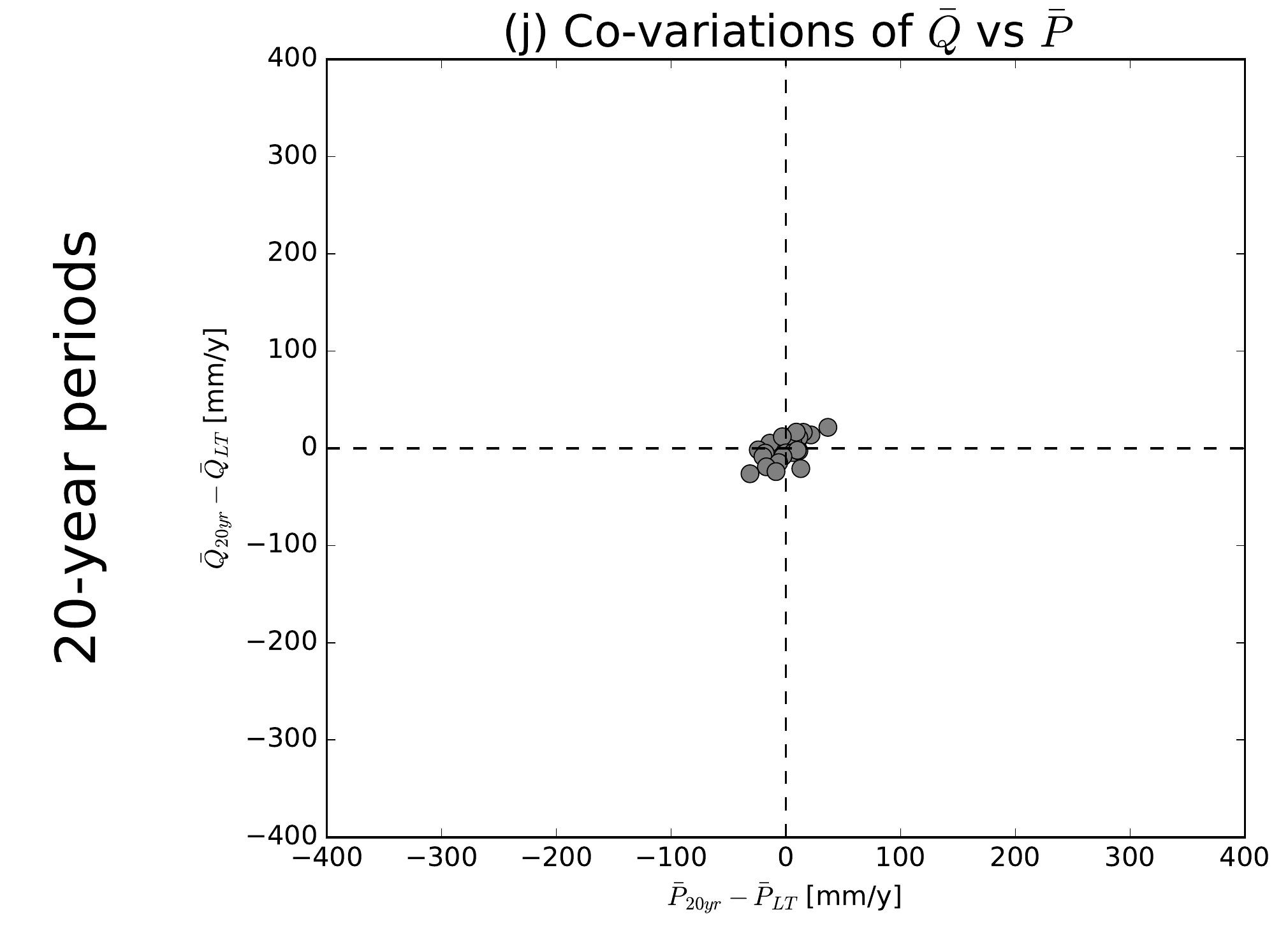

(b) Co-variations of $\bar{Q}$ vs $\overline{E 0}$

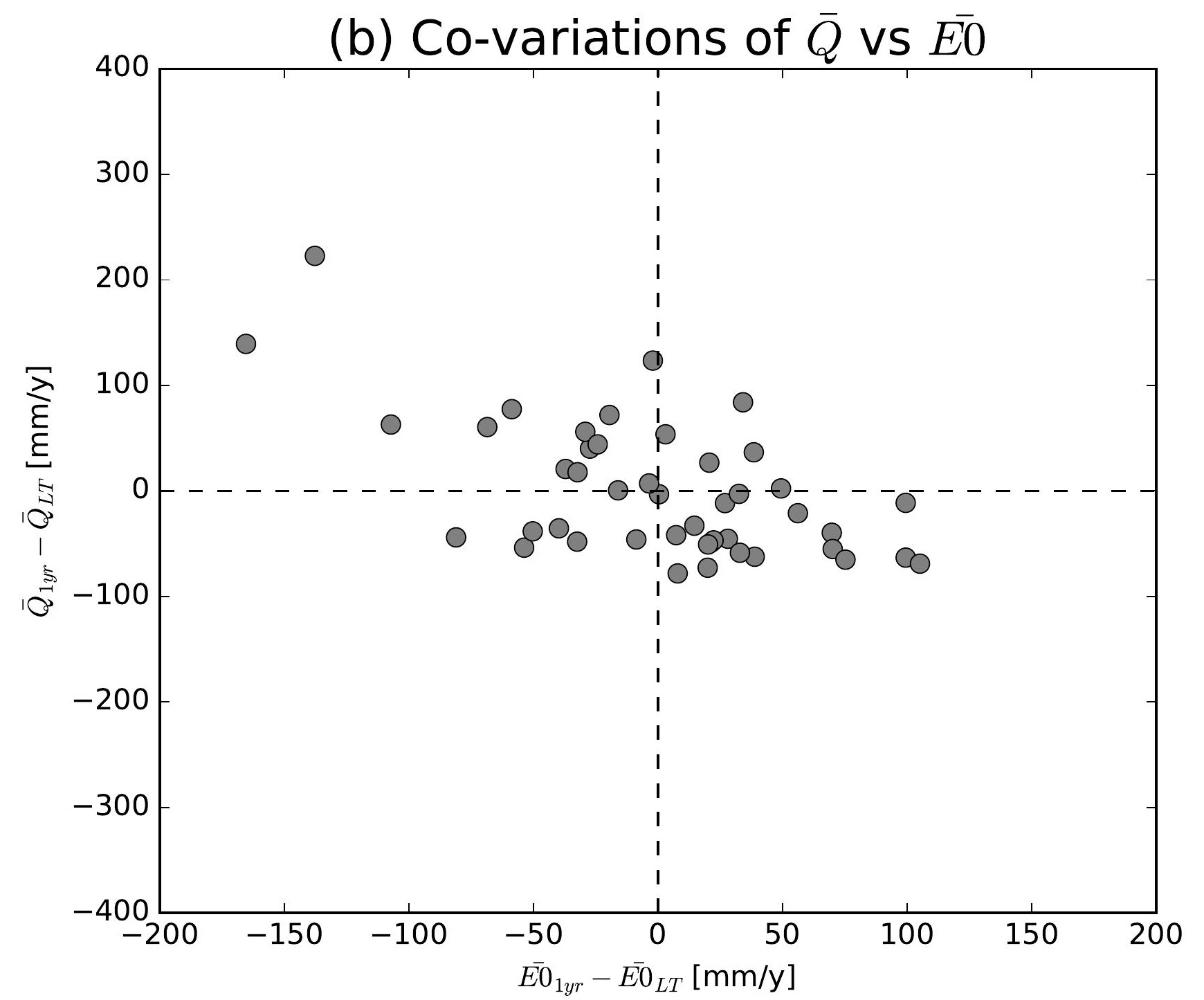

(e) Co-variations of $\bar{Q}$ vs $\overline{E 0}$

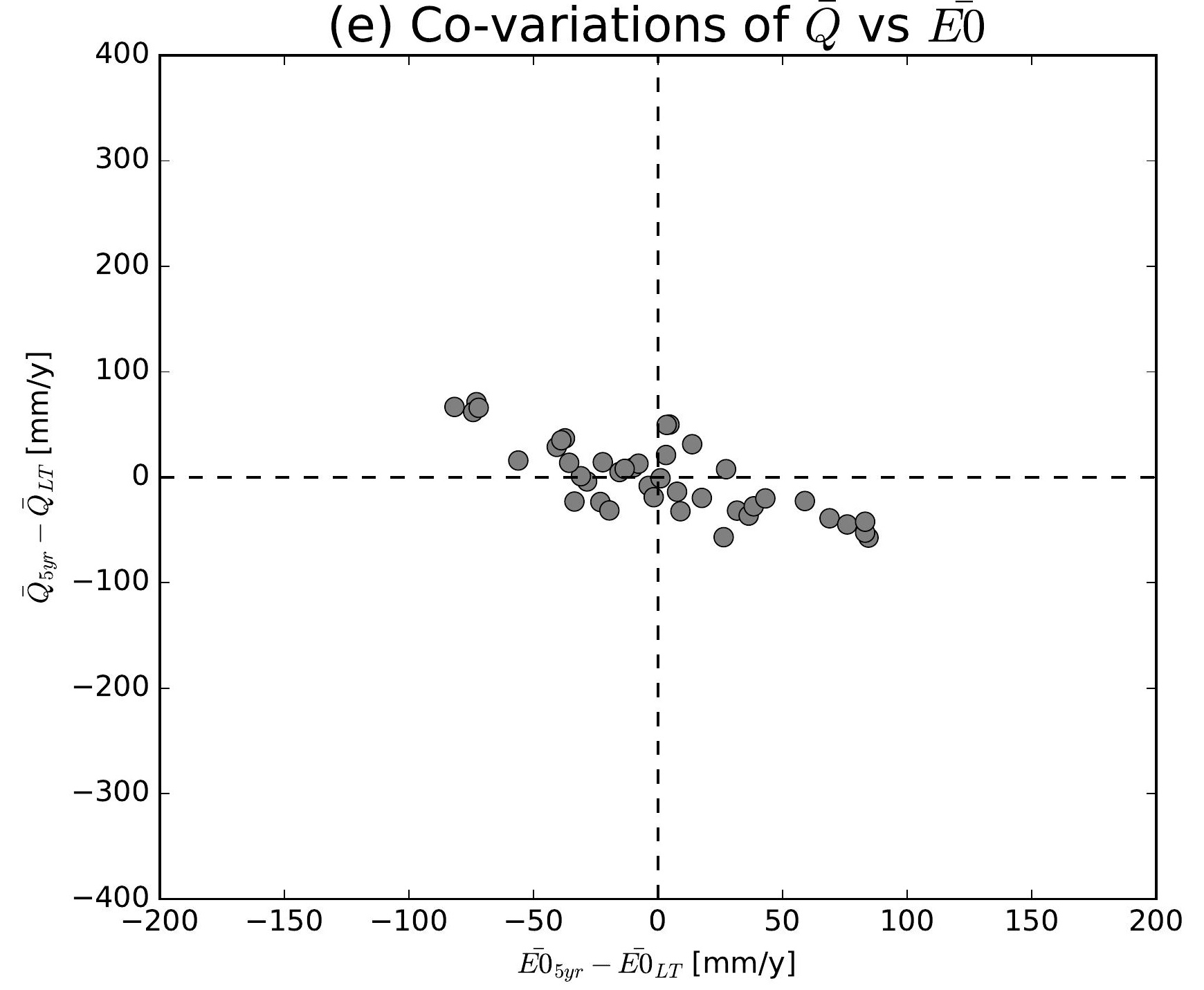

(h) Co-variations of $\bar{Q}$ vs $\overline{E 0}$

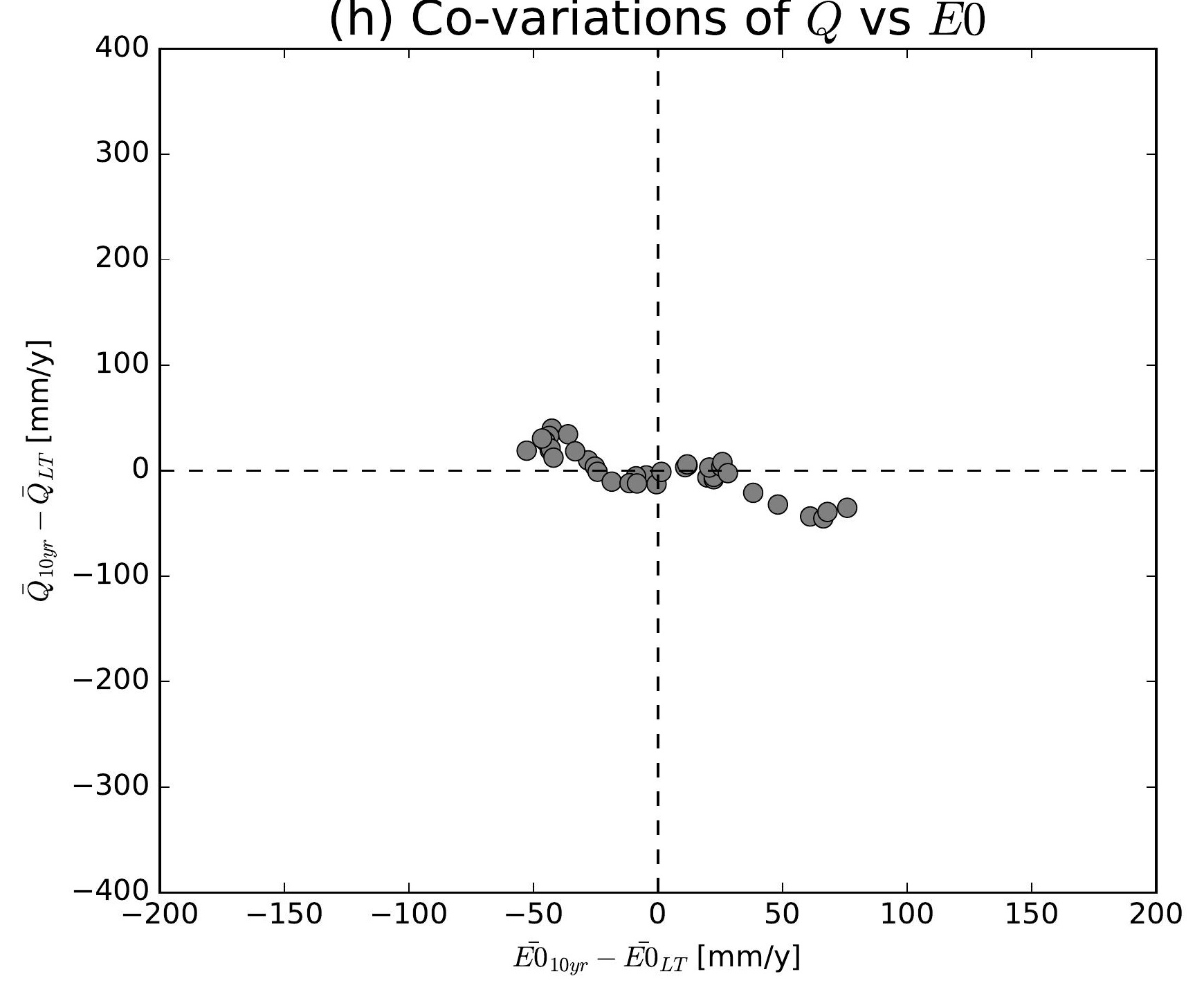

(k) Co-variations of $\bar{Q}$ vs $\overline{E 0}$

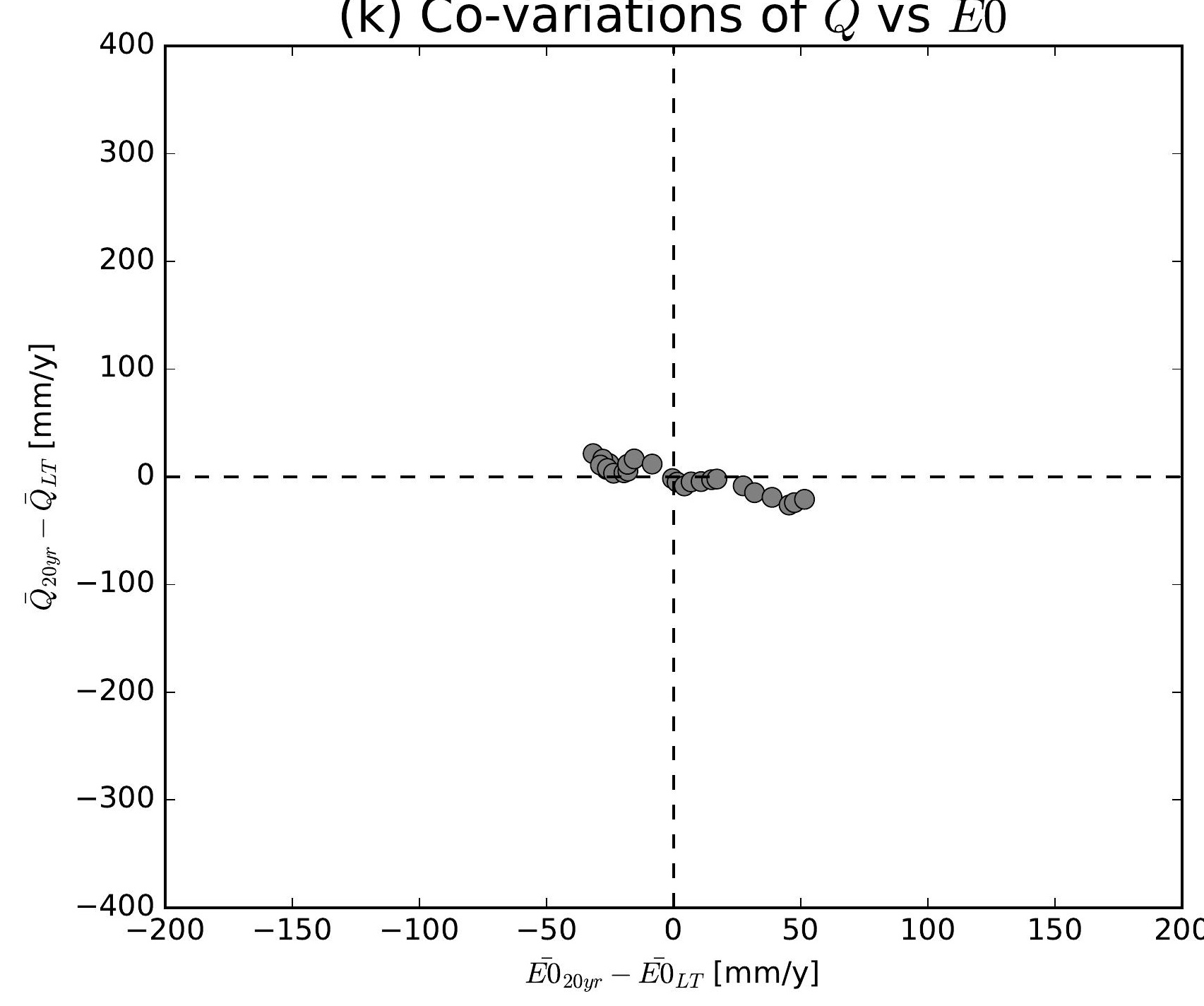

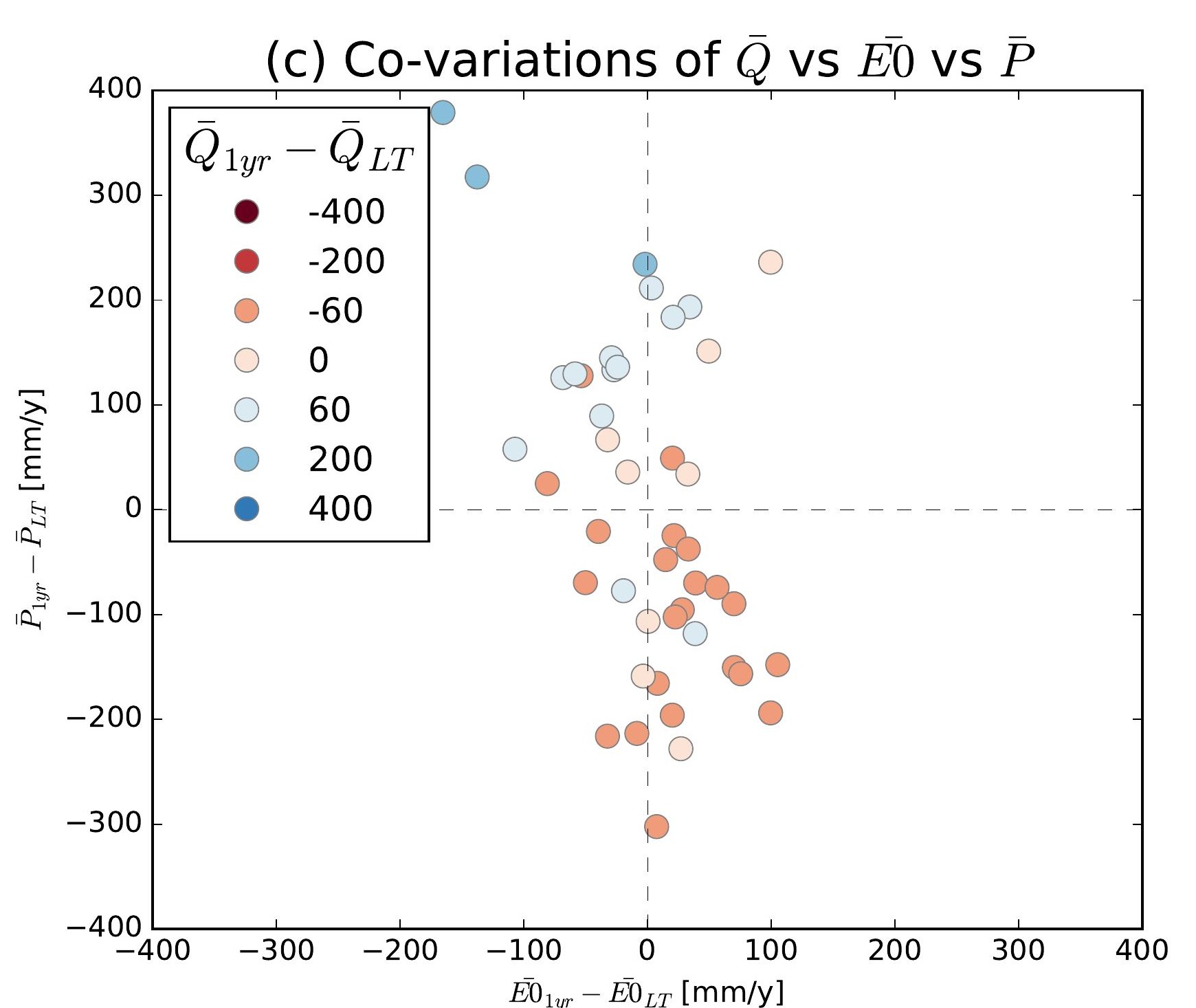
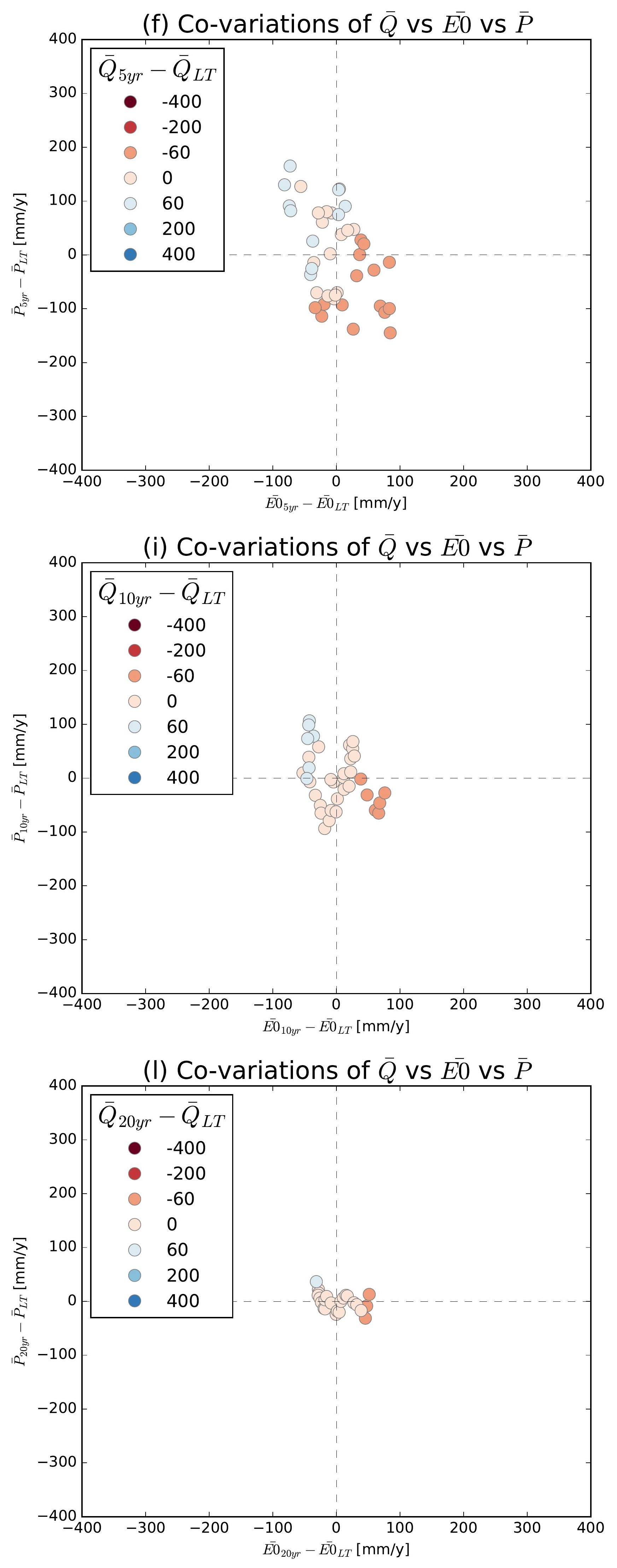

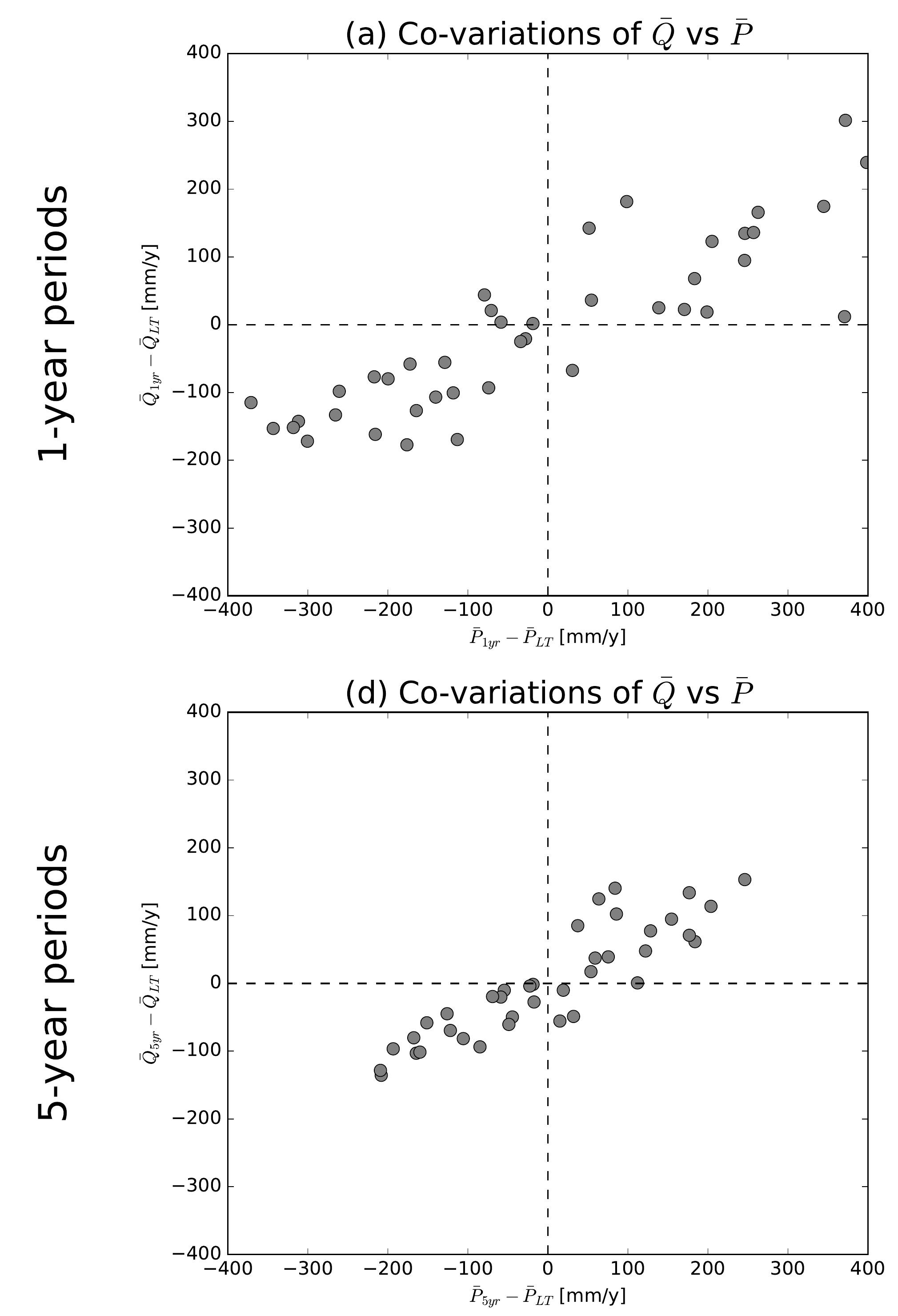

(g) Co-variations of $\bar{Q}$ vs $\bar{P}$
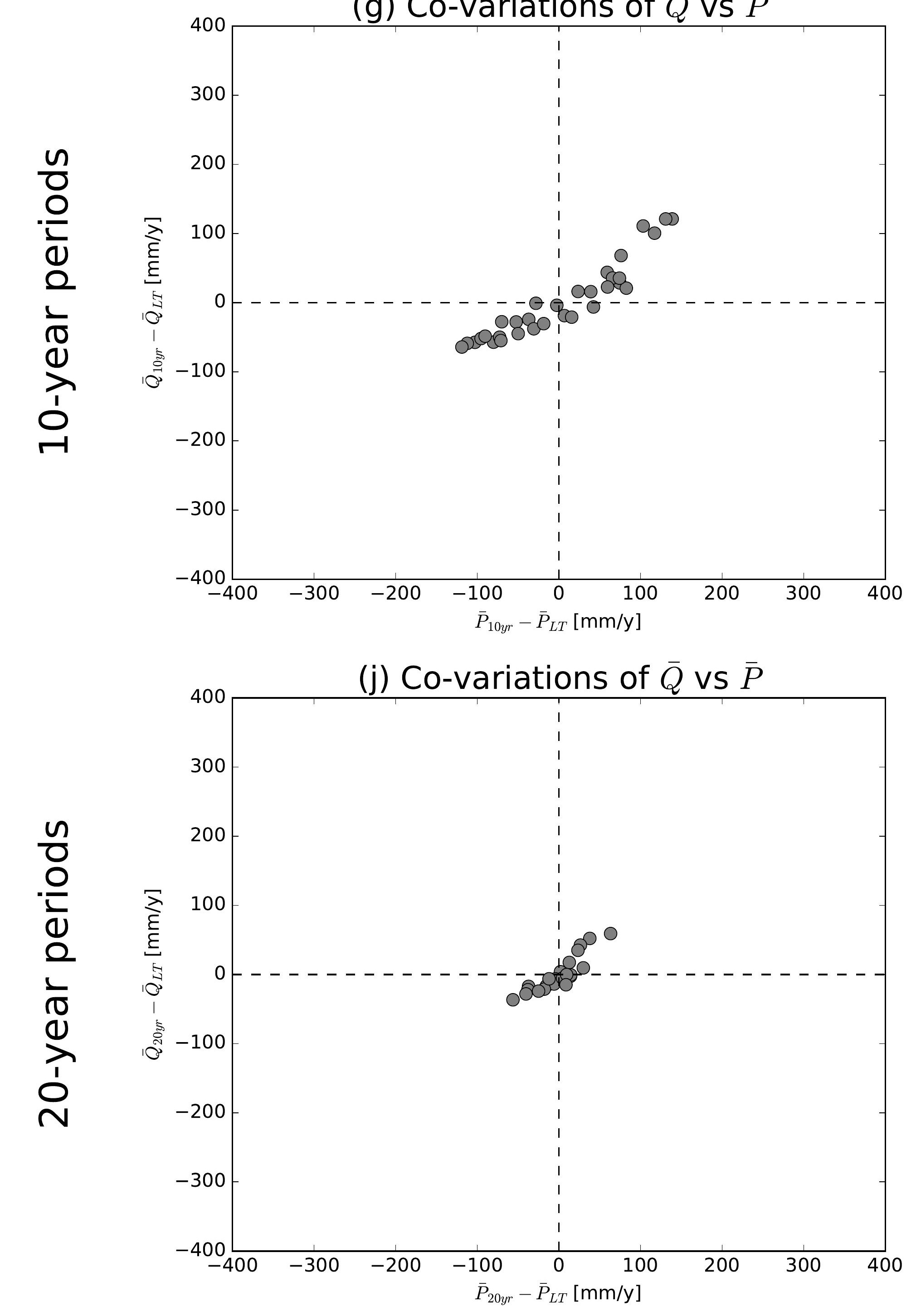

(b) Co-variations of $\bar{Q}$ vs $\overline{E 0}$

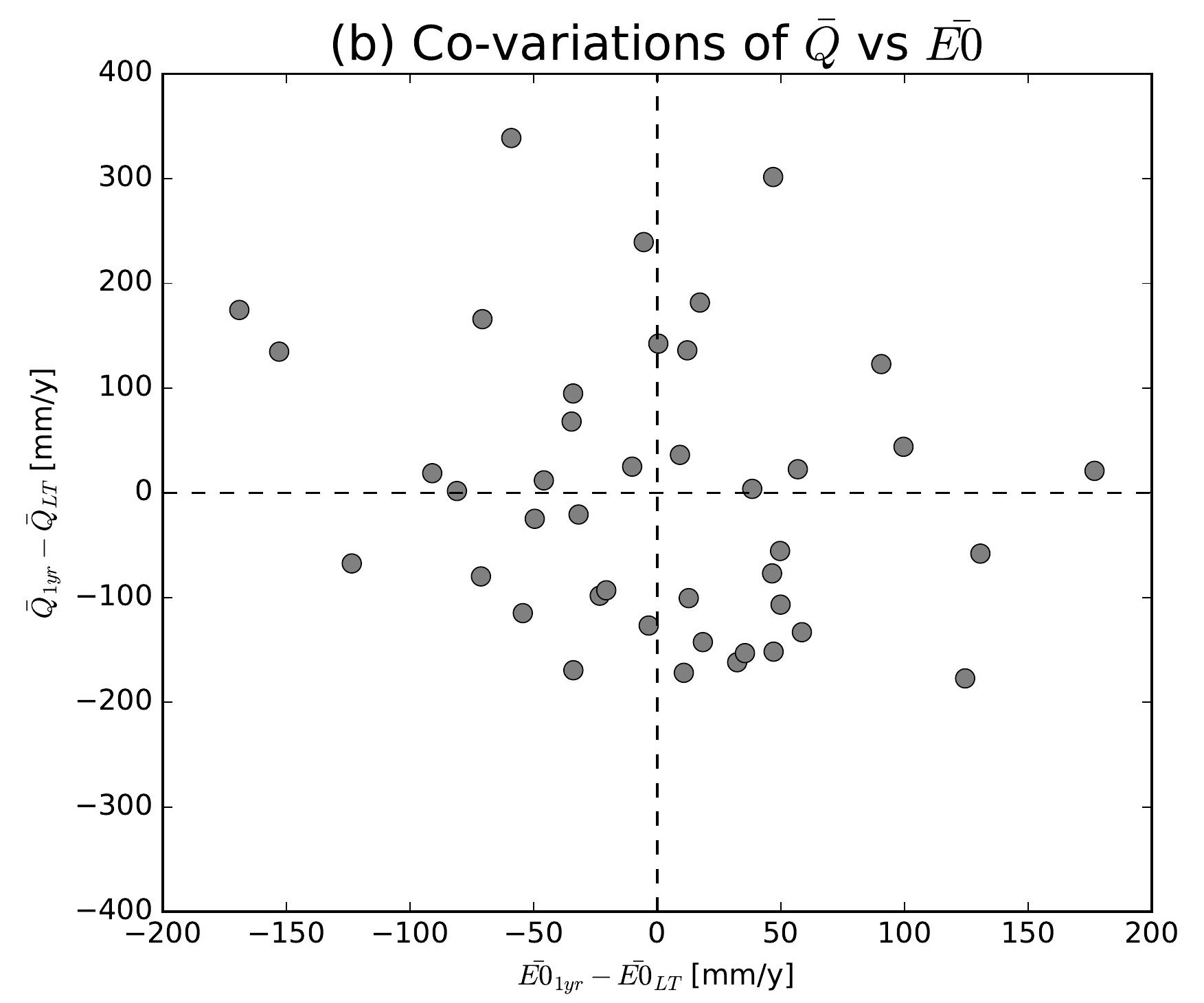

(e) Co-variations of $\bar{Q}$ vs $\overline{E 0}$

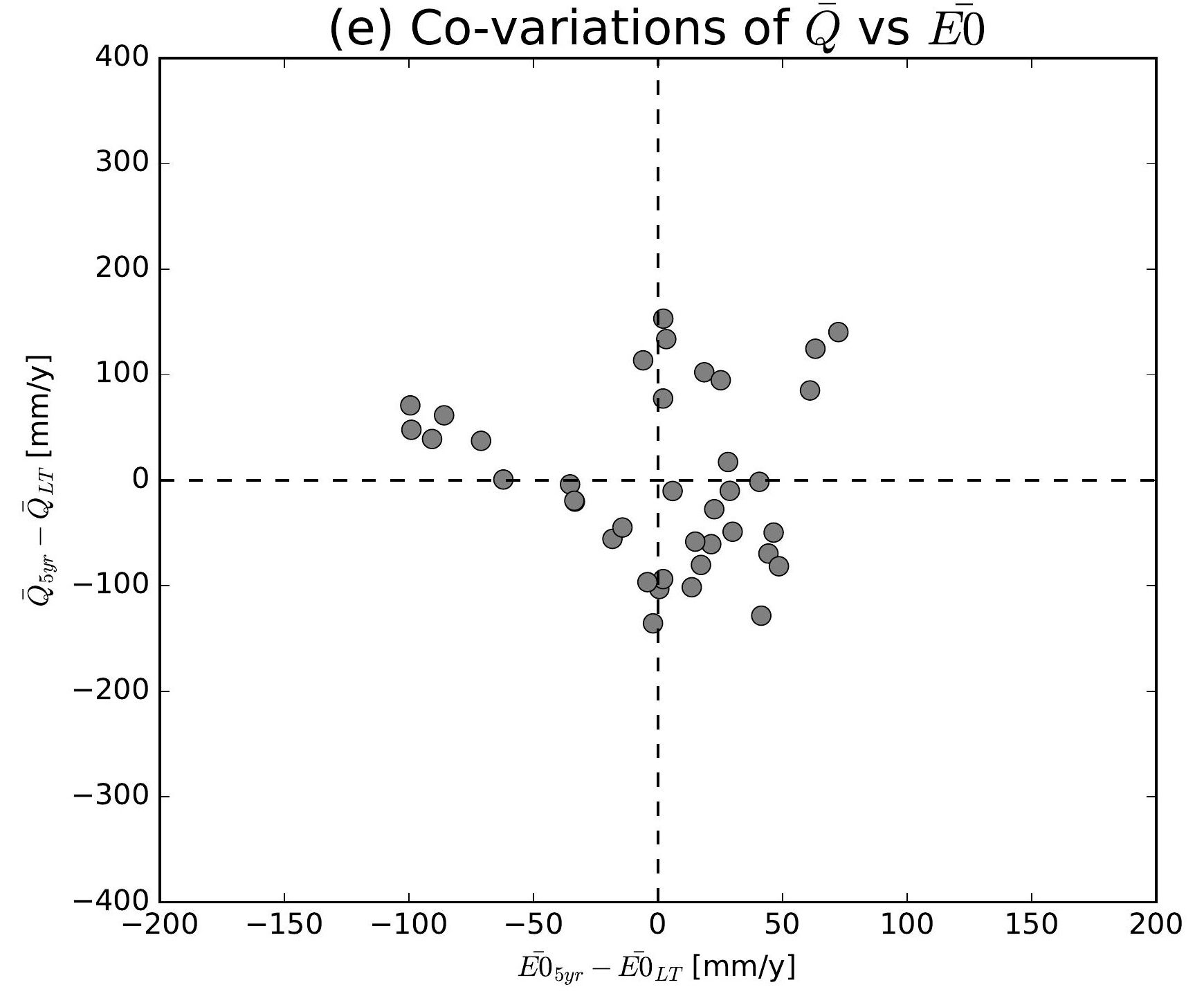

(h) Co-variations of $\bar{Q}$ vs $\overline{E 0}$

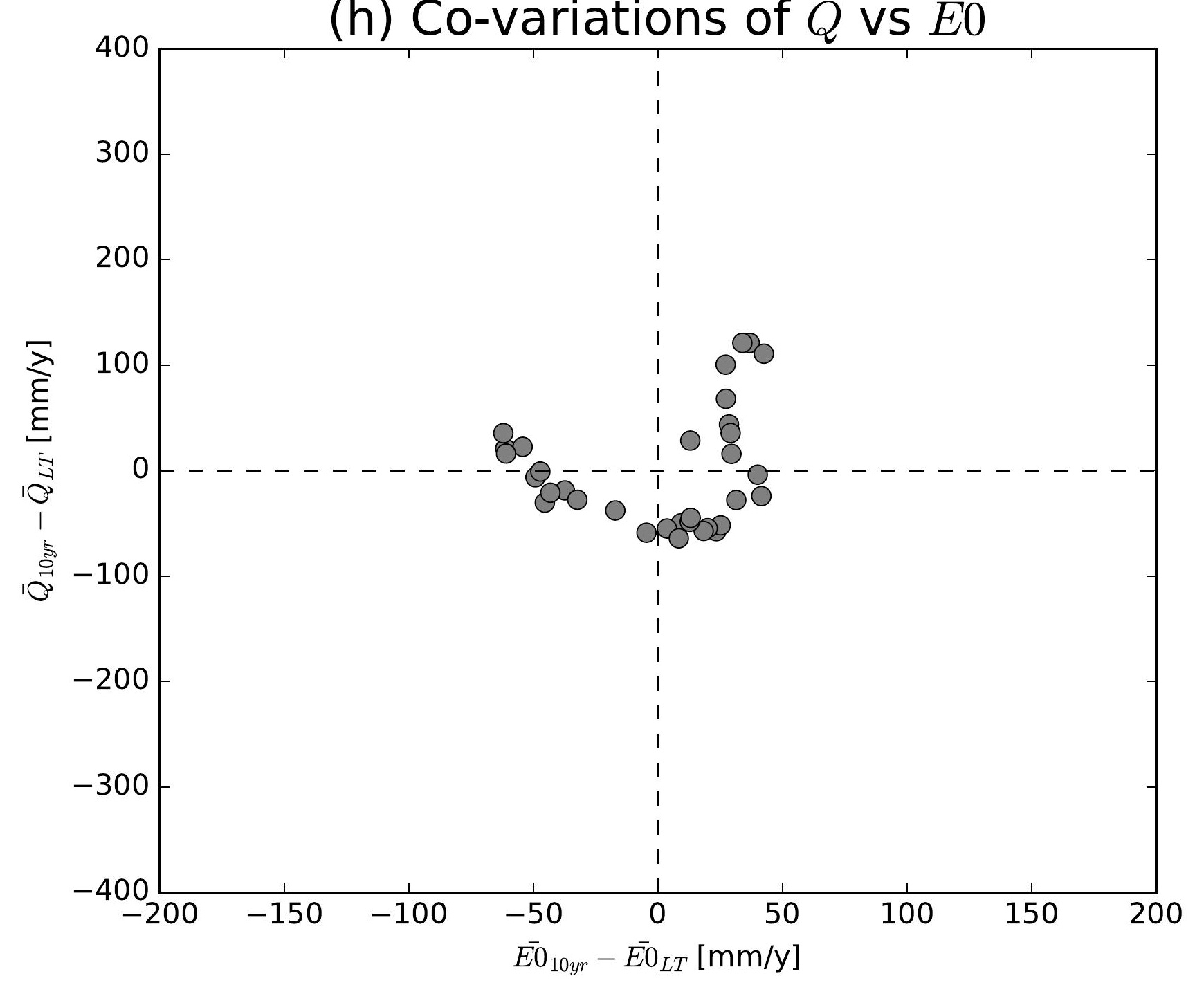

(k) Co-variations of $\bar{Q}$ vs $\overline{E 0}$

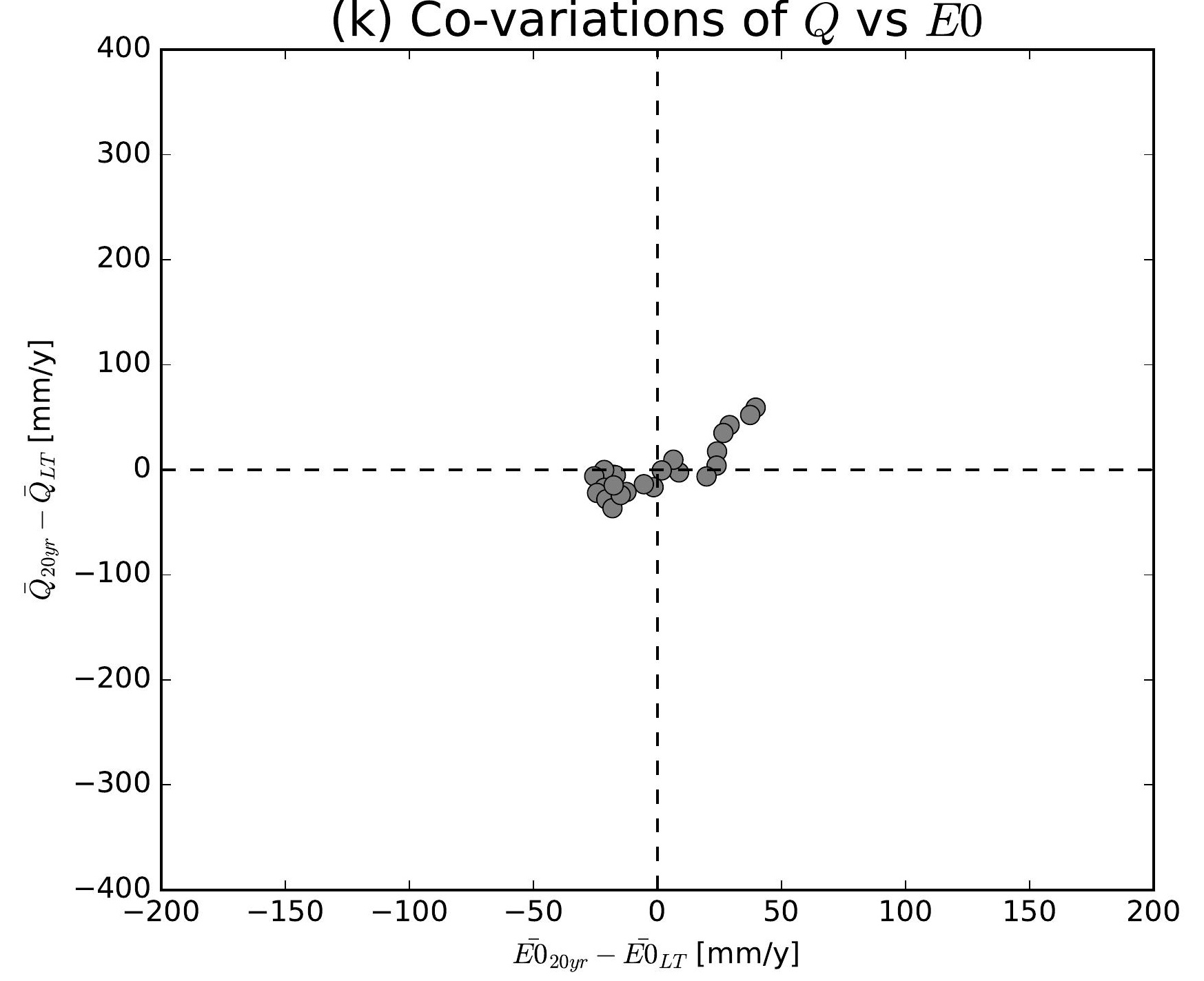

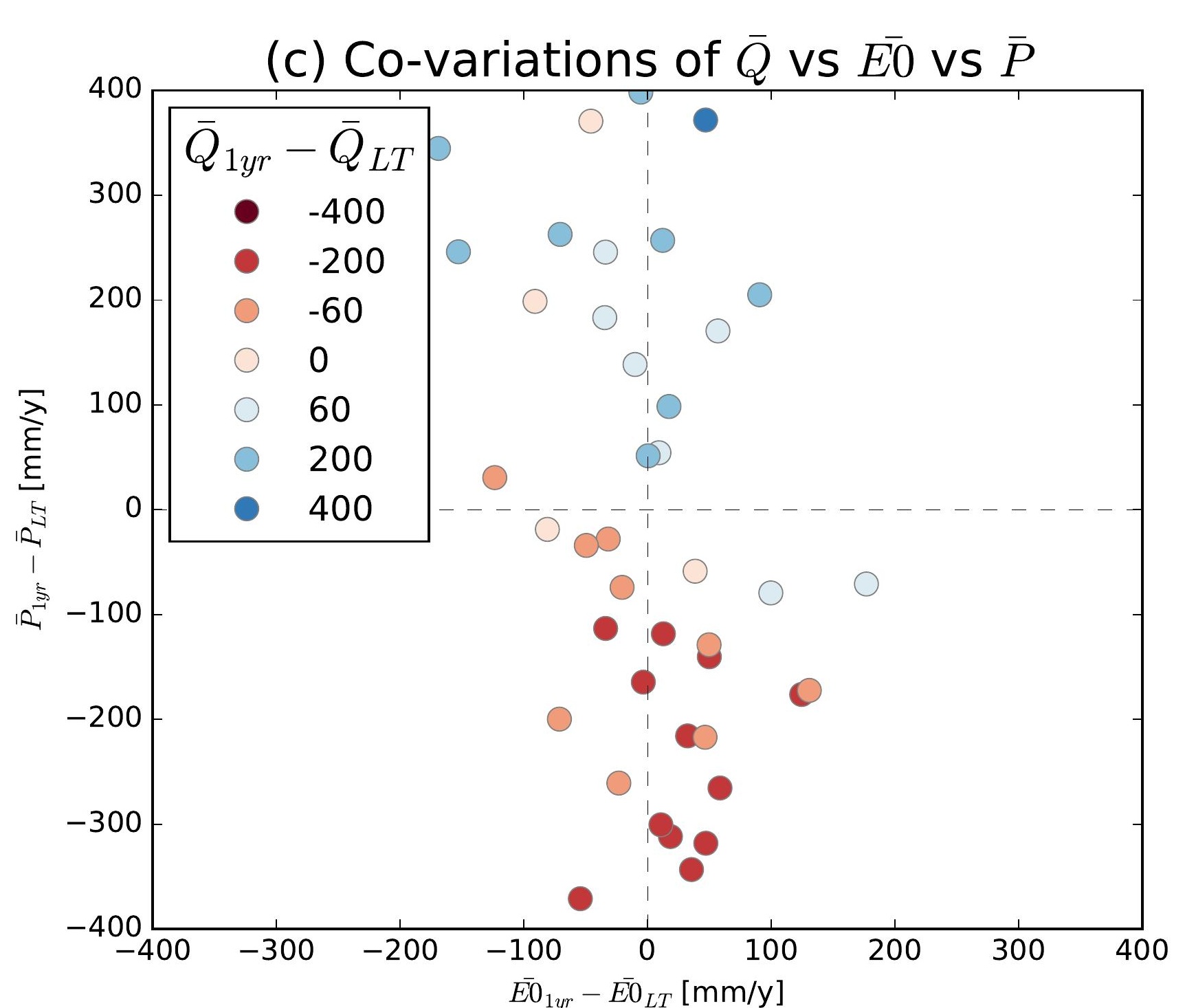
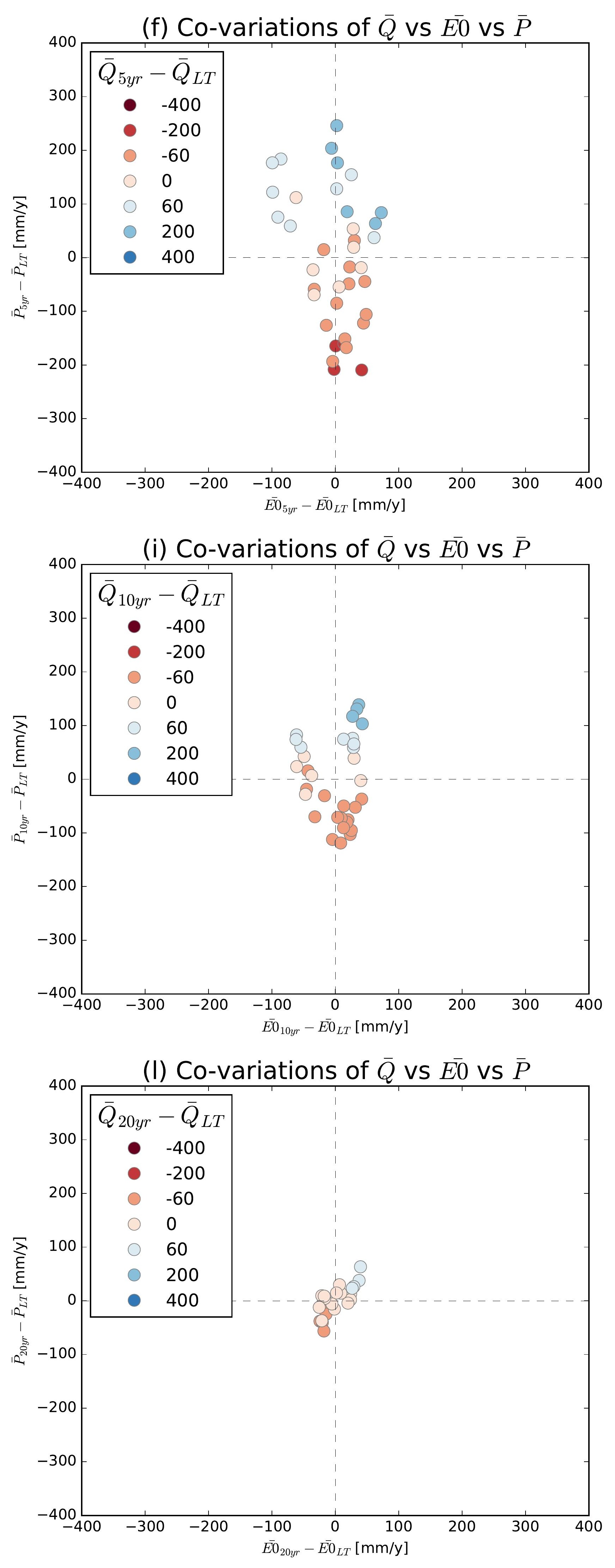

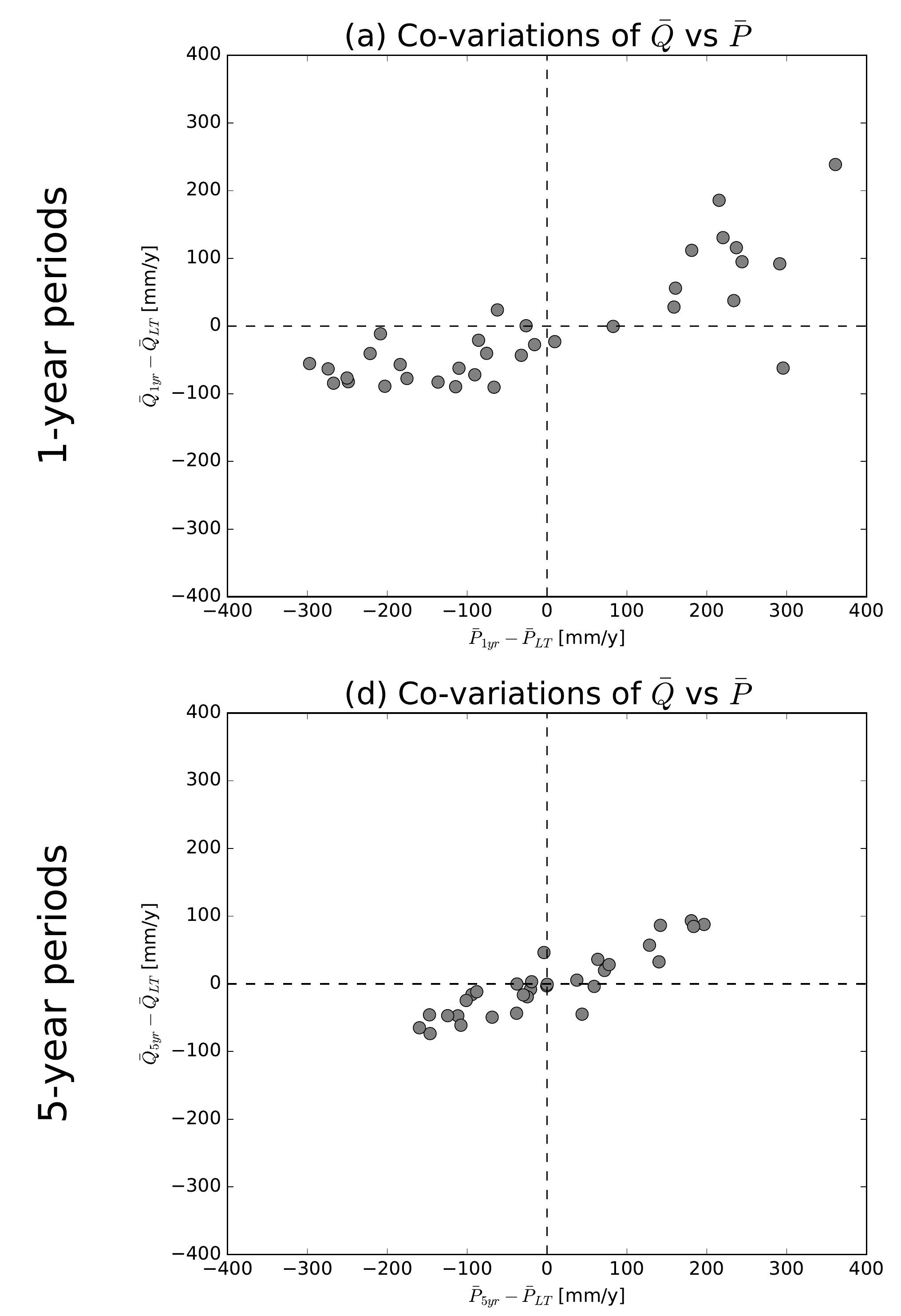

(g) Co-variations of $\bar{Q}$ vs $\bar{P}$

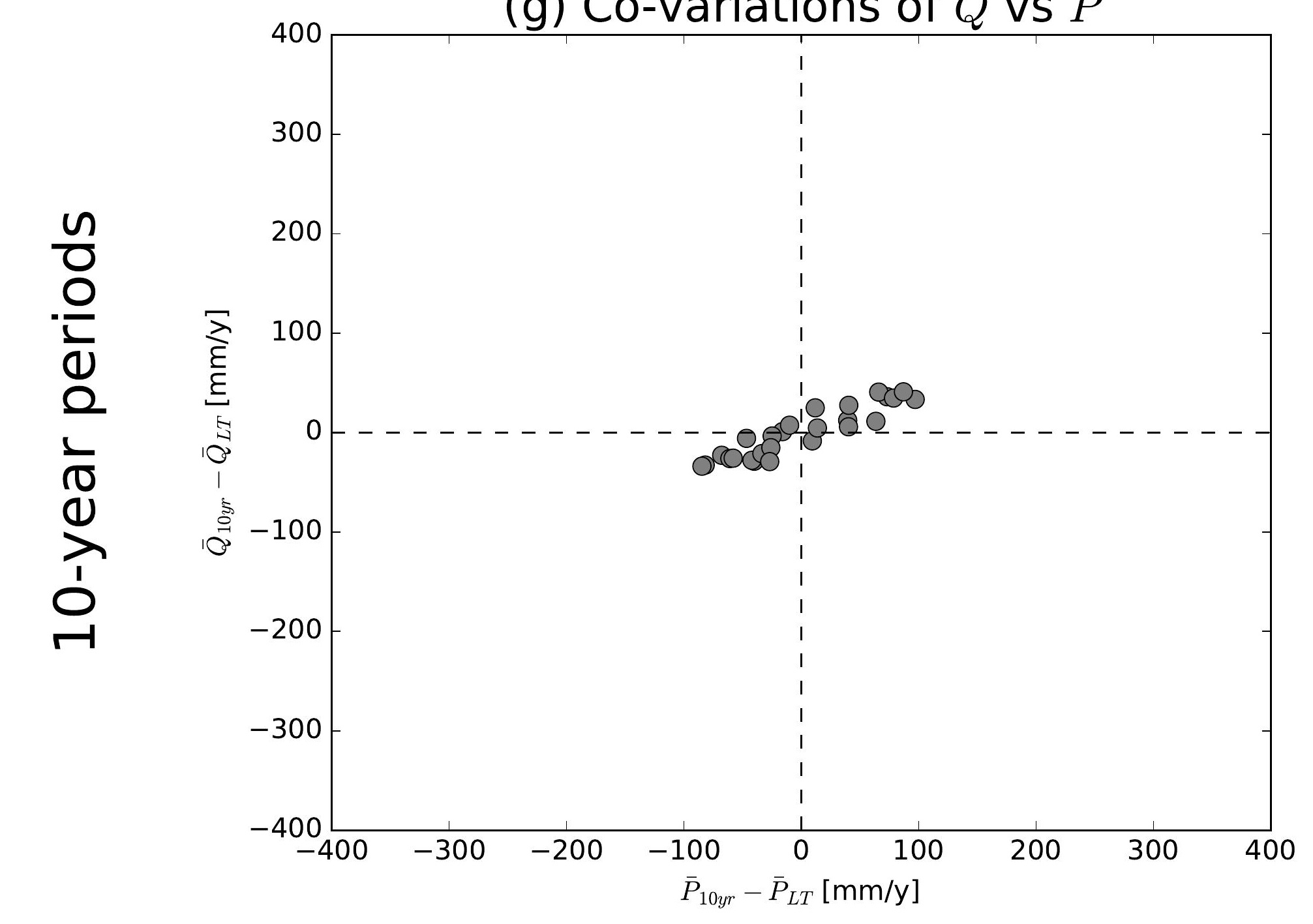

(j) Co-variations of $\bar{Q}$ vs $\bar{P}$

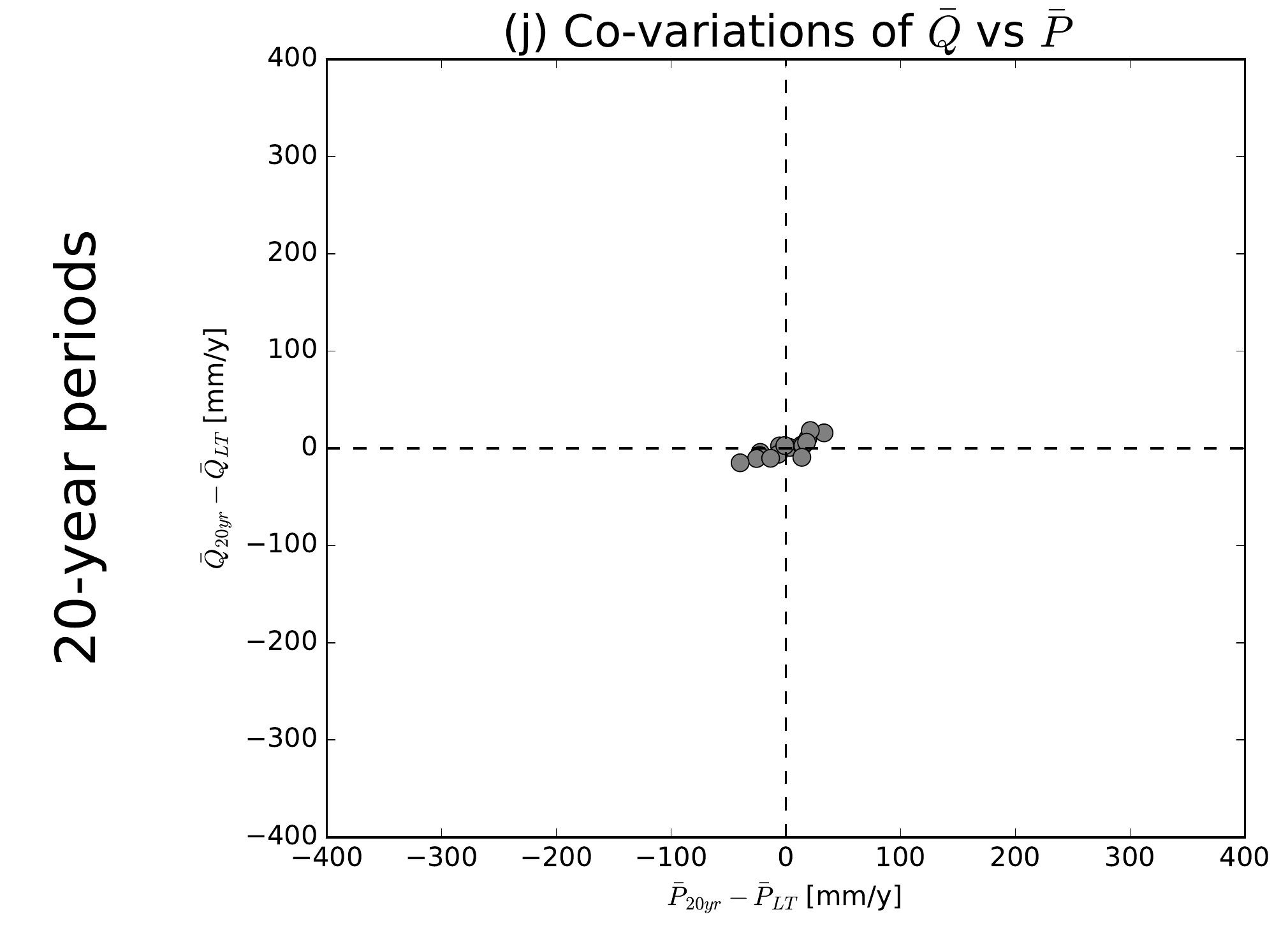

(b) Co-variations of $\bar{Q}$ vs $\overline{E 0}$

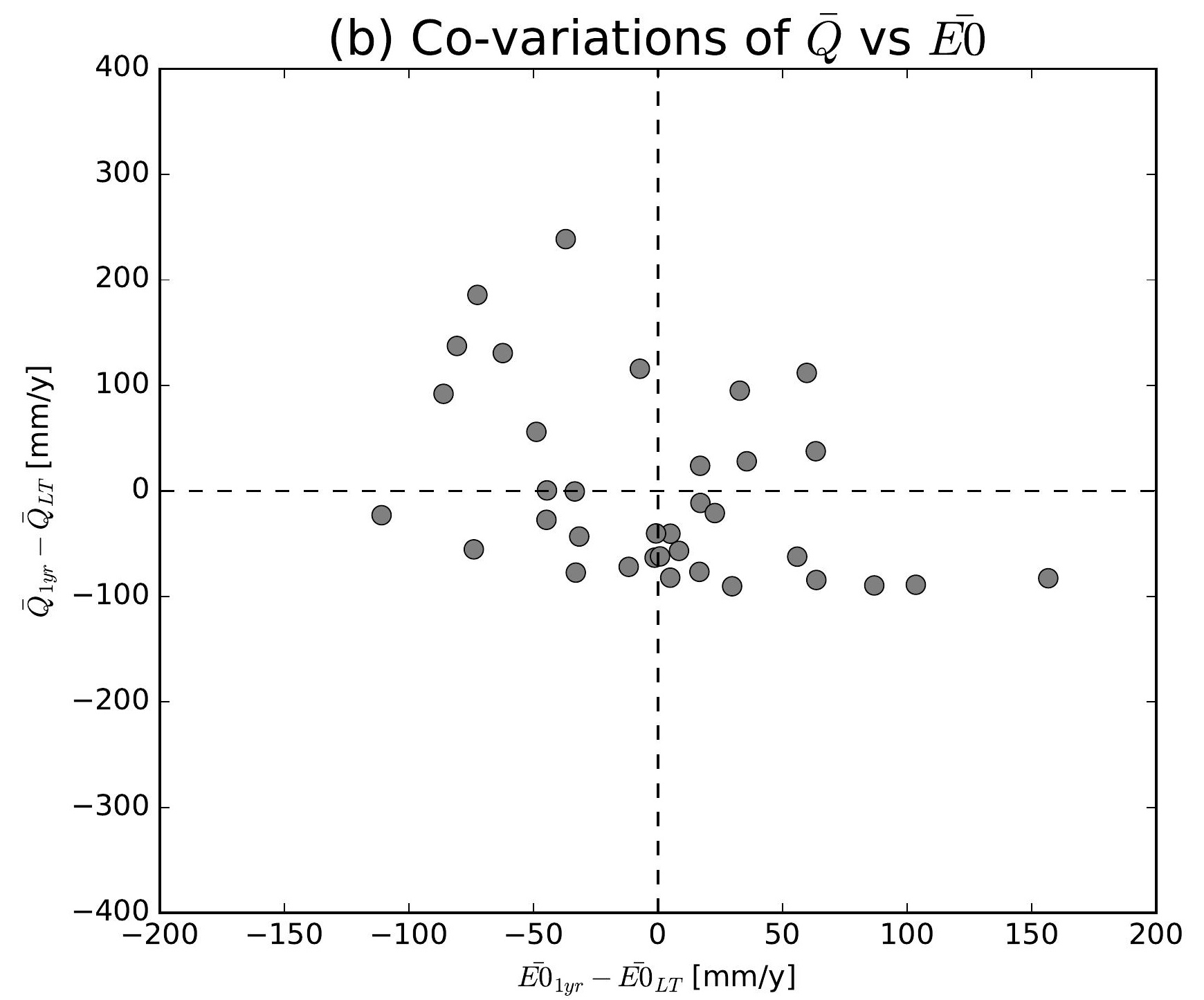

(e) Co-variations of $\bar{Q}$ vs $\overline{E 0}$

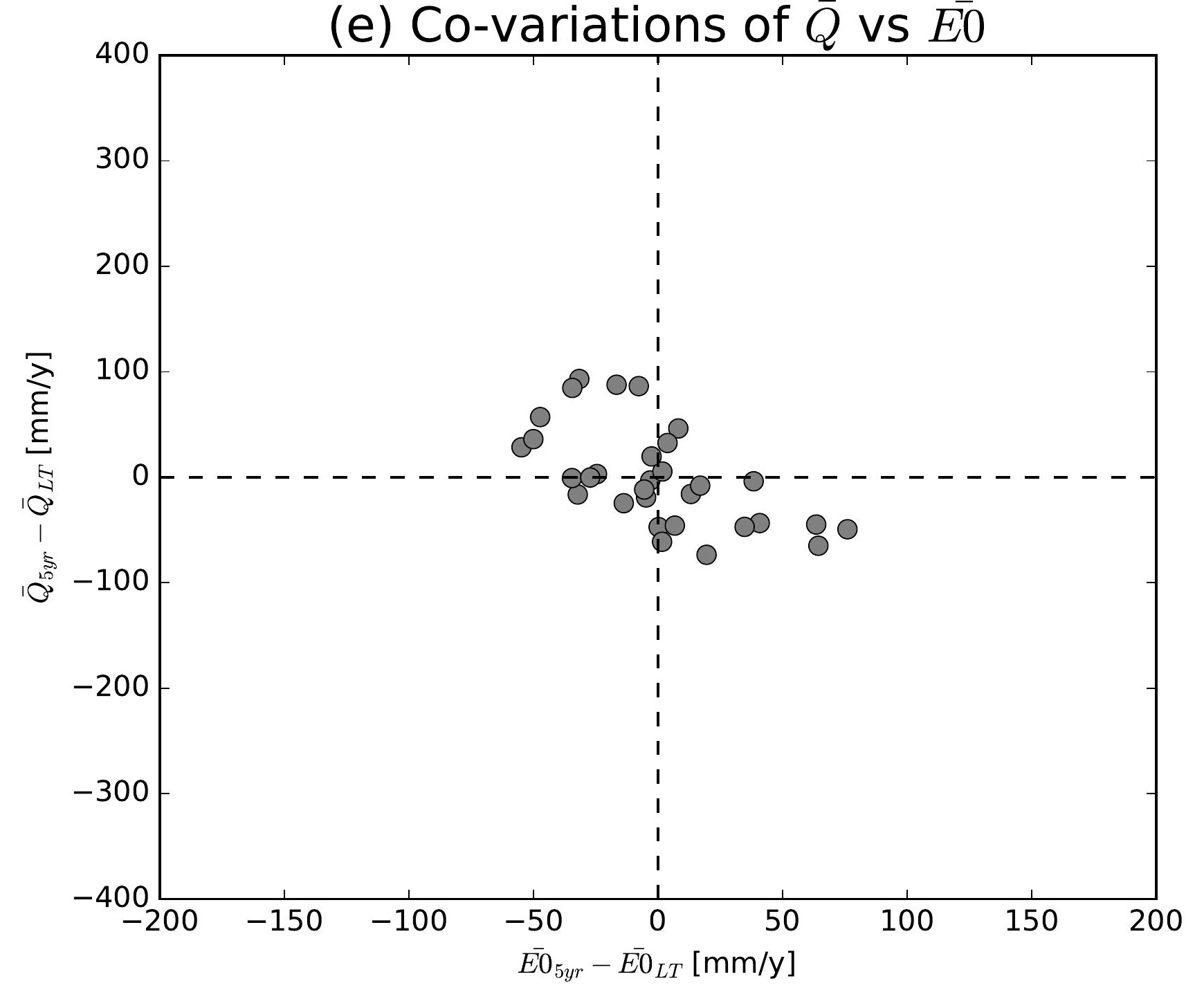

(h) Co-variations of $\bar{Q}$ vs $\overline{E 0}$

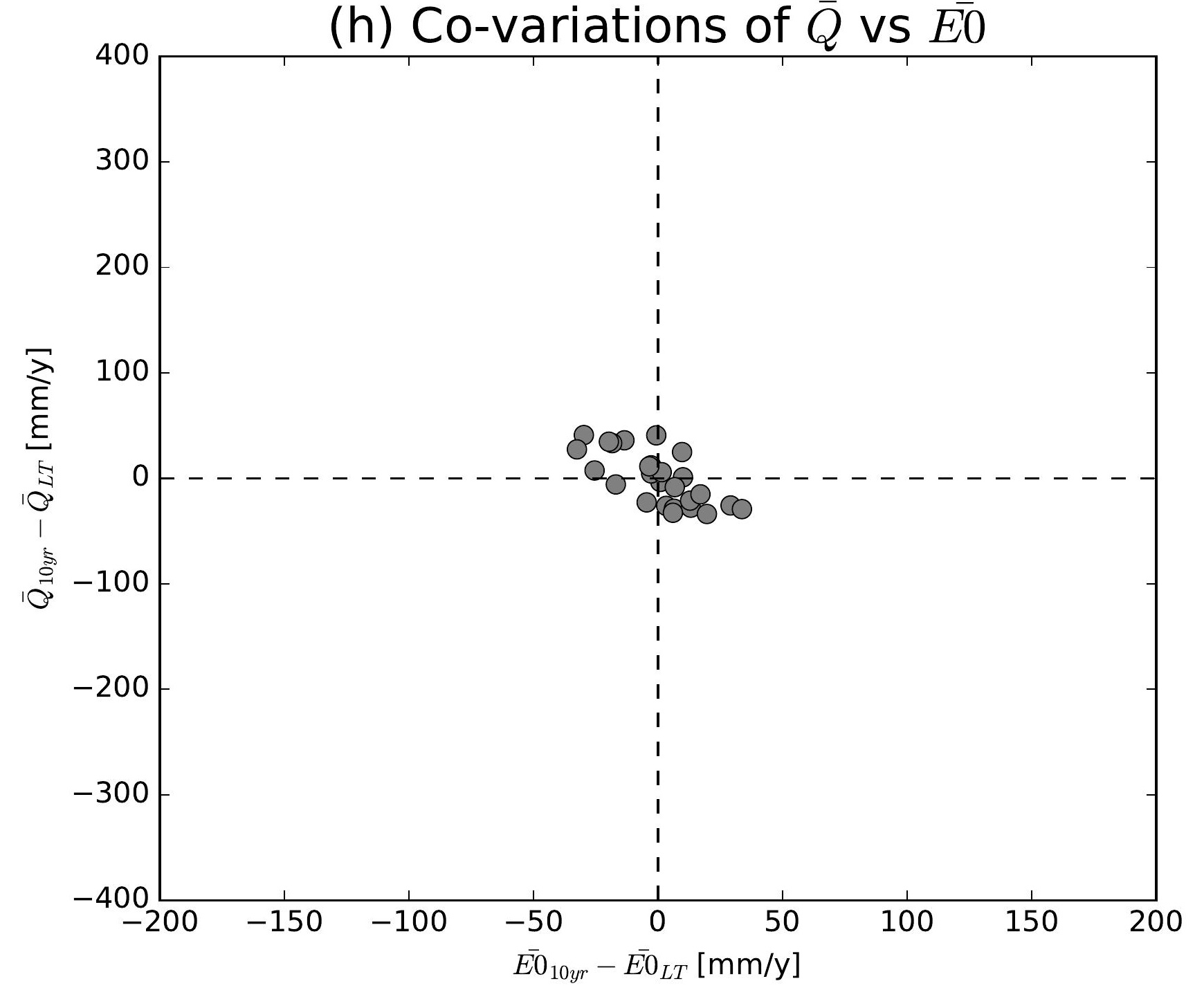

(k) Co-variations of $\bar{Q}$ vs $\overline{E 0}$

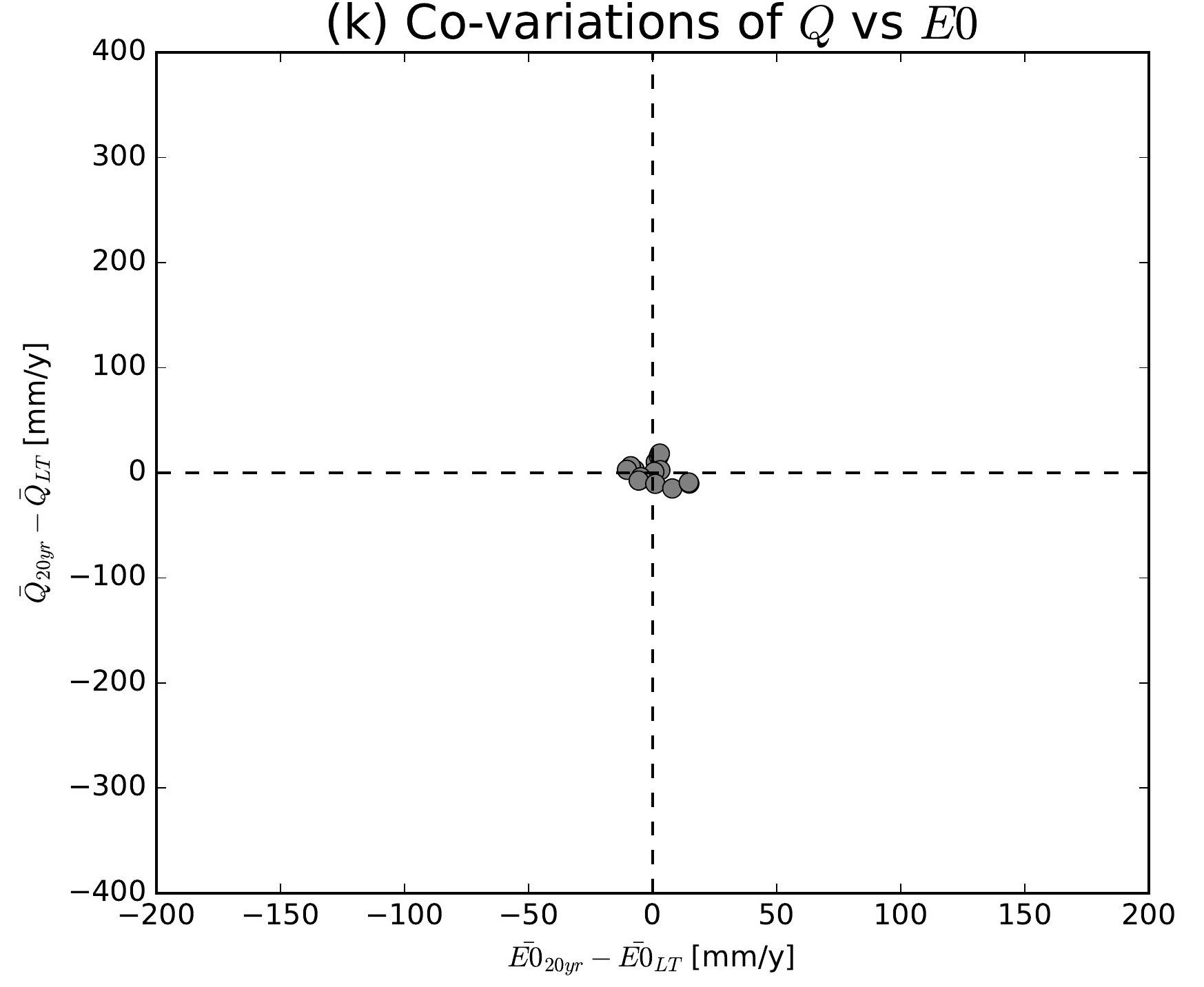

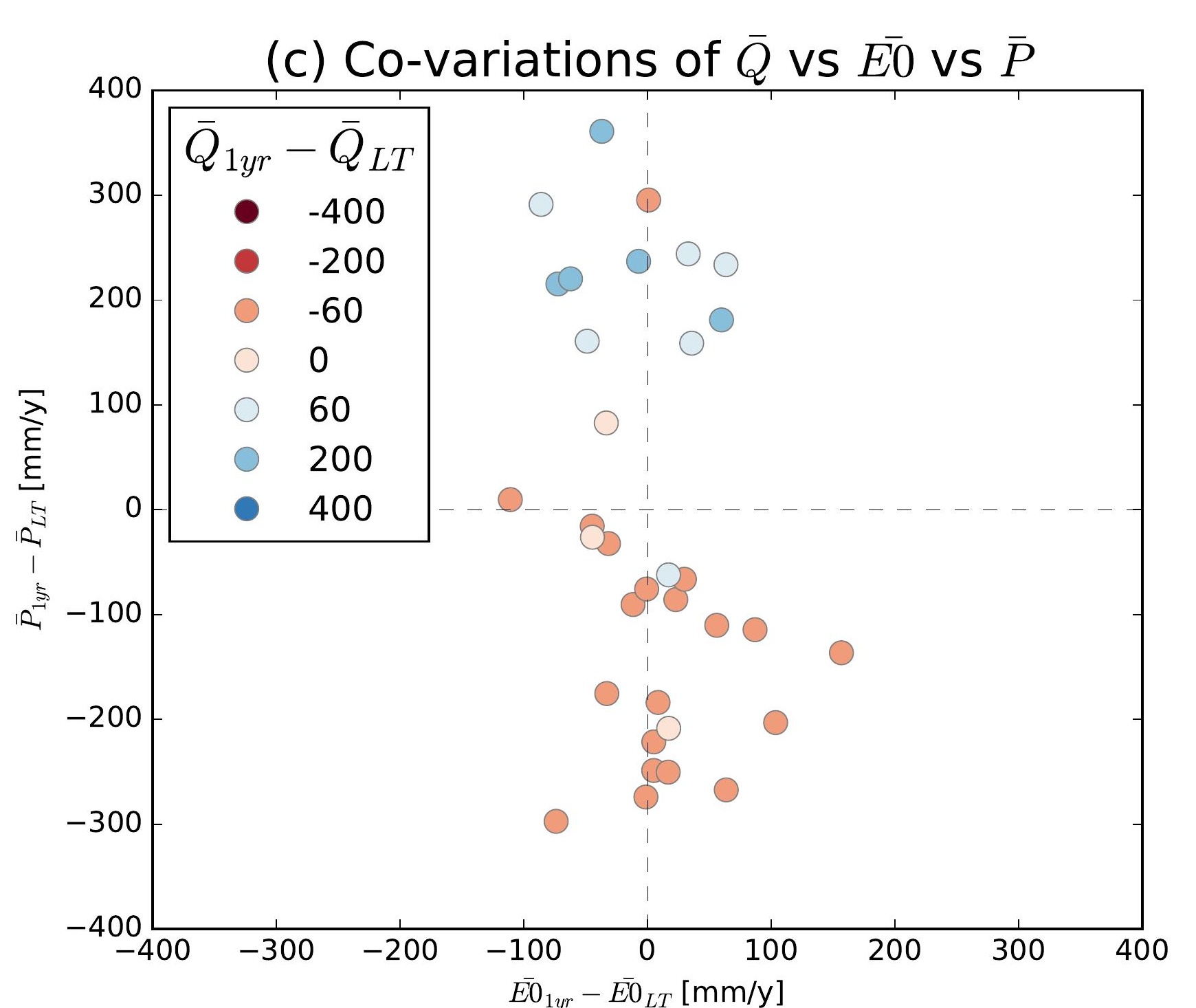
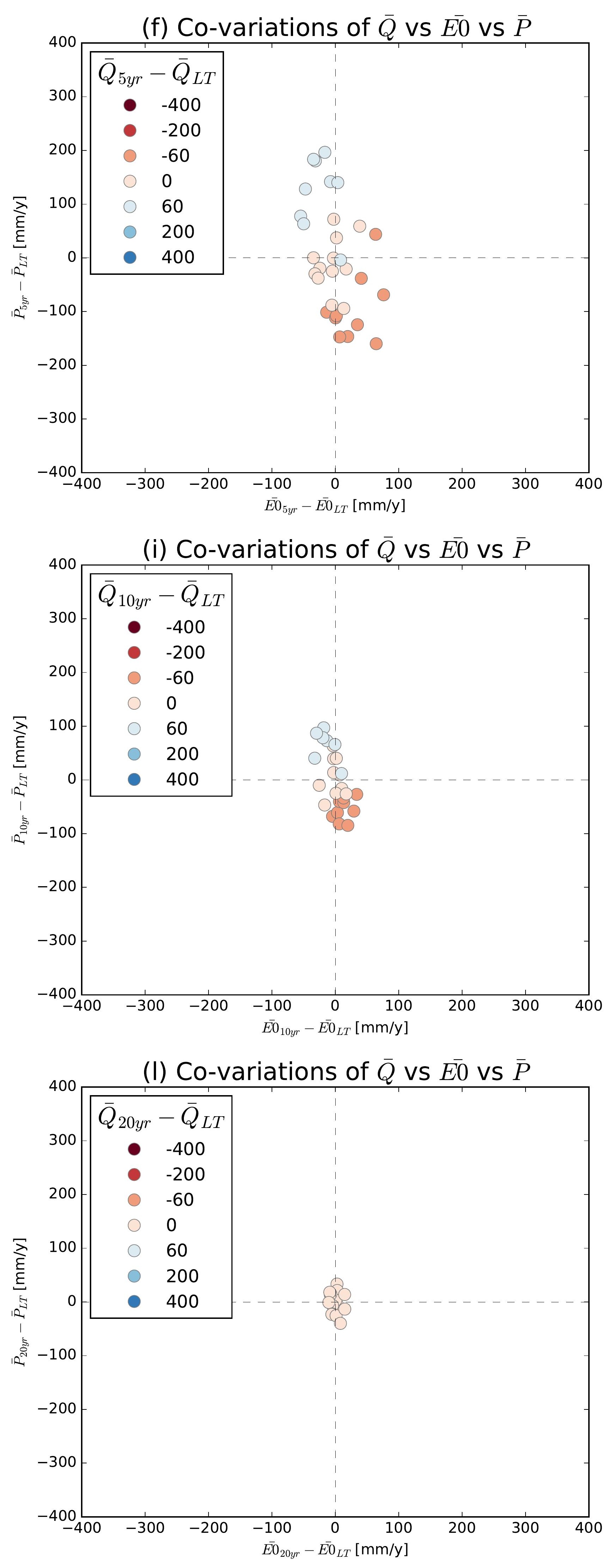

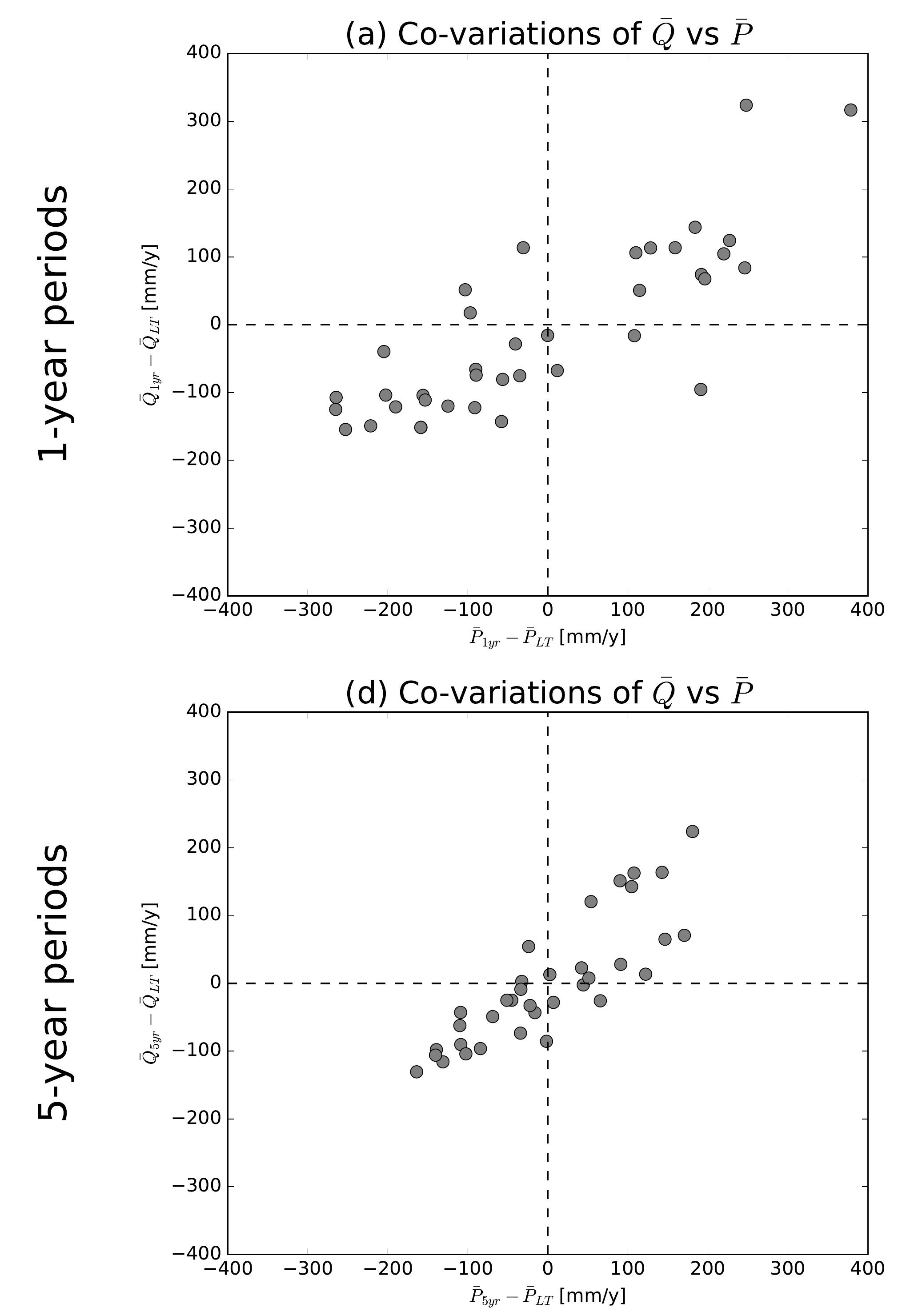

(g) Co-variations of $\bar{Q}$ vs $\bar{P}$
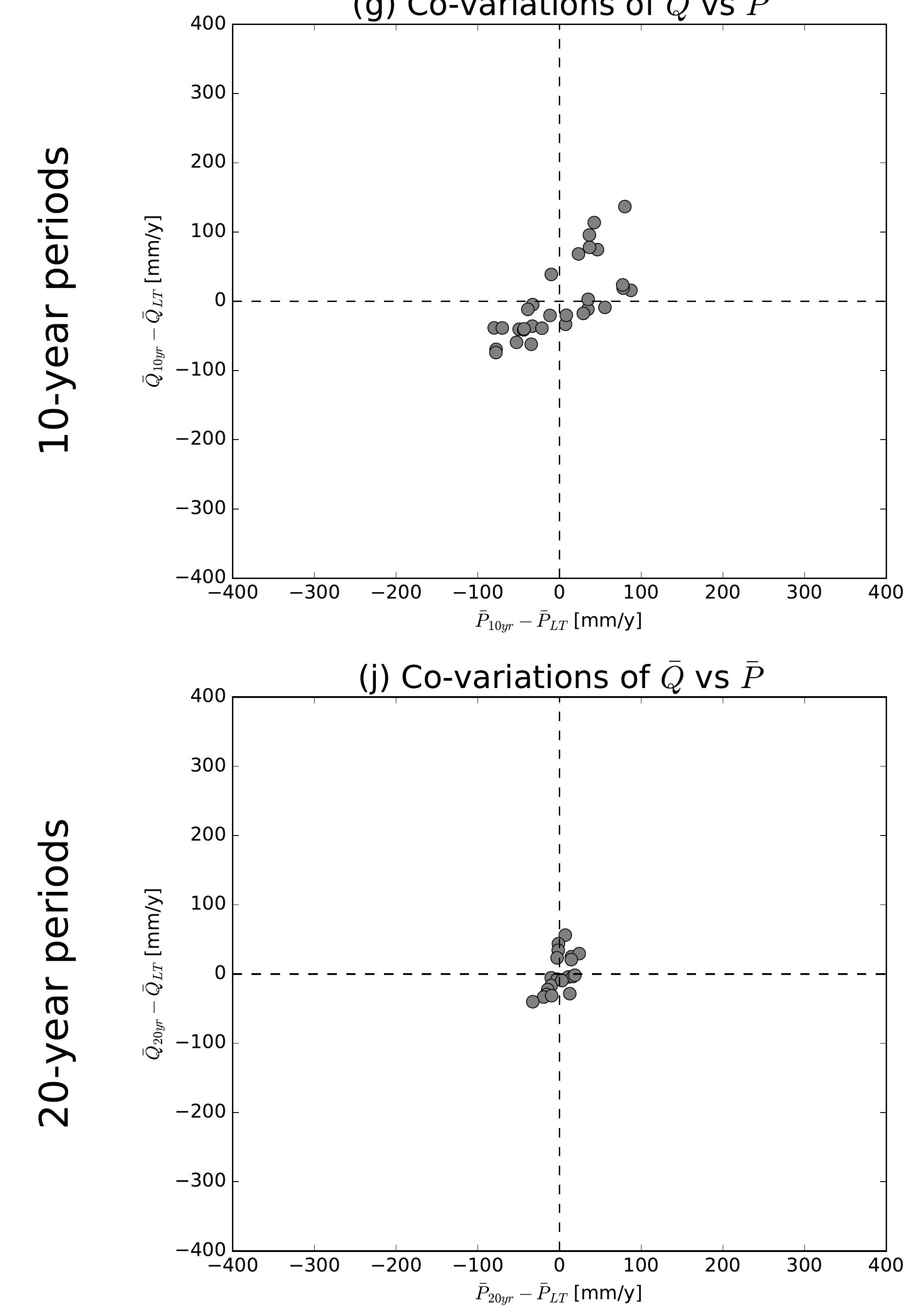

(b) Co-variations of $\bar{Q}$ vs $\overline{E 0}$

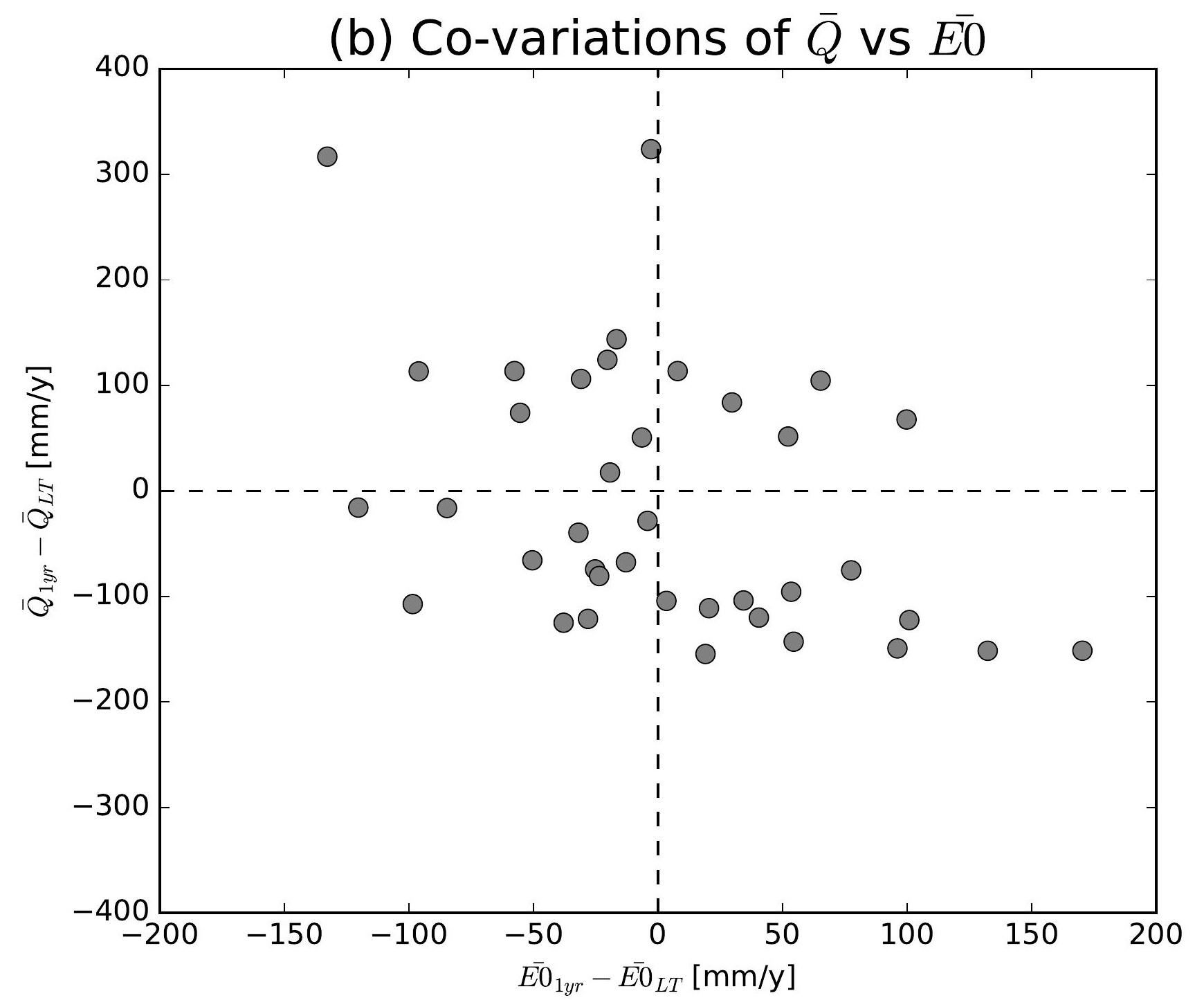

(e) Co-variations of $\bar{Q}$ vs $\overline{E 0}$

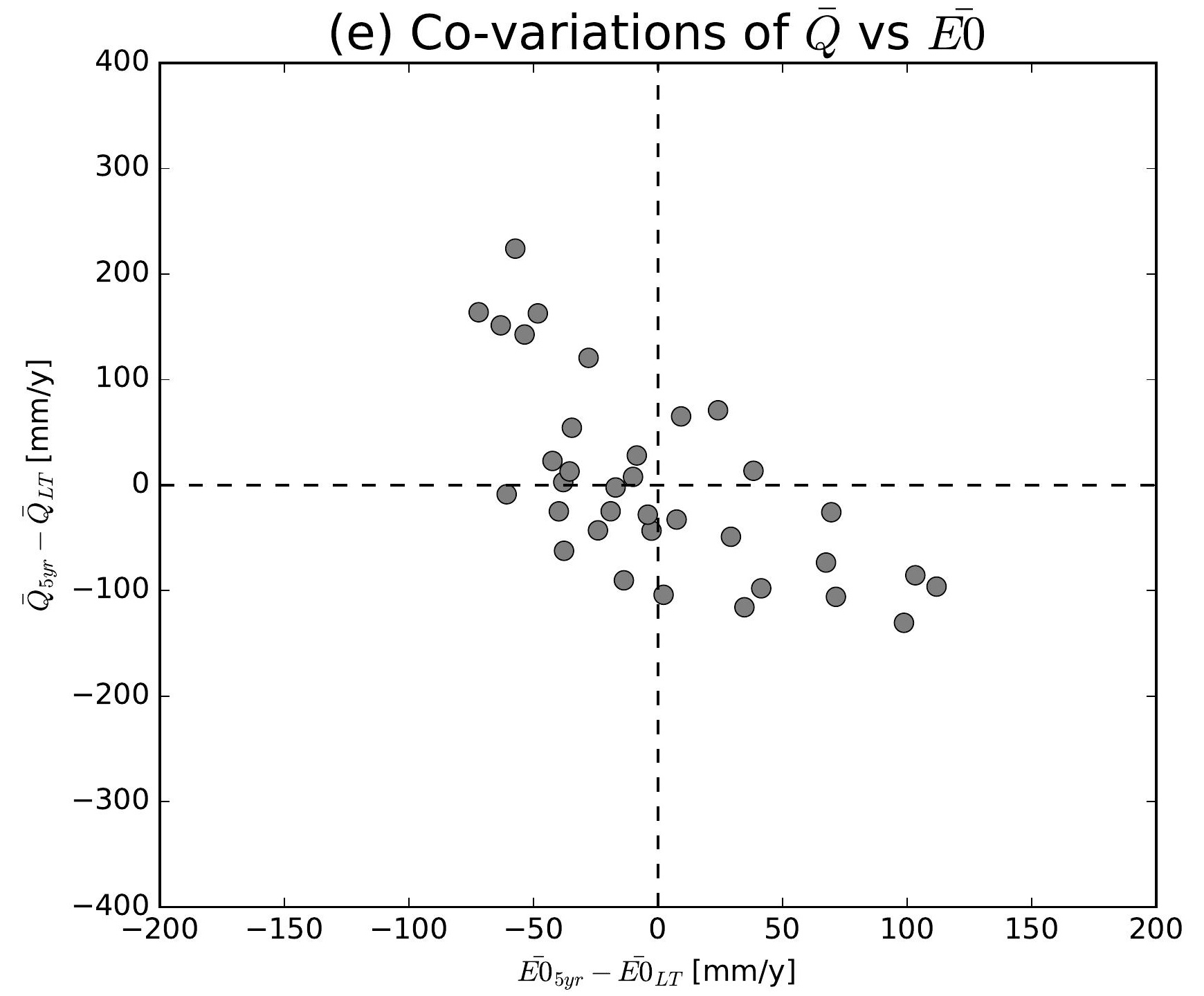

(h) Co-variations of $\bar{Q}$ vs $\overline{E 0}$

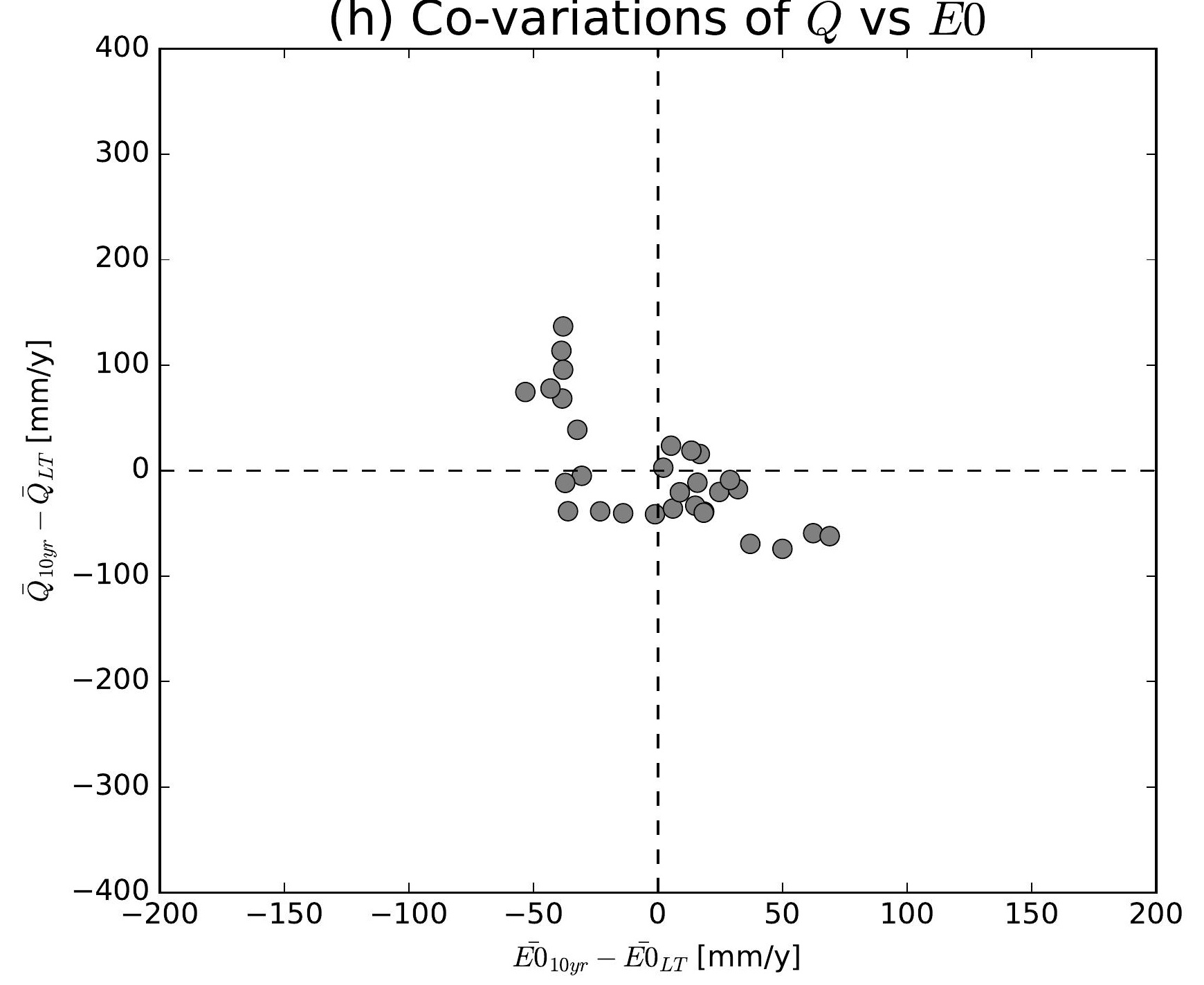

(k) Co-variations of $\bar{Q}$ vs $\overline{E 0}$

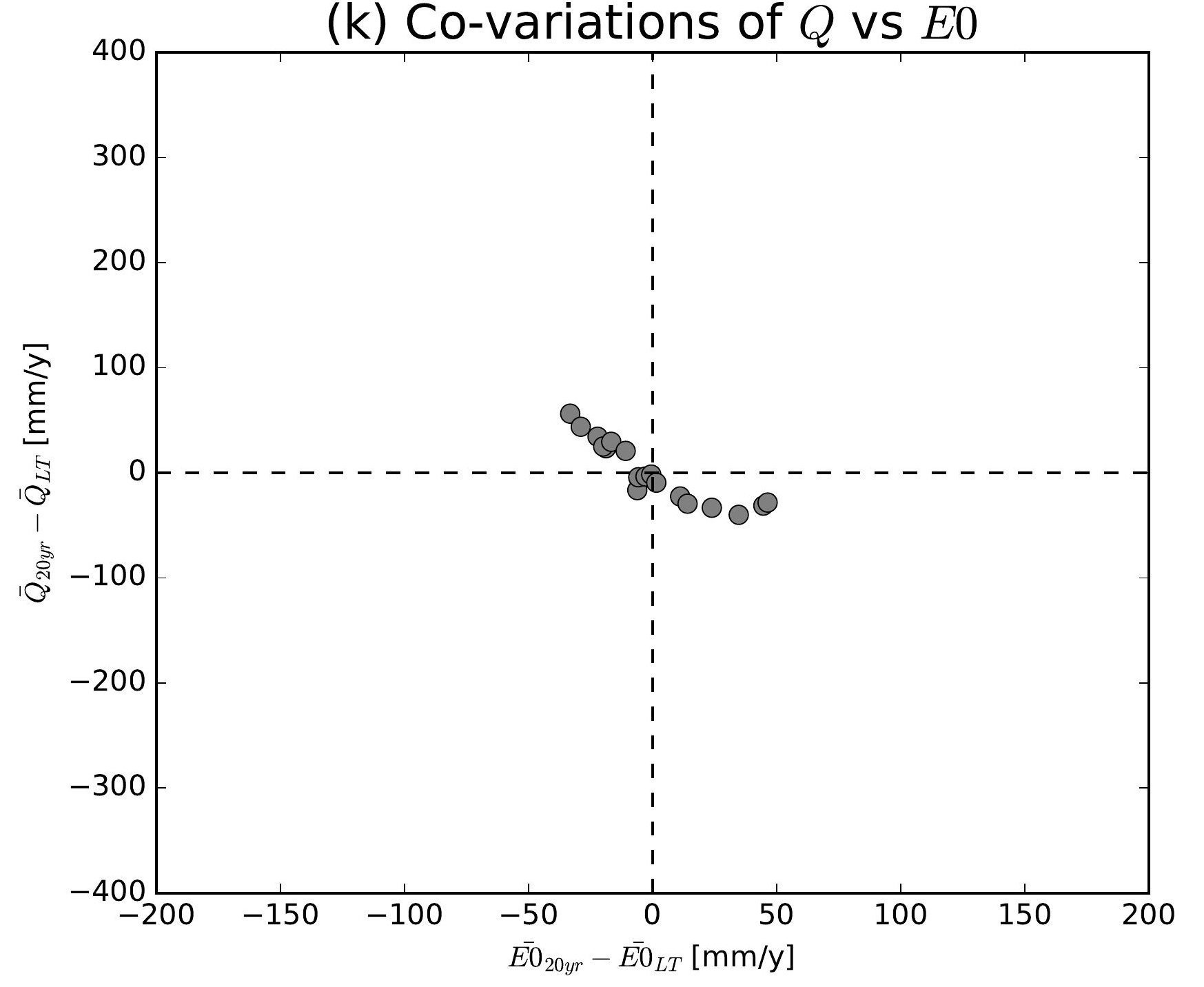

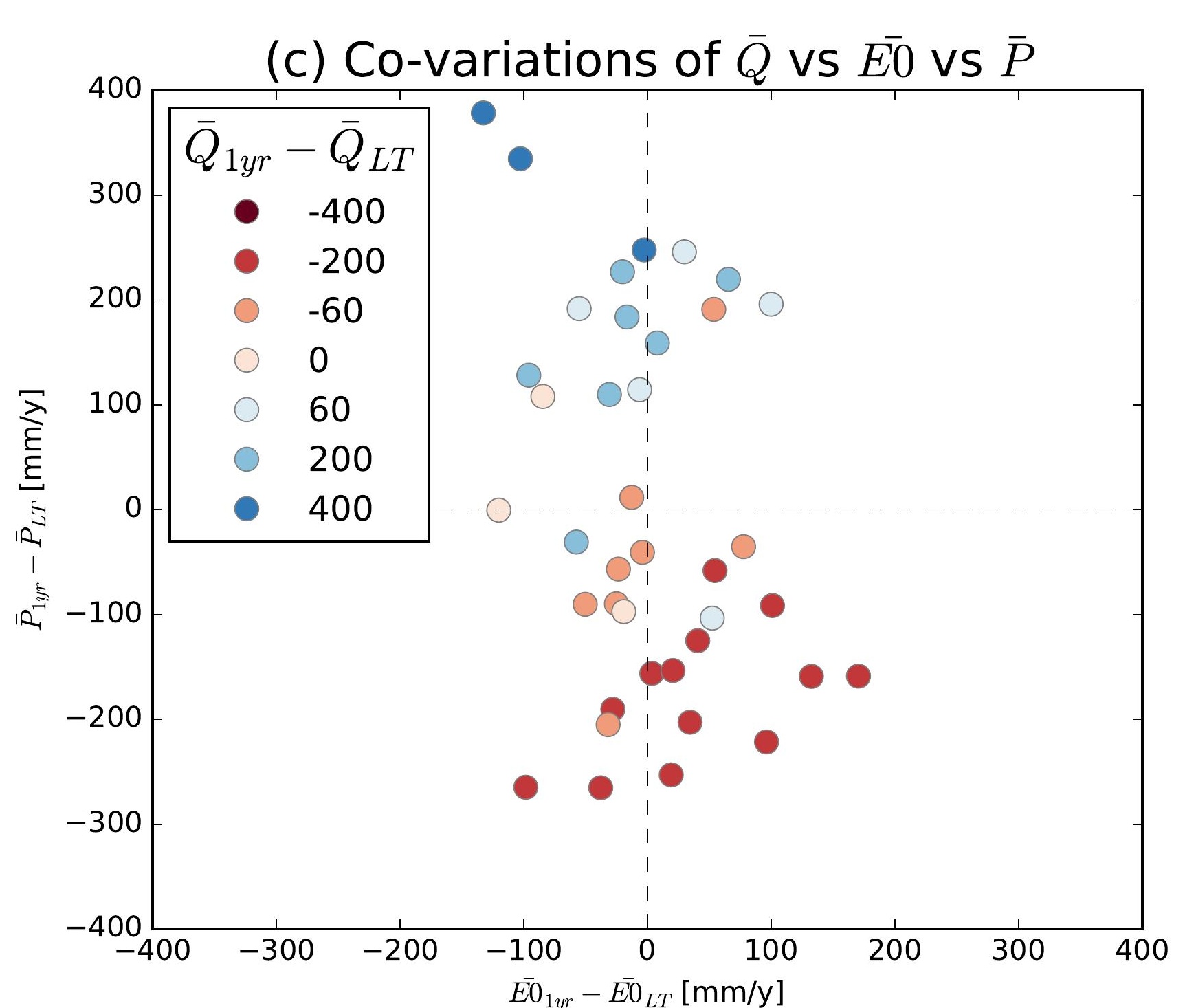
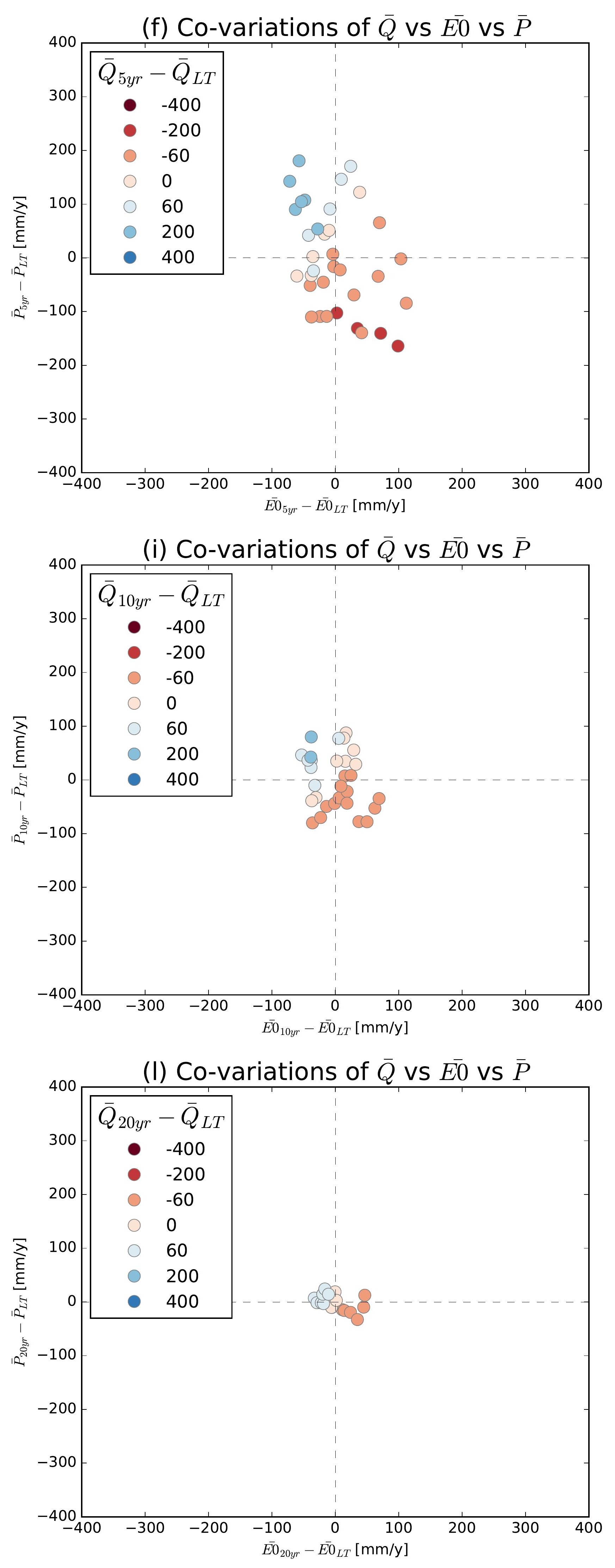

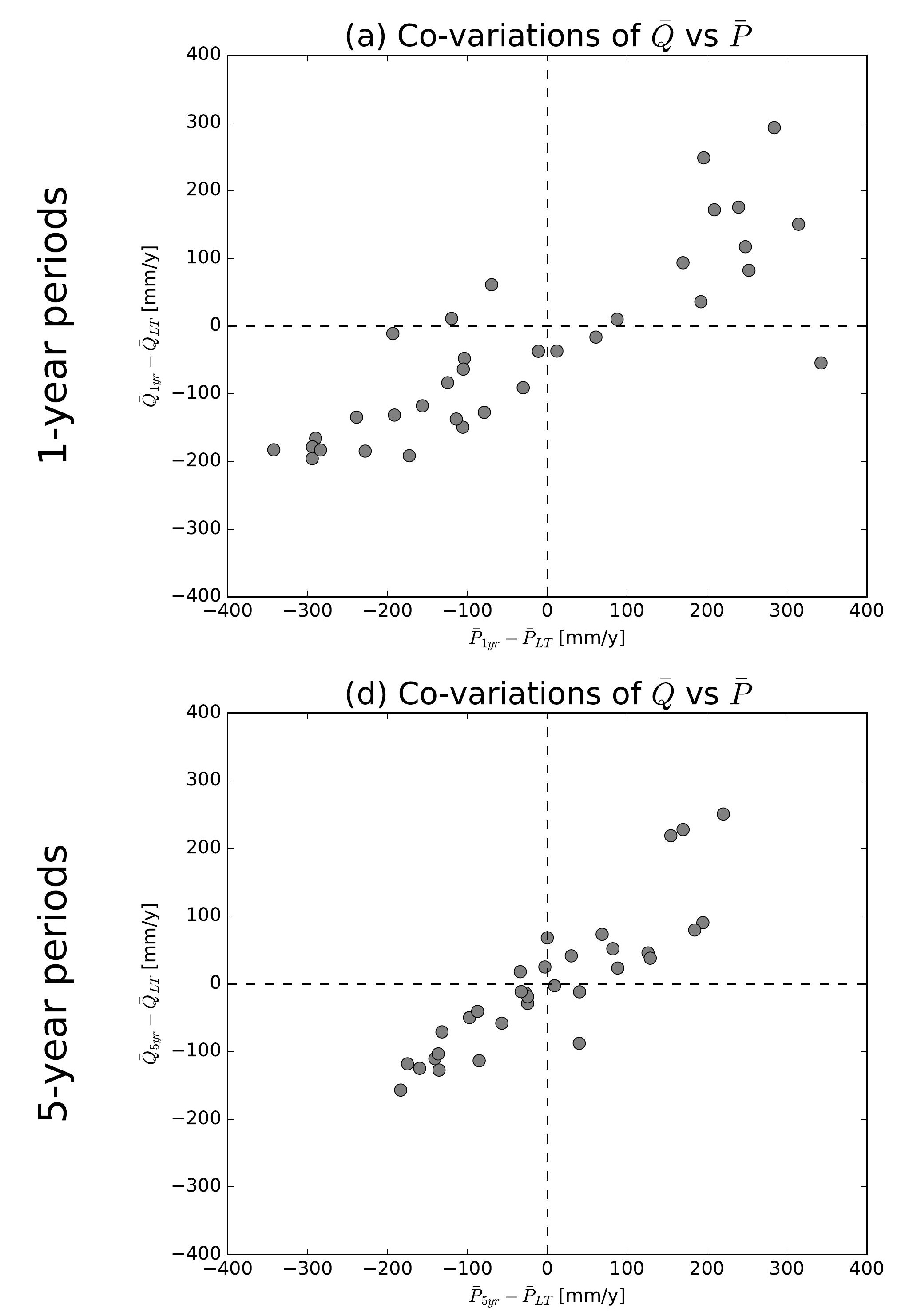

(g) Co-variations of $\bar{Q}$ vs $\bar{P}$

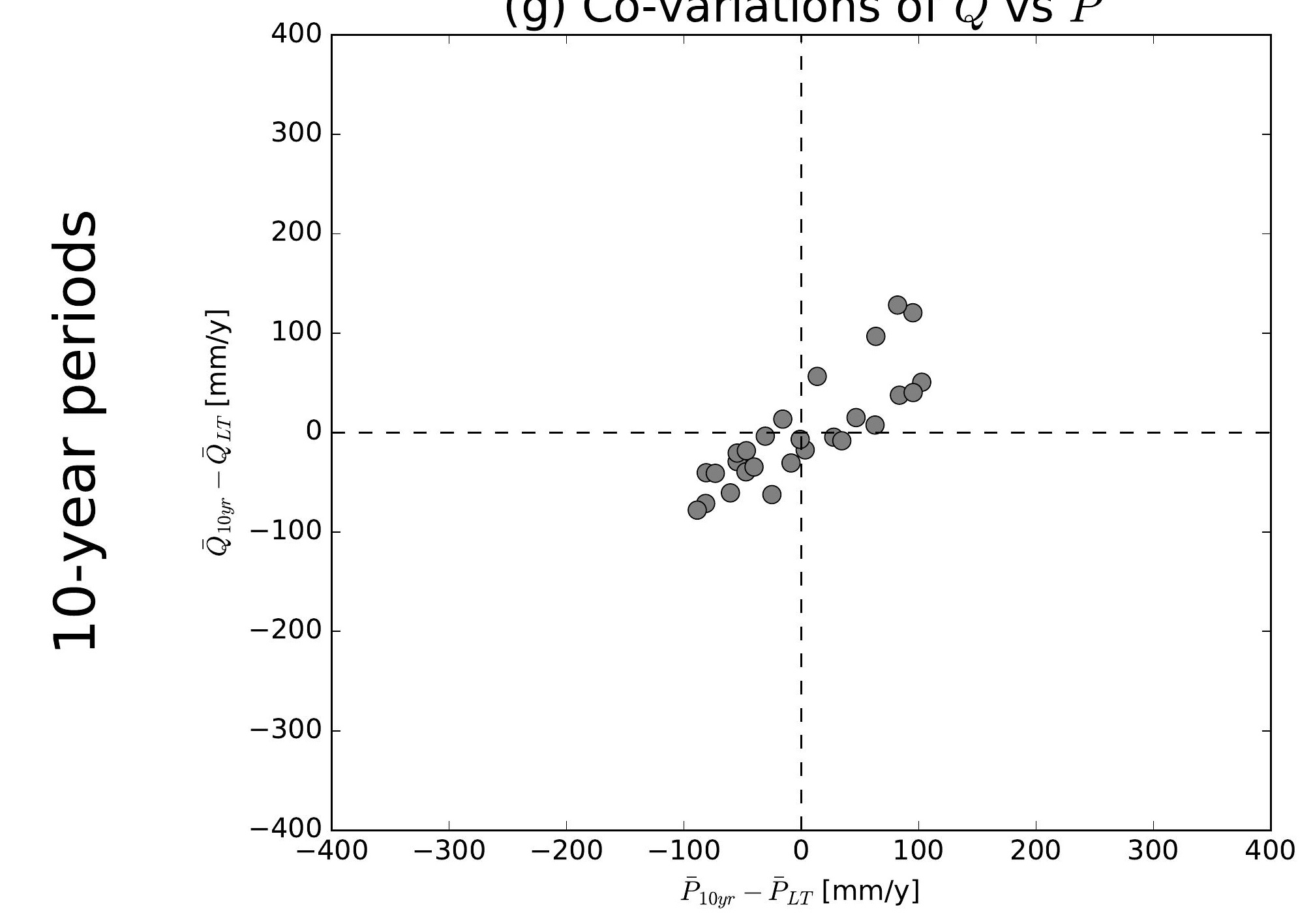

(j) Co-variations of $\bar{Q}$ vs $\bar{P}$

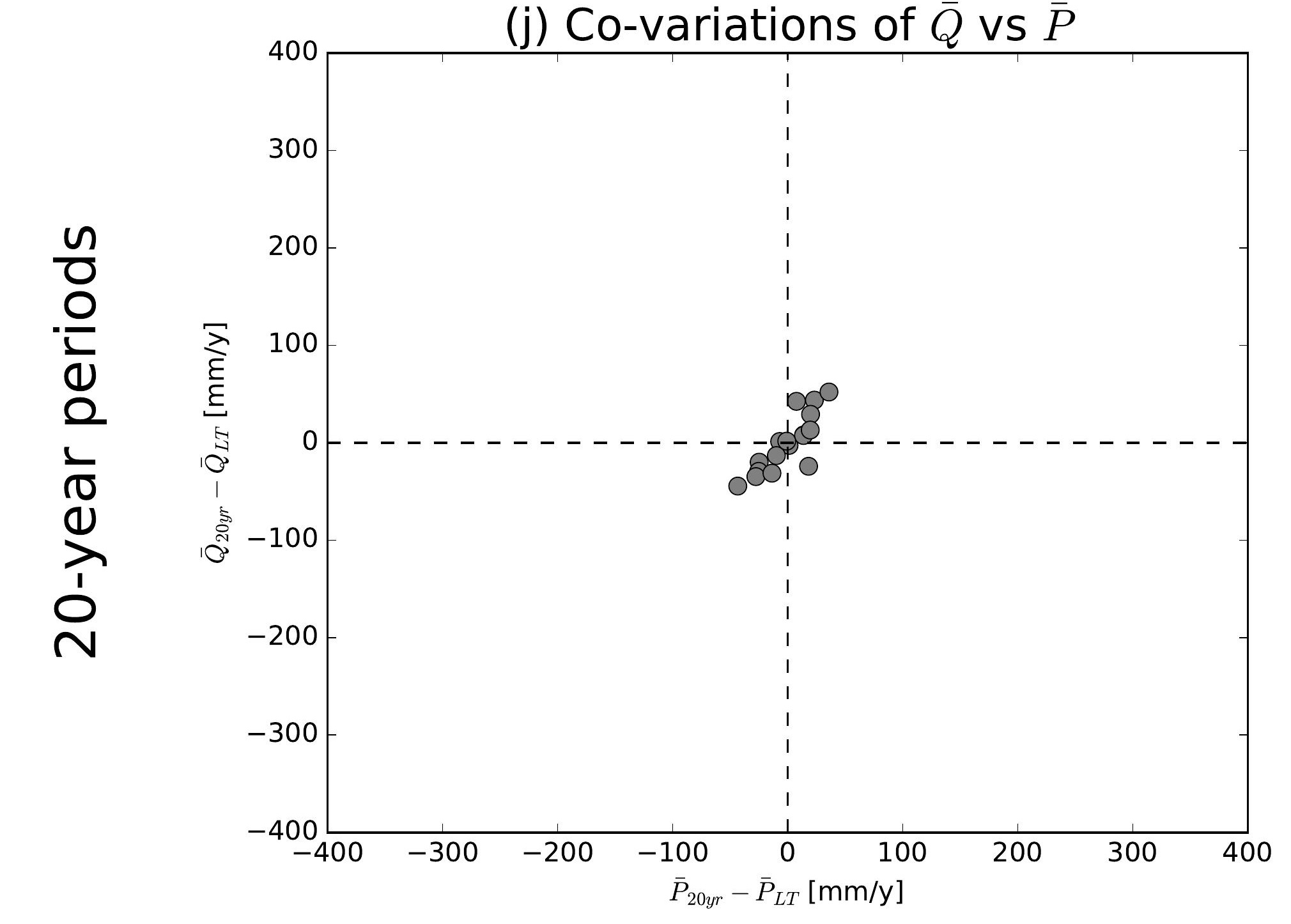

(b) Co-variations of $\bar{Q}$ vs $\overline{E 0}$

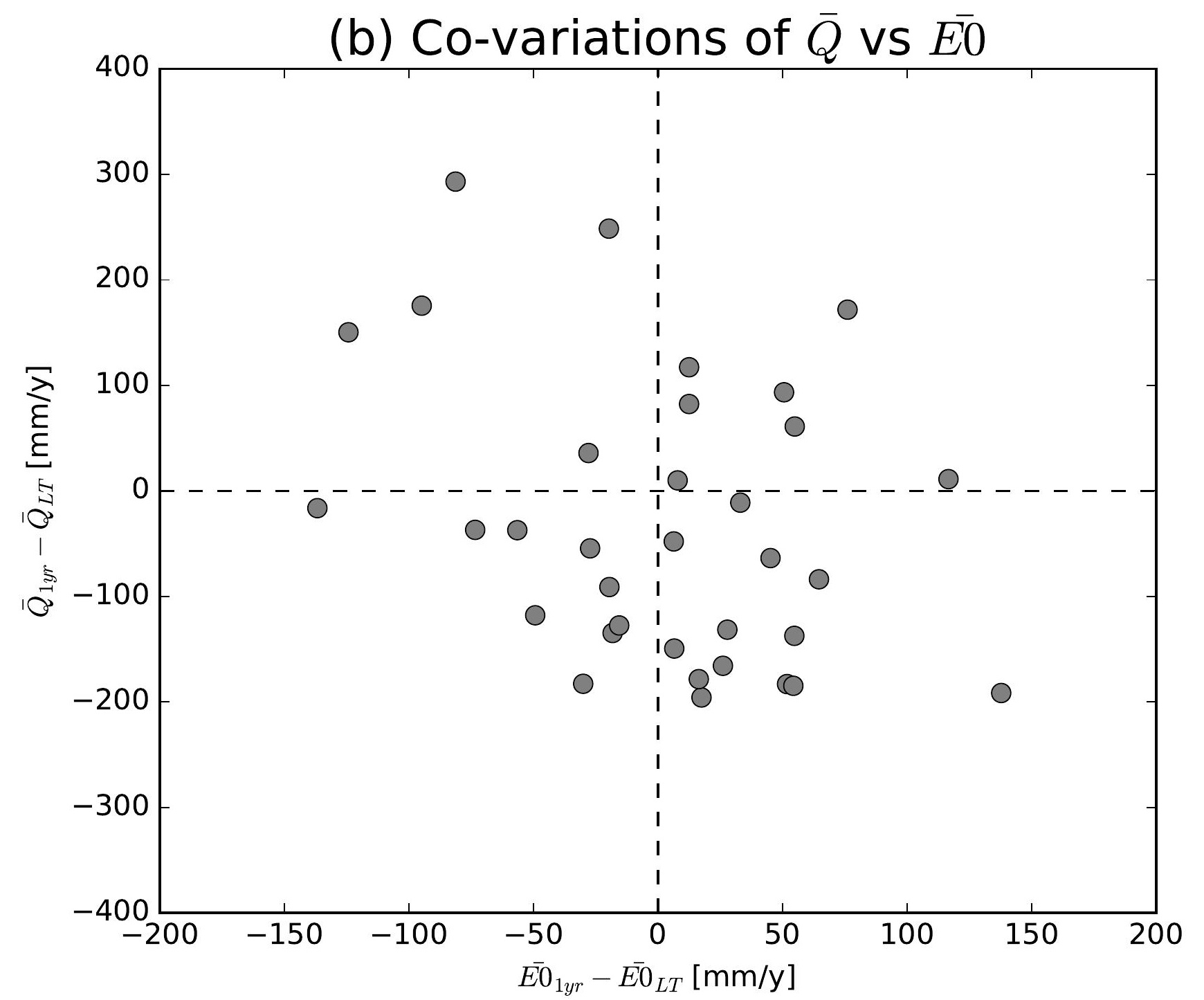

(e) Co-variations of $\bar{Q}$ vs $\overline{E 0}$

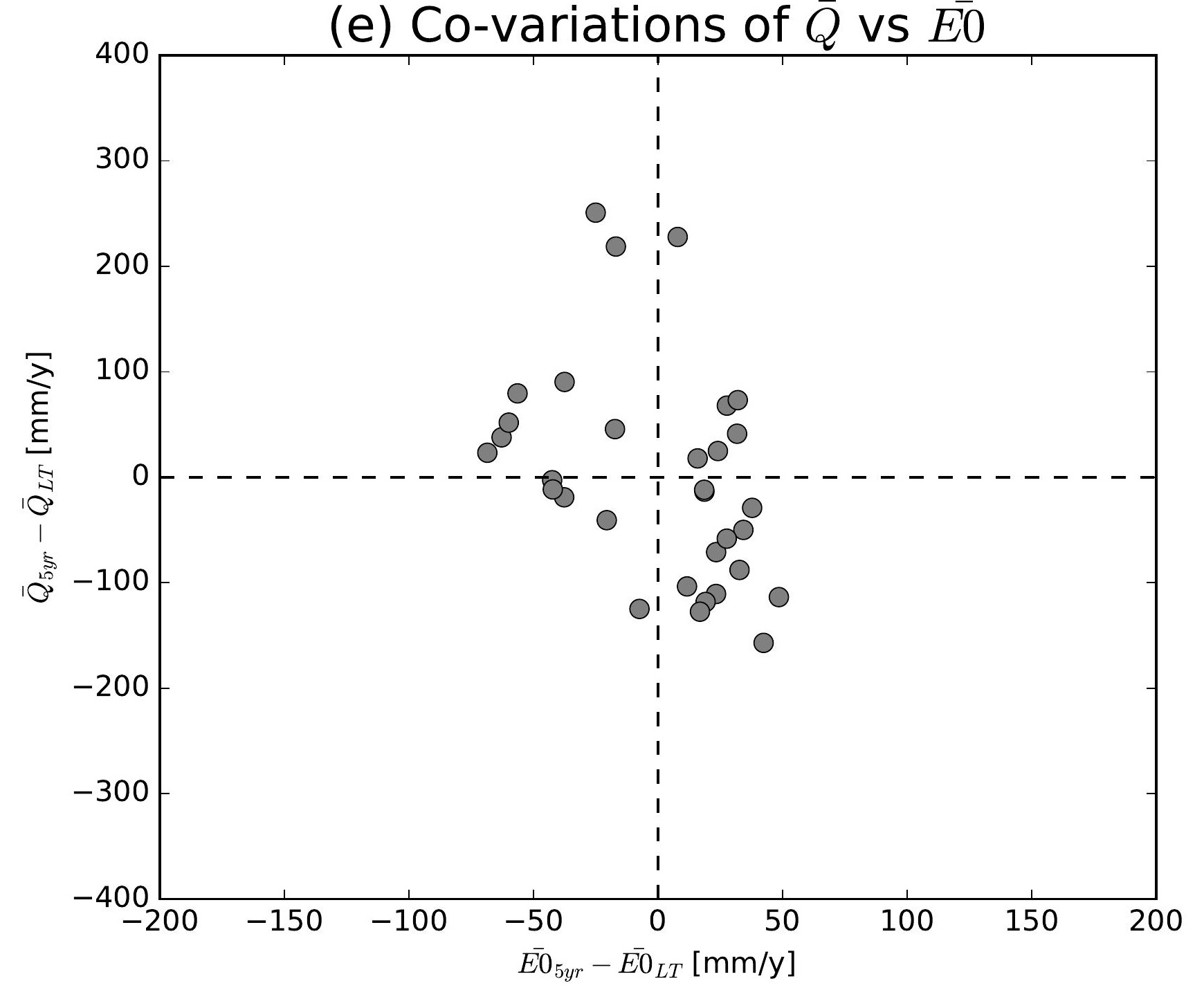

(h) Co-variations of $\bar{Q}$ vs $\overline{E 0}$

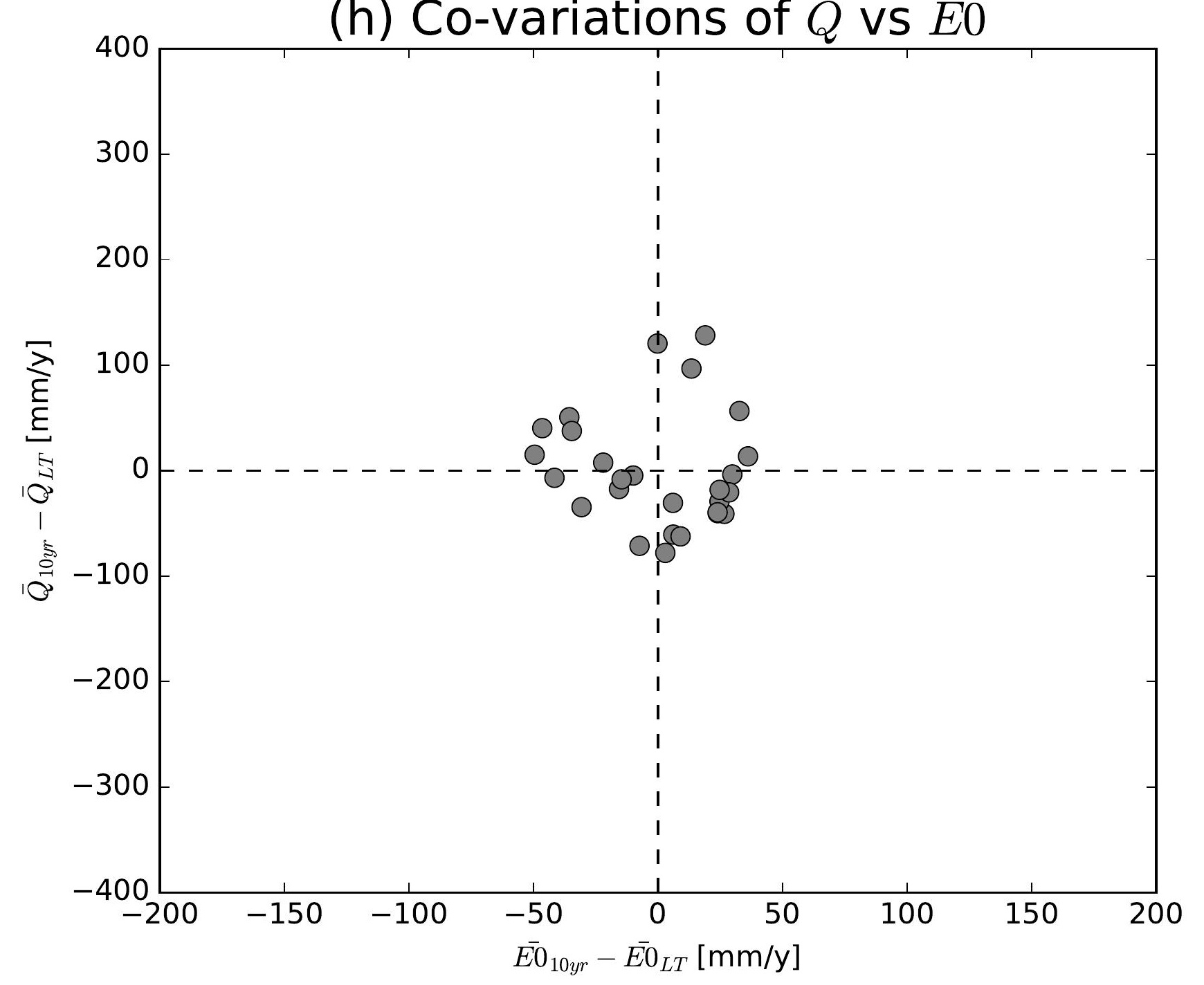

(k) Co-variations of $\bar{Q}$ vs $\overline{E 0}$

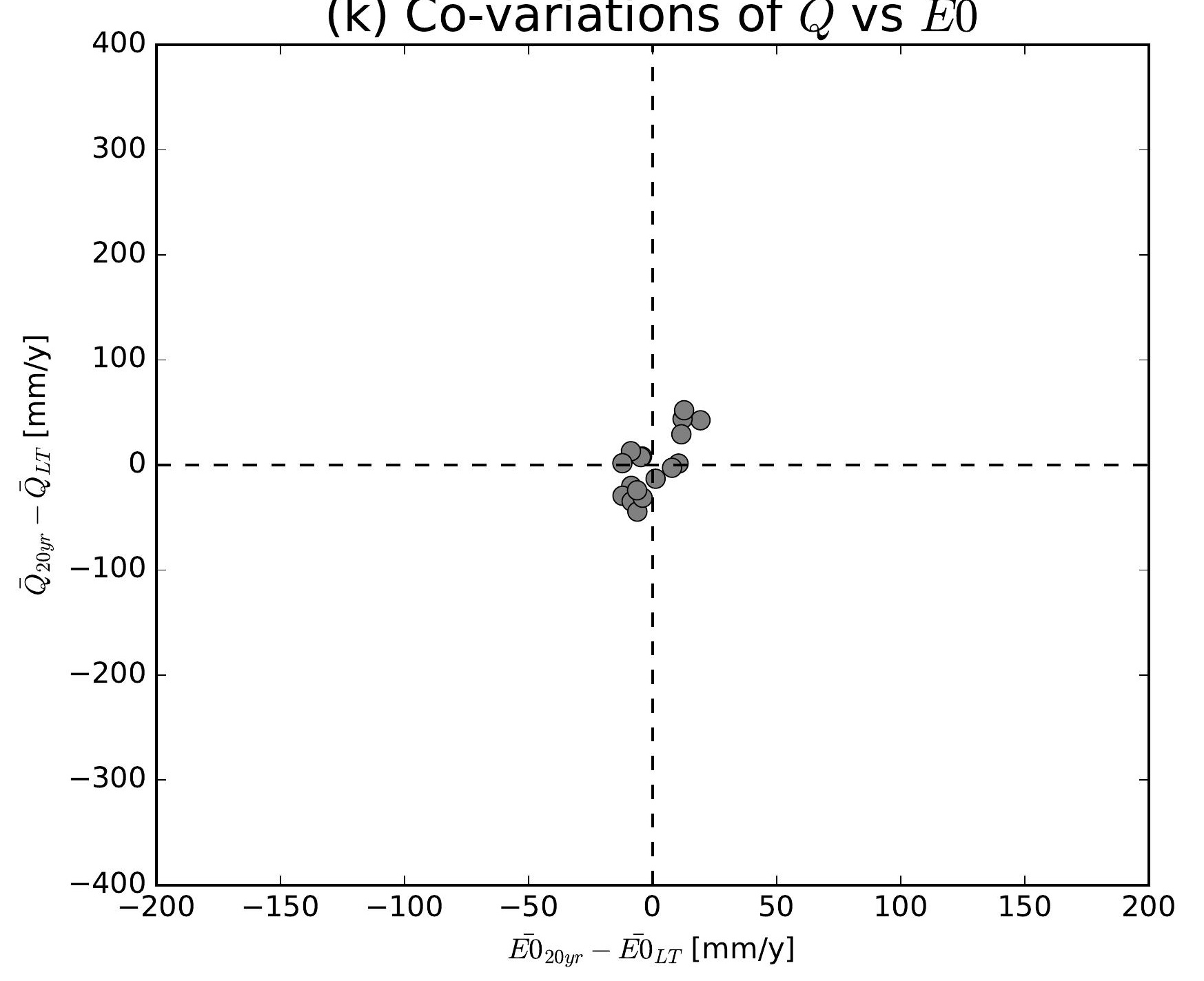

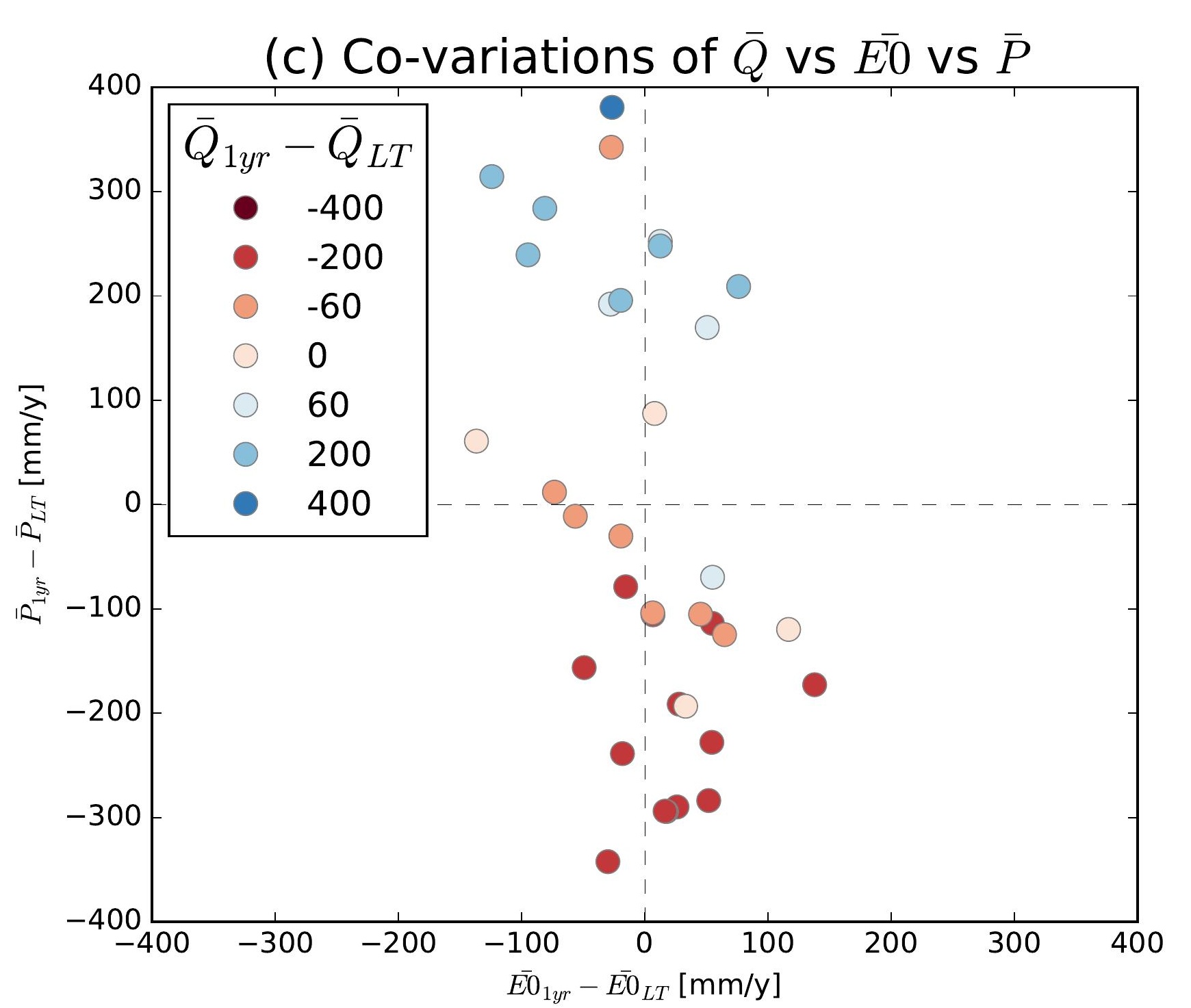
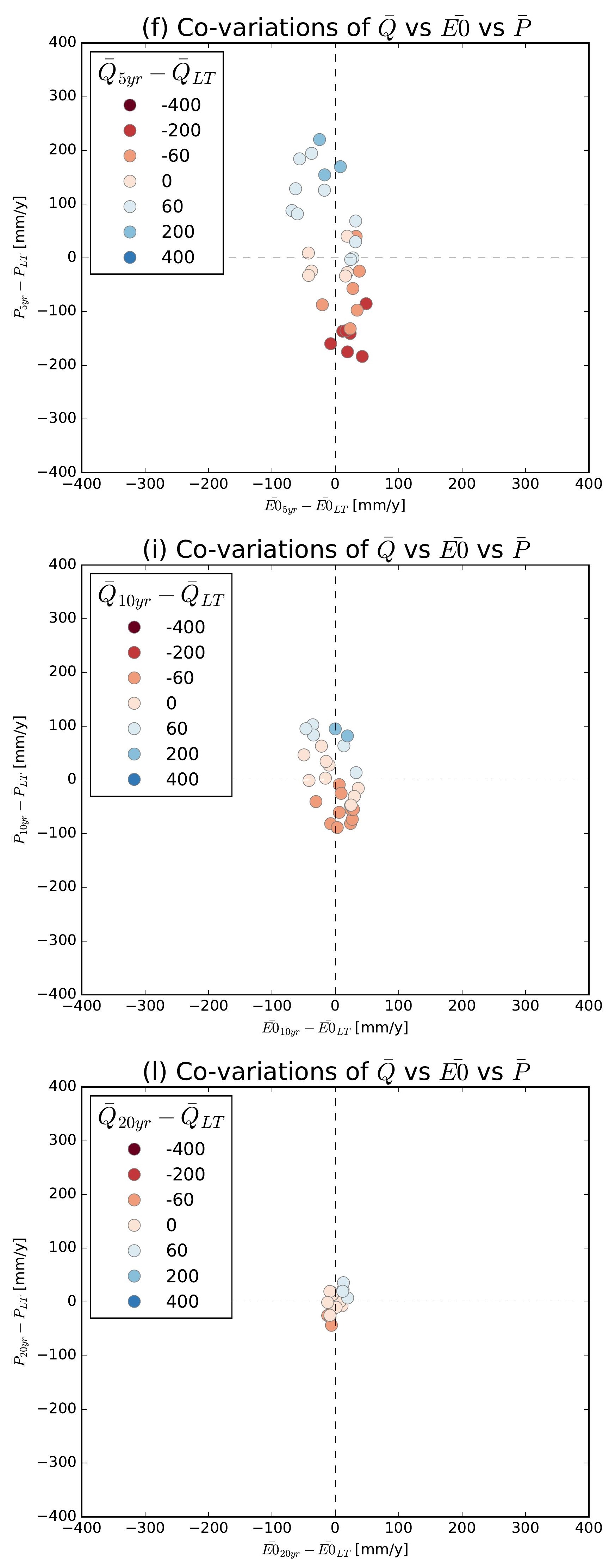

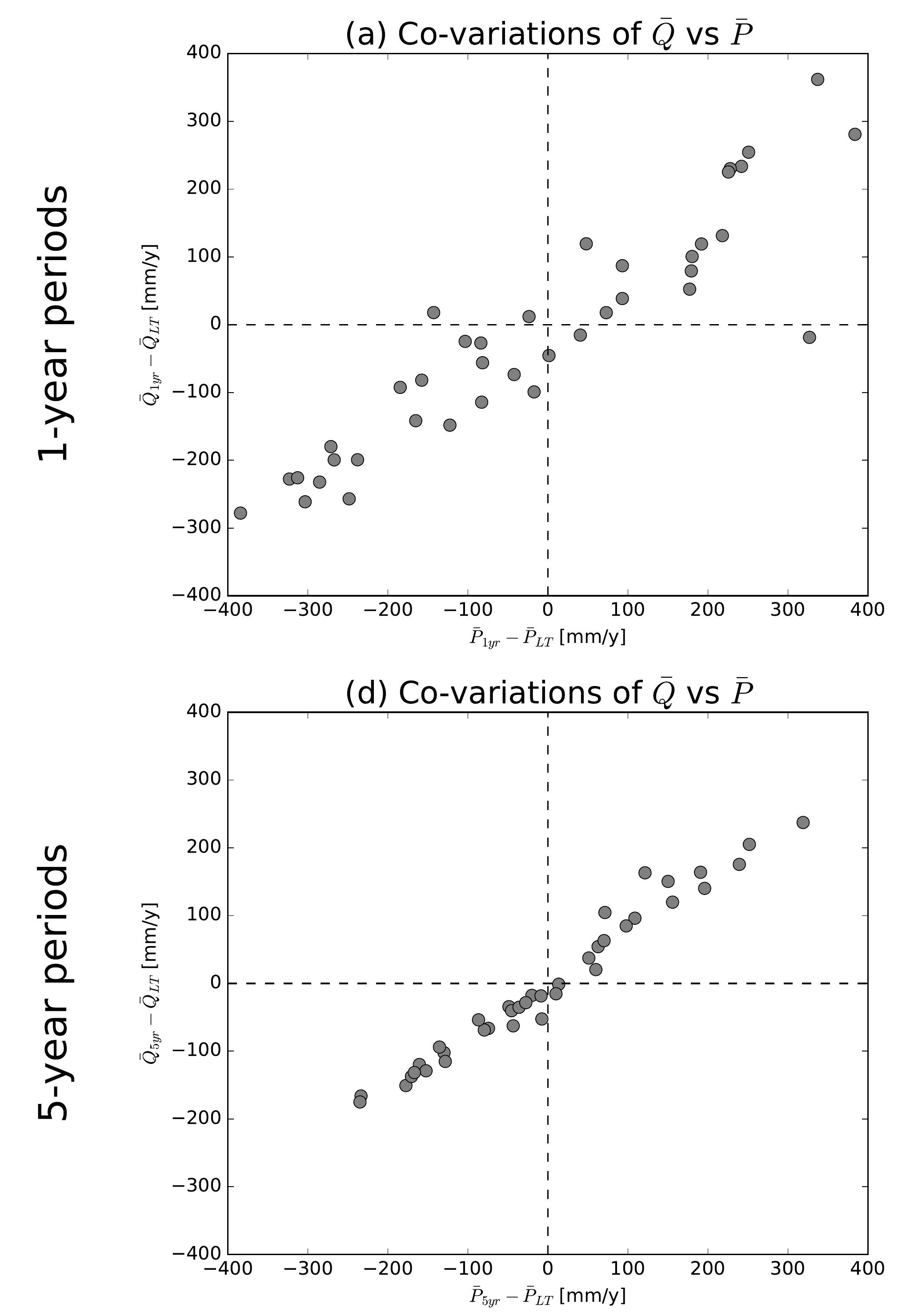

(g) Co-variations of $\bar{Q}$ vs $\bar{P}$
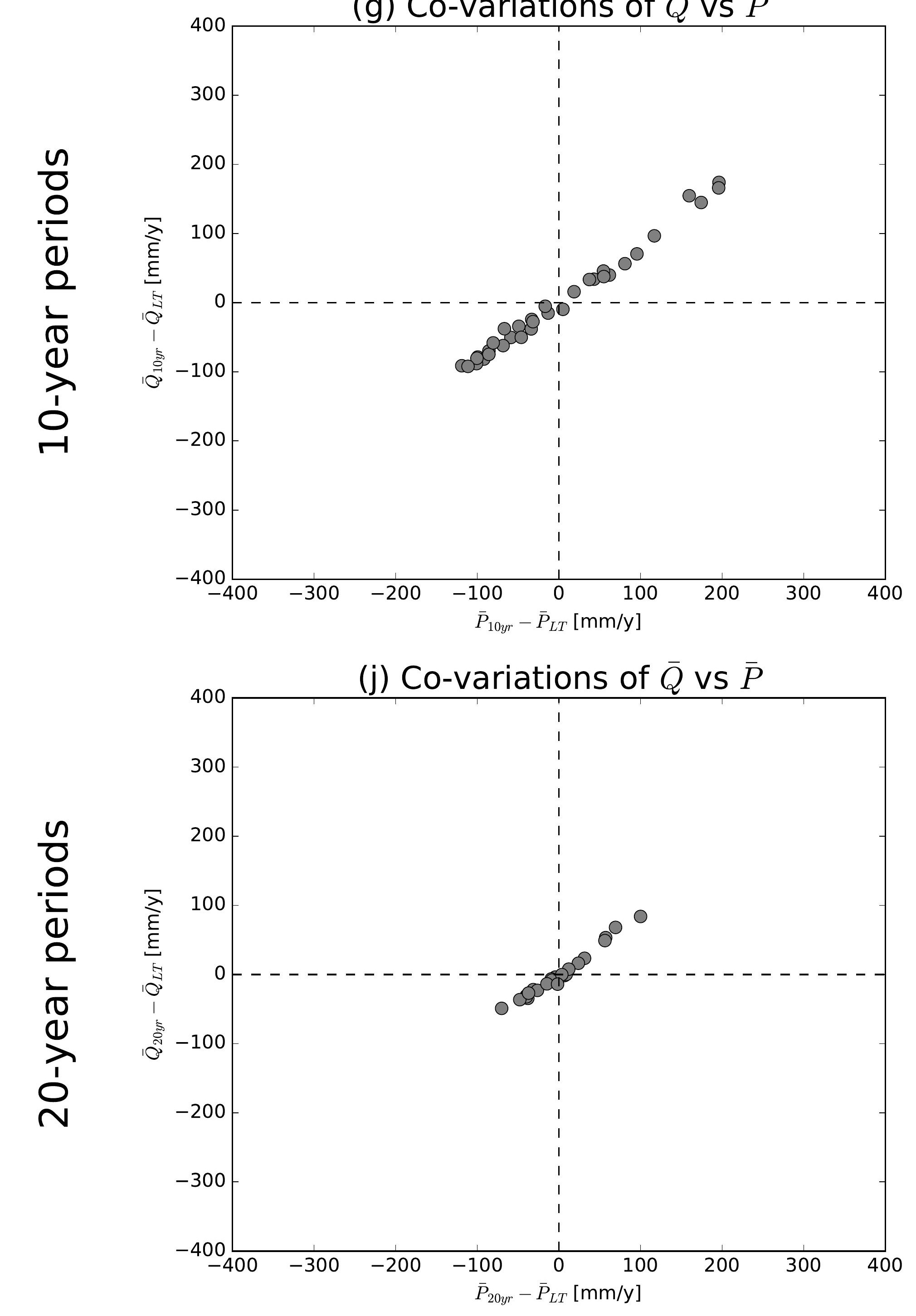

(b) Co-variations of $\bar{Q}$ vs $\overline{E 0}$

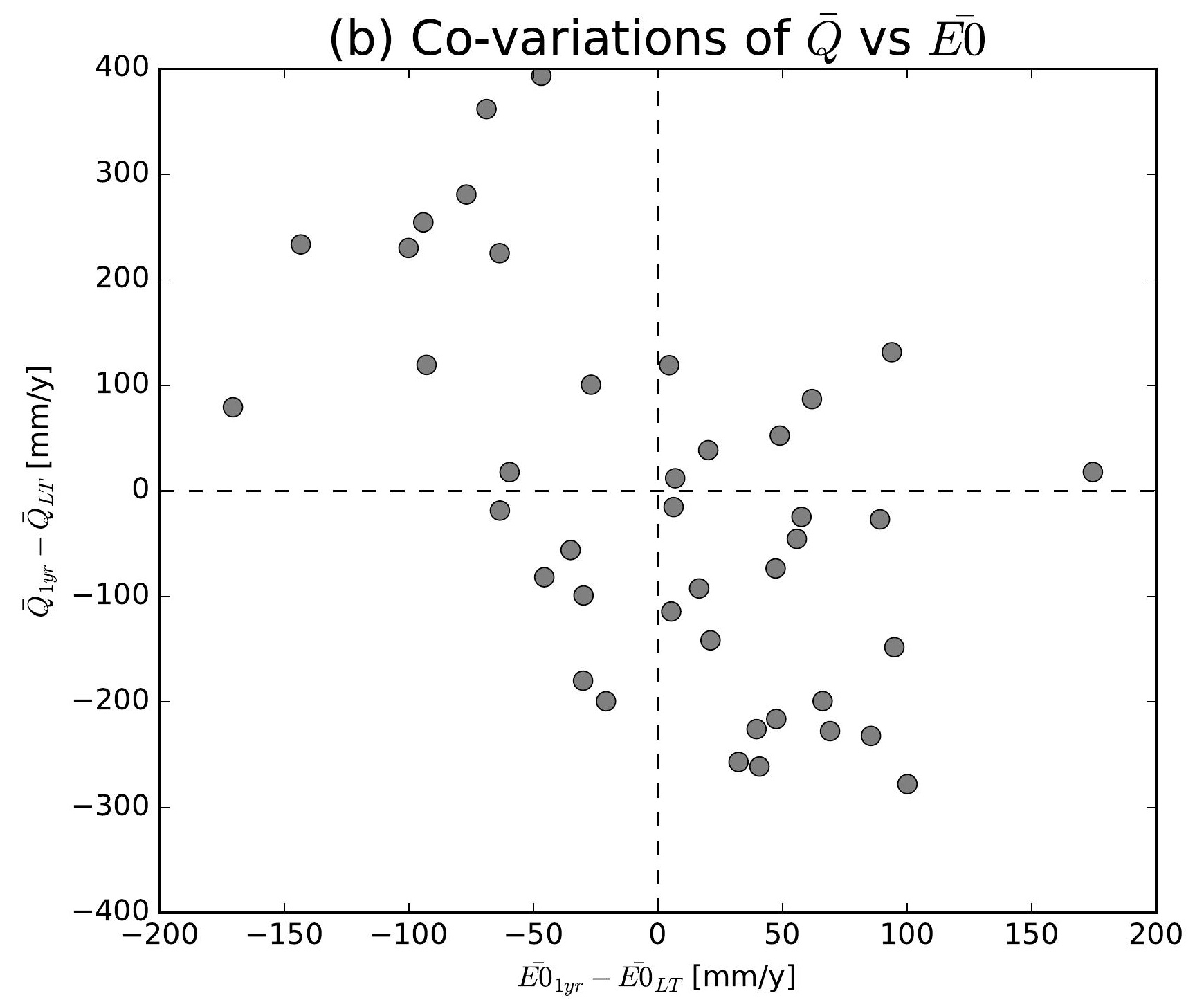

(e) Co-variations of $\bar{Q}$ vs $\overline{E 0}$

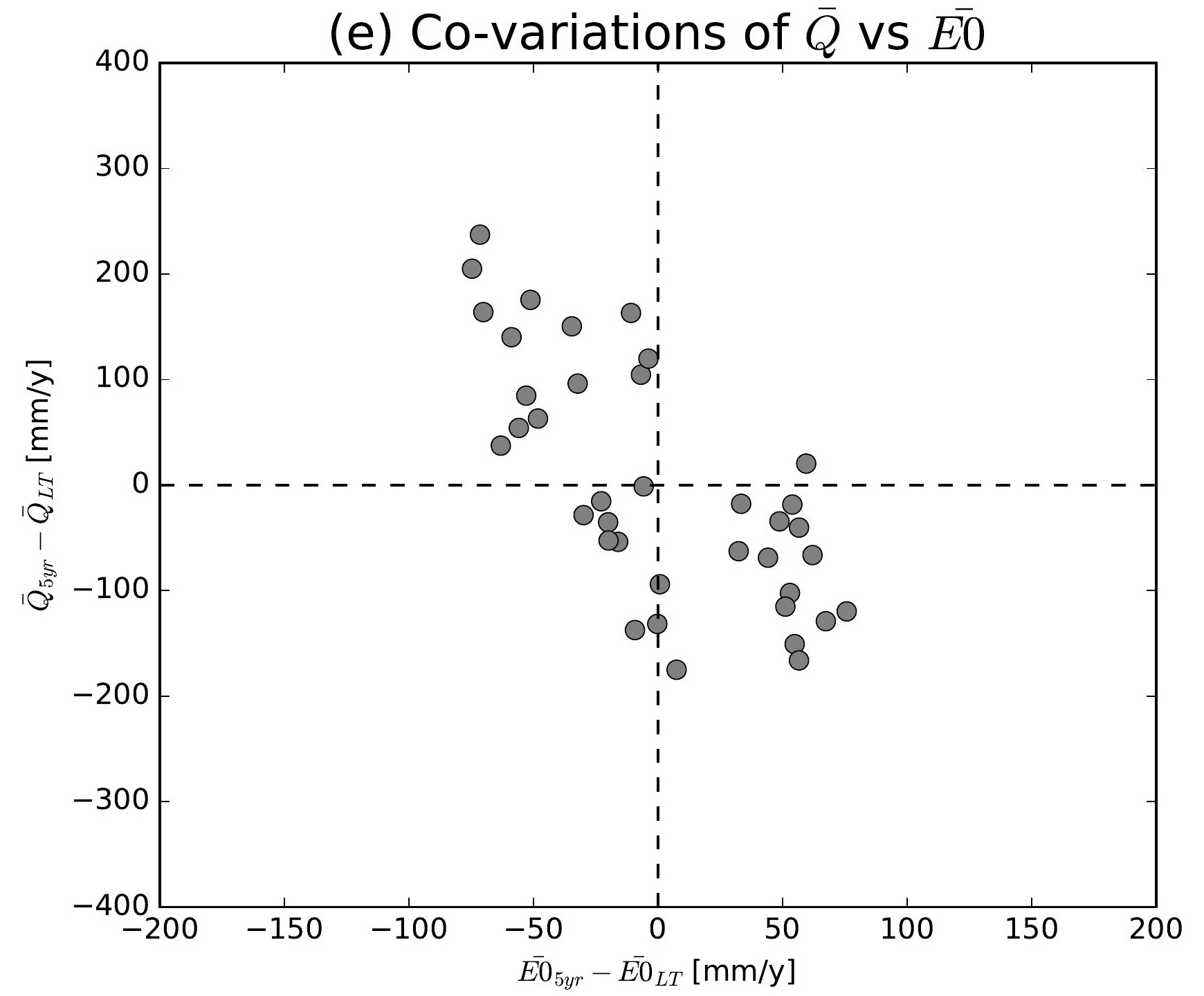

(h) Co-variations of $\bar{Q}$ vs $\overline{E 0}$

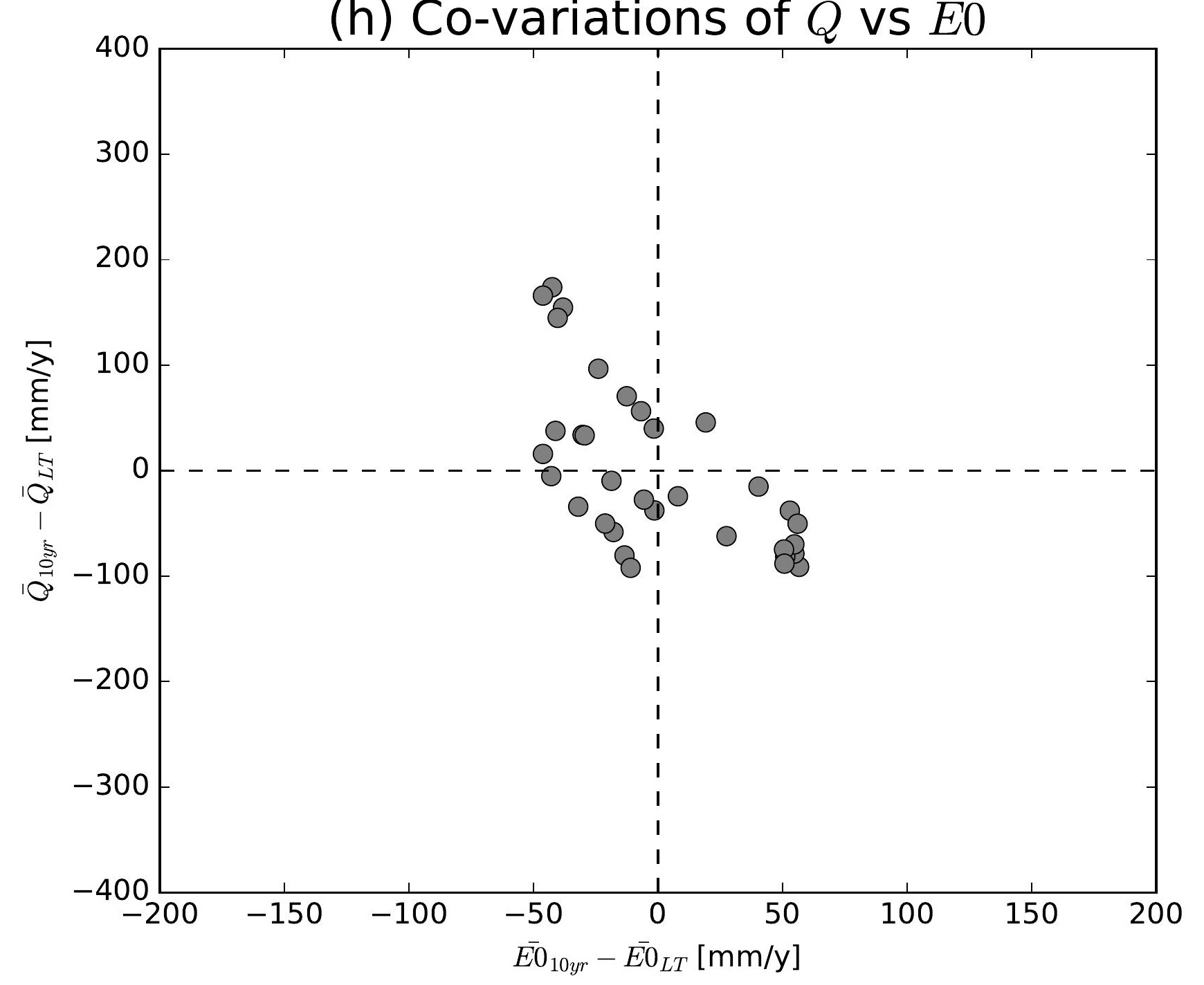

(k) Co-variations of $\bar{Q}$ vs $\overline{E 0}$

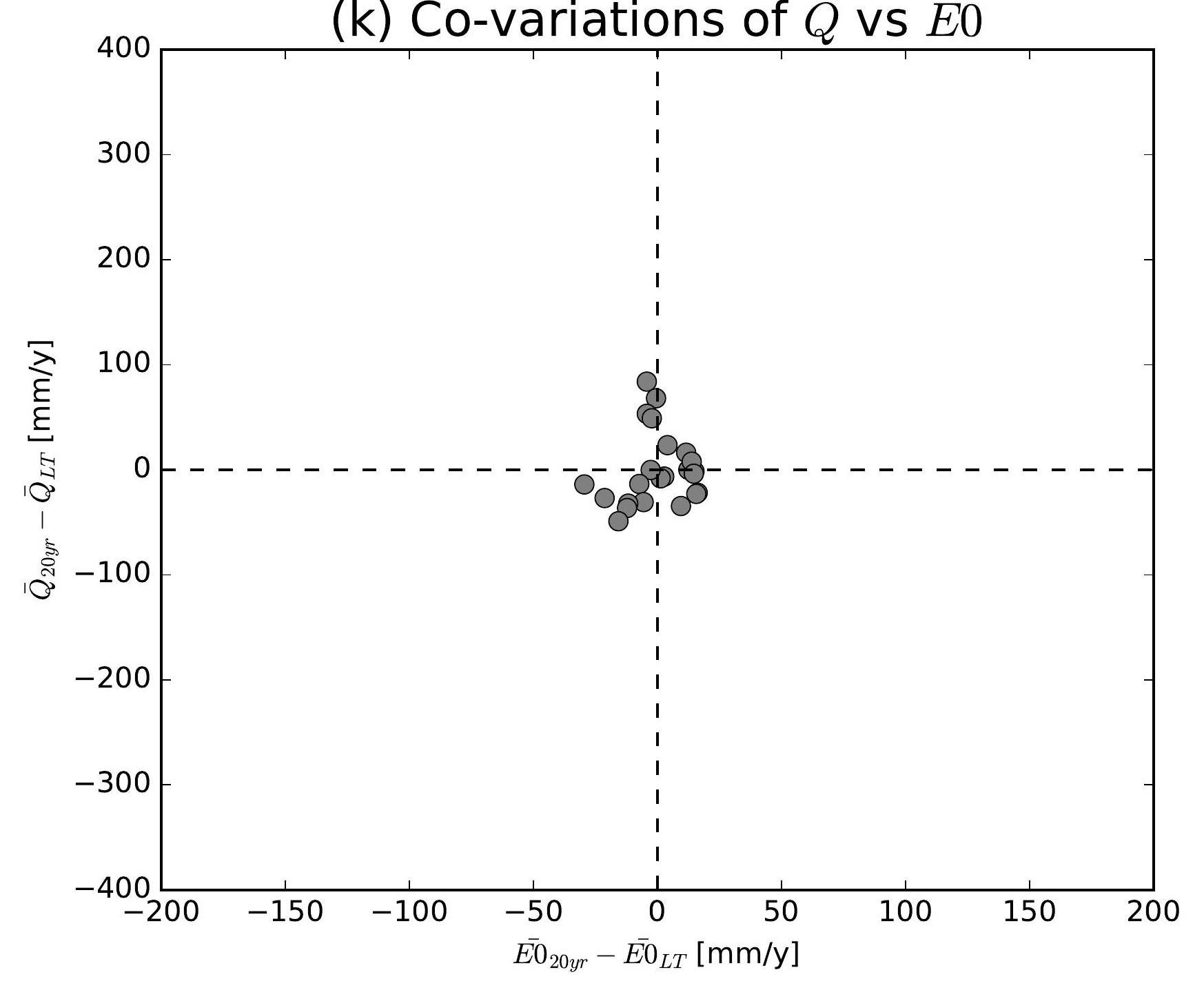

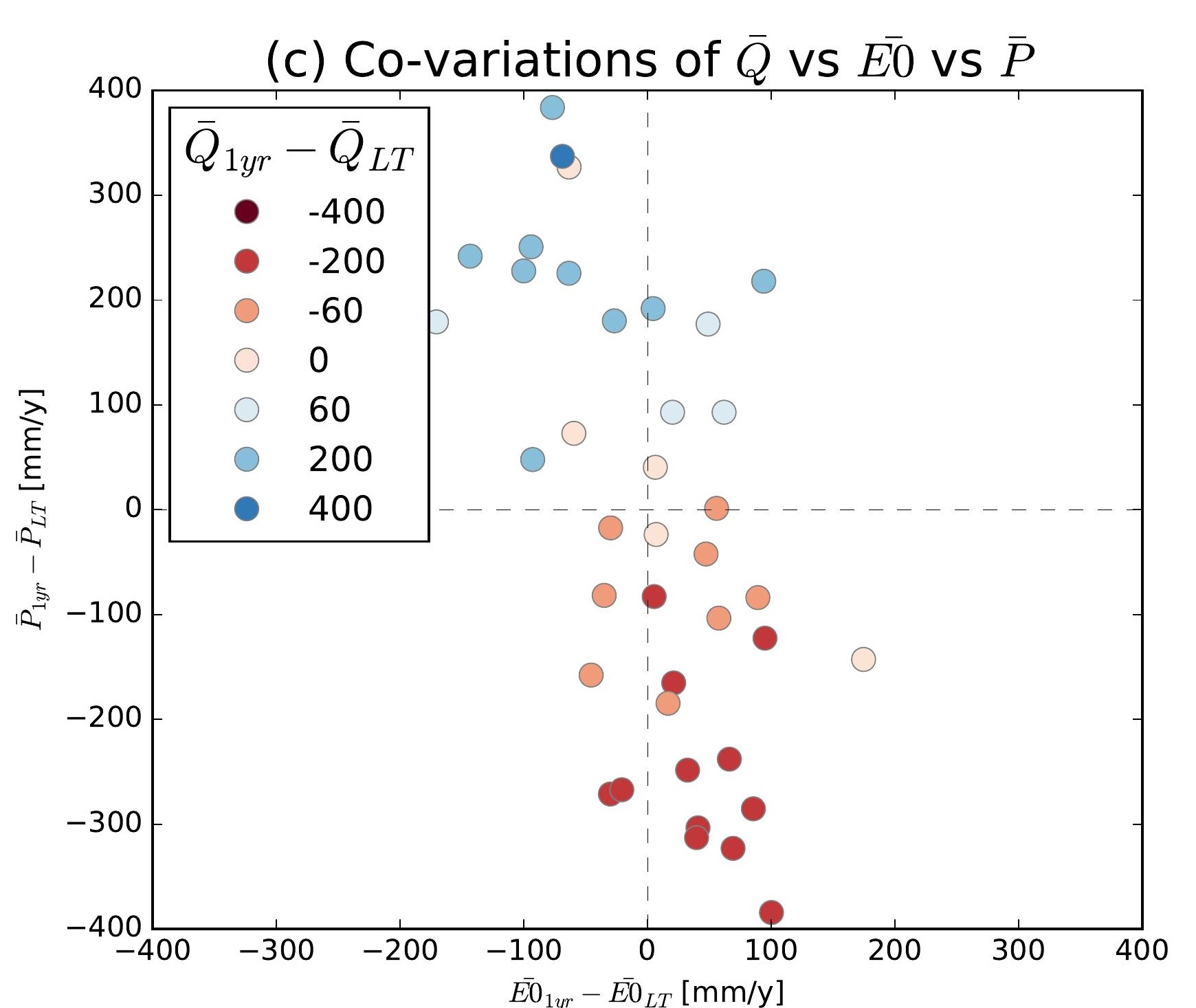
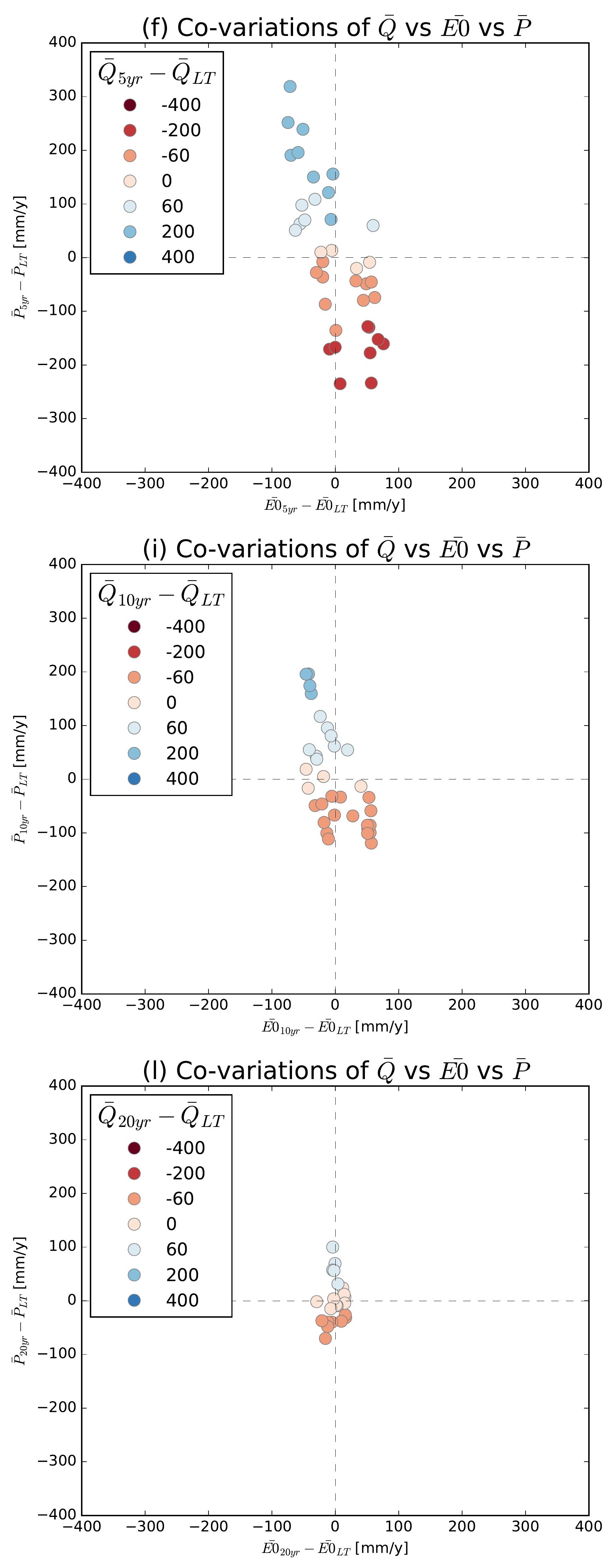

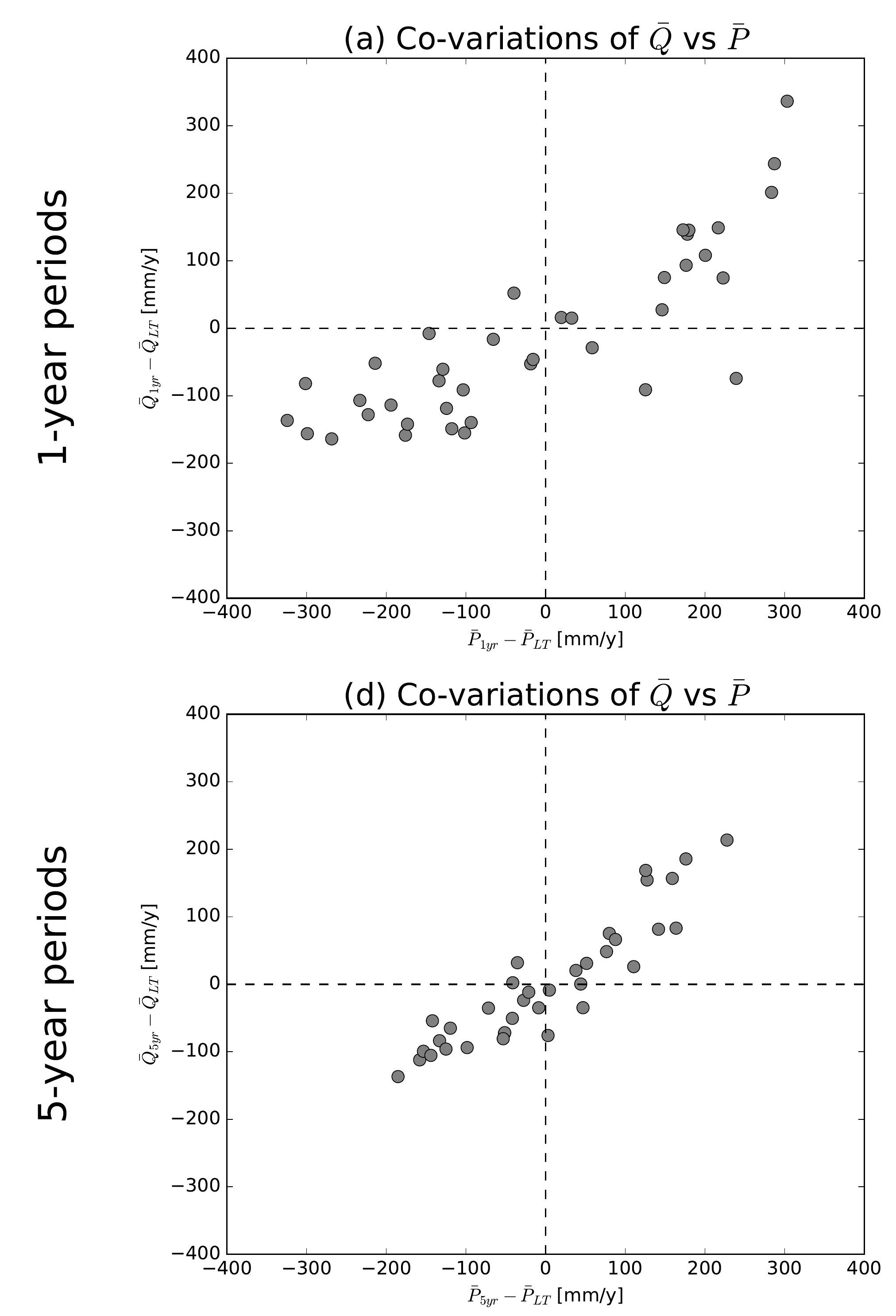

(g) Co-variations of $\bar{Q}$ vs $\bar{P}$

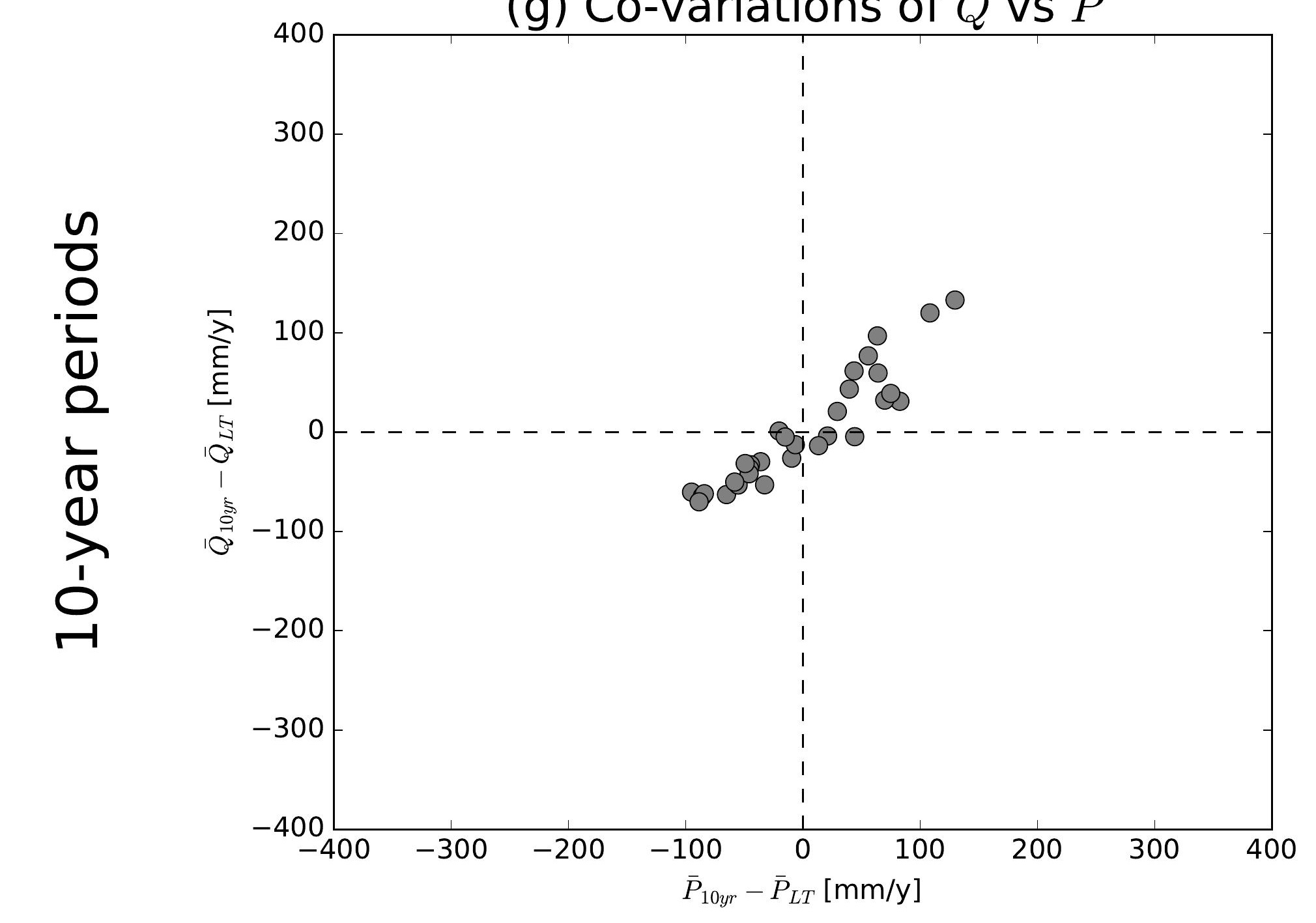

(j) Co-variations of $\bar{Q}$ vs $\bar{P}$

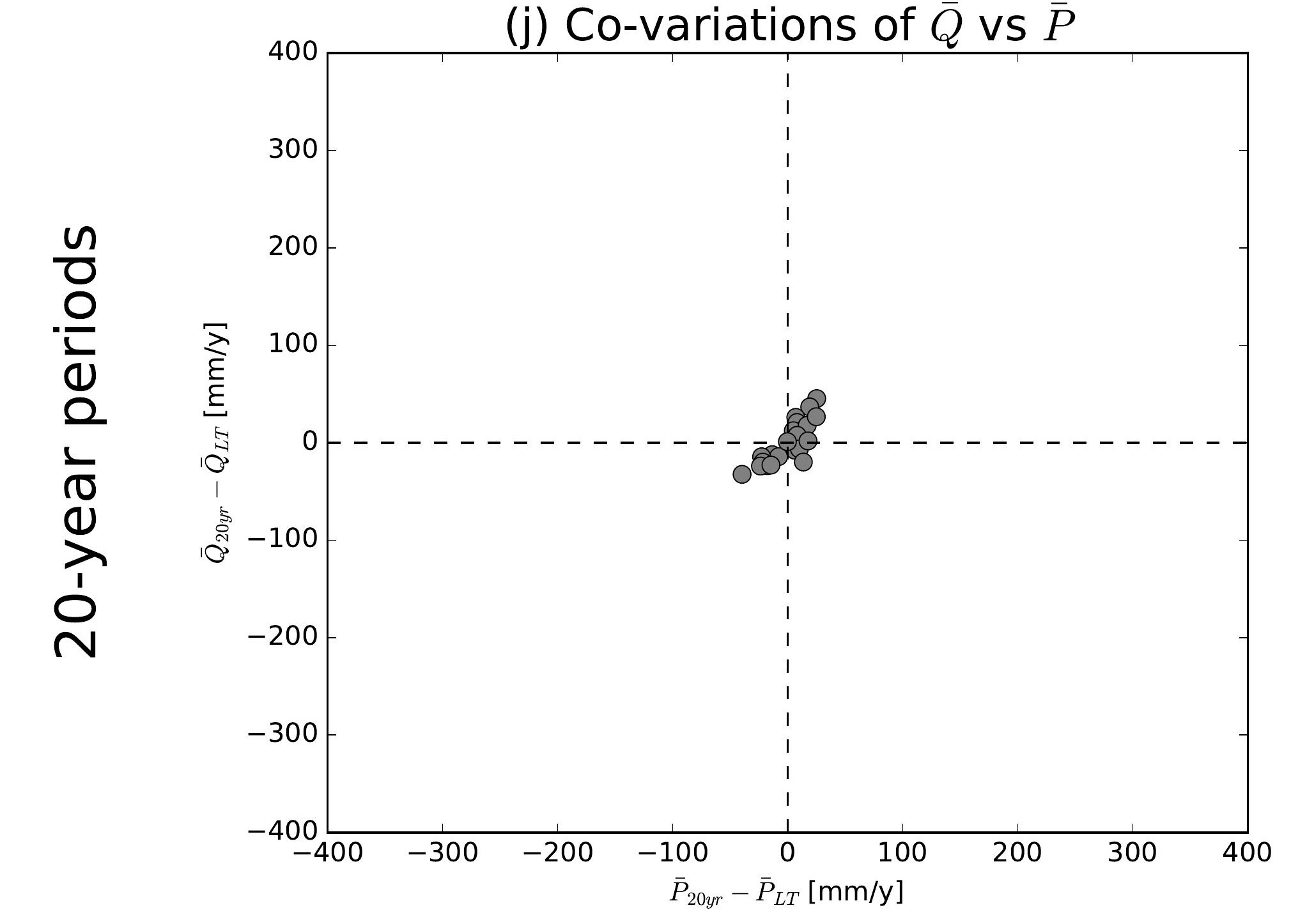

(b) Co-variations of $\bar{Q}$ vs $\overline{E 0}$

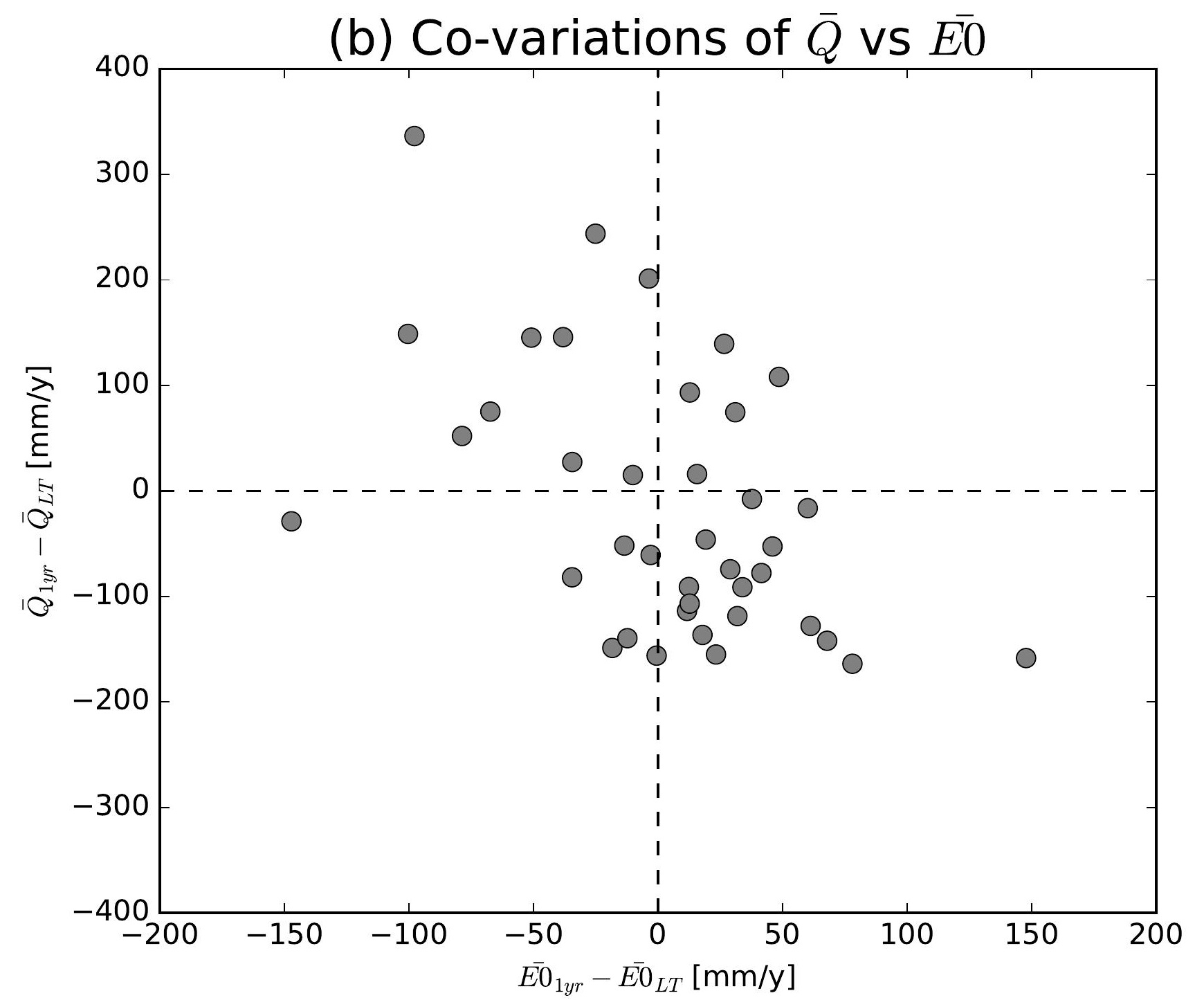

(e) Co-variations of $\bar{Q}$ vs $\overline{E 0}$

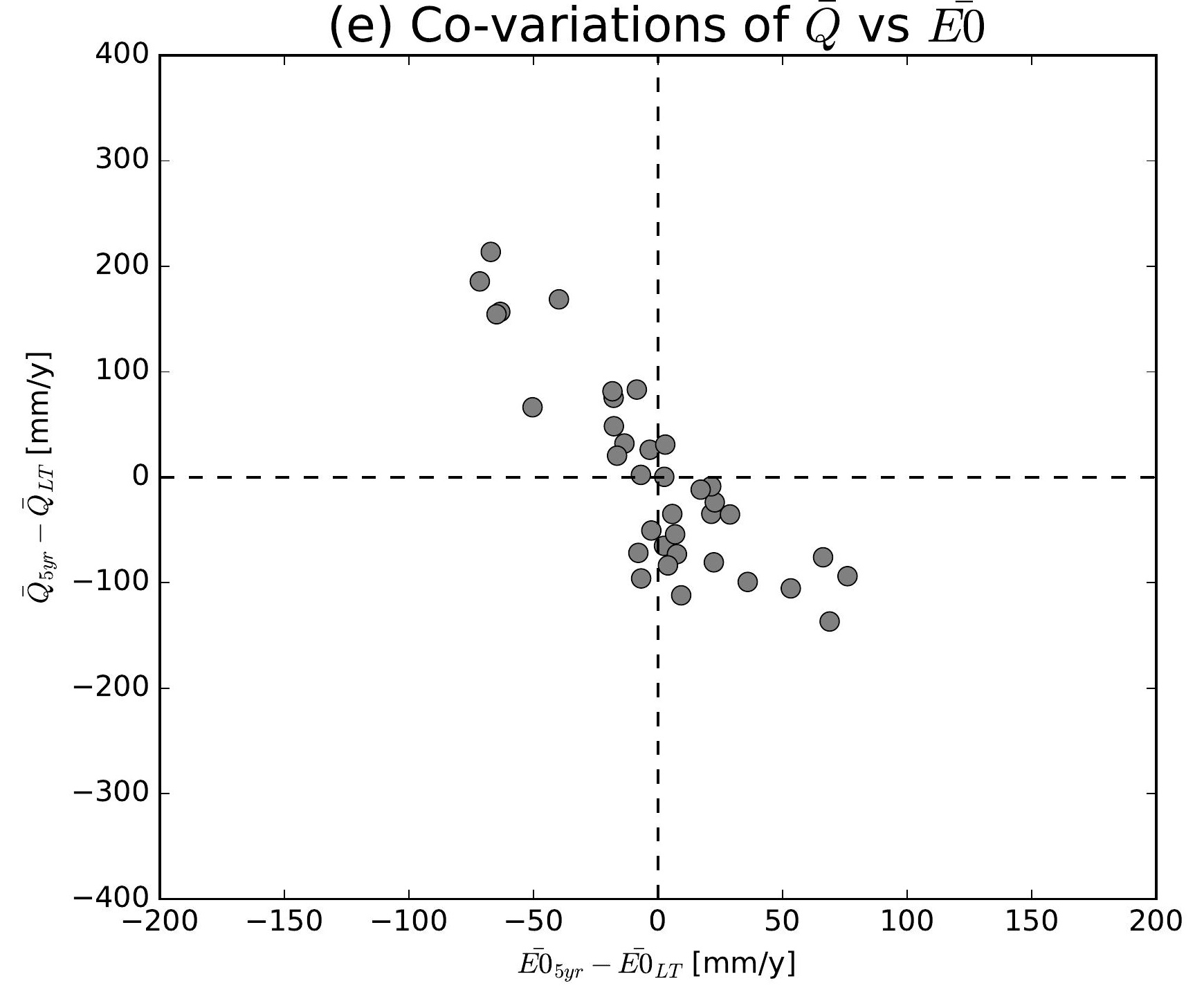

(h) Co-variations of $\bar{Q}$ vs $\overline{E 0}$

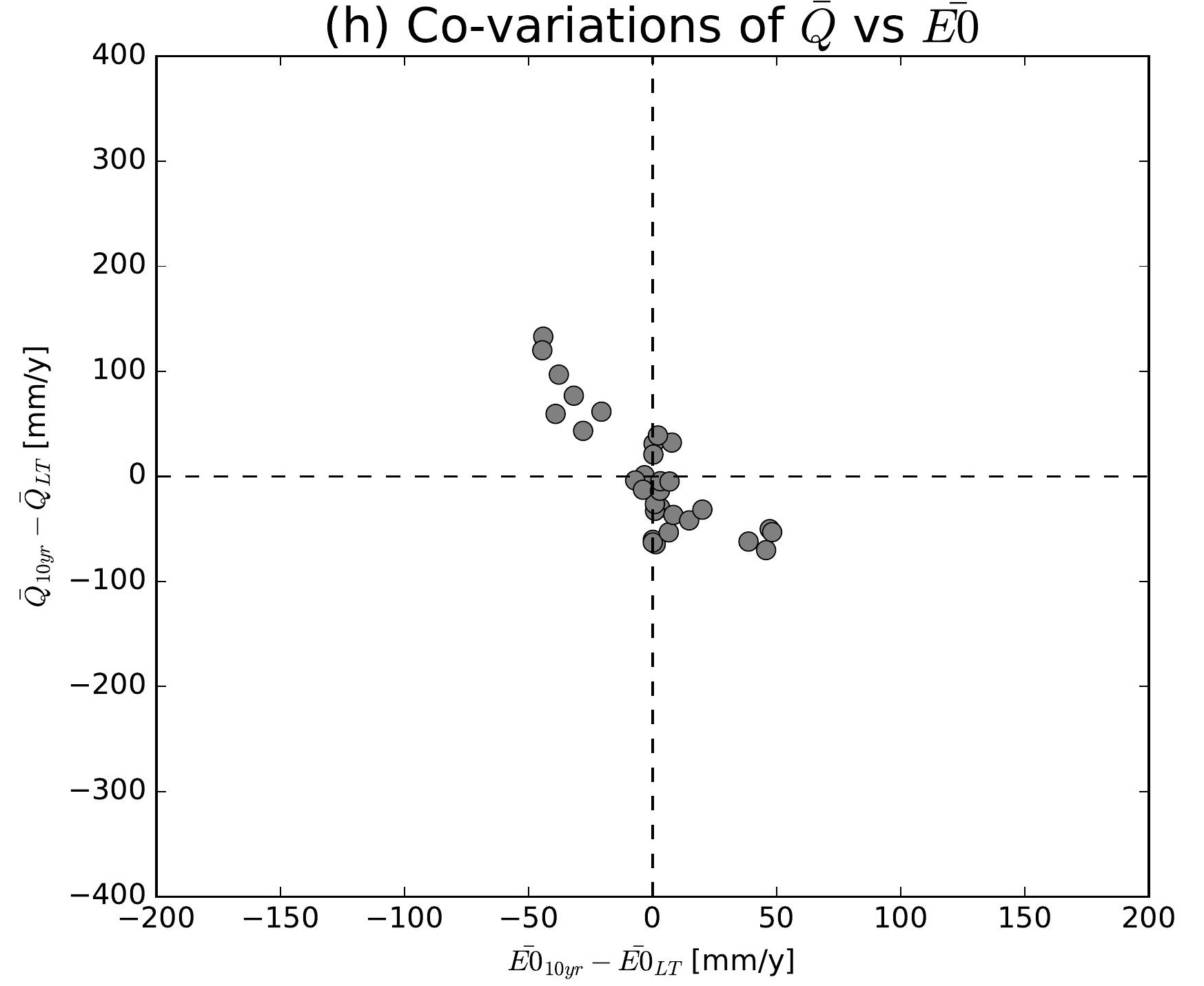

(k) Co-variations of $\bar{Q}$ vs $\overline{E 0}$

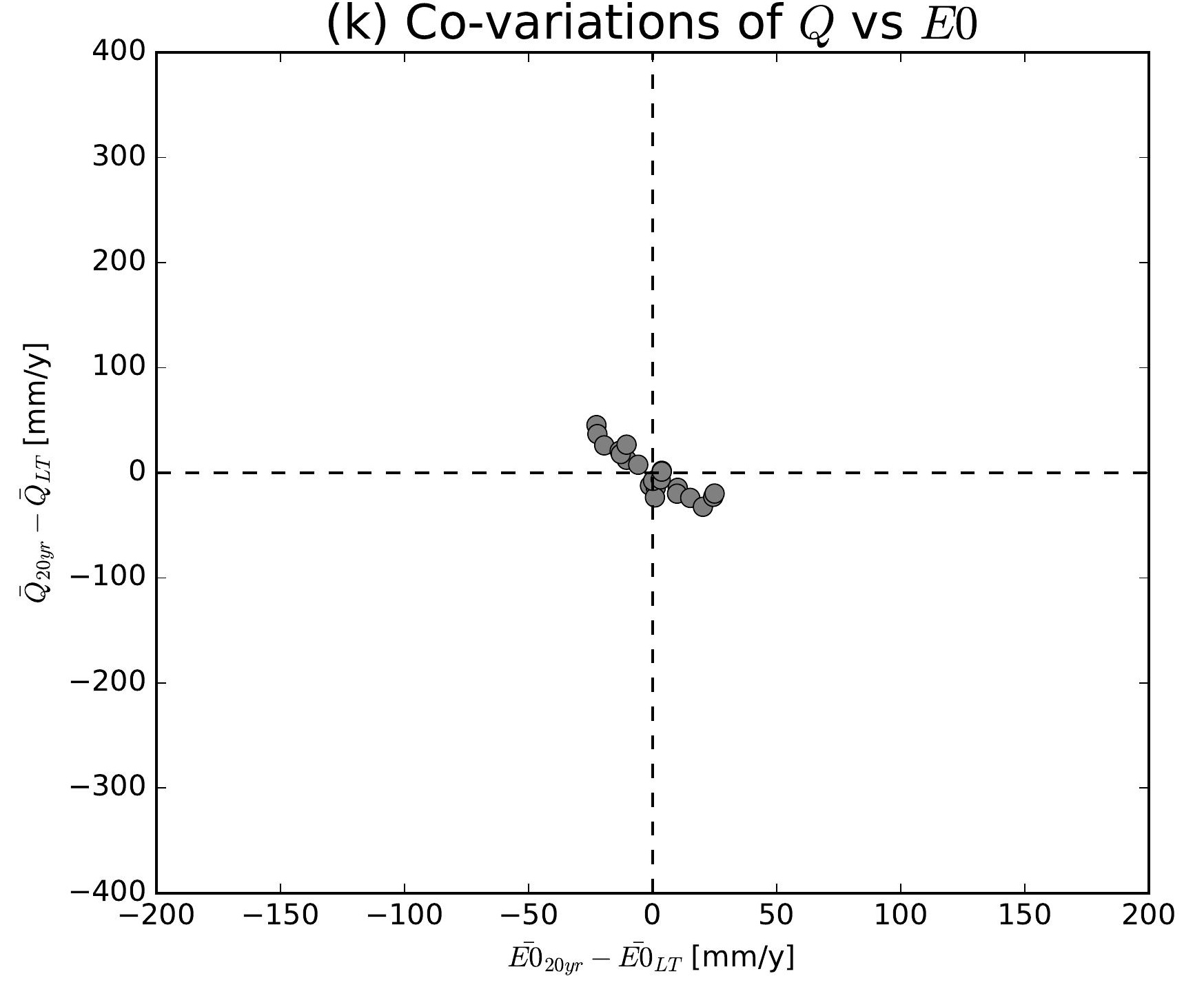

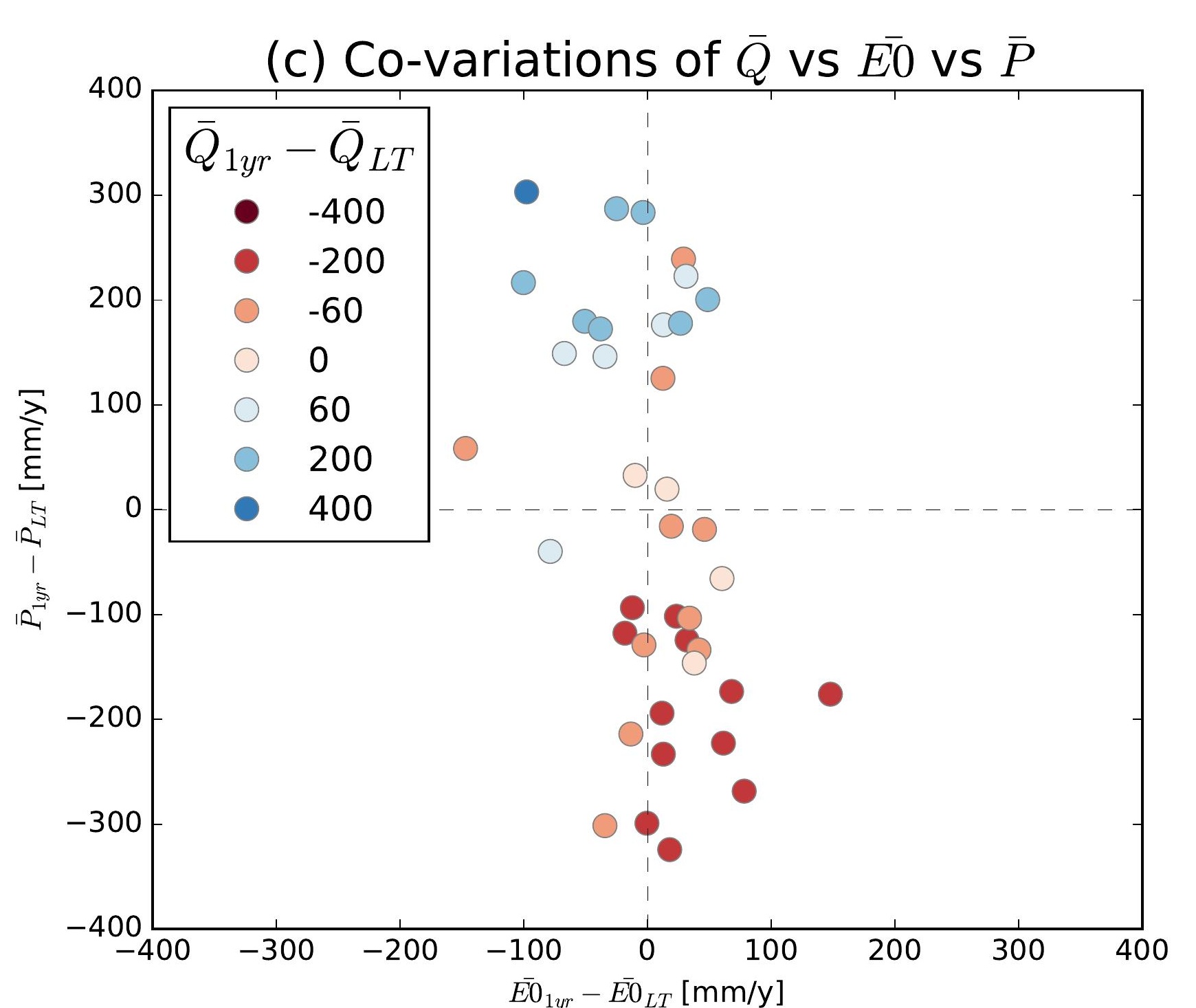
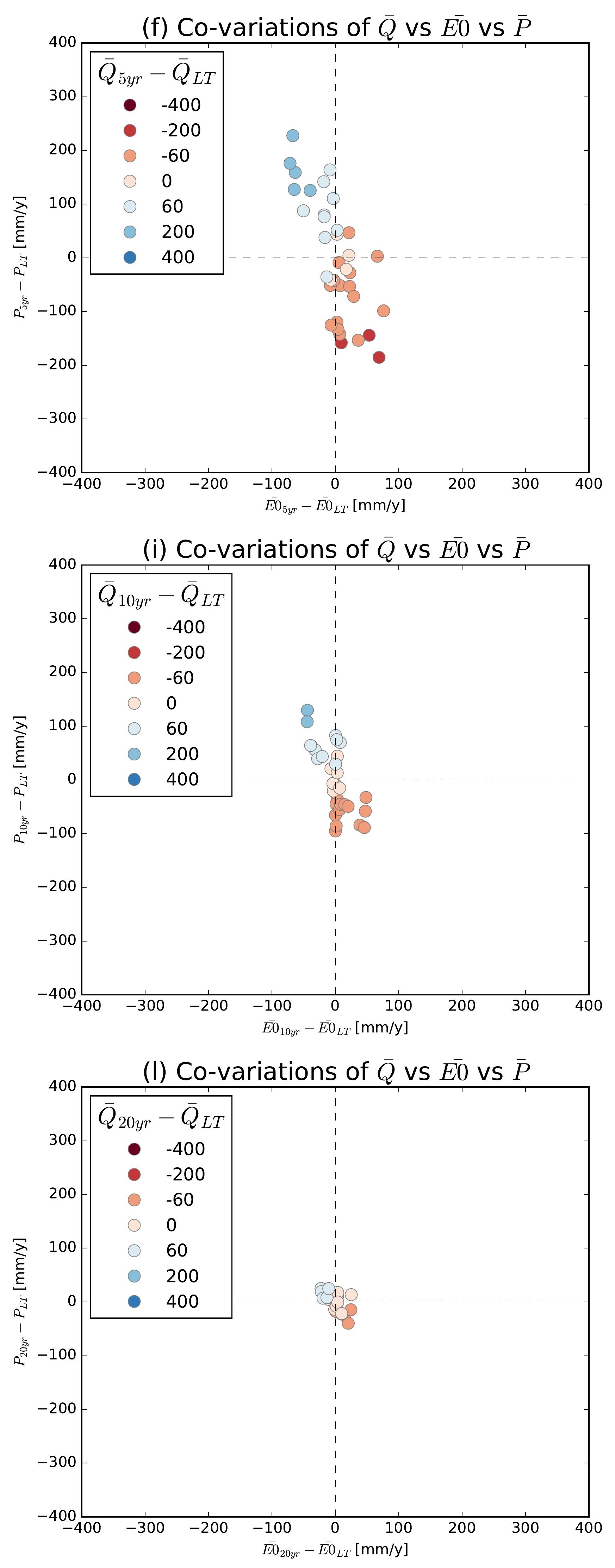
Catchment Y5615020

(a) Co-variations of $\bar{Q}$ vs $\bar{P}$

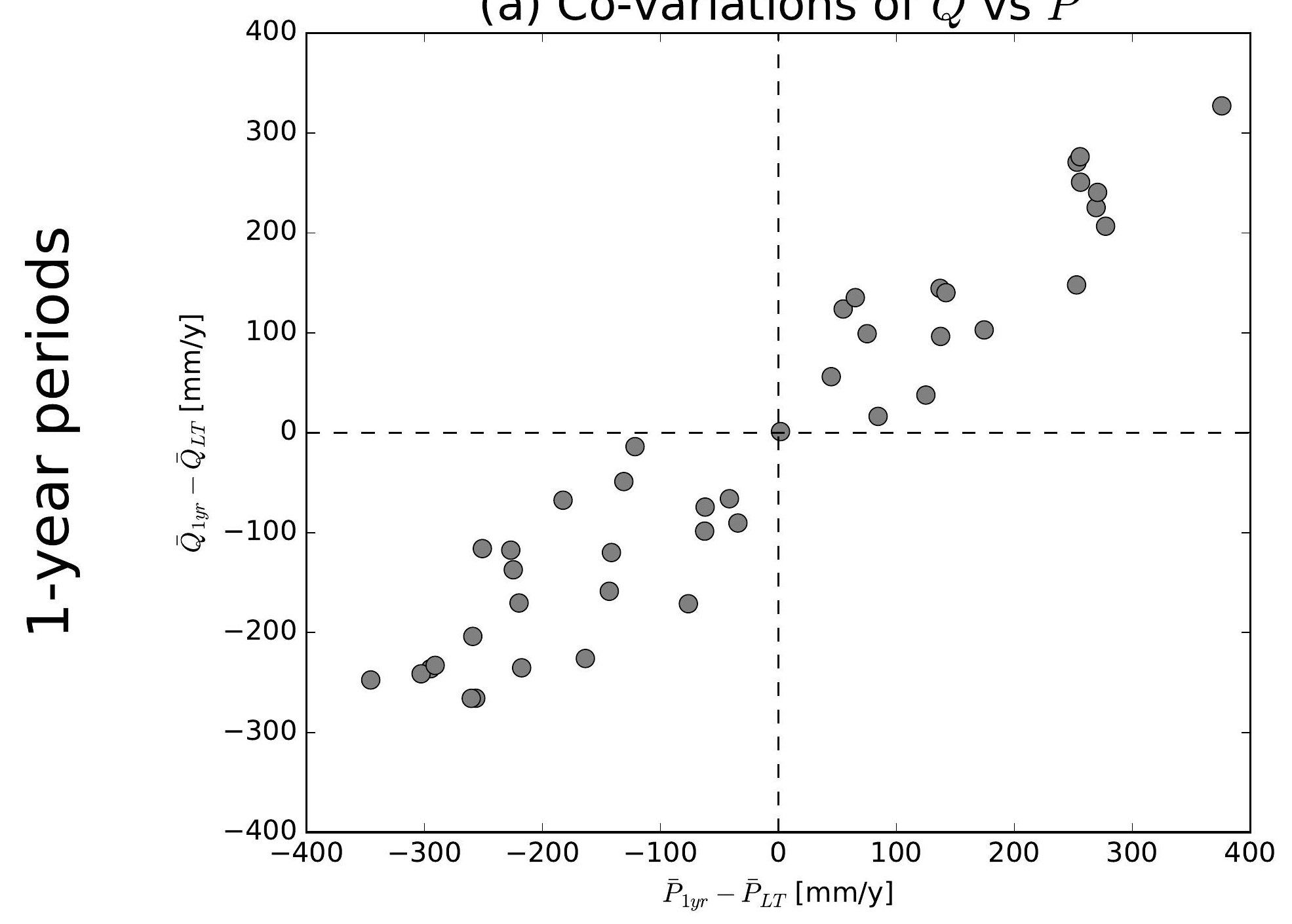

(d) Co-variations of $\bar{Q}$ vs $\bar{P}$

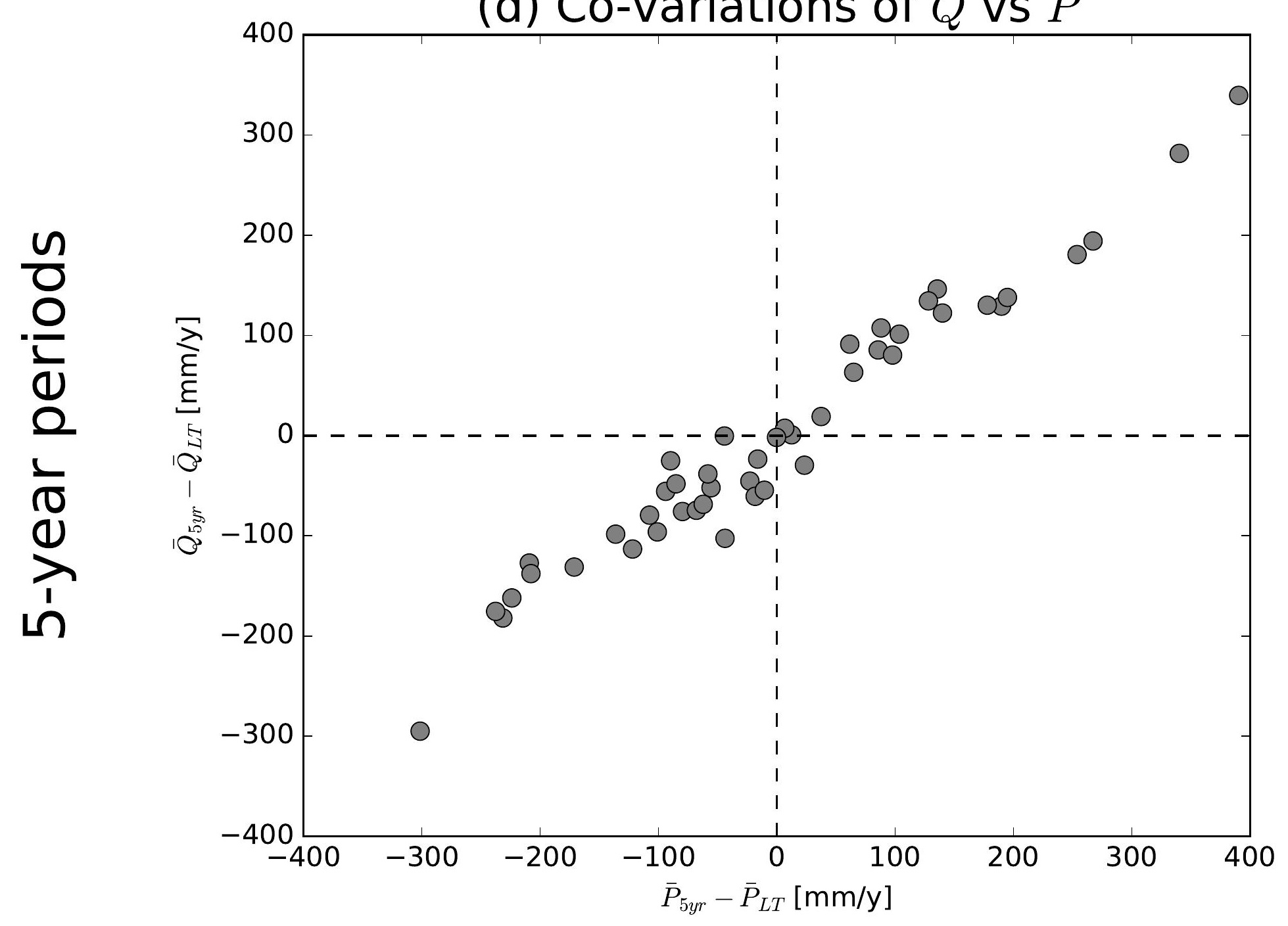

(g) Co-variations of $\bar{Q}$ vs $\bar{P}$

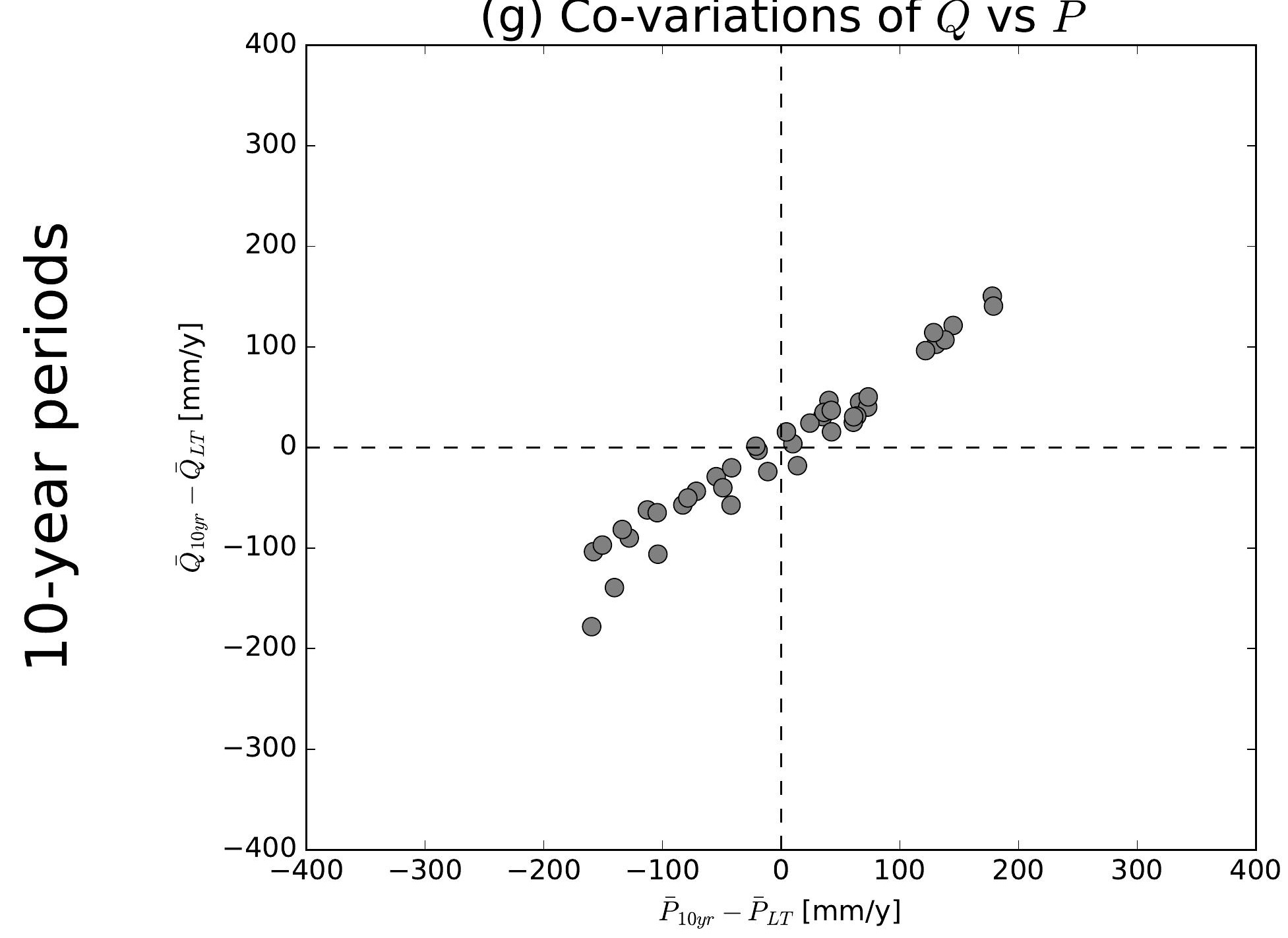

(j) Co-variations of $\bar{Q}$ vs $\bar{P}$

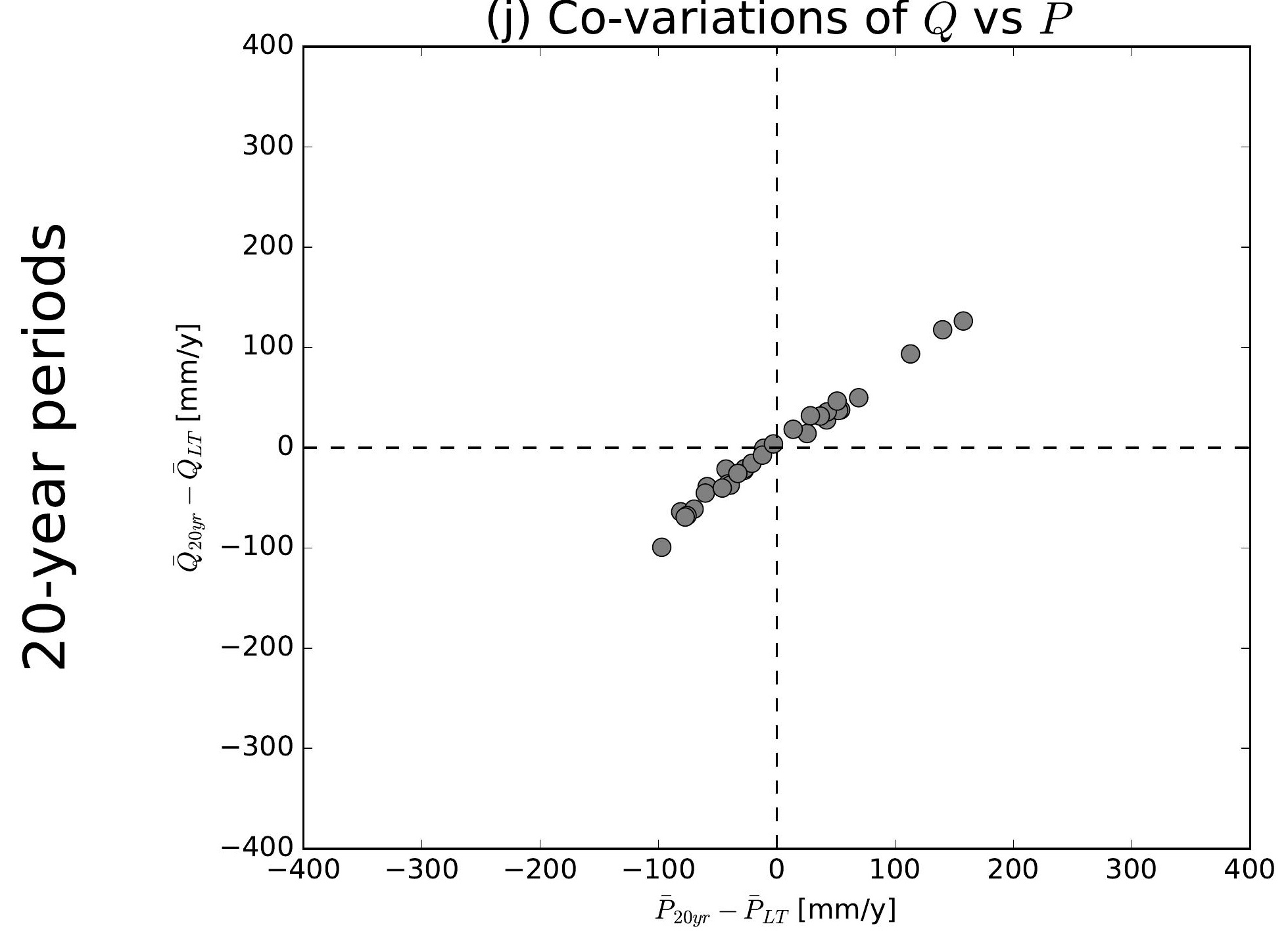

(b) Co-variations of $\bar{Q}$ vs $\overline{E 0}$

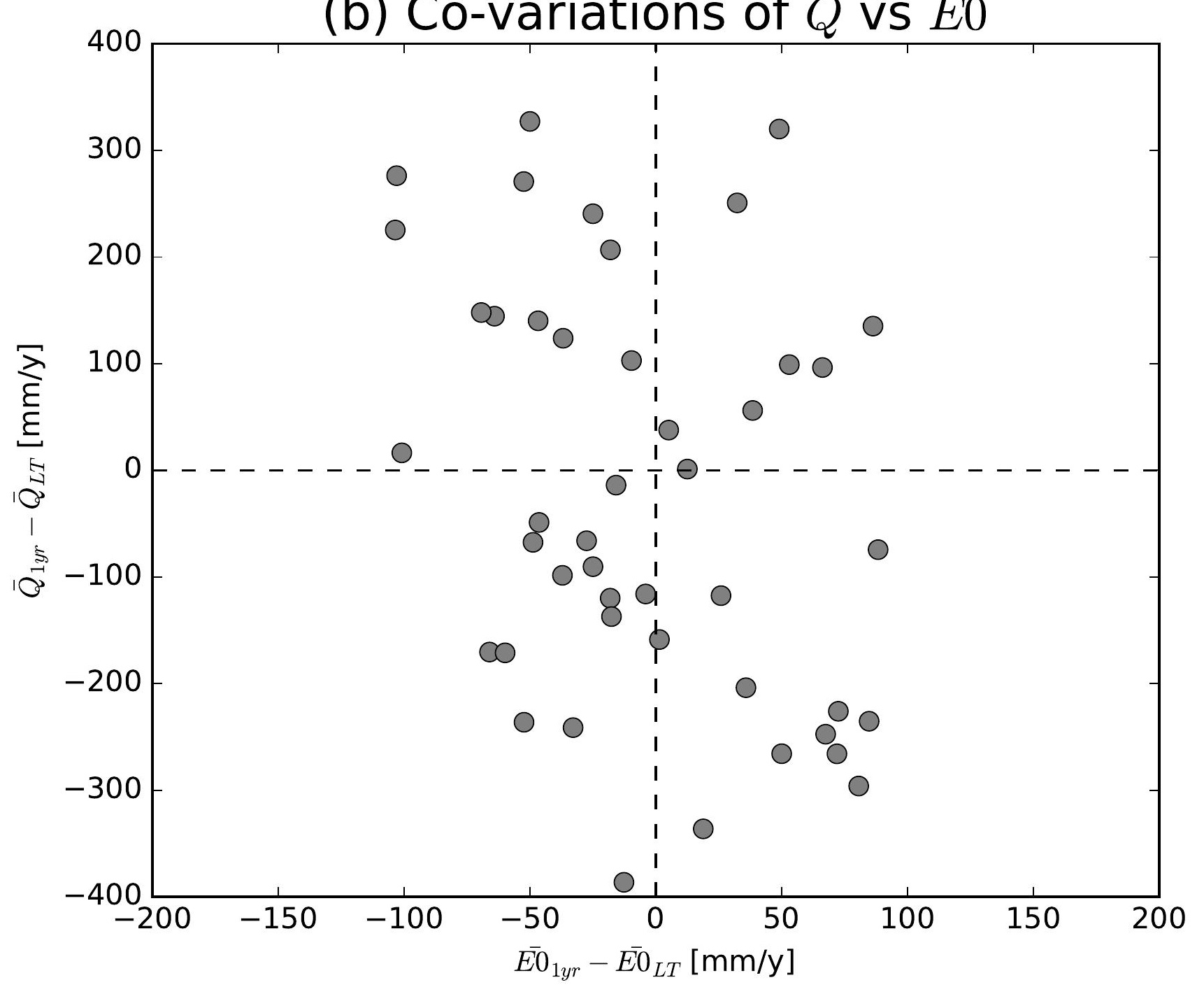

(e) Co-variations of $\bar{Q}$ vs $\overline{E 0}$

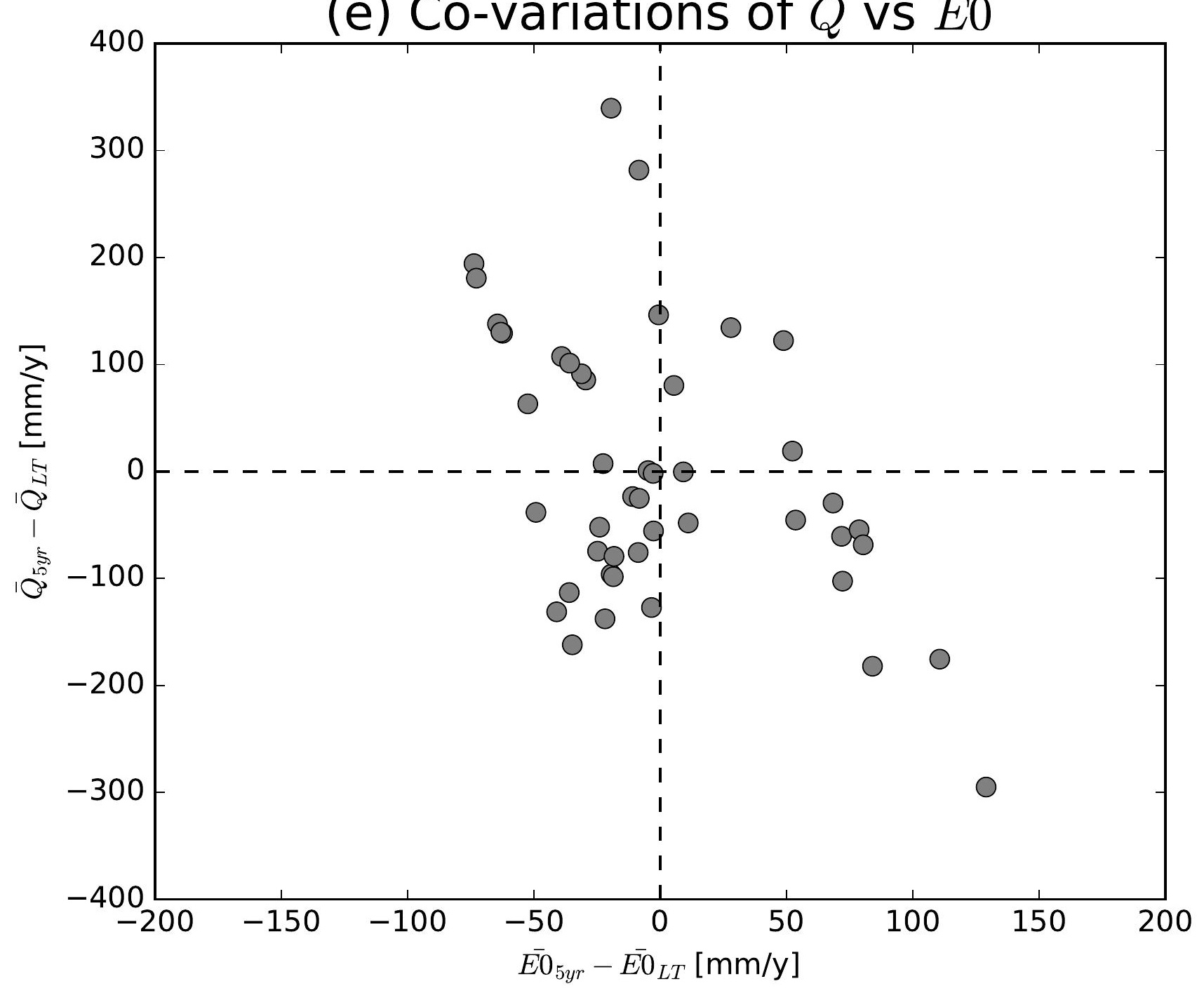

(h) Co-variations of $\bar{Q}$ vs $\overline{E 0}$

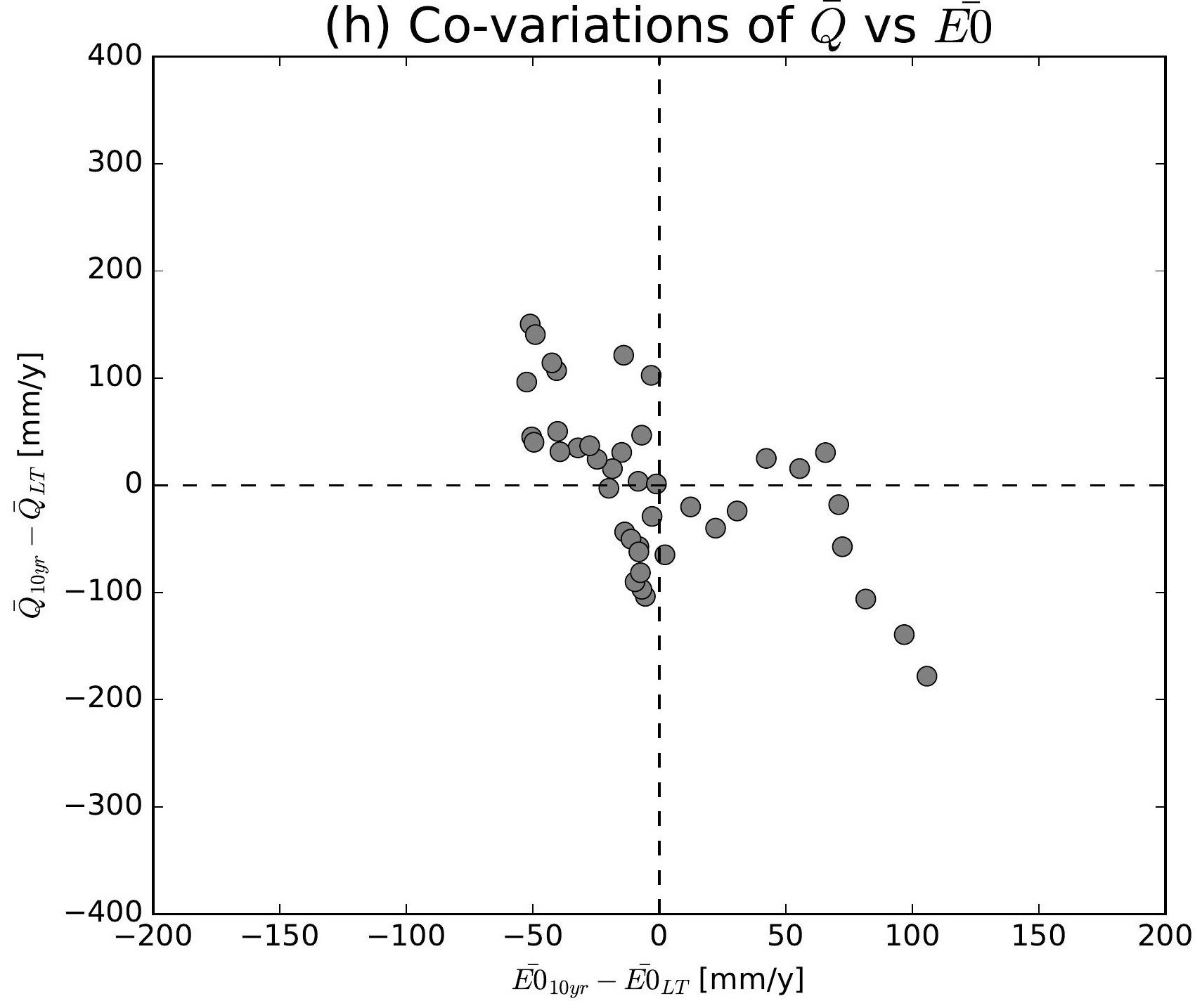

(k) Co-variations of $\bar{Q}$ vs $\overline{E 0}$

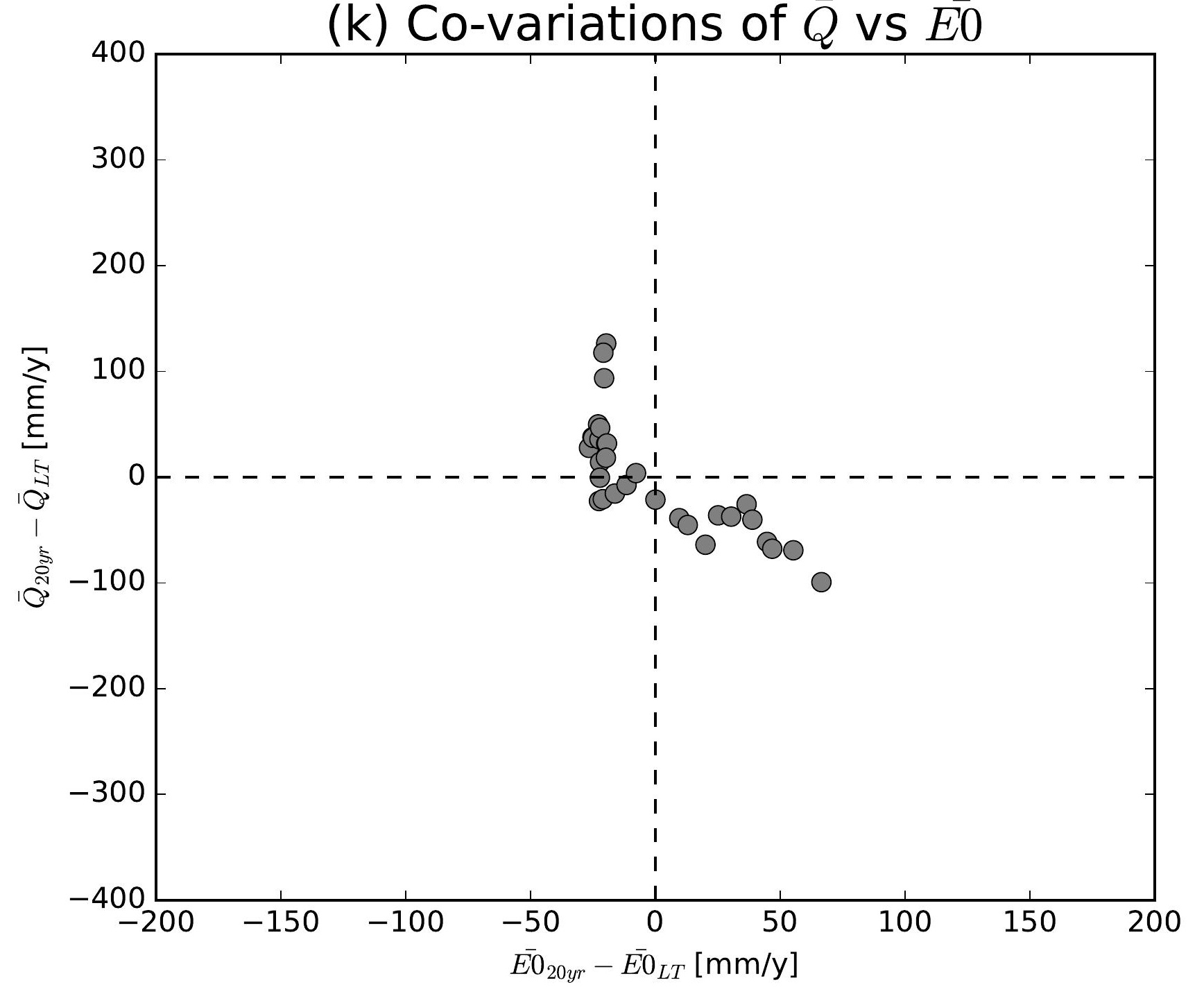

(c) Co-variations of $\bar{Q}$ vs $\overline{E 0}$ vs $\bar{P}$
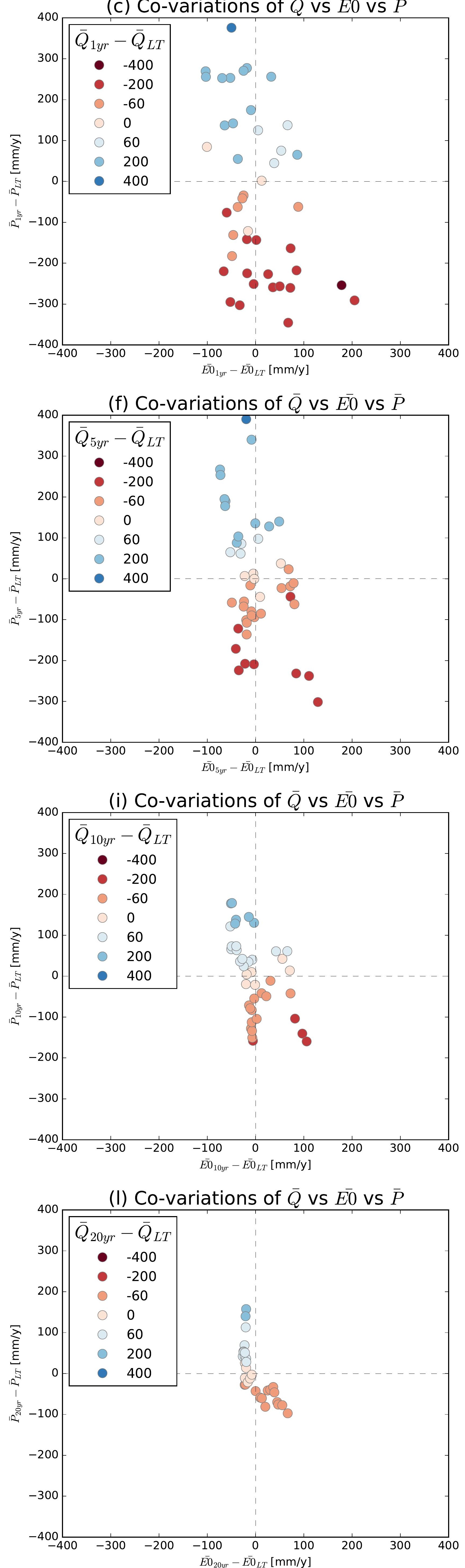

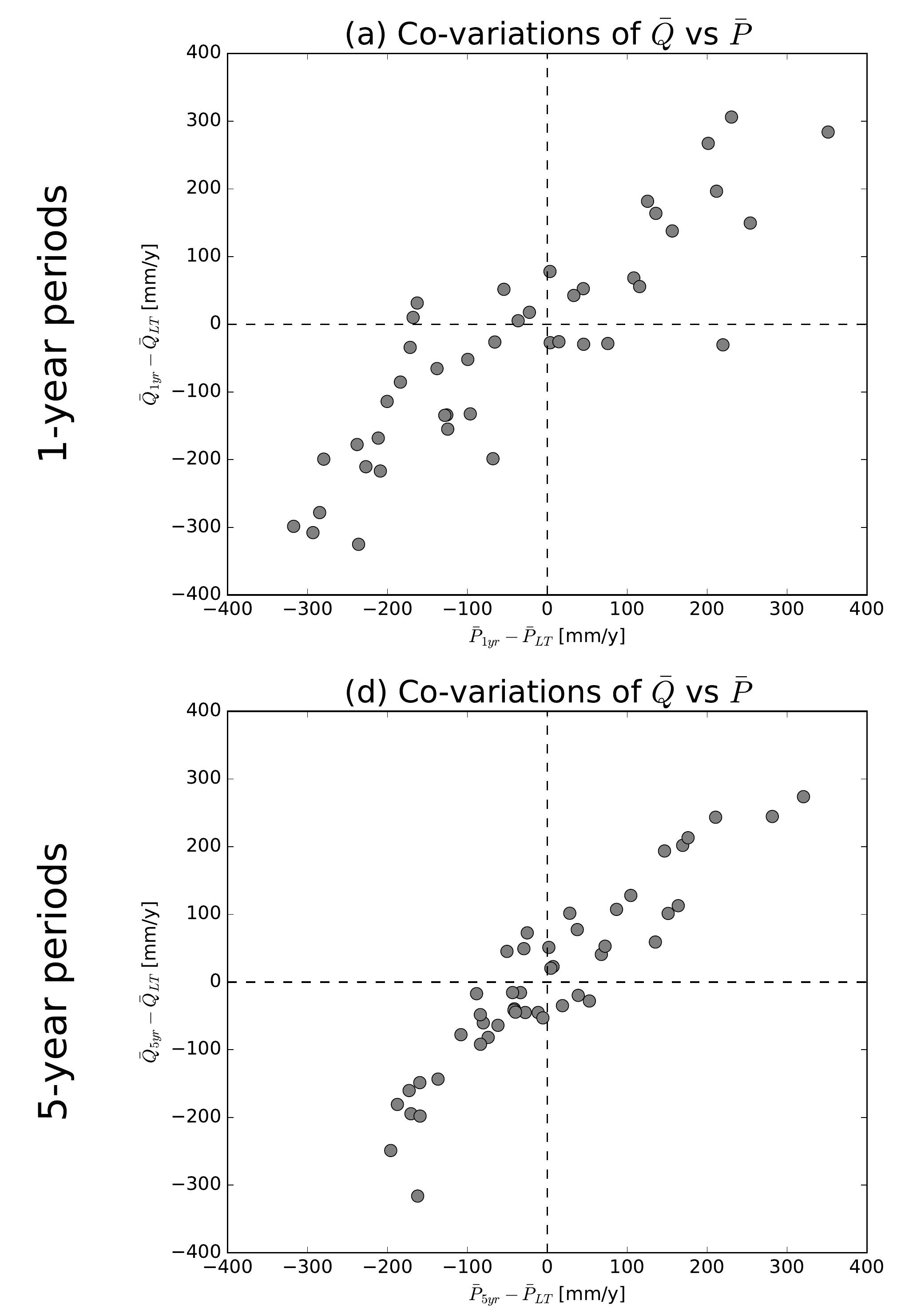

(g) Co-variations of $\bar{Q}$ vs $\bar{P}$

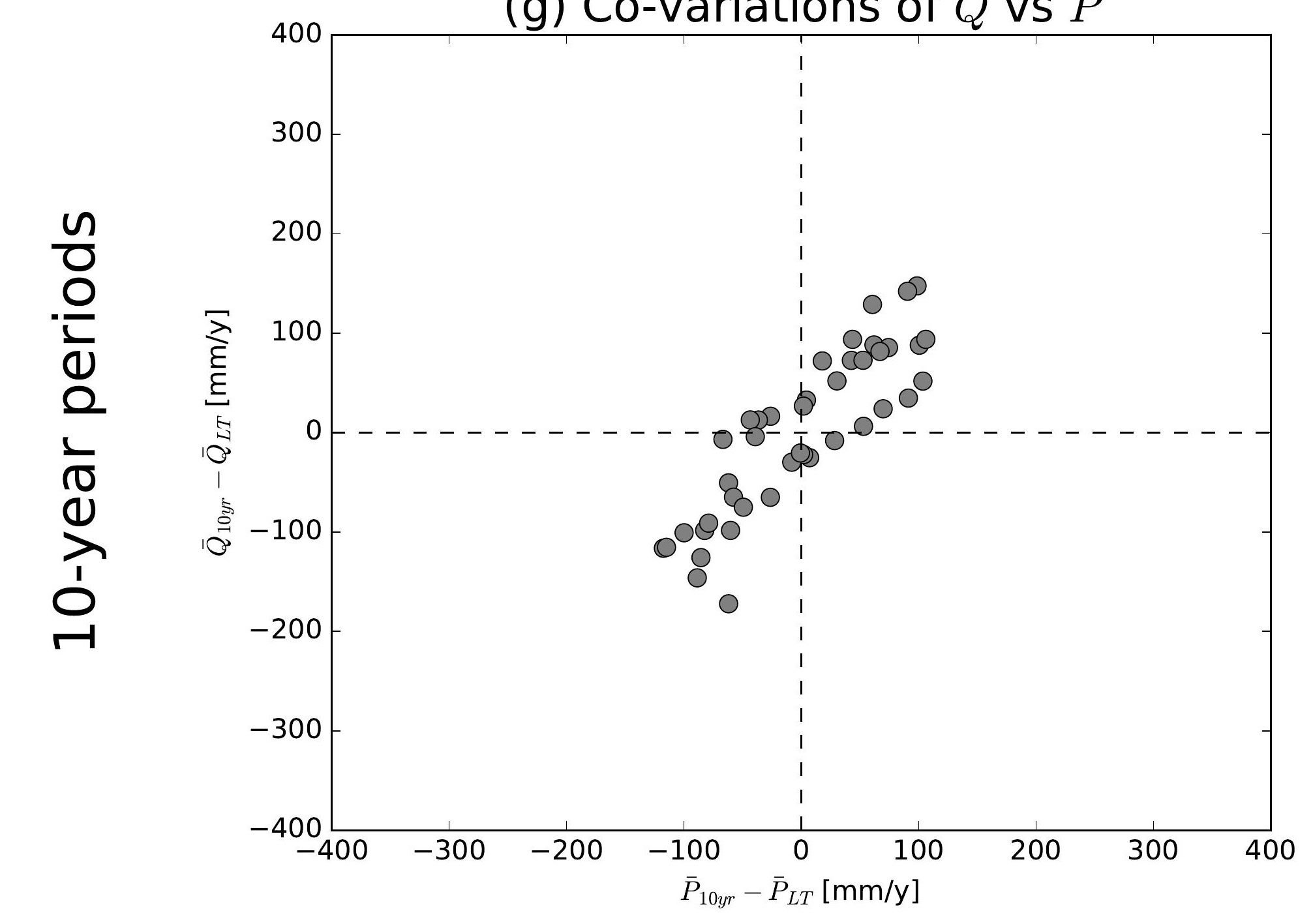

(j) Co-variations of $\bar{Q}$ vs $\bar{P}$

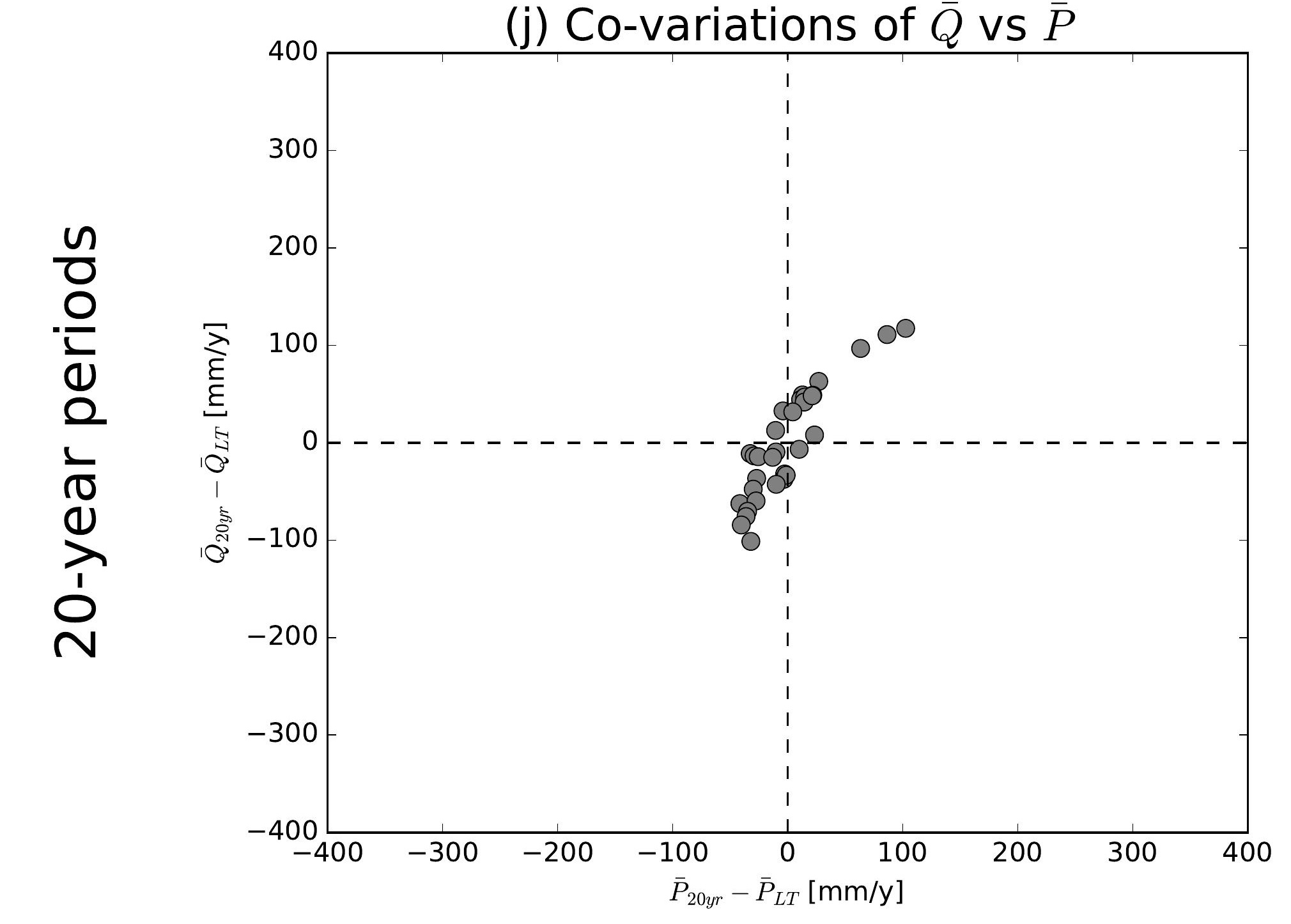

(b) Co-variations of $\bar{Q}$ vs $\overline{E 0}$

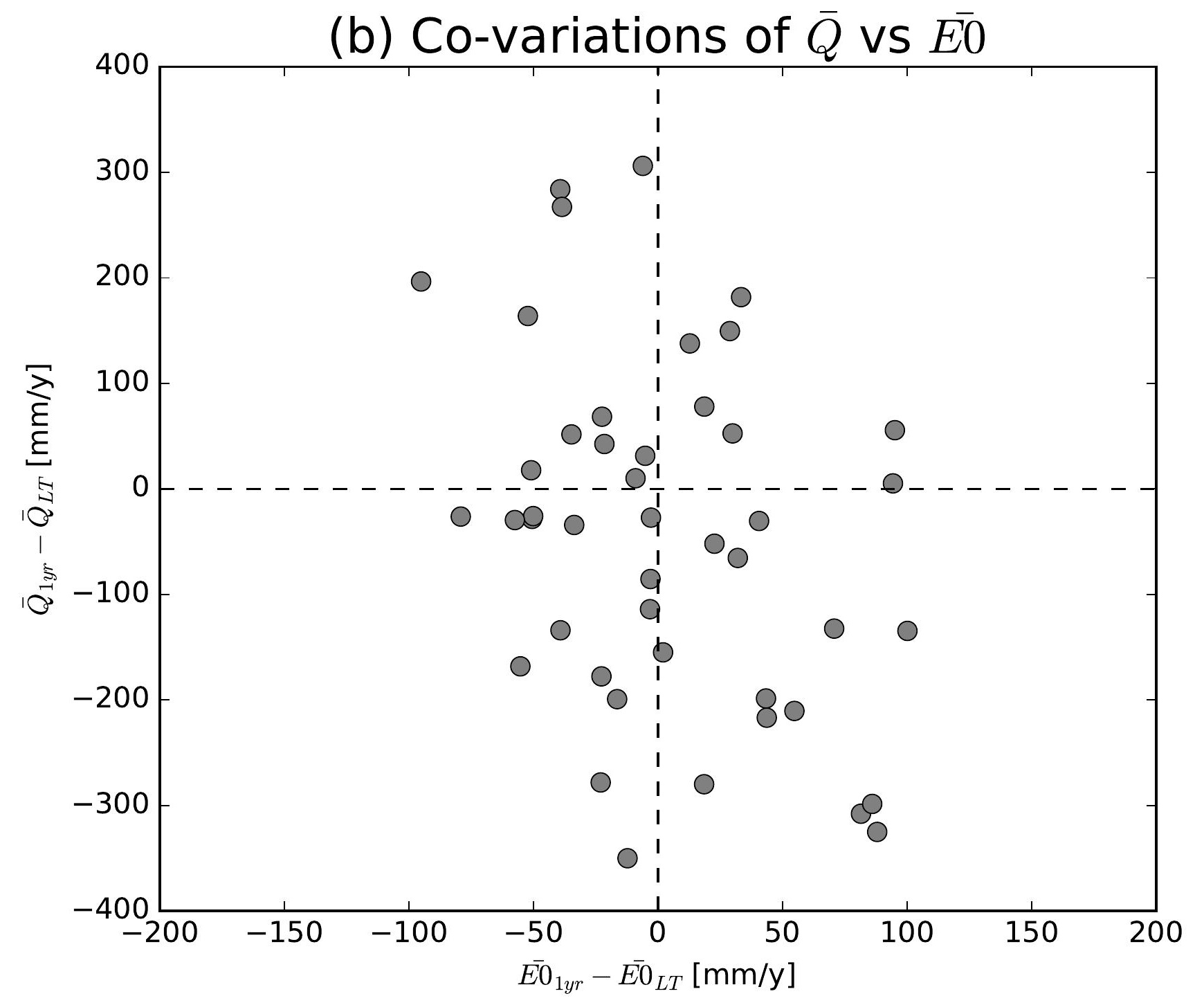

(e) Co-variations of $\bar{Q}$ vs $\overline{E 0}$

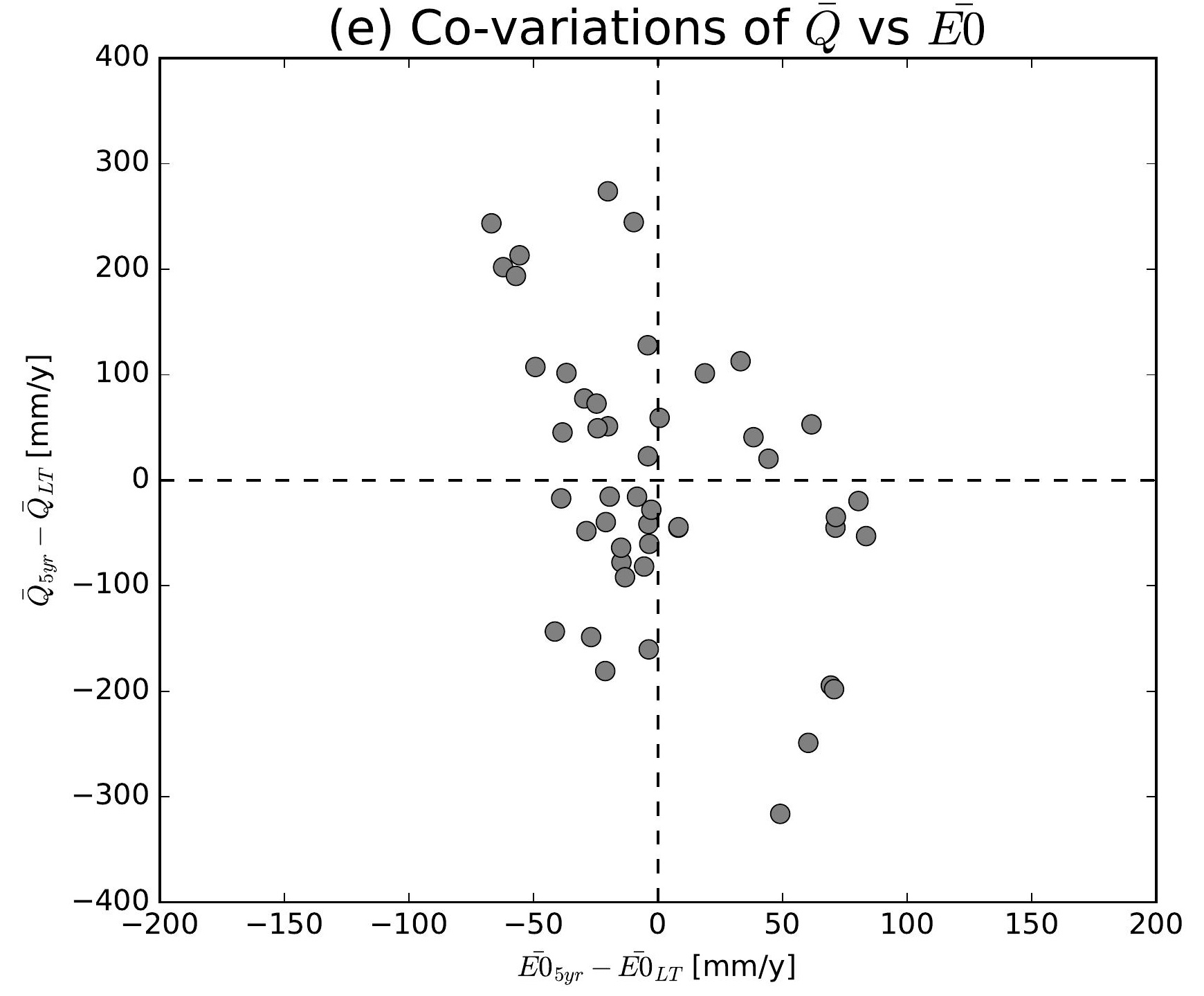

(h) Co-variations of $\bar{Q}$ vs $\overline{E 0}$

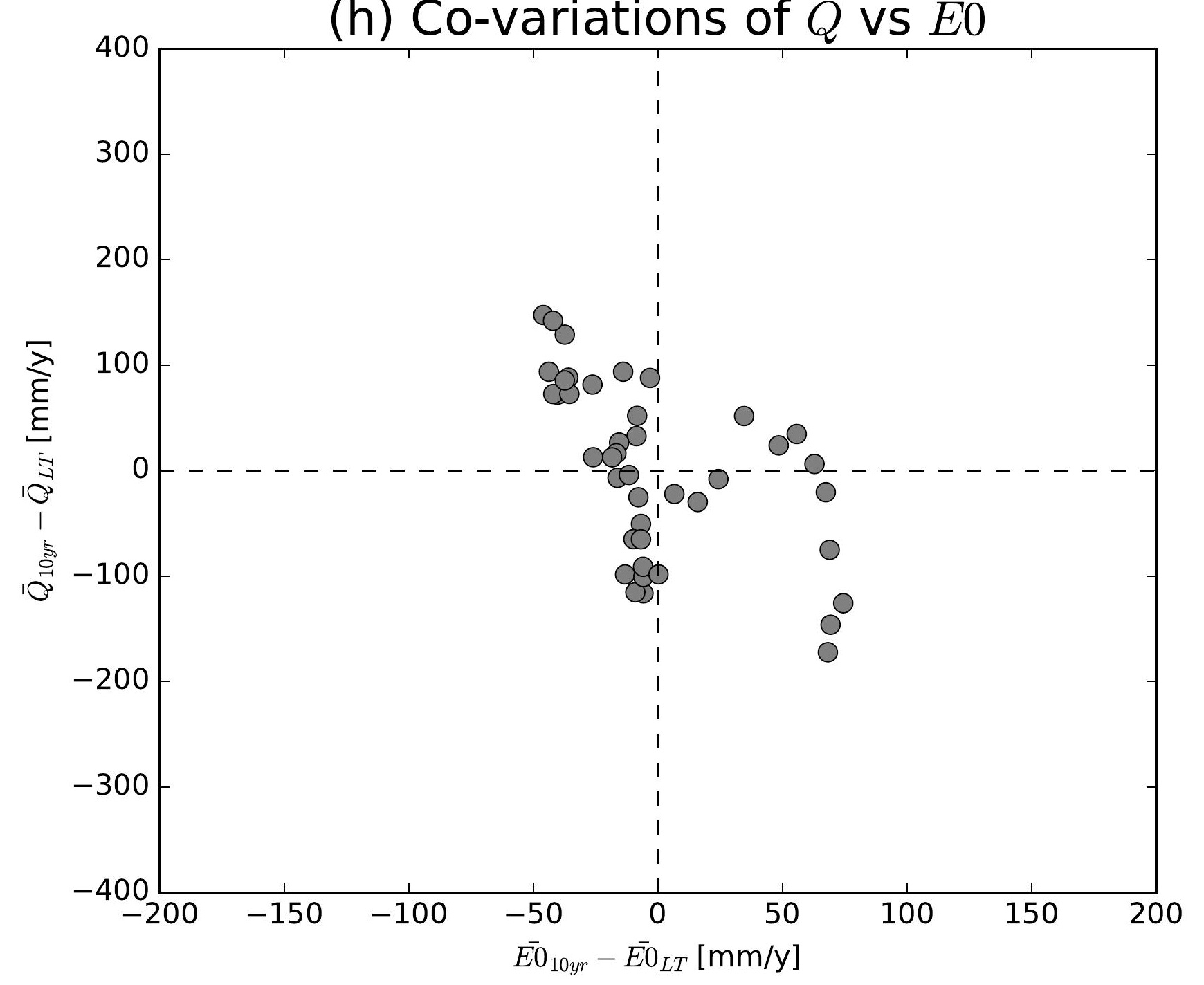

(k) Co-variations of $\bar{Q}$ vs $\overline{E 0}$

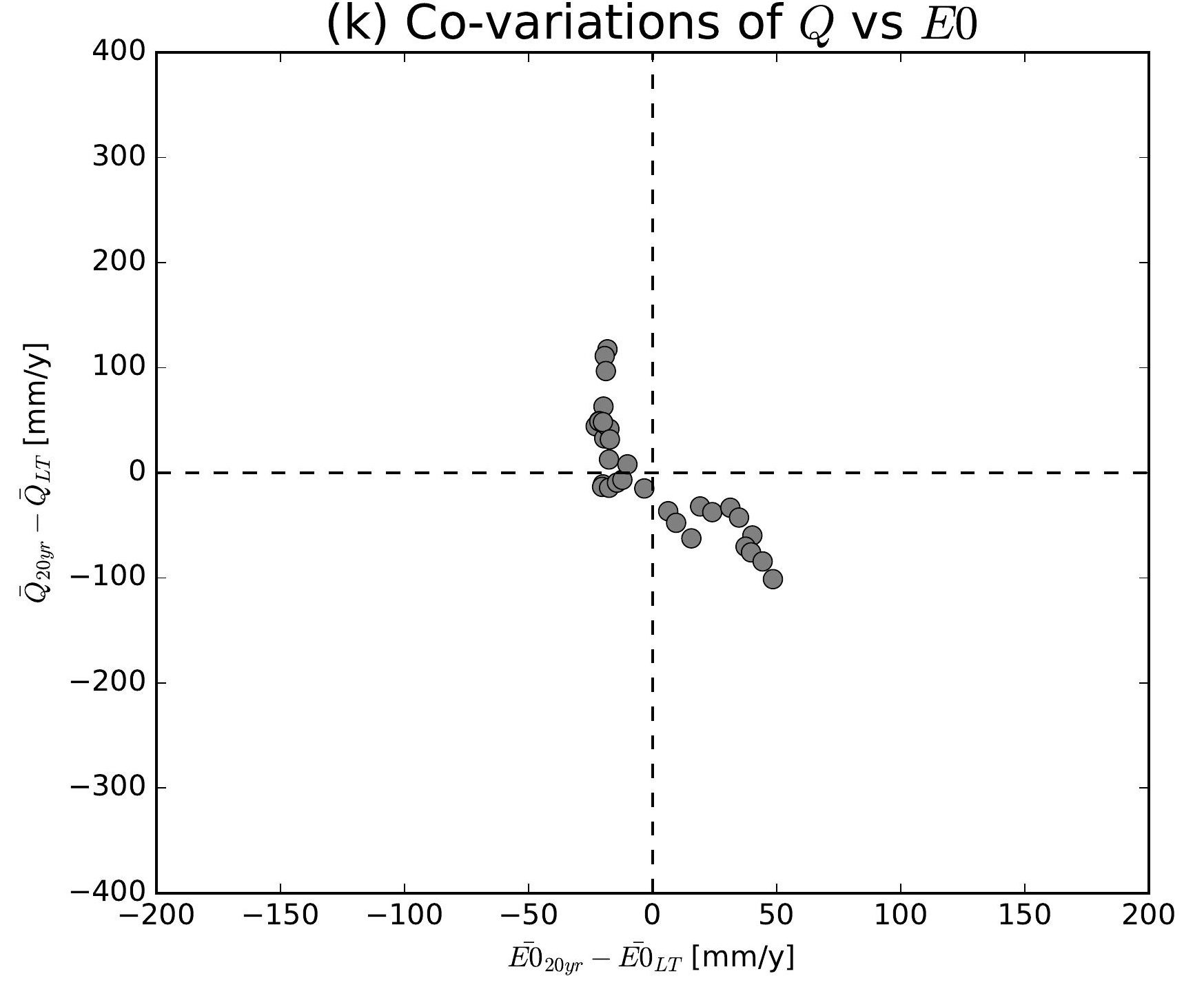

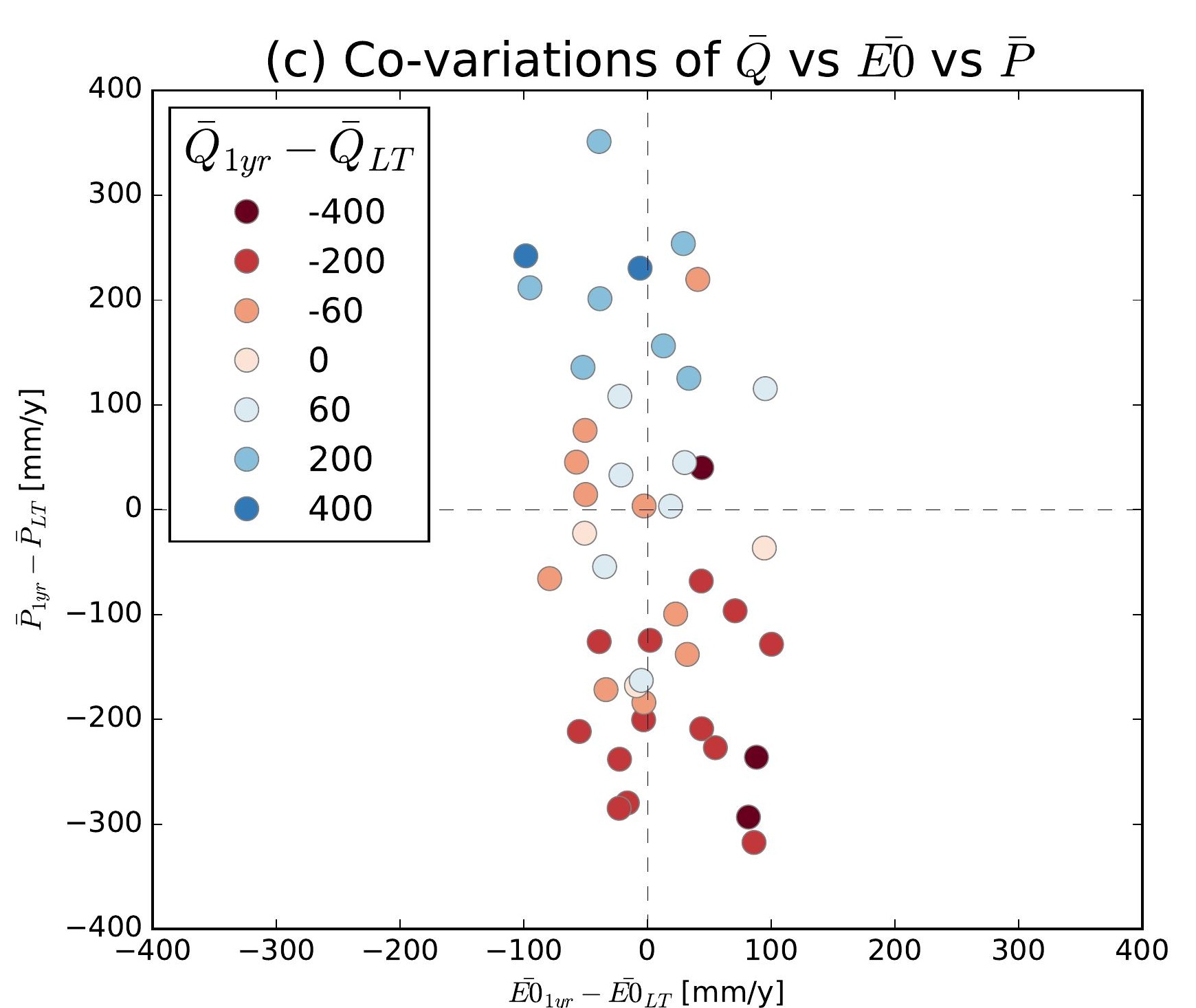
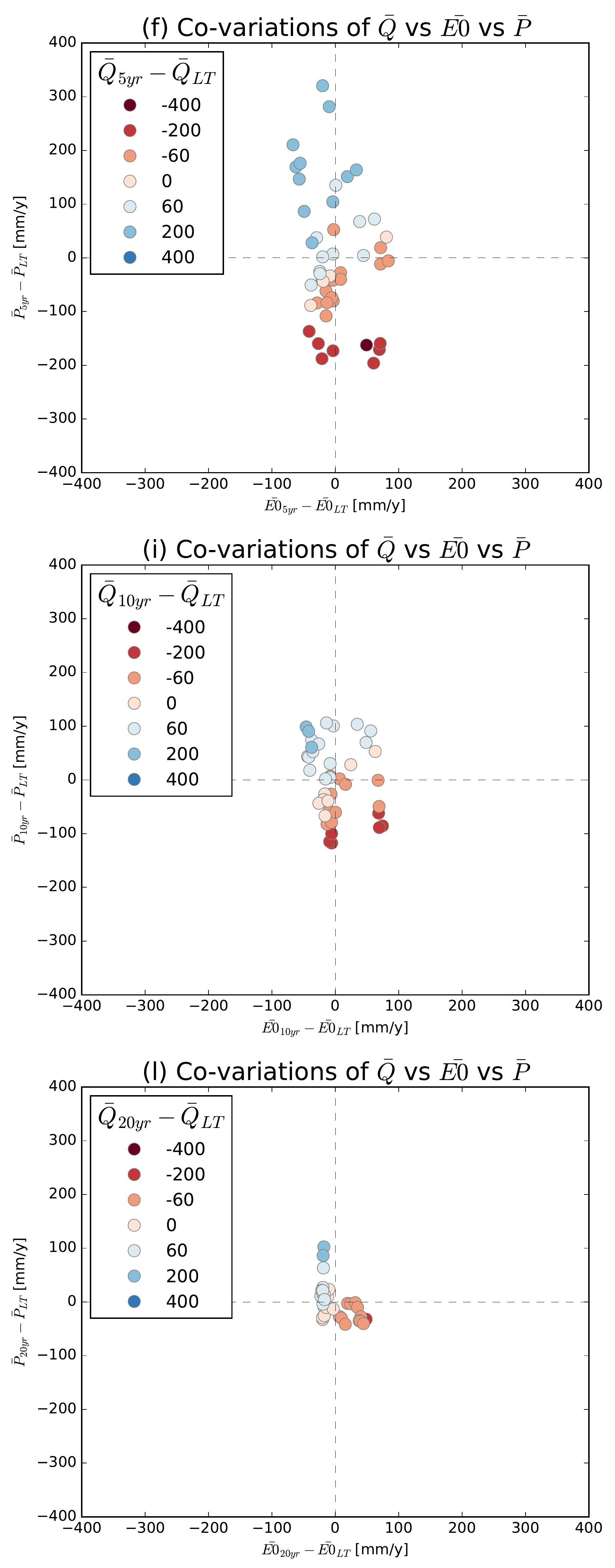
